\title{
$\left.{ }_{\text {SSCIENCEE }}^{\text {MATRIX }}\right\}$ Mascot Search Results
}

Results Generated by a Script Modified from Mascot Pepitde View

by Newman Sze, School of Biological Sciences, Nanyang Technological University

Spectrum No: 1; Query: 1525; Rank: 1

\section{Peptide View}

MS/MS Fragmentation of SNSTAGELDALQAEAGSLDK

Found in IPI00365542, Tax_Id=10116 Gene_Symbol=Lamb1_predicted laminin, beta 1

Match to Query 1525: 1976.923288 from(989.468920,2+)

Title: 100101RatKid_NS_deglyco_19.3877.3877.2.dta

Data file K:INewmanPaper|Piliang|3SubProteomes\Piliang3SP\mgf5ppm\ERLIC_3SubProteomes5ppm.mgf
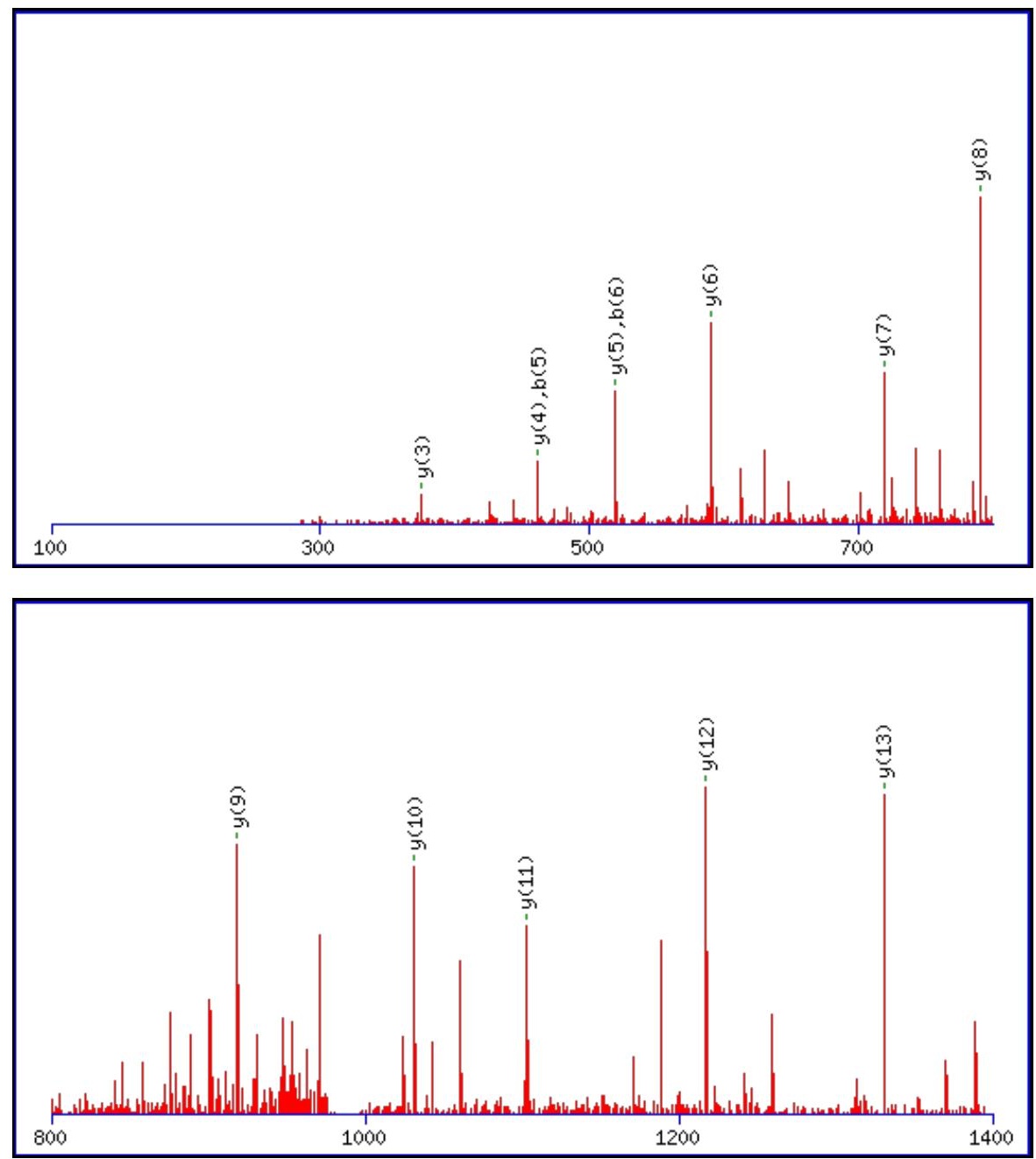


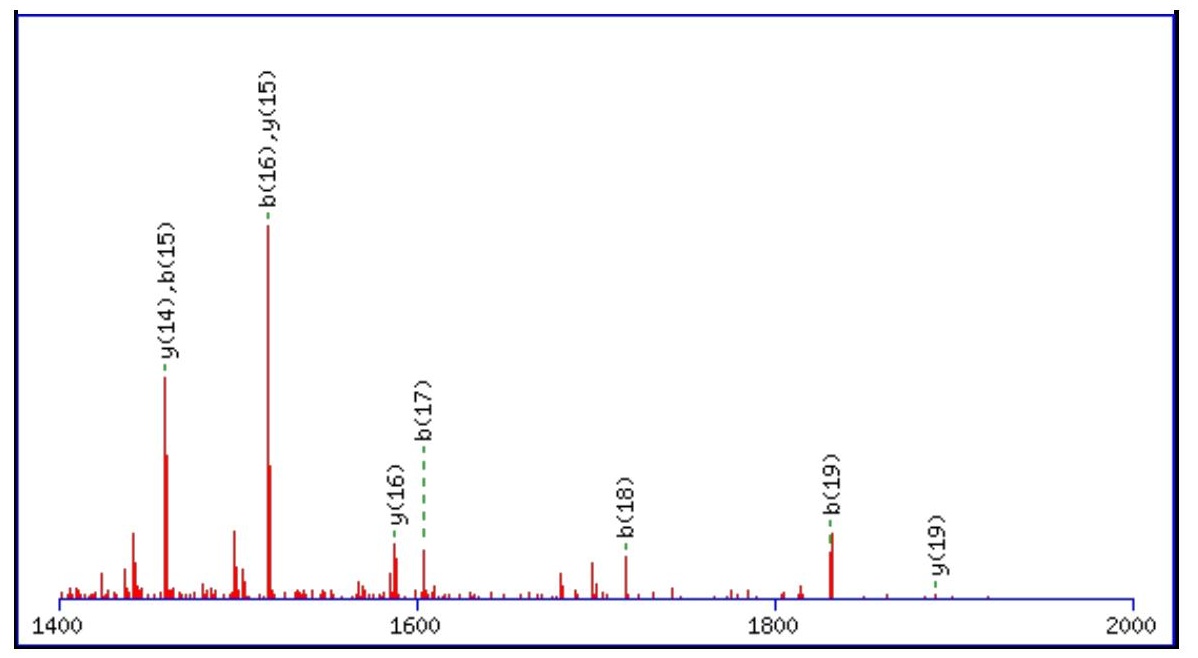

Monoisotopic mass of neutral peptide $\operatorname{Mr}($ calc): 1976.9174

Fixed modifications: Carbamidomethyl (C)

Variable modifications:

N2 : Deamidated $\mathrm{N}(\mathrm{N})$

Ions Score: 161 Expect: $1.7 \mathrm{e}-014$

Matches (Bold Red): 22/224 fragment ions using 20 most intense peaks

\begin{tabular}{|c|c|c|c|c|c|c|c|c|c|c|c|c|c|c|}
\hline \# & b & $\mathbf{b}^{++}$ & b* & $\mathbf{b}^{*^{++}}$ & $\mathbf{b}^{\mathbf{0}}$ & $\mathbf{b}^{0++}$ & Seq. & $\mathbf{y}$ & $\mathbf{y}^{++}$ & $\mathbf{y}^{*}$ & $\mathrm{y}^{*^{++}}$ & $\mathbf{y}^{0}$ & $\mathbf{y}^{0++}$ & \# \\
\hline 1 & 88.0393 & 44.5233 & & & 70.0287 & 35.5180 & S & & & & & & & 20 \\
\hline 2 & 203.0662 & 102.0368 & 186.0397 & 93.5235 & 185.0557 & 93.0315 & $\mathbf{N}$ & 1890.8927 & 945.9500 & 1873.8662 & 937.4367 & 1872.8821 & 936.9447 & 19 \\
\hline 3 & 290.0983 & 145.5528 & 273.0717 & 137.0395 & 272.0877 & 136.5475 & S & 1775.8658 & 888.4365 & 1758.8392 & 879.9232 & 1757.8552 & 879.4312 & 18 \\
\hline 4 & 391.1459 & 196.0766 & 374.1194 & 187.5633 & 373.1354 & 187.0713 & $\mathbf{T}$ & 1688.8337 & 844.9205 & 1671.8072 & 836.4072 & 1670.8232 & 835.9152 & 17 \\
\hline 5 & 462.1831 & 231.5952 & 445.1565 & 223.0819 & 444.1725 & 222.5899 & A & 1587.7861 & 794.3967 & 1570.7595 & 785.8834 & 1569.7755 & 785.3914 & 16 \\
\hline 6 & 519.2045 & 260.1059 & 502.1780 & 251.5926 & 501.1940 & 251.1006 & G & 1516.7489 & 758.8781 & 1499.7224 & 750.3648 & 1498.7384 & 749.8728 & 15 \\
\hline 7 & 648.2471 & 324.6272 & 631.2206 & 316.1139 & 630.2366 & 315.6219 & $\mathbf{E}$ & 1459.7275 & 730.3674 & 1442.7009 & 721.8541 & 1441.7169 & 721.3621 & 14 \\
\hline 8 & 761.3312 & 381.1692 & 744.3046 & 372.6560 & 743.3206 & 372.1639 & $\mathbf{L}$ & 1330.6849 & 665.8461 & 1313.6583 & 657.3328 & 1312.6743 & 656.8408 & 13 \\
\hline 9 & 876.3581 & 438.6827 & 859.3316 & 430.1694 & 858.3476 & 429.6774 & D & 1217.6008 & 609.3040 & 1200.5743 & 600.7908 & 1199.5903 & 600.2988 & 12 \\
\hline 10 & 947.3952 & 474.2013 & 930.3687 & 465.6880 & 929.3847 & 465.1960 & A & 1102.5739 & 551.7906 & 1085.5473 & 543.2773 & 1084.5633 & 542.7853 & 11 \\
\hline 11 & 1060.4793 & 530.7433 & 1043.4528 & 522.2300 & 1042.4687 & 521.7380 & $\mathbf{L}$ & 1031.5368 & 516.2720 & 1014.5102 & 507.7587 & 1013.5262 & 507.2667 & 10 \\
\hline 12 & 1188.5379 & 594.7726 & 1171.5113 & 586.2593 & 1170.5273 & 585.7673 & $\mathbf{Q}$ & 918.4527 & 459.7300 & 901.4262 & 451.2167 & 900.4421 & 450.7247 & 9 \\
\hline 13 & 1259.5750 & 630.2911 & 1242.5484 & 621.7779 & 1241.5644 & 621.2859 & A & 790.3941 & 395.7007 & 773.3676 & 387.1874 & 772.3836 & 386.6954 & 8 \\
\hline 14 & 1388.6176 & 694.8124 & 1371.5910 & 686.2992 & 1370.6070 & 685.8071 & $\mathbf{E}$ & 719.3570 & 360.1821 & 702.3305 & 351.6689 & 701.3464 & 351.1769 & 7 \\
\hline 15 & 1459.6547 & 730.3310 & 1442.6282 & 721.8177 & 1441.6441 & 721.3257 & A & 590.3144 & 295.6608 & 573.2879 & 287.1476 & 572.3039 & 286.6556 & 6 \\
\hline 16 & 1516.6762 & 758.8417 & 1499.6496 & 750.3284 & 1498.6656 & 749.8364 & G & 519.2773 & 260.1423 & 502.2508 & 251.6290 & 501.2667 & 251.1370 & 5 \\
\hline 17 & 1603.7082 & 802.3577 & 1586.6816 & 793.8445 & 1585.6976 & 793.3525 & S & 462.2558 & 231.6316 & 445.2293 & 223.1183 & 444.2453 & 222.6263 & 4 \\
\hline 18 & 1716.7923 & 858.8998 & 1699.7657 & 850.3865 & 1698.7817 & 849.8945 & $\mathbf{L}$ & 375.2238 & 188.1155 & 358.1973 & 179.6023 & 357.2132 & 179.1103 & 3 \\
\hline 19 & 1831.8192 & 916.4132 & 1814.7927 & 907.9000 & 1813.8086 & 907.4080 & D & 262.1397 & 131.5735 & 245.1132 & 123.0602 & 244.1292 & 122.5682 & 2 \\
\hline 20 & & & & & & & $\mathbf{K}$ & 147.1128 & 74.0600 & 130.0863 & 65.5468 & & & 1 \\
\hline
\end{tabular}

$$
\text { 五 }
$$$$
\text { RMS }
$$

\section{All matches to this query}

\begin{tabular}{|l|l|c|c|}
\hline Score & Mr(calc): & Delta & Sequence \\
\hline 161.4 & 1976.9174 & 0.0059 & SNSTAGELDALQAEAGSLDK \\
\hline & & &
\end{tabular}

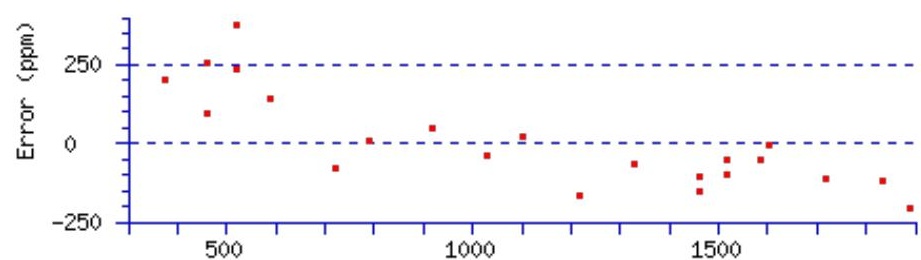

RNS error 150 ppm 


\begin{tabular}{|l|l|l|l|}
134.8 & 1975.9334 & 0.9899 & SNSTAGELDALQAEAGSLDK \\
\hline 6.4 & 1976.9327 & -0.0094 & QSLDGFLDETNKENLPR \\
\hline 5.3 & 1976.9035 & 0.0198 & YMRQQCIKQELICK \\
\hline 4.8 & 1976.9206 & 0.0027 & YMVLVPSQLYTETPEK \\
\hline 4.8 & 1976.9206 & 0.0027 & YMVLVPSQLYTETPEK \\
\hline 4.1 & 1976.9196 & 0.0037 & ILMHHIPEQTTQLLK \\
\hline 3.9 & 1976.9206 & 0.0027 & YMVLVPSQLYTETPEK \\
\hline 2.4 & 1974.9201 & 2.0032 & SDWIHDTPVSLGKADVR \\
\hline 2.0 & 1976.9258 & -0.0025 & NPPRWXTAGLAGSLTWR \\
\hline
\end{tabular}

Spectrum No: 2; Query: 1414; Rank: 1

\section{Peptide View}

MS/MS Fragmentation of IYAGMVSLLDEAVGNVTK

Found in IPI00198405, Tax_Id=10116 Gene_Symbol=Arsb Arylsulfatase B

Match to Query 1414: 1895.951128 from(948.982840,2+)

Title: 091008RatKidney_NH4Format01_23.3772.3772.2.dta

Data file K:INewmanPaper|Piliang|3SubProteomes\Piliang3SP\mgf5ppm\ERLIC_3SubProteomes5ppm.mgf
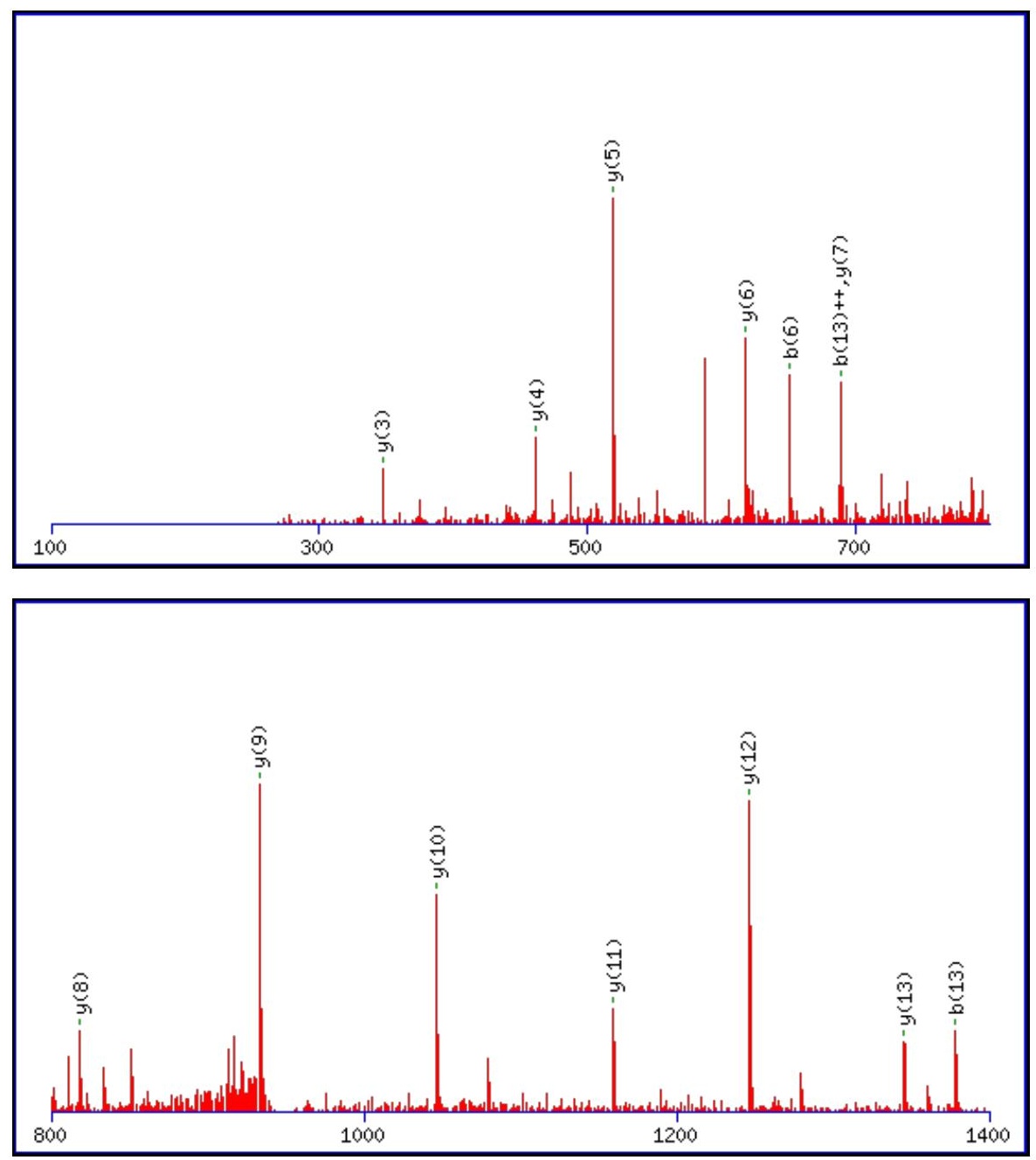


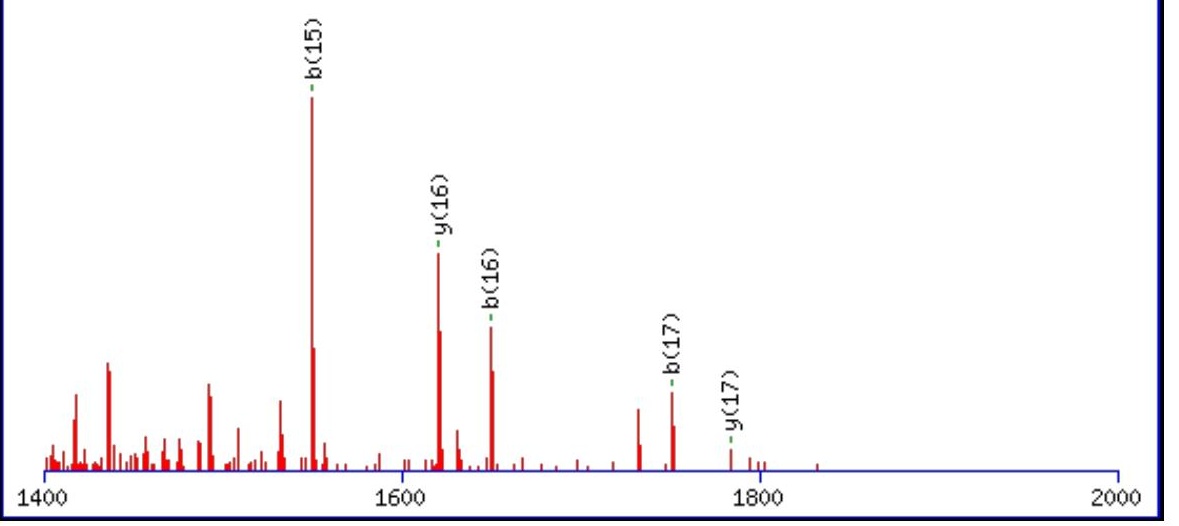

Monoisotopic mass of neutral peptide $\operatorname{Mr}($ calc): 1895.9550

Fixed modifications: Carbamidomethyl (C)

Variable modifications:

M5 : Oxidation (M)

N15 : Deamidated_N (N)

Ions Score: 145 Expect: $6.2 \mathrm{e}-013$

Matches (Bold Red): 19/162 fragment ions using 23 most intense peaks

\begin{tabular}{|c|c|c|c|c|c|c|c|c|c|c|c|c|c|c|}
\hline \# & b & $\mathbf{b}^{++}$ & $\mathbf{b}^{*}$ & $\mathbf{b}^{*^{++}}$ & $\mathbf{b}^{0}$ & $\mathbf{b}^{\mathbf{0 + +}}$ & Seq. & $\mathbf{y}$ & $y^{++}$ & $\mathbf{y}^{*}$ & $\mathrm{y}^{*^{++}}$ & $\mathbf{y}^{0}$ & $\mathbf{y}^{0++}$ & \# \\
\hline 1 & 114.0913 & 57.5493 & & & & & I & & & & & & & 1 \\
\hline 2 & 277.1547 & 139.0810 & & & & & $\mathbf{Y}$ & 1783.8782 & 892.4428 & 1766.8517 & 883.9295 & 1765.8677 & 883.4375 & 17 \\
\hline 3 & 348.1918 & 174.5995 & & & & & A & 1620.8149 & 810.9111 & 1603.7884 & 802.3978 & 1602.8043 & 801.9058 & \\
\hline 4 & 405.2132 & 203.1103 & & & & & $\mathbf{G}$ & 1549.7778 & 775.3925 & 1532.7513 & 766.8793 & 1531.7672 & 766.3873 & 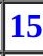 \\
\hline 5 & 552.2487 & 276.6280 & & & & & $\mathbf{M}$ & 1492.7563 & 746.8818 & 1475.7298 & 738.3685 & 1474.7458 & 737.8765 & 1 \\
\hline 6 & 651.3171 & 326.1622 & & & & & $\mathbf{V}$ & 1345.7209 & 673.3641 & 1328.6944 & 664.8508 & 1327.7104 & 664.3588 & 13 \\
\hline 7 & 738.3491 & 369.6782 & & & 720.3385 & 360.6729 & $\mathrm{~S}$ & 1246.6525 & 623.8299 & 1229.6260 & 615.3166 & 1228.6420 & 614.8246 & 12 \\
\hline 8 & 851.4332 & 426.2202 & & & 833.4226 & 417.2149 & $\mathbf{L}$ & 1159.6205 & 580.3139 & 1142.5939 & 571.8006 & 1141.6099 & 571.3086 & 11 \\
\hline 9 & 964.5172 & 482.7622 & & & 946.5067 & 473.7570 & $\mathbf{L}$ & 1046.5364 & 523.7719 & 1029.5099 & 515.2586 & 1028.5259 & 514.7666 & 10 \\
\hline 10 & 1079.5442 & 540.2757 & & & 1061.5336 & 531.2704 & D & 933.4524 & 467.2298 & 916.4258 & 458.7165 & 915.4418 & 458.2245 & \\
\hline 11 & 1208.5868 & 604.7970 & & & 1190.5762 & 595.7917 & $\mathbf{E}$ & 818.4254 & 409.7163 & 801.3989 & 401.2031 & 800.4149 & 400.7111 & 8 \\
\hline 12 & 1279.6239 & 640.3156 & & & 1261.6133 & 631.3103 & $\mathbf{A}$ & 689.3828 & 345.1951 & 672.3563 & 336.6818 & 671.3723 & 336.1898 & . \\
\hline 13 & 1378.6923 & 689.8498 & & & 1360.6817 & 680.8445 & V & 618.3457 & 309.6765 & 601.3192 & 301.1632 & 600.3351 & 300.6712 & \\
\hline 14 & 1435.7137 & 718.3605 & & & 1417.7032 & 709.3552 & G & 519.2773 & 260.1423 & 502.2507 & 251.6290 & 501.2667 & 251.1370 & \\
\hline 15 & 1550.7407 & 775.8740 & \begin{tabular}{|l|l}
1533.7141 \\
\end{tabular} & 767.3607 & 1532.7301 & 766.8687 & $\mathbf{N}$ & 462.2558 & 231.6316 & 445.2293 & 223.1183 & 444.2453 & 222.6263 & 4 \\
\hline 16 & 1649.8091 & 825.4082 & 1632.7826 & 816.8949 & 1631.7985 & 816.4029 & V & 347.2289 & 174.1181 & 330.2023 & 165.6048 & 329.2183 & 165.1128 & \\
\hline 17 & 1750.8568 & 875.9320 & 1733.8302 & 867.4188 & 1732.8462 & 866.9267 & $T$ & 248.1605 & 124.5839 & 231.1339 & 116.0706 & 230.1499 & 115.5786 & \\
\hline 18 & & & & & & & $\mathbf{K}$ & 147.1128 & 74.0600 & 130.0863 & 65.5468 & & & \\
\hline
\end{tabular}

然$$
\text { 蒠 }
$$

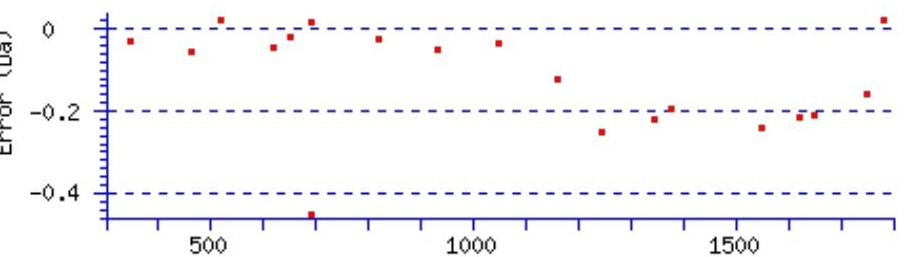

RMS error $181 \mathrm{ppm}$

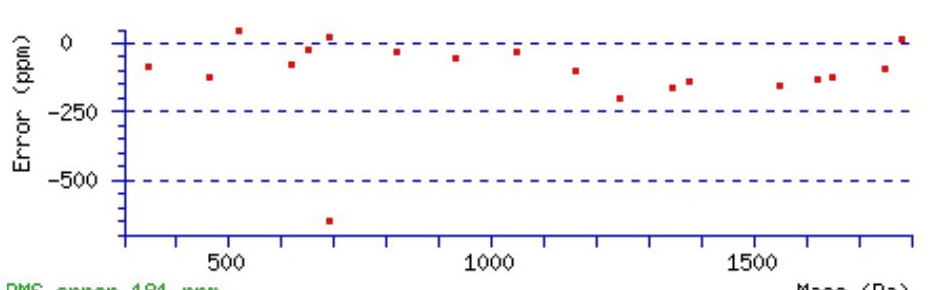

RMS error $181 \mathrm{ppm}$

\section{All matches to this query}

\begin{tabular}{|l|l|c|c|}
\hline Score & Mr(calc): & Delta & Sequence \\
\hline 145.0 & 1895.9550 & -0.0039 & IYAGMVSLLDEAVGNVTK \\
\hline 0.4 & 1895.9332 & 0.0179 & CTLSANLVASGELMSSKK \\
\hline
\end{tabular}


Spectrum No: 3; Query: 2284; Rank: 1

\section{Peptide View}

MS/MS Fragmentation of LVQDVANNTNEEAGDGTTTATVLAR

Found in IPI00339148, Tax_Id=10116 Gene_Symbol=Hspd1 60 kDa heat shock protein, mitochondrial precursor

Match to Query 2284: 2560.230988 from(1281.122770,2+)

Title: 091008RatKidney_NoSalt_21.2179.2179.2.dta

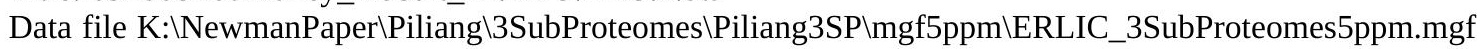
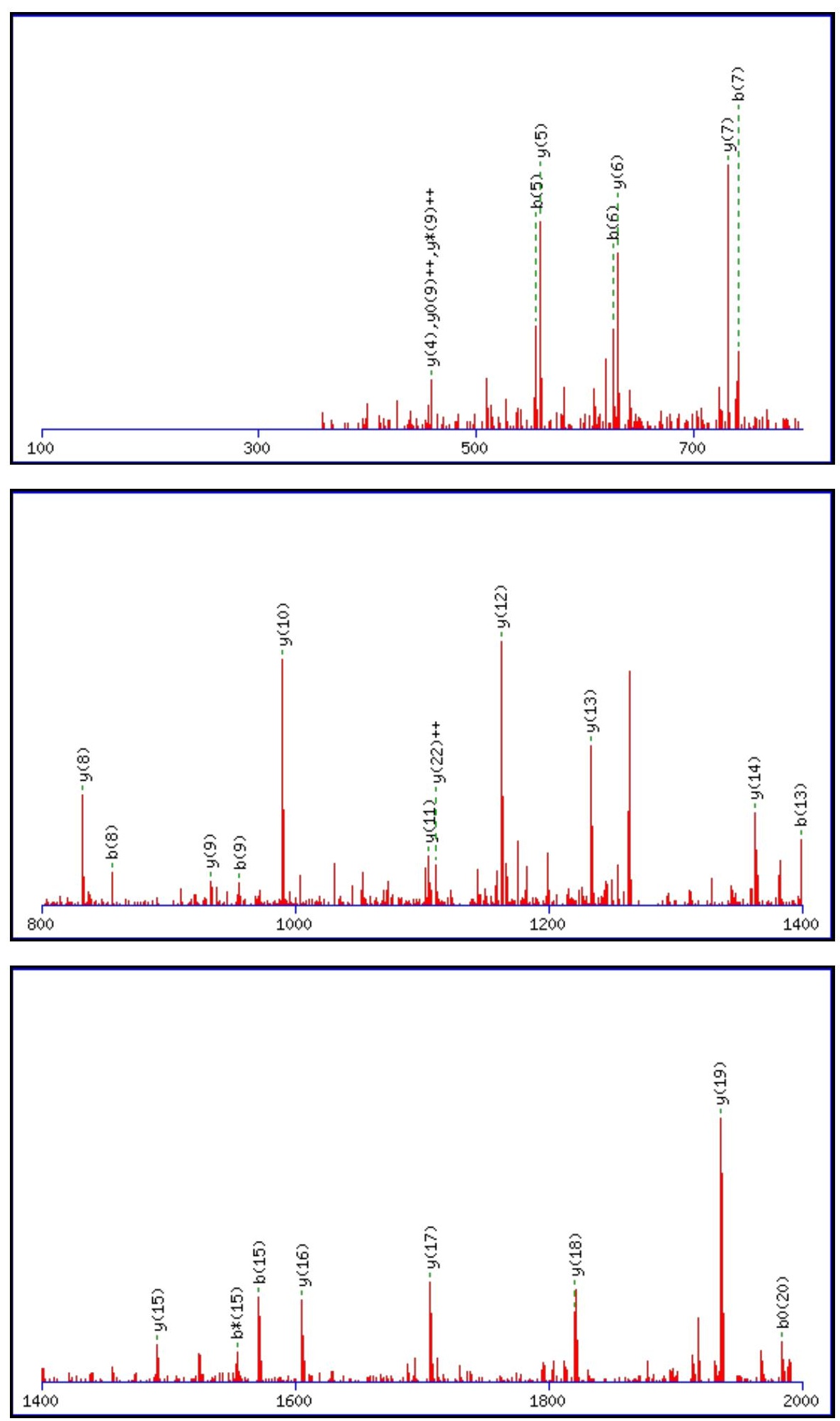
Monoisotopic mass of neutral peptide $\operatorname{Mr}($ calc): 2560.2253

Fixed modifications: Carbamidomethyl (C)

Variable modifications:

N7 : Deamidated_N (N)

Ions Score: 139 Expect: $3.6 \mathrm{e}-012$

Matches (Bold Red): 28/270 fragment ions using 37 most intense peaks

\begin{tabular}{|c|c|c|c|c|c|c|c|c|c|c|c|c|c|c|}
\hline \# & b & $\mathbf{b}^{++}$ & $\mathbf{b}^{*}$ & $\mathbf{b}^{*^{++}}$ & $\mathbf{b}^{\mathbf{0}}$ & $\mathbf{b}^{\mathbf{0 + +}}$ & Seq. & $\mathbf{y}$ & $y^{++}$ & $\mathbf{y}^{*}$ & $\mathrm{y}^{\mathrm{*}^{++}}$ & $\mathbf{y}^{0}$ & $\mathbf{y}^{0++}$ & $\#$ \\
\hline 1 & 114.0913 & 57.5493 & & & & & $\mathbf{L}$ & & & & & & & 25 \\
\hline 2 & 213.1598 & 107.0835 & & & & & $\bar{V}$ & 2448.1485 & 1224.5779 & 2431.1219 & 1216.0646 & 2430.1379 & 1215.5726 & 24 \\
\hline 3 & 341.2183 & 171.1128 & 324.1918 & 162.5995 & & & $\mathbf{Q}$ & 2349.0801 & 1175.0437 & 2332.0535 & 1166.5304 & 2331.0695 & 1166.0384 & 23 \\
\hline 4 & 456.2453 & 228.6263 & 439.2187 & 220.1130 & 438.2347 & 219.6210 & D & 2221.0215 & 1111.0144 & 2203.9949 & 1102.5011 & 2203.0109 & 1102.0091 & 22 \\
\hline 5 & 555.3137 & 278.1605 & 538.2871 & 269.6472 & 537.3031 & 269.1552 & V & 2105.9945 & 1053.5009 & 2088.9680 & 1044.9876 & 2087.9840 & 1044.4956 & 21 \\
\hline 6 & 626.3508 & 313.6790 & 609.3243 & 305.1658 & 608.3402 & 304.6738 & A & 2006.9261 & 1003.9667 & 1989.8996 & 995.4534 & 1988.9156 & 994.9614 & 20 \\
\hline 7 & 741.3777 & 371.1925 & 724.3512 & 362.6792 & 723.3672 & 362.1872 & $\mathbf{N}$ & 1935.8890 & 968.4481 & 1918.8625 & 959.9349 & 1917.8784 & 959.4429 & 19 \\
\hline 8 & 855.4207 & 428.2140 & 838.3941 & 419.7007 & 837.4101 & 419.2087 & $\mathbf{N}$ & 1820.8621 & 910.9347 & 1803.8355 & 902.4214 & 1802.8515 & 901.9294 & 18 \\
\hline 9 & 956.4683 & 478.7378 & 939.4418 & 470.2245 & 938.4578 & 469.7325 & $\mathbf{T}$ & 1706.8192 & 853.9132 & 1689.7926 & 845.3999 & 1688.8086 & 844.9079 & 17 \\
\hline 10 & 1070.5113 & 535.7593 & 1053.4847 & 527.2460 & 1052.5007 & 526.7540 & $\mathbf{N}$ & 1605.7715 & 803.3894 & 1588.7449 & 794.8761 & 1587.7609 & 794.3841 & 16 \\
\hline 11 & 1199.5539 & 600.2806 & 1182.5273 & 591.7673 & 1181.5433 & 591.2753 & $\mathbf{E}$ & 1491.7285 & 746.3679 & 1474.7020 & 737.8546 & 1473.7180 & 737.3626 & 15 \\
\hline 12 & 1328.5965 & 664.8019 & 1311.5699 & 656.2886 & 1310.5859 & 655.7966 & $\mathbf{E}$ & 1362.6860 & 681.8466 & 1345.6594 & 673.3333 & 1344.6754 & 672.8413 & 14 \\
\hline 13 & 1399.6336 & 700.3204 & 1382.6070 & 691.8071 & 1381.6230 & 691.3151 & $\mathbf{A}$ & 1233.6434 & 617.3253 & 1216.6168 & 608.8120 & 1215.6328 & 608.3200 & 13 \\
\hline 14 & 1456.6550 & 728.8312 & 1439.6285 & 720.3179 & 1438.6445 & 719.8259 & G & 1162.6062 & 581.8068 & 1145.5797 & 573.2935 & 1144.5957 & 572.8015 & 12 \\
\hline 15 & $\mathbf{1 5 7 1 . 6 8 2 0}$ & 786.3446 & 1554.6554 & 777.8314 & 1553.6714 & 777.3393 & D & 1105.5848 & 553.2960 & 1088.5582 & 544.7828 & 1087.5742 & 544.2907 & 11 \\
\hline 16 & 1628.7034 & 814.8554 & 1611.6769 & 806.3421 & 1610.6929 & 805.8501 & $\mathbf{G}$ & 990.5578 & 495.7826 & 973.5313 & 487.2693 & 972.5473 & 486.7773 & 10 \\
\hline 17 & 1729.7511 & 865.3792 & 1712.7246 & 856.8659 & 1711.7406 & 856.3739 & $T$ & 933.5364 & 467.2718 & 916.5098 & 458.7586 & 915.5258 & 458.2665 & 9 \\
\hline 18 & 1830.7988 & 915.9030 & 1813.7723 & 907.3898 & 1812.7882 & 906.8978 & $\mathbf{T}$ & 832.4887 & 416.7480 & 815.4621 & 408.2347 & 814.4781 & 407.7427 & 8 \\
\hline 19 & 1931.8465 & 966.4269 & 1914.8199 & 957.9136 & 1913.8359 & 957.4216 & $T$ & 731.4410 & 366.2241 & 714.4145 & 357.7109 & 713.4305 & 357.2189 & 7 \\
\hline 20 & 2002.8836 & 1001.9454 & 1985.8570 & 993.4322 & \begin{tabular}{|l|}
1984.8730 \\
\end{tabular} & 992.9402 & $\mathbf{A}$ & 630.3933 & 315.7003 & 613.3668 & 307.1870 & 612.3828 & 306.6950 & 6 \\
\hline 21 & 2103.9313 & 1052.4693 & 2086.9047 & 1043.9560 & 2085.9207 & 1043.4640 & $T$ & 559.3562 & 280.1817 & 542.3297 & 271.6685 & 541.3457 & 271.1765 & 5 \\
\hline 22 & 2202.9997 & 1102.0035 & 2185.9731 & 1093.4902 & 2184.9891 & 1092.9982 & $\mathrm{~V}$ & 458.3085 & 229.6579 & 441.2820 & 221.1446 & & & 4 \\
\hline 23 & 2316.0838 & 1158.5455 & 2299.0572 & 1150.0322 & 2298.0732 & 1149.5402 & $\mathbf{L}$ & 359.2401 & 180.1237 & 342.2136 & 171.6104 & & & 3 \\
\hline 24 & 2387.1209 & 1194.0641 & 2370.0943 & 1185.5508 & 2369.1103 & 1185.0588 & $\mathbf{A}$ & 246.1561 & 123.5817 & 229.1295 & 115.0684 & & & 2 \\
\hline 25 & & & & & & & $\mathbf{R}$ & 175.1190 & 88.0631 & 158.0924 & 79.5498 & & & 1 \\
\hline
\end{tabular}
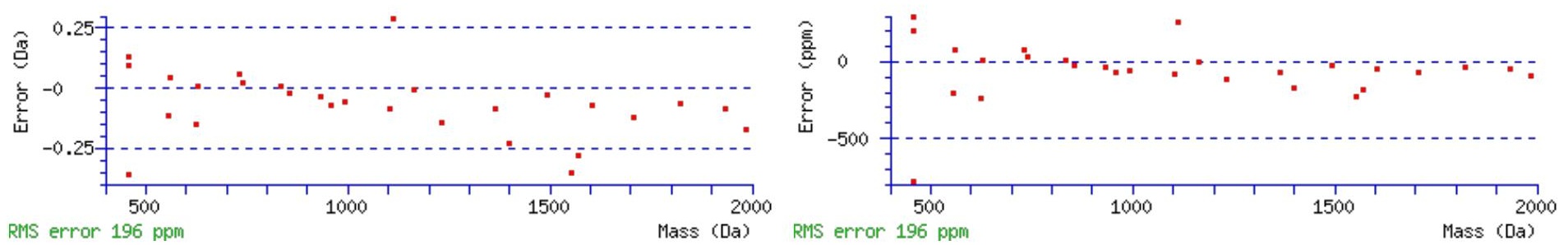

\section{All matches to this query}

\begin{tabular}{|l|l|l|l|}
\hline Score & Mr(calc): & Delta & \multicolumn{1}{c|}{ Sequence } \\
\hline 138.9 & 2560.2253 & 0.0057 & LVQDVANNTNEEAGDGTTTATVLAR \\
\hline 135.4 & 2560.2253 & 0.0057 & LVQDVANNTNEEAGDGTTTATVLAR \\
\hline 113.3 & 2560.2253 & 0.0057 & LVQDVANNTNEEAGDGTTTATVLAR \\
\hline 103.0 & 2559.2413 & 0.9897 & LVQDVANNTNEEAGDGTTTATVLAR \\
\hline 1.9 & 2559.2080 & 1.0230 & QYLFVSNKTGTLHEACEQLLK \\
\hline
\end{tabular}

Spectrum No: 4; Query: 1901; Rank: 1 


\section{Peptide View}

MS/MS Fragmentation of DGGSTTAGNSSQVSDGAAAVLLAR

Found in IPI00370596, Tax_Id=10116 Gene_Symbol=RGD1562373_predicted 3-ketoacyl-CoA thiolase B, peroxisomal precursor

Match to Query 1901: 2205.050968 from(1103.532760,2+)

Title: 091008RatKidney_NoSalt_22.2961.2961.2.dta

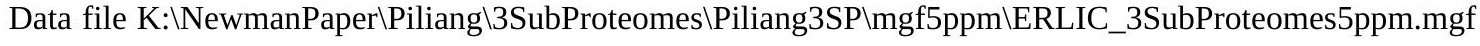
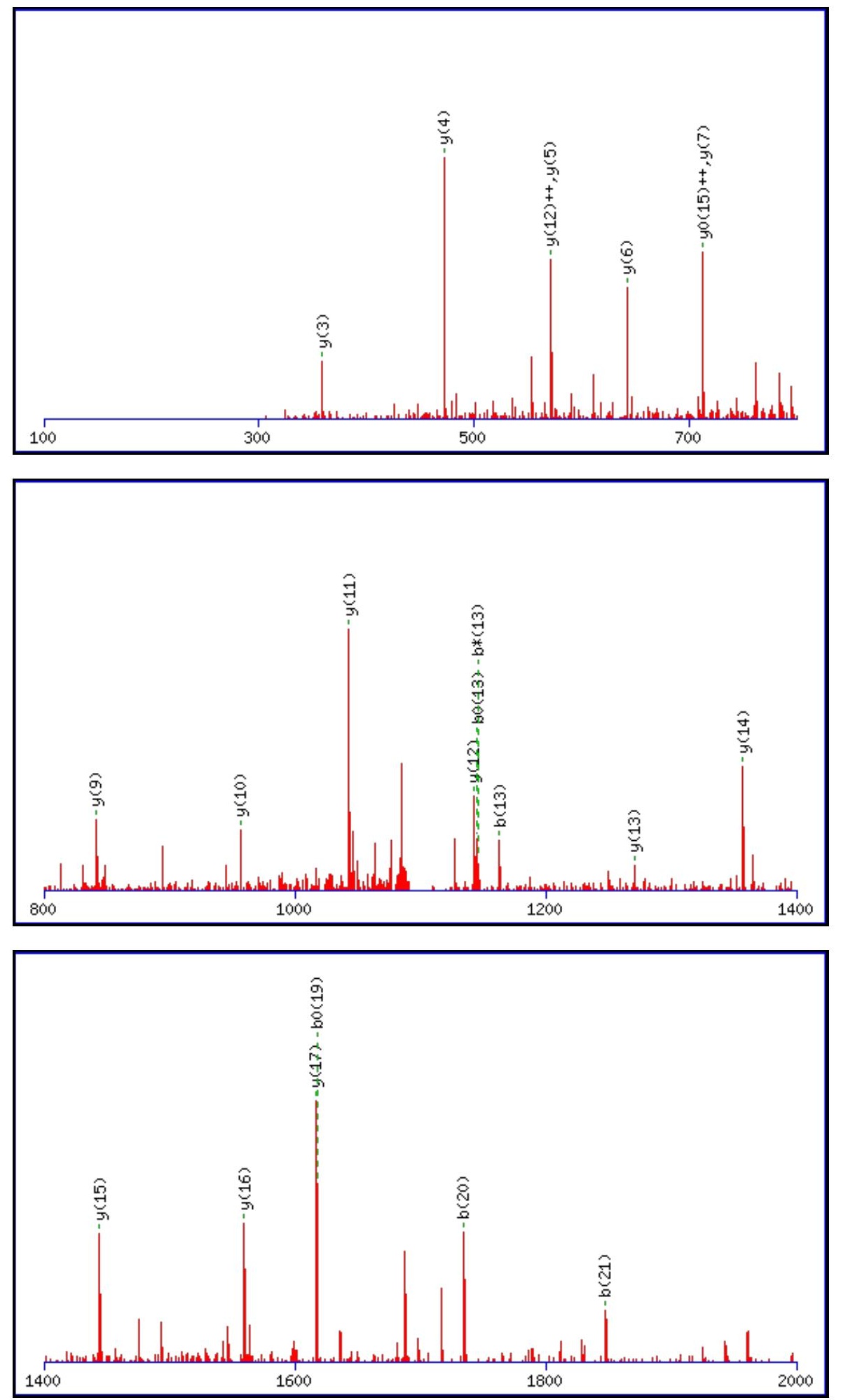

Monoisotopic mass of neutral peptide $\operatorname{Mr}$ (calc): 2205.0509

Fixed modifications: Carbamidomethyl (C)

Variable modifications:

N9 : Deamidated_N (N)

Ions Score: 139 Expect: $3.4 \mathrm{e}-012$

Matches (Bold Red): 22/242 fragment ions using 25 most intense peaks 


\begin{tabular}{|c|c|c|c|c|c|c|c|c|c|c|c|c|c|c|}
\hline \# & b & $\mathbf{b}^{++}$ & $\mathbf{b}^{*}$ & $\mathbf{b}^{*^{++}}$ & $\mathbf{b}^{\mathbf{0}}$ & $\mathbf{b}^{\mathbf{0 + +}}$ & Seq. & $\mathbf{y}$ & $\mathbf{y}^{++}$ & $\mathrm{y}^{*}$ & $\mathrm{y}^{\mathrm{*}^{++}}$ & $\mathbf{y}^{\mathbf{0}}$ & $y^{0++}$ & \# \\
\hline 1 & 116.0342 & 58.5207 & & & 98.0237 & 49.5155 & D & & & & & & & 24 \\
\hline 2 & 173.0557 & 87.0315 & & & 155.0451 & 78.0262 & G & 2091.0313 & 1046.0193 & 2074.0047 & 1037.5060 & 2073.0207 & 1037.0140 & 23 \\
\hline 3 & 230.0771 & 115.5422 & & & 212.0666 & 106.5369 & G & 2034.0098 & 1017.5085 & 2016.9832 & 1008.9953 & 2015.9992 & 1008.5033 & 22 \\
\hline 4 & 317.1092 & 159.0582 & & & 299.0986 & 150.0529 & $\mathrm{~S}$ & 1976.9883 & 988.9978 & 1959.9618 & 980.4845 & 1958.9778 & 979.9925 & 21 \\
\hline 5 & 418.1569 & 209.5821 & & & 400.1463 & 200.5768 & $T$ & 1889.9563 & 945.4818 & 1872.9298 & 936.9685 & 1871.9457 & 936.4765 & 20 \\
\hline 6 & 519.2045 & 260.1059 & & & 501.1940 & 251.1006 & $T$ & 1788.9086 & 894.9580 & 1771.8821 & 886.4447 & 1770.8981 & 885.9527 & 19 \\
\hline 7 & 590.2416 & 295.6245 & & & 572.2311 & 286.6192 & A & 1687.8609 & 844.4341 & 1670.8344 & 835.9208 & 1669.8504 & 835.4288 & 18 \\
\hline 8 & 647.2631 & 324.1352 & & & 629.2525 & 315.1299 & G & \begin{tabular}{|l|}
1616.8238 \\
\end{tabular} & 808.9156 & 1599.7973 & 800.4023 & 1598.8133 & 799.9103 & 17 \\
\hline 9 & 762.2900 & 381.6487 & 745.2635 & 373.1354 & 744.2795 & 372.6434 & $\mathbf{N}$ & 1559.8024 & 780.4048 & 1542.7758 & 771.8915 & 1541.7918 & 771.3995 & 16 \\
\hline 10 & 849.3221 & 425.1647 & 832.2955 & 416.6514 & 831.3115 & 416.1594 & $\mathrm{~S}$ & 1444.7754 & 722.8914 & 1427.7489 & 714.3781 & 1426.7649 & 713.8861 & 15 \\
\hline 11 & 936.3541 & 468.6807 & 919.3276 & 460.1674 & 918.3435 & 459.6754 & $\mathrm{~S}$ & 1357.7434 & 679.3753 & 1340.7169 & 670.8621 & 1339.7328 & 670.3701 & 14 \\
\hline 12 & 1064.4127 & 532.7100 & 1047.3861 & 524.1967 & 1046.4021 & 523.7047 & $\mathbf{Q}$ & 1270.7114 & 635.8593 & 1253.6848 & 627.3461 & 1252.7008 & 626.8540 & 13 \\
\hline 13 & 1163.4811 & 582.2442 & 1146.4545 & 573.7309 & 1145.4705 & 573.2389 & $\mathbf{V}$ & 1142.6528 & 571.8300 & 1125.6263 & 563.3168 & 1124.6422 & 562.8248 & 12 \\
\hline 14 & 1250.5131 & 625.7602 & 1233.4866 & 617.2469 & 1232.5026 & 616.7549 & $\mathrm{~S}$ & 1043.5844 & 522.2958 & 1026.5578 & 513.7826 & 1025.5738 & 513.2905 & 11 \\
\hline 15 & 1365.5401 & 683.2737 & 1348.5135 & 674.7604 & 1347.5295 & 674.2684 & D & 956.5524 & 478.7798 & 939.5258 & 470.2665 & 938.5418 & 469.7745 & 10 \\
\hline 16 & 1422.5615 & 711.7844 & 1405.5350 & 703.2711 & 1404.5510 & 702.7791 & G & 841.5254 & 421.2663 & 824.4989 & 412.7531 & & & 9 \\
\hline 17 & 1493.5986 & 747.3030 & 1476.5721 & 738.7897 & 1475.5881 & 738.2977 & $\mathbf{A}$ & 784.5040 & 392.7556 & 767.4774 & 384.2423 & & & 8 \\
\hline 18 & 1564.6358 & 782.8215 & 1547.6092 & 774.3082 & 1546.6252 & 773.8162 & A & 713.4668 & 357.2371 & 696.4403 & 348.7238 & & & 7 \\
\hline 19 & 1635.6729 & 818.3401 & 1618.6463 & 809.8268 & 1617.6623 & 809.3348 & A & 642.4297 & 321.7185 & 625.4032 & 313.2052 & & & 6 \\
\hline 20 & 1734.7413 & 867.8743 & 1717.7147 & 859.3610 & 1716.7307 & 858.8690 & $\mathbf{V}$ & 571.3926 & 286.1999 & 554.3661 & 277.6867 & & & 5 \\
\hline 21 & 1847.8254 & 924.4163 & 1830.7988 & 915.9030 & 1829.8148 & 915.4110 & $\mathbf{L}$ & 472.3242 & 236.6657 & 455.2976 & 228.1525 & & & 4 \\
\hline 22 & 1960.9094 & 980.9583 & 1943.8829 & \begin{tabular}{|l|}
972.4451 \\
\end{tabular} & 1942.8988 & 971.9531 & $\mathbf{L}$ & 359.2401 & 180.1237 & 342.2136 & 171.6104 & & & 3 \\
\hline 23 & 2031.9465 & 1016.4769 & 2014.9200 & 1007.9636 & 2013.9360 & 1007.4716 & A & 246.1561 & 123.5817 & 229.1295 & 115.0684 & & & 2 \\
\hline 24 & & & & & & & $\mathbf{R}$ & 175.1190 & 88.0631 & 158.0924 & 79.5498 & & & 1 \\
\hline
\end{tabular}
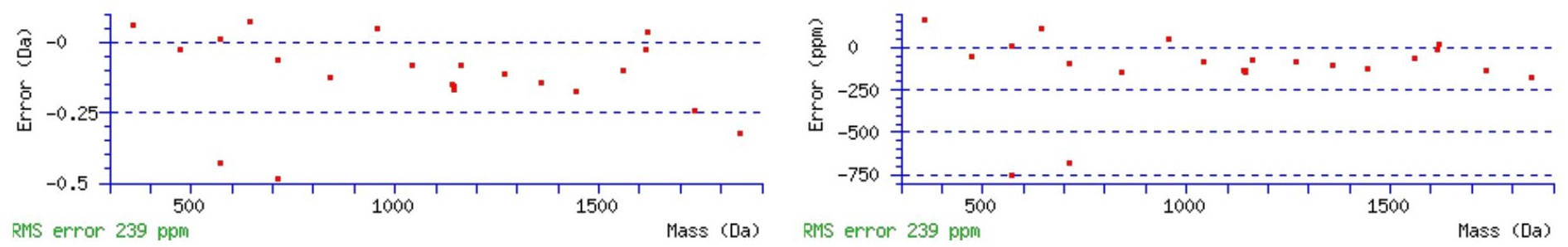

\section{All matches to this query}

\begin{tabular}{|l|l|l|l|}
\hline Score & Mr(calc): & Delta & \multicolumn{1}{|c|}{ Sequence } \\
\hline 138.8 & 2205.0509 & 0.0000 & DGGSTTAGNSSQVSDGAAAVLLAR \\
\hline 91.7 & 2204.0669 & 0.9840 & DGGSTTAGNSSQVSDGAAAVLLAR \\
\hline 12.8 & 2203.0438 & 2.0072 & GFSWWPAMVVSWKATSKR \\
\hline 12.2 & 2203.0438 & 2.0072 & GFSWWPAMVVSWKATSKR \\
\hline 3.7 & 2205.0428 & 0.0082 & IMASFGNFANDNEIKVLLK \\
\hline 2.5 & 2204.0588 & 0.9922 & IMASFGNFANDNEIKVLLK \\
\hline 2.5 & 2204.0588 & 0.9922 & IMASFGNFANDNEIKVLLK \\
\hline 2.0 & 2205.0496 & 0.0014 & ESPKMQMMAVSITSILALK \\
\hline 1.6 & 2203.0270 & 2.0239 & NFLLQGSSTNRPTASTSNTK \\
\hline 1.6 & 2203.0270 & 2.0239 & NFLLQGSSTNRPTASTSNTK \\
\hline
\end{tabular}

Spectrum No: 5; Query: 2792; Rank: 1 


\section{Peptide View}

\section{MS/MS Fragmentation of VSGILFNDEMDDFSSPNFTNQFGVAPSPANFIKPGK}

Found in IPI00206254, Tax_Id=10116 Gene_Symbol=Ggt1 Gamma-glutamyltranspeptidase 1 precursor

Match to Query 2792: 3887.852742 from(1296.958190,3+)

Title: 100101RatKid_NS_deglyco_14.6003.6003.3.dta

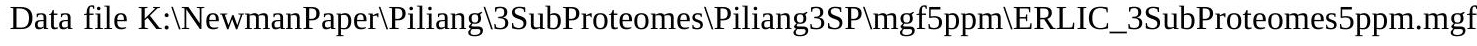
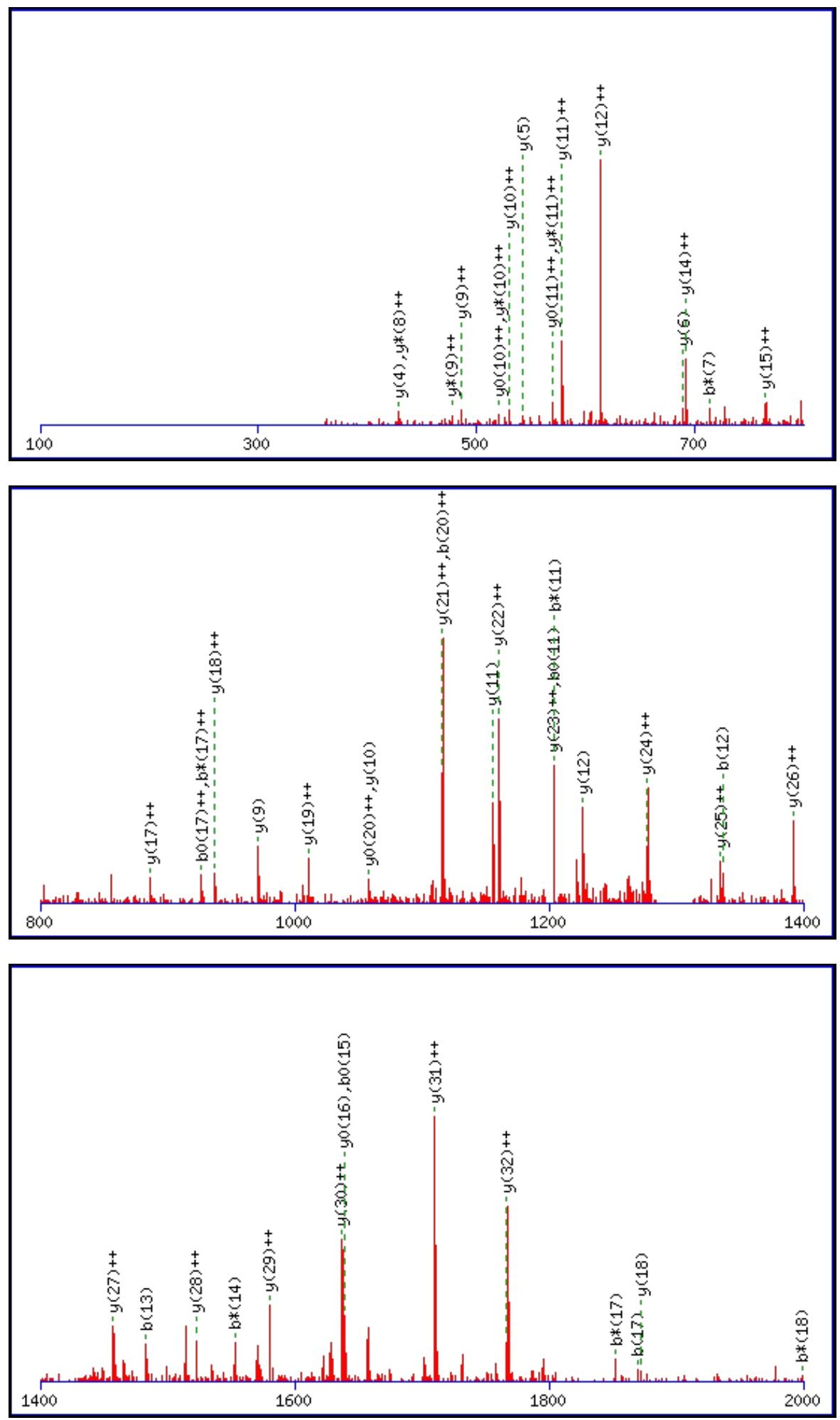

Monoisotopic mass of neutral peptide $\operatorname{Mr}($ calc): 3887.8353

Fixed modifications: Carbamidomethyl (C)

Variable modifications:

N17 : Deamidated_N (N)

Ions Score: 136 Expect: 1e-011

Matches (Bold Red): 50/388 fragment ions using 71 most intense peaks 


\begin{tabular}{|c|c|c|c|c|c|c|c|c|c|c|c|c|c|c|}
\hline \# & b & $\mathbf{b}^{++}$ & $\mathbf{b}^{*}$ & $\mathbf{b}^{*++}$ & $\mathbf{b}^{\mathbf{0}}$ & $\mathbf{b}^{\mathbf{0 + +}}$ & Seq. & $\mathbf{y}$ & $\mathbf{y}^{++}$ & $\mathbf{y}^{*}$ & $\mathrm{y}^{\mathrm{*}^{++}}$ & $\mathbf{y}^{0}$ & $\mathbf{y}^{\mathbf{0 + +}^{++}}$ & \# \\
\hline 1 & 100.0757 & 50.5415 & & & & & $\mathbf{V}$ & & & & & & & 36 \\
\hline 2 & 187.1077 & 94.0575 & & & 169.0972 & 85.0522 & $\mathrm{~S}$ & 3789.7741 & 1895.3907 & 3772.7476 & 1886.8774 & 3771.7635 & 1886.3854 & 35 \\
\hline 3 & 244.1292 & 122.5682 & & & 226.1186 & 113.5629 & $\mathbf{G}$ & 3702.7421 & 1851.8747 & 3685.7155 & 1843.3614 & 3684.7315 & 1842.8694 & 34 \\
\hline 4 & 357.2132 & 179.1103 & & & 339.2027 & 170.1050 & I & 3645.7206 & 1823.3639 & 3628.6941 & 1814.8507 & 3627.7101 & 1814.3587 & 33 \\
\hline 5 & 470.2973 & 235.6523 & & & 452.2867 & 226.6470 & $\mathbf{L}$ & 3532.6366 & 1766.8219 & 3515.6100 & 1758.3086 & 3514.6260 & 1757.8166 & 32 \\
\hline 6 & 617.3657 & 309.1865 & & & 599.3552 & 300.1812 & $\mathbf{F}$ & 3419.5525 & 1710.2799 & 3402.5259 & 1701.7666 & 3401.5419 & 1701.2746 & 31 \\
\hline 7 & 731.4087 & 366.2080 & 714.3821 & 357.6947 & 713.3981 & 357.2027 & $\mathbf{N}$ & 3272.4841 & 1636.7457 & 3255.4575 & 1628.2324 & 3254.4735 & 1627.7404 & 30 \\
\hline 8 & 846.4356 & 423.7214 & 829.4090 & 415.2082 & 828.4250 & 414.7162 & D & 3158.4412 & 1579.7242 & 3141.4146 & 1571.2109 & 3140.4306 & 1570.7189 & 29 \\
\hline 9 & 975.4782 & 488.2427 & 958.4516 & 479.7295 & 957.4676 & 479.2374 & $\mathbf{E}$ & 3043.4142 & 1522.2107 & 3026.3877 & 1513.6975 & 3025.4036 & 1513.2055 & 28 \\
\hline 10 & 1106.5187 & 553.7630 & 1089.4921 & 545.2497 & 1088.5081 & 544.7577 & $\mathbf{M}$ & 2914.3716 & 1457.6894 & 2897.3451 & 1449.1762 & 2896.3611 & 1448.6842 & 27 \\
\hline 11 & 1221.5456 & 611.2764 & 1204.5191 & 602.7632 & 1203.5351 & 602.2712 & D & 2783.3311 & 1392.1692 & 2766.3046 & 1383.6559 & 2765.3206 & 1383.1639 & 26 \\
\hline 12 & 1336.5726 & 668.7899 & 1319.5460 & 660.2766 & 1318.5620 & 659.7846 & D & 2668.3042 & 1334.6557 & 2651.2776 & 1326.1425 & 2650.2936 & 1325.6504 & 25 \\
\hline 13 & 1483.6410 & 742.3241 & 1466.6144 & 733.8108 & 1465.6304 & 733.3188 & $F$ & 2553.2772 & 1277.1423 & 2536.2507 & 1268.6290 & 2535.2667 & 1268.1370 & 24 \\
\hline 14 & 1570.6730 & 785.8401 & 1553.6465 & 777.3269 & 1552.6624 & 776.8349 & S & 2406.2088 & 1203.6081 & 2389.1823 & 1195.0948 & 2388.1983 & 1194.6028 & 23 \\
\hline 15 & 1657.7050 & 829.3562 & 1640.6785 & 820.8429 & 1639.6945 & 820.3509 & $\mathrm{~S}$ & 2319.1768 & 1160.0920 & 2302.1503 & 1151.5788 & 2301.1662 & 1151.0868 & 22 \\
\hline 16 & 1754.7578 & 877.8825 & 1737.7312 & 869. & 1736.7472 & 868.8773 & $\mathbf{P}$ & 2232.1448 & 1116 & 2215.1182 & 1108.0628 & 1342 & 1107.5707 & 21 \\
\hline 17 & 1869.7847 & 935.3960 & 1852.7582 & 926.8827 & 1851.7742 & 926.3907 & $\mathbf{N}$ & 2135.0920 & 1068.0496 & 2118.0655 & 1059.5364 & 2117.0814 & 1059.0444 & 20 \\
\hline 18 & 2016.8531 & 1008.9302 & 1999.8266 & 1000.4169 & 1998.8426 & 999.9249 & $\mathbf{F}$ & 2020.0651 & 1010.5362 & 2003.0385 & 1002.0229 & 2002.0545 & 1001.5309 & 19 \\
\hline 19 & 2117.9008 & 1059.4540 & 2100.8743 & 1050.9408 & 2099.8903 & 1050.4488 & $\mathbf{T}$ & 1872.9967 & 937.0020 & 1855.9701 & 928.4887 & 1854.9861 & 927.9967 & 18 \\
\hline 20 & 2231.9437 & 1116.4755 & 2214.9172 & 1107.9622 & 2213.9332 & 1107.4702 & $\mathbf{N}$ & 1771.9490 & 886.4781 & 1754.9224 & 877.9649 & 1753.9384 & 877.4728 & 17 \\
\hline 21 & 2360.0023 & 1180.5048 & 2342.9758 & 1171.9915 & 2341.9918 & 1171.4995 & $\mathbf{Q}$ & 1657.9061 & 829.4567 & 1640.8795 & 820.9434 & 1639.8955 & 820.4514 & 16 \\
\hline 22 & 2507.0707 & 1254.0390 & 2490.0442 & 1245.5257 & 2489.0602 & 1245.0337 & $\mathbf{F}$ & 1529.8475 & 765.4274 & 1512.8209 & 756.9141 & 1511.8369 & 756.4221 & 15 \\
\hline 23 & 2564.0922 & 1282.5497 & 2547.0657 & 1274.0365 & 2546.0816 & 1273.5445 & G & 1382.7791 & 691.8932 & 1365.7525 & 683.3799 & 1364.7685 & 682.8879 & 14 \\
\hline 24 & 2663.1606 & 1332.0839 & 2646.1341 & 1323.5707 & 2645.1501 & 1323.0787 & $\mathbf{V}$ & 1325.7576 & 663.3824 & 1308.7310 & 654.8692 & 1307.7470 & 654.3772 & 13 \\
\hline 25 & 2734.1977 & 1367.6025 & 2717.1712 & 1359.0892 & 2716.1872 & 1358.5972 & $\mathbf{A}$ & 1226.6892 & 613.8482 & 1209.6626 & 605.3350 & 1208.6786 & 604.8429 & 12 \\
\hline 26 & 2831.2505 & 1416.1289 & 2814.2239 & 1407.6156 & 2813.2399 & 1407.1236 & $\mathbf{P}$ & 1155.6521 & 578.3297 & 1138.6255 & 569.8164 & 1137.6415 & 569.3244 & 11 \\
\hline 27 & 2918.2825 & 1459.6449 & 2901.2560 & 1451.1316 & 2900.2720 & 1450.6396 & $\mathrm{~S}$ & 1058.5993 & 529.8033 & 1041.5728 & 521.2900 & 1040.5887 & 520.7980 & 10 \\
\hline 28 & 3015.3353 & 1508.1713 & 2998.3087 & 1499.6580 & 2997.3247 & 1499.1660 & $\mathbf{P}$ & 971.5673 & 486.2873 & 954.5407 & 477.7740 & & & 9 \\
\hline 29 & 3086.3724 & 1543.6898 & 3069.3459 & 1535.1766 & 3068.3618 & 1534.6846 & A & 874.5145 & 437.7609 & 857.4880 & 429.2476 & & & 8 \\
\hline 30 & 3200.4153 & 1600.7113 & 3183.3888 & 1592.1980 & 3182.4048 & 1591.7060 & $\mathbf{N}$ & 803.4774 & 402.2423 & 786.4509 & 393.7291 & & & 7 \\
\hline 31 & 3347.4837 & 1674.2455 & 3330.4572 & 1665.7322 & 3329.4732 & 1665.2402 & F & 689.4345 & 345.2209 & 672.4079 & 336.7076 & & & 6 \\
\hline 32 & 3460.5678 & 1730.7875 & 3443.5413 & 1722.2743 & 3442.5572 & 1721.7823 & $\mathbf{I}$ & 542.3661 & 271.6867 & 525.3395 & 263.1734 & & & 5 \\
\hline 33 & 3588.6628 & 1794.8350 & 3571.6362 & 1786.3217 & 3570.6522 & 1785.8297 & $\mathbf{K}$ & 429.2820 & 215.1446 & 412.2554 & 206.6314 & & & 4 \\
\hline 34 & 3685.7155 & 1843.3614 & 3668.6890 & 1834.8481 & 3667.7050 & 1834.3561 & $\mathbf{P}$ & 301.1870 & 151.0972 & 284.1605 & 142.5839 & & & 3 \\
\hline 35 & 3742.7370 & 1871.8721 & 3725.7104 & 1863.3589 & 3724.7264 & 1862.8669 & G & 204.1343 & 102.5708 & 187.1077 & 94.0575 & & & 2 \\
\hline 36 & & & & & & & $\mathbf{K}$ & 147.1128 & 74.0600 & 130.0863 & 65.5468 & & & 1 \\
\hline
\end{tabular}
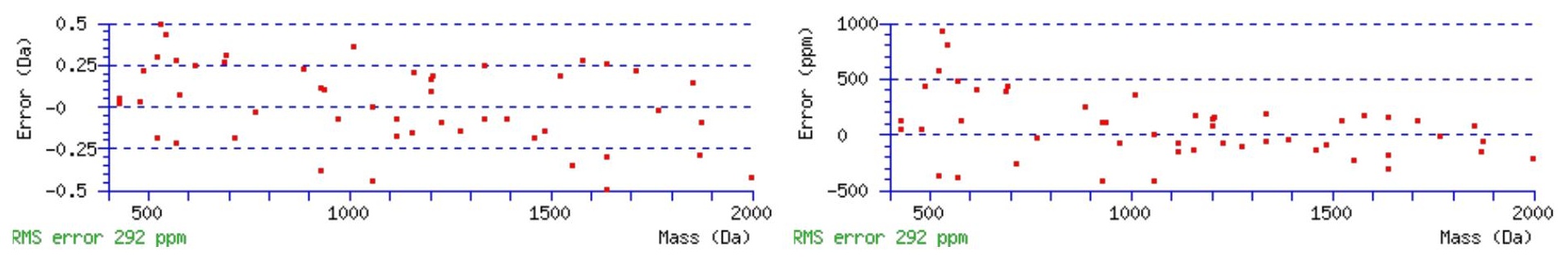

\section{All matches to this query}

\begin{tabular}{|l|l|l|l|}
\hline Score & Mr(calc): & Delta & \multicolumn{1}{c|}{ Sequence } \\
\hline 135.6 & 3887.8353 & 0.0175 & VSGILFNDEMDDFSSPNFTNQFGVAPSPANFIKPGK \\
\hline 123.7 & 3887.8353 & 0.0175 & VSGILFNDEMDDFSSPNFTNQFGVAPSPANFIKPGK \\
\hline
\end{tabular}




\begin{tabular}{|l|l|l|l|}
98.7 & 3887.8353 & 0.0175 & VSGILFNDEMDDFSSPNFTNQFGVAPSPANFIKPGK \\
\hline 69.5 & 3887.8353 & 0.0175 & VSGILFNDEMDDFSSPNFTNQFGVAPSPANFIKPGK \\
\hline 55.8 & 3886.8513 & 1.0015 & VSGILFNDEMDDFSSPNFTNQFGVAPSPANFIKPGK \\
\hline 3.8 & 3885.8155 & 2.0373 & NCRSLMNQVQNIAELATLSLERLTGILGHSGNAK \\
\hline 3.6 & 3885.8099 & 2.0428 & EEKPMPTASLLSPVVLAPVPSRRNPSGGMSSLILN \\
\hline 2.5 & 3887.8436 & 0.0091 & NVSIPALNDSKYIGMSVYNVGIMCIIGAAVSFLTR \\
\hline 2.1 & 3885.8747 & 1.9780 & LAALKDEPQTVPDVPSFGDSPPLSPIDMDTQERIK \\
\hline 1.9 & 3886.8790 & 0.9737 & TGPMKEINLSPDSTSVIVSGLMVATKYEVSVYALK \\
\hline
\end{tabular}

Spectrum No: 6; Query: 1942; Rank: 1

\section{Peptide View}

MS/MS Fragmentation of YGYENDTEIANLYDEMVAK

Found in IPI00191437, Tax_Id=10116 Gene_Symbol=- 64 kDa protein

Match to Query 1942: 2237.971288 from(1119.992920,2+)

Title: 091008RatKidney_NoSalt_21.5392.5392.2.dta

Data file K:INewmanPaper|Piliangl3SubProteomes\Piliang3SP\mgf5ppm\ERLIC_3SubProteomes5ppm.mgf
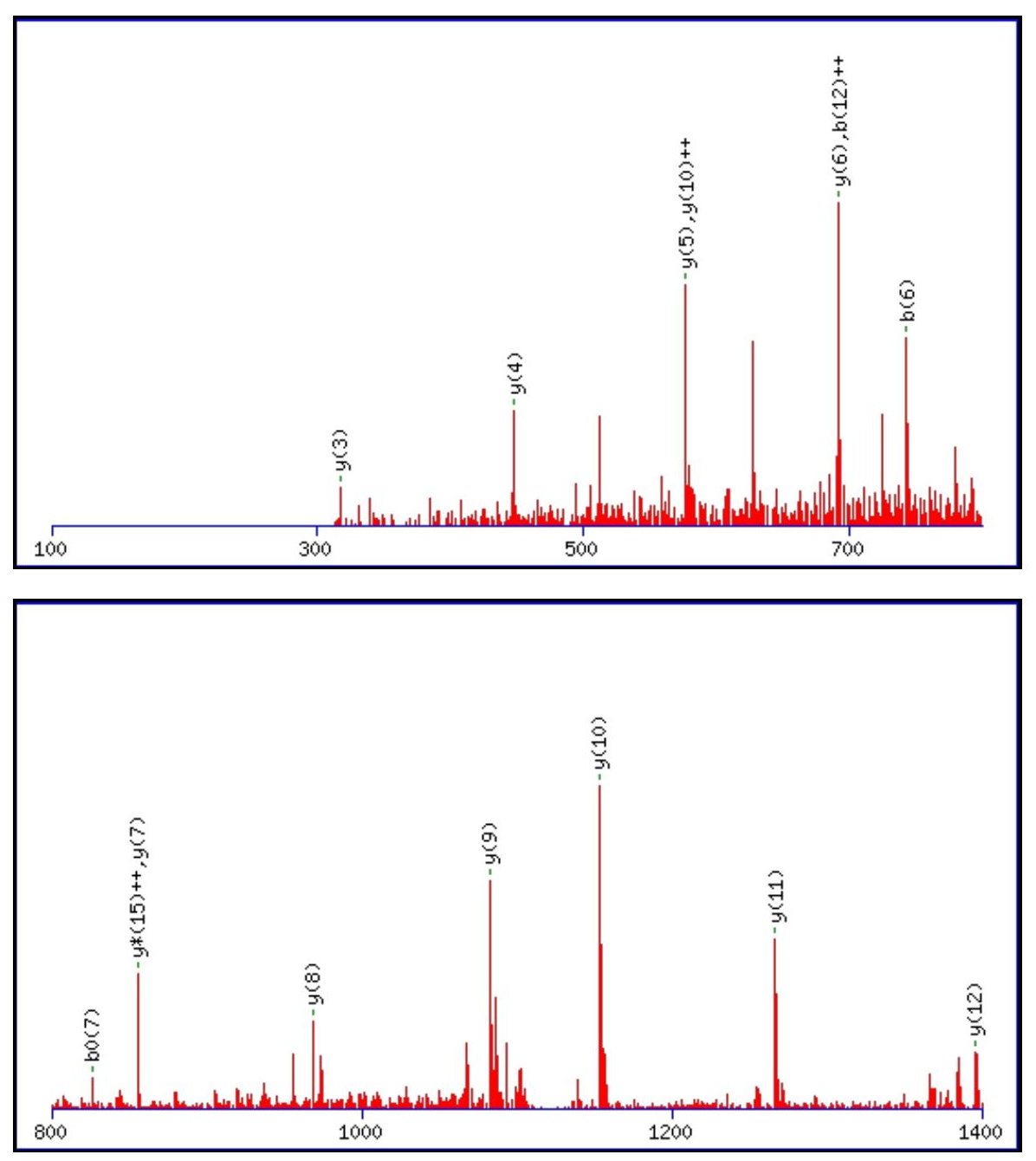


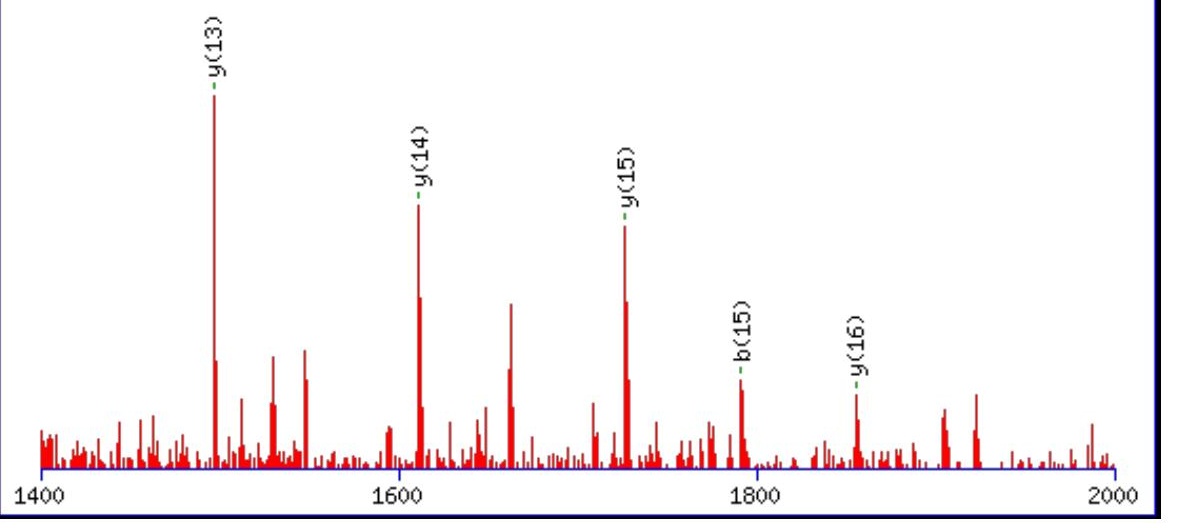

Monoisotopic mass of neutral peptide $\operatorname{Mr}($ calc): 2237.9674

Fixed modifications: Carbamidomethyl (C)

Variable modifications:

N5: : Deamidated N (N)

Ions Score: 132 Expect: $1.8 \mathrm{e}-011$

Matches (Bold Red): 20/194 fragment ions using 25 most intense peaks

\begin{tabular}{|c|c|c|c|c|c|c|c|c|c|c|c|c|c|c|}
\hline \# & b & $\mathbf{b}^{++}$ & $\mathbf{b}^{*}$ & $\mathbf{b}^{*^{++}}$ & $\mathbf{b}^{\mathbf{0}}$ & & Seq. & $\mathbf{y}$ & $\mathbf{y}^{++}$ & $\mathbf{y}^{*}$ & $\mathrm{y}^{\mathrm{*}^{++}}$ & $\mathbf{y}^{0}$ & & \# \\
\hline 1 & 164.0706 & 82.5389 & & & & & $\mathbf{Y}$ & & & & & & & 19 \\
\hline 2 & 221.0921 & 111.0497 & & & & & $\mathbf{G}$ & 2075.9114 & 1038.4593 & 2058.8848 & 1029.9461 & 2057.9008 & 1029.4540 & 18 \\
\hline 3 & 384.1554 & 192.5813 & & & & & $\mathbf{Y}$ & 2018.8899 & 1009.9486 & 2001.8634 & 1001.4353 & 2000.8794 & 1000.9433 & 17 \\
\hline 4 & 513.1980 & 257.1026 & & & 495.1874 & & $\mathbf{E}$ & 1855.8266 & 928.4169 & 1838.8000 & 919.9037 & 1837.8160 & 919.4117 & 16 \\
\hline 5 & 628.2249 & 314.6161 & 611.1984 & 306.1028 & 610.2144 & 305.6108 & $\mathbf{N}$ & 1726.7840 & 863.8956 & 1709.7575 & 855.3824 & 1708.7734 & 854.8904 & 15 \\
\hline 6 & 743.2519 & 372.1296 & 726.2253 & 363.6163 & 725.2413 & 363.1243 & D & 1611.7571 & 806.3822 & 1594.7305 & 797.8689 & 1593.7465 & 797.3769 & 14 \\
\hline 7 & 844.2995 & 422.6534 & 827.2730 & 414.1401 & 826.2890 & 413.6481 & $\mathbf{T}$ & 1496.7301 & 748.8687 & 1479.7036 & 740.3554 & 1478.7196 & 739.8634 & 13 \\
\hline 8 & 973.3421 & 487.1747 & 956.3156 & 478.6614 & 955.3316 & 478.1694 & $\mathbf{E}$ & 1395.6824 & 698.3449 & 1378.6559 & 689.8316 & 1377.6719 & 689.3396 & 12 \\
\hline 9 & 1086.4262 & 543.7167 & 1069.3997 & 535.2035 & 1068.4156 & 534.7115 & I & 1266.6399 & 633.8236 & 1249.6133 & 625.3103 & 1248.6293 & 624.8183 & 11 \\
\hline 10 & 1157.4633 & 579.2353 & 1140.4368 & 570.7220 & 1139.4528 & 570.2300 & A & 1153.5558 & 577.2815 & 1136.5292 & 568.7683 & 1135.5452 & 568.2762 & 10 \\
\hline 11 & 1271.5062 & 636.2568 & 1254.4797 & 627.7435 & 1253.4957 & 627.2515 & $\mathbf{N}$ & 1082.5187 & 541.7630 & 1065.4921 & 533.2497 & 1064.5081 & 532.7577 & 9 \\
\hline 12 & 1384.5903 & 692.7988 & 1367.5638 & 684.2855 & 1366.5797 & 683.7935 & $\mathbf{L}$ & 968.4757 & 484.7415 & 951.4492 & 476.2282 & 950.4652 & 475.7362 & 8 \\
\hline 13 & 1547.6536 & 774.3305 & 1530.6271 & 765.8172 & 1529.6431 & 765.3252 & $\mathbf{Y}$ & 855.3917 & 428.1995 & 838.3651 & 419.6862 & 837.3811 & 419.1942 & 7 \\
\hline 14 & 1662.6806 & 831.8439 & 1645.6540 & 823.3307 & 1644.6700 & 822.8386 & D & 692.3284 & 346.6678 & 675.3018 & 338.1545 & 674.3178 & 337.6625 & 6 \\
\hline 15 & 1791.7232 & 896.3652 & 1774.6966 & 887.8520 & 1773.7126 & 887.3599 & $\mathbf{E}$ & 577.3014 & 289.1543 & 560.2749 & 280.6411 & 559.2908 & 280.1491 & 5 \\
\hline 16 & 1922.7637 & 961.8855 & 1905.7371 & 953.3722 & 1904.7531 & 952.8802 & $\mathbf{M}$ & 448.2588 & 224.6330 & 431.2323 & 216.1198 & & & 4 \\
\hline 17 & 2021.8321 & 1011.4197 & 2004.8055 & 1002.9064 & 2003.8215 & 1002.4144 & V & 317.2183 & 159.1128 & 300.1918 & 150.5995 & & & 3 \\
\hline 18 & 2092.8692 & 1046.9382 & 2075.8426 & 1038.4250 & 2074.8586 & 1037.9329 & A & 218.1499 & 109.5786 & 201.1234 & 101.0653 & & & 2 \\
\hline 19 & & & & & & & $\mathbf{K}$ & 147.1128 & 74.0600 & 130.0863 & 65.5468 & & & 1 \\
\hline
\end{tabular}$$
\text { 要 }
$$
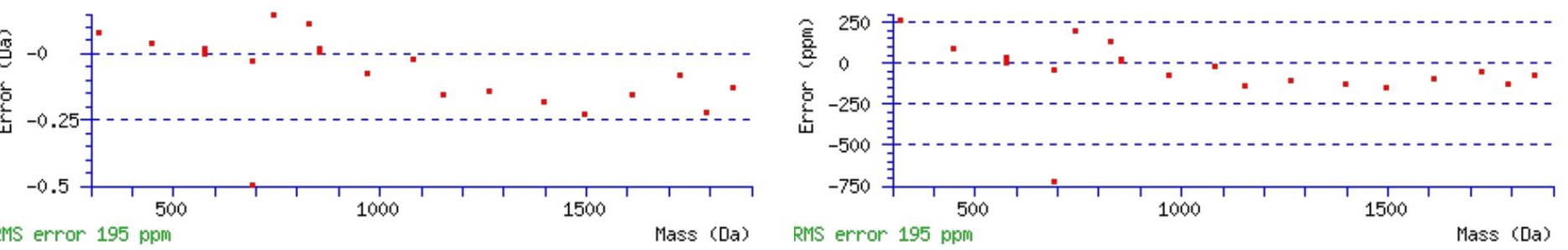

\section{All matches to this query}

\begin{tabular}{|l|l|c|l|}
\hline Score & Mr(calc): & Delta & \multicolumn{1}{c|}{ Sequence } \\
\hline 132.0 & 2237.9674 & 0.0039 & YGYENDTEIANLYDEMVAK \\
\hline 98.7 & 2237.9674 & 0.0039 & YGYENDTEIANLYDEMVAK \\
\hline & & & \\
\hline
\end{tabular}




\begin{tabular}{|l|l|l|l|}
96.6 & 2236.9834 & 0.9879 & YGYENDTEIANLYDEMVAK \\
\hline 2.4 & 2235.9618 & 2.0095 & KSASGDSSQRSSHSDTHVQR \\
\hline 2.0 & 2237.9858 & -0.0145 & EQLEYTIEEEVAKQTIR \\
\hline 1.2 & 2235.9844 & 1.9869 & KATGAVSSNVLQWGSHXKK \\
\hline 0.7 & 2235.9792 & 1.9920 & SMLGNGNYDVNVIMAALQTK \\
\hline 0.4 & 2236.9729 & 0.9984 & TCTNPAPLNGGAFCEGQSVQK \\
\hline
\end{tabular}

Spectrum No: 7; Query: 1942; Rank: 1

\section{Peptide View}

MS/MS Fragmentation of YGYENDTEIANLYDEMVAK

Found in IPI00191437, Tax_Id=10116 Gene_Symbol=- 64 kDa protein

Match to Query 1942: 2237.971288 from(1119.992920,2+)

Title: 091008RatKidney_NoSalt_21.5392.5392.2.dta

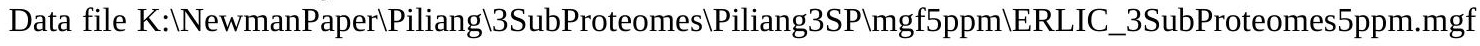
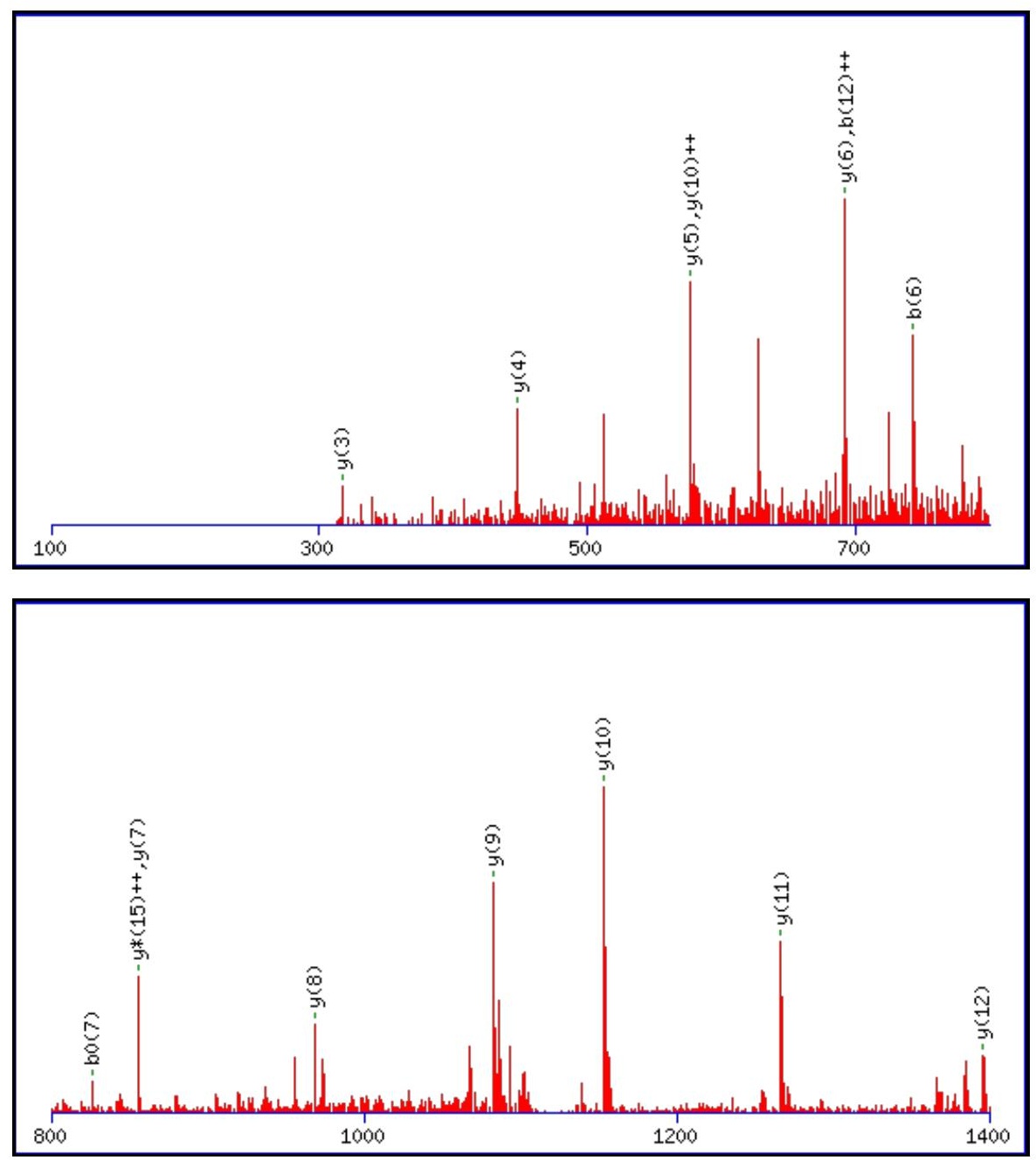


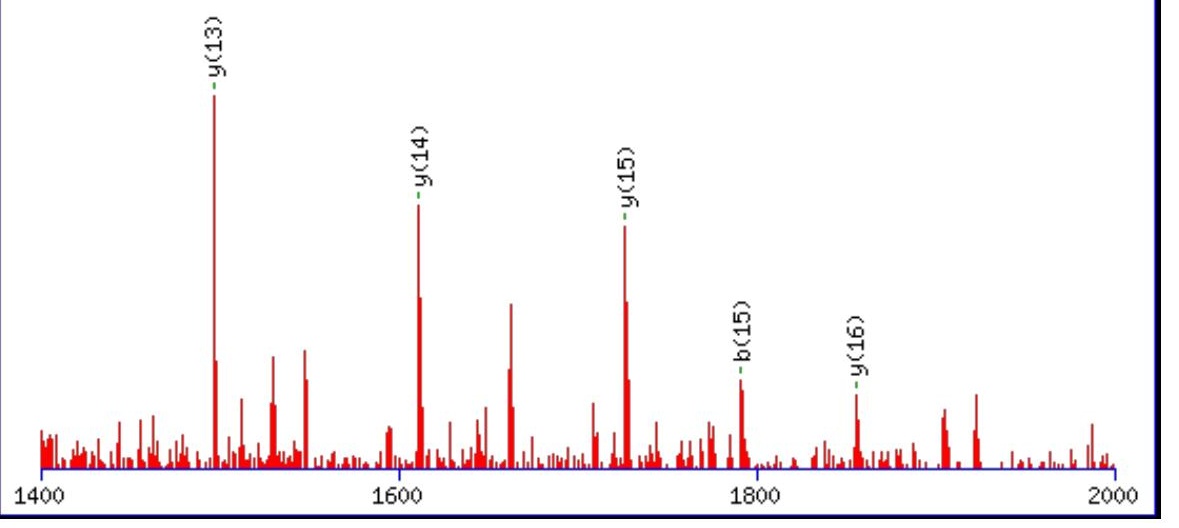

Monoisotopic mass of neutral peptide $\operatorname{Mr}($ calc): 2237.9674

Fixed modifications: Carbamidomethyl (C)

Variable modifications:

N5: : Deamidated N (N)

Ions Score: 132 Expect: $1.8 \mathrm{e}-011$

Matches (Bold Red): 20/194 fragment ions using 25 most intense peaks

\begin{tabular}{|c|c|c|c|c|c|c|c|c|c|c|c|c|c|c|}
\hline \# & b & $\mathbf{b}^{++}$ & $\mathbf{b}^{*}$ & $\mathbf{b}^{*^{++}}$ & $\mathbf{b}^{\mathbf{0}}$ & & Seq. & $\mathbf{y}$ & $\mathbf{y}^{++}$ & $\mathbf{y}^{*}$ & $\mathrm{y}^{\mathrm{*}^{++}}$ & $\mathbf{y}^{0}$ & & \# \\
\hline 1 & 164.0706 & 82.5389 & & & & & $\mathbf{Y}$ & & & & & & & 19 \\
\hline 2 & 221.0921 & 111.0497 & & & & & $\mathbf{G}$ & 2075.9114 & 1038.4593 & 2058.8848 & 1029.9461 & 2057.9008 & 1029.4540 & 18 \\
\hline 3 & 384.1554 & 192.5813 & & & & & $\mathbf{Y}$ & 2018.8899 & 1009.9486 & 2001.8634 & 1001.4353 & 2000.8794 & 1000.9433 & 17 \\
\hline 4 & 513.1980 & 257.1026 & & & 495.1874 & & $\mathbf{E}$ & 1855.8266 & 928.4169 & 1838.8000 & 919.9037 & 1837.8160 & 919.4117 & 16 \\
\hline 5 & 628.2249 & 314.6161 & 611.1984 & 306.1028 & 610.2144 & 305.6108 & $\mathbf{N}$ & 1726.7840 & 863.8956 & 1709.7575 & 855.3824 & 1708.7734 & 854.8904 & 15 \\
\hline 6 & 743.2519 & 372.1296 & 726.2253 & 363.6163 & 725.2413 & 363.1243 & D & 1611.7571 & 806.3822 & 1594.7305 & 797.8689 & 1593.7465 & 797.3769 & 14 \\
\hline 7 & 844.2995 & 422.6534 & 827.2730 & 414.1401 & 826.2890 & 413.6481 & $\mathbf{T}$ & 1496.7301 & 748.8687 & 1479.7036 & 740.3554 & 1478.7196 & 739.8634 & 13 \\
\hline 8 & 973.3421 & 487.1747 & 956.3156 & 478.6614 & 955.3316 & 478.1694 & $\mathbf{E}$ & 1395.6824 & 698.3449 & 1378.6559 & 689.8316 & 1377.6719 & 689.3396 & 12 \\
\hline 9 & 1086.4262 & 543.7167 & 1069.3997 & 535.2035 & 1068.4156 & 534.7115 & I & 1266.6399 & 633.8236 & 1249.6133 & 625.3103 & 1248.6293 & 624.8183 & 11 \\
\hline 10 & 1157.4633 & 579.2353 & 1140.4368 & 570.7220 & 1139.4528 & 570.2300 & A & 1153.5558 & 577.2815 & 1136.5292 & 568.7683 & 1135.5452 & 568.2762 & 10 \\
\hline 11 & 1271.5062 & 636.2568 & 1254.4797 & 627.7435 & 1253.4957 & 627.2515 & $\mathbf{N}$ & 1082.5187 & 541.7630 & 1065.4921 & 533.2497 & 1064.5081 & 532.7577 & 9 \\
\hline 12 & 1384.5903 & 692.7988 & 1367.5638 & 684.2855 & 1366.5797 & 683.7935 & $\mathbf{L}$ & 968.4757 & 484.7415 & 951.4492 & 476.2282 & 950.4652 & 475.7362 & 8 \\
\hline 13 & 1547.6536 & 774.3305 & 1530.6271 & 765.8172 & 1529.6431 & 765.3252 & $\mathbf{Y}$ & 855.3917 & 428.1995 & 838.3651 & 419.6862 & 837.3811 & 419.1942 & 7 \\
\hline 14 & 1662.6806 & 831.8439 & 1645.6540 & 823.3307 & 1644.6700 & 822.8386 & D & 692.3284 & 346.6678 & 675.3018 & 338.1545 & 674.3178 & 337.6625 & 6 \\
\hline 15 & 1791.7232 & 896.3652 & 1774.6966 & 887.8520 & 1773.7126 & 887.3599 & $\mathbf{E}$ & 577.3014 & 289.1543 & 560.2749 & 280.6411 & 559.2908 & 280.1491 & 5 \\
\hline 16 & 1922.7637 & 961.8855 & 1905.7371 & 953.3722 & 1904.7531 & 952.8802 & $\mathbf{M}$ & 448.2588 & 224.6330 & 431.2323 & 216.1198 & & & 4 \\
\hline 17 & 2021.8321 & 1011.4197 & 2004.8055 & 1002.9064 & 2003.8215 & 1002.4144 & V & 317.2183 & 159.1128 & 300.1918 & 150.5995 & & & 3 \\
\hline 18 & 2092.8692 & 1046.9382 & 2075.8426 & 1038.4250 & 2074.8586 & 1037.9329 & A & 218.1499 & 109.5786 & 201.1234 & 101.0653 & & & 2 \\
\hline 19 & & & & & & & $\mathbf{K}$ & 147.1128 & 74.0600 & 130.0863 & 65.5468 & & & 1 \\
\hline
\end{tabular}$$
\text { 要 }
$$
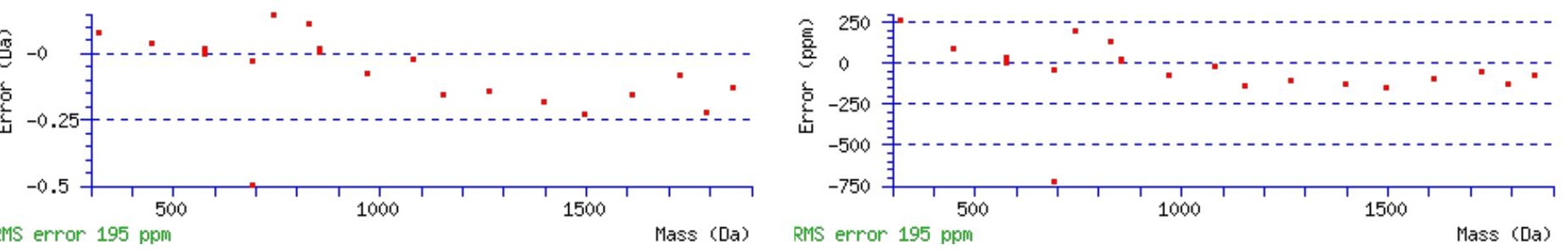

\section{All matches to this query}

\begin{tabular}{|l|l|c|l|}
\hline Score & Mr(calc): & Delta & \multicolumn{1}{c|}{ Sequence } \\
\hline 132.0 & 2237.9674 & 0.0039 & YGYENDTEIANLYDEMVAK \\
\hline 98.7 & 2237.9674 & 0.0039 & YGYENDTEIANLYDEMVAK \\
\hline & & & \\
\hline
\end{tabular}




\begin{tabular}{|l|l|l|l|}
96.6 & 2236.9834 & 0.9879 & YGYENDTEIANLYDEMVAK \\
\hline 2.4 & 2235.9618 & 2.0095 & KSASGDSSQRSSHSDTHVQR \\
\hline 2.0 & 2237.9858 & -0.0145 & EQLEYTIEEEVAKQTIR \\
\hline 1.2 & 2235.9844 & 1.9869 & KATGAVSSNVLQWGSHXKK \\
\hline 0.7 & 2235.9792 & 1.9920 & SMLGNGNYDVNVIMAALQTK \\
\hline 0.4 & 2236.9729 & 0.9984 & TCTNPAPLNGGAFCEGQSVQK \\
\hline
\end{tabular}

Spectrum No: 8; Query: 1425; Rank: 1

\section{Peptide View}

MS/MS Fragmentation of TLAGENQTALEIEELNR

Found in IPI00363849, Tax_Id=10116 Gene_Symbol=Lamc1 similar to Laminin gamma-1 chain precursor

Match to Query 1425: 1900.944208 from(951.479380,2+)

Title: 100101RatKid_NS_deglyco_25.3189.3189.2.dta

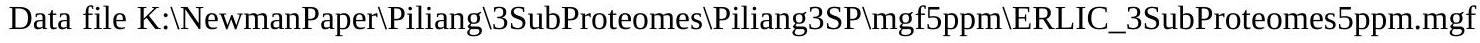
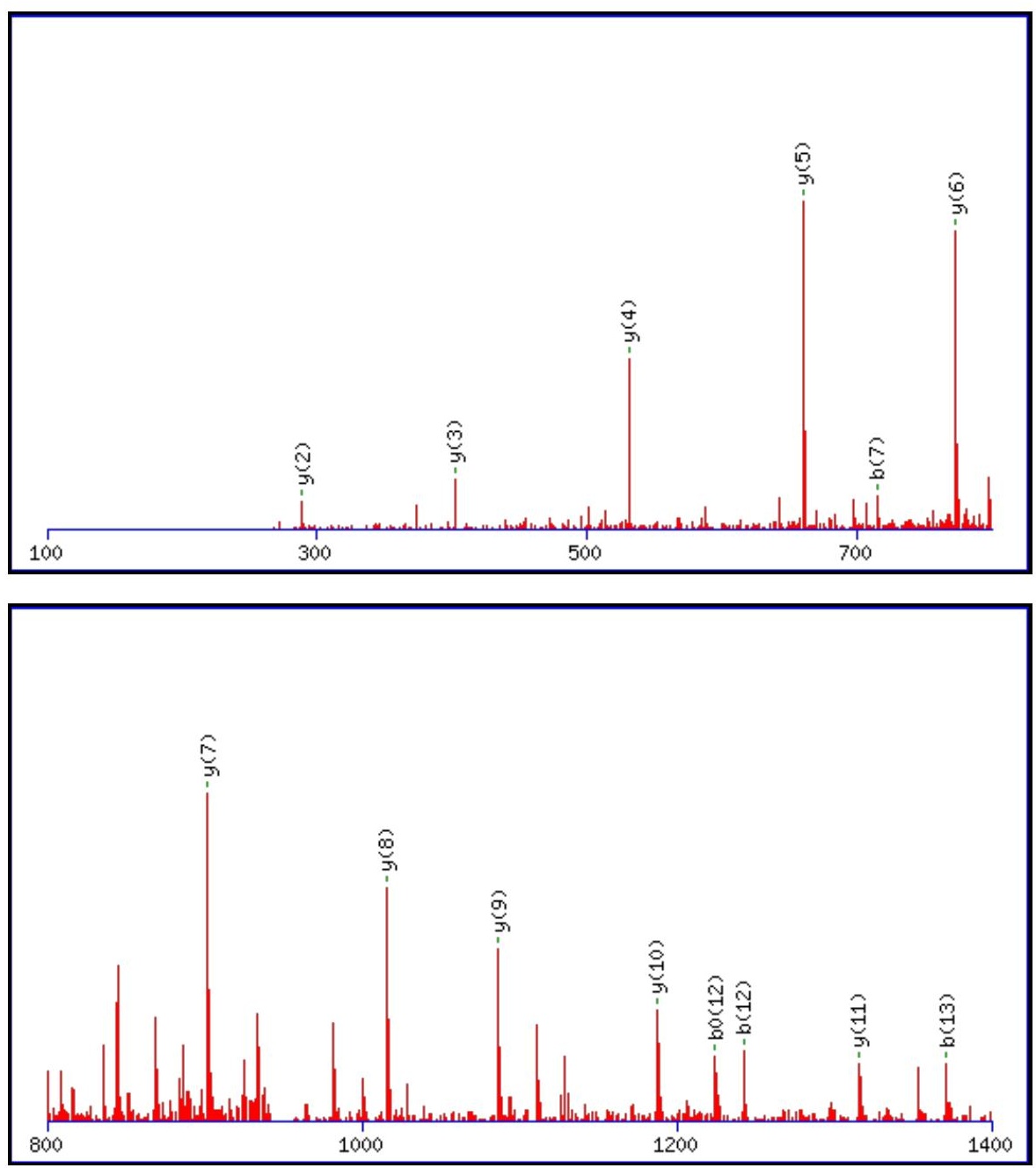


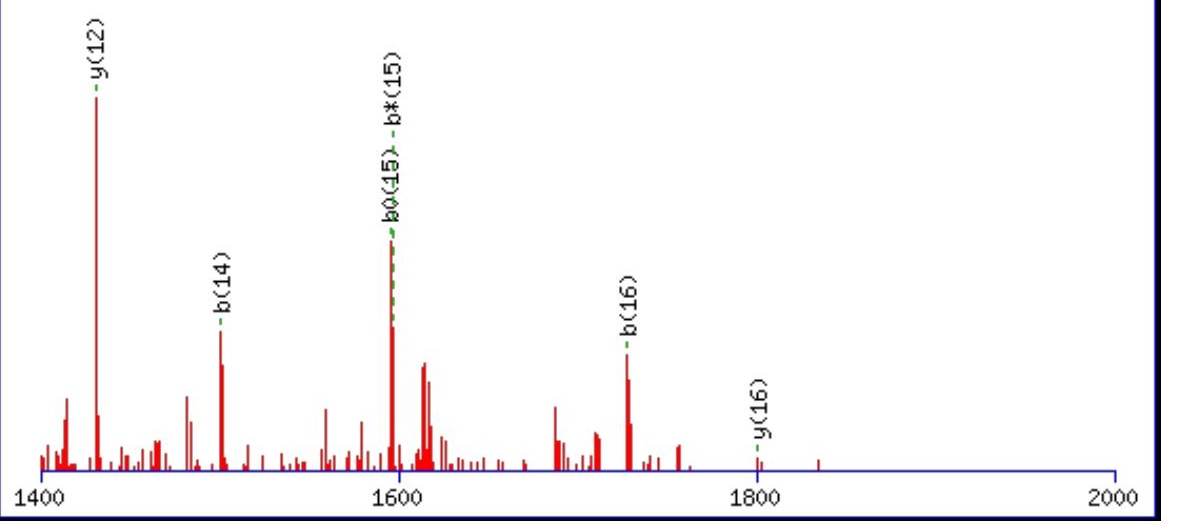

Monoisotopic mass of neutral peptide $\operatorname{Mr}($ calc): 1900.9377

Fixed modifications: Carbamidomethyl (C)

Variable modifications:

N6 : Deamidated $\mathrm{N}(\mathrm{N})$

Ions Score: 130 Expect: 2.1e-011

Matches (Bold Red): 20/176 fragment ions using 27 most intense peaks

\begin{tabular}{|c|c|c|c|c|c|c|c|c|c|c|c|c|c|c|}
\hline \# & b & $\mathbf{b}^{++}$ & $\mathbf{b}^{*}$ & $\mathbf{b}^{*^{++}}$ & $\mathbf{b}^{0}$ & $\mathbf{b}^{0++}$ & Seq. & $\mathbf{y}$ & $y^{++}$ & $\mathrm{y}^{*}$ & $y^{*^{++}}$ & $\mathbf{y}^{\mathbf{0}}$ & $y^{0++}$ & $\#$ \\
\hline 1 & 102.0550 & 51.5311 & & & 84.0444 & 42.5258 & $T$ & & & & & & & 17 \\
\hline 2 & 215.1390 & 108.0731 & & & 197.1285 & 99.0679 & $\mathbf{L}$ & 1800.8974 & 900.9523 & 1783.8708 & 892.4391 & 1782.8868 & 891.9471 & 16 \\
\hline 3 & 286.1761 & 143.5917 & & & 268.1656 & 134.5864 & A & 1687.8133 & 844.4103 & 1670.7868 & 835.8970 & 1669.8028 & 835.4050 & 15 \\
\hline 4 & 343.1976 & 172.1024 & & & 325.1870 & 163.0972 & G & 1616.7762 & 808.8917 & \begin{tabular}{|c|c|}
1599.7497 \\
\end{tabular} & 800.3785 & 1598.7656 & 799.8865 & 14 \\
\hline 5 & 472.2402 & 236.6237 & & & 454.2296 & 227.6185 & $E$ & 1559.7548 & 780.3810 & 1542.7282 & 771.8677 & 1541.7442 & 771.3757 & 13 \\
\hline 6 & 587.2671 & 294.1372 & 570.2406 & 285.6239 & 569.2566 & 285.1319 & $\mathbf{N}$ & 1430.7122 & 715.8597 & 1413.6856 & 707.3464 & 1412.7016 & 706.8544 & 12 \\
\hline 7 & 715.3257 & 358.1665 & 698.2992 & 349.6532 & 697.3151 & 349.1612 & $\mathbf{Q}$ & 1315.6852 & 658.3462 & 1298.6587 & 649.8330 & 1297.6747 & 649.3410 & 11 \\
\hline 8 & 816.3734 & 408.6903 & 799.3468 & 400.1771 & 798.3628 & 399.6850 & $T$ & 1187.6266 & 594.3170 & 1170.6001 & 585.8037 & 1169.6161 & 585.3117 & 10 \\
\hline 9 & 887.4105 & 444.2089 & 870.3839 & 435.6956 & 869.3999 & 435.2036 & A & 1086.5790 & 543.7931 & 1069.5524 & 535.2798 & 1068.5684 & 534.7878 & 9 \\
\hline 101 & 1000.4946 & 500.7509 & 983.4680 & 492.2376 & 982.4840 & 491.7456 & $\mathbf{L}$ & 1015.5419 & 508.2746 & 998.5153 & 499.7613 & 997.5313 & 499.2693 & 8 \\
\hline 11 & 1129.5372 & 565.2722 & 1112.5106 & 556.7589 & 1111.5266 & 556.2669 & $\mathbf{E}$ & 902.4578 & 451.7325 & 885.4312 & 443.2193 & 884.4472 & 442.7272 & 7 \\
\hline 121 & 1242.6212 & 621.8142 & 1225.5947 & 613.3010 & 1224.6107 & 612.8090 & I & 773.4152 & 387.2112 & 756.3886 & 378.6980 & 755.4046 & 378.2060 & 6 \\
\hline 13 & \begin{tabular}{|l|}
1371.6638 \\
\end{tabular} & 686.3355 & 1354.6373 & 677.8223 & 1353.6532 & 677.3303 & $\mathbf{E}$ & 660.3311 & 330.6692 & 643.3046 & 322.1559 & 642.3206 & 321.6639 & 5 \\
\hline 14 & 1500.7064 & 750.8568 & 1483.6799 & 742.3436 & 1482.6958 & 741.8516 & $\mathbf{E}$ & 531.2885 & 266.1479 & 514.2620 & 257.6346 & 513.2780 & 257.1426 & 4 \\
\hline 15 & 1613.7905 & 807.3989 & 1596.7639 & 798.8856 & 1595.7799 & 798.3936 & $\mathbf{L}$ & 402.2459 & 201.6266 & 385.2194 & 193.1133 & & & 3 \\
\hline 16 & \begin{tabular}{|l|}
1727.8334 \\
\end{tabular} & 864.4203 & 1710.8068 & 855.9071 & 1709.8228 & 855.4151 & $\mathbf{N}$ & 289.1619 & 145.0846 & 272.1353 & 136.5713 & & & 2 \\
\hline 17 & & & & & & & $\mathbf{R}$ & 175.1190 & 88.0631 & 158.0924 & 79.5498 & & & 1 \\
\hline
\end{tabular}
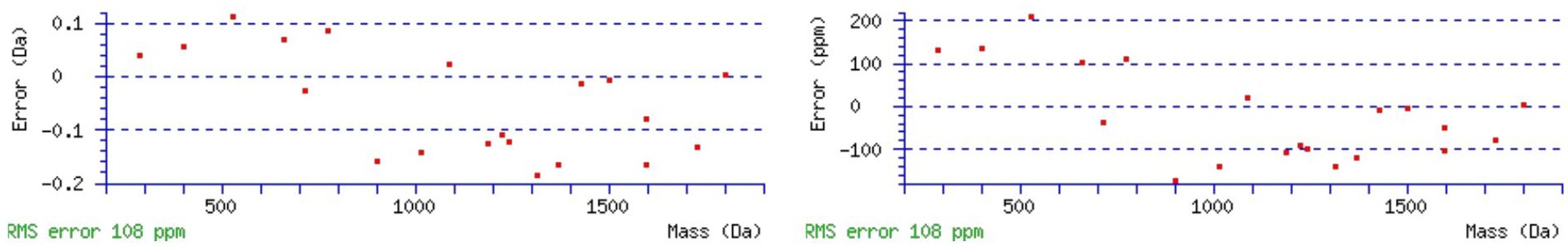

\section{All matches to this query}

\begin{tabular}{|l|l|l|l|}
\hline Score & Mr(calc): & Delta & \multicolumn{1}{c|}{ Sequence } \\
\hline 129.8 & 1900.9377 & 0.0065 & TLAGENQTALEIEELNR \\
\hline 73.4 & 1899.9537 & 0.9905 & TLAGENQTALEIEELNR \\
\hline 58.1 & 1900.9377 & 0.0065 & TLAGENQTALEIEELNR \\
\hline 9.8 & 1899.9482 & 0.9960 & TELDSFLIEITANILK \\
\hline & & &
\end{tabular}




\begin{tabular}{|l|l|l|l|}
9.8 & 1899.9482 & 0.9960 & TELDSFLIEITANILK \\
\hline 8.2 & 1900.9274 & 0.0168 & SVSTMLYLMALQELNR \\
\hline 5.4 & 1900.9457 & -0.0015 & GFLMTKIFIGCPPGKR \\
\hline 3.5 & 1900.9376 & 0.0066 & EDVFWLPLLEKAYAK \\
\hline 2.3 & 1900.9560 & -0.0118 & VYTLTPFLANNREKR \\
\hline 2.3 & 1900.9560 & -0.0118 & VYTLTPFLANNREKR \\
\hline
\end{tabular}

Spectrum No: 9; Query: 2109; Rank: 1

\section{Peptide View}

MS/MS Fragmentation of FKLDWLGNCSGLNDESYGYK

Found in IPI00339124, Tax_Id=10116 Gene_Symbol=Atp1b1 Sodium/potassium-transporting ATPase subunit beta-1

Match to Query 2109: 2366.063148 from(1184.038850,2+)

Title: 100101RatKid_NS_deglyco_14.4549.4549.2.dta

Data file K:\NewmanPaper\Piliang \3SubProteomes\Piliang3SP \mgf5ppm\ERLIC_3SubProteomes5ppm.mgf
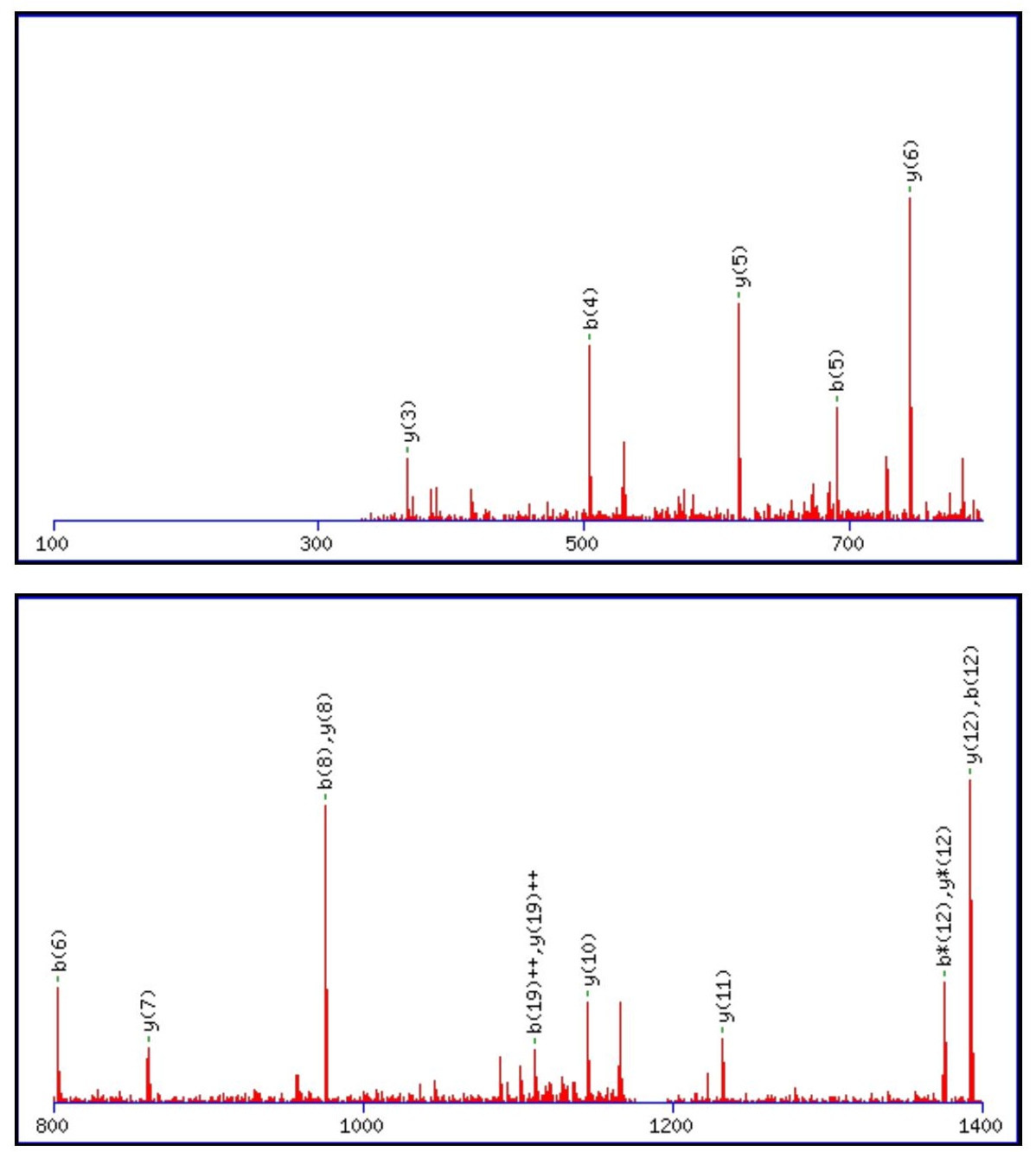


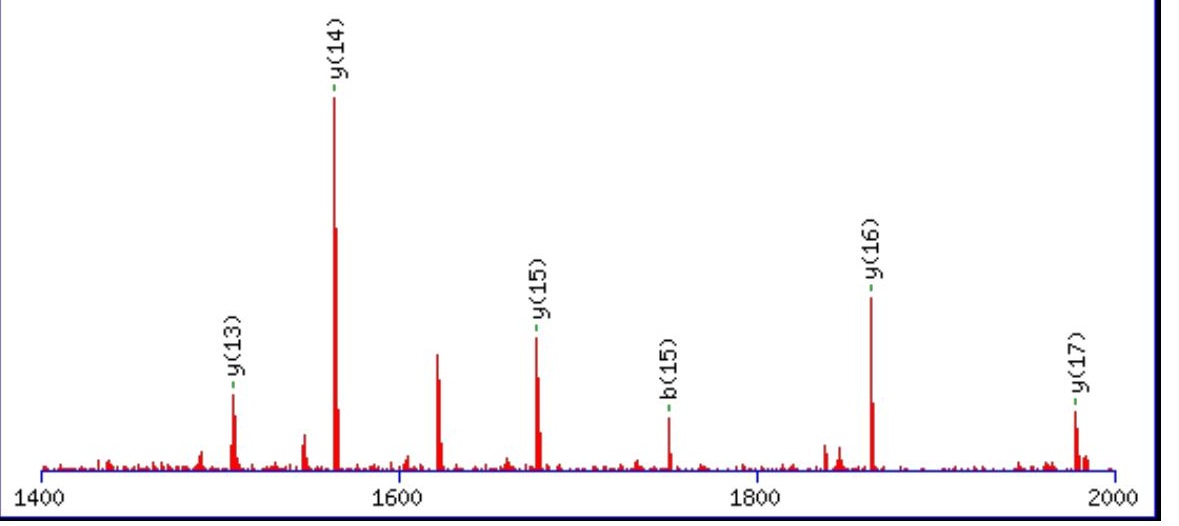

Monoisotopic mass of neutral peptide $\operatorname{Mr}($ calc): 2366.0525

Fixed modifications: Carbamidomethyl (C)

Variable modifications:

N8 : Deamidated $\mathrm{N}(\mathrm{N})$

Ions Score: 129 Expect: $3.7 \mathrm{e}-011$

Matches (Bold Red): 23/212 fragment ions using 26 most intense peaks

\begin{tabular}{|c|c|c|c|c|c|c|c|c|c|c|c|c|c|c|}
\hline \# & b & $\mathbf{b}^{++}$ & b* & $\mathbf{b}^{*++}$ & $\mathbf{b}^{0}$ & $\mathbf{b}^{0++}$ & Seq. & $\mathbf{y}$ & $y^{++}$ & $\mathbf{y}^{*}$ & $\mathbf{y}^{*^{++}}$ & $\mathbf{y}^{0}$ & $\mathbf{y}^{0++}$ & \# \\
\hline 1 & 148.0757 & 74.5415 & & & & & $\mathbf{F}$ & & & & & & & 20 \\
\hline 2 & 276.1707 & 138.5890 & 259.1441 & 130.0757 & & & $\mathbf{K}$ & 2219.9914 & 1110.4993 & 2202.9648 & 1101.9860 & 2201.9808 & 1101.4940 & 19 \\
\hline 3 & 389.2547 & 195.1310 & 372.2282 & 186.6177 & & & $\mathbf{L}$ & 2091.8964 & 1046.4518 & 2074.8699 & 1037.9386 & 2073.8858 & 1037.4466 & 18 \\
\hline 4 & 504.2817 & 252.6445 & 487.2551 & 244.1312 & 486.2711 & 243.6392 & D & 1978.8123 & 989.9098 & 1961.7858 & 981.3965 & 1960.8018 & 980.9045 & 17 \\
\hline 5 & 690.3610 & 345.6841 & 673.3344 & 337.1709 & 672.3504 & 336.6788 & $\mathbf{W}$ & 1863.7854 & 932.3963 & 1846.7589 & 923.8831 & 1845.7748 & 923.3911 & 16 \\
\hline 6 & 803.4450 & 402.2262 & 786.4185 & 393.7129 & 785.4345 & 393.2209 & $\mathbf{L}$ & 1677.7061 & 839.3567 & 1660.6795 & 830.8434 & 1659.6955 & 830.3514 & 15 \\
\hline 7 & 860.4665 & 430.7369 & 843.4400 & 422.2236 & 842.4559 & 421.7316 & $\mathbf{G}$ & 1564.6220 & 782.8146 & 1547.5955 & 774.3014 & 1546.6115 & 773.8094 & 14 \\
\hline 8 & 975.4934 & 488.2504 & 958.4669 & 479.7371 & 957.4829 & 479.2451 & $\mathbf{N}$ & 1507.6006 & 754.3039 & 1490.5740 & 745.7906 & 1489.5900 & 745.2986 & 13 \\
\hline 9 & 1135.5241 & 568.2657 & 1118.4975 & 559.7524 & 1117.5135 & 559.2604 & C & 1392.5736 & 696.7904 & 1375.5471 & 688.2772 & 1374.5631 & 687.7852 & 12 \\
\hline 10 & 1222.5561 & 611.7817 & 1205.5296 & 603.2684 & 1204.5455 & 602.7764 & $S$ & 1232.5430 & 616.7751 & 1215.5164 & 608.2619 & 1214.5324 & 607.7698 & 11 \\
\hline 11 & 1279.5776 & 640.2924 & 1262.5510 & 631.7792 & 1261.5670 & 631.2871 & G & 1145.5109 & 573.2591 & 1128.4844 & 564.7458 & 1127.5004 & 564.2538 & 10 \\
\hline 12 & 1392.6616 & 696.8345 & 1375.6351 & 688.3212 & 1374.6511 & 687.8292 & $\mathbf{L}$ & 1088.4895 & 544.7484 & 1071.4629 & 536.2351 & 1070.4789 & 535.7431 & 9 \\
\hline 13 & 1506.7046 & 753.8559 & 1489.6780 & 745.3426 & 1488.6940 & 744.8506 & $\mathbf{N}$ & 975.4054 & 488.2063 & 958.3789 & 479.6931 & 957.3949 & 479.2011 & 8 \\
\hline 14 & 1621.7315 & 811.3694 & 1604.7050 & 802.8561 & 1603.7209 & 802.3641 & D & 861.3625 & 431.1849 & 844.3359 & 422.6716 & 843.3519 & 422.1796 & 7 \\
\hline 15 & 1750.7741 & 875.8907 & 1733.7476 & 867.3774 & 1732.7635 & 866.8854 & $\mathbf{E}$ & 746.3355 & 373.6714 & 729.3090 & 365.1581 & 728.3250 & 364.6661 & 6 \\
\hline 16 & 1837.8061 & 919.4067 & 1820.7796 & 910.8934 & 1819.7956 & 910.4014 & $\mathrm{~S}$ & 617.2930 & 309.1501 & 600.2664 & 300.6368 & 599.2824 & 300.1448 & 5 \\
\hline 17 & 2000.8695 & 1000.9384 & 1983.8429 & 992.4251 & 1982.8589 & 991.9331 & $\mathbf{Y}$ & 530.2609 & 265.6341 & 513.2344 & 257.1208 & & & 4 \\
\hline 18 & 2057.8909 & 1029.4491 & 2040.8644 & 1020.9358 & 2039.8804 & 1020.4438 & G & 367.1976 & 184.1024 & 350.1710 & 175.5892 & & & 3 \\
\hline 19 & 2220.9543 & 1110.9808 & 2203.9277 & 1102.4675 & 2202.9437 & 1101.9755 & $\mathbf{Y}$ & 310.1761 & 155.5917 & 293.1496 & 147.0784 & & & 2 \\
\hline 20 & & & & & & & $\mathbf{K}$ & 147.1128 & 74.0600 & 130.0863 & 65.5468 & & & 1 \\
\hline
\end{tabular}
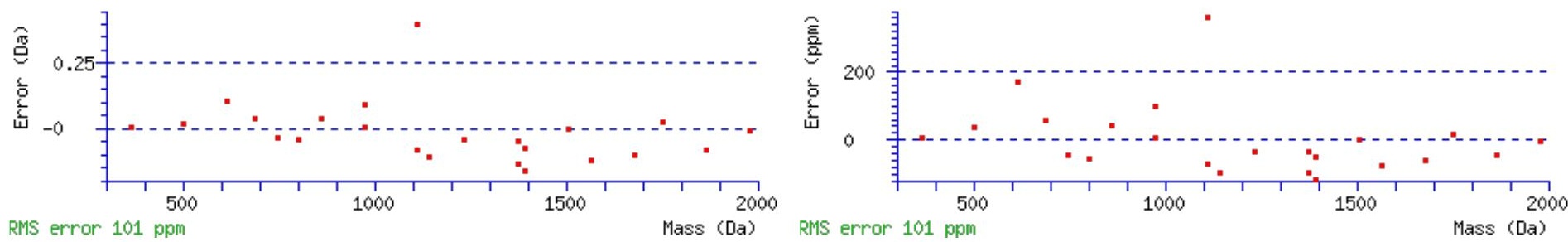

\section{All matches to this query}

\begin{tabular}{|c|c|c|c|}
\hline Score & Mr(calc): & Delta & \multicolumn{1}{c|}{ Sequence } \\
\hline 129.2 & 2366.0525 & 0.0107 & FKLDWLGNCSGLNDESYGYK \\
\hline & & &
\end{tabular}




\begin{tabular}{|l|l|l|l||}
92.3 & 2366.0525 & 0.0107 & FKLDWLGNCSGLNDESYGYK \\
\hline 55.0 & 2365.0685 & 0.9947 & FKLDWLGNCSGLNDESYGYK \\
\hline 9.2 & 2366.0630 & 0.0001 & LQGGKDFNMPLTISSLKDGGK \\
\hline 9.2 & 2366.0630 & 0.0001 & LQGGKDFNMPLTISSLKDGGK \\
\hline 9.2 & 2366.0630 & 0.0001 & LQGGKDFNMPLTISSLKDGGK \\
\hline 5.3 & 2366.0759 & -0.0127 & SFSFSVNWNEIINFPLEIK \\
\hline 5.3 & 2366.0759 & -0.0127 & SFSFSVNWNEIINFPLEIK \\
\hline 5.3 & 2366.0759 & -0.0127 & SFSFSVNWNEIINFPLEIK \\
\hline 4.6 & 2366.0776 & -0.0145 & MGANNLERMGLERMGANSLER \\
\hline
\end{tabular}

Spectrum No: 10; Query: 2162; Rank: 1

\section{Peptide View}

MS/MS Fragmentation of IYYSDFSNQTINSMAEDGSNR

Found in IPI00205325, Tax_Id=10116 Gene_Symbol=Lrp2 Low-density lipoprotein receptor-related protein 2 precursor

Match to Query 2162: 2412.025568 from(1207.020060,2+)

Title: 100101RatKid_NS_deglyco_18.3791.3791.2.dta

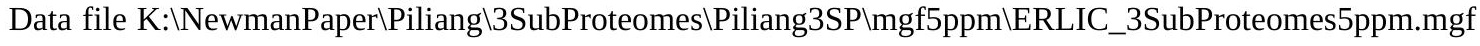
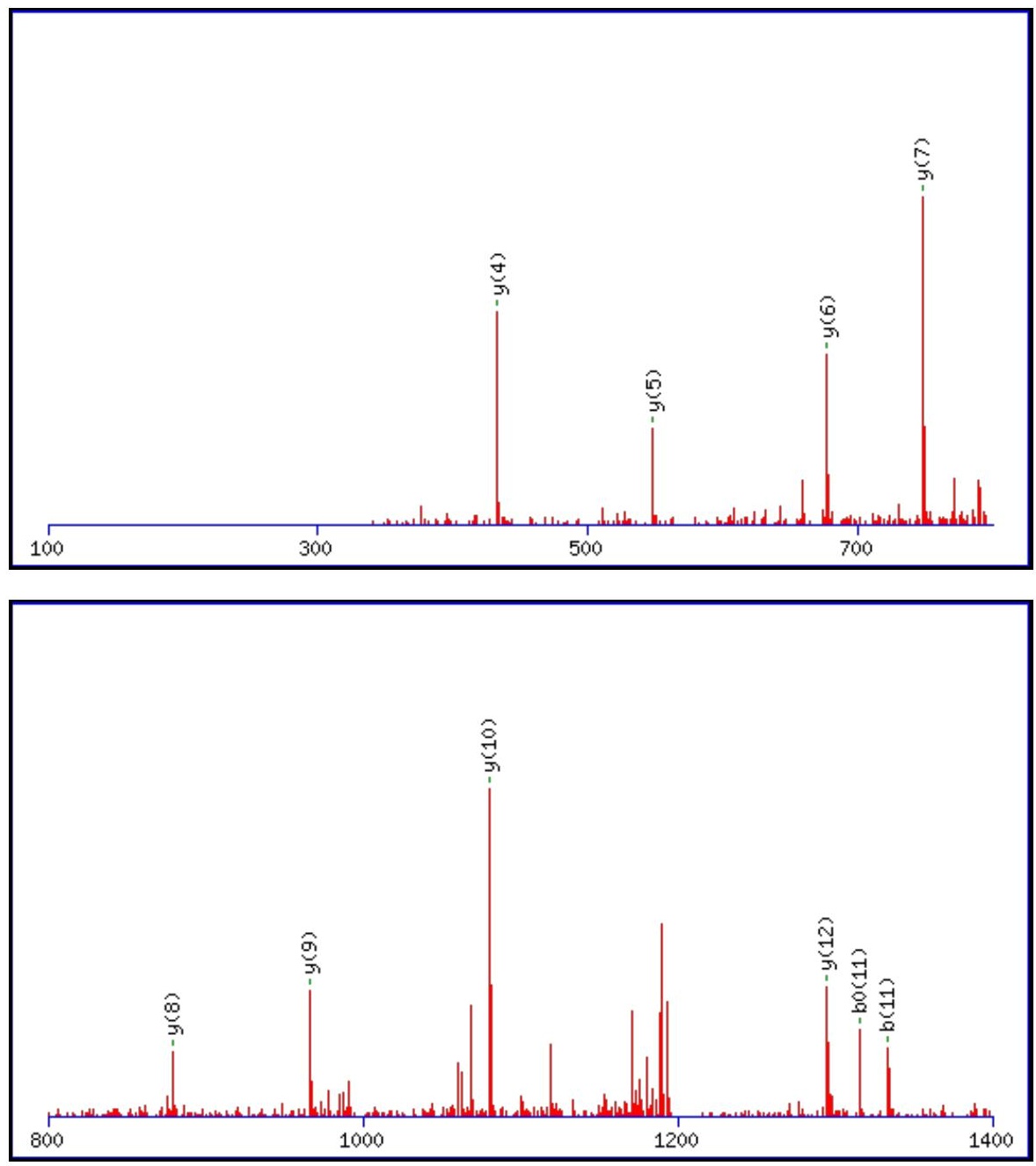


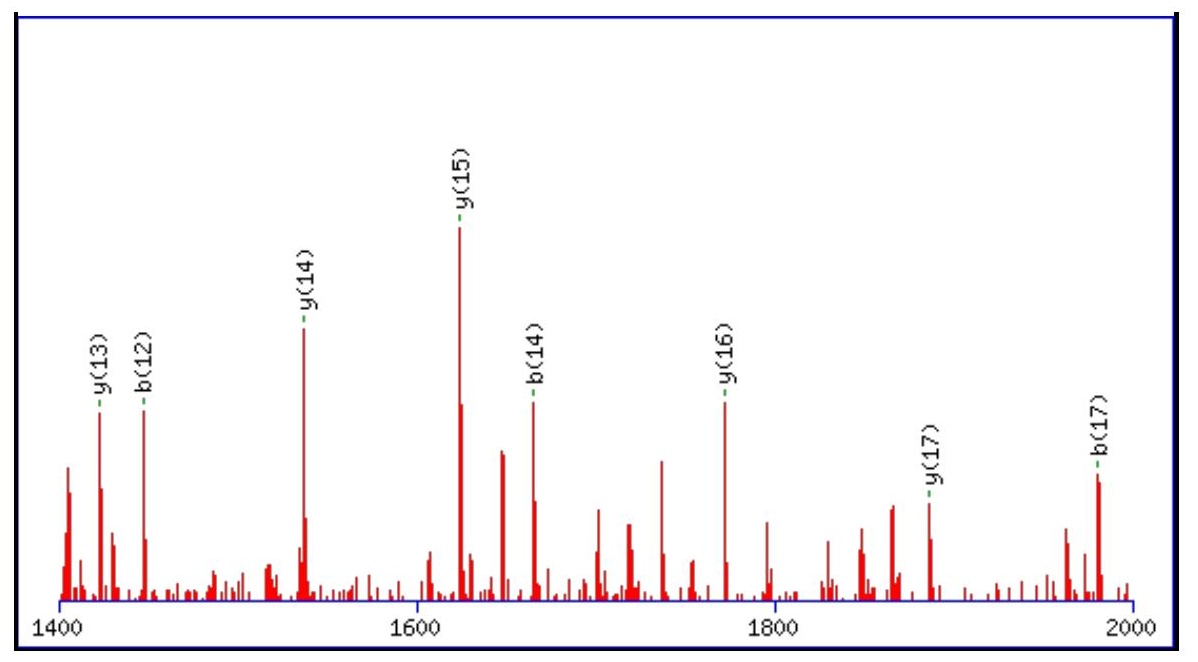

Monoisotopic mass of neutral peptide $\operatorname{Mr}($ calc): 2412.0175

Fixed modifications: Carbamidomethyl (C)

Variable modifications:

N8 : Deamidated_N (N)

Ions Score: 129 Expect: $4 \mathrm{e}-011$

Matches (Bold Red): 18/216 fragment ions using 23 most intense peaks

\begin{tabular}{|c|c|c|c|c|c|c|c|c|c|c|c|c|c|c|}
\hline \# & b & $\mathbf{b}^{++}$ & $\mathbf{b}^{*}$ & $\mathbf{b}^{*++}$ & $\mathbf{b}^{0}$ & $\mathbf{b}^{0++}$ & Seq. & $\mathbf{y}$ & $\mathbf{y}^{++}$ & $\mathbf{y}^{*}$ & $\mathbf{y}^{*^{++}}$ & $\mathbf{y}^{0}$ & $\mathbf{y}^{0++}$ & \# \\
\hline 1 & 114.0913 & 57.5493 & & & & & I & & & & & & & 21 \\
\hline 2 & 277.1547 & 139.0810 & & & & & $\mathbf{Y}$ & 2299.9408 & 1150.4740 & 2282.9142 & 1141.9608 & 2281.9302 & 1141.4687 & 20 \\
\hline 3 & 440.2180 & 220.6126 & & & & & $\mathbf{Y}$ & 2136.8775 & 1068.9424 & 2119.8509 & 1060.4291 & 2118.8669 & 1059.9371 & 19 \\
\hline 4 & 527.2500 & 264.1287 & & & 509.2395 & 255.1234 & $S$ & 1973.8141 & 987.4107 & 1956.7876 & 978.8974 & 1955.8036 & 978.4054 & 18 \\
\hline 5 & 642.2770 & 321.6421 & & & 624.2664 & 312.6368 & D & 1886.7821 & 943.8947 & 1869.7556 & 935.3814 & 1868.7715 & 934.8894 & 17 \\
\hline 6 & 789.3454 & 395.1763 & & & 771.3348 & 386.1710 & $\mathbf{F}$ & 1771.7552 & 886.3812 & 1754.7286 & 877.8679 & 1753.7446 & 877.3759 & 16 \\
\hline 7 & 876.3774 & 438.6923 & & & 858.3668 & 429.6871 & S & 1624.6867 & 812.8470 & 1607.6602 & 804.3337 & 1606.6762 & 803.8417 & 15 \\
\hline 8 & 991.4043 & 496.2058 & 974.3778 & 487.6925 & 973.3938 & 487.2005 & $\mathbf{N}$ & 1537.6547 & 769.3310 & 1520.6282 & 760.8177 & 1519.6442 & 760.3257 & 14 \\
\hline 9 & 1119.4629 & 560.2351 & 1102.4364 & 551.7218 & 1101.4524 & 551.2298 & $\mathbf{Q}$ & 1422.6278 & 711.8175 & 1405.6012 & 703.3043 & 1404.6172 & 702.8122 & 13 \\
\hline 10 & 1220.5106 & 610.7589 & 1203.4841 & 602.2457 & 1202.5000 & 601.7537 & $T$ & 1294.5692 & 647.7882 & 1277.5427 & 639.2750 & 1276.5586 & 638.7830 & 12 \\
\hline 11 & 1333.5947 & 667.3010 & 1316.5681 & 658.7877 & 1315.5841 & 658.2957 & I & 1193.5215 & 597.2644 & 1176.4950 & 588.7511 & 1175.5110 & 588.2591 & 11 \\
\hline 121 & 1447.6376 & 724.3 & 1430.6110 & 715.8 & 1429.6270 & 715.3172 & $\mathbf{N}$ & 1080.4375 & 540.7224 & 1063.4109 & 532.2091 & 1062.4269 & 531.7171 & 10 \\
\hline 131 & 1534.6696 & 767.8384 & 1517.6431 & 759.3252 & 1516.6591 & 758.8332 & $\mathrm{~S}$ & 966.3945 & 483.7009 & 949.3680 & 475.1876 & 948.3840 & 474.6956 & 9 \\
\hline 14 & 1665.7101 & 833.3587 & 1648.6836 & 824.8454 & 1647.6995 & 824.3534 & $\mathbf{M}$ & 879.3625 & 440.1849 & 862.3360 & 431.6716 & 861.3519 & 431.1796 & 8 \\
\hline 151 & 1736.7472 & 868.8772 & 1719.7207 & 860.3640 & 1718.7367 & 859.8720 & A & 748.3220 & 374.6646 & 731.2955 & 366.1514 & 730.3115 & 365.6594 & 7 \\
\hline 16 & 1865.7898 & 933.3985 & 1848.7633 & 924.8853 & 1847.7792 & 924.3933 & $\mathbf{E}$ & 677.2849 & 339.1461 & 660.2584 & 330.6328 & 659.2743 & 330.1408 & 6 \\
\hline 17 & 1980.8168 & 990.9120 & 1963.7902 & 982.3987 & 1962.8062 & 981.9067 & D & 548.2423 & 274.6248 & 531.2158 & 266.1115 & 530.2317 & 265.6195 & 5 \\
\hline 182 & 2037.8382 & 1019.4227 & 2020.8117 & 1010.9095 & 2019.8277 & 1010.4175 & $\mathbf{G}$ & 433.2154 & 217.1113 & 416.1888 & 208.5980 & 415.2048 & 208.1060 & 4 \\
\hline 192 & 2124.8702 & 1062.9388 & 2107.8437 & 1054.4255 & 2106.8597 & 1053.9335 & S & 376.1939 & 188.6006 & 359.1674 & 180.0873 & 358.1833 & 179.5953 & 3 \\
\hline 202 & 2238.9132 & 1119.9602 & 2221.8866 & 1111.4470 & 2220.9026 & 1110.9549 & $\mathbf{N}$ & 289.1619 & 145.0846 & 272.1353 & 136.5713 & & & 2 \\
\hline 21 & & & & & & & $\mathbf{R}$ & 175.1190 & 88.0631 & 158.0924 & 79.5498 & & & 1 \\
\hline
\end{tabular}
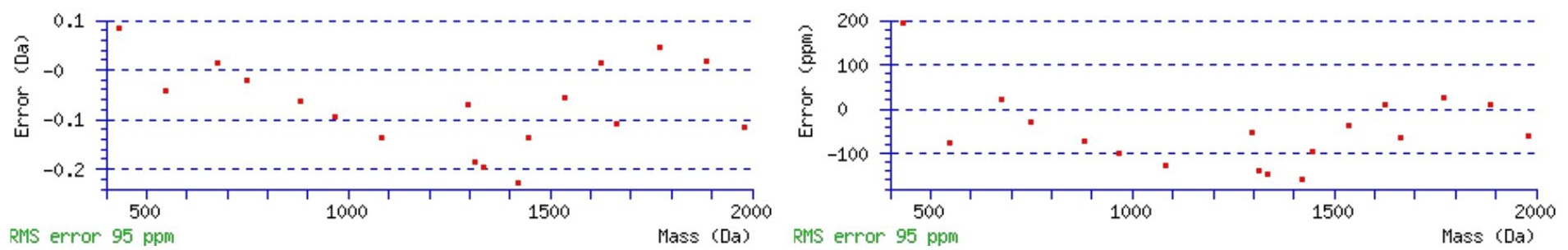

\section{All matches to this query}

\begin{tabular}{|l|l|l|}
\hline Score & Mr(calc): & Delta \\
\hline
\end{tabular}

Sequence 


\begin{tabular}{|l|l|l|l|}
128.8 & 2412.0175 & 0.0080 & IYYSDFSNQTINSMAEDGSNR \\
\hline 88.6 & 2412.0175 & 0.0080 & IYYSDFSNQTINSMAEDGSNR \\
\hline 56.9 & 2412.0175 & 0.0080 & $\underline{\text { IYYSDFSNQTINSMAEDGSNR }}$ \\
\hline 50.9 & 2411.0335 & 0.9920 & $\underline{\text { IYYSDFSNQTINSMAEDGSNR }}$ \\
\hline 3.3 & 2410.0299 & 1.9957 & DSRAVSEQTAPNSNSNGSWAPR \\
\hline 2.3 & 2412.0403 & -0.0147 & EGICGSCAMNINGGNTLACTRR \\
\hline 1.9 & 2411.0287 & 0.9969 & QSEWHKMTVSKNCPDQDLK \\
\hline 0.8 & 2412.0279 & -0.0024 & TNYLLSSTFPEXMALCWNR \\
\hline 0.8 & 2412.0279 & -0.0024 & TNYLLSSTFPEXMALCWNR \\
\hline 0.8 & 2412.0279 & -0.0024 & TNYLLSSTFPEXMALCWNR \\
\hline
\end{tabular}

Spectrum No: 11; Query: 1176; Rank: 1

\section{Peptide View}

MS/MS Fragmentation of MESDLANTTAGFTAALK

Found in IPI00392894, Tax_Id=10116 Gene_Symbol=Mgam_predicted 89 kDa protein

Match to Query 1176: 1756.816828 from(879.415690,2+)

Title: 091008RatKidney_NoSalt_30.2788.2788.2.dta

Data file K:\NewmanPaper\Piliang \3SubProteomes \Piliang3SP $\backslash$ mgf5ppm\ERLIC_3SubProteomes5ppm.mgf
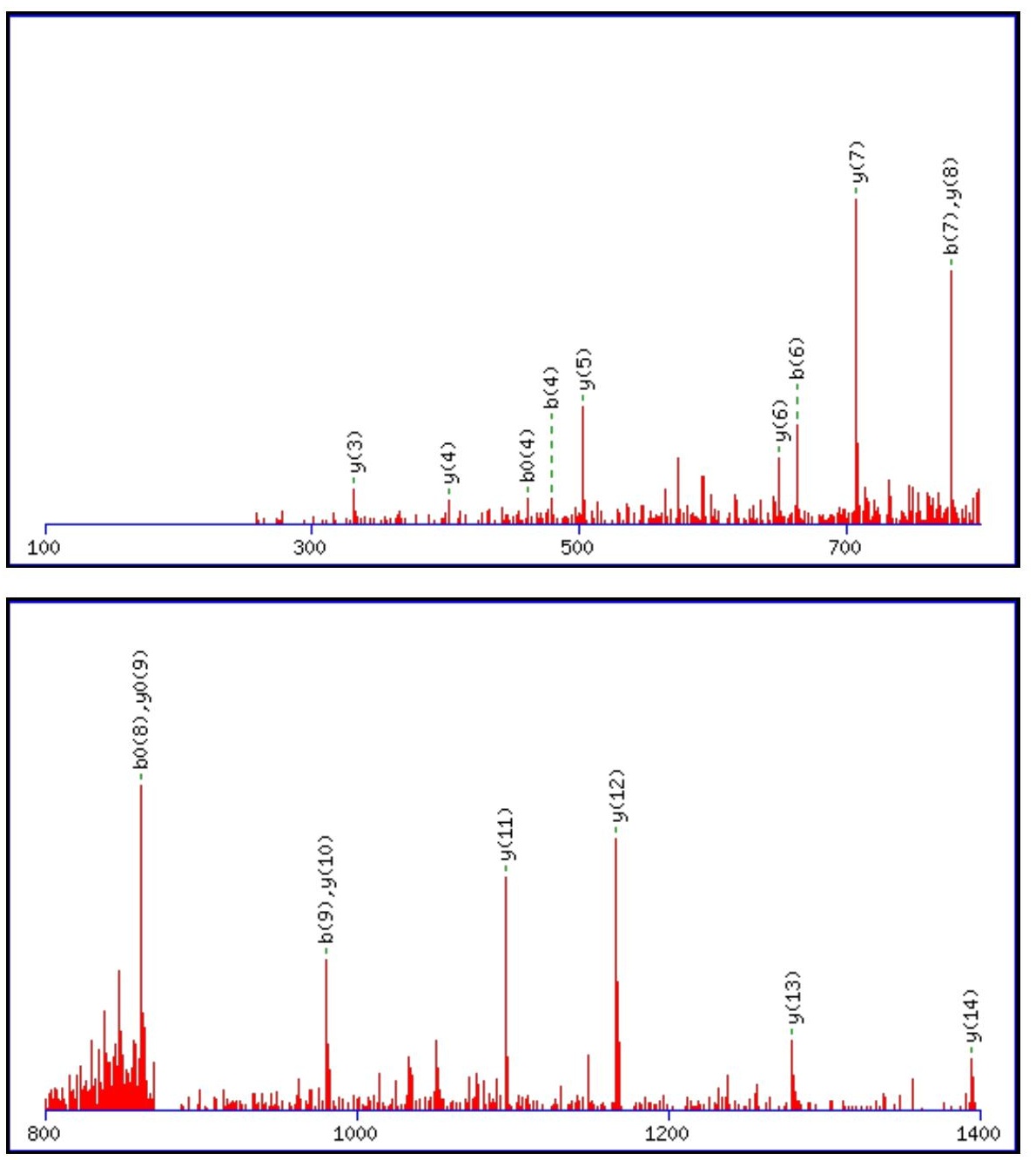


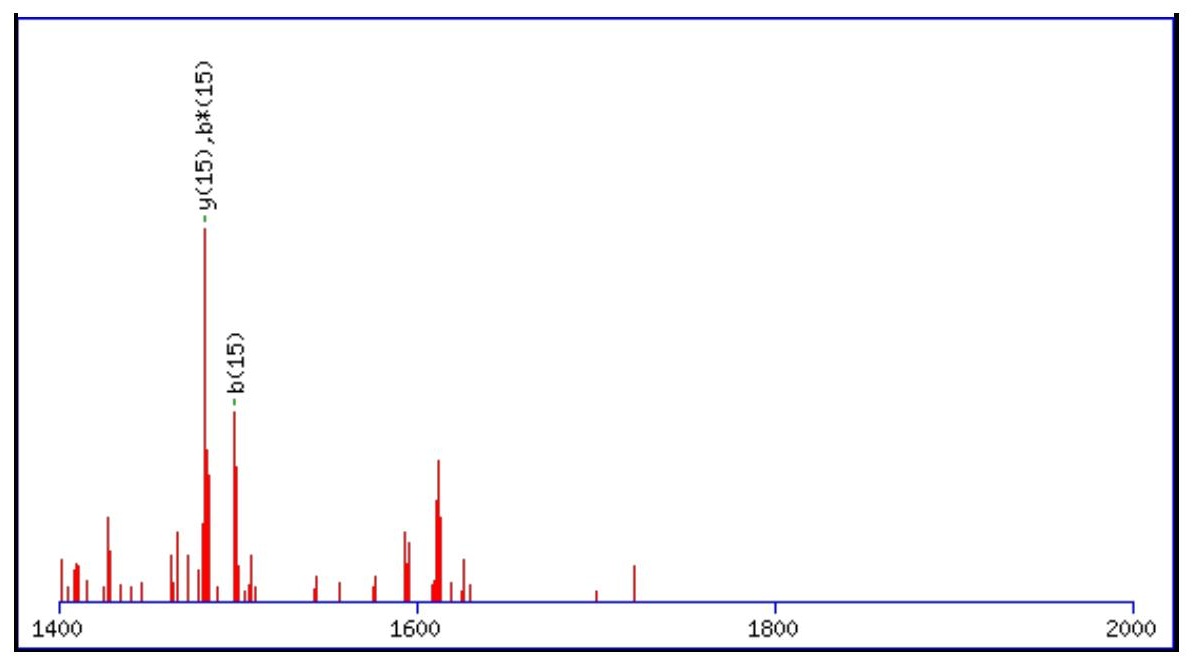

Monoisotopic mass of neutral peptide $\operatorname{Mr}($ calc): 1756.8189

Fixed modifications: Carbamidomethyl (C)

Variable modifications:

M1 : Oxidation (M)

N7 : Deamidated_N (N)

Ions Score: 125 Expect: $7 \mathrm{e}-011$

Matches (Bold Red): 21/170 fragment ions using 23 most intense peaks

\begin{tabular}{|c|c|c|c|c|c|c|c|c|c|c|c|c|c|c|}
\hline \# & b & $\mathbf{b}^{++}$ & $\mathbf{b}^{*}$ & $\mathbf{b}^{*^{++}}$ & $\mathbf{b}^{0}$ & $\mathbf{b}^{0++}$ & Seq. & $\mathbf{y}$ & $y^{++}$ & $\mathbf{y}^{*}$ & $\mathbf{y}^{*^{++}}$ & $\mathbf{y}^{0}$ & $\mathbf{y}^{0++}$ & \# \\
\hline 1 & 148.0427 & 74.5250 & & & & & $\mathbf{M}$ & & & & & & & 17 \\
\hline 2 & 277.0853 & 139.0463 & & & 259.0747 & 130.0410 & $\mathbf{E}$ & 1610.7908 & 805.8990 & 1593.7643 & 797.3858 & 1592.7802 & 796.8938 & 16 \\
\hline 3 & 364.1173 & 182.5623 & & & 346.1067 & 173.5570 & S & 1481.7482 & 741.3777 & 1464.7217 & 732.8645 & 1463.7376 & 732.3725 & 15 \\
\hline 4 & 479.1442 & 240.0758 & & & 461.1337 & 231.0705 & D & 1394.7162 & 697.8617 & 1377.6896 & 689.3485 & 1376.7056 & 688.8564 & 14 \\
\hline 5 & 592.2283 & 296.6178 & & & 574.2177 & 287.6125 & $\mathbf{L}$ & 1279.6892 & 640.3483 & 1262.6627 & 631.8350 & 1261.6787 & 631.3430 & 13 \\
\hline 6 & 663.2654 & 332.1363 & & & 645.2549 & 323.1311 & A & 1166.6052 & 583.8062 & 1149.5786 & 575.2930 & 1148.5946 & 574.8009 & 12 \\
\hline 7 & 778.2924 & 389.6498 & 761.2658 & 381.1365 & 760.2818 & 380.6445 & $\mathbf{N}$ & 1095.5681 & 548.2877 & 1078.5415 & 539.7744 & 1077.5575 & 539.2824 & 11 \\
\hline 8 & 879.3400 & 440.1737 & 862.3135 & 431.6604 & 861.3295 & 431.1684 & $\mathbf{T}$ & 980.5411 & 490.7742 & 963.5146 & 482.2609 & 962.5306 & 481.7689 & 10 \\
\hline 9 & 980.3877 & 490.6975 & 963.3612 & 482.1842 & 962.3772 & 481.6922 & $\mathbf{T}$ & 879.4934 & 440.2504 & 862.4669 & 431.7371 & 861.4829 & 431.2451 & 9 \\
\hline 10 & 1051.4248 & 526.2161 & 1034.3983 & 517.7028 & 1033.4143 & 517.2108 & A & 778.4458 & 389.7265 & 761.4192 & 381.2132 & 760.4352 & 380.7212 & 8 \\
\hline 11 & 1108.4463 & 554.7268 & 1091.4197 & 546.2135 & 1090.4357 & 545.7215 & G & 707.4087 & 354.2080 & 690.3821 & 345.6947 & 689.3981 & 345.2027 & 7 \\
\hline 12 & 1255.5147 & 628.2610 & 1238.4882 & 619.7477 & 1237.5041 & 619.2557 & $\mathbf{F}$ & 650.3872 & 325.6972 & 633.3606 & 317.1840 & 632.3766 & 316.6920 & 6 \\
\hline 13 & 1356.5624 & 678.7848 & 1339.5358 & 670.2716 & 1338.5518 & 669.7795 & $\mathbf{T}$ & 503.3188 & 252.1630 & 486.2922 & 243.6498 & 485.3082 & 243.1577 & 5 \\
\hline 14 & 1427.5995 & 714.3034 & 1410.5730 & 705.7901 & 1409.5889 & 705.2981 & A & 402.2711 & 201.6392 & 385.2445 & 193.1259 & & & 4 \\
\hline 15 & 1498.6366 & 749.8219 & 1481.6101 & 741.3087 & 1480.6260 & 740.8167 & A & 331.2340 & 166.1206 & 314.2074 & 157.6074 & & & 3 \\
\hline 16 & 1611.7207 & 806.3640 & 1594.6941 & 797.8507 & 1593.7101 & 797.3587 & $\mathbf{L}$ & 260.1969 & 130.6021 & 243.1703 & 122.0888 & & & 2 \\
\hline 17 & & & & & & & $\mathbf{K}$ & 147.1128 & 74.0600 & 130.0863 & 65.5468 & & & 1 \\
\hline
\end{tabular}
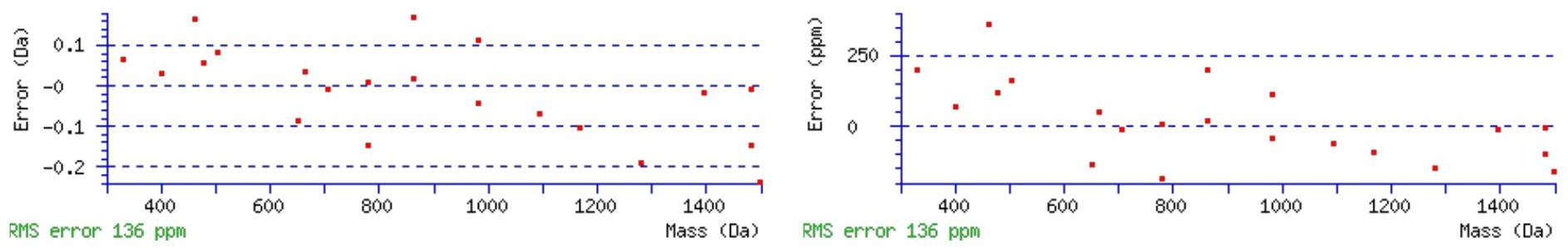

\section{All matches to this query}

\begin{tabular}{|l|l|l|l|}
\hline Score & Mr(calc): & Delta & \multicolumn{1}{c|}{ Sequence } \\
\hline 124.8 & 1756.8189 & -0.0021 & MESDLANTTAGFTAALK \\
\hline 5.9 & 1756.8045 & 0.0123 & $\underline{\text { TLLNTSCDNMLMAIK }}$ \\
\hline 5.4 & 1754.7997 & 2.0171 & $\underline{\text { MMSFGKEHRPPSKK }}$ \\
\hline 5.4 & 1754.7997 & 2.0171 & $\underline{\text { MMSFGKEHRPPSKK }}$ \\
\hline
\end{tabular}




\begin{tabular}{|l|l|l|l|}
3.5 & 1756.8318 & -0.0149 & TSILINALGSNTCLAK \\
\hline 3.2 & 1756.8219 & -0.0051 & LKVTHQAESNLYMK \\
\hline 3.1 & 1756.8050 & 0.0118 & GSSARPSDDVPPMNQAK \\
\hline 2.8 & 1756.8318 & -0.0149 & TSILINALGSNTCLAK \\
\hline 2.8 & 1756.8318 & -0.0149 & TSILINALGSNTCLAK \\
\hline 1.5 & 1755.7975 & 1.0193 & APQAAKACXQTSVSTR \\
\hline
\end{tabular}

Spectrum No: 12; Query: 714; Rank: 1

\section{Peptide View}

MS/MS Fragmentation of VFGANQSLSAAQLGR

Found in IPI00392474, Tax_Id=10116 Gene_Symbol=Slc39a8 Zinc transporter ZIP8

Match to Query 714: 1518.780108 from(760.397330,2+)

Title: 100101RatKid_NS_deglyco_25.2513.2513.2.dta

Data file K:INewmanPaper\Piliangl3SubProteomes\Piliang3SP\mgf5ppm|ERLIC_3SubProteomes5ppm.mgf
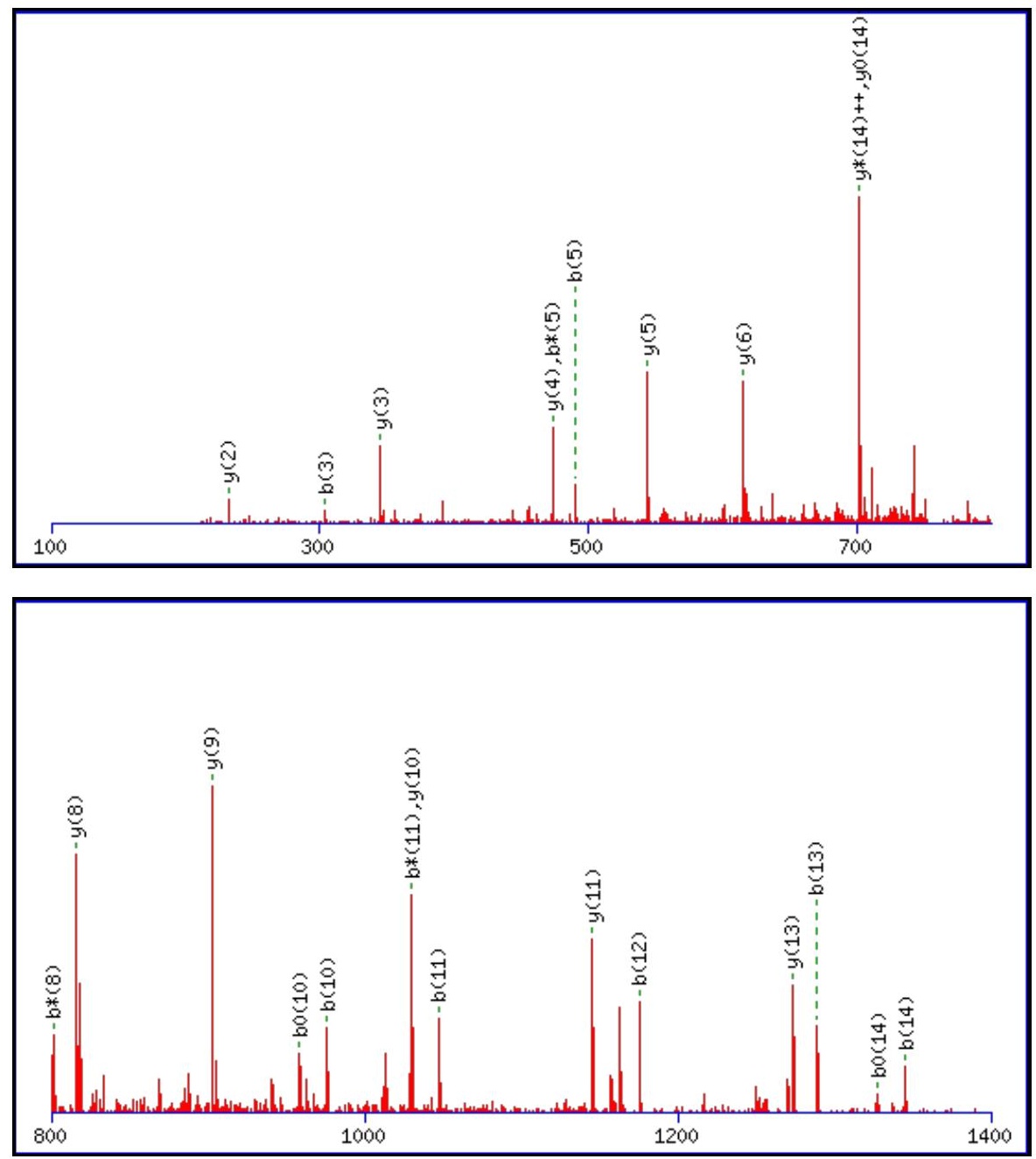


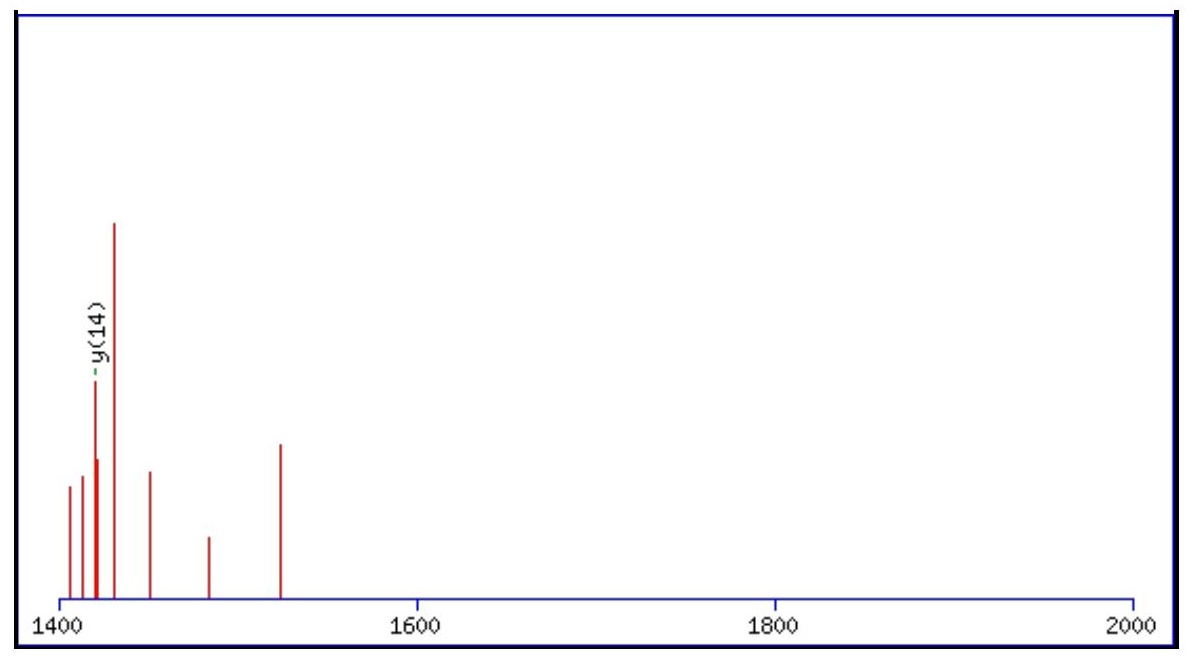

Monoisotopic mass of neutral peptide $\operatorname{Mr}($ calc): 1518.7790

Fixed modifications: Carbamidomethyl (C)

Variable modifications:

N5 : Deamidated $\mathrm{N}(\mathrm{N})$

Ions Score: 123 Expect: $6.4 \mathrm{e}-011$

Matches (Bold Red): 26/136 fragment ions using 32 most intense peaks

\begin{tabular}{|r|c|c|c|c|c|c|c|c|c|c|c|c|c|c|}
\hline$\#$ & $\mathbf{b}$ & $\mathbf{b}^{++}$ & $\mathbf{b}^{*}$ & $\mathbf{b}^{\mathbf{*}^{++}}$ & $\mathbf{b}^{\mathbf{0}}$ & $\mathbf{b}^{\mathbf{0 + +}}$ & $\mathbf{S e q}$ & $\mathbf{y}$ & $\mathbf{y}^{++}$ & $\mathbf{y}^{\mathbf{*}}$ & $\mathbf{y}^{\mathbf{*}^{++}}$ & $\mathbf{y}^{\mathbf{0}}$ & $\mathbf{y}^{\mathbf{0 + +}}$ & $\#$ \\
\hline $\mathbf{1}$ & 100.0757 & 50.5415 & & & & & $\mathbf{V}$ & & & & & & & $\mathbf{1 5}$ \\
\hline $\mathbf{2}$ & 247.1441 & 124.0757 & & & & & $\mathbf{F}$ & $\mathbf{1 4 2 0 . 7 1 7 9}$ & 710.8626 & 1403.6914 & $\mathbf{7 0 2 . 3 4 9 3}$ & 1402.7073 & $\mathbf{7 0 1 . 8 5 7 3}$ & $\mathbf{1 4}$ \\
\hline $\mathbf{3}$ & $\mathbf{3 0 4 . 1 6 5 6}$ & 152.5864 & & & & & $\mathbf{G}$ & $\mathbf{1 2 7 3 . 6 4 9 5}$ & 637.3284 & 1256.6229 & 628.8151 & 1255.6389 & 628.3231 & $\mathbf{1 3}$ \\
\hline $\mathbf{4}$ & 375.2027 & 188.1050 & & & & & $\mathbf{A}$ & 1216.6280 & 608.8177 & 1199.6015 & 600.3044 & 1198.6175 & 599.8124 & $\mathbf{1 2}$ \\
\hline $\mathbf{5}$ & $\mathbf{4 9 0 . 2 2 9 6}$ & 245.6184 & 473.2031 & 237.1052 & & & $\mathbf{N}$ & $\mathbf{1 1 4 5 . 5 9 0 9}$ & 573.2991 & 1128.5644 & 564.7858 & 1127.5804 & 564.2938 & $\mathbf{1 1}$ \\
\hline $\mathbf{6}$ & 618.2882 & 309.6477 & 601.2616 & 301.1345 & & & $\mathbf{Q}$ & $\mathbf{1 0 3 0 . 5 6 4 0}$ & 515.7856 & 1013.5374 & 507.2724 & 1012.5534 & 506.7803 & $\mathbf{1 0}$ \\
\hline $\mathbf{7}$ & 705.3202 & 353.1637 & 688.2937 & 344.6505 & 687.3097 & 344.1585 & $\mathbf{S}$ & $\mathbf{9 0 2 . 5 0 5 4}$ & 451.7563 & 885.4789 & 443.2431 & 884.4948 & 442.7511 & $\mathbf{9}$ \\
\hline $\mathbf{8}$ & 818.4043 & 409.7058 & $\mathbf{8 0 1 . 3 7 7 7}$ & 401.1925 & 800.3937 & 400.7005 & $\mathbf{L}$ & $\mathbf{8 1 5 . 4 7 3 4}$ & 408.2403 & 798.4468 & 399.7271 & 797.4628 & 399.2350 & $\mathbf{8}$ \\
\hline $\mathbf{9}$ & 905.4363 & 453.2218 & 888.4098 & 444.7085 & 887.4258 & 444.2165 & $\mathbf{S}$ & $\mathbf{7 0 2 . 3 8 9 3}$ & 351.6983 & 685.3628 & 343.1850 & 684.3787 & 342.6930 & $\mathbf{7}$ \\
\hline $\mathbf{1 0}$ & $\mathbf{9 7 6 . 4 7 3 4}$ & 488.7404 & 959.4469 & 480.2271 & $\mathbf{9 5 8 . 4 6 2 9}$ & 479.7351 & $\mathbf{A}$ & $\mathbf{6 1 5 . 3 5 7 3}$ & 308.1823 & 598.3307 & 299.6690 & & & $\mathbf{6}$ \\
\hline $\mathbf{1 1}$ & $\mathbf{1 0 4 7 . 5 1 0 5}$ & 524.2589 & $\mathbf{1 0 3 0 . 4 8 4 0}$ & 515.7456 & 1029.5000 & 515.2536 & $\mathbf{A}$ & $\mathbf{5 4 4 . 3 2 0 2}$ & 272.6637 & 527.2936 & 264.1504 & & & $\mathbf{5}$ \\
\hline $\mathbf{1 2}$ & $\mathbf{1 1 7 5 . 5 6 9 1}$ & 588.2882 & 1158.5426 & 579.7749 & 1157.5586 & 579.2829 & $\mathbf{Q}$ & $\mathbf{4 7 3 . 2 8 3 1}$ & 237.1452 & 456.2565 & 228.6319 & & & $\mathbf{4}$ \\
\hline $\mathbf{1 3}$ & $\mathbf{1 2 8 8 . 6 5 3 2}$ & 644.8302 & 1271.6266 & 636.3170 & 1270.6426 & 635.8249 & $\mathbf{L}$ & $\mathbf{3 4 5 . 2 2 4 5}$ & 173.1159 & 328.1979 & 164.6026 & & & $\mathbf{3}$ \\
\hline $\mathbf{1 4}$ & $\mathbf{1 3 4 5 . 6 7 4 6}$ & 673.3410 & 1328.6481 & 664.8277 & $\mathbf{1 3 2 7 . 6 6 4 1}$ & 664.3357 & $\mathbf{G}$ & $\mathbf{2 3 2 . 1 4 0 4}$ & 116.5738 & 215.1139 & 108.0606 & & & $\mathbf{2}$ \\
\hline $\mathbf{1 5}$ & & & & & & & $\mathbf{R}$ & 175.1190 & 88.0631 & 158.0924 & 79.5498 & & & $\mathbf{1}$ \\
\hline
\end{tabular}
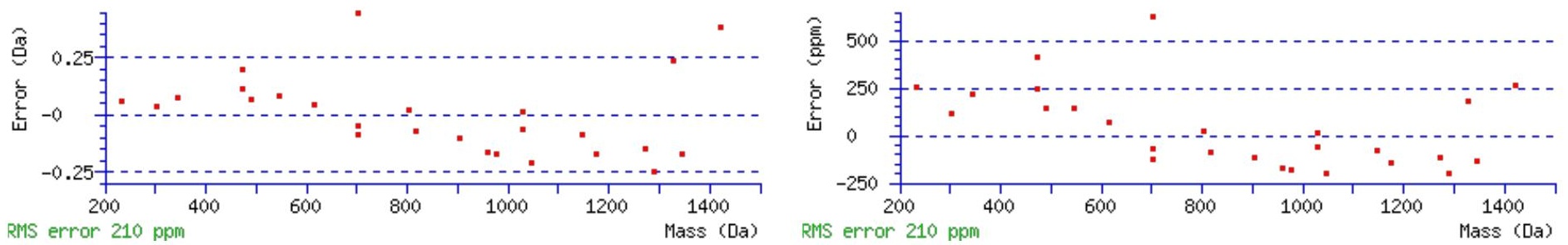

\section{All matches to this query}

\begin{tabular}{|l|l|l|l|}
\hline Score & Mr(calc): & Delta & \multicolumn{1}{|c|}{ Sequence } \\
\hline 123.5 & 1518.7790 & 0.0011 & VFGANQSLSAAQLGR \\
\hline 11.4 & 1518.7903 & -0.0102 & VEFEGRVLSRNGR \\
\hline 10.4 & 1517.7755 & 1.0046 & NLFSRYGKVVGAK \\
\hline 7.1 & 1518.7748 & 0.0053 & FASIWYAKKLGR \\
\hline 7.1 & 1518.7748 & 0.0053 & FASIWYAKKLGR \\
\hline 6.5 & 1518.7807 & -0.0006 & VKASNPLEQQVVK \\
\hline & & & \\
\hline
\end{tabular}




\begin{tabular}{|l|l|l|l|}
5.8 & 1517.7616 & 1.0185 & INFSHPKAAARAR \\
\hline 5.5 & 1517.7732 & 1.0069 & SKAGAGAASRLMNER \\
\hline 5.1 & 1518.7711 & 0.0090 & NKTAGEEEMIKIR \\
\hline 4.9 & 1517.7912 & 0.9889 & VMLTNVLWTDLGR \\
\hline
\end{tabular}

Spectrum No: 13; Query: 1841; Rank: 1

\section{Peptide View}

MS/MS Fragmentation of ISASGAELEALEAQVLNLSLK

Found in IPI00188541, Tax_Id=10116 Gene_Symbol=Itih4 inter-alpha-inhibitor H4 heavy chain

Match to Query 1841: 2156.161228 from(1079.087890,2+)

Title: 091008RatKidney_NoSalt_26.5458.5458.2.dta

Data file K:INewmanPaper|Piliang|3SubProteomes\Piliang3SP\mgf5ppm\ERLIC_3SubProteomes5ppm.mgf
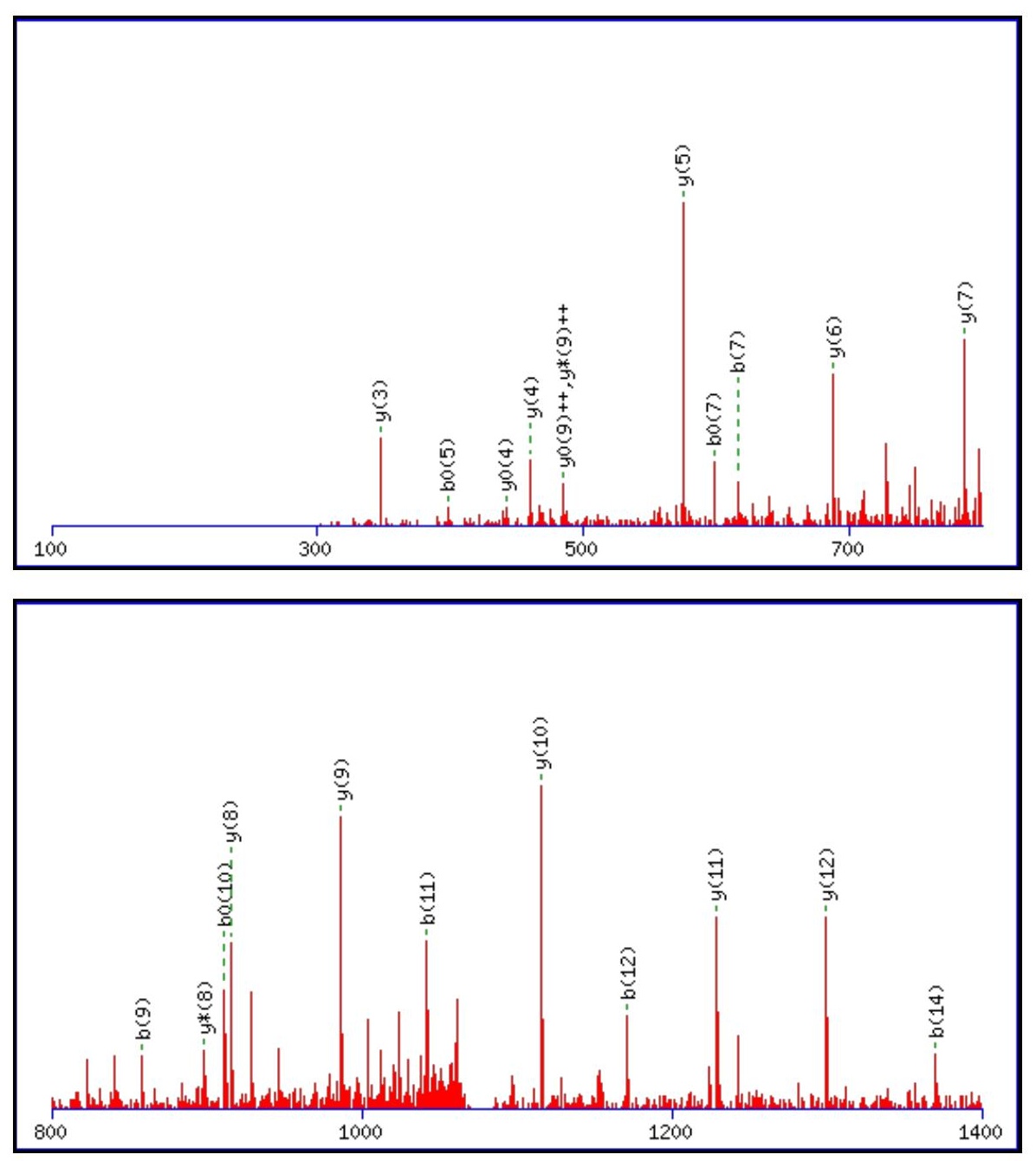


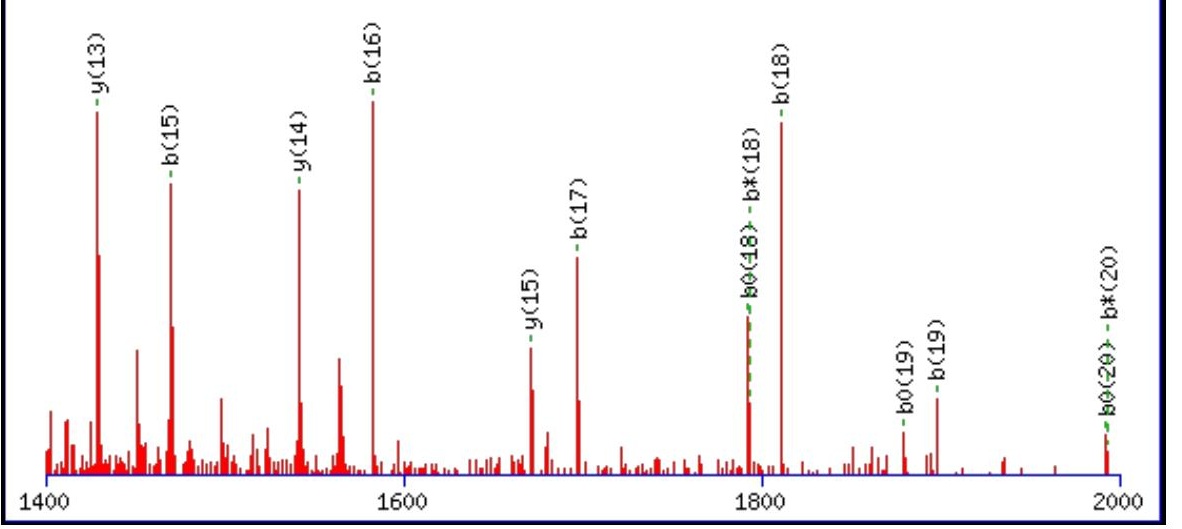

Monoisotopic mass of neutral peptide $\operatorname{Mr}($ calc): 2156.1576

Fixed modifications: Carbamidomethyl (C)

Variable modifications:

N17 : Deamidated $\mathrm{N}(\mathrm{N})$

Ions Score: 123 Expect: $3.3 \mathrm{e}-011$

Matches (Bold Red): 35/208 fragment ions using 42 most intense peaks

\begin{tabular}{|c|c|c|c|c|c|c|c|c|c|c|c|c|c|c|}
\hline \# & b & $\mathbf{b}^{++}$ & $\mathbf{b}^{*}$ & $\mathbf{b}^{*^{++}}$ & $\mathbf{b}^{0}$ & $\mathbf{b}^{\mathbf{0 + +}}$ & Seq. & $\mathbf{y}$ & $\mathrm{y}^{++}$ & $\mathbf{y}^{*}$ & $\mathbf{y}^{*^{++}}$ & $\mathbf{y}^{0}$ & $\mathbf{y}^{\mathbf{0 + +}}$ & \# \\
\hline 1 & 114.0913 & 57.5493 & & & & & I & & & & & & & 21 \\
\hline 2 & 201.1234 & 101.0653 & & & 183.1128 & 92.0600 & S & 2044.0808 & 1022.5441 & 2027.0543 & 1014.0308 & 2026.0703 & 1013.5388 & 20 \\
\hline 3 & 272.1605 & 136.5839 & & & 254.1499 & 127.5786 & A & 1957.0488 & 979.0280 & 1940.0223 & 970.5148 & 1939.0382 & 970.0228 & 19 \\
\hline 4 & 359.1925 & 180.0999 & & & 341.1819 & 171.0946 & S & 1886.0117 & 943.5095 & 1868.9851 & 934.9962 & 1868.0011 & 934.5042 & 18 \\
\hline 5 & 416.2140 & 208.6106 & & & 398.2034 & 199.6053 & G & 1798.9797 & 899.9935 & 1781.9531 & 891.4802 & 1780.9691 & 890.9882 & 17 \\
\hline 6 & 487.2511 & 244.1292 & & & 469.2405 & 235.1239 & A & 1741.9582 & 871.4827 & 1724.9317 & 862.9695 & 1723.9476 & 862.4775 & 16 \\
\hline 7 & 616.2937 & 308.6505 & & & 598.2831 & 299.6452 & $\mathbf{E}$ & 1670.9211 & 835.9642 & 1653.8945 & 827.4509 & 1652.9105 & 826.9589 & 15 \\
\hline 8 & 729.3777 & 365.1925 & & & 711.3672 & 356.1872 & $\mathbf{L}$ & 1541.8785 & 771.4429 & 1524.8519 & 762.9296 & 1523.8679 & 762.4376 & 14 \\
\hline 9 & 858.4203 & 429.7138 & & & 840.4098 & 420.7085 & $\mathbf{E}$ & 1428.7944 & 714.9009 & 1411.7679 & 706.3876 & 1410.7839 & 705.8956 & 13 \\
\hline 10 & 929.4575 & 465.2324 & & & 911.4469 & 456.2271 & A & 1299.7518 & 650.3796 & 1282.7253 & 641.8663 & 1281.7413 & 641.3743 & 12 \\
\hline 11 & 1042.5415 & 521.7744 & & & 1024.5310 & 512.7691 & $\mathbf{L}$ & 1228.7147 & 614.8610 & 1211.6882 & 606.3477 & 1210.7042 & 605.8557 & 11 \\
\hline 12 & 1171.5841 & 586.2957 & & & 1153.5735 & 577.2904 & $\mathbf{E}$ & 1115.6307 & 558.3190 & 1098.6041 & 549.8057 & 1097.6201 & 549.3137 & 10 \\
\hline 13 & 1242.6212 & 621.8142 & & & 1224.6107 & 612.8090 & A & 986.5881 & 493.7977 & 969.5615 & 485.2844 & 968.5775 & 484.7924 & 9 \\
\hline 14 & 1370.6798 & 685.8435 & 1353.6533 & 677.3303 & 1352.6692 & 676.8383 & $\mathbf{Q}$ & 915.5510 & 458.2791 & 898.5244 & 449.7658 & 897.5404 & 449.2738 & 8 \\
\hline 15 & 1469.7482 & 735.3777 & 1452.7217 & 726.8645 & 1451.7377 & 726.3725 & $\mathrm{~V}$ & 787.4924 & 394.2498 & 770.4658 & 385.7366 & 769.4818 & 385.2445 & 7 \\
\hline 16 & 1582.8323 & 791.9198 & 1565.8057 & 783.4065 & 1564.8217 & 782.9145 & $\mathbf{L}$ & 688.4240 & 344.7156 & 671.3974 & 336.2023 & 670.4134 & 335.7103 & 6 \\
\hline 17 & 1697.8592 & 849.4332 & 1680.8327 & 840.9200 & 1679.8486 & 840.4280 & $\mathbf{N}$ & 575.3399 & 288.1736 & 558.3133 & 279.6603 & 557.3293 & 279.1683 & 5 \\
\hline 18 & 1810.9433 & 905.9753 & 1793.9167 & 897.4620 & 1792.9327 & 896.9700 & $\mathbf{L}$ & 460.3130 & 230.6601 & 443.2864 & 222.1468 & 442.3024 & 221.6548 & 4 \\
\hline 19 & 1897.9753 & 949.4913 & 1880.9488 & 940.9780 & 1879.9647 & 940.4860 & S & 347.2289 & 174.1181 & 330.2023 & 165.6048 & 329.2183 & 165.1128 & 3 \\
\hline 20 & 2011.0594 & 1006.0333 & 1994.0328 & 997.5200 & 1993.0488 & 997.0280 & $\mathbf{L}$ & 260.1969 & 130.6021 & 243.1703 & 122.0888 & & & 2 \\
\hline 21 & & & & & & & $\mathbf{K}$ & 147.1128 & 74.0600 & 130.0863 & 65.5468 & & & 1 \\
\hline
\end{tabular}

$$
\text { 音 }
$$

$$
\text { RMS }
$$

RIS error 244 ppm
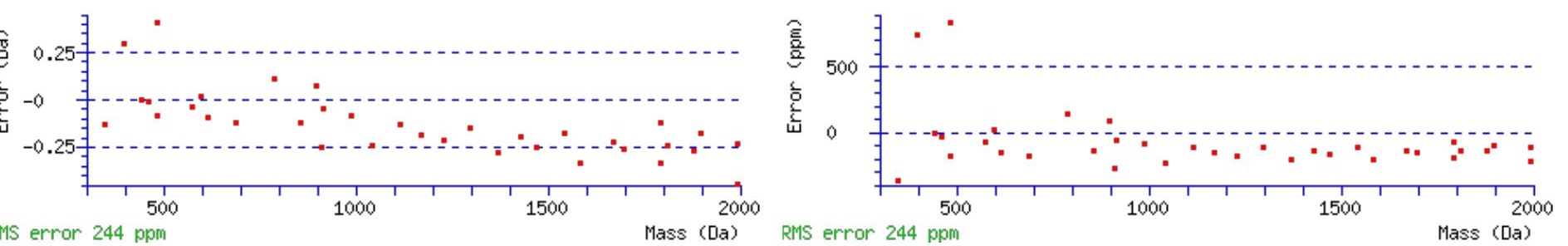

\section{All matches to this query}

\section{Score $\operatorname{Mr}$ (calc): $\quad$ Delta}

Sequence 


\begin{tabular}{|l|l|l|l|}
123.4 & 2156.1576 & 0.0037 & $\underline{\text { ISASGAELEALEAQVLNLSLK }}$ \\
\hline 26.8 & 2155.1736 & 0.9877 & ISASGAELEALEAQVLNLSLK \\
\hline 7.2 & 2156.1801 & -0.0188 & TVQIEASTVEIEERGVKLR \\
\hline 5.7 & 2156.1412 & 0.0201 & WCGDPALLNSLKQKSALVR \\
\hline 1.8 & 2156.1549 & 0.0063 & IINLTVDGNALGREAGGTRLK \\
\hline 0.6 & 2156.1814 & -0.0202 & NAEIFLALQNQRGGRISLR \\
\hline
\end{tabular}

Spectrum No: 14; Query: 458; Rank: 1

\section{Peptide View}

MS/MS Fragmentation of VSLTNVSISDEGR

Found in IPI00464765, Tax_Id=10116 Gene_Symbol=Igsf4a Immunoglobulin superfamily, member 4A

Match to Query 458: 1376.680908 from(689.347730,2+)

Title: 100101RatKid_NS_deglyco_27.2032.2032.2.dta

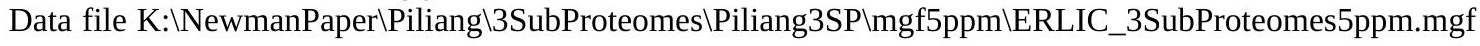
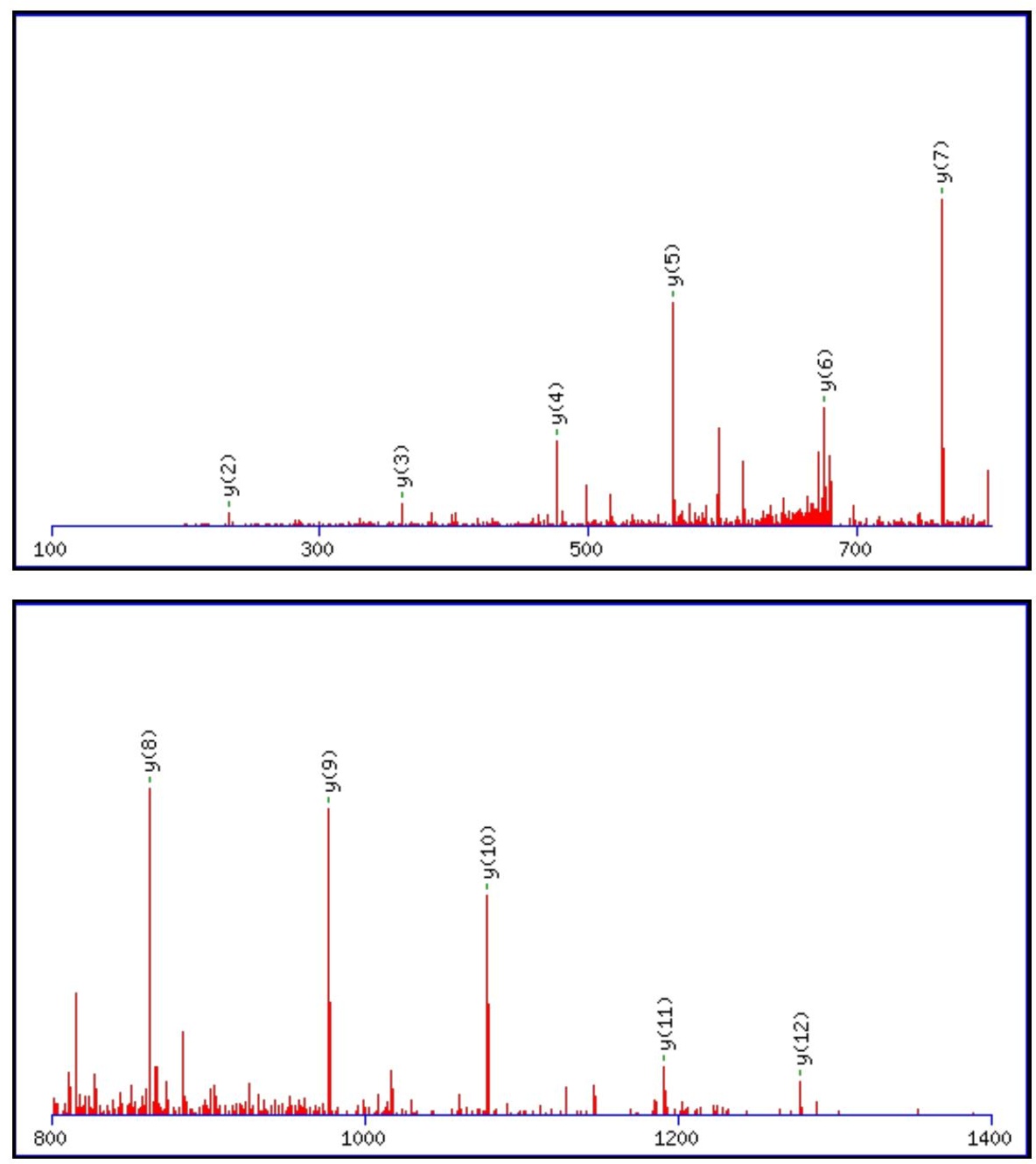
Monoisotopic mass of neutral peptide $\operatorname{Mr}($ calc): 1376.6783

Fixed modifications: Carbamidomethyl (C)

Variable modifications:

N5 : Deamidated $\mathrm{N}(\mathrm{N})$

Ions Score: 122 Expect: 8.7e-011

Matches (Bold Red): 11/130 fragment ions using 15 most intense peaks

\begin{tabular}{|c|c|c|c|c|c|c|c|c|c|c|c|c|c|c|}
\hline \# & b & $\mathbf{b}^{++}$ & b* & $\mathbf{b}^{*^{++}}$ & $\mathbf{b}^{0}$ & $\mathbf{b}^{0++}$ & Seq. & $\mathbf{y}$ & $\mathbf{y}^{++}$ & $\mathbf{y}^{*}$ & $\mathrm{y}^{*^{++}}$ & $\mathbf{y}^{0}$ & $\mathbf{y}^{0++}$ & \# \\
\hline 1 & 100.0757 & 50.5415 & & & & & $\mathbf{V}$ & & & & & & & 13 \\
\hline 2 & 187.1077 & 94.0575 & & & 169.0972 & 85.0522 & S & 1278.6172 & 639.8122 & 1261.5906 & 631.2990 & 1260.6066 & 630.8070 & 12 \\
\hline 3 & 300.1918 & 150.5995 & & & 282.1812 & 141.5942 & $\mathbf{L}$ & 1191.5852 & 596.2962 & 1174.5586 & 587.7829 & 1173.5746 & 587.2909 & 11 \\
\hline 4 & 401.2395 & 201.1234 & & & 383.2289 & 192.1181 & $\mathbf{T}$ & 1078.5011 & 539.7542 & 1061.4746 & 531.2409 & 1060.4905 & 530.7489 & 10 \\
\hline 5 & 516.2664 & 258.6368 & 499.2398 & 250.1236 & 498.2558 & 249.6316 & $\mathbf{N}$ & 977.4534 & 489.2303 & 960.4269 & 480.7171 & 959.4429 & 480.2251 & 9 \\
\hline 6 & 615.3348 & 308.1710 & 598.3083 & 299.6578 & 597.3242 & 299.1658 & V & 862.4265 & 431.7169 & 845.3999 & 423.2036 & 844.4159 & 422.7116 & 8 \\
\hline 7 & 702.3668 & 351.6871 & 685.3403 & 343.1738 & 684.3563 & 342.6818 & S & 763.3581 & 382.1827 & 746.3315 & 373.6694 & 745.3475 & 373.1774 & 7 \\
\hline 8 & 815.4509 & 408.2291 & 798.4244 & 399.7158 & 797.4403 & 399.2238 & I & 676.3260 & 338.6667 & 659.2995 & 330.1534 & 658.3155 & 329.6614 & 6 \\
\hline 9 & 902.4829 & 451.7451 & 885.4564 & 443.2318 & 884.4724 & 442.7398 & S & 563.2420 & 282.1246 & 546.2154 & 273.6114 & 545.2314 & 273.1193 & 5 \\
\hline 10 & 1017.5099 & 509.2586 & 1000.4833 & 500.7453 & 999.4993 & 500.2533 & D & 476.2100 & 238.6086 & 459.1834 & 230.0953 & 458.1994 & 229.6033 & 4 \\
\hline 11 & 1146.5525 & 573.7799 & 1129.5259 & 565.2666 & 1128.5419 & 564.7746 & $\mathbf{E}$ & 361.1830 & 181.0951 & 344.1565 & 172.5819 & 343.1724 & 172.0899 & 3 \\
\hline 12 & 1203.5739 & 602.2906 & 1186.5474 & 593.7773 & 1185.5634 & 593.2853 & G & 232.1404 & 116.5738 & 215.1139 & 108.0606 & & & 2 \\
\hline 13 & & & & & & & $\mathbf{R}$ & 175.1190 & 88.0631 & 158.0924 & 79.5498 & & & 1 \\
\hline
\end{tabular}

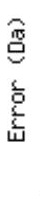

\section{All matches to this query}

\begin{tabular}{|l|l|l|l|}
\hline Score & Mr(calc): & Delta & \multicolumn{1}{|c|}{ Sequence } \\
\hline 122.0 & 1376.6783 & 0.0026 & VSLTNVSISDEGR \\
\hline 3.6 & 1376.6871 & -0.0061 & IFKSSDHIQMR \\
\hline 3.2 & 1376.6837 & -0.0028 & DAPFFRESPVGR \\
\hline 2.1 & 1376.6718 & 0.0091 & KNIEEKSGMEGR \\
\hline 2.0 & 1376.6693 & 0.0116 & MHIASLQYMAGR \\
\hline 1.8 & 1374.6853 & 1.9956 & YLELMIVNDHK \\
\hline 1.8 & 1376.6909 & -0.0100 & RGHPIDSQSPQR \\
\hline 1.1 & 1375.6732 & 1.0077 & SHFNKVSTNVDK \\
\hline & & & \\
\hline
\end{tabular}

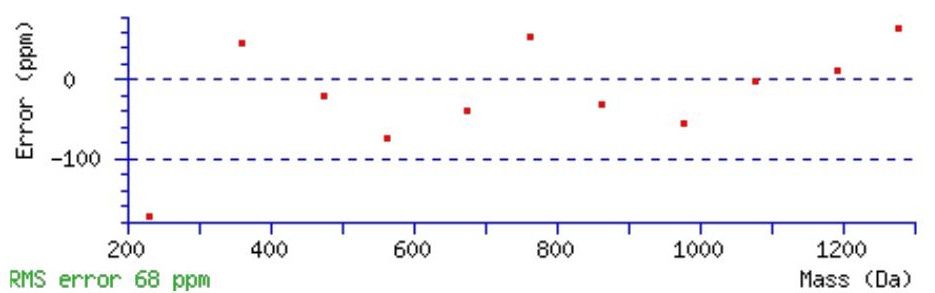




\section{\begin{tabular}{|l|l|l|l|}
0.3 & 1375.6766 & 1.0043 & TSEPKAMVVGEGR \\
\hline
\end{tabular}}

Spectrum No: 15; Query: 2222; Rank: 1

\section{Peptide View}

MS/MS Fragmentation of LVSDDLDSSLANLVGNLGIGNGTTK

Found in IPI00194958, Tax_Id=10116 Gene_Symbol=Picalm Isoform 2 of Phosphatidylinositol-binding clathrin assembly protein

Match to Query 2222: 2473.261408 from(1237.637980,2+)

Title: 091008RatKidney_NoSalt_17.6783.6783.2.dta

Data file K:\NewmanPaper\Piliang \3SubProteomes \Piliang3SP $\backslash$ mgf5ppm\ERLIC_3SubProteomes5ppm.mgf
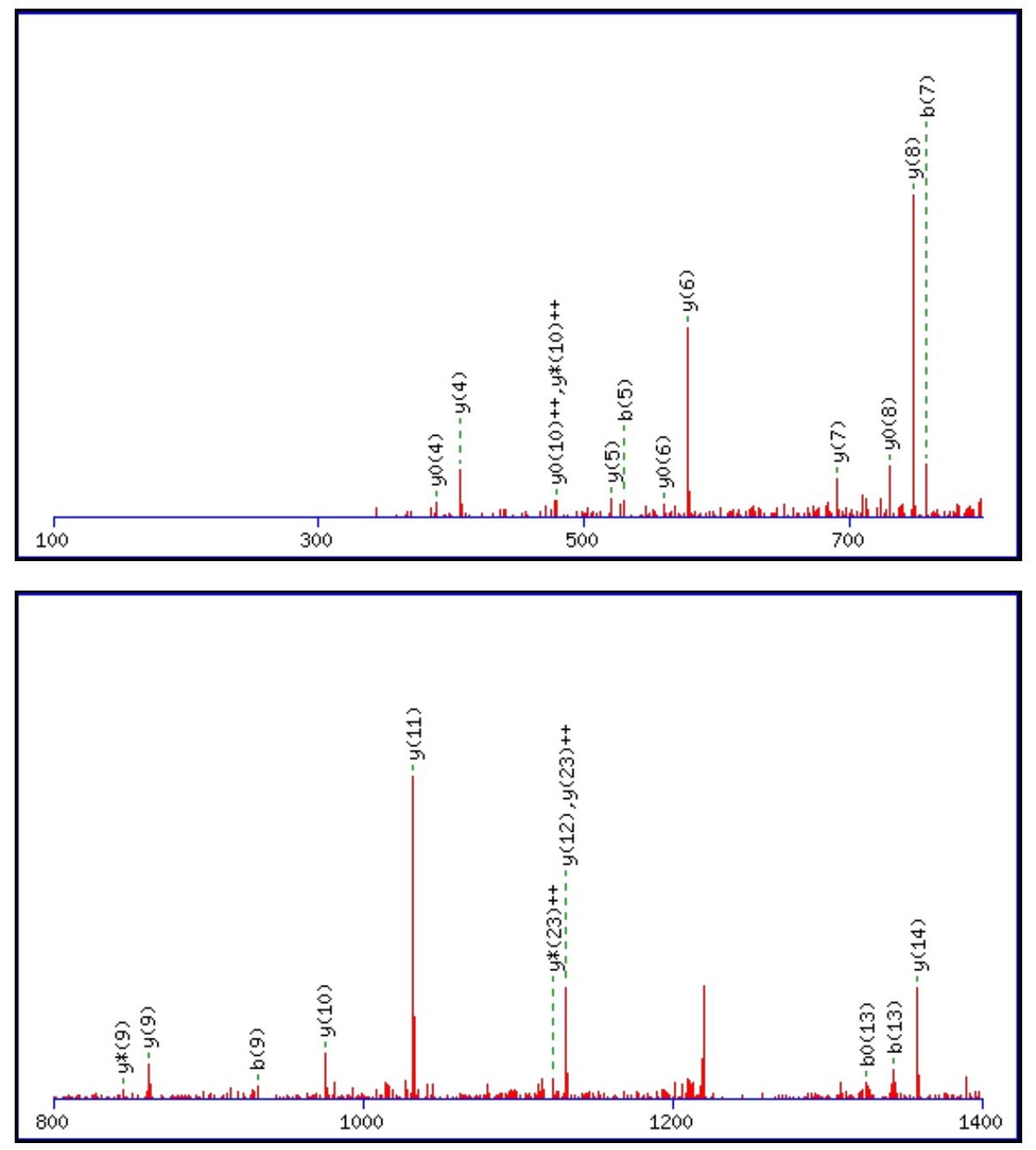


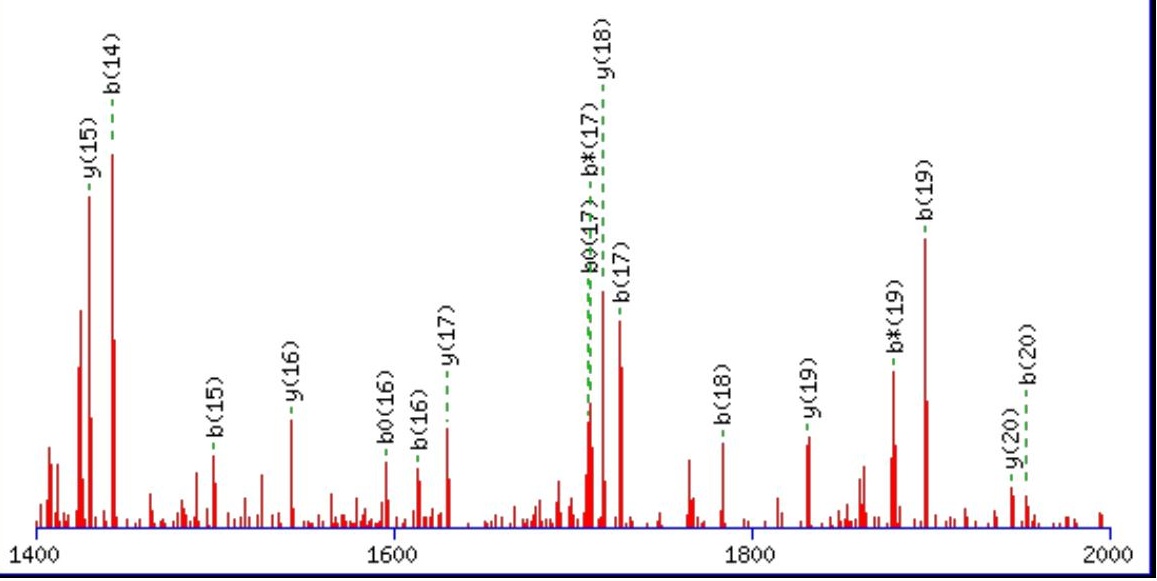

Monoisotopic mass of neutral peptide $\operatorname{Mr}($ calc): 2473.2548

Fixed modifications: Carbamidomethyl (C)

Variable modifications:

N21 : Deamidated $\mathrm{N}(\mathrm{N})$

Ions Score: 122 Expect: $1.2 \mathrm{e}-010$

Matches (Bold Red): 40/260 fragment ions using 58 most intense peaks

\begin{tabular}{|c|c|c|c|c|c|c|c|c|c|c|c|c|c|c|}
\hline \# & b & $\mathbf{b}^{++}$ & b* & $\mathbf{b}^{*^{++}}$ & $\mathbf{b}^{\mathbf{0}}$ & $\mathbf{b}^{\mathbf{0 + +}}$ & Seq. & $\mathbf{y}$ & $\mathbf{y}^{++}$ & $\mathbf{y}^{*}$ & $\mathrm{y}^{*^{++}}$ & $\mathbf{y}^{\mathbf{0}}$ & & \# \\
\hline 1 & 114.0913 & 57.5493 & & & & & $\mathbf{L}$ & & & & & & & 25 \\
\hline 2 & 213.1598 & 107.0835 & & & & & $\mathbf{V}$ & 2361.1780 & 1181.0926 & 2344.1514 & 1172.5794 & 2343.1674 & 1172.0873 & 24 \\
\hline 3 & 300.1918 & 150.5995 & & & 282.1812 & 141.5942 & $S$ & 2262.1096 & 1131.5584 & 2245.0830 & 1123.0451 & 2244.0990 & 1122.5531 & 23 \\
\hline 4 & 415.2187 & 208.1130 & & & 397.2082 & 199.1077 & D & 2175.0775 & 1088.0424 & 2158.0510 & 1079.5291 & 2157.0670 & 1079.0371 & 22 \\
\hline 5 & 530.2457 & 265.6265 & & & 512.2351 & 256.6212 & D & 2060.0506 & 1030.5289 & 2043.0240 & 1022.0157 & 2042.0400 & 1021.5237 & 21 \\
\hline 6 & 643.3297 & 322.1685 & & & 625.3192 & 313.1632 & $\mathbf{L}$ & 1945.0237 & 973.0155 & 1927.9971 & 964.5022 & 1927.0131 & 964.0102 & 20 \\
\hline 7 & 758.3567 & 379.6820 & & & 740.3461 & 370.6767 & D & 1831.9396 & 916.4734 & 1814.9130 & 907.9602 & 1813.9290 & 907.4682 & 19 \\
\hline 8 & 845.3887 & 423.1980 & & & 827.3781 & 414.1927 & S & 1716.9126 & 858.9600 & 1699.8861 & 850.4467 & 1698.9021 & 849.9547 & 18 \\
\hline 9 & 932.4207 & 466.7140 & & & 914.4102 & 457.7087 & S & 1629.8806 & 815.4439 & 1612.8541 & 806.9307 & 1611.8701 & 806.4387 & 17 \\
\hline 10 & 1045.5048 & 523.2560 & & & 1027.4942 & 514.2508 & $\mathbf{L}$ & 1542.8486 & 771.9279 & 1525.8220 & 763.4147 & 1524.8380 & 762.9227 & 16 \\
\hline 11 & 1116.5419 & 558.7746 & & & 1098.5313 & 549.7693 & A & 1429.7645 & 715.3859 & 1412.7380 & 706.8726 & 1411.7540 & 706.3806 & 15 \\
\hline 12 & 1230.5848 & 615.7961 & 1213.5583 & & 1212.5743 & 606.7908 & $\mathbf{N}$ & 1358.7274 & 679.8673 & 1341.7009 & 671.3541 & 1340.7168 & 670.8621 & 14 \\
\hline 13 & 1343.6689 & 672.3381 & 1326.6424 & 663.8248 & 1325.6583 & 663.3328 & $\mathbf{L}$ & 1244.6845 & 622.8459 & 1227.6579 & 614.3326 & 1226.6739 & 613.8406 & 13 \\
\hline 14 & 1442.7373 & 721.8723 & 1425.7108 & 713.3590 & 1424.7267 & 712.8670 & V & 1131.6004 & 566.3038 & 1114.5739 & 557.7906 & 1113.5899 & 557.2986 & 12 \\
\hline 15 & 1499.7588 & 750.3830 & 1482.7322 & 741.8698 & 1481.7482 & 741.3777 & G & 1032.5320 & 516.7696 & 1015.5055 & 508.2564 & 1014.5214 & 507.7644 & 11 \\
\hline 16 & 1613.8017 & 807.4045 & 1596.7752 & 798.8912 & 1595.7911 & 798.3992 & $\mathbf{N}$ & 975.5105 & 488.2589 & 958.4840 & 479.7456 & 957.5000 & 479.2536 & 10 \\
\hline 17 & 1726.8858 & 863.9465 & 1709.8592 & 855.4332 & 1708.8752 & 854.9412 & $\mathbf{L}$ & 861.4676 & 431.2374 & 844.4411 & 422.7242 & 843.4571 & 422.2322 & 9 \\
\hline 18 & 1783.9072 & 892.4573 & 1766.8807 & 883.9440 & 1765.8967 & 883.4520 & G & 748.3836 & 374.6954 & 731.3570 & 366.1821 & 730.3730 & 365.6901 & 8 \\
\hline 19 & 1896.9913 & 948.9993 & 1879.9647 & 940.4860 & 1878.9807 & 939.9940 & I & 691.3621 & 346.1847 & 674.3355 & 337.6714 & 673.3515 & 337.1794 & 7 \\
\hline 20 & 1954.0128 & 977.5100 & 1936.9862 & 968.9967 & 1936.0022 & 968.5047 & G & 578.2780 & 289.6427 & 561.2515 & 281.1294 & 560.2675 & 280.6374 & 6 \\
\hline 21 & 2069.0397 & 1035.0235 & 2052.0131 & 1026.5102 & 2051.0291 & 1026.0182 & $\mathbf{N}$ & 521.2566 & 261.1319 & 504.2300 & 252.6186 & 503.2460 & 252.1266 & 5 \\
\hline 22 & 2126.0612 & 1063.5342 & 2109.0346 & 1055.0209 & 2108.0506 & 1054.5289 & G & 406.2296 & 203.6185 & 389.2031 & 195.1052 & 388.2191 & 194.6132 & 4 \\
\hline 23 & 2227.1088 & 1114.0581 & 2210.0823 & 1105.5448 & 2209.0983 & 1105.0528 & $\mathbf{T}$ & 349.2082 & 175.1077 & 332.1816 & 166.5944 & 331.1976 & 166.1024 & 3 \\
\hline 24 & 2328.1565 & 1164.5819 & 2311.1300 & 1156.0686 & 2310.1460 & 1155.5766 & $\mathbf{T}$ & 248.1605 & 124.5839 & 231.1339 & 116.0706 & 230.1499 & 115.5786 & 2 \\
\hline 25 & & & & & & & $\mathbf{K}$ & 147.1128 & 74.0600 & 130.0863 & 65.5468 & & & 1 \\
\hline
\end{tabular}

$$
\text { 告 }
$$
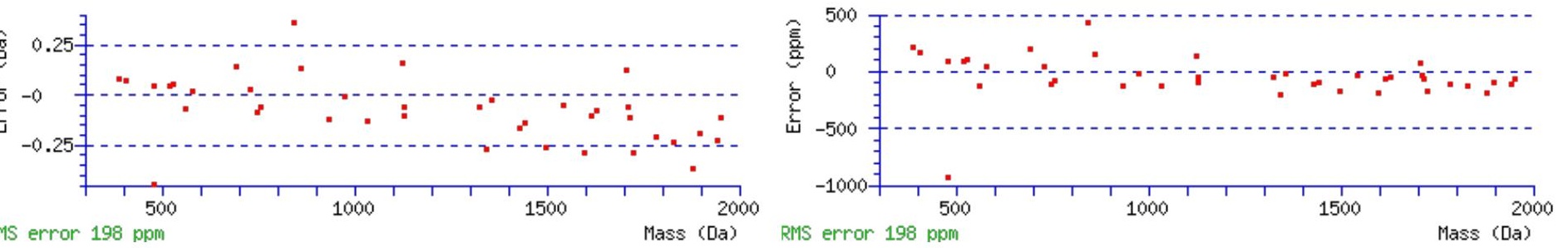


\section{All matches to this query}

\begin{tabular}{|l|l|l|l|}
\hline Score & Mr(calc): & Delta & \multicolumn{1}{|c|}{ Sequence } \\
\hline 121.5 & 2473.2548 & 0.0066 & LVSDDLDSSLANLVGNLGIGNGTTK \\
\hline 63.7 & 2473.2548 & 0.0066 & LVSDDLDSSLANLVGNLGIGNGTTK \\
\hline 44.7 & 2473.2548 & 0.0066 & LVSDDLDSSLANLVGNLGIGNGTTK \\
\hline 38.5 & 2472.2708 & 0.9906 & LVSDDLDSSLANLVGNLGIGNGTTK \\
\hline 0.8 & 2473.2617 & -0.0003 & SSADPPITPNPERILNVFAVKK \\
\hline 0.8 & 2473.2617 & -0.0003 & SSADPPITPNPERILNVFAVKK \\
\hline 0.5 & 2473.2804 & -0.0190 & RNLLLGTACAIYLGFLVSQVGK \\
\hline 0.1 & 2472.2348 & 1.0266 & CLEGTSDLRLVYTHHPLLLR \\
\hline
\end{tabular}

Spectrum No: 16; Query: 1889; Rank: 1

\section{Peptide View}

MS/MS Fragmentation of DQCIVDDITYNVNDTFHK

Found in IPI00200757, Tax_Id=10116 Gene_Symbol=Fn1 Isoform 1 of Fibronectin precursor

Match to Query 1889: 2196.970848 from(1099.492700,2+)

Title: 100101RatKid_NS_deglyco_08.4368.4368.2.dta

Data file K:INewmanPaper|Piliang|3SubProteomes\Piliang3SP\mgf5ppm\ERLIC_3SubProteomes5ppm.mgf
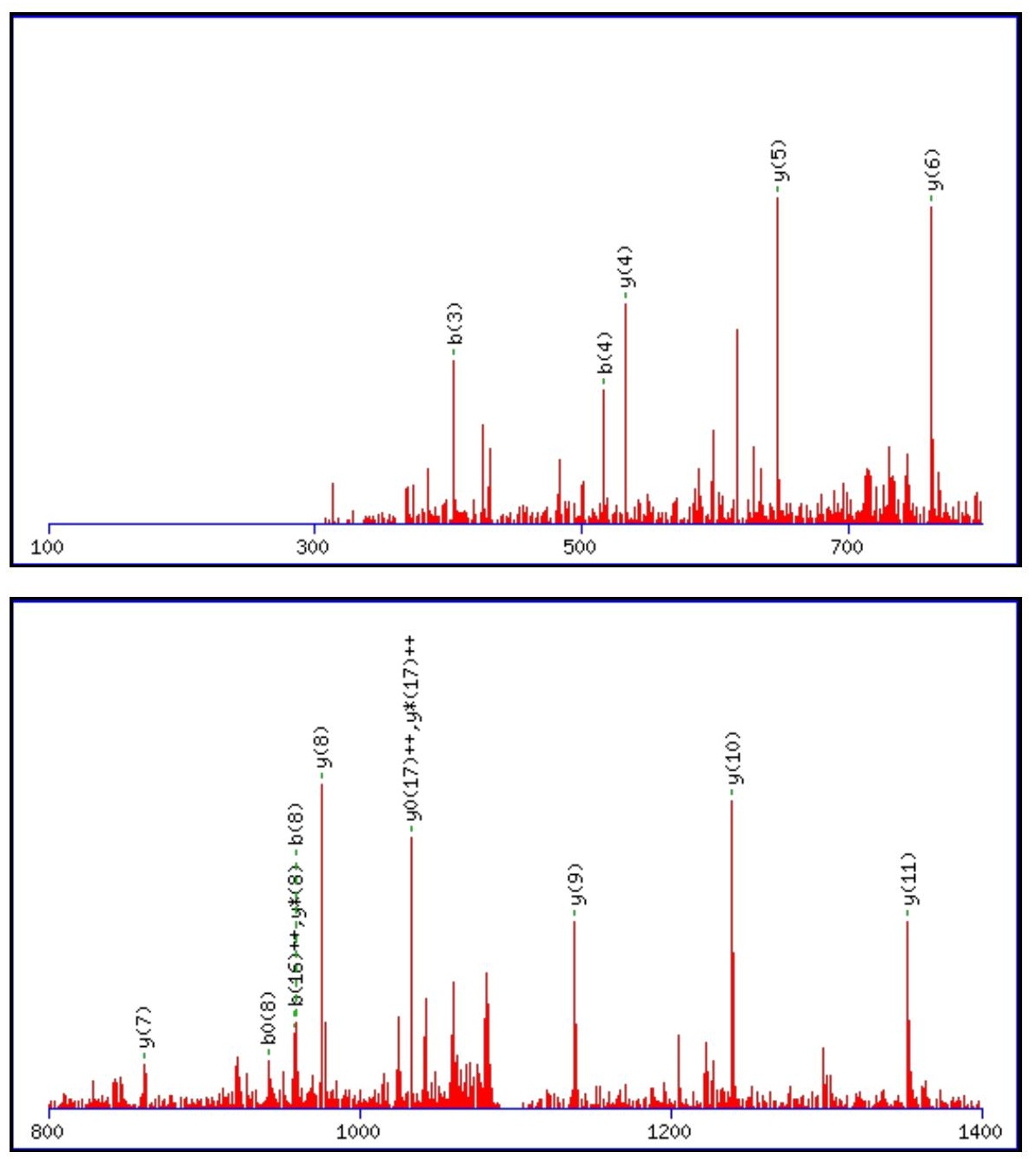


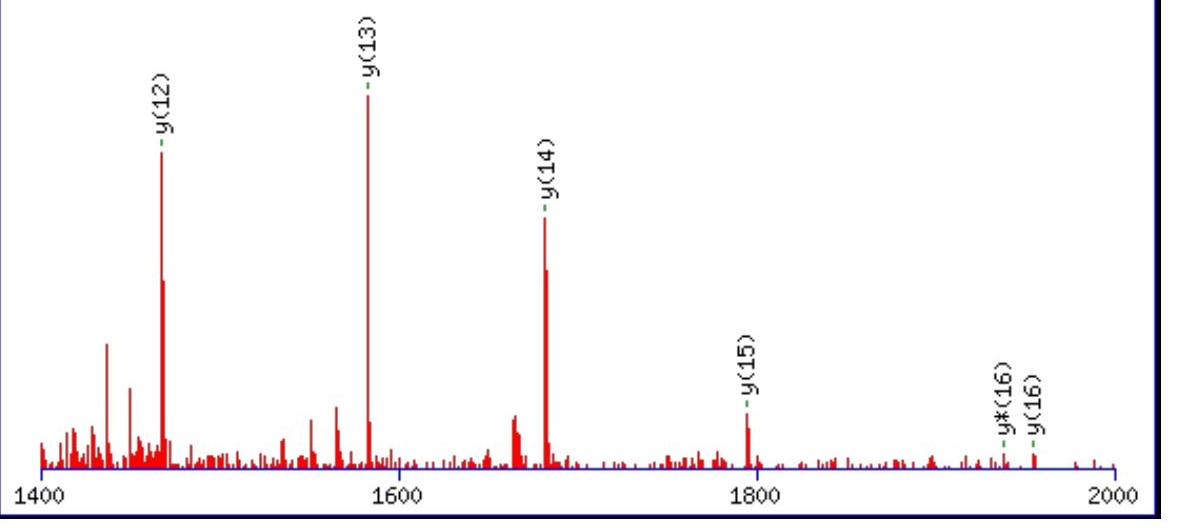

Monoisotopic mass of neutral peptide $\operatorname{Mr}($ calc): 2196.9634

Fixed modifications: Carbamidomethyl (C)

Variable modifications:

N13 : Deamidated $\mathrm{N}(\mathrm{N})$

Ions Score: 121 Expect: $2.3 e-010$

Matches (Bold Red): 22/196 fragment ions using 26 most intense peaks

\begin{tabular}{|c|c|c|c|c|c|c|c|c|c|c|c|c|c|c|}
\hline \# & b & $\mathbf{b}^{++}$ & $\mathbf{b}^{*}$ & & $\mathbf{b}^{0}$ & & Seq. & $\mathbf{y}$ & & $\mathbf{y}^{*}$ & $\mathbf{y}^{*-}$ & $\mathbf{y}^{0}$ & & \# \\
\hline 1 & 116.0342 & 58.5207 & & & 98.0237 & 49.5155 & D & & & & & & & 18 \\
\hline 2 & 244.0928 & 122.5500 & 227.0662 & 368 & 226.0822 & 113.5448 & $\mathbf{Q}$ & & & 171 & 4622 & 9331 & 1032.9702 & 17 \\
\hline 3 & 404.1234 & 202.5654 & 387.0969 & 194.0 & 386.1129 & 193.5601 & $\mathrm{C}$ & 1954.8851 & 977.9462 & 1937.8586 & 969.4329 & 1936.8745 & 968.9409 & 16 \\
\hline 4 & 517.2075 & 259.1 & 500.1810 & 250. & 499.1 & & I & 1794.8545 & 897.9309 & 1777.8279 & & 1776.8439 & 888.9256 & 15 \\
\hline 5 & 616.2759 & 308.6416 & 599.2494 & 300.1283 & 598.2654 & 299.6363 & V & 1681.7704 & 841.3888 & 1664.7439 & 832.8756 & 1663.7598 & 832.3836 & 14 \\
\hline 6 & 731.3029 & 366.1551 & 714.2763 & 357.6418 & 713.2923 & 357.1498 & D & 1582.7020 & 791.8546 & 1565. & 783.3414 & 1564.6914 & 782.8493 & 13 \\
\hline 7 & 846.3298 & 423.6685 & 829.3033 & 415.1553 & 828.3192 & 414.6633 & D & 1467.6750 & 3412 & 1450. & 725.8279 & 1449.6645 & 725.3359 & 12 \\
\hline 8 & 959.4139 & 480.2106 & 942.3873 & 471.6973 & 941.4033 & 471.2053 & I & 1352.6481 & 676.8277 & 1335.6216 & 668.3144 & 1334.6375 & 667.8224 & 11 \\
\hline 9 & 1060.4616 & 530.7344 & 1043.4350 & 522.2211 & 042.4510 & 521.7291 & $\mathbf{T}$ & 1239.5640 & 620.2857 & 1222. & 611.7724 & 5535 & 611.2804 & 10 \\
\hline 10 & 1223.5249 & 612.2661 & 1206.4983 & 603.7528 & 1205.5143 & 603.2608 & $\mathbf{Y}$ & 1138.5164 & 569.7618 & 1121.4898 & 561.2485 & 1120.5058 & 560.7565 & 9 \\
\hline 11 & 1337.5678 & 669.2875 & 1320.5413 & 660.7743 & 1319.5572 & 660.2823 & $\mathbf{N}$ & 975.4530 & 488.2302 & 958.4265 & 479.7169 & 957.4425 & 479.2249 & 8 \\
\hline 12 & 1436.6362 & 718.8217 & 1419.6097 & 710.3085 & 1418.6257 & 709.8165 & V & 861.4101 & 431.2087 & 844.3836 & 422.6954 & 843.3995 & 422.2034 & 7 \\
\hline 13 & 1551.6632 & 776.3352 & 1534.6366 & 767.8219 & 1533.6526 & 767.3299 & $\mathbf{N}$ & 762.3417 & 381.6745 & 745.3151 & 373.1612 & 744.3311 & 372.6692 & 6 \\
\hline 14 & 1666.6901 & 833.8487 & 1649.6636 & 825.3354 & 1648.6795 & 824.8434 & D & 647.3148 & 324.1610 & 630.2882 & 315.6477 & 629.3042 & 315.1557 & 5 \\
\hline 15 & 1767.7378 & 884.3725 & 1750.7112 & 875.8593 & 1749.7272 & 875.3672 & $\mathbf{T}$ & 532.2878 & 266.6475 & 515.2613 & 258.1343 & 514.2772 & 257.6423 & 4 \\
\hline 16 & 1914.8062 & 957.9067 & 1897.7796 & 949.3935 & 1896.7956 & 948.9015 & $\mathbf{F}$ & 431.2401 & 216.1237 & 414.2136 & 207.6104 & & & 3 \\
\hline 17 & 2051.8651 & 1026.4362 & 2034.8386 & 1017.9229 & 2033.8545 & 1017.4309 & $\mathbf{H}$ & 284.1717 & 142.5895 & 267.1452 & 134.0762 & & & 2 \\
\hline 18 & & & & & & & $\mathbf{K}$ & 147.1128 & 74.0600 & 130.0863 & 65.5468 & & & 1 \\
\hline
\end{tabular}
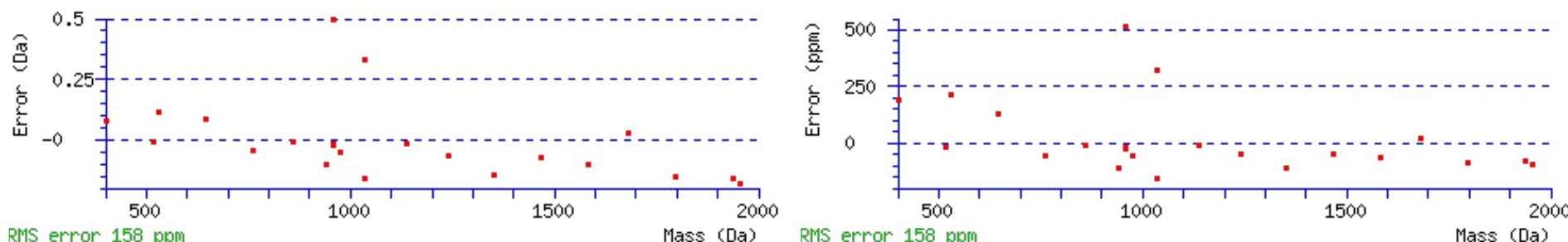

RMS error 158 ppm

Mass (Da) RMS error 158 ppm

\section{All matches to this query}

\begin{tabular}{|l|l|l|l|}
\hline Score & Mr(calc): & Delta & \multicolumn{1}{c|}{ Sequence } \\
\hline 120.9 & 2196.9634 & 0.0075 & DQCIVDDITYNVNDTFHK \\
\hline 86.5 & 2196.9634 & 0.0075 & DQCIVDDITYNVNDTFHK \\
\hline 21.0 & 2195.9794 & 0.9915 & DQCIVDDITYNVNDTFHK \\
\hline & & &
\end{tabular}




\begin{tabular}{|l|l|l|l|}
12.9 & 2196.9752 & -0.0044 & HGSLXLSVTPTLRGSMHNK \\
\hline 10.4 & 2196.9583 & 0.0126 & DDQGKNQSNRNVSSAICPK \\
\hline 8.6 & 2196.9752 & -0.0044 & HGSLXLSVTPTLRGSMHNK \\
\hline 6.7 & 2196.9752 & -0.0044 & HGSLXLSVTPTLRGSMHNK \\
\hline 6.1 & 2196.9857 & -0.0149 & VIHLSNLPHSGYSDSAVLK \\
\hline 4.4 & 2196.9752 & -0.0044 & HGSLXLSVTPTLRGSMHNK \\
\hline 4.3 & 2196.9752 & -0.0044 & HGSLXLSVTPTLRGSMHNK \\
\hline
\end{tabular}

Spectrum No: 17; Query: 1778; Rank: 1

\section{Peptide View}

MS/MS Fragmentation of MYVTNDTEVAENNYQALK

Found in IPI00464785, Tax_Id=10116 Gene_Symbol=Ppgb Protective protein for beta-galactosidase

Match to Query 1778: 2118.945948 from(1060.480250,2+)

Title: 091008RatKidney_NoSalt_21.2270.2270.2.dta

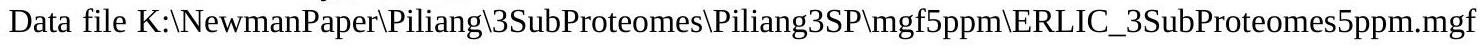
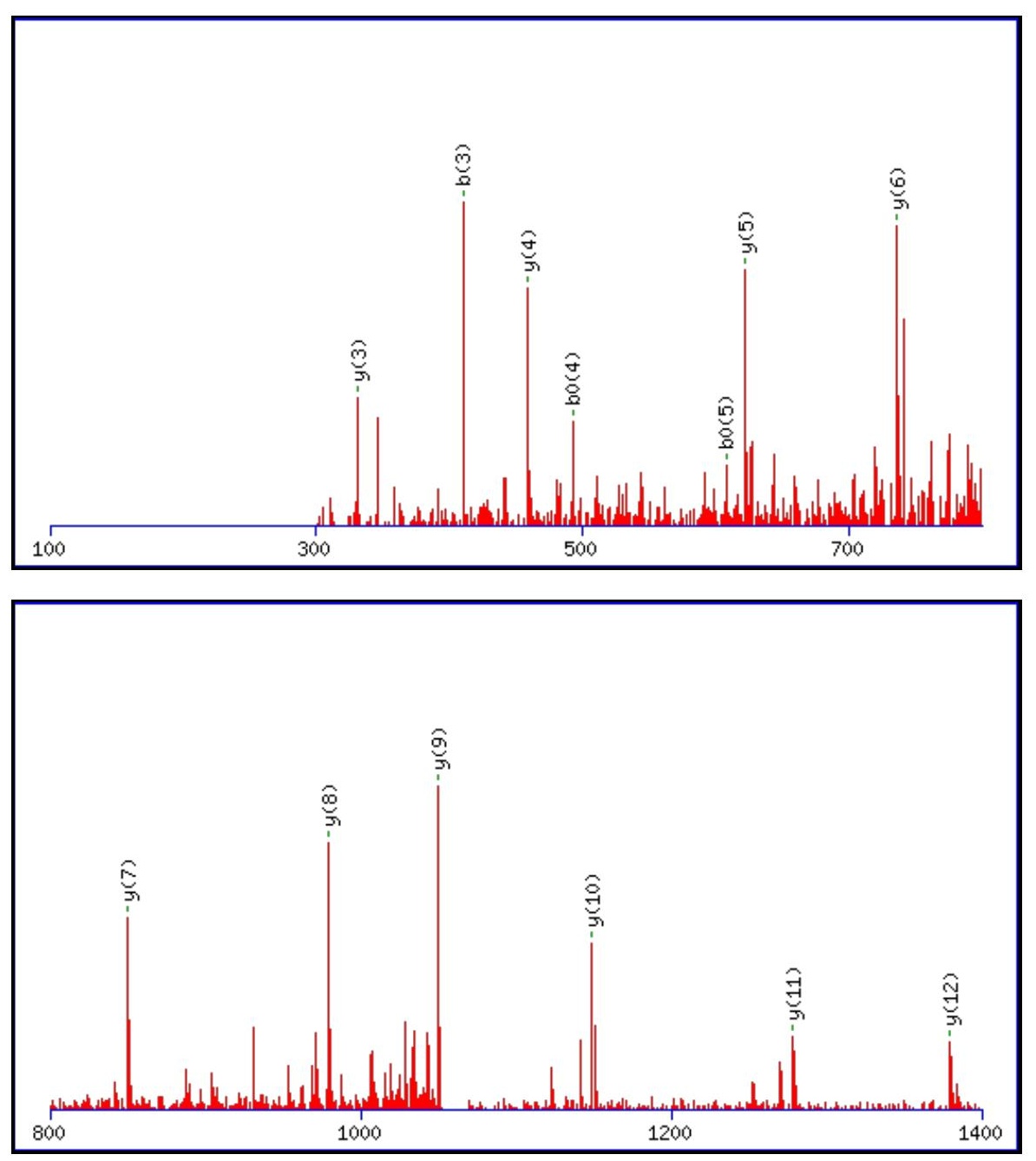


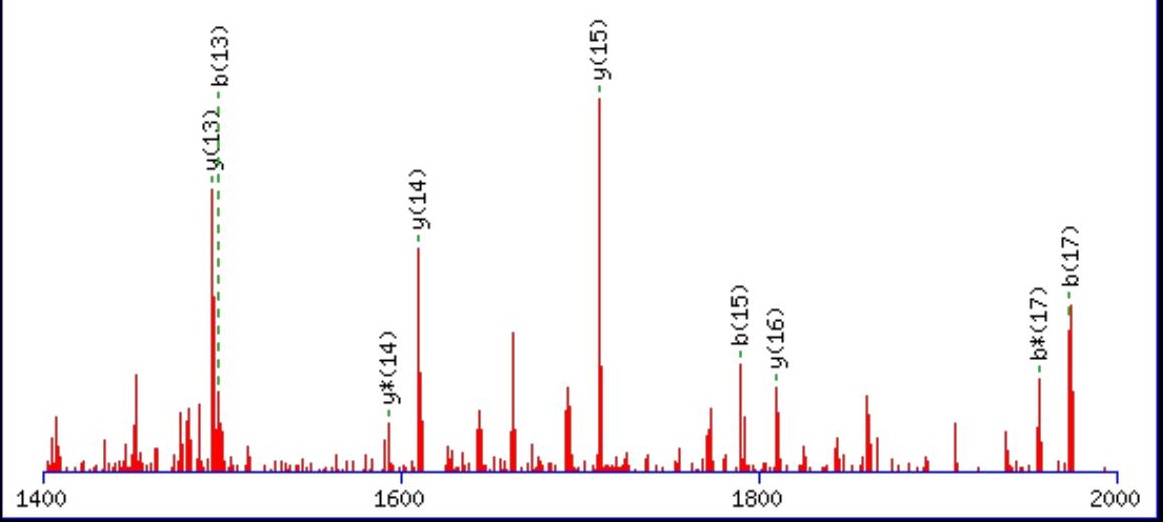

Monoisotopic mass of neutral peptide $\operatorname{Mr}($ calc): 2118.9415

Fixed modifications: Carbamidomethyl (C)

Variable modifications:

M1 : Oxidation (M)

N5 : Deamidated_N (N)

Ions Score: 121 Expect: $2.2 \mathrm{e}-010$

Matches (Bold Red): 22/176 fragment ions using 30 most intense peaks

\begin{tabular}{|c|c|c|c|c|c|c|c|c|c|c|c|c|c|c|}
\hline$\#$ & b & $\mathbf{b}^{++}$ & $\mathbf{b}^{*}$ & $\mathbf{b}^{*^{++}}$ & $\mathbf{b}^{0}$ & $\mathbf{b}^{\mathbf{0 + +}}$ & Seq. & $\mathbf{y}$ & $\mathbf{y}^{++}$ & $\mathbf{y}^{*}$ & $\mathrm{y}^{*^{++}}$ & $\mathbf{y}^{0}$ & $\mathbf{y}^{\mathbf{0 + +}}$ & \# \\
\hline 1 & 148.0427 & 74.5250 & & & & & $\mathbf{M}$ & & & & & & & 1 \\
\hline 2 & 311.1060 & 156.0566 & & & & & $\mathbf{Y}$ & 1972.9134 & 986.9604 & 1955.8869 & 978.4471 & 1954.9029 & 977.9551 & 17 \\
\hline 3 & 410.1744 & 205.5908 & & & & & $\mathbf{V}$ & 1809.8501 & 905.4287 & 1792.8236 & 896.9154 & 1791.8395 & 896.4234 & 16 \\
\hline 4 & 511.2221 & 256.1147 & & & 493.2115 & 247.1094 & $T$ & 1710.7817 & 855.8945 & 1693.7551 & 847.3812 & 1692.7711 & 846.8892 & 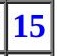 \\
\hline 5 & 626.2490 & 313.6282 & 609.2225 & 305.1149 & 608.2385 & 304.6229 & $\mathbf{N}$ & 1609.7340 & 805.3706 & 1592.7075 & 796.8574 & 1591.7235 & 796.3654 & \\
\hline 6 & 741.2760 & 371.1416 & 724.2494 & 362.6284 & 723.2654 & 362.1363 & D & 1494.7071 & 747.8572 & 1477.6805 & 739.3439 & 1476.6965 & 738.8519 & \\
\hline 7 & 842.3237 & 421.6655 & 825.2971 & 413.1522 & 824.3131 & 412.6602 & $\mathbf{T}$ & 1379.6801 & 690.3437 & 1362.6536 & 681.8304 & 1361.6696 & 681.3384 & 12 \\
\hline 8 & 971.3663 & 486.1868 & 954.3397 & 477.6735 & 953.3557 & 477.1815 & $\mathbf{E}$ & 1278.6325 & 639.8199 & 1261.6059 & 631.3066 & 1260.6219 & 630.8146 & 11 \\
\hline 9 & 1070.4347 & 535.7210 & 1053.4081 & 527.2077 & 1052.4241 & 526.7157 & $\bar{V}$ & 1149.5899 & 575.2986 & 1132.5633 & 566.7853 & 1131.5793 & 566.2933 & 10 \\
\hline 10 & 1141.4718 & 571.2395 & 1124.4452 & 562.7263 & 1123.4612 & 562.2342 & A & 1050.5215 & 525.7644 & 1033.4949 & 517.2511 & 1032.5109 & 516.7591 & \\
\hline 11 & 1270.5144 & 635.7608 & 1253.4878 & 627.2476 & 1252.5038 & 626.7555 & $E$ & 979.4843 & 490.2458 & 962.4578 & 481.7325 & 961.4738 & 481.2405 & r \\
\hline 12 & 1384.5573 & 692.7823 & 1367.5308 & 684.2690 & 1366.5467 & 683.7770 & $\mathbf{N}$ & 850.4417 & 425.7245 & 833.4152 & 417.2112 & & & \\
\hline 13 & 1498.6002 & 749.8038 & \begin{tabular}{|l|l}
1481.5737 \\
\end{tabular} & 741.2905 & 1480.5897 & 740.7985 & $\mathbf{N}$ & 736.3988 & 368.7030 & 719.3723 & 360.1898 & & & \\
\hline 14 & 1661.6636 & 831.3354 & 1644.6370 & 822.8221 & 1643.6530 & 822.3301 & $\mathbf{Y}$ & 622.3559 & 311.6816 & 605.3293 & 303.1683 & & & \\
\hline 15 & 1789.7221 & 895.3647 & 1772.6956 & 886.8514 & 1771.7116 & 886.3594 & $\mathbf{Q}$ & 459.2926 & 230.1499 & 442.2660 & 221.6366 & & & \\
\hline 16 & 1860.7592 & 930.8833 & 1843.7327 & 922.3700 & 1842.7487 & 921.8780 & A & 331.2340 & 166.1206 & 314.2074 & 157.6074 & & & \\
\hline 17 & 1973.8433 & 987.4253 & 1956.8168 & 978.9120 & 1955.8327 & 978.4200 & $\mathbf{L}$ & 260.1969 & 130.6021 & 243.1703 & 122.0888 & & & \\
\hline 18 & & & & & & & $\mathbf{K}$ & 147.1128 & 74.0600 & 130.0863 & 65.5468 & & & \\
\hline
\end{tabular}
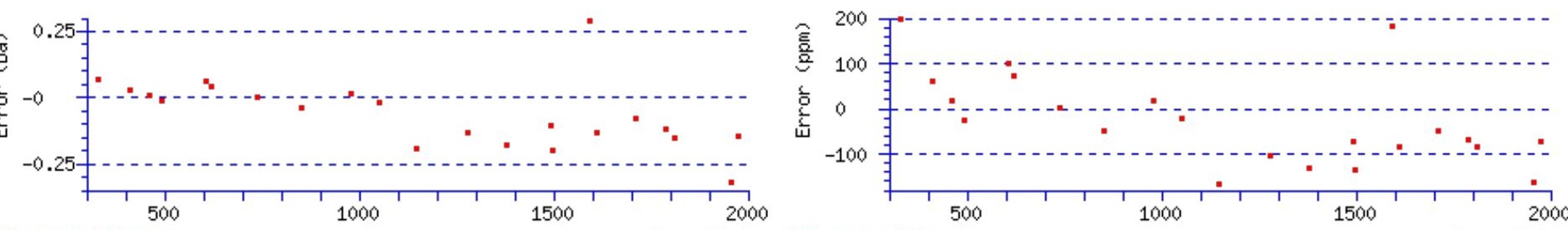

RNS error 101 ppm

Mass (Da) RMS error 101 ppm

Mass (Da)

\section{All matches to this query}

\begin{tabular}{|l|l|l|l|}
\hline Score & Mr(calc): & Delta & \multicolumn{1}{c|}{ Sequence } \\
\hline 120.8 & 2118.9415 & 0.0044 & MYVTNDTEVAENNYQALK \\
\hline 86.9 & 2118.9415 & 0.0044 & $\underline{\text { MYVTNDTEVAENNYQALK }}$ \\
\hline 72.3 & 2118.9415 & 0.0044 & $\underline{\text { MYVTNDTEVAENNYQALK }}$ \\
\hline
\end{tabular}




\begin{tabular}{|l|l|l|l|}
67.3 & 2117.9575 & 0.9884 & MYVTNDTEVAENNYQALK \\
\hline 3.7 & 2117.9493 & 0.9967 & AGSKPFNPVLGETYECIR \\
\hline 0.1 & 2117.9560 & 0.9899 & KTVXNLLGMAGMTPNVNSK \\
\hline
\end{tabular}

Spectrum No: 18; Query: 2181; Rank: 1

\section{Peptide View}

MS/MS Fragmentation of IYYSDFSNQTINSMAEDGSNR

Found in IPI00205325, Tax_Id=10116 Gene_Symbol=Lrp2 Low-density lipoprotein receptor-related protein 2 precursor

Match to Query 2181: 2428.017308 from(1215.015930,2+)

Title: 100101RatKid_NS_deglyco_19.2718.2718.2.dta

Data file K:INewmanPaper|Piliang|3SubProteomes\Piliang3SP\mgf5ppm|ERLIC_3SubProteomes5ppm.mgf
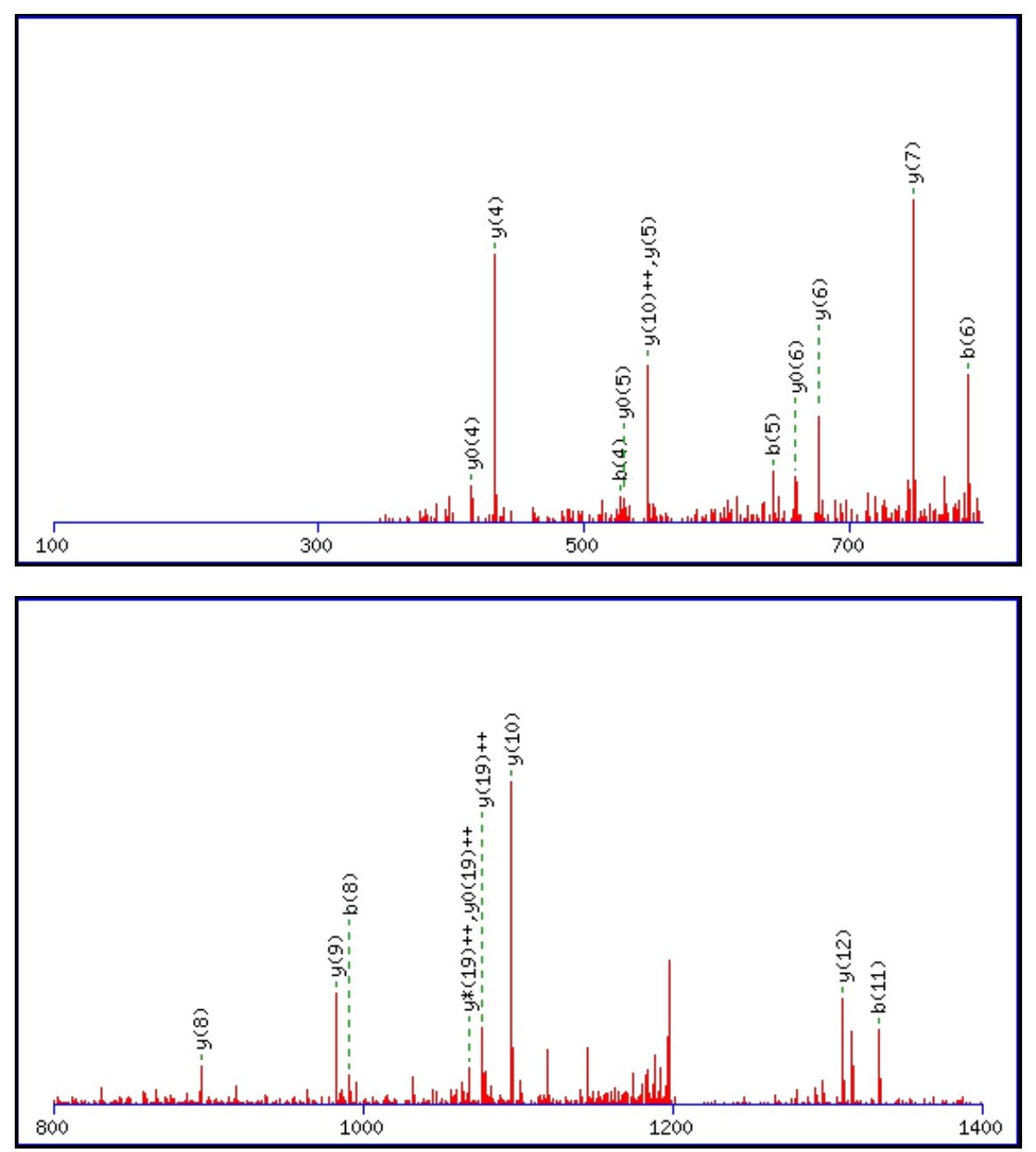


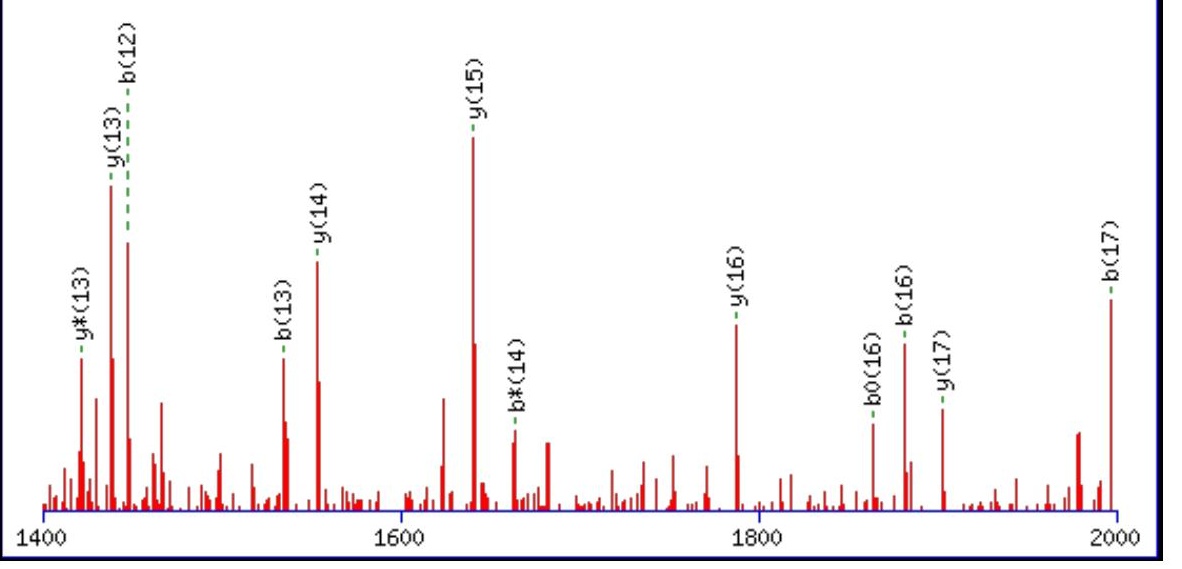

Monoisotopic mass of neutral peptide $\operatorname{Mr}($ calc): 2428.0125

Fixed modifications: Carbamidomethyl (C)

Variable modifications:

N8 : Deamidated_N (N)

M14 : Oxidation (M)

Ions Score: 118 Expect: $5.5 \mathrm{e}-010$

Matches (Bold Red): 32/216 fragment ions using 38 most intense peaks

\begin{tabular}{|c|c|c|c|c|c|c|c|c|c|c|c|c|c|c|}
\hline \# & b & $\mathbf{b}^{++}$ & b* & $\mathbf{b}^{*^{++}}$ & $\mathbf{b}^{\mathbf{0}}$ & $\mathbf{b}^{0++}$ & Seq. & $\mathbf{y}$ & $\mathbf{y}^{++}$ & $\mathbf{y}^{*}$ & $\mathbf{y}^{*^{++}}$ & $\mathbf{y}^{\mathbf{0}}$ & $\mathbf{y}^{\mathbf{0 + +}}$ & \# \\
\hline 1 & 114.0913 & 57.5493 & & & & & I & & & & & & & 21 \\
\hline 2 & 277.1547 & 139.0810 & & & & & $\mathbf{Y}$ & 2315.9357 & 1158.4715 & 2298.9092 & 1149.9582 & 2297.9251 & 1149.4662 & 20 \\
\hline 3 & 440.2180 & 220.6126 & & & & & $\mathbf{Y}$ & 2152.8724 & 1076.9398 & 2135.8458 & 1068.4266 & 2134.8618 & 1067.9345 & 19 \\
\hline 4 & 527.2500 & 264.1287 & & & 509.2395 & 255.1234 & $S$ & 1989.8091 & 995.4082 & 1972.7825 & 986.8949 & 1971.7985 & 986.4029 & 18 \\
\hline 5 & 642.2770 & 321.6421 & & & 624.2664 & 312.6368 & D & 1902.7770 & 951.8921 & 1885.7505 & 943.3789 & 1884.7665 & 942.8869 & 17 \\
\hline 6 & 789.3454 & 395.1763 & & & 771.3348 & 386.1710 & $\mathbf{F}$ & 1787.7501 & 894.3787 & 1770.7235 & 885.8654 & 1769.7395 & 885.3734 & 16 \\
\hline 7 & 876.3774 & 438.6923 & & & 858.3668 & 429.6871 & $S$ & 1640.6817 & 820.8445 & 1623.6551 & 812.3312 & 1622.6711 & 811.8392 & 15 \\
\hline 8 & 991.4043 & 496.2058 & 974.3778 & 487.6925 & 973.3938 & 487.2005 & $\mathbf{N}$ & 1553.6496 & 777.3285 & 1536.6231 & 768.8152 & 1535.6391 & 768.3232 & 14 \\
\hline 9 & 1119.4629 & 560.2351 & 1102.4364 & 551.7218 & 1101.4524 & 551.2298 & $\mathbf{Q}$ & 1438.6227 & 719.8150 & 1421.5962 & 711.3017 & 1420.6121 & 710.8097 & 13 \\
\hline 10 & 1220.5106 & 610.7589 & 1203.4841 & 602.2457 & 1202.5000 & 601.7537 & $\mathbf{T}$ & 1310.5641 & 655.7857 & 1293.5376 & 647.2724 & 1292.5536 & 646.7804 & 12 \\
\hline 11 & 1333.5947 & 667.3010 & 1316.5681 & 658.7877 & 1315.5841 & 658.2957 & I & 1209.5164 & 605.2619 & 1192.4899 & 596.7486 & 1191.5059 & 596.2566 & 11 \\
\hline 12 & 1447.6376 & 724.3224 & 1430.6110 & 715.8092 & 1429.6270 & 715.3172 & $\mathbf{N}$ & 1096.4324 & 548.7198 & 1079.4058 & 540.2066 & 1078.4218 & 539.7145 & 10 \\
\hline 13 & 1534.6696 & 767.8384 & 1517.6431 & 759.3252 & 1516.6591 & 758.8332 & $\mathrm{~S}$ & 982.3895 & 491.6984 & 965.3629 & 483.1851 & 964.3789 & 482.6931 & 9 \\
\hline 14 & 1681.7050 & 841.3562 & 1664.6785 & 832.8429 & 1663.6945 & 832.3509 & $\mathbf{M}$ & 895.3574 & 448.1824 & 878.3309 & 439.6691 & 877.3469 & 439.1771 & 8 \\
\hline 15 & 1752.7421 & 876.8747 & 1735.7156 & 868.3614 & 1734.7316 & 867.8694 & A & 748.3220 & 374.6646 & 731.2955 & 366.1514 & 730.3115 & 365.6594 & 7 \\
\hline 16 & 1881.7847 & 941.3960 & 1864.7582 & 932.8827 & 1863.7742 & 932.3907 & $\mathbf{E}$ & 677.2849 & 339.1461 & 660.2584 & 330.6328 & 659.2743 & 330.1408 & 6 \\
\hline 17 & 1996.8117 & 998.9095 & 1979.7851 & 990.3962 & 1978.8011 & 989.9042 & D & 548.2423 & 274.6248 & 531.2158 & 266.1115 & 530.2317 & 265.6195 & 5 \\
\hline 18 & 2053.8331 & 1027.4202 & 2036.8066 & 1018.9069 & 2035.8226 & 1018.4149 & G & 433.2154 & 217.1113 & 416.1888 & 208.5980 & 415.2048 & 208.1060 & 4 \\
\hline 19 & 2140.8652 & 1070.9362 & 2123.8386 & 1062.4229 & 2122.8546 & 1061.9309 & $S$ & 376.1939 & 188.6006 & 359.1674 & 180.0873 & 358.1833 & 179.5953 & 3 \\
\hline 20 & 2254.9081 & 1127.9577 & 2237.8815 & 1119.4444 & 2236.8975 & 1118.9524 & $\mathbf{N}$ & 289.1619 & 145.0846 & 272.1353 & 136.5713 & & & 2 \\
\hline 21 & & & & & & & $\mathbf{R}$ & 175.1190 & 88.0631 & 158.0924 & 79.5498 & & & 1 \\
\hline
\end{tabular}
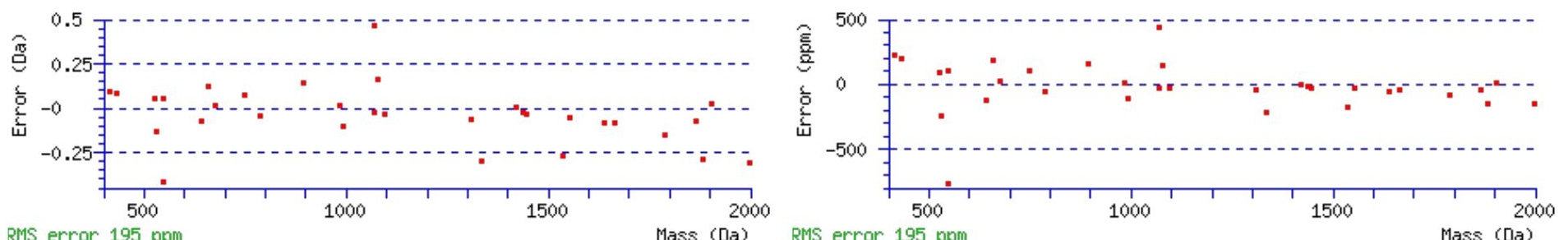

All matches to this query 


\begin{tabular}{|l|l|l|l|}
117.8 & 2428.0125 & 0.0048 & $\underline{\text {IYYSDFSNQTINSMAEDGSNR }}$ \\
\hline 64.9 & 2428.0125 & 0.0048 & $\underline{\text { IYYSDFSNQTINSMAEDGSNR }}$ \\
\hline 51.3 & 2427.0285 & 0.9889 & IYYSDFSNQTINSMAEDGSNR \\
\hline 31.3 & 2428.0125 & 0.0048 & IYYSDFSNQTINSMAEDGSNR \\
\hline 6.3 & 2428.0352 & -0.0179 & EGICGSCAMNINGGNTLACTRR \\
\hline 4.4 & 2428.0302 & -0.0129 & KAYSFAMGCWPKNGLLDMNK \\
\hline 3.0 & 2428.0397 & -0.0224 & MSYREVKYFSFPGELLMR \\
\hline 2.4 & 2428.0325 & -0.0151 & KLSKATQDSLAEATQLAEER \\
\hline 1.5 & 2428.0391 & -0.0218 & ENGINGELTSADRETAEEVSAR \\
\hline 1.5 & 2428.0391 & -0.0218 & ENGINGELTSADRETAEEVSAR \\
\hline
\end{tabular}

Spectrum No: 19; Query: 1299; Rank: 1

\section{Peptide View}

MS/MS Fragmentation of GVGIISEGNETVEDIAAR

Found in IPI00205693, Tax_Id=10116 Gene_Symbol=Atp1a2 Sodium/potassium-transporting ATPase subunit alpha-2 precursor

Match to Query 1299: 1829.903548 from(915.959050,2+)

Title: 091008RatKidney_NoSalt_18.2651.2651.2.dta

Data file K:\NewmanPaper\Piliang \3SubProteomes \Piliang3SP $\backslash$ mgf5ppm\ERLIC_3SubProteomes5ppm.mgf
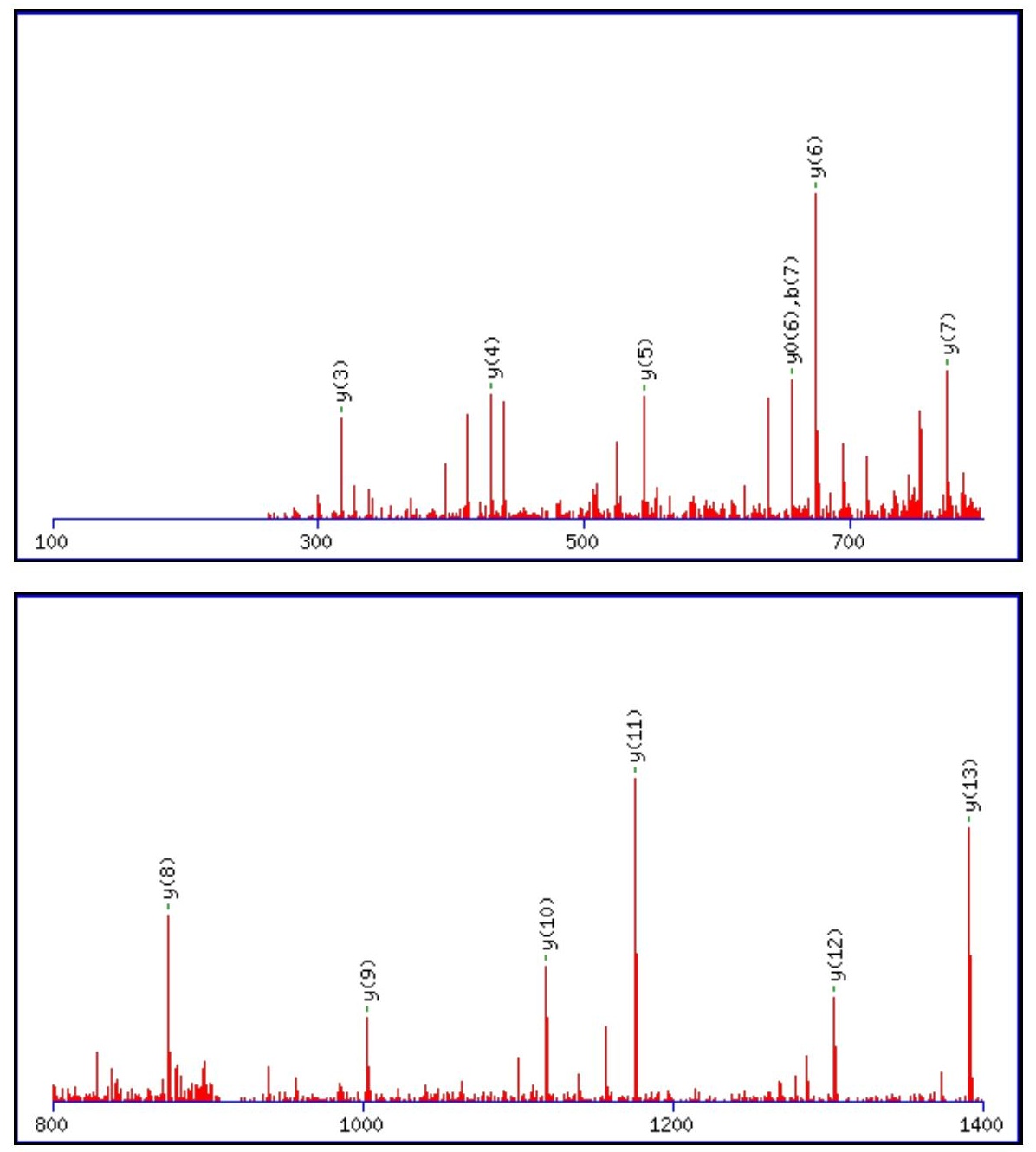


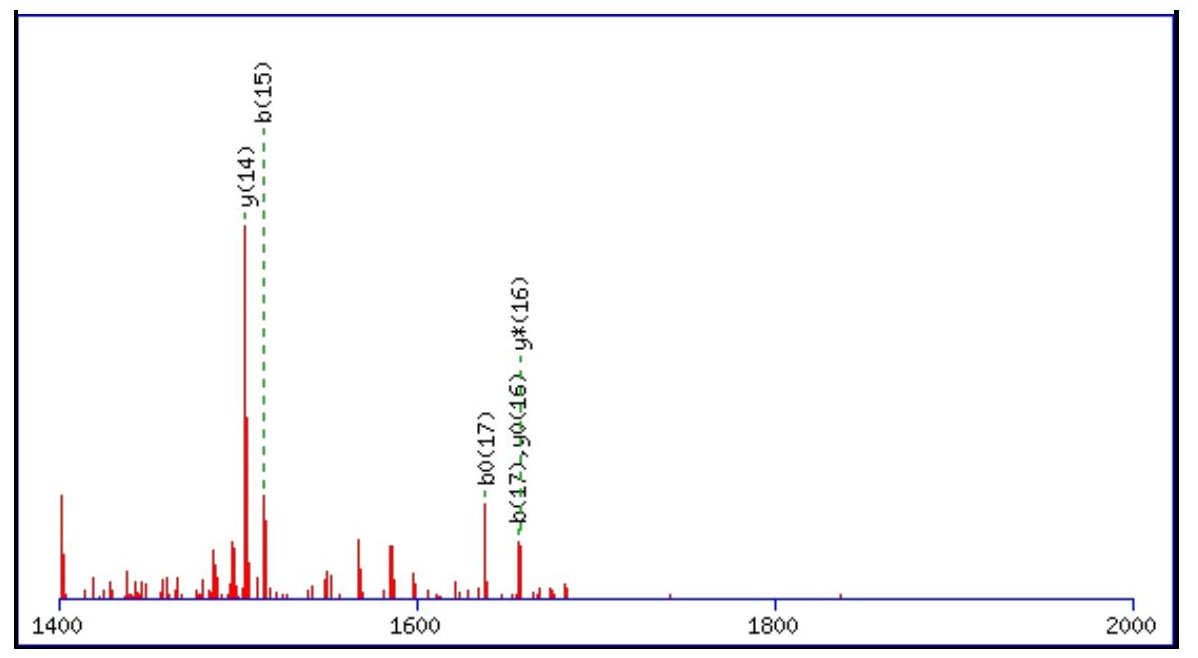

Monoisotopic mass of neutral peptide $\operatorname{Mr}($ calc): 1829.9007

Fixed modifications: Carbamidomethyl (C)

Variable modifications:

N9 : Deamidated $\mathrm{N}(\mathrm{N})$

Ions Score: 117 Expect: $3.4 \mathrm{e}-010$

Matches (Bold Red): 19/170 fragment ions using 23 most intense peaks

\begin{tabular}{|c|c|c|c|c|c|c|c|c|c|c|c|c|c|c|}
\hline \# & b & $\mathbf{b}^{++}$ & $\mathbf{b}^{*}$ & $\mathbf{b}^{*^{++}}$ & $\mathbf{b}^{0}$ & $\mathbf{b}^{0++}$ & Seq. & $\mathbf{y}$ & $y^{++}$ & $\mathrm{y}^{*}$ & $y^{*^{++}}$ & $\mathbf{y}^{0}$ & $y^{0++}$ & $\#$ \\
\hline 1 & 58.0287 & 29.5180 & & & & & G & & & & & & & 18 \\
\hline 2 & 157.0972 & 79.0522 & & & & & V & 1773.8865 & 887.4469 & 1756.8599 & 878.9336 & 1755.8759 & 878.4416 & 17 \\
\hline 3 & 214.1186 & 107.5629 & & & & & G & 1674.8181 & 837.9127 & 1657.7915 & 829.3994 & 1656.8075 & 828.9074 & 16 \\
\hline 4 & 327.2027 & 164.1050 & & & & & $\mathbf{I}$ & 1617.7966 & 809.4019 & 1600.7701 & 800.8887 & 1599.7861 & 800.3967 & 15 \\
\hline 5 & 440.2867 & 220.6470 & & & & & I & 1504.7126 & 752.8599 & 1487.6860 & 744.3466 & 1486.7020 & 743.8546 & 14 \\
\hline 6 & 527.3188 & 264.1630 & & & 509.3082 & 255.1577 & $S$ & 1391.6285 & 696.3179 & 1374.6019 & 687.8046 & 1373.6179 & 687.3126 & 13 \\
\hline 7 & 656.3614 & 328.6843 & & & 638.3508 & 319.6790 & $\mathbf{E}$ & 1304.5965 & 652.8019 & 1287.5699 & 644.2886 & 1286.5859 & 643.7966 & 12 \\
\hline 8 & 713.3828 & 357.1951 & & & 695.3723 & 348.1898 & G & 1175.5539 & 588.2806 & 1158.5273 & 579.7673 & 1157.5433 & 579.2753 & 11 \\
\hline 9 & 828.4098 & 414.7085 & 811.3832 & 406.1952 & 810.3992 & 405.7032 & $\mathbf{N}$ & 1118.5324 & 559.7698 & 1101.5059 & 551.2566 & 1100.5218 & 550.7646 & 10 \\
\hline 10 & 957.4524 & 479.2298 & 940.4258 & 470.7165 & 939.4418 & 470.2245 & $\mathbf{E}$ & 1003.5055 & 502.2564 & 986.4789 & 493.7431 & 985.4949 & 493.2511 & 9 \\
\hline 11 & 1058.5000 & 529.7537 & 1041.4735 & 521.2404 & 1040.4895 & 520.7484 & $T$ & 874.4629 & 437.7351 & 857.4363 & 429.2218 & 856.4523 & 428.7298 & 8 \\
\hline 121 & 1157.5685 & 579.2879 & 1140.5419 & 570.7746 & 1139.5579 & 570.2826 & $\mathbf{V}$ & 773.4152 & 387.2112 & 756.3886 & 378.6980 & 755.4046 & 378.2060 & 7 \\
\hline 13 & 1286.6110 & 643.8092 & 1269.5845 & 635.2959 & 1268.6005 & 634.8039 & $\mathbf{E}$ & 674.3468 & 337.6770 & 657.3202 & 329.1638 & 656.3362 & 328.6717 & 6 \\
\hline 14 & 1401.6380 & 701.3226 & 1384.6114 & 692.8094 & 1383.6274 & 692.3174 & D & 545.3042 & 273.1557 & 528.2776 & 264.6425 & 527.2936 & 264.1504 & 5 \\
\hline 151 & 1514.7221 & 757.8647 & 1497.6955 & 749.3514 & 1496.7115 & 748.8594 & I & 430.2772 & 215.6423 & 413.2507 & 207.1290 & & & 4 \\
\hline 16 & 1585.7592 & 793.3832 & 1568.7326 & 784.8699 & 1567.7486 & 784.3779 & A & 317.1932 & 159.1002 & 300.1666 & 150.5870 & & & 3 \\
\hline 17 & 1656.7963 & 828.9018 & 1639.7697 & 820.3885 & 1638.7857 & 819.8965 & A & 246.1561 & 123.5817 & 229.1295 & 115.0684 & & & 2 \\
\hline 18 & & & & & & & $\mathbf{R}$ & 175.1190 & 88.0631 & 158.0924 & 79.5498 & & & 1 \\
\hline
\end{tabular}
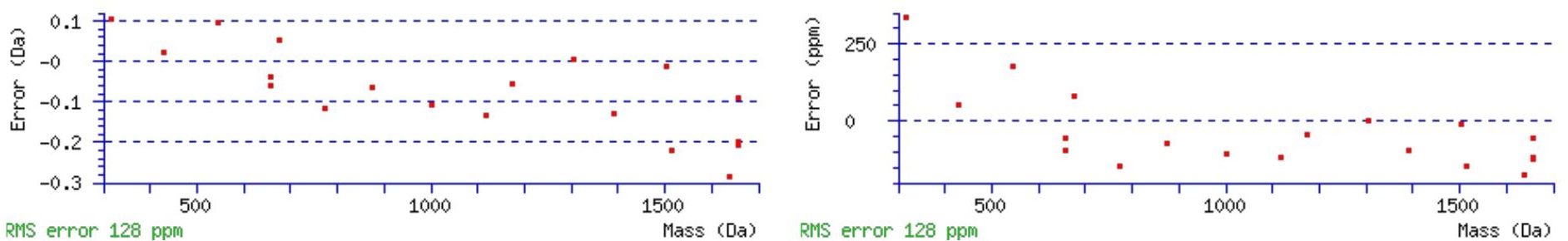

All matches to this query

\begin{tabular}{|l|l|l|l|}
\hline Score & Mr(calc): & Delta & \multicolumn{1}{c|}{ Sequence } \\
\hline 117.4 & 1829.9007 & 0.0029 & GVGIISEGNETVEDIAAR \\
\hline 36.0 & 1828.9167 & 0.9869 & GVGIISEGNETVEDIAAR \\
\hline 2.1 & 1829.9119 & -0.0083 & ARSQVNGNNGTLIVEEK \\
\hline & & & \\
\hline
\end{tabular}




\begin{tabular}{|l|l|l|l|}
1.6 & 1829.9149 & -0.0113 & RTISALEVELQAQHR \\
\hline 0.8 & 1827.9053 & 1.9983 & SDLKVILMSATLNAEK \\
\hline 0.8 & 1827.8871 & 2.0164 & KSMIIGFFLRTSLR \\
\hline 0.8 & 1827.8871 & 2.0164 & KSMIIGFFLRTSLR \\
\hline 0.3 & 1827.8963 & 2.0073 & TSQIASAGVPAPGGSQSADK \\
\hline
\end{tabular}

Spectrum No: 20; Query: 1071; Rank: 1

\section{Peptide View}

MS/MS Fragmentation of AITCGGIYNESSGILR

Found in IPI00196620, Tax_Id=10116 Gene_Symbol=Cubn Cubilin precursor

Match to Query 1071: 1710.825448 from(856.420000,2+)

Title: 091008RatKidney_NoSalt_25.2890.2890.2.dta

Data file K:\NewmanPaper\Piliang\3SubProteomes\Piliang3SP\mgf5ppm\ERLIC_3SubProteomes5ppm.mgf
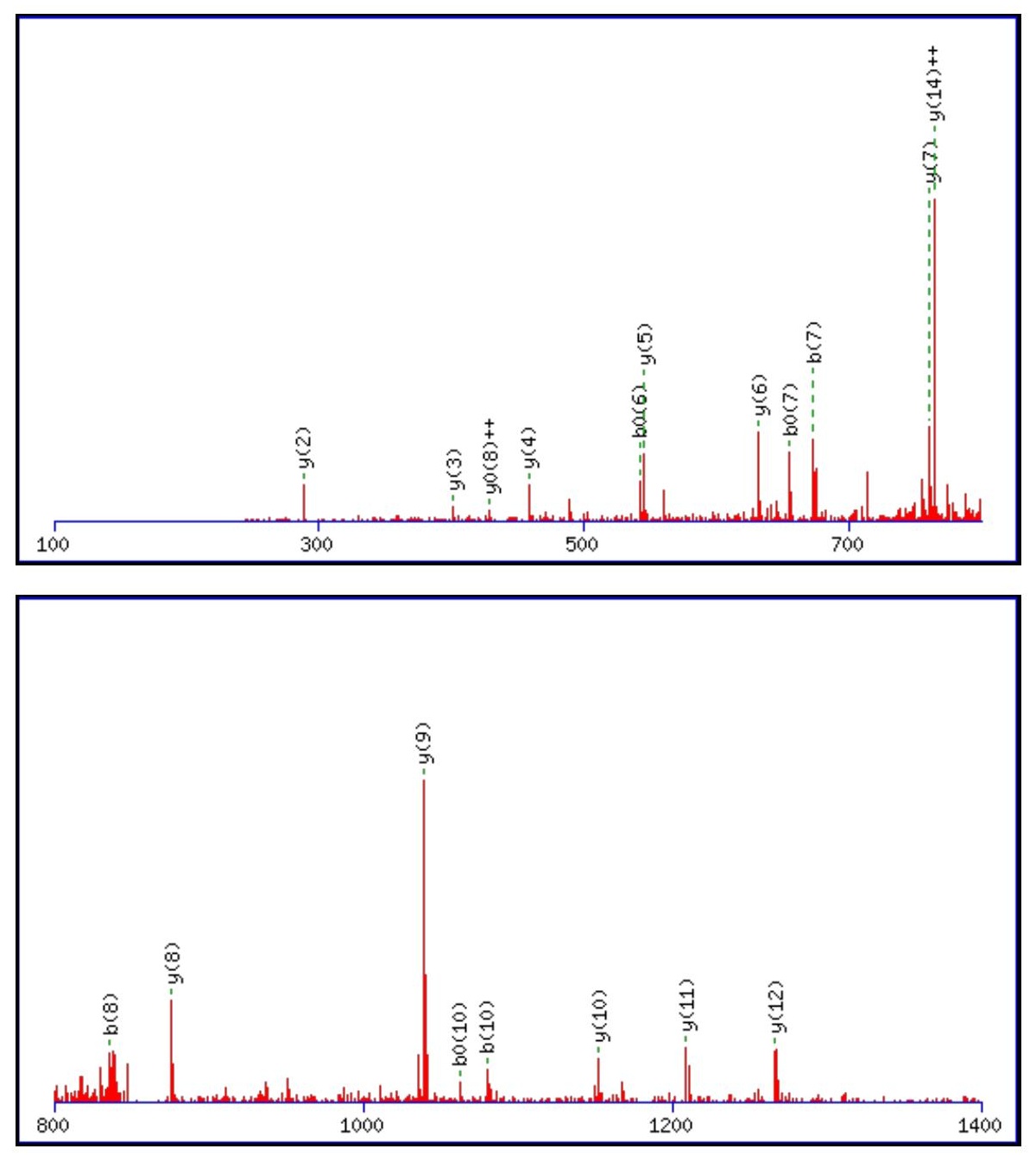


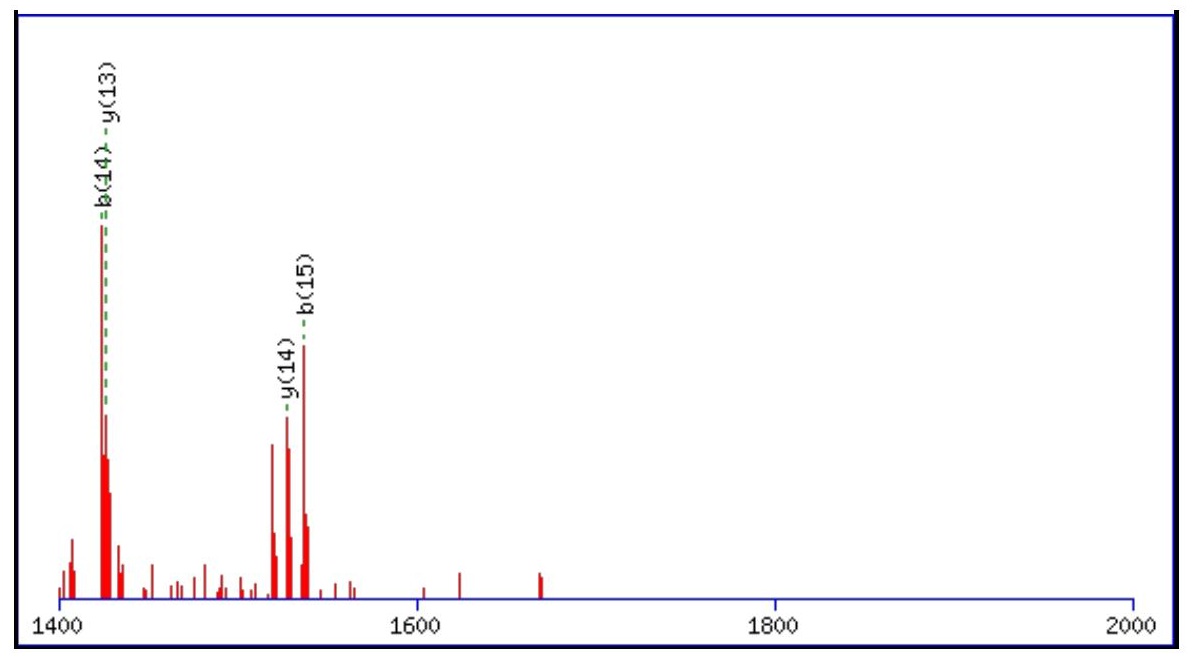

Monoisotopic mass of neutral peptide $\operatorname{Mr}($ calc): 1710.8247

Fixed modifications: Carbamidomethyl (C)

Variable modifications:

N9: Deamidated $\mathrm{N}(\mathrm{N})$

Ions Score: 117 Expect: $3.7 \mathrm{e}-010$

Matches (Bold Red): 23/152 fragment ions using 36 most intense peaks

\begin{tabular}{|c|c|c|c|c|c|c|c|c|c|c|c|c|c|c|}
\hline \# & b & $\mathbf{b}^{++}$ & $\mathbf{b}^{*}$ & $\mathbf{b}^{*^{++}}$ & $\mathbf{b}^{\mathbf{0}}$ & $\mathbf{b}^{0++}$ & Seq. & $\mathbf{y}$ & $y^{++}$ & $\mathbf{y}^{*}$ & $\mathrm{y}^{\mathrm{*}^{++}}$ & $\mathbf{y}^{\mathbf{0}}$ & $\mathbf{y}^{0++}$ & \# \\
\hline 1 & 72.0444 & 36.5258 & & & & & A & & & & & & & 16 \\
\hline 2 & 185.1285 & 93.0679 & & & & & I & 1640.7948 & 820.9011 & 1623.7683 & 812.3878 & 1622.7843 & 811.8958 & 15 \\
\hline 3 & 286.1761 & 143.5917 & & & 268.1656 & 134.5864 & $\mathbf{T}$ & 1527.7108 & 764.3590 & 1510.6842 & 755.8458 & 1509.7002 & 755.3537 & 14 \\
\hline 4 & 446.2068 & 223.6070 & & & 428.1962 & 214.6017 & C & 1426.6631 & 713.8352 & 1409.6365 & 705.3219 & 1408.6525 & 704.8299 & 13 \\
\hline 5 & 503.2282 & 252.1178 & & & 485.2177 & 243.1125 & G & 1266.6324 & 633.8199 & 1249.6059 & 625.3066 & 1248.6219 & 624.8146 & 12 \\
\hline 6 & 560.2497 & 280.6285 & & & 542.2391 & 271.6232 & G & 1209.6110 & 605.3091 & 1192.5844 & 596.7959 & 1191.6004 & 596.3038 & 11 \\
\hline 7 & 673.3338 & 337.1705 & & & 655.3232 & 328.1652 & I & 1152.5895 & 576.7984 & 1135.5630 & 568.2851 & 1134.5790 & 567.7931 & 10 \\
\hline 8 & 836.3971 & 418.7022 & & & 818.3865 & 409.6969 & $\mathbf{Y}$ & 1039.5055 & 520.2564 & 1022.4789 & 511.7431 & 1021.4949 & 511.2511 & 9 \\
\hline 9 & 951.4240 & 476.2157 & 934.3975 & 467.7024 & 933.4135 & 467.2104 & $\mathbf{N}$ & 876.4421 & 438.7247 & 859.4156 & 430.2114 & 858.4316 & 429.7194 & 8 \\
\hline 10 & 1080.4666 & 540.7370 & 1063.4401 & 532.2237 & 1062.4561 & 531.7317 & $\mathbf{E}$ & 761.4152 & 381.2112 & 744.3886 & 372.6980 & 743.4046 & 372.2060 & 7 \\
\hline 11 & 1167.4987 & 584.2530 & 1150.4721 & 575.7397 & 1149.4881 & 575.2477 & $\mathbf{S}$ & 632.3726 & 316.6899 & 615.3461 & 308.1767 & 614.3620 & 307.6847 & 6 \\
\hline 12 & 1254.5307 & 627.7690 & 1237.5041 & 619.2557 & 1236.5201 & 618.7637 & $\mathrm{~S}$ & 545.3406 & 273.1739 & 528.3140 & 264.6606 & 527.3300 & 264.1686 & 5 \\
\hline 13 & 1311.5522 & 656.2797 & 1294.5256 & 647.7664 & 1293.5416 & 647.2744 & G & 458.3085 & 229.6579 & 441.2820 & 221.1446 & & & 4 \\
\hline 14 & 1424.6362 & 712.8217 & 1407.6097 & 704.3085 & 1406.6257 & 703.8165 & I & 401.2871 & 201.1472 & 384.2605 & 192.6339 & & & 3 \\
\hline 15 & 1537.7203 & 769.3638 & 1520.6937 & 760.8505 & 1519.7097 & 760.3585 & $\mathbf{L}$ & 288.2030 & 144.6051 & 271.1765 & 136.0919 & & & 2 \\
\hline 16 & & & & & & & $\mathbf{R}$ & 175.1190 & 88.0631 & 158.0924 & 79.5498 & & & 1 \\
\hline
\end{tabular}
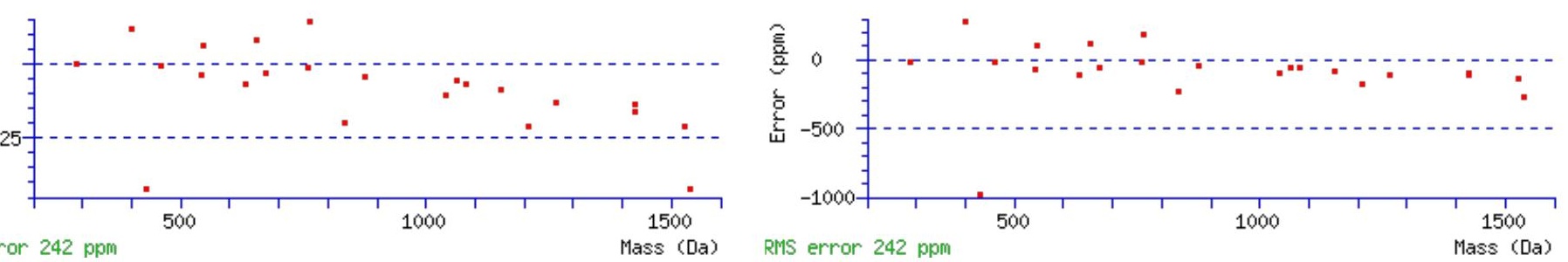

\section{All matches to this query}

\begin{tabular}{|l|l|l|l|}
\hline Score & Mr(calc): & Delta & \multicolumn{1}{c|}{ Sequence } \\
\hline 117.4 & 1710.8247 & 0.0008 & AITCGGIYNESSGILR \\
\hline 8.7 & 1710.8375 & -0.0121 & KSSEQLLGSMPVSLR \\
\hline 6.2 & 1710.8103 & 0.0152 & LMELNMEIRDMIR \\
\hline 6.0 & 1710.8375 & -0.0121 & KSSEQLLGSMPVSLR \\
\hline 5.0 & 1710.8237 & 0.0018 & VVMLLTAGSGGGAGGGRR \\
\hline & & & \\
\hline
\end{tabular}




\begin{tabular}{|l|l|l|l|}
\hline 3.6 & 1710.8124 & 0.0131 & EVVVNMLNSLSRNR \\
\hline 3.6 & 1710.8124 & 0.0131 & EVVVNMLNSLSRNR \\
\hline 2.9 & 1709.8250 & 1.0005 & RQGASTAXAATPIPAR \\
\hline 2.7 & 1710.8189 & 0.0066 & QELILSNSEDKSIR \\
\hline 2.6 & 1708.8172 & 2.0083 & GETLETLVLLNPSDK \\
\hline
\end{tabular}

Spectrum No: 21; Query: 1956; Rank: 1

\section{Peptide View}

MS/MS Fragmentation of YGYENDTEIANLYDEMVAK

Found in IPI00191437, Tax_Id=10116 Gene_Symbol=- 64 kDa protein

Match to Query 1956: 2253.969528 from(1127.992040,2+)

Title: 100101RatKid_NS_deglyco_25.3594.3594.2.dta

Data file K:\NewmanPaper|Piliang|3SubProteomes\Piliang3SP\mgf5ppm\ERLIC_3SubProteomes5ppm.mgf
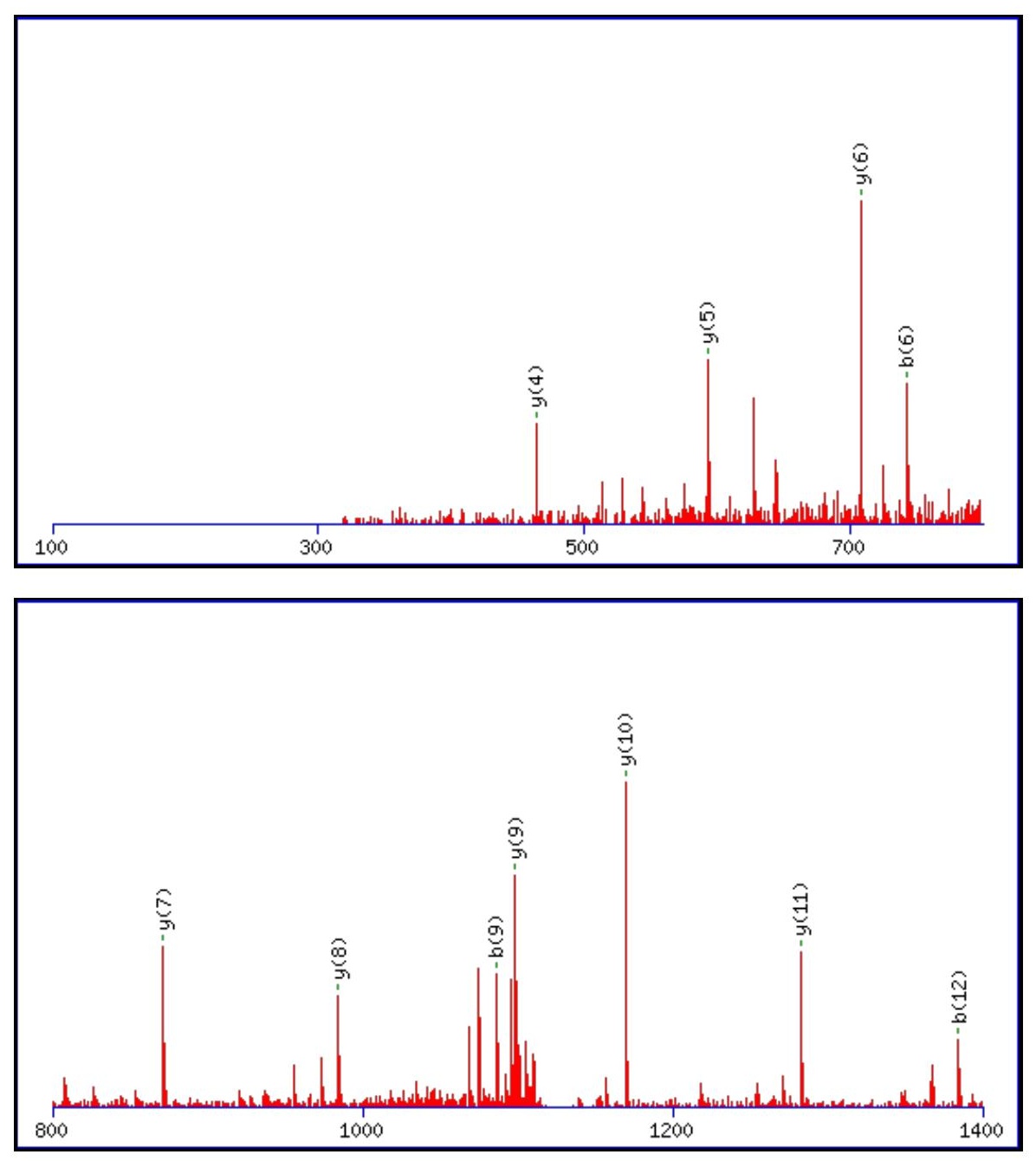


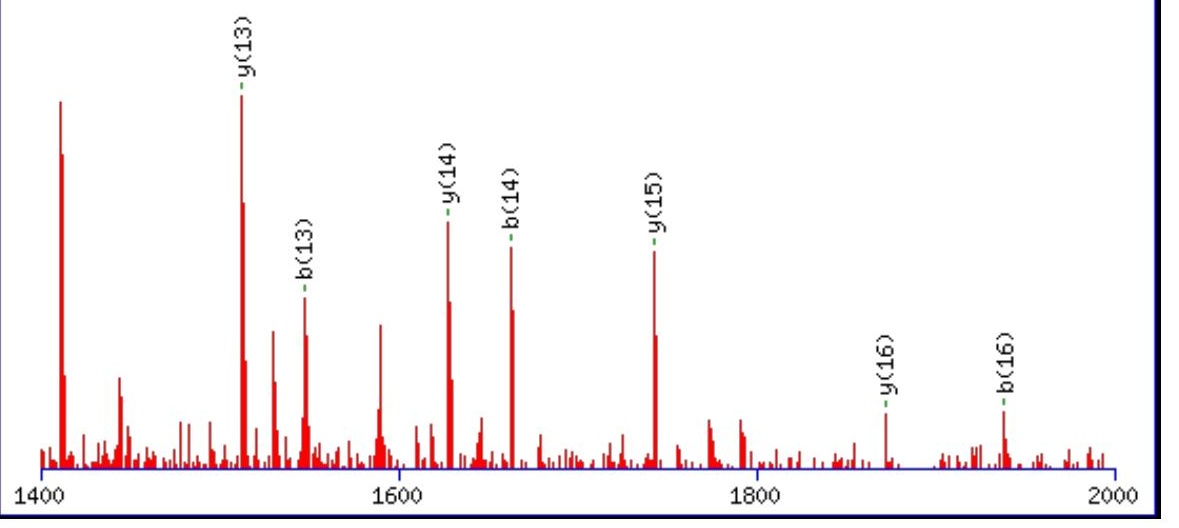

Monoisotopic mass of neutral peptide $\operatorname{Mr}($ calc): 2253.9623

Fixed modifications: Carbamidomethyl (C)

Variable modifications:

N5 : Deamidated_N (N)

M16 : Oxidation (M)

Ions Score: 117 Expect: $5.5 \mathrm{e}-010$

Matches (Bold Red): 18/194 fragment ions using 25 most intense peaks

\begin{tabular}{|c|c|c|c|c|c|c|c|c|c|c|c|c|c|c|}
\hline \# & b & $\mathbf{b}^{++}$ & $\mathbf{b}^{*}$ & $\mathbf{b}^{*^{++}}$ & $\mathbf{b}^{\mathbf{0}}$ & $\mathbf{b}^{0++}$ & Seq. & $\mathbf{y}$ & $y^{++}$ & $\mathbf{y}^{*}$ & $\mathbf{y}^{*^{++}}$ & $\mathbf{y}^{0}$ & $\mathbf{y}^{0++}$ & \# \\
\hline 1 & 164.0706 & 82.5389 & & & & & $\mathbf{Y}$ & & & & & & & 19 \\
\hline 2 & 221.0921 & 111.0497 & & & & & G & 2091.9063 & 1046.4568 & 2074.8798 & 1037.9435 & 2073.8957 & 1037.4515 & 18 \\
\hline 3 & 384.1554 & 192.5813 & & & & & $\mathbf{Y}$ & 2034.8848 & 1017.9461 & 2017.8583 & 1009.4328 & 2016.8743 & 1008.9408 & 17 \\
\hline 4 & 513.1980 & 257.1026 & & & 495.1874 & 248.0974 & $\mathbf{E}$ & 1871.8215 & 936.4144 & 1854.7950 & 927.9011 & 1853.8109 & 927.4091 & 16 \\
\hline 5 & 628.2249 & 314.6161 & 611.1984 & 306.1028 & 610.2144 & 305.6108 & $\mathbf{N}$ & 1742.7789 & 871.8931 & 1725.7524 & 863.3798 & 1724.7684 & 862.8878 & 15 \\
\hline 6 & 743.2519 & 372.1296 & 726.2253 & 363.6163 & 725.2413 & 363.1243 & D & 1627.7520 & 814.3796 & 1610.7254 & 805.8664 & 1609.7414 & 805.3743 & 14 \\
\hline 7 & 844.2995 & 422.6534 & 827.2730 & 414.1401 & 826.2890 & 413.6481 & $\mathbf{T}$ & 1512.7250 & 756.8662 & 1495.6985 & 748.3529 & 1494.7145 & 747.8609 & 13 \\
\hline 8 & 973.3421 & 487.1747 & 956.3156 & 478.6614 & 955.3316 & 478.1694 & $\mathbf{E}$ & 1411.6774 & 706.3423 & 1394.6508 & 697.8290 & 1393.6668 & 697.3370 & 12 \\
\hline 9 & 1086.4262 & 543.7167 & 1069.3997 & 535.2035 & 1068.4156 & 534.7115 & I & 1282.6348 & 641.8210 & 1265.6082 & 633.3077 & 1264.6242 & 632.8157 & 11 \\
\hline 10 & 1157.4633 & 579.2353 & 1140.4368 & 570.7220 & 1139.4528 & 570.2300 & $\mathbf{A}$ & 1169.5507 & 585.2790 & 1152.5242 & 576.7657 & 1151.5401 & 576.2737 & 10 \\
\hline 11 & 1271.5062 & 636.2568 & 1254.4797 & 627.7435 & 1253.4957 & 627.2515 & $\mathbf{N}$ & 1098.5136 & 549.7604 & 1081.4870 & 541.2472 & 1080.5030 & 540.7552 & 9 \\
\hline 12 & 1384.5903 & 692.7988 & 1367.5638 & 684.2855 & 1366.5797 & 683.7935 & $\mathbf{L}$ & 984.4707 & 492.7390 & 967.4441 & 484.2257 & 966.4601 & 483.7337 & 8 \\
\hline 13 & 1547.6536 & 774.3305 & 1530.6271 & 765.8172 & 1529.6431 & 765.3252 & $\mathbf{Y}$ & 871.3866 & 436.1969 & 854.3601 & 427.6837 & 853.3760 & 427.1917 & 7 \\
\hline 14 & 1662.6806 & 831.8439 & 1645.6540 & 823.3307 & 1644.6700 & 822.8386 & D & 708.3233 & 354.6653 & 691.2967 & 346.1520 & 690.3127 & 345.6600 & 6 \\
\hline 15 & 1791.7232 & 896.3652 & 1774.6966 & 887.8520 & 1773.7126 & 887.3599 & $\mathbf{E}$ & 593.2963 & 297.1518 & 576.2698 & 288.6385 & 575.2858 & 288.1465 & 5 \\
\hline 16 & 1938.7586 & 969.8829 & 1921.7320 & 961.3697 & 1920.7480 & 960.8776 & $\mathbf{M}$ & 464.2537 & 232.6305 & 447.2272 & 224.1172 & & & 4 \\
\hline 17 & 2037.8270 & 1019.4171 & 2020.8004 & 1010.9039 & 2019.8164 & 1010.4119 & V & 317.2183 & 159.1128 & 300.1918 & 150.5995 & & & 3 \\
\hline 18 & 2108.8641 & 1054.9357 & 2091.8376 & 1046.4224 & 2090.8535 & 1045.9304 & A & 218.1499 & 109.5786 & 201.1234 & 101.0653 & & & 2 \\
\hline 19 & & & & & & & $\mathbf{K}$ & 147.1128 & 74.0600 & 130.0863 & 65.5468 & & & 1 \\
\hline
\end{tabular}

$$
\text { 蒠 }
$$

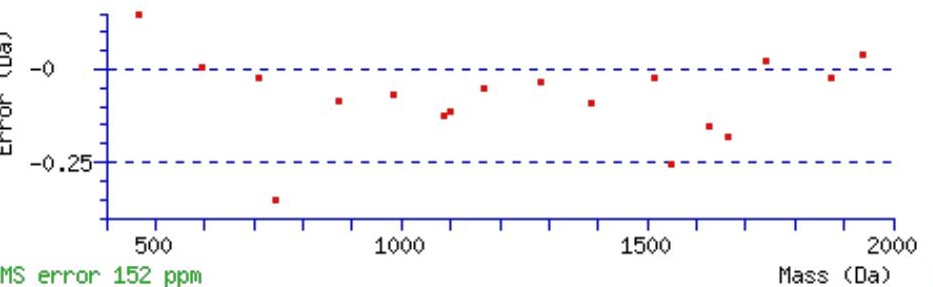

All matches to this query

\begin{tabular}{|l|l|l|l|}
\hline Score & Mr(calc): & Delta & Sequence \\
\hline 117.0 & 2253.9623 & 0.0072 & YGYENDTEIANLYDEMVAK \\
\hline 66.0 & 2252.9783 & 0.9912 & YGYENDTEIANLYDEMVAK \\
\hline
\end{tabular}

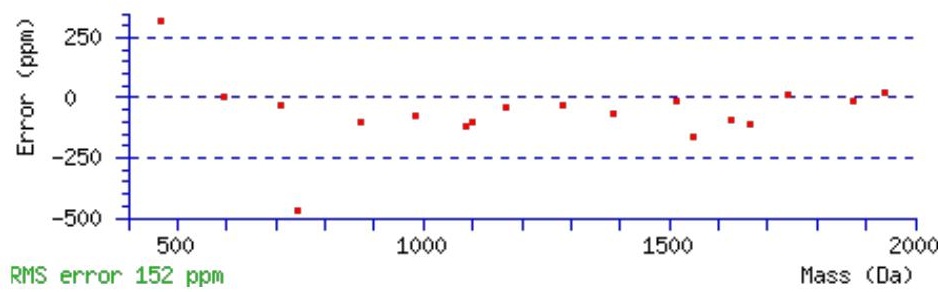

file:///R//wwwroot/SupportingData/3SubProteomes/ERLIC_3SubProteo_Glyco/ERLIC_3SubProteo_Glyco_001.html[4/27/2010 11:11:06 AM] 


\begin{tabular}{|l|l|l|l|}
54.6 & 2253.9623 & 0.0072 & YGYENDTEIANLYDEMVAK \\
\hline 8.4 & 2251.9482 & 2.0214 & GNQSDEGSDIDSEPDLPLKR \\
\hline 6.3 & 2251.9482 & 2.0214 & GNQSDEGSDIDSEPDLPLKR \\
\hline 4.8 & 2253.9596 & 0.0099 & $\underline{\text { LDSTAASLISNGPGGSVSFWK }}$ \\
\hline 3.9 & 2251.9763 & 1.9932 & $\underline{\text { GKKDDNGIGTAIDFVLSNAR }}$ \\
\hline 3.8 & 2252.9464 & 1.0231 & HPSNPKVRSSPSGNTQSSPK \\
\hline 3.1 & 2252.9500 & 1.0195 & GKFYSCNDLSKMTEEECR \\
\hline 2.7 & 2251.9829 & 1.9866 & YSMYNSVSQKLMAKMGFR \\
\hline
\end{tabular}

Spectrum No: 22; Query: 1956; Rank: 1

\section{Peptide View}

MS/MS Fragmentation of YGYENDTEIANLYDEMVAK

Found in IPI00191437, Tax_Id=10116 Gene_Symbol=- 64 kDa protein

Match to Query 1956: 2253.969528 from(1127.992040,2+)

Title: 100101RatKid_NS_deglyco_25.3594.3594.2.dta

Data file K:INewmanPaper|Piliangl3SubProteomes\Piliang3SP\mgf5ppm\ERLIC_3SubProteomes5ppm.mgf
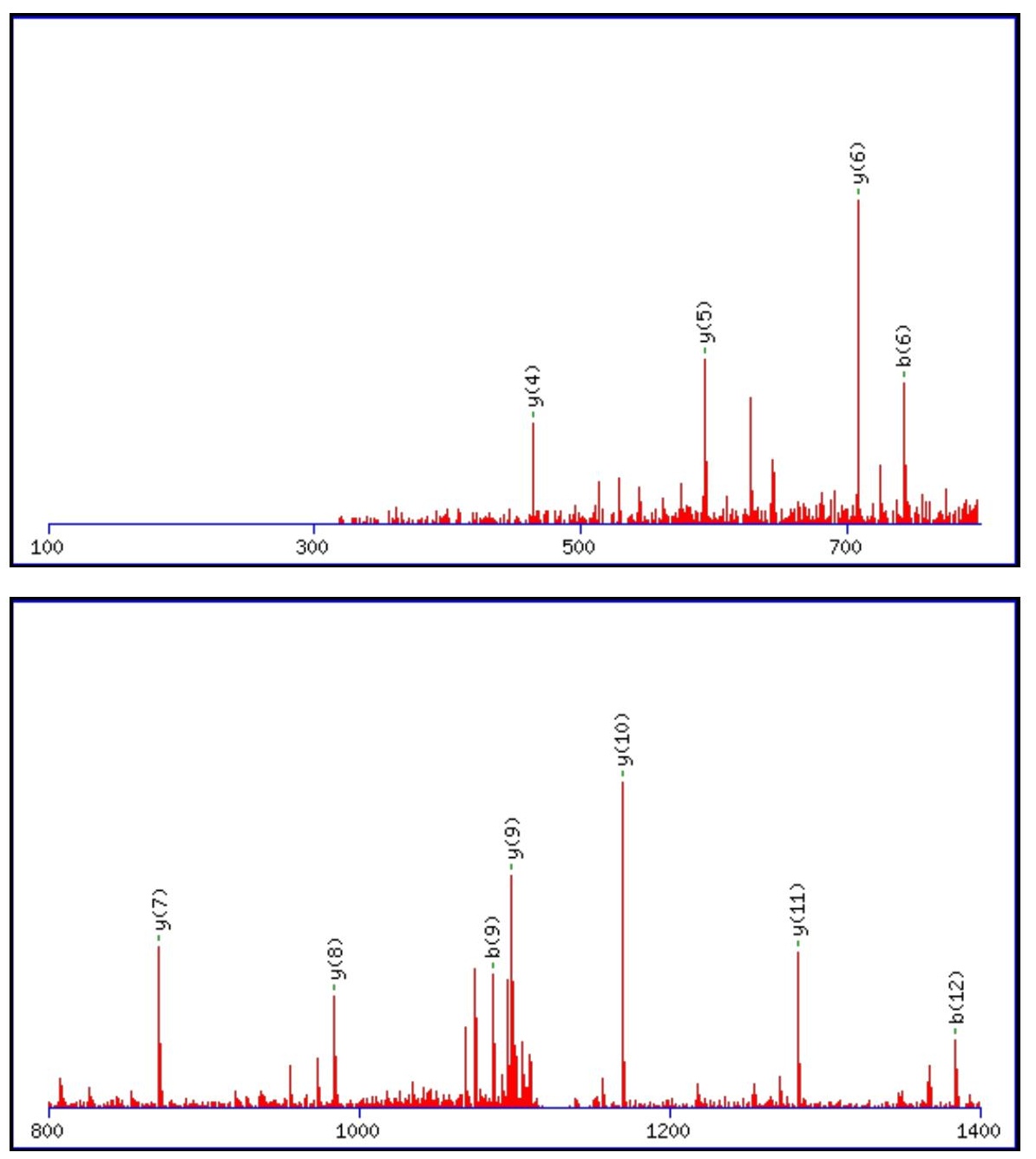


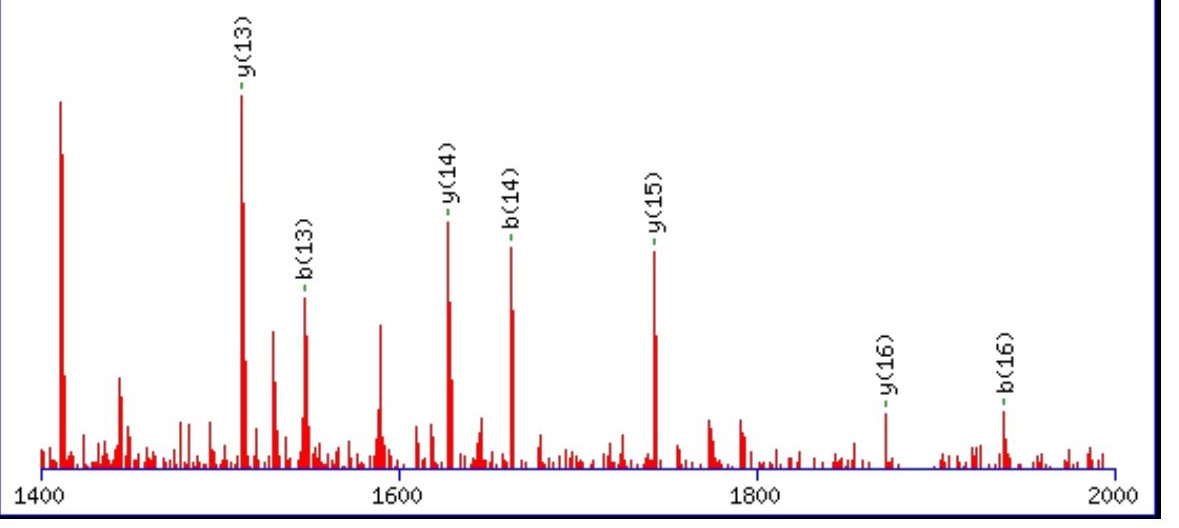

Monoisotopic mass of neutral peptide $\operatorname{Mr}($ calc): 2253.9623

Fixed modifications: Carbamidomethyl (C)

Variable modifications:

N5 : Deamidated_N (N)

M16 : Oxidation (M)

Ions Score: 117 Expect: $5.5 \mathrm{e}-010$

Matches (Bold Red): 18/194 fragment ions using 25 most intense peaks

\begin{tabular}{|c|c|c|c|c|c|c|c|c|c|c|c|c|c|c|}
\hline \# & b & $\mathbf{b}^{++}$ & $\mathbf{b}^{*}$ & $\mathbf{b}^{*^{++}}$ & $\mathbf{b}^{\mathbf{0}}$ & $\mathbf{b}^{0++}$ & Seq. & $\mathbf{y}$ & $y^{++}$ & $\mathbf{y}^{*}$ & $\mathbf{y}^{*^{++}}$ & $\mathbf{y}^{0}$ & $\mathbf{y}^{0++}$ & \# \\
\hline 1 & 164.0706 & 82.5389 & & & & & $\mathbf{Y}$ & & & & & & & 19 \\
\hline 2 & 221.0921 & 111.0497 & & & & & G & 2091.9063 & 1046.4568 & 2074.8798 & 1037.9435 & 2073.8957 & 1037.4515 & 18 \\
\hline 3 & 384.1554 & 192.5813 & & & & & $\mathbf{Y}$ & 2034.8848 & 1017.9461 & 2017.8583 & 1009.4328 & 2016.8743 & 1008.9408 & 17 \\
\hline 4 & 513.1980 & 257.1026 & & & 495.1874 & 248.0974 & $\mathbf{E}$ & 1871.8215 & 936.4144 & 1854.7950 & 927.9011 & 1853.8109 & 927.4091 & 16 \\
\hline 5 & 628.2249 & 314.6161 & 611.1984 & 306.1028 & 610.2144 & 305.6108 & $\mathbf{N}$ & 1742.7789 & 871.8931 & 1725.7524 & 863.3798 & 1724.7684 & 862.8878 & 15 \\
\hline 6 & 743.2519 & 372.1296 & 726.2253 & 363.6163 & 725.2413 & 363.1243 & D & 1627.7520 & 814.3796 & 1610.7254 & 805.8664 & 1609.7414 & 805.3743 & 14 \\
\hline 7 & 844.2995 & 422.6534 & 827.2730 & 414.1401 & 826.2890 & 413.6481 & $\mathbf{T}$ & 1512.7250 & 756.8662 & 1495.6985 & 748.3529 & 1494.7145 & 747.8609 & 13 \\
\hline 8 & 973.3421 & 487.1747 & 956.3156 & 478.6614 & 955.3316 & 478.1694 & $\mathbf{E}$ & 1411.6774 & 706.3423 & 1394.6508 & 697.8290 & 1393.6668 & 697.3370 & 12 \\
\hline 9 & 1086.4262 & 543.7167 & 1069.3997 & 535.2035 & 1068.4156 & 534.7115 & I & 1282.6348 & 641.8210 & 1265.6082 & 633.3077 & 1264.6242 & 632.8157 & 11 \\
\hline 10 & 1157.4633 & 579.2353 & 1140.4368 & 570.7220 & 1139.4528 & 570.2300 & $\mathbf{A}$ & 1169.5507 & 585.2790 & 1152.5242 & 576.7657 & 1151.5401 & 576.2737 & 10 \\
\hline 11 & 1271.5062 & 636.2568 & 1254.4797 & 627.7435 & 1253.4957 & 627.2515 & $\mathbf{N}$ & 1098.5136 & 549.7604 & 1081.4870 & 541.2472 & 1080.5030 & 540.7552 & 9 \\
\hline 12 & 1384.5903 & 692.7988 & 1367.5638 & 684.2855 & 1366.5797 & 683.7935 & $\mathbf{L}$ & 984.4707 & 492.7390 & 967.4441 & 484.2257 & 966.4601 & 483.7337 & 8 \\
\hline 13 & 1547.6536 & 774.3305 & 1530.6271 & 765.8172 & 1529.6431 & 765.3252 & $\mathbf{Y}$ & 871.3866 & 436.1969 & 854.3601 & 427.6837 & 853.3760 & 427.1917 & 7 \\
\hline 14 & 1662.6806 & 831.8439 & 1645.6540 & 823.3307 & 1644.6700 & 822.8386 & D & 708.3233 & 354.6653 & 691.2967 & 346.1520 & 690.3127 & 345.6600 & 6 \\
\hline 15 & 1791.7232 & 896.3652 & 1774.6966 & 887.8520 & 1773.7126 & 887.3599 & $\mathbf{E}$ & 593.2963 & 297.1518 & 576.2698 & 288.6385 & 575.2858 & 288.1465 & 5 \\
\hline 16 & 1938.7586 & 969.8829 & 1921.7320 & 961.3697 & 1920.7480 & 960.8776 & $\mathbf{M}$ & 464.2537 & 232.6305 & 447.2272 & 224.1172 & & & 4 \\
\hline 17 & 2037.8270 & 1019.4171 & 2020.8004 & 1010.9039 & 2019.8164 & 1010.4119 & V & 317.2183 & 159.1128 & 300.1918 & 150.5995 & & & 3 \\
\hline 18 & 2108.8641 & 1054.9357 & 2091.8376 & 1046.4224 & 2090.8535 & 1045.9304 & A & 218.1499 & 109.5786 & 201.1234 & 101.0653 & & & 2 \\
\hline 19 & & & & & & & $\mathbf{K}$ & 147.1128 & 74.0600 & 130.0863 & 65.5468 & & & 1 \\
\hline
\end{tabular}

$$
\text { 蒠 }
$$

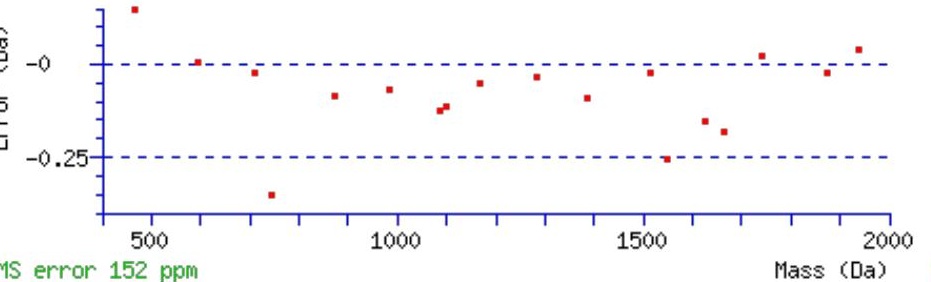

All matches to this query

\begin{tabular}{|l|l|l|l|}
\hline Score & Mr(calc): & Delta & Sequence \\
\hline 117.0 & 2253.9623 & 0.0072 & YGYENDTEIANLYDEMVAK \\
\hline 66.0 & 2252.9783 & 0.9912 & YGYENDTEIANLYDEMVAK \\
\hline
\end{tabular}

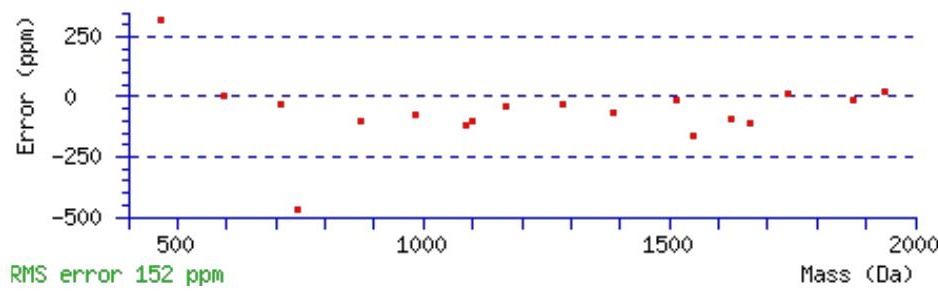

file:///R//wwwroot/SupportingData/3SubProteomes/ERLIC_3SubProteo_Glyco/ERLIC_3SubProteo_Glyco_001.html[4/27/2010 11:11:06 AM] 


\begin{tabular}{|l|l|l|l|}
54.6 & 2253.9623 & 0.0072 & YGYENDTEIANLYDEMVAK \\
\hline 8.4 & 2251.9482 & 2.0214 & GNQSDEGSDIDSEPDLPLKR \\
\hline 6.3 & 2251.9482 & 2.0214 & GNQSDEGSDIDSEPDLPLKR \\
\hline 4.8 & 2253.9596 & 0.0099 & $\underline{\text { LDSTAASLISNGPGGSVSFWK }}$ \\
\hline 3.9 & 2251.9763 & 1.9932 & $\underline{\text { GKKDDNGIGTAIDFVLSNAR }}$ \\
\hline 3.8 & 2252.9464 & 1.0231 & HPSNPKVRSSPSGNTQSSPK \\
\hline 3.1 & 2252.9500 & 1.0195 & GKFYSCNDLSKMTEEECR \\
\hline 2.7 & 2251.9829 & 1.9866 & YSMYNSVSQKLMAKMGFR \\
\hline
\end{tabular}

Spectrum No: 23; Query: 1912; Rank: 1

\section{Peptide View}

MS/MS Fragmentation of IGCFALSEPGNGSDAGAASTTAR

Found in IPI00231359, Tax_Id=10116 Gene_Symbol=Acads Acetyl-Coenzyme A dehydrogenase, short chain

Match to Query 1912: 2209.988948 from(1106.001750,2+)

Title: 091008RatKidney_NH4Format02_24.2775.2775.2.dta

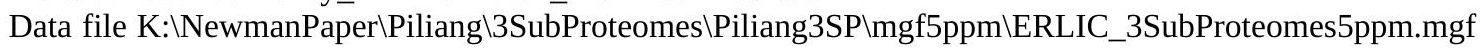
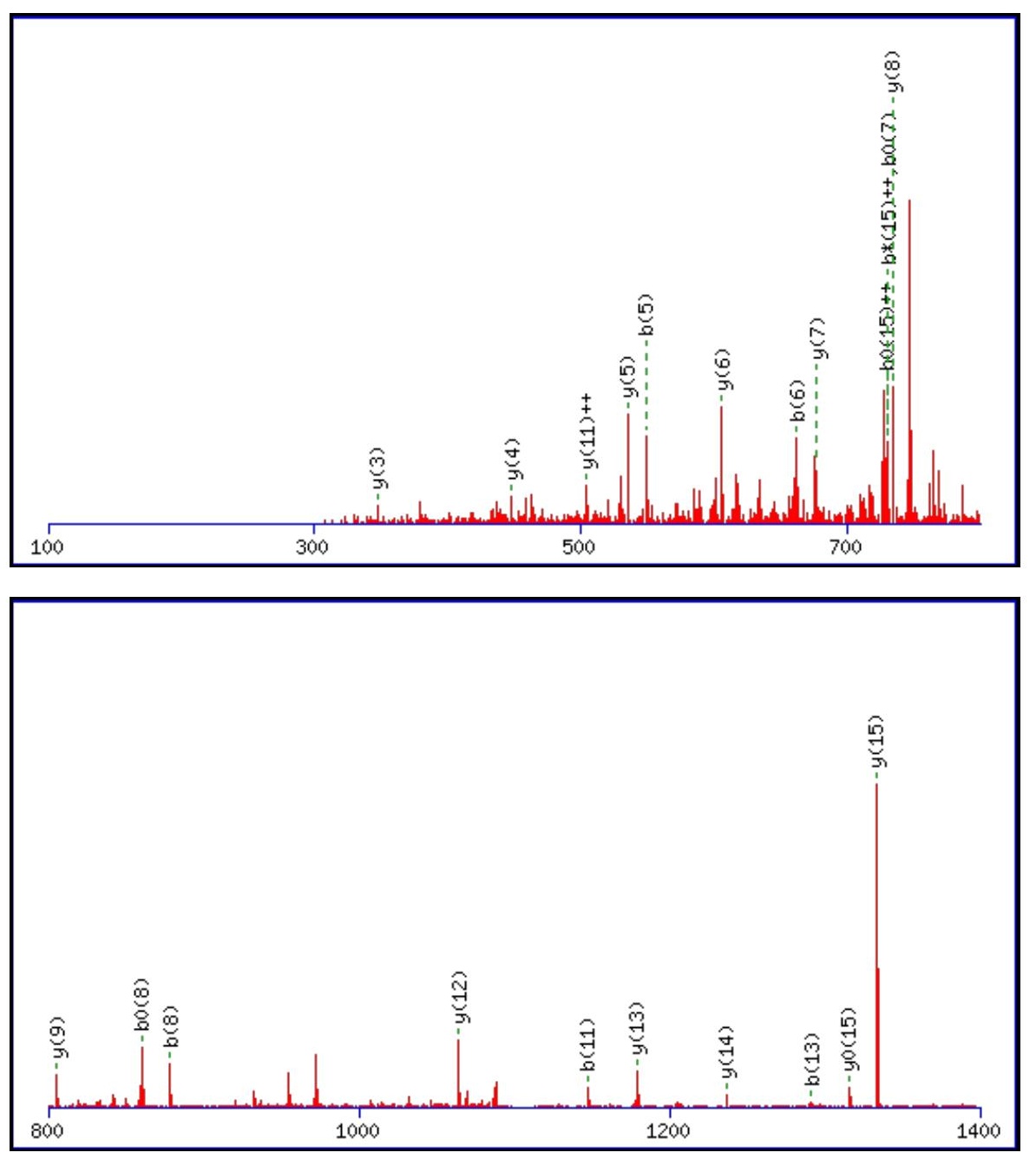


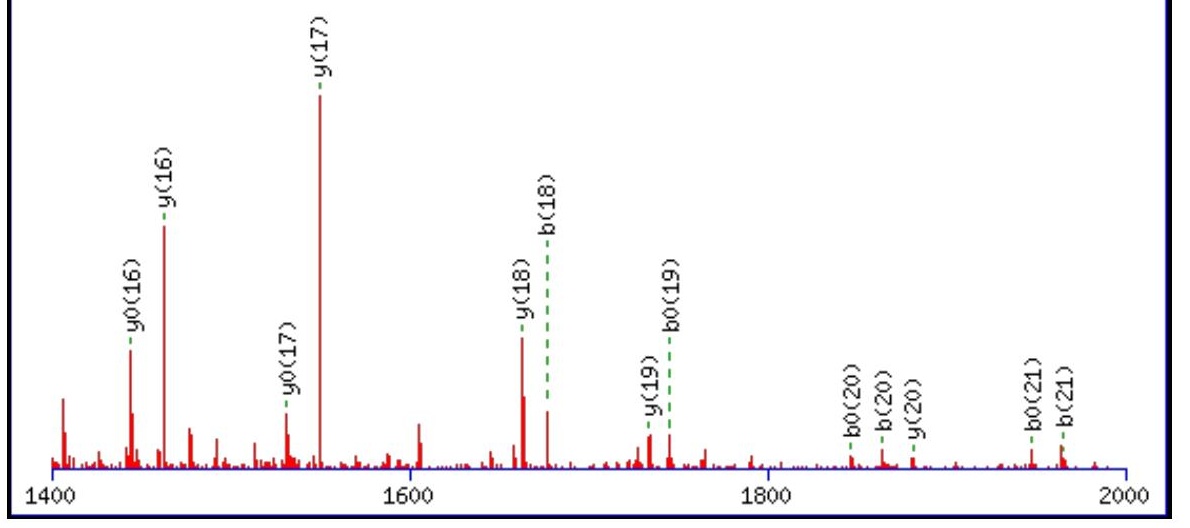

Monoisotopic mass of neutral peptide $\operatorname{Mr}($ calc): 2209.9910

Fixed modifications: Carbamidomethyl (C)

Variable modifications:

N11 : Deamidated $\mathrm{N}(\mathrm{N})$

Ions Score: 116 Expect: $6.5 \mathrm{e}-010$

Matches (Bold Red): 35/228 fragment ions using 63 most intense peaks

\begin{tabular}{|c|c|c|c|c|c|c|c|c|c|c|c|c|c|c|}
\hline \# & b & $\mathbf{b}^{++}$ & b* & $\mathbf{b}^{*++}$ & $\mathbf{b}^{0}$ & $\mathbf{b}^{0++}$ & Seq. & $\mathbf{y}$ & $y^{++}$ & $\mathbf{y}^{*}$ & $\mathbf{y}^{*^{++}}$ & $\mathbf{y}^{0}$ & $\mathbf{y}^{0++}$ & \# \\
\hline 1 & 114.0913 & 57.5493 & & & & & I & & & & & & & 23 \\
\hline 2 & 171.1128 & 86.0600 & & & & & $\mathbf{G}$ & 2097.9142 & 1049.4607 & 2080.8876 & 1040.9475 & 2079.9036 & 1040.4554 & 22 \\
\hline 3 & 331.1435 & 166.0754 & & & & & C & 2040.8927 & 1020.9500 & 2023.8662 & 1012.4367 & 2022.8822 & 1011.9447 & 21 \\
\hline 4 & 478.2119 & 239.6096 & & & & & $\mathbf{F}$ & 1880.8621 & 940.9347 & 1863.8355 & 932.4214 & 1862.8515 & 931.9294 & 20 \\
\hline 5 & 549.2490 & 275.1281 & & & & & A & 1733.7937 & 867.4005 & 1716.7671 & 858.8872 & 1715.7831 & 858.3952 & 19 \\
\hline 6 & 662.3330 & 331.6702 & & & & & $\mathbf{L}$ & 1662.7565 & 831.8819 & 1645.7300 & 823.3686 & 1644.7460 & 822.8766 & 18 \\
\hline 7 & 749.3651 & 375.1862 & & & 731.3545 & 366.1809 & $S$ & 1549.6725 & 775.3399 & 1532.6459 & 766.8266 & 1531.6619 & 766.3346 & 17 \\
\hline 8 & 878.4077 & 439.7075 & & & 860.3971 & 430.7022 & $\mathbf{E}$ & 1462.6405 & 731.8239 & 1445.6139 & 723.3106 & 1444.6299 & 722.8186 & 16 \\
\hline 9 & 975.4604 & 488.2339 & & & 957.4499 & 479.2286 & $\mathbf{P}$ & 1333.5979 & 667.3026 & 1316.5713 & 658.7893 & 1315.5873 & 658.2973 & 15 \\
\hline 10 & 1032.4819 & 516.7446 & & & 1014.4713 & 507.7393 & G & 1236.5451 & 618.7762 & 1219.5185 & 610.2629 & 1218.5345 & 609.7709 & 14 \\
\hline 11 & 1147.5088 & 574.2581 & 1130.4823 & 565.7448 & 1129.4983 & 565.2528 & $\mathbf{N}$ & 1179.5236 & 590.2655 & 1162.4971 & 581.7522 & 1161.5131 & 581.2602 & 13 \\
\hline 12 & 1204.5303 & 602.7688 & 1187.5037 & 594.2555 & 1186.5197 & 593.7635 & $\mathbf{G}$ & 1064.4967 & 532.7520 & 1047.4701 & 524.2387 & 1046.4861 & 523.7467 & 12 \\
\hline 13 & 1291.5623 & 646.2848 & 1274.5358 & 637.7715 & 1273.5518 & 637.2795 & $S$ & 1007.4752 & 504.2413 & 990.4487 & 495.7280 & 989.4647 & 495.2360 & 11 \\
\hline 14 & 1406.5893 & 703.7983 & 1389.5627 & 695.2850 & 1388.5787 & 694.7930 & D & 920.4432 & 460.7252 & 903.4167 & 452.2120 & 902.4326 & 451.7200 & 10 \\
\hline 15 & 1477.6264 & 739.3168 & 1460.5998 & 730.8036 & 1459.6158 & 730.3115 & A & 805.4163 & 403.2118 & 788.3897 & 394.6985 & 787.4057 & 394.2065 & 9 \\
\hline 16 & 1534.6478 & 767.8276 & 1517.6213 & 759.3143 & 1516.6373 & 758.8223 & $\mathbf{G}$ & 734.3791 & 367.6932 & 717.3526 & 359.1799 & 716.3686 & 358.6879 & 8 \\
\hline 17 & 1605.6850 & 803.3461 & 1588.6584 & 794.8328 & 1587.6744 & 794.3408 & A & 677.3577 & 339.1825 & 660.3311 & 330.6692 & 659.3471 & 330.1772 & 7 \\
\hline 18 & 1676.7221 & 838.8647 & 1659.6955 & 830.3514 & 1658.7115 & 829.8594 & A & 606.3206 & 303.6639 & 589.2940 & 295.1506 & 588.3100 & 294.6586 & 6 \\
\hline 19 & 1763.7541 & 882.3807 & 1746.7276 & 873.8674 & 1745.7435 & 873.3754 & S & 535.2835 & 268.1454 & 518.2569 & 259.6321 & 517.2729 & 259.1401 & 5 \\
\hline 20 & 1864.8018 & 932.9045 & 1847.7752 & 924.3913 & 1846.7912 & 923.8992 & $\mathbf{T}$ & 448.2514 & 224.6294 & 431.2249 & 216.1161 & 430.2409 & 215.6241 & 4 \\
\hline 21 & 1965.8495 & 983.4284 & 1948.8229 & 974.9151 & 1947.8389 & 974.4231 & $\mathbf{T}$ & 347.2037 & 174.1055 & 330.1772 & 165.5922 & 329.1932 & 165.1002 & 3 \\
\hline 22 & 2036.8866 & 1018.9469 & 2019.8600 & 1010.4336 & 2018.8760 & 1009.9416 & A & 246.1561 & 123.5817 & 229.1295 & 115.0684 & & & 2 \\
\hline 23 & & & & & & & $\mathbf{R}$ & 175.1190 & 88.0631 & 158.0924 & 79.5498 & & & 1 \\
\hline
\end{tabular}

$$
\text { 要 }
$$

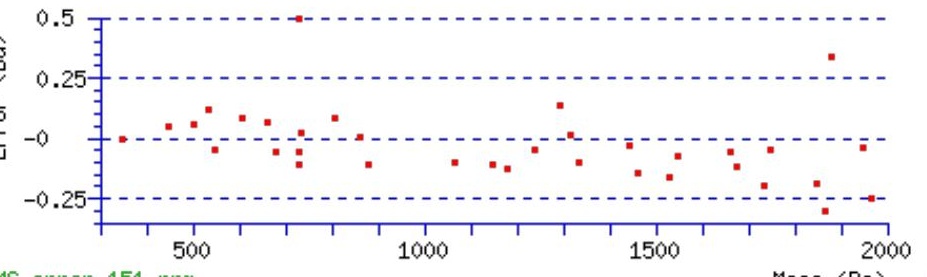

RMS error 151 ppm

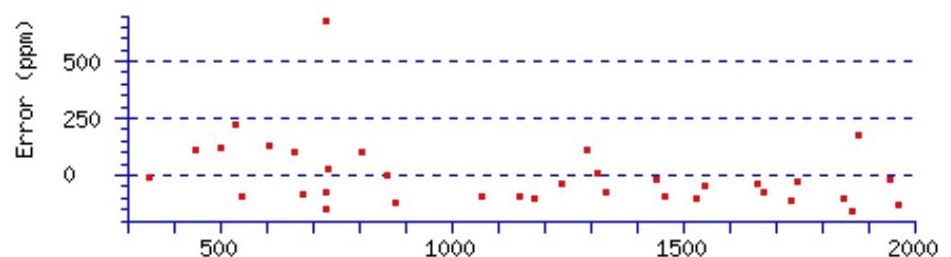

Mass (Da)

\section{All matches to this query}




\begin{tabular}{|l|l|l|l|}
\hline Score & Mr(calc): & Delta & \multicolumn{1}{c|}{ Sequence } \\
\hline 116.2 & 2209.9910 & -0.0020 & IGCFALSEPGNGSDAGAASTTAR \\
\hline 26.3 & 2209.0070 & 0.9820 & IGCFALSEPGNGSDAGAASTTAR \\
\hline 11.2 & 2208.9673 & 1.0216 & LNATTDVMRGLLSNVLCR \\
\hline 2.3 & 2207.9613 & 2.0277 & EHLSLSIEENVQPRSPGR \\
\hline 1.7 & 2209.0007 & 0.9882 & SLAEMLMSKNTRTSDTLSK \\
\hline 1.0 & 2209.9674 & 0.0215 & EPRKSAEEALAAAGDMDNAGK \\
\hline 0.7 & 2209.9711 & 0.0178 & KGTGPVQIPPGEWHSSFXR \\
\hline 0.4 & 2207.9683 & 2.0206 & SPWRPGSSKVWTPISKR \\
\hline 0.3 & 2208.9704 & 1.0185 & NQINIRNSETDTIIFIR \\
\hline 0.1 & 2209.9787 & 0.0103 & TRPRAESDDPENEAKMIR \\
\hline
\end{tabular}

Spectrum No: 24; Query: 2727; Rank: 1

\section{Peptide View}

MS/MS Fragmentation of QAEVADQQTTDLPAENGETENQSPASEEEKEAK

Found in IPI00373052, Tax_Id=10116 Gene_Symbol=Hmgn1 High mobility group nucleosomal binding domain 1

Match to Query 2727: 3573.575502 from(1192.199110,3+)

Title: 091008RatKidney_NoSalt_21.1314.1314.3.dta

Data file K:\NewmanPaper\Piliang\3SubProteomes\Piliang3SP\mgf5ppm\ERLIC_3SubProteomes5ppm.mgf
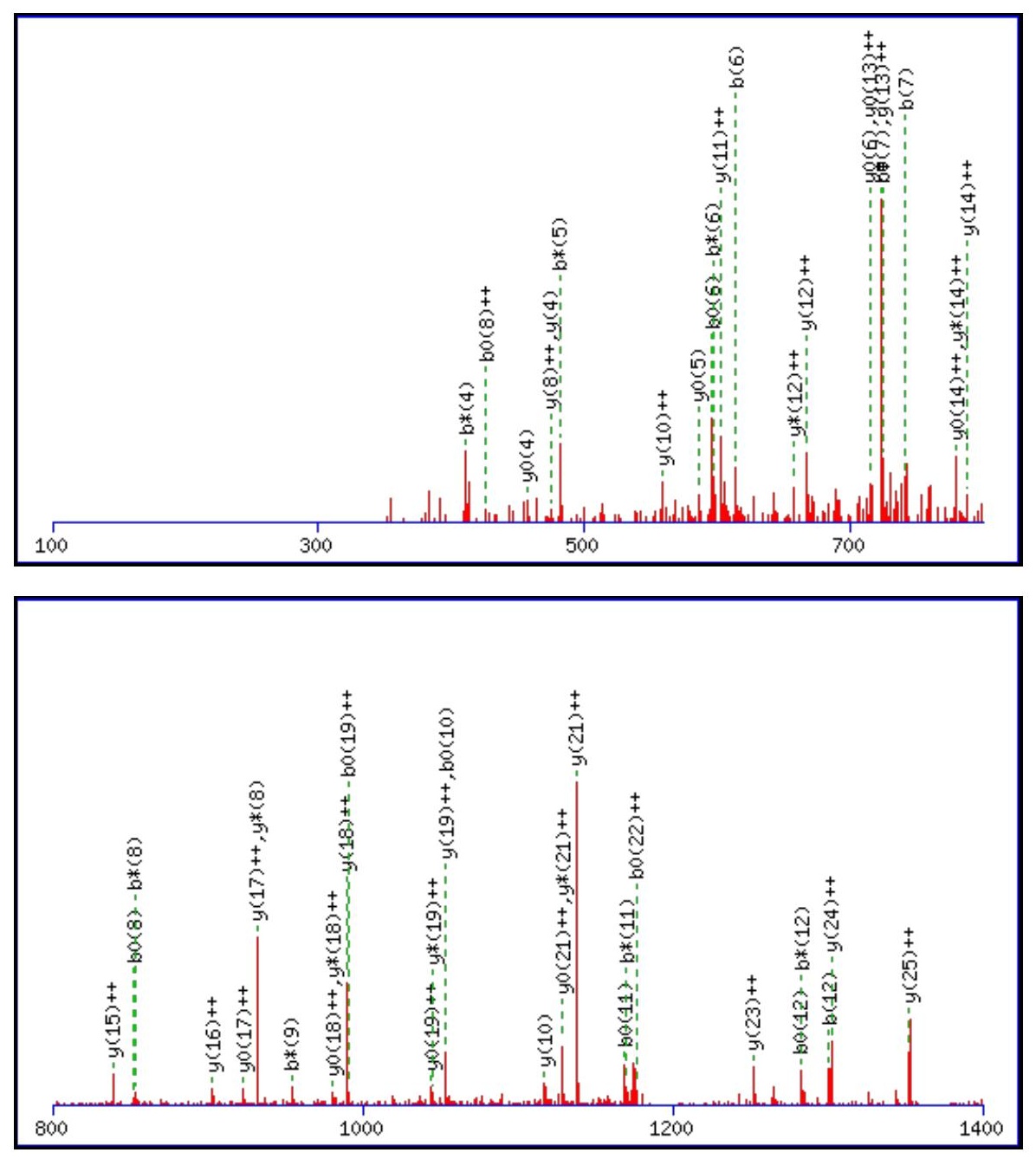


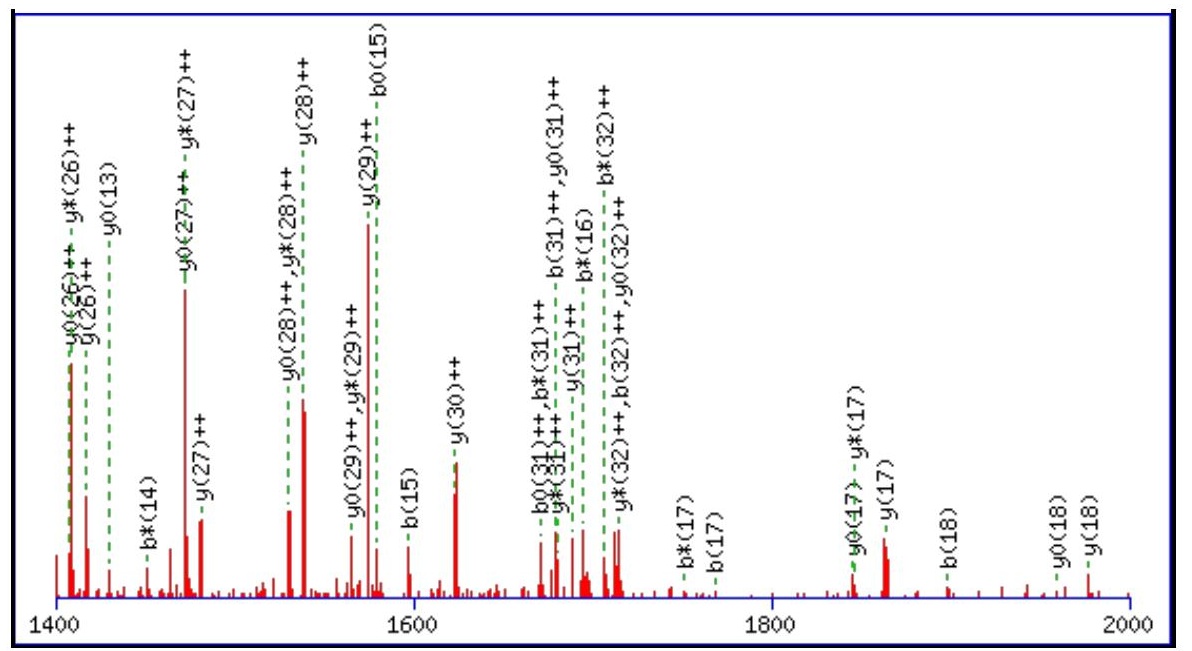

Monoisotopic mass of neutral peptide $\operatorname{Mr}($ calc): 3573.5714

Fixed modifications: Carbamidomethyl (C)

Variable modifications:

N21 : Deamidated_N (N)

Ions Score: 115 Expect: $1.4 \mathrm{e}-009$

Matches (Bold Red): 88/376 fragment ions using 142 most intense peaks

\begin{tabular}{|c|c|c|c|c|c|c|c|c|c|c|c|c|c|c|}
\hline & b & $\mathbf{b}^{++}$ & $\mathbf{b}^{*}$ & $\mathbf{b}^{*^{++}}$ & $\mathbf{b}^{\mathbf{0}}$ & $\mathbf{b}^{0++}$ & Seq. & $\mathbf{y}$ & $\mathbf{y}^{++}$ & $\mathbf{y}^{*}$ & $\mathrm{y}^{\mathrm{*}^{++}}$ & $\mathbf{y}^{0}$ & $\mathbf{y}^{0++}$ & \# \\
\hline 1 & 129.0659 & 65.0366 & 112.0393 & 56.5233 & & & $\mathbf{Q}$ & & & & & & & 33 \\
\hline 2 & 200.1030 & 100.5551 & 183.0 & 92.0418 & & & A & 201 & 37 & 36 & & & 7584 & 32 \\
\hline 3 & 329.1456 & 165.0764 & 312. & 56.5631 & & & $\mathbf{E}$ & 830 & 451 & 565 & 319 & 724 & 399 & 31 \\
\hline 4 & 428.2140 & 214.6106 & 411.1874 & 206.0974 & 410.2034 & 205.6053 & V & 3246.4404 & 1623.7238 & 3229.4139 & 1615.2106 & 3228.4299 & & 30 \\
\hline 5 & 499.2511 & 250.1 & 482.2 & 241. & 105 & 241 & A & 720 & 896 & 455 & & 614 & & 29 \\
\hline & 614.2780 & 307.6427 & 597.2515 & 299. & 596. & 298. & D & 3076. & 1538 & 3059 & 578 & 3058 & 3658 & 28 \\
\hline 7 & 742.3366 & 371.6719 & 725.3101 & 363.1587 & 260 & 362.6667 & $\mathbf{Q}$ & 2961.3079 & 1481 & 2944.2814 & 1472.6443 & 974 & & 27 \\
\hline 8 & 870.3952 & 435.7 & 853.3 & 427. & & & $\mathbf{Q}$ & & 283 & 2816 & & & 230 & 26 \\
\hline 9 & 971.4429 & 486.2251 & 954.4163 & 477.7118 & 953.4323 & 477.2198 & $\mathbf{T}$ & 2705.1908 & 1353.0990 & 2688.1642 & 1344.5858 & 2687.1802 & 0938 & 25 \\
\hline 10 & 1072.4905 & 536.7489 & 55.4640 & 528.2356 & 800 & 527.7 & $T$ & 2604 & 1302 & 2587 & 619 & 325 & & \\
\hline 1 & 1187.5175 & 594.2624 & 70.4909 & 491 & 069 & 585. & D & 954 & 514 & 589 & & 849 & & 23 \\
\hline 12 & 1300.6016 & 650.8044 & 1283.5750 & 642.2911 & 1282.5910 & 641.7991 & $\mathbf{L}$ & 2388.0685 & 1194.5379 & 2371.0419 & 1186.0246 & 2370.0579 & .5326 & 22 \\
\hline 13 & 97.6543 & 699.3308 & 380.6278 & 690. & 438 & & $\mathbf{P}$ & 2274 & 1137 & 2257 & & & & 21 \\
\hline 4 & 1 & 734.8 & 49 & & & & $\mathbf{A}$ & & & & & & & 20 \\
\hline 15 & 1597.7340 & 799.3707 & 1580.7075 & 790.8574 & 1579.7235 & 790.3654 & $\mathbf{E}$ & 2106.8946 & 1053.9509 & 2089.8680 & 1045.4376 & 2088.8840 & 1044.9456 & 19 \\
\hline 16 & 11.7770 & 856.3 & 94.7 & 847. & 664 & 847. & $\mathbf{N}$ & 1977. & & 1960 & & 1959 & 243 & 18 \\
\hline 7 & 17 & 884. & 19 & & 50.7878 & 875.8 & G & 1863. & & 1846 & & 1845. & & 17 \\
\hline 18 & 1897.8410 & 949.4241 & 1880.8145 & 940.9109 & 1879.8304 & 940.4189 & $\mathbf{E}$ & 1806.7876 & 903.8974 & 1789.7610 & 895.3841 & 1788.7770 & 894.8921 & 16 \\
\hline 19 & 98.8887 & 999.9 & 21 & 47 & 81 & 27 & $\mathbf{T}$ & 1677. & & 166 & & 1659 & 3708 & 15 \\
\hline 20 & 2127.9313 & 1064.4693 & 2110.9047 & 9560 & 2109. & 1055. & $\mathbf{E}$ & 1576.6 & & 1559 & & 1558 & 470 & 14 \\
\hline 21 & 2242.9582 & 1121.9827 & 2225.9317 & 13.4695 & 2224.9477 & 1112.9775 & $\mathbf{N}$ & 1447.6547 & 724.3310 & 1430.6282 & 715.8177 & \begin{tabular}{|l}
1429.6441 \\
\end{tabular} & 715.3257 & 13 \\
\hline 22 & 71.0168 & 86.0120 & 2353.9902 & 77.4988 & 353.0062 & 1177.0068 & $\mathbf{Q}$ & 1332.6278 & 666.8175 & 1315.6012 & 658.3042 & 1314.6172 & 657.8122 & 12 \\
\hline 23 & 2458.0488 & 1229.5280 & 2441.0223 & 21.0148 & 2440.0383 & 1220.5228 & $\mathrm{~s}$ & 1204.5692 & 602. & 1187 & & 1186. & 7829 & 11 \\
\hline 24 & 2555.1016 & 1278.0544 & 2538.0750 & 1269.5412 & 2537.0910 & 1269.0491 & $\mathbf{P}$ & 1117.5372 & 559.2722 & 1100.5106 & 550.7589 & 1099.5266 & 550.2669 & 10 \\
\hline 25 & 2626.1387 & 1313.5730 & 2609.1122 & 5997 & 281 & 1304.5677 & A & 4844 & 7458 & 1003.4578 & 502.2326 & 1002.4738 & 501.7406 & 9 \\
\hline 26 & 2713.1707 & 1357.0890 & 2696.1442 & 348.5757 & 2695.1602 & 1348.0837 & $\mathrm{~S}$ & 949.4473 & & & & 931 & 2220 & \\
\hline 27 & 2842.2133 & 1421.6103 & 2825.1868 & 1413.0970 & 2824.2028 & 1412.6050 & $\mathbf{E}$ & 862.4153 & 431.7113 & 845.3887 & 423.1980 & 844.4047 & 422.7060 & 7 \\
\hline 28 & 2971.2559 & 1486.1316 & 2954.2294 & 1477.6183 & 2953.2453 & 1477.1263 & $\mathbf{E}$ & 733.3727 & 367.1900 & 716.3461 & 6767 & 715.3621 & 358.1847 & \\
\hline 29 & 3100.2985 & 1550.6529 & 3083.2720 & 1542.1396 & 3082.2879 & 1541.6476 & $E$ & 604.3301 & 302.6687 & & & 586.3195 & 293.6634 & 5 \\
\hline 30 & 3228.3935 & 1614.7004 & 3211.3669 & 506.1871 & 3210.3829 & 1605.6951 & $\mathbf{K}$ & 475.2875 & 238.1474 & 458.2609 & 229.6341 & 457.2769 & 229.1421 & 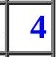 \\
\hline 31 & 3357.4361 & 1679.2217 & 3340.4095 & 1670.7084 & 3339.4255 & 1670.2164 & $\mathbf{E}$ & 347.1925 & 174.0999 & 330.1660 & 165.5866 & 329.1819 & 165.0946 & 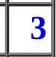 \\
\hline 32 & 428.4732 & 1714.7402 & 411.4466 & 706.2270 & 410.4626 & 705.7349 & $\mathbf{A}$ & 218.1499 & 109.5786 & 201.1234 & 101.0653 & & & \\
\hline
\end{tabular}




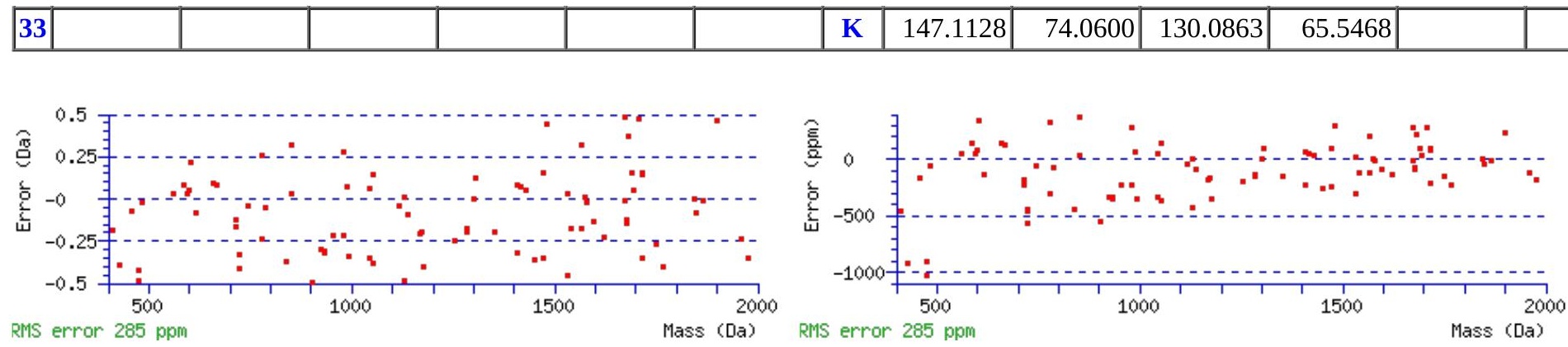

\section{All matches to this query}

\begin{tabular}{|l|l|l|l|}
\hline Score & Mr(calc): & Delta & \multicolumn{1}{|c|}{ Sequence } \\
\hline 115.4 & 3573.5714 & 0.0041 & QAEVADQQTTDLPAENGETENQSPASEEEKEAK \\
\hline 112.1 & 3573.5714 & 0.0041 & QAEVADQQTTDLPAENGETENQSPASEEEKEAK \\
\hline 54.8 & 3572.5874 & 0.9881 & QAEVADQQTTDLPAENGETENQSPASEEEKEAK \\
\hline 12.2 & 3573.5809 & -0.0054 & EETFALSSTKTLEDAVNNIITFLGMQPCER \\
\hline 12.2 & 3573.5809 & -0.0054 & EETFALSSTKTLEDAVNNIITFLGMQPCER \\
\hline 12.2 & 3573.5809 & -0.0054 & EETFALSSTKTLEDAVNNIITFLGMQPCER \\
\hline 12.2 & 3573.5809 & -0.0054 & EETFALSSTKTLEDAVNNIITFLGMQPCER \\
\hline 8.9 & 3573.5684 & 0.0071 & CXHSQNTSLACQTLAPSVWQLESGWSARAR \\
\hline 8.9 & 3573.5684 & 0.0071 & CXHSQNTSLACQTLAPSVWQLESGWSARAR \\
\hline 8.4 & 3573.5677 & 0.0078 & MALFPAFAGVSEASGDGASRKELDWLSNPSFR \\
\hline
\end{tabular}

Spectrum No: 25; Query: 1309; Rank: 1

\section{Peptide View}

\section{MS/MS Fragmentation of GTFTDCALANMTQQIR}

Found in IPI00372839, Tax_Id=10116 Gene_Symbol=Col6a2 similar to procollagen, type VI, alpha 2

Match to Query 1309: 1842.819008 from(922.416780,2+)

Title: 091008RatKidney_NH4Format01_23.2093.2093.2.dta

Data file K:INewmanPaper|Piliang|3SubProteomes\Piliang3SP\mgf5ppm\ERLIC_3SubProteomes5ppm.mgf

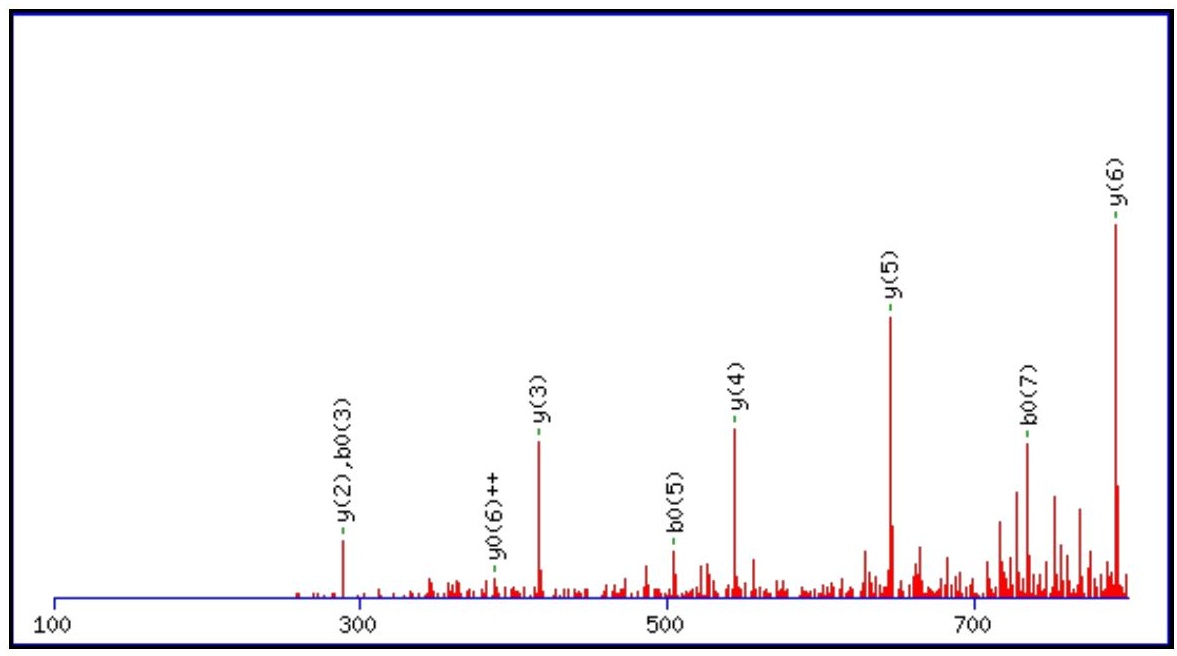



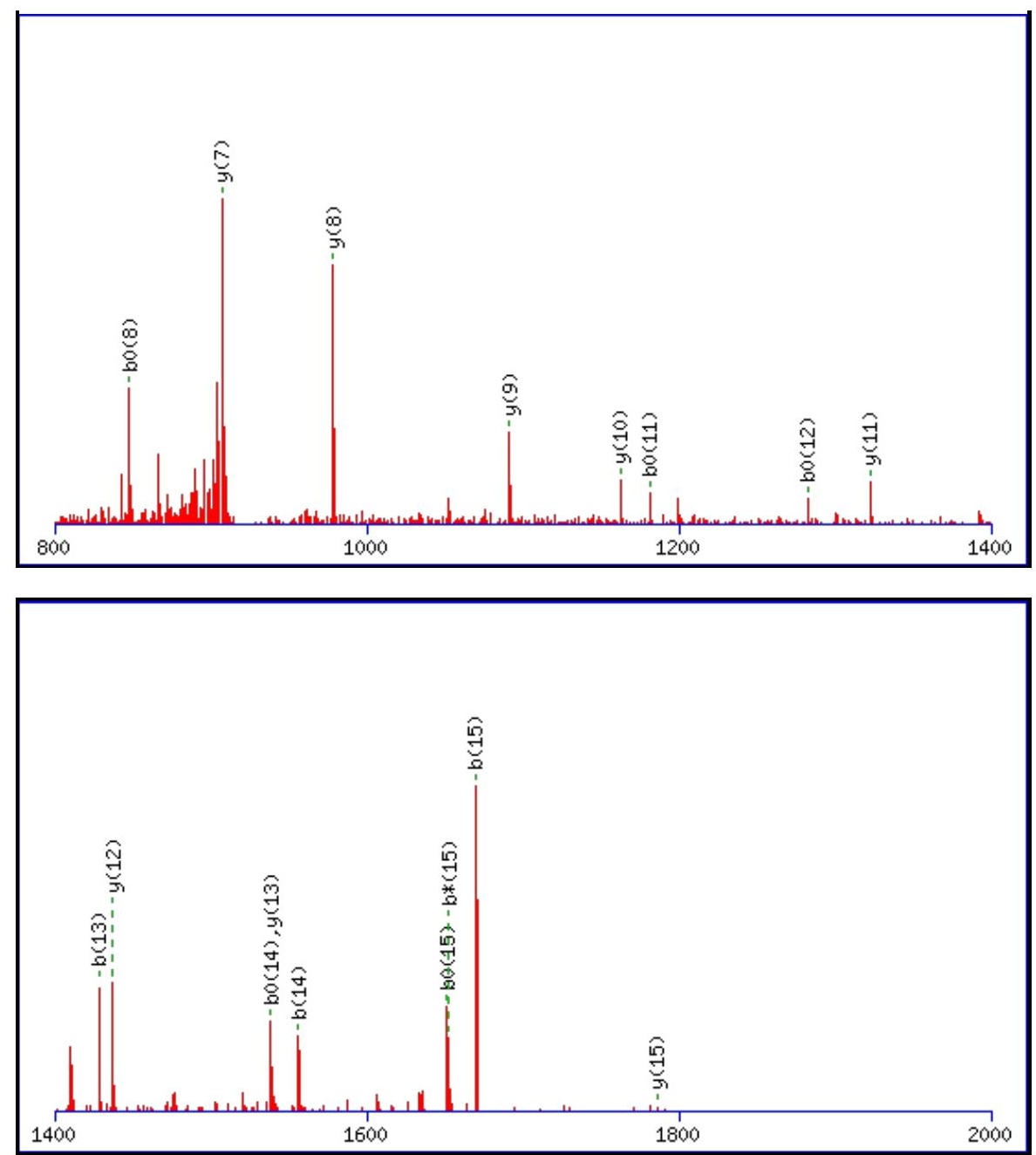

Monoisotopic mass of neutral peptide $\operatorname{Mr}($ calc): 1842.8240

Fixed modifications: Carbamidomethyl (C)

Variable modifications:

N10 : Deamidated_N (N)

M11 : Oxidation $(M)$

Ions Score: 115 Expect: $6.2 \mathrm{e}-010$

Matches (Bold Red): 26/152 fragment ions using 36 most intense peaks

\begin{tabular}{|c|c|c|c|c|c|c|c|c|c|c|c|c|c|c|}
\hline \# & b & $\mathbf{b}^{++}$ & b* & $\mathbf{b}^{*^{++}}$ & $\mathbf{b}^{0}$ & $\mathbf{b}^{0++}$ & Seq. & $\mathbf{y}$ & $\mathbf{y}^{++}$ & $\mathbf{y}^{*}$ & $\mathbf{y}^{*^{++}}$ & $\mathbf{y}^{0}$ & $\mathbf{y}^{0++}$ & \# \\
\hline 1 & 58.0287 & 29.5180 & & & & & G & & & & & & & 16 \\
\hline 2 & 159.0764 & 80.0418 & & & 141.0659 & 71.0366 & $\mathbf{T}$ & 1786.8098 & 893.9086 & 1769.7833 & 885.3953 & 1768.7993 & 884.9033 & 15 \\
\hline 3 & 306.1448 & 153.5761 & & & 288.1343 & 144.5708 & $\mathbf{F}$ & 1685.7622 & 843.3847 & 1668.7356 & 834.8714 & 1667.7516 & 834.3794 & 14 \\
\hline 4 & 407.1925 & 204.0999 & & & 389.1819 & 195.0946 & $\mathbf{T}$ & 1538.6938 & 769.8505 & 1521.6672 & 761.3372 & 1520.6832 & 760.8452 & 13 \\
\hline 5 & 522.2195 & 261.6134 & & & 504.2089 & 252.6081 & D & 1437.6461 & 719.3267 & 1420.6195 & 710.8134 & 1419.6355 & 710.3214 & 12 \\
\hline 6 & 682.2501 & 341.6287 & & & 664.2395 & 332.6234 & C & 1322.6191 & 661.8132 & 1305.5926 & 653.2999 & 1304.6086 & 652.8079 & 11 \\
\hline 7 & 753.2872 & 377.1472 & & & 735.2767 & 368.1420 & A & 1162.5885 & 581.7979 & 1145.5619 & 573.2846 & 1144.5779 & 572.7926 & 10 \\
\hline 8 & 866.3713 & 433.6893 & & & 848.3607 & 424.6840 & $\mathbf{L}$ & 1091.5514 & 546.2793 & 1074.5248 & 537.7660 & 1073.5408 & 537.2740 & 9 \\
\hline 9 & 937.4084 & 469.2078 & & & 919.3978 & 460.2026 & A & 978.4673 & 489.7373 & 961.4408 & 481.2240 & 960.4567 & 480.7320 & 8 \\
\hline 10 & 1052.4353 & 526.7213 & 1035.4088 & 518.2080 & 1034.4248 & 517.7160 & $\mathbf{N}$ & 907.4302 & 454.2187 & 890.4036 & 445.7055 & 889.4196 & 445.2135 & 7 \\
\hline 11 & 1199.4707 & 600.2390 & 1182.4442 & 591.7257 & 1181.4602 & 591.2337 & $\mathbf{M}$ & 792.4033 & 396.7053 & 775.3767 & 388.1920 & 774.3927 & 387.7000 & 6 \\
\hline 12 & 1300.5184 & 650.7628 & 1283.4919 & 642.2496 & 1282.5079 & 641.7576 & $\mathbf{T}$ & 645.3679 & 323.1876 & 628.3413 & 314.6743 & 627.3573 & 314.1823 & 5 \\
\hline 13 & 1428.5770 & 714.7921 & 1411.5504 & 706.2789 & 1410.5664 & 705.7869 & $\mathbf{Q}$ & 544.3202 & 272.6637 & 527.2936 & 264.1504 & & & 4 \\
\hline 14 & 1556.6356 & 778.8214 & 1539.6090 & 770.3081 & 1538.6250 & 769.8161 & $\mathbf{Q}$ & 416.2616 & 208.6344 & 399.2350 & 200.1212 & & & 3 \\
\hline 15 & 1669.7196 & 835.3635 & 1652.6931 & 826.8502 & 1651.7091 & 826.3582 & I & 288.2030 & 144.6051 & 271.1765 & 136.0919 & & & 2 \\
\hline 16 & & & & & & & $\mathbf{R}$ & 175.1190 & 88.0631 & 158.0924 & 79.5498 & & & 1 \\
\hline
\end{tabular}



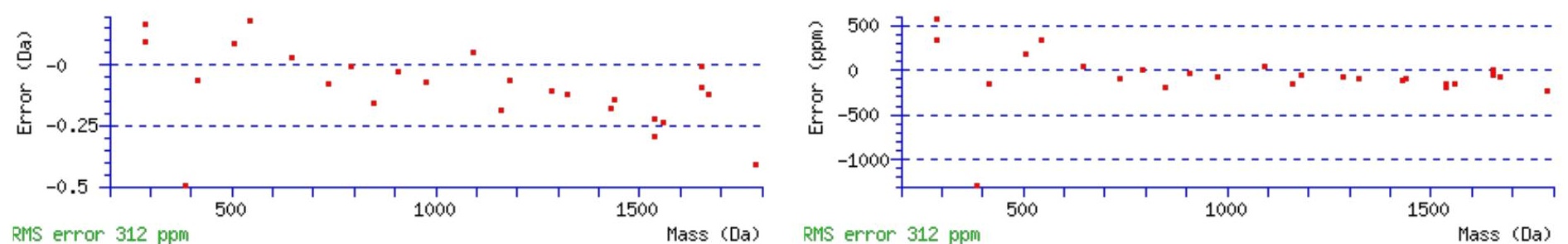

\section{All matches to this query}

\begin{tabular}{|l|l|c|l|}
\hline Score & Mr(calc): & Delta & \multicolumn{1}{|c|}{ Sequence } \\
\hline 115.1 & 1842.8240 & -0.0050 & GTFTDCALANMTQQIR \\
\hline 9.5 & 1842.8278 & -0.0088 & SSLQDTNVRSPIPIR \\
\hline 8.6 & 1842.8278 & -0.0088 & SSLQDTNVRSPIPIR \\
\hline 8.6 & 1842.8278 & -0.0088 & SSLQDTNVRSPIPIR \\
\hline 6.8 & 1842.8374 & -0.0184 & TSRAVHPTQPSSNPAGR \\
\hline 5.8 & 1842.8067 & 0.0124 & FQLSNGHLLSPLGSGR \\
\hline 5.6 & 1842.8067 & 0.0124 & FQLSNGHLLSPLGSGR \\
\hline 4.7 & 1842.8070 & 0.0120 & LMQVNDTLTSEDAGLR \\
\hline 4.4 & 1840.8025 & 2.0165 & AVLINNLGTIAKSGTK \\
\hline 4.2 & 1842.8118 & 0.0073 & GATLCVICQDRNSLR \\
\hline
\end{tabular}

Spectrum No: 26; Query: 516; Rank: 1

\section{Peptide View}

\section{MS/MS Fragmentation of AFYNGSLTAQIVK}

Found in IPI00206254, Tax_Id=10116 Gene_Symbol=Ggt1 Gamma-glutamyltranspeptidase 1 precursor

Match to Query 516: 1411.741148 from(706.877850,2+)

Title: 091008RatKidney_NoSalt_24.3462.3462.2.dta

Data file K:INewmanPaper|Piliang\3SubProteomes\Piliang3SP\mgf5ppm\ERLIC_3SubProteomes5ppm.mgf

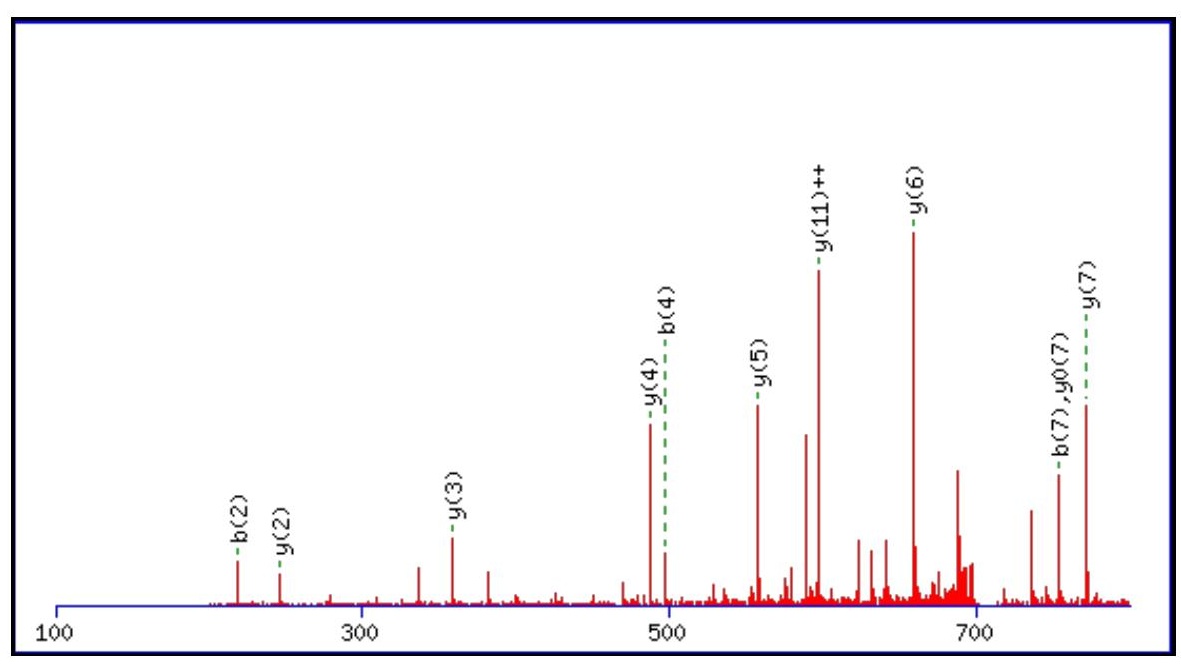



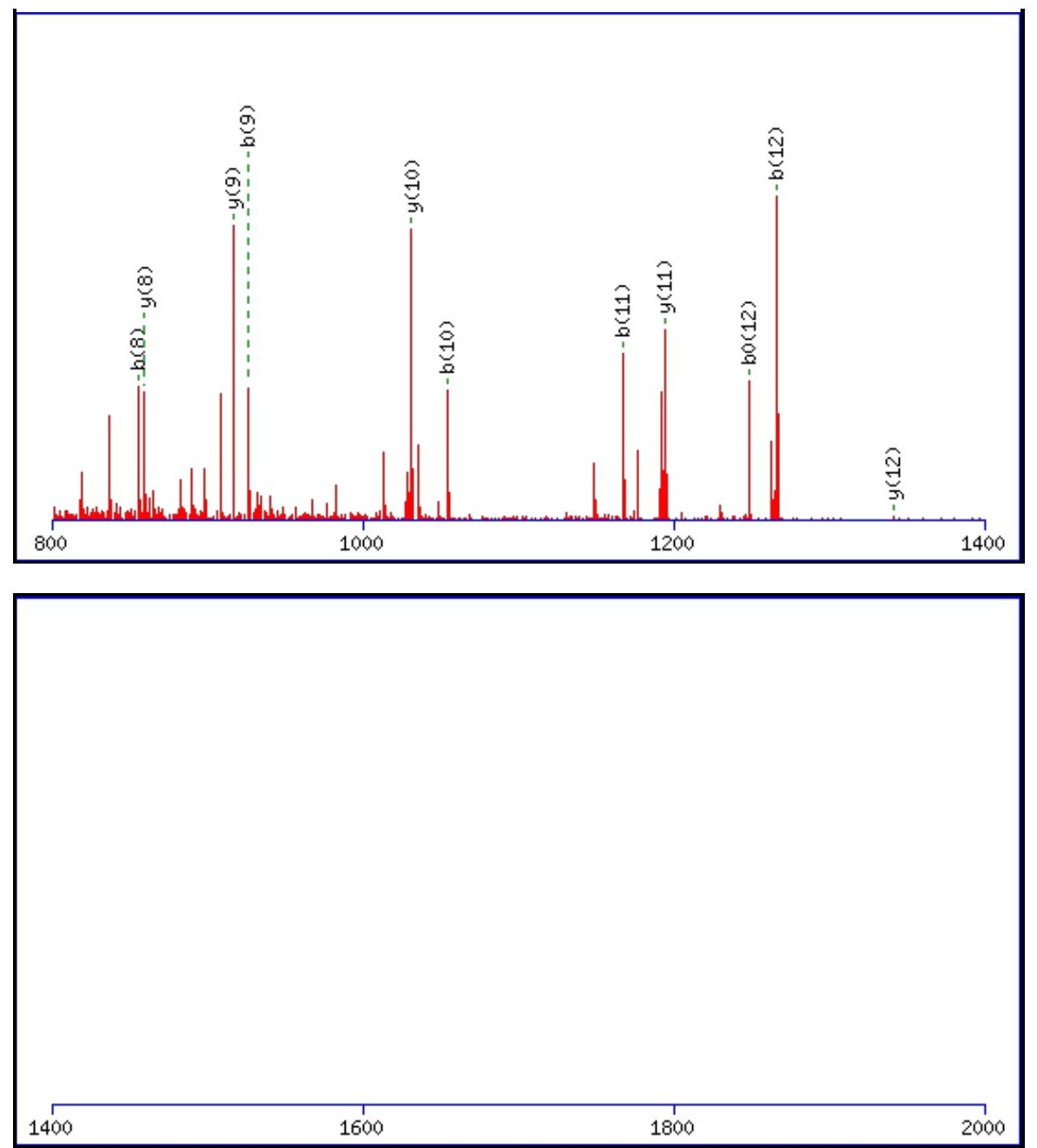

Monoisotopic mass of neutral peptide $\operatorname{Mr}($ calc): 1411.7347

Fixed modifications: Carbamidomethyl (C)

Variable modifications:

N4 : Deamidated $\mathrm{N}(\mathrm{N})$

Ions Score: 115 Expect: $3.1 \mathrm{e}-010$

Matches (Bold Red): 22/118 fragment ions using 25 most intense peaks

\begin{tabular}{|r|c|c|c|c|c|c|c|c|c|c|c|c|c|c|}
\hline$\#$ & $\mathbf{b}$ & $\mathbf{b}^{++}$ & $\mathbf{b}^{*}$ & $\mathbf{b}^{\mathbf{*}^{++}}$ & $\mathbf{b}^{\mathbf{0}}$ & $\mathbf{b}^{\mathbf{0 + +}}$ & $\mathbf{S e q}$ & $\mathbf{y}$ & $\mathbf{y}^{++}$ & $\mathbf{y}^{*}$ & $\mathbf{y}^{\mathbf{*}^{++}}$ & $\mathbf{y}^{\mathbf{0}}$ & $\mathbf{y}^{\mathbf{0 + +}}$ & $\#^{\#}$ \\
\hline $\mathbf{1}$ & 72.0444 & 36.5258 & & & & & $\mathbf{A}$ & & & & & & & $\mathbf{1 3}$ \\
\hline $\mathbf{2}$ & $\mathbf{2 1 9 . 1 1 2 8}$ & 110.0600 & & & & & $\mathbf{F}$ & $\mathbf{1 3 4 1 . 7 0 4 9}$ & 671.3561 & 1324.6783 & 662.8428 & 1323.6943 & 662.3508 & $\mathbf{1 2}$ \\
\hline $\mathbf{3}$ & 382.1761 & 191.5917 & & & & & $\mathbf{Y}$ & $\mathbf{1 1 9 4 . 6 3 6 5}$ & $5 \mathbf{9 7 . 8 2 1 9}$ & 1177.6099 & 589.3086 & 1176.6259 & 588.8166 & $\mathbf{1 1}$ \\
\hline $\mathbf{4}$ & $\mathbf{4 9 7 . 2 0 3 1}$ & 249.1052 & 480.1765 & 240.5919 & & & $\mathbf{N}$ & $\mathbf{1 0 3 1 . 5 7 3 1}$ & 516.2902 & 1014.5466 & 507.7769 & 1013.5626 & 507.2849 & $\mathbf{1 0}$ \\
\hline $\mathbf{5}$ & 554.2245 & 277.6159 & 537.1980 & 269.1026 & & & $\mathbf{G}$ & $\mathbf{9 1 6 . 5 4 6 2}$ & 458.7767 & 899.5197 & 450.2635 & 898.5356 & 449.7715 & $\mathbf{9}$ \\
\hline $\mathbf{6}$ & 641.2566 & 321.1319 & 624.2300 & 312.6186 & 623.2460 & 312.1266 & $\mathbf{S}$ & $\mathbf{8 5 9 . 5 2 4 7}$ & 430.2660 & 842.4982 & 421.7527 & 841.5142 & 421.2607 & $\mathbf{8}$ \\
\hline $\mathbf{7}$ & $\mathbf{7 5 4 . 3 4 0 6}$ & 377.6740 & 737.3141 & 369.1607 & 736.3301 & 368.6687 & $\mathbf{L}$ & 772.4927 & 386.7500 & 755.4662 & 378.2367 & 754.4822 & 377.7447 & $\mathbf{7}$ \\
\hline $\mathbf{8}$ & $\mathbf{8 5 5 . 3 8 8 3}$ & 428.1978 & 838.3618 & 419.6845 & 837.3777 & 419.1925 & $\mathbf{T}$ & $\mathbf{6 5 9 . 4 0 8 7}$ & 330.2080 & 642.3821 & 321.6947 & 641.3981 & 321.2027 & $\mathbf{6}$ \\
\hline $\mathbf{9}$ & $\mathbf{9 2 6 . 4 2 5 4}$ & 463.7163 & 909.3989 & 455.2031 & 908.4149 & 454.7111 & $\mathbf{A}$ & $\mathbf{5 5 8 . 3 6 1 0}$ & 279.6841 & 541.3344 & 271.1709 & & & $\mathbf{5}$ \\
\hline $\mathbf{1 0}$ & $\mathbf{1 0 5 4 . 4 8 4 0}$ & 527.7456 & 1037.4574 & 519.2324 & 1036.4734 & 518.7404 & $\mathbf{Q}$ & $\mathbf{4 8 7 . 3 2 3 9}$ & 244.1656 & 470.2973 & 235.6523 & & & $\mathbf{4}$ \\
\hline $\mathbf{1 1}$ & $\mathbf{1 1 6 7 . 5 6 8 1}$ & 584.2877 & 1150.5415 & 575.7744 & 1149.5575 & 575.2824 & $\mathbf{I}$ & 359.2653 & 180.1363 & 342.2387 & 171.6230 & & & 3 \\
\hline $\mathbf{1 2}$ & $\mathbf{1 2 6 6 . 6 3 6 5}$ & 633.8219 & 1249.6099 & 625.3086 & $\mathbf{1 2 4 8 . 6 2 5 9}$ & 624.8166 & $\mathbf{V}$ & $\mathbf{2 4 6 . 1 8 1 2}$ & 123.5942 & 229.1547 & 115.0810 & & & $\mathbf{2}$ \\
\hline $\mathbf{1 3}$ & & & & & & & $\mathbf{K}$ & 147.1128 & 74.0600 & 130.0863 & 65.5468 & & & $\mathbf{1}$ \\
\hline
\end{tabular}



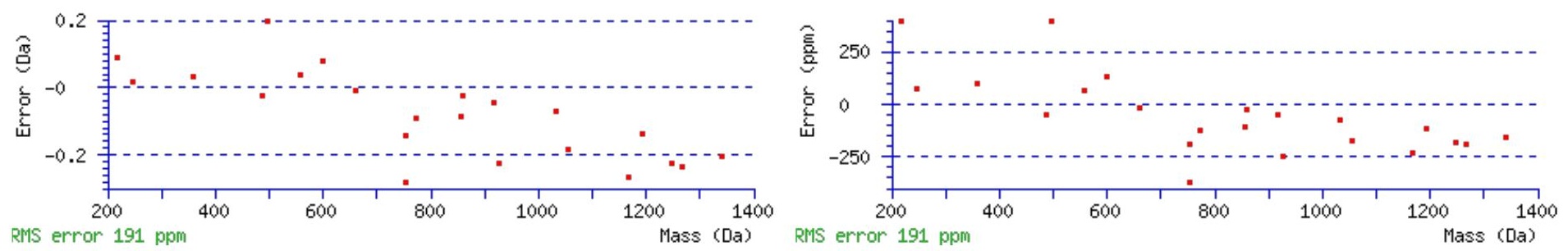

\section{All matches to this query}

\begin{tabular}{|l|l|l|l|}
\hline Score & Mr(calc): & Delta & \multicolumn{1}{|c|}{ Sequence } \\
\hline 114.9 & 1411.7347 & 0.0064 & AFYNGSLTAQIVK \\
\hline 45.8 & 1410.7507 & 0.9904 & AFYNGSLTAQIVK \\
\hline 9.7 & 1411.7476 & -0.0065 & LTGQIFLGGSIVK \\
\hline 8.9 & 1411.7320 & 0.0091 & AFSDHSSLRKHK \\
\hline 4.8 & 1411.7364 & 0.0048 & FLISDLTLSPVK \\
\hline 4.6 & 1411.7298 & 0.0113 & FMTDPIRILVK \\
\hline 3.1 & 1409.7375 & 2.0037 & KDLQHLSREER \\
\hline 2.5 & 1411.7476 & -0.0065 & LTGQIFLGGSIVK \\
\hline 1.5 & 1411.7363 & 0.0048 & NLDLYLEILVK \\
\hline 1.2 & 1409.7263 & 2.0149 & LHNAIEGTAQLSR \\
\hline
\end{tabular}

Spectrum No: 27; Query: 356; Rank: 1

\section{Peptide View}

MS/MS Fragmentation of LNLSGGEVSATEK

Found in IPI00326462, Tax_Id=10116 Gene_Symbol=Enpp3 Ectonucleotide pyrophosphatase/phosphodiesterase family member 3

Match to Query 356: 1304.643548 from(653.329050,2+)

Title: 091008RatKidney_NH4Format01_25.1340.1340.2.dta

Data file K:INewmanPaper|Piliangl3SubProteomes\Piliang3SP\mgf5ppmlERLIC_3SubProteomes5ppm.mgf

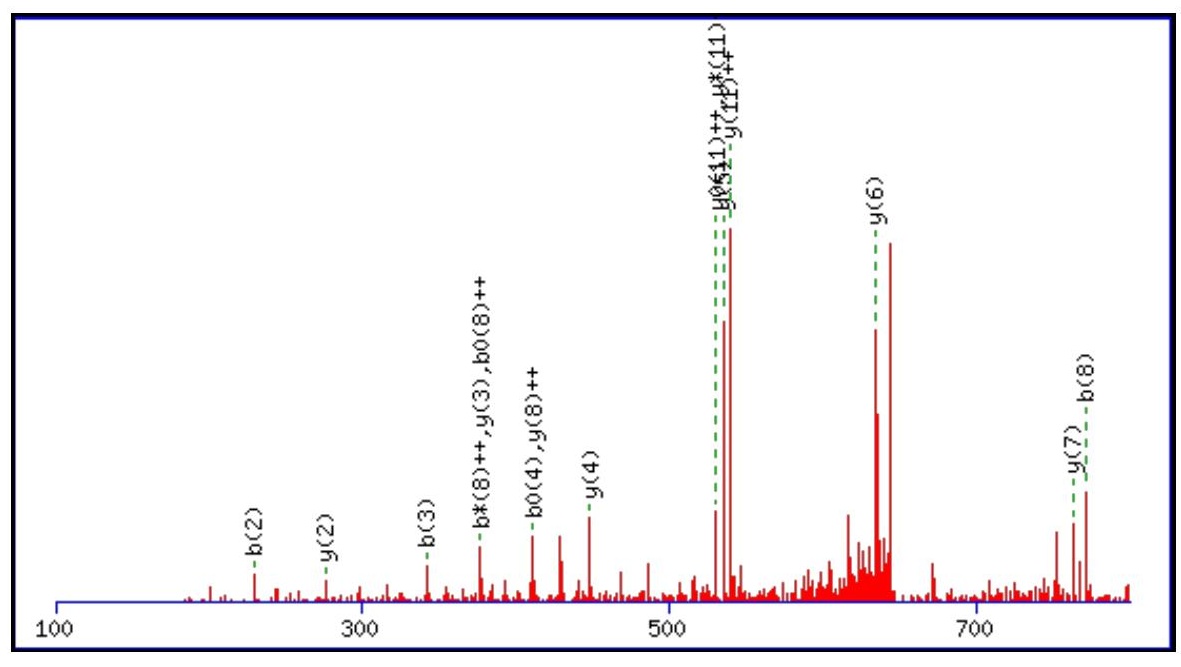



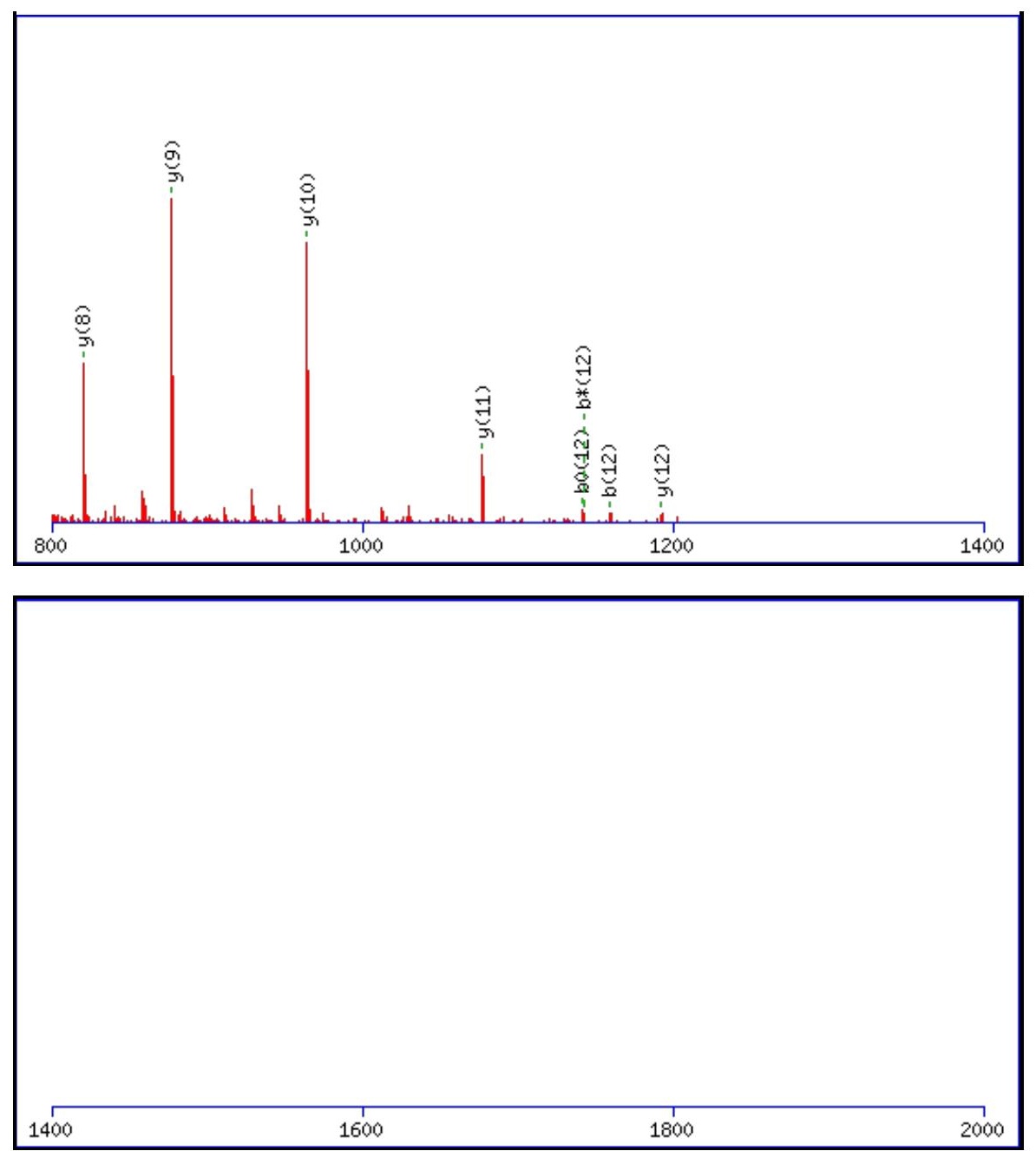

Monoisotopic mass of neutral peptide $\operatorname{Mr}($ calc): 1304.6460

Fixed modifications: Carbamidomethyl (C)

Variable modifications:

N2 : Deamidated $\mathrm{N}(\mathrm{N})$

Ions Score: 114 Expect: $4.3 \mathrm{e}-010$

Matches (Bold Red): 24/134 fragment ions using 25 most intense peaks

\begin{tabular}{|c|c|c|c|c|c|c|c|c|c|c|c|c|c|c|}
\hline \# & b & $\mathbf{b}^{++}$ & $\mathbf{b}^{*}$ & $\mathbf{b}^{*^{++}}$ & $\mathbf{b}^{0}$ & $\mathbf{b}^{0++}$ & Seq. & $\mathbf{y}$ & $\mathbf{y}^{++}$ & $\mathbf{y}^{*}$ & $\mathrm{y}^{*^{++}}$ & $\mathbf{y}^{0}$ & $\mathbf{y}^{0++}$ & \# \\
\hline 1 & 114.0913 & 57.5493 & & & & & $\mathbf{L}$ & & & & & & & 13 \\
\hline 2 & 229.1183 & 115.0628 & 212.0917 & 106.5495 & & & $\mathbf{N}$ & 1192.5692 & 596.7882 & 1175.5426 & 588.2750 & 1174.5586 & 587.7829 & 12 \\
\hline 3 & 342.2023 & 171.6048 & 325.1758 & 163.0915 & & & $\mathbf{L}$ & 1077.5422 & 539.2748 & 1060.5157 & 530.7615 & 1059.5317 & 530.2695 & 11 \\
\hline 4 & 429.2344 & 215.1208 & 412.2078 & 206.6075 & 411.2238 & 206.1155 & S & 964.4582 & 482.7327 & 947.4316 & 474.2195 & 946.4476 & 473.7274 & 10 \\
\hline 5 & 486.2558 & 243.6316 & 469.2293 & 235.1183 & 468.2453 & 234.6263 & G & 877.4262 & 439.2167 & 860.3996 & 430.7034 & 859.4156 & 430.2114 & 9 \\
\hline 6 & 543.2773 & 272.1423 & 526.2507 & 263.6290 & 525.2667 & 263.1370 & G & 820.4047 & 410.7060 & 803.3781 & 402.1927 & 802.3941 & 401.7007 & 8 \\
\hline 7 & 672.3199 & 336.6636 & 655.2933 & 328.1503 & 654.3093 & 327.6583 & $\mathbf{E}$ & 763.3832 & 382.1953 & 746.3567 & 373.6820 & 745.3727 & 373.1900 & 7 \\
\hline 8 & 771.3883 & 386.1978 & 754.3618 & 377.6845 & 753.3777 & 377.1925 & V & 634.3406 & 317.6740 & 617.3141 & 309.1607 & 616.3301 & 308.6687 & 6 \\
\hline 9 & 858.4203 & 429.7138 & 841.3938 & 421.2005 & 840.4098 & 420.7085 & S & 535.2722 & 268.1397 & 518.2457 & 259.6265 & 517.2617 & 259.1345 & 5 \\
\hline 10 & 929.4574 & 465.2324 & 912.4309 & 456.7191 & 911.4469 & 456.2271 & A & 448.2402 & 224.6237 & 431.2136 & 216.1105 & 430.2296 & 215.6185 & 4 \\
\hline 11 & 1030.5051 & 515.7562 & 1013.4786 & 507.2429 & 1012.4946 & 506.7509 & $\mathbf{T}$ & 377.2031 & 189.1052 & 360.1765 & 180.5919 & 359.1925 & 180.0999 & 3 \\
\hline 12 & 1159.5477 & 580.2775 & 1142.5212 & 571.7642 & 1141.5372 & 571.2722 & $\mathbf{E}$ & 276.1554 & 138.5813 & 259.1288 & 130.0681 & 258.1448 & 129.5761 & 2 \\
\hline 13 & & & & & & & $\mathbf{K}$ & 147.1128 & 74.0600 & 130.0863 & 65.5468 & & & 1 \\
\hline
\end{tabular}



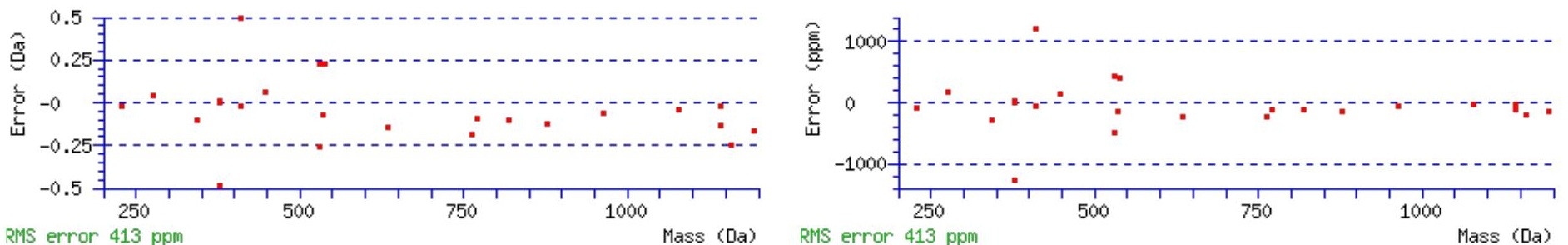

\section{All matches to this query}

\begin{tabular}{|l|l|l|l|}
\hline Score & Mr(calc): & Delta & \multicolumn{1}{|c|}{ Sequence } \\
\hline 114.4 & 1304.6460 & -0.0024 & LNLSGGEVSATEK \\
\hline 7.2 & 1304.6401 & 0.0034 & DVTGLPNWFEK \\
\hline 4.9 & 1304.6394 & 0.0041 & DLEALSKQCNK \\
\hline 4.8 & 1304.6312 & 0.0124 & ILAMIQGSHQK \\
\hline 4.8 & 1304.6394 & 0.0041 & IVQNCGTEKEK \\
\hline 4.6 & 1304.6312 & 0.0124 & LTHTHTIKMK \\
\hline 4.5 & 1304.6473 & -0.0037 & NVQSLAAAHHEK \\
\hline 3.9 & 1304.6312 & 0.0124 & LTHTHTIKMK \\
\hline 3.9 & 1304.6489 & -0.0054 & TVINNKAHLSK \\
\hline 3.7 & 1304.6460 & -0.0024 & LSVELEASDSQK \\
\hline
\end{tabular}

Spectrum No: 28; Query: 813; Rank: 1

\section{Peptide View}

MS/MS Fragmentation of TNGSVDLGEEEEAAR

Found in IPI00464886, Tax_Id=10116 Gene_Symbol=LOC652956 p55 protein

Match to Query 813: 1576.679608 from(789.347080,2+)

Title: 091008RatKidney_NoSalt_23.1182.1182.2.dta

Data file K:INewmanPaper|Piliang|3SubProteomes\Piliang3SP\mgf5ppm|ERLIC_3SubProteomes5ppm.mgf

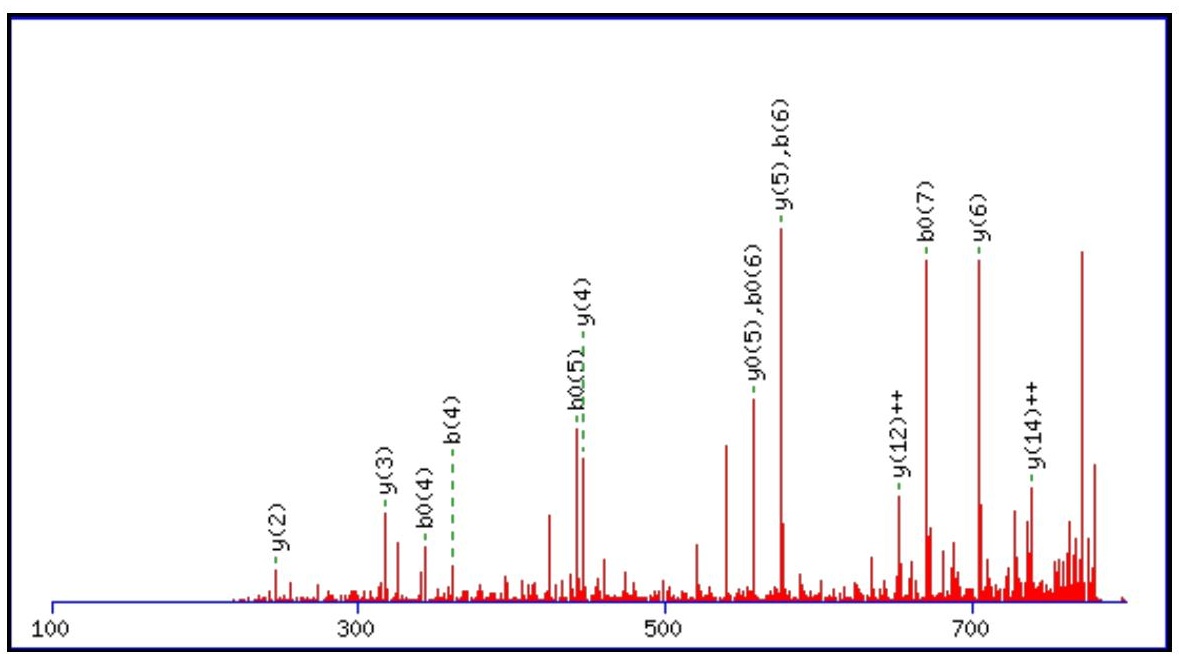



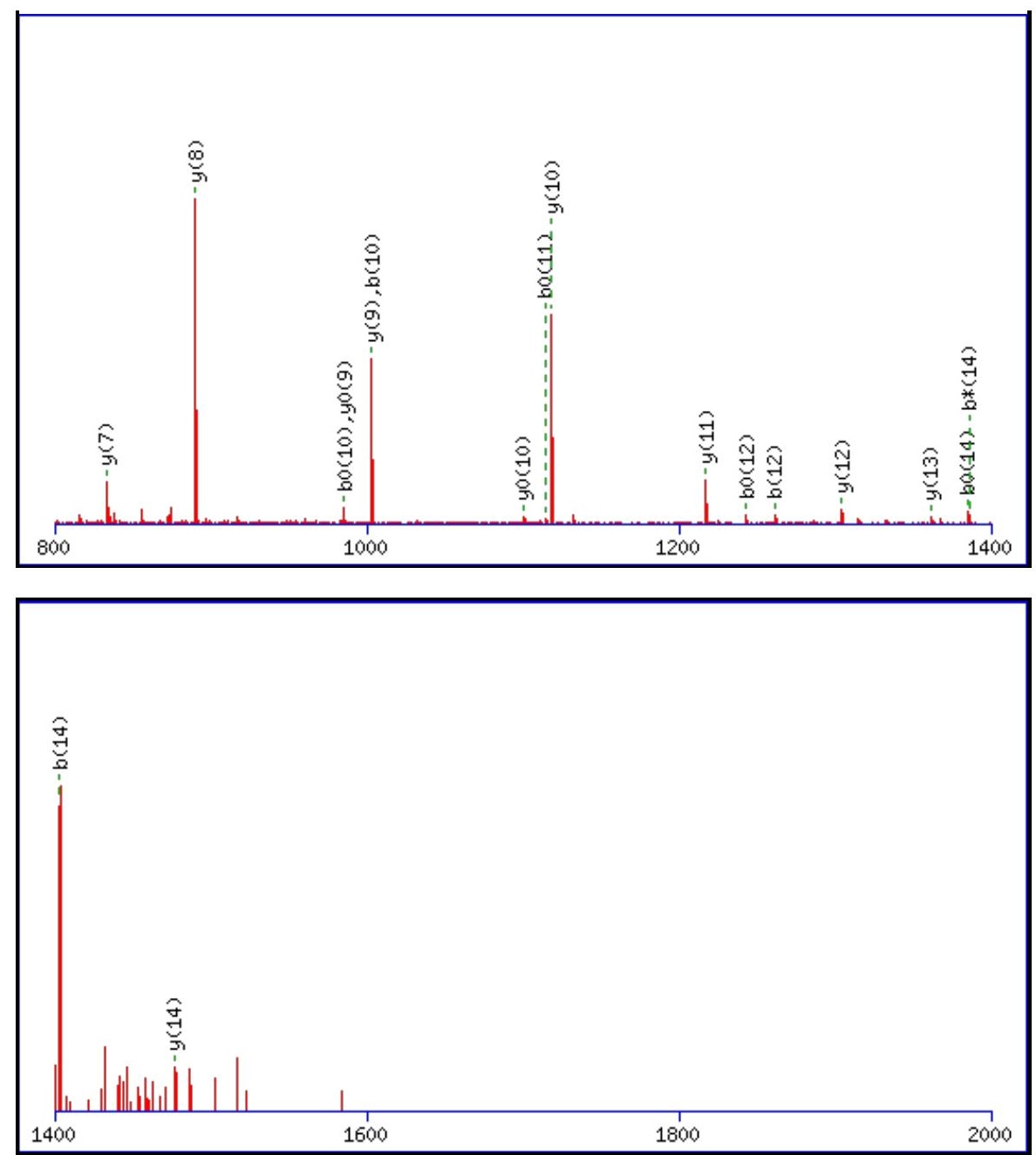

Monoisotopic mass of neutral peptide $\operatorname{Mr}($ calc): 1576.6852

Fixed modifications: Carbamidomethyl (C)

Variable modifications:

N2 : Deamidated $\mathrm{N}(\mathrm{N})$

Ions Score: 114 Expect: $6.2 \mathrm{e}-010$

Matches (Bold Red): 32/160 fragment ions using 43 most intense peaks

\begin{tabular}{|c|c|c|c|c|c|c|c|c|c|c|c|c|c|c|}
\hline \# & b & $\mathbf{b}^{++}$ & b* & $\mathbf{b}^{*^{++}}$ & $\mathbf{b}^{0}$ & $\mathbf{b}^{0++}$ & Seq. & $\mathbf{y}$ & $\mathbf{y}^{++}$ & $\mathbf{y}^{*}$ & $\mathrm{y}^{*^{++}}$ & $\mathbf{y}^{0}$ & $y^{0++}$ & \# \\
\hline 1 & 102.0550 & 51.5311 & & & 84.0444 & 42.5258 & $\mathbf{T}$ & & & & & & & 15 \\
\hline 2 & 217.0819 & 109.0446 & 200.0553 & 100.5313 & 199.0713 & 100.0393 & $\mathbf{N}$ & 1476.6449 & 738.8261 & 1459.6183 & 730.3128 & 1458.6343 & 729.8208 & 14 \\
\hline 3 & 274.1034 & 137.5553 & 257.0768 & 129.0420 & 256.0928 & 128.5500 & $\mathbf{G}$ & 1361.6179 & 681.3126 & 1344.5914 & 672.7993 & 1343.6074 & 672.3073 & 13 \\
\hline 4 & 361.1354 & 181.0713 & 344.1088 & 172.5581 & 343.1248 & 172.0660 & S & 1304.5965 & 652.8019 & 1287.5699 & 644.2886 & 1286.5859 & 643.7966 & 12 \\
\hline 5 & 460.2038 & 230.6055 & 443.1772 & 222.0923 & 442.1932 & 221.6003 & $\mathbf{V}$ & 1217.5644 & 609.2859 & 1200.5379 & 600.7726 & 1199.5539 & 600.2806 & 11 \\
\hline 6 & 575.2307 & 288.1190 & 558.2042 & 279.6057 & 557.2202 & 279.1137 & D & 1118.4960 & 559.7516 & 1101.4695 & 551.2384 & 1100.4855 & 550.7464 & 10 \\
\hline 7 & 688.3148 & 344.6610 & 671.2883 & 336.1478 & 670.3042 & 335.6558 & $\mathbf{L}$ & 1003.4691 & 502.2382 & 986.4425 & 493.7249 & 985.4585 & 493.2329 & 9 \\
\hline 8 & 745.3363 & 373.1718 & 728.3097 & 364.6585 & 727.3257 & 364.1665 & $\mathbf{G}$ & 890.3850 & 445.6961 & 873.3585 & 437.1829 & 872.3745 & 436.6909 & 8 \\
\hline 9 & 874.3789 & 437.6931 & 857.3523 & 429.1798 & 856.3683 & 428.6878 & $\mathbf{E}$ & 833.3636 & 417.1854 & 816.3370 & 408.6721 & 815.3530 & 408.1801 & 7 \\
\hline 10 & 1003.4215 & 502.2144 & 986.3949 & 493.7011 & 985.4109 & 493.2091 & $\mathbf{E}$ & 704.3210 & 352.6641 & 687.2944 & 344.1508 & 686.3104 & 343.6588 & 6 \\
\hline 11 & 1132.4640 & 566.7357 & 1115.4375 & 558.2224 & 1114.4535 & 557.7304 & $\mathbf{E}$ & 575.2784 & 288.1428 & 558.2518 & 279.6295 & 557.2678 & 279.1375 & 5 \\
\hline 12 & 1261.5066 & 631.2570 & 1244.4801 & 622.7437 & 1243.4961 & 622.2517 & $\mathbf{E}$ & 446.2358 & 223.6215 & 429.2092 & 215.1083 & 428.2252 & 214.6162 & 4 \\
\hline 13 & 1332.5438 & 666.7755 & 1315.5172 & 658.2622 & 1314.5332 & 657.7702 & A & 317.1932 & 159.1002 & 300.1666 & 150.5870 & & & 3 \\
\hline 14 & 1403.5809 & 702.2941 & 1386.5543 & 693.7808 & 1385.5703 & 693.2888 & A & 246.1561 & 123.5817 & 229.1295 & 115.0684 & & & 2 \\
\hline 15 & & & & & & & $\mathbf{R}$ & 175.1190 & 88.0631 & 158.0924 & 79.5498 & & & 1 \\
\hline
\end{tabular}



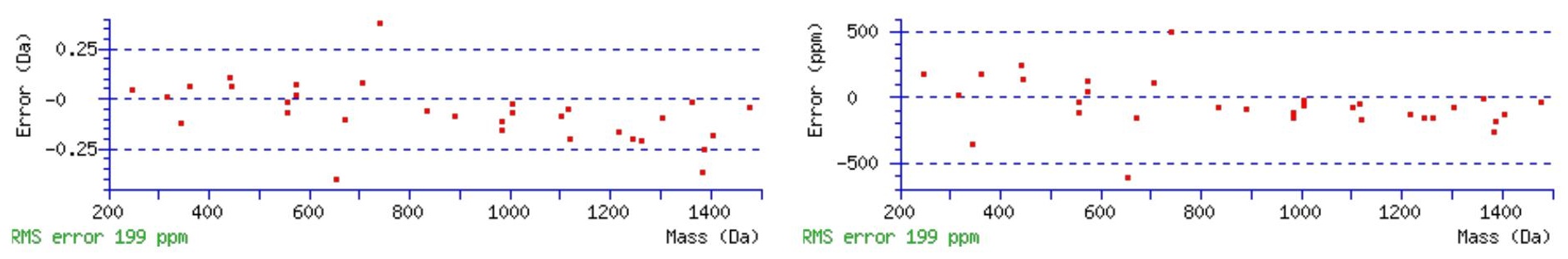

\section{All matches to this query}

\begin{tabular}{|l|l|l|l|}
\hline Score & Mr(calc): & Delta & \multicolumn{1}{|c|}{ Sequence } \\
\hline 114.0 & 1576.6852 & -0.0056 & TNGSVDLGEEEEAAR \\
\hline 9.6 & 1576.6658 & 0.0139 & STQSVYQENLAEK \\
\hline 9.6 & 1576.6658 & 0.0139 & STQSVYQENLAEK \\
\hline 4.7 & 1576.6844 & -0.0047 & MKAEDGENYAIKK \\
\hline 4.5 & 1576.6658 & 0.0139 & STQSVYQENLAEK \\
\hline 4.5 & 1576.6658 & 0.0139 & STQSVYQENLAEK \\
\hline 2.4 & 1576.6891 & -0.0095 & CSFIVRDCVSVR \\
\hline 2.0 & 1576.6770 & 0.0026 & SPEQIESHEVTNK \\
\hline 1.3 & 1576.6779 & 0.0017 & NTPFMGIMDKTAR \\
\hline 1.3 & 1576.6779 & 0.0017 & NTPFMGIMDKTAR \\
\hline
\end{tabular}

Spectrum No: 29; Query: 1377; Rank: 1

\section{Peptide View}

MS/MS Fragmentation of FTSDTSVAGTGFNASFHK

Found in IPI00196620, Tax_Id=10116 Gene_Symbol=Cubn Cubilin precursor

Match to Query 1377: 1873.852428 from(937.933490,2+)

Title: 100101RatKid_NS_deglyco_13.2504.2504.2.dta

Data file K:INewmanPaper|Piliangl3SubProteomes\Piliang3SP\mgf5ppm\ERLIC_3SubProteomes5ppm.mgf

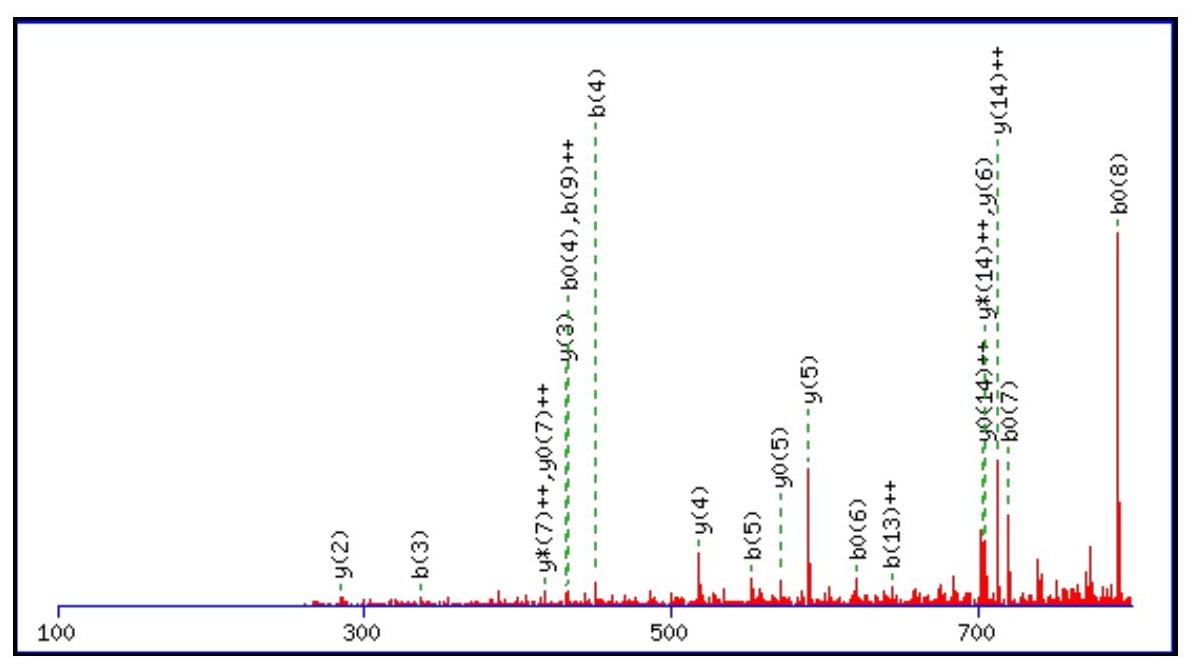



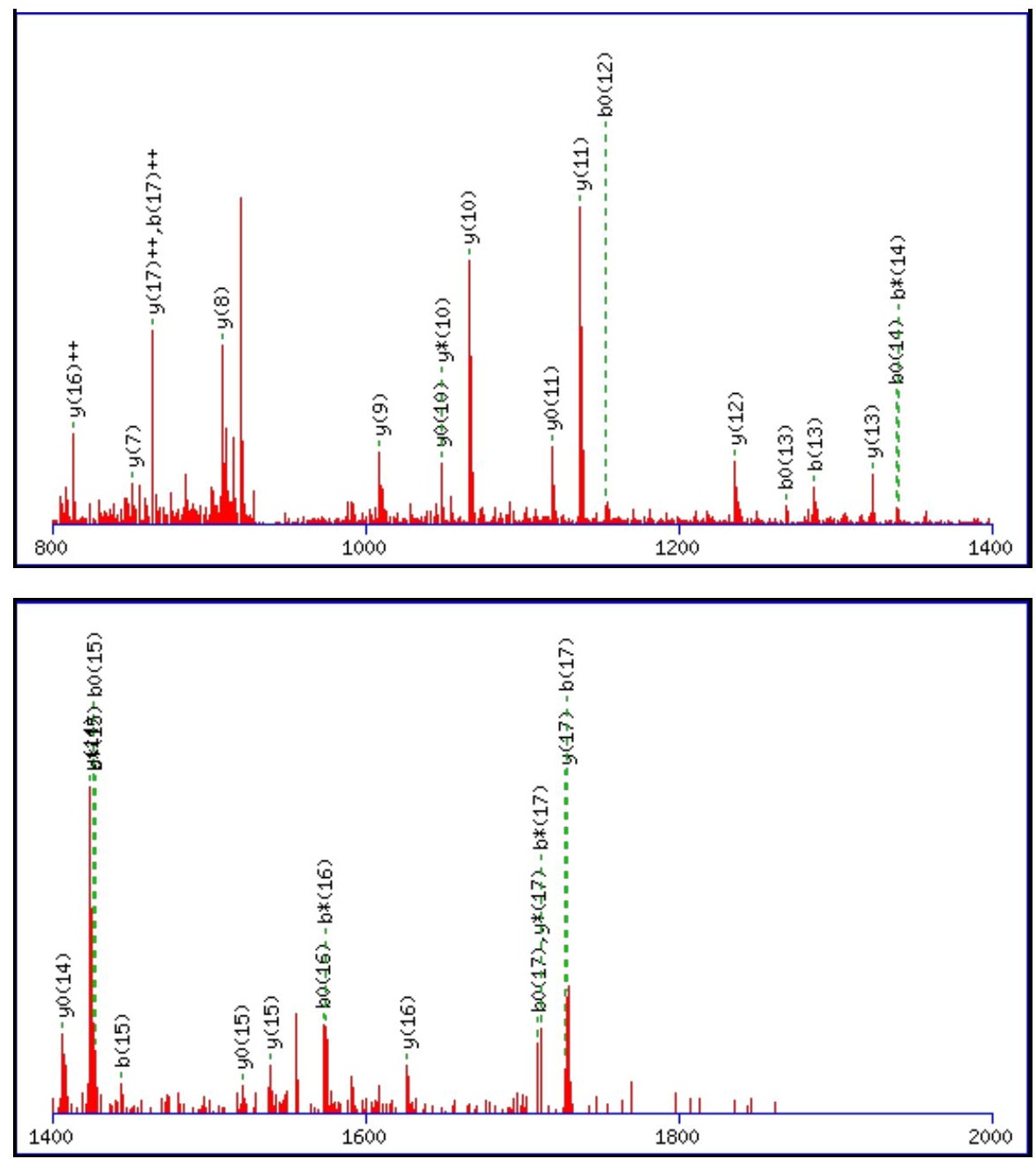

Monoisotopic mass of neutral peptide $\operatorname{Mr}($ calc): 1873.8483

Fixed modifications: Carbamidomethyl (C)

Variable modifications:

N13 : Deamidated $\mathrm{N}(\mathrm{N})$

Ions Score: 114 Expect: $8.9 \mathrm{e}-010$

Matches (Bold Red): 53/172 fragment ions using 95 most intense peaks

\begin{tabular}{|c|c|c|c|c|c|c|c|c|c|c|c|c|c|c|}
\hline \# & b & $\mathbf{b}^{++}$ & b* & $\mathbf{b}^{*^{++}}$ & $\mathbf{b}^{0}$ & $\mathbf{b}^{0++}$ & Seq. & $\mathbf{y}$ & $\mathbf{y}^{++}$ & $\mathbf{y}^{*}$ & $\mathbf{y}^{*^{++}}$ & $\mathbf{y}^{0}$ & $y^{0++}$ & \# \\
\hline 1 & 148.0757 & 74.5415 & & & & & $\mathbf{F}$ & & & & & & & 18 \\
\hline 2 & 249.1234 & 125.0653 & & & 231.1128 & 116.0600 & $\mathbf{T}$ & 1727.7871 & 864.3972 & 1710.7606 & 855.8839 & 1709.7765 & 855.3919 & 17 \\
\hline 3 & 336.1554 & 168.5813 & & & 318.1448 & 159.5761 & $\mathbf{S}$ & 1626.7394 & 813.8734 & 1609.7129 & 805.3601 & 1608.7289 & 804.8681 & 16 \\
\hline 4 & 451.1823 & 226.0948 & & & 433.1718 & 217.0895 & D & 1539.7074 & 770.3573 & 1522.6809 & 761.8441 & 1521.6968 & 761.3521 & 15 \\
\hline 5 & 552.2300 & 276.6186 & & & 534.2195 & 267.6134 & $\mathbf{T}$ & 1424.6805 & 712.8439 & 1407.6539 & 704.3306 & 1406.6699 & 703.8386 & 14 \\
\hline 6 & 639.2620 & 320.1347 & & & 621.2515 & 311.1294 & S & 1323.6328 & 662.3200 & 1306.6062 & 653.8068 & 1305.6222 & 653.3147 & 13 \\
\hline 7 & 738.3305 & 369.6689 & & & 720.3199 & 360.6636 & $\mathbf{V}$ & 1236.6008 & 618.8040 & 1219.5742 & 610.2907 & 1218.5902 & 609.7987 & 12 \\
\hline 8 & 809.3676 & 405.1874 & & & 791.3570 & 396.1821 & A & 1137.5323 & 569.2698 & 1120.5058 & 560.7565 & 1119.5218 & 560.2645 & 11 \\
\hline 9 & 866.3890 & 433.6982 & & & 848.3785 & 424.6929 & G & 1066.4952 & 533.7513 & 1049.4687 & 525.2380 & 1048.4847 & 524.7460 & 10 \\
\hline 10 & 967.4367 & 484.2220 & & & 949.4262 & 475.2167 & $\mathbf{T}$ & 1009.4738 & 505.2405 & 992.4472 & 496.7272 & 991.4632 & 496.2352 & 9 \\
\hline 11 & 1024.4582 & 512.7327 & & & 1006.4476 & 503.7274 & G & 908.4261 & 454.7167 & 891.3995 & 446.2034 & 890.4155 & 445.7114 & 8 \\
\hline 12 & 1171.5266 & 586.2669 & & & 1153.5160 & 577.2617 & $\mathbf{F}$ & 851.4046 & 426.2059 & 834.3781 & 417.6927 & 833.3941 & 417.2007 & 7 \\
\hline 13 & 1286.5535 & 643.7804 & 1269.5270 & 635.2671 & 1268.5430 & 634.7751 & $\mathbf{N}$ & 704.3362 & 352.6717 & 687.3097 & 344.1585 & 686.3256 & 343.6665 & 6 \\
\hline 14 & 1357.5906 & 679.2990 & 1340.5641 & 670.7857 & 1339.5801 & 670.2937 & A & 589.3093 & 295.1583 & 572.2827 & 286.6450 & 571.2987 & 286.1530 & 5 \\
\hline 15 & 1444.6227 & 722.8150 & 1427.5961 & 714.3017 & 1426.6121 & 713.8097 & $\mathrm{~S}$ & 518.2722 & 259.6397 & 501.2456 & 251.1264 & 500.2616 & 250.6344 & 4 \\
\hline 16 & 1591.6911 & 796.3492 & 1574.6645 & 787.8359 & 1573.6805 & 787.3439 & $\mathbf{F}$ & 431.2401 & 216.1237 & 414.2136 & 207.6104 & & & 3 \\
\hline 17 & 1728.7500 & 864.8786 & 1711.7235 & 856.3654 & 1710.7394 & 855.8734 & H & 284.1717 & 142.5895 & 267.1452 & 134.0762 & & & 2 \\
\hline 18 & & & & & & & $\mathbf{K}$ & 147.1128 & 74.0600 & 130.0863 & 65.5468 & & & 1 \\
\hline
\end{tabular}



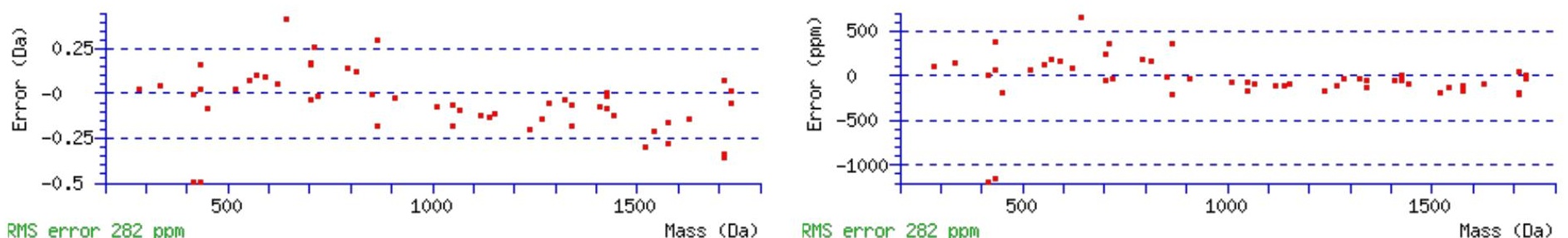

RMS error 282 ppm

Mass (Da) RMS error 282 ppm

\section{All matches to this query}

\begin{tabular}{|l|l|l|l|}
\hline Score & Mr(calc): & Delta & \multicolumn{1}{c|}{ Sequence } \\
\hline 114.0 & 1873.8483 & 0.0041 & FTSDTSVAGTGFNASFHK \\
\hline 14.3 & 1872.8643 & 0.9882 & FTSDTSVAGTGFNASFHK \\
\hline 7.3 & 1872.8492 & 1.0032 & MERTSVMERTSVMER \\
\hline 4.3 & 1873.8628 & -0.0104 & EFYRGRCVLNEDSTK \\
\hline 2.8 & 1873.8628 & -0.0104 & APSTPVPPSPAPTPGLTK \\
\hline 2.1 & 1871.8349 & 2.0175 & MSHPSPAGKPSNSKNPR \\
\hline 1.2 & 1872.8492 & 1.0032 & MERTSVMERTSVMER \\
\hline
\end{tabular}

Spectrum No: 30; Query: 2355; Rank: 1

\section{Peptide View}

MS/MS Fragmentation of YEVDWNQSTLDCVDPLSSLAADR

Found in IPI00188881, Tax_Id=10116 Gene_Symbol=Slc22a2 Organic cation transporter OCT2

Match to Query 2355: 2654.186168 from(1328.100360,2+)

Title: 100101RatKid_NS_deglyco_25.4833.4833.2.dta

Data file K:\NewmanPaper\Piliang \3SubProteomes\Piliang3SP \mgf5ppm\ERLIC_3SubProteomes5ppm.mgf

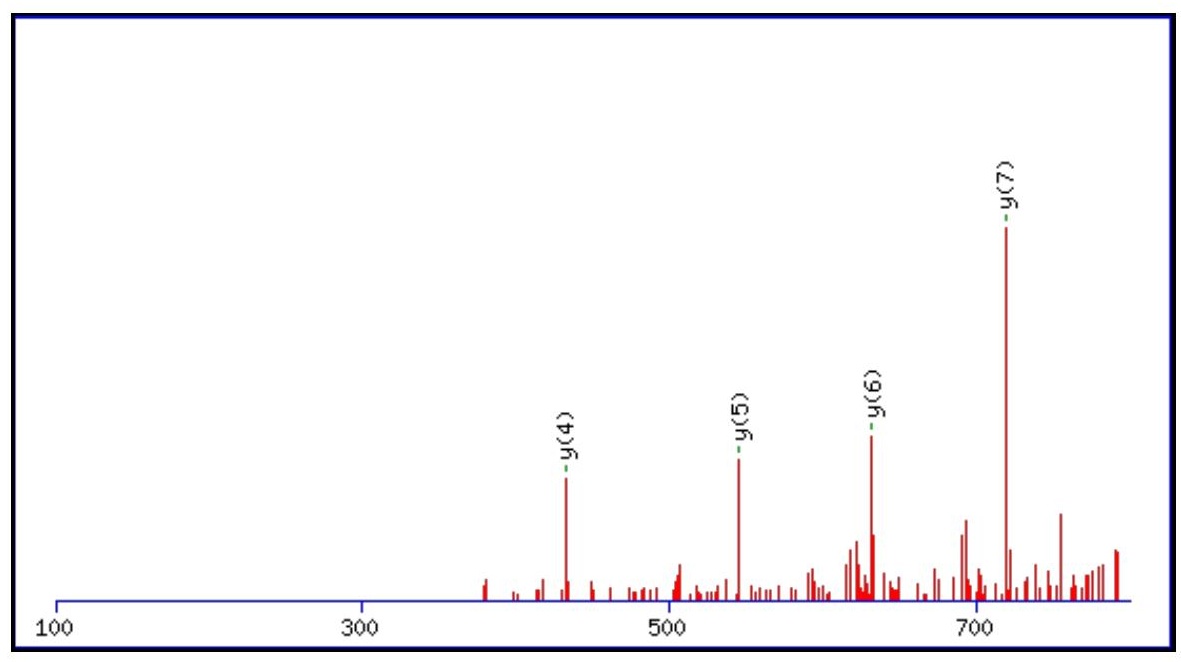



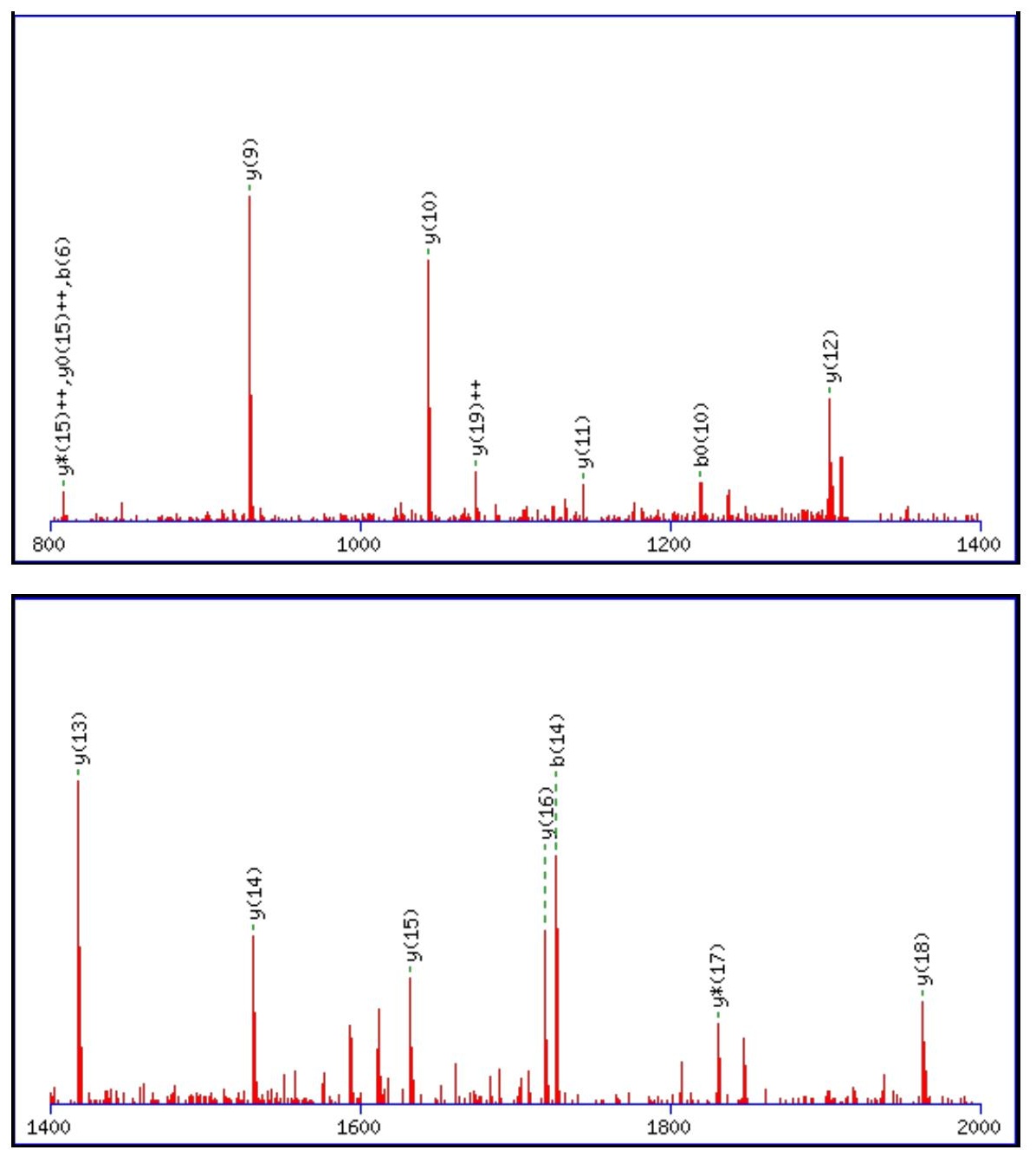

Monoisotopic mass of neutral peptide $\operatorname{Mr}($ calc): 2654.1806

Fixed modifications: Carbamidomethyl (C)

Variable modifications:

N6 : Deamidated $\mathrm{N}(\mathrm{N})$

Ions Score: 113 Expect: $1.6 \mathrm{e}-009$

Matches (Bold Red): 20/250 fragment ions using 24 most intense peaks

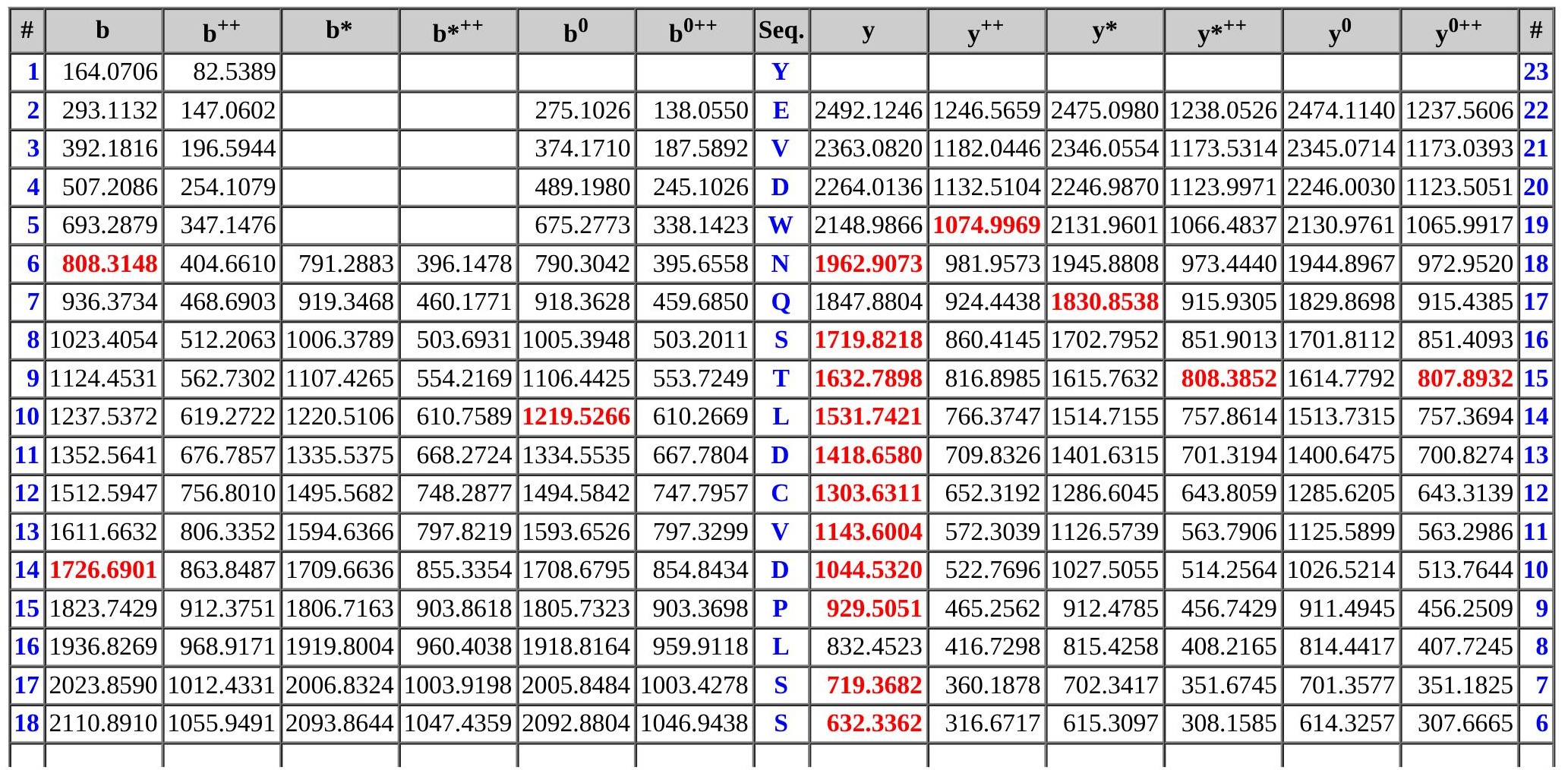




\begin{tabular}{|l|r|r|r|r|r|r|r|r|r|r|r|r|r|r|}
$\mathbf{1 9}$ & 2223.9751 & 1112.4912 & 2206.9485 & 1103.9779 & 2205.9645 & 1103.4859 & L & 545.3042 & 273.1557 & 528.2776 & 264.6425 & 527.2936 & 264.1504 & $\mathbf{5}$ \\
\hline $\mathbf{2 0}$ & 2295.0122 & 1148.0097 & 2277.9856 & 1139.4964 & 2277.0016 & 1139.0044 & A & 432.2201 & 216.6137 & 415.1936 & 208.1004 & 414.2096 & 207.6084 & $\mathbf{4}$ \\
\hline $\mathbf{2 1}$ & 2366.0493 & 1183.5283 & 2349.0227 & 1175.0150 & 2348.0387 & 1174.5230 & A & 361.1830 & 181.0951 & 344.1565 & 172.5819 & 343.1724 & 172.0899 & $\mathbf{3}$ \\
\hline $\mathbf{2 2}$ & 2481.0762 & 1241.0417 & 2464.0497 & 1232.5285 & 2463.0657 & 1232.0365 & D & 290.1459 & 145.5766 & 273.1193 & 137.0633 & 272.1353 & 136.5713 & $\mathbf{2}$ \\
\hline $\mathbf{2 3}$ & & & & & & & R & 175.1190 & 88.0631 & 158.0924 & 79.5498 & & & $\mathbf{1}$ \\
\hline
\end{tabular}
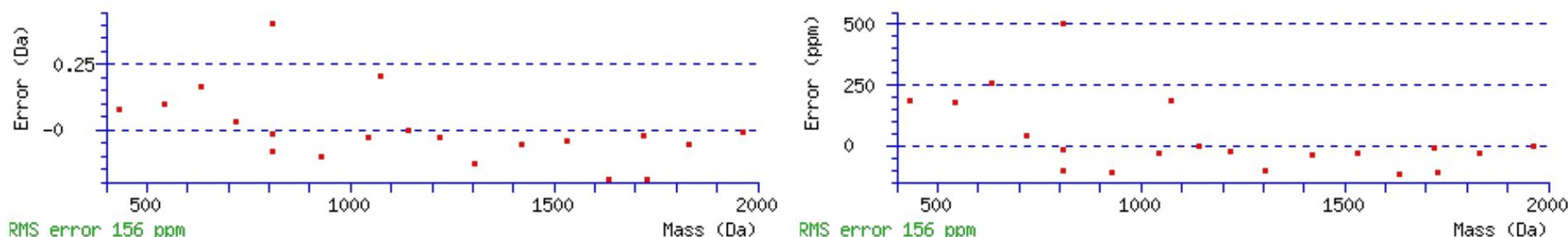

\section{All matches to this query}

\begin{tabular}{|l|l|l|l|}
\hline Score & Mr(calc): & Delta & \multicolumn{1}{c|}{ Sequence } \\
\hline 113.4 & 2654.1806 & 0.0056 & YEVDWNQSTLDCVDPLSSLAADR \\
\hline 85.8 & 2653.1966 & 0.9896 & YEVDWNQSTLDCVDPLSSLAADR \\
\hline 3.4 & 2653.2014 & 0.9847 & VISLFYTVVTPMFNPMVYSLR \\
\hline 2.8 & 2652.1737 & 2.0125 & SISIAALLDVNKEEPNKELNSK \\
\hline 2.8 & 2652.1737 & 2.0125 & SISIAALLDVNKEEPNKELNSK \\
\hline 2.8 & 2653.1577 & 1.0285 & SISIAALLDVNKEEPNKELNSK \\
\hline 2.8 & 2652.1737 & 2.0125 & SISIAALLDVNKEEPNKELNSK \\
\hline 2.8 & 2653.1577 & 1.0285 & SISIAALLDVNKEEPNKELNSK \\
\hline 2.8 & 2653.1577 & 1.0285 & SISIAALLDVNKEEPNKELNSK \\
\hline 0.8 & 2653.2056 & 0.9806 & TMSKSDMLNALEEVINNPFYKK \\
\hline
\end{tabular}

Spectrum No: 31; Query: 1004; Rank: 1

\section{Peptide View}

MS/MS Fragmentation of TANETSAEAYNLLLR

Found in IPI00363849, Tax_Id=10116 Gene_Symbol=Lamc1 similar to Laminin gamma-1 chain precursor

Match to Query 1004: 1665.820168 from(833.917360,2+)

Title: 091008RatKidney_NH4Format01_23.2929.2929.2.dta

Data file K:INewmanPaper|Piliang|3SubProteomes\Piliang3SP\mgf5ppm\ERLIC_3SubProteomes5ppm.mgf

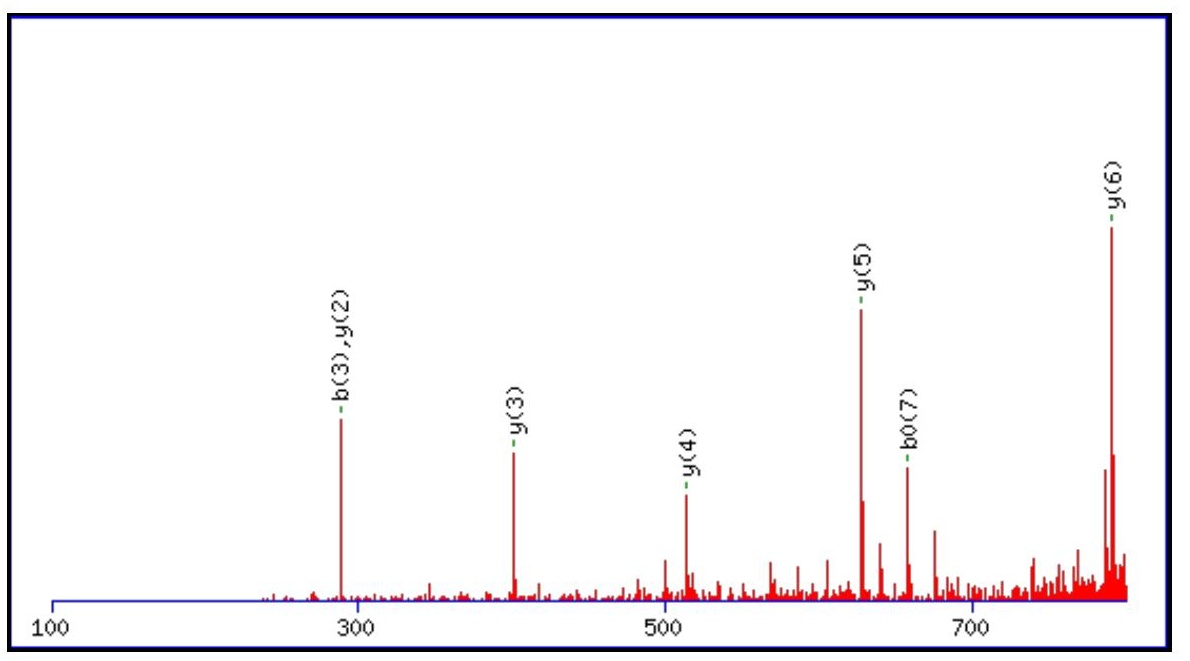



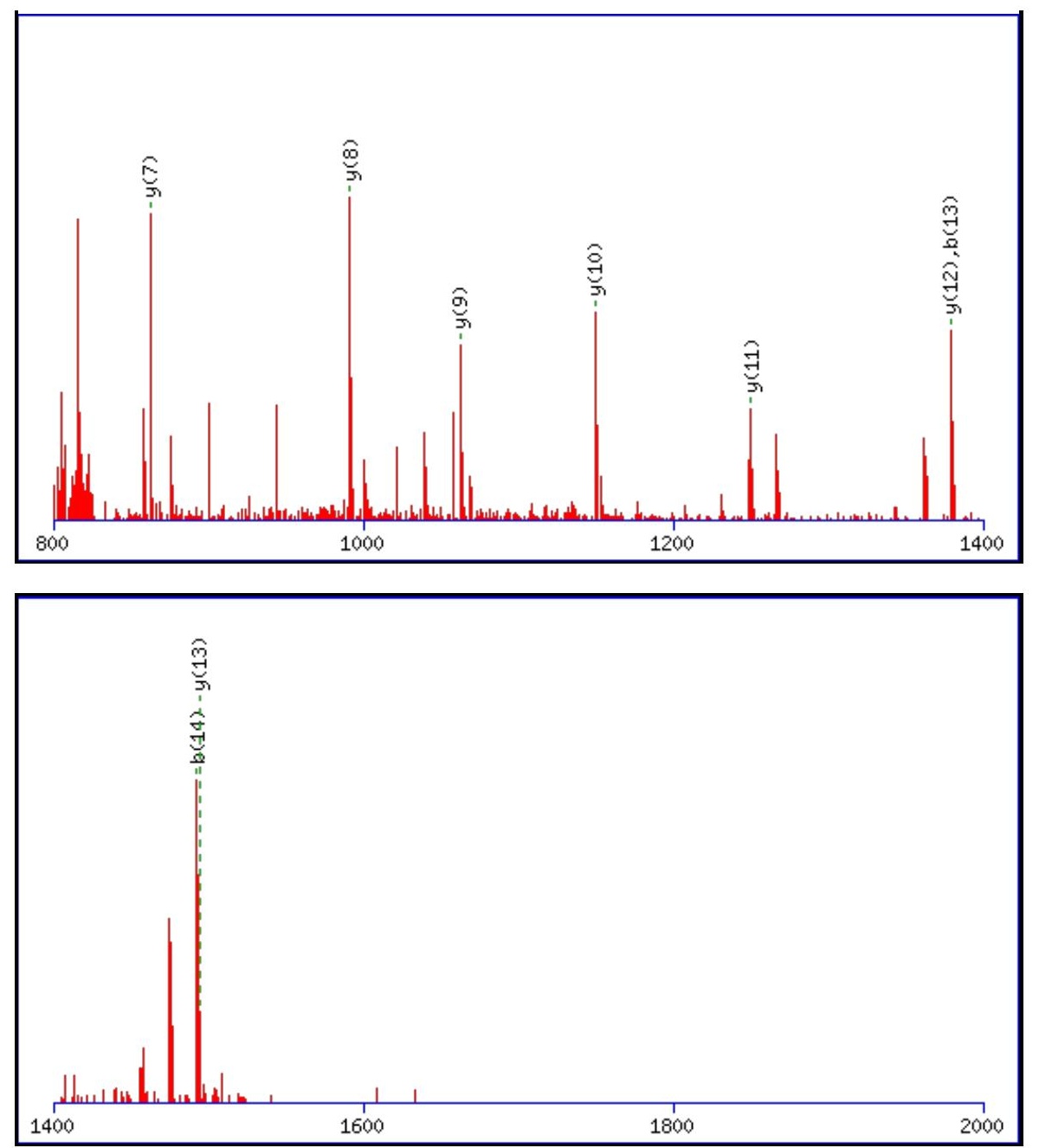

Monoisotopic mass of neutral peptide $\operatorname{Mr}($ calc): 1665.8209

Fixed modifications: Carbamidomethyl (C)

Variable modifications:

N3 : Deamidated $\mathrm{N}(\mathrm{N})$

Ions Score: 113 Expect: $8.3 \mathrm{e}-010$

Matches (Bold Red): 16/150 fragment ions using 19 most intense peaks

\begin{tabular}{|c|c|c|c|c|c|c|c|c|c|c|c|c|c|c|}
\hline \# & b & $\mathbf{b}^{++}$ & $\mathbf{b}^{*}$ & $\mathbf{b}^{*^{++}}$ & $\mathbf{b}^{0}$ & $\mathbf{b}^{0++}$ & Seq. & $\mathbf{y}$ & $\mathbf{y}^{++}$ & $\mathbf{y}^{*}$ & $\mathbf{y}^{*^{++}}$ & $\mathbf{y}^{\mathbf{0}}$ & $\mathbf{y}^{\mathbf{0 + +}}$ & \# \\
\hline 1 & 102.0550 & 51.5311 & & & 84.0444 & 42.5258 & $\mathbf{T}$ & & & & & & & 15 \\
\hline 2 & 173.0921 & 87.0497 & & & 155.0815 & 78.0444 & A & 1565.7806 & 783.3939 & 1548.7540 & 774.8806 & 1547.7700 & 774.3886 & 14 \\
\hline 3 & 288.1190 & 144.5631 & 271.0925 & 136.0499 & 270.1084 & 135.5579 & $\mathbf{N}$ & 1494.7435 & 747.8754 & 1477.7169 & 739.3621 & 1476.7329 & 738.8701 & 13 \\
\hline 4 & 417.1616 & 209.0844 & 400.1350 & 200.5712 & 399.1510 & 200.0792 & $\mathbf{E}$ & 1379.7165 & 690.3619 & 1362.6900 & 681.8486 & 1361.7060 & 681.3566 & 12 \\
\hline 5 & 518.2093 & 259.6083 & 501.1827 & 251.0950 & 500.1987 & 250.6030 & $\mathbf{T}$ & 1250.6739 & 625.8406 & 1233.6474 & 617.3273 & 1232.6634 & 616.8353 & 11 \\
\hline 6 & 605.2413 & 303.1243 & 588.2148 & 294.6110 & 587.2307 & 294.1190 & S & 1149.6262 & 575.3168 & 1132.5997 & 566.8035 & 1131.6157 & 566.3115 & 10 \\
\hline 7 & 676.2784 & 338.6428 & 659.2519 & 330.1296 & 658.2679 & 329.6376 & A & 1062.5942 & 531.8007 & 1045.5677 & 523.2875 & 1044.5837 & 522.7955 & 9 \\
\hline 8 & 805.3210 & 403.1641 & 788.2945 & 394.6509 & 787.3104 & 394.1589 & $\mathbf{E}$ & 991.5571 & 496.2822 & 974.5306 & 487.7689 & 973.5465 & 487.2769 & 8 \\
\hline 9 & 876.3581 & 438.6827 & 859.3316 & 430.1694 & 858.3476 & 429.6774 & A & 862.5145 & 431.7609 & 845.4880 & 423.2476 & & & 7 \\
\hline 10 & 1039.4215 & 520.2144 & 1022.3949 & 511.7011 & 1021.4109 & 511.2091 & $\mathbf{Y}$ & 791.4774 & 396.2423 & 774.4509 & 387.7291 & & & 6 \\
\hline 11 & 1153.4644 & 577.2358 & 1136.4378 & 568.7226 & 1135.4538 & 568.2305 & $\mathbf{N}$ & 628.4141 & 314.7107 & 611.3875 & 306.1974 & & & 5 \\
\hline 12 & 1266.5484 & 633.7779 & 1249.5219 & 625.2646 & 1248.5379 & 624.7726 & $\mathbf{L}$ & 514.3711 & 257.6892 & 497.3446 & 249.1759 & & & 4 \\
\hline 13 & 1379.6325 & 690.3199 & 1362.6060 & 681.8066 & 1361.6219 & 681.3146 & $\mathbf{L}$ & 401.2871 & 201.1472 & 384.2605 & 192.6339 & & & 3 \\
\hline 14 & 1492.7166 & 746.8619 & 1475.6900 & 738.3487 & 1474.7060 & 737.8566 & $\mathbf{L}$ & 288.2030 & 144.6051 & 271.1765 & 136.0919 & & & 2 \\
\hline 15 & & & & & & & $\mathbf{R}$ & 175.1190 & 88.0631 & 158.0924 & 79.5498 & & & 1 \\
\hline
\end{tabular}



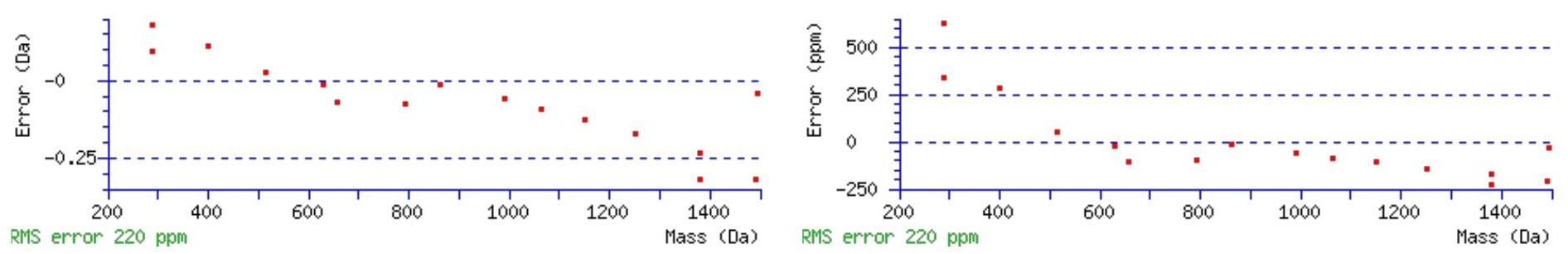

\section{All matches to this query}

\begin{tabular}{|l|l|l|l|}
\hline Score & Mr(calc): & Delta & \multicolumn{1}{|c|}{ Sequence } \\
\hline 113.2 & 1665.8209 & -0.0008 & TANETSAEAYNLLLR \\
\hline 66.1 & 1665.8209 & -0.0008 & TANETSAEAYNLLLR \\
\hline 19.9 & 1665.8087 & 0.0115 & NNSTPLNISETKLR \\
\hline 12.0 & 1665.8199 & 0.0002 & DGKQQTNQTKGLLR \\
\hline 8.8 & 1664.8022 & 1.0180 & GDVENTILDILGGLR \\
\hline 7.7 & 1665.8087 & 0.0115 & EIEQKDSEIARLR \\
\hline 7.5 & 1665.8352 & -0.0150 & SVSRSLWRTPELR \\
\hline 7.1 & 1663.8182 & 2.0020 & GDVENTILDILGGLR \\
\hline 5.9 & 1665.8239 & -0.0037 & NPDYRSNKPIILR \\
\hline 5.3 & 1665.8239 & -0.0037 & NPDYRSNKPIILR \\
\hline
\end{tabular}

Spectrum No: 32; Query: 1653; Rank: 1

\section{Peptide View}

MS/MS Fragmentation of ADNLYSQVVGLSAAQANLSK

Found in IPI00204504, Tax_Id=10116 Gene_Symbol=Plvap Plasmalemma vesicle-associated protein

Match to Query 1653: 2049.044068 from(1025.529310,2+)

Title: 100101RatKid_NS_deglyco_08.4414.4414.2.dta

Data file K:INewmanPaper|Piliangl3SubProteomes\Piliang3SP\mgf5ppm\ERLIC_3SubProteomes5ppm.mgf

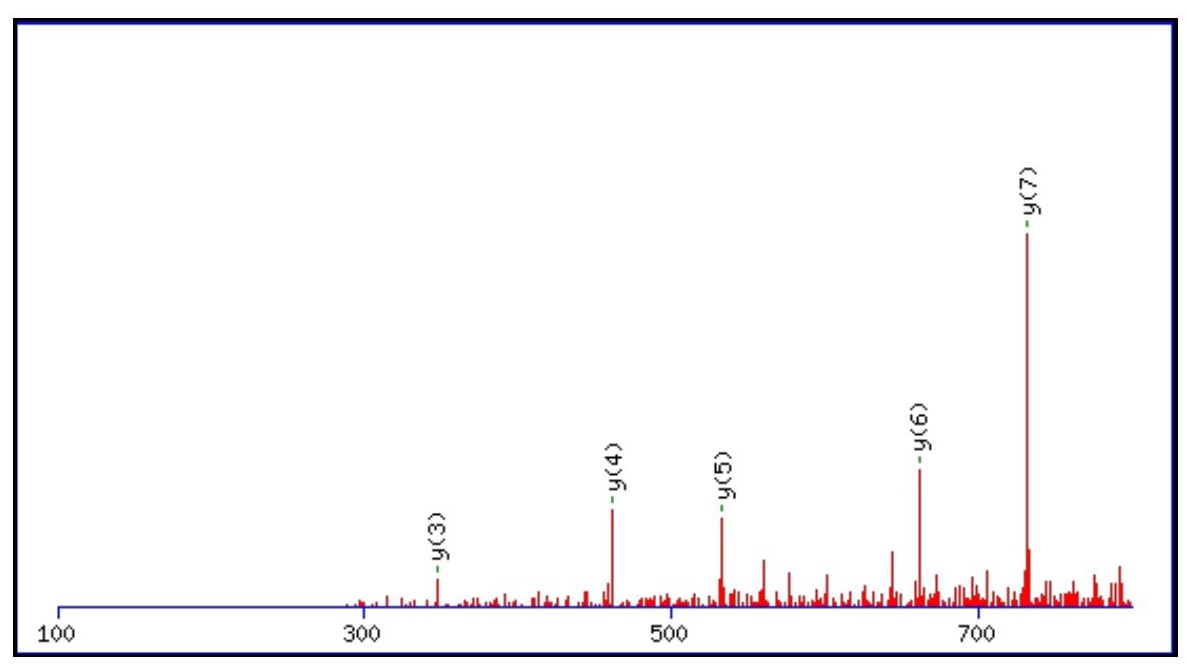



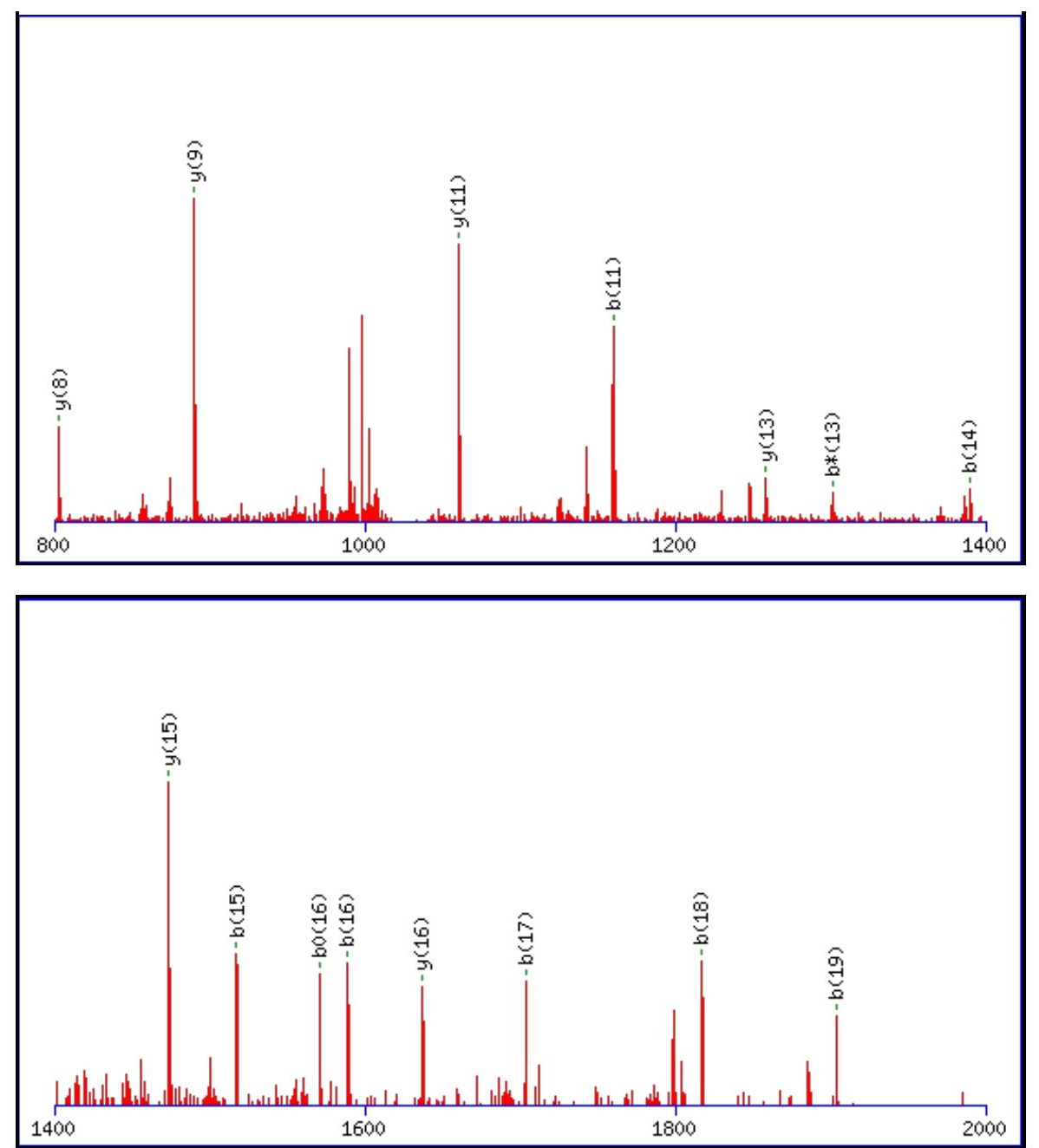

Monoisotopic mass of neutral peptide $\operatorname{Mr}($ calc): 2049.0378

Fixed modifications: Carbamidomethyl (C)

Variable modifications:

N17 : Deamidated $\mathrm{N}(\mathrm{N})$

Ions Score: 113 Expect: $9.2 \mathrm{e}-010$

Matches (Bold Red): 20/220 fragment ions using 23 most intense peaks

\begin{tabular}{|c|c|c|c|c|c|c|c|c|c|c|c|c|c|c|}
\hline \# & b & $\mathbf{b}^{++}$ & b* & $\mathbf{b}^{*^{++}}$ & $\mathbf{b}^{0}$ & $\mathbf{b}^{0++}$ & Seq. & $\mathbf{y}$ & $\mathbf{y}^{++}$ & $\mathbf{y}^{*}$ & $\mathrm{y}^{*^{++}}$ & $\mathbf{y}^{0}$ & $y^{0++}$ & \# \\
\hline 1 & 72.0444 & 36.5258 & & & & & A & & & & & & & 20 \\
\hline 2 & 187.0713 & 94.0393 & & & 169.0608 & 85.0340 & D & 1979.0080 & 990.0076 & 1961.9815 & 981.4944 & 1960.9974 & 981.0024 & 19 \\
\hline 3 & 301.1143 & 151.0608 & 284.0877 & 142.5475 & 283.1037 & 142.0555 & $\mathbf{N}$ & 1863.9811 & 932.4942 & 1846.9545 & 923.9809 & 1845.9705 & 923.4889 & 18 \\
\hline 4 & 414.1983 & 207.6028 & 397.1718 & 199.0895 & 396.1878 & 198.5975 & $\mathbf{L}$ & 1749.9381 & 875.4727 & 1732.9116 & 866.9594 & 1731.9276 & 866.4674 & 17 \\
\hline 5 & 577.2617 & 289.1345 & 560.2351 & 280.6212 & 559.2511 & 280.1292 & $\mathbf{Y}$ & 1636.8541 & 818.9307 & 1619.8275 & 810.4174 & 1618.8435 & 809.9254 & 16 \\
\hline 6 & 664.2937 & 332.6505 & 647.2671 & 324.1372 & 646.2831 & 323.6452 & S & 1473.7907 & 737.3990 & 1456.7642 & 728.8857 & 1455.7802 & 728.3937 & 15 \\
\hline 7 & 792.3523 & 396.6798 & 775.3257 & 388.1665 & 774.3417 & 387.6745 & $\mathbf{Q}$ & 1386.7587 & 693.8830 & 1369.7322 & 685.3697 & 1368.7481 & 684.8777 & 14 \\
\hline 8 & 891.4207 & 446.2140 & 874.3941 & 437.7007 & 873.4101 & 437.2087 & V & 1258.7001 & 629.8537 & 1241.6736 & 621.3404 & 1240.6896 & 620.8484 & 13 \\
\hline 9 & 990.4891 & 495.7482 & 973.4625 & 487.2349 & 972.4785 & 486.7429 & $\mathbf{V}$ & 1159.6317 & 580.3195 & 1142.6052 & 571.8062 & 1141.6212 & 571.3142 & 12 \\
\hline 10 & 1047.5106 & 524.2589 & 1030.4840 & 515.7456 & 1029.5000 & 515.2536 & G & 1060.5633 & 530.7853 & 1043.5368 & 522.2720 & 1042.5527 & 521.7800 & 11 \\
\hline 11 & 1160.5946 & 580.8009 & 1143.5681 & 572.2877 & 1142.5840 & 571.7957 & $\mathbf{L}$ & 1003.5418 & 502.2746 & 986.5153 & 493.7613 & 985.5313 & 493.2693 & 10 \\
\hline 12 & 1247.6266 & 624.3170 & 1230.6001 & 615.8037 & 1229.6161 & 615.3117 & $\mathrm{~S}$ & 890.4578 & 445.7325 & 873.4312 & 437.2193 & 872.4472 & 436.7272 & 9 \\
\hline 13 & 1318.6638 & 659.8355 & 1301.6372 & 651.3222 & 1300.6532 & 650.8302 & A & 803.4258 & 402.2165 & 786.3992 & 393.7032 & 785.4152 & 393.2112 & 8 \\
\hline 14 & 1389.7009 & 695.3541 & 1372.6743 & 686.8408 & 1371.6903 & 686.3488 & A & 732.3886 & 366.6980 & 715.3621 & 358.1847 & 714.3781 & 357.6927 & 7 \\
\hline 15 & 1517.7594 & 759.3834 & 1500.7329 & 750.8701 & 1499.7489 & 750.3781 & $\mathbf{Q}$ & 661.3515 & 331.1794 & 644.3250 & 322.6661 & 643.3410 & 322.1741 & 6 \\
\hline 16 & 1588.7966 & 794.9019 & 1571.7700 & 786.3886 & 1570.7860 & 785.8966 & A & 533.2929 & 267.1501 & 516.2664 & 258.6368 & 515.2824 & 258.1448 & 5 \\
\hline 17 & 1703.8235 & 852.4154 & 1686.7969 & 843.9021 & 1685.8129 & 843.4101 & $\mathbf{N}$ & 462.2558 & 231.6316 & 445.2293 & 223.1183 & 444.2453 & 222.6263 & 4 \\
\hline 18 & 1816.9076 & 908.9574 & 1799.8810 & 900.4441 & 1798.8970 & 899.9521 & $\mathbf{L}$ & 347.2289 & 174.1181 & 330.2023 & 165.6048 & 329.2183 & 165.1128 & 3 \\
\hline
\end{tabular}




\begin{tabular}{|c|c|c|c|c|c|c|c|c|c|c|c|c|c|c|}
\hline $\mid 19$ & |1903.9396 & 952.4734 & 1886.9130 & 943.9602 & |1885.9290 & 943.4682 & S & 234.1448 & 117.5761 & 217.1183 & |109.0628 & 216.1343 & |108.5708 & 2 \\
\hline 20 & & & & & & & $\mathbf{K}$ & 147.1128 & 74.0600 & 130.0863 & 65.5468 & & & 1 \\
\hline
\end{tabular}
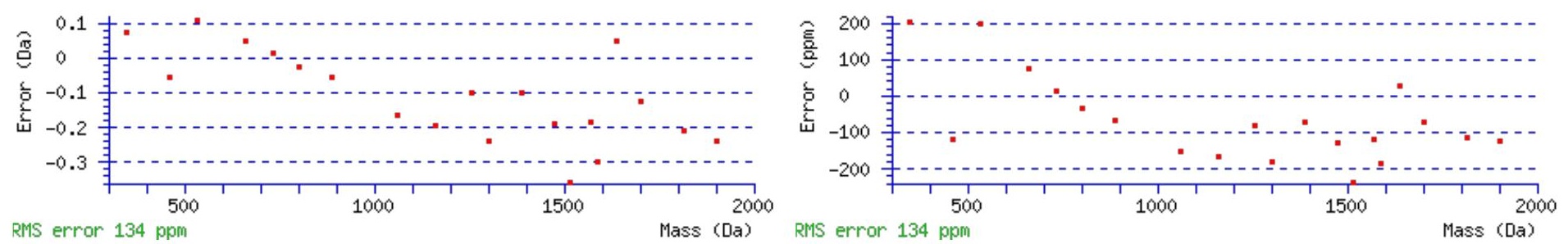

\section{All matches to this query}

\begin{tabular}{|l|l|l|l|}
\hline Score & Mr(calc): & Delta & \multicolumn{1}{c|}{ Sequence } \\
\hline 112.6 & 2049.0378 & 0.0063 & ADNLYSQVVGLSAAQANLSK \\
\hline 28.8 & 2048.0538 & 0.9903 & ADNLYSQVVGLSAAQANLSK \\
\hline 19.1 & 2049.0378 & 0.0063 & ADNLYSQVVGLSAAQANLSK \\
\hline 8.7 & 2047.0474 & 1.9967 & FTATAGIQVVGDDLTVTNPK \\
\hline 3.3 & 2048.0249 & 1.0192 & FTATAGIQVVGDDLTMANPK \\
\hline 1.6 & 2049.0565 & -0.0124 & ELRLYNTGLTEVPSGICK \\
\hline
\end{tabular}

Spectrum No: 33; Query: 590; Rank: 1

\section{Peptide View}

\section{MS/MS Fragmentation of SLGNVNFSVSAEAR}

Found in IPI00201262, Tax_Id=10116 Gene_Symbol=LOC297568;Mug2;Mug1 Alpha-1-inhibitor 3 precursor

Match to Query 590: 1450.708868 from(726.361710,2+)

Title: 100101RatKid_NS_deglyco_21.3904.3904.2.dta

Data file K:INewmanPaper|Piliangl3SubProteomes\Piliang3SP\mgf5ppm\ERLIC_3SubProteomes5ppm.mgf

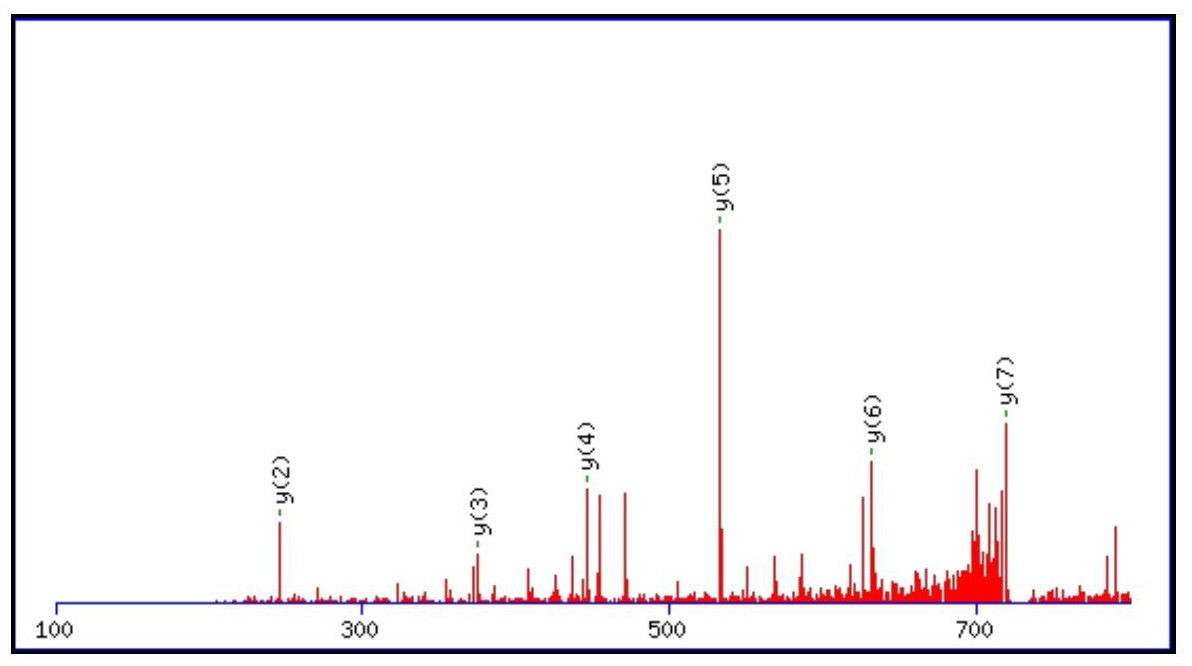



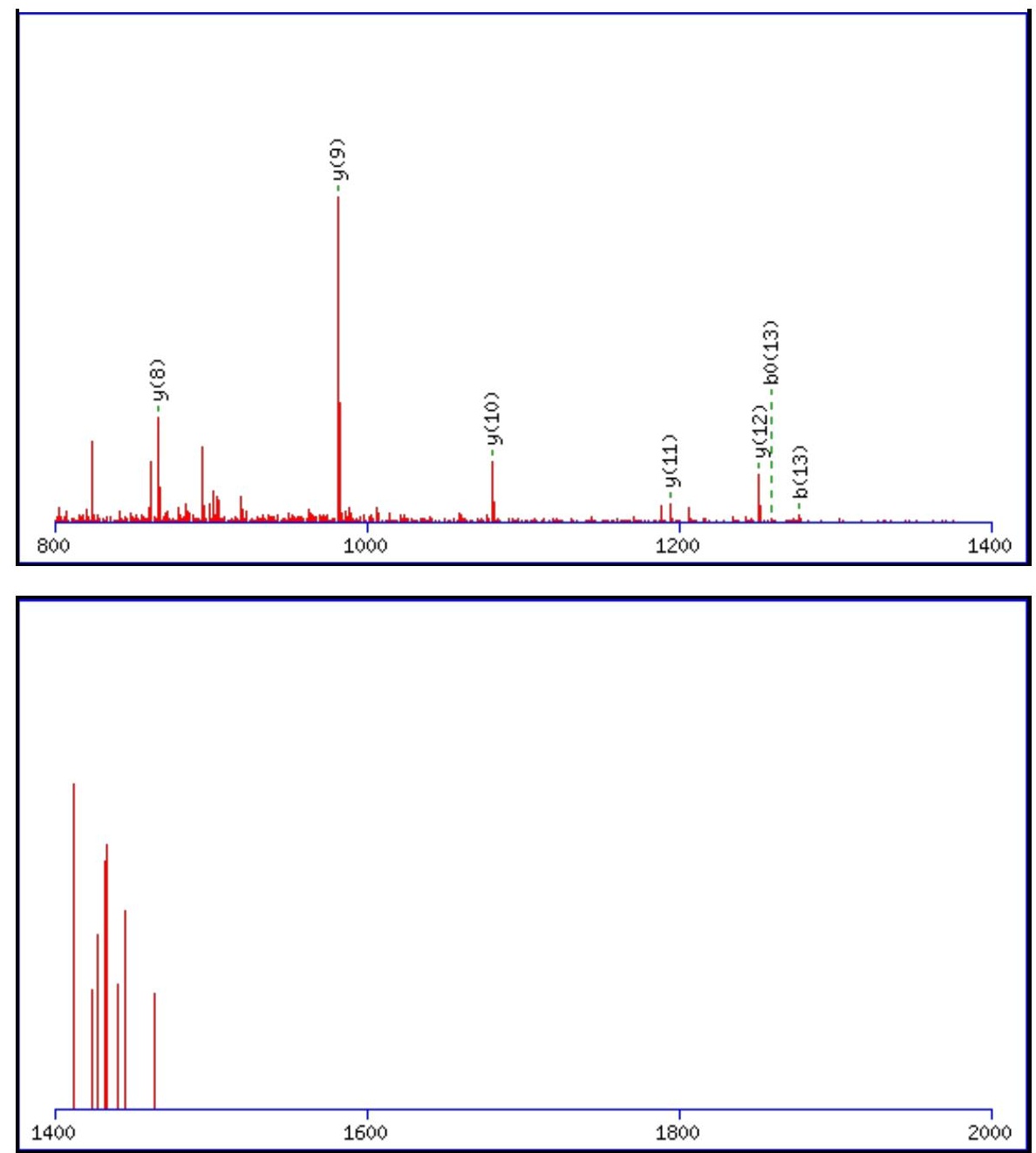

Monoisotopic mass of neutral peptide $\operatorname{Mr}($ calc): 1450.7052

Fixed modifications: Carbamidomethyl (C)

Variable modifications:

N6 : Deamidated $\mathrm{N}(\mathrm{N})$

Ions Score: 112 Expect: $8.8 \mathrm{e}-010$

Matches (Bold Red): 13/146 fragment ions using 22 most intense peaks

\begin{tabular}{|c|c|c|c|c|c|c|c|c|c|c|c|c|c|c|}
\hline \# & b & $\mathbf{b}^{++}$ & $\mathbf{b}^{*}$ & $\mathbf{b}^{*^{++}}$ & $\mathbf{b}^{0}$ & $\mathbf{b}^{0++}$ & Seq. & $\mathbf{y}$ & $y^{++}$ & $\mathbf{y}^{*}$ & $\mathbf{y}^{*^{++}}$ & $\mathbf{y}^{\mathbf{0}}$ & $\mathbf{y}^{0++}$ & \# \\
\hline 1 & 88.0393 & 44.5233 & & & 70.0287 & 35.5180 & S & & & & & & & 14 \\
\hline 2 & 201.1234 & 101.0653 & & & 183.1128 & 92.0600 & $\mathbf{L}$ & 1364.6805 & 682.8439 & 1347.6539 & 674.3306 & 1346.6699 & 673.8386 & 13 \\
\hline 3 & 258.1448 & 129.5761 & & & 240.1343 & 120.5708 & G & 1251.5964 & 626.3018 & 1234.5698 & 617.7886 & 1233.5858 & 617.2966 & 12 \\
\hline 4 & 372.1878 & 186.5975 & 355.1612 & 178.0842 & 354.1772 & 177.5922 & $\mathbf{N}$ & 1194.5749 & 597.7911 & 1177.5484 & 589.2778 & 1176.5644 & 588.7858 & 11 \\
\hline 5 & 471.2562 & 236.1317 & 454.2296 & 227.6185 & 453.2456 & 227.1264 & $\mathbf{V}$ & 1080.5320 & 540.7696 & 1063.5055 & 532.2564 & 1062.5214 & 531.7644 & 10 \\
\hline 6 & 586.2831 & 293.6452 & 569.2566 & 285.1319 & 568.2725 & 284.6399 & $\mathbf{N}$ & 981.4636 & 491.2354 & 964.4370 & 482.7222 & 963.4530 & 482.2302 & 9 \\
\hline 7 & 733.3515 & 367.1794 & 716.3250 & 358.6661 & 715.3410 & 358.1741 & $\mathbf{F}$ & 866.4367 & 433.7220 & 849.4101 & 425.2087 & 848.4261 & 424.7167 & 8 \\
\hline 8 & 820.3836 & 410.6954 & 803.3570 & 402.1821 & 802.3730 & 401.6901 & $\mathrm{~S}$ & 719.3682 & 360.1878 & 702.3417 & 351.6745 & 701.3577 & 351.1825 & 7 \\
\hline 9 & 919.4520 & 460.2296 & 902.4254 & 451.7163 & 901.4414 & 451.2243 & $\mathbf{V}$ & 632.3362 & 316.6717 & 615.3097 & 308.1585 & 614.3256 & 307.6665 & 6 \\
\hline 10 & 1006.4840 & 503.7456 & 989.4574 & 495.2324 & 988.4734 & 494.7404 & $S$ & 533.2678 & 267.1375 & 516.2413 & 258.6243 & 515.2572 & 258.1323 & 5 \\
\hline 11 & 1077.5211 & 539.2642 & 1060.4946 & 530.7509 & 1059.5105 & 530.2589 & A & 446.2358 & 223.6215 & 429.2092 & 215.1082 & 428.2252 & 214.6162 & 4 \\
\hline 12 & 1206.5637 & 603.7855 & 1189.5372 & 595.2722 & 1188.5531 & 594.7802 & $\mathbf{E}$ & 375.1987 & 188.1030 & 358.1721 & 179.5897 & 357.1881 & 179.0977 & 3 \\
\hline 13 & 1277.6008 & 639.3040 & 1260.5743 & 630.7908 & 1259.5902 & 630.2988 & A & 246.1561 & 123.5817 & 229.1295 & 115.0684 & & & 2 \\
\hline 14 & & & & & & & $\mathbf{R}$ & 175.1190 & 88.0631 & 158.0924 & 79.5498 & & & 1 \\
\hline
\end{tabular}



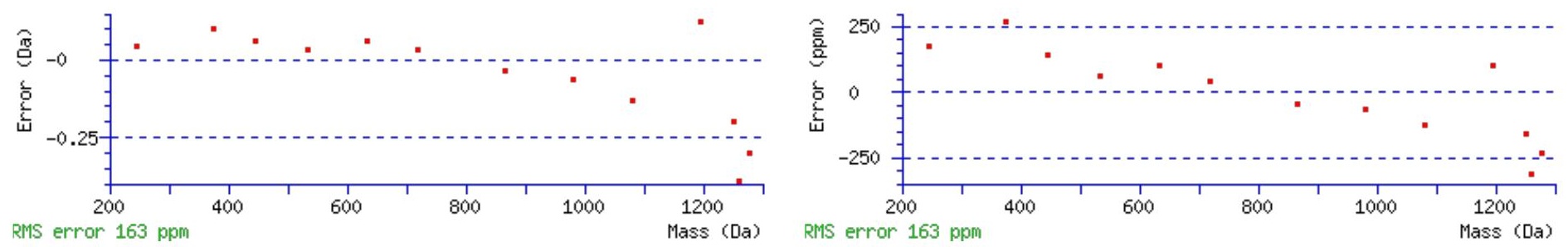

\section{All matches to this query}

\begin{tabular}{|l|l|c|c|}
\hline Score & Mr(calc): & Delta & Sequence \\
\hline 112.2 & 1450.7052 & 0.0037 & SLGNVNFSVSAEAR \\
\hline 73.0 & 1450.7052 & 0.0037 & SLGNVNFSVSAEAR \\
\hline 0.1 & 1450.7181 & -0.0092 & TSTKARGPTPVEK \\
\hline
\end{tabular}

Spectrum No: 34; Query: 1729; Rank: 1

\section{Peptide View}

MS/MS Fragmentation of AEYAVANDTGFVDIPQQEK

Found in IPI00326070, Tax_Id=10116 Gene_Symbol=Ctsl Cathepsin L1 precursor

Match to Query 1729: 2094.973008 from(1048.493780,2+)

Title: 091008RatKidney_NH4Format01_24.2755.2755.2.dta

Data file K:INewmanPaper|Piliang\3SubProteomes\Piliang3SP\mgf5ppm|ERLIC_3SubProteomes5ppm.mgf
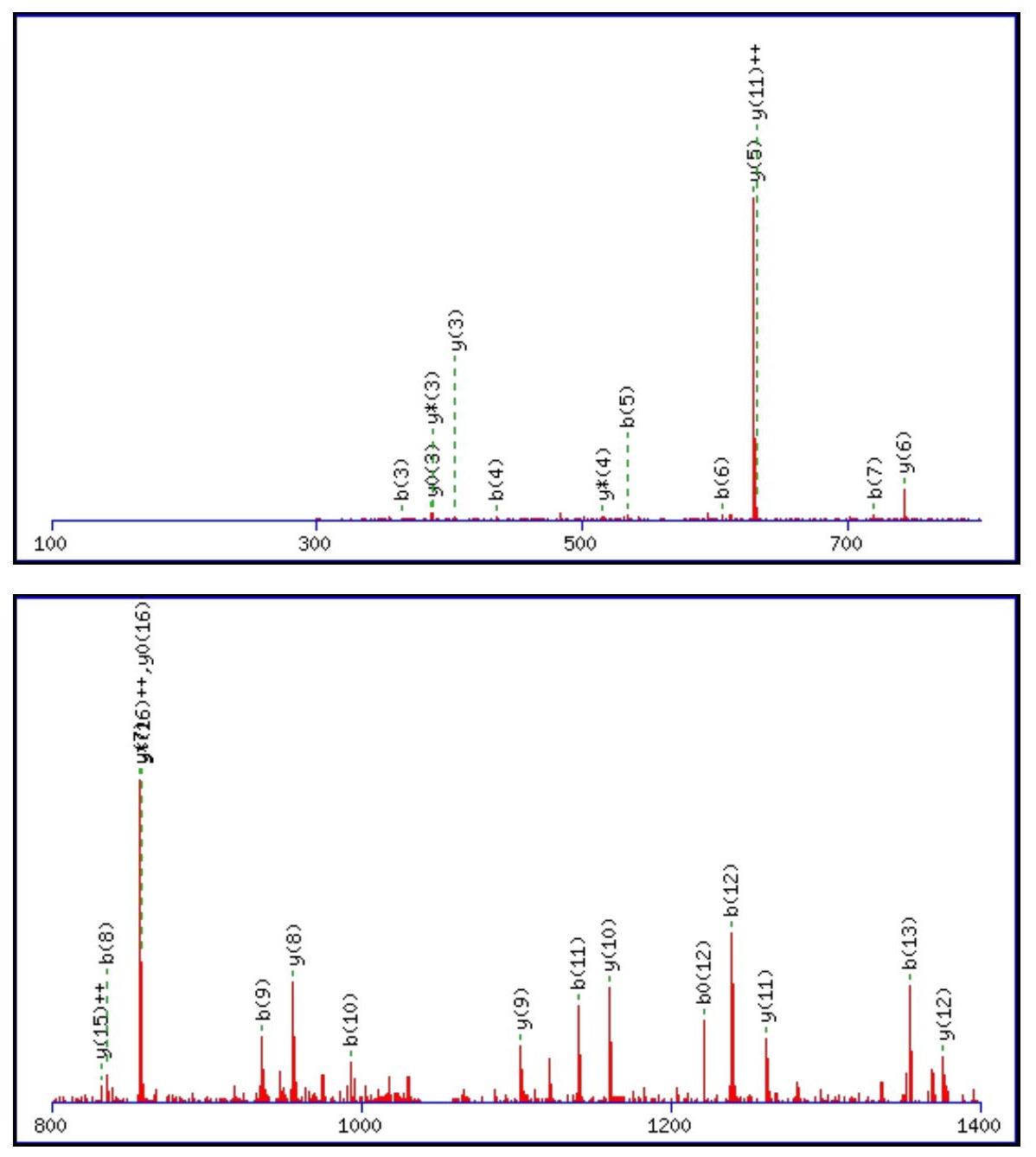


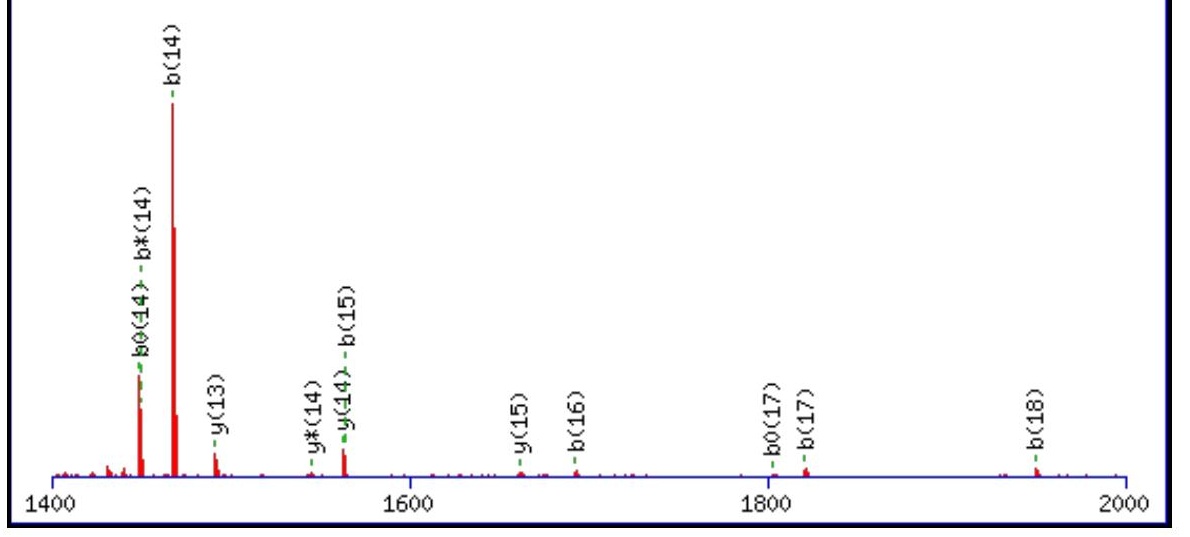

Monoisotopic mass of neutral peptide $\operatorname{Mr}($ calc): 2094.9746

Fixed modifications: Carbamidomethyl (C)

Variable modifications:

N7 : Deamidated_N (N)

Ions Score: 112 Expect: $1.6 \mathrm{e}-009$

Matches (Bold Red): 40/200 fragment ions using 78 most intense peaks

\begin{tabular}{|c|c|c|c|c|c|c|c|c|c|c|c|c|c|c|}
\hline \# & b & $\mathbf{b}^{++}$ & b* & $\mathbf{b}^{*^{++}}$ & $\mathbf{b}^{0}$ & $\mathbf{b}^{\mathbf{0 + +}}$ & Seq. & $\mathbf{y}$ & $\mathbf{y}^{++}$ & $\mathbf{y}^{*}$ & $\mathbf{y}^{*^{++}}$ & $\mathbf{y}^{0}$ & $y^{0++}$ & \# \\
\hline 1 & 72.0444 & 36.5258 & & & & & A & & & & & & & 19 \\
\hline 2 & 201.0870 & 101.0471 & & & 183.0764 & 92.0418 & $\mathbf{E}$ & 2024.9447 & 1012.9760 & 2007.9182 & 1004.4627 & 2006.9342 & 1003.9707 & 18 \\
\hline 3 & 364.1503 & 182.5788 & & & 346.1397 & 173.5735 & $\mathbf{Y}$ & 1895.9021 & 948.4547 & 1878.8756 & 939.9414 & 1877.8916 & 939.4494 & 17 \\
\hline 4 & 435.1874 & 218.0974 & & & 417.1769 & 209.0921 & A & 1732.8388 & 866.9230 & 1715.8123 & 858.4098 & 1714.8283 & 857.9178 & 16 \\
\hline 5 & 534.2558 & 267.6316 & & & 516.2453 & 258.6263 & $\mathbf{V}$ & 1661.8017 & 831.4045 & 1644.7752 & 822.8912 & 1643.7911 & 822.3992 & 15 \\
\hline 6 & 605.2930 & 303.1501 & & & 587.2824 & 294.1448 & A & 1562.7333 & 781.8703 & 1545.7067 & 773.3570 & 1544.7227 & 772.8650 & 14 \\
\hline 7 & 720.3199 & 360.6636 & 703.2933 & 352.1503 & 702.3093 & 351.6583 & $\mathbf{N}$ & 1491.6962 & 746.3517 & 1474.6696 & 737.8385 & 1473.6856 & 737.3464 & 13 \\
\hline 8 & 835.3468 & 418.1771 & 818.3203 & 409.6638 & 817.3363 & 409.1718 & D & 1376.6692 & 688.8383 & 1359.6427 & 680.3250 & 1358.6587 & 679.8330 & 12 \\
\hline 9 & 936.3945 & 468.7009 & 919.3680 & 460.1876 & 918.3839 & 459.6956 & $\mathbf{T}$ & 1261.6423 & 631.3248 & 1244.6157 & 622.8115 & 1243.6317 & 622.3195 & 11 \\
\hline 10 & 993.4160 & 497.2116 & 976.3894 & 488.6984 & 975.4054 & 488.2063 & G & 1160.5946 & 580.8009 & 1143.5681 & 572.2877 & 1142.5841 & 571.7957 & 10 \\
\hline 11 & 1140.4844 & 570.7458 & 1123.4578 & 562.2326 & 1122.4738 & 561.7406 & $\mathbf{F}$ & 1103.5732 & 552.2902 & 1086.5466 & 543.7769 & 1085.5626 & 543.2849 & 9 \\
\hline 12 & 1239.5528 & 620.2800 & 1222.5263 & 611.7668 & 1221.5422 & 611.2748 & $\mathbf{V}$ & 956.5047 & 478.7560 & 939.4782 & 470.2427 & 938.4942 & 469.7507 & 8 \\
\hline 13 & 1354.5797 & 677.7935 & 1337.5532 & 669.2802 & 1336.5692 & 668.7882 & D & 857.4363 & 429.2218 & 840.4098 & 420.7085 & 839.4258 & 420.2165 & 7 \\
\hline 14 & 1467.6638 & 734.3355 & 1450.6373 & 725.8223 & 1449.6532 & 725.3303 & I & 742.4094 & 371.7083 & 725.3828 & 363.1951 & 724.3988 & 362.7030 & 6 \\
\hline 15 & 1564.7166 & 782.8619 & 1547.6900 & 774.3487 & 1546.7060 & 773.8566 & $\mathbf{P}$ & 629.3253 & 315.1663 & 612.2988 & 306.6530 & 611.3148 & 306.1610 & 5 \\
\hline 16 & 1692.7752 & 846.8912 & 1675.7486 & 838.3779 & 1674.7646 & 837.8859 & $\mathbf{Q}$ & 532.2726 & 266.6399 & 515.2460 & 258.1266 & 514.2620 & 257.6346 & 4 \\
\hline 17 & 1820.8337 & 910.9205 & 1803.8072 & 902.4072 & 1802.8232 & 901.9152 & $\mathbf{Q}$ & 404.2140 & 202.6106 & 387.1874 & 194.0974 & 386.2034 & 193.6053 & 3 \\
\hline 18 & 1949.8763 & 975.4418 & 1932.8498 & 966.9285 & 1931.8658 & 966.4365 & $\mathbf{E}$ & 276.1554 & 138.5813 & 259.1288 & 130.0681 & 258.1448 & 129.5761 & 2 \\
\hline 19 & & & & & & & $\mathbf{K}$ & 147.1128 & 74.0600 & 130.0863 & 65.5468 & & & 1 \\
\hline
\end{tabular}
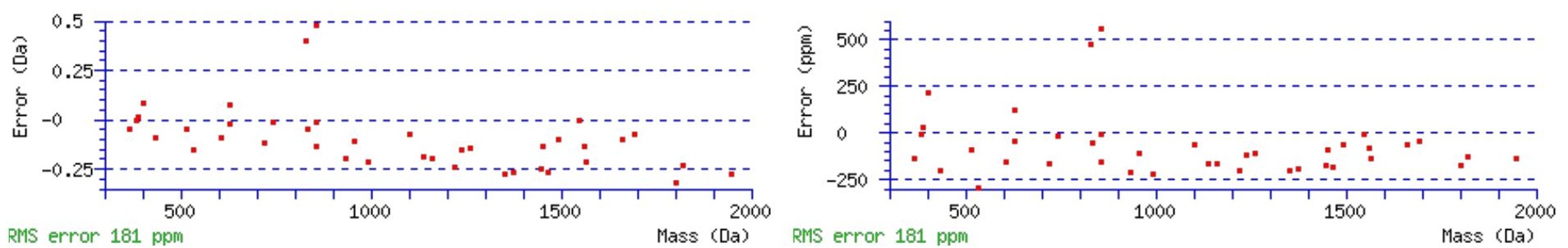

\section{All matches to this query}

\begin{tabular}{|l|l|l|l|}
\hline Score & Mr(calc): & Delta & Sequence \\
\hline 112.1 & 2094.9746 & -0.0016 & AEYAVANDTGFVDIPQQEK \\
\hline 34.5 & 2093.9906 & 0.9825 & AEYAVANDTGFVDIPQQEK \\
\hline
\end{tabular}




\begin{tabular}{|l|l|l|l|}
13.7 & 2094.9760 & -0.0030 & FKGPMTVLRLNNLMASK \\
\hline 12.0 & 2092.9764 & 1.9966 & TISSYTAFSRLILILR \\
\hline 11.1 & 2094.9834 & -0.0104 & KEIEELQSQAQALSQEGK \\
\hline 5.3 & 2094.9623 & 0.0107 & VTGNQGANIAYPPGSTPTIR \\
\hline 5.3 & 2094.9623 & 0.0107 & VTGNQGANIAYPPGSTPTIR \\
\hline 5.3 & 2094.9623 & 0.0107 & VTGNQGANIAYPPGSTPTIR \\
\hline 5.2 & 2094.9623 & 0.0107 & VTGNQGANIAYPPGSTPTIR \\
\hline 5.0 & 2094.9834 & -0.0104 & KEIEELQSQAQALSQEGK \\
\hline
\end{tabular}

Spectrum No: 35; Query: 1507; Rank: 1

\section{Peptide View}

MS/MS Fragmentation of ALASETNGTDSNGSNSSNIQ

Found in IPI00201410, Tax_Id=10116 Gene_Symbol=Ppp3ca Isoform 1 of Serine/threonine-protein phosphatase 2B catalytic subunit alpha isoform

Match to Query 1507: 1967.810968 from(984.912760,2+)

Title: 091008RatKidney_NoSalt_29.797.797.2.dta

Data file K:\NewmanPaper|Piliang|3SubProteomes\Piliang3SP\mgf5ppm\ERLIC_3SubProteomes5ppm.mgf
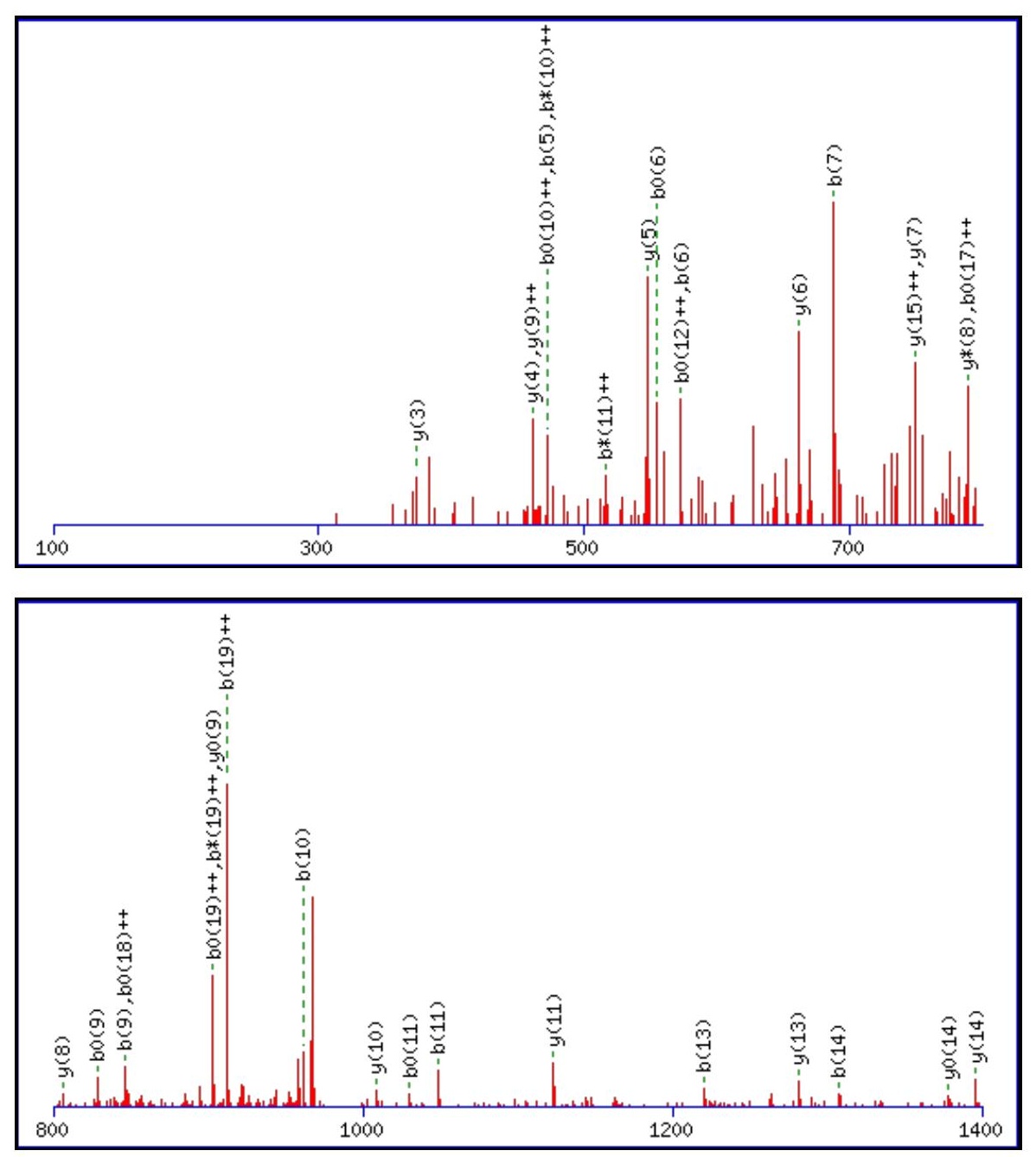


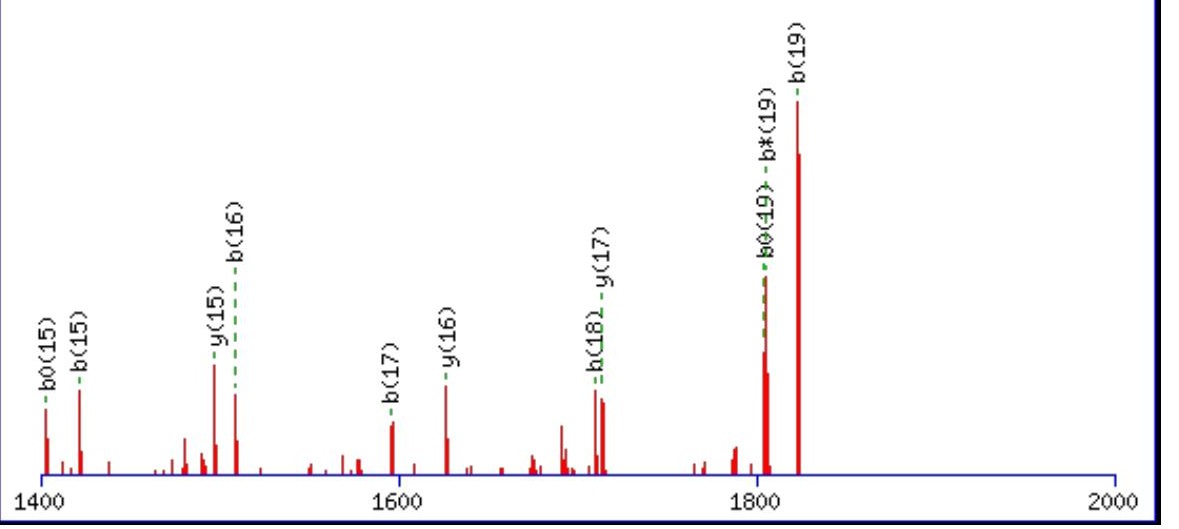

Monoisotopic mass of neutral peptide $\operatorname{Mr}($ calc): 1967.8192

Fixed modifications: Carbamidomethyl (C)

Variable modifications:

N7 : Deamidated_N (N)

N12 : Deamidated $\mathrm{N}(\mathrm{N})$

Ions Score: 112 Expect: $1.2 \mathrm{e}-009$

Matches (Bold Red): 46/204 fragment ions using 54 most intense peaks

\begin{tabular}{|c|c|c|c|c|c|c|c|c|c|c|c|c|c|c|}
\hline \# & b & $\mathbf{b}^{++}$ & b* & $\mathbf{b}^{*^{++}}$ & $\mathbf{b}^{0}$ & $\mathbf{b}^{\mathbf{0 + +}}$ & Seq. & $\mathbf{y}$ & $y^{++}$ & $\mathbf{y}^{*}$ & $\mathbf{y}^{*^{++}}$ & $\mathbf{y}^{\mathbf{0}}$ & $\mathbf{y}^{0++}$ & \# \\
\hline 1 & 72.0444 & 36.5258 & & & & & A & & & & & & & 20 \\
\hline 2 & 185.1285 & 93.0679 & & & & & $\mathbf{L}$ & 1897.7893 & 949.3983 & 1880.7628 & 940.8850 & 1879.7788 & 940.3930 & 19 \\
\hline 3 & 256.1656 & 128.5864 & & & & & A & 1784.7053 & 892.8563 & 1767.6787 & 884.3430 & 1766.6947 & 883.8510 & 18 \\
\hline 4 & 343.1976 & 172.1024 & & & 325.1870 & 163.0972 & $S$ & 1713.6682 & 857.3377 & 1696.6416 & 848.8244 & 1695.6576 & 848.3324 & 17 \\
\hline 5 & 472.2402 & 236.6237 & & & 454.2296 & 227.6185 & $\mathbf{E}$ & 1626.6361 & 813.8217 & 1609.6096 & 805.3084 & 1608.6256 & 804.8164 & 16 \\
\hline 6 & 573.2879 & 287.1476 & & & 555.2773 & 278.1423 & $\mathbf{T}$ & 1497.5936 & 749.3004 & 1480.5670 & 740.7871 & 1479.5830 & 740.2951 & 15 \\
\hline 7 & 688.3148 & 344.6610 & 671.2883 & 336.1478 & 670.3042 & 335.6558 & $\mathbf{N}$ & 1396.5459 & 698.7766 & 1379.5193 & 690.2633 & 1378.5353 & 689.7713 & 14 \\
\hline 8 & 745.3363 & 373.1718 & 728.3097 & 364.6585 & 727.3257 & 364.1665 & G & 1281.5189 & 641.2631 & 1264.4924 & 632.7498 & 1263.5084 & 632.2578 & 13 \\
\hline 9 & 846.3839 & 423.6956 & 829.3574 & 415.1823 & 828.3734 & 414.6903 & $\mathbf{T}$ & 1224.4975 & 612.7524 & 1207.4709 & 604.2391 & 1206.4869 & 603.7471 & 12 \\
\hline 10 & 961.4109 & 481.2091 & 944.3843 & 472.6958 & 943.4003 & 472.2038 & D & 1123.4498 & 562.2285 & 1106.4232 & 553.7153 & 1105.4392 & 553.2233 & 11 \\
\hline 11 & 1048.4429 & 524.7251 & 1031.4164 & 516.2118 & 1030.4324 & 515.7198 & $\mathrm{~S}$ & 1008.4228 & 504.7151 & 991.3963 & 496.2018 & 990.4123 & 495.7098 & 10 \\
\hline 12 & 1163.4699 & 582.2386 & 1146.4433 & 573.7253 & 1145.4593 & 573.2333 & $\mathbf{N}$ & 921.3908 & 461.1990 & 904.3643 & 452.6858 & 903.3803 & 452.1938 & 9 \\
\hline 13 & 1220.4913 & 610.7493 & 1203.4648 & 602.2360 & 1202.4808 & 601.7440 & G & 806.3639 & 403.6856 & 789.3373 & 395.1723 & 788.3533 & 394.6803 & 8 \\
\hline 14 & 1307.5233 & 654.2653 & 1290.4968 & 645.7520 & 1289.5128 & 645.2600 & $\mathbf{S}$ & 749.3424 & 375.1748 & 732.3159 & 366.6616 & 731.3319 & 366.1696 & 7 \\
\hline 15 & 1421.5663 & 711.2868 & 1404.5397 & 702.7735 & 1403.5557 & 702.2815 & $\mathbf{N}$ & 662.3104 & 331.6588 & 645.2838 & 323.1456 & 644.2998 & 322.6536 & 6 \\
\hline 16 & 1508.5983 & 754.8028 & 1491.5718 & 746.2895 & 1490.5877 & 745.7975 & $S$ & 548.2675 & 274.6374 & 531.2409 & 266.1241 & 530.2569 & 265.6321 & 5 \\
\hline 17 & 1595.6303 & 798.3188 & 1578.6038 & 789.8055 & 1577.6198 & 789.3135 & $S$ & 461.2354 & 231.1214 & 444.2089 & 222.6081 & 443.2249 & 222.1161 & 4 \\
\hline 18 & 1709.6733 & 855.3403 & 1692.6467 & 846.8270 & 1691.6627 & 846.3350 & $\mathbf{N}$ & 374.2034 & 187.6053 & 357.1769 & 179.0921 & & & 3 \\
\hline 19 & 1822.7573 & 911.8823 & 1805.7308 & 903.3690 & 1804.7468 & 902.8770 & $\mathbf{I}$ & 260.1605 & 130.5839 & 243.1339 & 122.0706 & & & 2 \\
\hline 20 & & & & & & & $\mathbf{Q}$ & 147.0764 & 74.0418 & 130.0499 & 65.5286 & & & 1 \\
\hline
\end{tabular}

要
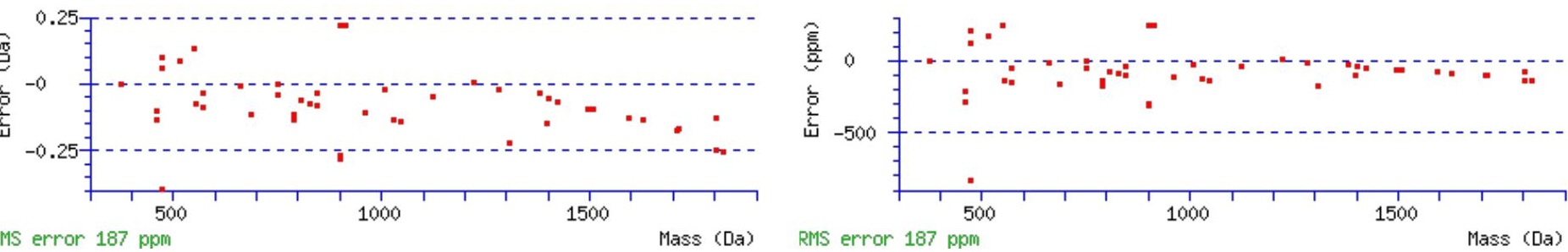

\section{All matches to this query}

\begin{tabular}{|l|l|c|c|}
\hline Score & Mr(calc): & Delta & Sequence \\
\hline 111.8 & 1967.8192 & -0.0082 & ALASETNGTDSNGSNSSNIQ \\
\hline
\end{tabular}




\begin{tabular}{|l|l|l|l|}
72.9 & 1967.8192 & -0.0082 & ALASETNGTDSNGSNSSNIQ \\
\hline 41.5 & 1967.8192 & -0.0082 & ALASETNGTDSNGSNSSNIQ \\
\hline 37.7 & 1967.8192 & -0.0082 & ALASETNGTDSNGSNSSNIQ \\
\hline 16.5 & 1967.8192 & -0.0082 & ALASETNGTDSNGSNSSNIQ \\
\hline 15.3 & 1967.8192 & -0.0082 & ALASETNGTDSNGSNSSNIQ \\
\hline 10.6 & 1967.8006 & 0.0104 & ENEFSTTGTRMDLQMK \\
\hline 8.3 & 1967.7923 & 0.0187 & MEALSMAHYSSPAILR \\
\hline 8.3 & 1967.7923 & 0.0187 & MEALSMAHYSSPAILR \\
\hline 8.1 & 1967.8093 & 0.0017 & CNICNKSFTWCASLK \\
\hline
\end{tabular}

Spectrum No: 36; Query: 1522; Rank: 1

\section{Peptide View}

MS/MS Fragmentation of AQEAGYTNATTTDQHGLAK

Found in IPI00212666, Tax_Id=10116 Gene_Symbol=Mug1 Isoform 1 of Murinoglobulin-1 precursor

Match to Query 1522: 1976.911128 from(989.462840,2+)

Title: 100101RatKid_NS_deglyco_24.855.855.2.dta

Data file K:INewmanPaper|Piliang|3SubProteomes\Piliang3SP\mgf5ppm\ERLIC_3SubProteomes5ppm.mgf
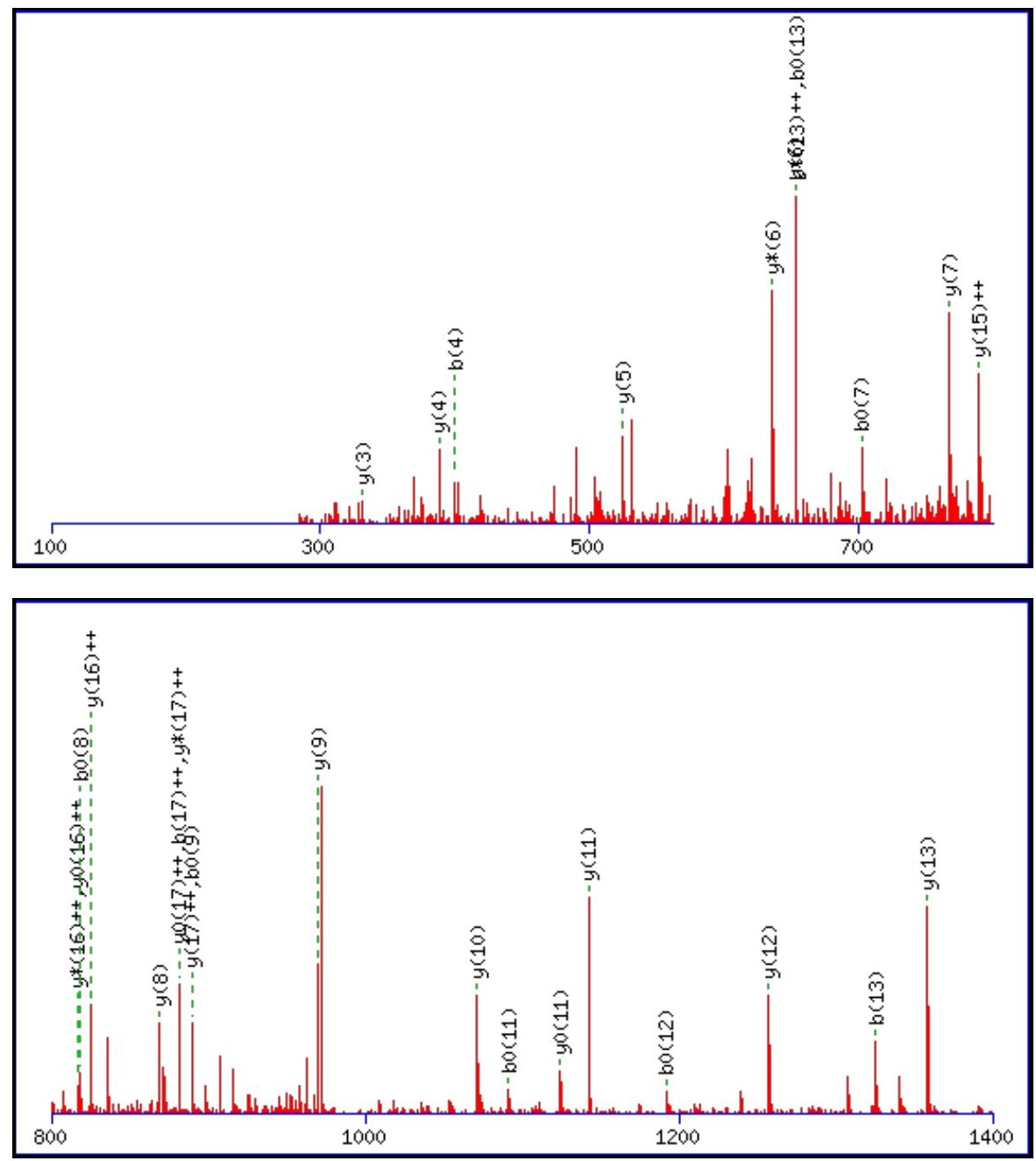


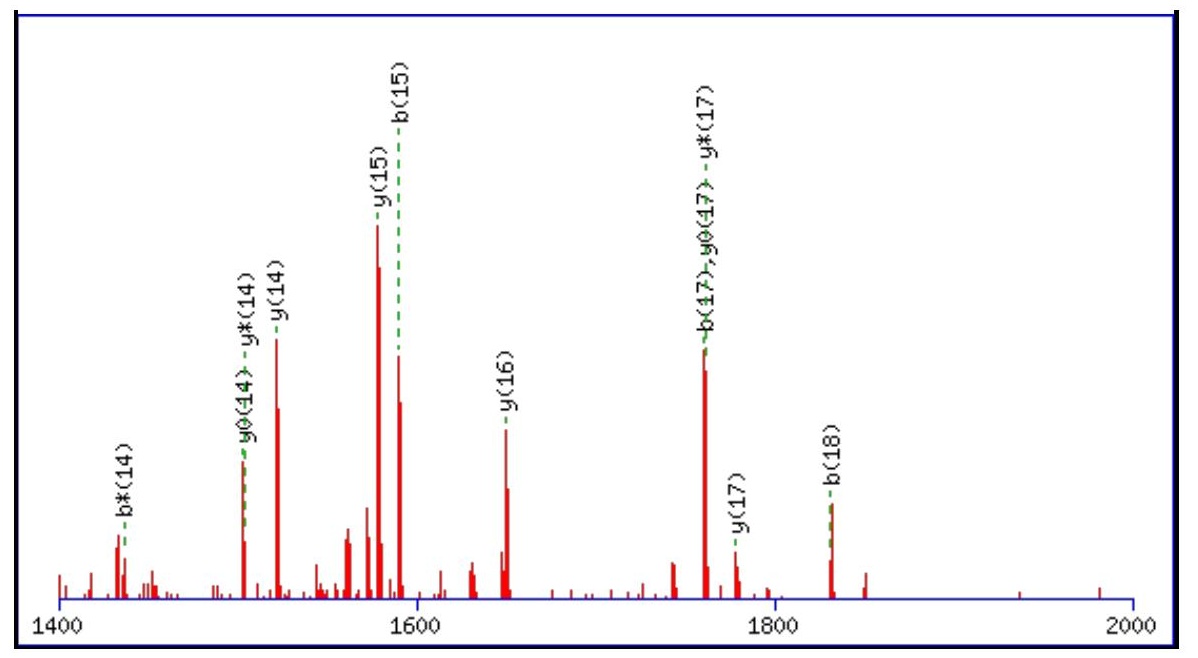

Monoisotopic mass of neutral peptide $\operatorname{Mr}($ calc): 1976.9075

Fixed modifications: Carbamidomethyl (C)

Variable modifications:

N8: Deamidated $\mathrm{N}(\mathrm{N})$

Ions Score: 112 Expect: $1.6 \mathrm{e}-009$

Matches (Bold Red): 42/198 fragment ions using 59 most intense peaks

\begin{tabular}{|c|c|c|c|c|c|c|c|c|c|c|c|c|c|c|}
\hline \# & b & $\mathbf{b}^{++}$ & $\mathbf{b}^{*}$ & $\mathbf{b}^{*^{++}}$ & $\mathbf{b}^{0}$ & $\mathbf{b}^{0++}$ & Seq. & $\mathbf{y}$ & $\mathbf{y}^{++}$ & $\mathbf{y}^{*}$ & $\mathbf{y}^{*^{++}}$ & $\mathbf{y}^{0}$ & $\mathbf{y}^{0++}$ & \# \\
\hline 1 & 72.0444 & 36.5258 & & & & & A & & & & & & & 19 \\
\hline 2 & 200.1030 & 100.5551 & 183.0764 & 92.0418 & & & $\mathbf{Q}$ & 1906.8777 & 953.9425 & 1889.8512 & 945.4292 & 1888.8672 & 944.9372 & 18 \\
\hline 3 & 329.1456 & 165.0764 & 312.1190 & 156.5631 & 311.1350 & 156.0711 & $\mathbf{E}$ & 1778.8191 & 889.9132 & 1761.7926 & 881.3999 & 1760.8086 & 880.9079 & 17 \\
\hline 4 & 400.1827 & 200.5950 & 383.1561 & 192.0817 & 382.1721 & 191.5897 & A & 1649.7766 & |825.3919| & 1632.7500 & 816.8786 & 1631.7660 & 816.3866 & 16 \\
\hline 5 & 457.2041 & 229.1057 & 440.1776 & 220.5924 & 439.1936 & 220.1004 & G & 1578.7394 & 789.8734 & 1561.7129 & 781.3601 & 1560.7289 & 780.8681 & 15 \\
\hline 6 & 620.2675 & 310.6374 & 603.2409 & 302.1241 & 602.2569 & 301.6321 & $\mathbf{Y}$ & 1521.7180 & 761.3626 & 1504.6914 & 752.8494 & 1503.7074 & 752.3573 & 14 \\
\hline 7 & 721.3151 & 361.1612 & 704.2886 & 352.6479 & 703.3046 & 352.1559 & $\mathbf{T}$ & 1358.6546 & 679.8310 & 1341.6281 & 671.3177 & 1340.6441 & 670.8257 & 13 \\
\hline 8 & 836.3421 & 418.6747 & 819.3155 & 410.1614 & 818.3315 & 409.6694 & $\mathbf{N}$ & 1257.6070 & 629.3071 & 1240.5804 & 620.7938 & 1239.5964 & 620.3018 & 12 \\
\hline 9 & 907.3792 & 454.1932 & 890.3526 & 445.6800 & 889.3686 & 445.1880 & A & 1142.5800 & 571.7937 & 1125.5535 & 563.2804 & 1124.5695 & 562.7884 & 11 \\
\hline 10 & 1008.4269 & 504.7171 & 991.4003 & 496.2038 & 990.4163 & 495.7118 & $\mathbf{T}$ & 1071.5429 & 536.2751 & 1054.5164 & 527.7618 & 1053.5324 & 527.2698 & 10 \\
\hline 11 & 1109.4746 & 555.2409 & 1092.4480 & 546.7276 & 1091.4640 & 546.2356 & $\mathbf{T}$ & 970.4952 & 485.7513 & 953.4687 & 477.2380 & 952.4847 & 476.7460 & 9 \\
\hline 12 & 1210.5222 & 605.7648 & 1193.4957 & 597.2515 & 1192.5117 & 596.7595 & $\mathbf{T}$ & 869.4476 & 435.2274 & 852.4210 & 426.7141 & 851.4370 & 426.2221 & 8 \\
\hline 13 & 1325.5492 & 663.2782 & 1308.5226 & 654.7650 & 1307.5386 & 654.2729 & D & 768.3999 & 384.7036 & 751.3733 & 376.1903 & 750.3893 & 375.6983 & 7 \\
\hline 14 & 1453.6078 & 727.3075 & 1436.5812 & 718.7942 & 1435.5972 & 718.3022 & $\mathbf{Q}$ & 653.3729 & 327.1901 & 636.3464 & 318.6768 & & & 6 \\
\hline 15 & 1590.6667 & 795.8370 & 1573.6401 & 787.3237 & 1572.6561 & 786.8317 & $\mathbf{H}$ & 525.3144 & 263.1608 & 508.2878 & 254.6475 & & & 5 \\
\hline 16 & 1647.6881 & 824.3477 & 1630.6616 & 815.8344 & 1629.6776 & 815.3424 & $\mathbf{G}$ & 388.2554 & 194.6314 & 371.2289 & 186.1181 & & & 4 \\
\hline 17 & 1760.7722 & 880.8897 & 1743.7456 & 872.3765 & 1742.7616 & 871.8845 & $\mathbf{L}$ & 331.2340 & 166.1206 & 314.2074 & 157.6074 & & & 3 \\
\hline 18 & 1831.8093 & 916.4083 & 1814.7828 & 907.8950 & 1813.7987 & 907.4030 & A & 218.1499 & 109.5786 & 201.1234 & 101.0653 & & & 2 \\
\hline 19 & & & & & & & $\mathbf{K}$ & 147.1128 & 74.0600 & 130.0863 & 65.5468 & & & 1 \\
\hline
\end{tabular}
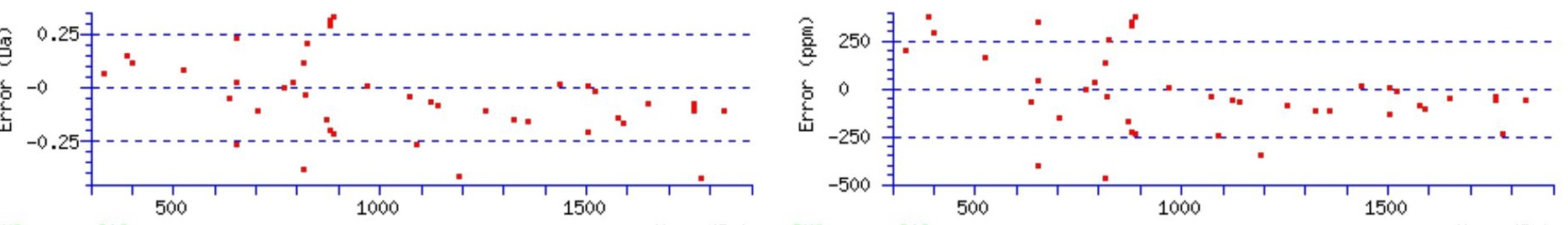

RMS error $203 \mathrm{ppm}$

Mass (Da)

RMS error $203 \mathrm{ppm}$

Mass (Da)

All matches to this query

\begin{tabular}{|l|l|l|l|}
\hline Score & Mr(calc): & Delta & Sequence \\
\hline 111.6 & 1976.9075 & 0.0036 & AQEAGYTNATTTDQHGLAK \\
\hline 30.5 & 1975.9235 & 0.9876 & AQEAGYTNATTTDQHGLAK \\
\hline & & & \\
\hline
\end{tabular}




\begin{tabular}{|l|l|l|l|}
5.8 & 1976.9050 & 0.0061 & TFGESHPFTKLVVDLK \\
\hline 2.7 & 1976.9027 & 0.0085 & STLDSHPMNISGIPERK \\
\hline 1.3 & 1976.8993 & 0.0118 & AQEALDFYGEVRTRDK \\
\hline
\end{tabular}

Spectrum No: 37; Query: 2170; Rank: 1

\section{Peptide View}

MS/MS Fragmentation of VILILDPAISGNETEPYPAFTR

Found in IPI00191437, Tax_Id=10116 Gene_Symbol=- 64 kDa protein

Match to Query 2170: 2416.261828 from(1209.138190,2+)

Title: 091008RatKidney_NoSalt_26.4795.4795.2.dta

Data file K:INewmanPaper\Piliang|3SubProteomes\Piliang3SP\mgf5ppm\ERLIC_3SubProteomes5ppm.mgf
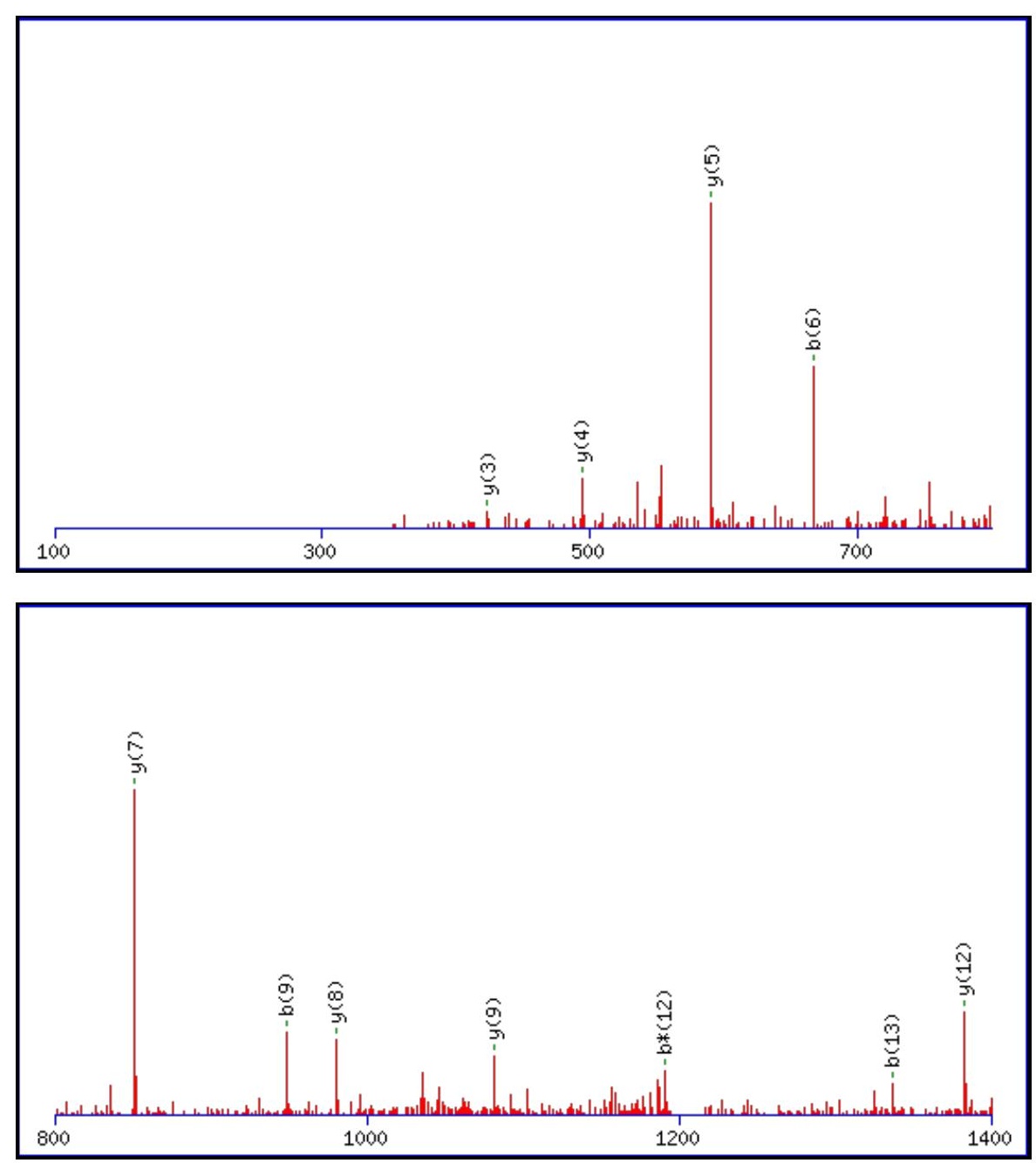


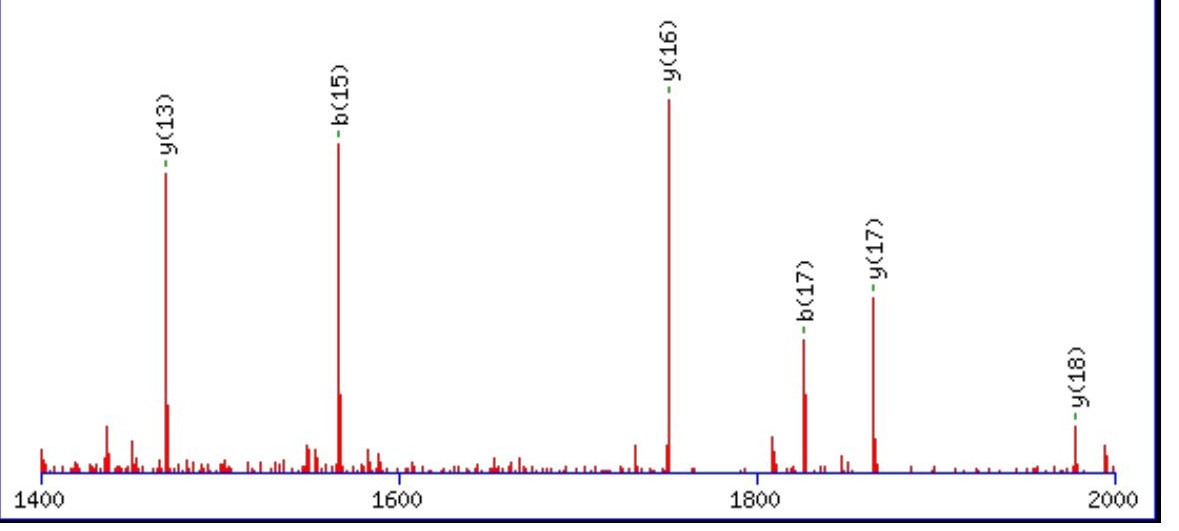

Monoisotopic mass of neutral peptide $\operatorname{Mr}($ calc): 2416.2526

Fixed modifications: Carbamidomethyl (C)

Variable modifications:

N12 : Deamidated $\mathrm{N}(\mathrm{N})$

Ions Score: 111 Expect: $8.1 \mathrm{e}-010$

Matches (Bold Red): 17/218 fragment ions using 18 most intense peaks

\begin{tabular}{|c|c|c|c|c|c|c|c|c|c|c|c|c|c|c|}
\hline \# & b & $\mathbf{b}^{++}$ & $\mathbf{b}^{*}$ & $\mathbf{b}^{*^{++}}$ & $\mathbf{b}^{\mathbf{0}}$ & $\mathbf{b}^{0++}$ & Seq. & $\mathbf{y}$ & $y^{++}$ & $\mathbf{y}^{*}$ & $\mathrm{y}^{\mathrm{*}^{++}}$ & $\mathbf{y}^{0}$ & $\mathbf{y}^{0++}$ & \# \\
\hline 1 & 100.0757 & 50.5415 & & & & & $\mathbf{V}$ & & & & & & & 22 \\
\hline 2 & 213.1598 & 107.0835 & & & & & I & 2318.1914 & 1159.5994 & 2301.1649 & 1151.0861 & 2300.1809 & 1150.5941 & 21 \\
\hline 3 & 326.2438 & 163.6255 & & & & & $\mathbf{L}$ & 2205.1074 & 1103.0573 & 2188.0808 & 1094.5441 & 2187.0968 & 1094.0520 & 20 \\
\hline 4 & 439.3279 & 220.1676 & & & & & I & 2092.0233 & 1046.5153 & 2074.9968 & 1038.0020 & 2074.0128 & 1037.5100 & 19 \\
\hline 5 & 552.4119 & 276.7096 & & & & & $\mathbf{L}$ & 1978.9393 & 989.9733 & 1961.9127 & 981.4600 & 1960.9287 & 980.9680 & 18 \\
\hline 6 & 667.4389 & 334.2231 & & & 649.4283 & 325.2178 & D & 1865.8552 & 933.4312 & 1848.8286 & 924.9180 & 1847.8446 & 924.4260 & 17 \\
\hline 7 & 764.4917 & 382.7495 & & & 746.4811 & 373.7442 & $\mathbf{P}$ & 1750.8283 & 875.9178 & 1733.8017 & 867.4045 & 1732.8177 & 866.9125 & 16 \\
\hline 8 & 835.5288 & 418.2680 & & & 817.5182 & 409.2627 & A & 1653.7755 & 827.3914 & 1636.7489 & 818.8781 & 1635.7649 & 818.3861 & 15 \\
\hline 9 & 948.6128 & 474.8101 & & & 930.6023 & 465.8048 & I & 1582.7384 & 791.8728 & 1565.7118 & 783.3596 & 1564.7278 & 782.8675 & 14 \\
\hline 10 & 1035.6449 & 518.3261 & & & 1017.6343 & 509.3208 & $\mathrm{~S}$ & 1469.6543 & 735.3308 & 1452.6278 & 726.8175 & 1451.6437 & 726.3255 & 13 \\
\hline 11 & 1092.6663 & 546.8368 & & & 1074.6558 & 537.8315 & G & 1382.6223 & 691.8148 & 1365.5957 & 683.3015 & 1364.6117 & 682.8095 & 12 \\
\hline 12 & 1207.6933 & 604.3503 & 1190.6667 & 595.8370 & 1189.6827 & 595.3450 & $\mathbf{N}$ & 1325.6008 & 663.3040 & 1308.5743 & 654.7908 & 1307.5903 & 654.2988 & 11 \\
\hline 13 & 1336.7359 & 668.8716 & 1319.7093 & 660.3583 & 1318.7253 & 659.8663 & $\mathbf{E}$ & 1210.5739 & 605.7906 & 1193.5473 & 597.2773 & 1192.5633 & 596.7853 & 10 \\
\hline 14 & 1437.7835 & 719.3954 & 1420.7570 & 710.8821 & 1419.7730 & 710.3901 & $T$ & 1081.5313 & 541.2693 & 1064.5047 & 532.7560 & 1063.5207 & 532.2640 & 9 \\
\hline 15 & \begin{tabular}{|l|}
1566.8261 \\
\end{tabular} & 783.9167 & 1549.7996 & 775.4034 & 1548.8156 & 774.9114 & $\mathbf{E}$ & 980.4836 & 490.7454 & 963.4571 & 482.2322 & 962.4730 & 481.7402 & 8 \\
\hline 16 & 1663.8789 & 832.4431 & 1646.8523 & 823.9298 & 1645.8683 & 823.4378 & $\mathbf{P}$ & 851.4410 & 426.2241 & 834.4145 & 417.7109 & 833.4305 & 417.2189 & 7 \\
\hline 17 & \begin{tabular}{|l|}
1826.9422 \\
\end{tabular} & 913.9747 & 1809.9157 & 905.4615 & 1808.9317 & 904.9695 & $\mathbf{Y}$ & 754.3883 & 377.6978 & 737.3617 & 369.1845 & 736.3777 & 368.6925 & 6 \\
\hline 18 & 1923.9950 & 962.5011 & 1906.9684 & 953.9879 & 1905.9844 & 953.4958 & $\mathbf{P}$ & 591.3249 & 296.1661 & 574.2984 & 287.6528 & 573.3144 & 287.1608 & 5 \\
\hline 19 & 1995.0321 & 998.0197 & 1978.0055 & 989.5064 & 1977.0215 & 989.0144 & A & 494.2722 & 247.6397 & 477.2456 & 239.1264 & 476.2616 & 238.6344 & 4 \\
\hline 20 & 2142.1005 & 1071.5539 & 2125.0740 & 1063.0406 & 2124.0899 & 1062.5486 & $\mathbf{F}$ & 423.2350 & 212.1212 & 406.2085 & 203.6079 & 405.2245 & 203.1159 & 3 \\
\hline 21 & 2243.1482 & 1122.0777 & 2226.1216 & 1113.5645 & 2225.1376 & 1113.0724 & $T$ & 276.1666 & 138.5870 & 259.1401 & 130.0737 & 258.1561 & 129.5817 & 2 \\
\hline 22 & & & & & & & $\mathbf{R}$ & 175.1190 & 88.0631 & 158.0924 & 79.5498 & & & \\
\hline
\end{tabular}
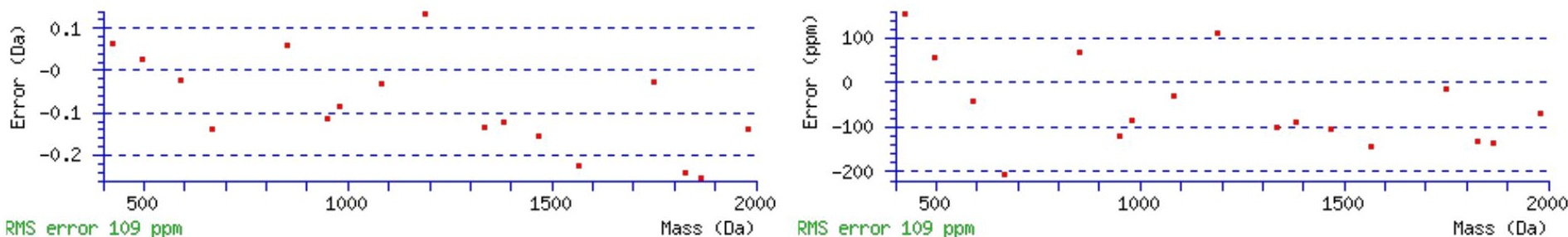

\section{All matches to this query}




\begin{tabular}{|l|l|l|l|} 
Score & Mr(calc): & Delta & \multicolumn{1}{|c|}{ Sequence } \\
\hline 111.4 & 2416.2526 & 0.0093 & VILILDPAISGNETEPYPAFTR \\
\hline 30.0 & 2415.2686 & 0.9933 & VILILDPAISGNETEPYPAFTR \\
\hline 5.8 & 2416.2515 & 0.0103 & EIQRLLPSPLKDAVEVSTWR \\
\hline 5.1 & 2416.2646 & -0.0028 & FAFQAEVNRMMKLIINSLYK \\
\hline 0.2 & 2414.2570 & 2.0048 & GLNLTSLSITHTNITAVPAAALR \\
\hline 0.2 & 2414.2570 & 2.0048 & GLNLTSLSITHTNITAVPAAALR \\
\hline
\end{tabular}

Spectrum No: 38; Query: 1722; Rank: 1

\section{Peptide View}

MS/MS Fragmentation of LDWLGNCSGLNDESYGYK

Found in IPI00339124, Tax_Id=10116 Gene_Symbol=Atp1b1 Sodium/potassium-transporting ATPase subunit beta-1

Match to Query 1722: 2090.894568 from(1046.454560,2+)

Title: 100101RatKid_NS_deglyco_27.3469.3469.2.dta

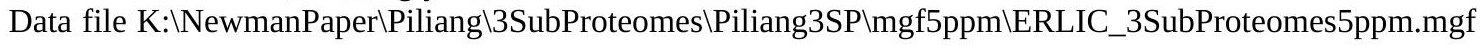
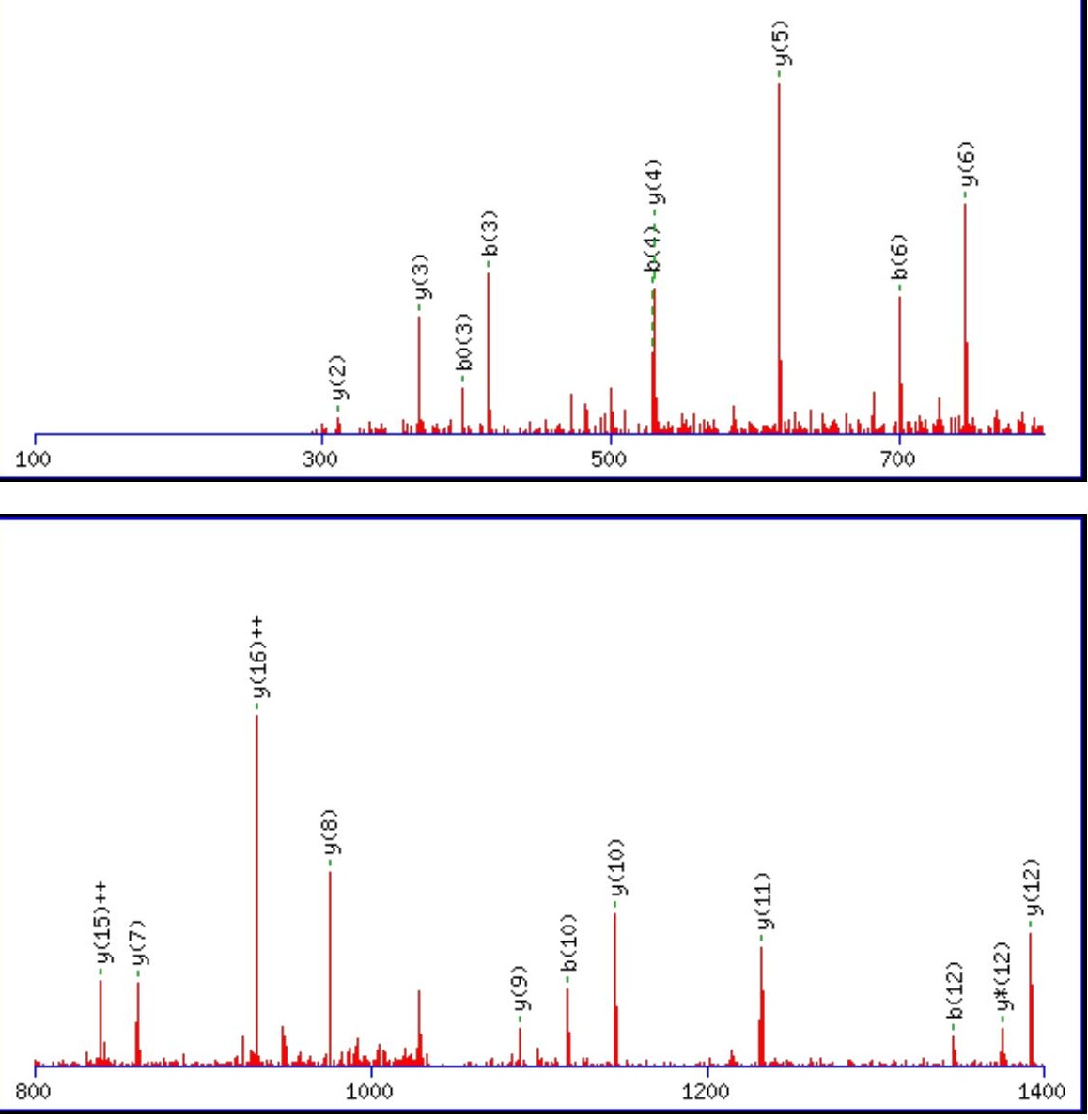


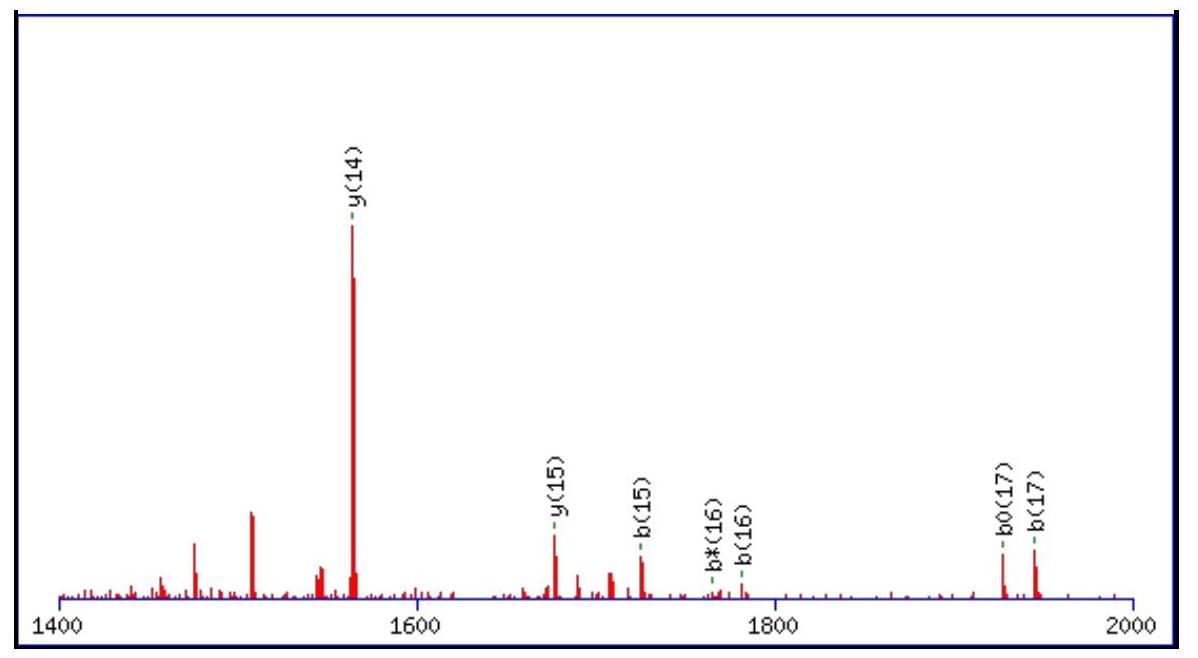

Monoisotopic mass of neutral peptide $\operatorname{Mr}($ calc): 2090.8891

Fixed modifications: Carbamidomethyl (C)

Variable modifications:

N6: Deamidated $\mathrm{N}(\mathrm{N})$

Ions Score: 111 Expect: $2.5 \mathrm{e}-009$

Matches (Bold Red): 27/184 fragment ions using 43 most intense peaks

\begin{tabular}{|c|c|c|c|c|c|c|c|c|c|c|c|c|c|c|}
\hline \# & b & $\mathbf{b}^{++}$ & $\mathbf{b}^{*}$ & $\mathbf{b}^{*^{++}}$ & $\mathbf{b}^{0}$ & $\mathbf{b}^{0++}$ & Seq. & $\mathbf{y}$ & $\mathbf{y}^{++}$ & $\mathbf{y}^{*}$ & $\mathbf{y}^{*^{++}}$ & $\mathbf{y}^{0}$ & $\mathbf{y}^{0++}$ & \# \\
\hline 1 & 114.0913 & 57.5493 & & & & & $\mathbf{L}$ & & & & & & & 18 \\
\hline 2 & 229.1183 & 115.0628 & & & 211.1077 & 106.0575 & D & 1978.8123 & 989.9098 & 1961.7858 & 981.3965 & 1960.8018 & 980.9045 & 17 \\
\hline 3 & 415.1976 & 208.1024 & & & 397.1870 & 199.0972 & $\mathbf{W}$ & 1863.7854 & 932.3963 & 1846.7589 & 923.8831 & 1845.7748 & 923.3911 & 16 \\
\hline 4 & 528.2817 & 264.6445 & & & 510.2711 & 255.6392 & $\mathbf{L}$ & 1677.7061 & 839.3567 & 1660.6795 & 830.8434 & 1659.6955 & 830.3514 & 15 \\
\hline 5 & 585.3031 & 293.1552 & & & 567.2926 & 284.1499 & G & 1564.6220 & 782.8146 & 1547.5955 & 774.3014 & 1546.6115 & 773.8094 & 14 \\
\hline 6 & 700.3301 & 350.6687 & 683.3035 & 342.1554 & 682.3195 & 341.6634 & $\mathbf{N}$ & 1507.6006 & 754.3039 & 1490.5740 & 745.7906 & 1489.5900 & 745.2986 & 13 \\
\hline 7 & 860.3607 & 430.6840 & 843.3342 & 422.1707 & 842.3501 & 421.6787 & C & 1392.5736 & 696.7904 & 1375.5471 & 688.2772 & 1374.5631 & 687.7852 & 12 \\
\hline 8 & 947.3927 & 474.2000 & 930.3662 & 465.6867 & 929.3822 & 465.1947 & S & 1232.5430 & 616.7751 & 1215.5164 & 608.2619 & 1214.5324 & 607.7698 & 11 \\
\hline 9 & 1004.4142 & 502.7107 & 987.3877 & 494.1975 & 986.4036 & 493.7055 & G & 1145.5109 & 573.2591 & 1128.4844 & 564.7458 & 1127.5004 & 564.2538 & 10 \\
\hline 10 & 1117.4983 & 559.2528 & 1100.4717 & 550.7395 & 1099.4877 & 550.2475 & $\mathbf{L}$ & 1088.4895 & 544.7484 & 1071.4629 & 536.2351 & 1070.4789 & 535.7431 & 9 \\
\hline 11 & 1231.5412 & 616.2742 & 1214.5146 & 607.7610 & 1213.5306 & 607.2690 & $\mathbf{N}$ & 975.4054 & 488.2063 & 958.3789 & 479.6931 & 957.3949 & 479.2011 & 8 \\
\hline 12 & 1346.5681 & 673.7877 & 1329.5416 & 665.2744 & 1328.5576 & 664.7824 & D & 861.3625 & 431.1849 & 844.3359 & 422.6716 & 843.3519 & 422.1796 & 7 \\
\hline 13 & 1475.6107 & 738.3090 & 1458.5842 & 729.7957 & 1457.6002 & 729.3037 & $\mathbf{E}$ & 746.3355 & 373.6714 & 729.3090 & 365.1581 & 728.3250 & 364.6661 & 6 \\
\hline 14 & 1562.6428 & 781.8250 & 1545.6162 & 773.3117 & 1544.6322 & 772.8197 & S & 617.2930 & 309.1501 & 600.2664 & 300.6368 & 599.2824 & 300.1448 & 5 \\
\hline 15 & 1725.7061 & 863.3567 & 1708.6795 & 854.8434 & 1707.6955 & 854.3514 & $\mathbf{Y}$ & 530.2609 & 265.6341 & 513.2344 & 257.1208 & & & 4 \\
\hline 16 & 1782.7275 & 891.8674 & 1765.7010 & 883.3541 & 1764.7170 & 882.8621 & $\mathbf{G}$ & 367.1976 & 184.1024 & 350.1710 & 175.5892 & & & 3 \\
\hline 17 & 1945.7909 & 973.3991 & 1928.7643 & 964.8858 & 1927.7803 & 964.3938 & $\mathbf{Y}$ & 310.1761 & 155.5917 & 293.1496 & 147.0784 & & & 2 \\
\hline 18 & & & & & & & $\mathbf{K}$ & 147.1128 & 74.0600 & 130.0863 & 65.5468 & & & 1 \\
\hline
\end{tabular}
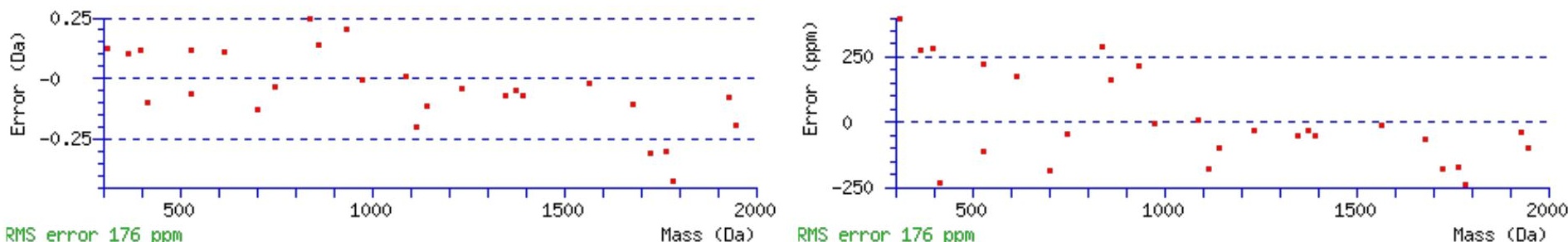

RMS error 176 ppm

Mass (Da) RMS error $176 \mathrm{ppm}$

\section{All matches to this query}

\begin{tabular}{|l|c|c|c|}
\hline Score & Mr(calc): & Delta & Sequence \\
\hline 110.8 & 2090.8891 & 0.0055 & LDWLGNCSGLNDESYGYK \\
\hline 82.5 & 2090.8891 & 0.0055 & LDWLGNCSGLNDESYGYK \\
\hline 63.0 & 2089.9051 & 0.9895 & LDWLGNCSGLNDESYGYK \\
\hline & & & \\
\hline
\end{tabular}


Mascot Search Results: Peptide View

\begin{tabular}{|l|l|l|l|}
\hline 5.7 & 2090.8979 & -0.0034 & EREEMQRQYEEDILK \\
\hline 3.6 & 2090.9132 & -0.0186 & IFHRYEALNSQTMNTGK \\
\hline 3.0 & 2090.8768 & 0.0177 & NIGGNKDWCKENFVNSK \\
\hline 1.4 & 2089.9065 & 0.9880 & ESSESTNNRFAANQGKLR \\
\hline 1.4 & 2089.9065 & 0.9880 & ESSESTNNRFAANQGKLR \\
\hline 1.4 & 2089.9065 & 0.9880 & ESSESTNNRFAANQGKLR \\
\hline 1.4 & 2089.9065 & 0.9880 & ESSESTNNRFAANQGKLR \\
\hline
\end{tabular}

Spectrum No: 39; Query: 1347; Rank: 1

\section{Peptide View}

MS/MS Fragmentation of VIDLWDLAQSANFTEK

Found in IPI00364124, Tax_Id=10116 Gene_Symbol=Lrpap1 Alpha-2-macroglobulin receptor-associated protein precursor

Match to Query 1347: 1849.912148 from(925.963350,2+)

Title: 091008RatKidney_NoSalt_20.5946.5946.2.dta

Data file K:\NewmanPaper \Piliang \3SubProteomes \Piliang3SP $\backslash$ mgf5ppm\ERLIC_3SubProteomes5ppm.mgf
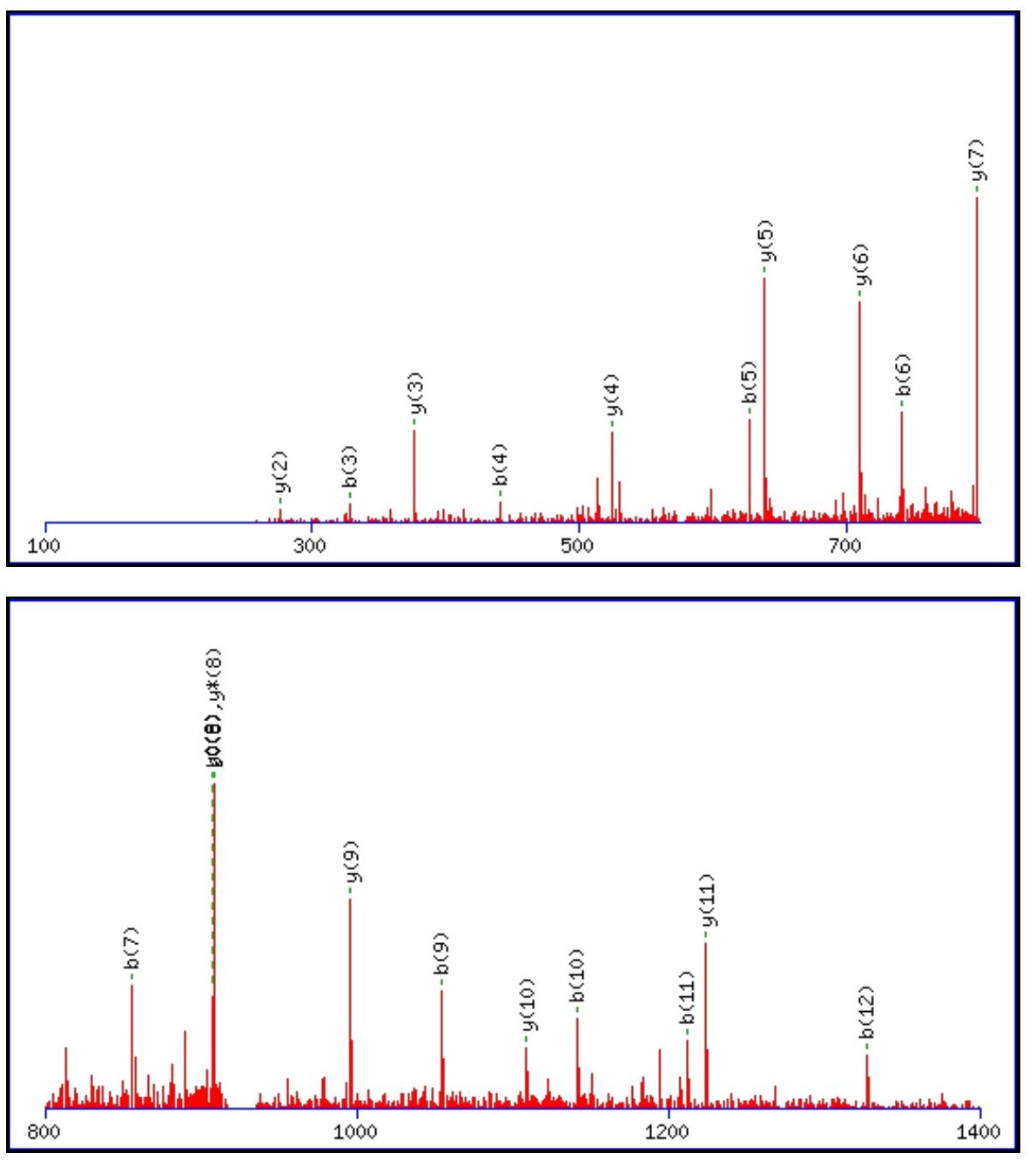


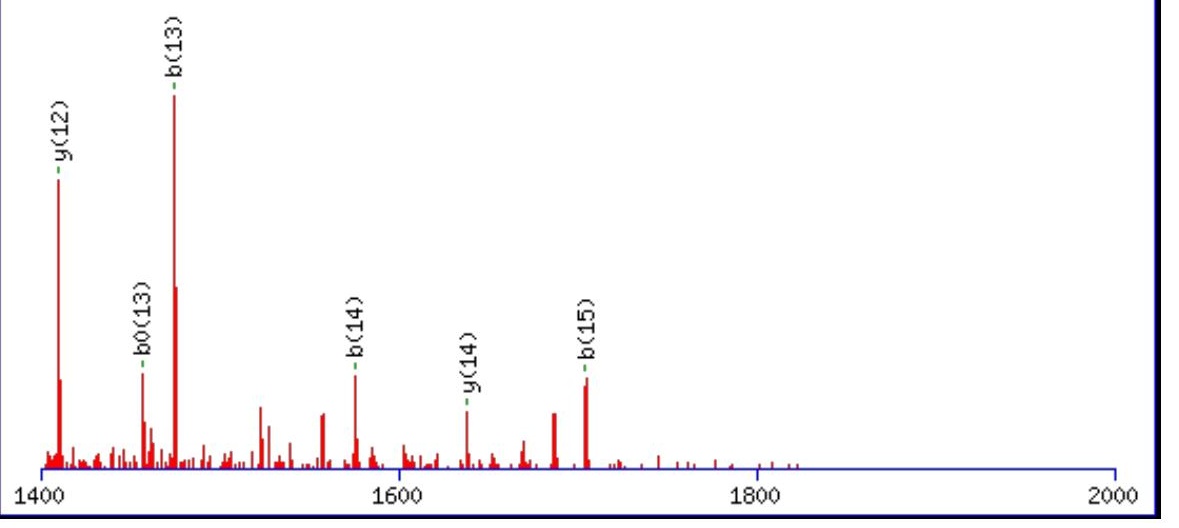

Monoisotopic mass of neutral peptide $\operatorname{Mr}($ calc): 1849.9098

Fixed modifications: Carbamidomethyl (C)

Variable modifications:

N12 : Deamidated_N (N)

Ions Score: 111 Expect: 2e-009

Matches (Bold Red): 27/158 fragment ions using 36 most intense peaks

\begin{tabular}{|c|c|c|c|c|c|c|c|c|c|c|c|c|c|c|}
\hline \# & b & $\mathbf{b}^{++}$ & b* & $\mathbf{b}^{*^{++}}$ & $\mathbf{b}^{0}$ & $\mathbf{b}^{0++}$ & Seq. & $\mathbf{y}$ & $\mathbf{y}^{++}$ & $\mathbf{y}^{*}$ & $\mathbf{y}^{*^{++}}$ & $\mathbf{y}^{0}$ & $\mathbf{y}^{0++}$ & \# \\
\hline 1 & 100.0757 & 50.5415 & & & & & $\mathbf{V}$ & & & & & & & 16 \\
\hline 2 & 213.1598 & 107.0835 & & & & & I & 1751.8487 & 876.4280 & 1734.8221 & 867.9147 & 1733.8381 & 867.4227 & 15 \\
\hline 3 & 328.1867 & 164.5970 & & & 310.1761 & 155.5917 & D & 1638.7646 & 819.8859 & 1621.7380 & 811.3727 & 1620.7540 & 810.8806 & 14 \\
\hline 4 & 441.2708 & 221.1390 & & & 423.2602 & 212.1337 & $\mathbf{L}$ & 1523.7376 & 762.3725 & 1506.7111 & 753.8592 & 1505.7271 & 753.3672 & 13 \\
\hline 5 & 627.3501 & 314.1787 & & & 609.3395 & 305.1734 & $\mathbf{W}$ & 1410.6536 & 705.8304 & 1393.6270 & 697.3172 & 1392.6430 & 696.8251 & 12 \\
\hline 6 & 742.3770 & 371.6921 & & & 724.3665 & 362.6869 & D & 1224.5743 & 612.7908 & 1207.5477 & 604.2775 & 1206.5637 & 603.7855 & 11 \\
\hline 7 & 855.4611 & 428.2342 & & & 837.4505 & 419.2289 & $\mathbf{L}$ & 1109.5473 & 555.2773 & 1092.5208 & 546.7640 & 1091.5368 & 546.2720 & 10 \\
\hline 8 & 926.4982 & 463.7527 & & & 908.4876 & 454.7475 & A & 996.4633 & 498.7353 & 979.4367 & 490.2220 & 978.4527 & 489.7300 & 9 \\
\hline 9 & 1054.5568 & 527.7820 & 1037.5302 & 519.2688 & 1036.5462 & 518.7767 & $\mathbf{Q}$ & 925.4261 & 463.2167 & 908.3996 & 454.7034 & 907.4156 & 454.2114 & 8 \\
\hline 10 & 1141.5888 & 571.2980 & 1124.5623 & 562.7848 & 1123.5782 & 562.2928 & $\mathbf{S}$ & 797.3676 & 399.1874 & 780.3410 & 390.6741 & 779.3570 & 390.1821 & 7 \\
\hline 11 & 1212.6259 & 606.8166 & 1195.5994 & 598.3033 & 1194.6154 & 597.8113 & A & 710.3355 & 355.6714 & 693.3090 & 347.1581 & 692.3250 & 346.6661 & 6 \\
\hline 12 & 1327.6529 & 664.3301 & 1310.6263 & 655.8168 & 1309.6423 & 655.3248 & $\mathbf{N}$ & 639.2984 & 320.1529 & 622.2719 & 311.6396 & 621.2879 & 311.1476 & 5 \\
\hline 13 & 1474.7213 & 737.8643 & 1457.6947 & 729.3510 & 1456.7107 & 728.8590 & $\mathbf{F}$ & 524.2715 & 262.6394 & 507.2449 & 254.1261 & 506.2609 & 253.6341 & 4 \\
\hline 14 & 1575.7689 & 788.3881 & 1558.7424 & 779.8748 & 1557.7584 & 779.3828 & $\mathbf{T}$ & 377.2031 & 189.1052 & 360.1765 & 180.5919 & 359.1925 & 180.0999 & 3 \\
\hline 15 & 1704.8115 & 852.9094 & 1687.7850 & 844.3961 & 1686.8010 & 843.9041 & $\mathbf{E}$ & 276.1554 & 138.5813 & 259.1288 & 130.0681 & 258.1448 & 129.5761 & 2 \\
\hline 16 & & & & & & & $\mathbf{K}$ & 147.1128 & 74.0600 & 130.0863 & 65.5468 & & & 1 \\
\hline
\end{tabular}

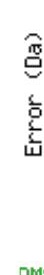<smiles>C1CCC2(C1)CC2</smiles><smiles>C1CC2C1C1C3CCC4C2C1C34</smiles>

S error $205 \mathrm{ppm}$

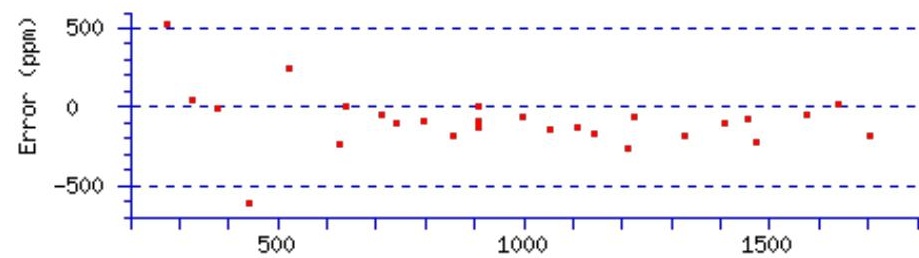

RMS error $205 \mathrm{ppm}$

\section{All matches to this query}

\begin{tabular}{|l|l|c|l|}
\hline Score & Mr(calc): & Delta & \multicolumn{1}{c|}{ Sequence } \\
\hline 110.6 & 1849.9098 & 0.0024 & VIDLWDLAQSANFTEK \\
\hline 34.5 & 1848.9258 & 0.9864 & VIDLWDLAQSANFTEK \\
\hline 5.4 & 1849.9244 & -0.0122 & NLVHYLTTVERMETK \\
\hline 4.0 & 1849.9267 & -0.0145 & VLYESLKYPSFPLSK \\
\hline 1.5 & 1849.9298 & -0.0177 & KDLRNDLNTIQELAK \\
\hline & & & \\
\hline
\end{tabular}




\section{\begin{tabular}{|l|l|l|l|}
0.6 & 1849.8959 & 0.0163 & DPYLAGPGSRPLSYSDR \\
\hline
\end{tabular}}

Spectrum No: 40; Query: 2776; Rank: 1

\section{Peptide View}

MS/MS Fragmentation of EGNCSVQSGLTWQDCDFKDAEEAATGECTTTLGK

Found in IPI00327182, Tax_Id=10116 Gene_Symbol=Kng1 T-kininogen 1 precursor

Match to Query 2776: 3765.583392 from(1256.201740,3+)

Title: 100101RatKid_NS_deglyco_24.4023.4023.3.dta

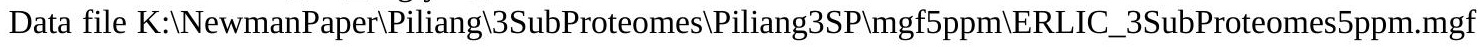
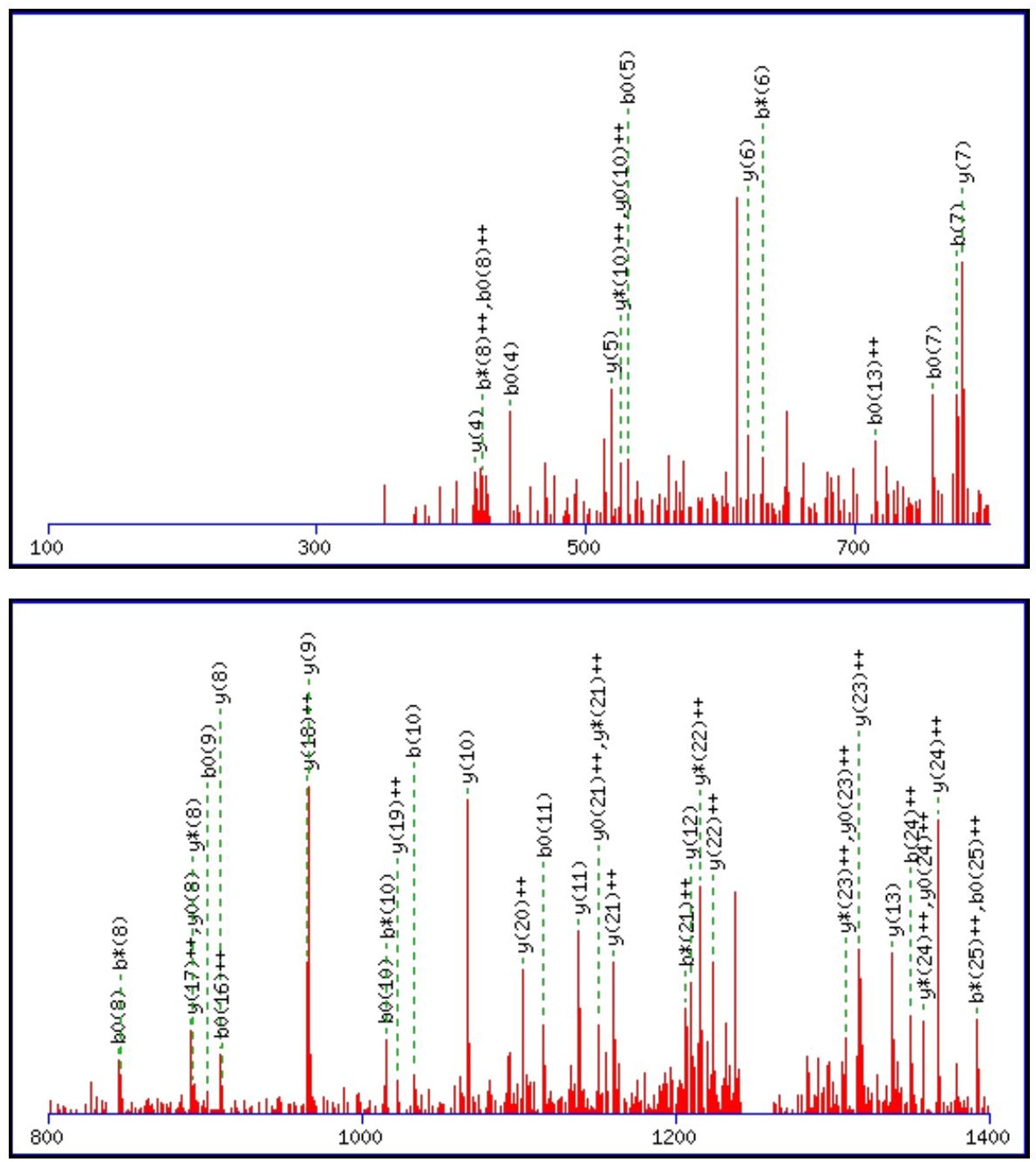


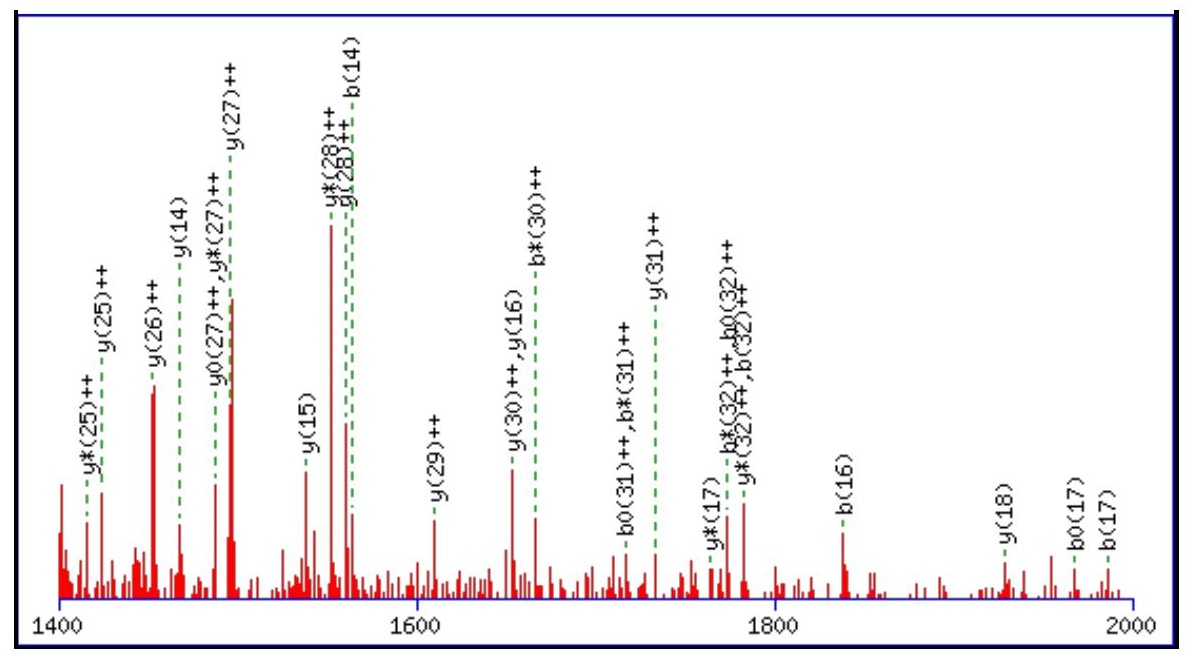

Monoisotopic mass of neutral peptide $\operatorname{Mr}($ calc): 3765.5717

Fixed modifications: Carbamidomethyl (C)

Variable modifications:

N3 : Deamidated $\mathrm{N}(\mathrm{N})$

Ions Score: 110 Expect: $4.9 \mathrm{e}-\odot 09$

Matches (Bold Red): 76/386 fragment ions using 121 most intense peaks

\begin{tabular}{|c|c|c|c|c|c|c|c|c|c|c|c|c|c|c|}
\hline \# & b & $\mathbf{b}^{++}$ & $\mathbf{b}^{*}$ & $\mathbf{b}^{*^{++}}$ & $\mathbf{b}^{\mathbf{0}}$ & $\mathbf{b}^{0++}$ & Seq. & $\mathbf{y}$ & $\mathbf{y}^{++}$ & $\mathbf{y}^{*}$ & $\mathrm{y}^{\mathrm{*}^{++}}$ & $\mathbf{y}^{0}$ & $y^{0++}$ & \# \\
\hline 1 & 130.0499 & 65.5286 & & & 112.0393 & 56.5233 & $\mathbf{E}$ & & & & & & & 34 \\
\hline 2 & 187.0713 & 94.0393 & & & 169.0608 & 85.0340 & G & 3637.5363 & 1819.2718 & 3620.5098 & & 3619.5258 & & 33 \\
\hline 3 & 302.0983 & 151.5528 & 285.0717 & 143.0395 & 284.0877 & 142.5475 & $\mathbf{N}$ & 3580.5149 & 1790.7611 & 3563.4883 & 1782.2478 & 3562.5043 & 1781.7558 & 32 \\
\hline 4 & 462.1289 & 231.5681 & 445.1024 & 223.0548 & 444.1184 & 222.5628 & C & 3465.4879 & 1733.2476 & |3448.4614 & 1724.7343 & 3447.4774 & 1724.2423 & 31 \\
\hline 5 & 549.1609 & 275.0841 & 532.1344 & 266.5708 & 531.1504 & 266.0788 & $S$ & 3305.4573 & 1653.2323 & 3288.4307 & 1644.7190 & 3287.4467 & 1644.2270 & 30 \\
\hline 6 & 648.2294 & 324.6183 & 631.2028 & 316.1050 & 630.2188 & 315.6130 & V & 3218.4253 & 1609.7163 & |3201.3987 & 1601.2030 & 3200.4147 & 1600.7110 & 29 \\
\hline 7 & 776.2879 & 388.6476 & 759.2614 & 380.1343 & 758.2774 & 379.6423 & $\mathbf{Q}$ & 3119.3568 & 1560.1821 & 3102.3303 & 1551.6688 & 3101.3463 & 1551.1768 & 28 \\
\hline 8 & 863.3200 & 432.1636 & 846.2934 & 423.6503 & 845.3094 & 423.1583 & $S$ & 2991.2983 & 1496.1528 & 2974.2717 & 1487.6395 & 2973.2877 & 1487.1475 & 27 \\
\hline 9 & 920.3414 & 460.6744 & 903.3149 & 452.1611 & 902.3309 & 451.6691 & G & 2904.2662 & 1452.6368 & 2887.2397 & 1444.1235 & 2886.2557 & 1443.6315 & 26 \\
\hline 10 & 1033.4255 & 517.2164 & 1016.3989 & 508.7031 & 1015.4149 & 508.2111 & $\mathbf{L}$ & 2847.2448 & 1424.1260 & 2830.2182 & 1415.6127 & 2829.2342 & 1415.1207 & 25 \\
\hline 11 & 1134.4732 & 567.7402 & 1117.4466 & 559.2270 & 1116.4626 & 558.7349 & $T$ & 2734.1607 & 1367.5840 & 2717.1342 & 1359.0707 & 2716.1501 & 1358.5787 & 24 \\
\hline 12 & 20.5525 & 660.7799 & 1303.5259 & 652.2666 & 1302.5419 & 651.7746 & $\mathbf{W}$ & 2633.1130 & 1317.0602 & 2616.0865 & 8469 & 2615 & 549 & 23 \\
\hline 13 & 1448.6111 & 724.8092 & 1431.5845 & 716.2959 & 1430.6005 & 715.8039 & $\mathbf{Q}$ & 2447.0337 & 1224.0205 & 2430.0072 & 1215.5072 & 2429.0231 & 1215.0152 & 22 \\
\hline 14 & 1563.6380 & 782.3226 & 1546.6115 & 773.8094 & 1545.6274 & 773.3174 & D & 2318.9751 & 1159.9912 & 2301.9486 & 1151.4779 & 2300.9646 & 1150.9859 & 21 \\
\hline 15 & 1723.6687 & 862.3 & 1706.6421 & & 1705.6581 & 853.3327 & C & 2203.9482 & 1102 & 9216 & & & 725 & 20 \\
\hline 16 & 1838.6956 & 919.8514 & 1821.6691 & 911.3382 & 1820.6850 & 910.8462 & D & 2043.9175 & 1022.4624 & 2026.8910 & 1013.9491 & 2025.9070 & 1013.4571 & 19 \\
\hline 17 & 1985.7640 & 993.3856 & 1968.7375 & 984.8724 & 1967.7534 & 984.3804 & $\mathbf{F}$ & 1928.8906 & 964.9489 & 1911.8641 & 956.4357 & 1910.8800 & 955.9437 & 18 \\
\hline 18 & 90 & 31 & 2096. & 1048 & 2095 & 4278 & $\mathbf{K}$ & 1781.8222 & & 1764.7956 & 015 & 1763 & 882.4094 & 17 \\
\hline 19 & 2228.8859 & 1114.9466 & 2211.8594 & 1106.4333 & 2210.8754 & 1105.9413 & D & 1653.7272 & 827.3673 & 1636.7007 & 818.8540 & 1635.7167 & 818.3620 & 16 \\
\hline 20 & 2299.9230 & 1150.4652 & 2282.8965 & 1141.9519 & 2281.9125 & 1141.4599 & A & 1538.7003 & 769.8538 & 1521.6737 & 761.3405 & 1520.6897 & 760.8485 & 15 \\
\hline 21 & 2428 & 1214. & 2411. & 1206.4732 & 2410.9551 & 1205.9812 & $\mathbf{E}$ & 1467.6632 & 734.3352 & 1450.6366 & 725.8219 & 1449.6526 & 725.3299 & 14 \\
\hline 22 & 2558.0082 & 1279.5077 & 2540.9817 & 1270.9945 & 2539.9977 & 1270.5025 & $\mathbf{E}$ & \begin{tabular}{|l|}
1338.6206 \\
\end{tabular} & 669.8139 & 1321.5940 & 661.3007 & 1320.6100 & 660.8086 & 13 \\
\hline 23 & 2629.0453 & 1315.0263 & 2612.0188 & 1306.5130 & 2611.0348 & 1306.0210 & $\mathbf{A}$ & 1209.5780 & 605.2926 & 1192.5514 & 596.7794 & 1191.5674 & 596.2873 & 12 \\
\hline 24 & 2700.0824 & 1350.5449 & 2683.0559 & 1342.0316 & 2682.0719 & 1341.5396 & A & \begin{tabular}{|l|}
1138.5409 \\
\end{tabular} & 569.7741 & 1121.5143 & 561.2608 & 1120.5303 & 560.7688 & 11 \\
\hline 25 & 2801.1301 & 1401.0687 & 2784.1036 & 1392.5554 & 2783.1196 & 1392.0634 & $\mathbf{T}$ & 1067.5038 & 534.2555 & 1050.4772 & 525.7422 & 1049.4932 & 525.2502 & 10 \\
\hline 26 & 2858.1516 & 1429.5794 & 2841.1250 & 1421.0662 & 2840.1410 & 1420.5742 & G & 966.4561 & 483.7317 & 949.4295 & 475.2184 & 948.4455 & 474.7264 & 9 \\
\hline 27 & 2987.1942 & 1494.1007 & 2970.1676 & 1485.5875 & 2969.1836 & 1485.0954 & $\mathbf{E}$ & 909.4346 & 455.2209 & 892.4081 & 446.7077 & 891.4240 & 446.2157 & 8 \\
\hline 28 & 3147.2248 & 1574.1161 & 3130.1983 & 1565.6028 & 3129.2143 & 1565.1108 & $\mathrm{C}$ & 780.3920 & 390.6996 & 763.3655 & 382.1864 & 762.3815 & 381.6944 & 7 \\
\hline 29 & 3248.2725 & 1624.6399 & 3231.2460 & 1616.1266 & 3230.2619 & 1615.6346 & $\mathbf{T}$ & 620.3614 & 310.6843 & 603.3348 & 302.1710 & 602.3508 & 301.6790 & 6 \\
\hline 30 & 3349.3202 & \begin{tabular}{|l|}
1675.1637 \\
\end{tabular} & 3332.2936 & 1666.6505 & 3331.3096 & 1666.1585 & $\mathbf{T}$ & 519.3137 & 260.1605 & 502.2871 & 251.6472 & 501.3031 & 251.1552 & 5 \\
\hline 31 & 3450.3679 & 1725.6876 & 3433.3413 & 1717.1743 & 3432.3573 & \begin{tabular}{|l|}
1716.6823 \\
\end{tabular} & $T$ & 418.2660 & 209.6366 & 401.2395 & 201.1234 & 400.2554 & 200.6314 & 4 \\
\hline 32 & |3563.4519| & 1782.2296 & $|3546.4254|$ & 1773.7163 & |3545.4414 & $\mid 1773.2243$ & $\mathbf{L}$ & 317.2183 & 159.1128 & |300.1918 & 150.5995 & & & 3 \\
\hline
\end{tabular}




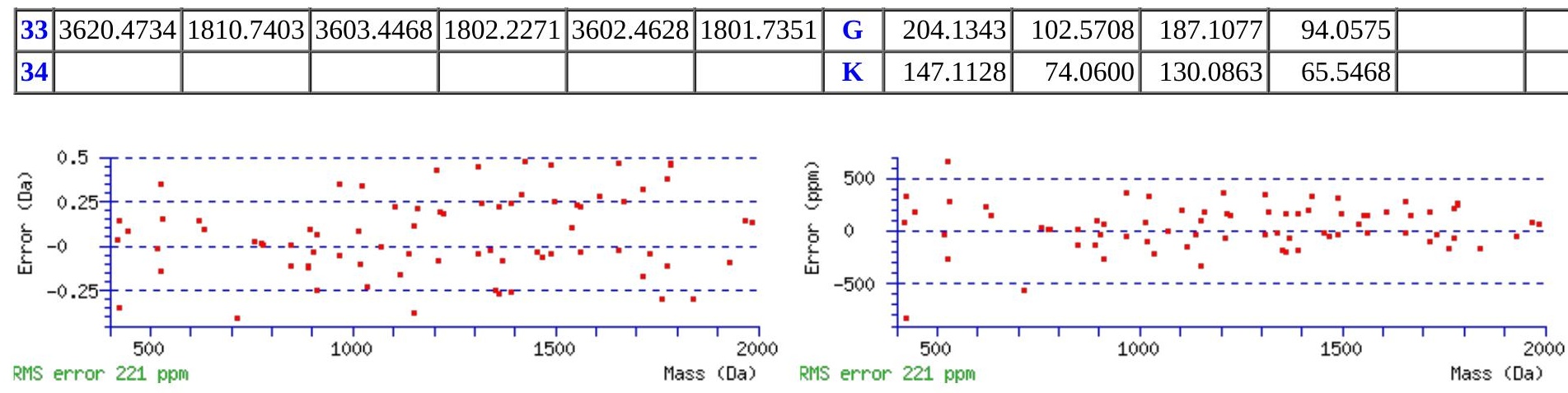

\section{All matches to this query}

\begin{tabular}{|l|l|l|l|}
\hline Score & Mr(calc): & Delta & \multicolumn{1}{c|}{ Sequence } \\
\hline 110.3 & 3765.5717 & 0.0117 & EGNCSVQSGLTWQDCDFKDAEEAATGECTTTLGK \\
\hline 79.5 & 3764.5877 & 0.9957 & EGNCSVQSGLTWODCDFKDAEEAATGECTTTLGK \\
\hline 5.5 & 3763.6105 & 1.9728 & DFHGSLLGFYENGELCDVTLKVGSKLISCHK \\
\hline 2.2 & 3763.5614 & 2.0220 & DSATGETEVSTPPKASMKLVNFQOSEPTSANEK \\
\hline 1.5 & 3764.5454 & 1.0380 & DSATGETEVSTPPKASMKLVNFQQSEPTSANEK \\
\hline 1.4 & 3763.6105 & 1.9728 & DFHGSLLGFYENGELCDVTLKVGSKLISCHK \\
\hline 1.2 & 3765.5914 & -0.0080 & IPDLESETWMSPLAWLEKGVNTSVMLQSLR \\
\hline 1.2 & 3764.6062 & 0.9772 & AGSMTVTVGETCSLECKVAGTPELSVEWYKDGK \\
\hline 1.1 & 3765.6107 & -0.0273 & NGSSNPLNWNSSKNGDWHLEANTSTLYYLVSGR \\
\hline 0.3 & 3763.5924 & 1.9910 & AFSTCSSHMVVVSITYESCMFMYMKTSAKER \\
\hline
\end{tabular}

Spectrum No: 41; Query: 1568; Rank: 1

\section{Peptide View}

MS/MS Fragmentation of MGAGEALGNASQLFEAWLK

Found in IPI00327398, Tax_Id=10116 Gene_Symbol=Enpep Isoform 1 of Glutamyl aminopeptidase

Match to Query 1568: 1992.966768 from(997.490660,2+)

Title: 100101RatKid_NS_deglyco_19.6506.6506.2.dta

Data file K:\NewmanPaper|Piliang\3SubProteomes\Piliang3SP\mgf5ppm\ERLIC_3SubProteomes5ppm.mgf

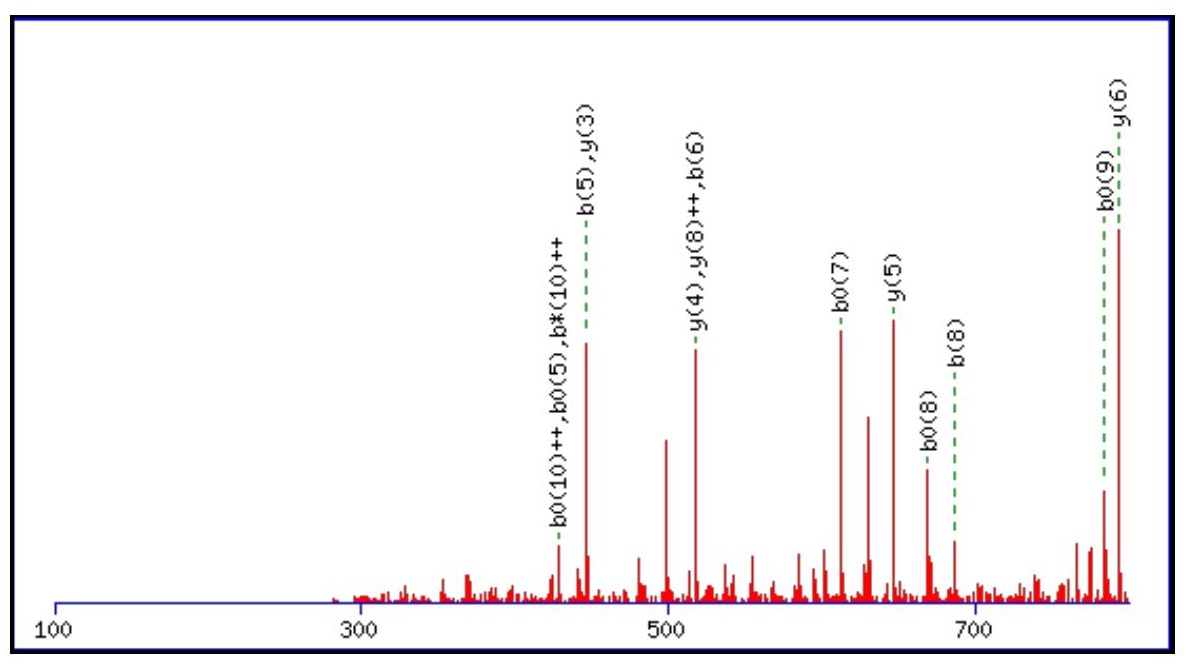



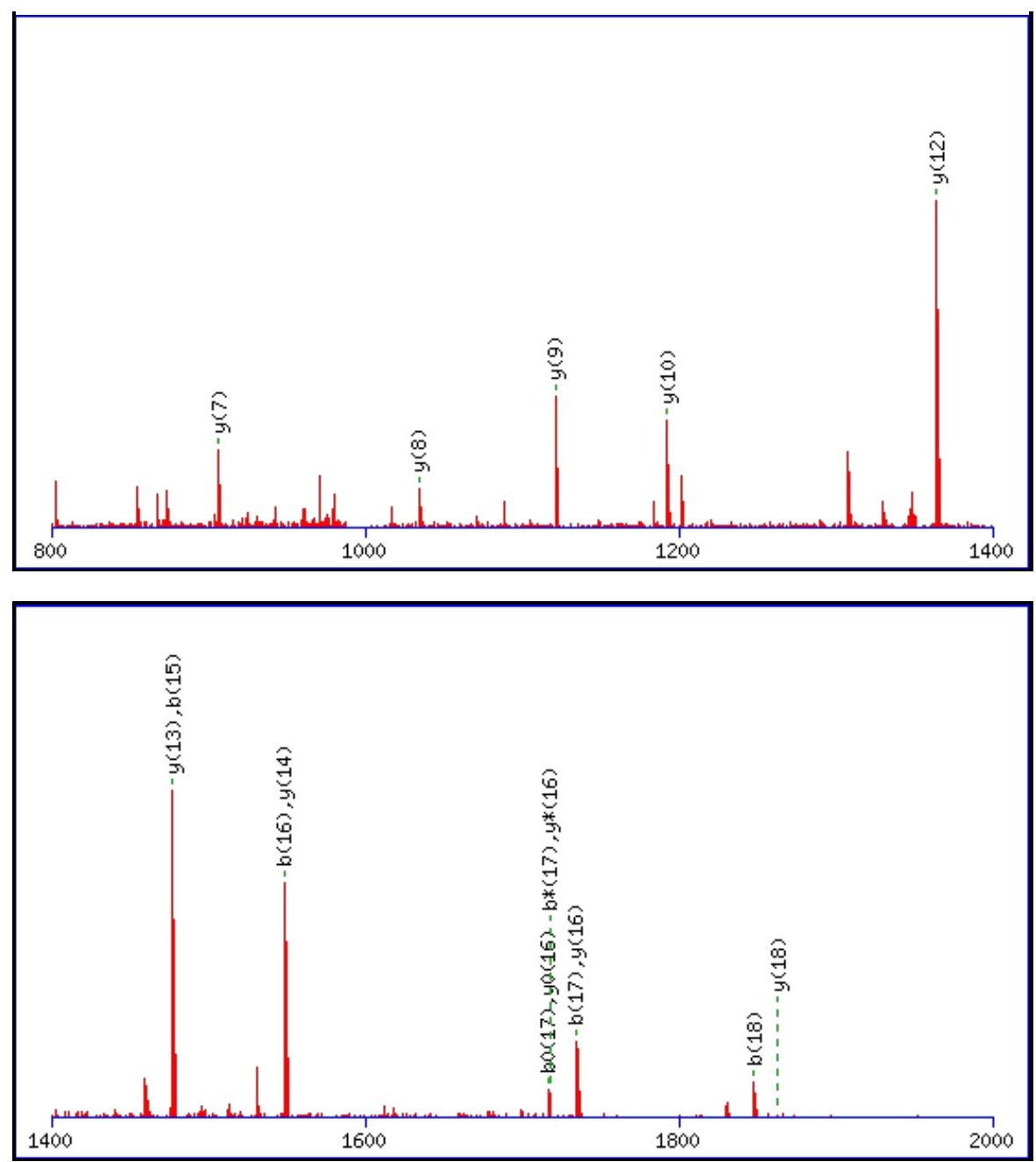

Monoisotopic mass of neutral peptide $\operatorname{Mr}($ calc): 1992.9615

Fixed modifications: Carbamidomethyl (C)

Variable modifications:

N9: Deamidated $\mathrm{N}(\mathrm{N})$

Ions Score: 110 Expect: $2.1 \mathrm{e}-009$

Matches (Bold Red): 31/184 fragment ions using 39 most intense peaks

\begin{tabular}{|c|c|c|c|c|c|c|c|c|c|c|c|c|c|c|}
\hline \# & b & $\mathbf{b}^{++}$ & $\mathbf{b}^{*}$ & $\mathbf{b}^{*^{++}}$ & $\mathbf{b}^{0}$ & $\mathbf{b}^{0++}$ & Seq. & $\mathbf{y}$ & $y^{++}$ & $\mathbf{y}^{*}$ & $\mathbf{y}^{*^{++}}$ & $\mathbf{y}^{\mathbf{0}}$ & $y^{0++}$ & \# \\
\hline 1 & 132.0478 & 66.5275 & & & & & $\mathbf{M}$ & & & & & & & 19 \\
\hline 2 & 189.0692 & 95.0383 & & & & & G & 1862.9283 & 931.9678 & 1845.9018 & 923.4545 & 1844.9177 & 922.9625 & 18 \\
\hline 3 & 260.1063 & 130.5568 & & & & & A & 1805.9068 & 903.4571 & 1788.8803 & 894.9438 & 1787.8963 & 894.4518 & 17 \\
\hline 4 & 317.1278 & 159.0675 & & & & & G & 1734.8697 & 867.9385 & 1717.8432 & 859.4252 & 1716.8592 & 858.9332 & 16 \\
\hline 5 & 446.1704 & 223.5888 & & & 428.1598 & 214.5836 & $\mathbf{E}$ & 1677.8483 & 839.4278 & 1660.8217 & 830.9145 & 1659.8377 & 830.4225 & 15 \\
\hline 6 & 517.2075 & 259.1074 & & & 499.1969 & 250.1021 & A & 1548.8057 & 774.9065 & 1531.7791 & 766.3932 & 1530.7951 & 765.9012 & 14 \\
\hline 7 & 630.2916 & 315.6494 & & & 612.2810 & 306.6441 & $\mathbf{L}$ & 1477.7686 & 739.3879 & 1460.7420 & 730.8746 & 1459.7580 & 730.3826 & 13 \\
\hline 8 & 687.3130 & 344.1602 & & & 669.3025 & 335.1549 & G & 1364.6845 & 682.8459 & 1347.6579 & 674.3326 & 1346.6739 & 673.8406 & 12 \\
\hline 9 & 802.3400 & 401.6736 & 785.3134 & 393.1604 & 784.3294 & 392.6683 & $\mathbf{N}$ & 1307.6630 & 654.3351 & 1290.6365 & 645.8219 & 1289.6525 & 645.3299 & 11 \\
\hline 10 & 873.3771 & 437.1922 & 856.3505 & 428.6789 & 855.3665 & 428.1869 & A & 1192.6361 & 596.8217 & 1175.6095 & 588.3084 & 1174.6255 & 587.8164 & 10 \\
\hline 11 & 960.4091 & 480.7082 & 943.3826 & 472.1949 & 942.3986 & 471.7029 & S & 1121.5990 & 561.3031 & 1104.5724 & 552.7898 & 1103.5884 & 552.2978 & 9 \\
\hline 12 & 1088.4677 & 544.7375 & 1071.4411 & 536.2242 & 1070.4571 & 535.7322 & $\mathbf{Q}$ & 1034.5669 & 517.7871 & 1017.5404 & 509.2738 & 1016.5564 & 508.7818 & 8 \\
\hline 13 & 1201.5518 & 601.2795 & 1184.5252 & 592.7662 & 1183.5412 & 592.2742 & $\mathbf{L}$ & 906.5084 & 453.7578 & 889.4818 & 445.2445 & 888.4978 & 444.7525 & 7 \\
\hline 14 & 1348.6202 & 674.8137 & 1331.5936 & 666.3004 & 1330.6096 & 665.8084 & $\mathbf{F}$ & 793.4243 & 397.2158 & 776.3978 & 388.7025 & 775.4137 & 388.2105 & 6 \\
\hline 15 & 1477.6628 & 739.3350 & 1460.6362 & 730.8217 & 1459.6522 & 730.3297 & $\mathbf{E}$ & 646.3559 & 323.6816 & 629.3293 & 315.1683 & 628.3453 & 314.6763 & 5 \\
\hline 16 & 1548.6999 & 774.8536 & 1531.6733 & 766.3403 & 1530.6893 & 765.8483 & A & 517.3133 & 259.1603 & 500.2867 & 250.6470 & & & 4 \\
\hline 17 & 1734.7792 & 867.8932 & 1717.7526 & 859.3800 & 1716.7686 & 858.8880 & W & 446.2762 & 223.6417 & 429.2496 & 215.1285 & & & 3 \\
\hline 18 & 1847.8633 & 924.4353 & 1830.8367 & 915.9220 & 1829.8527 & 915.4300 & $\mathbf{L}$ & 260.1969 & 130.6021 & 243.1703 & 122.0888 & & & 2 \\
\hline
\end{tabular}




\section{\begin{tabular}{l|l||}
19 & 19
\end{tabular}}
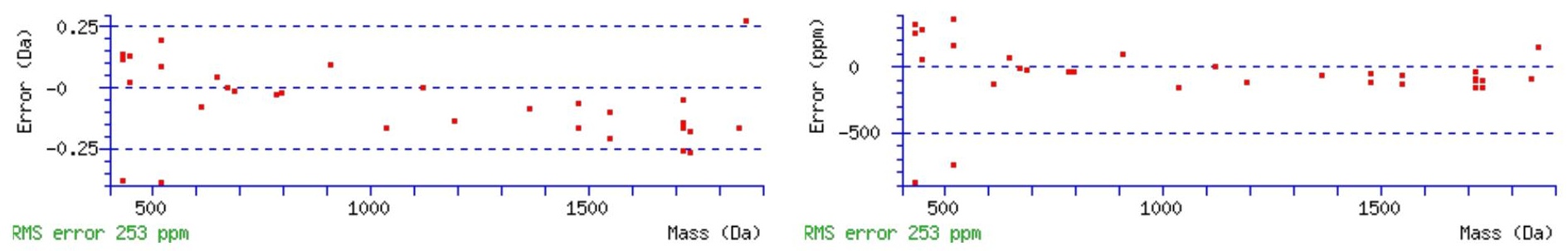

\section{All matches to this query}

\begin{tabular}{|l|l|l|l|}
\hline Score & Mr(calc): & Delta & \multicolumn{1}{|c|}{ Sequence } \\
\hline 110.1 & 1992.9615 & 0.0053 & MGAGEALGNASQLFEAWLK \\
\hline 43.2 & 1991.9775 & 0.9893 & MGAGEALGNASQLFEAWLK \\
\hline 12.7 & 1992.9629 & 0.0038 & DLNTLREAQENINKQK \\
\hline 12.0 & 1992.9686 & -0.0019 & KVTVTTYNTSITIHKK \\
\hline 10.3 & 1992.9686 & -0.0019 & KVTVTTYNTSITIHKK \\
\hline 7.1 & 1992.9856 & -0.0188 & VCKEIANIPSKNIWNK \\
\hline 4.3 & 1992.9526 & 0.0142 & SATICLAYLMRTNRVK \\
\hline 4.0 & 1992.9686 & -0.0019 & KVTVTTYNTSITIHKK \\
\hline 3.3 & 1992.9686 & -0.0019 & KVTVTTYNTSITIHKK \\
\hline 2.7 & 1991.9679 & 0.9988 & GSILQFMPFTTVSELVK \\
\hline
\end{tabular}

Spectrum No: 42; Query: 1670; Rank: 1

\section{Peptide View}

\section{MS/MS Fragmentation of YCGNSTPSSVDTSSNVASVK}

Found in IPI00196620, Tax_Id=10116 Gene_Symbol=Cubn Cubilin precursor

Match to Query 1670: 2059.892388 from(1030.953470,2+)

Title: 091008RatKidney_NoSalt_19.1023.1023.2.dta

Data file K:INewmanPaper|Piliangl3SubProteomes\Piliang3SP\mgf5ppm\ERLIC_3SubProteomes5ppm.mgf

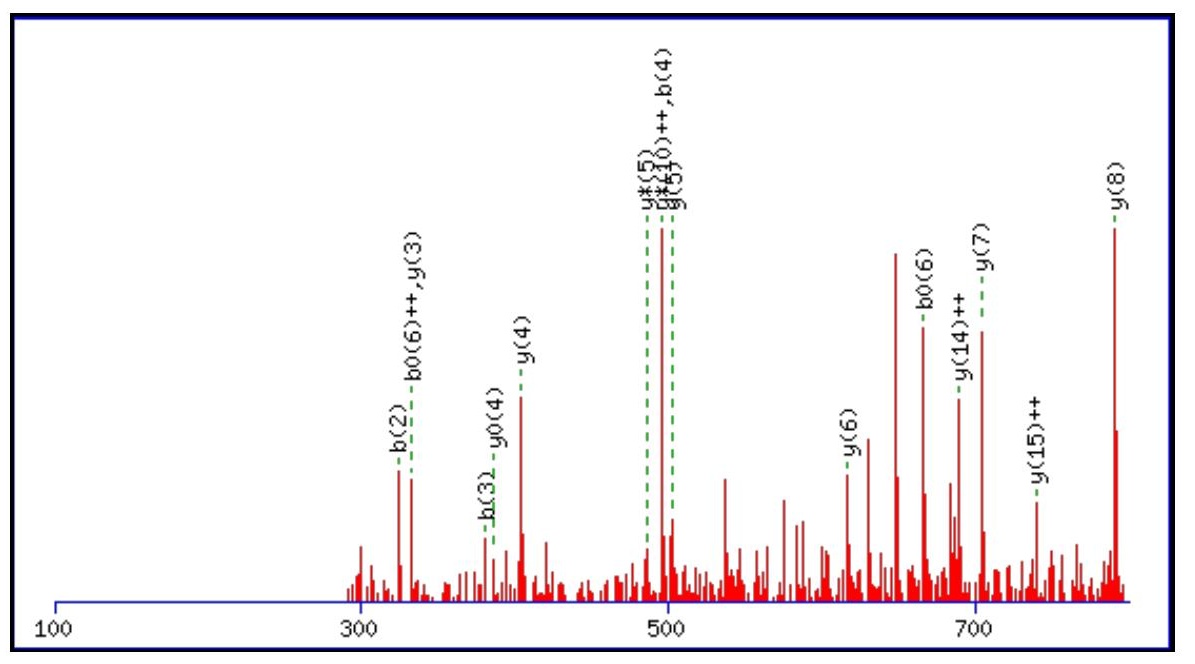



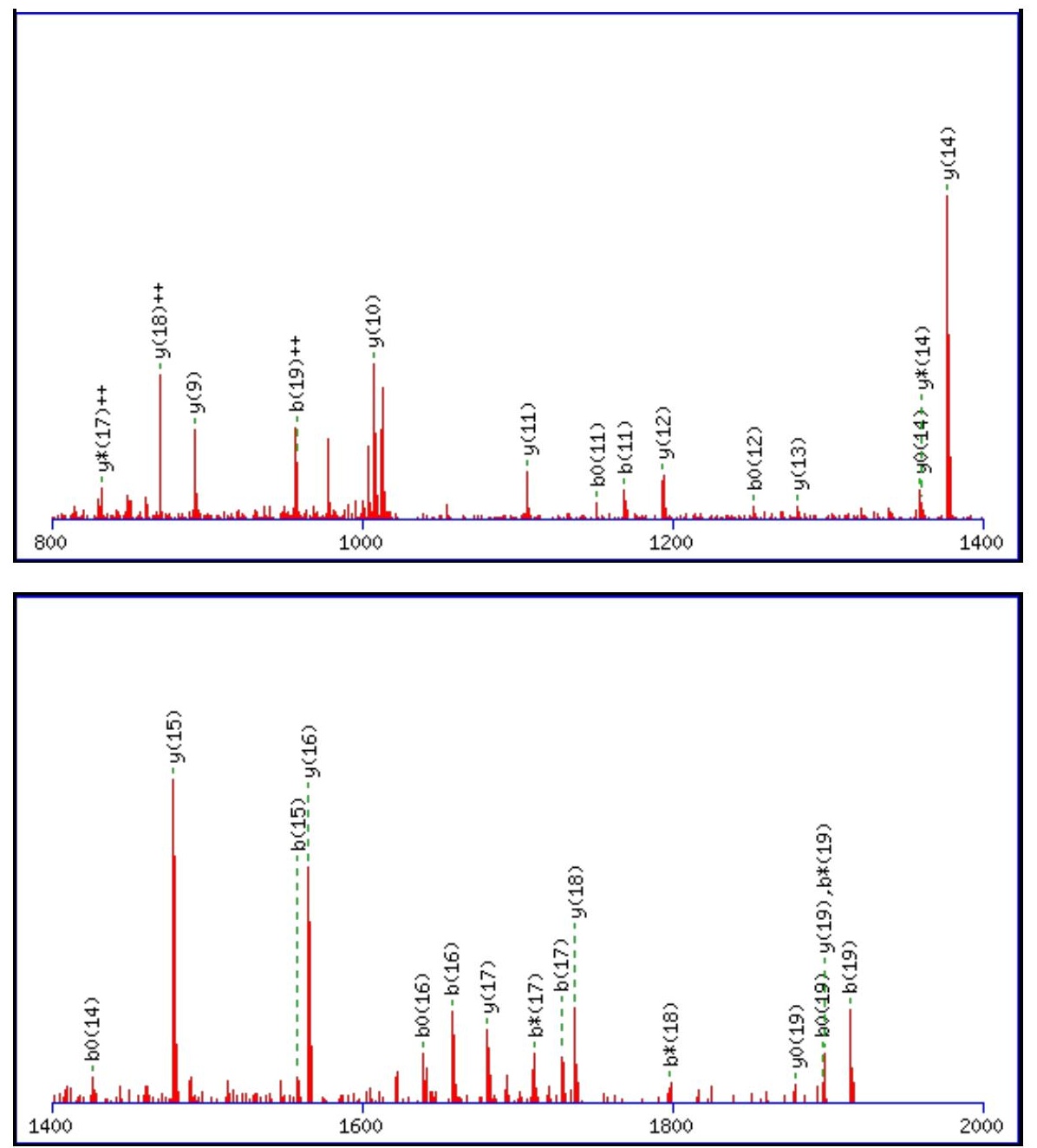

Monoisotopic mass of neutral peptide $\operatorname{Mr}($ calc): 2059.9004

Fixed modifications: Carbamidomethyl (C)

Variable modifications:

N4 : Deamidated $\mathrm{N}(\mathrm{N})$

Ions Score: 110 Expect: $2.6 \mathrm{e}-009$

Matches (Bold Red): 46/210 fragment ions using 87 most intense peaks

\begin{tabular}{|c|c|c|c|c|c|c|c|c|c|c|c|c|c|c|}
\hline \# & b & $\mathbf{b}^{++}$ & b* & $\mathbf{b}^{*^{++}}$ & $\mathbf{b}^{\mathbf{0}}$ & $\mathbf{b}^{0++}$ & Seq. & $\mathbf{y}$ & $\mathbf{y}^{++}$ & $\mathbf{y}^{*}$ & $\mathrm{y}^{*^{++}}$ & $\mathbf{y}^{0}$ & $\mathbf{y}^{0++}$ & \# \\
\hline 1 & 164.0706 & 82.5389 & & & & & $\mathbf{Y}$ & & & & & & & 20 \\
\hline 2 & 324.1013 & 162.5543 & & & & & C & 1897.8444 & 949.4258 & 1880.8178 & 940.9125 & 1879.8338 & 940.4205 & 19 \\
\hline 3 & 381.1227 & 191.0650 & & & & & G & 1737.8137 & 869.4105 & 1720.7872 & 860.8972 & 1719.8032 & 860.4052 & 18 \\
\hline 4 & 496.1497 & 248.5785 & 479.1231 & 240.0652 & & & $\mathbf{N}$ & 1680.7923 & 840.8998 & 1663.7657 & 832.3865 & 1662.7817 & 831.8945 & 17 \\
\hline 5 & 583.1817 & 292.0945 & 566.1551 & 283.5812 & 565.1711 & 283.0892 & S & 1565.7653 & 783.3863 & 1548.7388 & 774.8730 & 1547.7548 & 774.3810 & 16 \\
\hline 6 & 684.2294 & 342.6183 & 667.2028 & 334.1050 & 666.2188 & 333.6130 & $\mathbf{T}$ & 1478.7333 & 739.8703 & 1461.7067 & 731.3570 & 1460.7227 & 730.8650 & 15 \\
\hline 7 & 781.2821 & 391.1447 & 764.2556 & 382.6314 & 763.2716 & 382.1394 & $\mathbf{P}$ & 1377.6856 & 689.3464 & 1360.6591 & 680.8332 & 1359.6750 & 680.3412 & 14 \\
\hline 8 & 868.3142 & 434.6607 & 851.2876 & 426.1474 & 850.3036 & 425.6554 & S & 1280.6328 & 640.8201 & 1263.6063 & 632.3068 & 1262.6223 & 631.8148 & 13 \\
\hline 9 & 955.3462 & 478.1767 & 938.3196 & 469.6635 & 937.3356 & 469.1714 & S & 1193.6008 & 597.3040 & 1176.5743 & 588.7908 & 1175.5903 & 588.2988 & 12 \\
\hline 10 & 1054.4146 & 527.7109 & 1037.3880 & 519.1977 & 1036.4040 & 518.7057 & $\mathbf{V}$ & 1106.5688 & 553.7880 & 1089.5422 & 545.2748 & 1088.5582 & 544.7828 & 11 \\
\hline 11 & 1169.4415 & 585.2244 & 1152.4150 & 576.7111 & 1151.4310 & 576.2191 & D & 1007.5004 & 504.2538 & 990.4738 & 495.7406 & 989.4898 & 495.2485 & 10 \\
\hline 12 & 1270.4892 & 635.7482 & 1253.4627 & 627.2350 & 1252.4787 & 626.7430 & $\mathbf{T}$ & 892.4734 & 446.7404 & 875.4469 & 438.2271 & 874.4629 & 437.7351 & 9 \\
\hline 13 & 1357.5212 & 679.2643 & 1340.4947 & 670.7510 & 1339.5107 & 670.2590 & S & 791.4258 & 396.2165 & 774.3992 & 387.7032 & 773.4152 & 387.2112 & 8 \\
\hline 14 & 1444.5533 & 722.7803 & 1427.5267 & 714.2670 & 1426.5427 & 713.7750 & $\mathrm{~S}$ & 704.3937 & 352.7005 & 687.3672 & 344.1872 & 686.3832 & 343.6952 & 7 \\
\hline 15 & 1558.5962 & 779.8017 & 1541.5697 & 771.2885 & 1540.5856 & 770.7965 & $\mathbf{N}$ & 617.3617 & 309.1845 & 600.3352 & 300.6712 & 599.3511 & 300.1792 & 6 \\
\hline 16 & 1657.6646 & 829.3359 & 1640.6381 & 820.8227 & 1639.6540 & 820.3307 & V & 503.3188 & 252.1630 & 486.2922 & 243.6498 & 485.3082 & 243.1577 & 5 \\
\hline 17 & 1728.7017 & 864.8545 & 1711.6752 & 856.3412 & 1710.6912 & 855.8492 & A & 404.2504 & 202.6288 & 387.2238 & 194.1155 & 386.2398 & 193.6235 & 4 \\
\hline 18 & 1815.7338 & 908.3705 & 1798.7072 & 899.8572 & 1797.7232 & 899.3652 & $\mathrm{~S}$ & 333.2132 & 167.1103 & 316.1867 & 158.5970 & 315.2027 & 158.1050 & 3 \\
\hline
\end{tabular}




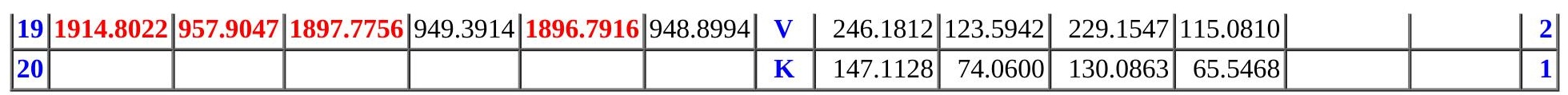
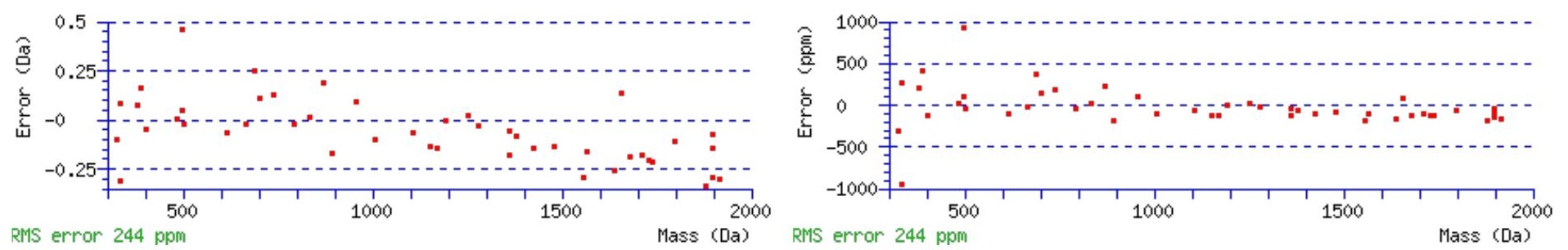

\section{All matches to this query}

\begin{tabular}{|l|l|l|l|}
\hline Score & Mr(calc): & Delta & \multicolumn{1}{|c|}{ Sequence } \\
\hline 109.8 & 2059.9004 & -0.0080 & YCGNSTPSSVDTSSNVASVK \\
\hline 72.0 & 2059.9004 & -0.0080 & YCGNSTPSSVDTSSNVASVK \\
\hline 4.9 & 2059.8985 & -0.0061 & RSGNMGIPGTMNQISPLK \\
\hline 4.1 & 2059.8985 & -0.0061 & RSGNMGIPGTMNQISPLK \\
\hline 1.0 & 2059.8750 & 0.0174 & MTSSSQLLLXKPKRR \\
\hline 0.4 & 2059.8951 & -0.0027 & LPLRSVSMNRGNGPSWK \\
\hline
\end{tabular}

Spectrum No: 43; Query: 2450; Rank: 1

\section{Peptide View}

\section{MS/MS Fragmentation of MCQLYPETFECVTNSSDILQAFR}

Found in IPI00327697, Tax_Id=10116 Gene_Symbol=Dpep1 Dipeptidase 1 precursor

Match to Query 2450: 2810.247608 from(1406.131080,2+)

Title: 100101RatKid_NS_deglyco_21.5660.5660.2.dta

Data file K:INewmanPaper|Piliangl3SubProteomes\Piliang3SP\mgf5ppm\ERLIC_3SubProteomes5ppm.mgf

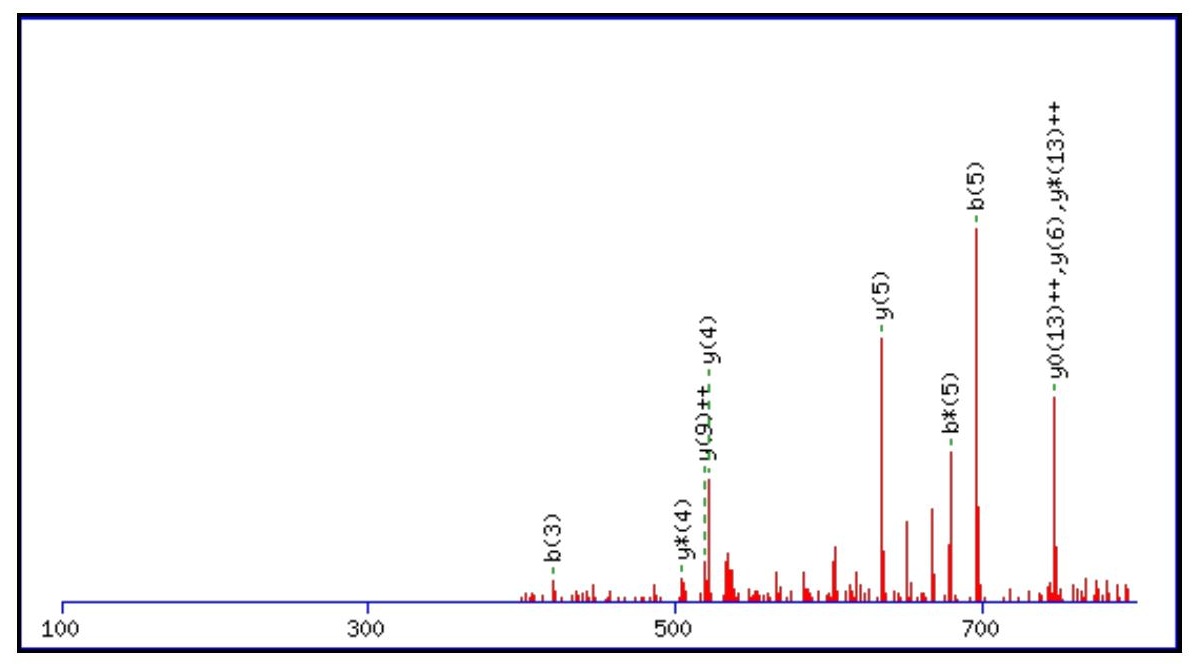



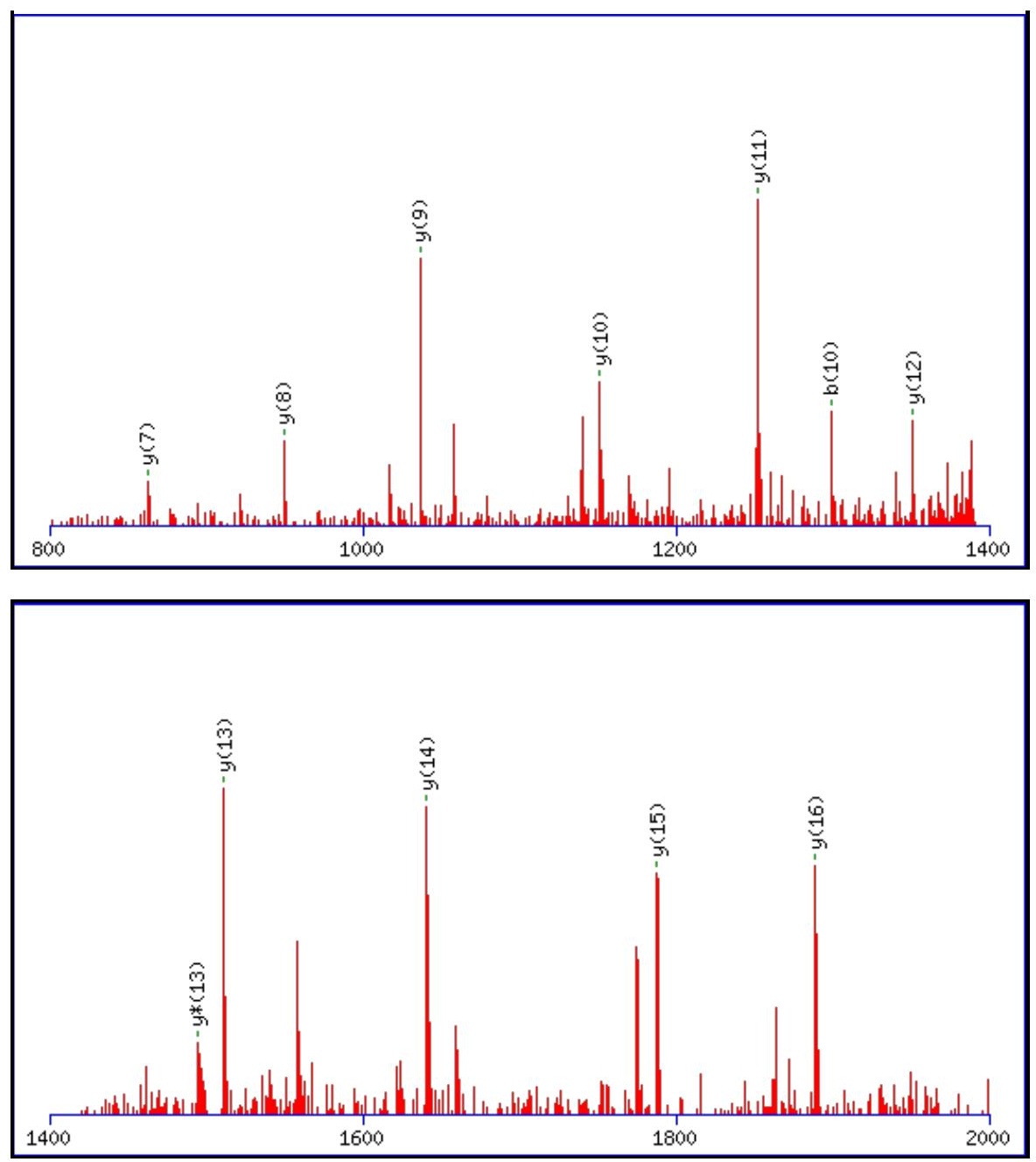

Monoisotopic mass of neutral peptide $\operatorname{Mr}($ calc): 2809.2397

Fixed modifications: Carbamidomethyl (C)

Variable modifications:

N14 : Deamidated $\mathrm{N}(\mathrm{N})$

Ions Score: 110 Expect: $3.8 \mathrm{e}-009$

Matches (Bold Red): 22/236 fragment ions using 27 most intense peaks

\begin{tabular}{|c|c|c|c|c|c|c|c|c|c|c|c|c|c|c|}
\hline \# & b & $\mathbf{b}^{++}$ & b* & $\mathbf{b}^{*^{++}}$ & $\mathbf{b}^{\mathbf{0}}$ & $\mathbf{b}^{\mathbf{0 + +}}$ & Seq. & $\mathbf{y}$ & $\mathbf{y}^{++}$ & $\mathbf{y}^{*}$ & $\mathrm{y}^{*^{++}}$ & $\mathbf{y}^{0}$ & $y^{0++}$ & \# \\
\hline 1 & 132.0478 & 66.5275 & & & & & $\mathbf{M}$ & & & & & & & 23 \\
\hline 2 & 292.0784 & 146.5428 & & & & & C & 2679.2065 & 1340.1069 & 2662.1800 & 1331.5936 & 2661.1960 & 1331.1016 & 22 \\
\hline 3 & 420.1370 & 210.5721 & 403.1104 & 202.0589 & & & $\mathbf{Q}$ & 2519.1759 & 1260.0916 & 2502.1493 & 1251.5783 & 2501.1653 & 1251.0863 & |21 \\
\hline 4 & 533.2211 & 267.1142 & 516.1945 & 258.6009 & & & $\mathbf{L}$ & 2391.1173 & 1196.0623 & 2374.0907 & 1187.5490 & 2373.1067 & 1187.0570 & 20 \\
\hline 5 & 696.2844 & 348.6458 & 679.2578 & 340.1326 & & & $\mathbf{Y}$ & 2278.0332 & 1139.5203 & 2261.0067 & 1131.0070 & 2260.0227 & 1130.5150 & 19 \\
\hline 6 & 793.3371 & 397.1722 & 776.3106 & 388.6589 & & & $\mathbf{P}$ & 2114.9699 & 1057.9886 & 2097.9434 & 1049.4753 & 2096.9593 & 1048.9833 & 18 \\
\hline 7 & 922.3797 & 461.6935 & 905.3532 & 453.1802 & 904.3692 & 452.6882 & $\mathbf{E}$ & 2017.9171 & 1009.4622 & 2000.8906 & 1000.9489 & 1999.9066 & 1000.4569 & 17 \\
\hline 8 & 1023.4274 & 512.2173 & 1006.4009 & 503.7041 & 1005.4169 & 503.2121 & $\mathbf{T}$ & 1888.8745 & 944.9409 & 1871.8480 & 936.4276 & 1870.8640 & 935.9356 & 16 \\
\hline 9 & 1170.4958 & 585.7516 & 1153.4693 & 577.2383 & 1152.4853 & 576.7463 & $\mathbf{F}$ & 1787.8269 & 894.4171 & 1770.8003 & 885.9038 & 1769.8163 & 885.4118 & 15 \\
\hline 10 & 1299.5384 & 650.2729 & 1282.5119 & 641.7596 & 1281.5279 & 641.2676 & $\mathbf{E}$ & 1640.7585 & 820.8829 & 1623.7319 & 812.3696 & 1622.7479 & 811.8776 & 14 \\
\hline 11 & 1459.5691 & 730.2882 & 1442.5425 & 721.7749 & 1441.5585 & 721.2829 & C & 1511.7159 & 756.3616 & 1494.6893 & 747.8483 & 1493.7053 & 747.3563 & 13 \\
\hline 12 & 1558.6375 & 779.8224 & 1541.6109 & 771.3091 & 1540.6269 & 770.8171 & V & 1351.6852 & 676.3462 & 1334.6587 & 667.8330 & 1333.6746 & 667.3410 & 12 \\
\hline 13 & 1659.6852 & 830.3462 & 1642.6586 & 821.8329 & 1641.6746 & 821.3409 & $\mathbf{T}$ & 1252.6168 & 626.8120 & 1235.5903 & 618.2988 & 1234.6062 & 617.8068 & 11 \\
\hline 14 & 1774.7121 & 887.8597 & 1757.6856 & 879.3464 & 1756.7015 & 878.8544 & $\mathbf{N}$ & 1151.5691 & 576.2882 & 1134.5426 & 567.7749 & 1133.5586 & 567.2829 & 10 \\
\hline 15 & 1861.7441 & 931.3757 & 1844.7176 & 922.8624 & 1843.7336 & 922.3704 & S & 1036.5422 & 518.7747 & 1019.5156 & 510.2615 & 1018.5316 & 509.7694 & 9 \\
\hline 16 & 1948.7762 & 974.8917 & 1931.7496 & 966.3784 & 1930.7656 & 965.8864 & S & 949.5102 & 475.2587 & 932.4836 & 466.7454 & 931.4996 & 466.2534 & 8 \\
\hline 17 & 2063.8031 & 1032.4052 & 2046.7766 & 1023.8919 & 2045.7925 & 1023.3999 & D & 862.4781 & 431.7427 & 845.4516 & 423.2294 & 844.4676 & 422.7374 & 7 \\
\hline 18 & 2176.8872 & 1088.9472 & 2159.8606 & 1080.4339 & 2158.8766 & 1079.9419 & I & 747.4512 & 374.2292 & 730.4246 & 365.7160 & & & 6 \\
\hline
\end{tabular}




\begin{tabular}{|r|r|r|r|r|r|r|r|r|r|r|r|r|r|r|r|r|}
$\mathbf{1 9}$ & 2289.9712 & 1145.4893 & 2272.9447 & 1136.9760 & 2271.9607 & 1136.4840 & L & $\mathbf{6 3 4 . 3 6 7 1}$ & 317.6872 & 617.3406 & 309.1739 & & & $\mathbf{5}$ \\
\hline $\mathbf{2 0}$ & 2418.0298 & 1209.5185 & 2401.0033 & 1201.0053 & 2400.0192 & 1200.5133 & $\mathbf{Q}$ & $\mathbf{5 2 1 . 2 8 3 1}$ & 261.1452 & $\mathbf{5 0 4 . 2 5 6 5}$ & 252.6319 & & & $\mathbf{4}$ \\
\hline $\mathbf{2 1}$ & 2489.0669 & 1245.0371 & 2472.0404 & 1236.5238 & 2471.0564 & 1236.0318 & A & 393.2245 & 197.1159 & 376.1979 & 188.6026 & & \\
\hline $\mathbf{2 2}$ & 2636.1353 & 1318.5713 & 2619.1088 & 1310.0580 & 2618.1248 & 1309.5660 & F & 322.1874 & 161.5973 & 305.1608 & 153.0840 & & \\
\hline $\mathbf{2 3}$ & & & & & & & R & 175.1190 & 88.0631 & 158.0924 & 79.5498 & & & $\mathbf{2}$ \\
\hline
\end{tabular}
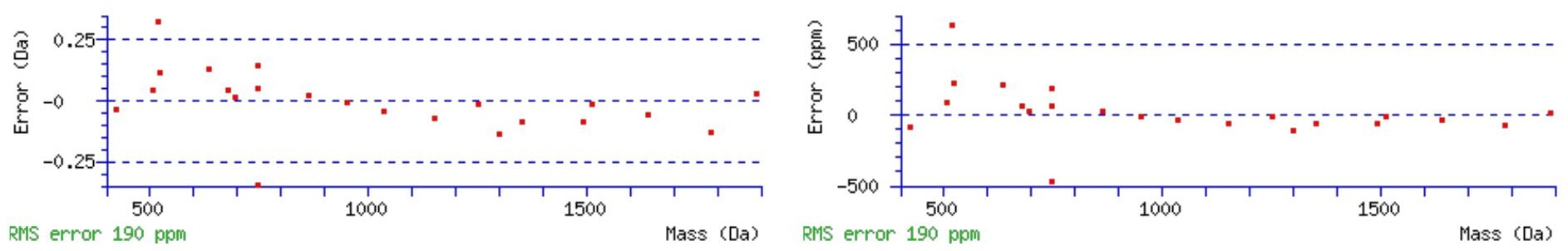

\section{All matches to this query}

\begin{tabular}{|l|l|l|l|}
\hline Score & Mr(calc): & Delta & \multicolumn{1}{|c|}{ Sequence } \\
\hline 109.7 & 2809.2397 & 1.0079 & MCQLYPETFECVTNSSDILQAFR \\
\hline 23.4 & 2808.2557 & 1.9919 & MCQLYPETFECVTNSSDILQAFR \\
\hline 2.3 & 2809.2541 & 0.9935 & FRDIPNTSSMENPAPNKNPWDTFK \\
\hline 2.0 & 2809.2477 & 0.9999 & QMAPVERLSOPDGEGVSSELTEENK \\
\hline 0.1 & 2810.2533 & -0.0057 & HTTDVIYAAKMISESGSRMDVLAR \\
\hline 0.1 & 2810.2533 & -0.0057 & HTTDVIYAAKMISESGSRMDVLAR \\
\hline 0.1 & 2810.2533 & -0.0057 & HTTDVIYAAKMISESGSRMDVLAR \\
\hline 0.1 & 2810.2533 & -0.0057 & HTTDVIYAAKMISESGSRMDVLAR \\
\hline
\end{tabular}

Spectrum No: 44; Query: 2062; Rank: 1

\section{Peptide View}

MS/MS Fragmentation of NNLSFIETSALDSTNVEEAFK

Found in IPI00210381, Tax_Id=10116 Gene_Symbol=Rab11b Ras-related protein Rab-11B

Match to Query 2062: 2329.107068 from(1165.560810,2+)

Title: 100101RatKid_NS_deglyco_13.6301.6301.2.dta

Data file K:INewmanPaper|Piliangl3SubProteomes\Piliang3SP\mgf5ppm\ERLIC_3SubProteomes5ppm.mgf

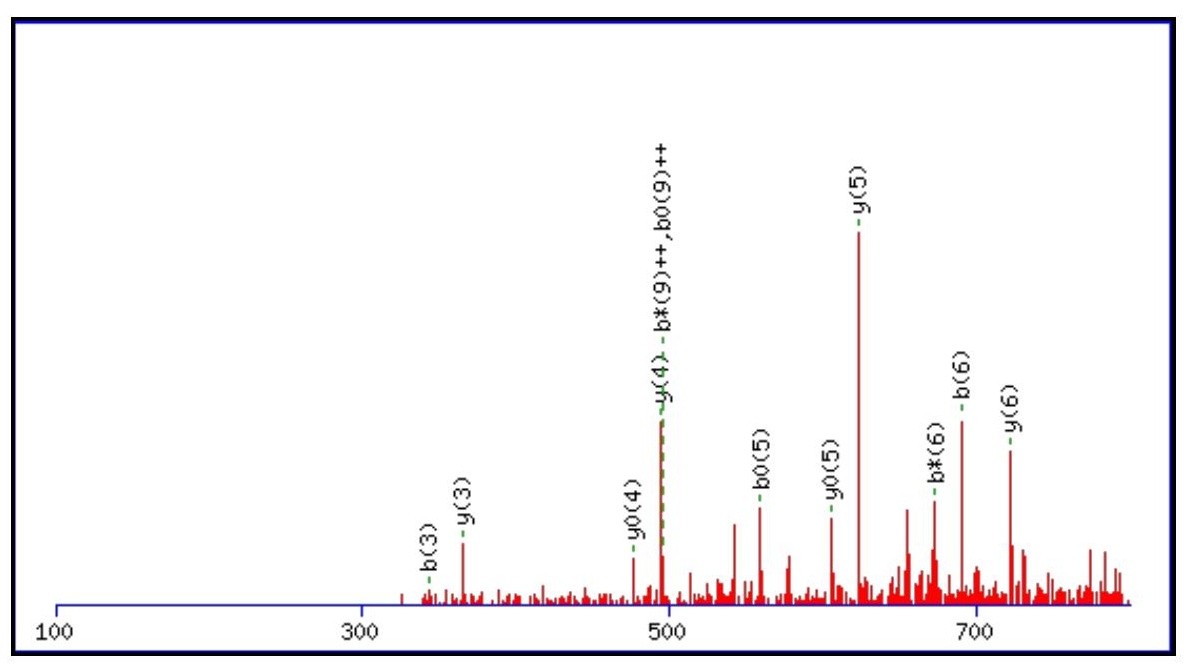




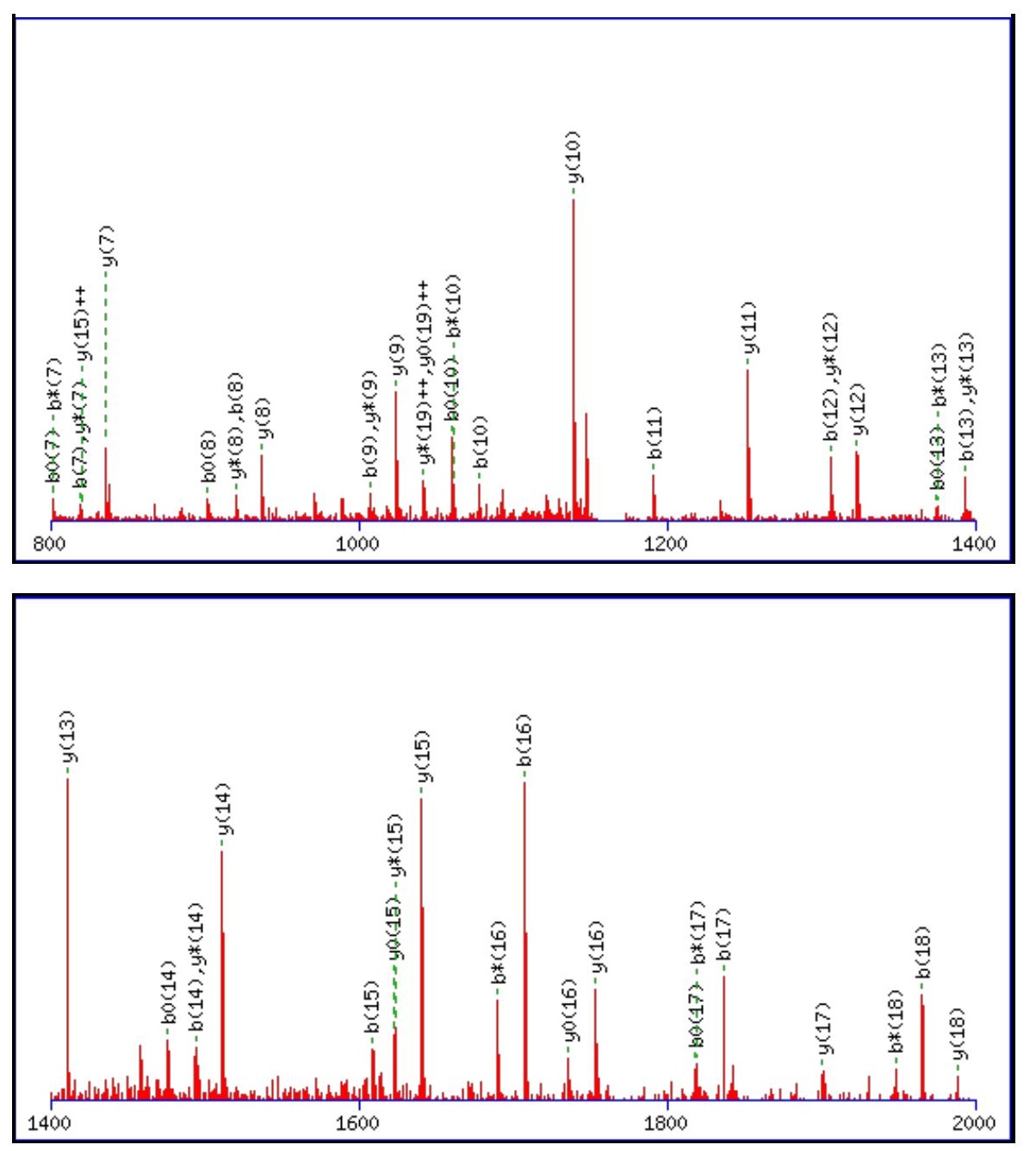

Monoisotopic mass of neutral peptide $\operatorname{Mr}($ calc): 2329.0961

Fixed modifications: Carbamidomethyl (C)

Variable modifications:

N2 : Deamidated $\mathrm{N}(\mathrm{N})$

Ions Score: 110 Expect: $3.1 \mathrm{e}-\odot 09$

Matches (Bold Red): 60/228 fragment ions using 86 most intense peaks

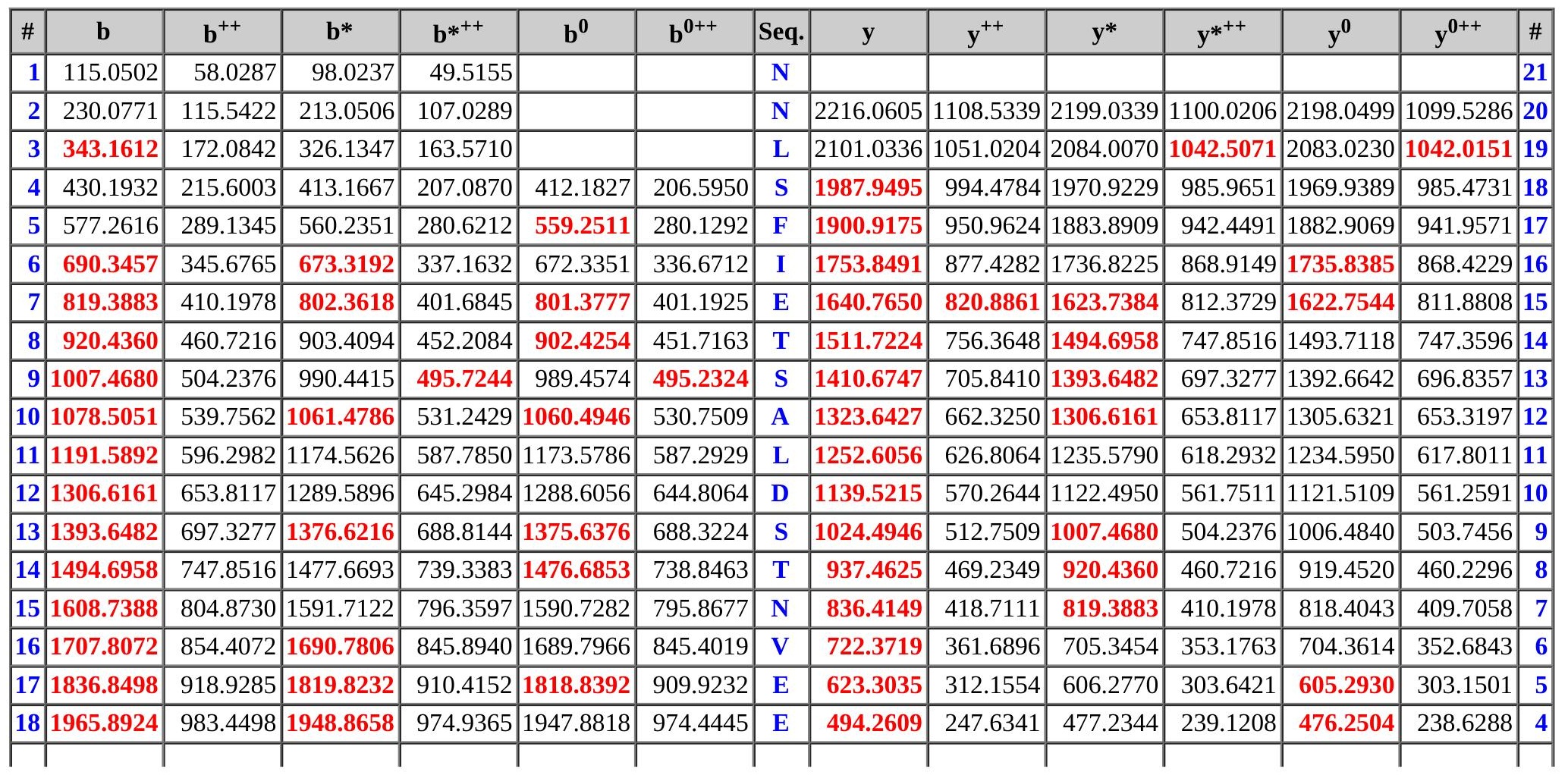




\begin{tabular}{|l|r|r|r|r|r|r|r|r|r|r|r|r|r|r|r|r|}
$\mathbf{1 9}$ & 2036.9295 & 1018.9684 & 2019.9029 & 1010.4551 & 2018.9189 & 1009.9631 & $\mathbf{A}$ & 365.2183 & 183.1128 & 348.1918 & 174.5995 & & & $\mathbf{3}$ \\
\hline $\mathbf{2 0}$ & 2183.9979 & 1092.5026 & 2166.9713 & 1083.9893 & 2165.9873 & 1083.4973 & $\mathbf{F}$ & 294.1812 & 147.5942 & 277.1547 & 139.0810 & & & $\mathbf{2}$ \\
\hline $\mathbf{2 1}$ & & & & & & & K & 147.1128 & 74.0600 & 130.0863 & 65.5468 & & & $\mathbf{1}$ \\
\hline
\end{tabular}
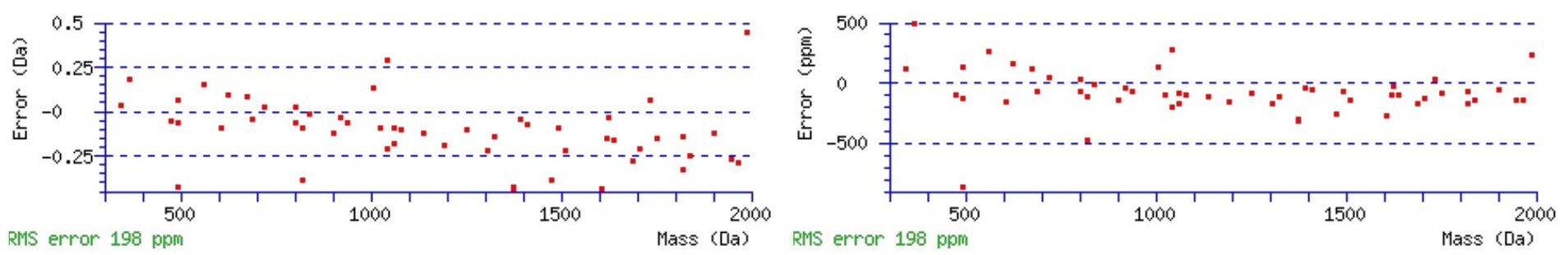

\section{All matches to this query}

\begin{tabular}{|l|c|c|l|}
\hline Score & Mr(calc): & Delta & \multicolumn{1}{c|}{ Sequence } \\
\hline 109.6 & 2329.0961 & 0.0110 & NNLSFIETSALDSTNVEEAFK \\
\hline 109.6 & 2329.0961 & 0.0110 & NNLSFIETSALDSTNVEEAFK \\
\hline 80.5 & 2328.1121 & 0.9950 & NNLSFIETSALDSTNVEEAFK \\
\hline 76.8 & 2329.0961 & 0.0110 & NNLSFIETSALDSTNVEEAFK \\
\hline 4.2 & 2329.1082 & -0.0012 & DIGNSAFITFICKGNCEILR \\
\hline 0.1 & 2327.1059 & 2.0012 & $\underline{\text { IRNIFHQNDIIYSLSSNGR }}$ \\
\hline 0.1 & 2328.0899 & 1.0171 & $\underline{\text { IRNIFHQNDIIYSLSSNGR }}$ \\
\hline 0.1 & 2327.1059 & 2.0012 & $\underline{\text { IRNIFHQNDIIYSLSSNGR }}$ \\
\hline 0.1 & 2328.0899 & 1.0171 & $\underline{\text { IRNIFHQNDIIYSLSSNGR }}$ \\
\hline 0.1 & 2327.1059 & 2.0012 & $\underline{\text { IRNIFHQNDIIYSLSSNGR }}$ \\
\hline
\end{tabular}

Spectrum No: 45; Query: 1741; Rank: 1

\section{Peptide View}

MS/MS Fragmentation of TTLVDNNTWNNSHIALVGK

Found in IPI00382320, Tax_Id=10116 Gene_Symbol=RGD1311563 93 kDa protein

Match to Query 1741: 2097.058928 from(1049.536740,2+)

Title: 100101RatKid_NS_deglyco_12.3324.3324.2.dta

Data file K:INewmanPaper|Piliangl3SubProteomes\Piliang3SP\mgf5ppm\ERLIC_3SubProteomes5ppm.mgf

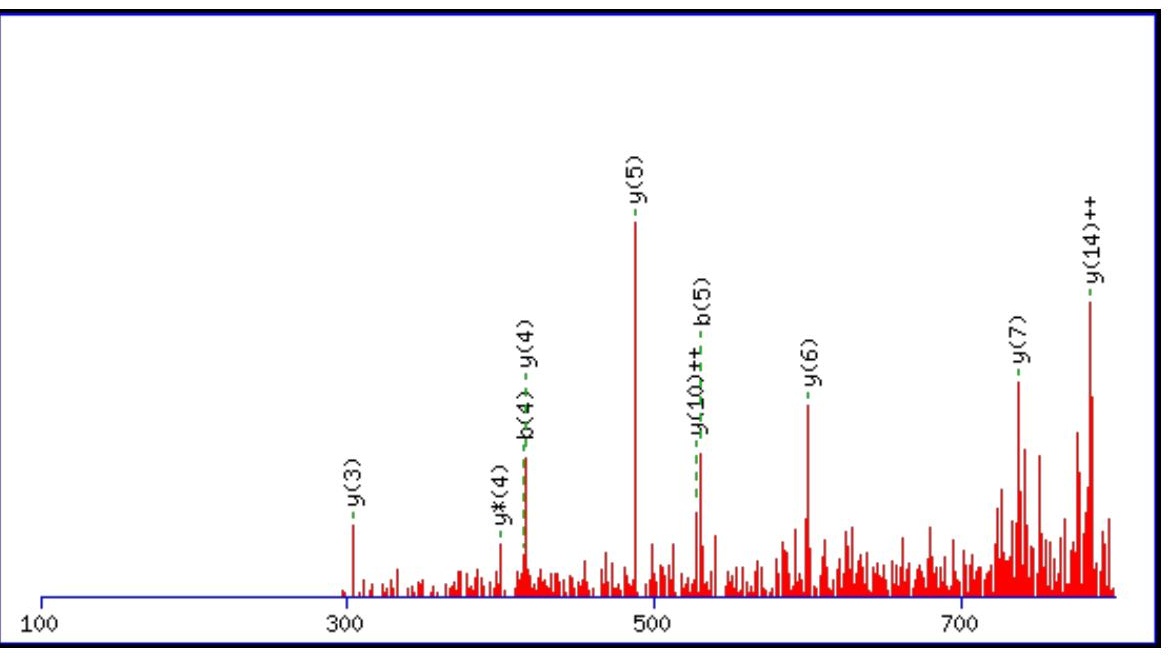



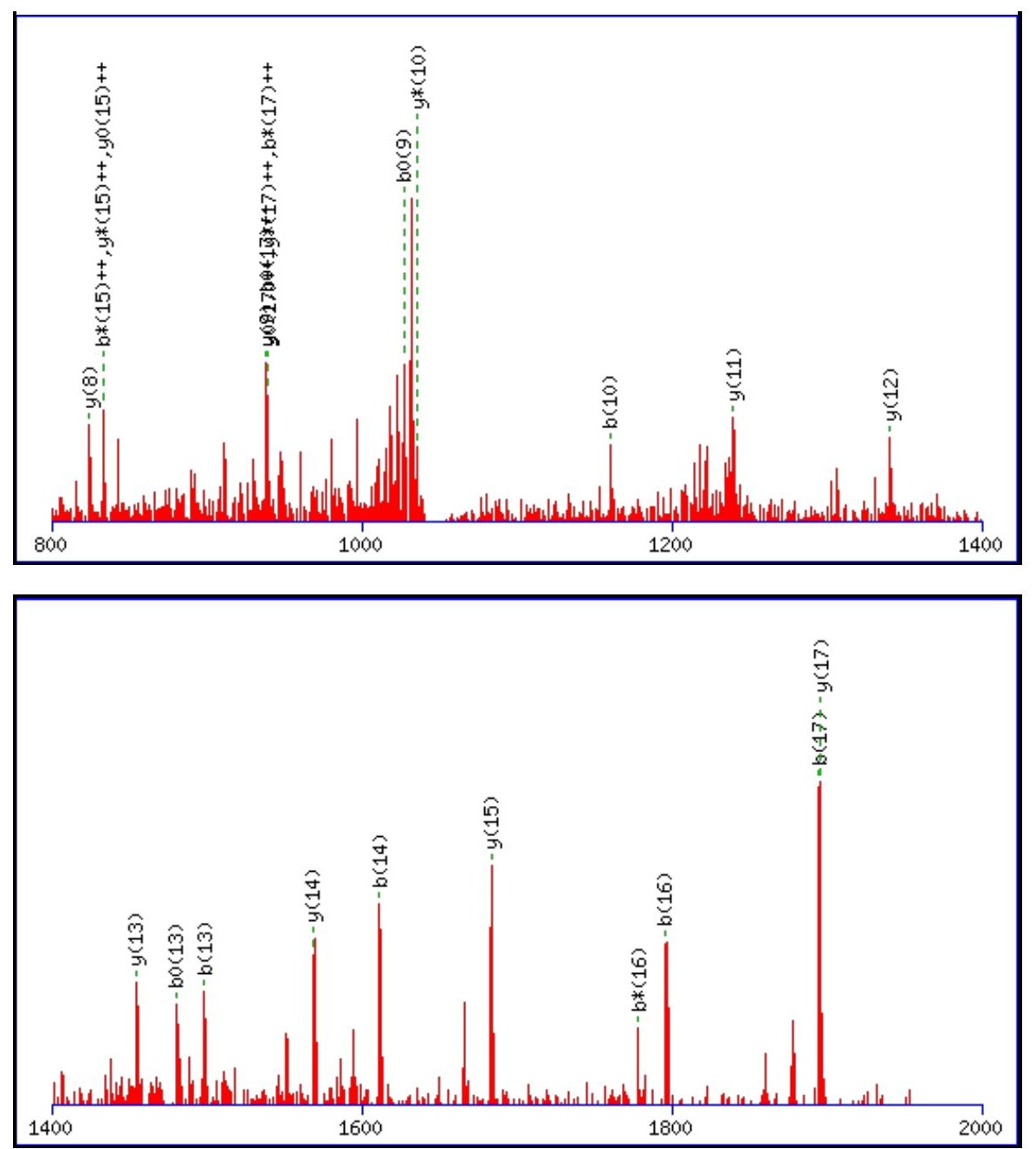

Monoisotopic mass of neutral peptide $\operatorname{Mr}($ calc): 2097.0491

Fixed modifications: Carbamidomethyl (C)

Variable modifications:

N10 : Deamidated $\mathrm{N}(\mathrm{N})$

Ions Score: 109 Expect: $2.2 \mathrm{e}-009$

Matches (Bold Red): 34/192 fragment ions using 46 most intense peaks

\begin{tabular}{|c|c|c|c|c|c|c|c|c|c|c|c|c|c|c|}
\hline \# & b & $\mathbf{b}^{++}$ & b* $^{*}$ & $\mathbf{b}^{*^{++}}$ & $\mathbf{b}^{0}$ & $\mathbf{b}^{\mathbf{0}^{++}}$ & Seq. & $\mathbf{y}$ & $\mathbf{y}^{++}$ & $\mathbf{y}^{*}$ & $\mathbf{y}^{*^{++}}$ & $\mathbf{y}^{0}$ & $\mathbf{y}^{\mathbf{0 + +}^{++}}$ & \# \\
\hline 1 & 102.0550 & 51.5311 & & & 84.0444 & 42.5258 & $\mathbf{T}$ & & & & & & & 19 \\
\hline 2 & 203.1026 & 102.0550 & & & 185.0921 & 93.0497 & $T$ & 1997.0087 & 999.0080 & |1979.9821 & 990.4947 & 1978.9981 & 990.0027 & 18 \\
\hline 3 & 316.1867 & 158.5970 & & & 298.1761 & 149.5917 & $\mathbf{L}$ & 1895.9610 & 948.4841 & 1878.9344 & 939.9709 & 1877.9504 & 939.4789 & 17 \\
\hline 4 & 415.2551 & 208.1312 & & & 397.2445 & 199.1259 & V & 1782.8769 & 891.9421 & 1765.8504 & 883.4288 & 1764.8664 & 882.9368 & 16 \\
\hline 5 & 530.2821 & 265.6447 & & & 512.2715 & 256.6394 & D & \begin{tabular}{|l}
1683.8085 \\
\end{tabular} & 842.4079 & 1666.7820 & 833.8946 & 1665.7980 & 833.4026 & 15 \\
\hline 6 & 644.3250 & 322.6661 & 627.2984 & 314.1529 & 626.3144 & 313.6608 & $\mathbf{N}$ & 1568.7816 & 784.8944 & 1551.7550 & 776.3812 & 1550.7710 & 775.8891 & 14 \\
\hline 7 & 758.3679 & 379.6876 & 741.3414 & 371.1743 & 740.3573 & 370.6823 & $\mathbf{N}$ & 1454.7386 & 727.8730 & 1437.7121 & 719.3597 & 1436.7281 & 718.8677 & 13 \\
\hline 8 & 859.4156 & 430.2114 & 842.3890 & 421.6982 & 841.4050 & 421.2061 & $\mathbf{T}$ & 1340.6957 & 670.8515 & 1323.6692 & 662.3382 & 1322.6852 & 661.8462 & 12 \\
\hline 9 & 1045.4949 & 523.2511 & 1028.4684 & 514.7378 & \begin{tabular}{|l}
1027.4843 \\
\end{tabular} & 514.2458 & $\mathbf{W}$ & \begin{tabular}{|l|l|}
1239.6480 \\
\end{tabular} & 620.3277 & 1222.6215 & 611.8144 & 1221.6375 & 611.3224 & 11 \\
\hline 10 & 1160.5218 & 580.7646 & 1143.4953 & 572.2513 & 1142.5113 & 571.7593 & $\mathbf{N}$ & 1053.5687 & 527.2880 & 1036.5422 & 518.7747 & 1035.5582 & 518.2827 & 10 \\
\hline 11 & 1274.5648 & 637.7860 & 1257.5382 & 629.2727 & 1256.5542 & 628.7807 & $\mathbf{N}$ & 938.5418 & 469.7745 & 921.5152 & 461.2613 & 920.5312 & 460.7693 & 9 \\
\hline 12 & 1361.5968 & 681.3020 & 1344.5702 & 672.7888 & 1343.5862 & 672.2968 & $\mathrm{~S}$ & 824.4989 & 412.7531 & 807.4723 & 404.2398 & 806.4883 & 403.7478 & 8 \\
\hline 13 & \begin{tabular}{|l|}
1498.6557 \\
\end{tabular} & 749.8315 & 1481.6292 & 741.3182 & 1480.6451 & 740.8262 & $\mathbf{H}$ & 737.4668 & 369.2371 & 720.4403 & 360.7238 & & & 7 \\
\hline 14 & 1611.7398 & 806.3735 & 1594.7132 & 797.8602 & 1593.7292 & 797.3682 & I & 600.4079 & 300.7076 & 583.3814 & 292.1943 & & & 6 \\
\hline 15 & 1682.7769 & 841.8921 & 1665.7503 & 833.3788 & 1664.7663 & 832.8868 & A & 487.3239 & 244.1656 & 470.2973 & 235.6523 & & & 5 \\
\hline 16 & 1795.8609 & 898.4341 & \begin{tabular}{|l}
1778.8344 \\
\end{tabular} & 889.9208 & 1777.8504 & 889.4288 & $\mathbf{L}$ & 416.2867 & 208.6470 & 399.2602 & 200.1337 & & & 4 \\
\hline 17 & \begin{tabular}{|l|}
1894.9294 \\
\end{tabular} & 947.9683 & 1877.9028 & 939.4550 & 1876.9188 & 938.9630 & V & 303.2027 & 152.1050 & 286.1761 & 143.5917 & & & 3 \\
\hline 18 & 1951.9508 & 976.4790 & 1934.9243 & 967.9658 & 1933.9403 & 967.4738 & G & 204.1343 & 102.5708 & 187.1077 & 94.0575 & & & 2 \\
\hline
\end{tabular}




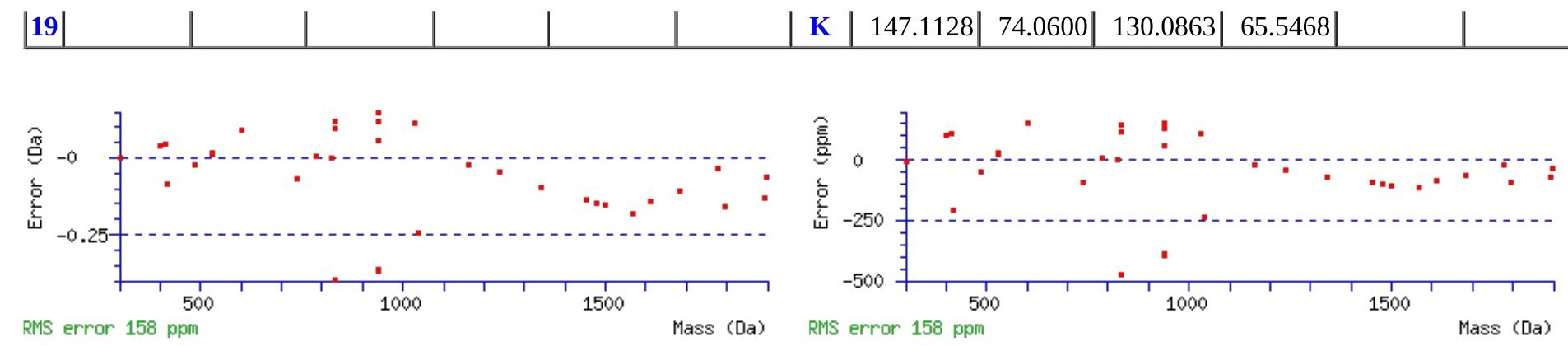

\section{All matches to this query}

\begin{tabular}{|l|c|c|c|}
\hline Score & Mr(calc): & Delta & \multicolumn{1}{|c|}{ Sequence } \\
\hline 108.9 & 2097.0491 & 0.0099 & TTLVDNNTWNNSHIALVGK \\
\hline 105.8 & 2097.0491 & 0.0099 & TTLVDNNTWNNSHIALVGK \\
\hline 74.4 & 2097.0491 & 0.0099 & TTLVDNNTWNNSHIALVGK \\
\hline 61.6 & 2097.0491 & 0.0099 & TTLVDNNTWNNSHIALVGK \\
\hline 21.2 & 2096.0651 & 0.9939 & TTLVDNNTWNNSHIALVGK \\
\hline 10.9 & 2097.0701 & -0.0112 & QLETQNNNLQAOILALQR \\
\hline 5.1 & 2097.0531 & 0.0058 & WDNASGDWDLILLLVPDR \\
\hline 3.1 & 2097.0660 & -0.0071 & FNOPLDLSLFSLVGSPRK \\
\hline 2.6 & 2097.0660 & -0.0071 & FNQPLDLSLFSLVGSPRK \\
\hline
\end{tabular}

Spectrum No: 46; Query: 2190; Rank: 1

\section{Peptide View}

MS/MS Fragmentation of SLGEVNFTATAEALQSPELCGNK

Found in IPI00326140, Tax_Id=10116 Gene_Symbol=Pzp Alpha-1-macroglobulin precursor

Match to Query 2190: 2436.156668 from(1219.085610,2+)

Title: 100101RatKid_NS_deglyco_21.4347.4347.2.dta

Data file K:INewmanPaper|Piliangl3SubProteomes\Piliang3SP\mgf5ppm\ERLIC_3SubProteomes5ppm.mgf

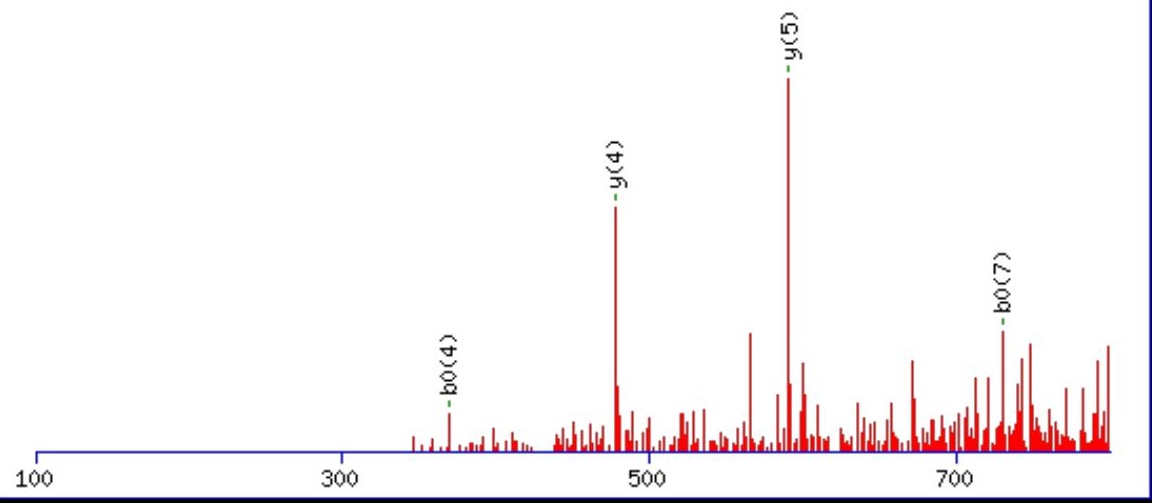



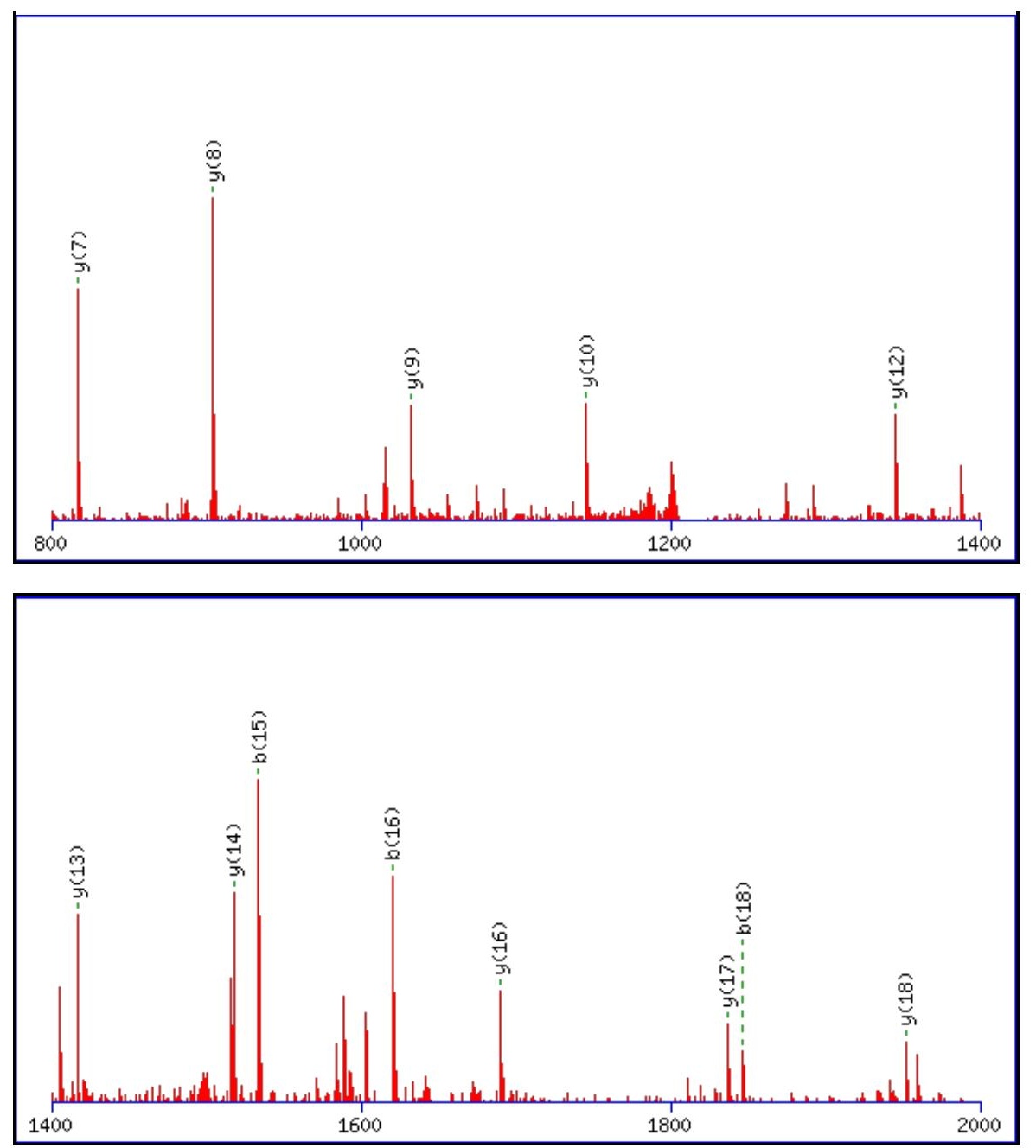

Monoisotopic mass of neutral peptide $\operatorname{Mr}($ calc): 2436.1478

Fixed modifications: Carbamidomethyl (C)

Variable modifications:

N6 : Deamidated $\mathrm{N}(\mathrm{N})$

Ions Score: 109 Expect: $3.8 \mathrm{e}-009$

Matches (Bold Red): 17/244 fragment ions using 18 most intense peaks

\begin{tabular}{|c|c|c|c|c|c|c|c|c|c|c|c|c|c|c|}
\hline \# & b & $\mathbf{b}^{++}$ & $\mathbf{b}^{*}$ & $\mathbf{b}^{*+}$ & $\mathbf{b}^{\mathbf{0}}$ & $\mathbf{b}^{\mathbf{0 + +}}$ & Seq. & $\mathbf{y}$ & $\mathbf{y}^{++}$ & $\mathbf{y}^{*}$ & $\mathrm{y}^{\mathrm{*}^{++}}$ & $y^{0}$ & $\mathbf{y}^{0++}$ & \# \\
\hline 1 & 88.0393 & 44.5233 & & & 70.0287 & 35.5180 & $\mathrm{~S}$ & & & & & & & 23 \\
\hline 2 & 201.1234 & 101.0653 & & & 183.1128 & 92.0600 & $\mathbf{L}$ & 2350.1231 & 1175.5652 & 2333.0966 & 1167.0519 & 2332.1125 & 1166.5599 & 22 \\
\hline 3 & 258.1448 & 129.5761 & & & 240.1343 & 120.5708 & $\mathbf{G}$ & 2237.0390 & 1119.0232 & 2220.0125 & 1110.5099 & 2219.0285 & 1110.0179 & 21 \\
\hline 4 & 387.1874 & 194.0974 & & & 369.1769 & 185.0921 & $\mathbf{E}$ & 2180.0176 & 1090.5124 & 2162.9910 & 1081.9992 & 2162.0070 & 1081.5071 & 20 \\
\hline 5 & 486.2558 & 243.6316 & & & 468.2453 & 234.6263 & $\mathbf{V}$ & 2050.9750 & 1025.9911 & 2033.9484 & 1017.4779 & 2032.9644 & 1016.9859 & 19 \\
\hline 6 & 601.2828 & 301.1450 & 584.2562 & & 583.2722 & 292.1397 & $\mathbf{N}$ & 1951.9066 & 976.4569 & 1934.8800 & 967.9437 & 1933.8960 & 967.4516 & 18 \\
\hline 7 & 748.3512 & 374.6792 & 731.3246 & 366.1660 & 730.3406 & 365.6739 & $\mathbf{F}$ & 1836.8796 & 918.9435 & 1819.8531 & 910.4302 & 1818.8691 & 909.9382 & 17 \\
\hline 8 & 849.3989 & 425.2031 & 832.3723 & 416.6898 & 831.3883 & 416.1978 & $\mathbf{T}$ & 1689.8112 & 845.4093 & 1672.7847 & 836.8960 & 1671.8007 & 836.4040 & 16 \\
\hline 9 & 920.4360 & 460.7216 & 903.4094 & 452.2084 & 902.4254 & 451.7163 & A & 1588.7635 & 794.8854 & 1571.7370 & 786.3721 & 1570.7530 & 785.8801 & 15 \\
\hline 10 & 1021.4837 & 511.2455 & 1004.4571 & 502.7322 & 1003.4731 & 502.2402 & $\mathbf{T}$ & 1517.7264 & 759.3669 & 1500.6999 & 750.8536 & 1499.7159 & 750.3616 & 14 \\
\hline 11 & 1092.5208 & 546.7640 & 1075.4942 & 538.2508 & 1074.5102 & 537.7587 & A & 1416.6788 & 708.8430 & 1399.6522 & 700.3297 & 1398.6682 & 699.8377 & 13 \\
\hline 12 & 1221.5634 & 611.2853 & 1204.5368 & 602.7720 & 1203.5528 & 602.2800 & $\mathbf{E}$ & 1345.6416 & 673.3245 & 1328.6151 & 664.8112 & 1327.6311 & 664.3192 & 12 \\
\hline 13 & 1292.6005 & 646.8039 & 1275.5739 & 638.2906 & 1274.5899 & 637.7986 & A & 1216.5990 & 608.8032 & 1199.5725 & 600.2899 & 1198.5885 & 599.7979 & 11 \\
\hline 14 & 1405.6845 & 703.3459 & 1388.6580 & 694.8326 & 1387.6740 & 694.3406 & $\mathbf{L}$ & 1145.5619 & 573.2846 & 1128.5354 & 564.7713 & 1127.5514 & 564.2793 & 10 \\
\hline 15 & 1533.7431 & 767.3752 & 1516.7166 & 758.8619 & 1515.7326 & 758.3699 & $\mathbf{Q}$ & 1032.4779 & 516.7426 & 1015.4513 & 508.2293 & 1014.4673 & 507.7373 & 9 \\
\hline 16 & 1620.7752 & 810.8912 & 1603.7486 & 802.3779 & 1602.7646 & 801.8859 & $\mathrm{~S}$ & 904.4193 & 452.7133 & 887.3927 & 444.2000 & 886.4087 & 443.7080 & 8 \\
\hline 17 & 1717.8279 & 859.4176 & 1700.8014 & 850.9043 & 1699.8174 & 850.4123 & $\mathbf{P}$ & 817.3873 & 409.1973 & 800.3607 & 400.6840 & 799.3767 & 400.1920 & 7 \\
\hline 18 & 1846.8705 & 923.9389 & 1829.8440 & 915.4256 & 1828.8599 & 914.9336 & $\mathbf{E}$ & 720.3345 & 360.6709 & 703.3080 & 352.1576 & 702.3239 & 351.6656 & 6 \\
\hline
\end{tabular}




\begin{tabular}{|r|r|r|r|r|r|r|r|r|r|r|r|r|r|r|r|}
$\mathbf{1 9}$ & 1959.9546 & 980.4809 & 1942.9280 & 971.9677 & 1941.9440 & 971.4756 & L & 591.2919 & 296.1496 & 574.2654 & 287.6363 & & & $\mathbf{5}$ \\
\hline $\mathbf{2 0}$ & 2119.9852 & 1060.4962 & 2102.9587 & 1051.9830 & 2101.9747 & 1051.4910 & C & $\mathbf{4 7 8 . 2 0 7 8}$ & 239.6076 & 461.1813 & 231.0943 & & & $\mathbf{4}$ \\
\hline $\mathbf{2 1}$ & 2177.0067 & 1089.0070 & 2159.9801 & 1080.4937 & 2158.9961 & 1080.0017 & G & 318.1772 & 159.5922 & 301.1506 & 151.0790 & & & $\mathbf{3}$ \\
\hline $\mathbf{2 2}$ & 2291.0496 & 1146.0284 & 2274.0231 & 1137.5152 & 2273.0390 & 1137.0232 & N & 261.1557 & 131.0815 & 244.1292 & 122.5682 & & & $\mathbf{2}$ \\
\hline $\mathbf{2 3}$ & & & & & & & K & 147.1128 & 74.0600 & 130.0863 & 65.5468 & & & $\mathbf{1}$ \\
\hline
\end{tabular}
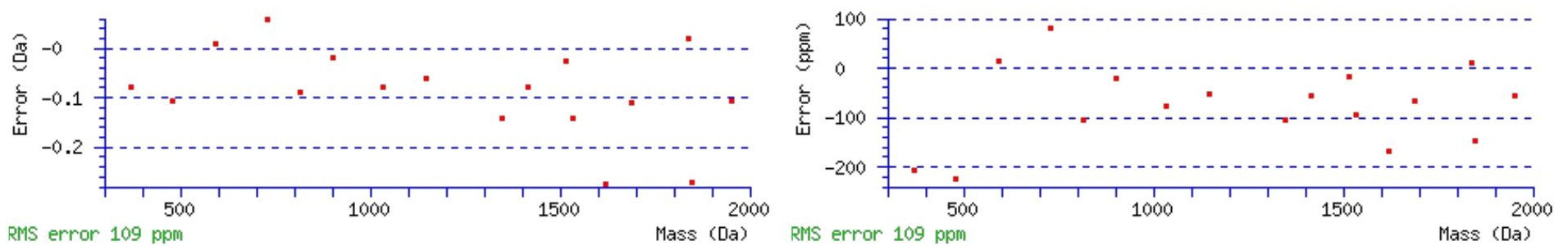

\section{All matches to this query}

\begin{tabular}{|l|l|l|l|}
\hline Score & Mr(calc): & Delta & \multicolumn{1}{c|}{ Sequence } \\
\hline 108.7 & 2436.1478 & 0.0088 & SLGEVNFTATAEALQSPELCGNK \\
\hline 64.0 & 2435.1638 & 0.9928 & SLGEVNFTATAEALQSPELCGNK \\
\hline 29.8 & 2436.1478 & 0.0088 & SLGEVNFTATAEALQSPELCGNK \\
\hline 10.7 & 2436.1394 & 0.0173 & VTSATQLNGRQAOPSSKPASGNR \\
\hline 10.7 & 2436.1394 & 0.0173 & VTSATQLNGRQAQPSSKPASGNR \\
\hline 10.7 & 2436.1394 & 0.0173 & VTSATQLNGRQAQPSSKPASGNR \\
\hline 3.9 & 2436.1378 & 0.0188 & LSSGTKWIIEQNLSQLPYAK \\
\hline 3.9 & 2436.1378 & 0.0188 & LSSGTKWIIEQNLSQLPYAK \\
\hline 3.9 & 2436.1378 & 0.0188 & LSSGTKWIIEQNLSQLPYAK \\
\hline 3.7 & 2434.1273 & 2.0293 & EMQRNSVCLAFVSIIISDEK \\
\hline
\end{tabular}

Spectrum No: 47; Query: 2436; Rank: 1

\section{Peptide View}

MS/MS Fragmentation of DLVVGLYSQWMNNSDNNPIHPNLR

Found in IPI00230862, Tax_Id=10116 Gene_Symbol=Anpep Aminopeptidase N

Match to Query 2436: 2796.332802 from(933.118210,3+)

Title: 100101RatKid_NS_deglyco_19.5135.5135.3.dta

Data file K:INewmanPaper|Piliang|3SubProteomes\Piliang3SP\mgf5ppm\ERLIC_3SubProteomes5ppm.mgf

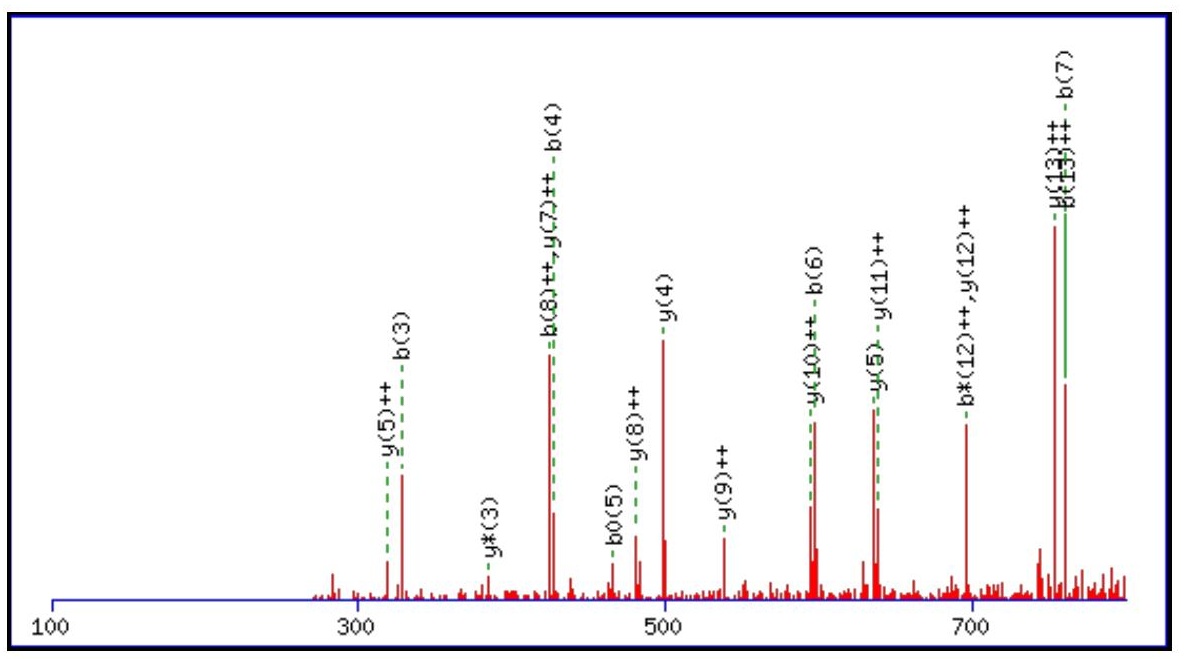



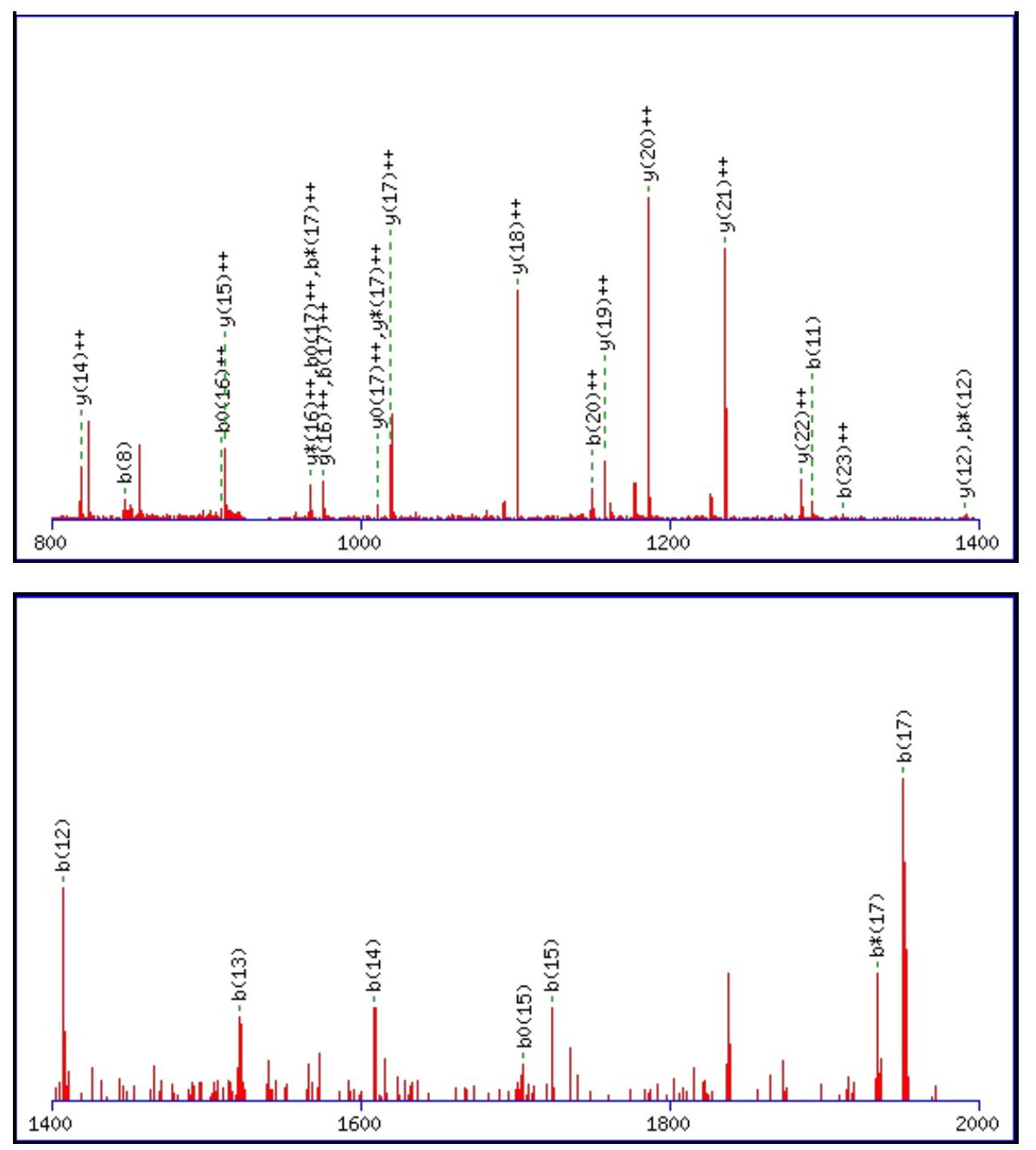

Monoisotopic mass of neutral peptide $\operatorname{Mr}($ calc): 2796.3289

Fixed modifications: Carbamidomethyl (C)

Variable modifications:

N12 : Deamidated $\mathrm{N}(\mathrm{N})$

Ions Score: 108 Expect: $4.5 \mathrm{e}-009$

Matches (Bold Red): 48/242 fragment ions using 74 most intense peaks

\begin{tabular}{|c|c|c|c|c|c|c|c|c|c|c|c|c|c|c|}
\hline \# & b & $\mathbf{b}^{++}$ & b* & $\mathbf{b}^{*^{++}}$ & $\mathbf{b}^{\mathbf{0}}$ & $\mathbf{b}^{0++}$ & Seq. & $\mathbf{y}$ & $\mathbf{y}^{++}$ & $\mathbf{y}^{*}$ & $\mathrm{y}^{*^{++}}$ & $\mathbf{y}^{0}$ & $\mathbf{y}^{0++}$ & \# \\
\hline 1 & 116.0342 & 58.5207 & & & 98.0237 & 49.5155 & D & & & & & & & 24 \\
\hline 2 & 229.1183 & 115.0628 & & & 211.1077 & 106.0575 & $\mathbf{L}$ & 2682.3093 & 1341.6583 & 2665.2827 & 1333.1450 & 2664.2987 & 1332.6530 & 23 \\
\hline 3 & 328.1867 & 164.5970 & & & 310.1761 & 155.5917 & V & 2569.2252 & 1285.1162 & 2552.1987 & 1276.6030 & 2551.2147 & 1276.1110 & 22 \\
\hline 4 & 427.2551 & 214.1312 & & & 409.2445 & 205.1259 & $\mathbf{V}$ & 2470.1568 & 1235.5820 & 2453.1303 & 1227.0688 & 2452.1462 & 1226.5768 & 21 \\
\hline 5 & 484.2766 & 242.6419 & & & 466.2660 & 233.6366 & G & 2371.0884 & 1186.0478 & 2354.0618 & 1177.5346 & 2353.0778 & 1177.0426 & 20 \\
\hline 6 & 597.3606 & 299.1840 & & & 579.3501 & 290.1787 & $\mathbf{L}$ & 2314.0669 & $\mid 1157.5371$ & 2297.0404 & 1149.0238 & 2296.0564 & 1148.5318 & 19 \\
\hline 7 & 760.4240 & 380.7156 & & & 742.4134 & 371.7103 & $\mathbf{Y}$ & 2200.9829 & 1100.9951 & 2183.9563 & 1092.4818 & 2182.9723 & 1091.9898 & 18 \\
\hline 8 & 847.4560 & 424.2316 & & & 829.4454 & 415.2264 & $\mathrm{~S}$ & 2037.9195 & 1019.4634 & 2020.8930 & 1010.9501 & 2019.9090 & 1010.4581 & 17 \\
\hline 9 & 975.5146 & 488.2609 & 958.4880 & 479.7477 & 957.5040 & 479.2556 & $\mathbf{Q}$ & 1950.8875 & 975.9474 & 1933.8610 & 967.4341 & 1932.8769 & 966.9421 & 16 \\
\hline 10 & 1161.5939 & 581.3006 & 1144.5673 & 572.7873 & 1143.5833 & 572.2953 & $\mathbf{W}$ & 1822.8289 & 911.9181 & 1805.8024 & 903.4048 & 1804.8184 & 902.9128 & 15 \\
\hline 11 & 1292.6344 & 646.8208 & 1275.6078 & 638.3075 & 1274.6238 & 637.8155 & $\mathbf{M}$ & 1636.7496 & 818.8784 & 1619.7231 & 810.3652 & 1618.7391 & 809.8732 & 14 \\
\hline 12 & 1407.6613 & 704.3343 & 1390.6348 & 695.8210 & 1389.6507 & 695.3290 & $\mathbf{N}$ & 1505.7091 & 753.3582 & 1488.6826 & 744.8449 & 1487.6986 & 744.3529 & 13 \\
\hline 13 & 1521.7042 & 761.3558 & 1504.6777 & 752.8425 & 1503.6937 & 752.3505 & $\mathbf{N}$ & 1390.6822 & 695.8447 & 1373.6557 & 687.3315 & 1372.6716 & 686.8395 & 12 \\
\hline 14 & 1608.7363 & 804.8718 & 1591.7097 & 796.3585 & 1590.7257 & 795.8665 & $\mathrm{~S}$ & 1276.6393 & 638.8233 & 1259.6127 & 630.3100 & 1258.6287 & 629.8180 & 11 \\
\hline 15 & 1723.7632 & 862.3852 & 1706.7367 & 853.8720 & 1705.7526 & 853.3800 & D & 1189.6072 & 595.3073 & 1172.5807 & 586.7940 & 1171.5967 & 586.3020 & 10 \\
\hline 16 & 1837.8061 & 919.4067 & 1820.7796 & 910.8934 & 1819.7956 & 910.4014 & $\mathbf{N}$ & 1074.5803 & 537.7938 & 1057.5538 & 529.2805 & & & 9 \\
\hline 17 & 1951.8491 & 976.4282 & 1934.8225 & 967.9149 & 1933.8385 & 967.4229 & $\mathbf{N}$ & 960.5374 & 480.7723 & 943.5108 & 472.2591 & & & 8 \\
\hline 18 & 2048.9018 & 1024.9545 & 2031.8753 & 1016.4413 & 2030.8913 & 1015.9493 & $\mathbf{P}$ & 846.4944 & 423.7509 & 829.4679 & 415.2376 & & & 7 \\
\hline
\end{tabular}




\begin{tabular}{|l|l|r|r|r|r|r|r|r|r|r|r|r|r|r|r|r|r|r|r|}
$\mathbf{1 9}$ & 2161.9859 & 1081.4966 & 2144.9593 & 1072.9833 & 2143.9753 & 1072.4913 & $\mathbf{I}$ & 749.4417 & 375.2245 & 732.4151 & 366.7112 & & & $\mathbf{6}$ \\
\hline $\mathbf{2 0}$ & 2299.0448 & $\mathbf{1 1 5 0 . 0 2 6 0}$ & 2282.0183 & 1141.5128 & 2281.0342 & 1141.0208 & $\mathbf{H}$ & $\mathbf{6 3 6 . 3 5 7 6}$ & $\mathbf{3 1 8 . 6 8 2 4}$ & 619.3311 & 310.1692 & & & $\mathbf{5}$ \\
\hline $\mathbf{2 1}$ & 2396.0976 & 1198.5524 & 2379.0710 & 1190.0391 & 2378.0870 & 1189.5471 & $\mathbf{P}$ & $\mathbf{4 9 9 . 2 9 8 7}$ & 250.1530 & 482.2722 & 241.6397 & & & $\mathbf{4}$ \\
\hline $\mathbf{2 2}$ & 2510.1405 & 1255.5739 & 2493.1139 & 1247.0606 & 2492.1299 & 1246.5686 & $\mathbf{N}$ & 402.2459 & 201.6266 & $\mathbf{3 8 5 . 2 1 9 4}$ & 193.1133 & & & $\mathbf{3}$ \\
\hline $\mathbf{2 3}$ & 2623.2246 & $\mathbf{1 3 1 2 . 1 1 5 9}$ & 2606.1980 & 1303.6026 & 2605.2140 & 1303.1106 & $\mathbf{L}$ & 288.2030 & 144.6051 & 271.1765 & 136.0919 & & & $\mathbf{2}$ \\
\hline $\mathbf{2 4}$ & & & & & & & $\mathbf{R}$ & 175.1190 & 88.0631 & 158.0924 & 79.5498 & & & $\mathbf{1}$ \\
\hline
\end{tabular}
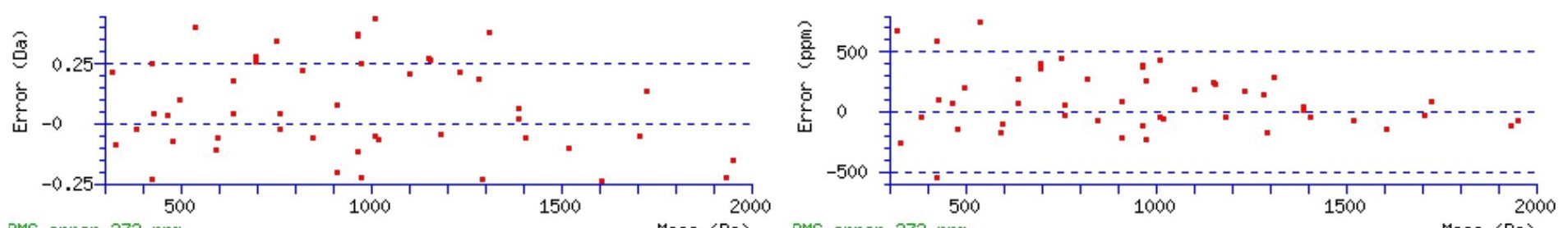

RMS error 273 ppm

\section{All matches to this query}

\begin{tabular}{|l|l|l|l|}
\hline Score & Mr(calc): & Delta & \multicolumn{1}{|c|}{ Sequence } \\
\hline 108.3 & 2796.3289 & 0.0039 & DLVVGLYSQWMNNSDNNPIHPNLR \\
\hline 108.3 & 2796.3289 & 0.0039 & DLVVGLYSQWMNNSDNNPIHPNLR \\
\hline 91.1 & 2796.3289 & 0.0039 & DLVVGLYSOWMNNSDNNPIHPNLR \\
\hline 89.5 & 2796.3289 & 0.0039 & DLVVGLYSQWMNNSDNNPIHPNLR \\
\hline 75.7 & 2796.3289 & 0.0039 & DLVVGLYSOWMNNSDNNPIHPNLR \\
\hline 29.9 & 2795.3449 & 0.9879 & DLVVGLYSQWMNNSDNNPIHPNLR \\
\hline 3.9 & 2794.3228 & 2.0100 & ALNSAANNVYQYGREWITHKLHK \\
\hline 3.9 & 2794.3228 & 2.0100 & ALNSAANNVYQYGREWITHKLHK \\
\hline 3.9 & 2794.3228 & 2.0100 & ALNSAANNVYQYGREWITHKLHK \\
\hline 2.6 & 2795.3493 & 0.9835 & NIPGIPLTGLVGFPAGFATMPTGEDVK \\
\hline
\end{tabular}

Spectrum No: 48; Query: 1113; Rank: 1

\section{Peptide View}

MS/MS Fragmentation of TALAAFNAQNNGTYFK

Found in IPI00327469, Tax_Id=10116 Gene_Symbol=Ahsg Alpha-2-HS-glycoprotein precursor

Match to Query 1113: 1730.824108 from(866.419330,2+)

Title: 091008RatKidney_NoSalt_34.2213.2213.2.dta

Data file K:INewmanPaper|Piliang|3SubProteomes\Piliang3SP\mgf5ppm\ERLIC_3SubProteomes5ppm.mgf

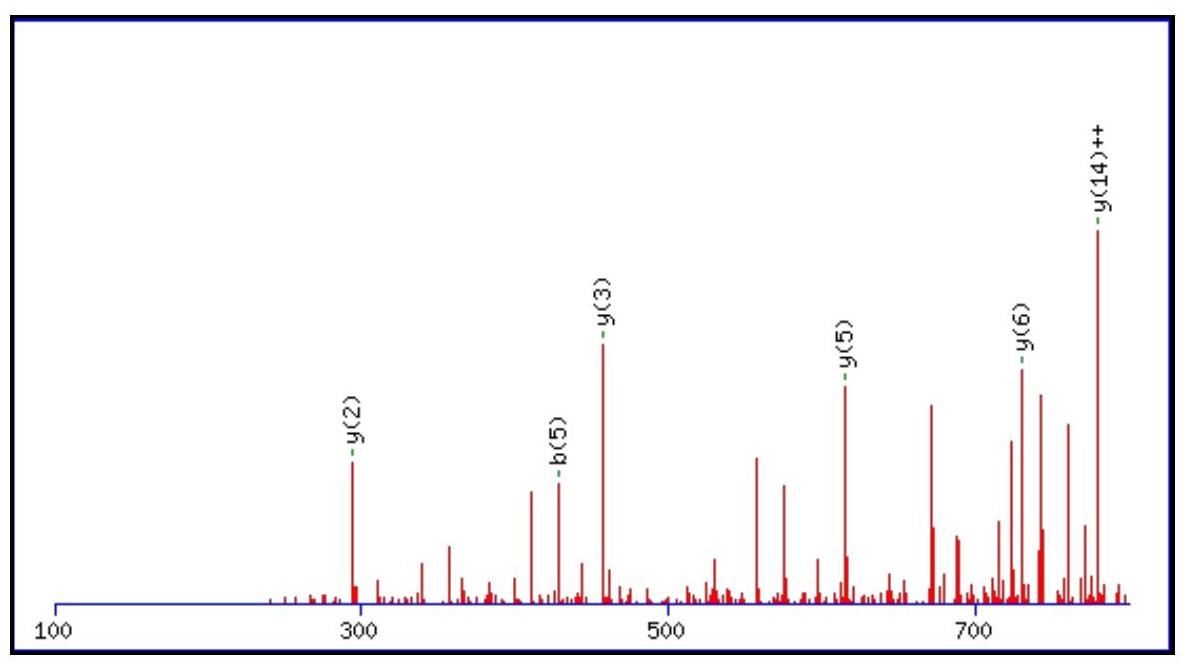



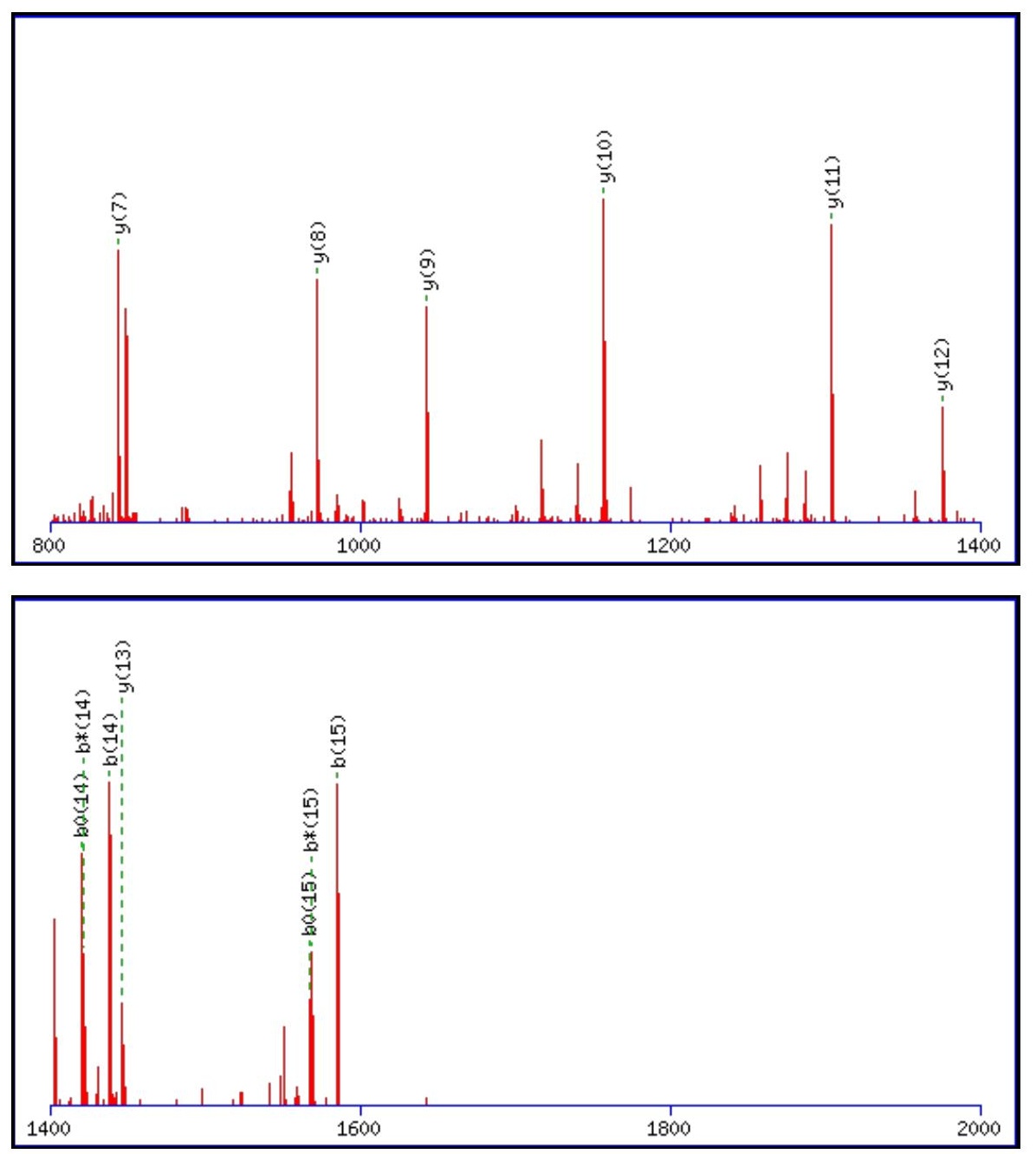

Monoisotopic mass of neutral peptide $\operatorname{Mr}$ (calc): 1730.8264

Fixed modifications: Carbamidomethyl (C)

Variable modifications:

N11 : Deamidated $\mathrm{N}(\mathrm{N})$

Ions Score: 108 Expect: $4.1 \mathrm{e}-009$

Matches (Bold Red): 20/162 fragment ions using 25 most intense peaks

\begin{tabular}{|c|c|c|c|c|c|c|c|c|c|c|c|c|c|c|}
\hline \# & b & $\mathbf{b}^{++}$ & $\mathbf{b}^{*}$ & $\mathbf{b}^{*^{++}}$ & $\mathbf{b}^{\mathbf{0}}$ & $\mathbf{b}^{0++}$ & Seq. & $\mathbf{y}$ & $\mathbf{y}^{++}$ & $\mathbf{y}^{*}$ & $\mathrm{y}^{*^{++}}$ & $\mathbf{y}^{\mathbf{0}}$ & $\mathbf{y}^{\mathbf{0}^{++}}$ & \# \\
\hline 1 & 102.0550 & 51.5311 & & & 84.0444 & 42.5258 & $\mathbf{T}$ & & & & & & & 16 \\
\hline 2 & 173.0921 & 87.0497 & & & 155.0815 & 78.0444 & A & $\mid 1630.7860$ & 815.8966 & 1613.7594 & 807.3834 & 1612.7754 & 806.8914 & 15 \\
\hline 3 & 286.1761 & 143.5917 & & & 268.1656 & 134.5864 & $\mathbf{L}$ & 1559.7489 & 780.3781 & 1542.7223 & 771.8648 & 1541.7383 & 771.3728 & 14 \\
\hline 4 & 357.2132 & 179.1103 & & & 339.2027 & 170.1050 & A & 1446.6648 & 723.8360 & 1429.6383 & 715.3228 & 1428.6542 & 714.8308 & 13 \\
\hline 5 & 428.2504 & 214.6288 & & & 410.2398 & 205.6235 & A & 1375.6277 & 688.3175 & 1358.6012 & 679.8042 & 1357.6171 & 679.3122 & 12 \\
\hline 6 & 575.3188 & 288.1630 & & & 557.3082 & 279.1577 & $\mathbf{F}$ & 1304.5906 & 652.7989 & 1287.5640 & 644.2857 & 1286.5800 & 643.7936 & 11 \\
\hline 7 & 689.3617 & 345.1845 & 672.3352 & 336.6712 & 671.3511 & 336.1792 & $\mathbf{N}$ & 1157.5222 & 579.2647 & 1140.4956 & 570.7514 & 1139.5116 & 570.2594 & 10 \\
\hline 8 & 760.3988 & 380.7030 & 743.3723 & 372.1898 & 742.3883 & 371.6978 & $\mathbf{A}$ & 1043.4792 & 522.2433 & 1026.4527 & 513.7300 & 1025.4687 & 513.2380 & 9 \\
\hline 9 & 888.4574 & 444.7323 & 871.4308 & 436.2191 & 870.4468 & 435.7271 & $\mathbf{Q}$ & 972.4421 & 486.7247 & 955.4156 & 478.2114 & 954.4316 & 477.7194 & 8 \\
\hline 10 & 1002.5003 & 501.7538 & 985.4738 & 493.2405 & 984.4898 & 492.7485 & $\mathbf{N}$ & 844.3836 & 422.6954 & 827.3570 & 414.1821 & 826.3730 & 413.6901 & 7 \\
\hline 11 & 1117.5273 & 559.2673 & 1100.5007 & 550.7540 & 1099.5167 & 550.2620 & $\mathbf{N}$ & 730.3406 & 365.6740 & 713.3141 & 357.1607 & 712.3301 & 356.6687 & 6 \\
\hline 12 & 1174.5487 & 587.7780 & 1157.5222 & 579.2647 & 1156.5382 & 578.7727 & G & 615.3137 & 308.1605 & 598.2871 & 299.6472 & 597.3031 & 299.1552 & 5 \\
\hline 13 & 1275.5964 & 638.3018 & 1258.5699 & 629.7886 & 1257.5858 & 629.2966 & $T$ & 558.2922 & 279.6498 & 541.2657 & 271.1365 & 540.2817 & 270.6445 & 4 \\
\hline 14 & \begin{tabular}{|l|}
1438.6597 \\
\end{tabular} & 719.8335 & \begin{tabular}{|l}
1421.6332 \\
\end{tabular} & 711.3202 & 1420.6492 & 710.8282 & $\mathbf{Y}$ & 457.2445 & 229.1259 & 440.2180 & 220.6126 & & & 3 \\
\hline 15 & 1585.7281 & 793.3677 & 1568.7016 & 784.8544 & 1567.7176 & 784.3624 & $\mathbf{F}$ & 294.1812 & 147.5942 & 277.1547 & 139.0810 & & & 2 \\
\hline 16 & & & & & & & $\mathbf{K}$ & 147.1128 & 74.0600 & 130.0863 & \begin{tabular}{|l|}
65.5468 \\
\end{tabular} & & & 1 \\
\hline
\end{tabular}



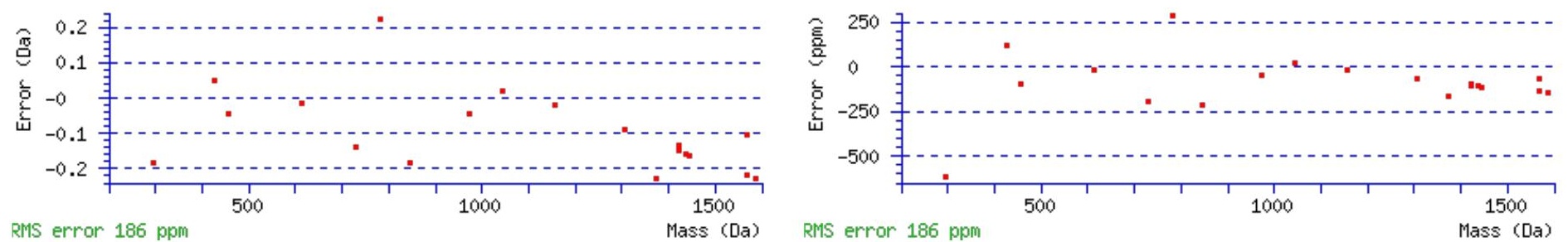

\section{All matches to this query}

\begin{tabular}{|l|l|l|l|}
\hline Score & Mr(calc): & Delta & \multicolumn{1}{|c|}{ Sequence } \\
\hline 107.7 & 1730.8264 & -0.0023 & TALAAFNAQNNGTYFK \\
\hline 89.2 & 1730.8264 & -0.0023 & TALAAFNAQNNGTYFK \\
\hline 46.5 & 1730.8264 & -0.0023 & TALAAFNAQNNGTYFK \\
\hline 7.4 & 1730.8253 & -0.0012 & TQTRAGSHSLRYFK \\
\hline 7.4 & 1730.8253 & -0.0012 & TQTRAGSHSLRYFK \\
\hline 5.7 & 1729.8036 & 1.0205 & DAAAEKPGAPGSPLNVR \\
\hline 3.1 & 1730.8322 & -0.0081 & KISQGTELQNPDNGTK \\
\hline 3.1 & 1730.8069 & 0.0172 & YHFKALDPEFGTVK \\
\hline 3.0 & 1730.8103 & 0.0139 & LFSSKSSAQFKMYK \\
\hline 2.9 & 1730.8158 & 0.0083 & MNINGQWEGEVNGRK \\
\hline
\end{tabular}

Spectrum No: 49; Query: 1118; Rank: 1

\section{Peptide View}

\section{MS/MS Fragmentation of LDAPTNLQFVNETDR}

Found in IPI00200757, Tax_Id=10116 Gene_Symbol=Fn1 Isoform 1 of Fibronectin precursor

Match to Query 1118: 1732.823828 from(867.419190,2+)

Title: 091008RatKidney_NoSalt_31.2396.2396.2.dta

Data file K:INewmanPaper|Piliang|3SubProteomes\Piliang3SP\mgf5ppm\ERLIC_3SubProteomes5ppm.mgf

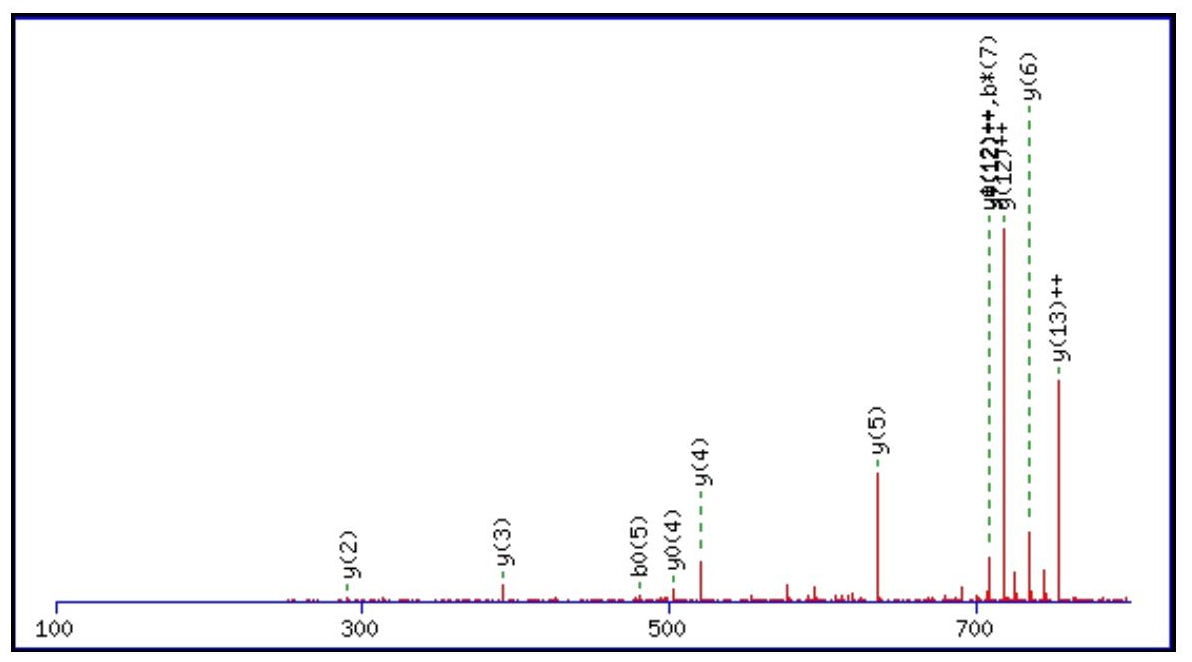



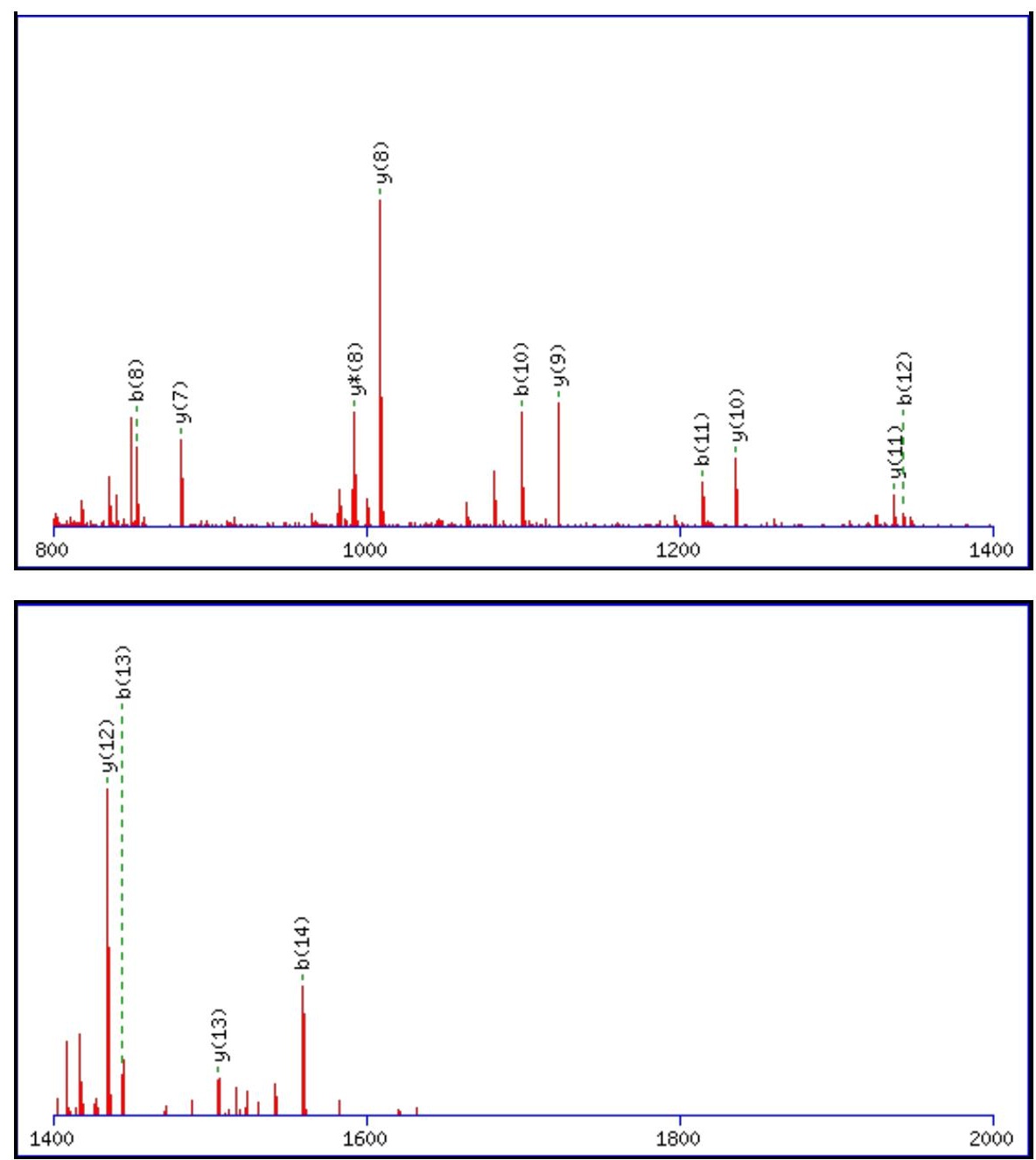

Monoisotopic mass of neutral peptide $\operatorname{Mr}($ calc): 1732.8268

Fixed modifications: Carbamidomethyl (C)

Variable modifications:

N11 : Deamidated $\mathrm{N}(\mathrm{N})$

Ions Score: 107 Expect: $4.5 \mathrm{e}-009$

Matches (Bold Red): 26/154 fragment ions using 37 most intense peaks

\begin{tabular}{|c|c|c|c|c|c|c|c|c|c|c|c|c|c|c|}
\hline \# & b & $\mathbf{b}^{++}$ & b* & $\mathbf{b}^{*^{++}}$ & $\mathbf{b}^{0}$ & $\mathbf{b}^{0++}$ & Seq. & $\mathbf{y}$ & $y^{++}$ & $\mathbf{y}^{*}$ & $\mathbf{y}^{*^{++}}$ & $\mathbf{y}^{\mathbf{0}}$ & $\mathbf{y}^{0++}$ & \# \\
\hline 1 & 114.0913 & 57.5493 & & & & & $\mathbf{L}$ & & & & & & & 15 \\
\hline 2 & 229.1183 & 115.0628 & & & 211.1077 & 106.0575 & D & 1620.7500 & 810.8786 & 1603.7235 & 802.3654 & 1602.7394 & 801.8734 & 14 \\
\hline 3 & 300.1554 & 150.5813 & & & 282.1448 & 141.5761 & A & 1505.7231 & 753.3652 & 1488.6965 & 744.8519 & 1487.7125 & 744.3599 & 13 \\
\hline 4 & 397.2082 & 199.1077 & & & 379.1976 & 190.1024 & $\mathbf{P}$ & 1434.6859 & 717.8466 & 1417.6594 & 709.3333 & 1416.6754 & 708.8413 & 12 \\
\hline 5 & 498.2558 & 249.6316 & & & 480.2453 & 240.6263 & $\mathbf{T}$ & 1337.6332 & 669.3202 & 1320.6066 & 660.8070 & 1319.6226 & 660.3149 & 11 \\
\hline 6 & 612.2988 & 306.6530 & 595.2722 & 298.1397 & 594.2882 & 297.6477 & $\mathbf{N}$ & 1236.5855 & 618.7964 & 1219.5590 & 610.2831 & 1218.5749 & 609.7911 & 10 \\
\hline 7 & 725.3828 & 363.1951 & 708.3563 & 354.6818 & 707.3723 & 354.1898 & $\mathbf{L}$ & 1122.5426 & 561.7749 & 1105.5160 & 553.2617 & 1104.5320 & 552.7696 & 9 \\
\hline 8 & 853.4414 & 427.2243 & 836.4149 & 418.7111 & 835.4308 & 418.2191 & $\mathbf{Q}$ & 1009.4585 & 505.2329 & 992.4320 & 496.7196 & 991.4479 & 496.2276 & 8 \\
\hline 9 & 1000.5098 & 500.7585 & 983.4833 & 492.2453 & 982.4993 & 491.7533 & $\mathbf{F}$ & 881.3999 & 441.2036 & 864.3734 & 432.6903 & 863.3894 & 432.1983 & 7 \\
\hline 10 & 1099.5782 & 550.2928 & 1082.5517 & 541.7795 & 1081.5677 & 541.2875 & V & 734.3315 & 367.6694 & 717.3050 & 359.1561 & 716.3210 & 358.6641 & 6 \\
\hline 11 & 1214.6052 & 607.8062 & 1197.5786 & 599.2930 & 1196.5946 & 598.8009 & $\mathbf{N}$ & 635.2631 & 318.1352 & 618.2366 & 309.6219 & 617.2525 & 309.1299 & 5 \\
\hline 12 & 1343.6478 & 672.3275 & 1326.6212 & 663.8142 & 1325.6372 & 663.3222 & $\mathbf{E}$ & 520.2362 & 260.6217 & 503.2096 & 252.1084 & 502.2256 & 251.6164 & 4 \\
\hline 13 & 1444.6954 & 722.8514 & 1427.6689 & 714.3381 & 1426.6849 & 713.8461 & $\mathbf{T}$ & 391.1936 & 196.1004 & 374.1670 & 187.5872 & 373.1830 & 187.0951 & 3 \\
\hline 14 & 1559.7224 & 780.3648 & 1542.6958 & 771.8516 & 1541.7118 & 771.3595 & D & 290.1459 & 145.5766 & 273.1193 & 137.0633 & 272.1353 & 136.5713 & 2 \\
\hline 15 & & & & & & & $\mathbf{R}$ & 175.1190 & 88.0631 & 158.0924 & 79.5498 & & & 1 \\
\hline
\end{tabular}



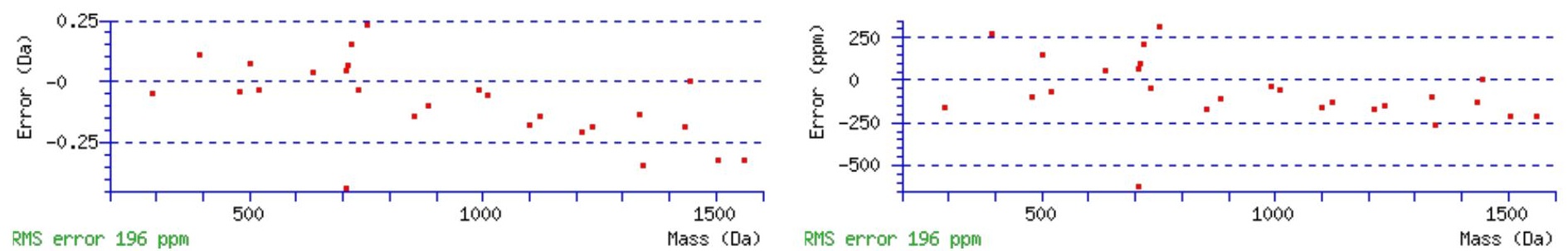

\section{All matches to this query}

\begin{tabular}{|l|l|l|l|}
\hline Score & Mr(calc): & Delta & \multicolumn{1}{|c|}{ Sequence } \\
\hline 107.2 & 1732.8268 & -0.0030 & LDAPTNLQFVNETDR \\
\hline 37.5 & 1732.8268 & -0.0030 & LDAPTNLQFVNETDR \\
\hline 18.0 & 1731.8080 & 1.0158 & VTSSGVLLLDNYTDR \\
\hline 18.0 & 1731.8080 & 1.0158 & VTSSGVLLLDNYTDR \\
\hline 13.3 & 1731.8080 & 1.0158 & VTSSGVLLLDNYTDR \\
\hline 7.9 & 1731.8120 & 1.0118 & EPNGLIVLYEVSYR \\
\hline 6.9 & 1732.8331 & -0.0093 & LLEAAVQQHSTMIGR \\
\hline 5.9 & 1732.8096 & 0.0142 & AVTVEGMLKISRNR \\
\hline 3.2 & 1732.8331 & -0.0093 & LLEAAVQQHSTMIGR \\
\hline 2.2 & 1732.8331 & -0.0093 & EASLLVSCRTSKFR \\
\hline
\end{tabular}

Spectrum No: 50; Query: 1830; Rank: 1

\section{Peptide View}

MS/MS Fragmentation of SVCADAPYVNVTAEDLDGPR

Found in IPI00358687, Tax_Id=10116 Gene_Symbol=Dsg2_predicted similar to desmoglein 2

Match to Query 1830: 2148.966048 from(1075.490300,2+)

Title: 100101RatKid_NS_deglyco_25.3198.3198.2.dta

Data file K:INewmanPaper|Piliangl3SubProteomes\Piliang3SP\mgf5ppm\ERLIC_3SubProteomes5ppm.mgf

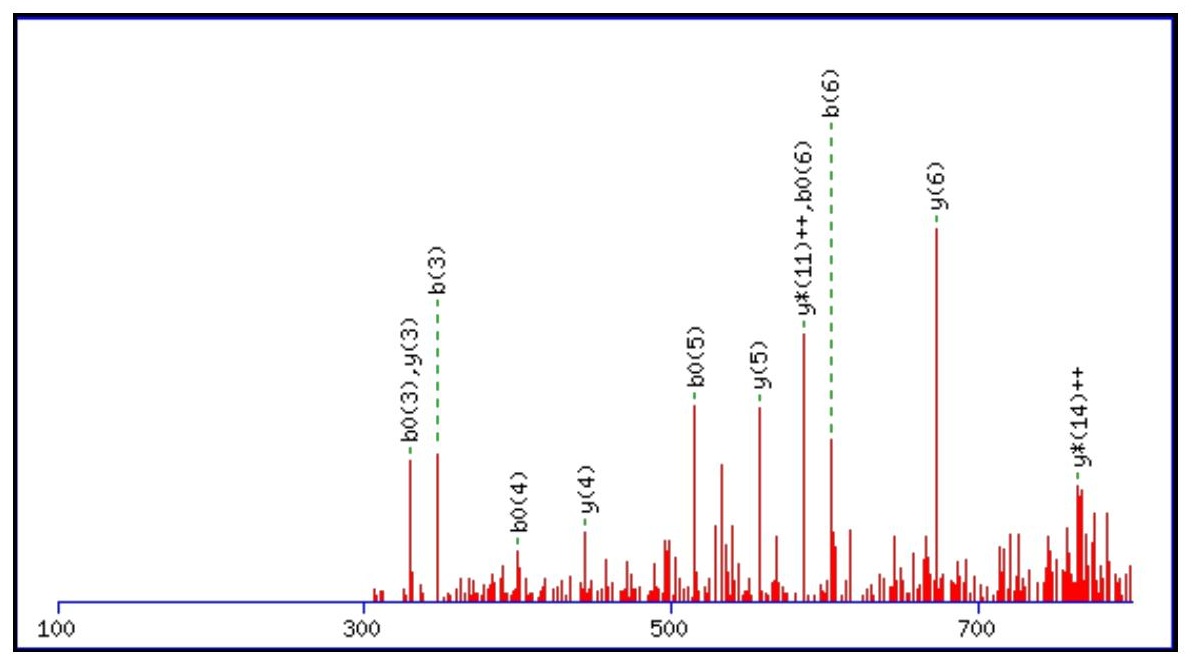



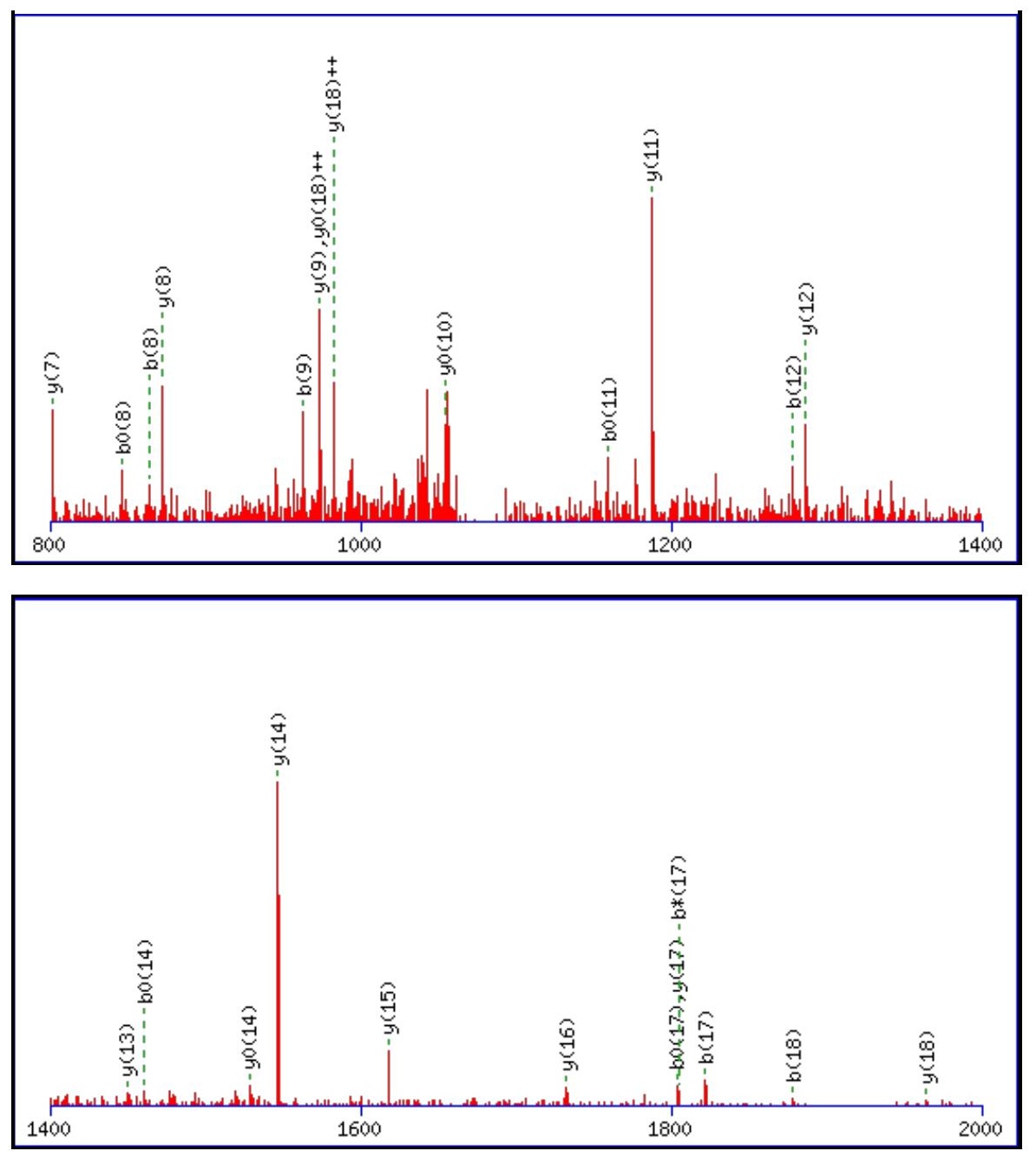

Monoisotopic mass of neutral peptide $\operatorname{Mr}($ calc): 2148.9634

Fixed modifications: Carbamidomethyl (C)

Variable modifications:

N10 : Deamidated_N (N)

Ions Score: 107 Expect: $5.2 \mathrm{e}-009$

Matches (Bold Red): 37/204 fragment ions using 59 most intense peaks

\begin{tabular}{|c|c|c|c|c|c|c|c|c|c|c|c|c|c|c|}
\hline$\#$ & b & $\mathbf{b}^{++}$ & $\mathbf{b}^{*}$ & $\mathbf{b}^{*^{++}}$ & $\mathbf{b}^{0}$ & $\mathbf{b}^{0++}$ & Seq. & $\mathbf{y}$ & $\mathbf{y}^{++}$ & $\mathbf{y}^{*}$ & $\mathrm{y}^{*^{++}}$ & $\mathbf{y}^{0}$ & $\mathbf{y}^{0++}$ & \# \\
\hline 1 & 88.0393 & 44.5233 & & & 70.0287 & 35.5180 & $S$ & & & & & & & 20 \\
\hline 2 & 187.1077 & 94.0575 & & & 169.0972 & 85.0522 & $\mathbf{V}$ & 2062.9386 & 1031.9729 & 2045.9121 & 1023.4597 & 2044.9280 & 1022.9677 & 19 \\
\hline 3 & 347.1384 & 174.0728 & & & 329.1278 & 165.0675 & $\mathrm{C}$ & 1963.8702 & 982.4387 & 1946.8436 & 973.9255 & 1945.8596 & 973.4335 & 18 \\
\hline 4 & 418.1755 & 209.5914 & & & 400.1649 & 200.5861 & A & 1803.8395 & 902.4234 & 1786.8130 & 893.9101 & 1785.8290 & 893.4181 & 17 \\
\hline 5 & 533.2024 & 267.1049 & & & 515.1919 & 258.0996 & D & 1732.8024 & 866.9049 & 1715.7759 & 858.3916 & 1714.7919 & 857.8996 & 16 \\
\hline 6 & 604.2395 & 302.6234 & & & 586.2290 & 293.6181 & A & 1617.7755 & 809.3914 & 1600.7489 & 800.8781 & 1599.7649 & 800.3861 & 15 \\
\hline 7 & 701.2923 & 351.1498 & & & 683.2817 & 342.1445 & $\mathbf{P}$ & 1546.7384 & 773.8728 & 1529.7118 & 765.3596 & 1528.7278 & 764.8675 & 14 \\
\hline 8 & 864.3556 & 432.6815 & & & 846.3451 & 423.6762 & $\mathbf{Y}$ & 1449.6856 & 725.3464 & 1432.6591 & 716.8332 & 1431.6750 & 716.3412 & 13 \\
\hline 9 & 963.4240 & 482.2157 & & & 945.4135 & 473.2104 & $\mathbf{V}$ & 1286.6223 & 643.8148 & 1269.5957 & 635.3015 & 1268.6117 & 634.8095 & 12 \\
\hline 10 & 1078.4510 & 539.7291 & 1061.4244 & 531.2159 & 1060.4404 & 530.7238 & $\mathbf{N}$ & 1187.5539 & 594.2806 & 1170.5273 & 585.7673 & 1169.5433 & 585.2753 & 11 \\
\hline 11 & 1177.5194 & 589.2633 & 1160.4928 & 580.7501 & 1159.5088 & 580.2581 & $\mathbf{V}$ & 1072.5269 & 536.7671 & 1055.5004 & 528.2538 & 1054.5164 & 527.7618 & 10 \\
\hline 12 & 1278.5671 & 639.7872 & 1261.5405 & 631.2739 & 1260.5565 & 630.7819 & $\mathbf{T}$ & 973.4585 & 487.2329 & 956.4320 & 478.7196 & 955.4480 & 478.2276 & 9 \\
\hline 13 & 1349.6042 & 675.3057 & 1332.5776 & 666.7925 & 1331.5936 & 666.3004 & A & 872.4108 & 436.7091 & 855.3843 & 428.1958 & 854.4003 & 427.7038 & 8 \\
\hline 14 & 1478.6468 & 739.8270 & 1461.6202 & 731.3138 & 1460.6362 & 730.8217 & $\mathbf{E}$ & 801.3737 & 401.1905 & 784.3472 & 392.6772 & 783.3632 & 392.1852 & 7 \\
\hline 15 & 1593.6737 & 797.3405 & 1576.6472 & 788.8272 & 1575.6632 & 788.3352 & D & 672.3311 & 336.6692 & 655.3046 & 328.1559 & 654.3206 & 327.6639 & 6 \\
\hline 16 & 1706.7578 & 853.8825 & 1689.7312 & 845.3693 & 1688.7472 & 844.8772 & $\mathbf{L}$ & 557.3042 & 279.1557 & 540.2776 & 270.6425 & 539.2936 & 270.1504 & 5 \\
\hline 17 & 1821.7847 & 911.3960 & 1804.7582 & 902.8827 & 1803.7742 & 902.3907 & D & 444.2201 & 222.6137 & 427.1936 & 214.1004 & 426.2096 & 213.6084 & 4 \\
\hline 18 & 1878.8062 & 939.9067 & 1861.7796 & 931.3935 & 1860.7956 & 930.9015 & G & 329.1932 & 165.1002 & 312.1666 & 156.5870 & & & 3 \\
\hline
\end{tabular}




\begin{tabular}{|r|r|r|r|r|r|r|r|r|r|r|r|r|r|}
$\mathbf{1 9}$ & 1975.8590 & 988.4331 & 1958.8324 & 979.9198 & 1957.8484 & 979.4278 & $\mathbf{P}$ & 272.1717 & 136.5895 & 255.1452 & 128.0762 & \\
\hline $\mathbf{2 0}$ & & & & & & & $\mathbf{R}$ & 175.1190 & 88.0631 & 158.0924 & 79.5498 & \\
\hline
\end{tabular}
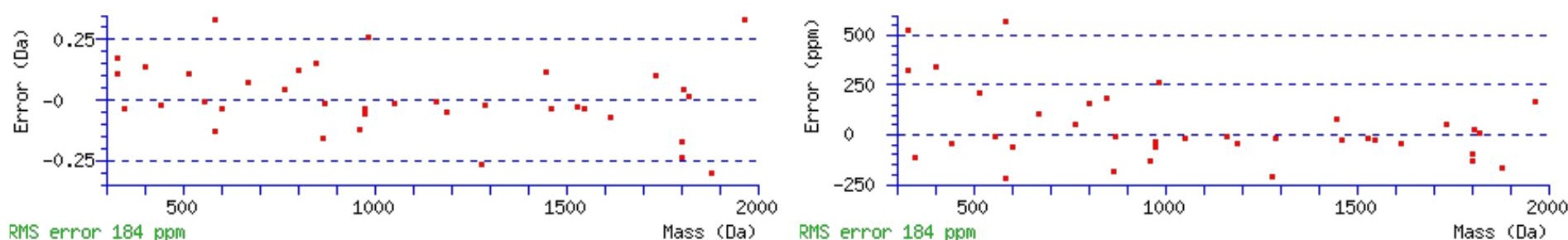

RMS error 184 ppm

\section{All matches to this query}

\begin{tabular}{|l|l|l|l|}
\hline Score & Mr(calc): & Delta & \multicolumn{1}{c|}{ Sequence } \\
\hline 106.8 & 2148.9634 & 0.0027 & SVCADAPYVNVTAEDLDGPR \\
\hline 31.1 & 2147.9794 & 0.9867 & SVCADAPYVNVTAEDLDGPR \\
\hline 3.0 & 2147.9671 & 0.9990 & QESVSALSASMHPQLSPQR \\
\hline 2.8 & 2148.9558 & 0.0102 & AVCGGAGFGSRSLCGVGSSKR \\
\hline 2.3 & 2147.9792 & 0.9868 & TDTLLYSVLPPSVANELR \\
\hline 2.3 & 2147.9792 & 0.9868 & TDTLLYSVLPPSVANELR \\
\hline 2.3 & 2147.9792 & 0.9868 & TDTLLYSVLPPSVANELR \\
\hline 2.3 & 2147.9792 & 0.9868 & TDTLLYSVLPPSVANELR \\
\hline 0.6 & 2147.9840 & 0.9821 & TINFQIECTPVKTGPRK \\
\hline 0.4 & 2148.9558 & 0.0102 & $\underline{\text { AVCGGAGFGSRSLCGVGSSKR }}$ \\
\hline
\end{tabular}

Spectrum No: 51; Query: 2508; Rank: 1

\section{Peptide View}

MS/MS Fragmentation of LLEFDSTNVSEGTQPPGKPYPPYSLAK

Found in IPI00196226, Tax_Id=10116 Gene_Symbol=RGD1303130 Uncharacterized protein C1orf85 homolog precursor

Match to Query 2508: 2935.453032 from(979.491620,3+)

Title: 100101RatKid_NS_deglyco_18.3782.3782.3.dta

Data file K:INewmanPaper|Piliangl3SubProteomes\Piliang3SP\mgf5ppm\ERLIC_3SubProteomes5ppm.mgf

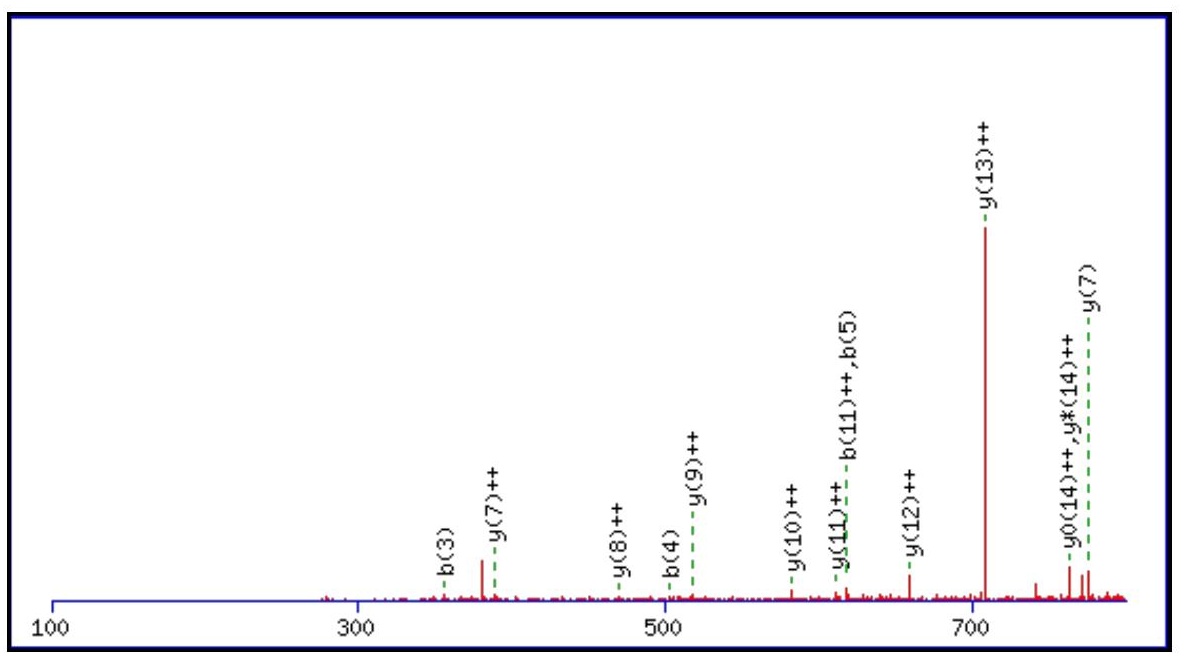



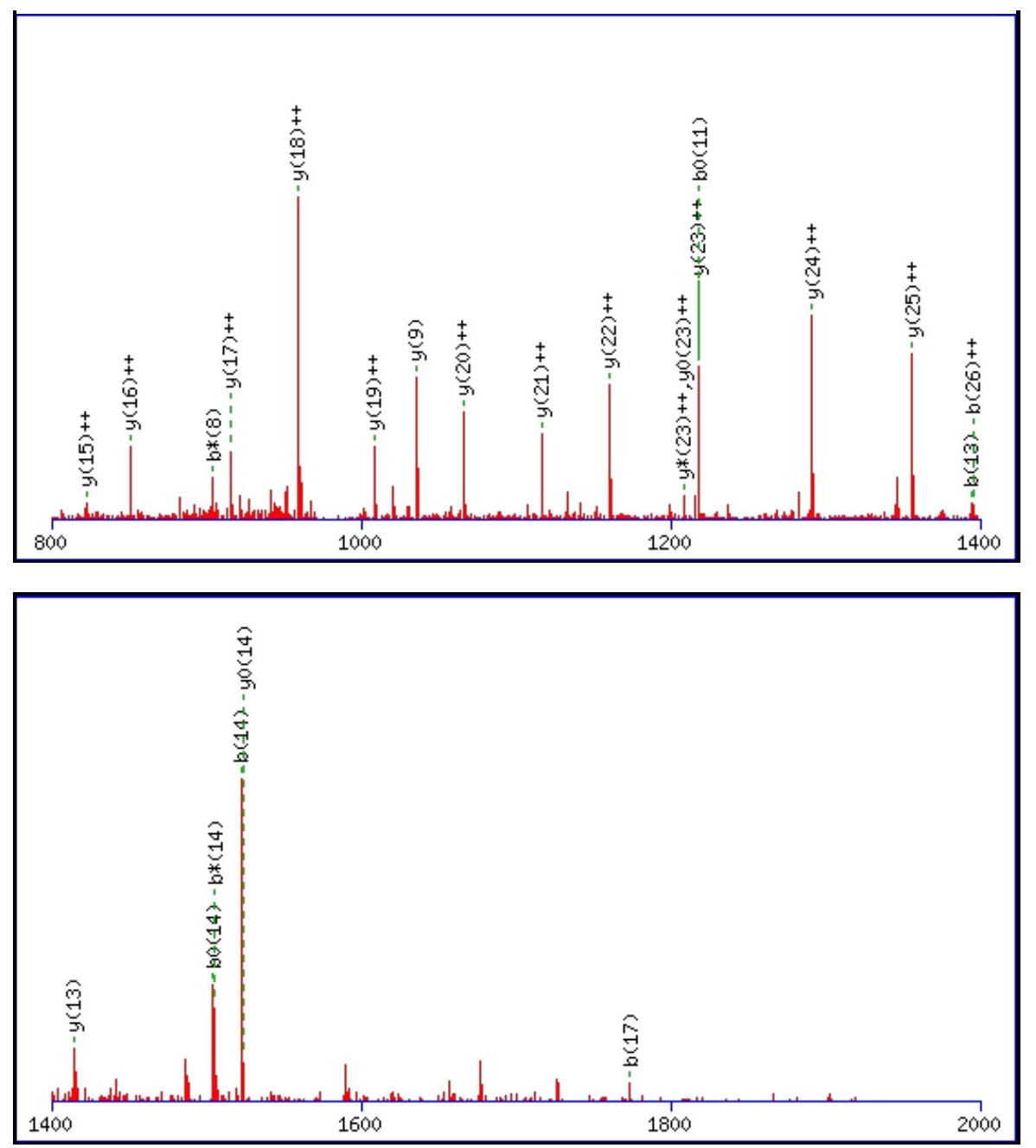

Monoisotopic mass of neutral peptide $\operatorname{Mr}($ calc): 2935.4491

Fixed modifications: Carbamidomethyl (C)

Variable modifications:

N8: Deamidated $\mathrm{N}(\mathrm{N})$

Ions Score: 106 Expect: $5.5 \mathrm{e}-009$

Matches (Bold Red): 38/288 fragment ions using 70 most intense peaks

\begin{tabular}{|c|c|c|c|c|c|c|c|c|c|c|c|c|c|c|}
\hline \# & b & $\mathbf{b}^{++}$ & $\mathbf{b}^{*}$ & $\mathbf{b}^{*^{++}}$ & $\mathbf{b}^{0}$ & $\mathbf{b}^{\mathbf{0 + +}}$ & Seq. & $\mathbf{y}$ & $\mathbf{y}^{++}$ & $\mathbf{y}^{*}$ & $\mathbf{y}^{*^{++}}$ & $\mathbf{y}^{0}$ & $\mathbf{y}^{0++}$ & \# \\
\hline 1 & 114.0913 & 57.5493 & & & & & $\mathbf{L}$ & & & & & & & 27 \\
\hline 2 & 227.1754 & 114.0913 & & & & & $\mathbf{L}$ & 2823.3723 & 1412.1898 & 2806.3458 & 1403.6765 & 2805.3618 & 1403.1845 & 26 \\
\hline 3 & 356.2180 & 178.6126 & & & 338.2074 & 169.6074 & $\mathbf{E}$ & 2710.2883 & 1355.6478 & 2693.2617 & 1347.1345 & |2692.2777 & 1346.6425 & 25 \\
\hline 4 & 503.2864 & 252.1468 & & & 485.2758 & 243.1416 & $\mathbf{F}$ & 2581.2457 & 1291.1265 & 2564.2191 & 1282.6132 & 2563.2351 & 1282.1212 & 24 \\
\hline 5 & 618.3134 & 309.6603 & & & 600.3028 & 300.6550 & D & 2434.1773 & 1217.5923 & 2417.1507 & 1209.0790 & 2416.1667 & 1208.5870 & 23 \\
\hline 6 & 705.3454 & 353.1763 & & & 687.3348 & 344.1710 & $\mathrm{~S}$ & 2319.1503 & 1160.0788 & 2302.1238 & 1151.5655 & 2301.1397 & 1151.0735 & 22 \\
\hline 7 & 806.3931 & 403.7002 & & & 788.3825 & 394.6949 & $T$ & 2232.1183 & 1116.5628 & 2215.0917 & 1108.0495 & 2214.1077 & 1107.5575 & 21 \\
\hline 8 & 921.4200 & 461.2136 & 904.3934 & 452.7004 & 903.4094 & 452.2084 & $\mathbf{N}$ & 2131.0706 & 1066.0389 & 2114.0441 & 1057.5257 & 2113.0600 & 1057.0337 & 20 \\
\hline 9 & 1020.4884 & 510.7478 & 1003.4619 & 502.2346 & 1002.4778 & 501.7426 & $\mathbf{V}$ & 2016.0437 & 1008.5255 & 1999.0171 & 1000.0122 & 1998.0331 & 999.5202 & 19 \\
\hline 10 & 1107.5204 & 554.2639 & 1090.4939 & 545.7506 & 1089.5099 & 545.2586 & $\mathrm{~S}$ & 1916.9753 & 958.9913 & 1899.9487 & 950.4780 & 1898.9647 & 949.9860 & 18 \\
\hline 11 & 1236.5630 & 618.7852 & 1219.5365 & 610.2719 & 1218.5525 & 609.7799 & $E$ & 1829.9432 & 915.4753 & 1812.9167 & 906.9620 & 1811.9327 & 906.4700 & 17 \\
\hline 12 & 1293.5845 & 647.2959 & 1276.5579 & 638.7826 & 1275.5739 & 638.2906 & $\mathbf{G}$ & 1700.9006 & 850.9540 & 1683.8741 & 842.4407 & 1682.8901 & 841.9487 & 16 \\
\hline 13 & \begin{tabular}{|l|l|}
1394.6322 \\
\end{tabular} & 697.8197 & 1377.6056 & 689.3065 & 1376.6216 & 688.8144 & $\mathbf{T}$ & 1643.8792 & 822.4432 & 1626.8526 & 813.9299 & 1625.8686 & 813.4379 & 15 \\
\hline 14 & 1522.6908 & 761.8490 & 1505.6642 & 753.3357 & 1504.6802 & 752.8437 & $\mathbf{Q}$ & 1542.8315 & 771.9194 & 1525.8049 & 763.4061 & 1524.8209 & 762.9141 & 14 \\
\hline 15 & 1619.7435 & 810.3754 & 1602.7170 & 801.8621 & 1601.7330 & 801.3701 & $\mathbf{P}$ & \begin{tabular}{|l}
1414.7729 \\
\end{tabular} & 707.8901 & 1397.7464 & 699.3768 & 1396.7624 & 698.8848 & 13 \\
\hline 16 & 1716.7963 & 858.9018 & 1699.7697 & 850.3885 & 1698.7857 & 849.8965 & $\mathbf{P}$ & 1317.7202 & 659.3637 & 1300.6936 & 650.8504 & 1299.7096 & 650.3584 & 12 \\
\hline 17 & 1773.8177 & 887.4125 & 1756.7912 & 878.8992 & 1755.8072 & 878.4072 & G & 1220.6674 & 610.8373 & 1203.6408 & 602.3241 & 1202.6568 & 601.8320 & 11 \\
\hline 18 & 1901.9127 & 951.4600 & 1884.8862 & 942.9467 & 1883.9021 & 942.4547 & $\mathbf{K}$ & 1163.6459 & 582.3266 & 1146.6194 & 573.8133 & 1145.6354 & 573.3213 & 10 \\
\hline
\end{tabular}




\begin{tabular}{|c|c|c|c|c|c|c|c|c|c|c|c|c|c|c|}
\hline 19 & |1998.9655 & 999.9864 & |1981.9389 & 991.4731 & 1980.9549 & 990.9811 & $\mathbf{P}$ & 1035.5510 & 518.2791 & 1018.5244 & 509.7658 & |1017.5404| & 509.2738 & 9 \\
\hline 20 & 2162.0288 & 1081.5180 & 2145.0023 & 1073.0048 & 2144.0182 & 1072.5128 & $\mathbf{Y}$ & 938.4982 & 469.7527 & 921.4716 & 461.2395 & 920.4876 & 460.7475 & 8 \\
\hline 21 & 2259.0816 & 1130.0444 & 2242.0550 & 1121.5311 & 2241.0710 & 1121.0391 & $\mathbf{P}$ & 775.4349 & 388.2211 & 758.4083 & 379.7078 & 757.4243 & 379.2158 & 7 \\
\hline 22 & 2356.1343 & 1178.5708 & 2339.1078 & 1170.0575 & 2338.1238 & 1169.5655 & $\mathbf{P}$ & 678.3821 & 339.6947 & 661.3556 & 331.1814 & 660.3715 & 330.6894 & 6 \\
\hline 23 & 2519.1977 & 1260.1025 & 2502.1711 & 1251.5892 & 2501.1871 & 1251.0972 & $\mathbf{Y}$ & 581.3293 & 291.1683 & 564.3028 & 282.6550 & 563.3188 & 282.1630 & 5 \\
\hline 24 & 2606.2297 & 1303.6185 & 2589.2031 & 1295.1052 & 2588.2191 & 1294.6132 & S & 418.2660 & 209.6366 & 401.2395 & 201.1234 & 400.2554 & 200.6314 & 4 \\
\hline 25 & 2719.3137 & 1360.1605 & 2702.2872 & 1351.6472 & 2701.3032 & 1351.1552 & $\mathbf{L}$ & 331.2340 & 166.1206 & 314.2074 & 157.6074 & & & 3 \\
\hline 26 & 2790.3509 & 1395.6791 & 2773.3243 & 1387.1658 & 2772.3403 & 1386.6738 & A & 218.1499 & 109.5786 & 201.1234 & 101.0653 & & & 2 \\
\hline 27 & & & & & & & $\mathbf{K}$ & 147.1128 & 74.0600 & 130.0863 & 65.5468 & & & 1 \\
\hline
\end{tabular}
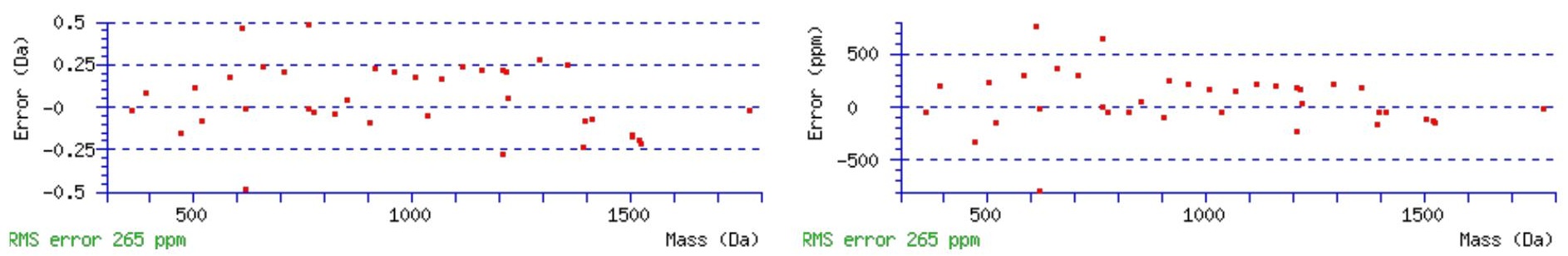

\section{All matches to this query}

\begin{tabular}{|l|l|l|l|}
\hline Score & Mr(calc): & Delta & \multicolumn{1}{|c|}{ Sequence } \\
\hline 106.4 & 2935.4491 & 0.0039 & LLEFDSTNVSEGTQPPGKPYPPYSLAK \\
\hline 40.6 & 2934.4651 & 0.9879 & LLEFDSTNVSEGTOPPGKPYPPYSLAK \\
\hline 9.5 & 2935.4613 & -0.0083 & EVILSGGAINSPQLLMLSGVGNADDLKK \\
\hline 5.5 & 2935.4375 & 0.0155 & NSAMLVQNPYITVRSDGSLHIERVR \\
\hline 3.7 & 2935.4553 & -0.0022 & EEAEAAPTPGLGGGPGPRQRTPLDILTR \\
\hline 1.9 & 2935.4613 & -0.0083 & EVILSGGAINSPQLLMLSGVGNADDLKK \\
\hline 1.5 & 2935.4493 & 0.0037 & LLQGGTLMYEQVPMVEIDGMNLVQTR \\
\hline 1.4 & 2935.4613 & -0.0083 & EVILSGGAINSPQLLMLSGVGNADDLKK \\
\hline 1.3 & 2935.4643 & -0.0113 & LLEPLIIQVTTLVNCPQNPSNRKK \\
\hline 1.3 & 2935.4643 & -0.0113 & LLEPLIIQVTTLVNCPQNPSNRKK \\
\hline
\end{tabular}

Spectrum No: 52; Query: 1603; Rank: 1

\section{Peptide View}

MS/MS Fragmentation of TQLTCFLNSSGIDIVGHR

Found in IPI00193425, Tax_Id=10116 Gene_Symbol=Bsg Isoform 2 of Basigin precursor

Match to Query 1603: 2017.994488 from(1010.004520,2+)

Title: 100101RatKid_NS_deglyco_26.4122.4122.2.dta

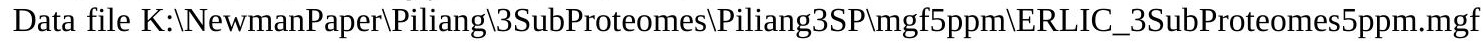



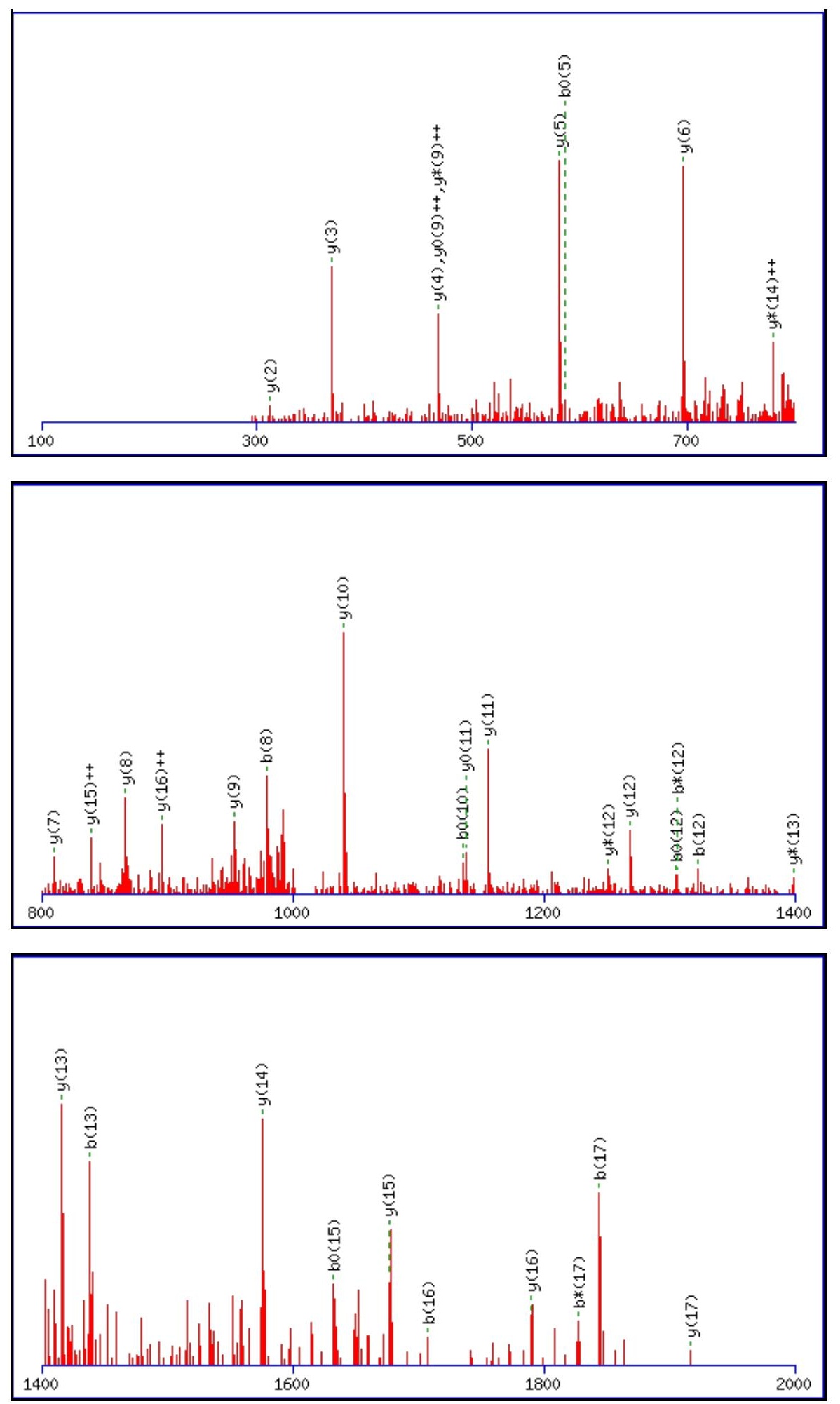

Monoisotopic mass of neutral peptide $\operatorname{Mr}($ calc): 2017.9891

Fixed modifications: Carbamidomethyl (C)

Variable modifications:

N8

Ions Score: 106 Expect: $5.5 \mathrm{e}-009$

Matches (Bold Red): 35/192 fragment ions using 72 most intense peaks

\begin{tabular}{|r|c|c|c|c|c|c|c|c|c|c|c|c|c|c|}
\hline$\#$ & $\mathbf{b}$ & $\mathbf{b}^{++}$ & $\mathbf{b}^{*}$ & $\mathbf{b}^{\boldsymbol{*}^{++}}$ & $\mathbf{b}^{\mathbf{0}}$ & $\mathbf{b}^{\mathbf{0 + +}}$ & Seq. & $\mathbf{y}$ & $\mathbf{y}^{++}$ & $\mathbf{y}^{\mathbf{*}}$ & $\mathbf{y}^{\boldsymbol{*}^{++}}$ & $\mathbf{y}^{\mathbf{0}}$ & $\mathbf{y}^{\mathbf{0 + +}}$ & $\#$ \\
\hline $\mathbf{1}$ & 102.0550 & 51.5311 & & & 84.0444 & 42.5258 & $\mathbf{T}$ & & & & & & & $\mathbf{1 8}$ \\
\hline $\mathbf{2}$ & 230.1135 & 115.5604 & 213.0870 & 107.0471 & 212.1030 & 106.5551 & $\mathbf{Q}$ & $\mathbf{1 9 1 7 . 9 4 8 7}$ & 959.4780 & 1900.9222 & 950.9647 & 1899.9382 & 950.4727 & $\mathbf{1 7}$ \\
\hline 3 & 343.1976 & 172.1024 & 326.1710 & 163.5892 & 325.1870 & 163.0972 & $\mathbf{L}$ & $\mathbf{1 7 8 9 . 8 9 0 1}$ & $\mathbf{8 9 5 . 4 4 8 7}$ & 1772.8636 & 886.9354 & 1771.8796 & 886.4434 & $\mathbf{1 6}$ \\
\hline $\mathbf{4}$ & 444.2453 & 222.6263 & 427.2187 & 214.1130 & 426.2347 & 213.6210 & $\mathbf{T}$ & $\mathbf{1 6 7 6 . 8 0 6 1}$ & $\mathbf{8 3 8 . 9 0 6 7}$ & 1659.7795 & 830.3934 & 1658.7955 & 829.9014 & $\mathbf{1 5}$ \\
\hline $\mathbf{5}$ & 604.2759 & 302.6416 & 587.2494 & 294.1283 & $5 \mathbf{5 8 6 . 2 6 5 4}$ & 293.6363 & $\mathbf{C}$ & $\mathbf{1 5 7 5 . 7 5 8 4}$ & 788.3828 & 1558.7318 & $\mathbf{7 7 9 . 8 6 9 6}$ & 1557.7478 & $\mathbf{7 7 9 . 3 7 7 6}$ & $\mathbf{1 4}$ \\
\hline
\end{tabular}




\begin{tabular}{|c|c|c|c|c|c|c|c|c|c|c|c|c|c|c|}
\hline 6 & 751.3443 & |376.1758 & 734.3178| & |367.6625 & 733.3338 & 367.1705 & $\mathbf{F}$ & 1415.7277| & |708.3675| & |1398.7012 & |699.8542 & |1397.7172| & 699.3622 & 13 \\
\hline 7 & 864.4284 & 432.7178 & 847.4019 & 424.2046 & 846.4178 & 423.7126 & $\mathbf{L}$ & 1268.6593 & 634.8333 & 1251.6328 & 626.3200 & 1250.6488 & 625.8280 & 12 \\
\hline 8 & 979.4553 & 490.2313 & 962.4288 & 481.7180 & 961.4448 & 481.2260 & $\mathbf{N}$ & 1155.5753 & 578.2913 & 1138.5487 & 569.7780 & 1137.5647 & 569.2860 & 11 \\
\hline 9 & 1066.4874 & 533.7473 & 1049.4608 & 525.2340 & 1048.4768 & 524.7420 & S & 1040.5483 & 520.7778 & 1023.5218 & 512.2645 & 1022.5378 & 511.7725 & 10 \\
\hline 10 & 1153.5194 & 577.2633 & 1136.4928 & 568.7501 & 1135.5088 & 568.2581 & S & 953.5163 & 477.2618 & 936.4898 & 468.7485 & 935.5057 & 468.2565 & 9 \\
\hline 11 & 1210.5409 & 605.7741 & 1193.5143 & 597.2608 & 1192.5303 & 596.7688 & G & 866.4843 & 433.7458 & 849.4577 & 425.2325 & 848.4737 & 424.7405 & 8 \\
\hline 12 & 1323.6249 & 662.3161 & 1306.5984 & 653.8028 & 1305.6144 & 653.3108 & I & 809.4628 & 405.2350 & 792.4363 & 396.7218 & 791.4522 & 396.2298 & 7 \\
\hline 13 & 1438.6519 & 719.8296 & 1421.6253 & 711.3163 & 1420.6413 & 710.8243 & D & 696.3787 & 348.6930 & 679.3522 & 340.1797 & 678.3682 & 339.6877 & 6 \\
\hline 14 & 1551.7359 & 776.3716 & 1534.7094 & 767.8583 & 1533.7254 & 767.3663 & I & 581.3518 & 291.1795 & 564.3253 & 282.6663 & & & 5 \\
\hline 15 & 1650.8043 & 825.9058 & 1633.7778 & 817.3925 & 1632.7938 & 816.9005 & V & 468.2677 & 234.6375 & 451.2412 & 226.1242 & & & 4 \\
\hline 16 & 1707.8258 & 854.4165 & 1690.7993 & 845.9033 & 1689.8152 & 845.4113 & $\mathbf{G}$ & 369.1993 & 185.1033 & 352.1728 & 176.5900 & & & 3 \\
\hline 17 & 1844.8847 & 922.9460 & 1827.8582 & 914.4327 & 1826.8742 & 913.9407 & H & 312.1779 & 156.5926 & 295.1513 & 148.0793 & & & 2 \\
\hline 18 & & & & & & & $\mathbf{R}$ & 175.1190 & 88.0631 & 158.0924 & 79.5498 & & & 1 \\
\hline
\end{tabular}

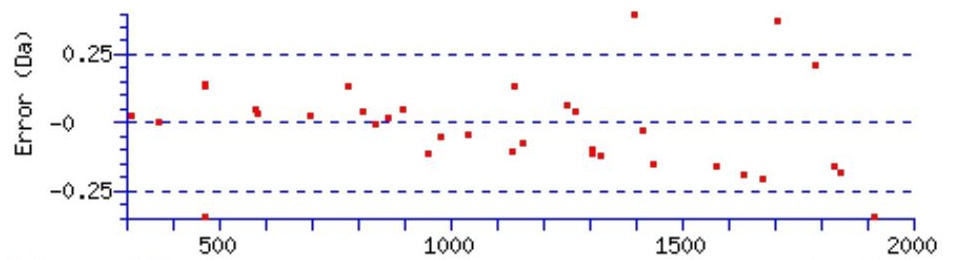

RMS error 176 ppm

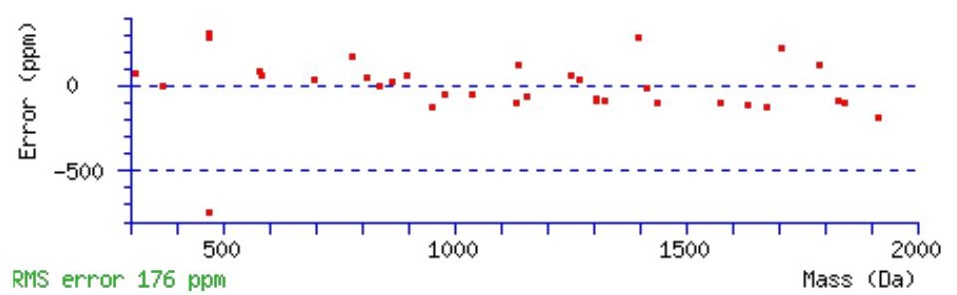

\section{All matches to this query}

\begin{tabular}{|l|l|l|l|}
\hline Score & Mr(calc): & Delta & \multicolumn{1}{|c|}{ Sequence } \\
\hline 106.0 & 2017.9891 & 0.0054 & TQLTCFLNSSGIDIVGHR \\
\hline 19.6 & 2017.0051 & 0.9894 & TQLTCFLNSSGIDIVGHR \\
\hline 4.8 & 2017.9850 & 0.0095 & NANVSITIASSALRTLVK \\
\hline 4.8 & 2017.9850 & 0.0095 & NANVSITIASSALRTLVK \\
\hline 1.9 & 2017.0010 & 0.9935 & NANVSITIASSALRTLVK \\
\hline
\end{tabular}

Spectrum No: 53; Query: 2405; Rank: 1

\section{Peptide View}

MS/MS Fragmentation of HIDCASVYGNETEIGEALKESVGAGK

Found in IPI00230859, Tax_Id=10116 Gene_Symbol=Akr1a1 Alcohol dehydrogenase

Match to Query 2405: 2734.286862 from(912.436230,3+)

Title: 091008RatKidney_NH4Format01_14.3949.3949.3.dta

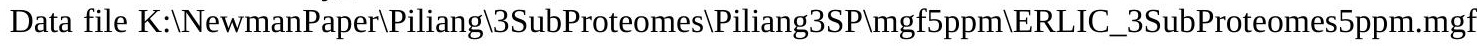



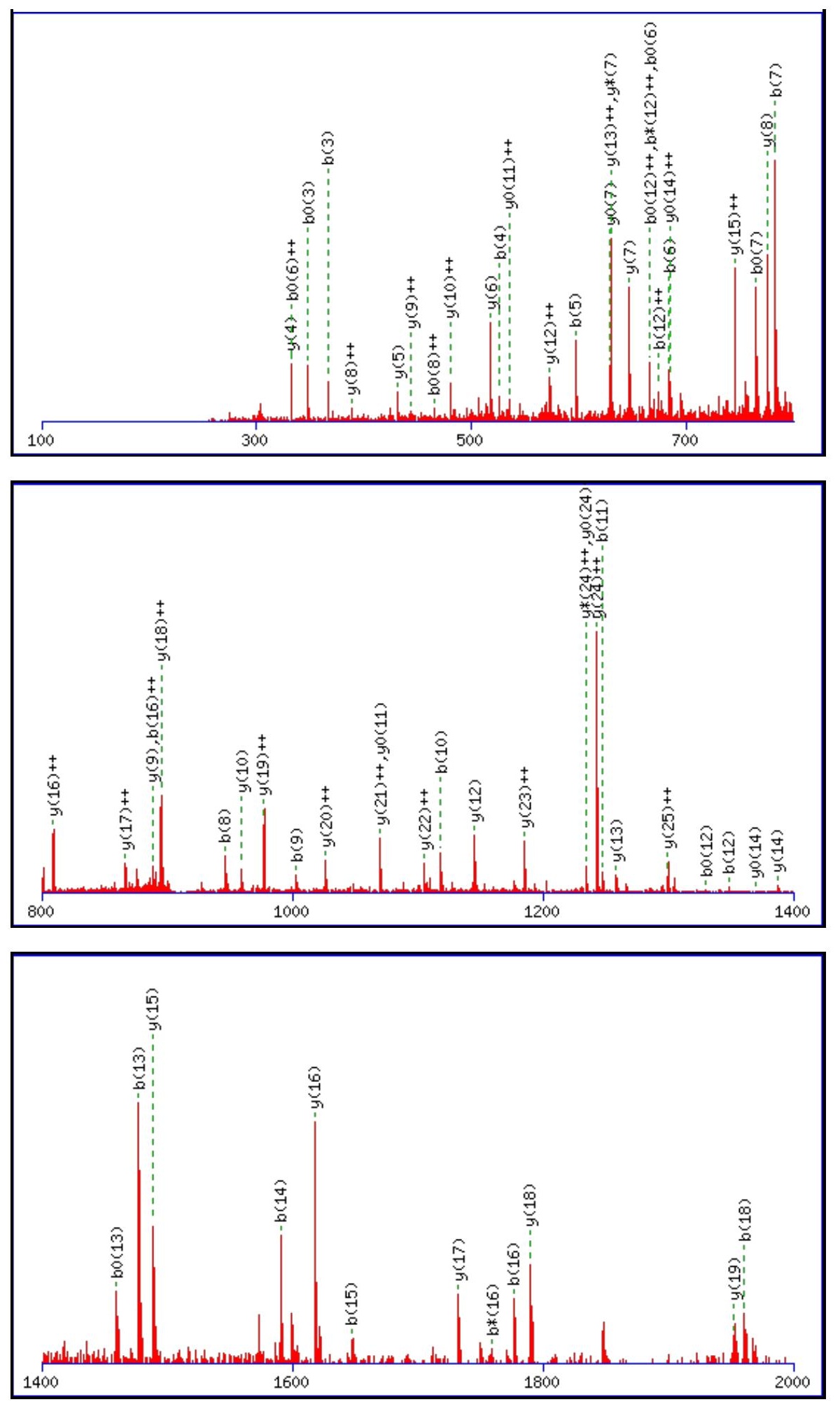

Monoisotopic mass of neutral peptide $\operatorname{Mr}($ calc): 2734.2756

Fixed modifications: Carbamidomethyl (C)

Variable modifications:

N10 : Deamidated $N(N)$

Ions Score: 106 Expect: $8.4 \mathrm{e}-009$

Matches (Bold Red): 66/268 fragment ions using 110 most intense peaks

\begin{tabular}{|c|c|c|c|c|c|c|c|c|c|c|c|c|c|c|}
\hline$\#$ & $\mathbf{b}$ & $\mathbf{b}^{++}$ & $\mathbf{b}^{*}$ & $\mathbf{b}^{\boldsymbol{*}^{++}}$ & $\mathbf{b}^{\mathbf{0}}$ & $\mathbf{b}^{\mathbf{0 + +}}$ & Seq. & $\mathbf{y}$ & $\mathbf{y}^{++}$ & $\mathbf{y}^{*}$ & $\mathbf{y}^{\mathbf{*}^{++}}$ & $\mathbf{y}^{\mathbf{0}}$ & $\mathbf{y}^{\mathbf{0 + +}}$ & $\#$ \\
\hline $\mathbf{1}$ & $\mathbf{1} 38.0662$ & 69.5367 & & & & & $\mathbf{H}$ & & & & & & & $\mathbf{2 6}$ \\
\hline $\mathbf{2}$ & 251.1503 & 126.0788 & & & & & $\mathbf{I}$ & 2598.2239 & $\mathbf{1 2 9 9 . 6 1 5 6}$ & $\mathbf{2 5 8 1 . 1 9 7 4}$ & 1291.1023 & 2580.2134 & 1290.6103 & $\mathbf{2 5}$ \\
\hline $\mathbf{3}$ & 366.1772 & 183.5922 & & & 348.1666 & 174.5870 & $\mathbf{D}$ & 2485.1399 & $\mathbf{1 2 4 3 . 0 7 3 6}$ & 2468.1133 & $\mathbf{1 2 3 4 . 5 6 0 3}$ & 2467.1293 & $\mathbf{1 2 3 4 . 0 6 8 3}$ & $\mathbf{2 4}$ \\
\hline $\mathbf{4}$ & $\mathbf{5 2 6 . 2 0 7 8}$ & 263.6076 & & & 508.1973 & 254.6023 & $\mathbf{C}$ & 2370.1129 & $\mathbf{1 1 8 5 . 5 6 0 1}$ & 2353.0864 & 1177.0468 & 2352.1024 & 1176.5548 & $\mathbf{2 3}$ \\
\hline $\mathbf{5}$ & $\mathbf{5 9 7 . 2 4 5 0}$ & 299.1261 & & & 579.2344 & 290.1208 & $\mathbf{A}$ & 2210.0823 & $\mathbf{1 1 0 5 . 5 4 4 8}$ & 2193.0557 & 1097.0315 & 2192.0717 & 1096.5395 & $\mathbf{2 2}$ \\
\hline
\end{tabular}




\begin{tabular}{|c|c|c|c|c|c|c|c|c|c|c|c|c|c|c|}
\hline 6 & 684.2770 & 342.6421 & & & 666.2664 & |333.6368 & S & 2139.0452 & 1070.0262 & |2122.0186 & 1061.5130 & 2121.0346 & 1061.0209 & 21 \\
\hline 7 & 783.3454 & 392.1763 & & & 765.3348 & 383.1711 & V & 2052.0132 & 1026.5102 & 2034.9866 & 1017.9969 & 2034.0026 & 1017.5049 & 20 \\
\hline 8 & 946.4087 & 473.7080 & & & 928.3982 & 464.7027 & $\mathbf{Y}$ & 1952.9447 & 976.9760 & 1935.9182 & 968.4627 & 1934.9342 & 967.9707 & \\
\hline 9 & 1003.4302 & 502.2187 & & & 985.4196 & 493.2135 & G & 1789.8814 & 895.4443 & 1772.8549 & 886.9311 & 1771.8708 & 886.4391 & 18 \\
\hline 10 & 1118.4571 & 559.7322 & 1101.4306 & 551.2189 & 1100.4466 & 550.7269 & $\mathbf{N}$ & 1732.8599 & 866.9336 & 1715.8334 & 858.4203 & 1714.8494 & 857.9283 & 17 \\
\hline 11 & 1247.4997 & 624.2535 & 1230.4732 & 615.7402 & 1229.4892 & 615.2482 & $\mathbf{E}$ & 1617.8330 & 809.4201 & 1600.8065 & 800.9069 & 1599.8224 & 800.4149 & 6 \\
\hline 12 & 1348.5474 & 674.7773 & 1331.5209 & 666.2641 & 1330.5368 & 665.7721 & $\mathbf{T}$ & 1488.7904 & 744.8988 & 1471.7639 & 736.3856 & 1470.7798 & 735.8936 & 15 \\
\hline 13 & 1477.5900 & 739.2986 & 1460.5634 & 730.7854 & 1459.5794 & 730.2934 & $\mathbf{E}$ & 1387.7427 & 694.3750 & 1370.7162 & 685.8617 & 1369.7322 & 685.3697 & 14 \\
\hline 14 & 1590.6741 & 795.8407 & 1573.6475 & 787.3274 & 1572.6635 & 786.8354 & I & 1258.7001 & 629.8537 & 1241.6736 & 621.3404 & 1240.6896 & 620.8484 & 13 \\
\hline 15 & 1647.6955 & 824.3514 & 1630.6690 & 815.8381 & 1629.6850 & 815.3461 & G & 1145.6161 & 573.3117 & 1128.5895 & 564.7984 & 1127.6055 & 564.3064 & \\
\hline 16 & 1776.7381 & $\mathbf{8 8 8 . 8 7 2 7}$ & 1759.7116 & 880.3594 & 1758.7275 & 879.8674 & $\mathbf{E}$ & 1088.5946 & 544.8009 & 1071.5681 & 536.2877 & 1070.5840 & 535.7957 & 11 \\
\hline 17 & 1847.7752 & 924.3913 & 1830.7487 & 915.8780 & 1829.7647 & 915.3860 & A & 959.5520 & 480.2796 & 942.5255 & 471.7664 & 941.5415 & 471.2744 & 10 \\
\hline 18 & 1960.8593 & 980.9333 & 1943.8327 & 972.4200 & 1942.8487 & 971.9280 & $\mathbf{L}$ & 888.5149 & 444.7611 & 871.4884 & 436.2478 & 870.5043 & 435.7558 & 9 \\
\hline 19 & 2088.9543 & 1044.9808 & 2071.9277 & 1036.4675 & 2070.9437 & 1035.9755 & $\mathbf{K}$ & 775.4308 & 388.2191 & 758.4043 & 379.7058 & 757.4203 & 379.2138 & 8 \\
\hline 20 & 2217.9968 & 1109.5021 & 2200.9703 & 1100.9888 & 2199.9863 & 1100.4968 & E & 647.3359 & 324.1716 & 630.3093 & 315.6583 & 629.3253 & 315.1663 & 7 \\
\hline 21 & 2305.0289 & 1153.0181 & 2288.0023 & 1144.5048 & 2287.0183 & 1144.0128 & $\mathrm{~S}$ & 518.2933 & 259.6503 & 501.2667 & 251.1370 & 500.2827 & 250.6450 & 6 \\
\hline 22 & 2404.0973 & 1202.5523 & 2387.0707 & 1194.0390 & 2386.0867 & 1193.5470 & V & 431.2613 & 216.1343 & 414.2347 & 207.6210 & & & 5 \\
\hline 23 & 2461.1188 & 1231.0630 & 2444.0922 & 1222.5497 & 2443.1082 & 1222.0577 & G & 332.1928 & 166.6001 & 315.1663 & 158.0868 & & & 4 \\
\hline 24 & 2532.1559 & 1266.5816 & 2515.1293 & 1258.0683 & 2514.1453 & 1257.5763 & A & 275.1714 & 138.0893 & 258.1448 & 129.5761 & & & 3 \\
\hline 25 & 2589.1773 & 1295.0923 & 2572.1508 & 1286.5790 & 2571.1668 & 1286.0870 & G & 204.1343 & 102.5708 & 187.1077 & 94.0575 & & & 2 \\
\hline 26 & & & & & & & $\mathbf{K}$ & 147.1128 & 74.0600 & 130.0863 & 65.5468 & & & 1 \\
\hline
\end{tabular}
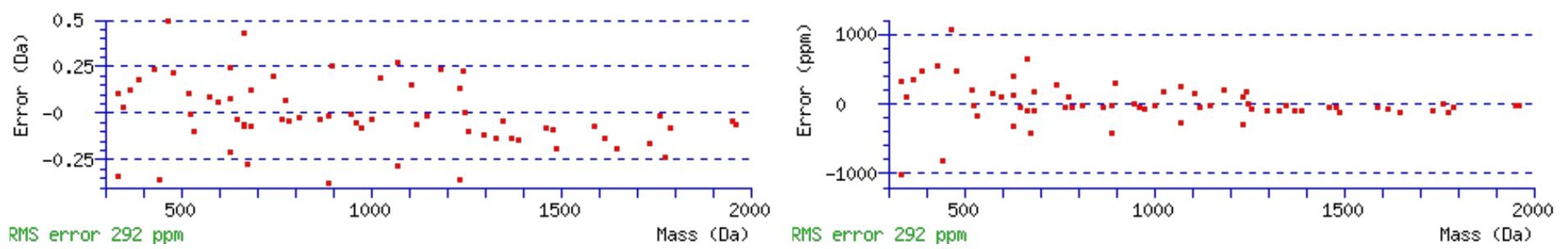

\section{All matches to this query}

\begin{tabular}{|l|l|c|l|}
\hline Score & Mr(calc): & Delta & \multicolumn{1}{c|}{ Sequence } \\
\hline 105.8 & 2734.2756 & 0.0113 & HIDCASVYGNETEIGEALKESVGAGK \\
\hline 51.7 & 2733.2915 & 0.9953 & HIDCASVYGNETEIGEALKESVGAGK \\
\hline 4.9 & 2732.2550 & 2.0318 & GPDLPNAIAGVSACVCALNPRLEEK \\
\hline 0.7 & 2734.2906 & -0.0038 & HLRGEASGSSSSSSSSDNELAPFARAK \\
\hline
\end{tabular}

Spectrum No: 54; Query: 2325; Rank: 1

\section{Peptide View}

MS/MS Fragmentation of LVIILDPAISNNSLSSNPYGPYDR

Found in IPI00193894, Tax_Id=10116 Gene_Symbol=Mgam_predicted 198 kDa protein

Match to Query 2325: 2618.331428 from(1310.172990,2+)

Title: 100101RatKid_NS_deglyco_21.4862.4862.2.dta

Data file K:INewmanPaper|Piliang|3SubProteomes\Piliang3SP\mgf5ppm\ERLIC_3SubProteomes5ppm.mgf 

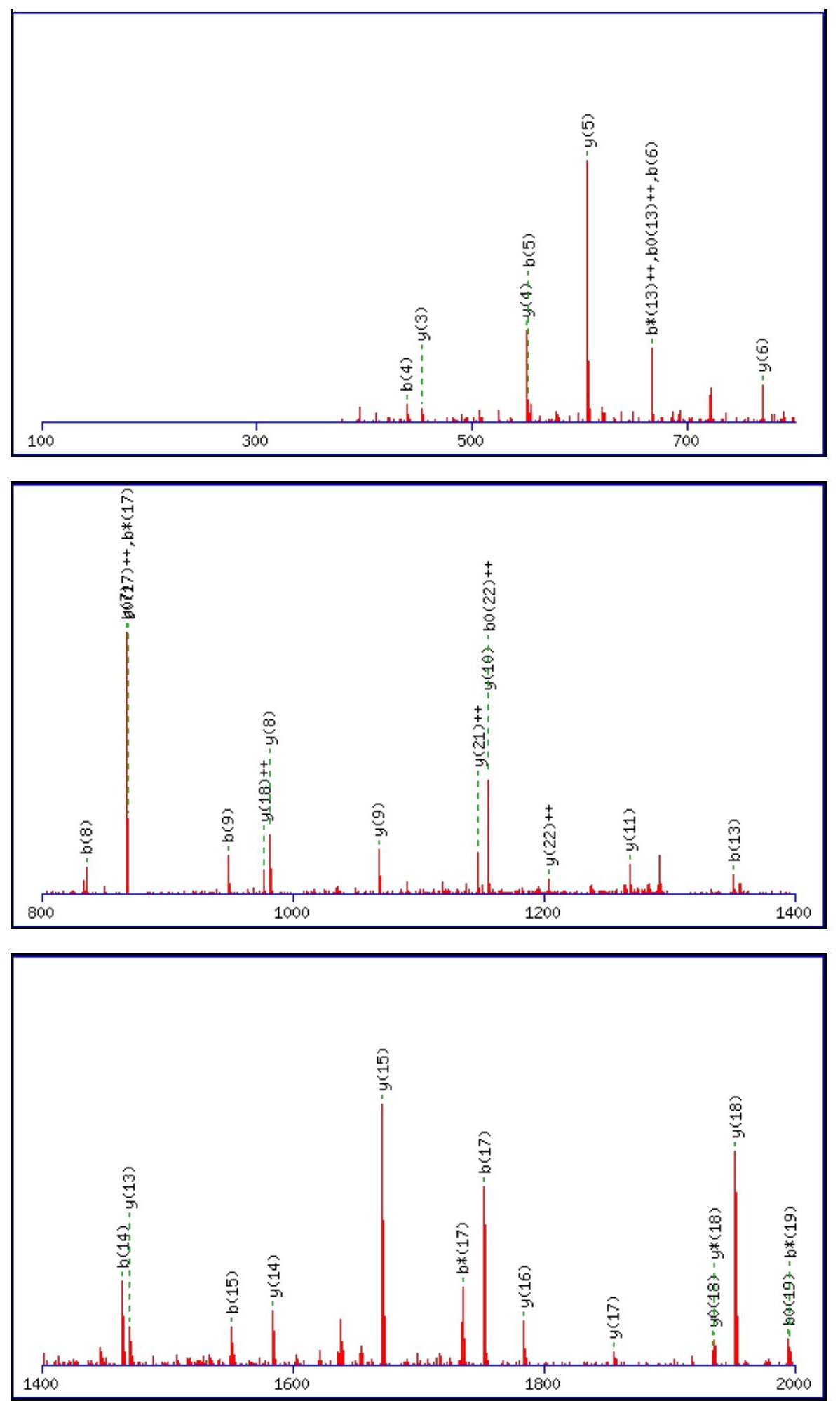

Monoisotopic mass of neutral peptide $\operatorname{Mr}($ calc): 2618.3228

Fixed modifications: Carbamidomethyl (C)

Variable modifications:

N11 : Deamidated N (N)

Ions Score: 106 Expect : $4.8 \mathrm{e}-009$

Matches (Bold Red): 37/244 fragment ions using 55 most intense peaks

\begin{tabular}{|r|c|c|c|c|c|c|c|c|c|c|c|c|c|c|}
\hline$\#$ & $\mathbf{b}$ & $\mathbf{b}^{++}$ & $\mathbf{b}^{*}$ & $\mathbf{b}^{\boldsymbol{*}^{++}}$ & $\mathbf{b}^{\mathbf{0}}$ & $\mathbf{b}^{\mathbf{0 + +}}$ & Seq. & $\mathbf{y}$ & $\mathbf{y}^{++}$ & $\mathbf{y}^{\mathbf{*}}$ & $\mathbf{y}^{\mathbf{*}^{++}}$ & $\mathbf{y}^{\mathbf{0}}$ & $\mathbf{y}^{\mathbf{0 + +}}$ & $\#$ \\
\hline $\mathbf{1}$ & 114.0913 & 57.5493 & & & & & $\mathbf{L}$ & & & & & & & $\mathbf{2 4}$ \\
\hline $\mathbf{2}$ & 213.1598 & 107.0835 & & & & & $\mathbf{V}$ & 2506.2460 & 1253.6266 & 2489.2195 & 1245.1134 & 2488.2354 & 1244.6214 & $\mathbf{2 3}$ \\
\hline $\mathbf{3}$ & 326.2438 & 163.6255 & & & & & $\mathbf{I}$ & 2407.1776 & $\mathbf{1 2 0 4 . 0 9 2 4}$ & 2390.1510 & 1195.5792 & 2389.1670 & 1195.0871 & $\mathbf{2 2}$ \\
\hline $\mathbf{4}$ & $\mathbf{4 3 9 . 3 2 7 9}$ & 220.1676 & & & & & $\mathbf{I}$ & 2294.0935 & $\mathbf{1 1 4 7 . 5 5 0 4}$ & 2277.0670 & 1139.0371 & 2276.0830 & 1138.5451 & $\mathbf{2 1}$ \\
\hline $\mathbf{5}$ & $\mathbf{5 5 2 . 4 1 1 9}$ & 276.7096 & & & & & $\mathbf{L}$ & 2181.0095 & 1091.0084 & 2163.9829 & 1082.4951 & 2162.9989 & 1082.0031 & $\mathbf{2 0}$ \\
\hline
\end{tabular}




\begin{tabular}{|c|c|c|c|c|c|c|c|c|c|c|c|c|c|c|}
\hline 6 & 667.4389 & 334.2231 & & & 649.4283 & 325.2178 & D & 2067.9254 & 1034.4663 & |2050.8988| & 1025.9531 & 2049.9148| & 1025.4611|1 & |19| \\
\hline 7 & 764.4917 & 382.7495 & & & 746.4811 & 373.7442 & $\mathbf{P}$ & 1952.8985 & 976.9529 & 1935.8719 & 968.4396 & 1934.8879 & 967.9476 & 18 \\
\hline 8 & 835.5288 & 418.2680 & & & 817.5182 & 409.2627 & A & 1855.8457 & 928.4265 & 1838.8191 & 919.9132 & 1837.8351 & 919.4212 & 17 \\
\hline 9 & 948.6128 & 474.8101 & & & 930.6023 & 465.8048 & I & 1784.8086 & 892.9079 & 1767.7820 & 884.3947 & 1766.7980 & 883.9026 & 16 \\
\hline 10 & 1035.6449 & 518.3261 & & & 1017.6343 & 509.3208 & $\mathrm{~S}$ & 1671.7245 & 836.3659 & 1654.6980 & 827.8526 & 1653.7139 & \begin{tabular}{l|l|l}
827.3606 & 1
\end{tabular} & 15 \\
\hline 11 & 1150.6718 & 575.8395 & 1133.6452 & 567.3263 & 1132.6612 & 566.8343 & $\mathbf{N}$ & \begin{tabular}{|l|}
1584.6925 \\
\end{tabular} & 792.8499 & 1567.6659 & 784.3366 & 1566.6819 & 783.8446 & 14 \\
\hline 12 & 1264.7147 & 632.8610 & 1247.6882 & 624.3477 & 1246.7042 & 623.8557 & $\mathbf{N}$ & 1469.6655 & 735.3364 & 1452.6390 & 726.8231 & 1451.6550 & 726.3311 & 13 \\
\hline 13 & 1351.7468 & 676.3770 & 1334.7202 & 667.8637 & 1333.7362 & 667.3717 & $S$ & 1355.6226 & 678.3149 & 1338.5961 & 669.8017 & 1337.6121 & 669.3097 & 12 \\
\hline 14 & 1464.8308 & 732.9190 & 1447.8043 & 724.4058 & 1446.8202 & 723.9138 & $\mathbf{L}$ & 1268.5906 & 634.7989 & 1251.5640 & 626.2857 & 1250.5800 & 625.7937 & 11 \\
\hline 15 & 1551.8628 & 776.4351 & 1534.8363 & 767.9218 & 1533.8523 & 767.4298 & $\mathrm{~S}$ & 1155.5065 & 578.2569 & 1138.4800 & 569.7436 & 1137.4960 & 569.2516 & 10 \\
\hline 16 & 1638.8949 & 819.9511 & 1621.8683 & 811.4378 & 1620.8843 & 810.9458 & $S$ & 1068.4745 & 534.7409 & 1051.4480 & 526.2276 & 1050.4639 & 525.7356 & 9 \\
\hline 17 & 1752.9378 & 876.9725 & 1735.9112 & 868.4593 & 1734.9272 & 867.9673 & $\mathbf{N}$ & 981.4425 & 491.2249 & 964.4159 & 482.7116 & 963.4319 & 482.2196 & 8 \\
\hline 18 & 1849.9906 & 925.4989 & 1832.9640 & 916.9856 & 1831.9800 & 916.4936 & $\mathbf{P}$ & 867.3995 & 434.2034 & 850.3730 & 425.6901 & 849.3890 & 425.1981 & 7 \\
\hline 19 & 2013.0539 & 1007.0306 & 1996.0273 & 998.5173 & 1995.0433 & 998.0253 & $\mathbf{Y}$ & 770.3468 & 385.6770 & 753.3202 & 377.1638 & 752.3362 & 376.6717 & 6 \\
\hline 20 & 2070.0754 & 1035.5413 & 2053.0488 & 1027.0280 & 2052.0648 & 1026.5360 & G & 607.2835 & 304.1454 & 590.2569 & 295.6321 & 589.2729 & 295.1401 & 5 \\
\hline 21 & 2167.1281 & 1084.0677 & 2150.1016 & 1075.5544 & 2149.1176 & 1075.0624 & $\mathbf{P}$ & 550.2620 & 275.6346 & 533.2354 & 267.1214 & 532.2514 & 266.6293 & 4 \\
\hline 22 & 2330.1914 & 1165.5994 & 2313.1649 & 1157.0861 & 2312.1809 & 1156.5941 & $\mathbf{Y}$ & 453.2092 & 227.1082 & 436.1827 & 218.5950 & 435.1987 & 218.1030 & 3 \\
\hline 23 & 2445.2184 & 1223.1128 & 2428.1918 & 1214.5996 & 2427.2078 & 1214.1076 & D & 290.1459 & 145.5766 & 273.1193 & 137.0633 & 272.1353 & 136.5713 & 2 \\
\hline 24 & & & & & & & $\mathbf{R}$ & 175.1190 & 88.0631 & 158.0924 & 79.5498 & & & 1 \\
\hline
\end{tabular}
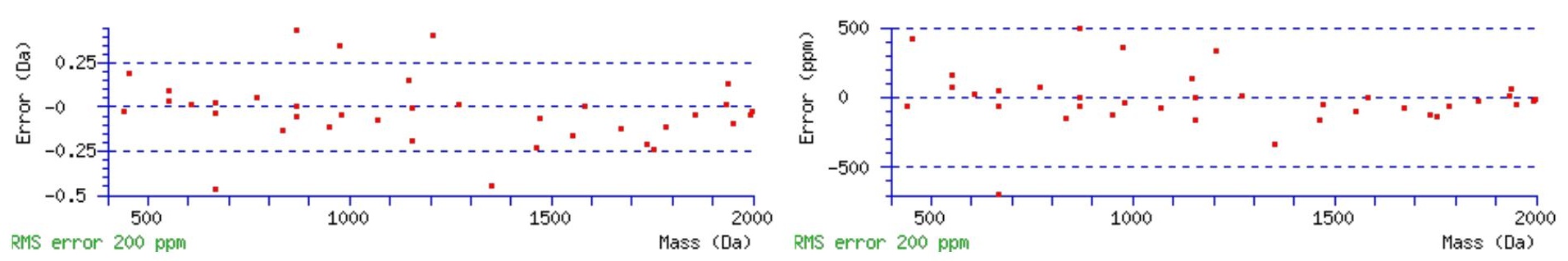

\section{All matches to this query}

\begin{tabular}{|l|c|c|l|}
\hline Score & Mr(calc): & Delta & \multicolumn{1}{|c|}{ Sequence } \\
\hline 105.6 & 2618.3228 & 0.0087 & $\underline{\text { LVIILDPAISNNSLSSNPYGPYDR }}$ \\
\hline 95.8 & 2618.3228 & 0.0087 & LVIILDPAISNNSLSSNPYGPYDR \\
\hline 81.3 & 2618.3228 & 0.0087 & LVIILDPAISNNSLSSNPYGPYDR \\
\hline 40.1 & 2617.3387 & 0.9927 & LVIILDPAISNNSLSSNPYGPYDR \\
\hline 7.8 & 2618.3551 & -0.0237 & QNLSQQLDVKLVPSSYSEAVASVR \\
\hline 3.2 & 2617.3460 & 0.9855 & EETVLATVQALHTASHLSQQADLR \\
\hline 0.9 & 2616.3080 & 2.0235 & $\underline{\text { SRAAYNVTLLNFMDPQKMPYLK }}$ \\
\hline
\end{tabular}

Spectrum No: 55; Query: 1924; Rank: 1

\section{Peptide View}

MS/MS Fragmentation of SCATSQEDFLIYSLNNSLR

Found in IPI00205325, Tax_Id=10116 Gene_Symbol=Lrp2 Low-density lipoprotein receptor-related protein 2 precursor

Match to Query 1924: 2219.018988 from(1110.516770,2+)

Title: 091008RatKidney_NH4Format01_24.4275.4275.2.dta

Data file K:INewmanPaper|Piliangl3SubProteomes\Piliang3SP\mgf5ppm\ERLIC_3SubProteomes5ppm.mgf 

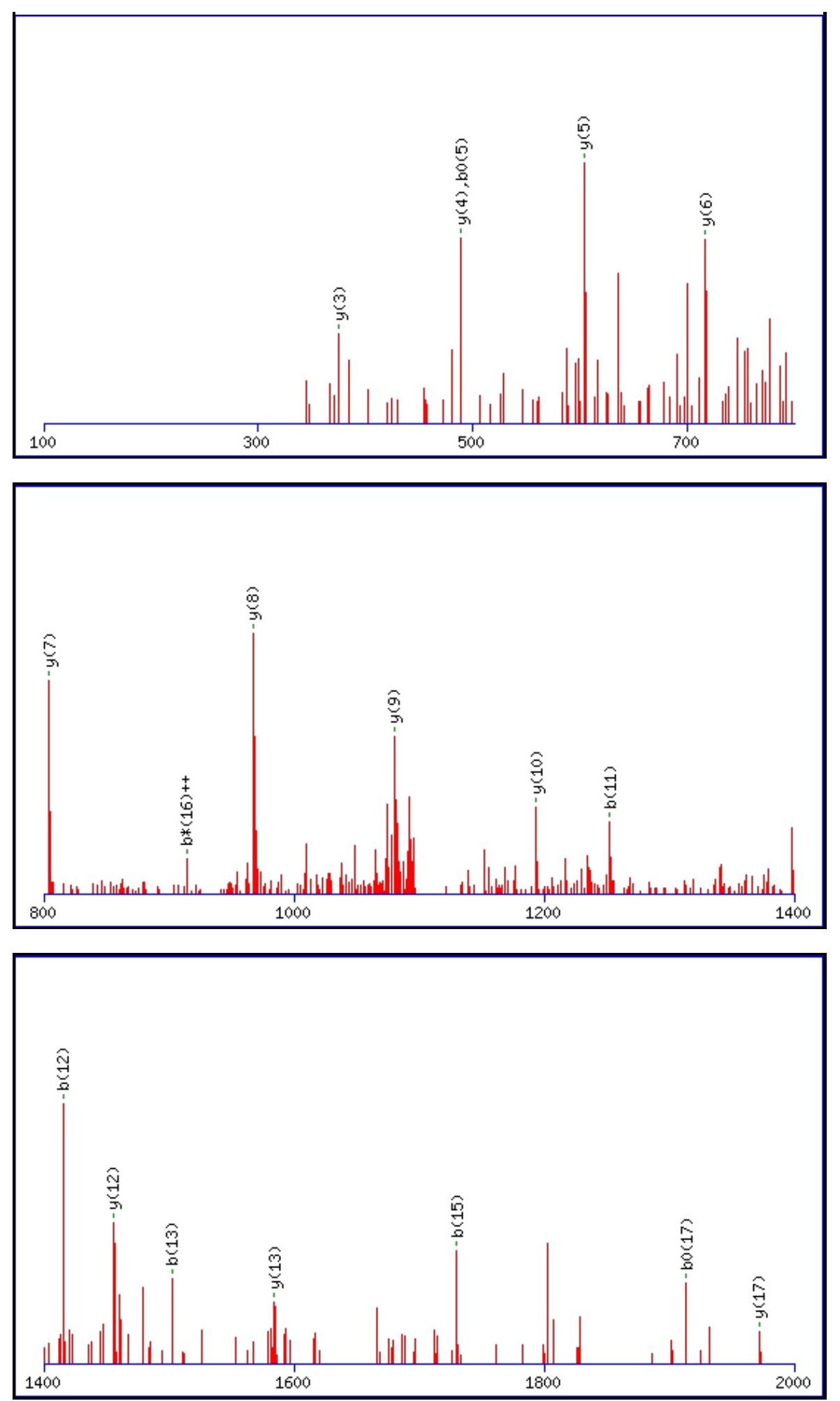

Monoisotopic mass of neutral peptide $\operatorname{Mr}($ calc): 2218.0212

Fixed modifications: Carbamidomethyl (C)

Variable modifications:

N15 : Deamidated $N(N)$

Ions Score: 105 Expect: $8.7 \mathrm{e}-009$

Matches (Bold Red): 18/202 fragment ions using 25 most intense peaks

\begin{tabular}{|c|c|c|c|c|c|c|c|c|c|c|c|c|c|c|}
\hline \# & b & $\mathbf{b}^{++}$ & $\mathbf{b}^{*}$ & $\mathbf{b}^{*^{++}}$ & $\mathbf{b}^{0}$ & $\mathbf{b}^{\mathbf{0 + +}}$ & Seq. & $\mathbf{y}$ & $y^{++}$ & $\mathbf{y}^{*}$ & $\mathrm{y}^{*^{++}}$ & $\mathbf{y}^{\mathbf{0}}$ & $y^{0++}$ & \# \\
\hline 1 & 88.0393 & 44.5233 & & & 70.0287 & 35.5180 & $\mathbf{S}$ & & & & & & & 19 \\
\hline 2 & 248.0700 & 124.5386 & & & 230.0594 & 115.5333 & C & 2131.9965 & 1066.5019 & 2114.9699 & 1057.9886 & 2113.9859 & 1057.4966 & 18 \\
\hline 3 & 319.1071 & 160.0572 & & & 301.0965 & 151.0519 & A & 1971.9658 & 986.4865 & |1954.9393 & 977.9733 & 1953.9552 & 977.4813 & 17 \\
\hline 4 & 420.1547 & 210.5810 & & & 402.1442 & 201.5757 & $T$ & 1900.9287 & 950.9680 & 1883.9021 & 942.4547 & 1882.9181 & 941.9627 & 16 \\
\hline 5 & 507.1868 & 254.0970 & & & 489.1762 & 245.0917 & $S$ & 1799.8810 & 900.4441 & 1782.8545 & 891.9309 & 1781.8704 & 891.4389 & 15 \\
\hline
\end{tabular}




\begin{tabular}{|c|c|c|c|c|c|c|c|c|c|c|c|c|c|c|}
\hline 6 & 635.2454 & 318.1263 & 618.2188 & 309.6130 & 617.2348 & 309.1210 & $\mathbf{Q}$ & |1712.8490 & 856.9281 & |1695.8224 & 848.4149 & |1694.8384| & 847.9228 & 14 \\
\hline 7 & 764.2879 & 382.6476 & 747.2614 & 374.1343 & 746.2774 & 373.6423 & $\mathbf{E}$ & 1584.7904 & 792.8988 & 1567.7639 & 784.3856 & 1566.7798 & 783.8936 & 13 \\
\hline 8 & 879.3149 & 440.1611 & 862.2883 & 431.6478 & 861.3043 & 431.1558 & D & 1455.7478 & 728.3775 & 1438.7213 & 719.8643 & 1437.7372 & 719.3723 & 12 \\
\hline 9 & 1026.3833 & 513.6953 & 1009.3568 & 505.1820 & 1008.3727 & 504.6900 & $\mathbf{F}$ & 1340.7209 & 670.8641 & 1323.6943 & 662.3508 & 1322.7103 & 661.8588 & 11 \\
\hline 10 & 1139.4674 & 570.2373 & 1122.4408 & 561.7240 & 1121.4568 & 561.2320 & $\mathbf{L}$ & 1193.6525 & 597.3299 & 1176.6259 & 588.8166 & 1175.6419 & 588.3246 & 10 \\
\hline 11 & 1252.5514 & 626.7794 & 1235.5249 & 618.2661 & 1234.5409 & 617.7741 & I & 1080.5684 & 540.7878 & 1063.5418 & 532.2746 & 1062.5578 & 531.7826 & 9 \\
\hline 12 & 1415.6148 & 708.3110 & 1398.5882 & 699.7977 & 1397.6042 & 699.3057 & $\mathbf{Y}$ & 967.4843 & 484.2458 & 950.4578 & 475.7325 & 949.4738 & 475.2405 & 8 \\
\hline 13 & 1502.6468 & 751.8270 & 1485.6202 & 743.3138 & 1484.6362 & 742.8217 & S & 804.4210 & 402.7141 & 787.3945 & 394.2009 & 786.4104 & 393.7089 & 7 \\
\hline 14 & 1615.7309 & 808.3691 & 1598.7043 & 799.8558 & 1597.7203 & 799.3638 & $\mathbf{L}$ & 717.3890 & 359.1981 & 700.3624 & 350.6848 & 699.3784 & 350.1928 & 6 \\
\hline 15 & 1730.7578 & 865.8825 & 1713.7312 & 857.3693 & 1712.7472 & 856.8772 & $\mathbf{N}$ & 604.3049 & 302.6561 & 587.2784 & 294.1428 & 586.2943 & 293.6508 & 5 \\
\hline 16 & 1844.8007 & 922.9040 & 1827.7742 & 914.3907 & 1826.7901 & 913.8987 & $\mathbf{N}$ & 489.2780 & 245.1426 & 472.2514 & 236.6293 & 471.2674 & 236.1373 & 4 \\
\hline 17 & 1931.8327 & 966.4200 & 1914.8062 & 957.9067 & 1913.8222 & 957.4147 & S & 375.2350 & 188.1212 & 358.2085 & 179.6079 & 357.2245 & 179.1159 & 3 \\
\hline 18 & 2044.9168 & 1022.9620 & 2027.8903 & 1014.4488 & 2026.9062 & 1013.9568 & $\mathbf{L}$ & 288.2030 & 144.6051 & 271.1765 & 136.0919 & & & 2 \\
\hline 19 & & & & & & & $\mathbf{R}$ & 175.1190 & 88.0631 & 158.0924 & 79.5498 & & & 1 \\
\hline
\end{tabular}
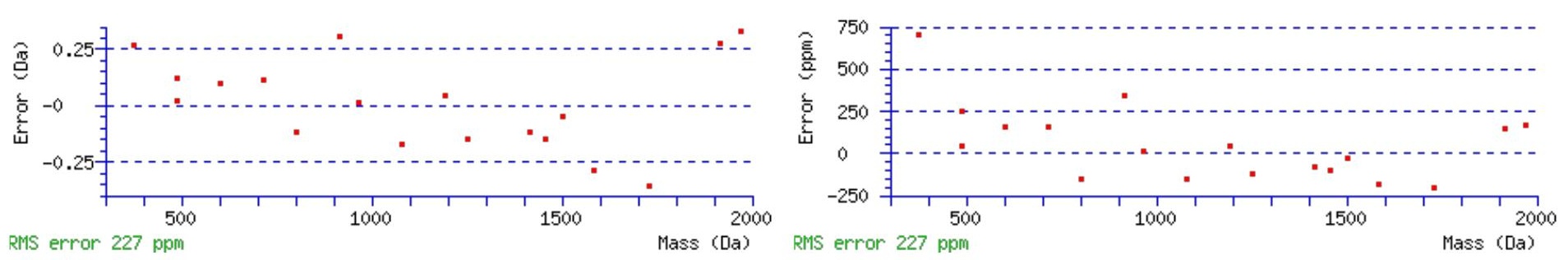

\section{All matches to this query}

\begin{tabular}{|l|l|l|l|}
\hline Score & Mr(calc): & Delta & \multicolumn{1}{c|}{ Sequence } \\
\hline 105.3 & 2218.0212 & 0.9978 & SCATSQEDFLIYSLNNSLR \\
\hline 87.3 & 2218.0212 & 0.9978 & SCATSQEDFLIYSLNNSLR \\
\hline 56.6 & 2219.0052 & 0.0138 & SCATSQEDFLIYSLNNSLR \\
\hline 6.0 & 2217.0191 & 1.9999 & TKGPSRRPSLISSDSNNLK \\
\hline 6.0 & 2217.0191 & 1.9999 & TKGPSRRPSLISSDSNNLK \\
\hline 5.0 & 2218.0031 & 1.0158 & TKGPSRRPSLISSDSNNLK \\
\hline 5.0 & 2218.0031 & 1.0158 & TKGPSRRPSLISSDSNNLK \\
\hline 5.0 & 2218.0031 & 1.0158 & TKGPSRRPSLISSDSNNLK \\
\hline 4.8 & 2219.0161 & 0.0029 & LPGASLSVHTWQGNGYSHTK \\
\hline 4.1 & 2219.0161 & 0.0029 & $\underline{\text { LPGASLSVHTWQGNGYSHTK }}$ \\
\hline
\end{tabular}

Spectrum No: 56; Query: 1915; Rank: 1

\section{Peptide View}

MS/MS Fragmentation of LDNNGCSTQEVNITEFQLK

Found in IPI00201262, Tax_Id=10116 Gene_Symbol=LOC297568;Mug2;Mug1 Alpha-1-inhibitor 3 precursor

Match to Query 1915: 2210.025308 from(1106.019930,2+)

Title: 100101RatKid_NS_deglyco_26.3319.3319.2.dta

Data file K:INewmanPaper|Piliangl3SubProteomes\Piliang3SP\mgf5ppm\ERLIC_3SubProteomes5ppm.mgf 

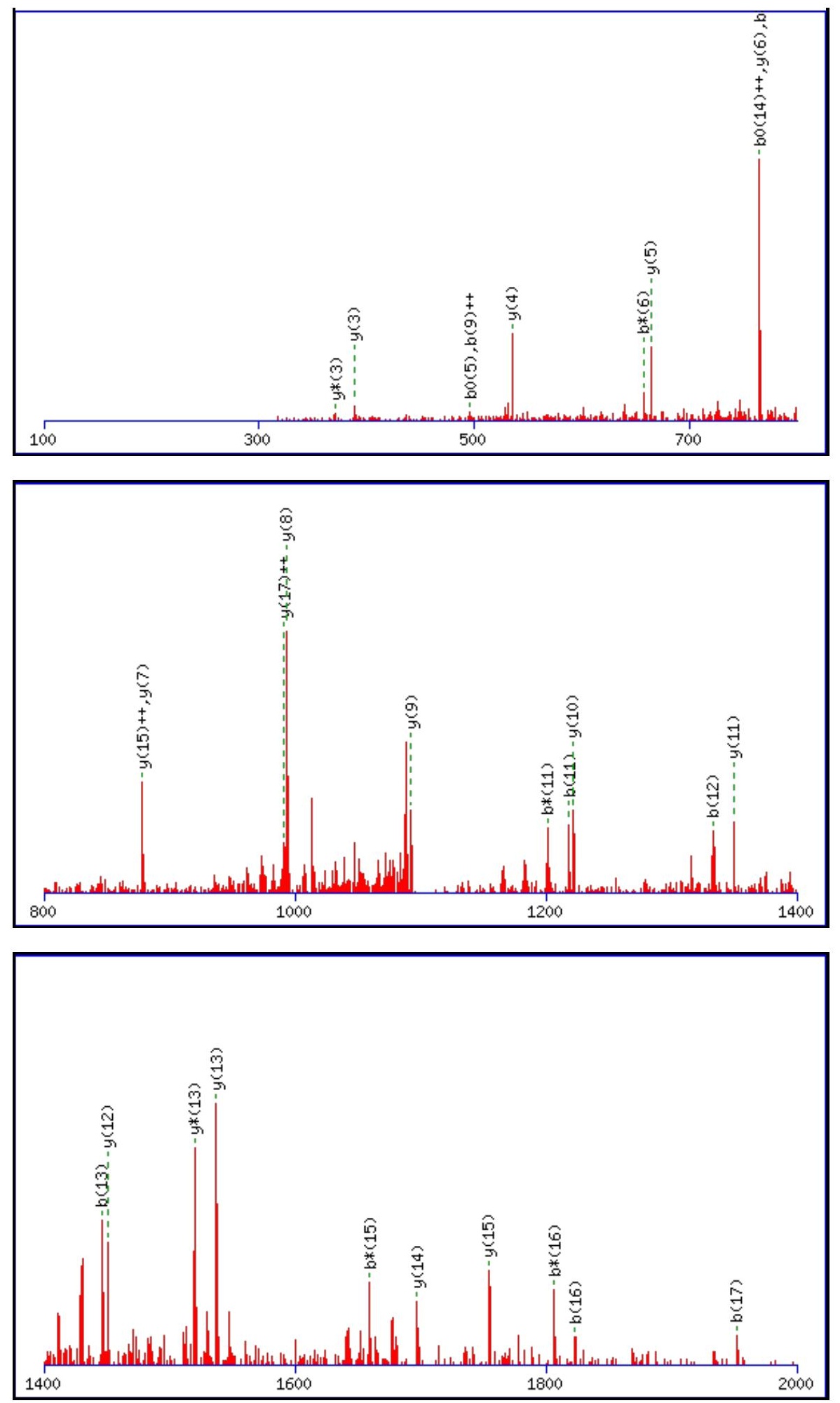

Monoisotopic mass of neutral peptide $\operatorname{Mr}($ calc): 2210.0161

Fixed modifications: Carbamidomethyl (C)

Variable modifications:

N12 : Deamidated $\mathrm{N}(\mathrm{N})$

Ions Score: 105 Expect: $8.8 \mathrm{e}-009$

Matches (Bold Red): $30 / 202$ fragment ions using 44 most intense peaks

\begin{tabular}{|c|c|c|c|c|c|c|c|c|c|c|c|c|c|c|}
\hline \# & b & $\mathbf{b}^{++}$ & $\mathbf{b}^{*}$ & $\mathbf{b}^{*^{++}}$ & $\mathbf{b}^{0}$ & $\mathbf{b}^{0++}$ & Seq. & $\mathbf{y}$ & $y^{++}$ & $\mathrm{y}^{*}$ & $\mathrm{y}^{*^{++}}$ & $\mathbf{y}^{0}$ & $y^{0++}$ & $\#$ \\
\hline 1 & 114.0913 & 57.5493 & & & & & $\mathbf{L}$ & & & & & & & 19 \\
\hline 2 & 229.1183 & 115.0628 & & & 211.1077 & 106.0575 & D & 2097.9393 & 1049.4733 & 2080.9128 & 1040.9600 & 2079.9288 & 1040.4680 & 18 \\
\hline 3 & 343.1612 & 172.0842 & 326.1347 & 163.5710 & 325.1506 & 163.0790 & $\mathbf{N}$ & 1982.9124 & 991.9598 & 1965.8858 & 983.4466 & 1964.9018 & 982.9546 & 17 \\
\hline 4 & 457.2041 & 229.1057 & 440.1776 & 220.5924 & 439.1936 & 220.1004 & $\mathbf{N}$ & 1868.8695 & 934.9384 & |1851.8429 & 926.4251 & 1850.8589 & 925.9331 & 16 \\
\hline 5 & 514.2256 & 257.6164 & 497.1991 & 249.1032 & 496.2150 & 248.6112 & G & 1754.8265 & 877.9169 & 1737.8000 & 869.4036 & 1736.8160 & 868.9116 & 15 \\
\hline
\end{tabular}




\begin{tabular}{|c|c|c|c|c|c|c|c|c|c|c|c|c|c|c|}
\hline 6 & 674.2563 & 337.6318 & 657.2297 & 329.1185 & 656.2457 & 328.6265 & C & $\mid 1697.8051$ & 849.4062 & 1680.7785 & 840.8929 & |1679.7945 & 840.4009 & 14 \\
\hline 7 & 761.2883 & 381.1478 & 744.2617 & 372.6345 & 743.2777 & 372.1425 & $\mathbf{S}$ & 1537.7744 & 769.3909 & 1520.7479 & 760.8776 & 1519.7639 & 760.3856 & 13 \\
\hline 8 & 862.3360 & 431.6716 & 845.3094 & 423.1583 & 844.3254 & 422.6663 & $\mathbf{T}$ & 1450.7424 & 725.8748 & 1433.7158 & 717.3616 & 1432.7318 & 716.8696 & 12 \\
\hline 9 & 990.3945 & 495.7009 & 973.3680 & 487.1876 & 972.3840 & 486.6956 & $\mathbf{Q}$ & 1349.6947 & 675.3510 & 1332.6682 & 666.8377 & 1331.6842 & 666.3457 & 11 \\
\hline 10 & 1119.4371 & 560.2222 & 1102.4106 & 551.7089 & 1101.4266 & 551.2169 & $\mathbf{E}$ & 1221.6361 & 611.3217 & 1204.6096 & 602.8084 & 1203.6256 & 602.3164 & 10 \\
\hline 11 & 1218.5055 & 609.7564 & 1201.4790 & 601.2431 & 1200.4950 & 600.7511 & V & 1092.5935 & 546.8004 & 1075.5670 & 538.2871 & 1074.5830 & 537.7951 & 9 \\
\hline 12 & 1333.5325 & 667.2699 & 1316.5059 & 658.7566 & 1315.5219 & 658.2646 & $\mathbf{N}$ & 993.5251 & 497.2662 & 976.4986 & 488.7529 & 975.5146 & 488.2609 & 8 \\
\hline 13 & 1446.6165 & 723.8119 & 1429.5900 & 715.2986 & 1428.6060 & 714.8066 & I & 878.4982 & 439.7527 & 861.4716 & 431.2395 & 860.4876 & 430.7475 & 7 \\
\hline 14 & 1547.6642 & 774.3357 & 1530.6377 & 765.8225 & 1529.6537 & 765.3305 & $\mathbf{T}$ & 765.4141 & 383.2107 & 748.3876 & 374.6974 & 747.4036 & 374.2054 & 6 \\
\hline 15 & 1676.7068 & 838.8570 & 1659.6803 & 830.3438 & 1658.6962 & 829.8518 & $\mathbf{E}$ & 664.3665 & 332.6869 & 647.3399 & 324.1736 & 646.3559 & 323.6816 & 5 \\
\hline 16 & 1823.7752 & 912.3913 & 1806.7487 & 903.8780 & 1805.7647 & 903.3860 & $\mathbf{F}$ & 535.3239 & 268.1656 & 518.2973 & 259.6523 & & & 4 \\
\hline 17 & 1951.8338 & 976.4205 & 1934.8073 & 967.9073 & 1933.8232 & 967.4153 & $\mathbf{Q}$ & 388.2554 & 194.6314 & 371.2289 & 186.1181 & & & 3 \\
\hline 18 & 2064.9179 & 1032.9626 & 2047.8913 & 1024.4493 & 2046.9073 & 1023.9573 & $\mathbf{L}$ & 260.1969 & 130.6021 & 243.1703 & 122.0888 & & & 2 \\
\hline 19 & & & & & & & K & 147.1128 & 74.0600 & 130.0863 & 65.5468 & & & 1 \\
\hline
\end{tabular}
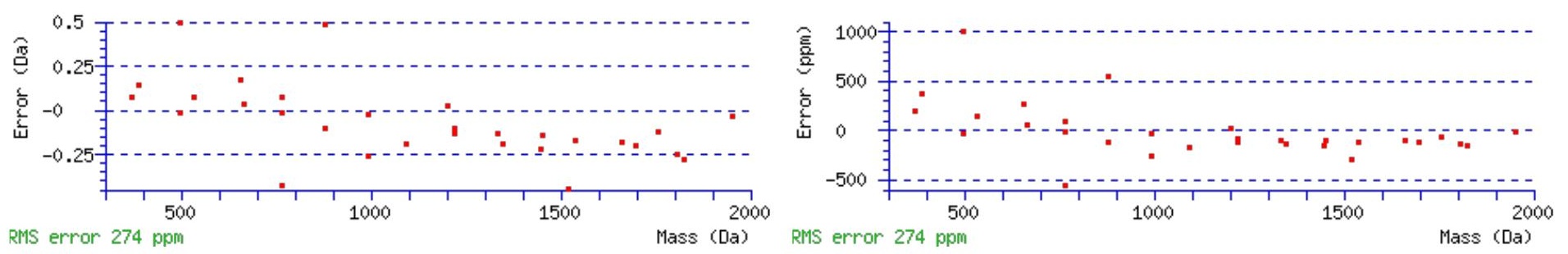

\section{All matches to this query}

\begin{tabular}{|l|r|r|l|}
\hline Score & Mr(calc): & Delta & \multicolumn{1}{|c|}{ Sequence } \\
\hline 104.8 & 2210.0161 & 0.0092 & LDNNGCSTQEVNITEFQLK \\
\hline 19.9 & 2210.0161 & 0.0092 & LDNNGCSTQEVNITEFQLK \\
\hline 19.9 & 2210.0161 & 0.0092 & LDNNGCSTQEVNITEFQLK \\
\hline 10.0 & 2209.0321 & 0.9932 & LDNNGCSTQEVNITEFQLK \\
\hline 6.3 & 2210.0248 & 0.0006 & LKSHPXTVMNLKNYFLK \\
\hline 2.6 & 2210.0402 & -0.0149 & QDLGGLINVNPETNKSMKR \\
\hline 2.5 & 2210.0157 & 0.0096 & EQLDTIWSDLSHHPLSPR \\
\hline 2.3 & 2208.0059 & 2.0194 & NRVTLTLDNDAASPAPETSR \\
\hline 2.1 & 2209.0126 & 1.0127 & DCNLVYLIPHAVGSSEDLK \\
\hline 1.7 & 2209.0126 & 1.0127 & DCNLVYLIPHAVGSSEDLK \\
\hline
\end{tabular}

Spectrum No: 57; Query: 1915; Rank: 1

\section{Peptide View}

MS/MS Fragmentation of LDNNGCSTQEVNITEFQLK

Found in IPI00201262, Tax_Id=10116 Gene_Symbol=LOC297568;Mug2;Mug1 Alpha-1-inhibitor 3 precursor

Match to Query 1915: 2210.025308 from(1106.019930,2+)

Title: 100101RatKid_NS_deglyco_26.3319.3319.2.dta

Data file K:INewmanPaper|Piliangl3SubProteomes\Piliang3SP\mgf5ppm\ERLIC_3SubProteomes5ppm.mgf 

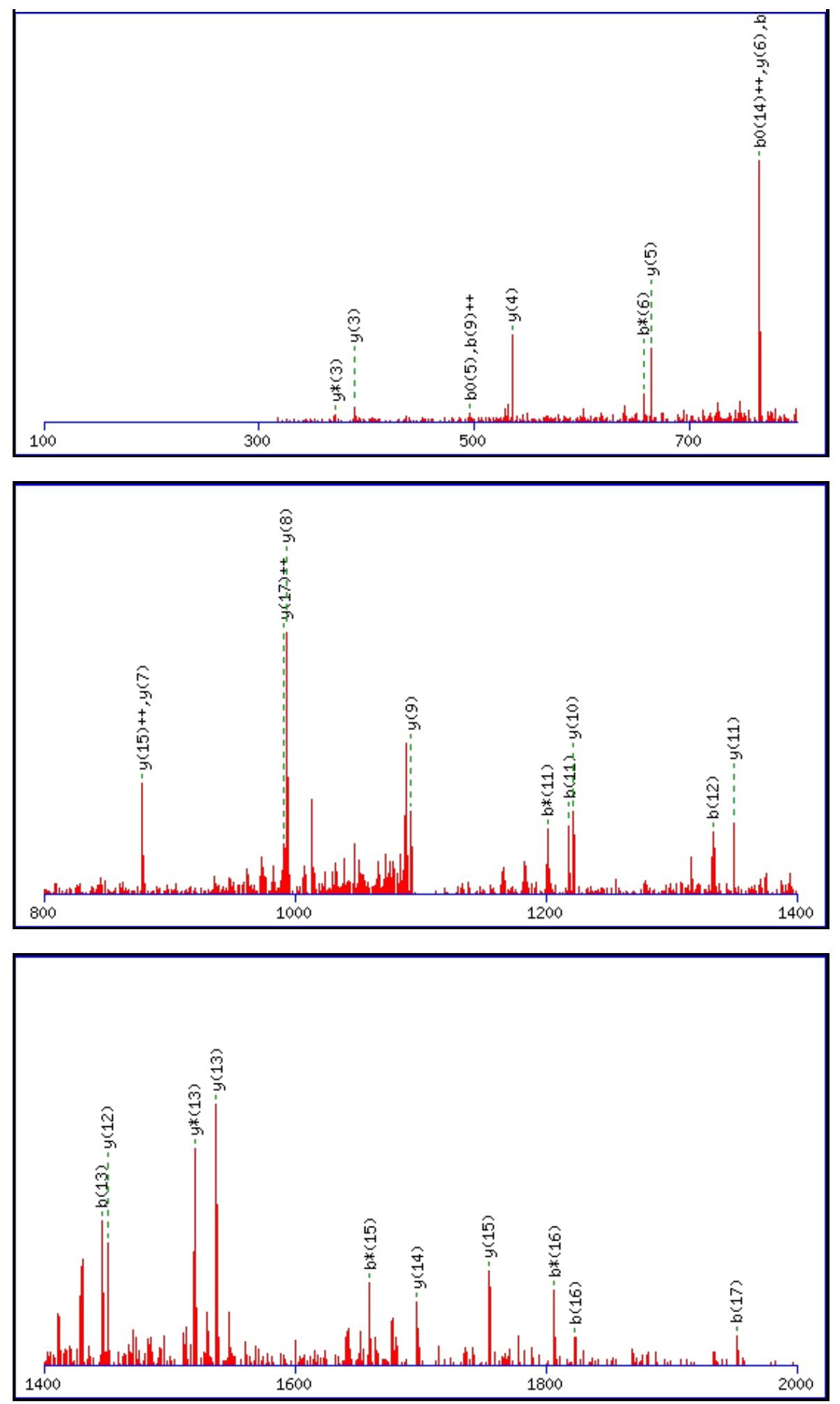

Monoisotopic mass of neutral peptide $\operatorname{Mr}($ calc): 2210.0161

Fixed modifications: Carbamidomethyl (C)

Variable modifications:

N12 : Deamidated $\mathrm{N}(\mathrm{N})$

Ions Score: 105 Expect: $8.8 \mathrm{e}-009$

Matches (Bold Red): $30 / 202$ fragment ions using 44 most intense peaks

\begin{tabular}{|c|c|c|c|c|c|c|c|c|c|c|c|c|c|c|}
\hline \# & b & $\mathbf{b}^{++}$ & $\mathbf{b}^{*}$ & $\mathbf{b}^{*^{++}}$ & $\mathbf{b}^{0}$ & $\mathbf{b}^{0++}$ & Seq. & $\mathbf{y}$ & $y^{++}$ & $\mathrm{y}^{*}$ & $\mathrm{y}^{*^{++}}$ & $\mathbf{y}^{0}$ & $y^{0++}$ & $\#$ \\
\hline 1 & 114.0913 & 57.5493 & & & & & $\mathbf{L}$ & & & & & & & 19 \\
\hline 2 & 229.1183 & 115.0628 & & & 211.1077 & 106.0575 & D & 2097.9393 & 1049.4733 & 2080.9128 & 1040.9600 & 2079.9288 & 1040.4680 & 18 \\
\hline 3 & 343.1612 & 172.0842 & 326.1347 & 163.5710 & 325.1506 & 163.0790 & $\mathbf{N}$ & 1982.9124 & 991.9598 & 1965.8858 & 983.4466 & 1964.9018 & 982.9546 & 17 \\
\hline 4 & 457.2041 & 229.1057 & 440.1776 & 220.5924 & 439.1936 & 220.1004 & $\mathbf{N}$ & 1868.8695 & 934.9384 & |1851.8429 & 926.4251 & 1850.8589 & 925.9331 & 16 \\
\hline 5 & 514.2256 & 257.6164 & 497.1991 & 249.1032 & 496.2150 & 248.6112 & G & 1754.8265 & 877.9169 & 1737.8000 & 869.4036 & 1736.8160 & 868.9116 & 15 \\
\hline
\end{tabular}




\begin{tabular}{|c|c|c|c|c|c|c|c|c|c|c|c|c|c|c|}
\hline 6 & 674.2563 & 337.6318 & 657.2297 & 329.1185 & 656.2457 & 328.6265 & C & |1697.8051 & 849.4062 & |1680.7785 & 840.8929 & |1679.7945 & 840.4009 & $\mid 14$ \\
\hline 7 & 761.2883 & 381.1478 & 744.2617 & 372.6345 & 743.2777 & 372.1425 & S & 1537.7744 & 769.3909 & 1520.7479 & 760.8776 & 1519.7639 & 760.3856 & 13 \\
\hline 8 & 862.3360 & 431.6716 & 845.3094 & 423.1583 & 844.3254 & 422.6663 & $\mathbf{T}$ & 1450.7424 & 725.8748 & 1433.7158 & 717.3616 & 1432.7318 & 716.8696 & 12 \\
\hline 9 & 990.3945 & 495.7009 & 973.3680 & 487.1876 & 972.3840 & 486.6956 & $\mathbf{Q}$ & 1349.6947 & 675.3510 & 1332.6682 & 666.8377 & 1331.6842 & 666.3457 & 11 \\
\hline 10 & 1119.4371 & 560.2222 & 1102.4106 & 551.7089 & 1101.4266 & 551.2169 & $\mathbf{E}$ & 1221.6361 & 611.3217 & 1204.6096 & 602.8084 & 1203.6256 & 602.3164 & 10 \\
\hline 11 & 1218.5055 & 609.7564 & 1201.4790 & 601.2431 & 1200.4950 & 600.7511 & V & 1092.5935 & 546.8004 & 1075.5670 & 538.2871 & 1074.5830 & 537.7951 & 9 \\
\hline 12 & 1333.5325 & 667.2699 & 1316.5059 & 658.7566 & 1315.5219 & 658.2646 & $\mathbf{N}$ & 993.5251 & 497.2662 & 976.4986 & 488.7529 & 975.5146 & 488.2609 & 8 \\
\hline 13 & 1446.6165 & 723.8119 & 1429.5900 & 715.2986 & 1428.6060 & 714.8066 & I & 878.4982 & 439.7527 & 861.4716 & 431.2395 & 860.4876 & 430.7475 & 7 \\
\hline 14 & 1547.6642 & 774.3357 & 1530.6377 & 765.8225 & 1529.6537 & 765.3305 & $\mathbf{T}$ & 765.4141 & 383.2107 & 748.3876 & 374.6974 & 747.4036 & 374.2054 & 6 \\
\hline 15 & 1676.7068 & 838.8570 & 1659.6803 & 830.3438 & 1658.6962 & 829.8518 & $\mathbf{E}$ & 664.3665 & 332.6869 & 647.3399 & 324.1736 & 646.3559 & 323.6816 & 5 \\
\hline 16 & 1823.7752 & 912.3913 & 1806.7487 & 903.8780 & 1805.7647 & 903.3860 & $\mathbf{F}$ & 535.3239 & 268.1656 & 518.2973 & 259.6523 & & & 4 \\
\hline 17 & 1951.8338 & 976.4205 & 1934.8073 & 967.9073 & 1933.8232 & 967.4153 & $\mathbf{Q}$ & 388.2554 & 194.6314 & 371.2289 & 186.1181 & & & 3 \\
\hline 18 & 2064.9179 & 1032.9626 & 2047.8913 & 1024.4493 & 2046.9073 & 1023.9573 & $\mathbf{L}$ & 260.1969 & 130.6021 & 243.1703 & 122.0888 & & & 2 \\
\hline 19 & & & & & & & $\mathbf{K}$ & 147.1128 & 74.0600 & 130.0863 & 65.5468 & & & 1 \\
\hline
\end{tabular}
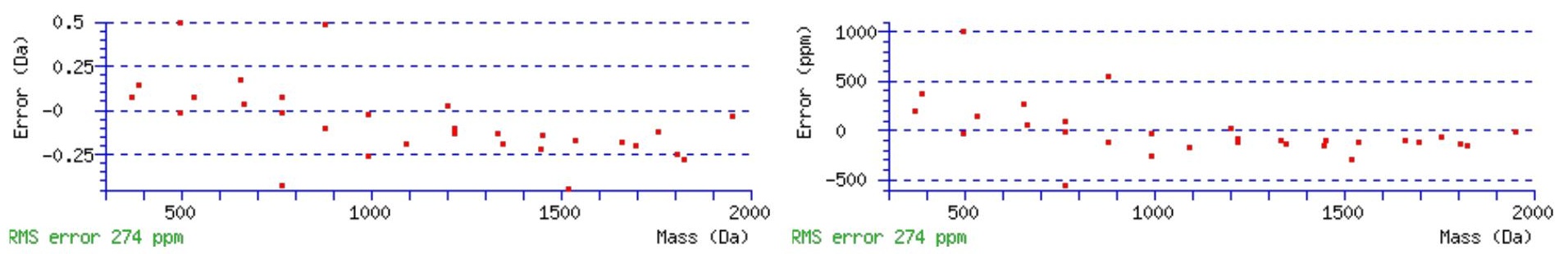

\section{All matches to this query}

\begin{tabular}{|l|r|r|l|}
\hline Score & Mr(calc): & Delta & \multicolumn{1}{|c|}{ Sequence } \\
\hline 104.8 & 2210.0161 & 0.0092 & LDNNGCSTQEVNITEFQLK \\
\hline 19.9 & 2210.0161 & 0.0092 & LDNNGCSTQEVNITEFQLK \\
\hline 19.9 & 2210.0161 & 0.0092 & LDNNGCSTQEVNITEFQLK \\
\hline 10.0 & 2209.0321 & 0.9932 & LDNNGCSTQEVNITEFQLK \\
\hline 6.3 & 2210.0248 & 0.0006 & LKSHPXTVMNLKNYFLK \\
\hline 2.6 & 2210.0402 & -0.0149 & QDLGGLINVNPETNKSMKR \\
\hline 2.5 & 2210.0157 & 0.0096 & EQLDTIWSDLSHHPLSPR \\
\hline 2.3 & 2208.0059 & 2.0194 & NRVTLTLDNDAASPAPETSR \\
\hline 2.1 & 2209.0126 & 1.0127 & DCNLVYLIPHAVGSSEDLK \\
\hline 1.7 & 2209.0126 & 1.0127 & DCNLVYLIPHAVGSSEDLK \\
\hline
\end{tabular}

Spectrum No: 58; Query: 1206; Rank: 1

\section{Peptide View}

MS/MS Fragmentation of TSVGNSSFYQDTVVFK

Found in IPI00195241, Tax_Id=10116 Gene_Symbol=Apoh Beta-2-glycoprotein 1 precursor

Match to Query 1206: 1778.837308 from(890.425930,2+)

Title: 100101RatKid_NS_deglyco_27.3109.3109.2.dta

Data file K:INewmanPaper|Piliangl3SubProteomes\Piliang3SP\mgf5ppm\ERLIC_3SubProteomes5ppm.mgf 

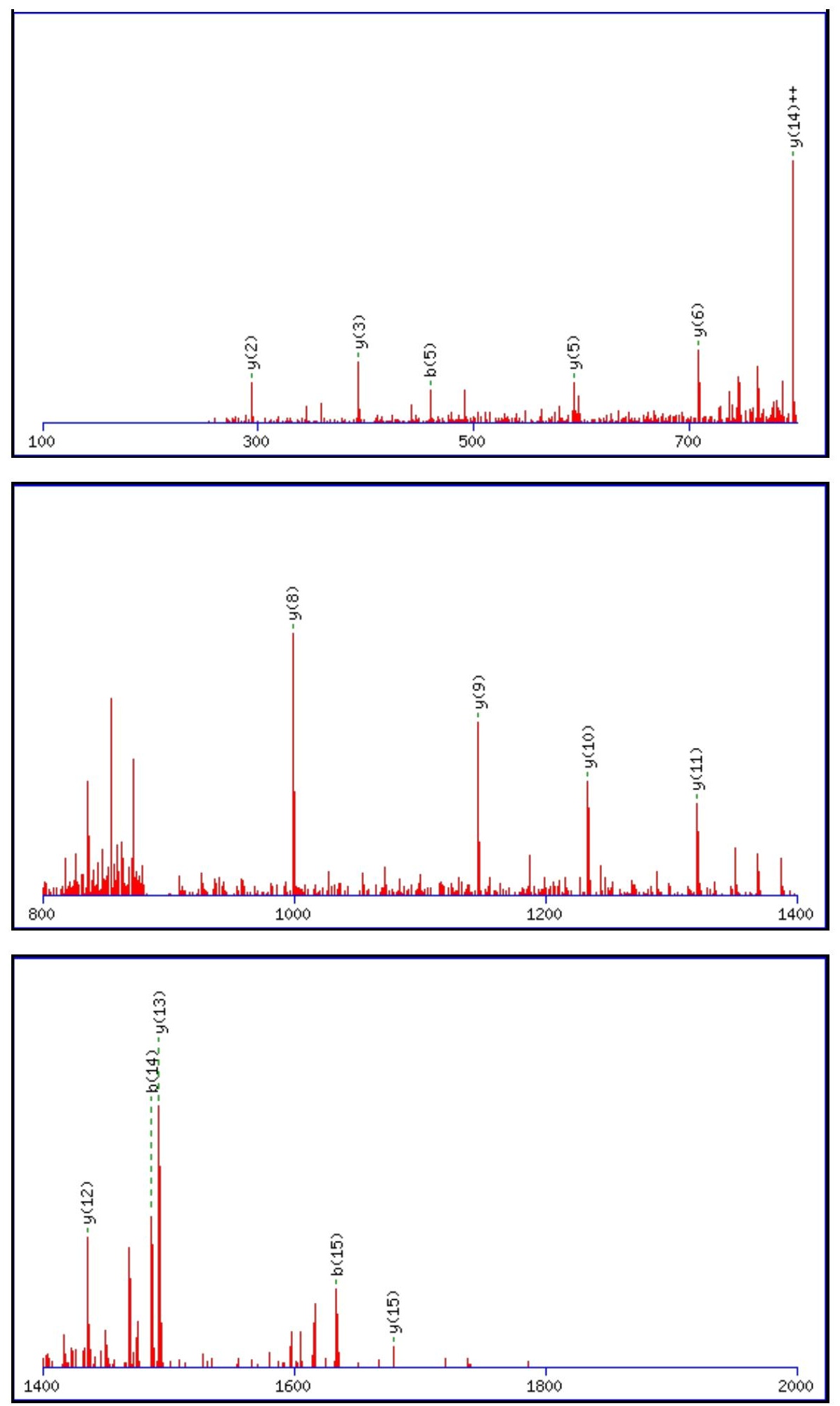

Monoisotopic mass of neutral peptide $\operatorname{Mr}($ calc): 1778.8363

Fixed modifications: Carbamidomethyl (C)

Variable modifications:

N5: : Deamidated N (N)

Ions Score: 105 Expect: $7 \mathrm{e}-009$

Matches (Bold Red): 15/164 fragment ions using 20 most intense peaks

\begin{tabular}{|c|c|c|c|c|c|c|c|c|c|c|c|c|c|c|}
\hline \# & b & $\mathbf{b}^{++}$ & $\mathbf{b}^{*}$ & $\mathbf{b}^{*^{++}}$ & $\mathbf{b}^{0}$ & $\mathbf{b}^{\mathbf{0 + +}}$ & Seq. & $\mathbf{y}$ & $\mathbf{y}^{++}$ & $\mathrm{y}^{*}$ & $\mathrm{y}^{*^{++}}$ & $\mathbf{y}^{0}$ & $y^{0++}$ & $\#$ \\
\hline 1 & 102.0550 & 51.5311 & & & 84.0444 & 42.5258 & $\mathbf{T}$ & & & & & & & 16 \\
\hline 2 & 189.0870 & 95.0471 & & & 171.0764 & 86.0418 & S & 1678.7959 & 839.9016 & 1661.7693 & 831.3883 & 1660.7853 & 830.8963 & 15 \\
\hline 3 & 288.1554 & 144.5813 & & & 270.1448 & 135.5761 & V & 1591.7639 & 796.3856 & 1574.7373 & 787.8723 & 1573.7533 & 787.3803 & 14 \\
\hline 4 & 345.1769 & 173.0921 & & & 327.1663 & 164.0868 & G & 1492.6954 & 746.8514 & 1475.6689 & 738.3381 & 1474.6849 & 737.8461 & 13 \\
\hline 5 & 460.2038 & 230.6055 & 443.1772 & 222.0923 & 442.1932 & 221.6003 & $\mathbf{N}$ & 1435.6740 & 718.3406 & 1418.6474 & 709.8274 & 1417.6634 & 709.3353 & 12 \\
\hline
\end{tabular}




\begin{tabular}{|r|r|r|r|r|r|r|r|r|r|r|r|r|r|r|r|r|r|}
$\mathbf{6}$ & 547.2358 & 274.1216 & 530.2093 & 265.6083 & 529.2253 & 265.1163 & S & $\mathbf{1 3 2 0 . 6 4 7 0}$ & 660.8272 & 1303.6205 & 652.3139 & 1302.6365 & 651.8219 & $\mathbf{1 1}$ \\
\hline $\mathbf{7}$ & 634.2679 & 317.6376 & 617.2413 & 309.1243 & 616.2573 & 308.6323 & S & $\mathbf{1 2 3 3 . 6 1 5 0}$ & 617.3111 & 1216.5885 & 608.7979 & 1215.6045 & 608.3059 & $\mathbf{1 0}$ \\
\hline $\mathbf{8}$ & 781.3363 & 391.1718 & 764.3097 & 382.6585 & 763.3257 & 382.1665 & F & $\mathbf{1 1 4 6 . 5 8 3 0}$ & 573.7951 & 1129.5564 & 565.2819 & 1128.5724 & 564.7899 & $\mathbf{9}$ \\
\hline $\mathbf{9}$ & 944.3996 & 472.7034 & 927.3730 & 464.1902 & 926.3890 & 463.6982 & Y & $\mathbf{9 9 9 . 5 1 4 6}$ & 500.2609 & 982.4880 & 491.7477 & 981.5040 & 491.2556 & $\mathbf{8}$ \\
\hline $\mathbf{1 0}$ & 1072.4582 & 536.7327 & 1055.4316 & 528.2195 & 1054.4476 & 527.7274 & Q & 836.4512 & 418.7293 & 819.4247 & 410.2160 & 818.4407 & 409.7240 & $\mathbf{7}$ \\
\hline $\mathbf{1 1}$ & 1187.4851 & 594.2462 & 1170.4586 & 585.7329 & 1169.4746 & 585.2409 & D & 708.3927 & 354.7000 & 691.3661 & 346.1867 & 690.3821 & 345.6947 & $\mathbf{6}$ \\
\hline $\mathbf{1 2}$ & 1288.5328 & 644.7700 & 1271.5062 & 636.2568 & 1270.5222 & 635.7648 & T & 593.3657 & 297.1865 & 576.3392 & 288.6732 & 575.3552 & 288.1812 & $\mathbf{5}$ \\
\hline $\mathbf{1 3}$ & 1387.6012 & 694.3042 & 1370.5747 & 685.7910 & 1369.5906 & 685.2990 & V & 492.3180 & 246.6627 & 475.2915 & 238.1494 & & & $\mathbf{4}$ \\
\hline $\mathbf{1 4}$ & $\mathbf{1 4 8 6 . 6 6 9 6}$ & 743.8385 & 1469.6431 & 735.3252 & 1468.6591 & 734.8332 & V & 393.2496 & 197.1285 & 376.2231 & 188.6152 & & & $\mathbf{3}$ \\
\hline $\mathbf{1 5}$ & $\mathbf{1 6 3 3 . 7 3 8 0}$ & 817.3727 & 1616.7115 & 808.8594 & 1615.7275 & 808.3674 & F & $\mathbf{2 9 4 . 1 8 1 2}$ & 147.5942 & 277.1547 & 139.0810 & & & \\
\hline $\mathbf{1 6}$ & & & & & & & K & 147.1128 & 74.0600 & 130.0863 & 65.5468 & & & $\mathbf{1}$ \\
\hline
\end{tabular}
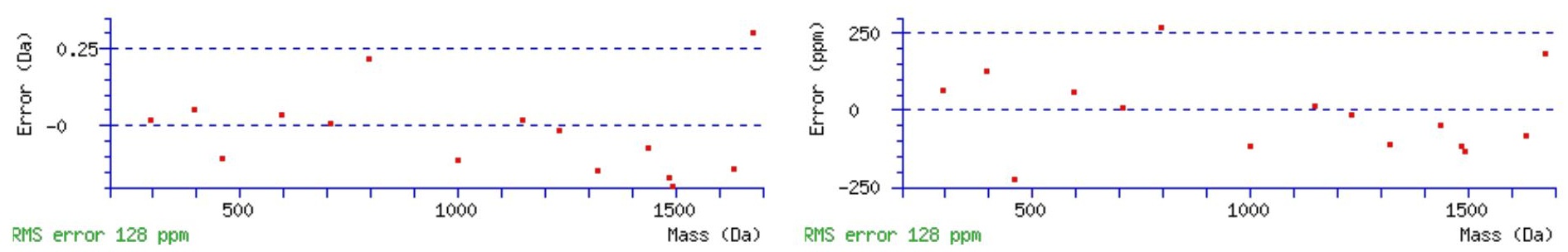

\section{All matches to this query}

\begin{tabular}{|l|l|l|l|}
\hline Score & Mr(calc): & Delta & \multicolumn{1}{|c|}{ Sequence } \\
\hline 104.7 & 1778.8363 & 0.0010 & TSVGNSSFYQDTVVFK \\
\hline 9.3 & 1777.8264 & 1.0109 & AGTKTSSASLSSLALPK \\
\hline 6.1 & 1778.8491 & -0.0118 & TLFINGISKYAESEK \\
\hline 5.9 & 1778.8543 & -0.0170 & TASSXGPALATGNVVVMK \\
\hline 5.6 & 1776.8369 & 2.0004 & SGSLSLTQFADMISLK \\
\hline 3.1 & 1778.8304 & 0.0069 & EDQRAFHRMMTGLR \\
\hline 1.4 & 1778.8509 & -0.0136 & TVNCSLWQENSLTVK \\
\hline 1.4 & 1778.8509 & -0.0136 & TVNCSLWQENSLTVK \\
\hline 1.4 & 1778.8491 & -0.0118 & TLFINGISKYAESEK \\
\hline 0.2 & 1778.8465 & -0.0092 & SVLHGVNDNRSQVFK \\
\hline
\end{tabular}

Spectrum No: 59; Query: 871; Rank: 1

\section{Peptide View}

MS/MS Fragmentation of MIENGSLSFLPTLR

Found in IPI00191090, Tax_Id=10116 Gene_Symbol=Bgn Biglycan precursor

Match to Query 871: 1593.807468 from(797.911010,2+)

Title: 091008RatKidney_NH4Format01_26.3451.3451.2.dta

Data file K:INewmanPaper|Piliang|3SubProteomes\Piliang3SP\mgf5ppm\ERLIC_3SubProteomes5ppm.mgf 

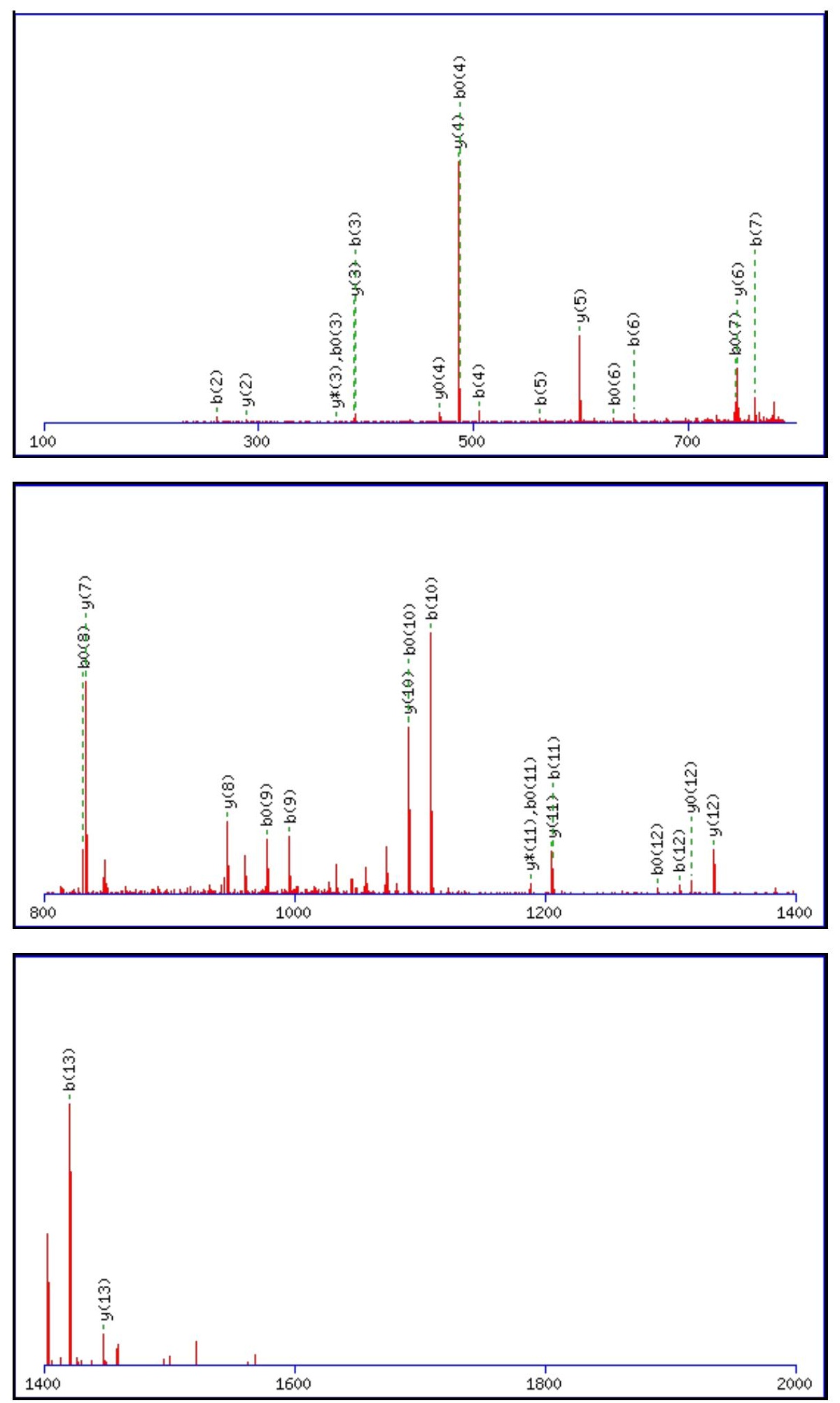

Monoisotopic mass of neutral peptide $\operatorname{Mr}($ calc): 1593.8072

Fixed modifications: Carbamidomethyl (C)

Variable modifications:

M1 : Oxidation (M)

N4 : Deamidated_N (N)

Ions Score: 105 Expect: $5 \mathrm{e}-009$

Matches (Bold Red): 35/142 fragment ions using 46 most intense peaks

\begin{tabular}{|c|c|c|c|c|c|c|c|c|c|c|c|c|c|c|}
\hline$\#$ & $\mathbf{b}$ & $\mathbf{b}^{++}$ & $\mathbf{b}^{*}$ & $\mathbf{b}^{\boldsymbol{*}_{++}}$ & $\mathbf{b}^{\mathbf{0}}$ & $\mathbf{b}^{\mathbf{0}+}$ & $\mathbf{S e q}$ & $\mathbf{y}$ & $\mathbf{y}^{++}$ & $\mathbf{y}^{\mathbf{*}}$ & $\mathbf{y}^{\mathbf{*}^{++}}$ & $\mathbf{y}^{\mathbf{0}}$ & $\mathbf{y}^{\mathbf{0}+}$ & $\#$ \\
\hline $\mathbf{1}$ & 148.0427 & 74.5250 & & & & & $\mathbf{M}$ & & & & & & & $\mathbf{1 4}$ \\
\hline $\mathbf{2}$ & $\mathbf{2 6 1 . 1 2 6 7}$ & 131.0670 & & & & & $\mathbf{I}$ & $\mathbf{1 4 4 7 . 7 7 9 1}$ & 724.3932 & 1430.7526 & 715.8799 & 1429.7685 & 715.3879 & $\mathbf{1 3}$ \\
\hline $\mathbf{3}$ & $\mathbf{3 9 0 . 1 6 9 3}$ & 195.5883 & & & 372.1588 & 186.5830 & $\mathbf{E}$ & $\mathbf{1 3 3 4 . 6 9 5 1}$ & 667.8512 & 1317.6685 & 659.3379 & $\mathbf{1 3 1 6 . 6 8 4 5}$ & 658.8459 & $\mathbf{1 2}$ \\
\hline $\mathbf{4}$ & $\mathbf{5 0 5 . 1 9 6 3}$ & 253.1018 & 488.1697 & 244.5885 & $\mathbf{4 8 7 . 1 8 5 7}$ & 244.0965 & $\mathbf{N}$ & $\mathbf{1 2 0 5 . 6 5 2 5}$ & 603.3299 & $\mathbf{1 1 8 8 . 6 2 5 9}$ & 594.8166 & 1187.6419 & 594.3246 & $\mathbf{1 1}$ \\
\hline & & & & & & & & & & & & & &
\end{tabular}




\begin{tabular}{|r|r|r|r|r|r|r|r|r|r|r|r|r|r|r|r|r|r|r|}
$\mathbf{5}$ & $\mathbf{5 6 2 . 2 1 7 7}$ & 281.6125 & 545.1912 & 273.0992 & 544.2072 & 272.6072 & G & $\mathbf{1 0 9 0 . 6 2 5 5}$ & 545.8164 & 1073.5990 & 537.3031 & 1072.6150 & 536.8111 & $\mathbf{1 0}$ \\
\hline $\mathbf{6}$ & $\mathbf{6 4 9 . 2 4 9 8}$ & 325.1285 & 632.2232 & 316.6152 & $\mathbf{6 3 1 . 2 3 9 2}$ & 316.1232 & S & 1033.6041 & 517.3057 & 1016.5775 & 508.7924 & 1015.5935 & 508.3004 & $\mathbf{9}$ \\
\hline $\mathbf{7}$ & $\mathbf{7 6 2 . 3 3 3 8}$ & 381.6706 & 745.3073 & 373.1573 & $\mathbf{7 4 4 . 3 2 3 3}$ & 372.6653 & L & $\mathbf{9 4 6 . 5 7 2 0}$ & $\mathbf{4 7 3 . 7 8 9 7}$ & 929.5455 & 465.2764 & 928.5615 & 464.7844 & $\mathbf{8}$ \\
\hline $\mathbf{8}$ & 849.3659 & 425.1866 & 832.3393 & 416.6733 & $\mathbf{8 3 1 . 3 5 5 3}$ & 416.1813 & S & $\mathbf{8 3 3 . 4 8 8 0}$ & 417.2476 & 816.4614 & 408.7343 & 815.4774 & 408.2423 & $\mathbf{7}$ \\
\hline $\mathbf{9}$ & $\mathbf{9 9 6 . 4 3 4 3}$ & 498.7208 & 979.4077 & 490.2075 & $\mathbf{9 7 8 . 4 2 3 7}$ & 489.7155 & F & $\mathbf{7 4 6 . 4 5 5 9}$ & 373.7316 & 729.4294 & 365.2183 & 728.4454 & 364.7263 & $\mathbf{6}$ \\
\hline $\mathbf{1 0}$ & $\mathbf{1 1 0 9 . 5 1 8 3}$ & 555.2628 & 1092.4918 & 546.7495 & $\mathbf{1 0 9 1 . 5 0 7 8}$ & 546.2575 & L & $5 \mathbf{5 9 . 3 8 7 5}$ & 300.1974 & 582.3610 & 291.6841 & 581.3770 & 291.1921 & $\mathbf{5}$ \\
\hline $\mathbf{1 1}$ & $\mathbf{1 2 0 6 . 5 7 1 1}$ & 603.7892 & 1189.5446 & 595.2759 & $\mathbf{1 1 8 8 . 5 6 0 5}$ & 594.7839 & P & $\mathbf{4 8 6 . 3 0 3 5}$ & 243.6554 & 469.2769 & 235.1421 & $\mathbf{4 6 8 . 2 9 2 9}$ & 234.6501 & $\mathbf{4}$ \\
\hline $\mathbf{1 2}$ & $\mathbf{1 3 0 7 . 6 1 8 8}$ & 654.3130 & 1290.5922 & 645.7998 & $\mathbf{1 2 8 9 . 6 0 8 2}$ & 645.3077 & T & $\mathbf{3 8 9 . 2 5 0 7}$ & 195.1290 & $\mathbf{3 7 2 . 2 2 4 1}$ & 186.6157 & 371.2401 & 186.1237 & $\mathbf{3}$ \\
\hline $\mathbf{1 3}$ & $\mathbf{1 4 2 0 . 7 0 2 8}$ & 710.8551 & 1403.6763 & $\mathbf{7 0 2 . 3 4 1 8}$ & $\mathbf{1 4 0 2 . 6 9 2 3}$ & $\mathbf{7 0 1 . 8 4 9 8}$ & L & $\mathbf{2 8 8 . 2 0 3 0}$ & 144.6051 & 271.1765 & 136.0919 & & & & $\mathbf{2}$ \\
\hline $\mathbf{1 4}$ & & & & & & & R & 175.1190 & 88.0631 & 158.0924 & 79.5498 & & & & $\mathbf{1}$ \\
\hline
\end{tabular}
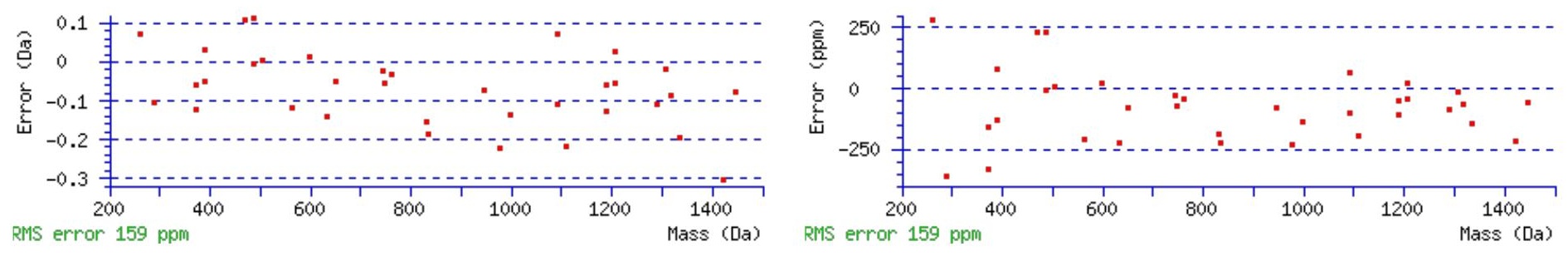

\section{All matches to this query}

\begin{tabular}{|l|l|l|l|}
\hline Score & Mr(calc): & Delta & \multicolumn{1}{|c|}{ Sequence } \\
\hline 104.6 & 1593.8072 & 0.0002 & MIENGSLSFLPTLR \\
\hline 16.6 & 1593.8223 & -0.0148 & SLRELHLQDNNVR \\
\hline 14.3 & 1593.8223 & -0.0148 & SLRELHLQDNNVR \\
\hline 12.2 & 1593.8055 & 0.0020 & FKEVIPISDPELK \\
\hline 10.3 & 1593.8106 & -0.0031 & LMQDKEEMIGKLK \\
\hline 9.2 & 1593.8223 & -0.0148 & RISELEHENAQLR \\
\hline 8.5 & 1592.8079 & 0.9995 & MTEEQQSLKNKIK \\
\hline 7.9 & 1593.8144 & -0.0070 & MLKAESSGERTTLR \\
\hline 7.6 & 1593.8184 & -0.0110 & FLEGNSMKLASLER \\
\hline 5.9 & 1593.8068 & 0.0006 & FYFIEAAKRTIR \\
\hline
\end{tabular}

Spectrum No: 60; Query: 992; Rank: 1

\section{Peptide View}

MS/MS Fragmentation of DLNISLDTDAPLSVGK

Found in IPI00326990, Tax_Id=10116 Gene_Symbol=Slc15a2 Oligopeptide transporter, kidney isoform

Match to Query 992: 1657.840788 from(829.927670,2+)

Title: 091008RatKidney_NH4Format01_27.2868.2868.2.dta

Data file K:INewmanPaper|Piliangl3SubProteomes\Piliang3SP\mgf5ppm\ERLIC_3SubProteomes5ppm.mgf 

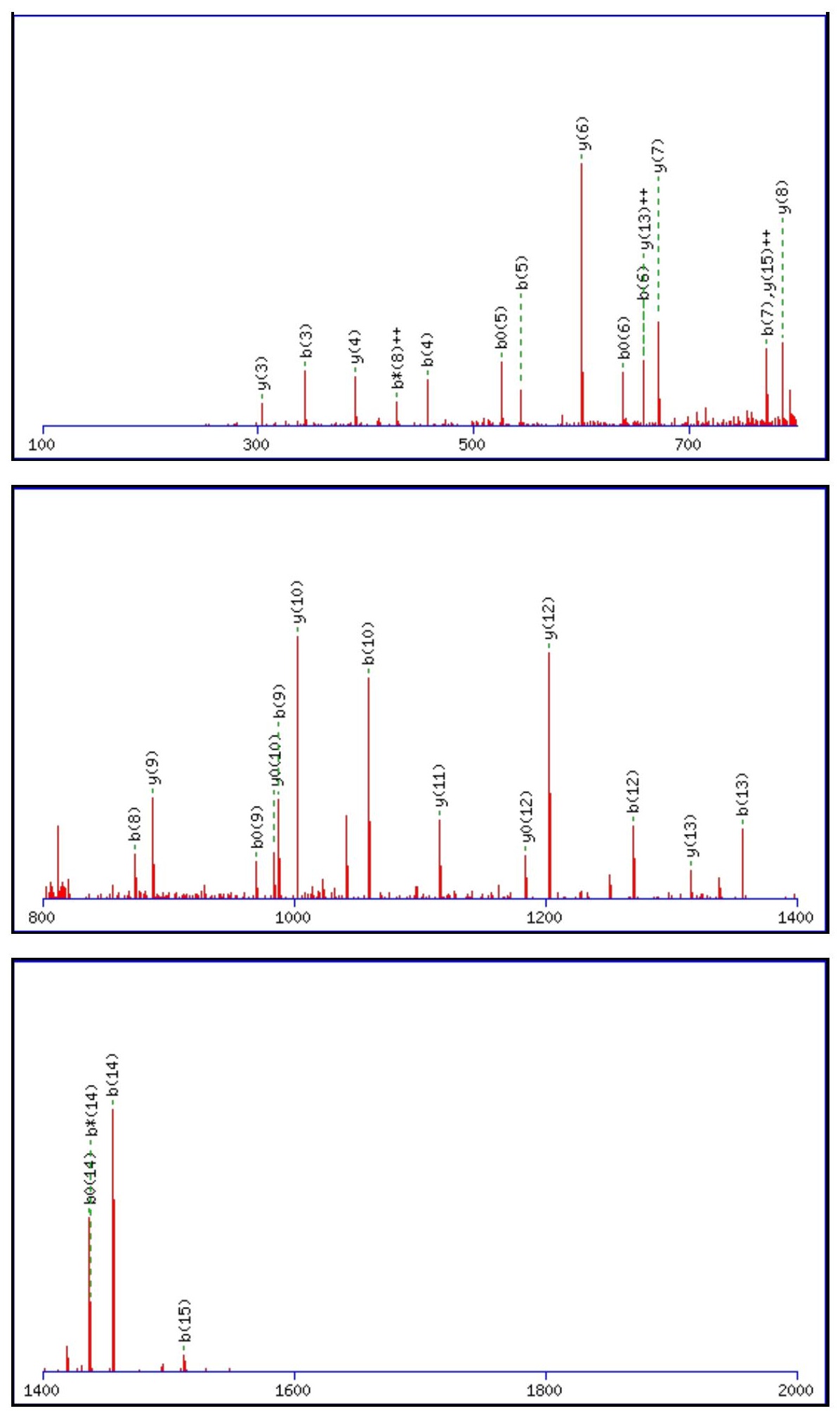

Monoisotopic mass of neutral peptide $\operatorname{Mr}($ calc): 1657.8410

Fixed modifications: Carbamidomethyl (C)

Variable modifications:

N3 : Deamidated $\mathrm{N}(\mathrm{N})$

Ions Score: 105 Expect: $5.2 \mathrm{e}-009$

Matches (Bold Red): 32/170 fragment ions using 41 most intense peaks

\begin{tabular}{|r|c|c|c|c|c|c|c|c|c|c|c|c|c|c|}
\hline$\#$ & $\mathbf{b}$ & $\mathbf{b}^{++}$ & $\mathbf{b}^{*}$ & $\mathbf{b}^{\mathbf{*}^{++}}$ & $\mathbf{b}^{\mathbf{0}}$ & $\mathbf{b}^{\mathbf{0}+}$ & Seq. & $\mathbf{y}$ & $\mathbf{y}^{++}$ & $\mathbf{y}^{*}$ & $\mathbf{y}^{\mathbf{*}^{++}}$ & $\mathbf{y}^{\mathbf{0}}$ & $\mathbf{y}^{\mathbf{0 + +}}$ & $\#$ \\
\hline $\mathbf{1}$ & 116.0342 & 58.5207 & & & 98.0237 & 49.5155 & $\mathbf{D}$ & & & & & & & $\mathbf{1 6}$ \\
\hline $\mathbf{2}$ & 229.1183 & 115.0628 & & & 211.1077 & 106.0575 & $\mathbf{L}$ & 1543.8214 & 772.4143 & 1526.7948 & 763.9011 & 1525.8108 & 763.4090 & $\mathbf{1 5}$ \\
\hline $\mathbf{3}$ & $\mathbf{3 4 4 . 1 4 5 2}$ & 172.5762 & 327.1187 & 164.0630 & 326.1347 & 163.5710 & $\mathbf{N}$ & 1430.7373 & 715.8723 & 1413.7108 & 707.3590 & 1412.7267 & 706.8670 & $\mathbf{1 4}$ \\
\hline $\mathbf{4}$ & $\mathbf{4 5 7 . 2 2 9 3}$ & 229.1183 & 440.2027 & 220.6050 & 439.2187 & 220.1130 & $\mathbf{I}$ & $\mathbf{1 3 1 5 . 7 1 0 4}$ & $\mathbf{6 5 8 . 3 5 8 8}$ & 1298.6838 & 649.8456 & 1297.6998 & 649.3535 & $\mathbf{1 3}$ \\
\hline $\mathbf{5}$ & $\mathbf{5 4 4 . 2 6 1 3}$ & 272.6343 & 527.2348 & 264.1210 & 526.2507 & 263.6290 & $\mathbf{S}$ & $\mathbf{1 2 0 2 . 6 2 6 3}$ & 601.8168 & 1185.5998 & 593.3035 & $\mathbf{1 1 8 4 . 6 1 5 7}$ & 592.8115 & $\mathbf{1 2}$ \\
\hline
\end{tabular}




\begin{tabular}{|c|c|c|c|c|c|c|c|c|c|c|c|c|c|c|}
\hline 6 & 657.3454 & |329.1763| & 640.3188 & |320.6631 & 639.3348 & 320.1710 & ـ & 1115.5943 & & & & & & 11 \\
\hline 7 & 772.3723 & 386.6898 & 755.3458 & 378.1765 & 754.3618 & 377.6845 & D & 1002.5102 & 501.7587 & 985.4837 & 493.2455 & 984.4997 & 492.7535 & 10 \\
\hline 8 & 873.4200 & 437.2136 & 856.3934 & 428.7004 & 855.4094 & 428.2084 & $\mathbf{T}$ & 887.4833 & 444.2453 & 870.4567 & 435.7320 & 869.4727 & 435.2400 & 9 \\
\hline 9 & 988.4469 & 494.7271 & 971.4204 & 486.2138 & 970.4364 & 485.7218 & D & 786.4356 & 393.7214 & 769.4090 & 385.2082 & 768.4250 & 384.7162 & 8 \\
\hline 10 & 1059.4841 & 530.2457 & 1042.4575 & 521.7324 & 1041.4735 & 521.2404 & A & 671.4087 & 336.2080 & 654.3821 & 327.6947 & 653.3981 & 327.2027 & 7 \\
\hline 11 & 1156.5368 & 578.7720 & 1139.5103 & 570.2588 & 1138.5263 & 569.7668 & $\mathbf{P}$ & 600.3715 & 300.6894 & 583.3450 & 292.1761 & 582.3610 & 291.6841 & 6 \\
\hline 12 & 1269.6209 & 635.3141 & 1252.5943 & 626.8008 & 1251.6103 & 626.3088 & $\mathbf{L}$ & 503.3188 & 252.1630 & 486.2922 & 243.6498 & 485.3082 & 243.1577 & 5 \\
\hline 13 & 1356.6529 & 678.8301 & 1339.6264 & 670.3168 & 1338.6423 & 669.8248 & S & 390.2347 & 195.6210 & 373.2082 & 187.1077 & 372.2241 & 186.6157 & 4 \\
\hline 14 & 1455.7213 & 728.3643 & 1438.6948 & 719.8510 & 1437.7108 & 719.3590 & V & 303.2027 & 152.1050 & 286.1761 & 143.5917 & & & 3 \\
\hline 15 & 1512.7428 & 756.8750 & 1495.7162 & 748.3618 & 1494.7322 & 747.8697 & $\mathbf{G}$ & 204.1343 & 102.5708 & 187.1077 & 94.0575 & & & 2 \\
\hline 16 & & & & & & & K & 147.1128 & 74.0600 & 130.0863 & 65.5468 & & & 1 \\
\hline
\end{tabular}
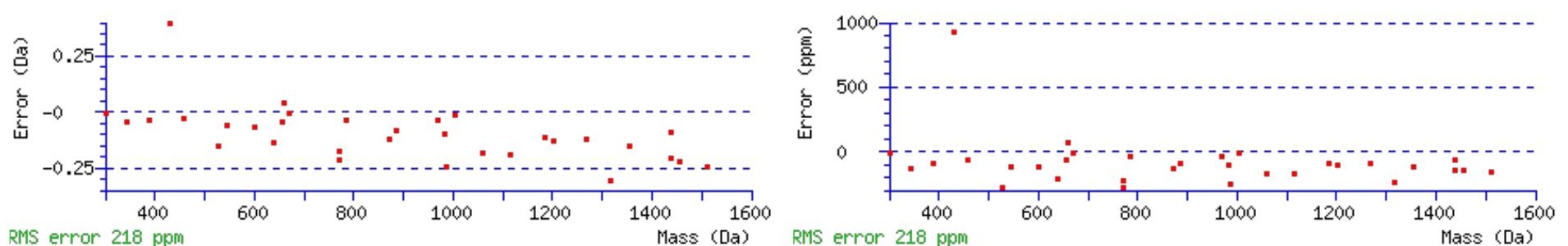

\section{All matches to this query}

\begin{tabular}{|l|l|l|l|}
\hline Score & Mr(calc): & Delta & \multicolumn{1}{|c|}{ Sequence } \\
\hline 104.5 & 1657.8410 & -0.0002 & DLNISLDTDAPLSVGK \\
\hline 14.8 & 1656.8488 & 0.9920 & GGGLFSVGETTAKILK \\
\hline 10.7 & 1657.8383 & 0.0025 & KASESPGGAASPAGTSKR \\
\hline 7.4 & 1657.8280 & 0.0128 & QHSSSNMVIMLIGNK \\
\hline 6.5 & 1657.8385 & 0.0023 & NAPFLLNDPVLGGMAK \\
\hline 3.9 & 1656.8220 & 1.0188 & GSPGNIGHPGSPGLPGEK \\
\hline 3.5 & 1657.8440 & -0.0032 & VSKGILKAFGNSTEK \\
\hline 3.4 & 1657.8497 & -0.0090 & MELSLRNIYGYVGK \\
\hline 2.9 & 1657.8311 & 0.0096 & QFINPAGDNVVLDEK \\
\hline 2.7 & 1655.8283 & 2.0124 & ALADFIRNNLSTIK \\
\hline
\end{tabular}

Spectrum No: 61; Query: 1268; Rank: 1

\section{Peptide View}

MS/MS Fragmentation of NEIQVNTSQIPDTVTR

Found in IPI00211648, Tax_Id=10116 Gene_Symbol=Slc3a1 Neutral and basic amino acid transport protein rBAT

Match to Query 1268: 1814.896088 from(908.455320,2+)

Title: 091008RatKidney_NoSalt_30.2060.2060.2.dta

Data file K:INewmanPaper|Piliang|3SubProteomes\Piliang3SP\mgf5ppm\ERLIC_3SubProteomes5ppm.mgf 

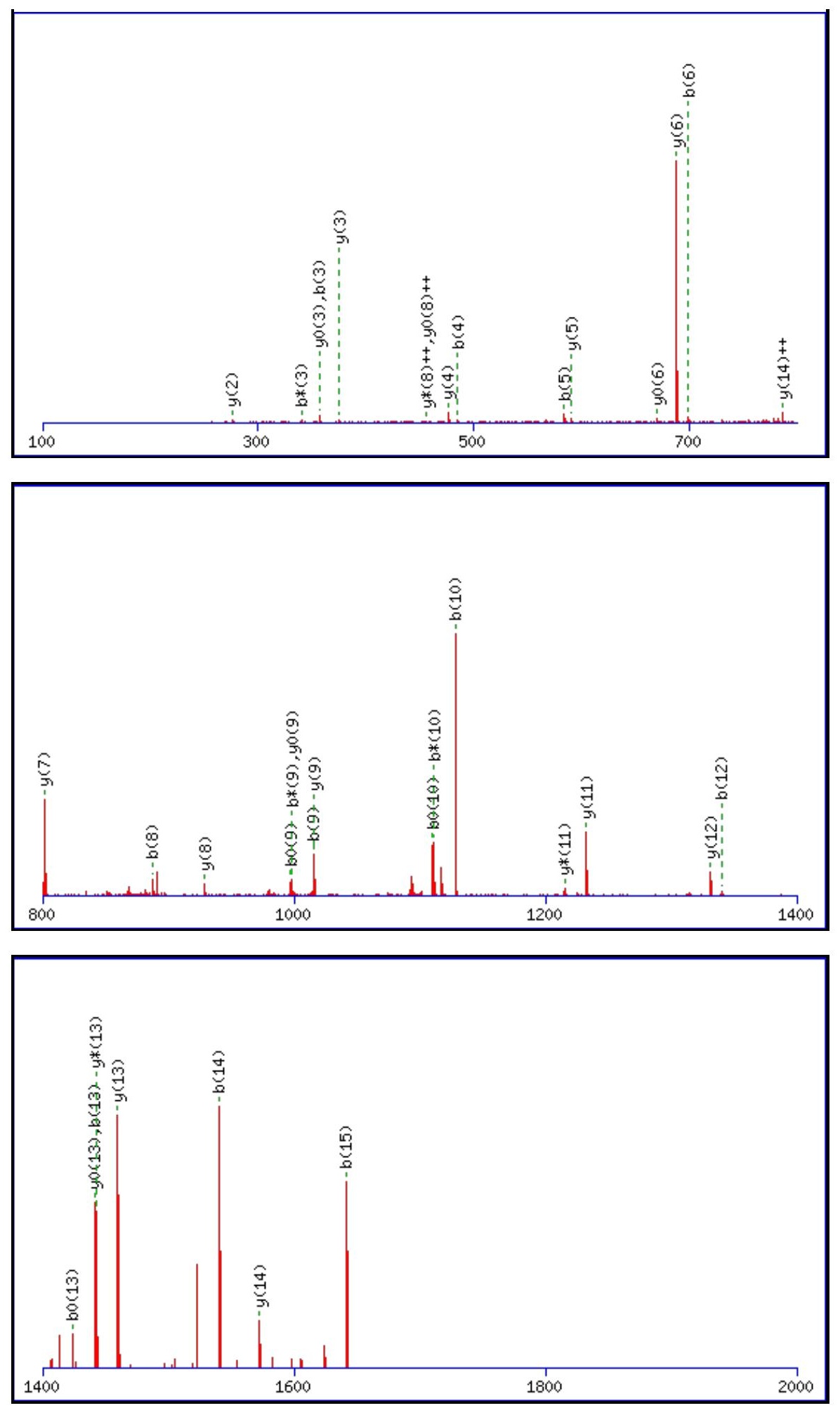

Monoisotopic mass of neutral peptide $\operatorname{Mr}($ calc): 1814.9010

Fixed modifications: Carbamidomethyl (C)

Variable modifications:

N6

Ions Score: 104 Expect: $6.9 \mathrm{e}-009$

Matches (Bold Red): 38/176 fragment ions using 46 most intense peaks

\begin{tabular}{|r|c|c|c|c|c|c|c|c|c|c|c|c|c|c|}
\hline$\#$ & $\mathbf{b}$ & $\mathbf{b}^{++}$ & $\mathbf{b}^{*}$ & $\mathbf{b}^{\boldsymbol{*}^{++}}$ & $\mathbf{b}^{\mathbf{0}}$ & $\mathbf{b}^{\mathbf{0 + +}}$ & $\mathbf{S e q}$ & $\mathbf{y}$ & $\mathbf{y}^{++}$ & $\mathbf{y}^{\mathbf{*}}$ & $\mathbf{y}^{\boldsymbol{*}^{++}}$ & $\mathbf{y}^{\mathbf{0}}$ & $\mathbf{y}^{\mathbf{0 + +}}$ & $\#$ \\
\hline $\mathbf{1}$ & 115.0502 & 58.0287 & 98.0237 & 49.5155 & & & $\mathbf{N}$ & & & & & & & $\mathbf{1 6}$ \\
\hline $\mathbf{2}$ & 244.0928 & 122.5500 & 227.0662 & 114.0368 & 226.0822 & 113.5448 & $\mathbf{E}$ & 1701.8654 & 851.4363 & 1684.8388 & 842.9230 & 1683.8548 & $\mathbf{8 4 2 . 4 3 1 0}$ & $\mathbf{1 5}$ \\
\hline $\mathbf{3}$ & $\mathbf{3 5 7 . 1 7 6 9}$ & 179.0921 & 340.1503 & 170.5788 & 339.1663 & 170.0868 & $\mathbf{I}$ & $\mathbf{1 5 7 2 . 8 2 2 8}$ & $\mathbf{7 8 6 . 9 1 5 0}$ & 1555.7962 & 778.4017 & 1554.8122 & 777.9097 & $\mathbf{1 4}$ \\
\hline $\mathbf{4}$ & $\mathbf{4 8 5 . 2 3 5 4}$ & 243.1214 & 468.2089 & 234.6081 & 467.2249 & 234.1161 & $\mathbf{Q}$ & $\mathbf{1 4 5 9 . 7 3 8 7}$ & 730.3730 & $\mathbf{1 4 4 2 . 7 1 2 2}$ & 721.8597 & $\mathbf{1 4 4 1 . 7 2 8 1}$ & $\mathbf{7 2 1 . 3 6 7 7}$ & $\mathbf{1 3}$ \\
\hline $\mathbf{5}$ & $\mathbf{5 8 4 . 3 0 3 9}$ & 292.6556 & 567.2773 & 284.1423 & 566.2933 & 283.6503 & $\mathbf{V}$ & $\mathbf{1 3 3 1 . 6 8 0 1}$ & 666.3437 & 1314.6536 & 657.8304 & 1313.6696 & 657.3384 & $\mathbf{1 2}$ \\
\hline
\end{tabular}




\begin{tabular}{|c|c|c|c|c|c|c|c|c|c|c|c|c|c|c|}
\hline 6 & 699.3308 & |350.1690 & 682.3042 & |341.6558 & 681.3202 & |341.1637 & & 1232.6117 & 616.8095 & & & & & \\
\hline 7 & 800.3785 & 400.6929 & 783.3519 & 392.1796 & 782.3679 & 391.6876 & $\mathbf{T}$ & 1117.5848 & 559.2960 & 1100.5582 & 550.7828 & 1099.5742 & 550.2907 & 10 \\
\hline 8 & 887.4105 & 444.2089 & 870.3839 & 435.6956 & 869.3999 & 435.2036 & J & 1016.5371 & 508.7722 & 999.5106 & 500.2589 & 998.5265 & 499.7669 & 9 \\
\hline 9 & 1015.4691 & 508.2382 & 998.4425 & 499.7249 & 997.4585 & 499.2329 & $\mathbf{Q}$ & & 465.2 & 912.4785 & 456. & 1.4945 & 456. & 8 \\
\hline 10 & 128.5531 & 564.7802 & 1111.5266 & 556.2669 & 1110.5426 & 555.7749 & 1 & 801.4465 & 401.2269 & 784.4199 & 392.7136 & 4359 & 392.2216 & 7 \\
\hline 11 & 1225.6059 & 613.3066 & 1208.5794 & 604.7933 & 1207.5953 & 604.3013 & $\mathbf{P}$ & 688.3624 & 344.6849 & 671.3359 & 336.1716 & 670.3519 & 335.6796 & 6 \\
\hline 12 & 1340.6328 & 670.8201 & 1323.6063 & 662.3068 & 1322.6223 & 661.8148 & $\mathbf{D}$ & & 296. & 574.2831 & 287.6452 & 573.2991 & 287.1532 & 5 \\
\hline 13 & 1441.6805 & 721.3439 & 1424.6540 & 712.8306 & 1423.6700 & 712.3386 & $\mathbf{T}$ & 476.2827 & 238.6450 & 459.2562 & 230.1317 & 458.2722 & 229.6397 & 4 \\
\hline 14 & 1540.7489 & 770.8781 & 1523.7224 & 762.3648 & 1522.7384 & 761.8728 & V & 375.2350 & 188.1212 & 358.2085 & 179.6079 & 357.2245 & 179.1159 & 3 \\
\hline 15 & 1641.7966 & 821.4019 & 1624.7701 & 812.8887 & 1623.7861 & 812.3967 & $\mathbf{T}$ & 276.1666 & 138.5870 & 259.1401 & 130.0737 & 258.1561 & 129.5817 & 2 \\
\hline 16 & & & & & & & $\mathbf{R}$ & 175.1190 & 88.0631 & 158.0924 & 79.5498 & & & 1 \\
\hline
\end{tabular}
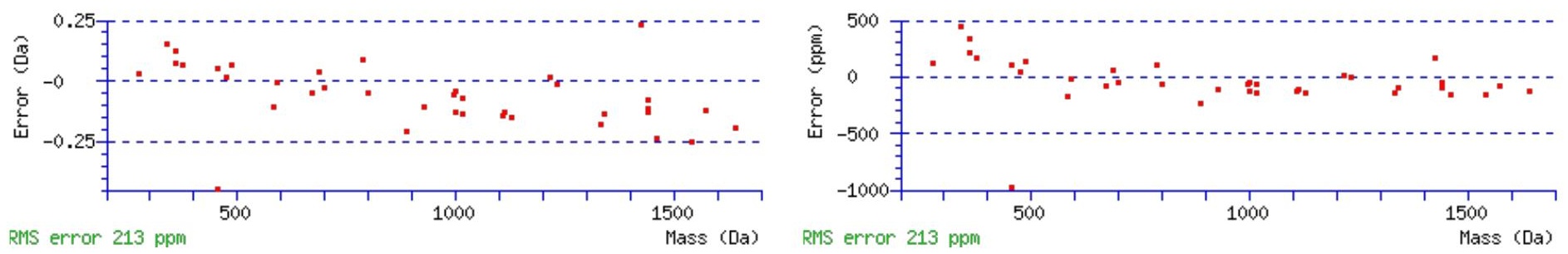

\section{All matches to this query}

\begin{tabular}{|l|l|l|l|}
\hline Score & Mr(calc): & Delta & \multicolumn{1}{|c|}{ Sequence } \\
\hline 104.3 & 1814.9010 & -0.0049 & NEIQVNTSQIPDTVTR \\
\hline 72.9 & 1814.9010 & -0.0049 & NEIQVNTSQIPDTVTR \\
\hline 18.0 & 1814.9040 & -0.0079 & AQSALHEQKTLPGVTR \\
\hline 16.2 & 1812.8870 & 2.0091 & EEKLSLTKSLDDVTR \\
\hline 14.8 & 1814.8788 & 0.0173 & SDLRHLRAVPTDEAR \\
\hline 10.1 & 1814.8985 & -0.0024 & LGPAQSGPCLFLSNPTR \\
\hline 7.4 & 1812.8870 & 2.0091 & EEKLSLTKSLDDVTR \\
\hline 6.5 & 1812.8870 & 2.0091 & EEKLSLTKSLDDVTR \\
\hline 6.1 & 1814.9131 & -0.0170 & MAGGPTRMEGNLPAKLR \\
\hline 4.6 & 1814.8981 & -0.0020 & AFRVWESATPLRFR \\
\hline
\end{tabular}

Spectrum No: 62; Query: 2817; Rank: 1

\section{Peptide View}

MS/MS Fragmentation of CDVDNDCGDYSDEPIDECTTAAYNCDNHTEFSCK

Found in IPI00205325, Tax_Id=10116 Gene_Symbol=Lrp2 Low-density lipoprotein receptor-related protein 2 precursor

Match to Query 2817: 4078.461192 from(1360.494340,3+)

Title: 100101RatKid_NS_deglyco_22.2718.2718.3.dta

Data file K:INewmanPaper|Piliang|3SubProteomes\Piliang3SP\mgf5ppm\ERLIC_3SubProteomes5ppm.mgf 

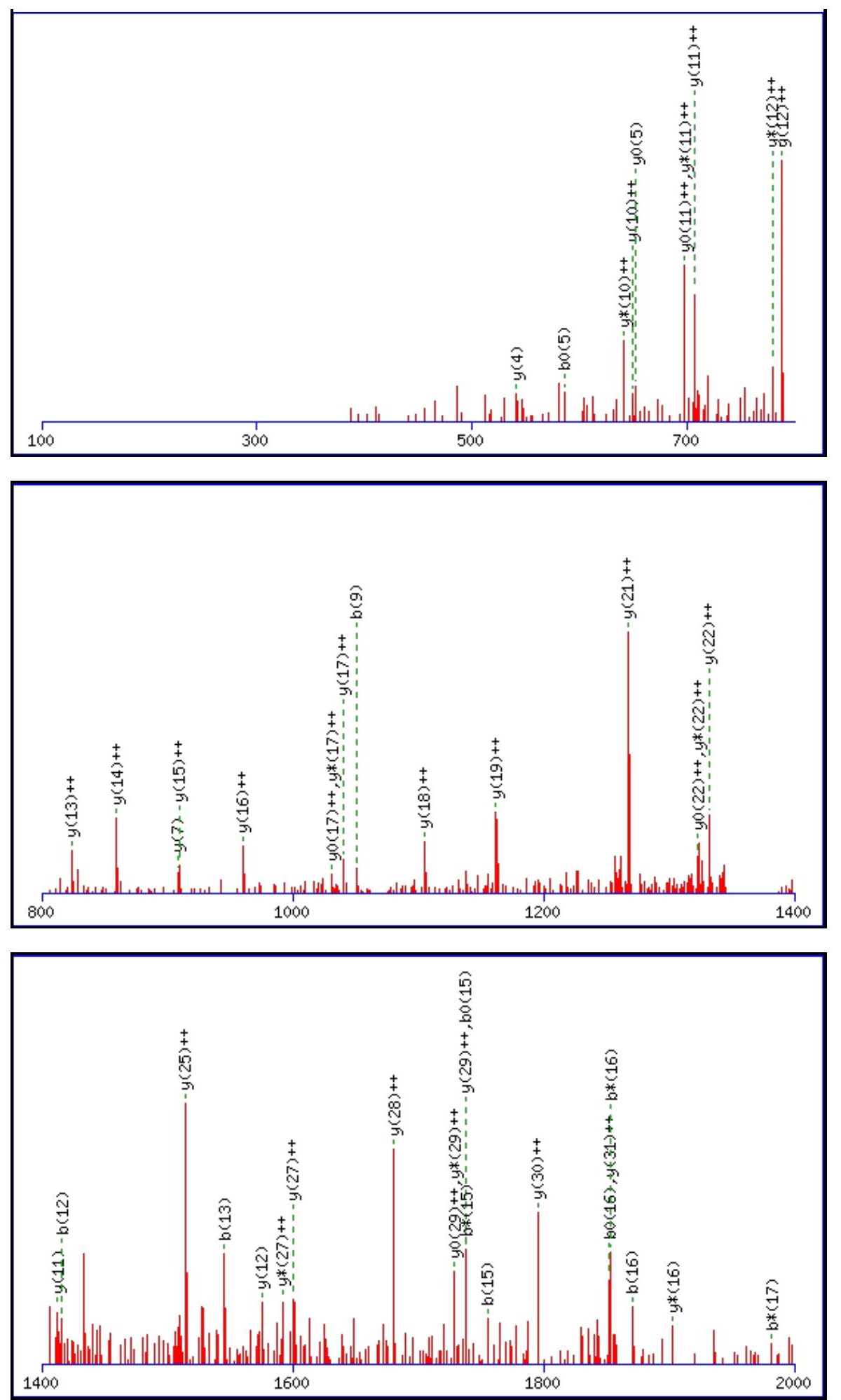

Monoisotopic mass of neutral peptide $\operatorname{Mr}($ calc): 4077.4500

Fixed modifications: Carbamidomethyl (C)

Variable modifications:

N27 : Deamidated_N (N)

Ions Score: 104 Expect: $8 \mathrm{e}-009$

Matches (Bold Red): 46/382 fragment ions using 71 most intense peaks

\begin{tabular}{|r|c|c|c|c|c|c|c|c|c|c|c|c|c|c|}
\hline$\#$ & $\mathbf{b}$ & $\mathbf{b}^{++}$ & $\mathbf{b}^{*}$ & $\mathbf{b}^{\boldsymbol{*}^{++}}$ & $\mathbf{b}^{\mathbf{0}}$ & $\mathbf{b}^{\mathbf{0 + +}}$ & $\mathbf{S e q}$ & $\mathbf{y}$ & $\mathbf{y}^{++}$ & $\mathbf{y}^{\mathbf{*}}$ & $\mathbf{y}^{\mathbf{*}^{++}}$ & $\mathbf{y}^{\mathbf{0}}$ & $\mathbf{y}^{\mathbf{0 + +}}$ & $\#$ \\
\hline $\mathbf{1}$ & 161.0379 & 81.0226 & & & & & $\mathbf{C}$ & & & & & & & 34 \\
\hline $\mathbf{2}$ & 276.0649 & 138.5361 & & & 258.0543 & 129.5308 & $\mathbf{D}$ & 3918.4266 & 1959.7169 & 3901.4000 & 1951.2037 & 3900.4160 & 1950.7116 & $\mathbf{3 3}$ \\
\hline $\mathbf{3}$ & 375.1333 & 188.0703 & & & 357.1227 & 179.0650 & $\mathbf{V}$ & 3803.3996 & 1902.2035 & 3786.3731 & 1893.6902 & 3785.3891 & 1893.1982 & 32 \\
\hline $\mathbf{4}$ & 490.1602 & 245.5838 & & & 472.1497 & 236.5785 & $\mathbf{D}$ & 3704.3312 & $\mathbf{1 8 5 2 . 6 6 9 2}$ & 3687.3047 & 1844.1560 & 3686.3207 & 1843.6640 & $\mathbf{3 1}$ \\
\hline $\mathbf{5}$ & 604.2032 & 302.6052 & 587.1766 & 294.0919 & $\mathbf{5 8 6 . 1 9 2 6}$ & 293.5999 & $\mathbf{N}$ & 3589.3043 & $\mathbf{1 7 9 5 . 1 5 5 8}$ & 3572.2777 & 1786.6425 & 3571.2937 & 1786.1505 & $\mathbf{3 0}$ \\
\hline
\end{tabular}




\begin{tabular}{|c|c|c|c|c|c|c|c|c|c|c|c|c|c|c|}
\hline & 719.2301 & 360.1187 & 702.2035 & 351.6054 & 701.2195 & 351.1134 & D & 3475.2614 & \begin{tabular}{|l|l|}
1738.1343 \\
\end{tabular} & 345 & 1729.6210 & 3457.2508 & |1729.1290 & 29 \\
\hline 7 & 879.2607 & 440.1340 & 862.2342 & 431.6207 & 861.2502 & 431.1287 & $\mathrm{C}$ & 3360.2344 & 1680.6208 & 3343.2079 & 1672.1076 & 3342.2238 & 1671.6156 & 28 \\
\hline 8 & 936.2822 & 468.6447 & 919.2557 & 460.1315 & 918.2716 & 459.6395 & G & 3200.2038 & 1600.6055 & 3183.1772 & 1592.0922 & 3182.1932 & 6002 & 27 \\
\hline 9 & 1051.3092 & 526.1582 & 1034.2826 & 517.6449 & 1033.2986 & 517.1529 & D & 3143.1823 & 1572.0948 & 3126.1557 & 1563.5815 & 3125.1717 & 1563.0895 & 26 \\
\hline 10 & 1214.3725 & 607.6899 & 1197.3459 & 599.1766 & |1196.3619 & 598.6846 & $\mathbf{Y}$ & 3028.1554 & 1514.5813 & 3011.1288 & 1506.0680 & 3010.1448 & 1505.5760 & 25 \\
\hline 11 & 1301.4045 & 651.2059 & 1284.3780 & 642.6926 & 1283.3939 & 642.2006 & $\mathrm{~S}$ & 2865.0920 & 1433.0497 & 2848.0655 & 1424.5364 & 2847.0815 & 1424.0444 & 24 \\
\hline 12 & 1416.4315 & 708.7194 & 1399.4049 & 700.2061 & 1398.4209 & 699.7141 & D & 2778.0600 & 1389.5336 & 2761.0334 & 1381.0204 & 0494 & 1380.5284 & 23 \\
\hline 13 & 1545.4740 & 773.2407 & 1528.4475 & 764.7274 & 1527.4635 & 764.2354 & E & 2663.0331 & 1332.0202 & 2646.0065 & 1323.5069 & 2645.0225 & 1323.0149 & 22 \\
\hline 14 & 1642.5268 & 821.7670 & 1625.5003 & 813.2538 & 1624.5162 & 812.7618 & $\mathbf{P}$ & 2533.9905 & 1267.4989 & 2516.9639 & 1258.9856 & 2515.9799 & 1258.4936 & 21 \\
\hline 5 & 1755.6109 & 878.3091 & 1738.5843 & 869.7958 & 1737.6003 & 869.3038 & I & 2436.9377 & 1218.9725 & 2419.9111 & 592 & 271 & 672 & 20 \\
\hline 16 & 1870.6378 & 935.8225 & 1853.6113 & 927.3093 & 1852.6273 & 926.8173 & D & 2323.8536 & 1162.4305 & 2306.8271 & 1153.9172 & 2305.8431 & 1153.4252 & 19 \\
\hline 17 & 1999.6804 & 1000.3438 & 1982.6539 & 991.8306 & 1981.6698 & 991.3386 & $E$ & 2208.8267 & 1104.9170 & 2191.8001 & 1096.4037 & 2190.8161 & 1095.9117 & 18 \\
\hline 18 & 2159.7111 & 1080.3592 & 2142.6845 & 071.8459 & 2141.7005 & 1071.3539 & $\mathrm{C}$ & 2079.7841 & 1040.3957 & 2062.7575 & 1031.8824 & 2061 & 3904 & 17 \\
\hline 19 & 2260.7587 & 1130.8830 & 2243.7322 & 1122.3697 & 2242.7482 & 1121.8777 & $\mathbf{T}$ & 1919.7534 & 960.3804 & 1902.7269 & 951.8671 & 1901.7429 & 951.3751 & 16 \\
\hline 20 & 2361.8064 & 1181.4068 & 2344.7799 & 1172.8936 & 2343.7958 & 1172.4016 & $\mathbf{T}$ & 1818.7058 & 909.8565 & 1801.6792 & 901.3432 & 1800.6952 & 900.8512 & 15 \\
\hline 21 & 2432.8435 & 1216.9254 & 2415.8170 & 1208.4121 & 2414.8330 & 1207.9201 & A & 1717.6581 & 859.3327 & 1700.6315 & 850.8194 & 1699.6475 & 3274 & 14 \\
\hline 22 & 2503.8806 & 1252.4440 & 2486.8541 & 1243.9307 & 2485.8701 & 1243.4387 & A & 1646.6210 & 823.8141 & 1629.5944 & 815.3009 & 1628.6104 & 814.8088 & 13 \\
\hline 23 & 2666.9440 & 1333.9756 & 2649.9174 & 1325.4623 & 2648.9334 & 1324.9703 & $\mathbf{Y}$ & 1575.5839 & 788.2956 & 1558.5573 & 779.7823 & 1557.5733 & 779.2903 & 12 \\
\hline 24 & 2780.9869 & 1390.9971 & 2763.9603 & 1382.4838 & 2762.9763 & 1381.9918 & $\mathbf{N}$ & 1412.5205 & 706.7639 & 1395.4940 & 698.2506 & 1394.5100 & 697.7586 & 11 \\
\hline 25 & 2941.0175 & 1471.0124 & 2923.9910 & 1462.4991 & 2923.0070 & 1462.0071 & $\mathrm{C}$ & 1298.4776 & 649.7424 & 1281.4511 & 641.2292 & 1280.4670 & 640.7372 & 10 \\
\hline 26 & 3056.0445 & 1528.5259 & 3039.0179 & 1520.0126 & 3038.0339 & 1519.5206 & D & 1138.4470 & 569.7271 & 1121.4204 & 561.2138 & 1120.4364 & 560.7218 & 9 \\
\hline 27 & 3171.0714 & 1586.0394 & 3154.0449 & 1577.5261 & 3153.0609 & 1577.0341 & $\mathbf{N}$ & 1023.4200 & 512.2136 & 1006.3935 & 503.7004 & 1005.4094 & 503.2084 & 8 \\
\hline 28 & 3308.1303 & 1654.5688 & 3291.1038 & 1646.0555 & 3290.1198 & 1645.5635 & $\mathbf{H}$ & 908.3931 & 454.7002 & 891.3665 & 446.1869 & 890.3825 & 445.6949 & 7 \\
\hline 29 & 3409.1780 & 1705.0926 & 3392.1515 & 1696.5794 & 3391.1675 & 1696.0874 & $\mathbf{T}$ & 771.3342 & 386.1707 & 754.3076 & 377.6574 & 753.3236 & 377.1654 & 6 \\
\hline 30 & 3538.2206 & 1769.6139 & 3521.1941 & 1761.1007 & 3520.2100 & 1760.6087 & $E$ & 670.2865 & 335.6469 & 653.2599 & 327.1336 & 652.2759 & 326.6416 & 5 \\
\hline 31 & 3685.2890 & 1843.1482 & 3668.2625 & 1834.6349 & 3667.2785 & 1834.1429 & $\mathbf{F}$ & 541.2439 & 271.1256 & 524.2173 & 262.6123 & 523.2333 & 262.1203 & 4 \\
\hline 32 & 3772.3211 & 1886.6642 & 3755.2945 & 1878.1509 & 3754.3105 & 1877.6589 & $\mathrm{~S}$ & 394.1755 & 197.5914 & 377.1489 & 189.0781 & 376.1649 & 188.5861 & 3 \\
\hline 33 & 3932.3517 & 1966.6795 & 3915.3252 & 1958.1662 & 3914.3411 & 1957.6742 & $\mathrm{C}$ & 307.1435 & 154.0754 & 290.1169 & 145.5621 & & & 2 \\
\hline 34 & & & & & & & $\mathbf{K}$ & 147.1128 & 74.0600 & 130.0863 & 65.5468 & & & 1 \\
\hline
\end{tabular}
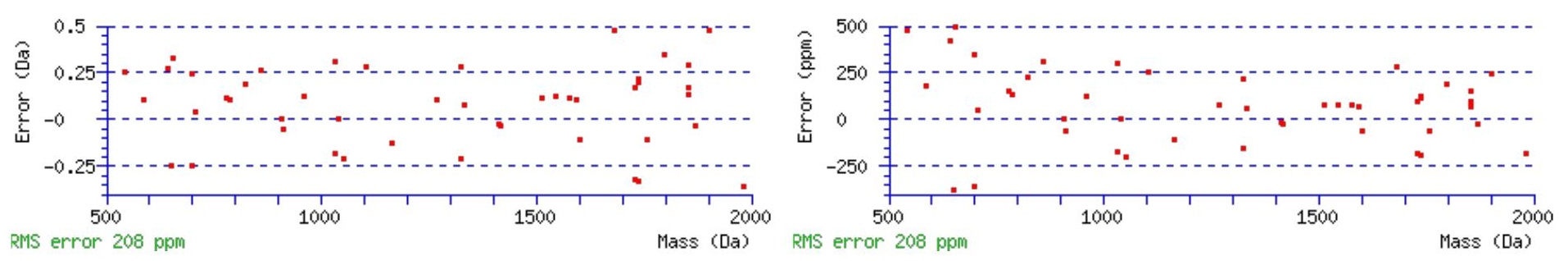

\section{All matches to this query}

\begin{tabular}{|l|l|l|l|}
\hline Score & Mr(calc): & Delta & \multicolumn{1}{|c|}{ Sequence } \\
\hline 104.3 & 4077.4500 & 1.0112 & CDVDNDCGDYSDEPIDECTTAAYNCDNHTEFSCK \\
\hline 102.6 & 4078.4340 & 0.0272 & CDVDNDCGDYSDEPIDECTTAAYNCDNHTEFSCK \\
\hline 102.5 & 4077.4500 & 1.0112 & CDVDNDCGDYSDEPIDECTTAAYNCDNHTEFSCK \\
\hline 89.5 & 4078.4340 & 0.0272 & CDVDNDCGDYSDEPIDECTTAAYNCDNHTEFSCK \\
\hline 87.6 & 4078.4340 & 0.0272 & CDVDNDCGDYSDEPIDECTTAAYNCDNHTEFSCK \\
\hline 9.7 & 4077.4218 & 1.0393 & NQDYMAPSEPNSPTKTNMEYPNTPGKQDLVSK \\
\hline 9.3 & 4078.5013 & -0.0401 & NDFISVPSIQPLDPISDSDSENSFQDSKPENQK \\
\hline 7.6 & 4078.5013 & -0.0401 & NDFISVPSIQPLDPISDSDSENSFQDSKPENQK \\
\hline 6.6 & 4076.4660 & 1.9952 & CDVDNDCGDYSDEPIDECTTAAYNCDNHTEFSCK \\
\hline 6.1 & 4078.4705 & -0.0093 & GTAGNALMEGASQLVGENRTMTIHNGMFFSTYDR \\
\hline
\end{tabular}


Spectrum No: 63; Query: 2377; Rank: 1

\section{Peptide View}

MS/MS Fragmentation of ALSCLESSWENIEQGGNGSFVYTK

Found in IPI00201262, Tax_Id=10116 Gene_Symbol=LOC297568;Mug2;Mug1 Alpha-1-inhibitor 3 precursor

Match to Query 2377: 2678.207088 from(1340.110820,2+)

Title: 100101RatKid_NS_deglyco_27.4299.4299.2.dta

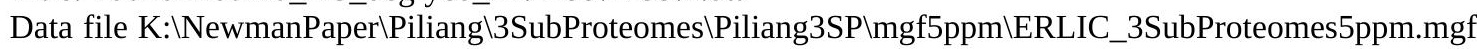
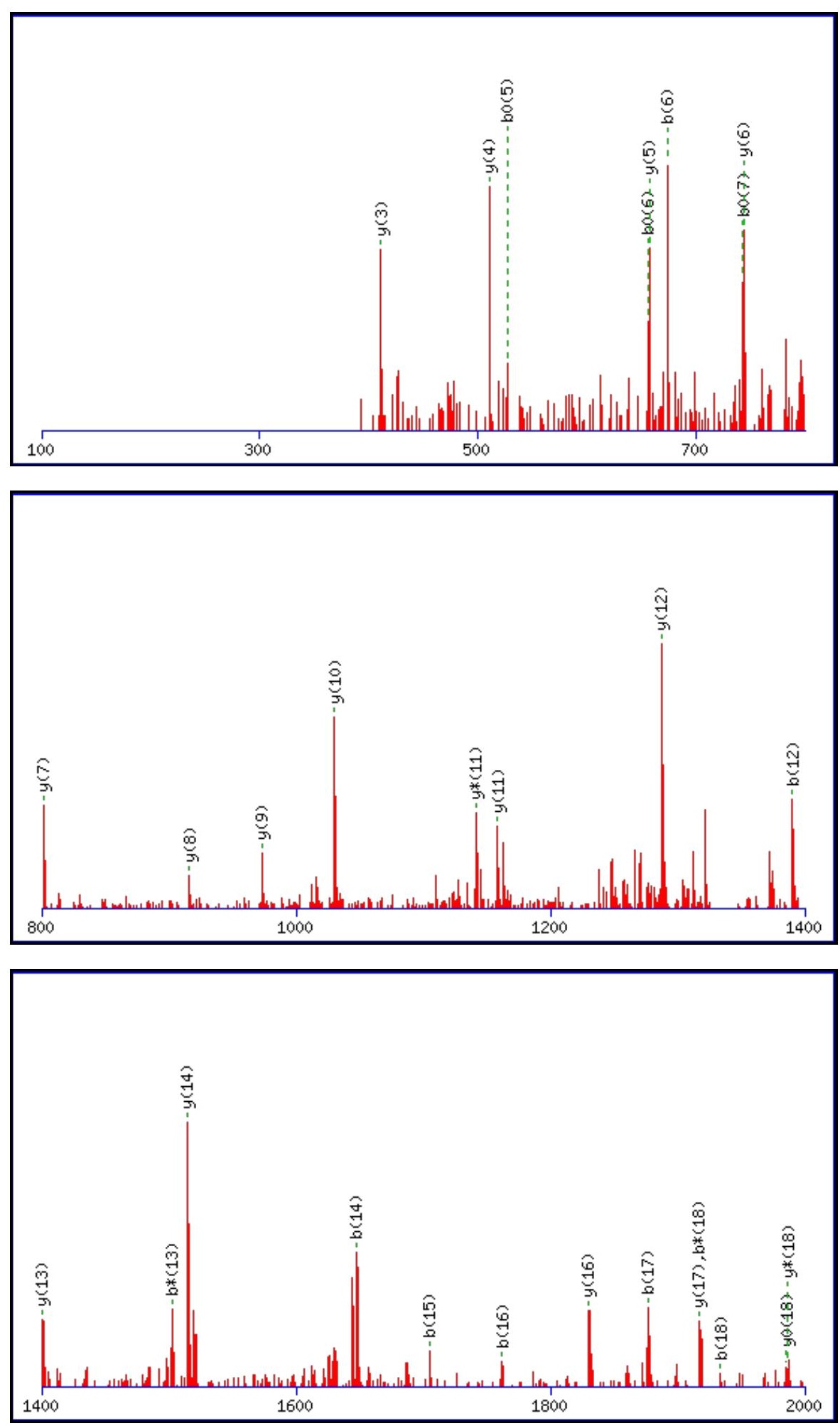
Monoisotopic mass of neutral peptide $\operatorname{Mr}($ calc): 2676.2013

Fixed modifications: Carbamidomethyl (C)

Variable modifications:

N17 : Deamidated_N (N)

Ions Score: 104 Expect: $1.3 \mathrm{e}-008$

Matches (Bold Red): 29/250 fragment ions using 51 most intense peaks

\begin{tabular}{|c|c|c|c|c|c|c|c|c|c|c|c|c|c|c|}
\hline \# & b & $\mathbf{b}^{++}$ & b* & & $\mathbf{b}^{\mathbf{0}}$ & & Seq. & $\mathbf{y}$ & & $\mathbf{y}^{*}$ & & $\mathbf{y}^{\mathbf{0}}$ & & $\#$ \\
\hline 1 & 72.0444 & 36.5258 & & & & & A & & & & & & & 24 \\
\hline 2 & 185.1285 & 93.0679 & & & & & $\mathbf{L}$ & 2606.1715 & 1303.5894 & 2589.1450 & 1295.0761 & 2588.1610 & 1294.5841 & 23 \\
\hline 3 & 272.1605 & 136.5839 & & & 254.1499 & & $\mathrm{~S}$ & 2493.0875 & 1247.0474 & 2476.0609 & 1238.5341 & 2475.0769 & 1238.0421 & 22 \\
\hline 4 & 432.1911 & 216.5992 & & & 414.1806 & 207.5939 & $\mathrm{C}$ & 2406.0554 & 1203.5314 & 2389.0289 & 1195.0181 & 2388.0449 & 1194.5261 & 21 \\
\hline 5 & 545.2752 & 273.1412 & & & 527.2646 & 264.1360 & $\mathbf{L}$ & 2246.0248 & 1123.5160 & 2228.9982 & 1115.0028 & 2228.0142 & 1114.5107 & 20 \\
\hline 6 & 674.3178 & 337.6625 & & & 656.3072 & 328.6572 & $\mathbf{E}$ & 2132.9407 & 1066.9740 & 2115.9142 & 1058.4607 & 2114.9301 & 1057.9687 & 19 \\
\hline 7 & 761.3498 & 381.1785 & & & 743.3393 & 372.1733 & $\mathrm{~S}$ & 2003.8981 & 1002.4527 & 1986.8716 & 993.9394 & 1985.8876 & 993.4474 & 18 \\
\hline 8 & 848.3818 & 424.6946 & & & 830.3713 & 415.6893 & $S$ & 1916.8661 & 958.9367 & 1899.8395 & 950.4234 & 1898.8555 & 949.9314 & 17 \\
\hline 9 & 1034.4612 & 517.7342 & & & 1016.4506 & 508.7289 & $\mathbf{W}$ & 1829.8341 & 915.4207 & 1812.8075 & 906.9074 & 1811.8235 & 906.4154 & 16 \\
\hline 10 & 1163.5038 & 582.2555 & & & 1145.4932 & 573.2502 & $\mathbf{E}$ & 1643.7548 & 822.3810 & 1626.7282 & 813.8677 & 1625.7442 & 813.3757 & 15 \\
\hline 11 & 1277.5467 & 639.2770 & 1260.5201 & 630.7637 & 1259.5361 & 630.2717 & $\mathbf{N}$ & 1514.7122 & 757.8597 & 1497.6856 & 749.3464 & 1496.7016 & 748.8544 & 14 \\
\hline 12 & 1390.6307 & 695.8190 & 1373.6042 & 687.3057 & 1372.6202 & 686.8137 & I & 1400.6692 & 700.8383 & 1383.6427 & 692.3250 & 1382.6587 & 691.8330 & 13 \\
\hline 13 & 1519.6733 & 760.3403 & 1502.6468 & 751.8270 & 1501.6628 & 751.3350 & $\mathbf{E}$ & 1287.5852 & 644.2962 & 1270.5586 & 635.7829 & 1269.5746 & 635.2909 & 12 \\
\hline 14 & 1647.7319 & 824.3696 & 1630.7054 & 815.8563 & 1629.7213 & 815.3643 & $\mathbf{Q}$ & 1158.5426 & 579.7749 & 1141.5160 & 571.2617 & 1140.5320 & 570.7696 & 11 \\
\hline 15 & 1704.7534 & 852.8803 & 1687.7268 & 844.3671 & 1686.7428 & 843.8750 & G & 1030.4840 & 515.7456 & 1013.4574 & 507.2324 & 1012.4734 & 506.7404 & 10 \\
\hline 16 & 1761.7748 & 881.3911 & 1744.7483 & 872.8778 & 1743.7643 & 872.3858 & G & 973.4625 & 487.2349 & 956.4360 & 478.7216 & 955.4520 & 478.2296 & 9 \\
\hline 17 & 1876.8018 & 938.9045 & 1859.7752 & 930.3913 & 1858.7912 & 929.8992 & $\mathbf{N}$ & 916.4411 & 458.7242 & 899.4145 & 450.2109 & 898.4305 & 449.7189 & 8 \\
\hline 18 & 1933.8232 & 967.4153 & 1916.7967 & 958.9020 & 1915.8127 & 958.4100 & G & 801.4141 & 401.2107 & 784.3876 & 392.6974 & 783.4036 & 392.2054 & 7 \\
\hline 19 & 2020.8553 & 1010.9313 & 2003.8287 & 1002.4180 & 2002.8447 & 1001.9260 & $S$ & 744.3927 & 372.7000 & 727.3661 & 364.1867 & 726.3821 & 363.6947 & 6 \\
\hline 20 & 2167.9237 & 1084.4655 & 2150.8971 & 1075.9522 & 2149.9131 & 1075.4602 & $\mathbf{F}$ & 657.3606 & 329.1840 & 640.3341 & 320.6707 & 639.3501 & 320.1787 & 5 \\
\hline 21 & 2266.9921 & 1133.9997 & 2249.9655 & 1125.4864 & 2248.9815 & 1124.9944 & $\mathbf{V}$ & 510.2922 & 255.6498 & 493.2657 & 247.1365 & 492.2817 & 246.6445 & 4 \\
\hline 22 & 2430.0554 & 1215.5314 & 2413.0289 & 1207.0181 & 2412.0449 & 1206.5261 & $\mathbf{Y}$ & 411.2238 & 206.1155 & 394.1973 & 197.6023 & 393.2132 & 197.1103 & 3 \\
\hline 23 & 2531.1031 & 1266.0552 & 2514.0766 & 1257.5419 & 2513.0925 & 1257.0499 & $\mathbf{T}$ & 248.1605 & 124.5839 & 231.1339 & 116.0706 & 230.1499 & 115.5786 & 2 \\
\hline 24 & & & & & & & $\mathbf{K}$ & 147.1128 & 74.0600 & 130.0863 & 65.5468 & & & 1 \\
\hline
\end{tabular}
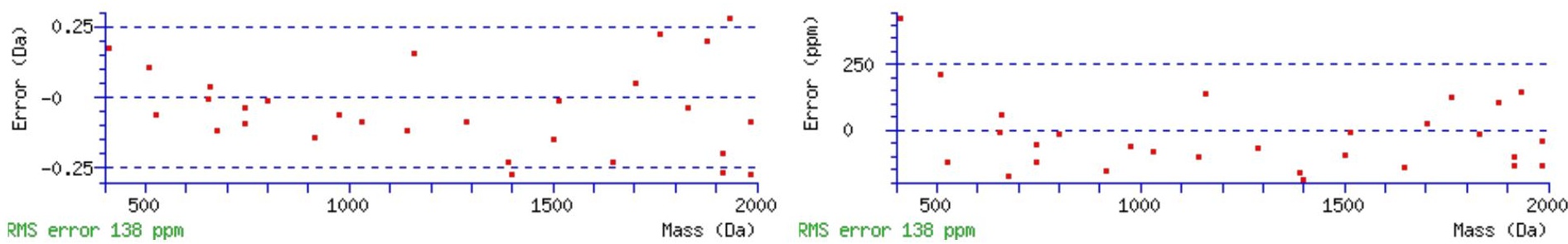

All matches to this query

\begin{tabular}{|l|l|l|l|}
\hline Score & Mr(calc): & Delta & \multicolumn{1}{|c|}{ Sequence } \\
\hline 104.2 & 2676.2013 & 2.0057 & ALSCLESSWENIEQGGNGSFVYTK \\
\hline 87.5 & 2677.1853 & 1.0217 & ALSCLESSWENIEQGGNGSFVYTK \\
\hline 36.8 & 2676.2013 & 2.0057 & ALSCLESSWENIEQGGNGSFVYTK \\
\hline 5.0 & 2677.2128 & 0.9943 & EKEVMSLLHNLEIKNTEHCQK \\
\hline 4.6 & 2677.1966 & 1.0105 & VTYDMTEGDRGFFEIDQVNGEVR \\
\hline 4.6 & 2677.1813 & 1.0258 & DNYVPEVSVLDQEITKVEPDTK \\
\hline 4.4 & 2676.2126 & 1.9945 & VTYDMTEGDRGFFEIDQVNGEVR \\
\hline 3.7 & 2677.2218 & 0.9853 & FKPEIMPDDSVDEFDETIHLER \\
\hline 2.1 & 2676.1847 & 2.0224 & APYHGSRAGIATQFSTANGQVNLR \\
\hline 2.1 & 2676.1847 & 2.0224 & APYHGSRAGIATOFSTANGOVNLR \\
\hline
\end{tabular}


Spectrum No: 64; Query: 1570; Rank: 1

\section{Peptide View}

MS/MS Fragmentation of TNSTCSPNLDACLVAVSGK

Found in IPI00195173, Tax_Id=10116 Gene_Symbol=Cd59 CD59 glycoprotein precursor

Match to Query 1570: 1993.913468 from(997.964010,2+)

Title: 100101RatKid_NS_deglyco_25.2956.2956.2.dta

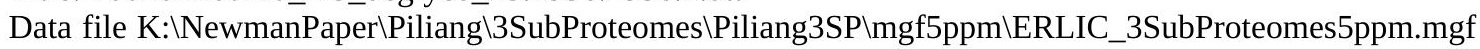
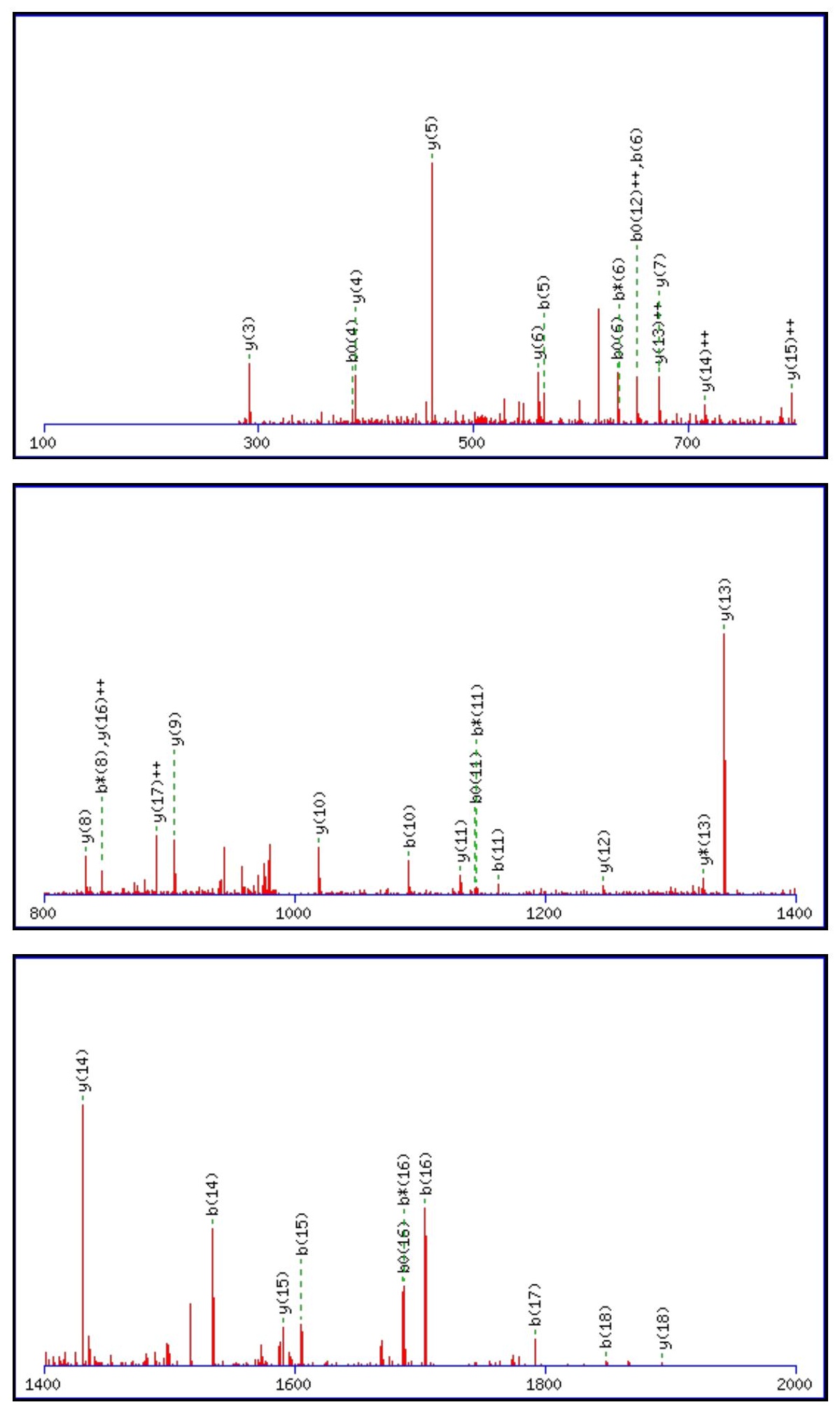
Monoisotopic mass of neutral peptide $\operatorname{Mr}($ calc): 1993.9085

Fixed modifications: Carbamidomethyl (C)

Variable modifications:

N2 : Deamidated_N (N)

Ions Score: 104 Expect: $1 \mathrm{e}-008$

Matches (Bold Red): 38/210 fragment ions using 53 most intense peaks

\begin{tabular}{|c|c|c|c|c|c|c|c|c|c|c|c|c|c|c|}
\hline \# & b & $\mathbf{b}^{++}$ & b* & & $\mathbf{b}^{0}$ & $\mathbf{b}^{0++}$ & Seq. & $\mathbf{y}$ & & $\mathbf{y}^{*}$ & & $\mathbf{y}^{\mathbf{0}}$ & & \# \\
\hline 1 & 102.0550 & 51.5311 & & & 84.0444 & 42.5258 & $\mathbf{T}$ & & & & & & & 19 \\
\hline 2 & 217.0819 & 109.0446 & 200.0553 & 100.5313 & 199.0713 & 100.0393 & $\mathbf{N}$ & 1893.8681 & 947.4377 & 1876.8415 & 938.9244 & 1875.8575 & 938.4324 & 18 \\
\hline 3 & 304.1139 & 152.5606 & 287.0874 & 144.0473 & 286.1034 & 143.5553 & $S$ & 1778.8411 & 889.9242 & 1761.8146 & 881.4109 & 1760.8306 & 880.9189 & 17 \\
\hline 4 & 405.1616 & 203.0844 & 388.1350 & 194.5712 & 387.1510 & 194.0792 & $\mathbf{T}$ & 1691.8091 & 846.4082 & 1674.7826 & 837.8949 & 1673.7986 & 837.4029 & 16 \\
\hline 5 & 565.1922 & 283.0998 & 548.1657 & 274.5865 & 547.1817 & 274.0945 & $\mathrm{C}$ & 1590.7614 & 795.8844 & 1573.7349 & 787.3711 & 1572.7509 & 786.8791 & 15 \\
\hline 6 & 652.2243 & 326.6158 & 635.1977 & 318.1025 & 634.2137 & 317.6105 & $\mathrm{~S}$ & 1430.7308 & 715.8690 & 1413.7042 & 707.3558 & 1412.7202 & 706.8638 & 14 \\
\hline 7 & 749.2770 & 375.1422 & 732.2505 & 366.6289 & 731.2665 & 366.1369 & $\mathbf{P}$ & 1343.6988 & 672.3530 & 1326.6722 & 663.8397 & 1325.6882 & 663.3477 & 13 \\
\hline 8 & 863.3200 & 432.1636 & 846.2934 & 423.6503 & 845.3094 & 423.1583 & $\mathbf{N}$ & 1246.6460 & 623.8266 & 1229.6195 & 615.3134 & 1228.6354 & 614.8214 & 12 \\
\hline 9 & 976.4040 & 488.7057 & 959.3775 & 480.1924 & 958.3935 & 479.7004 & $\mathbf{L}$ & 1132.6031 & 566.8052 & 1115.5765 & 558.2919 & 1114.5925 & 557.7999 & 11 \\
\hline 10 & 1091.4310 & 546.2191 & 1074.4044 & 537.7059 & 1073.4204 & 537.2138 & D & 1019.5190 & 510.2631 & 1002.4925 & 501.7499 & 1001.5084 & 501.2579 & 10 \\
\hline 11 & 1162.4681 & 581.7377 & 1145.4415 & 573.2244 & 1144.4575 & 572.7324 & A & 904.4921 & 452.7497 & 887.4655 & 444.2364 & 886.4815 & 443.7444 & 9 \\
\hline 12 & 1322.4987 & 661.7530 & 1305.4722 & 653.2397 & 1304.4882 & 652.7477 & $\mathrm{C}$ & 833.4550 & 417.2311 & 816.4284 & 408.7178 & 815.4444 & 408.2258 & 8 \\
\hline 13 & 1435.5828 & 718.2950 & 1418.5563 & 709.7818 & 1417.5722 & 709.2898 & $\mathbf{L}$ & 673.4243 & 337.2158 & 656.3978 & 328.7025 & 655.4137 & 328.2105 & 7 \\
\hline 14 & 1534.6512 & 767.8292 & 1517.6247 & 759.3160 & 1516.6406 & 758.8240 & $\mathbf{V}$ & 560.3402 & 280.6738 & 543.3137 & 272.1605 & 542.3297 & 271.6685 & 6 \\
\hline 15 & 1605.6883 & 803.3478 & 1588.6618 & 794.8345 & 1587.6778 & 794.3425 & A & 461.2718 & 231.1396 & 444.2453 & 222.6263 & 443.2613 & 222.1343 & 5 \\
\hline 16 & 1704.7567 & 852.8820 & 1687.7302 & 844.3687 & 1686.7462 & 843.8767 & $\mathbf{V}$ & 390.2347 & 195.6210 & 373.2082 & 187.1077 & 372.2241 & 186.6157 & 4 \\
\hline 17 & $\mathbf{1 7 9 1 . 7 8 8 8}$ & 896.3980 & 1774.7622 & 887.8847 & 1773.7782 & 887.3927 & $\mathrm{~S}$ & 291.1663 & 146.0868 & 274.1397 & 137.5735 & 273.1557 & 137.0815 & 3 \\
\hline 18 & 1848.8102 & 924.9088 & 1831.7837 & 916.3955 & 1830.7997 & 915.9035 & G & 204.1343 & 102.5708 & 187.1077 & 94.0575 & & & 2 \\
\hline 19 & & & & & & & $\mathbf{K}$ & 147.1128 & 74.0600 & 130.0863 & 65.5468 & & & 1 \\
\hline
\end{tabular}
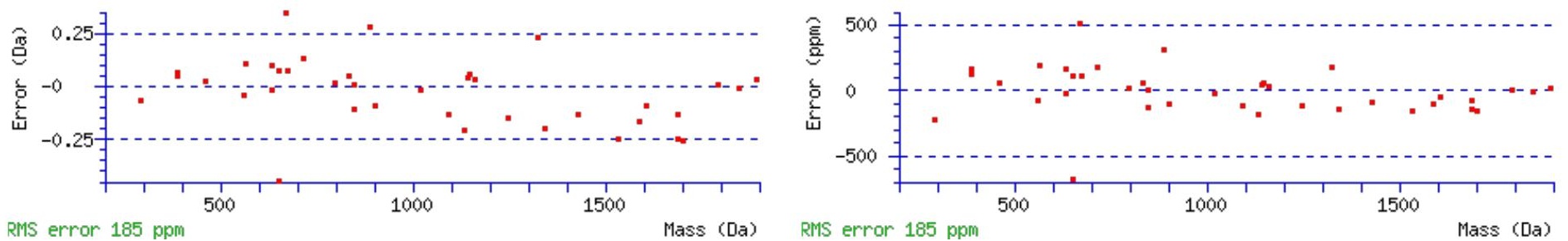

All matches to this query

\begin{tabular}{|l|l|l|l|}
\hline Score & Mr(calc): & Delta & \multicolumn{1}{c|}{ Sequence } \\
\hline 104.0 & 1993.9085 & 0.0050 & TNSTCSPNLDACLVAVSGK \\
\hline 84.1 & 1993.9085 & 0.0050 & TNSTCSPNLDACLVAVSGK \\
\hline 70.5 & 1992.9245 & 0.9890 & TNSTCSPNLDACLVAVSGK \\
\hline 3.2 & 1991.8924 & 2.0211 & NLHDAICAVNSLHRYK \\
\hline 0.8 & 1993.9285 & -0.0151 & EGDATPDNIPADIVFVLK \\
\hline 0.1 & 1993.9267 & -0.0132 & $\underline{\text { RHLLEALWLSCGGAGMK }}$ \\
\hline
\end{tabular}

Spectrum No: 65; Query: 1494; Rank: 1

\section{Peptide View}

MS/MS Fragmentation of DQDPASFGEDSLLLNSSR

Found in IPI00193894, Tax_Id=10116 Gene_Symbol=Mgam_predicted 198 kDa protein

Match to Query 1494: 1950.886088 from(976.450320,2+) 
Title: 100101RatKid_NS_deglyco_20.3676.3676.2.dta

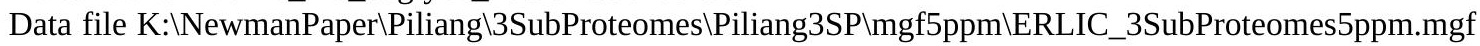
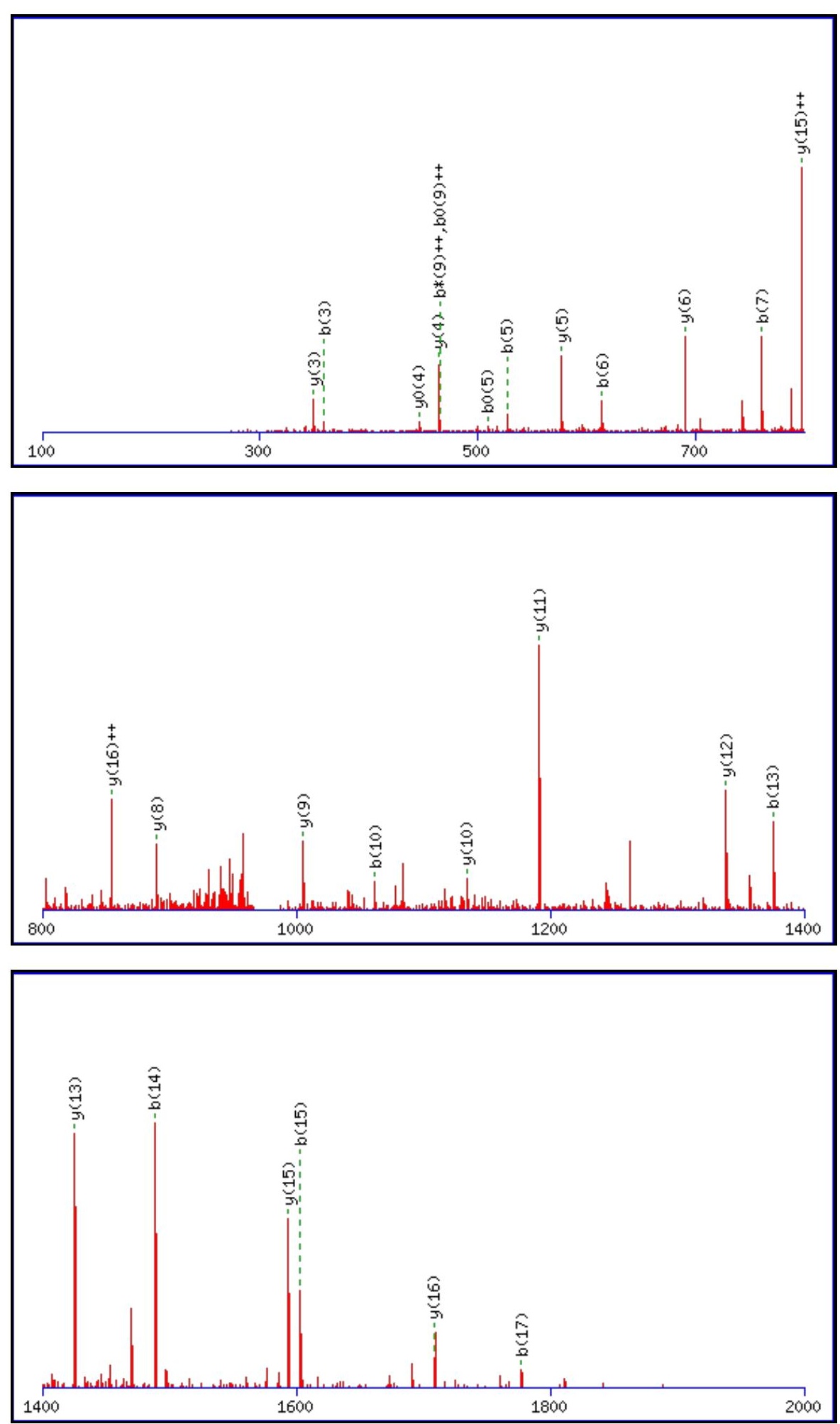

Monoisotopic mass of neutral peptide $\operatorname{Mr}($ calc): 1950.8807

Fixed modifications: Carbamidomethyl (C)

Variable modifications:

N15 : Deamidated $\mathrm{N}(\mathrm{N})$

Ions Score: 103 Expect: $1.3 \mathrm{e}-008$

Matches (Bold Red): 27/200 fragment ions using 39 most intense peaks

\begin{tabular}{|c|c|c|c|c|r|r|r|r|c|c|c|c|c|c|}
\hline$\#$ & $\mathbf{b}$ & $\mathbf{b}^{++}$ & $\mathbf{b}^{*}$ & $\mathbf{b}^{\boldsymbol{*}^{++}}$ & \multicolumn{1}{|c|}{$\mathbf{b}^{\mathbf{0}}$} & $\mathbf{b}^{\mathbf{0}+}$ & Seq. & $\mathbf{y}$ & $\mathbf{y}^{++}$ & $\mathbf{y}^{*}$ & $\mathbf{y}^{\boldsymbol{*}^{++}}$ & $\mathbf{y}^{\mathbf{0}}$ & $\mathbf{y}^{\mathbf{0}++}$ & $\#$ \\
\hline $\mathbf{1}$ & 116.0342 & 58.5207 & & & 98.0237 & 49.5155 & $\mathbf{D}$ & & & & & & & $\mathbf{1 8}$ \\
\hline $\mathbf{2}$ & 244.0928 & 122.5500 & 227.0662 & 114.0368 & 226.0822 & 113.5448 & $\mathbf{Q}$ & 1836.8610 & 918.9341 & 1819.8345 & 910.4209 & 1818.8504 & 909.9289 & $\mathbf{1 7}$ \\
\hline $\mathbf{3}$ & 359.1197 & 180.0635 & 342.0932 & 171.5502 & 341.1092 & 171.0582 & $\mathbf{D}$ & $\mathbf{1 7 0 8 . 8 0 2 4}$ & $\mathbf{8 5 4 . 9 0 4 9}$ & 1691.7759 & 846.3916 & 1690.7919 & 845.8996 & $\mathbf{1 6}$ \\
\hline
\end{tabular}




\begin{tabular}{|r|r|r|r|r|r|r|r|r|r|r|r|r|r|r|r|r|r|}
$\mathbf{4}$ & 456.1725 & 228.5899 & 439.1460 & 220.0766 & 438.1619 & 219.5846 & $\mathbf{P}$ & $\mathbf{1 5 9 3 . 7 7 5 5}$ & $\mathbf{7 9 7 . 3 9 1 4}$ & 1576.7489 & 788.8781 & 1575.7649 & 788.3861 & $\mathbf{1 5}$ \\
\hline $\mathbf{5}$ & $\mathbf{5 2 7 . 2 0 9 6}$ & 264.1084 & 510.1831 & 255.5952 & $\mathbf{5 0 9 . 1 9 9 1}$ & 255.1032 & A & 1496.7227 & 748.8650 & 1479.6962 & 740.3517 & 1478.7122 & 739.8597 & $\mathbf{1 4}$ \\
\hline $\mathbf{6}$ & $\mathbf{6 1 4 . 2 4 1 6}$ & 307.6245 & 597.2151 & 299.1112 & 596.2311 & 298.6192 & S & $\mathbf{1 4 2 5 . 6 8 5 6}$ & 713.3464 & 1408.6591 & 704.8332 & 1407.6750 & 704.3412 & $\mathbf{1 3}$ \\
\hline $\mathbf{7}$ & $\mathbf{7 6 1 . 3 1 0 1}$ & 381.1587 & 744.2835 & 372.6454 & 743.2995 & 372.1534 & F & $\mathbf{1 3 3 8 . 6 5 3 6}$ & 669.8304 & 1321.6270 & 661.3172 & 1320.6430 & 660.8251 & $\mathbf{1 2}$ \\
\hline $\mathbf{8}$ & 818.3315 & 409.6694 & 801.3050 & 401.1561 & 800.3210 & 400.6641 & G & $\mathbf{1 1 9 1 . 5 8 5 2}$ & 596.2962 & 1174.5586 & 587.7829 & 1173.5746 & 587.2909 & $\mathbf{1 1}$ \\
\hline $\mathbf{9}$ & 947.3741 & 474.1907 & 930.3476 & $\mathbf{4 6 5 . 6 7 7 4}$ & 929.3636 & $\mathbf{4 6 5 . 1 8 5 4}$ & E & $\mathbf{1 1 3 4 . 5 6 3 7}$ & 567.7855 & 1117.5372 & 559.2722 & 1116.5531 & 558.7802 & $\mathbf{1 0}$ \\
\hline $\mathbf{1 0}$ & $\mathbf{1 0 6 2 . 4 0 1 1}$ & 531.7042 & 1045.3745 & 523.1909 & 1044.3905 & 522.6989 & D & $\mathbf{1 0 0 5 . 5 2 1 1}$ & 503.2642 & 988.4946 & 494.7509 & 987.5105 & 494.2589 & $\mathbf{9 9}$ \\
\hline $\mathbf{1 1}$ & 1149.4331 & 575.2202 & 1132.4065 & 566.7069 & 1131.4225 & 566.2149 & S & $\mathbf{8 9 0 . 4 9 4 2}$ & 445.7507 & 873.4676 & 437.2374 & 872.4836 & 436.7454 & $\mathbf{8}$ \\
\hline $\mathbf{1 2}$ & 1262.5172 & 631.7622 & 1245.4906 & 623.2489 & 1244.5066 & 622.7569 & L & 803.4621 & 402.2347 & 786.4356 & 393.7214 & 785.4516 & 393.2294 & $\mathbf{7}$ \\
\hline $\mathbf{1 3}$ & $\mathbf{1 3 7 5 . 6 0 1 2}$ & 688.3042 & 1358.5747 & 679.7910 & 1357.5907 & 679.2990 & L & $\mathbf{6 9 0 . 3 7 8}$ & 345.6927 & 673.3515 & 337.1794 & 672.3675 & 336.6874 & $\mathbf{6}$ \\
\hline $\mathbf{1 4}$ & $\mathbf{1 4 8 8 . 6 8 5 3}$ & 744.8463 & 1471.6587 & 736.3330 & 1470.6747 & 735.8410 & L & 577.2940 & 289.1506 & 560.2675 & 280.6374 & 559.2834 & 280.1454 & $\mathbf{5}$ \\
\hline $\mathbf{1 5}$ & $\mathbf{1 6 0 3 . 7 1 2 2}$ & 802.3597 & 1586.6857 & 793.8465 & 1585.7017 & 793.3545 & N & $\mathbf{4 6 4 . 2 0 9 9}$ & 232.6086 & 447.1834 & 224.0953 & $\mathbf{4 4 6 . 1 9 9 4}$ & 223.6033 & $\mathbf{4}$ \\
\hline $\mathbf{1 6}$ & 1690.7442 & 845.8758 & 1673.7177 & 837.3625 & 1672.7337 & 836.8705 & S & 349.1830 & 175.0951 & 332.1565 & 166.5819 & 331.1724 & 166.0899 & $\mathbf{3}$ \\
\hline $\mathbf{1 7}$ & $\mathbf{1 7 7 7 . 7 7 6 3}$ & 889.3918 & 1760.7497 & 880.8785 & 1759.7657 & 880.3865 & S & 262.1510 & 131.5791 & 245.1244 & 123.0659 & 244.1404 & 122.5738 & $\mathbf{2}$ \\
\hline $\mathbf{1 8}$ & & & & & & & R & 175.1190 & 88.0631 & 158.0924 & 79.5498 & & & $\mathbf{1}$ \\
\hline
\end{tabular}
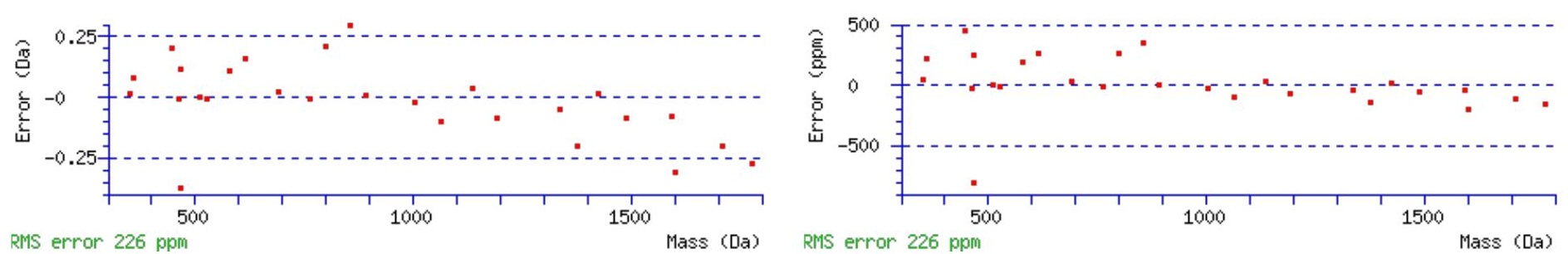

\section{All matches to this query}

\begin{tabular}{|l|l|l|l|}
\hline Score & Mr(calc): & Delta & \multicolumn{1}{|c|}{ Sequence } \\
\hline 103.1 & 1950.8807 & 0.0054 & DQDPASFGEDSLLLNSSR \\
\hline 24.8 & 1949.8967 & 0.9894 & DQDPASFGEDSLLLNSSR \\
\hline 7.9 & 1949.8983 & 0.9878 & QDAIVAVTGDGVNDSPALK \\
\hline 7.3 & 1950.9040 & -0.0179 & SSSMALKGHGGSFIQECR \\
\hline 4.5 & 1950.8740 & 0.0120 & SEYIIIPKSTLQEDR \\
\hline 4.5 & 1950.8740 & 0.0120 & SEYIIIPKSTLQEDR \\
\hline 3.5 & 1950.8838 & 0.0023 & EFIGSFLEMFGPEGALK \\
\hline 3.5 & 1949.8983 & 0.9878 & QDAIVAVTGDGVNDSPALK \\
\hline 3.0 & 1950.8714 & 0.0147 & TPGNPVNSARKPGSAGGPK \\
\hline 2.6 & 1950.8737 & 0.0123 & RSLSPWGYSINHDPSR \\
\hline
\end{tabular}

Spectrum No: 66; Query: 2745; Rank: 1

\section{Peptide View}

MS/MS Fragmentation of NSTPSEPDSGQGPPPEEEEGEEEAAKEEAEAQGVR

Found in IPI00326566, Tax_Id=10116 Gene_Symbol=Hdgf Hepatoma-derived growth factor

Match to Query 2745: 3666.571992 from(1223.197940,3+)

Title: 100101RatKid_NS_deglyco_20.2758.2758.3.dta

Data file K:INewmanPaper|Piliang|3SubProteomes\Piliang3SP\mgf5ppm\ERLIC_3SubProteomes5ppm.mgf 

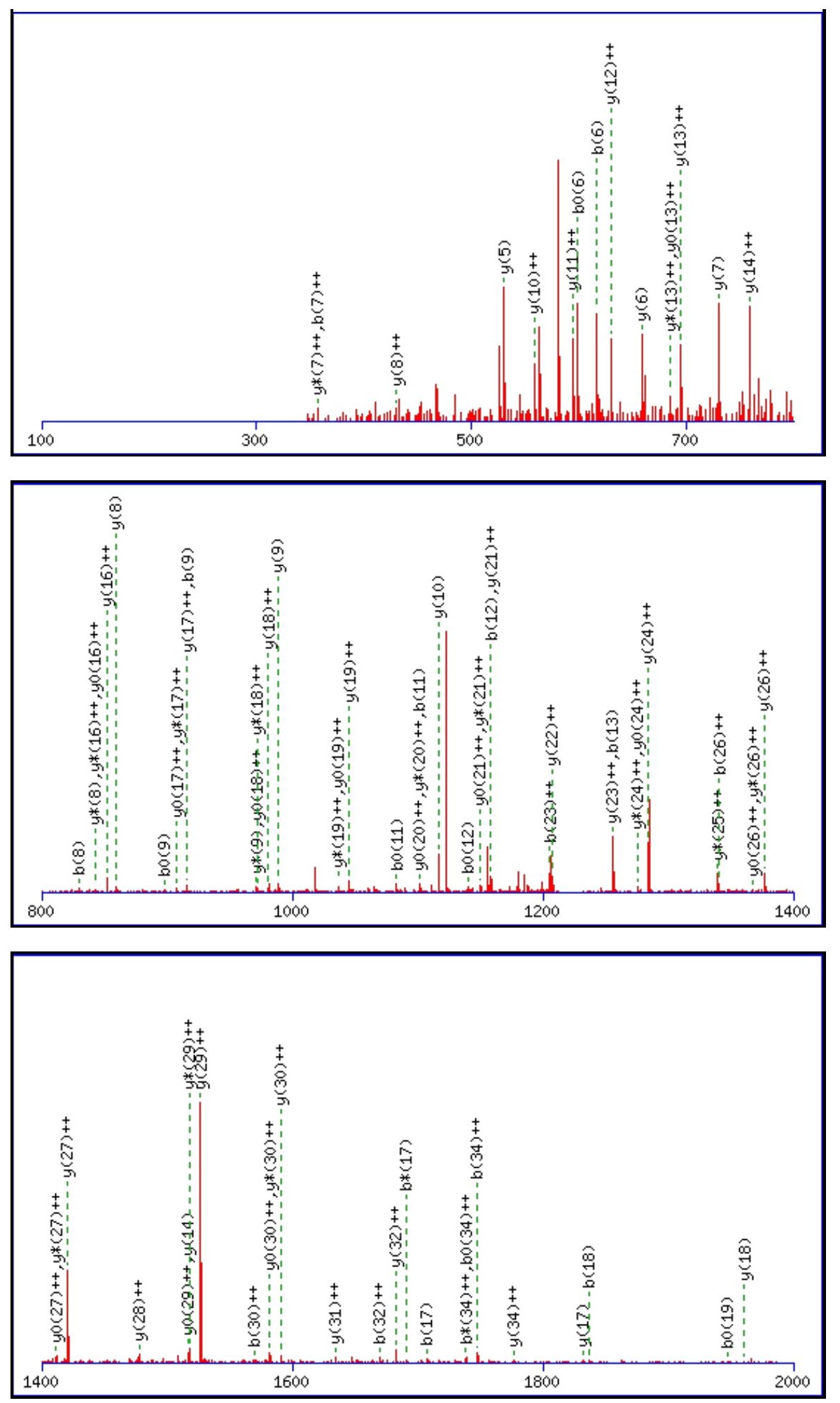

Monoisotopic mass of neutral peptide $\operatorname{Mr}($ calc): 3666.5564

Fixed modifications: Carbamidomethyl (C)

Variable modifications:

N1 : Deamidated $\mathrm{N}(\mathrm{N})$

Ions Score: 103 Expect: $2.7 \mathrm{e}-008$

Matches (Bold Red): 81/396 fragment ions using 140 most intense peaks

\begin{tabular}{|c|c|c|c|c|c|c|c|c|c|c|c|c|c|c|}
\hline \# & b & $\mathbf{b}^{++}$ & $\mathbf{b}^{*}$ & $\mathbf{b}^{*^{++}}$ & $\mathbf{b}^{0}$ & $\mathbf{b}^{0++}$ & Seq. & $\mathbf{y}$ & $y^{++}$ & $\mathrm{y}^{*}$ & $\mathrm{y}^{\mathrm{*}^{++}}$ & $\mathbf{y}^{0}$ & $y^{0++}$ & $\#$ \\
\hline 1 & 116.0342 & 58.5207 & 99.0077 & 50.0075 & & & $\mathbf{N}$ & & & & & & & 35 \\
\hline 2 & 203.0662 & 102.0368 & 186.0397 & 93.5235 & 185.0557 & 93.0315 & $S$ & 3552.5368 & 1776.7721 & 3535.5103 & 1768.2588 & 3534.5263 & 1767.7668 & 34 \\
\hline 3 & 304.1139 & 152.5606 & 287.0874 & 144.0473 & 286.1034 & 143.5553 & $\mathbf{T}$ & 3465.5048 & 1733.2560 & 3448.4783 & 1724.7428 & 3447.4943 & 1724.2508 & 33 \\
\hline 4 & 401.1667 & 201.0870 & 384.1401 & 192.5737 & 383.1561 & 192.0817 & $\mathbf{P}$ & 3364.4571 & 1682.7322 & 3347.4306 & 1674.2189 & 3346.4466 & 1673.7269 & 32 \\
\hline 5 & 488.1987 & 244.6030 & 471.1722 & 236.0897 & 470.1881 & 235.5977 & $S$ & 3267.4044 & 1634.2058 & 3250.3778 & 1625.6926 & 3249.3938 & 1625.2005 & 31 \\
\hline
\end{tabular}




\begin{tabular}{|c|c|c|c|c|c|c|c|c|c|c|c|c|c|c|}
\hline 6 & 617.2413 & 309.1243 & 600.2148 & 300.6110 & 599.2307 & 300.1190 & $\mathbf{E}$ & 3180.3723 & $\mid$ & 3163.3458 & |1582.1765 & |3162.3618 & |1581.6845 & 30 \\
\hline 7 & 714.2941 & 357.6507 & 697.2675 & 349.1374 & 696.2835 & 348.6454 & $\mathbf{P}$ & 3051.3298 & 1526.1685 & 3034.3032 & 1517.6552 & 3033.3192 & 1517.1632 & 29 \\
\hline 8 & 829.3210 & 415.1641 & 812.2945 & 406.6509 & 811.3104 & 406.1589 & D & 2954.2770 & 1477.6421 & 2937.2504 & 1469.1289 & 2936.2664 & 1468.6369 & 28 \\
\hline 9 & 916.3530 & 458.6802 & 899.3265 & 450.1669 & 898.3425 & 449.6749 & S & 2839.2500 & 1420.1287 & 2822.2235 & 1411.6154 & 2821.2395 & 1411.1234 & 27 \\
\hline 10 & 973.3745 & 487.1909 & 956.3480 & 478.6776 & 955.3639 & 478.1856 & G & 2752.2180 & 1376.6126 & 2735.1915 & 1368.0994 & 2734.2075 & 1367.6074 & 26 \\
\hline 1 & 1101.4331 & 551.2202 & 1084.4065 & 542.7069 & 1083.4225 & 542.2149 & $\mathbf{Q}$ & 2695.1966 & 1348.1019 & 2678.1700 & 1339.5886 & 2677.1860 & 1339.0966 & 25 \\
\hline 12 & 1158.4545 & 579.7309 & 1141.4280 & 571.2176 & 1140.4440 & 570.7256 & G & 2567.1380 & 1284.0726 & 2550.1114 & 1275.5594 & 2549.1274 & 1275.0673 & 24 \\
\hline 3 & 1255.5073 & 628.2573 & 1238.4808 & 619.7440 & 1237.4967 & 619.2520 & $\mathbf{P}$ & 2510.1165 & 1255.5619 & 2493.0900 & 1247.0486 & 2492.1059 & 1246.5566 & 23 \\
\hline 14 & 1352.5601 & 676.7837 & 1335.5335 & 668.2704 & 1334.5495 & 667.7784 & $\mathbf{P}$ & 2413.0637 & 1207.0355 & 2396.0372 & 1198.5222 & 2395.0532 & 1198.0302 & 22 \\
\hline 15 & 1449.6128 & 725.3101 & 1432.5863 & 716.7968 & 1431.6023 & 716.3048 & $\mathbf{P}$ & 2316.0110 & 1158.5091 & 2298.9844 & 1149.9959 & 2298.0004 & 1149.5038 & 21 \\
\hline 16 & 1578.6554 & 789.8314 & 1561.6289 & 781.3181 & 1560.6449 & 780.8261 & $\mathbf{E}$ & 2218.9582 & 1109.9827 & 2201.9317 & 1101.4695 & 2200.9477 & 1100.9775 & 20 \\
\hline 17 & 1707.6980 & 854.3526 & 1690.6715 & 845.8394 & 1689.6875 & 845.3474 & $E$ & 2089.9156 & 1045.4615 & 2072.8891 & 1036.9482 & 2071.9051 & 1036.4562 & 19 \\
\hline 18 & 1836.7406 & 918.8739 & 1819.7141 & 910.3607 & 1818.7301 & 909.8687 & $\mathbf{E}$ & 1960.8730 & 980.9402 & 1943.8465 & 972.4269 & 1942.8625 & 971.9349 & 18 \\
\hline 19 & 1965.7832 & 983.3952 & 1948.7567 & 974.8820 & 1947.7726 & 974.3900 & $\mathbf{E}$ & 1831.8304 & 916.4189 & 1814.8039 & 907.9056 & 1813.8199 & 907.4136 & 17 \\
\hline 20 & 2022.8047 & 1011.9060 & 2005.7781 & 1003.3927 & 2004.7941 & 1002.9007 & $\mathbf{G}$ & 1702.7878 & 851.8976 & 1685.7613 & 843.3843 & 1684.7773 & 842.8923 & 16 \\
\hline 21 & 2151.8473 & 1076.4273 & 2134.8207 & 1067.9140 & 2133.8367 & 1067.4220 & $\mathbf{E}$ & 1645.7664 & 823.3868 & 1628.7398 & 814.8736 & 1627.7558 & 814.3815 & 15 \\
\hline 22 & 2280.8899 & 1140.9486 & 2263.8633 & 1132.4353 & 2262.8793 & 1131.9433 & $\mathbf{E}$ & 1516.7238 & 758.8655 & 1499.6972 & 750.3523 & 1498.7132 & 749.8603 & 14 \\
\hline 23 & 2409.9325 & 1205.4699 & 2392.9059 & 1196.9566 & 2391.9219 & 1196.4646 & $E$ & 1387.6812 & 694.3442 & 1370.6547 & 685.8310 & 1369.6706 & 685.3390 & 13 \\
\hline 24 & 2480.9696 & 1240.9884 & 2463.9430 & 1232.4751 & 2462.9590 & 1231.9831 & $\mathbf{A}$ & 1258.6386 & 629.8229 & 1241.6121 & 621.3097 & 1240.6280 & 620.8177 & 12 \\
\hline 25 & 2552.0067 & 1276.5070 & 2534.9801 & 1267.9937 & 2533.9961 & 1267.5017 & A & 1187.6015 & 594.3044 & 1170.5749 & 585.7911 & 1169.5909 & 585.2991 & 11 \\
\hline 26 & 2680.1016 & 1340.5545 & 2663.0751 & 1332.0412 & 2662.0911 & 1331.5492 & K & 1116.5644 & 558.7858 & 1099.5378 & 550.2726 & 1098.5538 & 549.7805 & 10 \\
\hline 27 & 2809.1442 & 1405.0758 & 2792.1177 & 1396.5625 & 2791.1337 & 1396.0705 & $\mathbf{E}$ & 988.4694 & 494.7383 & 971.4429 & 486.2251 & 970.4589 & 485.7331 & 9 \\
\hline 28 & 2938.1868 & 1469.5971 & 2921.1603 & 1461.0838 & 2920.1763 & 1460.5918 & $\mathbf{E}$ & 859.4268 & 430.2170 & 842.4003 & 421.7038 & 841.4163 & 421.2118 & 8 \\
\hline 29 & 3009.2239 & 1505.1156 & 2992.1974 & 1496.6023 & 2991.2134 & 1496.1103 & A & 730.3842 & 365.6958 & 713.3577 & 357.1825 & 712.3737 & 356.6905 & 7 \\
\hline 30 & 3138.2665 & \begin{tabular}{|l}
1569.6369 \\
\end{tabular} & 3121.2400 & 1561.1236 & 3120.2560 & 1560.6316 & $\mathbf{E}$ & 659.3471 & 330.1772 & 642.3206 & 321.6639 & 641.3366 & 321.1719 & 6 \\
\hline 31 & 3209.3037 & 1605.1555 & 3192.2771 & 1596.6422 & 3191.2931 & 1596.1502 & $\mathbf{A}$ & 530.3045 & 265.6559 & 513.2780 & 257.1426 & & & 5 \\
\hline 32 & 3337.3622 & 1669.1848 & 3320.3357 & 1660.6715 & 3319.3517 & 1660.1795 & $\mathbf{Q}$ & 459.2674 & 230.1373 & 442.2409 & 221.6241 & & & 4 \\
\hline 33 & 3394.3837 & 1697.6955 & 3377.3571 & 1689.1822 & 3376.3731 & 1688.6902 & $\mathbf{G}$ & 331.2088 & 166.1081 & 314.1823 & 157.5948 & & & 3 \\
\hline 34 & 3493.4521 & 1747.2297 & 3476.4256 & 1738.7164 & 3475.4415 & 1738.2244 & $\mathbf{V}$ & 274.1874 & 137.5973 & 257.1608 & 129.0840 & & & 2 \\
\hline 35 & & & & & & & $\mathbf{R}$ & 175.1190 & 88.0631 & 158.0924 & 79.5498 & & & 1 \\
\hline
\end{tabular}
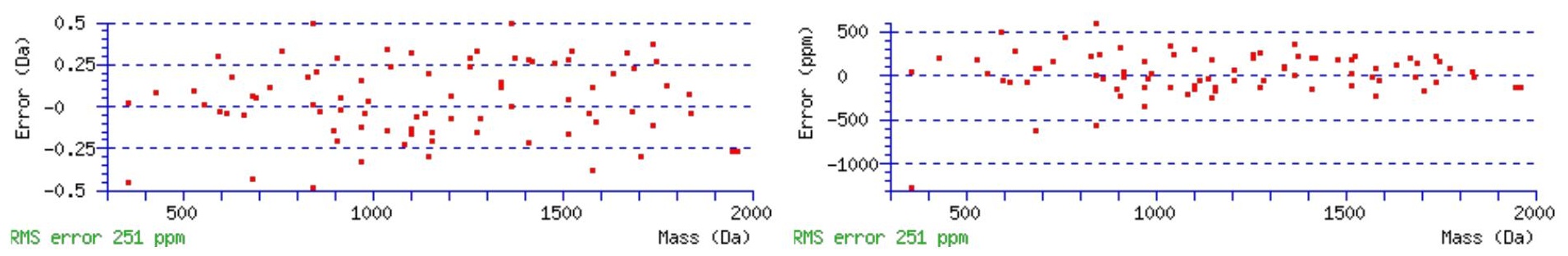

\section{All matches to this query}

\begin{tabular}{|l|l|l|l|}
\hline Score & Mr(calc): & Delta & \multicolumn{1}{c|}{ Sequence } \\
\hline 102.6 & 3666.5564 & 0.0156 & NSTPSEPDSGQGPPPEEEEGEEEAAKEEAEAQGVR \\
\hline 86.6 & 3665.5724 & 0.9996 & NSTPSEPDSGQGPPPEEEEGEEEAAKEEAEAQGVR \\
\hline 8.4 & 3665.5727 & 0.9993 & YFLTAVSRPGLGEPWFIIVGYVDDTEFVR \\
\hline 8.4 & 3665.5727 & 0.9993 & YFLTAVSRPGLGEPWFIIVGYVDDTEFVR \\
\hline 8.3 & 3666.5677 & 0.0043 & VYLIRGLSFETTDESLKTMENTSNFLVMR \\
\hline 8.2 & 3665.5836 & 0.9883 & VYLIRGLSFETTDESLKTMENTSNFLVMR \\
\hline 8.2 & 3666.5677 & 0.0043 & VYLIRGLSFETTDESLKTMENTSNFLVMR \\
\hline 6.9 & 3666.5723 & -0.0004 & EVGGTNPQQHRGQKPESENVGDETSDIENLLK \\
\hline 5.5 & 3666.5779 & -0.0059 & IYYIHTAEDEVKMDSFEFQVTDGRNPVFR \\
\hline 5.4 & 3665.5940 & 0.9780 & YGDLLPADGVLIQGNDLKIDESSLTGESDHVR \\
\hline
\end{tabular}


Spectrum No: 67; Query: 2088; Rank: 1

\section{Peptide View}

MS/MS Fragmentation of YLQPLLAVQFTNLTLDTEIR

Found in IPI00339124, Tax_Id=10116 Gene_Symbol=Atp1b1 Sodium/potassium-transporting ATPase subunit beta-1

Match to Query 2088: 2348.267428 from(1175.140990,2+)

Title: 091008RatKidney_NH4Format01_25.4494.4494.2.dta

Data file K:INewmanPaper|Piliang|3SubProteomes\Piliang3SP\mgf5ppm\ERLIC_3SubProteomes5ppm.mgf
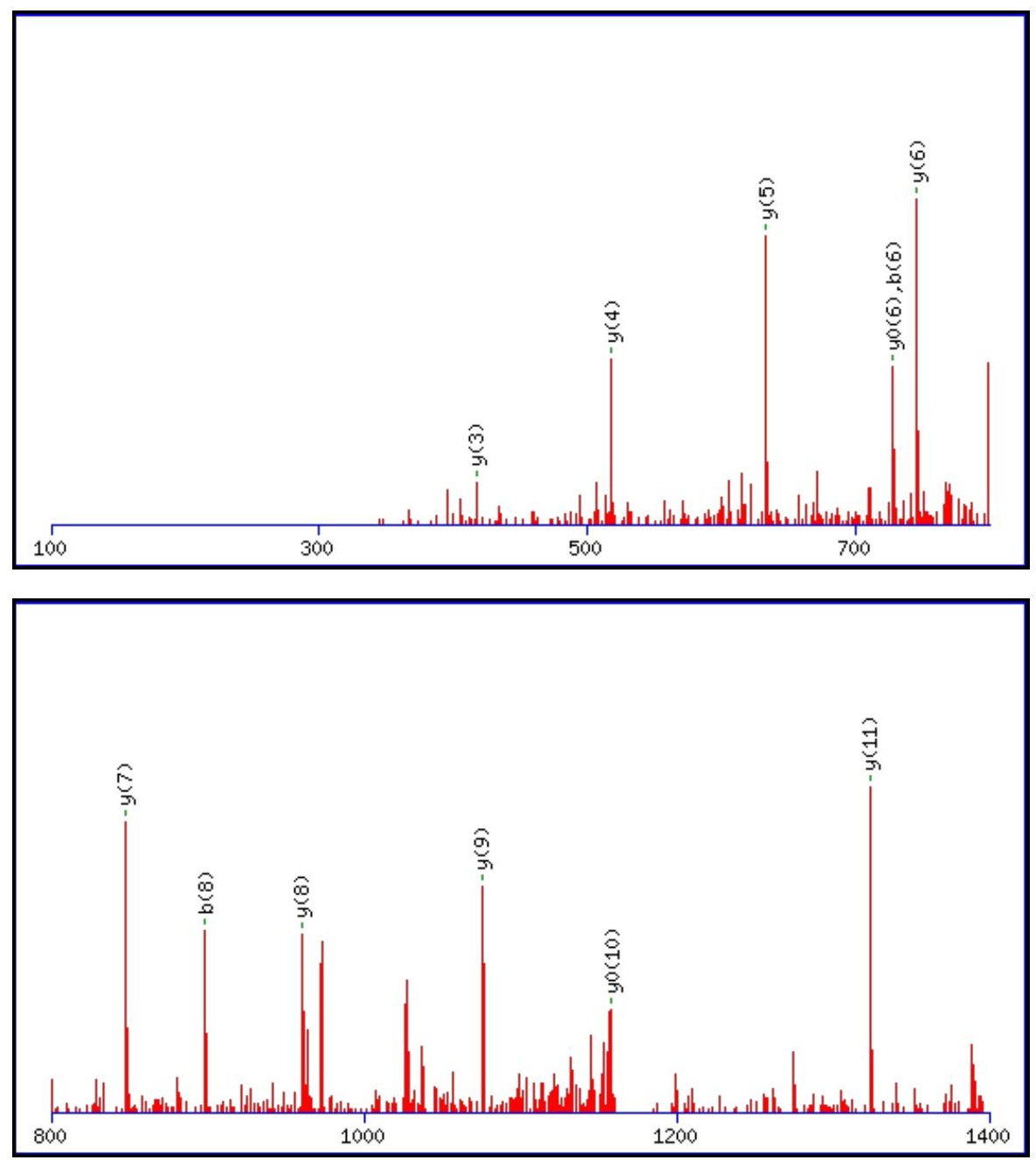


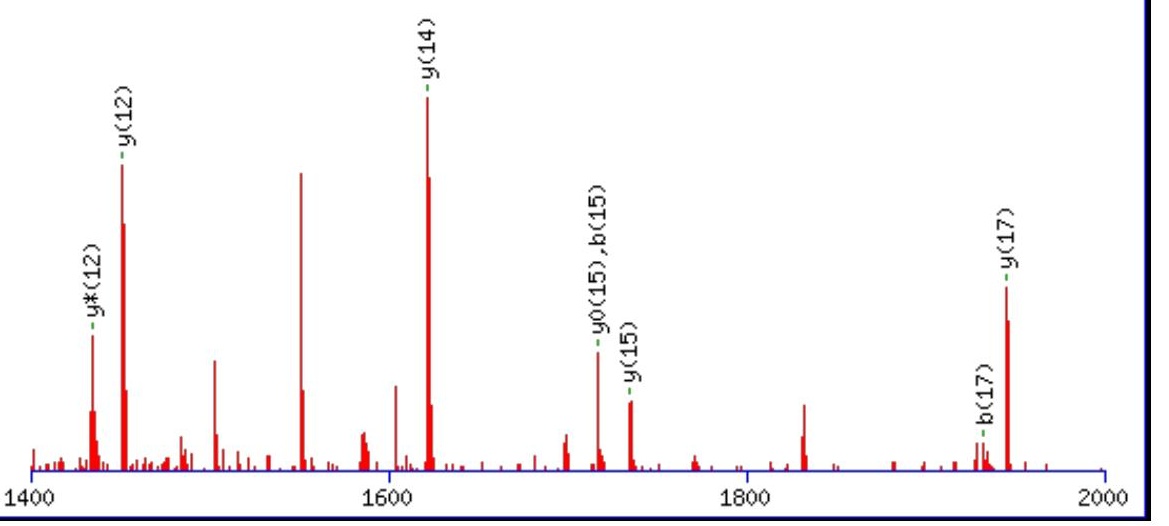

Monoisotopic mass of neutral peptide $\operatorname{Mr}($ calc): 2348.2627

Fixed modifications: Carbamidomethyl (C)

Variable modifications:

N12 : Deamidated $\mathrm{N}(\mathrm{N})$

Ions Score: 102 Expect: $3.6 \mathrm{e}-009$

Matches (Bold Red): 20/200 fragment ions using 23 most intense peaks

\begin{tabular}{|c|c|c|c|c|c|c|c|c|c|c|c|c|c|c|}
\hline \# & b & $\mathbf{b}^{++}$ & b* & $\mathbf{b}^{*++}$ & $\mathbf{b}^{\mathbf{0}}$ & $\mathbf{b}^{0++}$ & Seq. & $\mathbf{y}$ & $y^{++}$ & $\mathbf{y}^{*}$ & $\mathbf{y}^{*^{++}}$ & $\mathbf{y}^{0}$ & $\mathbf{y}^{0++}$ & \# \\
\hline 1 & 164.0706 & 82.5389 & & & & & $\mathbf{Y}$ & & & & & & & 20 \\
\hline 2 & 277.1547 & 139.0810 & & & & & $\mathbf{L}$ & 2186.2067 & 1093.6070 & 2169.1802 & 1085.0937 & 2168.1961 & 1084.6017 & 19 \\
\hline 3 & 405.2132 & 203.1103 & 388.1867 & 194.5970 & & & $\mathbf{Q}$ & 2073.1226 & 1037.0650 & 2056.0961 & 1028.5517 & 2055.1121 & 1028.0597 & 18 \\
\hline 4 & 502.2660 & 251.6366 & 485.2395 & 243.1234 & & & $\mathbf{P}$ & 1945.0641 & 973.0357 & 1928.0375 & 964.5224 & 1927.0535 & 964.0304 & 17 \\
\hline 5 & 615.3501 & 308.1787 & 598.3235 & 299.6654 & & & $\mathbf{L}$ & 1848.0113 & 924.5093 & 1830.9848 & 915.9960 & 1830.0007 & 915.5040 & 16 \\
\hline 6 & 728.4341 & 364.7207 & 711.4076 & 356.2074 & & & $\mathbf{L}$ & 1734.9272 & 867.9673 & 1717.9007 & 859.4540 & 1716.9167 & 858.9620 & 15 \\
\hline 7 & 799.4713 & 400.2393 & 782.4447 & 391.7260 & & & A & 1621.8432 & 811.4252 & 1604.8166 & 802.9120 & 1603.8326 & 802.4199 & 14 \\
\hline 8 & 898.5397 & 449.7735 & 881.5131 & 441.2602 & & & $\mathbf{V}$ & 1550.8061 & 775.9067 & 1533.7795 & 767.3934 & 1532.7955 & 766.9014 & 13 \\
\hline 9 & 1026.5982 & 513.8028 & 1009.5717 & 505.2895 & & & $\mathbf{Q}$ & 1451.7376 & 726.3725 & 1434.7111 & 717.8592 & 1433.7271 & 717.3672 & 12 \\
\hline 10 & 1173.6667 & 587.3370 & 1156.6401 & 578.8237 & & & $\mathbf{F}$ & 1323.6791 & 662.3432 & 1306.6525 & 653.8299 & 1305.6685 & 653.3379 & 11 \\
\hline 11 & 1274.7143 & 637.8608 & 1257.6878 & 629.3475 & 1256.7038 & 628.8555 & $\mathbf{T}$ & 1176.6107 & 588.8090 & 1159.5841 & 580.2957 & 1158.6001 & 579.8037 & 10 \\
\hline 12 & 1389.7413 & 695.3743 & 1372.7147 & 686.8610 & 1371.7307 & 686.3690 & $\mathbf{N}$ & 1075.5630 & 538.2851 & 1058.5364 & 529.7719 & 1057.5524 & 529.2798 & 9 \\
\hline 13 & 1502.8253 & 751.9163 & 1485.7988 & 743.4030 & 1484.8148 & 742.9110 & $\mathbf{L}$ & 960.5360 & 480.7717 & 943.5095 & 472.2584 & 942.5255 & 471.7664 & 8 \\
\hline 14 & 1603.8730 & 802.4401 & 1586.8465 & 793.9269 & 1585.8625 & 793.4349 & $\mathbf{T}$ & 847.4520 & 424.2296 & 830.4254 & 415.7164 & 829.4414 & 415.2243 & 7 \\
\hline 15 & 1716.9571 & 858.9822 & 1699.9305 & 850.4689 & 1698.9465 & 849.9769 & $\mathbf{L}$ & 746.4043 & 373.7058 & 729.3777 & 365.1925 & 728.3937 & 364.7005 & 6 \\
\hline 16 & 1831.9840 & 916.4956 & 1814.9575 & 907.9824 & 1813.9735 & 907.4904 & D & 633.3202 & 317.1638 & 616.2937 & 308.6505 & 615.3097 & 308.1585 & 5 \\
\hline 17 & 1933.0317 & 967.0195 & 1916.0052 & 958.5062 & 1915.0211 & 958.0142 & $\mathbf{T}$ & 518.2933 & 259.6503 & 501.2667 & 251.1370 & 500.2827 & 250.6450 & 4 \\
\hline 18 & 2062.0743 & 1031.5408 & 2045.0477 & 1023.0275 & 2044.0637 & 1022.5355 & $\mathbf{E}$ & 417.2456 & 209.1264 & 400.2191 & 200.6132 & 399.2350 & 200.1212 & 3 \\
\hline 19 & 2175.1584 & 1088.0828 & 2158.1318 & 1079.5695 & 2157.1478 & 1079.0775 & I & 288.2030 & 144.6051 & 271.1765 & 136.0919 & & & 2 \\
\hline 20 & & & & & & & $\mathbf{R}$ & 175.1190 & 88.0631 & 158.0924 & 79.5498 & & & 1 \\
\hline
\end{tabular}

告$$
\text { 要 }
$$
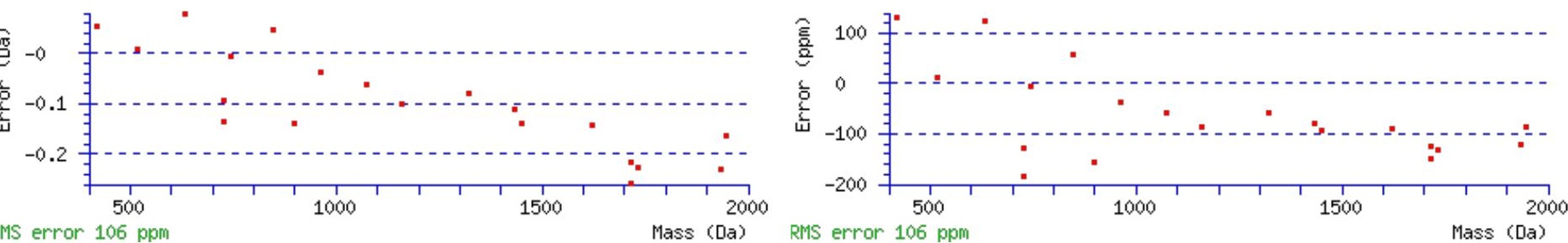

\section{All matches to this query}

\begin{tabular}{|l|l|l|l|}
\hline Score & Mr(calc): & Delta & Sequence \\
\hline 102.5 & 2348.2627 & 0.0047 & YLQPLLAVQFTNLTLDTEIR \\
\hline & & &
\end{tabular}




\section{\begin{tabular}{|l|l|l|l|}
23.2 & 2347.2787 & 0.9887 & YLQPLLAVQFTNLTLDTEIR \\
\hline 4.7 & 2348.2577 & 0.0098 & TLTSILVGLOGAARRESDLLR \\
\hline
\end{tabular}

\begin{tabular}{|l|l|l|l|}
\hline 4.7 & 2348.2577 & 0.0098 & TLTSILVGLQGAARRESDLLR \\
\hline
\end{tabular}

Spectrum No: 68; Query: 964; Rank: 1

\section{Peptide View}

MS/MS Fragmentation of NCNSTTEFTCSNGR

Found in IPI00205325, Tax_Id=10116 Gene_Symbol=Lrp2 Low-density lipoprotein receptor-related protein 2 precursor

Match to Query 964: 1647.632248 from(824.823400,2+)

Title: 100101RatKid_NS_deglyco_24.766.766.2.dta

Data file K:INewmanPaper|Piliang|3SubProteomes\Piliang3SP\mgf5ppm|ERLIC_3SubProteomes5ppm.mgf
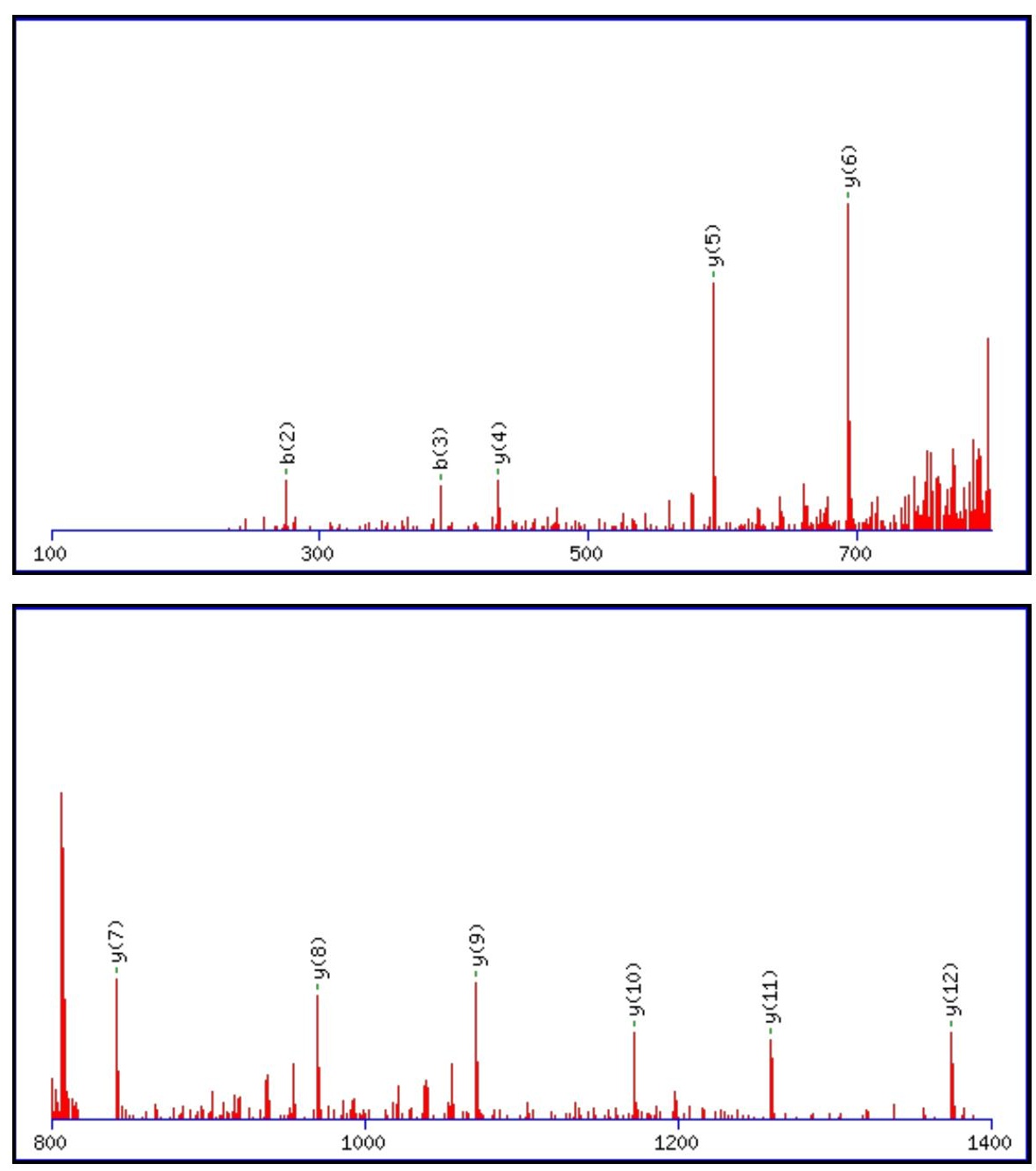


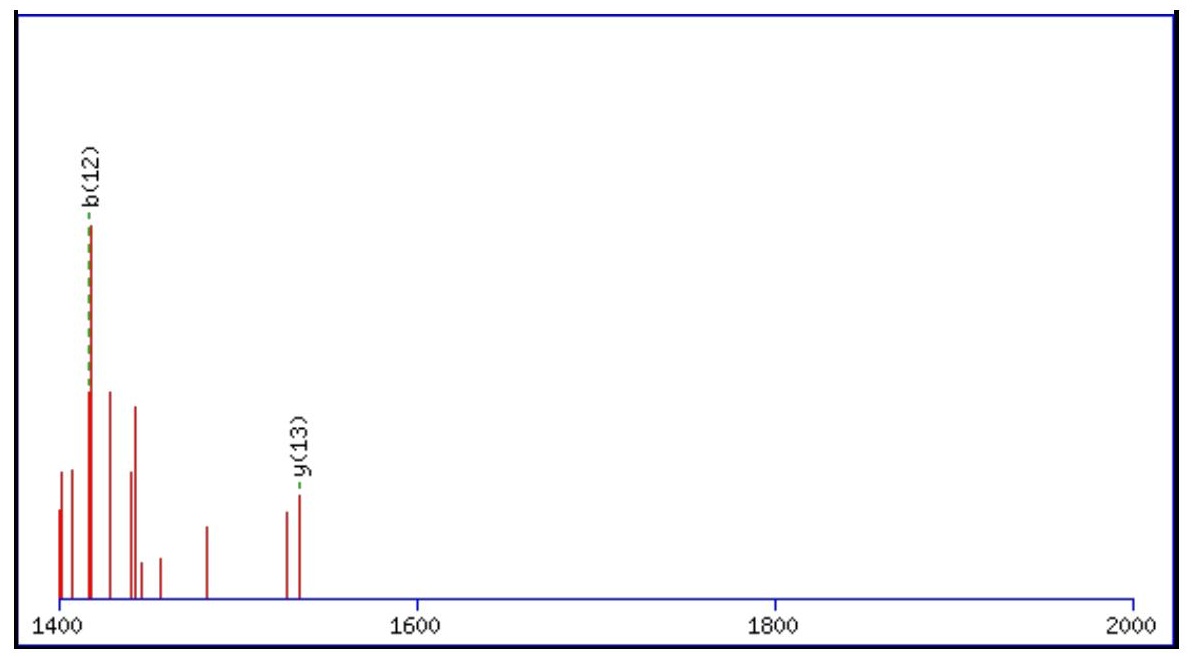

Monoisotopic mass of neutral peptide $\operatorname{Mr}($ calc): 1647.6253

Fixed modifications: Carbamidomethyl (C)

Variable modifications:

N3 : Deamidated $\mathrm{N}(\mathrm{N})$

Ions Score: 102 Expect: $7.4 \mathrm{e}-009$

Matches (Bold Red): 13/144 fragment ions using 21 most intense peaks

\begin{tabular}{|c|c|c|c|c|c|c|c|c|c|c|c|c|c|c|}
\hline \# & b & $\mathbf{b}^{++}$ & b* & $\mathbf{b}^{*^{++}}$ & $\mathbf{b}^{0}$ & $\mathbf{b}^{0++}$ & Seq. & $\mathbf{y}$ & $y^{++}$ & $\mathbf{y}^{*}$ & $\mathrm{y}^{\mathrm{*}^{++}}$ & $\mathbf{y}^{0}$ & $y^{0++}$ & \# \\
\hline 1 & 115.0502 & 58.0287 & 98.0237 & 49.5155 & & & $\mathbf{N}$ & & & & & & & 14 \\
\hline 2 & 275.0809 & 138.0441 & 258.0543 & 129.5308 & & & C & 1534.5897 & 767.7985 & 1517.5631 & 759.2852 & 1516.5791 & 758.7932 & 13 \\
\hline 3 & 390.1078 & 195.5575 & 373.0812 & 187.0443 & & & $\mathbf{N}$ & 1374.5590 & 687.7832 & 1357.5325 & 679.2699 & 1356.5485 & 678.7779 & 12 \\
\hline 4 & 477.1398 & 239.0735 & 460.1133 & 230.5603 & 459.1293 & 230.0683 & $\mathrm{~S}$ & 1259.5321 & 630.2697 & 1242.5055 & 621.7564 & 1241.5215 & 621.2644 & 11 \\
\hline 5 & 578.1875 & 289.5974 & 561.1609 & 281.0841 & 560.1769 & 280.5921 & $\mathbf{T}$ & 1172.5001 & 586.7537 & 1155.4735 & 578.2404 & 1154.4895 & 577.7484 & 10 \\
\hline 6 & 679.2352 & 340.1212 & 662.2086 & 331.6080 & 661.2246 & 331.1159 & $\mathbf{T}$ & 1071.4524 & 536.2298 & 1054.4258 & 527.7166 & 1053.4418 & 527.2245 & 9 \\
\hline 7 & 808.2778 & 404.6425 & 791.2512 & 396.1292 & 790.2672 & 395.6372 & $\mathbf{E}$ & 970.4047 & 485.7060 & 953.3782 & 477.1927 & 952.3941 & 476.7007 & 8 \\
\hline 8 & 955.3462 & 478.1767 & 938.3196 & 469.6635 & 937.3356 & 469.1714 & $\mathbf{F}$ & 841.3621 & 421.1847 & 824.3356 & 412.6714 & 823.3515 & 412.1794 & 7 \\
\hline 9 & 1056.3939 & 528.7006 & 1039.3673 & 520.1873 & 1038.3833 & 519.6953 & $\mathbf{T}$ & 694.2937 & 347.6505 & 677.2672 & 339.1372 & 676.2831 & 338.6452 & 6 \\
\hline 10 & 1216.4245 & 608.7159 & 1199.3980 & 600.2026 & 1198.4139 & 599.7106 & $\mathrm{C}$ & 593.2460 & 297.1266 & 576.2195 & 288.6134 & 575.2355 & 288.1214 & 5 \\
\hline 11 & 1303.4565 & 652.2319 & 1286.4300 & 643.7186 & 1285.4460 & 643.2266 & $\mathrm{~S}$ & 433.2154 & 217.1113 & 416.1888 & 208.5980 & 415.2048 & 208.1060 & 4 \\
\hline 12 & 1417.4995 & 709.2534 & 1400.4729 & 700.7401 & 1399.4889 & 700.2481 & $\mathbf{N}$ & 346.1833 & 173.5953 & 329.1568 & 165.0820 & & & 3 \\
\hline 13 & 1474.5209 & 737.7641 & 1457.4944 & 729.2508 & 1456.5104 & 728.7588 & G & 232.1404 & 116.5738 & 215.1139 & 108.0606 & & & 2 \\
\hline 14 & & & & & & & $\mathbf{R}$ & 175.1190 & 88.0631 & 158.0924 & 79.5498 & & & 1 \\
\hline
\end{tabular}
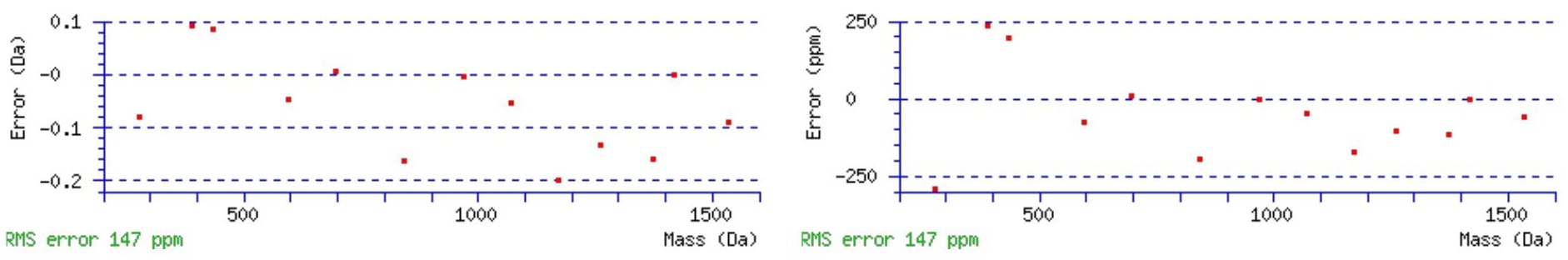

\section{All matches to this query}

\begin{tabular}{|l|l|l|l|}
\hline Score & Mr(calc): & Delta & \multicolumn{1}{c|}{ Sequence } \\
\hline 101.5 & 1647.6253 & 0.0069 & NCNSTTEFTCSNGR \\
\hline 62.0 & 1646.6413 & 0.9909 & NCNSTTEFTCSNGR \\
\hline 54.7 & 1647.6253 & 0.0069 & NCNSTTEFTCSNGR \\
\hline 42.8 & 1647.6253 & 0.0069 & $\underline{\text { NCNSTTEFTCSNGR }}$ \\
\hline 1.0 & 1647.6331 & -0.0008 & $\underline{\text { IFSGNSHQNLNQK }}$ \\
\hline 1.0 & 1647.6331 & -0.0008 & $\underline{\text { IFSGNSHQNLNQK }}$ \\
\hline 0.2 & 1647.6365 & -0.0042 & MSKQTARASDSYK \\
\hline & & & \\
\hline
\end{tabular}




\section{|0.2 |1647.6365|-0.0042|MSKQTARASDSYK}

Spectrum No: 69; Query: 687; Rank: 1

\section{Peptide View}

\section{MS/MS Fragmentation of TAFQNGTESTIITK}

Found in IPI00204808, Tax_Id=10116 Gene_Symbol=Mep1b Meprin A subunit beta precursor

Match to Query 687: 1510.749568 from(756.382060,2+)

Title: 091008RatKidney_NH4Format01_27.1460.1460.2.dta

Data file K:\NewmanPaper\Piliang \3SubProteomes\Piliang3SP \mgf5ppm\ERLIC_3SubProteomes5ppm.mgf
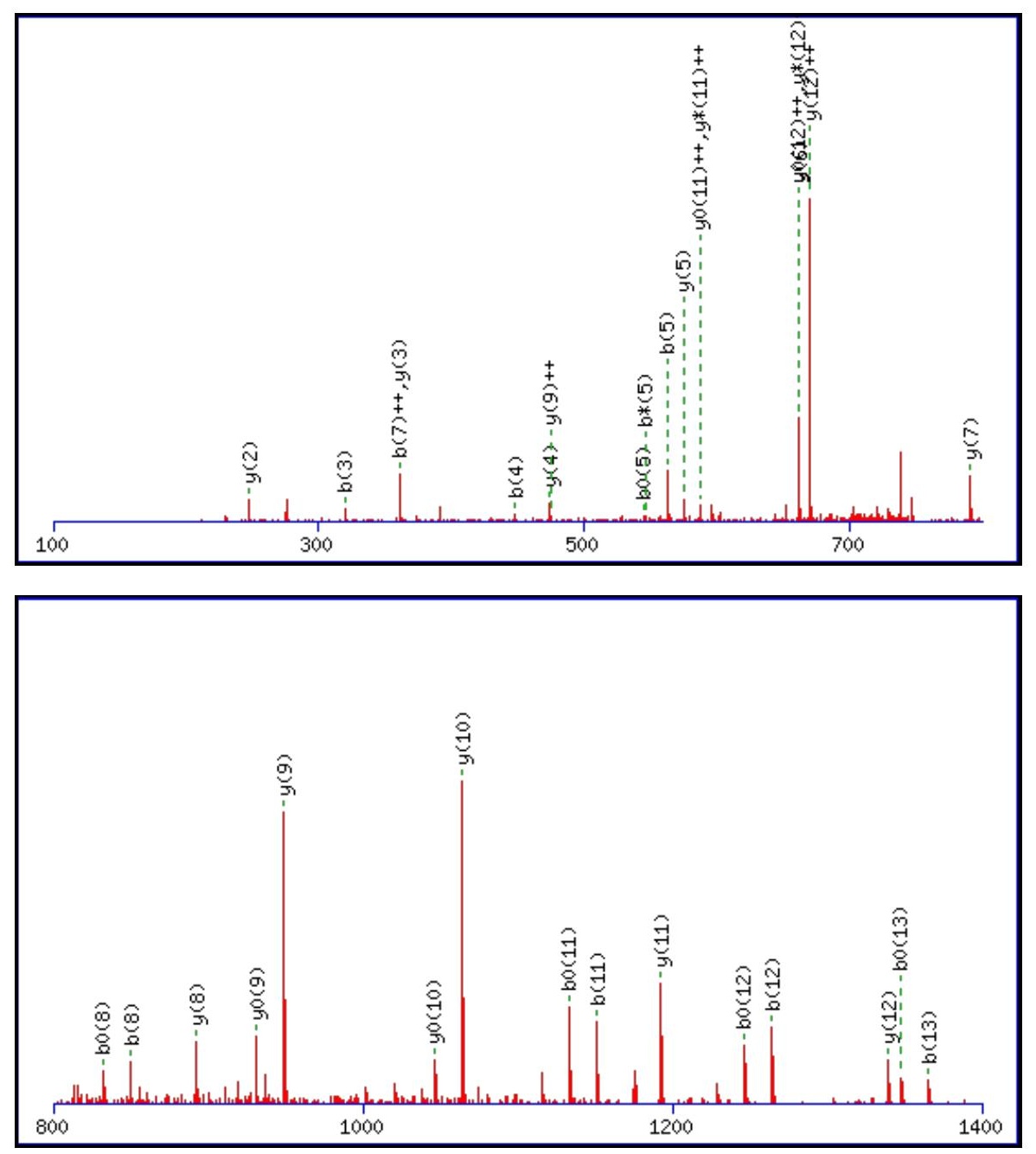


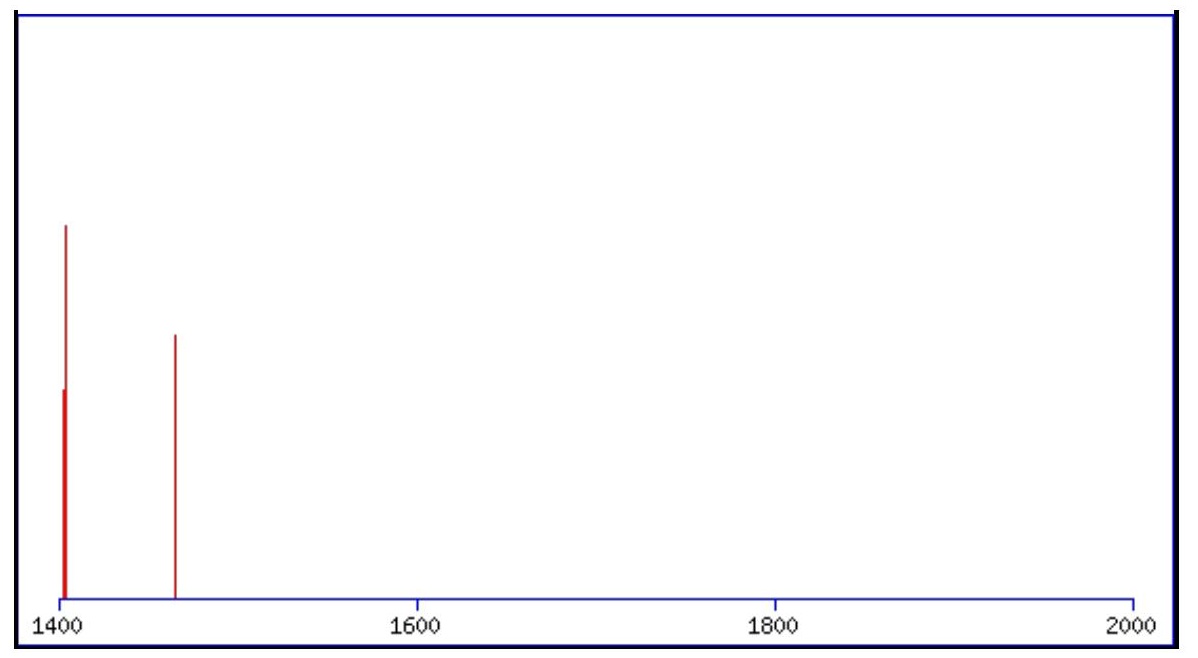

Monoisotopic mass of neutral peptide $\operatorname{Mr}($ calc): 1510.7515

Fixed modifications: Carbamidomethyl (C)

Variable modifications:

N5 : Deamidated $\mathrm{N}(\mathrm{N})$

Ions Score: 101 Expect: $1.1 \mathrm{e}-008$

Matches (Bold Red): 33/148 fragment ions using 43 most intense peaks

\begin{tabular}{|c|c|c|c|c|c|c|c|c|c|c|c|c|c|c|}
\hline \# & b & $\mathbf{b}^{++}$ & b* & $\mathbf{b}^{*^{++}}$ & $\mathbf{b}^{0}$ & $\mathbf{b}^{0++}$ & Seq. & $\mathbf{y}$ & $\mathbf{y}^{++}$ & $\mathbf{y}^{*}$ & $\mathbf{y}^{*^{++}}$ & $\mathbf{y}^{0}$ & $\mathbf{y}^{0++}$ & \# \\
\hline 1 & 102.0550 & 51.5311 & & & 84.0444 & 42.5258 & $\mathbf{T}$ & & & & & & & 14 \\
\hline 2 & 173.0921 & 87.0497 & & & 155.0815 & 78.0444 & A & 1410.7111 & 705.8592 & 1393.6845 & 697.3459 & 1392.7005 & 696.8539 & 13 \\
\hline 3 & 320.1605 & 160.5839 & & & 302.1499 & 151.5786 & $\mathbf{F}$ & 1339.6740 & 670.3406 & 1322.6474 & 661.8274 & 1321.6634 & 661.3353 & 12 \\
\hline 4 & 448.2191 & 224.6132 & 431.1925 & 216.0999 & 430.2085 & 215.6079 & $\mathbf{Q}$ & 1192.6056 & 596.8064 & 1175.5790 & 588.2931 & 1174.5950 & 587.8011 & 11 \\
\hline 5 & 563.2460 & 282.1266 & 546.2194 & 273.6134 & 545.2354 & 273.1214 & $\mathbf{N}$ & 1064.5470 & 532.7771 & 1047.5204 & 524.2639 & 1046.5364 & 523.7719 & 10 \\
\hline 6 & 620.2675 & 310.6374 & 603.2409 & 302.1241 & 602.2569 & 301.6321 & G & 949.5201 & 475.2637 & 932.4935 & 466.7504 & 931.5095 & 466.2584 & 9 \\
\hline 7 & 721.3151 & 361.1612 & 704.2886 & 352.6479 & 703.3046 & 352.1559 & $\mathbf{T}$ & 892.4986 & 446.7529 & 875.4720 & 438.2397 & 874.4880 & 437.7477 & 8 \\
\hline 8 & 850.3577 & 425.6825 & 833.3312 & 417.1692 & 832.3472 & 416.6772 & $\mathbf{E}$ & 791.4509 & 396.2291 & 774.4244 & 387.7158 & 773.4403 & 387.2238 & 7 \\
\hline 9 & 937.3898 & 469.1985 & 920.3632 & 460.6852 & 919.3792 & 460.1932 & $S$ & 662.4083 & 331.7078 & 645.3818 & 323.1945 & 644.3978 & 322.7025 & 6 \\
\hline 10 & 1038.4374 & 519.7224 & 1021.4109 & 511.2091 & 1020.4269 & 510.7171 & $\mathbf{T}$ & 575.3763 & 288.1918 & 558.3497 & 279.6785 & 557.3657 & 279.1865 & 5 \\
\hline 11 & 1151.5215 & 576.2644 & 1134.4950 & 567.7511 & 1133.5109 & 567.2591 & I & 474.3286 & 237.6679 & 457.3021 & 229.1547 & 456.3180 & 228.6627 & 4 \\
\hline 12 & 1264.6056 & 632.8064 & 1247.5790 & 624.2931 & 1246.5950 & 623.8011 & I & 361.2445 & 181.1259 & 344.2180 & 172.6126 & 343.2340 & 172.1206 & 3 \\
\hline 13 & 1365.6532 & 683.3303 & 1348.6267 & 674.8170 & 1347.6427 & 674.3250 & $\mathbf{T}$ & 248.1605 & 124.5839 & 231.1339 & 116.0706 & 230.1499 & 115.5786 & 2 \\
\hline 14 & & & & & & & $\mathbf{K}$ & 147.1128 & 74.0600 & 130.0863 & 65.5468 & & & 1 \\
\hline
\end{tabular}
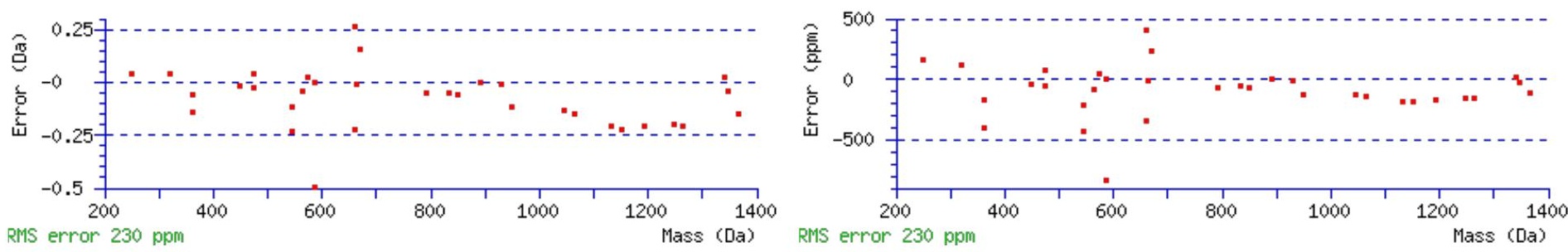

\section{All matches to this query}

\begin{tabular}{|l|l|l|l|}
\hline Score & Mr(calc): & Delta & \multicolumn{1}{c|}{ Sequence } \\
\hline 101.4 & 1510.7515 & -0.0019 & TAFQNGTESTIITK \\
\hline 9.7 & 1508.7501 & 1.9995 & FQGPQPSSRAVKK \\
\hline 5.8 & 1508.7501 & 1.9995 & FQGPQPSSRAVKK \\
\hline 5.7 & 1510.7371 & 0.0125 & MQTSLDEVMKTTK \\
\hline 5.3 & 1510.7456 & 0.0039 & TAFQEEFVHYLK \\
\hline 4.2 & 1510.7502 & -0.0006 & TVPHQGTEGHHRR \\
\hline 2.2 & 1510.7504 & -0.0009 & TELKQRLNSQSK \\
\hline & & & \\
\hline
\end{tabular}




\begin{tabular}{|l||l|l|l|}
\hline 1.9 & 1508.7545 & 1.9951 & GLFCDVTLIAGDTK \\
\hline 1.8 & 1508.7505 & 1.9991 & $\underline{\text { SGNVTTVMETIGRK }}$ \\
\hline
\end{tabular}

Spectrum No: 70; Query: 1748; Rank: 1

\section{Peptide View}

MS/MS Fragmentation of MYVTNDTEVAENNYQALK

Found in IPI00464785, Tax_Id=10116 Gene_Symbol=Ppgb Protective protein for beta-galactosidase

Match to Query 1748: 2102.955528 from(1052.485040,2+)

Title: 100101RatKid_NS_deglyco_23.2834.2834.2.dta

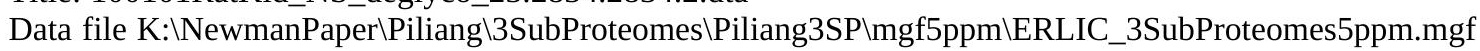
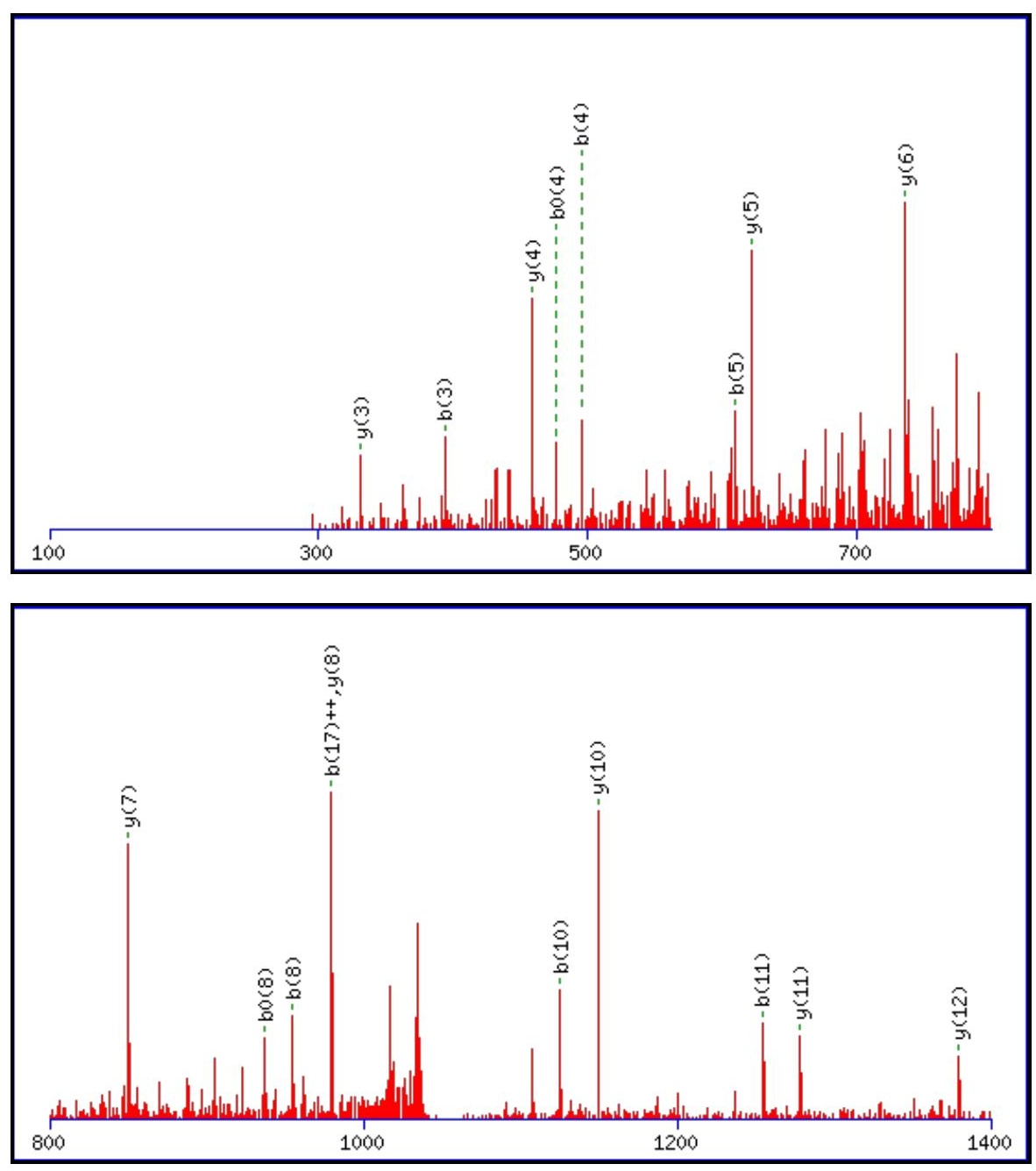


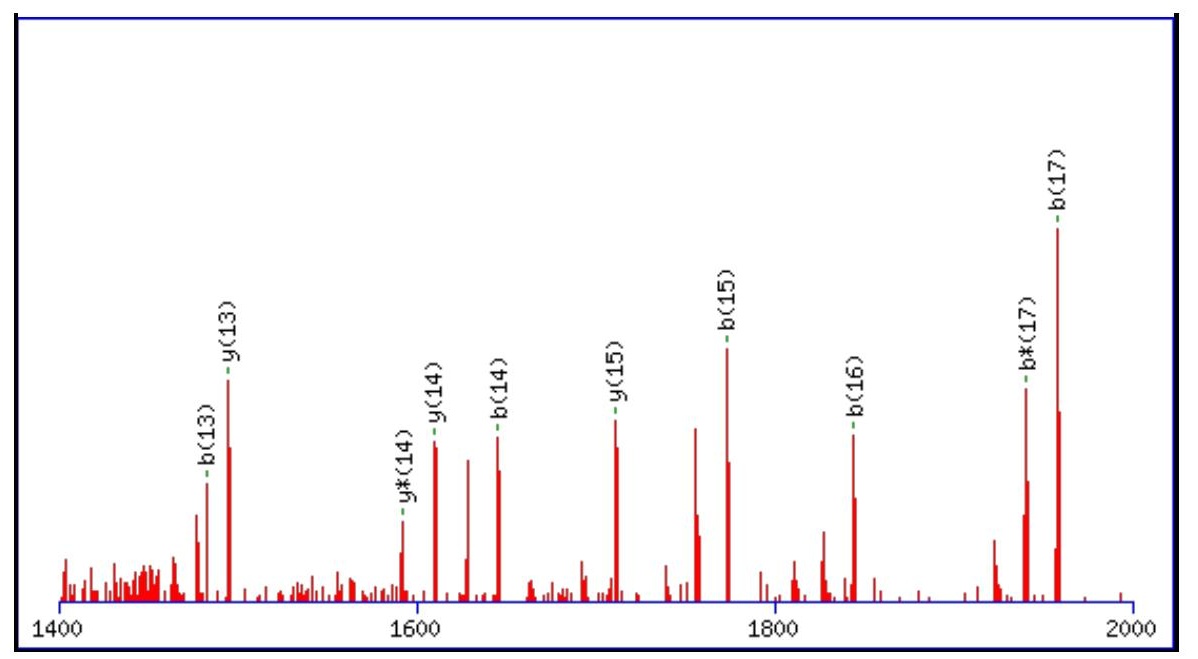

Monoisotopic mass of neutral peptide $\operatorname{Mr}($ calc): 2102.9466

Fixed modifications: Carbamidomethyl (C)

Variable modifications:

N5 : Deamidated $\mathrm{N}(\mathrm{N})$

Ions Score: 101 Expect: $1.9 \mathrm{e}-008$

Matches (Bold Red): 28/176 fragment ions using 38 most intense peaks

\begin{tabular}{|c|c|c|c|c|c|c|c|c|c|c|c|c|c|c|}
\hline \# & b & $\mathbf{b}^{++}$ & $\mathbf{b}^{*}$ & $\mathbf{b}^{*^{++}}$ & $\mathbf{b}^{0}$ & $\mathbf{b}^{0++}$ & Seq. & $\mathbf{y}$ & $y^{++}$ & $\mathrm{y}^{*}$ & $y^{*^{++}}$ & $\mathbf{y}^{0}$ & $y^{0++}$ & $\#$ \\
\hline 1 & 132.0478 & 66.5275 & & & & & $\mathbf{M}$ & & & & & & & 18 \\
\hline 2 & 295.1111 & 148.0592 & & & & & $\mathbf{Y}$ & 1972.9134 & 986.9604 & 1955.8869 & 978.4471 & 1954.9029 & 977.9551 & 17 \\
\hline 3 & 394.1795 & 197.5934 & & & & & V & 1809.8501 & 905.4287 & 1792.8236 & 896.9154 & 1791.8395 & 896.4234 & 16 \\
\hline 4 & 495.2272 & 248.1172 & & & 477.2166 & 239.1119 & $T$ & 1710.7817 & 855.8945 & 1693.7551 & 847.3812 & 1692.7711 & 846.8892 & 15 \\
\hline 5 & 610.2541 & 305.6307 & 593.2276 & 297.1174 & 592.2436 & 296.6254 & $\mathbf{N}$ & 1609.7340 & 805.3706 & 1592.7075 & 796.8574 & 1591.7234 & 796.3654 & 14 \\
\hline 6 & 725.2811 & 363.1442 & 708.2545 & 354.6309 & 707.2705 & 354.1389 & D & 1494.7071 & 747.8572 & 1477.6805 & 739.3439 & 1476.6965 & 738.8519 & 13 \\
\hline 7 & 826.3287 & 413.6680 & 809.3022 & 405.1547 & 808.3182 & 404.6627 & $\mathbf{T}$ & 1379.6801 & 690.3437 & 1362.6536 & 681.8304 & 1361.6696 & 681.3384 & 12 \\
\hline 8 & 955.3713 & 478.1893 & 938.3448 & 469.6760 & 937.3608 & 469.1840 & $\mathbf{E}$ & 1278.6325 & 639.8199 & 1261.6059 & 631.3066 & 1260.6219 & 630.8146 & 11 \\
\hline 9 & 1054.4397 & 527.7235 & 1037.4132 & 519.2102 & 1036.4292 & 518.7182 & $\mathbf{V}$ & 1149.5899 & 575.2986 & 1132.5633 & 566.7853 & 1131.5793 & 566.2933 & 10 \\
\hline 101 & \begin{tabular}{|l|}
1125.4769 \\
\end{tabular} & 563.2421 & 1108.4503 & 554.7288 & 1107.4663 & 554.2368 & A & 1050.5215 & 525.7644 & 1033.4949 & 517.2511 & 1032.5109 & 516.7591 & 9 \\
\hline 11 & 1254.5195 & 627.7634 & 1237.4929 & 619.2501 & 1236.5089 & 618.7581 & $\mathbf{E}$ & \begin{tabular}{|l|l|}
979.4843 \\
\end{tabular} & 490.2458 & 962.4578 & 481.7325 & 961.4738 & 481.2405 & 8 \\
\hline 121 & 1368.5624 & 684.7848 & 1351.5358 & 676.2716 & 1350.5518 & 675.7795 & $\mathbf{N}$ & 850.4417 & 425.7245 & 833.4152 & 417.2112 & & & 7 \\
\hline 13 & \begin{tabular}{|l|}
1482.6053 \\
\end{tabular} & 741.8063 & 1465.5788 & 733.2930 & 1464.5947 & 732.8010 & $\mathbf{N}$ & 736.3988 & 368.7030 & 719.3723 & 360.1898 & & & 6 \\
\hline 14 & 1645.6686 & 823.3380 & 1628.6421 & 814.8247 & 1627.6581 & 814.3327 & $\mathbf{Y}$ & 622.3559 & 311.6816 & 605.3293 & 303.1683 & & & 5 \\
\hline 151 & \begin{tabular}{|l|}
1773.7272 \\
\end{tabular} & 887.3672 & 1756.7007 & 878.8540 & 1755.7167 & 878.3620 & $\mathbf{Q}$ & 459.2926 & 230.1499 & 442.2660 & 221.6366 & & & 4 \\
\hline 16 & 1844.7643 & 922.8858 & 1827.7378 & 914.3725 & 1826.7538 & 913.8805 & A & 331.2340 & 166.1206 & 314.2074 & 157.6074 & & & 3 \\
\hline 17 & 1957.8484 & 979.4278 & 1940.8218 & 970.9146 & 1939.8378 & 970.4226 & $\mathbf{L}$ & 260.1969 & 130.6021 & 243.1703 & 122.0888 & & & 2 \\
\hline 18 & & & & & & & $\mathbf{K}$ & 147.1128 & 74.0600 & 130.0863 & 65.5468 & & & 1 \\
\hline
\end{tabular}

$$
\text { 舀 }
$$

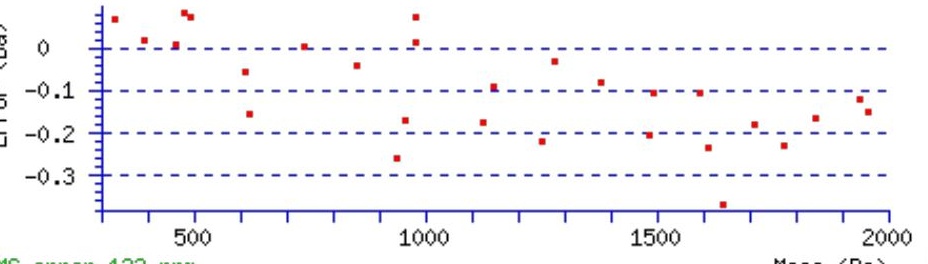

RMS error $132 \mathrm{ppm}$

\section{All matches to this query}

\begin{tabular}{|l|l|c|c|}
\hline Score & Mr(calc): & Delta & Sequence \\
\hline 101.4 & 2102.9466 & 0.0089 & MYVTNDTEVAENNYQALK \\
\hline 68.3 & 2102.9466 & 0.0089 & MYVTNDTEVAENNYQALK \\
\hline 56.1 & 2102.9466 & 0.0089 & MYVTNDTEVAENNYQALK \\
\hline & & &
\end{tabular}




\begin{tabular}{|l|l|l|l|}
50.8 & 2101.9626 & 0.9929 & MYVTNDTEVAENNYQALK \\
\hline 6.7 & 2102.9762 & -0.0207 & RPTSELGDARKTLDSVAK \\
\hline 5.4 & 2102.9551 & 0.0004 & GAPSASASPTEAGPLRPHIK \\
\hline 5.4 & 2102.9551 & 0.0004 & GAPSASASPTEAGPLRPHIK \\
\hline 4.2 & 2102.9551 & 0.0004 & GAPSASASPTEAGPLRPHIK \\
\hline 4.2 & 2102.9551 & 0.0004 & GAPSASASPTEAGPLRPHIK \\
\hline 3.8 & 2102.9496 & 0.0060 & $\underline{\text { SAQYPFVASLTVVAVALK }}$ \\
\hline
\end{tabular}

Spectrum No: 71; Query: 1265; Rank: 1

\section{Peptide View}

MS/MS Fragmentation of QGAIVAVTGDGVNDSPALK

Found in IPI00205693, Tax_Id=10116 Gene_Symbol=Atp1a2 Sodium/potassium-transporting ATPase subunit alpha-2 precursor

Match to Query 1265: 1811.933048 from(906.973800,2+)

Title: 091008RatKidney_NoSalt_17.2326.2326.2.dta

Data file K:INewmanPaper|Piliang|3SubProteomes\Piliang3SP\mgf5ppm\ERLIC_3SubProteomes5ppm.mgf
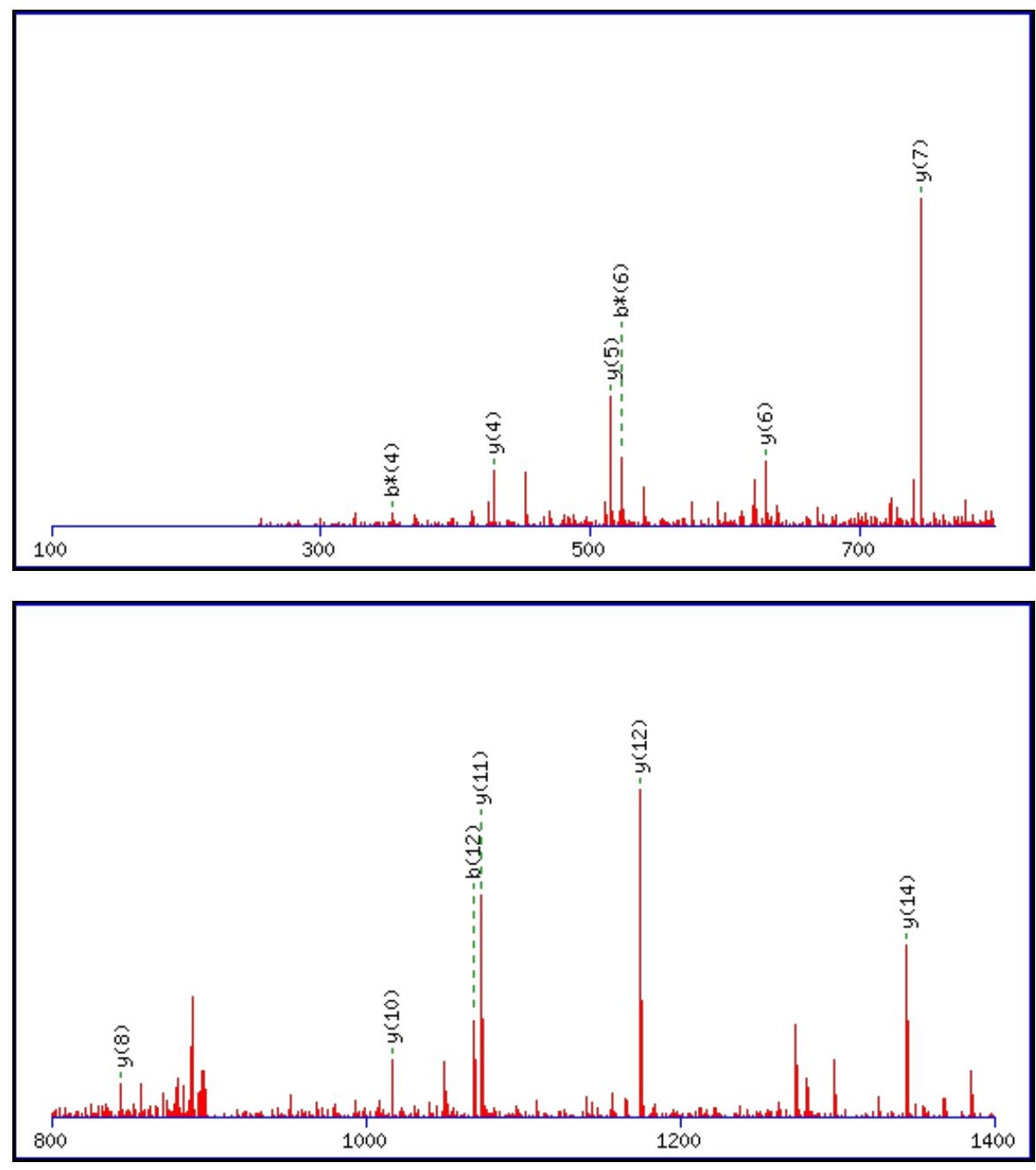


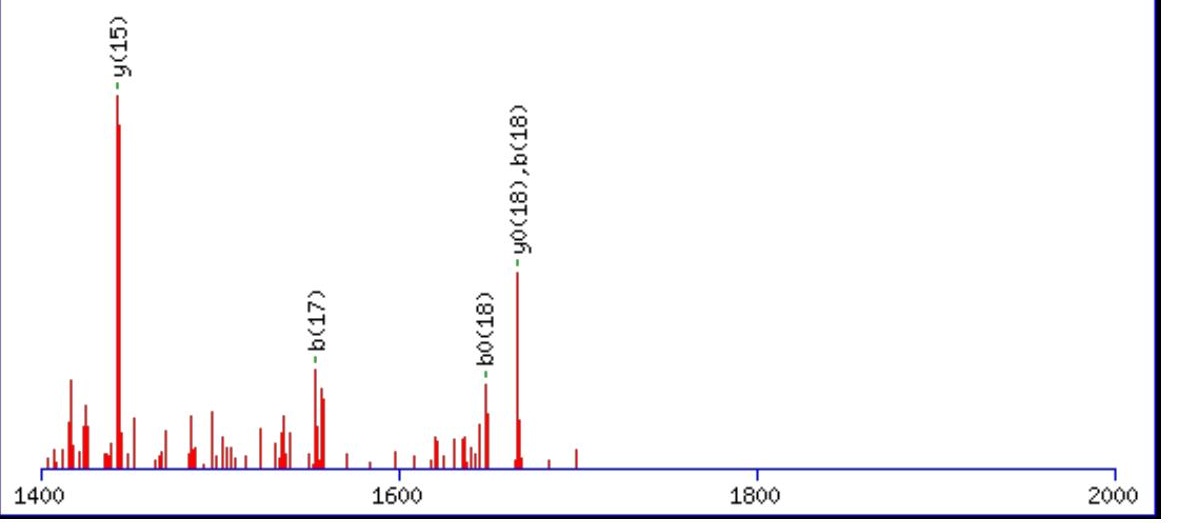

Monoisotopic mass of neutral peptide $\operatorname{Mr}($ calc): 1811.9265

Fixed modifications: Carbamidomethyl (C)

Variable modifications:

N13 : Deamidated $\mathrm{N}(\mathrm{N})$

Ions Score: 101 Expect: $1.1 \mathrm{e}-008$

Matches (Bold Red): 17/194 fragment ions using 25 most intense peaks

\begin{tabular}{|c|c|c|c|c|c|c|c|c|c|c|c|c|c|c|}
\hline \# & b & $\mathbf{b}^{++}$ & b* & $\mathbf{b}^{*^{++}}$ & $\mathbf{b}^{0}$ & $\mathbf{b}^{\mathbf{0 + +}}$ & Seq. & $\mathbf{y}$ & $\mathbf{y}^{++}$ & $\mathbf{y}^{*}$ & $\mathbf{y}^{*^{++}}$ & $\mathbf{y}^{0}$ & $\mathbf{y}^{0++}$ & \# \\
\hline 1 & 129.0659 & 65.0366 & 112.0393 & 56.5233 & & & $\mathbf{Q}$ & & & & & & & 19 \\
\hline 2 & 186.0873 & 93.5473 & 169.0608 & 85.0340 & & & G & 1684.8752 & 842.9412 & 1667.8487 & 834.4280 & 1666.8646 & 833.9360 & 18 \\
\hline 3 & 257.1244 & 129.0659 & 240.0979 & 120.5526 & & & A & 1627.8537 & 814.4305 & 1610.8272 & 805.9172 & 1609.8432 & 805.4252 & 17 \\
\hline 4 & 370.2085 & 185.6079 & 353.1819 & 177.0946 & & & I & 1556.8166 & 778.9119 & 1539.7901 & 770.3987 & 1538.8061 & 769.9067 & 16 \\
\hline 5 & 469.2769 & 235.1421 & 452.2504 & 226.6288 & & & $\mathbf{V}$ & 1443.7326 & 722.3699 & 1426.7060 & 713.8566 & 1425.7220 & 713.3646 & 15 \\
\hline 6 & 540.3140 & 270.6607 & 523.2875 & 262.1474 & & & A & 1344.6641 & 672.8357 & 1327.6376 & 664.3224 & 1326.6536 & 663.8304 & 14 \\
\hline 7 & 639.3824 & 320.1949 & 622.3559 & 311.6816 & & & $\mathbf{V}$ & 1273.6270 & 637.3172 & 1256.6005 & 628.8039 & 1255.6165 & 628.3119 & 13 \\
\hline 8 & 740.4301 & 370.7187 & 723.4036 & 362.2054 & 722.4196 & 361.7134 & $\mathbf{T}$ & 1174.5586 & 587.7829 & 1157.5321 & 579.2697 & 1156.5481 & 578.7777 & 12 \\
\hline 9 & 797.4516 & 399.2294 & 780.4250 & 390.7162 & 779.4410 & 390.2241 & G & 1073.5109 & 537.2591 & 1056.4844 & 528.7458 & 1055.5004 & 528.2538 & 11 \\
\hline 10 & 912.4785 & 456.7429 & 895.4520 & 448.2296 & 894.4680 & 447.7376 & D & 1016.4895 & 508.7484 & 999.4629 & 500.2351 & 998.4789 & 499.7431 & 10 \\
\hline 11 & 969.5000 & 485.2536 & 952.4734 & 476.7404 & 951.4894 & 476.2483 & $\mathbf{G}$ & 901.4625 & 451.2349 & 884.4360 & 442.7216 & 883.4520 & 442.2296 & 9 \\
\hline 12 & 1068.5684 & 534.7878 & 1051.5419 & 526.2746 & 1050.5578 & 525.7826 & $\mathbf{V}$ & 844.4411 & 422.7242 & 827.4145 & 414.2109 & 826.4305 & 413.7189 & 8 \\
\hline 13 & 1183.5953 & 592.3013 & 1166.5688 & 583.7880 & 1165.5848 & 583.2960 & $\mathbf{N}$ & 745.3727 & 373.1900 & 728.3461 & 364.6767 & 727.3621 & 364.1847 & 7 \\
\hline 14 & 1298.6223 & 649.8148 & 1281.5957 & 641.3015 & 1280.6117 & 640.8095 & D & 630.3457 & 315.6765 & 613.3192 & 307.1632 & 612.3352 & 306.6712 & 6 \\
\hline 15 & 1385.6543 & 693.3308 & 1368.6278 & 684.8175 & 1367.6437 & 684.3255 & S & 515.3188 & 258.1630 & 498.2922 & 249.6498 & 497.3082 & 249.1577 & 5 \\
\hline 16 & 1482.7071 & 741.8572 & 1465.6805 & 733.3439 & 1464.6965 & 732.8519 & $\mathbf{P}$ & 428.2867 & 214.6470 & 411.2602 & 206.1337 & & & 4 \\
\hline 17 & 1553.7442 & 777.3757 & 1536.7176 & 768.8625 & 1535.7336 & 768.3704 & A & 331.2340 & 166.1206 & 314.2074 & 157.6074 & & & 3 \\
\hline 18 & 1666.8283 & 833.9178 & 1649.8017 & 825.4045 & 1648.8177 & 824.9125 & $\mathbf{L}$ & 260.1969 & 130.6021 & 243.1703 & 122.0888 & & & 2 \\
\hline 19 & & & & & & & $\mathbf{K}$ & 147.1128 & 74.0600 & 130.0863 & 65.5468 & & & 1 \\
\hline
\end{tabular}

$$
\text { 鱼 }
$$$$
\begin{gathered}
0.1 \text { 寿 } \\
-0.2 \text { 寿 } \\
-0.1
\end{gathered}
$$

RMS error $119 \mathrm{ppm}$

\section{All matches to this query}

\begin{tabular}{|l|l|l|l|}
\hline Score & Mr(calc): & Delta & Sequence \\
\hline 101.3 & 1811.9265 & 0.0065 & QGAIVAVTGDGVNDSPALK \\
\hline 8.4 & 1810.9425 & 0.9905 & QGAIVAVTGDGVNDSPALK \\
\hline & & & \\
\hline
\end{tabular}

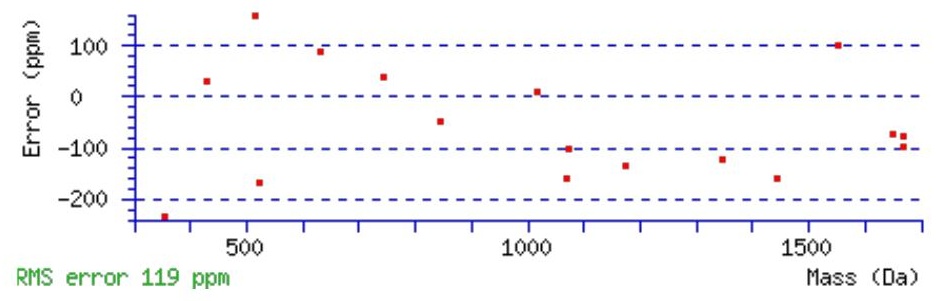




\begin{tabular}{|l|l|l|l|}
6.5 & 1811.9166 & 0.0164 & EKGLSGRGPDTGPDWLK \\
\hline 3.5 & 1811.9264 & 0.0066 & APSPEKAEEESERLLK \\
\hline 1.1 & 1810.9188 & 1.0142 & YFLQGMGYIAHLKDR \\
\hline 0.3 & 1810.9230 & 1.0100 & ATTPSVTVKNSAAPIFK \\
\hline 0.3 & 1810.9230 & 1.0100 & ATTPSVTVKNSAAPIFK \\
\hline
\end{tabular}

Spectrum No: 72; Query: 1151; Rank: 1

\section{Peptide View}

MS/MS Fragmentation of DYYPINESLASLPLR

Found in IPI00193869, Tax_Id=10116 Gene_Symbol=Clptm1_predicted cleft lip and palate associated transmembrane protein 1

Match to Query 1151: 1750.875988 from(876.445270,2+)

Title: 091008RatKidney_NH4Format01_23.3892.3892.2.dta

Data file K:INewmanPaper|Piliang|3SubProteomes\Piliang3SP\mgf5ppm\ERLIC_3SubProteomes5ppm.mgf
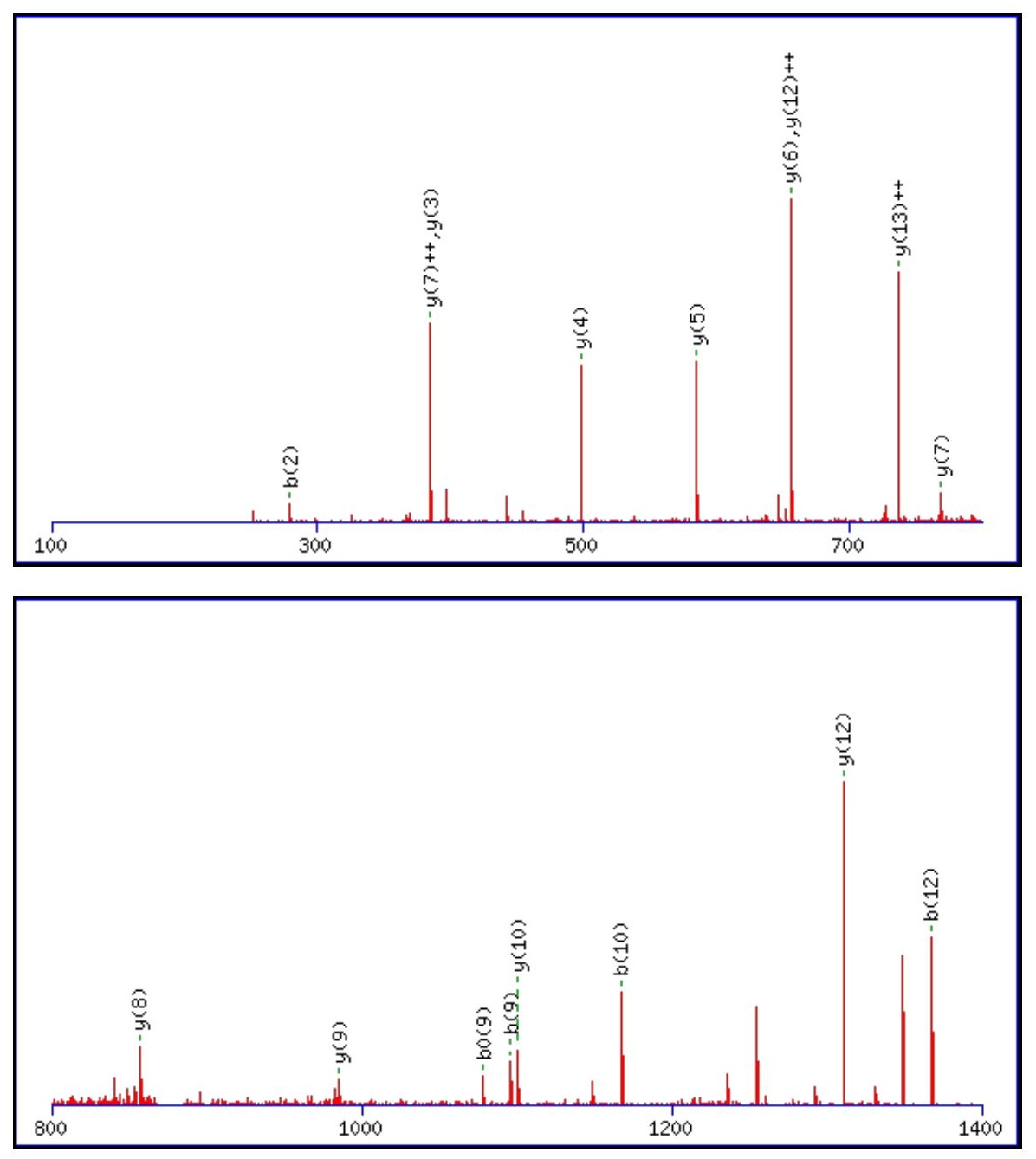


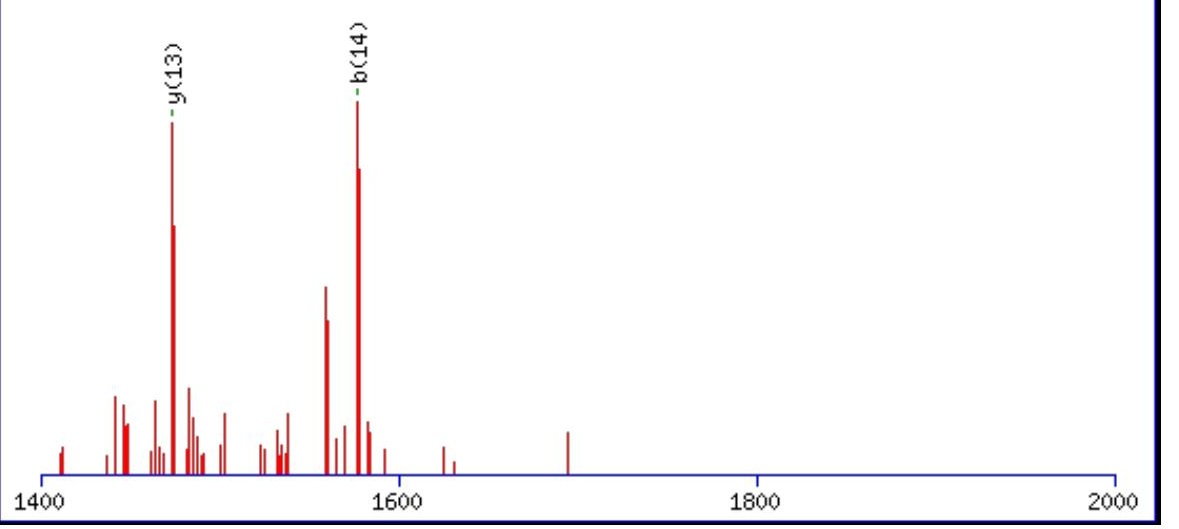

Monoisotopic mass of neutral peptide $\operatorname{Mr}($ calc): 1750.8777

Fixed modifications: Carbamidomethyl (C)

Variable modifications:

N6: Deamidated $\mathrm{N}(\mathrm{N})$

Ions Score: 101 Expect: $1.4 \mathrm{e}-008$

Matches (Bold Red): 19/150 fragment ions using 24 most intense peaks

\begin{tabular}{|c|c|c|c|c|c|c|c|c|c|c|c|c|c|c|}
\hline \# & b & $\mathbf{b}^{++}$ & $\mathbf{b}^{*}$ & $\mathbf{b}^{*^{++}}$ & $\mathbf{b}^{0}$ & $\mathbf{b}^{\mathbf{0 + +}}$ & Seq. & $\mathbf{y}$ & $\mathbf{y}^{++}$ & $\mathbf{y}^{*}$ & $\mathbf{y}^{*^{++}}$ & $\mathbf{y}^{0}$ & $\mathbf{y}^{0++}$ & \# \\
\hline 1 & 116.0342 & 58.5207 & & & 98.0237 & 49.5155 & D & & & & & & & 15 \\
\hline 2 & 279.0975 & 140.0524 & & & 261.0870 & 131.0471 & $\mathbf{Y}$ & 1636.8581 & 818.9327 & 1619.8315 & 810.4194 & 1618.8475 & 809.9274 & 14 \\
\hline 3 & 442.1609 & 221.5841 & & & 424.1503 & 212.5788 & $\mathbf{Y}$ & 1473.7948 & 737.4010 & 1456.7682 & 728.8877 & 1455.7842 & 728.3957 & 13 \\
\hline 4 & 539.2136 & 270.1105 & & & 521.2031 & 261.1052 & $\mathbf{P}$ & 1310.7314 & 655.8694 & 1293.7049 & 647.3561 & 1292.7209 & 646.8641 & 12 \\
\hline 5 & 652.2977 & 326.6525 & & & 634.2871 & 317.6472 & I & 1213.6787 & 607.3430 & 1196.6521 & 598.8297 & 1195.6681 & 598.3377 & 11 \\
\hline 6 & 767.3246 & 384.1660 & 750.2981 & 375.6527 & 749.3141 & 375.1607 & $\mathbf{N}$ & 1100.5946 & 550.8009 & 1083.5681 & 542.2877 & 1082.5840 & 541.7957 & 10 \\
\hline 7 & 896.3672 & 448.6873 & 879.3407 & 440.1740 & 878.3567 & 439.6820 & $\mathbf{E}$ & 985.5677 & 493.2875 & 968.5411 & 484.7742 & 967.5571 & 484.2822 & 9 \\
\hline 8 & 983.3993 & 492.2033 & 966.3727 & 483.6900 & 965.3887 & 483.1980 & S & 856.5251 & 428.7662 & 839.4985 & 420.2529 & 838.5145 & 419.7609 & 8 \\
\hline 9 & 1096.4833 & 548.7453 & 1079.4568 & 540.2320 & 1078.4728 & 539.7400 & $\mathbf{L}$ & 769.4930 & 385.2502 & 752.4665 & 376.7369 & 751.4825 & 376.2449 & 7 \\
\hline 10 & 1167.5204 & 584.2639 & 1150.4939 & 575.7506 & 1149.5099 & 575.2586 & A & 656.4090 & 328.7081 & 639.3824 & 320.1949 & 638.3984 & 319.7028 & 6 \\
\hline 11 & 1254.5525 & 627.7799 & 1237.5259 & 619.2666 & 1236.5419 & 618.7746 & S & 585.3719 & 293.1896 & 568.3453 & 284.6763 & 567.3613 & 284.1843 & 5 \\
\hline 12 & 1367.6365 & 684.3219 & 1350.6100 & 675.8086 & 1349.6260 & 675.3166 & $\mathbf{L}$ & 498.3398 & 249.6736 & 481.3133 & 241.1603 & & & 4 \\
\hline 13 & 1464.6893 & 732.8483 & 1447.6627 & 724.3350 & 1446.6787 & 723.8430 & $\mathbf{P}$ & 385.2558 & 193.1315 & 368.2292 & 184.6183 & & & 3 \\
\hline 14 & 1577.7734 & 789.3903 & 1560.7468 & 780.8770 & 1559.7628 & 780.3850 & $\mathbf{L}$ & 288.2030 & 144.6051 & 271.1765 & 136.0919 & & & 2 \\
\hline 15 & & & & & & & $\mathbf{R}$ & 175.1190 & 88.0631 & 158.0924 & 79.5498 & & & 1 \\
\hline
\end{tabular}
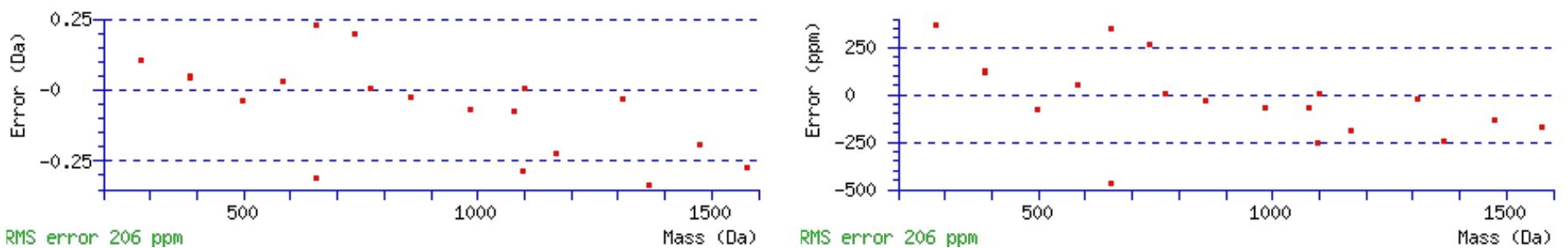

\section{All matches to this query}

\begin{tabular}{|l|l|l|l|}
\hline Score & Mr(calc): & Delta & \multicolumn{1}{c|}{ Sequence } \\
\hline 100.7 & 1750.8777 & -0.0017 & DYYPINESLASLPLR \\
\hline 8.7 & 1750.8879 & -0.0120 & RQGYDLRVDAIPIR \\
\hline 7.7 & 1750.8614 & 0.0146 & $\underline{\text { ELPASSAVSSALDRIR }}$ \\
\hline 7.7 & 1750.8614 & 0.0146 & ELPASSAVSSALDRIR \\
\hline 7.5 & 1750.8615 & 0.0145 & VDGLGLGVVTRQNSQK \\
\hline 7.2 & 1749.8662 & 1.0098 & DLLVQTLENSGVLNR \\
\hline & & & \\
\hline
\end{tabular}




\begin{tabular}{|l|l|l|l|}
4.5 & 1750.8866 & -0.0106 & EASPLSSNKLILRDK \\
\hline 4.2 & 1748.8822 & 1.9938 & NVAGEVVLITGAGSGLGR \\
\hline 3.8 & 1749.8597 & 1.0163 & RATLPQAAMGVATVER \\
\hline 3.5 & 1749.8662 & 1.0098 & NVAGEVVLITGAGSGLGR \\
\hline
\end{tabular}

Spectrum No: 73; Query: 2137; Rank: 1

\section{Peptide View}

MS/MS Fragmentation of LDPPCTNTTAPSTYLNNPYVR

Found in IPI00464785, Tax_Id=10116 Gene_Symbol=Ppgb Protective protein for beta-galactosidase

Match to Query 2137: 2394.113488 from(1198.064020,2+)

Title: 091008RatKidney_NH4Format01_24.2946.2946.2.dta

Data file K:INewmanPaper|Piliang|3SubProteomes\Piliang3SP\mgf5ppm\ERLIC_3SubProteomes5ppm.mgf
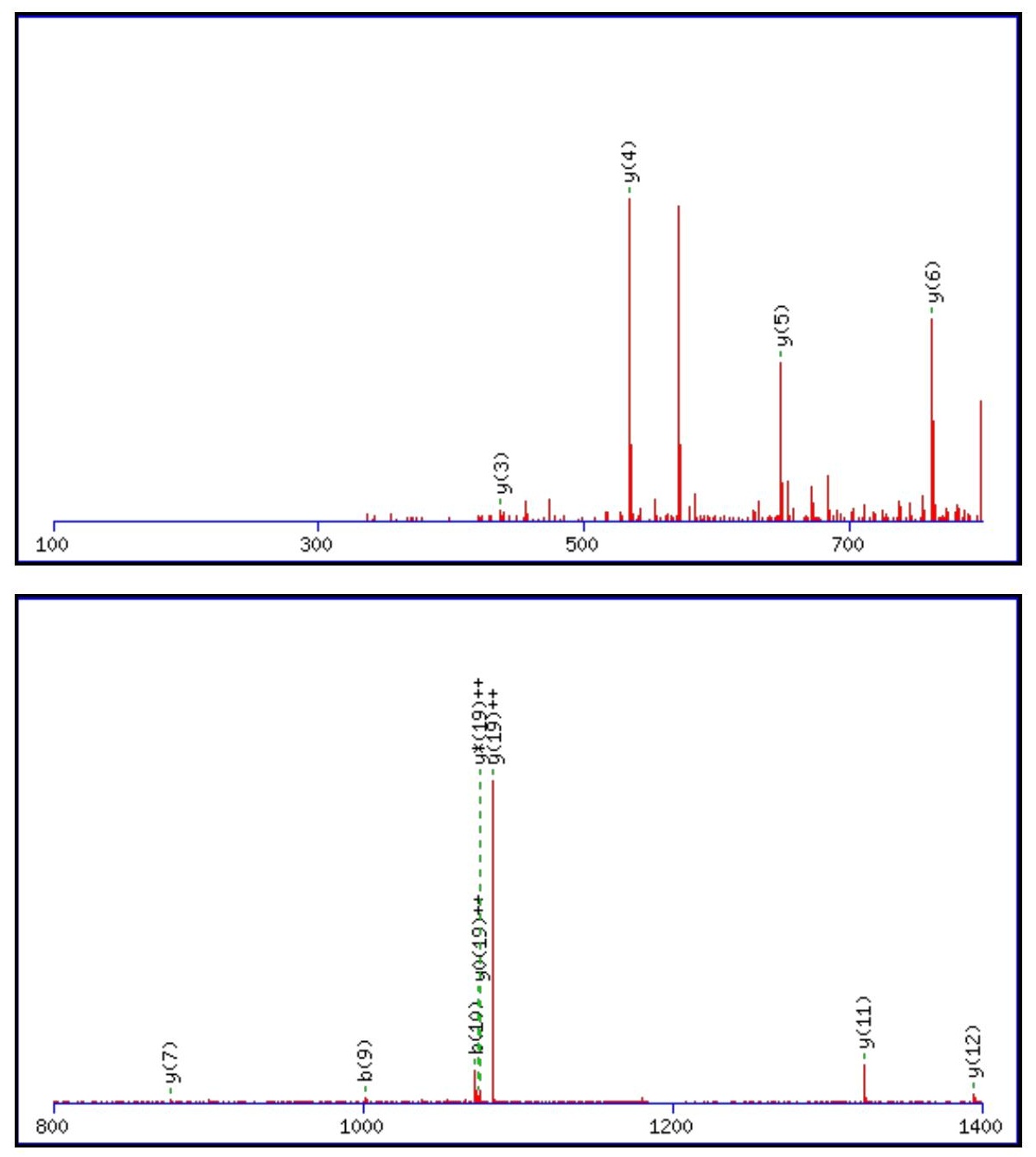


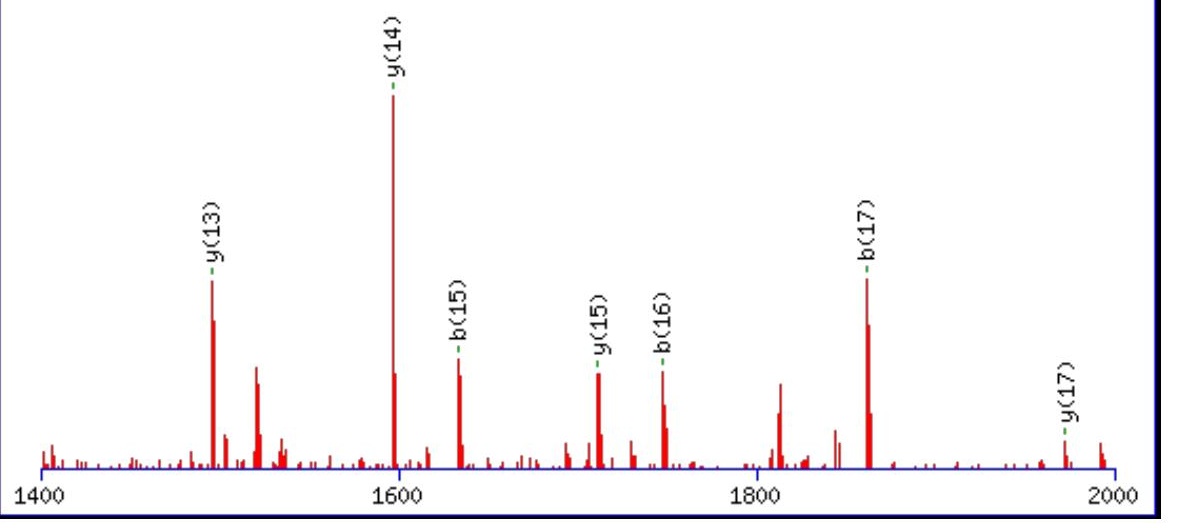

Monoisotopic mass of neutral peptide $\operatorname{Mr}($ calc): 2394.1162

Fixed modifications: Carbamidomethyl (C)

Variable modifications:

N7 : Deamidated $\mathrm{N}(\mathrm{N})$

Ions Score: 101 Expect: $2.5 \mathrm{e}-008$

Matches (Bold Red): 19/210 fragment ions using 30 most intense peaks

\begin{tabular}{|c|c|c|c|c|c|c|c|c|c|c|c|c|c|c|}
\hline \# & b & $\mathbf{b}^{++}$ & $\mathbf{b}^{*}$ & $\mathbf{b}^{*^{++}}$ & $\mathbf{b}^{0}$ & $\mathbf{b}^{0++}$ & Seq. & $\mathbf{y}$ & $y^{++}$ & $\mathbf{y}^{*}$ & $\mathbf{y}^{*^{++}}$ & $\mathbf{y}^{0}$ & $\mathbf{y}^{0++}$ & \# \\
\hline 1 & 114.0913 & 57.5493 & & & & & $\mathbf{L}$ & & & & & & & 21 \\
\hline 2 & 229.1183 & 115.0628 & & & 211.1077 & 106.0575 & D & 2282.0394 & 1141.5233 & 2265.0128 & 1133.0101 & 2264.0288 & 1132.5180 & 20 \\
\hline 3 & 326.1710 & 163.5892 & & & 308.1605 & 154.5839 & $\mathbf{P}$ & 2167.0124 & 1084.0099 & 2149.9859 & 1075.4966 & 2149.0019 & 1075.0046 & 19 \\
\hline 4 & 423.2238 & 212.1155 & & & 405.2132 & 203.1103 & $\mathbf{P}$ & 2069.9597 & 1035.4835 & 2052.9331 & 1026.9702 & 2051.9491 & 1026.4782 & 18 \\
\hline 5 & 583.2545 & 292.1309 & & & 565.2439 & 283.1256 & C & 1972.9069 & 986.9571 & 1955.8804 & 978.4438 & 1954.8963 & 977.9518 & 17 \\
\hline 6 & 684.3021 & 342.6547 & & & 666.2916 & 333.6494 & $\mathbf{T}$ & 1812.8763 & 906.9418 & 1795.8497 & 898.4285 & 1794.8657 & 897.9365 & 16 \\
\hline 7 & 799.3291 & 400.1682 & 782.3025 & 391.6549 & 781.3185 & 391.1629 & $\mathbf{N}$ & 1711.8286 & 856.4179 & 1694.8020 & 847.9047 & 1693.8180 & 847.4126 & 15 \\
\hline 8 & 900.3768 & 450.6920 & 883.3502 & 442.1787 & 882.3662 & 441.6867 & $\mathbf{T}$ & 1596.8016 & 798.9045 & 1579.7751 & 790.3912 & 1578.7911 & 789.8992 & 14 \\
\hline 9 & 1001.4244 & 501.2159 & 984.3979 & 492.7026 & 983.4139 & 492.2106 & $\mathbf{T}$ & 1495.7540 & 748.3806 & 1478.7274 & 739.8673 & 1477.7434 & 739.3753 & 13 \\
\hline 10 & 1072.4615 & 536.7344 & 1055.4350 & 528.2211 & 1054.4510 & 527.7291 & A & 1394.7063 & 697.8568 & 1377.6797 & 689.3435 & 1376.6957 & 688.8515 & 12 \\
\hline 11 & 1169.5143 & 585.2608 & 1152.4878 & 576.7475 & 1151.5037 & 576.2555 & $\mathbf{P}$ & 1323.6692 & 662.3382 & 1306.6426 & 653.8250 & 1305.6586 & 653.3329 & 11 \\
\hline 12 & 1256.5463 & 628.7768 & 1239.5198 & 620.2635 & 1238.5358 & 619.7715 & $S$ & 1226.6164 & 613.8118 & 1209.5899 & 605.2986 & 1208.6058 & 604.8066 & 10 \\
\hline 13 & 1357.5940 & 679.3006 & 1340.5675 & 670.7874 & 1339.5835 & 670.2954 & $\mathbf{T}$ & 1139.5844 & 570.2958 & 1122.5578 & 561.7826 & 1121.5738 & 561.2905 & 9 \\
\hline 14 & 1520.6573 & 760.8323 & 1503.6308 & 752.3190 & 1502.6468 & 751.8270 & $\mathbf{Y}$ & 1038.5367 & 519.7720 & 1021.5102 & 511.2587 & & & 8 \\
\hline 15 & 1633.7414 & 817.3743 & 1616.7149 & 808.8611 & 1615.7308 & 808.3691 & $\mathbf{L}$ & 875.4734 & 438.2403 & 858.4468 & 429.7271 & & & 7 \\
\hline 16 & 1747.7843 & 874.3958 & 1730.7578 & 865.8825 & 1729.7738 & 865.3905 & $\mathbf{N}$ & 762.3893 & 381.6983 & 745.3628 & 373.1850 & & & 6 \\
\hline 17 & 1861.8273 & 931.4173 & 1844.8007 & 922.9040 & 1843.8167 & 922.4120 & $\mathbf{N}$ & 648.3464 & 324.6768 & 631.3198 & 316.1636 & & & 5 \\
\hline 18 & 1958.8800 & 979.9437 & 1941.8535 & 971.4304 & 1940.8695 & 970.9384 & $\mathbf{P}$ & 534.3035 & 267.6554 & 517.2769 & 259.1421 & & & 4 \\
\hline 19 & 2121.9434 & 1061.4753 & 2104.9168 & 1052.9620 & 2103.9328 & 1052.4700 & $\mathbf{Y}$ & 437.2507 & 219.1290 & 420.2241 & 210.6157 & & & 3 \\
\hline 20 & 2221.0118 & 1111.0095 & 2203.9852 & 1102.4962 & 2203.0012 & 1102.0042 & $\mathbf{V}$ & 274.1874 & 137.5973 & 257.1608 & 129.0840 & & & 2 \\
\hline 21 & & & & & & & $\mathbf{R}$ & 175.1190 & 88.0631 & 158.0924 & 79.5498 & & & 1 \\
\hline
\end{tabular}

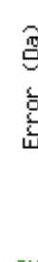
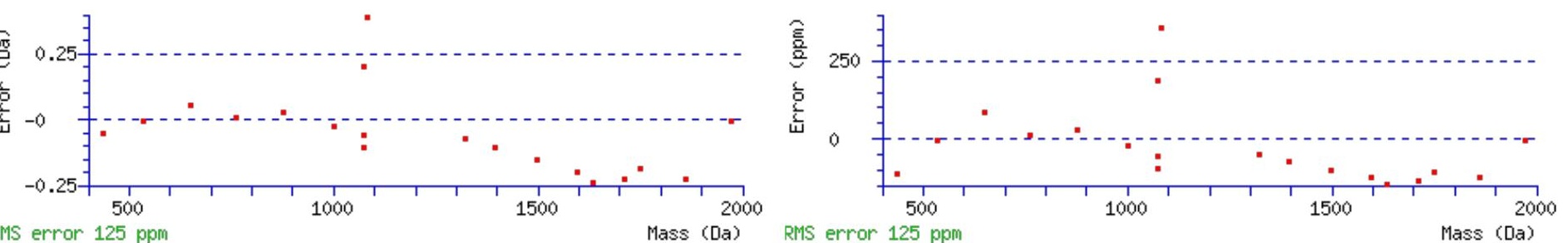

All matches to this query

\begin{tabular}{|l|l|l|}
\hline Score & Mr(calc): & Delta \\
\hline
\end{tabular}

Sequence 


\begin{tabular}{|l|l|l|l|}
100.6 & 2394.1162 & -0.0027 & LDPPCTNTTAPSTYLNNPYVR \\
\hline 55.5 & 2394.1162 & -0.0027 & LDPPCTNTTAPSTYLNNPYVR \\
\hline 53.1 & 2394.1162 & -0.0027 & $\underline{\text { LDPPCTNTTAPSTYLNNPYVR }}$ \\
\hline 48.0 & 2393.1322 & 0.9813 & $\underline{\text { LDPPCTNTTAPSTYLNNPYVR }}$ \\
\hline 5.1 & 2394.1104 & 0.0031 & EFQENIIKQVEAHKEESQK \\
\hline 5.0 & 2394.1121 & 0.0014 & AHETNLLIATSVVEEGVDIPK \\
\hline 5.0 & 2394.1121 & 0.0014 & AHETNLLIATSVVEEGVDIPK \\
\hline 5.0 & 2394.1121 & 0.0014 & AHETNLLIATSVVEEGVDIPK \\
\hline 4.3 & 2393.1182 & 0.9953 & AGVSFALGITTIDRGNHTYLK \\
\hline 4.3 & 2393.1182 & 0.9953 & $\underline{\text { AGVSFALGITTIDRGNHTYLK }}$ \\
\hline
\end{tabular}

Spectrum No: 74; Query: 735; Rank: 1

\section{Peptide View}

MS/MS Fragmentation of IYGGILNLSEITNK

Found in IPI00203384, Tax_Id=10116 Gene_Symbol=Klkb1 Plasma kallikrein precursor

Match to Query 735: 1534.822188 from(768.418370,2+)

Title: 091008RatKidney_NoSalt_29.3574.3574.2.dta

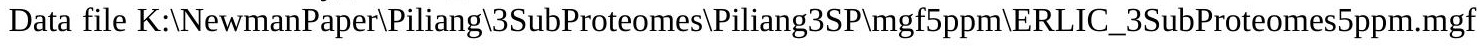
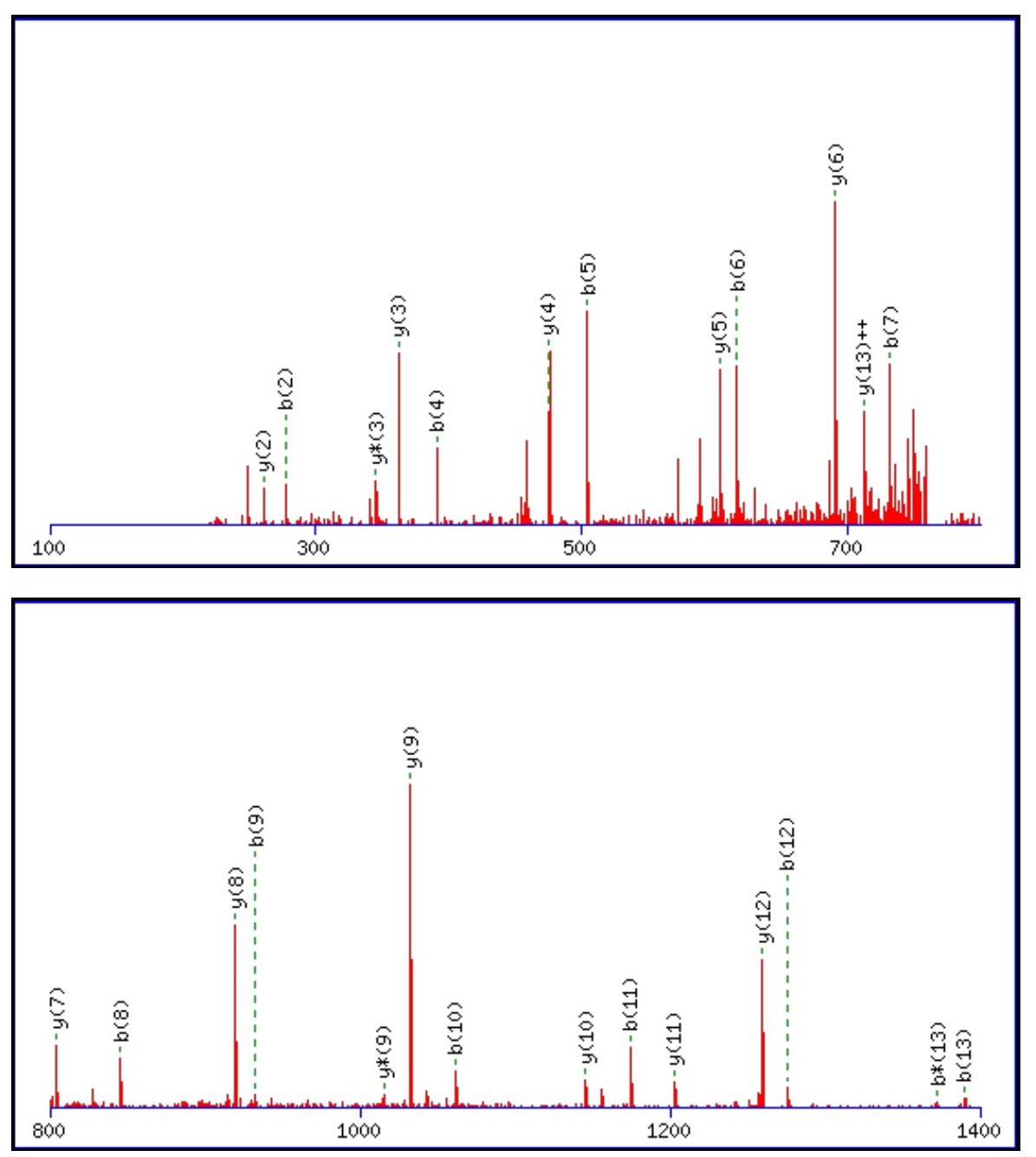


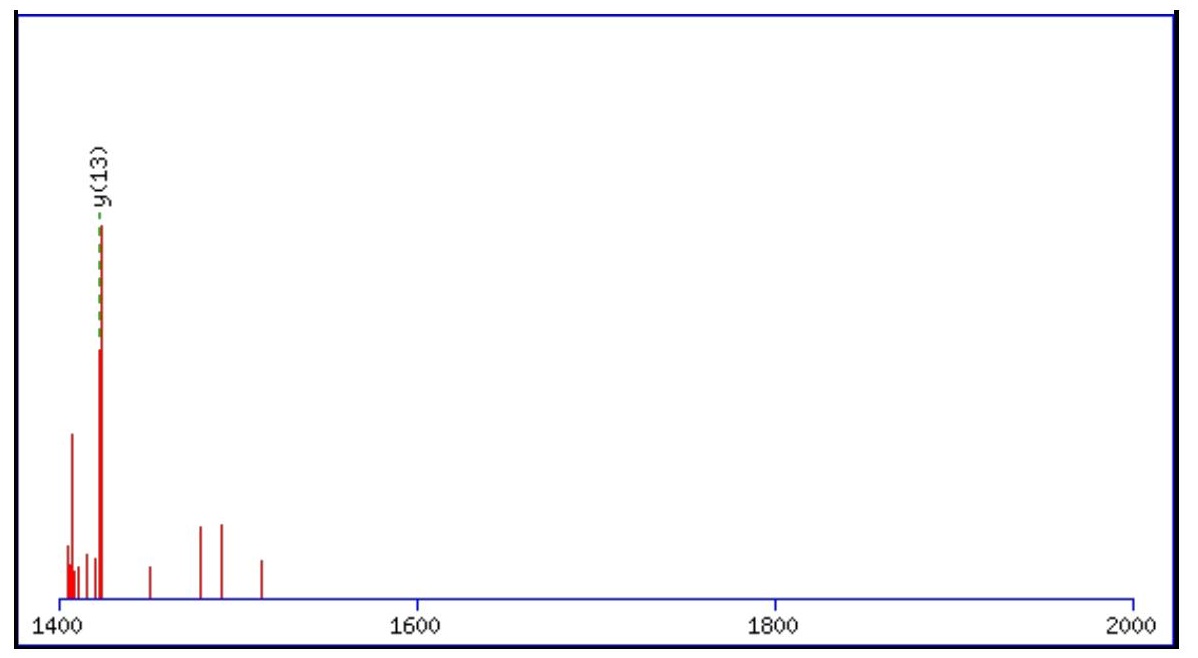

Monoisotopic mass of neutral peptide $\operatorname{Mr}($ calc): 1534.8242

Fixed modifications: Carbamidomethyl (C)

Variable modifications:

N7 : Deamidated $\mathrm{N}(\mathrm{N})$

Ions Score: 101 Expect: $8.3 \mathrm{e}-009$

Matches (Bold Red): 27/124 fragment ions using 43 most intense peaks

\begin{tabular}{|r|c|c|c|c|c|c|c|c|c|c|c|c|c|c|}
\hline$\#$ & $\mathbf{b}$ & $\mathbf{b}^{++}$ & $\mathbf{b}^{*}$ & $\mathbf{b}^{\mathbf{*}^{++}}$ & $\mathbf{b}^{\mathbf{0}}$ & $\mathbf{b}^{\mathbf{0 + +}}$ & $\mathbf{S e q}$ & $\mathbf{y}$ & $\mathbf{y}^{++}$ & $\mathbf{y}^{\mathbf{*}}$ & $\mathbf{y}^{\mathbf{*}^{++}}$ & $\mathbf{y}^{\mathbf{0}}$ & $\mathbf{y}^{\mathbf{0 + +}}$ & $\#$ \\
\hline $\mathbf{1}$ & 114.0913 & 57.5493 & & & & & $\mathbf{I}$ & & & & & & & $\mathbf{1 4}$ \\
\hline $\mathbf{2}$ & $\mathbf{2 7 7 . 1 5 4 7}$ & 139.0810 & & & & & $\mathbf{Y}$ & $\mathbf{1 4 2 2 . 7 4 7 5}$ & $\mathbf{7 1 1 . 8 7 7 4}$ & 1405.7209 & 703.3641 & 1404.7369 & $\mathbf{7 0 2 . 8 7 2 1}$ & $\mathbf{1 3}$ \\
\hline $\mathbf{3}$ & 334.1761 & 167.5917 & & & & & $\mathbf{G}$ & $\mathbf{1 2 5 9 . 6 8 4 2}$ & 630.3457 & 1242.6576 & 621.8324 & 1241.6736 & 621.3404 & $\mathbf{1 2}$ \\
\hline $\mathbf{4}$ & $\mathbf{3 9 1 . 1 9 7 6}$ & 196.1024 & & & & & $\mathbf{G}$ & $\mathbf{1 2 0 2 . 6 6 2 7}$ & 601.8350 & 1185.6361 & 593.3217 & 1184.6521 & 592.8297 & $\mathbf{1 1}$ \\
\hline $\mathbf{5}$ & $\mathbf{5 0 4 . 2 8 1 7}$ & 252.6445 & & & & & $\mathbf{I}$ & $\mathbf{1 1 4 5 . 6 4 1 2}$ & 573.3242 & 1128.6147 & 564.8110 & 1127.6307 & 564.3190 & $\mathbf{1 0}$ \\
\hline $\mathbf{6}$ & $\mathbf{6 1 7 . 3 6 5 7}$ & 309.1865 & & & & & $\mathbf{L}$ & $\mathbf{1 0 3 2 . 5 5 7 2}$ & 516.7822 & $\mathbf{1 0 1 5 . 5 3 0 6}$ & 508.2689 & 1014.5466 & 507.7769 & $\mathbf{9}$ \\
\hline $\mathbf{7}$ & $\mathbf{7 3 2 . 3 9 2 7}$ & 366.7000 & 715.3661 & 358.1867 & & & $\mathbf{N}$ & $\mathbf{9 1 9 . 4 7 3 1}$ & 460.2402 & 902.4465 & 451.7269 & 901.4625 & 451.2349 & $\mathbf{8}$ \\
\hline $\mathbf{8}$ & $\mathbf{8 4 5 . 4 7 6 7}$ & 423.2420 & 828.4502 & 414.7287 & & & $\mathbf{L}$ & $\mathbf{8 0 4 . 4 4 6 2}$ & 402.7267 & 787.4196 & 394.2134 & 786.4356 & 393.7214 & $\mathbf{7}$ \\
\hline $\mathbf{9}$ & $\mathbf{9 3 2 . 5 0 8 8}$ & 466.7580 & 915.4822 & 458.2447 & 914.4982 & 457.7527 & $\mathbf{S}$ & $\mathbf{6 9 1 . 3 6 2 1}$ & 346.1847 & 674.3355 & 337.6714 & 673.3515 & 337.1794 & $\mathbf{6}$ \\
\hline $\mathbf{1 0}$ & $\mathbf{1 0 6 1 . 5 5 1 3}$ & 531.2793 & 1044.5248 & 522.7660 & 1043.5408 & 522.2740 & $\mathbf{E}$ & $\mathbf{6 0 4 . 3 3 0 1}$ & 302.6687 & 587.3035 & 294.1554 & 586.3195 & 293.6634 & $\mathbf{5}$ \\
\hline $\mathbf{1 1}$ & $\mathbf{1 1 7 4 . 6 3 5 4}$ & 587.8213 & 1157.6089 & 579.3081 & 1156.6248 & 578.8161 & $\mathbf{I}$ & $\mathbf{4 7 5 . 2 8 7 5}$ & 238.1474 & 458.2609 & 229.6341 & 457.2769 & 229.1421 & $\mathbf{4}$ \\
\hline $\mathbf{1 2}$ & $\mathbf{1 2 7 5 . 6 8 3 1}$ & 638.3452 & 1258.6565 & 629.8319 & 1257.6725 & 629.3399 & $\mathbf{T}$ & $\mathbf{3 6 2 . 2 0 3 4}$ & 181.6053 & 345.1769 & 173.0921 & 344.1928 & 172.6001 & $\mathbf{3}$ \\
\hline $\mathbf{1 3}$ & $\mathbf{1 3 8 9 . 7 2 6 0}$ & 695.3666 & $\mathbf{1 3 7 2 . 6 9 9 5}$ & 686.8534 & 1371.7155 & 686.3614 & $\mathbf{N}$ & $\mathbf{2 6 1 . 1 5 5 7}$ & 131.0815 & 244.1292 & 122.5682 & & & $\mathbf{2}$ \\
\hline $\mathbf{1 4}$ & & & & & & & $\mathbf{K}$ & 147.1128 & 74.0600 & 130.0863 & 65.5468 & & & $\mathbf{1}$ \\
\hline
\end{tabular}
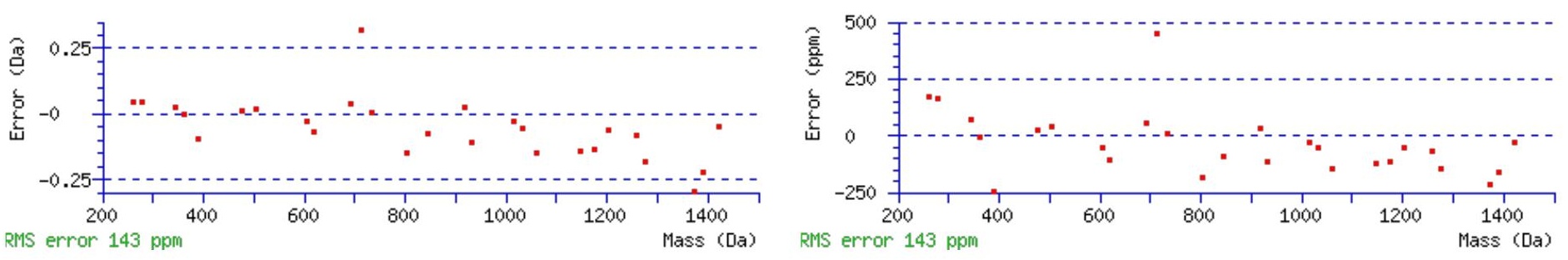

\section{All matches to this query}

\begin{tabular}{|l|l|l|l|}
\hline Score & Mr(calc): & Delta & \multicolumn{1}{c|}{ Sequence } \\
\hline 100.5 & 1534.8242 & -0.0020 & IYGGILNLSEITNK \\
\hline 46.9 & 1534.8242 & -0.0020 & IYGGILNLSEITNK \\
\hline 14.8 & 1534.8355 & -0.0133 & KFNLDATELSIRK \\
\hline 10.6 & 1534.8242 & -0.0020 & LLNYLLDNLSGTAK \\
\hline 9.8 & 1533.8072 & 1.0150 & IMNKNISLVDNKK \\
\hline 7.4 & 1534.8251 & -0.0029 & YLMVPALQGALTMK \\
\hline 7.2 & 1534.8355 & -0.0133 & DGYLELLKEATRK \\
\hline & & & \\
\hline
\end{tabular}




\begin{tabular}{|l|l|l|l|}
\hline 5.9 & 1534.8355 & -0.0133 & FLSQESGVAQTLKK \\
\hline 5.2 & 1534.8218 & 0.0004 & NFTAFPSLPGLIMK \\
\hline 3.6 & 1533.8072 & 1.0150 & IMNKNISLVDNKK \\
\hline
\end{tabular}

Spectrum No: 75; Query: 917; Rank: 1

\section{Peptide View}

\section{MS/MS Fragmentation of VDNSSLTGESEPQTR}

Found in IPI00205693, Tax_Id=10116 Gene_Symbol=Atp1a2 Sodium/potassium-transporting ATPase subunit alpha-2 precursor

Match to Query 917: 1619.724248 from(810.869400,2+)

Title: 091008RatKidney_NH4Format01_18.591.591.2.dta

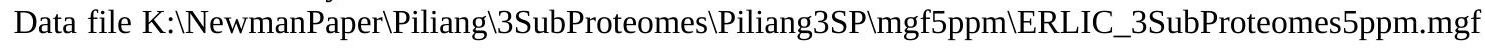
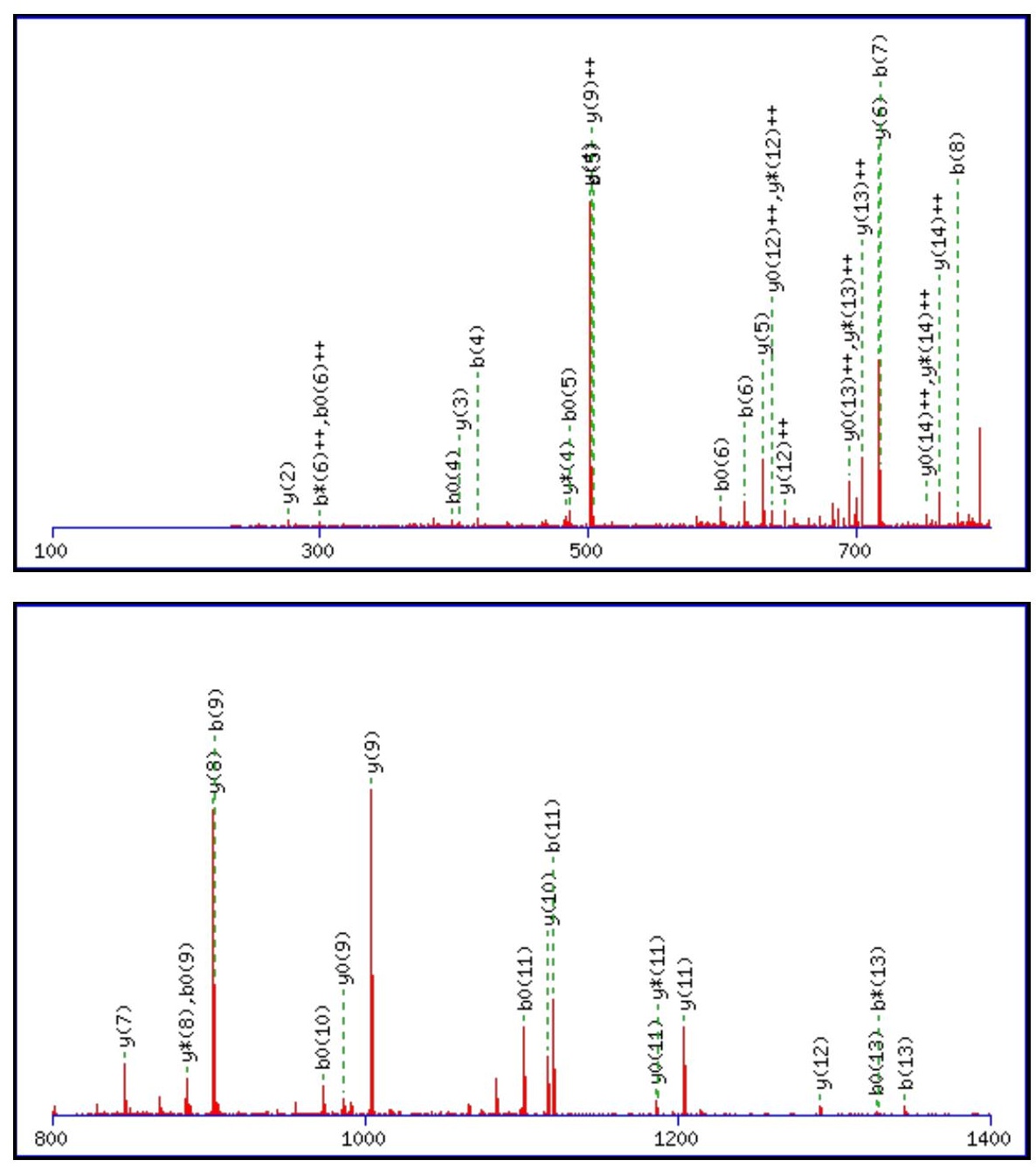


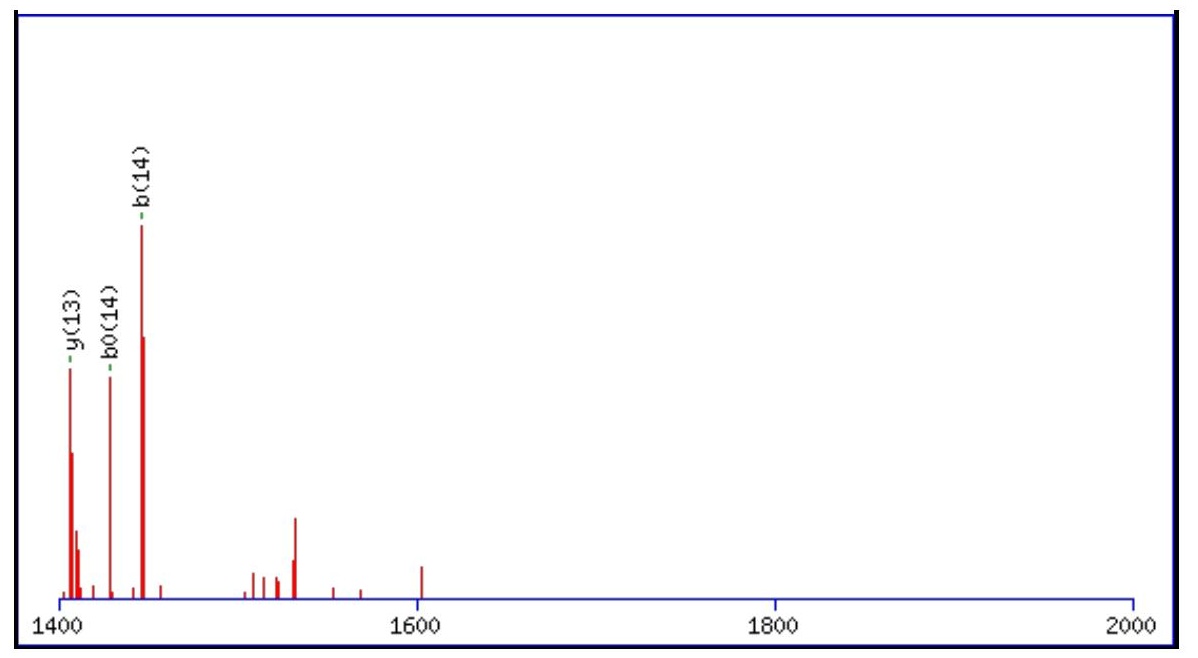

Monoisotopic mass of neutral peptide $\operatorname{Mr}($ calc): 1619.7275

Fixed modifications: Carbamidomethyl (C)

Variable modifications:

N3 : Deamidated $\mathrm{N}(\mathrm{N})$

Ions Score: 100 Expect: $1.8 \mathrm{e}-008$

Matches (Bold Red): 47/160 fragment ions using 63 most intense peaks

\begin{tabular}{|c|c|c|c|c|c|c|c|c|c|c|c|c|c|c|}
\hline \# & b & $\mathbf{b}^{++}$ & $\mathbf{b}^{*}$ & $\mathbf{b}^{*^{++}}$ & $\mathbf{b}^{\mathbf{0}}$ & $\mathbf{b}^{0++}$ & Seq. & $\mathbf{y}$ & $\mathbf{y}^{++}$ & $\mathbf{y}^{*}$ & $\mathbf{y}^{*^{++}}$ & $\mathbf{y}^{\mathbf{0}}$ & $\mathbf{y}^{0++}$ & \# \\
\hline 1 & 100.0757 & 50.5415 & & & & & $\mathbf{V}$ & & & & & & & 15 \\
\hline 2 & 215.1026 & 108.0550 & & & 197.0921 & 99.0497 & D & 1521.6663 & 761.3368 & 1504.6398 & 752.8235 & 1503.6558 & 752.3315 & 14 \\
\hline 3 & 330.1296 & 165.5684 & 313.1030 & 157.0551 & 312.1190 & 156.5631 & $\mathbf{N}$ & 1406.6394 & 703.8233 & 1389.6128 & 695.3101 & 1388.6288 & 694.8180 & 13 \\
\hline 4 & 417.1616 & 209.0844 & 400.1350 & 200.5712 & 399.1510 & 200.0792 & S & 1291.6124 & 646.3099 & 1274.5859 & 637.7966 & 1273.6019 & 637.3046 & 12 \\
\hline 5 & 504.1936 & 252.6005 & 487.1671 & 244.0872 & 486.1831 & 243.5952 & $\mathbf{S}$ & 1204.5804 & 602.7938 & 1187.5539 & 594.2806 & 1186.5699 & 593.7886 & 11 \\
\hline 6 & 617.2777 & 309.1425 & 600.2511 & 300.6292 & 599.2671 & 300.1372 & $\mathbf{L}$ & 1117.5484 & 559.2778 & 1100.5218 & 550.7646 & 1099.5378 & 550.2726 & 10 \\
\hline 7 & 718.3254 & 359.6663 & 701.2988 & 351.1530 & 700.3148 & 350.6610 & $\mathbf{T}$ & 1004.4643 & 502.7358 & 987.4378 & 494.2225 & 986.4538 & 493.7305 & 9 \\
\hline 8 & 775.3468 & 388.1771 & 758.3203 & 379.6638 & 757.3363 & 379.1718 & G & 903.4167 & 452.2120 & 886.3901 & 443.6987 & 885.4061 & 443.2067 & 8 \\
\hline 9 & 904.3894 & 452.6984 & 887.3629 & 444.1851 & 886.3789 & 443.6931 & $\mathbf{E}$ & 846.3952 & 423.7012 & 829.3686 & 415.1880 & 828.3846 & 414.6959 & 7 \\
\hline 10 & 991.4215 & 496.2144 & 974.3949 & 487.7011 & 973.4109 & 487.2091 & $\mathbf{S}$ & 717.3526 & 359.1799 & 700.3260 & 350.6667 & 699.3420 & 350.1747 & 6 \\
\hline 11 & 1120.4640 & 560.7357 & 1103.4375 & 552.2224 & 1102.4535 & 551.7304 & $\mathbf{E}$ & 630.3206 & 315.6639 & 613.2940 & 307.1506 & 612.3100 & 306.6586 & 5 \\
\hline 12 & 1217.5168 & 609.2620 & 1200.4903 & 600.7488 & 1199.5062 & 600.2568 & $\mathbf{P}$ & 501.2780 & 251.1426 & 484.2514 & 242.6293 & 483.2674 & 242.1373 & 4 \\
\hline 13 & 1345.5754 & 673.2913 & 1328.5488 & 664.7781 & 1327.5648 & 664.2860 & $\mathbf{Q}$ & 404.2252 & 202.6162 & 387.1987 & 194.1030 & 386.2146 & 193.6110 & 3 \\
\hline 14 & 1446.6231 & 723.8152 & 1429.5965 & 715.3019 & 1428.6125 & 714.8099 & $\mathbf{T}$ & 276.1666 & 138.5870 & 259.1401 & 130.0737 & 258.1561 & 129.5817 & 2 \\
\hline 15 & & & & & & & $\mathbf{R}$ & 175.1190 & 88.0631 & 158.0924 & 79.5498 & & & 1 \\
\hline
\end{tabular}
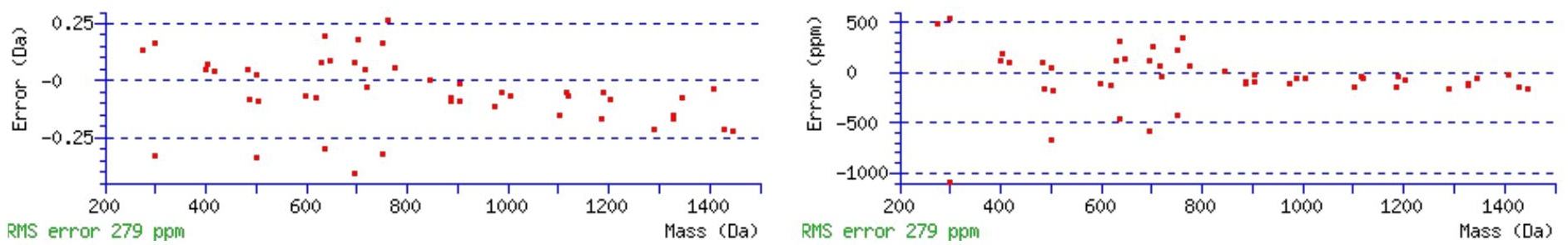

\section{All matches to this query}

\begin{tabular}{|l|l|l|l|}
\hline Score & Mr(calc): & Delta & \multicolumn{1}{c|}{ Sequence } \\
\hline 100.2 & 1619.7275 & -0.0032 & VDNSSLTGESEPQTR \\
\hline 11.3 & 1619.7165 & 0.0077 & RNSIPQXSGSSSHR \\
\hline 7.1 & 1619.7345 & -0.0102 & $\underline{\text { EKYSSAPNAVAFTR }}$ \\
\hline 6.8 & 1618.7321 & 0.9922 & $\underline{\text { KMRVSGVMTQGASR }}$ \\
\hline 4.1 & 1619.7279 & -0.0037 & $\underline{\text { FNHHSAMVLAAGLR }}$ \\
\hline 2.7 & 1619.7226 & 0.0016 & $\underline{\text { ILDGTGTSGTMKSTR }}$ \\
\hline & & & \\
\hline
\end{tabular}




\begin{tabular}{|l|l|l|l|}
2.4 & 1619.7153 & 0.0089 & IEYECKLNTLEK \\
\hline 2.1 & 1619.7127 & 0.0116 & GNGKNYMGNLSKTR \\
\hline 2.1 & 1619.7127 & 0.0116 & GNGKNYMGNLSKTR \\
\hline 1.9 & 1618.7313 & 0.9929 & $\underline{\text { IEYECKLNTLEK }}$ \\
\hline
\end{tabular}

Spectrum No: 76; Query: 1132; Rank: 1

\section{Peptide View}

MS/MS Fragmentation of MESDLANTTAGFTAALK

Found in IPI00392894, Tax_Id=10116 Gene_Symbol=Mgam_predicted 89 kDa protein

Match to Query 1132: 1740.824148 from(871.419350,2+)

Title: 091008RatKidney_NoSalt_26.3424.3424.2.dta

Data file K:\NewmanPaper\Piliang \3SubProteomes \Piliang3SP $\backslash$ mgf5ppm\ERLIC_3SubProteomes5ppm.mgf
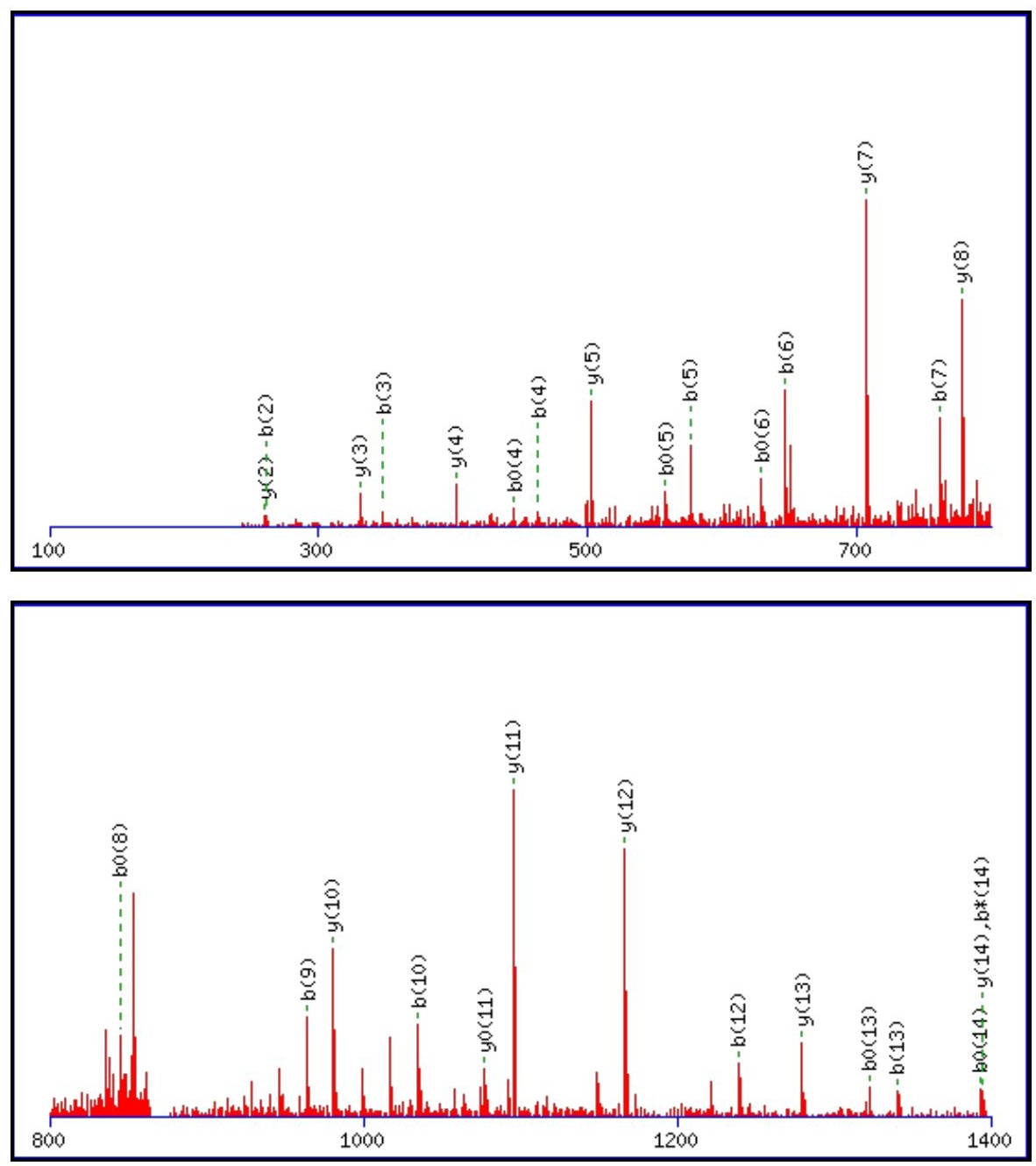


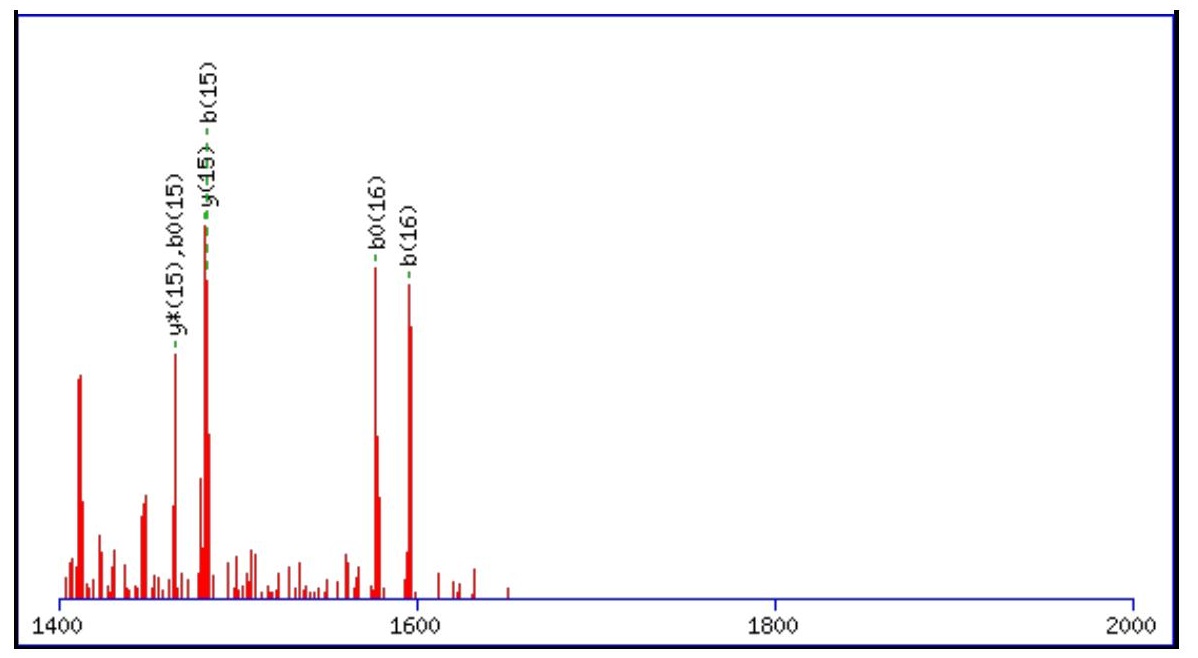

Monoisotopic mass of neutral peptide $\operatorname{Mr}($ calc): 1740.8240

Fixed modifications: Carbamidomethyl (C)

Variable modifications:

N7 : Deamidated $\mathrm{N}(\mathrm{N})$

Ions Score: 100 Expect: $1.8 \mathrm{e}-008$

Matches (Bold Red): 35/170 fragment ions using 53 most intense peaks

\begin{tabular}{|c|c|c|c|c|c|c|c|c|c|c|c|c|c|c|}
\hline \# & b & $\mathbf{b}^{++}$ & b* & $\mathbf{b}^{*++}$ & $\mathbf{b}^{\mathbf{0}}$ & $\mathbf{b}^{0++}$ & Seq. & $\mathbf{y}$ & $y^{++}$ & $\mathbf{y}^{*}$ & $\mathrm{y}^{\mathrm{*}^{++}}$ & $\mathbf{y}^{\mathbf{0}}$ & $\mathbf{y}^{0++}$ & \# \\
\hline 1 & 132.0478 & 66.5275 & & & & & $\mathbf{M}$ & & & & & & & 17 \\
\hline 2 & 261.0904 & 131.0488 & & & 243.0798 & 122.0435 & $\mathbf{E}$ & 1610.7908 & 805.8990 & 1593.7643 & 797.3858 & 1592.7802 & 796.8938 & 16 \\
\hline 3 & 348.1224 & 174.5648 & & & 330.1118 & 165.5595 & S & 1481.7482 & 741.3777 & 1464.7217 & 732.8645 & 1463.7376 & 732.3725 & 15 \\
\hline 4 & 463.1493 & 232.0783 & & & 445.1388 & 223.0730 & D & 1394.7162 & 697.8617 & 1377.6896 & 689.3485 & 1376.7056 & 688.8564 & 14 \\
\hline 5 & 576.2334 & 288.6203 & & & 558.2228 & 279.6151 & $\mathbf{L}$ & 1279.6892 & 640.3483 & 1262.6627 & 631.8350 & 1261.6787 & 631.3430 & 13 \\
\hline 6 & 647.2705 & 324.1389 & & & 629.2599 & 315.1336 & A & 1166.6052 & 583.8062 & 1149.5786 & 575.2930 & 1148.5946 & 574.8009 & 12 \\
\hline 7 & 762.2974 & 381.6524 & 745.2709 & 373.1391 & 744.2869 & 372.6471 & $\mathbf{N}$ & 1095.5681 & 548.2877 & 1078.5415 & 539.7744 & 1077.5575 & 539.2824 & 11 \\
\hline 8 & 863.3451 & 432.1762 & 846.3186 & 423.6629 & 845.3346 & 423.1709 & $\mathbf{T}$ & 980.5411 & 490.7742 & 963.5146 & 482.2609 & 962.5306 & 481.7689 & 10 \\
\hline 9 & 964.3928 & 482.7000 & 947.3662 & 474.1868 & 946.3822 & 473.6948 & $\mathbf{T}$ & 879.4934 & 440.2504 & 862.4669 & 431.7371 & 861.4829 & 431.2451 & 9 \\
\hline 10 & 1035.4299 & 518.2186 & 1018.4034 & 509.7053 & 1017.4193 & 509.2133 & A & 778.4458 & 389.7265 & 761.4192 & 381.2132 & 760.4352 & 380.7212 & 8 \\
\hline 11 & 1092.4514 & 546.7293 & 1075.4248 & 538.2161 & 1074.4408 & 537.7240 & G & 707.4087 & 354.2080 & 690.3821 & 345.6947 & 689.3981 & 345.2027 & 7 \\
\hline 12 & 1239.5198 & 620.2635 & 1222.4932 & 611.7503 & 1221.5092 & 611.2582 & $\mathbf{F}$ & 650.3872 & 325.6972 & 633.3606 & 317.1840 & 632.3766 & 316.6920 & 6 \\
\hline 13 & 1340.5675 & 670.7874 & 1323.5409 & 662.2741 & 1322.5569 & 661.7821 & $\mathbf{T}$ & 503.3188 & 252.1630 & 486.2922 & 243.6498 & 485.3082 & 243.1577 & 5 \\
\hline 14 & 1411.6046 & 706.3059 & 1394.5780 & 697.7927 & 1393.5940 & 697.3006 & A & 402.2711 & 201.6392 & 385.2445 & 193.1259 & & & 4 \\
\hline 15 & 1482.6417 & 741.8245 & 1465.6151 & 733.3112 & 1464.6311 & 732.8192 & A & 331.2340 & 166.1206 & 314.2074 & 157.6074 & & & 3 \\
\hline 16 & 1595.7258 & 798.3665 & 1578.6992 & 789.8532 & 1577.7152 & 789.3612 & $\mathbf{L}$ & 260.1969 & 130.6021 & 243.1703 & 122.0888 & & & 2 \\
\hline 17 & & & & & & & $\mathbf{K}$ & 147.1128 & 74.0600 & 130.0863 & 65.5468 & & & 1 \\
\hline
\end{tabular}

$$
\text { 跣 }
$$

$$
\text { RI }
$$$$
\text { (1) }
$$

\section{A}

\section{All matches to this query}

\begin{tabular}{|l|l|l|l|}
\hline Score & Mr(calc): & Delta & \multicolumn{1}{c|}{ Sequence } \\
\hline 100.1 & 1740.8240 & 0.0002 & $\underline{\text { MESDLANTTAGFTAALK }}$ \\
\hline 6.3 & 1739.8213 & 1.0028 & INSENSFSEASNLSLK \\
\hline 5.5 & 1739.8213 & 1.0028 & $\underline{\text { INSENSFSEASNLSLK }}$ \\
\hline 4.1 & 1740.8240 & 0.0002 & TSLMVDNQELGYLNK \\
\hline & & &
\end{tabular}

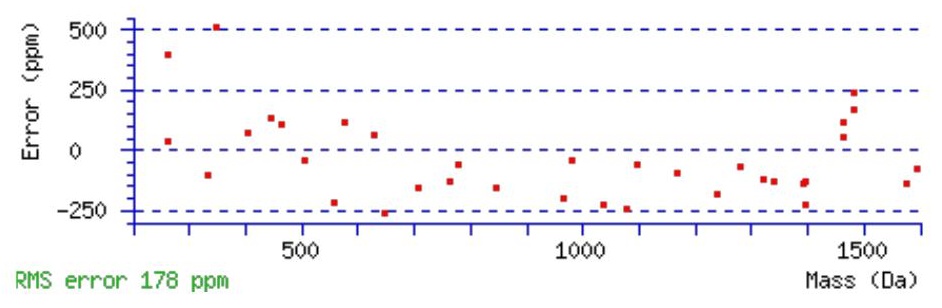

RMS error 178 ppm

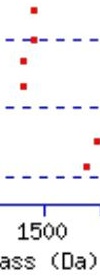

Mass (Da) 


\begin{tabular}{|l|l|l|l|}
3.9 & 1740.8270 & -0.0028 & LKVTHQAESNLYMK \\
\hline 3.5 & 1739.8188 & 1.0053 & EWMGYQGNKASVELK \\
\hline 3.4 & 1740.8270 & -0.0028 & LKVTHQAESNLYMK \\
\hline 3.4 & 1740.8196 & 0.0045 & GFVDDIIQPSSTRAR \\
\hline 2.7 & 1740.8196 & 0.0045 & GFVDDIIQPSSTRAR \\
\hline 2.0 & 1739.8213 & 1.0028 & INSENSFSEASNLSLK \\
\hline
\end{tabular}

Spectrum No: 77; Query: 1044; Rank: 1

\section{Peptide View}

MS/MS Fragmentation of YTGNASALFILPDQGK

Found in IPI00200591, Tax_Id=10116 Gene_Symbol=LOC299282 Serine protease inhibitor A3L precursor

Match to Query 1044: 1694.850428 from(848.432490,2+)

Title: 091008RatKidney_NoSalt_30.2971.2971.2.dta

Data file K:\NewmanPaper\Piliang \3SubProteomes \Piliang3SP \mgf5ppm\ERLIC_3SubProteomes5ppm.mgf
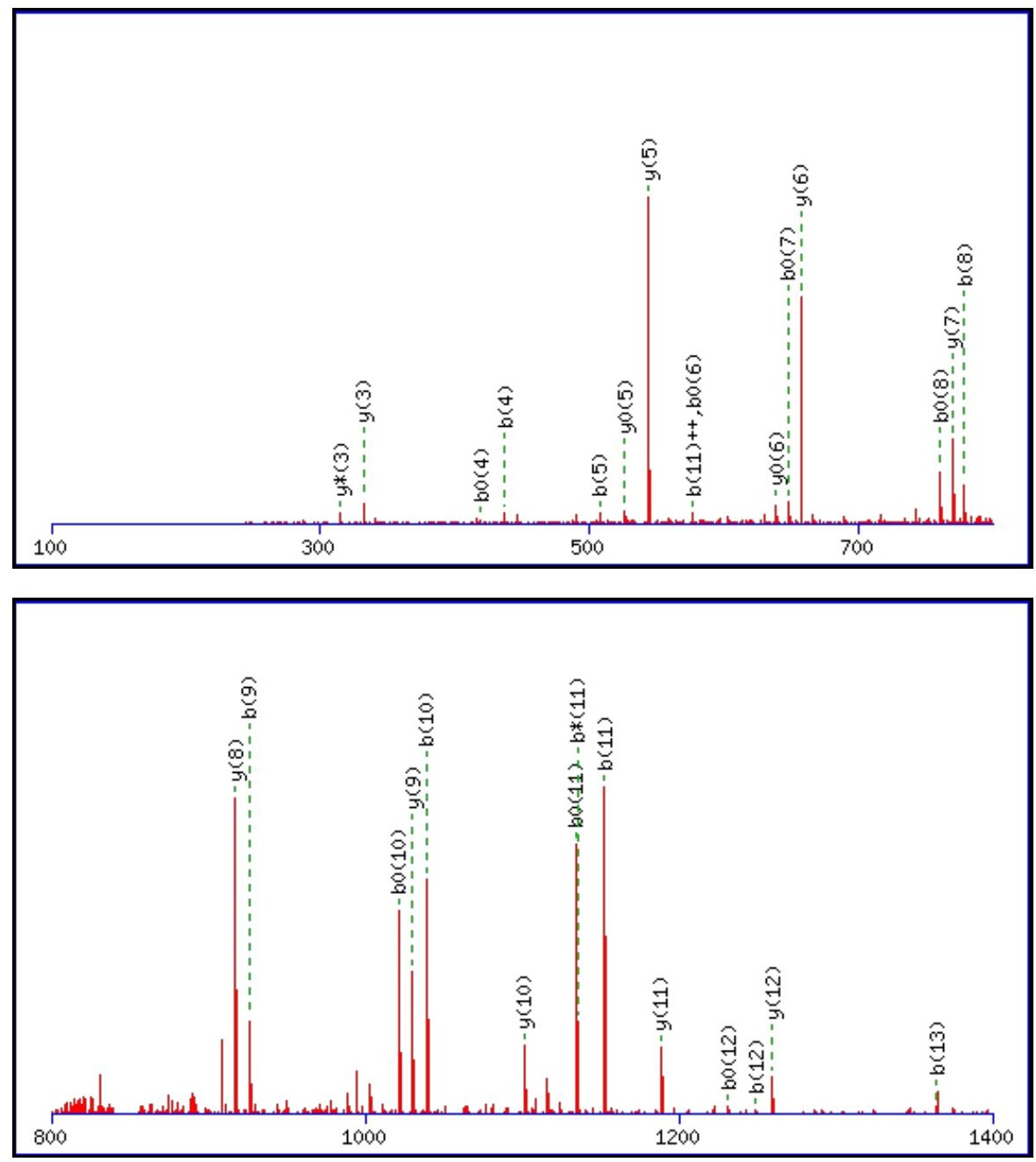


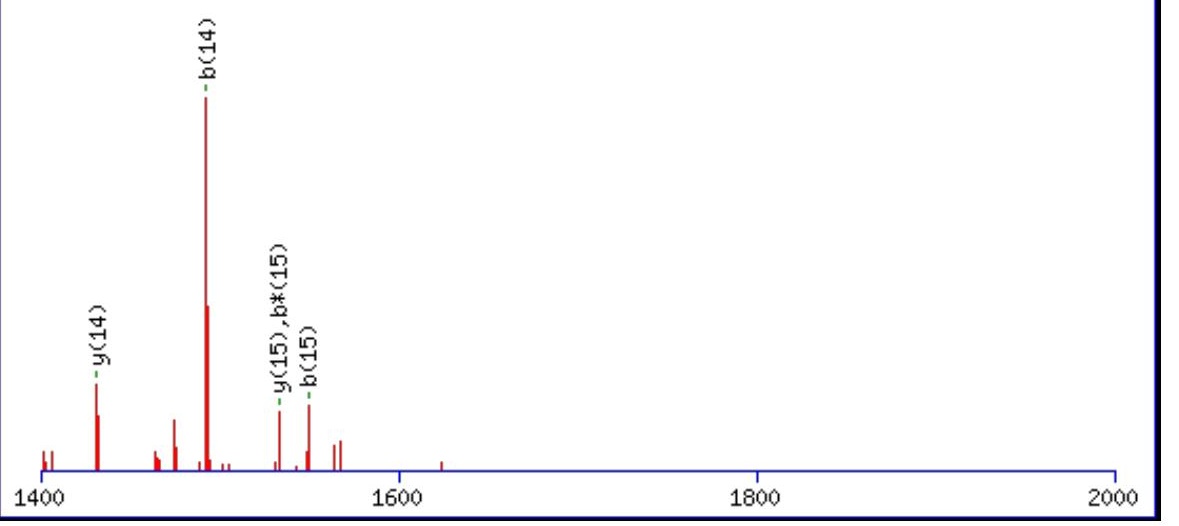

Monoisotopic mass of neutral peptide $\operatorname{Mr}($ calc): 1694.8515

Fixed modifications: Carbamidomethyl (C)

Variable modifications:

N4 : Deamidated $\mathrm{N}(\mathrm{N})$

Ions Score: 100 Expect: $1.7 \mathrm{e}-008$

Matches (Bold Red): 34/166 fragment ions using 48 most intense peaks

\begin{tabular}{|c|c|c|c|c|c|c|c|c|c|c|c|c|c|c|}
\hline \# & b & $\mathbf{b}^{++}$ & $\mathbf{b}^{*}$ & $\mathbf{b}^{*^{++}}$ & $\mathbf{b}^{0}$ & $\mathbf{b}^{\mathbf{0 + +}}$ & Seq. & $\mathbf{y}$ & $\mathbf{y}^{++}$ & $\mathbf{y}^{*}$ & $\mathbf{y}^{*^{++}}$ & $\mathbf{y}^{0}$ & $\mathbf{y}^{0++}$ & \# \\
\hline 1 & 164.0706 & 82.5389 & & & & & $\mathbf{Y}$ & & & & & & & 16 \\
\hline 2 & 265.1183 & 133.0628 & & & 247.1077 & 124.0575 & $\mathbf{T}$ & 1532.7955 & 766.9014 & 1515.7689 & 758.3881 & 1514.7849 & 757.8961 & 15 \\
\hline 3 & 322.1397 & 161.5735 & & & 304.1292 & 152.5682 & G & 1431.7478 & 716.3775 & 1414.7213 & 707.8643 & 1413.7372 & 707.3723 & 14 \\
\hline 4 & 437.1667 & 219.0870 & 420.1401 & 210.5737 & 419.1561 & 210.0817 & $\mathbf{N}$ & 1374.7264 & 687.8668 & 1357.6998 & 679.3535 & 1356.7158 & 678.8615 & 13 \\
\hline 5 & 508.2038 & 254.6055 & 491.1772 & 246.0923 & 490.1932 & 245.6003 & A & 1259.6994 & 630.3533 & 1242.6729 & 621.8401 & 1241.6888 & 621.3481 & 12 \\
\hline 6 & 595.2358 & 298.1216 & 578.2093 & 289.6083 & 577.2253 & 289.1163 & S & 1188.6623 & 594.8348 & 1171.6358 & 586.3215 & 1170.6517 & 585.8295 & 11 \\
\hline 7 & 666.2729 & 333.6401 & 649.2464 & 325.1268 & 648.2624 & 324.6348 & A & 1101.6303 & 551.3188 & 1084.6037 & 542.8055 & 1083.6197 & 542.3135 & 10 \\
\hline 8 & 779.3570 & 390.1821 & 762.3305 & 381.6689 & 761.3464 & 381.1769 & $\mathbf{L}$ & 1030.5932 & 515.8002 & 1013.5666 & 507.2869 & 1012.5826 & 506.7949 & 9 \\
\hline 9 & 926.4254 & 463.7163 & 909.3989 & 455.2031 & 908.4149 & 454.7111 & $\mathbf{F}$ & 917.5091 & 459.2582 & 900.4825 & 450.7449 & 899.4985 & 450.2529 & 8 \\
\hline 10 & 1039.5095 & 520.2584 & 1022.4829 & 511.7451 & 1021.4989 & 511.2531 & I & 770.4407 & 385.7240 & 753.4141 & 377.2107 & 752.4301 & 376.7187 & 7 \\
\hline 11 & 1152.5935 & 576.8004 & 1135.5670 & 568.2871 & 1134.5830 & 567.7951 & $\mathbf{L}$ & 657.3566 & 329.1819 & 640.3301 & 320.6687 & 639.3461 & 320.1767 & 6 \\
\hline 12 & |1249.6463 & 625.3268 & 1232.6198 & 616.8135 & 1231.6357 & 616.3215 & $\mathbf{P}$ & 544.2726 & 272.6399 & 527.2460 & 264.1266 & 526.2620 & 263.6346 & 5 \\
\hline 13 & 1364.6733 & 682.8403 & 1347.6467 & 674.3270 & 1346.6627 & 673.8350 & D & 447.2198 & 224.1135 & 430.1932 & 215.6003 & 429.2092 & 215.1082 & 4 \\
\hline 14 & 1492.7318 & 746.8696 & 1475.7053 & 738.3563 & 1474.7213 & 737.8643 & $\mathbf{Q}$ & 332.1928 & 166.6001 & 315.1663 & 158.0868 & & & 3 \\
\hline 15 & 1549.7533 & 775.3803 & 1532.7267 & 766.8670 & 1531.7427 & 766.3750 & G & 204.1343 & 102.5708 & 187.1077 & 94.0575 & & & 2 \\
\hline 16 & & & & & & & $\mathbf{K}$ & 147.1128 & 74.0600 & 130.0863 & 65.5468 & & & 1 \\
\hline
\end{tabular}
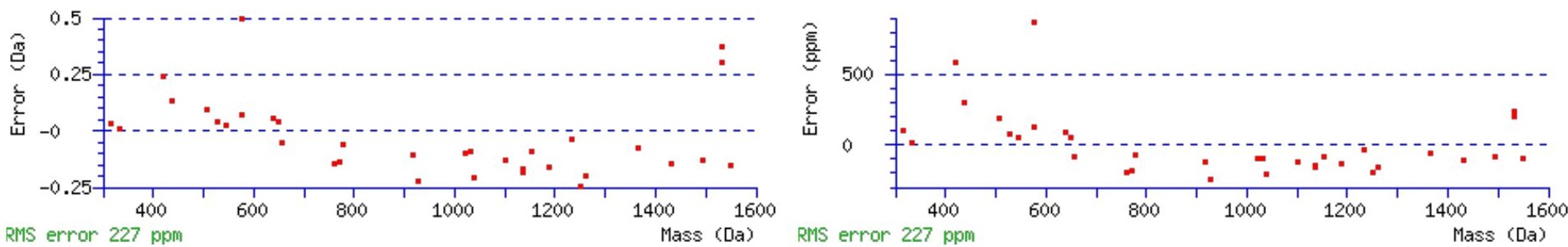

\section{All matches to this query}

\begin{tabular}{|l|l|l|l|}
\hline Score & Mr(calc): & Delta & \multicolumn{1}{c|}{ Sequence } \\
\hline 100.0 & 1694.8515 & -0.0011 & YTGNASALFILPDQGK \\
\hline 10.9 & 1693.8440 & 1.0064 & FAENQGTPLLVIANK \\
\hline 9.7 & 1694.8662 & -0.0157 & SLITFEGGSMIQIQR \\
\hline 8.7 & 1694.8362 & 0.0142 & VLEPEAAEEALENGPK \\
\hline 8.6 & 1694.8662 & -0.0157 & SQDNVIKVCASVTFK \\
\hline & & & \\
\hline
\end{tabular}




\begin{tabular}{|l|l|l|l|}
5.6 & 1694.8628 & -0.0124 & SDGGNLNFEFQIVKK \\
\hline 5.5 & 1694.8426 & 0.0078 & VLPDTMRNLLSTQK \\
\hline 4.0 & 1694.8379 & 0.0125 & LETEILNKEAVEVK \\
\hline 3.6 & 1694.8426 & 0.0078 & VLPDTMRNLLSTQK \\
\hline 2.8 & 1694.8406 & 0.0098 & $\underline{\text { IHHRIHTGEKPYK }}$ \\
\hline
\end{tabular}

Spectrum No: 78; Query: 1084; Rank: 1

\section{Peptide View}

MS/MS Fragmentation of DSSDEANCTTLCSQK

Found in IPI00205325, Tax_Id=10116 Gene_Symbol=Lrp2 Low-density lipoprotein receptor-related protein 2 precursor

Match to Query 1084: 1715.660968 from(858.837760,2+)

Title: 091008RatKidney_NoSalt_26.531.531.2.dta

Data file K:INewmanPaper|Piliangl3SubProteomes\Piliang3SP\mgf5ppm\ERLIC_3SubProteomes5ppm.mgf
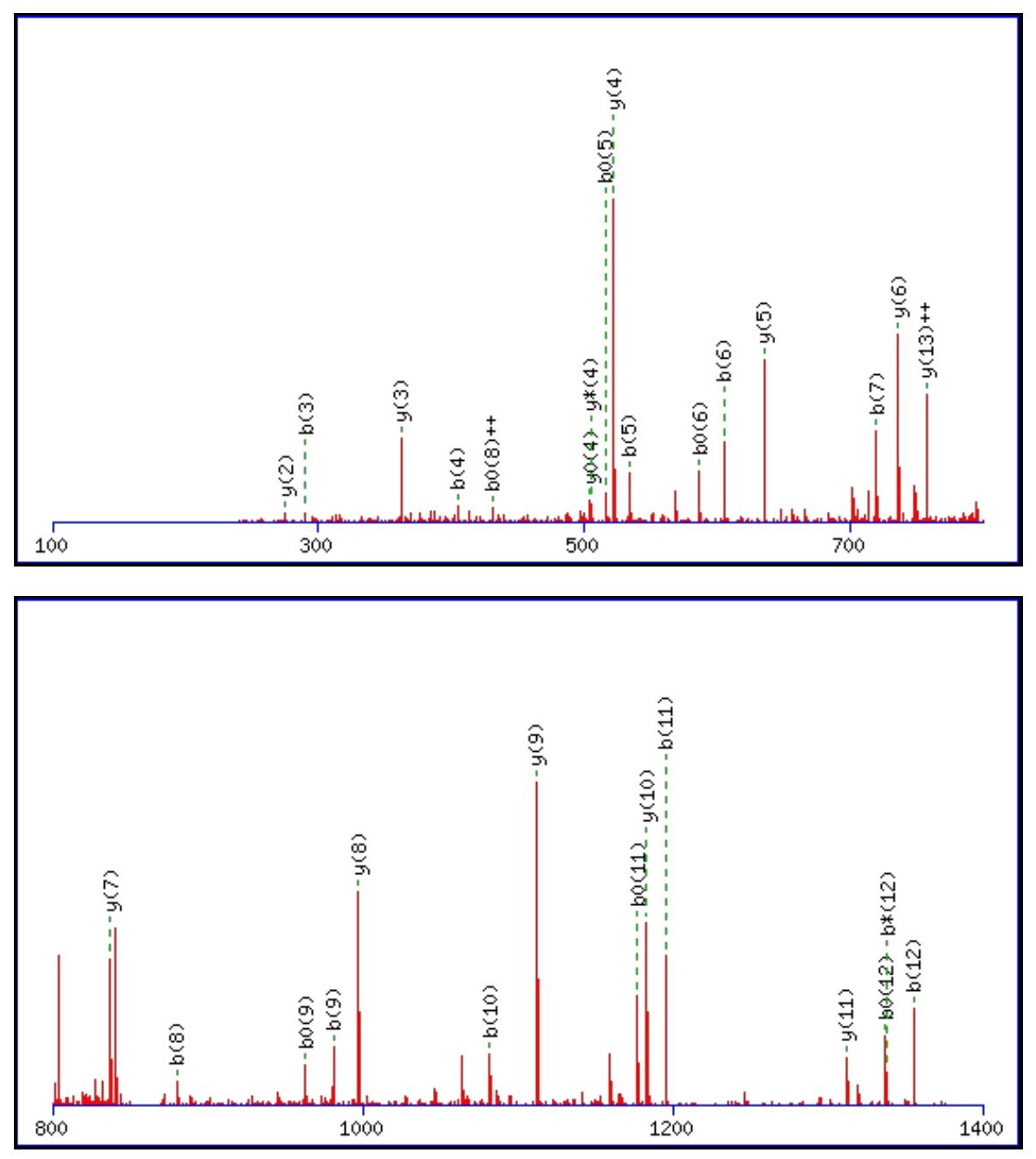


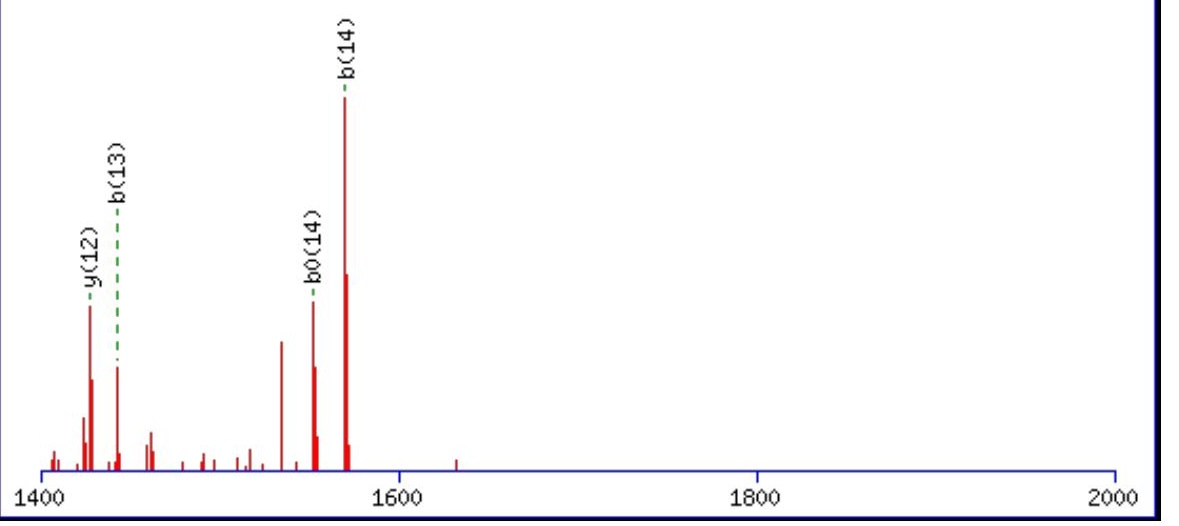

Monoisotopic mass of neutral peptide $\operatorname{Mr}($ calc): 1715.6614

Fixed modifications: Carbamidomethyl (C)

Variable modifications:

N7 : Deamidated $\mathrm{N}(\mathrm{N})$

Ions Score: 100 Expect: $1.3 \mathrm{e}-008$

Matches (Bold Red): 34/152 fragment ions using 48 most intense peaks

\begin{tabular}{|c|c|c|c|c|c|c|c|c|c|c|c|c|c|c|}
\hline \# & b & $\mathbf{b}^{++}$ & $\mathbf{b}^{*}$ & $\mathbf{b}^{*^{++}}$ & $\mathbf{b}^{0}$ & $\mathbf{b}^{\mathbf{0 + +}}$ & Seq. & $\mathbf{y}$ & $\mathbf{y}^{++}$ & $\mathbf{y}^{*}$ & $\mathbf{y}^{*^{++}}$ & $\mathbf{y}^{0}$ & $\mathbf{y}^{0++}$ & \# \\
\hline 1 & 116.0342 & 58.5207 & & & 98.0237 & 49.5155 & D & & & & & & & 15 \\
\hline 2 & 203.0662 & 102.0368 & & & 185.0557 & 93.0315 & $\mathbf{S}$ & 1601.6418 & 801.3245 & 1584.6152 & 792.8112 & 1583.6312 & 792.3192 & 14 \\
\hline 3 & 290.0983 & 145.5528 & & & 272.0877 & 136.5475 & $\mathbf{S}$ & 1514.6097 & 757.8085 & 1497.5832 & 749.2952 & 1496.5992 & 748.8032 & 13 \\
\hline 4 & 405.1252 & 203.0662 & & & 387.1147 & 194.0610 & D & 1427.5777 & 714.2925 & 1410.5512 & 705.7792 & 1409.5672 & 705.2872 & 12 \\
\hline 5 & 534.1678 & 267.5875 & & & 516.1572 & 258.5823 & $\mathbf{E}$ & 1312.5508 & 656.7790 & 1295.5242 & 648.2658 & 1294.5402 & 647.7737 & 11 \\
\hline 6 & 605.2049 & 303.1061 & & & 587.1944 & 294.1008 & A & 1183.5082 & 592.2577 & 1166.4816 & 583.7445 & 1165.4976 & 583.2524 & 10 \\
\hline 7 & 720.2319 & 360.6196 & 703.2053 & 352.1063 & 702.2213 & 351.6143 & $\mathbf{N}$ & 1112.4711 & 556.7392 & 1095.4445 & 548.2259 & 1094.4605 & 547.7339 & 9 \\
\hline 8 & 880.2625 & 440.6349 & 863.2360 & 432.1216 & 862.2519 & 431.6296 & C & 997.4441 & 499.2257 & 980.4176 & 490.7124 & 979.4336 & 490.2204 & 8 \\
\hline 9 & 981.3102 & 491.1587 & 964.2836 & 482.6455 & 963.2996 & 482.1535 & $\mathbf{T}$ & 837.4135 & 419.2104 & 820.3869 & 410.6971 & 819.4029 & 410.2051 & 7 \\
\hline 10 & 1082.3579 & 541.6826 & 1065.3313 & 533.1693 & 1064.3473 & 532.6773 & $\mathbf{T}$ & 736.3658 & 368.6865 & 719.3393 & 360.1733 & 718.3552 & 359.6813 & 6 \\
\hline 11 & 1195.4419 & 598.2246 & 1178.4154 & 589.7113 & 1177.4314 & 589.2193 & $\mathbf{L}$ & 635.3181 & 318.1627 & 618.2916 & 309.6494 & 617.3076 & 309.1574 & 5 \\
\hline 12 & 1355.4726 & 678.2399 & 1338.4460 & 669.7267 & 1337.4620 & 669.2346 & $\mathrm{C}$ & 522.2341 & 261.6207 & 505.2075 & 253.1074 & 504.2235 & 252.6154 & 4 \\
\hline 13 & 1442.5046 & 721.7559 & 1425.4781 & 713.2427 & 1424.4940 & 712.7507 & $\mathbf{S}$ & 362.2034 & 181.6053 & 345.1769 & 173.0921 & 344.1928 & 172.6001 & 3 \\
\hline 14 & 1570.5632 & 785.7852 & 1553.5366 & 777.2720 & 1552.5526 & 776.7799 & $\mathbf{Q}$ & 275.1714 & 138.0893 & 258.1448 & 129.5761 & & & 2 \\
\hline 15 & & & & & & & $\mathbf{K}$ & 147.1128 & 74.0600 & 130.0863 & 65.5468 & & & 1 \\
\hline
\end{tabular}

.

RMS error 202 ppm

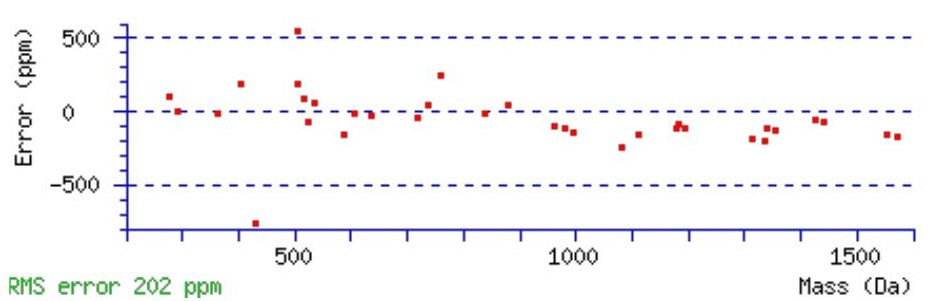

\section{All matches to this query}

\begin{tabular}{|l|l|l|l|}
\hline Score & Mr(calc): & Delta & \multicolumn{1}{c|}{ Sequence } \\
\hline 99.9 & 1715.6614 & -0.0005 & $\underline{\text { DSSDEANCTTLCSQK }}$ \\
\hline 7.8 & 1715.6701 & -0.0091 & $\underline{\text { SADFTCSNLLAMVK }}$ \\
\hline 7.3 & 1715.6548 & 0.0062 & $\underline{\text { MTVTNLLSRNMDK }}$ \\
\hline 5.8 & 1715.6706 & -0.0096 & $\underline{\text { RFYRGDSPTDSQK }}$ \\
\hline 4.6 & 1715.6481 & 0.0129 & $\underline{\text { YTHAANTVVYSSNK }}$ \\
\hline 4.6 & 1715.6481 & 0.0129 & $\underline{\text { YTHAANTVVYSSNK }}$ \\
\hline & & & \\
\hline
\end{tabular}




\begin{tabular}{|l|l|l|l|}
3.8 & 1715.6593 & 0.0017 & NQNFNESFTISQK \\
\hline 3.8 & 1715.6706 & -0.0096 & RFYRGDSPTDSQK \\
\hline 3.3 & 1715.6706 & -0.0096 & RFYRGDSPTDSQK \\
\hline 3.3 & 1715.6457 & 0.0152 & TDSLVTGTKPDSQK \\
\hline
\end{tabular}

Spectrum No: 79; Query: 2372; Rank: 1

\section{Peptide View}

MS/MS Fragmentation of DGQQNISLLYTEPGASQTQTGASFR

Found in IPI00214859, Tax_Id=10116 Gene_Symbol=Col18a1 similar to Collagen alpha-1(XVIII) chain precursor

Match to Query 2372: 2669.267428 from(1335.640990,2+)

Title: 100101RatKid_NS_deglyco_18.3864.3864.2.dta

Data file K:INewmanPaper|Piliangl3SubProteomes\Piliang3SP\mgf5ppm\ERLIC_3SubProteomes5ppm.mgf
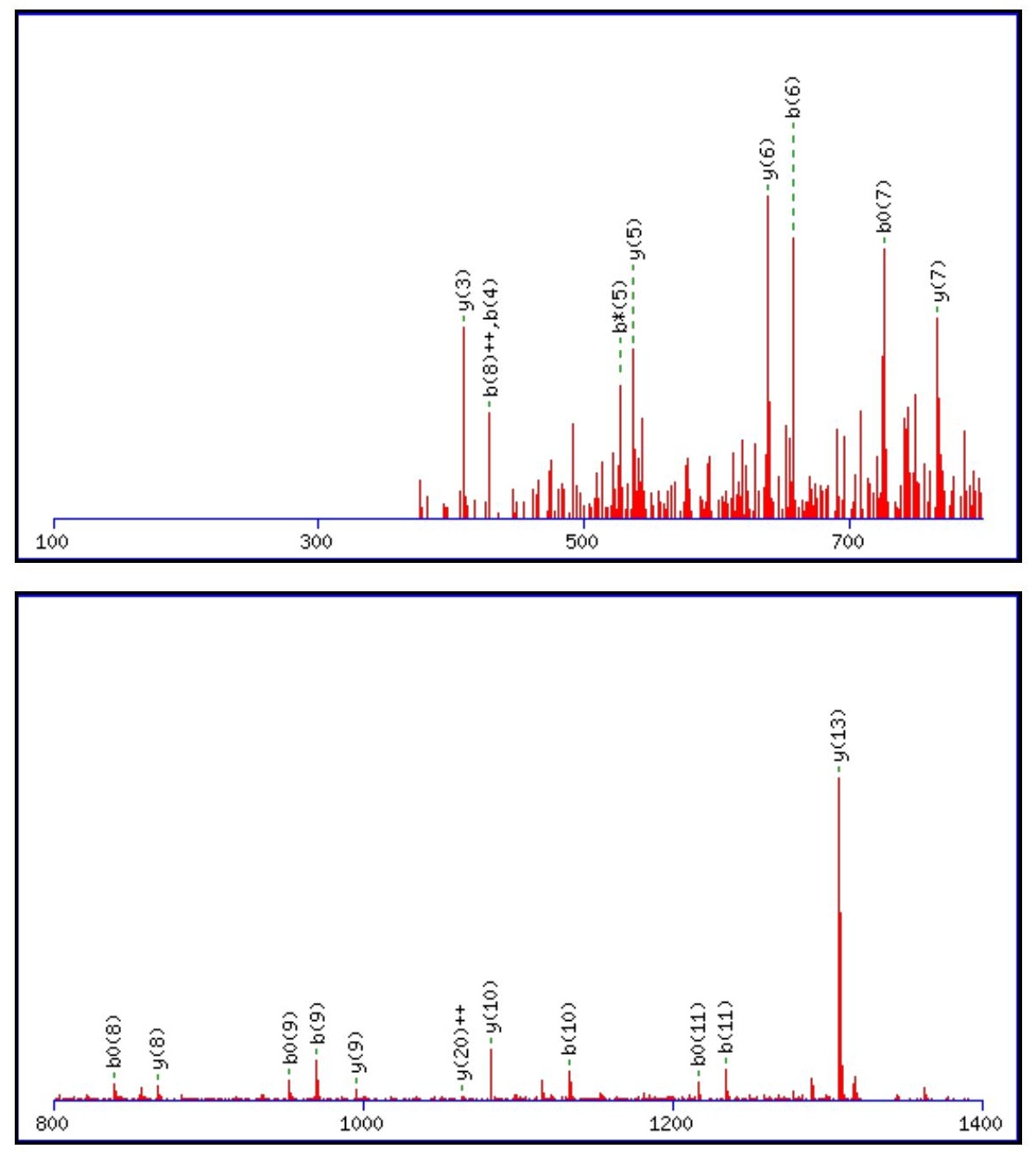


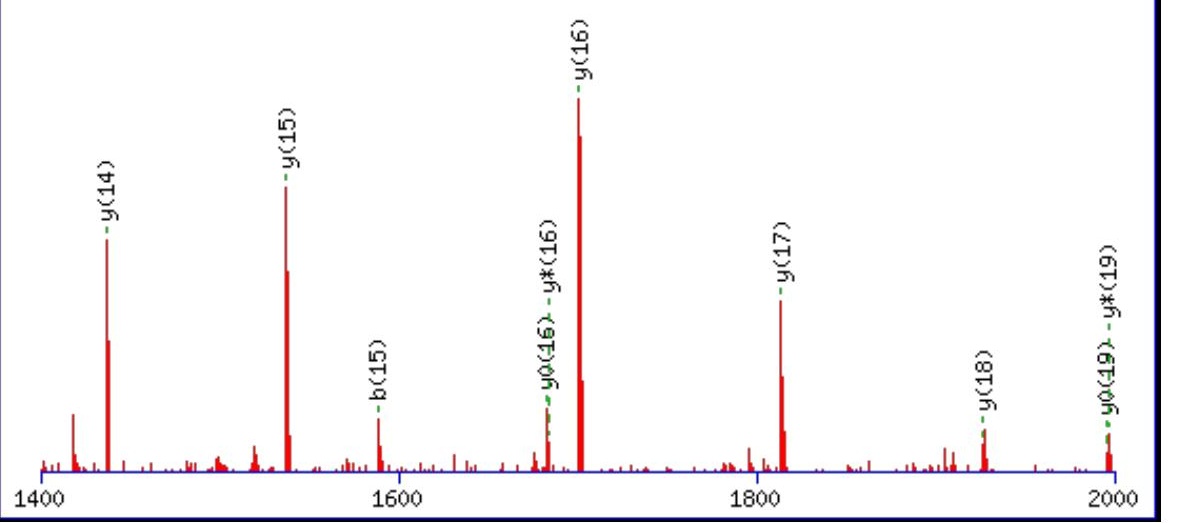

Monoisotopic mass of neutral peptide $\operatorname{Mr}($ calc): 2669.2569

Fixed modifications: Carbamidomethyl (C)

Variable modifications:

N5: Deamidated N (N)

Ions Score: 100 Expect: $3 e-008$

Matches (Bold Red): 30/280 fragment ions using 38 most intense peaks

\begin{tabular}{|c|c|c|c|c|c|c|c|c|c|c|c|c|c|c|}
\hline \# & b & $\mathbf{b}^{++}$ & b* & & $\mathbf{b}^{\mathbf{0}}$ & & Seq. & $\mathbf{y}$ & & $\mathbf{y}^{*}$ & & $\mathbf{y}^{\mathbf{0}}$ & & \# \\
\hline 1 & 116.0342 & 58.5207 & & & 98.0237 & 49.5155 & D & & & & & & & 25 \\
\hline 2 & 173.0557 & 87.0315 & & & 155.0451 & & G & & & & & 2537.2267 & 1170 & 24 \\
\hline 3 & 301.1143 & 151.0608 & & & 283.1037 & 142.0555 & $\mathbf{Q}$ & 2158 & 115 & 1892 & 982 & 2052 & 062 & 23 \\
\hline 4 & 429.1728 & 215.0 & 412.1463 & 206.5768 & 411.1623 & 206.0848 & $\mathbf{Q}$ & 2370.1572 & 1185.5822 & 2353.1306 & 1177.0690 & 2352.1466 & 1176.5770 & 22 \\
\hline 5 & 544.1998 & 272.6035 & 527.1732 & 264.0903 & 526.1892 & 263.5982 & $\mathbf{N}$ & 2242.0986 & 1121.5529 & 2225.0721 & 1113.0397 & 2224.0880 & 5477 & 21 \\
\hline 6 & 657.2838 & 329.1456 & 640.2 & & 639.2 & 320.1 & I & 2127.0717 & & 2110.0451 & & 2109.0611 & 1055.0342 & 20 \\
\hline 7 & 744.3159 & 372.6616 & 727.2893 & 364. & 726.3053 & 363.6563 & S & 2013.9876 & 1007.4974 & .9611 & 9842 & 1995.9770 & 998.4922 & 19 \\
\hline 8 & 857.3999 & 429.2036 & 840.3734 & 420.6903 & 839.3894 & 420.1983 & $\mathbf{L}$ & 1926.9556 & & 1909 & 955.4682 & 1908.9450 & 9761 & 18 \\
\hline 9 & 970.4840 & 485.7456 & 953.4574 & 477.2324 & 952.4734 & 476.7404 & $\mathbf{L}$ & 1813.8715 & 907.4394 & 1796 & 9261 & 1795.8610 & 4341 & 17 \\
\hline 10 & 1133.5473 & 567.2773 & 1116.5208 & 558.7640 & 1115.5368 & 558.2720 & $\mathbf{Y}$ & 1700.7875 & 850.8974 & 1683.7609 & 842.3841 & 1682.7769 & 841.8921 & 16 \\
\hline 11 & 234.5950 & 617.8011 & 217.5685 & 609.2879 & 1216.5844 & 608.7959 & $\mathbf{T}$ & 1537.7241 & 3657 & 6976 & 8524 & 1519.7136 & 3604 & 15 \\
\hline 12 & 1363.6376 & 682.3224 & 1346.6110 & 673.8092 & 1345.6270 & 673.3172 & $\mathbf{E}$ & 1436.6764 & 718.8419 & 1419.6499 & 710.3286 & 1418.6659 & 709.8366 & 14 \\
\hline 13 & 460.6904 & 730.8488 & 443.6638 & 722.3355 & 1442.6798 & 721.8435 & $\mathbf{P}$ & 1307.6339 & 654.3206 & 1290 & 3073 & 1289. & 153 & 13 \\
\hline 14 & 1517.7118 & 759.3596 & 1500.6853 & 750.8463 & 1499.7013 & 750.3543 & G & 1210.5811 & 605.7942 & 1193.5545 & 597.2809 & 1192.5705 & 596.7889 & 12 \\
\hline 15 & 588.7489 & 794.8781 & 571.7224 & 786.3648 & 1570.7384 & 785.8728 & A & 1153.5596 & 577.2835 & 1136. & 568.7702 & 1135.5491 & 568.2782 & 11 \\
\hline 16 & 675.7810 & 838.3941 & 1658.7544 & 829.8808 & 1657.7704 & 829.3888 & $\mathbf{S}$ & 1082.5225 & 541.7649 & 1065.4960 & 533.2516 & 1064.5119 & 532.7596 & 10 \\
\hline 17 & 1803.8395 & 902.4234 & 786.8130 & 893.9101 & 1785.8290 & 893.4181 & $\mathbf{Q}$ & 995.4905 & 498.2489 & 978.4639 & 489.7356 & 977.4799 & 489.2436 & 9 \\
\hline 18 & 1904.8872 & 952.9472 & 1887.8607 & 944.4340 & 1886.8767 & 943.9420 & $\mathbf{T}$ & 867.4319 & 434.2196 & 850.4054 & 425.7063 & 849.4213 & 425.2143 & 8 \\
\hline 19 & 2032.9458 & 1016.9765 & 2015.9193 & 1008.4633 & 2014.9352 & 1007.9713 & $\mathbf{Q}$ & 766.3842 & 383.6958 & 749.3577 & 375.1825 & 748.3737 & 374.6905 & 7 \\
\hline 20 & 2133.9935 & 1067.5004 & 2116.9669 & 1058.9871 & 2115.9829 & 1058.4951 & $\mathbf{T}$ & 638.3257 & 319.6665 & 621.2991 & 311.1532 & 620.3151 & 310.6612 & 6 \\
\hline 21 & 2191.0149 & 1096.0111 & 2173.9884 & 1087.4978 & 2173.0044 & 1087.0058 & G & 537.2780 & 269.1426 & 520.2514 & 260.6293 & 519.2674 & 260.1373 & 5 \\
\hline 22 & 2262.0521 & 1131.5297 & 2245.0255 & 1123.0164 & 2244.0415 & 1122.5244 & A & 480.2565 & 240.6319 & 463.2300 & 232.1186 & 462.2459 & 231.6266 & 4 \\
\hline 23 & 2349.0841 & 175.0457 & 2332.0575 & 1166.5324 & 2331.0735 & 1166.0404 & S & 409.2194 & 205.1133 & 392.1928 & 196.6001 & 391.2088 & 196.1081 & 3 \\
\hline 24 & 2496.1525 & 1248.5799 & 2479.1260 & 1240.0666 & 2478.1419 & 1239.5746 & $\mathbf{F}$ & 322.1874 & 161.5973 & 305.1608 & 153.0840 & & & 2 \\
\hline 25 & & & & & & & $\mathbf{R}$ & 175.1190 & 88.0631 & 158.0924 & 79.5498 & & & 1 \\
\hline
\end{tabular}
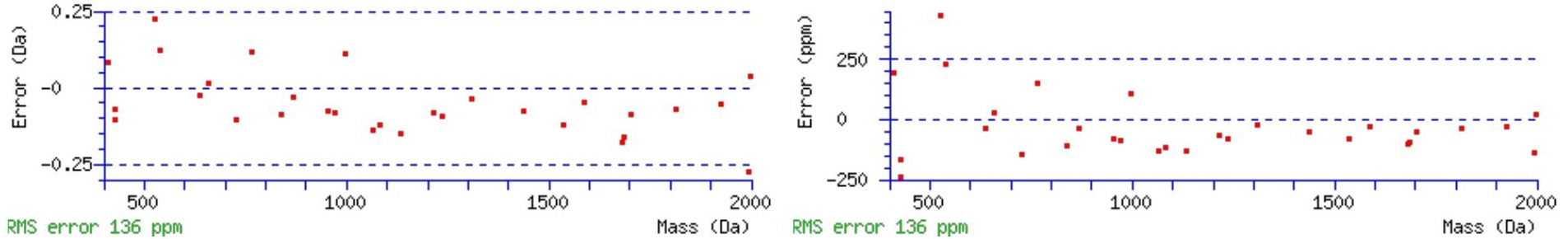


\section{All matches to this query}

\begin{tabular}{|l|l|l|l|}
\hline Score & Mr(calc): & Delta & \multicolumn{1}{|c|}{ Sequence } \\
\hline 99.9 & 2669.2569 & 0.0105 & $\underline{\text { DGQQNISLLYTEPGASQTQTGASFR }}$ \\
\hline 75.2 & 2668.2729 & 0.9945 & DGQQNISLLYTEPGASQTQTGASFR \\
\hline 7.8 & 2667.2370 & 2.0304 & $\underline{\text { TVSVWSLINSNKDEFTNPFYAR }}$ \\
\hline 7.3 & 2669.2808 & -0.0133 & YIAWYQQKPGKAPGLLIGYTSR \\
\hline 7.3 & 2669.2808 & -0.0133 & YIAWYQQKPGKAPGLLIGYTSR \\
\hline 7.3 & 2669.2808 & -0.0133 & $\underline{\text { YIAWYQQKPGKAPGLLIGYTSR }}$ \\
\hline 4.5 & 2667.2458 & 2.0216 & $\underline{\text { FNFNSDLRPRITTAIETVREK }}$ \\
\hline 4.5 & 2667.2458 & 2.0216 & $\underline{\text { FNFNSDLRPRITTAIETVREK }}$ \\
\hline 3.5 & 2667.2458 & 2.0216 & $\underline{\text { FNFNSDLRPRITTAIETVREK }}$ \\
\hline 3.4 & 2667.2370 & 2.0304 & $\underline{\text { TVSVWSLINSNKDEFTNPFYAR }}$ \\
\hline
\end{tabular}

Spectrum No: 80; Query: 1822; Rank: 1

\section{Peptide View}

MS/MS Fragmentation of EDAQINTTIGSVAAQDPDAAR

Found in IPI00215357, Tax_Id=10116 Gene_Symbol=Cdh6 Cadherin-6 precursor

Match to Query 1822: 2143.007088 from(1072.510820,2+)

Title: 100101RatKid_NS_deglyco_21.2614.2614.2.dta

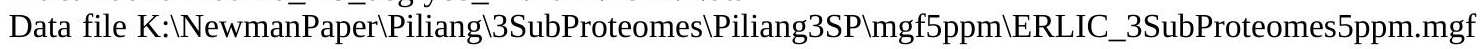

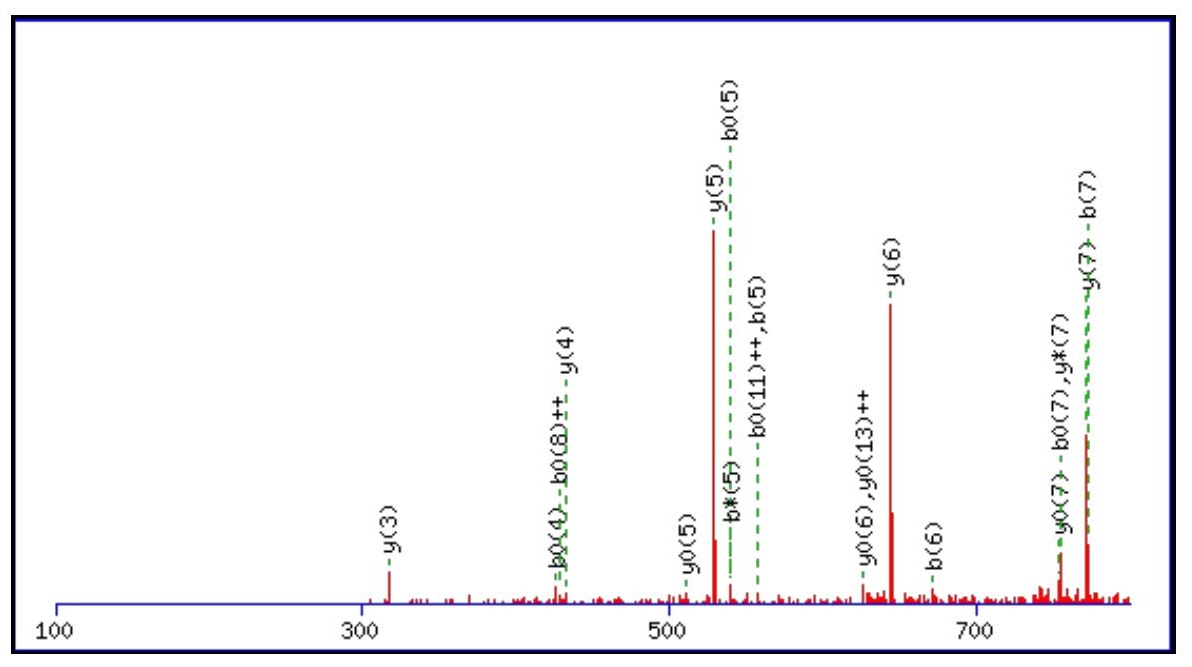



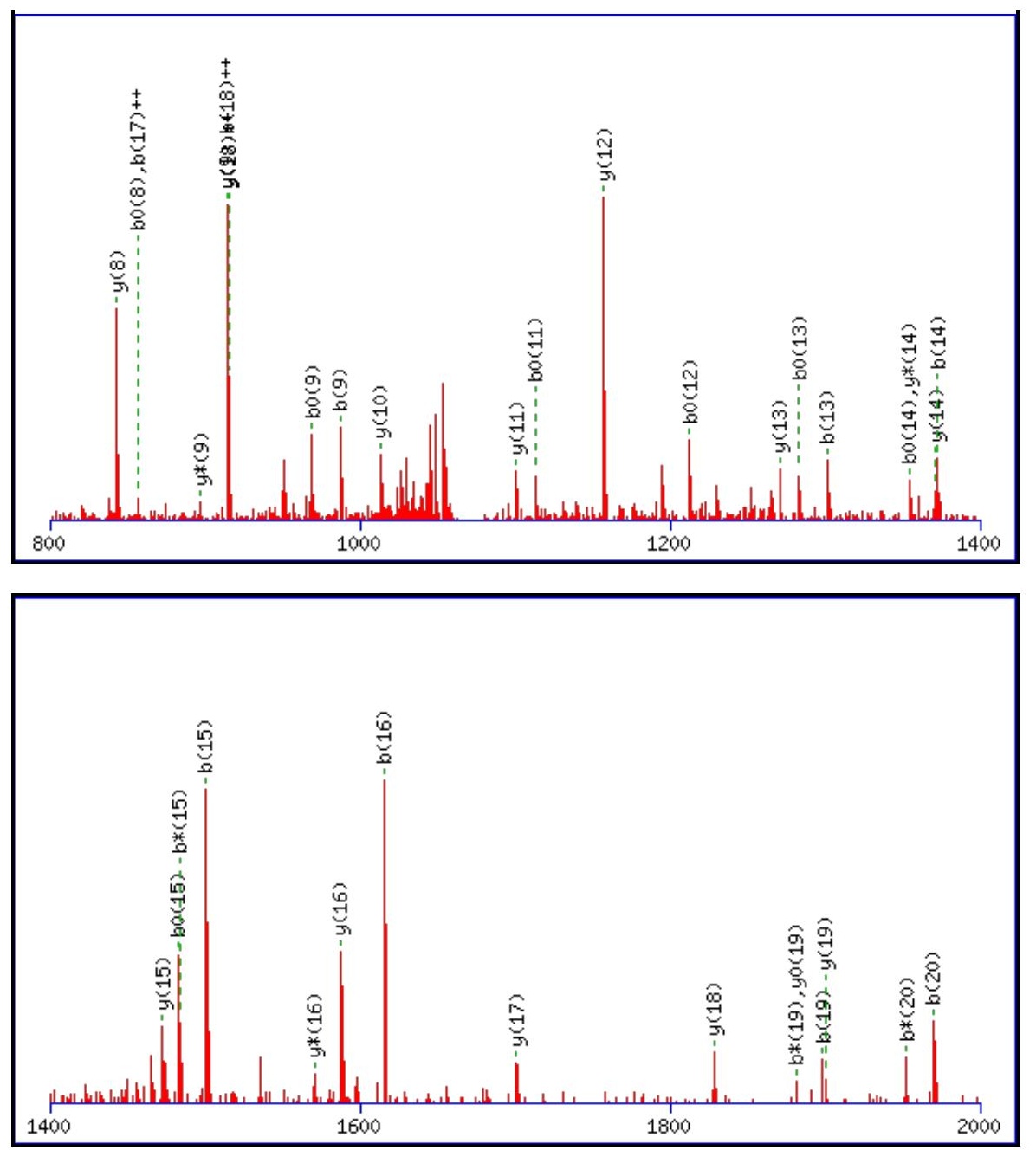

Monoisotopic mass of neutral peptide $\operatorname{Mr}($ calc): 2143.0029

Fixed modifications: Carbamidomethyl (C)

Variable modifications:

N6 : Deamidated $\mathrm{N}(\mathrm{N})$

Ions Score: 100 Expect: $2.7 \mathrm{e}-008$

Matches (Bold Red): 55/228 fragment ions using 94 most intense peaks

\begin{tabular}{|c|c|c|c|c|c|c|c|c|c|c|c|c|c|c|}
\hline \# & b & $\mathbf{b}^{++}$ & $\mathbf{b}^{*}$ & $\mathbf{b}^{*^{++}}$ & $\mathbf{b}^{0}$ & $\mathbf{b}^{\mathbf{0 + +}}$ & Seq. & $\mathbf{y}$ & $\mathbf{y}^{++}$ & $\mathbf{y}^{*}$ & $\mathrm{y}^{*^{++}}$ & $y^{0}$ & $\mathbf{y}^{\mathbf{0 + +}}$ & \# \\
\hline 1 & 130.0499 & 65.5286 & & & 112.0393 & 56.5233 & $\mathbf{E}$ & & & & & & & 21 \\
\hline 2 & 245.0768 & 123.0420 & & & 227.0662 & 114.0368 & D & 2014.9676 & 1007.9874 & 1997.9411 & 999.4742 & 1996.9570 & 998.9822 & 20 \\
\hline 3 & 316.1139 & 158.5606 & & & 298.1034 & 149.5553 & A & 1899.9407 & 950.4740 & 1882.9141 & 941.9607 & 1881.9301 & 941.4687 & 19 \\
\hline 4 & 444.1725 & 222.5899 & 427.1460 & 214.0766 & 426.1619 & 213.5846 & $\mathbf{Q}$ & 1828.9035 & 914.9554 & 1811.8770 & 906.4421 & |1810.8930 & 905.9501 & 18 \\
\hline 5 & 557.2566 & 279.1319 & 540.2300 & 270.6186 & 539.2460 & 270.1266 & I & 1700.8450 & 850.9261 & 1683.8184 & 842.4128 & 1682.8344 & 841.9208 & 17 \\
\hline 6 & 672.2835 & 336.6454 & 655.2570 & 328.1321 & 654.2729 & 327.6401 & $\mathbf{N}$ & 1587.7609 & 794.3841 & 1570.7344 & 785.8708 & 1569.7503 & 785.3788 & 16 \\
\hline 7 & 773.3312 & 387.1692 & 756.3046 & 378.6560 & 755.3206 & 378.1639 & $\mathbf{T}$ & 1472.7340 & 736.8706 & 1455.7074 & 728.3573 & 1454.7234 & 727.8653 & 15 \\
\hline 8 & 874.3789 & 437.6931 & 857.3523 & 429.1798 & 856.3683 & 428.6878 & $\mathbf{T}$ & 1371.6863 & 686.3468 & 1354.6597 & 677.8335 & 1353.6757 & 677.3415 & 14 \\
\hline 9 & 987.4629 & 494.2351 & 970.4364 & 485.7218 & 969.4524 & 485.2298 & I & 1270.6386 & 635.8229 & 1253.6121 & 627.3097 & 1252.6280 & 626.8177 & 13 \\
\hline 10 & 1044.4844 & 522.7458 & 1027.4578 & 514.2326 & 1026.4738 & 513.7406 & G & 1157.5545 & 579.2809 & 1140.5280 & 570.7676 & 1139.5440 & 570.2756 & 12 \\
\hline 11 & 1131.5164 & 566.2618 & 1114.4899 & 557.7486 & 1113.5059 & 557.2566 & S & 1100.5331 & 550.7702 & 1083.5065 & 542.2569 & 1082.5225 & 541.7649 & 11 \\
\hline 12 & 1230.5848 & 615.7961 & 1213.5583 & 607.2828 & 1212.5743 & 606.7908 & V & 1013.5011 & 507.2542 & 996.4745 & 498.7409 & 995.4905 & 498.2489 & 10 \\
\hline 13 & 1301.6219 & 651.3146 & 1284.5954 & 642.8013 & 1283.6114 & 642.3093 & A & 914.4326 & 457.7200 & 897.4061 & 449.2067 & 896.4221 & 448.7147 & 9 \\
\hline 14 & 1372.6591 & 686.8332 & 1355.6325 & 678.3199 & 1354.6485 & 677.8279 & A & 843.3955 & 422.2014 & 826.3690 & 413.6881 & 825.3850 & 413.1961 & 8 \\
\hline 15 & 1500.7176 & 750.8625 & 1483.6911 & 742.3492 & 1482.7071 & 741.8572 & $\mathbf{Q}$ & 772.3584 & 386.6828 & 755.3319 & 378.1696 & 754.3478 & 377.6776 & 7 \\
\hline 16 & 1615.7446 & 808.3759 & 1598.7180 & 799.8627 & 1597.7340 & 799.3706 & D & 644.2998 & 322.6536 & 627.2733 & 314.1403 & 626.2893 & 313.6483 & 6 \\
\hline 17 & 1712.7973 & 856.9023 & 1695.7708 & 848.3890 & 1694.7868 & 847.8970 & $\mathbf{P}$ & 529.2729 & 265.1401 & 512.2463 & 256.6268 & 511.2623 & 256.1348 & 5 \\
\hline 18 & 1827.8243 & 914.4158 & 1810.7977 & 905.9025 & 1809.8137 & 905.4105 & D & 432.2201 & 216.6137 & 415.1936 & 208.1004 & 414.2096 & 207.6084 & 4 \\
\hline
\end{tabular}




\begin{tabular}{|c|c|c|c|c|c|c|c|c|c|c|c|c|}
\hline 19| & |1898.8614 & |949.9343| & |1881.8349 & |941.4211 & |1880.8508| & |940.9291 & $\mathbf{A}$ & 317.1932 & 159.1002 & 300.1666 & |150.5870 & 3 \\
\hline 20 & 1969.8985 & 985.4529 & 1952.8720 & 976.9396 & 1951.8880 & 976.4476 & A & 246.1561 & 123.5817 & 229.1295 & 115.0684 & 2 \\
\hline 21 & & & & & & & $\mathbf{R}$ & 175.1190 & 88.0631 & 158.0924 & 79.5498 & 1 \\
\hline
\end{tabular}
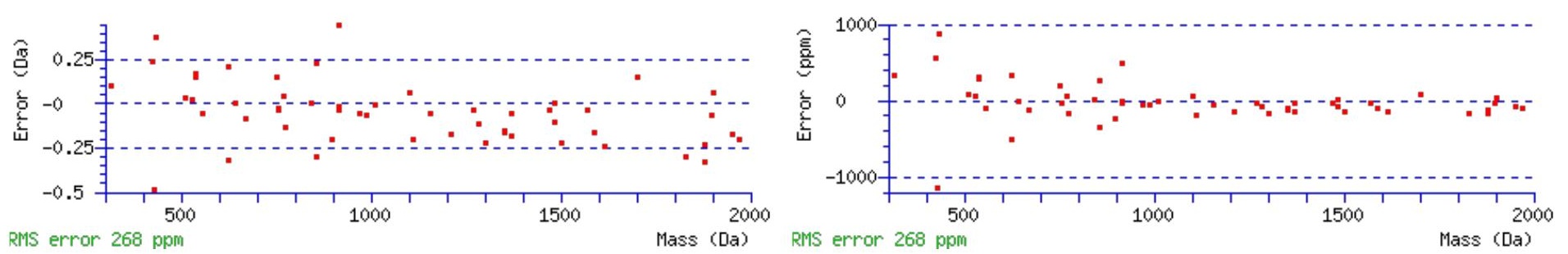

\section{All matches to this query}

\begin{tabular}{|l|l|l|l|}
\hline Score & Mr(calc): & Delta & \multicolumn{1}{|c|}{ Sequence } \\
\hline 99.8 & 2143.0029 & 0.0042 & EDAQINTTIGSVAAQDPDAAR \\
\hline 51.1 & 2142.0189 & 0.9882 & EDAQINTTIGSVAAQDPDAAR \\
\hline 11.6 & 2143.0089 & -0.0018 & LRSSGLRGGGEAALGAWLGR \\
\hline 9.3 & 2142.0106 & 0.9965 & KANGIFSTATLPENAERSR \\
\hline 6.5 & 2140.9830 & 2.0241 & WLNTHFDTENLKSSIQK \\
\hline 5.8 & 2142.9868 & 0.0203 & AAASLDTAALSATDMALALNR \\
\hline 5.8 & 2142.9868 & 0.0203 & AAASLDTAALSATDMALALNR \\
\hline 4.5 & 2142.9858 & 0.0213 & SLDVNQDSELKFNEYWR \\
\hline 4.1 & 2142.9946 & 0.0125 & NIRDSLNDYEAKLQDLR \\
\hline 4.1 & 2142.9946 & 0.0125 & NIRDSLNDYEAKLQDLR \\
\hline
\end{tabular}

Spectrum No: 81; Query: 413; Rank: 1

\section{Peptide View}

MS/MS Fragmentation of KLDFIVLNETR

Found in IPI00208422, Tax_Id=10116 Gene_Symbol=Dpp4 Dipeptidyl peptidase 4

Match to Query 413: 1347.736688 from(674.875620,2+)

Title: 091008RatKidney_NH4Format01_23.2662.2662.2.dta

Data file K:\NewmanPaper \Piliang \3SubProteomes\Piliang3SP \mgf5ppm\ERLIC_3SubProteomes5ppm.mgf

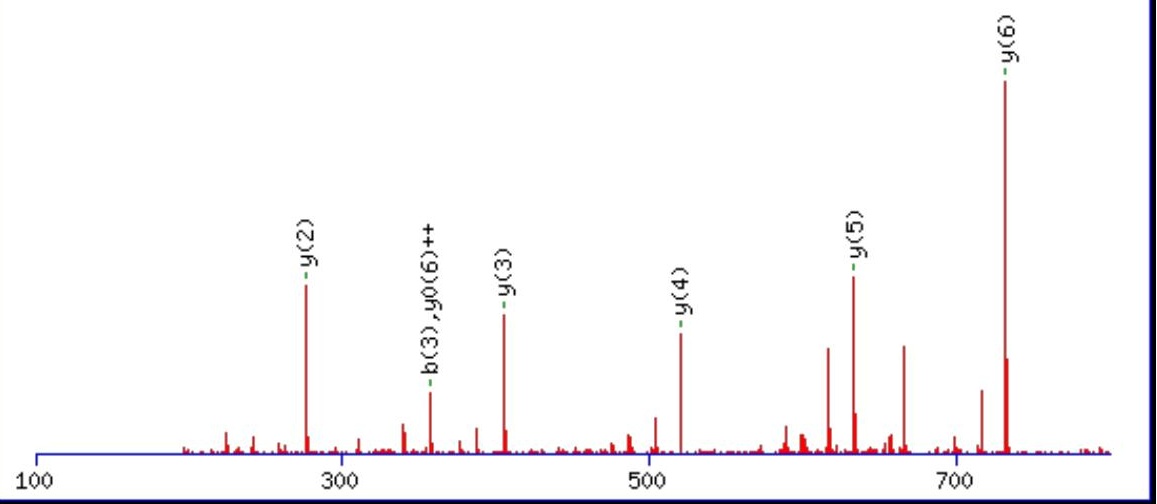



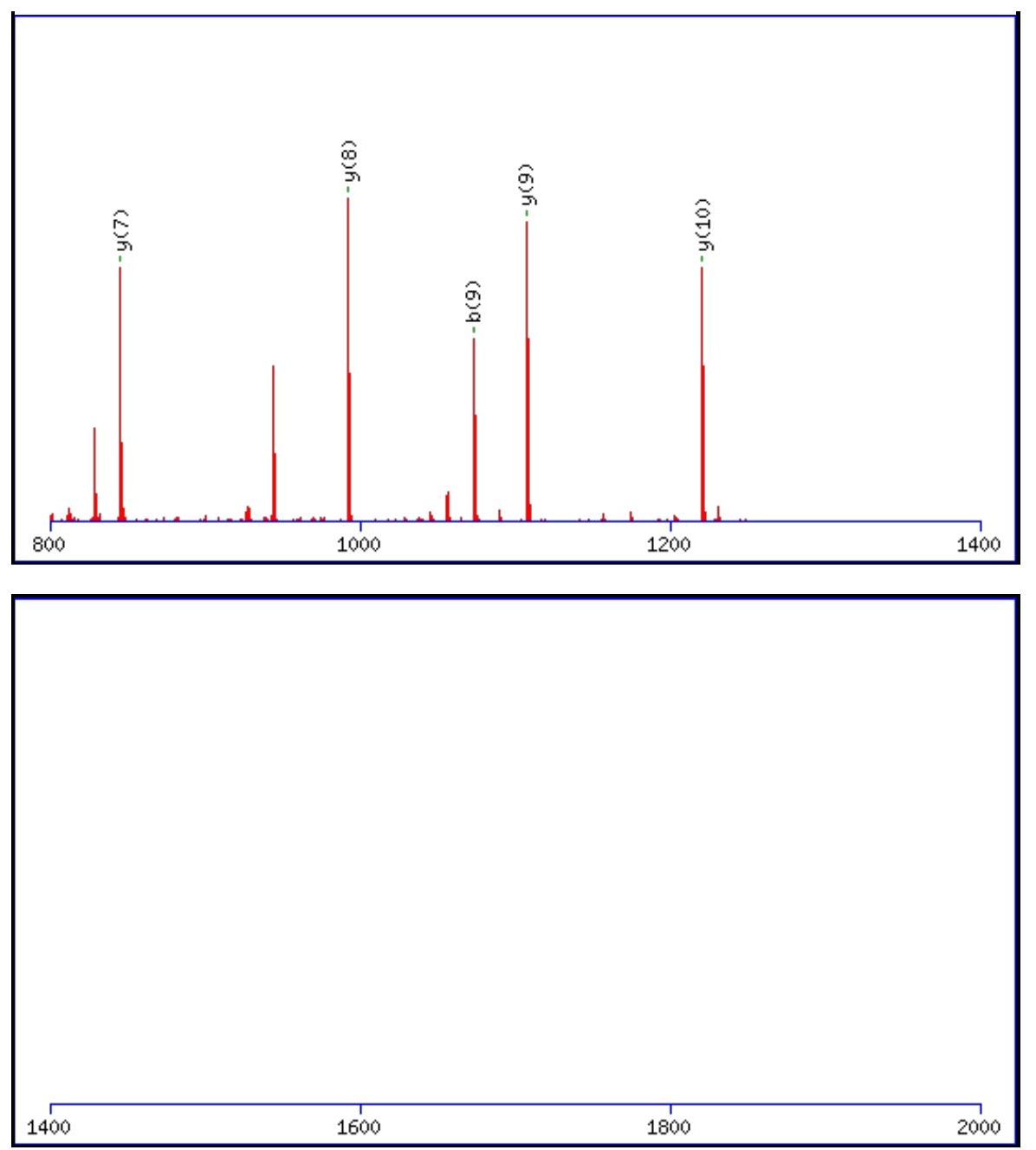

Monoisotopic mass of neutral peptide $\operatorname{Mr}($ calc): 1347.7398

Fixed modifications: Carbamidomethyl (C)

Variable modifications:

N8: Deamidated $\mathrm{N}(\mathrm{N})$

Ions Score: 100 Expect: $8.9 \mathrm{e}-009$

Matches (Bold Red): 12/114 fragment ions using 11 most intense peaks

\begin{tabular}{|r|c|c|c|c|c|c|c|c|c|c|c|c|c|c|}
\hline$\#$ & $\mathbf{b}$ & $\mathbf{b}^{++}$ & $\mathbf{b}^{*}$ & $\mathbf{b}^{\mathbf{*}^{++}}$ & $\mathbf{b}^{\mathbf{0}}$ & $\mathbf{b}^{\mathbf{0}+}$ & $\mathbf{S e q}$ & $\mathbf{y}$ & $\mathbf{y}^{++}$ & $\mathbf{y}^{*}$ & $\mathbf{y}^{\mathbf{*}^{++}}$ & $\mathbf{y}^{\mathbf{0}}$ & $\mathbf{y}^{\mathbf{0 + +}}$ & $\#$ \\
\hline $\mathbf{1}$ & 129.1022 & 65.0548 & 112.0757 & 56.5415 & & & $\mathbf{K}$ & & & & & & & $\mathbf{1 1}$ \\
\hline $\mathbf{2}$ & 242.1863 & 121.5968 & 225.1598 & 113.0835 & & & $\mathbf{L}$ & $\mathbf{1 2 2 0 . 6 5 2 1}$ & 610.8297 & 1203.6256 & 602.3164 & 1202.6416 & 601.8244 & $\mathbf{1 0}$ \\
\hline $\mathbf{3}$ & $\mathbf{3 5 7 . 2 1 3 2}$ & 179.1103 & 340.1867 & 170.5970 & 339.2027 & 170.1050 & $\mathbf{D}$ & $\mathbf{1 1 0 7 . 5 6 8 1}$ & 554.2877 & 1090.5415 & 545.7744 & 1089.5575 & 545.2824 & $\mathbf{9}$ \\
\hline $\mathbf{4}$ & 504.2817 & 252.6445 & 487.2551 & 244.1312 & 486.2711 & 243.6392 & $\mathbf{F}$ & $\mathbf{9 9 2 . 5 4 1 1}$ & 496.7742 & 975.5146 & 488.2609 & 974.5306 & 487.7689 & $\mathbf{8}$ \\
\hline $\mathbf{5}$ & 617.3657 & 309.1865 & 600.3392 & 300.6732 & 599.3552 & 300.1812 & $\mathbf{I}$ & $\mathbf{8 4 5 . 4 7 2 7}$ & 423.2400 & 828.4462 & 414.7267 & 827.4621 & 414.2347 & $\mathbf{7}$ \\
\hline $\mathbf{6}$ & 716.4341 & 358.7207 & 699.4076 & 350.2074 & 698.4236 & 349.7154 & $\mathbf{V}$ & $\mathbf{7 3 2 . 3 8 8 6}$ & 366.6980 & 715.3621 & 358.1847 & 714.3781 & $\mathbf{3 5 7 . 6 9 2 7}$ & $\mathbf{6}$ \\
\hline $\mathbf{7}$ & 829.5182 & 415.2627 & 812.4917 & 406.7495 & 811.5076 & 406.2575 & $\mathbf{L}$ & $\mathbf{6 3 3 . 3 2 0 2}$ & 317.1638 & 616.2937 & 308.6505 & 615.3097 & 308.1585 & $\mathbf{5}$ \\
\hline $\mathbf{8}$ & 944.5451 & 472.7762 & 927.5186 & 464.2629 & 926.5346 & 463.7709 & $\mathbf{N}$ & $\mathbf{5 2 0 . 2 3 6 2}$ & 260.6217 & 503.2096 & 252.1084 & 502.2256 & 251.6164 & $\mathbf{4}$ \\
\hline $\mathbf{9}$ & $\mathbf{1 0 7 3 . 5 8 7 7}$ & 537.2975 & 1056.5612 & 528.7842 & 1055.5772 & 528.2922 & $\mathbf{E}$ & $\mathbf{4 0 5 . 2 0 9 2}$ & 203.1082 & 388.1827 & 194.5950 & 387.1987 & 194.1030 & $\mathbf{3}$ \\
\hline $\mathbf{1 0}$ & 1174.6354 & 587.8213 & 1157.6089 & 579.3081 & 1156.6248 & 578.8161 & $\mathbf{T}$ & $\mathbf{2 7 6 . 1 6 6 6}$ & 138.5870 & 259.1401 & 130.0737 & 258.1561 & 129.5817 & $\mathbf{2}$ \\
\hline $\mathbf{1 1}$ & & & & & & & $\mathbf{R}$ & 175.1190 & 88.0631 & 158.0924 & 79.5498 & & & $\mathbf{1}$ \\
\hline
\end{tabular}
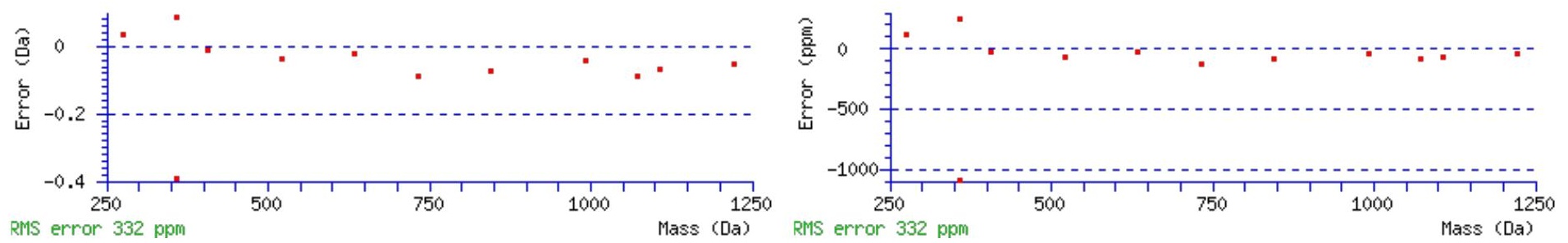


\section{All matches to this query}

\begin{tabular}{|l|l|l|l|}
\hline Score & Mr(calc): & Delta & \multicolumn{1}{|c|}{ Sequence } \\
\hline 99.7 & 1347.7398 & -0.0031 & KLDFIVLNETR \\
\hline 9.1 & 1347.7431 & -0.0065 & KITNLKITCEK \\
\hline 8.3 & 1347.7428 & -0.0061 & ITQPLVKKWR \\
\hline 7.7 & 1347.7431 & -0.0065 & QIVSLKEKMEK \\
\hline 5.5 & 1347.7398 & -0.0031 & QNIEDVKKVFK \\
\hline 5.4 & 1347.7387 & -0.0021 & QLLSIPRASKR \\
\hline 5.1 & 1347.7431 & -0.0065 & KMKLVESQLEK \\
\hline 3.5 & 1346.7228 & 1.0139 & QLDMELVSIKR \\
\hline 3.0 & 1347.7398 & -0.0031 & QIYEKGLELQK \\
\hline 2.6 & 1346.7405 & 0.9962 & VSSTKEVAAEAKK \\
\hline
\end{tabular}

Spectrum No: 82; Query: 2815; Rank: 1

\section{Peptide View}

MS/MS Fragmentation of SSPSLSILAGGQCNESVGYFVEPCIIESKDPQEPIMK

Found in IPI00475676, Tax_Id=10116 Gene_Symbol=Tas1r2 Delta-1-pyrroline-5-carboxylate dehydrogenase, mitochondrial precursor

Match to Query 2815: 4066.915662 from(1356.645830,3+)

Title: 100101RatKid_NS_deglyco_07.5803.5803.3.dta

Data file K:INewmanPaper|Piliangl3SubProteomes\Piliang3SP\mgf5ppm\ERLIC_3SubProteomes5ppm.mgf
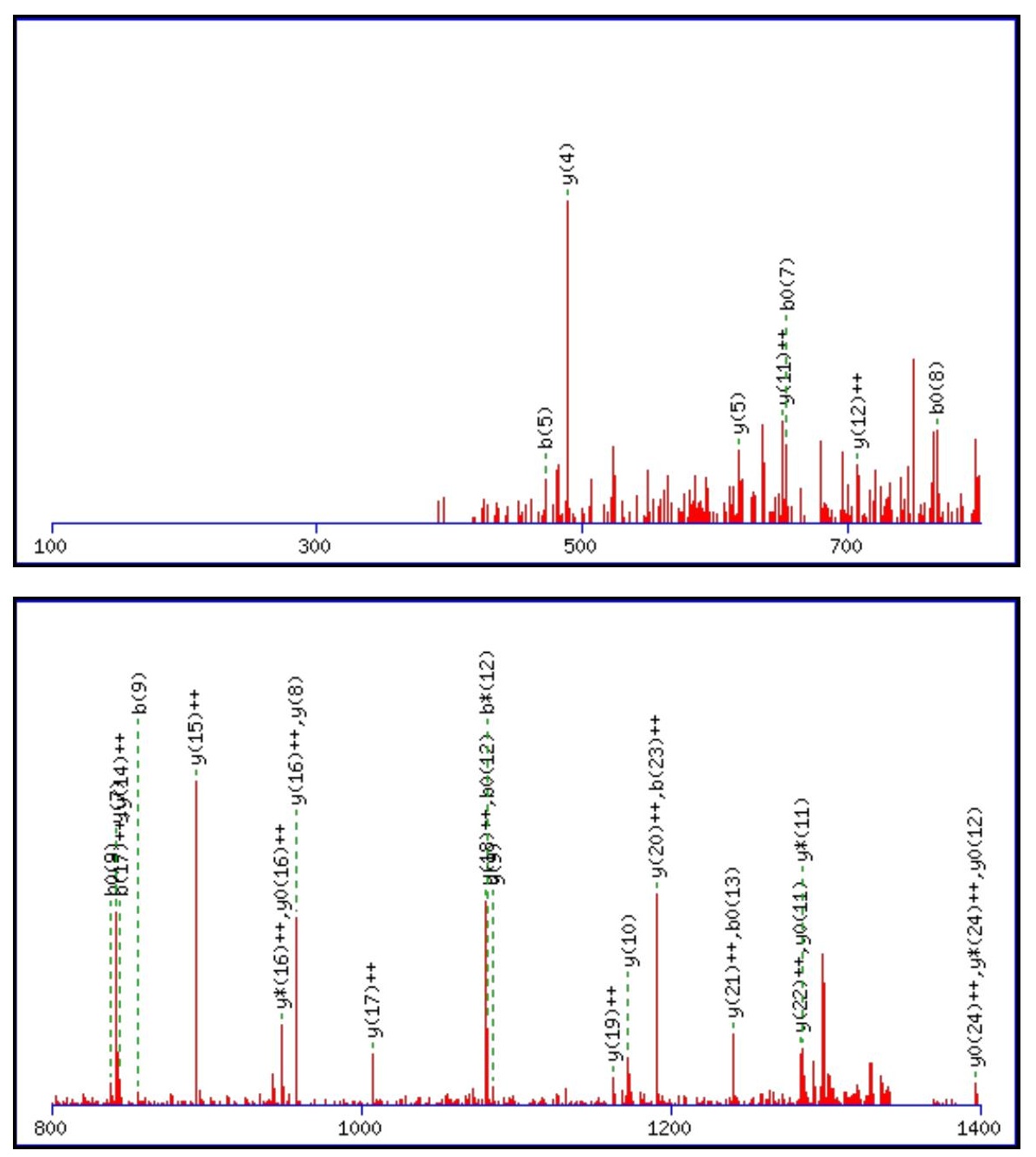


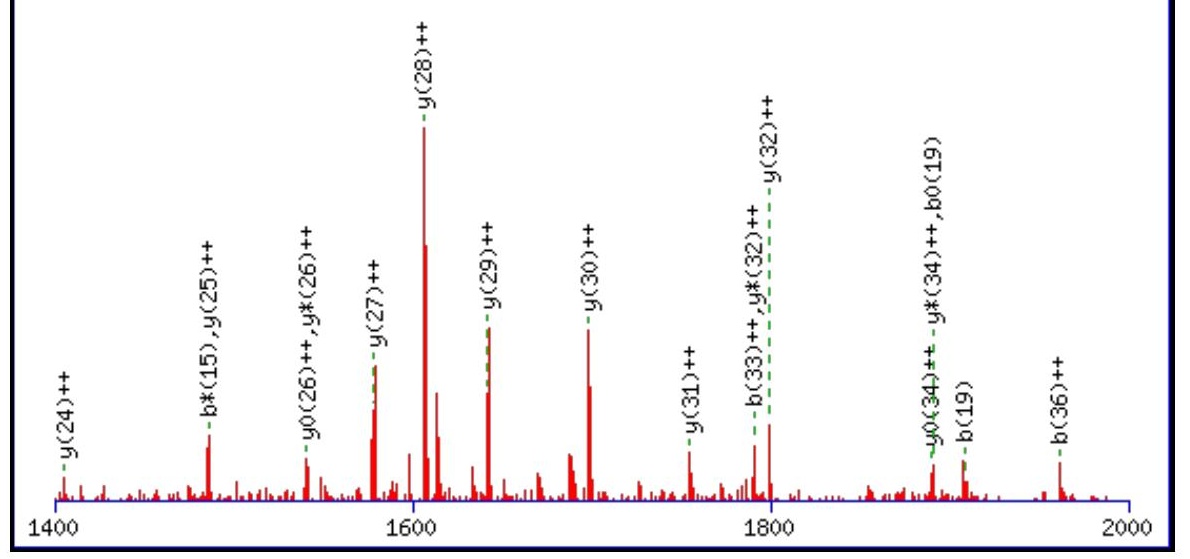

Monoisotopic mass of neutral peptide $\operatorname{Mr}($ calc): 4066.9213

Fixed modifications: Carbamidomethyl (C)

Variable modifications:

N14 : Deamidated_N (N)

Ions Score: 99 Expect: $4.8 \mathrm{e}-008$

Matches (Bold Red): 52/402 fragment ions using 83 most intense peaks

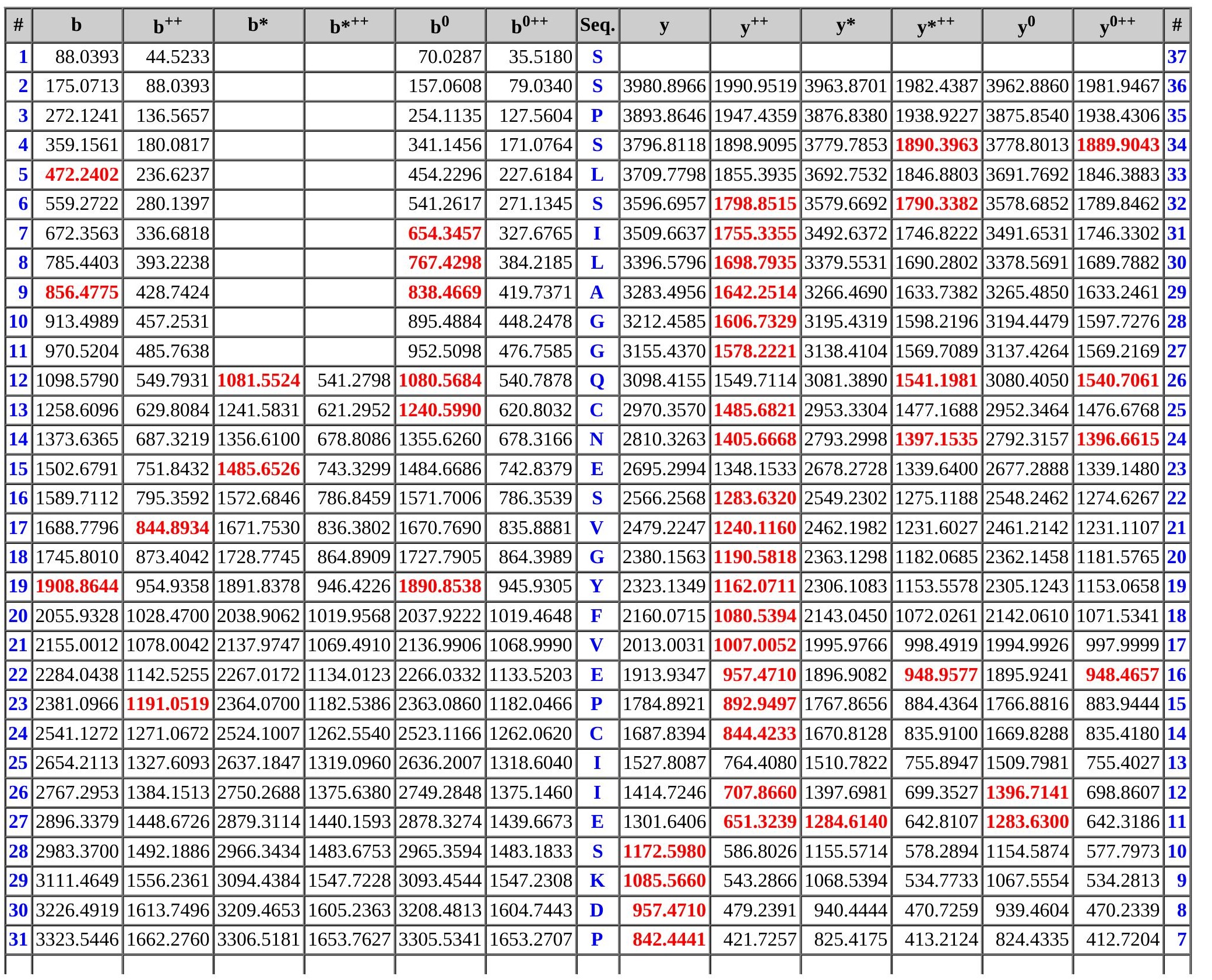




\begin{tabular}{|r|r|r|r|r|r|r|r|r|r|r|r|r|r|r|r|r|r|}
$\mathbf{3 2}$ & 3451.6032 & 1726.3052 & 3434.5767 & 1717.7920 & 3433.5926 & 1717.3000 & $\mathbf{Q}$ & 745.3913 & 373.1993 & 728.3647 & 364.6860 & 727.3807 & 364.1940 & $\mathbf{6}$ \\
\hline $\mathbf{3 3}$ & 3580.6458 & $\mathbf{1 7 9 0 . 8 2 6 5}$ & 3563.6193 & 1782.3133 & 3562.6352 & 1781.8213 & $\mathbf{E}$ & $\mathbf{6 1 7 . 3 3 2 7}$ & 309.1700 & 600.3062 & 300.6567 & 599.3221 & 300.1647 & $\mathbf{5}$ \\
\hline $\mathbf{3 4}$ & 3677.6986 & 1839.3529 & 366.6720 & 1830.8396 & 3659.6880 & 1830.3476 & $\mathbf{P}$ & $\mathbf{4 8 8 . 2 9 0 1}$ & 244.6487 & 471.2636 & 236.1354 & & & & $\mathbf{4}$ \\
\hline $\mathbf{3 5}$ & 3790.7826 & 1895.8950 & 3773.7561 & 1887.3817 & 3772.7721 & 1886.8897 & $\mathbf{I}$ & 391.2374 & 196.1223 & 374.2108 & 187.6090 & & & $\mathbf{3}$ \\
\hline $\mathbf{3 6}$ & 3921.8231 & $\mathbf{1 9 6 1 . 4 1 5 2}$ & 3904.7966 & 1952.9019 & 3903.8125 & 1952.4099 & $\mathbf{M}$ & 278.1533 & 139.5803 & 261.1267 & 131.0670 & & & $\mathbf{2}$ \\
\hline $\mathbf{3 7}$ & & & & & & & $\mathbf{K}$ & 147.1128 & 74.0600 & 130.0863 & 65.5468 & & & $\mathbf{1}$ \\
\hline
\end{tabular}
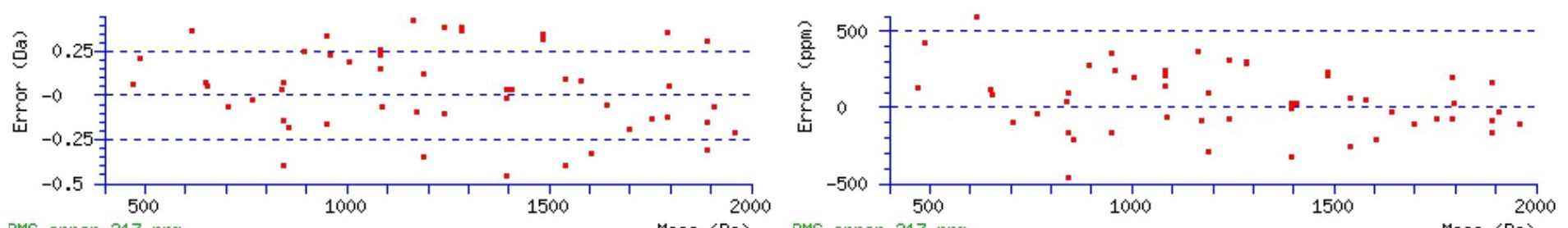

RMS error 217 ppm

Mass (Da) RMS error 217 ppm

Mass (Da)

\section{All matches to this query}

\begin{tabular}{|l|l|l|l|}
\hline Score & Mr(calc): & Delta & \multicolumn{1}{c|}{ Sequence } \\
\hline 99.5 & 4066.9213 & -0.0057 & SSPSLSILAGGQCNESVGYFVEPCIIESKDPQEPIMK \\
\hline 74.0 & 4065.9373 & 0.9783 & SSPSLSILAGGQCNESVGYFVEPCIIESKDPQEPIMK \\
\hline 4.4 & 4065.9240 & 0.9916 & VFPVKVIALEDGDLEATVTVWVTGYCYNIEVHLR \\
\hline 4.4 & 4066.8846 & 0.0311 & TYFAQEGCSFLYEKLPPSPSSPGNGDCTLLALAQLR \\
\hline 4.0 & 4066.9055 & 0.0101 & RSENTPEPSSTQTPNPLSTLVPTPPSTETDNLMRHR \\
\hline 4.0 & 4066.9055 & 0.0101 & RSENTPEPSSTQTPNPLSTLVPTPPSTETDNLMRHR \\
\hline 4.0 & 4066.9055 & 0.0101 & RSENTPEPSSTQTPNPLSTLVPTPPSTETDNLMRHR \\
\hline 3.5 & 4066.8846 & 0.0311 & TYFAQEGCSFLYEKLPPSPSSPGNGDCTLLALAQLR \\
\hline 3.4 & 4066.9086 & 0.0070 & SIKATPMAVADLIPVDTVVNLTIAVGWYTAVHRPK \\
\hline 3.4 & 4065.8829 & 1.0327 & EGAGDTGALETLGRMGDSMQLNQETAFQGVLLKLDIK \\
\hline
\end{tabular}

Spectrum No: 83; Query: 966; Rank: 1

\section{Peptide View}

MS/MS Fragmentation of EGITLNANLSGDGFLK

Found in IPI00327398, Tax_Id=10116 Gene_Symbol=Enpep Isoform 1 of Glutamyl aminopeptidase

Match to Query 966: 1648.832468 from(825.423510,2+)

Title: 091008RatKidney_NH4Format01_25.3412.3412.2.dta

Data file K:INewmanPaper|Piliangl3SubProteomes\Piliang3SP\mgf5ppm|ERLIC_3SubProteomes5ppm.mgf

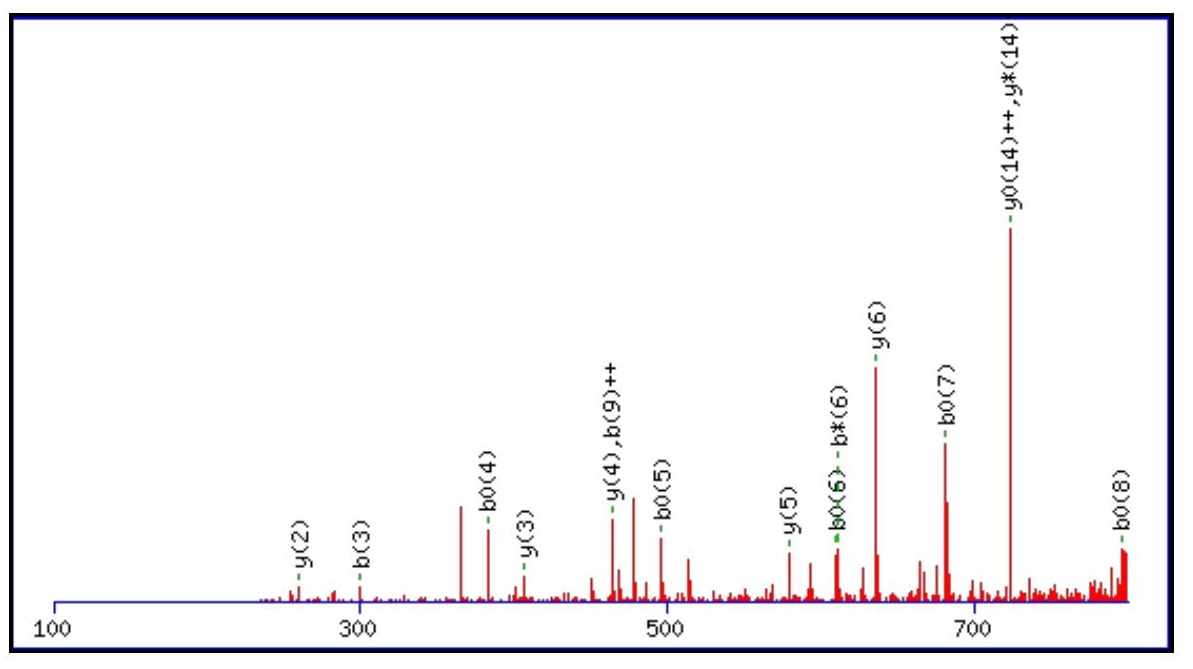



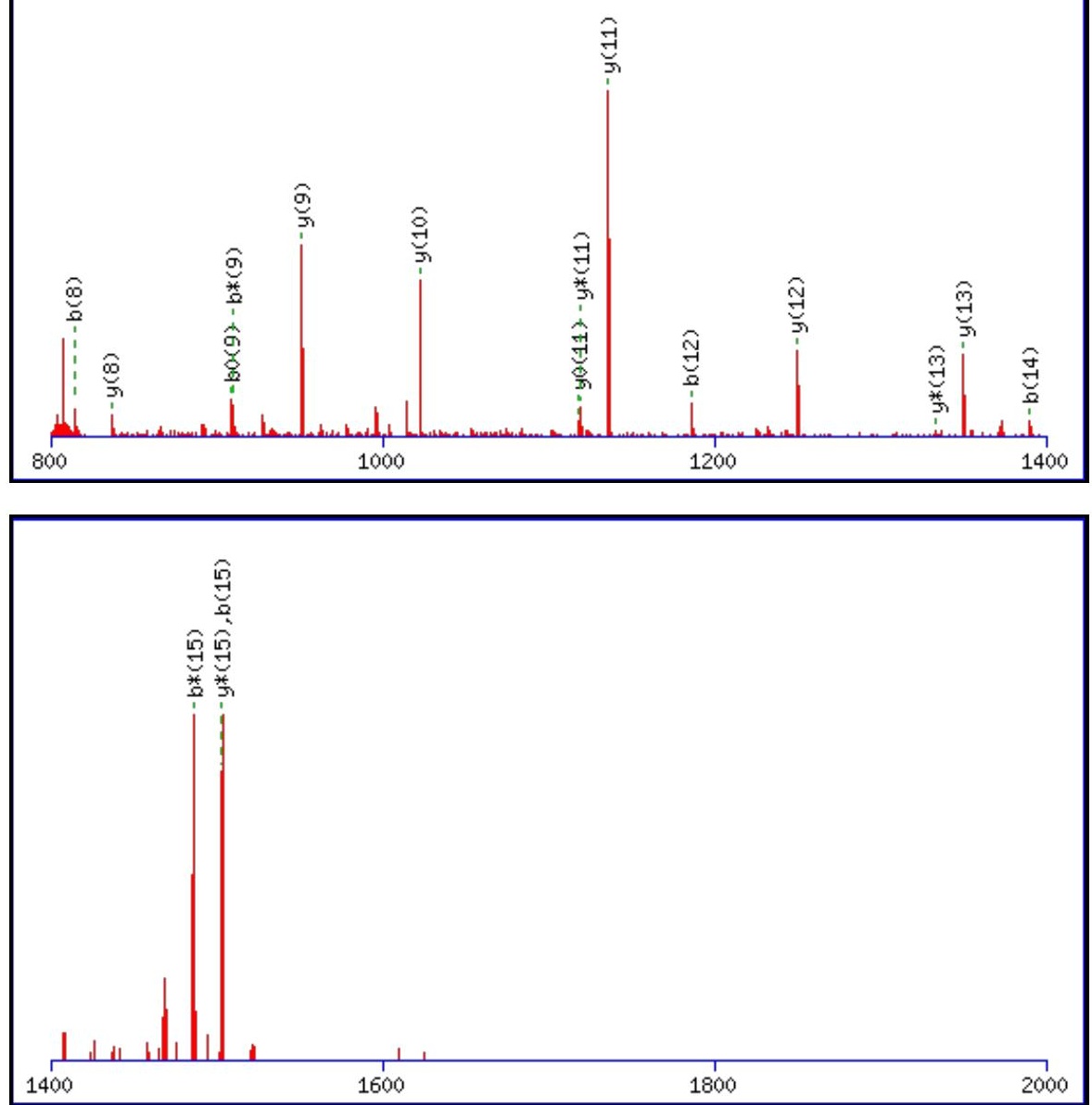

Monoisotopic mass of neutral peptide $\operatorname{Mr}($ calc): 1648.8308

Fixed modifications: Carbamidomethyl (C)

Variable modifications:

N8

: Deamidated $\mathrm{N}(\mathrm{N})$

Ions Score: 100 Expect: $1.9 \mathrm{e}-008$

Matches (Bold Red): 33/162 fragment ions using 46 most intense peaks

\begin{tabular}{|c|c|c|c|c|c|c|c|c|c|c|c|c|c|c|}
\hline \# & b & $\mathbf{b}^{++}$ & $\mathbf{b}^{*}$ & $\mathbf{b}^{*^{++}}$ & $\mathbf{b}^{0}$ & $\mathbf{b}^{0++}$ & Seq. & $\mathbf{y}$ & $y^{++}$ & $\mathbf{y}^{*}$ & $\mathrm{y}^{*^{++}}$ & $\mathbf{y}^{0}$ & $\mathbf{y}^{0++}$ & \# \\
\hline 1 & 130.0499 & 65.5286 & & & 112.0393 & 56.5233 & $\mathbf{E}$ & & & & & & & 16 \\
\hline 2 & 187.0713 & 94.0393 & & & 169.0608 & 85.0340 & G & 1520.7955 & 760.9014 & 1503.7689 & 752.3881 & 1502.7849 & 751.8961 & 15 \\
\hline 3 & 300.1554 & 150.5813 & & & 282.1448 & 141.5761 & I & 1463.7740 & 732.3907 & 1446.7475 & 723.8774 & 1445.7635 & 723.3854 & 14 \\
\hline 4 & 401.2031 & 201.1052 & & & 383.1925 & 192.0999 & $\mathbf{T}$ & 1350.6900 & 675.8486 & 1333.6634 & 667.3353 & 1332.6794 & 666.8433 & 13 \\
\hline 5 & 514.2871 & 257.6472 & & & 496.2766 & 248.6419 & $\mathbf{L}$ & 1249.6423 & 625.3248 & 1232.6157 & 616.8115 & 1231.6317 & 616.3195 & 12 \\
\hline 6 & 628.3301 & 314.6687 & 611.3035 & 306.1554 & 610.3195 & 305.6634 & $\mathbf{N}$ & 1136.5582 & 568.7827 & 1119.5317 & 560.2695 & 1118.5477 & 559.7775 & 11 \\
\hline 7 & 699.3672 & 350.1872 & 682.3406 & 341.6740 & 681.3566 & 341.1819 & A & 1022.5153 & 511.7613 & 1005.4887 & 503.2480 & 1004.5047 & 502.7560 & 10 \\
\hline 8 & 814.3941 & 407.7007 & 797.3676 & 399.1874 & 796.3836 & 398.6954 & $\mathbf{N}$ & 951.4782 & 476.2427 & 934.4516 & 467.7295 & 933.4676 & 467.2374 & 9 \\
\hline 9 & 927.4782 & 464.2427 & 910.4516 & 455.7295 & 909.4676 & 455.2374 & $\mathbf{L}$ & 836.4512 & 418.7293 & 819.4247 & 410.2160 & 818.4407 & 409.7240 & 8 \\
\hline 10 & 1014.5102 & 507.7587 & 997.4837 & 499.2455 & 996.4996 & 498.7535 & $\mathrm{~S}$ & 723.3672 & 362.1872 & 706.3406 & 353.6740 & 705.3566 & 353.1819 & 7 \\
\hline 11 & 1071.5317 & 536.2695 & 1054.5051 & 527.7562 & 1053.5211 & 527.2642 & G & 636.3352 & 318.6712 & 619.3086 & 310.1579 & 618.3246 & 309.6659 & 6 \\
\hline 12 & 1186.5586 & 593.7829 & 1169.5321 & 585.2697 & 1168.5481 & 584.7777 & D & 579.3137 & 290.1605 & 562.2871 & 281.6472 & 561.3031 & 281.1552 & 5 \\
\hline 13 & 1243.5801 & 622.2937 & 1226.5535 & 613.7804 & 1225.5695 & 613.2884 & G & 464.2867 & 232.6470 & 447.2602 & 224.1337 & & & 4 \\
\hline 14 & 1390.6485 & 695.8279 & 1373.6219 & 687.3146 & 1372.6379 & 686.8226 & $\mathbf{F}$ & 407.2653 & 204.1363 & 390.2387 & 195.6230 & & & 3 \\
\hline 15 & 1503.7326 & 752.3699 & 1486.7060 & 743.8566 & 1485.7220 & 743.3646 & $\mathbf{L}$ & 260.1969 & 130.6021 & 243.1703 & 122.0888 & & & 2 \\
\hline 16 & & & & & & & $\mathbf{K}$ & 147.1128 & 74.0600 & 130.0863 & 65.5468 & & & 1 \\
\hline
\end{tabular}



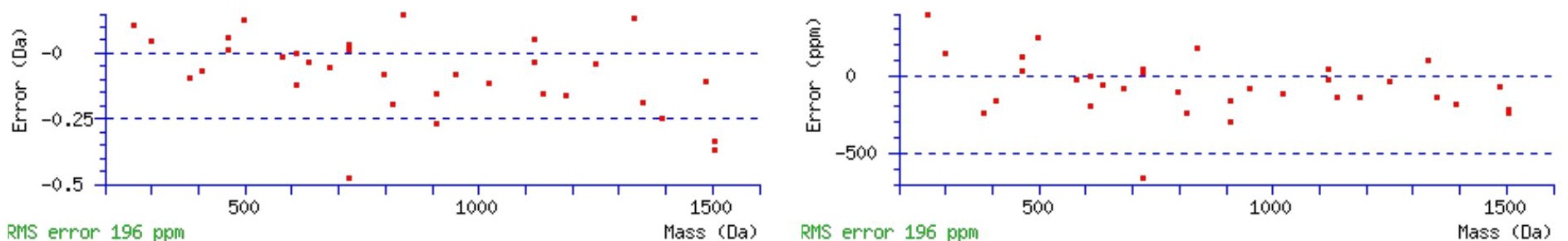

RMS error 196 ppm

Mass (Da) RMS error 196 ppm

Mass (Da)

\section{All matches to this query}

\begin{tabular}{|l|l|l|l|}
\hline Score & Mr(calc): & Delta & \multicolumn{1}{c|}{ Sequence } \\
\hline 99.5 & 1648.8308 & 0.0017 & EGITLNANLSGDGFLK \\
\hline 66.5 & 1648.8308 & 0.0017 & EGITLNANLSGDGFLK \\
\hline 5.0 & 1648.8466 & -0.0142 & ALSYLIRAVSLKR \\
\hline 0.2 & 1648.8243 & 0.0082 & MFNSPNNQALVSALK \\
\hline 0.1 & 1647.8167 & 1.0157 & SMTLGSLPPKPRER \\
\hline 0.0 & 1648.8308 & 0.0017 & EADENGALKKEGFIK \\
\hline
\end{tabular}

Spectrum No: 84; Query: 868; Rank: 1

\section{Peptide View}

MS/MS Fragmentation of YLNETQQLTENIK

Found in IPI00564327, Tax_Id=10116 Gene_Symbol=Mug2 Murinoglobulin-2 precursor

Match to Query 868: 1593.788508 from(797.901530,2+)

Title: 091008RatKidney_NoSalt_30.1986.1986.2.dta

Data file K:INewmanPaper|Piliangl3SubProteomes\Piliang3SP\mgf5ppm\ERLIC_3SubProteomes5ppm.mgf

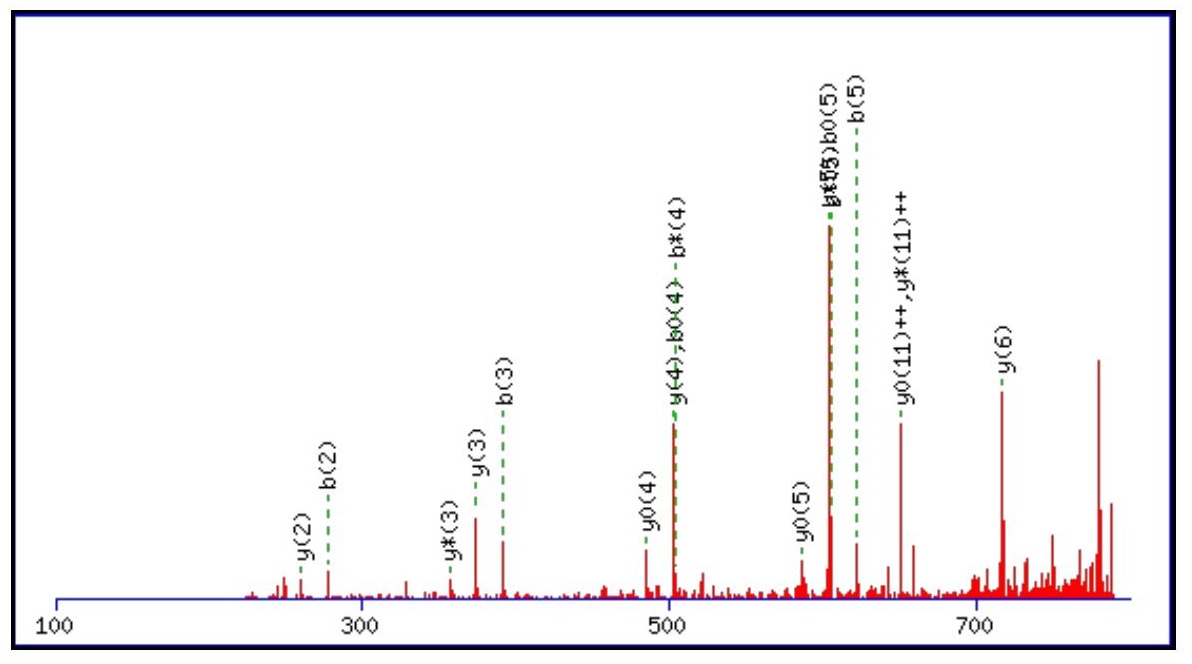



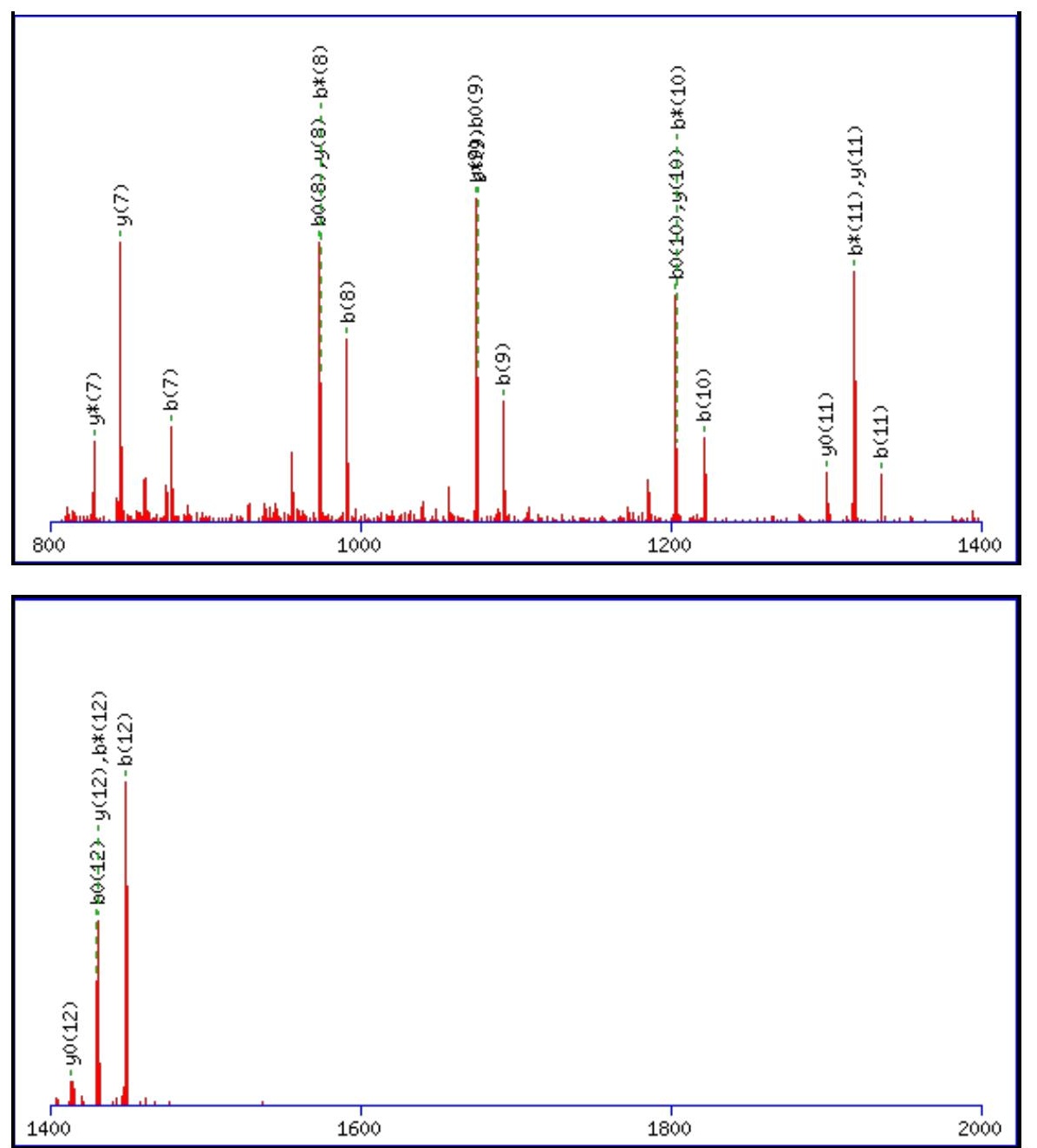

Monoisotopic mass of neutral peptide $\operatorname{Mr}($ calc): 1593.7886

Fixed modifications: Carbamidomethyl (C)

Variable modifications:

N3 : Deamidated $\mathrm{N}(\mathrm{N})$

Ions Score: 99 Expect: $2 \mathrm{e}-008$

Matches (Bold Red): 41/128 fragment ions using 45 most intense peaks

\begin{tabular}{|r|c|c|c|c|c|c|c|c|c|c|c|c|c|c|}
\hline$\#$ & $\mathbf{b}$ & $\mathbf{b}^{++}$ & $\mathbf{b}^{*}$ & $\mathbf{b}^{\mathbf{*}^{++}}$ & $\mathbf{b}^{\mathbf{0}}$ & $\mathbf{b}^{\mathbf{0 + +}}$ & $\mathbf{S e q}$ & $\mathbf{y}$ & $\mathbf{y}^{++}$ & $\mathbf{y}^{\mathbf{*}}$ & $\mathbf{y}^{\mathbf{*}^{++}}$ & $\mathbf{y}^{\mathbf{0}}$ & $\mathbf{y}^{\mathbf{0 + +}}$ & $\#$ \\
\hline $\mathbf{1}$ & 164.0706 & 82.5389 & & & & & $\mathbf{Y}$ & & & & & & & $\mathbf{1 3}$ \\
\hline $\mathbf{2}$ & $\mathbf{2 7 7 . 1 5 4 7}$ & 139.0810 & & & & & $\mathbf{L}$ & $\mathbf{1 4 3 1 . 7 3 2 6}$ & 716.3699 & 1414.7060 & 707.8566 & $\mathbf{1 4 1 3 . 7 2 2 0}$ & $\mathbf{7 0 7 . 3 6 4 6}$ & $\mathbf{1 2}$ \\
\hline $\mathbf{3}$ & $\mathbf{3 9 2 . 1 8 1 6}$ & 196.5944 & 375.1551 & 188.0812 & & & $\mathbf{N}$ & $\mathbf{1 3 1 8 . 6 4 8 5}$ & 659.8279 & 1301.6219 & $\mathbf{6 5 1 . 3 1 4 6}$ & $\mathbf{1 3 0 0 . 6 3 7 9}$ & $\mathbf{6 5 0 . 8 2 2 6}$ & $\mathbf{1 1}$ \\
\hline $\mathbf{4}$ & 521.2242 & 261.1157 & $\mathbf{5 0 4 . 1 9 7 6}$ & 252.6025 & $\mathbf{5 0 3 . 2 1 3 6}$ & 252.1105 & $\mathbf{E}$ & $\mathbf{1 2 0 3 . 6 2 1 6}$ & 602.3144 & 1186.5950 & 593.8011 & 1185.6110 & 593.3091 & $\mathbf{1 0}$ \\
\hline $\mathbf{5}$ & $\mathbf{6 2 2 . 2 7 1 9}$ & 311.6396 & $\mathbf{6 0 5 . 2 4 5 3}$ & 303.1263 & $\mathbf{6 0 4 . 2 6 1 3}$ & 302.6343 & $\mathbf{T}$ & $\mathbf{1 0 7 4 . 5 7 9 0}$ & 537.7931 & 1057.5524 & 529.2798 & 1056.5684 & 528.7878 & $\mathbf{9}$ \\
\hline $\mathbf{6}$ & 750.3305 & 375.6689 & 733.3039 & 367.1556 & 732.3199 & 366.6636 & $\mathbf{Q}$ & $\mathbf{9 7 3 . 5 3 1 3}$ & 487.2693 & 956.5047 & 478.7560 & 955.5207 & 478.2640 & $\mathbf{8}$ \\
\hline $\mathbf{7}$ & $\mathbf{8 7 8 . 3 8 9 0}$ & 439.6982 & 861.3625 & 431.1849 & 860.3785 & 430.6929 & $\mathbf{Q}$ & $\mathbf{8 4 5 . 4 7 2 7}$ & 423.2400 & $\mathbf{8 2 8 . 4 4 6 2}$ & 414.7267 & 827.4621 & 414.2347 & $\mathbf{7}$ \\
\hline $\mathbf{8}$ & $\mathbf{9 9 1 . 4 7 3 1}$ & 496.2402 & $\mathbf{9 7 4 . 4 4 6 5}$ & 487.7269 & $\mathbf{9 7 3 . 4 6 2 5}$ & 487.2349 & $\mathbf{L}$ & $\mathbf{7 1 7 . 4 1 4 1}$ & 359.2107 & 700.3876 & 350.6974 & 699.4036 & 350.2054 & $\mathbf{6}$ \\
\hline $\mathbf{9}$ & $\mathbf{1 0 9 2 . 5 2 0 8}$ & 546.7640 & $\mathbf{1 0 7 5 . 4 9 4 2}$ & 538.2508 & $\mathbf{1 0 7 4 . 5 1 0 2}$ & 537.7587 & $\mathbf{T}$ & $\mathbf{6 0 4 . 3 3 0 1}$ & 302.6687 & 587.3035 & 294.1554 & $\mathbf{5 8 6 . 3 1 9 5}$ & 293.6634 & $\mathbf{5}$ \\
\hline $\mathbf{1 0}$ & $\mathbf{1 2 2 1 . 5 6 3 4}$ & 611.2853 & $\mathbf{1 2 0 4 . 5 3 6 8}$ & 602.7720 & $\mathbf{1 2 0 3 . 5 5 2 8}$ & 602.2800 & $\mathbf{E}$ & $\mathbf{5 0 3 . 2 8 2 4}$ & 252.1448 & 486.2558 & 243.6316 & $\mathbf{4 8 5 . 2 7 1 8}$ & 243.1395 & $\mathbf{4}$ \\
\hline $\mathbf{1 1}$ & $\mathbf{1 3 3 5 . 6 0 6 3}$ & 668.3068 & $\mathbf{1 3 1 8 . 5 7 9 7}$ & 659.7935 & 1317.5957 & 659.3015 & $\mathbf{N}$ & $\mathbf{3 7 4 . 2 3 9 8}$ & 187.6235 & 357.2132 & 179.1103 & & & $\mathbf{3}$ \\
\hline $\mathbf{1 2}$ & $\mathbf{1 4 4 8 . 6 9 0 4}$ & 724.8488 & $\mathbf{1 4 3 1 . 6 6 3 8}$ & $\mathbf{7 1 6 . 3 3 5 5}$ & $\mathbf{1 4 3 0 . 6 7 9 8}$ & $\mathbf{7 1 5 . 8 4 3 5}$ & $\mathbf{I}$ & $\mathbf{2 6 0 . 1 9 6 9}$ & 130.6021 & 243.1703 & 122.0888 & & & $\mathbf{2}$ \\
\hline $\mathbf{1 3}$ & & & & & & & $\mathbf{K}$ & 147.1128 & 74.0600 & 130.0863 & 65.5468 & & & $\mathbf{1}$ \\
\hline
\end{tabular}



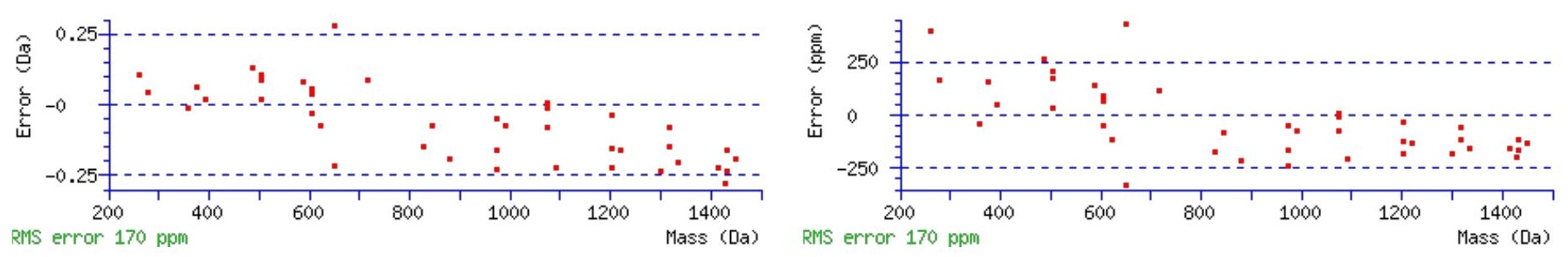

\section{All matches to this query}

\begin{tabular}{|l|l|l|l|}
\hline Score & Mr(calc): & Delta & \multicolumn{1}{|c|}{ Sequence } \\
\hline 99.0 & 1593.7886 & -0.0001 & YLNETQQLTENIK \\
\hline 66.3 & 1593.7886 & -0.0001 & YLNETQQLTENIK \\
\hline 29.1 & 1593.7763 & 0.0122 & EQARISLLNDGGLK \\
\hline 12.9 & 1593.7738 & 0.0147 & QNMQHQLYVILK \\
\hline 9.8 & 1592.7923 & 0.9962 & EQARISLLNDGGLK \\
\hline 9.6 & 1593.7928 & -0.0043 & IICTTQMLENMIK \\
\hline 7.3 & 1592.7976 & 0.9909 & MKLELNTMQLPMK \\
\hline 7.1 & 1592.7981 & 0.9905 & KMPETFSNLPRTR \\
\hline 5.9 & 1593.7988 & -0.0103 & LTRGNRESVQINK \\
\hline 5.8 & 1593.7933 & -0.0048 & GNDAVMTRGTFANIK \\
\hline
\end{tabular}

Spectrum No: 85; Query: 859; Rank: 1

\section{Peptide View}

MS/MS Fragmentation of ESNSTSLTQAALLEK

Found in IPI00199867, Tax_Id=10116 Gene_Symbol=Emilin1_predicted elastin microfibril interfacer 1

Match to Query 859: 1591.793788 from(796.904170,2+)

Title: 091008RatKidney_NH4Format01_24.2113.2113.2.dta

Data file K:INewmanPaper\Piliang\3SubProteomes\Piliang3SP\mgf5ppm\ERLIC_3SubProteomes5ppm.mgf

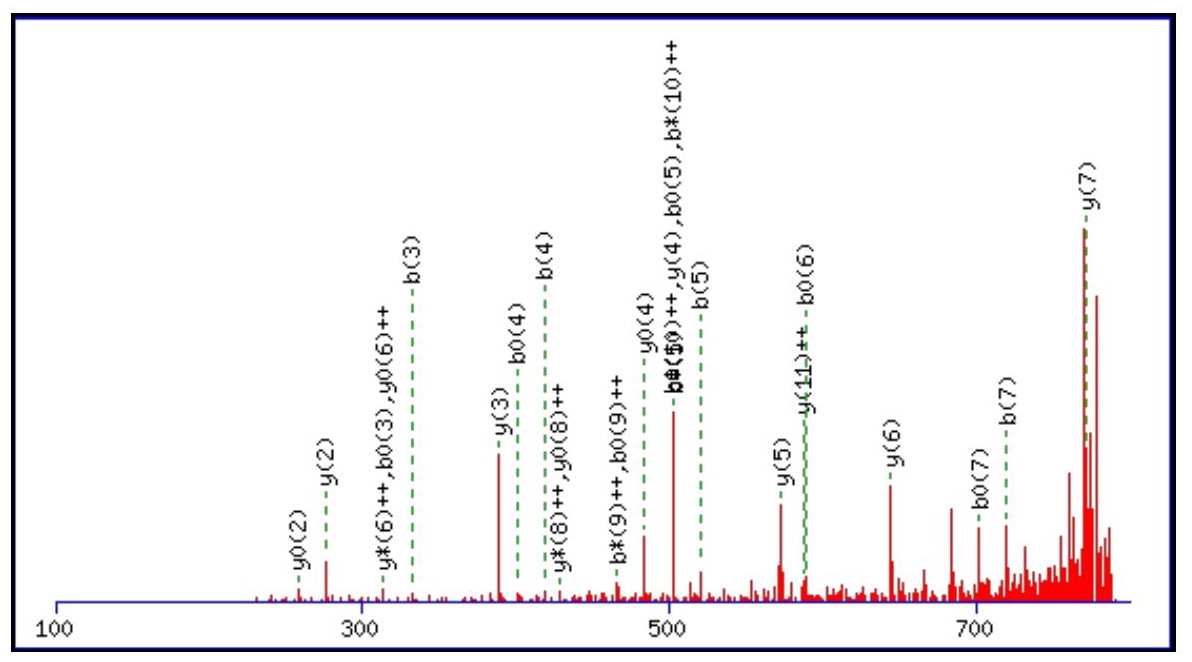



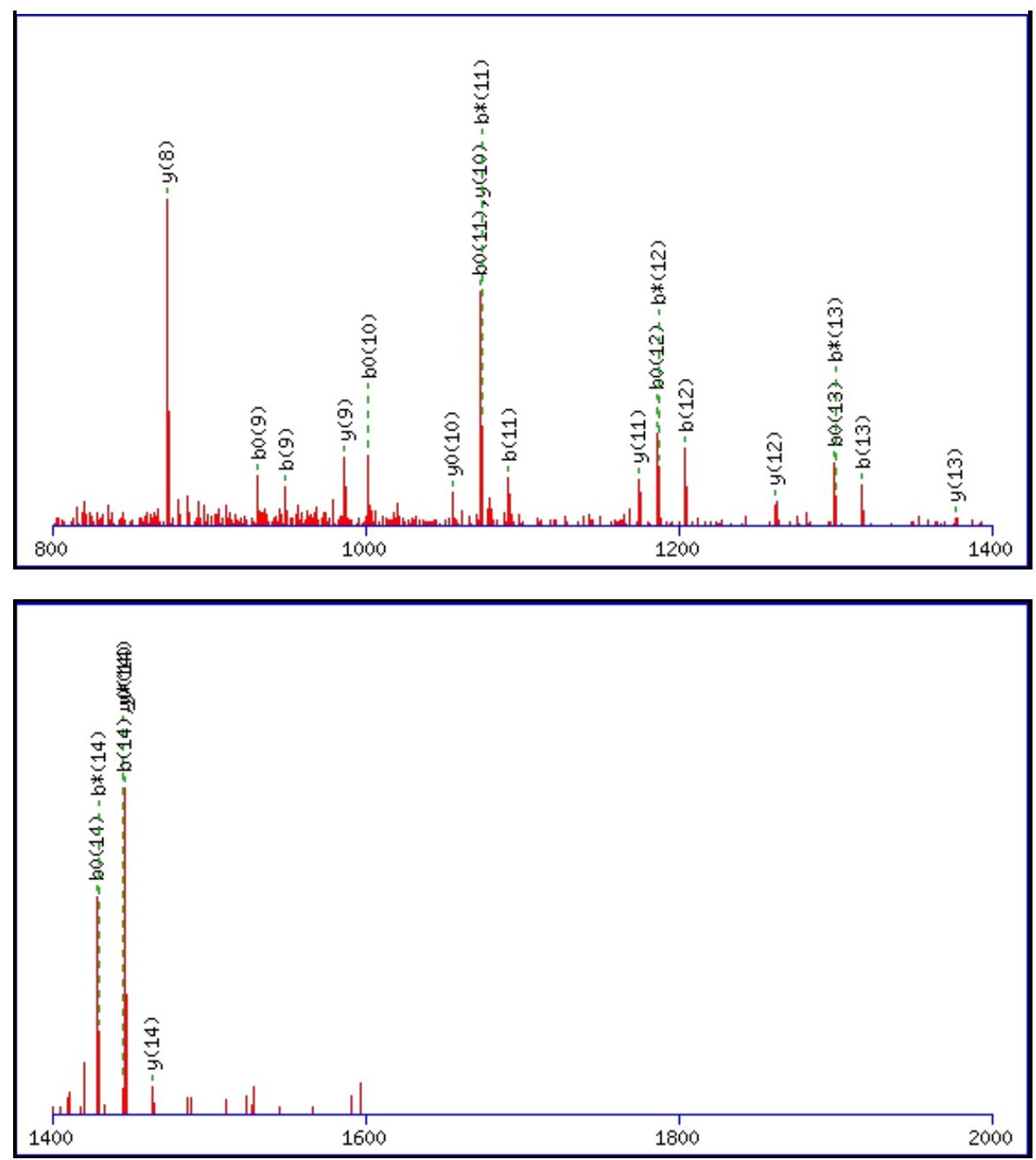

Monoisotopic mass of neutral peptide $\operatorname{Mr}($ calc): 1591.7941

Fixed modifications: Carbamidomethyl (C)

Variable modifications:

N3 : Deamidated $\mathrm{N}(\mathrm{N})$

Ions Score: 99 Expect: $2.1 \mathrm{e}-008$

Matches (Bold Red): 52/162 fragment ions using 75 most intense peaks

\begin{tabular}{|c|c|c|c|c|c|c|c|c|c|c|c|c|c|c|}
\hline \# & b & $\mathbf{b}^{++}$ & b* & $\mathbf{b}^{*^{++}}$ & $\mathbf{b}^{0}$ & $\mathbf{b}^{\mathbf{0 + +}}$ & Seq. & $\mathbf{y}$ & $\mathbf{y}^{++}$ & $\mathbf{y}^{*}$ & $\mathrm{y}^{*^{++}}$ & $\mathbf{y}^{\mathbf{0}}$ & $y^{0++}$ & \# \\
\hline 1 & 130.0499 & 65.5286 & & & 112.0393 & 56.5233 & $\mathbf{E}$ & & & & & & & 15 \\
\hline 2 & 217.0819 & 109.0446 & & & 199.0713 & 100.0393 & S & 1463.7588 & 732.3830 & 1446.7322 & 723.8697 & 1445.7482 & 723.3777 & 14 \\
\hline 3 & 332.1088 & 166.5581 & 315.0823 & 158.0448 & 314.0983 & 157.5528 & $\mathbf{N}$ & 1376.7267 & 688.8670 & 1359.7002 & 680.3537 & 1358.7162 & 679.8617 & 13 \\
\hline 4 & 419.1409 & 210.0741 & 402.1143 & 201.5608 & 401.1303 & 201.0688 & S & 1261.6998 & 631.3535 & 1244.6733 & 622.8403 & 1243.6892 & 622.3483 & 12 \\
\hline 5 & 520.1885 & 260.5979 & 503.1620 & 252.0846 & 502.1780 & 251.5926 & $\mathbf{T}$ & 1174.6678 & 587.8375 & 1157.6412 & 579.3243 & 1156.6572 & 578.8322 & 11 \\
\hline 6 & 607.2206 & 304.1139 & 590.1940 & 295.6006 & 589.2100 & 295.1086 & S & 1073.6201 & 537.3137 & 1056.5936 & 528.8004 & 1055.6095 & 528.3084 & 10 \\
\hline 7 & 720.3046 & 360.6560 & 703.2781 & 352.1427 & 702.2941 & 351.6507 & $\mathbf{L}$ & 986.5881 & 493.7977 & 969.5615 & 485.2844 & 968.5775 & 484.7924 & 9 \\
\hline 8 & 821.3523 & 411.1798 & 804.3258 & 402.6665 & 803.3417 & 402.1745 & $\mathbf{T}$ & 873.5040 & 437.2556 & 856.4775 & 428.7424 & 855.4934 & 428.2504 & 8 \\
\hline 9 & 949.4109 & 475.2091 & 932.3843 & 466.6958 & 931.4003 & 466.2038 & $\mathbf{Q}$ & 772.4563 & 386.7318 & 755.4298 & 378.2185 & 754.4458 & 377.7265 & 7 \\
\hline 10 & 1020.4480 & 510.7276 & 1003.4215 & 502.2144 & 1002.4374 & 501.7224 & A & 644.3978 & 322.7025 & 627.3712 & 314.1892 & 626.3872 & 313.6972 & 6 \\
\hline 11 & 1091.4851 & 546.2462 & 1074.4586 & 537.7329 & 1073.4746 & 537.2409 & A & 573.3606 & 287.1840 & 556.3341 & 278.6707 & 555.3501 & 278.1787 & 5 \\
\hline 12 & 1204.5692 & 602.7882 & 1187.5426 & 594.2750 & 1186.5586 & 593.7829 & $\mathbf{L}$ & 502.3235 & 251.6654 & 485.2970 & 243.1521 & 484.3130 & 242.6601 & 4 \\
\hline 13 & 1317.6532 & 659.3303 & 1300.6267 & 650.8170 & 1299.6427 & 650.3250 & $\mathbf{L}$ & 389.2395 & 195.1234 & 372.2129 & 186.6101 & 371.2289 & 186.1181 & 3 \\
\hline 14 & 1446.6958 & 723.8516 & 1429.6693 & 715.3383 & 1428.6853 & 714.8463 & $\mathbf{E}$ & 276.1554 & 138.5813 & 259.1288 & 130.0681 & 258.1448 & 129.5761 & 2 \\
\hline 15 & & & & & & & K & 147.1128 & 74.0600 & 130.0863 & 65.5468 & & & 1 \\
\hline
\end{tabular}



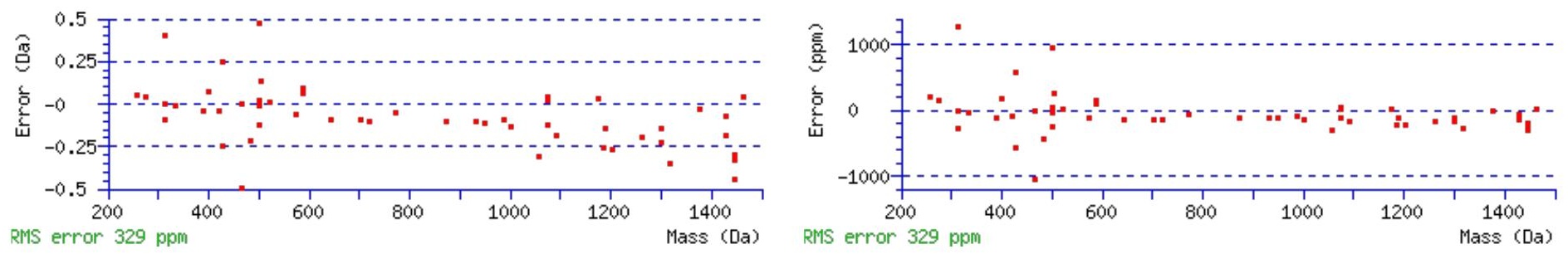

\section{All matches to this query}

\begin{tabular}{|l|l|l|l|}
\hline Score & Mr(calc): & Delta & \multicolumn{1}{|c|}{ Sequence } \\
\hline 98.8 & 1591.7941 & -0.0003 & ESNSTSLTQAALLEK \\
\hline 26.5 & 1591.7828 & 0.0110 & TSSLLILTENENEK \\
\hline 18.7 & 1589.7970 & 1.9968 & GESMALNEKQILEK \\
\hline 16.0 & 1589.7970 & 1.9968 & GEAMALNEKQILEK \\
\hline 11.9 & 1591.8062 & -0.0124 & MKLMRSEGPDVSVK \\
\hline 11.7 & 1591.7945 & -0.0008 & GNYRFMKLLLTR \\
\hline 10.5 & 1591.8053 & -0.0115 & EKVAGEDVGTSSSAKK \\
\hline 10.3 & 1590.7988 & 0.9950 & TSSLLILTENENEK \\
\hline 7.5 & 1591.7810 & 0.0128 & CNKVKPMEGRTEK \\
\hline 6.5 & 1590.8018 & 0.9920 & LALSLEPSQPSLTR \\
\hline
\end{tabular}

Spectrum No: 86; Query: 820; Rank: 1

\section{Peptide View}

\section{MS/MS Fragmentation of KNSTLCDLCIGPAK}

Found in IPI00196656, Tax_Id=10116 Gene_Symbol=Srprb Ba1-667

Match to Query 820: 1576.762428 from(789.388490,2+)

Title: 100101RatKid_NS_deglyco_24.2039.2039.2.dta

Data file K:INewmanPaper|Piliangl3SubProteomes\Piliang3SP\mgf5ppm\ERLIC_3SubProteomes5ppm.mgf

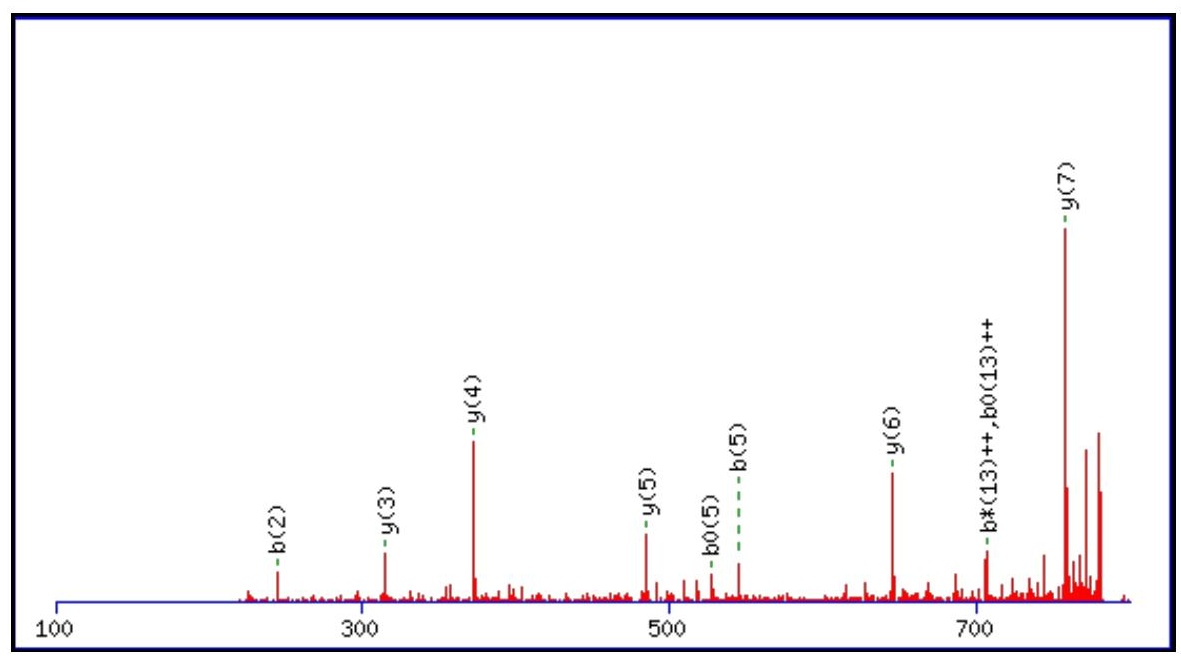



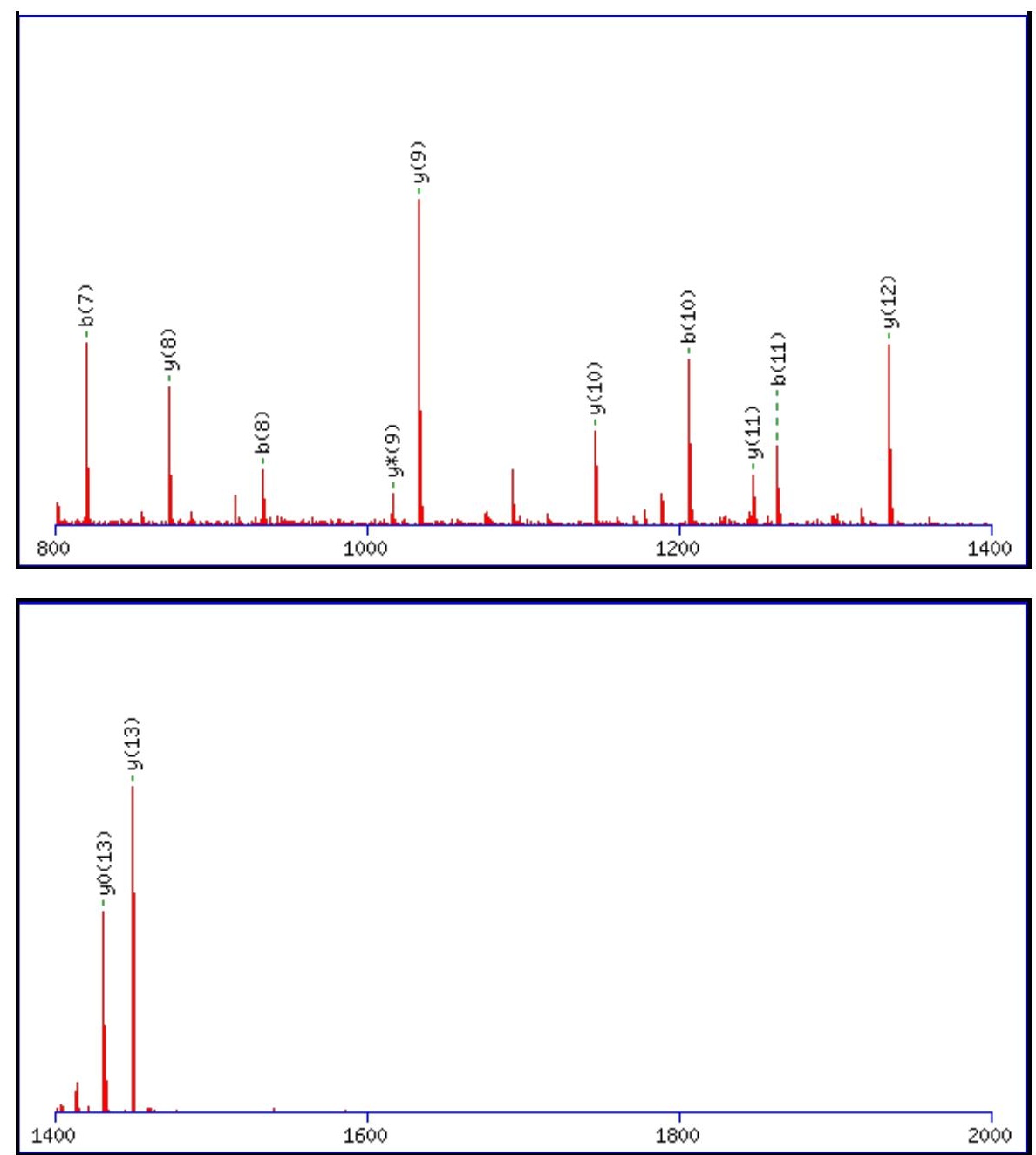

Monoisotopic mass of neutral peptide $\operatorname{Mr}($ calc): 1576.7589

Fixed modifications: Carbamidomethyl (C)

Variable modifications:

N2 : Deamidated $\mathrm{N}(\mathrm{N})$

Ions Score: 99 Expect: $2.4 \mathrm{e}-008$

Matches (Bold Red): 23/138 fragment ions using 30 most intense peaks

\begin{tabular}{|c|c|c|c|c|c|c|c|c|c|c|c|c|c|c|}
\hline \# & b & $\mathbf{b}^{++}$ & $\mathbf{b}^{*}$ & $\mathbf{b}^{*^{++}}$ & $\mathbf{b}^{0}$ & $\mathbf{b}^{0++}$ & Seq. & $\mathbf{y}$ & $y^{++}$ & $\mathbf{y}^{*}$ & $\mathbf{y}^{*^{++}}$ & $\mathbf{y}^{0}$ & $\mathbf{y}^{\mathbf{0 + +}}$ & \# \\
\hline 1 & 129.1022 & 65.0548 & 112.0757 & 56.5415 & & & $\mathbf{K}$ & & & & & & & 14 \\
\hline 2 & 244.1292 & 122.5682 & 227.1026 & 114.0550 & & & $\mathbf{N}$ & 1449.6712 & 725.3392 & 1432.6447 & 716.8260 & 1431.6607 & 716.3340 & 13 \\
\hline 3 & 331.1612 & 166.0842 & 314.1347 & 157.5710 & 313.1506 & 157.0790 & S & 1334.6443 & 667.8258 & 1317.6177 & 659.3125 & 1316.6337 & 658.8205 & 12 \\
\hline 4 & 432.2089 & 216.6081 & 415.1823 & 208.0948 & 414.1983 & 207.6028 & $\mathbf{T}$ & 1247.6123 & 624.3098 & 1230.5857 & 615.7965 & 1229.6017 & 615.3045 & 11 \\
\hline 5 & 545.2929 & 273.1501 & 528.2664 & 264.6368 & 527.2824 & 264.1448 & $\mathbf{L}$ & 1146.5646 & 573.7859 & 1129.5380 & 565.2727 & 1128.5540 & 564.7806 & 10 \\
\hline 6 & 705.3236 & 353.1654 & 688.2970 & 344.6522 & 687.3130 & 344.1602 & C & 1033.4805 & 517.2439 & 1016.4540 & 508.7306 & 1015.4699 & 508.2386 & 9 \\
\hline 7 & 820.3505 & 410.6789 & 803.3240 & 402.1656 & 802.3400 & 401.6736 & D & 873.4499 & 437.2286 & 856.4233 & 428.7153 & 855.4393 & 428.2233 & 8 \\
\hline 8 & 933.4346 & 467.2209 & 916.4081 & 458.7077 & 915.4240 & 458.2157 & $\mathbf{L}$ & 758.4229 & 379.7151 & 741.3964 & 371.2018 & & & 7 \\
\hline 9 & 1093.4653 & 547.2363 & 1076.4387 & 538.7230 & 1075.4547 & 538.2310 & C & 645.3389 & 323.1731 & 628.3123 & 314.6598 & & & 6 \\
\hline 10 & 1206.5493 & 603.7783 & 1189.5228 & 595.2650 & 1188.5387 & 594.7730 & I & 485.3082 & 243.1577 & 468.2817 & 234.6445 & & & 5 \\
\hline 11 & 1263.5708 & 632.2890 & 1246.5442 & 623.7758 & 1245.5602 & 623.2837 & G & 372.2241 & 186.6157 & 355.1976 & 178.1024 & & & 4 \\
\hline 12 & 1360.6235 & 680.8154 & 1343.5970 & 672.3021 & 1342.6130 & 671.8101 & $\mathbf{P}$ & 315.2027 & 158.1050 & 298.1761 & 149.5917 & & & 3 \\
\hline 13 & 1431.6607 & 716.3340 & 1414.6341 & 707.8207 & 1413.6501 & 707.3287 & A & 218.1499 & 109.5786 & 201.1234 & 101.0653 & & & 2 \\
\hline 14 & & & & & & & $\mathbf{K}$ & 147.1128 & 74.0600 & 130.0863 & 65.5468 & & & 1 \\
\hline
\end{tabular}



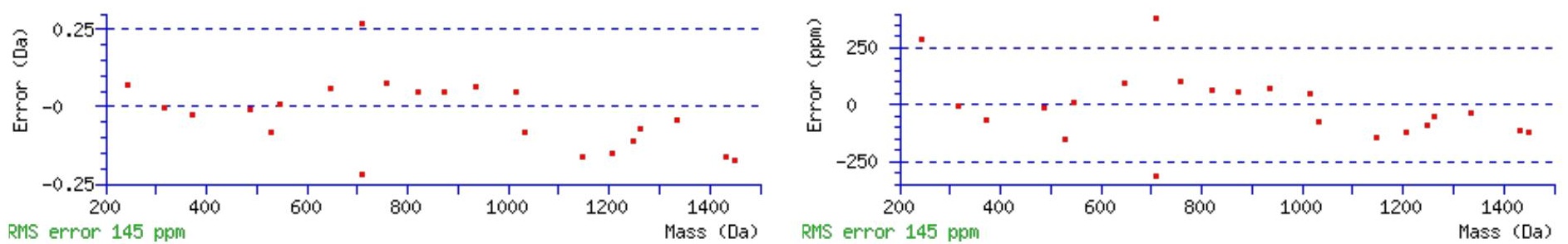

\section{All matches to this query}

\begin{tabular}{|l|l|l|l|}
\hline Score & Mr(calc): & Delta & \multicolumn{1}{|c|}{ Sequence } \\
\hline 98.5 & 1576.7589 & 0.0035 & KNSTLCDLCIGPAK \\
\hline 9.6 & 1576.7722 & -0.0098 & KDQSNLRQLNGPK \\
\hline 8.3 & 1576.7498 & 0.0126 & QNSVADEVGLLPQK \\
\hline 8.3 & 1576.7571 & 0.0053 & NSVAILMKNLYSK \\
\hline 7.2 & 1576.7668 & -0.0043 & GGQELSWVSRPMSK \\
\hline 6.4 & 1576.7780 & -0.0155 & KNEGVNWLRMASR \\
\hline 5.2 & 1576.7498 & 0.0126 & FSGSSSGTTAILTIR \\
\hline 5.2 & 1576.7538 & 0.0087 & QYEKSIVNYKPK \\
\hline 4.7 & 1576.7538 & 0.0087 & QYEKSIVNYKPK \\
\hline 4.5 & 1576.7555 & 0.0069 & ELEEEKMWAEKR \\
\hline
\end{tabular}

Spectrum No: 87; Query: 1961; Rank: 1

\section{Peptide View}

MS/MS Fragmentation of EDSCQLNYSEGPCLGMQQK

Found in IPI00210900, Tax_Id=10116 Gene_Symbol=Ambp AMBP protein precursor

Match to Query 1961: 2259.910208 from(1130.962380,2+)

Title: 091008RatKidney_NoSalt_31.1573.1573.2.dta

Data file K:INewmanPaper|Piliang|3SubProteomes\Piliang3SP\mgf5ppm\ERLIC_3SubProteomes5ppm.mgf

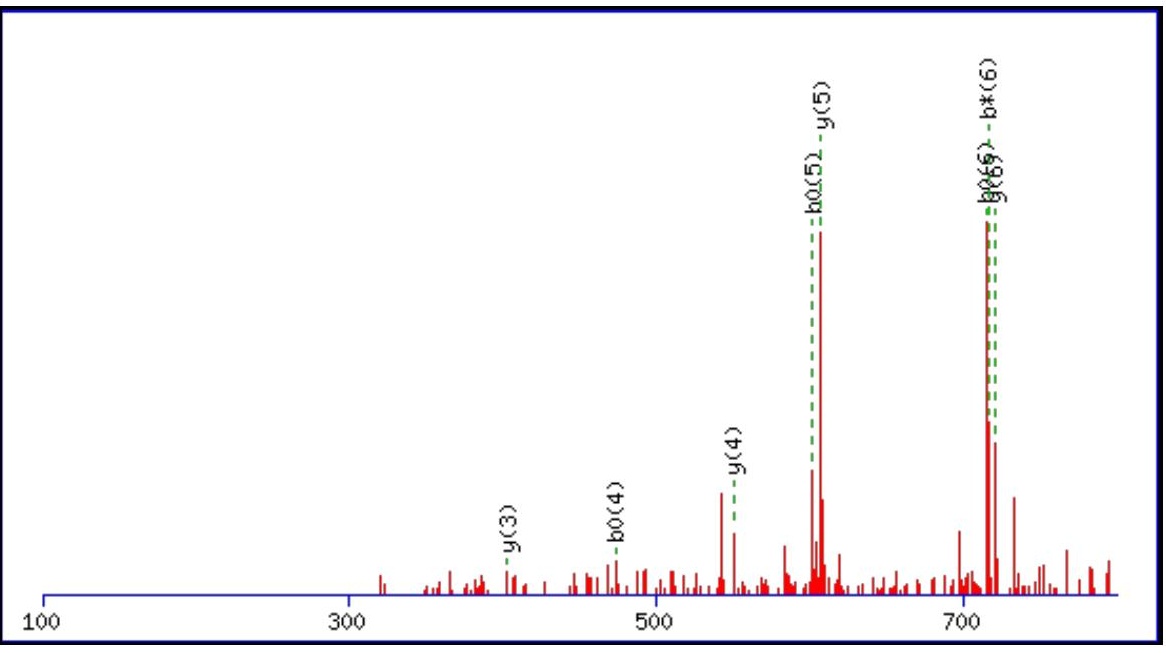



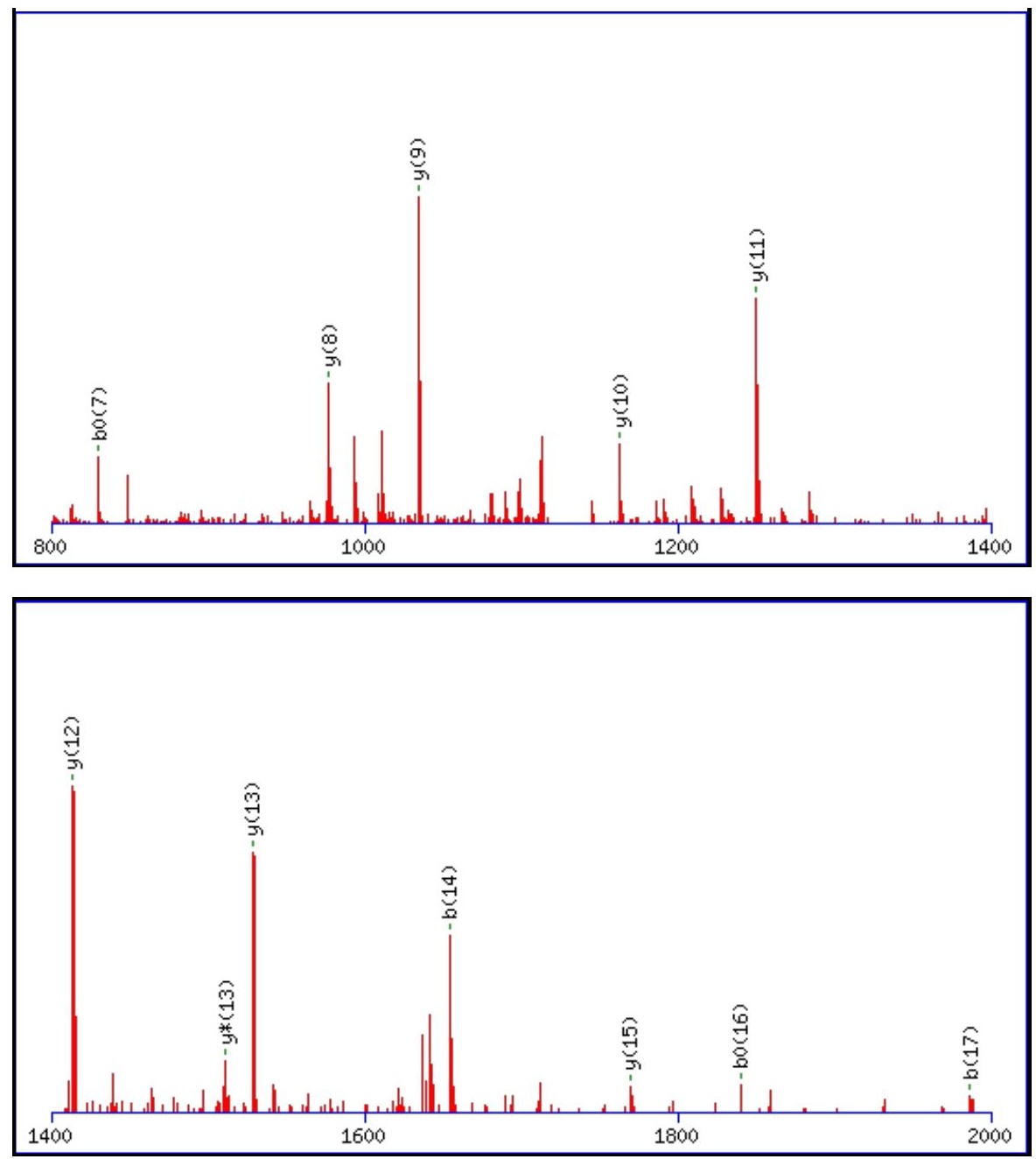

Monoisotopic mass of neutral peptide $\operatorname{Mr}($ calc): 2259.9082

Fixed modifications: Carbamidomethyl (C)

Variable modifications:

N7 : Deamidated_N (N)

M16 : Oxidation (M)

Ions Score: 98 Expect: $3 \mathrm{e}-008$

Matches (Bold Red): 20/190 fragment ions using 35 most intense peaks

\begin{tabular}{|c|c|c|c|c|c|c|c|c|c|c|c|c|c|c|}
\hline \# & b & $\mathbf{b}^{++}$ & b* & $\mathbf{b}^{*^{++}}$ & $\mathbf{b}^{0}$ & $\mathbf{b}^{0++}$ & Seq. & $\mathbf{y}$ & $y^{++}$ & $\mathbf{y}^{*}$ & $y^{*^{++}}$ & $\mathbf{y}^{0}$ & $y^{0++}$ & $\#$ \\
\hline 1 & 130.0499 & 65.5286 & & & 112.0393 & 56.5233 & $\mathbf{E}$ & & & & & & & 19 \\
\hline 2 & 245.0768 & 123.0420 & & & 227.0662 & 114.0368 & D & 2131.8729 & 1066.4401 & 2114.8464 & 1057.9268 & 2113.8624 & 1057.4348 & 18 \\
\hline 3 & 332.1088 & 166.5581 & & & 314.0983 & 157.5528 & $\mathrm{~S}$ & 2016.8460 & 1008.9266 & 1999.8194 & 1000.4134 & 1998.8354 & 999.9213 & 17 \\
\hline 4 & 492.1395 & 246.5734 & & & 474.1289 & 237.5681 & $\mathrm{C}$ & 1929.8139 & 965.4106 & 1912.7874 & 956.8973 & 1911.8034 & 956.4053 & 16 \\
\hline 5 & 620.1981 & 310.6027 & 603.1715 & 302.0894 & 602.1875 & 301.5974 & $\mathbf{Q}$ & 1769.7833 & 885.3953 & 1752.7567 & 876.8820 & 1751.7727 & 876.3900 & 15 \\
\hline 6 & 733.2821 & 367.1447 & 716.2556 & 358.6314 & 715.2716 & 358.1394 & $\mathbf{L}$ & 1641.7247 & 821.3660 & 1624.6982 & 812.8527 & 1623.7142 & 812.3607 & 14 \\
\hline 7 & 848.3091 & 424.6582 & 831.2825 & 416.1449 & 830.2985 & 415.6529 & $\mathbf{N}$ & 1528.6407 & 764.8240 & 1511.6141 & 756.3107 & 1510.6301 & 755.8187 & 13 \\
\hline 8 & 1011.3724 & 506.1898 & 994.3458 & 497.6766 & 993.3618 & 497.1846 & $\mathbf{Y}$ & 1413.6137 & 707.3105 & 1396.5872 & 698.7972 & 1395.6032 & 698.3052 & 12 \\
\hline 9 & 1098.4044 & 549.7059 & 1081.3779 & 541.1926 & 1080.3939 & 540.7006 & $S$ & 1250.5504 & 625.7788 & 1233.5238 & 617.2656 & 1232.5398 & 616.7736 & 11 \\
\hline 10 & 1227.4470 & 614.2271 & 1210.4205 & 605.7139 & 1209.4365 & 605.2219 & $\mathbf{E}$ & 1163.5184 & 582.2628 & 1146.4918 & 573.7495 & 1145.5078 & 573.2575 & 10 \\
\hline 11 & 1284.4685 & 642.7379 & 1267.4419 & 634.2246 & 1266.4579 & 633.7326 & $\mathbf{G}$ & 1034.4758 & 517.7415 & 1017.4492 & 509.2282 & & & 9 \\
\hline 12 & 1381.5212 & 691.2643 & 1364.4947 & 682.7510 & 1363.5107 & 682.2590 & $\mathbf{P}$ & 977.4543 & 489.2308 & 960.4278 & 480.7175 & & & 8 \\
\hline 13 & 1541.5519 & 771.2796 & 1524.5253 & 762.7663 & 1523.5413 & 762.2743 & $\mathrm{C}$ & 880.4015 & 440.7044 & 863.3750 & 432.1911 & & & 7 \\
\hline 14 & 1654.6360 & 827.8216 & 1637.6094 & 819.3083 & 1636.6254 & 818.8163 & $\mathbf{L}$ & 720.3709 & 360.6891 & 703.3443 & 352.1758 & & & 6 \\
\hline 15 & 1711.6574 & 856.3323 & 1694.6309 & 847.8191 & 1693.6469 & 847.3271 & G & 607.2868 & 304.1471 & 590.2603 & 295.6338 & & & 5 \\
\hline 16 & 1858.6928 & 929.8501 & 1841.6663 & 921.3368 & 1840.6823 & 920.8448 & $\mathbf{M}$ & 550.2654 & 275.6363 & 533.2388 & 267.1230 & & & 4 \\
\hline 17 & 1986.7514 & 993.8793 & 1969.7249 & 985.3661 & 1968.7408 & 984.8741 & $\mathbf{Q}$ & 403.2300 & 202.1186 & 386.2034 & 193.6053 & & & 3 \\
\hline 18 & 2114.8100 & 1057.9086 & 2097.7834 & 1049.3954 & 2096.7994 & 1048.9033 & $\mathbf{Q}$ & 275.1714 & 138.0893 & 258.1448 & 129.5761 & & & 2 \\
\hline
\end{tabular}




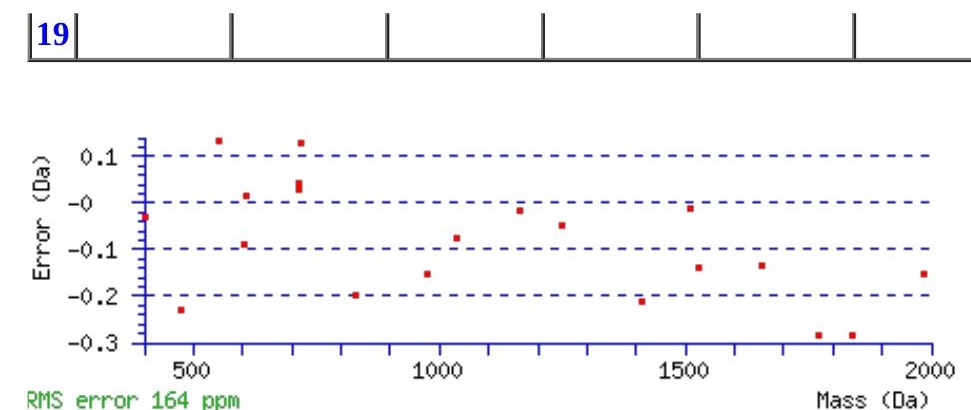

\section{All matches to this query}

\begin{tabular}{|l|l|c|l|}
\hline Score & Mr(calc): & Delta & \multicolumn{1}{|c|}{ Sequence } \\
\hline 98.3 & 2259.9082 & 0.0020 & EDSCQLNYSEGPCLGMQQK \\
\hline 40.3 & 2258.9242 & 0.9860 & EDSCQLNYSEGPCLGMQQK \\
\hline 11.0 & 2259.9046 & 0.0056 & RPEEGTVDAQSSELSENPR \\
\hline 7.1 & 2259.8951 & 0.0151 & MDSQNGDTEGEGRAEEGLIR \\
\hline 4.5 & 2259.9281 & -0.0179 & ETPLPNNNHVASDAGETEER \\
\hline 2.7 & 2259.8951 & 0.0151 & MDSQNGDTEGEGRAEEGLIR \\
\hline 1.8 & 2257.9213 & 1.9889 & KPRPSCQGSAMALLKSCK \\
\hline 1.0 & 2257.9157 & 1.9945 & QLSTEQAALQESLEKESK \\
\hline 0.9 & 2258.9139 & 0.9963 & RPTSLPLNTKNSTKEPR \\
\hline 0.7 & 2259.8997 & 0.0105 & STATVQICSGSVNLRGNVR \\
\hline
\end{tabular}

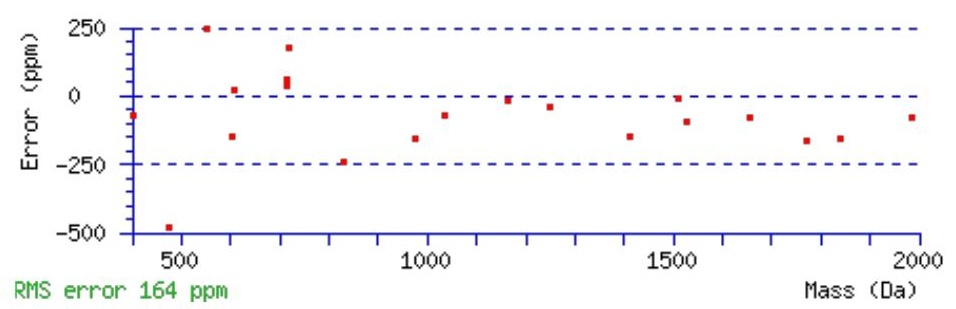

Spectrum No: 88; Query: 2241; Rank: 1

\section{Peptide View}

MS/MS Fragmentation of YGMQNSGNEAAWNYTLEQYQK

Found in IPI00327398, Tax_Id=10116 Gene_Symbol=Enpep Isoform 1 of Glutamyl aminopeptidase

Match to Query 2241: 2495.079988 from(1248.547270,2+)

Title: 100101RatKid_NS_deglyco_23.3857.3857.2.dta

Data file K:INewmanPaper|Piliangl3SubProteomes\Piliang3SP\mgf5ppm\ERLIC_3SubProteomes5ppm.mgf

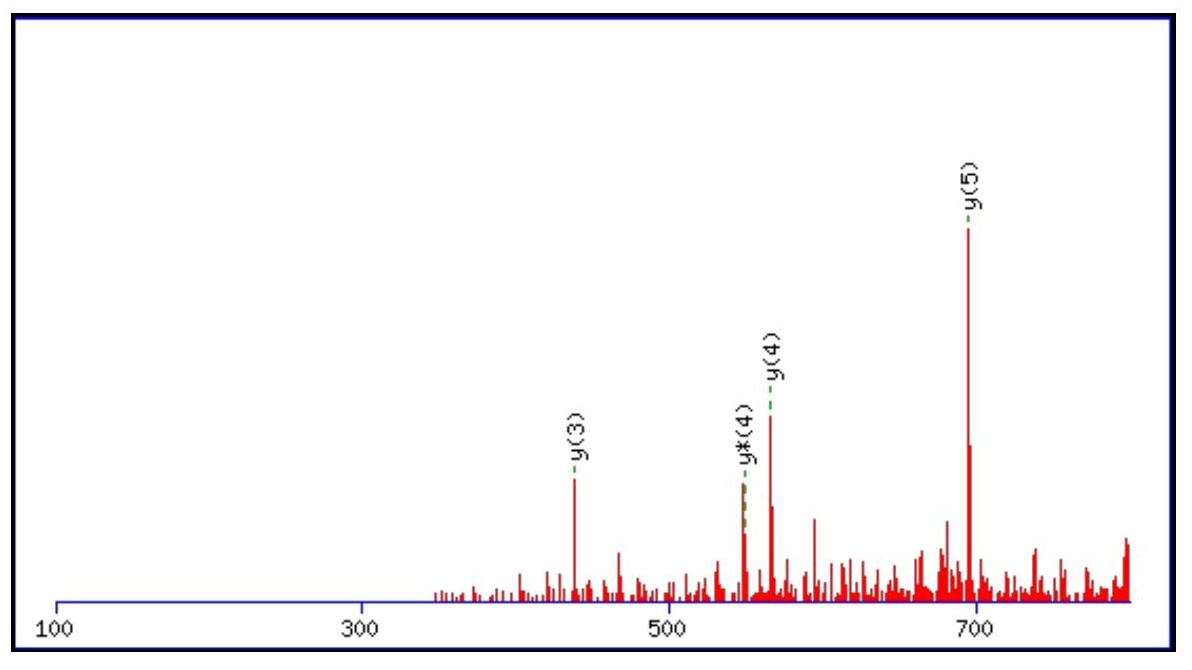




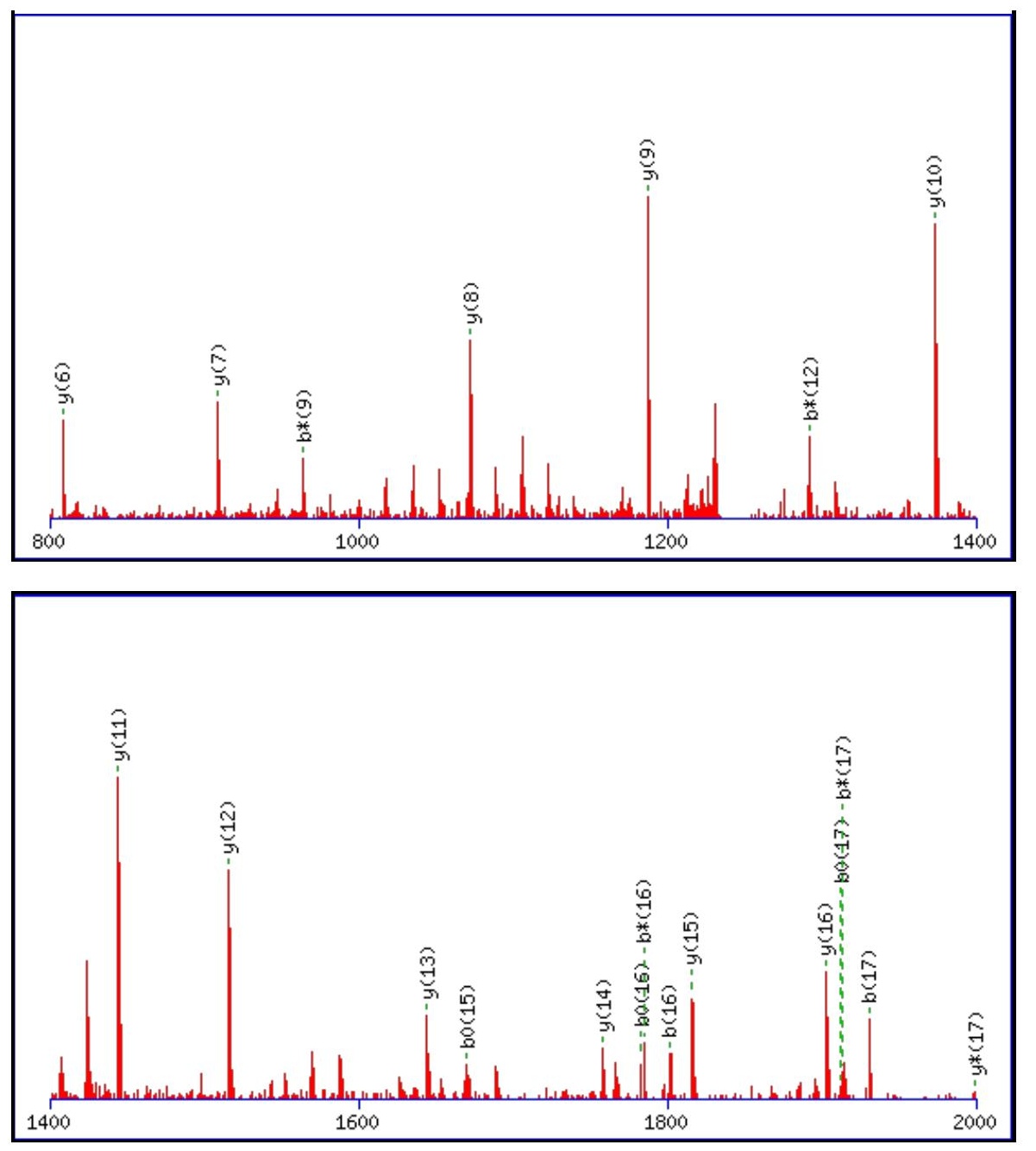

Monoisotopic mass of neutral peptide $\operatorname{Mr}($ calc): 2495.0699

Fixed modifications: Carbamidomethyl (C)

Variable modifications:

N13 : Deamidated_N (N)

Ions Score: 98 Expect: $4.5 \mathrm{e}-008$

Matches (Bold Red): 25/216 fragment ions using 42 most intense peaks

\begin{tabular}{|c|c|c|c|c|c|c|c|c|c|c|c|c|c|c|}
\hline \# & b & $\mathbf{b}^{++}$ & $\mathbf{b}^{*}$ & $\mathbf{b}^{*^{++}}$ & $\mathbf{b}^{\mathbf{0}}$ & $\mathbf{b}^{\mathbf{0 + +}}$ & Seq. & $\mathbf{y}$ & $y^{++}$ & $\mathbf{y}^{*}$ & $\mathbf{y}^{*^{++}}$ & $\mathbf{y}^{0}$ & $\mathbf{y}^{0++}$ & \# \\
\hline 1 & 164.0706 & 82.5389 & & & & & $\mathbf{Y}$ & & & & & & & 21 \\
\hline 2 & 221.0921 & 111.0497 & & & & & G & 2333.0139 & 1167.0106 & 2315.9873 & 1158.4973 & 2315.0033 & 1158.0053 & 20 \\
\hline 4 & 480.1911 & 240.5992 & 463.1646 & 232.0859 & & & $\mathbf{Q}$ & 2144.9519 & 1072.9796 & 2127.9254 & 1064.4663 & 2126.9414 & 1063.9743 & 18 \\
\hline 5 & 594.2341 & 297.6207 & 577.2075 & 289.1074 & & & $\mathbf{N}$ & 2016.8934 & 1008.9503 & 1999.8668 & 1000.4370 & 1998.8828 & 999.9450 & 17 \\
\hline 6 & 681.2661 & 341.1367 & 664.2395 & 332.6234 & 663.2555 & 332.1314 & S & 1902.8504 & 951.9289 & 1885.8239 & 943.4156 & 1884.8399 & 942.9236 & 16 \\
\hline 7 & 738.2876 & 369.6474 & 721.2610 & 361.1341 & 720.2770 & 360.6421 & G & 1815.8184 & 908.4128 & 1798.7919 & 899.8996 & 1797.8079 & 899.4076 & 15 \\
\hline 8 & 852.3305 & 426.6689 & 835.3039 & 418.1556 & 834.3199 & 417.6636 & $\mathbf{N}$ & 1758.7970 & 879.9021 & 1741.7704 & 871.3888 & 1740.7864 & 870.8968 & 14 \\
\hline 9 & 981.3731 & 491.1902 & 964.3465 & 482.6769 & 963.3625 & 482.1849 & $\mathbf{E}$ & 1644.7540 & 822.8807 & 1627.7275 & 814.3674 & 1626.7435 & 813.8754 & 13 \\
\hline 10 & 1052.4102 & 526.7087 & 1035.3836 & 518.1955 & 1034.3996 & 517.7034 & A & 1515.7114 & 758.3594 & 1498.6849 & 749.8461 & 1497.7009 & 749.3541 & 12 \\
\hline 11 & 1123.4473 & 562.2273 & 1106.4208 & 553.7140 & 1105.4367 & 553.2220 & A & 1444.6743 & 722.8408 & 1427.6478 & 714.3275 & 1426.6638 & 713.8355 & 11 \\
\hline 12 & 1309.5266 & 655.2669 & 1292.5001 & 646.7537 & 1291.5160 & 646.2617 & $\mathbf{W}$ & 1373.6372 & 687.3222 & 1356.6107 & 678.8090 & 1355.6266 & 678.3170 & 10 \\
\hline 14 & 1587.6169 & 794.3121 & 1570.5903 & 785.7988 & 1569.6063 & 785.3068 & $\mathbf{Y}$ & 1072.5310 & 536.7691 & 1055.5044 & 528.2558 & 1054.5204 & 527.7638 & 8 \\
\hline 15 & 1688.6646 & 844.8359 & 1671.6380 & 836.3226 & 1670.6540 & 835.8306 & $\mathbf{T}$ & 909.4676 & 455.2375 & 892.4411 & 446.7242 & 891.4571 & 446.2322 & 7 \\
\hline 16 & 1801.7486 & 901.3779 & 1784.7221 & 892.8647 & 1783.7381 & 892.3727 & $\mathbf{L}$ & 808.4199 & 404.7136 & 791.3934 & 396.2003 & 790.4094 & 395.7083 & 6 \\
\hline 17 & 1930.7912 & 965.8992 & 1913.7647 & 957.3860 & 1912.7806 & 956.8940 & $\mathbf{E}$ & 695.3359 & 348.1716 & 678.3093 & 339.6583 & 677.3253 & 339.1663 & 5 \\
\hline 18 & 2058.8498 & 1029.9285 & 2041.8232 & 1021.4153 & 2040.8392 & 1020.9233 & $\mathbf{Q}$ & 566.2933 & 283.6503 & 549.2667 & 275.1370 & & & 4 \\
\hline
\end{tabular}




\begin{tabular}{|r|r|r|r|r|r|r|r|r|r|r|r|r|r|r|r|r|r|r|r|r|}
$\mathbf{1 9}$ & 2221.9131 & 1111.4602 & 2204.8866 & 1102.9469 & 2203.9026 & 1102.4549 & $\mathbf{Y}$ & $\mathbf{4 3 8 . 2 3 4 7}$ & 219.6210 & $\mathbf{4 2 1 . 2 0 8 2}$ & $\mathbf{2 1 1 . 1 0 7 7}$ & $\mathbf{3}$ \\
\hline $\mathbf{2 0}$ & 2349.9717 & 1175.4895 & 2332.9451 & 1166.9762 & 2331.9611 & 1166.4842 & $\mathbf{Q}$ & 275.1714 & 138.0893 & 258.1448 & 129.5761 & \\
\hline $\mathbf{2 1}$ & & & & & & & $\mathbf{K}$ & 147.1128 & 74.0600 & 130.0863 & 65.5468 & & & $\mathbf{1}$ \\
\hline
\end{tabular}
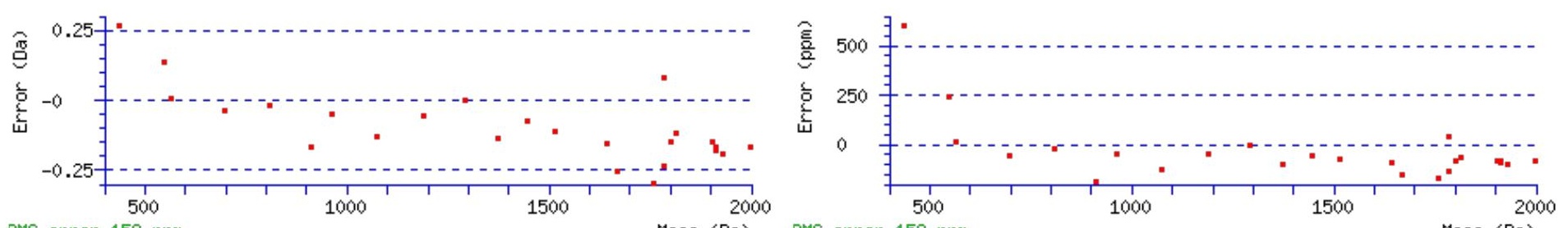

RMS error 158 ppm

Mass (Da) RMS error 158 ppm

Mass (Da)

\section{All matches to this query}

\begin{tabular}{|l|l|l|l|}
\hline Score & Mr(calc): & Delta & \multicolumn{1}{c|}{ Sequence } \\
\hline 98.2 & 2495.0699 & 0.0101 & YGMQNSGNEAAWNYTLEQYQK \\
\hline 39.4 & 2495.0699 & 0.0101 & YGMQNSGNEAAWNYTLEQYQK \\
\hline 18.4 & 2495.0699 & 0.0101 & YGMQNSGNEAAWNYTLEQYQK \\
\hline 16.4 & 2494.0859 & 0.9941 & YGMQNSGNEAAWNYTLEOYQK \\
\hline 7.1 & 2495.1013 & -0.0213 & AFDMISQRSSGGHPGSGPQLGTGR \\
\hline 0.8 & 2495.0553 & 0.0247 & GVHSGKMDENRFVAVTSTNAAK \\
\hline 0.8 & 2495.0553 & 0.0247 & GVHSGKMDENRFVAVTSTNAAK \\
\hline 0.8 & 2495.0553 & 0.0247 & GVHSGKMDENRFVAVTSTNAAK \\
\hline
\end{tabular}

Spectrum No: 89; Query: 432; Rank: 1

\section{Peptide View}

MS/MS Fragmentation of AFINSSYTIDPK

Found in IPI00200067, Tax_Id=10116 Gene_Symbol=F11r Junctional adhesion molecule A precursor

Match to Query 432: 1355.658988 from(678.836770,2+)

Title: 091008RatKidney_NoSalt_30.2083.2083.2.dta

Data file K:INewmanPaper|Piliangl3SubProteomes\Piliang3SP\mgf5ppm\ERLIC_3SubProteomes5ppm.mgf

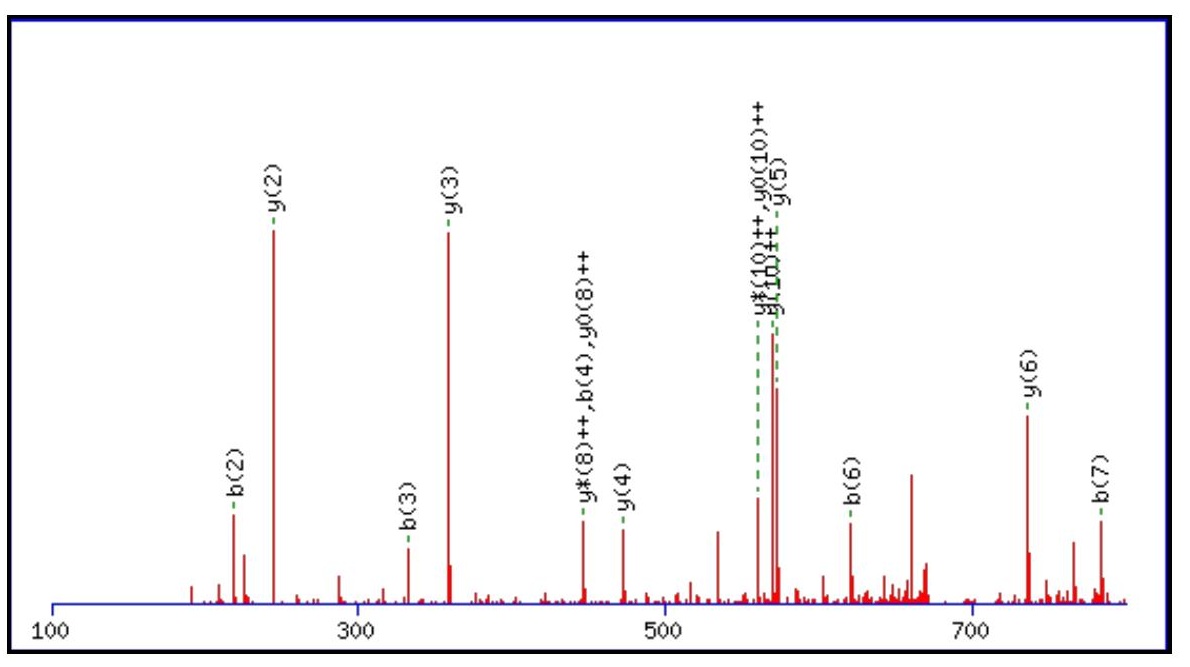



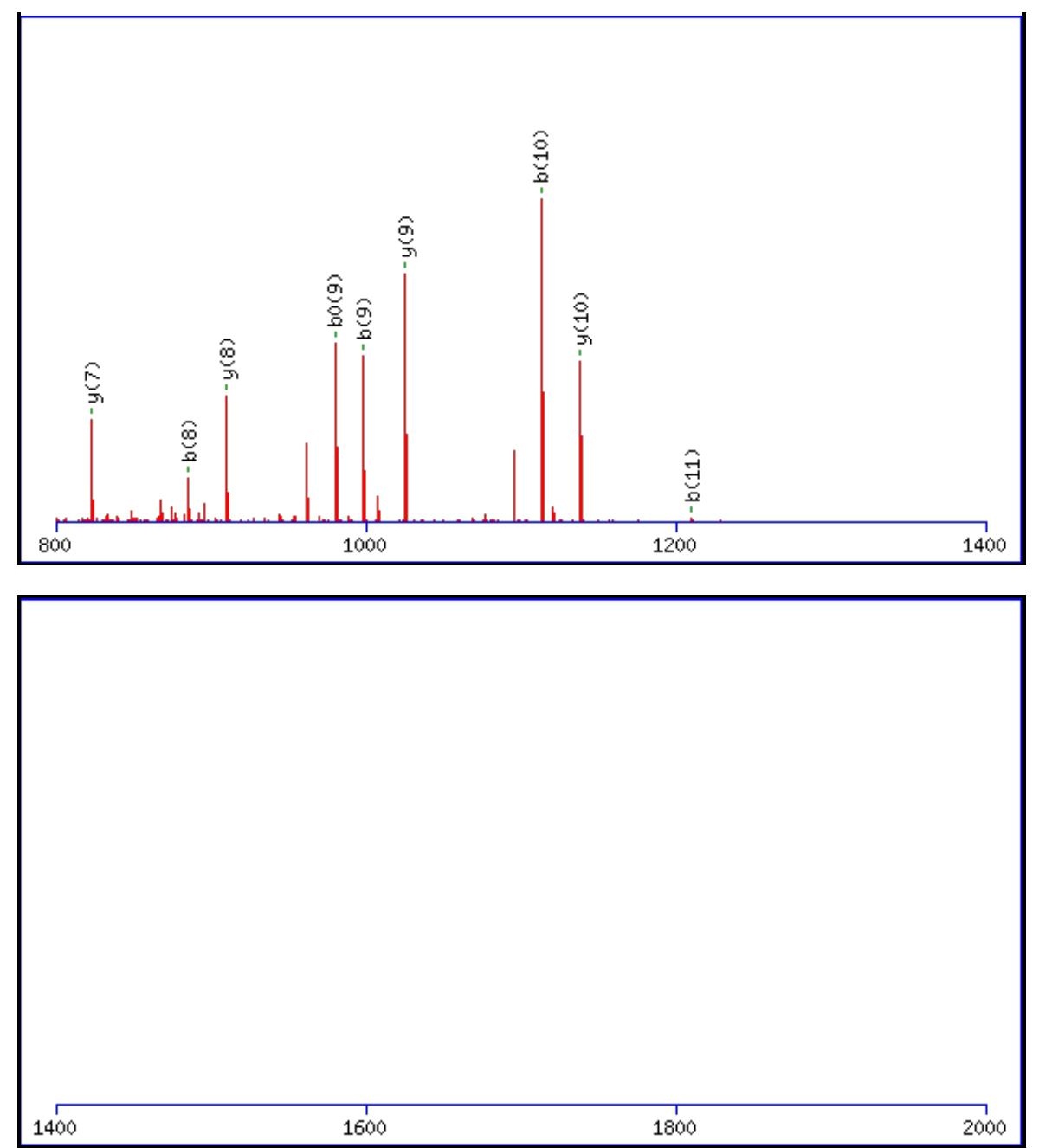

Monoisotopic mass of neutral peptide $\operatorname{Mr}($ calc): 1355.6609

Fixed modifications: Carbamidomethyl (C)

Variable modifications:

N4 : Deamidated $\mathrm{N}(\mathrm{N})$

Ions Score: 98 Expect: $2.2 \mathrm{e}-008$

Matches (Bold Red): 24/114 fragment ions using 24 most intense peaks

\begin{tabular}{|c|c|c|c|c|c|c|c|c|c|c|c|c|c|c|}
\hline$\#$ & b & $\mathbf{b}^{++}$ & $\mathbf{b}^{*}$ & $\mathbf{b}^{*^{++}}$ & $\mathbf{b}^{0}$ & $\mathbf{b}^{0++}$ & Seq. & $\mathbf{y}$ & $y^{++}$ & $\mathbf{y}^{*}$ & $\mathrm{y}^{*^{++}}$ & $\mathbf{y}^{0}$ & $\mathbf{y}^{0++}$ & \# \\
\hline 1 & 72.0444 & 36.5258 & & & & & A & & & & & & & 12 \\
\hline 2 & 219.1128 & 110.0600 & & & & & $\mathbf{F}$ & 1285.6311 & 643.3192 & 1268.6045 & 634.8059 & 1267.6205 & 634.3139 & 11 \\
\hline 3 & 332.1969 & 166.6021 & & & & & I & 1138.5626 & 569.7850 & 1121.5361 & 561.2717 & 1120.5521 & 560.7797 & 10 \\
\hline 4 & 447.2238 & 224.1155 & 430.1973 & 215.6023 & & & $\mathbf{N}$ & 1025.4786 & 513.2429 & 1008.4520 & 504.7297 & 1007.4680 & 504.2376 & 9 \\
\hline 5 & 534.2558 & 267.6316 & 517.2293 & 259.1183 & 516.2453 & 258.6263 & S & 910.4516 & 455.7295 & 893.4251 & 447.2162 & 892.4411 & 446.7242 & 8 \\
\hline 6 & 621.2879 & 311.1476 & 604.2613 & 302.6343 & 603.2773 & 302.1423 & $\mathrm{~S}$ & 823.4196 & 412.2134 & 806.3931 & 403.7002 & 805.4090 & 403.2082 & 7 \\
\hline 7 & 784.3512 & 392.6792 & 767.3246 & 384.1660 & 766.3406 & 383.6739 & $\mathbf{Y}$ & 736.3876 & 368.6974 & 719.3610 & 360.1842 & 718.3770 & 359.6921 & 6 \\
\hline 8 & 885.3989 & 443.2031 & 868.3723 & 434.6898 & 867.3883 & 434.1978 & $\mathbf{T}$ & 573.3243 & 287.1658 & 556.2977 & 278.6525 & 555.3137 & 278.1605 & 5 \\
\hline 9 & 998.4829 & 499.7451 & 981.4564 & 491.2318 & 980.4724 & 490.7398 & I & 472.2766 & 236.6419 & 455.2500 & 228.1287 & 454.2660 & 227.6366 & 4 \\
\hline 10 & 1113.5099 & 557.2586 & 1096.4833 & 548.7453 & 1095.4993 & 548.2533 & D & 359.1925 & 180.0999 & 342.1660 & 171.5866 & 341.1819 & 171.0946 & 3 \\
\hline 11 & 1210.5626 & 605.7850 & 1193.5361 & 597.2717 & 1192.5521 & 596.7797 & $\mathbf{P}$ & 244.1656 & 122.5864 & 227.1390 & 114.0731 & & & 2 \\
\hline 12 & & & & & & & $\mathbf{K}$ & 147.1128 & 74.0600 & 130.0863 & 65.5468 & & & 1 \\
\hline
\end{tabular}



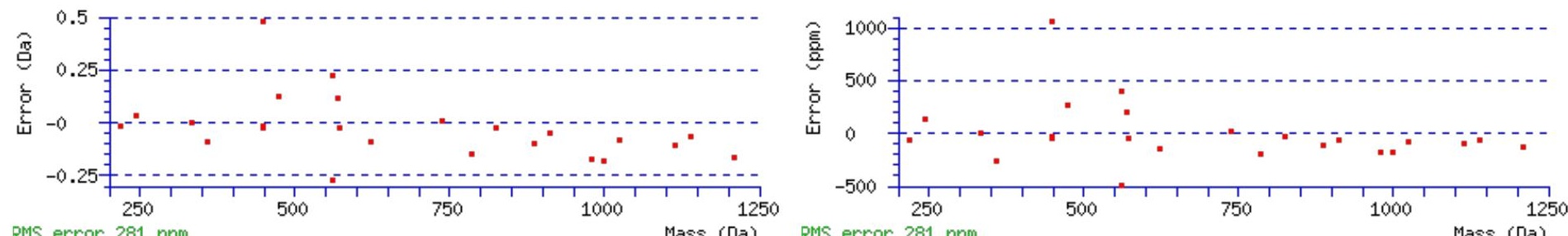

RIS error 281 ppm

Mass (Da) RMS error 281 ppm

Mass (Da)

\section{All matches to this query}

\begin{tabular}{|l|l|l|l|}
\hline Score & Mr(calc): & Delta & \multicolumn{1}{|c|}{ Sequence } \\
\hline 98.2 & 1355.6609 & -0.0019 & AFINSSYTIDPK \\
\hline 12.7 & 1355.6520 & 0.0070 & KDMPTTKNTLK \\
\hline 11.9 & 1355.6486 & 0.0104 & YDLSGRPLNIK \\
\hline 11.4 & 1355.6520 & 0.0070 & KDMPTTKNTLK \\
\hline 10.9 & 1355.6569 & 0.0021 & QNDVEIPSPTQK \\
\hline 9.0 & 1355.6681 & -0.0091 & KNNLKDHEETK \\
\hline 8.7 & 1355.6503 & 0.0087 & AKHTVSVCNNPK \\
\hline 6.8 & 1355.6469 & 0.0120 & DHRXFLAENPK \\
\hline 6.2 & 1355.6711 & -0.0121 & KTESHHKPKGK \\
\hline 5.7 & 1355.6681 & -0.0091 & KNNKAEDEPSPK \\
\hline
\end{tabular}

Spectrum No: 90; Query: 1234; Rank: 1

\section{Peptide View}

MS/MS Fragmentation of LDNTSMSTVIDGVVLPK

Found in IPI00195148, Tax_Id=10116 Gene_Symbol=Es2 Liver carboxylesterase 1 precursor

Match to Query 1234: 1788.923948 from(895.469250,2+)

Title: 091008RatKidney_NoSalt_30.3470.3470.2.dta

Data file K:INewmanPaper|Piliang|3SubProteomes\Piliang3SP\mgf5ppm\ERLIC_3SubProteomes5ppm.mgf

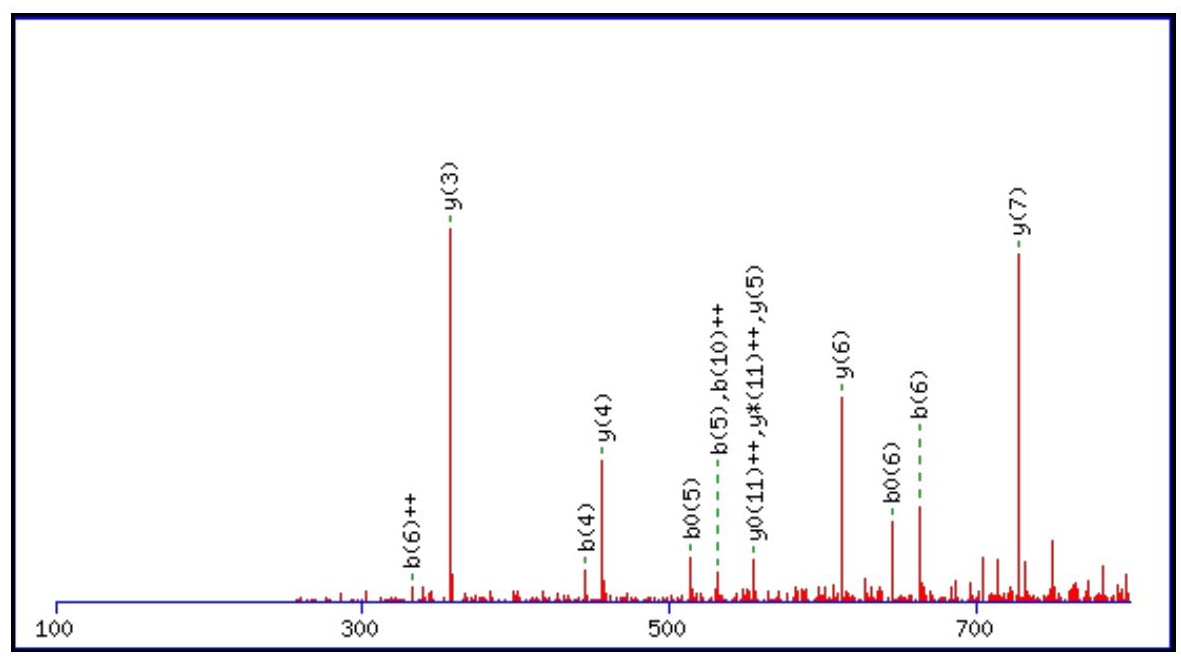



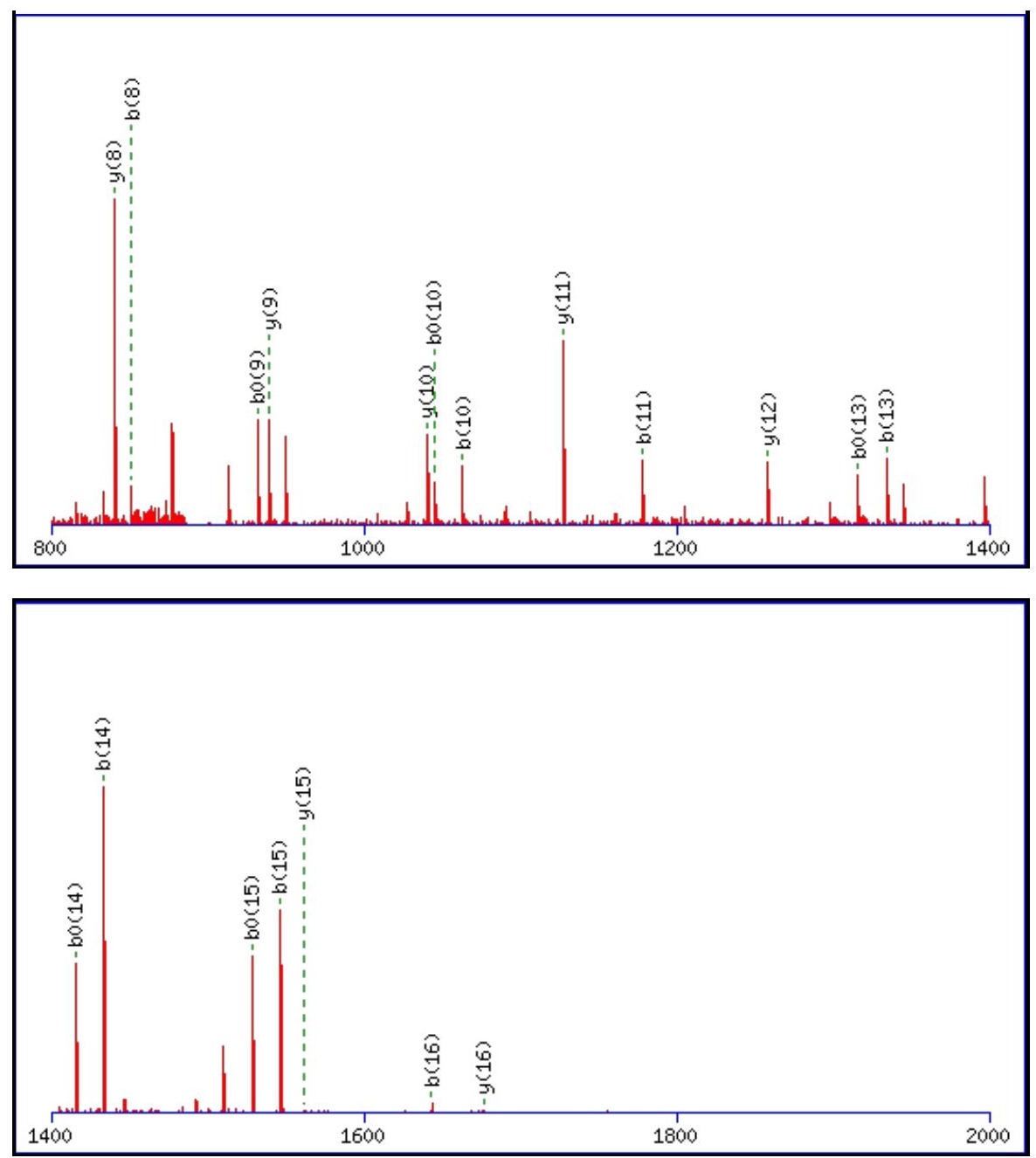

Monoisotopic mass of neutral peptide $\operatorname{Mr}($ calc): 1788.9179

Fixed modifications: Carbamidomethyl (C)

Variable modifications:

N3: Deamidated $\mathrm{N}(\mathrm{N})$

Ions Score: 98 Expect: $2.3 \mathrm{e}-008$

Matches (Bold Red): 33/174 fragment ions using 46 most intense peaks

\begin{tabular}{|c|c|c|c|c|c|c|c|c|c|c|c|c|c|c|}
\hline \# & b & $\mathbf{b}^{++}$ & b* & $\mathbf{b}^{*^{++}}$ & $\mathbf{b}^{\mathbf{0}}$ & $\mathbf{b}^{0++}$ & Seq. & $\mathbf{y}$ & $\mathbf{y}^{++}$ & $\mathbf{y}^{*}$ & $\mathrm{y}^{*^{++}}$ & $\mathbf{y}^{\mathbf{0}}$ & $\mathbf{y}^{\mathbf{0 + +}}$ & \# \\
\hline 1 & 114.0913 & 57.5493 & & & & & $\mathbf{L}$ & & & & & & & 17 \\
\hline 2 & 229.1183 & 115.0628 & & & 211.1077 & 106.0575 & D & 1676.8411 & 838.9242 & 1659.8146 & 830.4109 & 1658.8306 & 829.9189 & 16 \\
\hline 3 & 344.1452 & 172.5762 & 327.1187 & 164.0630 & 326.1347 & 163.5710 & $\mathbf{N}$ & 1561.8142 & 781.4107 & 1544.7876 & 772.8975 & 1543.8036 & 772.4054 & 15 \\
\hline 4 & 445.1929 & 223.1001 & 428.1663 & 214.5868 & 427.1823 & 214.0948 & $\mathbf{T}$ & 1446.7872 & 723.8973 & 1429.7607 & 715.3840 & 1428.7767 & 714.8920 & 14 \\
\hline 5 & 532.2249 & 266.6161 & 515.1984 & 258.1028 & 514.2144 & 257.6108 & S & 1345.7396 & 673.3734 & 1328.7130 & 664.8601 & 1327.7290 & 664.3681 & 13 \\
\hline 6 & 663.2654 & 332.1363 & 646.2389 & 323.6231 & 645.2548 & 323.1311 & $\mathbf{M}$ & 1258.7075 & 629.8574 & 1241.6810 & 621.3441 & 1240.6970 & 620.8521 & 12 \\
\hline 7 & 750.2974 & 375.6524 & 733.2709 & 367.1391 & 732.2869 & 366.6471 & $\mathbf{S}$ & 1127.6671 & 564.3372 & 1110.6405 & 555.8239 & 1109.6565 & 555.3319 & 11 \\
\hline 8 & 851.3451 & 426.1762 & 834.3186 & 417.6629 & 833.3346 & 417.1709 & $\mathbf{T}$ & 1040.6350 & 520.8212 & 1023.6085 & 512.3079 & 1022.6245 & 511.8159 & 10 \\
\hline 9 & 950.4135 & 475.7104 & 933.3870 & 467.1971 & 932.4030 & 466.7051 & $\mathbf{V}$ & 939.5873 & 470.2973 & 922.5608 & 461.7840 & 921.5768 & 461.2920 & 9 \\
\hline 10 & 1063.4976 & 532.2524 & 1046.4710 & 523.7392 & 1045.4870 & 523.2472 & I & 840.5189 & 420.7631 & 823.4924 & 412.2498 & 822.5084 & 411.7578 & 8 \\
\hline 11 & 1178.5245 & 589.7659 & 1161.4980 & 581.2526 & 1160.5140 & 580.7606 & D & 727.4349 & 364.2211 & 710.4083 & 355.7078 & 709.4243 & 355.2158 & 7 \\
\hline 12 & 1235.5460 & 618.2766 & 1218.5195 & 609.7634 & 1217.5354 & 609.2714 & G & 612.4079 & 306.7076 & 595.3814 & 298.1943 & & & 6 \\
\hline 13 & 1334.6144 & 667.8108 & 1317.5879 & 659.2976 & 1316.6039 & 658.8056 & $\mathbf{V}$ & 555.3865 & 278.1969 & 538.3599 & 269.6836 & & & 5 \\
\hline 14 & 1433.6828 & 717.3451 & 1416.6563 & 708.8318 & 1415.6723 & 708.3398 & V & 456.3180 & 228.6627 & 439.2915 & 220.1494 & & & 4 \\
\hline 15 & 1546.7669 & 773.8871 & 1529.7403 & 765.3738 & 1528.7563 & 764.8818 & $\mathbf{L}$ & 357.2496 & 179.1285 & 340.2231 & 170.6152 & & & 3 \\
\hline 16 & 1643.8197 & 822.4135 & 1626.7931 & 813.9002 & 1625.8091 & 813.4082 & $\mathbf{P}$ & 244.1656 & 122.5864 & 227.1390 & 114.0731 & & & 2 \\
\hline 17 & & & & & & & $\mathbf{K}$ & 147.1128 & 74.0600 & 130.0863 & 65.5468 & & & 1 \\
\hline
\end{tabular}



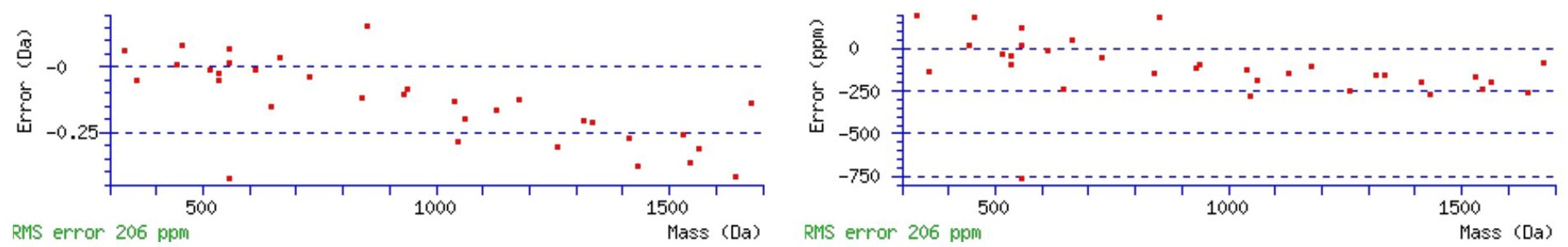

\section{All matches to this query}

\begin{tabular}{|l|l|r|l|}
\hline Score & Mr(calc): & Delta & \multicolumn{1}{c|}{ Sequence } \\
\hline 97.8 & 1788.9179 & 0.0060 & LDNTSMSTVIDGVVLPK \\
\hline 45.7 & 1787.9339 & 0.9900 & LDNTSMSTVIDGVVLPK \\
\hline 5.8 & 1788.9217 & 0.0023 & ILEQQNSSRTLEKNK \\
\hline 1.5 & 1788.9291 & -0.0052 & DQLALAIGTLGENMSLK \\
\hline
\end{tabular}

Spectrum No: 91; Query: 575; Rank: 1

\section{Peptide View}

\section{MS/MS Fragmentation of VANLTSVVNSLDSK}

Found in IPI00324102, Tax_Id=10116 Gene_Symbol=Fgl2 fibrinogen-like 2

Match to Query 575: 1446.759988 from(724.387270,2+)

Title: 100101RatKid_NS_deglyco_23.3766.3766.2.dta

Data file K:INewmanPaper|Piliangl3SubProteomes\Piliang3SP\mgf5ppm\ERLIC_3SubProteomes5ppm.mgf

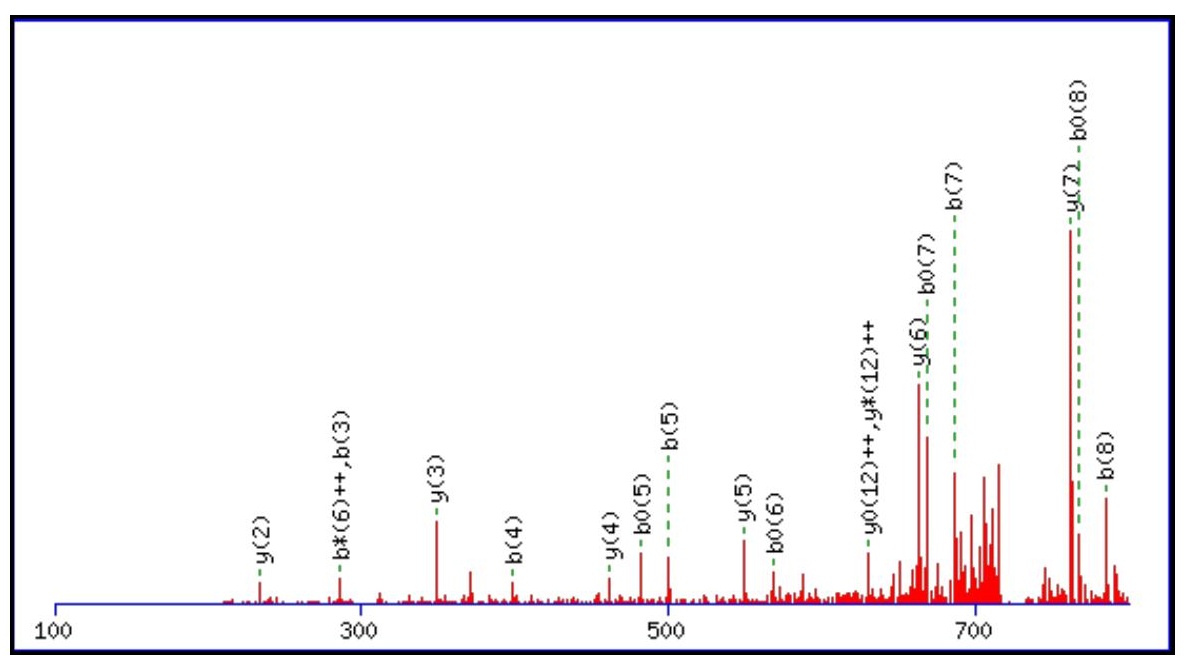



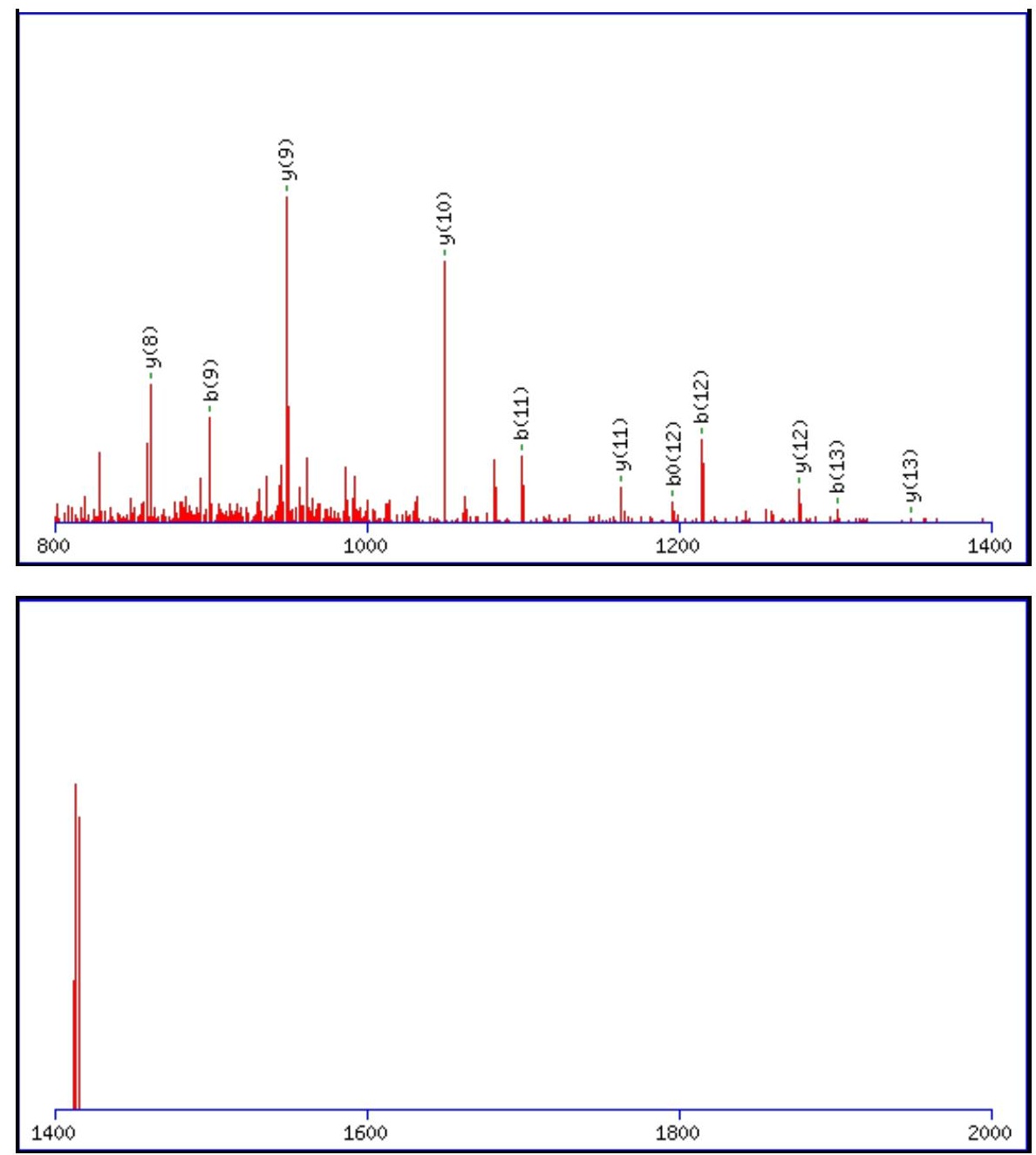

Monoisotopic mass of neutral peptide $\operatorname{Mr}($ calc): 1446.7566

Fixed modifications: Carbamidomethyl (C)

Variable modifications:

N3 : Deamidated $\mathrm{N}(\mathrm{N})$

Ions Score: 98 Expect: $2 \mathrm{e}-008$

Matches (Bold Red): 29/142 fragment ions using 55 most intense peaks

\begin{tabular}{|r|c|c|c|c|c|c|c|c|c|c|c|c|c|c|}
\hline$\#$ & $\mathbf{b}$ & $\mathbf{b}^{++}$ & $\mathbf{b}^{*}$ & $\mathbf{b}^{\mathbf{*}^{++}}$ & $\mathbf{b}^{\mathbf{0}}$ & $\mathbf{b}^{\mathbf{0 + +}}$ & $\mathbf{S e q}$ & $\mathbf{y}$ & $\mathbf{y}^{++}$ & $\mathbf{y}^{\mathbf{*}}$ & $\mathbf{y}^{\mathbf{*}^{++}}$ & $\mathbf{y}^{\mathbf{0}}$ & $\mathbf{y}^{\mathbf{0 + +}}$ & $\#$ \\
\hline $\mathbf{1}$ & 100.0757 & 50.5415 & & & & & $\mathbf{V}$ & & & & & & & $\mathbf{1 4}$ \\
\hline $\mathbf{2}$ & 171.1128 & 86.0600 & & & & & $\mathbf{A}$ & $\mathbf{1 3 4 8 . 6 9 5 4}$ & 674.8514 & 1331.6689 & 666.3381 & 1330.6849 & 665.8461 & $\mathbf{1 3}$ \\
\hline $\mathbf{3}$ & $\mathbf{2 8 6 . 1 3 9 7}$ & 143.5735 & 269.1132 & 135.0602 & & & $\mathbf{N}$ & $\mathbf{1 2 7 7 . 6 5 8 3}$ & 639.3328 & 1260.6318 & $\mathbf{6 3 0 . 8 1 9 5}$ & 1259.6478 & $\mathbf{6 3 0 . 3 2 7 5}$ & $\mathbf{1 2}$ \\
\hline $\mathbf{4}$ & $\mathbf{3 9 9 . 2 2 3 8}$ & 200.1155 & 382.1973 & 191.6023 & & & $\mathbf{L}$ & $\mathbf{1 1 6 2 . 6 3 1 4}$ & 581.8193 & 1145.6048 & 573.3061 & 1144.6208 & 572.8141 & $\mathbf{1 1}$ \\
\hline $\mathbf{5}$ & $\mathbf{5 0 0 . 2 7 1 5}$ & 250.6394 & 483.2449 & 242.1261 & $\mathbf{4 8 2 . 2 6 0 9}$ & 241.6341 & $\mathbf{T}$ & $\mathbf{1 0 4 9 . 5 4 7 3}$ & 525.2773 & 1032.5208 & 516.7640 & 1031.5368 & 516.2720 & $\mathbf{1 0}$ \\
\hline $\mathbf{6}$ & 587.3035 & 294.1554 & 570.2770 & 285.6421 & $\mathbf{5 6 9 . 2 9 2 9}$ & 285.1501 & $\mathbf{S}$ & $\mathbf{9 4 8 . 4 9 9 7}$ & 474.7535 & 931.4731 & 466.2402 & 930.4891 & 465.7482 & $\mathbf{9}$ \\
\hline $\mathbf{7}$ & $\mathbf{6 8 6 . 3 7 1 9}$ & 343.6896 & 669.3454 & 335.1763 & $\mathbf{6 6 8 . 3 6 1 4}$ & 334.6843 & $\mathbf{V}$ & $\mathbf{8 6 1 . 4 6 7 6}$ & 431.2374 & 844.4411 & 422.7242 & 843.4571 & 422.2322 & $\mathbf{8}$ \\
\hline $\mathbf{8}$ & $\mathbf{7 8 5 . 4 4 0 3}$ & 393.2238 & 768.4138 & 384.7105 & $\mathbf{7 6 7 . 4 2 9 8}$ & 384.2185 & $\mathbf{V}$ & $\mathbf{7 6 2 . 3 9 9 2}$ & 381.7032 & 745.3727 & 373.1900 & 744.3886 & 372.6980 & $\mathbf{7}$ \\
\hline $\mathbf{9}$ & $\mathbf{8 9 9 . 4 8 3 3}$ & 450.2453 & 882.4567 & 441.7320 & 881.4727 & 441.2400 & $\mathbf{N}$ & $\mathbf{6 6 3 . 3 3 0 8}$ & 332.1690 & 646.3042 & 323.6558 & 645.3202 & 323.1638 & $\mathbf{6}$ \\
\hline $\mathbf{1 0}$ & $\mathbf{9 8 6 . 5 1 5 3}$ & 493.7613 & 969.4887 & 485.2480 & 968.5047 & 484.7560 & $\mathbf{S}$ & 549.2879 & 275.1476 & 532.2613 & 266.6343 & 531.2773 & 266.1423 & $\mathbf{5}$ \\
\hline $\mathbf{1 1}$ & $\mathbf{1 0 9 9 . 5 9 9 4}$ & 550.3033 & 1082.5728 & 541.7900 & 1081.5888 & 541.2980 & $\mathbf{L}$ & $\mathbf{4 6 2 . 2 5 5 8}$ & 231.6316 & 445.2293 & 223.1183 & 444.2453 & 222.6263 & $\mathbf{4}$ \\
\hline $\mathbf{1 2}$ & $\mathbf{1 2 1 4 . 6 2 6 3}$ & 607.8168 & 1197.5998 & 599.3035 & $\mathbf{1 1 9 6 . 6 1 5 7}$ & 598.8115 & $\mathbf{D}$ & $\mathbf{3 4 9 . 1 7 1 8}$ & 175.0895 & 332.1452 & 166.5763 & 331.1612 & 166.0842 & $\mathbf{3}$ \\
\hline $\mathbf{1 3}$ & $\mathbf{1 3 0 1 . 6 5 8 3}$ & 651.3328 & 1284.6318 & 642.8195 & 1283.6478 & 642.3275 & $\mathbf{S}$ & $\mathbf{2 3 4 . 1 4 4 8}$ & 117.5761 & 217.1183 & 109.0628 & 216.1343 & 108.5708 & $\mathbf{2}$ \\
\hline $\mathbf{1 4}$ & & & & & & & $\mathbf{K}$ & 147.1128 & 74.0600 & 130.0863 & 65.5468 & & & $\mathbf{1}$ \\
\hline
\end{tabular}



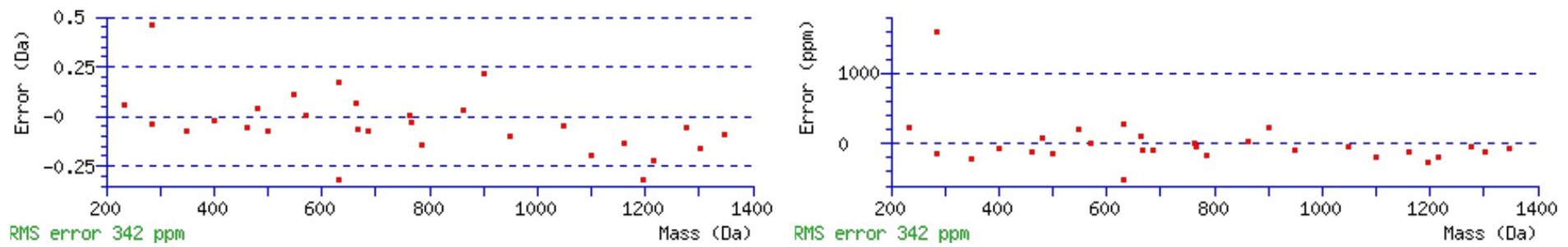

\section{All matches to this query}

\begin{tabular}{|l|l|l|l|}
\hline Score & Mr(calc): & Delta & \multicolumn{1}{|c|}{ Sequence } \\
\hline 97.7 & 1446.7566 & 0.0034 & VANLTSVVNSLDSK \\
\hline 58.4 & 1446.7566 & 0.0034 & VANLTSVVNSLDSK \\
\hline 10.6 & 1446.7541 & 0.0059 & VALDSVTCVWGIK \\
\hline 7.8 & 1444.7522 & 2.0078 & GLILTSNDSQQAAK \\
\hline 3.5 & 1445.7436 & 1.0164 & MTDLSKGVIEEPK \\
\hline 2.1 & 1446.7500 & 0.0100 & NISGKQSSLPAMSK \\
\hline
\end{tabular}

Spectrum No: 92; Query: 1760; Rank: 1

\section{Peptide View}

MS/MS Fragmentation of HIDCASVYGNETEIGEALK

Found in IPI00230859, Tax_Id=10116 Gene_Symbol=Akr1a1 Alcohol dehydrogenase

Match to Query 1760: 2105.963128 from(1053.988840,2+)

Title: 091008RatKidney_NH4Format02_22.2946.2946.2.dta

Data file K:INewmanPaper|Piliangl3SubProteomes\Piliang3SP\mgf5ppm\ERLIC_3SubProteomes5ppm.mgf

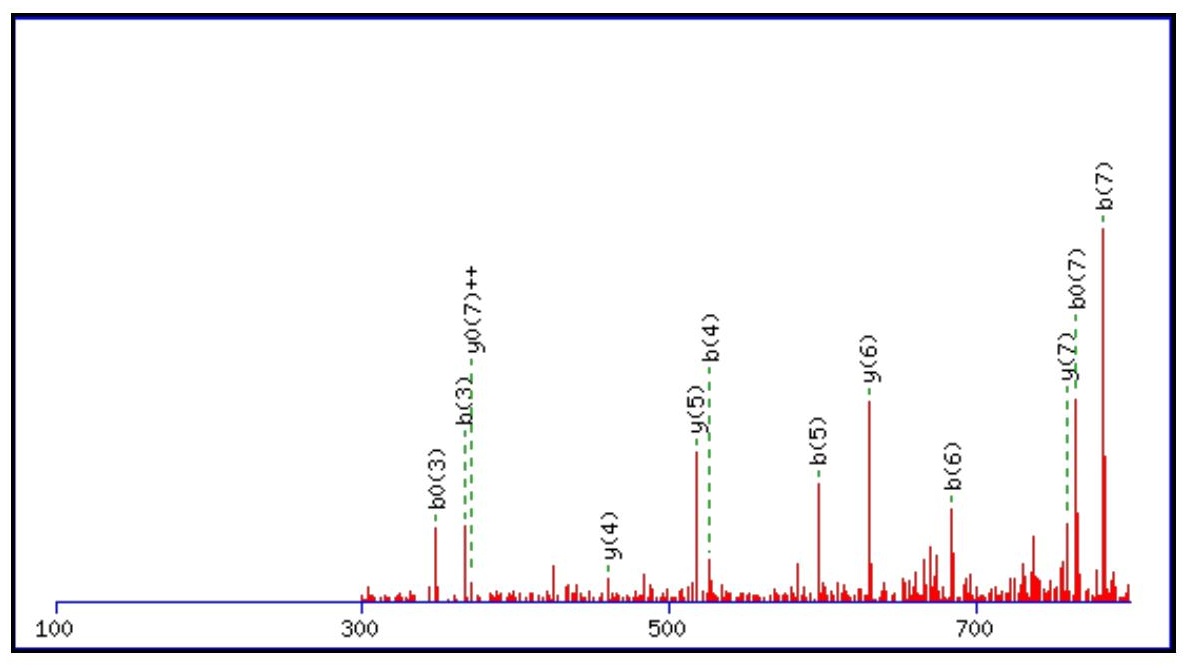



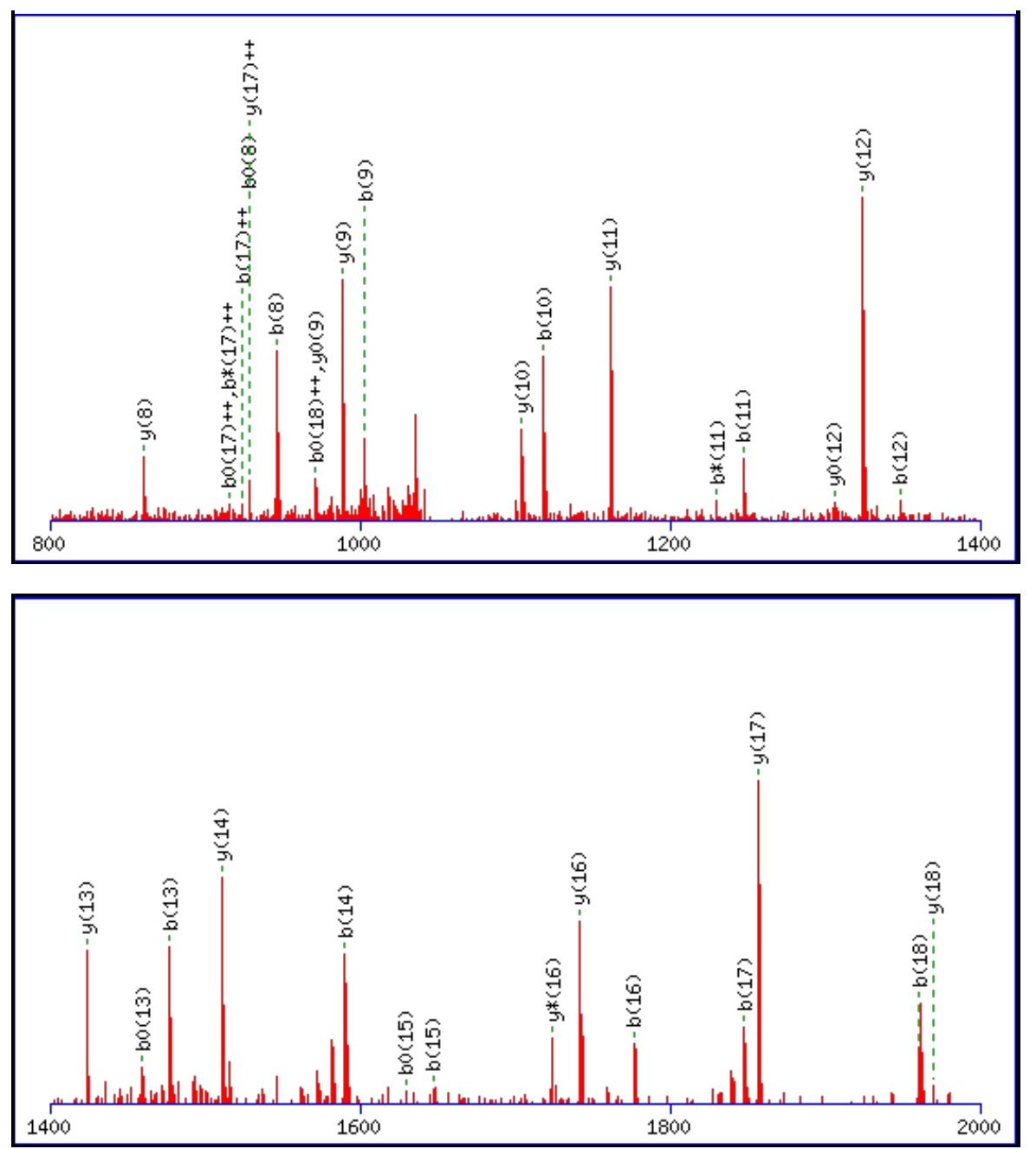

Monoisotopic mass of neutral peptide $\operatorname{Mr}($ calc): 2105.9575

Fixed modifications: Carbamidomethyl (C)

Variable modifications:

N10 : Deamidated_N (N)

Ions Score: 97 Expect: $5 \mathrm{e}-008$

Matches (Bold Red): 45/188 fragment ions using 96 most intense peaks

\begin{tabular}{|c|c|c|c|c|c|c|c|c|c|c|c|c|c|c|}
\hline \# & b & $\mathbf{b}^{++}$ & $\mathbf{b}^{*}$ & $\mathbf{b}^{*^{++}}$ & $\mathbf{b}^{0}$ & $\mathbf{b}^{0++}$ & Seq. & $\mathbf{y}$ & $\mathbf{y}^{++}$ & $\mathbf{y}^{*}$ & $\mathbf{y}^{*^{++}}$ & $\mathbf{y}^{\mathbf{0}}$ & $\mathbf{y}^{\mathbf{0}^{++}}$ & \# \\
\hline 1 & 138.0662 & 69.5367 & & & & & H & & & & & & & 19 \\
\hline 2 & 251.1503 & 126.0788 & & & & & I & 1969.9059 & 985.4566 & 1952.8794 & 976.9433 & 1951.8953 & 976.4513 & 18 \\
\hline 3 & 366.1772 & |183.5922 & & & 348.1666 & 174.5870 & D & 1856.8218 & 928.9146 & 1839.7953 & 920.4013 & 1838.8113 & 919.9093 & 17 \\
\hline 4 & 526.2078 & 263.6076 & & & 508.1973 & 254.6023 & C & 1741.7949 & 871.4011 & | 1724.7684 & 862.8878 & 1723.7843 & 862.3958 & 16 \\
\hline 5 & 597.2450 & 299.1261 & & & 579.2344 & 290.1208 & $\overline{\mathbf{A}}$ & 1581.7643 & 791.3858 & 1564.7377 & 782.8725 & 1563.7537 & 782.3805 & 15 \\
\hline 6 & 684.2770 & 342.6421 & & & 666.2664 & 333.6368 & $\mathrm{~S}$ & 1510.7271 & 755.8672 & 1493.7006 & 747.3539 & 1492.7166 & 746.8619 & 14 \\
\hline 7 & 783.3454 & 392.1763 & & & 765.3348 & 383.1711 & V & 1423.6951 & 712.3512 & 1406.6686 & 703.8379 & 1405.6845 & 703.3459 & 13 \\
\hline 8 & 946.4087 & 473.7080 & & & 928.3982 & 464.7027 & $\mathbf{Y}$ & 1324.6267 & 662.8170 & 1307.6001 & 654.3037 & 1306.6161 & 653.8117 & 12 \\
\hline 9 & 1003.4302 & 502.2187 & & & 985.4196 & 493.2135 & G & 1161.5634 & 581.2853 & 1144.5368 & 572.7720 & 1143.5528 & 572.2800 & 11 \\
\hline 10 & 1118.4571 & 559.7322 & 1101.4306 & 551.2189 & 1100.4466 & 550.7269 & $\mathbf{N}$ & 1104.5419 & 552.7746 & 1087.5154 & 544.2613 & 1086.5313 & 543.7693 & 10 \\
\hline 11 & 1247.4997 & 624.2535 & 1230.4732 & 615.7402 & 1229.4892 & 615.2482 & $\mathbf{E}$ & 989.5150 & 495.2611 & 972.4884 & 486.7478 & 971.5044 & 486.2558 & 9 \\
\hline 12 & 1348.5474 & 674.7773 & 1331.5209 & 666.2641 & 1330.5368 & 665.7721 & $\mathbf{T}$ & 860.4724 & 430.7398 & 843.4458 & 422.2266 & 842.4618 & 421.7345 & 8 \\
\hline 13 & 1477.5900 & 739.2986 & 1460.5634 & 730.7854 & 1459.5794 & 730.2934 & $\mathbf{E}$ & 759.4247 & 380.2160 & 742.3981 & 371.7027 & 741.4141 & 371.2107 & 7 \\
\hline 14 & 1590.6741 & 795.8407 & 1573.6475 & 787.3274 & 1572.6635 & 786.8354 & I & 630.3821 & 315.6947 & 613.3556 & 307.1814 & 612.3715 & 306.6894 & 6 \\
\hline 15 & 1647.6955 & 824.3514 & 1630.6690 & 815.8381 & 1629.6850 & 815.3461 & G & 517.2980 & 259.1527 & 500.2715 & 250.6394 & 499.2875 & 250.1474 & 5 \\
\hline 16 & 1776.7381 & 888.8727 & 1759.7116 & 880.3594 & 1758.7275 & 879.8674 & $E$ & 460.2766 & 230.6419 & 443.2500 & 222.1287 & 442.2660 & 221.6366 & 4 \\
\hline 17 & 1847.7752 & 924.3913 & 1830.7487 & 915.8780 & 1829.7647 & 915.3860 & A & 331.2340 & 166.1206 & 314.2074 & 157.6074 & & & 3 \\
\hline 18 & 1960.8593 & 980.9333 & 1943.8327 & 972.4200 & 1942.8487 & 971.9280 & $\mathbf{L}$ & 260.1969 & 130.6021 & 243.1703 & 122.0888 & & & 2 \\
\hline
\end{tabular}




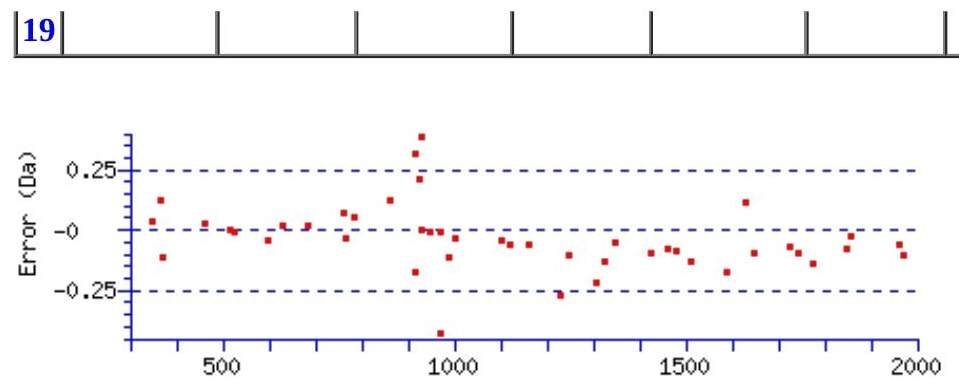

RNS error 149 ppm

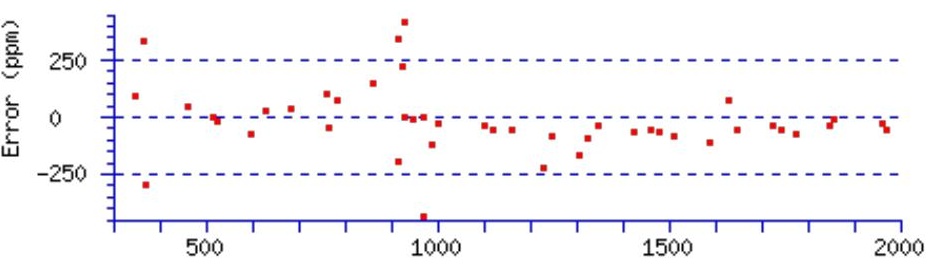

All matches to this query

\begin{tabular}{|l|l|l|l|}
\hline Score & Mr(calc): & Delta & Sequence \\
\hline 97.3 & 2105.9575 & 0.0056 & HIDCASVYGNETEIGEALK \\
\hline 25.3 & 2104.9735 & 0.9896 & HIDCASVYGNETEIGEALK \\
\hline
\end{tabular}

Spectrum No: 93; Query: 2020; Rank: 1

\section{Peptide View}

MS/MS Fragmentation of CGTLQDLYATVDFGTTGNITR

Found in IPI00371132, Tax_Id=10116 Gene_Symbol=Glb1_predicted galactosidase, beta 1

Match to Query 2020: 2303.085108 from(1152.549830,2+)

Title: 100101RatKid_NS_deglyco_15.5409.5409.2.dta

Data file K:\NewmanPaper\Piliang \3SubProteomes\Piliang3SP \mgf5ppm\ERLIC_3SubProteomes5ppm.mgf

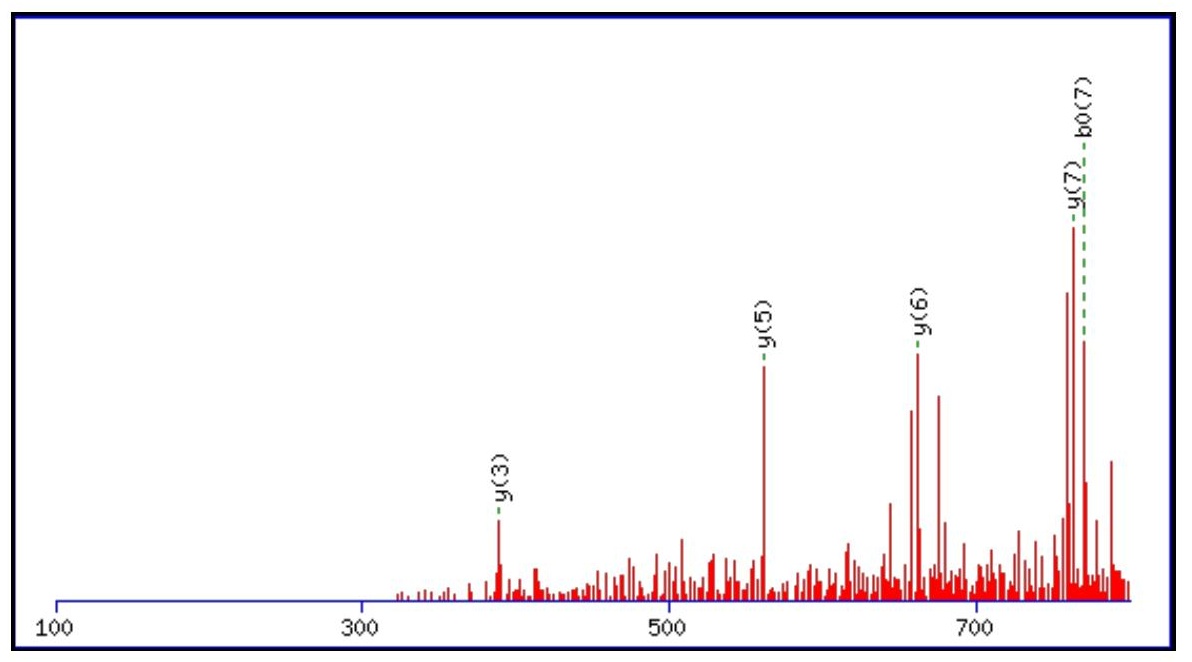



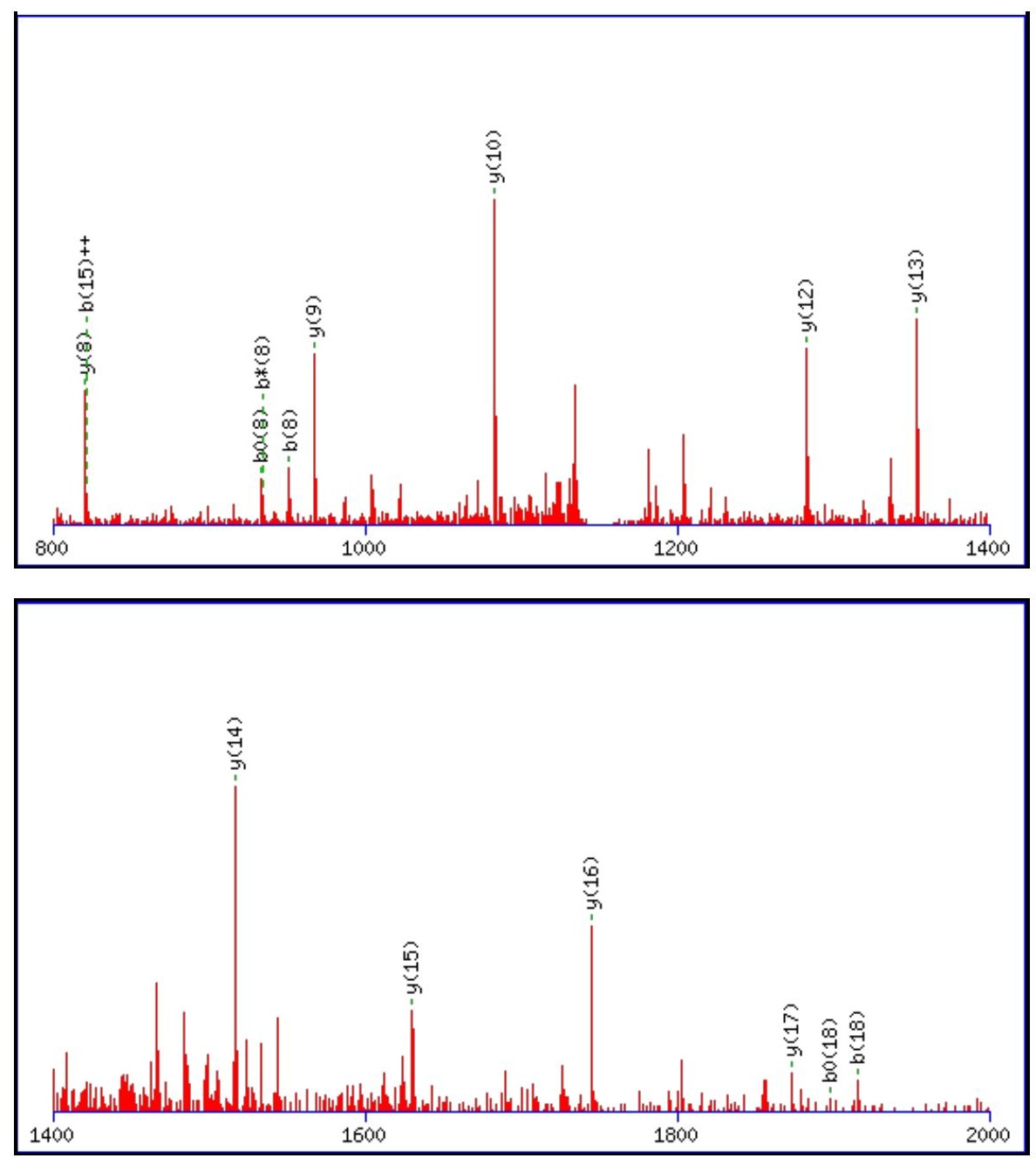

Monoisotopic mass of neutral peptide $\operatorname{Mr}($ calc): 2303.0740

Fixed modifications: Carbamidomethyl (C)

Variable modifications:

N18 : Deamidated_N (N)

Ions Score: 97 Expect: $5.7 \mathrm{e}-008$

Matches (Bold Red): 20/226 fragment ions using 32 most intense peaks

\begin{tabular}{|c|c|c|c|c|c|c|c|c|c|c|c|c|c|c|}
\hline \# & b & $\mathbf{b}^{++}$ & $\mathbf{b}^{*}$ & $\mathbf{b}^{*^{++}}$ & $\mathbf{b}^{\mathbf{0}}$ & $\mathbf{b}^{\mathbf{0 + +}}$ & Seq. & $\mathbf{y}$ & $y^{++}$ & $\mathbf{y}^{*}$ & $\mathbf{y}^{*^{++}}$ & $\mathbf{y}^{0}$ & $\mathbf{y}^{0++}$ & \# \\
\hline 1 & 161.0379 & 81.0226 & & & & & $\mathrm{C}$ & & & & & & & 21 \\
\hline 2 & 218.0594 & 109.5333 & & & & & G & 2144.0506 & 1072.5289 & 2127.0241 & 1064.0157 & 2126.0400 & 1063.5237 & 20 \\
\hline 3 & 319.1071 & 160.0572 & & & 301.0965 & 151.0519 & $\mathbf{T}$ & 2087.0291 & 1044.0182 & 2070.0026 & 1035.5049 & 2069.0186 & 1035.0129 & 19 \\
\hline 4 & 432.1911 & 216.5992 & & & 414.1806 & 207.5939 & $\mathbf{L}$ & 1985.9815 & 993.4944 & 1968.9549 & 984.9811 & 1967.9709 & 984.4891 & 18 \\
\hline 5 & 560.2497 & 280.6285 & 543.2232 & 272.1152 & 542.2391 & 271.6232 & $\mathbf{Q}$ & 1872.8974 & 936.9523 & 1855.8708 & 928.4391 & 1854.8868 & 927.9471 & 17 \\
\hline 6 & 675.2767 & 338.1420 & 658.2501 & 329.6287 & 657.2661 & 329.1367 & D & 1744.8388 & 872.9230 & 1727.8123 & 864.4098 & 1726.8283 & 863.9178 & 16 \\
\hline 7 & 788.3607 & 394.6840 & 771.3342 & 386.1707 & 770.3502 & 385.6787 & $\mathbf{L}$ & 1629.8119 & 815.4096 & 1612.7853 & 806.8963 & 1611.8013 & 806.4043 & 15 \\
\hline 8 & 951.4240 & 476.2157 & 934.3975 & 467.7024 & 933.4135 & 467.2104 & $\mathbf{Y}$ & 1516.7278 & 758.8675 & 1499.7013 & 750.3543 & 1498.7172 & 749.8623 & 14 \\
\hline 9 & 1022.4612 & 511.7342 & 1005.4346 & 503.2209 & 1004.4506 & 502.7289 & A & 1353.6645 & 677.3359 & 1336.6379 & 668.8226 & 1335.6539 & 668.3306 & 13 \\
\hline 10 & 1123.5088 & 562.2581 & 1106.4823 & 553.7448 & 1105.4983 & 553.2528 & $\mathbf{T}$ & 1282.6274 & 641.8173 & 1265.6008 & 633.3040 & 1264.6168 & 632.8120 & 12 \\
\hline 11 & 1222.5773 & 611.7923 & 1205.5507 & 603.2790 & 1204.5667 & 602.7870 & $\mathbf{V}$ & 1181.5797 & 591.2935 & 1164.5531 & 582.7802 & 1163.5691 & 582.2882 & 11 \\
\hline 12 & 1337.6042 & 669.3057 & 1320.5776 & 660.7925 & 1319.5936 & 660.3005 & D & 1082.5113 & 541.7593 & 1065.4847 & 533.2460 & 1064.5007 & 532.7540 & 10 \\
\hline 13 & 1484.6726 & 742.8399 & 1467.6461 & 734.3267 & 1466.6620 & 733.8347 & $\mathbf{F}$ & 967.4843 & 484.2458 & 950.4578 & 475.7325 & 949.4738 & 475.2405 & 9 \\
\hline 14 & 1541.6941 & 771.3507 & 1524.6675 & 762.8374 & 1523.6835 & 762.3454 & G & 820.4159 & 410.7116 & 803.3894 & 402.1983 & 802.4054 & 401.7063 & 8 \\
\hline 15 & 1642.7418 & 821.8745 & 1625.7152 & 813.3612 & 1624.7312 & 812.8692 & $\mathbf{T}$ & 763.3945 & 382.2009 & 746.3679 & 373.6876 & 745.3839 & 373.1956 & 7 \\
\hline 16 & 1743.7894 & 872.3984 & 1726.7629 & 863.8851 & 1725.7789 & 863.3931 & $\mathbf{T}$ & 662.3468 & 331.6770 & 645.3202 & 323.1638 & 644.3362 & 322.6717 & 6 \\
\hline 17 & 1800.8109 & 900.9091 & 1783.7843 & 892.3958 & 1782.8003 & 891.9038 & G & 561.2991 & 281.1532 & 544.2725 & 272.6399 & 543.2885 & 272.1479 & 5 \\
\hline 18 & 1915.8378 & 958.4226 & 1898.8113 & 949.9093 & 1897.8273 & 949.4173 & $\mathbf{N}$ & 504.2776 & 252.6425 & 487.2511 & 244.1292 & 486.2671 & 243.6372 & 4 \\
\hline
\end{tabular}




\begin{tabular}{|r|r|r|r|r|r|r|r|r|r|r|r|r|r|r|r|r|r|r|r|}
$\mathbf{1 9}$ & 2028.9219 & 1014.9646 & 2011.8953 & 1006.4513 & 2010.9113 & 1005.9593 & $\mathbf{I}$ & $\mathbf{3 8 9 . 2 5 0 7}$ & 195.1290 & 372.2241 & 186.6157 & 371.2401 & 186.1237 & $\mathbf{3}$ \\
\hline $\mathbf{2 0}$ & 2129.9696 & 1065.4884 & 2112.9430 & 1056.9752 & 2111.9590 & 1056.4831 & $\mathbf{T}$ & 276.1666 & 138.5870 & 259.1401 & 130.0737 & 258.1561 & 129.5817 & $\mathbf{2}$ \\
\hline $\mathbf{2 1}$ & & & & & & & $\mathbf{R}$ & 175.1190 & 88.0631 & 158.0924 & $\mathbf{7 9 . 5 4 9 8}$ & & & $\mathbf{1}$ \\
\hline
\end{tabular}
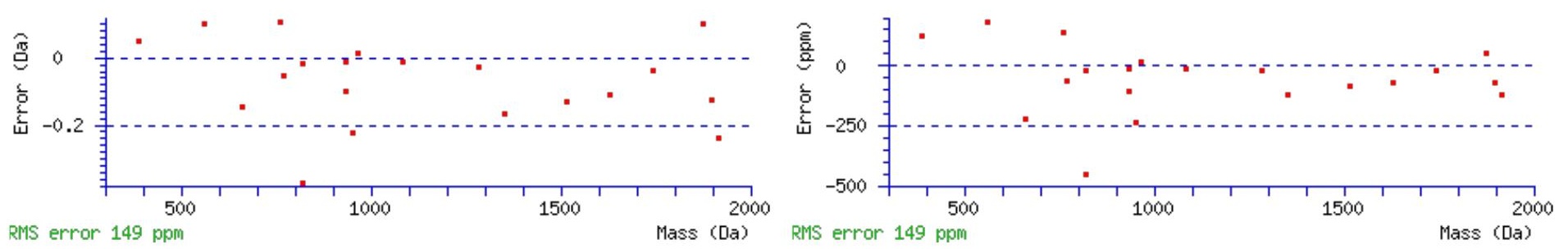

\section{All matches to this query}

\begin{tabular}{|l|l|l|l|}
\hline Score & Mr(calc): & Delta & Sequence \\
\hline 97.1 & 2303.0740 & 0.0111 & CGTLQDLYATVDFGTTGNITR \\
\hline 15.4 & 2302.0900 & 0.9951 & CGTLQDLYATVDFGTTGNITR \\
\hline 2.5 & 2301.0589 & 2.0262 & DLANMTGLLHLSLSRNTIR \\
\hline
\end{tabular}

Spectrum No: 94; Query: 1036; Rank: 1

\section{Peptide View}

MS/MS Fragmentation of TINISNLYIGGLPEGK

Found in IPI00363534, Tax_Id=10116 Gene_Symbol=Lama1 laminin, alpha 1

Match to Query 1036: 1688.901988 from(845.458270,2+)

Title: 100101RatKid_NS_deglyco_13.4688.4688.2.dta

Data file K:INewmanPaper|Piliangl3SubProteomes\Piliang3SP\mgf5ppm\ERLIC_3SubProteomes5ppm.mgf

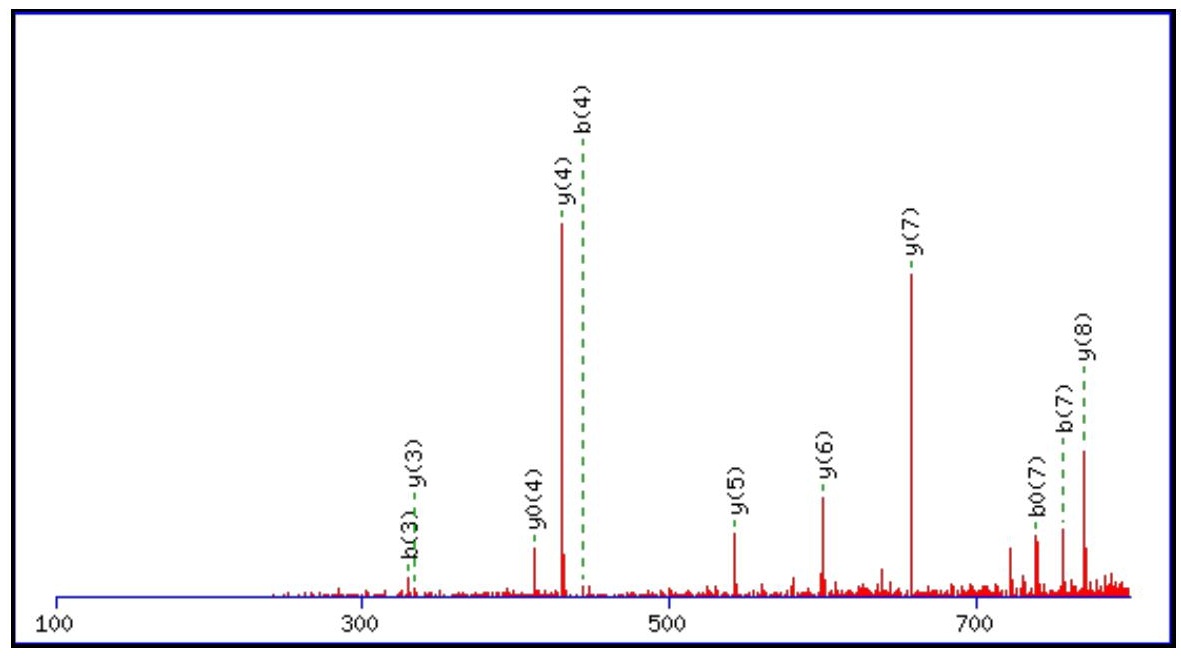



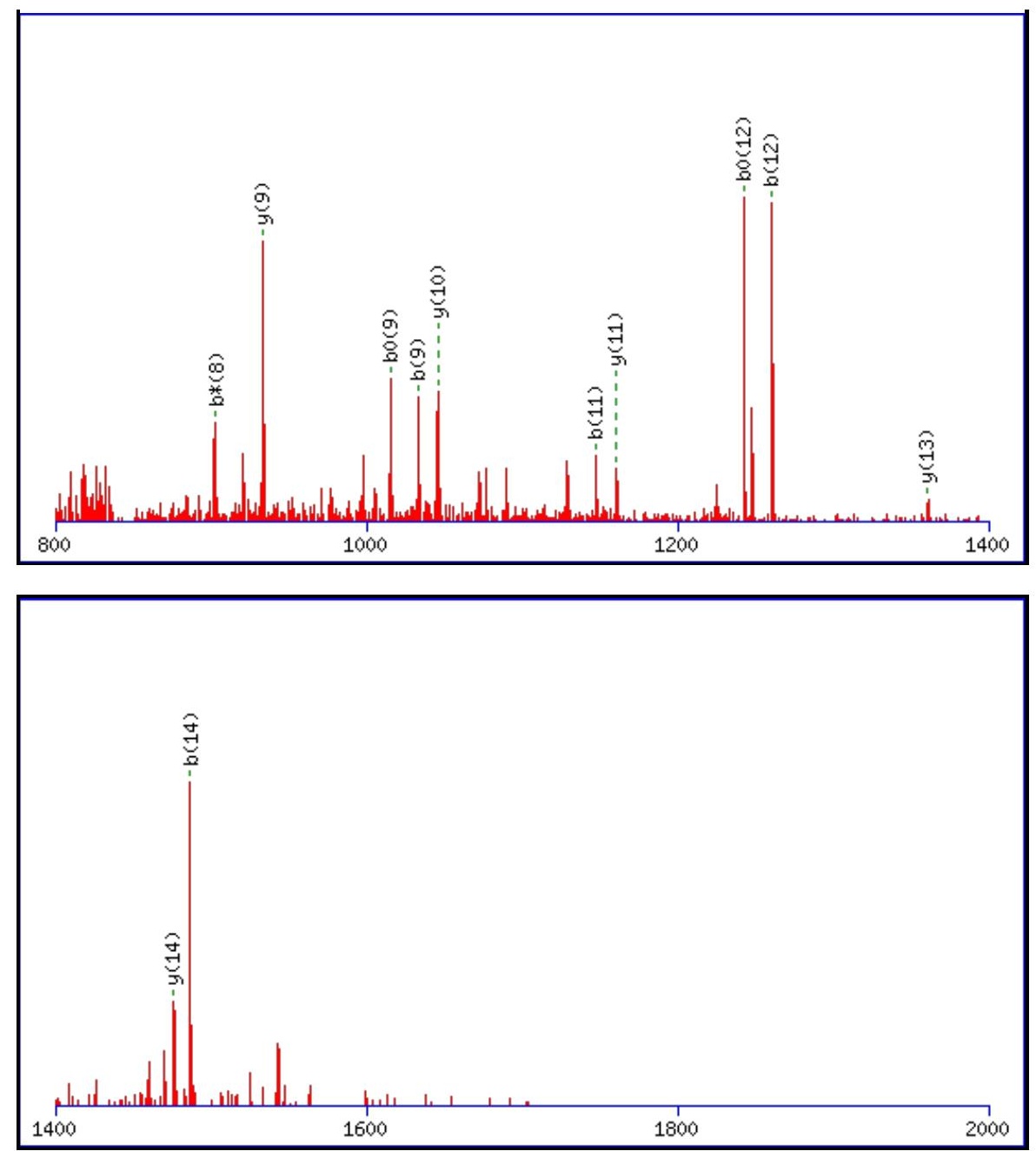

Monoisotopic mass of neutral peptide $\operatorname{Mr}($ calc): 1688.8985

Fixed modifications: Carbamidomethyl (C)

Variable modifications:

N3 : Deamidated $\mathrm{N}(\mathrm{N})$

Ions Score: 97 Expect: $2.2 \mathrm{e}-008$

Matches (Bold Red): 23/172 fragment ions using 39 most intense peaks

\begin{tabular}{|c|c|c|c|c|c|c|c|c|c|c|c|c|c|c|}
\hline \# & b & $\mathbf{b}^{++}$ & $\mathbf{b}^{*}$ & $\mathbf{b}^{*^{++}}$ & $\mathbf{b}^{0}$ & $\mathbf{b}^{\mathbf{0 + +}}$ & Seq. & $\mathbf{y}$ & $\mathbf{y}^{++}$ & $\mathbf{y}^{*}$ & & $\mathbf{y}^{0}$ & $\mathbf{y}^{0++}$ & \# \\
\hline 1 & 102.0550 & 51.5311 & & & 84.0444 & 42.5258 & $\mathbf{T}$ & & & & & & & 16 \\
\hline 2 & 215.1390 & 108.0731 & & & 197.1285 & 99.0679 & I & 1588.8581 & 794.9327 & 1571.8315 & 786.4194 & 1570.8475 & 785.9274 & 15 \\
\hline 3 & 330.1660 & 165.5866 & 313.1394 & & 312.1554 & 156.5813 & $\mathbf{N}$ & 1475.7740 & 738.3907 & 1458.7475 & 729.8774 & 1457.7635 & 729.3854 & 14 \\
\hline 4 & 443.2500 & 222.1286 & 426.2235 & 213.6154 & 425.2395 & 213.1234 & I & 1360.7471 & 680.8772 & 1343.7205 & 672.3639 & 1342.7365 & 671.8719 & 13 \\
\hline 5 & 530.2820 & 265.6447 & 513.2555 & 257.1314 & 512.2715 & 256.6394 & S & 1247.6630 & 624.3352 & 1230.6365 & 615.8219 & 1229.6525 & 615.3299 & 12 \\
\hline 6 & 644.3250 & 322.6661 & 627.2984 & 314.1529 & 626.3144 & 313.6608 & $\mathbf{N}$ & 1160.6310 & 580.8191 & 1143.6045 & 572.3059 & 1142.6204 & 571.8139 & 11 \\
\hline 7 & 757.4090 & 379.2082 & 740.3825 & 370.6949 & 739.3985 & 370.2029 & $\mathbf{L}$ & 1046.5881 & 523.7977 & 1029.5615 & 515.2844 & 1028.5775 & 514.7924 & 10 \\
\hline 8 & 920.4724 & 460.7398 & 903.4458 & 452.2265 & 902.4618 & 451.7345 & $\mathbf{Y}$ & 933.5040 & 467.2556 & 916.4775 & 458.7424 & 915.4934 & 458.2504 & 9 \\
\hline 9 & 1033.5564 & 517.2819 & 1016.5299 & 508.7686 & 1015.5459 & 508.2766 & I & 770.4407 & 385.7240 & 753.4141 & 377.2107 & 752.4301 & 376.7187 & 8 \\
\hline 10 & 1090.5779 & 545.7926 & 1073.5513 & 537.2793 & 1072.5673 & 536.7873 & G & 657.3566 & 329.1819 & 640.3301 & 320.6687 & 639.3461 & 320.1767 & 7 \\
\hline 11 & 1147.5994 & 574.3033 & 1130.5728 & 565.7900 & 1129.5888 & 565.2980 & G & 600.3352 & 300.6712 & 583.3086 & 292.1579 & 582.3246 & 291.6659 & 6 \\
\hline 12 & 1260.6834 & 630.8453 & 1243.6569 & 622.3321 & 1242.6729 & 621.8401 & $\mathbf{L}$ & 543.3137 & 272.1605 & 526.2871 & 263.6472 & 525.3031 & 263.1552 & 5 \\
\hline 13 & 1357.7362 & 679.3717 & 1340.7096 & 670.8585 & 1339.7256 & 670.3664 & $\mathbf{P}$ & 430.2296 & 215.6185 & 413.2031 & 207.1052 & 412.2191 & 206.6132 & 4 \\
\hline 14 & 1486.7788 & 743.8930 & 1469.7522 & 735.3798 & 1468.7682 & 734.8877 & $\mathbf{E}$ & 333.1769 & 167.0921 & 316.1503 & 158.5788 & 315.1663 & 158.0868 & 3 \\
\hline 15 & 1543.8002 & 772.4038 & 1526.7737 & 763.8905 & 1525.7897 & 763.3985 & G & 204.1343 & 102.5708 & 187.1077 & 94.0575 & & & 2 \\
\hline 16 & & & & & & & $\mathbf{K}$ & 147.1128 & 74.0600 & 130.0863 & 65.5468 & & & 1 \\
\hline
\end{tabular}



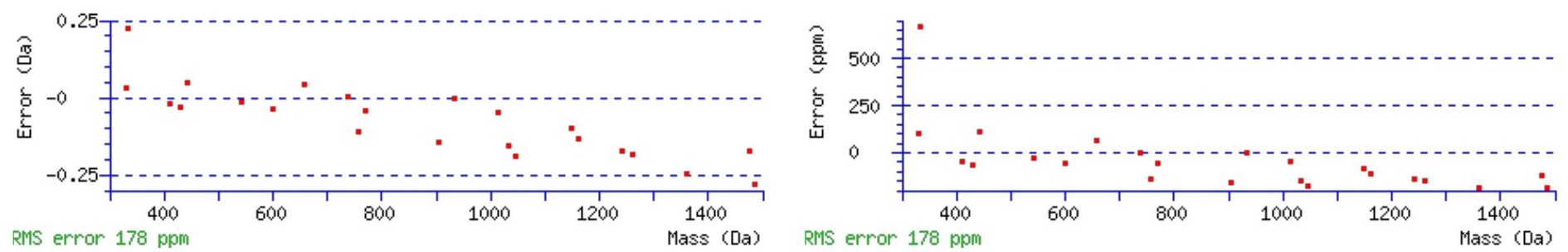

\section{All matches to this query}

\begin{tabular}{|l|l|l|l|}
\hline Score & Mr(calc): & Delta & \multicolumn{1}{c|}{ Sequence } \\
\hline 96.7 & 1688.8985 & 0.0035 & TINISNLYIGGLPEGK \\
\hline 76.1 & 1688.8985 & 0.0035 & TINISNLYIGGLPEGK \\
\hline 50.3 & 1687.9145 & 0.9875 & TINISNLYIGGLPEGK \\
\hline 1.5 & 1686.9043 & 1.9977 & LRASGGPLGAAGLGVRR \\
\hline 1.3 & 1686.8957 & 2.0063 & SKITILKTFNASGVK \\
\hline
\end{tabular}

Spectrum No: 95; Query: 2093; Rank: 1

\section{Peptide View}

MS/MS Fragmentation of TEAGAFEYVADPTFENFTGGVK

Found in IPI00370450, Tax_Id=10116 Gene_Symbol=Plxnb2 plexin B2

Match to Query 2093: 2350.075808 from(1176.045180,2+)

Title: 100101RatKid_NS_deglyco_16.5101.5101.2.dta

Data file K:INewmanPaper|Piliangl3SubProteomes\Piliang3SP\mgf5ppm\ERLIC_3SubProteomes5ppm.mgf

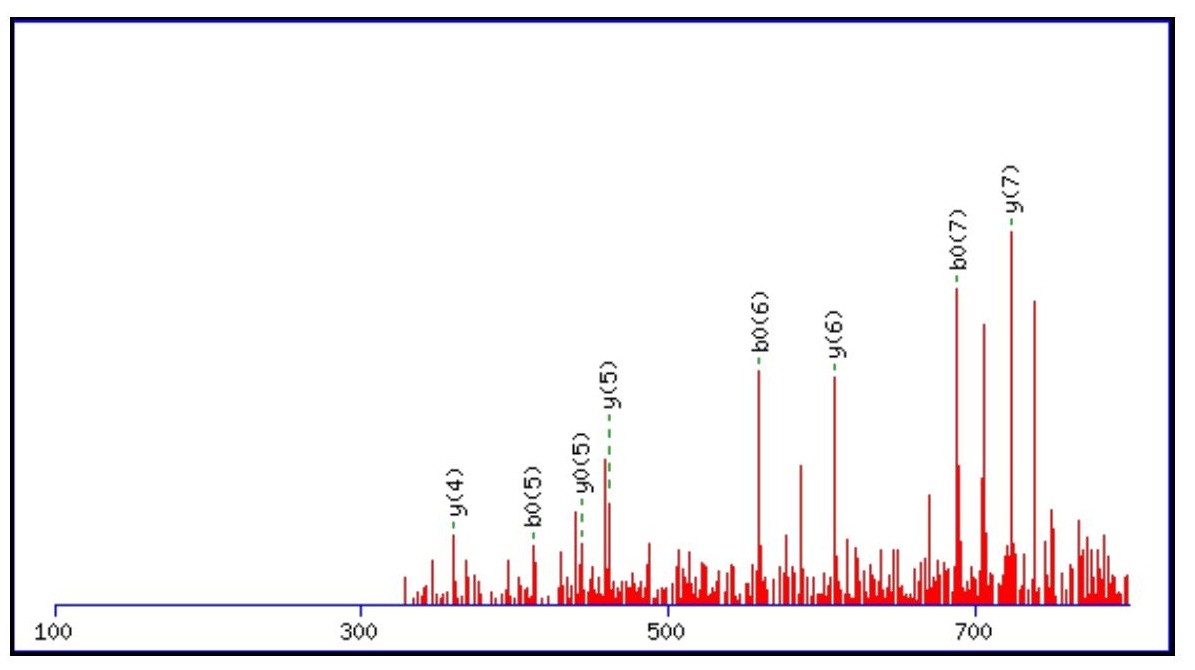



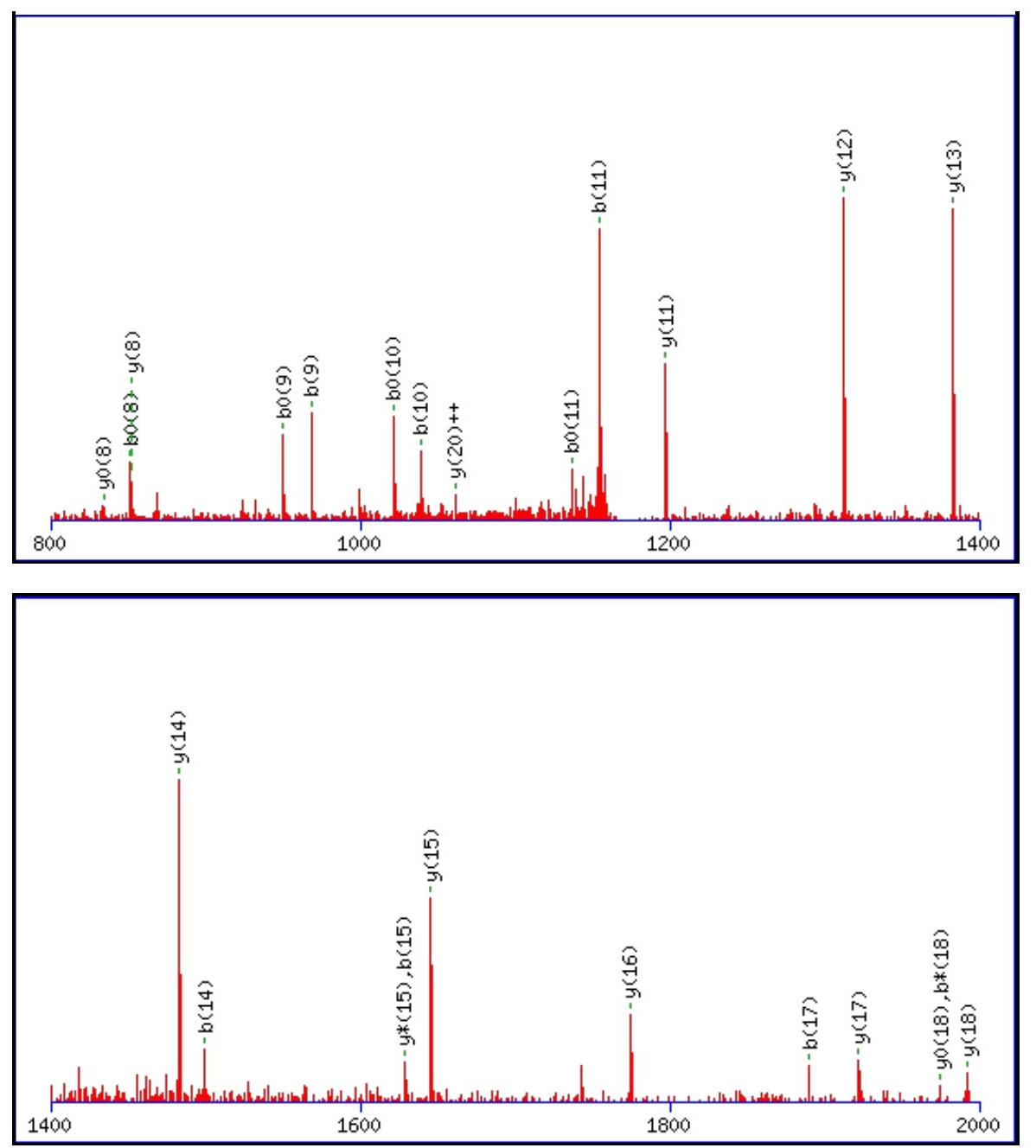

Monoisotopic mass of neutral peptide $\operatorname{Mr}($ calc): 2350.0641

Fixed modifications: Carbamidomethyl (C)

Variable modifications:

N16 : Deamidated_N (N)

Ions Score: 96 Expect: $6.9 \mathrm{e}-008$

Matches (Bold Red): 32/214 fragment ions using 48 most intense peaks

\begin{tabular}{|c|c|c|c|c|c|c|c|c|c|c|c|c|c|c|}
\hline \# & b & $\mathbf{b}^{++}$ & b* & $\mathbf{b}^{*^{++}}$ & $\mathbf{b}^{0}$ & $\mathbf{b}^{0++}$ & Seq. & $\mathbf{y}$ & $y^{++}$ & $\mathbf{y}^{*}$ & $\mathrm{y}^{*^{++}}$ & $\mathbf{y}^{0}$ & $\mathbf{y}^{\mathbf{0 + +}}$ & \# \\
\hline 1 & 102.0550 & 51.5311 & & & 84.0444 & 42.5258 & $\mathbf{T}$ & & & & & & & 22 \\
\hline 2 & 231.0975 & 116.0524 & & & 213.0870 & 107.0471 & $\mathbf{E}$ & 2250.0237 & 1125.5155 & 2232.9972 & 1117.0022 & 2232.0132 & 1116.5102 & 21 \\
\hline 3 & 302.1347 & 151.5710 & & & 284.1241 & 142.5657 & A & 2120.9811 & 1060.9942 & 2103.9546 & 1052.4809 & 2102.9706 & 1051.9889 & 20 \\
\hline 4 & 359.1561 & 180.0817 & & & 341.1456 & 171.0764 & G & 2049.9440 & 1025.4756 & 2032.9175 & 1016.9624 & 2031.9334 & 1016.4704 & 19 \\
\hline 5 & 430.1932 & 215.6003 & & & 412.1827 & 206.5950 & $\mathbf{A}$ & 1992.9225 & 996.9649 & 1975.8960 & 988.4516 & 1974.9120 & 987.9596 & 18 \\
\hline 6 & 577.2617 & 289.1345 & & & 559.2511 & 280.1292 & $\mathbf{F}$ & 1921.8854 & 961.4464 & 1904.8589 & 952.9331 & 1903.8749 & 952.4411 & 17 \\
\hline 7 & 706.3042 & 353.6558 & & & 688.2937 & 344.6505 & $\mathbf{E}$ & 1774.8170 & 887.9121 & 1757.7905 & 879.3989 & 1756.8065 & 878.9069 & 16 \\
\hline 8 & 869.3676 & 435.1874 & & & 851.3570 & 426.1821 & $\mathbf{Y}$ & 1645.7744 & 823.3909 & 1628.7479 & 814.8776 & 1627.7639 & 814.3856 & 15 \\
\hline 9 & 968.4360 & 484.7216 & & & 950.4254 & 475.7164 & $\mathbf{V}$ & 1482.7111 & 741.8592 & 1465.6845 & 733.3459 & 1464.7005 & 732.8539 & 14 \\
\hline 10 & 1039.4731 & 520.2402 & & & 1021.4625 & 511.2349 & A & 1383.6427 & 692.3250 & 1366.6161 & 683.8117 & 1365.6321 & 683.3197 & 13 \\
\hline 11 & 1154.5000 & 577.7537 & & & 1136.4895 & 568.7484 & D & 1312.6056 & 656.8064 & 1295.5790 & 648.2931 & 1294.5950 & 647.8011 & 12 \\
\hline 12 & 1251.5528 & 626.2800 & & & 1233.5422 & 617.2748 & $\mathbf{P}$ & 1197.5786 & 599.2930 & 1180.5521 & 590.7797 & 1179.5681 & 590.2877 & 11 \\
\hline 13 & 1352.6005 & 676.8039 & & & 1334.5899 & 667.7986 & $\mathbf{T}$ & 1100.5259 & 550.7666 & 1083.4993 & 542.2533 & 1082.5153 & 541.7613 & 10 \\
\hline 14 & 1499.6689 & 750.3381 & & & 1481.6583 & 741.3328 & $\mathbf{F}$ & 999.4782 & 500.2427 & 982.4516 & 491.7295 & 981.4676 & 491.2374 & 9 \\
\hline 15 & 1628.7115 & 814.8594 & & & 1610.7009 & 805.8541 & $\mathbf{E}$ & 852.4098 & 426.7085 & 835.3832 & 418.1952 & 834.3992 & 417.7032 & 8 \\
\hline 16 & 1743.7384 & 872.3729 & 1726.7119 & 863.8596 & 1725.7279 & 863.3676 & $\mathbf{N}$ & 723.3672 & 362.1872 & 706.3406 & 353.6740 & 705.3566 & 353.1819 & 7 \\
\hline 17 & 1890.8068 & 945.9071 & 1873.7803 & 937.3938 & 1872.7963 & 936.9018 & $\mathbf{F}$ & 608.3402 & 304.6738 & 591.3137 & 296.1605 & 590.3297 & 295.6685 & 6 \\
\hline 18 & 1991.8545 & 996.4309 & 1974.8280 & 987.9176 & 1973.8440 & 987.4256 & $\mathbf{T}$ & 461.2718 & 231.1396 & 444.2453 & 222.6263 & 443.2613 & 222.1343 & 5 \\
\hline
\end{tabular}




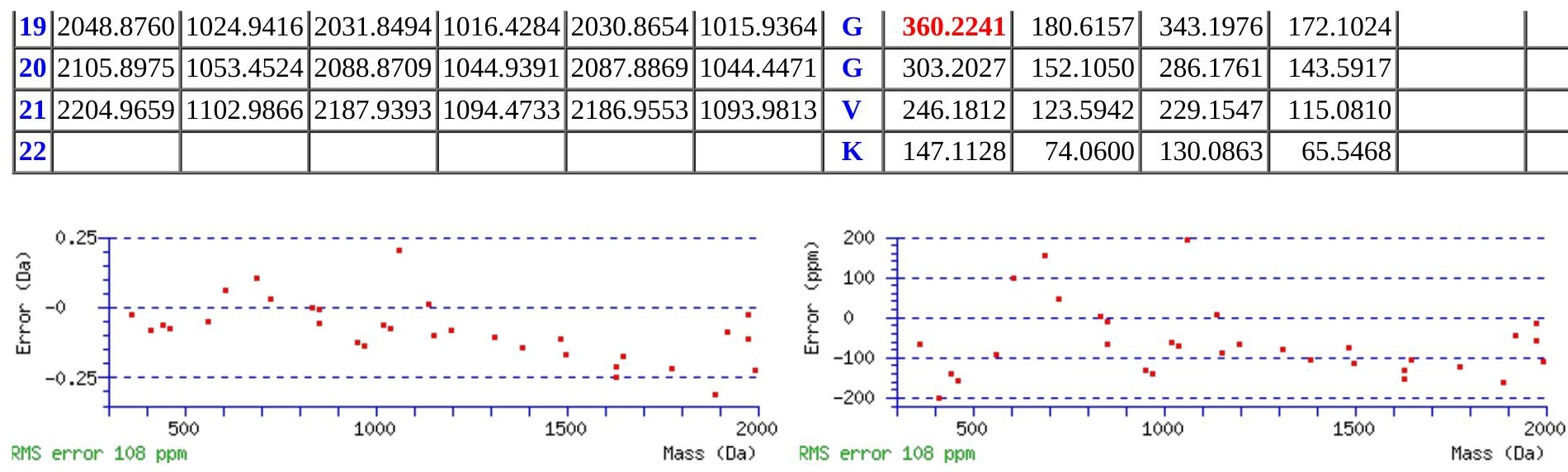

\section{All matches to this query}

\begin{tabular}{|l|l|l|l|}
\hline Score & Mr(calc): & Delta & \multicolumn{1}{c|}{ Sequence } \\
\hline 96.3 & 2350.0641 & 0.0117 & TEAGAFEYVADPTFENFTGGVK \\
\hline 25.2 & 2349.0801 & 0.9957 & TEAGAFEYVADPTFENFTGGVK \\
\hline 2.9 & 2350.0755 & 0.0003 & NVFELGPPVMLDAATLKTMK \\
\hline 2.6 & 2348.0787 & 1.9971 & SGRPPRRSVAAPADSSVPEPR \\
\hline 2.6 & 2348.0787 & 1.9971 & SGRPPRRSVAAPADSSVPEPR \\
\hline 2.1 & 2348.0474 & 2.0284 & EHAAVNQKGIYDDAISSFFR \\
\hline 2.1 & 2348.0474 & 2.0284 & EHAAVNQKGIYDDAISSFFR \\
\hline 1.3 & 2348.0686 & 2.0073 & RLSESPVFDAPPSPPDSLSDR \\
\hline 0.2 & 2350.0621 & 0.0138 & GLRPQEGYFVRVTAVNDGGR \\
\hline 0.1 & 2348.0703 & 2.0055 & LMDAWAGSARVPDEENTTGTAR \\
\hline
\end{tabular}

Spectrum No: 96; Query: 2571; Rank: 1

\section{Peptide View}

MS/MS Fragmentation of ENGTDAVQEEEESPAEGSKDEPAEQGELK

Found in IPI00210975, Tax_Id=10116 Gene_Symbol=Hyou1 Hypoxia up-regulated protein 1 precursor

Match to Query 2571: 3102.333702 from(1035.118510,3+)

Title: 100101RatKid_NS_deglyco_20.1634.1634.3.dta

Data file K:INewmanPaper|Piliangl3SubProteomes\Piliang3SP\mgf5ppm\ERLIC_3SubProteomes5ppm.mgf

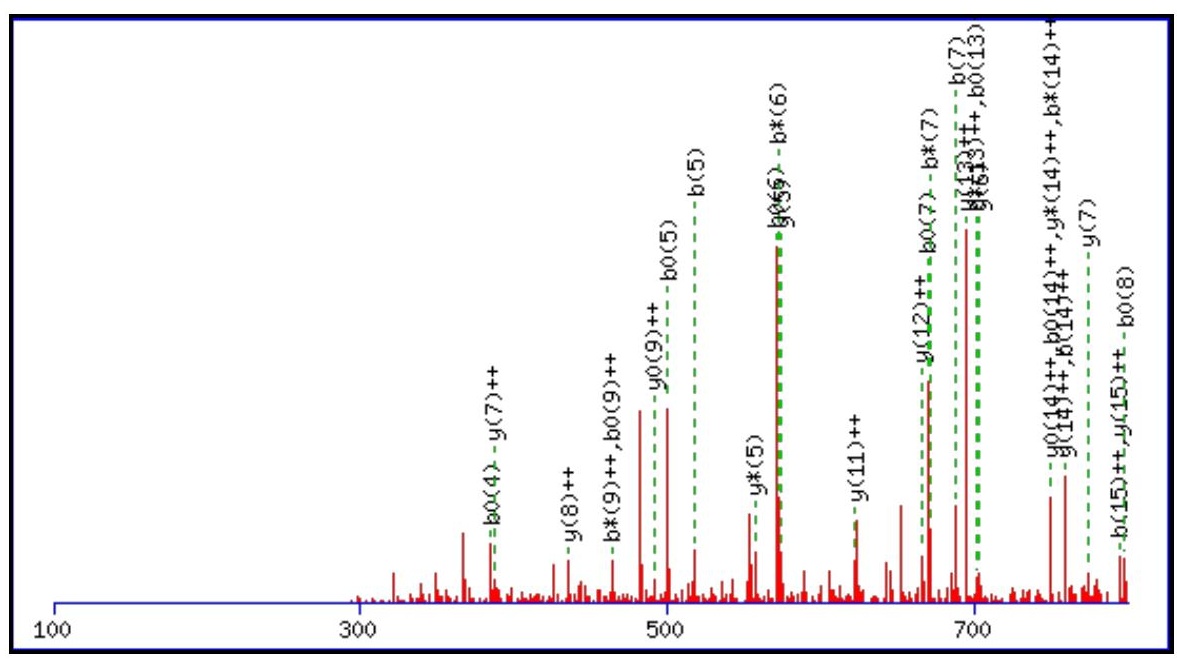



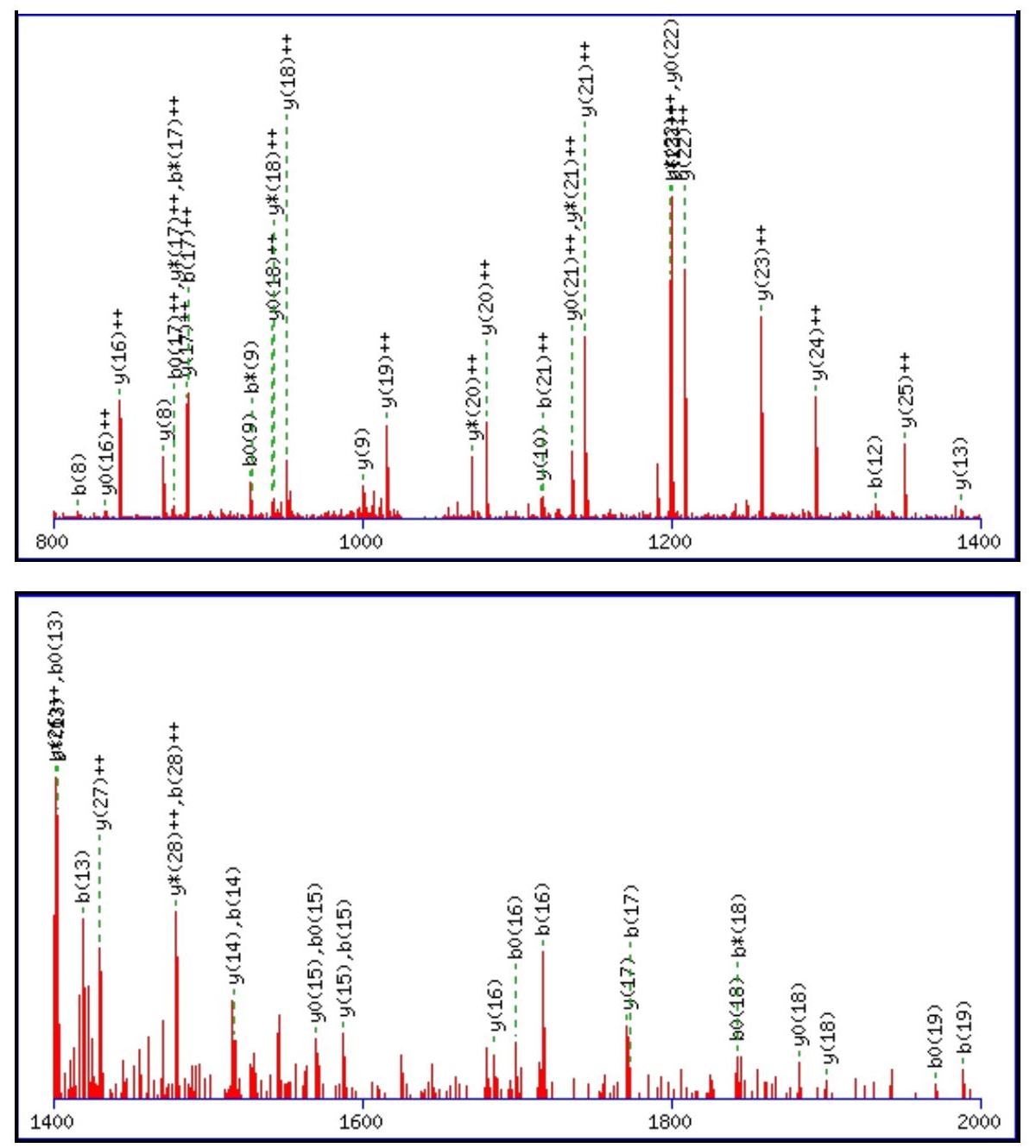

Monoisotopic mass of neutral peptide $\operatorname{Mr}($ calc): 3102.3272

Fixed modifications: Carbamidomethyl (C)

Variable modifications:

N2 : Deamidated $\mathrm{N}(\mathrm{N})$

Ions Score: 96 Expect: $1.1 \mathrm{e}-007$

Matches (Bold Red): 87/330 fragment ions using 127 most intense peaks

\begin{tabular}{|c|c|c|c|c|c|c|c|c|c|c|c|c|c|c|}
\hline \# & b & $\mathbf{b}^{++}$ & b* & $\mathbf{b}^{*^{++}}$ & $\mathbf{b}^{0}$ & $\mathbf{b}^{0++}$ & Seq. & $\mathbf{y}$ & $y^{++}$ & $\mathbf{y}^{*}$ & $\mathbf{y}^{*^{++}}$ & $\mathbf{y}^{0}$ & $y^{0++}$ & \# \\
\hline 1 & 130.0499 & 65.5286 & & & 112.0393 & 56.5233 & $\mathbf{E}$ & & & & & & & 29 \\
\hline 2 & 245.0768 & 23.0420 & 228.0503 & 114.5288 & 227.0662 & 114.0368 & $\mathbf{N}$ & & 1487.6496 & 2957.2654 & 1479.1363 & 2956.2814 & 1478.6443 & 28 \\
\hline 3 & 302.0983 & 151.5528 & 285.0717 & 143.0395 & 284.0877 & 142.5475 & G & 2859.2650 & 1430.1362 & 2842.2385 & 1421.6229 & 2841.2545 & 1421.1309 & 27 \\
\hline 4 & 403.1459 & 202.0766 & 386.1194 & 193.5633 & 385.1354 & 193.0713 & $\mathbf{T}$ & 2802.2436 & 1401.6254 & 2785.2170 & 1393.1121 & 2784.2330 & |1392.6201 & 26 \\
\hline 5 & 518.1729 & 259.5901 & 501.1463 & 251.0768 & 500.1623 & 250.5848 & D & 2701.1959 & 1351.1016 & 2684.1693 & 1342.5883 & 2683.1853 & 1342.0963 & 25 \\
\hline 6 & 589.2100 & 295.1086 & 572.1835 & 286.5954 & 571.1994 & 286.1034 & A & 2586.1689 & 1293.5881 & 2569.1424 & 1285.0748 & 2568.1584 & 1284.5828 & 24 \\
\hline 7 & 688.2784 & 344.6428 & 671.2519 & 336.1296 & 670.2679 & 335.6376 & V & 2515.1318 & 1258.0696 & 2498.1053 & 1249.5563 & 2497.1213 & 1249.0643 & 23 \\
\hline 8 & 816.3370 & 408.6721 & 799.3104 & 400.1589 & 798.3264 & 399.6669 & $\mathbf{Q}$ & 2416.0634 & 1208.5353 & 2399.0369 & 1200.0221 & 2398.0528 & 1199.5301 & 22 \\
\hline 9 & 945.3796 & 473.1934 & 928.3530 & 464.6802 & 927.3690 & 464.1882 & $\mathbf{E}$ & 2288.0048 & 1144. & 2270.9783 & 1135.9928 & 2269.9943 & 1135.5008 & 21 \\
\hline 10 & 1074.4222 & 537.7147 & 1057.3956 & 529.2015 & 1056.4116 & 528.7094 & $\mathbf{E}$ & 2158.9622 & 1079.9848 & 2141.9357 & 1071.4715 & 2140.9517 & 1070.9795 & 20 \\
\hline 11 & 1203.4648 & 602.2360 & 1186.4382 & 593.7228 & 1185.4542 & 593.2307 & $\mathbf{E}$ & 2029.9197 & 1015.4635 & 2012.8931 & 1006.9502 & 2011.9091 & 1006.4582 & 19 \\
\hline 12 & 1332.5074 & 666.7573 & 1315.4808 & 658.2440 & 1314.4968 & 657.7520 & $\mathbf{E}$ & 1900.8771 & 950.9422 & 1883.8505 & 942.4289 & 1882.8665 & 941.9369 & 18 \\
\hline 13 & 1419.5394 & 710.2733 & 1402.5128 & 701.7601 & 1401.5288 & 701.2681 & S & 1771.8345 & 886.4209 & 1754.8079 & 877.9076 & 1753.8239 & 877.4156 & 17 \\
\hline 14 & 1516.5922 & 758.7997 & 1499.5656 & 750.2864 & 1498.5816 & 749.7944 & $\mathbf{P}$ & 1684.8024 & 842.9049 & 1667.7759 & 834.3916 & 1666.7919 & 833.8996 & 16 \\
\hline 15 & 1587.6293 & 794.3183 & 1570.6027 & 785.8050 & 1569.6187 & 785.3130 & A & 1587.7497 & 794.3785 & 1570.7231 & 785.8652 & 1569.7391 & 785.3732 & 15 \\
\hline 16 & 1716.6719 & 858.8396 & 1699.6453 & 850.3263 & 1698.6613 & 849.8343 & $\mathbf{E}$ & 1516.7126 & 758.8599 & 1499.6860 & 750.3466 & 1498.7020 & 749.8546 & 14 \\
\hline 17 & 1773.6933 & 887.3503 & 1756.6668 & 878.8370 & 1755.6828 & 878.3450 & G & 1387.6700 & 694.3386 & 1370.6434 & 685.8253 & 1369.6594 & 685.3333 & 13 \\
\hline 18 & 1860.7254 & 930.8663 & 1843.6988 & 922.3530 & 1842.7148 & 921.8610 & S & 1330.6485 & 665.8279 & 1313.6220 & 657.3146 & 1312.6379 & 656.8226 & 12 \\
\hline
\end{tabular}




\begin{tabular}{|c|c|c|c|c|c|c|c|c|c|c|c|c|c|c|}
\hline 19 & 1988.8203 & 994.9138 & |1971.7938 & 986.4005 & $\mid 1970.8098$ & 985.9085 & $\mathbf{K}$ & 1243.6165 & 622.3119 & |1226.5899 & 613.7986 & 1225.6059 & 613.3066 & 11 \\
\hline 20 & 2103.8473 & 1052.4273 & 2086.8207 & 1043.9140 & 2085.8367 & 1043.4220 & D & 1115.5215 & 558.2644 & 1098.4950 & 549.7511 & 1097.5109 & 549.2591 & 10 \\
\hline 21 & 2232.8899 & 1116.9486 & 2215.8633 & 1108.4353 & 2214.8793 & 1107.9433 & $\mathbf{E}$ & 1000.4946 & 500.7509 & 983.4680 & 492.2376 & 982.4840 & 491.7456 & 9 \\
\hline 22 & 2329.9426 & 1165.4749 & 2312.9161 & 1156.9617 & 2311.9321 & 1156.4697 & $\mathbf{P}$ & 871.4520 & 436.2296 & 854.4254 & 427.7164 & 853.4414 & 427.2243 & 8 \\
\hline 23 & 2400.9797 & 1200.9935 & 2383.9532 & 1192.4802 & 2382.9692 & 1191.9882 & A & 774.3992 & 387.7032 & 757.3727 & 379.1900 & 756.3886 & 378.6980 & 7 \\
\hline 24 & 2530.0223 & 1265.5148 & 2512.9958 & 1257.0015 & 2512.0118 & 1256.5095 & E & 703.3621 & 352.1847 & 686.3355 & 343.6714 & 685.3515 & 343.1794 & 6 \\
\hline 25 & 2658.0809 & 1329.5441 & 2641.0544 & 1321.0308 & 2640.0703 & 1320.5388 & $\mathbf{Q}$ & 574.3195 & 287.6634 & 557.2930 & 279.1501 & 556.3089 & 278.6581 & 5 \\
\hline 26 & 2715.1024 & 1358.0548 & 2698.0758 & 1349.5415 & 2697.0918 & 1349.0495 & G & 446.2609 & 223.6341 & 429.2344 & 215.1208 & 428.2504 & 214.6288 & 4 \\
\hline 27 & 2844.1450 & 1422.5761 & 2827.1184 & 1414.0628 & 2826.1344 & 1413.5708 & E & 389.2395 & 195.1234 & 372.2129 & 186.6101 & 371.2289 & 186.1181 & 3 \\
\hline 28 & 2957.2290 & 1479.1182 & 2940.2025 & 1470.6049 & 2939.2185 & 1470.1129 & L & 260.1969 & 130.6021 & 243.1703 & 122.0888 & & & 2 \\
\hline 29 & & & & & & & $\mathbf{K}$ & 147.1128 & 74.0600 & 130.0863 & 65.5468 & & & 1 \\
\hline
\end{tabular}
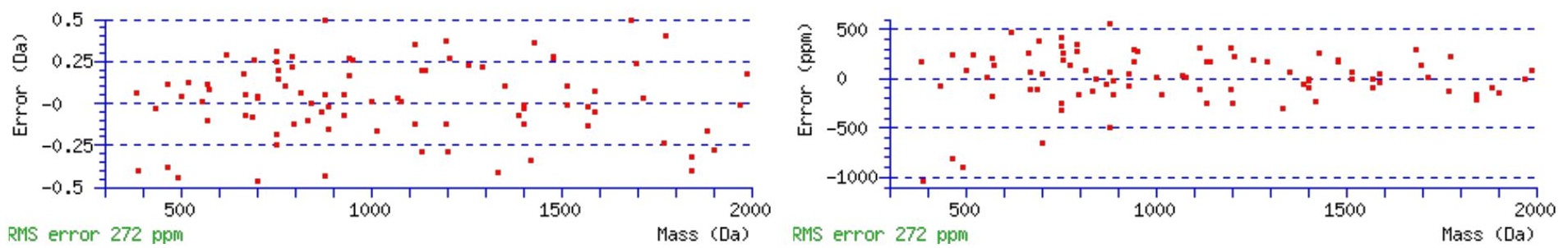

\section{All matches to this query}

\begin{tabular}{|l|l|l|l|}
\hline Score & Mr(calc): & Delta & \multicolumn{1}{|c|}{ Sequence } \\
\hline 96.2 & 3102.3272 & 0.0065 & ENGTDAVQEEEESPAEGSKDEPAEQGELK \\
\hline 73.0 & 3101.3432 & 0.9905 & ENGTDAVQEEEESPAEGSKDEPAEQGELK \\
\hline 7.4 & 3102.3522 & -0.0185 & NPSPGMSAKNAEKPNMQRNNTLGISTTK \\
\hline 7.4 & 3102.3522 & -0.0185 & NPSPGMSAKNAEKPNMQRNNTLGISTTK \\
\hline 7.4 & 3102.3522 & -0.0185 & NPSPGMSAKNAEKPNMQRNNTLGISTTK \\
\hline 6.4 & 3101.3359 & 0.9978 & DLDFDMDSSMNGAGPLGPAEPSAKVALER \\
\hline 5.2 & 3102.3196 & 0.0141 & TEQSGSELSSRLDTLNAEKEALSGAIR \\
\hline 4.7 & 3102.3406 & -0.0069 & KEMVSQSPKYEEAVLPVHGEGQEESR \\
\hline 4.4 & 3102.3199 & 0.0138 & DLDFDMDSSMNGAGPLGPAEPSAKVALER \\
\hline 4.0 & 3102.3415 & -0.0078 & MNFLTNLELNFAECILDGGRATMGVR \\
\hline
\end{tabular}

Spectrum No: 97; Query: 1879; Rank: 1

\section{Peptide View}

MS/MS Fragmentation of VPFNPNDTFESEFYLDEK

Found in IPI00200591, Tax_Id=10116 Gene_Symbol=LOC299282 Serine protease inhibitor A3L precursor

Match to Query 1879: 2190.966228 from(1096.490390,2+)

Title: 091008RatKidney_NoSalt_28.3850.3850.2.dta

Data file K:INewmanPaper|Piliang|3SubProteomes\Piliang3SP\mgf5ppm\ERLIC_3SubProteomes5ppm.mgf 

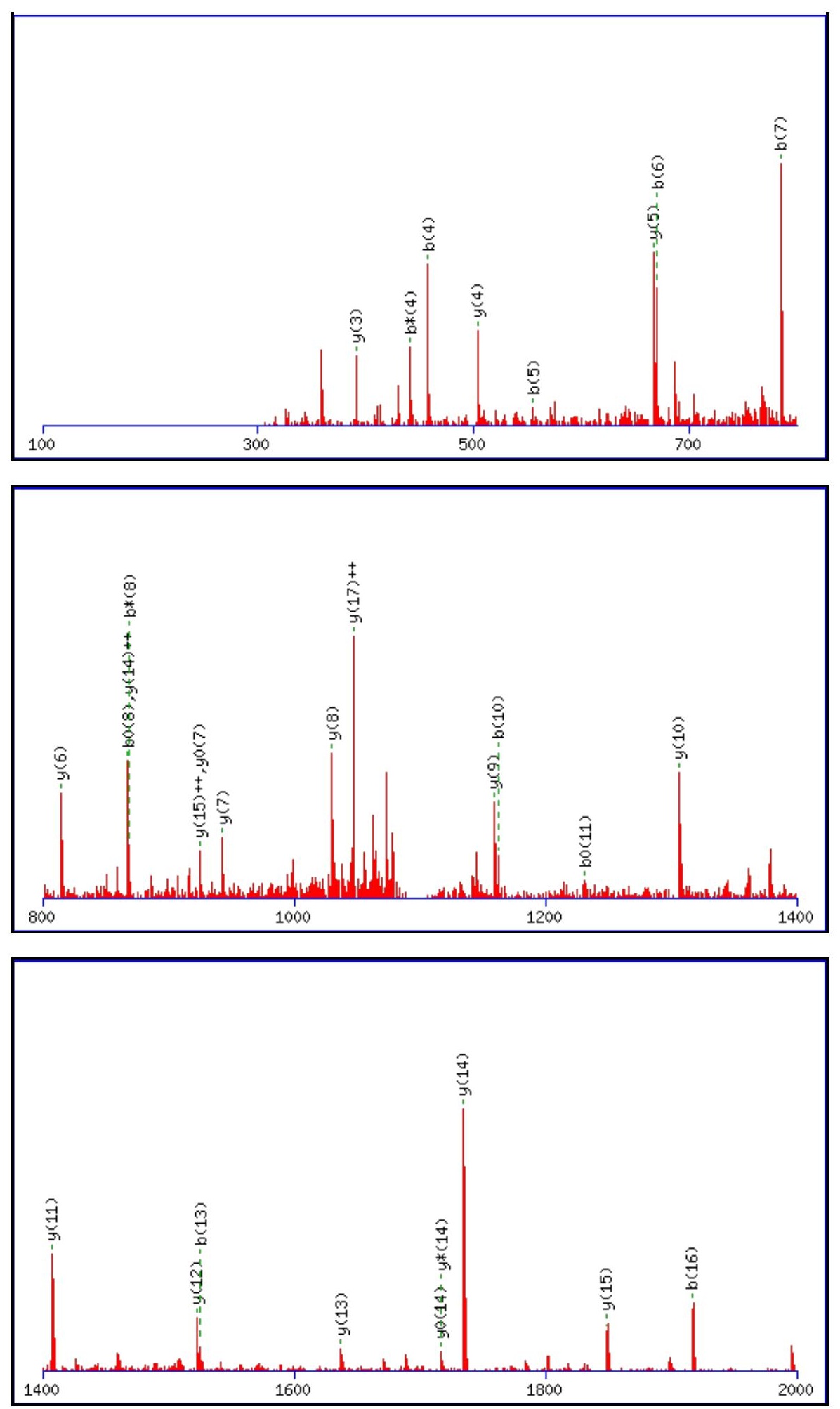

Monoisotopic mass of neutral peptide $\operatorname{Mr}($ calc): 2190.9633

Fixed modifications: Carbamidomethyl (C)

Variable modifications:

N6 : Deamidated $\mathrm{N}(\mathrm{N})$

Ions Score: 96 Expect : $7.3 \mathrm{e}-008$

Matches (Bold Red): 30/184 fragment ions using 44 most intense peaks

\begin{tabular}{|c|c|c|c|c|c|c|c|c|c|c|c|c|c|c|}
\hline \# & b & $\mathbf{b}^{++}$ & $\mathbf{b}^{*}$ & $\mathbf{b}^{*^{++}}$ & $\mathbf{b}^{\mathbf{0}}$ & $\mathbf{b}^{\mathbf{0 + +}}$ & Seq. & $\mathbf{y}$ & $y^{++}$ & $\mathbf{y}^{*}$ & $\mathrm{y}^{\mathrm{*}^{++}}$ & $\mathbf{y}^{\mathbf{0}}$ & $\mathbf{y}^{\mathbf{0 + +}}$ & \# \\
\hline 1 & 100.0757 & 50.5415 & & & & & V & & & & & & & 18 \\
\hline 2 & 197.1285 & 99.0679 & & & & & $\mathbf{P}$ & 2092.9022 & 1046.9547 & 2075.8757 & 1038.4415 & 2074.8916 & 1037.9495 & 17 \\
\hline 3 & 344.1969 & 172.6021 & & & & & $\mathbf{F}$ & 1995.8494 & 998.4284 & |1978.8229 & 989.9151 & 1977.8389 & 989.4231 & 16 \\
\hline 4 & 458.2398 & 229.6235 & 441.2132 & 221.1103 & & & $\mathbf{N}$ & 1848.7810 & 924.8942 & 1831.7545 & 916.3809 & 1830.7705 & 915.8889 & 15 \\
\hline 5 & 555.2926 & 278.1499 & 538.2660 & 269.6366 & & & $\mathbf{P}$ & 1734.7381 & 867.8727 & 1717.7115 & 859.3594 & 1716.7275 & 858.8674 & 14 \\
\hline
\end{tabular}




\begin{tabular}{|c|c|c|c|c|c|c|c|c|c|c|c|c|c|c|}
\hline 6 & 670.3195 & 335.6634 & 653.2929 & 327.1501 & & & $\mathbf{N}$ & |1637.6853 & 819.3463 & |1620.6588 & 810.8330 & |1619.6748 & 810.3410 & 13 \\
\hline 7 & 785.3464 & 393.1769 & 768.3199 & 384.6636 & 767.3359 & 384.1716 & D & 1522.6584 & 761.8328 & 1505.6318 & 753.3196 & 1504.6478 & 752.8276 & 12 \\
\hline 8 & 886.3941 & 443.7007 & 869.3676 & 435.1874 & 868.3836 & 434.6954 & $\mathbf{T}$ & 1407.6315 & 704.3194 & 1390.6049 & 695.8061 & 1389.6209 & 695.3141 & 11 \\
\hline 9 & 1033.4625 & 517.2349 & 1016.4360 & 508.7216 & 1015.4520 & 508.2296 & $\mathbf{F}$ & 1306.5838 & 653.7955 & 1289.5572 & 645.2823 & 1288.5732 & 644.7902 & 10 \\
\hline 10 & 1162.5051 & 581.7562 & 1145.4786 & 573.2429 & 1144.4946 & 572.7509 & $\mathbf{E}$ & 1159.5154 & 580.2613 & 1142.4888 & 571.7480 & 1141.5048 & 571.2560 & 9 \\
\hline 11 & 1249.5372 & 625.2722 & 1232.5106 & 616.7589 & 1231.5266 & 616.2669 & S & 1030.4728 & 515.7400 & 1013.4462 & 507.2267 & 1012.4622 & 506.7347 & 8 \\
\hline 12 & 1378.5797 & 689.7935 & 1361.5532 & 681.2802 & 1360.5692 & 680.7882 & $\mathbf{E}$ & 943.4407 & 472.2240 & 926.4142 & 463.7107 & 925.4302 & 463.2187 & 7 \\
\hline 13 & 1525.6482 & 763.3277 & 1508.6216 & 754.8144 & 1507.6376 & 754.3224 & $\mathbf{F}$ & 814.3981 & 407.7027 & 797.3716 & 399.1894 & 796.3876 & 398.6974 & 6 \\
\hline 14 & 1688.7115 & 844.8594 & 1671.6849 & 836.3461 & 1670.7009 & 835.8541 & $\mathbf{Y}$ & 667.3297 & 334.1685 & 650.3032 & 325.6552 & 649.3192 & 325.1632 & 5 \\
\hline 15 & 1801.7956 & 901.4014 & 1784.7690 & 892.8881 & 1783.7850 & 892.3961 & $\mathbf{L}$ & 504.2664 & 252.6368 & 487.2399 & 244.1236 & 486.2558 & 243.6316 & 4 \\
\hline 16 & 1916.8225 & 958.9149 & 1899.7959 & 950.4016 & 1898.8119 & 949.9096 & D & 391.1823 & 196.0948 & 374.1558 & 187.5815 & 373.1718 & 187.0895 & 3 \\
\hline 17 & 2045.8651 & 1023.4362 & 2028.8385 & 1014.9229 & 2027.8545 & 1014.4309 & $\mathbf{E}$ & 276.1554 & 138.5813 & 259.1288 & 130.0681 & 258.1448 & 129.5761 & 2 \\
\hline 18 & & & & & & & $\mathbf{K}$ & 147.1128 & 74.0600 & 130.0863 & 65.5468 & & & 1 \\
\hline
\end{tabular}
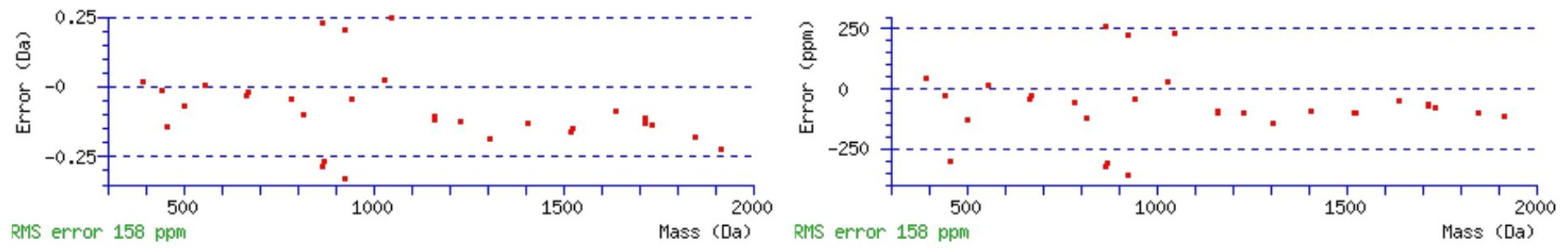

\section{All matches to this query}

\begin{tabular}{|l|l|l|l|}
\hline Score & Mr(calc): & Delta & \multicolumn{1}{|c|}{ Sequence } \\
\hline 96.2 & 2190.9633 & 0.0029 & VPFNPNDTFESEFYLDEK \\
\hline 63.7 & 2190.9633 & 0.0029 & VPFNPNDTFESEFYLDEK \\
\hline 46.5 & 2189.9793 & 0.9869 & VPFNPNDTFESEFYLDEK \\
\hline 8.3 & 2190.9745 & -0.0083 & $\underline{\text { EVMNTDGTGRRVLVEDK }}$ \\
\hline 7.2 & 2188.9654 & 2.0008 & ESVATVALTVLDKNDNSPR \\
\hline 7.2 & 2189.9494 & 1.0168 & ESVATVALTVLDKNDNSPR \\
\hline 7.2 & 2188.9654 & 2.0008 & ESVATVALTVLDKNDNSPR \\
\hline 7.2 & 2189.9494 & 1.0168 & ESVATVALTVLDKNDNSPR \\
\hline 4.0 & 2190.9810 & -0.0148 & RSNLTIIGIEESEDSQLK \\
\hline 3.3 & 2190.9731 & -0.0068 & EFNLMYDGTKEVPMNPVK \\
\hline
\end{tabular}

Spectrum No: 98; Query: 1267; Rank: 1

\section{Peptide View}

MS/MS Fragmentation of LSPNCCNSSTVADAYR

Found in IPI00203176, Tax_Id=10116 Gene_Symbol=Tpcn1 Two pore calcium channel protein 1

Match to Query 1267: 1814.761648 from(908.388100,2+)

Title: 100101RatKid_NS_deglyco_25.1508.1508.2.dta

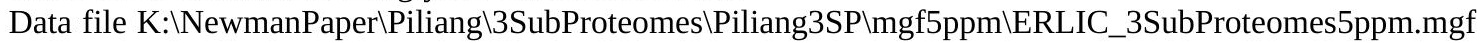



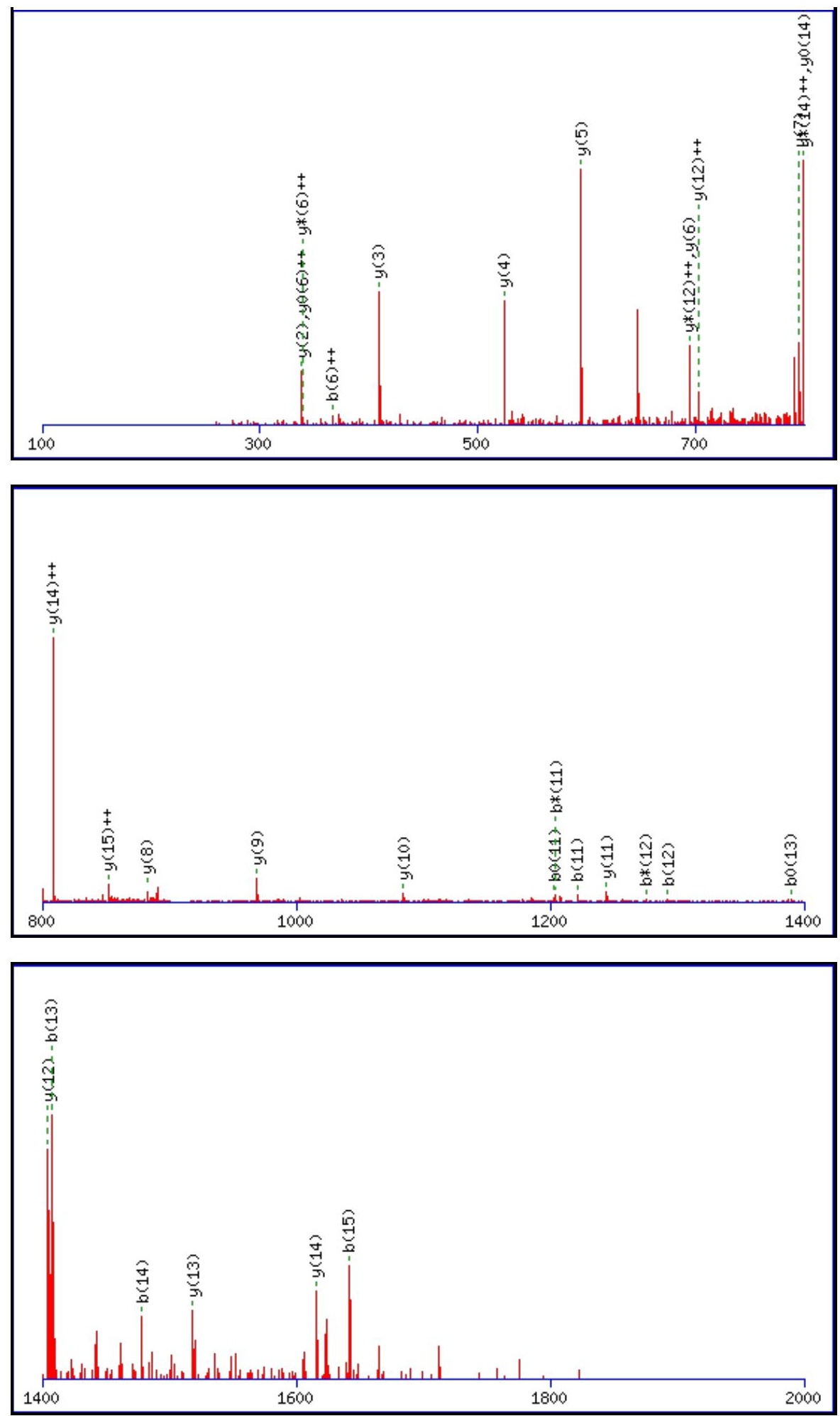

Monoisotopic mass of neutral peptide $\operatorname{Mr}($ calc): 1814.7563

Fixed modifications: Carbamidomethyl (C)

Variable modifications:

N7 : Deamidated $\mathrm{N}(\mathrm{N})$

Ions Score: 96 Expect: $4.7 \mathrm{e}-008$

Matches (Bold Red): 31/166 fragment ions using 51 most intense peaks

\begin{tabular}{|r|c|c|c|c|c|c|c|c|c|c|c|c|c|c|}
\hline$\#$ & $\mathbf{b}$ & $\mathbf{b}^{++}$ & $\mathbf{b}^{*}$ & $\mathbf{b}^{\boldsymbol{*}^{++}}$ & $\mathbf{b}^{\mathbf{0}}$ & $\mathbf{b}^{\mathbf{0 + +}}$ & Seq. & $\mathbf{y}$ & $\mathbf{y}^{++}$ & $\mathbf{y}^{\mathbf{*}}$ & $\mathbf{y}^{\boldsymbol{*}^{++}}$ & $\mathbf{y}^{\mathbf{0}}$ & $\mathbf{y}^{\mathbf{0 + +}}$ & $\#$ \\
\hline $\mathbf{1}$ & 114.0913 & 57.5493 & & & & & $\mathbf{L}$ & & & & & & & $\mathbf{1 6}$ \\
\hline $\mathbf{2}$ & 201.1234 & 101.0653 & & & 183.1128 & 92.0600 & S & 1702.6796 & $\mathbf{8 5 1 . 8 4 3 4}$ & 1685.6530 & 843.3301 & 1684.6690 & $\mathbf{8 4 2 . 8 3 8 1}$ & $\mathbf{1 5}$ \\
\hline $\mathbf{3}$ & 298.1761 & 149.5917 & & & 280.1656 & 140.5864 & $\mathbf{P}$ & $\mathbf{1 6 1 5 . 6 4 7 5}$ & $\mathbf{8 0 8 . 3 2 7 4}$ & 1598.6210 & $\mathbf{7 9 9 . 8 1 4 1}$ & 1597.6370 & $\mathbf{7 9 9 . 3 2 2 1}$ & $\mathbf{1 4}$ \\
\hline $\mathbf{4}$ & 412.2191 & 206.6132 & 395.1925 & 198.0999 & 394.2085 & 197.6079 & $\mathbf{N}$ & $\mathbf{1 5 1 8 . 5 9 4 8}$ & 759.8010 & 1501.5682 & 751.2877 & 1500.5842 & 750.7957 & $\mathbf{1 3}$ \\
\hline $\mathbf{5}$ & 572.2497 & 286.6285 & 555.2232 & 278.1152 & 554.2391 & 277.6232 & $\mathbf{C}$ & $\mathbf{1 4 0 4 . 5 5 1 8}$ & $\mathbf{7 0 2 . 7 7 9 6}$ & 1387.5253 & $\mathbf{6 9 4 . 2 6 6 3}$ & 1386.5413 & 693.7743 & $\mathbf{1 2}$ \\
\hline
\end{tabular}




\begin{tabular}{|c|c|c|c|c|c|c|c|c|c|c|c|c|c|c|}
\hline 6 & 732.2804 & |366.6438 & 715.2538 & 358.1305 & 714.2698 & |357.6385 & C & 1244.5212 & |622.7642 & 1227.4946 & 614.2510 & 1226.5106 & |613.7589 & 11 \\
\hline 7 & 847.3073 & 424.1573 & 830.2807 & 415.6440 & 829.2967 & 415.1520 & $\mathbf{N}$ & 1084.4905 & 542.7489 & 1067.4640 & 534.2356 & 1066.4800 & 533.7436 & 10 \\
\hline 8 & 934.3393 & 467.6733 & 917.3128 & 459.1600 & 916.3288 & 458.6680 & S & 969.4636 & 485.2354 & 952.4371 & 476.7222 & 951.4530 & 476.2302 & 9 \\
\hline 9 & 1021.3713 & 511.1893 & 1004.3448 & 502.6760 & 1003.3608 & 502.1840 & $\mathrm{~J}$ & 882.4316 & 441.7194 & 865.4050 & 433.2062 & 864.4210 & 432.7141 & 8 \\
\hline 10 & 1122.4190 & 561.7132 & 1105.3925 & 553.1999 & 1104.4085 & 552.7079 & $\mathbf{T}$ & 795.3995 & 398.2034 & 778.3730 & 389.6901 & 777.3890 & 389.1981 & 7 \\
\hline 11 & 1221.4874 & 611.2474 & 1204.4609 & 602.7341 & 1203.4769 & 602.2421 & V & 694.3519 & 347.6796 & 677.3253 & 339.1663 & 676.3413 & 338.6743 & 6 \\
\hline 12 & 1292.5246 & 646.7659 & 1275.4980 & 638.2526 & 1274.5140 & 637.7606 & A & 595.2835 & 298.1454 & 578.2569 & 289.6321 & 577.2729 & 289.1401 & 5 \\
\hline 13 & 1407.5515 & 704.2794 & 1390.5250 & 695.7661 & 1389.5409 & 695.2741 & D & 524.2463 & 262.6268 & 507.2198 & 254.1135 & 506.2358 & 253.6215 & 4 \\
\hline 14 & 1478.5886 & 739.7979 & 1461.5621 & 731.2847 & 1460.5780 & 730.7927 & A & 409.2194 & 205.1133 & 392.1928 & 196.6001 & & & 3 \\
\hline 15 & 1641.6519 & 821.3296 & 1624.6254 & 812.8163 & 1623.6414 & 812.3243 & $\mathbf{Y}$ & 338.1823 & 169.5948 & 321.1557 & 161.0815 & & & 2 \\
\hline 16 & & & & & & & $\mathbf{R}$ & 175.1190 & 88.0631 & 158.0924 & 79.5498 & & & 1 \\
\hline
\end{tabular}
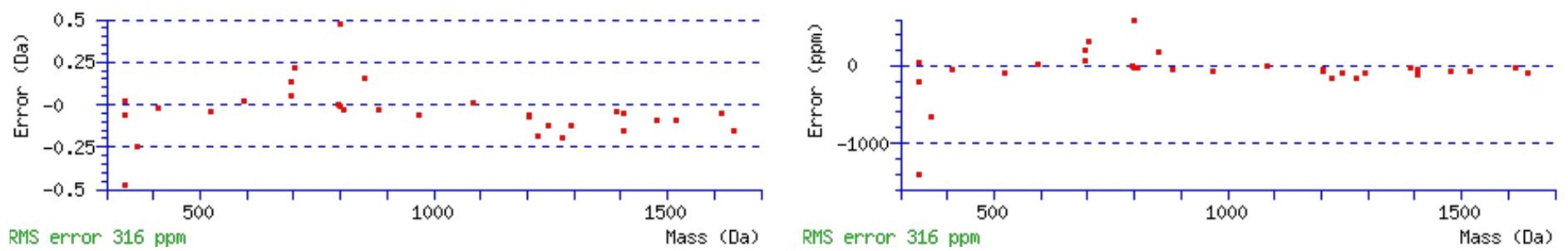

\section{All matches to this query}

\begin{tabular}{|l|l|l|l|}
\hline Score & Mr(calc): & Delta & \multicolumn{1}{|c|}{ Sequence } \\
\hline 96.0 & 1814.7563 & 0.0053 & $\underline{\text { LSPNCCNSSTVADAYR }}$ \\
\hline 51.3 & 1814.7563 & 0.0053 & $\underline{\text { LSPNCCNSSTVADAYR }}$ \\
\hline 25.2 & 1813.7723 & 0.9893 & $\underline{\text { LSPNCCNSSTVADAYR }}$ \\
\hline 10.3 & 1813.7416 & 1.0201 & TELFCSINCLSAYR \\
\hline 5.7 & 1814.7724 & -0.0107 & $\underline{\text { INTTSDEKDPTNPFR }}$ \\
\hline 3.8 & 1814.7665 & -0.0048 & HYTPDNNFHSLYVK \\
\hline 3.8 & 1814.7665 & -0.0048 & $\underline{\text { HYTPDNNFHSLYVK }}$ \\
\hline 3.6 & 1812.7689 & 1.9928 & VWQCGGSVEVLPCSR \\
\hline 3.1 & 1812.7567 & 2.0049 & $\underline{\text { TTYVNTNDEVVSHPR }}$ \\
\hline 2.7 & 1812.7567 & 2.0049 & TTYVNTNDEVVSHPR \\
\hline
\end{tabular}

Spectrum No: 99; Query: 2101; Rank: 1

\section{Peptide View}

MS/MS Fragmentation of GPNVPISTEVNATQFLALVQEK

Found in IPI00471666, Tax_Id=10116 Gene_Symbol=MGC94145 Uncharacterized protein C1orf179 homolog

Match to Query 2101: 2355.243168 from(1178.628860,2+)

Title: 100101RatKid_NS_deglyco_19.5544.5544.2.dta

Data file K:INewmanPaper|Piliang|3SubProteomes\Piliang3SP\mgf5ppm\ERLIC_3SubProteomes5ppm.mgf 

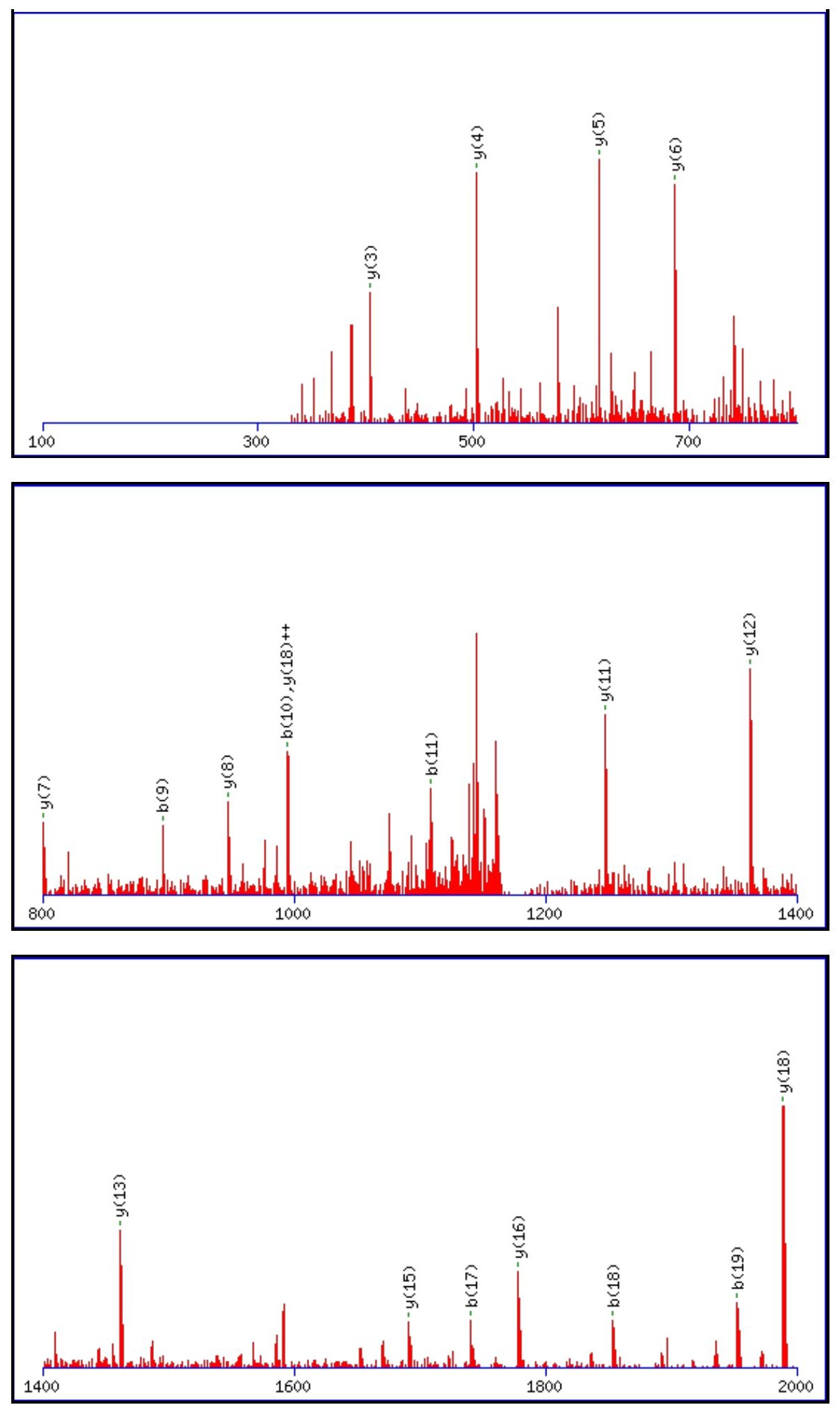

Monoisotopic mass of neutral peptide $\operatorname{Mr}$ (calc): 2355.2322

Fixed modifications: Carbamidomethyl (C)

Variable modifications:

N11 : Deamidated $\mathrm{N}(\mathrm{N})$

Ions Score: 96 Expect: $2.5 \mathrm{e}-008$

Matches (Bold Red): 19/234 fragment ions using 28 most intense peaks

\begin{tabular}{|r|r|r|c|c|c|r|r|r|r|r|r|r|r|r|}
\hline$\#$ & $\mathbf{b}$ & $\mathbf{b}^{++}$ & $\mathbf{b}^{*}$ & $\mathbf{b}^{\boldsymbol{*}^{++}}$ & $\mathbf{b}^{\mathbf{0}}$ & $\mathbf{b}^{\mathbf{0 + +}}$ & $\mathbf{S e q}$ & $\mathbf{y}$ & $\mathbf{y}^{++}$ & $\mathbf{y}^{\mathbf{*}}$ & $\mathbf{y}^{\mathbf{*}^{++}}$ & $\mathbf{y}^{\mathbf{0}}$ & $\mathbf{y}^{\mathbf{0 + +}}$ & $\#$ \\
\hline $\mathbf{1}$ & 58.0287 & 29.5180 & & & & & $\mathbf{G}$ & & & & & & & $\mathbf{2 2}$ \\
\hline $\mathbf{2}$ & 155.0815 & 78.0444 & & & & & $\mathbf{P}$ & 2299.2180 & 1150.1126 & 2282.1914 & 1141.5994 & 2281.2074 & 1141.1074 & $\mathbf{2 1}$ \\
\hline 3 & 269.1244 & 135.0659 & 252.0979 & 126.5526 & & & $\mathbf{N}$ & 2202.1652 & 1101.5863 & 2185.1387 & 1093.0730 & 2184.1547 & 1092.5810 & $\mathbf{2 0}$ \\
\hline $\mathbf{4}$ & 368.1928 & 184.6001 & 351.1663 & 176.0868 & & & $\mathbf{V}$ & 2088.1223 & 1044.5648 & 2071.0958 & 1036.0515 & 2070.1117 & 1035.5595 & $\mathbf{1 9}$ \\
\hline $\mathbf{5}$ & 465.2456 & 233.1264 & 448.2191 & 224.6132 & & & $\mathbf{P}$ & $\mathbf{1 9 8 9 . 0 5 3 9}$ & $\mathbf{9 9 5 . 0 3 0 6}$ & 1972.0273 & 986.5173 & 1971.0433 & 986.0253 & $\mathbf{1 8}$ \\
\hline
\end{tabular}




\begin{tabular}{|c|c|c|c|c|c|c|c|c|c|c|c|c|c|c|}
\hline 6 & 578.3297| & 289.6685 & 561.3031 & 281.1552 & & & I & |1892.0011 & 946.5042 & |1874.9746 & 937.9909 & |1873.9906 & 937.4989| & 17 \\
\hline 7 & 665.3617 & 333.1845 & 648.3352 & 324.6712 & 647.3511 & 324.1792 & S & 1778.9171 & 889.9622 & 1761.8905 & 881.4489 & 1760.9065 & 880.9569 & 16 \\
\hline 8 & 766.4094 & 383.7083 & 749.3828 & 375.1951 & 748.3988 & 374.7030 & $\mathbf{T}$ & 1691.8850 & 846.4462 & 1674.8585 & 837.9329 & 1673.8745 & 837.4409 & 15 \\
\hline 9 & 895.4520 & 448.2296 & 878.4254 & 439.7164 & 877.4414 & 439.2243 & $\mathbf{E}$ & 1590.8374 & 795.9223 & 1573.8108 & 787.4090 & 1572.8268 & 786.9170 & 4 \\
\hline 10 & 994.5204 & 497.7638 & 977.4938 & 489.2506 & 976.5098 & 488.7585 & $\mathbf{V}$ & 1461.7948 & 731.4010 & 1444.7682 & 722.8877 & 1443.7842 & 722.3957 & 13 \\
\hline 11 & 1109.5473 & 555.2773 & 1092.5208 & 546.7640 & 1091.5368 & 546.2720 & $\mathbf{N}$ & 1362.7264 & 681.8668 & 1345.6998 & 673.3535 & 1344.7158 & 672.8615 & 12 \\
\hline 12 & 1180.5844 & 590.7959 & 1163.5579 & 582.2826 & 1162.5739 & 581.7906 & A & 1247.6994 & 624.3533 & 1230.6729 & 615.8401 & 1229.6889 & 615.3481 & 11 \\
\hline 13 & 1281.6321 & 641.3197 & 1264.6056 & 632.8064 & 1263.6216 & 632.3144 & $\mathbf{T}$ & 1176.6623 & 588.8348 & 1159.6358 & 580.3215 & 1158.6517 & 579.8295 & 10 \\
\hline 14 & 1409.6907 & 705.3490 & 1392.6641 & 696.8357 & 1391.6801 & 696.3437 & $\mathbf{Q}$ & 1075.6146 & 538.3109 & 1058.5881 & 529.7977 & 1057.6041 & 529.3057 & 9 \\
\hline 15 & 1556.7591 & 778.8832 & 1539.7326 & 770.3699 & 1538.7485 & 769.8779 & $\mathbf{F}$ & 947.5560 & 474.2817 & 930.5295 & 465.7684 & 929.5455 & 465.2764 & 8 \\
\hline 16 & 1669.8432 & 835.4252 & 1652.8166 & 826.9119 & 1651.8326 & 826.4199 & $\mathbf{L}$ & 800.4876 & 400.7475 & 783.4611 & 392.2342 & 782.4771 & 391.7422 & 7 \\
\hline 17 & 1740.8803 & 870.9438 & 1723.8537 & 862.4305 & 1722.8697 & 861.9385 & A & 687.4036 & 344.2054 & 670.3770 & 335.6921 & 669.3930 & 335.2001 & 6 \\
\hline 18 & 1853.9644 & 927.4858 & 1836.9378 & 918.9725 & 1835.9538 & 918.4805 & $\mathbf{L}$ & 616.3665 & 308.6869 & 599.3399 & 300.1736 & 598.3559 & 299.6816 & 5 \\
\hline 19 & 1953.0328 & 977.0200 & 1936.0062 & 968.5067 & 1935.0222 & 968.0147 & $\mathbf{V}$ & 503.2824 & 252.1448 & 486.2558 & 243.6316 & 485.2718 & 243.1396 & 4 \\
\hline 21 & 2210.1339 & 1105.5706 & 2193.1074 & 1097.0573 & 2192.1234 & 1096.5653 & $\mathbf{E}$ & 276.1554 & 138.5813 & 259.1288 & 130.0681 & 258.1448 & 129.5761 & 2 \\
\hline 22 & & & & & & & $\mathbf{K}$ & 147.1128 & 74.0600 & 130.0863 & 65.5468 & & & 1 \\
\hline
\end{tabular}
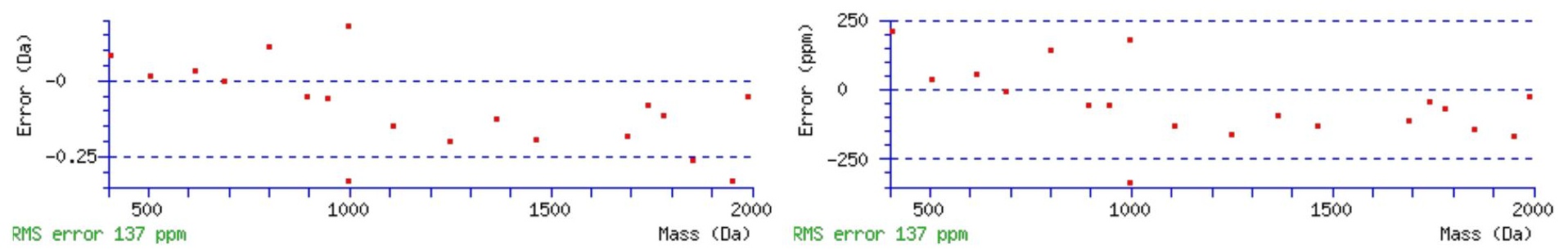

\section{All matches to this query}

\begin{tabular}{|l|l|l|l|}
\hline Score & Mr(calc): & Delta & Sequence \\
\hline 96.0 & 2355.2322 & 0.0110 & GPNVPISTEVNATQFLALVQEK \\
\hline 20.2 & 2355.2322 & 0.0110 & GPNVPISTEVNATQFLALVQEK \\
\hline 19.6 & 2354.2482 & 0.9950 & GPNVPISTEVNATQFLALVQEK \\
\hline
\end{tabular}

Spectrum No: 100; Query: 1396; Rank: 1

\section{Peptide View}

MS/MS Fragmentation of IYAGMVSLLDEAVGNVTK

Found in IPI00198405, Tax_Id=10116 Gene_Symbol=Arsb Arylsulfatase B

Match to Query 1396: 1879.961068 from(940.987810,2+)

Title: 091008RatKidney_NH4Format02_25.5366.5366.2.dta

Data file K:INewmanPaper|Piliangl3SubProteomes\Piliang3SP\mgf5ppm\ERLIC_3SubProteomes5ppm.mgf 

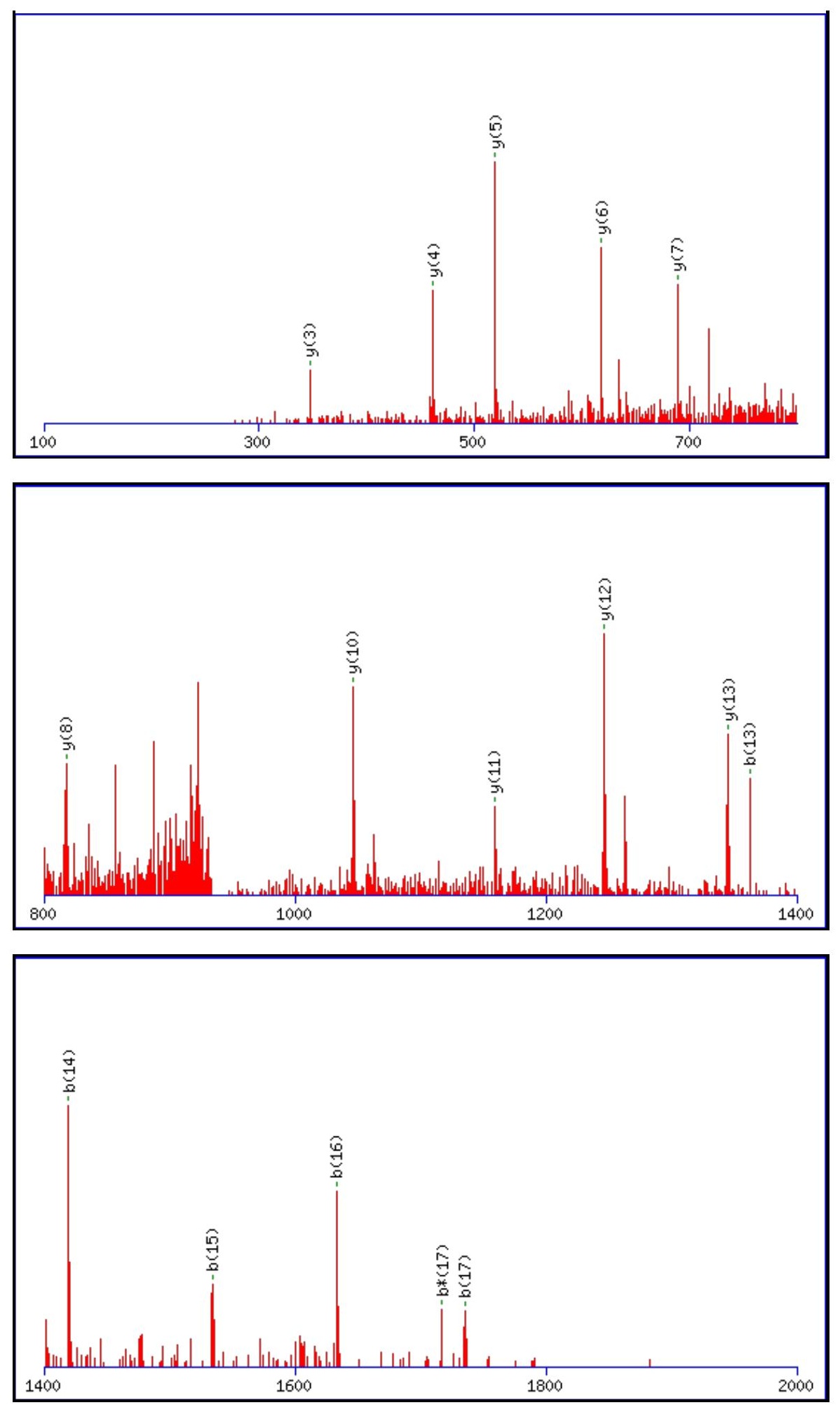

Monoisotopic mass of neutral peptide $\operatorname{Mr}($ calc): 1879.9601

Fixed modifications: Carbamidomethyl (C)

Variable modifications:

N15 : Deamidated N (N)

Ions Score: 96 Expect: $4 \mathrm{e}-008$

Matches (Bold Red): 16/162 fragment ions using 26 most intense peaks

\begin{tabular}{|r|c|c|c|c|c|c|c|c|c|c|c|c|c|c|}
\hline$\#$ & $\mathbf{b}$ & $\mathbf{b}^{++}$ & $\mathbf{b}^{*}$ & $\mathbf{b}^{\boldsymbol{*}^{++}}$ & $\mathbf{b}^{\mathbf{0}}$ & $\mathbf{b}^{\mathbf{0 + +}}$ & Seq. & $\mathbf{y}$ & $\mathbf{y}^{++}$ & $\mathbf{y}^{\mathbf{*}}$ & $\mathbf{y}^{\boldsymbol{*}^{++}}$ & $\mathbf{y}^{\mathbf{0}}$ & $\mathbf{y}^{\mathbf{0 + +}}$ & $\#$ \\
\hline $\mathbf{1}$ & 114.0913 & 57.5493 & & & & & $\mathbf{I}$ & & & & & & & $\mathbf{1 8}$ \\
\hline $\mathbf{2}$ & 277.1547 & 139.0810 & & & & & $\mathbf{Y}$ & 1767.8833 & 884.4453 & 1750.8568 & 875.9320 & 1749.8728 & 875.4400 & $\mathbf{1 7}$ \\
\hline 3 & 348.1918 & 174.5995 & & & & & A & 1604.8200 & 802.9136 & 1587.7934 & 794.4004 & 1586.8094 & 793.9084 & $\mathbf{1 6}$ \\
\hline $\mathbf{4}$ & 405.2132 & 203.1103 & & & & & $\mathbf{G}$ & 1533.7829 & 767.3951 & 1516.7563 & 758.8818 & 1515.7723 & 758.3898 & $\mathbf{1 5}$ \\
\hline $\mathbf{5}$ & 536.2537 & 268.6305 & & & & & $\mathbf{M}$ & 1476.7614 & 738.8843 & 1459.7349 & 730.3711 & 1458.7509 & 729.8791 & $\mathbf{1 4}$ \\
\hline
\end{tabular}




\begin{tabular}{|c|c|c|c|c|c|c|c|c|c|c|c|c|c|c|}
\hline 6 & 635.3221 & |318.1647 & & & & & V & $\mid 1345.7209$ & |673.3641 & |1328.6944 & 664.8508 & 1327.7104 & 664.3588 & 13 \\
\hline 7 & 722.3542 & 361.6807 & & & 704.3436 & 352.6754 & $\mathbf{S}$ & 1246.6525 & 623.8299 & 1229.6260 & 615.3166 & 1228.6420 & 614.8246 & 12 \\
\hline 8 & 835.4382 & 418.2228 & & & 817.4277 & |409.2175 & L & 1159.6205 & 580.3139 & 1142.5939 & 571.8006 & 1141.6099 & 571.3086 & 11 \\
\hline 9 & 948.5223 & 474.7648 & & & 930.5117 & 465.7595 & $\mathbf{L}$ & 1046.5364 & 523.7719 & 1029.5099 & 515.2586 & 1028.5259 & 514.7666 & 10 \\
\hline 10 & 1063.5492 & 532.2783 & & & 1045.5387 & 523.2730 & D & 933.4524 & 467.2298 & 916.4258 & 458.7165 & 915.4418 & 458.2245 & 9 \\
\hline 11 & 1192.5918 & 596.7996 & & & 1174.5813 & 587.7943 & $\mathbf{E}$ & 818.4254 & 409.7163 & 801.3989 & 401.2031 & 800.4149 & 400.7111 & 8 \\
\hline 12 & 1263.6290 & 632.3181 & & & 1245.6184 & 623.3128 & A & 689.3828 & 345.1951 & 672.3563 & 336.6818 & 671.3723 & 336.1898 & 7 \\
\hline 13 & 1362.6974 & 681.8523 & & & 1344.6868 & 672.8470 & $\mathbf{V}$ & 618.3457 & 309.6765 & 601.3192 & 301.1632 & 600.3351 & 300.6712 & 6 \\
\hline 14 & 1419.7188 & 710.3631 & & & 1401.7083 & 701.3578 & G & 519.2773 & 260.1423 & 502.2507 & 251.6290 & 501.2667 & 251.1370 & 5 \\
\hline 15 & \begin{tabular}{|l|}
1534.7458 \\
\end{tabular} & 767.8765 & 1517.7192 & 759.3632 & 1516.7352 & 758.8712 & $\mathbf{N}$ & 462.2558 & 231.6316 & 445.2293 & 223.1183 & 444.2453 & 222.6263 & 4 \\
\hline 16 & 1633.8142 & 817.4107 & 1616.7876 & 808.8975 & 1615.8036 & 808.4054 & V & 347.2289 & 174.1181 & 330.2023 & 165.6048 & 329.2183 & 165.1128 & 3 \\
\hline 17 & \begin{tabular}{|l|}
1734.8619 \\
\end{tabular} & 867.9346 & 1717.8353 & 859.4213 & 1716.8513 & 858.9293 & $T$ & 248.1605 & 124.5839 & 231.1339 & 116.0706 & 230.1499 & 115.5786 & 2 \\
\hline 18 & & & & & & & K & 147.1128 & 74.0600 & 130.0863 & 65.5468 & & & 1 \\
\hline
\end{tabular}
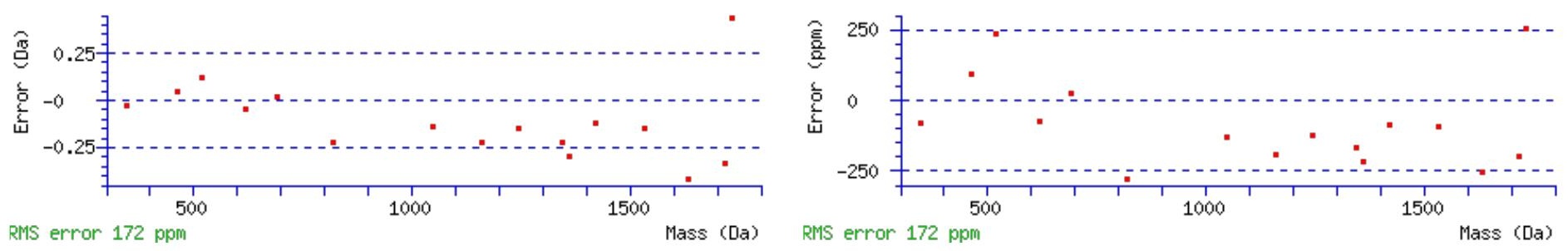

\section{All matches to this query}

\begin{tabular}{|l|l|c|l|}
\hline Score & Mr(calc): & Delta & \multicolumn{1}{c|}{ Sequence } \\
\hline 95.7 & 1879.9601 & 0.0010 & IYAGMVSLLDEAVGNVTK \\
\hline 24.8 & 1878.9761 & 0.9850 & IYAGMVSLLDEAVGNVTK \\
\hline 8.9 & 1879.9536 & 0.0075 & SWVQCGISMKILNSLK \\
\hline 5.1 & 1879.9502 & 0.0109 & AWEDMLIQFLRNSLK \\
\hline 3.0 & 1878.9588 & 1.0022 & RTYVTAPGTGFLPGDTAR \\
\hline 3.0 & 1877.9557 & 2.0054 & NFLQAVCTLLNSGGGVVK \\
\hline 2.5 & 1879.9462 & 0.0149 & QFDKVREDMELSLVR \\
\hline 1.6 & 1879.9639 & -0.0028 & FQSRTSEAAKLEAEVSK \\
\hline 0.8 & 1877.9595 & 2.0015 & RSSGLVQNIDQLYSGGGK \\
\hline 0.8 & 1878.9696 & 0.9915 & SWVQCGISMKILNSLK \\
\hline
\end{tabular}

Spectrum No: 101; Query: 1885; Rank: 1

\section{Peptide View}

MS/MS Fragmentation of TGEANLTQLYLQEALDFIR

Found in IPI00359847, Tax_Id=10116 Gene_Symbol=Galns N-acetylgalactosamine-6-sulfatase precursor

Match to Query 1885: 2195.118008 from(1098.566280,2+)

Title: 100101RatKid_NS_deglyco_11.7671.7671.2.dta

Data file K:INewmanPaper|Piliangl3SubProteomes\Piliang3SP\mgf5ppm\ERLIC_3SubProteomes5ppm.mgf 

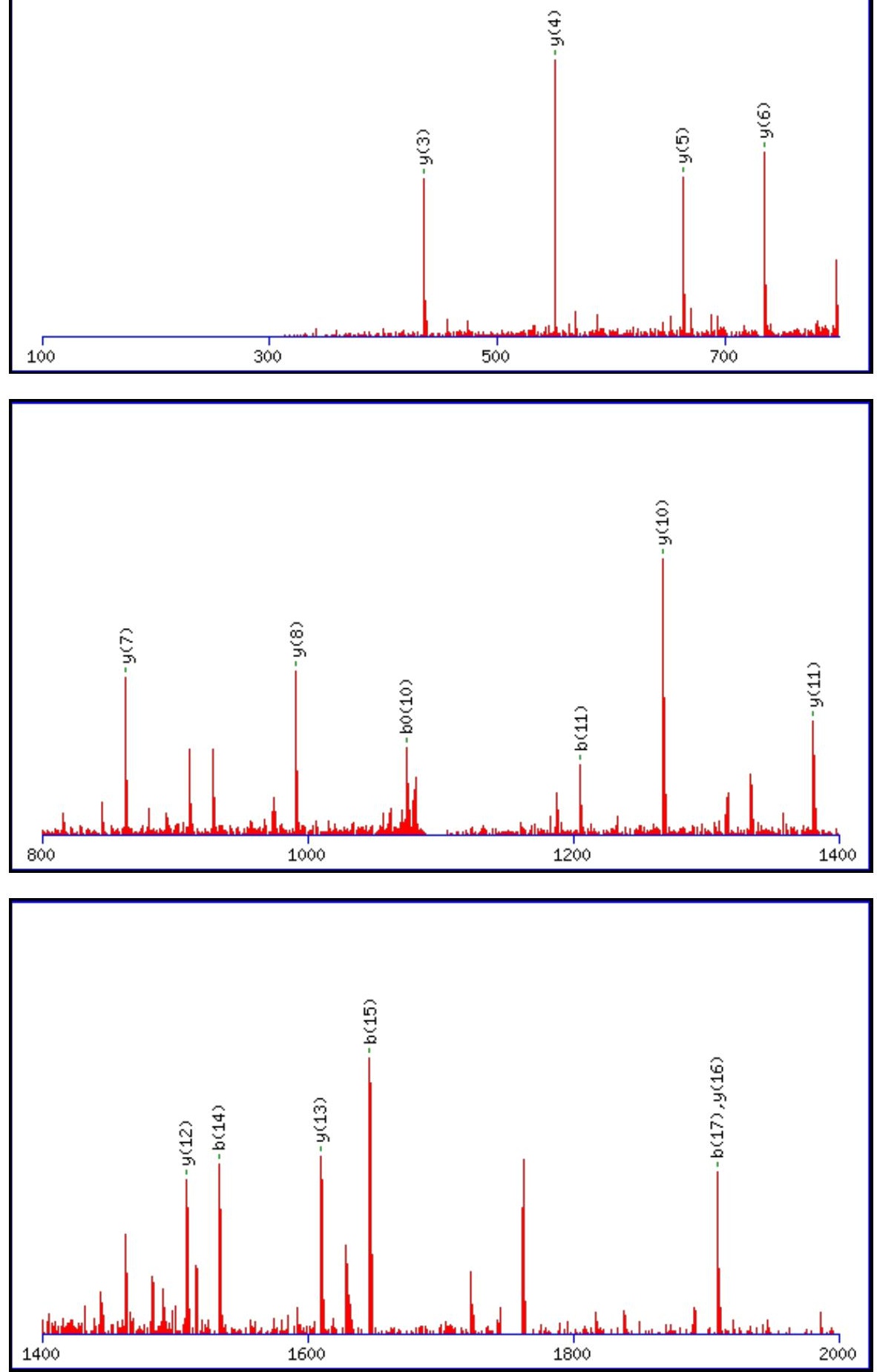

Monoisotopic mass of neutral peptide $\operatorname{Mr}($ calc): 2195.1110

Fixed modifications: Carbamidomethyl (C)

Variable modifications:

N5

Ions Score: 96 Expect: $4.5 \mathrm{e}-008$

Matches (Bold Red): 16/202 fragment ions using 23 most intense peaks

\begin{tabular}{|r|c|c|c|c|r|r|r|r|r|r|r|r|r|r|}
\hline$\#$ & $\mathbf{b}$ & $\mathbf{b}^{++}$ & $\mathbf{b}^{*}$ & $\mathbf{b}^{\boldsymbol{*}^{++}}$ & \multicolumn{1}{|c|}{$\mathbf{b}^{\mathbf{0}}$} & $\mathbf{b}^{\mathbf{0 + +}}$ & Seq. & $\mathbf{y}$ & $\mathbf{y}^{++}$ & $\mathbf{y}^{\mathbf{*}}$ & $\mathbf{y}^{\mathbf{*}^{++}}$ & $\mathbf{y}^{\mathbf{0}}$ & $\mathbf{y}^{\mathbf{0 + +}}$ & $\#$ \\
\hline $\mathbf{1}$ & 102.0550 & 51.5311 & & & 84.0444 & 42.5258 & $\mathbf{T}$ & & & & & & & $\mathbf{1 9}$ \\
\hline $\mathbf{2}$ & 159.0764 & 80.0418 & & & 141.0659 & 71.0366 & $\mathbf{G}$ & 2095.0706 & 1048.0389 & 2078.0441 & 1039.5257 & 2077.0600 & 1039.0337 & $\mathbf{1 8}$ \\
\hline $\mathbf{3}$ & 288.1190 & 144.5631 & & & 270.1084 & 135.5579 & $\mathbf{E}$ & 2038.0491 & 1019.5282 & 2021.0226 & 1011.0149 & 2020.0386 & 1010.5229 & $\mathbf{1 7}$ \\
\hline $\mathbf{4}$ & 359.1561 & 180.0817 & & & 341.1456 & 171.0764 & $\mathbf{A}$ & $\mathbf{1 9 0 9 . 0 0 6 6}$ & 955.0069 & 1891.9800 & 946.4936 & 1890.9960 & 946.0016 & $\mathbf{1 6}$ \\
\hline $\mathbf{5}$ & 474.1831 & 237.5952 & 457.1565 & 229.0819 & 456.1725 & 228.5899 & $\mathbf{N}$ & 1837.9694 & 919.4884 & 1820.9429 & 910.9751 & 1819.9589 & 910.4831 & $\mathbf{1 5}$ \\
\hline
\end{tabular}




\begin{tabular}{|c|c|c|c|c|c|c|c|c|c|c|c|c|c|c|}
\hline 6 & 587.2671 & 294.1372 & 570.2406 & 285.6239 & 569.2566 & 285.1319 & $\mathbf{L}$ & |1722.9425 & 861.9749 & 1705.9160 & 853.4616 & |1704.9319 & 852.9696 & 14 \\
\hline 7 & 688.3148 & 344.6610 & 671.2883 & 336.1478 & 670.3042 & 335.6558 & $\mathbf{T}$ & 1609.8584 & 805.4329 & 1592.8319 & 796.9196 & 1591.8479 & 796.4276 & 13 \\
\hline 8 & 816.3734 & 408.6903 & 799.3468 & 400.1771 & 798.3628 & 399.6850 & $\mathbf{Q}$ & 1508.8108 & 754.9090 & 1491.7842 & 746.3957 & 1490.8002 & 745.9037 & 12 \\
\hline 9 & 929.4574 & 465.2324 & 912.4309 & 456.7191 & 911.4469 & 456.2271 & $\mathbf{L}$ & 1380.7522 & 690.8797 & 1363.7256 & 682.3665 & 1362.7416 & 681.8744 & 11 \\
\hline 10 & 1092.5208 & 546.7640 & 1075.4942 & 538.2508 & 1074.5102 & 537.7587 & $\mathbf{Y}$ & 1267.6681 & 634.3377 & 1250.6416 & 625.8244 & 1249.6576 & 625.3324 & 10 \\
\hline 11 & 1205.6048 & 603.3061 & 1188.5783 & 594.7928 & 1187.5943 & 594.3008 & $\mathbf{L}$ & 1104.6048 & 552.8060 & 1087.5782 & 544.2928 & 1086.5942 & 543.8007 & 9 \\
\hline 12 & 1333.6634 & 667.3353 & 1316.6369 & 658.8221 & 1315.6529 & 658.3301 & $\mathbf{Q}$ & 991.5207 & 496.2640 & 974.4942 & 487.7507 & 973.5102 & 487.2587 & 8 \\
\hline 14 & 1533.7431 & 767.3752 & 1516.7166 & 758.8619 & 1515.7326 & 758.3699 & A & 734.4196 & 367.7134 & 717.3930 & 359.2001 & 716.4090 & 358.7081 & 6 \\
\hline 15 & 1646.8272 & 823.9172 & 1629.8006 & 815.4040 & 1628.8166 & 814.9119 & $\mathbf{L}$ & 663.3824 & 332.1949 & 646.3559 & 323.6816 & 645.3719 & 323.1896 & 5 \\
\hline 16 & 1761.8541 & 881.4307 & 1744.8276 & 872.9174 & 1743.8436 & 872.4254 & D & 550.2984 & 275.6528 & 533.2718 & 267.1396 & 532.2878 & 266.6475 & 4 \\
\hline 17 & 1908.9225 & 954.9649 & 1891.8960 & 946.4516 & 1890.9120 & 945.9596 & $\mathbf{F}$ & 435.2714 & 218.1394 & 418.2449 & 209.6261 & & & 3 \\
\hline 18 & 2022.0066 & 1011.5069 & 2004.9801 & 1002.9937 & 2003.9960 & 1002.5017 & I & 288.2030 & 144.6051 & 271.1765 & 136.0919 & & & 2 \\
\hline 19 & & & & & & & $\mathbf{R}$ & 175.1190 & 88.0631 & 158.0924 & 79.5498 & & & 1 \\
\hline
\end{tabular}
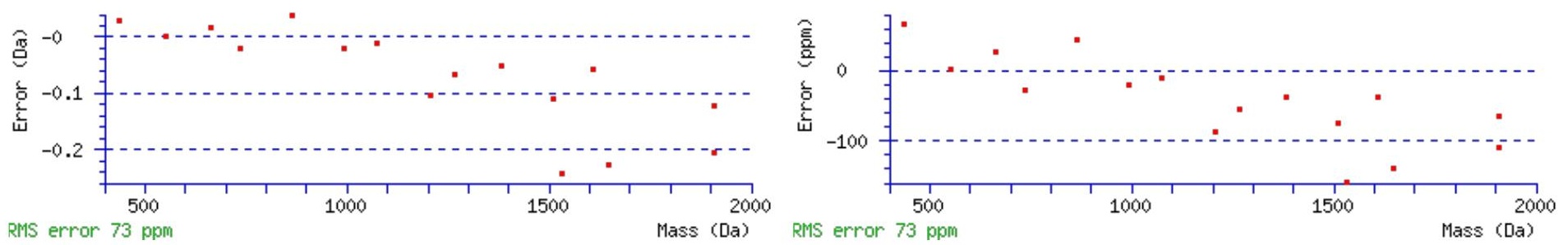

\section{All matches to this query}

\begin{tabular}{|l|l|c|c|}
\hline Score & Mr(calc): & Delta & Sequence \\
\hline 95.7 & 2195.1110 & 0.0070 & TGEANLTQLYLQEALDFIR \\
\hline 67.2 & 2194.1270 & 0.9910 & TGEANLTQLYLQEALDFIR \\
\hline 2.9 & 2195.1395 & -0.0215 & TLAKVNTFMSETGNILNIVK \\
\hline
\end{tabular}

Spectrum No: 102; Query: 952; Rank: 1

\section{Peptide View}

\section{MS/MS Fragmentation of KFINDYVSNQTQGK}

Found in IPI00200593, Tax_Id=10116 Gene_Symbol=Serpina3k Serine protease inhibitor A3K precursor

Match to Query 952: 1641.799808 from(821.907180,2+)

Title: 091008RatKidney_NoSalt_31.1289.1289.2.dta

Data file K:INewmanPaper\Piliang\3SubProteomes\Piliang3SP\mgf5ppm\ERLIC_3SubProteomes5ppm.mgf 

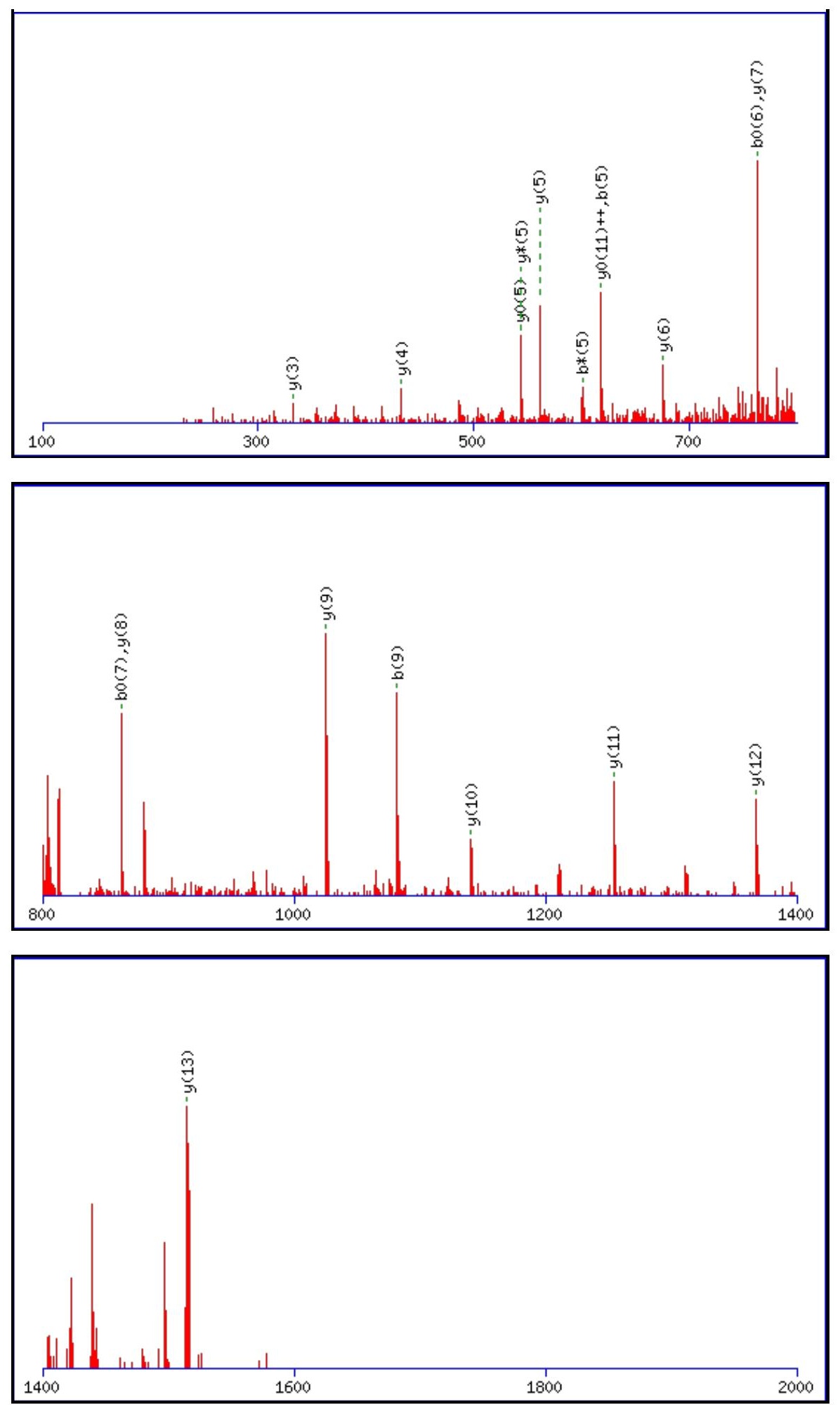

Monoisotopic mass of neutral peptide $\operatorname{Mr}($ calc): 1641.7998

Fixed modifications: Carbamidomethyl (C)

Variable modifications:

N9: Deamidated $\mathrm{N}(\mathrm{N})$

Ions Score: 96 Expect: 5 e- -008

Matches (Bold Red): 19/142 fragment ions using 22 most intense peaks

\begin{tabular}{|r|c|c|c|c|c|c|c|c|c|c|c|c|c|c|}
\hline$\#$ & $\mathbf{b}$ & $\mathbf{b}^{++}$ & $\mathbf{b}^{*}$ & $\mathbf{b}^{\boldsymbol{*}_{++}}$ & $\mathbf{b}^{\mathbf{0}}$ & $\mathbf{b}^{\mathbf{0 + +}}$ & $\mathbf{S e q}$ & $\mathbf{y}$ & $\mathbf{y}^{++}$ & $\mathbf{y}^{\mathbf{*}}$ & $\mathbf{y}^{\boldsymbol{*}^{++}}$ & $\mathbf{y}^{\mathbf{0}}$ & $\mathbf{y}^{\mathbf{0 + +}}$ & $\#$ \\
\hline $\mathbf{1}$ & 129.1022 & 65.0548 & 112.0757 & 56.5415 & & & $\mathbf{K}$ & & & & & & & $\mathbf{1 4}$ \\
\hline $\mathbf{2}$ & 276.1707 & 138.5890 & 259.1441 & 130.0757 & & & $\mathbf{F}$ & $\mathbf{1 5 1 4 . 7 1 2 2}$ & 757.8597 & 1497.6856 & 749.3464 & 1496.7016 & $\mathbf{7 4 8 . 8 5 4 4}$ & $\mathbf{1 3}$ \\
\hline $\mathbf{3}$ & 389.2547 & 195.1310 & 372.2282 & 186.6177 & & & $\mathbf{I}$ & $\mathbf{1 3 6 7 . 6 4 3 7}$ & 684.3255 & 1350.6172 & 675.8122 & 1349.6332 & 675.3202 & $\mathbf{1 2}$ \\
\hline $\mathbf{4}$ & 503.2976 & 252.1525 & 486.2711 & 243.6392 & & & $\mathbf{N}$ & $\mathbf{1 2 5 4 . 5 5 9 7}$ & 627.7835 & 1237.5331 & 619.2702 & 1236.5491 & $\mathbf{6 1 8 . 7 7 8 2}$ & $\mathbf{1 1}$ \\
\hline $\mathbf{5}$ & $\mathbf{6 1 8 . 3 2 4 6}$ & 309.6659 & $\mathbf{6 0 1 . 2 9 8 0}$ & 301.1527 & 600.3140 & 300.6606 & $\mathbf{D}$ & $\mathbf{1 1 4 0 . 5 1 6 8}$ & 570.7620 & 1123.4902 & 562.2487 & 1122.5062 & 561.7567 & $\mathbf{1 0}$ \\
\hline
\end{tabular}




\begin{tabular}{|c|c|c|c|c|c|c|c|c|c|c|c|c|c|c|}
\hline 6 & 781.3879 & |391.1976| & 764.3614 & |382.6843 & 763.3774 & |382.1923 & $\mathbf{Y}$ & |1025.4898| & |513.2485| & |1008.4633 & |504.7353 & 1007.4792 & |504.2433 & 9 \\
\hline 7 & 880.4563 & 440.7318 & 863.4298 & 432.2185 & 862.4458 & 431.7265 & V & 862.4265 & 431.7169 & 845.3999 & 423.2036 & 844.4159 & 422.7116 & 8 \\
\hline 8 & 967.4884 & 484.2478 & 950.4618 & 475.7345 & 949.4778 & 475.2425 & $\mathbf{S}$ & 763.3581 & 382.1827 & 746.3315 & 373.6694 & 745.3475 & 373.1774 & 7 \\
\hline 9 & 1082.5153 & 541.7613 & 1065.4887 & 533.2480 & 1064.5047 & 532.7560 & $\mathbf{N}$ & 676.3260 & 338.6667 & 659.2995 & 330.1534 & 658.3155 & 329.6614 & 6 \\
\hline 10 & 1210.5739 & 605.7906 & 1193.5473 & 597.2773 & 1192.5633 & 596.7853 & $\mathbf{Q}$ & 561.2991 & 281.1532 & 544.2726 & 272.6399 & 543.2885 & 272.1479 & 5 \\
\hline 11 & 1311.6216 & 656.3144 & 1294.5950 & 647.8011 & 1293.6110 & 647.3091 & $\mathbf{T}$ & 433.2405 & 217.1239 & 416.2140 & 208.6106 & 415.2300 & 208.1186 & 4 \\
\hline 12 & 1439.6801 & 720.3437 & 1422.6536 & 711.8304 & 1421.6696 & 711.3384 & $\mathbf{Q}$ & 332.1928 & 166.6001 & 315.1663 & 158.0868 & & & 3 \\
\hline 13 & 1496.7016 & 748.8544 & 1479.6750 & 740.3412 & 1478.6910 & 739.8492 & G & 204.1343 & 102.5708 & 187.1077 & 94.0575 & & & 2 \\
\hline 14 & & & & & & & K & 147.1128 & 74.0600 & 130.0863 & 65.5468 & & & 1 \\
\hline
\end{tabular}
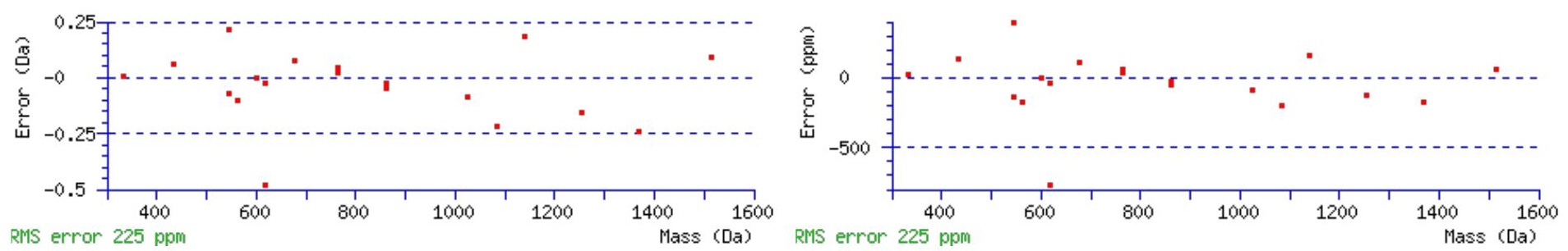

\section{All matches to this query}

\begin{tabular}{|l|l|l|l|}
\hline Score & Mr(calc): & Delta & \multicolumn{1}{c|}{ Sequence } \\
\hline 95.5 & 1641.7998 & -0.0000 & KFINDYVSNQTQGK \\
\hline 44.4 & 1641.7998 & -0.0000 & KFINDYVSNOTOGK \\
\hline 17.5 & 1641.8032 & -0.0034 & MENIGAHLSNAVLEK \\
\hline 7.0 & 1640.7957 & 1.0042 & QELDLNSSMRLKK \\
\hline 6.5 & 1639.8093 & 1.9905 & VFLEEEDSFSALVR \\
\hline 5.8 & 1641.8015 & -0.0017 & LTVGDPVTVEYITR \\
\hline 5.5 & 1641.7988 & 0.0010 & SRKNRPAASGGFVSK \\
\hline 4.9 & 1641.7875 & 0.0123 & WTTNXRSLNSNKK \\
\hline 4.9 & 1641.7875 & 0.0123 & WTTNXRSLNSNKK \\
\hline 4.8 & 1640.7866 & 1.0132 & RTGERQNLSPDDPR \\
\hline
\end{tabular}

Spectrum No: 103; Query: 894; Rank: 1

\section{Peptide View}

MS/MS Fragmentation of VTINNWVANKTEGR

Found in IPI00372372, Tax_Id=10116 Gene_Symbol=Serpinc1 Serine (Or cysteine) peptidase inhibitor, clade C (Antithrombin), member 1

Match to Query 894: 1601.819008 from(801.916780,2+)

Title: 091008RatKidney_NoSalt_27.1969.1969.2.dta

Data file K:INewmanPaper|Piliang $\mid 3 S$ SubProteomes\Piliang3SP\mgf5ppm\ERLIC_3SubProteomes5ppm.mgf 

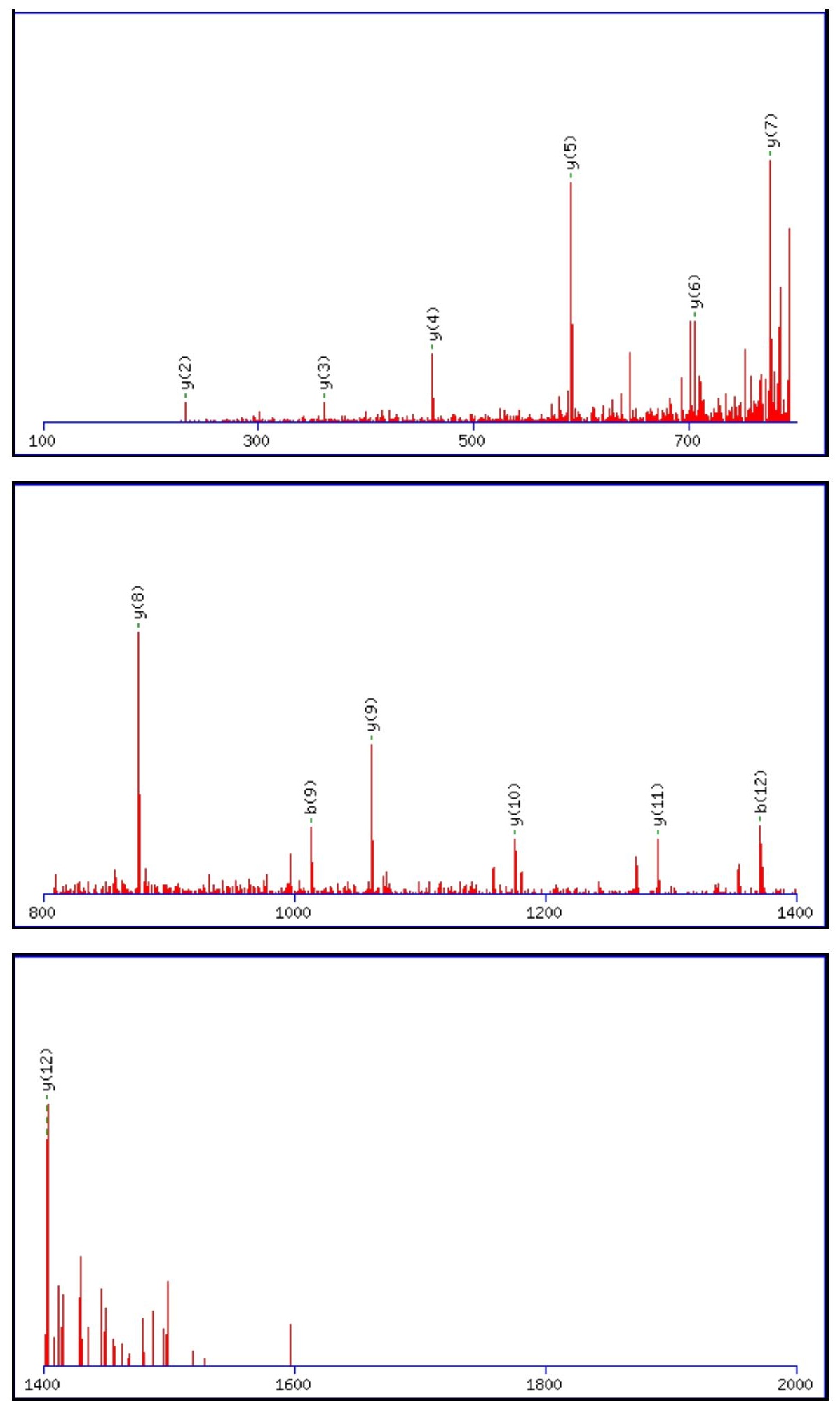

Monoisotopic mass of neutral peptide $\operatorname{Mr}($ calc): 1601.8161

Fixed modifications: Carbamidomethyl (C)

Variable modifications:

N9 : Deamidated $\mathrm{N}(\mathrm{N})$

Ions Score: 96 Expect: $3.7 \mathrm{e}-008$

Matches (Bold Red): 13/144 fragment ions using 24 most intense peaks

\begin{tabular}{|r|c|c|c|c|c|c|c|c|c|c|c|c|c|c|}
\hline$\#$ & $\mathbf{b}$ & $\mathbf{b}^{++}$ & $\mathbf{b}^{*}$ & $\mathbf{b}^{\boldsymbol{*}^{++}}$ & $\mathbf{b}^{\mathbf{0}}$ & $\mathbf{b}^{\mathbf{0 + +}}$ & Seq. & $\mathbf{y}$ & $\mathbf{y}^{++}$ & $\mathbf{y}^{\mathbf{*}}$ & $\mathbf{y}^{\boldsymbol{*}^{++}}$ & $\mathbf{y}^{\mathbf{0}}$ & $\mathbf{y}^{\mathbf{0 + +}}$ & $\#$ \\
\hline $\mathbf{1}$ & 100.0757 & 50.5415 & & & & & $\mathbf{V}$ & & & & & & & $\mathbf{1 4}$ \\
\hline $\mathbf{2}$ & 201.1234 & 101.0653 & & & 183.1128 & 92.0600 & $\mathbf{T}$ & 1503.7550 & 752.3812 & 1486.7285 & 743.8679 & 1485.7445 & 743.3759 & $\mathbf{1 3}$ \\
\hline 3 & 314.2074 & 157.6074 & & & 296.1969 & 148.6021 & $\mathbf{I}$ & $\mathbf{1 4 0 2 . 7 0 7 3}$ & 701.8573 & 1385.6808 & 693.3440 & 1384.6968 & 692.8520 & $\mathbf{1 2}$ \\
\hline $\mathbf{4}$ & 428.2504 & 214.6288 & 411.2238 & 206.1155 & 410.2398 & 205.6235 & $\mathbf{N}$ & $\mathbf{1 2 8 9 . 6 2 3 3}$ & 645.3153 & 1272.5967 & 636.8020 & 1271.6127 & 636.3100 & $\mathbf{1 1}$ \\
\hline $\mathbf{5}$ & 542.2933 & 271.6503 & 525.2667 & 263.1370 & 524.2827 & 262.6450 & $\mathbf{N}$ & $\mathbf{1 1 7 5 . 5 8 0 4}$ & 588.2938 & 1158.5538 & 579.7805 & 1157.5698 & 579.2885 & $\mathbf{1 0}$ \\
\hline
\end{tabular}




\begin{tabular}{|c|c|c|c|c|c|c|c|c|c|c|c|c|c|c|}
\hline 6 & 728.3726 & |364.6899 & 711.3461 & |356.1767| & 710.3620 & |355.6847| & $\mathbf{W}$ & 1061.5374 & |531.2724| & 1044.5109| & |522.7591| & |1043.5269| & 522.2671 & 9 \\
\hline 7 & 827.4410 & 414.2241 & 810.4145 & 405.7109 & 809.4304 & 405.2189 & $\mathbf{V}$ & 875.4581 & 438.2327 & 858.4316 & 429.7194 & 857.4475 & 429.2274 & 8 \\
\hline 8 & 898.4781 & 449.7427 & 881.4516 & 441.2294 & 880.4676 & 440.7374 & A & 776.3897 & 388.6985 & 759.3632 & 380.1852 & 758.3791 & 379.6932 & 7 \\
\hline 9 & 1013.5051 & 507.2562 & 996.4785 & 498.7429 & 995.4945 & 498.2509 & $\mathbf{N}$ & 705.3526 & 353.1799 & 688.3260 & 344.6667 & 687.3420 & 344.1746 & 6 \\
\hline 10 & 1141.6000 & 571.3037 & 1124.5735 & 562.7904 & 1123.5895 & 562.2984 & $\mathbf{K}$ & 590.3257 & 295.6665 & 573.2991 & 287.1532 & 572.3151 & 286.6612 & 5 \\
\hline 11 & 1242.6477 & 621.8275 & 1225.6212 & 613.3142 & 1224.6371 & 612.8222 & $\mathbf{T}$ & 462.2307 & 231.6190 & 445.2041 & 223.1057 & 444.2201 & 222.6137 & 4 \\
\hline 12 & 1371.6903 & 686.3488 & 1354.6638 & 677.8355 & 1353.6797 & 677.3435 & $\mathbf{E}$ & 361.1830 & 181.0951 & 344.1565 & 172.5819 & 343.1724 & 172.0899 & 3 \\
\hline 13 & 1428.7118 & 714.8595 & 1411.6852 & 706.3462 & 1410.7012 & 705.8542 & G & 232.1404 & 116.5738 & 215.1139 & 108.0606 & & & 2 \\
\hline 14 & & & & & & & $\mathbf{R}$ & 175.1190 & 88.0631 & 158.0924 & 79.5498 & & & 1 \\
\hline
\end{tabular}
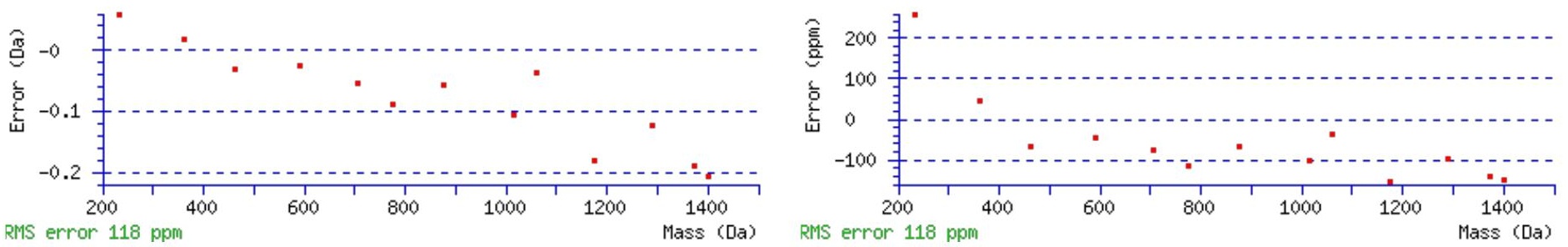

\section{All matches to this query}

\begin{tabular}{|l|l|l|l|}
\hline Score & Mr(calc): & Delta & \multicolumn{1}{c|}{ Sequence } \\
\hline 95.5 & 1601.8161 & 0.0029 & $\underline{\text { VTINNWVANKTEGR }}$ \\
\hline 31.0 & 1601.8161 & 0.0029 & VTINNWVANKTEGR \\
\hline 19.6 & 1601.8161 & 0.0029 & $\underline{\text { VTINNWVANKTEGR }}$ \\
\hline 2.6 & 1601.8168 & 0.0022 & $\underline{\text { GAAGGNLSSRCVRAAR }}$ \\
\hline
\end{tabular}

Spectrum No: 104; Query: 1254; Rank: 1

\section{Peptide View}

MS/MS Fragmentation of LDNTSMSTVIDGVVLPK

Found in IPI00195148, Tax_Id=10116 Gene_Symbol=Es2 Liver carboxylesterase 1 precursor

Match to Query 1254: 1804.911988 from(903.463270,2+)

Title: 091008RatKidney_NoSalt_30.2570.2570.2.dta

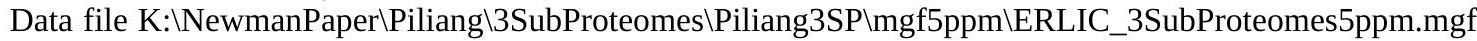

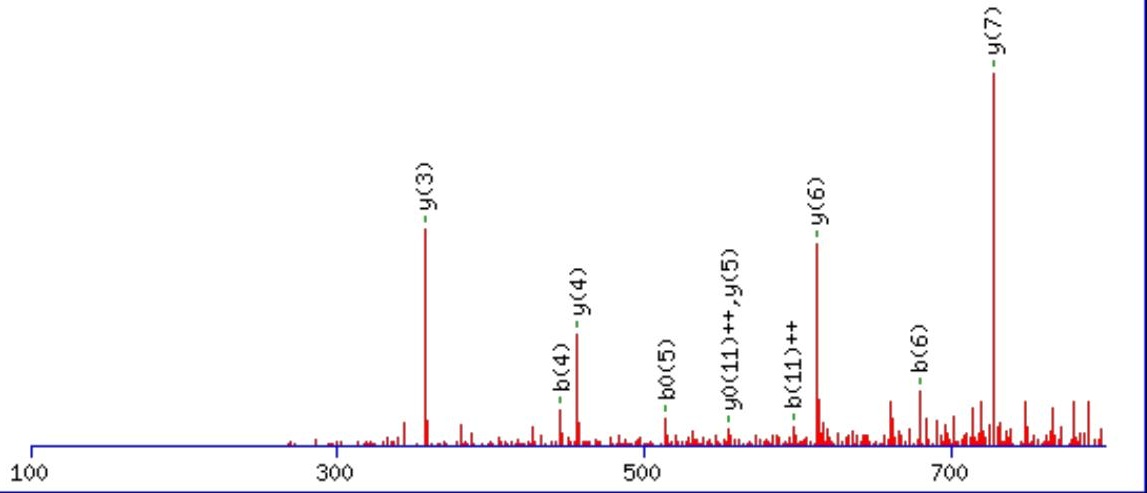




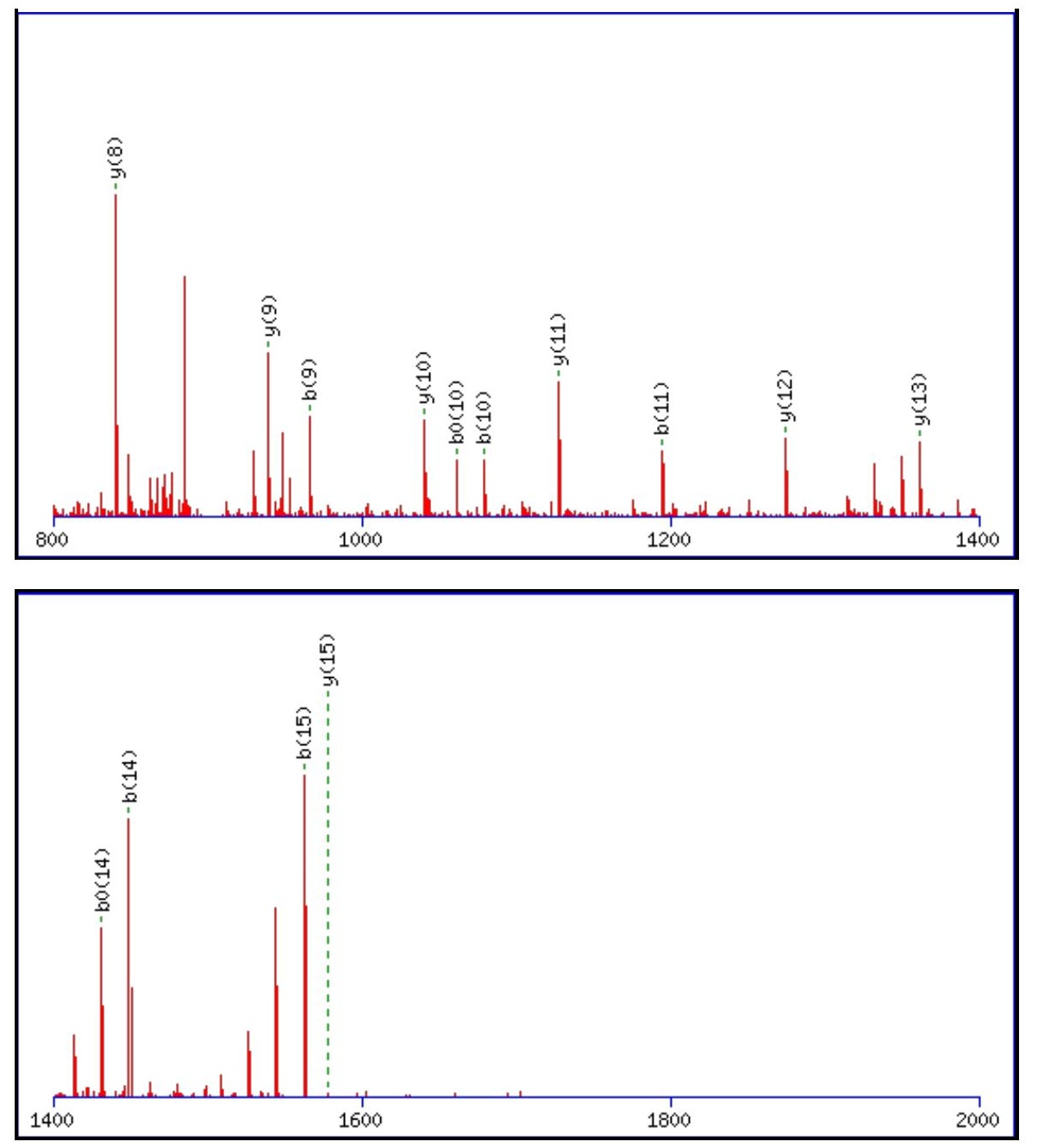

Monoisotopic mass of neutral peptide $\operatorname{Mr}($ calc): 1804.9128

Fixed modifications: Carbamidomethyl (C)

Variable modifications:

N3 : Deamidated_N (N)

M6 : Oxidation (M)

Ions Score: 95 Expect : $4.8 \mathrm{e}-008$

Matches (Bold Red): 24/174 fragment ions using 36 most intense peaks

\begin{tabular}{|c|c|c|c|c|c|c|c|c|c|c|c|c|c|c|}
\hline \# & b & $\mathbf{b}^{++}$ & $\mathbf{b}^{*}$ & $\mathbf{b}^{*^{++}}$ & $\mathbf{b}^{\mathbf{0}}$ & $\mathbf{b}^{\mathbf{0 + +}}$ & Seq. & $\mathbf{y}$ & $\mathbf{y}^{++}$ & $\mathbf{y}^{*}$ & $\mathrm{y}^{*^{++}}$ & $\mathbf{y}^{\mathbf{0}}$ & $\mathbf{y}^{\mathbf{0 + +}}$ & \# \\
\hline 1 & 114.0913 & 57.5493 & & & & & $\mathbf{L}$ & & & & & & & 17 \\
\hline 2 & 229.1183 & 115.0628 & & & 211.1077 & 106.0575 & D & 1692.8360 & 846.9217 & 1675.8095 & 838.4084 & 1674.8255 & 837.9164 & 16 \\
\hline 3 & 344.1452 & 172.5762 & 327.1187 & 164.0630 & 326.1347 & 163.5710 & $\mathbf{N}$ & 1577.8091 & 789.4082 & 1560.7826 & 780.8949 & 1559.7985 & 780.4029 & 15 \\
\hline 4 & 445.1929 & 223.1001 & 428.1663 & 214.5868 & 427.1823 & 214.0948 & $\mathbf{T}$ & 1462.7822 & 731.8947 & 1445.7556 & 723.3814 & 1444.7716 & 722.8894 & 14 \\
\hline 5 & 532.2249 & 266.6161 & 515.1984 & 258.1028 & 514.2144 & 257.6108 & S & 1361.7345 & 681.3709 & 1344.7079 & 672.8576 & 1343.7239 & 672.3656 & 13 \\
\hline 6 & 679.2603 & 340.1338 & 662.2338 & 331.6205 & 661.2498 & 331.1285 & $\mathbf{M}$ & 1274.7025 & 637.8549 & 1257.6759 & 629.3416 & 1256.6919 & 628.8496 & 12 \\
\hline 7 & 766.2924 & 383.6498 & 749.2658 & 375.1365 & 748.2818 & 374.6445 & S & 1127.6671 & 564.3372 & 1110.6405 & 555.8239 & 1109.6565 & 555.3319 & 11 \\
\hline 8 & 867.3400 & 434.1737 & 850.3135 & 425.6604 & 849.3295 & 425.1684 & $\mathbf{T}$ & 1040.6350 & 520.8212 & 1023.6085 & 512.3079 & 1022.6245 & 511.8159 & 10 \\
\hline 9 & 966.4085 & 483.7079 & 949.3819 & 475.1946 & 948.3979 & 474.7026 & $\mathbf{V}$ & 939.5873 & 470.2973 & 922.5608 & 461.7840 & 921.5768 & 461.2920 & 9 \\
\hline 10 & 1079.4925 & 540.2499 & 1062.4660 & 531.7366 & 1061.4819 & 531.2446 & I & 840.5189 & 420.7631 & 823.4924 & 412.2498 & 822.5084 & 411.7578 & 8 \\
\hline 11 & 1194.5195 & 597.7634 & 1177.4929 & 589.2501 & 1176.5089 & 588.7581 & D & 727.4349 & 364.2211 & 710.4083 & 355.7078 & 709.4243 & 355.2158 & 7 \\
\hline 12 & 1251.5409 & 626.2741 & 1234.5144 & 617.7608 & 1233.5304 & 617.2688 & G & 612.4079 & 306.7076 & 595.3814 & 298.1943 & & & 6 \\
\hline 13 & 1350.6093 & 675.8083 & 1333.5828 & 667.2950 & 1332.5988 & 666.8030 & $\mathbf{V}$ & 555.3865 & 278.1969 & 538.3599 & 269.6836 & & & 5 \\
\hline 14 & 1449.6778 & 725.3425 & 1432.6512 & 716.8292 & 1431.6672 & 716.3372 & $\mathbf{V}$ & 456.3180 & 228.6627 & 439.2915 & 220.1494 & & & 4 \\
\hline 15 & 1562.7618 & 781.8845 & 1545.7353 & 773.3713 & 1544.7512 & 772.8793 & $\mathbf{L}$ & 357.2496 & 179.1285 & 340.2231 & 170.6152 & & & 3 \\
\hline 16 & 1659.8146 & 830.4109 & 1642.7880 & 821.8977 & 1641.8040 & 821.4056 & $\mathbf{P}$ & 244.1656 & 122.5864 & 227.1390 & 114.0731 & & & 2 \\
\hline 17 & & & & & & & $\mathbf{K}$ & 147.1128 & 74.0600 & 130.0863 & 65.5468 & & & 1 \\
\hline
\end{tabular}



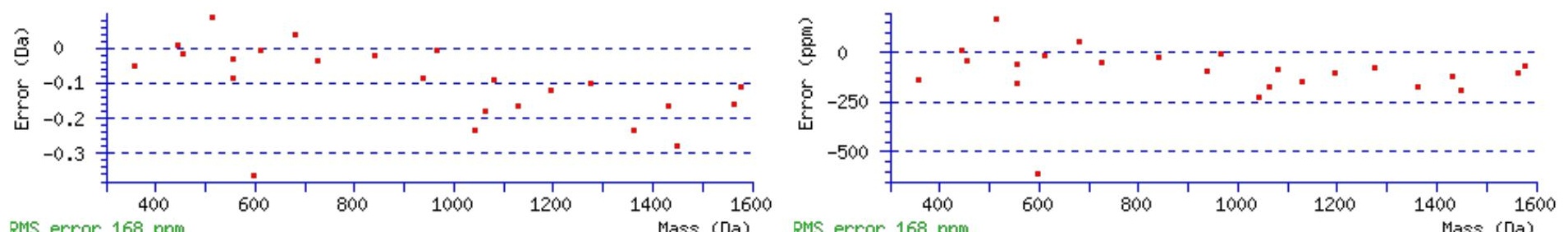

RIS error 168 ppm

\section{All matches to this query}

\begin{tabular}{|l|l|l|l|}
\hline Score & Mr(calc): & Delta & \multicolumn{1}{c|}{ Sequence } \\
\hline 95.3 & 1804.9128 & -0.0009 & LDNTSMSTVIDGVVLPK \\
\hline 8.1 & 1804.9100 & 0.0020 & LKKLSLSSLETASLR \\
\hline 8.1 & 1804.9100 & 0.0020 & LKKLSLSSLETASLR \\
\hline 4.5 & 1803.9132 & 0.9988 & DVLKGVDASNALVLPGR \\
\hline 2.8 & 1803.9189 & 0.9931 & LECLHVPANGTTGPPIK \\
\hline 2.2 & 1803.8992 & 1.0128 & RTRSPSPTLGESLAPR \\
\hline 0.4 & 1803.8920 & 1.0200 & $\underline{\text { IYKEISVTGPPHDR }}$ \\
\hline 0.2 & 1804.9100 & 0.0020 & LKKLSLSSLETASLR \\
\hline 0.2 & 1804.9100 & 0.0020 & LKKLSLSSLETASLR \\
\hline
\end{tabular}

Spectrum No: 105; Query: 1311; Rank: 1

\section{Peptide View}

MS/MS Fragmentation of LENLSSTESGYTATLTR

Found in IPI00400579, Tax_Id=10116 Gene_Symbol=Gaa Lysosomal alpha-glucosidase precursor

Match to Query 1311: 1842.890108 from(922.452330,2+)

Title: 100101RatKid_NS_deglyco_14.2819.2819.2.dta

Data file K:INewmanPaper|Piliangl3SubProteomes\Piliang3SP\mgf5ppm\ERLIC_3SubProteomes5ppm.mgf

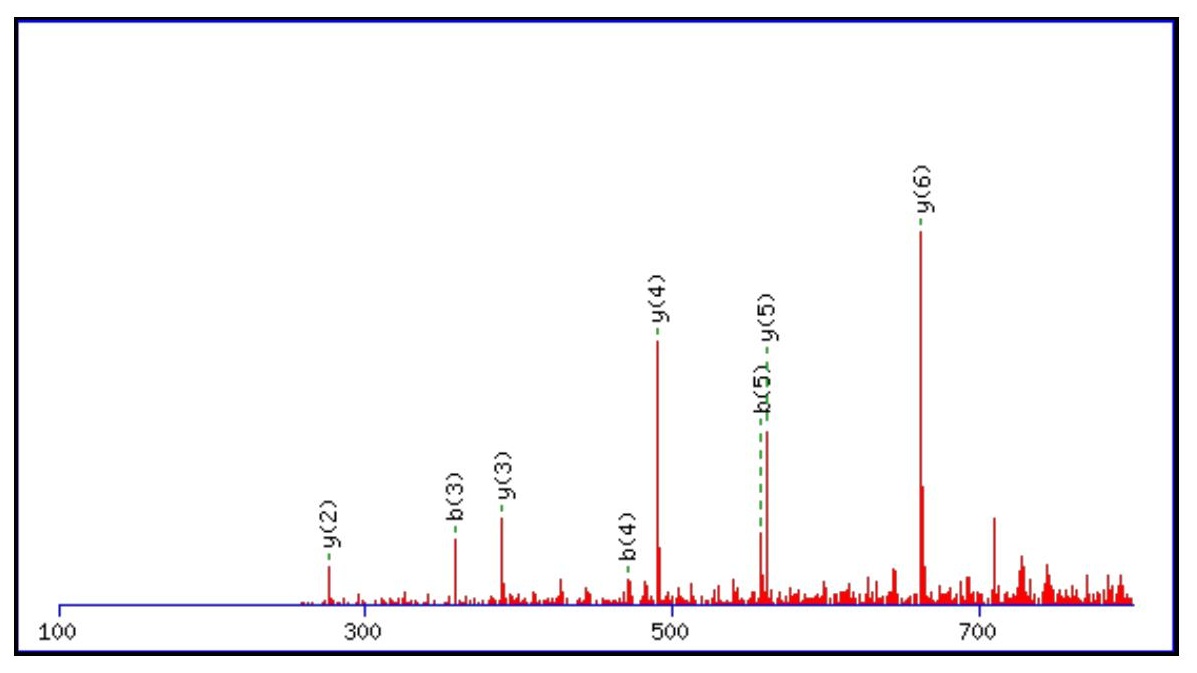




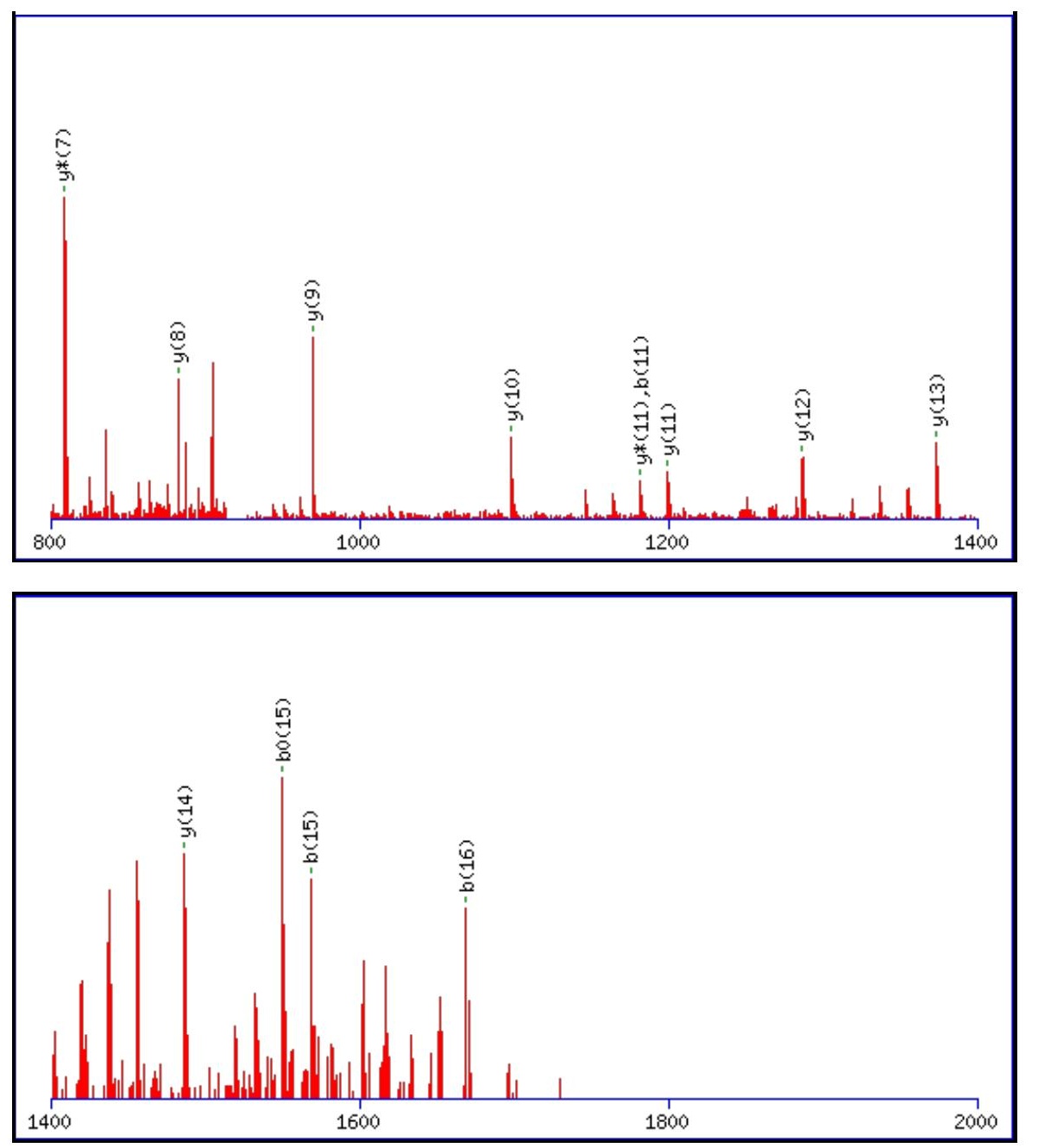

Monoisotopic mass of neutral peptide $\operatorname{Mr}($ calc): 1842.8847

Fixed modifications: Carbamidomethyl (C)

Variable modifications:

N3 : Deamidated N (N)

Ions Score: 95 Expect: $6.7 \mathrm{e}-008$

Matches (Bold Red): 21/184 fragment ions using 31 most intense peaks

\begin{tabular}{|c|c|c|c|c|c|c|c|c|c|c|c|c|c|c|}
\hline \# & b & $\mathbf{b}^{++}$ & $\mathbf{b}^{*}$ & & $\mathbf{b}^{0}$ & & Seq. & $\mathbf{y}$ & & $\mathbf{y}^{*}$ & & $\mathbf{y}^{0}$ & & \# \\
\hline 1 & 114.05 & 57.5493 & & & & & $\mathbf{L}$ & & & & & & & 17 \\
\hline 2 & 243.1339 & 122.0706 & & & & & $\mathbf{E}$ & & & & & 1712.7973 & 856.9023 & 16 \\
\hline 3 & 358.1609 & 179.5841 & & & & & $\mathbf{N}$ & & & & & 1583.7548 & & 15 \\
\hline 4 & 471.2449 & 236.1261 & 454.2184 & 227.6128 & 453.2344 & 227.1208 & $\mathbf{L}$ & 1486.7384 & 743.8728 & 1469.7118 & 735.3596 & 1468.7278 & 734.8675 & 14 \\
\hline 5 & 558.2770 & 279.6421 & 541.2504 & 271.1288 & 540.2664 & 270.6368 & S & 1373.6543 & 687.3308 & 1356.6278 & 678.8175 & 1355.6438 & 678.3255 & 13 \\
\hline 6 & 645.3090 & 323.1581 & 628.2824 & 314.6449 & 627.2984 & 314.1529 & S & 1286.6223 & 643.8148 & 1269.5957 & 635.3015 & 1268.6117 & 634.8095 & 12 \\
\hline 7 & 746.3567 & 373.6820 & 729.3301 & 365.1687 & 728.3461 & 364.6767 & $\mathbf{T}$ & 1199.5903 & 600.2988 & 1182.5637 & 591.7855 & 1181.5797 & 591.2935 & 11 \\
\hline 8 & 875.3993 & 438.2033 & 858.3727 & 429.6900 & 857.3887 & 429.1980 & $\mathbf{E}$ & 1098.5426 & 549.7749 & 1081.5160 & 541.2617 & 1080.5320 & 540.7696 & 10 \\
\hline 9 & 962.4313 & 481.7193 & 945.4047 & 473.2060 & 944.4207 & 472.7140 & S & 969.5000 & 485.2536 & 952.4734 & 476.7404 & 951.4894 & 476.2483 & 9 \\
\hline 10 & 019.4528 & 510.2300 & 1002.4262 & 501.7167 & 1001.4422 & 501.2247 & G & 882.4680 & 441.7376 & 865.4414 & 433.2243 & 864.4574 & 432.7323 & 8 \\
\hline 11 & 182.5161 & 591.7617 & 165.4895 & 583.2484 & 1164.5055 & 582.7564 & $\mathbf{Y}$ & 825.4465 & 413.2269 & 808.4199 & 404.7136 & 807.4359 & 404.2216 & 7 \\
\hline 12 & 1283.5638 & 642.2855 & 1266.5372 & 633.7722 & 1265.5532 & 633.2802 & $\mathbf{T}$ & 662.3832 & 331.6952 & 645.3566 & 323.1819 & 644.3726 & 322.6899 & 6 \\
\hline 13 & 1354.6009 & 677.8041 & 1337.5743 & 669.2908 & 1336.5903 & 668.7988 & A & 561.3355 & 281.1714 & 544.3089 & 272.6581 & 543.3249 & 272.1661 & 5 \\
\hline 14 & 1455.6486 & 728.3279 & 1438.6220 & 719.8146 & 1437.6380 & 719.3226 & $\mathbf{T}$ & 490.2984 & 245.6528 & 473.2718 & 237.1396 & 472.2878 & 236.6475 & 4 \\
\hline 15 & 1568.7326 & 784.8699 & 1551.7061 & 776.3567 & 1550.7221 & 775.8647 & $\mathbf{L}$ & 389.2507 & 195.1290 & 372.2241 & 186.6157 & 371.2401 & 186.1237 & 3 \\
\hline 16 & 1669.7803 & 835.3938 & 1652.7537 & 826.8805 & 1651.7697 & 826.3885 & $\mathbf{T}$ & 276.1666 & 138.5870 & 259.1401 & 130.0737 & 258.1561 & 129.5817 & 2 \\
\hline 17 & & & & & & & $\mathbf{R}$ & 175.1190 & 88.0631 & 158.0924 & 79.5498 & & & 1 \\
\hline
\end{tabular}



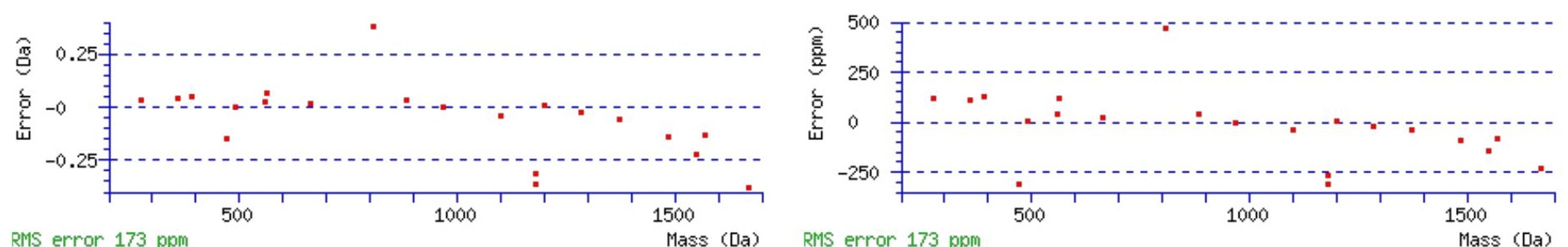

\section{All matches to this query}

\begin{tabular}{|l|l|l|l|}
\hline Score & Mr(calc): & Delta & \multicolumn{1}{|c|}{ Sequence } \\
\hline 94.7 & 1842.8847 & 0.0054 & LENLSSTESGYTATLTR \\
\hline 77.3 & 1841.9007 & 0.9894 & LENLSSTESGYTATLTR \\
\hline 8.7 & 1842.8950 & -0.0049 & MKDYQLNALLPALTR \\
\hline 5.2 & 1842.8877 & 0.0024 & FKSDQNLQTALELTR \\
\hline 3.3 & 1842.8911 & -0.0010 & QTKVTEISLVVSGCSR \\
\hline 2.9 & 1842.8794 & 0.0107 & VKNFLHSIVSGLDVR \\
\hline 2.8 & 1842.8876 & 0.0025 & LVEERTQAYLEEKR \\
\hline 2.6 & 1842.8887 & 0.0014 & LNEDYTKEYVQALEK \\
\hline 2.3 & 1842.8798 & 0.0103 & DIESKMLSAILTGVNR \\
\hline 1.5 & 1842.8959 & -0.0058 & $\underline{\text { LDEELQIQAQEDREK }}$ \\
\hline
\end{tabular}

Spectrum No: 106; Query: 2827; Rank: 1

\section{Peptide View}

MS/MS Fragmentation of ALAGLVYNSSGMEPCFDIYQMYQSCADPTGCGTGSNAR

Found in IPI00230946, Tax_Id=10116 Gene_Symbol=Dpp7 Dipeptidyl-peptidase 2 precursor

Match to Query 2827: 4149.743652 from(1384.255160,3+)

Title: 091008RatKidney_NoSalt_20.5990.5990.3.dta

Data file K:INewmanPaper|Piliang|3SubProteomes\Piliang3SP\mgf5ppm\ERLIC_3SubProteomes5ppm.mgf

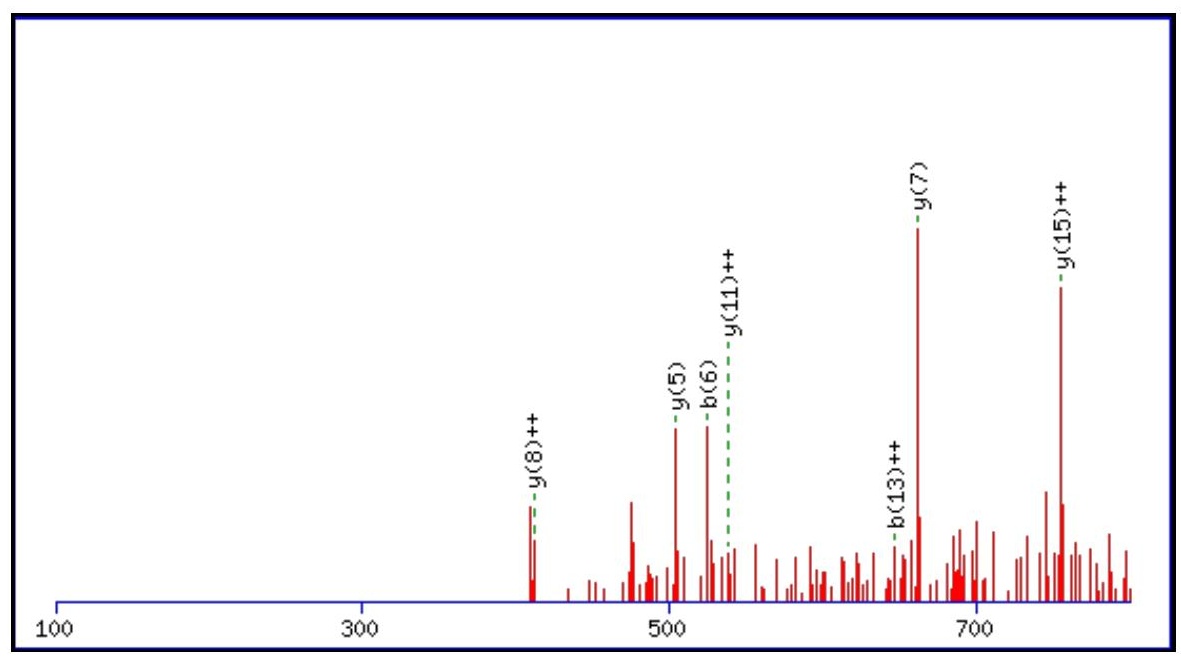



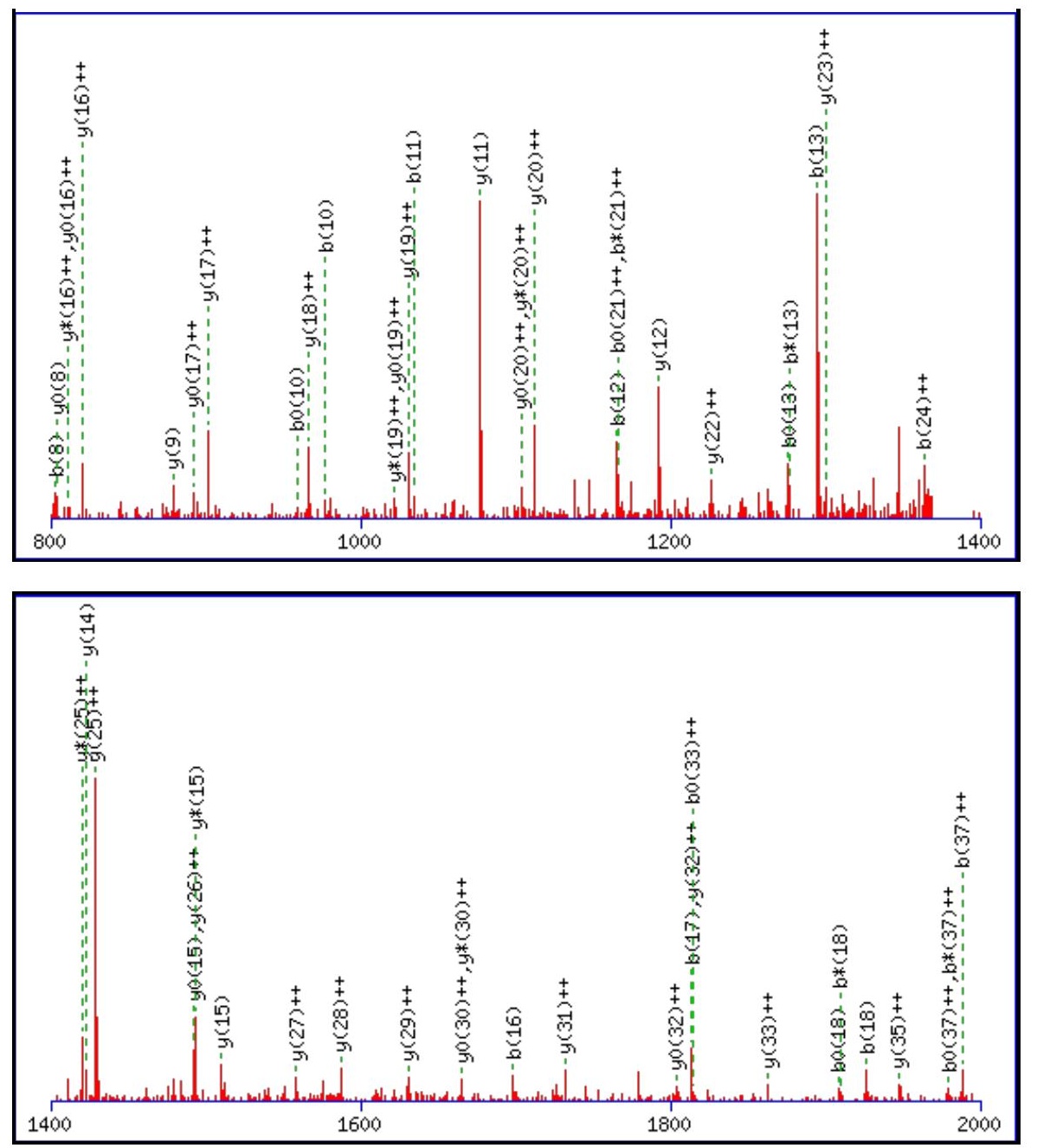

Monoisotopic mass of neutral peptide $\operatorname{Mr}($ calc): 4148.7319

Fixed modifications: Carbamidomethyl (C)

Variable modifications:

N8: Deamidated $\mathrm{N}(\mathrm{N})$

Ions Score: 94 Expect: $1.7 \mathrm{e}-007$

Matches (Bold Red): 62/408 fragment ions using 100 most intense peaks

\begin{tabular}{|c|c|c|c|c|c|c|c|c|c|c|c|c|c|c|}
\hline \# & b & $\mathbf{b}^{++}$ & $\mathbf{b}^{*}$ & $\mathbf{b}^{*^{++}}$ & $\mathbf{b}^{0}$ & $\mathbf{b}^{\mathbf{0 + +}}$ & Seq. & $\mathbf{y}$ & $\mathbf{y}^{++}$ & $\mathbf{y}^{*}$ & $\mathrm{y}^{*^{++}}$ & $\mathbf{y}^{\mathbf{0}}$ & $\mathbf{y}^{\mathbf{0 + +}}$ & \# \\
\hline 1 & 72.0444 & 36.5258 & & & & & A & & & & & & & 38 \\
\hline 2 & 185.1285 & 93.0679 & & & & & L & 4078.7020 & 2039.8547 & 4061.6755 & 2031.3414 & 4060.6915 & 2030.8494 & 37 \\
\hline 3 & 256.1656 & 128.5864 & & & & & A & 3965.6180 & 1983.3126 & 3948.5914 & 1974.7993 & 3947.6074 & 1974.3073 & 36 \\
\hline 4 & 313.1870 & 157.0972 & & & & & G & 3894.5809 & 1947.7941 & 3877.5543 & 1939.2808 & 3876.5703 & 1938.7888 & 35 \\
\hline 5 & 426.2711 & 213.6392 & & & & & $\mathbf{L}$ & 3837.5594 & 1919.2833 & 3820.5328 & 1910.7701 & 3819.5488 & 1910.2781 & 34 \\
\hline 6 & 525.3395 & 263.1734 & & & & & $\mathbf{V}$ & 3724.4753 & 1862.7413 & 3707.4488 & 1854.2280 & 3706.4648 & 1853.7360 & 33 \\
\hline 7 & 688.4028 & 344.7051 & & & & & $\mathbf{Y}$ & 3625.4069 & 1813.2071 & 3608.3804 & 1804.6938 & 3607.3963 & 1804.2018 & 32 \\
\hline 8 & 803.4298 & 402.2185 & 786.4032 & 393.7053 & & & $\mathbf{N}$ & 3462.3436 & 1731.6754 & 3445.3170 & 1723.1622 & 3444.3330 & 1722.6701 & 31 \\
\hline 9 & 890.4618 & 445.7345 & 873.4353 & 437.2213 & 872.4512 & 436.7293 & S & 3347.3166 & 1674.1620 & 3330.2901 & 1665.6487 & 3329.3061 & 1665.1567 & 30 \\
\hline 10 & 977.4938 & 489.2506 & 960.4673 & 480.7373 & 959.4833 & 480.2453 & S & 3260.2846 & 1630.6459 & 3243.2581 & 1622.1327 & 3242.2741 & 1621.6407 & 29 \\
\hline 11 & 1034.5153 & 517.7613 & 1017.4887 & 509.2480 & 1016.5047 & 508.7560 & $\mathbf{G}$ & 3173.2526 & 1587.1299 & 3156.2260 & 1578.6167 & 3155.2420 & 1578.1247 & 28 \\
\hline 12 & 1165.5558 & 583.2815 & 1148.5292 & 574.7683 & 1147.5452 & 574.2762 & $\mathbf{M}$ & 3116.2311 & 1558.6192 & 3099.2046 & 1550.1059 & 3098.2206 & 1549.6139 & 27 \\
\hline 13 & 1294.5984 & 647.8028 & 1277.5718 & 639.2896 & \begin{tabular}{|l|}
1276.5878 \\
\end{tabular} & 638.7975 & $\mathbf{E}$ & 2985.1906 & 1493.0990 & 2968.1641 & 1484.5857 & 2967.1801 & 1484.0937 & 26 \\
\hline 14 & 1391.6511 & 696.3292 & 1374.6246 & 687.8159 & 1373.6406 & 687.3239 & $\mathbf{P}$ & 2856.1481 & 1428.5777 & 2839.1215 & 1420.0644 & 2838.1375 & 1419.5724 & 425 \\
\hline 15 & 1551.6818 & 776.3445 & 1534.6552 & 767.8313 & 1533.6712 & 767.3392 & $\mathrm{C}$ & 2759.0953 & 1380.0513 & 2742.0687 & 1371.5380 & 2741.0847 & 1371.0460 & 24 \\
\hline 16 & \begin{tabular}{|l|}
1698.7502 \\
\end{tabular} & 849.8787 & 1681.7237 & 841.3655 & 1680.7396 & 840.8735 & $\mathbf{F}$ & 2599.0646 & 1300.0360 & 2582.0381 & 1291.5227 & 2581.0541 & 1291.0307 & 723 \\
\hline 17 & 1813.7771 & 907.3922 & 1796.7506 & 898.8789 & 1795.7666 & 898.3869 & D & 2451.9962 & 1226.5017 & 2434.9697 & 1217.9885 & 2433.9857 & 1217.4965 & 52 \\
\hline 18 & 1926.8612 & 963.9342 & 1909.8347 & 955.4210 & 1908.8506 & 954.9290 & I & 2336.9693 & 1168.9883 & 2319.9427 & 1160.4750 & 2318.9587 & 1159.9830 & 21 \\
\hline
\end{tabular}




\begin{tabular}{|c|c|c|c|c|c|c|c|c|c|c|c|c|c|c|}
\hline 19 & 2089.9245 & & & & & & 1 & & & & & & & 20 \\
\hline 20 & 2217.9831 & 1109.4952 & 2200.9566 & 1100.9819 & 2199.9725 & 1100.4899 & $\mathbf{Q}$ & 2060.8219 & 1030.9146 & 2043.7953 & 1022.4013 & 2042.8113 & 1021.9093 & 19 \\
\hline 21 & 2349.0236 & 1175.0154 & 2331.9970 & 1166.5022 & 2331.0130 & 1166.0102 & M & 1932.7633 & 966.8853 & 1915.7368 & 958.3720 & 1914.7527 & 957.8800 & 18 \\
\hline 22 & 2512.0869 & 1256.5471 & 2495.0604 & 1248.0338 & 2494.0764 & 1247.5418 & $\mathbf{Y}$ & 1801.7228 & 901.3651 & 1784.6963 & 892.8518 & 1783.7123 & 892.3598 & 17 \\
\hline 23 & 2640.1455 & 1320.5764 & 2623.1190 & 1312.0631 & 2622.1349 & 1311.5711 & $\mathbf{Q}$ & 1638.6595 & 819.8334 & 1621.6329 & 811.3201 & 1620.6489 & 810.8281 & 16 \\
\hline 24 & 2727.1775 & 1364.0924 & 2710.1510 & 1355.5791 & 2709.1670 & 1355.0871 & S & 1510.6009 & 755.8041 & 1493.5744 & 747.2908 & 1492.5904 & 746.7988 & 15 \\
\hline 25 & 2887.2082 & 1444.1077 & 2870.1816 & 1435.5945 & 2869.1976 & 1435.1024 & $\mathrm{C}$ & 1423.5689 & 712.2881 & 1406.5423 & 703.7748 & 1405.5583 & 703.2828 & 14 \\
\hline 26 & 2958.2453 & 1479.6263 & 2941.2187 & 1471.1130 & 2940.2347 & 1470.6210 & A & 1263.5382 & 632.2728 & 1246.5117 & 623.7595 & 1245.5277 & 623.2675 & 13 \\
\hline 27 & 3073.2722 & 1537 & 3056.2457 & 1528.6265 & 2617 & 1528.1345 & D & 1192.5011 & 596.7542 & 1175.4746 & 588.2409 & 1174.4906 & 587.7489 & 12 \\
\hline 28 & 3170.3250 & 1585.6661 & 3153.2985 & 1577.1529 & 3152.3144 & 1576.6609 & $\mathbf{P}$ & 1077.4742 & 539.2407 & 1060.4476 & 530.7275 & 1059.4636 & 530.2354 & 11 \\
\hline 29 & 3271.3727 & 1636.1900 & 3254.3461 & 1627.6767 & 3253.3621 & 1627.1847 & $T$ & 980.4214 & 490.7143 & 963.3949 & 482.2011 & 962.4109 & 481.7091 & 10 \\
\hline 30 & 3328.3941 & 1664.7007 & 3311.3676 & 1656.1874 & 3310.3836 & 1655.6954 & G & 879.3737 & 440.1905 & 862.3472 & 431.6772 & 861.3632 & 431.1852 & 9 \\
\hline 31 & 3488.4248 & 1744.7160 & 3471.3982 & 1736.2028 & 3470.4142 & 1735.7108 & C & 822.3523 & 411.6798 & 805.3257 & 403.1665 & 804.3417 & 402.6745 & 8 \\
\hline 32 & 3545.4463 & 1773.2268 & 3528.4197 & 1764.7135 & 3527.4357 & 1764.2215 & G & 662.3216 & 331.6645 & 645.2951 & 323.1512 & 644.3111 & 322.6592 & 7 \\
\hline 33 & 3646.4939 & 1823.7506 & 3629.4674 & 1815.2373 & 3628.4834 & \begin{tabular}{|l|}
1814.7453 \\
\end{tabular} & $\mathbf{T}$ & 605.3002 & 303.1537 & 588.2736 & 294.6404 & 587.2896 & 294.1484 & 6 \\
\hline 34 & 3703.5154 & 1852.2613 & 3686.4889 & 1843.7481 & 3685.5048 & 1843.2561 & G & 504.2525 & 252.6299 & 487.2259 & 244.1166 & 486.2419 & 243.6246 & 5 \\
\hline 35 & 3790.5474 & \begin{tabular}{|l}
1895.7774 \\
\end{tabular} & 3773.5209 & 1887.2641 & 3772.5369 & 1886.7721 & $\mathrm{~S}$ & 447.2310 & 224.1191 & 430.2045 & 215.6059 & 429.2205 & 215.1139 & 4 \\
\hline 36 & 3904.5904 & 1952.7988 & 3887.5638 & 1944.2855 & 3886.5798 & 1943.7935 & $\mathbf{N}$ & 360.1990 & 180.6031 & 343.1724 & 172.0899 & & & 3 \\
\hline 37 & 3975.6275 & 1988.3174 & 3958.6009 & 1979.8041 & 3957.6169 & 1979.3121 & A & 246.1561 & 123.5817 & 229.1295 & 115.0684 & & & 2 \\
\hline 38 & & & & & & & $\mathbf{R}$ & 175.1190 & 88.0631 & 158.0924 & 79.5498 & & & 1 \\
\hline
\end{tabular}
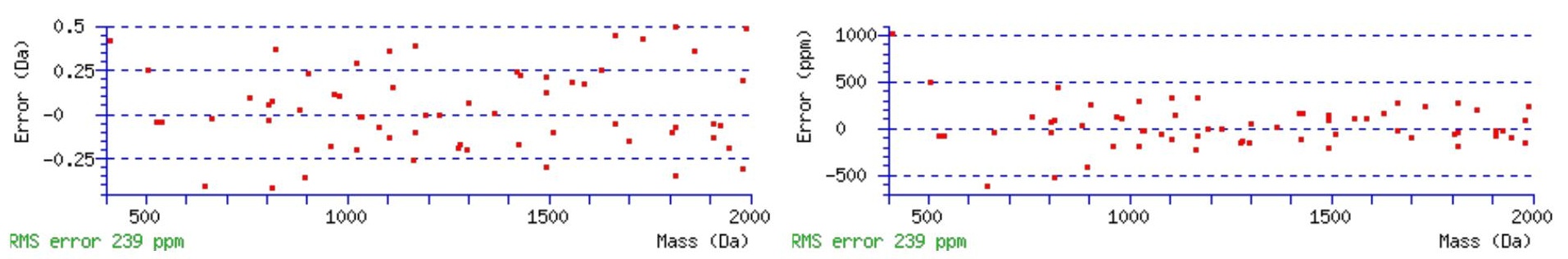

\section{All matches to this query}

\begin{tabular}{|l|l|l|l|}
\hline Score & Mr(calc): & Delta & \multicolumn{1}{c|}{ Sequence } \\
\hline 94.4 & 4148.7319 & 1.0118 & ALAGLVYNSSGMEPCFDIYQMYQSCADPTGCGTGSNAR \\
\hline 80.1 & 4149.7159 & 0.0278 & ALAGLVYNSSGMEPCFDIYQMYQSCADPTGCGTGSNAR \\
\hline 63.9 & 4148.7319 & 1.0118 & ALAGLVYNSSGMEPCFDIYQMYQSCADPTGCGTGSNAR \\
\hline 54.1 & 4147.7479 & 1.9958 & ALAGLVYNSSGMEPCFDIYQMYQSCADPTGCGTGSNAR \\
\hline 7.1 & 4147.7044 & 2.0393 & TKSESMGSLLCDEGSKESDPMTWTSPYIPEVCGNSR \\
\hline 7.1 & 4147.7044 & 2.0393 & TKSESMGSLLCDEGSKESDPMTWTSPYIPEVCGNSR \\
\hline 7.1 & 4147.7044 & 2.0393 & TKSESMGSLLCDEGSKESDPMTWTSPYIPEVCGNSR \\
\hline 5.9 & 4149.7494 & -0.0057 & ELORYLFELNPNAEDEQEKTSNLQTQQNVVGTSS \\
\hline 5.5 & 4149.7747 & -0.0311 & YTNGESFQPNCRYNCTCIDGTVGCTPLCLSPRPPR \\
\hline 5.3 & 4148.7768 & 0.9668 & FPYLSESYFITTDAAGSIATATPDGGIVLISGTGSNCR \\
\hline
\end{tabular}

Spectrum No: 107; Query: 1218; Rank: 1

\section{Peptide View}

MS/MS Fragmentation of ATEPCLDGWIYNSTR

Found in IPI00207702, Tax_Id=10116 Gene_Symbol=Slc22a8 Solute carrier family 22 member 8

Match to Query 1218: 1782.787828 from(892.401190,2+)

Title: 091008RatKidney_NoSalt_26.3448.3448.2.dta 

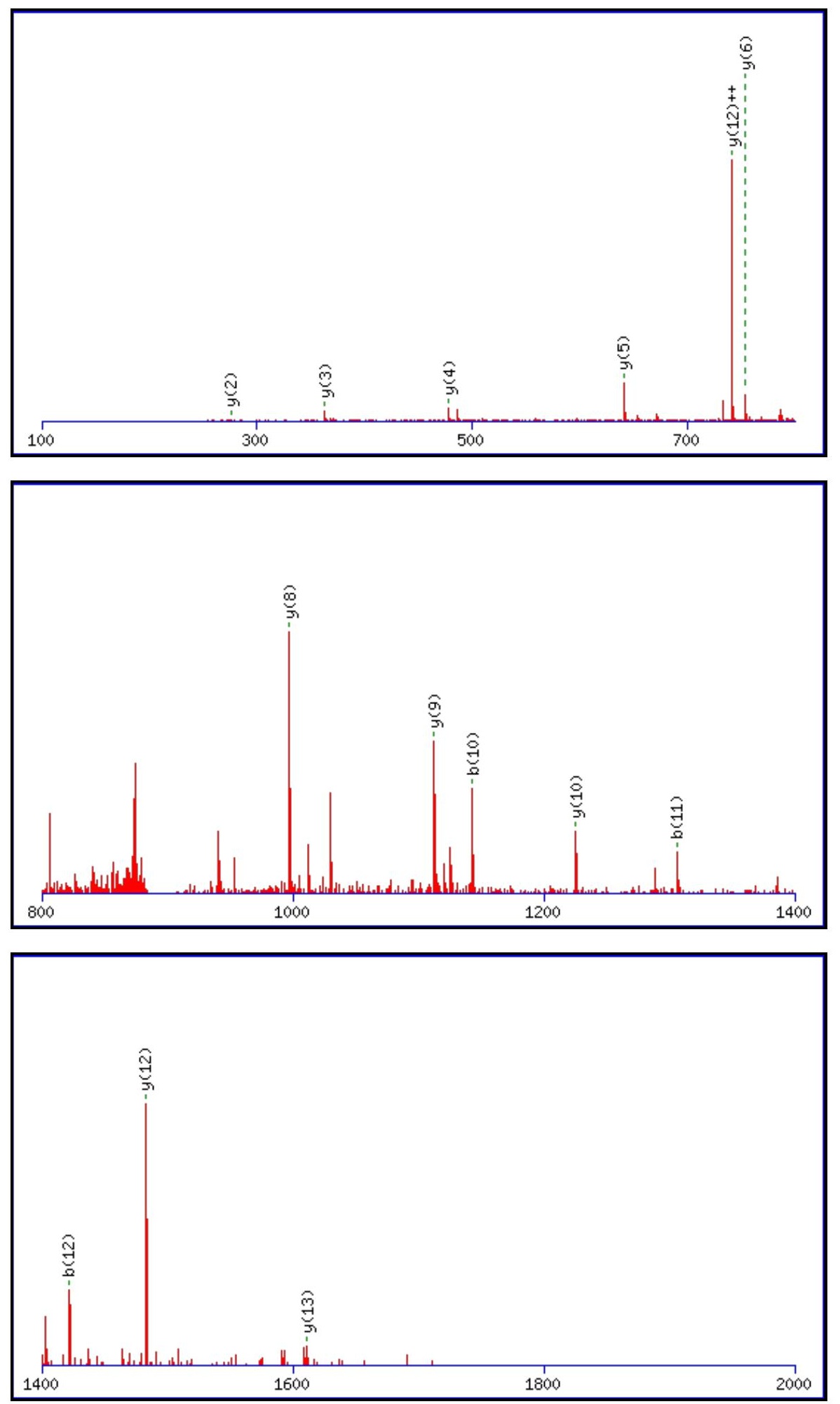

Monoisotopic mass of neutral peptide $\operatorname{Mr}($ calc): 1782.7883

Fixed modifications: Carbamidomethyl (C)

Variable modifications:

N12 : Deamidated_N (N)

Ions Score: 94 Expect: $7.5 \mathrm{e}-008$

Matches (Bold Red): 14/142 fragment ions using 18 most intense peaks

\begin{tabular}{|r|c|c|c|c|c|c|c|c|c|c|c|c|c|c|}
\hline$\#$ & $\mathbf{b}$ & $\mathbf{b}^{++}$ & $\mathbf{b}^{*}$ & $\mathbf{b}^{\boldsymbol{*}^{++}}$ & $\mathbf{b}^{\mathbf{0}}$ & $\mathbf{b}^{\mathbf{0}+}$ & Seq. & $\mathbf{y}$ & $\mathbf{y}^{++}$ & $\mathbf{y}^{\mathbf{*}}$ & $\mathbf{y}^{\boldsymbol{*}^{++}}$ & $\mathbf{y}^{\mathbf{0}}$ & $\mathbf{y}^{\mathbf{0 + +}}$ & $\#$ \\
\hline $\mathbf{1}$ & 72.0444 & 36.5258 & & & & & $\mathbf{A}$ & & & & & & & $\mathbf{1 5}$ \\
\hline $\mathbf{2}$ & 173.0921 & 87.0497 & & & 155.0815 & 78.0444 & $\mathbf{T}$ & 1712.7585 & 856.8829 & 1695.7319 & 848.3696 & 1694.7479 & $\mathbf{8 4 7 . 8 7 7 6}$ & $\mathbf{1 4}$ \\
\hline $\mathbf{3}$ & 302.1347 & 151.5710 & & & 284.1241 & 142.5657 & $\mathbf{E}$ & $\mathbf{1 6 1 1 . 7 1 0 8}$ & 806.3590 & 1594.6842 & 797.8458 & 1593.7002 & 797.3537 & $\mathbf{1 3}$ \\
\hline $\mathbf{4}$ & 399.1874 & 200.0974 & & & 381.1769 & 191.0921 & $\mathbf{P}$ & $\mathbf{1 4 8 2 . 6 6 8 2}$ & $\mathbf{7 4 1 . 8 3 7 7}$ & 1465.6416 & 733.3245 & 1464.6576 & 732.8324 & $\mathbf{1 2}$
\end{tabular}




\begin{tabular}{|c|c|c|c|c|c|c|c|c|c|c|c|c|c|c|}
\hline 5 & 559.2181 & 280.1127 & & & 541.2075 & 271.1074 & C & 1385.6154 & 693.3113 & 1368.5889 & 684.7981 & 1367.6049 & 684.3061 & 11 \\
\hline 6 & 672.3021 & 336.6547 & & & 654.2916 & 327.6494 & $\mathbf{L}$ & 1225.5848 & 613.2960 & 1208.5582 & 604.7827 & 1207.5742 & 604.2907 & 10 \\
\hline 7 & 787.3291 & 394.1682 & & & 769.3185 & 385.1629 & D & 1112.5007 & 556.7540 & 1095.4742 & 548.2407 & 1094.4901 & 547.7487 & 9 \\
\hline 8 & 844.3505 & 422.6789 & & & 826.3400 & 413.6736 & G & 997.4738 & 499.2405 & 980.4472 & 490.7272 & 979.4632 & 490.2352 & 8 \\
\hline 9 & 1030.4299 & 515.7186 & & & 1012.4193 & 506.7133 & $\mathbf{W}$ & 940.4523 & 470.7298 & 923.4258 & 462.2165 & 922.4417 & 461.7245 & 7 \\
\hline 10 & 1143.5139 & 572.2606 & & & 1125.5034 & 563.2553 & I & 754.3730 & 377.6901 & 737.3464 & 369.1769 & 736.3624 & 368.6848 & 6 \\
\hline 11 & 1306.5773 & 653.7923 & & & 1288.5667 & 644.7870 & $\mathbf{Y}$ & 641.2889 & 321.1481 & 624.2624 & 312.6348 & 623.2784 & 312.1428 & 5 \\
\hline 12 & 1421.6042 & 711.3057 & 1404.5776 & 702.7925 & 1403.5936 & 702.3004 & $\mathbf{N}$ & 478.2256 & 239.6164 & 461.1990 & 231.1032 & 460.2150 & 230.6112 & 4 \\
\hline 13 & 1508.6362 & 754.8217 & 1491.6097 & 746.3085 & 1490.6257 & 745.8165 & S & 363.1987 & 182.1030 & 346.1721 & 173.5897 & 345.1881 & 173.0977 & 3 \\
\hline 14 & 1609.6839 & 805.3456 & 1592.6573 & 796.8323 & 1591.6733 & 796.3403 & $\mathbf{T}$ & 276.1666 & 138.5870 & 259.1401 & 130.0737 & 258.1561 & 129.5817 & 2 \\
\hline 15 & & & & & & & $\mathbf{R}$ & 175.1190 & 88.0631 & 158.0924 & 79.5498 & & & 1 \\
\hline
\end{tabular}
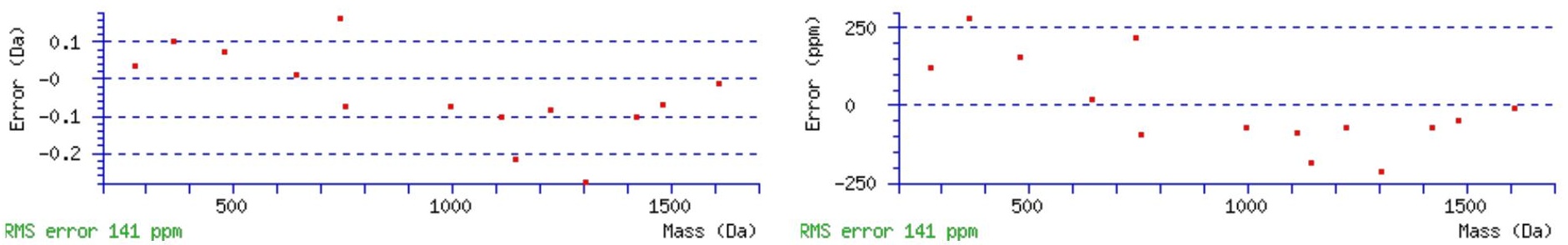

\section{All matches to this query}

\begin{tabular}{|l|l|l|l|}
\hline Score & Mr(calc): & Delta & \multicolumn{1}{c|}{ Sequence } \\
\hline 94.3 & 1782.7883 & -0.0005 & ATEPCLDGWIYNSTR \\
\hline 7.2 & 1781.7695 & 1.0183 & GQLCTYFLNTDLTR \\
\hline 7.2 & 1780.7637 & 2.0241 & MSGPNGDLGMPVDAGRK \\
\hline 4.3 & 1781.7729 & 1.0149 & DVMNGEQTGLLMLHK \\
\hline 4.0 & 1782.8036 & -0.0158 & EKGPNNTANSSEVKVK \\
\hline 4.0 & 1782.8036 & -0.0158 & EKGPNNTANSSEVKVK \\
\hline 3.7 & 1782.8050 & -0.0172 & SNTRYYTGTQSRIR \\
\hline 2.2 & 1780.7920 & 1.9958 & VEAEFHLVTAEEAEK \\
\hline 1.5 & 1782.8050 & -0.0172 & SNTRYYTGTQSRIR \\
\hline 1.3 & 1782.8036 & -0.0158 & EKGPNNTANSSEVKVK \\
\hline
\end{tabular}

Spectrum No: 108; Query: 1835; Rank: 1

\section{Peptide View}

MS/MS Fragmentation of YPVPFQNENVTAAFVELGR

Found in IPI00213569, Tax_Id=10116 Gene_Symbol=Ugt1a1 UDP-glucuronosyltransferase 1-1 precursor

Match to Query 1835: 2151.070828 from(1076.542690,2+)

Title: 100101RatKid_NS_deglyco_12.5821.5821.2.dta

Data file K:\NewmanPaper\Piliang \3SubProteomes \Piliang3SP $\backslash$ mgf5ppm\ERLIC_3SubProteomes5ppm.mgf 

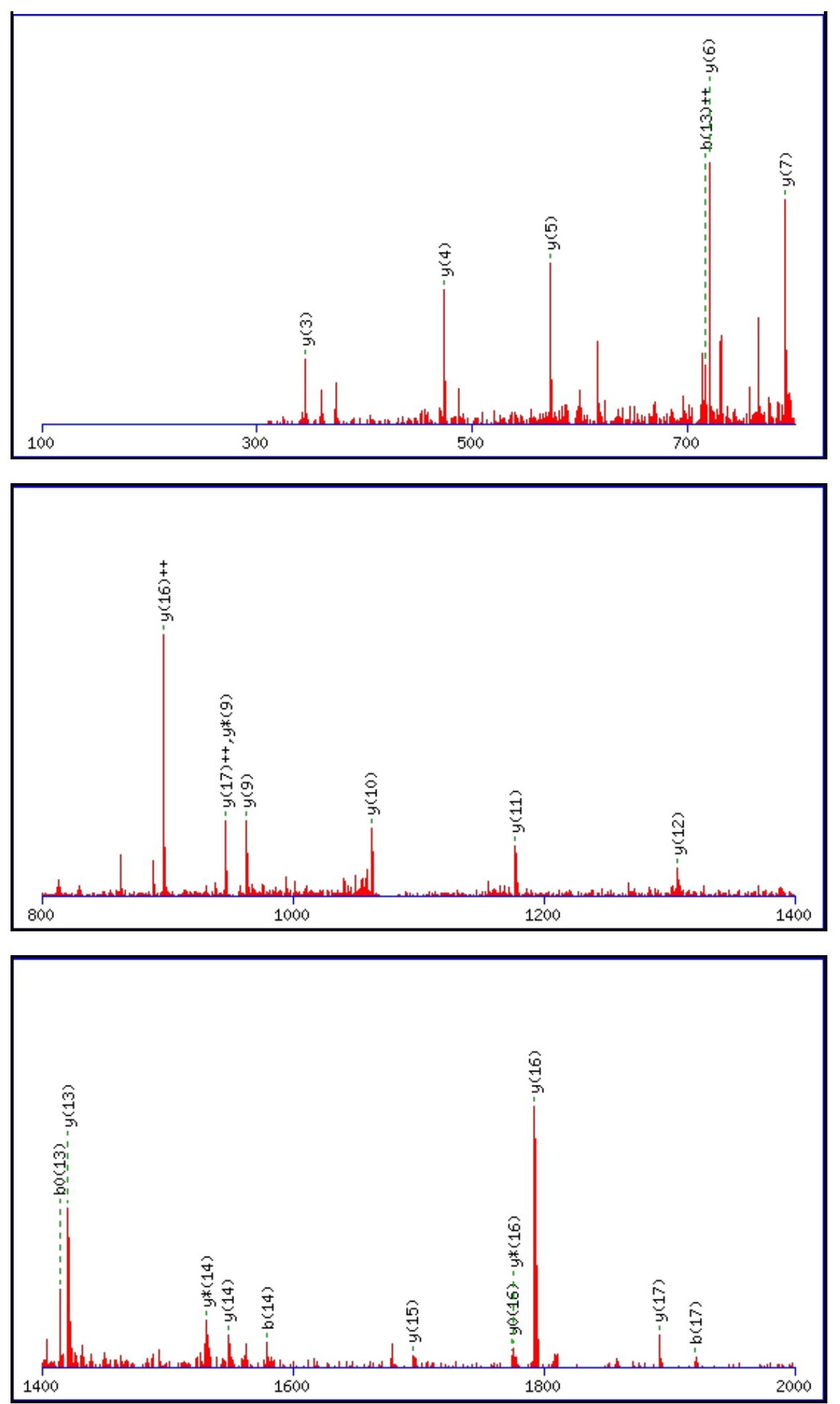

Monoisotopic mass of neutral peptide $\operatorname{Mr}($ calc): 2151.0637

Fixed modifications: Carbamidomethyl (C)

Variable modifications:

N9 : Deamidated $\mathrm{N}(\mathrm{N})$

Ions Score: 94 Expect: $8.2 \mathrm{e}-008$

Matches (Bold Red): 24/186 fragment ions using 43 most intense peaks

\begin{tabular}{|r|c|c|c|c|c|c|c|c|c|c|c|c|c|c|}
\hline$\#$ & $\mathbf{b}$ & $\mathbf{b}^{++}$ & $\mathbf{b}^{*}$ & $\mathbf{b}^{\boldsymbol{*}^{++}}$ & $\mathbf{b}^{\mathbf{0}}$ & $\mathbf{b}^{\mathbf{0 + +}}$ & Seq. & $\mathbf{y}$ & $\mathbf{y}^{++}$ & $\mathbf{y}^{\mathbf{*}}$ & $\mathbf{y}^{\boldsymbol{*}^{++}}$ & $\mathbf{y}^{\mathbf{0}}$ & $\mathbf{y}^{\mathbf{0 + +}}$ & $\#$ \\
\hline $\mathbf{1}$ & 164.0706 & 82.5389 & & & & & $\mathbf{Y}$ & & & & & & & $\mathbf{1 9}$ \\
\hline $\mathbf{2}$ & 261.1234 & 131.0653 & & & & & $\mathbf{P}$ & 1989.0076 & 995.0074 & 1971.9811 & 986.4942 & 1970.9970 & 986.0022 & $\mathbf{1 8}$ \\
\hline 3 & 360.1918 & 180.5995 & & & & & $\mathbf{V}$ & $\mathbf{1 8 9 1 . 9 5 4 8}$ & $\mathbf{9 4 6 . 4 8 1 1}$ & 1874.9283 & 937.9678 & 1873.9443 & 937.4758 & $\mathbf{1 7}$ \\
\hline $\mathbf{4}$ & 457.2445 & 229.1259 & & & & & $\mathbf{P}$ & $\mathbf{1 7 9 2 . 8 8 6 4}$ & $\mathbf{8 9 6 . 9 4 6 9}$ & $\mathbf{1 7 7 5 . 8 5 9 9}$ & 888.4336 & $\mathbf{1 7 7 4 . 8 7 5 9}$ & 887.9416 & $\mathbf{1 6}$ \\
\hline $\mathbf{5}$ & 604.3130 & 302.6601 & & & & & $\mathbf{F}$ & $\mathbf{1 6 9 5 . 8 3 3 7}$ & 848.4205 & 1678.8071 & 839.9072 & 1677.8231 & 839.4152 & $\mathbf{1 5}$ \\
\hline
\end{tabular}




\begin{tabular}{|c|c|c|c|c|c|c|c|c|c|c|c|c|c|c|}
\hline 6 & 732.3715 & |366.6894 & 715.3450 & |358.1761 & & & $\mathbf{Q}$ & & & & 766.3 & 15307547 & 765.8810 & 14 \\
\hline 7 & 846.4145 & |423.7109 & 829.3879 & 415.1976 & & & $\mathbf{N}$ & 1420.7067 & 710.8570 & 1403.6801 & 702.3437 & 1402.6961 & 701.8517 & 13 \\
\hline 8 & 975.4571 & 488.2322 & 958.4305 & |479.7189 & 957.4465 & 479.2269 & $\mathbf{E}$ & 1306.6638 & 653.8355 & |1289.6372 & 645.3222 & 1288.6532 & 644.8302 & 12 \\
\hline 9 & 1090.4840 & 545.7456 & 1073.4574 & 537.2324 & 1072.4734 & 536.7404 & $\mathbf{N}$ & 1177.6212 & 589.3142 & 1160.5946 & 580.8009 & 1159.6106 & 580.3089 & 11 \\
\hline 10 & 189.5524 & 595.2798 & 1172.5259 & 586.7666 & 1171.5418 & 586.2746 & V & 1062.5942 & 531.8007 & 1045.5677 & 523.2875 & 1044.5837 & 522.7955 & 10 \\
\hline 11 & 1290.6001 & 645.8037 & 1273.5735 & 637.2904 & 1272.5895 & 636.7984 & $\mathbf{T}$ & 963.5258 & 482.2665 & 946.4993 & 473.7533 & 945.5152 & 473.2613 & 9 \\
\hline 12 & 1361.6372 & 681.3222 & 1344.6107 & 672.8090 & 1343.6266 & 672.3170 & A & 862.4781 & 431.7427 & 845.4516 & 423.2294 & 844.4676 & 422.7374 & 8 \\
\hline 13 & 1432.6743 & 716.8408 & 1415.6478 & 708.3275 & 1414.6638 & 707.8355 & A & 791.4410 & 396.2241 & 774.4145 & 387.7109 & 773.4305 & 387.2189 & 7 \\
\hline 14 & 1579.7427 & 790.3750 & 1562.7162 & 781.8617 & 1561.7322 & 781.3697 & $\mathbf{F}$ & 720.4039 & 360.7056 & 703.3774 & 352.1923 & 702.3933 & 351.7003 & 6 \\
\hline 15 & 1678.8111 & 839.9092 & 1661.7846 & 831.3959 & 1660.8006 & 830.9039 & V & 573.3355 & 287.1714 & 556.3089 & 278.6581 & 555.3249 & 278.1661 & 5 \\
\hline 16 & 1807.8537 & 904.4305 & 1790.8272 & 895.9172 & 1789.8432 & 895.4252 & E & 474.2671 & 237.6372 & 457.2405 & 229.1239 & 456.2565 & |228.6319 & 4 \\
\hline 17 & 1920.9378 & 960.9725 & 1903.9113 & 952.4593 & 1902.9272 & 951.9673 & $\mathbf{L}$ & 345.2245 & 173.1159 & 328.1979 & 164.6026 & & & 3 \\
\hline 18 & 1977.9593 & 989.4833 & 1960.9327 & 980.9700 & 1959.9487 & 980.4780 & G & 232.1404 & 116.5738 & 215.1139 & 108.0606 & & & 2 \\
\hline 19 & & & & & & & $\mathbf{R}$ & 175.1190 & 88.0631 & 158.0924 & 79.5498 & & & \\
\hline
\end{tabular}
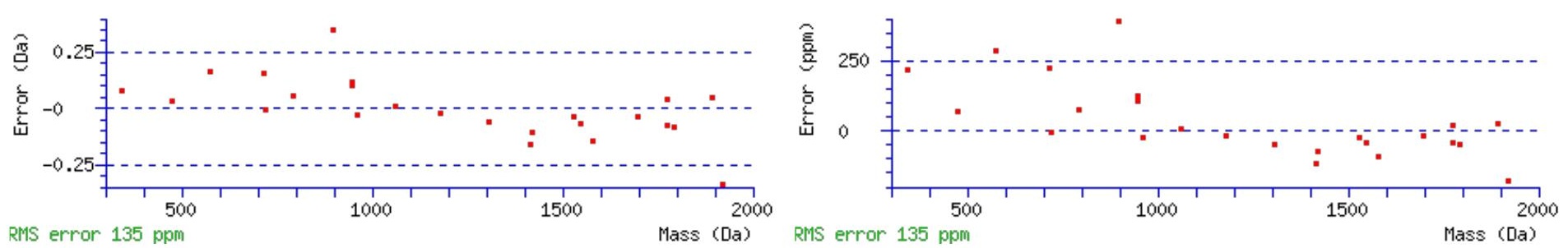

\section{All matches to this query}

\begin{tabular}{|l|l|l|l|}
\hline Score & Mr(calc): & Delta & \multicolumn{1}{|c|}{ Sequence } \\
\hline 94.2 & 2151.0637 & 0.0072 & YPVPFQNENVTAAFVELGR \\
\hline 67.4 & 2151.0637 & 0.0072 & YPVPFQNENVTAAFVELGR \\
\hline 17.2 & 2150.0797 & 0.9912 & YPVPFQNENVTAAFVELGR \\
\hline 3.3 & 2151.0583 & 0.0126 & DDEIIKKAVNEYDETLEK \\
\hline 3.1 & 2150.0578 & 1.0131 & YDILPYSIRVLLEAAVR \\
\hline 0.7 & 2149.0539 & 2.0169 & WINGSTLNSDVLSITGDTEK \\
\hline 0.4 & 2151.0590 & 0.0118 & NSTGSTLAAMVGAGGSSLSVAASR \\
\hline
\end{tabular}

Spectrum No: 109; Query: 1754; Rank: 1

\section{Peptide View}

MS/MS Fragmentation of LAGGTNFNNSIQADPQTVTR

Found in IPI00192495, Tax_Id=10116 Gene_Symbol=Ncstn Isoform 1 of Nicastrin precursor

Match to Query 1754: 2104.023548 from(1053.019050,2+)

Title: 100101RatKid_NS_deglyco_25.2200.2200.2.dta

Data file K:INewmanPaper|Piliangl3SubProteomes\Piliang3SP\mgf5ppm\ERLIC_3SubProteomes5ppm.mgf 

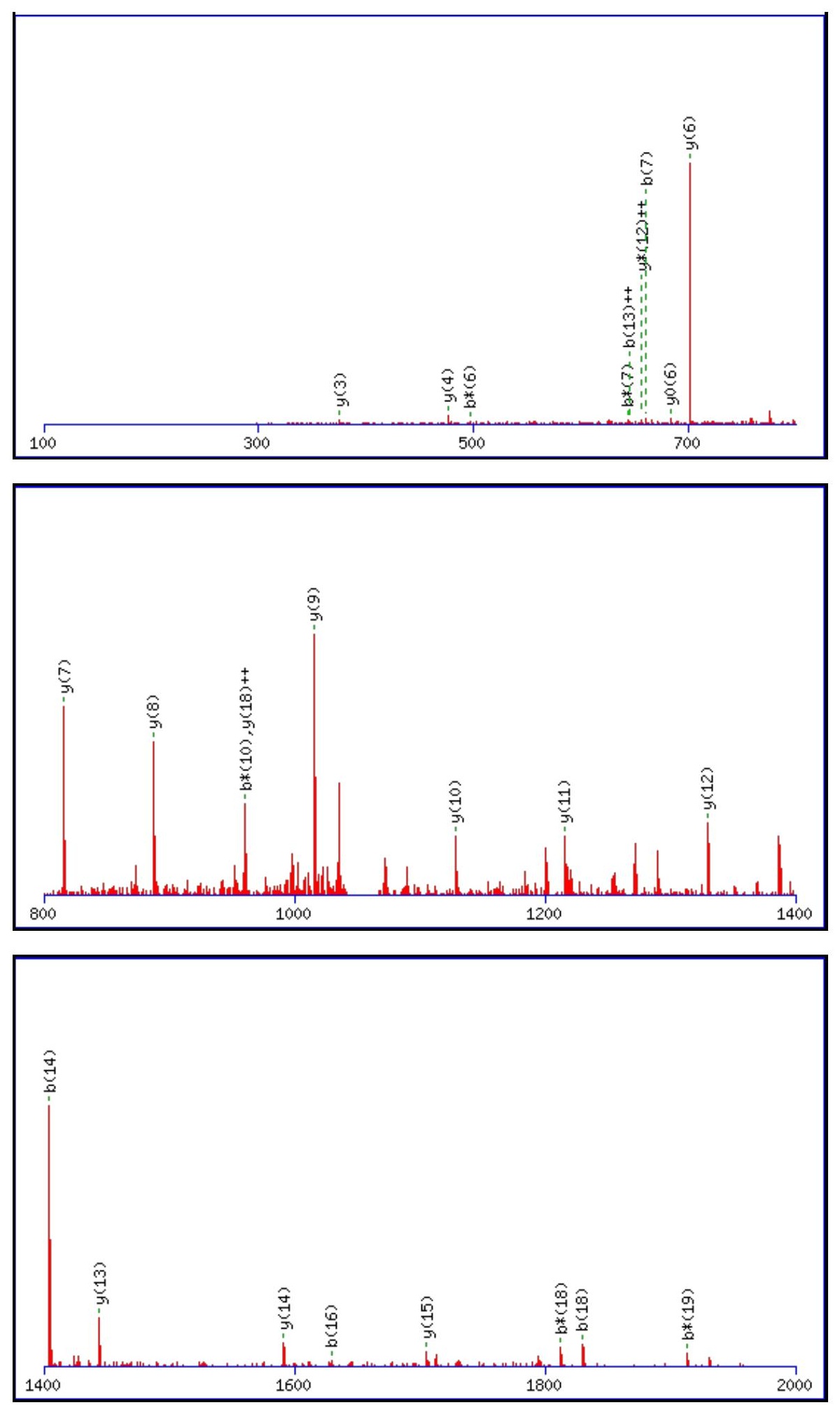

Monoisotopic mass of neutral peptide $\operatorname{Mr}($ calc): 2104.0185

Fixed modifications: Carbamidomethyl (C)

Variable modifications:

N8: Deamidated_N (N)

Ions Score: 94 Expect: $9.2 \mathrm{e}-008$

Matches (Bold Red): 25/208 fragment ions using 37 most intense peaks

\begin{tabular}{|r|c|c|c|c|c|c|c|c|c|c|c|c|c|c|}
\hline$\#$ & $\mathbf{b}$ & $\mathbf{b}^{++}$ & $\mathbf{b}^{*}$ & $\mathbf{b}^{\boldsymbol{*}^{++}}$ & $\mathbf{b}^{\mathbf{0}}$ & $\mathbf{b}^{\mathbf{0 + +}}$ & Seq. & $\mathbf{y}$ & $\mathbf{y}^{++}$ & $\mathbf{y}^{\mathbf{*}}$ & $\mathbf{y}^{\boldsymbol{*}^{++}}$ & $\mathbf{y}^{\mathbf{0}}$ & $\mathbf{y}^{\mathbf{0 + +}}$ & $\#$ \\
\hline $\mathbf{1}$ & 114.0913 & 57.5493 & & & & & $\mathbf{L}$ & & & & & & & $\mathbf{2 0}$ \\
\hline $\mathbf{2}$ & 185.1285 & 93.0679 & & & & & A & 1991.9417 & 996.4745 & 1974.9152 & 987.9612 & 1973.9312 & 987.4692 & $\mathbf{1 9}$ \\
\hline 3 & 242.1499 & 121.5786 & & & & & G & 1920.9046 & $\mathbf{9 6 0 . 9 5 5 9}$ & 1903.8781 & 952.4427 & 1902.8940 & 951.9507 & $\mathbf{1 8}$ \\
\hline $\mathbf{4}$ & 299.1714 & 150.0893 & & & & & G & 1863.8831 & 932.4452 & 1846.8566 & 923.9319 & 1845.8726 & 923.4399 & $\mathbf{1 7}$ \\
\hline $\mathbf{5}$ & 400.2191 & 200.6132 & & & 382.2085 & 191.6079 & $\mathbf{T}$ & 1806.8617 & 903.9345 & 1789.8351 & 895.4212 & 1788.8511 & 894.9292 & $\mathbf{1 6}$ \\
\hline
\end{tabular}




\begin{tabular}{|r|r|r|r|r|r|r|r|r|r|r|r|r|r|r|r|r|}
$\mathbf{6}$ & 514.2620 & 257.6346 & 497.2354 & 249.1214 & 496.2514 & 248.6293 & $\mathbf{N}$ & $\mathbf{1 7 0 5 . 8 1 4 0}$ & 853.4106 & 1688.7874 & 844.8974 & 1687.8034 & 844.4054 & $\mathbf{1 5}$ \\
\hline $\mathbf{7}$ & $\mathbf{6 6 1 . 3 3 0 4}$ & 331.1688 & $\mathbf{6 4 4 . 3 0 3 9}$ & 322.6556 & 643.3198 & 322.1636 & $\mathbf{F}$ & $\mathbf{1 5 9 1 . 7 7 1 1}$ & 796.3892 & 1574.7445 & 787.8759 & 1573.7605 & 787.3839 & $\mathbf{1 4}$ \\
\hline $\mathbf{8}$ & 776.3573 & 388.6823 & 759.3308 & 380.1690 & 758.3468 & 379.6770 & $\mathbf{N}$ & $\mathbf{1 4 4 4 . 7 0 2 7}$ & 722.8550 & 1427.6761 & 714.3417 & 1426.6921 & 713.8497 & $\mathbf{1 3}$ \\
\hline $\mathbf{9}$ & 890.4003 & 445.7038 & 873.3737 & 437.1905 & 872.3897 & 436.6985 & $\mathbf{N}$ & $\mathbf{1 3 2 9 . 6 7 5 7}$ & 665.3415 & 1312.6492 & $\mathbf{6 5 6 . 8 2 8 2}$ & 1311.6652 & 656.3362 & $\mathbf{1 2}$ \\
\hline $\mathbf{1 0}$ & 977.4323 & 489.2198 & $\mathbf{9 6 0 . 4 0 5 7}$ & 480.7065 & 959.4217 & 480.2145 & S & $\mathbf{1 2 1 5 . 6 3 2 8}$ & 608.3200 & 1198.6062 & 599.8068 & 1197.6222 & 599.3148 & $\mathbf{1 1}$ \\
\hline $\mathbf{1 1}$ & 1090.5164 & 545.7618 & 1073.4898 & 537.2485 & 1072.5058 & 536.7565 & I & $\mathbf{1 1 2 8 . 6 0 0 8}$ & 564.8040 & 1111.5742 & 556.2907 & 1110.5902 & 555.7987 & $\mathbf{1 0}$ \\
\hline $\mathbf{1 2}$ & 1218.5749 & 609.7911 & 1201.5484 & 601.2778 & 1200.5644 & 600.7858 & $\mathbf{Q}$ & $\mathbf{1 0 1 5 . 5 1 6 7}$ & 508.2620 & 998.4902 & 499.7487 & 997.5061 & 499.2567 & $\mathbf{9 9}$ \\
\hline $\mathbf{1 3}$ & 1289.6120 & $\mathbf{6 4 5 . 3 0 9 7}$ & 1272.5855 & 636.7964 & 1271.6015 & 636.3044 & $\mathbf{A}$ & $\mathbf{8 8 7 . 4 5 8 1}$ & 444.2327 & 870.4316 & 435.7194 & 869.4476 & 435.2274 & $\mathbf{8}$ \\
\hline $\mathbf{1 4}$ & $\mathbf{1 4 0 4 . 6 3 9 0}$ & 702.8231 & 1387.6124 & 694.3099 & 1386.6284 & 693.8179 & $\mathbf{D}$ & $\mathbf{8 1 6 . 4 2 1 0}$ & 408.7141 & 799.3945 & 400.2009 & 798.4104 & 399.7089 & $\mathbf{7}$ \\
\hline $\mathbf{1 5}$ & 1501.6918 & 751.3495 & 1484.6652 & 742.8362 & 1483.6812 & 742.3442 & $\mathbf{P}$ & $\mathbf{7 0 1 . 3 9 4 1}$ & 351.2007 & 684.3675 & 342.6874 & $\mathbf{6 8 3 . 3 8 3 5}$ & 342.1954 & $\mathbf{6}$ \\
\hline $\mathbf{1 6}$ & $\mathbf{1 6 2 9 . 7 5 0 3}$ & 815.3788 & 1612.7238 & 806.8655 & 1611.7398 & 806.3735 & $\mathbf{Q}$ & 604.3413 & 302.6743 & 587.3148 & 294.1610 & 586.3307 & 293.6690 & $\mathbf{5}$ \\
\hline $\mathbf{1 7}$ & 1730.7980 & 865.9026 & 1713.7715 & 857.3894 & 1712.7874 & 856.8974 & $\mathbf{T}$ & $\mathbf{4 7 6 . 2 8 2 7}$ & 238.6450 & 459.2562 & 230.1317 & 458.2722 & 229.6397 & $\mathbf{4}$ \\
\hline $\mathbf{1 8}$ & $\mathbf{1 8 2 9 . 8 6 6 4}$ & 915.4369 & $\mathbf{1 8 1 2 . 8 3 9 9}$ & 906.9236 & 1811.8559 & 906.4316 & $\mathbf{V}$ & $\mathbf{3 7 5 . 2 3 5 0}$ & 188.1212 & 358.2085 & 179.6079 & 357.2245 & 179.1159 & $\mathbf{3}$ \\
\hline $\mathbf{1 9}$ & 1930.9141 & 965.9607 & $\mathbf{1 9 1 3 . 8 8 7 6}$ & 957.4474 & 1912.9035 & 956.9554 & $\mathbf{T}$ & 276.1666 & 138.5870 & 259.1401 & 130.0737 & 258.1561 & 129.5817 & $\mathbf{2}$ \\
\hline $\mathbf{2 0}$ & & & & & & & $\mathbf{R}$ & 175.1190 & 88.0631 & 158.0924 & 79.5498 & & & \\
\hline
\end{tabular}
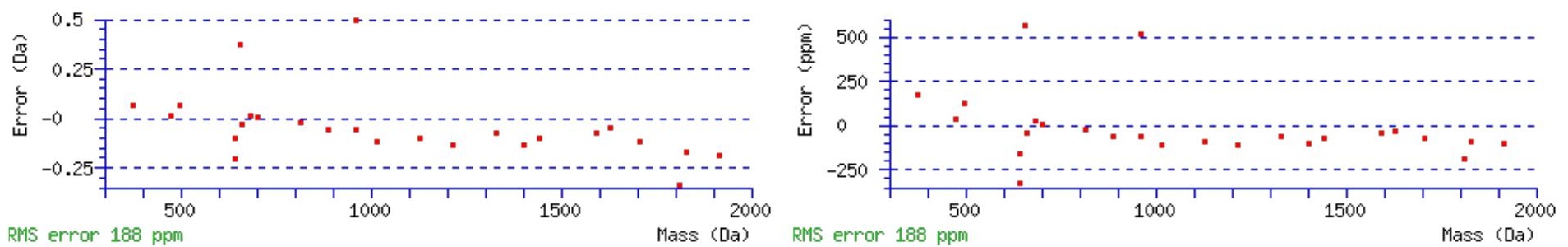

\section{All matches to this query}

\begin{tabular}{|l|l|l|l|}
\hline Score & Mr(calc): & Delta & \multicolumn{1}{|c|}{ Sequence } \\
\hline 94.1 & 2104.0185 & 0.0050 & $\underline{\text { LAGGTNFNNSIQADPQTVTR }}$ \\
\hline 77.0 & 2104.0185 & 0.0050 & $\underline{\text { LAGGTNFNNSIQADPQTVTR }}$ \\
\hline 76.9 & 2104.0185 & 0.0050 & $\underline{\text { LAGGTNFNNSIQADPQTVTR }}$ \\
\hline 45.2 & 2103.0345 & 0.9890 & $\underline{\text { LAGGTNFNNSIQADPQTVTR }}$ \\
\hline 4.3 & 2104.0354 & -0.0118 & SLFQDKNRTLEAFSLVR \\
\hline 2.1 & 2102.0092 & 2.0144 & CSQLLRENAKAGHTPALR \\
\hline 1.6 & 2104.0201 & 0.0034 & DASSSSKLFSAKLEQLASR \\
\hline 1.1 & 2103.0080 & 1.0156 & $\underline{\text { KKSSDTAGSGAPNSDSOLPEK }}$ \\
\hline 0.6 & 2102.0296 & 1.9939 & $\underline{\text { ELHIALLKLSADLDTNXE }}$ \\
\hline
\end{tabular}

Spectrum No: 110; Query: 2068; Rank: 1

\section{Peptide View}

MS/MS Fragmentation of FFNDSIVDPVDSEWFGFYR

Found in IPI00208382, Tax_Id=10116 Gene_Symbol=Ppt1 Palmitoyl-protein thioesterase 1 precursor

Match to Query 2068: 2340.045288 from(1171.029920,2+)

Title: 091008RatKidney_NoSalt_18.6114.6114.2.dta

Data file K:INewmanPaper|Piliangl3SubProteomes\Piliang3SP\mgf5ppm\ERLIC_3SubProteomes5ppm.mgf 

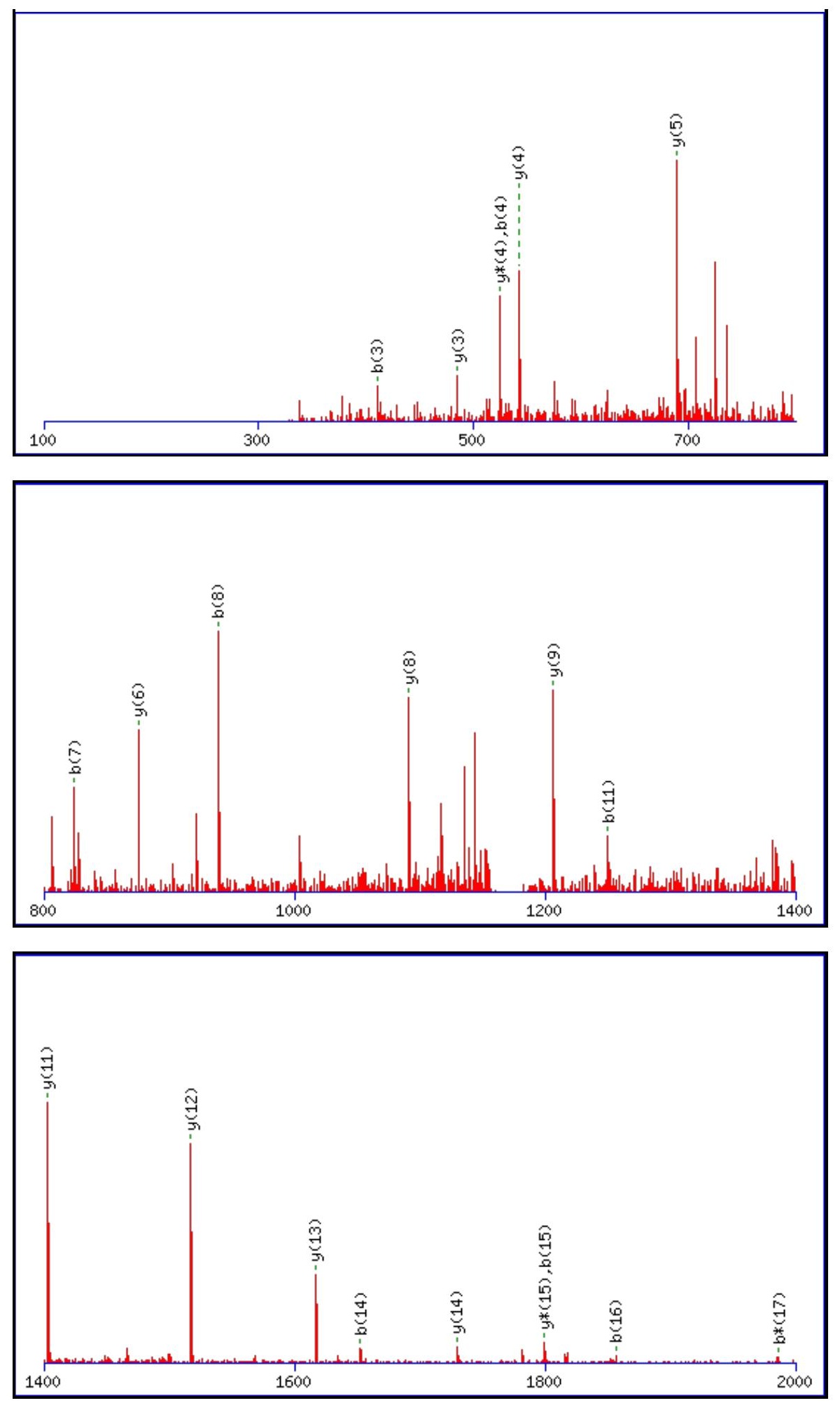

Monoisotopic mass of neutral peptide $\operatorname{Mr}($ calc): 2340.0375

Fixed modifications: Carbamidomethyl (C)

Variable modifications:

N3 : Deamidated $\mathrm{N}(\mathrm{N})$

Ions Score: 94 Expect: $1.3 \mathrm{e}-007$

Matches (Bold Red): 21/194 fragment ions using 26 most intense peaks

\begin{tabular}{|r|c|c|c|c|c|c|c|c|c|c|c|c|c|c|}
\hline$\#$ & $\mathbf{b}$ & $\mathbf{b}^{++}$ & $\mathbf{b}^{*}$ & $\mathbf{b}^{\boldsymbol{*}^{++}}$ & $\mathbf{b}^{\mathbf{0}}$ & $\mathbf{b}^{\mathbf{0 + +}}$ & $\mathbf{S e q}$ & $\mathbf{y}$ & $\mathbf{y}^{++}$ & $\mathbf{y}^{\mathbf{*}}$ & $\mathbf{y}^{\mathbf{*}^{++}}$ & $\mathbf{y}^{\mathbf{0}}$ & $\mathbf{y}^{\mathbf{0 + +}}$ & $\#$ \\
\hline $\mathbf{1}$ & 148.0757 & 74.5415 & & & & & $\mathbf{F}$ & & & & & & & $\mathbf{1 9}$ \\
\hline $\mathbf{2}$ & 295.1441 & 148.0757 & & & & & $\mathbf{F}$ & 2193.9764 & 1097.4918 & 2176.9498 & 1088.9785 & 2175.9658 & 1088.4865 & $\mathbf{1 8}$ \\
\hline $\mathbf{3}$ & $\mathbf{4 1 0 . 1 7 1 0}$ & 205.5892 & 393.1445 & 197.0759 & & & $\mathbf{N}$ & 2046.9080 & 1023.9576 & 2029.8814 & 1015.4443 & 2028.8974 & 1014.9523 & $\mathbf{1 7}$ \\
\hline $\mathbf{4}$ & $\mathbf{5 2 5 . 1 9 8 0}$ & 263.1026 & 508.1714 & 254.5894 & 507.1874 & 254.0973 & $\mathbf{D}$ & 1931.8810 & 966.4441 & 1914.8545 & 957.9309 & 1913.8705 & 957.4389 & $\mathbf{1 6}$ \\
\hline $\mathbf{5}$ & 612.2300 & 306.6186 & 595.2035 & 298.1054 & 594.2194 & 297.6134 & $\mathbf{S}$ & 1816.8541 & 908.9307 & $\mathbf{1 7 9 9 . 8 2 7 5}$ & 900.4174 & 1798.8435 & 899.9254 & $\mathbf{1 5}$ \\
\hline
\end{tabular}




\begin{tabular}{|c|c|c|c|c|c|c|c|c|c|c|c|c|c|c|}
\hline 6 & 725.3141 & 363.1607 & 708.2875 & 354.6474 & 707.3035 & 354.1554 & I & $\mid 1729.8220$ & 865.4147 & |1712.7955 & 856.9014 & 1711.8115 & 856.4094 & 14 \\
\hline 7 & 824.3825 & 412.6949 & 807.3559 & 404.1816 & 806.3719 & 403.6896 & $\mathbf{V}$ & 1616.7380 & 808.8726 & 1599.7114 & 800.3594 & 1598.7274 & 799.8673 & 13 \\
\hline 8 & 939.4094 & 470.2084 & 922.3829 & 461.6951 & 921.3989 & 461.2031 & D & 1517.6696 & 759.3384 & 1500.6430 & 750.8251 & 1499.6590 & 750.3331 & 12 \\
\hline 9 & 1036.4622 & 518.7347 & 1019.4356 & 510.2215 & 1018.4516 & 509.7295 & $\mathbf{P}$ & 1402.6426 & 701.8250 & 1385.6161 & 693.3117 & 1384.6321 & 692.8197 & 11 \\
\hline 10 & 1135.5306 & 568.2689 & 1118.5041 & 559.7557 & 1117.5200 & 559.2637 & V & 1305.5899 & 653.2986 & 1288.5633 & 644.7853 & 1287.5793 & 644.2933 & 10 \\
\hline 11 & 1250.5576 & 625.7824 & 1233.5310 & 617.2691 & 1232.5470 & 616.7771 & D & 1206.5215 & 603.7644 & 1189.4949 & 595.2511 & 1188.5109 & 594.7591 & 9 \\
\hline 12 & 1337.5896 & 669.2984 & 1320.5630 & 660.7852 & 1319.5790 & 660.2931 & S & 1091.4945 & 546.2509 & 1074.4680 & 537.7376 & 1073.4839 & 537.2456 & 8 \\
\hline 14 & 1652.7115 & 826.8594 & 1635.6849 & 818.3461 & 1634.7009 & 817.8541 & W & 875.4199 & 438.2136 & 858.3933 & 429.7003 & & & 6 \\
\hline 15 & 1799.7799 & 900.3936 & 1782.7534 & 891.8803 & 1781.7693 & 891.3883 & $\mathbf{F}$ & 689.3406 & 345.1739 & 672.3140 & 336.6606 & & & 5 \\
\hline 16 & 1856.8014 & 928.9043 & 1839.7748 & 920.3910 & 1838.7908 & 919.8990 & G & 542.2722 & 271.6397 & 525.2456 & 263.1264 & & & 4 \\
\hline 17 & 2003.8698 & 1002.4385 & 1986.8432 & 993.9253 & 1985.8592 & 993.4332 & $\mathbf{F}$ & 485.2507 & 243.1290 & 468.2241 & 234.6157 & & & 3 \\
\hline 18 & 2166.9331 & 1083.9702 & 2149.9066 & 1075.4569 & 2148.9225 & 1074.9649 & $\mathbf{Y}$ & 338.1823 & 169.5948 & 321.1557 & 161.0815 & & & 2 \\
\hline 19 & & & & & & & $\mathbf{R}$ & 175.1190 & 88.0631 & 158.0924 & 79.5498 & & & 1 \\
\hline
\end{tabular}
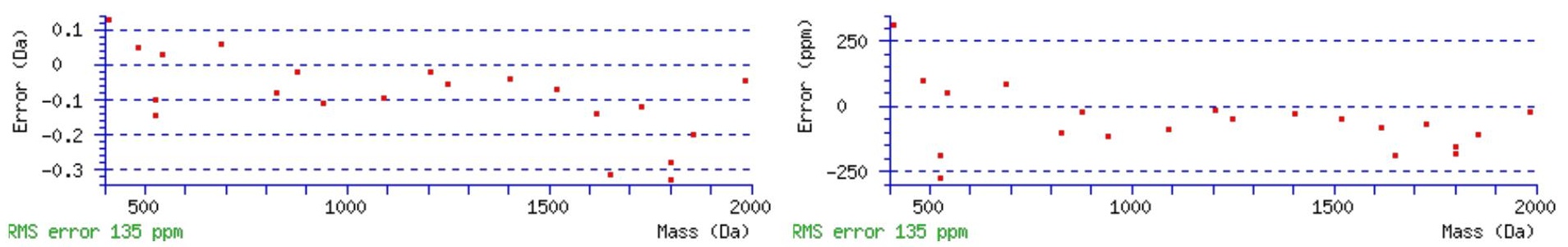

\section{All matches to this query}

\begin{tabular}{|l|l|l|l|}
\hline Score & Mr(calc): & Delta & \multicolumn{1}{|c|}{ Sequence } \\
\hline 94.0 & 2340.0375 & 0.0078 & FFNDSIVDPVDSEWFGFYR \\
\hline 46.8 & 2339.0535 & 0.9918 & FFNDSIVDPVDSEWFGFYR \\
\hline 11.5 & 2340.0512 & -0.0059 & LLSASSSPTLPPSPGGGGGSKGNR \\
\hline 3.8 & 2340.0341 & 0.0112 & TYWESNGTAGSHFITIRIK \\
\hline 2.9 & 2339.0569 & 0.9884 & RAPQTGIVDECCFRSCDLR \\
\hline 2.1 & 2338.0607 & 1.9846 & APGTLVGQISANDLDSPASPIR \\
\hline 1.9 & 2340.0512 & -0.0060 & NTVNGTGTPVHISTLQVGETR \\
\hline 1.8 & 2340.0512 & -0.0060 & NTVNGTGTPVHISTLQVGETR \\
\hline 1.4 & 2340.0512 & -0.0059 & LLSASSSPTLPPSPGGGGGSKGNR \\
\hline 1.2 & 2340.0552 & -0.0099 & WPEPXALSAAVQAGLQVTGER \\
\hline
\end{tabular}

Spectrum No: 111; Query: 2233; Rank: 1

\section{Peptide View}

MS/MS Fragmentation of HYSCACPSGWNLSDDSVNCVR

Found in IPI00205325, Tax_Id=10116 Gene_Symbol=Lrp2 Low-density lipoprotein receptor-related protein 2 precursor

Match to Query 2233: 2484.000108 from(1243.007330,2+)

Title: 100101RatKid_NS_deglyco_14.2681.2681.2.dta

Data file K:INewmanPaper|Piliangl3SubProteomes\Piliang3SP\mgf5ppm\ERLIC_3SubProteomes5ppm.mgf 

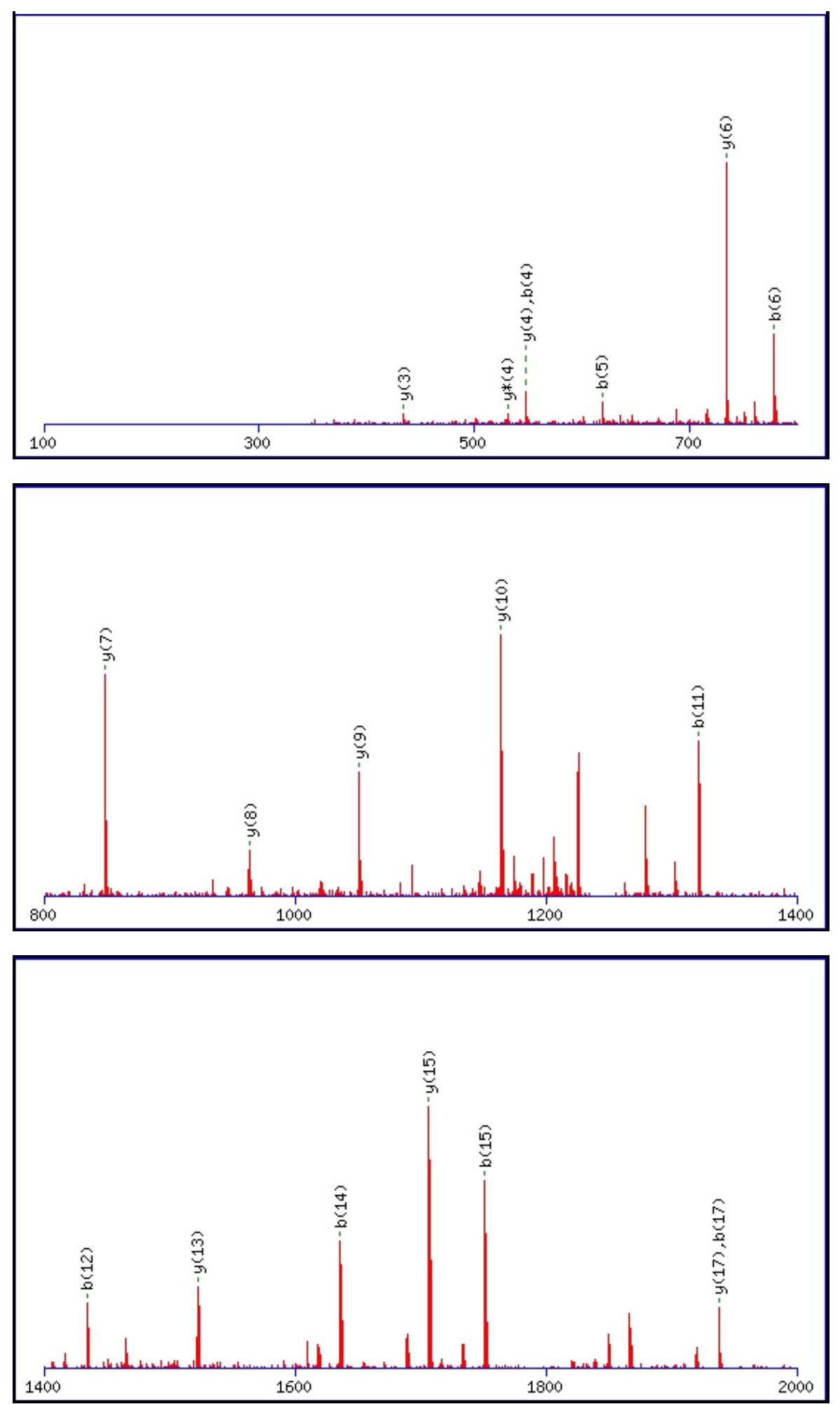

Monoisotopic mass of neutral peptide $\operatorname{Mr}($ calc): 2483.9893

Fixed modifications: Carbamidomethyl (C)

Variable modifications:

N11 : Deamidated N (N)

Ions Score: 94 Expect: $9.8 \mathrm{e}-008$

Matches (Bold Red): 19/206 fragment ions using 25 most intense peaks

\begin{tabular}{|r|c|c|c|c|c|r|r|r|r|r|r|r|r|r|}
\hline$\#$ & $\mathbf{b}$ & $\mathbf{b}^{++}$ & $\mathbf{b}^{*}$ & $\mathbf{b}^{\boldsymbol{*}^{++}}$ & $\mathbf{b}^{\mathbf{0}}$ & $\mathbf{b}^{\mathbf{0 + +}}$ & $\mathbf{S e q}$ & $\mathbf{y}$ & $\mathbf{y}^{++}$ & $\mathbf{y}^{\mathbf{*}}$ & $\mathbf{y}^{\mathbf{*}^{++}}$ & $\mathbf{y}^{\mathbf{0}}$ & $\mathbf{y}^{\mathbf{0 + +}}$ & $\#$ \\
\hline $\mathbf{1}$ & 138.0662 & 69.5367 & & & & & $\mathbf{H}$ & & & & & & & $\mathbf{2 1}$ \\
\hline $\mathbf{2}$ & 301.1295 & 151.0684 & & & & & $\mathbf{Y}$ & 2347.9376 & 1174.4725 & 2330.9111 & 1165.9592 & 2329.9271 & 1165.4672 & $\mathbf{2 0}$ \\
\hline $\mathbf{3}$ & 388.1615 & 194.5844 & & & 370.1510 & 185.5791 & S & 2184.8743 & 1092.9408 & 2167.8478 & 1084.4275 & 2166.8637 & 1083.9355 & $\mathbf{1 9}$ \\
\hline $\mathbf{4}$ & $\mathbf{5 4 8 . 1 9 2 2}$ & 274.5997 & & & 530.1816 & 265.5945 & $\mathbf{C}$ & 2097.8423 & 1049.4248 & 2080.8157 & 1040.9115 & 2079.8317 & 1040.4195 & $\mathbf{1 8}$ \\
\hline $\mathbf{5}$ & $\mathbf{6 1 9 . 2 2 9 3}$ & 310.1183 & & & 601.2187 & 301.1130 & $\mathbf{A}$ & $\mathbf{1 9 3 7 . 8 1 1 6}$ & 969.4095 & 1920.7851 & 960.8962 & 1919.8011 & 960.4042 & $\mathbf{1 7}$ \\
\hline
\end{tabular}




\begin{tabular}{|c|c|c|c|c|c|c|c|c|c|c|c|c|c|c|}
\hline 6 & 779.2600 & 390.1336 & & & 761.2494| & 381.1283 & C & 1866.7745 & 933.8909| & 1849.7480 & 925.3776 & |1848.7640| & 924.8856 & 16 \\
\hline 7 & 876.3127 & 438.6600 & & & 858.3022 & 429.6547 & $\mathbf{P}$ & 1706.7439 & 853.8756 & 1689.7173 & 845.3623 & 1688.7333 & 844.8703 & 15 \\
\hline 8 & 963.3447 & 482.1760 & & & 945.3342 & 473.1707 & $S$ & 1609.6911 & 805.3492 & 1592.6646 & 796.8359 & 1591.6805 & 796.3439 & 14 \\
\hline 9 & 1020.3662 & 510.6867 & & & 1002.3556 & 501.6815 & G & 1522.6591 & 761.8332 & 1505.6325 & 753.3199 & 1504.6485 & 752.8279 & 13 \\
\hline 10 & 1206.4455 & 603.7264 & & & 1188.4350 & 594.7211 & W & 1465.6376 & 733.3224 & 1448.6111 & 724.8092 & 1447.6270 & 724.3172 & 12 \\
\hline 11 & 1321.4725 & 661.2399 & 1304.4459 & 652.7266 & 1303.4619 & 652.2346 & $\mathbf{N}$ & 1279.5583 & 640.2828 & 1262.5317 & 631.7695 & 1261.5477 & 631.2775 & 11 \\
\hline 12 & 1434.5565 & 717.7819 & 1417.5300 & 709.2686 & 1416.5460 & 708.7766 & $\mathbf{L}$ & 1164.5314 & 582.7693 & 1147.5048 & 574.2560 & 1146.5208 & 573.7640 & 10 \\
\hline 13 & 1521.5886 & 761.2979 & 1504.5620 & 752.7846 & 1503.5780 & 752.2926 & $\mathrm{~S}$ & 1051.4473 & 526.2273 & 1034.4207 & 517.7140 & 1033.4367 & 517.2220 & 9 \\
\hline 14 & 1636.6155 & 818.8114 & 1619.5889 & 810.2981 & 1618.6049 & 809.8061 & D & 964.4153 & 482.7113 & 947.3887 & 474.1980 & 946.4047 & 473.7060 & 8 \\
\hline 15 & 1751.6424 & 876.3249 & 1734.6159 & 867.8116 & 1733.6319 & 867.3196 & D & 849.3883 & 425.1978 & 832.3618 & 416.6845 & 831.3778 & 416.1925 & 7 \\
\hline 16 & 1838.6745 & 919.8409 & 1821.6479 & 911.3276 & 1820.6639 & 910.8356 & $S$ & 734.3614 & 367.6843 & 717.3348 & 359.1711 & 716.3508 & 358.6790 & 6 \\
\hline 17 & 1937.7429 & 969.3751 & 1920.7163 & 960.8618 & 1919.7323 & 960.3698 & $\mathbf{V}$ & 647.3294 & 324.1683 & 630.3028 & 315.6550 & & & 5 \\
\hline 18 & 2051.7858 & 1026.3965 & 2034.7593 & 1017.8833 & 2033.7752 & 1017.3913 & $\mathbf{N}$ & 548.2609 & 274.6341 & 531.2344 & 266.1208 & & & 4 \\
\hline 19 & 2211.8165 & 1106.4119 & 2194.7899 & 1097.8986 & 2193.8059 & 1097.4066 & C & 434.2180 & 217.6126 & 417.1915 & 209.0994 & & & 3 \\
\hline 20 & 2310.8849 & 1155.9461 & 2293.8583 & 1147.4328 & 2292.8743 & 1146.9408 & $\mathrm{~V}$ & 274.1874 & 137.5973 & 257.1608 & 129.0840 & & & 2 \\
\hline 21 & & & & & & & $\mathbf{R}$ & 175.1190 & 88.0631 & 158.0924 & 79.5498 & & & 1 \\
\hline
\end{tabular}
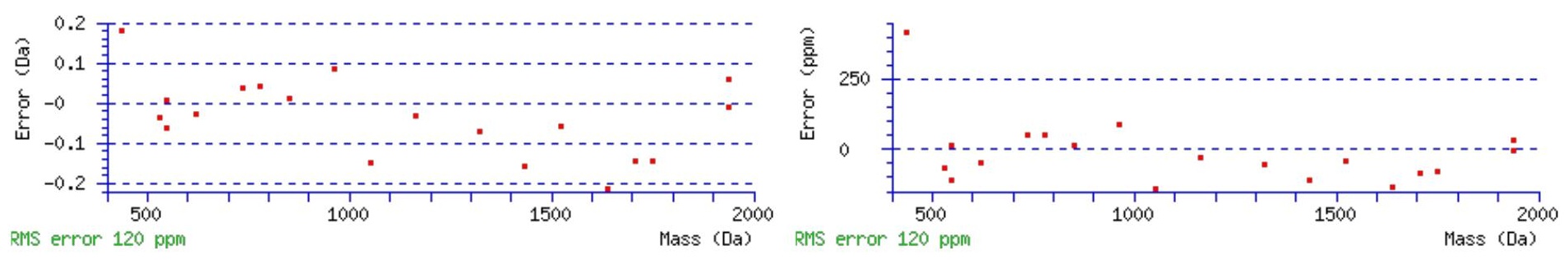

\section{All matches to this query}

\begin{tabular}{|l|l|l|l|}
\hline Score & Mr(calc): & Delta & \multicolumn{1}{|c|}{ Sequence } \\
\hline 94.0 & 2483.9893 & 0.0108 & HYSCACPSGWNLSDDSVNCVR \\
\hline 59.4 & 2483.9893 & 0.0108 & HYSCACPSGWNLSDDSVNCVR \\
\hline 32.7 & 2483.0053 & 0.9948 & HYSCACPSGWNLSDDSVNCVR \\
\hline 14.4 & 2481.9938 & 2.0063 & TTSSGVGGSTESNTDLFTYRNK \\
\hline 9.6 & 2482.9778 & 1.0223 & TTSSGVGGSTESNTDLFTYRNK \\
\hline 9.1 & 2481.9938 & 2.0063 & TTSSGVGGSTESNTDLFTYRNK \\
\hline 9.1 & 2481.9938 & 2.0063 & TTSSGVGGSTESNTDLFTYRNK \\
\hline 8.5 & 2481.9938 & 2.0063 & TTSSGVGGSTESNTDLFTYRNK \\
\hline 2.5 & 2482.9989 & 1.0012 & TEGSDGAVSLSSADAQDSLEKEK \\
\hline 2.5 & 2481.9938 & 2.0063 & TTSSGVGGSTESNTDLFTYRNK \\
\hline
\end{tabular}

Spectrum No: 112; Query: 791; Rank: 1

\section{Peptide View}

MS/MS Fragmentation of SEELDFLGNSSLQK

Found in IPI00766673, Tax_Id=10116 Gene_Symbol=Tlr3 similar to toll-like receptor 3

Match to Query 791: 1566.746768 from(784.380660,2+)

Title: 100101RatKid_NS_deglyco_23.3535.3535.2.dta

Data file K:INewmanPaper|Piliangl3SubProteomes\Piliang3SP\mgf5ppm\ERLIC_3SubProteomes5ppm.mgf 

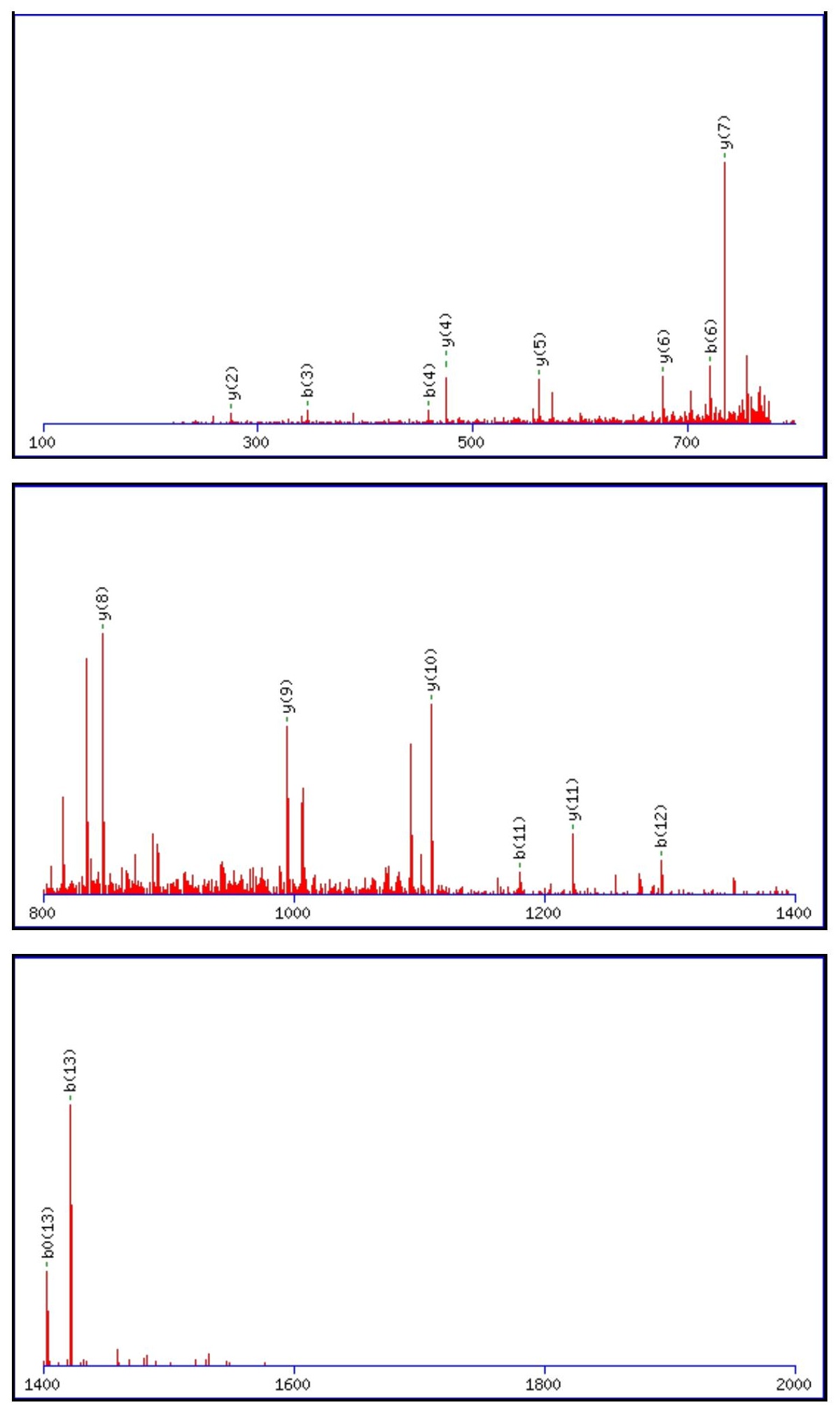

Monoisotopic mass of neutral peptide $\operatorname{Mr}($ calc): 1566.7413

Fixed modifications: Carbamidomethyl (C)

Variable modifications:

N9 : Deamidated $\mathrm{N}(\mathrm{N})$

Ions Score: 94 Expect : $7.3 \mathrm{e}-008$

Matches (Bold Red): 16/134 fragment ions using 21 most intense peaks

\begin{tabular}{|c|c|c|c|c|c|c|c|c|c|c|c|c|c|c|}
\hline \# & b & $\mathbf{b}^{++}$ & $\mathbf{b}^{*}$ & $\mathbf{b}^{*^{++}}$ & $\mathbf{b}^{0}$ & $\mathbf{b}^{\mathbf{0 + +}}$ & Seq. & $\mathbf{y}$ & $\mathbf{y}^{++}$ & $\mathrm{y}^{*}$ & $\mathrm{y}^{*^{++}}$ & $\mathbf{y}^{0}$ & $y^{0++}$ & $\#$ \\
\hline 1 & 88.0393 & 44.5233 & & & 70.0287 & 35.5180 & $\mathbf{S}$ & & & & & & & 14 \\
\hline 2 & 217.0819 & 109.0446 & & & 199.0713 & 100.0393 & E & 1480.7166 & 740.8619 & 1463.6900 & 732.3487 & 1462.7060 & 731.8566 & 13 \\
\hline 3 & 346.1245 & 173.5659 & & & 328.1139 & 164.5606 & $\mathbf{E}$ & 1351.6740 & 676.3406 & 1334.6474 & 667.8274 & 1333.6634 & 667.3353 & 12 \\
\hline 4 & 459.2086 & 230.1079 & & & 441.1980 & 221.1026 & L & 1222.6314 & 611.8193 & 1205.6048 & 603.3061 & 1204.6208 & 602.8140 & 11 \\
\hline 5 & 574.2355 & 287.6214 & & & 556.2249 & 278.6161 & D & 1109.5473 & 555.2773 & 1092.5208 & 546.7640 & 1091.5368 & 546.2720 & 10 \\
\hline
\end{tabular}




\begin{tabular}{|c|c|c|c|c|c|c|c|c|c|c|c|c|c|c|}
\hline 6 & 721.3039 & 361.1556 & & & 703.2933 & |352.1503| & $\mathbf{F}$ & 994.5204 & |497.7638 & 977.4938 & 489.2506 & 976.5098 & |488.7585 & 9 \\
\hline 7 & 834.3880 & 417.6976 & & & 816.3774 & 408.6923 & $\mathbf{L}$ & 847.4520 & 424.2296 & 830.4254 & 415.7163 & 829.4414 & 415.2243 & 8 \\
\hline 8 & 891.4094 & 446.2084 & & & 873.3989 & 437.2031 & G & 734.3679 & 367.6876 & 717.3414 & 359.1743 & 716.3573 & 358.6823 & 7 \\
\hline 9 & 1006.4364 & 503.7218 & 989.4098 & 495.2086 & 988.4258 & 494.7165 & $\mathbf{N}$ & 677.3464 & 339.1769 & 660.3199 & 330.6636 & 659.3359 & 330.1716 & 6 \\
\hline 10 & 1093.4684 & 547.2378 & 1076.4419 & 538.7246 & 1075.4578 & 538.2326 & $\mathrm{~S}$ & 562.3195 & 281.6634 & 545.2930 & 273.1501 & 544.3089 & 272.6581 & 5 \\
\hline 11 & 1180.5004 & 590.7539 & 1163.4739 & 582.2406 & 1162.4899 & 581.7486 & S & 475.2875 & 238.1474 & 458.2609 & 229.6341 & 457.2769 & 229.1421 & 4 \\
\hline 12 & 1293.5845 & 647.2959 & 1276.5579 & 638.7826 & 1275.5739 & 638.2906 & $\mathbf{L}$ & 388.2554 & 194.6314 & 371.2289 & 186.1181 & & & 3 \\
\hline 13 & 1421.6431 & |711.3252 & 1404.6165 & 702.8119 & 1403.6325 & 702.3199 & $\mathbf{Q}$ & 275.1714 & 138.0893 & 258.1448 & 129.5761 & & & 2 \\
\hline 14 & & & & & & & $\mathbf{K}$ & 147.1128 & 74.0600 & 130.0863 & 65.5468 & & & 1 \\
\hline
\end{tabular}
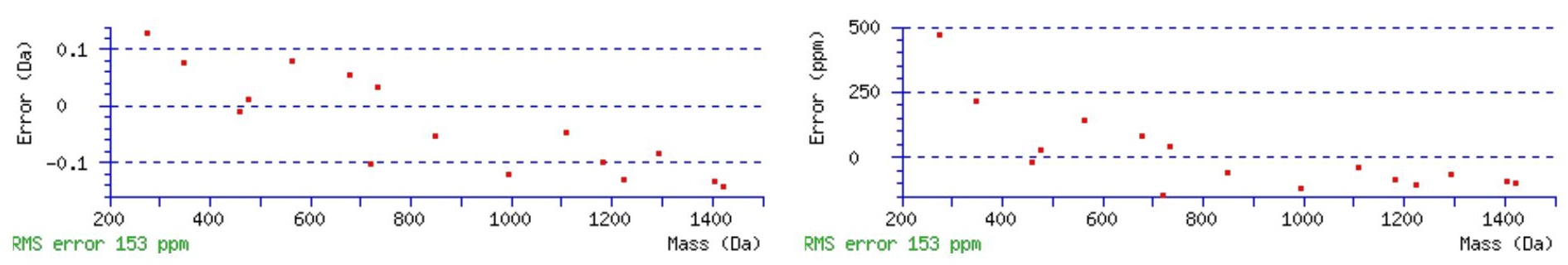

\section{All matches to this query}

\begin{tabular}{|l|l|l|l|}
\hline Score & Mr(calc): & Delta & \multicolumn{1}{|c|}{ Sequence } \\
\hline 93.9 & 1566.7413 & 0.0055 & SEELDFLGNSSLQK \\
\hline 22.7 & 1565.7573 & 0.9895 & SEELDFLGNSSLQK \\
\hline 10.3 & 1566.7542 & -0.0074 & EESLEVVNSILQK \\
\hline 9.4 & 1566.7460 & 0.0008 & SSVAGCYIRNPTNK \\
\hline 9.3 & 1566.7477 & -0.0009 & SMTVTLGAHNIKAK \\
\hline 9.3 & 1566.7477 & -0.0009 & SMTVTLGAHNIKAK \\
\hline 9.3 & 1566.7477 & -0.0009 & SMTVTLGAHNIKAK \\
\hline 7.7 & 1565.7459 & 1.0009 & ITXLNCIRIHSK \\
\hline 7.1 & 1566.7599 & -0.0132 & YVELTEGVECIQK \\
\hline 6.3 & 1566.7365 & 0.0103 & VIVYSGKATMSTSK \\
\hline
\end{tabular}

Spectrum No: 113; Query: 2813; Rank: 1

\section{Peptide View}

MS/MS Fragmentation of QAIDDDCNQTGQMTAGLLDWPQGTFASQVTLEGDKVK

Found in IPI00364321, Tax_Id=10116 Gene_Symbol=Etfb Electron transfer flavoprotein subunit beta

Match to Query 2813: 4037.882382 from(1346.968070,3+)

Title: 091008RatKidney_NoSalt_17.5765.5765.3.dta

Data file K:INewmanPaper|Piliang|3SubProteomes\Piliang3SP\mgf5ppm\ERLIC_3SubProteomes5ppm.mgf 

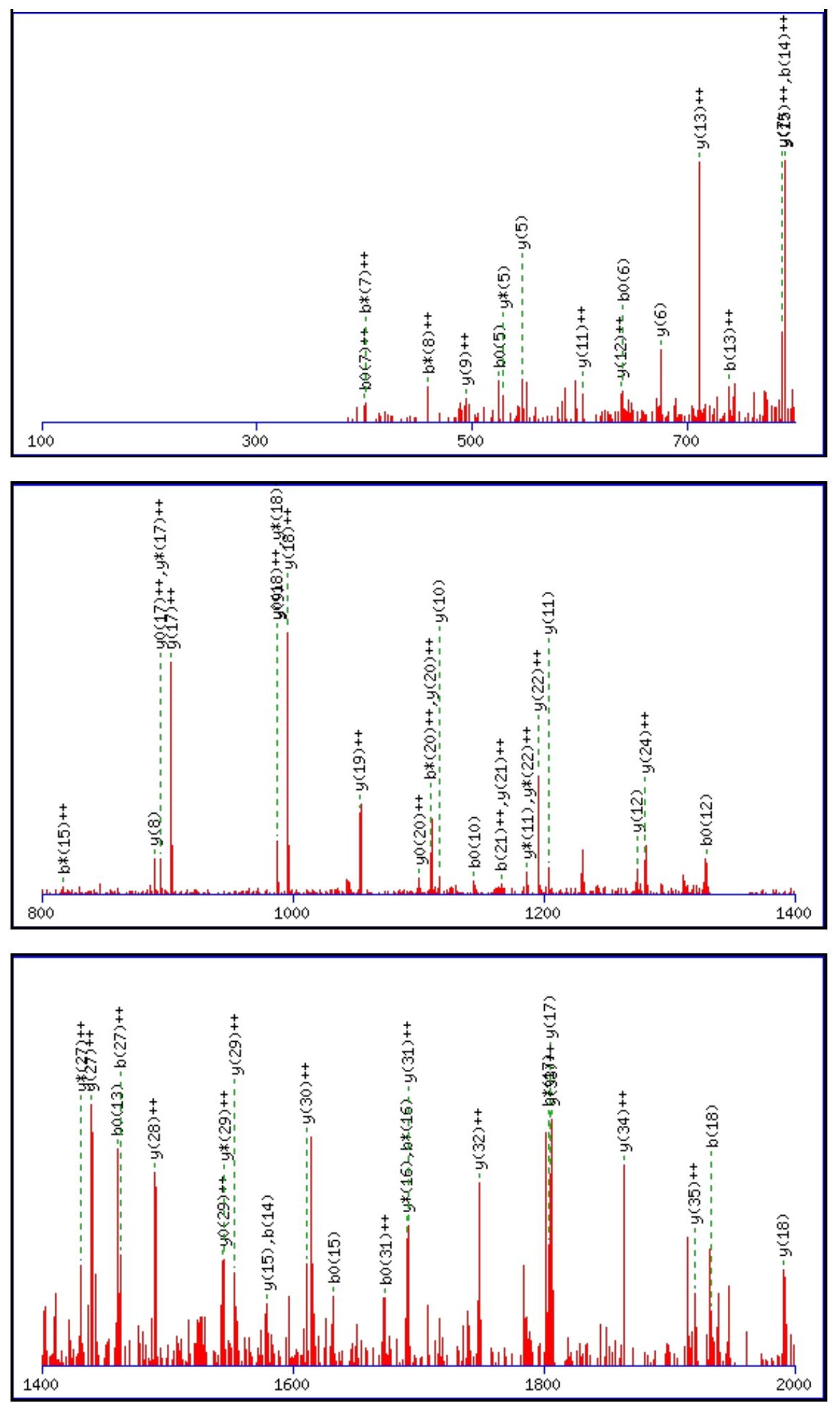

Monoisotopic mass of neutral peptide $\operatorname{Mr}($ calc): 4037.8623

Fixed modifications: Carbamidomethyl (C)

Variable modifications:

N8: Deamidated $\mathrm{N}(\mathrm{N})$

Ions Score: 94 Expect: $1.8 \mathrm{e}-007$

Matches (Bold Red): 64/420 fragment ions using 104 most intense peaks

\begin{tabular}{|r|c|c|c|c|c|c|c|c|c|c|c|c|c|c|}
\hline$\#$ & $\mathbf{b}$ & \multicolumn{1}{|c|}{$\mathbf{b}^{++}$} & $\mathbf{b}^{*}$ & $\mathbf{b}^{\boldsymbol{*}^{++}}$ & $\mathbf{b}^{\mathbf{0}}$ & $\mathbf{b}^{\mathbf{0 + +}}$ & $\mathbf{S e q}$ & $\mathbf{y}$ & $\mathbf{y}^{++}$ & $\mathbf{y}^{\mathbf{*}}$ & $\mathbf{y}^{\mathbf{*}^{++}}$ & $\mathbf{y}^{\mathbf{0}}$ & $\mathbf{y}^{\mathbf{0 + +}}$ & $\#$ \\
\hline $\mathbf{1}$ & 129.0659 & 65.0366 & 112.0393 & 56.5233 & & & $\mathbf{Q}$ & & & & & & & $\mathbf{3 7}$ \\
\hline $\mathbf{2}$ & 200.1030 & 100.5551 & 183.0764 & 92.0418 & & & $\mathbf{A}$ & 3910.8110 & 1955.9091 & 3893.7844 & 1947.3959 & 3892.8004 & 1946.9038 & $\mathbf{3 6}$ \\
\hline $\mathbf{3}$ & 313.1870 & 157.0972 & 296.1605 & 148.5839 & & & $\mathbf{I}$ & 3839.7739 & $\mathbf{1 9 2 0 . 3 9 0 6}$ & 3822.7473 & 1911.8773 & 3821.7633 & 1911.3853 & $\mathbf{3 5}$ \\
\hline $\mathbf{4}$ & 428.2140 & 214.6106 & 411.1874 & 206.0974 & 410.2034 & 205.6053 & $\mathbf{D}$ & 3726.6898 & $\mathbf{1 8 6 3 . 8 4 8 5}$ & 3709.6632 & 1855.3353 & 3708.6792 & 1854.8433 & $\mathbf{3 4}$ \\
\hline $\mathbf{5}$ & 543.2409 & 272.1241 & 526.2144 & 263.6108 & 525.2304 & 263.1188 & $\mathbf{D}$ & 3611.6629 & $\mathbf{1 8 0 6 . 3 3 5 1}$ & 3594.6363 & 1797.8218 & 3593.6523 & 1797.3298 & $\mathbf{3 3}$ \\
\hline
\end{tabular}




\begin{tabular}{|c|c|c|c|c|c|c|c|c|c|c|c|c|c|c|}
\hline & 658.2679 & 329.6376 & 641.2413 & 321.1243 & 640.2573 & 320.6323 & D & 3496.6359 & |1748.8216 & |3479.6094 & 1740.3083 & |3478.6253 & |1739.8163 & |32 \\
\hline 7 & 818.2985 & 409.6529 & 801.2720 & 401.1396 & 800.2879 & 400.6476 & $\mathrm{C}$ & 3381.6090 & 1691.3081 & 3364.5824 & 1682.7948 & 3363.5984 & 1682.3028 & 31 \\
\hline 8 & 933.3254 & 467.1664 & 916.2989 & 458.6531 & 915.3149 & 458.1611 & $\mathbf{N}$ & 3221.5783 & 1611.2928 & 3204.5518 & 1602.7795 & 3203.5678 & 1602.2875 & 30 \\
\hline 9 & 1061.3840 & 531.1957 & 1044.3575 & 522.6824 & 1043.3735 & 522.1904 & $\mathbf{Q}$ & 3106.5514 & 1553.7793 & 3089.5248 & 1545.2661 & 3088.5408 & 1544.7740 & 29 \\
\hline 10 & 1162.4317 & 581.7195 & 1145.4052 & 573.2062 & 1144.4211 & 572.7142 & $\mathbf{T}$ & 2978.4928 & 1489.7500 & 2961.4663 & 1481.2368 & 2960.4822 & 1480.7448 & 28 \\
\hline 11 & 1219.4532 & 610.2302 & 1202.4266 & 601.7169 & 1201.4426 & 601.2249 & G & 2877.4451 & 1439.2262 & 2860.4186 & 1430.7129 & 2859.4346 & 1430.2209 & 27 \\
\hline 12 & 1347.5117 & 674.2595 & 1330.4852 & 665.7462 & 1329.5012 & 665.2542 & $\mathbf{Q}$ & 2820.4237 & 1410.7155 & 2803.3971 & 1402.2022 & 2802.4131 & 1401.7102 & 26 \\
\hline 13 & 1478.5522 & 739.7798 & 1461.5257 & 731.2665 & 1460.5417 & 730.7745 & M & 2692.3651 & 1346.6862 & 2675.3385 & 1338.1729 & 2674.3545 & 1337.6809 & 25 \\
\hline 14 & 1579.5999 & 790.3036 & 1562.5734 & 781.7903 & 1561.5893 & 781.2983 & $\mathbf{T}$ & 2561.3246 & 1281.1659 & 2544.2980 & 1272.6527 & 2543.3140 & 1272.1607 & 24 \\
\hline 15 & 1650.6370 & 825.8221 & 1633.6105 & 817.3089 & 1632.6265 & 816.8169 & $\mathbf{A}$ & 2460.2769 & 1230.6421 & 2443.2504 & 1222.1288 & 2442.2664 & 1221.6368 & 23 \\
\hline 16 & 1707.6585 & 854.3329 & 1690.6319 & 845.8196 & 1689.6479 & 845.3276 & G & 2389.2398 & 1195.1235 & 2372.2133 & 1186.6103 & 2371.2292 & 1186.1183 & 22 \\
\hline 17 & 1820.7426 & 910.8749 & 1803.7160 & 902.3616 & 1802.7320 & 901.8696 & $\mathbf{L}$ & 2332.2183 & 1166.6128 & 2315.1918 & 1158.0995 & 2314.2078 & 1157.6075 & 21 \\
\hline 18 & 1933.8266 & 967.4169 & 1916.8001 & 958.9037 & 1915.8160 & 958.4117 & $\mathbf{L}$ & 2219.1343 & 1110.0708 & 2202.1077 & 5575 & 2201.1237 & 1101.0655 & 20 \\
\hline 19 & 2048.8536 & 1024.9304 & 2031.8270 & 1016.4171 & 2030.8430 & 1015.9251 & D & 2106.0502 & 1053.5287 & 2089.0237 & 1045.0155 & 2088.0396 & 1044.5235 & 19 \\
\hline 20 & 2234.9329 & 1117.9701 & 2217.9063 & 1109.4568 & 2216.9223 & 1108.9648 & $\mathbf{W}$ & 1991.0233 & 996.0153 & 1973.9967 & 987.5020 & 1973.0127 & 987.0100 & 18 \\
\hline 21 & 2331.9856 & 1166.4965 & 2314.9591 & 1157.9832 & 2313.9751 & 1157.4912 & $\mathbf{P}$ & 1804.9440 & 902.9756 & 1787.9174 & & 1786.9334 & 9703 & 17 \\
\hline 22 & 2460.0442 & 1230.5257 & 2443.0177 & 1222.0125 & 2442.0336 & 1221.5205 & $\bar{Q}$ & 1707.8912 & 854.4492 & 1690.8646 & 845.9360 & 1689.8806 & 845.4440 & 16 \\
\hline 23 & 2517.0657 & 1259.0365 & 2500.0391 & 1250.5232 & 2499.0551 & 1250.0312 & G & 1579.8326 & 790.4199 & 1562.8061 & 781.9067 & 1561.8220 & 781.4147 & 15 \\
\hline 24 & 2618.1134 & 1309.5603 & 2601.0868 & 1301.0470 & 2600.1028 & 1300.5550 & $\mathbf{T}$ & 1522.8112 & 761.9092 & 1505.7846 & 753.3959 & 1504.8006 & 752.9039 & 14 \\
\hline 25 & 2765.1818 & 1383.0945 & 2748.1552 & 1374.5812 & 2747.1712 & 1374.0892 & $\mathbf{F}$ & 1421.7635 & 711.3854 & 1404.7369 & 702.8721 & 1403.7529 & 702.3801 & 13 \\
\hline 26 & 2836.2189 & 1418.6131 & 2819.1923 & 1410.0998 & 2818.2083 & 1409.6078 & $\mathbf{A}$ & 1274.6951 & 637.8512 & 1257.6685 & 629.3379 & 1256.6845 & 628.8459 & 12 \\
\hline 27 & 2923.2509 & 1462.1291 & 2906.2244 & 1453.6158 & 2905.2403 & 1453.1238 & $S$ & 1203.6579 & 602.3326 & 1186.6314 & 593.8193 & 1185.6474 & 593.3273 & 11 \\
\hline 28 & 3051.3095 & 1526.1584 & 3034.2829 & 1517.6451 & 3033.2989 & 1517.1531 & $\mathbf{Q}$ & 1116.6259 & 558.8166 & 1099.5994 & 550.3033 & 1098.6154 & 549.8113 & 10 \\
\hline 29 & 3150.3779 & 1575.6926 & 3133.3514 & 1567.1793 & 3132.3673 & 1566.6873 & V & 988.5673 & 494.7873 & 971.5408 & 486.2740 & 970.5568 & 485.7820 & 9 \\
\hline 30 & 3251.4256 & 1626.2164 & 3234.3990 & 1617.7032 & 3233.4150 & 1617.2111 & $\mathbf{T}$ & 889.4989 & 445.2531 & 872.4724 & 436.7398 & 871.4884 & 436.2478 & 8 \\
\hline 31 & 3364.5096 & 1682.7585 & 3347.4831 & 1674.2452 & 3346.4991 & 1673.7532 & $\mathbf{L}$ & 788.4512 & 394.7293 & 771.4247 & 386.2160 & 770.4407 & 385.7240 & 7 \\
\hline 32 & 3493.5522 & 1747.2798 & 3476.5257 & 1738.7665 & 3475.5417 & 1738.2745 & $E$ & 675.3672 & 338.1872 & 658.3406 & 329.6740 & 657.3566 & 329.1819 & 6 \\
\hline 33 & 3550.5737 & 1775.7905 & 3533.5472 & 1767.2772 & 3532.5631 & 1766.7852 & G & 546.3246 & 273.6659 & 529.2980 & 265.1527 & 528.3140 & 264.6606 & 5 \\
\hline 34 & 3665.6006 & 1833.3040 & 3648.5741 & 1824.7907 & 3647.5901 & 1824.2987 & D & 489.3031 & 245.1552 & 472.2766 & 236.6419 & 471.2926 & 236.1499 & 4 \\
\hline 35 & 3793.6956 & 1897.3514 & 3776.6691 & 1888.8382 & 3775.6850 & 1888.3462 & $\mathbf{K}$ & 374.2762 & 187.6417 & 357.2496 & 179.1285 & & & 3 \\
\hline 36 & 3892.7640 & 1946.8856 & 3875.7375 & 1938.3724 & 3874.7535 & 1937.8804 & $\bar{V}$ & 246.1812 & 123.5942 & 229.1547 & 115.0810 & & & 2 \\
\hline 37 & & & & & & & $\mathbf{K}$ & 147.1128 & 74.0600 & 130.0863 & $\begin{array}{l}65.5468 \\
\end{array}$ & & & 1 \\
\hline
\end{tabular}
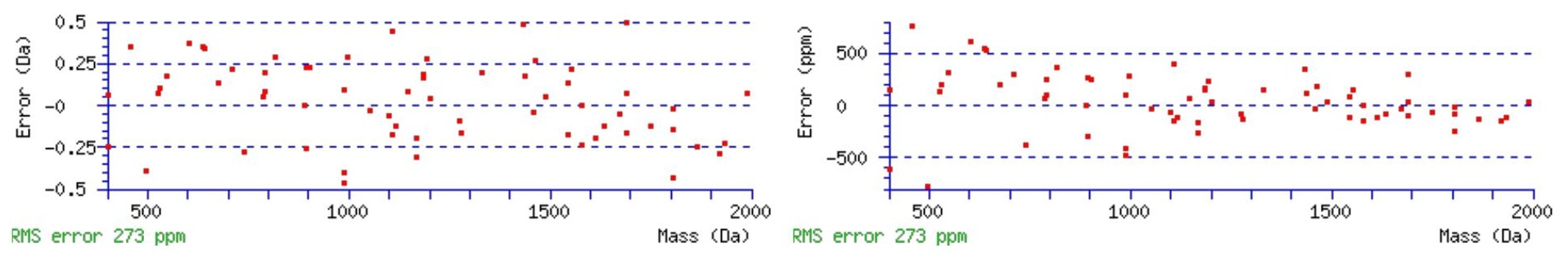

\section{All matches to this query}

\begin{tabular}{|l|l|l|l|}
\hline Score & Mr(calc): & Delta & \multicolumn{1}{|c|}{ Sequence } \\
\hline 93.8 & 4037.8623 & 0.0201 & QAIDDDCNQTGQMTAGLLDWPQGTFASQVTLEGDKVK \\
\hline 90.4 & 4036.8783 & 1.0041 & QAIDDDCNQTGQMTAGLLDWPQGTFASQVTLEGDKVK \\
\hline 8.1 & 4037.8601 & 0.0222 & ESNTAGNDIFHKFSAFIKNPVPTQDDALYQQLLR \\
\hline 8.1 & 4037.8601 & 0.0222 & ESNTAGNDIFHKFSAFIKNPVPTQDDALYQQLLR \\
\hline 8.1 & 4037.8601 & 0.0222 & ESNTAGNDIFHKFSAFIKNPVPTQDDALYQQLLR \\
\hline 7.8 & 4037.8601 & 0.0222 & ESNTAGNDIFHKFSAFIKNPVPTQDDALYQQLLR \\
\hline 7.5 & 4037.8587 & 0.0236 & AFAEKNNLSFIETSALDSTNVEEAFKNILTEIYR \\
\hline 7.0 & 4036.8389 & 1.0435 & YRVYIVIPLLPGFEGDISTGGGNALQAIMHFNYR \\
\hline
\end{tabular}




\begin{tabular}{|l|l|l|l|}
6.6 & 4037.8587 & 0.0236 & AFAEKNNLSFIETSALDSTNVEEAFKNILTEIYR \\
\hline 6.3 & 4036.9088 & 0.9735 & DNSNIILLGDSQGDLRMADGVANVEHILKIGYLNDR \\
\hline
\end{tabular}

Spectrum No: 114; Query: 2447; Rank: 1

\section{Peptide View}

MS/MS Fragmentation of HTGPGILSMANAGPNTNGSQFFICTAK

Found in IPI00364439, Tax_Id=10116 Gene_Symbol=RGD1559682_predicted similar to Peptidyl-prolyl cis-trans isomerase A

Match to Query 2447: 2807.290152 from(936.770660,3+)

Title: 091008RatKidney_NH4Format01_10.3633.3633.3.dta

Data file K:INewmanPaper|Piliangl3SubProteomes\Piliang3SP\mgf5ppm\ERLIC_3SubProteomes5ppm.mgf
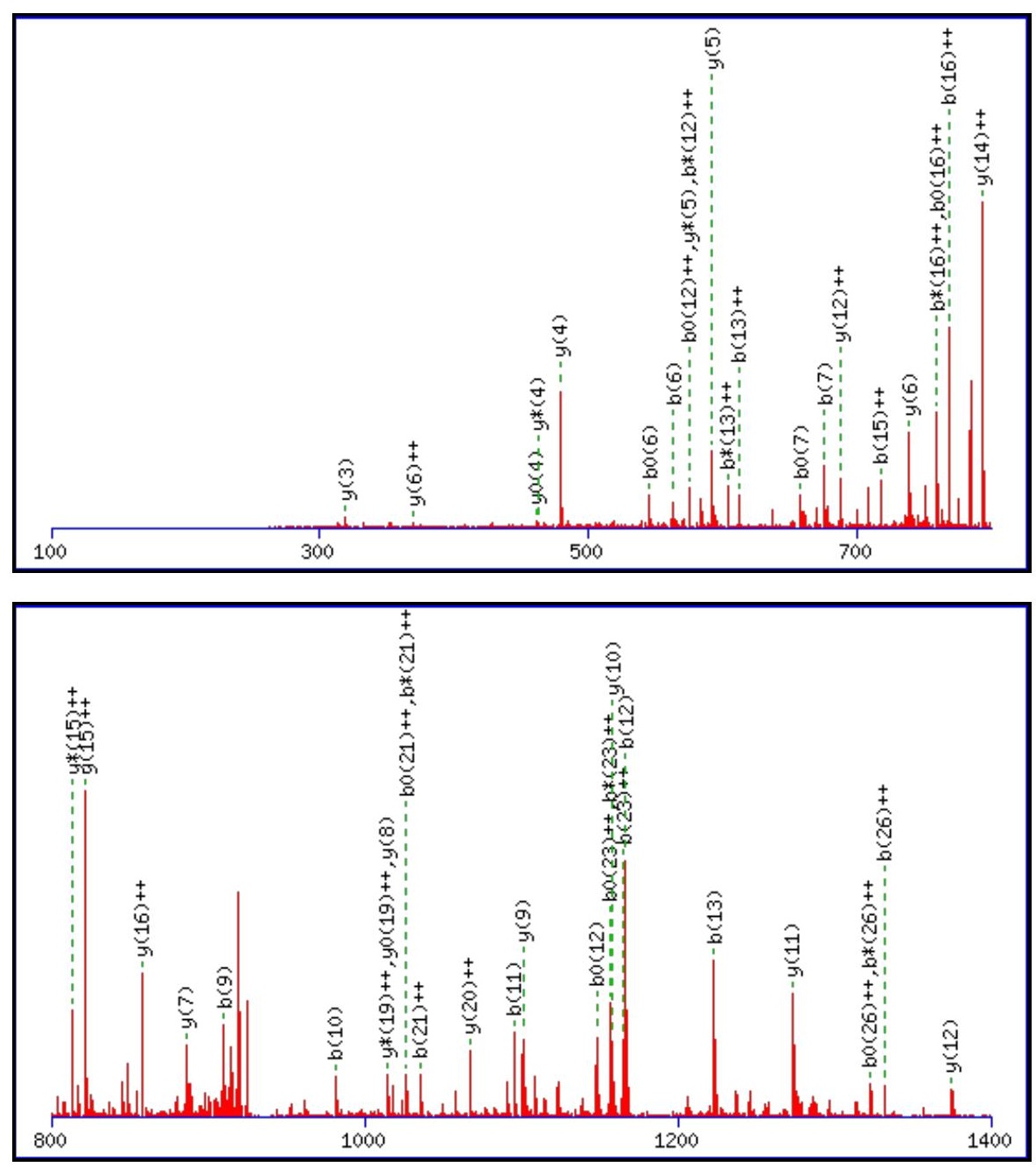


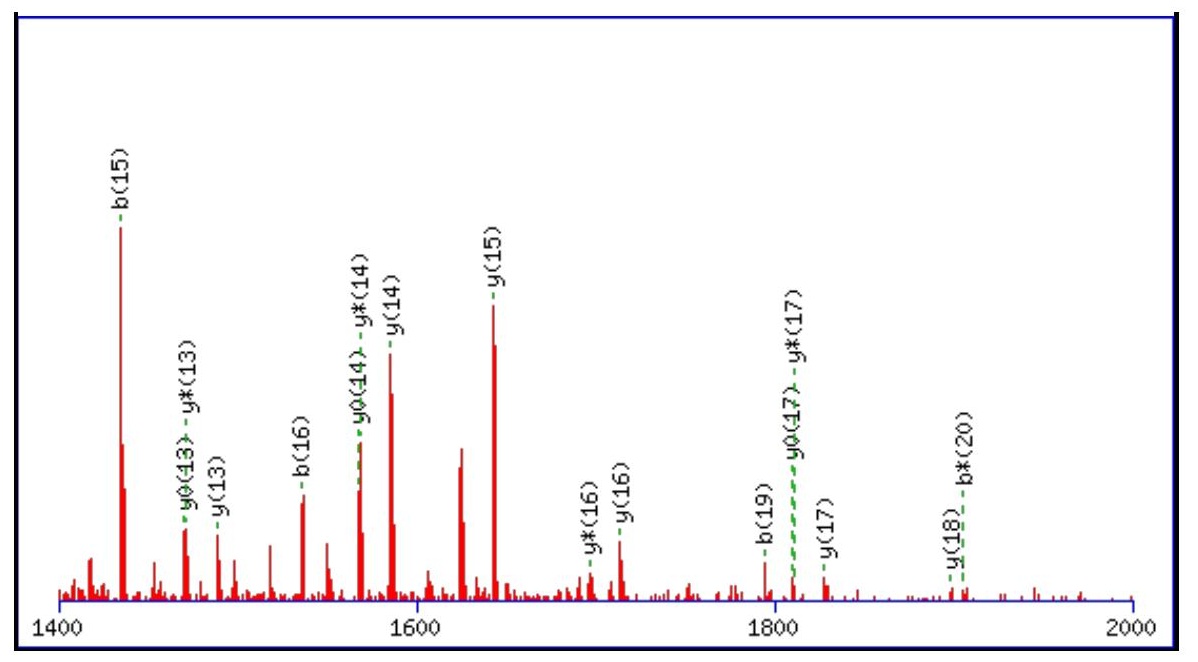

Monoisotopic mass of neutral peptide $\operatorname{Mr}($ calc): 2807.3007

Fixed modifications: Carbamidomethyl (C)

Variable modifications:

M9 : Oxidation $(M)$

N17 : Deamidated $\mathrm{N}(\mathrm{N})$

Ions Score: 94 Expect : $1.6 \mathrm{e}-007$

Matches (Bold Red): 66/286 fragment ions using 102 most intense peaks

\begin{tabular}{|c|c|c|c|c|c|c|c|c|c|c|c|c|c|c|}
\hline \# & b & $\mathbf{b}^{++}$ & $\mathbf{b}^{*}$ & $\mathbf{b}^{*^{++}}$ & $\mathbf{b}^{\mathbf{0}}$ & $\mathbf{b}^{0++}$ & Seq. & $\mathbf{y}$ & $\mathbf{y}^{++}$ & $\mathbf{y}^{*}$ & $\mathbf{y}^{*^{++}}$ & $\mathbf{y}^{0}$ & $y^{0++}$ & \# \\
\hline 1 & 138.0662 & 69.5367 & & & & & $\mathbf{H}$ & & & & & & & 27 \\
\hline 2 & 239.1139 & 120.0606 & & & 221.1033 & 111.0553 & $\mathbf{T}$ & 2671.2491 & 1336.1282 & 2654.2225 & 1327.6149 & 2653.2385 & 1327.1229 & 26 \\
\hline 3 & 296.1353 & 148.5713 & & & 278.1248 & 139.5660 & G & 2570.2014 & 1285.6043 & 2553.1748 & 1277.0911 & 2552.1908 & 1276.5990 & 25 \\
\hline 4 & 393.1881 & 197.0977 & & & 375.1775 & 188.0924 & $\mathbf{P}$ & 2513.1799 & 1257.0936 & 2496.1534 & 1248.5803 & 2495.1694 & 1248.0883 & 24 \\
\hline 5 & 450.2096 & 225.6084 & & & 432.1990 & 216.6031 & G & 2416.1272 & 1208.5672 & 2399.1006 & 1200.0539 & 2398.1166 & 1199.5619 & 23 \\
\hline 6 & 563.2936 & 282.1504 & & & 545.2831 & 273.1452 & I & 2359.1057 & 1180.0565 & 2342.0791 & 1171.5432 & 2341.0951 & 1171.0512 & 22 \\
\hline 7 & 676.3777 & 338.6925 & & & 658.3671 & 329.6872 & $\mathbf{L}$ & 2246.0216 & 1123.5145 & 2228.9951 & 1115.0012 & 2228.0111 & 1114.5092 & 21 \\
\hline 8 & 763.4097 & 382.2085 & & & 745.3991 & 373.2032 & S & 2132.9376 & 1066.9724 & 2115.9110 & 1058.4591 & 2114.9270 & 1057.9671 & 20 \\
\hline 9 & 910.4451 & 455.7262 & & & 892.4346 & 446.7209 & $\mathbf{M}$ & 2045.9055 & 1023.4564 & 2028.8790 & 1014.9431 & 2027.8950 & 1014.4511 & 19 \\
\hline 10 & 981.4822 & 491.2448 & & & 963.4717 & 482.2395 & A & 1898.8701 & 949.9387 & 1881.8436 & 941.4254 & 1880.8596 & 940.9334 & 18 \\
\hline 11 & 1095.5252 & 548.2662 & 1078.4986 & 539.7529 & 1077.5146 & 539.2609 & $\mathbf{N}$ & 1827.8330 & 914.4201 & 1810.8065 & 905.9069 & 1809.8225 & 905.4149 & 17 \\
\hline 12 & 1166.5623 & 583.7848 & 1149.5357 & 575.2715 & 1148.5517 & 574.7795 & A & 1713.7901 & 857.3987 & 1696.7635 & 848.8854 & 1695.7795 & 848.3934 & 16 \\
\hline 13 & 1223.5837 & 612.2955 & 1206.5572 & 603.7822 & 1205.5732 & 603.2902 & G & 1642.7530 & 821.8801 & 1625.7264 & 813.3669 & 1624.7424 & 812.8748 & 15 \\
\hline 14 & 1320.6365 & 660.8219 & 1303.6100 & 652.3086 & 1302.6259 & 651.8166 & $\mathbf{P}$ & 1585.7315 & 793.3694 & 1568.7050 & 784.8561 & 1567.7209 & 784.3641 & 14 \\
\hline 15 & 1434.6794 & 717.8434 & 1417.6529 & 709.3301 & 1416.6689 & 708.8381 & $\mathbf{N}$ & 1488.6787 & 744.8430 & 1471.6522 & 736.3297 & 1470.6682 & 735.8377 & 13 \\
\hline 16 & 1535.7271 & 768.3672 & 1518.7006 & 759.8539 & 1517.7165 & 759.3619 & $\mathbf{T}$ & 1374.6358 & 687.8215 & 1357.6093 & 679.3083 & 1356.6253 & 678.8163 & 12 \\
\hline 17 & 1650.7540 & 825.8807 & 1633.7275 & 817.3674 & 1632.7435 & 816.8754 & $\mathbf{N}$ & 1273.5881 & 637.2977 & 1256.5616 & 628.7844 & 1255.5776 & 628.2924 & 11 \\
\hline 18 & 1707.7755 & 854.3914 & 1690.7490 & 845.8781 & 1689.7649 & 845.3861 & G & 1158.5612 & 579.7842 & 1141.5347 & 571.2710 & 1140.5506 & 570.7790 & 10 \\
\hline 19 & 1794.8075 & 897.9074 & 1777.7810 & 889.3941 & 1776.7970 & 888.9021 & S & 1101.5397 & 551.2735 & 1084.5132 & 542.7602 & 1083.5292 & 542.2682 & 9 \\
\hline 20 & 1922.8661 & 961.9367 & 1905.8396 & 953.4234 & 1904.8555 & 952.9314 & $\mathbf{Q}$ & 1014.5077 & 507.7575 & 997.4812 & 499.2442 & 996.4972 & 498.7522 & 8 \\
\hline 21 & 2069.9345 & 1035.4709 & 2052.9080 & 1026.9576 & 2051.9240 & 1026.4656 & $\mathbf{F}$ & 886.4491 & 443.7282 & 869.4226 & 435.2149 & 868.4386 & 434.7229 & 7 \\
\hline 22 & 2217.0029 & 1109.0051 & 2199.9764 & 1100.4918 & 2198.9924 & 1099.9998 & $\mathbf{F}$ & 739.3807 & 370.1940 & 722.3542 & 361.6807 & 721.3702 & 361.1887 & 6 \\
\hline 23 & 2330.0870 & 1165.5471 & 2313.0605 & 1157.0339 & 2312.0764 & 1156.5419 & I & 592.3123 & 296.6598 & 575.2858 & 288.1465 & 574.3017 & 287.6545 & 5 \\
\hline 24 & 2490.1177 & 1245.5625 & 2473.0911 & 1237.0492 & 2472.1071 & 1236.5572 & $\mathrm{C}$ & 479.2282 & 240.1178 & 462.2017 & 231.6045 & 461.2177 & 231.1125 & 4 \\
\hline 25 & 2591.1653 & 1296.0863 & 2574.1388 & 1287.5730 & 2573.1548 & 1287.0810 & $\mathbf{T}$ & 319.1976 & 160.1024 & 302.1710 & 151.5892 & 301.1870 & 151.0972 & 3 \\
\hline 26 & 2662.2024 & 1331.6049 & 2645.1759 & 1323.0916 & 2644.1919 & 1322.5996 & A & 218.1499 & 109.5786 & 201.1234 & 101.0653 & & & 2 \\
\hline 27 & & & & & & & $\mathrm{~K}$ & 147.1128 & 74.0600 & 130.0863 & 65.5468 & & & 1 \\
\hline
\end{tabular}



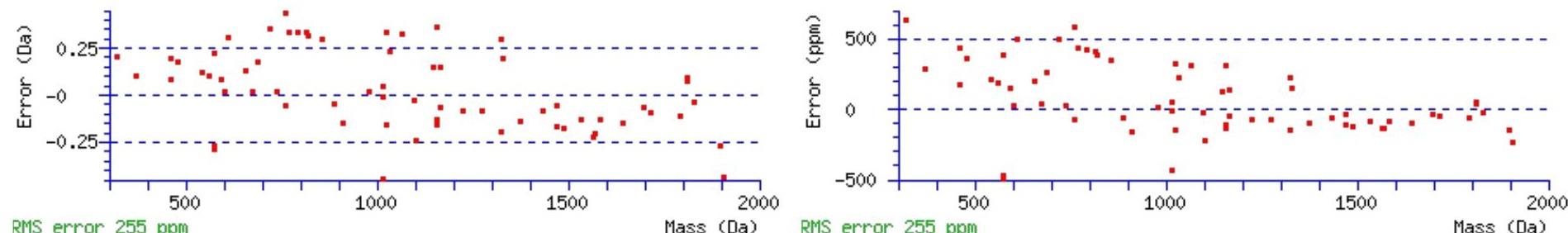

RMS error 255 ppm Mass (Da) RMS error 255 ppm

Mass (Da)

\section{All matches to this query}

\begin{tabular}{|l|l|l|l|}
\hline Score & Mr(calc): & Delta & \multicolumn{1}{|c|}{ Sequence } \\
\hline 93.7 & 2807.3007 & -0.0106 & HTGPGILSMANAGPNTNGSQFFICTAK \\
\hline 70.9 & 2807.3007 & -0.0106 & $\underline{\text { HTGPGILSMANAGPNTNGSQFFICTAK }}$ \\
\hline 39.0 & 2807.3007 & -0.0106 & $\underline{\text { HTGPGILSMANAGPNTNGSQFFICTAK }}$ \\
\hline 6.1 & 2807.2967 & -0.0066 & $\underline{\text { DASMLGSLAYNVLLIALCTLYAFK }}$ \\
\hline 4.5 & 2805.3060 & 1.9841 & $\underline{\text { LNSLLKNLKFMNPGGDISLNPSEK }}$ \\
\hline 3.8 & 2805.2793 & 2.0109 & $\underline{\text { QLHLPERSPDDSPPGAPAPGPMSASAK }}$ \\
\hline 2.9 & 2807.2919 & -0.0017 & MANYTNLTQGAKEHEEAENITEGKK \\
\hline 1.9 & 2807.2919 & -0.0017 & MANYTNLTQGAKEHEEAENITEGKK \\
\hline 1.4 & 2807.3062 & -0.0160 & QPSASAPAQSSTAPCLSSXPALPRQR \\
\hline 1.4 & 2807.3062 & -0.0160 & QPSASAPAQSSTAPCLSSXPALPRQR \\
\hline
\end{tabular}

Spectrum No: 115; Query: 1893; Rank: 1

\section{Peptide View}

MS/MS Fragmentation of EGNSALINCSAFGIPDLEYK

Found in IPI00188728, Tax_Id=10116 Gene_Symbol=Mfap3l Microfibrillar-associated protein 3-like precursor

Match to Query 1893: 2199.022968 from(1100.518760,2+)

Title: 100101RatKid_NS_deglyco_27.4337.4337.2.dta

Data file K:INewmanPaper|Piliangl3SubProteomes\Piliang3SP\mgf5ppm|ERLIC_3SubProteomes5ppm.mgf

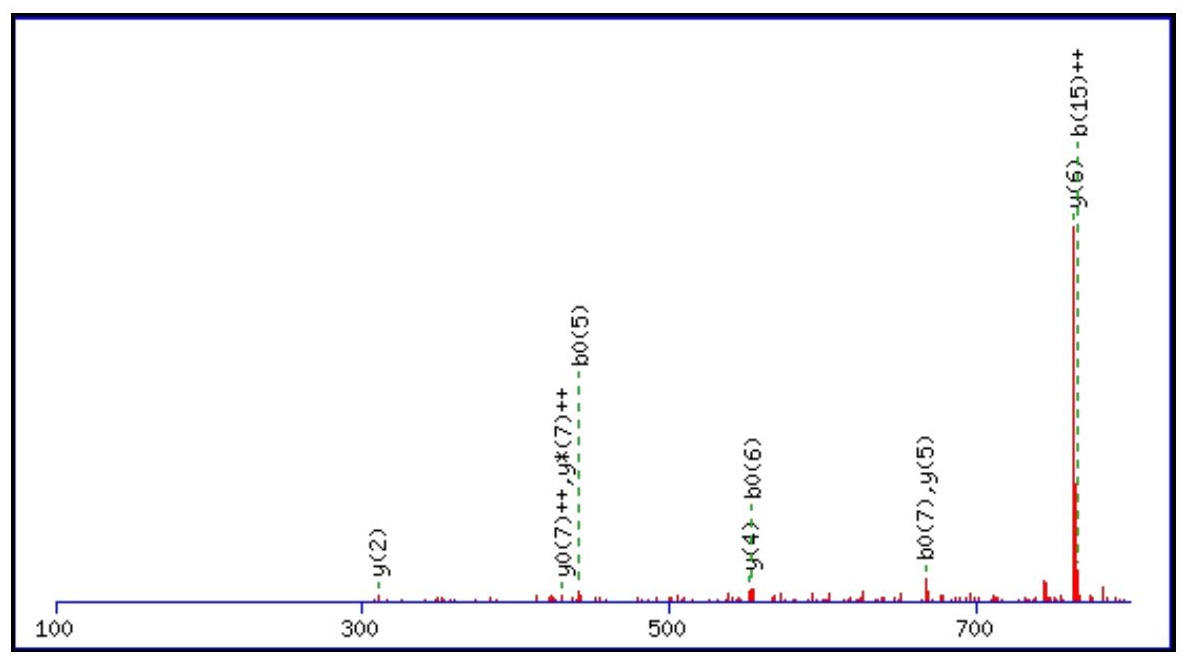




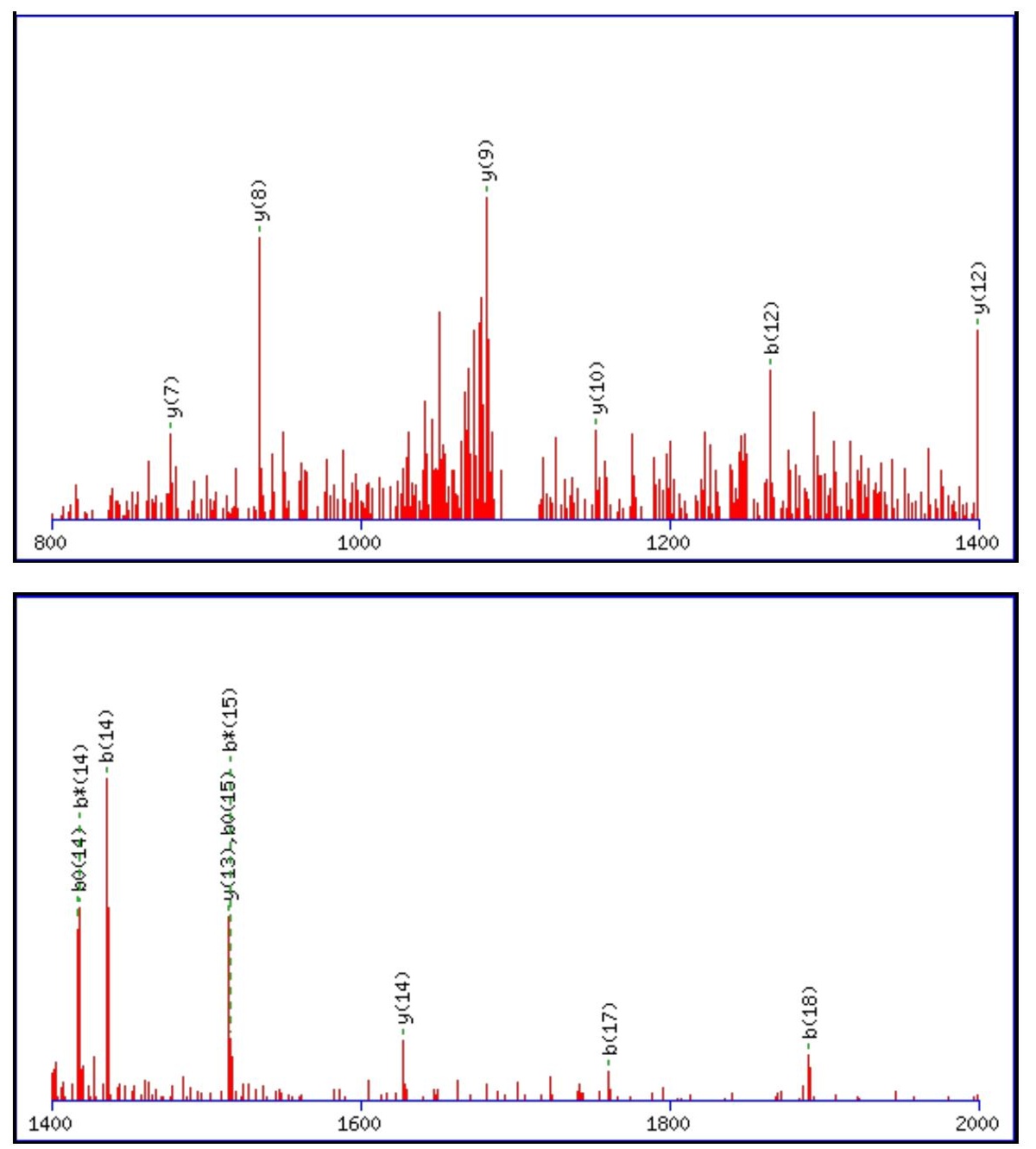

Monoisotopic mass of neutral peptide $\operatorname{Mr}($ calc): 2198.0201

Fixed modifications: Carbamidomethyl (C)

Variable modifications:

N8: : Deamidated $\mathrm{N}(\mathrm{N})$

Ions Score: 93 Expect: $1.3 \mathrm{e}-007$

Matches (Bold Red): 25/220 fragment ions using 30 most intense peaks

\begin{tabular}{|c|c|c|c|c|c|c|c|c|c|c|c|c|c|c|}
\hline \# & b & $\mathbf{b}^{++}$ & $\mathbf{b}^{*}$ & $\mathbf{b}^{*^{++}}$ & $\mathbf{b}^{\mathbf{0}}$ & $\mathbf{b}^{0++}$ & Seq. & $\mathbf{y}$ & $y^{++}$ & $\mathbf{y}^{*}$ & $\mathrm{y}^{\mathrm{*}^{++}}$ & $\mathbf{y}^{0}$ & $\mathbf{y}^{0++}$ & \# \\
\hline 1 & 130.0499 & 65.5286 & & & 112.0393 & 56.5233 & $\mathbf{E}$ & & & & & & & 20 \\
\hline 2 & 187.0713 & 94.0393 & & & 169.0608 & 85.0340 & G & 2069.9848 & 1035.4961 & 2052.9583 & 1026.9828 & 2051.9743 & 1026.4908 & 19 \\
\hline 4 & 388.1463 & 194.5768 & 371.1197 & 186.0635 & 370.1357 & 185.5715 & $\mathrm{~S}$ & 1898.9204 & 949.9639 & 1881.8939 & 941.4506 & 1880.9099 & 940.9586 & 17 \\
\hline 5 & 459.1834 & 230.0953 & 442.1569 & 221.5821 & 441.1728 & 221.0901 & A & 1811.8884 & 906.4478 & 1794.8619 & 897.9346 & 1793.8778 & 897.4426 & 16 \\
\hline 6 & 572.2675 & 286.6374 & 555.2409 & 278.1241 & 554.2569 & 277.6321 & $\mathbf{L}$ & 1740.8513 & 870.9293 & 1723.8247 & 862.4160 & 1722.8407 & 861.9240 & 15 \\
\hline 7 & 685.3515 & 343.1794 & 668.3250 & 334.6661 & 667.3410 & 334.1741 & $\mathbf{I}$ & 1627.7672 & 814.3873 & 1610.7407 & 805.8740 & 1609.7567 & 805.3820 & 14 \\
\hline 8 & 800.3785 & 400.6929 & 783.3519 & 392.1796 & 782.3679 & 391.6876 & $\mathbf{N}$ & 1514.6832 & 757.8452 & 1497.6566 & 749.3319 & 1496.6726 & 748.8399 & 13 \\
\hline 9 & 960.4091 & 480.7082 & 943.3826 & 472.1949 & 942.3986 & 471.7029 & $\mathrm{C}$ & 1399.6562 & 700.3318 & 1382.6297 & 691.8185 & 1381.6457 & 691.3265 & 12 \\
\hline 10 & 1047.4411 & 524.2242 & 1030.4146 & 515.7109 & 1029.4306 & 515.2189 & $\mathrm{~S}$ & 1239.6256 & 620.3164 & 1222.5990 & 611.8032 & 1221.6150 & 611.3111 & 11 \\
\hline 11 & 1118.4783 & 559.7428 & 1101.4517 & 551.2295 & 1100.4677 & 550.7375 & A & 1152.5936 & 576.8004 & 1135.5670 & 568.2871 & 1134.5830 & 567.7951 & 10 \\
\hline 12 & 1265.5467 & 633.2770 & 1248.5201 & 624.7637 & 1247.5361 & 624.2717 & $\mathbf{F}$ & 1081.5564 & 541.2819 & 1064.5299 & 532.7686 & 1063.5459 & 532.2766 & 9 \\
\hline 14 & 1435.6522 & 718.3297 & 1418.6257 & 709.8165 & 1417.6416 & 709.3245 & I & 877.4666 & 439.2369 & 860.4400 & 430.7236 & 859.4560 & 430.2316 & 7 \\
\hline 15 & 1532.7050 & 766.8561 & 1515.6784 & 758.3428 & 1514.6944 & 757.8508 & $\mathbf{P}$ & 764.3825 & 382.6949 & 747.3559 & 374.1816 & 746.3719 & 373.6896 & 6 \\
\hline 16 & 1647.7319 & 824.3696 & 1630.7054 & 815.8563 & 1629.7213 & 815.3643 & D & 667.3297 & 334.1685 & 650.3032 & 325.6552 & 649.3192 & 325.1632 & 5 \\
\hline 17 & 1760.8160 & 880.9116 & 1743.7894 & 872.3983 & 1742.8054 & 871.9063 & $\mathbf{L}$ & 552.3028 & 276.6550 & 535.2762 & 268.1418 & 534.2922 & 267.6498 & 4 \\
\hline 18 & 1889.8586 & 945.4329 & 1872.8320 & 936.9196 & 1871.8480 & 936.4276 & $\mathbf{E}$ & 439.2187 & 220.1130 & 422.1922 & 211.5997 & 421.2082 & 211.1077 & 3 \\
\hline
\end{tabular}




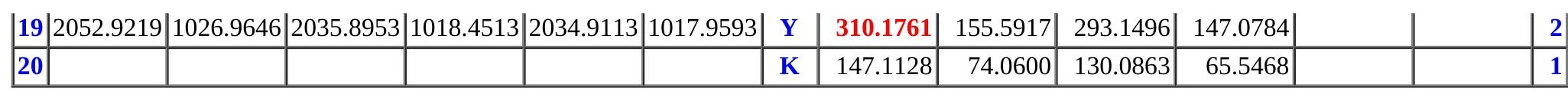
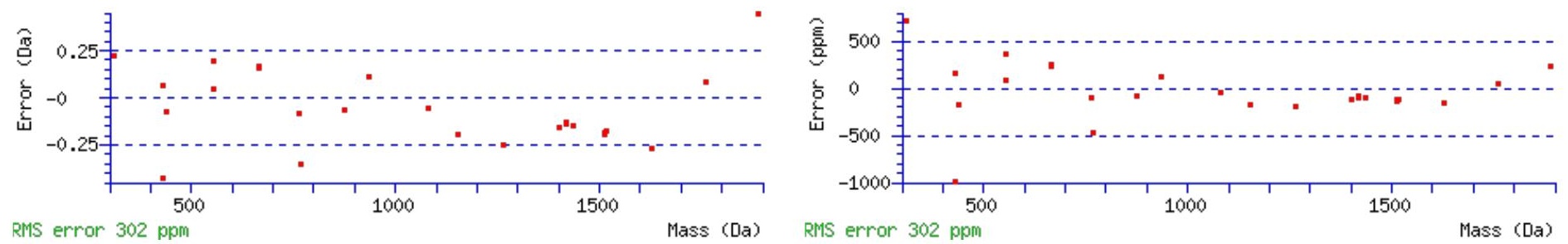

\section{All matches to this query}

\begin{tabular}{|l|l|l|l|}
\hline Score & Mr(calc): & Delta & \multicolumn{1}{c|}{ Sequence } \\
\hline 93.1 & 2198.0201 & 1.0029 & EGNSALINCSAFGIPDLEYK \\
\hline 66.8 & 2199.0041 & 0.0188 & EGNSALINCSAFGIPDLEYK \\
\hline 57.1 & 2198.0201 & 1.0029 & EGNSALINCSAFGIPDLEYK \\
\hline 40.3 & 2197.0361 & 1.9869 & EGNSALINCSAFGIPDLEYK \\
\hline 5.4 & 2197.0142 & 2.0087 & LCTVNSVEEKILAAAKYK \\
\hline 4.5 & 2199.0395 & -0.0165 & LSMKPLSDGPPDPKSHELR \\
\hline 3.3 & 2197.0069 & 2.0161 & $\underline{\text { SRNDLPLLPAOTTATPDVK }}$ \\
\hline 3.3 & 2197.0069 & 2.0161 & $\underline{\text { SRNDLPLLPAOTTATPDVK }}$ \\
\hline 3.3 & 2197.0069 & 2.0161 & $\underline{\text { SRNDLPLLPAOTTATPDVK }}$ \\
\hline 0.9 & 2198.0320 & 0.9910 & $\underline{\text { ATGIRKGISPMNRPPLSDK }}$ \\
\hline
\end{tabular}

Spectrum No: 116; Query: 1378; Rank: 1

\section{Peptide View}

MS/MS Fragmentation of TSLQPMVSALNISLGGTGK

Found in IPI00198021, Tax_Id=10116 Gene_Symbol=Umod Uromodulin precursor

Match to Query 1378: 1873.990808 from(938.002680,2+)

Title: 100101RatKid_NS_deglyco_20.4676.4676.2.dta

Data file K:INewmanPaper|Piliang 3 SubProteomes\Piliang3SP\mgf5ppm\ERLIC_3SubProteomes5ppm.mgf

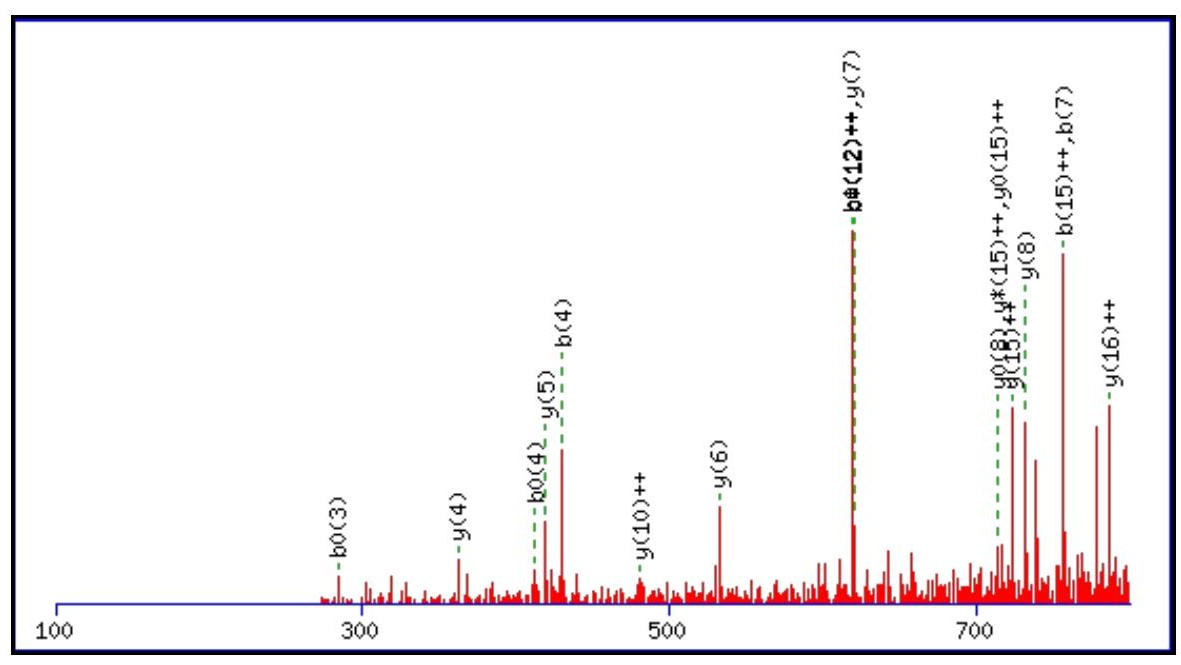



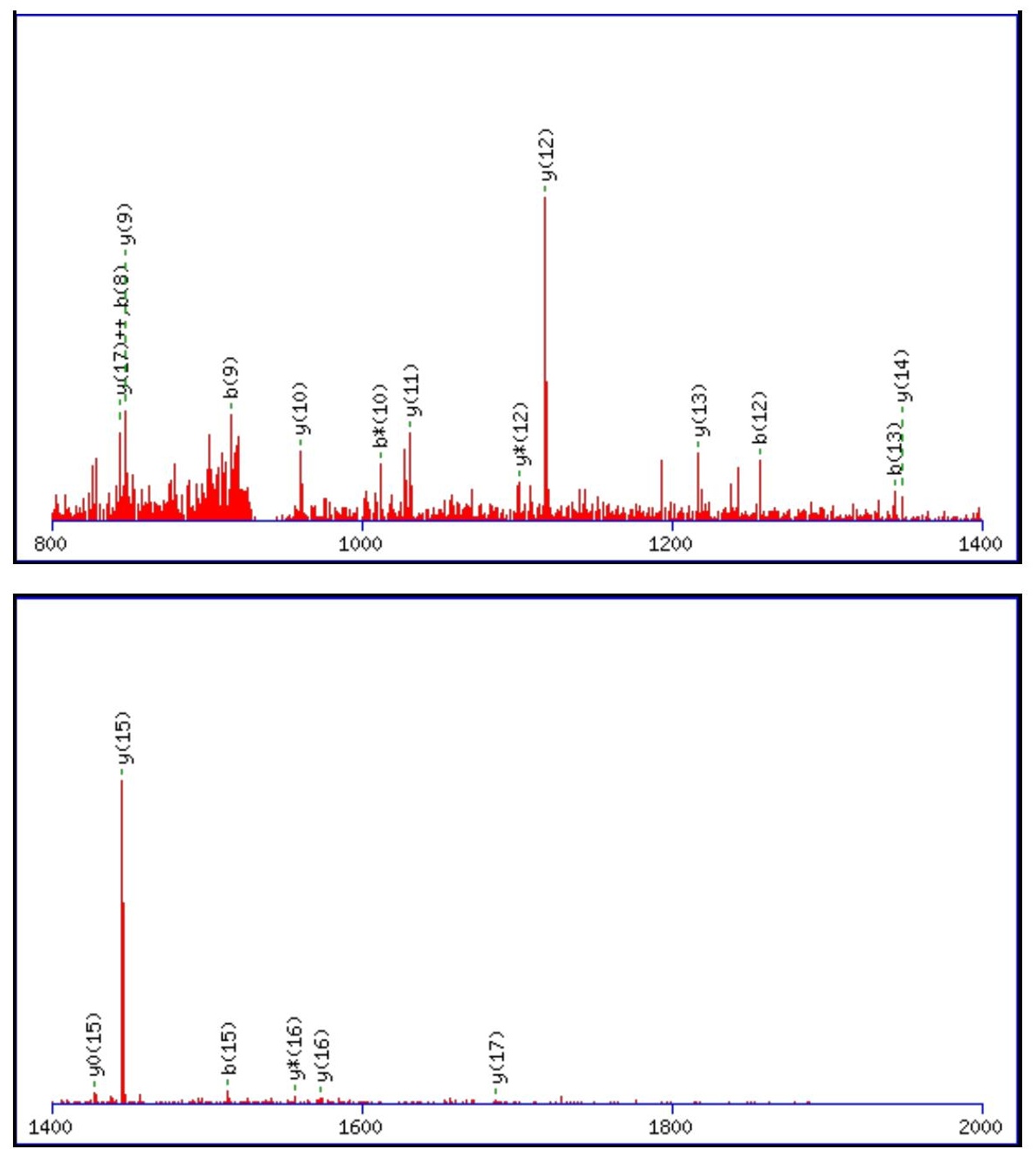

Monoisotopic mass of neutral peptide $\operatorname{Mr}($ calc): 1873.9819

Fixed modifications: Carbamidomethyl (C)

Variable modifications:

N11 : Deamidated_N (N)

Ions Score: 93 Expect: $6 \mathrm{e}-008$

Matches (Bold Red): 37/206 fragment ions using 54 most intense peaks

\begin{tabular}{|c|c|c|c|c|c|c|c|c|c|c|c|c|c|c|}
\hline \# & b & $\mathbf{b}^{++}$ & $\mathbf{b}^{*}$ & $\mathbf{b}^{*^{++}}$ & $\mathbf{b}^{0}$ & $\mathbf{b}^{0++}$ & Seq. & $\mathbf{y}$ & $\mathbf{y}^{++}$ & $\mathbf{y}^{*}$ & $\mathrm{y}^{*^{++}}$ & $\mathbf{y}^{\mathbf{0}}$ & $y^{0++}$ & \# \\
\hline 1 & 102.0550 & 51.5311 & & & 84.0444 & 42.5258 & $\mathbf{T}$ & & & & & & & 19 \\
\hline 2 & 189.0870 & 95.0471 & & & 171.0764 & 86.0418 & S & 1773.9415 & 887.4744 & 1756.9150 & 878.9611 & 1755.9309 & 878.4691 & 18 \\
\hline 3 & 302.1710 & 151.5892 & & & 284.1605 & 142.5839 & $\mathbf{L}$ & 1686.9095 & 843.9584 & 1669.8829 & 835.4451 & 1668.8989 & 834.9531 & 17 \\
\hline 4 & 430.2296 & 215.6185 & 413.2031 & 207.1052 & 412.2191 & 206.6132 & $\mathbf{Q}$ & 1573.8254 & 787.4163 & 1556.7989 & 778.9031 & 1555.8148 & 778.4111 & 16 \\
\hline 5 & 527.2824 & 264.1448 & 510.2558 & 255.6316 & 509.2718 & 255.1396 & $\mathbf{P}$ & 1445.7668 & 723.3871 & 1428.7403 & 714.8738 & 1427.7563 & 714.3818 & 15 \\
\hline 6 & 658.3229 & 329.6651 & 641.2963 & 321.1518 & 640.3123 & 320.6598 & $\mathbf{M}$ & 1348.7141 & 674.8607 & 1331.6875 & 666.3474 & 1330.7035 & 665.8554 & 14 \\
\hline 7 & 757.3913 & 379.1993 & 740.3647 & 370.6860 & 739.3807 & 370.1940 & $\mathbf{V}$ & 1217.6736 & 609.3404 & 1200.6470 & 600.8272 & 1199.6630 & 600.3351 & 13 \\
\hline 8 & 844.4233 & 422.7153 & 827.3968 & 414.2020 & 826.4128 & 413.7100 & S & 1118.6052 & 559.8062 & 1101.5786 & 551.2929 & 1100.5946 & 550.8009 & 12 \\
\hline 9 & 915.4604 & 458.2339 & 898.4339 & 449.7206 & 897.4499 & 449.2286 & A & 1031.5731 & 516.2902 & 1014.5466 & 507.7769 & 1013.5626 & 507.2849 & 11 \\
\hline 10 & 1028.5445 & 514.7759 & 1011.5179 & 506.2626 & 1010.5339 & 505.7706 & $\mathbf{L}$ & 960.5360 & 480.7717 & 943.5095 & 472.2584 & 942.5255 & 471.7664 & 10 \\
\hline 11 & 1143.5714 & 572.2894 & 1126.5449 & 563.7761 & 1125.5609 & 563.2841 & $\mathbf{N}$ & 847.4520 & 424.2296 & 830.4254 & 415.7163 & 829.4414 & 415.2243 & 9 \\
\hline 12 & 1256.6555 & 628.8314 & 1239.6289 & 620.3181 & 1238.6449 & 619.8261 & I & 732.4250 & 366.7162 & 715.3985 & 358.2029 & 714.4145 & 357.7109 & 8 \\
\hline 13 & 1343.6875 & 672.3474 & 1326.6610 & 663.8341 & 1325.6770 & 663.3421 & S & 619.3410 & 310.1741 & 602.3144 & 301.6608 & 601.3304 & 301.1688 & 7 \\
\hline 14 & 1456.7716 & 728.8894 & 1439.7450 & 720.3762 & 1438.7610 & 719.8841 & $\mathbf{L}$ & 532.3089 & 266.6581 & 515.2824 & 258.1448 & 514.2984 & 257.6528 & 6 \\
\hline 15 & 1513.7930 & 757.4002 & 1496.7665 & 748.8869 & 1495.7825 & 748.3949 & G & 419.2249 & 210.1161 & 402.1983 & 201.6028 & 401.2143 & 201.1108 & 5 \\
\hline 16 & 1570.8145 & 785.9109 & 1553.7880 & 777.3976 & 1552.8039 & 776.9056 & $\mathbf{G}$ & 362.2034 & 181.6053 & 345.1769 & 173.0921 & 344.1928 & 172.6001 & 4 \\
\hline 17 & 1671.8622 & 836.4347 & 1654.8356 & 827.9215 & 1653.8516 & 827.4295 & $\mathbf{T}$ & 305.1819 & 153.0946 & 288.1554 & 144.5813 & 287.1714 & 144.0893 & 3 \\
\hline 18 & 1728.8837 & 864.9455 & 1711.8571 & 856.4322 & 1710.8731 & 855.9402 & G & 204.1343 & 102.5708 & 187.1077 & 94.0575 & & & 2 \\
\hline
\end{tabular}




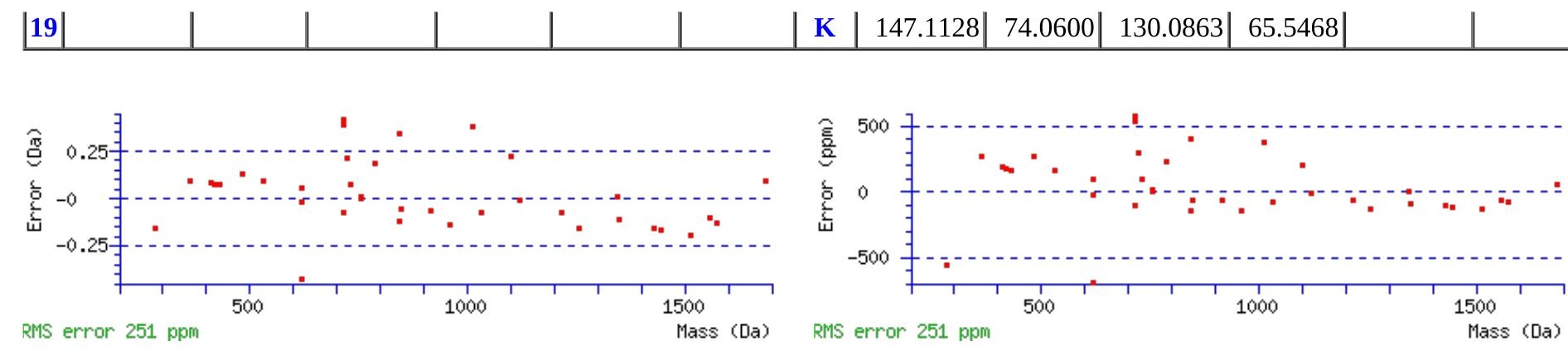

\section{All matches to this query}

\begin{tabular}{|l|l|l|l|}
\hline Score & Mr(calc): & Delta & \multicolumn{1}{c|}{ Sequence } \\
\hline 92.8 & 1873.9819 & 0.0089 & TSLQPMVSALNISLGGTGK \\
\hline 21.0 & 1872.9979 & 0.9929 & TSLQPMVSALNISLGGTGK \\
\hline 6.0 & 1872.9913 & 0.9995 & QNGKLCLVIQVANMRK \\
\hline 1.1 & 1871.9788 & 2.0120 & NGKGAGTHCLKLNSFLR \\
\hline
\end{tabular}

Spectrum No: 117; Query: 763; Rank: 1

\section{Peptide View}

MS/MS Fragmentation of NGSEADIDESLYSR

Found in IPI00368347, Tax_Id=10116 Gene_Symbol=Ube1x Ubiquitin-activating enzyme E1, Chr X

Match to Query 763: 1555.665028 from(778.839790,2+)

Title: 091008RatKidney_NoSalt_23.1775.1775.2.dta

Data file K:INewmanPaper|Piliang|3SubProteomes\Piliang3SP\mgf5ppm\ERLIC_3SubProteomes5ppm.mgf

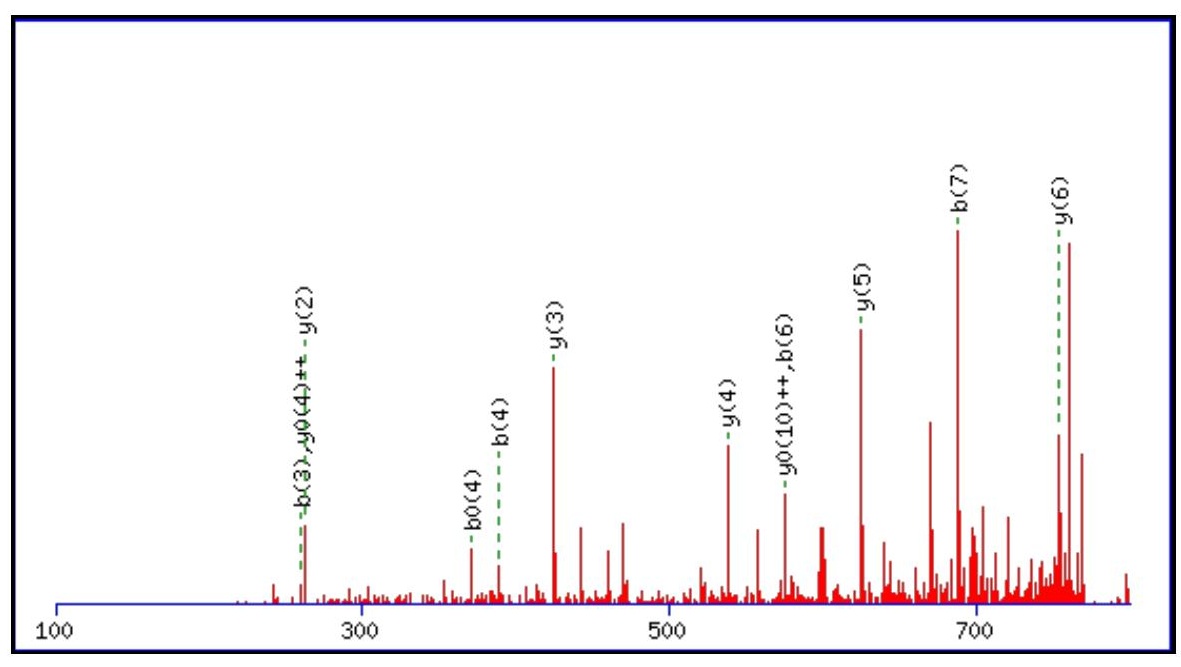



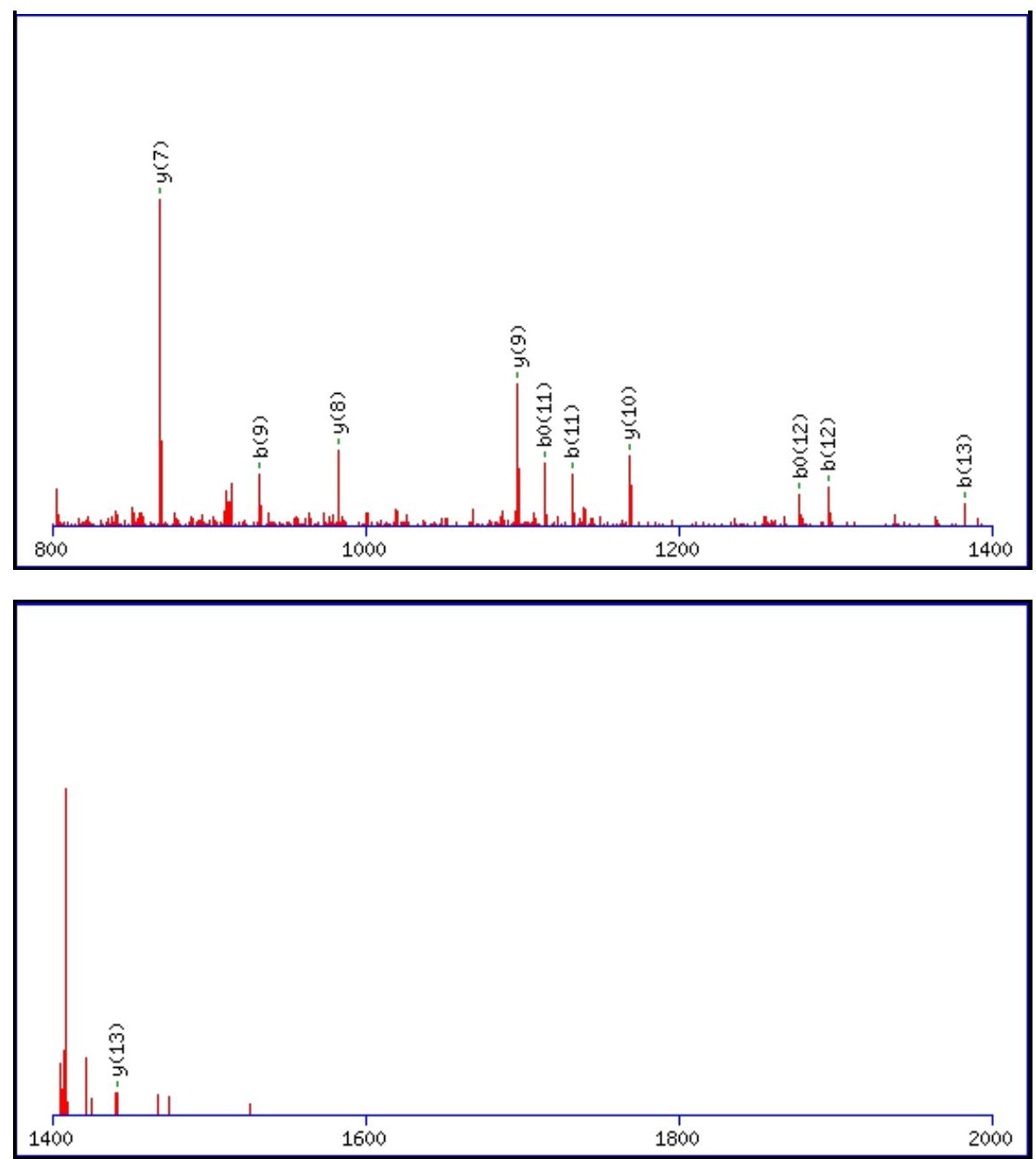

Monoisotopic mass of neutral peptide $\operatorname{Mr}($ calc): 1555.6638

Fixed modifications: Carbamidomethyl (C)

Variable modifications:

N1 : Deamidated $\mathrm{N}(\mathrm{N})$

Ions Score: 93 Expect: $7.6 \mathrm{e}-008$

Matches (Bold Red): 23/150 fragment ions using 31 most intense peaks

\begin{tabular}{|c|c|c|c|c|c|c|c|c|c|c|c|c|c|c|}
\hline \# & b & $\mathbf{b}^{++}$ & $\mathbf{b}^{*}$ & $\mathbf{b}^{*^{++}}$ & $\mathbf{b}^{0}$ & $\mathbf{b}^{0++}$ & Seq. & $\mathbf{y}$ & $\mathbf{y}^{++}$ & $\mathbf{y}^{*}$ & $\mathrm{y}^{*^{++}}$ & $\mathbf{y}^{\mathbf{0}}$ & $\mathbf{y}^{\mathbf{0 + +}}$ & \# \\
\hline 1 & 116.0342 & 58.5207 & 99.0077 & 50.0075 & & & $\mathbf{N}$ & & & & & & & 14 \\
\hline 2 & 173.0557 & 87.0315 & 156.0291 & 78.5182 & & & $\mathbf{G}$ & 1441.6441 & 721.3257 & 1424.6176 & 712.8124 & 1423.6336 & 712.3204 & 13 \\
\hline 3 & 260.0877 & 130.5475 & 243.0612 & 122.0342 & 242.0771 & & $\mathbf{S}$ & 1384.6227 & 692.8150 & 1367.5961 & 684.3017 & 1366.6121 & 683.8097 & 12 \\
\hline 4 & 389.1303 & 195.0688 & 372.1037 & 186.5555 & 371.1197 & 186.0635 & $\mathbf{E}$ & 1297.5907 & 649.2990 & 1280.5641 & 640.7857 & 1279.5801 & 640.2937 & 11 \\
\hline 5 & 460.1674 & 230.5873 & 443.1409 & 222.0741 & 442.1568 & 221.5821 & A & 1168.5481 & 584.7777 & 1151.5215 & 576.2644 & 1150.5375 & 575.7724 & 10 \\
\hline 6 & 575.1944 & 288.1008 & 558.1678 & 279.5875 & 557.1838 & 279.0955 & D & 1097.5109 & 549.2591 & 1080.4844 & 540.7458 & 1079.5004 & 540.2538 & 9 \\
\hline 7 & 688.2784 & 344.6428 & 671.2519 & 336.1296 & 670.2679 & 335.6376 & I & 982.4840 & 491.7456 & 965.4575 & 483.2324 & 964.4734 & 482.7404 & 8 \\
\hline 8 & 803.3054 & 402.1563 & 786.2788 & 393.6430 & 785.2948 & 393.1510 & D & 869.3999 & 435.2036 & 852.3734 & 426.6903 & 851.3894 & 426.1983 & 7 \\
\hline 9 & 932.3480 & 466.6776 & 915.3214 & 458.1643 & 914.3374 & 457.6723 & $\mathbf{E}$ & 754.3730 & 377.6901 & 737.3464 & 369.1769 & 736.3624 & 368.6849 & 6 \\
\hline 10 & 1019.3800 & 510.1936 & 1002.3534 & 501.6804 & 1001.3694 & 501.1883 & $\mathrm{~S}$ & 625.3304 & 313.1688 & 608.3039 & 304.6556 & 607.3198 & 304.1636 & 5 \\
\hline 11 & 1132.4640 & 566.7357 & 1115.4375 & 558.2224 & 1114.4535 & 557.7304 & $\mathbf{L}$ & 538.2984 & 269.6528 & 521.2718 & 261.1396 & 520.2878 & 260.6475 & 4 \\
\hline 12 & 1295.5274 & 648.2673 & 1278.5008 & 639.7541 & 1277.5168 & 639.2620 & $\mathbf{Y}$ & 425.2143 & 213.1108 & 408.1878 & 204.5975 & 407.2037 & 204.1055 & 3 \\
\hline 13 & 1382.5594 & 691.7833 & 1365.5329 & 683.2701 & 1364.5488 & 682.7781 & $S$ & 262.1510 & 131.5791 & 245.1244 & 123.0659 & 244.1404 & 122.5738 & 2 \\
\hline 14 & & & & & & & $\mathbf{R}$ & 175.1190 & 88.0631 & 158.0924 & 79.5498 & & & 1 \\
\hline
\end{tabular}



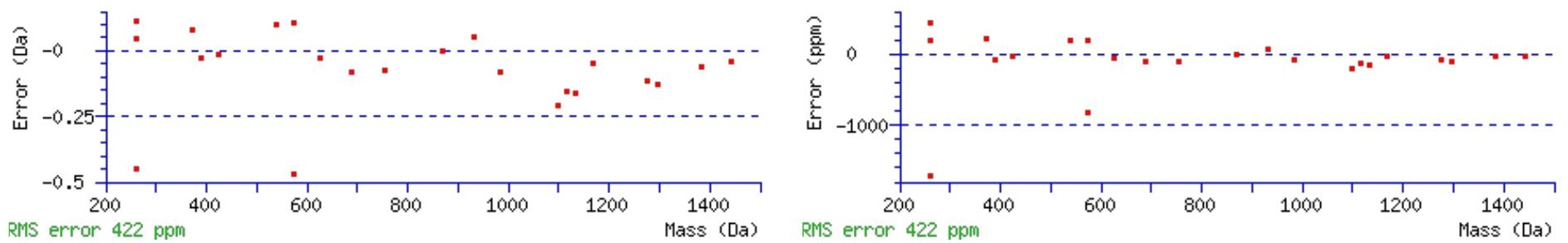

\section{All matches to this query}

\begin{tabular}{|l|l|l|l|}
\hline Score & Mr(calc): & Delta & \multicolumn{1}{|c|}{ Sequence } \\
\hline 92.8 & 1555.6638 & 0.0013 & NGSEADIDESLYSR \\
\hline 16.4 & 1553.6507 & 2.0144 & MKMFESLDSSATK \\
\hline 16.3 & 1555.6784 & -0.0134 & QLETSETSSMGQSR \\
\hline 14.6 & 1555.6780 & -0.0130 & YQPRDEEVRER \\
\hline 13.6 & 1553.6507 & 2.0144 & MKMFESLDSSATK \\
\hline 13.6 & 1553.6507 & 2.0144 & MKMFESLDSSATK \\
\hline 7.9 & 1555.6799 & -0.0149 & TLNMDMFTWEPR \\
\hline 6.3 & 1555.6603 & 0.0047 & SHRLGGTMPFDSR \\
\hline 4.8 & 1555.6780 & -0.0130 & KESNEGRHYISR \\
\hline 3.9 & 1554.6637 & 1.0013 & TAMGPSSTTNLIEGP \\
\hline
\end{tabular}

Spectrum No: 118; Query: 2252; Rank: 1

\section{Peptide View}

MS/MS Fragmentation of YGMQNSGNEAAWNYTLEQYQK

Found in IPI00327398, Tax_Id=10116 Gene_Symbol=Enpep Isoform 1 of Glutamyl aminopeptidase

Match to Query 2252: 2511.072288 from(1256.543420,2+)

Title: 100101RatKid_NS_deglyco_21.3338.3338.2.dta

Data file K:INewmanPaper|Piliangl3SubProteomes\Piliang3SP\mgf5ppm|ERLIC_3SubProteomes5ppm.mgf

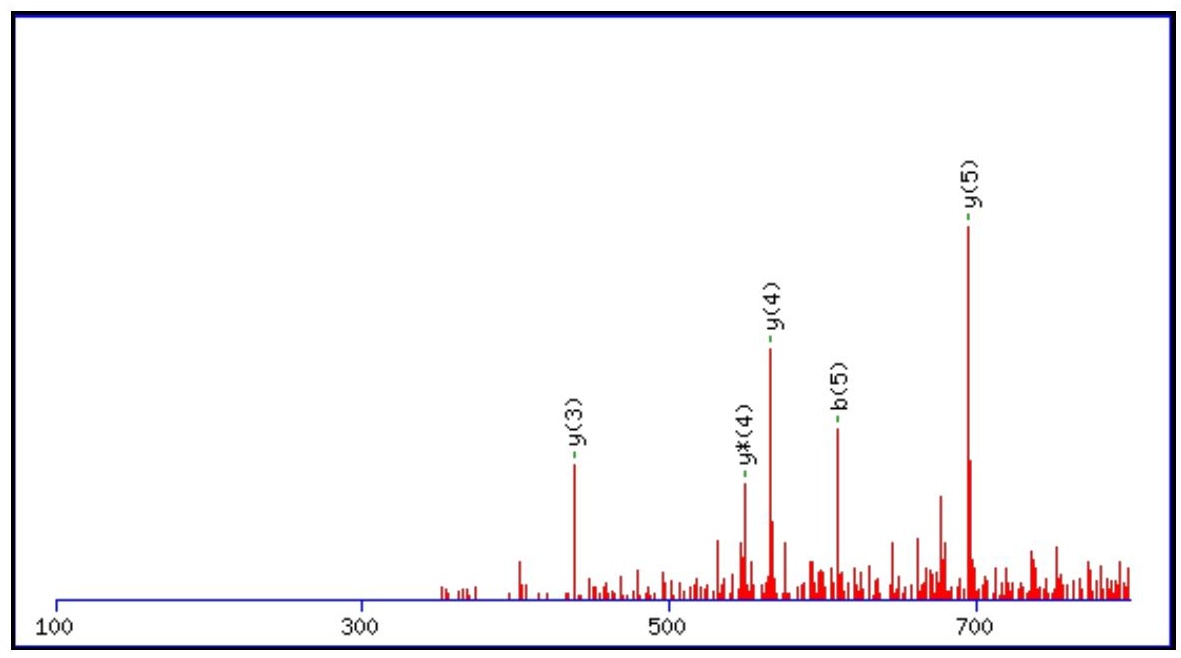




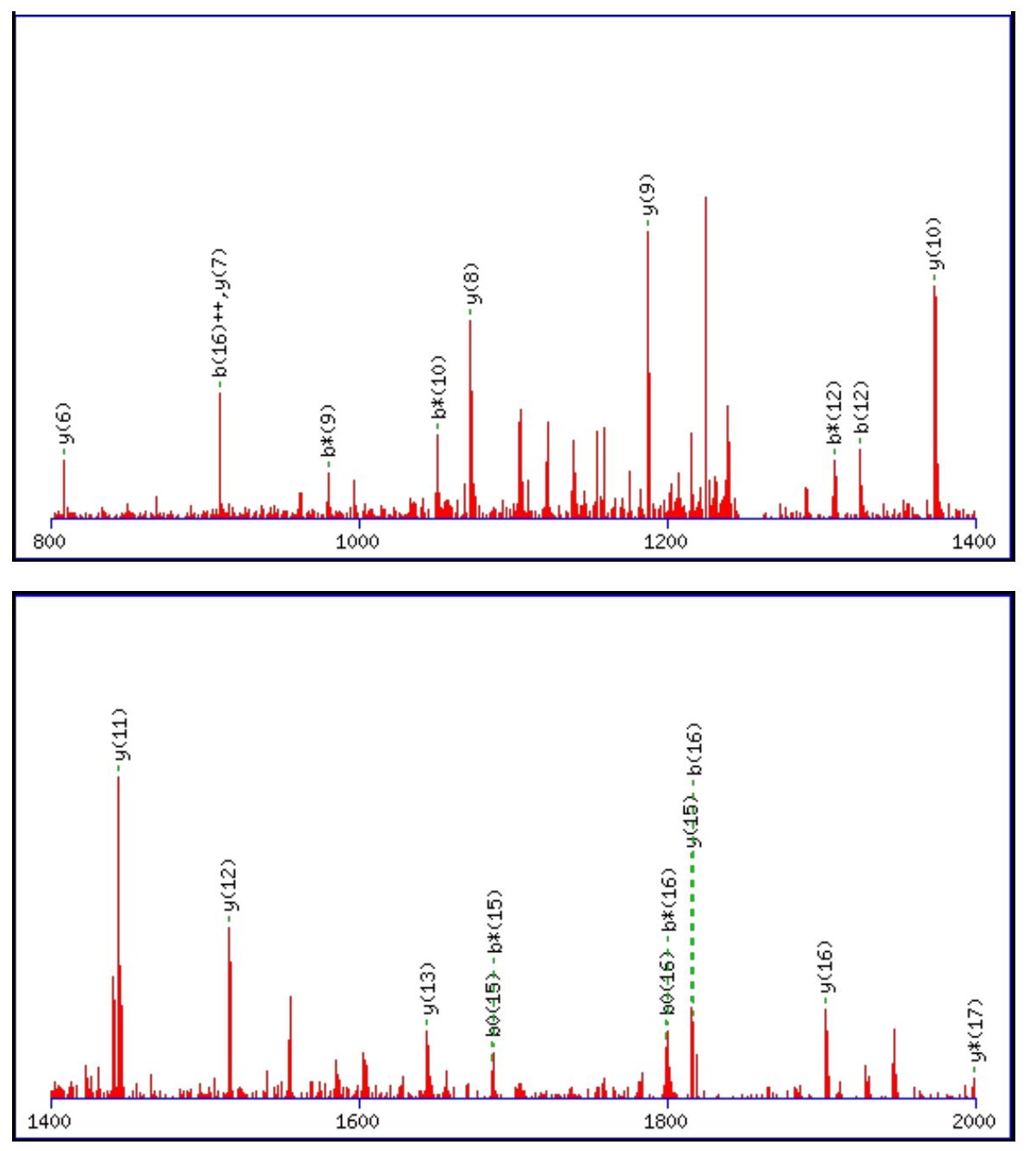

Monoisotopic mass of neutral peptide $\operatorname{Mr}($ calc): 2511.0648

Fixed modifications: Carbamidomethyl (C)

Variable modifications:

M3 : Oxidation (M)

N13 : Deamidated_N (N)

Ions Score: 93 Expect: $1.5 \mathrm{e}-007$

Matches (Bold Red): 26/216 fragment ions using 41 most intense peaks

\begin{tabular}{|c|c|c|c|c|c|c|c|c|c|c|c|c|c|c|}
\hline \# & b & $\mathbf{b}^{++}$ & $\mathbf{b}^{*}$ & $\mathbf{b}^{*^{++}}$ & $\mathbf{b}^{\mathbf{0}}$ & $\mathbf{b}^{\mathbf{0 + +}}$ & Seq. & $\mathbf{y}$ & $\mathbf{y}^{++}$ & $\mathbf{y}^{*}$ & $\mathrm{y}^{*^{++}}$ & $\mathbf{y}^{0}$ & $\mathbf{y}^{0++}$ & \# \\
\hline 1 & 164.0706 & 82.5389 & & & & & $\mathbf{Y}$ & & & & & & & 21 \\
\hline 2 & 221.0921 & 111.0497 & & & & & G & 2349.0088 & 1175.0080 & 2331.9823 & 1166.4948 & 2330.9983 & 1166.0028 & 20 \\
\hline 3 & 368.1275 & 184.5674 & & & & & $\mathbf{M}$ & 2291.9874 & 1146.4973 & 2274.9608 & 1137.9840 & 2273.9768 & 1137.4920 & 19 \\
\hline 4 & 496.1861 & 248.5967 & 479.1595 & 240.0834 & & & $\mathbf{Q}$ & 2144.9519 & 1072.9796 & 2127.9254 & 1064.4663 & 2126.9414 & 1063.9743 & 18 \\
\hline 5 & 610.2290 & 305.6181 & 593.2024 & 297.1049 & & & $\mathbf{N}$ & 2016.8934 & 1008.9503 & 1999.8668 & 1000.4370 & 1998.8828 & 999.9450 & 17 \\
\hline 6 & 697.2610 & 349.1341 & 680.2345 & 340.6209 & 679.2504 & 340.1289 & $\mathrm{~S}$ & 1902.8504 & 951.9289 & 1885.8239 & 943.4156 & 1884.8399 & 942.9236 & 16 \\
\hline 7 & 754.2825 & 377.6449 & 737.2559 & 369.1316 & 736.2719 & 368.6396 & G & 1815.8184 & 908.4128 & 1798.7919 & 899.8996 & 1797.8079 & 899.4076 & 15 \\
\hline 8 & 868.3254 & 434.6663 & 851.2988 & 426.1531 & 850.3148 & 425.6611 & $\mathbf{N}$ & 1758.7970 & 879.9021 & 1741.7704 & 871.3888 & 1740.7864 & 870.8968 & 14 \\
\hline 9 & 997.3680 & 499.1876 & 980.3414 & 490.6744 & 979.3574 & 490.1824 & $\mathbf{E}$ & 1644.7540 & 822.8807 & 1627.7275 & 814.3674 & 1626.7435 & 813.8754 & 13 \\
\hline 10 & 1068.4051 & 534.7062 & 1051. & & 1050. & 525.7009 & A & 1515.7114 & 758.3594 & 1498.6849 & 749.8461 & 1497.7009 & 749.3541 & 12 \\
\hline 11 & 1139.4422 & 570.2247 & 1122.4157 & 561.7115 & 1121.4317 & 561.2195 & $\mathbf{A}$ & 1444.6743 & 722.8408 & 1427.6478 & 714.3275 & 1426.6638 & 713.8355 & 11 \\
\hline 12 & 1325.5215 & 663.2644 & 1308.4950 & 654.7511 & 1307.5110 & 654.2591 & $\mathbf{W}$ & 1373.6372 & 687.3222 & 1356.6107 & 678.8090 & 1355.6266 & 678.3170 & 10 \\
\hline 13 & 1440.5485 & 720.7779 & 1423.5219 & 712.2646 & 1422.5379 & 711.7726 & $\mathbf{N}$ & \begin{tabular}{|l}
1187.5579 \\
\end{tabular} & 594.2826 & 1170.5313 & 585.7693 & 1169.5473 & 585.2773 & 9 \\
\hline 14 & 1603.6118 & 802.3095 & 1586.5852 & 793.7963 & 1585.6012 & 793.3043 & $\mathbf{Y}$ & 1072.5310 & 536.7691 & 1055.5044 & 528.2558 & 1054.5204 & 527.7638 & 8 \\
\hline 15 & 1704.6595 & 852.8334 & \begin{tabular}{|l|l|}
1687.6329 \\
\end{tabular} & 844.3201 & 1686.6489 & 843.8281 & $T$ & 909.4676 & 455.2375 & 892.4411 & 446.7242 & 891.4571 & 446.2322 & 7 \\
\hline 16 & \begin{tabular}{|l|l|}
1817.745 \\
\end{tabular} & 909.3754 & \begin{tabular}{|l|l|}
1800.7170 \\
\end{tabular} & 900.8621 & 1799.7330 & 900.3701 & $\mathbf{L}$ & 808.4199 & 404.7136 & 791.3934 & 396.2003 & 790.4094 & 395.7083 & 6 \\
\hline 17 & 1946.7861 & 973.8967 & 1929.7596 & 965.3834 & 1928.7756 & 964.8914 & $\mathbf{E}$ & 695.3359 & 348.1716 & 678.3093 & 339.6583 & 677.3253 & 339.1663 & 5 \\
\hline 18 & 2074.8447 & 1037.9260 & 2057.8182 & 1029.4127 & 2056.8341 & 1028.9207 & $\mathbf{Q}$ & 566.2933 & 283.6503 & 549.2667 & 275.1370 & & & 4 \\
\hline
\end{tabular}




\begin{tabular}{|c|c|c|c|c|c|c|c|c|c|c|c|c|}
\hline |19| & |2237.9080| & |1119.4577 & |2220.8815 & |1110.9444 & |2219.8975 & |1110.4524 & $\mathbf{Y}$ & 438.2347 & 219.6210 & 421.2082 & 211.1077 & 3 \\
\hline 20 & 2365.9666 & 1183.4869 & 2348.9401 & 1174.9737 & 2347.9561 & 1174.4817 & $\mathbf{Q}$ & 275.1714 & 138.0893 & 258.1448 & 129.5761 & 2 \\
\hline 21 & & & & & & & $\mathbf{K}$ & 147.1128 & 74.0600 & 130.0863 & 65.5468 & 1 \\
\hline
\end{tabular}
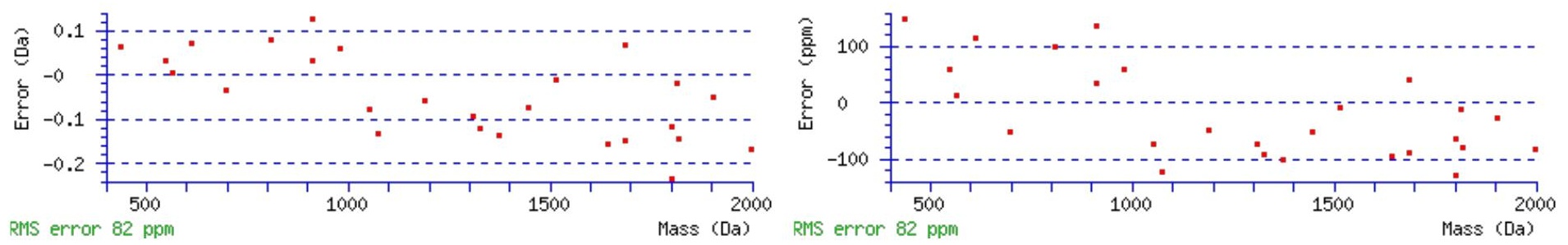

\section{All matches to this query}

\begin{tabular}{|l|l|c|l|}
\hline Score & Mr(calc): & Delta & \multicolumn{1}{|c|}{ Sequence } \\
\hline 92.8 & 2511.0648 & 0.0075 & YGMQNSGNEAAWNYTLEQYQK \\
\hline 41.6 & 2511.0648 & 0.0075 & YGMQNSGNEAAWNYTLEQYQK \\
\hline 19.8 & 2511.0648 & 0.0075 & YGMQNSGNEAAWNYTLEQYQK \\
\hline 17.9 & 2510.0808 & 0.9915 & YGMQNSGNEAAWNYTLEQYQK \\
\hline 1.9 & 2511.0913 & -0.0190 & KGYFGGGTAAVYLAGEDFYIPR \\
\hline 1.0 & 2511.0809 & -0.0086 & ELQGTGSQLGGTTGEKLPAGRSK \\
\hline 1.0 & 2511.0809 & -0.0086 & ELQGTGSQLGGTTGEKLPAGRSK \\
\hline 0.8 & 2511.0551 & 0.0172 & EGESYNESTVNGTPVNHQALER \\
\hline
\end{tabular}

Spectrum No: 119; Query: 1997; Rank: 1

\section{Peptide View}

\section{MS/MS Fragmentation of IVNDNPYGNGTAIFTTNGAIAR}

Found in IPI00205018, Tax_Id=10116 Gene_Symbol=Aldh6a1 Methylmalonate-semialdehyde dehydrogenase [acylating], mitochondrial precursor

Match to Query 1997: 2279.128108 from(1140.571330,2+)

Title: 100101RatKid_NS_deglyco_09.3724.3724.2.dta

Data file K:\NewmanPaper\Piliang \3SubProteomes\Piliang3SP \mgf5ppm\ERLIC_3SubProteomes5ppm.mgf

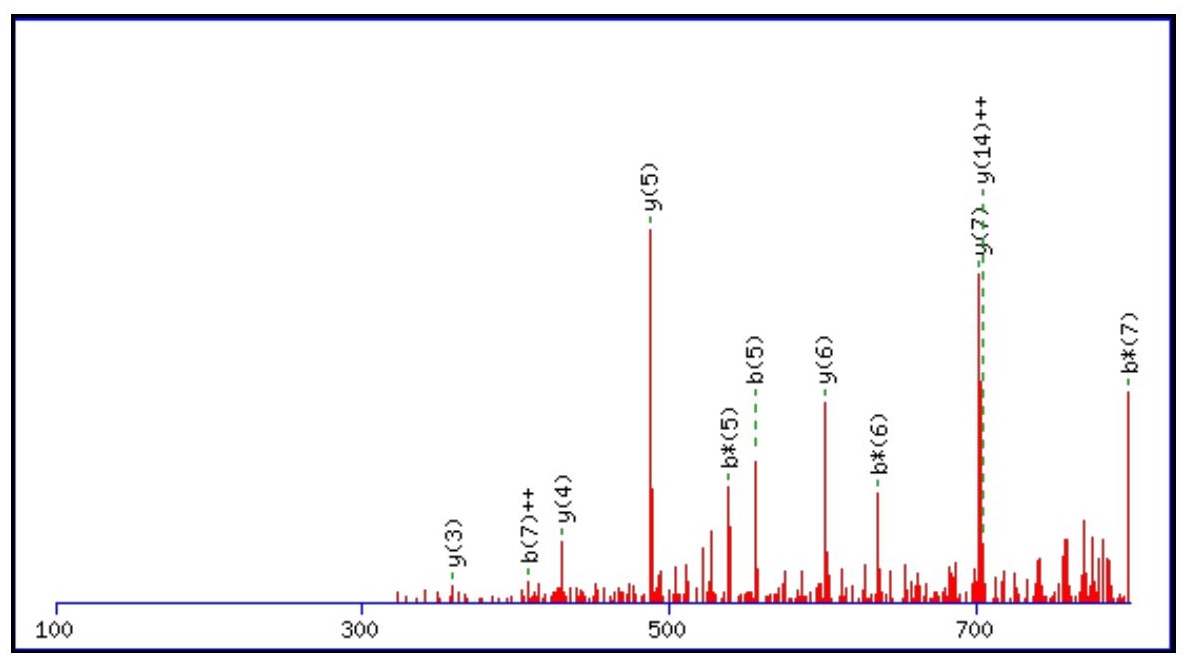



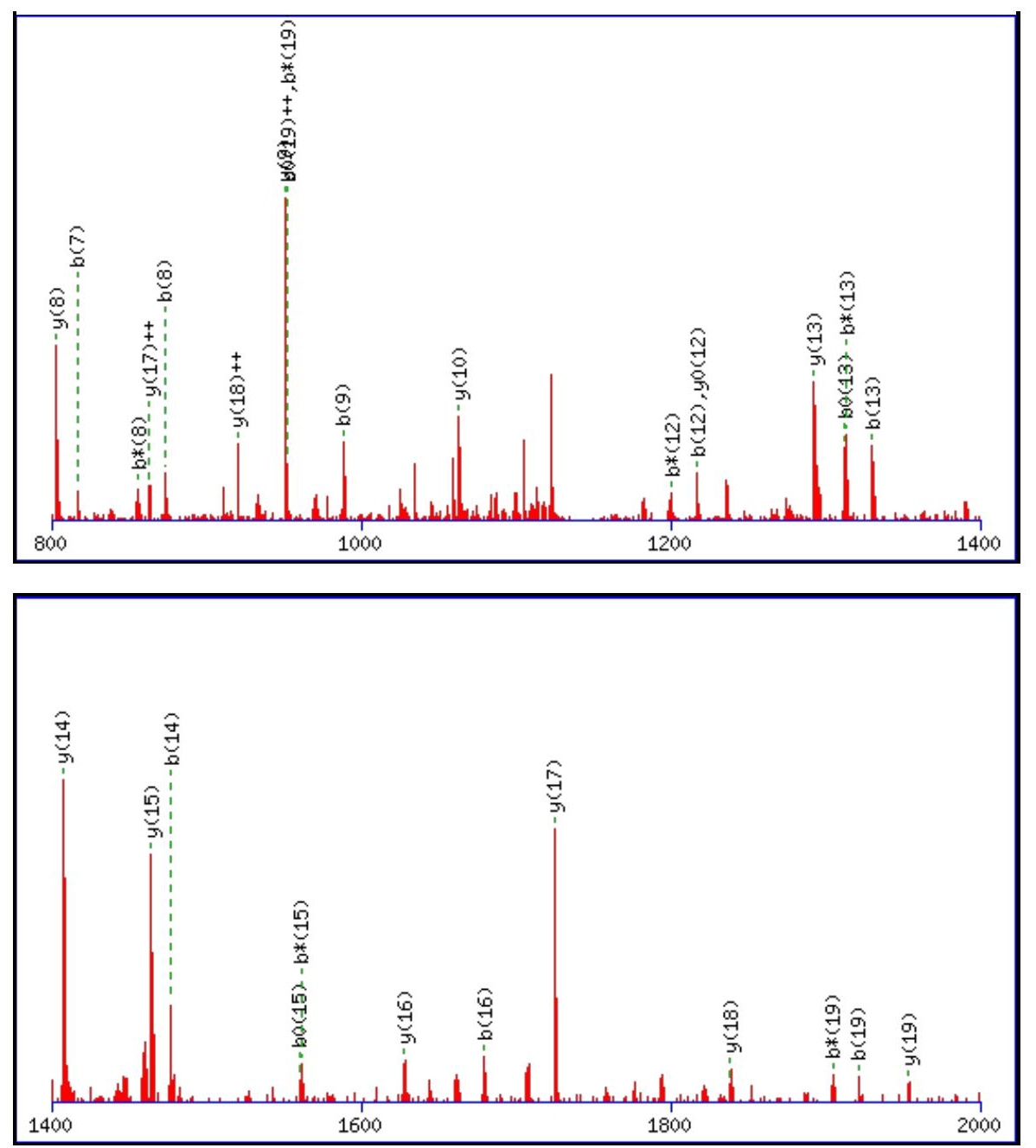

Monoisotopic mass of neutral peptide $\operatorname{Mr}($ calc): 2279.1182

Fixed modifications: Carbamidomethyl (C)

Variable modifications:

N9: Deamidated $\mathrm{N}(\mathrm{N})$

Ions Score: 93 Expect: $1.2 \mathrm{e}-007$

Matches (Bold Red): 41/230 fragment ions using 78 most intense peaks

\begin{tabular}{|c|c|c|c|c|c|c|c|c|c|c|c|c|c|c|}
\hline \# & b & $\mathbf{b}^{++}$ & b* & $\mathbf{b}^{*^{++}}$ & $\mathbf{b}^{\mathbf{0}}$ & $\mathbf{b}^{0++}$ & Seq. & $\mathbf{y}$ & $\mathbf{y}^{++}$ & $\mathbf{y}^{*}$ & $\mathbf{y}^{*^{++}}$ & $\mathbf{y}^{\mathbf{0}}$ & $y^{0++}$ & \# \\
\hline 1 & 114.0913 & 57.5493 & & & & & I & & & & & & & 22 \\
\hline 2 & 213.1598 & 107.0835 & & & & & V & 2167.0414 & 1084.0244 & 2150.0149 & 1075.5111 & 2149.0309 & 1075.0191 & 21 \\
\hline 4 & 442.2296 & 221.6185 & 425.2031 & 213.1052 & 424.2191 & 212.6132 & D & 1953.9301 & 977.4687 & 1936.9035 & 968.9554 & 1935.9195 & 968.4634 & 19 \\
\hline 5 & 556.2726 & 278.6399 & 539.2460 & 270.1266 & 538.2620 & 269.6346 & $\mathbf{N}$ & 1838.9031 & 919.9552 & 1821.8766 & 911.4419 & 1820.8926 & 910.9499 & 18 \\
\hline 6 & 653.3253 & 327.1663 & 636.2988 & 318.6530 & 635.3148 & 318.1610 & $\mathbf{P}$ & 1724.8602 & 862.9337 & 1707.8337 & 854.4205 & 1706.8497 & 853.9285 & 17 \\
\hline 7 & 816.3886 & 408.6980 & 799.3621 & 400.1847 & 798.3781 & 399.6927 & $\mathbf{Y}$ & 1627.8075 & 814.4074 & 1610.7809 & 805.8941 & 1609.7969 & 805.4021 & 16 \\
\hline 8 & 873.4101 & 437.2087 & 856.3836 & 428.6954 & 855.3995 & 428.2034 & G & 1464.7441 & 732.8757 & 1447.7176 & 724.3624 & 1446.7336 & 723.8704 & 15 \\
\hline 9 & 988.4370 & 494.7222 & 971.4105 & 486.2089 & 970.4265 & 485.7169 & $\mathbf{N}$ & 1407.7227 & 704.3650 & 1390.6961 & 695.8517 & 1389.7121 & 695.3597 & 14 \\
\hline 10 & 1045.4585 & 523.2329 & 1028.4320 & 514.7196 & 1027.4479 & 514.2276 & G & 1292.6957 & 646.8515 & 1275.6692 & 638.3382 & 1274.6852 & 637.8462 & 13 \\
\hline 11 & 1146.5062 & 573.7567 & 1129.4796 & 565.2435 & 1128.4956 & 564.7514 & $\mathbf{T}$ & 1235.6743 & 618.3408 & 1218.6477 & 609.8275 & 1217.6637 & 609.3355 & 12 \\
\hline 12 & 1217.5433 & 609.2753 & 1200.5168 & 600.7620 & 1199.5327 & 600.2700 & A & 1134.6266 & 567.8169 & 1117.6000 & 559.3037 & 1116.6160 & 558.8116 & 11 \\
\hline 14 & 1477.6958 & 739.3515 & 1460.6692 & 730.8383 & 1459.6852 & 730.3462 & $\mathbf{F}$ & 950.5054 & 475.7563 & 933.4789 & 467.2431 & 932.4948 & 466.7511 & 9 \\
\hline 15 & 1578.7435 & 789.8754 & 1561.7169 & 781.3621 & 1560.7329 & 780.8701 & $\mathbf{T}$ & 803.4370 & 402.2221 & 786.4104 & 393.7089 & 785.4264 & 393.2169 & 8 \\
\hline 16 & 1679.7911 & 840.3992 & 1662.7646 & 831.8859 & 1661.7806 & 831.3939 & $\mathbf{T}$ & 702.3893 & 351.6983 & 685.3628 & 343.1850 & 684.3787 & 342.6930 & 7 \\
\hline 17 & 1793.8341 & 897.4207 & 1776.8075 & 888.9074 & 1775.8235 & 888.4154 & $\mathbf{N}$ & 601.3416 & 301.1745 & 584.3151 & 292.6612 & & & 6 \\
\hline 18 & 1850.8555 & 925.9314 & 1833.8290 & 917.4181 & 1832.8450 & 916.9261 & $\mathbf{G}$ & 487.2987 & 244.1530 & 470.2722 & 235.6397 & & & 5 \\
\hline
\end{tabular}




\begin{tabular}{|r|r|r|r|r|r|r|r|r|r|r|r|r|r|r|r|}
$\mathbf{1 9}$ & $\mathbf{1 9 2 1 . 8 9 2 6}$ & $\mathbf{9 6 1 . 4 5 0 0}$ & $\mathbf{1 9 0 4 . 8 6 6 1}$ & $\mathbf{9 5 2 . 9 3 6 7}$ & $\mathbf{1 9 0 3 . 8 8 2 1}$ & $\mathbf{9 5 2 . 4 4 4 7}$ & $\mathbf{A}$ & $\mathbf{4 3 0 . 2 7 7 2}$ & 215.6423 & 413.2507 & 207.1290 & & & $\mathbf{4}$ \\
\hline $\mathbf{2 0}$ & 2034.9767 & 1017.9920 & 2017.9502 & 1009.4787 & 2016.9661 & 1008.9867 & $\mathbf{I}$ & $\mathbf{3 5 9 . 2 4 0 1}$ & 180.1237 & 342.2136 & 171.6104 & & & $\mathbf{3}$ \\
\hline $\mathbf{2 1}$ & 2106.0138 & 1053.5105 & 2088.9873 & 1044.9973 & 2088.0033 & 1044.5053 & $\mathbf{A}$ & 246.1561 & 123.5817 & 229.1295 & 115.0684 & & & $\mathbf{2}$ \\
\hline $\mathbf{2 2}$ & & & & & & & $\mathbf{R}$ & 175.1190 & 88.0631 & 158.0924 & 79.5498 & & & $\mathbf{1}$ \\
\hline
\end{tabular}

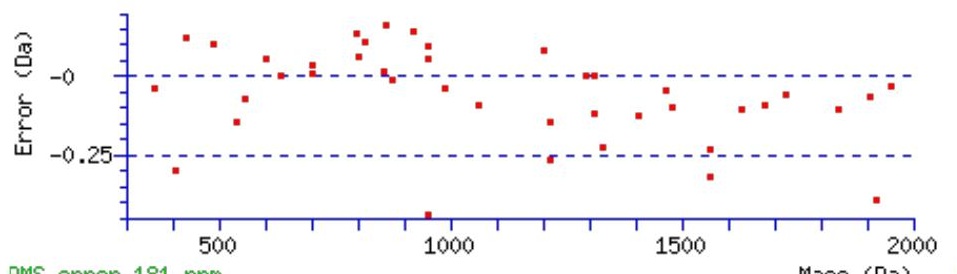

RMS error 181 ppm

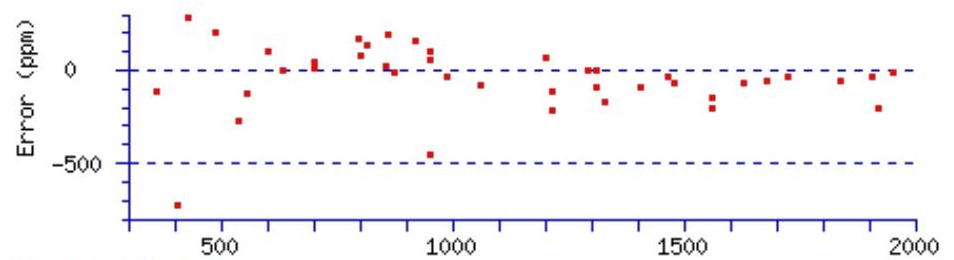

Mass (Da)

\section{All matches to this query}

\begin{tabular}{|l|l|l|l|}
\hline Score & Mr(calc): & Delta & \multicolumn{1}{|c|}{ Sequence } \\
\hline 92.7 & 2279.1182 & 0.0099 & $\underline{\text { IVNDNPYGNGTAIFTTNGAIAR }}$ \\
\hline 78.6 & 2279.1182 & 0.0099 & $\underline{\text { IVNDNPYGNGTAIFTTNGAIAR }}$ \\
\hline 42.9 & 2279.1182 & 0.0099 & $\underline{\text { IVNDNPYGNGTAIFTTNGAIAR }}$ \\
\hline 32.0 & 2279.1182 & 0.009 & $\underline{\text { IVNDNPYGNGTAIFTTNGAIAR }}$ \\
\hline 19.8 & 2278.1342 & 0.9939 & $\underline{\text { IVNDNPYGNGTAIFTTNGAIAR }}$ \\
\hline 2.5 & 2278.1090 & 1.0191 & SGANDQETLADNIRAFSRWK \\
\hline 1.2 & 2278.1407 & 0.9874 & $\underline{\text { ARSLVMFFLPKLDYMVPR }}$ \\
\hline
\end{tabular}

Spectrum No: 120; Query: 914; Rank: 1

\section{Peptide View}

MS/MS Fragmentation of DYGGNCLIYDADNK

Found in IPI00371386, Tax_Id=10116 Gene_Symbol=Ptdss2_predicted phosphatidylserine synthase 2

Match to Query 914: 1617.665548 from(809.840050,2+)

Title: 100101RatKid_NS_deglyco_21.2307.2307.2.dta

Data file K:INewmanPaper|Piliangl3SubProteomes\Piliang3SP\mgf5ppm\ERLIC_3SubProteomes5ppm.mgf

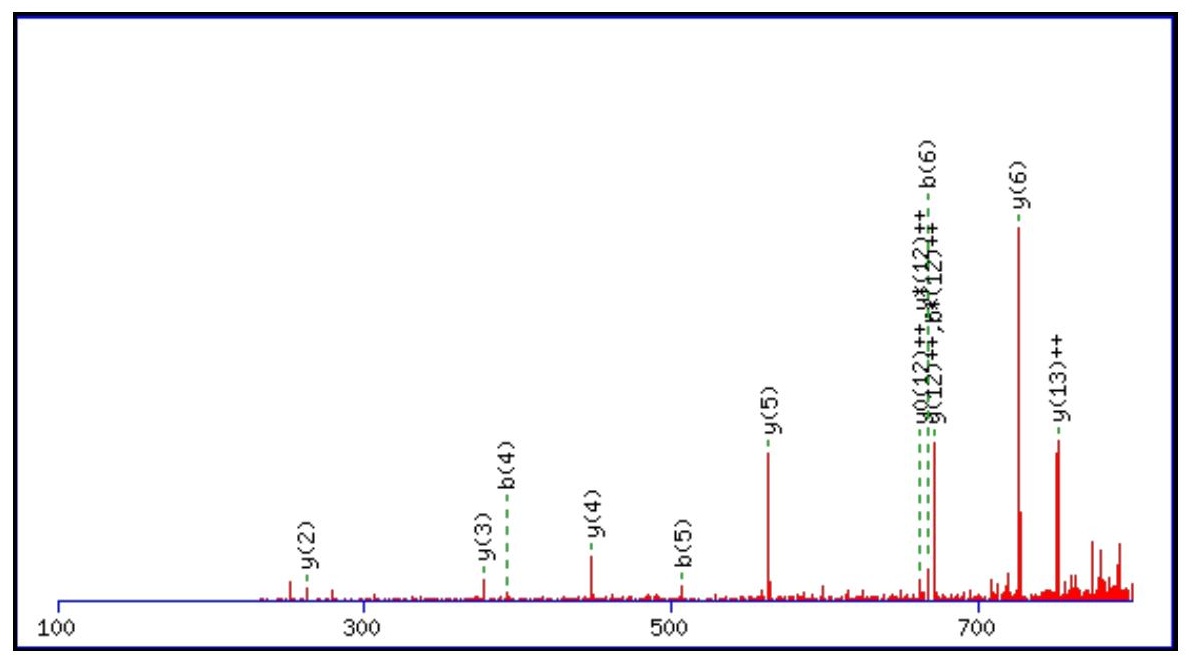



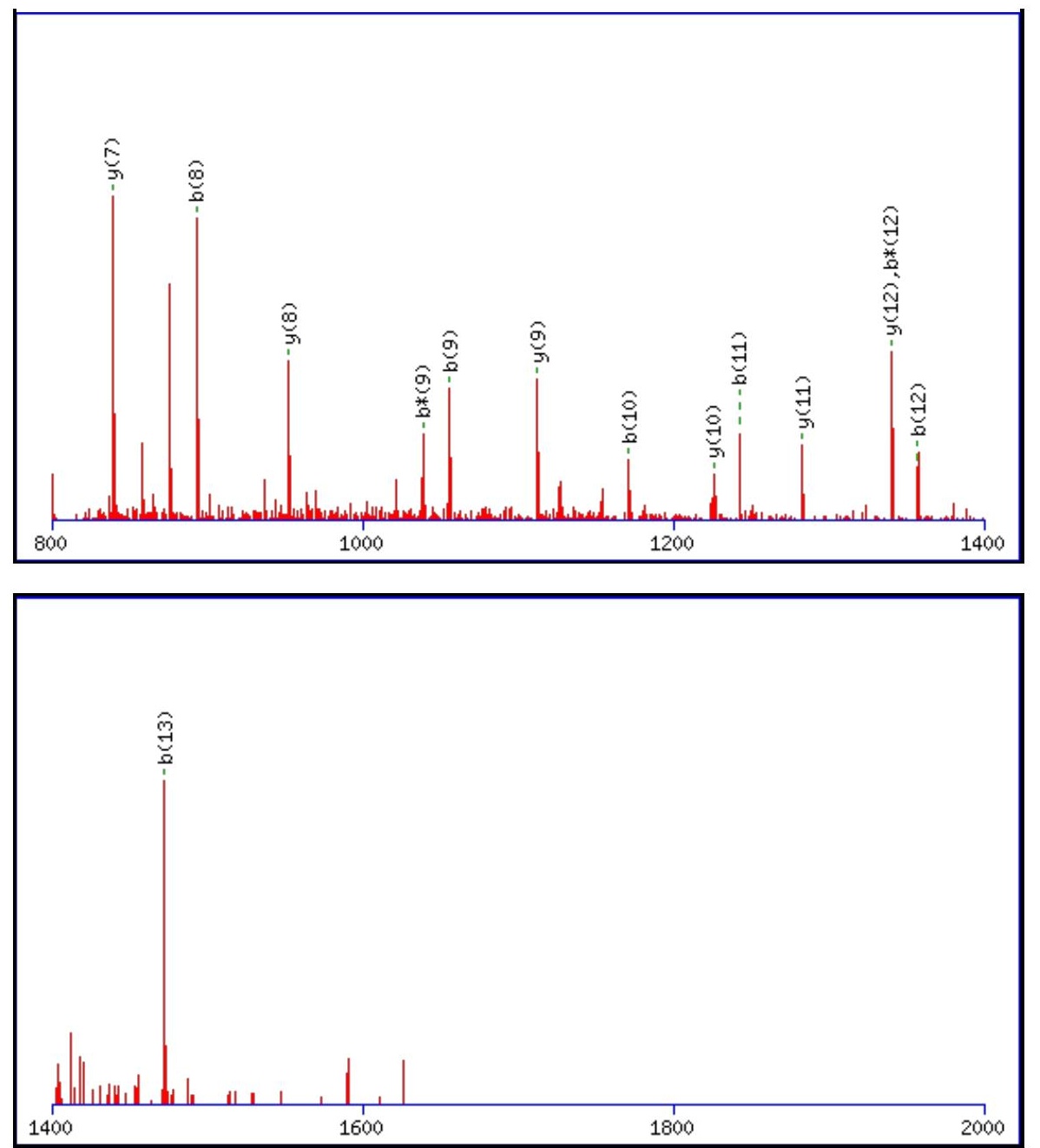

Monoisotopic mass of neutral peptide $\operatorname{Mr}($ calc): 1617.6617

Fixed modifications: Carbamidomethyl (C)

Variable modifications:

N13 : Deamidated $\mathrm{N}(\mathrm{N})$

Ions Score: 93 Expect: $7 \mathrm{e}-008$

Matches (Bold Red): 27/144 fragment ions using 34 most intense peaks

\begin{tabular}{|c|c|c|c|c|c|c|c|c|c|c|c|c|c|c|}
\hline \# & b & $\mathbf{b}^{++}$ & b* & $\mathbf{b}^{*^{++}}$ & $\mathbf{b}^{0}$ & $\mathbf{b}^{0++}$ & Seq. & $\mathbf{y}$ & $y^{++}$ & $\mathbf{y}^{*}$ & $\mathbf{y}^{*^{++}}$ & $\mathbf{y}^{\mathbf{0}}$ & $\mathbf{y}^{0++}$ & \# \\
\hline 1 & 116.0342 & 58.5207 & & & 98.0237 & 49.5155 & D & & & & & & & 14 \\
\hline 2 & 279.0975 & 140.0524 & & & 261.0870 & 131.0471 & $\mathbf{Y}$ & 1503.6420 & 752.3247 & 1486.6155 & 743.8114 & 1485.6315 & 743.3194 & 13 \\
\hline 3 & 336.1190 & 168.5631 & & & 318.1084 & 159.5579 & G & 1340.5787 & 670.7930 & 1323.5522 & 662.2797 & 1322.5681 & 661.7877 & 12 \\
\hline 4 & 393.1405 & 197.0739 & & & 375.1299 & 188.0686 & G & 1283.5572 & 642.2823 & 1266.5307 & 633.7690 & 1265.5467 & 633.2770 & 11 \\
\hline 5 & 507.1834 & 254.0953 & 490.1569 & 245.5821 & 489.1728 & 245.0901 & $\mathbf{N}$ & 1226.5358 & 613.7715 & 1209.5092 & 605.2583 & 1208.5252 & 604.7662 & 10 \\
\hline 6 & 667.2141 & 334.1107 & 650.1875 & 325.5974 & 649.2035 & 325.1054 & C & 1112.4928 & 556.7501 & 1095.4663 & 548.2368 & 1094.4823 & 547.7448 & 9 \\
\hline 7 & 780.2981 & 390.6527 & 763.2716 & 382.1394 & 762.2876 & 381.6474 & $\mathbf{L}$ & 952.4622 & 476.7347 & 935.4356 & 468.2215 & 934.4516 & 467.7295 & 8 \\
\hline 8 & 893.3822 & 447.1947 & 876.3556 & 438.6815 & 875.3716 & 438.1894 & I & 839.3781 & 420.1927 & 822.3516 & 411.6794 & 821.3676 & 411.1874 & 7 \\
\hline 9 & 1056.4455 & 528.7264 & 1039.4190 & 520.2131 & 1038.4349 & 519.7211 & $\mathbf{Y}$ & 726.2941 & 363.6507 & 709.2675 & 355.1374 & 708.2835 & 354.6454 & 6 \\
\hline 10 & 1171.4725 & 586.2399 & 1154.4459 & 577.7266 & 1153.4619 & 577.2346 & D & 563.2307 & 282.1190 & 546.2042 & 273.6057 & 545.2202 & 273.1137 & 5 \\
\hline 11 & 1242.5096 & 621.7584 & 1225.4830 & 613.2451 & 1224.4990 & 612.7531 & A & 448.2038 & 224.6055 & 431.1772 & 216.0923 & 430.1932 & 215.6003 & 4 \\
\hline 12 & 1357.5365 & 679.2719 & 1340.5100 & 670.7586 & 1339.5259 & 670.2666 & D & 377.1667 & 189.0870 & 360.1401 & 180.5737 & 359.1561 & 180.0817 & 3 \\
\hline 13 & 1472.5634 & 736.7854 & 1455.5369 & 728.2721 & 1454.5529 & 727.7801 & $\mathbf{N}$ & 262.1397 & 131.5735 & 245.1132 & 123.0602 & & & 2 \\
\hline 14 & & & & & & & $\mathbf{K}$ & 147.1128 & 74.0600 & 130.0863 & 65.5468 & & & 1 \\
\hline
\end{tabular}



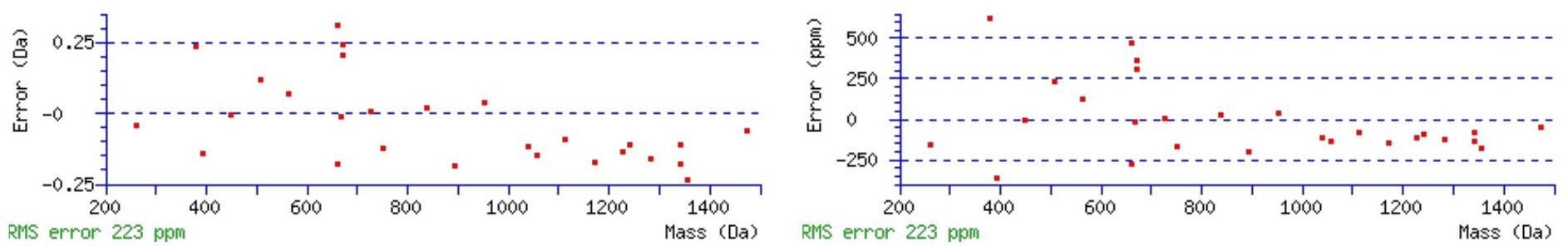

\section{All matches to this query}

\begin{tabular}{|l|l|l|l|}
\hline Score & Mr(calc): & Delta & \multicolumn{1}{|c|}{ Sequence } \\
\hline 92.7 & 1617.6617 & 0.0039 & DYGGNCLIYDADNK \\
\hline 40.3 & 1616.6777 & 0.9879 & DYGGNCLIYDADNK \\
\hline 26.1 & 1617.6617 & 0.0039 & DYGGNCLIYDADNK \\
\hline 12.8 & 1617.6623 & 0.0032 & ASNGCLQLNGTVK \\
\hline 9.9 & 1615.6745 & 1.9910 & DCMEEISLFTENK \\
\hline 4.1 & 1617.6682 & -0.0026 & NYENLDQLSYDNK \\
\hline 3.6 & 1617.6519 & 0.0137 & VSNSEEGSRSKNDK \\
\hline 3.6 & 1617.6519 & 0.0137 & VSNSEEGSRSKNDK \\
\hline 2.5 & 1617.6746 & -0.0090 & KLGEMWSSTAADDK \\
\hline 2.5 & 1617.6746 & -0.0090 & KLGEMWSSTAADDK \\
\hline
\end{tabular}

Spectrum No: 121; Query: 2766; Rank: 1

\section{Peptide View}

MS/MS Fragmentation of CISPLYVCDGDKDCADGSDEAGCVLNCTSAQFK

Found in IPI00205325, Tax_Id=10116 Gene_Symbol=Lrp2 Low-density lipoprotein receptor-related protein 2 precursor

Match to Query 2766: 3712.499112 from(1238.506980,3+)

Title: 091008RatKidney_NoSalt_26.3560.3560.3.dta

Data file K:INewmanPaper|Piliang|3SubProteomes\Piliang3SP\mgf5ppm\ERLIC_3SubProteomes5ppm.mgf

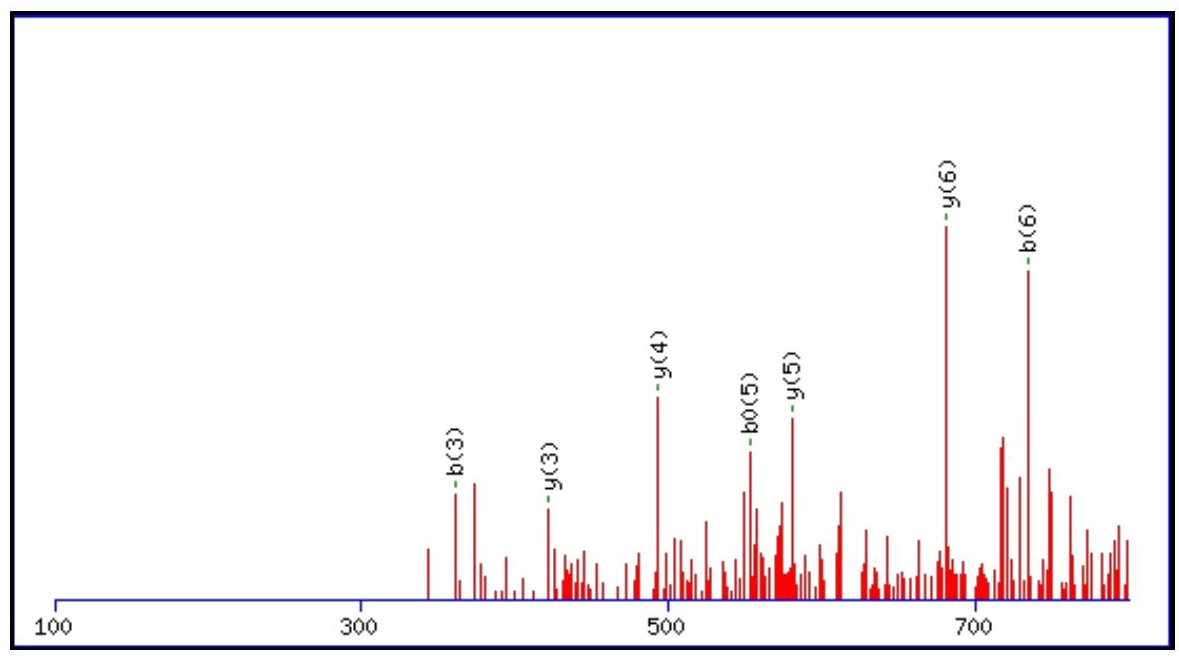



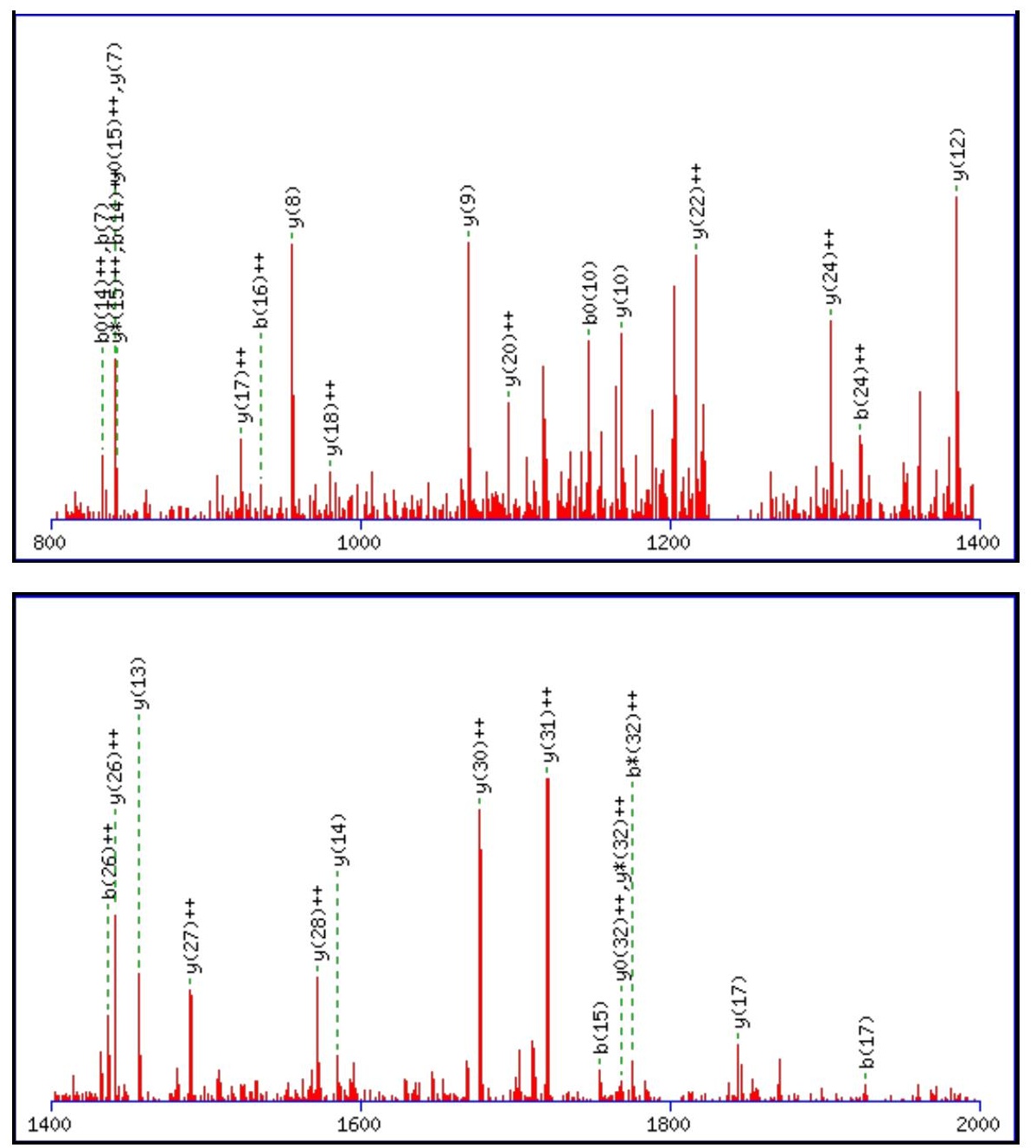

Monoisotopic mass of neutral peptide $\operatorname{Mr}($ calc): 3712.5096

Fixed modifications: Carbamidomethyl (C)

Variable modifications:

N26 : Deamidated $\mathrm{N}(\mathrm{N})$

Ions Score: 93 Expect: $2.2 \mathrm{e}-007$

Matches (Bold Red): 39/350 fragment ions using 55 most intense peaks

\begin{tabular}{|c|c|c|c|c|c|c|c|c|c|c|c|c|c|c|}
\hline$\#$ & b & $\mathbf{b}^{++}$ & $\mathbf{b}^{*}$ & $\mathbf{b}^{*^{++}}$ & $\mathbf{b}^{0}$ & $\mathbf{b}^{0++}$ & Seq. & $\mathbf{y}$ & $\mathbf{y}^{++}$ & $\mathbf{y}^{*}$ & $\mathrm{y}^{*^{++}}$ & $\mathbf{y}^{0}$ & $y^{0++}$ & \# \\
\hline 1 & 161.0379 & 81.0226 & & & & & C & & & & & & & 33 \\
\hline 2 & 274.1220 & 137.5646 & & & & & I & 3553.4862 & 1777.2467 & 3536.4597 & 1768.7335 & 3535.4756 & $\mid 1768.2415$ & 32 \\
\hline 3 & 361.1540 & 181.0806 & & & 343.1435 & 172.0754 & $\mathbf{S}$ & 3440.4021 & $\mid 1720.7047$ & 3423.3756 & 1712.1914 & 3422.3916 & |1711.6994 & 31 \\
\hline 4 & 458.2068 & 229.6070 & & & 440.1962 & 220.6017 & $\mathbf{P}$ & 3353.3701 & 1677.1887 & 3336.3436 & 1668.6754 & 3335.3596 & 1668.1834 & 30 \\
\hline 5 & 571.2908 & 286.1491 & & & 553.2803 & 277.1438 & $\mathbf{L}$ & 3256.3174 & 1628.6623 & 3239.2908 & 1620.1490 & 3238.3068 & 1619.6570 & 29 \\
\hline 6 & 734.3542 & 367.6807 & & & 716.3436 & 358.6754 & $\mathbf{Y}$ & 3143.2333 & 1572.1203 & 3126.2067 & 1563.6070 & 3125.2227 & 1563.1150 & 28 \\
\hline 7 & 833.4226 & 417.2149 & & & 815.4120 & 408.2096 & $\mathbf{V}$ & 2980.1700 & 1490.5886 & 2963.1434 & 1482.0753 & 2962.1594 & 1481.5833 & 27 \\
\hline 8 & 993.4532 & 497.2303 & & & 975.4427 & 488.2250 & C & 2881.1015 & 1441.0544 & 2864.0750 & 1432.5411 & 2863.0910 & 1432.0491 & 26 \\
\hline 9 & 1108.4802 & 554.7437 & & & 1090.4696 & 545.7384 & D & 2721.0709 & 1361.0391 & 2704.0443 & 1352.5258 & 2703.0603 & 1352.0338 & 25 \\
\hline 10 & 1165.5016 & 583.2545 & & & 1147.4911 & 574.2492 & G & 2606.0440 & 1303.5256 & 2589.0174 & 1295.0123 & 2588.0334 & 1294.5203 & 24 \\
\hline 11 & 1280.5286 & 640.7679 & & & 1262.5180 & 631.7626 & D & 2549.0225 & 1275.0149 & 2531.9959 & 1266.5016 & 2531.0119 | & 1266.0096 & 23 \\
\hline 12 & 1408.6236 & 704.8154 & 1391.5970 & 696.3021 & 1390.6130 & 695.8101 & $\mathbf{K}$ & 2433.9955 & 1217.5014 & 2416.9690 & 1208.9881 & 2415.9850 & 1208.4961 & 22 \\
\hline 13 & 1523.6505 & 762.3289 & 1506.6239 & 753.8156 & 1505.6399 & 753.3236 & D & 2305.9006 & 1153.4539 & 2288.8740 & 1144.9407 & 2287.8900 & 1144.4486 & 21 \\
\hline 14 & 1683.6811 & 842.3442 & 1666.6546 & 833.8309 & 1665.6706 & 833.3389 & C & 2190.8736 & 1095.9405 & 2173.8471 & 1087.4272 & 2172.8631 & 1086.9352 & 20 \\
\hline 15 & 1754.7183 & 877.8628 & 1737.6917 & 869.3495 & 1736.7077 & 868.8575 & A & 2030.8430 & 1015.9251 & 2013.8164 & 1007.4119 & 2012.8324 & 1006.9199 & 19 \\
\hline 16 & 1869.7452 & 935.3762 & 1852.7187 & 926.8630 & 1851.7346 & 926.3710 & D & 1959.8059 & 980.4066 & 1942.7793 & 971.8933 & 1941.7953 & 971.4013 & 18 \\
\hline 17 & 1926.7667 & 963.8870 & 1909.7401 & 955.3737 & 1908.7561 & 954.8817 & G & 1844.7789 & 922.8931 & 1827.7524 & 914.3798 & 1826.7684 & 913.8878 & 17 \\
\hline 18 & 2013.7987 & 1007.4030 & 1996.7721 & 998.8897 & 1995.7881 & 998.3977 & $S$ & 1787.7575 & 894.3824 & 1770.7309 & 885.8691 & 1769.7469 & 885.3771 & 16 \\
\hline
\end{tabular}




\begin{tabular}{|c|c|c|c|c|c|c|c|c|c|c|c|c|c|c|}
\hline 19 & 2128.8256 & |1064.9165 & |2111.7991 & | 1056.4032 & |2110.8151| & 1055.9112 & D & & 850.8664 & 1683.6989 & 842.3531 & & 841.8611 & 15 \\
\hline 20 & 2257.8682 & 1129.4378 & 2240.8417 & 1120.9245 & 2239.8577 & 1120.4325 & $\mathbf{E}$ & 1585.6985 & 793.3529 & 1568.6720 & 784.8396 & 1567.6879 & 784.3476 & 14 \\
\hline 21 & 2328.9053 & 1164.9563 & |2311.8788 & 1156.4430 & 2310.8948 & 1155.9510 & A & 1456.6559 & 728.8316 & 1439.6294 & 720.3183 & 1438.6453 & 719.8263 & 13 \\
\hline 22 & 2385.9268 & 1193.4670 & 2368.9003 & 1184.9538 & 2367.9162 & 1184.4618 & G & 1385.6188 & 693.3130 & 1368.5922 & 684.7998 & 1367.6082 & 684.3078 & 12 \\
\hline 23 & 2545.9575 & 1273.4824 & 2528.9309 & 1264.9691 & 2527.9469 & 1264.4771 & C & 1328.5973 & 664.8023 & 1311.5708 & 656.2890 & 1310.5868 & 655.7970 & 11 \\
\hline 24 & 2645.0259 & 1323.0166 & 2627.9993 & 1314.5033 & 2627.0153 & 1314.0113 & V & 1168.5667 & 584.7870 & 1151.5401 & 576.2737 & 1150.5561 & 575.7817 & 10 \\
\hline 25 & 2758.1099 & 1379.5586 & 2741.0834 & 1371.0453 & 2740.0994 & 1370.5533 & L & 1069.4983 & 535.2528 & 1052.4717 & 526.7395 & 1051.4877 & 526.2475 & 9 \\
\hline 26 & 2873.1369 & 1437.0721 & 2856.1103 & 1428.5588 & 2855.1263 & 1428.0668 & $\mathbf{N}$ & 956.4142 & 478.7107 & 939.3877 & 470.1975 & 938.4036 & 469.7055 & 8 \\
\hline 27 & 3033.1675 & 1517.0874 & 3016.1410 & 1508.5741 & 3015.1570 & 1508.0821 & C & 841.3873 & 421.1973 & 824.3607 & 412.6840 & 823.3767 & 412.1920 & 7 \\
\hline 28 & 3134.2152 & 1567.6112 & 3117.1886 & 1559.0980 & 3116.2046 & 1558.6060 & $T$ & 681.3566 & 341.1819 & 664.3301 & 332.6687 & 663.3461 & 332.1767 & 6 \\
\hline 29 & 3221.2472 & 1611.1272 & 3204.2207 & 1602.6140 & 3203.2367 & 1602.1220 & S & 580.3089 & 290.6581 & 563.2824 & 282.1448 & 562.2984 & 281.6528 & 5 \\
\hline 30 & 3292.2843 & 1646.6458 & 3275.2578 & 1638.1325 & 3274.2738 & 1637.6405 & A & 493.2769 & 247.1421 & 476.2504 & 238.6288 & & & 4 \\
\hline 31 & 3420.3429 & 1710.6751 & 3403.3164 & 1702.1618 & 3402.3324 & 1701.6698 & $\mathbf{Q}$ & 422.2398 & 211.6235 & 405.2132 & 203.1103 & & & 3 \\
\hline 32 & 3567.4113 & 1784.2093 & 3550.3848 & 1775.6960 & 3549.4008 & 1775.2040 & $\mathbf{F}$ & 294.1812 & 147.5942 & 277.1547 & 139.0810 & & & 2 \\
\hline 33 & & & & & & & $\mathbf{K}$ & 147.1128 & 74.0600 & 130.0863 & 65.5468 & & & 1 \\
\hline
\end{tabular}
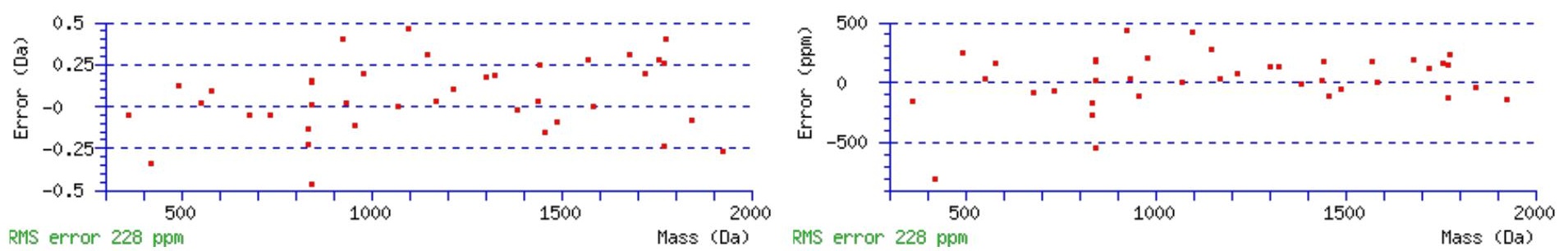

\section{All matches to this query}

\begin{tabular}{|l|l|l|l|}
\hline Score & Mr(calc): & Delta & \multicolumn{1}{|c|}{ Sequence } \\
\hline 92.6 & 3712.5096 & -0.0105 & CISPLYVCDGDKDCADGSDEAGCVLNCTSAQFK \\
\hline 3.5 & 3710.4940 & 2.0051 & ELPGAKASDDSAVPLTSLAGEAAGASEGTSAGNHPR \\
\hline 2.1 & 3711.4733 & 1.0258 & NAGIYNDGKDGQDWEVNYENCYYKTEESNR \\
\hline 2.1 & 3711.4733 & 1.0258 & NAGIYNDGKDGQDWEVNYENCYYKTEESNR \\
\hline 2.1 & 3711.4733 & 1.0258 & NAGIYNDGKDGQDWEVNYENCYYKTEESNR \\
\hline 2.1 & 3711.4733 & 1.0258 & NAGIYNDGKDGQDWEVNYENCYYKTEESNR \\
\hline 1.9 & 3711.4733 & 1.0258 & NAGIYNDGKDGQDWEVNYENCYYKTEESNR \\
\hline 1.9 & 3711.4733 & 1.0258 & NAGIYNDGKDGQDWEVNYENCYYKTEESNR \\
\hline 1.9 & 3711.4733 & 1.0258 & NAGIYNDGKDGQDWEVNYENCYYKTEESNR \\
\hline 1.9 & 3711.4733 & 1.0258 & NAGIYNDGKDGQDWEVNYENCYYKTEESNR \\
\hline
\end{tabular}

Spectrum No: 122; Query: 2778; Rank: 1

\section{Peptide View}

MS/MS Fragmentation of MLVSNIDLGPTILDLAGYDLNKTQMDGTSLLPILK

Found in IPI00366226, Tax_Id=10116 Gene_Symbol=Gns Glucosamine (N-acetyl)-6-sulfatase

Match to Query 2778: 3772.991622 from(1258.671150,3+)

Title: 091008RatKidney_NoSalt_15.7354.7354.3.dta

Data file K:INewmanPaper|Piliang $\mid 3 S$ SubProteomes\Piliang3SP\mgf5ppm\ERLIC_3SubProteomes5ppm.mgf 

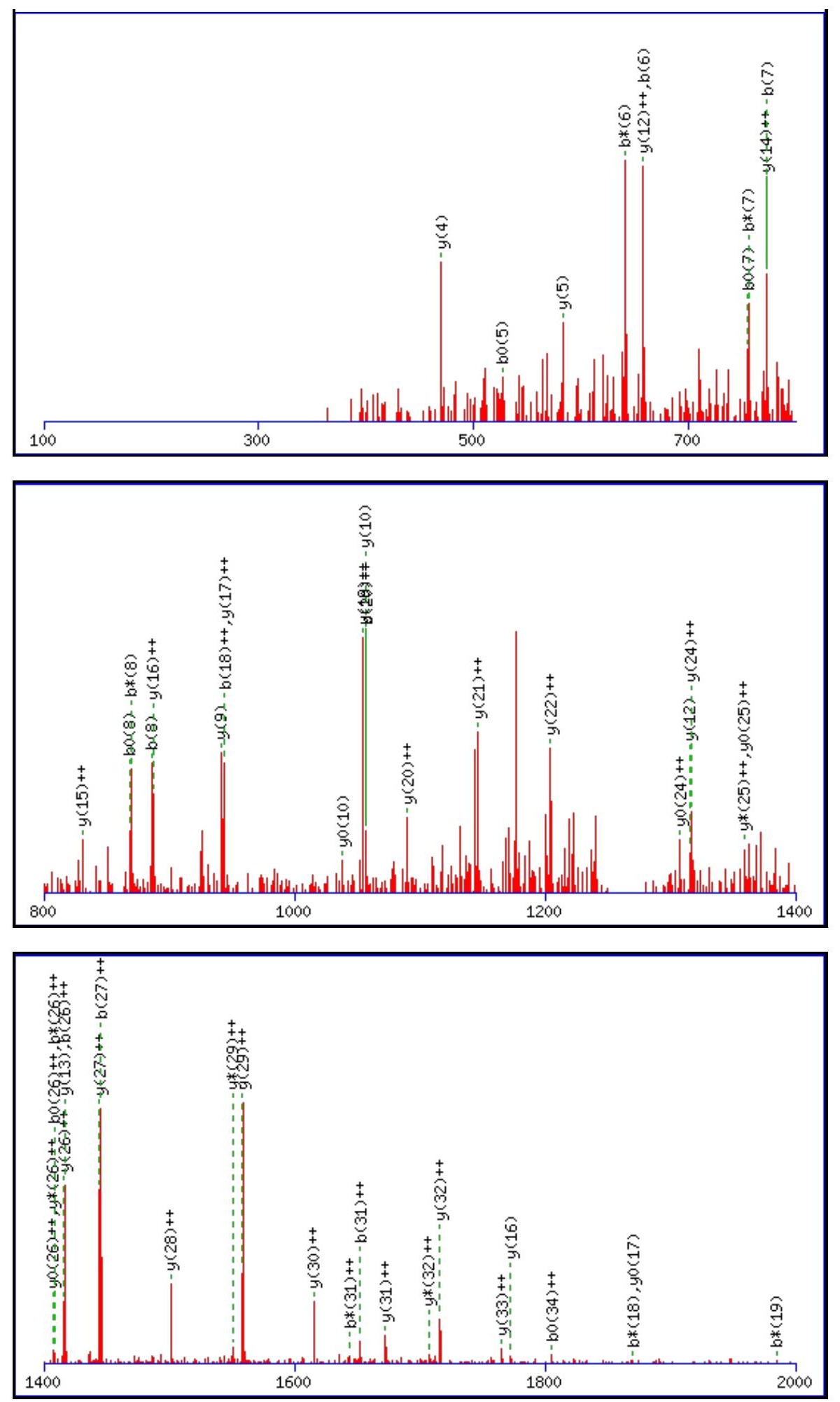

Monoisotopic mass of neutral peptide $\operatorname{Mr}($ calc): 3772.9882

Fixed modifications: Carbamidomethyl (C)

Variable modifications:

N21 : Deamidated_N (N)

Ions Score: 93 Expect: $3.9 \mathrm{e}-008$

Matches (Bold Red): 54/382 fragment ions using 96 most intense peaks

\begin{tabular}{|c|c|c|c|c|c|c|c|c|c|c|c|c|c|c|}
\hline$\#$ & $\mathbf{b}$ & $\mathbf{b}^{++}$ & $\mathbf{b}^{*}$ & $\mathbf{b}^{\boldsymbol{*}^{++}}$ & $\mathbf{b}^{\mathbf{0}}$ & $\mathbf{b}^{\mathbf{0 + +}}$ & $\mathbf{S e q}$ & $\mathbf{y}$ & $\mathbf{y}^{++}$ & $\mathbf{y}^{*}$ & $\mathbf{y}^{\mathbf{*}^{++}}$ & $\mathbf{y}^{\mathbf{0}}$ & $\mathbf{y}^{\mathbf{0 + +}}$ & $\#$ \\
\hline $\mathbf{1}$ & 132.0478 & 66.5275 & & & & & $\mathbf{M}$ & & & & & & & 35 \\
\hline $\mathbf{2}$ & 245.1318 & 123.0696 & & & & & $\mathbf{L}$ & 3642.9550 & 1821.9812 & 3625.9285 & 1813.4679 & 3624.9445 & 1812.9759 & $\mathbf{3 4}$ \\
\hline $\mathbf{3}$ & 344.2002 & 172.6038 & & & & & $\mathbf{V}$ & 3529.8710 & $\mathbf{1 7 6 5 . 4 3 9 1}$ & 3512.8444 & 1756.9259 & 3511.8604 & 1756.4338 & 33 \\
\hline $\mathbf{4}$ & 431.2323 & 216.1198 & & & 413.2217 & 207.1145 & $\mathbf{S}$ & 3430.8026 & $\mathbf{1 7 1 5 . 9 0 4 9}$ & 3413.7760 & $\mathbf{1 7 0 7 . 3 9 1 6}$ & 3412.7920 & 1706.8996 & $\mathbf{3 2}$ \\
\hline $\mathbf{5}$ & 545.2752 & 273.1412 & 528.2486 & 264.6280 & 527.2646 & 264.1360 & $\mathbf{N}$ & 3343.7705 & $\mathbf{1 6 7 2 . 3 8 8 9}$ & 3326.7440 & 1663.8756 & 3325.7600 & 1663.3836 & $\mathbf{3 1}$ \\
\hline
\end{tabular}




\begin{tabular}{|c|c|c|c|c|c|c|c|c|c|c|c|c|c|c|}
\hline 6 & 658.3593 & 329.6833 & 641.3327 & 321.1700 & 640.3487| & 320.6780 & I & 3229.7276 & 1615.3674 & 3212.7011 & 1606.8542 & |3211.7170| & 1606.3622 & $|30|$ \\
\hline 7 & 773.3862 & 387.1967 & 756.3597 & 378.6835 & 755.3756 & 378.1915 & D & 3116.6435 & 1558.8254 & 3099.6170 & 1550.3121 & 3098.6330 & 1549.8201 & 29 \\
\hline 8 & 886.4703 & 443.7388 & 869.4437 & 435.2255 & 868.4597 & 434.7335 & $\mathbf{L}$ & 3001.6166 & 1501.3119 & 2984.5900 & 1492.7987 & 2983.6060 & 1492.3067 & 28 \\
\hline 9 & 943.4917 & 472.2495 & 926.4652 & 463.7362 & 925.4812 & 463.2442 & G & 2888.5325 & 1444.7699 & 2871.5060 & 1436.2566 & 2870.5220 & 1435.7646 & 27 \\
\hline 101 & 1040.5445 & 520.7759 & 1023.5179 & 512.2626 & 1022.5339 & 511.7706 & $\mathbf{P}$ & 2831.5111 & 1416.2592 & 2814.4845 & 1407.7459 & 2813.5005 & 1407.2539 & 26 \\
\hline 111 & 1141.5922 & 571.2997 & 1124.5656 & 562.7864 & 1123.5816 & 562.2944 & $\mathbf{T}$ & 2734.4583 & 1367.7328 & 2717.4318 & 1359.2195 & 2716.4477 & 1358.7275 & 25 \\
\hline 12 1 & 1254.6762 & 627.8418 & 1237.6497 & 619.3285 & 1236.6657 & 618.8365 & I & 2633.4106 & 1317.2090 & 2616.3841 & 1308.6957 & 2615.4001 & 1308.2037 & 24 \\
\hline 13 & 1367.7603 & 684.3838 & 1350.7338 & 675.8705 & 1349.7497 & 675.3785 & $\mathbf{L}$ & 2520.3266 & 1260.6669 & 2503.3000 & 1252.1536 & 2502.3160 & 1251.6616 & 23 \\
\hline 141 & 1482.7872 & 741.8973 & 1465.7607 & 733.3840 & 1464.7767 & 732.8920 & D & 2407.2425 & 1204.1249 & 2390.2160 & 1195.6116 & 2389.2319 & 1195.1196 & 22 \\
\hline 151 & 1595.8713 & 798.4393 & 1578.8448 & 789.9260 & 1577.8607 & 789.4340 & $\mathbf{L}$ & 2292.2156 & 1146.6114 & 2275.1890 & 1138.0981 & 2274.2050 & 1137.6061 & 21 \\
\hline 161 & 1666.9084 & 833.9578 & 1649.8819 & 825.4446 & 1648.8979 & 824.9526 & A & 2179.1315 & 1090.0694 & 2162.1049 & 1081.5561 & 2161.1209 & 1081.0641 & 20 \\
\hline 171 & 1723.9299 & 862.4686 & 1706.9033 & 853.9553 & 1705.9193 & 853.4633 & G & 2108.0944 & 1054.5508 & 2091.0678 & 1046.0376 & 2090.0838 & 1045.5455 & 19 \\
\hline 18 & 1886.9932 & 944.0002 & 1869.9667 & 935.4870 & 1868.9826 & 934.9950 & $\mathbf{Y}$ & 2051.0729 & 1026.0401 & 2034.0464 & 1017.5268 & 2033.0623 & 1017.0348 & 18 \\
\hline 192 & 2002.0202 & 1001.5137 & 1984.9936 & 993.0004 & 1984.0096 & 992.5084 & D & 1888.0096 & 944.5084 & 1870.9830 & 935.9952 & 1869.9990 & 935.5031 & 17 \\
\hline 202 & 2115.1042 & 1058.0557 & 2098.0777 & 1049.5425 & 2097.0937 & 1049.0505 & $\mathbf{L}$ & 1772.9826 & 886.9950 & 1755.9561 & 878.4817 & 1754.9721 & 877.9897 & 16 \\
\hline 21 & 2230.1312 & 1115.5692 & 2213.1046 & 1107.0559 & 2212.1206 & 1106.5639 & $\mathbf{N}$ & 1659.8986 & 830.4529 & 1642.8720 & 821.9397 & 1641.8880 & 821.4476 & 15 \\
\hline 222 & 2358.2261 & 1179.6167 & 2341.1996 & 1171.1034 & 2340.2156 & 1170.6114 & $\mathbf{K}$ & 1544.8716 & 772.9395 & 1527.8451 & 764.4262 & 1526.8611 & 763.9342 & 14 \\
\hline 232 & 2459.2738 & 1230.1405 & 2442.2472 & 1221.6273 & 2441.2632 & 1221.1353 & $\mathbf{T}$ & \begin{tabular}{|l|}
1416.7767 \\
\end{tabular} & 708.8920 & 1399.7501 & 700.3787 & 1398.7661 & 699.8867 & 13 \\
\hline 242 & 2587.3324 & 1294.1698 & 2570.3058 & 1285.6566 & 2569.3218 & 1285.1645 & $\bar{Q}$ & 1315.7290 & 658.3681 & 1298.7025 & 649.8549 & 1297.7184 & 649.3629 & 12 \\
\hline 252 & 2718.3729 & 1359.6901 & 2701.3463 & 1351.1768 & 2700.3623 & 1350.6848 & $\mathbf{M}$ & 1187.6704 & 594.3388 & 1170.6439 & 585.8256 & 1169.6599 & 585.3336 & 11 \\
\hline 26 & 2833.3998 & 1417.2035 & 2816.3733 & 1408.6903 & 2815.3892 & 1408.1983 & D & 1056.6299 & 528.8186 & 1039.6034 & 520.3053 & 1038.6194 & 519.8133 & 10 \\
\hline 27 & 2890.4213 & 1445.7143 & 2873.3947 & 1437.2010 & 2872.4107 & 1436.7090 & G & 941.6030 & 471.3051 & 924.5764 & 462.7919 & 923.5924 & 462.2999 & 9 \\
\hline 282 & 2991.4689 & 1496.2381 & 2974.4424 & 1487.7248 & 2973.4584 & 1487.2328 & $T$ & 884.5815 & 442.7944 & 867.5550 & 434.2811 & 866.5710 & 433.7891 & 8 \\
\hline 293 & 3078.5010 & 1539.7541 & 3061.4744 & 1531.2409 & 3060.4904 & 1530.7488 & $S$ & 783.5339 & 392.2706 & 766.5073 & 383.7573 & 765.5233 & 383.2653 & 7 \\
\hline 303 & 3191.5850 & 1596.2962 & 3174.5585 & 1587.7829 & 3173.5745 & 1587.2909 & $\mathbf{L}$ & 696.5018 & 348.7546 & 679.4753 & 340.2413 & & & 6 \\
\hline 313 & 3304.6691 & 1652.8382 & 3287.6426 & \begin{tabular}{|l|}
1644.3249 \\
\end{tabular} & 3286.6585 & 1643.8329 & $\mathbf{L}$ & 583.4178 & 292.2125 & 566.3912 & 283.6992 & & & 5 \\
\hline 323 & 3401.7219 & 1701.3646 & 3384.6953 & 1692.8513 & 3383.7113 & 1692.3593 & $\mathbf{P}$ & 470.3337 & 235.6705 & 453.3071 & 227.1572 & & & 4 \\
\hline 333 & 3514.8059 & 1757.9066 & 3497.7794 & 1749.3933 & 3496.7954 & 1748.9013 & $\mathbf{I}$ & 373.2809 & 187.1441 & 356.2544 & 178.6308 & & & 3 \\
\hline 343 & 3627.8900 & 1814.4486 & 3610.8634 & 1805.9354 & 3609.8794 & 1805.4434 & $\mathbf{L}$ & 260.1969 & 130.6021 & 243.1703 & 122.0888 & & & 2 \\
\hline 35 & & & & & & & $\mathbf{K}$ & 147.1128 & 74.0600 & 130.0863 & 65.5468 & & & 1 \\
\hline
\end{tabular}
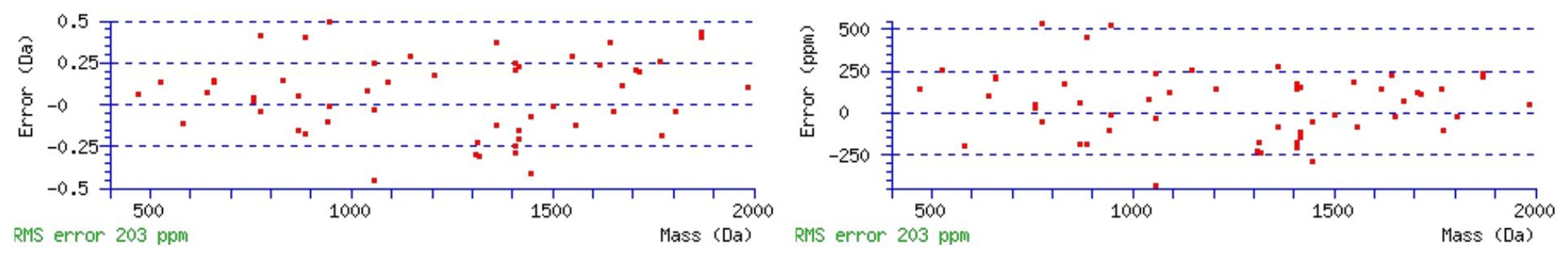

\section{All matches to this query}

\begin{tabular}{|l|l|l|l|}
\hline Score & Mr(calc): & Delta & \multicolumn{1}{|c|}{ Sequence } \\
\hline 92.6 & 3772.9882 & 0.0034 & MLVSNIDLGPTILDLAGYDLNKTQMDGTSLLPILK \\
\hline 39.0 & 3772.9882 & 0.0034 & MLVSNIDLGPTILDLAGYDLNKTQMDGTSLLPILK \\
\hline 18.5 & 3772.0042 & 0.9874 & MLVSNIDLGPTILDLAGYDLNKTQMDGTSLLPILK \\
\hline 6.2 & 3772.9783 & 0.0133 & EKKDLGTLGYVLGVTMTVIIIAIGVGIVLGYTYK \\
\hline 6.2 & 3772.9783 & 0.0133 & EKKDLGTLGYVLGVTMTVIIIAIGVGIVLGYTYK \\
\hline 2.5 & 3770.9762 & 2.0154 & ITDVQLAIFANMLGVSLFLLVVLYHYVAVNNPK \\
\hline 1.8 & 3772.9783 & 0.0133 & EKKDLGTLGYVLGVTMTVIIIAIGVGIVLGYTYK \\
\hline 1.8 & 3772.9783 & 0.0133 & EKKDLGTLGYVLGVTMTVIIIAIGVGIVLGYTYK \\
\hline 1.8 & 3772.9783 & 0.0133 & EKKDLGTLGYVLGVTMTVIIIAIGVGIVLGYTYK \\
\hline 1.8 & 3772.9783 & 0.0133 & EKKDLGTLGYVLGVTMTVIIIAIGVGIVLGYTYK \\
\hline
\end{tabular}


Spectrum No: 123; Query: 491; Rank: 1

\section{Peptide View}

MS/MS Fragmentation of ALGYENATQALSR

Found in IPI00199448, Tax_Id=10116 Gene_Symbol=Lgals3bp Mama

Match to Query 491: 1393.685488 from(697.850020,2+)

Title: 100101RatKid_NS_deglyco_22.2533.2533.2.dta

Data file K:\NewmanPaper\Piliang \3SubProteomes \Piliang3SP \mgf5ppm\ERLIC_3SubProteomes5ppm.mgf
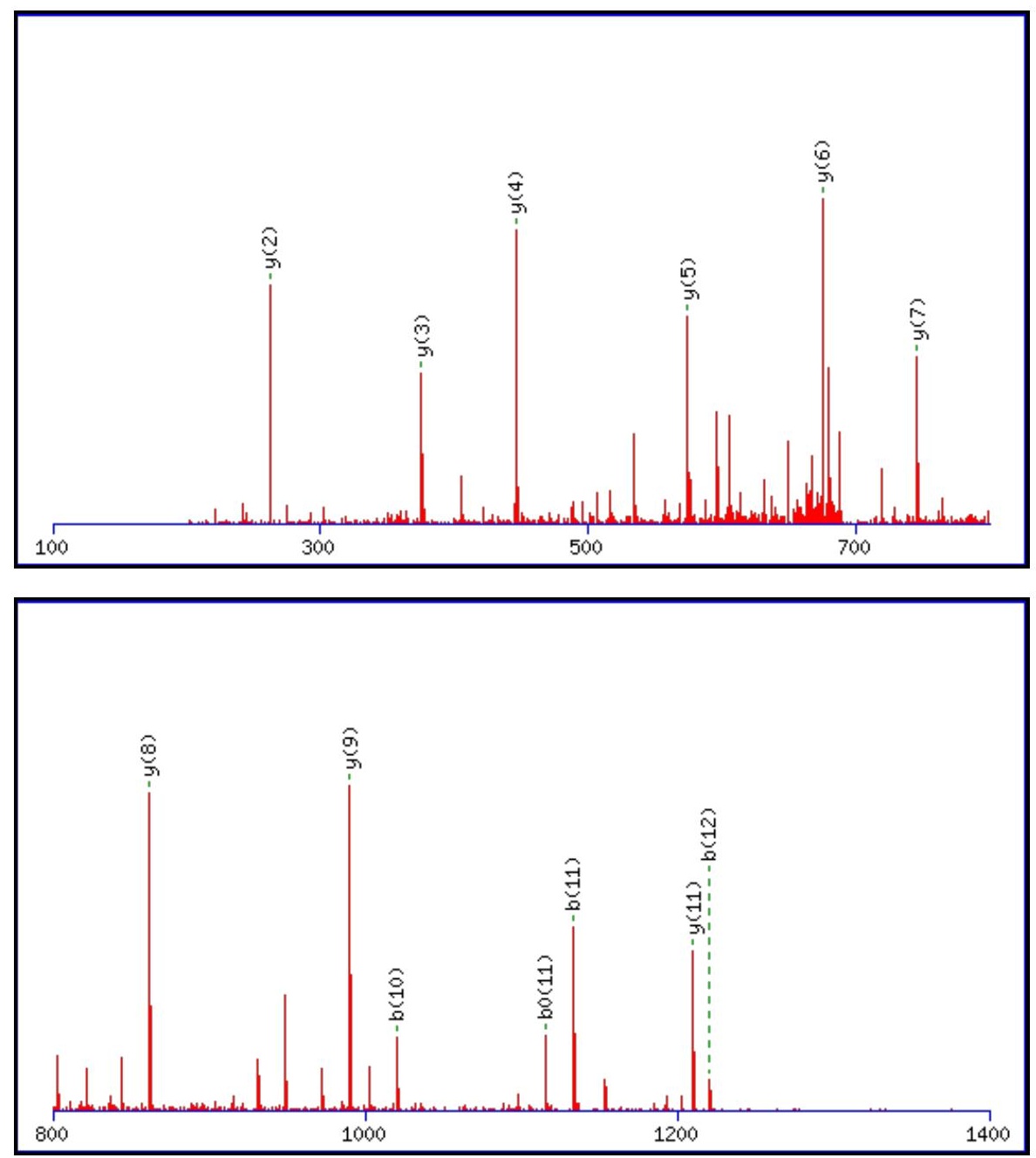
Monoisotopic mass of neutral peptide $\operatorname{Mr}($ calc): 1393.6837

Fixed modifications: Carbamidomethyl (C)

Variable modifications:

N6 : Deamidated_N (N)

Ions Score: 93 Expect: 7.8e-008

Matches (Bold Red): 13/124 fragment ions using 17 most intense peaks

\begin{tabular}{|r|c|c|c|c|c|c|c|c|c|c|c|c|c|c|}
\hline$\#$ & $\mathbf{b}$ & $\mathbf{b}^{++}$ & $\mathbf{b}^{*}$ & $\mathbf{b}^{\mathbf{*}^{++}}$ & $\mathbf{b}^{\mathbf{0}}$ & $\mathbf{b}^{\mathbf{0 + +}}$ & $\mathbf{S e q}$ & $\mathbf{y}$ & $\mathbf{y}^{++}$ & $\mathbf{y}^{\mathbf{*}}$ & $\mathbf{y}^{\boldsymbol{*}^{++}}$ & $\mathbf{y}^{\mathbf{0}}$ & $\mathbf{y}^{\mathbf{0 + +}}$ & $\#$ \\
\hline $\mathbf{1}$ & 72.0444 & 36.5258 & & & & & $\mathbf{A}$ & & & & & & & $\mathbf{1 3}$ \\
\hline $\mathbf{2}$ & 185.1285 & 93.0679 & & & & & $\mathbf{L}$ & 1323.6539 & 662.3306 & 1306.6274 & 653.8173 & 1305.6434 & 653.3253 & $\mathbf{1 2}$ \\
\hline $\mathbf{3}$ & 242.1499 & 121.5786 & & & & & $\mathbf{G}$ & $\mathbf{1 2 1 0 . 5 6 9 9}$ & 605.7886 & 1193.5433 & 597.2753 & 1192.5593 & 596.7833 & $\mathbf{1 1}$ \\
\hline $\mathbf{4}$ & 405.2132 & 203.1103 & & & & & $\mathbf{Y}$ & 1153.5484 & 577.2778 & 1136.5218 & 568.7646 & 1135.5378 & 568.2725 & $\mathbf{1 0}$ \\
\hline $\mathbf{5}$ & 534.2558 & 267.6316 & & & 516.2453 & 258.6263 & $\mathbf{E}$ & $\mathbf{9 9 0 . 4 8 5 1}$ & 495.7462 & 973.4585 & 487.2329 & 972.4745 & 486.7409 & $\mathbf{9}$ \\
\hline $\mathbf{6}$ & 649.2828 & 325.1450 & 632.2562 & 316.6318 & 631.2722 & 316.1397 & $\mathbf{N}$ & $\mathbf{8 6 1 . 4 4 2 5}$ & 431.2249 & 844.4159 & 422.7116 & 843.4319 & 422.2196 & $\mathbf{8}$ \\
\hline $\mathbf{7}$ & 720.3199 & 360.6636 & 703.2933 & 352.1503 & 702.3093 & 351.6583 & $\mathbf{A}$ & $\mathbf{7 4 6 . 4 1 5 5}$ & 373.7114 & 729.3890 & 365.1981 & 728.4050 & 364.7061 & $\mathbf{7}$ \\
\hline $\mathbf{8}$ & 821.3676 & 411.1874 & 804.3410 & 402.6741 & 803.3570 & 402.1821 & $\mathbf{T}$ & $\mathbf{6 7 5 . 3 7 8 4}$ & 338.1928 & 658.3519 & 329.6796 & 657.3679 & 329.1876 & $\mathbf{6}$ \\
\hline $\mathbf{9}$ & 949.4261 & 475.2167 & 932.3996 & 466.7034 & 931.4156 & 466.2114 & $\mathbf{Q}$ & $\mathbf{5 7 4 . 3 3 0 7}$ & 287.6690 & 557.3042 & 279.1557 & 556.3202 & 278.6637 & $\mathbf{5}$ \\
\hline $\mathbf{1 0}$ & $\mathbf{1 0 2 0 . 4 6 3 3}$ & 510.7353 & 1003.4367 & 502.2220 & 1002.4527 & 501.7300 & $\mathbf{A}$ & $\mathbf{4 4 6 . 2 7 2 2}$ & 223.6397 & 429.2456 & 215.1264 & 428.2616 & 214.6344 & $\mathbf{4}$ \\
\hline $\mathbf{1 1}$ & $\mathbf{1 1 3 3 . 5 4 7 3}$ & 567.2773 & 1116.5208 & 558.7640 & $\mathbf{1 1 1 5 . 5 3 6 8}$ & 558.2720 & $\mathbf{L}$ & $\mathbf{3 7 5 . 2 3 5 0}$ & 188.1212 & 358.2085 & 179.6079 & 357.2245 & $\mathbf{1 7 9 . 1 1 5 9}$ & $\mathbf{3}$ \\
\hline $\mathbf{1 2}$ & $\mathbf{1 2 2 0 . 5 7 9 4}$ & 610.7933 & 1203.5528 & 602.2800 & 1202.5688 & 601.7880 & $\mathbf{S}$ & $\mathbf{2 6 2 . 1 5 1 0}$ & 131.5791 & 245.1244 & 123.0659 & 244.1404 & 122.5738 & $\mathbf{2}$ \\
\hline $\mathbf{1 3}$ & & & & & & & $\mathbf{R}$ & 175.1190 & 88.0631 & 158.0924 & 79.5498 & & & $\mathbf{1}$ \\
\hline
\end{tabular}$$
\text { 年 }
$$

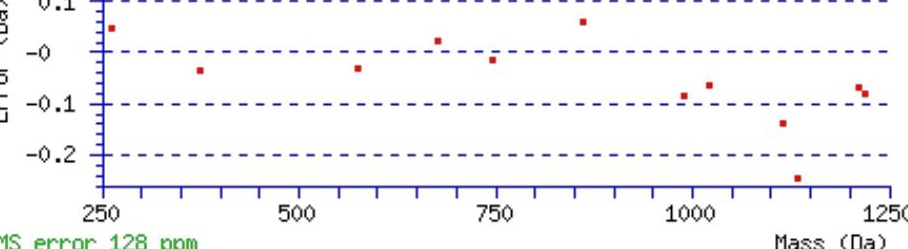

RMS error $128 \mathrm{ppm}$

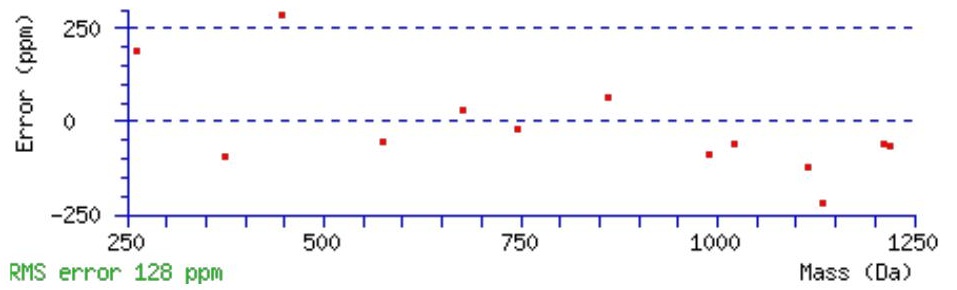

All matches to this query

\begin{tabular}{|l|l|l|l|}
\hline Score & Mr(calc): & Delta & \multicolumn{1}{|c|}{ Sequence } \\
\hline 92.5 & 1393.6837 & 0.0018 & ALGYENATQALSR \\
\hline 8.1 & 1393.6949 & -0.0095 & $\underline{\text { IQENYNTRLSR }}$ \\
\hline 7.9 & 1393.6949 & -0.0095 & $\underline{\text { IQENYNTRLSR }}$ \\
\hline 6.1 & 1393.6827 & 0.0028 & NSQQRRVALDK \\
\hline 5.5 & 1393.6741 & 0.0114 & LTPEKEEILNK \\
\hline 5.5 & 1393.6827 & 0.0028 & ASPSNARKQISR \\
\hline 5.5 & 1393.6827 & 0.0028 & ASPSNARKQISR \\
\hline 4.0 & 1393.6936 & -0.0081 & $\underline{\text { SDATASISLSSNLK }}$ \\
\hline & & & \\
\hline
\end{tabular}




\begin{tabular}{|l|l|l|l|}
3.6 & 1393.6759 & 0.0096 & AGTSTLTECVINK \\
\hline 2.9 & 1393.6837 & 0.0018 & YQQLEEASASLR \\
\hline
\end{tabular}

Spectrum No: 124; Query: 1184; Rank: 1

\section{Peptide View}

MS/MS Fragmentation of DAGYTNATTTDQHGLAK

Found in IPI00201262, Tax_Id=10116 Gene_Symbol=LOC297568;Mug2;Mug1 Alpha-1-inhibitor 3 precursor

Match to Query 1184: 1763.798508 from(882.906530,2+)

Title: 091008RatKidney_NH4Format01_26.609.609.2.dta

Data file K:INewmanPaper|Piliang \3SubProteomes\Piliang3SP\mgf5ppm\ERLIC_3SubProteomes5ppm.mgf
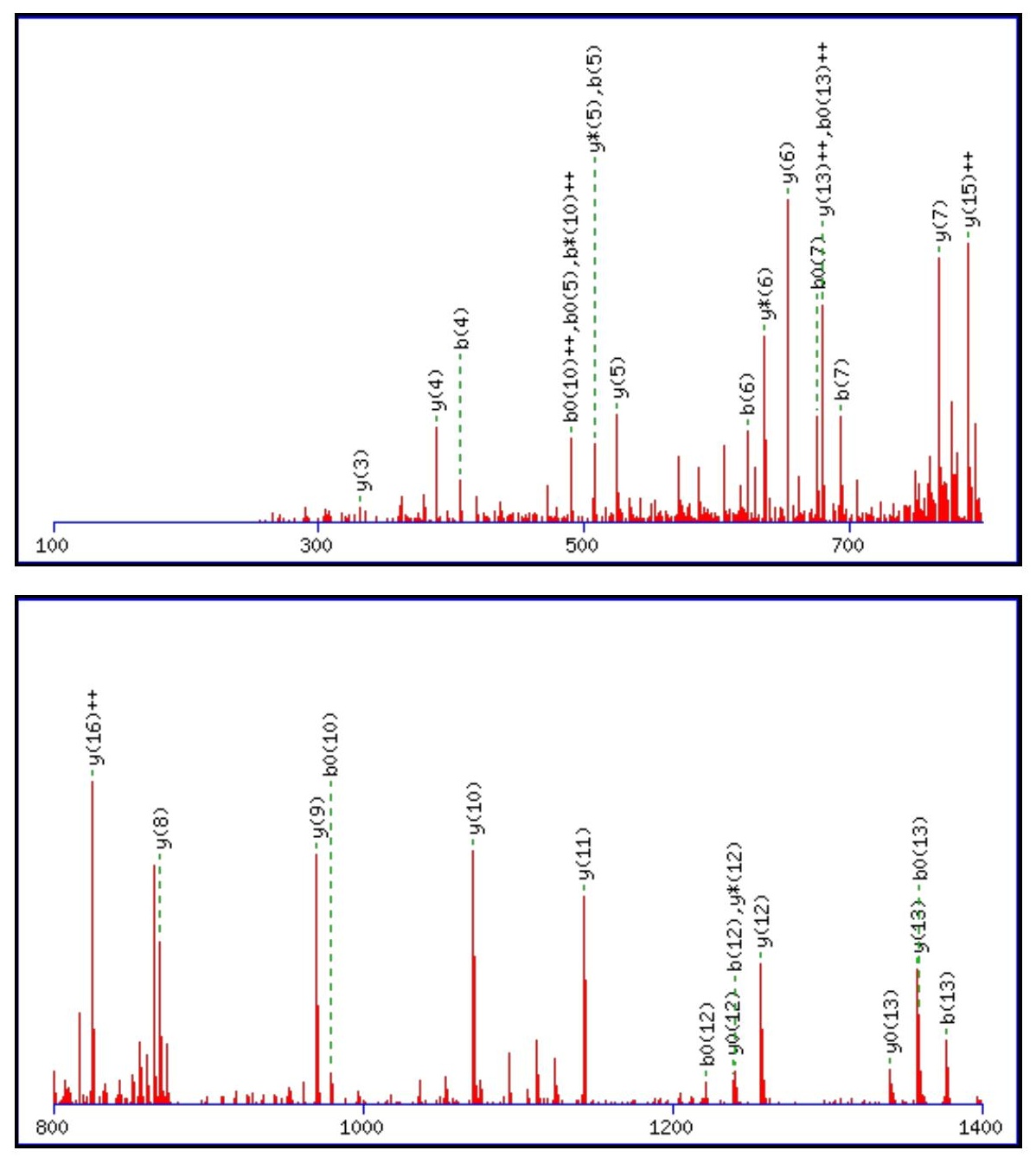


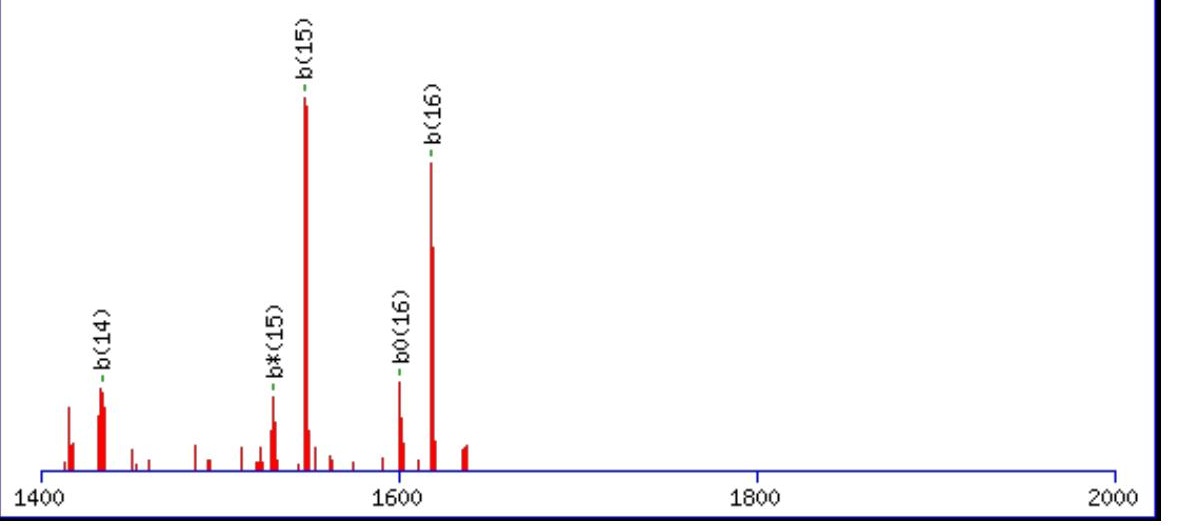

Monoisotopic mass of neutral peptide $\operatorname{Mr}($ calc): 1763.7962

Fixed modifications: Carbamidomethyl (C)

Variable modifications:

N6: : Deamidated $\mathrm{N}(\mathrm{N})$

Ions Score: 92 Expect: $1.3 \mathrm{e}-007$

Matches (Bold Red): 38/170 fragment ions using 50 most intense peaks

\begin{tabular}{|c|c|c|c|c|c|c|c|c|c|c|c|c|c|c|}
\hline \# & b & $\mathbf{b}^{++}$ & $\mathbf{b}^{*}$ & $\mathbf{b}^{*^{++}}$ & $\mathbf{b}^{\mathbf{0}}$ & $\mathbf{b}^{0++}$ & Seq. & $\mathbf{y}$ & $\mathbf{y}^{++}$ & $\mathbf{y}^{*}$ & $\mathbf{y}^{*^{++}}$ & $\mathbf{y}^{\mathbf{0}}$ & $\mathbf{y}^{0++}$ & \# \\
\hline 1 & 116.0342 & 58.5207 & & & 98.0237 & 49.5155 & D & & & & & & & 17 \\
\hline 2 & 187.0713 & 94.0393 & & & 169.0608 & 85.0340 & A & 1649.7766 & 825.3919 & 1632.7500 & 816.8786 & 1631.7660 & 816.3866 & 16 \\
\hline 3 & 244.0928 & 122.5500 & & & 226.0822 & 113.5448 & $\mathbf{G}$ & 1578.7394 & 789.8734 & 1561.7129 & 781.3601 & 1560.7289 & 780.8681 & 15 \\
\hline 4 & 407.1561 & 204.0817 & & & 389.1456 & 195.0764 & $\mathbf{Y}$ & 1521.7180 & 761.3626 & 1504.6914 & 752.8494 & 1503.7074 & 752.3573 & 14 \\
\hline 5 & 508.2038 & 254.6055 & & & 490.1932 & 245.6003 & $\mathbf{T}$ & 1358.6546 & 679.8310 & 1341.6281 & 671.3177 & 1340.6441 & 670.8257 & 13 \\
\hline 6 & 623.2307 & 312.1190 & 606.2042 & 303.6057 & 605.2202 & 303.1137 & $\mathbf{N}$ & 1257.6070 & 629.3071 & 1240.5804 & 620.7938 & 1239.5964 & 620.3018 & 12 \\
\hline 7 & 694.2679 & 347.6376 & 677.2413 & 339.1243 & 676.2573 & 338.6323 & A & 1142.5800 & 571.7937 & 1125.5535 & 563.2804 & 1124.5695 & 562.7884 & 11 \\
\hline 8 & 795.3155 & 398.1614 & 778.2890 & 389.6481 & 777.3050 & 389.1561 & $\mathbf{T}$ & 1071.5429 & 536.2751 & 1054.5164 & 527.7618 & 1053.5324 & 527.2698 & 10 \\
\hline 9 & 896.3632 & 448.6852 & 879.3367 & 440.1720 & 878.3526 & 439.6800 & $\mathbf{T}$ & 970.4952 & 485.7513 & 953.4687 & 477.2380 & 952.4847 & 476.7460 & 9 \\
\hline 10 & 997.4109 & 499.2091 & 980.3843 & 490.6958 & 979.4003 & 490.2038 & $\mathbf{T}$ & 869.4476 & 435.2274 & 852.4210 & 426.7141 & 851.4370 & 426.2221 & 8 \\
\hline 11 & 1112.4378 & 556.7226 & 1095.4113 & 548.2093 & 1094.4273 & 547.7173 & D & 768.3999 & 384.7036 & 751.3733 & 376.1903 & 750.3893 & 375.6983 & 7 \\
\hline 12 & 1240.4964 & 620.7518 & 1223.4699 & 612.2386 & 1222.4858 & 611.7466 & $\mathbf{Q}$ & 653.3729 & 327.1901 & 636.3464 & 318.6768 & & & 6 \\
\hline 13 & 1377.5553 & 689.2813 & 1360.5288 & 680.7680 & 1359.5448 & 680.2760 & $\mathbf{H}$ & 525.3144 & 263.1608 & 508.2878 & 254.6475 & & & 5 \\
\hline 14 & 1434.5768 & 717.7920 & 1417.5502 & 709.2788 & 1416.5662 & 708.7867 & $\mathbf{G}$ & 388.2554 & 194.6314 & 371.2289 & 186.1181 & & & 4 \\
\hline 15 & 1547.6609 & 774.3341 & 1530.6343 & 765.8208 & 1529.6503 & 765.3288 & $\mathbf{L}$ & 331.2340 & 166.1206 & 314.2074 & 157.6074 & & & 3 \\
\hline 16 & 1618.6980 & 809.8526 & 1601.6714 & 801.3393 & 1600.6874 & 800.8473 & A & 218.1499 & 109.5786 & 201.1234 & 101.0653 & & & 2 \\
\hline 17 & & & & & & & $\mathbf{K}$ & 147.1128 & 74.0600 & 130.0863 & 65.5468 & & & 1 \\
\hline
\end{tabular}
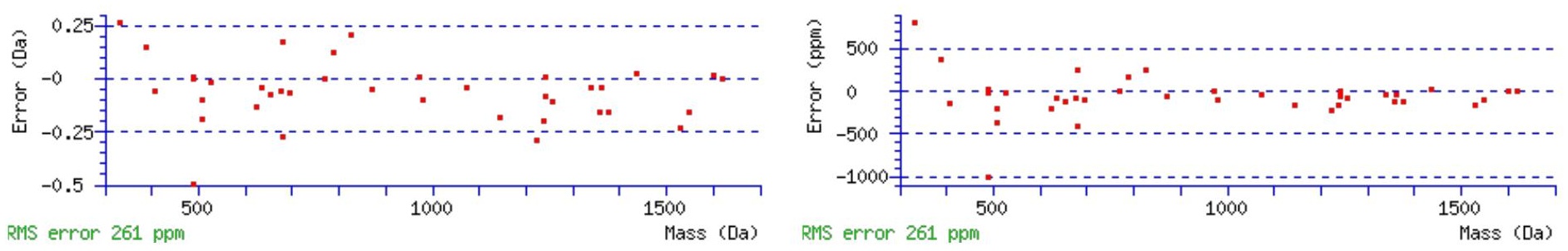

\section{All matches to this query}

\begin{tabular}{|l|l|l|l|}
\hline Score & Mr(calc): & Delta & \multicolumn{1}{c|}{ Sequence } \\
\hline 92.0 & 1763.7962 & 0.0023 & DAGYTNATTTDQHGLAK \\
\hline 48.1 & 1762.8122 & 0.9863 & $\underline{\text { DAGYTNATTTDQHGLAK }}$ \\
\hline 7.0 & 1762.7791 & 1.0194 & $\underline{\text { KSTKQTELTPEDVK }}$ \\
\hline 6.1 & 1763.8008 & -0.0023 & EGPDRSVSRLTFLK \\
\hline & & &
\end{tabular}




\begin{tabular}{|l|l|l|l|}
\hline 5.0 & 1763.8043 & -0.0058 & MNETMATDSPRRPSR \\
\hline 4.7 & 1763.8043 & -0.0058 & MNETMATDSPRRPSR \\
\hline 3.2 & 1762.7888 & 1.0097 & $\underline{\text { IPFSMENLKVNFNK }}$ \\
\hline 2.5 & 1763.7987 & -0.0002 & GATSKEVMCFNAKLK \\
\hline 2.4 & 1762.7862 & 1.0123 & SDSSLFMFRSHNKK \\
\hline 2.0 & 1762.7815 & 1.0170 & $\underline{\text { ISVEYDTSLSDWR }}$ \\
\hline
\end{tabular}

Spectrum No: 125; Query: 837; Rank: 1

\section{Peptide View}

MS/MS Fragmentation of TEPPLNASAGDQEEK

Found in IPI00210975, Tax_Id=10116 Gene_Symbol=Hyou1 Hypoxia up-regulated protein 1 precursor

Match to Query 837: 1585.705668 from(793.860110,2+)

Title: 091008RatKidney_NH4Format01_25.789.789.2.dta

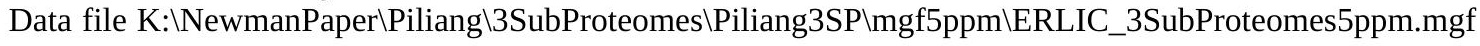
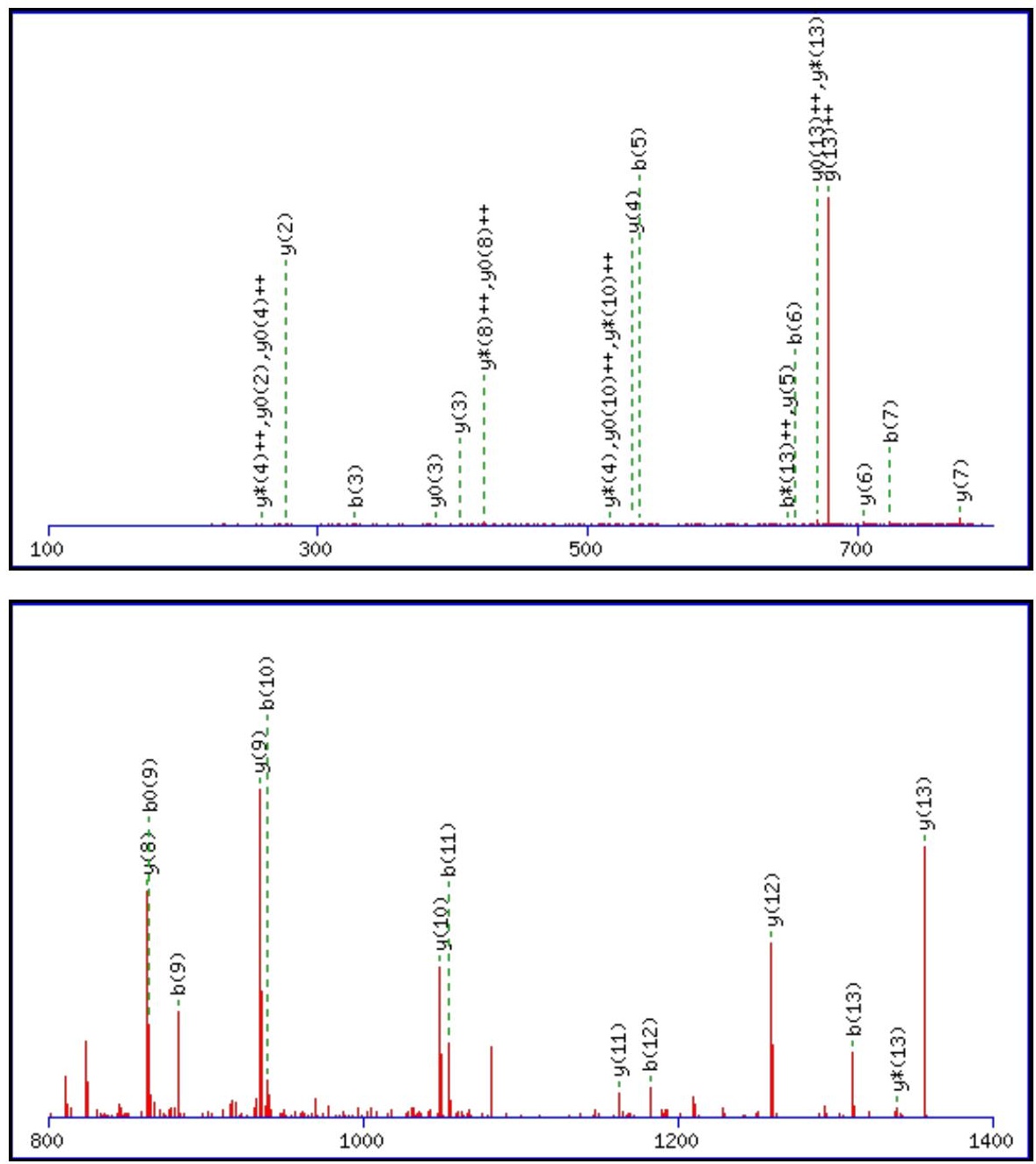


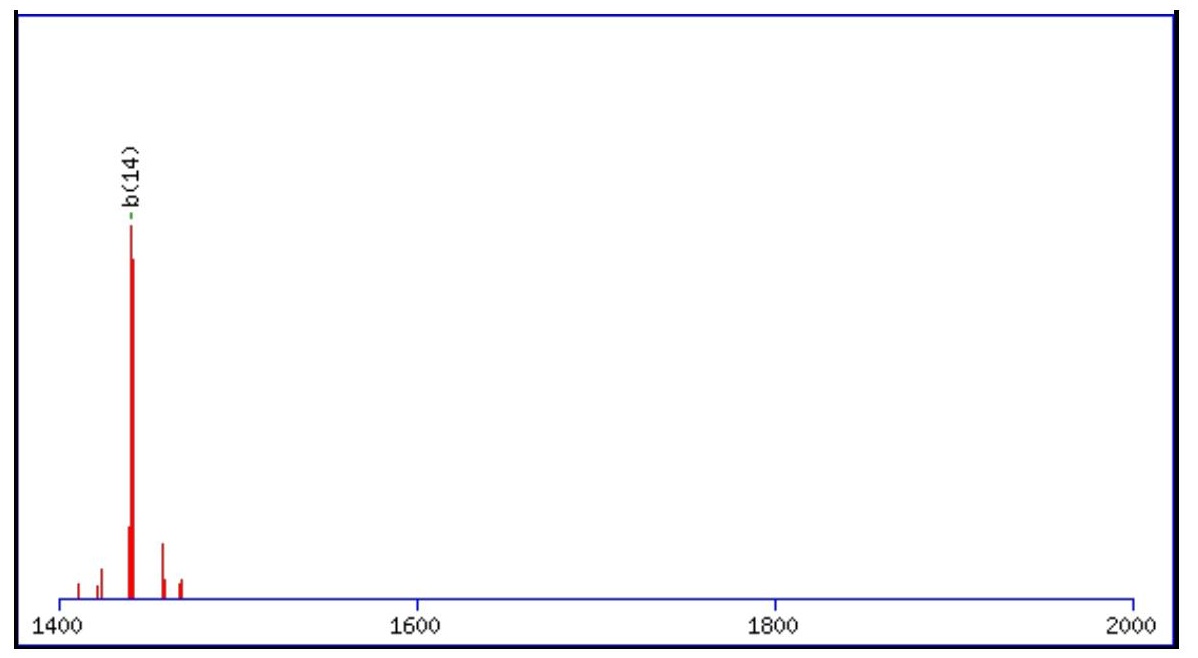

Monoisotopic mass of neutral peptide $\operatorname{Mr}($ calc): 1585.7107

Fixed modifications: Carbamidomethyl (C)

Variable modifications:

N6 : Deamidated_N (N)

Ions Score: 92 Expect: $1.1 \mathrm{e}-007$

Matches (Bold Red): 37/156 fragment ions using 42 most intense peaks

\begin{tabular}{|c|c|c|c|c|c|c|c|c|c|c|c|c|c|c|}
\hline \# & b & $\mathbf{b}^{++}$ & $\mathbf{b}^{*}$ & $\mathbf{b}^{*^{++}}$ & $\mathbf{b}^{0}$ & $\mathbf{b}^{0++}$ & Seq. & $\mathbf{y}$ & $y^{++}$ & $\mathbf{y}^{*}$ & $y^{*^{++}}$ & $\mathbf{y}^{0}$ & $\mathbf{y}^{0++}$ & \# \\
\hline 1 & 102.0550 & 51.5311 & & & 84.0444 & 42.5258 & $\mathbf{T}$ & & & & & & & 15 \\
\hline 2 & 231.0975 & 116.0524 & & & 213.0870 & 107.0471 & $\mathbf{E}$ & 1485.6704 & 743.3388 & 1468.6438 & 734.8255 & 1467.6598 & 734.3335 & 14 \\
\hline 3 & 328.1503 & 164.5788 & & & 310.1397 & 155.5735 & $\mathbf{P}$ & 1356.6278 & 678.8175 & 1339.6012 & 670.3042 & 1338.6172 & 669.8122 & 13 \\
\hline 4 & 425.2031 & 213.1052 & & & 407.1925 & 204.0999 & $\mathbf{P}$ & 1259.5750 & 630.2911 & 1242.5484 & 621.7779 & 1241.5644 & 621.2859 & 12 \\
\hline 5 & 538.2871 & 269.6472 & & & 520.2766 & 260.6419 & $\mathbf{L}$ & 1162.5222 & 581.7648 & 1145.4957 & 573.2515 & 1144.5117 & 572.7595 & 11 \\
\hline 6 & 653.3141 & 327.1607 & 636.2875 & 318.6474 & 635.3035 & 318.1554 & $\mathbf{N}$ & 1049.4382 & 525.2227 & 1032.4116 & 516.7094 & 1031.4276 & 516.2174 & 10 \\
\hline 7 & 724.3512 & 362.6792 & 707.3246 & 354.1660 & 706.3406 & 353.6740 & A & 934.4112 & 467.7093 & 917.3847 & 459.1960 & 916.4007 & 458.7040 & 9 \\
\hline 8 & 811.3832 & 406.1952 & 794.3567 & 397.6820 & 793.3727 & 397.1900 & S & 863.3741 & 432.1907 & 846.3476 & 423.6774 & 845.3636 & 423.1854 & 8 \\
\hline 9 & 882.4203 & 441.7138 & 865.3938 & 433.2005 & 864.4098 & 432.7085 & A & 776.3421 & 388.6747 & 759.3155 & 380.1614 & 758.3315 & 379.6694 & 7 \\
\hline 10 & 939.4418 & 470.2245 & 922.4152 & 461.7113 & 921.4312 & 461.2193 & G & 705.3050 & 353.1561 & 688.2784 & 344.6429 & 687.2944 & 344.1508 & 6 \\
\hline 11 & 1054.4687 & 527.7380 & 1037.4422 & 519.2247 & 1036.4582 & 518.7327 & D & 648.2835 & 324.6454 & 631.2570 & 316.1321 & 630.2729 & 315.6401 & 5 \\
\hline 12 & 1182.5273 & 591.7673 & 1165.5008 & 583.2540 & 1164.5168 & 582.7620 & $\mathbf{Q}$ & 533.2566 & 267.1319 & 516.2300 & 258.6186 & 515.2460 & 258.1266 & 4 \\
\hline 13 & 1311.5699 & 656.2886 & 1294.5434 & 647.7753 & 1293.5593 & 647.2833 & $\mathbf{E}$ & 405.1980 & 203.1026 & 388.1714 & 194.5894 & 387.1874 & 194.0974 & 3 \\
\hline 14 & 1440.6125 & 720.8099 & 1423.5860 & 712.2966 & 1422.6019 & 711.8046 & $\mathbf{E}$ & 276.1554 & 138.5813 & 259.1288 & 130.0681 & 258.1448 & 129.5761 & 2 \\
\hline 15 & & & & & & & $\mathbf{K}$ & 147.1128 & 74.0600 & 130.0863 & 65.5468 & & & 1 \\
\hline
\end{tabular}
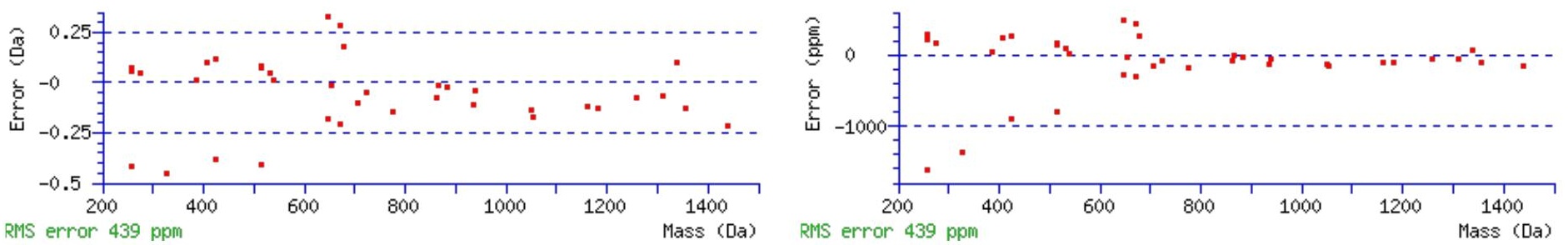

\section{All matches to this query}

\begin{tabular}{|l|l|l|l|}
\hline Score & Mr(calc): & Delta & \multicolumn{1}{c|}{ Sequence } \\
\hline 91.7 & 1585.7107 & -0.0051 & TEPPLNASAGDQEEK \\
\hline 15.1 & 1584.7007 & 1.0050 & MSSKNYASHPAAIK \\
\hline 11.8 & 1585.7059 & -0.0002 & VLDCDTISQAKEK \\
\hline 11.4 & 1585.6943 & 0.0114 & ETRYTVVNLFGK \\
\hline 10.8 & 1585.7059 & -0.0002 & VLDCDTISQAKEK \\
\hline 10.2 & 1585.7004 & 0.0053 & VLCEVFSNVNMEK \\
\hline & & & \\
\hline
\end{tabular}




\begin{tabular}{|l|l|l|l|}
9.7 & 1585.7137 & -0.0080 & QTEFAPESGKREK \\
\hline 9.4 & 1583.6942 & 2.0114 & ECPEATFAIPIEK \\
\hline 9.2 & 1584.6903 & 1.0153 & NPPDLEEEETQER \\
\hline 9.0 & 1584.7146 & 0.9910 & $\underline{\text { MPEFLEDPSVLTK }}$ \\
\hline
\end{tabular}

Spectrum No: 126; Query: 2730; Rank: 1

\section{Peptide View}

MS/MS Fragmentation of ETIGPQQGCGGYLTEDNQSFVSPDSDSNGRYDK

Found in IPI00196620, Tax_Id=10116 Gene_Symbol=Cubn Cubilin precursor

Match to Query 2730: 3621.559902 from(1208.193910,3+)

Title: 091008RatKidney_NoSalt_20.2568.2568.3.dta

Data file K:INewmanPaper|Piliang|3SubProteomes\Piliang3SP\mgf5ppm\ERLIC_3SubProteomes5ppm.mgf
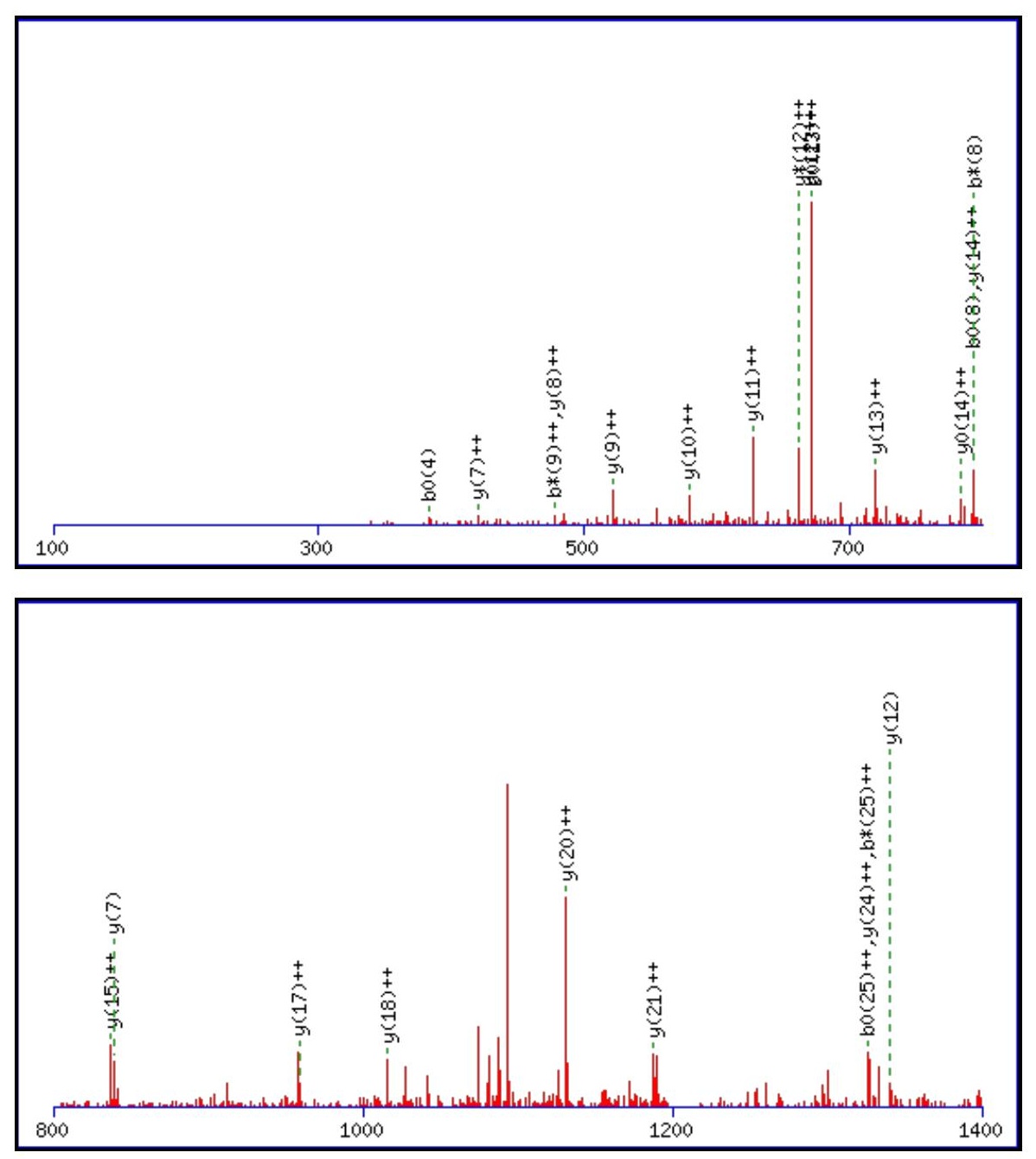


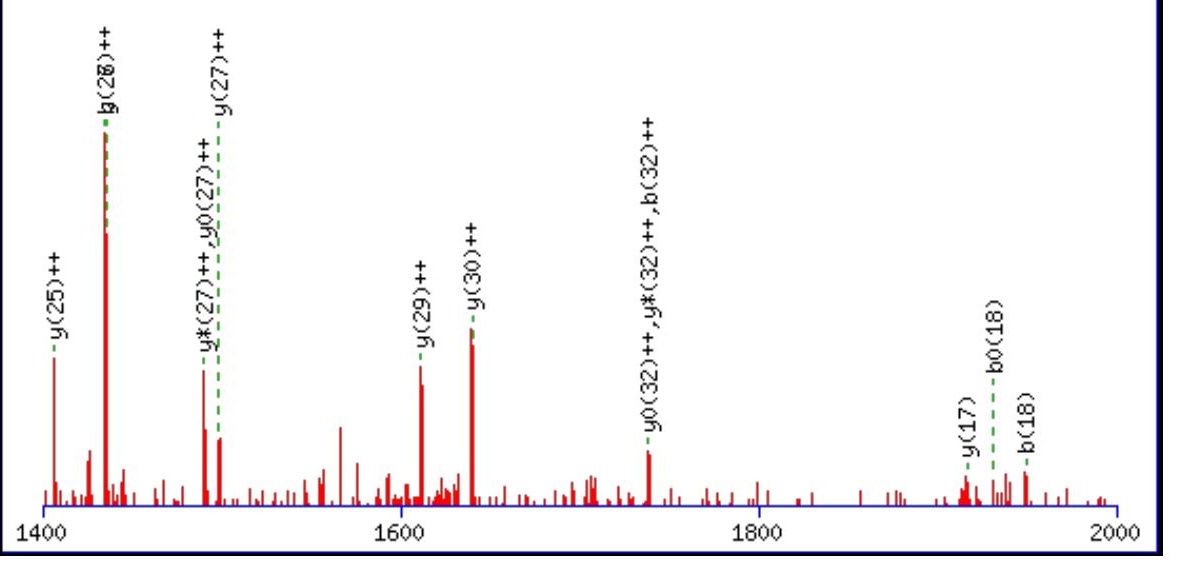

Monoisotopic mass of neutral peptide $\operatorname{Mr}($ calc): 3621.5438

Fixed modifications: Carbamidomethyl (C)

Variable modifications:

N17 : Deamidated_N (N)

Ions Score: 92 Expect: $3.3 \mathrm{e}-007$

Matches (Bold Red): 39/372 fragment ions using 69 most intense peaks

\begin{tabular}{|c|c|c|c|c|c|c|c|c|c|c|c|c|c|c|}
\hline & b & $\mathbf{b}^{++}$ & $\mathbf{b}^{*}$ & + & $\mathbf{b}^{0}$ & $\mathbf{b}^{0++}$ & Seq. & $\mathbf{y}$ & & $\mathbf{y}^{*}$ & & $\mathbf{y}^{0}$ & & \\
\hline 1 & 130.0499 & 65.5286 & & & & & $\mathbf{E}$ & & & & & & & 33 \\
\hline 2 & 231.0975 & 116.0524 & & & 213.0870 & & $\mathbf{T}$ & & & & & & & 32 \\
\hline 3 & 344.1816 & 172. & & & 326. & 392 & 1 & & 340 & 342 & & 502 & 287 & 31 \\
\hline 4 & 401.2031 & 201. & & & 383. & 192. & G & 3767 & & 3262.3502 & 6787 & 3661 & & 30 \\
\hline 5 & 498.2558 & 249.6316 & & & 480.2453 & & $\mathbf{P}$ & 3222.3553 & & & & & & 29 \\
\hline 6 & 626.3144 & 313.6 & 9 & & 608.3 & 304 & $\mathbf{Q}$ & 025 & & 759 & & 919 & 496 & 28 \\
\hline 7 & 754.3730 & 377.6901 & 737.3 & 369. & 736.3624 & 368.6849 & $\mathbf{Q}$ & 2997.2439 & 1499.1256 & 2980.2174 & 6123 & 2979.2333 & 1203 & 27 \\
\hline 8 & 811.3945 & 406.2009 & 794.3 & 397. & 793.3839 & 397. & G & 2869. & 963 & 2852.1588 & & 748 & & 26 \\
\hline 9 & 971.4251 & 486.2162 & 954 & & 953.4 & 477 & $\mathrm{C}$ & 639 & 856 & 373 & 723 & 533 & 803 & 25 \\
\hline 10 & 1028.4466 & 514.7269 & 011.4200 & 506. & 1010.4360 & 505.7216 & G & 2652.1332 & 8702 & 2635.1067 & .0570 & 2634.1227 & 5650 & 24 \\
\hline 11 & 1085.4680 & 543.2377 & 415 & & 75 & & G & 2595. & & & & & & 23 \\
\hline 12 & 1248.5314 & 624.7693 & 48 & 616. & 230.5208 & 615. & $\mathbf{Y}$ & 903 & 126 & 637 & 355 & 252 & & 22 \\
\hline 13 & 1361.6154 & 681.3114 & 1344.5889 & 672. & 1343.6049 & $672.3^{3}$ & $\mathbf{L}$ & 2375.0270 & 1188 & 2358.0004 & 5038 & 0164 & 0118 & 21 \\
\hline 4 & 62.6631 & 731.8 & 66 & & 25 & & $T$ & 2261 & 113 & 2244 & & 323 & & 20 \\
\hline 5 & 1591.7057 & 796.3565 & 92 & 787. & 1573.6951 & 787.3 & $\mathbf{E}$ & 2160. & 512 & 687 & 380 & 847 & 460 & 19 \\
\hline 16 & 1706.7326 & 853.8700 & 589.7061 & 845. & 1688.7221 & 844.8647 & D & 2031.8526 & 1016.4300 & 2014.8261 & 1007.9167 & 2013.8421 & 4247 & 18 \\
\hline 7 & 21.7596 & 34 & 30 & & 90 & & $\mathbf{N}$ & & & 1899 & & 189 & 112 & 17 \\
\hline 8 & 49.8182 & 975.4127 & 1932.7 & 94 & 1931.8076 & 966.4074 & $\mathbf{Q}$ & 1801.7987 & & 1784 & & 1783 & 977 & 16 \\
\hline 19 & 2036.8502 & 18.9287 & 2019.8236 & 155 & 2018.8396 & 1009.9234 & $S$ & 1673.7402 & 3737 & 1656.7136 & 8604 & 1655 & 3684 & 15 \\
\hline 20 & 83.9186 & 92.4629 & 21 & & 165.9080 & 77 & $\mathbf{F}$ & 1586.7081 & & 1569. & & 1568.6976 & 8524 & 14 \\
\hline 1 & 2282.9870 & 41.9971 & 2265.9605 & 339 & 2264.9764 & 1132. & $\mathrm{~V}$ & 1439.6397 & 3235 & 1422 & & 1421 & 3182 & 13 \\
\hline 22 & 2370.0190 & 1185.5132 & 2352.9925 & 176.9999 & 2352.0085 & 1176.5079 & $S$ & 1340.5713 & 670.7893 & 1323.5448 & 662.2760 & 1322.5607 & 661.7840 & 12 \\
\hline 23 & 467.0718 & 395 & 2450 & 25.5263 & 2449.0612 & 1225.0343 & $\mathbf{P}$ & 1253.5393 & 2733 & 1236.5127 & 618.7600 & 1235.5287 & 2680 & 11 \\
\hline 24 & 2582.0987 & 291.5530 & 2565.0722 & 283.0397 & 2564.0882 & 1282.5477 & D & 1156.4865 & 7469 & 1139.4600 & & 1138.4760 & 7416 & 10 \\
\hline 25 & 2669.1308 & 1335.0690 & 2652.1042 & 1326.5558 & 2651.1202 & 1326.0637 & S & 1041.4596 & 2334 & 1024.4330 & 512.7202 & 1023.4490 & 512.2281 & 9 \\
\hline 26 & 2784.1577 & 1392.5825 & 2767.1312 & 1384.0692 & 2766.1472 & 1383.5772 & D & 954.4275 & 7174 & 937.4010 & 469.2041 & 936.4170 & 468.7121 & 8 \\
\hline 27 & 2871.1897 & 1436.0985 & 2854.1632 & 1427.5852 & 2853.1792 & 1427.0932 & $\mathrm{~S}$ & 839.4006 & 420.2039 & 822.3741 & & 821.3900 & 1987 & 7 \\
\hline 28 & 2985.2327 & 1493.1200 & 2968.2061 & 1484.6067 & 2967.2221 & 1484.1147 & $\mathbf{N}$ & 752.3686 & 376.6879 & 735.3420 & 1747 & 734.3580 & 6826 & 6 \\
\hline 29 & 3042.2541 & 1521.6307 & 3025.2276 & 1513.1174 & 3024.2436 & 1512.6254 & G & 638.3257 & 319.6665 & 621.2991 & 311.1532 & 620.3151 & 310.6612 & 5 \\
\hline 30 & 3198.3553 & 1599.6813 & 3181.3287 & 1591.1680 & 3180.3447 & 1590.6760 & $\mathbf{R}$ & 581.3042 & 291.1557 & 564.2776 & 282.6425 & 563.2936 & 282.1504 & 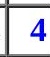 \\
\hline 31 & 3361.4186 & 1681.2129 & 3344.3920 & 1672.6997 & 3343.4080 & 1672.2076 & $\mathbf{Y}$ & 425.2031 & 213.1052 & 408.1765 & 204.5919 & 407.1925 & 204.0999 & 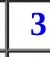 \\
\hline 32 & 3476.4455 & 1738.7264 & 3459.4190 & 1730.2131 & 3458.4350 & 1729.7211 & D & 262.1397 & 131.5735 & 245.1132 & 123.0602 & 244.1292 & 122.5682 & \\
\hline
\end{tabular}




\begin{tabular}{|l|l|l|l|l|l|l|l|l|l|l|l|l|l|}
\hline 33 & & & & & & & K & 147.1128 & 74.0600 & 130.0863 & 65.5468 & & \\
\hline
\end{tabular}
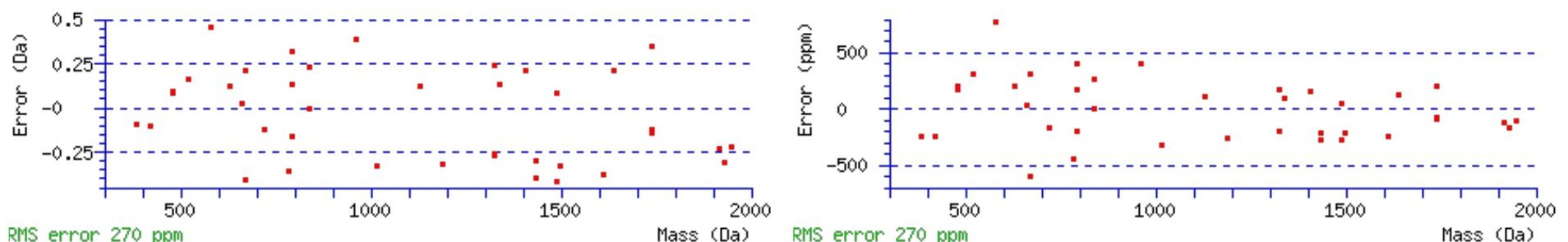

RMS error 270 ppm Mass (Da) RMS error 270 ppm

\section{All matches to this query}

\begin{tabular}{|l|l|l|l|}
\hline Score & Mr(calc): & Delta & \multicolumn{1}{|c|}{ Sequence } \\
\hline 91.6 & 3621.5438 & 0.0161 & ETIGPQQGCGGYLTEDNQSFVSPDSDSNGRYDK \\
\hline 88.5 & 3621.5438 & 0.0161 & ETIGPQQGCGGYLTEDNQSFVSPDSDSNGRYDK \\
\hline 85.7 & 3620.5598 & 1.0001 & ETIGPQQGCGGYLTEDNQSFVSPDSDSNGRYDK \\
\hline 9.1 & 3621.5791 & -0.0192 & DLTRLQPQNLVGTSTVNSDSPASDPTVFHKR \\
\hline 6.8 & 3621.5791 & -0.0192 & DLTRLQPQNLVGTSTVNSDSPASDPTVFHKR \\
\hline 6.1 & 3620.5823 & 0.9776 & DWYEQASPAPLLVNPEALEPSLSVNGSDGMFK \\
\hline 4.0 & 3619.5388 & 2.0211 & LYSAVKEDELMEFSDLESDSEEKPCIKPR \\
\hline 3.8 & 3619.5651 & 1.9948 & QNPAEGLQTLGAQMQGGFGGGNSQLPKTDGGSETK \\
\hline 3.1 & 3620.5674 & 0.9925 & WTGTGERGYMISAGARATGTAQTFIHIPSTAR \\
\hline 3.1 & 3620.5674 & 0.9925 & WTGTGERGYMISAGARATGTAQTFIHIPSTAR \\
\hline
\end{tabular}

Spectrum No: 127; Query: 1780; Rank: 1

\section{Peptide View}

MS/MS Fragmentation of NGSLILVQDSDSDDNSLLSK

Found in IPI00363032, Tax_Id=10116 Gene_Symbol=Usp40 similar to Ubiquitin carboxyl-terminal hydrolase 40

Match to Query 1780: 2120.016628 from(1061.015590,2+)

Title: 100101RatKid_NS_deglyco_20.3794.3794.2.dta

Data file K:INewmanPaper|Piliangl3SubProteomes|Piliang3SP\mgf5ppm|ERLIC_3SubProteomes5ppm.mgf

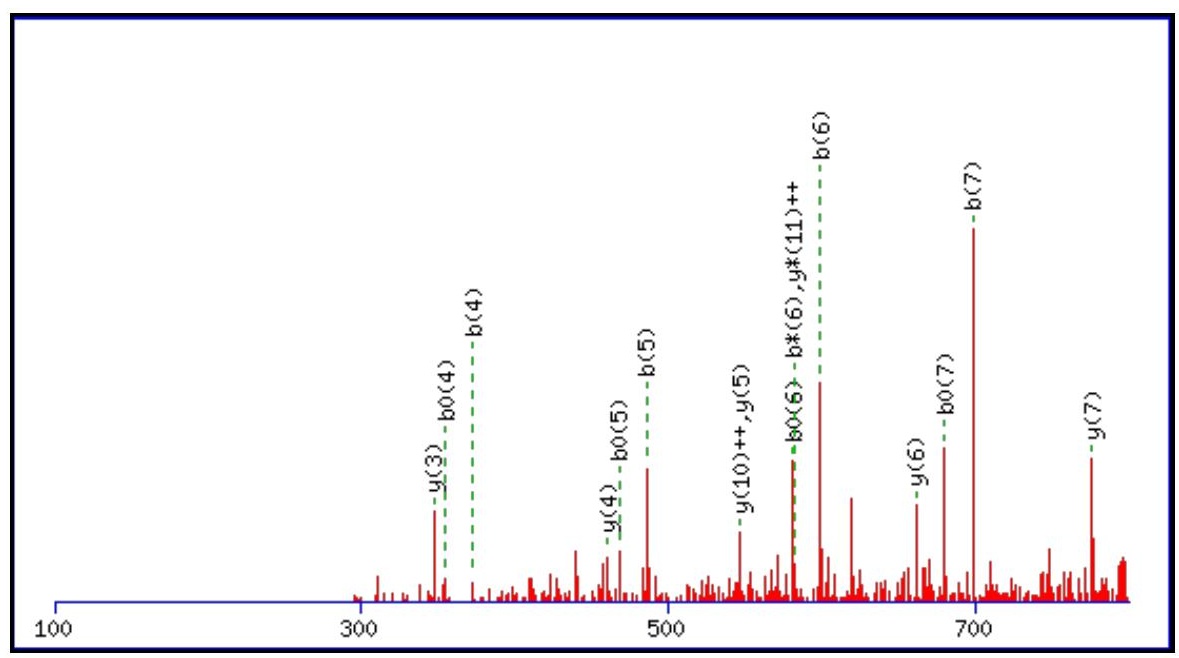



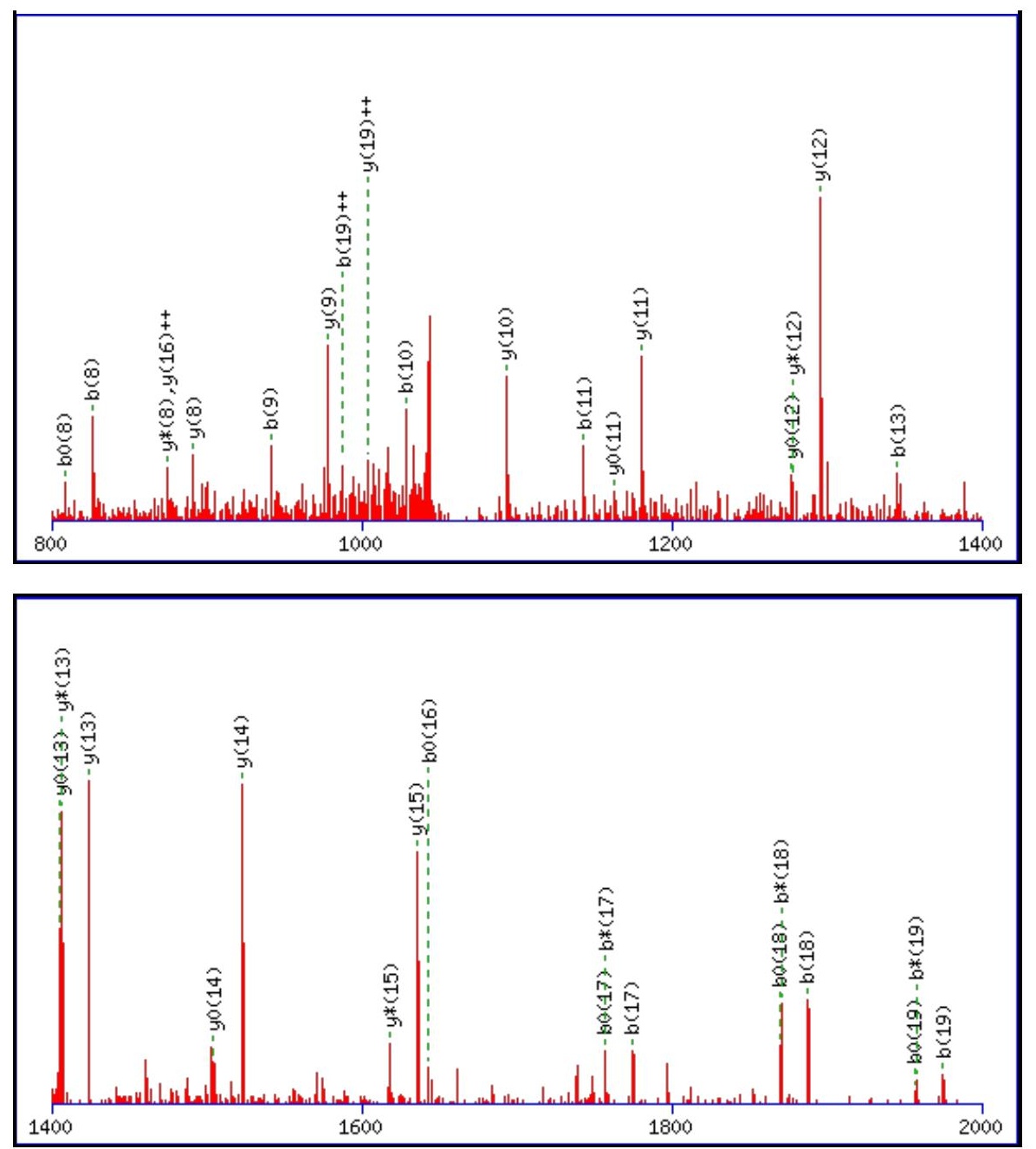

Monoisotopic mass of neutral peptide $\operatorname{Mr}($ calc): 2120.0121

Fixed modifications: Carbamidomethyl (C)

Variable modifications:

N1 : Deamidated $\mathrm{N}(\mathrm{N})$

Ions Score: 92 Expect: $1.7 \mathrm{e}-007$

Matches (Bold Red): 51/222 fragment ions using 78 most intense peaks

\begin{tabular}{|c|c|c|c|c|c|c|c|c|c|c|c|c|c|c|}
\hline \# & b & $\mathbf{b}^{++}$ & $\mathbf{b}^{*}$ & $\mathbf{b}^{*^{++}}$ & $\mathbf{b}^{0}$ & $\mathbf{b}^{\mathbf{0 + +}}$ & Seq. & $\mathbf{y}$ & $\mathbf{y}^{++}$ & $\mathbf{y}^{*}$ & $\mathrm{y}^{\mathrm{*}^{++}}$ & $\mathbf{y}^{0}$ & $\mathbf{y}^{0++}$ & \# \\
\hline 1 & 116.0342 & 58.5207 & 99.0077 & 50.0075 & & & $\mathbf{N}$ & & & & & & & 20 \\
\hline 2 & 173.0557 & 87.0315 & 156.0291 & 78.5182 & & & G & & 1003 & & & 1987 & 994.4946 & 19 \\
\hline 3 & 260.0877 & 130.5475 & 243.0612 & 122.0342 & 242.0771 & 121.5422 & S & 1948.9710 & 974.9891 & 1931.9444 & 966.4758 & 1930.9604 & 965.9838 & 18 \\
\hline 4 & 373.1718 & 187.0895 & 356.1452 & 178.5762 & 355.1612 & 178.0842 & $\mathbf{L}$ & 1861.9389 & 931.4731 & 1844.9124 & 598 & 1843.9284 & 922.4678 & 17 \\
\hline 5 & 486.2558 & 243.6316 & 469.2293 & 235.1183 & 468.2453 & 234.6263 & I & 1748.8549 & 874.9311 & 1731.8283 & 4178 & 1730.8443 & 865.9258 & 16 \\
\hline 6 & 599.3399 & 300.1736 & 582.3133 & 291.6603 & 581.3293 & 291.1683 & $\mathbf{L}$ & 1635.7708 & 818.3890 & 1618.7443 & 809.8758 & 1617.7602 & 809.3838 & 15 \\
\hline 7 & 698.4083 & 349.7078 & 681.3818 & 341.1945 & 680.3977 & 340.7025 & V & 1522.6867 & 761.8470 & 1505.6602 & 3337 & 1504.6762 & 752.8417 & 14 \\
\hline 8 & 826.4669 & 413.7371 & 809.4403 & 405.2238 & 808.4563 & 404.7318 & $\mathbf{Q}$ & 1423.6183 & 712.3128 & 1406.5918 & 703.7995 & 1405.6078 & 703.3075 & 13 \\
\hline 9 & 941.4938 & 471.2506 & 924.4673 & 462.7373 & 923.4833 & 462.2453 & D & 1295.5597 & 648.2835 & 1278.5332 & 639.7702 & 1277.5492 & 639.2782 & 12 \\
\hline 10 & 1028.5259 & 514.7666 & 1011.4993 & 506.2533 & 1010.5153 & 505.7613 & S & 1180.5328 & 590.7700 & 1163.5063 & 582.2568 & 1162.5222 & 581.7648 & 11 \\
\hline 11 & 1143.5528 & 572.2800 & 1126.5263 & 563.7668 & 1125.5422 & 563.2748 & D & 1093.5008 & 547.2540 & 1076.4742 & 538.7407 & 1075.4902 & 538.2487 & 10 \\
\hline 12 & 1230.5848 & 615.7961 & 1213.5583 & 607.2828 & 1212.5743 & 606.7908 & $\mathbf{S}$ & 978.4738 & 489.7406 & 961.4473 & 481.2273 & 960.4633 & 480.7353 & 9 \\
\hline 13 & 1345.6118 & 673.3095 & 1328.5852 & 664.7963 & 1327.6012 & 664.3042 & D & 891.4418 & 446.2245 & 874.4153 & 437.7113 & 873.4312 & 437.2193 & 8 \\
\hline 14 & 1460.6387 & 730.8230 & 1443.6122 & 722.3097 & 1442.6282 & 721.8177 & D & 776.4149 & 388.7111 & 759.3883 & 380.1978 & 758.4043 & 379.7058 & 7 \\
\hline 15 & 1574.6816 & 787.8445 & 1557.6551 & 779.3312 & 1556.6711 & 778.8392 & $\mathbf{N}$ & 661.3879 & 331.1976 & 644.3614 & 322.6843 & 643.3774 & 322.1923 & 6 \\
\hline 16 & 1661.7137 & 831.3605 & 1644.6871 & 822.8472 & 1643.7031 & 822.3552 & S & 547.3450 & 274.1761 & 530.3184 & 265.6629 & 529.3344 & 265.1708 & 5 \\
\hline 17 & 1774.7977 & 887.9025 & 1757.7712 & 879.3892 & 1756.7872 & 878.8972 & $\mathbf{L}$ & 460.3130 & 230.6601 & 443.2864 & 222.1468 & 442.3024 & 221.6548 & 4 \\
\hline 18 & 1887.8818 & 944.4445 & 1870.8553 & 935.9313 & 1869.8712 & 935.4393 & $\mathbf{L}$ & 347.2289 & 174.1181 & 330.2023 & 165.6048 & 329.2183 & 165.1128 & 3 \\
\hline
\end{tabular}




\begin{tabular}{|r|r|r|r|r|r|r|r|r|r|r|r|r|r|r|r|}
\hline $\mathbf{1 9}$ & $\mathbf{1 9 7 4 . 9 1 3 8}$ & $\mathbf{9 8 7 . 9 6 0 6}$ & $\mathbf{1 9 5 7 . 8 8 7 3}$ & $\mathbf{9 7 9 . 4 4 7 3}$ & $\mathbf{1 9 5 6 . 9 0 3 3}$ & $\mathbf{9 7 8 . 9 5 5 3}$ & $\mathrm{S}$ & 234.1448 & 117.5761 & 217.1183 & 109.0628 & 216.1343 & 108.5708 & $\mathbf{2}$ \\
\hline $\mathbf{2 0}$ & & & & & & & K & 147.1128 & 74.0600 & 130.0863 & 65.5468 & & & $\mathbf{1}$ \\
\hline
\end{tabular}
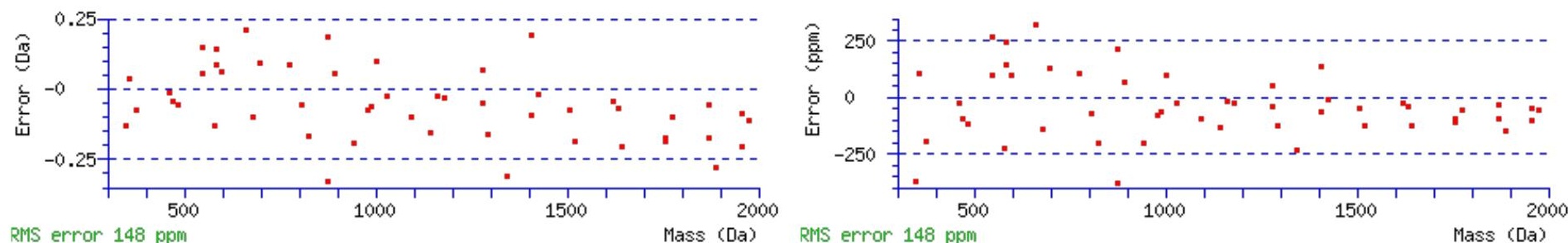

RMS error 148 ppm

\section{All matches to this query}

\begin{tabular}{|l|l|l|l|}
\hline Score & Mr(calc): & Delta & \multicolumn{1}{|c|}{ Sequence } \\
\hline 91.5 & 2120.0121 & 0.0046 & NGSLILVQDSDSDDNSLLSK \\
\hline 52.0 & 2119.0281 & 0.9886 & NGSLILVQDSDSDDNSLLSK \\
\hline 25.3 & 2120.0121 & 0.0046 & NGSLILVQDSDSDDNSLLSK \\
\hline 8.8 & 2119.9989 & 0.0177 & $\underline{\text { INRIEEEKISMASLLSK }}$ \\
\hline 6.7 & 2118.0051 & 2.0116 & ALADIVIPQEYGITKAEK \\
\hline 5.1 & 2120.0207 & -0.0041 & FKASLSENVLGSPKELLK \\
\hline 3.8 & 2120.0191 & -0.0025 & ERPDTGVYVKDLSSFVTK \\
\hline 3.8 & 2120.0191 & -0.0025 & ERPDTGVYVKDLSSFVTK \\
\hline 2.8 & 2120.0282 & -0.0116 & MKLAQQYVMTSLQQEYK \\
\hline 1.4 & 2120.0198 & -0.0031 & RLLTDDEVLAVTRHCSR \\
\hline
\end{tabular}

Spectrum No: 128; Query: 2400; Rank: 1

\section{Peptide View}

MS/MS Fragmentation of NGTNLDWATAETLALGSLLAQGFNVR

Found in IPI00364925, Tax_Id=10116 Gene_Symbol=Dhtkd1 Probable 2-oxoglutarate dehydrogenase E1 component DHKTD1, mitochondrial precursor

Match to Query 2400: 2732.376192 from(911.799340,3+)

Title: 091008RatKidney_NH4Format02_14.5093.5093.3.dta

Data file K:\NewmanPaper\Piliang\3SubProteomes\Piliang3SP\mgf5ppm\ERLIC_3SubProteomes5ppm.mgf

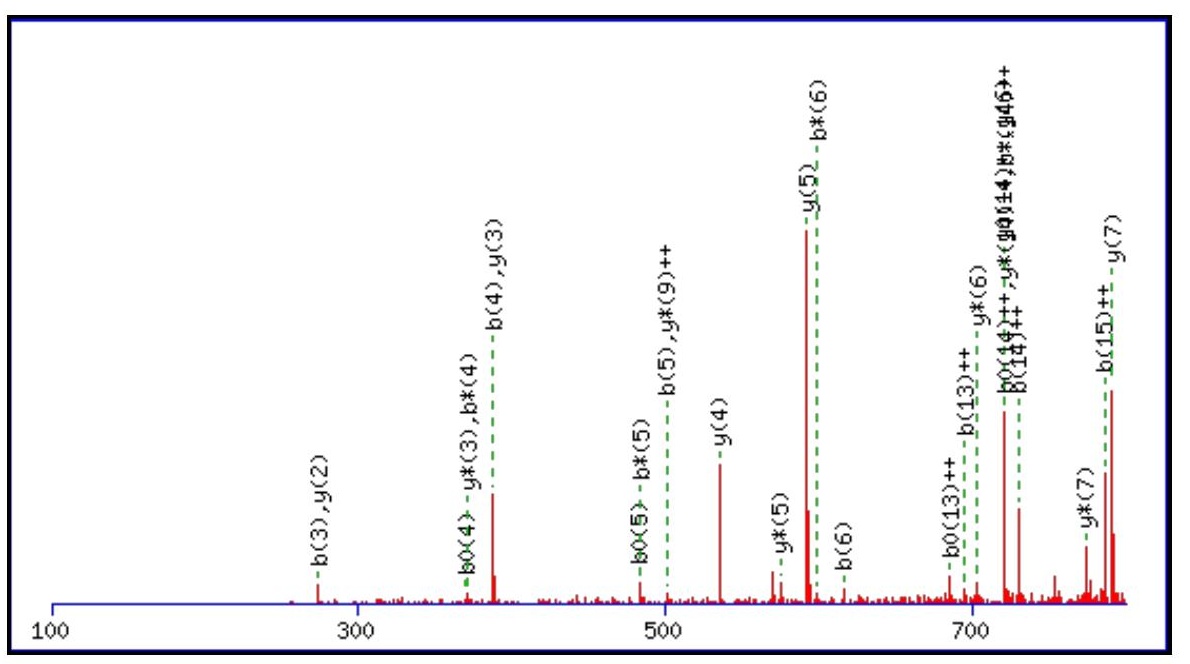



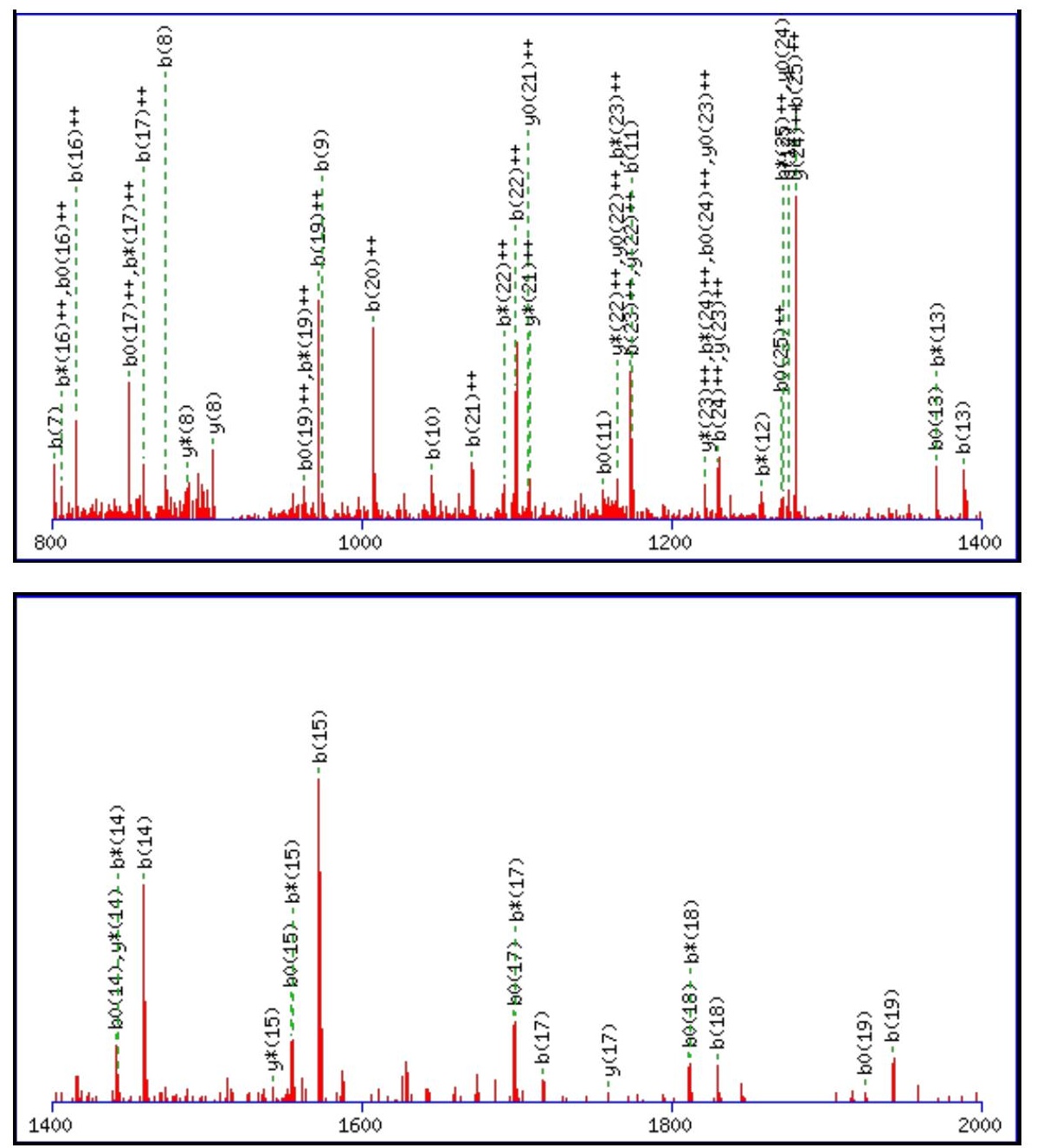

Monoisotopic mass of neutral peptide $\operatorname{Mr}($ calc): 2732.3769

Fixed modifications: Carbamidomethyl (C)

Variable modifications:

N1 : Deamidated $\mathrm{N}(\mathrm{N})$

Ions Score: 91 Expect: $1.3 \mathrm{e}-007$

Matches (Bold Red): 89/278 fragment ions using 144 most intense peaks

\begin{tabular}{|c|c|c|c|c|c|c|c|c|c|c|c|c|c|c|}
\hline \# & b & $\mathbf{b}^{++}$ & $\mathbf{b}^{*}$ & $\mathbf{b}^{*^{++}}$ & $\mathbf{b}^{\mathbf{0}}$ & $\mathbf{b}^{\mathbf{0 + +}}$ & Seq. & $\mathbf{y}$ & $\mathbf{y}^{++}$ & $\mathbf{y}^{*}$ & $\mathbf{y}^{*^{++}}$ & $\mathbf{y}^{0}$ & $\mathbf{y}^{\mathbf{0 + +}}$ & \# \\
\hline 1 & 116.0342 & 58.5207 & 99.0077 & 50.0075 & & & $\mathbf{N}$ & & & & & & & 26 \\
\hline 2 & 173.0557 & 87.0315 & 156.0291 & 78.5182 & & & G & 2618.3573 & 1309.6823 & 2601.3307 & 1301.1690 & 2600.3467 & 1300.6770 & 25 \\
\hline 3 & 274.1034 & 137.5553 & 257.0768 & 129.0420 & 256.0928 & 128.5500 & $\mathbf{T}$ & 2561.3358 & 1281.1716 & 2544.3093 & 1272.6583 & 2543.3253 & 1272.1663 & 24 \\
\hline 4 & 388.1463 & 194.5768 & 371.1197 & 186.0635 & 370.1357 & 185.5715 & $\mathbf{N}$ & 2460.2882 & 1230.6477 & 2443.2616 & 1222.1344 & 2442.2776 & 1221.6424 & 23 \\
\hline 5 & 501.2303 & 251.1188 & 484.2038 & 242.6055 & 483.2198 & 242.1135 & $\mathbf{L}$ & 2346.2452 & 1173.6262 & 2329.2187 & 1165.1130 & 2328.2347 & 1164.6210 & 22 \\
\hline 6 & 616.2573 & 308.6323 & 599.2307 & 300.1190 & 598.2467 & 299.6270 & D & 2233.1612 & 1117.0842 & 2216.1346 & 1108.5709 & 2215.1506 & 1108.0789 & 21 \\
\hline 7 & 802.3366 & 401.6719 & 785.3101 & 393.1587 & 784.3260 & 392.6667 & $\mathbf{W}$ & 2118.1342 & 1059.5707 & 2101.1077 & 1051.0575 & 2100.1237 & 1050.5655 & 20 \\
\hline 8 & 873.3737 & 437.1905 & 856.3472 & 428.6772 & 855.3632 & 428.1852 & A & 1932.0549 & 966.5311 & 1915.0284 & 958.0178 & 1914.0443 & 957.5258 & 19 \\
\hline 9 & 974.4214 & 487.7143 & 957.3948 & 479.2011 & 956.4108 & 478.7091 & $\mathbf{T}$ & 1861.0178 & 931.0125 & 1843.9912 & 922.4993 & 1843.0072 & 922.0073 & 18 \\
\hline 10 & 1045.4585 & 523.2329 & 1028.4320 & 514.7196 & 1027.4479 & 514.2276 & A & 1759.9701 & 880.4887 & 1742.9436 & 871.9754 & 1741.9595 & 871.4834 & 17 \\
\hline 11 & 1174.5011 & 587.7542 & 1157.4746 & 579.2409 & 1156.4905 & 578.7489 & $\mathbf{E}$ & 1688.9330 & 844.9701 & 1671.9064 & 836.4569 & 1670.9224 & 835.9649 & 16 \\
\hline 12 & 1275.5488 & 638.2780 & 1258.5222 & 629.7648 & 1257.5382 & 629.2727 & $\mathbf{T}$ & 1559.8904 & 780.4488 & 1542.8639 & 771.9356 & 1541.8798 & 771.4436 & 15 \\
\hline 13 & 1388.6328 & 694.8201 & 1371.6063 & 686.3068 & 1370.6223 & 685.8148 & $\mathbf{L}$ & 1458.8427 & 729.9250 & 1441.8162 & 721.4117 & 1440.8322 & 720.9197 & 14 \\
\hline 14 & 1459.6700 & 730.3386 & 1442.6434 & 721.8253 & 1441.6594 & 721.3333 & A & 1345.7587 & 673.3830 & 1328.7321 & 664.8697 & 1327.7481 & 664.3777 & 13 \\
\hline 15 & 1572.7540 & 786.8806 & 1555.7275 & 778.3674 & 1554.7435 & 777.8754 & $\mathbf{L}$ & 1274.7215 & 637.8644 & 1257.6950 & 629.3511 & 1256.7110 & 628.8591 & 12 \\
\hline 16 & 1629.7755 & 815.3914 & 1612.7489 & 806.8781 & 1611.7649 & 806.3861 & $\mathbf{G}$ & 1161.6375 & 581.3224 & 1144.6109 & 572.8091 & 1143.6269 & 572.3171 & 11 \\
\hline 17 & 1716.8075 & 858.9074 & 1699.7810 & 850.3941 & 1698.7969 & 849.9021 & $\mathbf{S}$ & 1104.6160 & 552.8116 & 1087.5895 & 544.2984 & 1086.6055 & 543.8064 & 10 \\
\hline 18 & 1829.8916 & 915.4494 & 1812.8650 & 906.9362 & 1811.8810 & 906.4441 & $\mathbf{L}$ & 1017.5840 & 509.2956 & 1000.5574 & 500.7824 & & & 9 \\
\hline
\end{tabular}




\begin{tabular}{|c|c|c|c|c|c|c|c|c|c|c|c|c|}
\hline 19 & |1942.9756 & 971.9915 & |1925.9491 & 963.4782 & |1924.9651 & 962.9862 & L & 904.4999 & 452.7536 & 887.4734 & 444.2403 & 8 \\
\hline 20 & 2014.0128 & 1007.5100 & 1996.9862 & 998.9967 & 1996.0022 & 998.5047 & A & 791.4159 & 396.2116 & 774.3893 & 387.6983 & 7 \\
\hline 21 & 2142.0713 & 1071.5393 & 2125.0448 & 1063.0260 & 2124.0608 & 1062.5340 & $\mathbf{Q}$ & 720.3787 & 360.6930 & 703.3522 & 352.1797 & 6 \\
\hline 22 & 2199.0928 & 1100.0500 & 2182.0662 & 1091.5368 & 2181.0822 & 1091.0448 & G & 592.3202 & 296.6637 & 575.2936 & 288.1504 & 5 \\
\hline 23 & 2346.1612 & 1173.5842 & 2329.1347 & 1165.0710 & 2328.1506 & 1164.5790 & $\mathbf{F}$ & 535.2987 & 268.1530 & 518.2722 & 259.6397 & 4 \\
\hline 24 & 2460.2041 & 1230.6057 & 2443.1776 & 1222.0924 & 2442.1936 & 1221.6004 & $\mathbf{N}$ & 388.2303 & 194.6188 & 371.2037 & 186.1055 & 3 \\
\hline 25 & 2559.2726 & 1280.1399 & 2542.2460 & 1271.6266 & 2541.2620 & 1271.1346 & V & 274.1874 & 137.5973 & 257.1608 & 129.0840 & 2 \\
\hline 26 & & & & & & & $\mathbf{R}$ & 175.1190 & 88.0631 & 158.0924 & 79.5498 & 1 \\
\hline
\end{tabular}
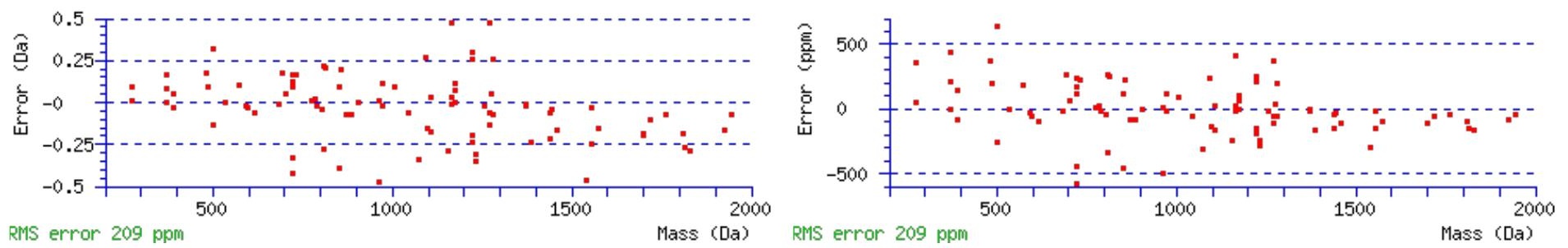

\section{All matches to this query}

\begin{tabular}{|l|l|l|l|}
\hline Score & Mr(calc): & Delta & \multicolumn{1}{|c|}{ Sequence } \\
\hline 91.5 & 2732.3769 & -0.0007 & NGTNLDWATAETLALGSLLAQGFNVR \\
\hline 83.9 & 2732.3769 & -0.0007 & NGTNLDWATAETLALGSLLAQGFNVR \\
\hline 24.9 & 2731.3929 & 0.9833 & NGTNLDWATAETLALGSLLAQGFNVR \\
\hline 9.7 & 2732.3769 & -0.0007 & NGTNLDWATAETLALGSLLAQGFNVR \\
\hline 5.5 & 2732.3981 & -0.0219 & SRSTNVKQFIAPEAGEGVSLQLGNTK \\
\hline 3.9 & 2732.3816 & -0.0054 & GESLWLNLRNRISQQMDGLAPYR \\
\hline 2.9 & 2730.3653 & 2.0109 & YSHFPDFSYSASSKIIISDVPSRK \\
\hline 2.2 & 2732.3938 & -0.0176 & LWASAFGGEIKSIAAKYSGSQLLQK \\
\hline 2.2 & 2732.3938 & -0.0176 & LWASAFGGEIKSIAAKYSGSQLLQK \\
\hline 2.1 & 2732.3608 & 0.0154 & ASLSALTMNTIVFNKLGGAVIFDDR \\
\hline
\end{tabular}

Spectrum No: 129; Query: 1279; Rank: 1

\section{Peptide View}

MS/MS Fragmentation of NETLGGTCLNVGCIPSK

Found in IPI00365545, Tax_Id=10116 Gene_Symbol=Dld Dihydrolipoyl dehydrogenase, mitochondrial precursor

Match to Query 1279: 1819.853708 from(910.934130,2+)

Title: 100101RatKid_NS_deglyco_12.3268.3268.2.dta

Data file K:INewmanPaper|Piliangl3SubProteomes\Piliang3SP\mgf5ppm\ERLIC_3SubProteomes5ppm.mgf 

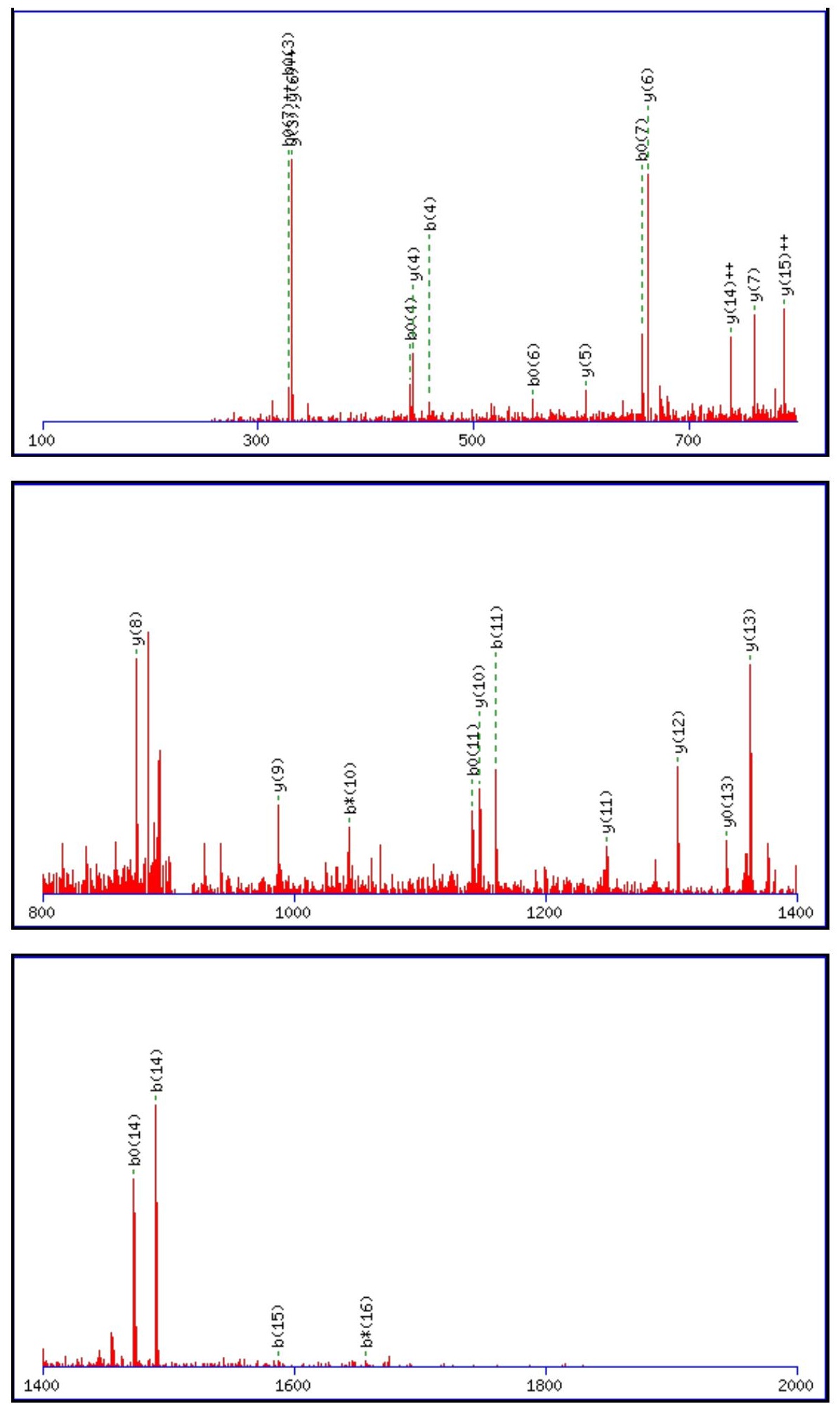

Monoisotopic mass of neutral peptide $\operatorname{Mr}($ calc): 1819.8444

Fixed modifications: Carbamidomethyl (C)

Variable modifications:

N1 : Deamidated $\mathrm{N}(\mathrm{N})$

Ions Score: 91 Expect: $1.6 \mathrm{e}-007$

Matches (Bold Red): 28/188 fragment ions using 43 most intense peaks

\begin{tabular}{|r|c|c|c|c|c|c|c|c|c|c|c|c|c|c|}
\hline$\#$ & $\mathbf{b}$ & $\mathbf{b}^{++}$ & $\mathbf{b}^{*}$ & $\mathbf{b}^{\boldsymbol{*}^{++}}$ & $\mathbf{b}^{\mathbf{0}}$ & $\mathbf{b}^{\mathbf{0 + +}}$ & Seq. & $\mathbf{y}$ & $\mathbf{y}^{++}$ & $\mathbf{y}^{\mathbf{*}}$ & $\mathbf{y}^{\boldsymbol{*}^{++}}$ & $\mathbf{y}^{\mathbf{0}}$ & $\mathbf{y}^{\mathbf{0 + +}}$ & $\#$ \\
\hline $\mathbf{1}$ & 116.0342 & 58.5207 & 99.0077 & 50.0075 & & & $\mathbf{N}$ & & & & & & & $\mathbf{1 7}$ \\
\hline $\mathbf{2}$ & 245.0768 & 123.0420 & 228.0503 & 114.5288 & 227.0662 & 114.0368 & $\mathbf{E}$ & 1705.8248 & 853.4160 & 1688.7982 & 844.9027 & 1687.8142 & 844.4107 & $\mathbf{1 6}$ \\
\hline $\mathbf{3}$ & 346.1245 & 173.5659 & 329.0979 & 165.0526 & 328.1139 & 164.5606 & $\mathbf{T}$ & 1576.7822 & 788.8947 & 1559.7556 & 780.3815 & 1558.7716 & 779.8894 & $\mathbf{1 5}$ \\
\hline $\mathbf{4}$ & $\mathbf{4 5 9 . 2 0 8 5}$ & 230.1079 & 442.1820 & 221.5946 & $\mathbf{4 4 1 . 1 9 8 0}$ & 221.1026 & $\mathbf{L}$ & 1475.7345 & 738.3709 & 1458.7079 & 729.8576 & 1457.7239 & 729.3656 & $\mathbf{1 4}$ \\
\hline $\mathbf{5}$ & 516.2300 & 258.6186 & 499.2035 & 250.1054 & 498.2194 & 249.6134 & $\mathbf{G}$ & $\mathbf{1 3 6 2 . 6 5 0 4}$ & 681.8289 & 1345.6239 & 673.3156 & $\mathbf{1 3 4 4 . 6 3 9 9}$ & 672.8236 & $\mathbf{1 3}$ \\
\hline
\end{tabular}




\begin{tabular}{|c|c|c|c|c|c|c|c|c|c|c|c|c|c|c|}
\hline 6 & 573.2515 & |287.1294 & 556.2249 & |278.6161 & 555.2409 & |278.1241 & & 1305.6290 & 653.3181 & |1288.6024 & 644.8048 & |1287.6184 & |644.3128 & 12 \\
\hline 7 & 674.2992 & 337.6532 & 657.2726 & 329.1399 & 656.2886 & 328.6479 & $\mathbf{T}$ & 1248.6075 & 624.8074 & 1231.5810 & 616.2941 & 1230.5969 & 615.8021 & 11 \\
\hline 8 & 834.3298 & 417.6685 & 817.3033 & 409.1 & 816.3 & 408.6633 & C & & 574.2836 & 1130.5333 & 565.7703 & 1129.5493 & 783 & \\
\hline 9 & 947.4139 & 474.2106 & 930.3873 & 465.6973 & 929.4033 & 465.2053 & $\mathbf{L}$ & 987.5292 & 494.2682 & 970.5026 & 485.7550 & 969.5186 & 485.2629 & 9 \\
\hline 10 & 1061.4568 & 531.2320 & 1044.4302 & 522.7188 & 1043.4462 & 522.2268 & $\mathbf{N}$ & 874.4451 & 437. & 857.4186 & 429. & 856.4345 & 428 & 8 \\
\hline 11 & 160.5252 & 580.7662 & 1143.2 & 572.2530 & 1142.5146 & 571.7610 & $v$ & 022 & 380.7 & 756 & 372. & 3916 & 371 & 7 \\
\hline 12 & 1217.5467 & 609.2770 & 1200.5201 & 600.7637 & 1199.5361 & 600.2717 & G & 661.3338 & 331.1705 & 644.3072 & 322.6573 & 643.3232 & 322.1652 & 6 \\
\hline 13 & 1377.5773 & 689.2923 & 1360.5508 & 680.7790 & 1359.5668 & 680.2870 & C & 604.3123 & 302.6598 & 587.2858 & 294.1465 & 6.3017 & 293. & 5 \\
\hline 14 & 1490.6614 & 745.8343 & 1473.6348 & 737.3211 & 1472.6508 & 736.8290 & 1 & 444.2817 & 222.6445 & 427.2551 & 214.1312 & 426.2711 & 5392 & 4 \\
\hline 15 & 1587.7142 & 794.3607 & 1570.6876 & 785.8474 & 1569.7036 & 785.3554 & $\mathbf{P}$ & 331.1976 & 166.1024 & 314.1710 & 157.5892 & 313.1870 & 157.0972 & 3 \\
\hline 16 & 1674.7462 & 837.8767 & 1657.7196 & 829.3635 & 1656.7356 & 828.8714 & $S$ & 234.1448 & 117.5761 & 217.1183 & 109.0628 & 216.1343 & 108.5708 & 2 \\
\hline 17 & & & & & & & T & 147.1128 & 74.0600 & 130.0863 & 65.5468 & & & 1 \\
\hline
\end{tabular}
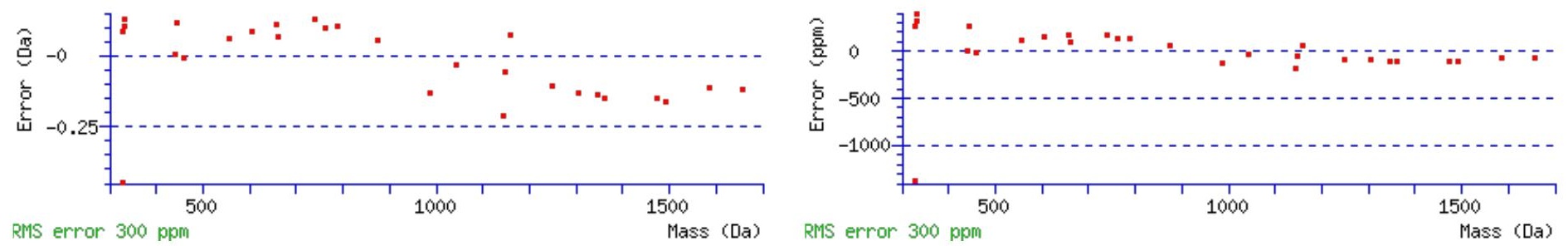

\section{All matches to this query}

\begin{tabular}{|l|c|c|l|}
\hline Score & Mr(calc): & Delta & \multicolumn{1}{|c|}{ Sequence } \\
\hline 91.3 & 1819.8444 & 0.0093 & NETLGGTCLNVGCIPSK \\
\hline 62.4 & 1818.8604 & 0.9933 & NETLGGTCLNVGCIPSK \\
\hline 47.3 & 1819.8444 & 0.0093 & NETLGGTCLNVGCIPSK \\
\hline 8.5 & 1819.8594 & -0.0057 & ATSSATTLASTPKLRR \\
\hline 8.1 & 1819.8651 & -0.0114 & SKMYLDLLSQSQRR \\
\hline 8.1 & 1819.8651 & -0.0114 & SKMYLDLLSQSQRR \\
\hline 5.8 & 1819.8687 & -0.0150 & NEITTLNEEDSISNLK \\
\hline 5.1 & 1819.8594 & -0.0057 & ATSSATTLASTPKLRR \\
\hline 5.0 & 1819.8594 & -0.0057 & ATSSATTLASTPKLRR \\
\hline 4.7 & 1817.8325 & 2.0212 & NLTLESRVQELQTK \\
\hline
\end{tabular}

Spectrum No: 130; Query: 1490; Rank: 1

\section{Peptide View}

MS/MS Fragmentation of LQVELDSVTGLLNQSDSK

Found in IPI00209113, Tax_Id=10116 Gene_Symbol=Myh9 Myosin-9

Match to Query 1490: 1945.981668 from(973.998110,2+)

Title: 100101RatKid_NS_deglyco_14.4576.4576.2.dta

Data file K:\NewmanPaper\Piliang \3SubProteomes\Piliang3SP \mgf5ppm\ERLIC_3SubProteomes5ppm.mgf 

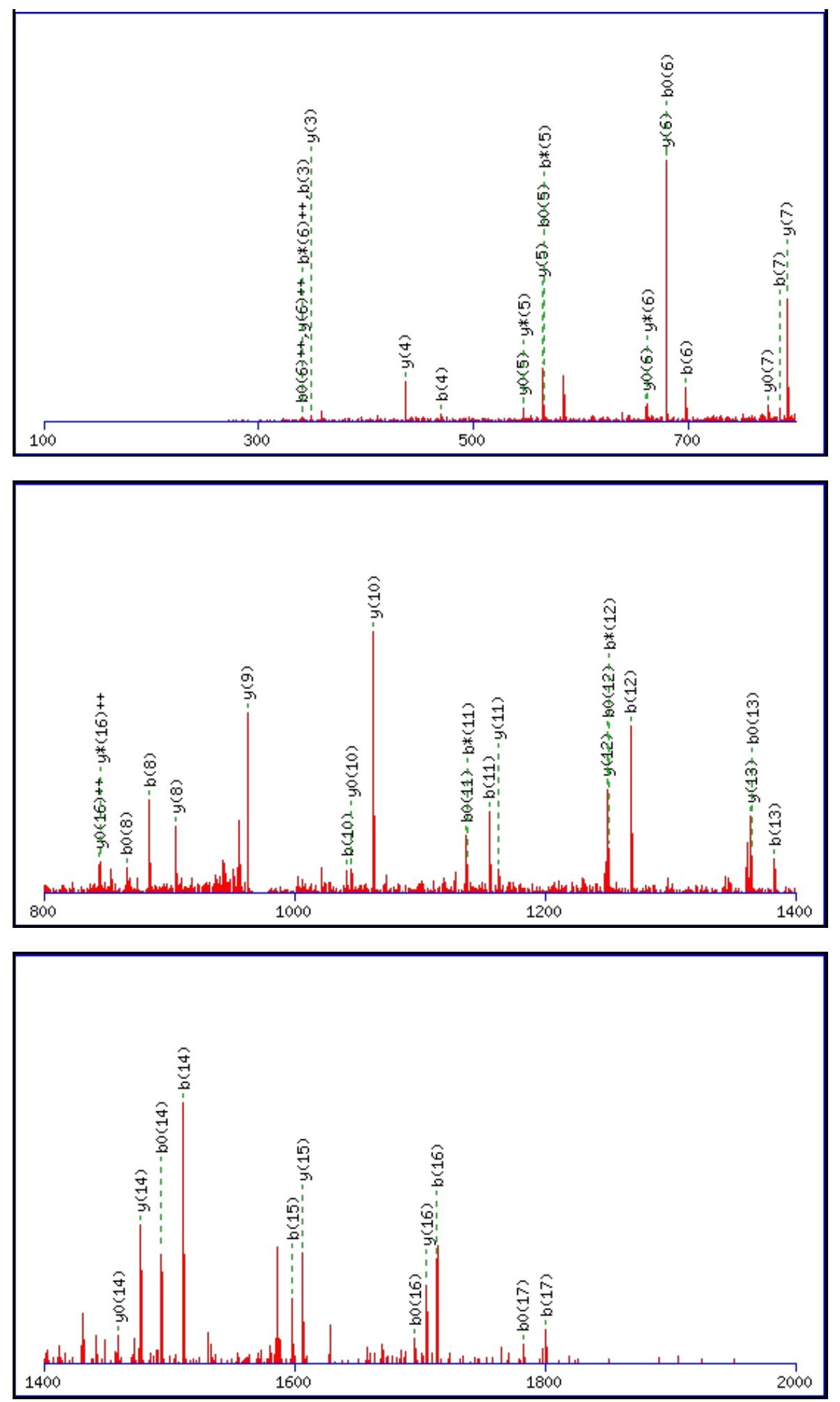

Monoisotopic mass of neutral peptide $\operatorname{Mr}($ calc): 1945.9844

Fixed modifications: Carbamidomethyl (C)

Variable modifications:

N13 : Deamidated $\mathrm{N}(\mathrm{N})$

Ions Score: 91 Expect: $1.5 \mathrm{e}-007$

Matches (Bold Red): 51/194 fragment ions using 87 most intense peaks

\begin{tabular}{|c|c|c|c|c|c|c|c|c|c|c|c|c|c|c|}
\hline \# & b & $\mathbf{b}^{++}$ & $\mathbf{b}^{*}$ & $\mathbf{b}^{*^{++}}$ & $\mathbf{b}^{0}$ & $\mathbf{b}^{\mathbf{0 + +}}$ & Seq. & $\mathbf{y}$ & $\mathbf{y}^{++}$ & $\mathrm{y}^{*}$ & $\mathrm{y}^{*^{++}}$ & $\mathbf{y}^{\mathbf{0}}$ & $y^{0++}$ & $\#$ \\
\hline 1 & 114.0913 & 57.5493 & & & & & $\mathbf{L}$ & & & & & & & 18 \\
\hline 2 & 242.1499 & 121.5786 & 225.1234 & 113.0653 & & & $\mathbf{Q}$ & 1833.9076 & 917.4574 & 1816.8811 & 908.9442 & 1815.8971 & 908.4522 & 17 \\
\hline 3 & 341.2183 & 171.1128 & 324.1918 & 162.5995 & & & $\mathbf{V}$ & 1705.8490 & 853.4282 & 1688.8225 & 844.9149 & 1687.8385 & 844.4229 & 16 \\
\hline 4 & 470.2609 & 235.6341 & 453.2344 & 227.1208 & 452.2504 & 226.6288 & $\mathbf{E}$ & 1606.7806 & 803.8940 & 1589.7541 & 795.3807 & 1588.7701 & 794.8887 & 15 \\
\hline 5 & 583.3450 & 292.1761 & 566.3184 & 283.6629 & 565.3344 & 283.1709 & $\mathbf{L}$ & 1477.7380 & 739.3727 & 1460.7115 & 730.8594 & 1459.7275 & 730.3674 & 14 \\
\hline
\end{tabular}




\begin{tabular}{|c|c|c|c|c|c|c|c|c|c|c|c|c|c|c|}
\hline 6 & 698.3719 & |349.6896| & 681.3454 & |341.1763 & 680.3614 & |340.6843 & D & |1364.6540 & 682.8306 & |1347.6274 & 674.3173 & |1346.6434 & |673.8253| & 13 \\
\hline 7 & 785.4040 & 393.2056 & 768.3774 & 384.6923 & 767.3934 & 384.2003 & $S$ & 1249.6270 & 625.3172 & 1232.6005 & 616.8039 & 1231.6165 & 616.3119 & 12 \\
\hline 8 & 884.4724 & 442.7398 & 867.4458 & 434.2266 & 866.4618 & 433.7345 & $\mathbf{V}$ & 1162.5950 & 581.8011 & 1145.5685 & 573.2879 & 1144.5844 & 572.7959 & 11 \\
\hline 9 & 985.5201 & 493.2637 & 968.4935 & 484.7504 & 967.5095 & 484.2584 & $\mathbf{T}$ & 1063.5266 & 532.2669 & 1046.5000 & 523.7537 & 1045.5160 & 523.2616 & 10 \\
\hline 10 & 1042.5415 & 521.7744 & 1025.5150 & 513.2611 & 1024.5310 & 512.7691 & G & 962.4789 & 481.7431 & 945.4524 & 473.2298 & 944.4683 & 472.7378 & 9 \\
\hline 11 & 1155.6256 & 578.3164 & 1138.5990 & 569.8032 & 1137.6150 & 569.3111 & $\mathbf{L}$ & 905.4574 & 453.2324 & 888.4309 & 444.7191 & 887.4469 & 444.2271 & 8 \\
\hline 12 & 1268.7096 & 634.8585 & 1251.6831 & 626.3452 & 1250.6991 & 625.8532 & $\mathbf{L}$ & 792.3734 & 396.6903 & 775.3468 & 388.1771 & 774.3628 & 387.6850 & 7 \\
\hline 13 & 1383.7366 & 692.3719 & 1366.7100 & 683.8587 & 1365.7260 & 683.3666 & $\mathbf{N}$ & 679.2893 & 340.1483 & 662.2628 & 331.6350 & 661.2788 & 331.1430 & 6 \\
\hline 14 & 1511.7952 & 756.4012 & 1494.7686 & 747.8879 & 1493.7846 & 747.3959 & $\mathbf{Q}$ & 564.2624 & 282.6348 & 547.2358 & 274.1216 & 546.2518 & 273.6295 & 5 \\
\hline 15 & 1598.8272 & 799.9172 & 1581.8006 & 791.4040 & 1580.8166 & 790.9119 & $S$ & 436.2038 & 218.6055 & 419.1773 & 210.0923 & 418.1932 & 209.6003 & 4 \\
\hline 16 & 1713.8541 & 857.4307 & 1696.8276 & 848.9174 & 1695.8436 & 848.4254 & D & 349.1718 & 175.0895 & 332.1452 & 166.5763 & 331.1612 & 166.0842 & 3 \\
\hline 17 & 1800.8862 & 900.9467 & 1783.8596 & 892.4334 & 1782.8756 & 891.9414 & $S$ & 234.1448 & 117.5761 & 217.1183 & 109.0628 & 216.1343 & 108.5708 & 2 \\
\hline 18 & & & & & & & K & 147.1128 & 74.0600 & 130.0863 & 65.5468 & & & 1 \\
\hline
\end{tabular}
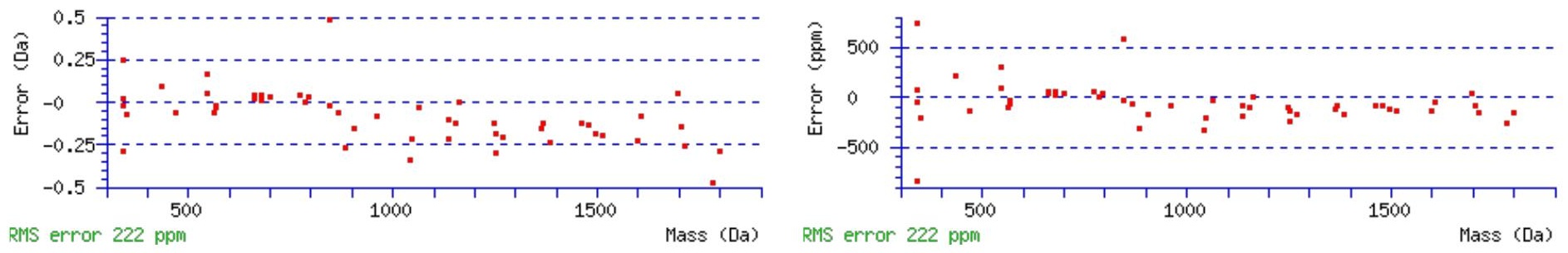

\section{All matches to this query}

\begin{tabular}{|l|l|c|c|}
\hline Score & Mr(calc): & Delta & Sequence \\
\hline 90.7 & 1945.9844 & -0.0027 & LQVELDSVTGLLNQSDSK \\
\hline 0.6 & 1945.9666 & 0.0151 & ELQVLDGANIQSIMASEK \\
\hline
\end{tabular}

Spectrum No: 131; Query: 1112; Rank: 1

\section{Peptide View}

MS/MS Fragmentation of NYTDDAIETDDLTIK

Found in IPI00215243, Tax_Id=10116 Gene_Symbol=Psma7 Isoform RC6-IL of Proteasome subunit alpha type-7

Match to Query 1112: 1726.785648 from(864.400100,2+)

Title: 100101RatKid_NS_deglyco_20.3282.3282.2.dta

Data file K:INewmanPaper|Piliangl3SubProteomes\Piliang3SP\mgf5ppm\ERLIC_3SubProteomes5ppm.mgf

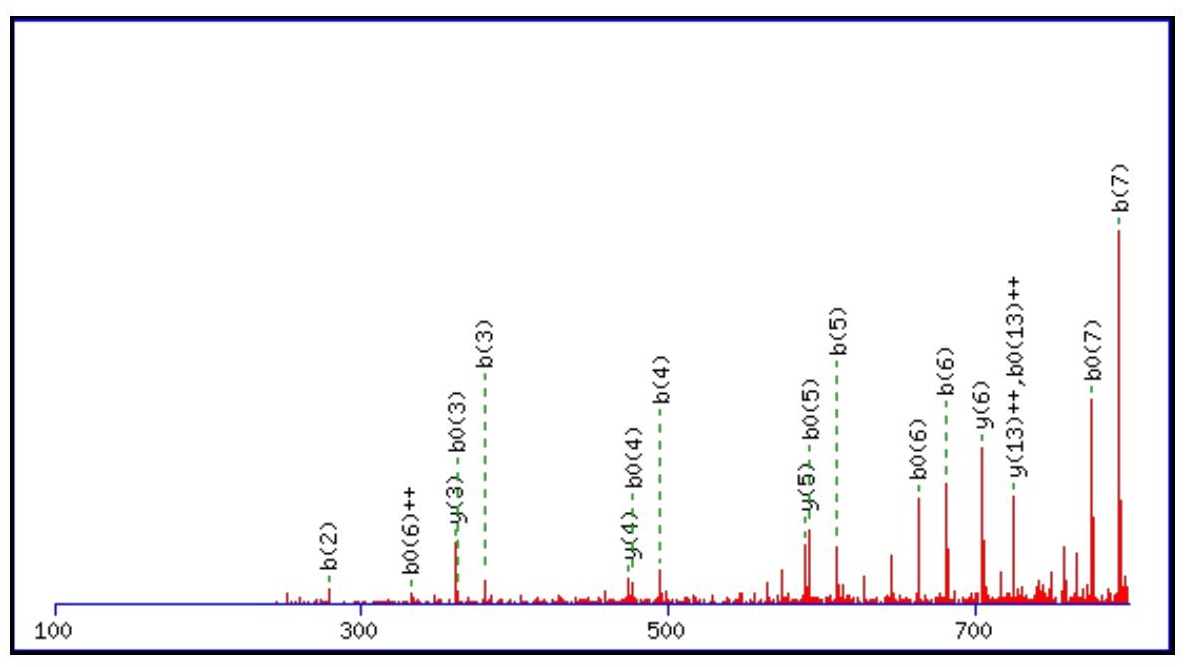



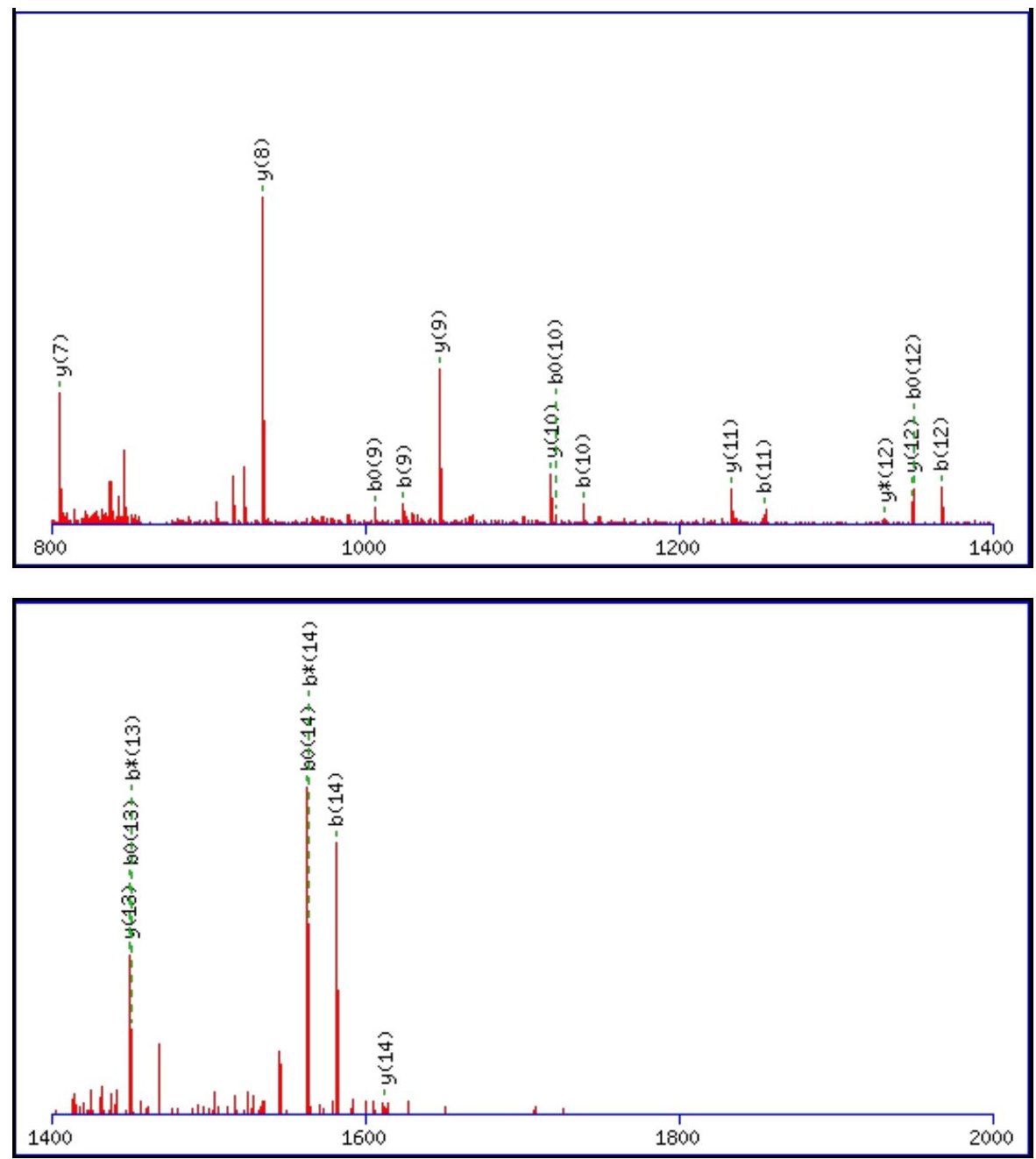

Monoisotopic mass of neutral peptide $\operatorname{Mr}($ calc): 1726.7785

Fixed modifications: Carbamidomethyl (C)

Variable modifications:

N1 : Deamidated $\mathrm{N}(\mathrm{N})$

Ions Score: 91 Expect: $1.8 \mathrm{e}-007$

Matches (Bold Red): 39/160 fragment ions using 59 most intense peaks

\begin{tabular}{|c|c|c|c|c|c|c|c|c|c|c|c|c|c|c|}
\hline \# & b & $\mathbf{b}^{++}$ & b* & $\mathbf{b}^{*^{++}}$ & $\mathbf{b}^{0}$ & $\mathbf{b}^{\mathbf{0 + +}}$ & Seq. & $\mathbf{y}$ & $\mathbf{y}^{++}$ & $\mathbf{y}^{*}$ & $\mathbf{y}^{*^{++}}$ & $\mathbf{y}^{\mathbf{0}}$ & $\mathbf{y}^{\mathbf{0 + +}}$ & \# \\
\hline 1 & 116.0342 & 58.5207 & 99.0077 & 50.0075 & & & $\mathbf{N}$ & & & & & & & 15 \\
\hline 2 & 279.0975 & 140.0524 & 262.0710 & 131.5391 & & & $\mathbf{Y}$ & 1612.7588 & 806.8831 & 1595.7323 & 798.3698 & 1594.7483 & 797.8778 & 14 \\
\hline 3 & 380.1452 & 190.5762 & 363.1187 & 182.0630 & 362.1347 & 181.5710 & $\mathbf{T}$ & 1449.6955 & 725.3514 & 1432.6690 & 716.8381 & 1431.6849 & 716.3461 & 13 \\
\hline 4 & 495.1722 & 248.0897 & 478.1456 & 239.5764 & 477.1616 & 239.0844 & D & 1348.6478 & 674.8276 & 1331.6213 & 666.3143 & 1330.6373 & 665.8223 & 12 \\
\hline 5 & 610.1991 & 305.6032 & 593.1726 & 297.0899 & 592.1885 & 296.5979 & D & 1233.6209 & 617.3141 & 1216.5943 & 608.8008 & 1215.6103 & 608.3088 & 11 \\
\hline 6 & 681.2362 & 341.1217 & 664.2097 & 332.6085 & 663.2257 & 332.1165 & A & 1118.5939 & 559.8006 & 1101.5674 & 551.2873 & 1100.5834 & 550.7953 & 10 \\
\hline 7 & 794.3203 & 397.6638 & 777.2937 & 389.1505 & 776.3097 & 388.6585 & I & 1047.5568 & 524.2821 & 1030.5303 & 515.7688 & 1029.5463 & 515.2768 & 9 \\
\hline 8 & 923.3629 & 462.1851 & 906.3363 & 453.6718 & 905.3523 & 453.1798 & $\mathbf{E}$ & 934.4728 & 467.7400 & 917.4462 & 459.2267 & 916.4622 & 458.7347 & 8 \\
\hline 9 & 1024.4106 & 512.7089 & 1007.3840 & 504.1956 & 1006.4000 & 503.7036 & $\mathbf{T}$ & 805.4302 & 403.2187 & 788.4036 & 394.7055 & 787.4196 & 394.2134 & 7 \\
\hline 10 & 1139.4375 & 570.2224 & 1122.4110 & 561.7091 & 1121.4269 & 561.2171 & D & 704.3825 & 352.6949 & 687.3559 & 344.1816 & 686.3719 & 343.6896 & 6 \\
\hline 11 & 1254.4644 & 627.7359 & 1237.4379 & 619.2226 & 1236.4539 & 618.7306 & D & 589.3556 & 295.1814 & 572.3290 & 286.6681 & 571.3450 & 286.1761 & 5 \\
\hline 12 & 1367.5485 & 684.2779 & 1350.5220 & 675.7646 & 1349.5379 & 675.2726 & $\mathbf{L}$ & 474.3286 & 237.6679 & 457.3021 & 229.1547 & 456.3180 & 228.6627 & 4 \\
\hline 13 & 1468.5962 & 734.8017 & 1451.5696 & 726.2885 & 1450.5856 & 725.7964 & $\mathbf{T}$ & 361.2445 & 181.1259 & 344.2180 & 172.6126 & 343.2340 & 172.1206 & 3 \\
\hline 14 & 1581.6802 & 791.3438 & 1564.6537 & 782.8305 & 1563.6697 & 782.3385 & I & 260.1969 & 130.6021 & 243.1703 & 122.0888 & & & 2 \\
\hline 15 & & & & & & & $\mathbf{K}$ & 147.1128 & 74.0600 & 130.0863 & 65.5468 & & & 1 \\
\hline
\end{tabular}



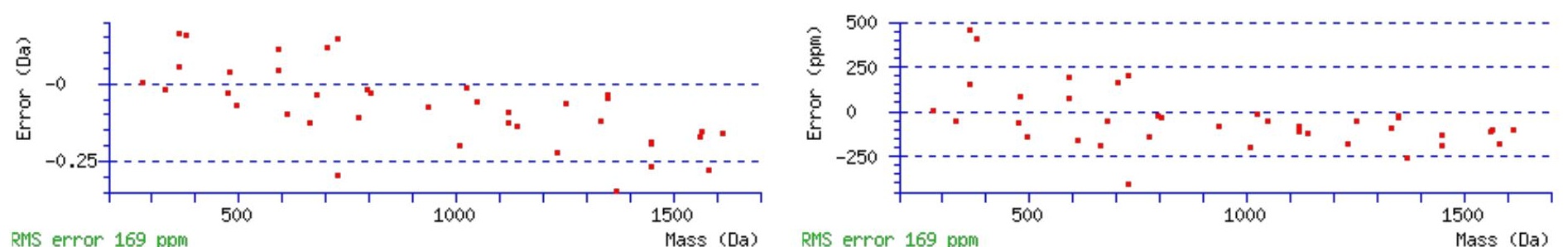

\section{All matches to this query}

\begin{tabular}{|l|l|c|l|}
\hline Score & Mr(calc): & Delta & \multicolumn{1}{c|}{ Sequence } \\
\hline 90.6 & 1726.7785 & 0.0072 & NYTDDAIETDDLTIK \\
\hline 48.8 & 1725.7945 & 0.9912 & NYTDDAIETDDLTIK \\
\hline 4.6 & 1726.7990 & -0.0134 & LPRRTHSNMLTIK \\
\hline 4.4 & 1726.7709 & 0.0147 & RMGPGATAGGAEKSNVK \\
\hline 4.3 & 1724.7706 & 2.0151 & QHQTPVYLGATAGMR \\
\hline 3.6 & 1725.7723 & 1.0133 & AASTNHLDTFDLSVR \\
\hline 1.2 & 1726.7749 & 0.0108 & APTYVKETLLKPK \\
\hline 1.0 & 1725.7767 & 1.0090 & MEKAEESEANIFAEK \\
\hline 0.6 & 1725.7949 & 0.9907 & NYXSKLSWIHPLK \\
\hline 0.6 & 1725.7949 & 0.9907 & NYXSKLSWIHPLK \\
\hline
\end{tabular}

Spectrum No: 132; Query: 1220; Rank: 1

\section{Peptide View}

MS/MS Fragmentation of EETLLYDNATSSVADR

Found in IPI00206434, Tax_Id=10116 Gene_Symbol=Lnpep Leucyl-cystinyl aminopeptidase

Match to Query 1220: 1783.813088 from(892.913820,2+)

Title: 100101RatKid_NS_deglyco_26.2553.2553.2.dta

Data file K:INewmanPaper|Piliangl3SubProteomes\Piliang3SP\mgf5ppm\ERLIC_3SubProteomes5ppm.mgf

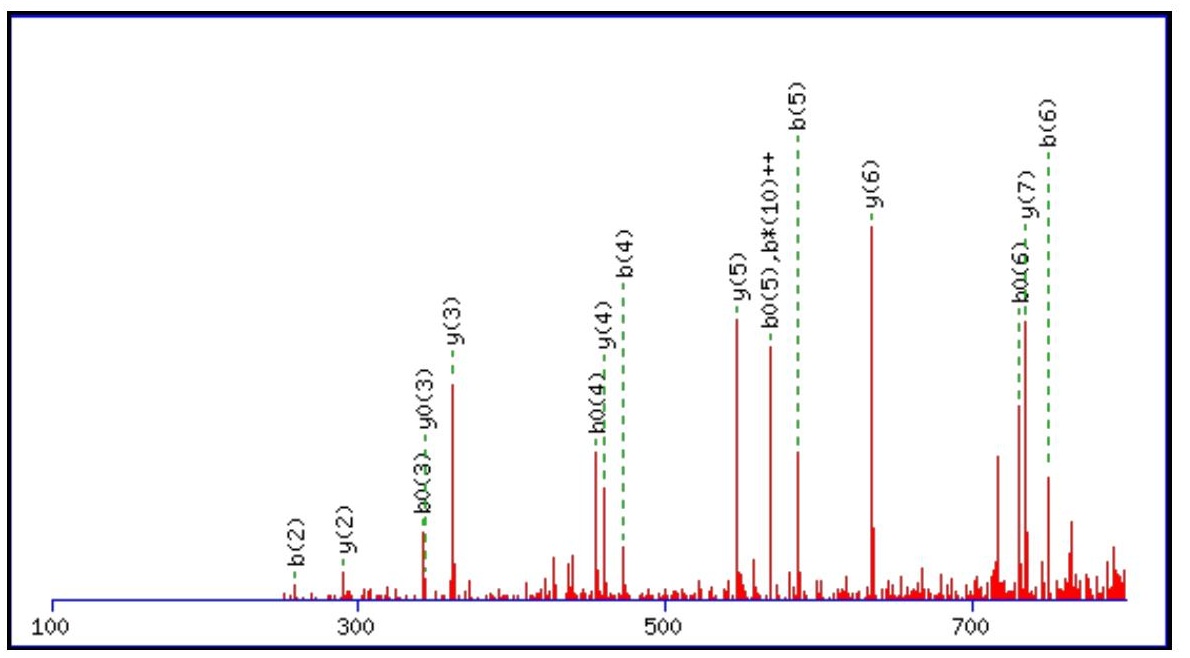



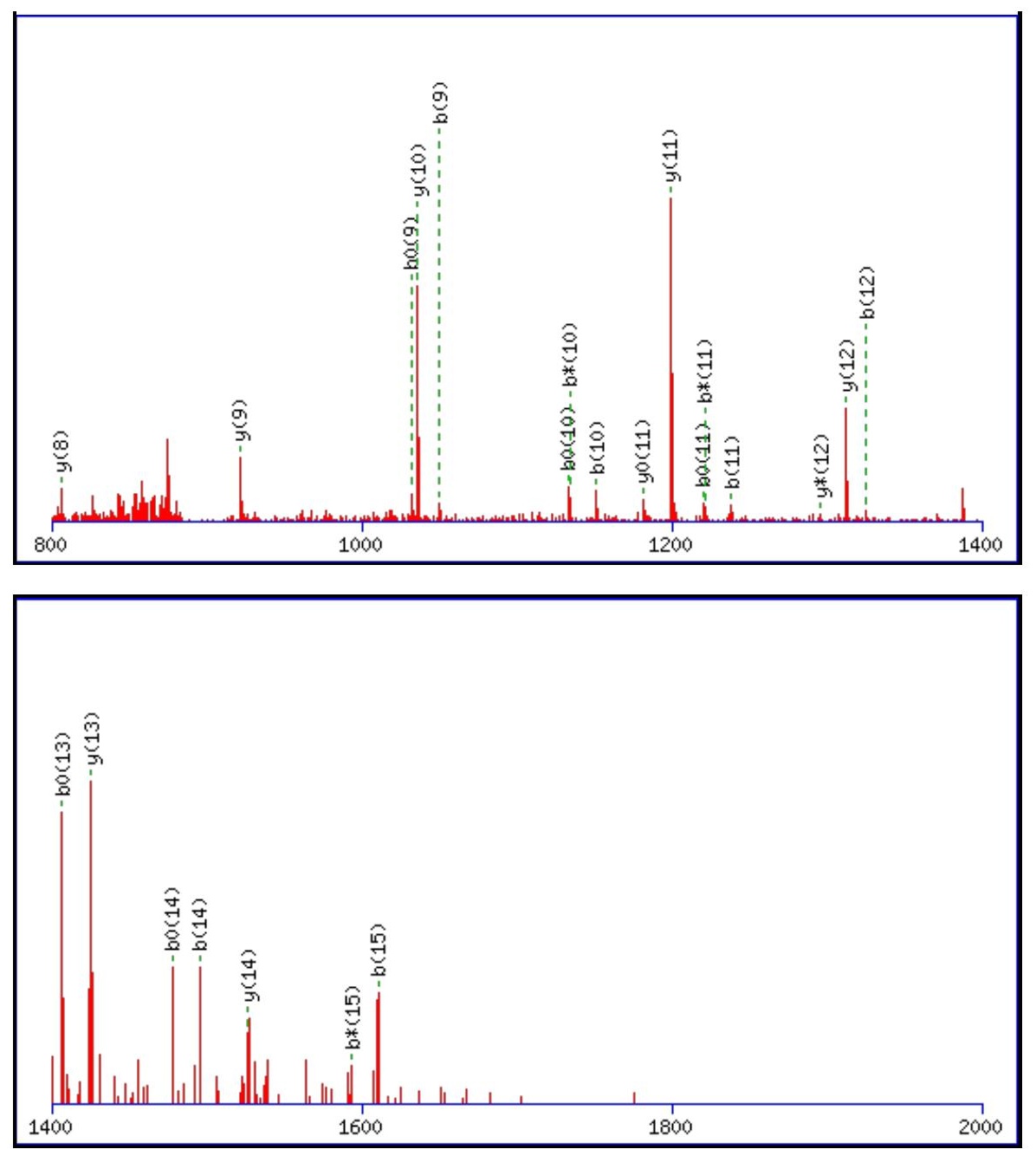

Monoisotopic mass of neutral peptide $\operatorname{Mr}($ calc): 1783.8112

Fixed modifications: Carbamidomethyl (C)

Variable modifications:

N8 : Deamidated_N (N)

Ions Score: 91 Expect: $2 \mathrm{e}-007$

Matches (Bold Red): 39/164 fragment ions using 72 most intense peaks

\begin{tabular}{|c|c|c|c|c|c|c|c|c|c|c|c|c|c|c|}
\hline \# & b & $\mathbf{b}^{++}$ & $\mathbf{b}^{*}$ & $\mathbf{b}^{*^{++}}$ & $\mathbf{b}^{0}$ & $\mathbf{b}^{0++}$ & Seq. & $\mathbf{y}$ & $\mathbf{y}^{++}$ & $\mathbf{y}^{*}$ & $\mathrm{y}^{*^{++}}$ & $\mathbf{y}^{\mathbf{0}}$ & $\mathbf{y}^{\mathbf{0 + +}}$ & \# \\
\hline 1 & 130.0499 & 65.5286 & & & 112.0393 & 56.5233 & $\mathbf{E}$ & & & & & & & 16 \\
\hline 2 & 259.0925 & 130.0499 & & & 241.0819 & 121.0446 & $\mathbf{E}$ & 1655.7759 & 828.3916 & 1638.7493 & 819.8783 & 1637.7653 & 819.3863 & 15 \\
\hline 3 & 360.1401 & 180.5737 & & & 342.1296 & 171.5684 & $\mathbf{T}$ & 1526.7333 & 763.8703 & 1509.7067 & 755.3570 & 1508.7227 & 754.8650 & 14 \\
\hline 4 & 473.2242 & 237.1157 & & & 455.2136 & 228.1105 & $\mathbf{L}$ & 1425.6856 & 713.3464 & 1408.6591 & 704.8332 & 1407.6750 & 704.3412 & 13 \\
\hline 5 & 586.3083 & 293.6578 & & & 568.2977 & 284.6525 & $\mathbf{L}$ & 1312.6015 & 656.8044 & 1295.5750 & 648.2911 & 1294.5910 & 647.7991 & 12 \\
\hline 6 & 749.3716 & 375.1894 & & & 731.3610 & 366.1842 & $\mathbf{Y}$ & 1199.5175 & 600.2624 & 1182.4909 & 591.7491 & 1181.5069 & 591.2571 & 11 \\
\hline 7 & 864.3985 & 432.7029 & & & 846.3880 & 423.6976 & D & 1036.4542 & 518.7307 & 1019.4276 & 510.2174 & 1018.4436 & 509.7254 & 10 \\
\hline 8 & 979.4255 & 490.2164 & 962.3989 & 481.7031 & 961.4149 & 481.2111 & $\mathbf{N}$ & 921.4272 & 461.2172 & 904.4007 & 452.7040 & 903.4166 & 452.2120 & 9 \\
\hline 9 & 1050.4626 & 525.7349 & 1033.4360 & 517.2217 & 1032.4520 & 516.7297 & A & 806.4003 & 403.7038 & 789.3737 & 395.1905 & 788.3897 & 394.6985 & 8 \\
\hline 10 & 1151.5103 & 576.2588 & 1134.4837 & 567.7455 & 1133.4997 & 567.2535 & $\mathbf{T}$ & 735.3632 & 368.1852 & 718.3366 & 359.6719 & 717.3526 & 359.1799 & 7 \\
\hline 11 & 1238.5423 & 619.7748 & 1221.5157 & 611.2615 & 1220.5317 & 610.7695 & $\mathrm{~S}$ & 634.3155 & 317.6614 & 617.2889 & 309.1481 & 616.3049 & 308.6561 & 6 \\
\hline 12 & 1325.5743 & 663.2908 & 1308.5478 & 654.7775 & 1307.5638 & 654.2855 & $\mathrm{~S}$ & 547.2835 & 274.1454 & 530.2569 & 265.6321 & 529.2729 & 265.1401 & 5 \\
\hline 13 & 1424.6427 & 712.8250 & 1407.6162 & 704.3117 & 1406.6322 & 703.8197 & V & 460.2514 & 230.6293 & 443.2249 & 222.1161 & 442.2409 & 221.6241 & 4 \\
\hline 14 & 1495.6799 & 748.3436 & 1478.6533 & 739.8303 & 1477.6693 & 739.3383 & A & 361.1830 & 181.0951 & 344.1565 & 172.5819 & 343.1724 & 172.0899 & 3 \\
\hline 15 & 1610.7068 & 805.8570 & 1593.6802 & 797.3438 & 1592.6962 & 796.8518 & D & 290.1459 & 145.5766 & 273.1193 & 137.0633 & 272.1353 & 136.5713 & 2 \\
\hline 16 & & & & & & & $\mathbf{R}$ & 175.1190 & 88.0631 & 158.0924 & 79.5498 & & & 1 \\
\hline
\end{tabular}



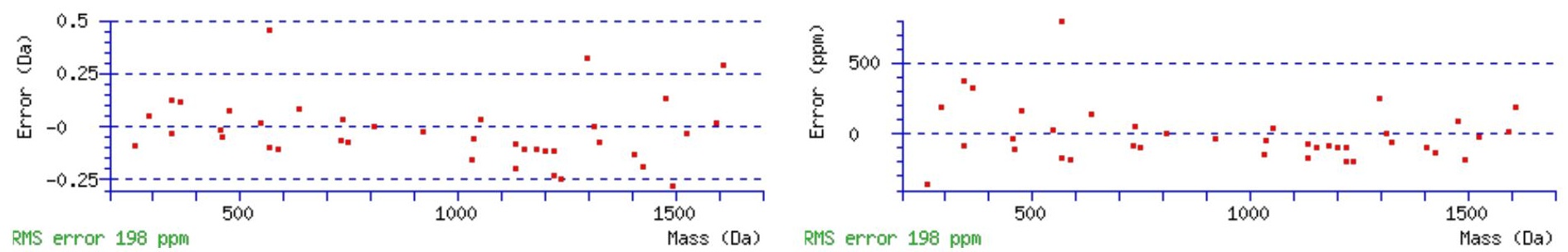

\section{All matches to this query}

\begin{tabular}{|l|l|c|l|}
\hline Score & Mr(calc): & Delta & \multicolumn{1}{|c|}{ Sequence } \\
\hline 90.6 & 1783.8112 & 0.0019 & EETLLYDNATSSVADR \\
\hline 29.7 & 1782.8272 & 0.9859 & EETLLYDNATSSVADR \\
\hline 5.4 & 1781.8057 & 2.0074 & DLESRQRSLEQANR \\
\hline 3.7 & 1783.8271 & -0.0140 & YCIKSNSXGEGARSER \\
\hline 3.5 & 1783.8270 & -0.0140 & SAPVINNGSPTILGKR \\
\hline 2.5 & 1783.8270 & -0.0140 & SAPVINNGSPTILGKR \\
\hline 2.5 & 1783.8270 & -0.0140 & SAPVINNGSPTILGKR \\
\hline 2.5 & 1783.8270 & -0.0140 & SAPVINNGSPTILGKR \\
\hline 2.1 & 1783.8047 & 0.0084 & YDSVNEKLSDSPSMGR \\
\hline 1.4 & 1783.8029 & 0.0102 & EIKYTSSVKYDSER \\
\hline
\end{tabular}

Spectrum No: 133; Query: 1716; Rank: 1

\section{Peptide View}

MS/MS Fragmentation of ESTGAQVQVAGDMLPNSTER

Found in IPI00464574, Tax_Id=10116 Gene_Symbol=Pcbp3 Poly(RC) binding protein 3

Match to Query 1716: 2089.968028 from(1045.991290,2+)

Title: 100101RatKid_NS_deglyco_15.2810.2810.2.dta

Data file K:INewmanPaper|Piliangl3SubProteomes\Piliang3SP\mgf5ppm|ERLIC_3SubProteomes5ppm.mgf

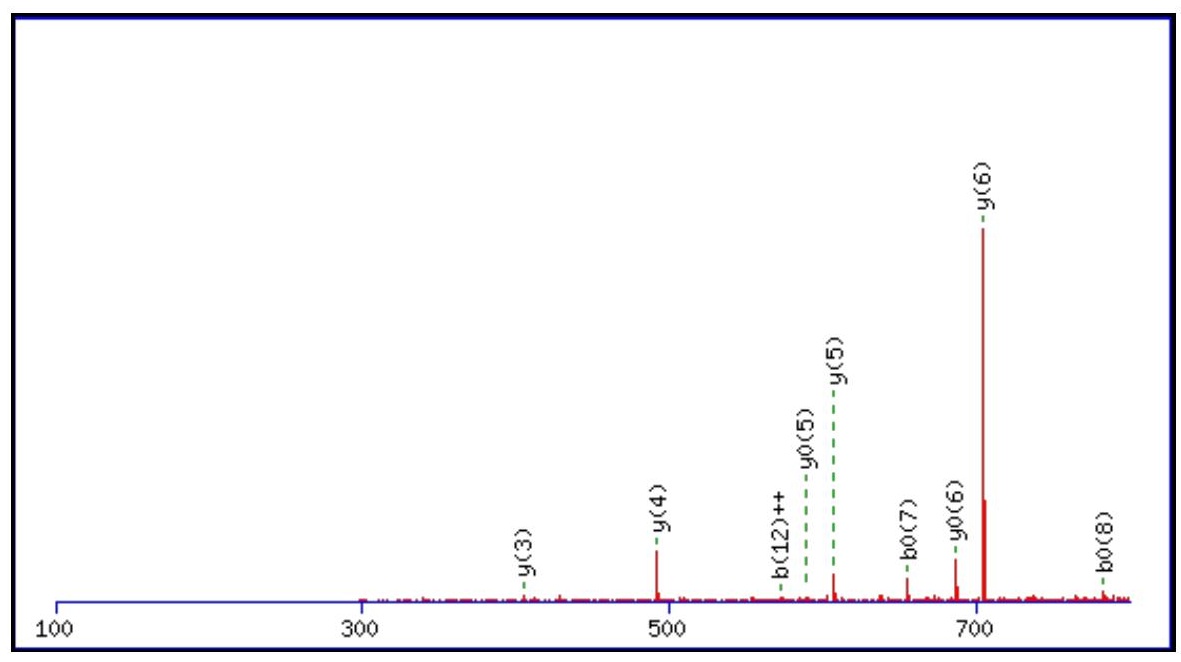



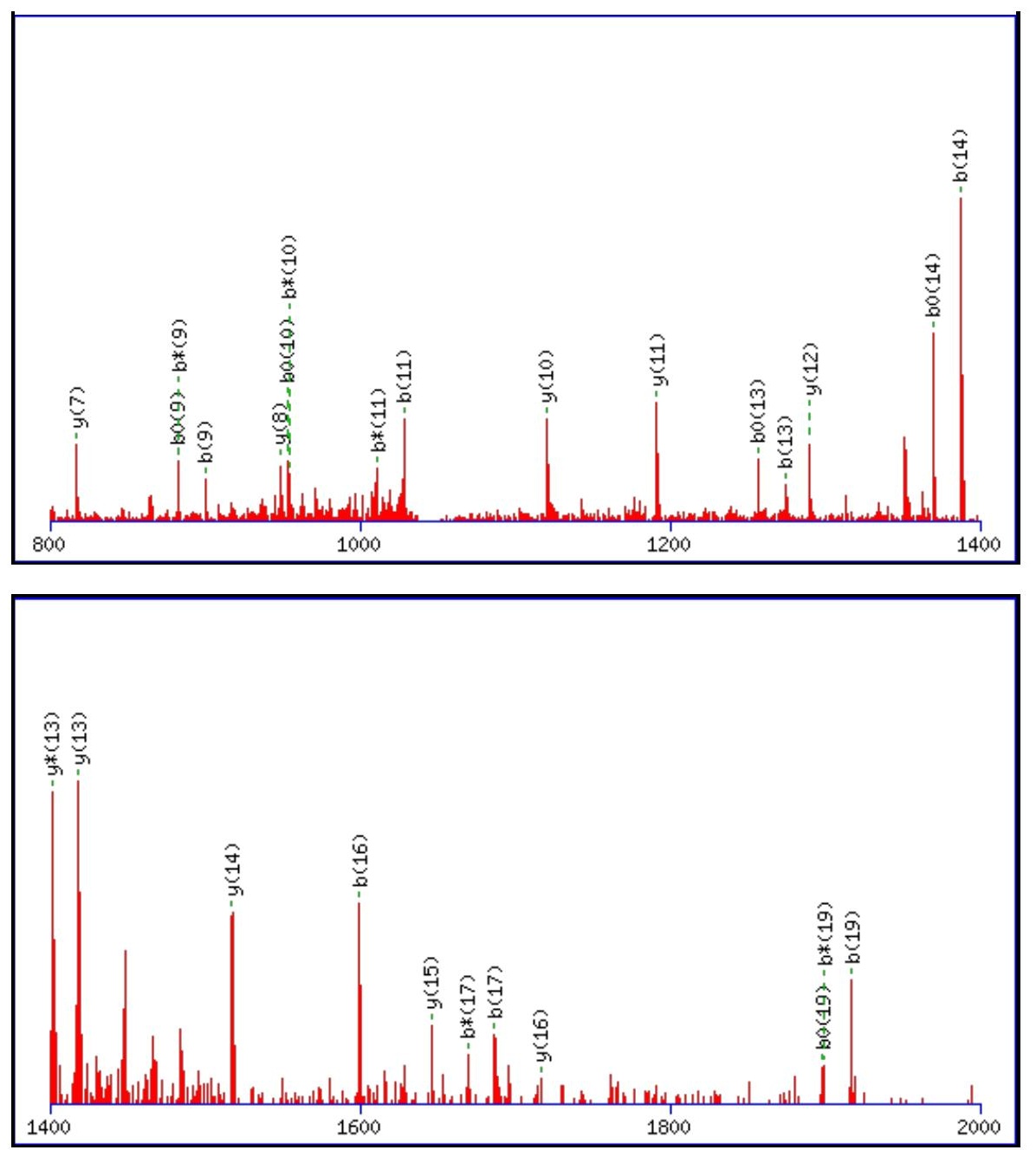

Monoisotopic mass of neutral peptide $\operatorname{Mr}($ calc): 2089.9586

Fixed modifications: Carbamidomethyl (C)

Variable modifications:

N16 : Deamidated $\mathrm{N}(\mathrm{N})$

Ions Score: 91 Expect: $2.1 \mathrm{e}-007$

Matches (Bold Red): 36/216 fragment ions using 61 most intense peaks

\begin{tabular}{|c|c|c|c|c|c|c|c|c|c|c|c|c|c|c|}
\hline \# & b & $\mathbf{b}^{++}$ & $\mathbf{b}^{*}$ & $\mathbf{b}^{*^{++}}$ & $\mathbf{b}^{0}$ & $\mathbf{b}^{0++}$ & Seq. & $\mathbf{y}$ & $\mathbf{y}^{++}$ & $\mathbf{y}^{*}$ & $\mathbf{y}^{*^{++}}$ & $\mathbf{y}^{\mathbf{0}}$ & $\mathbf{y}^{\mathbf{0 + +}}$ & \# \\
\hline 1 & 130.0499 & 65.5286 & & & 112.0393 & 56.5233 & $\mathbf{E}$ & & & & & & & 20 \\
\hline 2 & 217.0819 & 109.0446 & & & 199.0713 & 100.0393 & S & 1961.9233 & 981.4653 & 1944.8967 & 972.9520 & 1943.9127 & 972.4600 & 19 \\
\hline 3 & 318.1296 & 159.5684 & & & 300.1190 & 150.5631 & $\mathbf{T}$ & 1874.8913 & 937.9493 & 1857.8647 & 929.4360 & 1856.8807 & 928.9440 & 18 \\
\hline 4 & 375.1510 & 188.0792 & & & 357.1405 & 179.0739 & $\mathbf{G}$ & 1773.8436 & 887.4254 & 1756.8170 & 878.9122 & 1755.8330 & 878.4201 & 17 \\
\hline 5 & 446.1882 & 223.5977 & & & 428.1776 & 214.5924 & A & 1716.8221 & 858.9147 & 1699.7956 & 850.4014 & 1698.8116 & 849.9094 & 16 \\
\hline 6 & 574.2467 & 287.6270 & 557.2202 & 279.1137 & 556.2362 & 278.6217 & $\mathbf{Q}$ & 1645.7850 & 823.3961 & 1628.7585 & 814.8829 & 1627.7744 & 814.3909 & 15 \\
\hline 7 & 673.3151 & 337.1612 & 656.2886 & 328.6479 & 655.3046 & 328.1559 & $\mathbf{V}$ & 1517.7264 & 759.3669 & 1500.6999 & 750.8536 & 1499.7159 & 750.3616 & 14 \\
\hline 8 & 801.3737 & 401.1905 & 784.3472 & 392.6772 & 783.3632 & 392.1852 & $\mathbf{Q}$ & 1418.6580 & 709.8326 & 1401.6315 & 701.3194 & 1400.6474 & 700.8274 & 13 \\
\hline 9 & 900.4421 & 450.7247 & 883.4156 & 442.2114 & 882.4316 & 441.7194 & $\mathbf{V}$ & 1290.5994 & 645.8034 & 1273.5729 & 637.2901 & 1272.5889 & 636.7981 & 12 \\
\hline 10 & 971.4793 & 486.2433 & 954.4527 & 477.7300 & 953.4687 & 477.2380 & A & 1191.5310 & 596.2691 & 1174.5045 & 587.7559 & 1173.5205 & 587.2639 & 11 \\
\hline 11 & 1028.5007 & 514.7540 & 1011.4742 & 506.2407 & 1010.4902 & 505.7487 & G & 1120.4939 & 560.7506 & 1103.4674 & 552.2373 & 1102.4833 & 551.7453 & 10 \\
\hline 12 & 1143.5277 & 572.2675 & 1126.5011 & 563.7542 & 1125.5171 & 563.2622 & D & 1063.4724 & 532.2399 & 1046.4459 & 523.7266 & 1045.4619 & 523.2346 & 9 \\
\hline 13 & 1274.5681 & 637.7877 & 1257.5416 & 629.2744 & 1256.5576 & 628.7824 & $\mathbf{M}$ & 948.4455 & 474.7264 & 931.4190 & 466.2131 & 930.4349 & 465.7211 & 8 \\
\hline 14 & 1387.6522 & 694.3297 & 1370.6257 & 685.8165 & 1369.6416 & 685.3245 & $\mathbf{L}$ & 817.4050 & 409.2061 & 800.3785 & 400.6929 & 799.3945 & 400.2009 & 7 \\
\hline 15 & 1484.7050 & 742.8561 & 1467.6784 & 734.3428 & 1466.6944 & 733.8508 & $\mathbf{P}$ & 704.3210 & 352.6641 & 687.2944 & 344.1508 & 686.3104 & 343.6588 & 6 \\
\hline 16 & 1599.7319 & 800.3696 & 1582.7054 & 791.8563 & 1581.7213 & 791.3643 & $\mathbf{N}$ & 607.2682 & 304.1377 & 590.2416 & 295.6245 & 589.2576 & 295.1324 & 5 \\
\hline 17 & 1686.7639 & 843.8856 & 1669.7374 & 835.3723 & 1668.7534 & 834.8803 & S & 492.2413 & 246.6243 & 475.2147 & 238.1110 & 474.2307 & 237.6190 & 4 \\
\hline 18 & 1787.8116 & 894.4094 & 1770.7851 & 885.8962 & 1769.8011 & 885.4042 & $\mathbf{T}$ & 405.2092 & 203.1082 & 388.1827 & 194.5950 & 387.1987 & 194.1030 & 3 \\
\hline
\end{tabular}




\begin{tabular}{|l|l|l|l|l|l|l|l|l|l|l|l|l|l|l|l|l|}
$\mathbf{1 9}$ & $\mathbf{1 9 1 6 . 8 5 4 2}$ & 958.9307 & $\mathbf{1 8 9 9 . 8 2 7 7}$ & 950.4175 & $\mathbf{1 8 9 8 . 8 4 3 6}$ & $\mathbf{9 4 9 . 9 2 5 5}$ & E & 304.1615 & 152.5844 & 287.1350 & 144.0711 & $\mathbf{2 8 6 . 1 5 1 0}$ & 143.5791 & $\mathbf{2}$ \\
\hline $\mathbf{2 0}$ & & & & & & & R & 175.1190 & 88.0631 & 158.0924 & 79.5498 & & & $\mathbf{1}$ \\
\hline
\end{tabular}
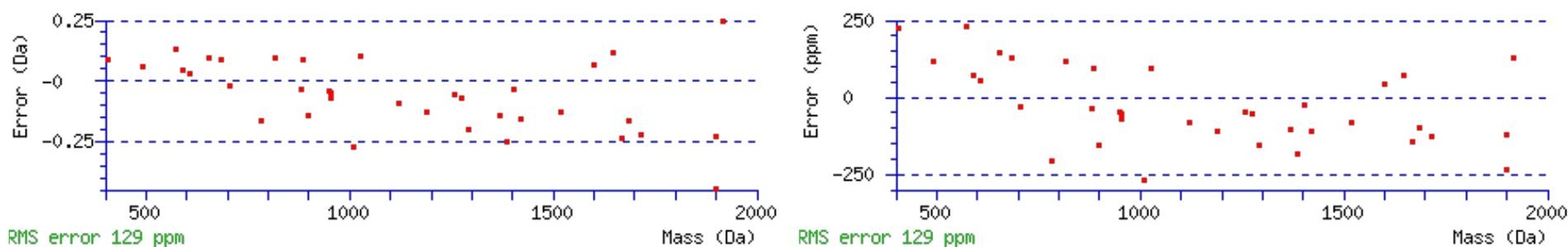

RMS error 129 ppm

\section{All matches to this query}

\begin{tabular}{|l|l|l|l|}
\hline Score & Mr(calc): & Delta & \multicolumn{1}{|c|}{ Sequence } \\
\hline 90.6 & 2089.9586 & 0.0094 & ESTGAQVQVAGDMLPNSTER \\
\hline 25.0 & 2088.9746 & 0.9934 & ESTGAQVQVAGDMLPNSTER \\
\hline 15.3 & 2089.9593 & 0.0088 & NIYTPPDSSTSFIQDYSR \\
\hline 10.2 & 2089.9601 & 0.0080 & SVGLMFKNSVYVTFLPK \\
\hline 4.0 & 2087.9458 & 2.0222 & KPSVPSTAPPGVKNLLSR \\
\hline 3.3 & 2089.9476 & 0.0204 & GRSHNAPCINTAAVAASRR \\
\hline 3.0 & 2089.9577 & 0.0103 & MSEMELKAFGSGVDIKPR \\
\hline 3.0 & 2089.9577 & 0.0103 & MSEMELKAFGSGVDIKPR \\
\hline 2.9 & 2089.9786 & -0.0105 & DAGFTVEDICMLRHRDR \\
\hline 2.6 & 2089.9745 & -0.0065 & ATGTAHFFNFLLNTTDYR \\
\hline
\end{tabular}

Spectrum No: 134; Query: 1565; Rank: 1

\section{Peptide View}

MS/MS Fragmentation of VNASTTDPNSTVEQSALTR

Found in IPI00365542, Tax_Id=10116 Gene_Symbol=Lamb1_predicted laminin, beta 1

Match to Query 1565: 1991.937868 from(996.976210,2+)

Title: 100101RatKid_NS_deglyco_21.1843.1843.2.dta

Data file K:INewmanPaper|Piliang 3 SSubProteomes\Piliang3SP\mgf5ppm\ERLIC_3SubProteomes5ppm.mgf

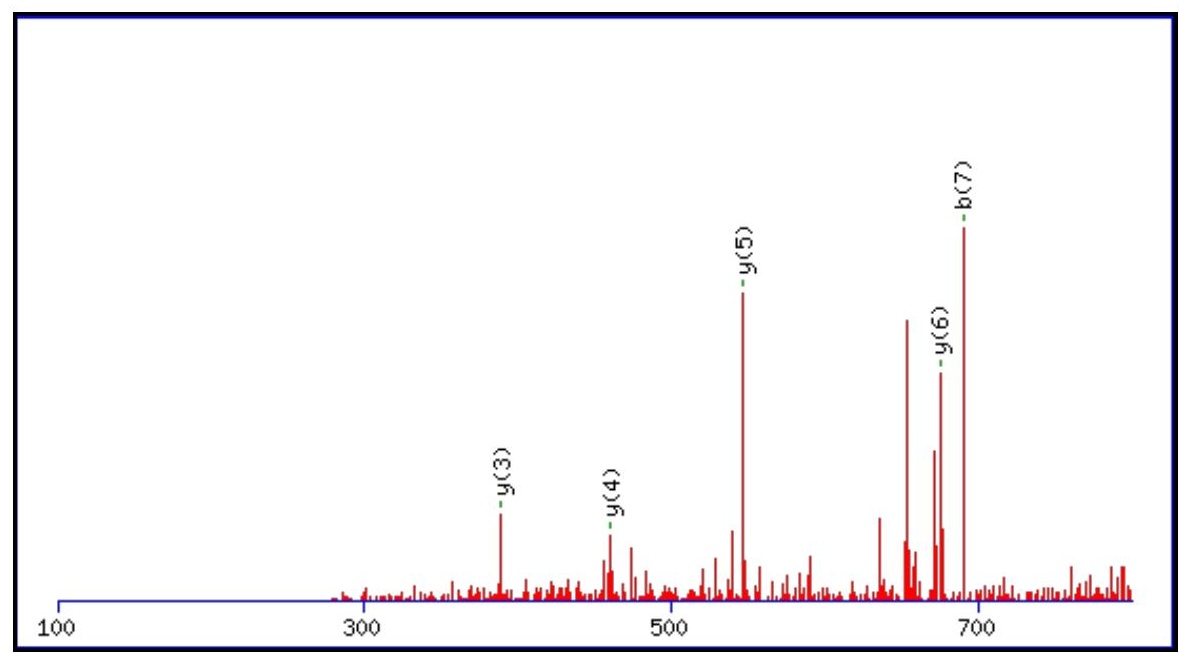



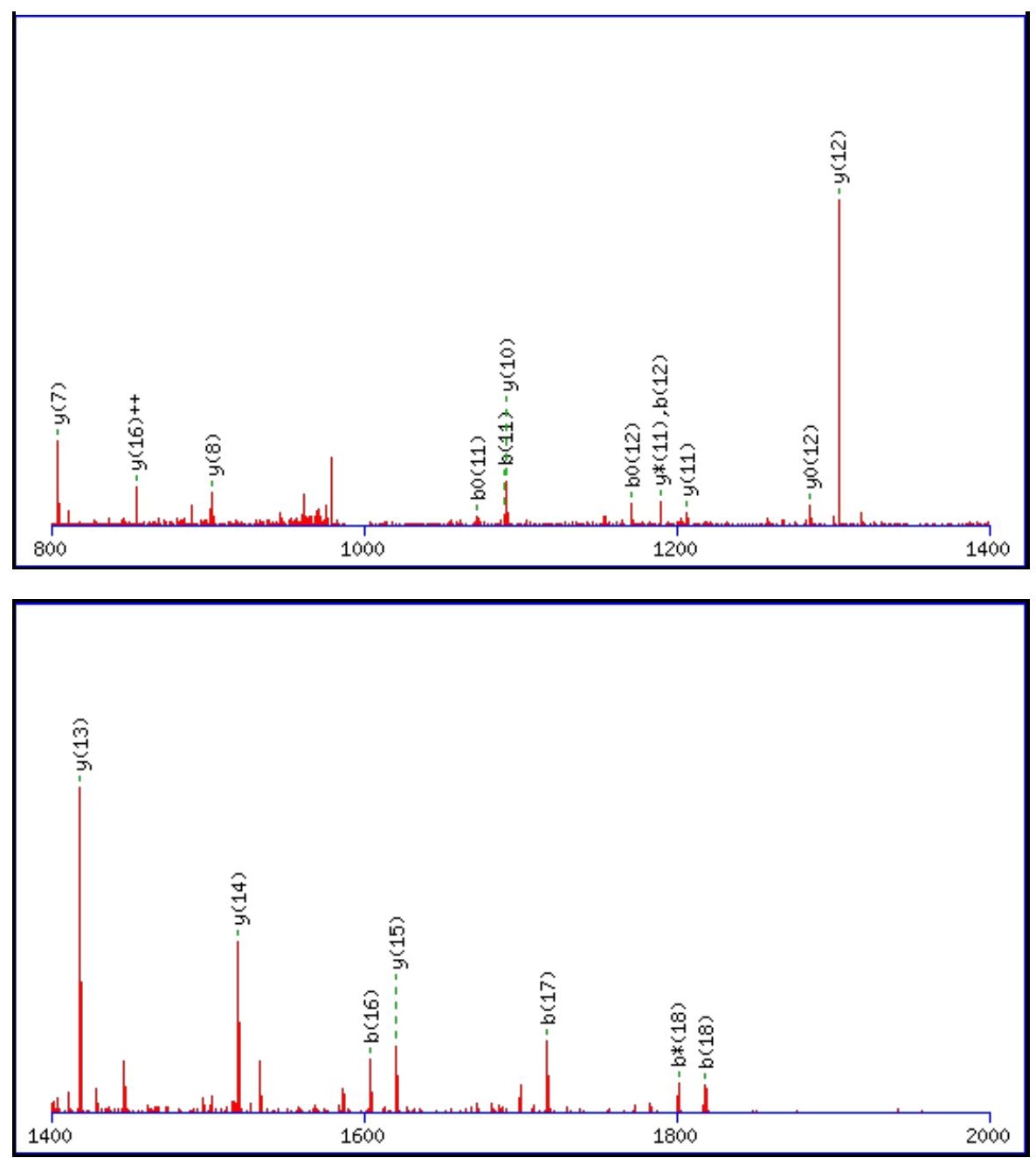

Monoisotopic mass of neutral peptide $\operatorname{Mr}($ calc): 1991.9283

Fixed modifications: Carbamidomethyl (C)

Variable modifications:

N2 : Deamidated_N (N)

N9 : Deamidated_N (N)

Ions Score: $9 \odot$ Expect : $2.2 \mathrm{e}-007$

Matches (Bold Red): 24/206 fragment ions using 37 most intense peaks

\begin{tabular}{|c|c|c|c|c|c|c|c|c|c|c|c|c|c|c|}
\hline \# & b & $\mathbf{b}^{++}$ & * & $\mathbf{b}^{*^{++}}$ & $\mathbf{b}^{0}$ & & eq. & $\mathbf{y}$ & & & & $\mathbf{y}^{\mathbf{0}}$ & & \# \\
\hline 1 & 100.0757 & 50.5415 & & & & & V & & & & & & & 19 \\
\hline 2 & 215.1026 & 108.0550 & 198.0761 & & & & $\mathbf{N}$ & & & & & & & 18 \\
\hline 3 & 286.1397 & 143.5735 & 269.1132 & 135.0602 & & & A & & & 1761.8137 & & 1760.8297 & & 17 \\
\hline 4 & 373.1718 & 187.0895 & 356.1452 & 178.5762 & & & S & 1707.8032 & 854.4052 & 1690.7766 & 845.8919 & 7926 & 845.3999 & 16 \\
\hline 5 & 474.2 & 237.6134 & 457 & 229. & 456 & 228. & $\mathbf{T}$ & & 392 & 446 & & 606 & 801.8839 & 15 \\
\hline 6 & 575.2671 & 288.1372 & 558.2406 & 279.6239 & 557.2566 & 279.1319 & $\mathbf{T}$ & 1519.7235 & 760.3654 & 1502.6969 & 751.8521 & 1501.7129 & 751.3601 & 14 \\
\hline 7 & 690.2941 & 345.6507 & 673.2675 & 337.1374 & 672.2835 & 454 & D & .6758 & 709. & 1401 & 701.3282 & 1400.6652 & 700.8362 & 13 \\
\hline 8 & 787.3468 & 394.1771 & 770.3203 & 385.6638 & 769.3363 & 385.1718 & $\mathbf{P}$ & 1303.6488 & 652.3281 & 1286.6223 & 643.8148 & 6383 & 643.3228 & 12 \\
\hline 9 & 902.3738 & 451.6905 & 885.3472 & 443.1772 & 884.3632 & 442.6852 & $\mathbf{N}$ & 1206.5961 & 603.8017 & 1189.5695 & 595.2884 & 1188.5855 & 594.7964 & 11 \\
\hline 10 & 989.4058 & 495.2065 & 972.3792 & 486.6933 & 971.3952 & 486.2013 & S & 691 & 546.2882 & 1074 & 7749 & 5586 & 537.2829 & 10 \\
\hline 11 & 1090.4535 & 545.7304 & 1073.4269 & 537.2171 & 1072.4429 & 536.7251 & $\mathbf{T}$ & 1004.5371 & 502.7722 & 987.5106 & 494.2589 & 986.5265 & 493.7669 & 9 \\
\hline 12 & 1189.5219 & 595.2646 & 1172.4953 & 586.7513 & 1171.5113 & 586.2 & $\mathbf{V}$ & 903.4894 & 452.2483 & 886.4629 & 443.7351 & 1789 & 443.2431 & 8 \\
\hline 13 & 1318.5645 & 659.7859 & 1301.5379 & 651.2726 & 1300.5539 & 650.7806 & $\mathbf{E}$ & 804.4210 & 402.7141 & 787.3945 & 394.2009 & 786.4104 & 393.7089 & 7 \\
\hline 14 & 1446.6231 & 723.8152 & 1429.5965 & 715.3019 & 1428.6125 & 714.8099 & $\mathbf{Q}$ & 675.3784 & 338.1928 & 658.3519 & 329.6796 & 657.3679 & 329.1876 & 6 \\
\hline 15 & 1533.6551 & 767.3312 & 1516.6285 & 758.8179 & 1515.6445 & 758.3259 & S & 547.3198 & 274.1636 & 530.2933 & 265.6503 & 529.3093 & 265.1583 & 5 \\
\hline 16 & 1604.6922 & 802.8497 & 1587.6657 & 794.3365 & 1586.6816 & 793.8445 & A & 460.2878 & 230.6475 & 443.2613 & 222.1343 & 442.2772 & 221.6423 & 4 \\
\hline 17 & 1717.7763 & 859.3918 & 1700.7497 & 850.8785 & 1699.7657 & 850.3865 & $\mathbf{L}$ & 389.2507 & 195.1290 & 372.2241 & 186.6157 & 371.2401 & 186.1237 & 3 \\
\hline 18 & 1818.8239 & 909.9156 & 1801.7974 & 901.4023 & 1800.8134 & 900.9103 & $\mathbf{T}$ & 276.1666 & 138.5870 & 259.1401 & 130.0737 & 258.1561 & 129.5817 & 2 \\
\hline
\end{tabular}




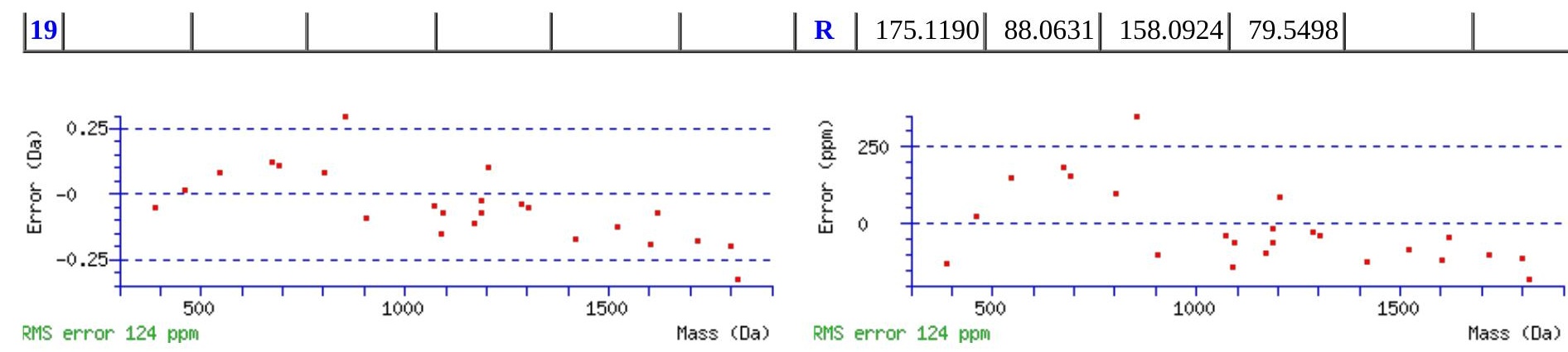

\section{All matches to this query}

\begin{tabular}{|l|l|l|l|}
\hline Score & Mr(calc): & Delta & \multicolumn{1}{|c|}{ Sequence } \\
\hline 90.5 & 1991.9283 & 0.0095 & VNASTTDPNSTVEQSALTR \\
\hline 64.4 & 1990.9443 & 0.9935 & VNASTTDPNSTVEQSALTR \\
\hline 16.0 & 1989.9296 & 2.0083 & SPSTYNALESGLTSLTIR \\
\hline 13.6 & 1989.9296 & 2.0083 & SPSTYNALESGLTSLTIR \\
\hline 13.2 & 1990.9443 & 0.9935 & VNASTTDPNSTVEQSALTR \\
\hline 11.4 & 1991.9201 & 0.0178 & QNSESAVSSTVNPITIHK \\
\hline 11.4 & 1991.9201 & 0.0178 & QNSESAVSSTVNPITIHK \\
\hline 10.4 & 1989.9296 & 2.0083 & SPSTYNALESGLTSLTIR \\
\hline 9.7 & 1989.9296 & 2.0083 & SPSTYNALESGLTSLTIR \\
\hline 8.2 & 1989.9296 & 2.0083 & SPSTYNALESGLTSLTIR \\
\hline
\end{tabular}

Spectrum No: 135; Query: 1932; Rank: 1

\section{Peptide View}

MS/MS Fragmentation of AGFAQCICPTLTCPEANSTK

Found in IPI00188885, Tax_Id=10116 Gene_Symbol=Agrn Isoform 1 of Agrin precursor

Match to Query 1932: 2225.983168 from(1113.998860,2+)

Title: 100101RatKid_NS_deglyco_21.2898.2898.2.dta

Data file K:INewmanPaper|Piliang|3SubProteomes\Piliang3SP\mgf5ppm\ERLIC_3SubProteomes5ppm.mgf

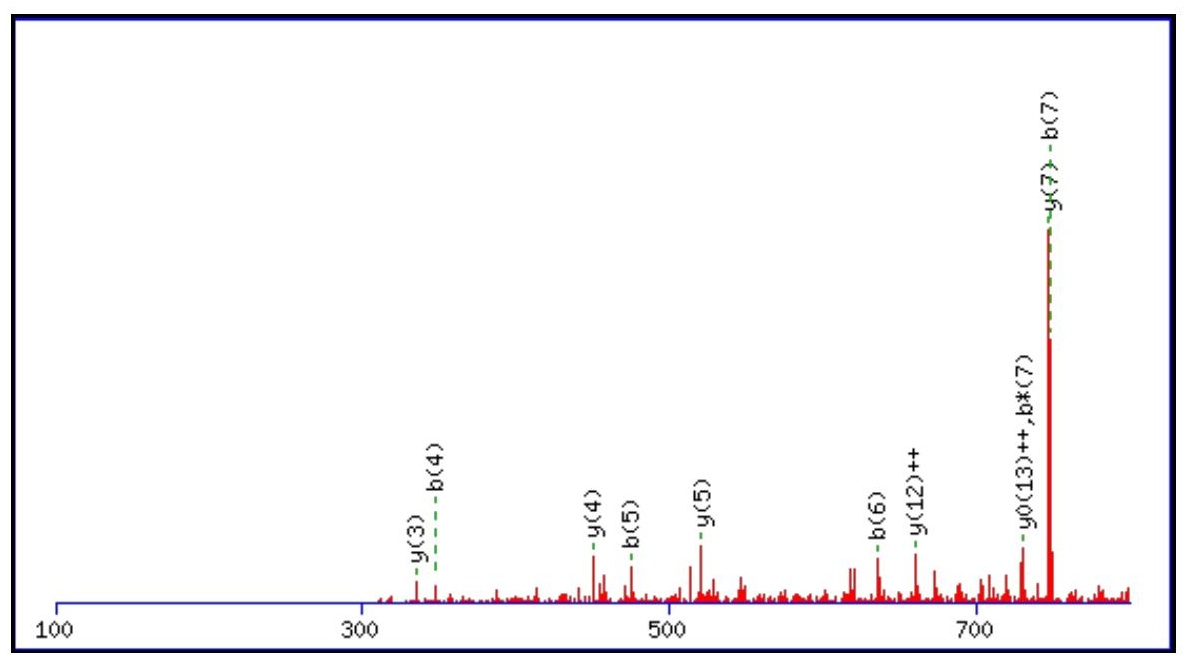



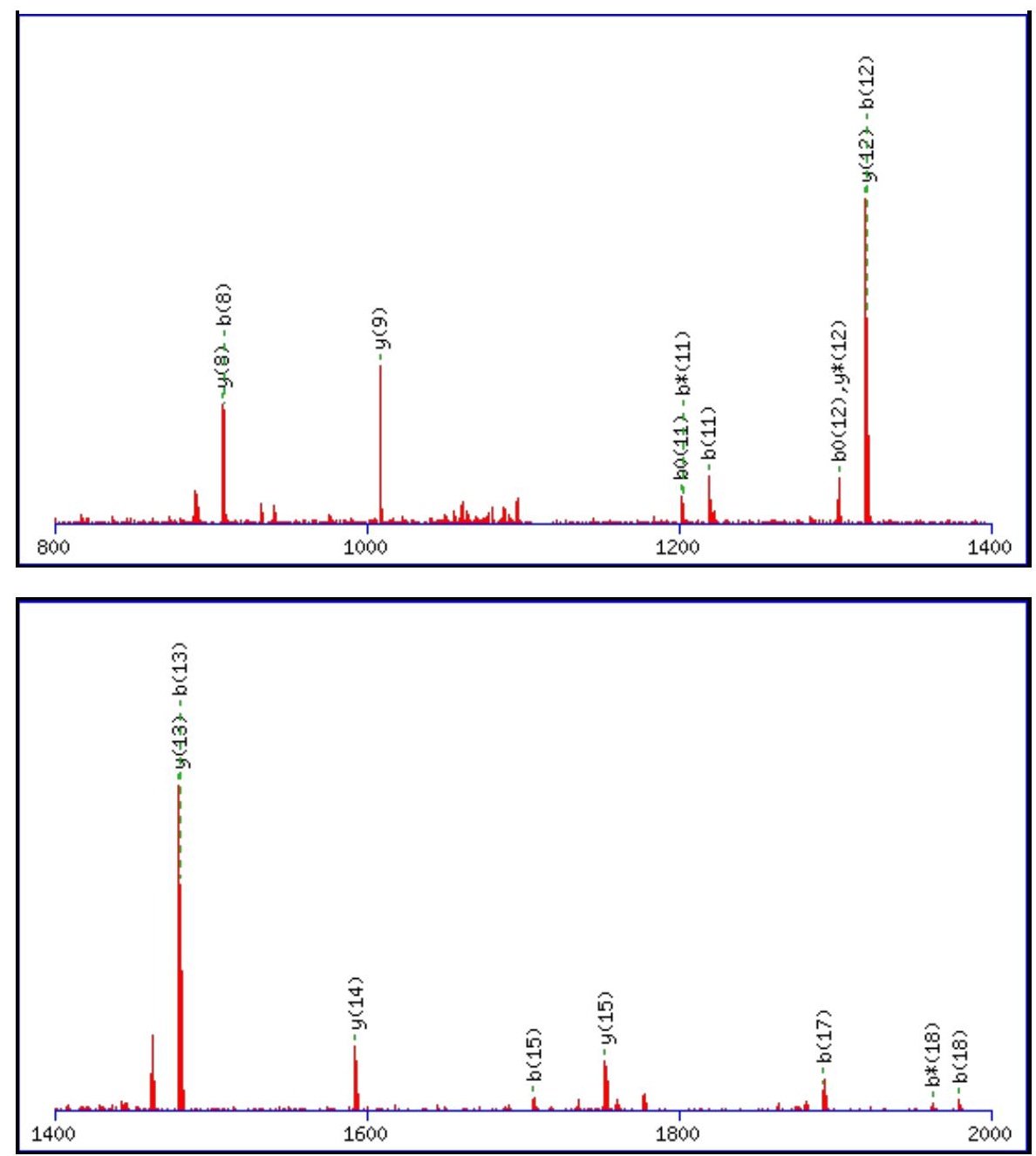

Monoisotopic mass of neutral peptide $\operatorname{Mr}($ calc): 2225.9755

Fixed modifications: Carbamidomethyl (C)

Variable modifications:

N17 : Deamidated $\mathrm{N}(\mathrm{N})$

Ions Score: 90 Expect: $2.6 \mathrm{e}-007$

Matches (Bold Red): 29/200 fragment ions using 39 most intense peaks

\begin{tabular}{|c|c|c|c|c|c|c|c|c|c|c|c|c|c|c|}
\hline \# & b & $\mathbf{b}^{++}$ & $\mathbf{b}^{*}$ & $\mathbf{b}^{*^{++}}$ & $\mathbf{b}^{\mathbf{0}}$ & $\mathbf{b}^{\mathbf{0 + +}}$ & Seq. & $\mathbf{y}$ & $y^{++}$ & $\mathbf{y}^{*}$ & $\mathbf{y}^{*^{++}}$ & $\mathbf{y}^{0}$ & $\mathbf{y}^{0++}$ & \# \\
\hline 1 & 72.0444 & 36.5258 & & & & & A & & & & & & & 20 \\
\hline 2 & 129.0659 & 65.0366 & & & & & G & 2155.9457 & 1078.4765 & 2138.9191 & 1069.9632 & 2137.9351 & 1069.4712 & 19 \\
\hline 3 & 276.1343 & 138.5708 & & & & & $\mathbf{F}$ & 2098.9242 & 1049.9657 & 2081.8977 & 1041.4525 & 2080.9137 & 1040.9605 & 18 \\
\hline 4 & 347.1714 & 174.0893 & & & & & A & 1951.8558 & 976.4315 & 1934.8293 & 967.9183 & 1933.8452 & 967.4263 & 17 \\
\hline 5 & 475.2300 & 238.1186 & 458.2034 & 229.6053 & & & $\mathbf{Q}$ & 1880.8187 & 940.9130 & 1863.7921 & 932.3997 & 1862.8081 & 931.9077 & 16 \\
\hline 6 & 635.2606 & 318.1339 & 618.2341 & 309.6207 & & & C & 1752.7601 & 876.8837 & 1735.7336 & 868.3704 & 1734.7496 & 867.8784 & 15 \\
\hline 7 & 748.3447 & 374.6760 & 731.3181 & 366.1627 & & & I & 1592.7295 & 796.8684 & 1575.7029 & 788.3551 & 1574.7189 & 787.8631 & 14 \\
\hline 8 & 908.3753 & 454.6913 & 891.3488 & 446.1780 & & & C & 1479.6454 & 740.3263 & 1462.6189 & 731.8131 & 1461.6348 & 731.3211 & 13 \\
\hline 9 & 1005.4281 & 503.2177 & 988.4015 & 494.7044 & & & $\mathbf{P}$ & 1319.6148 & 660.3110 & 1302.5882 & 651.7977 & 1301.6042 & 651.3057 & 12 \\
\hline 10 & 1106.4758 & 553.7415 & 1089.4492 & 545.2282 & 1088.4652 & 544.7362 & $\mathbf{T}$ & 1222.5620 & 611.7846 & 1205.5354 & 603.2714 & 1204.5514 & 602.7794 & 11 \\
\hline 11 & 1219.5598 & 610.2836 & 1202.5333 & 601.7703 & 1201.5493 & 601.2783 & $\mathbf{L}$ & 1121.5143 & 561.2608 & 1104.4878 & 552.7475 & 1103.5037 & 552.2555 & 10 \\
\hline 12 & 1320.6075 & 660.8074 & 1303.5810 & 652.2941 & 1302.5969 & 651.8021 & $\mathbf{T}$ & 1008.4302 & 504.7188 & 991.4037 & 496.2055 & 990.4197 & 495.7135 & 9 \\
\hline 13 & 1480.6382 & 740.8227 & 1463.6116 & 732.3094 & 1462.6276 & 731.8174 & $\mathrm{C}$ & 907.3826 & 454.1949 & 890.3560 & 445.6816 & 889.3720 & 445.1896 & 8 \\
\hline 14 & 1577.6909 & 789.3491 & 1560.6644 & 780.8358 & 1559.6804 & 780.3438 & $\mathbf{P}$ & 747.3519 & 374.1796 & 730.3254 & 365.6663 & 729.3414 & 365.1743 & 7 \\
\hline 15 & 1706.7335 & 853.8704 & 1689.7070 & 845.3571 & 1688.7229 & 844.8651 & $\mathbf{E}$ & 650.2992 & 325.6532 & 633.2726 & 317.1399 & 632.2886 & 316.6479 & 6 \\
\hline 16 & 1777.7706 & 889.3890 & 1760.7441 & 880.8757 & 1759.7601 & 880.3837 & A & 521.2566 & 261.1319 & 504.2300 & 252.6186 & 503.2460 & 252.1266 & 5 \\
\hline 17 & 1892.7976 & 946.9024 & 1875.7710 & 938.3891 & 1874.7870 & 937.8971 & $\mathbf{N}$ & 450.2194 & 225.6134 & 433.1929 & 217.1001 & 432.2089 & 216.6081 & 4 \\
\hline 18 & 1979.8296 & 990.4184 & 1962.8030 & 981.9052 & 1961.8190 & 981.4132 & $\mathrm{~S}$ & 335.1925 & 168.0999 & 318.1660 & 159.5866 & 317.1819 & 159.0946 & 3 \\
\hline
\end{tabular}




\begin{tabular}{|r|r|r|r|r|r|r|r|r|r|r|r|r|r|r|}
$\mathbf{1 9}$ & 2080.8773 & 1040.9423 & 2063.8507 & 1032.4290 & 2062.8667 & 1031.9370 & $\mathbf{T}$ & 248.1605 & 124.5839 & 231.1339 & 116.0706 & 230.1499 & 115.5786 & $\mathbf{2}$ \\
\hline $\mathbf{2 0}$ & & & & & & & $\mathbf{K}$ & 147.1128 & 74.0600 & 130.0863 & 65.5468 & & & $\mathbf{1}$ \\
\hline
\end{tabular}
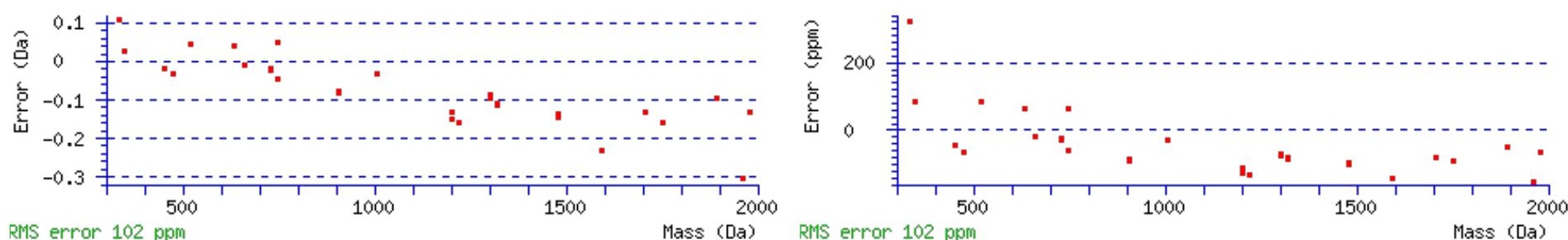

\section{All matches to this query}

\begin{tabular}{|l|c|c|l|}
\hline Score & $\mathbf{M r}$ (calc): & Delta & \multicolumn{1}{c|}{ Sequence } \\
\hline 90.3 & 2225.9755 & 0.0076 & AGFAQCICPTLTCPEANSTK \\
\hline 34.3 & 2224.9915 & 0.9917 & AGFAQCICPTLTCPEANSTK \\
\hline 9.1 & 2225.9720 & 0.0111 & NAWEESQITLFLDILMK \\
\hline 5.9 & 2225.9892 & -0.0061 & LMENRSCDQELSEKGTEGK \\
\hline 4.0 & 2223.9660 & 2.0172 & FSNWTNGAFLAOGSLLNMR \\
\hline 1.9 & 2224.9581 & 1.0250 & TNPGCPAEVRLGMGHCFVK \\
\hline 0.5 & 2223.9829 & 2.0003 & EYLKNFPLLTKYCGYR \\
\hline 0.3 & 2224.9956 & 0.9875 & SLAEMLMSKNTRTSDTLSK \\
\hline 0.2 & 2224.9612 & 1.0220 & THYGQKAILFLPLPVSSD \\
\hline 0.2 & 2224.9612 & 1.0220 & THYGQKAILFLPLPVSSD \\
\hline
\end{tabular}

Spectrum No: 136; Query: 1438; Rank: 1

\section{Peptide View}

MS/MS Fragmentation of NPCTSEQNCTSPFSYK

Found in IPI00191681, Tax_Id=10116 Gene_Symbol=Itgb1 Integrin beta-1 precursor

Match to Query 1438: 1919.774568 from(960.894560,2+)

Title: 100101RatKid_NS_deglyco_23.1805.1805.2.dta

Data file K:INewmanPaper|Piliangl3SubProteomes\Piliang3SP\mgf5ppm\ERLIC_3SubProteomes5ppm.mgf

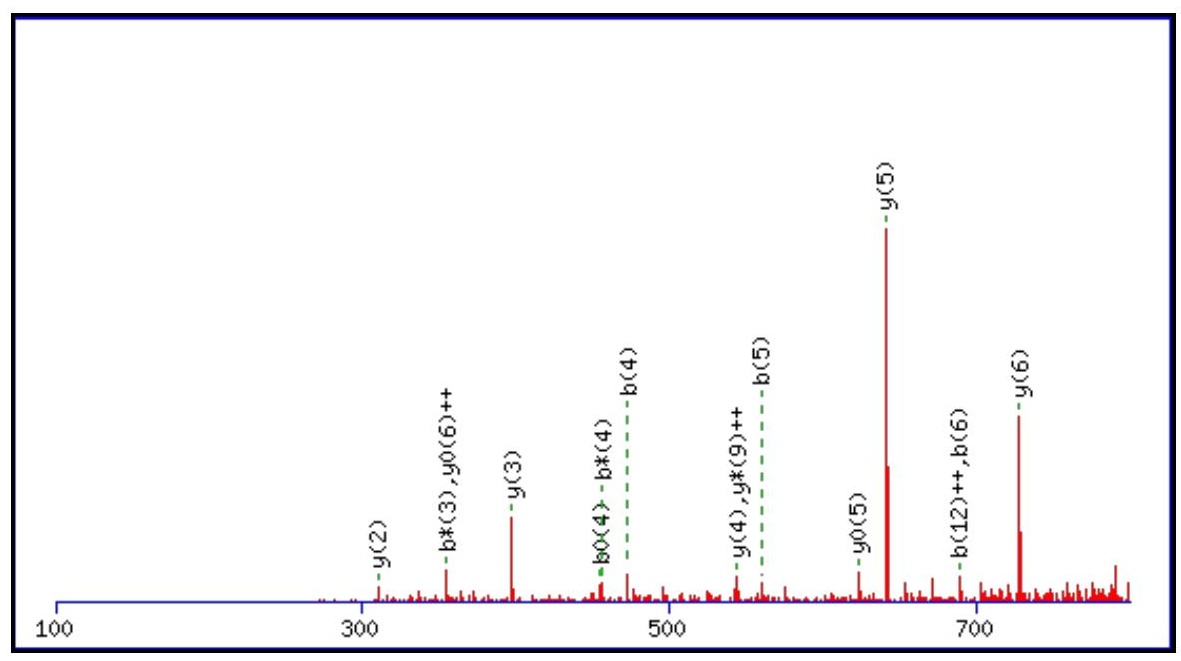



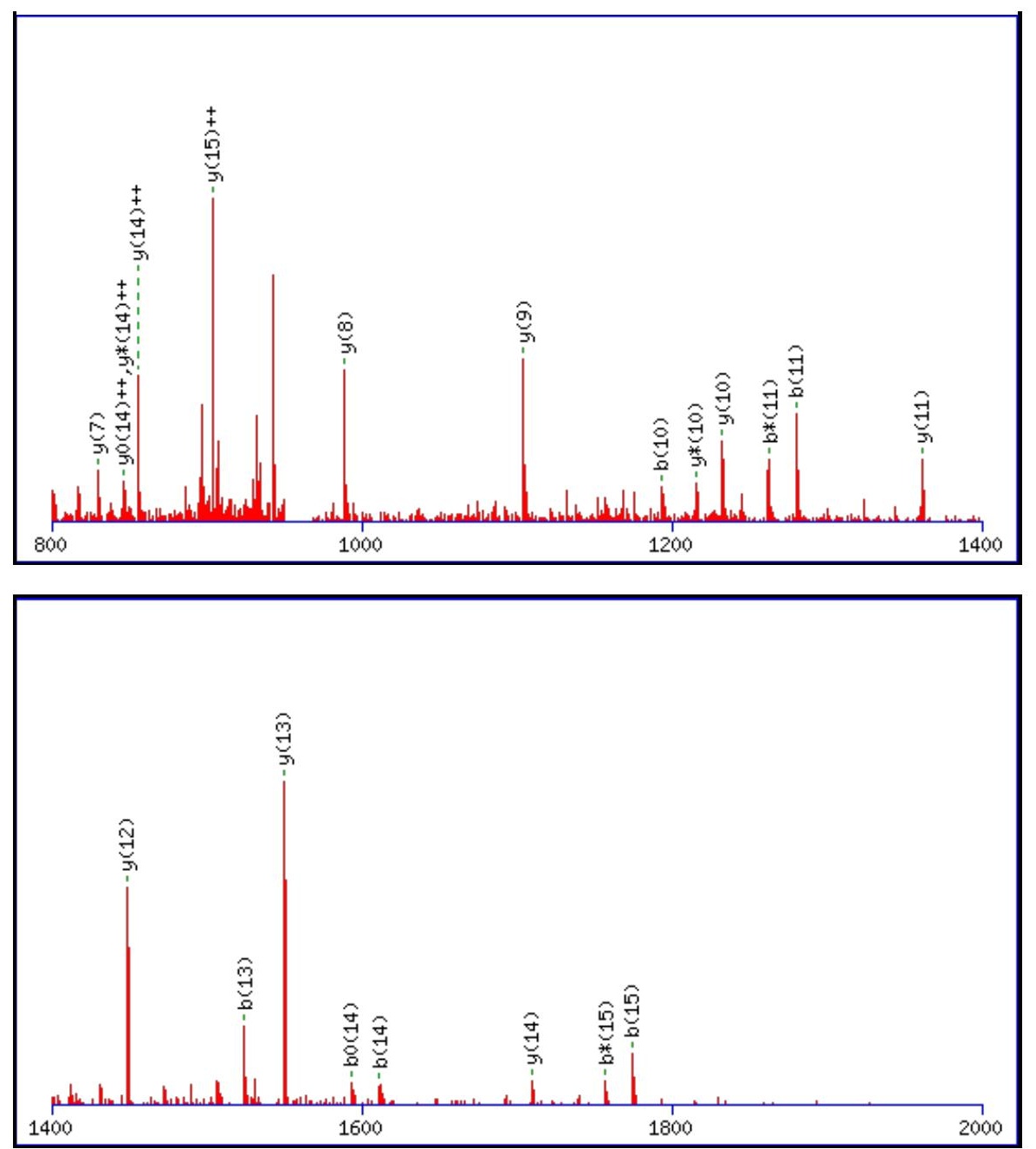

Monoisotopic mass of neutral peptide $\operatorname{Mr}($ calc): 1919.7666

Fixed modifications: Carbamidomethyl (C)

Variable modifications:

N8: Deamidated $\mathrm{N}(\mathrm{N})$

Ions Score: 90 Expect : $1.9 \mathrm{e}-007$

Matches (Bold Red): 36/170 fragment ions using 59 most intense peaks

\begin{tabular}{|c|c|c|c|c|c|c|c|c|c|c|c|c|c|c|}
\hline \# & b & $\mathbf{b}^{++}$ & $\mathbf{b}^{*}$ & $\mathbf{b}^{*^{++}}$ & $\mathbf{b}^{\mathbf{0}}$ & & Seq. & $\mathbf{y}$ & $\mathbf{y}^{++}$ & $\mathbf{y}^{*}$ & $\mathbf{y}^{*^{++}}$ & $\mathbf{y}^{\mathbf{0}}$ & $\mathbf{y}^{\mathbf{0 + +}}$ & \# \\
\hline 1 & 115.0502 & 58.0287 & 98.0237 & 49.5155 & & & $\mathbf{N}$ & & & & & & & 16 \\
\hline 2 & 212.1030 & 106.5551 & 195.0764 & 98.0418 & & & $\mathbf{P}$ & 1806.7309 & 903.8691 & 1789.7044 & & 1788.7204 & 894.8638 & 15 \\
\hline 3 & 372.1336 & 186.5704 & 355.1071 & 178.0572 & & & C & 1709.6782 & 855.3427 & 1692.6516 & 846.8294 & 1691.6676 & 846.3374 & 14 \\
\hline 4 & 473.1813 & 237.0943 & 456.1547 & 228.5810 & 455.1707 & 228.0890 & $\mathbf{T}$ & 1549.6475 & 775.3274 & 1532.6210 & 766.8141 & 1531.6369 & 766.3221 & 13 \\
\hline 5 & 560.2133 & 280.6103 & 543.1868 & 272.0970 & 542.2028 & 271.6050 & S & 1448.5998 & 724.8036 & 1431.5733 & 716.2903 & 1430.5893 & 715.7983 & 12 \\
\hline 6 & 689.2559 & 345.1316 & 672.2294 & 336.6183 & 671.2454 & 336.1263 & $\mathbf{E}$ & 1361.5678 & 681.2875 & 1344.5413 & 672.7743 & 1343.5572 & 672.2823 & 11 \\
\hline 7 & 817.3145 & 409.1609 & 800.2879 & 400.6476 & 799.3039 & 400.1556 & $\mathbf{Q}$ & 1232.5252 & 616.7662 & 1215.4987 & 608.2530 & 1214.5146 & 607.7610 & 10 \\
\hline 8 & 932.3414 & 466.6744 & 915.3149 & 458.1611 & 914.3309 & 457.6691 & $\mathbf{N}$ & 1104.4666 & 552.7370 & 1087.4401 & 544.2237 & 1086.4561 & 543.7317 & 9 \\
\hline 9 & 1092.3721 & 546.6897 & 1075.3455 & 538.1764 & 1074.3615 & 537.6844 & C & 989.4397 & 495.2235 & 972.4131 & 486.7102 & 971.4291 & 486.2182 & 8 \\
\hline 10 & 1193.4198 & 597.2135 & 1176.3932 & 588.7002 & 1175.4092 & 588.2082 & $\mathbf{T}$ & 829.4090 & 415.2082 & 812.3825 & 406.6949 & 811.3985 & 406.2029 & 7 \\
\hline 11 & 1280.4518 & 640.7295 & 1263.4252 & 632.2163 & 1262.4412 & 631.7242 & $\mathbf{S}$ & 728.3614 & 364.6843 & 711.3348 & 356.1710 & 710.3508 & 355.6790 & 6 \\
\hline 12 & 1377.5046 & 689.2559 & 1360.4780 & 680.7426 & 1359.4940 & 680.2506 & $\mathbf{P}$ & 641.3293 & 321.1683 & 624.3028 & 312.6550 & 623.3188 & 312.1630 & 5 \\
\hline 13 & 1524.5730 & 762.7901 & 1507.5464 & 754.2768 & 1506.5624 & 753.7848 & $\mathbf{F}$ & 544.2766 & 272.6419 & 527.2500 & 264.1287 & 526.2660 & 263.6366 & 4 \\
\hline 14 & 1611.6050 & 806.3061 & 1594.5784 & 797.7929 & 1593.5944 & 797.3009 & $\mathbf{S}$ & 397.2082 & 199.1077 & 380.1816 & 190.5944 & 379.1976 & 190.1024 & 3 \\
\hline 15 & 1774.6683 & 887.8378 & 1757.6418 & 879.3245 & 1756.6578 & 878.8325 & $\mathbf{Y}$ & 310.1761 & 155.5917 & 293.1496 & 147.0784 & & & 2 \\
\hline 16 & & & & & & & $\mathbf{K}$ & 147.1128 & 74.0600 & 130.0863 & 65.5468 & & & 1 \\
\hline
\end{tabular}



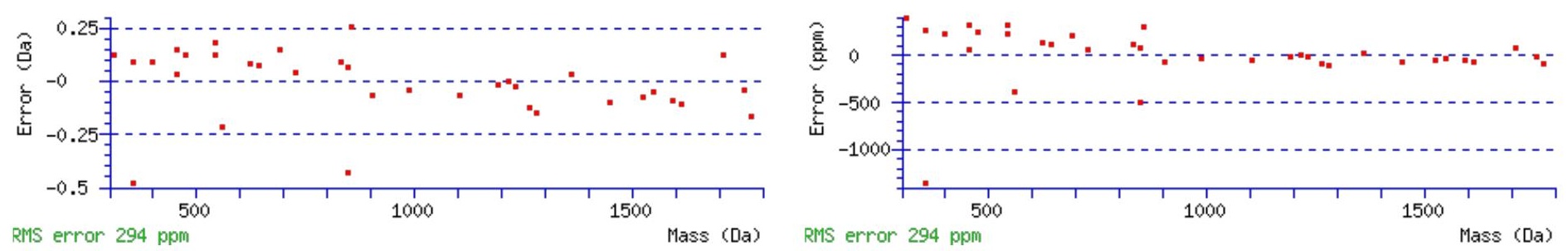

\section{All matches to this query}

\begin{tabular}{|l|l|l|l|}
\hline Score & Mr(calc): & Delta & \multicolumn{1}{c|}{ Sequence } \\
\hline 89.8 & 1919.7666 & 0.0080 & NPCTSEQNCTSPFSYK \\
\hline 22.8 & 1918.7826 & 0.9920 & NPCTSEQNCTSPFSYK \\
\hline 20.1 & 1919.7666 & 0.0080 & NPCTSEQNCTSPFSYK \\
\hline 6.9 & 1919.7614 & 0.0131 & KTSNVQSTAGMSVRAK \\
\hline 6.9 & 1919.7614 & 0.0131 & KTSNVQSTAGMSVRAK \\
\hline 4.8 & 1919.7614 & 0.0131 & KTSNVQSTAGMSVRAK \\
\hline 4.2 & 1919.7802 & -0.0056 & ATELSEEAQLEAVGDAK \\
\hline 1.3 & 1919.7720 & 0.0026 & ASGPGTPPAAEPSVLTTK \\
\hline 0.7 & 1919.7614 & 0.0131 & KTSNVQSTAGMSVRAK \\
\hline 0.7 & 1919.7614 & 0.0131 & KTSNVQSTAGMSVRAK \\
\hline
\end{tabular}

Spectrum No: 137; Query: 2806; Rank: 1

\section{Peptide View}

MS/MS Fragmentation of NGTPGGSFVSPSPLSTSSPILSADSTSVGSFPSAGSSDQGPR

Found in IPI00362755, Tax_Id=10116 Gene_Symbol=Tns similar to tensin

Match to Query 2806: 3980.847432 from(1327.956420,3+)

Title: 100101RatKid_NS_deglyco_08.4830.4830.3.dta

Data file K:INewmanPaper|Piliangl3SubProteomes\Piliang3SP\mgf5ppm|ERLIC_3SubProteomes5ppm.mgf

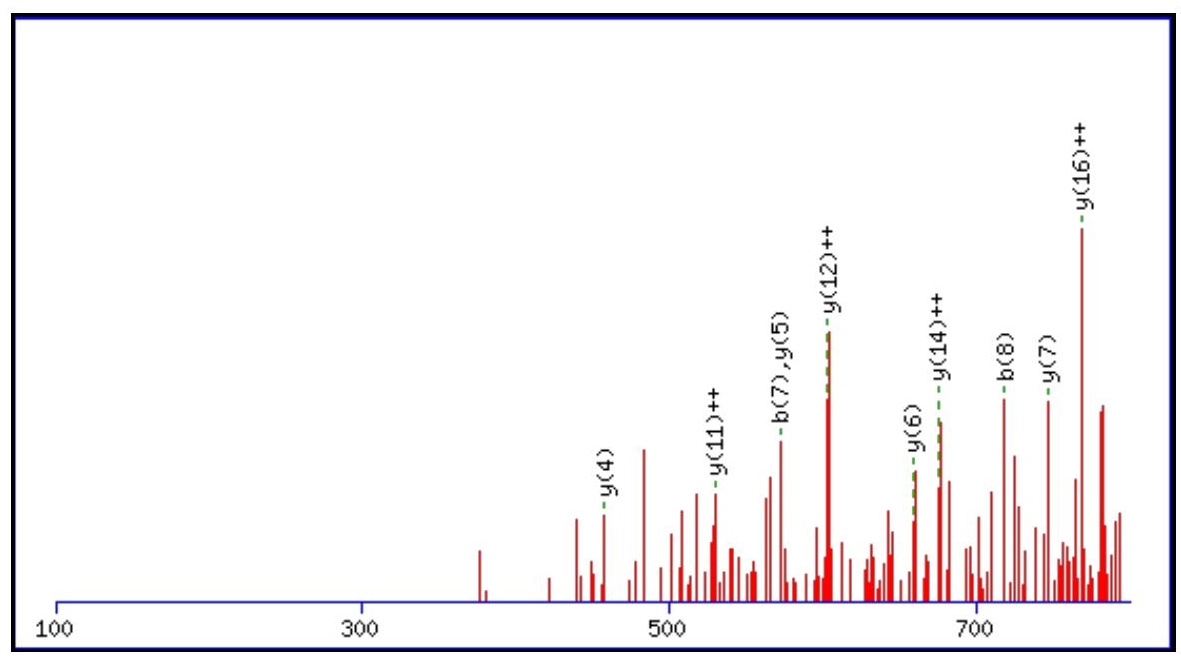



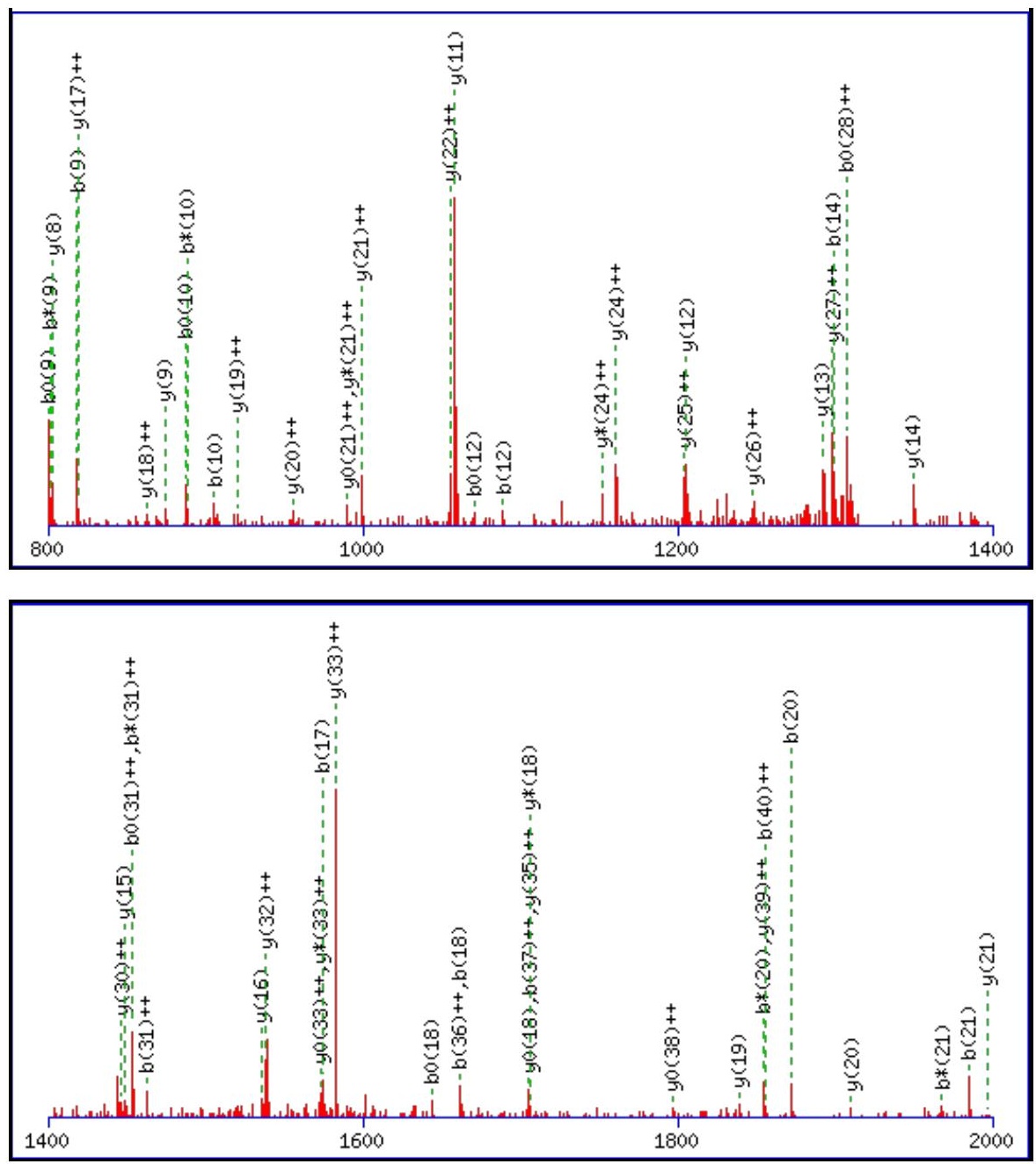

Monoisotopic mass of neutral peptide $\operatorname{Mr}($ calc): 3979.8560

Fixed modifications: Carbamidomethyl (C)

Variable modifications:

N1 : Deamidated_N (N)

Ions Score: 90 Expect : $4.5 \mathrm{e}-007$

Matches (Bold Red): 67/480 fragment ions using 122 most intense peaks

\begin{tabular}{|c|c|c|c|c|c|c|c|c|c|c|c|c|c|c|}
\hline \# & b & $\mathbf{b}^{++}$ & $\mathbf{b}^{*}$ & $\mathbf{b}^{*^{++}}$ & $\mathbf{b}^{\mathbf{0}}$ & $\mathbf{b}^{\mathbf{0 + +}}$ & Seq. & $\mathbf{y}$ & $\mathbf{y}^{++}$ & $\mathbf{y}^{*}$ & $\mathbf{y}^{*^{++}}$ & $\mathbf{y}^{0}$ & $\mathbf{y}^{0++}$ & \# \\
\hline 1 & 116.0342 & 58.5207 & 99.0077 & 50.0075 & & & $\mathbf{N}$ & & & & & & & 42 \\
\hline 2 & 173.0557 & 87.0315 & 156.0291 & 78.5182 & & & G & 3865.8363 & 1933.4218 & 3848.8097 & 1924.9085 & 3847.8257 & 1924.4165 & 41 \\
\hline 3 & 274.1034 & 137.5553 & 257.0768 & 129.0420 & 256.0928 & 128.5500 & $\mathbf{T}$ & 3808.8148 & 1904.9110 & 3791.7882 & 1896.3978 & 3790.8042 & 1895.9058 & 40 \\
\hline 4 & 371.1561 & 186.0817 & 354.1296 & 177.5684 & 353.1456 & 177.0764 & $\mathbf{P}$ & 3707.7671 & 1854.3872 & 3690.7406 & 1845.8739 & 3689.7566 & 1845.3819 & 39 \\
\hline 5 & 428.1776 & 214.5924 & 411.1510 & 206.0792 & 410.1670 & 205.5871 & G & 3610.7144 & 1805.8608 & 3593.6878 & 1797.3475 & 3592.7038 & 1796.8555 & 38 \\
\hline 6 & 485.1990 & 243.1032 & 468.1725 & 234.5899 & 467.1885 & 234.0979 & G & 3553.6929 & 1777.3501 & 3536.6663 & 1768.8368 & 3535.6823 & 1768.3448 & 37 \\
\hline 7 & 572.2311 & 286.6192 & 555.2045 & 278.1059 & 554.2205 & 277.6139 & S & 3496.6714 & 1748.8394 & 3479.6449 & 1740.3261 & 3478.6609 & 1739.8341 & 36 \\
\hline 8 & 719.2995 & 360.1534 & 702.2729 & 351.6401 & 701.2889 & 351.1481 & $\mathbf{F}$ & 3409.6394 & 1705.3233 & 3392.6128 & 1696.8101 & 3391.6288 & 1696.3181 & 35 \\
\hline 9 & 818.3679 & 409.6876 & 801.3414 & 401.1743 & 800.3573 & 400.6823 & V & 3262.5710 & 1631.7891 & 3245.5444 & 1623.2759 & 3244.5604 & 1622.7838 & 34 \\
\hline 10 & 905.3999 & 453.2036 & 888.3734 & 444.6903 & 887.3894 & 444.1983 & S & 3163.5026 & 1582.2549 & 3146.4760 & 1573.7416 & 3145.4920 & 1573.2496 & 33 \\
\hline 11 & 1002.4527 & 501.7300 & 985.4261 & 493.2167 & 984.4421 & 492.7247 & $\mathbf{P}$ & 3076.4705 & 1538.7389 & 3059.4440 & 1530.2256 & 3058.4600 & 1529.7336 & 32 \\
\hline 12 & 1089.4847 & 545.2460 & 1072.4582 & 536.7327 & 1071.4742 & 536.2407 & S & 2979.4178 & 1490.2125 & 2962.3912 & 1481.6993 & 2961.4072 & 1481.2072 & 31 \\
\hline 13 & 1186.5375 & 593.7724 & 1169.5109 & 585.2591 & 1168.5269 & 584.7671 & $\mathbf{P}$ & 2892.3857 & 1446.6965 & 2875.3592 & 1438.1832 & 2874.3752 & 1437.6912 & 30 \\
\hline 14 & 1299.6216 & 650.3144 & 1282.5950 & 641.8011 & 1281.6110 & 641.3091 & $\mathbf{L}$ & 2795.3330 & 1398.1701 & 2778.3064 & 1389.6569 & 2777.3224 & 1389.1648 & 29 \\
\hline 15 & 1386.6536 & 693.8304 & 1369.6270 & 685.3172 & 1368.6430 & 684.8251 & S & 2682.2489 & 1341.6281 & 2665.2224 & 1333.1148 & 2664.2384 & 1332.6228 & 28 \\
\hline 16 & 1487.7013 & 744.3543 & 1470.6747 & 735.8410 & 1469.6907 & 735.3490 & $\mathbf{T}$ & 2595.2169 & 1298.1121 & 2578.1903 & 1289.5988 & 2577.2063 & 1289.1068 & 27 \\
\hline 17 & 1574.7333 & 787.8703 & 1557.7067 & 779.3570 & 1556.7227 & 778.8650 & S & 2494.1692 & 1247.5882 & 2477.1427 & 1239.0750 & 2476.1586 & 1238.5830 & 26 \\
\hline 18 & 1661.7653 & 831.3863 & 1644.7388 & 822.8730 & 1643.7547 & 822.3810 & S & 2407.1372 & 1204.0722 & 2390.1106 & 1195.5590 & 2389.1266 & 1195.0669 & 25 \\
\hline
\end{tabular}




\begin{tabular}{|c|c|c|c|c|c|c|c|c|c|c|c|c|c|c|}
\hline 19| & |1758.8181 & 879.9127 & |1741.7915 & 871.3994 & |1740.8075 & 870.9074 & $\mathbf{P}$ & 2320.1052 & 1160.5562 & |2303.0786 | & $\mid 1152.0429$ & |2302.0946 & |1151.5509 & 24 \\
\hline 20 & 1871.9021 & 936.4547 & 1854.8756 & 927.9414 & 1853.8916 & 927.4494 & I & 2223.0524 & 1112.0298 & 2206.0258 & 1103.5166 & 2205.0418 & 1103.0246 & 23 \\
\hline 21 & 1984.9862 & 992.9967 & 1967.9597 & 984.4835 & 1966.9756 & 983.9915 & $\mathbf{L}$ & 2109.9683 & 1055.4878 & 2092.9418 & 1046.9745 & 2091.9578 & 1046.4825 & 22 \\
\hline 22 & 2072.0182 & 1036.5128 & 2054.9917 & 1027.9995 & 2054.0077 & 1027.5075 & $\mathrm{~S}$ & 1996.8843 & 998.9458 & 1979.8577 & 990.4325 & 1978.8737 & 989.9405 & 21 \\
\hline 23 & 2143.0553 & 1072.0313 & 2126.0288 & 1063.5180 & 2125.0448 & 1063.0260 & A & 1909.8522 & 955.4298 & |1892.8257 & 946.9165 & 1891.8417 & 946.4245 & 20 \\
\hline 24 & 2258.0823 & 1129.5448 & 2241.0557 & 1121.0315 & 2240.0717 & 1120.5395 & D & 1838.8151 & 919.9112 & 1821.7886 & 911.3979 & 1820.8046 & 910.9059 & 19 \\
\hline 25 & 2345.1143 & 1173.0608 & 2328.0878 & 1164.5475 & 2327.1038 & 1164.0555 & S & 1723.7882 & 862.3977 & 1706.7616 & 853.8845 & 1705.7776 & 853.3924 & 18 \\
\hline 26 & 2446.1620 & 1223.5846 & 2429.1354 & 1215.0714 & 2428.1514 & 1214.5794 & $T$ & 1636.7562 & 818.8817 & 1619.7296 & 810.3684 & 1618.7456 & 809.8764 & 17 \\
\hline 27 & 2533.1940 & 1267.1007 & 2516.1675 & 1258.5874 & 2515.1835 & 1258.0954 & S & 1535.7085 & 768.3579 & 1518.6819 & 759.8446 & 1517.6979 & 759.3526 & 16 \\
\hline 28 & 2632.2624 & 1316.6349 & 2615.2359 & 1308.1216 & 2614.2519 & 1307.6296 & $\mathbf{V}$ & 1448.6764 & 724.8419 & 1431.6499 & 716.3286 & 1430.6659 & 715.8366 & 15 \\
\hline 29 & 2689.2839 & 1345.1456 & 2672.2574 & 1336.6323 & 2671.2733 & 1336.1403 & G & 1349.6080 & 675.3077 & 1332.5815 & 666.7944 & 1331.5975 & 666.3024 & 14 \\
\hline 30 & 2776.3159 & 1388.6616 & 2759.2894 & 1380.1483 & 2758.3054 & 1379.6563 & S & 1292.5866 & 646.7969 & 1275.5600 & 638.2836 & 1274.5760 & 637.7916 & 13 \\
\hline 31 & 2923.3843 & 1462.1958 & 2906.3578 & 1453.6825 & 2905.3738 & 1453.1905 & $\mathbf{F}$ & 1205.5545 & 603.2809 & 1188.5280 & 594.7676 & 1187.5440 & 594.2756 & 12 \\
\hline 32 & 3020.4371 & 1510.7222 & 3003.4106 & 1502.2089 & 3002.4265 & 1501.7169 & $\mathbf{P}$ & 1058.4861 & 529.7467 & 1041.4596 & 521.2334 & 1040.4756 & 520.7414 & 11 \\
\hline 33 & 3107.4691 & 1554.2382 & 3090.4426 & 1545.7249 & 3089.4586 & 1545.2329 & S & 961.4334 & 481.2203 & 944.4068 & 472.7070 & 943.4228 & 472.2150 & 10 \\
\hline 34 & 3178.5063 & 1589.7568 & 3161.4797 & 1581.2435 & 3160.4957 & 1580.7515 & A & 874.4013 & 437.7043 & 857.3748 & 429.1910 & 856.3908 & 428.6990 & 9 \\
\hline 35 & 3235.5277 & 1618.2675 & 3218.5012 & 1609.7542 & 3217.5172 & 1609.2622 & G & 803.3642 & 402.1857 & 786.3377 & 393.6725 & 785.3537 & 393.1805 & 8 \\
\hline 36 & 3322.5597 & 1661.7835 & 3305.5332 & 1653.2702 & 3304.5492 & 1652.7782 & $\mathrm{~S}$ & 746.3428 & 373.6750 & 729.3162 & 365.1617 & 728.3322 & 364.6697 & 7 \\
\hline 37 & 3409.5918 & \begin{tabular}{|l|l|}
1705.2995 \\
\end{tabular} & 3392.5652 & 1696.7862 & 3391.5812 & 1696.2942 & $\mathrm{~S}$ & 659.3107 & 330.1590 & 642.2842 & 321.6457 & 641.3002 & 321.1537 & 6 \\
\hline 38 & 3524.6187 & 1762.8130 & 3507.5922 & 1754.2997 & 3506.6081 & 1753.8077 & D & 572.2787 & 286.6430 & 555.2522 & 278.1297 & 554.2681 & 277.6377 & 5 \\
\hline 39 & 3652.6773 & 1826.8423 & 3635.6507 & 1818.3290 & 3634.6667 & 1817.8370 & $\mathbf{Q}$ & 457.2518 & 229.1295 & 440.2252 & 220.6162 & & & 4 \\
\hline 40 & 3709.6988 & 1855.3530 & 3692.6722 & 1846.8397 & |3691.6882 & 1846.3477 & G & 329.1932 & 165.1002 & 312.1666 & 156.5870 & & & 3 \\
\hline 41 & 3806.7515 & 1903.8794 & 3789.7250 & 1895.3661 & 3788.7410 & 1894.8741 & $\mathbf{P}$ & 272.1717 & 136.5895 & 255.1452 & 128.0762 & & & 2 \\
\hline 42 & & & & & & & $\mathbf{R}$ & 175.1190 & 88.0631 & 158.0924 & 79.5498 & & & 1 \\
\hline
\end{tabular}
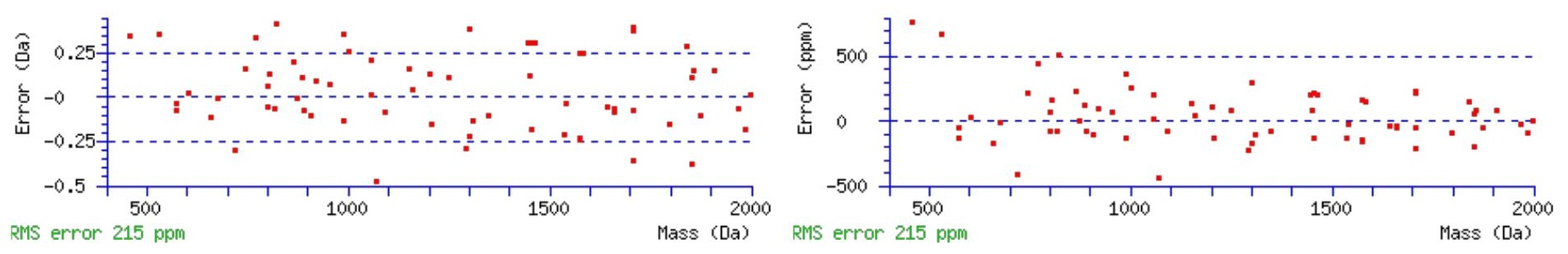

\section{All matches to this query}

\begin{tabular}{|l|l|c|l|}
\hline Score & Mr(calc): & Delta & \multicolumn{1}{c|}{ Sequence } \\
\hline 89.7 & 3979.8560 & 0.9915 & NGTPGGSFVSPSPLSTSSPILSADSTSVGSFPSAGSSDQGPR \\
\hline 71.4 & 3978.8720 & 1.9755 & NGTPGGSFVSPSPLSTSSPILSADSTSVGSFPSAGSSDQGPR \\
\hline 8.4 & 3978.8178 & 2.0297 & HYLTAFYVVECIAMSNSMINTLCFVTVRNNTSK \\
\hline 7.3 & 3978.8489 & 1.9986 & MVIIDHGLQTLTHEVIVPHSGWEREPNEDSKPR \\
\hline 7.3 & 3978.8489 & 1.9986 & MVIIDHGLQTLTHEVIVPHSGWEREPNEDSKPR \\
\hline 6.5 & 3979.8774 & 0.9700 & YSNDVTSLPFLLEILTVLPEEVHSRSLRIGANR \\
\hline 6.5 & 3979.8774 & 0.9700 & YSNDVTSLPFLLEILTVLPEEVHSRSLRIGANR \\
\hline 6.0 & 3979.8815 & 0.9660 & $\underline{\text { LAAVSDENLGNITSVVEAIPEFHKKPSLLSFWTK }}$ \\
\hline 5.6 & 3978.8605 & 1.9870 & SSYFVNSTFPVTLASGQESFLGQLPLYSSGYADPLR \\
\hline 4.3 & 3980.8503 & -0.0029 & FSSGSNIPKKSVVMQSPGTSYSSWEPVISAQEVDTR \\
\hline
\end{tabular}

Spectrum No: 138; Query: 649; Rank: 1

\section{Peptide View}




\section{MS/MS Fragmentation of LINDYVSNQTQGK}

Found in IPI00200591, Tax_Id=10116 Gene_Symbol=LOC299282 Serine protease inhibitor A3L precursor

Match to Query 649: 1479.728028 from(740.871290,2+)

Title: 091008RatKidney_NoSalt_28.1315.1315.2.dta

Data file K:INewmanPaper|Piliang|3SubProteomes\Piliang3SP\mgf5ppm\ERLIC_3SubProteomes5ppm.mgf
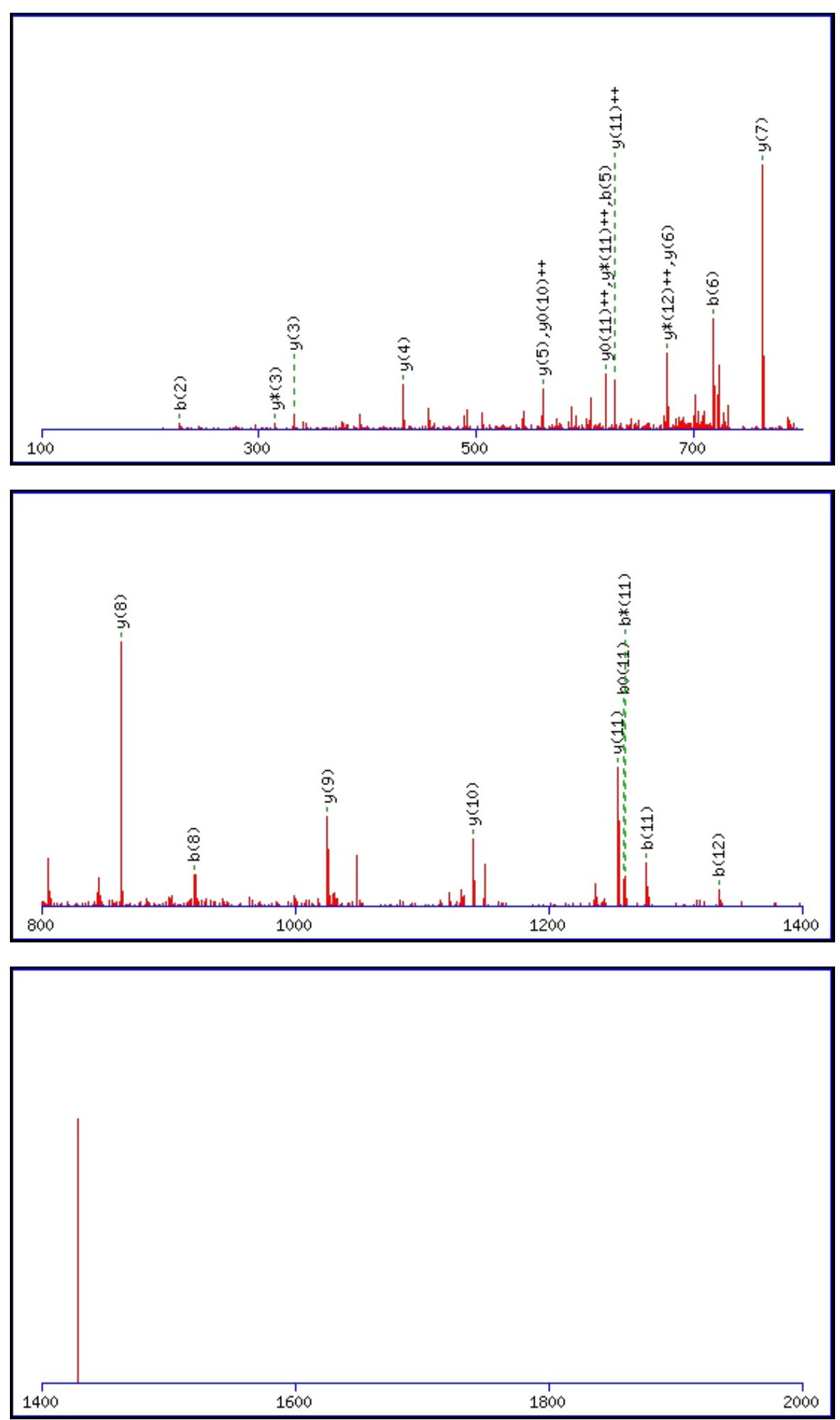

Monoisotopic mass of neutral peptide $\operatorname{Mr}($ calc): 1479.7205

Fixed modifications: Carbamidomethyl (C)

Variable modifications:

N8 : Deamidated $\mathrm{N}(\mathrm{N})$

Ions Score: 90 Expect : $1.6 \mathrm{e}-007$

Matches (Bold Red): 23/128 fragment ions using 25 most intense peaks

\begin{tabular}{|l|l|l|l|l|l|l|l|l|l|l|l|l|l|l|l|}
\hline$\#$ & $\mathbf{b}$ & $\mathbf{b}^{++}$ & $\mathbf{b}^{*}$ & $\mathbf{b}^{*^{++}}$ & $\mathbf{b}^{\mathbf{0}}$ & $\mathbf{b}^{\mathbf{0 + +}}$ & Seq. & $\mathbf{y}$ & $\mathbf{y}^{++}$ & $\mathbf{y}^{*}$ & $\mathbf{y}^{*^{++}}$ & $\mathbf{y}^{\mathbf{0}}$ & $\mathbf{y}^{\mathbf{0 + +}}$ & $\#$ \\
\hline
\end{tabular}




\begin{tabular}{|c|c|c|c|c|c|c|c|c|c|c|c|c|c|c|}
\hline 1 & 114.0913 & 57.5493 & & & & & L & & & & & & & 13 \\
\hline 2 & 227.1754 & 114.0913 & & & & & I & |1367.6437 & 684.3255 & 1350.6172 & 675.8122 & 1349.6332 & 675.3202 & 12 \\
\hline 3 & 341.2183 & |171.1128 & 324.1918 & 162.5995 & & & $\mathbf{N}$ & 1254.5597 & 627.7835 & 1237.5331 & 619.2702 & 1236.5491 & 618.7782 & 11 \\
\hline 4 & 456.2453 & 228.6263 & 439.2187 & 220.1130 & 438.2347 & 219.6210 & D & 1140.5168 & 570.7620 & 1123.4902 & 562.2487 & 1122.5062 & 561.7567 & 10 \\
\hline 5 & 619.3086 & 310.1579 & 602.2821 & 301.6447 & 601.2980 & 301.1527 & $\mathbf{Y}$ & 1025.4898 & 513.2485 & 1008.4633 & 504.7353 & 1007.4792 & 504.2433 & 9 \\
\hline 6 & 718.3770 & 359.6921 & 701.3505 & 351.1789 & 700.3665 & 350.6869 & V & 862.4265 & 431.7169 & 845.3999 & 423.2036 & 844.4159 & 422.7116 & 8 \\
\hline 7 & 805.4090 & 403.2082 & 788.3825 & |394.6949 & 787.3985 & 394.2029 & $\mathbf{S}$ & 763.3581 & |382.1827 & 746.3315 & 373.6694 & 745.3475 & 373.1774 & 7 \\
\hline 8 & 920.4360 & 460.7216 & 903.4094 & 452.2084 & 902.4254 & 451.7163 & $\mathbf{N}$ & 676.3260 & 338.6667 & 659.2995 & 330.1534 & 658.3155 & 329.6614 & 6 \\
\hline 9 & 1048.4946 & 524.7509 & 1031.4680 & 516.2376 & 1030.4840 & 515.7456 & $\mathbf{Q}$ & 561.2991 & 281.1532 & 544.2726 & 272.6399 & 543.2885 & 272.1479 & 5 \\
\hline 10 & 1149.5422 & 575.2748 & 1132.5157 & 566.7615 & 1131.5317 & 566.2695 & $\mathbf{T}$ & 433.2405 & 217.1239 & 416.2140 & 208.6106 & 415.2300 & 208.1186 & 4 \\
\hline 11 & 1277.6008 & 639.3040 & 1260.5743 & 630.7908 & 1259.5903 & 630.2988 & $\mathbf{Q}$ & 332.1928 & 166.6001 & 315.1663 & 158.0868 & & & 3 \\
\hline 12 & \begin{tabular}{|l|}
1334.6223 \\
\end{tabular} & 667.8148 & 1317.5957 & 659.3015 & 1316.6117 & 658.8095 & G & 204.1343 & 102.5708 & 187.1077 & 94.0575 & & & 2 \\
\hline 13 & & & & & & & K & 147.1128 & 74.0600 & 130.0863 & 65.5468 & & & 1 \\
\hline
\end{tabular}
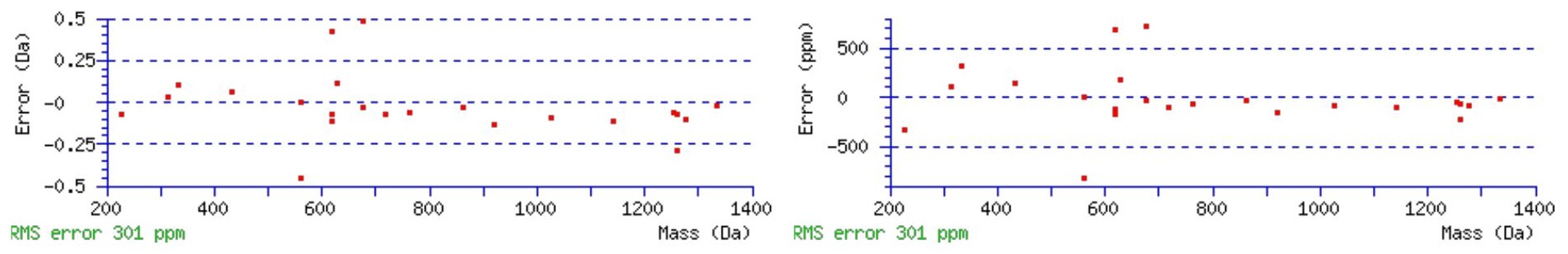

\section{All matches to this query}

\begin{tabular}{|l|c|c|l|}
\hline Score & Mr(calc): & Delta & \multicolumn{1}{c|}{ Sequence } \\
\hline 89.5 & 1479.7205 & 0.0075 & LINDYVSNQTQGK \\
\hline 36.6 & 1479.7205 & 0.0075 & LINDYVSNQTQGK \\
\hline 8.2 & 1478.7365 & 0.9915 & LINDYVSNQTQGK \\
\hline 7.5 & 1479.7334 & -0.0053 & KNPDSLELIRSK \\
\hline 6.0 & 1479.7425 & -0.0145 & QMTLLDMAKGTQK \\
\hline 4.4 & 1479.7309 & -0.0029 & LLSQGLAWPKCK \\
\hline 4.0 & 1479.7334 & -0.0054 & QGGGGVGTILSNVLK \\
\hline 3.1 & 1479.7334 & -0.0053 & KNPDSLELIRSK \\
\hline 1.8 & 1479.7425 & -0.0145 & QMTLLDMAKGTQK \\
\hline 1.0 & 1479.7205 & 0.0075 & FEETLKGDEREK \\
\hline
\end{tabular}

Spectrum No: 139; Query: 1998; Rank: 1

\section{Peptide View}

MS/MS Fragmentation of KYPVPFQNENVTAAFVELGR

Found in IPI00213569, Tax_Id=10116 Gene_Symbol=Ugt1a1 UDP-glucuronosyltransferase 1-1 precursor

Match to Query 1998: 2279.158328 from(1140.586440,2+)

Title: 091008RatKidney_NH4Format01_23.3987.3987.2.dta

Data file K:INewmanPaper|Piliangl3SubProteomes\Piliang3SP\mgf5ppm\ERLIC_3SubProteomes5ppm.mgf 

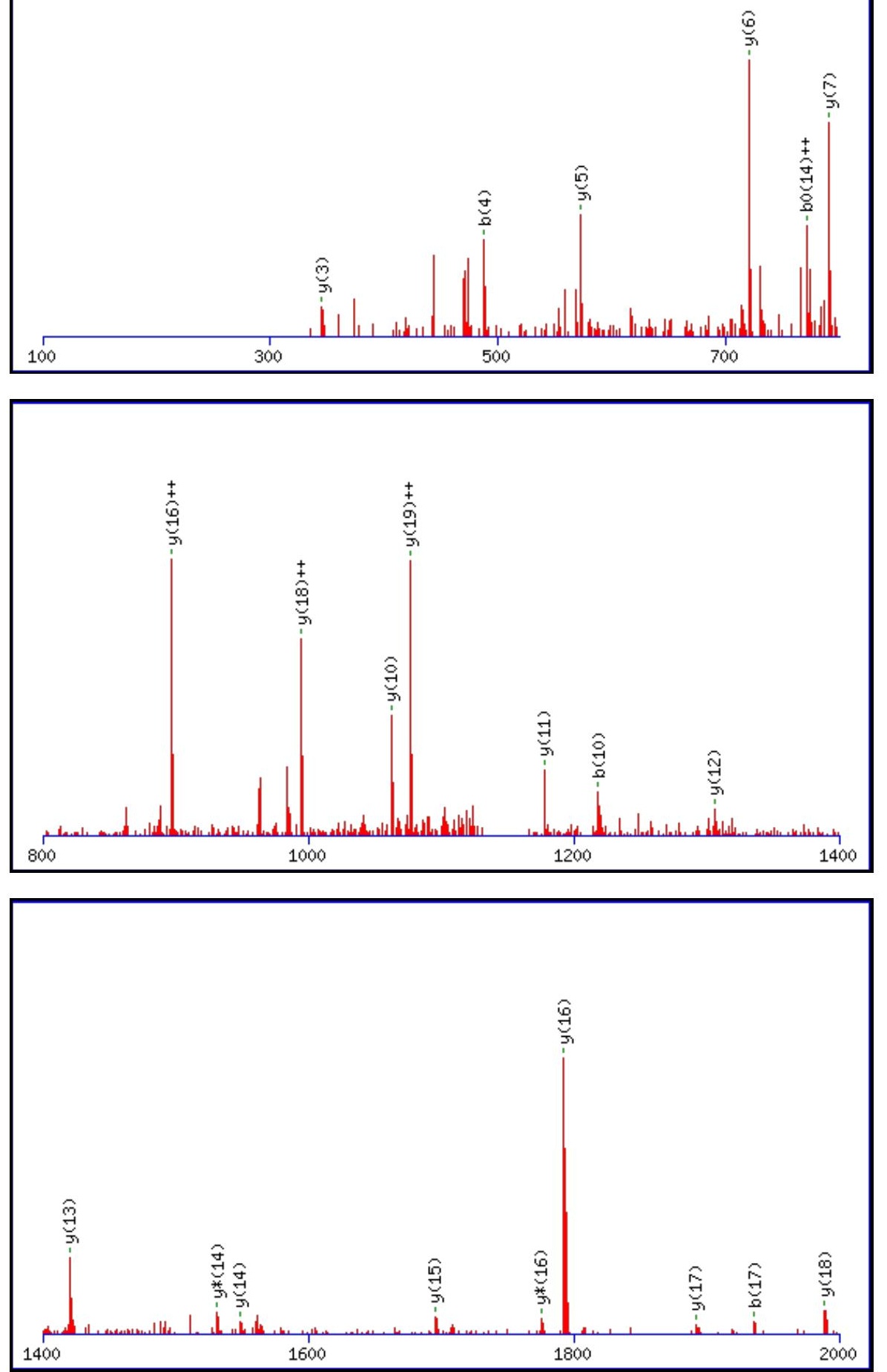

Monoisotopic mass of neutral peptide $\operatorname{Mr}($ calc): 2279.1586

Fixed modifications: Carbamidomethyl (C)

Variable modifications:

N10 : Deamidated $\mathrm{N}(\mathrm{N})$

Ions Score: $9 \odot$ Expect: $1.8 \mathrm{e}-007$

Matches (Bold Red): 22/206 fragment ions using 45 most intense peaks

\begin{tabular}{|r|c|c|c|c|c|r|r|r|r|r|r|r|r|r|}
\hline$\#$ & $\mathbf{b}$ & \multicolumn{1}{|c|}{$\mathbf{b}^{++}$} & $\mathbf{b}^{*}$ & $\mathbf{b}^{\boldsymbol{*}^{++}}$ & $\mathbf{b}^{\mathbf{0}}$ & $\mathbf{b}^{\mathbf{0 + +}}$ & $\mathbf{S e q}$ & $\mathbf{y}$ & $\mathbf{y}^{++}$ & $\mathbf{y}^{\mathbf{*}}$ & $\mathbf{y}^{\mathbf{*}^{++}}$ & $\mathbf{y}^{\mathbf{0}}$ & $\mathbf{y}^{\mathbf{0}++}$ & $\#$ \\
\hline $\mathbf{1}$ & 129.1022 & 65.0548 & 112.0757 & 56.5415 & & & $\mathbf{K}$ & & & & & & & $\mathbf{2 0}$ \\
\hline $\mathbf{2}$ & 292.1656 & 146.5864 & 275.1390 & 138.0731 & & & $\mathbf{Y}$ & 2152.0709 & $\mathbf{1 0 7 6 . 5 3 9 1}$ & 2135.0444 & 1068.0258 & 2134.0604 & 1067.5338 & $\mathbf{1 9}$ \\
\hline $\mathbf{3}$ & 389.2183 & 195.1128 & 372.1918 & 186.5995 & & & $\mathbf{P}$ & $\mathbf{1 9 8 9 . 0 0 7 6}$ & $\mathbf{9 9 5 . 0 0 7 4}$ & 1971.9811 & 986.4942 & 1970.9970 & 986.0022 & $\mathbf{1 8}$ \\
\hline $\mathbf{4}$ & $\mathbf{4 8 8 . 2 8 6 7}$ & 244.6470 & 471.2602 & 236.1337 & & & $\mathbf{V}$ & $\mathbf{1 8 9 1 . 9 5 4 8}$ & 946.4811 & 1874.9283 & 937.9678 & 1873.9443 & 937.4758 & $\mathbf{1 7}$ \\
\hline $\mathbf{5}$ & 585.3395 & 293.1734 & 568.3130 & 284.6601 & & & $\mathbf{P}$ & $\mathbf{1 7 9 2 . 8 8 6 4}$ & $\mathbf{8 9 6 . 9 4 6 9}$ & $\mathbf{1 7 7 5 . 8 5 9 9}$ & 888.4336 & $\mathbf{1 7 7 4 . 8 7 5 9}$ & 887.9416 & $\mathbf{1 6}$ \\
\hline
\end{tabular}




\begin{tabular}{|c|c|c|c|c|c|c|c|c|c|c|c|c|c|c|}
\hline 6 & 732.4079 & 366.7076 & 715.3814 & 358.1943 & & & $\mathbf{F}$ & |1695.8337 & 848.4205 & |1678.8071 & 839.9072 & |1677.8231 & 839.4152 & 15 \\
\hline 7 & 860.4665 & 430.7369 & 843.4400 & 422.2236 & & & $\mathbf{Q}$ & 1548.7653 & 774.8863 & 1531.7387 & 766.3730 & 1530.7547 & 765.8810 & 14 \\
\hline 8 & 974.5094 & 487.7584 & 957.4829 & 479.2451 & & & $\mathbf{N}$ & 1420.7067 & 710.8570 & 1403.6801 & 702.3437 & 1402.6961 & 701.8517 & 13 \\
\hline 9 & 1103.5520 & 552.2796 & 1086.5255 & 543.7664 & 1085.5415 & 543.2744 & $\mathbf{E}$ & 1306.6638 & 653.8355 & 1289.6372 & 645.3222 & 1288.6532 & 644.8302 & 12 \\
\hline 10 & 1218.5790 & 609.7931 & 1201.5524 & 601.2798 & 1200.5684 & 600.7878 & $\mathbf{N}$ & 1177.6212 & 589.3142 & 1160.5946 & 580.8009 & 1159.6106 & 580.3089 & 11 \\
\hline 11 & 1317.6474 & 659.3273 & 1300.6208 & 650.8140 & 1299.6368 & 650.3220 & $\mathbf{V}$ & 1062.5942 & 531.8007 & 1045.5677 & 523.2875 & 1044.5837 & 522.7955 & 10 \\
\hline 12 & 1418.6951 & 709.8512 & 1401.6685 & 701.3379 & 1400.6845 & 700.8459 & $\mathbf{T}$ & 963.5258 & 482.2665 & 946.4993 & 473.7533 & 945.5152 & 473.2613 & 9 \\
\hline 13 & 1489.7322 & 745.3697 & 1472.7056 & 736.8564 & 1471.7216 & 736.3644 & A & 862.4781 & 431.7427 & 845.4516 & 423.2294 & 844.4676 & 422.7374 & 8 \\
\hline 14 & 1560.7693 & 780.8883 & 1543.7427 & 772.3750 & 1542.7587 & 771.8830 & A & 791.4410 & 396.2241 & 774.4145 & 387.7109 & 773.4305 & 387.2189 & 7 \\
\hline 15 & 1707.8377 & 854.4225 & 1690.8111 & 845.9092 & 1689.8271 & 845.4172 & $\mathbf{F}$ & 720.4039 & 360.7056 & 703.3774 & 352.1923 & 702.3933 & 351.7003 & 6 \\
\hline 16 & 1806.9061 & 903.9567 & 1789.8796 & 895.4434 & 1788.8955 & 894.9514 & $\mathbf{V}$ & 573.3355 & 287.1714 & 556.3089 & 278.6581 & 555.3249 & 278.1661 & 5 \\
\hline 17 & 1935.9487 & 968.4780 & 1918.9222 & 959.9647 & 1917.9381 & 959.4727 & $\mathbf{E}$ & 474.2671 & 237.6372 & 457.2405 & 229.1239 & 456.2565 & 228.6319 & 4 \\
\hline 18 & 2049.0328 & 1025.0200 & 2032.0062 & 1016.5067 & 2031.0222 & 1016.0147 & $\mathbf{L}$ & 345.2245 & 173.1159 & 328.1979 & 164.6026 & & & 3 \\
\hline 19 & 2106.0542 & 1053.5308 & 2089.0277 & 1045.0175 & 2088.0437 & 1044.5255 & G & 232.1404 & 116.5738 & 215.1139 & 108.0606 & & & 2 \\
\hline 20 & & & & & & & $\mathbf{R}$ & 175.1190 & 88.0631 & 158.0924 & 79.5498 & & & 1 \\
\hline
\end{tabular}
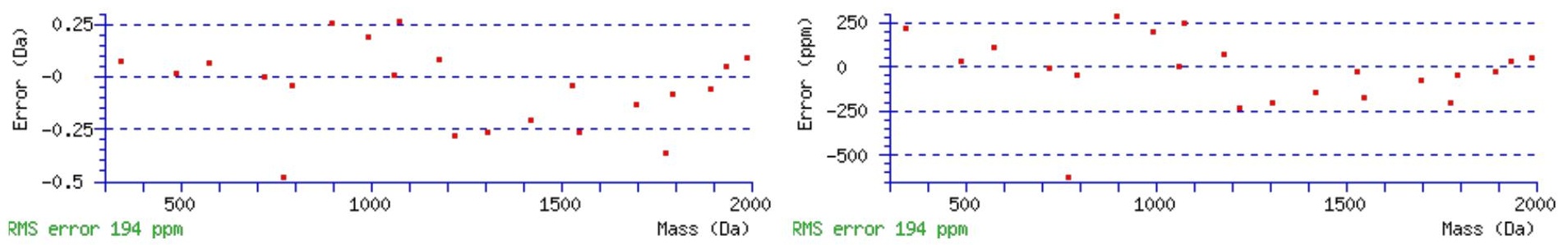

\section{All matches to this query}

\begin{tabular}{|l|c|c|l|}
\hline Score & Mr(calc): & Delta & \multicolumn{1}{|c|}{ Sequence } \\
\hline 89.6 & 2279.1586 & -0.0003 & KYPVPFQNENVTAAFVELGR \\
\hline 63.8 & 2279.1586 & -0.0003 & KYPVPFQNENVTAAFVELGR \\
\hline 4.9 & 2277.1366 & 2.0218 & ATATPVKASAPSANTKASATQPK \\
\hline 2.0 & 2277.1366 & 2.0218 & ATATPVKASAPSANTKASATQPK \\
\hline 1.6 & 2279.1433 & 0.0150 & DAGENNFINVDLKLARDVFK \\
\hline 1.3 & 2277.1518 & 2.0065 & TIINQVLSQTQIEFASHLR \\
\hline 0.5 & 2279.1490 & 0.0093 & KVFLNKAADFIEELSSLFK \\
\hline 0.4 & 2279.1490 & 0.0093 & KVFLNKAADFIEELSSLFK \\
\hline 0.3 & 2279.1433 & 0.0150 & DAGENNFINVDLKLARDVFK \\
\hline
\end{tabular}

Spectrum No: 140; Query: 1103; Rank: 1

\section{Peptide View}

MS/MS Fragmentation of VLPFNVTDYCQLVR

Found in IPI00204006, Tax_Id=10116 Gene_Symbol=Fbn1 Fibrillin-1

Match to Query 1103: 1723.856768 from(862.935660,2+)

Title: 091008RatKidney_NH4Format01_23.3770.3770.2.dta

Data file K:INewmanPaper|Piliangl3SubProteomes\Piliang3SP\mgf5ppm\ERLIC_3SubProteomes5ppm.mgf 

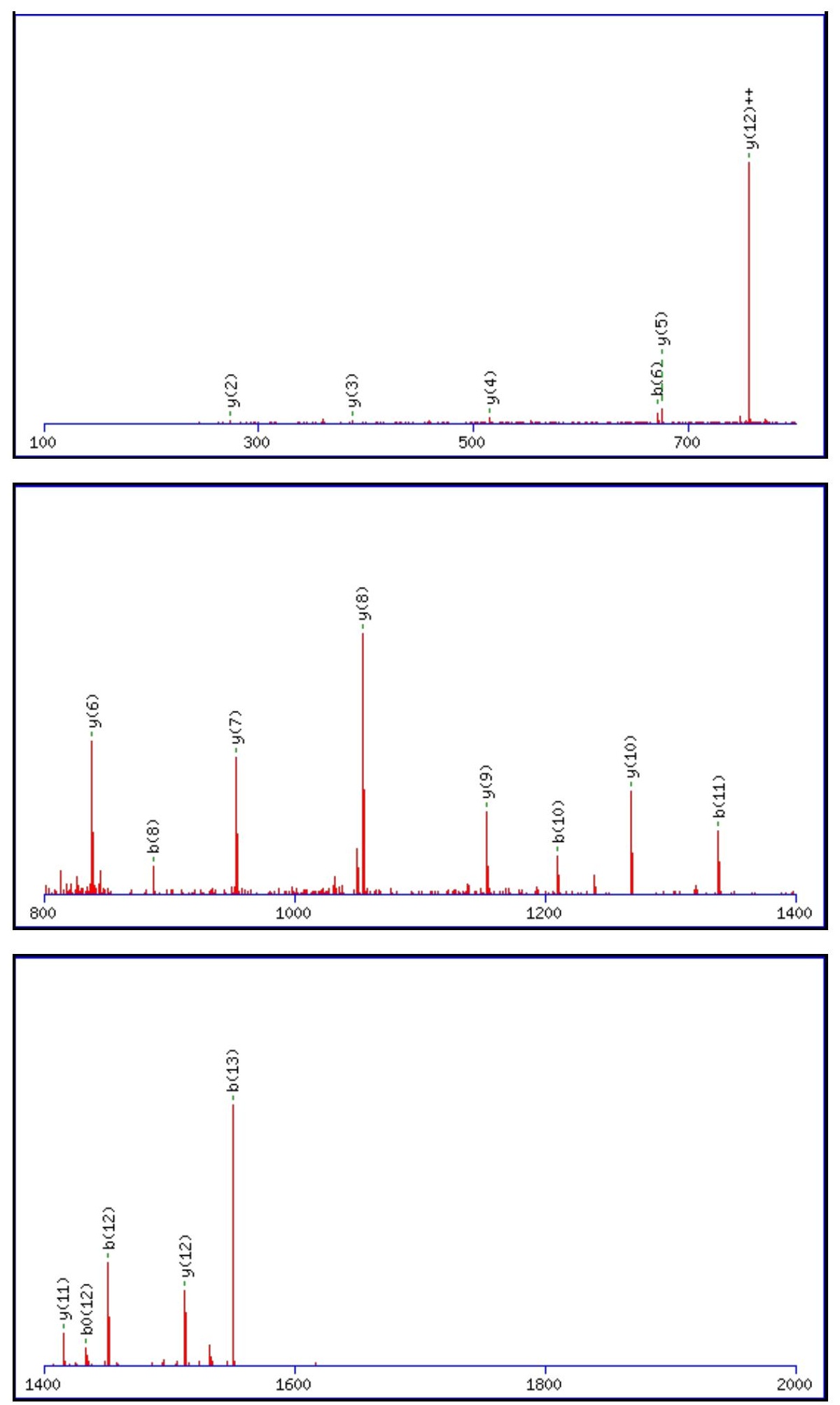

Monoisotopic mass of neutral peptide $\operatorname{Mr}($ calc): 1723.8604

Fixed modifications: Carbamidomethyl (C)

Variable modifications:

N5 : Deamidated $\mathrm{N}(\mathrm{N})$

Ions Score: 89 Expect: $2.1 \mathrm{e}-007$

Matches (Bold Red): 19/124 fragment ions using 30 most intense peaks

\begin{tabular}{|r|c|c|c|c|c|c|c|c|c|c|c|c|c|c|}
\hline$\#$ & $\mathbf{b}$ & $\mathbf{b}^{++}$ & $\mathbf{b}^{*}$ & $\mathbf{b}^{\boldsymbol{*}^{++}}$ & $\mathbf{b}^{\mathbf{0}}$ & $\mathbf{b}^{\mathbf{0 + +}}$ & Seq. & $\mathbf{y}$ & $\mathbf{y}^{++}$ & $\mathbf{y}^{\mathbf{*}}$ & $\mathbf{y}^{\boldsymbol{*}^{++}}$ & $\mathbf{y}^{\mathbf{0}}$ & $\mathbf{y}^{\mathbf{0 + +}}$ & $\#$ \\
\hline $\mathbf{1}$ & 100.0757 & 50.5415 & & & & & $\mathbf{V}$ & & & & & & & $\mathbf{1 4}$ \\
\hline $\mathbf{2}$ & 213.1598 & 107.0835 & & & & & $\mathbf{L}$ & 1625.7992 & 813.4032 & 1608.7727 & 804.8900 & 1607.7886 & 804.3980 & $\mathbf{1 3}$ \\
\hline 3 & 310.2125 & 155.6099 & & & & & $\mathbf{P}$ & $\mathbf{1 5 1 2 . 7 1 5 1}$ & 756.8612 & 1495.6886 & 748.3479 & 1494.7046 & $\mathbf{7 4 7 . 8 5 5 9}$ & $\mathbf{1 2}$ \\
\hline $\mathbf{4}$ & 457.2809 & 229.1441 & & & & & $\mathbf{F}$ & $\mathbf{1 4 1 5 . 6 6 2 4}$ & 708.3348 & 1398.6358 & 699.8215 & 1397.6518 & 699.3295 & $\mathbf{1 1}$ \\
\hline $\mathbf{5}$ & 572.3079 & 286.6576 & 555.2813 & 278.1443 & & & $\mathbf{N}$ & $\mathbf{1 2 6 8 . 5 9 4 0}$ & 634.8006 & 1251.5674 & 626.2873 & 1250.5834 & 625.7953 & $\mathbf{1 0}$ \\
\hline
\end{tabular}




\begin{tabular}{|r|r|r|r|r|r|r|r|r|r|r|r|r|r|r|r|r|r|r|}
$\mathbf{6}$ & $\mathbf{6 7 1 . 3 7 6 3}$ & 336.1918 & 654.3497 & 327.6785 & & & $\mathbf{V}$ & $\mathbf{1 1 5 3 . 5 6 7 0}$ & 577.2871 & 1136.5405 & 568.7739 & 1135.5565 & 568.2819 & $\mathbf{9}$ \\
\hline $\mathbf{7}$ & 772.4240 & 386.7156 & 755.3974 & 378.2023 & 754.4134 & 377.7103 & T & $\mathbf{1 0 5 4 . 4 9 8 6}$ & 527.7529 & 1037.4721 & 519.2397 & 1036.4880 & 518.7477 & $\mathbf{8}$ \\
\hline $\mathbf{8}$ & $\mathbf{8 8 7 . 4 5 0 9}$ & 444.2291 & 870.4244 & 435.7158 & 869.4403 & 435.2238 & D & $\mathbf{9 5 3 . 4 5 0 9}$ & 477.2291 & 936.4244 & 468.7158 & 935.4404 & 468.2238 & $\mathbf{7}$ \\
\hline $\mathbf{9}$ & 1050.5142 & 525.7608 & 1033.4877 & 517.2475 & 1032.5037 & 516.7555 & $\mathbf{Y}$ & $\mathbf{8 3 8 . 4 2 4 0}$ & 419.7156 & 821.3974 & 411.2024 & & & \\
\hline $\mathbf{1 0}$ & $\mathbf{1 2 1 0 . 5 4 4 9}$ & 605.7761 & 1193.5183 & 597.2628 & 1192.5343 & 596.7708 & $\mathbf{C}$ & $\mathbf{6 7 5 . 3 6 0 7}$ & 338.1840 & 658.3341 & 329.6707 & & & & $\mathbf{5}$ \\
\hline $\mathbf{1 1}$ & $\mathbf{1 3 3 8 . 6 0 3 5}$ & 669.8054 & 1321.5769 & 661.2921 & 1320.5929 & 660.8001 & $\mathbf{Q}$ & $\mathbf{5 1 5 . 3 3 0 0}$ & 258.1686 & 498.3035 & 249.6554 & & & & $\mathbf{4}$ \\
\hline $\mathbf{1 2}$ & $\mathbf{1 4 5 1 . 6 8 7 5}$ & 726.3474 & 1434.6610 & 717.8341 & $\mathbf{1 4 3 3 . 6 7 7 0}$ & 717.3421 & $\mathbf{L}$ & $\mathbf{3 8 7 . 2 7 1 4}$ & 194.1394 & 370.2449 & 185.6261 & & & & $\mathbf{3}$ \\
\hline $\mathbf{1 3}$ & $\mathbf{1 5 5 0 . 7 5 5 9}$ & 775.8816 & 1533.7294 & 767.3683 & 1532.7454 & 766.8763 & $\mathbf{V}$ & $\mathbf{2 7 4 . 1 8 7 4}$ & 137.5973 & 257.1608 & 129.0840 & & & $\mathbf{2}$ \\
\hline $\mathbf{1 4}$ & & & & & & & $\mathbf{R}$ & 175.1190 & 88.0631 & 158.0924 & 79.5498 & & & $\mathbf{1}$ \\
\hline
\end{tabular}
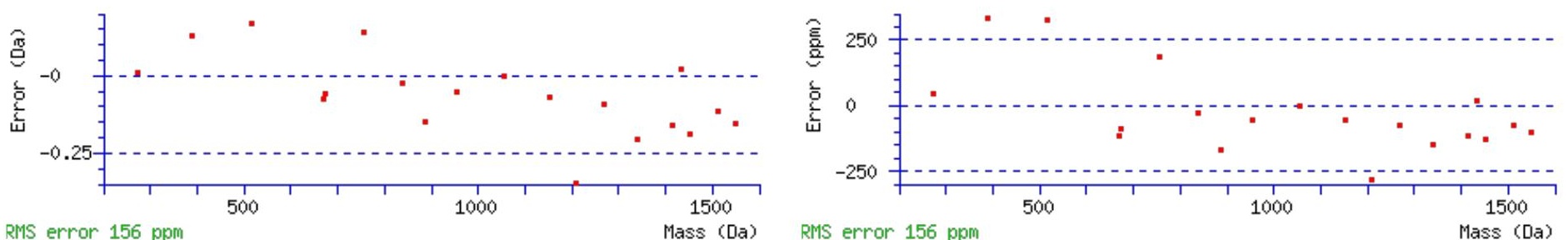

RMS error $156 \mathrm{ppm}$

RMS error 156 ppm

\section{All matches to this query}

\begin{tabular}{|l|l|l|l|}
\hline Score & Mr(calc): & Delta & \multicolumn{1}{|c|}{ Sequence } \\
\hline 89.3 & 1723.8604 & -0.0036 & VLPFNVTDYCQLVR \\
\hline 13.3 & 1723.8645 & -0.0077 & VISQTNLITTVTPEK \\
\hline 8.5 & 1723.8463 & 0.0105 & IPKASAKFYATIVR \\
\hline 8.4 & 1723.8514 & 0.0053 & LCLSPLTNLCGKLR \\
\hline 8.2 & 1723.8514 & 0.0053 & LCLSPLTNLCGKLR \\
\hline 5.7 & 1723.8645 & -0.0077 & VISQTNLITTVTPEK \\
\hline 5.0 & 1722.8665 & 0.9902 & LVAGELRLSKEGTDR \\
\hline 3.6 & 1722.8665 & 0.9902 & GAGTLDGTLIRNIVSR \\
\hline 3.5 & 1723.8553 & 0.0015 & MRKPDGSTVTQRIR \\
\hline 3.5 & 1723.8553 & 0.0015 & MRKPDGSTVTQRIR \\
\hline
\end{tabular}

Spectrum No: 141; Query: 469; Rank: 1

\section{Peptide View}

MS/MS Fragmentation of SAVFTVENGSTIR

Found in IPI00214373, Tax_Id=10116 Gene_Symbol=Aadat Kynurenine/alpha-aminoadipate aminotransferase mitochondrial precursor

Match to Query 469: 1380.694828 from(691.354690,2+)

Title: 091008RatKidney_NoSalt_08.1562.1562.2.dta

Data file K:INewmanPaper|Piliang|3SubProteomes\Piliang3SP\mgf5ppm\ERLIC_3SubProteomes5ppm.mgf 

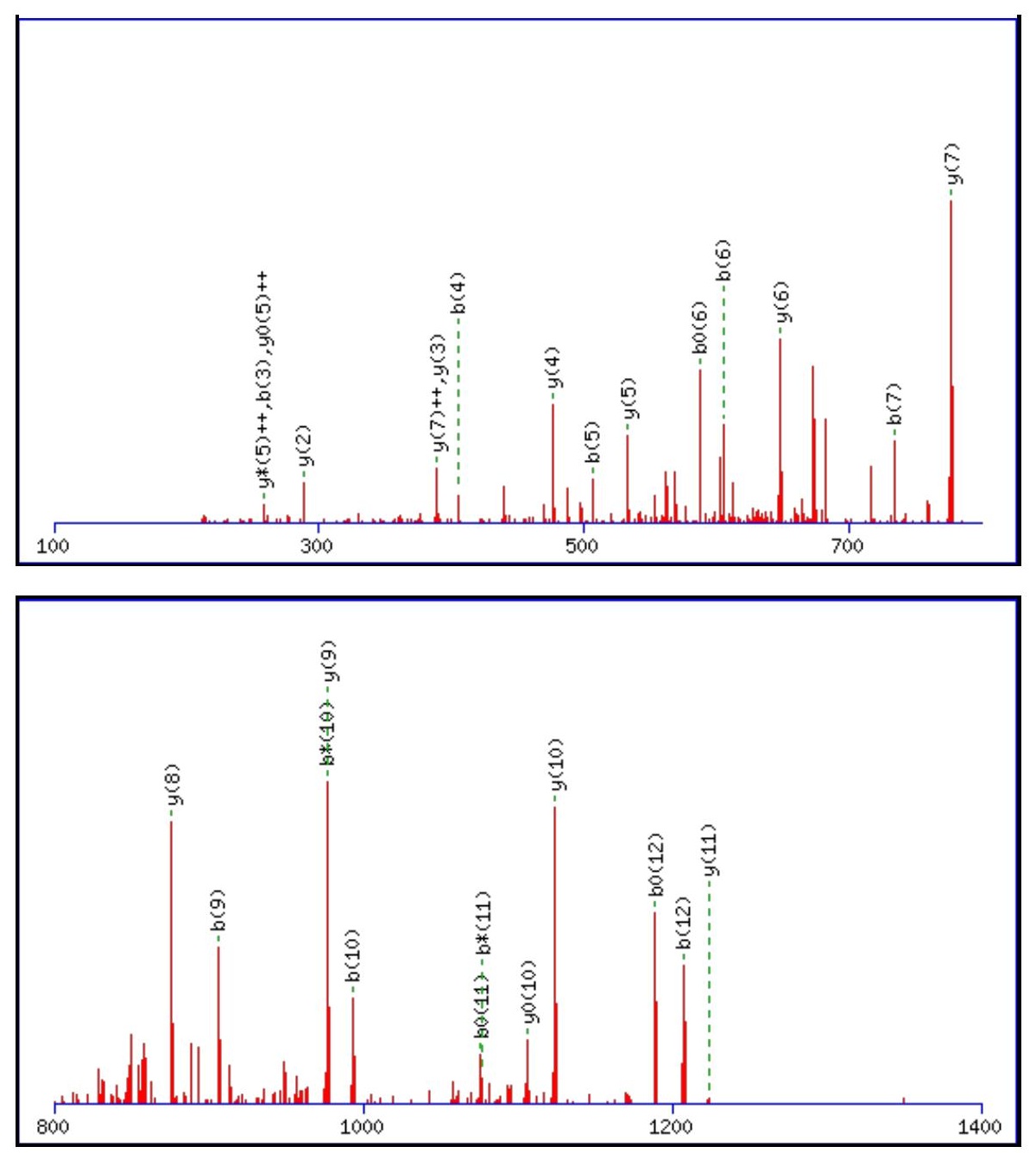

\begin{tabular}{rrrr}
\hline 00 & 1600 & 1800 & 2000 \\
\hline
\end{tabular}

Monoisotopic mass of neutral peptide $\operatorname{Mr}($ calc): 1380.6885

Fixed modifications: Carbamidomethyl (C)

Variable modifications:

N8 : Deamidated $\mathrm{N}(\mathrm{N})$

Ions Score: 89 Expect: $1.7 \mathrm{e}-007$

Matches (Bold Red): 27/126 fragment ions using 35 most intense peaks

\begin{tabular}{|c|c|c|c|c|c|c|c|c|c|c|c|c|c|c|}
\hline \# & b & $\mathbf{b}^{++}$ & $\mathbf{b}^{*}$ & $\mathbf{b}^{*^{++}}$ & $\mathbf{b}^{0}$ & $\mathbf{b}^{\mathbf{0 + +}}$ & Seq. & $\mathbf{y}$ & $\mathbf{y}^{++}$ & $\mathrm{y}^{*}$ & $\mathrm{y}^{*^{++}}$ & $\mathbf{y}^{0}$ & $y^{0++}$ & $\#$ \\
\hline 1 & 88.0393 & 44.5233 & & & 70.0287 & 35.5180 & $\mathbf{S}$ & & & & & & & 13 \\
\hline 2 & 159.0764 & 80.0418 & & & 141.0659 & 71.0366 & A & 1294.6638 & 647.8355 & 1277.6372 & 639.3222 & 1276.6532 & 638.8302 & 12 \\
\hline 3 & 258.1448 & 129.5761 & & & 240.1343 & 120.5708 & $\mathbf{V}$ & 1223.6266 & 612.3170 & 1206.6001 & 603.8037 & 1205.6161 & 603.3117 & 11 \\
\hline 4 & 405.2132 & 203.1103 & & & 387.2027 & 194.1050 & $\mathbf{F}$ & 1124.5582 & 562.7827 & 1107.5317 & 554.2695 & 1106.5477 & 553.7775 & 10 \\
\hline 5 & 506.2609 & 253.6341 & & & 488.2504 & 244.6288 & $\mathbf{T}$ & 977.4898 & 489.2485 & 960.4633 & 480.7353 & 959.4792 & 480.2433 & 9 \\
\hline
\end{tabular}




\begin{tabular}{|c|c|c|c|c|c|c|c|c|c|c|c|c|c|c|}
\hline 6 & 605.3293 & |303.1683 & & & 587.3188 & |294.1630 & $\mathbf{V}$ & 876.4421 & |438.7247 & 859.4156 & |430.2114 & 858.4316 & |429.7194| & 8 \\
\hline 7 & 734.3719 & 367.6896 & & & 716.3614 & 358.6843 & $\mathbf{E}$ & 777.3737 & 389.1905 & 760.3472 & 380.6772 & 759.3632 & 380.1852 & 7 \\
\hline 8 & 849.3989 & 425.2031 & 832.3723 & 416.6898 & 831.3883 & 416.1978 & $\mathbf{N}$ & 648.3311 & 324.6692 & 631.3046 & 316.1559 & 630.3206 & 315.6639 & 6 \\
\hline 9 & 906.4203 & 453.7138 & 889.3938 & 445.2005 & 888.4098 & 444.7085 & $\mathbf{G}$ & 533.3042 & 267.1557 & 516.2776 & 258.6425 & 515.2936 & 258.1504 & 5 \\
\hline 10 & 993.4524 & 497.2298 & 976.4258 & 488.7165 & 975.4418 & 488.2245 & S & 476.2827 & 238.6450 & 459.2562 & 230.1317 & 458.2722 & 229.6397 & 4 \\
\hline 11 & 1094.5000 & 547.7537 & 1077.4735 & 539.2404 & 1076.4895 & 538.7484 & $\mathbf{T}$ & 389.2507 & 195.1290 & 372.2241 & 186.6157 & 371.2401 & 186.1237 & 3 \\
\hline 12 & 1207.5841 & 604.2957 & 1190.5576 & 595.7824 & 1189.5735 & 595.2904 & I & 288.2030 & 144.6051 & 271.1765 & 136.0919 & & & 2 \\
\hline 13 & & & & & & & $\mathbf{R}$ & 175.1190 & 88.0631 & 158.0924 & 79.5498 & & & 1 \\
\hline
\end{tabular}
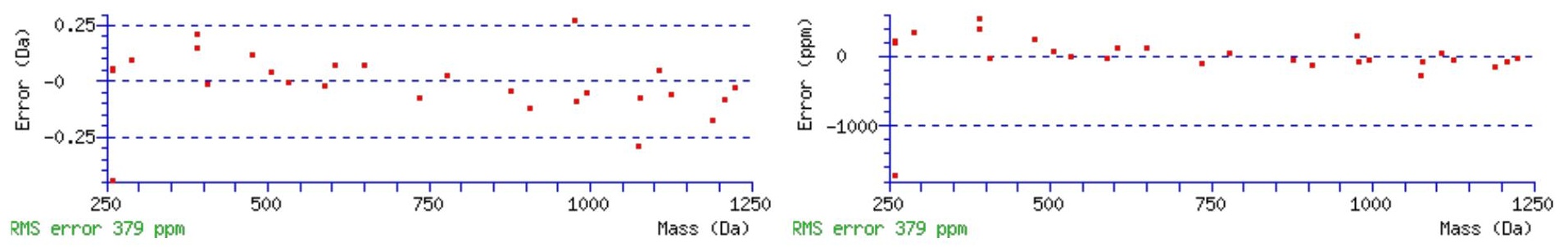

\section{All matches to this query}

\begin{tabular}{|l|l|c|l|}
\hline Score & Mr(calc): & Delta & \multicolumn{1}{c|}{ Sequence } \\
\hline 89.3 & 1380.6885 & 0.0063 & SAVFTVENGSTIR \\
\hline 39.7 & 1379.7045 & 0.9903 & SAVFTVENGSTIR \\
\hline 12.2 & 1380.6820 & 0.0129 & DNSFRICVLTR \\
\hline 10.8 & 1380.6997 & -0.0049 & EKFQSRTSEAAK \\
\hline 10.2 & 1380.7071 & -0.0123 & MESKEEFVKVR \\
\hline 9.0 & 1380.6867 & 0.0082 & QERHMIMHKR \\
\hline 8.7 & 1380.6820 & 0.0129 & RTFKEGMDELR \\
\hline 8.5 & 1380.6915 & 0.0034 & TGRLPEAAFLAR \\
\hline 8.1 & 1380.6845 & 0.0103 & SSVDSKRSSVDSK \\
\hline 7.4 & 1380.6932 & 0.0016 & $\underline{\text { MLNTGLHNPNVR }}$ \\
\hline
\end{tabular}

Spectrum No: 142; Query: 675; Rank: 1

\section{Peptide View}

MS/MS Fragmentation of TVANLSGCEQVDSK

Found in IPI00557299, Tax_Id=10116 Gene_Symbol=LOC498940 Similar to Carboxylesterase 2

Match to Query 675: 1507.684948 from(754.849750,2+)

Title: 100101RatKid_NS_deglyco_24.1123.1123.2.dta

Data file K:INewmanPaper|Piliang|3SubProteomes\Piliang3SP\mgf5ppm\ERLIC_3SubProteomes5ppm.mgf 

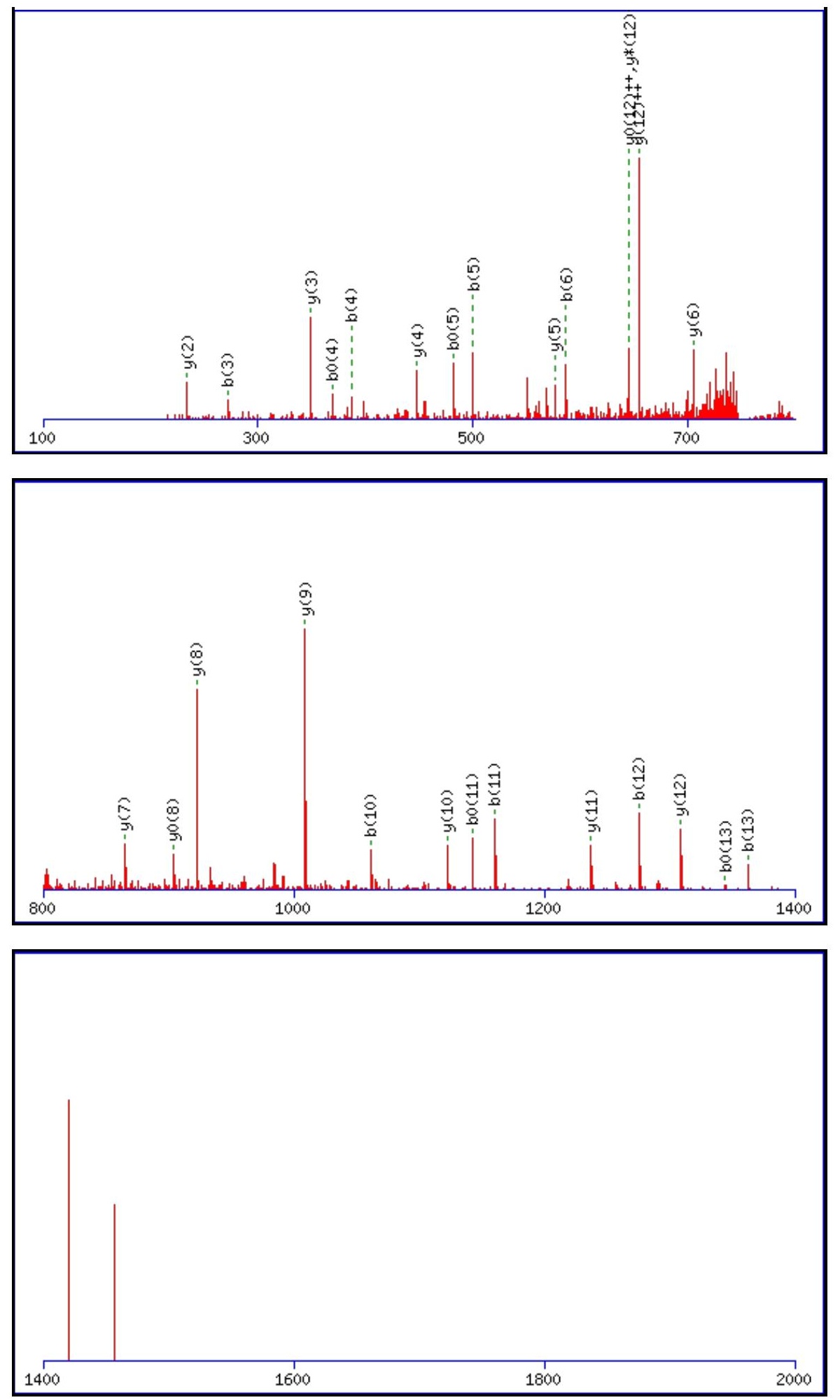

Monoisotopic mass of neutral peptide $\operatorname{Mr}($ calc): 1507.6824

Fixed modifications: Carbamidomethyl (C)

Variable modifications:

N4: Deamidated $\mathrm{N}(\mathrm{N})$

Ions Score: 89 Expect: $2 \mathrm{e}-007$

Matches (Bold Red): 27/148 fragment ions using 41 most intense peaks

\begin{tabular}{|c|c|c|c|c|c|c|c|c|c|c|c|c|c|c|}
\hline \# & b & $\mathbf{b}^{++}$ & $\mathbf{b}^{*}$ & $\mathbf{b}^{*^{++}}$ & $\mathbf{b}^{0}$ & $\mathbf{b}^{\mathbf{0 + +}}$ & Seq. & $\mathbf{y}$ & $\mathbf{y}^{++}$ & $\mathrm{y}^{*}$ & $\mathrm{y}^{*^{++}}$ & $\mathbf{y}^{\mathbf{0}}$ & $y^{0++}$ & $\#$ \\
\hline 1 & 102.0550 & 51.5311 & & & 84.0444 & 42.5258 & $\mathbf{T}$ & & & & & & & 14 \\
\hline 2 & 201.1234 & 101.0653 & & & 183.1128 & 92.0600 & V & 1407.6420 & 704.3247 & 1390.6155 & 695.8114 & 1389.6315 & 695.3194 & 13 \\
\hline 3 & 272.1605 & 136.5839 & & & 254.1499 & 127.5786 & A & 1308.5736 & 654.7904 & 1291.5471 & 646.2772 & 1290.5631 & 645.7852 & 12 \\
\hline 4 & 387.1874 & 194.0973 & 370.1609 & 185.5841 & 369.1769 & 185.0921 & $\mathbf{N}$ & 1237.5365 & 619.2719 & | 1220.5100 & 610.7586 & 1219.5259 & 610.2666 & 11 \\
\hline 5 & 500.2715 & 250.6394 & 483.2449 & 242.1261 & 482.2609 & 241.6341 & $\mathbf{L}$ & 1122.5096 & 561.7584 & 1105.4830 & 553.2451 & 1104.4990 & 552.7531 & 10 \\
\hline
\end{tabular}




\begin{tabular}{|c|c|c|c|c|c|c|c|c|c|c|c|c|c|c|}
\hline 6 & 587.3035 & |294.1554| & 570.2770 & |285.6421 & 569.2929 & |285.1501 & $S$ & |1009.4255| & |505.2164| & 992.3990 & |496.7031 & 991.4149 & 2111 & 9 \\
\hline 7 & 644.3250 & 322.6661 & 627.2984 & 314.1529 & 626.3144 & 313.6608 & G & 922.3935 & 461.7004 & 905.3669 & 453.1871 & 904.3829 & 452.6951 & 8 \\
\hline 8 & 804.3556 & 402.6815 & 787.3291 & 394.1682 & 786.3451 & 393.6762 & C & 865.3720 & 433.1896 & 848.3455 & 424.6764 & 847.3614 & 424.1844 & 7 \\
\hline 9 & 933.3982 & 467.2027 & 916.3717 & 458.6895 & 915.3877 & 458.1975 & $\mathbf{E}$ & 705.3414 & 353.1743 & 688.3148 & 344.6610 & 687.3308 & 344.1690 & 6 \\
\hline 10 & 1061.4568 & 531.2320 & 1044.4302 & 522.7188 & 1043.4462 & 522.2268 & $\mathbf{Q}$ & 576.2988 & 288.6530 & 559.2722 & 280.1397 & 558.2882 & 279.6477 & 5 \\
\hline 11 & 1160.5252 & 580.7662 & 1143.4987 & 572.2530 & 1142.5146 & 571.7610 & V & 448.2402 & 224.6237 & 431.2136 & 216.1105 & 430.2296 & 215.6185 & 4 \\
\hline 12 & 1275.5522 & 638.2797 & 1258.5256 & 629.7664 & 1257.5416 & 629.2744 & D & 349.1718 & 175.0895 & 332.1452 & 166.5763 & 331.1612 & 166.0842 & 3 \\
\hline 13 & 1362.5842 & 681.7957 & 1345.5576 & 673.2825 & 1344.5736 & 672.7904 & S & 234.1448 & 117.5761 & 217.1183 & 109.0628 & 216.1343 & 108.5708 & 2 \\
\hline 14 & & & & & & & $\mathbf{K}$ & 147.1128 & 74.0600 & 130.0863 & 65.5468 & & & 1 \\
\hline
\end{tabular}
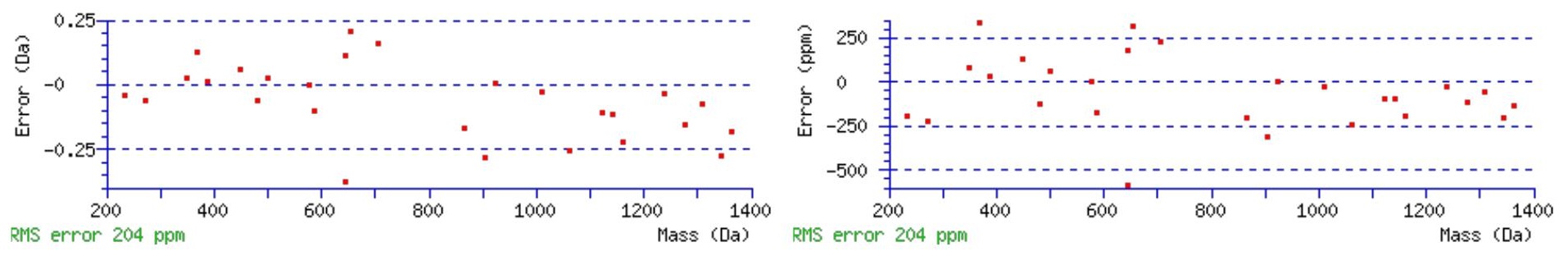

\section{All matches to this query}

\begin{tabular}{|l|l|l|l|}
\hline Score & Mr(calc): & Delta & \multicolumn{1}{|c|}{ Sequence } \\
\hline 89.2 & 1507.6824 & 0.0025 & TVANLSGCEQVDSK \\
\hline 6.5 & 1507.6782 & 0.0067 & CVLPYTQFSVSK \\
\hline 6.0 & 1507.6960 & -0.0110 & GGSFPLSIVSSYSK \\
\hline 6.0 & 1507.6960 & -0.0110 & GGSFPLSIVSSYSK \\
\hline 6.0 & 1507.6960 & -0.0110 & GGSFPLSIVSSYSK \\
\hline 5.1 & 1507.6960 & -0.0110 & GGSFPLSIVSSYSK \\
\hline 4.6 & 1507.6937 & -0.0087 & VCASDTRAGIEDSK \\
\hline 2.3 & 1506.6666 & 1.0183 & GAAVSLNIRTMSK \\
\hline 2.0 & 1507.6919 & -0.0070 & VNTASILGHSSTQL \\
\hline 0.4 & 1507.6807 & 0.0043 & AEESLPLPAAESSK \\
\hline
\end{tabular}

Spectrum No: 143; Query: 2794; Rank: 1

\section{Peptide View}

MS/MS Fragmentation of VSGILFNDEMDDFSSPNFTNQFGVAPSPANFIKPGK

Found in IPI00206254, Tax_Id=10116 Gene_Symbol=Ggt1 Gamma-glutamyltranspeptidase 1 precursor

Match to Query 2794: 3903.826362 from(1302.282730,3+)

Title: 100101RatKid_NS_deglyco_19.5285.5285.3.dta

Data file K:\NewmanPaper|Piliang|3SubProteomes\Piliang3SP\mgf5ppm\ERLIC_3SubProteomes5ppm.mgf 

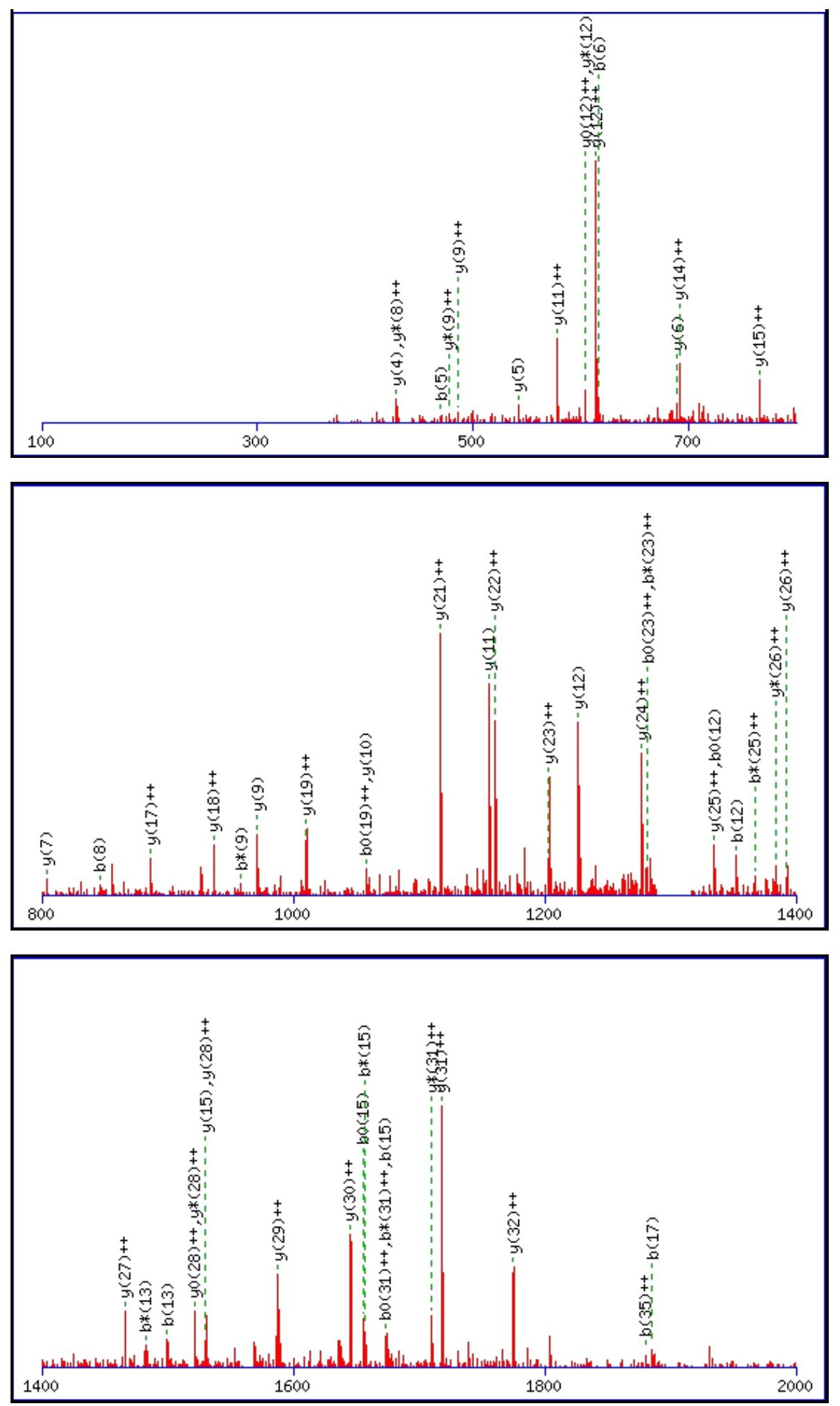

Monoisotopic mass of neutral peptide $\operatorname{Mr}($ calc): 3903.8302

Fixed modifications: Carbamidomethyl (C)

Variable modifications:

M10 : Oxidation $(M)$

N17 : Deamidated_N (N)

Ions Score: 89 Expect: $5.5 \mathrm{e}-007$

Matches (Bold Red): 56/388 fragment ions using 110 most intense peaks

\begin{tabular}{|c|c|c|c|c|c|c|c|c|c|c|c|c|c|c|}
\hline$\#$ & b & $\mathbf{b}^{++}$ & $\mathbf{b}^{*}$ & $\mathbf{b}^{*^{++}}$ & $\mathbf{b}^{0}$ & $\mathbf{b}^{0++}$ & Seq. & $\mathbf{y}$ & $y^{++}$ & $\mathbf{y}^{*}$ & $y^{*^{++}}$ & $\mathbf{y}^{\mathbf{0}}$ & $y^{0++}$ & \# \\
\hline 1 & 100.0757 & 50.5415 & & & & & V & & & & & & & 36 \\
\hline 2 & 187.1077 & 94.0575 & & & 169.0972 & 85.0522 & S & 3805.7690 & 1903.3882 & 3788.7425 & 1894.8749 & |3787.7585 & 1894.3829 & 35 \\
\hline 3 & 244.1292 & 122.5682 & & & 226.1186 & 113.5629 & G & 3718.7370 & 1859.8721 & 3701.7105 & 1851.3589 & 3700.7264 & 1850.8669 & 34 \\
\hline 4 & 357.2132 & 179.1103 & & & 339.2027 & 170.1050 & I & 3661.7155 & 1831.3614 & 3644.6890 & 1822.8481 & 3643.7050 & 1822.3561 & 33 \\
\hline
\end{tabular}




\begin{tabular}{|c|c|c|c|c|c|c|c|c|c|c|c|c|c|c|}
\hline & 470.2973 & 235.6523 & & & 2867 & & $\mathbf{L}$ & & 194 & & & 3530 & |1765.8141 & 32 \\
\hline 6 & 617.3657 & 309.1865 & & & 599.3552 & 300.1812 & $\mathbf{F}$ & 3435.5474 & 1718.2773 & 3418.5209 & 1709.7641 & 3417.5368 & 1709.2721 & 31 \\
\hline 7 & 731.4087 & 366.2080 & 714.3821 & 357.6947 & 713.3981 & 357.2027 & $\mathbf{N}$ & 3288.4790 & 1644.7431 & 3271.4524 & 1636.2299 & 3270.4684 & 7379 & \\
\hline 8 & 846.4356 & 423.7214 & 829.4090 & 415.2082 & 828.4250 & 414.7162 & D & 3174.4361 & 1587.7217 & 3157.4095 & 1579.2084 & 3156.4255 & 7164 & \\
\hline 9 & 975.4782 & 488.2427 & 958.4516 & 479.7295 & 957.4676 & 479.2374 & $\mathbf{E}$ & 3059.4091 & 1530.2082 & 3042.3826 & 1521.6949 & 3041.3986 & 1521.2029 & \\
\hline 10 & 1122.5136 & 561.7604 & 1105.4870 & 553.2472 & 1104.5030 & 552.7552 & $\mathbf{M}$ & 2930.3665 & 1465.6869 & 2913.3400 & 1457.1736 & 2912.3560 & 1456.6816 & \\
\hline 11 & 1237.5405 & 619.2739 & 1220.5140 & 610.7606 & 1219.5300 & 610.2686 & D & 2783.3311 & 1392.1692 & 2766.3046 & 1383.6559 & 2765.3206 & 1639 & \\
\hline 12 & 1352.5675 & 676.7874 & 1335.5409 & 668.2741 & 1334.5569 & 667.7821 & D & 2668.3042 & 1334.6557 & 2651.2776 & 1326.1425 & 2650.2936 & 1325.6504 & \\
\hline 13 & 1499.6359 & 750.3216 & 1482.6093 & 741.8083 & 1481.6253 & 741.3163 & $\mathbf{F}$ & 2553.2772 & 1277.1423 & 2536.2507 & 1268.6290 & 2535.2667 & 1268.1370 & \\
\hline 14 & 1586.6679 & 793.8376 & 569.6414 & 785.3243 & 1568.6574 & 784.8323 & S & 2406.2088 & & 2389.1823 & 1195.0948 & 1983 & 6028 & \\
\hline 15 & 1673.6999 & 837.3536 & 1656.6734 & 828.8403 & 1655.6894 & 828.3483 & $\mathrm{~S}$ & 2319.1768 & 1160.0920 & 2302.1503 & 1151.5788 & 2301.1662 & 1151.0868 & \\
\hline 16 & 1770.7527 & 885.8800 & 1753.7262 & 877.3667 & 1752.7421 & 876.8747 & $\mathbf{P}$ & 2232.1448 & 1116.5760 & 2215.1182 & 1108.0628 & 2214.1342 & 1107.5707 & \\
\hline 17 & 1885.7796 & 943.3 & 1868.7 & 934 & 1867.7691 & 934. & $\mathbf{N}$ & 2135 & & 0655 & & 814 & 444 & \\
\hline 182 & 2032.8481 & 1016.9277 & 2015.8215 & 1008.4144 & 2014.8375 & 1007.9224 & $\mathbf{F}$ & 2020.0651 & 1010.5362 & 2003.0385 & 1002.0229 & 2002.0545 & 1001.5309 & 1 \\
\hline 19 & 2133.8957 & 1067.4515 & 2116.8692 & 1058.9382 & 2115.8852 & 1058.4462 & $\mathbf{T}$ & 1872.9967 & 937.0020 & 1855.9701 & 928.4887 & 1854.9861 & 927.9967 & \\
\hline 20 & 2247.9387 & 24.4730 & 2230. & & 2229.9281 & 1115.4677 & $\mathbf{N}$ & 1771 & & 175 & & 175 & 1728 & \\
\hline 212 & 2375.9972 & 1188.5023 & 2358.9707 & 1179.9890 & 2357.9867 & 1179.4970 & $\bar{Q}$ & 1657.9061 & 829.4567 & 1640.8795 & 820.9434 & 1639.8955 & 820.4514 & 6 \\
\hline 222 & 2523.0657 & 1262.0365 & 2506.0391 & 1253.5232 & 2505.0551 & 1253.0312 & $\mathbf{F}$ & 1529.8475 & 765.4274 & 1512.8209 & 756.9141 & 1511.8369 & 756.4221 & 15 \\
\hline 232 & 2580. & 90.5472 & 2563. & 339 & 2562.0766 & 1281.5419 & $\mathbf{G}$ & 1382.7791 & 691.8932 & 1365.7525 & 683.3799 & 1364.7685 & 682.8879 & \\
\hline 242 & 2679.1555 & 1340.0814 & 2662.1290 & 1331.5681 & 2661.1450 & 1331.0761 & $\mathrm{~V}$ & 1325.7576 & 663.3824 & 1308.7310 & 654.8692 & 1307.7470 & 654.3772 & 13 \\
\hline 25 & 2750.1927 & 1375.6000 & 2733.1661 & 1367.0867 & 2732.1821 & 1366.5947 & A & 1226.6892 & 613.8482 & 1209.6626 & 605.3350 & 1208.6786 & 604.8429 & 2 \\
\hline 26 & 2847.2454 & 1424.1263 & 2830.2189 & 1415.6131 & 2829.2348 & 1415.1211 & $\mathbf{P}$ & 1155.6521 & 578.3297 & 1138.6255 & 569.8164 & 1137.6415 & 569.3244 & \\
\hline 272 & 2934.2774 & 1467.6424 & 2917.2509 & 1459.1291 & 2916.2669 & 1458.6371 & $\mathrm{~S}$ & 1058.5993 & 529.8033 & 1041.5728 & 521.2900 & .5887 & 520.7980 & 10 \\
\hline 283 & 3031.3302 & 1516.1687 & 3014.3037 & 1507.6555 & 3013.3196 & 1507.1635 & $\mathbf{P}$ & 971.5673 & 486.2873 & 954.5407 & 477.7740 & & & \\
\hline 293 & 3102.3673 & 1551.6873 & 3085.3408 & 1543.1740 & 3084.3568 & 1542.6820 & A & 874.5145 & 437.7609 & 857.4880 & 429.2476 & & & \\
\hline 303 & 3216.4102 & 1608.7088 & 3199.3837 & 1600.1955 & 3198.3997 & 1599.7035 & $\mathbf{N}$ & 803.4774 & 402.2423 & 786.4509 & 393.7291 & & & 7 \\
\hline 313 & 3363.4787 & 1682.2430 & 3346.4521 & 1673.7297 & 3345.4681 & 1673.2377 & $\mathbf{F}$ & 689.4345 & 345.2209 & 672.4079 & 336.7076 & & & 6 \\
\hline 323 & 3476.5627 & 1738.7850 & 3459.5362 & 1730.2717 & 3458.5522 & 1729.7797 & I & 542.3661 & 271.6867 & 525.3395 & 263.1734 & & & 0 \\
\hline 33 & 3604.6577 & 1802.8325 & 3587.6311 & 1794.3192 & 3586.6471 & 1793.8272 & K & 429.2820 & 215.1446 & 412.2554 & 206.6314 & & & 4 \\
\hline 343 & 3701.7105 & 1851.3589 & 3684.6839 & 1842.8456 & 3683.6999 & 1842.3536 & $\mathbf{P}$ & 301.1870 & 151.0972 & 284.1605 & 142.5839 & & & 3 \\
\hline 353 & 3758.7319 & 1879.8696 & 3741.7054 & 1871.3563 & 3740.7214 & 1870.8643 & G & 204.1343 & 102.5708 & 187.1077 & 94.0575 & & & 2 \\
\hline 36 & & & & & & & $\mathbf{K}$ & 147.1128 & 74.0600 & 130.0863 & 65.5468 & & & \\
\hline
\end{tabular}
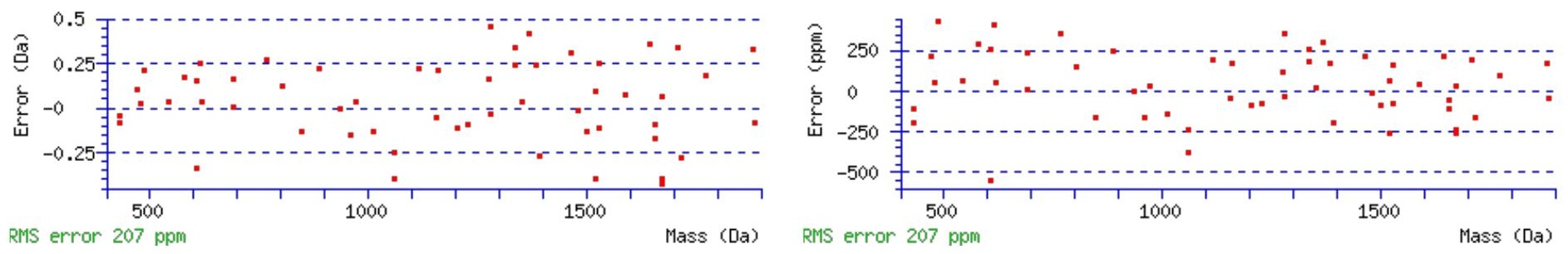

\section{All matches to this query}

\begin{tabular}{|l|l|l|l|}
\hline Score & Mr(calc): & Delta & \multicolumn{1}{|c|}{ Sequence } \\
\hline 89.1 & 3903.8302 & -0.0038 & VSGILFNDEMDDFSSPNFTNQFGVAPSPANFIKPGK \\
\hline 88.9 & 3903.8302 & -0.0038 & VSGILFNDEMDDFSSPNFTNQFGVAPSPANFIKPGK \\
\hline 87.7 & 3903.8302 & -0.0038 & VSGILFNDEMDDFSSPNFTNQFGVAPSPANFIKPGK \\
\hline 39.0 & 3903.8302 & -0.0038 & VSGILFNDEMDDFSSPNFTNQFGVAPSPANFIKPGK \\
\hline 30.4 & 3902.8462 & 0.9802 & VSGILFNDEMDDFSSPNFTNQFGVAPSPANFIKPGK \\
\hline 7.3 & 3901.7841 & 2.0422 & ELQNEVEKQMNMSPPPGNAGPVIMSLEEKMEADAR \\
\hline 6.5 & 3903.8148 & 0.0116 & IALYIGNLTWWTTDEDLTEAVHSLGVNDILEIK \\
\hline 5.5 & 3903.8424 & -0.0160 & QLLDMGGIYDTERNVWKSIQDLSLIAACTPSAGGK \\
\hline
\end{tabular}




\begin{tabular}{|l|l|l|l|l|}
5.5 & 3903.8424 & -0.0160 & QLLDMGGIYDTERNVWKSIODLSLIAACTPSAGGK \\
\hline 4.8 & 3901.7849 & 2.0415 & MDPRTPYRPPAAGVGAVPAAAAGSVGAAASLGNATRGTR \\
\hline
\end{tabular}

Spectrum No: 144; Query: 1392; Rank: 1

\section{Peptide View}

MS/MS Fragmentation of KQEEFDIANNGSSQANK

Found in IPI00213579, Tax_Id=10116 Gene_Symbol=Tpp2 Tripeptidyl-peptidase 2

Match to Query 1392: 1879.856128 from(940.935340,2+)

Title: 091008RatKidney_NH4Format01_19.893.893.2.dta

Data file K:INewmanPaper|Piliang|3SubProteomes\Piliang3SP\mgf5ppm\ERLIC_3SubProteomes5ppm.mgf
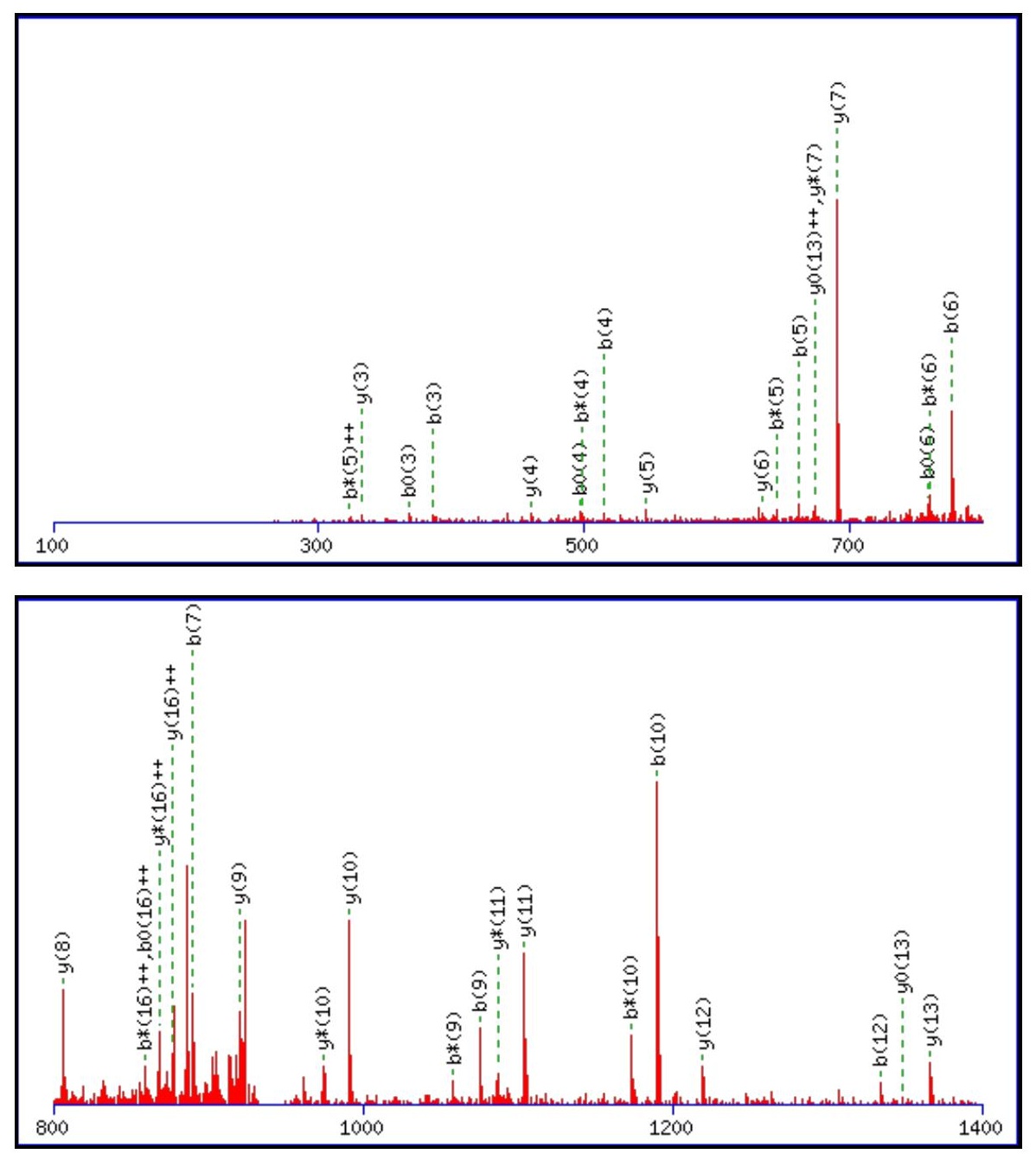


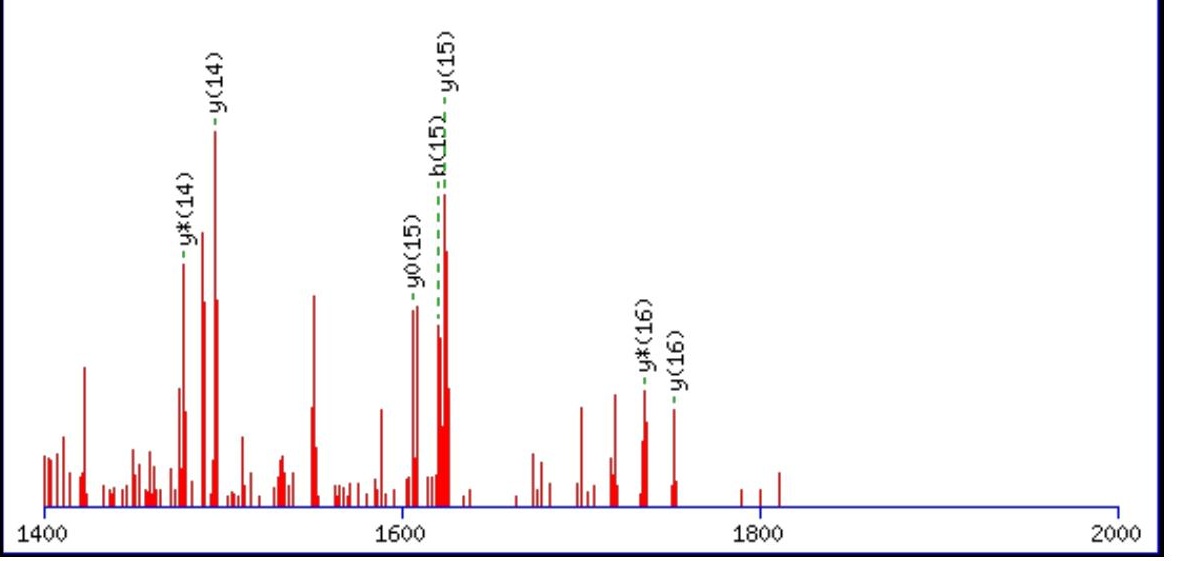

Monoisotopic mass of neutral peptide $\operatorname{Mr}($ calc): 1879.8548

Fixed modifications: Carbamidomethyl (C)

Variable modifications:

N10 : Deamidated N ( N)

Ions Score: 89 Expect: 2.8e-007

Matches (Bold Red): 44/180 fragment ions using 84 most intense peaks

\begin{tabular}{|c|c|c|c|c|c|c|c|c|c|c|c|c|c|c|}
\hline \# & b & $\mathbf{b}^{++}$ & b* & $\mathbf{b}^{*++}$ & $\mathbf{b}^{\mathbf{0}}$ & & Seq. & $\mathbf{y}$ & $\mathbf{y}^{++}$ & $\mathbf{y}^{*}$ & $\mathbf{y}^{*^{++}}$ & $\mathbf{y}^{\mathbf{0}}$ & $\mathbf{y}^{0++}$ & \# \\
\hline 1 & 129.1022 & 65.0548 & 112.0757 & 56.5415 & & & $\mathbf{K}$ & & & & & & & 17 \\
\hline 2 & 257.1608 & 129.0840 & 240.1343 & 120.5708 & & & $\mathbf{Q}$ & & 876.8872 & 1735.7406 & & 1734.7565 & 867.8819 & 16 \\
\hline 3 & 386.2034 & 193.6053 & 369.1769 & 185.0921 & 368.1928 & 184.6001 & $\mathbf{E}$ & 1624.7085 & 812.8579 & 1607.6820 & 804.3446 & 1606.6980 & 803.8526 & 15 \\
\hline 4 & 515.2460 & 258.1266 & 498.2195 & 249.6134 & 497.2354 & 249.1214 & $\mathbf{E}$ & 1495.6659 & 748.3366 & 1478.6394 & 739.8233 & 1477.6554 & 739.3313 & 14 \\
\hline 5 & 662.3144 & 331.6608 & 645.2879 & 323.1476 & 644.3039 & 322.6556 & $\mathbf{F}$ & 1366.6233 & 683.8153 & 1349.5968 & 675.3020 & 1348.6128 & 674.8100 & 13 \\
\hline 6 & 777.3414 & 389.1743 & 760.3148 & 380.6610 & 759.3308 & 380.1690 & D & 1219.5549 & 610.2811 & 1202.5284 & 601.7678 & 1201.5444 & 601.2758 & 12 \\
\hline 7 & 890.4254 & 445.7164 & 873.3989 & 437.2031 & 872.4149 & 436.7111 & I & 1104.5280 & 552.7676 & 1087.5014 & 544.2544 & 1086.5174 & 543.7623 & 11 \\
\hline 8 & 961.4625 & 481.2349 & 944.4360 & 472.7216 & 943.4520 & 472.2296 & A & 991.4439 & 496.2256 & 974.4174 & 487.7123 & 973.4334 & 487.2203 & 10 \\
\hline 9 & 1075.5055 & 538.2564 & 1058.4789 & 529.7431 & 1057.4949 & 529.2511 & $\mathbf{N}$ & 920.4068 & 460.7070 & 903.3803 & 452.1938 & 902.3962 & 451.7018 & 9 \\
\hline 10 & 1190.5324 & 595.7698 & 1173.5059 & 587.2566 & 1172.5218 & 586.7646 & $\mathbf{N}$ & 806.3639 & 403.6856 & 789.3373 & 395.1723 & 788.3533 & 394.6803 & 8 \\
\hline 11 & 1247.5539 & 624.2806 & 1230.5273 & 615.7673 & 1229.5433 & 615.2753 & G & 691.3369 & 346.1721 & 674.3104 & 337.6588 & 673.3264 & 337.1668 & 7 \\
\hline 12 & 1334.5859 & 667.7966 & 1317.5593 & 659.2833 & 1316.5753 & 658.7913 & S & 634.3155 & 317.6614 & 617.2889 & 309.1481 & 616.3049 & 308.6561 & 6 \\
\hline 13 & 1421.6179 & 711.3126 & 1404.5914 & 702.7993 & 1403.6074 & 702.3073 & S & 547.2835 & 274.1454 & 530.2569 & 265.6321 & 529.2729 & 265.1401 & 5 \\
\hline 14 & 1549.6765 & \begin{tabular}{|l|l|}
775.3419 \\
\end{tabular} & 1532.6500 & 766.8286 & 1531.6659 & 766.3366 & $\mathbf{Q}$ & 460.2514 & 230.6293 & 443.2249 & 222.1161 & & & 4 \\
\hline 15 & 1620.7136 & 810.8604 & 1603.6871 & 802.3472 & 1602.7030 & 801.8552 & A & 332.1928 & 166.6001 & 315.1663 & 158.0868 & & & 3 \\
\hline 16 & 1734.7565 & 867.8819 & 1717.7300 & 859.3686 & 1716.7460 & 858.8766 & $\mathbf{N}$ & 261.1557 & 131.0815 & 244.1292 & 122.5682 & & & 2 \\
\hline 17 & & & & & & & $\mathbf{K}$ & 147.1128 & 74.0600 & 130.0863 & 65.5468 & & & 1 \\
\hline
\end{tabular}

$$
\text { 跑 }
$$$$
-0.25
$$

RMS error 195 ppm
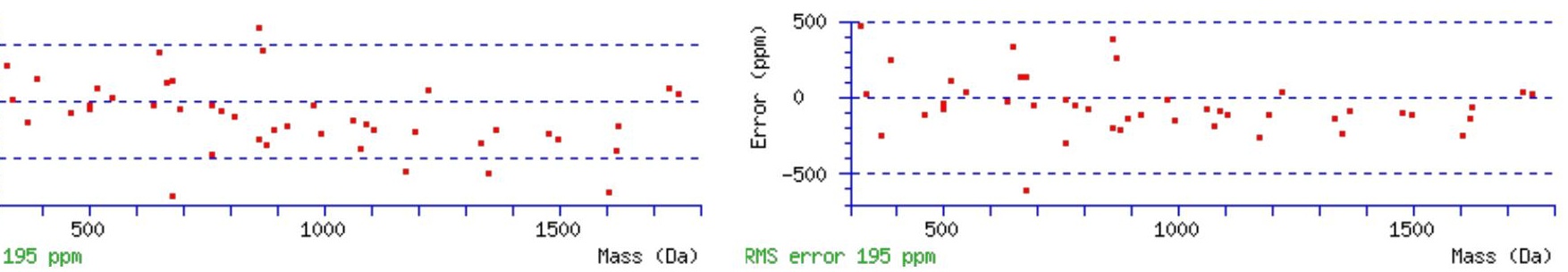

\section{All matches to this query}

\begin{tabular}{|l|l|l|l|}
\hline Score & Mr(calc): & Delta & Sequence \\
\hline 89.0 & 1879.8548 & 0.0014 & $\underline{\text { KQEEFDIANNGSSQANK }}$ \\
\hline 76.2 & 1879.8548 & 0.0014 & $\underline{\text { KQEEFDIANNGSSQANK }}$ \\
\hline 39.5 & 1879.8548 & 0.0014 & $\underline{\text { KQEEFDIANNGSSQANK }}$ \\
\hline 16.7 & 1878.8707 & 0.9854 & $\underline{\text { KQEEFDIANNGSSQANK }}$ \\
\hline & & &
\end{tabular}




\begin{tabular}{|l|l|l|l|}
10.4 & 1879.8427 & 0.0134 & TGFEFNIMVVGQSGLGK \\
\hline 4.0 & 1879.8638 & -0.0077 & ATVPLQSNVIEASPDMK \\
\hline 2.8 & 1879.8717 & -0.0155 & VTQYKNGKDSLYAQGK \\
\hline 2.5 & 1877.8594 & 1.9967 & RMNKVGSESLNFTVAK \\
\hline 1.4 & 1879.8638 & -0.0077 & ATVPLQSNVIEASPDMK \\
\hline 0.0 & 1878.8360 & 1.0201 & DHTVSRLEEANNALTK \\
\hline
\end{tabular}

Spectrum No: 145; Query: 625; Rank: 1

\section{Peptide View}

\section{MS/MS Fragmentation of IDVNSWIENFTK}

Found in IPI00391769, Tax_Id=10116 Gene_Symbol=Cacna2d1 Voltage-gated calcium channel alpha2/delta-1 subunit

Match to Query 625: 1465.711508 from(733.863030,2+)

Title: 091008RatKidney_NoSalt_20.5733.5733.2.dta

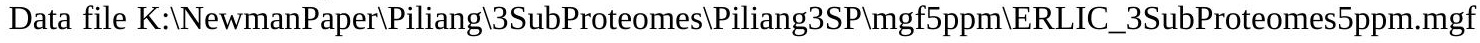
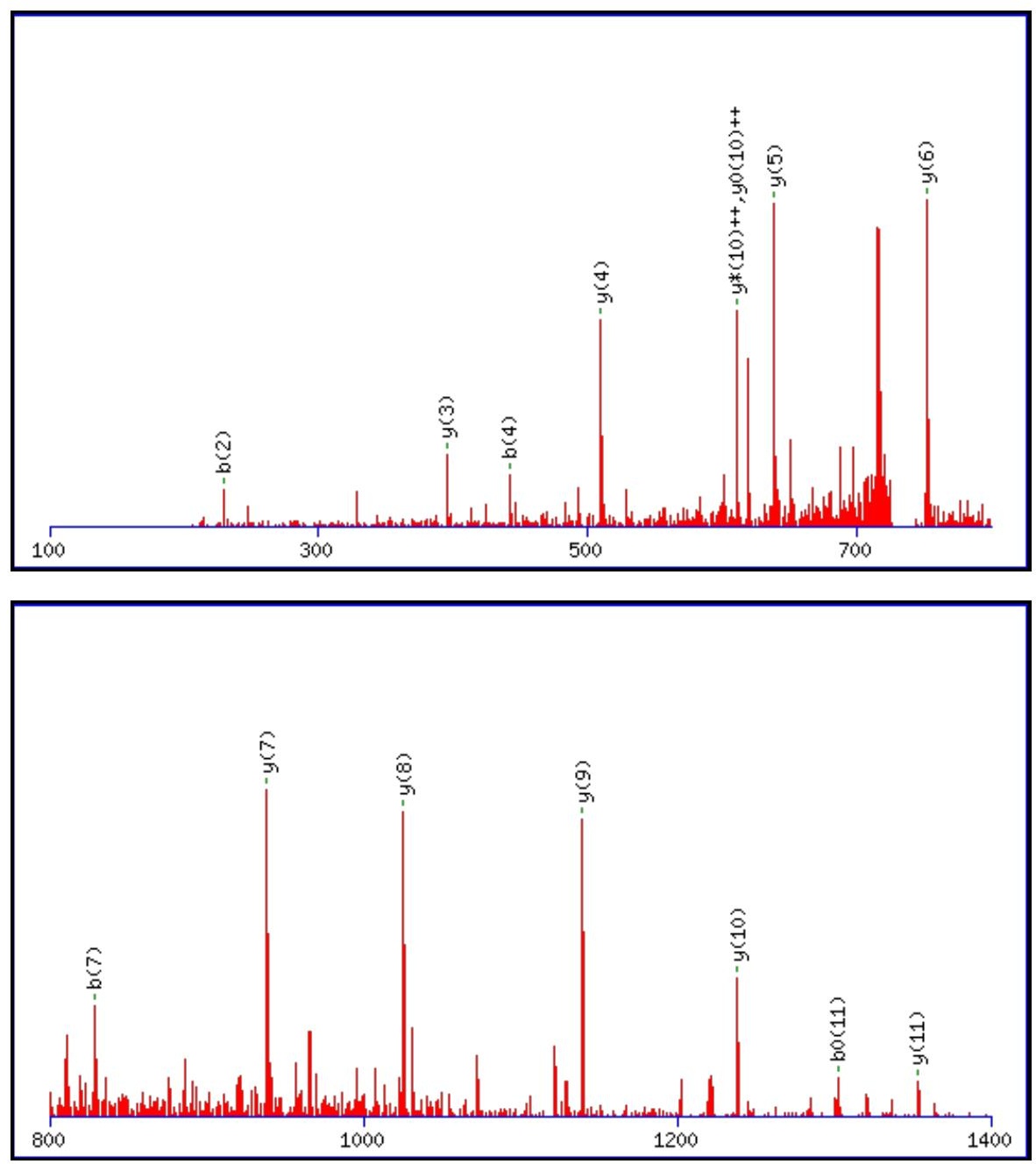


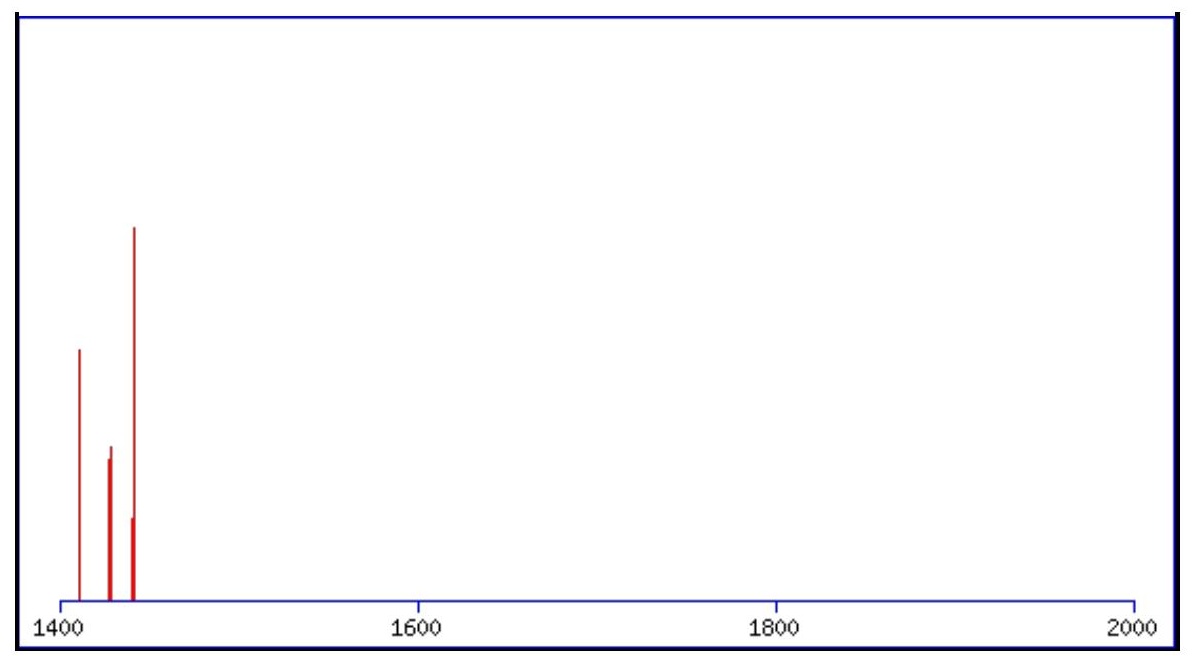

Monoisotopic mass of neutral peptide $\operatorname{Mr}($ calc): 1465.7089

Fixed modifications: Carbamidomethyl (C)

Variable modifications:

N9 : Deamidated_N (N)

Ions Score: 89 Expect: $2.2 \mathrm{e}-007$

Matches (Bold Red): 15/122 fragment ions using 21 most intense peaks

\begin{tabular}{|c|c|c|c|c|c|c|c|c|c|c|c|c|c|c|}
\hline \# & b & $\mathbf{b}^{++}$ & b* & $\mathbf{b}^{*^{++}}$ & $\mathbf{b}^{0}$ & $\mathbf{b}^{\mathbf{0 + +}}$ & Seq. & $\mathbf{y}$ & $\mathbf{y}^{++}$ & $\mathbf{y}^{*}$ & $\mathrm{y}^{*^{++}}$ & $\mathbf{y}^{0}$ & $\mathbf{y}^{0++}$ & \# \\
\hline 1 & 114.0913 & 57.5493 & & & & & I & & & & & & & 12 \\
\hline 2 & 229.1183 & 115.0628 & & & 211.1077 & 106.0575 & D & 1353.6321 & 677.3197 & 1336.6056 & 668.8064 & 1335.6216 & 668.3144 & 11 \\
\hline 3 & 328.1867 & 164.5970 & & & 310.1761 & 155.5917 & $\mathbf{V}$ & 1238.6052 & 619.8062 & 1221.5786 & 611.2929 & 1220.5946 & 610.8009 & 10 \\
\hline 4 & 442.2296 & 221.6185 & 425.2031 & 213.1052 & 424.2191 & 212.6132 & $\mathbf{N}$ & 1139.5368 & 570.2720 & 1122.5102 & 561.7587 & 1121.5262 & 561.2667 & 9 \\
\hline 5 & 529.2617 & 265.1345 & 512.2351 & 256.6212 & 511.2511 & 256.1292 & S & 1025.4938 & 513.2506 & 1008.4673 & 504.7373 & 1007.4833 & 504.2453 & 8 \\
\hline 6 & 715.3410 & 358.1741 & 698.3144 & 349.6608 & 697.3304 & 349.1688 & W & 938.4618 & 469.7345 & 921.4353 & 461.2213 & 920.4512 & 460.7293 & 7 \\
\hline 7 & 828.4250 & 414.7162 & 811.3985 & 406.2029 & 810.4145 & 405.7109 & I & 752.3825 & 376.6949 & 735.3559 & 368.1816 & 734.3719 & 367.6896 & 6 \\
\hline 8 & 957.4676 & 479.2374 & 940.4411 & 470.7242 & 939.4571 & 470.2322 & $\mathbf{E}$ & 639.2984 & 320.1529 & 622.2719 & 311.6396 & 621.2879 & 311.1476 & 5 \\
\hline 9 & 1072.4946 & 536.7509 & 1055.4680 & 528.2376 & 1054.4840 & 527.7456 & $\mathbf{N}$ & 510.2558 & 255.6316 & 493.2293 & 247.1183 & 492.2453 & 246.6263 & 4 \\
\hline 10 & 1219.5630 & 610.2851 & 1202.5364 & 601.7718 & 1201.5524 & 601.2798 & $\mathbf{F}$ & 395.2289 & 198.1181 & 378.2023 & 189.6048 & 377.2183 & 189.1128 & 3 \\
\hline 11 & 1320.6107 & 660.8090 & 1303.5841 & 652.2957 & 1302.6001 & 651.8037 & $\mathbf{T}$ & 248.1605 & 124.5839 & 231.1339 & 116.0706 & 230.1499 & 115.5786 & 2 \\
\hline 12 & & & & & & & $\mathbf{K}$ & 147.1128 & 74.0600 & 130.0863 & 65.5468 & & & 1 \\
\hline
\end{tabular}
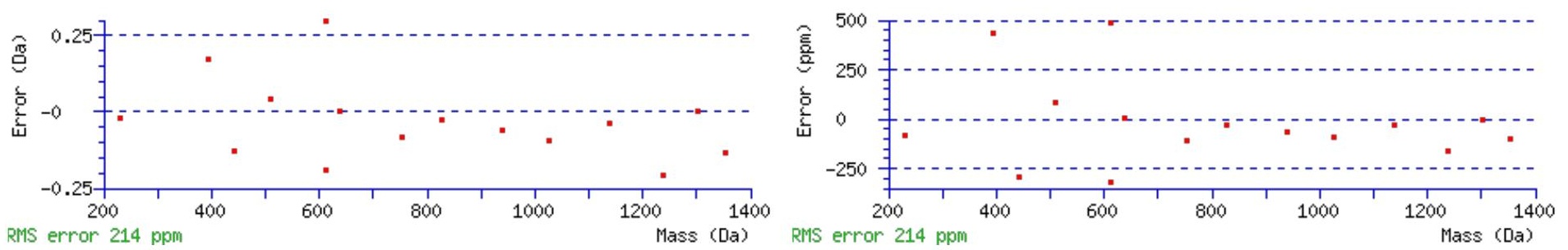

\section{All matches to this query}

\begin{tabular}{|l|l|l|l|}
\hline Score & Mr(calc): & Delta & \multicolumn{1}{|c|}{ Sequence } \\
\hline 88.9 & 1465.7089 & 0.0026 & $\underline{\text { IDVNSWIENFTK }}$ \\
\hline 19.6 & 1465.7089 & 0.0026 & $\underline{\text { IDVNSWIENFTK }}$ \\
\hline 11.7 & 1465.6983 & 0.0132 & $\underline{\text { YVSELTLVRVK }}$ \\
\hline 7.3 & 1465.7191 & -0.0076 & $\underline{\text { LNVKWGRSQAAR }}$ \\
\hline 5.2 & 1465.7177 & -0.0062 & $\underline{\text { TKPSNAVTPEKSK }}$ \\
\hline 4.1 & 1465.7161 & -0.0046 & $\underline{\text { LDNGQPVSAPSEPR }}$ \\
\hline 2.8 & 1465.7000 & 0.0115 & $\underline{\text { KVCNVAPIAGETK }}$ \\
\hline 1.3 & 1464.7086 & 1.0030 & $\underline{\text { LNNSLLPRSQSR }}$ \\
\hline
\end{tabular}


Spectrum No: 146; Query: 2505; Rank: 1

\section{Peptide View}

MS/MS Fragmentation of KGAGLENPVAVTIFFGANDSTLKDENPK

Found in IPI00421610, Tax_Id=10116 Gene_Symbol=Harpb64 Isoamyl acetate-hydrolyzing esterase 1 homolog

Match to Query 2505: 2932.485042 from(978.502290,3+)

Title: 091008RatKidney_NH4Format01_09.4337.4337.3.dta

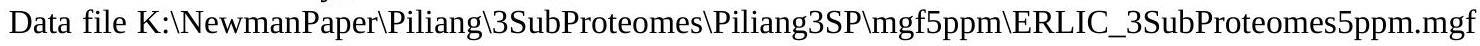
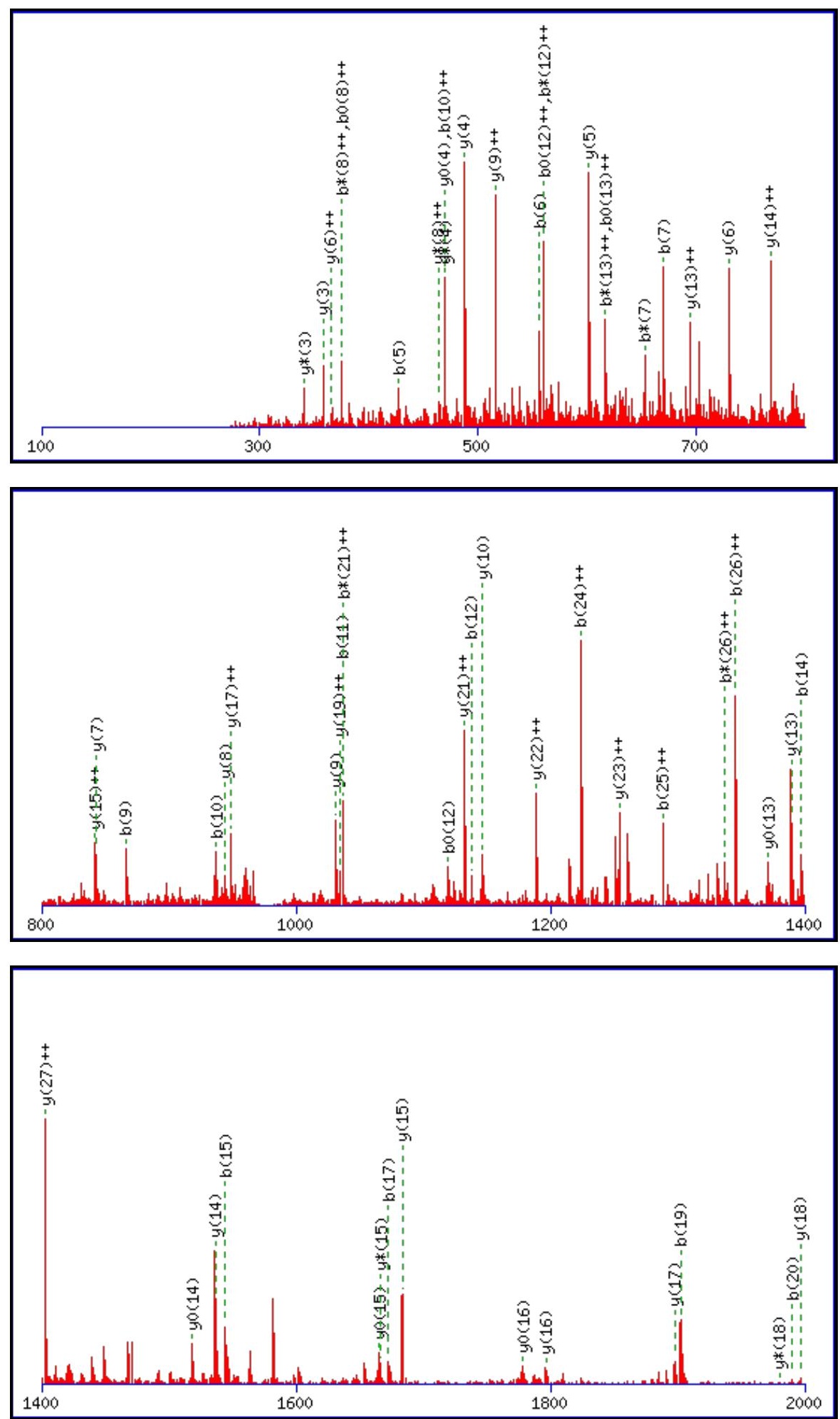

Monoisotopic mass of neutral peptide $\operatorname{Mr}($ calc): 2932.4818 
Fixed modifications: Carbamidomethyl (C)

Variable modifications:

N18 : Deamidated N (N)

Ions Score: 89 Expect: $2.3 \mathrm{e}-007$

Matches (Bold Red): 61/308 fragment ions using 104 most intense peaks

\begin{tabular}{|c|c|c|c|c|c|c|c|c|c|c|c|c|c|c|}
\hline \# & b & $\mathbf{b}^{++}$ & $\mathbf{b}^{*}$ & $\mathbf{b}^{*^{++}}$ & $\mathbf{b}^{0}$ & $\mathbf{b}^{\mathbf{0}^{++}}$ & Seq. & $\mathbf{y}$ & $\mathbf{y}^{++}$ & $\mathbf{y}^{*}$ & $y^{*^{++}}$ & $\mathbf{y}^{0}$ & $\mathbf{y}^{\mathbf{0 + +}}$ & \# \\
\hline 1 & 129.1022 & 65.0548 & 112.0757 & 56.5415 & & & $\mathbf{K}$ & & & & & & & 28 \\
\hline 2 & 186.1237 & 93.5655 & 169.0972 & 85.0522 & & & G & 2805.3941 & 1403.2007 & 2788.3676 & 1394.6874 & 2787.3836 & 1394.1954 & 27 \\
\hline 3 & 257.1608 & 129.0840 & 240.1343 & 120.5708 & & & A & 2748.3727 & 1374.6900 & 2731.3461 & 1366.1767 & 2730.3621 & 1365.6847 & 26 \\
\hline 4 & 314.1823 & 157.5948 & 297.1557 & 149.0815 & & & G & 2677.3355 & 1339.1714 & 2660.3090 & 1330.6581 & 2659.3250 & 1330.1661 & 25 \\
\hline 5 & 427.2663 & 214.1368 & 410.2398 & 205.6235 & & & $\mathbf{L}$ & 2620.3141 & 1310.6607 & 2603.2875 & 1302.1474 & 2602.3035 & 1301.6554 & 24 \\
\hline 6 & 556.3089 & 278.6581 & 539.2824 & 270.1448 & 538.2984 & 269.6528 & $\mathbf{E}$ & 2507.2300 & 1254.1186 & 2490.2035 & 1245.6054 & 2489.2195 & 1245.1134 & 23 \\
\hline 7 & 670.3519 & 335.6796 & 653.3253 & 327.1663 & 652.3413 & 326.6743 & $\mathbf{N}$ & 2378.1874 & \begin{tabular}{|l|l|}
1189.5974 \\
\end{tabular} & 2361.1609 & 1181.0841 & 2360.1769 & 1180.5921 & 22 \\
\hline 8 & 767.4046 & 384.2060 & 750.3781 & 375.6927 & 749.3941 & 375.2007 & $\mathbf{P}$ & 2264.1445 & 1132.5759 & 2247.1179 & 1124.0626 & 2246.1339 & 1123.5706 & 21 \\
\hline 9 & 866.4730 & 433.7402 & 849.4465 & 425.2269 & 848.4625 & 424.7349 & $\mathrm{~V}$ & 2167.0917 & 1084.0495 & 2150.0652 & 1075.5362 & 2149.0812 & 1075.0442 & 20 \\
\hline 10 & 937.5102 & 469.2587 & 920.4836 & 460.7454 & 919.4996 & 460.2534 & $\mathbf{A}$ & 2068.0233 & 1034.5153 & 2050.9968 & 1026.0020 & 2050.0128 & 1025.5100 & 19 \\
\hline 11 & 1036.5786 & 518.7929 & 1019.5520 & 510.2796 & 1018.5680 & 509.7876 & $\mathbf{V}$ & 1996.9862 & 998.9967 & 1979.9597 & 990.4835 & 1978.9756 & 989.9915 & 18 \\
\hline 12 & 1137.6263 & 569. & 1120. & & 1119. & & $T$ & 178 & 625 & 1880 & & 9072 & 940.4573 & 17 \\
\hline 13 & 1250.7103 & 625.8588 & 1233.6838 & 617.3455 & 1232.6997 & 616.8535 & I & \begin{tabular}{|l|}
1796.8701 \\
\end{tabular} & 898.9387 & 1779.8436 & 890.4254 & \begin{tabular}{|l}
1778.8595 \\
\end{tabular} & 889.9334 & 16 \\
\hline 14 & 1397.7787 & 699.3930 & 1380.7522 & 690.8797 & 1379.7682 & 690.3877 & $\mathbf{F}$ & 1683.7861 & 842.3967 & 1666.7595 & 833.8834 & 1665.7755 & 833.3914 & 15 \\
\hline 15 & 1544.8 & 772.9 & 1527. & 764 & 1526.8 & 763.9219 & $\mathbf{F}$ & 1536.7176 & 768.8625 & 1519.6911 & 760.3492 & \begin{tabular}{|l|}
1518.7071 \\
\end{tabular} & 759.8572 & 14 \\
\hline 16 & 1601.8686 & 801.4379 & 1584.8421 & 792.9247 & 1583.8580 & 792.4327 & G & 1389.6492 & 695.3282 & 1372.6227 & 686.8150 & 1371.6387 & 686.3230 & 13 \\
\hline 17 & 1672.9057 & 836.9565 & 1655.8792 & 828.4432 & 1654.8952 & 827.9512 & A & 1332.6278 & 666.8175 & 1315.6012 & 658.3042 & 1314.6172 & 657.8122 & 12 \\
\hline 18 & 1787.9327 & 894.4700 & 1770.9061 & 885.9567 & 1769.9221 & 885.4647 & $\mathbf{N}$ & 1261.5906 & 631.2990 & 1244.5641 & 622.7857 & 1243.5801 & 622.2937 & 11 \\
\hline 19 & 1902.9596 & 951.9834 & 1885.9331 & 943.4702 & 1884.9490 & 942.9782 & D & 1146.5637 & 573.7855 & 1129.5372 & 565.2722 & 1128.5531 & 564.7802 & 10 \\
\hline 20 & 1989.9916 & 995.4995 & 1972.9651 & 986.9862 & 1971.9811 & 986.4942 & $\mathrm{~S}$ & 1031.5368 & 516.2720 & 1014.5102 & 507.7587 & 1013.5262 & 507.2667 & 9 \\
\hline 21 & 2091.0393 & 1046.0233 & 2074.0128 & 1037.5100 & 2073.0287 & 1037.0180 & $T$ & 944.5047 & 472.7560 & 927.4782 & 464.2427 & 926.4942 & 463.7507 & 8 \\
\hline 22 & 2204.1234 & 1102.5653 & 2187.0968 & 1094.0520 & 2186.1128 & 1093.5600 & $\mathbf{L}$ & 843.4571 & 422.2322 & 826.4305 & 413.7189 & 825.4465 & 413.2269 & 7 \\
\hline 23 & 2332.2183 & 1166.6128 & 2315.1918 & 1158.0995 & 2314.2078 & 1157.6075 & $\mathbf{K}$ & 730.3730 & 365.6901 & 713.3464 & 357.1769 & 712.3624 & 356.6849 & 6 \\
\hline 24 & 2447.2453 & 1224.1263 & 2430.2187 & 1215.6130 & 2429.2347 & 1215.1210 & D & 602.2780 & 301.6427 & 585.2515 & 293.1294 & 584.2675 & 292.6374 & 5 \\
\hline 25 & 2576.2879 & 1288.6476 & 2559.2613 & 1280.1343 & 2558.2773 & 1279.6423 & $E$ & 487.2511 & 244.1292 & 470.2245 & 235.6159 & 469.2405 & 235.1239 & 4 \\
\hline 26 & 2690.3308 & 1345.6690 & 2673.3042 & \begin{tabular}{|l|l|}
1337.1558 \\
\end{tabular} & 2672.3202 & 1336.6638 & $\mathbf{N}$ & 358.2085 & 179.6079 & 341.1819 & 171.0946 & & & 3 \\
\hline 27 & 2787.3836 & 1394.1954 & 2770.3570 & 1385.6821 & 2769.3730 & 1385.1901 & $\mathbf{P}$ & 244.1656 & 122.5864 & 227.1390 & 114.0731 & & & 2 \\
\hline 28 & & & & & & & $\mathbf{K}$ & 147.1128 & 74.0600 & 130.0863 & 65.5468 & & & 1 \\
\hline
\end{tabular}
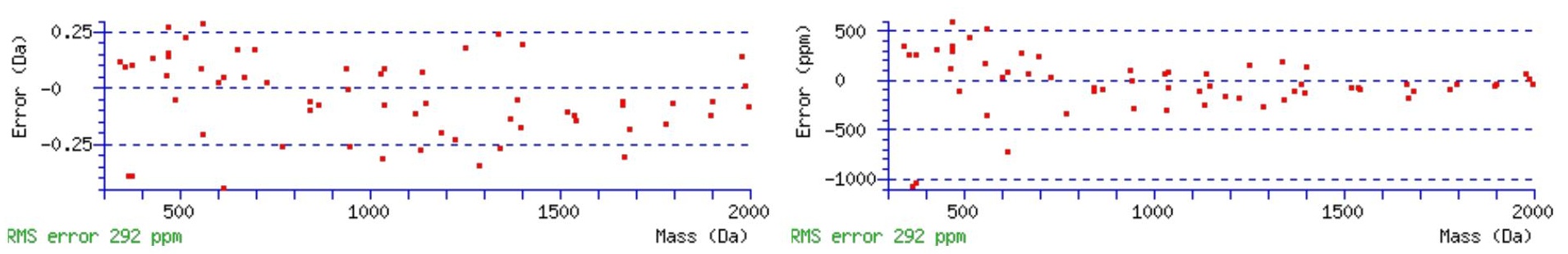

\section{All matches to this query}

\begin{tabular}{|l|l|l|l|}
\hline Score & Mr(calc): & Delta & \multicolumn{1}{|c|}{ Sequence } \\
\hline 88.8 & 2932.4818 & 0.0032 & KGAGLENPVAVTIFFGANDSTLKDENPK \\
\hline 84.7 & 2932.4818 & 0.0032 & KGAGLENPVAVTIFFGANDSTLKDENPK \\
\hline 84.0 & 2931.4978 & 0.9873 & KGAGLENPVAVTIFFGANDSTLKDENPK \\
\hline 71.1 & 2932.4818 & 0.0032 & KGAGLENPVAVTIFFGANDSTLKDENPK \\
\hline
\end{tabular}

Spectrum No: 147; Query: 2012; Rank: 1 


\section{Peptide View}

MS/MS Fragmentation of VGPVDPSLNTTYVFFDTFFK

Found in IPI00464518, Tax_Id=10116 Gene_Symbol=Hexb Beta-hexosaminidase beta chain precursor

Match to Query 2012: 2294.119908 from(1148.067230,2+)

Title: 091008RatKidney_NoSalt_14.7271.7271.2.dta

Data file K:\NewmanPaper\Piliang \3SubProteomes $\backslash$ Piliang3SP $\backslash$ mgf5ppm\ERLIC_3SubProteomes5ppm.mgf
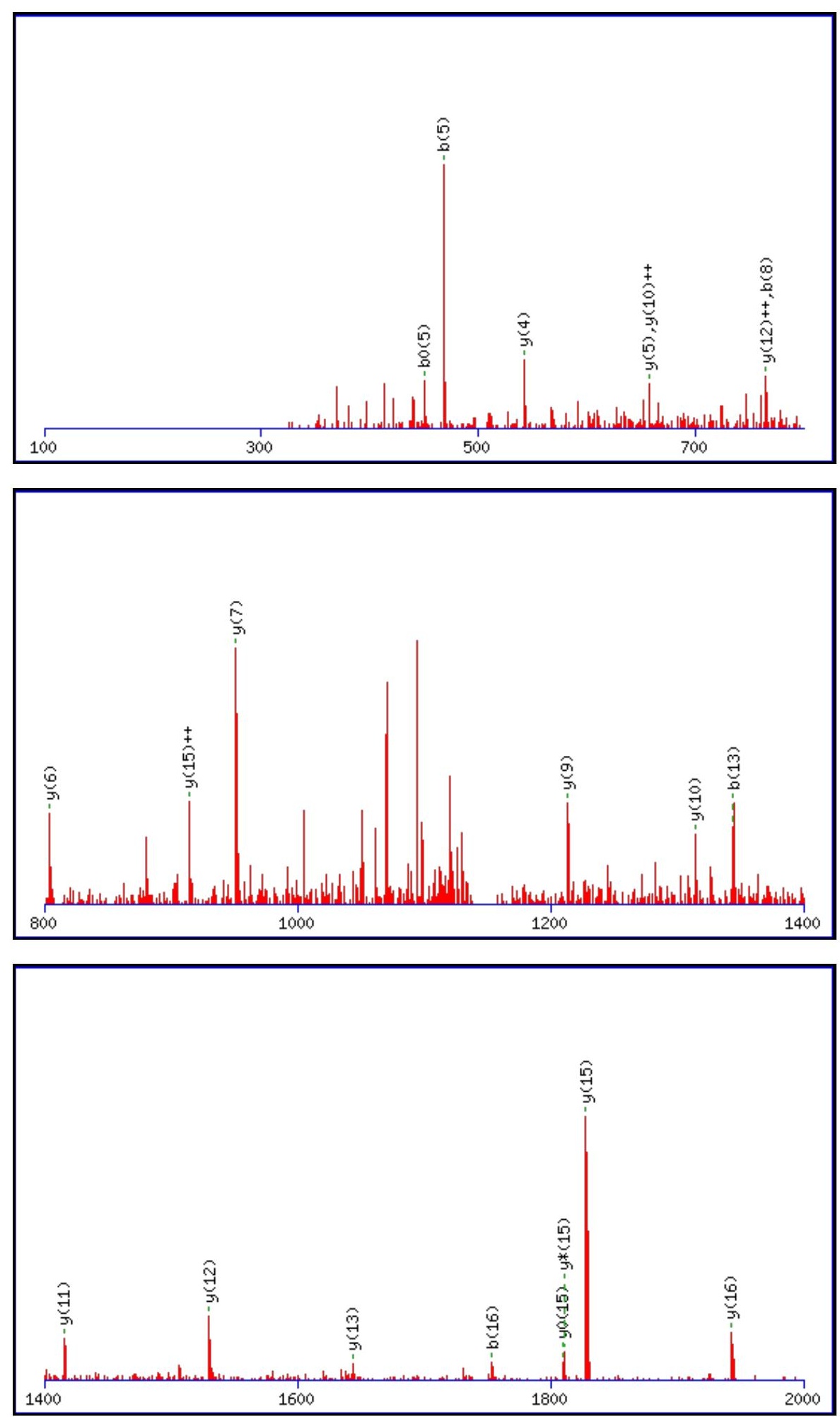

Monoisotopic mass of neutral peptide $\operatorname{Mr}($ calc): 2294.1147

Fixed modifications: Carbamidomethyl (C)

Variable modifications:

N9 : Deamidated_N (N) 
Ions Score: 89 Expect: $3.2 \mathrm{e}-007$

Matches (Bold Red): 21/198 fragment ions using 30 most intense peaks

\begin{tabular}{|c|c|c|c|c|c|c|c|c|c|c|c|c|c|c|}
\hline$\#$ & b & $\mathbf{b}^{++}$ & $\mathbf{b}^{*}$ & $\mathbf{b}^{*^{++}}$ & $\mathbf{b}^{0}$ & $\mathbf{b}^{0++}$ & Seq. & $\mathbf{y}$ & $y^{++}$ & $\mathbf{y}^{*}$ & $\mathrm{y}^{\mathrm{*}^{++}}$ & $\mathbf{y}^{0}$ & $y^{0++}$ & \# \\
\hline 1 & 100.0757 & 50.5415 & & & & & $\mathbf{V}$ & & & & & & & 20 \\
\hline 2 & 157.0972 & 79.0522 & & & & & G & 2196.0536 & 1098.5304 & 2179.0270 & 1090.0171 & 2178.0430 & 1089.5251 & 19 \\
\hline 3 & 254.1499 & 127.5786 & & & & & $\mathbf{P}$ & 2139.0321 & 1070.0197 & 2122.0055 & 1061.5064 & 2121.0215 & 1061.0144 & 18 \\
\hline 4 & 353.2183 & 177.1128 & & & & & V & 2041.9793 & 1021.4933 & 2024.9528 & 1012.9800 & 2023.9688 & 1012.4880 & 17 \\
\hline 5 & 468.2453 & 234.6263 & & & 450.2347 & 225.6210 & D & 1942.9109 & 971.9591 & 1925.8844 & 963.4458 & 1924.9004 & 962.9538 & 16 \\
\hline 6 & 565.2980 & 283.1527 & & & 547.2875 & 274.1474 & $\mathbf{P}$ & 1827.8840 & 914.4456 & 1810.8574 & 905.9324 & 1809.8734 & 905.4403 & 15 \\
\hline 7 & 652.3301 & 326.6687 & & & 634.3195 & 317.6634 & $S$ & 1730.8312 & 865.9192 & 1713.8047 & 857.4060 & 1712.8206 & 856.9140 & 14 \\
\hline 8 & 765.4141 & 383.2107 & & & 747.4036 & 374.2054 & $\mathbf{L}$ & 1643.7992 & 822.4032 & 1626.7726 & 813.8900 & 1625.7886 & 813.3979 & 13 \\
\hline 9 & 880.4411 & 440.7242 & 863.4145 & 432.2109 & 862.4305 & 431.7189 & $\mathbf{N}$ & 1530.7151 & 765.8612 & 1513.6886 & 757.3479 & 1512.7046 & 756.8559 & 12 \\
\hline 10 & 981.4887 & 491.2480 & \begin{tabular}{|l|}
964.4622 \\
\end{tabular} & 482.7347 & 963.4782 & 482.2427 & $T$ & 1415.6882 & 708.3477 & 1398.6616 & 699.8345 & 1397.6776 & 699.3424 & 11 \\
\hline 11 & 1082.5364 & 541.7719 & 1065.5099 & 533.2586 & 1064.5259 & 532.7666 & $T$ & 1314.6405 & 657.8239 & 1297.6140 & 649.3106 & 1296.6299 & 648.8186 & 10 \\
\hline 12 & 1245.5998 & 623.3035 & 1228.5732 & 614.7902 & 1227.5892 & 614.2982 & $\mathbf{Y}$ & 1213.5928 & 607.3001 & 1196.5663 & 598.7868 & 1195.5823 & 598.2948 & 9 \\
\hline 13 & 1344.6682 & 672.8377 & 1327.6416 & 664.3244 & 1326.6576 & 663.8324 & V & 1050.5295 & 525.7684 & 1033.5029 & 517.2551 & 1032.5189 & 516.7631 & 8 \\
\hline 14 & 1491.7366 & 746.3719 & 1474.7100 & 737.8587 & 1473.7260 & 737.3666 & $\mathbf{F}$ & 951.4611 & 476.2342 & 934.4345 & 467.7209 & 933.4505 & 467.2289 & 7 \\
\hline 15 & 1638.8050 & 819.9061 & 1621.7784 & 811.3929 & 1620.7944 & 810.9009 & $\mathbf{F}$ & 804.3927 & 402.7000 & 787.3661 & 394.1867 & 786.3821 & 393.6947 & 6 \\
\hline 16 & 1753.8319 & 877.4196 & 1736.8054 & 868.9063 & 1735.8214 & 868.4143 & D & 657.3243 & 329.1658 & 640.2977 & 320.6525 & 639.3137 & 320.1605 & 5 \\
\hline 17 & 1854.8796 & 927.9434 & 1837.8531 & 919.4302 & 1836.8691 & 918.9382 & $T$ & 542.2973 & 271.6523 & 525.2708 & 263.1390 & 524.2867 & 262.6470 & 4 \\
\hline 18 & 2001.9480 & \begin{tabular}{|l|l}
1001.4777 \\
\end{tabular} & 1984.9215 & 992.9644 & 1983.9375 & 992.4724 & $\mathbf{F}$ & 441.2496 & 221.1285 & 424.2231 & 212.6152 & & & 3 \\
\hline 19 & 2149.0164 & 1075.0119 & 2131.9899 & 1066.4986 & 2131.0059 & 1066.0066 & $\mathbf{F}$ & 294.1812 & 147.5942 & 277.1547 & 139.0810 & & & 2 \\
\hline 20 & & & & & & & $\mathbf{K}$ & 147.1128 & 74.0600 & 130.0863 & 65.5468 & & & 1 \\
\hline
\end{tabular}
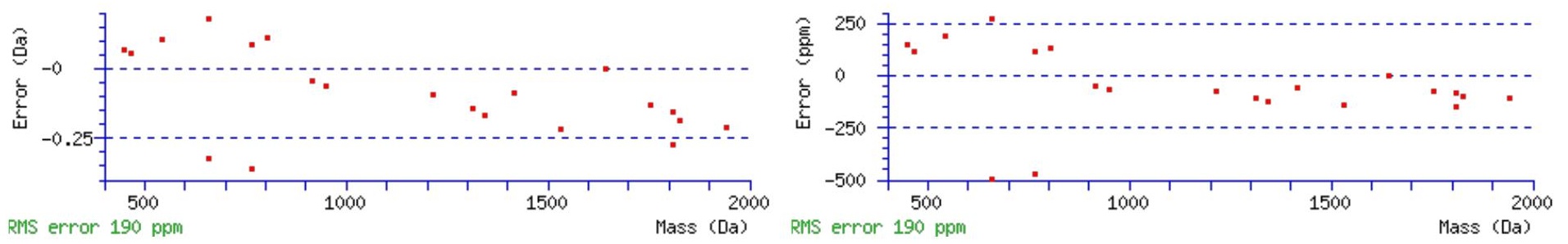

\section{All matches to this query}

\begin{tabular}{|l|c|c|c|}
\hline Score & Mr(calc): & Delta & Sequence \\
\hline 88.7 & 2294.1147 & 0.0052 & VGPVDPSLNTTYVFFDTFFK \\
\hline 28.6 & 2293.1307 & 0.9892 & VGPVDPSLNTTYVFFDTFFK \\
\hline
\end{tabular}

Spectrum No: 148; Query: 1406; Rank: 1

\section{Peptide View}

MS/MS Fragmentation of GTAGNALMEGASQLVGENR

Found in IPI00205389, Tax_Id=10116 Gene_Symbol=Fgb Isoform 1 of Fibrinogen beta chain precursor

Match to Query 1406: 1890.872308 from(946.443430,2+)

Title: 091008RatKidney_NH4Format01_27.2142.2142.2.dta

Data file K:INewmanPaper|Piliang|3SubProteomes\Piliang3SP\mgf5ppm\ERLIC_3SubProteomes5ppm.mgf 

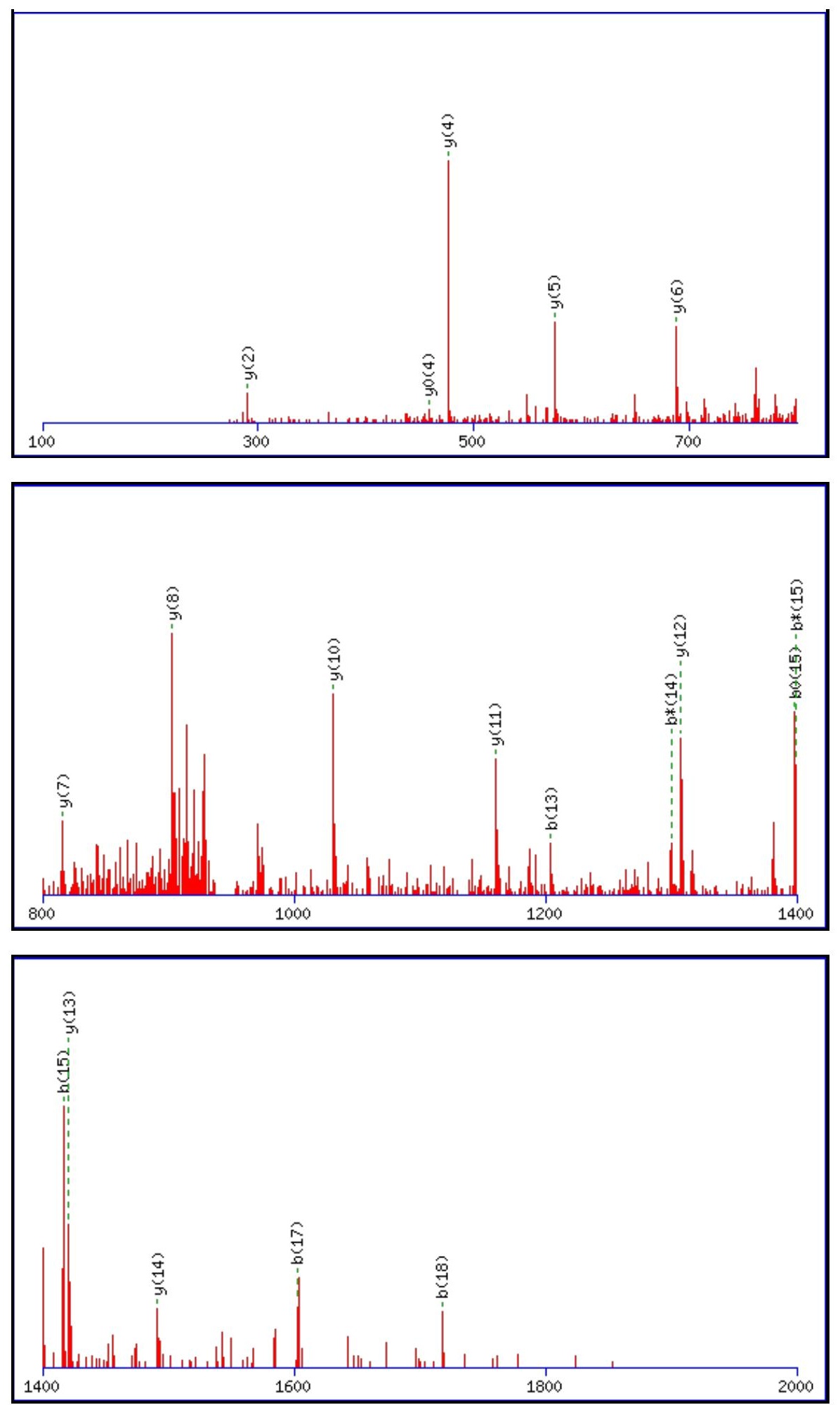

Monoisotopic mass of neutral peptide $\operatorname{Mr}($ calc): 1890.8741

Fixed modifications: Carbamidomethyl (C)

Variable modifications:

M8 : Oxidation $(M)$

N18 : Deamidated_N (N)

Ions Score: 89 Expect: $3 e-007$

Matches (Bold Red): 19/202 fragment ions using 30 most intense peaks

\begin{tabular}{|r|c|c|c|c|c|c|c|c|c|c|c|c|c|c|}
\hline$\#$ & $\mathbf{b}$ & $\mathbf{b}^{++}$ & $\mathbf{b}^{*}$ & $\mathbf{b}^{\boldsymbol{*}_{+}^{++}}$ & $\mathbf{b}^{\mathbf{0}}$ & $\mathbf{b}^{\mathbf{0 + +}}$ & Seq. & $\mathbf{y}$ & $\mathbf{y}^{++}$ & $\mathbf{y}^{\mathbf{*}}$ & $\mathbf{y}^{\mathbf{*}^{++}}$ & $\mathbf{y}^{\mathbf{0}}$ & $\mathbf{y}^{\mathbf{0}++}$ & $\#$ \\
\hline $\mathbf{1}$ & 58.0287 & 29.5180 & & & & & $\mathbf{G}$ & & & & & & & $\mathbf{1 9}$ \\
\hline $\mathbf{2}$ & 159.0764 & 80.0418 & & & 141.0659 & 71.0366 & $\mathbf{T}$ & 1834.8600 & 917.9336 & 1817.8334 & 909.4203 & 1816.8494 & 908.9283 & $\mathbf{1 8}$ \\
\hline $\mathbf{3}$ & 230.1135 & 115.5604 & & & 212.1030 & 106.5551 & $\mathbf{A}$ & 1733.8123 & 867.4098 & 1716.7857 & 858.8965 & 1715.8017 & 858.4045 & $\mathbf{1 7}$ \\
\hline $\mathbf{4}$ & 287.1350 & 144.0711 & & & 269.1244 & 135.0659 & $\mathbf{G}$ & 1662.7752 & 831.8912 & 1645.7486 & 823.3780 & 1644.7646 & 822.8859 & $\mathbf{1 6}$ \\
\hline & & & & & & & & & & & & & &
\end{tabular}




\begin{tabular}{|c|c|c|c|c|c|c|c|c|c|c|c|c|c|c|}
\hline & 401.1779 & 201.0926 & 384.1514 & |192.5793 & 383.1674 & 192.0873 & $\mathbf{N}$ & & 803.3805 & .7272 & |794.8672 & 1587.7431 & 794.3752 & 15 \\
\hline 6 & 472.2150 & 236.6112 & 455.1885 & 228.0979 & 454.2045 & 227.6059 & A & 1491.7108 & 746.3590 & 1474.6842 & 737.8458 & 1473.7002 & 737.3537 & 14 \\
\hline 7 & 585.2991 & 293.1532 & 568.2726 & 284.6399 & 567.2885 & 284.1479 & $\mathbf{L}$ & 1420.6737 & 710.8405 & 1403.6471 & 702.3272 & 1402.6631 & 701.8352 & 13 \\
\hline 8 & 732.3345 & |366.6709 & 715.3080 & 358.1576 & 714.3239 & 357.6656 & M & 1307.5896 & 654.2984 & 1290.5631 & 645.7852 & 1289.5790 & 645.2932 & 12 \\
\hline 9 & 861.3771 & 431.1922 & 844.3506 & 422.6789 & 843.3665 & 422.1869 & $\mathbf{E}$ & 1160.5542 & 580.7807 & 1143.5277 & 572.2675 & 1142.5436 & 571.7755 & 11 \\
\hline 10 & 918.3986 & 459.7029 & 901.3720 & 451.1896 & 900.3880 & 450.6976 & G & 1031.5116 & 516.2594 & 1014.4851 & 507.7462 & 1013.5010 & 507.2542 & 10 \\
\hline 11 & 989.4357 & 495.2215 & 972.4091 & 486.7082 & 971.4251 & 486.2162 & A & 974.4901 & 487.7487 & 957.4636 & 479.2354 & 956.4796 & 478.7434 & 9 \\
\hline 12 & 1076. & 538.7 & 1059. & 530. & 1058 & 529. & $\mathrm{~S}$ & & 452.2302 & & 443 & 4425 & 443.2249 & 8 \\
\hline 13 & 1204.5263 & 602.7668 & 1187.4997 & 594.2535 & 1186.5157 & 593.7615 & $\mathbf{Q}$ & 816.4210 & 408.7141 & 799.3945 & 400.2009 & 798.4104 & 399.7089 & 7 \\
\hline 14 & 1317.6103 & 659.3088 & 1300.5838 & 650.7955 & 1299.5998 & 650.3035 & $\mathbf{L}$ & 688.3624 & 344.6848 & 671.3359 & 336.1716 & 670.3519 & 335.6796 & 6 \\
\hline 15 & \begin{tabular}{|l|}
1416.6788 \\
\end{tabular} & 708.8430 & \begin{tabular}{|l}
1399.6522 \\
\end{tabular} & 700.3297 & 1398.6682 & 699.8377 & V & 575.2784 & 288.1428 & 558.2518 & 279.6295 & 557.2678 & 279.1375 & 5 \\
\hline 16 & 1473.7002 & 737.3538 & 1456.6737 & 728.8405 & 1455.6897 & 728.3485 & G & 476.2099 & 238.6086 & 459.1834 & 230.0953 & 458.1994 & 229.6033 & 4 \\
\hline 17 & 1602.7428 & 801.8750 & 1585.7163 & 793.3618 & 1584.7323 & 792.8698 & $\mathbf{E}$ & 419.1885 & 210.0979 & 402.1619 & 201.5846 & 401.1779 & 201.0926 & 3 \\
\hline 18 & \begin{tabular}{|l|}
1717.7698 \\
\end{tabular} & 859.3885 & 1700.7432 & 850.8752 & 1699.7592 & 850.3832 & $\mathbf{N}$ & 290.1459 & 145.5766 & 273.1193 & 137.0633 & & & 2 \\
\hline 19 & & & & & & & $\mathbf{R}$ & 175.1190 & 88.0631 & 158.0924 & 79.5498 & & & 1 \\
\hline
\end{tabular}
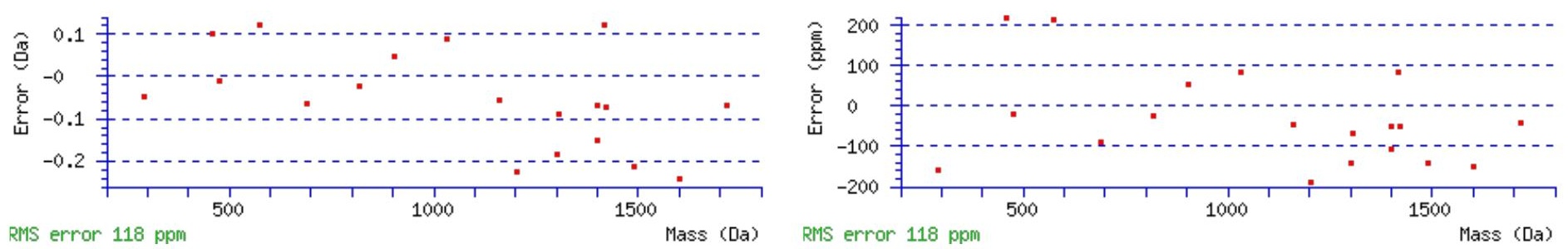

\section{All matches to this query}

\begin{tabular}{|l|l|l|l|}
\hline Score & Mr(calc): & Delta & \multicolumn{1}{|c|}{ Sequence } \\
\hline 88.7 & 1890.8741 & -0.0018 & GTAGNALMEGASQLVGENR \\
\hline 10.7 & 1889.8594 & 1.0129 & VRTMFSEQQLGELEK \\
\hline 3.0 & 1890.8625 & 0.0098 & AFRSSTIQDQQLFDR \\
\hline 3.0 & 1890.8625 & 0.0098 & AFRSSTIQDQQLFDR \\
\hline 2.4 & 1890.8625 & 0.0098 & AFRSSTIQDQQLFDR \\
\hline 1.0 & 1889.8611 & 1.0112 & EHMDEVCSSQLLTSVR \\
\hline
\end{tabular}

Spectrum No: 149; Query: 2629; Rank: 1

\section{Peptide View}

MS/MS Fragmentation of TSLDWWTTDTCNMINGTDGDSFHPLISK

Found in IPI00231478, Tax_Id=10116 Gene_Symbol=Scarb2 Lysosome membrane protein 2

Match to Query 2629: 3212.404452 from(1071.808760,3+)

Title: 091008RatKidney_NoSalt_20.5734.5734.3.dta

Data file K:INewmanPaper|Piliangl3SubProteomes\Piliang3SP\mgf5ppm\ERLIC_3SubProteomes5ppm.mgf 

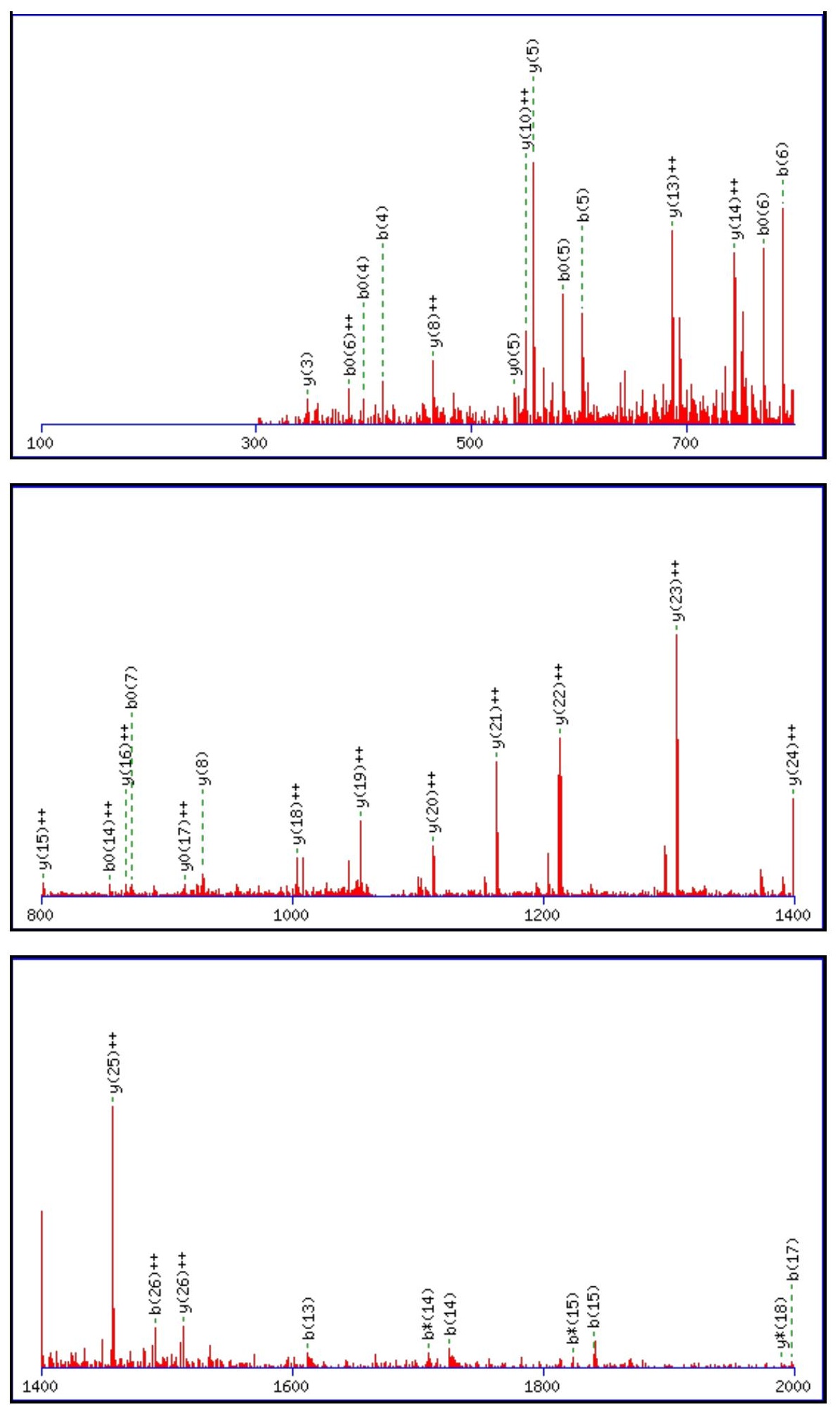

Monoisotopic mass of neutral peptide $\operatorname{Mr}($ calc): 3212.4067

Fixed modifications: Carbamidomethyl (C)

Variable modifications:

N15 : Deamidated_N (N)

Ions Score: 88 Expect: $6.2 \mathrm{e}-007$

Matches (Bold Red): $37 / 300$ fragment ions using 65 most intense peaks

\begin{tabular}{|c|c|c|c|c|c|c|c|c|c|c|c|c|c|c|}
\hline \# & b & $\mathbf{b}^{++}$ & $\mathbf{b}^{*}$ & $\mathbf{b}^{*^{++}}$ & $\mathbf{b}^{\mathbf{0}}$ & $\mathbf{b}^{\mathbf{0 + +}}$ & Seq. & $\mathbf{y}$ & $y^{++}$ & $\mathbf{y}^{*}$ & $\mathrm{y}^{\mathrm{*}^{++}}$ & $\mathbf{y}^{\mathbf{0}}$ & $\mathbf{y}^{\mathbf{0 + +}}$ & \# \\
\hline 1 & 102.0550 & 51.5311 & & & 84.0444 & 42.5258 & $\mathbf{T}$ & & & & & & & 28 \\
\hline 2 & 189.0870 & 95.0471 & & & 171.0764 & 86.0418 & S & 3112.3663 & 1556.6868 & 3095.3397 & 1548.1735 & 3094.3557 & 1547.6815 & 27 \\
\hline 3 & 302.1710 & 151.5892 & & & 284.1605 & 142.5839 & $\mathbf{L}$ & 3025.3342 & 1513.1708 & 3008.3077 & 1504.6575 & 3007.3237 & 1504.1655 & 26 \\
\hline 4 & 417.1980 & 209.1026 & & & 399.1874 & 200.0974 & D & 2912.2502 & 1456.6287 & 2895.2236 & 1448.1155 & 2894.2396 & 1447.6234 & 25 \\
\hline 5 & 603.2773 & 302.1423 & & & 585.2667 & 293.1370 & $\mathbf{W}$ & 2797.2232 & 1399.1153 & 2780.1967 & 1390.6020 & 2779.2127 & 1390.1100 & 24 \\
\hline
\end{tabular}




\begin{tabular}{|c|c|c|c|c|c|c|c|c|c|c|c|c|c|c|}
\hline 6 & 789.3566 & 395.1819 & & & 771.3461 & 386.1767 & $\mathbf{W}$ & 2611.1439 & |1306.0756 & 2594.1174 & 1297.5623 & 2593.1334 & |1297.0703 & \\
\hline 7 & 890.4043 & 445.7058 & & & 872.3937 & 436.7005 & $\mathbf{T}$ & 2425.0646 & 1213.0359 & 2408.0381 & 1204.5227 & 2407.0540 & 1204.0307 & 22 \\
\hline 8 & 991.4520 & 496.2296 & & & 973.4414 & 487.2243 & $\mathbf{T}$ & 2324.0169 & 1162.5121 & 2306.9904 & 1153.9988 & 2306.0064 & 1153.5068 & \\
\hline 9 & 1106.4789 & 553.7431 & & & 1088.4684 & 544.7378 & D & 2222.9693 & 1111.9883 & 2205.9427 & 1103.4750 & 2204.9587 & 1102.9830 & \\
\hline 10 & 1207.5266 & 604.2669 & & & 1189.5160 & 595.2617 & $\mathbf{T}$ & 2107.9423 & 1054.4748 & 2090.9158 & 1045.9615 & 2089.9317 & 1045.4695 & \\
\hline 11 & 1367.5572 & 684.2823 & & & 1349.5467 & 675.2770 & C & 2006.8946 & 1003.9510 & 1989.8681 & 995.4377 & 1988.8841 & 994.9457 & 8 \\
\hline 12 & 1481.6002 & 741.3037 & 1464.5736 & & 1463.5896 & 732.2984 & $\mathbf{N}$ & 1846.8640 & 923.9356 & 1829.8374 & 915.4224 & 1828.8534 & 914.9303 & 7 \\
\hline 13 & 1612.6407 & 806.8240 & 1595.6141 & 798.3107 & 1594.6301 & 797.8187 & M & 1732.8211 & 866.9142 & 1715.7945 & 858.4009 & 1714.8105 & 857.9089 & \\
\hline 14 & 1725.7247 & 863.3660 & 1708.6982 & 854.8527 & 1707.7142 & 854.3607 & I & 1601.7806 & 801.3939 & 1584.7540 & 792.8806 & 1583.7700 & 792.3886 & 5 \\
\hline 15 & 1840.7517 & 920.8795 & 1823.7251 & 912.3662 & 1822.7411 & 911.8742 & $\mathbf{N}$ & 1488.6965 & 744.8519 & 1471.6700 & 736.3386 & 1470.6859 & 735.8466 & \\
\hline 16 & 1897.7731 & 949.3902 & 1880.7466 & 940.8769 & 1879.7626 & 940.3849 & $\mathbf{G}$ & 1373.6696 & 687.3384 & 1356.6430 & 678.8251 & 1355.6590 & 678.3331 & 3 \\
\hline 17 & 1998.8208 & 999.9140 & 1981.7943 & 991.4008 & 1980.8102 & 990.9088 & $\mathbf{T}$ & 1316.6481 & 658.8277 & 1299.6216 & 650.3144 & 1298.6375 & 649.8224 & 12 \\
\hline 18 & 2113.8477 & 1057.4275 & 2096.8212 & 1048.9142 & 2095.8372 & 1048.4222 & D & 1215.6004 & 608.3039 & 1198.5739 & 599.7906 & 1197.5899 & 599.2986 & 1 \\
\hline 19 & 2170.8692 & 1085.9382 & 2153.8427 & 1077.4250 & 2152.8586 & 1076.9330 & G & 1100.5735 & 550.7904 & 1083.5469 & 542.2771 & 1082.5629 & 541.7851 & 10 \\
\hline 20 & 2285.8961 & 1143.4517 & 2268.8696 & 1134.9384 & 2267.8856 & 1134.4464 & D & 1043.5520 & 522.2796 & 1026.5255 & 513.7664 & 1025.5415 & 513.2744 & 9 \\
\hline 21 & 2372.9282 & 1186.9677 & 2355.9016 & 1178.4545 & 2354.9176 & 1177.9624 & $\mathrm{~S}$ & 928.5251 & 464.7662 & 911.4985 & 456.2529 & 910.5145 & 455.7609 & 8 \\
\hline 22 & 2519.9966 & 1260.5019 & 2502.9700 & 1251.9887 & 2501.9860 & 1251.4967 & $\mathbf{F}$ & 841.4931 & 421.2502 & 824.4665 & 412.7369 & 823.4825 & 412.2449 & 7 \\
\hline 23 & 2657.0555 & 1329.0314 & 2640.0290 & 1320.5181 & 2639.0449 & 1320.0261 & $\mathbf{H}$ & 694.4246 & 347.7160 & 677.3981 & 339.2027 & 676.4141 & 338.7107 & 6 \\
\hline 24 & 2754.1083 & 1377.5578 & 2737.0817 & 1369.0445 & 2736.0977 & 1368.5525 & $\mathbf{P}$ & 557.3657 & 279.1865 & 540.3392 & 270.6732 & 539.3552 & 270.1812 & 5 \\
\hline 25 & 2867.1923 & 1434.0998 & 2850.1658 & 1425.5865 & 2849.1818 & 1425.0945 & $\mathbf{L}$ & 460.3130 & 230.6601 & 443.2864 & 222.1468 & 442.3024 & 221.6548 & 4 \\
\hline 26 & 2980.2764 & 1490.6418 & 2963.2498 & 1482.1286 & 2962.2658 & 1481.6366 & I & 347.2289 & 174.1181 & 330.2023 & 165.6048 & 329.2183 & 165.1128 & 3 \\
\hline 27 & 3067.3084 & 1534.1578 & 3050.2819 & 1525.6446 & 3049.2979 & 1525.1526 & S & 234.1448 & 117.5761 & 217.1183 & 109.0628 & 216.1343 & 108.5708 & 2 \\
\hline 28 & & & & & & & $\mathbf{K}$ & 147.1128 & 74.0600 & 130.0863 & 65.5468 & & & 1 \\
\hline
\end{tabular}
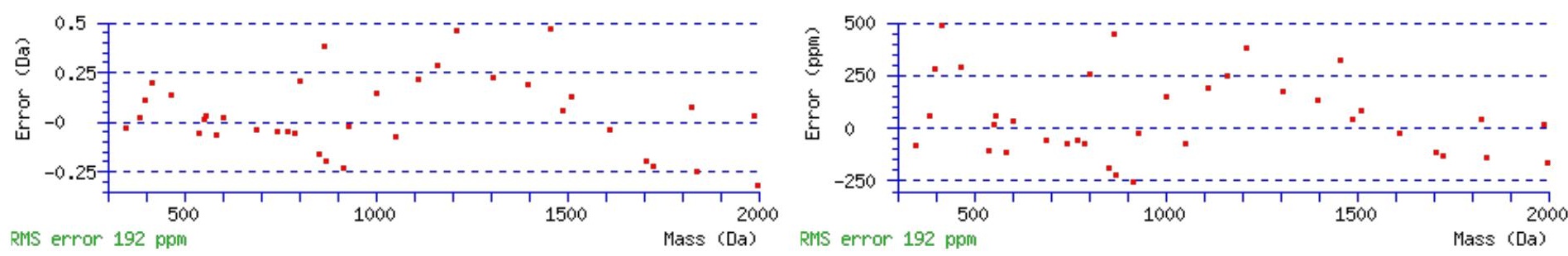

\section{All matches to this query}

\begin{tabular}{|l|l|l|l|}
\hline Score & Mr(calc): & Delta & \multicolumn{1}{c|}{ Sequence } \\
\hline 88.4 & 3212.4067 & -0.0023 & TSLDWWTTDTCNMINGTDGDSFHPLISK \\
\hline 73.0 & 3212.4067 & -0.0023 & TSLDWWTTDTCNMINGTDGDSFHPLISK \\
\hline 13.8 & 3211.4227 & 0.9818 & $\underline{\text { TSLDWWTTDTCNMINGTDGDSFHPLISK }}$ \\
\hline 3.5 & 3211.4002 & 1.0042 & $\underline{\text { GSFSGRLSPAYSLGSLTGASPRQSPHAQR }}$ \\
\hline 3.4 & 3212.4080 & -0.0035 & $\underline{\text { NAININELFIEISRRLPSTDASPASGGK }}$ \\
\hline 1.8 & 3212.4308 & -0.0263 & $\underline{\text { GLEWMGVMWSGGNTDYNSALKSRLSISR }}$ \\
\hline 1.2 & 3212.4113 & -0.0068 & $\underline{\text { MIYLVTEYASGGEIFDHLVAHGRMAEK }}$ \\
\hline 0.8 & 3212.4308 & -0.0263 & GLEWMGVMWSGGNTDYNSALKSRLSISR \\
\hline 0.6 & 3212.4308 & -0.0263 & $\underline{\text { GLEWMGVMWSGGNTDYNSALKSRLSISR }}$ \\
\hline 0.4 & 3210.3698 & 2.0346 & $\underline{\text { IGSENNMTSCHRPVCRKEGVLLDYSK }}$ \\
\hline
\end{tabular}

Spectrum No: 150; Query: 1128; Rank: 1

\section{Peptide View}

MS/MS Fragmentation of VISNNCTSYGVLDIGK 
Found in IPI00231197, Tax_Id=10116 Gene_Symbol=Cd36 Platelet glycoprotein 4

Match to Query 1128: 1739.842028 from(870.928290,2+)

Title: 100101RatKid_NS_deglyco_25.2853.2853.2.dta

Data file K:INewmanPaper|Piliang|3SubProteomes\Piliang3SP\mgf5ppm\ERLIC_3SubProteomes5ppm.mgf
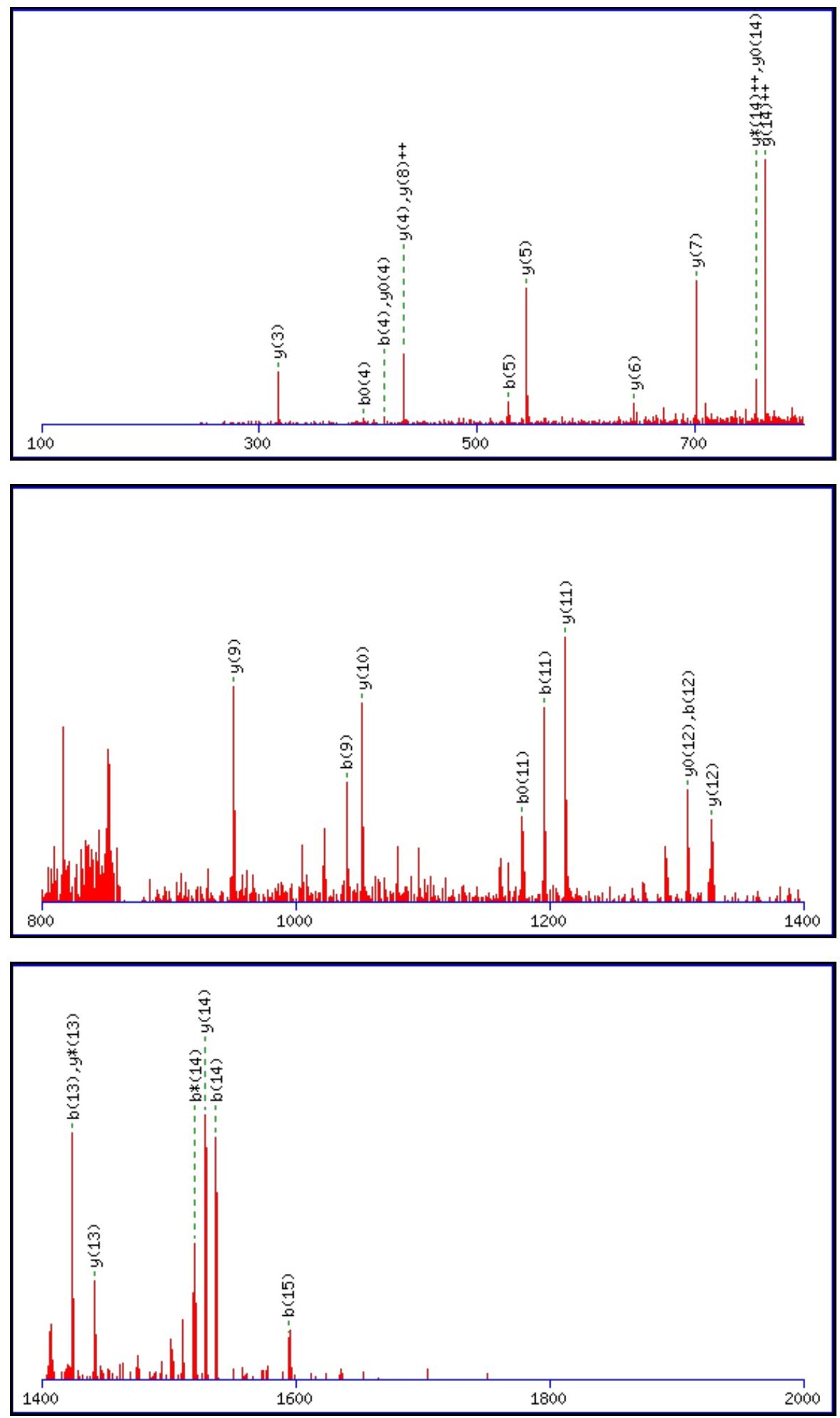

Monoisotopic mass of neutral peptide $\operatorname{Mr}($ calc): 1739.8400

Fixed modifications: Carbamidomethyl (C)

Variable modifications:

N5 : Deamidated $\mathrm{N}(\mathrm{N})$

Ions Score: 88 Expect: $2.8 \mathrm{e}-007$

Matches (Bold Red): 29/164 fragment ions using 39 most intense peaks

\begin{tabular}{|c|c|c|c|c|c|c|c|c|c|c|c|c|c|c|}
\hline$\#$ & $\mathbf{b}$ & $\mathbf{b}^{++}$ & $\mathbf{b}^{*}$ & $\mathbf{b}^{*^{++}}$ & $\mathbf{b}^{\mathbf{0}}$ & $\mathbf{b}^{\mathbf{0 + +}}$ & Seq. & $\mathbf{y}$ & $\mathbf{y}^{++}$ & $\mathbf{y}^{\mathbf{*}}$ & $\mathbf{y}^{\boldsymbol{*}^{++}}$ & $\mathbf{y}^{\mathbf{0}}$ & $\mathbf{y}^{\mathbf{0 + +}}$ & $\#$ \\
\hline $\mathbf{1}$ & 100.0757 & 50.5415 & & & & & $\mathbf{V}$ & & & & & & & $\mathbf{1 6}$ \\
\hline
\end{tabular}




\begin{tabular}{|c|c|c|c|c|c|c|c|c|c|c|c|c|c|c|}
\hline 2 & 213.1598 & 107.0835 & & & & & I & 1641.7789 & 821.3931 & 1624.7523 & 812.8798 & 1623.7683 & 812.3878 & 15 \\
\hline 3 & 300.1918 & 150.5995 & & & 282.1812 & 141.5942 & $\mathbf{S}$ & 1528.6948 & 764.8510 & 1511.6682 & 756.3378 & 1510.6842 & 755.8458 & 14 \\
\hline 4 & 414.2347 & 207.6210 & 397.2082 & 199.1077 & 396.2241 & 198.6157 & $\mathbf{N}$ & 1441.6628 & 721.3350 & 1424.6362 & 712.8217 & 1423.6522 & $\mid 712.3297$ & 13 \\
\hline 5 & 529.2616 & 265.1345 & 512.2351 & 256.6212 & 511.2511 & 256.1292 & $\mathbf{N}$ & 1327.6198 & 664.3136 & 1310.5933 & 655.8003 & 1309.6093 & 655.3083 & 12 \\
\hline 6 & 689.2923 & 345.1498 & 672.2657 & 336.6365 & 671.2817 & 336.1445 & C & 1212.5929 & 606.8001 & 1195.5664 & 598.2868 & 1194.5823 & 597.7948 & 11 \\
\hline 7 & 790.3400 & 395.6736 & 773.3134 & 387.1604 & 772.3294 & 386.6683 & $\mathbf{T}$ & 1052.5623 & 526.7848 & 1035.5357 & 518.2715 & 1034.5517 & 517.7795 & 10 \\
\hline 8 & 877.3720 & 439.1896 & 860.3455 & 430.6764 & 859.3614 & 430.1844 & $\mathbf{S}$ & 951.5146 & 476.2609 & 934.4880 & 467.7477 & 933.5040 & 467.2556 & 9 \\
\hline 9 & 1040.4353 & 520.7213 & 1023.4088 & 512.2080 & 1022.4248 & 511.7160 & $\mathbf{Y}$ & 864.4825 & 432.7449 & 847.4560 & 424.2316 & 846.4720 & 423.7396 & 8 \\
\hline 10 & 1097.4568 & 549.2320 & 1080.4302 & 540.7188 & 1079.4462 & 540.2268 & G & 701.4192 & 351.2132 & 684.3927 & 342.7000 & 683.4087 & 342.2080 & 7 \\
\hline 11. & 1196.5252 & 598.7662 & 1179.4987 & 590.2530 & 1178.5146 & 589.7610 & V & 644.3978 & 322.7025 & 627.3712 & 314.1892 & 626.3872 & 313.6972 & 6 \\
\hline 12 & 1309.6093 & 655.3083 & 1292.5827 & 646.7950 & 1291.5987 & 646.3030 & $\mathbf{L}$ & 545.3293 & 273.1683 & 528.3028 & 264.6550 & 527.3188 & 264.1630 & 5 \\
\hline 13 & 1424.6362 & 712.8217 & 1407.6097 & 704.3085 & 1406.6257 & 703.8165 & D & 432.2453 & 216.6263 & 415.2187 & 208.1130 & 414.2347 & 207.6210 & 4 \\
\hline 14 & 1537.7203 & 769.3638 & 1520.6937 & 760.8505 & 1519.7097 & 760.3585 & I & 317.2183 & 159.1128 & 300.1918 & 150.5995 & & & 3 \\
\hline 15 & 1594.7417 & 797.8745 & 1577.7152 & 789.3612 & 1576.7312 & 788.8692 & G & 204.1343 & 102.5708 & 187.1077 & 94.0575 & & & 2 \\
\hline 16 & & & & & & & $\mathbf{K}$ & 147.1128 & 74.0600 & 130.0863 & 65.5468 & & & 1 \\
\hline
\end{tabular}
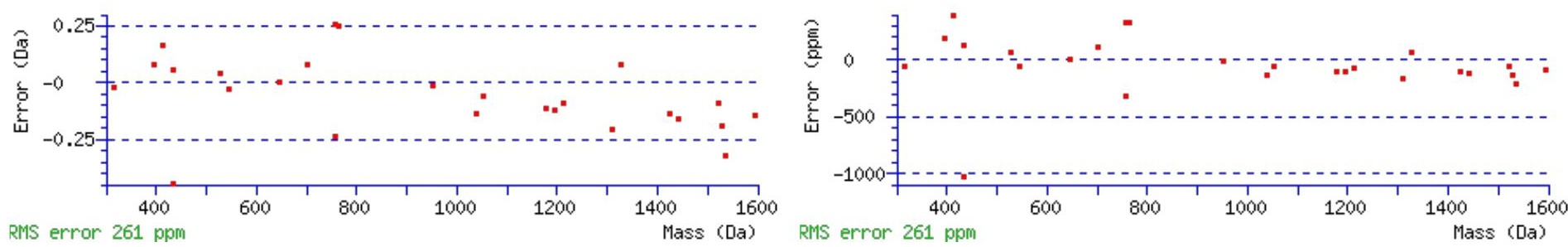

\section{All matches to this query}

\begin{tabular}{|l|l|l|l|}
\hline Score & Mr(calc): & Delta & \multicolumn{1}{|c|}{ Sequence } \\
\hline 88.2 & 1739.8400 & 0.0020 & VISNNCTSYGVLDIGK \\
\hline 74.1 & 1739.8400 & 0.0020 & VISNNCTSYGVLDIGK \\
\hline 39.8 & 1738.8560 & 0.9860 & VISNNCTSYGVLDIGK \\
\hline 6.6 & 1739.8502 & -0.0082 & MRKPDGSTVTQRIR \\
\hline 5.6 & 1739.8502 & -0.0082 & $\underline{\text { MRKPDGSTVTQRIR }}$ \\
\hline 5.6 & 1739.8502 & -0.0082 & $\underline{\text { MRKPDGSTVTQRIR }}$ \\
\hline 1.9 & 1738.8291 & 1.0129 & SSPPPPLPAEERPGTK \\
\hline 0.3 & 1738.8443 & 0.9977 & LHLWTNGSVAYSIAK \\
\hline 0.1 & 1739.8593 & -0.0173 & $\underline{\text { IKSEIEEELKSLDK }}$ \\
\hline
\end{tabular}

Spectrum No: 151; Query: 2462; Rank: 1

\section{Peptide View}

MS/MS Fragmentation of TVVTEAGNLLKDNATEEEILHYLEK

Found in IPI00195160, Tax_Id=10116 Gene_Symbol=Psap Sulfated glycoprotein 1 precursor

Match to Query 2462: 2829.433782 from(944.151870,3+)

Title: 091008RatKidney_NH4Format01_18.5332.5332.3.dta

Data file K:INewmanPaper|Piliang|3SubProteomes\Piliang3SP\mgf5ppm\ERLIC_3SubProteomes5ppm.mgf 

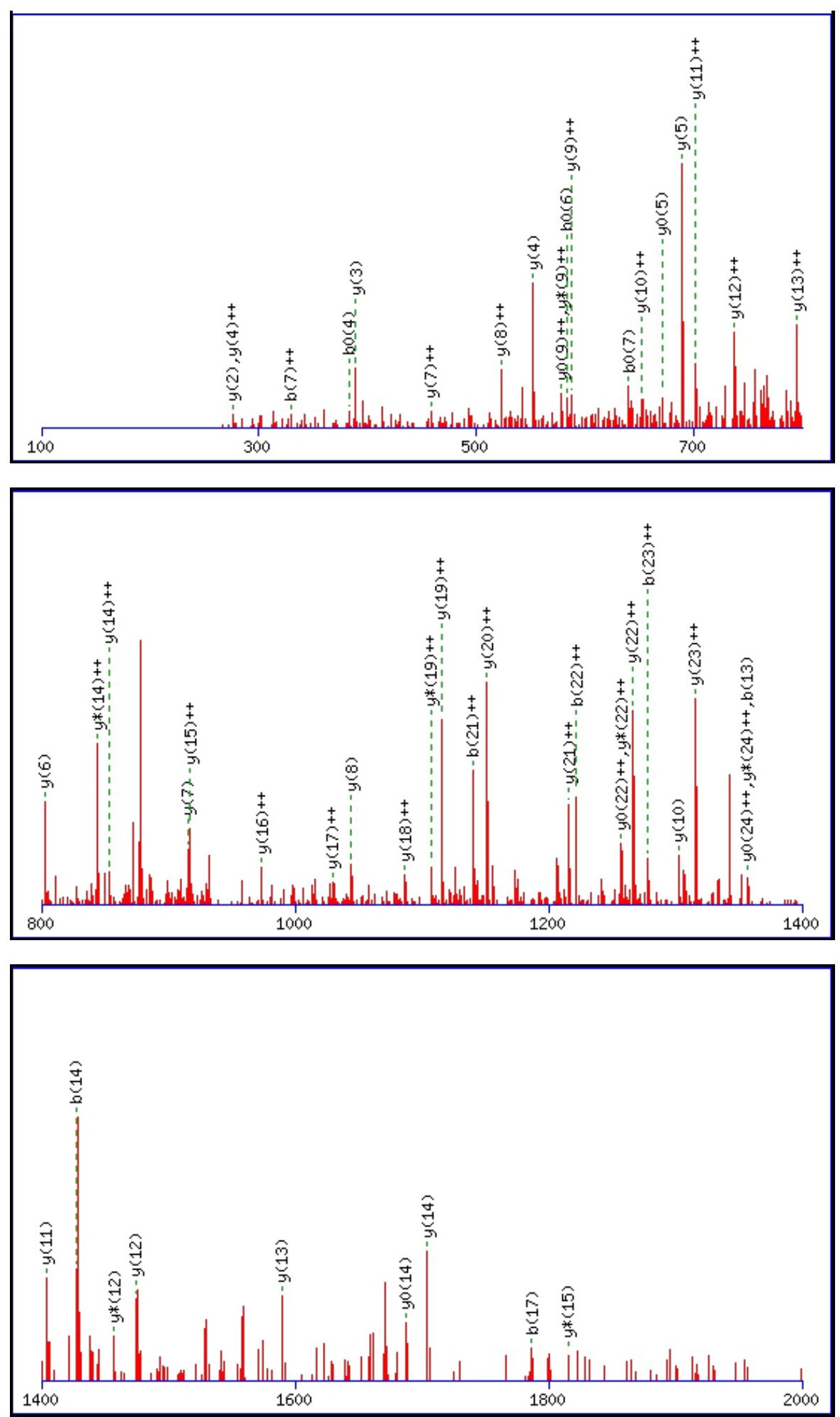

Monoisotopic mass of neutral peptide $\operatorname{Mr}($ calc): 2829.4283

Fixed modifications: Carbamidomethyl (C)

Variable modifications:

N13 : Deamidated_N (N)

Ions Score: 88 Expect: $2.7 \mathrm{e}-007$

Matches (Bold Red): 52/272 fragment ions using 96 most intense peaks

\begin{tabular}{|c|c|c|c|c|c|c|c|c|c|c|c|c|c|c|}
\hline \# & b & $\mathbf{b}^{++}$ & $\mathbf{b}^{*}$ & $\mathbf{b}^{*^{++}}$ & $\mathbf{b}^{0}$ & $\mathbf{b}^{\mathbf{0 + +}}$ & Seq. & $\mathbf{y}$ & $y^{++}$ & $\mathbf{y}^{*}$ & $\mathrm{y}^{*^{++}}$ & $\mathbf{y}^{\mathbf{0}}$ & $y^{0++}$ & \# \\
\hline 1 & 102.0550 & 51.5311 & & & 84.0444 & 42.5258 & $\mathbf{T}$ & & & & & & & 25 \\
\hline 2 & 201.1234 & 101.0653 & & & 183.1128 & 92.0600 & V & 2729.3880 & 1365.1976 & 2712.3614 & 1356.6844 & 2711.3774 & 1356.1923 & 24 \\
\hline 3 & 300.1918 & 150.5995 & & & 282.1812 & 141.5942 & V & 2630.3196 & 1315.6634 & 2613.2930 & 1307.1501 & 2612.3090 & 1306.6581 & 23 \\
\hline 4 & 401.2395 & 201.1234 & & & 383.2289 & 192.1181 & $T$ & 2531.2511 & 1266.1292 & 2514.2246 & 1257.6159 & 2513.2406 & 1257.1239 & 22 \\
\hline 5 & 530.2821 & 265.6447 & & & 512.2715 & 256.6394 & $\mathbf{E}$ & 2430.2035 & 1215.6054 & 2413.1769 & 1207.0921 & 2412.1929 & 1206.6001 & 21 \\
\hline
\end{tabular}




\begin{tabular}{|c|c|c|c|c|c|c|c|c|c|c|c|c|c|c|}
\hline 6 & 601.3192 & 301.1632 & & & 583.3086 & 292.1579| & A & 2301.1609| & $|1151.0841|$ & 2284.1343 & 1142.5708 & 2283.1503 & 1142.0788 & $20 \mid$ \\
\hline 7 & 658.3406 & 329.6740 & & & 640.3301 & 320.6687 & G & 2230.1238 & 1115.5655 & 2213.0972 & 1107.0522 & 2212.1132 & 1106.5602 & 19 \\
\hline 8 & 772.3836 & 386.6954 & 755.3570 & 378.1821 & 754.3730 & 377.6901 & $\mathbf{N}$ & 2173.1023 & 1087.0548 & 2156.0758 & 1078.5415 & 2155.0917 & 1078.0495 & 18 \\
\hline 9 & 885.4676 & 443.2375 & 868.4411 & 434.7242 & 867.4571 & 434.2322 & $\mathbf{L}$ & 2059.0594 & 1030.0333 & 2042.0328 & 1021.5200 & 2041.0488 & 1021.0280 & 17 \\
\hline 10 & 998.5517 & 499.7795 & 981.5251 & 491.2662 & 980.5411 & 490.7742 & $\mathbf{L}$ & 1945.9753 & 973.4913 & 1928.9488 & 964.9780 & 1927.9647 & 964.4860 & 16 \\
\hline 11 1 & 1126.6467 & 563.8270 & 1109.6201 & 555.3137 & 1108.6361 & 554.8217 & $\mathbf{K}$ & 1832.8912 & 916.9493 & 1815.8647 & 908.4360 & 1814.8807 & 907.9440 & 15 \\
\hline 12 & 1241.6736 & 621.3404 & 1224.6470 & 612.8272 & 1223.6630 & 612.3352 & D & 1704.7963 & 852.9018 & 1687.7697 & 844.3885 & 1686.7857 & 843.8965 & 14 \\
\hline 13 & 1356.7005 & 678.8539 & 1339.6740 & 670.3406 & 1338.6900 & 669.8486 & $\mathbf{N}$ & 1589.7693 & 795.3883 & 1572.7428 & 786.8750 & 1571.7588 & 786.3830 & 13 \\
\hline 14 & 1427.7376 & 714.3725 & 1410.7111 & 705.8592 & 1409.7271 & 705.3672 & A & 1474.7424 & 737.8748 & 1457.7159 & 729.3616 & 1456.7318 & 728.8696 & 12 \\
\hline 15 & 1528.7853 & 764.8963 & 1511.7588 & 756.3830 & 1510.7748 & 755.8910 & $T$ & 1403.7053 & 702.3563 & 1386.6787 & 693.8430 & 1385.6947 & 693.3510 & 11 \\
\hline 16 & 1657.8279 & 829.4176 & 1640.8014 & 820. & 1639.8174 & 820.4123 & $\mathbf{E}$ & 1302.6576 & 651.8324 & 1285.6311 & 643.3192 & 1284.6470 & 642.8272 & 10 \\
\hline 17 & 1786.8705 & 893.9389 & 1769.8440 & 885.4256 & 1768.8599 & 884.9336 & $\mathbf{E}$ & 1173.6150 & 587.3111 & 1156.5885 & 578.7979 & 1155.6045 & 578.3059 & 9 \\
\hline 18 & 1915.9131 & 958.4602 & 1898.8866 & 949.9469 & 1897.9025 & 949.4549 & $\mathbf{E}$ & 1044.5724 & 522.7898 & 1027.5459 & 514.2766 & 1026.5619 & 513.7846 & 8 \\
\hline 192 & 2028.9972 & 1015.0022 & 2011.9706 & 1006.4889 & 2010.9866 & 1005.9969 & I & 915.5298 & 458.2686 & 898.5033 & 449.7553 & 897.5193 & 449.2633 & 7 \\
\hline 202 & 2142.0812 & 1071.5443 & 2125.0547 & 1063.0310 & 2124.0707 & 1062.5390 & $\mathbf{L}$ & 802.4458 & 401.7265 & 785.4192 & 393.2132 & 784.4352 & 392.7212 & 6 \\
\hline 212 & 2279.1401 & 1140.0737 & 2262.1136 & 1131.5604 & 2261.1296 & 1131.0684 & H & 689.3617 & 345.1845 & 672.3352 & 336.6712 & 671.3511 & 336.1792 & 5 \\
\hline 222 & 2442.2035 & 1221.6054 & 2425.1769 & 1213.0921 & 2424.1929 & 1212.6001 & $\mathbf{Y}$ & 552.3028 & 276.6550 & 535.2762 & 268.1418 & 534.2922 & 267.6498 & 4 \\
\hline 232 & 2555.2875 & 1278.1474 & 2538.2610 & 1269.6341 & 2537.2770 & 1269.1421 & $\mathbf{L}$ & 389.2395 & 195.1234 & 372.2129 & 186.6101 & 371.2289 & 186.1181 & 3 \\
\hline 242 & 2684.3301 & 1342.6687 & 2667.3036 & 1334.1554 & 2666.3196 & 1333.6634 & $\mathbf{E}$ & 276.1554 & 138.5813 & 259.1288 & 130.0681 & 258.1448 & 129.5761 & 2 \\
\hline 25 & & & & & & & $\mathbf{K}$ & 147.1128 & 74.0600 & 130.0863 & 65.5468 & & & 1 \\
\hline
\end{tabular}
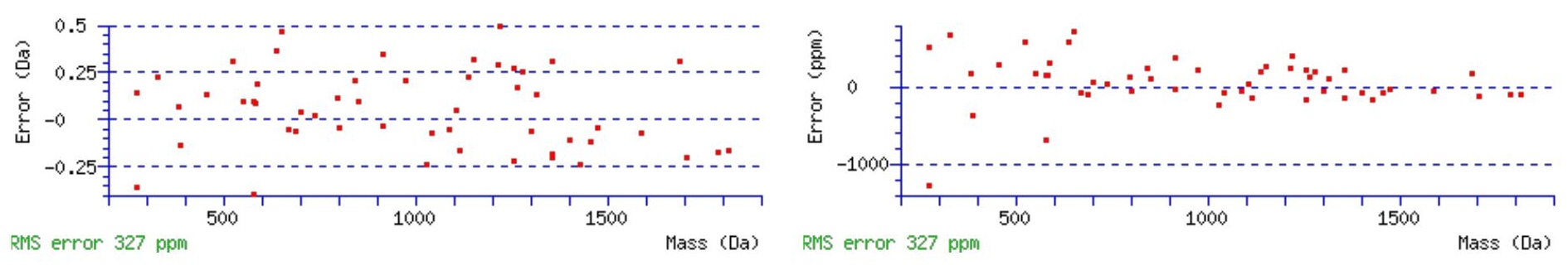

\section{All matches to this query}

\begin{tabular}{|l|l|l|l|}
\hline Score & Mr(calc): & Delta & \multicolumn{1}{|c|}{ Sequence } \\
\hline 88.0 & 2829.4283 & 0.0055 & TVVTEAGNLLKDNATEEEILHYLEK \\
\hline 48.1 & 2829.4283 & 0.0055 & TVVTEAGNLLKDNATEEEILHYLEK \\
\hline 18.8 & 2828.4443 & 0.9895 & TVVTEAGNLLKDNATEEEILHYLEK \\
\hline
\end{tabular}

Spectrum No: 152; Query: 1811; Rank: 1

\section{Peptide View}

MS/MS Fragmentation of GQIIGISCQSVNGTAPITYR

Found in IPI00206467, Tax_Id=10116 Gene_Symbol=Pecam Platelet endothelial cell adhesion molecule precursor

Match to Query 1811: 2135.075068 from(1068.544810,2+)

Title: 100101RatKid_NS_deglyco_21.3138.3138.2.dta

Data file K:INewmanPaper|Piliangl3SubProteomes|Piliang3SP\mgf5ppm|ERLIC_3SubProteomes5ppm.mgf 

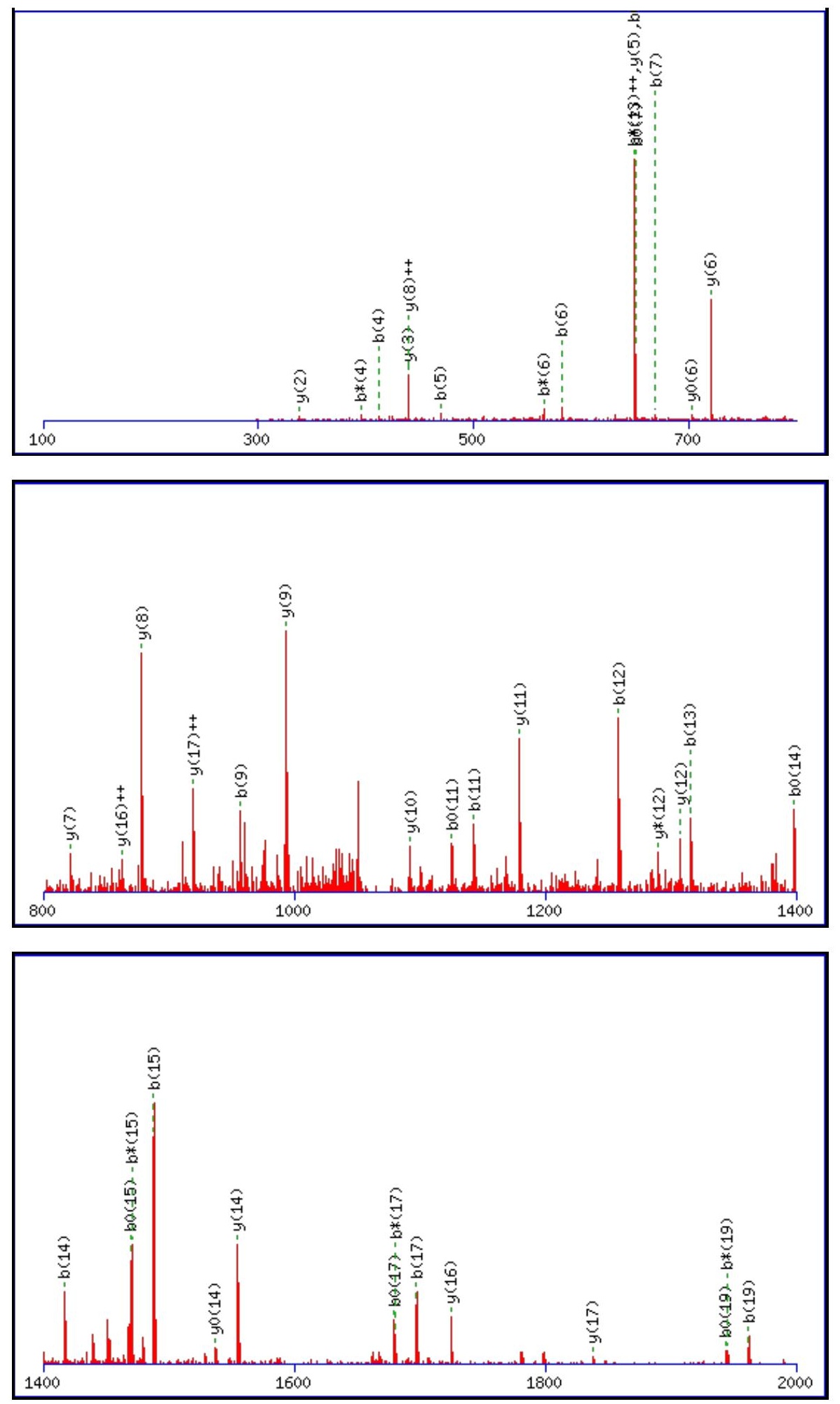

Monoisotopic mass of neutral peptide $\operatorname{Mr}($ calc): 2135.0681

Fixed modifications: Carbamidomethyl (C)

Variable modifications:

N12 : Deamidated_N (N)

Ions Score: 88 Expect: $3.2 \mathrm{e}-007$

Matches (Bold Red): 44/210 fragment ions using 73 most intense peaks

\begin{tabular}{|r|r|r|c|c|c|r|r|r|r|r|r|r|r|r|}
\hline$\#$ & $\mathbf{b}$ & $\mathbf{b}^{++}$ & $\mathbf{b}^{*}$ & $\mathbf{b}^{\boldsymbol{*}^{++}}$ & $\mathbf{b}^{\mathbf{0}}$ & $\mathbf{b}^{\mathbf{0 + +}}$ & Seq. & $\mathbf{y}$ & $\mathbf{y}^{++}$ & $\mathbf{y}^{\mathbf{*}}$ & $\mathbf{y}^{\boldsymbol{*}^{++}}$ & $\mathbf{y}^{\mathbf{0}}$ & $\mathbf{y}^{\mathbf{0 + +}}$ & $\#$ \\
\hline $\mathbf{1}$ & 58.0287 & 29.5180 & & & & & $\mathbf{G}$ & & & & & & & $\mathbf{2 0}$ \\
\hline $\mathbf{2}$ & 186.0873 & 93.5473 & 169.0608 & 85.0340 & & & $\mathbf{Q}$ & 2079.0539 & 1040.0306 & 2062.0274 & 1031.5173 & 2061.0433 & 1031.0253 & $\mathbf{1 9}$ \\
\hline $\mathbf{3}$ & 299.1714 & 150.0893 & 282.1448 & 141.5761 & & & $\mathbf{I}$ & 1950.9953 & 976.0013 & 1933.9688 & 967.4880 & 1932.9848 & 966.9960 & $\mathbf{1 8}$ \\
\hline $\mathbf{4}$ & $\mathbf{4 1 2 . 2 5 5 4}$ & 206.6314 & 395.2289 & 198.1181 & & & $\mathbf{I}$ & $\mathbf{1 8 3 7 . 9 1 1 3}$ & $\mathbf{9 1 9 . 4 5 9 3}$ & 1820.8847 & 910.9460 & 1819.9007 & 910.4540 & $\mathbf{1 7}$ \\
\hline $\mathbf{5}$ & $\mathbf{4 6 9 . 2 7 6 9}$ & 235.1421 & 452.2504 & 226.6288 & & & $\mathbf{G}$ & $\mathbf{1 7 2 4 . 8 2 7 2}$ & $\mathbf{8 6 2 . 9 1 7 2}$ & 1707.8007 & 854.4040 & 1706.8166 & 853.9120 & $\mathbf{1 6}$ \\
\hline
\end{tabular}




\begin{tabular}{|c|c|c|c|c|c|c|c|c|c|c|c|c|c|c|}
\hline & 582.3610 & |291.6841 & 565.3344 & 283.1709 & & & I & |1667.8057| & 834.4065 & 1650.7792 & 825.8932 & 1649.7952 & 825.4012 & $\mid 15$ \\
\hline 7 & 669.3930 & 335.2001 & 652.3665 & 326.6869 & 651.3824 & 326.1949 & S & 1554.7217 & 777.8645 & 1537.6951 & 769.3512 & 1536.7111 & 768.8592 & 14 \\
\hline 8 & 829.4237 & 415.2155 & 812.3971 & 406.7022 & 811.4131 & 406.2102 & C & 1467.6897 & 734.3485 & 1450.6631 & 725.8352 & 1449.6791 & 725.3432 & 13 \\
\hline 9 & 957.4822 & 479.2448 & 940.4557 & 470.7315 & 939.4717 & 470.2395 & $\mathbf{Q}$ & 1307.6590 & 654.3331 & 1290.6325 & 645.8199 & 1289.6484 & 645.3279 & 12 \\
\hline 10 & 044.5143 & 522.7608 & 1027.4877 & 514.2475 & 1026.5037 & 513.7555 & & 1179.6004 & 590.3038 & 1162.5739 & 581.7906 & 1161.5899 & 581.2986 & 11 \\
\hline 11 & 1143.5827 & 572.2950 & 1126.5561 & 563.7817 & 1125.5721 & 563.2897 & V & 1092.5684 & 546.7878 & 1075.5418 & 538.2746 & 1074.5578 & 537.7826 & 10 \\
\hline 12 & 1258.6096 & 629.8084 & 1241.5831 & 621.2952 & 1240.5990 & 620.8032 & $\mathbf{N}$ & 993.5000 & 497.2536 & 976.4734 & 488.7404 & 975.4894 & 488.2483 & 9 \\
\hline 13 & 1315.6311 & 658.3192 & 1298.6045 & 649.8059 & 1297. & 649.3139 & G & 878.4730 & 439.7402 & 861.4465 & 431.2269 & 860.4625 & 430.7349 & 8 \\
\hline 14 & \begin{tabular}{|l|l|}
1416.6788 \\
\end{tabular} & 708.8430 & 1399.6522 & 700.3297 & 1398.6682 & 699.8377 & $T$ & 821.4516 & 411.2294 & 804.4250 & 402.7162 & 803.4410 & 402.2241 & 7 \\
\hline 15 & 1487.7159 & 744.3616 & 1470.6893 & 735.8483 & 1469.7053 & 735.3563 & A & 720.4039 & 360.7056 & 703.3774 & 352.1923 & 702.3933 & 351.7003 & 6 \\
\hline 16 & 1584.7686 & 792.8880 & 1567.7421 & 784.3747 & 1566.7581 & 783.8827 & $\mathbf{P}$ & 649.3668 & 325.1870 & 632.3402 & 316.6738 & 631.3562 & 316.1817 & 5 \\
\hline 17 & 1697.8527 & 849.4300 & 1680.8261 & 840.9167 & 1679.8421 & 840.4247 & 1 & 552.3140 & 276.6607 & 535.2875 & 268.1474 & 534.3035 & 267.6554 & 4 \\
\hline 18 & 1798.9004 & 899.9538 & 1781.8738 & 891.4405 & 1780.8898 & 890.9485 & $\mathbf{T}$ & 439.2300 & 220.1186 & 422.2034 & 211.6053 & 421.2194 & 211.1133 & 3 \\
\hline 19 & 1961.9637 & 981.4855 & 1944.9372 & 972.9722 & 1943.9531 & 972.4802 & $\mathbf{Y}$ & 338.1823 & 169.5948 & 321.1557 & 161.0815 & & & 2 \\
\hline 20 & & & & & & & $\mathbf{R}$ & 175.1190 & 88.0631 & 158.0924 & 79.5498 & & & 1 \\
\hline
\end{tabular}
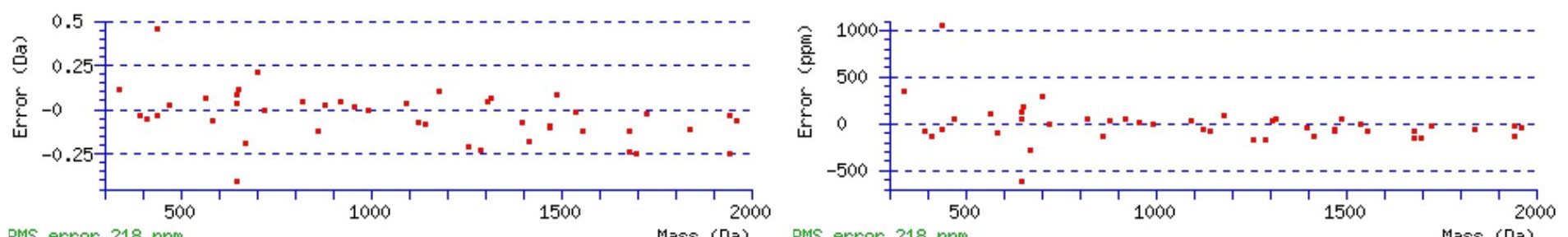

RMS error $218 \mathrm{ppm}$

Mass (Da) RMS error 218 ppm

\section{All matches to this query}

\begin{tabular}{|l|l|l|l|}
\hline Score & Mr(calc): & Delta & \multicolumn{1}{c|}{ Sequence } \\
\hline 88.0 & 2135.0681 & 0.0070 & GQIIGISCQSVNGTAPITYR \\
\hline 22.7 & 2134.0841 & 0.9910 & GQIIGISCQSVNGTAPITYR \\
\hline 0.2 & 2135.0697 & 0.0054 & LEPVLPSVAALSSAGTMERK \\
\hline 0.2 & 2135.0697 & 0.0054 & LEPVLPSVAALSSAGTMERK \\
\hline
\end{tabular}

Spectrum No: 153; Query: 937; Rank: 1

\section{Peptide View}

MS/MS Fragmentation of VDLEDFENNTAYAK

Found in IPI00369539, Tax_Id=10116 Gene_Symbol=Mfap4 Microfibrillar-associated protein 4

Match to Query 937: 1628.722468 from(815.368510,2+)

Title: 100101RatKid_NS_deglyco_26.2984.2984.2.dta

Data file K:INewmanPaper|Piliang|3SubProteomes\Piliang3SP\mgf5ppm\ERLIC_3SubProteomes5ppm.mgf 

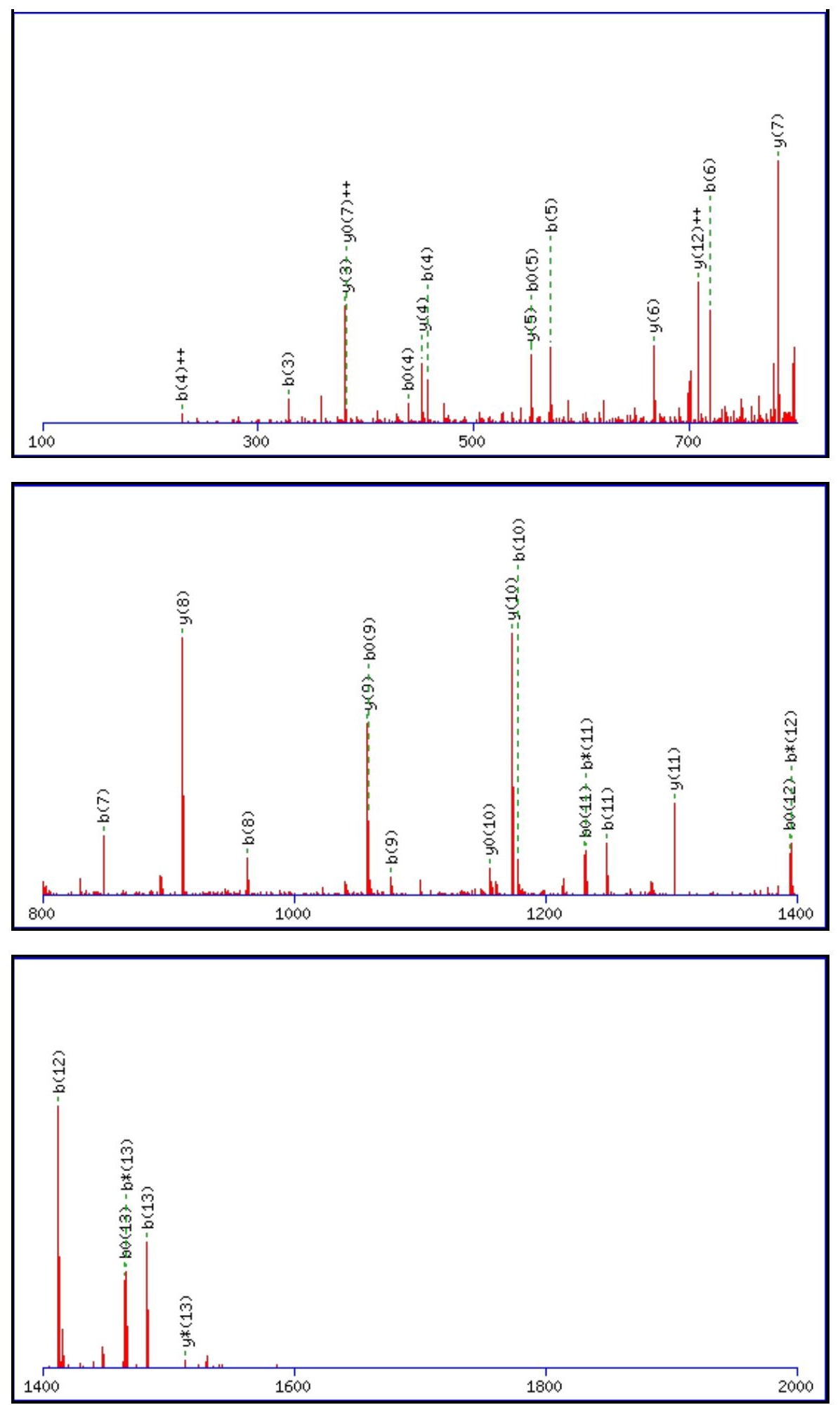

Monoisotopic mass of neutral peptide $\operatorname{Mr}($ calc): 1628.7206

Fixed modifications: Carbamidomethyl (C)

Variable modifications:

N8: : Deamidated N (N)

Ions Score: 88 Expect: $3.4 \mathrm{e}-007$

Matches (Bold Red): 34/132 fragment ions using 48 most intense peaks

\begin{tabular}{|r|c|c|c|c|c|c|c|c|c|c|c|c|c|c|}
\hline$\#$ & $\mathbf{b}$ & $\mathbf{b}^{++}$ & $\mathbf{b}^{*}$ & $\mathbf{b}^{\boldsymbol{*}^{++}}$ & $\mathbf{b}^{\mathbf{0}}$ & $\mathbf{b}^{\mathbf{0 + +}}$ & Seq. & $\mathbf{y}$ & $\mathbf{y}^{++}$ & $\mathbf{y}^{\mathbf{*}}$ & $\mathbf{y}^{\boldsymbol{*}^{++}}$ & $\mathbf{y}^{\mathbf{0}}$ & $\mathbf{y}^{\mathbf{0 + +}}$ & $\#$ \\
\hline $\mathbf{1}$ & 100.0757 & 50.5415 & & & & & $\mathbf{V}$ & & & & & & & $\mathbf{1 4}$ \\
\hline $\mathbf{2}$ & 215.1026 & 108.0550 & & & 197.0921 & 99.0497 & $\mathbf{D}$ & 1530.6595 & 765.8334 & $\mathbf{1 5 1 3 . 6 3 2 9}$ & 757.3201 & 1512.6489 & $\mathbf{7 5 6 . 8 2 8 1}$ & $\mathbf{1 3}$ \\
\hline $\mathbf{3}$ & $\mathbf{3 2 8 . 1 8 6 7}$ & 164.5970 & & & 310.1761 & 155.5917 & $\mathbf{L}$ & 1415.6325 & 708.3199 & 1398.6060 & 699.8066 & 1397.6219 & 699.3146 & $\mathbf{1 2}$ \\
\hline $\mathbf{4}$ & $\mathbf{4 5 7 . 2 2 9 3}$ & $\mathbf{2 2 9 . 1 1 8 3}$ & & & $\mathbf{4 3 9 . 2 1 8 7}$ & 220.1130 & $\mathbf{E}$ & $\mathbf{1 3 0 2 . 5 4 8 4}$ & 651.7779 & 1285.5219 & 643.2646 & 1284.5379 & 642.7726 & $\mathbf{1 1}$ \\
\hline $\mathbf{5}$ & $\mathbf{5 7 2 . 2 5 6 2}$ & 286.6318 & & & 554.2457 & 277.6265 & $\mathbf{D}$ & $\mathbf{1 1 7 3 . 5 0 5 9}$ & 587.2566 & 1156.4793 & 578.7433 & $\mathbf{1 1 5 5 . 4 9 5 3}$ & 578.2513 & $\mathbf{1 0}$ \\
\hline
\end{tabular}




\begin{tabular}{|r|r|r|r|r|r|r|r|r|r|r|r|r|r|r|r|r|r|r|}
$\mathbf{6}$ & $\mathbf{7 1 9 . 3 2 4 6}$ & 360.1660 & & & 701.3141 & 351.1607 & $\mathbf{F}$ & $\mathbf{1 0 5 8 . 4 7 8 9}$ & 529.7431 & 1041.4524 & 521.2298 & 1040.4683 & 520.7378 & $\mathbf{9}$ \\
\hline $\mathbf{7}$ & $\mathbf{8 4 8 . 3 6 7 2}$ & 424.6873 & & & 830.3567 & 415.6820 & $\mathbf{E}$ & $\mathbf{9 1 1 . 4 1 0 5}$ & 456.2089 & 894.3839 & 447.6956 & 893.3999 & 447.2036 & $\mathbf{8}$ \\
\hline $\mathbf{8}$ & $\mathbf{9 6 3 . 3 9 4 2}$ & 482.2007 & 946.3676 & $\mathbf{4 7 3 . 6 8 7 5}$ & 945.3836 & 473.1954 & $\mathbf{N}$ & $\mathbf{7 8 2 . 3 6 7 9}$ & 391.6876 & 765.3414 & 383.1743 & 764.3573 & $\mathbf{3 8 2 . 6 8 2 3}$ & $\mathbf{7}$ \\
\hline $\mathbf{9}$ & $\mathbf{1 0 7 7 . 4 3 7 1}$ & 539.2222 & 1060.4106 & 530.7089 & $\mathbf{1 0 5 9 . 4 2 6 5}$ & 530.2169 & $\mathbf{N}$ & $\mathbf{6 6 7 . 3 4 1 0}$ & 334.1741 & 650.3144 & 325.6608 & 649.3304 & 325.1688 & $\mathbf{6}$ \\
\hline $\mathbf{1 0}$ & $\mathbf{1 1 7 8 . 4 8 4 8}$ & 589.7460 & 1161.4582 & 581.2328 & 1160.4742 & 580.7407 & $\mathbf{T}$ & $\mathbf{5 5 3 . 2 9 8 0}$ & 277.1527 & 536.2715 & 268.6394 & 535.2875 & 268.1474 & $\mathbf{5}$ \\
\hline $\mathbf{1 1}$ & $\mathbf{1 2 4 9 . 5 2 1 9}$ & 625.2646 & $\mathbf{1 2 3 2 . 4 9 5 3}$ & 616.7513 & $\mathbf{1 2 3 1 . 5 1 1 3}$ & 616.2593 & $\mathbf{A}$ & $\mathbf{4 5 2 . 2 5 0 4}$ & 226.6288 & 435.2238 & 218.1155 & & & & $\mathbf{4}$ \\
\hline $\mathbf{1 2}$ & $\mathbf{1 4 1 2 . 5 8 5 2}$ & $\mathbf{7 0 6 . 7 9 6 3}$ & $\mathbf{1 3 9 5 . 5 5 8 7}$ & 698.2830 & $\mathbf{1 3 9 4 . 5 7 4 7}$ & 697.7910 & $\mathbf{Y}$ & $\mathbf{3 8 1 . 2 1 3 2}$ & 191.1103 & 364.1867 & 182.5970 & & & & $\mathbf{3}$ \\
\hline $\mathbf{1 3}$ & $\mathbf{1 4 8 3 . 6 2 2 3}$ & $\mathbf{7 4 2 . 3 1 4 8}$ & $\mathbf{1 4 6 6 . 5 9 5 8}$ & $\mathbf{7 3 3 . 8 0 1 5}$ & $\mathbf{1 4 6 5 . 6 1 1 8}$ & $\mathbf{7 3 3 . 3 0 9 5}$ & $\mathbf{A}$ & 218.1499 & 109.5786 & 201.1234 & 101.0653 & & & $\mathbf{2}$ \\
\hline $\mathbf{1 4}$ & & & & & & & $\mathbf{K}$ & 147.1128 & 74.0600 & 130.0863 & 65.5468 & & & $\mathbf{1}$ \\
\hline
\end{tabular}
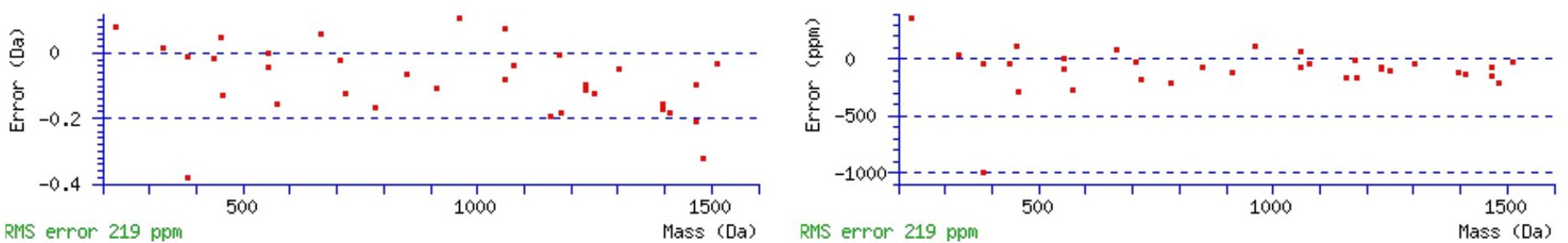

All matches to this query

\begin{tabular}{|l|l|c|l|}
\hline Score & Mr(calc): & Delta & \multicolumn{1}{|c|}{ Sequence } \\
\hline 87.8 & 1628.7206 & 0.0019 & VDLEDFENNTAYAK \\
\hline 85.7 & 1628.7206 & 0.0019 & VDLEDFENNTAYAK \\
\hline 9.4 & 1628.7083 & 0.0141 & SQPDFGSEIVTGNAK \\
\hline 7.2 & 1627.7131 & 1.0094 & KVVEDEVYSESHK \\
\hline 7.1 & 1628.7294 & -0.0070 & ADVNGDLNTVSTKSK \\
\hline 5.7 & 1628.7382 & -0.0157 & NNVVNTMRLWSAK \\
\hline 5.6 & 1628.7157 & 0.0068 & THNTLYTPEMLTK \\
\hline 2.4 & 1627.7131 & 1.0094 & KVVEDEVYSESHK \\
\hline 2.4 & 1627.7131 & 1.0094 & KVVEDEVYSESHK \\
\hline 0.6 & 1627.7065 & 1.0159 & MSPTERLEHAFSK \\
\hline
\end{tabular}

Spectrum No: 154; Query: 1765; Rank: 1

\section{Peptide View}

MS/MS Fragmentation of QQSQEDGDYSPNGSAQPGDK

Found in IPI00364925, Tax_Id=10116 Gene_Symbol=Dhtkd1 Probable 2-oxoglutarate dehydrogenase E1 component DHKTD1, mitochondrial precursor

Match to Query 1765: 2107.862948 from(1054.938750,2+)

Title: 100101RatKid_NS_deglyco_21.670.670.2.dta

Data file K:INewmanPaper|Piliangl3SubProteomes\Piliang3SP\mgf5ppm\ERLIC_3SubProteomes5ppm.mgf 

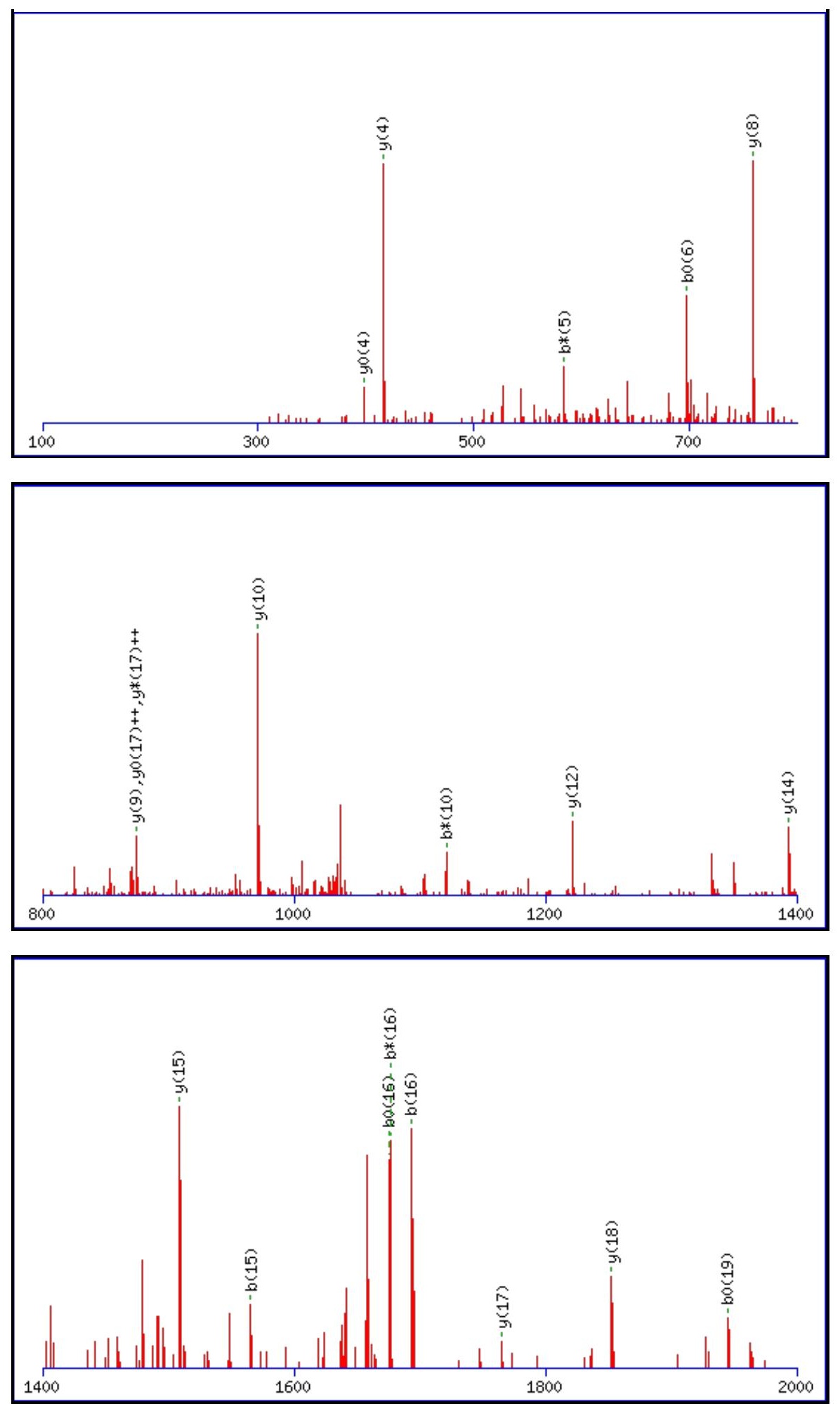

Monoisotopic mass of neutral peptide $\operatorname{Mr}($ calc): 2107.8566

Fixed modifications: Carbamidomethyl (C)

Variable modifications:

N12 : Deamidated_N (N)

Ions Score: 88 Expect: $3.8 \mathrm{e}-007$

Matches (Bold Red): 20/222 fragment ions using 28 most intense peaks

\begin{tabular}{|r|c|c|c|c|c|c|c|c|c|c|c|c|c|c|}
\hline$\#$ & $\mathbf{b}$ & $\mathbf{b}^{++}$ & $\mathbf{b}^{*}$ & $\mathbf{b}^{\boldsymbol{*}_{++}}$ & $\mathbf{b}^{\mathbf{0}}$ & $\mathbf{b}^{\mathbf{0 + +}}$ & $\mathbf{S e q}$ & $\mathbf{y}$ & $\mathbf{y}^{++}$ & $\mathbf{y}^{\mathbf{*}}$ & $\mathbf{y}^{\boldsymbol{*}^{++}}$ & $\mathbf{y}^{\mathbf{0}}$ & $\mathbf{y}^{\mathbf{0 + +}}$ & $\#$ \\
\hline $\mathbf{1}$ & 129.0659 & 65.0366 & 112.0393 & 56.5233 & & & $\mathbf{Q}$ & & & & & & & $\mathbf{2 0}$ \\
\hline $\mathbf{2}$ & 257.1244 & 129.0659 & 240.0979 & 120.5526 & & & $\mathbf{Q}$ & 1980.8053 & 990.9063 & 1963.7788 & 982.3930 & 1962.7948 & 981.9010 & $\mathbf{1 9}$ \\
\hline 3 & 344.1565 & 172.5819 & 327.1299 & 164.0686 & 326.1459 & 163.5766 & S & $\mathbf{1 8 5 2 . 7 4 6 8}$ & 926.8770 & 1835.7202 & 918.3637 & 1834.7362 & 917.8717 & $\mathbf{1 8}$ \\
\hline $\mathbf{4}$ & 472.2150 & 236.6112 & 455.1885 & 228.0979 & 454.2045 & 227.6059 & $\mathbf{Q}$ & $\mathbf{1 7 6 5 . 7 1 4 7}$ & 883.3610 & 1748.6882 & $\mathbf{8 7 4 . 8 4 7 7}$ & 1747.7042 & $\mathbf{8 7 4 . 3 5 5 7}$ & $\mathbf{1 7}$ \\
\hline $\mathbf{5}$ & 601.2576 & 301.1325 & 584.2311 & 292.6192 & 583.2471 & 292.1272 & $\mathbf{E}$ & 1637.6562 & 819.3317 & 1620.6296 & 810.8184 & 1619.6456 & 810.3264 & $\mathbf{1 6}$ \\
\hline
\end{tabular}




\begin{tabular}{|c|c|c|c|c|c|c|c|c|c|c|c|c|c|c|}
\hline & 716.2846 & & & & 698.2740 & 349.6406 & D & 136 & & & |746.2971 & 1490.6030 & & \\
\hline 7 & 773.3060 & |387.1567 & 756.2795 & |378.6434 & 755.2955 & 378.1514 & G & 1393.5866 & 697.2969 & 1376.5601 & 688.7837 & 1375.5761 & 688.2917 & 14 \\
\hline 8 & 888.3330 & 444.6701 & 871.3064 & 436.1569 & 870.3224 & 435.6648 & D & 1336.5652 & 668.7862 & 1319.5386 & 660.2729 & 1318.5546 & 659.7809 & 13 \\
\hline 9 & 1051.3963 & 526.2018 & 1034.3698 & 517.6885 & 1033.3857 & 517.1965 & $\mathbf{Y}$ & 1221.5382 & 611.2727 & 1204.5117 & 602.7595 & 1203.5277 & 602.2675 & 12 \\
\hline 10 & 38.4283 & 59.7178 & 018 & 561.2045 & 1120.4178 & 560.7125 & S & 1058.4749 & 529.7411 & 1041.4483 & 521.2278 & 1040.4643 & 520.7358 & \\
\hline 11 & 35.4811 & 618.2442 & 1218.4546 & 609.7309 & 1217.4705 & 609.2389 & $\mathbf{P}$ & 971.4429 & 486.2251 & 954.4163 & 477.7118 & 953.4323 & 477.2198 & 10 \\
\hline 12 & 1350.5080 & 675.7577 & 1333.4815 & 667.2444 & 1332.4975 & 666.7524 & $\mathbf{N}$ & 874.3901 & 437.6987 & 857.3635 & 429.1854 & 856.3795 & 428.6934 & 9 \\
\hline 13 & 1407.5295 & 704.2684 & 1390.5030 & 695.7551 & 1389.5189 & 695.2631 & G & 759.3632 & 380.1852 & 742.3366 & 371.6719 & 741.3526 & 371.1799 & 8 \\
\hline 14 & 494.5615 & 747.7844 & 1477.5350 & 739.2711 & 1476.5510 & 738.7791 & S & & 351.6745 & 685.3151 & 343.1612 & 684.3311 & 342.6692 & \\
\hline 15 & 1565.5986 & 783.3030 & 1548.5721 & 774.7897 & 1547.5881 & 774.2977 & A & 615.3097 & 308.1585 & 598.2831 & 299.6452 & 597.2991 & 299.1532 & 6 \\
\hline 16 & 1693.6572 & 847.3322 & \begin{tabular}{|l|}
1676.6307 \\
\end{tabular} & 838.8190 & 1675.6467 & 838.3270 & $\mathbf{Q}$ & 544.2726 & 272.6399 & 527.2460 & 264.1266 & 526.2620 & 263.6346 & 5 \\
\hline 17 & 1790.7100 & 895.8586 & 1773.6834 & 887.3454 & 1772.6994 & 886.8533 & $\mathbf{P}$ & 416.2140 & 208.6106 & 399.1874 & 200.0974 & 398.2034 & 199.6053 & 4 \\
\hline 18 & 1847.7315 & 924.3694 & 1830.7049 & 915.8561 & 1829.7209 & 915.3641 & G & 319.1612 & 160.0842 & 302.1347 & 151.5710 & 301.1506 & 151.0790 & 3 \\
\hline 19 & 1962.7584 & 981.8828 & 1945.7318 & 973.3696 & 1944.7478 & 972.8776 & D & 262.1397 & 131.5735 & 245.1132 & 123.0602 & 244.1292 & 122.5682 & 2 \\
\hline 20 & & & & & & & $\mathbf{K}$ & 147.1128 & 74.0600 & 130.0863 & 65.5468 & & & 1 \\
\hline
\end{tabular}
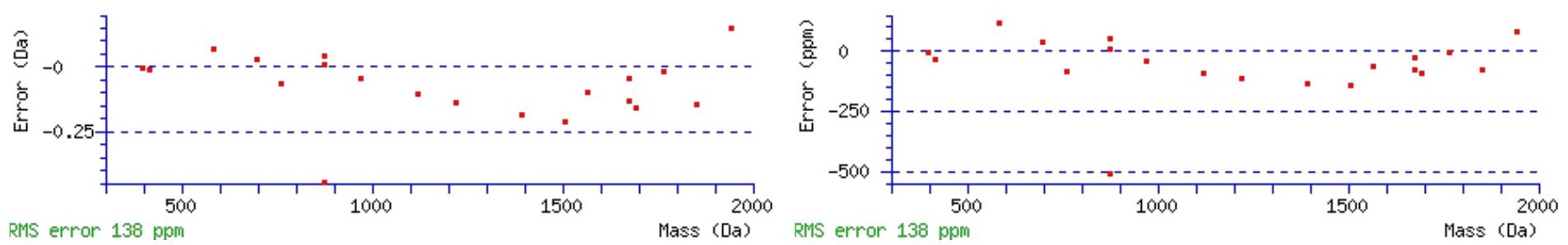

\section{All matches to this query}

\begin{tabular}{|l|l|l|l|}
\hline Score & Mr(calc): & Delta & \multicolumn{1}{|c|}{ Sequence } \\
\hline 87.6 & 2107.8566 & 0.0063 & QQSQEDGDYSPNGSAQPGDK \\
\hline 2.7 & 2107.8815 & -0.0186 & TGDMVLKSVNGSYTIRK \\
\hline 2.7 & 2107.8815 & -0.0186 & TGDMVLKSVNGSYTIRK \\
\hline 2.4 & 2107.8815 & -0.0186 & TGDMVLKSVNGSYTIRK \\
\hline 2.4 & 2107.8815 & -0.0186 & TGDMVLKSVNGSYTIRK \\
\hline 0.4 & 2106.8726 & 0.9903 & QQSQEDGDYSPNGSAQPGDK \\
\hline 0.2 & 2105.8643 & 1.9987 & STTNPGSFVLTGMHNGQAK \\
\hline 0.2 & 2105.8643 & 1.9987 & STTNPGSFVLTGMHNGQAK \\
\hline 0.2 & 2105.8643 & 1.9987 & STTNPGSFVLTGMHNGQAK \\
\hline 0.2 & 2105.8643 & 1.9987 & $\underline{\text { STTNPGSFVLTGMHNGQAK }}$ \\
\hline
\end{tabular}

Spectrum No: 155; Query: 874; Rank: 1

\section{Peptide View}

MS/MS Fragmentation of CSGDGLDSDGNQTLR

Found in IPI00364764, Tax_Id=10116 Gene_Symbol=RGD1559717_predicted similar to R17.3

Match to Query 874: 1594.655628 from(798.335090,2+)

Title: 100101RatKid_NS_deglyco_24.1065.1065.2.dta

Data file K:\NewmanPaper|Piliang|3SubProteomes\Piliang3SP\mgf5ppm\ERLIC_3SubProteomes5ppm.mgf 

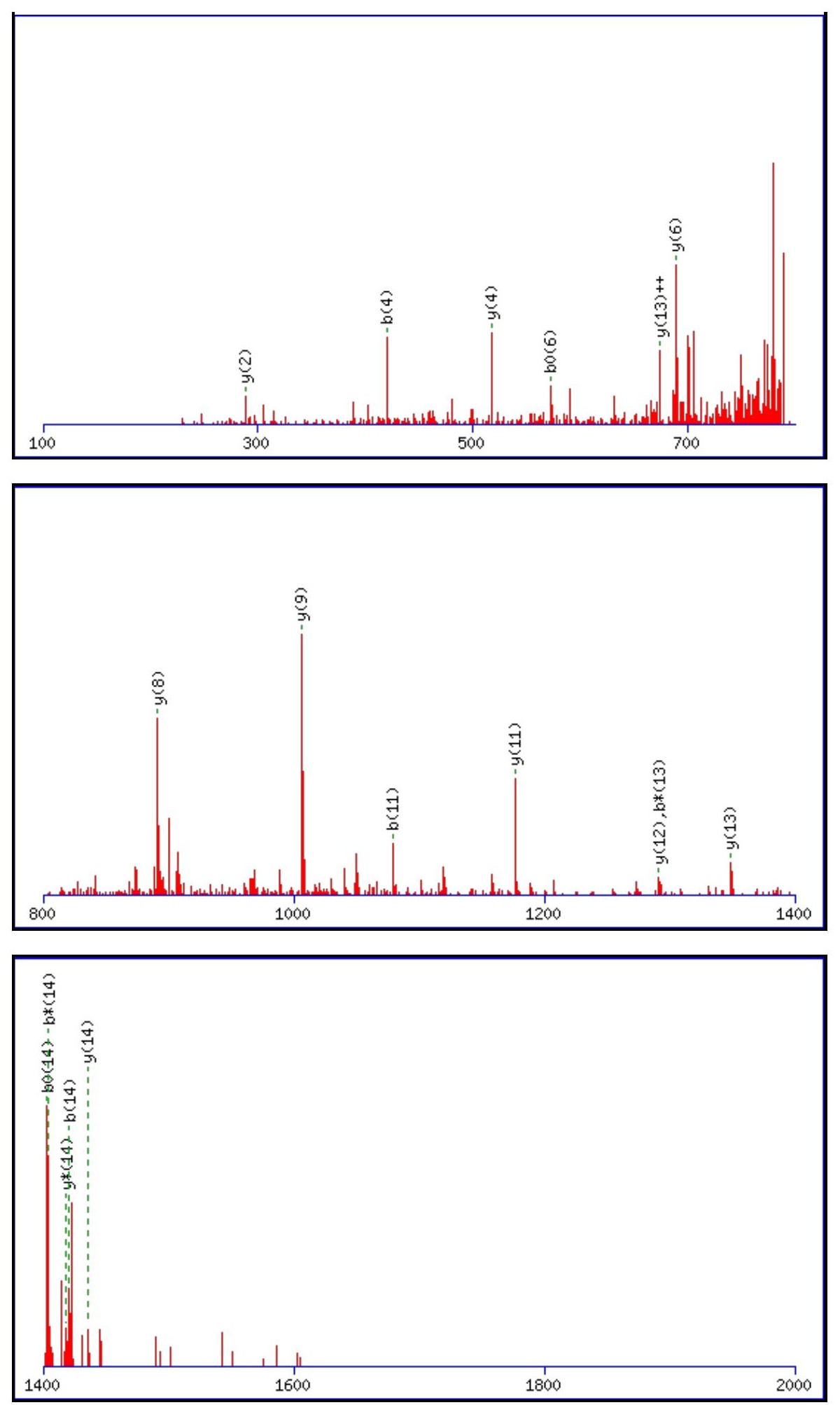

Monoisotopic mass of neutral peptide $\operatorname{Mr}($ calc): 1594.6529

Fixed modifications: Carbamidomethyl (C)

Variable modifications:

N11 : Deamidated $\mathrm{N}(\mathrm{N})$

Ions Score: 88 Expect: $2.3 \mathrm{e}-007$

Matches (Bold Red): 18/142 fragment ions using 34 most intense peaks

\begin{tabular}{|r|c|c|c|c|c|c|c|c|c|c|c|c|c|c|}
\hline$\#$ & $\mathbf{b}$ & $\mathbf{b}^{++}$ & $\mathbf{b}^{*}$ & $\mathbf{b}^{\boldsymbol{*}^{++}}$ & $\mathbf{b}^{\mathbf{0}}$ & $\mathbf{b}^{\mathbf{0 + +}}$ & Seq. & $\mathbf{y}$ & $\mathbf{y}^{++}$ & $\mathbf{y}^{\mathbf{*}}$ & $\mathbf{y}^{\boldsymbol{*}^{++}}$ & $\mathbf{y}^{\mathbf{0}}$ & $\mathbf{y}^{\mathbf{0 + +}}$ & $\#$ \\
\hline $\mathbf{1}$ & 161.0379 & 81.0226 & & & & & $\mathbf{C}$ & & & & & & & $\mathbf{1 5}$ \\
\hline $\mathbf{2}$ & 248.0700 & 124.5386 & & & 230.0594 & 115.5333 & S & $\mathbf{1 4 3 5 . 6 2 9 5}$ & 718.3184 & $\mathbf{1 4 1 8 . 6 0 3 0}$ & 709.8051 & 1417.6190 & 709.3131 & $\mathbf{1 4}$ \\
\hline $\mathbf{3}$ & 305.0914 & 153.0493 & & & 287.0809 & 144.0441 & $\mathbf{G}$ & $\mathbf{1 3 4 8 . 5 9 7 5}$ & $\mathbf{6 7 4 . 8 0 2 4}$ & 1331.5710 & 666.2891 & 1330.5870 & 665.7971 & $\mathbf{1 3}$ \\
\hline $\mathbf{4}$ & $\mathbf{4 2 0 . 1 1 8 4}$ & 210.5628 & & & 402.1078 & 201.5575 & $\mathbf{D}$ & $\mathbf{1 2 9 1 . 5 7 6 1}$ & 646.2917 & 1274.5495 & 637.7784 & 1273.5655 & 637.2864 & $\mathbf{1 2}$ \\
\hline $\mathbf{5}$ & 477.1398 & 239.0735 & & & 459.1293 & 230.0683 & $\mathbf{G}$ & $\mathbf{1 1 7 6 . 5 4 9 1}$ & 588.7782 & 1159.5226 & 580.2649 & 1158.5385 & 579.7729 & $\mathbf{1 1}$ \\
\hline
\end{tabular}


Mascot Search Results: Peptide View

\begin{tabular}{|r|r|r|r|r|r|r|r|r|r|r|r|r|r|r|r|r|r|}
$\mathbf{6}$ & 590.2239 & 295.6156 & & & 572.2133 & 286.6103 & L & 1119.5277 & 560.2675 & 1102.5011 & 551.7542 & 1101.5171 & 551.2622 & $\mathbf{1 0}$ \\
\hline $\mathbf{7}$ & 705.2508 & 353.1291 & & & 687.2403 & 344.1238 & D & $\mathbf{1 0 0 6 . 4 4 3 6}$ & 503.7254 & 989.4170 & 495.2122 & 988.4330 & 494.7201 & $\mathbf{9 9}$ \\
\hline $\mathbf{8}$ & 792.2829 & 396.6451 & & & 774.2723 & 387.6398 & S & $\mathbf{8 9 1 . 4 1 6 6}$ & 446.2120 & 874.3901 & 437.6987 & 873.4061 & 437.2067 & $\mathbf{8}$ \\
\hline $\mathbf{9}$ & 907.3098 & 454.1585 & & & 889.2992 & 445.1533 & D & 804.3846 & 402.6959 & 787.3581 & 394.1827 & 786.3741 & 393.6907 & $\mathbf{7}$ \\
\hline $\mathbf{1 0}$ & 964.3313 & 482.6693 & & & 946.3207 & 473.6640 & G & $\mathbf{6 8 9 . 3 5 7 7}$ & 345.1825 & 672.3311 & 336.6692 & 671.3471 & 336.1772 & $\mathbf{6}$ \\
\hline $\mathbf{1 1}$ & $\mathbf{1 0 7 9 . 3 5 8 2}$ & 540.1827 & 1062.3317 & 531.6695 & 1061.3476 & 531.1775 & N & 632.3362 & 316.6717 & 615.3097 & 308.1585 & 614.3256 & 307.6665 & $\mathbf{5}$ \\
\hline $\mathbf{1 2}$ & 1207.4168 & 604.2120 & 1190.3902 & 595.6988 & 1189.4062 & 595.2067 & Q & 517.3093 & 259.1583 & 500.2827 & 250.6450 & 499.2987 & 250.1530 & $\mathbf{4}$ \\
\hline $\mathbf{1 3}$ & 1308.4645 & 654.7359 & $\mathbf{1 2 9 1 . 4 3 7 9}$ & 646.2226 & 1290.4539 & 645.7306 & T & 389.2507 & 195.1290 & 372.2241 & 186.6157 & 371.2401 & 186.1237 & $\mathbf{3}$ \\
\hline $\mathbf{1 4}$ & $\mathbf{1 4 2 1 . 5 4 8 5}$ & 711.2779 & $\mathbf{1 4 0 4 . 5 2 2 0}$ & $\mathbf{7 0 2 . 7 6 4 6}$ & $\mathbf{1 4 0 3 . 5 3 8 0}$ & $\mathbf{7 0 2 . 2 7 2 6}$ & $\mathbf{L}$ & $\mathbf{2 8 8 . 2 0 3 0}$ & 144.6051 & 271.1765 & 136.0919 & & & & $\mathbf{2}$ \\
\hline $\mathbf{1 5}$ & & & & & & & R & 175.1190 & 88.0631 & 158.0924 & 79.5498 & & & \\
\hline
\end{tabular}
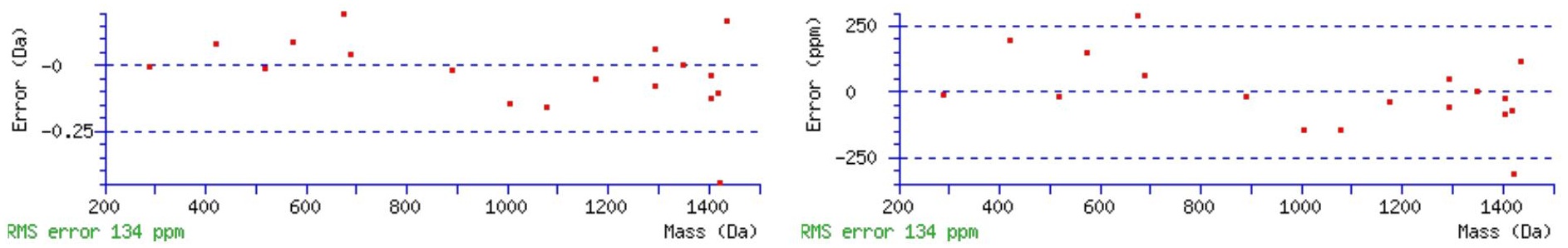

\section{All matches to this query}

\begin{tabular}{|l|l|l|c|}
\hline Score & Mr(calc): & Delta & Sequence \\
\hline 87.6 & 1594.6529 & 0.0027 & CSGDGLDSDGNQTLR \\
\hline
\end{tabular}

Spectrum No: 156; Query: 1367; Rank: 1

\section{Peptide View}

MS/MS Fragmentation of VGLIGSCTNSSYEDMGR

Found in IPI00421539, Tax_Id=10116 Gene_Symbol=Aco2 Aconitate hydratase, mitochondrial precursor

Match to Query 1367: 1861.776928 from(931.895740,2+)

Title: 091008RatKidney_NH4Format01_14.1882.1882.2.dta

Data file K:INewmanPaper|Piliang|3SubProteomes\Piliang3SP\mgf5ppm\ERLIC_3SubProteomes5ppm.mgf

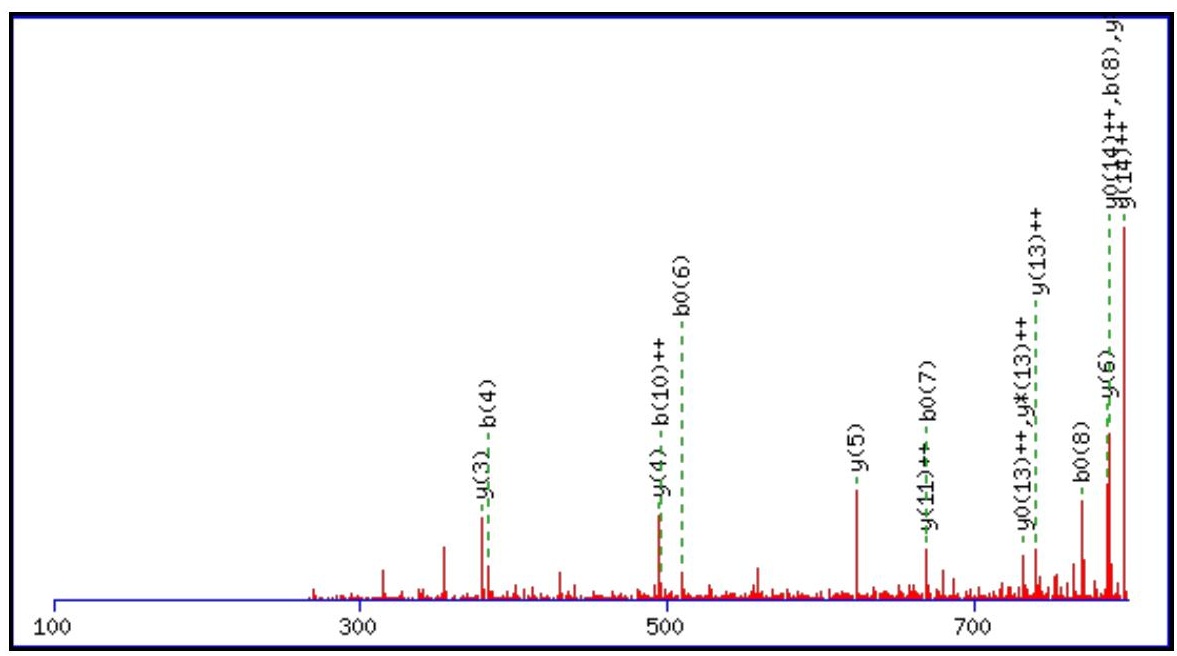



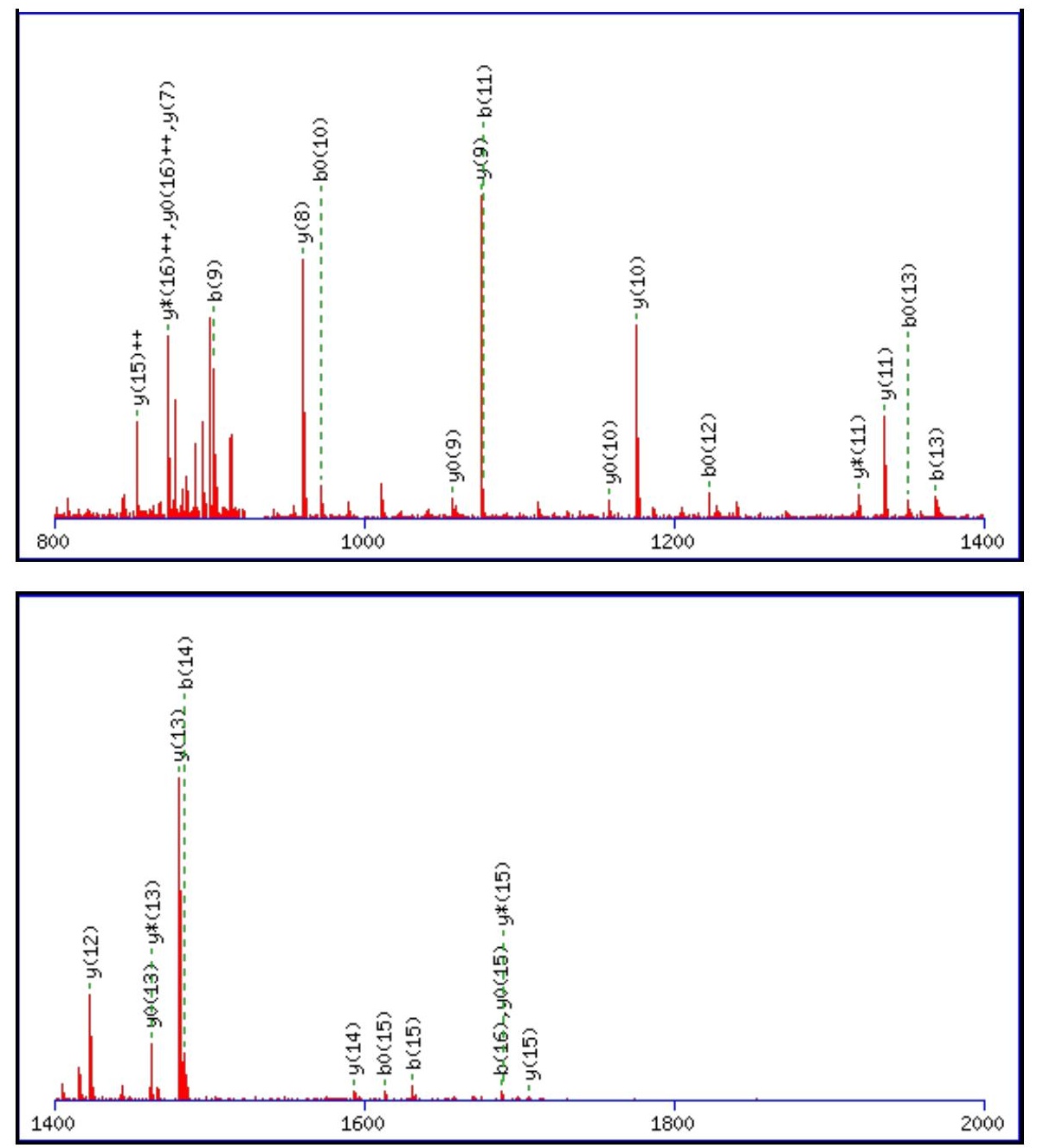

Monoisotopic mass of neutral peptide $\operatorname{Mr}($ calc): 1861.7822

Fixed modifications: Carbamidomethyl (C)

variable modifications:

N9 : Deamidated_N (N)

M15 : Oxidation (M)

Ions Score: 87 Expect : $3.3 \mathrm{e}-007$

Matches (Bold Red): 46/160 fragment ions using 77 most intense peaks

\begin{tabular}{|c|c|c|c|c|c|c|c|c|c|c|c|c|c|c|}
\hline \# & b & $\mathbf{b}^{++}$ & $\mathbf{b}^{*}$ & $\mathbf{b}^{*^{++}}$ & $\mathbf{b}^{0}$ & $\mathbf{b}^{\mathbf{0 + +}}$ & Seq. & $\mathbf{y}$ & $y^{++}$ & $\mathbf{y}^{*}$ & $\mathrm{y}^{*^{++}}$ & $\mathbf{y}^{\mathbf{0}}$ & $\mathbf{y}^{\mathbf{0 + +}}$ & \# \\
\hline 1 & 100.0757 & 50.5415 & & & & & $\mathbf{V}$ & & & & & & & 17 \\
\hline 2 & 157.0972 & 79.0522 & & & & & $\mathbf{G}$ & 1763.7211 & 882.3642 & 1746.6945 & 873.8509 & 1745.7105 & 873.3589 & 16 \\
\hline 3 & 270.1812 & 135.5942 & & & & & $\mathbf{L}$ & 1706.6996 & 853.8535 & 1689.6731 & 845.3402 & 1688.6891 & 844.8482 & 15 \\
\hline 4 & 383.2653 & 192.1363 & & & & & I & 1593.6156 & 797.3114 & 1576.5890 & 788.7981 & 1575.6050 & 788.3061 & 14 \\
\hline 5 & 440.2867 & 220.6470 & & & & & G & 1480.5315 & 740.7694 & 1463.5049 & 732.2561 & 1462.5209 & 731.7641 & 13 \\
\hline 6 & 527.3188 & 264.1630 & & & 509.3082 & 255.1577 & S & 1423.5100 & 712.2587 & 1406.4835 & 703.7454 & 1405.4995 & 703.2534 & 12 \\
\hline 7 & 687.3494 & 344.1783 & & & 669.3389 & 335.1731 & C & 1336.4780 & 668.7426 & 1319.4515 & 660.2294 & 1318.4674 & 659.7374 & 11 \\
\hline 8 & 788.3971 & 394.7022 & & & 770.3865 & 385.6969 & $\mathbf{T}$ & 1176.4474 & 588.7273 & 1159.4208 & 580.2140 & 1158.4368 & 579.7220 & 10 \\
\hline 9 & 903.4240 & 452.2157 & 886.3975 & 443.7024 & 885.4135 & 443.2104 & $\mathbf{N}$ & 1075.3997 & 538.2035 & 1058.3731 & 529.6902 & 1057.3891 & 529.1982 & 9 \\
\hline 10 & 990.4561 & 495.7317 & 973.4295 & 487.2184 & 972.4455 & 486.7264 & $\mathrm{~S}$ & 960.3727 & 480.6900 & 943.3462 & 472.1767 & 942.3622 & 471.6847 & 8 \\
\hline 11 & 1077.4881 & 539.2477 & 1060.4615 & 530.7344 & 1059.4775 & 530.2424 & S & 873.3407 & 437.1740 & 856.3142 & 428.6607 & 855.3301 & 428.1687 & 7 \\
\hline 12 & 1240.5514 & 620.7793 & 1223.5249 & 612.2661 & 1222.5409 & 611.7741 & $\mathbf{Y}$ & 786.3087 & 393.6580 & 769.2821 & 385.1447 & 768.2981 & 384.6527 & 6 \\
\hline 13 & 1369.5940 & 685.3006 & 1352.5675 & 676.7874 & 1351.5835 & 676.2954 & $\mathbf{E}$ & 623.2454 & 312.1263 & 606.2188 & 303.6130 & 605.2348 & 303.1210 & 5 \\
\hline 14 & 1484.6210 & 742.8141 & 1467.5944 & 734.3008 & 1466.6104 & 733.8088 & D & 494.2028 & 247.6050 & 477.1762 & 239.0917 & 476.1922 & 238.5997 & 4 \\
\hline 15 & 1631.6564 & 816.3318 & 1614.6298 & 807.8185 & 1613.6458 & 807.3265 & $\mathbf{M}$ & 379.1758 & 190.0915 & 362.1493 & 181.5783 & & & 3 \\
\hline 16 & 1688.6778 & 844.8426 & 1671.6513 & 836.3293 & 1670.6673 & 835.8373 & $\mathbf{G}$ & 232.1404 & 116.5738 & 215.1139 & 108.0606 & & & 2 \\
\hline 17 & & & & & & & $\mathbf{R}$ & 175.1190 & 88.0631 & 158.0924 & 79.5498 & & & 1 \\
\hline
\end{tabular}



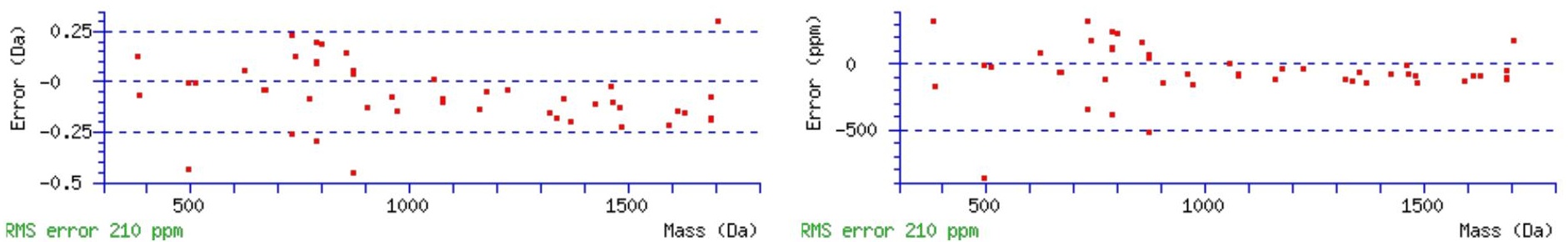

\section{All matches to this query}

\begin{tabular}{|l|l|c|c|}
\hline Score & Mr(calc): & Delta & Sequence \\
\hline 87.5 & 1861.7822 & -0.0053 & VGLIGSCTNSSYEDMGR \\
\hline
\end{tabular}

Spectrum No: 157; Query: 2292; Rank: 1

\section{Peptide View}

\section{MS/MS Fragmentation of DLNISLDTDAPLSVGKDYGVSAYR}

Found in IPI00326990, Tax_Id=10116 Gene_Symbol=Slc15a2 Oligopeptide transporter, kidney isoform

Match to Query 2292: 2569.263972 from(857.428600,3+)

Title: 100101RatKid_NS_deglyco_24.4219.4219.3.dta

Data file K:INewmanPaper|Piliangl3SubProteomes\Piliang3SP\mgf5ppm\ERLIC_3SubProteomes5ppm.mgf
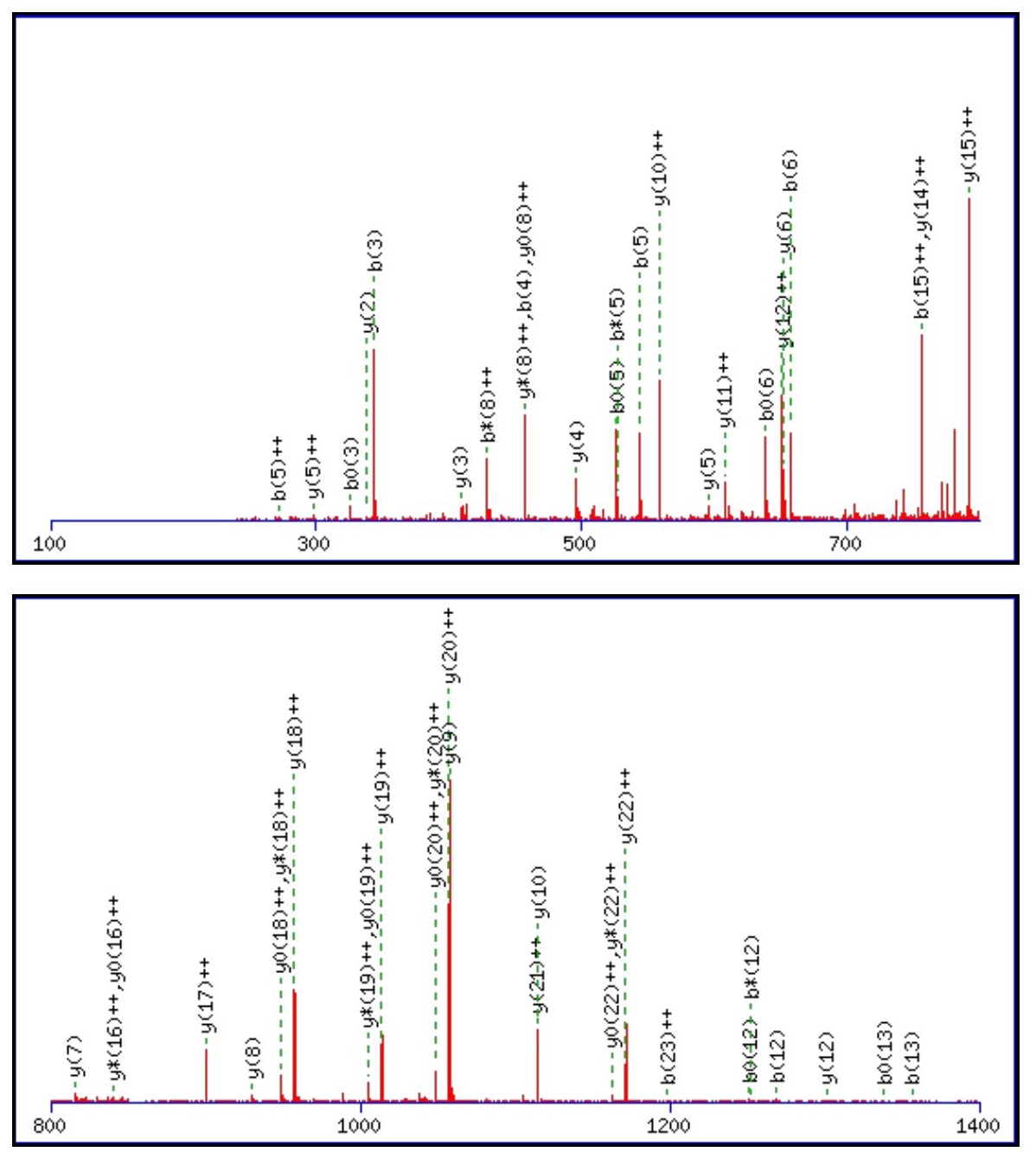


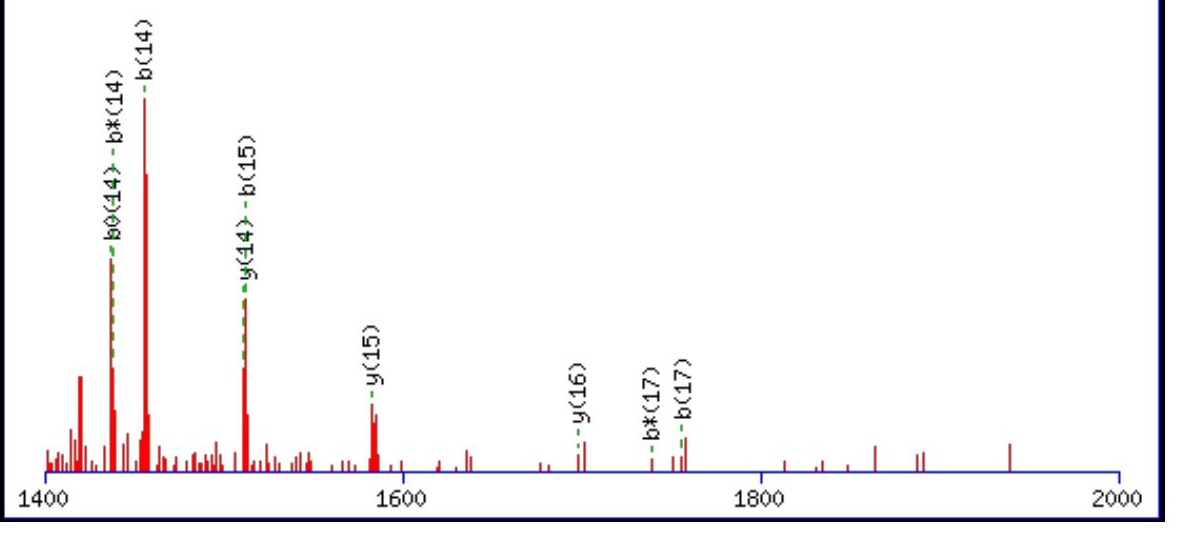

Monoisotopic mass of neutral peptide $\operatorname{Mr}($ calc): 2569.2548

Fixed modifications: Carbamidomethyl (C)

Variable modifications:

N3 : Deamidated_N (N)

Ions Score: 87 Expect : 4.6e- 007

Matches (Bold Red): 60/266 fragment ions using 103 most intense peaks

\begin{tabular}{|c|c|c|c|c|c|c|c|c|c|c|c|c|c|c|}
\hline \# & b & $\mathbf{b}^{++}$ & $\mathbf{b}^{*}$ & $\mathbf{b}^{*^{++}}$ & $\mathbf{b}^{\mathbf{0}}$ & $\mathbf{b}^{\mathbf{0 + +}}$ & Seq. & $\mathbf{y}$ & $\mathbf{y}^{++}$ & $\mathbf{y}^{*}$ & $\mathrm{y}^{\mathrm{*}^{++}}$ & $\mathbf{y}^{0}$ & & \# \\
\hline 1 & 116.0342 & 58.5207 & & & 98.0237 & 49.5155 & D & & & & & & & 24 \\
\hline 2 & 229.1183 & 628 & & & & 106.0575 & $\mathbf{L}$ & & & & & 2245 & & 23 \\
\hline 3 & 344.1452 & 172.5762 & 327.1187 & 164.0630 & 326.1347 & 163.5710 & $\mathbf{N}$ & 2342.1510 & 1171.5792 & 2325.1245 & 1163.0659 & 2324.1405 & 1162.5739 & 22 \\
\hline 4 & 457.2293 & 229.1183 & 440.2027 & & 439.2187 & 220.1130 & I & & & 2210.0976 & & 2209.1135 & 1105.0604 & 21 \\
\hline 5 & 544.2613 & 272.6343 & 527.2348 & 264.1210 & 526.2507 & 263.6290 & S & 2114.0400 & 1057.5237 & 2097.0135 & 0104 & 2096.0295 & 1048.5184 & 20 \\
\hline 6 & 657.3454 & 329.1763 & 640.3188 & 320.6631 & 639.3348 & 320.1710 & $\mathbf{L}$ & 2027.0080 & 0076 & 2009 & 4944 & 9974 & 1005.0024 & 19 \\
\hline 7 & 772.3723 & 386.6898 & 755.3458 & 378.1765 & 754.3618 & 377.6845 & D & 1913.9239 & 4656 & 1896 & 948.9523 & 1895.9134 & 948.4603 & 18 \\
\hline 8 & 873.4200 & 437.2136 & 856.3934 & 428.7004 & 855.4094 & 428.2084 & $\mathbf{T}$ & 1798.8970 & 9521 & 1781. & 891.4389 & 1780.8864 & 890.9469 & 17 \\
\hline 9 & 988.4469 & 494.7271 & 971.4204 & 486.2138 & 970.4364 & 485.7218 & D & 1697.8493 & 849.4283 & 1680.8228 & 840.9150 & 1679.8388 & 840.4230 & 16 \\
\hline 10 & 1059.4841 & 530.2457 & 1042.4575 & 521.7324 & 1041.4735 & 521.2404 & A & 1582.8224 & 791.9148 & 1565.7958 & 783.4016 & 1564.8118 & 782.9095 & 15 \\
\hline 11 & 156.5368 & 578.7720 & 139.5103 & 570.2588 & 1138.5263 & 569.7668 & $\mathbf{P}$ & 1511.7853 & 756.3963 & 1494.7587 & 747.8830 & 1493.7747 & 747.3910 & 14 \\
\hline 12 & 1269.6209 & 635.3141 & 1252.5943 & 626.8008 & 1251.6103 & 626.3088 & $\mathbf{L}$ & 1414.7325 & 707.8699 & 1397.7060 & 699.3566 & 1396.7219 & 698.8646 & 13 \\
\hline 13 & 356.6529 & 678.8301 & 1339.6264 & 670.3168 & 1338.6423 & 669.8248 & $\mathrm{~S}$ & 1301.6484 & 651.3279 & 1284.6219 & 642.8146 & 1283.6379 & 642.3226 & 12 \\
\hline 14 & 1455.7213 & 728.3643 & 438.6948 & 719.8510 & 1437.7108 & 719.3590 & V & 1214.6164 & 607.8118 & 1197.5899 & 599.2986 & 1196.6058 & 598.8066 & 11 \\
\hline 15 & 1512.7428 & 756.8750 & 1495.7162 & 748.3618 & 1494.7322 & 747.8697 & G & 1115.5480 & 558.2776 & 1098.5215 & 549.7644 & 1097.5374 & 549.2724 & 10 \\
\hline 16 & 1640.8378 & 820.9225 & 623.8112 & 812.4092 & 622.8272 & 811.9172 & $\mathbf{K}$ & 1058.5265 & 529.7669 & 1041.5000 & 521.2536 & 1040.5160 & 520.7616 & 9 \\
\hline 17 & 1755.8647 & 878.4360 & 1738.8381 & 869.9227 & 1737.8541 & 869.4307 & D & 930.4316 & 465.7194 & 913.4050 & 457.2062 & 912.4210 & 456.7141 & 8 \\
\hline 18 & 1918.9280 & 959.9676 & 1901.9015 & 951.4544 & 1900.9175 & 950.9624 & $\mathbf{Y}$ & 815.4046 & 408.2060 & 798.3781 & 399.6927 & 797.3941 & 399.2007 & 7 \\
\hline 19 & 1975.9495 & 988.4784 & 1958.9229 & 979.9651 & 1957.9389 & 979.4731 & G & 652.3413 & 326.6743 & 635.3148 & 318.1610 & 634.3307 & 317.6690 & 6 \\
\hline 20 & 2075.0179 & 1038.0126 & 2057.9914 & 1029.4993 & 2057.0073 & 1029.0073 & V & 595.3198 & 298.1636 & 578.2933 & 289.6503 & 577.3093 & 289.1583 & 5 \\
\hline 21 & 2162.0499 & 1081.5286 & 2145.0234 & 1073.0153 & 2144.0394 & 1072.5233 & S & 496.2514 & 248.6293 & 479.2249 & 240.1161 & 478.2409 & 239.6241 & 4 \\
\hline 22 & 2233.0870 & 1117.0472 & 2216.0605 & 1108.5339 & 2215.0765 & 1108.0419 & A & 409.2194 & 205.1133 & 392.1928 & 196.6001 & & & 3 \\
\hline 23 & 2396.1504 & 1198.5788 & 2379.1238 & 1190.0655 & 2378.1398 & 1189.5735 & $\mathbf{Y}$ & 338.1823 & 169.5948 & 321.1557 & 161.0815 & & & 2 \\
\hline 24 & & & & & & & $\mathbf{R}$ & 175.1190 & 88.0631 & 158.0924 & 79.5498 & & & 1 \\
\hline
\end{tabular}
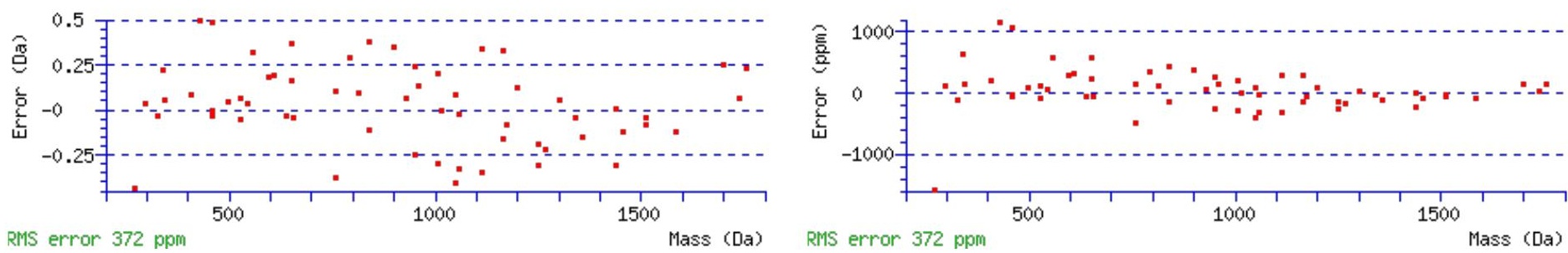
Mascot Search Results: Peptide View

\section{All matches to this query}

\begin{tabular}{|l|c|c|l|}
\hline Score & Mr(calc): & Delta & \multicolumn{1}{|c|}{ Sequence } \\
\hline 87.2 & 2569.2548 & 0.0092 & DLNISLDTDAPLSVGKDYGVSAYR \\
\hline 54.2 & 2568.2708 & 0.9932 & DLNISLDTDAPLSVGKDYGVSAYR \\
\hline 6.1 & 2568.2585 & 1.0055 & NIDRTDLQTITNDPRLVFTVR \\
\hline 4.7 & 2567.2649 & 1.9991 & DKPTETLLNTVKDNLPIQIQK \\
\hline 3.5 & 2568.2479 & 1.0160 & TQSLVCPETVSRVSSVLNRNSR \\
\hline 2.5 & 2568.2585 & 1.0055 & NIDRTDLQTITNDPRLVFTVR \\
\hline 2.5 & 2568.2672 & 0.9968 & IITNTNCWSPRKAQSRPAFK \\
\hline 2.5 & 2569.2512 & 0.0128 & IITNTNCWSPRKAQSRPAFK \\
\hline 2.3 & 2569.2693 & -0.0054 & VTINDLNENSVTLIQKNCHLNK \\
\hline 2.1 & 2567.2745 & 1.9895 & NIDRTDLQTITNDPRLVFTVR \\
\hline
\end{tabular}

Spectrum No: 158; Query: 1009; Rank: 1

\section{Peptide View}

MS/MS Fragmentation of FSLVNDSEGWLCIK

Found in IPI00471800, Tax_Id=10116 Gene_Symbol=Cdh16 Cadherin 16

Match to Query 1009: 1667.791228 from(834.902890,2+)

Title: 100101RatKid_NS_deglyco_25.5196.5196.2.dta

Data file K:INewmanPaper|Piliang|3SubProteomes\Piliang3SP\mgf5ppm\ERLIC_3SubProteomes5ppm.mgf
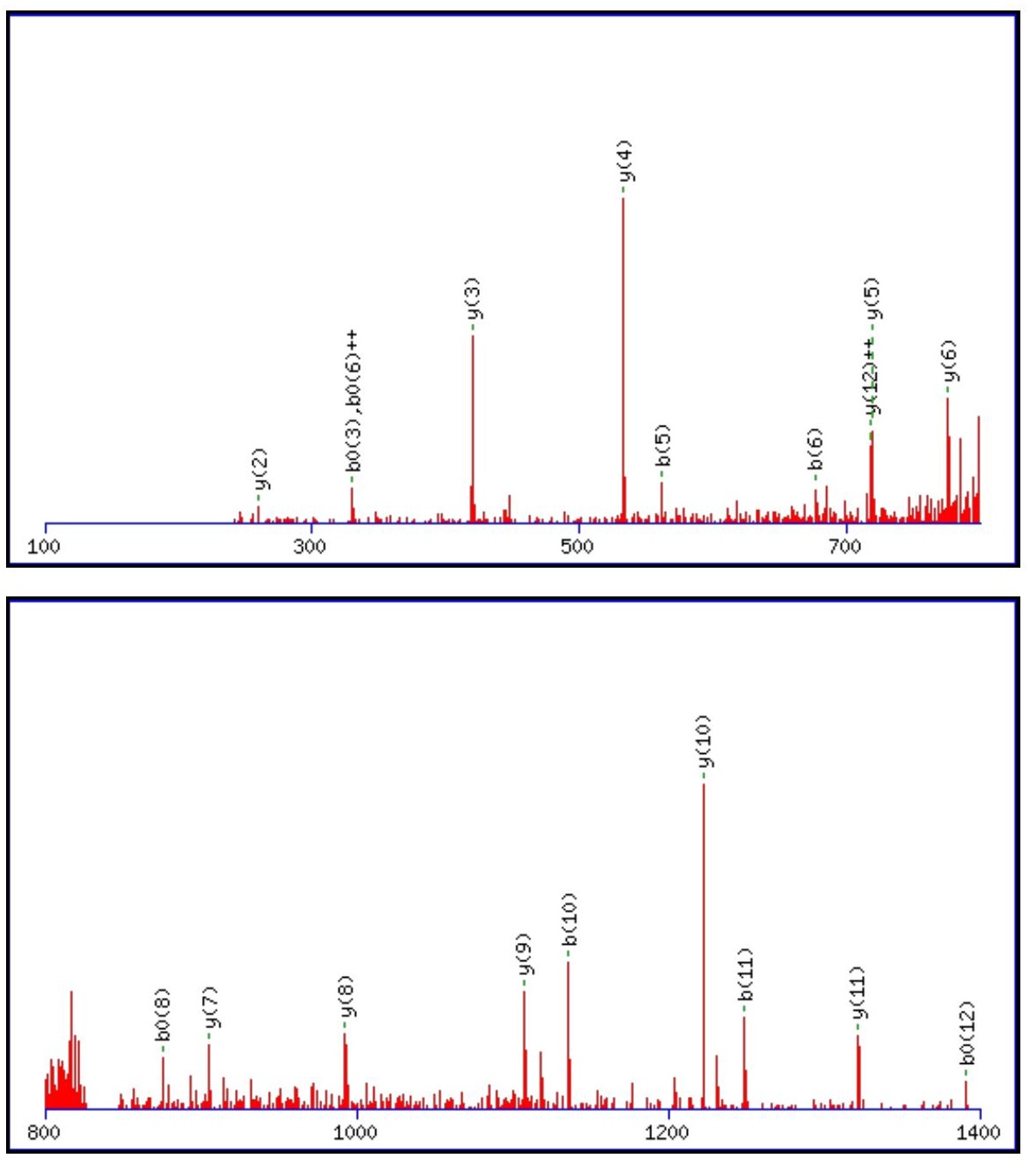


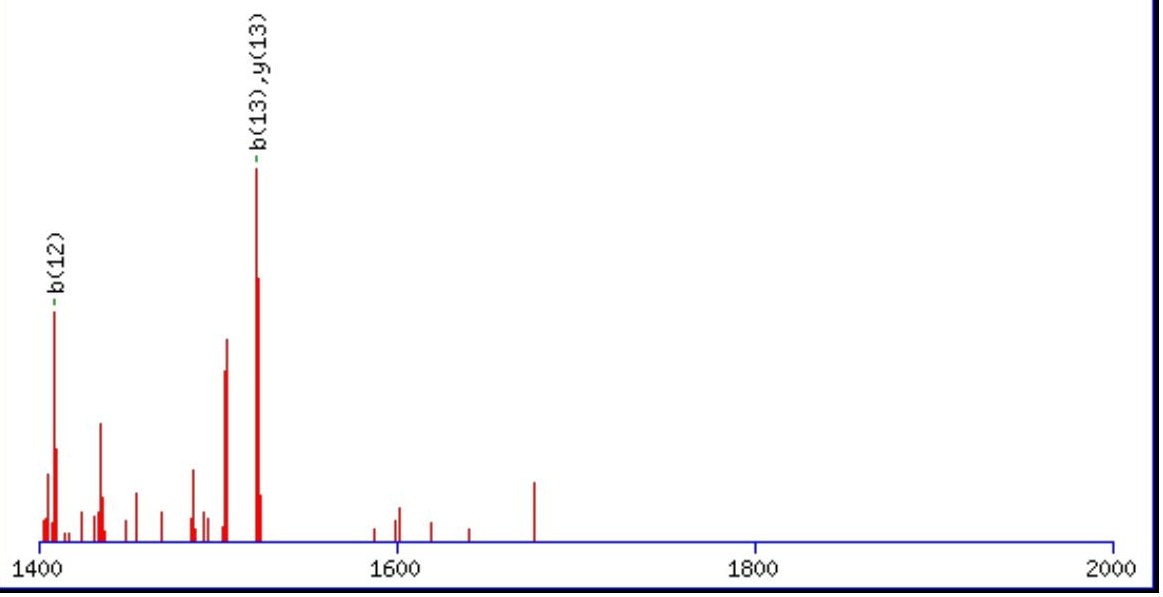

Monoisotopic mass of neutral peptide $\operatorname{Mr}($ calc): 1667.7865

Fixed modifications: Carbamidomethyl (C)

Variable modifications:

N5 : Deamidated_N (N)

Ions Score: 87 Expect : $3.7 \mathrm{e}-007$

Matches (Bold Red): 22/134 fragment ions using 37 most intense peaks

\begin{tabular}{|c|c|c|c|c|c|c|c|c|c|c|c|c|c|c|}
\hline \# & b & $\mathbf{b}^{++}$ & $\mathbf{b}^{*}$ & $\mathbf{b}^{*^{++}}$ & $\mathbf{b}^{\mathbf{0}}$ & $\mathbf{b}^{\mathbf{0 + +}}$ & Seq. & $\mathbf{y}$ & $\mathbf{y}^{++}$ & $\mathbf{y}^{*}$ & $\mathbf{y}^{*^{++}}$ & $\mathbf{y}^{\mathbf{0}}$ & $\mathbf{y}^{0++}$ & \# \\
\hline 1 & 148.0757 & 74.5415 & & & & & $\mathbf{F}$ & & & & & & & 14 \\
\hline 2 & 235.1077 & 118.0575 & & & 217.0972 & 109.0522 & $\mathrm{~S}$ & 1521.7254 & 761.3663 & 1504.6988 & 752.8530 & 1503.7148 & 752.3610 & 13 \\
\hline 3 & 348.1918 & 174.5995 & & & 330.1812 & 165.5942 & $\mathbf{L}$ & 1434.6933 & 717.8503 & 1417.6668 & 709.3370 & 1416.6828 & 708.8450 & 12 \\
\hline 4 & 447.2602 & 224.1337 & & & 429.2496 & 215.1285 & $\mathbf{V}$ & 1321.6093 & 661.3083 & 1304.5827 & 652.7950 & 1303.5987 & 652.3030 & 11 \\
\hline 5 & 562.2871 & 281.6472 & 545.2606 & 273.1339 & 544.2766 & 272.6419 & $\mathbf{N}$ & 1222.5409 & 611.7741 & 1205.5143 & 603.2608 & 1204.5303 & 602.7688 & 10 \\
\hline 6 & 677.3141 & 339.1607 & 660.2875 & 330.6474 & 659.3035 & 330.1554 & D & 1107.5139 & 554.2606 & 1090.4874 & 545.7473 & 1089.5034 & 545.2553 & 9 \\
\hline 7 & 764.3461 & 382.6767 & 747.3196 & 374.1634 & 746.3355 & 373.6714 & S & 992.4870 & 496.7471 & 975.4604 & 488.2339 & 974.4764 & 487.7418 & 8 \\
\hline 8 & 893.3887 & 447.1980 & 876.3621 & 438.6847 & 875.3781 & 438.1927 & $\mathbf{E}$ & 905.4550 & 453.2311 & 888.4284 & 444.7178 & 887.4444 & 444.2258 & 7 \\
\hline 9 & 950.4102 & 475.7087 & 933.3836 & 467.1954 & 932.3996 & 466.7034 & $\mathbf{G}$ & 776.4124 & 388.7098 & 759.3858 & 380.1965 & & & 6 \\
\hline 10 & 1136.4895 & 568.7484 & 1119.4629 & 560.2351 & 1118.4789 & 559.7431 & $\mathbf{W}$ & 719.3909 & 360.1991 & 702.3643 & 351.6858 & & & 5 \\
\hline 11 & 1249.5735 & 625.2904 & 1232.5470 & 616.7771 & 1231.5630 & 616.2851 & $\mathbf{L}$ & 533.3116 & 267.1594 & 516.2850 & 258.6462 & & & 4 \\
\hline 12 & 1409.6042 & 705.3057 & 1392.5776 & 696.7925 & 1391.5936 & 696.3004 & C & 420.2275 & 210.6174 & 403.2010 & 202.1041 & & & 3 \\
\hline 13 & 1522.6882 & 761.8478 & 1505.6617 & 753.3345 & 1504.6777 & 752.8425 & I & 260.1969 & 130.6021 & 243.1703 & 122.0888 & & & 2 \\
\hline 14 & & & & & & & $\mathbf{K}$ & 147.1128 & 74.0600 & 130.0863 & 65.5468 & & & 1 \\
\hline
\end{tabular}
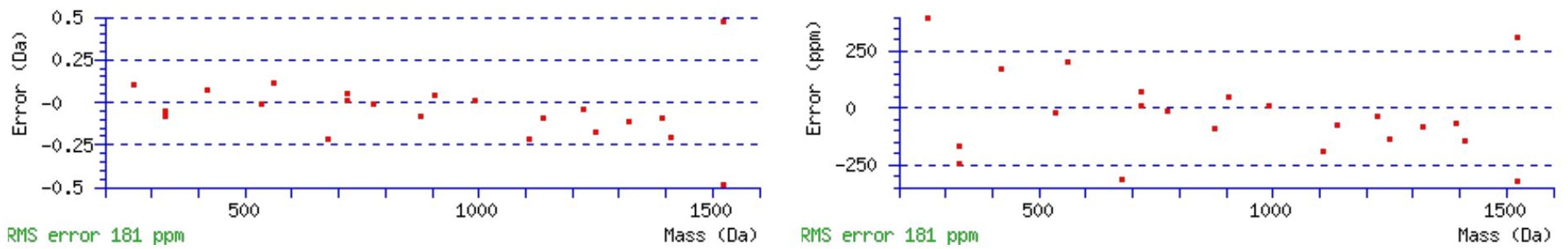

\section{All matches to this query}

\begin{tabular}{|l|l|l|l|}
\hline Score & Mr(calc): & Delta & \multicolumn{1}{c|}{ Sequence } \\
\hline 87.1 & 1667.7865 & 0.0047 & FSLVNDSEGWLCIK \\
\hline 28.7 & 1666.8025 & 0.9887 & FSLVNDSEGWLCIK \\
\hline 10.8 & 1666.7832 & 1.0080 & SLAMLGSSEDNTALSR \\
\hline 8.8 & 1666.7953 & 0.9959 & EMDCSVLKRLMNR \\
\hline 7.7 & 1667.7841 & 0.0071 & GILCDVTLIAEEQK \\
\hline 7.1 & 1666.7872 & 1.0040 & EEADSFNQKMVQLK \\
\hline 7.0 & 1667.7920 & -0.0008 & TKPSLSFHTGIGSEK \\
\hline & & & \\
\hline
\end{tabular}




\begin{tabular}{|l|l|l|l|}
\hline 7.0 & 1667.7920 & -0.0008 & TKPSLSFHTGIGSEK \\
\hline 5.8 & 1667.7920 & -0.0008 & TKPSLSFHTGIGSEK \\
\hline 5.8 & 1667.7920 & -0.0008 & TKPSLSFHTGIGSEK \\
\hline
\end{tabular}

Spectrum No: 159; Query: 2479; Rank: 1

\section{Peptide View}

MS/MS Fragmentation of GNSMEEILEGLKFNLTETPETEIHR

Found in IPI00211075, Tax_Id=10116 Gene_Symbol=Serpina3n Serine protease inhibitor A3N precursor

Match to Query 2479: 2887.396092 from(963.472640,3+)

Title: 100101RatKid_NS_deglyco_23.5737.5737.3.dta

Data file K:INewmanPaper|Piliang|3SubProteomes\Piliang3SP\mgf5ppm\ERLIC_3SubProteomes5ppm.mgf
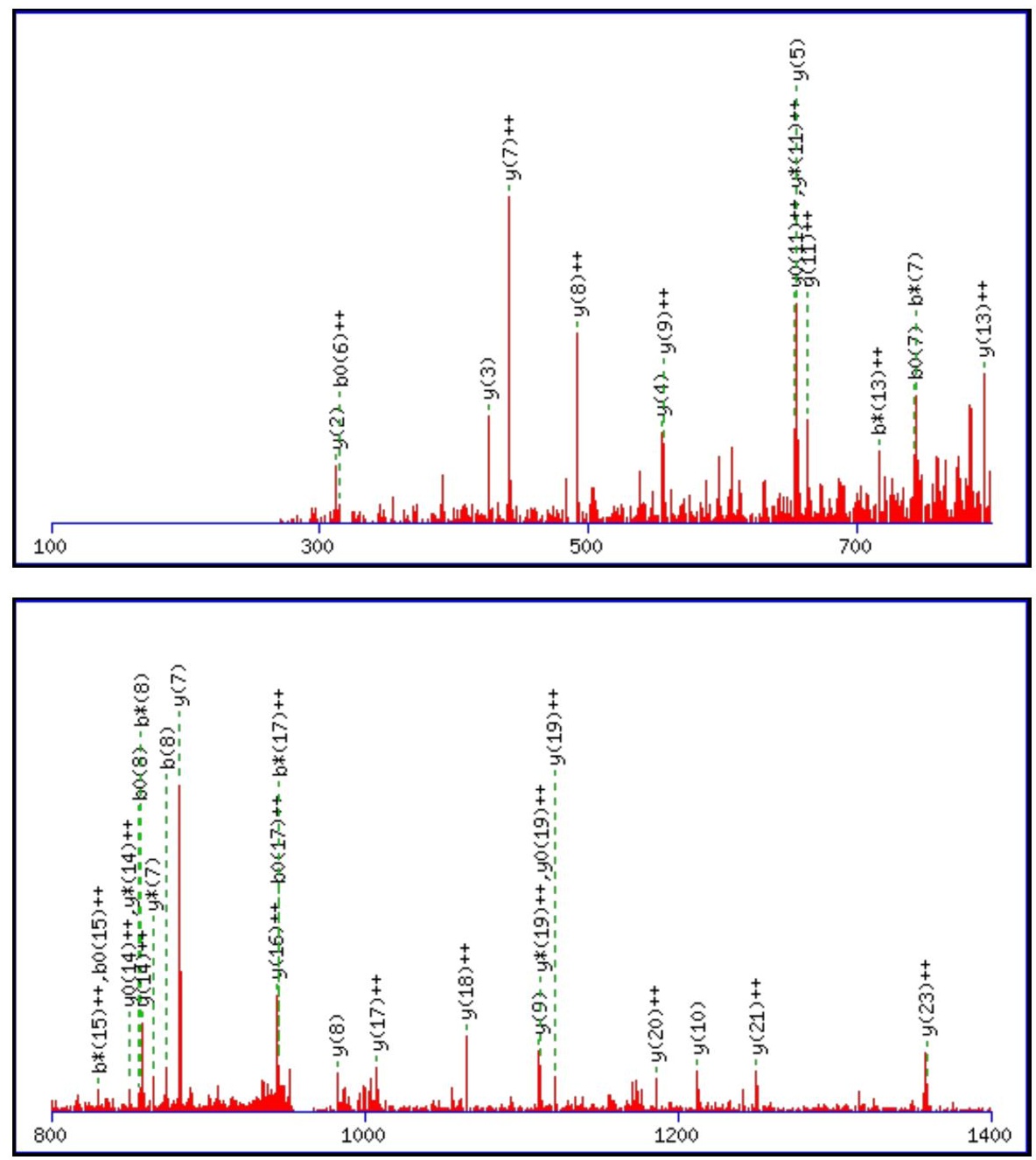


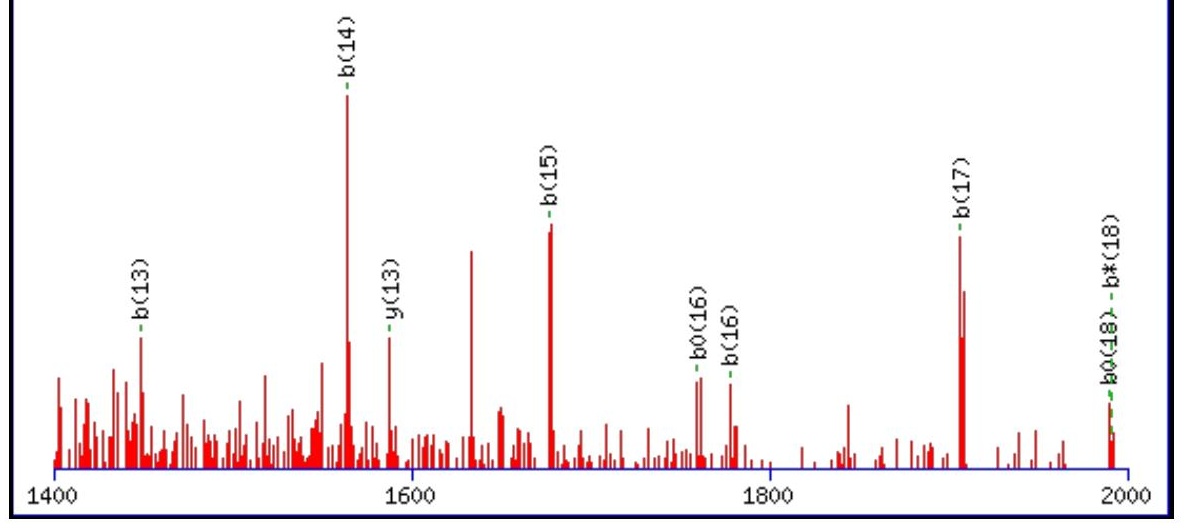

Monoisotopic mass of neutral peptide $\operatorname{Mr}($ calc): 2887.3909

Fixed modifications: Carbamidomethyl (C)

Variable modifications:

N14 : Deamidated_N (N)

Ions Score: 87 Expect: $5.9 \mathrm{e}-007$

Matches (Bold Red): 48/276 fragment ions using 65 most intense peaks

\begin{tabular}{|c|c|c|c|c|c|c|c|c|c|c|c|c|c|c|}
\hline \# & b & $\mathbf{b}^{++}$ & b* & & $\mathbf{b}^{\mathbf{0}}$ & & Seq. & $\mathbf{y}$ & & $\mathbf{y}^{*}$ & & $\mathbf{y}^{\mathbf{0}}$ & & \# \\
\hline 1 & 58.0287 & 29.5180 & & & & & G & & & & & & & 25 \\
\hline 2 & 172.0717 & 86.5395 & 155.0451 & & & & $\mathbf{N}$ & & & 2814.3502 & 6787 & 2813.3662 & 1407.1867 & 24 \\
\hline 3 & 259.1037 & 130.0555 & 242.0771 & 121.5422 & 241.0931 & & $S$ & 2717.3338 & 1359.1706 & 2700.3073 & 1350.6573 & 2699.3233 & 1653 & 23 \\
\hline 4 & 390.1442 & 195.5757 & 373.1176 & 187.0625 & 372.1336 & 186.5704 & $\mathbf{M}$ & 2630.3018 & 1315.6545 & 2613.2753 & 1307.1413 & 2612.2912 & 1306.6493 & 22 \\
\hline 5 & 519.1868 & 260.0970 & 502.1602 & 251.5838 & 501.1762 & 251.0917 & $\mathbf{E}$ & 2499.2613 & 1250.1343 & 2482.2348 & 1241.6210 & 2481.2508 & .1290 & 21 \\
\hline 6 & 648.2294 & 324.6183 & 631.2 & & & & $\mathbf{E}$ & 2370.2187 & & 922 & 997 & 2352.2082 & 1176.6077 & 20 \\
\hline 7 & 761.3134 & 381.1604 & 744.2869 & 372.6471 & 743. & 372.1551 & I & 2241.1761 & 1121.0917 & 1496 & 1112.5784 & 2223.1656 & 1112.0864 & 19 \\
\hline 8 & 874.3975 & 437.7024 & 857.3709 & 429.1891 & 869 & 428.6971 & $\mathbf{L}$ & 2128. & 1064.5497 & 655 & 1056.0364 & 2110.0815 & 1055.5444 & 18 \\
\hline 9 & 1003.4401 & 502.2237 & 986.4135 & 493.7104 & 985.4295 & 493.2184 & $\mathbf{E}$ & 2015.0080 & 1008.0076 & 1997.9815 & 999.4944 & 1996.9974 & 999.0024 & 17 \\
\hline 10 & 1060.4616 & 530.7344 & 1043.4350 & 522.2211 & 1042.4510 & 521.7291 & $\mathbf{G}$ & 1885.9654 & 943.4863 & 1868.9389 & 934.9731 & 1867.9548 & 934.4811 & 16 \\
\hline 11 & 173.5456 & 587.2764 & 156.5191 & 578.7632 & 1155.5 & 578.2712 & $\mathbf{L}$ & 1828.9439 & 9756 & 9174 & 4623 & 1810.9334 & 9703 & 15 \\
\hline 12 & 1301.6406 & 651.3239 & 1284.6140 & 642.8107 & 1283.6300 & 642.3186 & $\mathbf{K}$ & 1715.8599 & 858.4336 & 1698.8333 & 849.9203 & 1697.8493 & 849.4283 & 14 \\
\hline 13 & 448.7090 & 724.8581 & 431.6824 & 716.3449 & 1430.6984 & 715.8529 & $\mathbf{F}$ & 1587.7649 & 794.3861 & 384 & 785.8728 & 1569.7544 & 3808 & 13 \\
\hline 14 & 1563.7359 & 782.3716 & 1546.7094 & 773.8583 & 1545.7254 & 773.3663 & $\mathbf{N}$ & 1440.6965 & 720.8519 & 1423.6700 & 712.3386 & 1422.6859 & 711.8466 & 12 \\
\hline 15 & 1676.8200 & 838.9136 & 659.7934 & 830.4004 & 1658.8094 & 829.9084 & $\mathbf{L}$ & 1325.6696 & 663.3384 & 1308. & 654.8251 & 1307.6590 & 654.3331 & 11 \\
\hline 16 & $\mathbf{1 7 7 7 . 8 6 7 7}$ & 889.4375 & 1760.8411 & 880.9242 & 1759.8571 & 880.4322 & $\mathbf{T}$ & 1212.5855 & 606.7964 & 1195.5590 & 598.2831 & 1194.5749 & 597.7911 & 10 \\
\hline 17 & 1906.9103 & 953.9588 & 1889.8837 & 945.4455 & 1888.8997 & 944.9535 & $\mathbf{E}$ & 1111.5378 & 556.2726 & 1094.5113 & 547.7593 & 1093.5273 & 547.2673 & 9 \\
\hline 18 & 2007.9579 & 1004.4826 & 1990.9314 & 995.9693 & 1989.9474 & 995.4773 & $\mathbf{T}$ & 982.4952 & 491.7513 & 965.4687 & 483.2380 & 964.4847 & 482.7460 & 8 \\
\hline 19 & 2105.0107 & 1053.0090 & 2087.9842 & 1044.4957 & 2087.0001 & 1044.0037 & $\mathbf{P}$ & 881.4476 & 441.2274 & 864.4210 & 432.7141 & 863.4370 & 432.2221 & 7 \\
\hline 20 & 2234.0533 & 1117.5303 & 2217.0268 & 1109.0170 & 2216.0427 & 1108.5250 & $\mathbf{E}$ & 784.3948 & 392.7010 & 767.3682 & 384.1878 & 766.3842 & 383.6958 & 6 \\
\hline 21 & 2335.1010 & 1168.0541 & 2318.0744 & 1159.5409 & 2317.0904 & 1159.0488 & $\mathbf{T}$ & 655.3522 & 328.1797 & 638.3257 & 319.6665 & 637.3416 & 319.1745 & 5 \\
\hline 22 & 2464.1436 & 1232.5754 & 2447.1170 & 1224.0621 & 2446.1330 & 1223.5701 & $\mathbf{E}$ & 554.3045 & 277.6559 & 537.2780 & 269.1426 & 536.2940 & 268.6506 & 4 \\
\hline 23 & 2577.2276 & 289.1175 & 2560.2011 & 1280.6042 & 2559.2171 & 1280.1122 & I & 425.2619 & 213.1346 & 408.2354 & 204.6213 & & & 3 \\
\hline 24 & 2714.2865 & 1357.6469 & 2697.2600 & 1349.1336 & 2696.2760 & 1348.6416 & $\mathbf{H}$ & 312.1779 & 156.5926 & 295.1513 & 148.0793 & & & 2 \\
\hline 25 & & & & & & & $\mathbf{R}$ & 175.1190 & 88.0631 & 158.0924 & 79.5498 & & & 1 \\
\hline
\end{tabular}
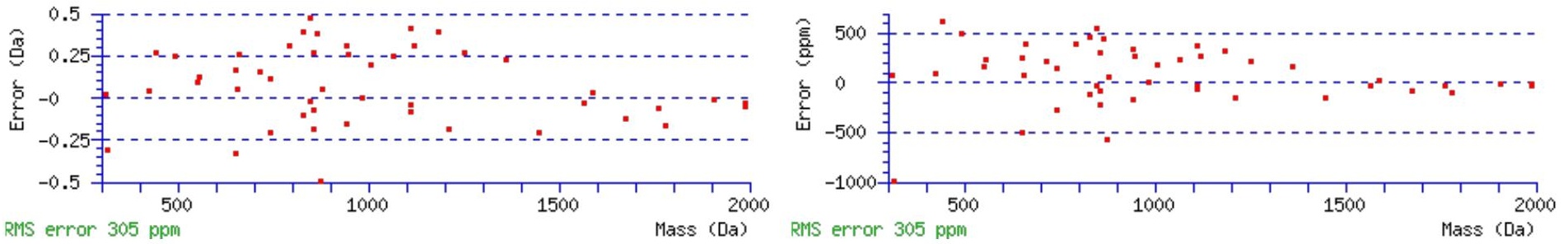


\section{All matches to this query}

\begin{tabular}{|l|l|l|l|}
\hline Score & Mr(calc): & Delta & \multicolumn{1}{c|}{ Sequence } \\
\hline 87.1 & 2887.3909 & 0.0052 & GNSMEEILEGLKFNLTETPETEIHR \\
\hline 29.7 & 2887.3909 & 0.0052 & GNSMEEILEGLKFNLTETPETEIHR \\
\hline 19.0 & 2886.4069 & 0.9892 & GNSMEEILEGLKFNLTETPETEIHR \\
\hline 3.8 & 2887.3688 & 0.0273 & HFSAASSLTVHHMLLRIXNGEEISK \\
\hline 3.8 & 2887.3687 & 0.0273 & HFSAASSLTVHHMLLRIXNGEEISK \\
\hline 2.9 & 2885.3711 & 2.0250 & MDSKNVHSPVFLVFPPEITSEYR \\
\hline 2.2 & 2885.3711 & 2.0250 & MDSKNVHSPVFLVFPPEITSEYR \\
\hline
\end{tabular}

Spectrum No: 160; Query: 1590; Rank: 1

\section{Peptide View}

MS/MS Fragmentation of MGAGEALGNASQLFEAWLK

Found in IPI00327398, Tax_Id=10116 Gene_Symbol=Enpep Isoform 1 of Glutamyl aminopeptidase

Match to Query 1590: 2008.964268 from(1005.489410,2+)

Title: 091008RatKidney_NH4Format01_23.4545.4545.2.dta

Data file K:INewmanPaper|Piliang|3SubProteomes\Piliang3SP\mgf5ppm\ERLIC_3SubProteomes5ppm.mgf
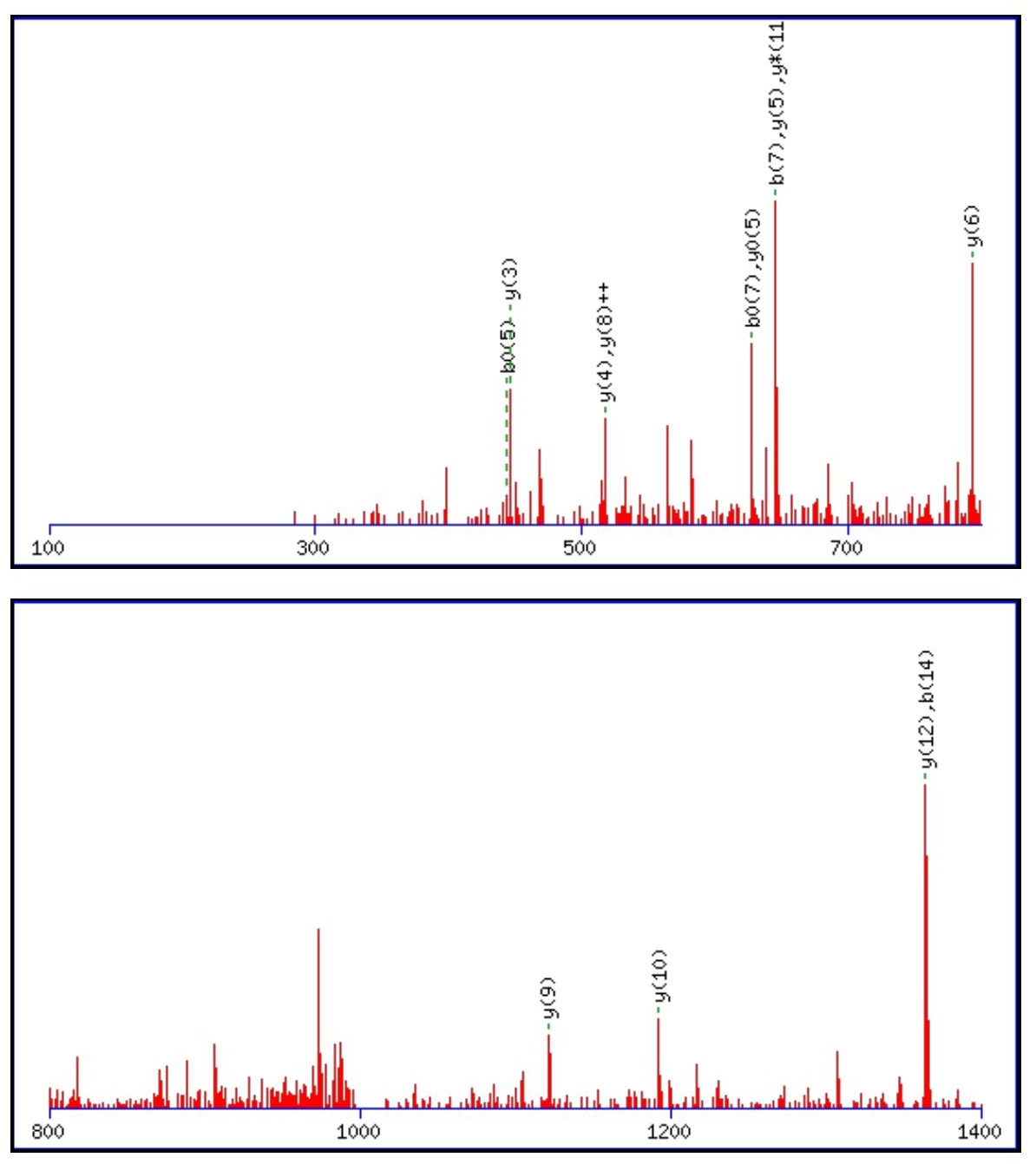


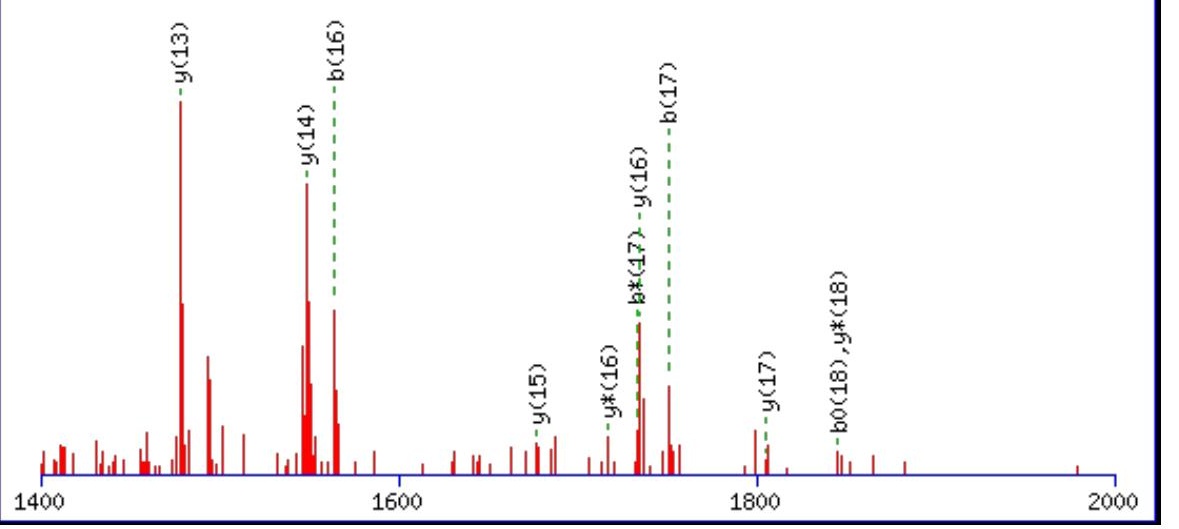

Monoisotopic mass of neutral peptide $\operatorname{Mr}($ calc): 2008.9564

Fixed modifications: Carbamidomethyl (C)

Variable modifications:

M1 : Oxidation (M)

N9 : Deamidated $\mathrm{N}(\mathrm{N})$

Ions Score: 87 Expect: $4.3 \mathrm{e}-007$

Matches (Bold Red): 25/184 fragment ions using 41 most intense peaks

\begin{tabular}{|c|c|c|c|c|c|c|c|c|c|c|c|c|c|c|}
\hline \# & b & $\mathbf{b}^{++}$ & $\mathbf{b}^{*}$ & $\mathbf{b}^{*^{++}}$ & $\mathbf{b}^{0}$ & $\mathbf{b}^{0++}$ & Seq. & $\mathbf{y}$ & $\mathbf{y}^{++}$ & $\mathbf{y}^{*}$ & $\mathbf{y}^{*^{++}}$ & $\mathbf{y}^{\mathbf{0}}$ & $\mathbf{y}^{0++}$ & \# \\
\hline 1 & 148.0427 & 74.5250 & & & & & $\mathbf{M}$ & & & & & & & 19 \\
\hline 2 & 205.0641 & 103.0357 & & & & & G & 1862.9283 & 931.9678 & 1845.9018 & 923.4545 & 1844.9177 & 922.9625 & 18 \\
\hline 3 & 276.1013 & 138.5543 & & & & & A & 1805.9068 & 903.4571 & 1788.8803 & 894.9438 & 1787.8963 & 894.4518 & 17 \\
\hline 4 & 333.1227 & 167.0650 & & & & & G & 1734.8697 & 867.9385 & 1717.8432 & 859.4252 & 1716.8592 & 858.9332 & 16 \\
\hline 5 & 462.1653 & 231.5863 & & & 444.1547 & 222.5810 & $\mathbf{E}$ & 1677.8483 & 839.4278 & 1660.8217 & 830.9145 & 1659.8377 & 830.4225 & 15 \\
\hline 6 & 533.2024 & 267.1049 & & & 515.1919 & 258.0996 & A & 1548.8057 & 774.9065 & 1531.7791 & 766.3932 & 1530.7951 & 765.9012 & 14 \\
\hline 7 & 646.2865 & 323.6469 & & & 628.2759 & 314.6416 & $\mathbf{L}$ & 1477.7686 & 739.3879 & 1460.7420 & 730.8746 & 1459.7580 & 730.3826 & 13 \\
\hline 8 & 703.3080 & 352.1576 & & & 685.2974 & 343.1523 & G & 1364.6845 & 682.8459 & 1347.6579 & 674.3326 & 1346.6739 & 673.8406 & 12 \\
\hline 9 & 818.3349 & 409.6711 & 801.3083 & 401.1578 & 800.3243 & 400.6658 & $\mathbf{N}$ & 1307.6630 & 654.3351 & 1290.6365 & 645.8219 & 1289.6525 & 645.3299 & 11 \\
\hline 10 & 889.3720 & 445.1896 & 872.3455 & 436.6764 & 871.3614 & 436.1844 & A & 1192.6361 & 596.8217 & 1175.6095 & 588.3084 & 1174.6255 & 587.8164 & 10 \\
\hline 11 & 976.4040 & 488.7057 & 959.3775 & 480.1924 & 958.3935 & 479.7004 & S & 1121.5990 & 561.3031 & 1104.5724 & 552.7898 & 1103.5884 & 552.2978 & 9 \\
\hline 12 & 1104.4626 & 552.7349 & 1087.4361 & 544.2217 & 1086.4520 & 543.7297 & $\mathbf{Q}$ & 1034.5669 & 517.7871 & 1017.5404 & 509.2738 & 1016.5564 & 508.7818 & 8 \\
\hline 13 & 1217.5467 & 609.2770 & 1200.5201 & 600.7637 & 1199.5361 & 600.2717 & $\mathbf{L}$ & 906.5084 & 453.7578 & 889.4818 & 445.2445 & 888.4978 & 444.7525 & 7 \\
\hline 14 & 1364.6151 & 682.8112 & 1347.5885 & 674.2979 & 1346.6045 & 673.8059 & $\mathbf{F}$ & 793.4243 & 397.2158 & 776.3978 & 388.7025 & 775.4137 & 388.2105 & 6 \\
\hline 15 & 1493.6577 & 747.3325 & 1476.6311 & 738.8192 & 1475.6471 & 738.3272 & $\mathbf{E}$ & 646.3559 & 323.6816 & 629.3293 & 315.1683 & 628.3453 & 314.6763 & 5 \\
\hline 16 & 1564.6948 & 782.8510 & 1547.6682 & 774.3378 & 1546.6842 & 773.8458 & A & 517.3133 & 259.1603 & 500.2867 & 250.6470 & & & 4 \\
\hline 17 & 1750.7741 & 875.8907 & 1733.7476 & 867.3774 & 1732.7635 & 866.8854 & $\mathbf{W}$ & 446.2762 & 223.6417 & 429.2496 & 215.1285 & & & 3 \\
\hline 18 & 1863.8582 & 932.4327 & 1846.8316 & 923.9195 & 1845.8476 & 923.4274 & $\mathbf{L}$ & 260.1969 & 130.6021 & 243.1703 & 122.0888 & & & 2 \\
\hline 19 & & & & & & & $\mathbf{K}$ & 147.1128 & 74.0600 & 130.0863 & 65.5468 & & & 1 \\
\hline
\end{tabular}

$$
\text { 옹 }
$$
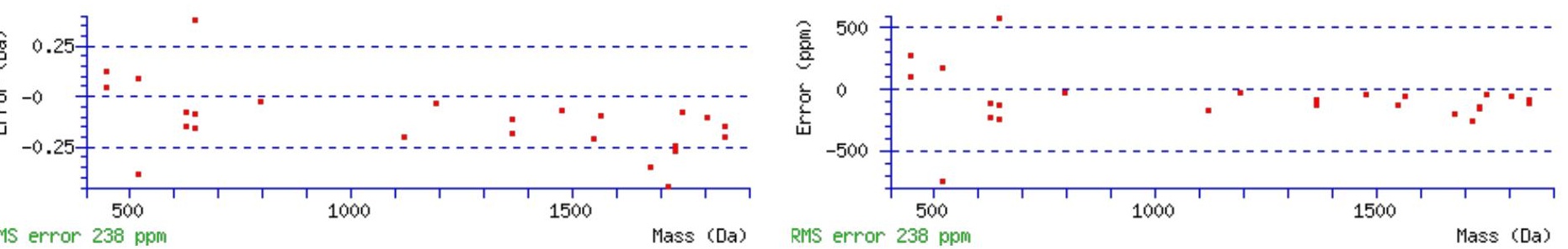

\section{All matches to this query}

\begin{tabular}{|l|c|c|c|}
\hline Score & Mr(calc): & Delta & Sequence \\
\hline 87.1 & 2008.9564 & 0.0079 & MGAGEALGNASQLFEAWLK \\
\hline 17.9 & 2007.9724 & 0.9919 & MGAGEALGNASQLFEAWLK \\
\hline
\end{tabular}




\begin{tabular}{|l|l|l|l|}
5.3 & 2008.9458 & 0.0185 & CVSFILRATVGSLLGEK \\
\hline 2.6 & 2008.9619 & 0.0024 & RQSSQWDPVSLISNALK \\
\hline 1.6 & 2007.9554 & 1.0089 & GNAYGFKISSLNKIADTK \\
\hline 1.6 & 2007.9554 & 1.0089 & GNAYGFKISSLNKIADTK \\
\hline 1.4 & 2006.9400 & 2.0242 & TLKTNTVGTLNMLELAK \\
\hline 0.4 & 2007.9796 & 0.9846 & TCGDVLGNRKGDGYQVLR \\
\hline
\end{tabular}

Spectrum No: 161; Query: 362; Rank: 1

\section{Peptide View}

MS/MS Fragmentation of ILNETLYENAK

Found in IPI00195931, Tax_Id=10116 Gene_Symbol=Tspan8 D6.1A protein

Match to Query 362: 1307.663228 from(654.838890,2+)

Title: 091008RatKidney_NoSalt_37.1416.1416.2.dta

Data file K:INewmanPaper|Piliangl3SubProteomes\Piliang3SP\mgf5ppm\ERLIC_3SubProteomes5ppm.mgf
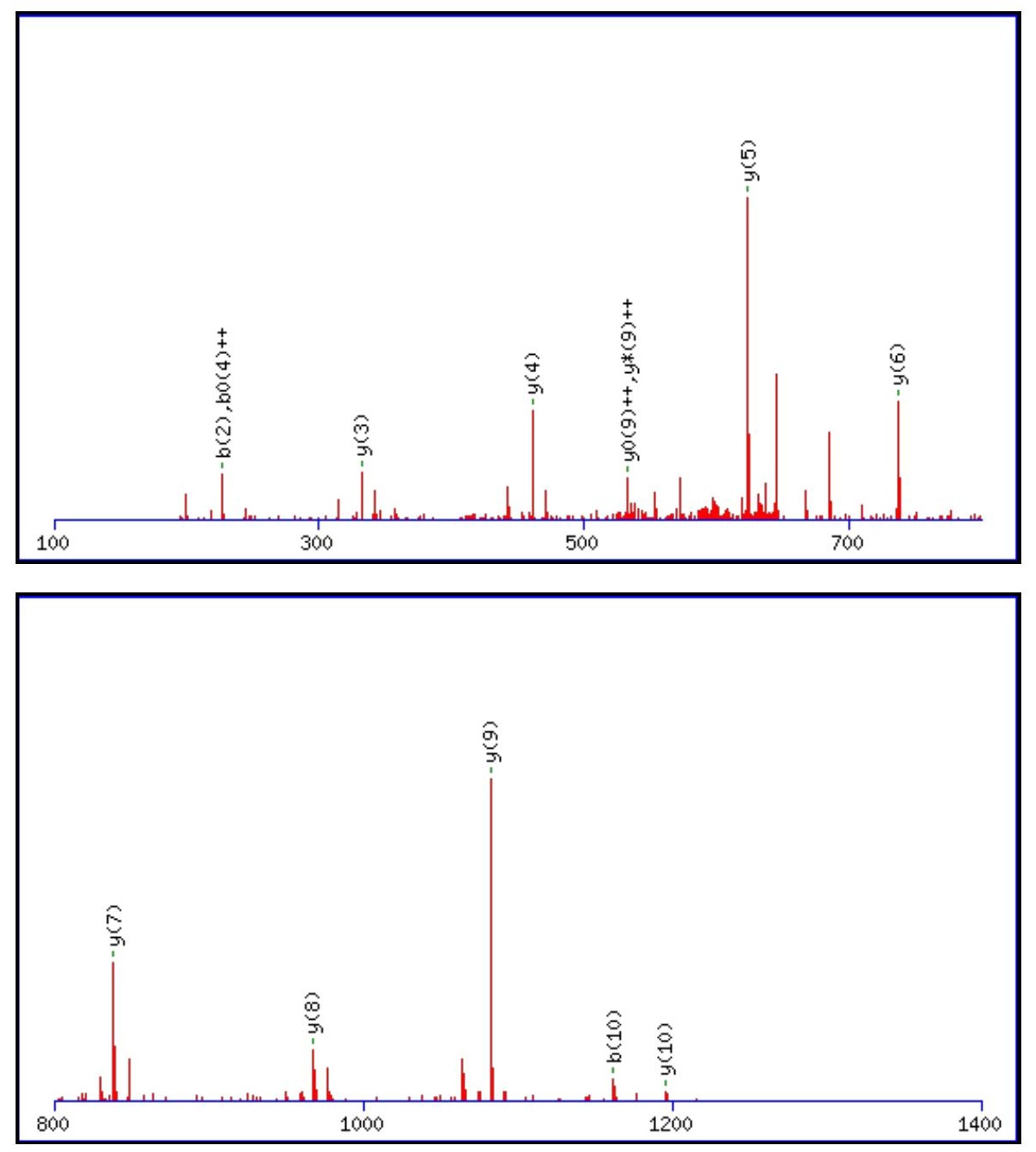


$1400 \quad 1600 \quad 1800 \quad 2000$

Monoisotopic mass of neutral peptide $\operatorname{Mr}($ calc): 1307.6608

Fixed modifications: Carbamidomethyl (C)

Variable modifications:

N3: Deamidated N (N)

Ions Score: 87 Expect: 2.4e- 007

Matches (Bold Red): 13/104 fragment ions using 16 most intense peaks

\begin{tabular}{|r|c|c|c|c|c|c|c|c|c|c|c|c|c|c|}
\hline$\#$ & $\mathbf{b}$ & $\mathbf{b}^{++}$ & $\mathbf{b}^{*}$ & $\mathbf{b}^{\mathbf{*}^{++}}$ & $\mathbf{b}^{\mathbf{0}}$ & $\mathbf{b}^{\mathbf{0 +}}$ & $\mathbf{S e q}$ & $\mathbf{y}$ & $\mathbf{y}^{++}$ & $\mathbf{y}^{\mathbf{*}}$ & $\mathbf{y}^{\mathbf{*}^{++}}$ & $\mathbf{y}^{\mathbf{0}}$ & $\mathbf{y}^{\mathbf{0 + +}}$ & $\#$ \\
\hline $\mathbf{1}$ & 114.0913 & 57.5493 & & & & & $\mathbf{I}$ & & & & & & & $\mathbf{1 1}$ \\
\hline $\mathbf{2}$ & $\mathbf{2 2 7 . 1 7 5 4}$ & 114.0913 & & & & & $\mathbf{L}$ & $\mathbf{1 1 9 5 . 5 8 4 1}$ & 598.2957 & 1178.5576 & 589.7824 & 1177.5735 & 589.2904 & $\mathbf{1 0}$ \\
\hline $\mathbf{3}$ & 342.2023 & 171.6048 & 325.1758 & 163.0915 & & & $\mathbf{N}$ & $\mathbf{1 0 8 2 . 5 0 0 0}$ & 541.7537 & 1065.4735 & 533.2404 & 1064.4895 & $\mathbf{5 3 2 . 7 4 8 4}$ & $\mathbf{9}$ \\
\hline $\mathbf{4}$ & 471.2449 & 236.1261 & 454.2184 & 227.6128 & 453.2344 & 227.1208 & $\mathbf{E}$ & $\mathbf{9 6 7 . 4 7 3 1}$ & 484.2402 & 950.4466 & 475.7269 & 949.4625 & 475.2349 & $\mathbf{8}$ \\
\hline $\mathbf{5}$ & 572.2926 & 286.6499 & 555.2661 & 278.1367 & 554.2820 & 277.6447 & $\mathbf{T}$ & $\mathbf{8 3 8 . 4 3 0 5}$ & 419.7189 & 821.4040 & 411.2056 & 820.4199 & 410.7136 & $\mathbf{7}$ \\
\hline $\mathbf{6}$ & 685.3767 & 343.1920 & 668.3501 & 334.6787 & 667.3661 & 334.1867 & $\mathbf{L}$ & $\mathbf{7 3 7 . 3 8 2 8}$ & 369.1951 & 720.3563 & 360.6818 & 719.3723 & 360.1898 & $\mathbf{6}$ \\
\hline $\mathbf{7}$ & 848.4400 & 424.7236 & 831.4135 & 416.2104 & 830.4294 & 415.7184 & $\mathbf{Y}$ & $\mathbf{6 2 4 . 2 9 8 8}$ & 312.6530 & 607.2722 & 304.1397 & 606.2882 & 303.6477 & $\mathbf{5}$ \\
\hline $\mathbf{8}$ & 977.4826 & 489.2449 & 960.4560 & 480.7317 & 959.4720 & 480.2397 & $\mathbf{E}$ & $\mathbf{4 6 1 . 2 3 5 4}$ & 231.1214 & 444.2089 & 222.6081 & 443.2249 & 222.1161 & $\mathbf{4}$ \\
\hline $\mathbf{9}$ & 1091.5255 & 546.2664 & 1074.4990 & 537.7531 & 1073.5150 & 537.2611 & $\mathbf{N}$ & 332.1928 & 166.6001 & 315.1663 & 158.0868 & & & $\mathbf{3}$ \\
\hline $\mathbf{1 0}$ & $\mathbf{1 1 6 2 . 5 6 2 6}$ & 581.7850 & 1145.5361 & 573.2717 & 1144.5521 & 572.7797 & $\mathbf{A}$ & 218.1499 & 109.5786 & 201.1234 & 101.0653 & & & $\mathbf{2}$ \\
\hline $\mathbf{1 1}$ & & & & & & & $\mathbf{K}$ & 147.1128 & 74.0600 & 130.0863 & 65.5468 & & & $\mathbf{1}$ \\
\hline
\end{tabular}$$
\text { 焉 }
$$
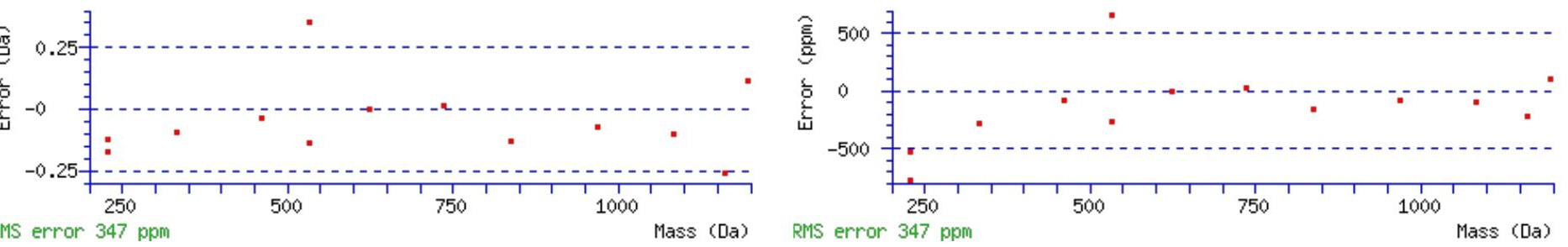

\section{All matches to this query}

\begin{tabular}{|l|l|l|l|}
\hline Score & Mr(calc): & Delta & \multicolumn{1}{c|}{ Sequence } \\
\hline 86.9 & 1307.6608 & 0.0024 & ILNETLYENAK \\
\hline 28.8 & 1307.6608 & 0.0024 & ILNETLYENAK \\
\hline 11.1 & 1307.6609 & 0.0024 & ILEDEGLYTQK \\
\hline 10.4 & 1307.6526 & 0.0106 & INVIHYINK \\
\hline 7.4 & 1307.6755 & -0.0122 & LLSREISSMEK \\
\hline 6.6 & 1307.6533 & 0.0099 & SRTXLRPVPR \\
\hline 4.2 & 1307.6526 & 0.0106 & IIYIVHDEVK \\
\hline 3.9 & 1307.6721 & -0.0089 & ITKQGNIYNEK \\
\hline 3.7 & 1307.6639 & -0.0006 & IISGQNFPKPK \\
\hline 3.7 & 1307.6761 & -0.0129 & $\underline{\text { ILYXEEKEAK }}$ \\
\hline
\end{tabular}


Spectrum No: 162; Query: 1584; Rank: 1

\section{Peptide View}

MS/MS Fragmentation of ATDDIIVNWVNGTLSEAGK

Found in IPI00210234, Tax_Id=10116 Gene_Symbol=Pls3 Plastin-3

Match to Query 1584: 2002.992748 from(1002.503650,2+)

Title: 100101RatKid_NS_deglyco_13.4731.4731.2.dta

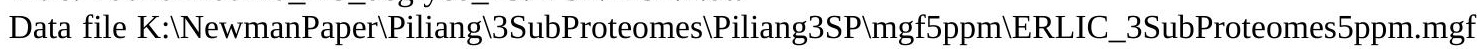
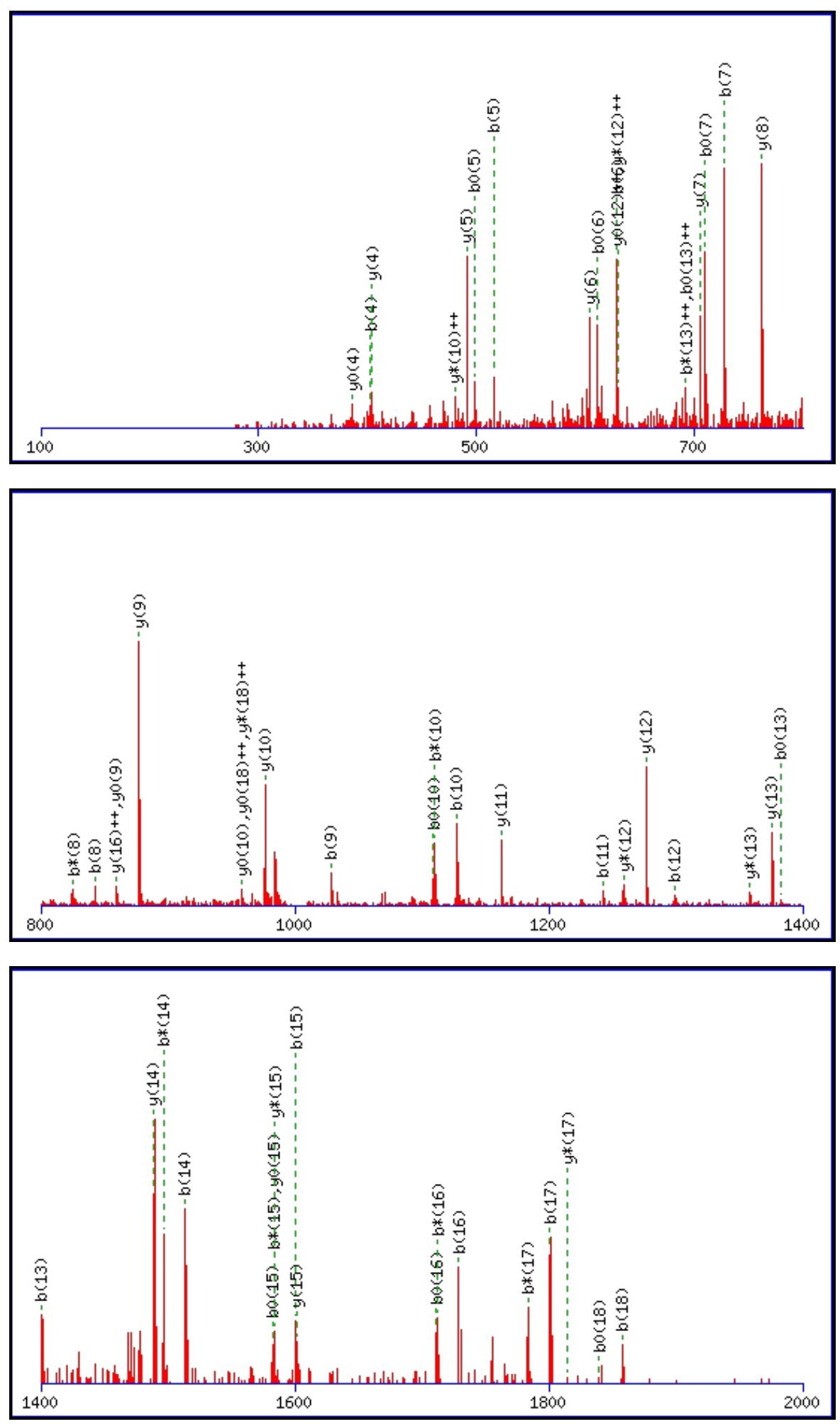
Monoisotopic mass of neutral peptide $\operatorname{Mr}($ calc): 2002.9847

Fixed modifications: Carbamidomethyl (C)

Variable modifications:

N11 : Deamidated_N (N)

Ions Score: 87 Expect: $4.3 e-007$

Matches (Bold Red): 57/194 fragment ions using 99 most intense peaks

\begin{tabular}{|c|c|c|c|c|c|c|c|c|c|c|c|c|c|c|}
\hline \# & b & $\mathbf{b}^{++}$ & b* & $\mathbf{b}^{*++}$ & $\mathbf{b}^{0}$ & & Seq. & $\mathbf{y}$ & $\mathbf{y}^{++}$ & $\mathrm{y}^{*}$ & & $\mathbf{y}^{0}$ & & \# \\
\hline 1 & 72.0444 & 36.5258 & & & & & A & & & & & & & 19 \\
\hline 2 & 173.0921 & 87.0497 & & & 155.0815 & 78.0444 & $\mathbf{T}$ & 1932.9549 & 966.9811 & 1915.9284 & 958.4678 & 1914.9443 & 957.9758 & 18 \\
\hline 3 & 288.1190 & 144.5631 & & & 270.1084 & 135.5579 & D & 1831.9072 & 916.4573 & 1814.8807 & 907.9440 & 1813.8967 & 907.4520 & 17 \\
\hline 4 & 403.1460 & 202.0766 & & & 385.1354 & 193.0713 & D & 1716.8803 & 858.9438 & 1699.8537 & 850.4305 & 1698.8697 & 849.9385 & 16 \\
\hline 5 & 516.2300 & 258.6186 & & & 498.2195 & 249.6134 & I & 1601.8533 & 801.4303 & 1584.8268 & 792.9170 & 1583.8428 & 792.4250 & 15 \\
\hline 6 & 629.3141 & 315.1607 & & & 611.3035 & 306.1554 & I & 1488.7693 & 744.8883 & 1471.7427 & 736.3750 & 1470.7587 & 735.8830 & 14 \\
\hline 7 & 728.3825 & 364.6949 & & & 710.3719 & 355.6896 & V & 1375.6852 & 688.3462 & 1358.6587 & 679.8330 & 1357.6746 & 679.3410 & 13 \\
\hline 8 & 842.4254 & 421.7164 & 825.3989 & 413.2031 & 824.4149 & 412.7111 & $\mathbf{N}$ & 1276.6168 & 638.8120 & 1259.5903 & 630.2988 & 1258.6062 & 629.8068 & 12 \\
\hline 9 & 1028.5047 & 514.7560 & 1011.4782 & 506.2427 & 1010.4942 & 505.7507 & W & 1162.5739 & 581.7906 & 1145.5473 & 573.2773 & 1144.5633 & 572.7853 & 11 \\
\hline 10 & 1127.5732 & 564.2902 & 1110.5466 & 555.7769 & 1109.5626 & 555.2849 & V & 976.4946 & 488.7509 & 959.4680 & 480.2376 & 958.4840 & 479.7456 & 10 \\
\hline 11 & 1242.6001 & 621.8037 & 1225.5735 & 613.2904 & 1224.5895 & 612.7984 & $\mathbf{N}$ & 877.4261 & 439.2167 & 860.3996 & 430.7034 & 859.4156 & 430.2114 & 9 \\
\hline 12 & 1299.6216 & 650.3144 & 1282.5950 & 641.8011 & 1281.6110 & 641.3091 & G & 762.3992 & 381.7032 & 745.3727 & 373.1900 & 744.3886 & 372.6980 & 8 \\
\hline 13 & 1400.6692 & 700.8383 & 1383.6427 & 692.3250 & 1382.6587 & 691.8330 & $\mathbf{T}$ & 705.3777 & 353.1925 & 688.3512 & 344.6792 & 687.3672 & 344.1872 & 7 \\
\hline 14 & 1513.7533 & 757.3803 & 1496.7267 & 748.8670 & 1495.7427 & 748.3750 & $\mathbf{L}$ & 604.3301 & 302.6687 & 587.3035 & 294.1554 & 586.3195 & 293.6634 & 6 \\
\hline 15 & 1600.7853 & 800.8963 & \begin{tabular}{|l}
1583.7588 \\
\end{tabular} & 792.3830 & $\mathbf{1 5 8 2 . 7 7 4 8}$ & 791.8910 & $\mathrm{~S}$ & 491.2460 & 246.1266 & 474.2195 & 237.6134 & 473.2354 & 237.1214 & 5 \\
\hline 16 & 1729.8279 & 865.4176 & 1712.8014 & 856.9043 & 1711.8174 & 856.4123 & $\mathbf{E}$ & 404.2140 & 202.6106 & 387.1874 & 194.0974 & 386.2034 & 193.6053 & 4 \\
\hline 17 & 1800.8650 & 900.9362 & 1783.8385 & 892.4229 & 1782.8545 & 891.9309 & A & 275.1714 & 138.0893 & 258.1448 & 129.5761 & & & 3 \\
\hline 18 & 1857.8865 & 929.4469 & 1840.8599 & 920.9336 & 1839.8759 & 920.4416 & G & 204.1343 & 102.5708 & 187.1077 & 94.0575 & & & 2 \\
\hline 19 & & & & & & & $\mathbf{K}$ & 147.1128 & 74.0600 & 130.0863 & 65.5468 & & & 1 \\
\hline
\end{tabular}
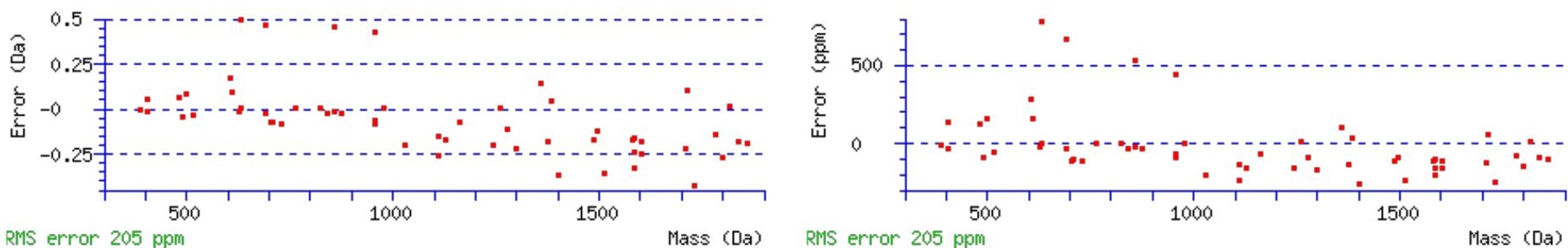

\section{All matches to this query}

\begin{tabular}{|l|l|l|l|}
\hline Score & Mr(calc): & Delta & \multicolumn{1}{c|}{ Sequence } \\
\hline 86.9 & 2002.9847 & 0.0080 & ATDDIIVNWVNGTLSEAGK \\
\hline 71.3 & 2002.9847 & 0.0080 & ATDDIIVNWVNGTLSEAGK \\
\hline 29.2 & 2002.0007 & 0.9920 & ATDDIIVNWVNGTLSEAGK \\
\hline 6.4 & 2002.9773 & 0.0154 & WGIILMMRYPEVQKK \\
\hline 5.3 & 2003.0000 & -0.0073 & $\underline{\text { FTFFADVLSVDNREKSK }}$ \\
\hline
\end{tabular}

Spectrum No: 163; Query: 347; Rank: 1

\section{Peptide View}

MS/MS Fragmentation of LDNYSTQELGR

Found in IPI00204348, Tax_Id=10116 Gene_Symbol=Nt5e 5'-nucleotidase precursor

Match to Query 347: 1295.601928 from(648.808240,2+)

Title: 100101RatKid_NS_deglyco_26.1488.1488.2.dta

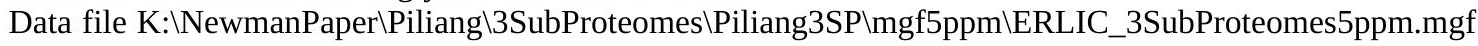



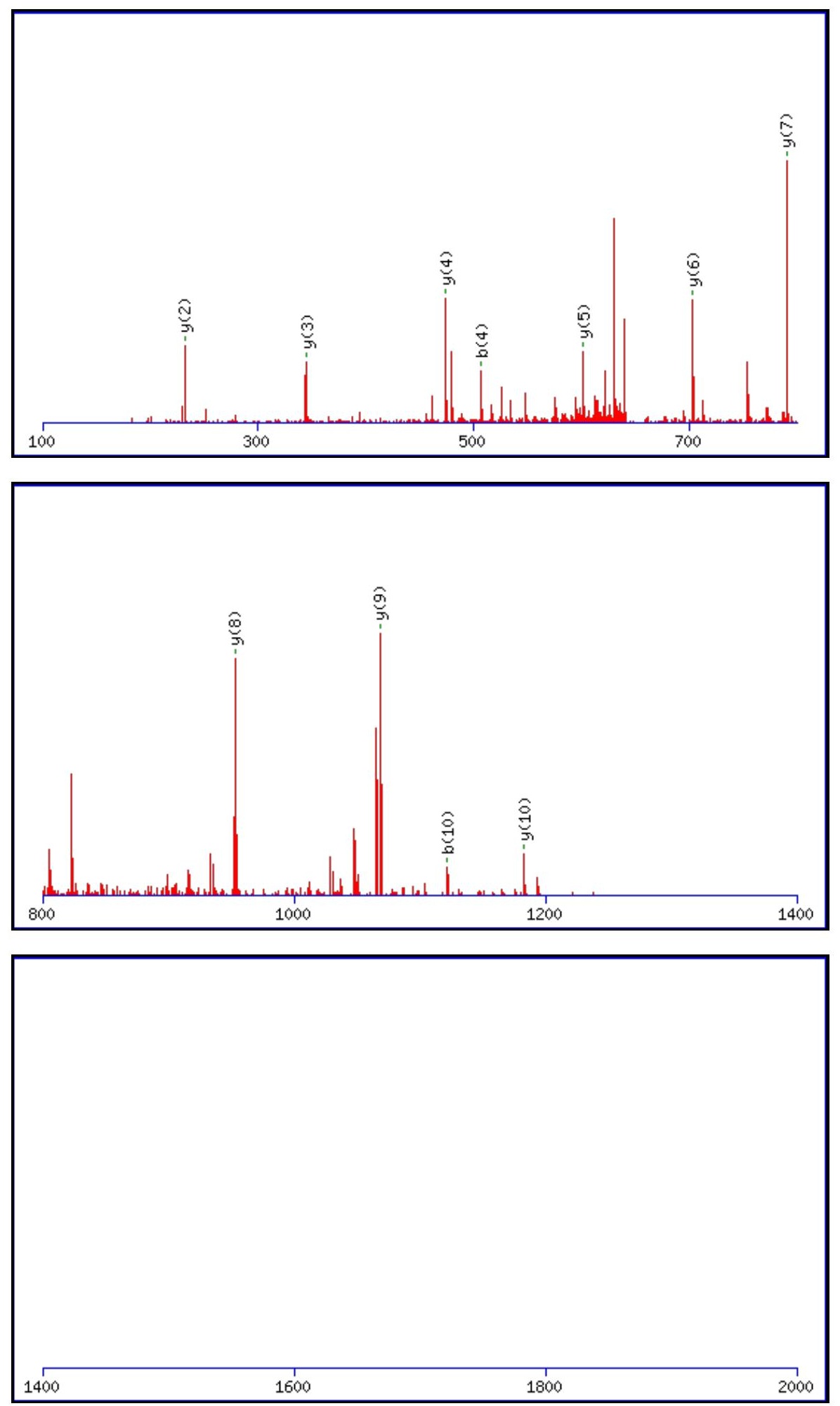

Monoisotopic mass of neutral peptide $\operatorname{Mr}($ calc): 1295.5993

Fixed modifications: Carbamidomethyl (C)

Variable modifications:

N3 : Deamidated_N (N)

Ions Score: 87 Expect: $2.9 \mathrm{e}-007$

Matches (Bold Red): 11/108 fragment ions using 15 most intense peaks

\begin{tabular}{|r|c|c|c|c|c|c|c|c|c|c|c|c|c|c|}
\hline$\#$ & $\mathbf{b}$ & $\mathbf{b}^{++}$ & $\mathbf{b}^{*}$ & $\mathbf{b}^{\boldsymbol{*}^{++}}$ & $\mathbf{b}^{\mathbf{0}}$ & $\mathbf{b}^{\mathbf{0}++}$ & $\mathbf{S e q}$ & $\mathbf{y}$ & $\mathbf{y}^{++}$ & $\mathbf{y}^{*}$ & $\mathbf{y}^{\mathbf{*}^{++}}$ & $\mathbf{y}^{\mathbf{0}}$ & $\mathbf{y}^{\mathbf{0 + +}}$ & $\#$ \\
\hline $\mathbf{1}$ & 114.0913 & 57.5493 & & & & & $\mathbf{L}$ & & & & & & & $\mathbf{1 1}$ \\
\hline $\mathbf{2}$ & 229.1183 & 115.0628 & & & 211.1077 & 106.0575 & $\mathbf{D}$ & $\mathbf{1 1 8 3 . 5 2 2 6}$ & 592.2649 & 1166.4960 & 583.7516 & 1165.5120 & 583.2596 & $\mathbf{1 0}$ \\
\hline $\mathbf{3}$ & 344.1452 & 172.5762 & 327.1187 & 164.0630 & 326.1347 & 163.5710 & $\mathbf{N}$ & $\mathbf{1 0 6 8 . 4 9 5 6}$ & 534.7514 & 1051.4691 & 526.2382 & 1050.4851 & 525.7462 & $\mathbf{9}$ \\
\hline $\mathbf{4}$ & $\mathbf{5 0 7 . 2 0 8 5}$ & 254.1079 & 490.1820 & 245.5946 & 489.1980 & 245.1026 & $\mathbf{Y}$ & $\mathbf{9 5 3 . 4 6 8 7}$ & 477.2380 & 936.4421 & 468.7247 & 935.4581 & 468.2327 & $\mathbf{8}$ \\
\hline $\mathbf{5}$ & 594.2406 & 297.6239 & 577.2140 & 289.1107 & 576.2300 & 288.6186 & $\mathbf{S}$ & $\mathbf{7 9 0 . 4 0 5 4}$ & 395.7063 & 773.3788 & 387.1930 & 772.3948 & 386.7010 & $\mathbf{7}$ \\
\hline
\end{tabular}




\begin{tabular}{|c|c|c|c|c|c|c|c|c|c|c|c|c|c|c|}
\hline 6 & 695.2883 & 348.1478 & 678.2617 & 339.6345 & 677.2777 & 339.1425 & $\mathbf{T}$ & 703.3733 & 352.1903 & 686.3468 & 343.6770 & 685.3628 & 343.1850 & 6 \\
\hline 7 & 823.3468 & 412.1771 & 806.3203 & 403.6638 & 805.3363 & 403.1718 & $\mathbf{Q}$ & 602.3257 & 301.6665 & 585.2991 & 293.1532 & 584.3151 & 292.6612 & 5 \\
\hline 8 & 952.3894 & 476.6984 & 935.3629 & 468.1851 & 934.3789 & 467.6931 & $\mathbf{E}$ & 474.2671 & 237.6372 & 457.2405 & 229.1239 & 456.2565 & 228.6319 & 4 \\
\hline 9 & 1065.4735 & 533.2404 & 1048.4469 & 524.7271 & 1047.4629 & 524.2351 & $\mathbf{L}$ & 345.2245 & 173.1159 & 328.1979 & 164.6026 & & & 3 \\
\hline 10 & 1122.4950 & 561.7511 & 1105.4684 & 553.2378 & 1104.4844 & 552.7458 & G & 232.1404 & 116.5738 & 215.1139 & 108.0606 & & & 2 \\
\hline 11 & & & & & & & $\mathbf{R}$ & 175.1190 & 88.0631 & 158.0924 & 79.5498 & & & 1 \\
\hline
\end{tabular}
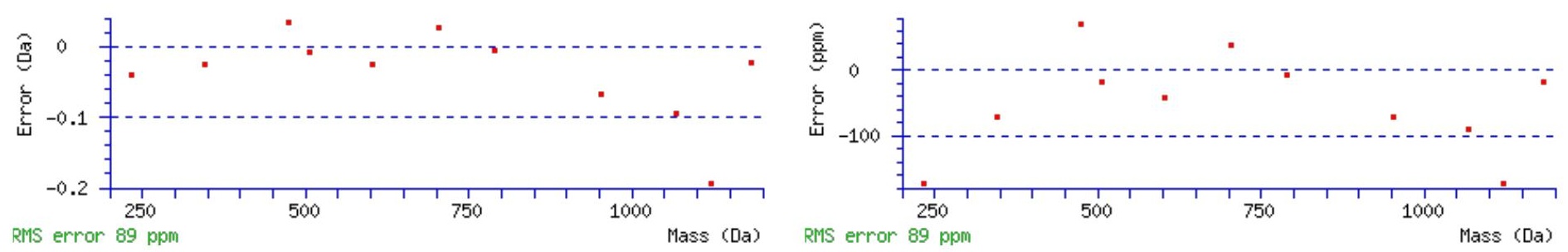

\section{All matches to this query}

\begin{tabular}{|l|l|l|l|}
\hline Score & Mr(calc): & Delta & \multicolumn{1}{|c|}{ Sequence } \\
\hline 86.6 & 1295.5993 & 0.0026 & LDNYSTQELGR \\
\hline 13.8 & 1295.6064 & -0.0044 & LSGWLXEIGR \\
\hline 8.1 & 1295.5983 & 0.0036 & GEKGQSRELGR \\
\hline 6.7 & 1295.5983 & 0.0036 & LETRAAAQGSGR \\
\hline 6.0 & 1295.5993 & 0.0026 & SYSLENEEAVR \\
\hline 5.7 & 1295.5983 & 0.0036 & DDLTREVRGR \\
\hline 4.3 & 1295.6115 & -0.0095 & MIVHMPPDQGR \\
\hline 4.3 & 1295.6002 & 0.0017 & VEFEICVMGGR \\
\hline 3.3 & 1295.6057 & -0.0038 & MQQSNVALLGR \\
\hline 3.1 & 1295.5993 & 0.0026 & LHDNPLTDENK \\
\hline
\end{tabular}

Spectrum No: 164; Query: 1354; Rank: 1

\section{Peptide View}

MS/MS Fragmentation of EGTEISHVLLCNVTQR

Found in IPI00191929, Tax_Id=10116 Gene_Symbol=Tmem27 Collectrin precursor

Match to Query 1354: 1855.917148 from(928.965850,2+)

Title: 100101RatKid_NS_deglyco_19.3401.3401.2.dta

Data file K:INewmanPaper|Piliangl3SubProteomes\Piliang3SP\mgf5ppm\ERLIC_3SubProteomes5ppm.mgf 

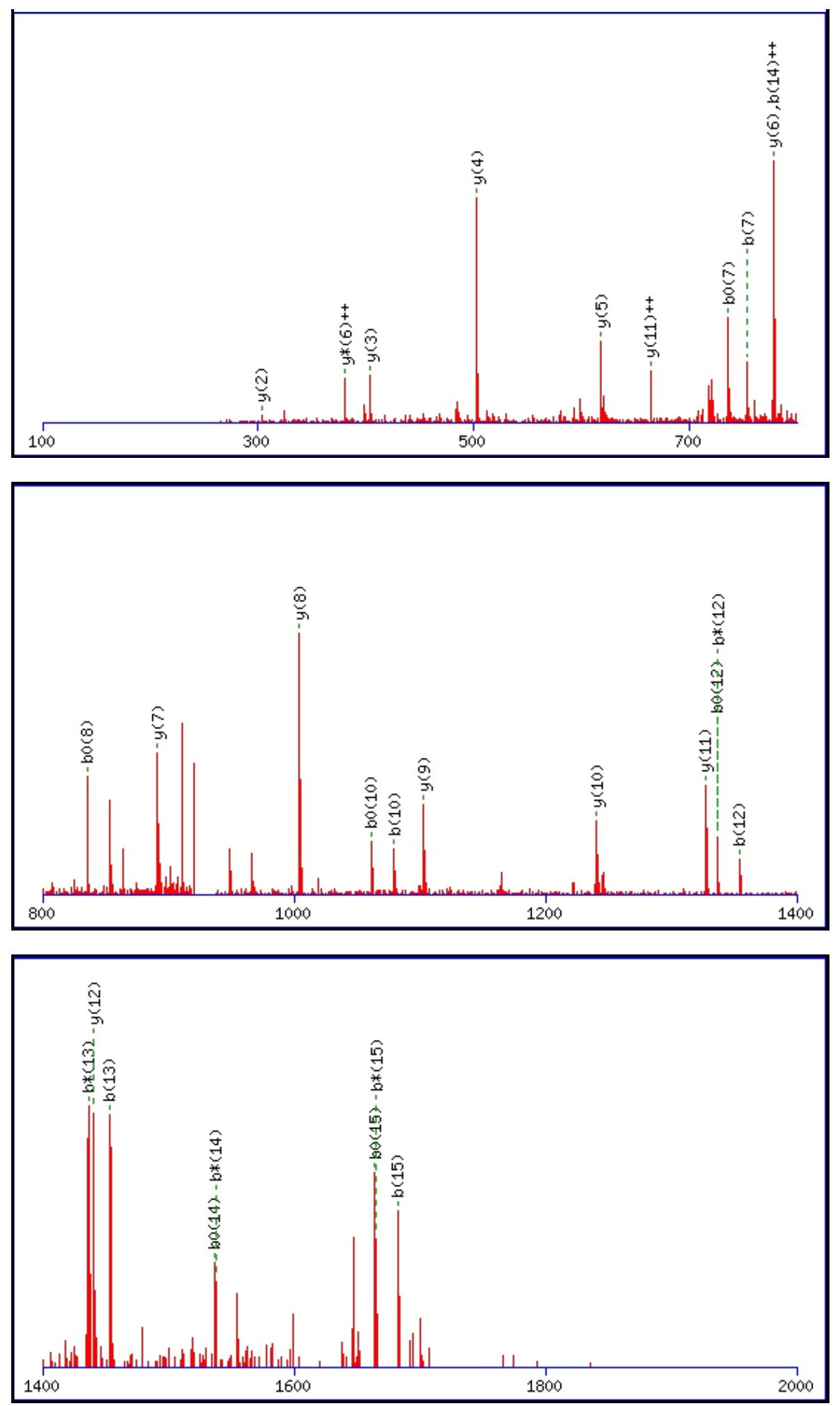

Monoisotopic mass of neutral peptide $\operatorname{Mr}($ calc): 1855.9098

Fixed modifications: Carbamidomethyl (C)

Variable modifications:

N12 : Deamidated $\mathrm{N}(\mathrm{N})$

Ions Score: 86 Expect: $4.1 \mathrm{e}-007$

Matches (Bold Red): 29/154 fragment ions using 45 most intense peaks

\begin{tabular}{|r|c|c|c|c|c|c|c|c|c|c|c|c|c|c|}
\hline$\#$ & $\mathbf{b}$ & $\mathbf{b}^{++}$ & $\mathbf{b}^{*}$ & $\mathbf{b}^{\boldsymbol{*}^{++}}$ & $\mathbf{b}^{\mathbf{0}}$ & $\mathbf{b}^{\mathbf{0 + +}}$ & Seq. & $\mathbf{y}$ & $\mathbf{y}^{++}$ & $\mathbf{y}^{\mathbf{*}}$ & $\mathbf{y}^{\boldsymbol{*}^{++}}$ & $\mathbf{y}^{\mathbf{0}}$ & $\mathbf{y}^{\mathbf{0 + +}}$ & $\#$ \\
\hline $\mathbf{1}$ & 130.0499 & 65.5286 & & & 112.0393 & 56.5233 & $\mathbf{E}$ & & & & & & & $\mathbf{1 6}$ \\
\hline $\mathbf{2}$ & 187.0713 & 94.0393 & & & 169.0608 & 85.0340 & $\mathbf{G}$ & 1727.8745 & 864.4409 & 1710.8479 & 855.9276 & 1709.8639 & 855.4356 & $\mathbf{1 5}$ \\
\hline $\mathbf{3}$ & 288.1190 & 144.5631 & & & 270.1084 & 135.5579 & $\mathbf{T}$ & 1670.8530 & 835.9302 & 1653.8265 & 827.4169 & 1652.8425 & 826.9249 & $\mathbf{1 4}$ \\
\hline $\mathbf{4}$ & 417.1616 & 209.0844 & & & 399.1510 & 200.0792 & $\mathbf{E}$ & 1569.8053 & 785.4063 & 1552.7788 & 776.8930 & 1551.7948 & 776.4010 & $\mathbf{1 3}$ \\
\hline $\mathbf{5}$ & 530.2457 & 265.6265 & & & 512.2351 & 256.6212 & $\mathbf{I}$ & $\mathbf{1 4 4 0 . 7 6 2 8}$ & 720.8850 & 1423.7362 & 712.3717 & 1422.7522 & 711.8797 & $\mathbf{1 2}$ \\
\hline
\end{tabular}




\begin{tabular}{|c|c|c|c|c|c|c|c|c|c|c|c|c|c|c|}
\hline 6 & 617.2777| & |309.1425 & & & 599.2671 & |300.1372| & S & |1327.6787 & 664.3430 & |1310.6521 & |655.8297 & |1309.6681 & |655.3377 & 11 \\
\hline 7 & 754.3366 & 377.6719 & & & 736.3260 & 368.6667 & $\mathbf{H}$ & 1240.6467 & 620.8270 & 1223.6201 & 612.3137 & 1222.6361 & 611.8217 & 10 \\
\hline 8 & 853.4050 & 427.2061 & & & 835.3945 & 418.2009 & $\mathbf{V}$ & 1103.5878 & 552.2975 & 1086.5612 & 543.7842 & 1085.5772 & 543.2922 & 9 \\
\hline 9 & 966.4891 & 483.7482 & & & 948.4785 & 474.7429 & $\mathbf{L}$ & 1004.5193 & 502.7633 & 987.4928 & 494.2500 & 986.5088 & 493.7580 & 8 \\
\hline 10 & 1079.5732 & 540.2902 & & & 1061.5626 & 531.2849 & $\mathbf{L}$ & 891.4353 & 446.2213 & 874.4087 & 437.7080 & 873.4247 & 437.2160 & 7 \\
\hline 11 & 1239.6038 & 620.3055 & & & 1221.5932 & 611.3003 & C & 778.3512 & 389.6792 & 761.3247 & 381.1660 & 760.3406 & 380.6740 & 6 \\
\hline 12 & 1354.6307 & 677.8190 & 1337.6042 & 669.3057 & 1336.6202 & 668.8137 & $\mathbf{N}$ & 618.3206 & 309.6639 & 601.2940 & 301.1506 & 600.3100 & 300.6586 & 5 \\
\hline 13 & 1453.6991 & 727.3532 & 1436.6726 & 718.8399 & 1435.6886 & 718.3479 & $\mathbf{V}$ & 503.2936 & 252.1504 & 486.2671 & 243.6372 & 485.2831 & 243.1452 & 4 \\
\hline 14 & 1554.7468 & 777.8771 & 1537.7203 & 769.3638 & 1536.7363 & 768.8718 & $\mathbf{T}$ & 404.2252 & 202.6162 & 387.1987 & 194.1030 & 386.2146 & 193.6110 & 3 \\
\hline 15 & 1682.8054 & 841.9063 & 1665.7789 & 833.3931 & 1664.7948 & 832.9011 & $\mathbf{Q}$ & 303.1775 & 152.0924 & 286.1510 & 143.5791 & & & 2 \\
\hline 16 & & & & & & & $\mathbf{R}$ & 175.1190 & 88.0631 & 158.0924 & 79.5498 & & & 1 \\
\hline
\end{tabular}
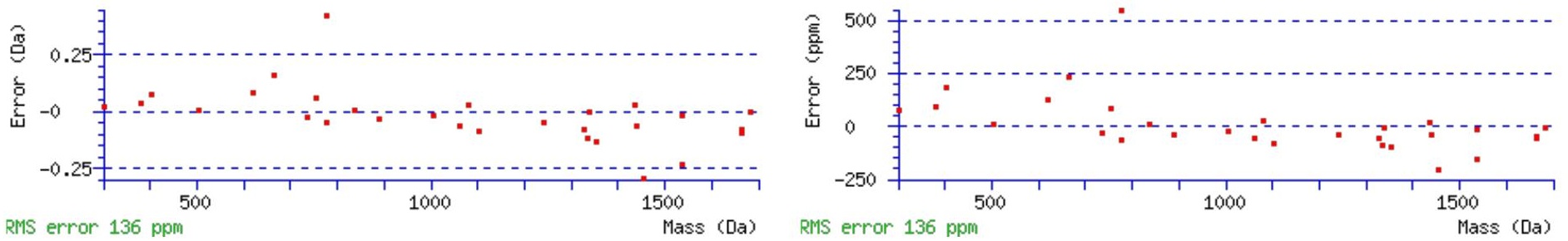

\section{All matches to this query}

\begin{tabular}{|l|l|l|l|}
\hline Score & Mr(calc): & Delta & \multicolumn{1}{c|}{ Sequence } \\
\hline 86.2 & 1855.9098 & 0.0073 & EGTEISHVLLCNVTQR \\
\hline 7.0 & 1854.9258 & 0.9914 & EGTEISHVLLCNVTQR \\
\hline 4.9 & 1855.9114 & 0.0057 & $\underline{\text { RTVETEAVQMLKDIK }}$ \\
\hline 4.2 & 1855.9114 & 0.0057 & $\underline{\text { RTVETEAVQMLKDIK }}$ \\
\hline 2.5 & 1855.9305 & -0.0134 & $\underline{\text { LADFGVSAKNTRTIQR }}$ \\
\hline 0.3 & 1855.9073 & 0.0098 & TKAMVACYPGNGLGYVR \\
\hline
\end{tabular}

Spectrum No: 165; Query: 2721; Rank: 1

\section{Peptide View}

MS/MS Fragmentation of QADEDIQGTQNLLTSIESETAASEETLTNASQR

Found in IPI00365542, Tax_Id=10116 Gene_Symbol=Lamb1_predicted laminin, beta 1

Match to Query 2721: 3550.647942 from(1184.556590,3+)

Title: 100101RatKid_NS_deglyco_25.5516.5516.3.dta

Data file K:\NewmanPaper\Piliang \3SubProteomes $\backslash P i l i a n g 3 S P \backslash m g f 5 p p m \backslash E R L I C \_3 S u b P r o t e o m e s 5 p p m . m g f$ 

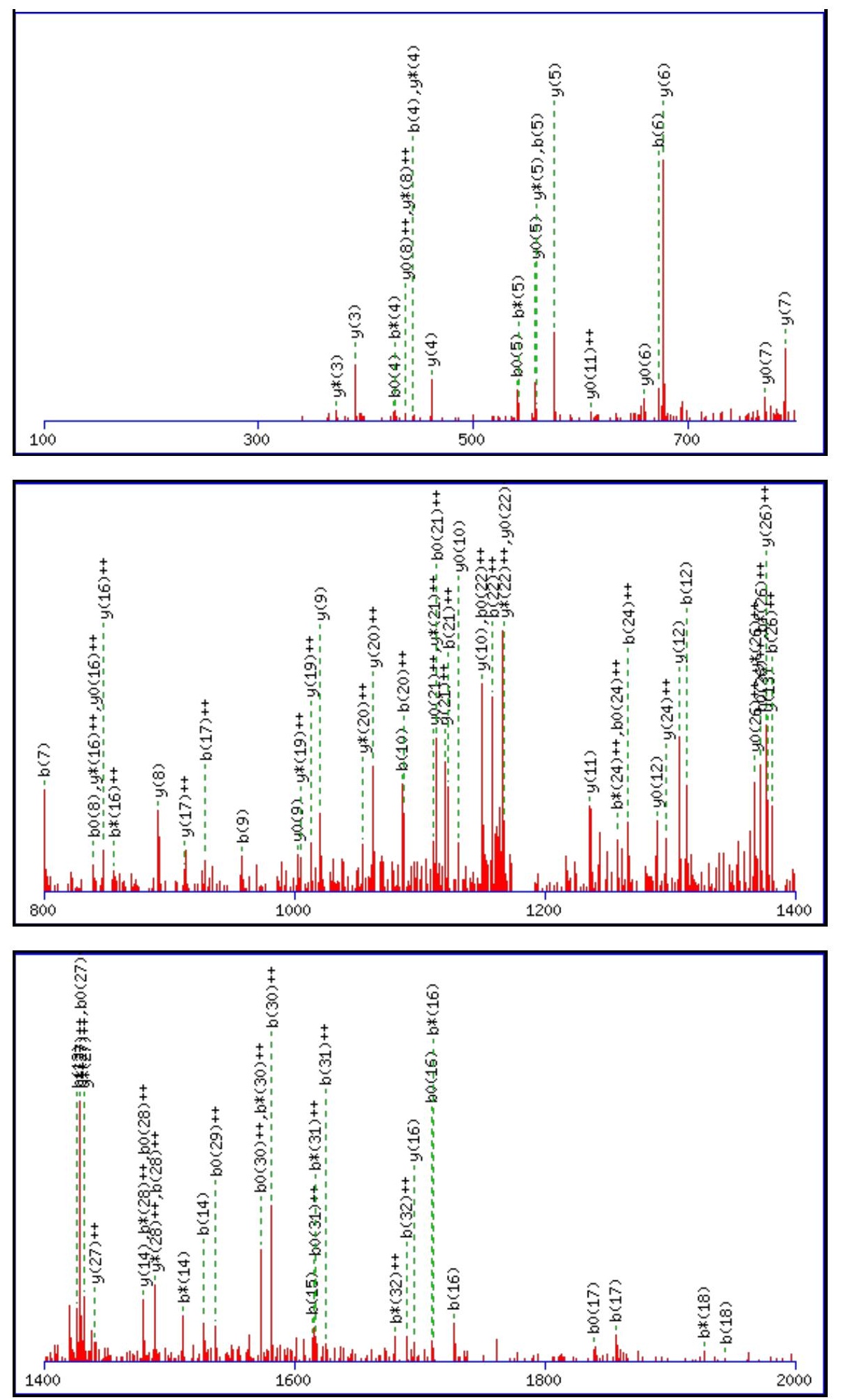

Monoisotopic mass of neutral peptide $\operatorname{Mr}($ calc): 3550.6394

Fixed modifications: Carbamidomethyl (C)

Variable modifications:

N29 : Deamidated_N (N)

Ions Score: 86 Expect: $9.8 \mathrm{e}-007$

Matches (Bold Red): 95/376 fragment ions using 163 most intense peaks

\begin{tabular}{|c|c|c|c|c|c|c|c|c|c|c|c|c|c|c|}
\hline$\#$ & $\mathbf{b}$ & \multicolumn{1}{|c|}{$\mathbf{b}^{++}$} & $\mathbf{b}^{*}$ & $\mathbf{b}^{\boldsymbol{*}^{++}}$ & $\mathbf{b}^{\mathbf{0}}$ & $\mathbf{b}^{\mathbf{0 + +}}$ & $\mathbf{S e q} \cdot$ & $\mathbf{y}$ & $\mathbf{y}^{++}$ & $\mathbf{y}^{\mathbf{*}}$ & $\mathbf{y}^{\mathbf{*}^{++}}$ & $\mathbf{y}^{\mathbf{0}}$ & $\mathbf{y}^{\mathbf{0 + +}}$ & $\#$ \\
\hline $\mathbf{1}$ & 129.0659 & 65.0366 & 112.0393 & 56.5233 & & & $\mathbf{Q}$ & & & & & & & 33 \\
\hline $\mathbf{2}$ & 200.1030 & 100.5551 & 183.0764 & 92.0418 & & & $\mathbf{A}$ & 3423.5881 & 1712.2977 & 3406.5616 & 1703.7844 & 3405.5776 & 1703.2924 & $\mathbf{3 2}$ \\
\hline $\mathbf{3}$ & 315.1299 & 158.0686 & 298.1034 & 149.5553 & 297.1193 & 149.0633 & $\mathbf{D}$ & 3352.5510 & 1676.7792 & 3335.5245 & 1668.2659 & 3334.5405 & 1667.7739 & 31 \\
\hline $\mathbf{4}$ & $\mathbf{4 4 4 . 1 7 2 5}$ & 222.5899 & $\mathbf{4 2 7 . 1 4 6 0}$ & 214.0766 & $\mathbf{4 2 6 . 1 6 1 9}$ & 213.5846 & $\mathbf{E}$ & 3237.5241 & 1619.2657 & 3220.4975 & 1610.7524 & 3219.5135 & 1610.2604 & $\mathbf{3 0}$ \\
\hline $\mathbf{5}$ & $\mathbf{5 5 9 . 1 9 9 4}$ & 280.1034 & 542.1729 & 271.5901 & $\mathbf{5 4 1 . 1 8 8 9}$ & 271.0981 & $\mathbf{D}$ & 3108.4815 & 1554.7444 & 3091.4549 & 1546.2311 & 3090.4709 & 1545.7391 & $\mathbf{2 9}$ \\
\hline
\end{tabular}




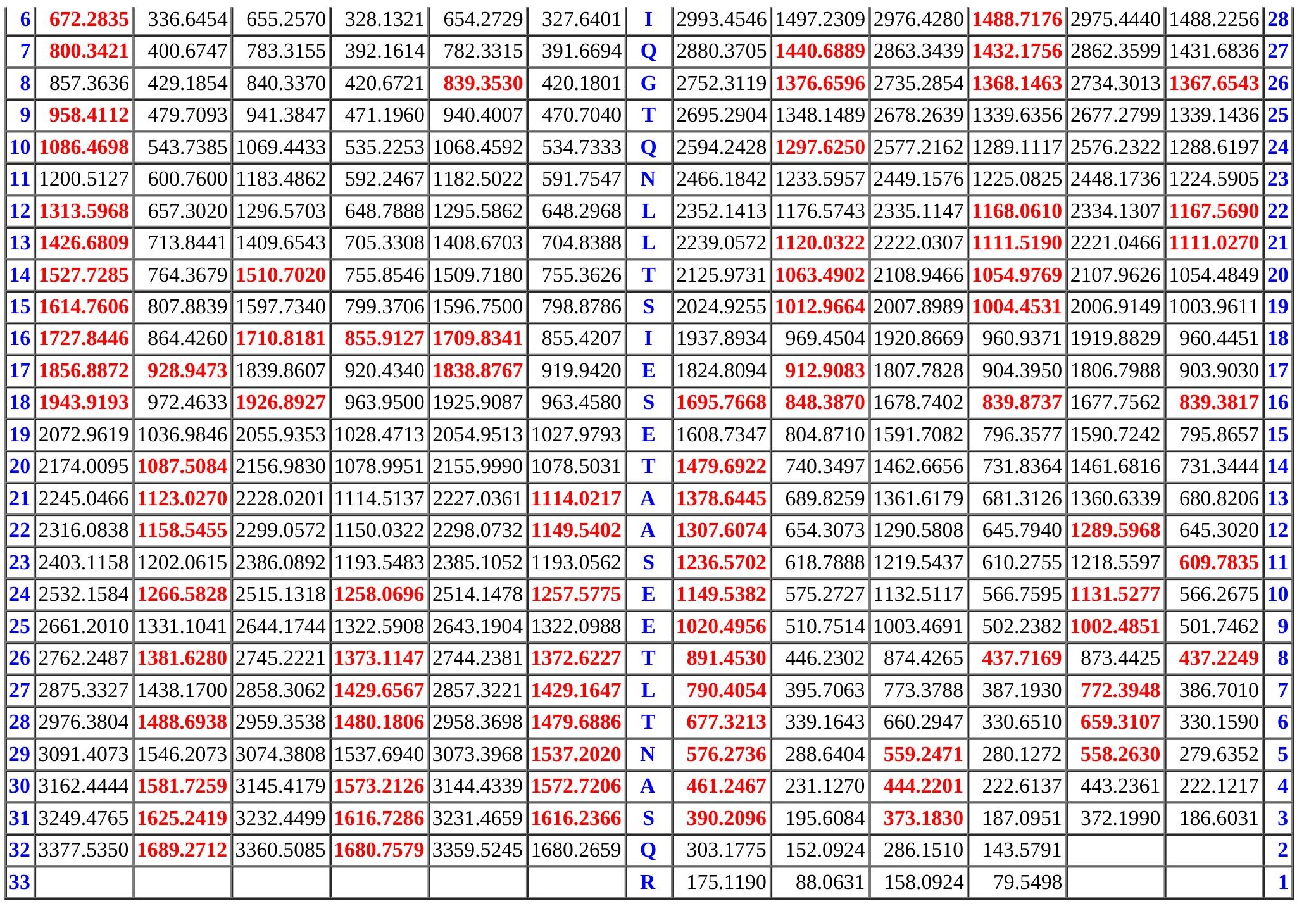
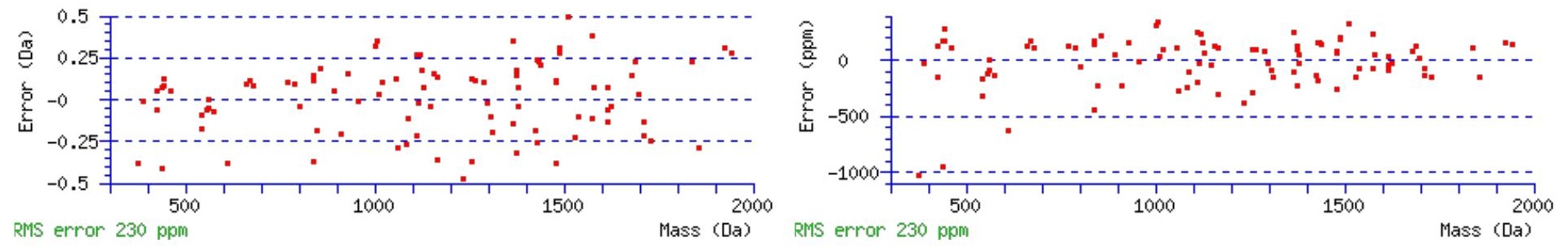

\section{All matches to this query}

\begin{tabular}{|l|r|r|l|}
\hline Score & Mr(calc): & Delta & \multicolumn{1}{c|}{ Sequence } \\
\hline 86.1 & 3550.6394 & 0.0085 & QADEDIQGTQNLLTSIESETAASEETLTNASQR \\
\hline 47.2 & 3549.6554 & 0.9925 & QADEDIQGTQNLLTSIESETAASEETLTNASQR \\
\hline 37.5 & 3550.6394 & 0.0085 & QADEDIQGTQNLLTSIESETAASEETLTNASQR \\
\hline 4.0 & 3549.6316 & 1.0164 & VVSAGNLLSHVGHTILGMNTVQLYMKVPGSR \\
\hline 4.0 & 3550.6278 & 0.0201 & MNELALVSVSDRYGSGSIYTLLPPMSFGSKR \\
\hline 4.0 & 3550.6278 & 0.0201 & MNELALVSVSDRYGSGSIYTLLPPMSFGSKR \\
\hline 3.3 & 3550.6278 & 0.0201 & MNELALVSVSDRYGSGSIYTLLPPMSFGSKR \\
\hline 2.8 & 3550.6278 & 0.0201 & MNELALVSVSDRYGSGSIYTLLPPMSFGSKR \\
\hline 2.8 & 3550.6278 & 0.0201 & MNELALVSVSDRYGSGSIYTLLPPMSFGSKR \\
\hline 2.0 & 3550.6528 & -0.0049 & SAIIETYVMDSRIDVSVAAGHTDRSGLGSVLR \\
\hline
\end{tabular}


Spectrum No: 166; Query: 900; Rank: 1

\section{Peptide View}

MS/MS Fragmentation of KLINDYVSNQTQGK

Found in IPI00200591, Tax_Id=10116 Gene_Symbol=LOC299282 Serine protease inhibitor A3L precursor

Match to Query 900: 1607.816888 from(804.915720,2+)

Title: 091008RatKidney_NoSalt_32.1068.1068.2.dta

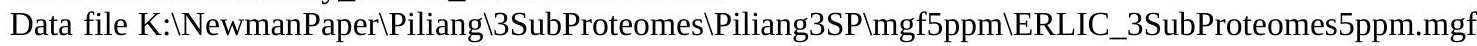
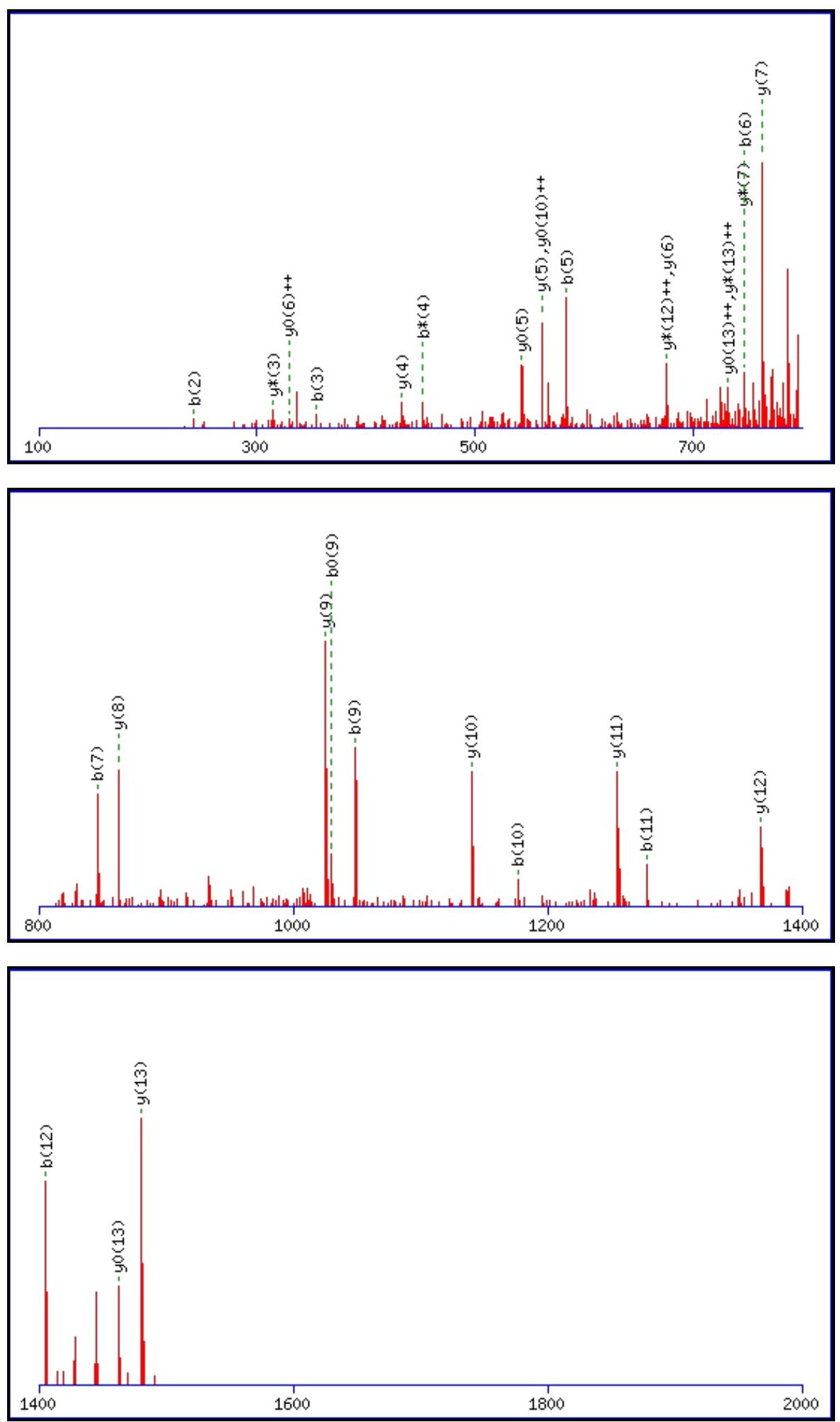

Monoisotopic mass of neutral peptide $\operatorname{Mr}$ (calc): 1607.8155

Fixed modifications: Carbamidomethyl (C) 
Variable modifications:

N9 : Deamidated_N (N)

Ions Score: 86 Expect: $3.6 \mathrm{e}-007$

Matches (Bold Red): 32/142 fragment ions using 42 most intense peaks

\begin{tabular}{|r|c|c|c|c|c|c|c|c|c|c|c|c|c|c|}
\hline$\#$ & $\mathbf{b}$ & $\mathbf{b}^{++}$ & $\mathbf{b}^{*}$ & $\mathbf{b}^{\mathbf{*}^{++}}$ & $\mathbf{b}^{\mathbf{0}}$ & $\mathbf{b}^{\mathbf{0 + +}}$ & $\mathbf{S e q}$ & $\mathbf{y}$ & $\mathbf{y}^{++}$ & $\mathbf{y}^{\mathbf{*}}$ & $\mathbf{y}^{\boldsymbol{*}^{++}}$ & $\mathbf{y}^{\mathbf{0}}$ & $\mathbf{y}^{\mathbf{0}+}$ & $\#$ \\
\hline $\mathbf{1}$ & 129.1022 & 65.0548 & 112.0757 & 56.5415 & & & $\mathbf{K}$ & & & & & & & $\mathbf{1 4}$ \\
\hline $\mathbf{2}$ & $\mathbf{2 4 2 . 1 8 6 3}$ & 121.5968 & 225.1598 & 113.0835 & & & $\mathbf{L}$ & $\mathbf{1 4 8 0 . 7 2 7 8}$ & 740.8675 & 1463.7013 & 732.3543 & $\mathbf{1 4 6 2 . 7 1 7 2}$ & $\mathbf{7 3 1 . 8 6 2 3}$ & $\mathbf{1 3}$ \\
\hline $\mathbf{3}$ & $\mathbf{3 5 5 . 2 7 0 4}$ & 178.1388 & 338.2438 & 169.6255 & & & $\mathbf{I}$ & $\mathbf{1 3 6 7 . 6 4 3 7}$ & 684.3255 & 1350.6172 & $\mathbf{6 7 5 . 8 1 2 2}$ & 1349.6332 & 675.3202 & $\mathbf{1 2}$ \\
\hline $\mathbf{4}$ & 469.3133 & 235.1603 & 452.2867 & 226.6470 & & & $\mathbf{N}$ & $\mathbf{1 2 5 4 . 5 5 9 7}$ & 627.7835 & 1237.5331 & 619.2702 & 1236.5491 & 618.7782 & $\mathbf{1 1}$ \\
\hline $\mathbf{5}$ & $\mathbf{5 8 4 . 3 4 0 2}$ & 292.6738 & 567.3137 & 284.1605 & 566.3297 & 283.6685 & $\mathbf{D}$ & $\mathbf{1 1 4 0 . 5 1 6 8}$ & 570.7620 & 1123.4902 & 562.2487 & 1122.5062 & $\mathbf{5 6 1 . 7 5 6 7}$ & $\mathbf{1 0}$ \\
\hline $\mathbf{6}$ & $\mathbf{7 4 7 . 4 0 3 6}$ & 374.2054 & 730.3770 & 365.6921 & 729.3930 & 365.2001 & $\mathbf{Y}$ & $\mathbf{1 0 2 5 . 4 8 9 8}$ & 513.2485 & 1008.4633 & 504.7353 & 1007.4792 & 504.2433 & $\mathbf{9}$ \\
\hline $\mathbf{7}$ & $\mathbf{8 4 6 . 4 7 2 0}$ & 423.7396 & 829.4454 & 415.2264 & 828.4614 & 414.7343 & $\mathbf{V}$ & $\mathbf{8 6 2 . 4 2 6 5}$ & 431.7169 & 845.3999 & 423.2036 & 844.4159 & 422.7116 & $\mathbf{8}$ \\
\hline $\mathbf{8}$ & 933.5040 & 467.2556 & 916.4775 & 458.7424 & 915.4934 & 458.2504 & $\mathbf{S}$ & $\mathbf{7 6 3 . 3 5 8 1}$ & 382.1827 & 746.3315 & 373.6694 & 745.3475 & 373.1774 & $\mathbf{7}$ \\
\hline $\mathbf{9}$ & $\mathbf{1 0 4 8 . 5 3 0 9}$ & 524.7691 & 1031.5044 & 516.2558 & $\mathbf{1 0 3 0 . 5 2 0 4}$ & 515.7638 & $\mathbf{N}$ & $\mathbf{6 7 6 . 3 2 6 0}$ & 338.6667 & 659.2995 & 330.1534 & 658.3155 & $\mathbf{3 2 9 . 6 6 1 4}$ & $\mathbf{6}$ \\
\hline $\mathbf{1 0}$ & $\mathbf{1 1 7 6 . 5 8 9 5}$ & 588.7984 & 1159.5630 & 580.2851 & 1158.5790 & 579.7931 & $\mathbf{Q}$ & $\mathbf{5 6 1 . 2 9 9 1}$ & 281.1532 & 544.2726 & 272.6399 & $\mathbf{5 4 3 . 2 8 8 5}$ & 272.1479 & $\mathbf{5}$ \\
\hline $\mathbf{1 1}$ & $\mathbf{1 2 7 7 . 6 3 7 2}$ & 639.3222 & 1260.6107 & 630.8090 & 1259.6266 & 630.3170 & $\mathbf{T}$ & $\mathbf{4 3 3 . 2 4 0 5}$ & 217.1239 & 416.2140 & 208.6106 & 415.2300 & 208.1186 & $\mathbf{4}$ \\
\hline $\mathbf{1 2}$ & $\mathbf{1 4 0 5 . 6 9 5 8}$ & 703.3515 & 1388.6692 & 694.8383 & 1387.6852 & 694.3462 & $\mathbf{Q}$ & 332.1928 & 166.6001 & 315.1663 & 158.0868 & & & $\mathbf{3}$ \\
\hline $\mathbf{1 3}$ & $\mathbf{1 4 6 2 . 7 1 7 2}$ & 731.8623 & 1445.6907 & 723.3490 & 1444.7067 & 722.8570 & $\mathbf{G}$ & 204.1343 & 102.5708 & 187.1077 & 94.0575 & & & $\mathbf{2}$ \\
\hline $\mathbf{1 4}$ & & & & & & & $\mathbf{K}$ & 147.1128 & 74.0600 & 130.0863 & 65.5468 & & & $\mathbf{1}$ \\
\hline
\end{tabular}
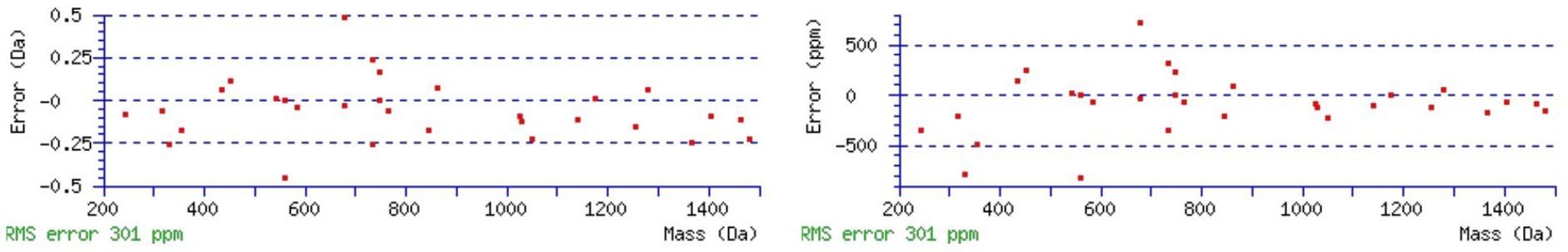

\section{All matches to this query}

\begin{tabular}{|l|l|l|l|}
\hline Score & Mr(calc): & Delta & \multicolumn{1}{|c|}{ Sequence } \\
\hline 85.8 & 1607.8155 & 0.0014 & KLINDYVSNQTQGK \\
\hline 35.3 & 1607.8155 & 0.0014 & KLINDYVSNQTQGK \\
\hline 7.8 & 1607.8073 & 0.0096 & TQLIFGSVTNIHAK \\
\hline 7.2 & 1607.8158 & 0.0011 & QLIRSSPAAHHRR \\
\hline 7.2 & 1607.8158 & 0.0011 & QLIRSSPAAHHRR \\
\hline 7.1 & 1607.8072 & 0.0097 & QLLAPGNSAGAFLIR \\
\hline 5.8 & 1607.8276 & -0.0107 & KLVENGGMFVCKAR \\
\hline 3.9 & 1607.8130 & 0.0039 & LGALNCTAFSIQWK \\
\hline 3.1 & 1607.8283 & -0.0114 & EKAILQATLREEK \\
\hline 1.8 & 1607.8283 & -0.0115 & KLQTLDLRDNALK \\
\hline
\end{tabular}

Spectrum No: 167; Query: 934; Rank: 1

\section{Peptide View}

MS/MS Fragmentation of NPDINMTFEQVYR

Found in IPI00325312, Tax_Id=10116 Gene_Symbol=Prom1 prominin 1 isoform 1

Match to Query 934: 1627.733728 from(814.874140,2+)

Title: 091008RatKidney_NoSalt_26.3064.3064.2.dta

Data file K:INewmanPaper|Piliang|3SubProteomes\Piliang3SP\mgf5ppm\ERLIC_3SubProteomes5ppm.mgf 

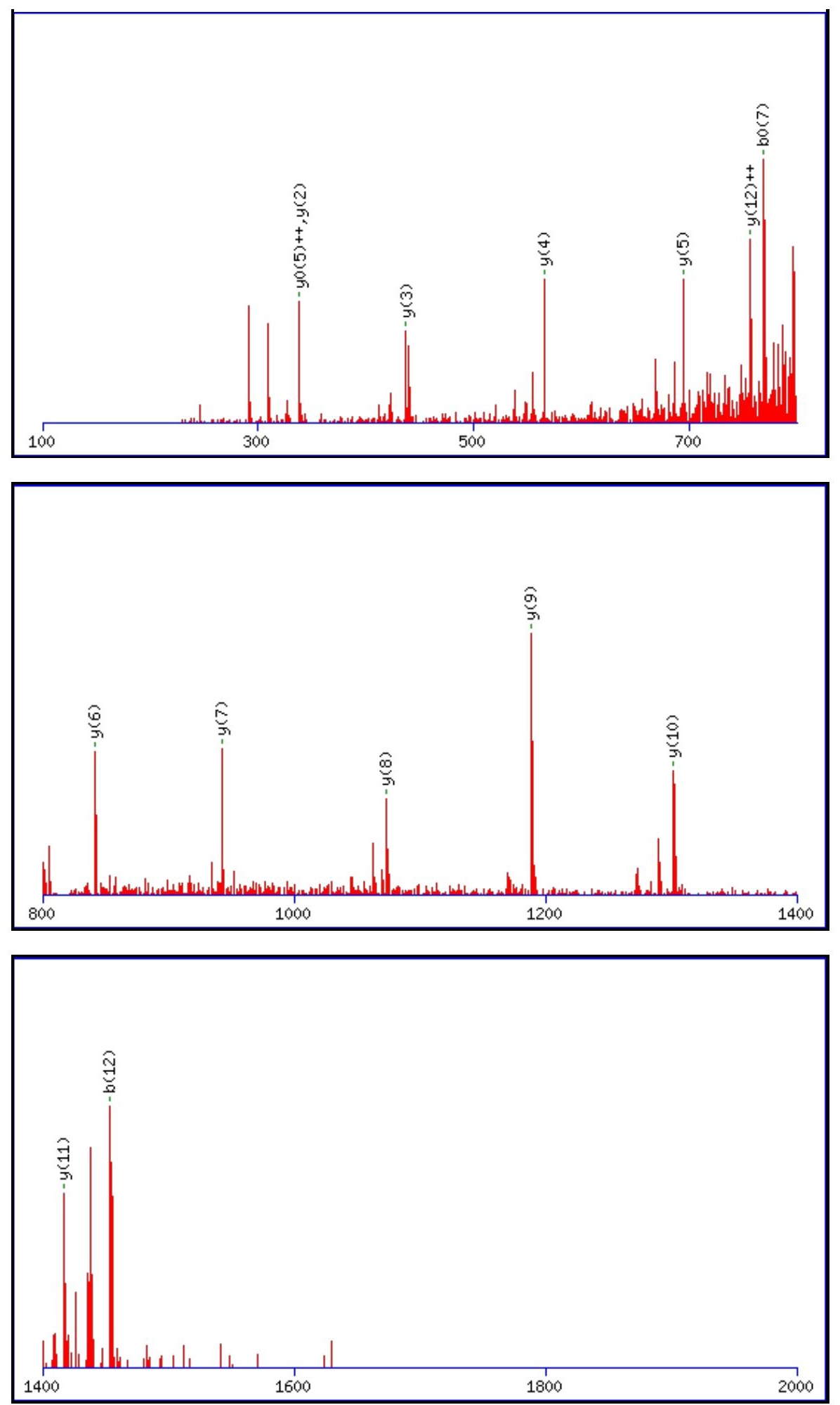

Monoisotopic mass of neutral peptide $\operatorname{Mr}($ calc): 1626.7348

Fixed modifications: Carbamidomethyl (C)

Variable modifications:

N5 : Deamidated $\mathrm{N}(\mathrm{N})$

Ions Score: 86 Expect: $4.7 \mathrm{e}-007$

Matches (Bold Red): 14/132 fragment ions using 22 most intense peaks

\begin{tabular}{|r|c|c|c|c|c|c|c|c|c|c|c|c|c|c|}
\hline$\#$ & $\mathbf{b}$ & $\mathbf{b}^{++}$ & $\mathbf{b}^{*}$ & $\mathbf{b}^{\boldsymbol{*}_{++}}$ & $\mathbf{b}^{\mathbf{0}}$ & $\mathbf{b}^{\mathbf{0 + +}}$ & Seq. & $\mathbf{y}$ & $\mathbf{y}^{++}$ & $\mathbf{y}^{\mathbf{*}}$ & $\mathbf{y}^{\boldsymbol{*}^{++}}$ & $\mathbf{y}^{\mathbf{0}}$ & $\mathbf{y}^{\mathbf{0 + +}}$ & $\#$ \\
\hline $\mathbf{1}$ & 115.0502 & 58.0287 & 98.0237 & 49.5155 & & & $\mathbf{N}$ & & & & & & & $\mathbf{1 3}$ \\
\hline $\mathbf{2}$ & 212.1030 & 106.5551 & 195.0764 & 98.0418 & & & $\mathbf{P}$ & 1513.6992 & 757.3532 & 1496.6726 & 748.8399 & 1495.6886 & 748.3479 & $\mathbf{1 2}$ \\
\hline 3 & 327.1299 & 164.0686 & 310.1034 & 155.5553 & 309.1193 & 155.0633 & $\mathbf{D}$ & $\mathbf{1 4 1 6 . 6 4 6 4}$ & 708.8268 & 1399.6198 & 700.3136 & 1398.6358 & 699.8215 & $\mathbf{1 1}$ \\
\hline $\mathbf{4}$ & 440.2140 & 220.6106 & 423.1874 & 212.0974 & 422.2034 & 211.6053 & $\mathbf{I}$ & $\mathbf{1 3 0 1 . 6 1 9 4}$ & 651.3134 & 1284.5929 & 642.8001 & 1283.6089 & 642.3081 & $\mathbf{1 0}$ \\
\hline $\mathbf{5}$ & 555.2409 & 278.1241 & 538.2144 & 269.6108 & 537.2303 & 269.1188 & $\mathbf{N}$ & $\mathbf{1 1 8 8 . 5 3 5 4}$ & 594.7713 & 1171.5088 & 586.2581 & 1170.5248 & 585.7660 & $\mathbf{9}$ \\
\hline
\end{tabular}




\begin{tabular}{|r|r|r|r|r|r|r|r|r|r|r|r|r|r|r|r|}
$\mathbf{6}$ & 686.2814 & 343.6443 & 669.2548 & 335.1311 & 668.2708 & 334.6391 & $\mathbf{M}$ & $\mathbf{1 0 7 3 . 5 0 8 4}$ & 537.2579 & 1056.4819 & 528.7446 & 1055.4979 & 528.2526 & $\mathbf{8}$ \\
\hline $\mathbf{7}$ & 787.3291 & 394.1682 & 770.3025 & 385.6549 & $\mathbf{7 6 9 . 3 1 8 5}$ & 385.1629 & $\mathbf{T}$ & $\mathbf{9 4 2 . 4 6 8 0}$ & $\mathbf{4 7 1 . 7 3 7 6}$ & 925.4414 & 463.2243 & 924.4574 & 462.7323 & $\mathbf{7}$ \\
\hline $\mathbf{8}$ & 934.3975 & 467.7024 & 917.3709 & 459.1891 & 916.3869 & 458.6971 & $\mathbf{F}$ & $\mathbf{8 4 1 . 4 2 0 3}$ & $\mathbf{4 2 1 . 2 1 3 8}$ & $\mathbf{8 2 4 . 3 9 3 7}$ & $\mathbf{4 1 2 . 7 0 0 5}$ & $\mathbf{8 2 3 . 4 0 9 7}$ & $\mathbf{4 1 2 . 2 0 8 5}$ & $\mathbf{6}$ \\
\hline $\mathbf{9}$ & 1063.4401 & 532.2237 & 1046.4135 & 523.7104 & 1045.4295 & 523.2184 & $\mathbf{E}$ & $\mathbf{6 9 4 . 3 5 1 9}$ & 347.6796 & 677.3253 & 339.1663 & 676.3413 & $\mathbf{3 3 8 . 6 7 4 3}$ & $\mathbf{5}$ \\
\hline $\mathbf{1 0}$ & 1191.4987 & 596.2530 & 1174.4721 & 587.7397 & 1173.4881 & 587.2477 & $\mathbf{Q}$ & $\mathbf{5 6 5 . 3 0 9 3}$ & 283.1583 & 548.2827 & 274.6450 & & & $\mathbf{4}$ \\
\hline $\mathbf{1 1}$ & 1290.5671 & 645.7872 & 1273.5405 & 637.2739 & 1272.5565 & 636.7819 & $\mathbf{V}$ & $\mathbf{4 3 7 . 2 5 0 7}$ & 219.1290 & $\mathbf{4 2 0 . 2 2 4 1}$ & 210.6157 & & & $\mathbf{3}$ \\
\hline $\mathbf{1 2}$ & $\mathbf{1 4 5 3 . 6 3 0 4}$ & 727.3188 & 1436.6039 & 718.8056 & 1435.6198 & 718.3136 & $\mathbf{Y}$ & $\mathbf{3 3 8 . 1 8 2 3}$ & $\mathbf{1 6 9 . 5 9 4 8}$ & 321.1557 & 161.0815 & & & $\mathbf{2}$ \\
\hline $\mathbf{1 3}$ & & & & & & & $\mathbf{R}$ & 175.1190 & 88.0631 & 158.0924 & 79.5498 & & & $\mathbf{1}$ \\
\hline
\end{tabular}
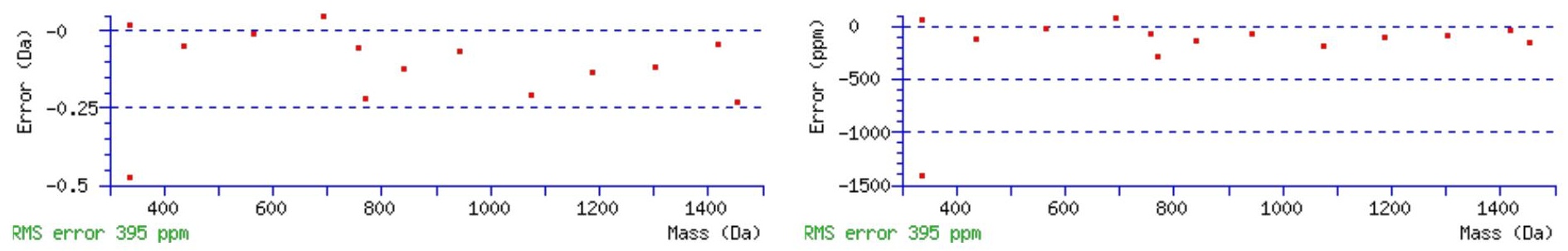

\section{All matches to this query}

\begin{tabular}{|l|l|l|l|}
\hline Score & Mr(calc): & Delta & Sequence \\
\hline 85.8 & 1626.7348 & 0.9989 & NPDINMTFEQVYR \\
\hline 85.1 & 1627.7188 & 0.0149 & NPDINMTFEQVYR \\
\hline 39.2 & 1626.7348 & 0.9989 & NPDINMTFEQVYR \\
\hline 5.8 & 1627.7290 & 0.0047 & NRSACTYLASHLR \\
\hline 5.0 & 1627.7243 & 0.0094 & VLDINDNAPTFSSR \\
\hline 5.0 & 1627.7243 & 0.0094 & VLDINDNAPTFSSR \\
\hline 4.5 & 1627.7243 & 0.0094 & VLDINDNAPTFSSR \\
\hline 1.2 & 1627.7274 & 0.0063 & GSFGTAVRHCDEHR \\
\hline 0.5 & 1627.7178 & 0.0160 & GFSQLSCLKSHER \\
\hline
\end{tabular}

Spectrum No: 168; Query: 1642; Rank: 1

\section{Peptide View}

MS/MS Fragmentation of SGPSDEGEEEMEDGTVTNGS

Found in IPI00192279, Tax_Id=10116 Gene_Symbol=- 28 kDa protein

Match to Query 1642: 2042.731788 from(1022.373170,2+)

Title: 091008RatKidney_NoSalt_31.613.613.2.dta

Data file K:INewmanPaper\Piliang\3SubProteomes\Piliang3SP\mgf5ppm\ERLIC_3SubProteomes5ppm.mgf 

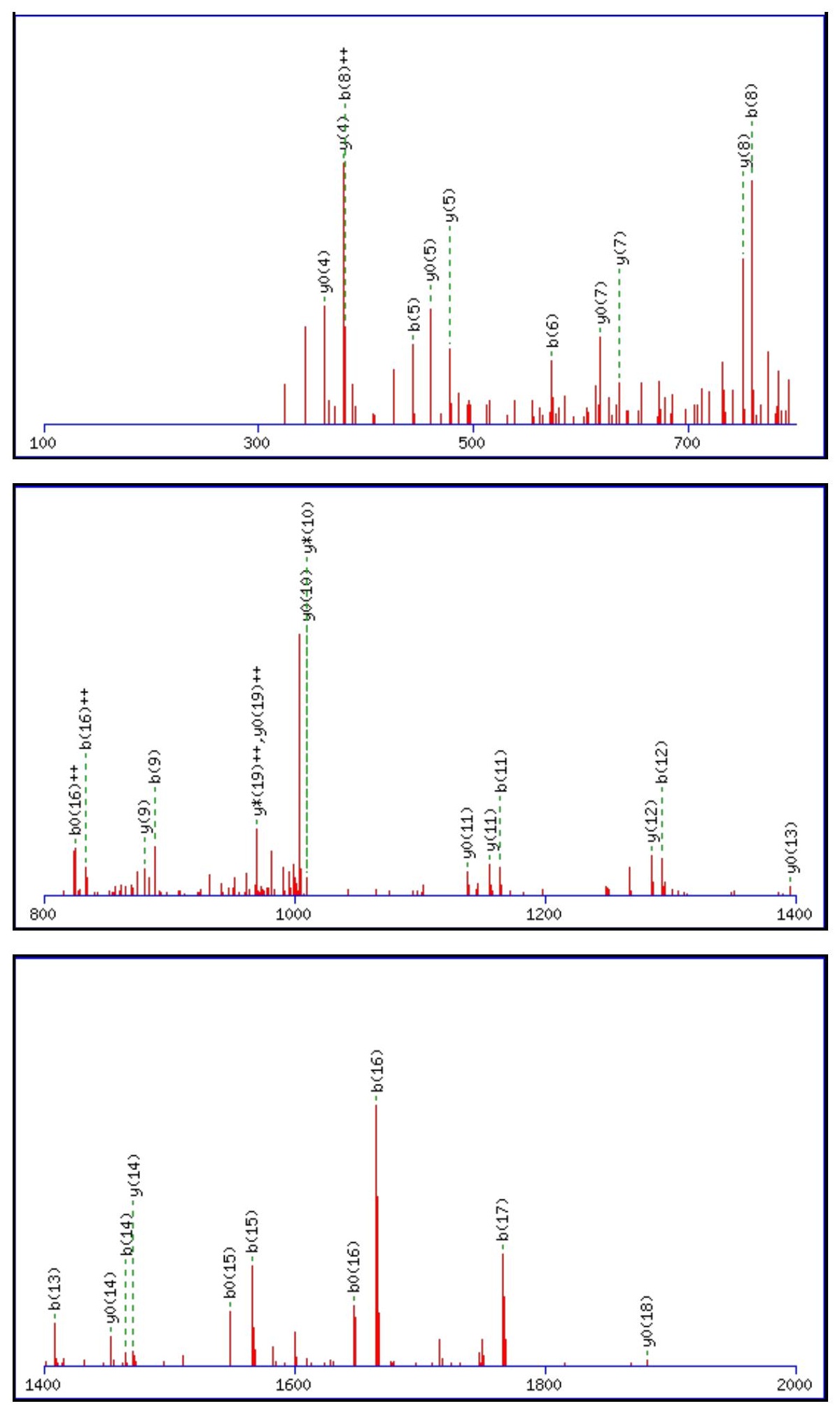

Monoisotopic mass of neutral peptide $\operatorname{Mr}($ calc): 2042.7382

Fixed modifications: Carbamidomethyl (C)

Variable modifications:

M11 : Oxidation (M)

N18: Deamidated_N (N)

Ions Score: 86 Expect: $2.7 \mathrm{e}-\odot \odot 7$

Matches (Bold Red): 37/190 fragment ions using 56 most intense peaks

\begin{tabular}{|c|c|c|c|c|c|c|c|c|c|c|c|c|c|c|}
\hline \# & b & $\mathbf{b}^{++}$ & $\mathbf{b}^{*}$ & $\mathbf{b}^{*^{++}}$ & $\mathbf{b}^{\mathbf{0}}$ & $\mathbf{b}^{0++}$ & Seq. & $\mathbf{y}$ & $\mathbf{y}^{++}$ & $\mathbf{y}^{*}$ & $\mathbf{y}^{*^{++}}$ & $\mathbf{y}^{0}$ & $y^{0++}$ & \# \\
\hline 1 & 88.0393 & 44.5233 & & & 70.0287 & 35.5180 & $\mathrm{~S}$ & & & & & & & 20 \\
\hline 2 & 145.0608 & 73.0340 & & & 127.0502 & 64.0287 & G & 1956.7135 & 978.8604 & 1939.6869 & 970.3471 & 1938.7029 & 969.8551 & 19 \\
\hline 3 & 242.1135 & 121.5604 & & & 224.1030 & 112.5551 & $\mathbf{P}$ & 1899.6920 & 950.3496 & 1882.6655 & 941.8364 & 1881.6815 & 941.3444 & 18 \\
\hline 4 & 329.1456 & 165.0764 & & & 311.1350 & 156.0711 & $S$ & 1802.6393 & 901.8233 & 1785.6127 & 893.3100 & 1784.6287 & 892.8180 & 17 \\
\hline
\end{tabular}




\begin{tabular}{|c|c|c|c|c|c|c|c|c|c|c|c|c|c|c|}
\hline & 444.1725 & |222.5899 & & & 426.1619 & |213.5846 & D & |1715.6072| & |858.3073 & |1698.5807 & |849.7940 & |1697.5967| & |849.3020| & 16 \\
\hline 6 & 573.2151 & 287.1112 & & & 555.2045 & 278.1059 & $\mathbf{E}$ & 1600.5803 & 800.7938 & 1583.5537 & 792.2805 & 1582.5697 & 791.7885 & 15 \\
\hline 7 & 630.2366 & 315.6219 & & & 612.2260 & 306.6166 & G & 1471.5377 & 736.2725 & 1454.5111 & 727.7592 & 1453.5271 & 727.2672 & 14 \\
\hline 8 & 759.2792 & 380.1432 & & & 741.2686 & 371.1379 & $\mathbf{E}$ & 1414.5162 & 707.7618 & 1397.4897 & 699.2485 & 1396.5057 & 698.7565 & 13 \\
\hline 9 & 888.3217 & 444.6645 & & & 870.3112 & 435.6592 & $\mathbf{E}$ & 1285.4736 & 643.2405 & 1268.4471 & 634.7272 & 1267.4631 & 634.2352 & 12 \\
\hline 10 & 1017.3643 & 509.1858 & & & 999.3538 & 500.1805 & $\mathbf{E}$ & 1156.4310 & 578.7192 & 1139.4045 & 570.2059 & 1138.4205 & 569.7139 & 11 \\
\hline 11 & 1164.3997 & 582.7035 & & & 1146.3892 & 573.6982 & $\mathbf{M}$ & 1027.3884 & 514.1979 & 1010.3619 & 505.6846 & 1009.3779 & 505.1926 & 10 \\
\hline 12 & 1293.4423 & 647.2248 & & & 1275.4318 & 638.2195 & $\mathbf{E}$ & 880.3530 & 440.6802 & 863.3265 & 432.1669 & 862.3425 & 431.6749 & 9 \\
\hline 13 & 1408.4693 & 704.7383 & & & 1390.4587 & 695.7330 & D & 751.3104 & 376.1589 & 734.2839 & 367.6456 & 733.2999 & 367.1536 & 8 \\
\hline 14 & 1465.4907 & 733.2490 & & & 1447.4802 & 724.2437 & G & 636.2835 & 318.6454 & 619.2570 & 310.1321 & 618.2729 & 309.6401 & 7 \\
\hline 15 & 1566.5384 & 783.7728 & & & 1548.5279 & 774.7676 & $\mathbf{T}$ & 579.2620 & 290.1347 & 562.2355 & 281.6214 & 561.2515 & 281.1294 & 6 \\
\hline 16 & 1665.6068 & 833.3071 & & & 1647.5963 & 824.3018 & $\mathrm{~V}$ & 478.2144 & 239.6108 & 461.1878 & 231.0975 & 460.2038 & 230.6055 & 5 \\
\hline 17 & 1766.6545 & 883.8309 & & & 1748.6440 & 874.8256 & $\mathbf{T}$ & 379.1459 & 190.0766 & 362.1194 & 181.5633 & 361.1354 & 181.0713 & 4 \\
\hline 18 & \begin{tabular}{|l|}
1881.6815 \\
\end{tabular} & 941.3444 & 1864.6549 & 932.8311 & 1863.6709 & 932.3391 & $\mathbf{N}$ & 278.0983 & 139.5528 & 261.0717 & 131.0395 & 260.0877 & 130.5475 & 3 \\
\hline 19 & 1938.7029 & 969.8551 & 1921.6764 & 961.3418 & 1920.6924 & 960.8498 & $\mathbf{G}$ & 163.0713 & 82.0393 & & & 145.0608 & 73.0340 & 2 \\
\hline 20 & & & & & & & $\mathrm{~S}$ & 106.0499 & 53.5286 & & & 88.0393 & 44.5233 & 1 \\
\hline
\end{tabular}
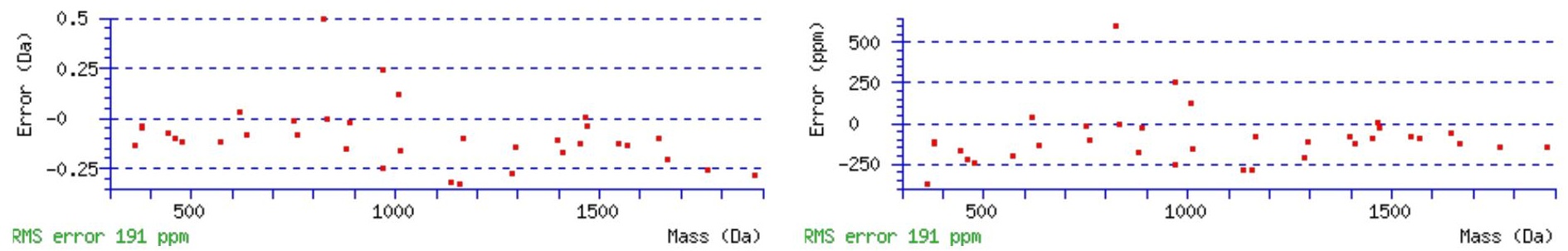

\section{All matches to this query}

\begin{tabular}{|l|c|c|c|}
\hline Score & Mr(calc): & Delta & Sequence \\
\hline 85.7 & 2042.7382 & -0.0064 & SGPSDEGEEEMEDGTVTNGS \\
\hline
\end{tabular}

Spectrum No: 169; Query: 1975; Rank: 1

\section{Peptide View}

\section{MS/MS Fragmentation of ETQSPAPLQNSSGDPAEEESPV}

Found in IPI00214787, Tax_Id=10116 Gene_Symbol=Slc16a1 Monocarboxylate transporter 1

Match to Query 1975: 2268.997148 from(1135.505850,2+)

Title: 100101RatKid_NS_deglyco_22.2416.2416.2.dta

Data file K:\NewmanPaper|Piliangl3SubProteomes\Piliang3SP\mgf5ppm\ERLIC_3SubProteomes5ppm.mgf 

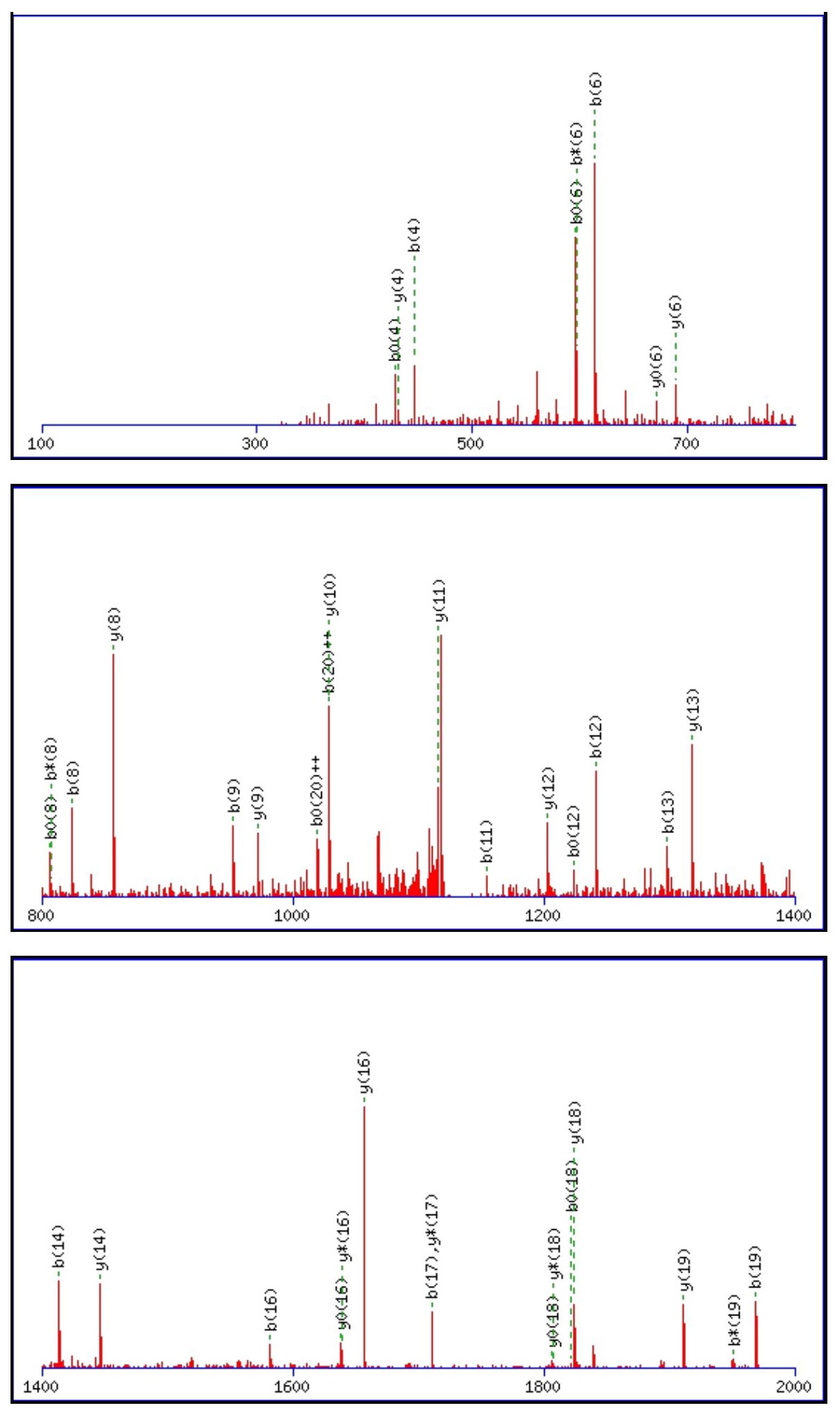

Monoisotopic mass of neutral peptide $\operatorname{Mr}($ calc): 2268.9870

Fixed modifications: Carbamidomethyl (C)

Variable modifications:

N10 : Deamidated N (N)

Ions Score: 86 Expect: $8 \mathrm{e}-007$

Matches (Bold Red): 39/220 fragment ions using 61 most intense peaks

\begin{tabular}{|r|c|c|c|c|c|r|r|r|r|r|r|r|r|r|}
\hline$\#$ & $\mathbf{b}$ & $\mathbf{b}^{++}$ & $\mathbf{b}^{*}$ & $\mathbf{b}^{\boldsymbol{*}^{++}}$ & $\mathbf{b}^{\mathbf{0}}$ & $\mathbf{b}^{\mathbf{0 + +}}$ & Seq. & $\mathbf{y}$ & $\mathbf{y}^{++}$ & $\mathbf{y}^{\mathbf{*}}$ & $\mathbf{y}^{\mathbf{*}^{++}}$ & $\mathbf{y}^{\mathbf{0}}$ & $\mathbf{y}^{\mathbf{0 + +}}$ & $\#$ \\
\hline $\mathbf{1}$ & 130.0499 & 65.5286 & & & 112.0393 & 56.5233 & $\mathbf{E}$ & & & & & & & $\mathbf{2 2}$ \\
\hline $\mathbf{2}$ & 231.0975 & 116.0524 & & & 213.0870 & 107.0471 & $\mathbf{T}$ & 2140.9517 & 1070.9795 & 2123.9251 & 1062.4662 & 2122.9411 & 1061.9742 & $\mathbf{2 1}$ \\
\hline 3 & 359.1561 & 180.0817 & 342.1296 & 171.5684 & 341.1456 & 171.0764 & $\mathbf{Q}$ & 2039.9040 & 1020.4556 & 2022.8774 & 1011.9424 & 2021.8934 & 1011.4504 & $\mathbf{2 0}$ \\
\hline $\mathbf{4}$ & 446.1882 & 223.5977 & 429.1616 & 215.0844 & 428.1776 & 214.5924 & S & $\mathbf{1 9 1 1 . 8 4 5 4}$ & 956.4263 & 1894.8189 & 947.9131 & 1893.8349 & 947.4211 & $\mathbf{1 9}$ \\
\hline $\mathbf{5}$ & 543.2409 & 272.1241 & 526.2144 & 263.6108 & 525.2304 & 263.1188 & $\mathbf{P}$ & $\mathbf{1 8 2 4 . 8 1 3 4}$ & 912.9103 & $\mathbf{1 8 0 7 . 7 8 6 8}$ & 904.3971 & $\mathbf{1 8 0 6 . 8 0 2 8}$ & 903.9050 & $\mathbf{1 8}$ \\
\hline
\end{tabular}




\begin{tabular}{|c|c|c|c|c|c|c|c|c|c|c|c|c|c|c|}
\hline 6 & 614.2780 & 307.6427 & 597.2515 & 299.1294 & 596.2675 & 298.6374 & A & 1727.7606 & 864.3839 & |1710.7341 & 855.8707 & |1709.7501| & 855.3787 & 17 \\
\hline 7 & 711.3308 & 356.1690 & 694.3042 & 347.6558 & 693.3202 & 347.1638 & $\mathbf{P}$ & 1656.7235 & 828.8654 & 1639.6970 & 820.3521 & 1638.7129 & 819.8601 & 16 \\
\hline 8 & 824.4149 & 412.7111 & $\mathbf{8 0 7 . 3 8 8 3}$ & 404.1978 & 806.4043 & 403.7058 & $\mathbf{L}$ & 1559.6707 & 780.3390 & 1542.6442 & 771.8257 & 1541.6602 & 771.3337 & 15 \\
\hline 9 & 952.4734 & 476.7404 & 935.4469 & 468.2271 & 934.4629 & 467.7351 & $\mathbf{Q}$ & 1446.5867 & 723.7970 & 1429.5601 & 715.2837 & 1428.5761 & 714.7917 & 14 \\
\hline 10 & 1067.5004 & 534.2538 & 1050.4738 & 525.7406 & 1049.4898 & 525.2485 & $\mathbf{N}$ & 1318.5281 & 659.7677 & 1301.5016 & 651.2544 & 1300.5175 & 650.7624 & 13 \\
\hline 11 & 1154.5324 & 577.7698 & 1137.5059 & 569.2566 & 1136.5218 & 568.7646 & $\mathrm{~S}$ & 1203.5012 & 602.2542 & & & 1185.4906 & 593.2489 & 12 \\
\hline 12 & 1241.5644 & 621.2859 & 1224.5379 & 612.7726 & 1223.5539 & 612.2806 & $\mathrm{~S}$ & 1116.4691 & 558.7382 & & & 1098.4586 & 549.7329 & 11 \\
\hline 13 & 1298.5859 & 649.7966 & 1281.5593 & 641.2833 & 1280.5753 & 640.7913 & G & 1029.4371 & 515.2222 & & & 1011.4265 & 506.2169 & 10 \\
\hline 14 & 1413.6128 & 707.3101 & 1396.5863 & 698.7968 & 1395.6023 & 698.3048 & D & 972.4156 & 486.7115 & & & 954.4051 & 477.7062 & 9 \\
\hline 15 & 1510.6656 & 755.8364 & 1493.6391 & 747.3232 & 1492.6550 & 746.8312 & $\mathbf{P}$ & 857.3887 & 429.1980 & & & 839.3781 & 420.1927 & 8 \\
\hline 16 & 1581.7027 & 791.3550 & 1564.6762 & 782.8417 & 1563.6922 & 782.3497 & A & 760.3359 & 380.6716 & & & 742.3254 & 371.6663 & 7 \\
\hline 17 & 1710.7453 & 855.8763 & 1693.7188 & 847.3630 & 1692.7347 & 846.8710 & $\mathbf{E}$ & 689.2988 & 345.1531 & & & 671.2883 & 336.1478 & 6 \\
\hline 18 & 1839.7879 & 920.3976 & 1822.7614 & 911.8843 & 1821.7773 & 911.3923 & $\mathbf{E}$ & 560.2562 & 280.6318 & & & 542.2457 & 271.6265 & 5 \\
\hline 19 & 1968.8305 & 984.9189 & 1951.8039 & 976.4056 & 1950.8199 & 975.9136 & $\mathbf{E}$ & 431.2136 & 216.1105 & & & 413.2031 & 207.1052 & 4 \\
\hline 20 & 2055.8625 & 1028.4349 & 2038.8360 & 1019.9216 & 2037.8520 & 1019.4296 & $S$ & 302.1710 & 151.5892 & & & 284.1605 & 142.5839 & 3 \\
\hline 21 & 2152.9153 & 1076.9613 & 2135.8887 & 1068.4480 & 2134.9047 & 1067.9560 & $\mathbf{P}$ & 215.1390 & 108.0731 & & & & & 2 \\
\hline 22 & & & & & & & V & 118.0863 & 59.5468 & & & & & 1 \\
\hline
\end{tabular}
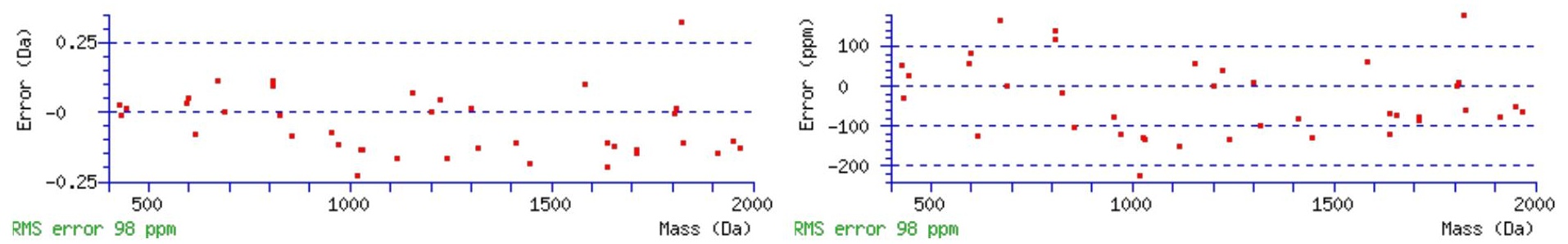

\section{All matches to this query}

\begin{tabular}{|l|l|c|l|}
\hline Score & Mr(calc): & Delta & \multicolumn{1}{|c|}{ Sequence } \\
\hline 85.7 & 2268.9870 & 0.0102 & ETQSPAPLQNSSGDPAEEESPV \\
\hline 22.9 & 2268.0030 & 0.9942 & ETQSPAPLQNSSGDPAEEESPV \\
\hline 6.8 & 2268.9821 & 0.0151 & NQGQSNLKDTYMLSSTVSSK \\
\hline 4.5 & 2267.9729 & 1.0243 & AKEPVSATILQTDQDLTAK \\
\hline 3.6 & 2267.9981 & 0.9991 & NQGQSNLKDTYMLSSTVSSK \\
\hline 2.5 & 2267.9729 & 1.0243 & AKEPVSATILQTDQDLTAK \\
\hline 2.5 & 2267.9729 & 1.0243 & AKEPVSATILQTDQDLTAK \\
\hline 1.5 & 2269.0089 & -0.0117 & HARRPAAPGCSPSRSLFSR \\
\hline 0.7 & 2269.0089 & -0.0117 & HARRPAAPGCSPSRSLFSR \\
\hline 0.6 & 2269.0089 & -0.0117 & $\underline{\text { HARRPAAPGCSPSRSLFSR }}$ \\
\hline
\end{tabular}

Spectrum No: 170; Query: 831; Rank: 1

\section{Peptide View}

MS/MS Fragmentation of CISDLTLNVSTTEK

Found in IPI00324585, Tax_Id=10116 Gene_Symbol=Itga1 Integrin alpha-1 precursor

Match to Query 831: 1580.767528 from(791.391040,2+)

Title: 100101RatKid_NS_deglyco_15.3149.3149.2.dta

Data file K:INewmanPaper|Piliangl3SubProteomes\Piliang3SP\mgf5ppm\ERLIC_3SubProteomes5ppm.mgf 

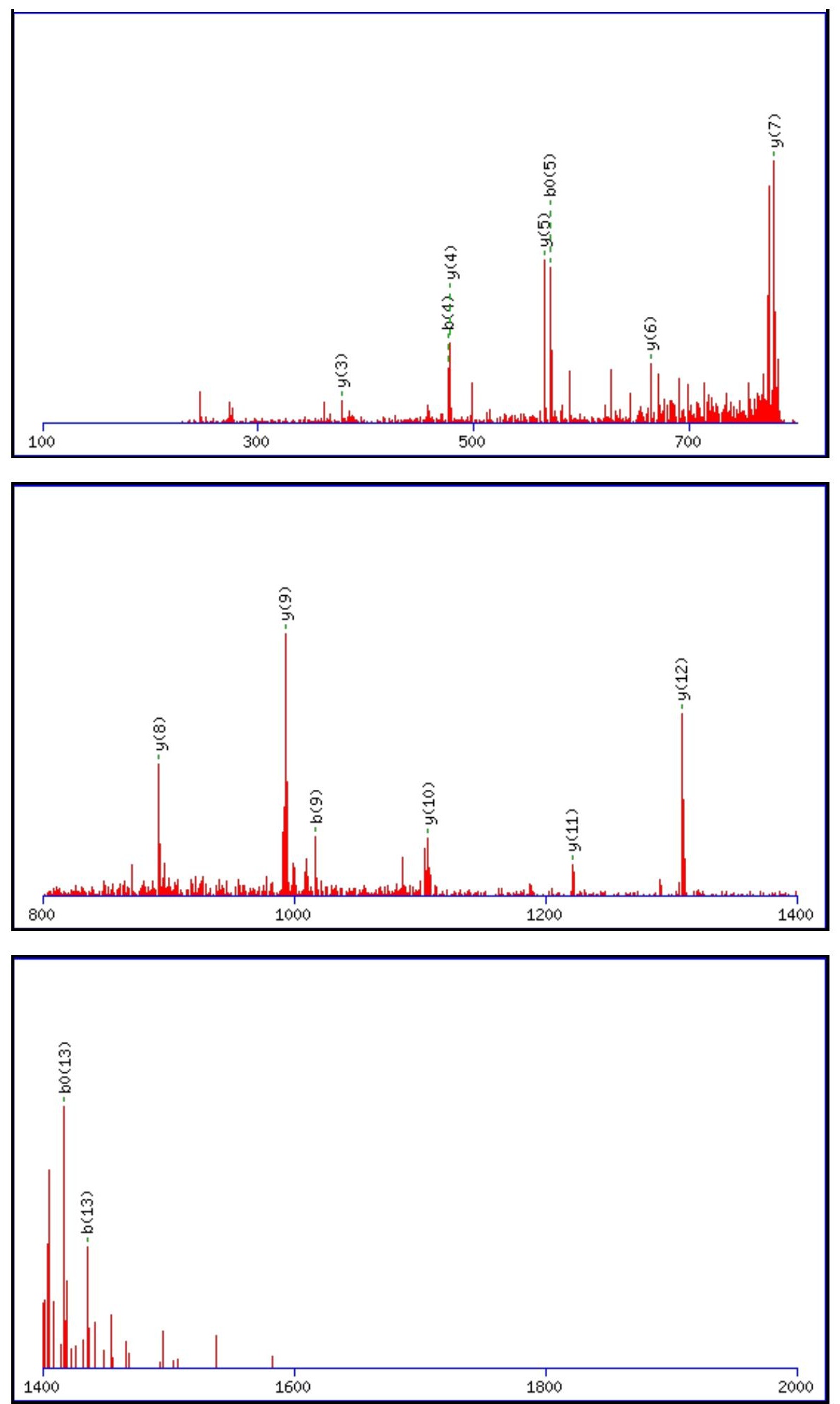

Monoisotopic mass of neutral peptide $\operatorname{Mr}($ calc): 1580.7603

Fixed modifications: Carbamidomethyl (C)

Variable modifications:

N8 : Deamidated $\mathrm{N}(\mathrm{N})$

Ions Score: 86 Expect: $4.2 \mathrm{e}-007$

Matches (Bold Red): 15/136 fragment ions using 29 most intense peaks

\begin{tabular}{|r|c|c|c|c|c|c|c|c|c|c|c|c|c|c|}
\hline$\#$ & $\mathbf{b}$ & $\mathbf{b}^{++}$ & $\mathbf{b}^{*}$ & $\mathbf{b}^{\boldsymbol{*}^{++}}$ & $\mathbf{b}^{\mathbf{0}}$ & $\mathbf{b}^{\mathbf{0 + +}}$ & Seq. & $\mathbf{y}$ & $\mathbf{y}^{++}$ & $\mathbf{y}^{\mathbf{*}}$ & $\mathbf{y}^{\boldsymbol{*}^{++}}$ & $\mathbf{y}^{\mathbf{0}}$ & $\mathbf{y}^{\mathbf{0 + +}}$ & $\#$ \\
\hline $\mathbf{1}$ & 161.0379 & 81.0226 & & & & & $\mathbf{C}$ & & & & & & & $\mathbf{1 4}$ \\
\hline $\mathbf{2}$ & 274.1220 & 137.5646 & & & & & $\mathbf{I}$ & 1421.7370 & 711.3721 & 1404.7104 & 702.8589 & 1403.7264 & 702.3668 & $\mathbf{1 3}$ \\
\hline 3 & 361.1540 & 181.0806 & & & 343.1435 & 172.0754 & S & $\mathbf{1 3 0 8 . 6 5 2 9}$ & 654.8301 & 1291.6264 & 646.3168 & 1290.6423 & 645.8248 & $\mathbf{1 2}$ \\
\hline $\mathbf{4}$ & $\mathbf{4 7 6 . 1 8 1 0}$ & 238.5941 & & & 458.1704 & 229.5888 & $\mathbf{D}$ & $\mathbf{1 2 2 1 . 6 2 0 9}$ & 611.3141 & 1204.5943 & 602.8008 & 1203.6103 & 602.3088 & $\mathbf{1 1}$ \\
\hline $\mathbf{5}$ & 589.2650 & 295.1362 & & & 571.2545 & 286.1309 & L & $\mathbf{1 1 0 6 . 5 9 3 9}$ & 553.8006 & 1089.5674 & 545.2873 & 1088.5834 & 544.7953 & $\mathbf{1 0}$ \\
\hline
\end{tabular}




\begin{tabular}{|r|r|r|r|r|r|r|r|r|r|r|r|r|r|r|r|r|}
$\mathbf{6}$ & 690.3127 & 345.6600 & & & 672.3021 & 336.6547 & $\mathbf{T}$ & $\mathbf{9 9 3 . 5 0 9 9}$ & 497.2586 & 976.4833 & 488.7453 & 975.4993 & 488.2533 & $\mathbf{9}$ \\
\hline $\mathbf{7}$ & 803.3968 & 402.2020 & & & 785.3862 & 393.1967 & $\mathbf{L}$ & $\mathbf{8 9 2 . 4 6 2 2}$ & 446.7347 & 875.4356 & 438.2215 & 874.4516 & 437.7295 & $\mathbf{8}$ \\
\hline $\mathbf{8}$ & 918.4237 & 459.7155 & 901.3972 & 451.2022 & 900.4131 & 450.7102 & $\mathbf{N}$ & 779.3781 & 390.1927 & 762.3516 & 381.6794 & 761.3676 & 381.1874 & $\mathbf{7}$ \\
\hline $\mathbf{9}$ & $\mathbf{1 0 1 7 . 4 9 2 1}$ & 509.2497 & 1000.4656 & 500.7364 & 999.4816 & 500.2444 & $\mathbf{V}$ & $\mathbf{6 6 4 . 3 5 1 2}$ & 332.6792 & 647.3246 & 324.1660 & 646.3406 & 323.6740 & $\mathbf{6}$ \\
\hline $\mathbf{1 0}$ & 1104.5241 & 552.7657 & 1087.4976 & 544.2524 & 1086.5136 & 543.7604 & $\mathbf{S}$ & $\mathbf{5 6 5 . 2 8 2 8}$ & 283.1450 & 548.2562 & 274.6318 & 547.2722 & 274.1397 & $\mathbf{5}$ \\
\hline $\mathbf{1 1}$ & 1205.5718 & 603.2896 & 1188.5453 & 594.7763 & 1187.5613 & 594.2843 & $\mathbf{T}$ & $\mathbf{4 7 8 . 2 5 0 8}$ & 239.6290 & 461.2242 & 231.1157 & 460.2402 & 230.6237 & $\mathbf{4}$ \\
\hline $\mathbf{1 2}$ & 1306.6195 & 653.8134 & 1289.5930 & 645.3001 & 1288.6089 & 644.8081 & $\mathbf{T}$ & 377.2031 & 189.1052 & 360.1765 & 180.5919 & 359.1925 & 180.0999 & $\mathbf{3}$ \\
\hline $\mathbf{1 3}$ & $\mathbf{1 4 3 5 . 6 6 2 1}$ & 718.3347 & 1418.6355 & 709.8214 & $\mathbf{1 4 1 7 . 6 5 1 5}$ & 709.3294 & $\mathbf{E}$ & 276.1554 & 138.5813 & 259.1288 & 130.0681 & 258.1448 & 129.5761 & $\mathbf{2}$ \\
\hline $\mathbf{1 4}$ & & & & & & & $\mathbf{K}$ & 147.1128 & 74.0600 & 130.0863 & 65.5468 & & & $\mathbf{1}$ \\
\hline
\end{tabular}
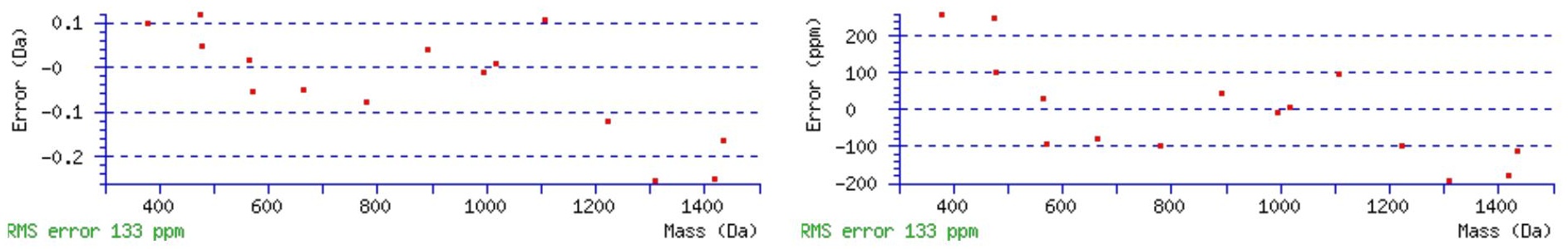

\section{All matches to this query}

\begin{tabular}{|l|l|l|l|}
\hline Score & Mr(calc): & Delta & \multicolumn{1}{c|}{ Sequence } \\
\hline 85.6 & 1580.7603 & 0.0072 & CISDLTLNVSTTEK \\
\hline 25.8 & 1579.7763 & 0.9912 & CISDLTLNVSTTEK \\
\hline 9.7 & 1580.7794 & -0.0119 & DSAIGQSPSVHNLEK \\
\hline 9.3 & 1580.7603 & 0.0072 & $\underline{\text { GLKDMELDTSSIEK }}$ \\
\hline 6.9 & 1580.7521 & 0.0155 & MPQLLNSILNVNK \\
\hline 6.8 & 1578.7654 & 2.0021 & $\underline{\text { LDSPGNLDTLQAKK }}$ \\
\hline 3.3 & 1580.7745 & -0.0070 & $\underline{\text { NLSMKARPEVNVK }}$ \\
\hline 2.9 & 1579.7746 & 0.9929 & $\underline{\text { AEIVPVTTTVLDDK }}$ \\
\hline 2.8 & 1579.7746 & 0.9929 & $\underline{\text { AEIVPVTTTVLDDK }}$ \\
\hline 1.6 & 1579.7607 & 1.0069 & $\underline{\text { GLITIAAQELSDNR }}$ \\
\hline
\end{tabular}

Spectrum No: 171; Query: 703; Rank: 1

\section{Peptide View}

MS/MS Fragmentation of LGALNSSLLLLEDR

Found in IPI00199867, Tax_Id=10116 Gene_Symbol=Emilin1_predicted elastin microfibril interfacer 1

Match to Query 703: 1513.832828 from(757.923690,2+)

Title: 091008RatKidney_NH4Format01_23.3825.3825.2.dta

Data file K:INewmanPaper|Piliangl3SubProteomes\Piliang3SP\mgf5ppm\ERLIC_3SubProteomes5ppm.mgf 

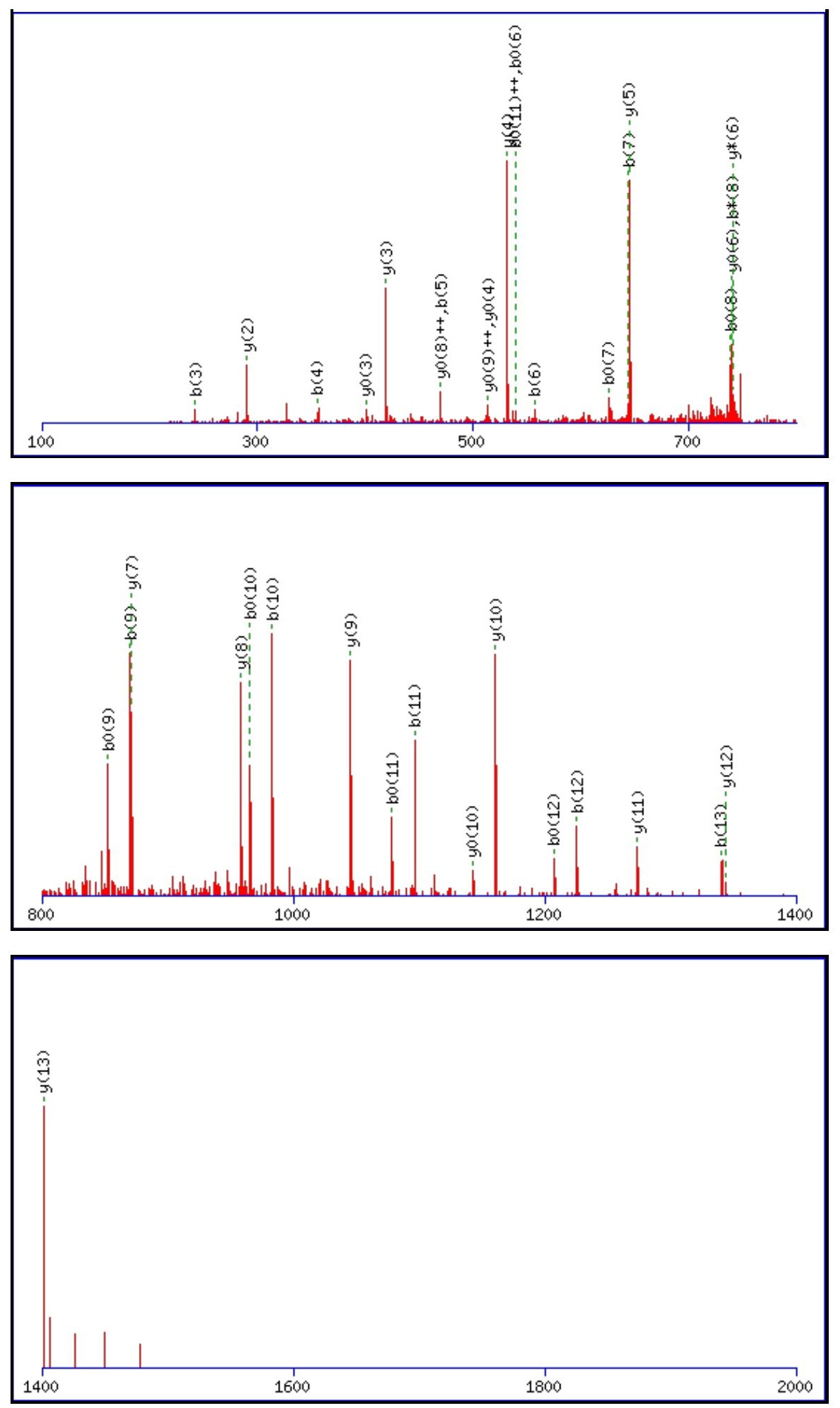

Monoisotopic mass of neutral peptide $\operatorname{Mr}($ calc): 1513.8351

Fixed modifications: Carbamidomethyl (C)

Variable modifications:

N5

Ions Score: 86 Expect: $1.9 \mathrm{e}-007$

Matches (Bold Red): 37/136 fragment ions using 53 most intense peaks

\begin{tabular}{|r|c|c|c|c|c|c|c|c|c|c|c|c|c|c|}
\hline$\#$ & $\mathbf{b}$ & $\mathbf{b}^{++}$ & $\mathbf{b}^{*}$ & $\mathbf{b}^{\boldsymbol{*}^{++}}$ & $\mathbf{b}^{\mathbf{0}}$ & $\mathbf{b}^{\mathbf{0 + +}}$ & Seq. & $\mathbf{y}$ & $\mathbf{y}^{++}$ & $\mathbf{y}^{\mathbf{*}}$ & $\mathbf{y}^{\boldsymbol{*}^{++}}$ & $\mathbf{y}^{\mathbf{0}}$ & $\mathbf{y}^{\mathbf{0 + +}}$ & $\#$ \\
\hline $\mathbf{1}$ & 114.0913 & 57.5493 & & & & & $\mathbf{L}$ & & & & & & & $\mathbf{1 4}$ \\
\hline $\mathbf{2}$ & 171.1128 & 86.0600 & & & & & $\mathbf{G}$ & $\mathbf{1 4 0 1 . 7 5 8 4}$ & 701.3828 & 1384.7318 & 692.8696 & 1383.7478 & 692.3775 & $\mathbf{1 3}$ \\
\hline 3 & $\mathbf{2 4 2 . 1 4 9 9}$ & 121.5786 & & & & & A & $\mathbf{1 3 4 4 . 7 3 6 9}$ & 672.8721 & 1327.7104 & 664.3588 & 1326.7263 & 663.8668 & $\mathbf{1 2}$ \\
\hline $\mathbf{4}$ & 355.2340 & 178.1206 & & & & & L & $\mathbf{1 2 7 3 . 6 9 9 8}$ & 637.3535 & 1256.6733 & 628.8403 & 1255.6892 & 628.3483 & $\mathbf{1 1}$ \\
\hline $\mathbf{5}$ & $\mathbf{4 7 0 . 2 6 0 9}$ & 235.6341 & 453.2344 & 227.1208 & & & $\mathbf{N}$ & $\mathbf{1 1 6 0 . 6 1 5 7}$ & 580.8115 & 1143.5892 & 572.2982 & $\mathbf{1 1 4 2 . 6 0 5 2}$ & 571.8062 & $\mathbf{1 0}$ \\
\hline
\end{tabular}




\begin{tabular}{|c|c|c|c|c|c|c|c|c|c|c|c|c|c|c|}
\hline 6 & 557.2929 & |279.1501 & 540.2664 & |270.6368 & 539.2824 & 270.1448 & $S$ & 1045.5888 & |523.2980| & |1028.5623 & & 1027.5782 & 9928 & 9 \\
\hline 7 & 644.3250 & 322.6661 & 627.2984 & 314.1529 & 626.3144 & 313.6608 & S & 958.5568 & 479.7820 & 941.5302 & 471.2687 & 940.5462 & 470.7767 & 8 \\
\hline 8 & 757.4090 & 379.2082 & 740.3825 & 370.6949 & 739.3985 & 370.2029 & $\mathbf{L}$ & 871.5247 & 436.2660 & 854.4982 & 427.7527 & 853.5142 & 427.2607 & 7 \\
\hline 9 & 870.4931 & 435.7502 & 853.4666 & 427.2369 & 852.4825 & 426.7449 & $\mathbf{L}$ & 758.4407 & 379.7240 & 741.4141 & 371.2107 & 740.4301 & 370.7187 & 6 \\
\hline 10 & 983.5772 & 492.2922 & 966.5506 & 483.7789 & 965.5666 & 483.2869 & $\mathbf{L}$ & 645.3566 & 323.1819 & 628.3301 & 314.6687 & 627.3461 & 314.1767 & 5 \\
\hline 11 & 1096.6612 & 548.8343 & 1079.6347 & 540.3210 & 1078.6507 & 539.8290 & $\mathbf{L}$ & 532.2726 & 266.6399 & 515.2460 & 258.1266 & 514.2620 & 257.6346 & 4 \\
\hline 12 & 1225.7038 & 613.3555 & 1208.6773 & 604.8423 & 1207.6933 & 604.3503 & $\mathbf{E}$ & 419.1885 & 210.0979 & 402.1619 & 201.5846 & 401.1779 & 201.0926 & 3 \\
\hline 13 & 1340.7308 & 670.8690 & 1323.7042 & 662.3557 & 1322.7202 & 661.8637 & D & 290.1459 & 145.5766 & 273.1193 & 137.0633 & 272.1353 & 136.5713 & 2 \\
\hline 14 & & & & & & & $\mathbf{R}$ & 175.1190 & 88.0631 & 158.0924 & 79.5498 & & & 1 \\
\hline
\end{tabular}
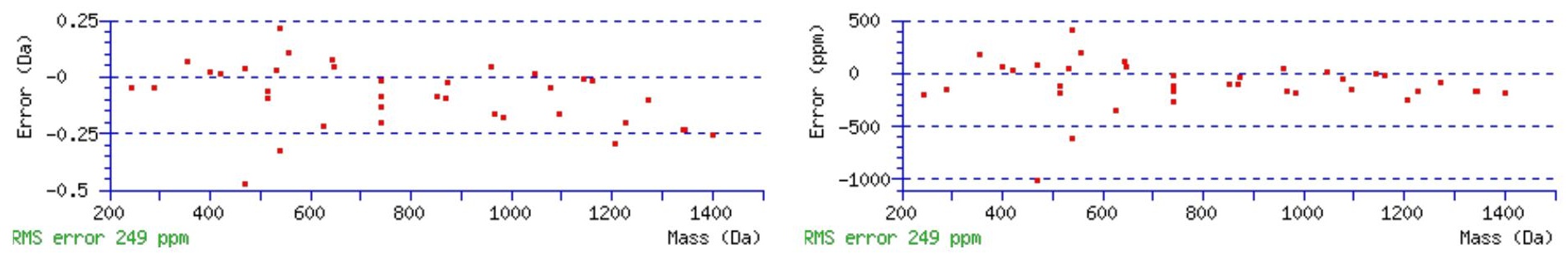

\section{All matches to this query}

\begin{tabular}{|l|l|l|l|}
\hline Score & Mr(calc): & Delta & \multicolumn{1}{c|}{ Sequence } \\
\hline 85.5 & 1513.8351 & -0.0023 & LGALNSSLLLLEDR \\
\hline 26.3 & 1513.8212 & 0.0116 & IQDLENRLTKQR \\
\hline 10.9 & 1513.8324 & 0.0004 & KNLISSXLQRGQR \\
\hline 5.7 & 1513.8212 & 0.0116 & LGAVAGANLSQLASSR \\
\hline 5.2 & 1513.8351 & -0.0023 & LQKVSENALPSSLK \\
\hline 2.5 & 1512.8412 & 0.9916 & KLLSDFQHIGSIR \\
\hline 1.3 & 1511.8321 & 2.0008 & GHREYVQINKIR \\
\hline 0.9 & 1512.8218 & 1.0111 & GIALLSPHPFLLR \\
\hline 0.1 & 1513.8212 & 0.0116 & AITSLRANGVEINR \\
\hline
\end{tabular}

Spectrum No: 172; Query: 291; Rank: 1

\section{Peptide View}

MS/MS Fragmentation of LANTSMFNNSK

Found in IPI00206254, Tax_Id=10116 Gene_Symbol=Ggt1 Gamma-glutamyltranspeptidase 1 precursor

Match to Query 291: 1243.538748 from(622.776650,2+)

Title: 091008RatKidney_NH4Format01_28.597.597.2.dta

Data file K:INewmanPaper|Piliang|3SubProteomes\Piliang3SP\mgf5ppm\ERLIC_3SubProteomes5ppm.mgf 

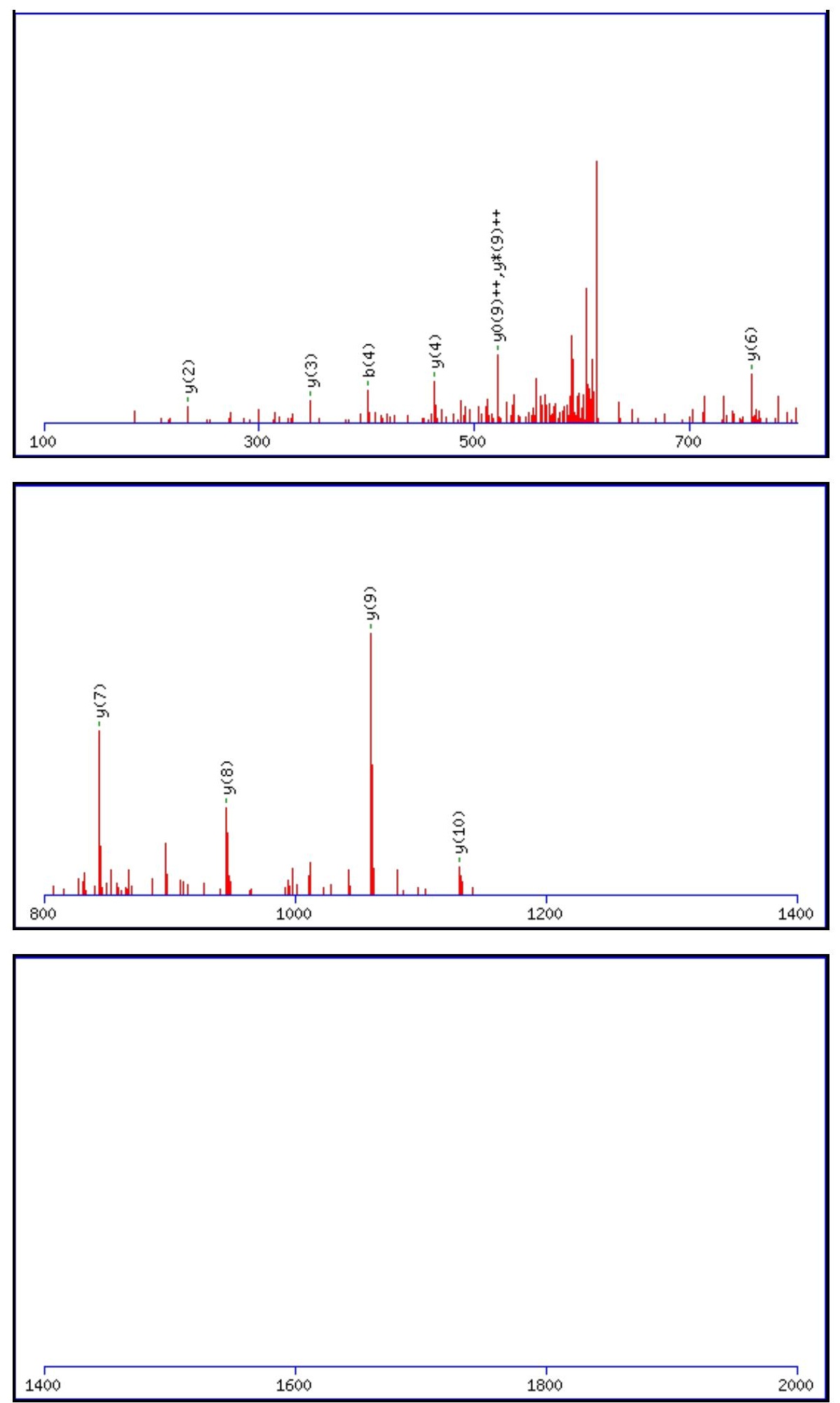

Monoisotopic mass of neutral peptide $\operatorname{Mr}($ calc): 1243.5390

Fixed modifications: Carbamidomethyl (C)

Variable modifications:

N3 : Deamidated_N (N)

M6 : Oxidation $(M)$

N8 : Deamidated_N (N)

Ions Score: 85 Expect: $3.3 \mathrm{e}-007$

Matches (Bold Red): 11/108 fragment ions using 15 most intense peaks

\begin{tabular}{|r|c|c|c|c|c|c|c|c|c|c|c|c|c|c|}
\hline$\#$ & $\mathbf{b}$ & $\mathbf{b}^{++}$ & $\mathbf{b}^{*}$ & $\mathbf{b}^{\boldsymbol{*}_{++}}$ & $\mathbf{b}^{\mathbf{0}}$ & $\mathbf{b}^{\mathbf{0}+}$ & Seq. & $\mathbf{y}$ & $\mathbf{y}^{++}$ & $\mathbf{y}^{\mathbf{*}}$ & $\mathbf{y}^{\boldsymbol{*}^{++}}$ & $\mathbf{y}^{\mathbf{0}}$ & $\mathbf{y}^{\mathbf{0}++}$ & $\#$ \\
\hline $\mathbf{1}$ & 114.0913 & 57.5493 & & & & & $\mathbf{L}$ & & & & & & & $\mathbf{1 1}$ \\
\hline $\mathbf{2}$ & 185.1285 & 93.0679 & & & & & $\mathbf{A}$ & $\mathbf{1 1 3 1 . 4 6 2 3}$ & 566.2348 & 1114.4357 & 557.7215 & 1113.4517 & 557.2295 & $\mathbf{1 0}$ \\
\hline $\mathbf{3}$ & 300.1554 & 150.5813 & 283.1288 & 142.0681 & & & $\mathbf{N}$ & $\mathbf{1 0 6 0 . 4 2 5 2}$ & 530.7162 & 1043.3986 & $\mathbf{5 2 2 . 2 0 2 9}$ & 1042.4146 & $\mathbf{5 2 1 . 7 1 0 9}$ & $\mathbf{9}$ \\
\hline $\mathbf{4}$ & $\mathbf{4 0 1 . 2 0 3 1}$ & 201.1052 & 384.1765 & 192.5919 & 383.1925 & 192.0999 & $\mathbf{T}$ & $\mathbf{9 4 5 . 3 9 8 2}$ & 473.2027 & 928.3717 & 464.6895 & 927.3877 & 464.1975 & $\mathbf{8}$ \\
\hline
\end{tabular}




\begin{tabular}{|c|c|c|c|c|c|c|c|c|c|c|c|c|c|c|}
\hline & 488.2351 & |244.6212 & 471.2085 & |236.1079 & 470.2245 & |235.6159 & S & 844.3505 & |422.6789 & 827.3240 & 414.1656 & 826.3400 & |413.6736 & 7 \\
\hline 6 & 635.2705 & 318.1389 & 618.2440 & |309.6256 & 617.2599 & 309.1336 & M & 757.3185 & |379.1629 & 740.2920 & 370.6496 & 739.3079 & 370.1576 & 6 \\
\hline 7 & 782.3389 & 391.6731 & 765.3124 & |383.1598 & 764.3284 & 382.6678 & $\mathbf{F}$ & 610.2831 & 305.6452 & 593.2566 & 297.1319 & 592.2725 & 296.6399 & 5 \\
\hline 8 & 897.3659 & 449.1866 & 880.3393 & 440.6733 & 879.3553 & 440.1813 & $\mathbf{N}$ & 463.2147 & 232.1110 & 446.1881 & 223.5977 & 445.2041 & 223.1057 & 4 \\
\hline 9 & 1011.4088 & 506.2080 & 994.3822 & 497.6948 & 993.3982 & 497.2027 & $\mathbf{N}$ & 348.1878 & |174.5975 & 331.1612 & 166.0842 & 330.1772 & 165.5922 & 3 \\
\hline 10 & 1098.4408 & 549.7240 & 1081.4143 & 541.2108 & 1080.4302 & 540.7188 & $\mathbf{S}$ & 234.1448 & 117.5761 & 217.1183 & 109.0628 & 216.1343 & 108.5708 & 2 \\
\hline 11 & & & & & & & $\mathbf{K}$ & 147.1128 & 74.0600 & 130.0863 & 65.5468 & & & 1 \\
\hline
\end{tabular}
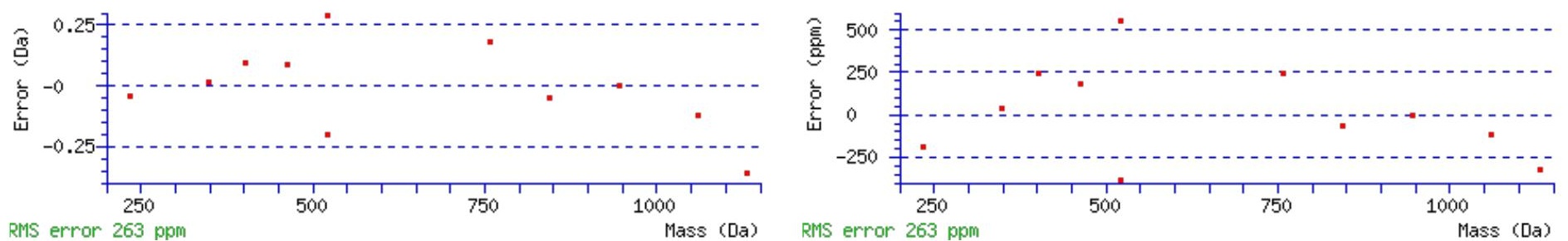

All matches to this query

\begin{tabular}{|l|l|l|l|}
\hline Score & Mr(calc): & Delta & \multicolumn{1}{|c|}{ Sequence } \\
\hline 85.4 & 1243.5390 & -0.0003 & LANTSMFNNSK \\
\hline 65.5 & 1243.5390 & -0.0003 & LANTSMFNNSK \\
\hline 23.7 & 1243.5390 & -0.0003 & LANTSMFNNSK \\
\hline 4.0 & 1243.5485 & -0.0098 & $\underline{\text { IATEAIENFR }}$ \\
\hline 3.5 & 1243.5454 & -0.0066 & ICGEASMKIR \\
\hline 3.2 & 1243.5268 & 0.0120 & $\underline{\text { LNAMNISETR }}$ \\
\hline 0.1 & 1243.5394 & -0.0006 & $\underline{\text { LAHARGCHSR }}$ \\
\hline
\end{tabular}

Spectrum No: 173; Query: 723; Rank: 1

\section{Peptide View}

MS/MS Fragmentation of EDNEGVFNGSWGGR

Found in IPI00208917, Tax_Id=10116 Gene_Symbol=Aldh7a1 similar to aldehyde dehydrogenase family 7, member A1

Match to Query 723: 1523.624948 from(762.819750,2+)

Title: 091008RatKidney_NoSalt_21.2096.2096.2.dta

Data file K:INewmanPaper|Piliang|3SubProteomes\Piliang3SP\mgf5ppm\ERLIC_3SubProteomes5ppm.mgf

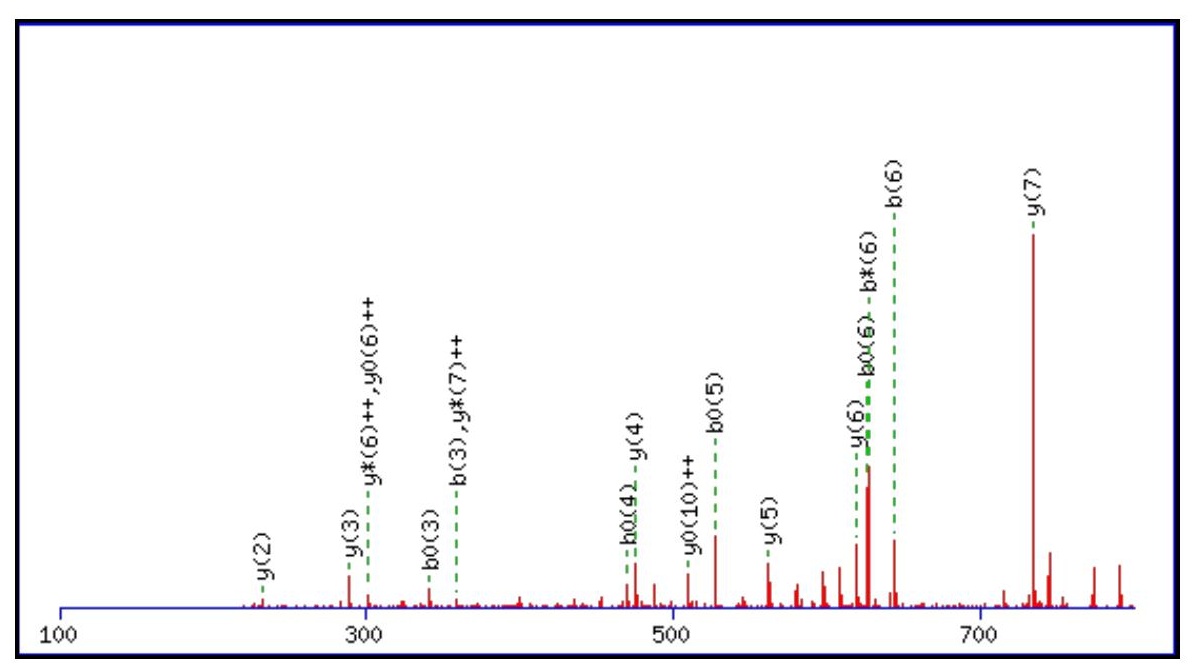



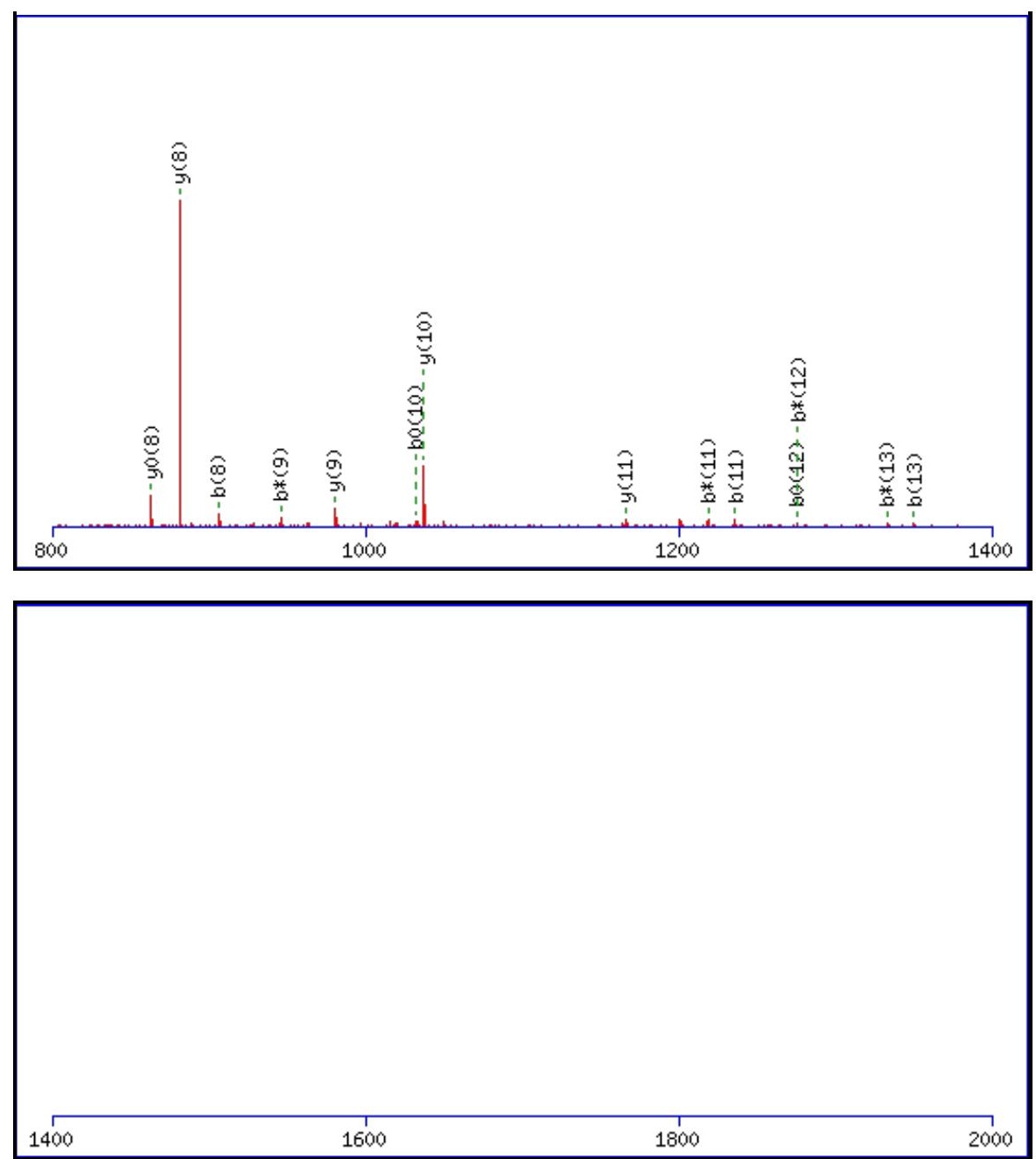

Monoisotopic mass of neutral peptide $\operatorname{Mr}($ calc): 1523.6277

Fixed modifications: Carbamidomethyl (C)

Variable modifications:

N8: : Deamidated $\mathrm{N}(\mathrm{N})$

Ions Score: 85 Expect: $3.9 \mathrm{e}-007$

Matches (Bold Red): 31/144 fragment ions using 39 most intense peaks

\begin{tabular}{|c|c|c|c|c|c|c|c|c|c|c|c|c|c|c|}
\hline \# & b & $\mathbf{b}^{++}$ & $\mathbf{b}^{*}$ & $\mathbf{b}^{*^{++}}$ & $\mathbf{b}^{0}$ & $\mathbf{b}^{0++}$ & Seq. & $\mathbf{y}$ & $\mathbf{y}^{++}$ & $\mathbf{y}^{*}$ & $\mathrm{y}^{*^{++}}$ & $\mathbf{y}^{\mathbf{0}}$ & $\mathbf{y}^{\mathbf{0 + +}}$ & \# \\
\hline 1 & 130.0499 & 65.5286 & & & 112.0393 & 56.5233 & $\mathbf{E}$ & & & & & & & 14 \\
\hline 2 & 245.0768 & 123.0420 & & & 227.0662 & 114.0368 & D & 1395.5924 & 698.2998 & 1378.5658 & 689.7866 & 1377.5818 & 689.2945 & 13 \\
\hline 3 & 359.1197 & 180.0635 & 342.0932 & 171.5502 & 341.1092 & 171.0582 & $\mathbf{N}$ & 1280.5654 & 640.7864 & 1263.5389 & 632.2731 & 1262.5549 & 631.7811 & 12 \\
\hline 4 & 488.1623 & 244.5848 & 471.1358 & 236.0715 & 470.1518 & 235.5795 & $\mathbf{E}$ & 1166.5225 & 583.7649 & 1149.4960 & 575.2516 & 1148.5119 & 574.7596 & 11 \\
\hline 5 & 545.1838 & 273.0955 & 528.1572 & 264.5823 & 527.1732 & 264.0903 & G & 1037.4799 & 519.2436 & 1020.4534 & 510.7303 & 1019.4693 & 510.2383 & 10 \\
\hline 6 & 644.2522 & 322.6297 & 627.2257 & 314.1165 & 626.2416 & 313.6245 & V & 980.4584 & 490.7329 & 963.4319 & 482.2196 & 962.4479 & 481.7276 & 9 \\
\hline 7 & 791.3206 & 396.1640 & 774.2941 & 387.6507 & 773.3101 & 387.1587 & $\mathbf{F}$ & 881.3900 & 441.1987 & 864.3635 & 432.6854 & 863.3795 & 432.1934 & 8 \\
\hline 8 & 906.3476 & 453.6774 & 889.3210 & 445.1641 & 888.3370 & 444.6721 & $\mathbf{N}$ & 734.3216 & 367.6644 & 717.2951 & 359.1512 & 716.3111 & 358.6592 & 7 \\
\hline 9 & 963.3690 & 482.1882 & 946.3425 & 473.6749 & 945.3585 & 473.1829 & G & 619.2947 & 310.1510 & 602.2681 & 301.6377 & 601.2841 & 301.1457 & 6 \\
\hline 10 & 1050.4011 & 525.7042 & 1033.3745 & 517.1909 & 1032.3905 & 516.6989 & $\mathrm{~S}$ & 562.2732 & 281.6402 & 545.2467 & 273.1270 & 544.2627 & 272.6350 & 5 \\
\hline 11 & 1236.4804 & 618.7438 & 1219.4538 & 610.2305 & 1218.4698 & 609.7385 & $\mathbf{W}$ & 475.2412 & 238.1242 & 458.2146 & 229.6110 & & & 4 \\
\hline 12 & 1293.5018 & 647.2546 & 1276.4753 & 638.7413 & 1275.4913 & 638.2493 & G & 289.1619 & 145.0846 & 272.1353 & 136.5713 & & & 3 \\
\hline 13 & 1350.5233 & 675.7653 & 1333.4967 & 667.2520 & 1332.5127 & 666.7600 & G & 232.1404 & 116.5738 & 215.1139 & 108.0606 & & & 2 \\
\hline 14 & & & & & & & $\mathbf{R}$ & 175.1190 & 88.0631 & 158.0924 & 79.5498 & & & 1 \\
\hline
\end{tabular}



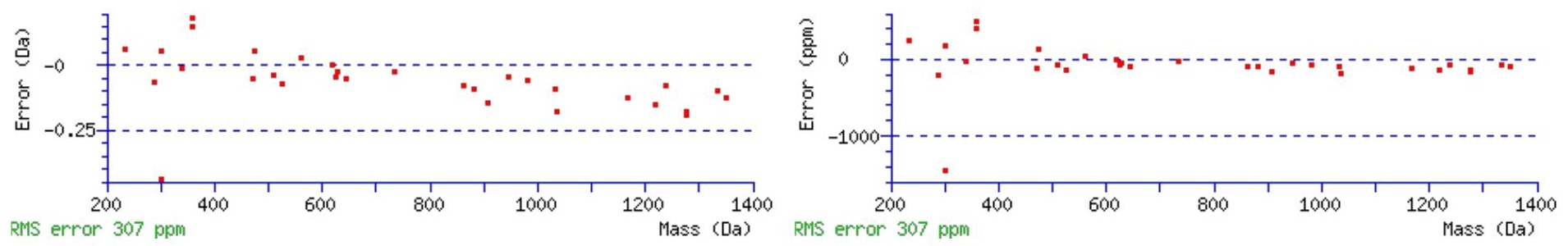

\section{All matches to this query}

\begin{tabular}{|l|l|l|l|}
\hline Score & Mr(calc): & Delta & \multicolumn{1}{c|}{ Sequence } \\
\hline 85.2 & 1523.6277 & -0.0028 & EDNEGVFNGSWGGR \\
\hline 15.6 & 1523.6277 & -0.0028 & EDNEGVFNGSWGGR \\
\hline 9.1 & 1523.6365 & -0.0116 & GQSSNAANVQQPSR \\
\hline 8.9 & 1523.6130 & 0.0119 & QRKSGSTSSSPSR \\
\hline 7.5 & 1523.6130 & 0.0119 & QRKSGSTSSSPSR \\
\hline 5.1 & 1523.6365 & -0.0116 & GQSSNAANVQQPSR \\
\hline 3.6 & 1523.6351 & -0.0101 & NGTMTIDWNEWR \\
\hline 3.5 & 1523.6365 & -0.0116 & GQSSNAANVQQPSR \\
\hline 3.3 & 1523.6365 & -0.0116 & GQSSNAANVQQPSR \\
\hline 2.6 & 1521.6137 & 2.0113 & STSYSSPSTXPSR \\
\hline
\end{tabular}

Spectrum No: 174; Query: 2590; Rank: 1

\section{Peptide View}

MS/MS Fragmentation of RNENFSSGPSFETISASGLNAALAHYSPTK

Found in IPI00197684, Tax_Id=10116 Gene_Symbol=Xpnpep2 Membrane-bound aminopeptidase P

Match to Query 2590: 3153.511662 from(1052.177830,3+)

Title: 100101RatKid_NS_deglyco_13.4729.4729.3.dta

Data file K:INewmanPaper|Piliangl3SubProteomes\Piliang3SP\mgf5ppm\ERLIC_3SubProteomes5ppm.mgf

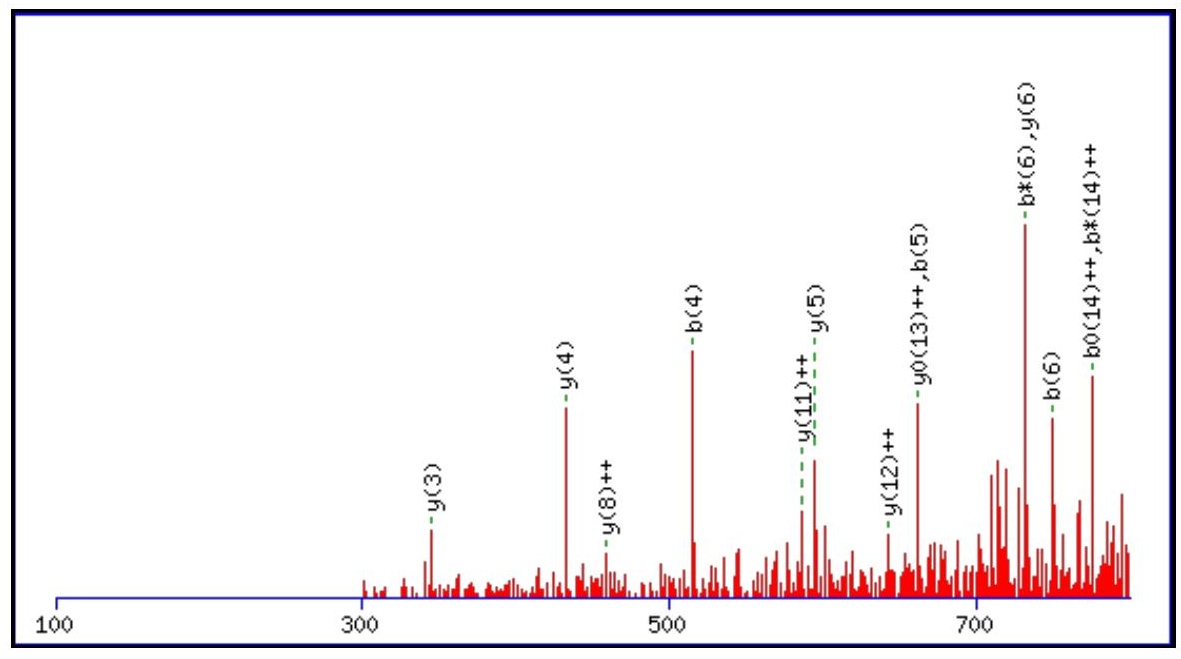



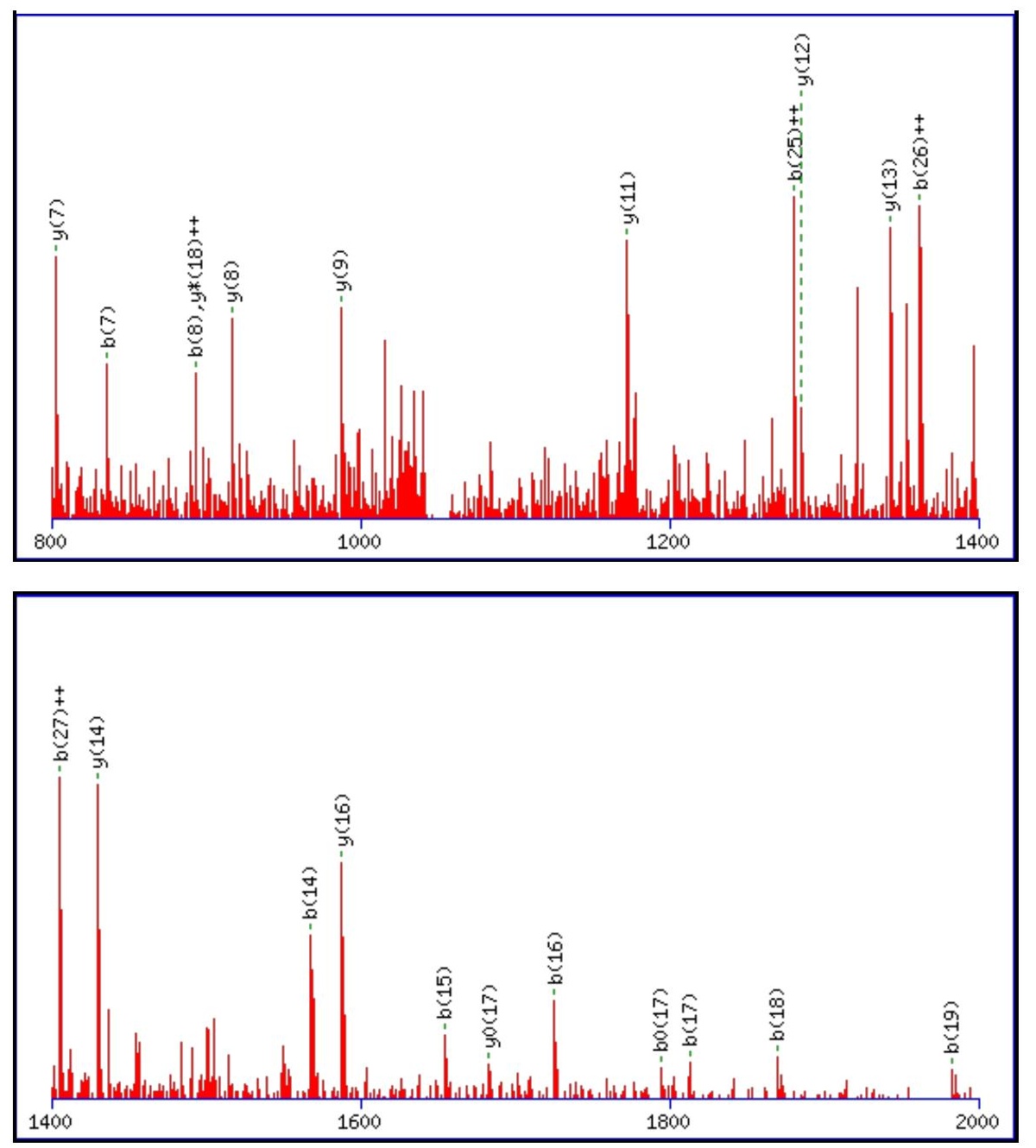

Monoisotopic mass of neutral peptide $\operatorname{Mr}($ calc): 3153.5003

Fixed modifications: Carbamidomethyl (C)

Variable modifications:

N4 : Deamidated_N (N)

Ions Score: 85 Expect: $9.9 \mathrm{e}-007$

Matches (Bold Red): 36/342 fragment ions using 54 most intense peaks

\begin{tabular}{|c|c|c|c|c|c|c|c|c|c|c|c|c|c|c|}
\hline \# & b & $\mathbf{b}^{++}$ & b* & $\mathbf{b}^{*^{++}}$ & $\mathbf{b}^{\mathbf{0}}$ & $\mathbf{b}^{\mathbf{0 + +}}$ & Seq. & $\mathbf{y}$ & $\mathbf{y}^{++}$ & $\mathbf{y}^{*}$ & $\mathbf{y}^{*^{++}}$ & $\mathbf{y}^{0}$ & $\mathbf{y}^{0++}$ & \# \\
\hline 1 & 157.1084 & 79.0578 & 140.0818 & 70.5446 & & & $\mathbf{R}$ & & & & & & & 30 \\
\hline 2 & 271.1513 & 136.0793 & 254.1248 & 127.5660 & & & $\mathbf{N}$ & 98.4065 & 7069 & 2981.3 & 1491.1 & 2980.3959 & 1490.7016 & 29 \\
\hline 3 & 400.1939 & 200.6006 & 383.1674 & 192.0873 & 382.1833 & & $\mathbf{E}$ & 2884.3636 & 1442.6854 & 2867.3370 & 1434.1721 & 2866.3530 & 1433.6801 & 28 \\
\hline 4 & 515.2208 & 258.1141 & 498.1943 & 249.6008 & 497.2103 & & $\mathbf{N}$ & 2755.3210 & 1378.1 & 2738. & 508 & 2737.3104 & 1369. & 27 \\
\hline 5 & 662.2893 & 331.6483 & 645.2627 & 323.1350 & 644.2787 & 322.6430 & $\mathbf{F}$ & 2640.2940 & 1320.6506 & 2623.2675 & 1312.1374 & 2622.2835 & 1311.6454 & 26 \\
\hline 6 & 749.3213 & 375.1643 & 732.2947 & 366.6510 & 731.3107 & 366.1590 & S & 2493.2256 & 1247.1164 & 2476.1991 & 1238.6032 & 2475.2150 & 1238.1112 & 25 \\
\hline 7 & 836.3533 & 418.6803 & 819.3268 & 410.1670 & 818.3427 & 409.6750 & S & 2406.1936 & 1203.6004 & 2389.1670 & 1195.0872 & 2388.1830 & 1194.5951 & 24 \\
\hline 8 & 893.3748 & 447.1910 & 876.3482 & 438.6778 & 875.3642 & 438.1857 & G & 2319.1616 & 1160.0844 & 2302.1350 & 1151.5711 & 2301.1510 & 1151.0791 & 23 \\
\hline 9 & 990.4275 & 495.7174 & 973.4010 & 487.2041 & 972.4170 & 486.7121 & $\mathbf{P}$ & 2262.1401 & 1131.5737 & 2245.1135 & 1123.0604 & 2244.1295 & 1122.5684 & 22 \\
\hline 10 & 1077.4596 & 539.2334 & 1060.4330 & 530.7201 & 1059.4490 & 530.2281 & S & 2165.0873 & 1083.0473 & 2148.0608 & 1074.5340 & 2147.0768 & 1074.0420 & 21 \\
\hline 11 & 1224.5280 & 612.7676 & 1207.5014 & 604.2544 & 1206.5174 & 603.7623 & $\mathbf{F}$ & 2078.0553 & 1039.5313 & 2061.0287 & 1031.0180 & 2060.0447 & 1030.5260 & 20 \\
\hline 12 & 1353.5706 & 677.2889 & 1336.5440 & 668.7757 & 1335.5600 & 668.2836 & $\mathbf{E}$ & 1930.9869 & 965.9971 & 1913.9603 & 957.4838 & 1912.9763 & 956.9918 & 19 \\
\hline 13 & 1454.6183 & 727.8128 & 1437.5917 & 719.2995 & 1436.6077 & 718.8075 & $\mathbf{T}$ & 1801.9443 & 901.4758 & 1784.9177 & 892.9625 & 1783.9337 & 892.4705 & 18 \\
\hline 14 & 1567.7023 & 784.3548 & 1550.6758 & 775.8415 & 1549.6918 & 775.3495 & I & 1700.8966 & 850.9519 & 1683.8701 & 842.4387 & 1682.8860 & 841.9467 & 17 \\
\hline 15 & 1654.7343 & 827.8708 & 1637.7078 & 819.3575 & 1636.7238 & 818.8655 & $S$ & 1587.8125 & 794.4099 & 1570.7860 & 785.8966 & 1569.8020 & 785.4046 & 16 \\
\hline 16 & 1725.7715 & 863.3894 & 1708.7449 & 854.8761 & 1707.7609 & 854.3841 & A & 1500.7805 & 750.8939 & 1483.7540 & 742.3806 & 1482.7700 & 741.8886 & 15 \\
\hline 17 & 1812.8035 & 906.9054 & 1795.7769 & 898.3921 & 1794.7929 & 897.9001 & S & 1429.7434 & 715.3753 & 1412.7169 & 706.8621 & 1411.7328 & 706.3701 & 14 \\
\hline 18 & 1869.8250 & 935.4161 & 1852.7984 & 926.9028 & 1851.8144 & 926.4108 & G & 1342.7114 & 671.8593 & 1325.6848 & 663.3461 & 1324.7008 & 662.8540 & 13 \\
\hline
\end{tabular}




\begin{tabular}{|c|c|c|c|c|c|c|c|c|c|c|c|c|c|c|}
\hline 19 & 1982.9090 & 991.9581 & 1965.8825 & 983.4449 & |1964.8985 & 982.9529 & L & 1285.6899 & 643.3486 & |1268.6634 & 634.8353 & 1267.6793 & 634.3433 & 12 \\
\hline 20 & 2096.9519 & 1048.9796 & 2079.9254 & 1040.4663 & 2078.9414 & 1039.9743 & $\mathbf{N}$ & 1172.6058 & 586.8066 & 1155.5793 & 578.2933 & 1154.5953 & 577.8013 & 11 \\
\hline 21 & 2167.9891 & 1084.4982 & 2150.9625 & 1075.9849 & 2149.9785 & 1075.4929 & A & 1058.5629 & 529.7851 & 1041.5364 & 521.2718 & 1040.5524 & 520.7798 & 10 \\
\hline 22 & 2239.0262 & 1120.0167 & 2221.9996 & 1111.5034 & 2221.0156 & 1111.0114 & A & 987.5258 & 494.2665 & 970.4993 & 485.7533 & 969.5152 & 485.2613 & 9 \\
\hline 23 & 2352.1102 & 1176.5588 & 2335.0837 & 1168.0455 & 2334.0997 & 1167.5535 & $\mathbf{L}$ & 916.4887 & 458.7480 & 899.4621 & 450.2347 & 898.4781 & 449.7427 & 8 \\
\hline 24 & 2423.1474 & 1212.0773 & 2406.1208 & 1203.5640 & 2405.1368 & 1203.0720 & A & 803.4046 & 402.2060 & 786.3781 & 393.6927 & 785.3941 & 393.2007 & 7 \\
\hline 25 & 2560.2063 & 1280.6068 & 2543.1797 & 1272.0935 & 2542.1957 & 1271.6015 & $\mathbf{H}$ & 732.3675 & 366.6874 & 715.3410 & 358.1741 & 714.3570 & 357.6821 & 6 \\
\hline 26 & 2723.2696 & 1362.1384 & 2706.2430 & 1353.6252 & 2705.2590 & 1353.1332 & $\mathbf{Y}$ & 595.3086 & 298.1579 & 578.2821 & 289.6447 & 577.2980 & 289.1527 & 5 \\
\hline 27 & 2810.3016 & 1405.6544 & 2793.2751 & 1397.1412 & 2792.2911 & 1396.6492 & S & 432.2453 & 216.6263 & 415.2187 & 208.1130 & 414.2347 & 207.6210 & 4 \\
\hline 28 & 2907.3544 & 1454.1808 & 2890.3278 & 1445.6676 & 2889.3438 & 1445.1755 & $\mathbf{P}$ & 345.2132 & 173.1103 & 328.1867 & 164.5970 & 327.2027 & 164.1050 & 3 \\
\hline 29 & 3008.4021 & 1504.7047 & 2991.3755 & 1496.1914 & 2990.3915 & 1495.6994 & $\mathbf{T}$ & 248.1605 & 124.5839 & 231.1339 & 116.0706 & 230.1499 & 115.5786 & 2 \\
\hline 30 & & & & & & & $\mathbf{K}$ & 147.1128 & 74.0600 & 130.0863 & 65.5468 & & & 1 \\
\hline
\end{tabular}
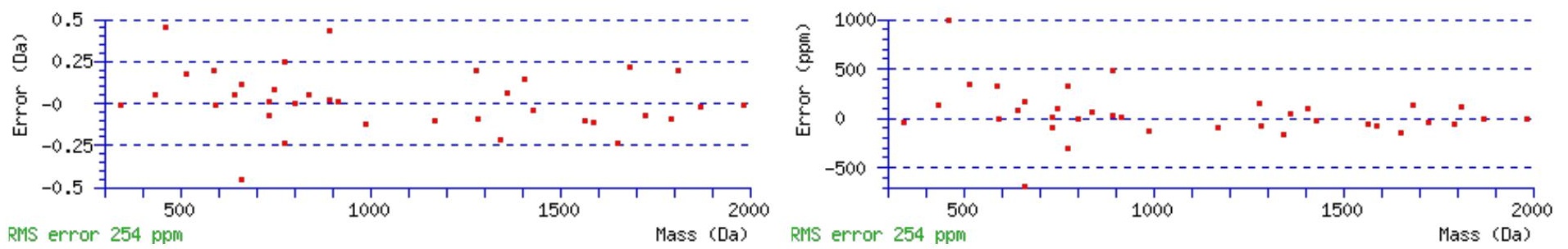

\section{All matches to this query}

\begin{tabular}{|l|l|l|l|}
\hline Score & Mr(calc): & Delta & \multicolumn{1}{|c|}{ Sequence } \\
\hline 85.2 & 3153.5003 & 0.0114 & RNENFSSGPSFETISASGLNAALAHYSPTK \\
\hline 85.2 & 3153.5003 & 0.0114 & RNENFSSGPSFETISASGLNAALAHYSPTK \\
\hline 39.5 & 3153.5003 & 0.0114 & RNENFSSGPSFETISASGLNAALAHYSPTK \\
\hline 39.3 & 3152.5163 & 0.9954 & RNENFSSGPSFETISASGLNAALAHYSPTK \\
\hline 0.9 & 3152.4930 & 1.0186 & EAASLTAAEPAPTKSIIAADSLTAAEPAPTK \\
\hline 0.4 & 3152.4807 & 1.0309 & TFSWASVTSKNLPPSGAVPVTGTPPHMVK \\
\hline 0.1 & 3151.5278 & 1.9839 & LSLRYTNKXRPLEEQSVSEVGCCSPVK \\
\hline 0.1 & 3151.5278 & 1.9839 & $\underline{\text { LSLRYTNKXRPLEEQSVSEVGCCSPVK }}$ \\
\hline
\end{tabular}

Spectrum No: 175; Query: 535; Rank: 1

\section{Peptide View}

MS/MS Fragmentation of IIYSIVQTNCSK

Found in IPI00327182, Tax_Id=10116 Gene_Symbol=Kng1 T-kininogen 1 precursor

Match to Query 535: 1425.721968 from(713.868260,2+)

Title: 091008RatKidney_NoSalt_37.1417.1417.2.dta

Data file K:INewmanPaper|Piliangl3SubProteomes\Piliang3SP\mgf5ppm\ERLIC_3SubProteomes5ppm.mgf 

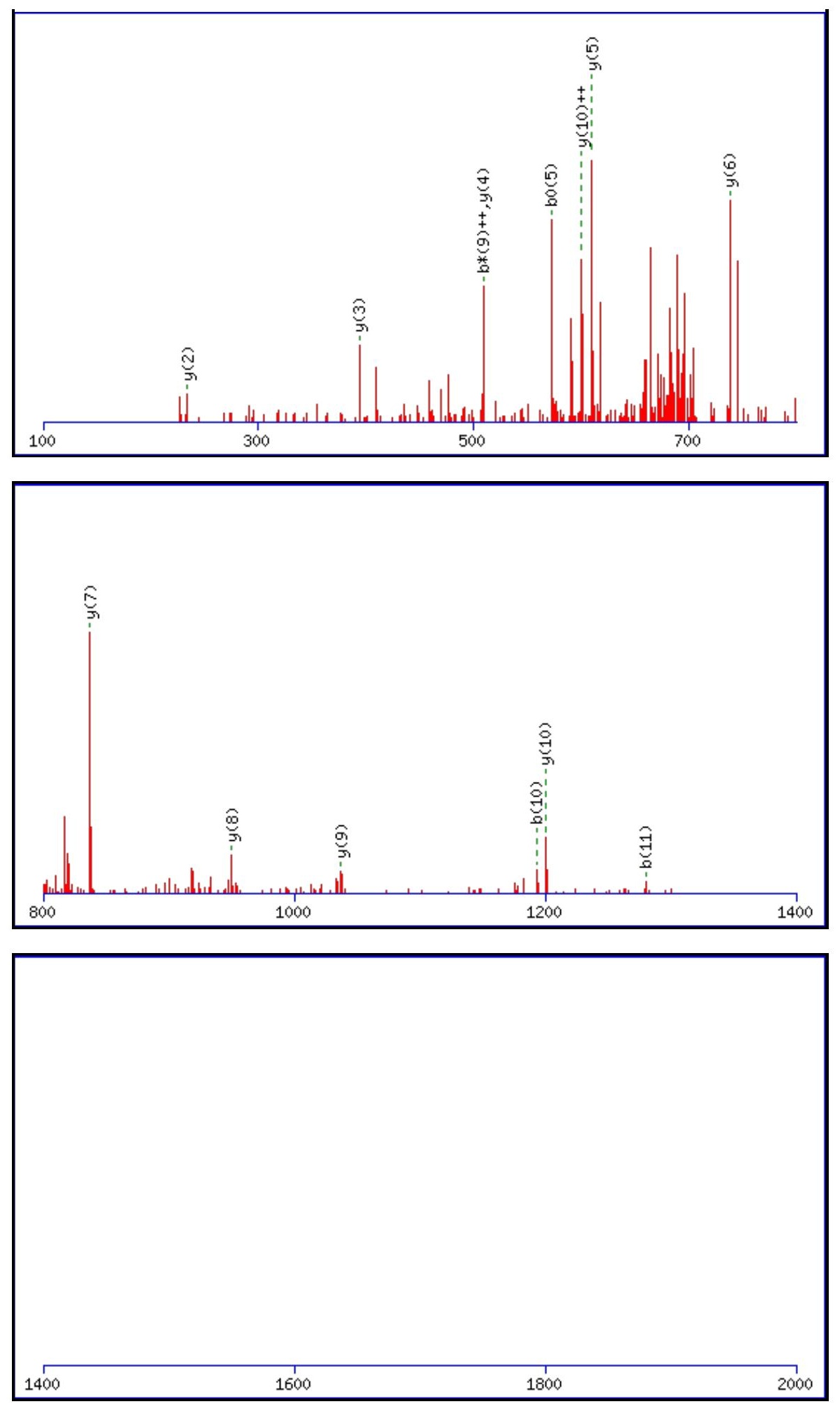

Monoisotopic mass of neutral peptide $\operatorname{Mr}($ calc): 1425.7173

Fixed modifications: Carbamidomethyl (C)

Variable modifications:

N9 : Deamidated $\mathrm{N}(\mathrm{N})$

Ions Score: 85 Expect: $3.9 \mathrm{e}-007$

Matches (Bold Red): 14/112 fragment ions using 16 most intense peaks

\begin{tabular}{|r|c|c|c|c|c|c|c|c|c|c|c|c|c|c|}
\hline$\#$ & $\mathbf{b}$ & $\mathbf{b}^{++}$ & $\mathbf{b}^{*}$ & $\mathbf{b}^{\boldsymbol{*}^{++}}$ & $\mathbf{b}^{\mathbf{0}}$ & $\mathbf{b}^{\mathbf{0 + +}}$ & Seq. & $\mathbf{y}$ & $\mathbf{y}^{++}$ & $\mathbf{y}^{\mathbf{*}}$ & $\mathbf{y}^{\boldsymbol{*}^{++}}$ & $\mathbf{y}^{\mathbf{0}}$ & $\mathbf{y}^{\mathbf{0 + +}}$ & $\#$ \\
\hline $\mathbf{1}$ & 114.0913 & 57.5493 & & & & & $\mathbf{I}$ & & & & & & & $\mathbf{1 2}$ \\
\hline $\mathbf{2}$ & 227.1754 & 114.0913 & & & & & $\mathbf{I}$ & 1313.6406 & 657.3239 & 1296.6140 & 648.8107 & 1295.6300 & 648.3186 & $\mathbf{1 1}$ \\
\hline 3 & 390.2387 & 195.6230 & & & & & $\mathbf{Y}$ & $\mathbf{1 2 0 0 . 5 5 6 5}$ & $\mathbf{6 0 0 . 7 8 1 9}$ & 1183.5300 & 592.2686 & 1182.5459 & 591.7766 & $\mathbf{1 0}$ \\
\hline $\mathbf{4}$ & 477.2708 & 239.1390 & & & 459.2602 & 230.1337 & S & $\mathbf{1 0 3 7 . 4 9 3 2}$ & 519.2502 & 1020.4666 & 510.7370 & 1019.4826 & 510.2449 & $\mathbf{9}$ \\
\hline $\mathbf{5}$ & 590.3548 & 295.6811 & & & 572.3443 & 286.6758 & $\mathbf{I}$ & $\mathbf{9 5 0 . 4 6 1 2}$ & 475.7342 & 933.4346 & 467.2209 & 932.4506 & 466.7289 & $\mathbf{8}$ \\
\hline
\end{tabular}




\begin{tabular}{|r|r|r|r|r|r|r|r|r|r|r|r|r|r|r|r|r|r|}
$\mathbf{6}$ & 689.4232 & 345.2153 & & & 671.4127 & 336.2100 & $\mathbf{V}$ & $\mathbf{8 3 7 . 3 7 7 1}$ & 419.1922 & 820.3505 & 410.6789 & 819.3665 & 410.1869 & $\mathbf{7}$ \\
\hline $\mathbf{7}$ & 817.4818 & 409.2445 & 800.4553 & 400.7313 & 799.4713 & 400.2393 & $\mathbf{Q}$ & $\mathbf{7 3 8 . 3 0 8 7}$ & 369.6580 & 721.2821 & 361.1447 & 720.2981 & 360.6527 & $\mathbf{6}$ \\
\hline $\mathbf{8}$ & 918.5295 & 459.7684 & 901.5029 & 451.2551 & 900.5189 & 450.7631 & $\mathbf{T}$ & $\mathbf{6 1 0 . 2 5 0 1}$ & 305.6287 & 593.2235 & 297.1154 & 592.2395 & 296.6234 & $\mathbf{5}$ \\
\hline $\mathbf{9}$ & 1033.5564 & 517.2819 & 1016.5299 & $5 \mathbf{5 0 8 . 7 6 8 6}$ & 1015.5459 & 508.2766 & $\mathbf{N}$ & $\mathbf{5 0 9 . 2 0 2 4}$ & 255.1048 & 492.1759 & 246.5916 & 491.1919 & 246.0996 & $\mathbf{4}$ \\
\hline $\mathbf{1 0}$ & $\mathbf{1 1 9 3 . 5 8 7 1}$ & 597.2972 & 1176.5605 & 588.7839 & 1175.5765 & 588.2919 & $\mathbf{C}$ & 394.1755 & 197.5914 & 377.1489 & 189.0781 & 376.1649 & 188.5861 & $\mathbf{3}$ \\
\hline $\mathbf{1 1}$ & $\mathbf{1 2 8 0 . 6 1 9 1}$ & 640.8132 & 1263.5926 & 632.2999 & 1262.6085 & 631.8079 & $\mathbf{S}$ & $\mathbf{2 3 4 . 1 4 4 8}$ & 117.5761 & 217.1183 & 109.0628 & 216.1343 & 108.5708 & $\mathbf{2}$ \\
\hline $\mathbf{1 2}$ & & & & & & & $\mathbf{K}$ & 147.1128 & 74.0600 & 130.0863 & 65.5468 & & & $\mathbf{1}$ \\
\hline
\end{tabular}
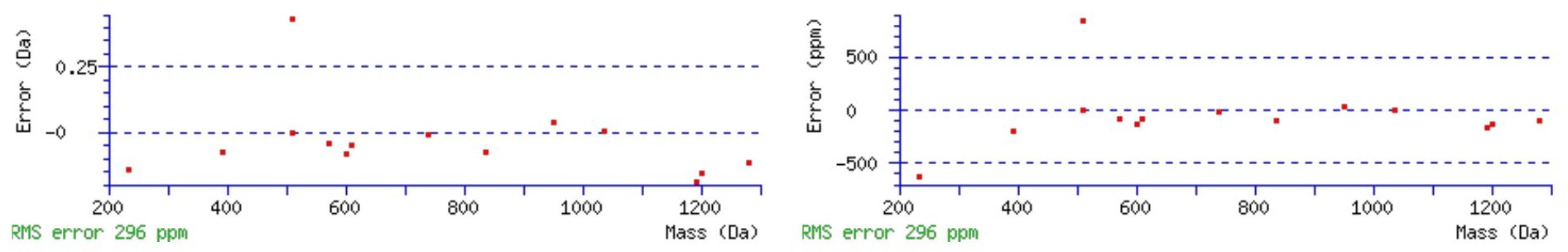

\section{All matches to this query}

\begin{tabular}{|l|l|l|l|}
\hline Score & Mr(calc): & Delta & \multicolumn{1}{c|}{ Sequence } \\
\hline 84.8 & 1425.7173 & 0.0046 & $\underline{\text { IYSIVQTNCSK }}$ \\
\hline 12.1 & 1424.7177 & 1.0043 & $\underline{\text { RSIWDVISKNK }}$ \\
\hline 11.6 & 1423.7194 & 2.0025 & TYEDQLSEAKIK \\
\hline 4.3 & 1425.7228 & -0.0009 & $\underline{\text { KPSKSEAKGTVSK }}$ \\
\hline 1.4 & 1424.7201 & 1.0019 & GFPPNPNILDWR \\
\hline 1.2 & 1423.7130 & 2.0090 & $\underline{\text { LNSCLVYVDVSR }}$ \\
\hline 1.0 & 1425.7228 & -0.0009 & $\underline{\text { KPSKSEAKGTVSK }}$ \\
\hline 0.9 & 1424.7115 & 1.0104 & $\underline{\text { KMTLSLADRCSK }}$ \\
\hline 0.5 & 1425.7228 & -0.0009 & $\underline{\text { SLLSLLQSRDSK }}$ \\
\hline
\end{tabular}

Spectrum No: 176; Query: 699; Rank: 1

\section{Peptide View}

MS/MS Fragmentation of FINDYVSNQTQGK

Found in IPI00200593, Tax_Id=10116 Gene_Symbol=Serpina3k Serine protease inhibitor A3K precursor

Match to Query 699: 1513.706228 from(757.860390,2+)

Title: 091008RatKidney_NoSalt_34.1214.1214.2.dta

Data file K:INewmanPaper|Piliangl3SubProteomes\Piliang3SP\mgf5ppm\ERLIC_3SubProteomes5ppm.mgf

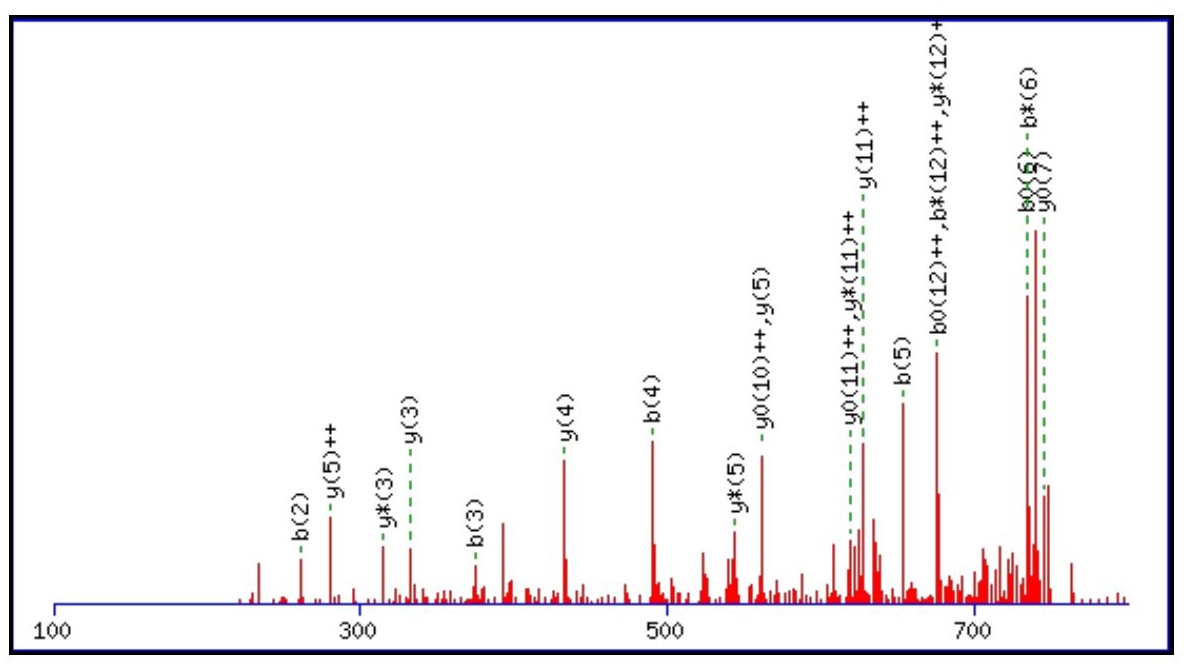




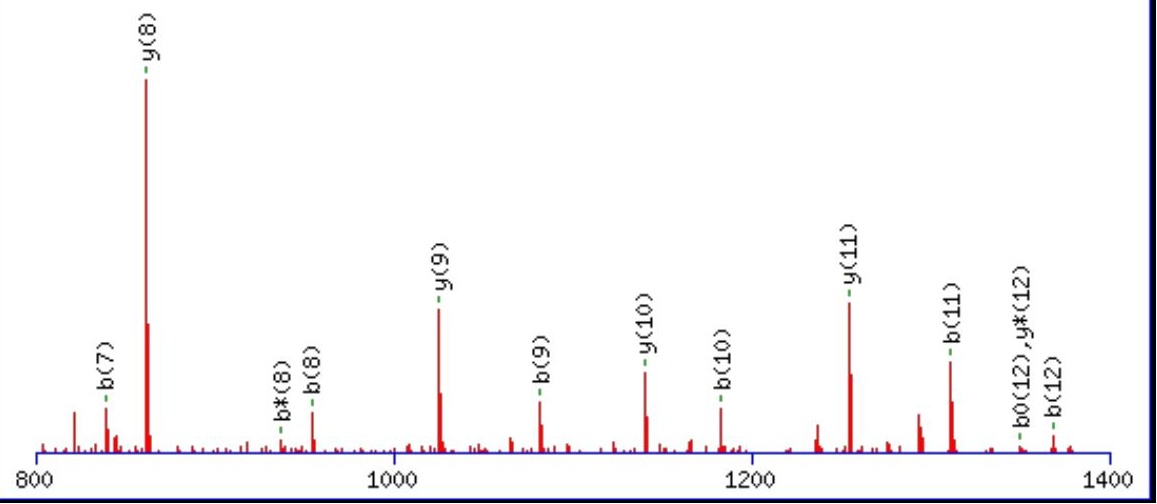

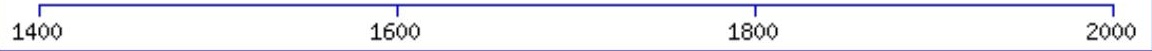

Monoisotopic mass of neutral peptide $\operatorname{Mr}$ (calc): 1513.7049

Fixed modifications: Carbamidomethyl (C)

Variable modifications:

N8 : Deamidated_N (N)

Ions Score: 85 Expect: $5.4 \mathrm{e}-007$

Matches (Bold Red): 34/128 fragment ions using 42 most intense peaks

\begin{tabular}{|r|c|c|c|c|c|c|c|c|c|c|c|c|c|c|}
\hline$\#$ & $\mathbf{b}$ & $\mathbf{b}^{++}$ & $\mathbf{b}^{*}$ & $\mathbf{b}^{\mathbf{*}^{++}}$ & $\mathbf{b}^{\mathbf{0}}$ & $\mathbf{b}^{\mathbf{0 + +}}$ & $\mathbf{S e q}$ & $\mathbf{y}$ & $\mathbf{y}^{++}$ & $\mathbf{y}^{\mathbf{*}}$ & $\mathbf{y}^{\boldsymbol{*}^{++}}$ & $\mathbf{y}^{\mathbf{0}}$ & $\mathbf{y}^{\mathbf{0 + +}}$ & $\#$ \\
\hline $\mathbf{1}$ & 148.0757 & 74.5415 & & & & & $\mathbf{F}$ & & & & & & & $\mathbf{1 3}$ \\
\hline $\mathbf{2}$ & $\mathbf{2 6 1 . 1 5 9 8}$ & 131.0835 & & & & & $\mathbf{I}$ & 1367.6437 & 684.3255 & $\mathbf{1 3 5 0 . 6 1 7 2}$ & $\mathbf{6 7 5 . 8 1 2 2}$ & 1349.6332 & 675.3202 & $\mathbf{1 2}$ \\
\hline $\mathbf{3}$ & $\mathbf{3 7 5 . 2 0 2 7}$ & 188.1050 & 358.1761 & 179.5917 & & & $\mathbf{N}$ & $\mathbf{1 2 5 4 . 5 5 9 7}$ & $\mathbf{6 2 7 . 7 8 3 5}$ & 1237.5331 & $\mathbf{6 1 9 . 2 7 0 2}$ & 1236.5491 & $\mathbf{6 1 8 . 7 7 8 2}$ & $\mathbf{1 1}$ \\
\hline $\mathbf{4}$ & $\mathbf{4 9 0 . 2 2 9 6}$ & 245.6185 & 473.2031 & 237.1052 & 472.2191 & 236.6132 & $\mathbf{D}$ & $\mathbf{1 1 4 0 . 5 1 6 8}$ & 570.7620 & 1123.4902 & 562.2487 & 1122.5062 & $\mathbf{5 6 1 . 7 5 6 7}$ & $\mathbf{1 0}$ \\
\hline $\mathbf{5}$ & $\mathbf{6 5 3 . 2 9 3 0}$ & 327.1501 & 636.2664 & 318.6368 & 635.2824 & 318.1448 & $\mathbf{Y}$ & $\mathbf{1 0 2 5 . 4 8 9 8}$ & 513.2485 & 1008.4633 & 504.7353 & 1007.4792 & 504.2433 & $\mathbf{9 9}$ \\
\hline $\mathbf{6}$ & 752.3614 & 376.6843 & 735.3348 & 368.1710 & 734.3508 & 367.6790 & $\mathbf{V}$ & $\mathbf{8 6 2 . 4 2 6 5}$ & 431.7169 & 845.3999 & 423.2036 & 844.4159 & 422.7116 & $\mathbf{8}$ \\
\hline $\mathbf{7}$ & $\mathbf{8 3 9 . 3 9 3 4}$ & 420.2003 & 822.3668 & 411.6871 & 821.3828 & 411.1951 & $\mathbf{S}$ & 763.3581 & 382.1827 & 746.3315 & 373.6694 & $\mathbf{7 4 5 . 3 4 7 5}$ & 373.1774 & $\mathbf{7}$ \\
\hline $\mathbf{8}$ & $\mathbf{9 5 4 . 4 2 0 3}$ & 477.7138 & $\mathbf{9 3 7 . 3 9 3 8}$ & 469.2005 & 936.4098 & 468.7085 & $\mathbf{N}$ & $\mathbf{6 7 6 . 3 2 6 0}$ & 338.6667 & 659.2995 & 330.1534 & 658.3155 & 329.6614 & $\mathbf{6}$ \\
\hline $\mathbf{9}$ & $\mathbf{1 0 8 2 . 4 7 8 9}$ & 541.7431 & 1065.4524 & 533.2298 & 1064.4683 & 532.7378 & $\mathbf{Q}$ & $\mathbf{5 6 1 . 2 9 9 1}$ & $\mathbf{2 8 1 . 1 5 3 2}$ & 544.2726 & 272.6399 & 543.2885 & 272.1479 & $\mathbf{5}$ \\
\hline $\mathbf{1 0}$ & $\mathbf{1 1 8 3 . 5 2 6 6}$ & 592.2669 & 1166.5000 & 583.7537 & 1165.5160 & 583.2616 & $\mathbf{T}$ & $\mathbf{4 3 3 . 2 4 0 5}$ & 217.1239 & 416.2140 & 208.6106 & 415.2300 & 208.1186 & $\mathbf{4}$ \\
\hline $\mathbf{1 1}$ & $\mathbf{1 3 1 1 . 5 8 5 2}$ & 656.2962 & 1294.5586 & 647.7829 & 1293.5746 & 647.2909 & $\mathbf{Q}$ & $\mathbf{3 3 2 . 1 9 2 8}$ & 166.6001 & $\mathbf{3 1 5 . 1 6 6 3}$ & 158.0868 & & & $\mathbf{3}$ \\
\hline $\mathbf{1 2}$ & $\mathbf{1 3 6 8 . 6 0 6 6}$ & 684.8070 & 1351.5801 & $\mathbf{6 7 6 . 2 9 3 7}$ & $\mathbf{1 3 5 0 . 5 9 6 1}$ & $\mathbf{6 7 5 . 8 0 1 7}$ & $\mathbf{G}$ & 204.1343 & 102.5708 & 187.1077 & 94.0575 & & & $\mathbf{2}$ \\
\hline $\mathbf{1 3}$ & & & & & & & $\mathbf{K}$ & 147.1128 & 74.0600 & 130.0863 & 65.5468 & & & $\mathbf{1}$ \\
\hline
\end{tabular}



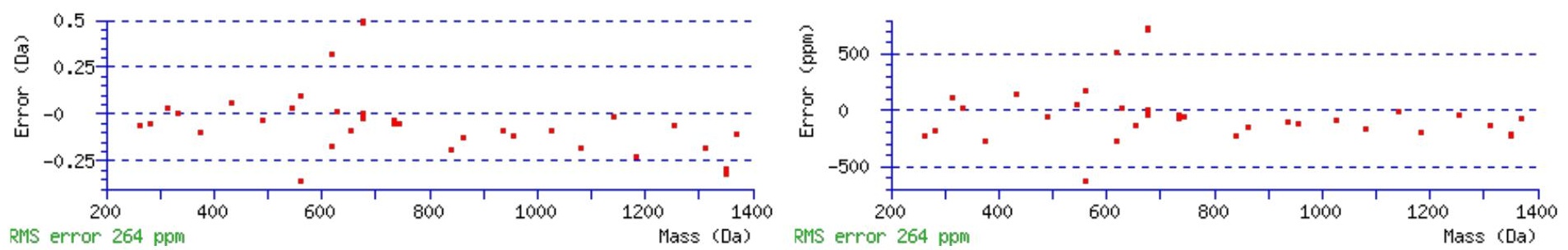

\section{All matches to this query}

\begin{tabular}{|l|l|l|l|}
\hline Score & Mr(calc): & Delta & \multicolumn{1}{|c|}{ Sequence } \\
\hline 84.7 & 1513.7049 & 0.0013 & FINDYVSNQTQGK \\
\hline 36.8 & 1513.7049 & 0.0013 & FINDYVSNQTQGK \\
\hline 11.9 & 1513.7153 & -0.0090 & FLNWIKTTMHK \\
\hline 11.9 & 1513.7153 & -0.0090 & FLNWIKTTMHK \\
\hline 11.9 & 1513.7057 & 0.0005 & AMRMADFWLTEK \\
\hline 10.9 & 1513.7211 & -0.0149 & KISCTNANLVKGK \\
\hline 9.2 & 1513.7137 & -0.0075 & NVKGTQASKSTQGK \\
\hline 7.2 & 1513.7055 & 0.0007 & DEIRHMRANAER \\
\hline 7.1 & 1513.6983 & 0.0079 & KGNSWMGLSYNNK \\
\hline 6.7 & 1511.7072 & 1.9990 & TETTKDMGRCLGK \\
\hline
\end{tabular}

Spectrum No: 177; Query: 1351; Rank: 1

\section{Peptide View}

MS/MS Fragmentation of ANVQFGENGTTISAVTNK

Found in IPI00231478, Tax_Id=10116 Gene_Symbol=Scarb2 Lysosome membrane protein 2

Match to Query 1351: 1850.907328 from(926.460940,2+)

Title: 100101RatKid_NS_deglyco_23.2492.2492.2.dta

Data file K:INewmanPaper|Piliangl3SubProteomes\Piliang3SP\mgf5ppm|ERLIC_3SubProteomes5ppm.mgf

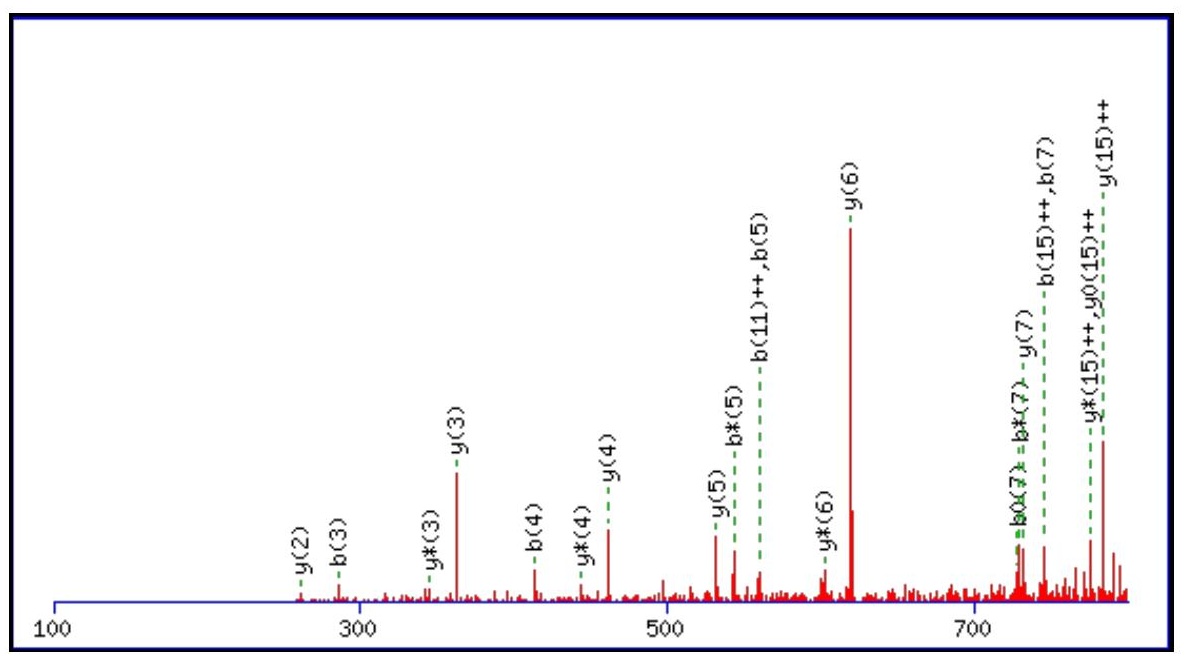



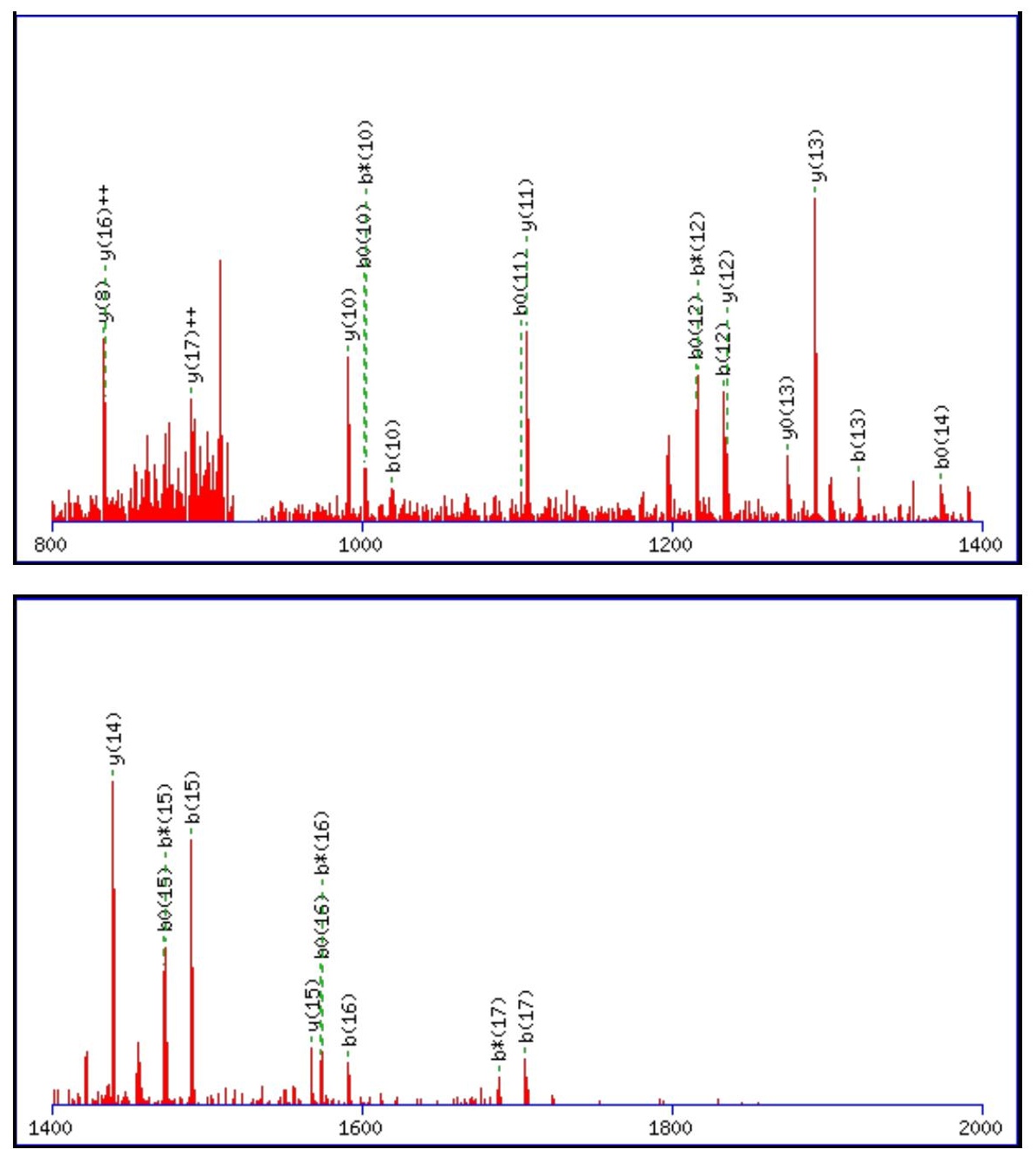

Monoisotopic mass of neutral peptide $\operatorname{Mr}($ calc): 1850.9010

Fixed modifications: Carbamidomethyl (C)

Variable modifications:

N8 : Deamidated_N (N)

Ions Score: 85 Expect: $7.5 \mathrm{e}-007$

Matches (Bold Red): 48/186 fragment ions using 75 most intense peaks

\begin{tabular}{|c|c|c|c|c|c|c|c|c|c|c|c|c|c|c|}
\hline \# & b & $\mathbf{b}^{++}$ & $\mathbf{b}^{*}$ & $\mathbf{b}^{*^{++}}$ & $\mathbf{b}^{0}$ & & Seq. & $\mathbf{y}$ & $\mathbf{y}^{++}$ & $\mathbf{y}^{*}$ & $\mathbf{y}^{*^{++}}$ & $\mathbf{y}^{0}$ & $y^{0++}$ & \# \\
\hline 1 & 72.0444 & 36.5258 & & & & & A & & & & & & & 18 \\
\hline 2 & 186.0873 & 93.5473 & 169.0608 & 85.0340 & & & $\mathbf{N}$ & 1780.8712 & 890.9392 & 1763.8446 & 882.4260 & 1762.8606 & 881.9339 & 17 \\
\hline 3 & 285.1557 & 143.0815 & 268.1292 & 134.5682 & & & $\mathbf{V}$ & 1666.8283 & 833.9178 & 1649.8017 & 825.4045 & 1648.8177 & 824.9125 & 16 \\
\hline 4 & 413.2143 & 207.1108 & 396.1878 & 198.5975 & & & $\mathbf{Q}$ & 1567.7598 & 784.3836 & 1550.7333 & 775.8703 & 1549.7493 & 775.3783 & 15 \\
\hline 5 & 560.2827 & 280.6450 & 543.2562 & 272.1317 & & & $\mathbf{F}$ & 1439.7013 & 720.3543 & 1422.6747 & 711.8410 & 1421.6907 & 711.3490 & 14 \\
\hline 6 & 617.3042 & 309.1557 & 600.2776 & 300.6425 & & & G & 1292.6328 & 646.8201 & 1275.6063 & 638.3068 & 1274.6223 & 637.8148 & 13 \\
\hline 7 & 746.3468 & 373.6770 & 729.3202 & 365.1638 & 728.3362 & 364.6717 & $\mathbf{E}$ & 1235.6114 & 618.3093 & 1218.5848 & 609.7961 & 1217.6008 & 609.3040 & 12 \\
\hline 8 & 861.3737 & 431.1905 & 844.3472 & 422.6772 & 843.3632 & 422.1852 & $\mathbf{N}$ & 1106.5688 & 553.7880 & 1089.5422 & 545.2748 & 1088.5582 & 544.7827 & 11 \\
\hline 9 & 918.3952 & 459.7012 & 901.3686 & 451.1880 & 900.3846 & 450.6959 & G & 991.5419 & 496.2746 & 974.5153 & 487.7613 & 973.5313 & 487.2693 & 10 \\
\hline 10 & 1019.4429 & 510.2251 & 1002.4163 & 501.7118 & 1001.4323 & 501.2198 & $\mathbf{T}$ & 934.5204 & 467.7638 & 917.4938 & 459.2506 & 916.5098 & 458.7585 & 9 \\
\hline 11 & 1120.4905 & 560.7489 & 1103.4640 & 552.2356 & 1102.4800 & 551.7436 & $\mathbf{T}$ & 833.4727 & 417.2400 & 816.4462 & 408.7267 & 815.4621 & 408.2347 & 8 \\
\hline 12 & 1233.5746 & 617.2909 & 1216.5481 & 608.7777 & 1215.5640 & 608.2857 & I & 732.4250 & 366.7162 & 715.3985 & 358.2029 & 714.4145 & 357.7109 & 7 \\
\hline 13 & 1320.6066 & 660.8070 & 1303.5801 & 652.2937 & 1302.5961 & 651.8017 & S & 619.3410 & 310.1741 & 602.3144 & 301.6608 & 601.3304 & 301.1688 & 6 \\
\hline 14 & 1391.6437 & 696.3255 & 1374.6172 & 687.8122 & 1373.6332 & 687.3202 & A & 532.3089 & 266.6581 & 515.2824 & 258.1448 & 514.2984 & 257.6528 & 5 \\
\hline 15 & 1490.7122 & 745.8597 & 1473.6856 & 737.3464 & 1472.7016 & 736.8544 & V & 461.2718 & 231.1396 & 444.2453 & 222.6263 & 443.2613 & 222.1343 & 4 \\
\hline 16 & 1591.7598 & 796.3836 & 1574.7333 & 787.8703 & 1573.7493 & 787.3783 & $\mathbf{T}$ & 362.2034 & 181.6053 & 345.1769 & 173.0921 & 344.1928 & 172.6001 & 3 \\
\hline 17 & 1705.8028 & 853.4050 & 1688.7762 & 844.8917 & 1687.7922 & 844.3997 & $\mathbf{N}$ & 261.1557 & 131.0815 & 244.1292 & 122.5682 & & & 2 \\
\hline 18 & & & & & & & $\mathbf{K}$ & 147.1128 & 74.0600 & 130.0863 & 65.5468 & & & 1 \\
\hline
\end{tabular}



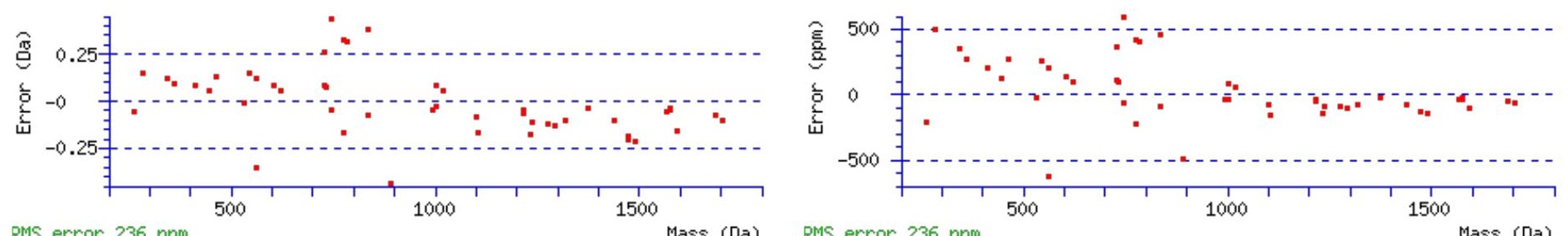

RMS error 236

\section{All matches to this query}

\begin{tabular}{|l|l|l|l|}
\hline Score & Mr(calc): & Delta & Sequence \\
\hline 84.5 & 1850.9010 & 0.0063 & ANVQFGENGTTISAVTNK \\
\hline 40.7 & 1850.9010 & 0.0063 & ANVQFGENGTTISAVTNK \\
\hline 36.6 & 1850.9010 & 0.0063 & ANVQFGENGTTISAVTNK \\
\hline 32.1 & 1849.9170 & 0.9903 & ANVQFGENGTTISAVTNK \\
\hline 2.3 & 1849.9138 & 0.9936 & SQSKITRLITAVVMK \\
\hline 0.6 & 1849.8905 & 1.0168 & ADNDDSLLTDISSSKLR \\
\hline
\end{tabular}

Spectrum No: 178; Query: 1359; Rank: 1

\section{Peptide View}

MS/MS Fragmentation of DGSQNFNQTWENYEK

Found in IPI00198903, Tax_Id=10116 Gene_Symbol=Angptl3 Angiopoietin-like 3

Match to Query 1359: 1859.762548 from(930.888550,2+)

Title: 100101RatKid_NS_deglyco_26.2770.2770.2.dta

Data file K:INewmanPaper|Piliangl3SubProteomes\Piliang3SP\mgf5ppm\ERLIC_3SubProteomes5ppm.mgf

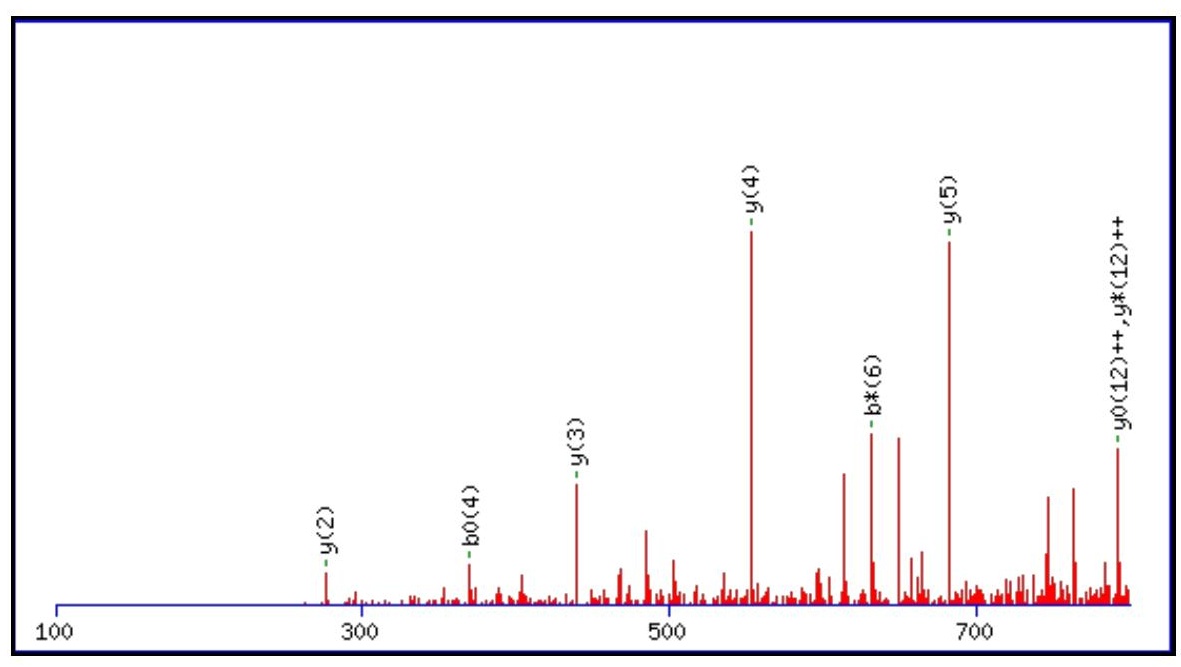



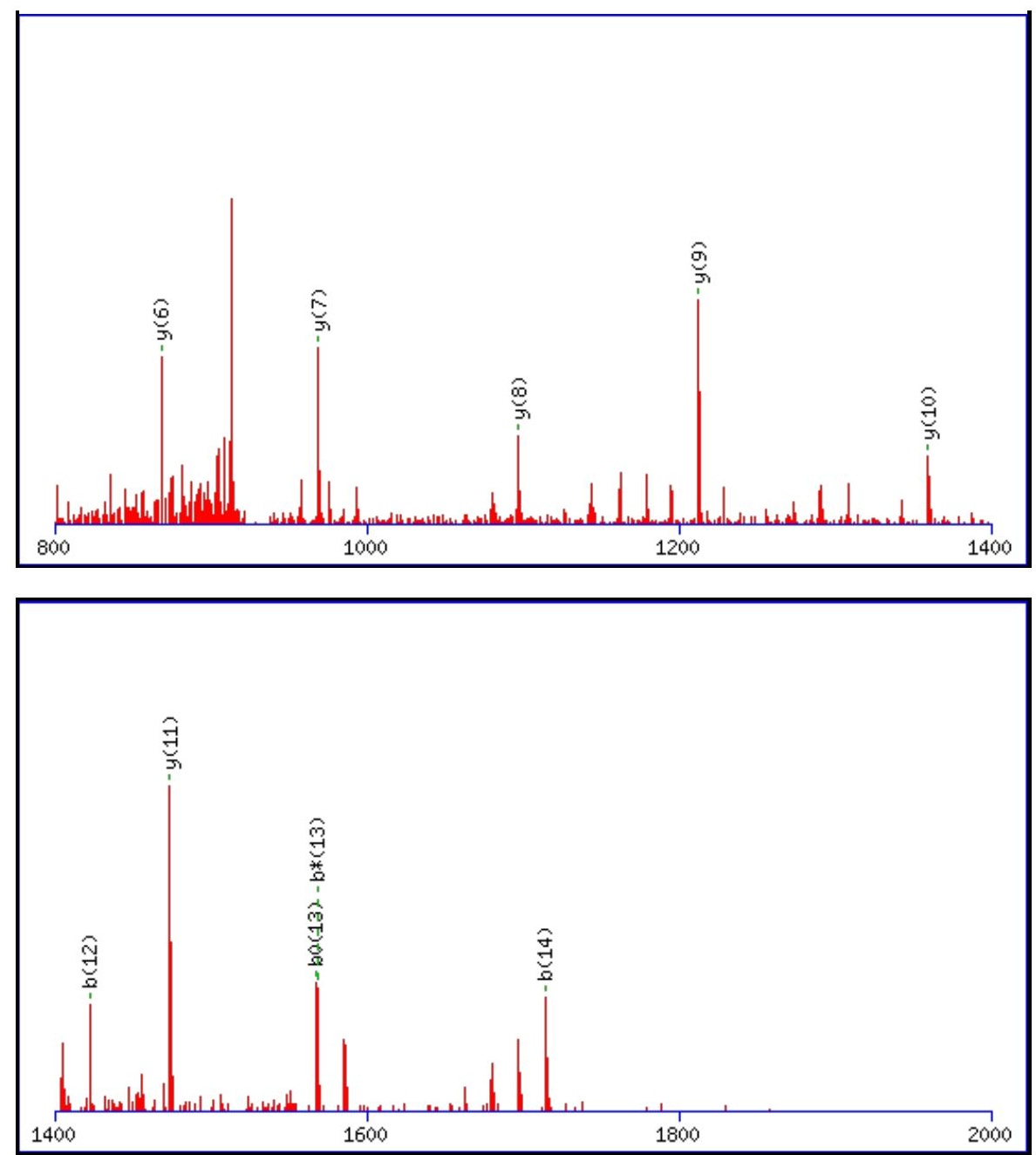

Monoisotopic mass of neutral peptide $\operatorname{Mr}($ calc): 1859.7598

Fixed modifications: Carbamidomethyl (C)

Variable modifications:

N7 : Deamidated $\mathrm{N}(\mathrm{N})$

Ions Score: 85 Expect: $6.3 \mathrm{e}-007$

Matches (Bold Red): 18/160 fragment ions using 31 most intense peaks

\begin{tabular}{|c|c|c|c|c|c|c|c|c|c|c|c|c|c|c|}
\hline \# & b & $\mathbf{b}^{++}$ & $\mathbf{b}^{*}$ & $\mathbf{b}^{*^{++}}$ & $\mathbf{b}^{0}$ & $\mathbf{b}^{0++}$ & Seq. & $\mathbf{y}$ & $y^{++}$ & $\mathbf{y}^{*}$ & $\mathbf{y}^{*^{++}}$ & $\mathbf{y}^{0}$ & $\mathbf{y}^{0++}$ & \# \\
\hline 1 & 116.0342 & 58.5207 & & & 98.0237 & 49.5155 & D & & & & & & & 15 \\
\hline 2 & 173.0557 & 87.0315 & & & 155.0451 & 78.0262 & G & 1745.7402 & & 1728.7136 & & 1727.7296 & & 14 \\
\hline 3 & 260.0877 & 130.5475 & & & 242.0771 & 121.5422 & S & 1688.7187 & 844.8630 & 1671.6922 & 836.3497 & 1670.7081 & 835.8577 & 13 \\
\hline 4 & 388.1463 & 194.5768 & 371.1197 & 186.0635 & 370.1357 & 185.5715 & $\mathbf{Q}$ & 1601.6867 & 801.3470 & 1584.6601 & 792.8337 & 1583.6761 & 792.3417 & 12 \\
\hline 5 & 502.1892 & 251.5982 & 485.1627 & 243.0850 & 484.1787 & 242.5930 & $\mathbf{N}$ & 1473.6281 & 737.3177 & 1456.6015 & 728.8044 & 1455.6175 & 728.3124 & 11 \\
\hline 6 & 649.2576 & 325.1325 & 632.2311 & 316.6192 & 631.2471 & 316.1272 & $\mathbf{F}$ & 1359.5852 & 680.2962 & 1342.5586 & 671.7829 & 1341.5746 & 671.2909 & 10 \\
\hline 7 & 764.2846 & 382.6459 & 747.2580 & 374.1326 & 746.2740 & 373.6406 & $\mathbf{N}$ & 1212.5168 & 606.7620 & 1195.4902 & 598.2487 & 1194.5062 & 597.7567 & 9 \\
\hline 8 & 892.3431 & 446.6752 & 875.3166 & 438.1619 & 874.3326 & 437.6699 & $\mathbf{Q}$ & 1097.4898 & 549.2485 & 1080.4633 & 540.7353 & 1079.4793 & 540.2433 & 8 \\
\hline 9 & 993.3908 & 497.1990 & 976.3643 & 488.6858 & 975.3803 & 488.1938 & $\mathbf{T}$ & 969.4312 & 485.2193 & 952.4047 & 476.7060 & 951.4207 & 476.2140 & 7 \\
\hline 10 & 1179.4701 & 590.2387 & 1162.4436 & 581.7254 & 1161.4596 & 581.2334 & $\mathbf{W}$ & 868.3836 & 434.6954 & 851.3570 & 426.1821 & 850.3730 & 425.6901 & 6 \\
\hline 11 & 1308.5127 & 654.7600 & 1291.4862 & 646.2467 & 1290.5022 & 645.7547 & $\mathbf{E}$ & 682.3042 & 341.6558 & 665.2777 & 333.1425 & 664.2937 & 332.6505 & 5 \\
\hline 12 & 1422.5557 & 711.7815 & 1405.5291 & 703.2682 & 1404.5451 & 702.7762 & $\mathbf{N}$ & 553.2617 & 277.1345 & 536.2351 & 268.6212 & 535.2511 & 268.1292 & 4 \\
\hline 13 & 1585.6190 & 793.3131 & 1568.5924 & 784.7999 & 1567.6084 & 784.3078 & $\mathbf{Y}$ & 439.2187 & 220.1130 & 422.1922 & 211.5997 & 421.2082 & 211.1077 & 3 \\
\hline 14 & 1714.6616 & 857.8344 & 1697.6350 & 849.3212 & 1696.6510 & 848.8291 & $\mathbf{E}$ & 276.1554 & 138.5813 & 259.1288 & 130.0681 & 258.1448 & 129.5761 & 2 \\
\hline 15 & & & & & & & $\mathbf{K}$ & 147.1128 & 74.0600 & 130.0863 & 65.5468 & & & 1 \\
\hline
\end{tabular}



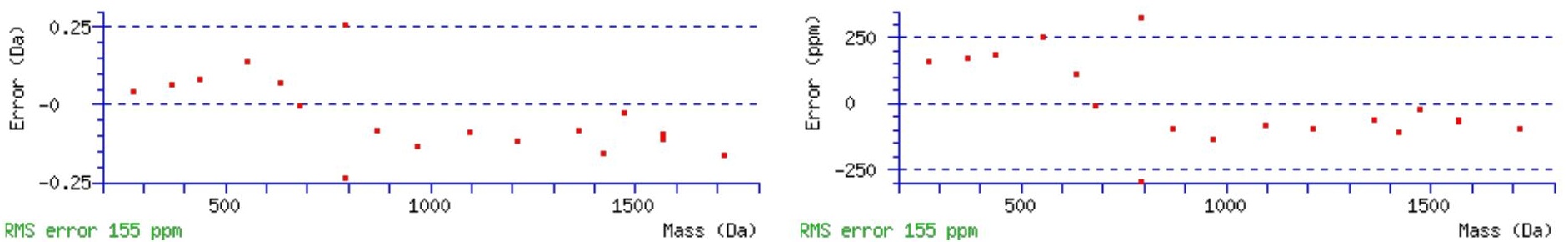

\section{All matches to this query}

\begin{tabular}{|l|l|c|l|}
\hline Score & Mr(calc): & Delta & \multicolumn{1}{|c|}{ Sequence } \\
\hline 84.5 & 1859.7598 & 0.0027 & DGSQNFNQTWENYEK \\
\hline 51.6 & 1859.7598 & 0.0027 & DGSQNFNQTWENYEK \\
\hline 43.7 & 1859.7598 & 0.0027 & DGSQNFNQTWENYEK \\
\hline 23.3 & 1858.7758 & 0.9867 & DGSQNFNQTWENYEK \\
\hline 8.0 & 1859.7484 & 0.0141 & QACFASCSGYFAXRK \\
\hline 3.7 & 1859.7808 & -0.0182 & MYQGHMQGKGSRAADK \\
\hline 2.8 & 1859.7743 & -0.0118 & GSDIFEYNSKGLLTR \\
\hline 2.3 & 1858.7498 & 1.0127 & AWENGEGGSWIWXTR \\
\hline 1.8 & 1859.7509 & 0.0116 & DSSMNNLKSYPXSHR \\
\hline 1.3 & 1859.7526 & 0.0100 & EDCNVHSAASILASVK \\
\hline
\end{tabular}

Spectrum No: 179; Query: 824; Rank: 1

\section{Peptide View}

MS/MS Fragmentation of MIENGSLSFLPTLR

Found in IPI00191090, Tax_Id=10116 Gene_Symbol=Bgn Biglycan precursor

Match to Query 824: 1577.818168 from(789.916360,2+)

Title: 100101RatKid_NS_deglyco_19.4623.4623.2.dta

Data file K:INewmanPaper|Piliangl3SubProteomes\Piliang3SP\mgf5ppm\ERLIC_3SubProteomes5ppm.mgf

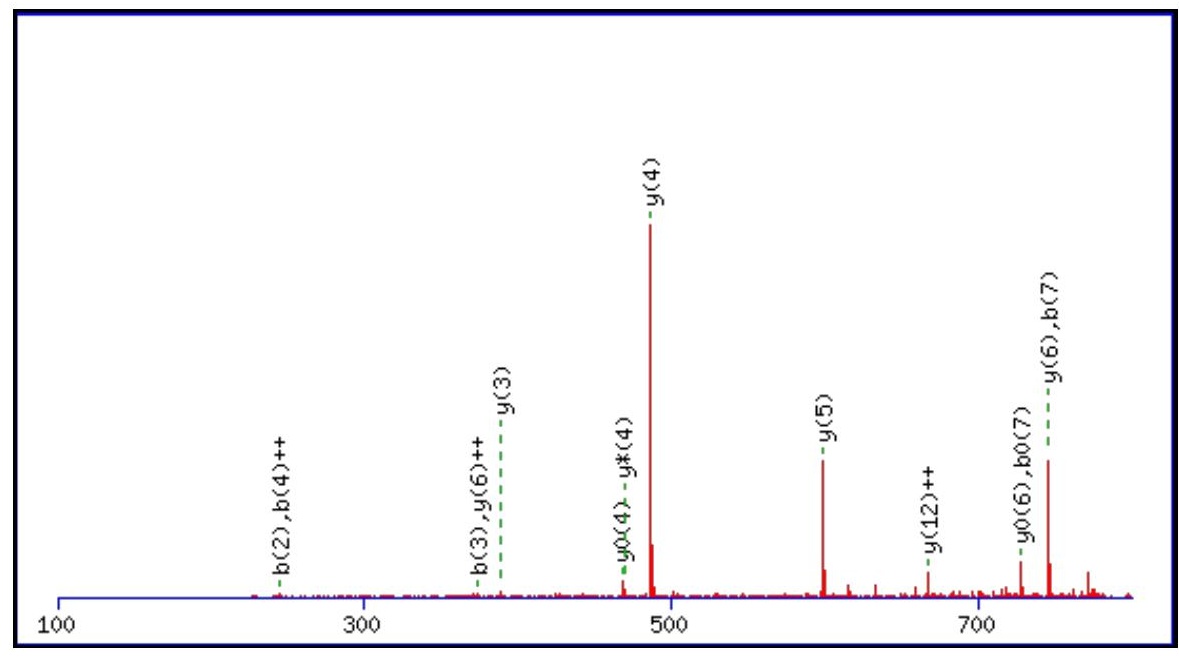



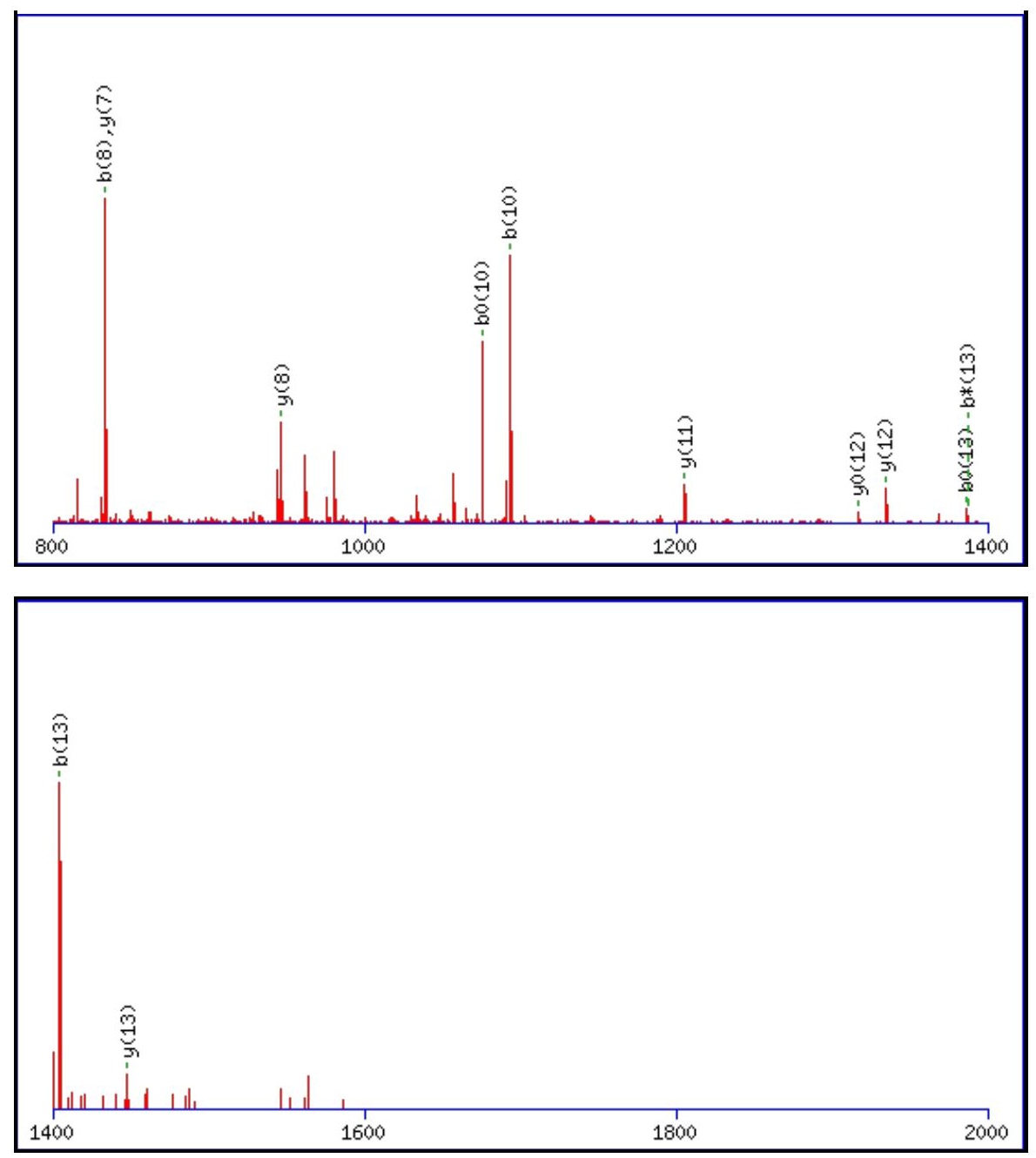

Monoisotopic mass of neutral peptide $\operatorname{Mr}($ calc): 1577.8123

Fixed modifications: Carbamidomethyl (C)

Variable modifications:

N4 : Deamidated $\mathrm{N}(\mathrm{N})$

Ions Score: 84 Expect: $5.1 \mathrm{e}-007$

Matches (Bold Red): 26/142 fragment ions using 30 most intense peaks

\begin{tabular}{|c|c|c|c|c|c|c|c|c|c|c|c|c|c|c|}
\hline \# & b & $\mathbf{b}^{++}$ & b* & $\mathbf{b}^{*^{++}}$ & $\mathbf{b}^{0}$ & $\mathbf{b}^{\mathbf{0 + +}}$ & Seq. & $\mathbf{y}$ & $y^{++}$ & $\mathbf{y}^{*}$ & $\mathrm{y}^{*^{++}}$ & $\mathbf{y}^{\mathbf{0}}$ & $\mathbf{y}^{\mathbf{0 + +}}$ & \# \\
\hline 1 & 132.0478 & 66.5275 & & & & & $\mathbf{M}$ & & & & & & & 14 \\
\hline 2 & 245.1318 & 123.0696 & & & & & I & 1447.7791 & 724.3932 & 1430.7526 & 715.8799 & 1429.7685 & 715.3879 & 13 \\
\hline 3 & 374.1744 & 187.5908 & & & 356.1639 & 178.5856 & $\mathbf{E}$ & 1334.6951 & 667.8512 & 1317.6685 & 659.3379 & 1316.6845 & 658.8459 & 12 \\
\hline 4 & 489.2014 & 245.1043 & 472.1748 & 236.5910 & 471.1908 & 236.0990 & $\mathbf{N}$ & 1205.6525 & 603.3299 & 1188.6259 & 594.8166 & 1187.6419 & 594.3246 & 11 \\
\hline 5 & 546.2228 & 273.6150 & 529.1963 & 265.1018 & 528.2123 & 264.6098 & $\mathbf{G}$ & 1090.6255 & 545.8164 & 1073.5990 & 537.3031 & 1072.6150 & 536.8111 & 10 \\
\hline 6 & 633.2548 & 317.1311 & 616.2283 & 308.6178 & 615.2443 & 308.1258 & $\mathrm{~S}$ & 1033.6041 & 517.3057 & 1016.5775 & 508.7924 & 1015.5935 & 508.3004 & 9 \\
\hline 7 & 746.3389 & 373.6731 & 729.3124 & 365.1598 & 728.3283 & 364.6678 & $\mathbf{L}$ & 946.5720 & 473.7897 & 929.5455 & 465.2764 & 928.5615 & 464.7844 & 8 \\
\hline 8 & 833.3709 & 417.1891 & 816.3444 & 408.6758 & 815.3604 & 408.1838 & S & 833.4880 & 417.2476 & 816.4614 & 408.7343 & 815.4774 & 408.2423 & 7 \\
\hline 9 & 980.4394 & 490.7233 & 963.4128 & 482.2100 & 962.4288 & 481.7180 & $\mathbf{F}$ & 746.4559 & 373.7316 & 729.4294 & 365.2183 & 728.4454 & 364.7263 & 6 \\
\hline 10 & 1093.5234 & 547.2653 & 1076.4969 & 538.7521 & 1075.5129 & 538.2601 & $\mathbf{L}$ & 599.3875 & 300.1974 & 582.3610 & 291.6841 & 581.3770 & 291.1921 & 5 \\
\hline 11 & 1190.5762 & 595.7917 & 1173.5496 & 587.2785 & 1172.5656 & 586.7864 & $\mathbf{P}$ & 486.3035 & 243.6554 & 469.2769 & 235.1421 & 468.2929 & 234.6501 & 4 \\
\hline 12 & 1291.6239 & 646.3156 & 1274.5973 & 637.8023 & 1273.6133 & 637.3103 & $\mathbf{T}$ & 389.2507 & 195.1290 & 372.2241 & 186.6157 & 371.2401 & 186.1237 & 3 \\
\hline 13 & 1404.7079 & 702.8576 & 1387.6814 & 694.3443 & 1386.6974 & 693.8523 & $\mathbf{L}$ & 288.2030 & 144.6051 & 271.1765 & 136.0919 & & & 2 \\
\hline 14 & & & & & & & $\mathbf{R}$ & 175.1190 & 88.0631 & 158.0924 & 79.5498 & & & 1 \\
\hline
\end{tabular}



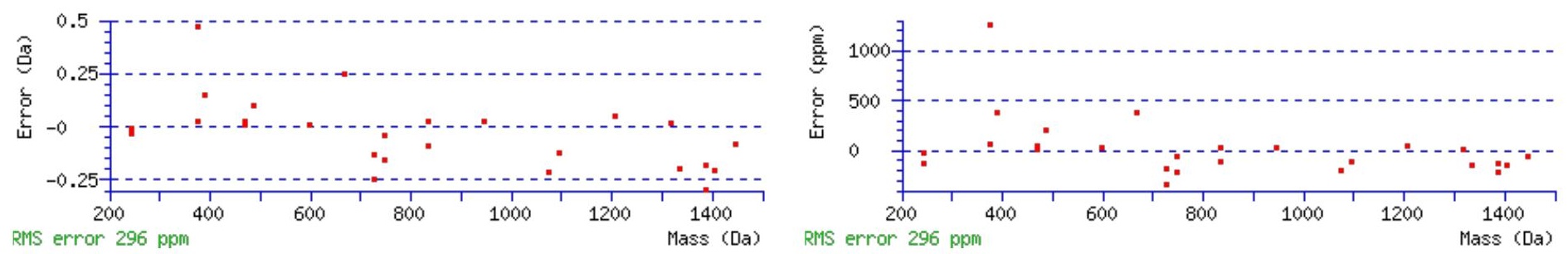

\section{All matches to this query}

\begin{tabular}{|l|l|l|l|}
\hline Score & Mr(calc): & Delta & \multicolumn{1}{|c|}{ Sequence } \\
\hline 84.3 & 1577.8123 & 0.0059 & MIENGSLSFLPTLR \\
\hline 33.0 & 1576.8283 & 0.9899 & MIENGSLSFLPTLR \\
\hline 8.6 & 1577.8161 & 0.0021 & IEELNRYRDTLR \\
\hline 7.8 & 1577.8309 & -0.0128 & FVPCISKMINLQK \\
\hline 7.3 & 1577.8300 & -0.0118 & KNEYLLSLLAEER \\
\hline 5.1 & 1577.8178 & 0.0004 & VKVDSELQLIQAR \\
\hline 4.5 & 1577.8039 & 0.0143 & ATNSIIKGPAQSRR \\
\hline 4.4 & 1577.8195 & -0.0013 & MLKAESSGERTTLR \\
\hline 3.2 & 1575.8178 & 2.0004 & MLLDNTALLGLGSNK \\
\hline 3.1 & 1577.8202 & -0.0020 & VGNLTPHLHNIFSK \\
\hline
\end{tabular}

Spectrum No: 180; Query: 2275; Rank: 1

\section{Peptide View}

MS/MS Fragmentation of NGSYCVLQMDQSYKPDENEVR

Found in IPI00393333, Tax_Id=10116 Gene_Symbol=Atic Bifunctional purine biosynthesis protein PURH

Match to Query 2275: 2532.090012 from(845.037280,3+)

Title: 091008RatKidney_NoSalt_18.2538.2538.3.dta

Data file K:INewmanPaper|Piliang\3SubProteomes\Piliang3SP\mgf5ppm\ERLIC_3SubProteomes5ppm.mgf

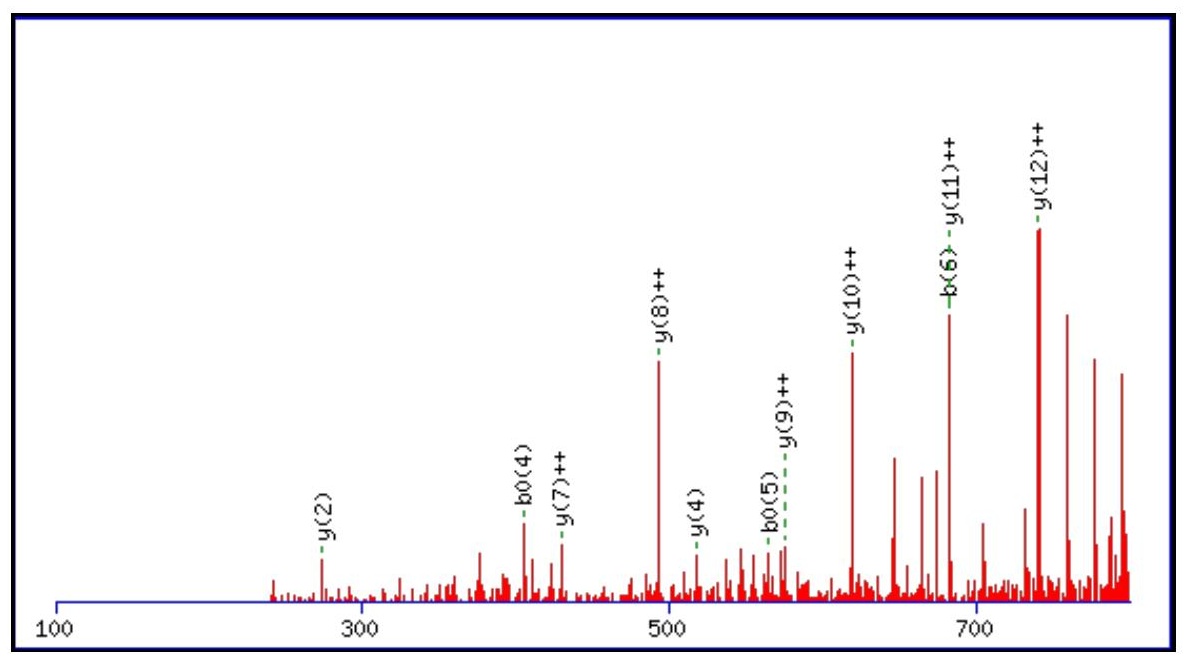



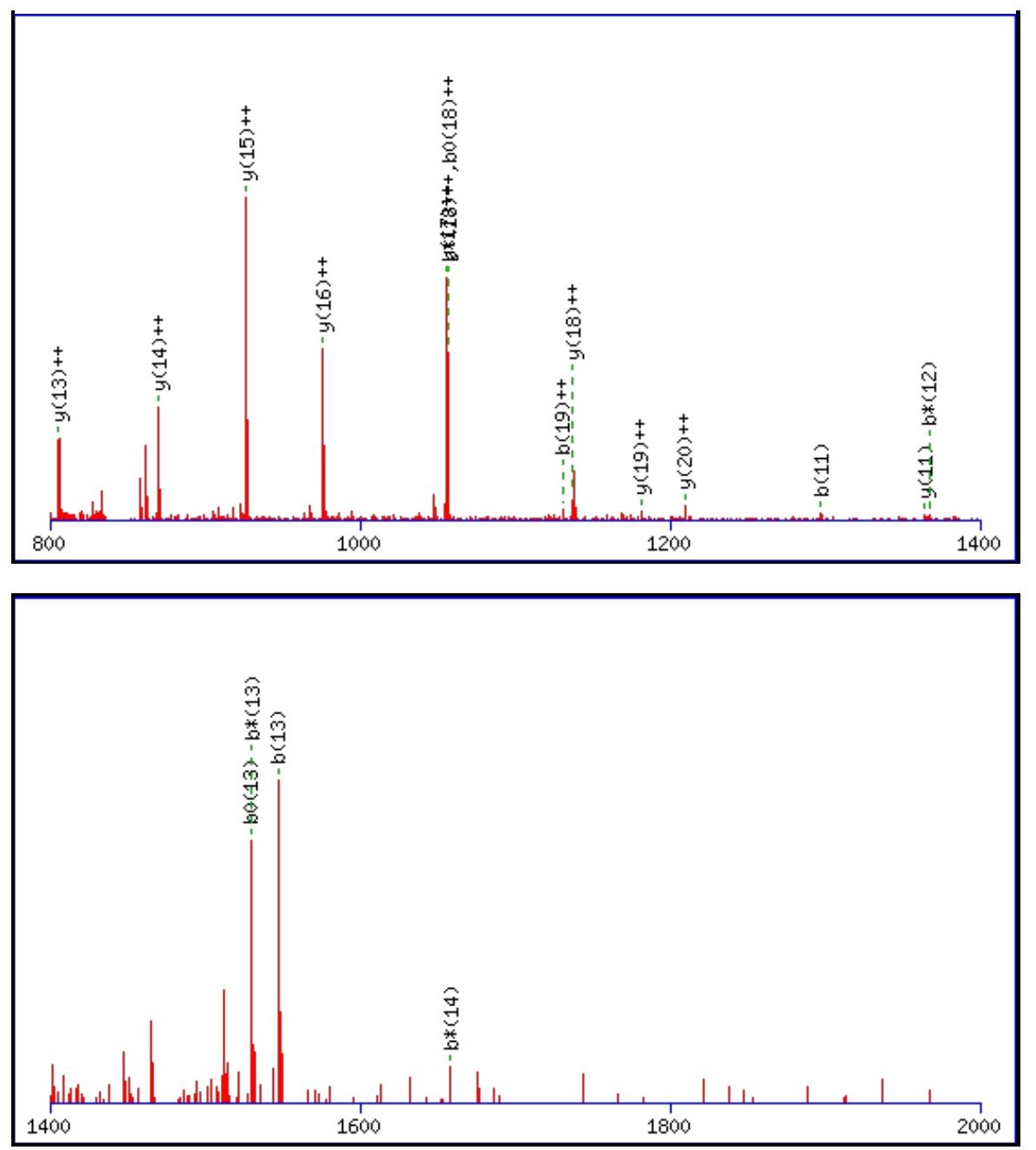

Monoisotopic mass of neutral peptide $\operatorname{Mr}($ calc): 2532.0897

Fixed modifications: Carbamidomethyl (C)

Variable modifications:

N1 : Deamidated $\mathrm{N}(\mathrm{N})$

Ions Score: 84 Expect: $1.3 \mathrm{e}-006$

Matches (Bold Red): 29/232 fragment ions using 52 most intense peaks

\begin{tabular}{|c|c|c|c|c|c|c|c|c|c|c|c|c|c|c|}
\hline \# & b & $\mathbf{b}^{++}$ & b* & $\mathbf{b}^{*^{++}}$ & $\mathbf{b}^{\mathbf{0}}$ & & Seq. & $\mathbf{y}$ & $\mathbf{y}^{++}$ & $\mathbf{y}^{*}$ & $\mathbf{y}^{*^{++}}$ & $y^{0}$ & $\mathbf{y}^{0++}$ & \# \\
\hline 1 & 116.0342 & 58.5207 & 99.0077 & 50.0075 & & & $\mathbf{N}$ & & & & & & & 21 \\
\hline 2 & 173.0557 & 87.0315 & 156.0291 & 78.5182 & & & G & 2418.0700 & 1209.5387 & 2401.0435 & 1201.0254 & 2400.0595 & 1200.5334 & 20 \\
\hline 3 & 260.0877 & 130.5475 & 243.0612 & 122.0342 & 242.0771 & 121.5422 & $\mathrm{~S}$ & 2361.0486 & 1181.0279 & 2344.0220 & 1172.5147 & 2343.0380 & 1172.0226 & 19 \\
\hline 4 & 423.1510 & 212.0792 & 406.1245 & 203.5659 & 405.1405 & 203.0739 & $\mathbf{Y}$ & 2274.0165 & 1137.5119 & 2256.9900 & 1128.9986 & 2256.0060 & 1128.5066 & 18 \\
\hline 5 & 583.1817 & 292.0945 & 566.1551 & 283.5812 & 565.1711 & 283.0892 & C & 2110.9532 & 1055.9802 & 2093.9267 & 1047.4670 & 2092.9427 & 1046.9750 & 17 \\
\hline 6 & 682.2501 & 341.6287 & 665.2235 & 333.1154 & 664.2395 & 332.6234 & V & 1950.9226 & 975.9649 & 1933.8960 & 967.4516 & 1932.9120 & 966.9596 & 16 \\
\hline 7 & 795.3342 & 398.1707 & 778.3076 & 389.6574 & 777.3236 & 389.1654 & $\mathbf{L}$ & 1851.8542 & 926.4307 & 1834.8276 & 917.9174 & 1833.8436 & 917.4254 & 15 \\
\hline 8 & 923.3927 & 462.2000 & 906.3662 & 453.6867 & 905.3822 & 453.1947 & $\mathbf{Q}$ & 1738.7701 & 869.8887 & 1721.7435 & 861.3754 & 1720.7595 & 860.8834 & 14 \\
\hline 9 & 1054.4332 & 527.7202 & 1037.4067 & 519.2070 & 1036.4227 & 518.7150 & $\mathbf{M}$ & 1610.7115 & 805.8594 & 1593.6850 & 797.3461 & 1592.7009 & 796.8541 & 13 \\
\hline 10 & 1169.4602 & 585.2337 & 1152.4336 & 576.7204 & 1151.4496 & 576.2284 & D & 1479.6710 & 740.3392 & 1462.6445 & 731.8259 & 1461.6605 & 731.3339 & 12 \\
\hline 11 & 1297.5187 & 649.2630 & 1280.4922 & 640.7497 & 1279.5082 & 640.2577 & $\mathbf{Q}$ & 1364.6441 & 682.8257 & 1347.6175 & 674.3124 & 1346.6335 & 673.8204 & 11 \\
\hline 12 & 1384.5508 & 692.7790 & 1367.5242 & 684.2657 & 1366.5402 & 683.7737 & $S$ & 1236.5855 & 618.7964 & 1219.5590 & 610.2831 & 1218.5749 & 609.7911 & 10 \\
\hline 13 & 1547.6141 & 774.3107 & 1530.5876 & 765.7974 & 1529.6035 & 765.3054 & $\bar{Y}$ & 1149.5535 & 575.2804 & 1132.5269 & 566.7671 & 1131.5429 & 566.2751 & 9 \\
\hline 14 & 1675.7091 & 838.3582 & 1658.6825 & 829.8449 & 1657.6985 & 829.3529 & $\mathbf{K}$ & 986.4901 & 493.7487 & 969.4636 & 485.2354 & 968.4796 & 484.7434 & 8 \\
\hline 15 & 1772.7618 & 886.8846 & 1755.7353 & 878.3713 & 1754.7513 & 877.8793 & $\mathbf{P}$ & 858.3952 & 429.7012 & 841.3686 & 421.1880 & 840.3846 & 420.6959 & 7 \\
\hline 16 & 1887.7888 & 944.3980 & 1870.7622 & 935.8847 & 1869.7782 & 935.3927 & D & 761.3424 & 381.1748 & 744.3159 & 372.6616 & 743.3319 & 372.1696 & 6 \\
\hline 17 & 2016.8314 & 1008.9193 & 1999.8048 & 1000.4060 & 1998.8208 & 999.9140 & $\mathbf{E}$ & 646.3155 & 323.6614 & 629.2889 & 315.1481 & 628.3049 & 314.6561 & 5 \\
\hline 18 & 2130.8743 & 1065.9408 & 2113.8477 & 1057.4275 & 2112.8637 & 1056.9355 & $\mathbf{N}$ & 517.2729 & 259.1401 & 500.2463 & 250.6268 & 499.2623 & 250.1348 & 4 \\
\hline
\end{tabular}




\begin{tabular}{|r|r|r|r|r|r|r|r|r|r|r|r|r|r|r|r|r|r|r|r|}
$\mathbf{1 9}$ & 2259.9169 & $\mathbf{1 1 3 0 . 4 6 2 1}$ & 2242.8903 & 1121.9488 & 2241.9063 & 1121.4568 & $\mathbf{E}$ & 403.2300 & 202.1186 & 386.2034 & 193.6053 & 385.2194 & 193.1133 & $\mathbf{3}$ \\
\hline $\mathbf{2 0}$ & 2358.9853 & 1179.9963 & 2341.9587 & 1171.4830 & 2340.9747 & 1170.9910 & $\mathbf{V}$ & $\mathbf{2 7 4 . 1 8 7 4}$ & 137.5973 & 257.1608 & 129.0840 & \\
\hline $\mathbf{2 1}$ & & & & & & & $\mathbf{R}$ & 175.1190 & 88.0631 & 158.0924 & $\mathbf{7 9 . 5 4 9 8}$ & & \\
\hline
\end{tabular}
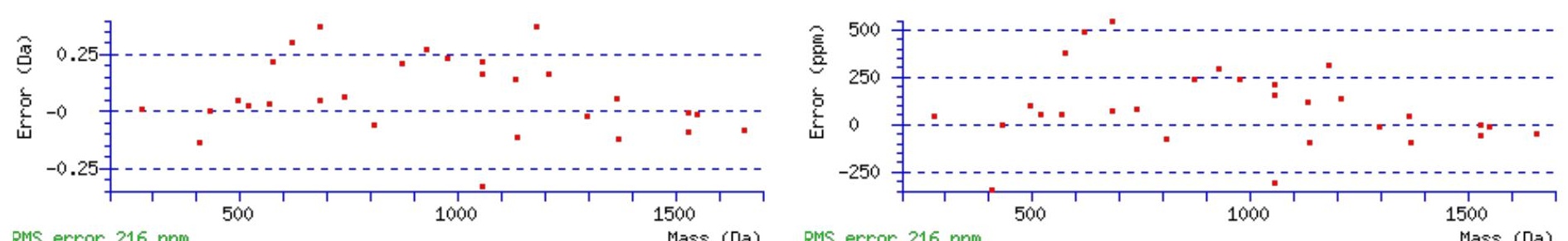

RMS error $216 \mathrm{ppm}$

\section{All matches to this query}

\begin{tabular}{|l|l|l|l|}
\hline Score & Mr(calc): & Delta & \multicolumn{1}{|c|}{ Sequence } \\
\hline 84.3 & 2532.0897 & 0.0003 & NGSYCVLQMDQSYKPDENEVR \\
\hline 66.0 & 2532.0897 & 0.0003 & NGSYCVLQMDQSYKPDENEVR \\
\hline 62.3 & 2531.1057 & 0.9843 & NGSYCVLQMDQSYKPDENEVR \\
\hline 1.6 & 2531.0934 & 0.9966 & MANRGATRPNGPNTGNKICOFK \\
\hline 1.5 & 2532.0700 & 0.0200 & VSHVCSQTNGSSAQQASAPPAPDR \\
\hline 1.5 & 2532.0700 & 0.0200 & VSHVCSOTNGSSAQQASAPPAPDR \\
\hline 1.1 & 2532.0951 & -0.0051 & LFNSLLLGPTASNSKTEGSSLR \\
\hline 0.9 & 2532.1047 & -0.0146 & LNQAAISQAFSNALHSLDGATSR \\
\hline 0.7 & 2531.0860 & 1.0040 & VSHVCSQTNGSSAQQASAPPAPDR \\
\hline 0.5 & 2531.0860 & 1.0040 & VSHVCSQTNGSSAQQASAPPAPDR \\
\hline
\end{tabular}

Spectrum No: 181; Query: 1903; Rank: 1

\section{Peptide View}

MS/MS Fragmentation of TNSSFVQGLVDHVKEDCDR

Found in IPI00195160, Tax_Id=10116 Gene_Symbol=Psap Sulfated glycoprotein 1 precursor

Match to Query 1903: 2205.996428 from(1104.005490,2+)

Title: 091008RatKidney_NH4Format02_24.3964.3964.2.dta

Data file K:INewmanPaper|Piliang|3SubProteomes\Piliang3SP\mgf5ppm\ERLIC_3SubProteomes5ppm.mgf

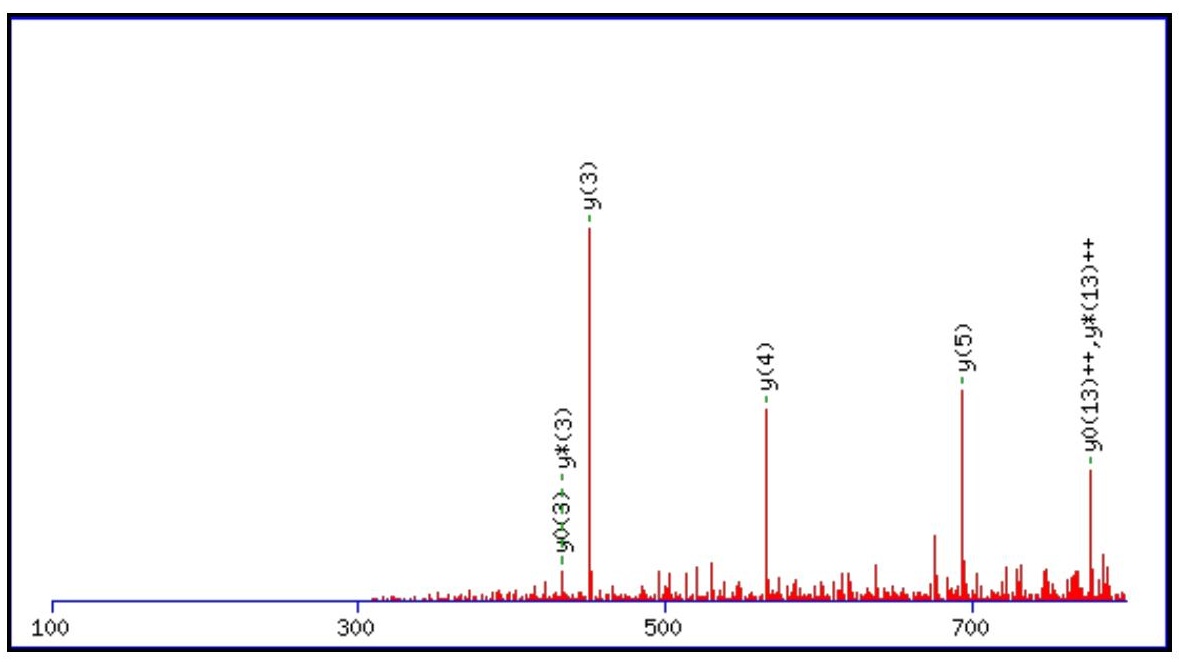



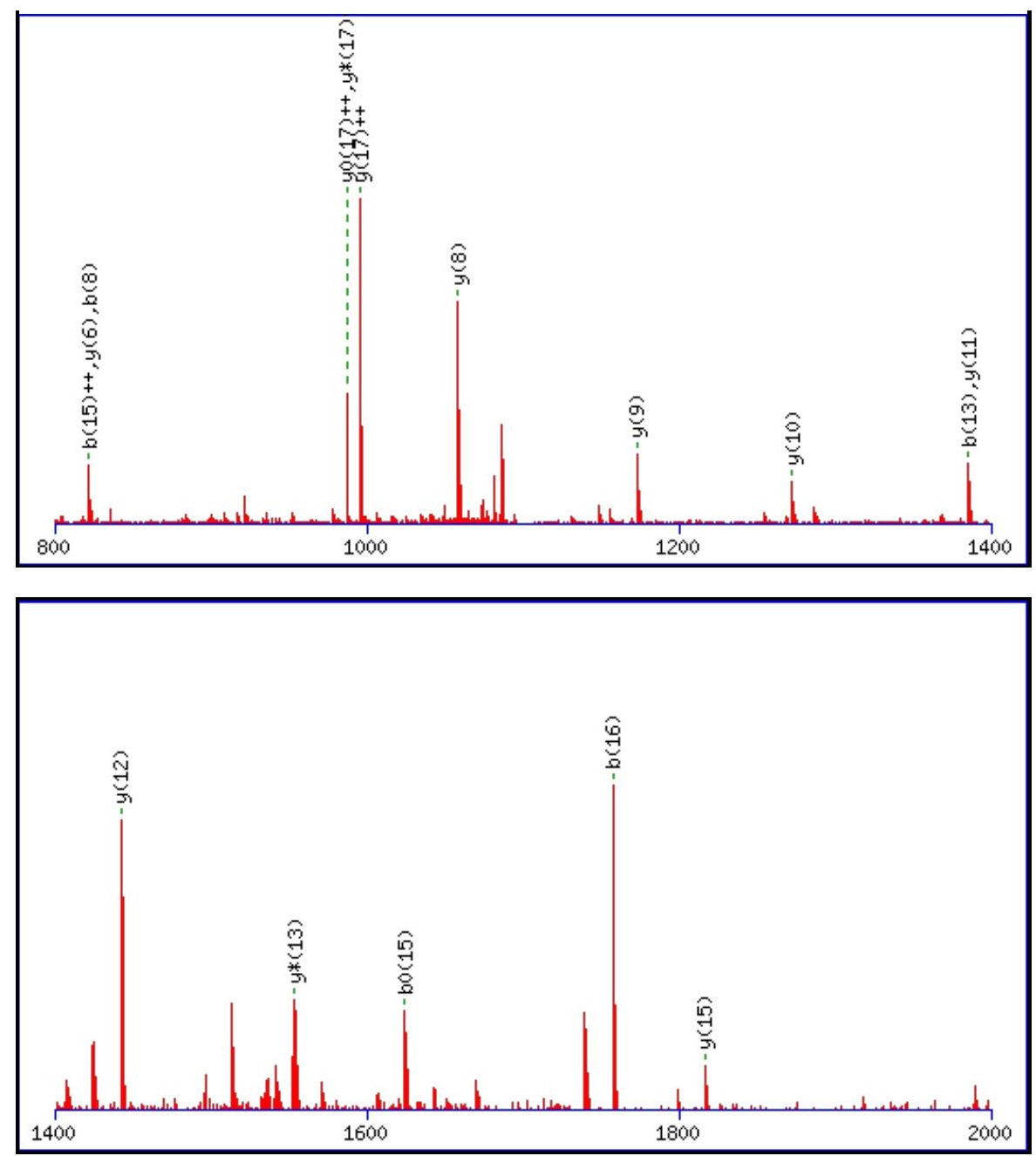

Monoisotopic mass of neutral peptide $\operatorname{Mr}($ calc): 2205.9961

Fixed modifications: Carbamidomethyl (C)

Variable modifications:

N2 : Deamidated_N (N)

Ions Score: 84 Expect: $1 \mathrm{e}-006$

Matches (Bold Red): 23/212 fragment ions using 30 most intense peaks

\begin{tabular}{|c|c|c|c|c|c|c|c|c|c|c|c|c|c|c|}
\hline \# & b & $\mathbf{b}^{++}$ & b* & $\mathbf{b}^{*+}$ & $\mathbf{b}^{0}$ & $\mathbf{b}^{0++}$ & Seq. & $\mathbf{y}$ & $\mathbf{y}^{++}$ & $\mathbf{y}^{*}$ & $\mathrm{y}^{\mathrm{*}^{++}}$ & $\mathbf{y}^{0}$ & $\mathbf{y}^{0++}$ & \# \\
\hline 1 & 102.0550 & 51.5311 & & & 84.0444 & 42.5258 & $\mathbf{T}$ & & & & & & & 19 \\
\hline 2 & 217.0819 & 109.0446 & 200.0553 & & 199.0713 & 100.0393 & $\mathbf{N}$ & & 1053.4815 & 2088.9291 & & 2087.9451 & 1044.4762 & 18 \\
\hline 3 & 304.1139 & 152.5606 & 287.0874 & 144.0473 & 286.1034 & 143.5553 & $\mathrm{~S}$ & 1990.9287 & 995.9680 & 1973.9022 & 987.4547 & 1972.9182 & 986.9627 & 17 \\
\hline 4 & 391.1459 & 196.0766 & 374.1194 & 187.5633 & 373.1354 & 187.0713 & $\mathbf{S}$ & 1903.8967 & 952.4520 & 1886.8701 & 943.9387 & 1885.8861 & 943.4467 & 16 \\
\hline 5 & 538.2144 & 269.6108 & 521.1878 & 261.0975 & 520.2038 & 260.6055 & $\mathbf{F}$ & 1816.8647 & 908.9360 & 1799.8381 & 4227 & 1798.8541 & 899.9307 & 15 \\
\hline 6 & 637.2828 & 319.1450 & 620.2562 & 310.6318 & 619.2722 & 310.1397 & $\mathbf{V}$ & 1669.7962 & 835.4018 & 1652.7697 & 826.8885 & 1651.7857 & 826.3965 & 14 \\
\hline 7 & 765.3414 & 383.1743 & 748.3148 & 374.6610 & 747.3308 & 374.1690 & $\mathbf{Q}$ & 1570.7278 & 785.8676 & 1553.7013 & 777.3543 & 1552.7173 & 776.8623 & 13 \\
\hline 8 & 822.3628 & 411.6850 & 805.3363 & 403.1718 & 804.3523 & 402.6798 & $\mathbf{G}$ & 1442.6693 & 721.8383 & 1425.6427 & 713.3250 & 1424.6587 & 712.8330 & 12 \\
\hline 9 & 935.4469 & 468.2271 & 918.4203 & 459.7138 & 917.4363 & 459.2218 & $\mathbf{L}$ & 1385.6478 & 693.3275 & 1368.6212 & 684.8143 & 1367.6372 & 684.3223 & 11 \\
\hline 10 & 1034.5153 & 517.7613 & 1017.4887 & 509.2480 & 1016.5047 & 508.7560 & $\mathbf{V}$ & 1272.5637 & 636.7855 & 1255.5372 & 628.2722 & 1254.5532 & 627.7802 & 10 \\
\hline 11 & 1149.5422 & 575.2748 & 1132.5157 & 566.7615 & 1131.5317 & 566.2695 & D & 1173.4953 & 587.2513 & 1156.4688 & 578.7380 & 1155.4847 & 578.2460 & 9 \\
\hline 12 & 1286.6012 & 643.8042 & 1269.5746 & 635.2909 & 1268.5906 & 634.7989 & H & 1058.4684 & 529.7378 & 1041.4418 & 521.2245 & 1040.4578 & 520.7325 & 8 \\
\hline 13 & 1385.6696 & 693.3384 & 1368.6430 & 684.8251 & 1367.6590 & 684.3331 & $\mathbf{V}$ & 921.4095 & 461.2084 & 904.3829 & 452.6951 & 903.3989 & 452.2031 & 7 \\
\hline 14 & 1513.7645 & 757.3859 & 1496.7380 & 748.8726 & 1495.7540 & 748.3806 & $\mathbf{K}$ & 822.3410 & 411.6742 & 805.3145 & 403.1609 & 804.3305 & 402.6689 & 6 \\
\hline 15 & 1642.8071 & 821.9072 & 1625.7806 & 813.3939 & 1624.7966 & 812.9019 & $\mathbf{E}$ & 694.2461 & 347.6267 & 677.2195 & 339.1134 & 676.2355 & 338.6214 & 5 \\
\hline 16 & 1757.8341 & 879.4207 & 1740.8075 & 870.9074 & 1739.8235 & 870.4154 & D & 565.2035 & 283.1054 & 548.1769 & 274.5921 & 547.1929 & 274.1001 & 4 \\
\hline 17 & 1917.8647 & 959.4360 & 1900.8382 & 950.9227 & 1899.8541 & 950.4307 & C & 450.1765 & 225.5919 & 433.1500 & 217.0786 & 432.1660 & 216.5866 & 3 \\
\hline 18 & 2032.8917 & 1016.9495 & 2015.8651 & 1008.4362 & 2014.8811 & 1007.9442 & D & 290.1459 & 145.5766 & 273.1193 & 137.0633 & 272.1353 & 136.5713 & 2 \\
\hline
\end{tabular}




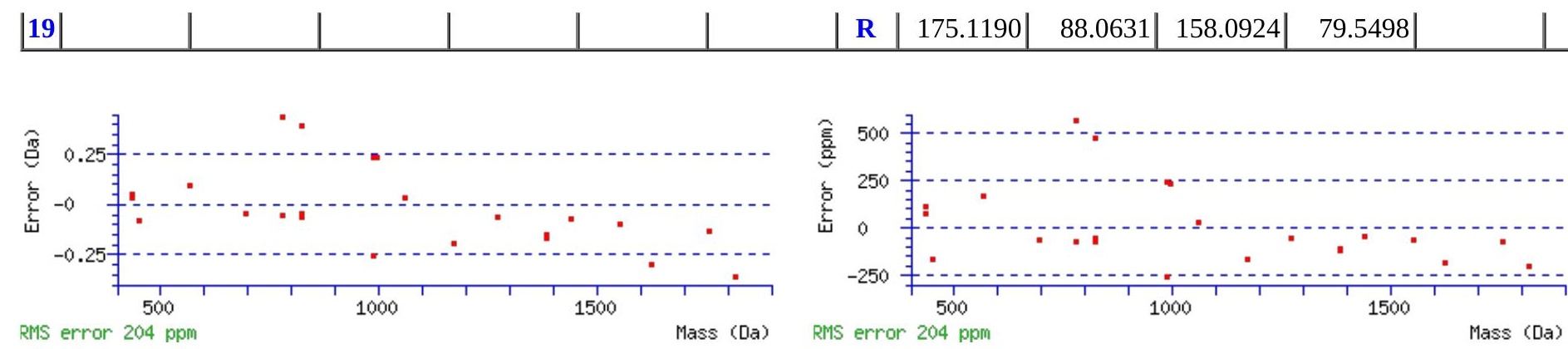

\section{All matches to this query}

\begin{tabular}{|l|l|l|l|}
\hline Score & Mr(calc): & Delta & \multicolumn{1}{|c|}{ Sequence } \\
\hline 84.2 & 2205.9961 & 0.0004 & TNSSFVQGLVDHVKEDCDR \\
\hline 75.9 & 2205.0121 & 0.9844 & TNSSFVQGLVDHVKEDCDR \\
\hline 4.7 & 2203.9816 & 2.0148 & TFKAWEGLHENSVRLTR \\
\hline 3.8 & 2203.9763 & 2.0201 & LENLTDTTVAQKAGGPSSVR \\
\hline 2.6 & 2204.9966 & 0.9999 & EYPQPMGQGVPSLEFRYK \\
\hline 0.9 & 2204.9724 & 1.0240 & TVAMPVRVVSVEENCTVR \\
\hline 0.3 & 2205.9933 & 0.0031 & IASSVQQRVRVHSSDSYK \\
\hline 0.3 & 2205.9933 & 0.0031 & IASSVQQRVRVHSSDSYK \\
\hline 0.3 & 2203.9884 & 2.0080 & LSAITMPSVARHTMQTIR \\
\hline 0.2 & 2204.9724 & 1.0240 & TVAMPVRVVSVEENCTVR \\
\hline
\end{tabular}

Spectrum No: 182; Query: 211; Rank: 1

\section{Peptide View}

MS/MS Fragmentation of ISSNETEIMR

Found in IPI00370427, Tax_Id=10116 Gene_Symbol=Tinag Tubulointerstitial nephritis antigen

Match to Query 211: 1195.535988 from(598.775270,2+)

Title: 091008RatKidney_NH4Format01_24.609.609.2.dta

Data file K:INewmanPaper|Piliangl3SubProteomes\Piliang3SP\mgf5ppm\ERLIC_3SubProteomes5ppm.mgf

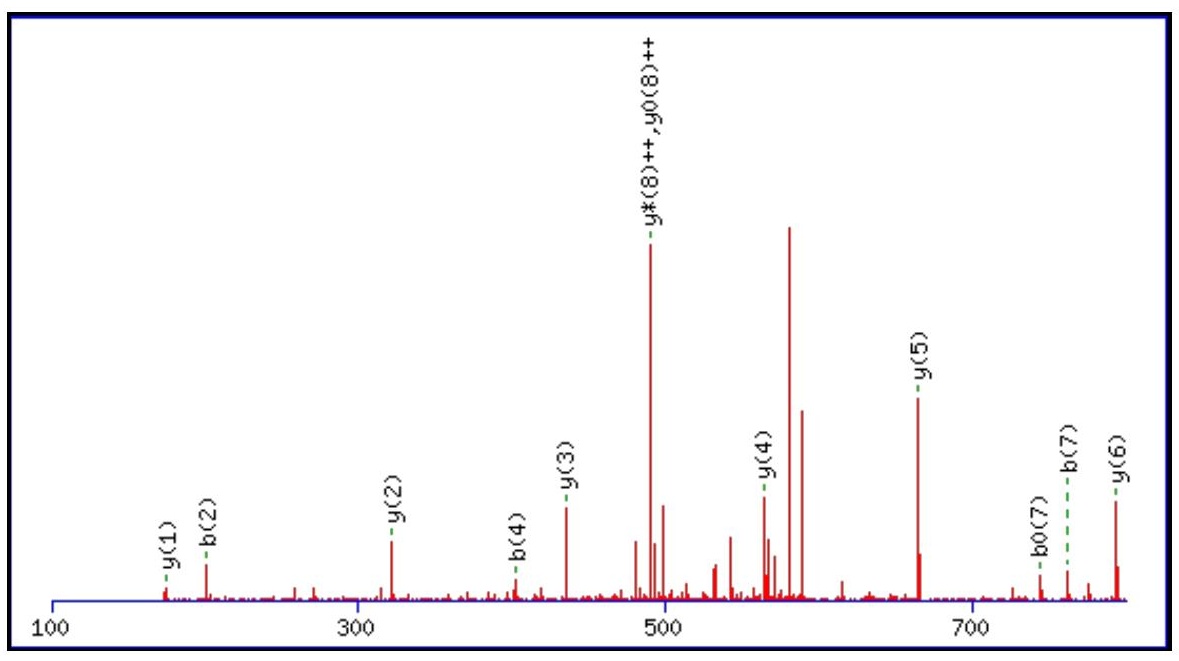



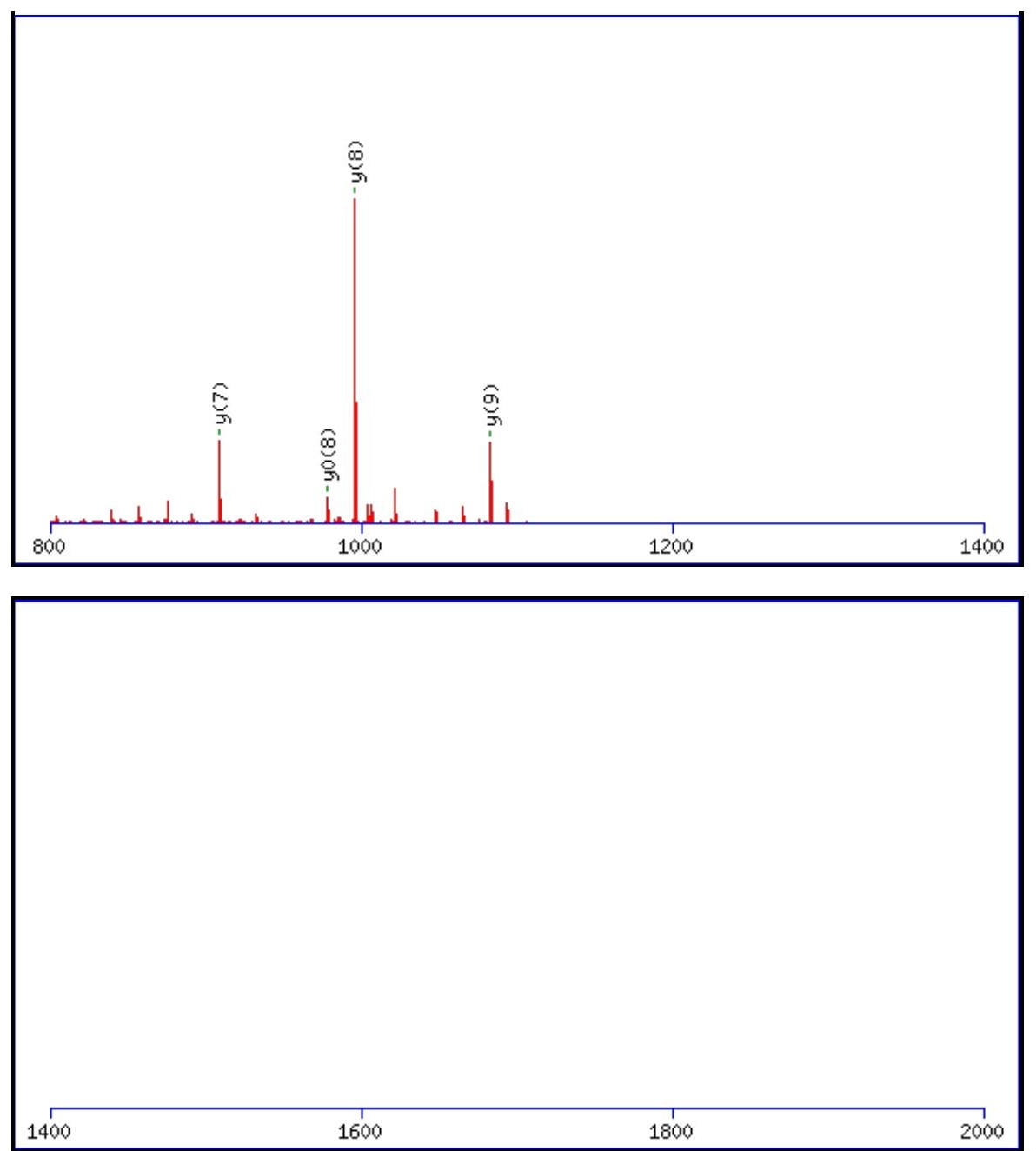

Monoisotopic mass of neutral peptide $\operatorname{Mr}($ calc): 1195.5390

Fixed modifications: Carbamidomethyl (C)

Variable modifications:

N4 : Deamidated_N (N)

M9 : Oxidation (M)

Ions Score: 84 Expect : $4.4 \mathrm{e}-007$

Matches (Bold Red): 16/94 fragment ions using 22 most intense peaks

\begin{tabular}{|r|c|c|c|c|c|c|c|c|c|c|c|c|c|c|}
\hline$\#$ & $\mathbf{b}$ & $\mathbf{b}^{++}$ & $\mathbf{b}^{*}$ & $\mathbf{b}^{\mathbf{*}^{++}}$ & $\mathbf{b}^{\mathbf{0}}$ & $\mathbf{b}^{\mathbf{0 + +}}$ & $\mathbf{S e q}$ & $\mathbf{y}$ & $\mathbf{y}^{++}$ & $\mathbf{y}^{\mathbf{*}}$ & $\mathbf{y}^{\boldsymbol{*}^{++}}$ & $\mathbf{y}^{\mathbf{0}}$ & $\mathbf{y}^{\mathbf{0 + +}}$ & $\#$ \\
\hline $\mathbf{1}$ & 114.0913 & 57.5493 & & & & & $\mathbf{I}$ & & & & & & & $\mathbf{1 0}$ \\
\hline $\mathbf{2}$ & $\mathbf{2 0 1 . 1 2 3 4}$ & 101.0653 & & & 183.1128 & 92.0600 & $\mathbf{S}$ & $\mathbf{1 0 8 3 . 4 6 2 3}$ & 542.2348 & 1066.4357 & 533.7215 & 1065.4517 & 533.2295 & $\mathbf{9}$ \\
\hline $\mathbf{3}$ & 288.1554 & 144.5813 & & & 270.1448 & 135.5761 & $\mathbf{S}$ & $\mathbf{9 9 6 . 4 3 0 2}$ & 498.7188 & 979.4037 & $\mathbf{4 9 0 . 2 0 5 5}$ & $\mathbf{9 7 8 . 4 1 9 7}$ & $\mathbf{4 8 9 . 7 1 3 5}$ & $\mathbf{8}$ \\
\hline $\mathbf{4}$ & $\mathbf{4 0 3 . 1 8 2 3}$ & 202.0948 & 386.1558 & 193.5815 & 385.1718 & 193.0895 & $\mathbf{N}$ & $\mathbf{9 0 9 . 3 9 8 2}$ & 455.2027 & 892.3717 & 446.6895 & 891.3877 & 446.1975 & $\mathbf{7}$ \\
\hline $\mathbf{5}$ & 532.2249 & 266.6161 & 515.1984 & 258.1028 & 514.2144 & 257.6108 & $\mathbf{E}$ & $\mathbf{7 9 4 . 3 7 1 3}$ & 397.6893 & 777.3447 & 389.1760 & 776.3607 & 388.6840 & $\mathbf{6}$ \\
\hline $\mathbf{6}$ & 633.2726 & 317.1399 & 616.2461 & 308.6267 & 615.2620 & 308.1347 & $\mathbf{T}$ & $\mathbf{6 6 5 . 3 2 8 7}$ & 333.1680 & 648.3021 & 324.6547 & 647.3181 & 324.1627 & $\mathbf{5}$ \\
\hline $\mathbf{7}$ & $\mathbf{7 6 2 . 3 1 5 2}$ & 381.6612 & 745.2886 & 373.1480 & 744.3046 & 372.6560 & $\mathbf{E}$ & $\mathbf{5 6 4 . 2 8 1 0}$ & 282.6441 & 547.2545 & 274.1309 & 546.2704 & 273.6389 & $\mathbf{4}$ \\
\hline $\mathbf{8}$ & $\mathbf{8 7 5 . 3 9 9 3}$ & 438.2033 & 858.3727 & 429.6900 & 857.3887 & 429.1980 & $\mathbf{I}$ & $\mathbf{4 3 5 . 2 3 8 4}$ & 218.1228 & 418.2119 & 209.6096 & & & $\mathbf{3}$ \\
\hline $\mathbf{9}$ & 1022.4347 & 511.7210 & 1005.4081 & 503.2077 & 1004.4241 & 502.7157 & $\mathbf{M}$ & $\mathbf{3 2 2 . 1 5 4 4}$ & 161.5808 & 305.1278 & 153.0675 & & & $\mathbf{2}$ \\
\hline $\mathbf{1 0}$ & & & & & & & $\mathbf{R}$ & $\mathbf{1 7 5 . 1 1 9 0}$ & 88.0631 & 158.0924 & 79.5498 & & & $\mathbf{1}$ \\
\hline
\end{tabular}



誉

RMS error $278 \mathrm{ppm}$

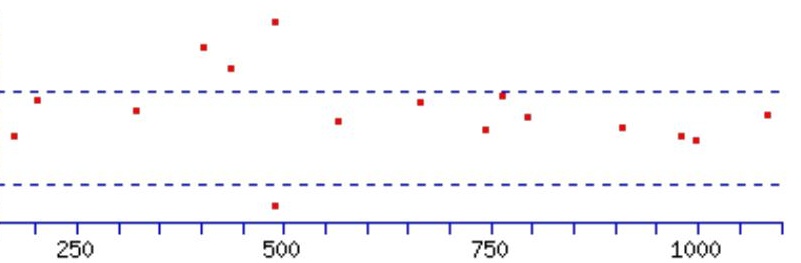

Mass (Da)

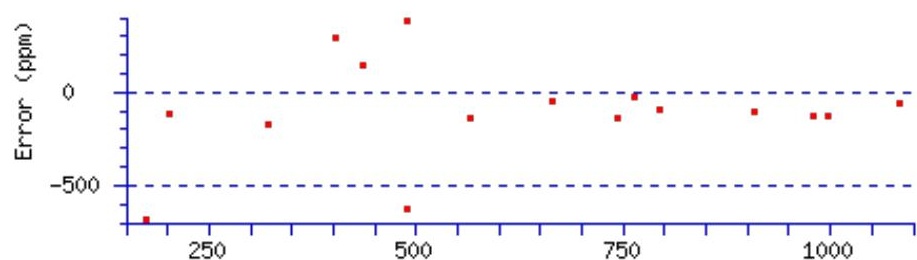

RMS error 278 ppm 


\section{All matches to this query}

\begin{tabular}{|l|l|l|l|}
\hline Score & Mr(calc): & Delta & \multicolumn{1}{|c|}{ Sequence } \\
\hline 84.1 & 1195.5390 & -0.0030 & $\underline{\text { SSNETEIMR }}$ \\
\hline 12.0 & 1195.5357 & 0.0003 & $\underline{\text { INSEFNSDLR }}$ \\
\hline 11.3 & 1194.5282 & 1.0078 & QASPTEVVER \\
\hline 10.4 & 1195.5292 & 0.0068 & EGTFPMTQNR \\
\hline 9.7 & 1195.5347 & 0.0013 & DGSAGLGRDLR \\
\hline 9.4 & 1195.5365 & -0.0006 & MGSEMEPLFR \\
\hline 9.2 & 1195.5258 & 0.0102 & $\underline{\text { SWYDNENR }}$ \\
\hline 8.4 & 1195.5258 & 0.0102 & $\underline{\text { SSFGLHNDYR }}$ \\
\hline 5.8 & 1195.5387 & -0.0027 & TGTVAFHDIR \\
\hline 5.5 & 1195.5478 & -0.0118 & $\underline{\text { SLWCGMGTIR }}$ \\
\hline
\end{tabular}

Spectrum No: 183; Query: 464; Rank: 1

\section{Peptide View}

MS/MS Fragmentation of CNVSEGVAQCTR

Found in IPI00231767, Tax_Id=10116 Gene_Symbol=Tpi1 Triosephosphate isomerase

Match to Query 464: 1380.580568 from(691.297560,2+)

Title: 100101RatKid_NS_deglyco_12.862.862.2.dta

Data file K:INewmanPaper|Piliangl3SubProteomes\Piliang3SP\mgf5ppm\ERLIC_3SubProteomes5ppm.mgf
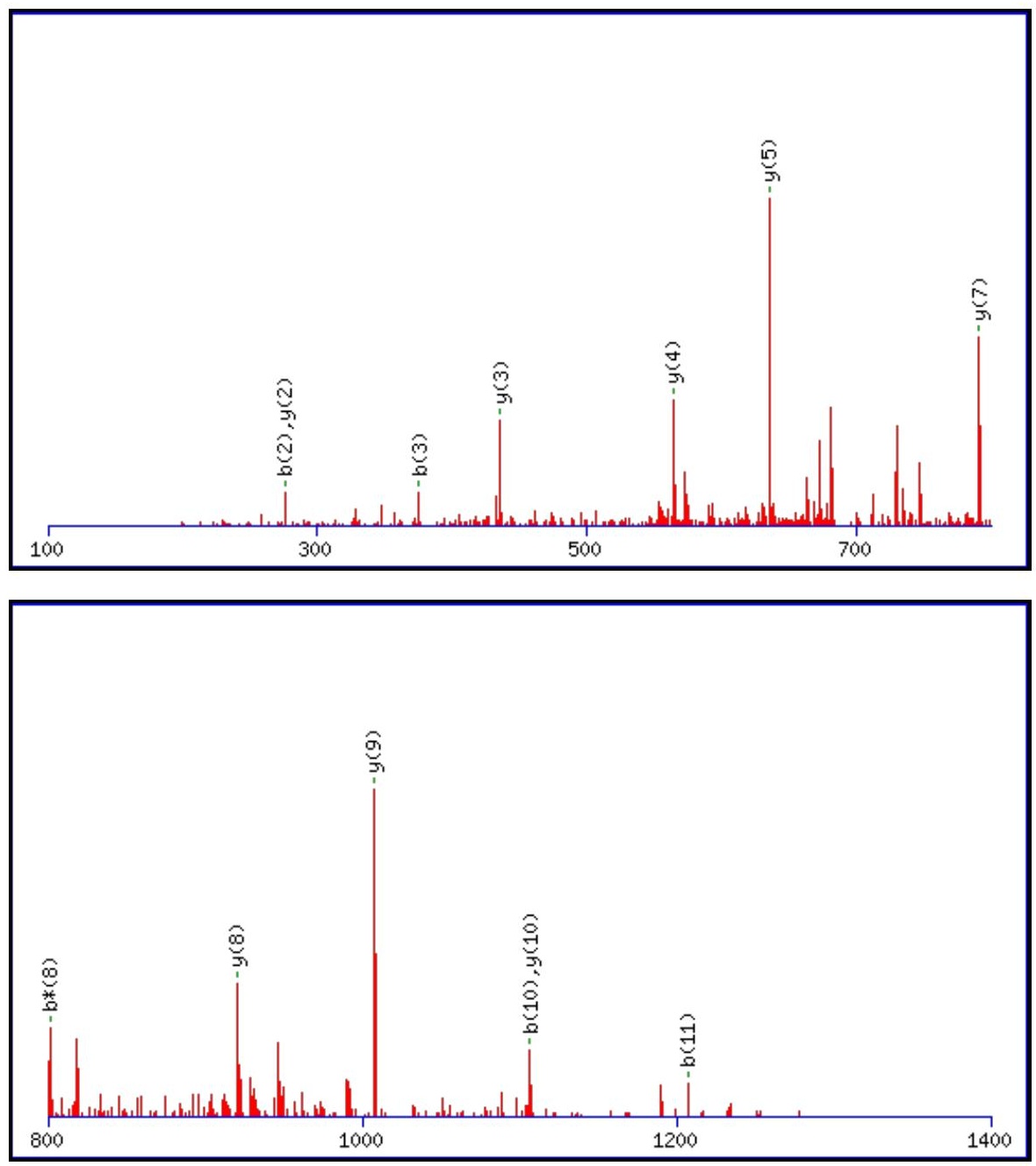


\begin{tabular}{|c|c|c|c|}
\hline 1400 & 1600 & 1800 & 2000 \\
\hline
\end{tabular}

Monoisotopic mass of neutral peptide $\operatorname{Mr}($ calc): 1380.5762

Fixed modifications: Carbamidomethyl (C)

Variable modifications:

N2 : Deamidated_N (N)

Ions Score: 84 Expect: $4.4 \mathrm{e}-007$

Matches (Bold Red): 13/122 fragment ions using 13 most intense peaks

\begin{tabular}{|c|c|c|c|c|c|c|c|c|c|c|c|c|c|c|}
\hline \# & b & $\mathbf{b}^{++}$ & b* & $\mathbf{b}^{*^{++}}$ & $\mathbf{b}^{0}$ & $\mathbf{b}^{\mathbf{0 + +}}$ & Seq. & $\mathbf{y}$ & $\mathbf{y}^{++}$ & $\mathbf{y}^{*}$ & $\mathbf{y}^{*^{++}}$ & $\mathbf{y}^{0}$ & $\mathbf{y}^{\mathbf{0 + +}}$ & \# \\
\hline 1 & 161.0379 & 81.0226 & & & & & $\mathrm{C}$ & & & & & & & 12 \\
\hline 2 & 276.0649 & 138.5361 & 259.0383 & 130.0228 & & & $\mathbf{N}$ & 1221.5528 & 611.2800 & 1204.5263 & 602.7668 & 1203.5423 & 602.2748 & 11 \\
\hline 3 & 375.1333 & 188.0703 & 358.1067 & 179.5570 & & & $\mathbf{V}$ & 1106.5259 & 553.7666 & 1089.4993 & 545.2533 & 1088.5153 & 544.7613 & 10 \\
\hline 4 & 462.1653 & 231.5863 & 445.1388 & 223.0730 & 444.1547 & 222.5810 & $\mathbf{S}$ & 1007.4575 & 504.2324 & 990.4309 & 495.7191 & 989.4469 & 495.2271 & 9 \\
\hline 5 & 591.2079 & 296.1076 & 574.1813 & 287.5943 & 573.1973 & 287.1023 & $\mathbf{E}$ & 920.4254 & 460.7164 & 903.3989 & 452.2031 & 902.4149 & 451.7111 & 8 \\
\hline 6 & 648.2294 & 324.6183 & 631.2028 & 316.1050 & 630.2188 & 315.6130 & G & 791.3828 & 396.1951 & 774.3563 & 387.6818 & 773.3723 & 387.1898 & 7 \\
\hline 7 & 747.2978 & 374.1525 & 730.2712 & 365.6393 & 729.2872 & 365.1472 & $\mathbf{V}$ & 734.3614 & 367.6843 & 717.3348 & 359.1711 & 716.3508 & 358.6790 & 6 \\
\hline 8 & 818.3349 & 409.6711 & 801.3083 & 401.1578 & 800.3243 & 400.6658 & A & 635.2930 & 318.1501 & 618.2664 & 309.6368 & 617.2824 & 309.1448 & 5 \\
\hline 9 & 946.3935 & 473.7004 & 929.3669 & 465.1871 & 928.3829 & 464.6951 & $\mathbf{Q}$ & 564.2559 & 282.6316 & 547.2293 & 274.1183 & 546.2453 & 273.6263 & 4 \\
\hline 10 & 1106.4241 & 553.7157 & 1089.3976 & 545.2024 & 1088.4136 & 544.7104 & C & 436.1973 & 218.6023 & 419.1707 & 210.0890 & 418.1867 & 209.5970 & 3 \\
\hline 11 & 1207.4718 & 604.2395 & 1190.4452 & 595.7263 & 1189.4612 & 595.2343 & $\mathbf{T}$ & 276.1666 & 138.5870 & 259.1401 & 130.0737 & 258.1561 & 129.5817 & 2 \\
\hline 12 & & & & & & & $\mathbf{R}$ & 175.1190 & 88.0631 & 158.0924 & 79.5498 & & & 1 \\
\hline
\end{tabular}
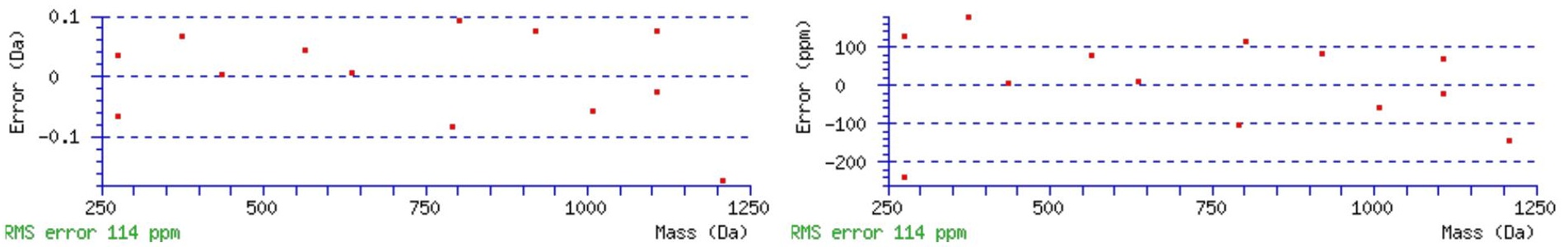

\section{All matches to this query}

\begin{tabular}{|l|l|l|l|}
\hline Score & Mr(calc): & Delta & \multicolumn{1}{|c|}{ Sequence } \\
\hline 84.1 & 1380.5762 & 0.0044 & CNVSEGVAQCTR \\
\hline 11.5 & 1380.5785 & 0.0021 & MQVLYEFEAR \\
\hline 8.1 & 1380.5836 & -0.0030 & MQNSTPMVCQGK \\
\hline 6.5 & 1380.5671 & 0.0135 & NESHSNGTVTVR \\
\hline 6.0 & 1380.5671 & 0.0135 & NESHSNGTVTVR \\
\hline 4.5 & 1380.5840 & -0.0034 & KFVNQTVTER \\
\hline 3.3 & 1380.5827 & -0.0021 & DSDGGRIEEMEK \\
\hline 3.2 & 1380.5940 & -0.0134 & DGSACVEGSGSSLR \\
\hline 3.1 & 1379.5680 & 1.0126 & TMGYSQEIVEK \\
\hline & & & \\
\hline
\end{tabular}




\section{|2.7 $\quad 1379.5809 \mid 0.9997$ MLLQSSVSVEK}

Spectrum No: 184; Query: 511; Rank: 1

\section{Peptide View}

MS/MS Fragmentation of SFSLISGSINQTR

Found in IPI00372786, Tax_Id=10116 Gene_Symbol=Nid2 similar to nidogen 2 isoform 2

Match to Query 511: 1409.717088 from(705.865820,2+)

Title: 100101RatKid_NS_deglyco_16.3339.3339.2.dta

Data file K:\NewmanPaper\Piliang \3SubProteomes \Piliang3SP $\backslash$ mgf5ppm\ERLIC_3SubProteomes5ppm.mgf
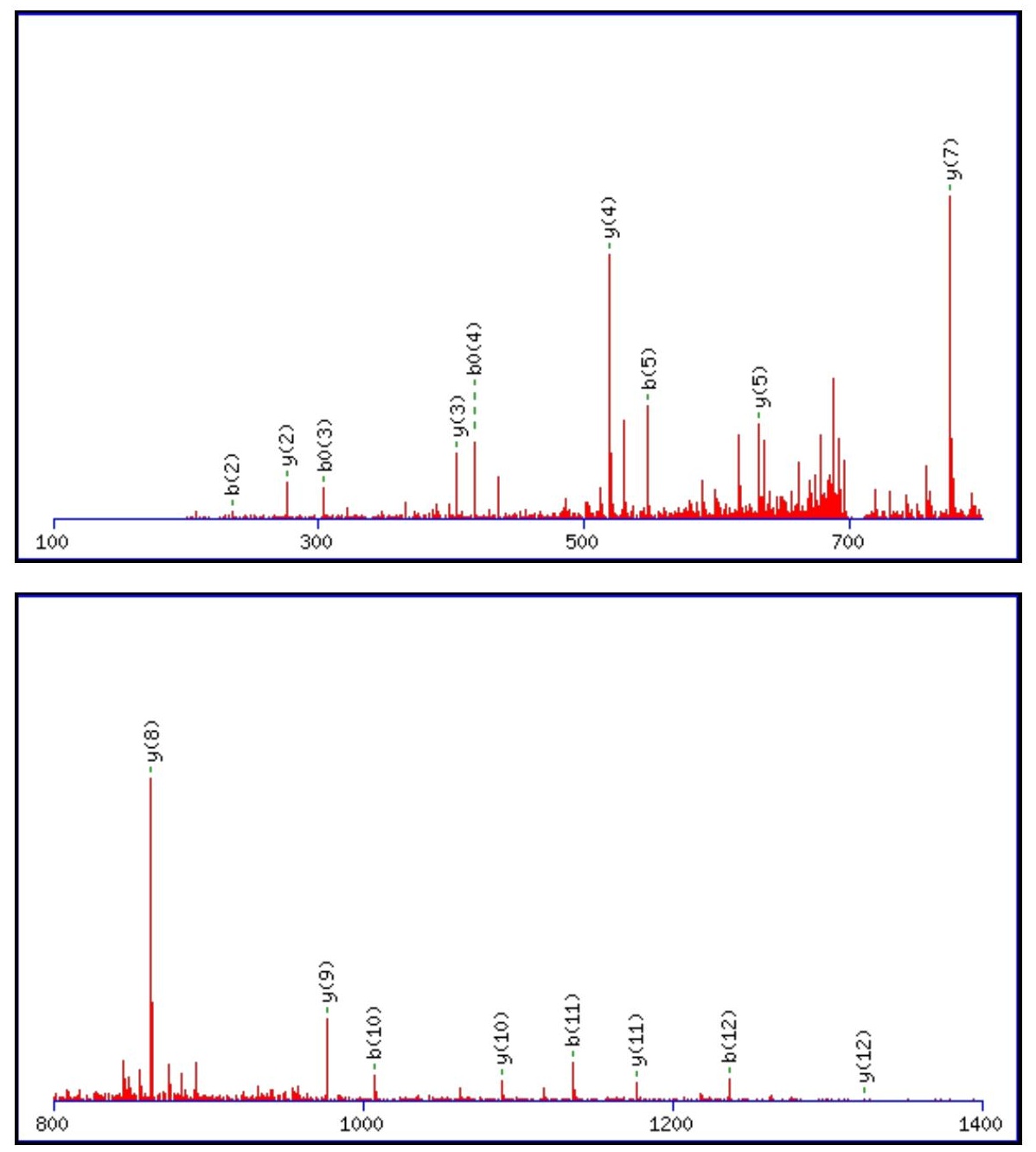


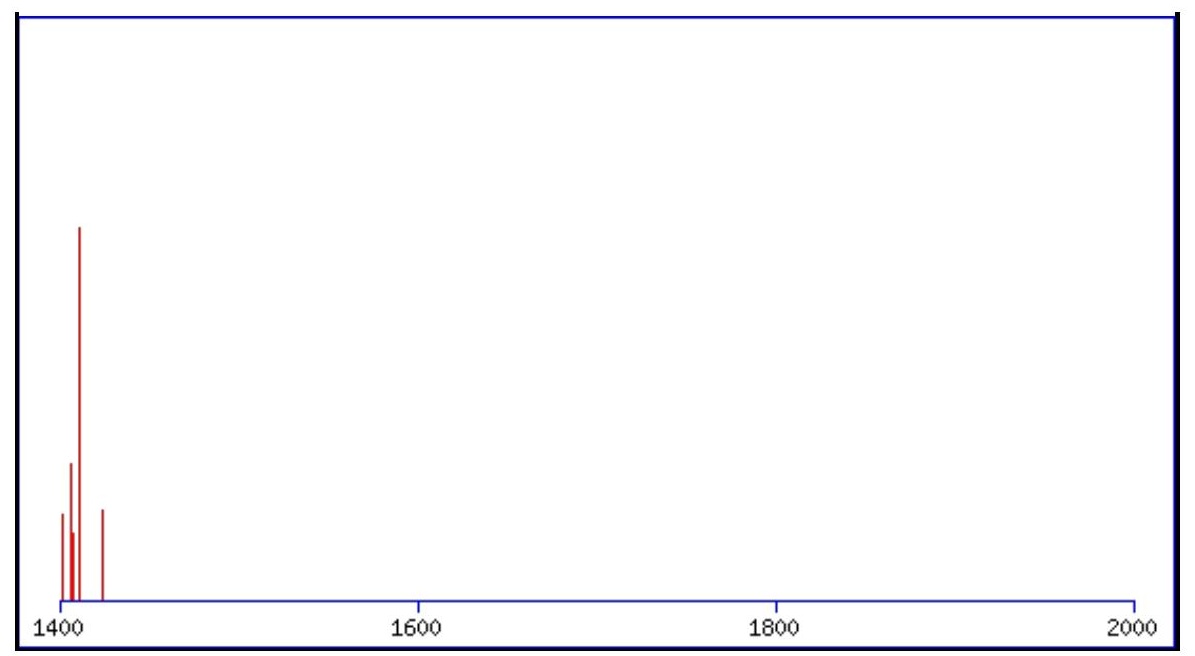

Monoisotopic mass of neutral peptide $\operatorname{Mr}($ calc): 1409.7150

Fixed modifications: Carbamidomethyl (C)

Variable modifications:

N10 : Deamidated_N (N)

Ions Score: 84 Expect : $5.1 \mathrm{e}-007$

Matches (Bold Red): 17/124 fragment ions using 35 most intense peaks

\begin{tabular}{|c|c|c|c|c|c|c|c|c|c|c|c|c|c|c|}
\hline \# & b & $\mathbf{b}^{++}$ & $\mathbf{b}^{*}$ & $\mathbf{b}^{*^{++}}$ & $\mathbf{b}^{0}$ & $\mathbf{b}^{0++}$ & Seq. & $\mathbf{y}$ & $y^{++}$ & $\mathbf{y}^{*}$ & $\mathbf{y}^{*^{++}}$ & $\mathbf{y}^{\mathbf{0}}$ & $\mathbf{y}^{0++}$ & \# \\
\hline 1 & 88.0393 & 44.5233 & & & 70.0287 & 35.5180 & S & & & & & & & 13 \\
\hline 2 & 235.1077 & 118.0575 & & & 217.0972 & 109.0522 & $\mathbf{F}$ & 1323.6903 & 662.3488 & 1306.6637 & 653.8355 & 1305.6797 & 653.3435 & 12 \\
\hline 3 & 322.1397 & 161.5735 & & & 304.1292 & 152.5682 & S & 1176.6219 & 588.8146 & 1159.5953 & 580.3013 & 1158.6113 & 579.8093 & 11 \\
\hline 4 & 435.2238 & 218.1155 & & & 417.2132 & 209.1103 & $\mathbf{L}$ & 1089.5899 & 545.2986 & 1072.5633 & 536.7853 & 1071.5793 & 536.2933 & 10 \\
\hline 5 & 548.3079 & 274.6576 & & & 530.2973 & 265.6523 & I & 976.5058 & 488.7565 & 959.4792 & 480.2433 & 958.4952 & 479.7513 & 9 \\
\hline 6 & 635.3399 & 318.1736 & & & 617.3293 & 309.1683 & $\mathbf{S}$ & 863.4217 & 432.2145 & 846.3952 & 423.7012 & 845.4112 & 423.2092 & 8 \\
\hline 7 & 692.3614 & 346.6843 & & & 674.3508 & 337.6790 & G & 776.3897 & 388.6985 & 759.3632 & 380.1852 & 758.3791 & 379.6932 & 7 \\
\hline 8 & 779.3934 & 390.2003 & & & 761.3828 & 381.1951 & $S$ & 719.3682 & 360.1878 & 702.3417 & 351.6745 & 701.3577 & 351.1825 & 6 \\
\hline 9 & 892.4775 & 446.7424 & & & 874.4669 & 437.7371 & I & 632.3362 & 316.6717 & 615.3097 & 308.1585 & 614.3256 & 307.6665 & 5 \\
\hline 10 & 1007.5044 & 504.2558 & 990.4778 & 495.7426 & 989.4938 & 495.2506 & $\mathbf{N}$ & 519.2521 & 260.1297 & 502.2256 & 251.6164 & 501.2416 & 251.1244 & 4 \\
\hline 11 & 1135.5630 & 568.2851 & 1118.5364 & 559.7718 & 1117.5524 & 559.2798 & $\mathbf{Q}$ & 404.2252 & 202.6162 & 387.1987 & 194.1030 & 386.2146 & 193.6110 & 3 \\
\hline 12 & 1236.6107 & 618.8090 & 1219.5841 & 610.2957 & 1218.6001 & 609.8037 & $\mathbf{T}$ & 276.1666 & 138.5870 & 259.1401 & 130.0737 & 258.1561 & 129.5817 & 2 \\
\hline 13 & & & & & & & $\mathbf{R}$ & 175.1190 & 88.0631 & 158.0924 & 79.5498 & & & 1 \\
\hline
\end{tabular}
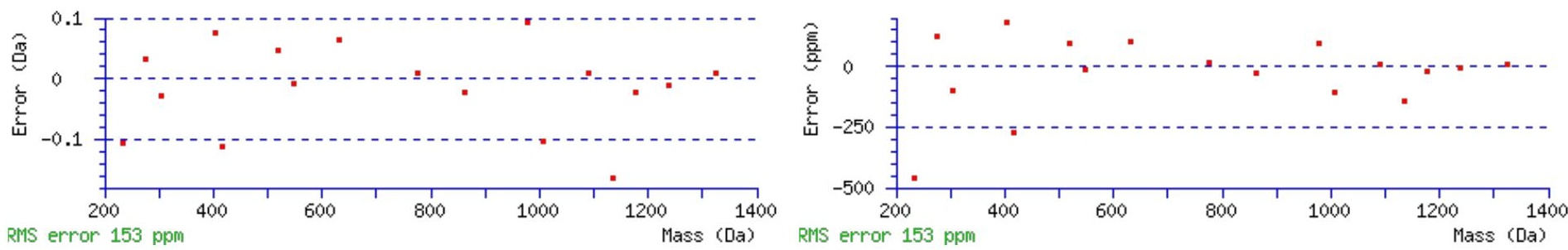

\section{All matches to this query}

\begin{tabular}{|l|l|l|l|}
\hline Score & Mr(calc): & Delta & \multicolumn{1}{c|}{ Sequence } \\
\hline 84.0 & 1409.7150 & 0.0020 & $\underline{\text { SFSLISGSINQTR }}$ \\
\hline 5.2 & 1409.7224 & -0.0053 & $\underline{\text { NEASALLYLLMR }}$ \\
\hline 3.6 & 1409.7263 & -0.0092 & $\underline{\text { SVSQETFRISTR }}$ \\
\hline 3.0 & 1409.7303 & -0.0132 & $\underline{\text { GLSNFYPDLTKR }}$ \\
\hline 2.0 & 1409.7038 & 0.0133 & $\underline{\text { TVFSDSQLSGLEK }}$ \\
\hline 1.5 & 1409.7108 & 0.0063 & $\underline{\text { KDIKFITYFR }}$ \\
\hline 1.0 & 1409.7159 & 0.0012 & $\underline{\text { KFMEKLDACIR }}$ \\
\hline 1.0 & 1409.7085 & 0.0086 & $\underline{\text { NMSIIDAFRSTR }}$ \\
\hline & & & \\
\hline
\end{tabular}




\section{\begin{tabular}{|l|l|l|l|}
0.5 & 1409.7085 & 0.0086 & MSSLPKPDGHGIR \\
\hline
\end{tabular}}

Spectrum No: 185; Query: 621; Rank: 1

\section{Peptide View}

MS/MS Fragmentation of QSNGSIAIISSMAGK

Found in IPI00211096, Tax_Id=10116 Gene_Symbol=Hsd11b1 Isoform 11-HSD1A of Corticosteroid 11-beta-dehydrogenase isozyme 1

Match to Query 621: 1463.732428 from(732.873490,2+)

Title: 100101RatKid_NS_deglyco_14.3201.3201.2.dta

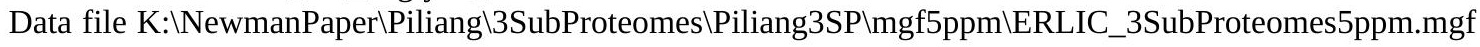
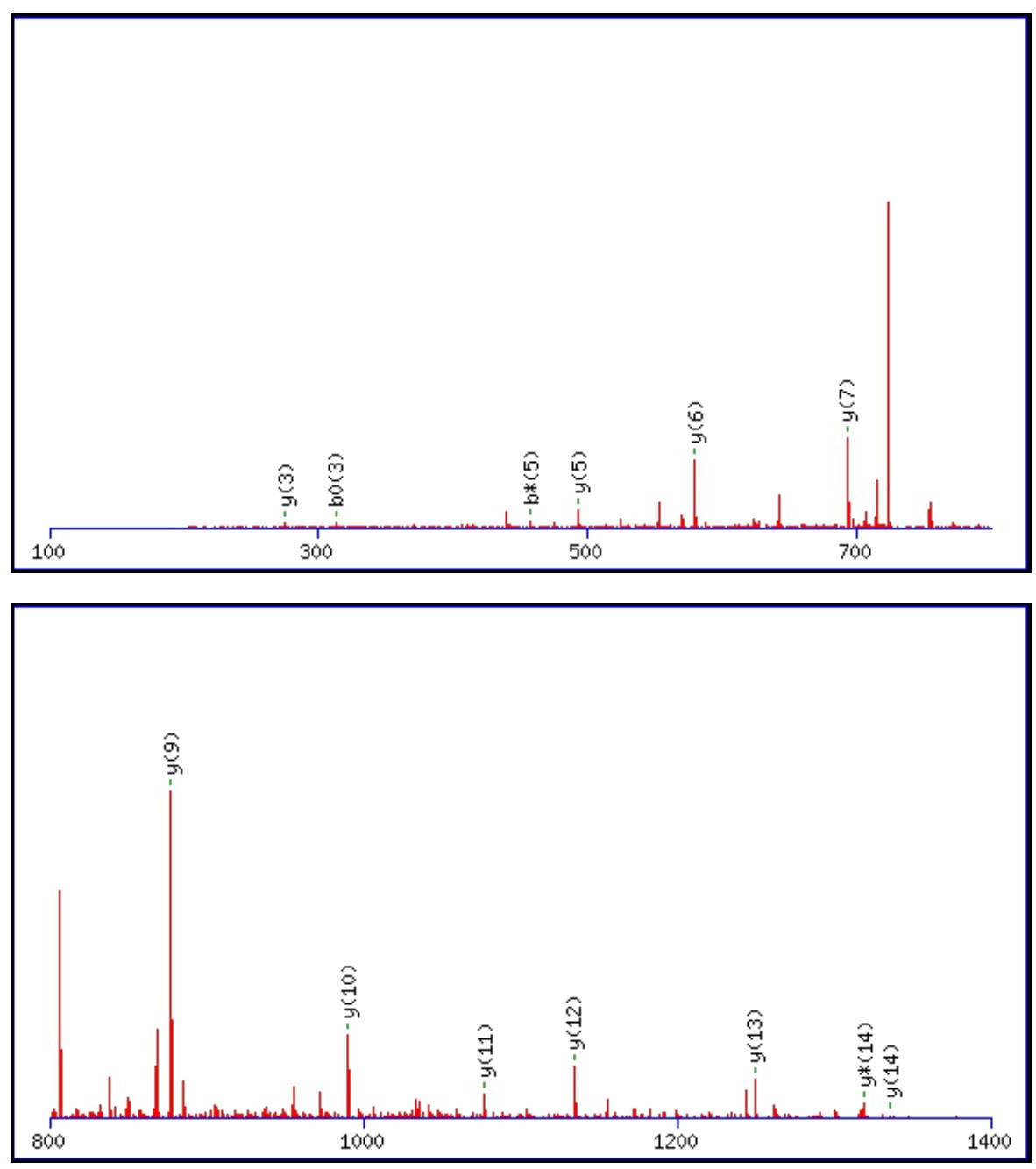


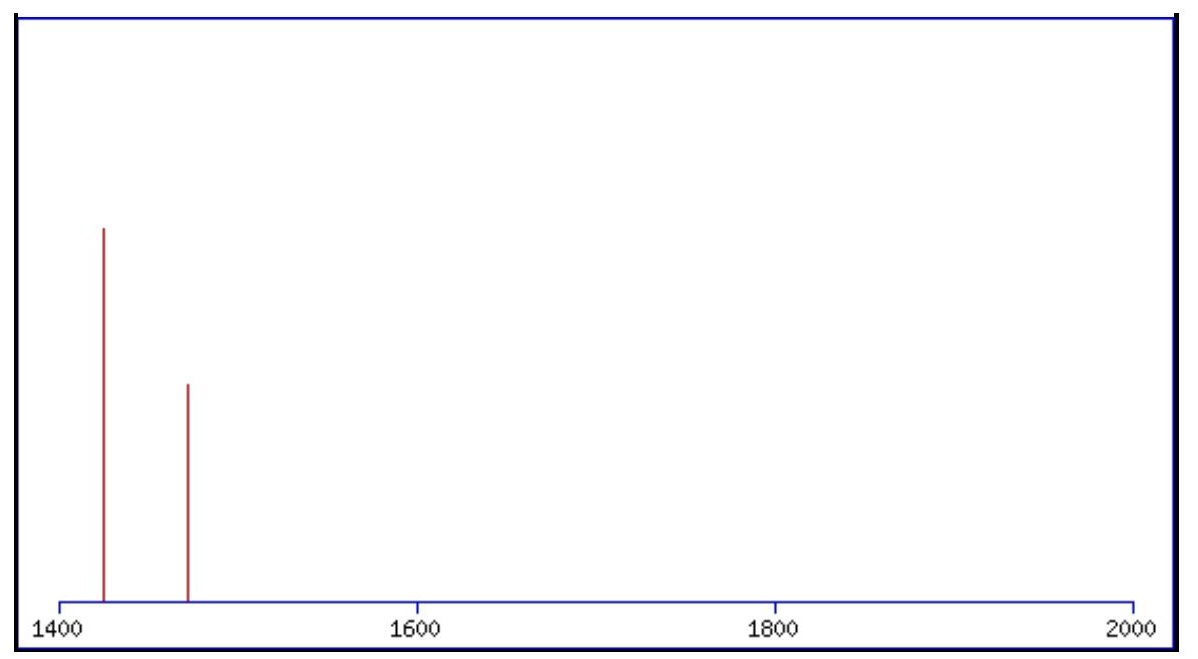

Monoisotopic mass of neutral peptide $\operatorname{Mr}($ calc): 1463.7290

Fixed modifications: Carbamidomethyl (C)

Variable modifications:

N3 : Deamidated $\mathrm{N}(\mathrm{N})$

Ions Score: 84 Expect: $5.6 \mathrm{e}-007$

Matches (Bold Red): 13/158 fragment ions using 22 most intense peaks

\begin{tabular}{|c|c|c|c|c|c|c|c|c|c|c|c|c|c|c|}
\hline \# & b & $\mathbf{b}^{++}$ & b* & $\mathbf{b}^{*^{++}}$ & $\mathbf{b}^{\mathbf{0}}$ & $\mathbf{b}^{0++}$ & Seq. & $\mathbf{y}$ & $\mathbf{y}^{++}$ & $\mathbf{y}^{*}$ & $\mathrm{y}^{\mathrm{*}^{++}}$ & $\mathbf{y}^{\mathbf{0}}$ & $\mathbf{y}^{0++}$ & \# \\
\hline 1 & 129.0659 & 65.0366 & 112.0393 & 56.5233 & & & $\mathbf{Q}$ & & & & & & & 15 \\
\hline 2 & 216.0979 & 108.5526 & 199.0713 & 100.0393 & 198.0873 & 99.5473 & $S$ & 1336.6777 & 668.8425 & 1319.6511 & 660.3292 & 1318.6671 & 659.8372 & 14 \\
\hline 3 & 331.1248 & 166.0660 & 314.0983 & 157.5528 & 313.1143 & 157.0608 & $\mathbf{N}$ & 1249.6457 & 625.3265 & 1232.6191 & 616.8132 & 1231.6351 & 616.3212 & 13 \\
\hline 4 & 388.1463 & 194.5768 & 371.1197 & 186.0635 & 370.1357 & 185.5715 & G & 1134.6187 & 567.8130 & 1117.5922 & 559.2997 & 1116.6082 & 558.8077 & 12 \\
\hline 5 & 475.1783 & 238.0928 & 458.1518 & 229.5795 & 457.1677 & 229.0875 & S & 1077.5973 & 539.3023 & 1060.5707 & 530.7890 & 1059.5867 & 530.2970 & 11 \\
\hline 6 & 588.2624 & 294.6348 & 571.2358 & 286.1216 & 570.2518 & 285.6295 & I & 990.5652 & 495.7863 & 973.5387 & 487.2730 & 972.5547 & 486.7810 & 10 \\
\hline 7 & 659.2995 & 330.1534 & 642.2729 & 321.6401 & 641.2889 & 321.1481 & A & 877.4812 & 439.2442 & 860.4546 & 430.7309 & 859.4706 & 430.2389 & 9 \\
\hline 8 & 772.3836 & 386.6954 & 755.3570 & 378.1821 & 754.3730 & 377.6901 & I & 806.4441 & 403.7257 & 789.4175 & 395.2124 & 788.4335 & 394.7204 & 8 \\
\hline 9 & 885.4676 & 443.2374 & 868.4411 & 434.7242 & 867.4571 & 434.2322 & I & 693.3600 & 347.1836 & 676.3334 & 338.6704 & 675.3494 & 338.1783 & 7 \\
\hline 10 & 972.4996 & 486.7535 & 955.4731 & 478.2402 & 954.4891 & 477.7482 & $\mathrm{~S}$ & 580.2759 & 290.6416 & 563.2494 & 282.1283 & 562.2654 & 281.6363 & 6 \\
\hline 11 & 1059.5317 & 530.2695 & 1042.5051 & 521.7562 & 1041.5211 & 521.2642 & S & 493.2439 & 247.1256 & 476.2173 & 238.6123 & 475.2333 & 238.1203 & 5 \\
\hline 12 & 1190.5722 & 595.7897 & 1173.5456 & 587.2764 & 1172.5616 & 586.7844 & $\mathbf{M}$ & 406.2119 & 203.6096 & 389.1853 & 195.0963 & & & 4 \\
\hline 13 & 1261.6093 & 631.3083 & 1244.5827 & 622.7950 & 1243.5987 & 622.3030 & A & 275.1714 & 138.0893 & 258.1448 & 129.5761 & & & 3 \\
\hline 14 & 1318.6307 & 659.8190 & 1301.6042 & 651.3057 & 1300.6202 & 650.8137 & G & 204.1343 & 102.5708 & 187.1077 & 94.0575 & & & 2 \\
\hline 15 & & & & & & & $\mathbf{K}$ & 147.1128 & 74.0600 & 130.0863 & 65.5468 & & & 1 \\
\hline
\end{tabular}
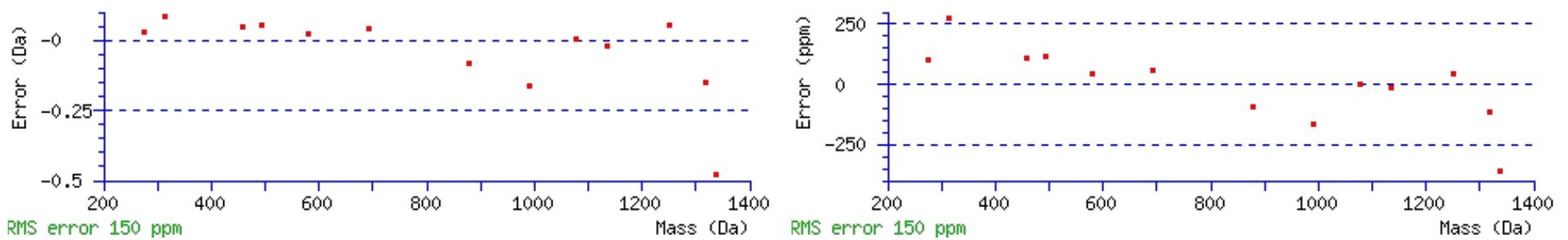

\section{All matches to this query}

\begin{tabular}{|l|l|l|l|}
\hline Score & Mr(calc): & Delta & \multicolumn{1}{c|}{ Sequence } \\
\hline 84.0 & 1463.7290 & 0.0035 & QSNGSIAIISSMAGK \\
\hline 14.9 & 1462.7272 & 1.0053 & RMEEKPLSSMQK \\
\hline 8.4 & 1463.7425 & -0.0101 & $\underline{\text { YEFLTKNTLKK }}$ \\
\hline 8.4 & 1463.7425 & -0.0101 & YEFLTKNTLKK \\
\hline 5.7 & 1462.7337 & 0.9987 & $\underline{\text { KAEEQLALATNMK }}$ \\
\hline 1.0 & 1463.7442 & -0.0118 & $\underline{\text { LKEVGYSTHMVGK }}$ \\
\hline & & & \\
\hline
\end{tabular}




\begin{tabular}{|l|l|l|l|}
0.8 & 1463.7319 & 0.0005 & HLLMSIDQRKK \\
\hline 0.8 & 1462.7238 & 1.0086 & KQNAPMTLEEFR \\
\hline 0.6 & 1463.7385 & -0.0061 & ATVPALQQKTEAK \\
\hline 0.6 & 1463.7231 & 0.0093 & WVNQLKYHMTK \\
\hline
\end{tabular}

Spectrum No: 186; Query: 1102; Rank: 1

\section{Peptide View}

MS/MS Fragmentation of VFDKEGNGTVMGAEIR

Found in IPI00365944, Tax_Id=10116 Gene_Symbol=Myl6_predicted Myosin light polypeptide 6

Match to Query 1102: 1722.830128 from(862.422340,2+)

Title: 100101RatKid_NS_deglyco_09.2141.2141.2.dta

Data file K:INewmanPaper|Piliangl3SubProteomes\Piliang3SP\mgf5ppm\ERLIC_3SubProteomes5ppm.mgf
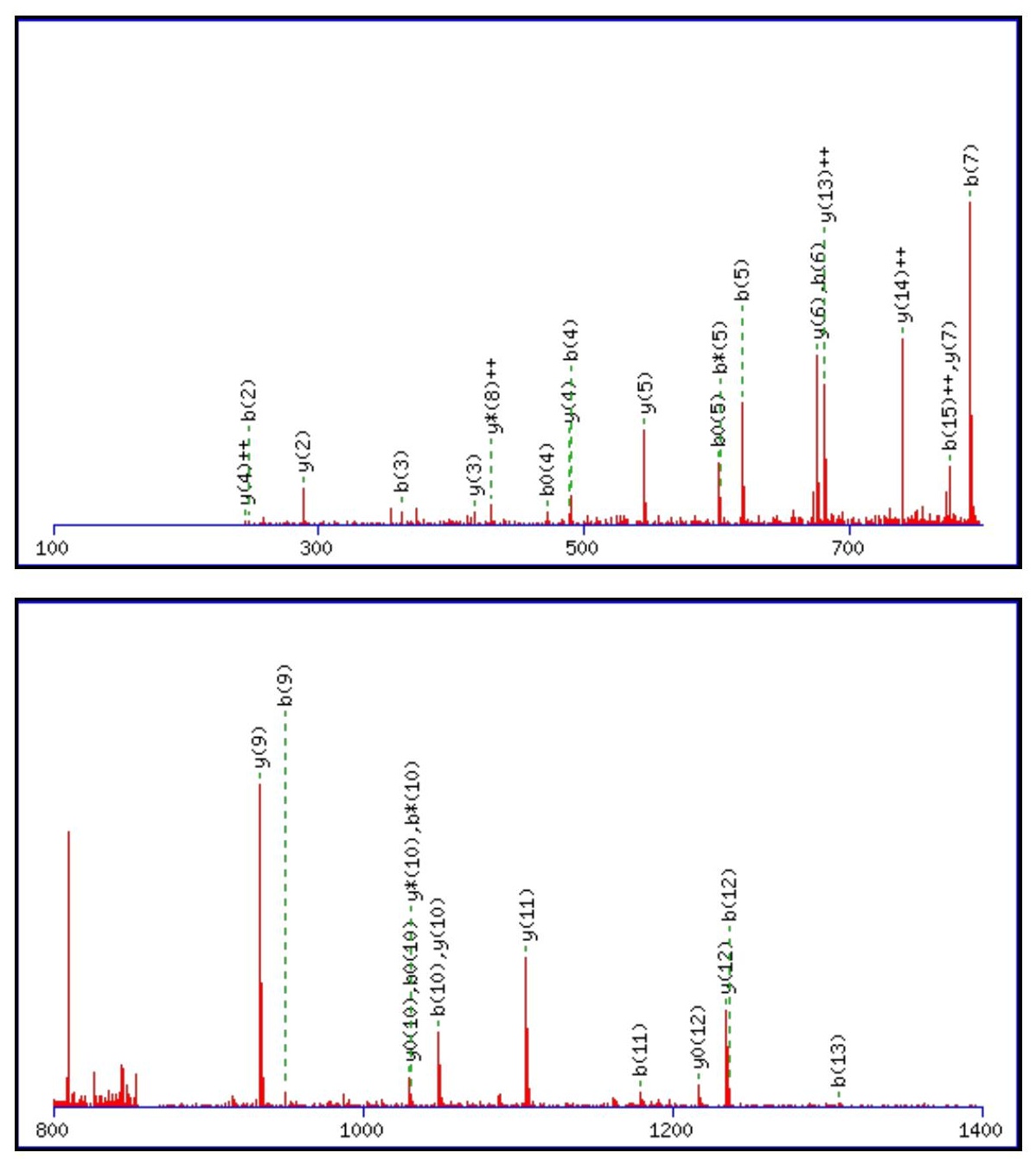


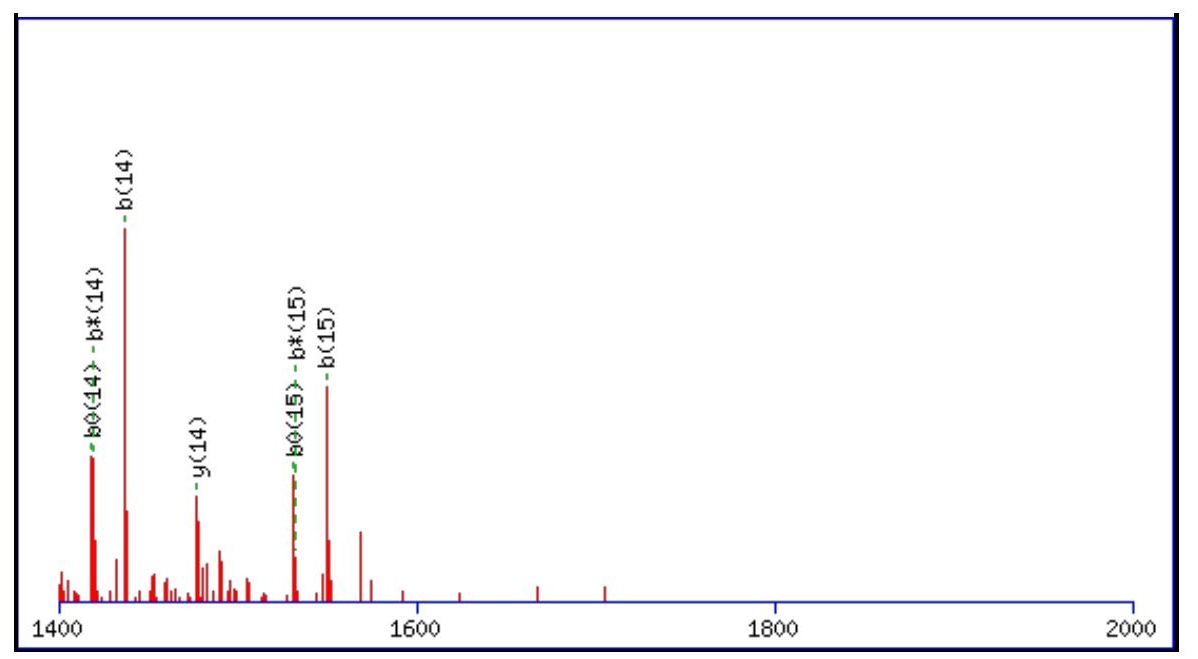

Monoisotopic mass of neutral peptide $\operatorname{Mr}($ calc): 1722.8247

Fixed modifications: Carbamidomethyl (C)

Variable modifications:

N7 : Deamidated_N (N)

Ions Score: 84 Expect: $8.8 \mathrm{e}-007$

Matches (Bold Red): 41/166 fragment ions using 73 most intense peaks

\begin{tabular}{|c|c|c|c|c|c|c|c|c|c|c|c|c|c|c|}
\hline \# & b & $\mathbf{b}^{++}$ & b* & $\mathbf{b}^{*++}$ & $\mathbf{b}^{\mathbf{0}}$ & $\mathbf{b}^{0++}$ & Seq. & $\mathbf{y}$ & $y^{++}$ & $\mathbf{y}^{*}$ & $\mathrm{y}^{\mathrm{*}^{++}}$ & $\mathbf{y}^{\mathbf{0}}$ & $\mathbf{y}^{0++}$ & \# \\
\hline 1 & 100.0757 & 50.5415 & & & & & V & & & & & & & 16 \\
\hline 2 & 247.1441 & 124.0757 & & & & & $\mathbf{F}$ & 1624.7635 & 812.8854 & 1607.7370 & 804.3721 & 1606.7530 & 803.8801 & 15 \\
\hline 3 & 362.1710 & 181.5892 & & & 344.1605 & 172.5839 & D & 1477.6951 & 739.3512 & 1460.6686 & 730.8379 & 1459.6846 & 730.3459 & 14 \\
\hline 4 & 490.2660 & 245.6366 & 473.2395 & 237.1234 & 472.2554 & 236.6314 & $\mathbf{K}$ & 1362.6682 & 681.8377 & 1345.6416 & 673.3245 & 1344.6576 & 672.8324 & 13 \\
\hline 5 & 619.3086 & 310.1579 & 602.2821 & 301.6447 & 601.2980 & 301.1527 & $\mathbf{E}$ & 1234.5732 & 617.7902 & 1217.5467 & 609.2770 & 1216.5627 & 608.7850 & 12 \\
\hline 6 & 676.3301 & 338.6687 & 659.3035 & 330.1554 & 658.3195 & 329.6634 & G & 1105.5306 & 553.2690 & 1088.5041 & 544.7557 & 1087.5201 & 544.2637 & 11 \\
\hline 7 & 791.3570 & 396.1821 & 774.3305 & 387.6689 & 773.3464 & 387.1769 & $\mathbf{N}$ & 1048.5092 & 524.7582 & 1031.4826 & 516.2449 & 1030.4986 & 515.7529 & 10 \\
\hline 8 & 848.3785 & 424.6929 & 831.3519 & 416.1796 & 830.3679 & 415.6876 & G & 933.4822 & 467.2448 & 916.4557 & 458.7315 & 915.4717 & 458.2395 & 9 \\
\hline 9 & 949.4261 & 475.2167 & 932.3996 & 466.7034 & 931.4156 & 466.2114 & $\mathbf{T}$ & 876.4608 & 438.7340 & 859.4342 & 430.2207 & 858.4502 & 429.7287 & 8 \\
\hline 10 & 1048.4946 & 524.7509 & 1031.4680 & 516.2376 & 1030.4840 & 515.7456 & V & 775.4131 & 388.2102 & 758.3865 & 379.6969 & 757.4025 & 379.2049 & 7 \\
\hline 11 & 1179.5350 & 590.2712 & 1162.5085 & 581.7579 & 1161.5245 & 581.2659 & $\mathbf{M}$ & 676.3447 & 338.6760 & 659.3181 & 330.1627 & 658.3341 & 329.6707 & 6 \\
\hline 12 & 1236.5565 & 618.7819 & 1219.5300 & 610.2686 & 1218.5459 & 609.7766 & G & 545.3042 & 273.1557 & 528.2776 & 264.6425 & 527.2936 & 264.1504 & 5 \\
\hline 13 & 1307.5936 & 654.3004 & 1290.5671 & 645.7872 & 1289.5831 & 645.2952 & A & 488.2827 & 244.6450 & 471.2562 & 236.1317 & 470.2722 & 235.6397 & 4 \\
\hline 14 & 1436.6362 & 718.8217 & 1419.6097 & 710.3085 & 1418.6257 & 709.8165 & $\mathbf{E}$ & 417.2456 & 209.1264 & 400.2191 & 200.6132 & 399.2350 & 200.1212 & 3 \\
\hline 15 & 1549.7203 & 775.3638 & 1532.6937 & 766.8505 & 1531.7097 & 766.3585 & I & 288.2030 & 144.6051 & 271.1765 & 136.0919 & & & 2 \\
\hline 16 & & & & & & & $\mathbf{R}$ & 175.1190 & 88.0631 & 158.0924 & 79.5498 & & & 1 \\
\hline
\end{tabular}

눈

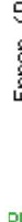

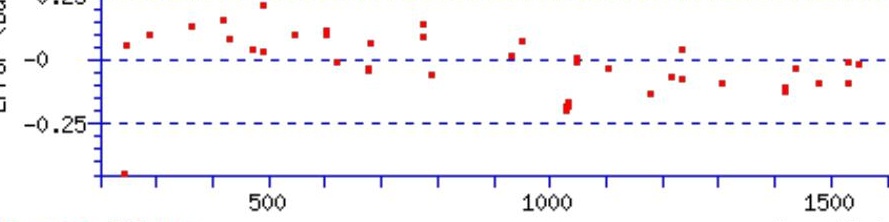

RMS error 333 ppm

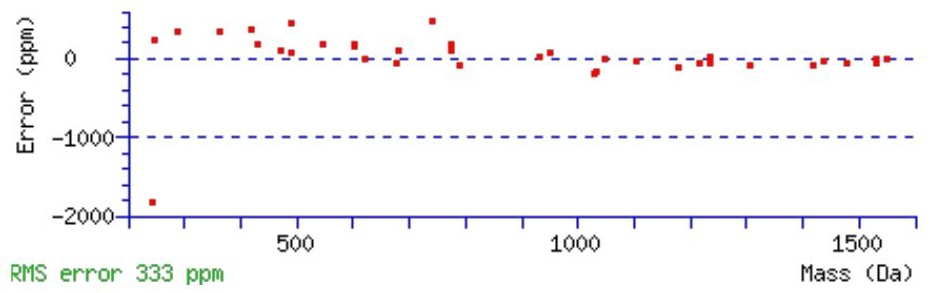

\section{All matches to this query}

\begin{tabular}{|l|l|l|l|}
\hline Score & Mr(calc): & Delta & \multicolumn{1}{c|}{ Sequence } \\
\hline 83.9 & 1722.8247 & 0.0055 & VFDKEGNGTVMGAEIR \\
\hline 83.9 & 1722.8247 & 0.0055 & VFDKEGNGTVMGAELR \\
\hline 24.7 & 1721.8407 & 0.9895 & $\underline{\text { VFDKEGNGTVMGAEIR }}$ \\
\hline 24.7 & 1721.8407 & 0.9895 & VFDKEGNGTVMGAELR \\
\hline 12.1 & 1722.8213 & 0.0088 & NEDLFSQPVSFNGIR \\
\hline & & & \\
\hline
\end{tabular}




\begin{tabular}{|l|l|l|l|}
7.3 & 1722.8280 & 0.0021 & DKEVAALTSQMDMLR \\
\hline 7.3 & 1722.8280 & 0.0021 & DKEVAALTSQMDMLR \\
\hline 7.0 & 1722.8414 & -0.0113 & GTAQGTPRNSXGTVLR \\
\hline 6.1 & 1722.8276 & 0.0025 & NKYHPDLRMVTIR \\
\hline 6.0 & 1722.8230 & 0.0072 & WDDDSTIVTPIILR \\
\hline
\end{tabular}

Spectrum No: 187; Query: 1102; Rank: 1

\section{Peptide View}

MS/MS Fragmentation of VFDKEGNGTVMGAEIR

Found in IPI00365944, Tax_Id=10116 Gene_Symbol=Myl6_predicted Myosin light polypeptide 6

Match to Query 1102: 1722.830128 from(862.422340,2+)

Title: 100101RatKid_NS_deglyco_09.2141.2141.2.dta

Data file K:INewmanPaper|Piliangl3SubProteomes\Piliang3SP\mgf5ppm\ERLIC_3SubProteomes5ppm.mgf
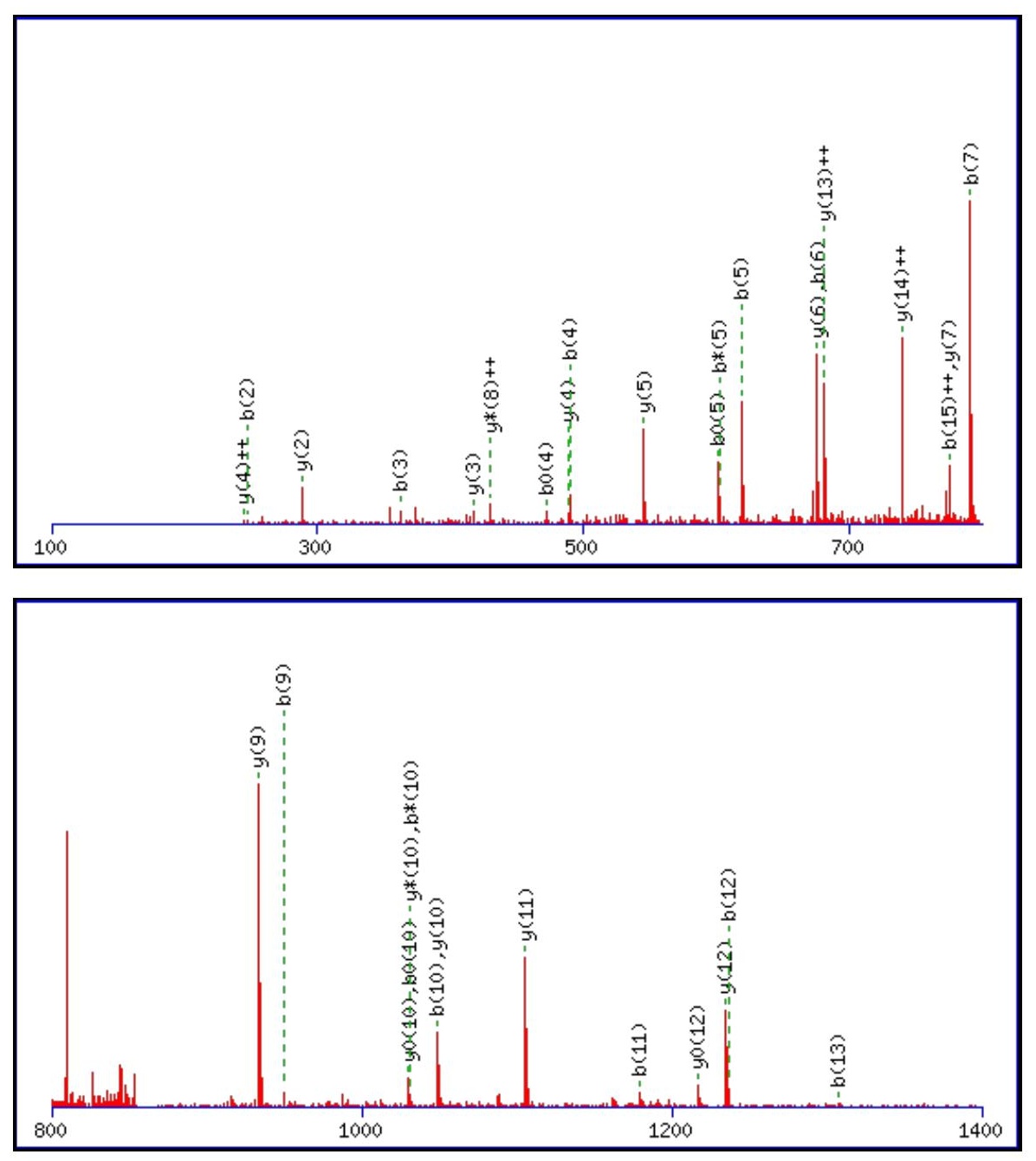


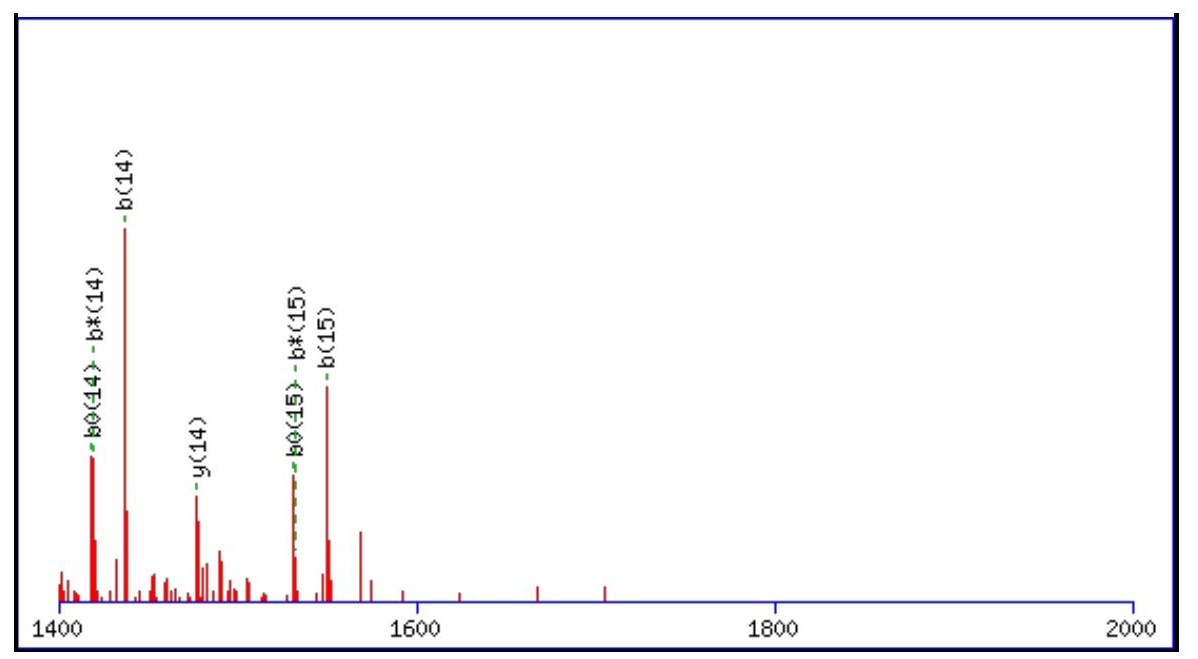

Monoisotopic mass of neutral peptide $\operatorname{Mr}($ calc): 1722.8247

Fixed modifications: Carbamidomethyl (C)

Variable modifications:

N7 : Deamidated_N (N)

Ions Score: 84 Expect: $8.8 \mathrm{e}-007$

Matches (Bold Red): 41/166 fragment ions using 73 most intense peaks

\begin{tabular}{|c|c|c|c|c|c|c|c|c|c|c|c|c|c|c|}
\hline \# & b & $\mathbf{b}^{++}$ & b* & $\mathbf{b}^{*++}$ & $\mathbf{b}^{\mathbf{0}}$ & $\mathbf{b}^{0++}$ & Seq. & $\mathbf{y}$ & $y^{++}$ & $\mathbf{y}^{*}$ & $\mathrm{y}^{\mathrm{*}^{++}}$ & $\mathbf{y}^{\mathbf{0}}$ & $\mathbf{y}^{0++}$ & \# \\
\hline 1 & 100.0757 & 50.5415 & & & & & V & & & & & & & 16 \\
\hline 2 & 247.1441 & 124.0757 & & & & & $\mathbf{F}$ & 1624.7635 & 812.8854 & 1607.7370 & 804.3721 & 1606.7530 & 803.8801 & 15 \\
\hline 3 & 362.1710 & 181.5892 & & & 344.1605 & 172.5839 & D & 1477.6951 & 739.3512 & 1460.6686 & 730.8379 & 1459.6846 & 730.3459 & 14 \\
\hline 4 & 490.2660 & 245.6366 & 473.2395 & 237.1234 & 472.2554 & 236.6314 & $\mathbf{K}$ & 1362.6682 & 681.8377 & 1345.6416 & 673.3245 & 1344.6576 & 672.8324 & 13 \\
\hline 5 & 619.3086 & 310.1579 & 602.2821 & 301.6447 & 601.2980 & 301.1527 & $\mathbf{E}$ & 1234.5732 & 617.7902 & 1217.5467 & 609.2770 & 1216.5627 & 608.7850 & 12 \\
\hline 6 & 676.3301 & 338.6687 & 659.3035 & 330.1554 & 658.3195 & 329.6634 & G & 1105.5306 & 553.2690 & 1088.5041 & 544.7557 & 1087.5201 & 544.2637 & 11 \\
\hline 7 & 791.3570 & 396.1821 & 774.3305 & 387.6689 & 773.3464 & 387.1769 & $\mathbf{N}$ & 1048.5092 & 524.7582 & 1031.4826 & 516.2449 & 1030.4986 & 515.7529 & 10 \\
\hline 8 & 848.3785 & 424.6929 & 831.3519 & 416.1796 & 830.3679 & 415.6876 & G & 933.4822 & 467.2448 & 916.4557 & 458.7315 & 915.4717 & 458.2395 & 9 \\
\hline 9 & 949.4261 & 475.2167 & 932.3996 & 466.7034 & 931.4156 & 466.2114 & $\mathbf{T}$ & 876.4608 & 438.7340 & 859.4342 & 430.2207 & 858.4502 & 429.7287 & 8 \\
\hline 10 & 1048.4946 & 524.7509 & 1031.4680 & 516.2376 & 1030.4840 & 515.7456 & V & 775.4131 & 388.2102 & 758.3865 & 379.6969 & 757.4025 & 379.2049 & 7 \\
\hline 11 & 1179.5350 & 590.2712 & 1162.5085 & 581.7579 & 1161.5245 & 581.2659 & $\mathbf{M}$ & 676.3447 & 338.6760 & 659.3181 & 330.1627 & 658.3341 & 329.6707 & 6 \\
\hline 12 & 1236.5565 & 618.7819 & 1219.5300 & 610.2686 & 1218.5459 & 609.7766 & G & 545.3042 & 273.1557 & 528.2776 & 264.6425 & 527.2936 & 264.1504 & 5 \\
\hline 13 & 1307.5936 & 654.3004 & 1290.5671 & 645.7872 & 1289.5831 & 645.2952 & A & 488.2827 & 244.6450 & 471.2562 & 236.1317 & 470.2722 & 235.6397 & 4 \\
\hline 14 & 1436.6362 & 718.8217 & 1419.6097 & 710.3085 & 1418.6257 & 709.8165 & $\mathbf{E}$ & 417.2456 & 209.1264 & 400.2191 & 200.6132 & 399.2350 & 200.1212 & 3 \\
\hline 15 & 1549.7203 & 775.3638 & 1532.6937 & 766.8505 & 1531.7097 & 766.3585 & I & 288.2030 & 144.6051 & 271.1765 & 136.0919 & & & 2 \\
\hline 16 & & & & & & & $\mathbf{R}$ & 175.1190 & 88.0631 & 158.0924 & 79.5498 & & & 1 \\
\hline
\end{tabular}$$
\text { ㄴ. }
$$

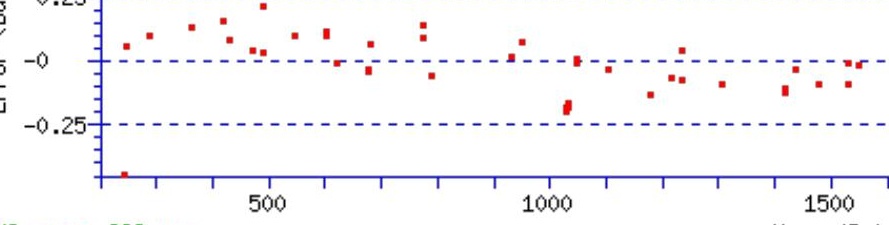

RMS error 333 ppm

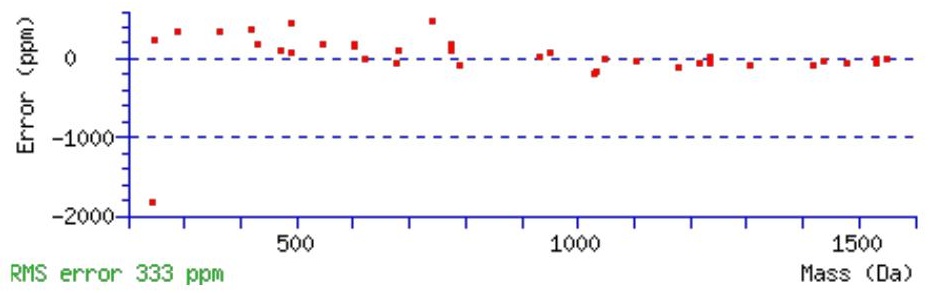

\section{All matches to this query}

\begin{tabular}{|l|l|c|l|}
\hline Score & $\mathbf{M r}($ calc): & Delta & \multicolumn{1}{c|}{ Sequence } \\
\hline 83.9 & 1722.8247 & 0.0055 & $\underline{\text { VFDKEGNGTVMGAEIR }}$ \\
\hline 83.9 & 1722.8247 & 0.0055 & VFDKEGNGTVMGAELR \\
\hline 24.7 & 1721.8407 & 0.9895 & VFDKEGNGTVMGAEIR \\
\hline 24.7 & 1721.8407 & 0.9895 & VFDKEGNGTVMGAELR \\
\hline 12.1 & 1722.8213 & 0.0088 & $\underline{\text { NEDLFSQPVSFNGIR }}$ \\
\hline & & & \\
\hline
\end{tabular}




\begin{tabular}{|l|l|l|l|}
7.3 & 1722.8280 & 0.0021 & DKEVAALTSQMDMLR \\
\hline 7.3 & 1722.8280 & 0.0021 & DKEVAALTSQMDMLR \\
\hline 7.0 & 1722.8414 & -0.0113 & GTAQGTPRNSXGTVLR \\
\hline 6.1 & 1722.8276 & 0.0025 & NKYHPDLRMVTIR \\
\hline 6.0 & 1722.8230 & 0.0072 & WDDDSTIVTPIILR \\
\hline
\end{tabular}

Spectrum No: 188; Query: 650; Rank: 1

\section{Peptide View}

MS/MS Fragmentation of VGPNGTLFVVDAYK

Found in IPI00382223, Tax_Id=10116 Gene_Symbol=RGD1308874 Ab2-305

Match to Query 650: 1479.756968 from(740.885760,2+)

Title: 091008RatKidney_NH4Format01_23.2960.2960.2.dta

Data file K:INewmanPaper|Piliangl3SubProteomes\Piliang3SP\mgf5ppm\ERLIC_3SubProteomes5ppm.mgf
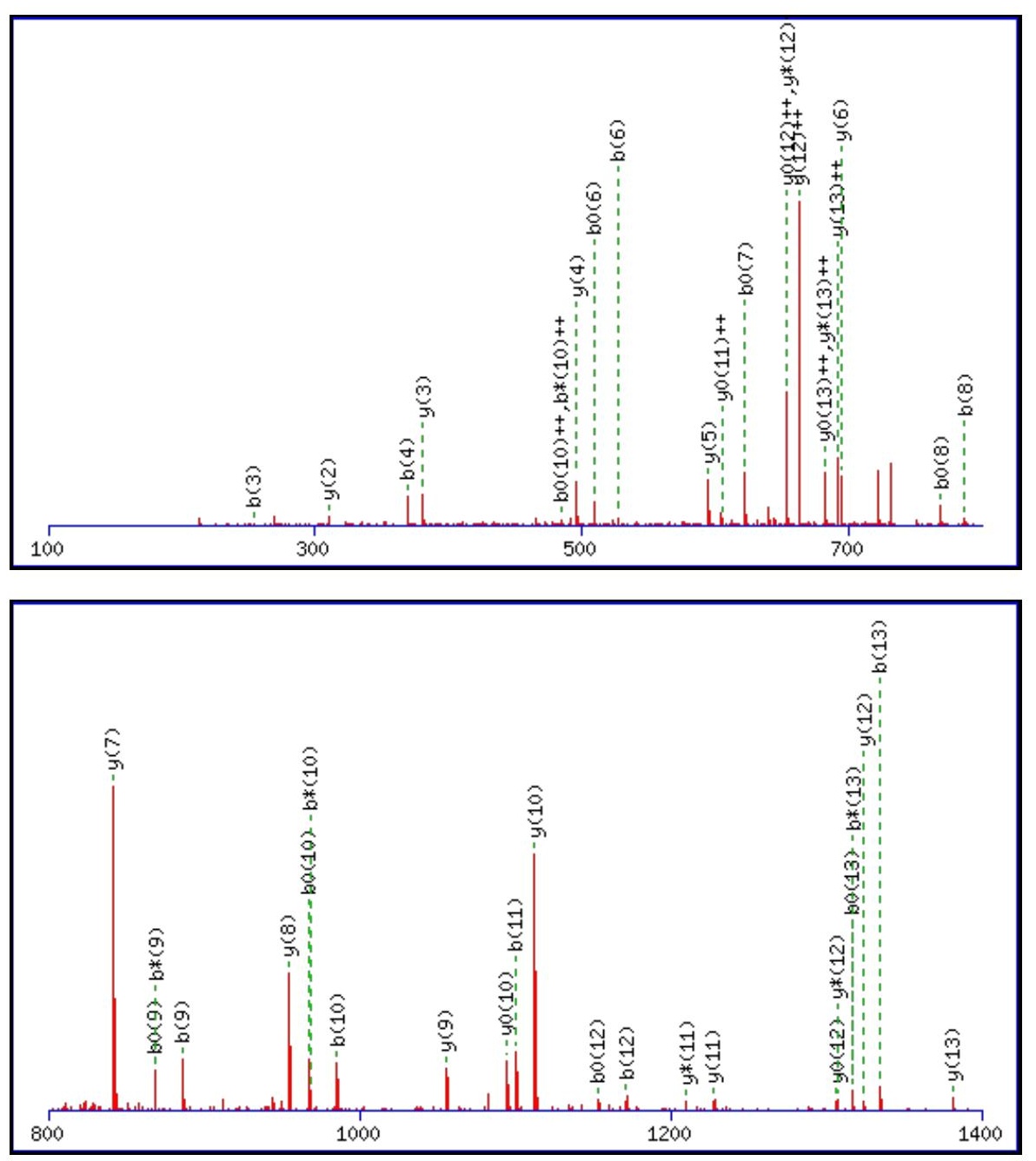
Monoisotopic mass of neutral peptide $\operatorname{Mr}($ calc): 1479.7610

Fixed modifications: Carbamidomethyl (C)

Variable modifications:

N4 : Deamidated N (N)

Ions Score: 84 Expect: $6.5 \mathrm{e}-\odot \odot 7$

Matches (Bold Red): 44/134 fragment ions using 72 most intense peaks

\begin{tabular}{|c|c|c|c|c|c|c|c|c|c|c|c|c|c|c|}
\hline \# & b & $\mathbf{b}^{++}$ & $\mathbf{b}^{*}$ & $\mathbf{b}^{*^{++}}$ & $\mathbf{b}^{0}$ & $\mathbf{b}^{0++}$ & Seq. & $\mathbf{y}$ & $y^{++}$ & $\mathbf{y}^{*}$ & $\mathrm{y}^{\mathrm{*}^{++}}$ & $\mathbf{y}^{0}$ & $\mathbf{y}^{0++}$ & \# \\
\hline 1 & 100.0757 & 50.5415 & & & & & V & & & & & & & 14 \\
\hline 2 & 157.0972 & 79.0522 & & & & & G & 1381.6998 & 691.3535 & 1364.6733 & 682.8403 & 1363.6892 & 682.3483 & 13 \\
\hline 3 & 254.1499 & 127.5786 & & & & & $\mathbf{P}$ & 1324.6783 & 662.8428 & 1307.6518 & 654.3295 & 1306.6678 & 653.8375 & 12 \\
\hline 4 & 369.1769 & 185.0921 & 352.1503 & 176.5788 & & & $\mathbf{N}$ & 1227.6256 & 614.3164 & 1210.5990 & 605.8032 & 1209.6150 & 605.3111 & 11 \\
\hline 5 & 426.1983 & 213.6028 & 409.1718 & 205.0895 & & & G & 1112.5986 & 556.8030 & 1095.5721 & 548.2897 & 1094.5881 & 547.7977 & 10 \\
\hline 6 & 527.2460 & 264.1266 & 510.2194 & 255.6134 & 509.2354 & 255.1214 & $\mathbf{T}$ & 1055.5772 & 528.2922 & 1038.5506 & 519.7790 & 1037.5666 & 519.2869 & 9 \\
\hline 7 & 640.3301 & 320.6687 & 623.3035 & 312.1554 & 622.3195 & 311.6634 & $\mathbf{L}$ & 954.5295 & 477.7684 & 937.5029 & 469.2551 & 936.5189 & 468.7631 & 8 \\
\hline 8 & 787.3985 & 394.2029 & 770.3719 & 385.6896 & 769.3879 & 385.1976 & $\mathbf{F}$ & 841.4454 & 421.2264 & 824.4189 & 412.7131 & 823.4349 & 412.2211 & 7 \\
\hline 9 & 886.4669 & 443.7371 & 869.4403 & 435.2238 & 868.4563 & 434.7318 & V & 694.3770 & 347.6921 & 677.3505 & 339.1789 & 676.3665 & 338.6869 & 6 \\
\hline 10 & 985.5353 & 493.2713 & 968.5088 & 484.7580 & 967.5247 & 484.2660 & V & 595.3086 & 298.1579 & 578.2821 & 289.6447 & 577.2980 & 289.1527 & 5 \\
\hline 11 & 1100.5622 & 550.7848 & 1083.5357 & 542.2715 & 1082.5517 & 541.7795 & D & 496.2402 & 248.6237 & 479.2136 & 240.1105 & 478.2296 & 239.6185 & 4 \\
\hline 12 & 1171.5994 & 586.3033 & 1154.5728 & 577.7900 & 1153.5888 & 577.2980 & A & 381.2132 & 191.1103 & 364.1867 & 182.5970 & & & 3 \\
\hline 13 & 1334.6627 & 667.8350 & 1317.6361 & 659.3217 & 1316.6521 & 658.8297 & $\mathbf{Y}$ & 310.1761 & 155.5917 & 293.1496 & 147.0784 & & & 2 \\
\hline 14 & & & & & & & $\mathbf{K}$ & 147.1128 & 74.0600 & 130.0863 & 65.5468 & & & 1 \\
\hline
\end{tabular}
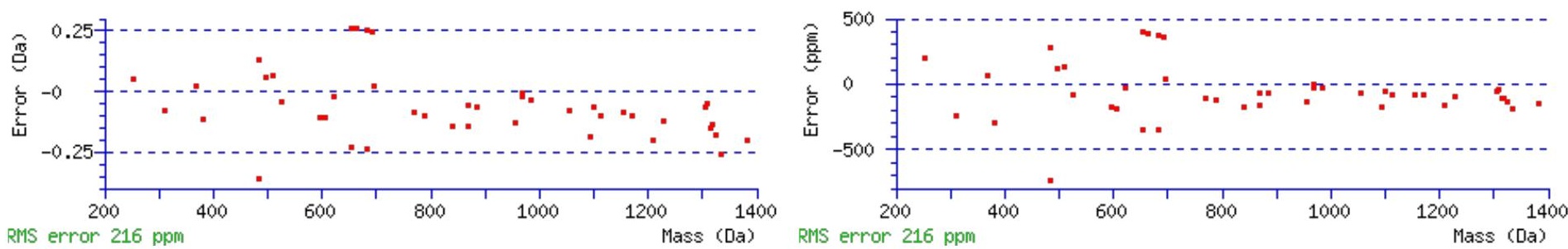

\section{All matches to this query}

\begin{tabular}{|l|l|l|l|}
\hline Score & Mr(calc): & Delta & \multicolumn{1}{c|}{ Sequence } \\
\hline 83.9 & 1479.7610 & -0.0040 & VGPGTLFVVDAYK \\
\hline 9.7 & 1479.7538 & 0.0032 & RLQTICLSGTGMK \\
\hline 6.7 & 1479.7711 & -0.0142 & RLGTLAFTTRHK \\
\hline 6.3 & 1479.7711 & -0.0142 & RLGTLAFTTRHK \\
\hline 5.6 & 1479.7711 & -0.0142 & RLGTLAFTTRHK \\
\hline 2.0 & 1479.7446 & 0.0124 & RLEAELEAVSRK \\
\hline 1.4 & 1477.7541 & 2.0028 & LRPTGIISADVEK \\
\hline & & & \\
\hline
\end{tabular}




\section{\begin{tabular}{|l||l|l|l|l|}
0.2 & 1478.7412 & 1.0157 & RLPSAAGPGPGCPSR \\
\hline
\end{tabular}}

Spectrum No: 189; Query: 776; Rank: 1

\section{Peptide View}

MS/MS Fragmentation of ITDIENGTFANIPR

Found in IPI00365784, Tax_Id=10116 Gene_Symbol=LOC306805 Similar to asporin

Match to Query 776: 1560.777088 from(781.395820,2+)

Title: 091008RatKidney_NoSalt_26.2794.2794.2.dta

Data file K:\NewmanPaper\Piliang \3SubProteomes \Piliang3SP $\backslash$ mgf5ppm\ERLIC_3SubProteomes5ppm.mgf
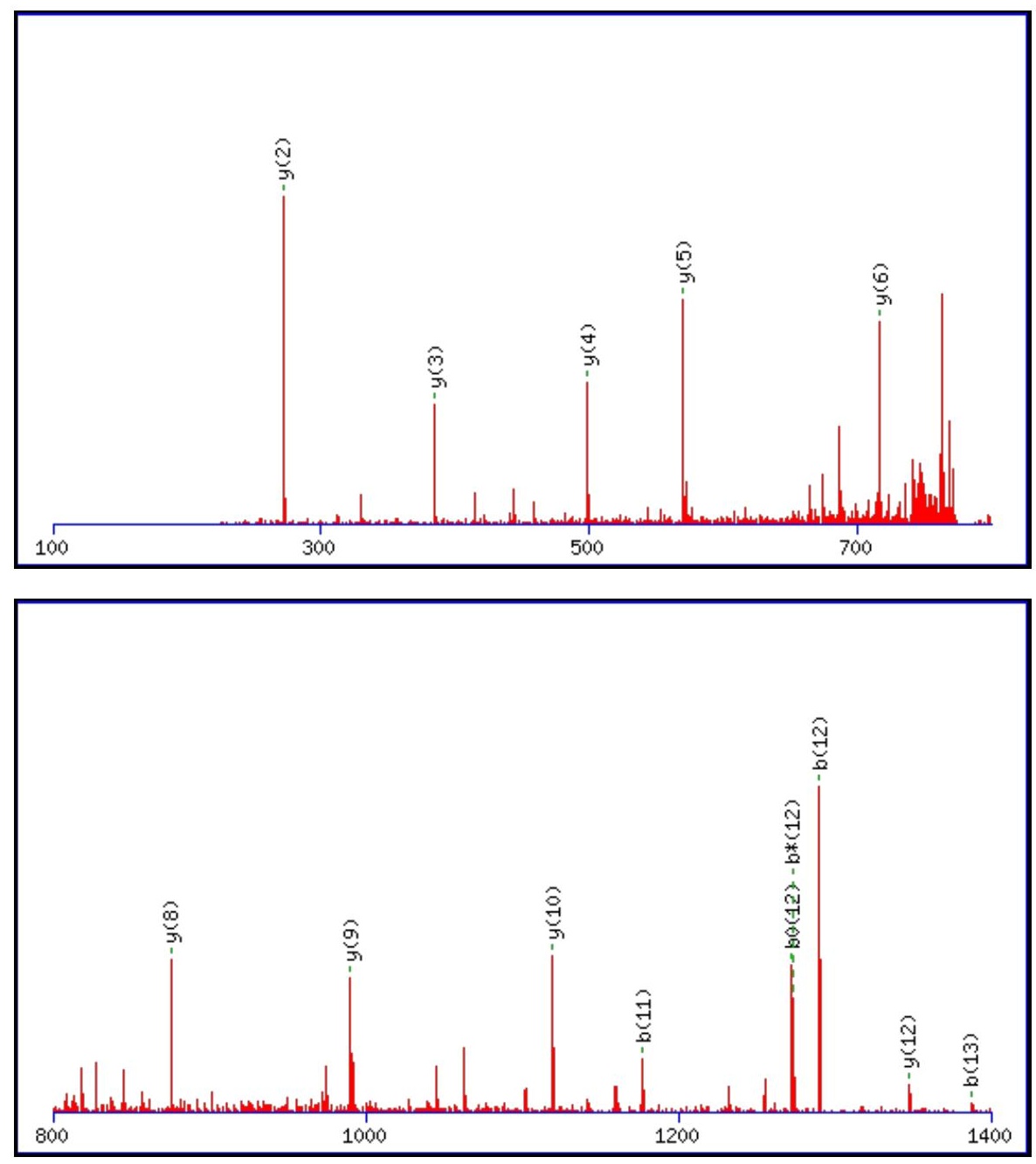


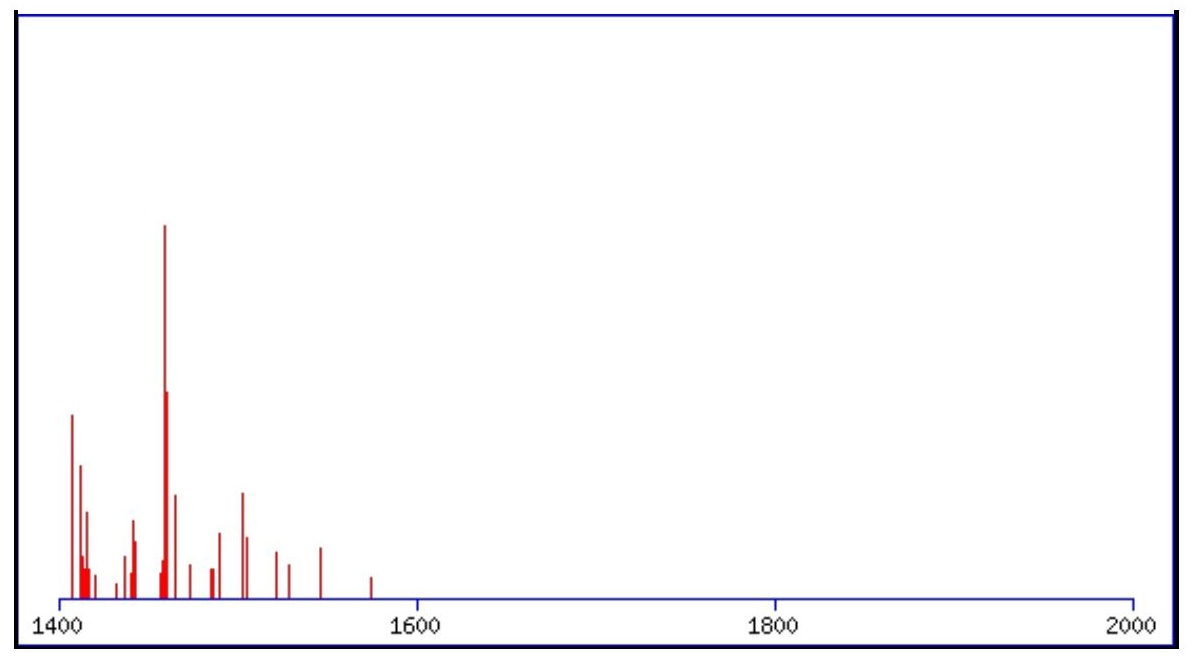

Monoisotopic mass of neutral peptide $\operatorname{Mr}($ calc): 1560.7784

Fixed modifications: Carbamidomethyl (C)

Variable modifications:

N6 : Deamidated $\mathrm{N}(\mathrm{N})$

Ions Score: 84 Expect: $7.1 \mathrm{e}-007$

Matches (Bold Red): 14/132 fragment ions using 26 most intense peaks

\begin{tabular}{|c|c|c|c|c|c|c|c|c|c|c|c|c|c|c|}
\hline \# & b & $\mathbf{b}^{++}$ & b* $^{*}$ & $\mathbf{b}^{*^{++}}$ & $\mathbf{b}^{0}$ & $\mathbf{b}^{0++}$ & Seq. & $\mathbf{y}$ & $y^{++}$ & $\mathrm{y}^{*}$ & $\mathrm{y}^{\mathrm{*}^{++}}$ & $\mathbf{y}^{0}$ & $y^{0++}$ & $\#$ \\
\hline 1 & 114.0913 & 57.5493 & & & & & I & & & & & & & 14 \\
\hline 2 & 215.1390 & 108.0731 & & & 197.1285 & 99.0679 & $\mathbf{T}$ & 1448.7016 & 724.8544 & 1431.6750 & 716.3412 & 1430.6910 & 715.8492 & 13 \\
\hline 3 & 330.1660 & 165.5866 & & & 312.1554 & 156.5813 & D & 1347.6539 & 674.3306 & 1330.6274 & 665.8173 & 1329.6433 & 665.3253 & 12 \\
\hline 4 & 443.2500 & 222.1287 & & & 425.2395 & 213.1234 & I & 1232.6270 & 616.8171 & 1215.6004 & 608.3038 & 1214.6164 & 607.8118 & 11 \\
\hline 5 & 572.2926 & 286.6499 & & & 554.2821 & 277.6447 & $\mathbf{E}$ & 1119.5429 & 560.2751 & 1102.5164 & 551.7618 & 1101.5323 & 551.2698 & 10 \\
\hline 6 & 687.3196 & 344.1634 & 670.2930 & 335.6501 & 669.3090 & 335.1581 & $\mathbf{N}$ & 990.5003 & 495.7538 & 973.4738 & 487.2405 & 972.4897 & 486.7485 & 9 \\
\hline 7 & 744.3410 & 372.6741 & 727.3145 & 364.1609 & 726.3305 & 363.6689 & G & 875.4734 & 438.2403 & 858.4468 & 429.7271 & 857.4628 & 429.2350 & 8 \\
\hline 8 & 845.3887 & 423.1980 & 828.3621 & 414.6847 & 827.3781 & 414.1927 & $T$ & 818.4519 & 409.7296 & 801.4254 & 401.2163 & 800.4413 & 400.7243 & 7 \\
\hline 9 & 992.4571 & 496.7322 & 975.4306 & 488.2189 & 974.4465 & 487.7269 & $\mathbf{F}$ & 717.4042 & 359.2058 & 700.3777 & 350.6925 & & & 6 \\
\hline 10 & 1063.4942 & 532.2508 & 1046.4677 & 523.7375 & 1045.4837 & 523.2455 & A & 570.3358 & 285.6715 & 553.3093 & 277.1583 & & & 5 \\
\hline 11 & 1177.5372 & 589.2722 & 1160.5106 & 580.7589 & 1159.5266 & 580.2669 & $\mathbf{N}$ & 499.2987 & 250.1530 & 482.2722 & 241.6397 & & & 4 \\
\hline 12 & 1290.6212 & 645.8142 & 1273.5947 & 637.3010 & 1272.6107 & 636.8090 & I & 385.2558 & 193.1315 & 368.2292 & 184.6183 & & & 3 \\
\hline 13 & 1387.6740 & 694.3406 & 1370.6474 & 685.8274 & 1369.6634 & 685.3353 & $\mathbf{P}$ & 272.1717 & 136.5895 & 255.1452 & 128.0762 & & & 2 \\
\hline 14 & & & & & & & $\mathbf{R}$ & 175.1190 & 88.0631 & 158.0924 & 79.5498 & & & 1 \\
\hline
\end{tabular}
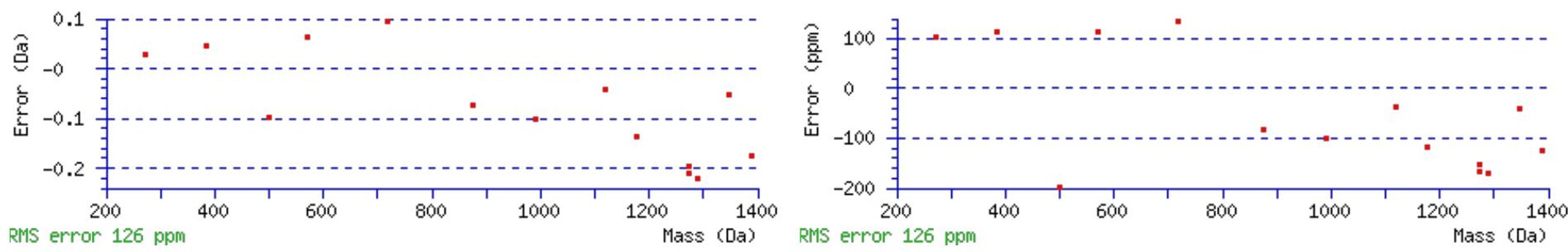

\section{All matches to this query}

\begin{tabular}{|l|l|l|l|}
\hline Score & Mr(calc): & Delta & \multicolumn{1}{c|}{ Sequence } \\
\hline 83.5 & 1560.7784 & -0.0013 & ITDIENGTFANIPR \\
\hline 38.6 & 1560.7784 & -0.0013 & ITDIENGTFANIPR \\
\hline 6.3 & 1560.7631 & 0.0140 & $\underline{\text { NSLKENSTSLNQPK }}$ \\
\hline 4.1 & 1558.7579 & 2.0192 & $\underline{\text { ATGGPLKSMTLFTR }}$ \\
\hline 3.0 & 1560.7661 & 0.0110 & $\underline{\text { TIIEYRSQKTSR }}$ \\
\hline 3.0 & 1560.7661 & 0.0110 & TIIEYRSQKTSR \\
\hline 2.5 & 1559.7878 & 0.9893 & $\underline{\text { RLXHTTMSNPAAAK }}$ \\
\hline & & & \\
\hline
\end{tabular}




\begin{tabular}{|l|l|l|l|}
1.9 & 1560.7864 & -0.0093 & DRARTQMMAEKPK \\
\hline 1.0 & 1558.7634 & 2.0137 & RLQGDKNGNMRPR \\
\hline 0.9 & 1560.7644 & 0.0127 & SPSWTKTRAEQNR \\
\hline
\end{tabular}

Spectrum No: 190; Query: 1421; Rank: 1

\section{Peptide View}

MS/MS Fragmentation of TILVDNNTWNNTHISR

Found in IPI00560990, Tax_Id=10116 Gene_Symbol=Stt3a_predicted similar to Oligosaccharyl transferase STT3 subunit homolog

Match to Query 1421: 1897.934048 from(949.974300,2+)

Title: 100101RatKid_NS_deglyco_12.2555.2555.2.dta

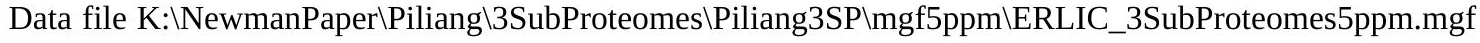
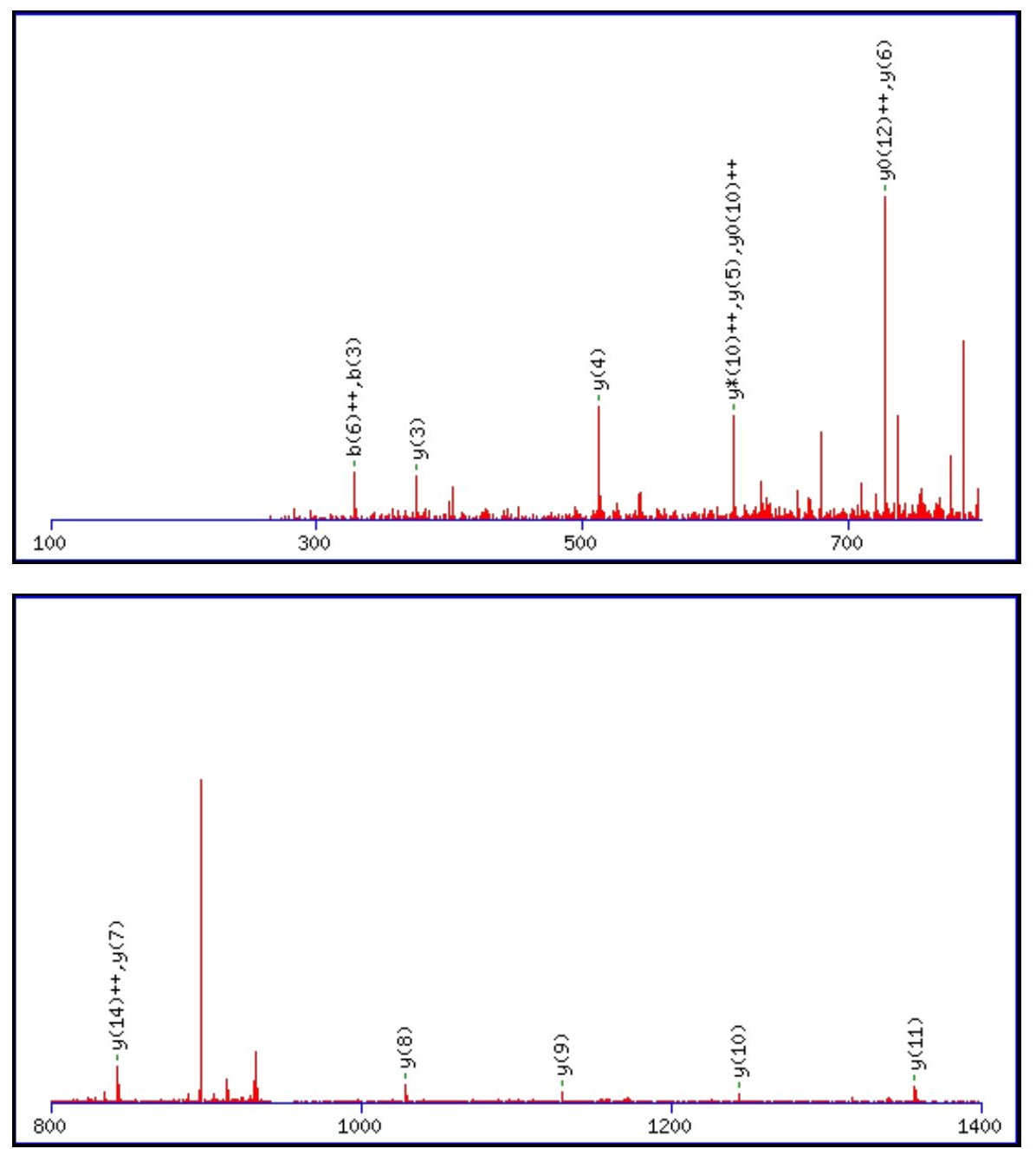


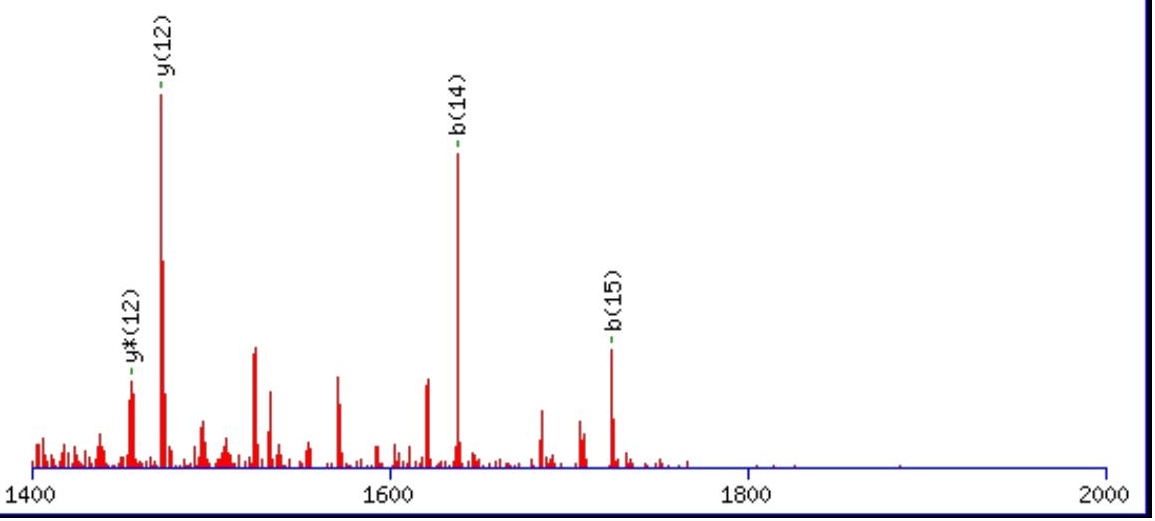

Monoisotopic mass of neutral peptide $\operatorname{Mr}($ calc): 1897.9282

Fixed modifications: Carbamidomethyl (C)

Variable modifications:

N10 : Deamidated_N (N)

Ions Score: 83 Expect: $8.3 \mathrm{e}-007$

Matches (Bold Red): 19/168 fragment ions using 29 most intense peaks

\begin{tabular}{|c|c|c|c|c|c|c|c|c|c|c|c|c|c|c|}
\hline \# & b & $\mathbf{b}^{++}$ & $\mathbf{b}^{*}$ & $\mathbf{b}^{*^{++}}$ & $\mathbf{b}^{0}$ & $\mathbf{b}^{\mathbf{0 + +}}$ & Seq. & $\mathbf{y}$ & $\mathbf{y}^{++}$ & $\mathbf{y}^{*}$ & $\mathbf{y}^{*^{++}}$ & $\mathbf{y}^{0}$ & $\mathbf{y}^{0++}$ & \# \\
\hline 1 & 102.0550 & 51.5311 & & & 84.0444 & 42.5258 & $\mathbf{T}$ & & & & & & & 16 \\
\hline 2 & 215.1390 & 108.0731 & & & 197.1285 & 99.0679 & I & 1797.8878 & 899.4476 & 1780.8613 & 890.9343 & 1779.8773 & 890.4423 & 15 \\
\hline 3 & 328.2231 & 164.6152 & & & 310.2125 & 155.6099 & $\mathbf{L}$ & 1684.8038 & 842.9055 & 1667.7772 & 834.3922 & 1666.7932 & 833.9002 & 14 \\
\hline 4 & 427.2915 & 214.1494 & & & 409.2809 & 205.1441 & $\mathbf{V}$ & 1571.7197 & 786.3635 & 1554.6932 & 777.8502 & 1553.7091 & 777.3582 & 13 \\
\hline 5 & 542.3184 & 271.6629 & & & 524.3079 & 262.6576 & D & 1472.6513 & 736.8293 & 1455.6247 & 728.3160 & 1454.6407 & 727.8240 & 12 \\
\hline 6 & 656.3614 & 328.6843 & 639.3348 & 320.1710 & 638.3508 & 319.6790 & $\mathbf{N}$ & 1357.6243 & 679.3158 & 1340.5978 & 670.8025 & 1339.6138 & 670.3105 & 11 \\
\hline 7 & 770.4043 & 385.7058 & 753.3777 & 377.1925 & 752.3937 & 376.7005 & $\mathbf{N}$ & 1243.5814 & 622.2943 & 1226.5549 & 613.7811 & 1225.5709 & 613.2891 & 10 \\
\hline 8 & 871.4520 & 436.2296 & 854.4254 & 427.7163 & 853.4414 & 427.2243 & $\mathbf{T}$ & 1129.5385 & 565.2729 & 1112.5119 & 556.7596 & 1111.5279 & 556.2676 & 9 \\
\hline 9 & 1057.5313 & 529.2693 & 1040.5047 & 520.7560 & 1039.5207 & 520.2640 & W & 1028.4908 & 514.7490 & 1011.4643 & 506.2358 & 1010.4802 & 505.7438 & 8 \\
\hline 10 & 1172.5582 & 586.7827 & 1155.5317 & 578.2695 & 1154.5477 & 577.7775 & $\mathbf{N}$ & 842.4115 & 421.7094 & 825.3849 & 413.1961 & 824.4009 & 412.7041 & 7 \\
\hline 11 & 1286.6011 & 643.8042 & 1269.5746 & 635.2909 & 1268.5906 & 634.7989 & $\mathbf{N}$ & 727.3846 & 364.1959 & 710.3580 & 355.6826 & 709.3740 & 355.1906 & 6 \\
\hline 12 & 1387.6488 & 694.3281 & 1370.6223 & 685.8148 & 1369.6383 & 685.3228 & $\mathbf{T}$ & 613.3416 & 307.1745 & 596.3151 & 298.6612 & 595.3311 & 298.1692 & 5 \\
\hline 13 & 1524.7077 & 762.8575 & 1507.6812 & 754.3442 & 1506.6972 & 753.8522 & $\mathbf{H}$ & 512.2940 & 256.6506 & 495.2674 & 248.1373 & 494.2834 & 247.6453 & 4 \\
\hline 14 & 1637.7918 & 819.3995 & 1620.7653 & 810.8863 & 1619.7812 & 810.3943 & I & 375.2350 & 188.1212 & 358.2085 & 179.6079 & 357.2245 & 179.1159 & 3 \\
\hline 15 & 1724.8238 & 862.9156 & 1707.7973 & 854.4023 & 1706.8133 & 853.9103 & S & 262.1510 & 131.5791 & 245.1244 & 123.0659 & 244.1404 & 122.5738 & 2 \\
\hline 16 & & & & & & & $\mathbf{R}$ & 175.1190 & 88.0631 & 158.0924 & 79.5498 & & & 1 \\
\hline
\end{tabular}

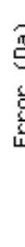
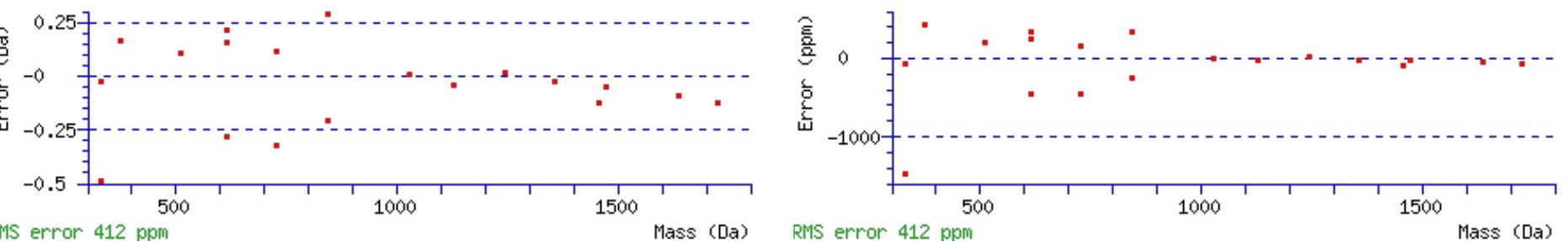

\section{All matches to this query}

\begin{tabular}{|l|c|c|c|}
\hline Score & Mr(calc): & Delta & Sequence \\
\hline 83.5 & 1897.9282 & 0.0058 & TILVDNNTWNNTHISR \\
\hline 71.5 & 1897.9282 & 0.0058 & TILVDNNTWNNTHISR \\
\hline 40.7 & 1897.9282 & 0.0058 & TILVDNNTWNNTHISR \\
\hline 30.9 & 1897.9282 & 0.0058 & TILVDNNTWNNTHISR \\
\hline 7.0 & 1897.9519 & -0.0178 & VDISMGLICVRLLSSR \\
\hline & & & \\
\hline
\end{tabular}




\begin{tabular}{|l|l|l|l|}
3.8 & 1896.9337 & 1.0004 & QRLNGQCXNKSETLHR \\
\hline 3.7 & 1896.9234 & 1.0107 & VLNASAIPEGQFIDSKK \\
\hline 3.1 & 1895.9434 & 1.9906 & GSDSLSILFSYVFILR \\
\hline 2.9 & 1897.9186 & 0.0154 & LLIYNTNNLQTGIPSR \\
\hline 2.9 & 1897.9186 & 0.0154 & LLIYNTNNLQTGIPSR \\
\hline
\end{tabular}

Spectrum No: 191; Query: 1111; Rank: 1

\section{Peptide View}

MS/MS Fragmentation of SCVTITDAFPQNMSR

Found in IPI00370450, Tax_Id=10116 Gene_Symbol=Plxnb2 plexin B2

Match to Query 1111: 1726.768668 from(864.391610,2+)

Title: 100101RatKid_NS_deglyco_25.2968.2968.2.dta

Data file K:INewmanPaper|Piliangl3SubProteomes\Piliang3SP\mgf5ppm\ERLIC_3SubProteomes5ppm.mgf
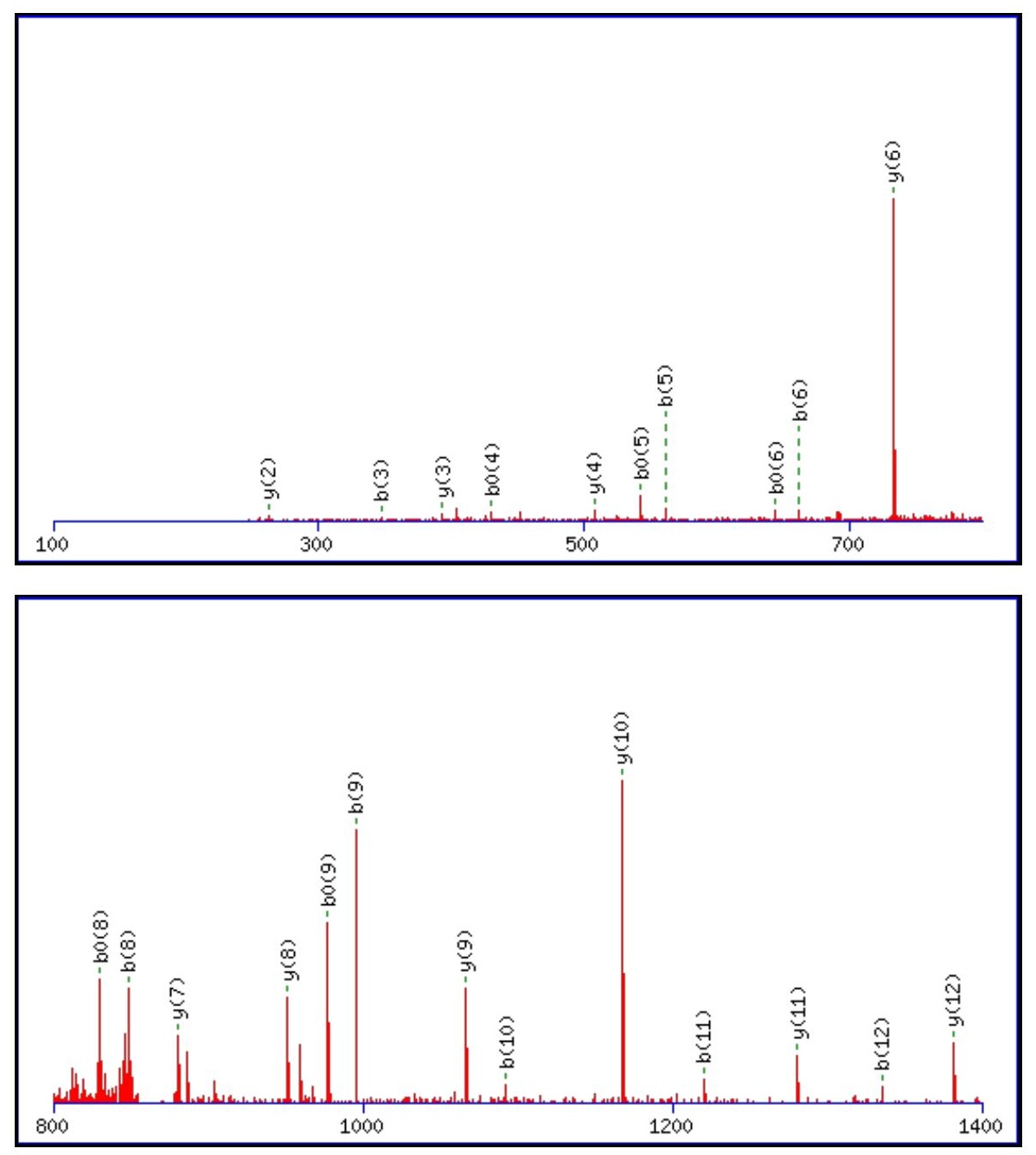


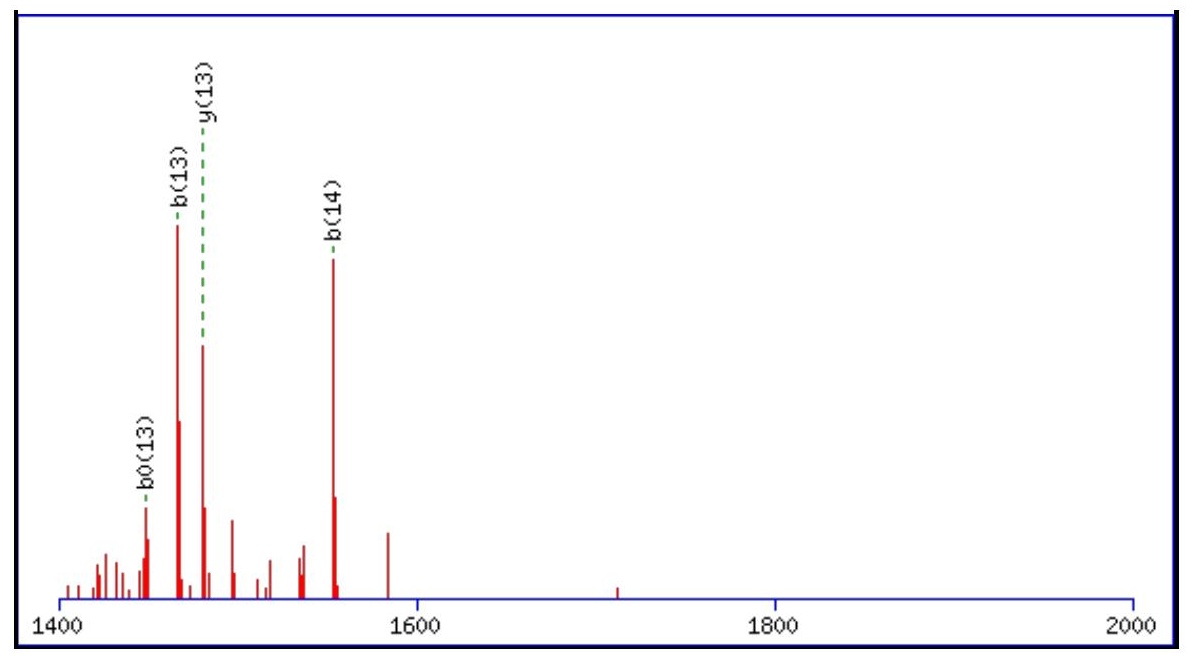

Monoisotopic mass of neutral peptide $\operatorname{Mr}($ calc): 1726.7655

Fixed modifications: Carbamidomethyl (C)

Variable modifications:

N12 : Deamidated_N (N)

Ions Score: 83 Expect: $9.6 \mathrm{e}-007$

Matches (Bold Red): 27/146 fragment ions using 45 most intense peaks

\begin{tabular}{|c|c|c|c|c|c|c|c|c|c|c|c|c|c|c|}
\hline \# & b & $\mathbf{b}^{++}$ & b* & $\mathbf{b}^{*^{++}}$ & $\mathbf{b}^{\mathbf{0}}$ & $\mathbf{b}^{0++}$ & Seq. & $\mathbf{y}$ & $y^{++}$ & $\mathbf{y}^{*}$ & $\mathrm{y}^{\mathrm{*}^{++}}$ & $\mathbf{y}^{\mathbf{0}}$ & $\mathbf{y}^{\mathbf{0 + +}}$ & \# \\
\hline 1 & 88.0393 & 44.5233 & & & 70.0287 & 35.5180 & S & & & & & & & 15 \\
\hline 2 & 248.0700 & 124.5386 & & & 230.0594 & 115.5333 & C & 1640.7407 & 820.8740 & 1623.7142 & 812.3607 & 1622.7301 & 811.8687 & 14 \\
\hline 3 & 347.1384 & 174.0728 & & & 329.1278 & 165.0675 & V & 1480.7100 & 740.8587 & 1463.6835 & 732.3454 & 1462.6995 & 731.8534 & 13 \\
\hline 4 & 448.1860 & 224.5967 & & & 430.1755 & 215.5914 & $\mathbf{T}$ & 1381.6416 & 691.3245 & 1364.6151 & 682.8112 & 1363.6311 & 682.3192 & 12 \\
\hline 5 & 561.2701 & 281.1387 & & & 543.2595 & 272.1334 & I & 1280.5940 & 640.8006 & 1263.5674 & 632.2873 & 1262.5834 & 631.7953 & 11 \\
\hline 6 & 662.3178 & 331.6625 & & & 644.3072 & 322.6573 & $\mathbf{T}$ & 1167.5099 & 584.2586 & 1150.4833 & 575.7453 & 1149.4993 & 575.2533 & 10 \\
\hline 7 & 777.3447 & 389.1760 & & & 759.3342 & 380.1707 & D & 1066.4622 & 533.7347 & 1049.4357 & 525.2215 & 1048.4516 & 524.7295 & 9 \\
\hline 8 & 848.3818 & 424.6946 & & & 830.3713 & 415.6893 & A & 951.4353 & 476.2213 & 934.4087 & 467.7080 & 933.4247 & 467.2160 & 8 \\
\hline 9 & 995.4503 & 498.2288 & & & 977.4397 & 489.2235 & $\mathbf{F}$ & 880.3982 & 440.7027 & 863.3716 & 432.1894 & 862.3876 & 431.6974 & 7 \\
\hline 10 & 1092.5030 & 546.7551 & & & 1074.4925 & 537.7499 & $\mathbf{P}$ & 733.3297 & 367.1685 & 716.3032 & 358.6552 & 715.3192 & 358.1632 & 6 \\
\hline 11 & 1220.5616 & 610.7844 & 1203.5351 & 602.2712 & 1202.5510 & 601.7792 & $\mathbf{Q}$ & 636.2770 & 318.6421 & 619.2504 & 310.1289 & 618.2664 & 309.6368 & 5 \\
\hline 12 & 1335.5885 & 668.2979 & 1318.5620 & 659.7846 & 1317.5780 & 659.2926 & $\mathbf{N}$ & 508.2184 & 254.6128 & 491.1919 & 246.0996 & 490.2078 & 245.6076 & 4 \\
\hline 13 & 1466.6290 & 733.8181 & 1449.6025 & 725.3049 & 1448.6185 & 724.8129 & $\mathbf{M}$ & 393.1915 & 197.0994 & 376.1649 & 188.5861 & 375.1809 & 188.0941 & 3 \\
\hline 14 & 1553.6611 & 777.3342 & 1536.6345 & 768.8209 & 1535.6505 & 768.3289 & $\mathrm{~S}$ & 262.1510 & 131.5791 & 245.1244 & 123.0659 & 244.1404 & 122.5738 & 2 \\
\hline 15 & & & & & & & $\mathbf{R}$ & 175.1190 & 88.0631 & 158.0924 & 79.5498 & & & 1 \\
\hline
\end{tabular}

$$
\text { RI }
$$

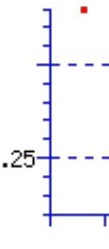

RMS error 165 ppm

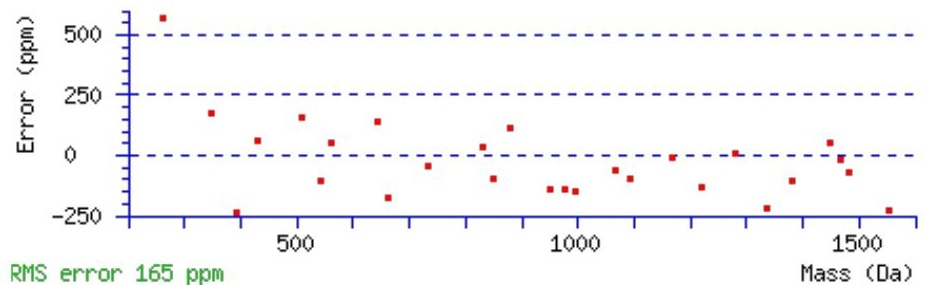

Mass (Da) RMS error 165 ppm

\section{All matches to this query}

\begin{tabular}{|l|l|l|l|}
\hline Score & Mr(calc): & Delta & \multicolumn{1}{c|}{ Sequence } \\
\hline 83.2 & 1726.7655 & 0.0032 & $\underline{\text { SCVTITDAFPQNMSR }}$ \\
\hline 20.3 & 1725.7814 & 0.9872 & SCVTITDAFPQNMSR \\
\hline 6.4 & 1726.7758 & -0.0071 & YRSAGSEETSESPTAR \\
\hline 3.2 & 1726.7727 & -0.0040 & ASAKCGTGGKQMEGXSR \\
\hline 3.2 & 1726.7644 & 0.0043 & NTMEKLRCVHTSR \\
\hline 2.6 & 1726.7603 & 0.0083 & FYQDTISALNFTAR \\
\hline & & & \\
\hline
\end{tabular}




\begin{tabular}{|l|l|l|l|}
2.6 & 1726.7603 & 0.0083 & FYQDTISALNFTAR \\
\hline 2.5 & 1724.7535 & 2.0151 & SLKPESGGNHIADLK \\
\hline 2.1 & 1726.7610 & 0.0076 & MPVSRGRDSYGGPPR \\
\hline 1.8 & 1726.7767 & -0.0080 & DGMNIVEAMERFGSR \\
\hline
\end{tabular}

Spectrum No: 192; Query: 263; Rank: 1

\section{Peptide View}

MS/MS Fragmentation of LDFIVLNETR

Found in IPI00208422, Tax_Id=10116 Gene_Symbol=Dpp4 Dipeptidyl peptidase 4

Match to Query 263: 1219.648868 from(610.831710,2+)

Title: 100101RatKid_NS_deglyco_12.4324.4324.2.dta

Data file K:\NewmanPaper\Piliang \3SubProteomes \Piliang3SP $\backslash$ mgf5ppm\ERLIC_3SubProteomes5ppm.mgf
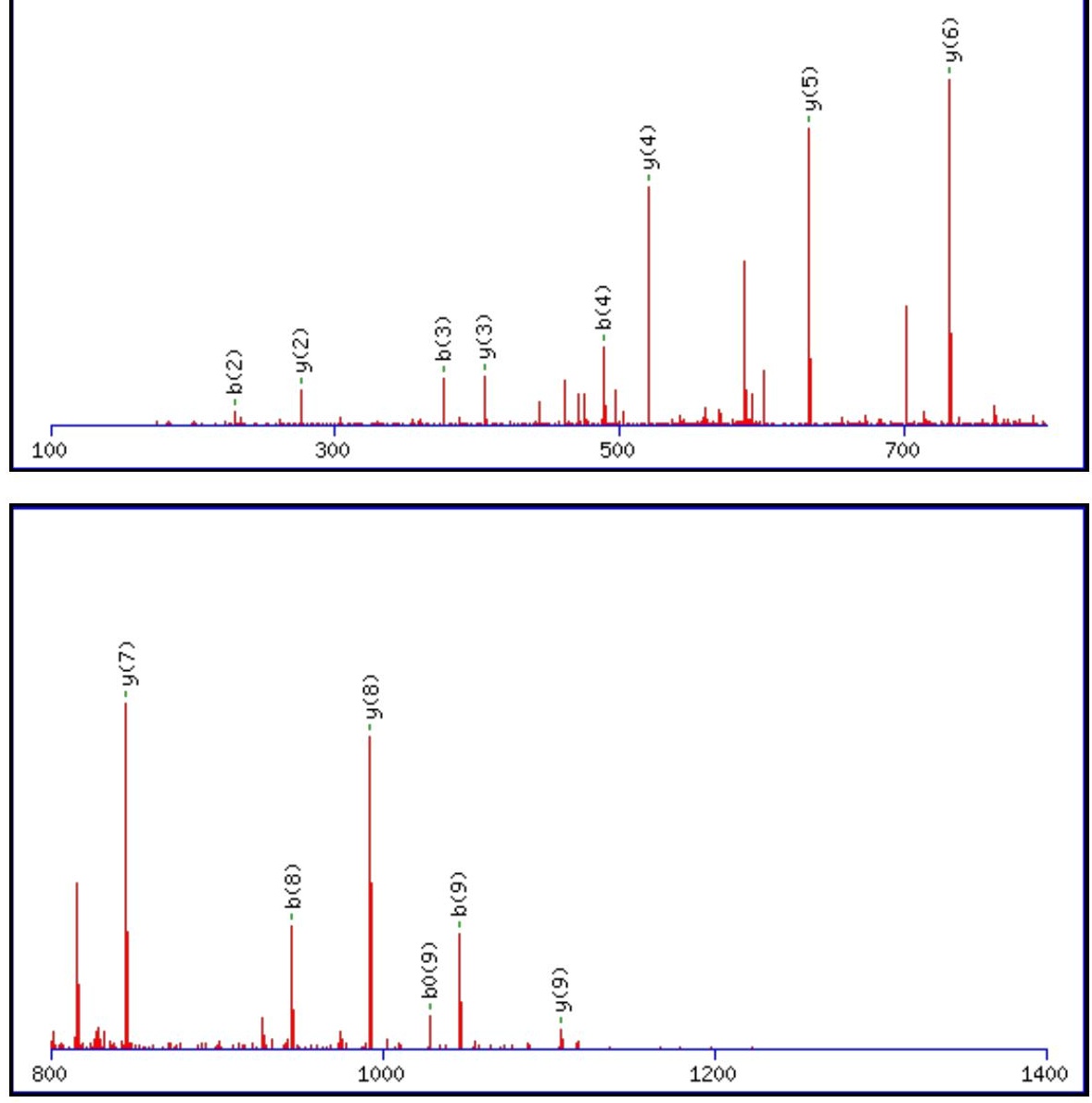


$1400 \quad 1600 \quad 1800 \quad 2000$

Monoisotopic mass of neutral peptide $\operatorname{Mr}($ calc): 1219.6448

Fixed modifications: Carbamidomethyl (C)

Variable modifications:

N7 : Deamidated_N (N)

Ions Score: 83 Expect : $6.1 \mathrm{e}-007$

Matches (Bold Red): 14/92 fragment ions using 24 most intense peaks

\begin{tabular}{|r|c|c|c|c|c|c|c|c|c|c|c|c|c|c|}
\hline$\#$ & $\mathbf{b}$ & $\mathbf{b}^{++}$ & $\mathbf{b}^{*}$ & $\mathbf{b}^{\mathbf{*}^{++}}$ & $\mathbf{b}^{\mathbf{0}}$ & $\mathbf{b}^{\mathbf{0 + +}}$ & $\mathbf{S e q}$ & $\mathbf{y}$ & $\mathbf{y}^{++}$ & $\mathbf{y}^{\mathbf{*}}$ & $\mathbf{y}^{\mathbf{*}^{++}}$ & $\mathbf{y}^{\mathbf{0}}$ & $\mathbf{y}^{\mathbf{0 + +}}$ & $\#$ \\
\hline $\mathbf{1}$ & 114.0913 & 57.5493 & & & & & $\mathbf{L}$ & & & & & & & $\mathbf{1 0}$ \\
\hline $\mathbf{2}$ & $\mathbf{2 2 9 . 1 1 8 3}$ & 115.0628 & & & 211.1077 & 106.0575 & $\mathbf{D}$ & $\mathbf{1 1 0 7 . 5 6 8}$ & 554.2877 & 1090.5415 & 545.7744 & 1089.5575 & 545.2824 & $\mathbf{9}$ \\
\hline $\mathbf{3}$ & $\mathbf{3 7 6 . 1 8 6 7}$ & 188.5970 & & & 358.1761 & 179.5917 & $\mathbf{F}$ & $\mathbf{9 9 2 . 5 4 1 1}$ & 496.7742 & 975.5146 & 488.2609 & 974.5306 & 487.7689 & $\mathbf{8}$ \\
\hline $\mathbf{4}$ & $\mathbf{4 8 9 . 2 7 0 8}$ & 245.1390 & & & 471.2602 & 236.1337 & $\mathbf{I}$ & $\mathbf{8 4 5 . 4 7 2 7}$ & 423.2400 & 828.4462 & 414.7267 & 827.4621 & 414.2347 & $\mathbf{7}$ \\
\hline $\mathbf{5}$ & 588.3392 & 294.6732 & & & 570.3286 & 285.6679 & $\mathbf{V}$ & $\mathbf{7 3 2 . 3 8 8 6}$ & 366.6980 & 715.3621 & 358.1847 & 714.3781 & 357.6927 & $\mathbf{6}$ \\
\hline $\mathbf{6}$ & 701.4232 & 351.2153 & & & 683.4127 & 342.2100 & $\mathbf{L}$ & $\mathbf{6 3 3 . 3 2 0 2}$ & 317.1638 & 616.2937 & 308.6505 & 615.3097 & 308.1585 & $\mathbf{5}$ \\
\hline $\mathbf{7}$ & 816.4502 & 408.7287 & 799.4236 & 400.2155 & 798.4396 & 399.7234 & $\mathbf{N}$ & $\mathbf{5 2 0 . 2 3 6 2}$ & 260.6217 & 503.2096 & 252.1084 & 502.2256 & 251.6164 & $\mathbf{4}$ \\
\hline $\mathbf{8}$ & $\mathbf{9 4 5 . 4 9 2 8}$ & 473.2500 & 928.4662 & 464.7367 & 927.4822 & 464.2447 & $\mathbf{E}$ & $\mathbf{4 0 5 . 2 0 9 2}$ & 203.1082 & 388.1827 & 194.5950 & 387.1987 & 194.1030 & $\mathbf{3}$ \\
\hline $\mathbf{9}$ & $\mathbf{1 0 4 6 . 5 4 0 4}$ & 523.7739 & 1029.5139 & 515.2606 & $\mathbf{1 0 2 8 . 5 2 9 9}$ & 514.7686 & $\mathbf{T}$ & $\mathbf{2 7 6 . 1 6 6 6}$ & 138.5870 & 259.1401 & 130.0737 & 258.1561 & 129.5817 & $\mathbf{2}$ \\
\hline $\mathbf{1 0}$ & & & & & & & $\mathbf{R}$ & 175.1190 & 88.0631 & 158.0924 & 79.5498 & & & $\mathbf{1}$ \\
\hline
\end{tabular}

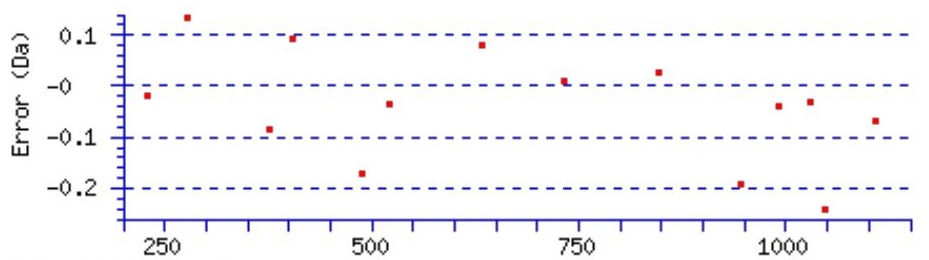

RNS error 204 ppm

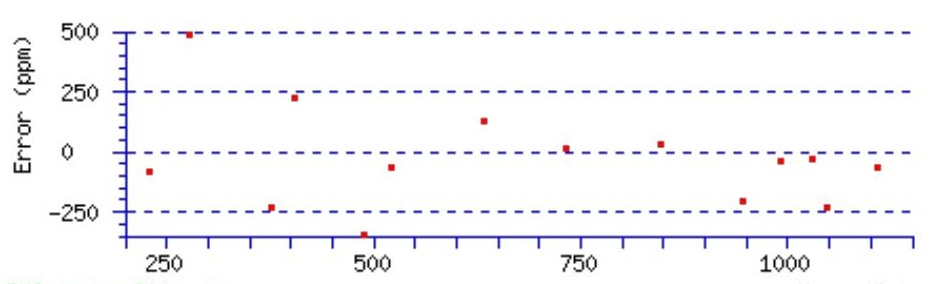

RMS error 204 ppm

\section{All matches to this query}

\begin{tabular}{|l|l|l|l|}
\hline Score & Mr(calc): & Delta & \multicolumn{1}{|c|}{ Sequence } \\
\hline 83.1 & 1219.6448 & 0.0040 & LDFIVLNETR \\
\hline 9.9 & 1218.6431 & 1.0058 & YQPVVDLVMR \\
\hline 4.9 & 1218.6456 & 1.0033 & TSSVLEGSALQK \\
\hline 4.0 & 1219.6482 & 0.0007 & NLEIKKSMEK \\
\hline 2.5 & 1218.6469 & 1.0020 & NLQNFITGRR \\
\hline 2.0 & 1218.6397 & 1.0092 & NLFLSNVFHK \\
\hline 1.4 & 1218.6413 & 1.0075 & VKFYIDVKK \\
\hline 0.8 & 1217.6438 & 2.0051 & LMDDLLNKTR \\
\hline 0.8 & 1219.6448 & 0.0040 & KFDQLLAEEK \\
\hline 0.6 & 1219.6449 & 0.0040 & VQSGVVINFEK \\
\hline
\end{tabular}


Spectrum No: 193; Query: 784; Rank: 1

\section{Peptide View}

MS/MS Fragmentation of TCNPETFPLNESR

Found in IPI00366496, Tax_Id=10116 Gene_Symbol=Slc44a2_predicted similar to CTL2 gene

Match to Query 784: 1564.680988 from(783.347770,2+)

Title: 091008RatKidney_NH4Format01_27.1596.1596.2.dta

Data file K:\NewmanPaper\Piliang \3SubProteomes \Piliang3SP $\backslash$ mgf5ppm\ERLIC_3SubProteomes5ppm.mgf
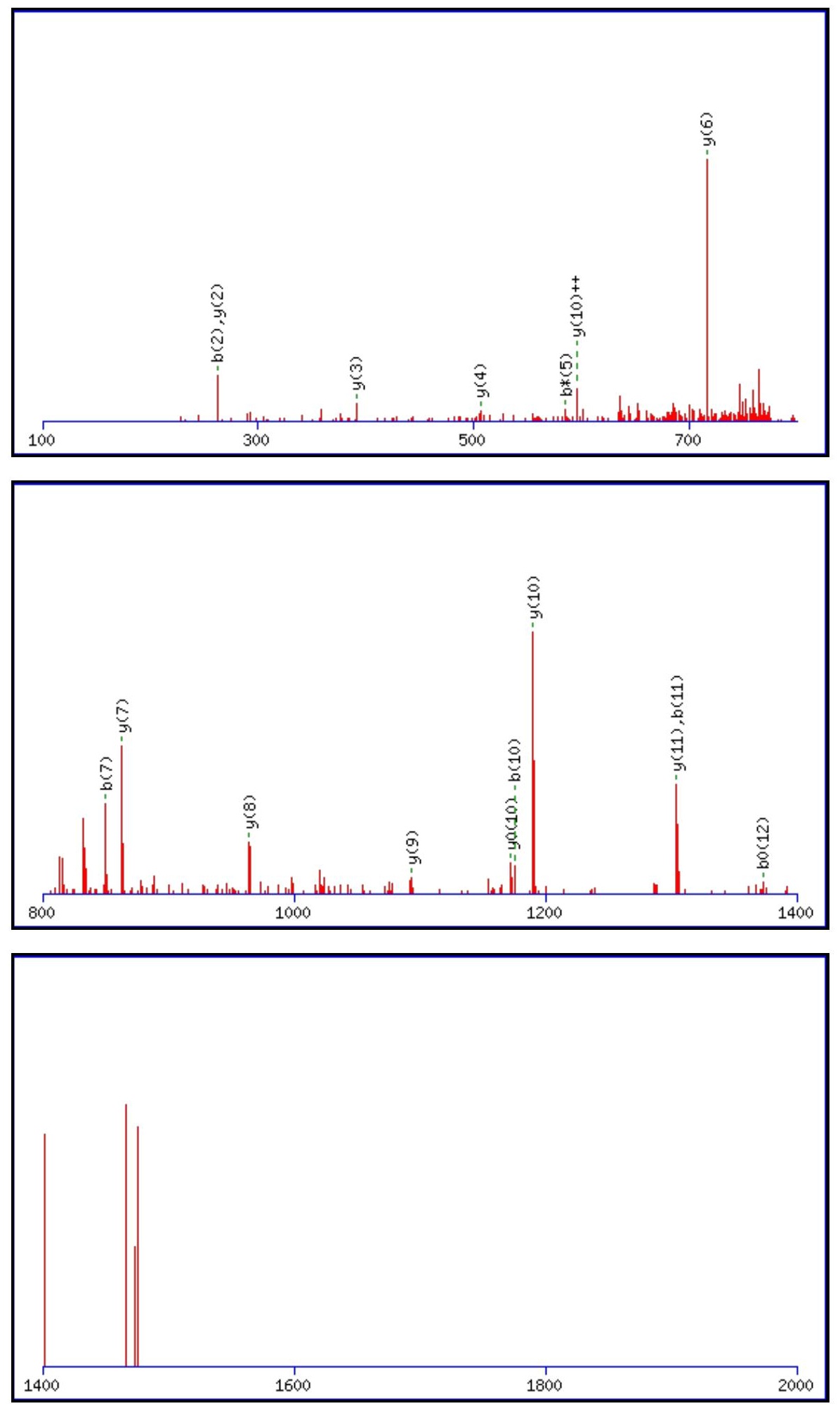

Monoisotopic mass of neutral peptide $\operatorname{Mr}($ calc): 1564.6828 
Fixed modifications: Carbamidomethyl (C)
Variable modifications:

N10 : Deamidated_N (N)

Ions Score: 83 Expect: $7.9 \mathrm{e}-007$

Matches (Bold Red): 17/138 fragment ions using 24 most intense peaks

\begin{tabular}{|r|c|c|c|c|r|r|r|r|c|c|c|c|c|c|}
\hline$\#$ & $\mathbf{b}$ & $\mathbf{b}^{++}$ & $\mathbf{b}^{*}$ & $\mathbf{b}^{\mathbf{*}^{++}}$ & $\mathbf{b}^{\mathbf{0}}$ & $\mathbf{b}^{\mathbf{0 + +}}$ & $\mathbf{S e q}$ & $\mathbf{y}$ & $\mathbf{y}^{++}$ & $\mathbf{y}^{\mathbf{*}}$ & $\mathbf{y}^{\mathbf{*}^{++}}$ & $\mathbf{y}^{\mathbf{0}}$ & $\mathbf{y}^{\mathbf{0 + +}}$ & $\#$ \\
\hline $\mathbf{1}$ & 102.0550 & 51.5311 & & & 84.0444 & 42.5258 & $\mathbf{T}$ & & & & & & & $\mathbf{1 3}$ \\
\hline $\mathbf{2}$ & $\mathbf{2 6 2 . 0 8 5 6}$ & 131.5464 & & & 244.0750 & 122.5412 & $\mathbf{C}$ & 1464.6424 & 732.8248 & 1447.6158 & 724.3115 & 1446.6318 & 723.8195 & $\mathbf{1 2}$ \\
\hline $\mathbf{3}$ & 376.1285 & 188.5679 & 359.1020 & 180.0546 & 358.1180 & 179.5626 & $\mathbf{N}$ & $\mathbf{1 3 0 4 . 6 1 1 7}$ & 652.8095 & 1287.5852 & 644.2962 & 1286.6011 & 643.8042 & $\mathbf{1 1}$ \\
\hline $\mathbf{4}$ & 473.1813 & 237.0943 & 456.1547 & 228.5810 & 455.1707 & 228.0890 & $\mathbf{P}$ & $\mathbf{1 1 9 0 . 5 6 8 8}$ & 595.7880 & 1173.5422 & 587.2748 & $\mathbf{1 1 7 2 . 5 5 8 2}$ & 586.7827 & $\mathbf{1 0}$ \\
\hline $\mathbf{5}$ & 602.2239 & 301.6156 & 585.1973 & 293.1023 & 584.2133 & 292.6103 & $\mathbf{E}$ & $\mathbf{1 0 9 3 . 5 1 6 0}$ & 547.2616 & 1076.4895 & 538.7484 & 1075.5055 & 538.2564 & $\mathbf{9}$ \\
\hline $\mathbf{6}$ & 703.2716 & 352.1394 & 686.2450 & 343.6261 & 685.2610 & 343.1341 & $\mathbf{T}$ & $\mathbf{9 6 4 . 4 7 3 4}$ & 482.7404 & 947.4469 & 474.2271 & 946.4629 & 473.7351 & $\mathbf{8}$ \\
\hline $\mathbf{7}$ & $\mathbf{8 5 0 . 3 4 0 0}$ & 425.6736 & 833.3134 & 417.1604 & 832.3294 & 416.6683 & $\mathbf{F}$ & $\mathbf{8 6 3 . 4 2 5 8}$ & 432.2165 & 846.3992 & 423.7032 & 845.4152 & 423.2112 & $\mathbf{7}$ \\
\hline $\mathbf{8}$ & 947.3927 & 474.2000 & 930.3662 & 465.6867 & 929.3822 & 465.1947 & $\mathbf{P}$ & $\mathbf{7 1 6 . 3 5 7 3}$ & 358.6823 & 699.3308 & 350.1690 & 698.3468 & 349.6770 & $\mathbf{6}$ \\
\hline $\mathbf{9}$ & 1060.4768 & 530.7420 & 1043.4503 & 522.2288 & 1042.4662 & 521.7368 & $\mathbf{L}$ & 619.3046 & 310.1559 & 602.2780 & 301.6426 & 601.2940 & 301.1506 & $\mathbf{5}$ \\
\hline $\mathbf{1 0}$ & $\mathbf{1 1 7 5 . 5 0 3 7}$ & 588.2555 & 1158.4772 & 579.7422 & 1157.4932 & 579.2502 & $\mathbf{N}$ & $\mathbf{5 0 6 . 2 2 0 5}$ & 253.6139 & 489.1940 & 245.1006 & 488.2099 & 244.6086 & $\mathbf{4}$ \\
\hline $\mathbf{1 1}$ & $\mathbf{1 3 0 4 . 5 4 6 3}$ & 652.7768 & 1287.5198 & 644.2635 & 1286.5358 & 643.7715 & $\mathbf{E}$ & $\mathbf{3 9 1 . 1 9 3 6}$ & 196.1004 & 374.1670 & 187.5871 & 373.1830 & 187.0951 & $\mathbf{3}$ \\
\hline $\mathbf{1 2}$ & 1391.5784 & 696.2928 & 1374.5518 & 687.7795 & $\mathbf{1 3 7 3 . 5 6 7 8}$ & 687.2875 & $\mathbf{S}$ & $\mathbf{2 6 2 . 1 5 1 0}$ & 131.5791 & 245.1244 & 123.0659 & 244.1404 & 122.5738 & $\mathbf{2}$ \\
\hline $\mathbf{1 3}$ & & & & & & & $\mathbf{R}$ & 175.1190 & 88.0631 & 158.0924 & 79.5498 & & & $\mathbf{1}$ \\
\hline
\end{tabular}
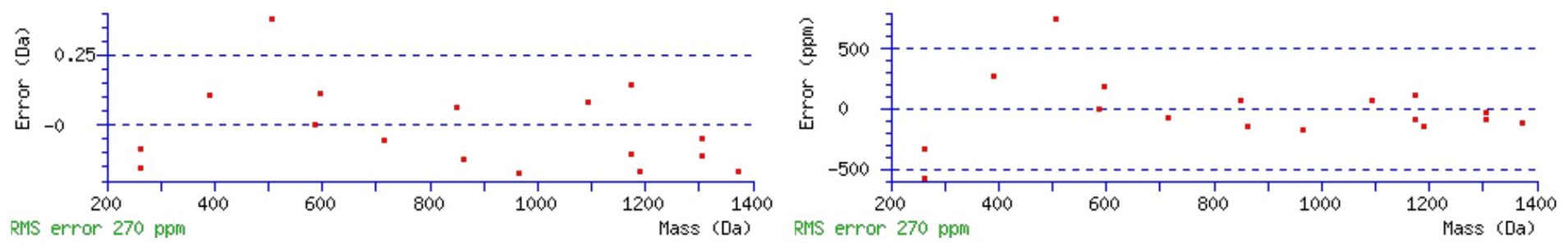

\section{All matches to this query}

\begin{tabular}{|l|c|c|l|}
\hline Score & Mr(calc): & Delta & \multicolumn{1}{|c|}{ Sequence } \\
\hline 83.0 & 1564.6828 & -0.0018 & TCNPETFPLNESR \\
\hline 10.1 & 1564.6884 & -0.0074 & EYTVDMPFKINK \\
\hline 9.1 & 1564.6810 & -0.0000 & QTYEQANKIFDK \\
\hline 9.1 & 1564.6810 & -0.0000 & QTYEQANKIFDK \\
\hline 7.0 & 1562.6800 & 2.0010 & CYLKLSSNGSPTR \\
\hline 7.0 & 1562.6800 & 2.0010 & CYLKLSSNGSPTR \\
\hline 6.8 & 1562.6654 & 2.0156 & SRYAELDFEPEK \\
\hline 4.1 & 1564.6658 & 0.0152 & LSSAYSLSASEQDK \\
\hline 3.2 & 1564.6828 & -0.0018 & TCNPETFPLNESR \\
\hline 2.9 & 1563.6698 & 1.0112 & DEEVAAFCHMLDK \\
\hline
\end{tabular}

Spectrum No: 194; Query: 1561; Rank: 1

\section{Peptide View}

MS/MS Fragmentation of VNASTTDPNSTVEQSALTR

Found in IPI00365542, Tax_Id=10116 Gene_Symbol=Lamb1_predicted laminin, beta 1

Match to Query 1561: 1990.942648 from(996.478600,2+)

Title: 091008RatKidney_NoSalt_22.1376.1376.2.dta

Data file K:INewmanPaper|Piliangl3SubProteomes\Piliang3SP\mgf5ppm\ERLIC_3SubProteomes5ppm.mgf 

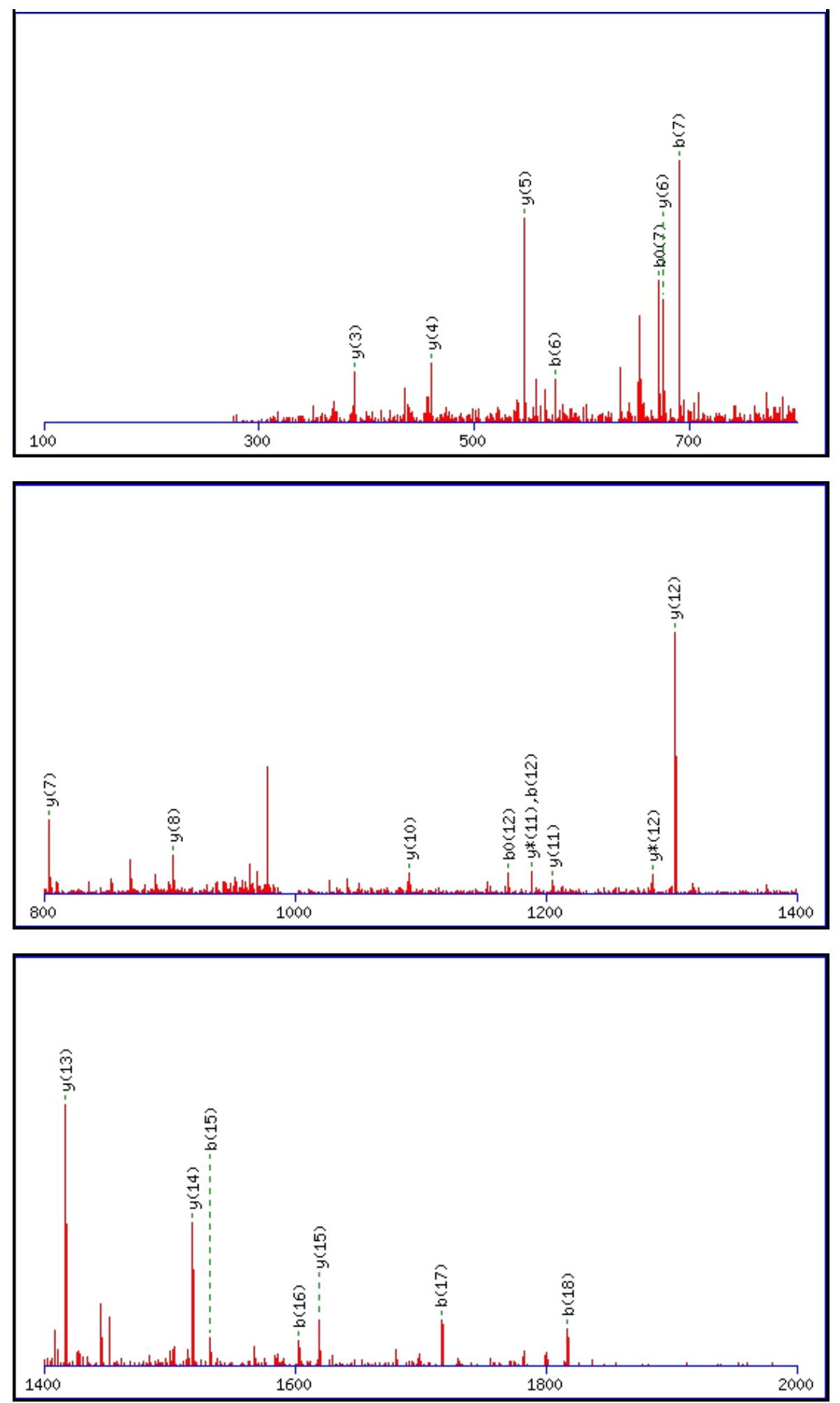

Monoisotopic mass of neutral peptide $\operatorname{Mr}($ calc): 1990.9443

Fixed modifications: Carbamidomethyl (C)

Variable modifications:

N2 : Deamidated $\mathrm{N}(\mathrm{N})$

Ions Score: 83 Expect: $1.2 \mathrm{e}-006$

Matches (Bold Red): 23/206 fragment ions using 43 most intense peaks

\begin{tabular}{|r|c|c|c|c|c|c|c|c|c|c|c|c|c|c|}
\hline$\#$ & $\mathbf{b}$ & $\mathbf{b}^{++}$ & $\mathbf{b}^{*}$ & $\mathbf{b}^{\boldsymbol{*}^{++}}$ & $\mathbf{b}^{\mathbf{0}}$ & $\mathbf{b}^{\mathbf{0 + +}}$ & Seq. & $\mathbf{y}$ & $\mathbf{y}^{++}$ & $\mathbf{y}^{\mathbf{*}}$ & $\mathbf{y}^{\boldsymbol{*}^{++}}$ & $\mathbf{y}^{\mathbf{0}}$ & $\mathbf{y}^{\mathbf{0 + +}}$ & $\#$ \\
\hline $\mathbf{1}$ & 100.0757 & 50.5415 & & & & & $\mathbf{V}$ & & & & & & & $\mathbf{1 9}$ \\
\hline $\mathbf{2}$ & 215.1026 & 108.0550 & 198.0761 & 99.5417 & & & $\mathbf{N}$ & 1892.8832 & 946.9452 & 1875.8567 & 938.4320 & 1874.8726 & 937.9400 & $\mathbf{1 8}$ \\
\hline 3 & 286.1397 & 143.5735 & 269.1132 & 135.0602 & & & A & 1777.8563 & 889.4318 & 1760.8297 & 880.9185 & 1759.8457 & 880.4265 & $\mathbf{1 7}$ \\
\hline $\mathbf{4}$ & 373.1718 & 187.0895 & 356.1452 & 178.5762 & 355.1612 & 178.0842 & S & 1706.8191 & 853.9132 & 1689.7926 & 845.3999 & 1688.8086 & 844.9079 & $\mathbf{1 6}$ \\
\hline $\mathbf{5}$ & 474.2194 & 237.6134 & 457.1929 & 229.1001 & 456.2089 & 228.6081 & $\mathbf{T}$ & $\mathbf{1 6 1 9 . 7 8 7 1}$ & 810.3972 & 1602.7606 & 801.8839 & 1601.7766 & 801.3919 & $\mathbf{1 5}$ \\
\hline
\end{tabular}




\begin{tabular}{|c|c|c|c|c|c|c|c|c|c|c|c|c|c|c|}
\hline 6 & 575.2671 & |288.1372| & 558.2406 & 279.6239 & 557.2566 & |279.1319 & $\mathbf{T}$ & 1518.7394 & |759.8734 & |1501.7129 & |751.3601 & |1500.7289 & |750.8681 & 14 \\
\hline 7 & 690.2941 & 345.6507 & 673.2675 & 337.1374 & 672.2835 & 336.6454 & D & 1417.6918 & 709.3495 & 1400.6652 & 700.8362 & 1399.6812 & 700.3442 & 13 \\
\hline 8 & 787.3468 & 394.1771 & 770.3203 & 385.6638 & 769.3363 & 385.1718 & $\mathbf{P}$ & 1302.6648 & 651.8360 & 1285.6383 & 643.3228 & 1284.6543 & 642.8308 & 12 \\
\hline 9 & 901.3898 & 451.1985 & 884.3632 & 442.6852 & 883.3792 & 442.1932 & $\mathbf{N}$ & 1205.6121 & 603.3097 & 1188.5855 & 594.7964 & 1187.6015 & 594.3044 & 11 \\
\hline 10 & 988.4218 & 494.7145 & 971.3952 & 486.2013 & 970.4112 & 485.7092 & S & 1091.5691 & 546.2882 & 1074.5426 & 537.7749 & 1073.5586 & 537.2829 & 10 \\
\hline 11 & 1089.4695 & 545.2384 & 1072.4429 & 536.7251 & 1071.4589 & 536.2331 & $\mathbf{T}$ & 1004.5371 & 502.7722 & 987.5106 & 494.2589 & 986.5265 & 493.7669 & 9 \\
\hline 12 & 1188.5379 & 594.7726 & 1171.5113 & 586.2593 & 1170.5273 & 585.7673 & V & 903.4894 & 452.2483 & 886.4629 & 443.7351 & 885.4789 & 443.2431 & 8 \\
\hline 13 & 1317.5805 & 659.2939 & 1300.5539 & 650.7806 & 1299.5699 & 650.2886 & $\mathbf{E}$ & 804.4210 & 402.7141 & 787.3945 & 394.2009 & 786.4104 & 393.7089 & 7 \\
\hline 14 & 1445.6391 & 723.3232 & 1428.6125 & 714.8099 & 1427.6285 & 714.3179 & $\mathbf{Q}$ & 675.3784 & 338.1928 & 658.3519 & 329.6796 & 657.3679 & 329.1876 & 6 \\
\hline 15 & 1532.6711 & 766.8392 & 1515.6445 & 758.3259 & 1514.6605 & 757.8339 & S & 547.3198 & 274.1636 & 530.2933 & 265.6503 & 529.3093 & 265.1583 & 5 \\
\hline 16 & 1603.7082 & 802.3577 & 1586.6816 & 793.8445 & 1585.6976 & 793.3525 & A & 460.2878 & 230.6475 & 443.2613 & 222.1343 & 442.2772 & 221.6423 & 4 \\
\hline 17 & 1716.7923 & 858.8998 & 1699.7657 & 850.3865 & 1698.7817 & 849.8945 & $\mathbf{L}$ & 389.2507 & 195.1290 & 372.2241 & 186.6157 & 371.2401 & 186.1237 & 3 \\
\hline 18 & 1817.8399 & 909.4236 & 1800.8134 & 900.9103 & 1799.8294 & 900.4183 & $\mathbf{T}$ & 276.1666 & 138.5870 & 259.1401 & 130.0737 & 258.1561 & 129.5817 & 2 \\
\hline 19 & & & & & & & $\mathbf{R}$ & 175.1190 & 88.0631 & 158.0924 & 79.5498 & & & 1 \\
\hline
\end{tabular}
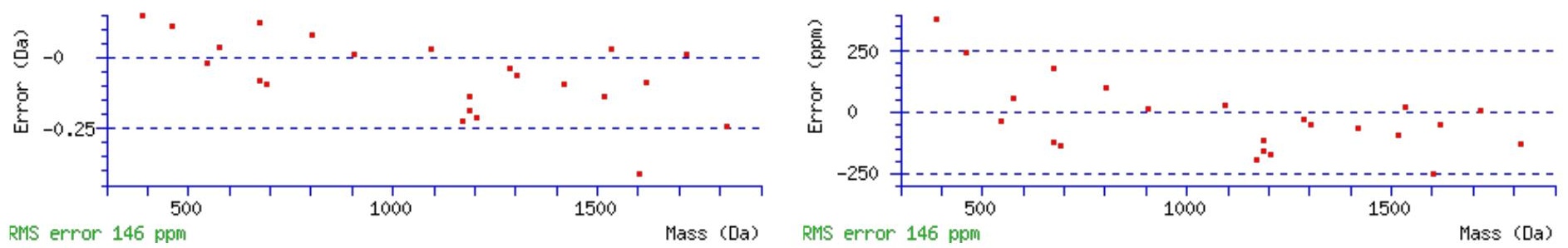

\section{All matches to this query}

\begin{tabular}{|l|l|l|l|}
\hline Score & Mr(calc): & Delta & \multicolumn{1}{|c|}{ Sequence } \\
\hline 83.0 & 1990.9443 & -0.0017 & VNASTTDPNSTVEQSALTR \\
\hline 56.2 & 1990.9443 & -0.0017 & VNASTTDPNSTVEQSALTR \\
\hline 19.9 & 1990.9249 & 0.0178 & SDTGLYSITAVNNLGTASK \\
\hline 19.9 & 1990.9249 & 0.0178 & SDTGLYSITAVNNLGTASK \\
\hline 13.3 & 1990.9249 & 0.0178 & $\underline{\text { SDTGLYSITAVNNLGTASK }}$ \\
\hline 11.9 & 1990.9249 & 0.0178 & SDTGLYSITAVNNLGTASK \\
\hline 11.9 & 1990.9249 & 0.0178 & SDTGLYSITAVNNLGTASK \\
\hline 3.7 & 1990.9408 & 0.0019 & LLCESSNRALSLQHAGR \\
\hline 1.8 & 1990.9458 & -0.0032 & QMVYFAISDQAFNIATR \\
\hline 1.3 & 1990.9297 & 0.0129 & IDMYAMGLVLWELVSR \\
\hline
\end{tabular}

Spectrum No: 195; Query: 146; Rank: 1

\section{Peptide View}

MS/MS Fragmentation of ALNVTLSSMGR

Found in IPI00213036, Tax_Id=10116 Gene_Symbol=C4a Complement C4 precursor

Match to Query 146: 1148.585048 from(575.299800,2+)

Title: 091008RatKidney_NoSalt_30.1930.1930.2.dta

Data file K:INewmanPaper|Piliang|3SubProteomes\Piliang3SP\mgf5ppm\ERLIC_3SubProteomes5ppm.mgf 

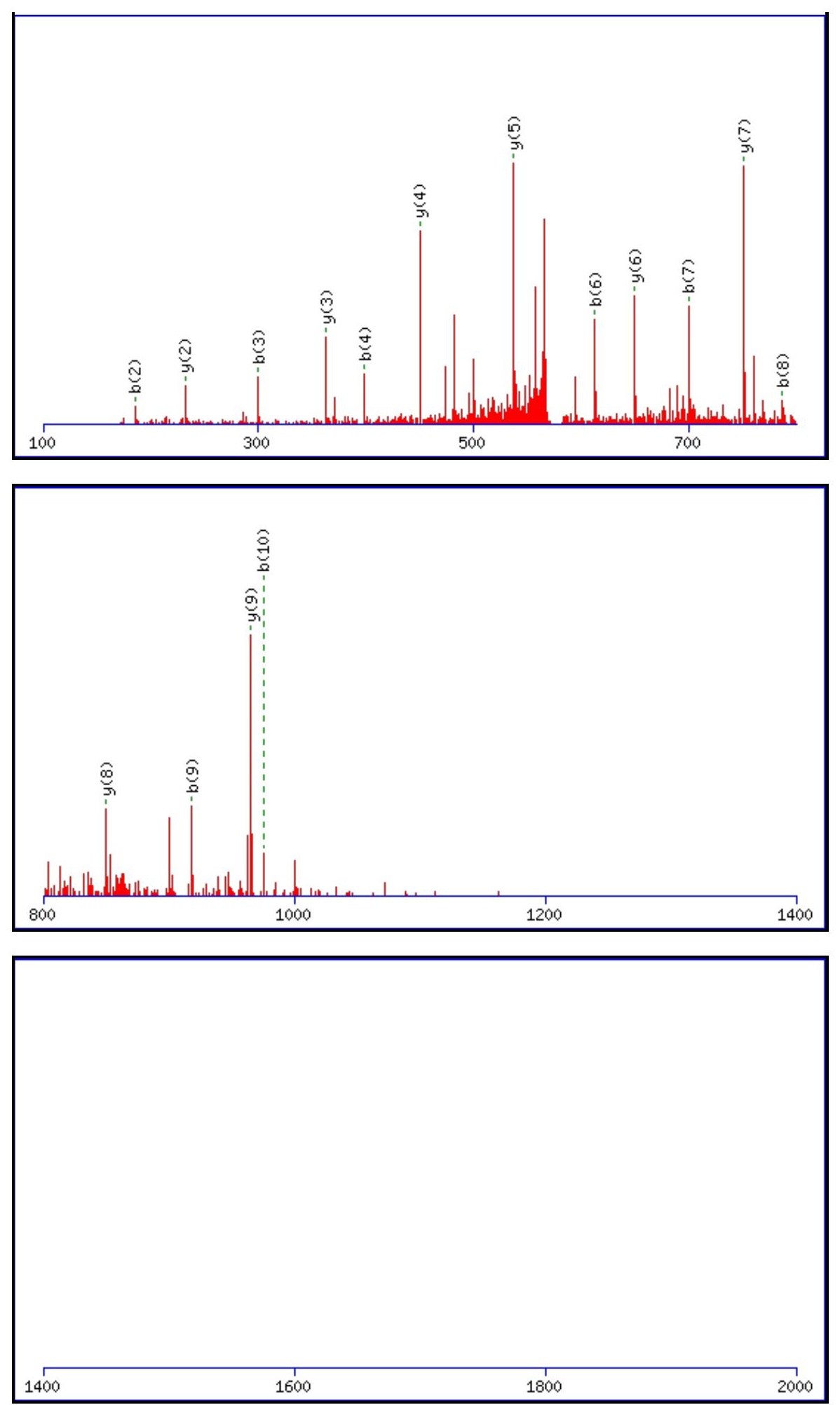

Monoisotopic mass of neutral peptide $\operatorname{Mr}($ calc): 1148.5859

Fixed modifications: Carbamidomethyl (C)

Variable modifications:

N3 : Deamidated $\mathrm{N}(\mathrm{N})$

Ions Score: 83 Expect: $7 \mathrm{e}-007$

Matches (Bold Red): 16/102 fragment ions using 23 most intense peaks

\begin{tabular}{|c|c|c|c|c|c|c|c|c|c|c|c|c|c|c|}
\hline$\#$ & $\mathbf{b}$ & $\mathbf{b}^{++}$ & $\mathbf{b}^{*}$ & $\mathbf{b}^{\boldsymbol{*}_{+}^{++}}$ & $\mathbf{b}^{\mathbf{0}}$ & $\mathbf{b}^{\mathbf{0 + +}}$ & $\mathbf{S e q}$ & $\mathbf{y}$ & $\mathbf{y}^{++}$ & $\mathbf{y}^{\mathbf{*}}$ & $\mathbf{y}^{\mathbf{*}^{++}}$ & $\mathbf{y}^{\mathbf{0}}$ & $\mathbf{y}^{\mathbf{0}++}$ & $\#$ \\
\hline $\mathbf{1}$ & $\mathbf{7 2 . 0 4 4 4}$ & 36.5258 & & & & & $\mathbf{A}$ & & & & & & & $\mathbf{1 1}$ \\
\hline $\mathbf{2}$ & $\mathbf{1 8 5 . 1 2 8 5}$ & 93.0679 & & & & & $\mathbf{L}$ & 1078.5561 & 539.7817 & 1061.5296 & 531.2684 & 1060.5455 & 530.7764 & $\mathbf{1 0}$ \\
\hline $\mathbf{3}$ & $\mathbf{3 0 0 . 1 5 5 4}$ & 150.5813 & 283.1288 & 142.0681 & & & $\mathbf{N}$ & $\mathbf{9 6 5 . 4 7 2 1}$ & 483.2397 & 948.4455 & 474.7264 & 947.4615 & 474.2344 & $\mathbf{9}$ \\
\hline $\mathbf{4}$ & $\mathbf{3 9 9 . 2 2 3 8}$ & 200.1155 & 382.1973 & 191.6023 & & & $\mathbf{V}$ & $\mathbf{8 5 0 . 4 4 5 1}$ & 425.7262 & 833.4186 & 417.2129 & 832.4345 & 416.7209 & $\mathbf{8}$ \\
\hline $\mathbf{5}$ & 500.2715 & 250.6394 & 483.2449 & 242.1261 & 482.2609 & 241.6341 & $\mathbf{T}$ & 751.3767 & 376.1920 & 734.3502 & 367.6787 & 733.3661 & 367.1867 & $\mathbf{7}$ \\
\hline
\end{tabular}




\begin{tabular}{|c|c|c|c|c|c|c|c|c|c|c|c|c|c|c|}
\hline 6 & |613.3555| & |307.1814 & |596.3290| & |298.6681 & |595.3450| & |298.1761 & $\mathbf{L}$ & 650.3290 & |325.6681 & 633.3025 & |317.1549| & 632.3185 & $\mid 316.6629$ & 6 \\
\hline 7 & 700.3876 & 350.6974 & 683.3610 & 342.1842 & 682.3770 & 341.6921 & $\mathrm{~S}$ & 537.2450 & 269.1261 & 520.2184 & 260.6128 & 519.2344 & 260.1208 & 5 \\
\hline 8 & 787.4196 & 394.2134 & 770.3931 & 385.7002 & 769.4090 & 385.2082 & $\mathrm{~S}$ & 450.2129 & 225.6101 & 433.1864 & 217.0968 & 432.2024 & 216.6048 & 4 \\
\hline 9 & \begin{tabular}{|l|}
918.4601 \\
\end{tabular} & |459.7337 & 901.4335 & 451.2204 & 900.4495 & 450.7284 & M & 363.1809 & 182.0941 & 346.1544 & 173.5808 & & & 3 \\
\hline 10 & 975.4816 & 488.2444 & 958.4550 & 479.7311 & 957.4710 & 479.2391 & G & 232.1404 & 116.5738 & 215.1139 & 108.0606 & & & 2 \\
\hline 11 & & & & & & & $\mathbf{R}$ & 175.1190 & 88.0631 & 158.0924 & 79.5498 & & & 1 \\
\hline
\end{tabular}
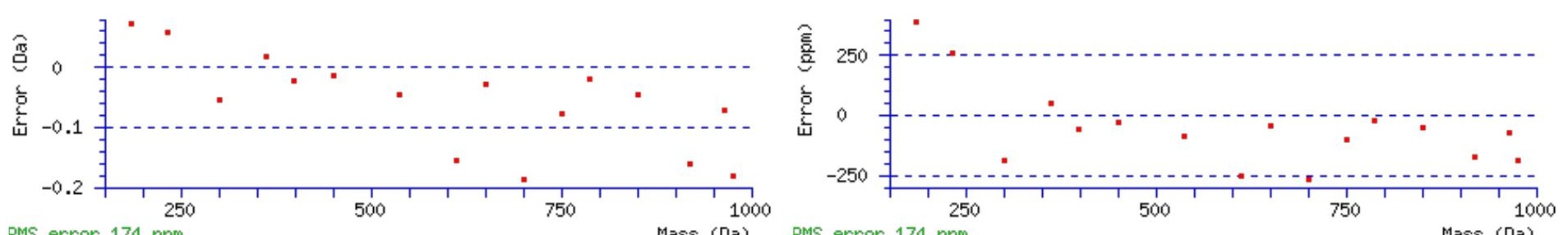

RMS error 174 ppm

Mass (Da) RMS error 174 ppm

Mass (Da)

\section{All matches to this query}

\begin{tabular}{|l|l|l|l|}
\hline Score & Mr(calc): & Delta & \multicolumn{1}{|c|}{ Sequence } \\
\hline 83.0 & 1148.5859 & -0.0009 & ALNVTLSSMGR \\
\hline 7.5 & 1148.5839 & 0.0011 & ALDRHWHSK \\
\hline 6.7 & 1147.5751 & 1.0100 & ALSGQLPAVGR \\
\hline 6.5 & 1148.5938 & -0.0087 & ALDPAEQHLR \\
\hline 6.0 & 1148.5859 & -0.0009 & VNREIVSGMK \\
\hline 5.8 & 1147.5833 & 1.0018 & VRELNSSNTK \\
\hline 5.6 & 1148.5760 & 0.0090 & MASPLRFDGR \\
\hline 4.5 & 1146.5768 & 2.0082 & SSPDILSDVSK \\
\hline 3.2 & 1146.5854 & 1.9997 & GGGSKRTQGSGR \\
\hline 3.0 & 1148.5794 & 0.0056 & NKDMKVAMGR \\
\hline
\end{tabular}

Spectrum No: 196; Query: 488; Rank: 1

\section{Peptide View}

MS/MS Fragmentation of AANYTSSLNLPDK

Found in IPI00366011, Tax_Id=10116 Gene_Symbol=Sema4d_predicted similar to semaphorin 4D

Match to Query 488: 1393.677128 from(697.845840,2+)

Title: 100101RatKid_NS_deglyco_25.2166.2166.2.dta

Data file K:INewmanPaper|Piliangl3SubProteomes|Piliang3SP\mgf5ppm|ERLIC_3SubProteomes5ppm.mgf

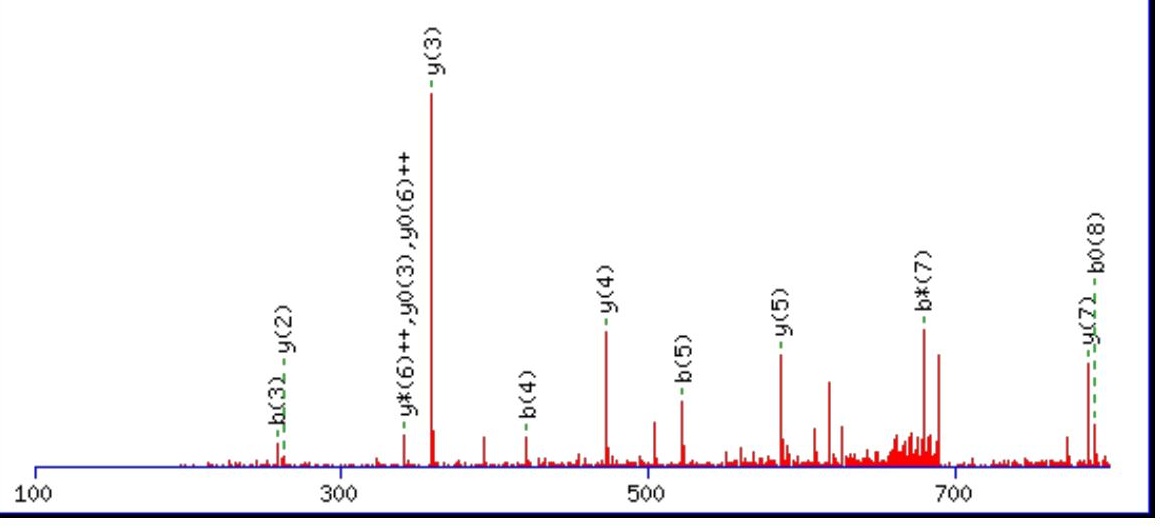




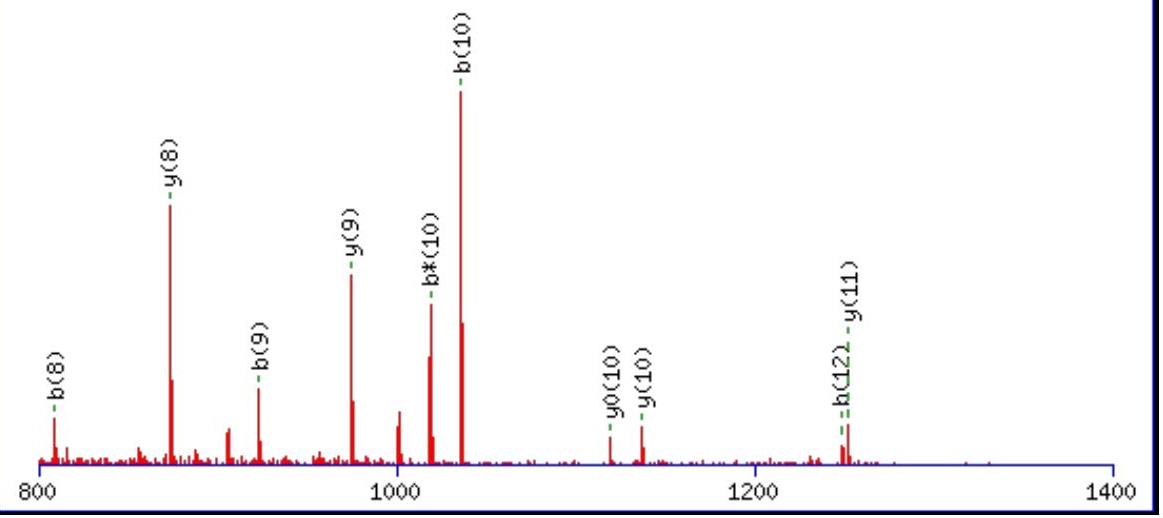

\begin{tabular}{rrr}
\hline 1600 & 1800 & 2000 \\
1400 & 1600
\end{tabular}

Monoisotopic mass of neutral peptide $\operatorname{Mr}($ calc): 1393.6725

Fixed modifications: Carbamidomethyl (C)

Variable modifications:

N3 : Deamidated_N (N)

Ions Score: 83 Expect: $7.5 \mathrm{e}-007$

Matches (Bold Red): 23/130 fragment ions using 31 most intense peaks

\begin{tabular}{|c|c|c|c|c|c|c|c|c|c|c|c|c|c|c|}
\hline \# & b & $\mathbf{b}^{++}$ & $\mathbf{b}^{*}$ & $\mathbf{b}^{*^{++}}$ & $\mathbf{b}^{\mathbf{0}}$ & $\mathbf{b}^{0++}$ & Seq. & $\mathbf{y}$ & $\mathbf{y}^{++}$ & $\mathbf{y}^{*}$ & $\mathbf{y}^{*^{++}}$ & $\mathbf{y}^{0}$ & $\mathbf{y}^{0++}$ & \# \\
\hline 1 & 72.0444 & 36.5258 & & & & & A & & & & & & & 13 \\
\hline 2 & 143.0815 & 72.0444 & & & & & A & 1323.6427 & 662.3250 & 1306.6161 & 653.8117 & 1305.6321 & 653.3197 & 12 \\
\hline 3 & 258.1084 & 129.5579 & 241.0819 & 121.0446 & & & $\mathbf{N}$ & 1252.6056 & 626.8064 & 1235.5790 & 618.2931 & 1234.5950 & 617.8011 & 11 \\
\hline 4 & 421.1718 & 211.0895 & 404.1452 & 202.5762 & & & $\mathbf{Y}$ & 1137.5786 & 569.2930 & 1120.5521 & 560.7797 & 1119.5681 & 560.2877 & 10 \\
\hline 5 & 522.2194 & 261.6134 & 505.1929 & 253.1001 & 504.2089 & 252.6081 & $\mathbf{T}$ & 974.5153 & 487.7613 & 957.4888 & 479.2480 & 956.5047 & 478.7560 & 9 \\
\hline 6 & 609.2515 & 305.1294 & 592.2249 & 296.6161 & 591.2409 & 296.1241 & $\mathbf{S}$ & 873.4676 & 437.2374 & 856.4411 & 428.7242 & 855.4571 & 428.2322 & 8 \\
\hline 7 & 696.2835 & 348.6454 & 679.2570 & 340.1321 & 678.2729 & 339.6401 & $\mathrm{~S}$ & 786.4356 & 393.7214 & 769.4090 & 385.2082 & 768.4250 & 384.7162 & 7 \\
\hline 8 & 809.3676 & 405.1874 & 792.3410 & 396.6741 & 791.3570 & 396.1821 & $\mathbf{L}$ & 699.4036 & 350.2054 & 682.3770 & 341.6921 & 681.3930 & 341.2001 & 6 \\
\hline 9 & 923.4105 & 462.2089 & 906.3839 & 453.6956 & 905.3999 & 453.2036 & $\mathbf{N}$ & 586.3195 & 293.6634 & 569.2930 & 285.1501 & 568.3089 & 284.6581 & 5 \\
\hline 10 & 1036.4946 & 518.7509 & 1019.4680 & 510.2376 & 1018.4840 & 509.7456 & $\mathbf{L}$ & 472.2766 & 236.6419 & 455.2500 & 228.1287 & 454.2660 & 227.6366 & 4 \\
\hline 11 & 1133.5473 & 567.2773 & 1116.5208 & 558.7640 & 1115.5368 & 558.2720 & $\mathbf{P}$ & 359.1925 & 180.0999 & 342.1660 & 171.5866 & 341.1819 & 171.0946 & 3 \\
\hline 12 & 1248.5743 & 624.7908 & 1231.5477 & 616.2775 & 1230.5637 & 615.7855 & D & 262.1397 & 131.5735 & 245.1132 & 123.0602 & 244.1292 & 122.5682 & 2 \\
\hline 13 & & & & & & & $\mathbf{K}$ & 147.1128 & 74.0600 & 130.0863 & 65.5468 & & & 1 \\
\hline
\end{tabular}



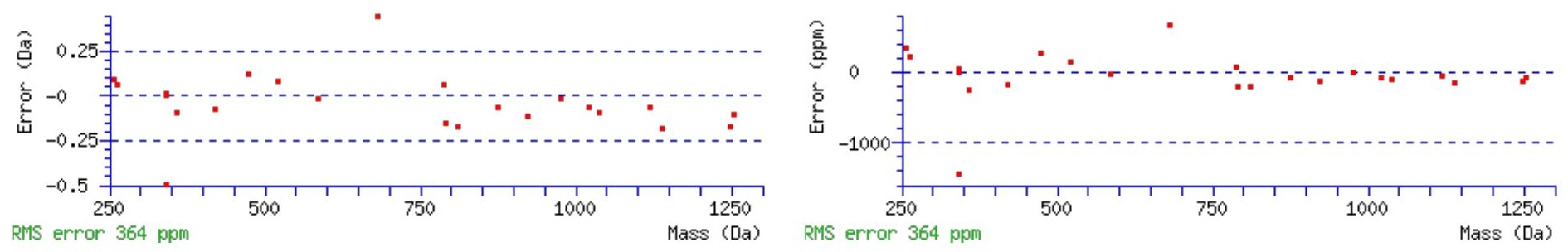

\section{All matches to this query}

\begin{tabular}{|l|l|l|l|}
\hline Score & Mr(calc): & Delta & \multicolumn{1}{|c|}{ Sequence } \\
\hline 82.9 & 1393.6725 & 0.0046 & AANYTSSLNLPDK \\
\hline 52.1 & 1393.6725 & 0.0046 & AANYTSSLNLPDK \\
\hline 9.6 & 1393.6854 & -0.0083 & EKTLETVPLER \\
\hline 8.9 & 1393.6837 & -0.0066 & GFGNIATNEDTKK \\
\hline 8.4 & 1393.6715 & 0.0057 & VQSKSAQARDPK \\
\hline 7.4 & 1392.6853 & 0.9918 & MLVIEQCKNSR \\
\hline 2.8 & 1393.6734 & 0.0037 & CPVMAGGLFSIDK \\
\hline 2.7 & 1393.6698 & 0.0073 & TRTFGNRAXEGR \\
\hline 2.7 & 1393.6715 & 0.0057 & VQSKSAQARDPK \\
\hline 0.4 & 1391.6681 & 2.0091 & DSGNIPEAIASYR \\
\hline
\end{tabular}

Spectrum No: 197; Query: 659; Rank: 1

\section{Peptide View}

\section{MS/MS Fragmentation of EVMSLLQPLNITK}

Found in IPI00388265, Tax_Id=10116 Gene_Symbol=Pgcp 48 kDa protein

Match to Query 659: 1485.812908 from(743.913730,2+)

Title: 100101RatKid_NS_deglyco_09.5128.5128.2.dta

Data file K:INewmanPaper|Piliangl3SubProteomes\Piliang3SP\mgf5ppm\ERLIC_3SubProteomes5ppm.mgf

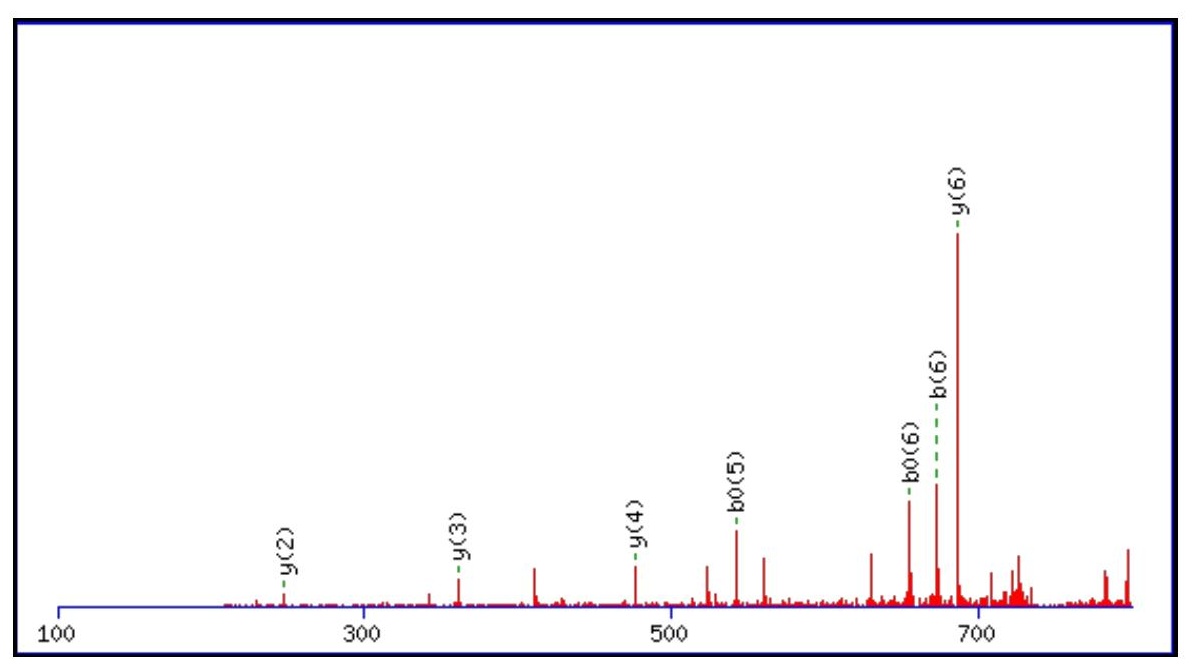



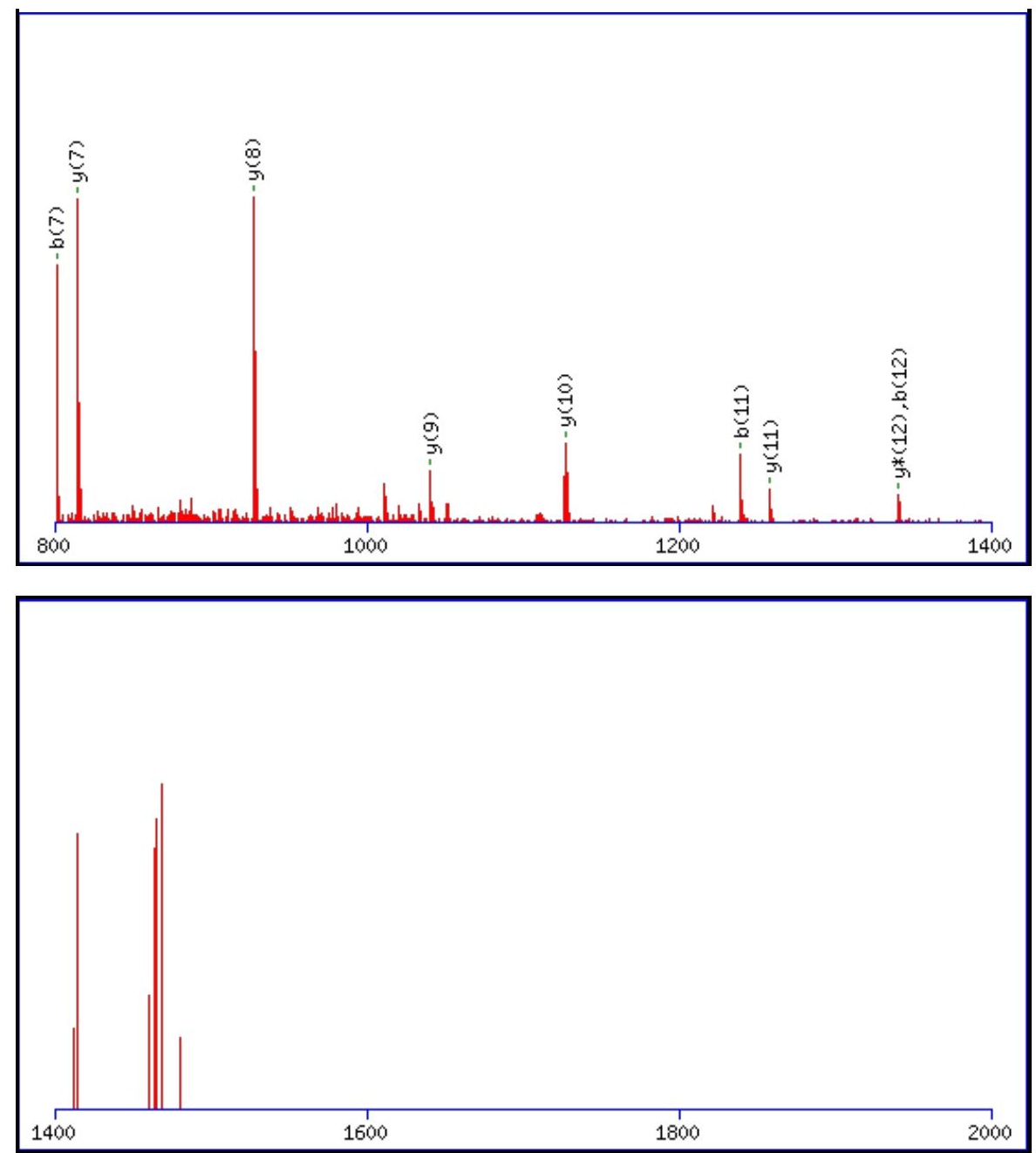

Monoisotopic mass of neutral peptide $\operatorname{Mr}($ calc): 1485.8112

Fixed modifications: Carbamidomethyl (C)

Variable modifications:

N10 : Deamidated_N (N)

Ions Score: 83 Expect: $4 \mathrm{e}-007$

Matches (Bold Red): 16/130 fragment ions using 20 most intense peaks

\begin{tabular}{|c|c|c|c|c|c|c|c|c|c|c|c|c|c|c|}
\hline \# & b & $\mathbf{b}^{++}$ & $\mathbf{b}^{*}$ & $\mathbf{b}^{*^{++}}$ & $\mathbf{b}^{0}$ & $\mathbf{b}^{\mathbf{0 + +}}$ & Seq. & $\mathbf{y}$ & $\mathbf{y}^{++}$ & $\mathbf{y}^{*}$ & $\mathrm{y}^{*^{++}}$ & $\mathbf{y}^{0}$ & $\mathbf{y}^{0++}$ & \# \\
\hline 1 & 130.0499 & 65.5286 & & & 112.0393 & 56.5233 & $\mathbf{E}$ & & & & & & & 13 \\
\hline 2 & 229.1183 & 115.0628 & & & 211.1077 & 106.0575 & V & 1357.7759 & 679.3916 & 1340.7494 & 670.8783 & 1339.7654 & 670.3863 & 12 \\
\hline 3 & 360.1588 & 180.5830 & & & 342.1482 & 171.5777 & $\mathbf{M}$ & 1258.7075 & 629.8574 & 1241.6810 & 621.3441 & 1240.6970 & 620.8521 & 11 \\
\hline 4 & 447.1908 & 224.0990 & & & 429.1802 & 215.0938 & $\mathbf{S}$ & 1127.6670 & 564.3372 & 1110.6405 & 555.8239 & 1109.6565 & 555.3319 & 10 \\
\hline 5 & 560.2749 & 280.6411 & & & 542.2643 & 271.6358 & $\mathbf{L}$ & 1040.6350 & 520.8211 & 1023.6085 & 512.3079 & 1022.6245 & 511.8159 & 9 \\
\hline 6 & 673.3589 & 337.1831 & & & 655.3484 & 328.1778 & $\mathbf{L}$ & 927.5510 & 464.2791 & 910.5244 & 455.7658 & 909.5404 & 455.2738 & 8 \\
\hline 7 & 801.4175 & 401.2124 & 784.3910 & 392.6991 & 783.4069 & 392.2071 & $\mathbf{Q}$ & 814.4669 & 407.7371 & 797.4403 & 399.2238 & 796.4563 & 398.7318 & 7 \\
\hline 8 & 898.4703 & 449.7388 & 881.4437 & 441.2255 & 880.4597 & 440.7335 & $\mathbf{P}$ & 686.4083 & 343.7078 & 669.3818 & 335.1945 & 668.3977 & 334.7025 & 6 \\
\hline 9 & 1011.5543 & 506.2808 & 994.5278 & 497.7675 & 993.5438 & 497.2755 & $\mathbf{L}$ & 589.3555 & 295.1814 & 572.3290 & 286.6681 & 571.3450 & 286.1761 & 5 \\
\hline 10 & 1126.5813 & 563.7943 & 1109.5547 & 555.2810 & 1108.5707 & 554.7890 & $\mathbf{N}$ & 476.2715 & 238.6394 & 459.2449 & 230.1261 & 458.2609 & 229.6341 & 4 \\
\hline 11 & 1239.6653 & 620.3363 & 1222.6388 & 611.8230 & 1221.6548 & 611.3310 & I & 361.2445 & 181.1259 & 344.2180 & 172.6126 & 343.2340 & 172.1206 & 3 \\
\hline 12 & 1340.7130 & 670.8601 & 1323.6865 & 662.3469 & 1322.7024 & 661.8549 & $\mathbf{T}$ & 248.1605 & 124.5839 & 231.1339 & 116.0706 & 230.1499 & 115.5786 & 2 \\
\hline 13 & & & & & & & $\mathbf{K}$ & 147.1128 & 74.0600 & 130.0863 & 65.5468 & & & 1 \\
\hline
\end{tabular}



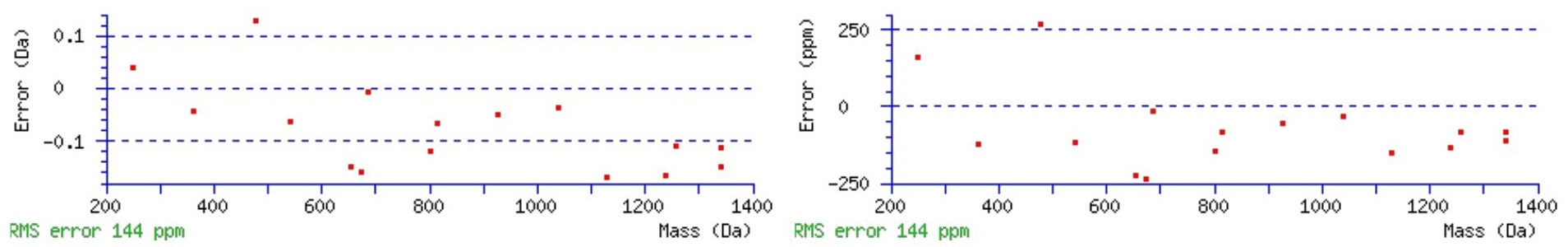

\section{All matches to this query}

\begin{tabular}{|l|c|c|l|}
\hline Score & Mr(calc): & Delta & \multicolumn{1}{|c|}{ Sequence } \\
\hline 82.9 & 1485.8112 & 0.0017 & EVMSLLQPLNITK \\
\hline 23.1 & 1485.8151 & -0.0022 & DILSRTKEGVGSPK \\
\hline 21.0 & 1485.8191 & -0.0062 & EFNPTKPLLSVNK \\
\hline 16.1 & 1485.8112 & 0.0017 & DLLSKMLVIDPDK \\
\hline 12.8 & 1483.8085 & 2.0045 & MLKSKLNVLTLK \\
\hline 8.1 & 1485.8224 & -0.0095 & VEMALEALRNVIK \\
\hline 6.0 & 1485.8225 & -0.0096 & DLINKMLTINPAK \\
\hline 5.8 & 1485.8151 & -0.0022 & TQKAKQVENVVDK \\
\hline 4.6 & 1484.8198 & 0.9931 & ENETIARLQALVK \\
\hline 4.4 & 1485.8052 & 0.0077 & YPANRAKGKPDAAK \\
\hline
\end{tabular}

Spectrum No: 198; Query: 2423; Rank: 1

\section{Peptide View}

MS/MS Fragmentation of GRADECALPYLGATCYCDLFCNR

Found in IPI00190428, Tax_Id=10116 Gene_Symbol=Lcn7 Tubulointerstitial nephritis antigen-like precursor

Match to Query 2423: 2782.169292 from(928.397040,3+)

Title: 100101RatKid_NS_deglyco_20.4510.4510.3.dta

Data file K:INewmanPaper|Piliangl3SubProteomes\Piliang3SP\mgf5ppm\ERLIC_3SubProteomes5ppm.mgf

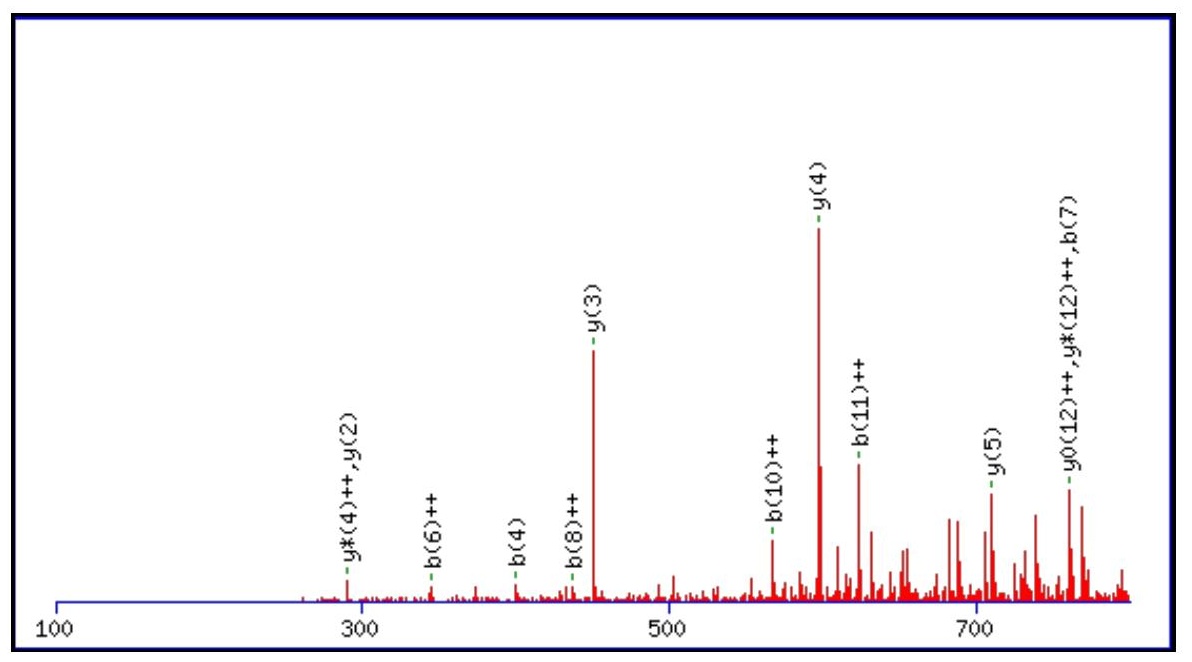



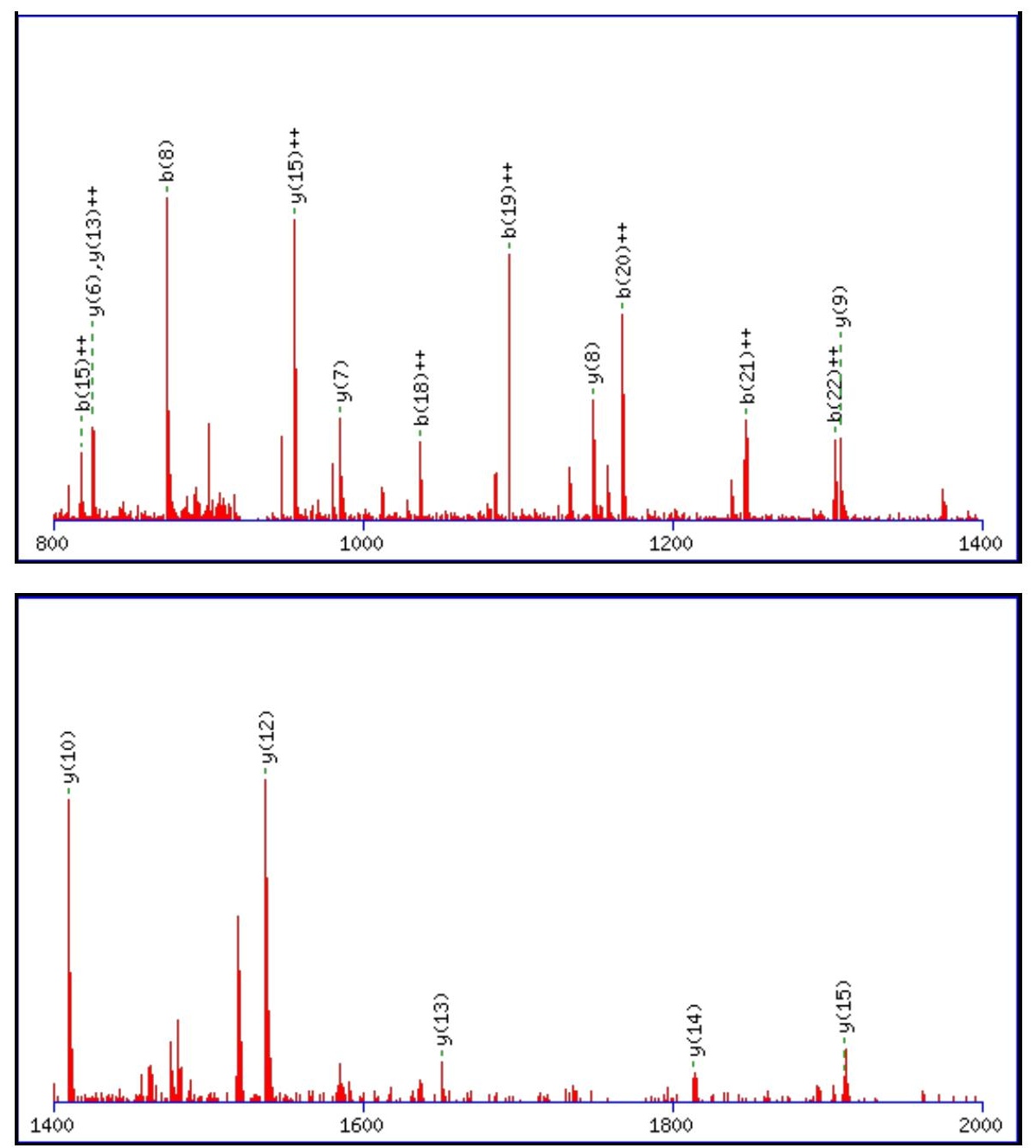

Monoisotopic mass of neutral peptide $\operatorname{Mr}($ calc): 2782.1608

Fixed modifications: Carbamidomethyl (C)

Variable modifications:

N22 : Deamidated_N (N)

Ions Score: 83 Expect: $1.8 \mathrm{e}-006$

Matches (Bold Red): 31/246 fragment ions using 52 most intense peaks

\begin{tabular}{|c|c|c|c|c|c|c|c|c|c|c|c|c|c|c|}
\hline \# & b & $\mathbf{b}^{++}$ & b* & $\mathbf{b}^{*^{++}}$ & $\mathbf{b}^{\mathbf{0}}$ & $\mathbf{b}^{0++}$ & Seq. & $\mathbf{y}$ & $\mathbf{y}^{++}$ & $\mathbf{y}^{*}$ & $\mathrm{y}^{*^{++}}$ & $\mathbf{y}^{0}$ & $\mathbf{y}^{0++}$ & \# \\
\hline 1 & 58.0287 & 29.5180 & & & & & G & & & & & & & 23 \\
\hline 2 & 214.1299 & 107.5686 & 197.1033 & 99.0553 & & & $\mathbf{R}$ & 2726.1466 & 1363.5769 & 2709.1200 & 1355.0637 & 2708.1360 & 5716 & 22 \\
\hline 3 & 285.1670 & 143.0871 & 268.1404 & 134.5738 & & & A & 2570.0455 & 1285.5264 & 2553.0189 & 1277.0131 & 2552.0349 & 1276.5211 & 21 \\
\hline 4 & 400.1939 & 200.6006 & 383.1674 & 192.0873 & 382.1833 & 191.5953 & D & 2499.0084 & 1250.0078 & 2481.9818 & 1241.4945 & 2480.9978 & 1241.0025 & 20 \\
\hline 5 & 529.2365 & 265.1219 & 512.2100 & 256.6086 & 511.2259 & 256.1166 & $\mathbf{E}$ & 2383.9814 & 1192.4943 & 2366.9549 & 1183.9811 & 2365.9709 & 1183.4891 & 19 \\
\hline 6 & 689.2672 & 345.1372 & 672.2406 & 336.6239 & 671.2566 & 336.1319 & C & 2254.9388 & |1127.9731 & 2237.9123 & 1119.4598 & 2236.9283 & 1118.9678 & 18 \\
\hline 7 & 760.3043 & 380.6558 & 743.2777 & 372.1425 & 742.2937 & 371.6505 & A & 2094.9082 & 1047.9577 & 2077.8816 & 1039.4445 & 2076.8976 & 1038.9524 & 17 \\
\hline 8 & 873.3883 & 437.1978 & 856.3618 & 428.6845 & 855.3778 & 428.1925 & $\mathbf{L}$ & 2023.8711 & 1012.4392 & 2006.8445 & 1003.9259 & 2005.8605 & 1003.4339 & 16 \\
\hline 9 & 970.4411 & 485.7242 & 953.4145 & 477.2109 & 952.4305 & 476.7189 & $\mathbf{P}$ & 1910.7870 & 955.8971 & 1893.7604 & 947.3839 & 1892.7764 & 946.8919 & 15 \\
\hline 10 & 1133.5044 & 567.2558 & 1116.4779 & 558.7426 & 1115.4939 & 558.2506 & $\mathbf{Y}$ & 1813.7342 & 907.3708 & 1796.7077 & 898.8575 & 1795.7237 & 898.3655 & 14 \\
\hline 11 & 1246.5885 & 623.7979 & 1229.5619 & 615.2846 & 1228.5779 & 614.7926 & $\mathbf{L}$ & 1650.6709 & 825.8391 & 1633.6444 & 817.3258 & 1632.6603 & 816.8338 & 13 \\
\hline 12 & 1303.6099 & 652.3086 & 1286.5834 & 643.7953 & 1285.5994 & 643.3033 & G & 1537.5868 & 769.2971 & 1520.5603 & 760.7838 & 1519.5763 & 760.2918 & 12 \\
\hline 13 & 1374.6471 & 687.8272 & 1357.6205 & 679.3139 & 1356.6365 & 678.8219 & A & 1480.5654 & 740.7863 & 1463.5388 & 732.2731 & 1462.5548 & 731.7810 & 11 \\
\hline 14 & 1475.6947 & 738.3510 & 1458.6682 & 729.8377 & 1457.6842 & 729.3457 & $\mathbf{T}$ & 1409.5283 & 705.2678 & 1392.5017 & 696.7545 & 1391.5177 & 696.2625 & 10 \\
\hline 15 & 1635.7254 & 818.3663 & 1618.6988 & 809.8531 & 1617.7148 & 809.3611 & C & 1308.4806 & 654.7439 & 1291.4540 & 646.2307 & 1290.4700 & 645.7386 & 9 \\
\hline 16 & 1798.7887 & 899.8980 & 1781.7622 & 891.3847 & 1780.7782 & 890.8927 & $\mathbf{Y}$ & 1148.4499 & 574.7286 & 1131.4234 & 566.2153 & 1130.4394 & 565.7233 & 8 \\
\hline 17 & 1958.8194 & 979.9133 & 1941.7928 & 971.4000 & 1940.8088 & 970.9080 & C & 985.3866 & 493.1969 & 968.3601 & 484.6837 & 967.3760 & 484.1917 & 7 \\
\hline 18 & 2073.8463 & 1037.4268 & 2056.8198 & 1028.9135 & 2055.8357 & 1028.4215 & D & 825.3560 & 413.1816 & 808.3294 & 404.6683 & 807.3454 & 404.1763 & 6 \\
\hline
\end{tabular}




\begin{tabular}{|l|r|r|r|r|r|r|r|r|r|r|r|r|r|r|r|r|}
$\mathbf{1 9}$ & 2186.9304 & $\mathbf{1 0 9 3 . 9 6 8 8}$ & 2169.9038 & 1085.4556 & 2168.9198 & 1084.9635 & $\mathbf{L}$ & $\mathbf{7 1 0 . 3 2 9 0}$ & 355.6681 & 693.3025 & 347.1549 & & $\mathbf{5}$ \\
\hline $\mathbf{2 0}$ & 2333.9988 & $\mathbf{1 1 6 7 . 5 0 3 0}$ & 2316.9722 & 1158.9898 & 2315.9882 & 1158.4978 & $\mathbf{F}$ & $\mathbf{5 9 7 . 2 4 5 0}$ & 299.1261 & 580.2184 & $\mathbf{2 9 0 . 6 1 2 8}$ & & & $\mathbf{4}$ \\
\hline $\mathbf{2 1}$ & 2494.0294 & $\mathbf{1 2 4 7 . 5 1 8 4}$ & 2477.0029 & 1239.0051 & 2476.0189 & 1238.5131 & $\mathbf{C}$ & $\mathbf{4 5 0 . 1 7 6 5}$ & 225.5919 & 433.1500 & 217.0786 & & & $\mathbf{3}$ \\
\hline $\mathbf{2 2}$ & 2609.0564 & $\mathbf{1 3 0 5 . 0 3 1 8}$ & 2592.0298 & 1296.5186 & 2591.0458 & 1296.0265 & $\mathbf{N}$ & $\mathbf{2 9 0 . 1 4 5 9}$ & 145.5766 & 273.1193 & 137.0633 & & & $\mathbf{2}$ \\
\hline $\mathbf{2 3}$ & & & & & & & $\mathbf{R}$ & 175.1190 & 88.0631 & 158.0924 & $\mathbf{7 9 . 5 4 9 8}$ & & & $\mathbf{1}$ \\
\hline
\end{tabular}
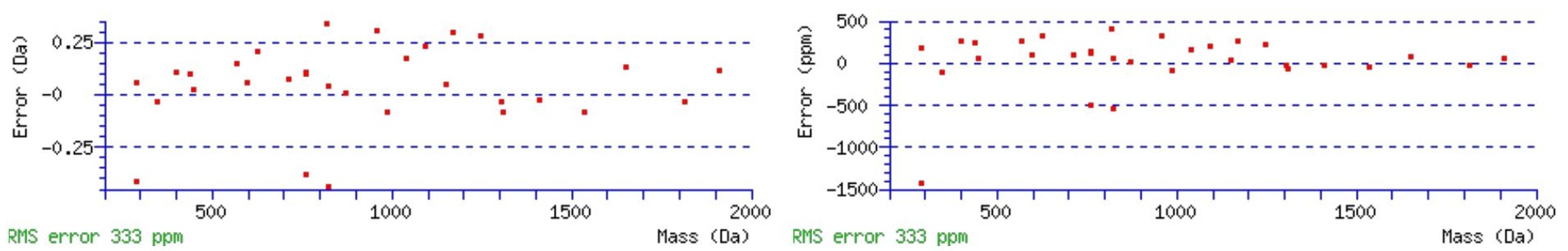

\section{All matches to this query}

\begin{tabular}{|l|l|r|l|}
\hline Score & Mr(calc): & Delta & \multicolumn{1}{c|}{ Sequence } \\
\hline 82.8 & 2782.1608 & 0.0085 & GRADECALPYLGATCYCDLFCNR \\
\hline 35.5 & 2781.1768 & 0.9925 & GRADECALPYLGATCYCDLFCNR \\
\hline 6.5 & 2780.1780 & 1.9913 & MVSIFYSIGTPFLNPLIYTLR \\
\hline 4.6 & 2781.1618 & 1.0075 & STRAVFYNSVNGIILVHDLTNK \\
\hline 4.6 & 2781.1618 & 1.0075 & STRAVFYNSVNGILVHDLTNK \\
\hline 4.0 & 2781.1493 & 1.0200 & DVKEMWNSSTELTEPSPELSLGR \\
\hline 3.3 & 2782.1879 & -0.0187 & SAIFLGPRGSLDLQVMYLDEYR \\
\hline 2.8 & 2780.1780 & 1.9913 & MVSIFYSIGTPFLNPLIYTLR \\
\hline 2.8 & 2780.1780 & 1.9913 & MVSIFYSIGTPFLNPLIYTLR \\
\hline 2.6 & 2782.1879 & -0.0187 & $\underline{\text { SAIFLGPRGSLDLQVMYLDEYR }}$ \\
\hline
\end{tabular}

Spectrum No: 199; Query: 398; Rank: 1

\section{Peptide View}

MS/MS Fragmentation of LLNLTNDGHSVR

Found in IPI00366665, Tax_Id=10116 Gene_Symbol=Car12 Membrane-bound carbonic anhydrase 12

Match to Query 398: 1338.687488 from(670.351020,2+)

Title: 091008RatKidney_NH4Format01_28.1177.1177.2.dta

Data file K:INewmanPaper|Piliang|3SubProteomes\Piliang3SP\mgf5ppm\ERLIC_3SubProteomes5ppm.mgf

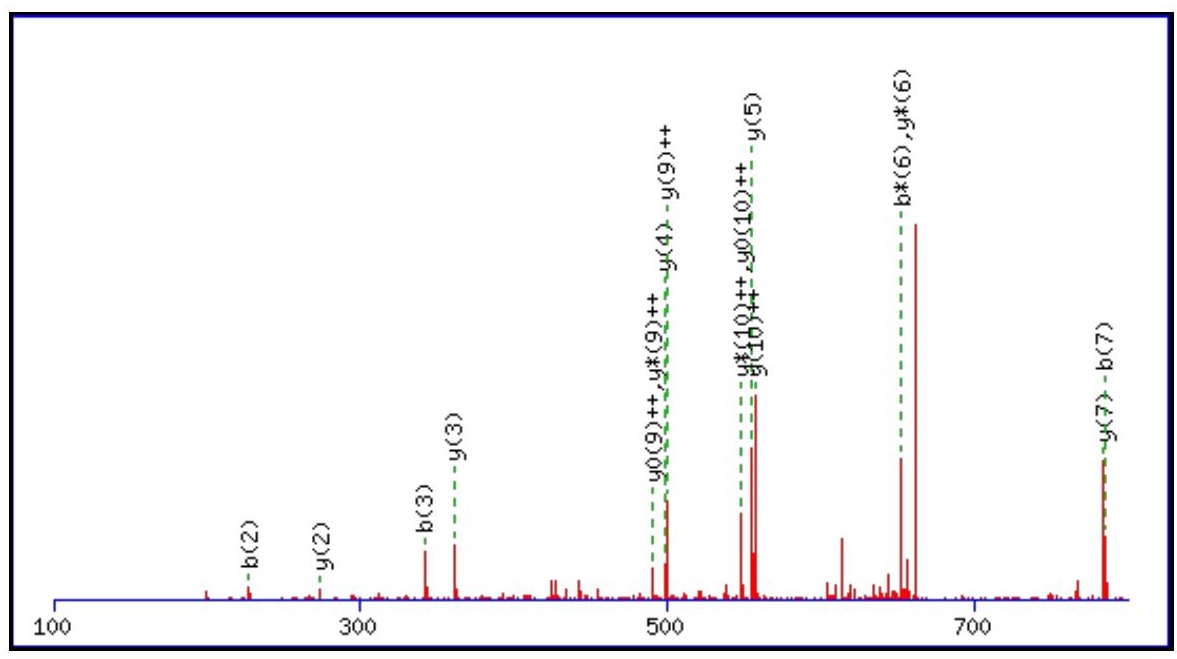



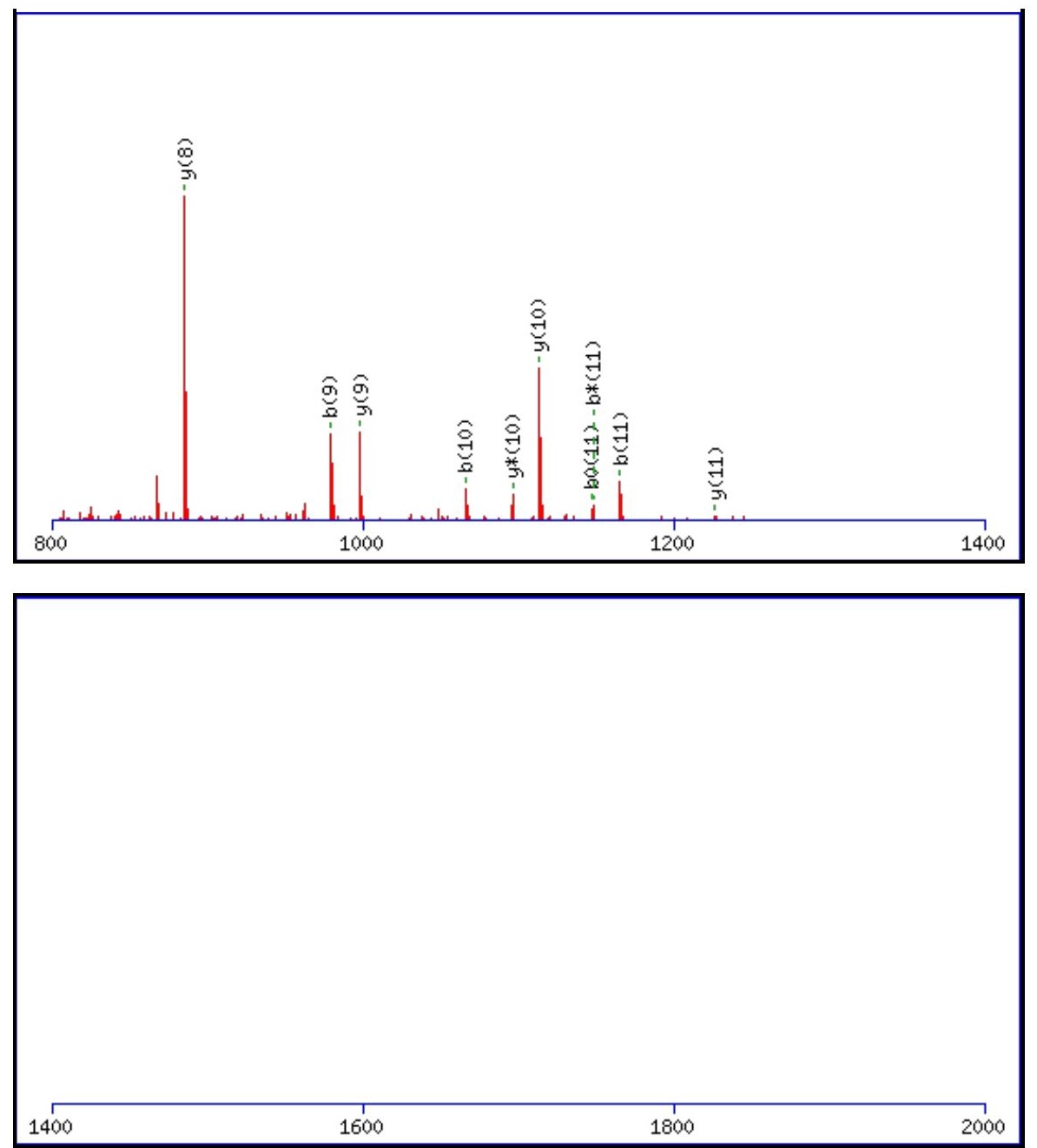

Monoisotopic mass of neutral peptide $\operatorname{Mr}($ calc): 1338.6892

Fixed modifications: Carbamidomethyl (C)

Variable modifications:

N3: Deamidated $\mathrm{N}(\mathrm{N})$

Ions Score: 83 Expect: $7.4 \mathrm{e}-007$

Matches (Bold Red): 26/116 fragment ions using 30 most intense peaks

\begin{tabular}{|c|c|c|c|c|c|c|c|c|c|c|c|c|c|c|}
\hline$\#$ & b & $\mathbf{b}^{++}$ & $\mathbf{b}^{*}$ & $\mathbf{b}^{*^{++}}$ & $\mathbf{b}^{0}$ & $\mathbf{b}^{0++}$ & Seq. & $\mathbf{y}$ & $y^{++}$ & $\mathbf{y}^{*}$ & $\mathrm{y}^{*^{++}}$ & $\mathbf{y}^{0}$ & $\mathbf{y}^{0++}$ & \# \\
\hline 1 & 114.0913 & 57.5493 & & & & & $\mathbf{L}$ & & & & & & & 12 \\
\hline 2 & 227.1754 & 114.0913 & & & & & $\mathbf{L}$ & 1226.6124 & 613.8098 & 1209.5858 & 605.2966 & 1208.6018 & 604.8045 & 11 \\
\hline 3 & 342.2023 & 171.6048 & 325.1758 & 163.0915 & & & $\mathbf{N}$ & 1113.5283 & 557.2678 & 1096.5018 & 548.7545 & 1095.5178 & 548.2625 & 10 \\
\hline 4 & 455.2864 & 228.1468 & 438.2599 & 219.6336 & & & $\mathbf{L}$ & 998.5014 & 499.7543 & 981.4748 & 491.2411 & 980.4908 & 490.7490 & 9 \\
\hline 5 & 556.3341 & 278.6707 & 539.3075 & 270.1574 & 538.3235 & 269.6654 & $\mathbf{T}$ & 885.4173 & 443.2123 & 868.3908 & 434.6990 & 867.4068 & 434.2070 & 8 \\
\hline 6 & 670.3770 & 335.6921 & 653.3505 & 327.1789 & 652.3664 & 326.6869 & $\mathbf{N}$ & 784.3696 & 392.6885 & 767.3431 & 384.1752 & 766.3591 & 383.6832 & 7 \\
\hline 7 & 785.4040 & 393.2056 & 768.3774 & 384.6923 & 767.3934 & 384.2003 & D & 670.3267 & 335.6670 & 653.3002 & 327.1537 & 652.3161 & 326.6617 & 6 \\
\hline 8 & 842.4254 & 421.7163 & 825.3989 & 413.2031 & 824.4149 & 412.7111 & G & 555.2998 & 278.1535 & 538.2732 & 269.6402 & 537.2892 & 269.1482 & 5 \\
\hline 9 & 979.4843 & 490.2458 & 962.4578 & 481.7325 & 961.4738 & 481.2405 & $\mathbf{H}$ & 498.2783 & 249.6428 & 481.2518 & 241.1295 & 480.2677 & 240.6375 & 4 \\
\hline 10 & 1066.5164 & 533.7618 & 1049.4898 & 525.2485 & 1048.5058 & 524.7565 & $\mathrm{~S}$ & 361.2194 & 181.1133 & 344.1928 & 172.6001 & 343.2088 & 172.1081 & 3 \\
\hline 11 & 1165.5848 & 583.2960 & 1148.5582 & 574.7827 & 1147.5742 & 574.2907 & V & 274.1874 & 137.5973 & 257.1608 & 129.0840 & & & 2 \\
\hline 12 & & & & & & & $\mathbf{R}$ & 175.1190 & 88.0631 & 158.0924 & 79.5498 & & & 1 \\
\hline
\end{tabular}



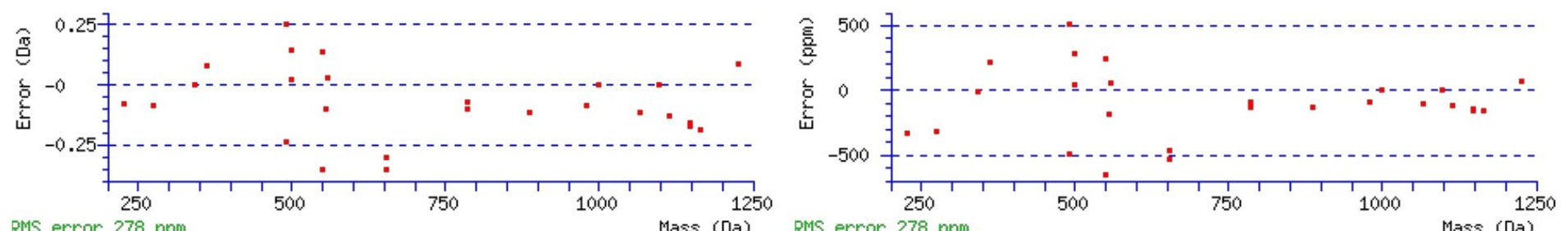

RMS error 278 ppm Mass (Da) RMS error 278 ppm

\section{All matches to this query}

\begin{tabular}{|l|l|l|l|}
\hline Score & Mr(calc): & Delta & \multicolumn{1}{|c|}{ Sequence } \\
\hline 82.7 & 1338.6892 & -0.0017 & LLNLTNDGHSVR \\
\hline 67.4 & 1338.6892 & -0.0017 & LLNLTNDGHSVR \\
\hline 10.9 & 1338.6769 & 0.0106 & LLGNSSRTRVR \\
\hline 9.9 & 1338.6796 & 0.0079 & QDKLISTVEVK \\
\hline 9.2 & 1338.6769 & 0.0106 & IRETARASLSR \\
\hline 8.7 & 1338.6769 & 0.0106 & LLGNSSRTRVR \\
\hline 8.7 & 1338.6769 & 0.0106 & LLGNSSRTRVR \\
\hline 7.7 & 1338.6932 & -0.0057 & LLFSSEKSSWR \\
\hline 7.7 & 1338.6820 & 0.0055 & LLDFVYNVDNK \\
\hline 7.1 & 1338.6853 & 0.0022 & IDILINYAMTR \\
\hline
\end{tabular}

Spectrum No: 200; Query: 570; Rank: 1

\section{Peptide View}

\section{MS/MS Fragmentation of AFNLTWISTDFK}

Found in IPI00197592, Tax_Id=10116 Gene_Symbol=F3 Tissue factor precursor

Match to Query 570: 1442.710708 from(722.362630,2+)

Title: 100101RatKid_NS_deglyco_21.5229.5229.2.dta

Data file K:INewmanPaper|Piliangl3SubProteomes\Piliang3SP\mgf5ppm\ERLIC_3SubProteomes5ppm.mgf

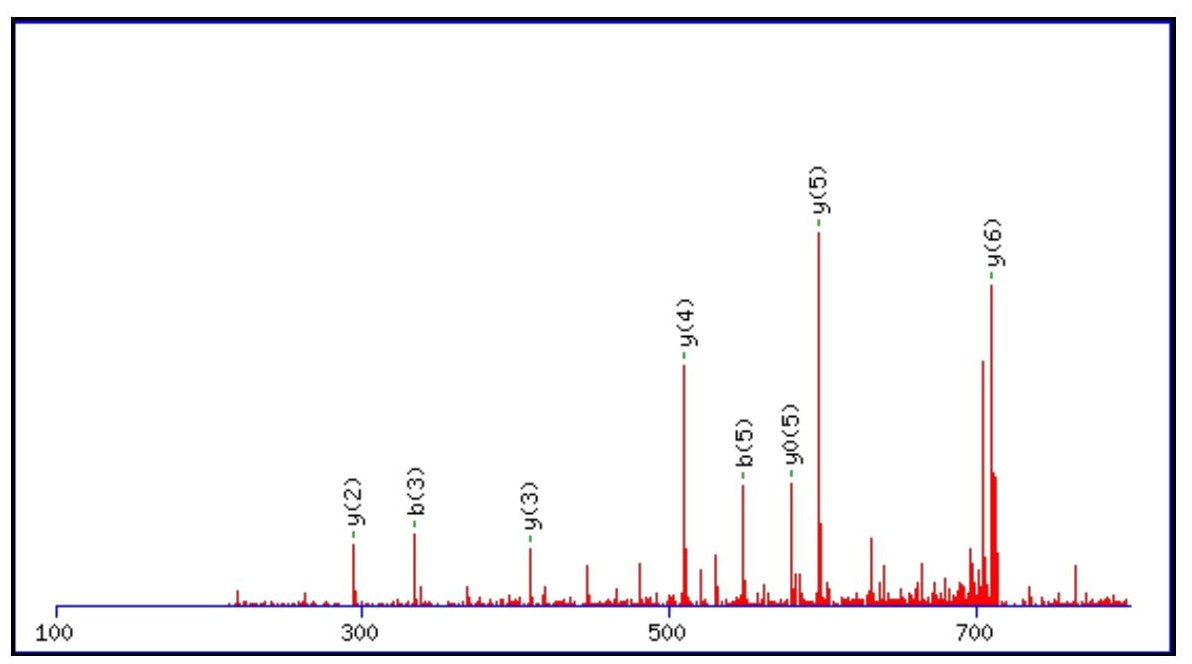



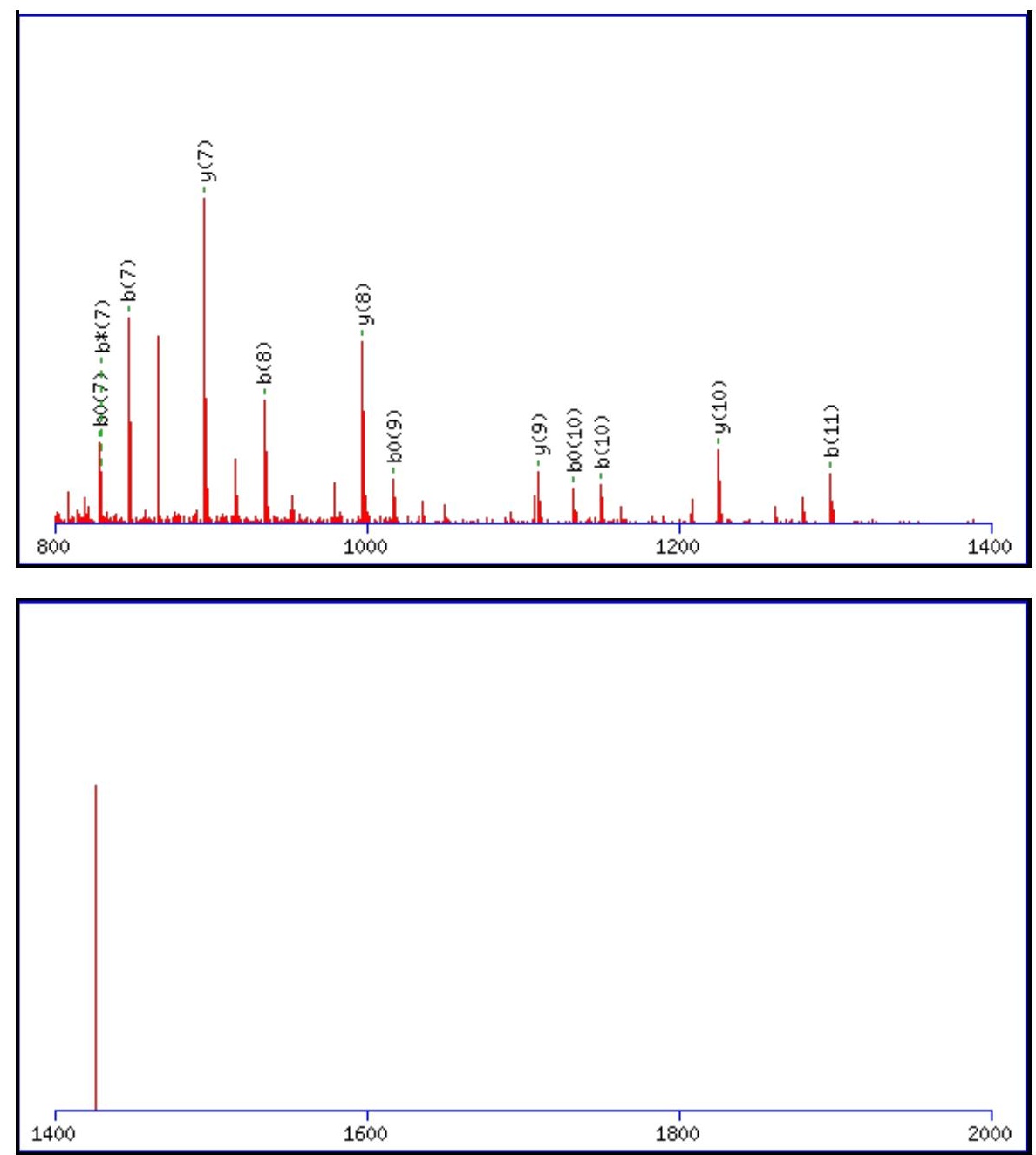

Monoisotopic mass of neutral peptide $\operatorname{Mr}($ calc): 1442.7082

Fixed modifications: Carbamidomethyl (C)

Variable modifications:

N3: Deamidated N (N)

Ions Score: 83 Expect: $7.9 \mathrm{e}-007$

Matches (Bold Red): 20/116 fragment ions using 33 most intense peaks

\begin{tabular}{|c|c|c|c|c|c|c|c|c|c|c|c|c|c|c|}
\hline$\#$ & b & $\mathbf{b}^{++}$ & $\mathbf{b}^{*}$ & $\mathbf{b}^{*^{++}}$ & $\mathbf{b}^{0}$ & $\mathbf{b}^{0++}$ & Seq. & $\mathbf{y}$ & $y^{++}$ & $\mathbf{y}^{*}$ & $\mathrm{y}^{*^{++}}$ & $\mathbf{y}^{0}$ & $\mathbf{y}^{0++}$ & \# \\
\hline 1 & 72.0444 & 36.5258 & & & & & A & & & & & & & 12 \\
\hline 2 & 219.1128 & 110.0600 & & & & & $\mathbf{F}$ & 1372.6783 & 686.8428 & 1355.6518 & 678.3295 & 1354.6678 & 677.8375 & 11 \\
\hline 3 & 334.1397 & 167.5735 & 317.1132 & 159.0602 & & & $\mathbf{N}$ & 1225.6099 & 613.3086 & 1208.5834 & 604.7953 & 1207.5994 & 604.3033 & 10 \\
\hline 4 & 447.2238 & 224.1155 & 430.1973 & 215.6023 & & & $\mathbf{L}$ & 1110.5830 & 555.7951 & 1093.5564 & 547.2819 & 1092.5724 & 546.7898 & 9 \\
\hline 5 & 548.2715 & 274.6394 & 531.2449 & 266.1261 & 530.2609 & 265.6341 & $\mathbf{T}$ & 997.4989 & 499.2531 & 980.4724 & 490.7398 & 979.4884 & 490.2478 & 8 \\
\hline 6 & 734.3508 & 367.6790 & 717.3242 & 359.1658 & 716.3402 & 358.6738 & W & 896.4512 & 448.7293 & 879.4247 & 440.2160 & 878.4407 & 439.7240 & 7 \\
\hline 7 & 847.4349 & 424.2211 & 830.4083 & 415.7078 & 829.4243 & 415.2158 & I & 710.3719 & 355.6896 & 693.3454 & 347.1763 & 692.3614 & 346.6843 & 6 \\
\hline 8 & 934.4669 & 467.7371 & 917.4403 & 459.2238 & 916.4563 & 458.7318 & $\mathrm{~S}$ & 597.2879 & 299.1476 & 580.2613 & 290.6343 & 579.2773 & 290.1423 & 5 \\
\hline 9 & 1035.5146 & 518.2609 & 1018.4880 & 509.7476 & 1017.5040 & 509.2556 & $\mathbf{T}$ & 510.2558 & 255.6316 & 493.2293 & 247.1183 & 492.2453 & 246.6263 & 4 \\
\hline 10 & 1150.5415 & 575.7744 & 1133.5150 & 567.2611 & 1132.5309 & 566.7691 & D & 409.2082 & 205.1077 & 392.1816 & 196.5944 & 391.1976 & 196.1024 & 3 \\
\hline 11 & 1297.6099 & 649.3086 & 1280.5834 & 640.7953 & 1279.5994 & 640.3033 & $\mathbf{F}$ & 294.1812 & 147.5942 & 277.1547 & 139.0810 & & & 2 \\
\hline 12 & & & & & & & $\mathbf{K}$ & 147.1128 & 74.0600 & 130.0863 & 65.5468 & & & 1 \\
\hline
\end{tabular}



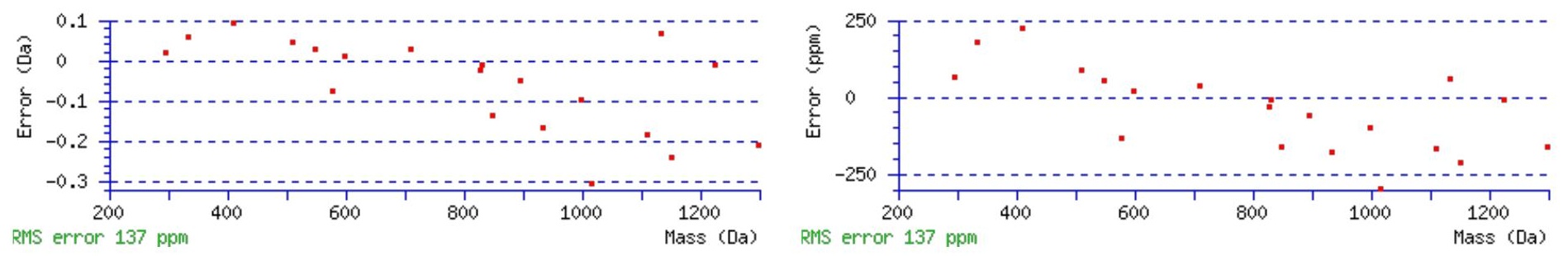

\section{All matches to this query}

\begin{tabular}{|l|l|l|l|}
\hline Score & Mr(calc): & Delta & \multicolumn{1}{|c|}{ Sequence } \\
\hline 82.5 & 1442.7082 & 0.0025 & AFNLTWISTDFK \\
\hline 7.5 & 1442.7179 & -0.0072 & GSLKMWLKCIK \\
\hline 5.6 & 1442.7065 & 0.0042 & RRLNXSSSIVMK \\
\hline 5.6 & 1442.7065 & 0.0042 & RRLNXSSSIVMK \\
\hline 5.4 & 1442.7228 & -0.0121 & WMLTTVPHESVK \\
\hline 5.3 & 1442.7122 & -0.0015 & VARSPAMNSVHMK \\
\hline 4.8 & 1442.7065 & 0.0042 & RRLNXSSSIVMK \\
\hline 1.5 & 1442.7058 & 0.0049 & LSLLGGDSVDIFK \\
\hline 1.4 & 1442.7228 & -0.0121 & GAFGKVYLAQDMK \\
\hline
\end{tabular}

Mascot: http://www.matrixscience.com/ 


\section{$\left.{ }_{S S C I E N C E E}^{\{M A T R I X}\right\}$ Mascot Search Results}

Results Generated by a Script Modified from Mascot Pepitde View

by Newman Sze, School of Biological Sciences, Nanyang Technological University

Spectrum No: 201; Query: 957; Rank: 1

\section{Peptide View}

MS/MS Fragmentation of NPDINMTFEQVYR

Found in IPI00325312, Tax_Id=10116 Gene_Symbol=Prom1 prominin 1 isoform 1

Match to Query 957: 1642.735788 from(822.375170,2+)

Title: 100101RatKid_NS_deglyco_21.3048.3048.2.dta

Data file K:INewmanPaper|Piliang|3SubProteomes\Piliang3SP\mgf5ppm\ERLIC_3SubProteomes5ppm.mgf
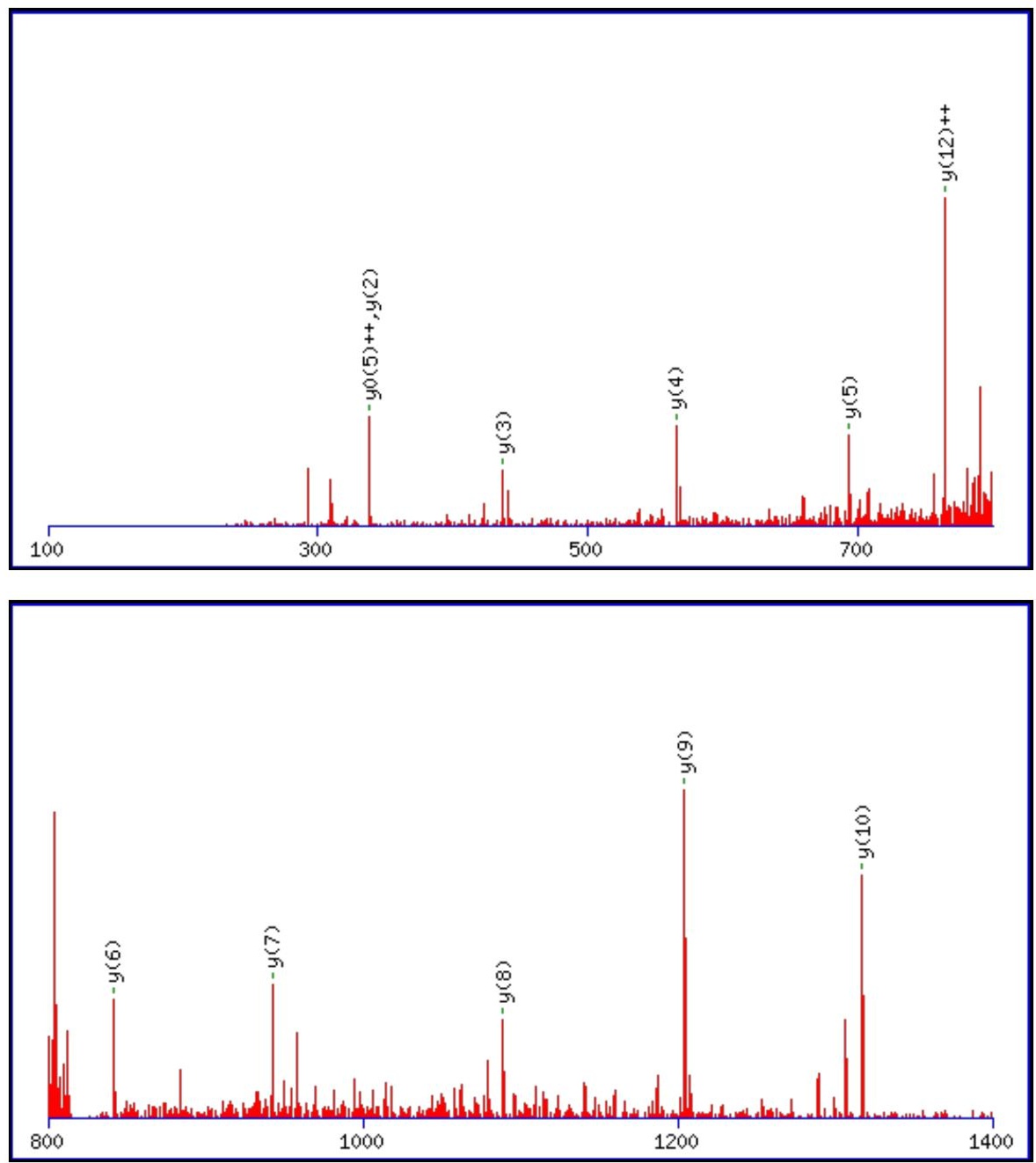


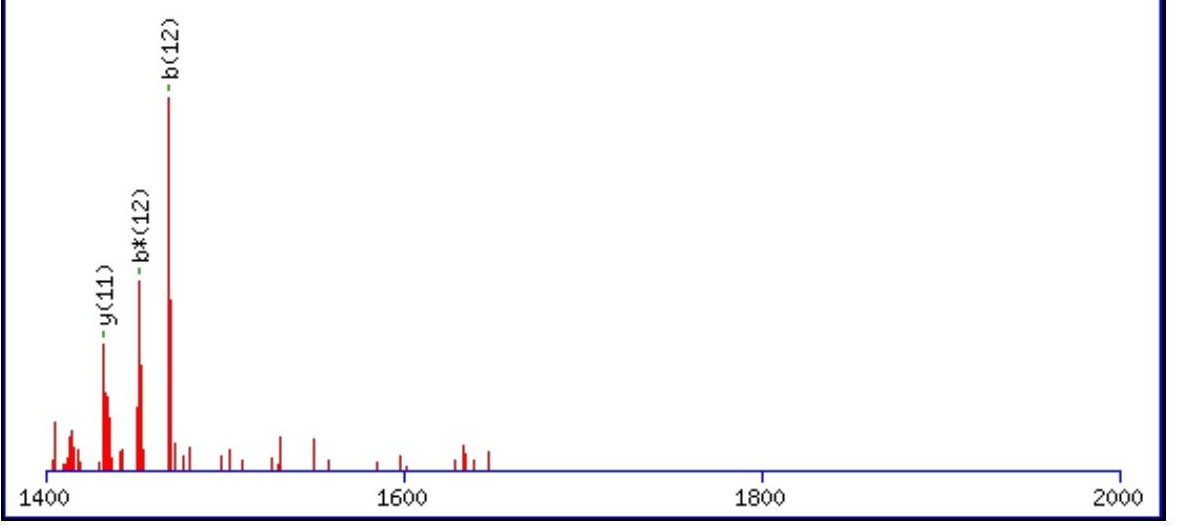

Monoisotopic mass of neutral peptide $\operatorname{Mr}($ calc): 1642.7297

Fixed modifications: Carbamidomethyl (C)

Variable modifications:

N5 : Deamidated_N (N)

M6 : Oxidation (M)

Ions Score: 82 Expect: $1.1 \mathrm{e}-006$

Matches (Bold Red): 14/132 fragment ions using 22 most intense peaks

\begin{tabular}{|c|c|c|c|c|c|c|c|c|c|c|c|c|c|c|}
\hline \# & b & $\mathbf{b}^{++}$ & $\mathbf{b}^{*}$ & $\mathbf{b}^{*^{++}}$ & $\mathbf{b}^{0}$ & $\mathbf{b}^{0++}$ & Seq. & $\mathbf{y}$ & $y^{++}$ & $\mathbf{y}^{*}$ & $\mathrm{y}^{*^{++}}$ & $\mathbf{y}^{0}$ & $\mathbf{y}^{0++}$ & \# \\
\hline 1 & 115.0502 & 58.0287 & 98.0237 & 49.5155 & & & $\mathbf{N}$ & & & & & & & 13 \\
\hline 2 & 212.1030 & 106.5551 & 195.0764 & 98.0418 & & & $\mathbf{P}$ & 1529.6941 & 765.3507 & 1512.6675 & 756.8374 & 1511.6835 & 756.3454 & 12 \\
\hline 3 & 327.1299 & 164.0686 & 310.1034 & 155.5553 & 309.1193 & 155.0633 & D & 1432.6413 & 716.8243 & 1415.6148 & 708.3110 & 1414.6307 & 707.8190 & 11 \\
\hline 4 & 440.2140 & 220.6106 & 423.1874 & 212.0974 & 422.2034 & 211.6053 & I & 1317.6144 & 659.3108 & 1300.5878 & 650.7975 & 1299.6038 & 650.3055 & 10 \\
\hline 5 & 555.2409 & 278.1241 & 538.2144 & 269.6108 & 537.2303 & 269.1188 & $\mathbf{N}$ & 1204.5303 & 602.7688 & 1187.5038 & 594.2555 & 1186.5197 & 593.7635 & 9 \\
\hline 6 & 702.2763 & 351.6418 & 685.2498 & 343.1285 & 684.2657 & 342.6365 & $\mathbf{M}$ & 1089.5034 & 545.2553 & 1072.4768 & 536.7420 & 1071.4928 & 536.2500 & 8 \\
\hline 7 & 803.3240 & 402.1656 & 786.2974 & 393.6524 & 785.3134 & 393.1604 & $\mathbf{T}$ & 942.4680 & 471.7376 & 925.4414 & 463.2243 & 924.4574 & 462.7323 & 7 \\
\hline 8 & 950.3924 & 475.6998 & 933.3659 & 467.1866 & 932.3818 & 466.6946 & $\mathbf{F}$ & 841.4203 & 421.2138 & 824.3937 & 412.7005 & 823.4097 & 412.2085 & 6 \\
\hline 9 & 1079.4350 & 540.2211 & 1062.4085 & 531.7079 & 1061.4244 & 531.2159 & $\mathbf{E}$ & 694.3519 & 347.6796 & 677.3253 & 339.1663 & 676.3413 & 338.6743 & 5 \\
\hline 10 & 1207.4936 & 604.2504 & 1190.4670 & 595.7372 & 1189.4830 & 595.2451 & $\mathbf{Q}$ & 565.3093 & 283.1583 & 548.2827 & 274.6450 & & & 4 \\
\hline 11 & 1306.5620 & 653.7846 & 1289.5354 & 645.2714 & 1288.5514 & 644.7794 & V & 437.2507 & 219.1290 & 420.2241 & 210.6157 & & & 3 \\
\hline 12 & 1469.6253 & 735.3163 & 1452.5988 & 726.8030 & 1451.6148 & 726.3110 & $\mathbf{Y}$ & 338.1823 & 169.5948 & 321.1557 & 161.0815 & & & 2 \\
\hline 13 & & & & & & & $\mathbf{R}$ & 175.1190 & 88.0631 & 158.0924 & 79.5498 & & & 1 \\
\hline
\end{tabular}

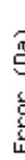
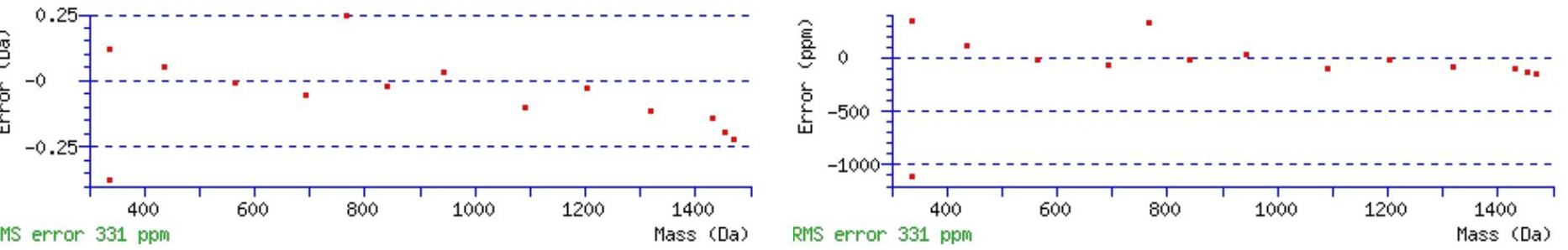

All matches to this query

\begin{tabular}{|l|l|l|l|}
\hline Score & Mr(calc): & Delta & Sequence \\
\hline 82.4 & 1642.7297 & 0.0061 & $\underline{\text { NPDINMTFEQVYR }}$ \\
\hline 35.6 & 1642.7297 & 0.0061 & $\underline{\text { NPDINMTFEQVYR }}$ \\
\hline 35.3 & 1641.7457 & 0.9901 & $\underline{\text { NPDINMTFEQVYR }}$ \\
\hline 0.1 & 1641.7334 & 1.0024 & $\underline{\text { LPNNFDIESAMRR }}$ \\
\hline
\end{tabular}

Spectrum No: 202; Query: 546; Rank: 1 


\section{Peptide View}

MS/MS Fragmentation of VNDNKTAAEEALR

Found in IPI00363849, Tax_Id=10116 Gene_Symbol=Lamc1 similar to Laminin gamma-1 chain precursor

Match to Query 546: 1430.703908 from(716.359230,2+)

Title: 100101RatKid_NS_deglyco_21.969.969.2.dta

Data file K:\NewmanPaper\Piliang $\backslash 3 S u b P r o t e o m e s \backslash P i l i a n g 3 S P \backslash m g f 5 p p m \backslash E R L I C \_3 S u b P r o t e o m e s 5 p p m . m g f$
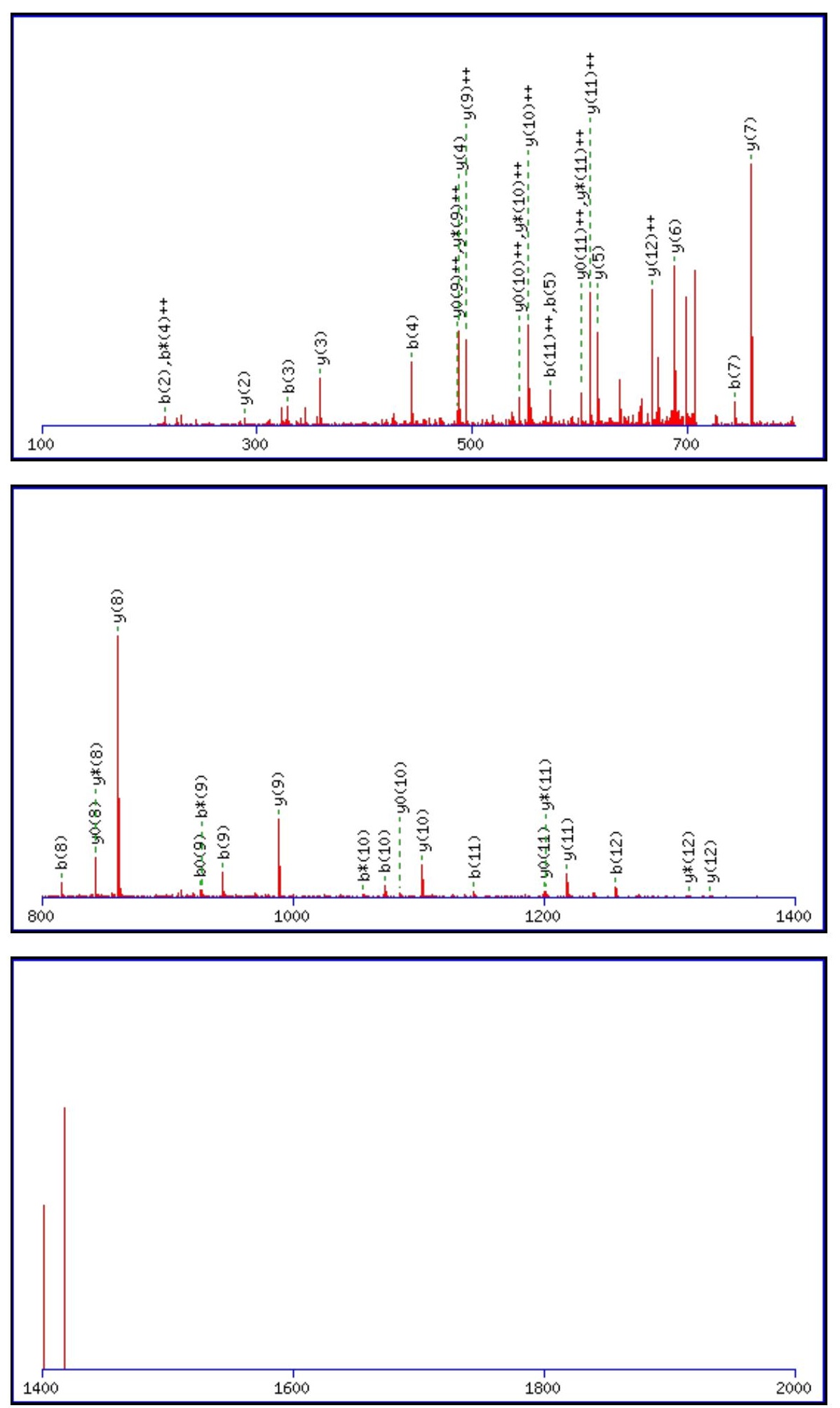

Monoisotopic mass of neutral peptide $\operatorname{Mr}($ calc): 1430.7001

Fixed modifications: Carbamidomethyl (C)

Variable modifications:

N4

: Deamidated_N (N)

Ions Score: 82 Expect: $7.6 \mathrm{e}-007$

Matches (Bold Red): 42/132 fragment ions using 68 most intense peaks 


\begin{tabular}{|c|c|c|c|c|c|c|c|c|c|c|c|c|c|c|}
\hline \# & b & $\mathbf{b}^{++}$ & $\mathbf{b}^{*}$ & $\mathbf{b}^{*^{++}}$ & $\mathbf{b}^{\mathbf{0}}$ & $\mathbf{b}^{0++}$ & Seq. & $\mathbf{y}$ & $\mathbf{y}^{++}$ & $\mathbf{y}^{*}$ & $\mathbf{y}^{*^{++}}$ & $\mathbf{y}^{0}$ & $\mathbf{y}^{0++}$ & \# \\
\hline 1 & 100.0757 & 50.5415 & & & & & $\mathbf{V}$ & & & & & & & 13 \\
\hline 2 & 214.1186 & 107.5629 & 197.0921 & 99.0497 & & & $\mathbf{N}$ & 1332.6390 & 666.8231 & 1315.6124 & 658.3099 & 1314.6284 & 657.8179 & 12 \\
\hline 3 & 329.1456 & 165.0764 & 312.1190 & 156.5631 & 311.1350 & 156.0711 & D & 1218.5961 & 609.8017 & 1201.5695 & 601.2884 & 1200.5855 & 600.7964 & 11 \\
\hline 4 & 444.1725 & 222.5899 & 427.1459 & 214.0766 & 426.1619 & 213.5846 & $\mathbf{N}$ & 1103.5691 & 552.2882 & 1086.5426 & 543.7749 & 1085.5586 & 543.2829 & 10 \\
\hline 5 & 572.2675 & 286.6374 & 555.2409 & 278.1241 & 554.2569 & 277.6321 & $\mathbf{K}$ & 988.5422 & 494.7747 & 971.5156 & 486.2615 & 970.5316 & 485.7694 & 9 \\
\hline 6 & 673.3151 & 337.1612 & 656.2886 & 328.6479 & 655.3046 & 328.1559 & $\mathbf{T}$ & 860.4472 & 430.7272 & 843.4207 & 422.2140 & 842.4367 & 421.7220 & 8 \\
\hline 7 & 744.3523 & 372.6798 & 727.3257 & 364.1665 & 726.3417 & 363.6745 & A & 759.3995 & 380.2034 & 742.3730 & 371.6901 & 741.3890 & 371.1981 & 7 \\
\hline 8 & 815.3894 & 408.1983 & 798.3628 & 399.6850 & 797.3788 & 399.1930 & A & 688.3624 & 344.6849 & 671.3359 & 336.1716 & 670.3519 & 335.6796 & 6 \\
\hline 9 & 944.4320 & 472.7196 & 927.4054 & 464.2063 & 926.4214 & 463.7143 & $\mathbf{E}$ & 617.3253 & 309.1663 & 600.2988 & 300.6530 & 599.3148 & 300.1610 & 5 \\
\hline 10 & 1073.4746 & 537.2409 & 1056.4480 & 528.7276 & 1055.4640 & 528.2356 & $\mathbf{E}$ & 488.2827 & 244.6450 & 471.2562 & 236.1317 & 470.2722 & 235.6397 & 4 \\
\hline 11 & 1144.5117 & 572.7595 & 1127.4851 & 564.2462 & 1126.5011 & 563.7542 & A & 359.2401 & 180.1237 & 342.2136 & 171.6104 & & & 3 \\
\hline 12 & 1257.5957 & 629.3015 & 1240.5692 & 620.7882 & 1239.5852 & 620.2962 & $\mathbf{L}$ & 288.2030 & 144.6051 & 271.1765 & 136.0919 & & & 2 \\
\hline 13 & & & & & & & $\mathbf{R}$ & 175.1190 & 88.0631 & 158.0924 & 79.5498 & & & 1 \\
\hline
\end{tabular}
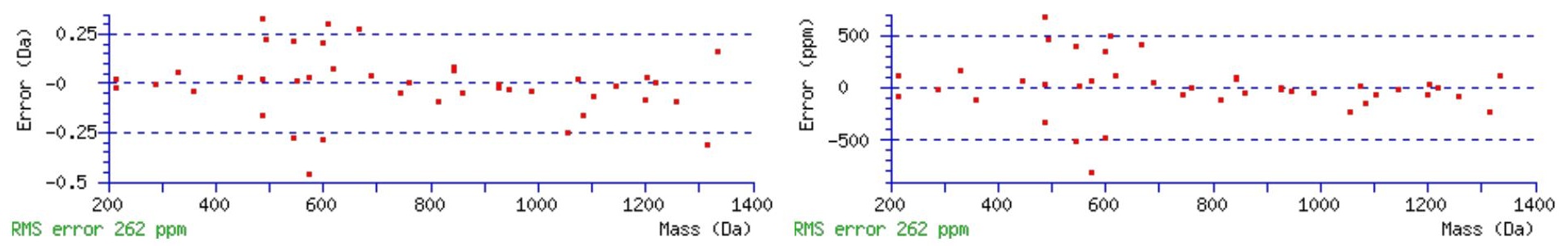

\section{All matches to this query}

\begin{tabular}{|l|l|l|l|}
\hline Score & Mr(calc): & Delta & \multicolumn{1}{|c|}{ Sequence } \\
\hline 82.4 & 1430.7001 & 0.0038 & VNDNKTAAEEALR \\
\hline 49.9 & 1430.7001 & 0.0038 & VNDNKTAAEEALR \\
\hline 7.5 & 1430.7048 & -0.0009 & GRNCTEKPTNVR \\
\hline 7.2 & 1428.6861 & 2.0178 & LTVXDKLSETTR \\
\hline 7.2 & 1428.6861 & 2.0178 & LTVXDKLSETTR \\
\hline 5.9 & 1430.7161 & -0.0122 & SRAACVXTRPSGR \\
\hline 5.8 & 1430.6975 & 0.0064 & VLPYILPSTLR \\
\hline 5.6 & 1430.6959 & 0.0080 & VINNFISAGFIR \\
\hline 5.5 & 1430.7089 & -0.0050 & VSRTHFDPMGIR \\
\hline 5.3 & 1430.6975 & 0.0064 & VLPYILPSTLR \\
\hline
\end{tabular}

Spectrum No: 203; Query: 750; Rank: 1

\section{Peptide View}

MS/MS Fragmentation of DLLTNQSDFSGNTK

Found in IPI00210824, Tax_Id=10116 Gene_Symbol=Serpina6 Corticosteroid-binding globulin precursor

Match to Query 750: 1539.702488 from(770.858520,2+)

Title: 091008RatKidney_NoSalt_41.1427.1427.2.dta

Data file K:\NewmanPaper\Piliang \3SubProteomes\Piliang3SP \mgf5ppm\ERLIC_3SubProteomes5ppm.mgf 

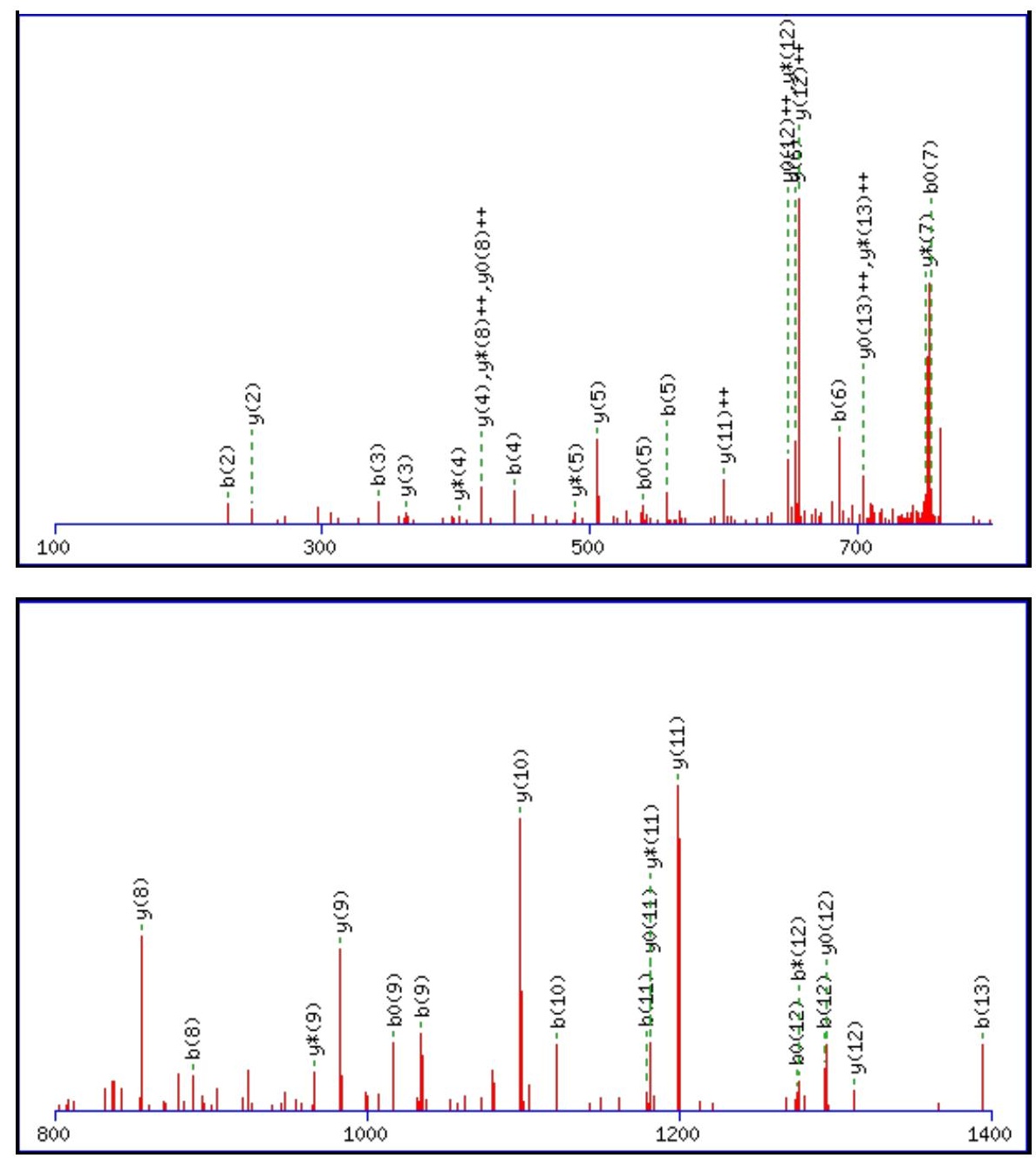

\begin{tabular}{rrrr}
\hline 00 & 1600 & 1800 & 2000 \\
\hline
\end{tabular}

Monoisotopic mass of neutral peptide $\operatorname{Mr}($ calc): 1539.7053

Fixed modifications: Carbamidomethyl (C)

Variable modifications:

N5 : Deamidated_N (N)

Ions Score: 82 Expect: $9.8 \mathrm{e}-007$

Matches (Bold Red): 41/146 fragment ions using 57 most intense peaks

\begin{tabular}{|r|c|c|c|c|r|r|r|r|c|c|c|c|c|c|}
\hline$\#$ & $\mathbf{b}$ & $\mathbf{b}^{++}$ & $\mathbf{b}^{*}$ & $\mathbf{b}^{\boldsymbol{*}^{++}}$ & $\mathbf{b}^{\mathbf{0}}$ & $\mathbf{b}^{\mathbf{0 + +}}$ & Seq. & $\mathbf{y}$ & $\mathbf{y}^{++}$ & $\mathbf{y}^{\mathbf{*}}$ & $\mathbf{y}^{\boldsymbol{*}^{++}}$ & $\mathbf{y}^{\mathbf{0}}$ & $\mathbf{y}^{\mathbf{0 + +}}$ & $\#$ \\
\hline $\mathbf{1}$ & 116.0342 & 58.5207 & & & 98.0237 & 49.5155 & $\mathbf{D}$ & & & & & & & $\mathbf{1 4}$ \\
\hline $\mathbf{2}$ & $\mathbf{2 2 9 . 1 1 8 3}$ & 115.0628 & & & 211.1077 & 106.0575 & $\mathbf{L}$ & 1425.6856 & 713.3464 & 1408.6591 & $\mathbf{7 0 4 . 8 3 3 2}$ & 1407.6750 & $\mathbf{7 0 4 . 3 4 1 2}$ & $\mathbf{1 3}$ \\
\hline 3 & $\mathbf{3 4 2 . 2 0 2 3}$ & 171.6048 & & & 324.1918 & 162.5995 & $\mathbf{L}$ & $\mathbf{1 3 1 2 . 6 0 1 5}$ & $\mathbf{6 5 6 . 8 0 4 4}$ & 1295.5750 & $\mathbf{6 4 8 . 2 9 1 1}$ & $\mathbf{1 2 9 4 . 5 9 1 0}$ & $\mathbf{6 4 7 . 7 9 9 1}$ & $\mathbf{1 2}$ \\
\hline $\mathbf{4}$ & $\mathbf{4 4 3 . 2 5 0 0}$ & 222.1287 & & & 425.2395 & 213.1234 & $\mathbf{T}$ & $\mathbf{1 1 9 9 . 5 1 7 5}$ & $\mathbf{6 0 0 . 2 6 2 4}$ & $\mathbf{1 1 8 2 . 4 9 0 9}$ & 591.7491 & $\mathbf{1 1 8 1 . 5 0 6 9}$ & 591.2571 & $\mathbf{1 1}$ \\
\hline $\mathbf{5}$ & $\mathbf{5 5 8 . 2 7 7 0}$ & 279.6421 & 541.2504 & 271.1288 & 540.2664 & 270.6368 & $\mathbf{N}$ & $\mathbf{1 0 9 8 . 4 6 9 8}$ & 549.7385 & 1081.4433 & 541.2253 & 1080.4592 & 540.7333 & $\mathbf{1 0}$ \\
\hline
\end{tabular}




\begin{tabular}{|c|c|c|c|c|c|c|c|c|c|c|c|c|c|c|}
\hline 6 & 686.3355 & |343.67 & 669.3090 & 335.1581 & 668.3250 & 334.6661 & $\mathbf{Q}$ & 983.4429 & |492.2251 & 966.4163 & 483.7118 & 965.4323 & |483.2198 & 9 \\
\hline 7 & 773.3676 & 387.1874 & 756.3410 & 378.6741 & 755.3570 & 378.1821 & S & 855.3843 & 428.1958 & 838.3577 & 419.6825 & 837.3737 & |419.1905 & 8 \\
\hline 8 & 888.3945 & 444.7009 & 871.3680 & 436.1876 & 870.3839 & 435.6956 & D & 768.3523 & 384.6798 & 751.3257 & 376.1665 & 750.3417 & 375.6745 & 7 \\
\hline 9 & 1035.4629 & 518.2351 & 1018.4364 & 509.7218 & 1017.4524 & 509.2298 & $\mathbf{F}$ & 653.3253 & 327.1663 & 636.2988 & 318.6530 & 635.3148 & |318.1610 & 6 \\
\hline 10 & 1122.4950 & 561.7511 & 1105.4684 & 553.2378 & 1104.4844 & 552.7458 & $\mathrm{~S}$ & 506.2569 & 253.6321 & 489.2304 & 245.1188 & 488.2463 & 244.6268 & 5 \\
\hline 11 & 1179.5164 & 590.2618 & 1162.4899 & 581.7486 & 1161.5059 & 581.2566 & G & 419.2249 & |210.1161 & 402.1983 & 201.6028 & 401.2143 & 201.1108 & 4 \\
\hline 12 & 1293.5593 & 647.2833 & 1276.5328 & 638.7700 & 1275.5488 & 638.2780 & $\mathbf{N}$ & 362.2034 & 181.6053 & 345.1769 & 173.0921 & 344.1928 & 172.6001 & 3 \\
\hline 13 & 1394.6070 & 697.8071 & 1377.5805 & 689.2939 & 1376.5965 & 688.8019 & $\mathbf{T}$ & 248.1605 & 124.5839 & 231.1339 & 116.0706 & 230.1499 & 115.5786 & 2 \\
\hline 14 & & & & & & & $\mathbf{K}$ & 147.1128 & 74.0600 & 130.0863 & 65.5468 & & & 1 \\
\hline
\end{tabular}
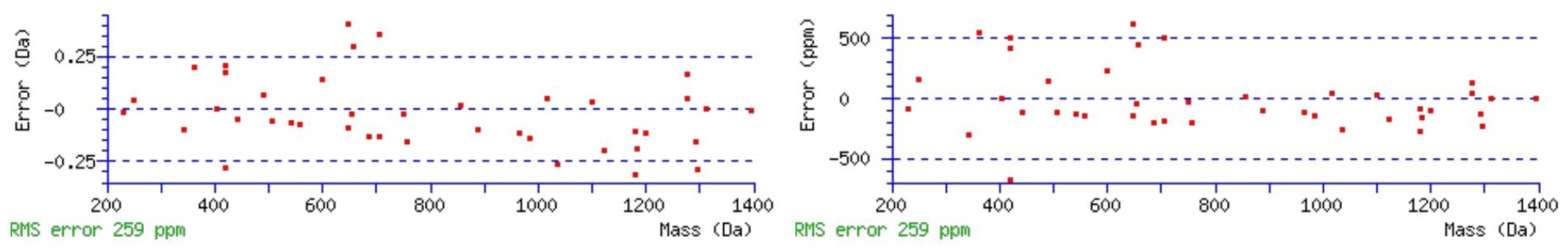

\section{All matches to this query}

\begin{tabular}{|l|l|l|l|}
\hline Score & Mr(calc): & Delta & \multicolumn{1}{|c|}{ Sequence } \\
\hline 82.3 & 1539.7053 & -0.0028 & DLLTNQSDFSGNTK \\
\hline 34.4 & 1539.7053 & -0.0028 & DLLTNQSDFSGNTK \\
\hline 14.5 & 1539.7043 & -0.0018 & EVLTGSRGDXSLGGR \\
\hline 13.7 & 1539.7083 & -0.0058 & GVGQETLLWNTSR \\
\hline 13.4 & 1539.7043 & -0.0018 & EVLTGSRGDXSLGGR \\
\hline 11.5 & 1538.6959 & 1.0066 & FITDKHFYFNK \\
\hline 8.0 & 1539.7069 & -0.0044 & DIINNTSLAELEK \\
\hline 8.0 & 1539.7069 & -0.0044 & DIINNTSLAELEK \\
\hline 7.5 & 1539.7083 & -0.0058 & GVGQETLLWNTSR \\
\hline 7.4 & 1539.7043 & -0.0018 & EVLTGSRGDXSLGGR \\
\hline
\end{tabular}

Spectrum No: 204; Query: 1947; Rank: 1

\section{Peptide View}

MS/MS Fragmentation of EDSCQLNYSEGPCLGMQQK

Found in IPI00210900, Tax_Id=10116 Gene_Symbol=Ambp AMBP protein precursor

Match to Query 1947: 2243.923648 from(1122.969100,2+)

Title: 100101RatKid_NS_deglyco_24.2363.2363.2.dta

Data file K:INewmanPaper|Piliang|3SubProteomes\Piliang3SP\mgf5ppm\ERLIC_3SubProteomes5ppm.mgf 

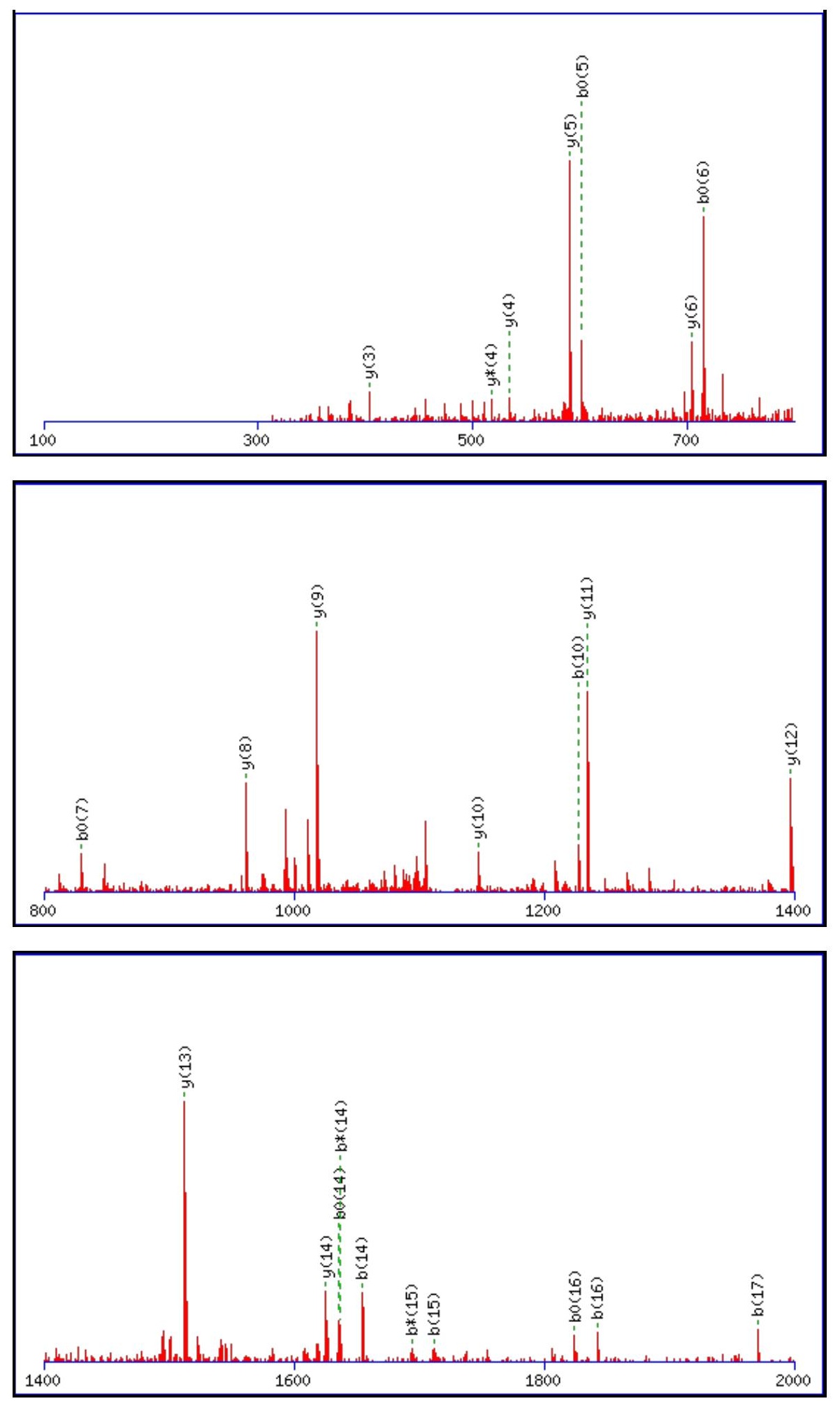

Monoisotopic mass of neutral peptide $\operatorname{Mr}($ calc): 2243.9133

Fixed modifications: Carbamidomethyl (C)

Variable modifications:

N7 : Deamidated $\mathrm{N}(\mathrm{N})$

Ions Score: 82 Expect: $1.3 \mathrm{e}-006$

Matches (Bold Red): 24/190 fragment ions using 35 most intense peaks

\begin{tabular}{|r|c|c|c|c|r|r|r|r|r|r|r|r|r|r|}
\hline$\#$ & $\mathbf{b}$ & $\mathbf{b}^{++}$ & $\mathbf{b}^{*}$ & $\mathbf{b}^{\boldsymbol{*}^{++}}$ & $\mathbf{b}^{\mathbf{0}}$ & $\mathbf{b}^{\mathbf{0 + +}}$ & Seq. & $\mathbf{y}$ & $\mathbf{y}^{++}$ & $\mathbf{y}^{\mathbf{*}}$ & $\mathbf{y}^{\mathbf{*}^{++}}$ & $\mathbf{y}^{\mathbf{0}}$ & $\mathbf{y}^{\mathbf{0 + +}}$ & $\#$ \\
\hline $\mathbf{1}$ & 130.0499 & 65.5286 & & & 112.0393 & 56.5233 & $\mathbf{E}$ & & & & & & & $\mathbf{1 9}$ \\
\hline $\mathbf{2}$ & 245.0768 & 123.0420 & & & 227.0662 & 114.0368 & $\mathbf{D}$ & 2115.8780 & 1058.4426 & 2098.8514 & 1049.9294 & 2097.8674 & 1049.4374 & $\mathbf{1 8}$ \\
\hline 3 & 332.1088 & 166.5581 & & & 314.0983 & 157.5528 & S & 2000.8511 & 1000.9292 & 1983.8245 & 992.4159 & 1982.8405 & 991.9239 & $\mathbf{1 7}$ \\
\hline $\mathbf{4}$ & 492.1395 & 246.5734 & & & 474.1289 & 237.5681 & $\mathbf{C}$ & 1913.8190 & 957.4132 & 1896.7925 & 948.8999 & 1895.8085 & 948.4079 & $\mathbf{1 6}$ \\
\hline $\mathbf{5}$ & 620.1981 & 310.6027 & 603.1715 & 302.0894 & $\mathbf{6 0 2 . 1 8 7 5}$ & 301.5974 & $\mathbf{Q}$ & 1753.7884 & 877.3978 & 1736.7618 & 868.8846 & 1735.7778 & 868.3925 & $\mathbf{1 5}$ \\
\hline
\end{tabular}




\begin{tabular}{|c|c|c|c|c|c|c|c|c|c|c|c|c|c|c|}
\hline 6 & 733.2821 & 367.1447 & 716.2556 & 358.6314 & 715.2716 & 358.1394 & L & 1625.7298 & 813.3685 & |1608.7033 & 804.8553 & 1607.7192 & 804.3633 & $\mid 14$ \\
\hline 7 & 848.3091 & 424.6582 & 831.2825 & 416.1449 & 830.2985 & 415.6529 & $\mathbf{N}$ & 1512.6457 & 756.8265 & 1495.6192 & 748.3132 & 1494.6352 & 747.8212 & 13 \\
\hline 8 & 1011.3724 & 506.1898 & 994.3458 & 497.6766 & 993.3618 & 497.1846 & $\mathbf{Y}$ & 1397.6188 & 699.3130 & 1380.5923 & 690.7998 & 1379.6082 & 690.3078 & 12 \\
\hline 9 & 1098.4044 & 549.7059 & 1081.3779 & 541.1926 & 1080.3939 & 540.7006 & $\mathrm{~S}$ & 1234.5555 & 617.7814 & 1217.5289 & 609.2681 & 1216.5449 & 608.7761 & 11 \\
\hline 10 & 1227.4470 & 614.2271 & 1210.4205 & 605.7139 & 1209.4365 & 605.2219 & E & 1147.5234 & 574.2654 & 1130.4969 & 565.7521 & 1129.5129 & 565.2601 & 10 \\
\hline 11 & 1284.4685 & 642.7379 & 1267.4419 & 634.2246 & 1266.4579 & 633.7326 & G & 1018.4809 & 509.7441 & 1001.4543 & 501.2308 & & & 9 \\
\hline 12 & 1381.5212 & 691.2643 & 1364.4947 & 682.7510 & |1363.5107 & 682.2590 & $\mathbf{P}$ & 961.4594 & 481.2333 & 944.4328 & 472.7201 & & & 8 \\
\hline 13 & 1541.5519 & 771.2796 & 1524.5253 & 762.7663 & 1523.5413 & 762.2743 & $\mathrm{C}$ & 864.4066 & 432.7069 & 847.3801 & 424.1937 & & & 7 \\
\hline 14 & 1654.6360 & 827.8216 & \begin{tabular}{|l|l|}
1637.6094 \\
\end{tabular} & 819.3083 & 1636.6254 & 818.8163 & $\mathbf{L}$ & 704.3760 & 352.6916 & 687.3494 & 344.1784 & & & 6 \\
\hline 15 & 1711.6574 & 856.3323 & 1694.6309 & 847.8191 & 1693.6469 & 847.3271 & G & 591.2919 & 296.1496 & 574.2654 & 287.6363 & & & 5 \\
\hline 16 & 1842.6979 & 921.8526 & 1825.6714 & 913.3393 & 1824.6873 & 912.8473 & M & 534.2704 & 267.6389 & 517.2439 & 259.1256 & & & 4 \\
\hline 17 & 1970.7565 & 985.8819 & 1953.7299 & 977.3686 & 1952.7459 & 976.8766 & $\mathbf{Q}$ & 403.2300 & 202.1186 & 386.2034 & 193.6053 & & & 3 \\
\hline 18 & 2098.8151 & 1049.9112 & 2081.7885 & 1041.3979 & 2080.8045 & 1040.9059 & $\mathbf{Q}$ & 275.1714 & 138.0893 & 258.1448 & 129.5761 & & & 2 \\
\hline 19 & & & & & & & $\mathbf{K}$ & 147.1128 & 74.0600 & 130.0863 & 65.5468 & & & 1 \\
\hline
\end{tabular}
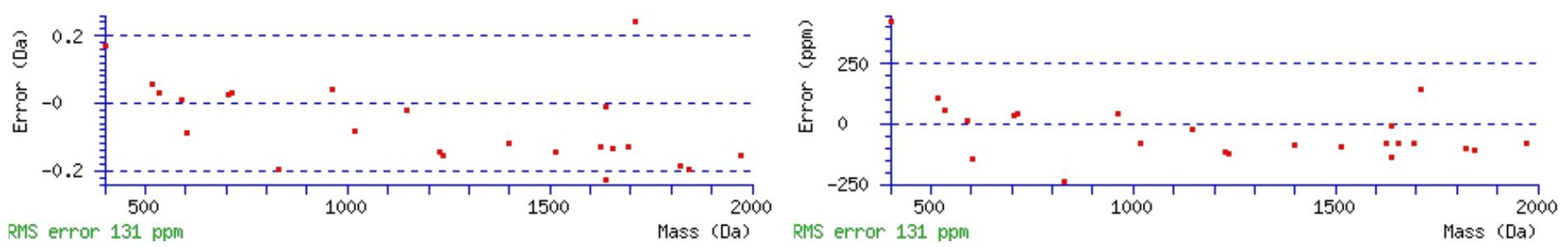

\section{All matches to this query}

\begin{tabular}{|l|l|c|l|}
\hline Score & Mr(calc): & Delta & \multicolumn{1}{|c|}{ Sequence } \\
\hline 82.3 & 2243.9133 & 0.0103 & EDSCQLNYSEGPCLGMQQK \\
\hline 40.8 & 2242.9293 & 0.9944 & EDSCQLNYSEGPCLGMQQK \\
\hline 9.3 & 2241.9245 & 1.9991 & SPGHXWEIPSLQSPYSQR \\
\hline 4.8 & 2241.9001 & 2.0236 & DDKNTMTDSTILLEDVQK \\
\hline 4.0 & 2241.9245 & 1.9991 & SPGHXWEIPSLQSPYSQR \\
\hline 3.8 & 2241.9175 & 2.0061 & NPQVASTWNEVNTNSNTQR \\
\hline 2.5 & 2241.9175 & 2.0061 & NPQVASTWNEVNTNSNTQR \\
\hline 2.5 & 2241.9175 & 2.0061 & NPQVASTWNEVNTNSNTQR \\
\hline 1.5 & 2241.9295 & 1.9941 & GSPIRITPDFSPETMKAR \\
\hline 0.3 & 2242.9079 & 1.0157 & AHSIPSEKDTGPQTGAGAGMR \\
\hline
\end{tabular}

Spectrum No: 205; Query: 945; Rank: 1

\section{Peptide View}

MS/MS Fragmentation of DDALLETTDGFNTTK

Found in IPI00212546, Tax_Id=10116 Gene_Symbol=Emb Embigin precursor

Match to Query 945: 1640.743508 from(821.379030,2+)

Title: 100101RatKid_NS_deglyco_28.2626.2626.2.dta

Data file K:INewmanPaper|Piliang 3 SubProteomes\Piliang3SP\mgf5ppm\ERLIC_3SubProteomes5ppm.mgf 

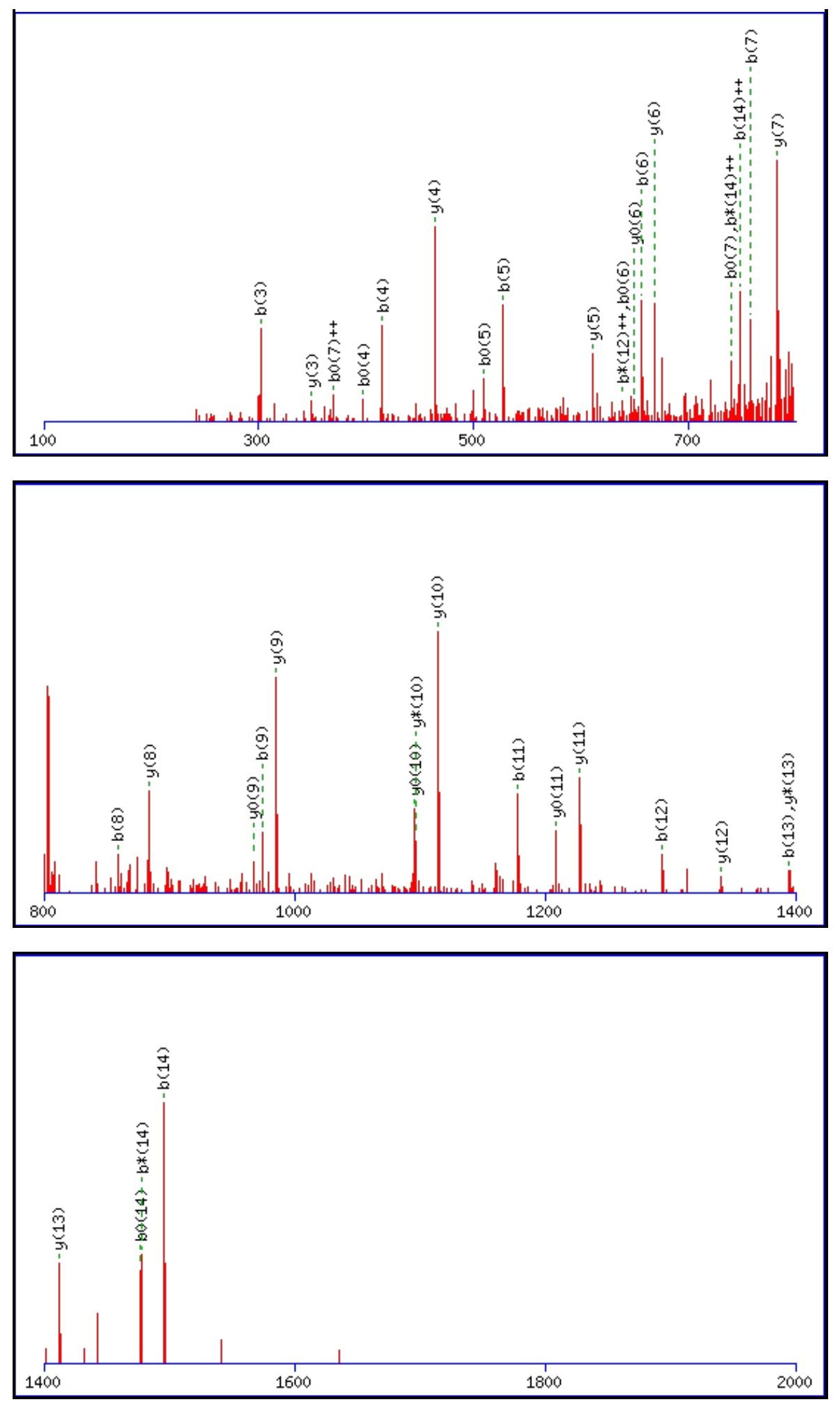

Monoisotopic mass of neutral peptide $\operatorname{Mr}($ calc): 1640.7417

Fixed modifications: Carbamidomethyl (C)

Variable modifications:

N12 : Deamidated $\mathrm{N}(\mathrm{N})$

Ions Score: 82 Expect: $1.1 \mathrm{e}-006$

Matches (Bold Red): 38/144 fragment ions using 60 most intense peaks

\begin{tabular}{|r|c|c|c|c|r|r|r|r|c|c|c|c|c|c|}
\hline$\#$ & $\mathbf{b}$ & $\mathbf{b}^{++}$ & $\mathbf{b}^{*}$ & $\mathbf{b}^{\mathbf{*}^{++}}$ & $\mathbf{b}^{\mathbf{0}}$ & $\mathbf{b}^{\mathbf{0 + +}}$ & Seq. & $\mathbf{y}$ & $\mathbf{y}^{++}$ & $\mathbf{y}^{\mathbf{*}}$ & $\mathbf{y}^{\boldsymbol{*}^{++}}$ & $\mathbf{y}^{\mathbf{0}}$ & $\mathbf{y}^{\mathbf{0 + +}}$ & $\#$ \\
\hline $\mathbf{1}$ & 116.0342 & 58.5207 & & & 98.0237 & 49.5155 & $\mathbf{D}$ & & & & & & & $\mathbf{1 5}$ \\
\hline $\mathbf{2}$ & 231.0612 & 116.0342 & & & 213.0506 & 107.0289 & $\mathbf{D}$ & 1526.7221 & 763.8647 & 1509.6955 & 755.3514 & 1508.7115 & 754.8594 & $\mathbf{1 4}$ \\
\hline $\mathbf{3}$ & $\mathbf{3 0 2 . 0 9 8 3}$ & 151.5528 & & & 284.0877 & 142.5475 & A & $\mathbf{1 4 1 1 . 6 9 5 1}$ & 706.3512 & $\mathbf{1 3 9 4 . 6 6 8 6}$ & 697.8379 & 1393.6845 & 697.3459 & $\mathbf{1 3}$ \\
\hline $\mathbf{4}$ & $\mathbf{4 1 5 . 1 8 2 3}$ & 208.0948 & & & 397.1718 & 199.0895 & L & $\mathbf{1 3 4 0 . 6 5 8 0}$ & 670.8326 & 1323.6314 & 662.3194 & 1322.6474 & 661.8274 & $\mathbf{1 2}$ \\
\hline $\mathbf{5}$ & $\mathbf{5 2 8 . 2 6 6 4}$ & 264.6368 & & & $\mathbf{5 1 0 . 2 5 5 8}$ & 255.6316 & $\mathbf{L}$ & $\mathbf{1 2 2 7 . 5 7 3 9}$ & 614.2906 & 1210.5474 & 605.7773 & $\mathbf{1 2 0 9 . 5 6 3 4}$ & 605.2853 & $\mathbf{1 1}$ \\
\hline
\end{tabular}




\begin{tabular}{|r|r|r|r|r|r|r|r|r|r|r|r|r|r|r|r|r|r|r|}
$\mathbf{6}$ & $\mathbf{6 5 7 . 3 0 9 0}$ & 329.1581 & & & $\mathbf{6 3 9 . 2 9 8 4}$ & 320.1529 & $\mathbf{E}$ & $\mathbf{1 1 1 4 . 4 8 9 9}$ & 557.7486 & $\mathbf{1 0 9 7 . 4 6 3 3}$ & 549.2353 & $\mathbf{1 0 9 6 . 4 7 9 3}$ & $\mathbf{5 4 8 . 7 4 3 3}$ & $\mathbf{1 0}$ \\
\hline $\mathbf{7}$ & $\mathbf{7 5 8 . 3 5 6 7}$ & 379.6820 & & & $\mathbf{7 4 0 . 3 4 6 1}$ & $\mathbf{3 7 0 . 6 7 6 7}$ & $\mathbf{T}$ & $\mathbf{9 8 5 . 4 4 7 3}$ & 493.2273 & 968.4207 & 484.7140 & $\mathbf{9 6 7 . 4 3 6 7}$ & 484.2220 & $\mathbf{9}$ \\
\hline $\mathbf{8}$ & $\mathbf{8 5 9 . 4 0 4 4}$ & 430.2058 & & & 841.3938 & 421.2005 & T & $\mathbf{8 8 4 . 3 9 9 6}$ & 442.7034 & 867.3730 & 434.1902 & 866.3890 & 433.6982 & $\mathbf{8}$ \\
\hline $\mathbf{9}$ & $\mathbf{9 7 4 . 4 3 1 3}$ & 487.7193 & & & 956.4207 & 478.7140 & D & $\mathbf{7 8 3 . 3 5 1 9}$ & 392.1796 & 766.3254 & 383.6663 & 765.3414 & 383.1743 & $\mathbf{7}$ \\
\hline $\mathbf{1 0}$ & 1031.4528 & 516.2300 & & & 1013.4422 & 507.2247 & G & $\mathbf{6 6 8 . 3 2 5 0}$ & 334.6661 & 651.2984 & 326.1529 & $\mathbf{6 5 0 . 3 1 4 4}$ & 325.6608 & $\mathbf{6}$ \\
\hline $\mathbf{1 1}$ & $\mathbf{1 1 7 8 . 5 2 1 2}$ & 589.7642 & & & 1160.5106 & 580.7589 & F & $\mathbf{6 1 1 . 3 0 3 5}$ & 306.1554 & 594.2770 & 297.6421 & 593.2929 & 297.1501 & $\mathbf{5}$ \\
\hline $\mathbf{1 2}$ & $\mathbf{1 2 9 3 . 5 4 8 1}$ & 647.2777 & 1276.5216 & $\mathbf{6 3 8 . 7 6 4 4}$ & 1275.5375 & 638.2724 & N & $\mathbf{4 6 4 . 2 3 5 1}$ & 232.6212 & 447.2085 & 224.1079 & 446.2245 & 223.6159 & $\mathbf{4}$ \\
\hline $\mathbf{1 3}$ & $\mathbf{1 3 9 4 . 5 9 5 8}$ & 697.8015 & 1377.5692 & 689.2883 & 1376.5852 & 688.7963 & T & 349.2082 & 175.1077 & 332.1816 & 166.5944 & 331.1976 & 166.1024 & $\mathbf{3}$ \\
\hline $\mathbf{1 4}$ & $\mathbf{1 4 9 5 . 6 4 3 5}$ & $\mathbf{7 4 8 . 3 2 5 4}$ & $\mathbf{1 4 7 8 . 6 1 6 9}$ & $\mathbf{7 3 9 . 8 1 2 1}$ & $\mathbf{1 4 7 7 . 6 3 2 9}$ & $\mathbf{7 3 9 . 3 2 0 1}$ & $\mathbf{T}$ & 248.1605 & 124.5839 & 231.1339 & 116.0706 & 230.1499 & 115.5786 & $\mathbf{2}$ \\
\hline $\mathbf{1 5}$ & & & & & & & K & 147.1128 & 74.0600 & 130.0863 & 65.5468 & & & \\
\hline
\end{tabular}
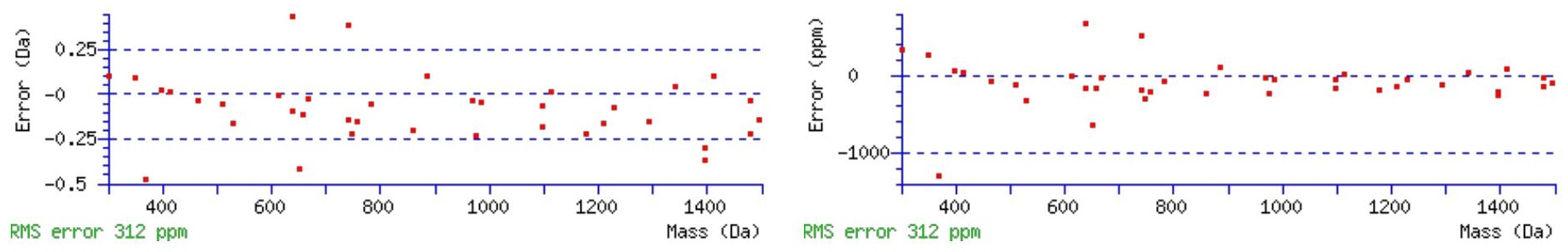

\section{All matches to this query}

\begin{tabular}{|l|l|l|l|}
\hline Score & Mr(calc): & Delta & Sequence \\
\hline 82.2 & 1640.7417 & 0.0018 & DDALLETTDGFNTTK \\
\hline 4.7 & 1639.7542 & 0.9893 & KTNLFSHVASMGPR \\
\hline 3.7 & 1640.7546 & -0.0111 & VLSLDTNITNQVNK \\
\hline 3.5 & 1640.7407 & 0.0028 & RNGLEGKQTTASADL \\
\hline 3.5 & 1640.7407 & 0.0028 & RNGLEGKQTTASADL \\
\hline 3.1 & 1640.7308 & 0.0127 & GYRPENSTAVTGGPR \\
\hline 2.2 & 1640.7308 & 0.0127 & GYRPENSTAVTGGPR \\
\hline 1.1 & 1638.7324 & 2.0111 & KANLHSSLENMGLK \\
\hline 1.1 & 1638.7324 & 2.0111 & KANLHSSLENMGLK \\
\hline
\end{tabular}

Spectrum No: 206; Query: 2749; Rank: 1

\section{Peptide View}

MS/MS Fragmentation of VFSTDINGLNTQEILNVSVDTPENLAVDWINNK

Found in IPI00205325, Tax_Id=10116 Gene_Symbol=Lrp2 Low-density lipoprotein receptor-related protein 2 precursor

Match to Query 2749: 3672.825342 from(1225.282390,3+)

Title: 100101RatKid_NS_deglyco_16.6878.6878.3.dta

Data file K:|NewmanPaper|Piliang|3SubProteomes\Piliang3SP\mgf5ppm\ERLIC_3SubProteomes5ppm.mgf 

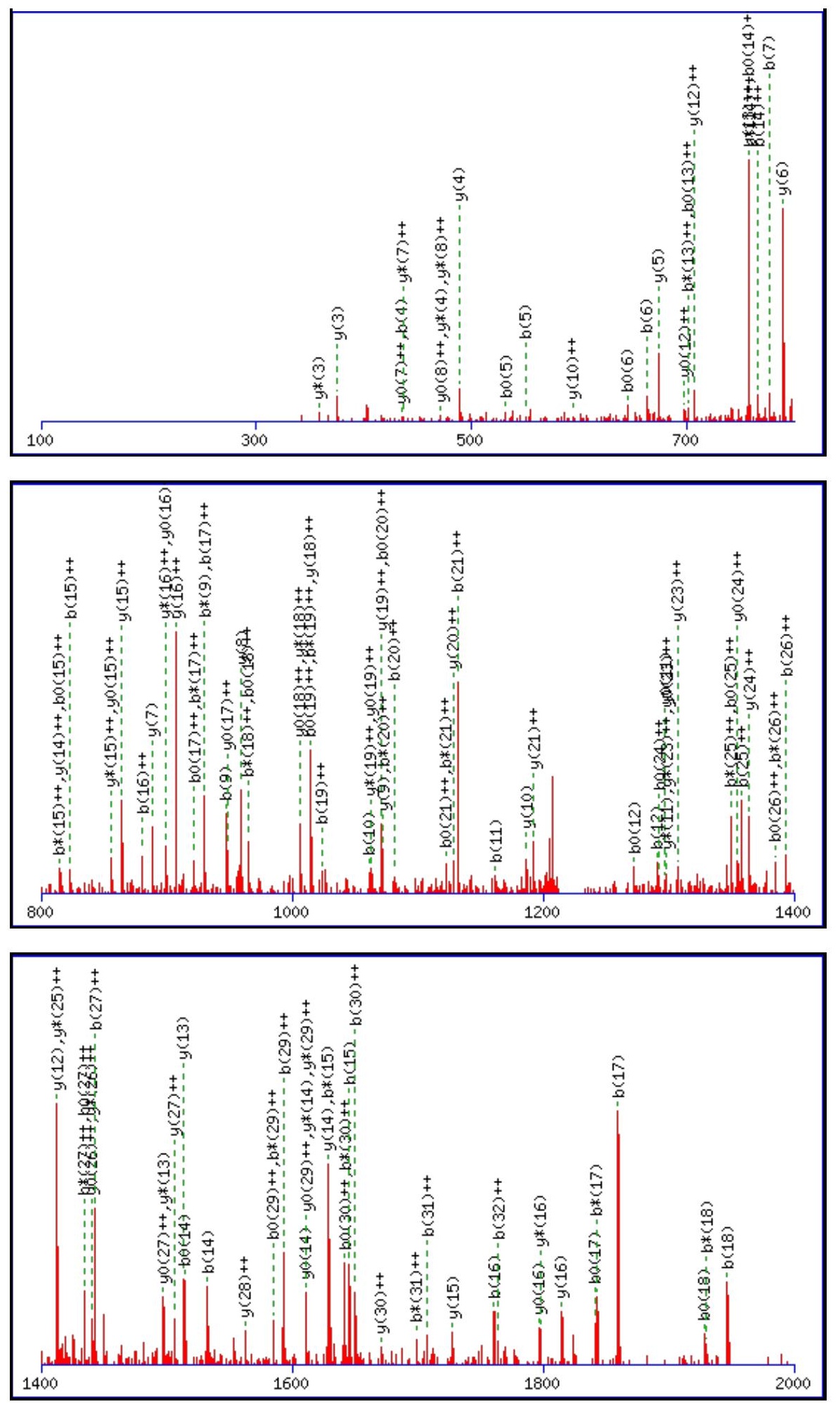

Monoisotopic mass of neutral peptide $\operatorname{Mr}($ calc): 3672.8159

Fixed modifications: Carbamidomethyl (C)

Variable modifications:

N16 : Deamidated_N (N)

Ions Score: 82 Expect: $1.4 \mathrm{e}-006$

Matches (Bold Red): 125/358 fragment ions using 169 most intense peaks

\begin{tabular}{|c|c|c|c|c|c|c|c|c|c|c|c|c|c|c|}
\hline \# & b & $\mathbf{b}^{++}$ & $\mathbf{b}^{*}$ & $\mathbf{b}^{*^{++}}$ & $\mathbf{b}^{0}$ & $\mathbf{b}^{\mathbf{0 + +}}$ & Seq. & $\mathbf{y}$ & $\mathbf{y}^{++}$ & $\mathbf{y}^{*}$ & $\mathbf{y}^{*^{++}}$ & $\mathbf{y}^{\mathbf{0}}$ & $\mathbf{y}^{0++}$ & \# \\
\hline 1 & 100.0757 & 50.5415 & & & & & V & & & & & & & 33 \\
\hline 2 & 247.1441 & 124.0757 & & & & & $\mathbf{F}$ & 3574.7548 & 1787.8810 & 3557.7282 & 1779.3677 & 3556.7442 & 1778.8757 & 32 \\
\hline 3 & 334.1761 & 167.5917 & & & 316.1656 & 158.5864 & S & 3427.6863 & 1714.3468 & 3410.6598 & 1705.8335 & 3409.6758 & 1705.3415 & 31 \\
\hline 4 & 435.2238 & 218.1155 & & & 417.2132 & 209.1103 & $\mathbf{T}$ & 3340.6543 & \begin{tabular}{|l|}
1670.8308 \\
\end{tabular} & 3323.6278 & 1662.3175 & 3322.6437 & 1661.8255 & 30 \\
\hline 5 & 550.2508 & 275.6290 & & & 532.2402 & 266.6237 & D & 3239.6066 & 1620.3070 & 3222.5801 & 1611.7937 & 3221.5961 & 1611.3017 & 29 \\
\hline
\end{tabular}




\begin{tabular}{|c|c|c|c|c|c|c|c|c|c|c|c|c|c|c|}
\hline & 663.3348 & 332.1710 & & & 645.3243 & 323.1658 & I & 3124.5797 & 1562.7935 & 3107.5531 & 1554.2802 & 3106.5691 & 1553.7882 & 28 \\
\hline 7 & 777.3777 & 389.1925 & 760.3512 & 380.6792 & 759.3672 & 380.1872 & $\mathbf{N}$ & 3011.4956 & 1506.2515 & 2994.4691 & 1497.7382 & 2993.4851 & 1497.2462 & 27 \\
\hline 8 & 834.3992 & 417.7032 & 817.3727 & 409.1900 & 816.3886 & 408.6980 & G & 2897.4527 & 1449.2300 & 2880.4262 & 1440.7167 & 2879.4421 & 1440.2247 & \\
\hline 9 & 947.4833 & 474.2453 & 930.4567 & 465.7320 & 929.4727 & 465.2400 & $\mathbf{L}$ & 2840.4312 & 1420.7193 & 2823.4047 & 1412.2060 & 2822.4207 & 1411.7140 & 25 \\
\hline 10 & 1061.5262 & 531.2667 & 1044.4997 & 522.7535 & 1043.5156 & 522.2615 & $\mathbf{N}$ & 2727.3472 & 1364.1772 & 2710.3206 & 1355.6639 & 2709.3366 & 1355.1719 & 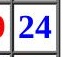 \\
\hline 11 & 1162.5739 & 581.7906 & 1145.5473 & 573.2773 & 1144.5633 & 572.7853 & $\mathbf{T}$ & 2613.3042 & 1307.1558 & 2596.2777 & 1298.6425 & 2595.2937 & 1298.1505 & 3 \\
\hline 12 & 1290.6325 & 645.8199 & 1273.6059 & 637.3066 & $\mid 1272.6219$ & 636.8146 & $\mathbf{Q}$ & 2512.2566 & 1256.6319 & 2495.2300 & 1248.1186 & 2494.2460 & 1247.6266 & 22 \\
\hline 13 & 1419.6751 & 710.3412 & 1402.6485 & 701.8279 & 1401.6645 & 701.3359 & $\mathbf{E}$ & 2384.1980 & 1192.6026 & 2367.1714 & 1184.0894 & 2366.1874 & 1183.5973 & 21 \\
\hline 14 & 1532.7591 & 766.8832 & 1515.7326 & 758.3699 & 1514.7485 & 757.8779 & I & 2255.1554 & 1128.0813 & 2238.1288 & 1119.5681 & 2237.1448 & 1119.0761 & 0 \\
\hline 15 & 1645.8432 & 823.4252 & 1628.8166 & 814.9120 & 1627.8326 & 814.4199 & L & 2142.0713 & 1071.5393 & 2125.0448 & 1063.0260 & 2124.0608 & 1062.5340 & 19 \\
\hline 16 & 1760.8701 & 880.9387 & 1743.8436 & 872.4254 & 1742.8595 & 871.9334 & $\mathbf{N}$ & 2028.9873 & 1014.9973 & 2011.9607 & 1006.4840 & 2010.9767 & 1005.9920 & 18 \\
\hline 17 & 1859.9385 & 930.4729 & 1842.9120 & 921.9596 & 1841.9280 & 921.4676 & V & 1913.9603 & 957.4838 & 1896.9338 & 948.9705 & 1895.9498 & 948.4785 & 17 \\
\hline 18 & 1946.9706 & 973.9889 & 1929.9440 & 965.4756 & 1928.9600 & 964.9836 & S & 1814. & 907.9496 & 1797.8654 & 899.4363 & 1796 & 9443 & 16 \\
\hline 19 & 2046.0390 & 1023.5231 & 2029.0124 & 1015.0098 & 2028.0284 & 1014.5178 & V & 1727.8599 & 864.4336 & 1710.8333 & 855.9203 & 1709.8493 & 855.4283 & 15 \\
\hline 20 & 2161.0659 & 1081.0366 & 2144.0394 & 1072.5233 & 2143.0553 & 1072.0313 & D & 1628.7915 & 814.8994 & 1611.7649 & 806.3861 & 1610.7809 & 805.8941 & 14 \\
\hline 21 & 2262.1136 & 1131.5604 & 2245.0870 & 1123.0472 & 2244.1030 & 1122.5552 & $\mathbf{T}$ & 1513.7645 & 757.3859 & 1496.7380 & 748.8726 & 1495.7540 & 748.3806 & \\
\hline 22 & 2359.1664 & 1180.0868 & 2342.1398 & 1171.5735 & 2341.1558 & 1171.0815 & $\mathbf{P}$ & 1412.7169 & 706.8621 & 1395.6903 & 698.3488 & 1394.7063 & 697.8568 & 12 \\
\hline 23 & 2488.2089 & 1244.6081 & 2471.1824 & 1236.0948 & 2470.1984 & 1235.6028 & $\mathbf{E}$ & 1315.6641 & 658.3357 & 1298.6375 & 649.8224 & 1297.6535 & 649.3304 & 11 \\
\hline 24 & 2602.2519 & 1301.6296 & 2585.2253 & 1293.1163 & 2584.2413 & 1292.6243 & $\mathbf{N}$ & 1186.6215 & 593.8144 & 1169.5949 & 585.3011 & 1168.6109 & 584.8091 & 10 \\
\hline 25 & 2715.3359 & 1358.1716 & 2698.3094 & 1349.6583 & 2697.3254 & 1349.1663 & $\mathbf{L}$ & 1072.5786 & 536.7929 & 1055.5520 & 528.2796 & 1054.5680 & 527.7876 & 9 \\
\hline 26 & 2786.3731 & 1393.6902 & 2769.3465 & 1385.1769 & 2768.3625 & 1384.6849 & A & 959.4945 & 480.2509 & 942.4680 & 471.7376 & 941.4839 & 471.2456 & 8 \\
\hline 27 & 2885.4415 & 1443.2244 & 2868.4149 & 1434.7111 & 2867.4309 & 1434.2191 & V & 888.4574 & 444.7323 & 871.4308 & 436.2191 & 870.4468 & 435.7271 & 7 \\
\hline 28 & 3000.4684 & 1500.7378 & 2983.4419 & 1492.2246 & 2982.4578 & 1491.7326 & D & 789.3890 & 395.1981 & 772.3624 & 386.6849 & 771.3784 & 386.1928 & 6 \\
\hline 29 & 3186.5477 & \begin{tabular}{|l|}
1593.7775 \\
\end{tabular} & 3169.5212 & 1585.2642 & 3168.5372 & 1584.7722 & W & 674.3620 & 337.6847 & 657.3355 & 329.1714 & & & 5 \\
\hline 30 & 3299.6318 & 1650.3195 & 3282.6052 & 1641.8063 & 3281.6212 & 1641.3142 & I & 488.2827 & 244.6450 & 471.2562 & 236.1317 & & & 4 \\
\hline 31 & 3413.6747 & 1707.3410 & 3396.6482 & 1698.8277 & 3395.6642 & 1698.3357 & $\mathbf{N}$ & 375.1987 & 188.1030 & 358.1721 & 179.5897 & & & 3 \\
\hline 323 & 3527.7176 & 1764.3625 & 3510.6911 & 1755.8492 & 3509.7071 & 1755.3572 & $\mathbf{N}$ & 261.1557 & 131.0815 & 244.1292 & 122.5682 & & & 2 \\
\hline 33 & & & & & & & $\mathbf{K}$ & 147.1128 & 74.0600 & 130.0863 & 65.5468 & & & 1 \\
\hline
\end{tabular}
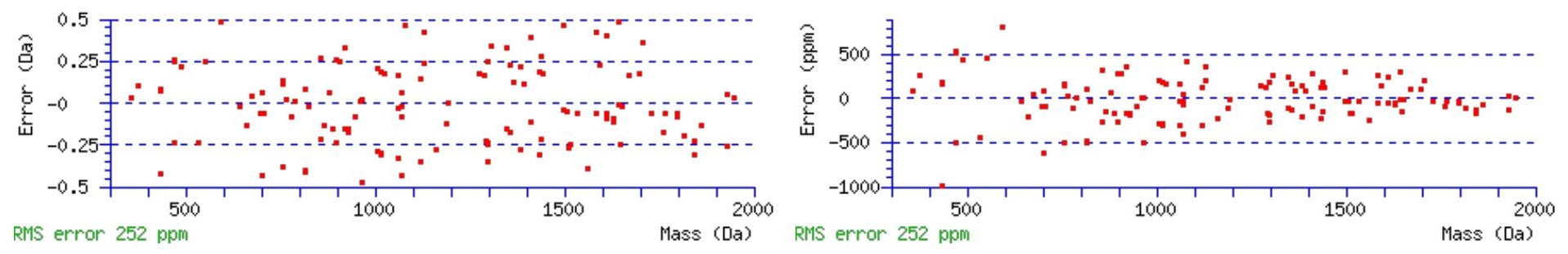

\section{All matches to this query}

\begin{tabular}{|l|l|l|l|}
\hline Score & Mr(calc): & Delta & \multicolumn{1}{|c|}{ Sequence } \\
\hline 82.1 & 3672.8159 & 0.0095 & VFSTDINGLNTQEILNVSVDTPENLAVDWINNK \\
\hline 70.4 & 3672.8159 & 0.0095 & VFSTDINGLNTQEILNVSVDTPENLAVDWINNK \\
\hline 70.2 & 3672.8159 & 0.0095 & VFSTDINGLNTQEILNVSVDTPENLAVDWINNK \\
\hline 62.6 & 3672.8159 & 0.0095 & VFSTDINGLNTQEILNVSVDTPENLAVDWINNK \\
\hline 59.6 & 3671.8319 & 0.9935 & VFSTDINGLNTQEILNVSVDTPENLAVDWINNK \\
\hline 50.4 & 3672.8159 & 0.0095 & VFSTDINGLNTQEILNVSVDTPENLAVDWINNK \\
\hline 49.1 & 3672.8159 & 0.0095 & VFSTDINGLNTQEILNVSVDTPENLAVDWINNK \\
\hline 8.4 & 3672.8007 & 0.0247 & TILMMGRYVEPIEDVPCGNIVGLVGVDQFLVK \\
\hline 6.3 & 3670.8412 & 1.9842 & LTVQPSAKGFRFTLVSCALSFFLFSFQVHEK \\
\hline 6.3 & 3670.8412 & 1.9842 & LTVQPSAKGFRFTLVSCALSFFLFSFQVHEK \\
\hline
\end{tabular}


Spectrum No: 207; Query: 748; Rank: 1

\section{Peptide View}

MS/MS Fragmentation of IKLDNYSTQELGR

Found in IPI00204348, Tax_Id=10116 Gene_Symbol=Nt5e 5'-nucleotidase precursor

Match to Query 748: 1536.785328 from(769.399940,2+)

Title: 100101RatKid_NS_deglyco_21.2056.2056.2.dta

Data file K:\NewmanPaper\Piliang \3SubProteomes $\backslash$ Piliang3SP $\backslash$ mgf5ppm\ERLIC_3SubProteomes5ppm.mgf
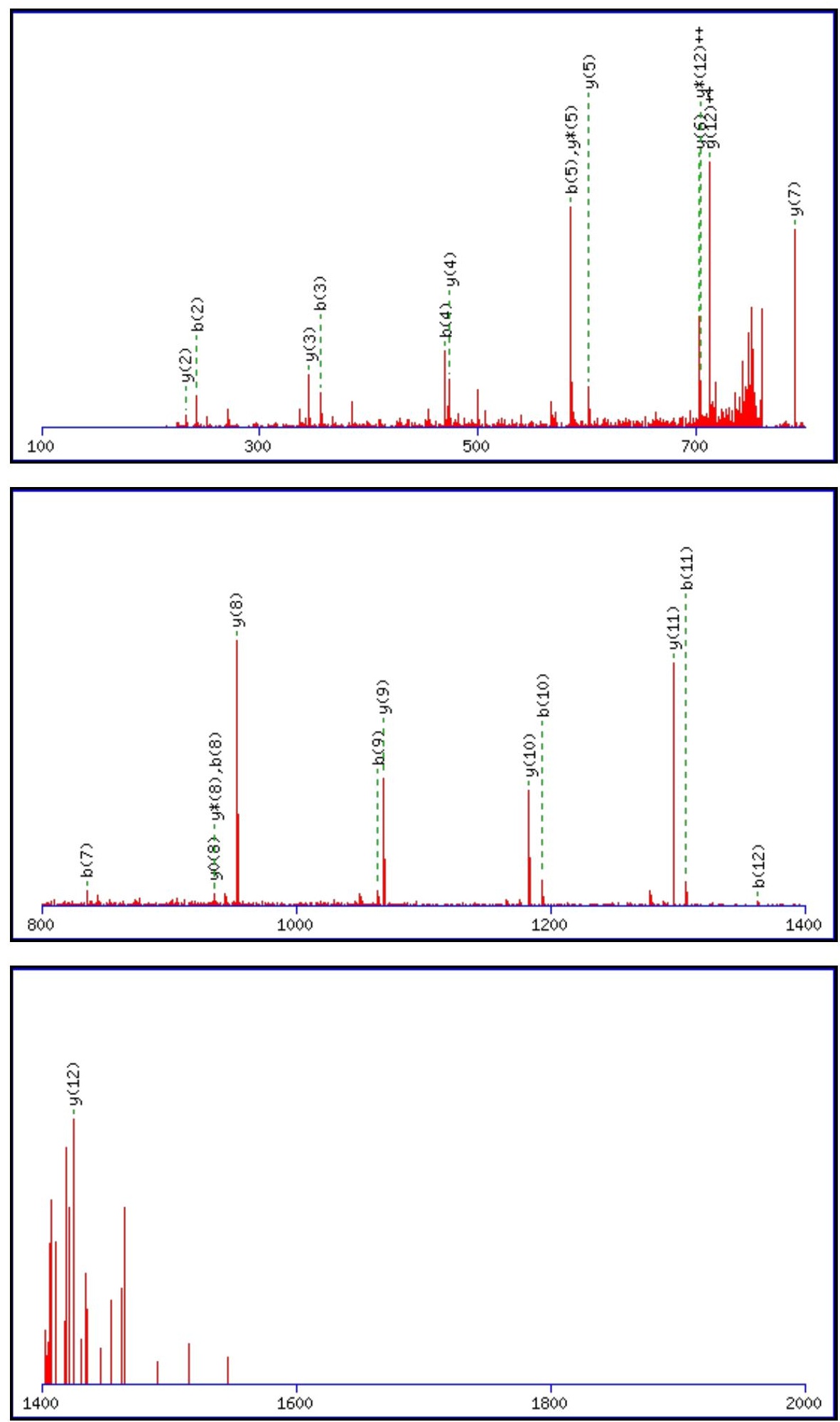

Monoisotopic mass of neutral peptide $\operatorname{Mr}($ calc): 1536.7784

Fixed modifications: Carbamidomethyl (C) 
Variable modifications:

N5 : Deamidated_N (N)

Ions Score: 82 Expect: 8.6e-007

Matches (Bold Red): 26/130 fragment ions using 50 most intense peaks

\begin{tabular}{|r|c|c|c|c|c|c|c|c|c|c|c|c|c|c|}
\hline$\#$ & $\mathbf{b}$ & $\mathbf{b}^{++}$ & $\mathbf{b}^{*}$ & $\mathbf{b}^{\mathbf{*}^{++}}$ & $\mathbf{b}^{\mathbf{0}}$ & $\mathbf{b}^{\mathbf{0 + +}}$ & $\mathbf{S e q}$ & $\mathbf{y}$ & $\mathbf{y}^{++}$ & $\mathbf{y}^{\mathbf{*}}$ & $\mathbf{y}^{\mathbf{*}^{++}}$ & $\mathbf{y}^{\mathbf{0}}$ & $\mathbf{y}^{\mathbf{0 + +}}$ & $\#$ \\
\hline $\mathbf{1}$ & 114.0913 & 57.5493 & & & & & $\mathbf{I}$ & & & & & & & $\mathbf{1 3}$ \\
\hline $\mathbf{2}$ & $\mathbf{2 4 2 . 1 8 6 3}$ & 121.5968 & 225.1598 & 113.0835 & & & $\mathbf{K}$ & $\mathbf{1 4 2 4 . 7 0 1 6}$ & $\mathbf{7 1 2 . 8 5 4 4}$ & 1407.6750 & $\mathbf{7 0 4 . 3 4 1 2}$ & 1406.6910 & 703.8492 & $\mathbf{1 2}$ \\
\hline $\mathbf{3}$ & $\mathbf{3 5 5 . 2 7 0 4}$ & 178.1388 & 338.2438 & 169.6255 & & & $\mathbf{L}$ & $\mathbf{1 2 9 6 . 6 0 6 6}$ & 648.8070 & 1279.5801 & 640.2937 & 1278.5961 & 639.8017 & $\mathbf{1 1}$ \\
\hline $\mathbf{4}$ & $\mathbf{4 7 0 . 2 9 7 3}$ & 235.6523 & 453.2708 & 227.1390 & 452.2867 & 226.6470 & $\mathbf{D}$ & $\mathbf{1 1 8 3 . 5 2 2 6}$ & 592.2649 & 1166.4960 & 583.7516 & 1165.5120 & 583.2596 & $\mathbf{1 0}$ \\
\hline $\mathbf{5}$ & $\mathbf{5 8 5 . 3 2 4 2}$ & 293.1658 & 568.2977 & 284.6525 & 567.3137 & 284.1605 & $\mathbf{N}$ & $\mathbf{1 0 6 8 . 4 9 5 6}$ & 534.7514 & 1051.4691 & 526.2382 & 1050.4851 & 525.7462 & $\mathbf{9}$ \\
\hline $\mathbf{6}$ & $\mathbf{7 4 8 . 3 8 7 6}$ & 374.6974 & 731.3610 & 366.1842 & 730.3770 & 365.6921 & $\mathbf{Y}$ & $\mathbf{9 5 3 . 4 6 8 7}$ & 477.2380 & $\mathbf{9 3 6 . 4 4 2 1}$ & 468.7247 & $\mathbf{9 3 5 . 4 5 8 1}$ & 468.2327 & $\mathbf{8}$ \\
\hline $\mathbf{7}$ & $\mathbf{8 3 5 . 4 1 9 6}$ & 418.2134 & 818.3931 & 409.7002 & 817.4090 & 409.2082 & $\mathbf{S}$ & $\mathbf{7 9 0 . 4 0 5 4}$ & 395.7063 & 773.3788 & 387.1930 & 772.3948 & 386.7010 & $\mathbf{7}$ \\
\hline $\mathbf{8}$ & $\mathbf{9 3 6 . 4 6 7 3}$ & 468.7373 & 919.4407 & 460.2240 & 918.4567 & 459.7320 & $\mathbf{T}$ & $\mathbf{7 0 3 . 3 7 3 3}$ & 352.1903 & 686.3468 & 343.6770 & 685.3628 & 343.1850 & $\mathbf{6}$ \\
\hline $\mathbf{9}$ & $\mathbf{1 0 6 4 . 5 2 5 9}$ & 532.7666 & 1047.4993 & 524.2533 & 1046.5153 & 523.7613 & $\mathbf{Q}$ & $\mathbf{6 0 2 . 3 2 5 7}$ & 301.6665 & $\mathbf{5 8 5 . 2 9 9 1}$ & 293.1532 & 584.3151 & 292.6612 & $\mathbf{5}$ \\
\hline $\mathbf{1 0}$ & $\mathbf{1 1 9 3 . 5 6 8 5}$ & 597.2879 & 1176.5419 & 588.7746 & 1175.5579 & 588.2826 & $\mathbf{E}$ & $\mathbf{4 7 4 . 2 6 7 1}$ & 237.6372 & 457.2405 & 229.1239 & 456.2565 & 228.6319 & $\mathbf{4}$ \\
\hline $\mathbf{1 1}$ & $\mathbf{1 3 0 6 . 6 5 2 5}$ & 653.8299 & 1289.6260 & 645.3166 & 1288.6420 & 644.8246 & $\mathbf{L}$ & 345.2245 & 173.1159 & 328.1979 & 164.6026 & & & $\mathbf{3}$ \\
\hline $\mathbf{1 2}$ & $\mathbf{1 3 6 3 . 6 7 4 0}$ & 682.3406 & 1346.6474 & 673.8274 & 1345.6634 & 673.3353 & $\mathbf{G}$ & $\mathbf{2 3 2 . 1 4 0 4}$ & 116.5738 & 215.1139 & 108.0606 & & & $\mathbf{2}$ \\
\hline $\mathbf{1 3}$ & & & & & & & $\mathbf{R}$ & 175.1190 & 88.0631 & 158.0924 & 79.5498 & & & $\mathbf{1}$ \\
\hline
\end{tabular}
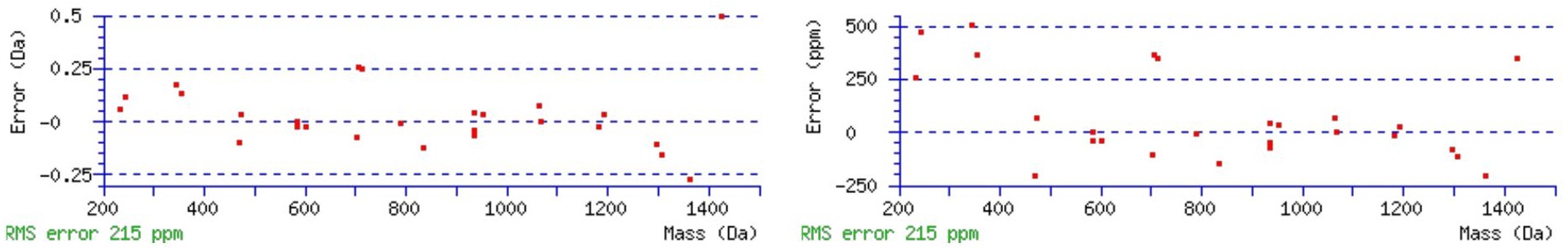

\section{All matches to this query}

\begin{tabular}{|l|l|l|l|}
\hline Score & Mr(calc): & Delta & \multicolumn{1}{|c|}{ Sequence } \\
\hline 81.9 & 1536.7784 & 0.0070 & IKLDNYSTQELGR \\
\hline 26.9 & 1535.7943 & 0.9910 & IKLDNYSTQELGR \\
\hline 4.6 & 1536.7752 & 0.0102 & IIKNTAMMREAAR \\
\hline 4.6 & 1536.7814 & 0.0040 & KLYVSNLGIGHTR \\
\hline 4.6 & 1536.7814 & 0.0040 & KLYVSNLGIGHTR \\
\hline 2.7 & 1536.7926 & -0.0072 & RPYGRNKPLISR \\
\hline 2.4 & 1536.7913 & -0.0059 & PLPTTLTSGSKSR \\
\hline 1.1 & 1536.7773 & 0.0080 & KQGRSAINEVVTR \\
\hline 0.9 & 1534.7878 & 1.9975 & KLLIDYDEEELR \\
\hline 0.8 & 1535.7821 & 1.0033 & AALEQRGLAKSGQK \\
\hline
\end{tabular}

Spectrum No: 208; Query: 183; Rank: 1

\section{Peptide View}

MS/MS Fragmentation of IVDVNLTSEGK

Found in IPI00192796, Tax_Id=10116 Gene_Symbol=RGD1564625_predicted similar to Transmembrane 9 superfamily protein member 3 precursor

Match to Query 183: 1174.604828 from(588.309690,2+)

Title: 091008RatKidney_NoSalt_31.1305.1305.2.dta

Data file K:INewmanPaper|Piliangl3SubProteomes\Piliang3SP\mgf5ppm\ERLIC_3SubProteomes5ppm.mgf 

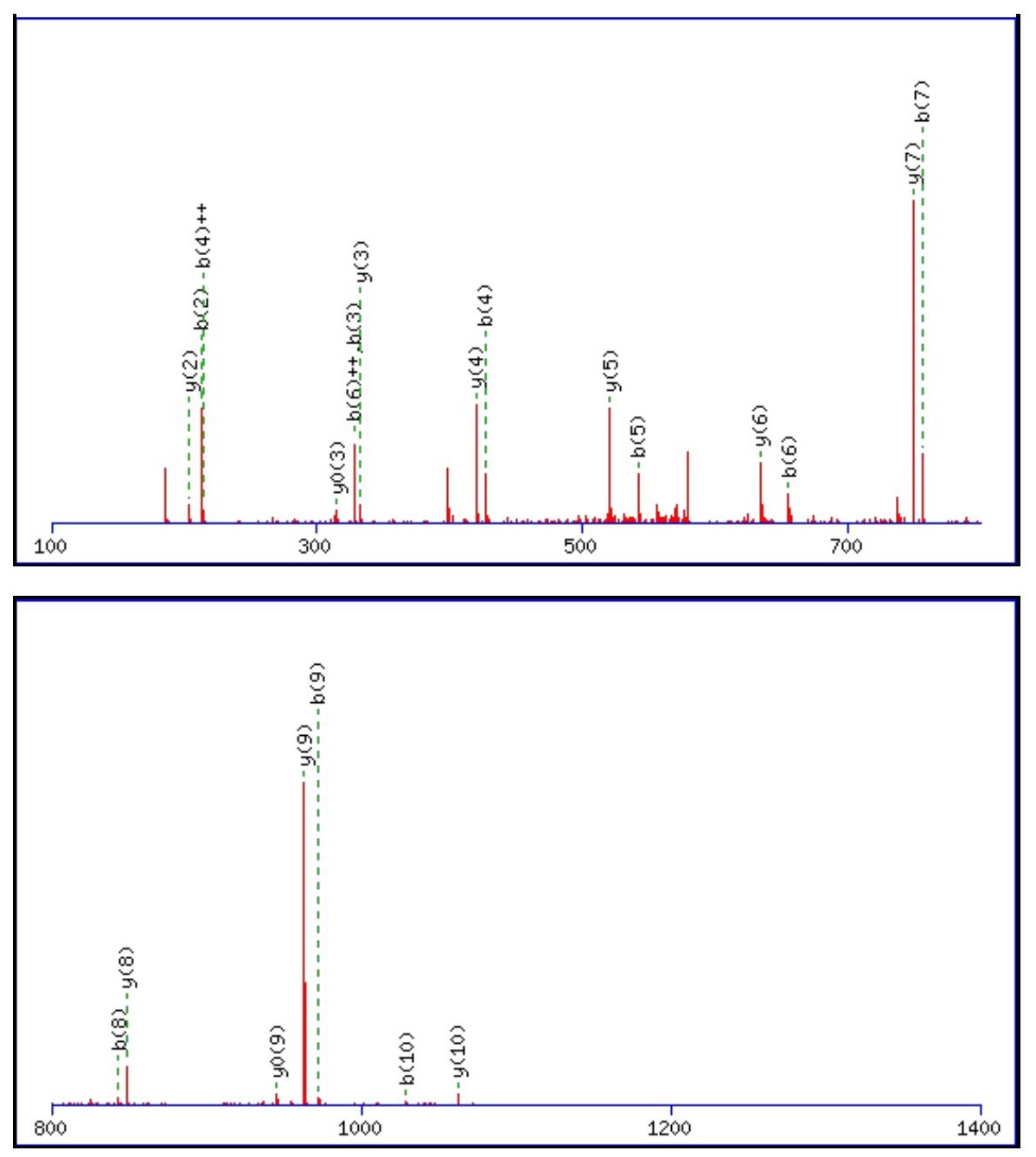

\begin{tabular}{rrrr}
\hline 00 & 1600 & 1800 & 2000 \\
\hline
\end{tabular}

Monoisotopic mass of neutral peptide $\operatorname{Mr}($ calc): 1174.6081

Fixed modifications: Carbamidomethyl (C)

Variable modifications:

N5 : Deamidated_N (N)

Ions Score: 82 Expect : $7.1 \mathrm{e}-007$

Matches (Bold Red): 22/104 fragment ions using 30 most intense peaks

\begin{tabular}{|r|c|c|c|c|c|c|c|c|c|c|c|c|c|c|}
\hline$\#$ & $\mathbf{b}$ & $\mathbf{b}^{++}$ & $\mathbf{b}^{*}$ & $\mathbf{b}^{\boldsymbol{*}^{++}}$ & $\mathbf{b}^{\mathbf{0}}$ & $\mathbf{b}^{\mathbf{0 + +}}$ & $\mathbf{S e q}$ & $\mathbf{y}$ & $\mathbf{y}^{++}$ & $\mathbf{y}^{\mathbf{*}}$ & $\mathbf{y}^{\boldsymbol{*}^{++}}$ & $\mathbf{y}^{\mathbf{0}}$ & $\mathbf{y}^{\mathbf{0 + +}}$ & $\#$ \\
\hline $\mathbf{1}$ & 114.0913 & 57.5493 & & & & & $\mathbf{I}$ & & & & & & & $\mathbf{1 1}$ \\
\hline $\mathbf{2}$ & $\mathbf{2 1 3 . 1 5 9 8}$ & 107.0835 & & & & & $\mathbf{V}$ & $\mathbf{1 0 6 2 . 5 3 1 3}$ & 531.7693 & 1045.5048 & 523.2560 & 1044.5208 & 522.7640 & $\mathbf{1 0}$ \\
\hline $\mathbf{3}$ & $\mathbf{3 2 8 . 1 8 6 7}$ & 164.5970 & & & 310.1761 & 155.5917 & $\mathbf{D}$ & $\mathbf{9 6 3 . 4 6 2 9}$ & 482.2351 & 946.4364 & 473.7218 & $\mathbf{9 4 5 . 4 5 2 4}$ & 473.2298 & $\mathbf{9}$ \\
\hline $\mathbf{4}$ & $\mathbf{4 2 7 . 2 5 5 1}$ & $\mathbf{2 1 4 . 1 3 1 2}$ & & & 409.2445 & 205.1259 & $\mathbf{V}$ & $\mathbf{8 4 8 . 4 3 6 0}$ & 424.7216 & 831.4094 & 416.2084 & 830.4254 & 415.7163 & $\mathbf{8}$ \\
\hline $\mathbf{5}$ & $\mathbf{5 4 2 . 2 8 2 0}$ & 271.6447 & 525.2555 & 263.1314 & 524.2715 & 262.6394 & $\mathbf{N}$ & $\mathbf{7 4 9 . 3 6 7 6}$ & 375.1874 & 732.3410 & 366.6741 & 731.3570 & 366.1821 & $\mathbf{7}$ \\
\hline
\end{tabular}




\begin{tabular}{|r|r|r|r|r|r|r|r|r|r|r|r|r|r|r|r|r|}
$\mathbf{6}$ & $\mathbf{6 5 5 . 3 6 6 1}$ & 328.1867 & 638.3396 & 319.6734 & 637.3555 & 319.1814 & L & $\mathbf{6 3 4 . 3 4 0 6}$ & 317.6740 & 617.3141 & 309.1607 & 616.3301 & 308.6687 & $\mathbf{6}$ \\
\hline $\mathbf{7}$ & $\mathbf{7 5 6 . 4 1 3 8}$ & 378.7105 & 739.3872 & 370.1973 & 738.4032 & 369.7053 & T & $\mathbf{5 2 1 . 2 5 6 6}$ & 261.1319 & 504.2300 & 252.6186 & 503.2460 & 252.1266 & $\mathbf{5}$ \\
\hline $\mathbf{8}$ & $\mathbf{8 4 3 . 4 4 5 8}$ & 422.2265 & 826.4193 & 413.7133 & 825.4353 & 413.2213 & S & $\mathbf{4 2 0 . 2 0 8 9}$ & 210.6081 & 403.1823 & 202.0948 & 402.1983 & 201.6028 & $\mathbf{4}$ \\
\hline $\mathbf{9}$ & $\mathbf{9 7 2 . 4 8 8 4}$ & 486.7478 & 955.4619 & 478.2346 & 954.4778 & 477.7426 & E & 333.1769 & 167.0921 & 316.1503 & 158.5788 & 315.1663 & 158.0868 & $\mathbf{3}$ \\
\hline $\mathbf{1 0}$ & $\mathbf{1 0 2 9 . 5 0 9 9}$ & 515.2586 & 1012.4833 & 506.7453 & 1011.4993 & 506.2533 & G & $\mathbf{2 0 4 . 1 3 4 3}$ & 102.5708 & 187.1077 & 94.0575 & & & \\
\hline $\mathbf{1 1}$ & & & & & & & K & 147.1128 & 74.0600 & 130.0863 & 65.5468 & & & & $\mathbf{1}$ \\
\hline
\end{tabular}
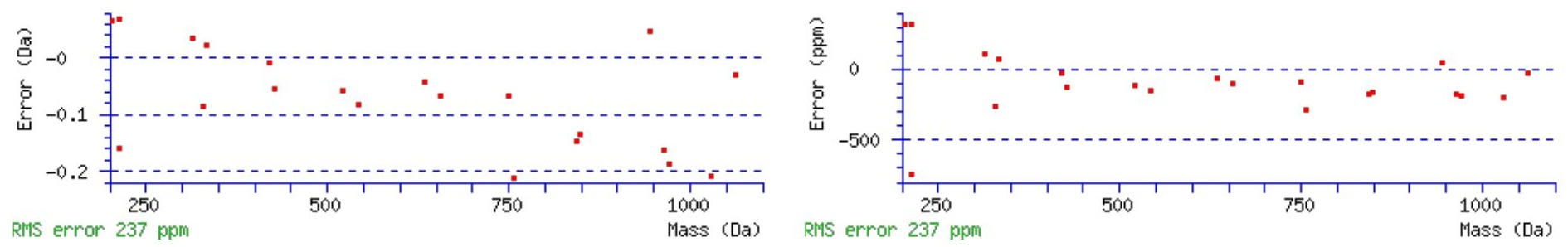

\section{All matches to this query}

\begin{tabular}{|l|l|l|l|}
\hline Score & Mr(calc): & Delta & \multicolumn{1}{c|}{ Sequence } \\
\hline 81.9 & 1174.6081 & -0.0033 & IVDVNLTSEGK \\
\hline 18.2 & 1174.5933 & 0.0115 & LVRGTKYMK \\
\hline 5.4 & 1174.6016 & 0.0032 & LVPTKDTMNR \\
\hline 5.2 & 1174.6127 & -0.0079 & IALKKTITK \\
\hline 5.0 & 1174.6111 & -0.0063 & VLEQHKLTK \\
\hline 5.0 & 1174.6016 & 0.0032 & VLNMAEIQTR \\
\hline 4.0 & 1174.5933 & 0.0115 & LVRGTKYMK \\
\hline 3.6 & 1174.6095 & -0.0046 & LNTGRTEAWK \\
\hline 1.2 & 1174.6016 & 0.0032 & IVQMTEAEVR \\
\hline 0.1 & 1174.6128 & -0.0080 & XQKQLSNCLK \\
\hline
\end{tabular}

Spectrum No: 209; Query: 448; Rank: 1

\section{Peptide View}

MS/MS Fragmentation of YLNETQQLTEK

Found in IPI00201262, Tax_Id=10116 Gene_Symbol=LOC297568;Mug2;Mug1 Alpha-1-inhibitor 3 precursor

Match to Query 448: 1366.659788 from(684.337170,2+)

Title: 091008RatKidney_NoSalt_26.1200.1200.2.dta

Data file K:INewmanPaper|Piliangl3SubProteomes\Piliang3SP\mgf5ppm|ERLIC_3SubProteomes5ppm.mgf

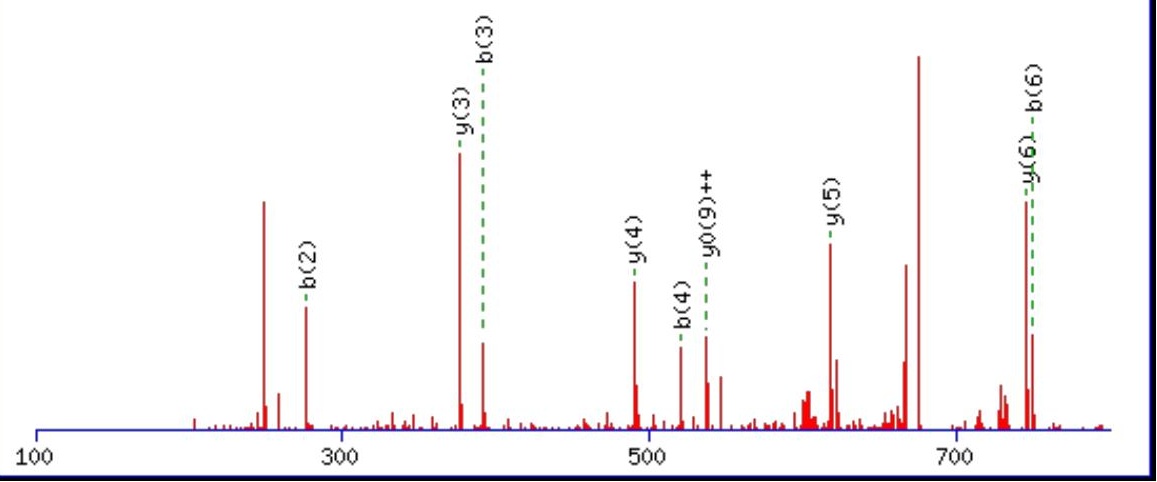




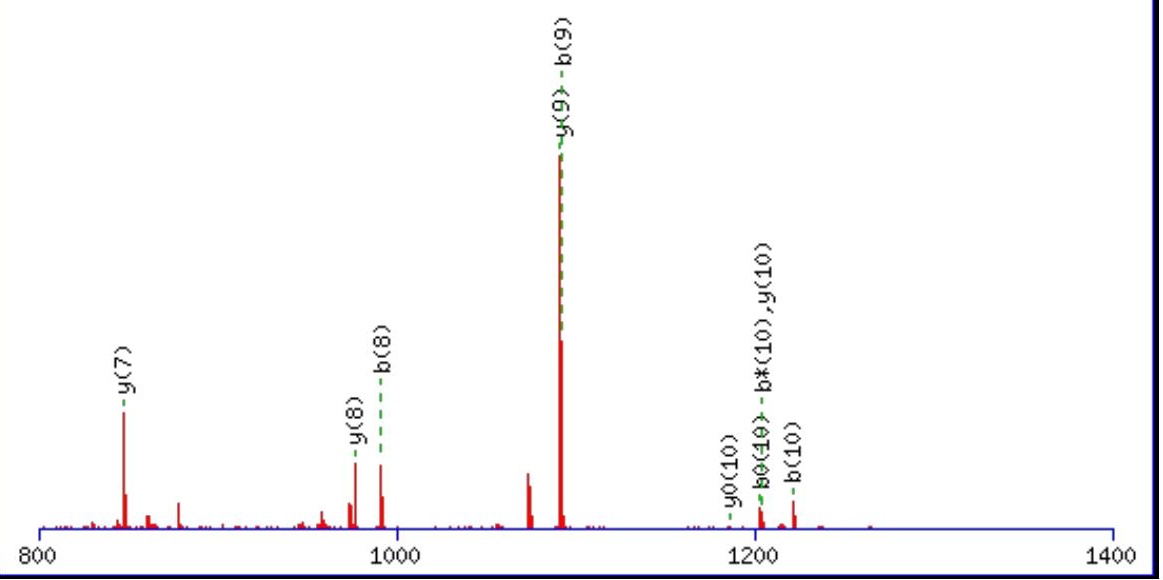

\begin{tabular}{rrrr}
\hline 1400 & 1600 & 1800 & 2000 \\
\hline
\end{tabular}

Monoisotopic mass of neutral peptide $\operatorname{Mr}($ calc): 1366.6616

Fixed modifications: Carbamidomethyl (C)

Variable modifications:

N3 : Deamidated_N (N)

Ions Score: 82 Expect: $1 \mathrm{e}-006$

Matches (Bold Red): 19/108 fragment ions using 23 most intense peaks

\begin{tabular}{|r|c|c|c|c|c|c|c|c|c|c|c|c|c|c|}
\hline$\#$ & $\mathbf{b}$ & $\mathbf{b}^{++}$ & $\mathbf{b}^{*}$ & $\mathbf{b}^{\mathbf{*}^{++}}$ & $\mathbf{b}^{\mathbf{0}}$ & $\mathbf{b}^{\mathbf{0 +}}$ & $\mathbf{S e q}$ & $\mathbf{y}$ & $\mathbf{y}^{++}$ & $\mathbf{y}^{*}$ & $\mathbf{y}^{\mathbf{*}^{++}}$ & $\mathbf{y}^{\mathbf{0}}$ & $\mathbf{y}^{\mathbf{0 + +}}$ & $\#$ \\
\hline $\mathbf{1}$ & 164.0706 & 82.5389 & & & & & $\mathbf{Y}$ & & & & & & & $\mathbf{1 1}$ \\
\hline $\mathbf{2}$ & $\mathbf{2 7 7 . 1 5 4 7}$ & 139.0810 & & & & & $\mathbf{L}$ & $\mathbf{1 2 0 4 . 6 0 5 6}$ & 602.8064 & 1187.5790 & 594.2931 & $\mathbf{1 1 8 6 . 5 9 5 0}$ & 593.8011 & $\mathbf{1 0}$ \\
\hline $\mathbf{3}$ & $\mathbf{3 9 2 . 1 8 1 6}$ & 196.5944 & 375.1551 & 188.0812 & & & $\mathbf{N}$ & $\mathbf{1 0 9 1 . 5 2 1 5}$ & 546.2644 & 1074.4950 & 537.7511 & 1073.5109 & $\mathbf{5 3 7 . 2 5 9 1}$ & $\mathbf{9}$ \\
\hline $\mathbf{4}$ & $\mathbf{5 2 1 . 2 2 4 2}$ & 261.1157 & 504.1976 & 252.6025 & 503.2136 & 252.1105 & $\mathbf{E}$ & $\mathbf{9 7 6 . 4 9 4 6}$ & 488.7509 & 959.4680 & 480.2376 & 958.4840 & 479.7456 & $\mathbf{8}$ \\
\hline $\mathbf{5}$ & 622.2719 & 311.6396 & 605.2453 & 303.1263 & 604.2613 & 302.6343 & $\mathbf{T}$ & $\mathbf{8 4 7 . 4 5 2 0}$ & 424.2296 & 830.4254 & 415.7164 & 829.4414 & 415.2243 & $\mathbf{7}$ \\
\hline $\mathbf{6}$ & $\mathbf{7 5 0 . 3 3 0 5}$ & 375.6689 & 733.3039 & 367.1556 & 732.3199 & 366.6636 & $\mathbf{Q}$ & $\mathbf{7 4 6 . 4 0 4 3}$ & 373.7058 & 729.3777 & 365.1925 & 728.3937 & 364.7005 & $\mathbf{6}$ \\
\hline $\mathbf{7}$ & $\mathbf{8 7 8 . 3 8 9 0}$ & 439.6982 & 861.3625 & 431.1849 & 860.3785 & 430.6929 & $\mathbf{Q}$ & $\mathbf{6 1 8 . 3 4 5 7}$ & 309.6765 & 601.3192 & 301.1632 & 600.3352 & 300.6712 & $\mathbf{5}$ \\
\hline $\mathbf{8}$ & $\mathbf{9 9 1 . 4 7 3 1}$ & 496.2402 & 974.4465 & 487.7269 & 973.4625 & 487.2349 & $\mathbf{L}$ & $\mathbf{4 9 0 . 2 8 7 1}$ & 245.6472 & 473.2606 & 237.1339 & 472.2766 & 236.6419 & $\mathbf{4}$ \\
\hline $\mathbf{9}$ & $\mathbf{1 0 9 2 . 5 2 0 8}$ & 546.7640 & 1075.4942 & 538.2508 & 1074.5102 & 537.7587 & $\mathbf{T}$ & $\mathbf{3 7 7 . 2 0 3 1}$ & 189.1052 & 360.1765 & 180.5919 & 359.1925 & 180.0999 & $\mathbf{3}$ \\
\hline $\mathbf{1 0}$ & $\mathbf{1 2 2 1 . 5 6 3 4}$ & 611.2853 & $\mathbf{1 2 0 4 . 5 3 6 8}$ & 602.7720 & $\mathbf{1 2 0 3 . 5 5 2 8}$ & 602.2800 & $\mathbf{E}$ & 276.1554 & 138.5813 & 259.1288 & 130.0681 & 258.1448 & 129.5761 & $\mathbf{2}$ \\
\hline $\mathbf{1 1}$ & & & & & & & $\mathbf{K}$ & 147.1128 & 74.0600 & 130.0863 & 65.5468 & & & $\mathbf{1}$ \\
\hline
\end{tabular}
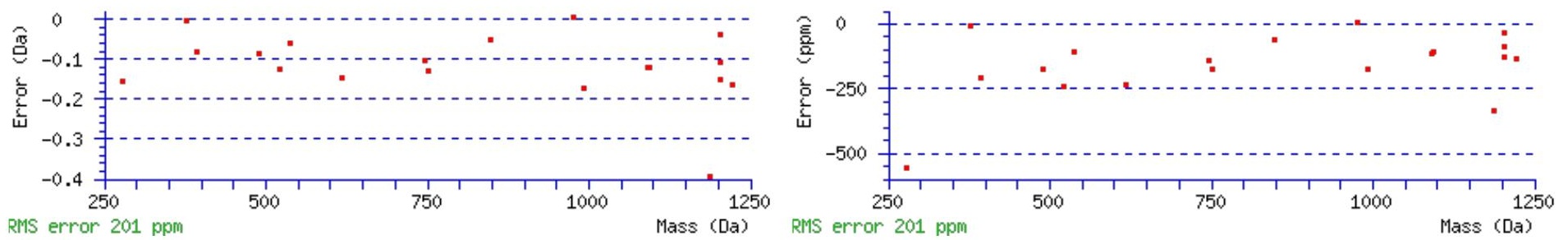


\section{All matches to this query}

\begin{tabular}{|l|l|l|l|}
\hline Score & Mr(calc): & Delta & \multicolumn{1}{c|}{ Sequence } \\
\hline 81.8 & 1366.6616 & -0.0018 & YLNETQQLTEK \\
\hline 14.6 & 1366.6728 & -0.0130 & YITENNRSEIK \\
\hline 13.3 & 1366.6680 & -0.0082 & ITDNLLQLMAR \\
\hline 12.2 & 1366.6728 & -0.0130 & YITENNRSEIK \\
\hline 9.7 & 1366.6517 & 0.0081 & ALFTSGWNNTEK \\
\hline 7.9 & 1365.6524 & 1.0074 & YNPNNSSAKITR \\
\hline 7.7 & 1366.6517 & 0.0081 & NNSTWFKLNDK \\
\hline 7.1 & 1365.6524 & 1.0074 & YNPNNSSAKITR \\
\hline 7.1 & 1366.6477 & 0.0121 & STSFNRVANNEK \\
\hline 6.9 & 1365.6524 & 1.0074 & YNPNNSSAKITR \\
\hline
\end{tabular}

Spectrum No: 210; Query: 668; Rank: 1

\section{Peptide View}

MS/MS Fragmentation of DASTQLSTNLTSVR

Found in IPI00325312, Tax_Id=10116 Gene_Symbol=Prom1 prominin 1 isoform 1

Match to Query 668: 1492.739668 from(747.377110,2+)

Title: 100101RatKid_NS_deglyco_24.2656.2656.2.dta

Data file K:INewmanPaper|Piliangl3SubProteomes\Piliang3SP\mgf5ppm\ERLIC_3SubProteomes5ppm.mgf

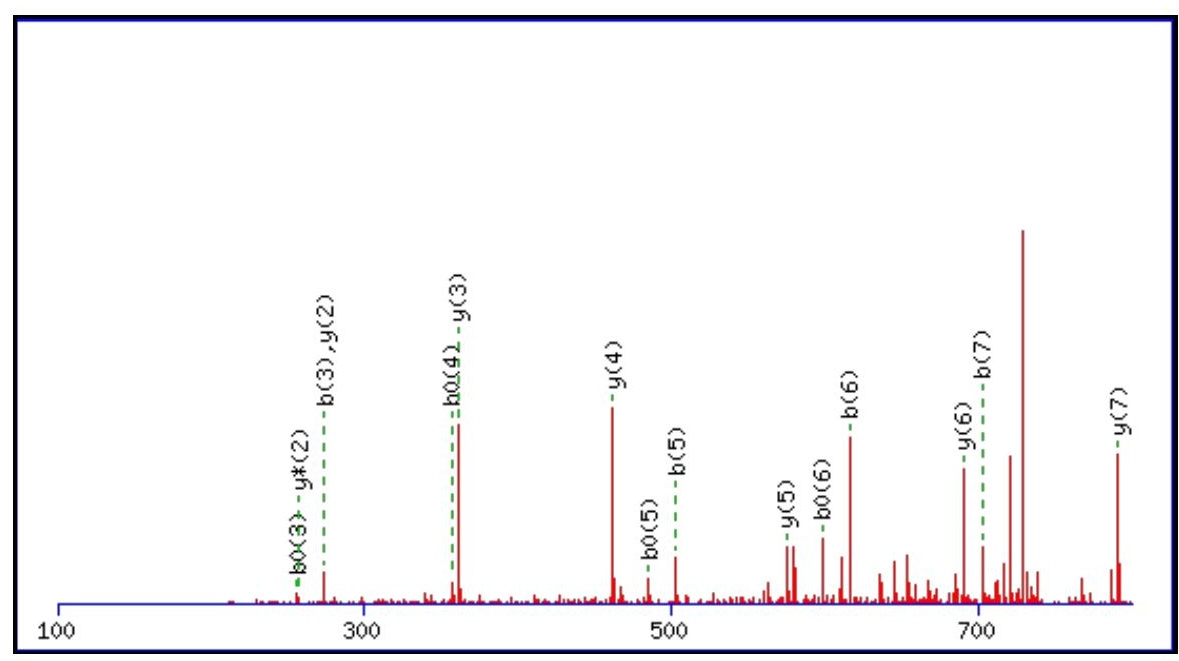



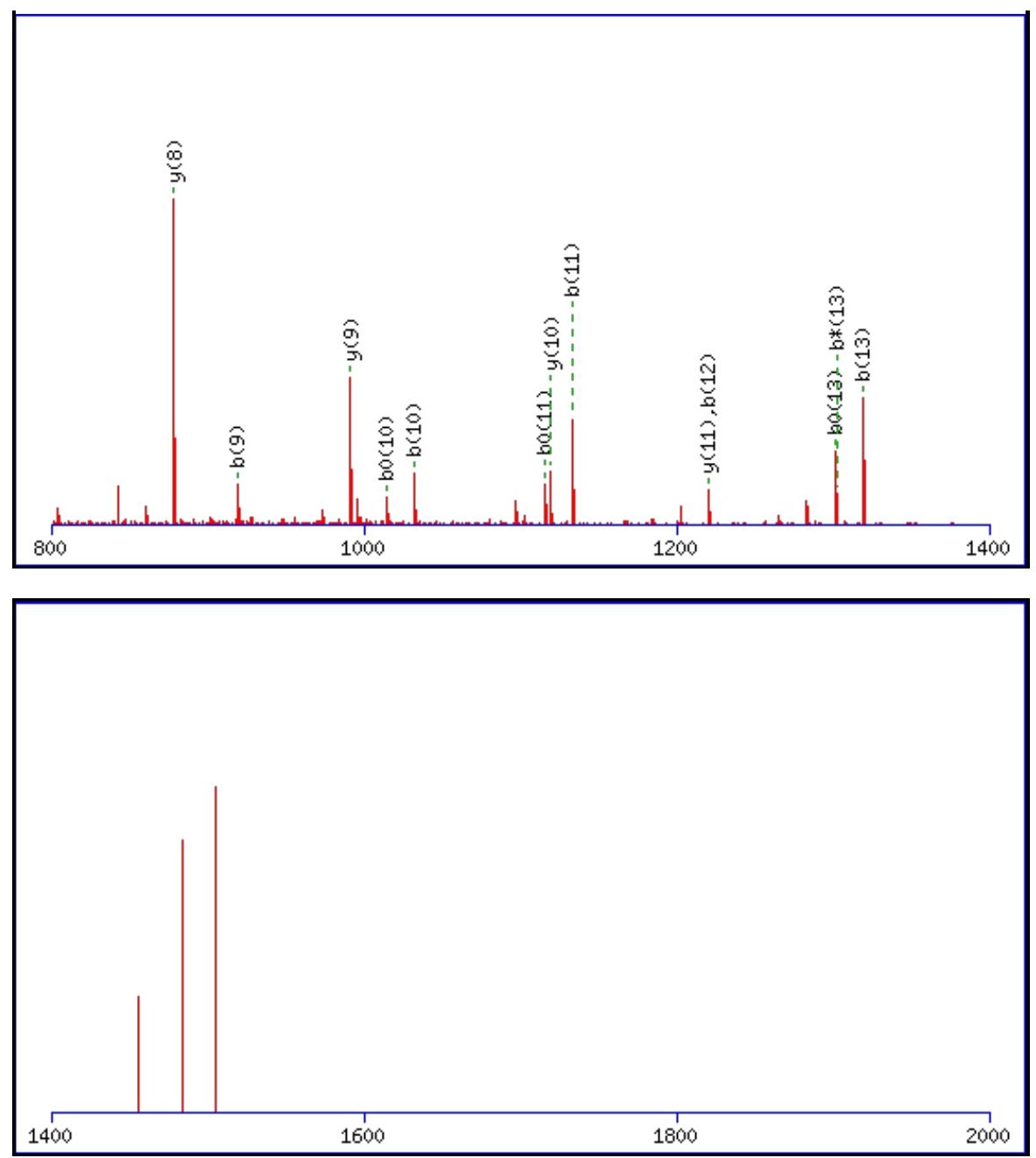

Monoisotopic mass of neutral peptide $\operatorname{Mr}($ calc): 1492.7369

Fixed modifications: Carbamidomethyl (C)

Variable modifications:

N9: Deamidated $\mathrm{N}(\mathrm{N})$

Ions Score: 82 Expect: $1.3 \mathrm{e}-006$

Matches (Bold Red): 28/144 fragment ions using 50 most intense peaks

\begin{tabular}{|c|c|c|c|c|c|c|c|c|c|c|c|c|c|c|}
\hline \# & b & $\mathbf{b}^{++}$ & $\mathbf{b}^{*}$ & $\mathbf{b}^{*^{++}}$ & $\mathbf{b}^{0}$ & $\mathbf{b}^{\mathbf{0 + +}}$ & Seq. & $\mathbf{y}$ & $y^{++}$ & $\mathbf{y}^{*}$ & $\mathbf{y}^{*^{++}}$ & $\mathbf{y}^{0}$ & $y^{0++}$ & \# \\
\hline 1 & 116.0342 & 58.5207 & & & 98.0237 & 49.5155 & D & & & & & & & 14 \\
\hline 2 & 187.0713 & 94.0393 & & & 169.0608 & 85.0340 & A & 1378.7172 & 689.8623 & 1361.6907 & 681.3490 & 1360.7067 & 680.8570 & 13 \\
\hline 3 & 274.1034 & 137.5553 & & & 256.0928 & 128.5500 & S & 1307.6801 & 654.3437 & 1290.6536 & 645.8304 & 1289.6696 & 645.3384 & 12 \\
\hline 4 & 375.1510 & 188.0792 & & & 357.1405 & 179.0739 & $\mathbf{T}$ & 1220.6481 & 610.8277 & 1203.6216 & 602.3144 & 1202.6375 & 601.8224 & 11 \\
\hline 5 & 503.2096 & 252.1084 & 486.1831 & 243.5952 & 485.1991 & 243.1032 & $\mathbf{Q}$ & 1119.6004 & 560.3038 & 1102.5739 & 551.7906 & 1101.5899 & 551.2986 & 10 \\
\hline 6 & 616.2937 & 308.6505 & 599.2671 & 300.1372 & 598.2831 & 299.6452 & $\mathbf{L}$ & 991.5418 & 496.2746 & 974.5153 & 487.7613 & 973.5313 & 487.2693 & 9 \\
\hline 7 & 703.3257 & 352.1665 & 686.2992 & 343.6532 & 685.3151 & 343.1612 & S & 878.4578 & 439.7325 & 861.4312 & 431.2193 & 860.4472 & 430.7272 & 8 \\
\hline 8 & 804.3734 & 402.6903 & 787.3468 & 394.1771 & 786.3628 & 393.6851 & $\mathbf{T}$ & 791.4258 & 396.2165 & 774.3992 & 387.7032 & 773.4152 & 387.2112 & 7 \\
\hline 9 & 919.4003 & 460.2038 & 902.3738 & 451.6905 & 901.3898 & 451.1985 & $\mathbf{N}$ & 690.3781 & 345.6927 & 673.3515 & 337.1794 & 672.3675 & 336.6874 & 6 \\
\hline 10 & 1032.4844 & 516.7458 & 1015.4578 & 508.2326 & 1014.4738 & 507.7405 & $\mathbf{L}$ & 575.3511 & 288.1792 & 558.3246 & 279.6659 & 557.3406 & 279.1739 & 5 \\
\hline 11 & 1133.5321 & 567.2697 & 1116.5055 & 558.7564 & 1115.5215 & 558.2644 & $\mathbf{T}$ & 462.2671 & 231.6372 & 445.2405 & 223.1239 & 444.2565 & 222.6319 & 4 \\
\hline 12 & 1220.5641 & 610.7857 & 1203.5375 & 602.2724 & 1202.5535 & 601.7804 & $S$ & 361.2194 & 181.1133 & 344.1928 & 172.6001 & 343.2088 & 172.1081 & 3 \\
\hline 13 & 1319.6325 & 660.3199 & 1302.6060 & 651.8066 & 1301.6219 & 651.3146 & $\mathbf{V}$ & 274.1874 & 137.5973 & 257.1608 & 129.0840 & & & 2 \\
\hline 14 & & & & & & & $\mathbf{R}$ & 175.1190 & 88.0631 & 158.0924 & 79.5498 & & & 1 \\
\hline
\end{tabular}



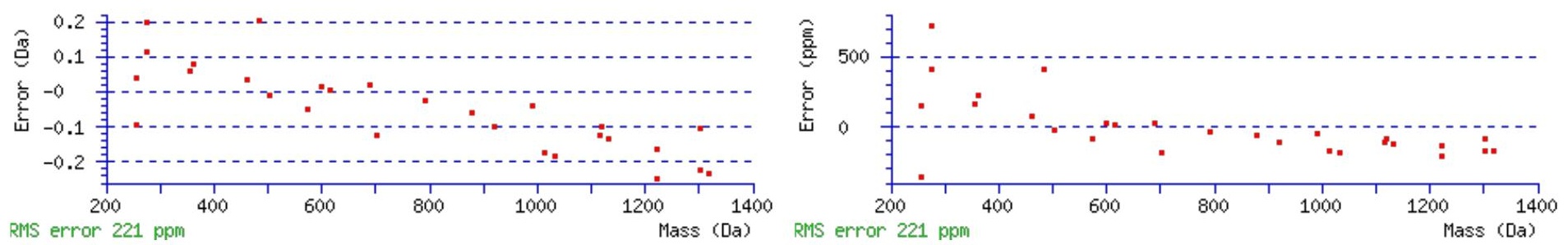

\section{All matches to this query}

\begin{tabular}{|l|l|l|l|}
\hline Score & Mr(calc): & Delta & \multicolumn{1}{|c|}{ Sequence } \\
\hline 81.8 & 1492.7369 & 0.0028 & DASTQLSTNLTSVR \\
\hline 11.7 & 1492.7286 & 0.0110 & AENGLGSPAIKSIR \\
\hline 7.2 & 1492.7522 & -0.0125 & VSENPYTSGIIASR \\
\hline 6.6 & 1492.7422 & -0.0026 & AAAAWEERFSSLR \\
\hline 6.0 & 1492.7490 & -0.0093 & CLSGSLQVMNKTR \\
\hline 6.0 & 1492.7344 & 0.0053 & NFAKKEMGTPDVR \\
\hline 5.6 & 1492.7286 & 0.0110 & EREVPISLEVSR \\
\hline 5.0 & 1490.7283 & 2.0114 & YTVGLRNXFSVK \\
\hline 5.0 & 1490.7220 & 2.0177 & MALSSLLLLATAK \\
\hline 4.1 & 1491.7219 & 1.0178 & TATSWSSGXRTWR \\
\hline
\end{tabular}

Spectrum No: 211; Query: 762; Rank: 1

\section{Peptide View}

MS/MS Fragmentation of LEDPTVLQVVNVTK

Found in IPI00360957, Tax_Id=10116 Gene_Symbol=Slc10a5 Sodium/bile acid cotransporter 5 precursor

Match to Query 762: 1554.852028 from(778.433290,2+)

Title: 100101RatKid_NS_deglyco_25.3485.3485.2.dta

Data file K:INewmanPaper|Piliangl3SubProteomes\Piliang3SP\mgf5ppm\ERLIC_3SubProteomes5ppm.mgf

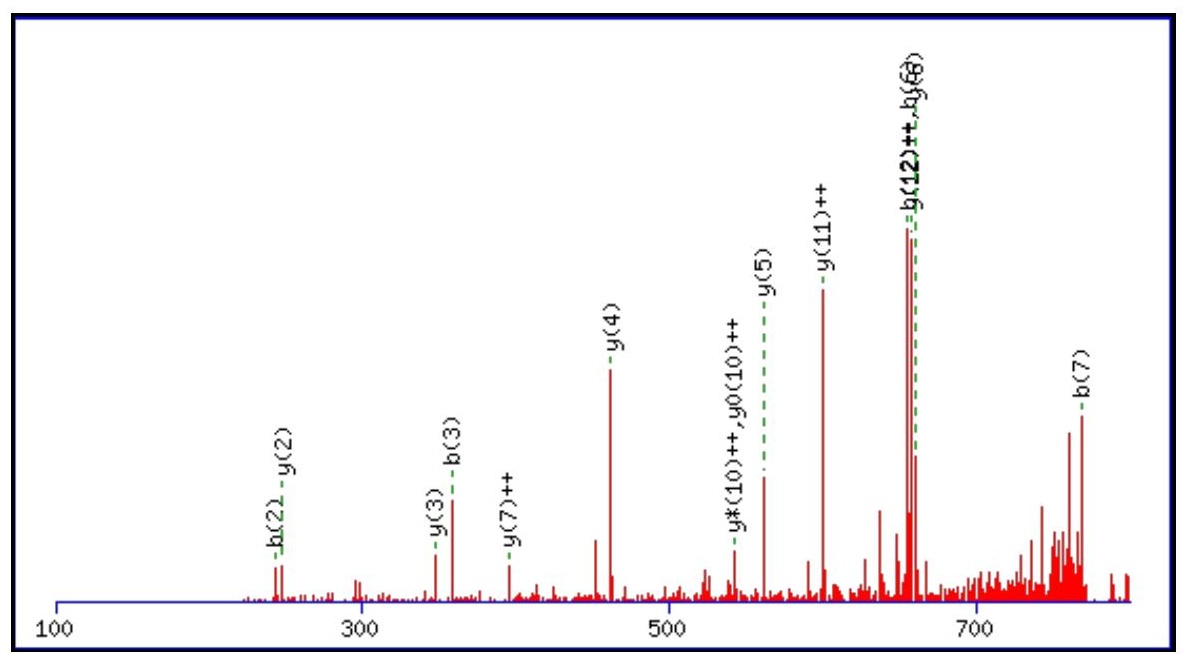



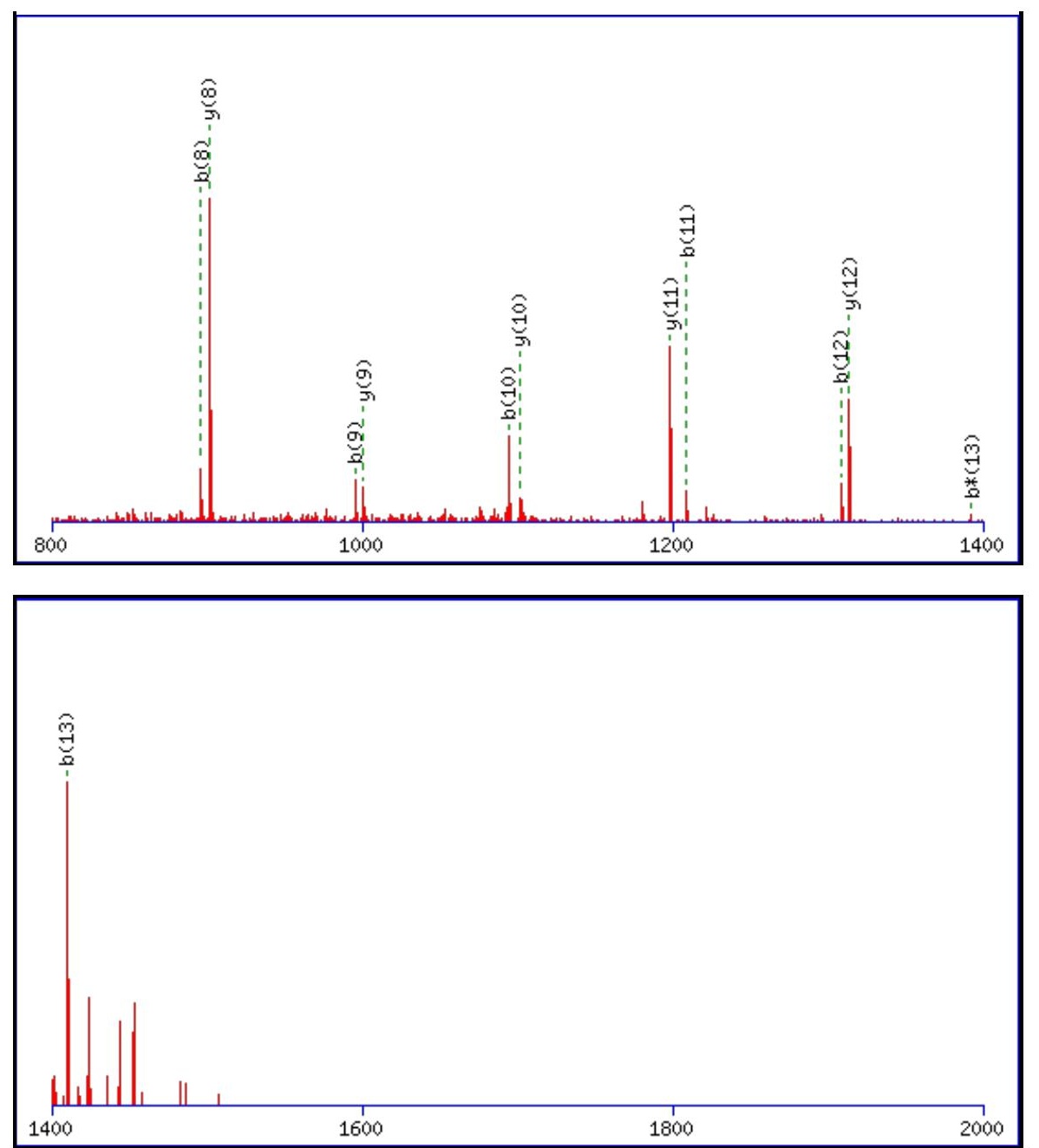

Monoisotopic mass of neutral peptide $\operatorname{Mr}($ calc): 1554.8505

Fixed modifications: Carbamidomethyl (C)

Variable modifications:

N11 : Deamidated_N (N)

Ions Score: 82 Expect: $4.7 \mathrm{e}-007$

Matches (Bold Red): 27/138 fragment ions using 43 most intense peaks

\begin{tabular}{|c|c|c|c|c|c|c|c|c|c|c|c|c|c|c|}
\hline \# & b & $\mathbf{b}^{++}$ & $\mathbf{b}^{*}$ & $\mathbf{b}^{*^{++}}$ & $\mathbf{b}^{0}$ & $\mathbf{b}^{\mathbf{0 + +}}$ & Seq. & $\mathbf{y}$ & $\mathrm{y}^{++}$ & $\mathbf{y}^{*}$ & $\mathrm{y}^{*^{++}}$ & $\mathbf{y}^{\mathbf{0}}$ & $y^{0++}$ & \# \\
\hline 1 & 114.0913 & 57.5493 & & & & & $\mathbf{L}$ & & & & & & & 14 \\
\hline 2 & 243.1339 & 122.0706 & & & 225.1234 & 113.0653 & $\mathbf{E}$ & 1442.7737 & 721.8905 & 1425.7471 & 713.3772 & 1424.7631 & 712.8852 & 13 \\
\hline 3 & 358.1609 & 179.5841 & & & 340.1503 & 170.5788 & D & 1313.7311 & 657.3692 & 1296.7046 & 648.8559 & 1295.7205 & 648.3639 & 12 \\
\hline 4 & 455.2136 & 228.1105 & & & 437.2031 & 219.1052 & $\mathbf{P}$ & 1198.7042 & 599.8557 & 1181.6776 & 591.3424 & 1180.6936 & 590.8504 & 11 \\
\hline 5 & 556.2613 & 278.6343 & & & 538.2508 & 269.6290 & $\mathbf{T}$ & 1101.6514 & 551.3293 & 1084.6248 & 542.8161 & 1083.6408 & 542.3241 & 10 \\
\hline 6 & 655.3297 & 328.1685 & & & 637.3192 & 319.1632 & $\mathbf{V}$ & 1000.6037 & 500.8055 & 983.5772 & 492.2922 & 982.5932 & 491.8002 & 9 \\
\hline 7 & 768.4138 & 384.7105 & & & 750.4032 & 375.7053 & $\mathbf{L}$ & 901.5353 & 451.2713 & 884.5088 & 442.7580 & 883.5247 & 442.2660 & 8 \\
\hline 8 & 896.4724 & 448.7398 & 879.4458 & 440.2266 & 878.4618 & 439.7345 & $\mathbf{Q}$ & 788.4512 & 394.7293 & 771.4247 & 386.2160 & 770.4407 & 385.7240 & 7 \\
\hline 9 & 995.5408 & 498.2740 & 978.5142 & 489.7608 & 977.5302 & 489.2688 & $\mathbf{V}$ & 660.3927 & 330.7000 & 643.3661 & 322.1867 & 642.3821 & 321.6947 & 6 \\
\hline 10 & 1094.6092 & 547.8082 & 1077.5827 & 539.2950 & 1076.5986 & 538.8030 & V & 561.3242 & 281.1658 & 544.2977 & 272.6525 & 543.3137 & 272.1605 & 5 \\
\hline 11 & 1209.6361 & 605.3217 & 1192.6096 & 596.8084 & 1191.6256 & 596.3164 & $\mathbf{N}$ & 462.2558 & 231.6316 & 445.2293 & 223.1183 & 444.2453 & 222.6263 & 4 \\
\hline 12 & 1308.7046 & 654.8559 & 1291.6780 & 646.3426 & 1290.6940 & 645.8506 & $\mathbf{V}$ & 347.2289 & 174.1181 & 330.2023 & 165.6048 & 329.2183 & 165.1128 & 3 \\
\hline 13 & 1409.7522 & 705.3798 & 1392.7257 & 696.8665 & 1391.7417 & 696.3745 & $\mathbf{T}$ & 248.1605 & 124.5839 & 231.1339 & 116.0706 & 230.1499 & 115.5786 & 2 \\
\hline 14 & & & & & & & $\mathbf{K}$ & 147.1128 & 74.0600 & 130.0863 & 65.5468 & & & 1 \\
\hline
\end{tabular}



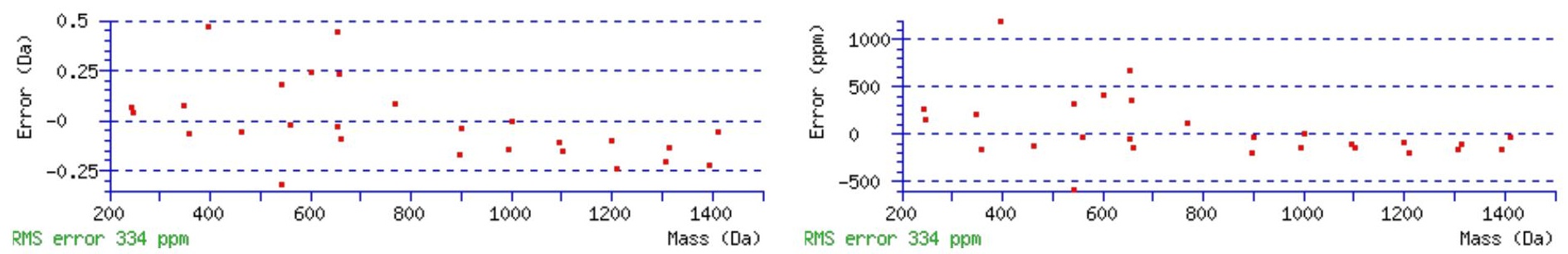

\section{All matches to this query}

\begin{tabular}{|l|l|c|l|}
\hline Score & Mr(calc): & Delta & \multicolumn{1}{c|}{ Sequence } \\
\hline 81.8 & 1554.8505 & 0.0015 & LEDPTVLQVVNVTK \\
\hline 3.5 & 1552.8514 & 2.0006 & AFHLPSWKLANLR \\
\hline 1.8 & 1552.8491 & 2.0030 & RTLIALVQVTFGR \\
\hline 1.8 & 1554.8365 & 0.0155 & IVNAPQNNKNLTTK \\
\hline 1.8 & 1554.8365 & 0.0155 & IVNAPQNNKNLTTK \\
\hline 1.8 & 1554.8365 & 0.0155 & IVNAPQNNKNLTTK \\
\hline 1.7 & 1553.8413 & 1.0107 & NKIPTVGEADELLR \\
\hline 0.9 & 1554.8365 & 0.0155 & IVNAPQNNKNLTTK \\
\hline
\end{tabular}

Spectrum No: 212; Query: 1003; Rank: 1

\section{Peptide View}

MS/MS Fragmentation of MSGDWELEVNGTEAK

Found in IPI00361686, Tax_Id=10116 Gene_Symbol=C1qbp Complement component 1 Q subcomponent-binding protein, mitochondrial precursor

Match to Query 1003: 1665.726188 from(833.870370,2+)

Title: 100101RatKid_NS_deglyco_14.2914.2914.2.dta

Data file K:INewmanPaper|Piliang|3SubProteomes\Piliang3SP\mgf5ppm\ERLIC_3SubProteomes5ppm.mgf

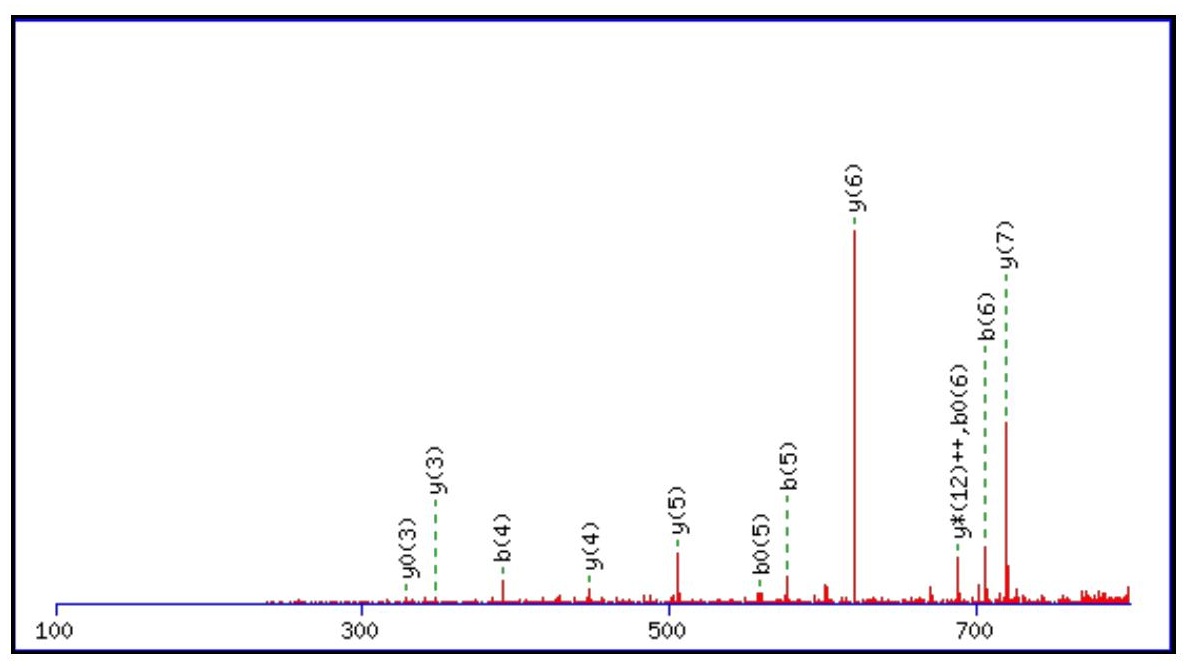



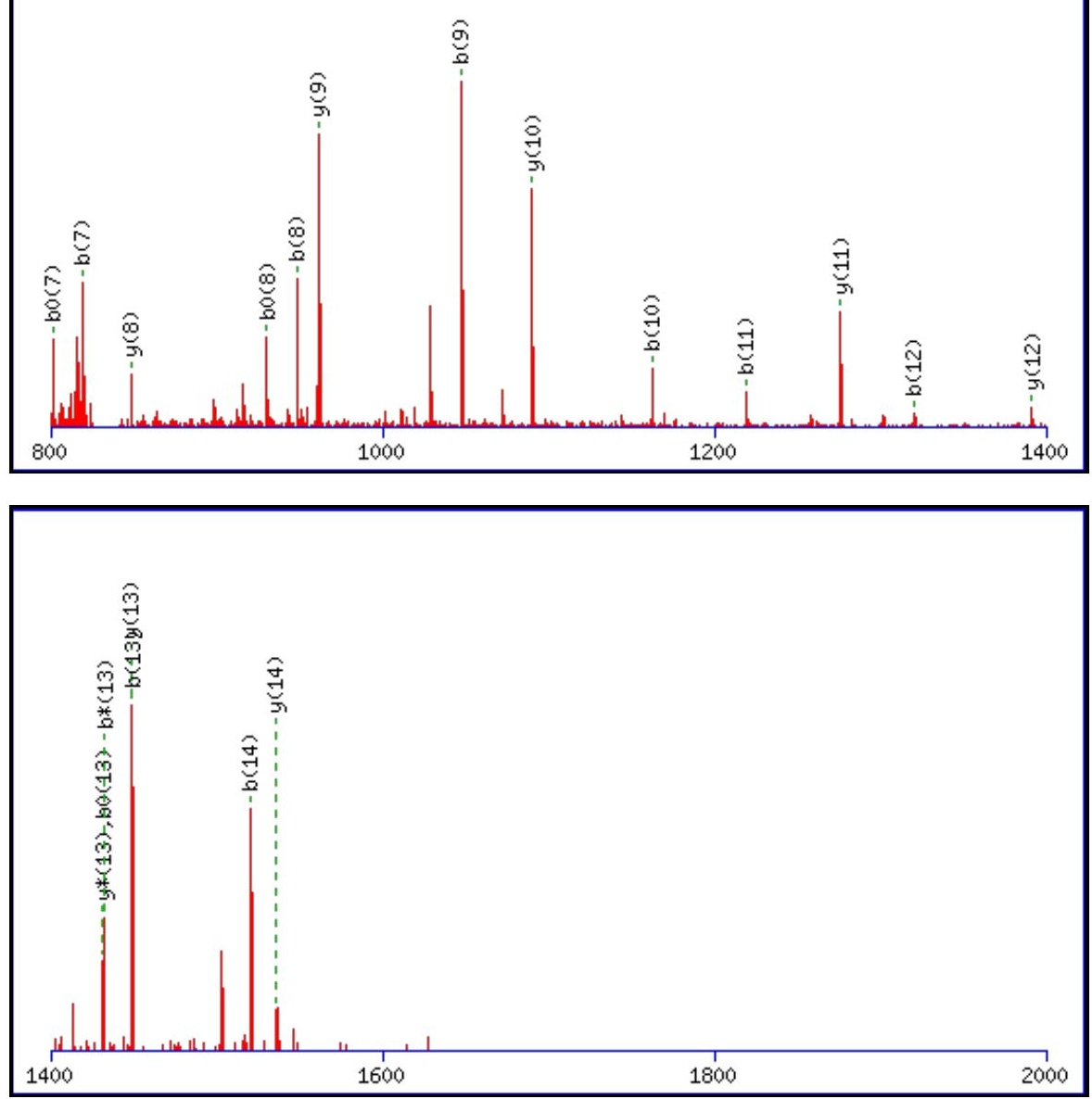

Monoisotopic mass of neutral peptide $\operatorname{Mr}($ calc): 1665.7192

Fixed modifications: Carbamidomethyl (C)

Variable modifications:

N10 : Deamidated $\mathrm{N}(\mathrm{N})$

Ions Score: 82 Expect: $1.1 \mathrm{e}-006$

Matches (Bold Red): 32/144 fragment ions using 51 most intense peaks

\begin{tabular}{|c|c|c|c|c|c|c|c|c|c|c|c|c|c|c|}
\hline \# & b & $\mathbf{b}^{++}$ & $\mathbf{b}^{*}$ & $\mathbf{b}^{*^{++}}$ & $\mathbf{b}^{0}$ & $\mathbf{b}^{\mathbf{0 + +}}$ & Seq. & $\mathbf{y}$ & $\mathbf{y}^{++}$ & $\mathbf{y}^{*}$ & $\mathrm{y}^{*^{++}}$ & $\mathbf{y}^{\mathbf{0}}$ & $\mathbf{y}^{0++}$ & \# \\
\hline 1 & 132.0478 & 66.5275 & & & & & M & & & & & & & 15 \\
\hline 2 & 219.0798 & 110.0435 & & & 201.0692 & 101.0382 & S & 1535.6860 & 768.3466 & 1518.6595 & 759.8334 & 1517.6754 & 759.3414 & 14 \\
\hline 3 & 276.1013 & 138.5543 & & & 258.0907 & 129.5490 & G & 1448.6540 & 724.8306 & 1431.6274 & 716.3174 & 1430.6434 & 715.8253 & 13 \\
\hline 4 & 391.1282 & 196.0677 & & & 373.1176 & 187.0625 & D & 1391.6325 & 696.3199 & 1374.6060 & 687.8066 & 1373.6219 & 687.3146 & 12 \\
\hline 5 & 577.2075 & 289.1074 & & & 559.1969 & 280.1021 & $\mathbf{W}$ & 1276.6056 & 638.8064 & 1259.5790 & 630.2931 & 1258.5950 & 629.8011 & 11 \\
\hline 6 & 706.2501 & 353.6287 & & & 688.2395 & 344.6234 & $\mathbf{E}$ & 1090.5263 & 545.7668 & 1073.4997 & 537.2535 & 1072.5157 & 536.7615 & 10 \\
\hline 7 & 819.3342 & 410.1707 & & & 801.3236 & 401.1654 & $\mathbf{L}$ & 961.4837 & 481.2455 & 944.4571 & 472.7322 & 943.4731 & 472.2402 & 9 \\
\hline 8 & 948.3768 & 474.6920 & & & 930.3662 & 465.6867 & $\mathbf{E}$ & 848.3996 & 424.7034 & 831.3730 & 416.1902 & 830.3890 & 415.6982 & 8 \\
\hline 9 & 1047.4452 & 524.2262 & & & 1029.4346 & 515.2209 & V & 719.3570 & 360.1821 & 702.3305 & 351.6689 & 701.3464 & 351.1769 & 7 \\
\hline 10 & 1162.4721 & 581.7397 & 1145.4456 & 573.2264 & 1144.4615 & 572.7344 & $\mathbf{N}$ & 620.2886 & 310.6479 & 603.2620 & 302.1347 & 602.2780 & 301.6427 & 6 \\
\hline 11 & 1219.4936 & 610.2504 & 1202.4670 & 601.7372 & 1201.4830 & 601.2451 & G & 505.2617 & 253.1345 & 488.2351 & 244.6212 & 487.2511 & 244.1292 & 5 \\
\hline 12 & 1320.5413 & 660.7743 & 1303.5147 & 652.2610 & 1302.5307 & 651.7690 & $\mathbf{T}$ & 448.2402 & 224.6237 & 431.2136 & 216.1105 & 430.2296 & 215.6185 & 4 \\
\hline 13 & 1449.5838 & 725.2956 & 1432.5573 & 716.7823 & 1431.5733 & 716.2903 & $\mathbf{E}$ & 347.1925 & 174.0999 & 330.1660 & 165.5866 & 329.1819 & 165.0946 & 3 \\
\hline 14 & 1520.6210 & 760.8141 & 1503.5944 & 752.3008 & 1502.6104 & 751.8088 & A & 218.1499 & 109.5786 & 201.1234 & 101.0653 & & & 2 \\
\hline 15 & & & & & & & $\mathbf{K}$ & 147.1128 & 74.0600 & 130.0863 & 65.5468 & & & 1 \\
\hline
\end{tabular}



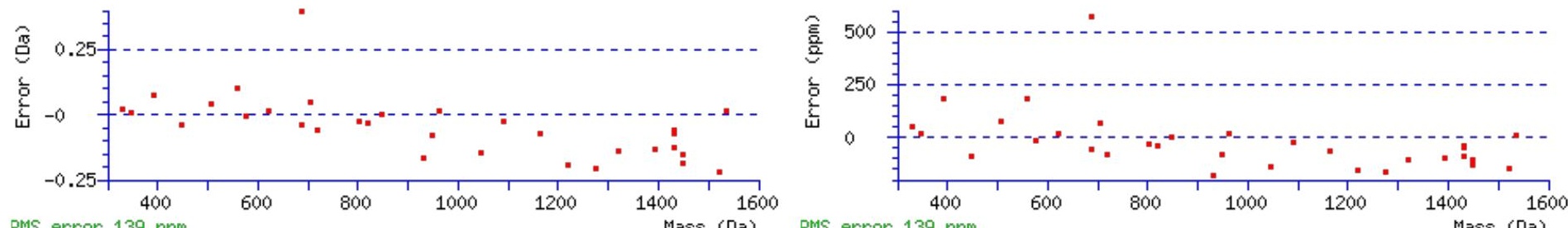

RIS error 139 ppm

Mass (Da) RMS error 139 ppm

Mass (Da)

\section{All matches to this query}

\begin{tabular}{|l|l|l|l|}
\hline Score & Mr(calc): & Delta & \multicolumn{1}{|c|}{ Sequence } \\
\hline 81.7 & 1665.7192 & 0.0070 & MSGDWELEVNGTEAK \\
\hline 28.3 & 1664.7352 & 0.9910 & MSGDWELEVNGTEAK \\
\hline 9.3 & 1665.7151 & 0.0111 & EENCSKAQESLENK \\
\hline 6.7 & 1665.7164 & 0.0098 & KHSAILASPNPDEK \\
\hline 6.4 & 1665.7205 & 0.0057 & FTVHTKSSLPNFK \\
\hline 6.3 & 1665.7143 & 0.0119 & MSEKFEVTNLNMK \\
\hline 5.9 & 1665.7205 & 0.0057 & FTVHTKSSLPNFK \\
\hline 5.8 & 1665.7124 & 0.0138 & TDRSKQQSQTVTK \\
\hline 2.5 & 1664.7311 & 0.9951 & EENCSKAQESLENK \\
\hline 1.5 & 1663.7350 & 1.9912 & MFSENKENVKVMK \\
\hline
\end{tabular}

Spectrum No: 213; Query: 2670; Rank: 1

\section{Peptide View}

MS/MS Fragmentation of RGVDVSQVAWQSQGDTPCSCCIVNNSNGSR

Found in IPI00211989, Tax_Id=10116 Gene_Symbol=Khk Isoform C of Ketohexokinase

Match to Query 2670: 3339.435792 from(1114.152540,3+)

Title: 100101RatKid_NS_deglyco_08.2862.2862.3.dta

Data file K:INewmanPaper|Piliangl3SubProteomes\Piliang3SP\mgf5ppm\ERLIC_3SubProteomes5ppm.mgf

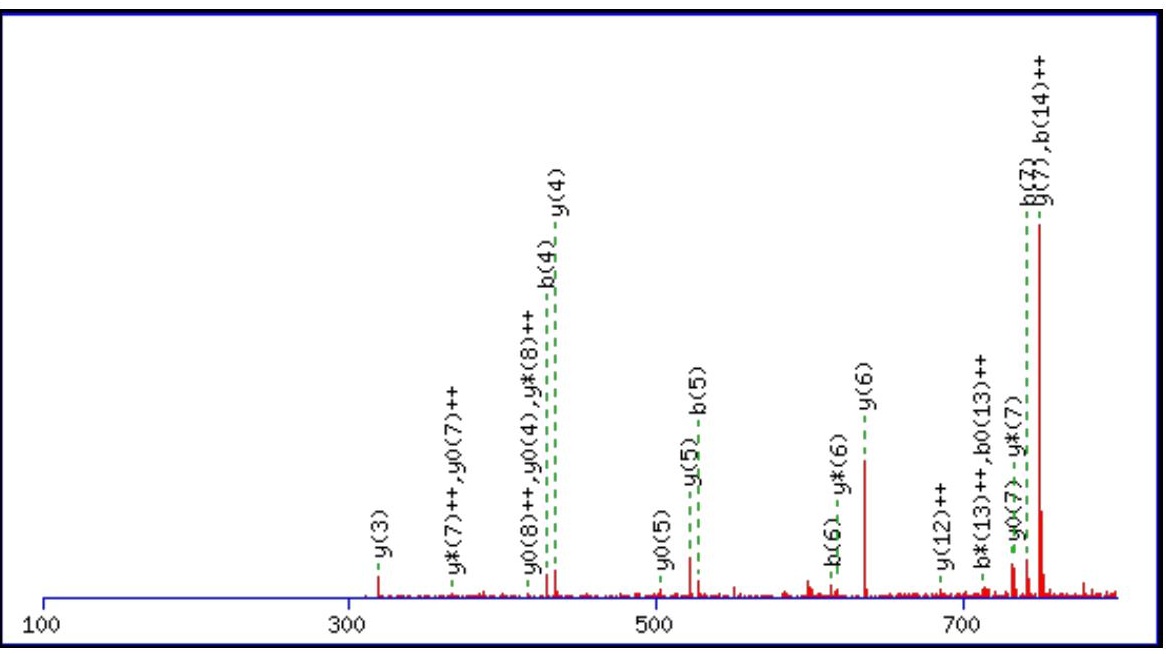



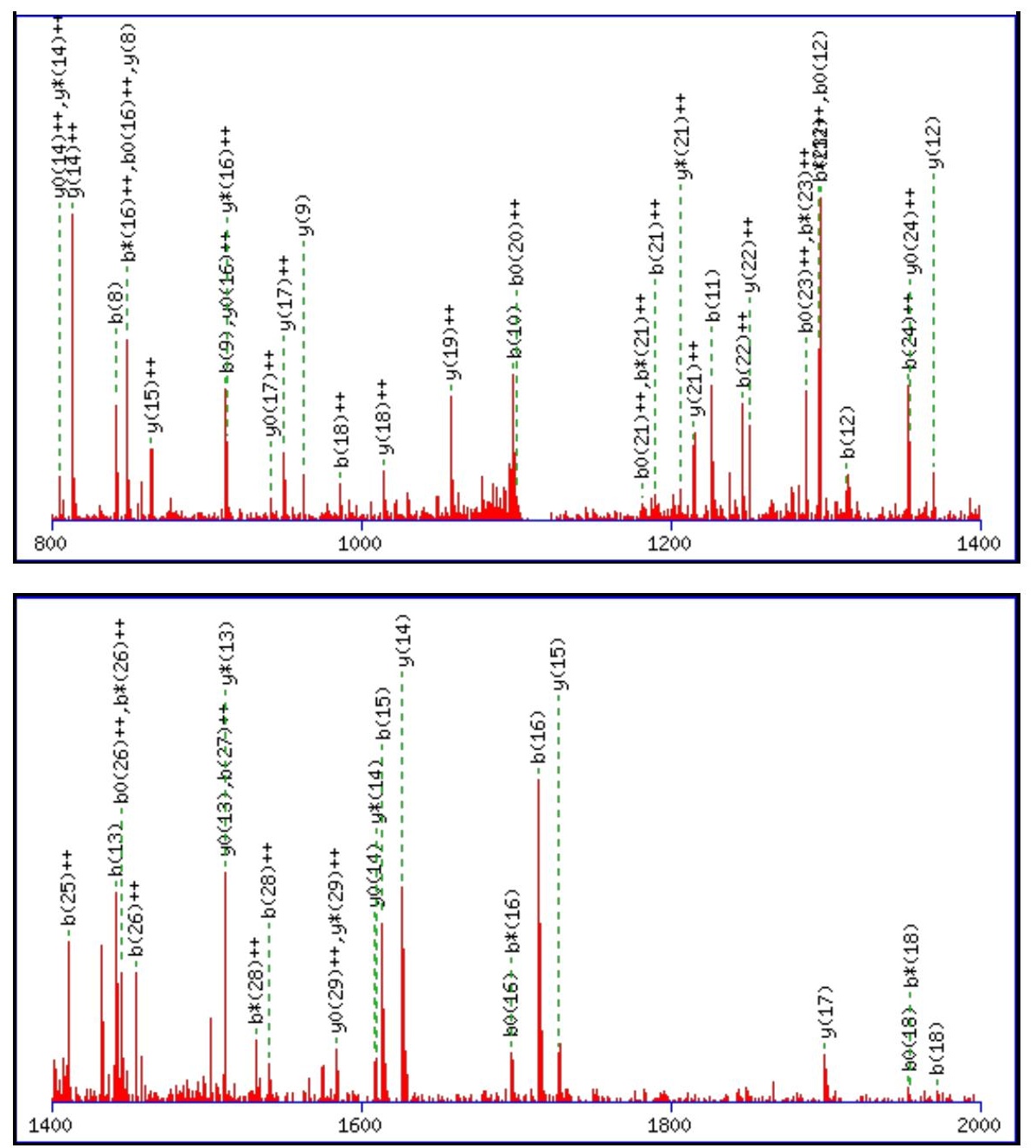

Monoisotopic mass of neutral peptide $\operatorname{Mr}($ calc): 3339.4303

Fixed modifications: Carbamidomethyl (C)

Variable modifications:

N24 : Deamidated_N (N)

N27 : Deamidated_N $(\mathrm{N})$

Ions Score: 82 Expect: $3.1 \mathrm{e}-006$

Matches (Bold Red): 83/340 fragment ions using 128 most intense peaks

\begin{tabular}{|c|c|c|c|c|c|c|c|c|c|c|c|c|c|c|}
\hline \# & b & $\mathbf{b}^{++}$ & $\mathbf{b}^{*}$ & $\mathbf{b}^{*^{++}}$ & $\mathbf{b}^{\mathbf{0}}$ & & eq. & $\mathbf{y}$ & & $\mathbf{y}^{*}$ & & $\mathbf{y}^{0}$ & & \# \\
\hline 1 & 157.1084 & 79.0578 & 140.0818 & 70.5446 & & & D & & & & & & & 30 \\
\hline 2 & 214.1299 & & & & & & $G$ & & & & & & & 29 \\
\hline 3 & 313.1983 & 028 & 296.1717 & & & & V & & & & & & & 28 \\
\hline 4 & 428.2252 & 162 & & & & & D & & & & & & & 27 \\
\hline 5 & 527.2936 & 504 & 510.2 & 372 & & & $\mathbf{V}$ & 196 & 134 & 931 & 002 & 2091 & 082 & 26 \\
\hline 6 & 614.3257 & 307.6665 & 597.2 & 299. & & & $\mathrm{~S}$ & & 1407 & 247 & 660 & 406 & 740 & 25 \\
\hline 7 & 742.3842 & 371.6958 & 725.3577 & 363.1825 & 724. & 362.6905 & $\mathbf{Q}$ & 2727.1192 & 632 & 2710 & 500 & 086 & 579 & 24 \\
\hline 8 & 841.4526 & 421.2300 & 824.4261 & 412. & & & $\mathbf{V}$ & 2599.0606 & 1300 & 2582 & & 2581. & 0287 & 23 \\
\hline 9 & 912.4898 & 456.7485 & 895.4632 & 448.2352 & & & A & 2499. & 1250 . & 2482 & 865 & 2481. & 1241 . & 22 \\
\hline 10 & 98.5691 & 549.7882 & 081.5425 & 541.2749 & 585 & 540 & $\mathbf{W}$ & 2428. & 1214 & 2411 & 679 & 2410. & 1205. & 21 \\
\hline 11 & 1226.6276 & 613.8175 & 1209.6011 & 605.3042 & 208.6171 & 604.8122 & $\mathbf{Q}$ & 2242.8758 & 1121. & 2225. & 1113.4282 & 2224.8652 & 1112.9362 & 20 \\
\hline 12 & 313.6597 & 657.3335 & 296.6331 & 648.8 & & & $S$ & 172 & 1057. & 2097. & 1049. & 2096.8066 & 1048. & 19 \\
\hline 13 & 1441.7183 & 721.3628 & 1424.6917 & 712.8495 & 1423.7077 & 712. & $\mathbf{Q}$ & 2027.7852 & 1014.3962 & 2010. & 1005.8829 & 2009.7746 & 1005.3909 & 18 \\
\hline 14 & 1498.7397 & 749.8735 & 1481.7132 & 741.3602 & 1480.7292 & & G & 1899. & 950.3669 & 1882. & 941.8537 & 1881. & 616 & 17 \\
\hline 15 & 1613.7667 & 807.3870 & 596.7401 & 798.8737 & 1595.7561 & 798.3817 & D & 1842.7051 & 921.8562 & 1825. & 913.3429 & 1824.6945 & 912. & 16 \\
\hline 16 & 1714.8143 & 857.9108 & 1697.7878 & 849.3975 & 1696.8038 & 848.9055 & $\mathbf{T}$ & 1727.6782 & 864.3427 & 1710.6516 & 855.8294 & 1709.6676 & 855.3374 & 15 \\
\hline 17 & 1811.8671 & 906.4372 & 1794.8406 & 897.9239 & 1793.8565 & 897.4319 & $\mathbf{P}$ & 1626.6305 & 813.8189 & 1609.6039 & 805.3056 & 1608.6199 & 804.8136 & 14 \\
\hline 18 & 1971.8978 & 986.4525 & 1954.8712 & 977.9392 & 1953.8872 & 977.4472 & $\mathrm{C}$ & 1529.5777 & 765.2925 & 1512.5512 & 756.7792 & 1511.5672 & 756.2872 & 13 \\
\hline
\end{tabular}




\begin{tabular}{|c|c|c|c|c|c|c|c|c|c|c|c|c|c|c|}
\hline 19 & 2058.9298 & |1029.9685 & |2041.9032 & | $1021.4553 \mid$ & |2040.9192 & |1020.9632 & S & 1369.5471 & 685.2772 & |1352.5205 & 676.7639 & 1351.5365 & 676.2719 & 12 \\
\hline 20 & 2218.9604 & 1109.9839 & 2201.9339 & 1101.4706 & 2200.9499 & 1100.9786 & C & 1282.5150 & 641.7612 & 1265.4885 & 633.2479 & 1264.5045 & 632.7559 & 11 \\
\hline 21 & 2378.9911 & 1189.9992 & 2361.9645 & 1181.4859 & 2360.9805 & 1180.9939 & C & 1122.4844 & 561.7458 & 1105.4578 & 553.2326 & 1104.4738 & 552.7406 & 10 \\
\hline 22 & 2492.0751 & 1246.5412 & 2475.0486 & 1238.0279 & 2474.0646 & 1237.5359 & I & 962.4537 & 481.7305 & 945.4272 & 473.2172 & 944.4432 & 472.7252 & 9 \\
\hline 23 & 2591.1436 & 1296.0754 & 2574.1170 & 1287.5621 & 2573.1330 & 1287.0701 & V & 849.3697 & 425.1885 & 832.3431 & 416.6752 & 831.3591 & 416.1832 & 8 \\
\hline 24 & 2706.1705 & 1353.5889 & 2689.1439 & 1345.0756 & 2688.1599 & 1344.5836 & $\mathbf{N}$ & 750.3013 & 375.6543 & 733.2747 & 367.1410 & 732.2907 & 366.6490 & 7 \\
\hline 25 & 2820.2134 & 1410.6103 & 2803.1869 & 1402.0971 & 2802.2029 & 1401.6051 & $\mathbf{N}$ & 635.2743 & 318.1408 & 618.2478 & 309.6275 & 617.2638 & 309.1355 & 6 \\
\hline 26 & 2907.2454 & 1454.1264 & 2890.2189 & 1445.6131 & 2889.2349 & 1445.1211 & S & 521.2314 & 261.1193 & 504.2049 & 252.6061 & 503.2208 & 252.1141 & 5 \\
\hline 27 & 3022.2724 & 1511.6398 & 3005.2458 & 1503.1266 & 3004.2618 & 1502.6345 & $\mathbf{N}$ & 434.1994 & 217.6033 & 417.1728 & 209.0901 & 416.1888 & 208.5980 & 4 \\
\hline 28 & 3079.2938 & 1540.1506 & 3062.2673 & 1531.6373 & 3061.2833 & 1531.1453 & G & 319.1724 & 160.0899 & 302.1459 & 151.5766 & 301.1619 & 151.0846 & 3 \\
\hline 29 & 3166.3259 & 1583.6666 & 3149.2993 & 1575.1533 & 3148.3153 & 1574.6613 & $\mathbf{S}$ & 262.1510 & 131.5791 & 245.1244 & 123.0659 & 244.1404 & 122.5738 & 2 \\
\hline 30 & & & & & & & $\mathbf{R}$ & 175.1190 & 88.0631 & 158.0924 & 79.5498 & & & 1 \\
\hline
\end{tabular}
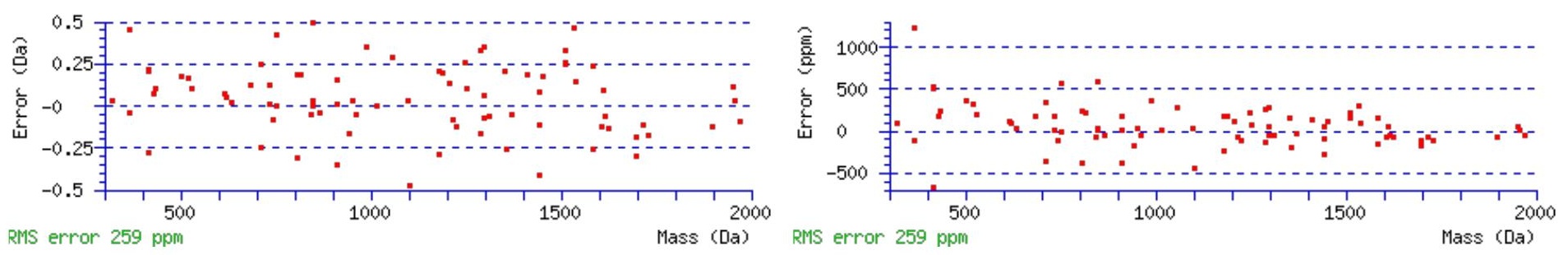

\section{All matches to this query}

\begin{tabular}{|l|l|l|l|}
\hline Score & Mr(calc): & Delta & \multicolumn{1}{|c|}{ Sequence } \\
\hline 81.6 & 3339.4303 & 0.0055 & RGVDVSOVAWQSQGDTPCSCCIVNNSNGSR \\
\hline 79.7 & 3339.4303 & 0.0055 & RGVDVSQVAWQSQGDTPCSCCIVNNSNGSR \\
\hline 76.3 & 3339.4303 & 0.0055 & RGVDVSQVAWQSQGDTPCSCCIVNNSNGSR \\
\hline 44.2 & 3338.4463 & 0.9895 & RGVDVSQVAWQSQGDTPCSCCIVNNSNGSR \\
\hline 39.6 & 3338.4463 & 0.9895 & RGVDVSQVAWQSQGDTPCSCCIVNNSNGSR \\
\hline 34.8 & 3338.4463 & 0.9895 & RGVDVSQVAWQSQGDTPCSCCIVNNSNGSR \\
\hline 32.1 & 3337.4623 & 1.9735 & RGVDVSQVAWQSQGDTPCSCCIVNNSNGSR \\
\hline 13.9 & 3337.4561 & 1.9797 & EEALQGVNVTIIDQDTTLSSLAITNQCDK \\
\hline 13.9 & 3337.4561 & 1.9797 & EEALQGVNVTIIDQDTTLSSLAITNQCDK \\
\hline 13.9 & 3337.4561 & 1.9797 & EEALQGVNVTIIDQDTTLSSLAITNQCDK \\
\hline
\end{tabular}

Spectrum No: 214; Query: 908; Rank: 1

\section{Peptide View}

MS/MS Fragmentation of FENQTCFPLPDSR

Found in IPI00190776, Tax_Id=10116 Gene_Symbol=Cspg2 Isoform V0 of Versican core protein precursor (Fragment)

Match to Query 908: 1610.706608 from(806.360580,2+)

Title: 100101RatKid_NS_deglyco_24.2754.2754.2.dta

Data file K:INewmanPaper|Piliangl3SubProteomes\Piliang3SP\mgf5ppm\ERLIC_3SubProteomes5ppm.mgf 

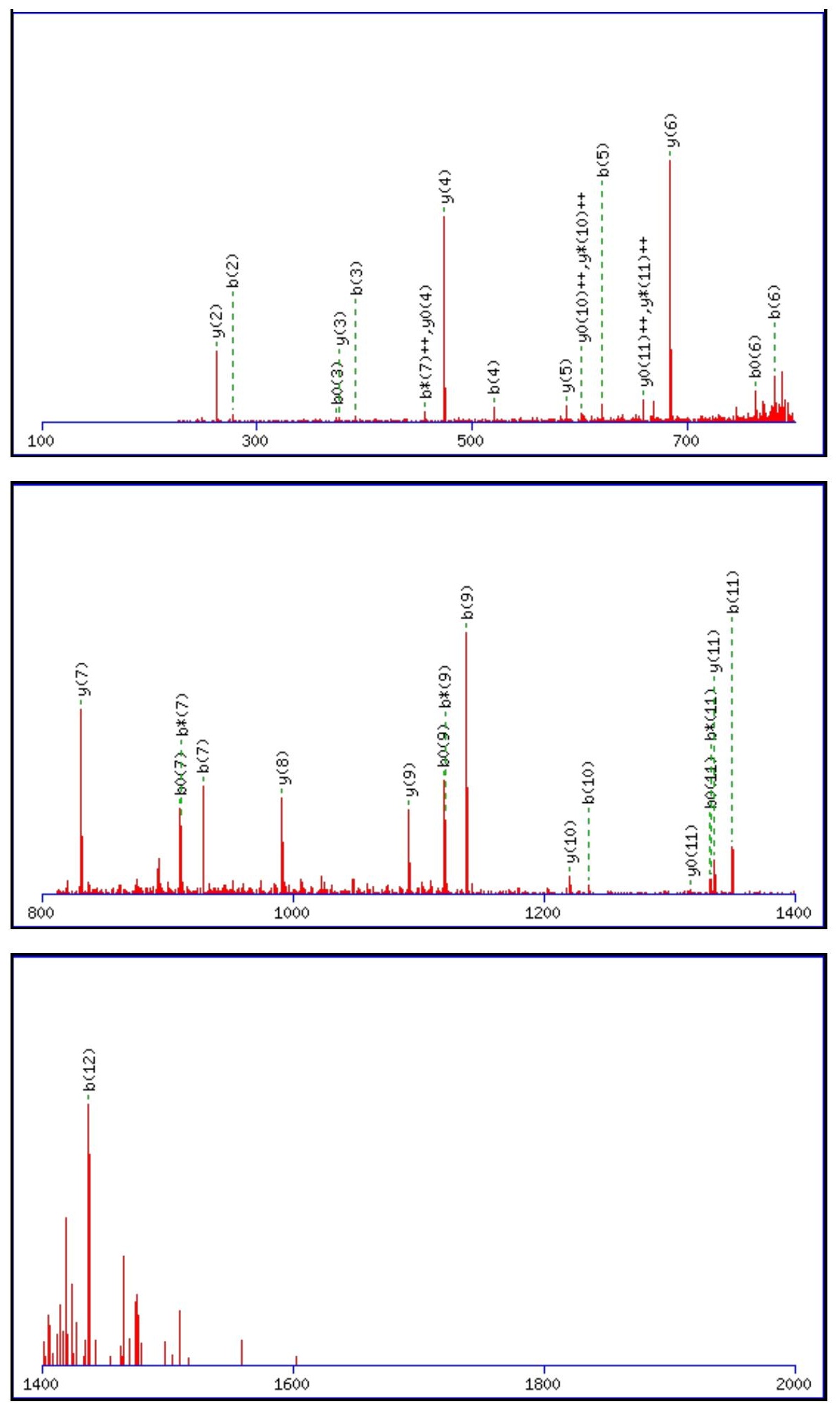

Monoisotopic mass of neutral peptide $\operatorname{Mr}($ calc): 1610.7035

Fixed modifications: Carbamidomethyl (C)

Variable modifications:

N3 : Deamidated $\mathrm{N}(\mathrm{N})$

Ions Score: 81 Expect: $1.3 \mathrm{e}-006$

Matches (Bold Red): 35/136 fragment ions using 50 most intense peaks

\begin{tabular}{|r|c|c|c|c|c|c|c|c|c|c|c|c|c|c|}
\hline$\#$ & $\mathbf{b}$ & $\mathbf{b}^{++}$ & $\mathbf{b}^{*}$ & $\mathbf{b}^{\boldsymbol{*}^{++}}$ & $\mathbf{b}^{\mathbf{0}}$ & $\mathbf{b}^{\mathbf{0 + +}}$ & $\mathbf{S e q}$ & $\mathbf{y}$ & $\mathbf{y}^{++}$ & $\mathbf{y}^{\mathbf{*}}$ & $\mathbf{y}^{\boldsymbol{*}^{++}}$ & $\mathbf{y}^{\mathbf{0}}$ & $\mathbf{y}^{\mathbf{0 + +}}$ & $\#$ \\
\hline $\mathbf{1}$ & 148.0757 & 74.5415 & & & & & $\mathbf{F}$ & & & & & & & $\mathbf{1 3}$ \\
\hline $\mathbf{2}$ & $\mathbf{2 7 7 . 1 1 8 3}$ & 139.0628 & & & 259.1077 & 130.0575 & $\mathbf{E}$ & 1464.6424 & 732.8248 & 1447.6158 & 724.3115 & 1446.6318 & 723.8195 & $\mathbf{1 2}$ \\
\hline $\mathbf{3}$ & $\mathbf{3 9 2 . 1 4 5 2}$ & 196.5762 & 375.1187 & 188.0630 & 374.1347 & 187.5710 & $\mathbf{N}$ & $\mathbf{1 3 3 5 . 5 9 9 8}$ & 668.3035 & 1318.5732 & $\mathbf{6 5 9 . 7 9 0 2}$ & $\mathbf{1 3 1 7 . 5 8 9 2}$ & $\mathbf{6 5 9 . 2 9 8 2}$ & $\mathbf{1 1}$ \\
\hline $\mathbf{4}$ & $\mathbf{5 2 0 . 2 0 3 8}$ & 260.6055 & 503.1772 & 252.0923 & 502.1932 & 251.6003 & $\mathbf{Q}$ & $\mathbf{1 2 2 0 . 5 7 2 8}$ & 610.7901 & 1203.5463 & $\mathbf{6 0 2 . 2 7 6 8}$ & 1202.5623 & $\mathbf{6 0 1 . 7 8 4 8}$ & $\mathbf{1 0}$ \\
\hline $\mathbf{5}$ & $\mathbf{6 2 1 . 2 5 1 5}$ & 311.1294 & 604.2249 & 302.6161 & 603.2409 & 302.1241 & $\mathbf{T}$ & $\mathbf{1 0 9 2 . 5 1 4 3}$ & 546.7608 & 1075.4877 & 538.2475 & 1074.5037 & 537.7555 & $\mathbf{9}$ \\
\hline
\end{tabular}




\begin{tabular}{|c|c|c|c|c|c|c|c|c|c|c|c|c|c|c|}
\hline 6 & 781.2821 & |391.1447 & 764.2556 & |382.6314 & 763.2716 & |382.1394| & $\mathrm{C}$ & 991.4666 & |496.2369 & 974.4400 & |487.7237 & 973.4560 & $\mid$ |487.2316 & 8 \\
\hline 7 & 928.3505 & 464.6789 & 911.3240 & 456.1656 & 910.3400 & 455.6736 & $\mathbf{F}$ & 831.4359 & 416.2216 & 814.4094 & 407.7083 & 813.4254 & 407.2163 & 7 \\
\hline 8 & 1025.4033 & 513.2053 & 1008.3768 & 504.6920 & 1007.3927 & 504.2000 & $\mathbf{P}$ & 684.3675 & 342.6874 & 667.3410 & 334.1741 & 666.3569 & 333.6821 & 6 \\
\hline 9 & 1138.4874 & 569.7473 & 1121.4608 & 561.2340 & 1120.4768 & 560.7420 & $\mathbf{L}$ & 587.3148 & 294.1610 & 570.2882 & 285.6477 & 569.3042 & 285.1557 & 5 \\
\hline 10 & 1235.5401 & 618.2737 & 1218.5136 & 609.7604 & 1217.5296 & 609.2684 & $\mathbf{P}$ & 474.2307 & 237.6190 & 457.2041 & 229.1057 & 456.2201 & 228.6137 & 4 \\
\hline 11 & 1350.5671 & 675.7872 & 1333.5405 & 667.2739 & 1332.5565 & 666.7819 & D & 377.1779 & 189.0926 & 360.1514 & 180.5793 & 359.1674 & 180.0873 & 3 \\
\hline 12 & 1437.5991 & 719.3032 & 1420.5726 & 710.7899 & 1419.5885 & 710.2979 & S & 262.1510 & 131.5791 & 245.1244 & 123.0659 & 244.1404 & 122.5738 & 2 \\
\hline 13 & & & & & & & $\mathbf{R}$ & 175.1190 & 88.0631 & 158.0924 & 79.5498 & & & 1 \\
\hline
\end{tabular}
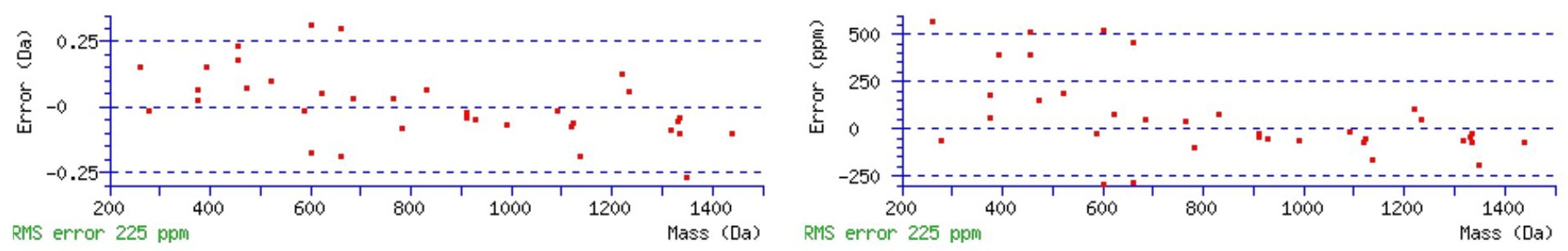

\section{All matches to this query}

\begin{tabular}{|l|l|l|l|}
\hline Score & Mr(calc): & Delta & \multicolumn{1}{c|}{ Sequence } \\
\hline 81.5 & 1610.7035 & 0.0031 & FENOTCFPLPDSR \\
\hline 23.7 & 1610.7028 & 0.0038 & EMDQTMAANAQKNK \\
\hline 10.4 & 1610.7076 & -0.0010 & TEKLNDELQSPEK \\
\hline 9.5 & 1610.7141 & -0.0075 & SMPSASSCSTRTPSR \\
\hline 3.7 & 1610.7049 & 0.0017 & RSASPPPAASSSSSSR \\
\hline 2.7 & 1610.7049 & 0.0017 & RSASPPPAASSSSSSR \\
\hline 2.7 & 1610.7049 & 0.0017 & RSASPPPAASSSSSSR \\
\hline 2.0 & 1610.7141 & -0.0075 & MTKSNGEEPRMGSR \\
\hline 1.5 & 1610.7076 & -0.0010 & KEDPEDEKSLVDK \\
\hline 0.5 & 1610.7214 & -0.0148 & LMKTMKPETTKK \\
\hline
\end{tabular}

Spectrum No: 215; Query: 2522; Rank: 1

\section{Peptide View}

MS/MS Fragmentation of LQAALDNEAGGRPAMEPGNGSLDLGGDAAGR

Found in IPI00210090, Tax_Id=10116 Gene_Symbol=Hnrpu SP120

Match to Query 2522: 2980.403352 from(994.475060,3+)

Title: 100101RatKid_NS_deglyco_12.3060.3060.3.dta

Data file K:INewmanPaper|Piliangl3SubProteomes\Piliang3SP\mgf5ppm\ERLIC_3SubProteomes5ppm.mgf 

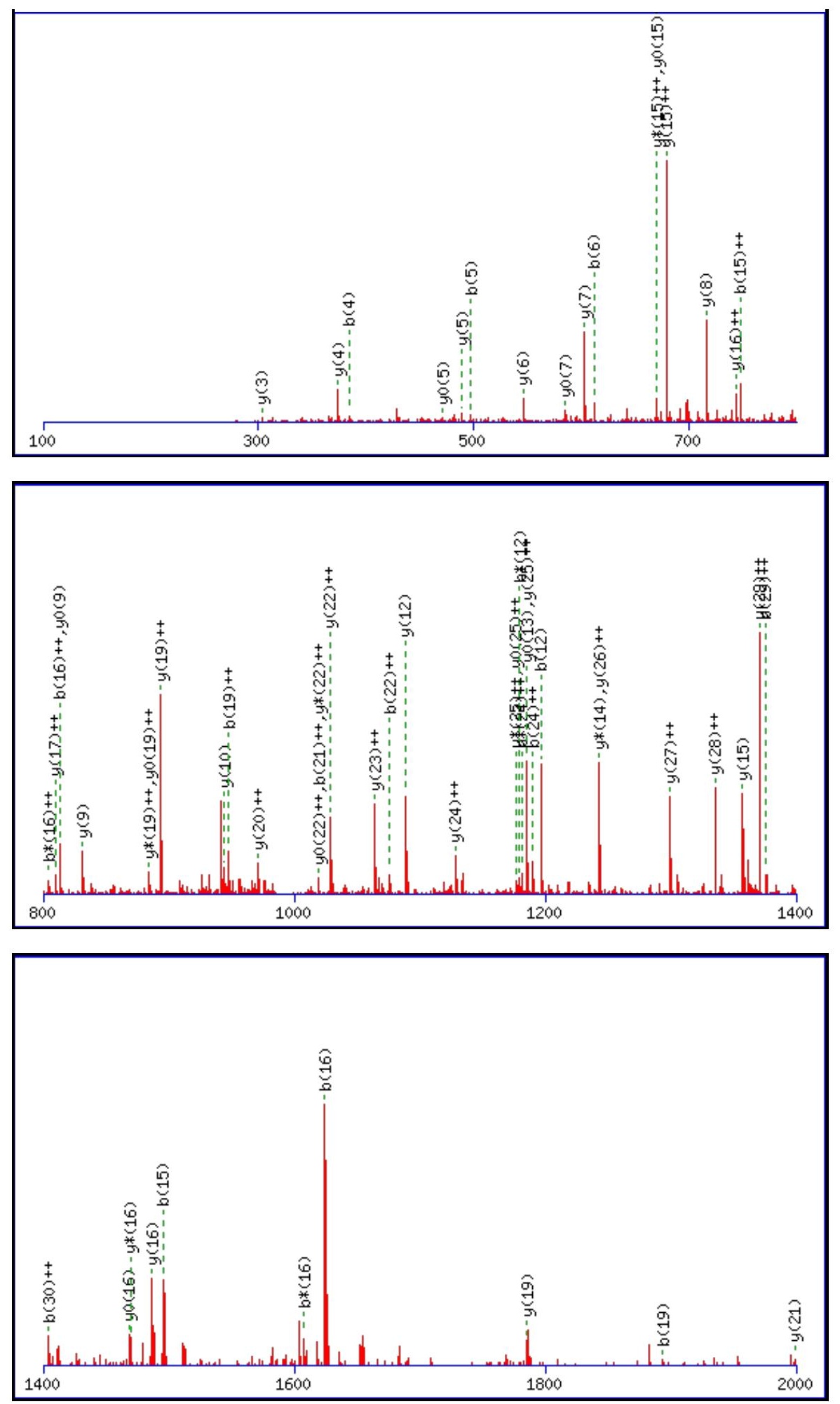

Monoisotopic mass of neutral peptide $\operatorname{Mr}($ calc): 2980.3944

Fixed modifications: Carbamidomethyl (C)

Variable modifications:

N19 : Deamidated_N (N)

Ions Score: 81 Expect: 2.6e-006

Matches (Bold Red): 60/340 fragment ions using 111 most intense peaks

\begin{tabular}{|c|c|c|c|c|c|c|c|c|c|c|c|c|c|c|}
\hline$\#$ & $\mathbf{b}$ & $\mathbf{b}^{++}$ & $\mathbf{b}^{*}$ & $\mathbf{b}^{\boldsymbol{*}^{++}}$ & $\mathbf{b}^{\mathbf{0}}$ & $\mathbf{b}^{\mathbf{0 + +}}$ & Seq. & $\mathbf{y}$ & $\mathbf{y}^{++}$ & $\mathbf{y}^{\mathbf{*}}$ & $\mathbf{y}^{\mathbf{*}^{++}}$ & $\mathbf{y}^{\mathbf{0}}$ & $\mathbf{y}^{\mathbf{0 + +}}$ & $\#$ \\
\hline $\mathbf{1}$ & 114.0913 & 57.5493 & & & & & $\mathbf{L}$ & & & & & & & $\mathbf{3 1}$ \\
\hline $\mathbf{2}$ & 242.1499 & 121.5786 & 225.1234 & 113.0653 & & & $\mathbf{Q}$ & 2868.3177 & 1434.6625 & 2851.2911 & 1426.1492 & 2850.3071 & 1425.6572 & $\mathbf{3 0}$ \\
\hline $\mathbf{3}$ & 313.1870 & 157.0972 & 296.1605 & 148.5839 & & & $\mathbf{A}$ & 2740.2591 & $\mathbf{1 3 7 0 . 6 3 3 2}$ & 2723.2326 & 1362.1199 & 2722.2485 & 1361.6279 & $\mathbf{2 9}$ \\
\hline $\mathbf{4}$ & $\mathbf{3 8 4 . 2 2 4 1}$ & 192.6157 & 367.1976 & 184.1024 & & & $\mathbf{A}$ & 2669.2220 & $\mathbf{1 3 3 5 . 1 1 4 6}$ & 2652.1954 & 1326.6014 & 2651.2114 & 1326.1094 & $\mathbf{2 8}$ \\
\hline $\mathbf{5}$ & $\mathbf{4 9 7 . 3 0 8 2}$ & 249.1577 & 480.2817 & 240.6445 & & & $\mathbf{L}$ & 2598.1849 & $\mathbf{1 2 9 9 . 5 9 6 1}$ & 2581.1583 & 1291.0828 & 2580.1743 & 1290.5908 & $\mathbf{2 7}$ \\
\hline
\end{tabular}




\begin{tabular}{|c|c|c|c|c|c|c|c|c|c|c|c|c|c|c|}
\hline & 612.3352 & 306.6712 & 595.3086 & 298.1579| & & & D & 2485.1008 & 1243.0540 & & & & 1234.0488 & 26 \\
\hline 7 & 726.3781 & 363.6927 & 709.3515 & 355.1794 & 708.3675 & 354.6874 & $\mathbf{N}$ & 2370.0739 & 1185.5406 & 2353.0473 & $\mid 1177.0273$ & 2352.0633 & 1176.5353 & 25 \\
\hline 8 & 855.4207 & 428.2140 & 838.3941 & 419.7007 & 837.4101 & 419.2087 & $\mathbf{E}$ & 2256.0309 & 1128.5191 & 2239.0044 & 1120.0058 & 2238.0204 & 1119.5138 & 24 \\
\hline 9 & 926.4578 & 463.7325 & 909.4312 & 455.2193 & 908.4472 & 454.7272 & A & 2126.9884 & 1063.9978 & 2109.9618 & 1055.4845 & 2108.9778 & 1054.9925 & 23 \\
\hline 10 & 983.4793 & 492.2433 & 966.4527 & 483.7300 & 965.4687 & 483.2380 & G & 2055.9512 & 1028.4793 & 2038.9247 & $\mid 1019.9660$ & 2037.9407 & 1019.4740 & 22 \\
\hline 111 & 1040.5007 & 520.7540 & 1023.4742 & 512.2407 & 1022.4901 & 511.7487 & G & 1998.9298 & 999.9685 & 1981.9032 & 991.4552 & 1980.9192 & 990.9632 & 21 \\
\hline 121 & 1196.6018 & 598.8046 & 1179.5753 & 590.2913 & 1178.5913 & 589.7993 & $\mathbf{R}$ & 1941.9083 & 971.4578 & 1924.8818 & 962.9445 & 1923.8977 & 962.4525 & 0 \\
\hline 13 & 1293.6546 & 647.3309 & 1276.6280 & 638.8177 & 1275.6440 & 638.3257 & $\mathbf{P}$ & 1785.8072 & 893.4072 & 1768.7806 & 884.8940 & 1767.7966 & 884.4020 & 19 \\
\hline 14 & 1364.6917 & 682.8495 & 1347.6652 & 674.3362 & 1346.6811 & 673.8442 & A & 1688.7544 & 844.8809 & 1671.7279 & 836.3676 & 1670.7439 & 835.8756 & 18 \\
\hline 15 & 1495.7322 & 748.3697 & 1478.7056 & 739.8565 & 1477.7216 & 739.3644 & $\mathbf{M}$ & 1617.7173 & 809.3623 & 1600.6908 & 800.8490 & 1599.7068 & 800.3570 & 17 \\
\hline 16 & 1624.7748 & 812.8910 & 1607.7482 & 804.3778 & 1606.7642 & 857 & $\mathbf{E}$ & 1486.6768 & 743.8421 & 1469.6 & 288 & 6663 & 368 & 16 \\
\hline 171 & 1721.8275 & 861.4174 & 1704.8010 & 852.9041 & 1703.8170 & 852.4121 & $\mathbf{P}$ & 1357.6342 & 679.3208 & 1340.6077 & $\mathbf{6 7 0 . 8 0 7 5}$ & 1339.6237 & 670.3155 & 15 \\
\hline 18 & 1778.8490 & 889.9281 & 1761.8225 & 881.4149 & 1760.8384 & 880.9229 & G & 1260.5815 & 630.7944 & 1243.5549 & 622.2811 & 1242.5709 & 621.7891 & 4 \\
\hline 191 & 1893.8759 & 947.4416 & 1876.8494 & 938.9283 & 1875.8654 & 363 & $\mathbf{N}$ & 1203.5600 & 602.2836 & 1186 & 704 & 1185.5494 & & 13 \\
\hline 201 & 1950.8974 & 975.9523 & 1933.8709 & 967.4391 & 1932.8868 & 966.9471 & G & 1088.5331 & 544.7702 & 1071.5065 & 536.2569 & 1070.5225 & 535.7649 & 12 \\
\hline 21 & 2037.9294 & 1019.4684 & 2020.9029 & 1010.9551 & 2019.9189 & 1010.4631 & $\mathrm{~S}$ & 1031.5116 & 516.2594 & 1014.4851 & 507.7462 & 1013.5010 & 507.2542 & 11 \\
\hline 222 & 2151.0135 & 1076.0104 & 2133.9870 & 1067.4971: & 2133.0029 & 1067.0051 & $\mathbf{L}$ & 944.4796 & 472.7434 & 927.4530 & 464.2302 & 926.4690 & 463.7381 & 10 \\
\hline \begin{tabular}{l|l}
23 & 2 \\
\end{tabular} & 2266.0404 & 1133.5239 & 2249.0139 & 1125.0106 & 2248.0299 & 1124.5186 & D & 831.3955 & 416.2014 & 814.3690 & 407.6881 & 813.3850 & 407.1961 & 9 \\
\hline 242 & 2379.1245 & 1190.0659 & 2362.0980 & 1181.5526 & 2361.1139 & 1181.0606 & $\mathbf{L}$ & 716.3686 & 358.6879 & 699.3420 & 350.1747 & 698.3580 & 349.6826 & 8 \\
\hline 252 & 2436.1460 & 1218.5766 & 2419.1194 & 1210.0633 & 2418.1354 & 1209.5713 & $\mathbf{G}$ & 603.2845 & 302.1459 & 586.2580 & 293.6326 & 585.2740 & 293.1406 & 7 \\
\hline \begin{tabular}{l|l}
26 & 2 \\
\end{tabular} & 2493.1674 & 1247.0874 & 2476.1409 & 1238.5741 & 2475.1569 & 1238.0821 & G & 546.2631 & 273.6352 & 529.2365 & 265.1219 & 528.2525 & 264.6299 & 6 \\
\hline 272 & 2608.1944 & 1304.6008 & 2591.1678 & 1296.0876 & 2590.1838 & 1295.5955 & D & 489.2416 & 245.1244 & 472.2150 & 236.6112 & 471.2310 & 236.1191 & 5 \\
\hline 282 & 2679.2315 & 1340.1194 & 2662.2049 & 1331.6061 & 2661.2209 & 1331.1141 & A & 374.2146 & 187.6110 & 357.1881 & 179.0977 & & & 4 \\
\hline 29 & 2750.2686 & 1375.6379 & 2733.2421 & 1367.1247 & 2732.2580 & 1366.6327 & A & 303.1775 & 152.0924 & 286.1510 & 143.5791 & & & 3 \\
\hline 302 & 2807.2901 & 1404.1487 & 2790.2635 & 1395.6354 & 2789.2795 & 1395.1434 & G & 232.1404 & 116.5738 & 215.1139 & 108.0606 & & & 2 \\
\hline 31 & & & & & & & $\mathbf{R}$ & 175.1190 & 88.0631 & 158.0924 & 79.5498 & & & 1 \\
\hline
\end{tabular}
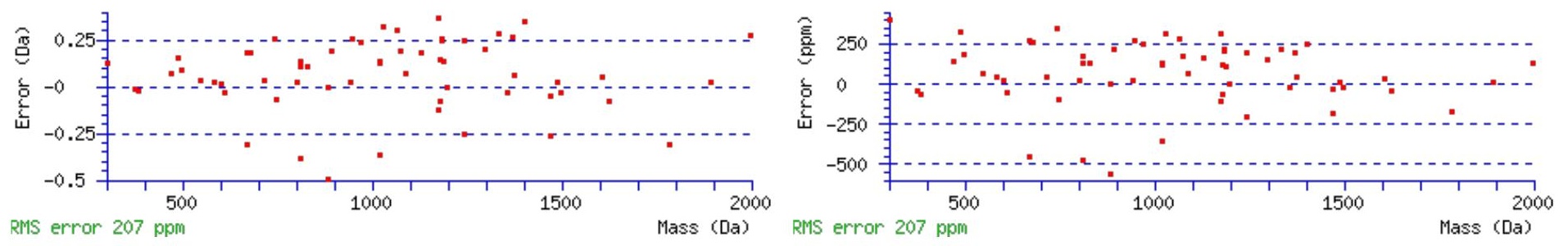

\section{All matches to this query}

\begin{tabular}{|l|c|c|l|}
\hline Score & Mr(calc): & Delta & \multicolumn{1}{|c|}{ Sequence } \\
\hline 81.3 & 2980.3944 & 0.0089 & LQAALDNEAGGRPAMEPGNGSLDLGGDAAGR \\
\hline 33.5 & 2980.3944 & 0.0089 & LQAALDNEAGGRPAMEPGNGSLDLGGDAAGR \\
\hline 27.0 & 2979.4104 & 0.9929 & LQAALDNEAGGRPAMEPGNGSLDLGGDAAGR \\
\hline 3.9 & 2980.4147 & -0.0114 & VQCAARAVNTNGNEGLELMSPIVTIAR \\
\hline 3.5 & 2980.3808 & 0.0225 & GSASLNLCIKETLRLRPPIMTMMR \\
\hline 3.5 & 2980.3808 & 0.0225 & GSASLNLCIKETLRLRPPIMTMMR \\
\hline 3.5 & 2980.3808 & 0.0225 & GSASLNLCIKETLRLRPPIMTMMR \\
\hline 3.3 & 2980.4147 & -0.0114 & VQCAARAVNTNGNEGLELMSPIVTIAR \\
\hline 3.1 & 2980.4147 & -0.0114 & VQCAARAVNTNGNEGLELMSPIVTIAR \\
\hline 2.9 & 2980.4147 & -0.0114 & VQCAARAVNTNGNEGLELMSPIVTIAR \\
\hline
\end{tabular}

Spectrum No: 216; Query: 2380; Rank: 1 


\section{Peptide View}

MS/MS Fragmentation of FQISPQLQFSPEEVLGMVLNYSR

Found in IPI00210975, Tax_Id=10116 Gene_Symbol=Hyou1 Hypoxia up-regulated protein 1 precursor

Match to Query 2380: 2682.335442 from(895.119090,3+)

Title: 091008RatKidney_NoSalt_18.6599.6599.3.dta

Data file K:\NewmanPaper\Piliang \3SubProteomes $\backslash$ Piliang3SP $\backslash$ mgf5ppm\ERLIC_3SubProteomes5ppm.mgf
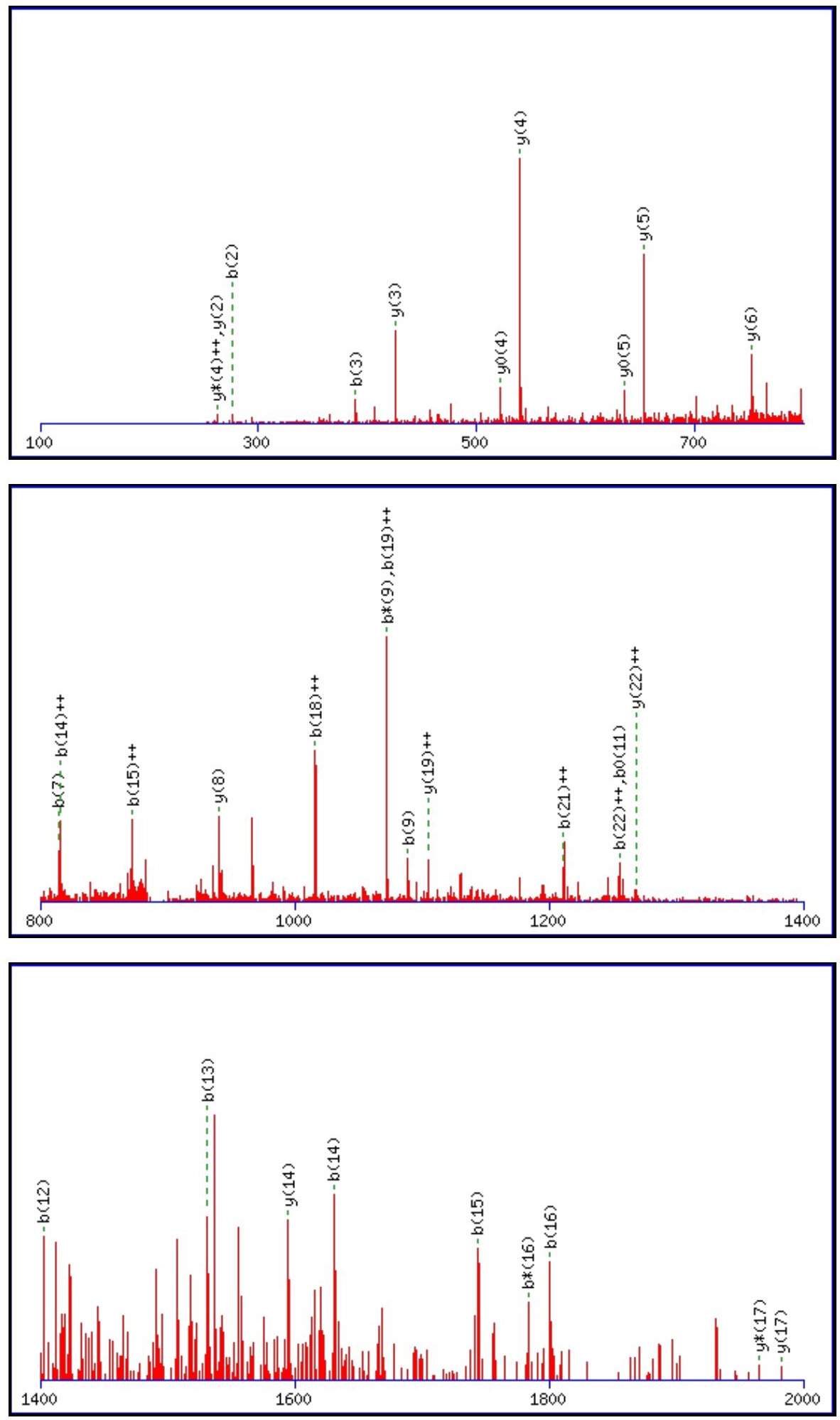

Monoisotopic mass of neutral peptide $\operatorname{Mr}($ calc): 2682.3363

Fixed modifications: Carbamidomethyl (C)

Variable modifications:

N20 : Deamidated_N (N)

Ions Score: 81 Expect: $1.6 \mathrm{e}-006$ 
Matches (Bold Red): 32/254 fragment ions using 41 most intense peaks

\begin{tabular}{|c|c|c|c|c|c|c|c|c|c|c|c|c|c|c|}
\hline \# & b & $\mathbf{b}^{++}$ & $\mathbf{b}^{*}$ & $\mathbf{b}^{*^{++}}$ & $\mathbf{b}^{0}$ & $\mathbf{b}^{0++}$ & Seq. & $\mathbf{y}$ & $\mathbf{y}^{++}$ & $\mathbf{y}^{*}$ & $\mathrm{y}^{*^{++}}$ & $\mathbf{y}^{\mathbf{0}}$ & $\mathbf{y}^{\mathbf{0 + +}}$ & \# \\
\hline 1 & 148.0757 & 74.5415 & & & & & $\mathbf{F}$ & & & & & & & 23 \\
\hline 2 & 276.1343 & 138.5708 & 259.1077 & 130.0575 & & & $\mathbf{Q}$ & 2536.2752 & 1268.6412 & 2519.2486 & 1260.1280 & 2518.2646 & 1259.6360 & 22 \\
\hline 3 & 389.2183 & 195.1128 & 372.1918 & 186.5995 & & & I & 2408.2166 & 1204.6119 & 2391.1901 & 1196.0987 & 2390.2061 & 1195.6067 & 21 \\
\hline 4 & 476.2504 & 238.6288 & 459.2238 & 230.1155 & 458.2398 & 229.6235 & $S$ & 2295.1326 & 1148.0699 & 2278.1060 & 1139.5566 & 2277.1220 & 1139.0646 & 20 \\
\hline 5 & 573.3031 & 287.1552 & 556.2766 & 278.6419 & 555.2926 & 278.1499 & $\mathbf{P}$ & 2208.1005 & 1104.5539 & 2191.0740 & 1096.0406 & 2190.0900 & 1095.5486 & 19 \\
\hline 6 & 701.3617 & 351.1845 & 684.3352 & 342.6712 & 683.3511 & 342.1792 & $\mathbf{Q}$ & 2111.0478 & 1056.0275 & 2094.0212 & 1047.5142 & 2093.0372 & 1047.0222 & 18 \\
\hline 7 & 814.4458 & 407.7265 & 797.4192 & 399.2132 & 796.4352 & 398.7212 & $\mathbf{L}$ & 1982.9892 & 991.9982 & 1965.9626 & 983.4850 & 1964.9786 & 982.9929 & 17 \\
\hline 8 & 942.5043 & 471.7558 & 925.4778 & 463. & 924. & 462.7505 & $\mathbf{Q}$ & 1869.9051 & 935.4562 & 1852.8786 & 926.9429 & 1851.8946 & 926.4509 & 16 \\
\hline 9 & 1089.5728 & 545.2900 & 1072.5462 & 536.7767 & 1071.5622 & 536.2847 & $\mathbf{F}$ & 1741.8465 & 871.4269 & 1724.8200 & 862.9136 & 1723.8360 & 862.4216 & 15 \\
\hline 10 & 1176.6048 & 588.8060 & 1159.5782 & 580.2928 & 1158.5942 & 579.8007 & $\mathrm{~S}$ & 1594.7781 & 797.8927 & 1577.7516 & 789.3794 & 1576.7676 & 788.8874 & 14 \\
\hline 11 & 1273 & 637. & 1256.6310 & 628.8191 & 1255.6470 & 628.3271 & $\mathbf{P}$ & 1507.7461 & 754.3767 & 1490.7196 & 745.8634 & 1489.7355 & & 13 \\
\hline 12 & 1402.7001 & 701.8537 & 1385.6736 & 693.3404 & 1384.6896 & 692.8484 & $\mathbf{E}$ & 1410.6933 & 705.8503 & 1393.6668 & 697.3370 & 1392.6828 & 696.8450 & 12 \\
\hline 13 & 1531.7427 & 766.3750 & 1514.7162 & 757.8617 & 1513.7322 & 757.3697 & $\mathbf{E}$ & 1281.6507 & 641.3290 & 1264.6242 & 632.8157 & 1263.6402 & 632.3237 & 11 \\
\hline 14 & \begin{tabular}{|l|l|}
1630.8112 \\
\end{tabular} & 815.9092 & 1613.7846 & 807.3959 & 1612.8006 & 806.9039 & $\mathrm{~V}$ & 1152.6081 & 576.8077 & 1135.5816 & 568.2944 & 1134.5976 & 567.8024 & 10 \\
\hline 15 & 1743.8952 & 872.4512 & 1726.8687 & 863.9380 & 1725.8846 & 863.4460 & $\mathbf{L}$ & 1053.5397 & 527.2735 & 1036.5132 & 518.7602 & 1035.5292 & 518.2682 & 9 \\
\hline 16 & \begin{tabular}{|l}
1800.9167 \\
\end{tabular} & 900.9620 & 1783.8901 & 892.4487 & 1782.9061 & 891.9567 & G & 940.4557 & 470.7315 & 923.4291 & 462.2182 & 922.4451 & 461.7262 & 8 \\
\hline 17 & 1931.9572 & 966.4822 & 1914.9306 & 957.9689 & 1913.9466 & 957.4769 & $\mathbf{M}$ & 883.4342 & 442.2207 & 866.4077 & 433.7075 & 865.4236 & 433.2155 & 7 \\
\hline 182 & 2031.0256 & 1016.0164 & 2013.9990 & 1007.5032 & 2013.0150 & 1007.0111 & $\mathrm{~V}$ & 752.3937 & 376.7005 & 735.3672 & 368.1872 & 734.3832 & 367.6952 & 6 \\
\hline 192 & 2144.1096 & 1072.5585 & 2127.0831 & 1064.0452 & 2126.0991 & 1063.5532 & $\mathbf{L}$ & 653.3253 & 327.1663 & 636.2988 & 318.6530 & 635.3147 & 318.1610 & 5 \\
\hline 202 & 2259.1366 & 1130.0719 & 2242.1100 & 1121.5587 & 2241.1260 & 1121.0666 & $\mathbf{N}$ & 540.2412 & 270.6243 & 523.2147 & 262.1110 & 522.2307 & 261.6190 & 4 \\
\hline 212 & 2422.1999 & 1211.6036 & 2405.1734 & 1203.0903 & 2404.1893 & 1202.5983 & $\mathbf{Y}$ & 425.2143 & 213.1108 & 408.1878 & 204.5975 & 407.2037 & 204.1055 & 3 \\
\hline 222 & 2509.2319 & 1255.1196 & 2492.2054 & 1246.6063 & 2491.2214 & 1246.1143 & $\mathrm{~S}$ & 262.1510 & 131.5791 & 245.1244 & 123.0659 & 244.1404 & 122.5738 & 2 \\
\hline 23 & & & & & & & $\mathbf{R}$ & 175.1190 & 88.0631 & 158.0924 & 79.5498 & & & 1 \\
\hline
\end{tabular}
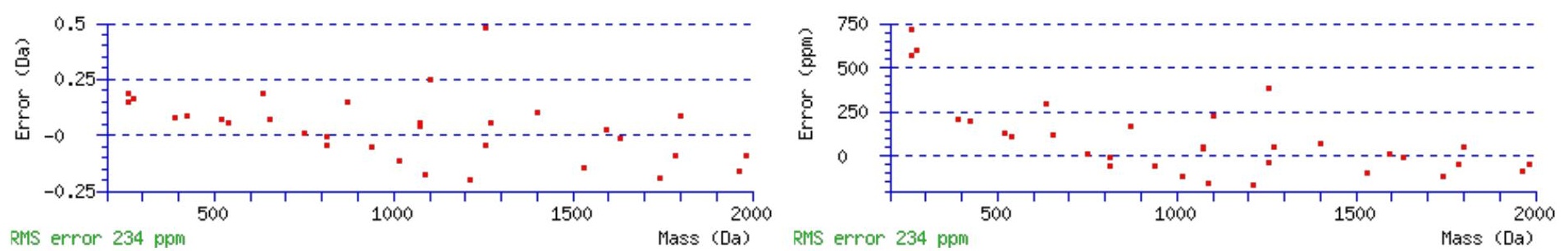

\section{All matches to this query}

\begin{tabular}{|l|l|l|l|}
\hline Score & Mr(calc): & Delta & \multicolumn{1}{|c|}{ Sequence } \\
\hline 81.3 & 2682.3363 & -0.0009 & FQISPQLQFSPEEVLGMVLNYSR \\
\hline 55.5 & 2681.3523 & 0.9831 & FQISPQLQFSPEEVLGMVLNYSR \\
\hline 5.4 & 2680.3077 & 2.0277 & DTVKVATVTGVGLAALGLIGVMLSR \\
\hline 5.3 & 2680.3077 & 2.0277 & DTVKVATVTGVGLAALGLIGVMLSR \\
\hline 5.3 & 2680.3077 & 2.0277 & DTVKVATVTGVGLAALGLIGVMLSR \\
\hline 2.8 & 2681.3516 & 0.9838 & VKTMNDLSSQLSPLDLHPSPKMSR \\
\hline 2.3 & 2680.3151 & 2.0203 & LKXGLLPTLPNTPMNTTLMDSCR \\
\hline 2.1 & 2681.3060 & 1.0294 & YTFKMNPGDVITFDNWRLLHGR \\
\hline 2.0 & 2682.3295 & 0.0059 & NALEVDLGYLITAARRLHEVR \\
\hline 1.4 & 2681.3191 & 1.0164 & TISLGAGDRQVIQTPLVDSLPVSR \\
\hline
\end{tabular}

Spectrum No: 217; Query: 499; Rank: 1 


\section{Peptide View}

MS/MS Fragmentation of SWPAVGNCTAALR

Found in IPI00195516, Tax_Id=10116 Gene_Symbol=Hpx Hemopexin precursor

Match to Query 499: 1402.662628 from(702.338590,2+)

Title: 091008RatKidney_NoSalt_30.2182.2182.2.dta

Data file K:\NewmanPaper\Piliang \3SubProteomes \Piliang3SP \mgf5ppm\ERLIC_3SubProteomes5ppm.mgf
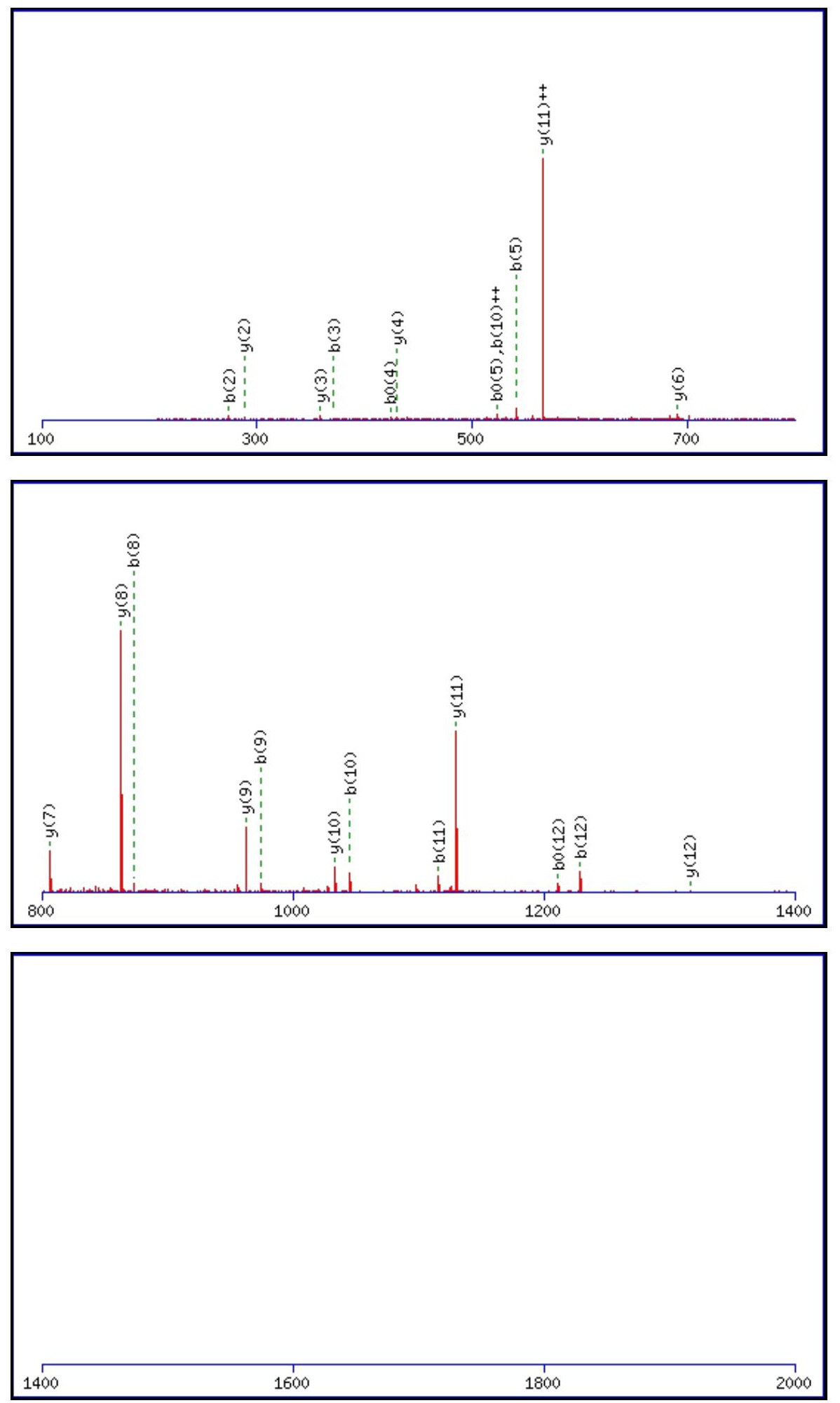

Monoisotopic mass of neutral peptide $\operatorname{Mr}($ calc): 1402.6663

Fixed modifications: Carbamidomethyl (C)

Variable modifications:

N7 : Deamidated_N (N)

Ions Score: 81 Expect: $1.1 \mathrm{e}-006$

Matches (Bold Red): 23/124 fragment ions using 37 most intense peaks 


\begin{tabular}{|r|c|c|c|c|r|r|r|r|c|c|c|c|c|c|}
\hline$\#$ & $\mathbf{b}$ & $\mathbf{b}^{++}$ & $\mathbf{b}^{*}$ & $\mathbf{b}^{\mathbf{*}^{++}}$ & $\mathbf{b}^{\mathbf{0}}$ & \multicolumn{1}{|c|}{$\mathbf{b}^{\mathbf{0 + +}}$} & $\mathbf{S e q}$ & $\mathbf{y}$ & $\mathbf{y}^{++}$ & $\mathbf{y}^{\mathbf{*}}$ & $\mathbf{y}^{\boldsymbol{*}^{++}}$ & $\mathbf{y}^{\mathbf{0}}$ & $\mathbf{y}^{\mathbf{0 + +}}$ & $\#$ \\
\hline $\mathbf{1}$ & $\mathbf{8 8 . 0 3 9 3}$ & 44.5233 & & & 70.0287 & 35.5180 & $\mathbf{S}$ & & & & & & & $\mathbf{1 3}$ \\
\hline $\mathbf{2}$ & $\mathbf{2 7 4 . 1 1 8 6}$ & 137.5629 & & & 256.1081 & 128.5577 & $\mathbf{W}$ & $\mathbf{1 3 1 6 . 6 4 1 6}$ & 658.8244 & 1299.6150 & 650.3112 & 1298.6310 & 649.8191 & $\mathbf{1 2}$ \\
\hline $\mathbf{3}$ & $\mathbf{3 7 1 . 1 7 1 4}$ & 186.0893 & & & 353.1608 & 177.0840 & $\mathbf{P}$ & $\mathbf{1 1 3 0 . 5 6 2 3}$ & 565.7848 & 1113.5357 & 557.2715 & 1112.5517 & 556.7795 & $\mathbf{1 1}$ \\
\hline $\mathbf{4}$ & 442.2085 & 221.6079 & & & $\mathbf{4 2 4 . 1 9 7 9}$ & 212.6026 & $\mathbf{A}$ & $\mathbf{1 0 3 3 . 5 0 9 5}$ & 517.2584 & 1016.4830 & 508.7451 & 1015.4989 & 508.2531 & $\mathbf{1 0}$ \\
\hline $\mathbf{5}$ & $\mathbf{5 4 1 . 2 7 6 9}$ & 271.1421 & & & 523.2663 & 262.1368 & $\mathbf{V}$ & $\mathbf{9 6 2 . 4 7 2 4}$ & 481.7398 & 945.4458 & 473.2266 & 944.4618 & 472.7345 & $\mathbf{9}$ \\
\hline $\mathbf{6}$ & 598.2984 & 299.6528 & & & 580.2878 & 290.6475 & $\mathbf{G}$ & $\mathbf{8 6 3 . 4 0 4 0}$ & 432.2056 & 846.3774 & 423.6923 & 845.3934 & 423.2003 & $\mathbf{8}$ \\
\hline $\mathbf{7}$ & 713.3253 & 357.1663 & 696.2988 & 348.6530 & 695.3147 & 348.1610 & $\mathbf{N}$ & $\mathbf{8 0 6 . 3 8 2 5}$ & 403.6949 & 789.3560 & 395.1816 & 788.3719 & 394.6896 & $\mathbf{7}$ \\
\hline $\mathbf{8}$ & $\mathbf{8 7 3 . 3 5 6 0}$ & 437.1816 & 856.3294 & 428.6683 & 855.3454 & 428.1763 & $\mathbf{C}$ & $\mathbf{6 9 1 . 3 5 5 6}$ & 346.1814 & 674.3290 & 337.6681 & 673.3450 & 337.1761 & $\mathbf{6}$ \\
\hline $\mathbf{9}$ & $\mathbf{9 7 4 . 4 0 3 6}$ & 487.7055 & 957.3771 & 479.1922 & 956.3931 & 478.7002 & $\mathbf{T}$ & 531.3249 & 266.1661 & 514.2984 & 257.6528 & 513.3144 & 257.1608 & $\mathbf{5}$ \\
\hline $\mathbf{1 0}$ & $\mathbf{1 0 4 5 . 4 4 0 8}$ & $\mathbf{5 2 3 . 2 2 4 0}$ & 1028.4142 & 514.7107 & 1027.4302 & 514.2187 & $\mathbf{A}$ & $\mathbf{4 3 0 . 2 7 7 2}$ & 215.6423 & 413.2507 & 207.1290 & & & $\mathbf{4}$ \\
\hline $\mathbf{1 1}$ & $\mathbf{1 1 1 6 . 4 7 7 9}$ & 558.7426 & 1099.4513 & 550.2293 & 1098.4673 & 549.7373 & $\mathbf{A}$ & $\mathbf{3 5 9 . 2 4 0 1}$ & 180.1237 & 342.2136 & 171.6104 & & & $\mathbf{3}$ \\
\hline $\mathbf{1 2}$ & $\mathbf{1 2 2 9 . 5 6 1 9}$ & 615.2846 & 1212.5354 & 606.7713 & $\mathbf{1 2 1 1 . 5 5 1 4}$ & 606.2793 & $\mathbf{L}$ & $\mathbf{2 8 8 . 2 0 3 0}$ & 144.6051 & 271.1765 & 136.0919 & & & $\mathbf{2}$ \\
\hline $\mathbf{1 3}$ & & & & & & & $\mathbf{R}$ & 175.1190 & 88.0631 & 158.0924 & 79.5498 & & & $\mathbf{1}$ \\
\hline
\end{tabular}
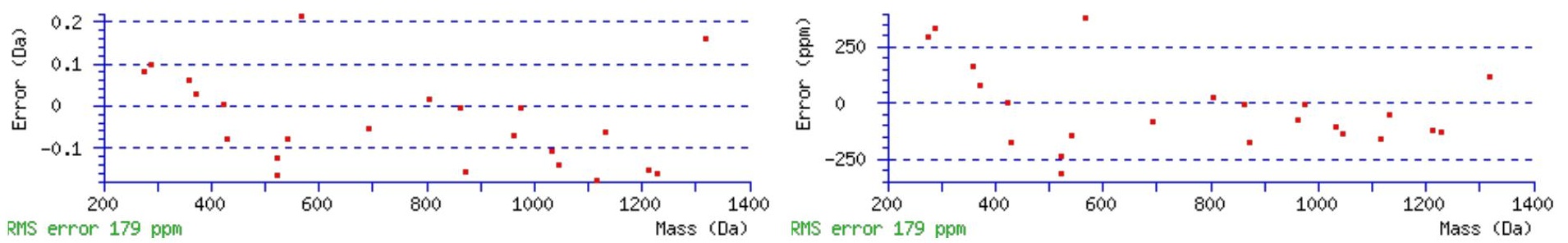

\section{All matches to this query}

\begin{tabular}{|c|c|c|c|}
\hline Score & Mr(calc): & Delta & Sequence \\
\hline 81.2 & 1402.6663 & -0.0037 & SWPAVGNCTAALR \\
\hline 16.8 & 1402.6688 & -0.0061 & SGENAANIASELAR \\
\hline 12.5 & 1402.6688 & -0.0061 & SGENAANIASELAR \\
\hline 10.7 & 1400.6523 & 2.0103 & XIDSLIGPPPPR \\
\hline 10.4 & 1401.6530 & 1.0096 & LRLGSGSNGLLR \\
\hline 9.0 & 1401.6571 & 1.0055 & YVKTHQVIVR \\
\hline 8.7 & 1402.6646 & -0.0020 & SDAFVPFSIGKR \\
\hline 8.2 & 1401.6653 & 0.9973 & HLSPLTVNSPTR \\
\hline 8.2 & 1401.6653 & 0.9973 & HLSPLTVNSPTR \\
\hline 8.1 & 1401.6653 & 0.9973 & SGKQYSIEAALR \\
\hline
\end{tabular}

Spectrum No: 218; Query: 165; Rank: 1

\section{Peptide View}

MS/MS Fragmentation of ALNVTLSSMGR

Found in IPI00213036, Tax_Id=10116 Gene_Symbol=C4a Complement C4 precursor

Match to Query 165: 1164.577928 from(583.296240,2+)

Title: 091008RatKidney_NH4Format01_30.1114.1114.2.dta

Data file K:INewmanPaper|Piliang|3SubProteomes\Piliang3SP\mgf5ppm\ERLIC_3SubProteomes5ppm.mgf 

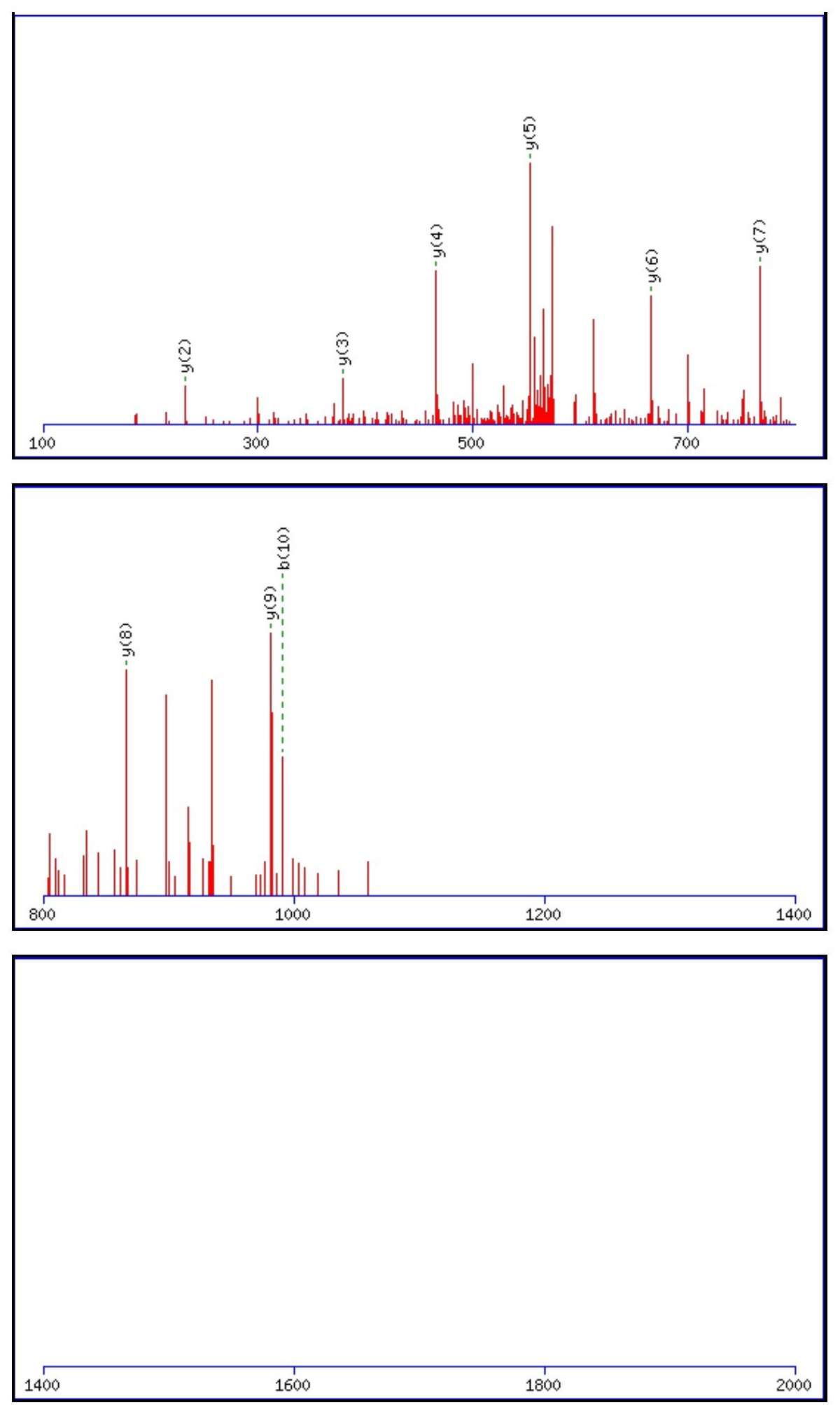

Monoisotopic mass of neutral peptide $\operatorname{Mr}($ calc): 1164.5809

Fixed modifications: Carbamidomethyl (C)

Variable modifications:

N3 : Deamidated_N (N)

M9 : Oxidation (M)

Ions Score: 81 Expect: $8.6 \mathrm{e}-007$

Matches (Bold Red): 9/102 fragment ions using 12 most intense peaks

\begin{tabular}{|c|c|c|c|c|c|c|c|c|c|c|c|c|c|c|}
\hline$\#$ & $\mathbf{b}$ & $\mathbf{b}^{++}$ & $\mathbf{b}^{*}$ & $\mathbf{b}^{\boldsymbol{*}^{++}}$ & $\mathbf{b}^{\mathbf{0}}$ & $\mathbf{b}^{\mathbf{0 + +}}$ & $\mathbf{S e q}$ & $\mathbf{y}$ & $\mathbf{y}^{++}$ & $\mathbf{y}^{\mathbf{*}}$ & $\mathbf{y}^{\mathbf{*}^{++}}$ & $\mathbf{y}^{\mathbf{0}}$ & $\mathbf{y}^{\mathbf{0}+}$ & $\#$ \\
\hline $\mathbf{1}$ & 72.0444 & 36.5258 & & & & & $\mathbf{A}$ & & & & & & & $\mathbf{1 1}$ \\
\hline $\mathbf{2}$ & 185.1285 & 93.0679 & & & & & $\mathbf{L}$ & 1094.5510 & 547.7792 & 1077.5245 & 539.2659 & 1076.5405 & 538.7739 & $\mathbf{1 0}$ \\
\hline $\mathbf{3}$ & 300.1554 & 150.5813 & 283.1288 & 142.0681 & & & $\mathbf{N}$ & $\mathbf{9 8 1 . 4 6 7 0}$ & 491.2371 & 964.4404 & 482.7238 & 963.4564 & 482.2318 & $\mathbf{9}$ \\
\hline $\mathbf{4}$ & 399.2238 & 200.1155 & 382.1973 & 191.6023 & & & $\mathbf{V}$ & $\mathbf{8 6 6 . 4 4 0 0}$ & 433.7237 & 849.4135 & 425.2104 & 848.4295 & 424.7184 & $\mathbf{8}$ \\
\hline & & & & & & & & & & & & & &
\end{tabular}




\begin{tabular}{|c|c|c|c|c|c|c|c|c|c|c|c|c|c|c|}
\hline & |500.2715 & |250.6394 & |483.2449 & |242.1261 & |482.2609 & |241.6341 & $T$ & 767.3716 & |384.1894 & 750.3451 & |375.6762 | & 749.3611 & |375.1842 | & 7 \\
\hline 6 & 613.3555 & 307.1814 & 596.3290 & |298.6681 & 595.3450 & 298.1761 & $\mathbf{L}$ & 666.3239 & |333.6656 & 649.2974 & 325.1523 & 648.3134 & 324.6603 & 6 \\
\hline 7 & 700.3876 & 350.6974 & 683.3610 & 342.1842 & 682.3770 & 341.6921 & $\mathrm{~S}$ & 553.2399 & 277.1236 & 536.2133 & 268.6103 & 535.2293 & 268.1183 & 5 \\
\hline 8 & 787.4196 & 394.2134 & 770.3931 & |385.7002 & 769.4090 & |385.2082 & S & 466.2078 & 233.6076 & 449.1813 & 225.0943 & 448.1973 & 224.6023 & 4 \\
\hline 9 & 934.4550 & 467.7311 & 917.4285 & 459.2179 & 916.4444 & 458.7259 & M & 379.1758 & 190.0915 & 362.1493 & 181.5783 & & & 3 \\
\hline 10 & 991.4765 & 496.2419 & 974.4499 & 487.7286 & 973.4659 & 487.2366 & G & 232.1404 & 116.5738 & 215.1139 & 108.0606 & & & 2 \\
\hline 11 & & & & & & & $\mathbf{R}$ & 175.1190 & 88.0631 & 158.0924 & 79.5498 & & & 1 \\
\hline
\end{tabular}
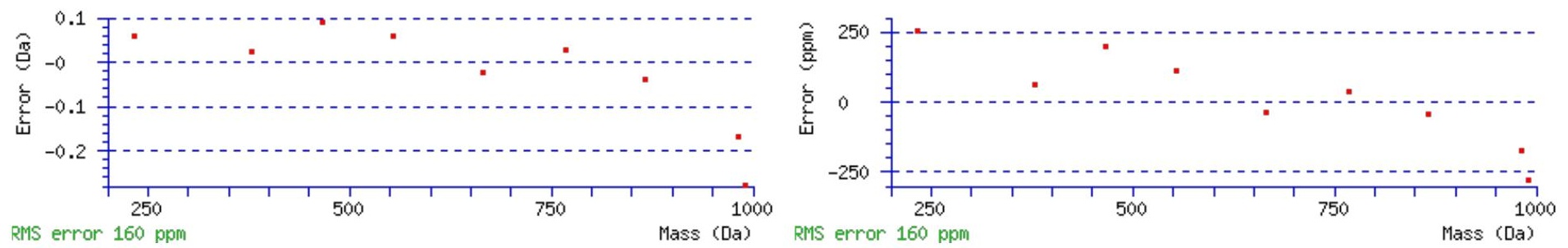

\section{All matches to this query}

\begin{tabular}{|l|l|l|l|}
\hline Score & Mr(calc): & Delta & \multicolumn{1}{c|}{ Sequence } \\
\hline 81.2 & 1164.5809 & -0.0029 & ALNVTLSSMGR \\
\hline 5.7 & 1164.5775 & 0.0005 & SPSEYKLEGR \\
\hline 5.3 & 1164.5743 & 0.0036 & NKDMKVAMGR \\
\hline 5.0 & 1164.5693 & 0.0087 & XVPGLWTGSR \\
\hline 5.0 & 1164.5693 & 0.0087 & XVPGLWTGSR \\
\hline 2.2 & 1162.5747 & 2.0032 & KTSGPPKGPSK \\
\hline 1.4 & 1164.5764 & 0.0015 & $\underline{\text { SRSQPVAKGR }}$ \\
\hline
\end{tabular}

Spectrum No: 219; Query: 1482; Rank: 1

\section{Peptide View}

MS/MS Fragmentation of LNLTAAQLSQLEGLLQAR

Found in IPI00199867, Tax_Id=10116 Gene_Symbol=Emilin1_predicted elastin microfibril interfacer 1

Match to Query 1482: 1939.078812 from(647.366880,3+)

Title: 100101RatKid_NS_deglyco_20.5386.5386.3.dta

Data file K:INewmanPaper|Piliangl3SubProteomes\Piliang3SP\mgf5ppm\ERLIC_3SubProteomes5ppm.mgf

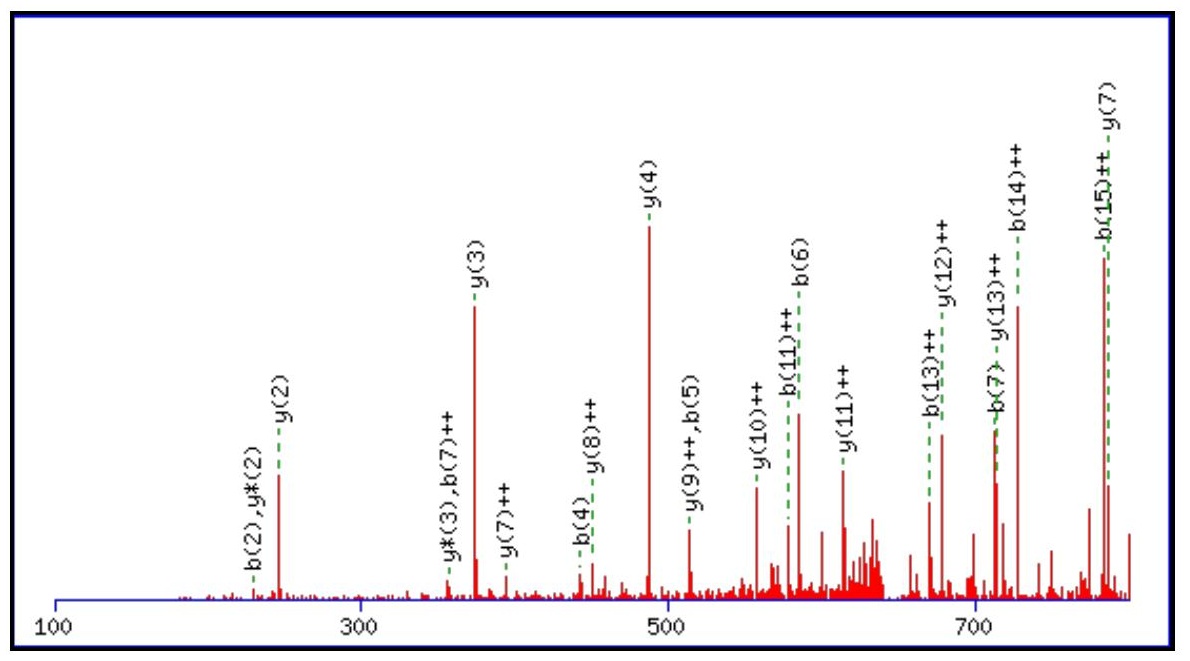



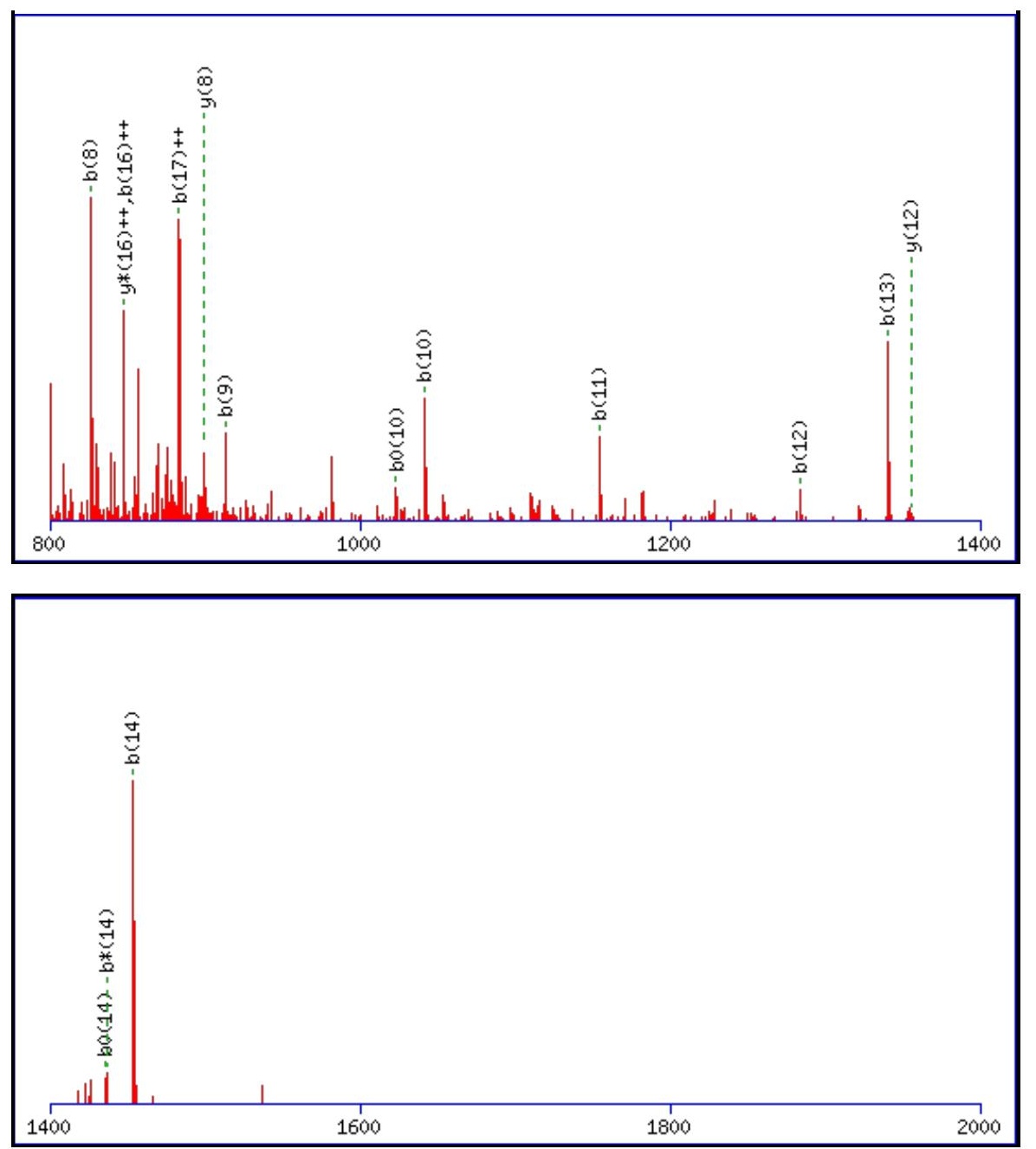

Monoisotopic mass of neutral peptide $\operatorname{Mr}($ calc): 1939.0738

Fixed modifications: Carbamidomethyl (C)

Variable modifications:

N2 : Deamidated $\mathrm{N}(\mathrm{N})$

Ions Score: 81 Expect: $3.1 \mathrm{e}-007$

Matches (Bold Red): 38/184 fragment ions using 57 most intense peaks

\begin{tabular}{|c|c|c|c|c|c|c|c|c|c|c|c|c|c|c|}
\hline \# & b & $\mathbf{b}^{++}$ & $\mathbf{b}^{*}$ & $\mathbf{b}^{*^{++}}$ & $\mathbf{b}^{0}$ & $\mathbf{b}^{\mathbf{0 + +}}$ & Seq. & $\mathbf{y}$ & $\mathbf{y}^{++}$ & $\mathbf{y}^{*}$ & $\mathbf{y}^{*^{++}}$ & $\mathbf{y}^{\mathbf{0}}$ & $\mathbf{y}^{\mathbf{0 + +}}$ & \# \\
\hline 1 & 114.0913 & 57.5493 & & & & & $\mathbf{L}$ & & & & & & & 18 \\
\hline 2 & 229.1183 & 115.0628 & 212.0917 & 106.5495 & & & $\mathbf{N}$ & 1826.9970 & 914.0022 & 1809.9705 & & 1808.9865 & 904.9969 & 17 \\
\hline 3 & 342.2023 & 171.6048 & 325.1758 & 163.0915 & & & $\mathbf{L}$ & 1711.9701 & 856.4887 & 1694.9436 & 847.9754 & 1693.9595 & 847.4834 & 16 \\
\hline 4 & 443.2500 & 222.1286 & 426.2235 & 213.6154 & 425.2395 & 213.1234 & $\mathbf{T}$ & 1598.8860 & 799.9467 & 1581.8595 & 791.4334 & 1580.8755 & 790.9414 & 15 \\
\hline 5 & 514.2871 & 257.6472 & 497.2606 & 249.1339 & 496.2766 & 248.6419 & A & 1497.8384 & 749.4228 & 1480.8118 & 740.9095 & 1479.8278 & 740.4175 & 14 \\
\hline 6 & 585.3242 & 293.1658 & 568.2977 & 284.6525 & 567.3137 & 284.1605 & A & 1426.8013 & 713.9043 & 1409.7747 & 705.3910 & 1408.7907 & 704.8990 & 13 \\
\hline 7 & 713.3828 & 357.1951 & 696.3563 & 348.6818 & 695.3723 & 348.1898 & $\mathbf{Q}$ & 1355.7641 & 678.3857 & 1338.7376 & 669.8724 & 1337.7536 & 669.3804 & 12 \\
\hline 8 & 826.4669 & 413.7371 & 809.4403 & 405.2238 & 808.4563 & 404.7318 & $\mathbf{L}$ & 1227.7056 & 614.3564 & 1210.6790 & 605.8431 & 1209.6950 & 605.3511 & 11 \\
\hline 9 & 913.4989 & 457.2531 & 896.4724 & 448.7398 & 895.4884 & 448.2478 & S & 1114.6215 & 557.8144 & 1097.5949 & 549.3011 & 1096.6109 & 548.8091 & 10 \\
\hline 10 & 1041.5575 & 521.2824 & 1024.5309 & 512.7691 & 1023.5469 & 512.2771 & $\mathbf{Q}$ & 1027.5895 & 514.2984 & 1010.5629 & 505.7851 & 1009.5789 & 505.2931 & 9 \\
\hline 11 & 1154.6416 & 577.8244 & 1137.6150 & 569.3111 & 1136.6310 & 568.8191 & $\mathbf{L}$ & 899.5309 & 450.2691 & 882.5043 & 441.7558 & 881.5203 & 441.2638 & 8 \\
\hline 12 & 1283.6842 & 642.3457 & 1266.6576 & 633.8324 & 1265.6736 & 633.3404 & $\mathbf{E}$ & 786.4468 & 393.7271 & 769.4203 & 385.2138 & 768.4363 & 384.7218 & 7 \\
\hline 13 & 1340.7056 & 670.8564 & 1323.6791 & 662.3432 & 1322.6951 & 661.8512 & $\mathbf{G}$ & 657.4042 & 329.2058 & 640.3777 & 320.6925 & & & 6 \\
\hline 14 & 1453.7897 & 727.3985 & 1436.7631 & 718.8852 & 1435.7791 & 718.3932 & $\mathbf{L}$ & 600.3828 & 300.6950 & 583.3562 & 292.1817 & & & 5 \\
\hline 15 & 1566.8737 & 783.9405 & 1549.8472 & 775.4272 & 1548.8632 & 774.9352 & $\mathbf{L}$ & 487.2987 & 244.1530 & 470.2722 & 235.6397 & & & 4 \\
\hline 16 & 1694.9323 & 847.9698 & 1677.9058 & 839.4565 & 1676.9218 & 838.9645 & $\mathbf{Q}$ & 374.2146 & 187.6110 & 357.1881 & 179.0977 & & & 3 \\
\hline 17 & 1765.9694 & 883.4884 & 1748.9429 & 874.9751 & 1747.9589 & 874.4831 & A & 246.1561 & 123.5817 & 229.1295 & 115.0684 & & & 2 \\
\hline 18 & & & & & & & $\mathbf{R}$ & 175.1190 & 88.0631 & 158.0924 & 79.5498 & & & 1 \\
\hline
\end{tabular}



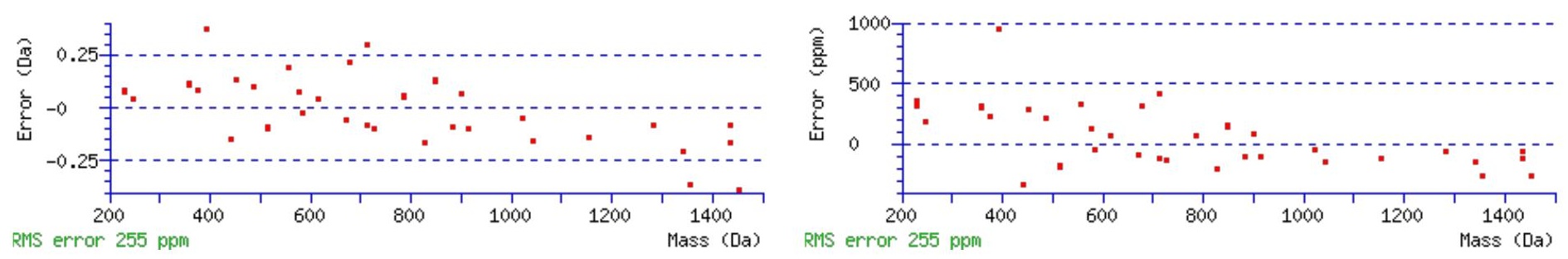

\section{All matches to this query}

\begin{tabular}{|l|l|c|l|}
\hline Score & Mr(calc): & Delta & \multicolumn{1}{|c|}{ Sequence } \\
\hline 81.1 & 1939.0738 & 0.0050 & LNLTAAQLSOLEGLLQAR \\
\hline 47.4 & 1938.0898 & 0.9890 & LNLTAAQLSQLEGLLQAR \\
\hline 8.4 & 1938.0734 & 1.0054 & WNPSLLGRPLGMLRRR \\
\hline 6.4 & 1939.0924 & -0.0136 & AQSSLKINTPPLLLRXK \\
\hline 6.1 & 1937.0894 & 1.9895 & WNPSLLGRPLGMLRRR \\
\hline 5.9 & 1938.0720 & 1.0068 & MGIAQKENINQKPVVLR \\
\hline 5.9 & 1938.0720 & 1.0068 & MGIAQKENINQKPVVLR \\
\hline 5.3 & 1938.0808 & 0.9980 & ILFIRMLHNKHVMVR \\
\hline 3.6 & 1939.0648 & 0.0140 & ILFIRMLHNKHVMVR \\
\hline 2.4 & 1939.0930 & -0.0142 & YIYPRKANGIYIINLK \\
\hline
\end{tabular}

Spectrum No: 220; Query: 1983; Rank: 1

\section{Peptide View}

MS/MS Fragmentation of NGSCGVSYIAQEPGNYEVSIK

Found in IPI00373752, Tax_Id=10116 Gene_Symbol=Flnb_predicted filamin, beta

Match to Query 1983: 2272.037728 from(1137.026140,2+)

Title: 091008RatKidney_NH4Format01_18.3482.3482.2.dta

Data file K:INewmanPaper|Piliangl3SubProteomes\Piliang3SP\mgf5ppm\ERLIC_3SubProteomes5ppm.mgf

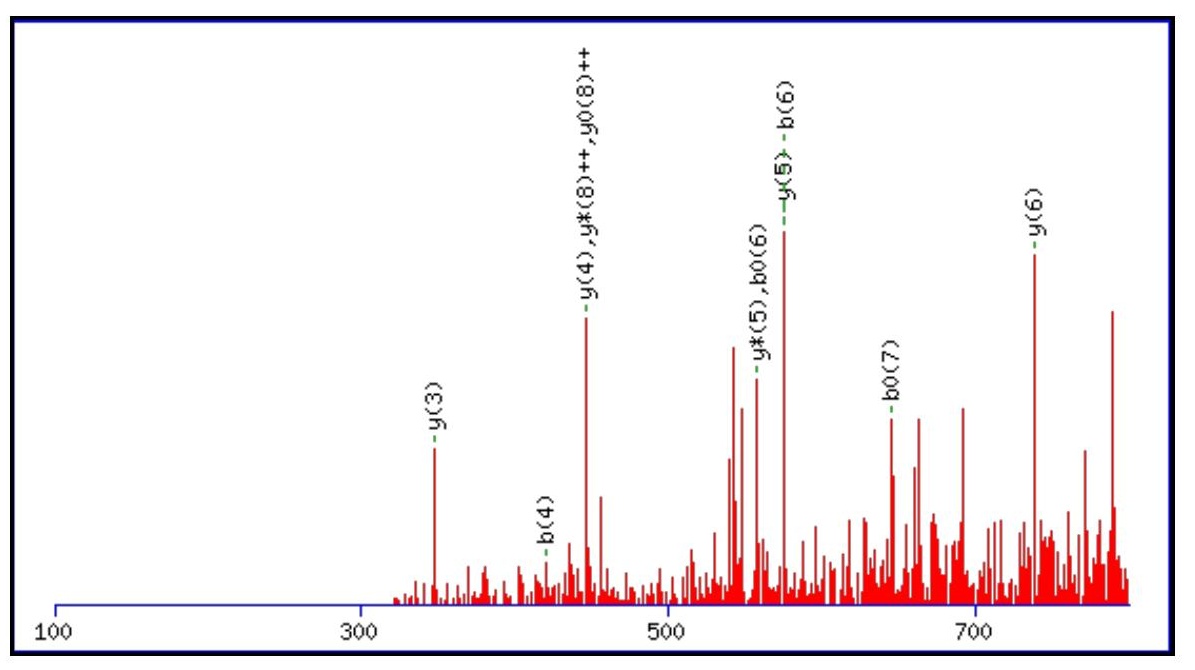



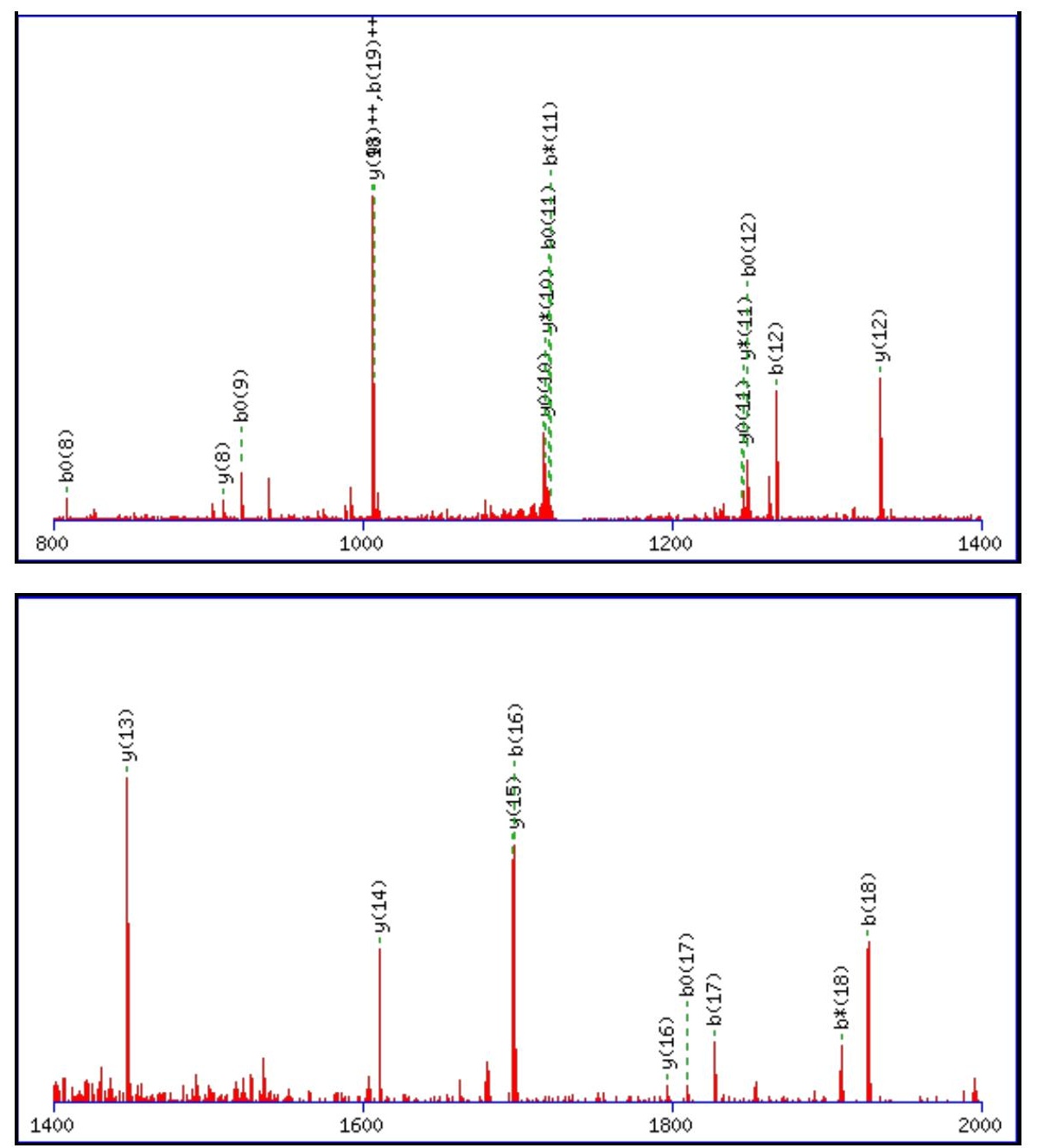

Monoisotopic mass of neutral peptide $\operatorname{Mr}($ calc): 2272.0318

Fixed modifications: Carbamidomethyl (C)

Variable modifications:

N1: Deamidated $\mathrm{N}(\mathrm{N})$

Ions Score: 81 Expect: $2.2 \mathrm{e}-006$

Matches (Bold Red): 35/232 fragment ions using 51 most intense peaks

\begin{tabular}{|c|c|c|c|c|c|c|c|c|c|c|c|c|c|c|}
\hline \# & b & $\mathbf{b}^{++}$ & $\mathbf{b}^{*}$ & $\mathbf{b}^{*^{++}}$ & $\mathbf{b}^{\mathbf{0}}$ & $\mathbf{b}^{0++}$ & Seq. & $\mathbf{y}$ & $\mathbf{y}^{++}$ & $\mathbf{y}^{*}$ & $\mathbf{y}^{*^{++}}$ & $\mathbf{y}^{\mathbf{0}}$ & $\mathbf{y}^{\mathbf{0 + +}}$ & \# \\
\hline 1 & 116.0342 & 58.5207 & 99.0077 & 50.0075 & & & $\mathbf{N}$ & & & & & & & 21 \\
\hline 2 & 173.0557 & 87.0315 & 156.0291 & 78.5182 & & & $\mathbf{G}$ & 2158.0121 & 1079.5097 & 2140.9856 & 1070.9964 & 2140.0015 & 1070.5044 & 20 \\
\hline 3 & 260.0877 & 130.5475 & 243.0612 & 122.0342 & 242.0771 & 121.5422 & $\mathbf{S}$ & 2100.9906 & 1050.9990 & 2083.9641 & 1042.4857 & 2082.9801 & 1041.9937 & 19 \\
\hline 4 & 420.1184 & 210.5628 & 403.0918 & 202.0495 & 402.1078 & 201.5575 & C & 2013.9586 & 1007.4829 & 1996.9321 & 998.9697 & 1995.9481 & 998.4777 & 18 \\
\hline 5 & 477.1398 & 239.0735 & 460.1133 & 230.5603 & 459.1293 & 230.0683 & G & 1853.9280 & 927.4676 & 1836.9014 & 918.9543 & 1835.9174 & 918.4623 & 17 \\
\hline 6 & 576.2082 & 288.6078 & 559.1817 & 280.0945 & 558.1977 & 279.6025 & $\mathbf{V}$ & 1796.9065 & 898.9569 & 1779.8800 & 890.4436 & 1778.8959 & 889.9516 & 16 \\
\hline 7 & 663.2403 & 332.1238 & 646.2137 & 323.6105 & 645.2297 & 323.1185 & $\mathbf{S}$ & 1697.8381 & 849.4227 & 1680.8115 & 840.9094 & 1679.8275 & 840.4174 & 15 \\
\hline 8 & 826.3036 & 413.6554 & 809.2770 & 405.1422 & 808.2930 & 404.6501 & $\mathbf{Y}$ & 1610.8061 & 805.9067 & 1593.7795 & 797.3934 & 1592.7955 & 796.9014 & 14 \\
\hline 9 & 939.3877 & 470.1975 & 922.3611 & 461.6842 & 921.3771 & 461.1922 & I & 1447.7427 & 724.3750 & 1430.7162 & 715.8617 & 1429.7322 & 715.3697 & 13 \\
\hline 10 & 1010.4248 & 505.7160 & 993.3982 & 497.2027 & 992.4142 & 496.7107 & A & 1334.6587 & 667.8330 & 1317.6321 & 659.3197 & 1316.6481 & 658.8277 & 12 \\
\hline 11 & 1138.4833 & 569.7453 & 1121.4568 & 561.2320 & 1120.4728 & 560.7400 & $\mathbf{Q}$ & 1263.6216 & 632.3144 & 1246.5950 & 623.8011 & 1245.6110 & 623.3091 & 11 \\
\hline 12 & 1267.5259 & 634.2666 & 1250.4994 & 625.7533 & 1249.5154 & 625.2613 & $\mathbf{E}$ & 1135.5630 & 568.2851 & 1118.5364 & 559.7719 & 1117.5524 & 559.2798 & 10 \\
\hline 13 & 1364.5787 & 682.7930 & 1347.5522 & 674.2797 & 1346.5681 & 673.7877 & $\mathbf{P}$ & 1006.5204 & 503.7638 & 989.4938 & 495.2506 & 988.5098 & 494.7585 & 9 \\
\hline 14 & 1421.6002 & 711.3037 & 1404.5736 & 702.7904 & 1403.5896 & 702.2984 & G & 909.4676 & 455.2374 & 892.4411 & 446.7242 & 891.4571 & 446.2322 & 8 \\
\hline 15 & 1535.6431 & 768.3252 & 1518.6165 & 759.8119 & 1517.6325 & 759.3199 & $\mathbf{N}$ & 852.4462 & 426.7267 & 835.4196 & 418.2134 & 834.4356 & 417.7214 & 7 \\
\hline 16 & 1698.7064 & 849.8568 & 1681.6799 & 841.3436 & 1680.6959 & 840.8516 & $\mathbf{Y}$ & 738.4032 & 369.7053 & 721.3767 & 361.1920 & 720.3927 & 360.7000 & 6 \\
\hline 17 & 1827.7490 & 914.3781 & 1810.7225 & 905.8649 & 1809.7384 & 905.3729 & $\mathbf{E}$ & 575.3399 & 288.1736 & 558.3134 & 279.6603 & 557.3293 & 279.1683 & 5 \\
\hline 18 & 1926.8174 & 963.9124 & 1909.7909 & 955.3991 & 1908.8069 & 954.9071 & V & 446.2973 & 223.6523 & 429.2708 & 215.1390 & 428.2867 & 214.6470 & 4 \\
\hline
\end{tabular}




\begin{tabular}{|r|r|r|r|r|r|r|r|r|r|r|r|r|r|r|r|r|r|}
$\mathbf{1 9}$ & 2013.8495 & $\mathbf{1 0 0 7 . 4 2 8 4}$ & 1996.8229 & 998.9151 & 1995.8389 & 998.4231 & $\mathbf{S}$ & $\mathbf{3 4 7 . 2 2 8 9}$ & 174.1181 & 330.2023 & 165.6048 & 329.2183 & 165.1128 & $\mathbf{3}$ \\
\hline $\mathbf{2 0}$ & 2126.9335 & 1063.9704 & 2109.9070 & 1055.4571 & 2108.9230 & 1054.9651 & $\mathbf{I}$ & 260.1969 & 130.6021 & 243.1703 & 122.0888 & \\
\hline $\mathbf{2 1}$ & & & & & & & $\mathbf{K}$ & 147.1128 & 74.0600 & 130.0863 & 65.5468 & & $\mathbf{2}$ \\
\hline
\end{tabular}
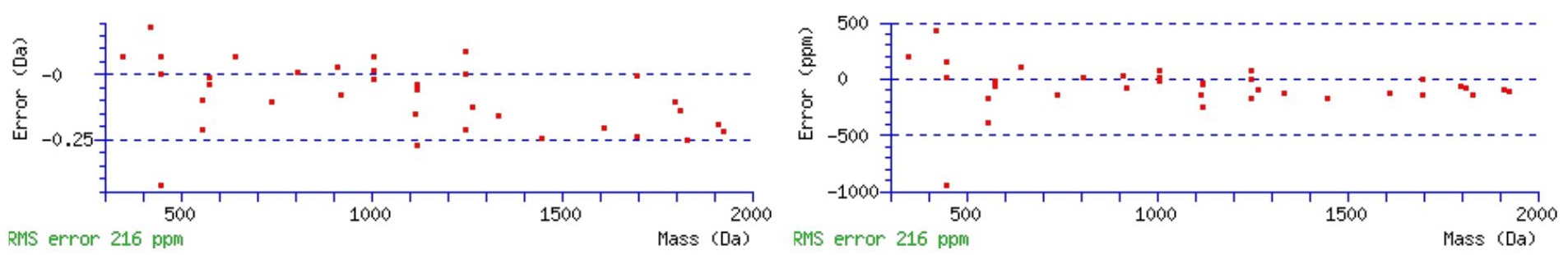

\section{All matches to this query}

\begin{tabular}{|l|l|l|l|}
\hline Score & Mr(calc): & Delta & \multicolumn{1}{|c|}{ Sequence } \\
\hline 81.0 & 2272.0318 & 0.0060 & NGSCGVSYIAQEPGNYEVSIK \\
\hline 64.3 & 2271.0477 & 0.9900 & NGSCGVSYIAQEPGNYEVSIK \\
\hline 45.2 & 2272.0318 & 0.0060 & NGSCGVSYIAQEPGNYEVSIK \\
\hline 3.5 & 2271.0186 & 1.0191 & LNSFNLIPAVGLNVYSMLK \\
\hline 3.5 & 2272.0582 & -0.0205 & ECSFWKWTTNXRSLNSNK \\
\hline 3.5 & 2272.0582 & -0.0205 & ECSFWKWTTNXRSLNSNK \\
\hline 3.2 & 2272.0419 & -0.0041 & ASXTVSLLSQSPRHPLKPK \\
\hline 1.2 & 2272.0534 & -0.0156 & RIVYFMEGRDSMQLIYR \\
\hline 0.3 & 2272.0208 & 0.0169 & QAFQIGSPWRTMDASERGR \\
\hline
\end{tabular}

Spectrum No: 221; Query: 1618; Rank: 1

\section{Peptide View}

MS/MS Fragmentation of SGPSDEGEEEMEDGTVTNGS

Found in IPI00192279, Tax_Id=10116 Gene_Symbol=- 28 kDa protein

Match to Query 1618: 2026.740048 from(1014.377300,2+)

Title: 091008RatKidney_NoSalt_29.1279.1279.2.dta

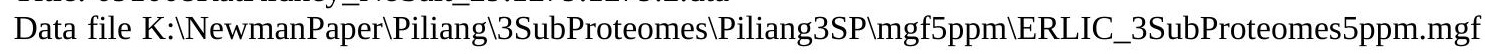

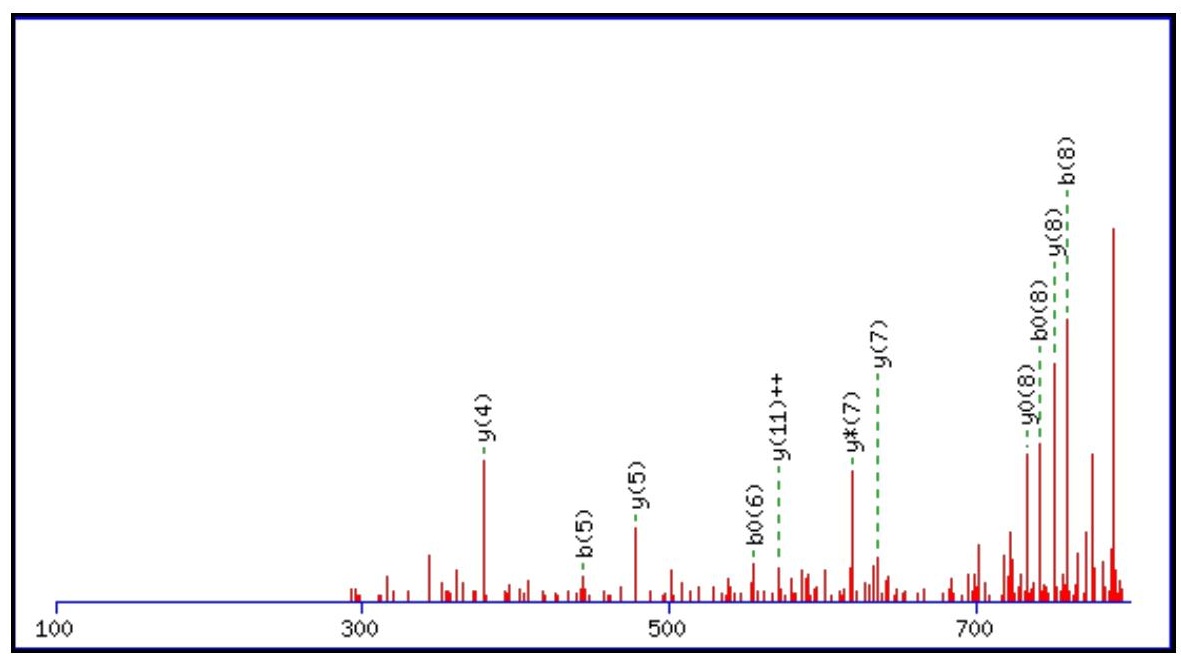



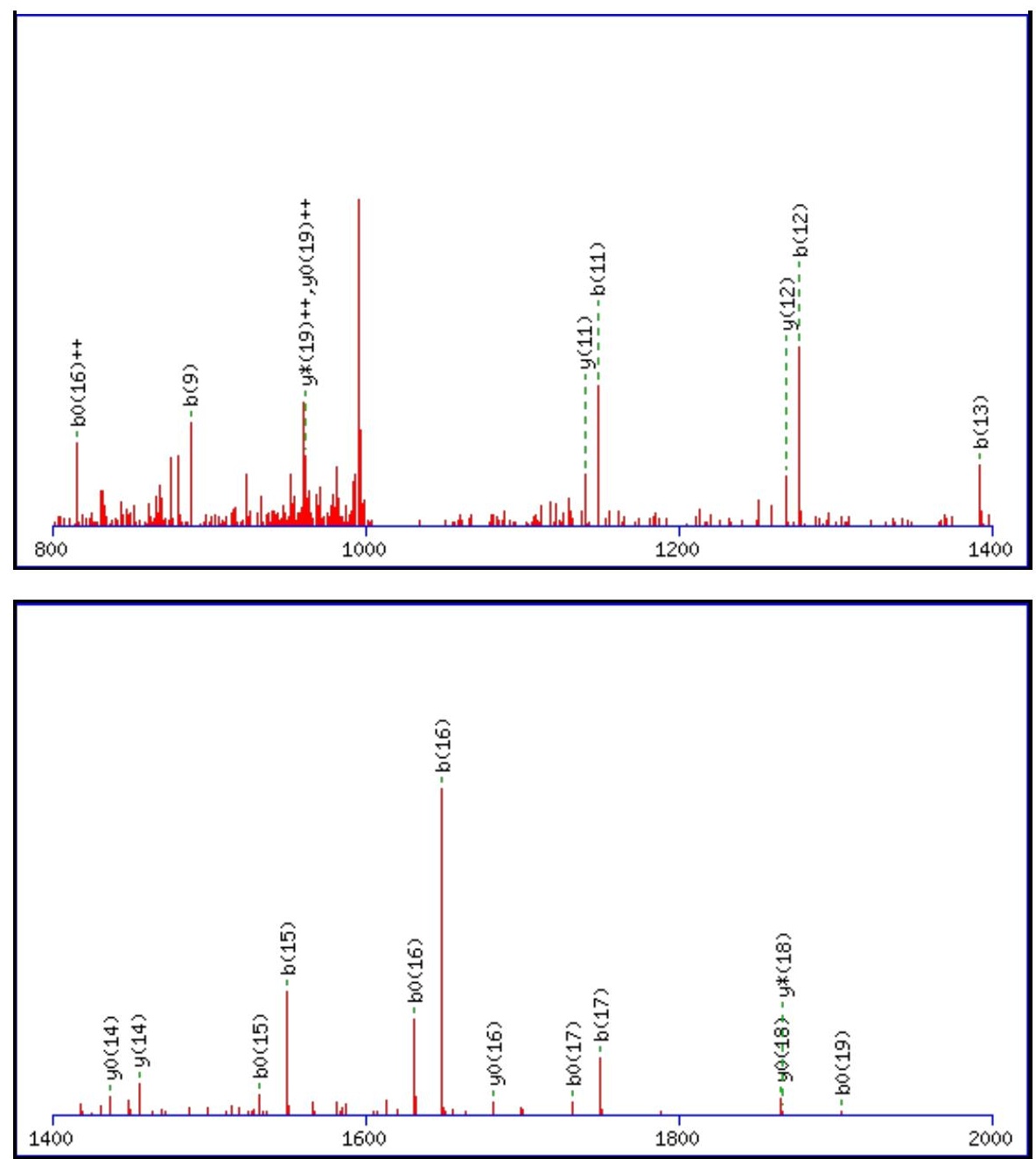

Monoisotopic mass of neutral peptide $\operatorname{Mr}($ calc): 2026.7433

Fixed modifications: Carbamidomethyl (C)

Variable modifications:

N18: Deamidated $\mathrm{N}(\mathrm{N})$

Ions Score: 81 Expect: $8.7 \mathrm{e}-007$

Matches (Bold Red): 34/190 fragment ions using 41 most intense peaks

\begin{tabular}{|c|c|c|c|c|c|c|c|c|c|c|c|c|c|c|}
\hline \# & b & $\mathbf{b}^{++}$ & $\mathbf{b}^{*}$ & $\mathbf{b}^{*^{++}}$ & $\mathbf{b}^{0}$ & $\mathbf{b}^{0++}$ & Seq. & $\mathbf{y}$ & $y^{++}$ & $\mathbf{y}^{*}$ & $\mathbf{y}^{*^{++}}$ & $\mathbf{y}^{\mathbf{0}}$ & $y^{0++}$ & \# \\
\hline 1 & 88.0393 & 44.5233 & & & 70.0287 & 35.5180 & S & & & & & & & 20 \\
\hline 2 & 145.0608 & 73.0340 & & & 127.0502 & 64.0287 & G & 1940.7186 & 970.8629 & 1923.6920 & 962.3496 & 1922.7080 & 961.8576 & 19 \\
\hline 3 & 242.1135 & 121.5604 & & & 224.1030 & 112.5551 & $\mathbf{P}$ & 1883.6971 & 942.3522 & 1866.6705 & 933.8389 & 1865.6865 & 933.3469 & 18 \\
\hline 4 & 329.1456 & 165.0764 & & & 311.1350 & 156.0711 & S & 1786.6443 & 893.8258 & 1769.6178 & 885.3125 & 1768.6338 & 884.8205 & 17 \\
\hline 5 & 444.1725 & 222.5899 & & & 426.1619 & 213.5846 & D & 1699.6123 & 850.3098 & 1682.5858 & 841.7965 & 1681.6017 & 841.3045 & 16 \\
\hline 6 & 573.2151 & 287.1112 & & & 555.2045 & 278.1059 & $\mathbf{E}$ & 1584.5854 & 792.7963 & 1567.5588 & 784.2830 & 1566.5748 & 783.7910 & 15 \\
\hline 7 & 630.2366 & 315.6219 & & & 612.2260 & 306.6166 & G & 1455.5428 & 728.2750 & 1438.5162 & 719.7617 & 1437.5322 & 719.2697 & 14 \\
\hline 8 & 759.2792 & 380.1432 & & & 741.2686 & 371.1379 & $\mathbf{E}$ & 1398.5213 & 699.7643 & 1381.4948 & 691.2510 & 1380.5107 & 690.7590 & 13 \\
\hline 9 & 888.3217 & 444.6645 & & & 870.3112 & 435.6592 & $\mathbf{E}$ & 1269.4787 & 635.2430 & 1252.4522 & 626.7297 & 1251.4681 & 626.2377 & 12 \\
\hline 10 & 1017.3643 & 509.1858 & & & 999.3538 & 500.1805 & $\mathbf{E}$ & 1140.4361 & 570.7217 & 1123.4096 & 562.2084 & 1122.4256 & 561.7164 & 11 \\
\hline 11 & 1148.4048 & 574.7061 & & & 1130.3943 & 565.7008 & $\mathbf{M}$ & 1011.3935 & 506.2004 & 994.3670 & 497.6871 & 993.3830 & 497.1951 & 10 \\
\hline 12 & 1277.4474 & 639.2273 & & & 1259.4369 & 630.2221 & $\mathbf{E}$ & 880.3530 & 440.6802 & 863.3265 & 432.1669 & 862.3425 & 431.6749 & 9 \\
\hline 13 & 1392.4744 & 696.7408 & & & 1374.4638 & 687.7355 & D & 751.3104 & 376.1589 & 734.2839 & 367.6456 & 733.2999 & 367.1536 & 8 \\
\hline 14 & 1449.4958 & 725.2516 & & & 1431.4853 & 716.2463 & G & 636.2835 & 318.6454 & 619.2570 & 310.1321 & 618.2729 & 309.6401 & 7 \\
\hline 15 & 1550.5435 & 775.7754 & & & 1532.5329 & 766.7701 & $\mathbf{T}$ & 579.2620 & 290.1347 & 562.2355 & 281.6214 & 561.2515 & 281.1294 & 6 \\
\hline 16 & 1649.6119 & 825.3096 & & & 1631.6014 & 816.3043 & $\mathbf{V}$ & 478.2144 & 239.6108 & 461.1878 & 231.0975 & 460.2038 & 230.6055 & 5 \\
\hline 17 & 1750.6596 & 875.8334 & & & 1732.6490 & 866.8282 & $\mathbf{T}$ & 379.1459 & 190.0766 & 362.1194 & 181.5633 & 361.1354 & 181.0713 & 4 \\
\hline 18 & 1865.6865 & 933.3469 & 1848.6600 & 924.8336 & 1847.6760 & 924.3416 & $\mathbf{N}$ & 278.0983 & 139.5528 & 261.0717 & 131.0395 & 260.0877 & 130.5475 & 3 \\
\hline
\end{tabular}




\begin{tabular}{|r|r|r|r|r|r|r|r|r|r|r|r|r|r|r|}
$\mathbf{1 9}$ & 1922.7080 & $\mathbf{9 6 1 . 8 5 7 6}$ & 1905.6814 & 953.3444 & $\mathbf{1 9 0 4 . 6 9 7 4}$ & 952.8524 & $\mathbf{G}$ & 163.0713 & $\mathbf{8 2 . 0 3 9 3}$ & & & 145.0608 & $\mathbf{7 3 . 0 3 4 0}$ & $\mathbf{2}$ \\
\hline $\mathbf{2 0}$ & & & & & & & S & 106.0499 & 53.5286 & & & 88.0393 & $\mathbf{4 4 . 5 2 3 3}$ & $\mathbf{1}$ \\
\hline
\end{tabular}
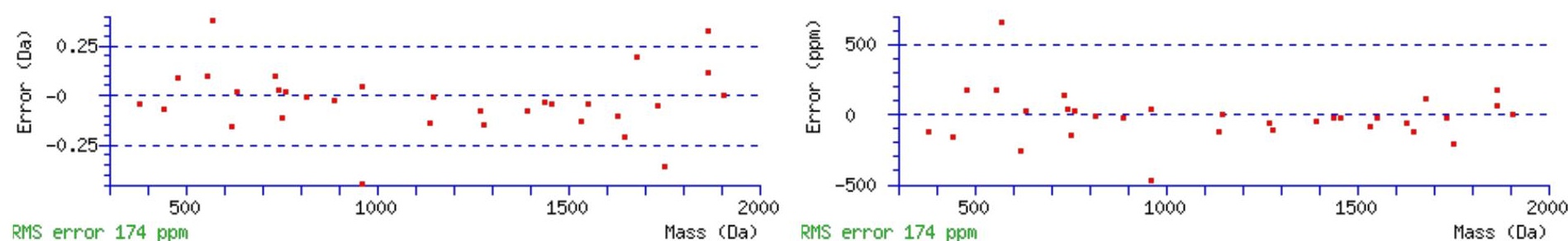

RMS error 174 ppm

\section{All matches to this query}

\begin{tabular}{|l|c|c|c|}
\hline Score & Mr(calc): & Delta & \multicolumn{1}{c|}{ Sequence } \\
\hline 80.9 & 2026.7433 & -0.0033 & SGPSDEGEEEMEDGTVTNGS \\
\hline 1.7 & 2026.7575 & -0.0174 & DGSGQVPSTAESSPIVEK \\
\hline
\end{tabular}

Spectrum No: 222; Query: 2058; Rank: 1

\section{Peptide View}

MS/MS Fragmentation of TDTTCLNNTEISENGSDLSQK

Found in IPI00372146, Tax_Id=10116 Gene_Symbol=Rai14 Isoform 2 of Ankycorbin

Match to Query 2058: 2327.017968 from(1164.516260,2+)

Title: 100101RatKid_NS_deglyco_08.2154.2154.2.dta

Data file K:\NewmanPaper\Piliang \3SubProteomes\Piliang3SP \mgf5ppm\ERLIC_3SubProteomes5ppm.mgf

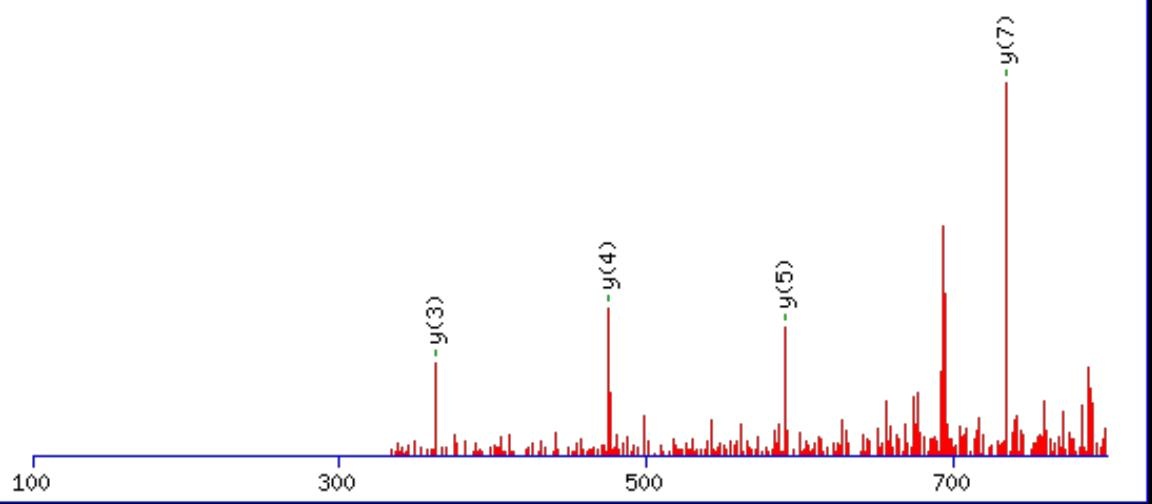



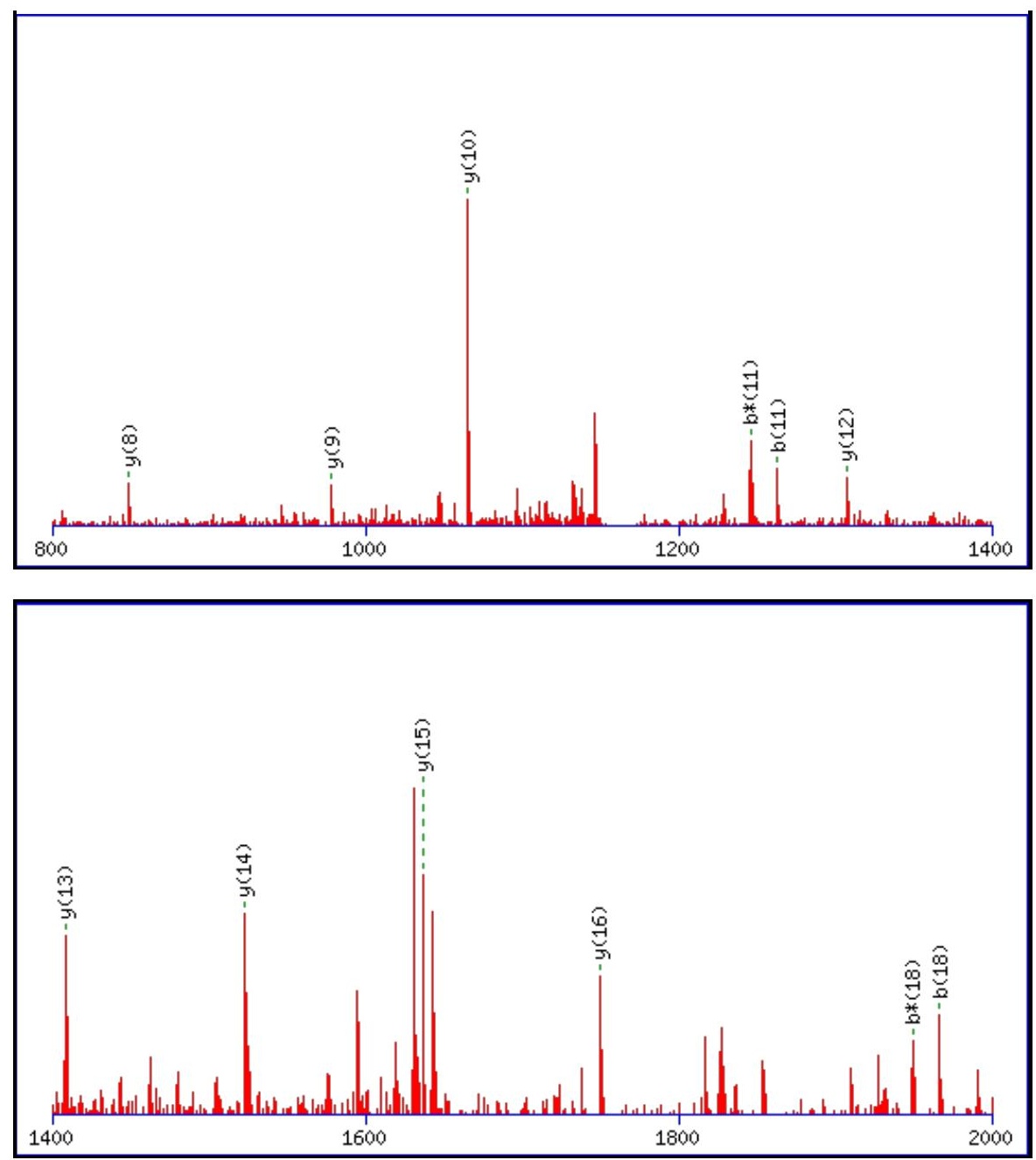

Monoisotopic mass of neutral peptide $\operatorname{Mr}($ calc): 2327.0071

Fixed modifications: Carbamidomethyl (C)

Variable modifications:

N14: Deamidated $\mathrm{N}(\mathrm{N})$

Ions Score: 81 Expect: $2.4 \mathrm{e}-006$

Matches (Bold Red): 16/224 fragment ions using 27 most intense peaks

\begin{tabular}{|c|c|c|c|c|c|c|c|c|c|c|c|c|c|c|}
\hline \# & b & $\mathbf{b}^{++}$ & $\mathbf{b}^{*}$ & $\mathbf{b}^{*^{++}}$ & $\mathbf{b}^{0}$ & $\mathbf{b}^{0++}$ & Seq. & $\mathbf{y}$ & $y^{++}$ & $\mathbf{y}^{*}$ & $\mathbf{y}^{*^{++}}$ & $\mathbf{y}^{0}$ & $\mathbf{y}^{0++}$ & \# \\
\hline 1 & 102.0550 & 51.5311 & & & 84.0444 & 42.5258 & $\mathbf{T}$ & & & & & & & 21 \\
\hline 2 & 217.0819 & 109.0446 & & & 199.0713 & 100.0393 & D & 2226.9667 & 9870 & 2209.9401 & 1105.4737 & 2208.9561 & 1104.9817 & 20 \\
\hline 3 & 318.1296 & 159.5684 & & & 300.1190 & 150.5631 & $\mathbf{T}$ & 2111.9397 & 1056.4735 & 2094.9132 & 1047.9602 & 2093.9292 & 1047.4682 & 19 \\
\hline 4 & 419.1773 & 210.0923 & & & 401.1667 & 201.0870 & $\mathbf{T}$ & 2010.8920 & 1005.9497 & 1993.8655 & 997.4364 & 1992.8815 & 996.9444 & 18 \\
\hline 5 & 579.2079 & 290.1076 & & & 561.1973 & 281.1023 & C & 1909.8444 & 955.4258 & 1892.8178 & 946.9125 & 1891.8338 & 946.4205 & 17 \\
\hline 6 & 692.2920 & 346.6496 & & & 674.2814 & 337.6443 & $\mathbf{L}$ & 1749.8137 & 875.4105 & 1732.7872 & 866.8972 & 1731.8032 & 866.4052 & 16 \\
\hline 7 & 806.3349 & 403.6711 & 789.3083 & 395.1578 & 788.3243 & 394.6658 & $\mathbf{N}$ & 1636.7297 & 818.8685 & 1619.7031 & 810.3552 & 1618.7191 & 809.8632 & 15 \\
\hline 8 & 920.3778 & 460.6925 & 903.3513 & 452.1793 & 902.3673 & 451.6873 & $\mathbf{N}$ & 1522.6867 & 761.8470 & 1505.6602 & 753.3337 & 1504.6762 & 752.8417 & 14 \\
\hline 9 & 1021.4255 & 511.2164 & 1004.3990 & 502.7031 & 1003.4149 & 502.2111 & $\mathbf{T}$ & 1408.6438 & 704.8255 & 1391.6173 & 696.3123 & 1390.6332 & 695.8203 & 13 \\
\hline 10 & 1150.4681 & 575.7377 & 1133.4415 & 567.2244 & 1132.4575 & 566.7324 & $\mathbf{E}$ & 1307.5961 & 654.3017 & 1290.5696 & 645.7884 & 1289.5856 & 645.2964 & 12 \\
\hline 11 & 1263.5522 & 632.2797 & 1246.5256 & 623.7664 & 1245.5416 & 623.2744 & I & 1178.5535 & 589.7804 & 1161.5270 & 581.2671 & 1160.5430 & 580.7751 & 11 \\
\hline 12 & 1350.5842 & 675.7957 & 1333.5576 & 667.2825 & 1332.5736 & 666.7904 & $\mathrm{~S}$ & 1065.4695 & 533.2384 & 1048.4429 & 524.7251 & 1047.4589 & 524.2331 & 10 \\
\hline 13 & 1479.6268 & 740.3170 & 1462.6002 & 731.8038 & 1461.6162 & 731.3117 & $\mathbf{E}$ & 978.4374 & 489.7224 & 961.4109 & 481.2091 & 960.4269 & 480.7171 & 9 \\
\hline 14 & 1594.6537 & 797.8305 & 1577.6272 & 789.3172 & 1576.6432 & 788.8252 & $\mathbf{N}$ & 849.3948 & 425.2011 & 832.3683 & 416.6878 & 831.3843 & 416.1958 & 8 \\
\hline 15 & 1651.6752 & 826.3412 & 1634.6486 & 817.8280 & 1633.6646 & 817.3359 & G & 734.3679 & 367.6876 & 717.3414 & 359.1743 & 716.3573 & 358.6823 & 7 \\
\hline 16 & 1738.7072 & 869.8572 & 1721.6807 & 861.3440 & 1720.6966 & 860.8520 & $S$ & 677.3464 & 339.1769 & 660.3199 & 330.6636 & 659.3359 & 330.1716 & 6 \\
\hline 17 & 1853.7342 & 927.3707 & 1836.7076 & 918.8574 & 1835.7236 & 918.3654 & D & 590.3144 & 295.6608 & 573.2879 & 287.1476 & 572.3039 & 286.6556 & 5 \\
\hline 18 & 1966.8182 & 983.9127 & 1949.7917 & 975.3995 & 1948.8076 & 974.9075 & $\mathbf{L}$ & 475.2875 & 238.1474 & 458.2609 & 229.6341 & 457.2769 & 229.1421 & 4 \\
\hline
\end{tabular}




\begin{tabular}{|r|r|r|r|r|r|r|r|r|r|r|r|r|r|r|r|r|r|r|r|r|r|r|r|}
$\mathbf{1 9}$ & 2053.8502 & 1027.4288 & 2036.8237 & 1018.9155 & 2035.8397 & 1018.4235 & $\mathbf{S}$ & 362.2034 & 181.6053 & 345.1769 & 173.0921 & 344.1928 & 172.6001 & $\mathbf{3}$ \\
\hline $\mathbf{2 0}$ & 2181.9088 & 1091.4580 & 2164.8823 & 1082.9448 & 2163.8983 & 1082.4528 & $\mathbf{Q}$ & 275.1714 & 138.0893 & 258.1448 & 129.5761 & \\
\hline $\mathbf{2 1}$ & & & & & & & $\mathbf{K}$ & 147.1128 & 74.0600 & 130.0863 & 65.5468 & & \\
\hline
\end{tabular}
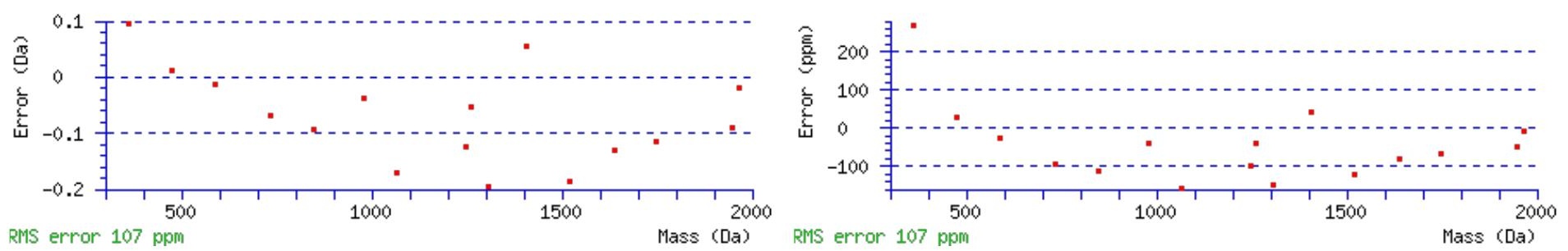

All matches to this query

\begin{tabular}{|l|l|l|l|}
\hline Score & Mr(calc): & Delta & \multicolumn{1}{|c|}{ Sequence } \\
\hline 80.9 & 2327.0071 & 0.0109 & TDTTCLNNTEISENGSDLSQK \\
\hline 30.2 & 2327.0071 & 0.0109 & TDTTCLNNTEISENGSDLSQK \\
\hline 22.6 & 2327.0071 & 0.0109 & TDTTCLNNTEISENGSDLSQK \\
\hline 7.5 & 2326.0231 & 0.9949 & TDTTCLNNTEISENGSDLSQK \\
\hline 2.2 & 2327.0222 & -0.0042 & SYLSLEVLDLSSNNITEIR \\
\hline 2.2 & 2327.0222 & -0.0042 & SYLSLEVLDLSSNNITEIR \\
\hline 2.2 & 2327.0222 & -0.0042 & SYLSLEVLDLSSNNITEIR \\
\hline 0.5 & 2326.0337 & 0.9843 & GWRNHSFVTEFILLGFSR \\
\hline 0.3 & 2327.0340 & -0.0161 & VVYPNNSMCHQDWKKVSR \\
\hline
\end{tabular}

Spectrum No: 223; Query: 1066; Rank: 1

\section{Peptide View}

MS/MS Fragmentation of DNATEEEILHYLEK

Found in IPI00195160, Tax_Id=10116 Gene_Symbol=Psap Sulfated glycoprotein 1 precursor

Match to Query 1066: 1703.789948 from(852.902250,2+)

Title: 091008RatKidney_NH4Format02_25.4379.4379.2.dta

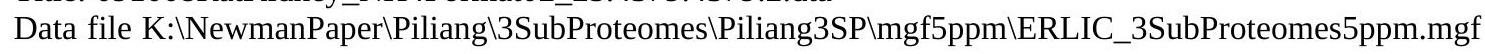

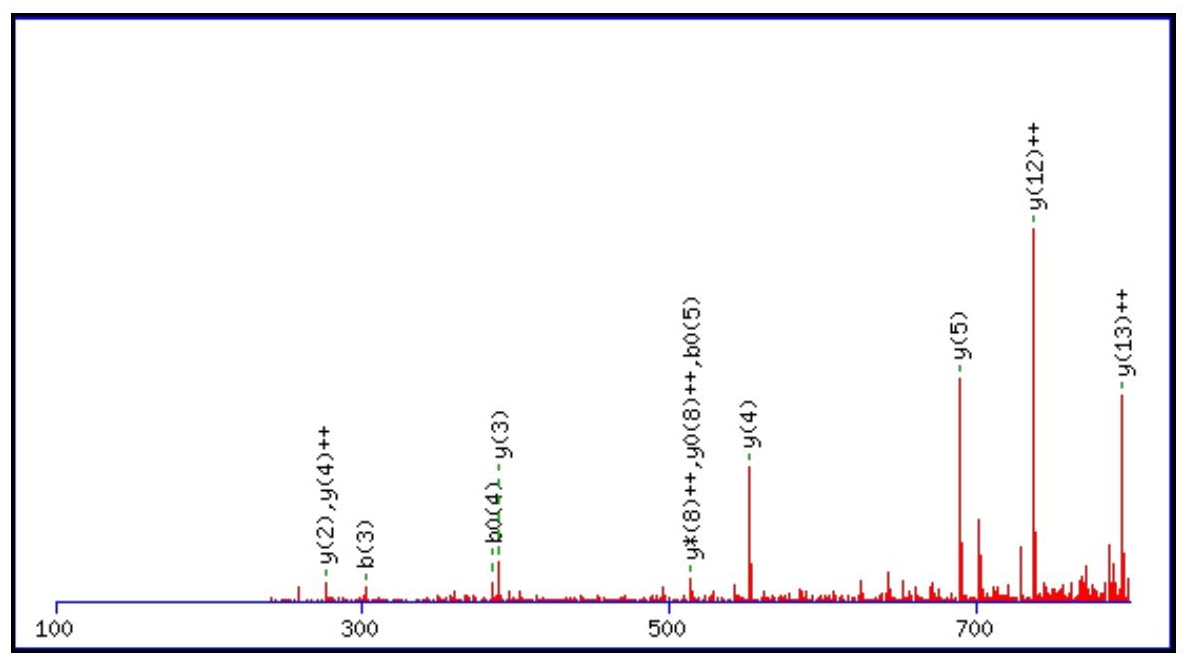



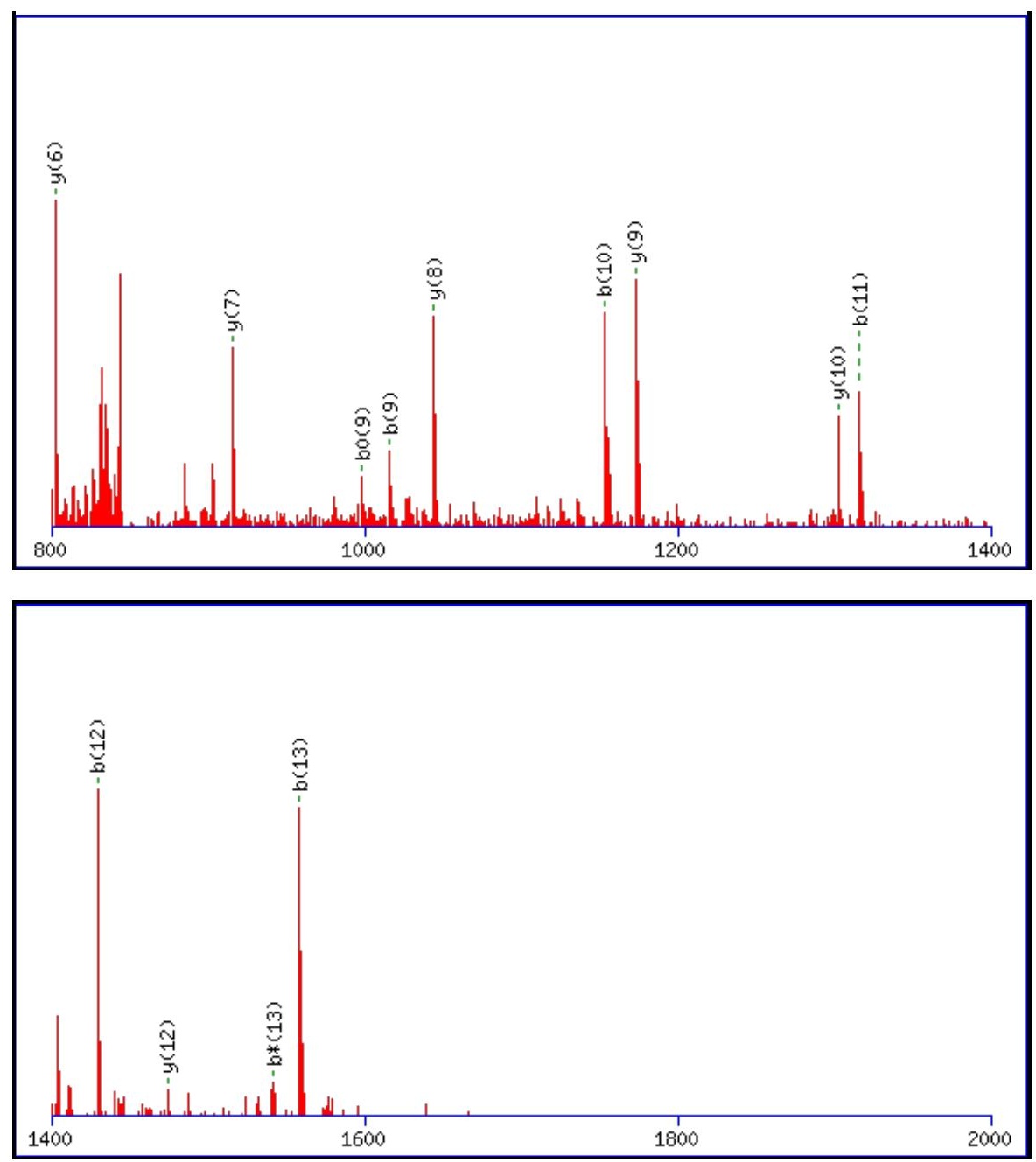

Monoisotopic mass of neutral peptide $\operatorname{Mr}($ calc): 1703.7890

Fixed modifications: Carbamidomethyl (C)

Variable modifications:

N2 : Deamidated $\mathrm{N}(\mathrm{N})$

Ions Score: 81 Expect: $1.6 \mathrm{e}-006$

Matches (Bold Red): 25/152 fragment ions using 44 most intense peaks

\begin{tabular}{|c|c|c|c|c|c|c|c|c|c|c|c|c|c|c|}
\hline \# & b & $\mathbf{b}^{++}$ & $\mathbf{b}^{*}$ & $\mathbf{b}^{*^{++}}$ & $\mathbf{b}^{0}$ & $\mathbf{b}^{\mathbf{0 + +}}$ & Seq. & $\mathbf{y}$ & $\mathbf{y}^{++}$ & $\mathbf{y}^{*}$ & $\mathbf{y}^{*^{++}}$ & $\mathbf{y}^{\mathbf{0}}$ & $\mathbf{y}^{\mathbf{0 + +}}$ & \# \\
\hline 1 & 116.0342 & 58.5207 & & & 98.0237 & 49.5155 & D & & & & & & & 14 \\
\hline 2 & 231.0612 & 116.0342 & 214.0346 & 107.5209 & 213.0506 & 107.0289 & $\mathbf{N}$ & 1589.7693 & 795.3883 & 1572.7428 & 786.8750 & 1571.7588 & 786.3830 & 13 \\
\hline 3 & 302.0983 & 151.5528 & 285.0717 & 143.0395 & 284.0877 & 142.5475 & A & 1474.7424 & 737.8748 & 1457.7159 & 729.3616 & 1456.7318 & 728.8696 & 12 \\
\hline 4 & 403.1459 & 202.0766 & 386.1194 & 193.5633 & 385.1354 & 193.0713 & $\mathbf{T}$ & 1403.7053 & 702.3563 & 1386.6787 & 693.8430 & 1385.6947 & 693.3510 & 11 \\
\hline 5 & 532.1885 & 266.5979 & 515.1620 & 258.0846 & 514.1780 & 257.5926 & $\mathbf{E}$ & 1302.6576 & 651.8324 & 1285.6311 & 643.3192 & 1284.6470 & 642.8272 & 10 \\
\hline 6 & 661.2311 & 331.1192 & 644.2046 & 322.6059 & 643.2206 & 322.1139 & $\mathbf{E}$ & 1173.6150 & 587.3111 & 1156.5885 & 578.7979 & 1155.6045 & 578.3059 & 9 \\
\hline 7 & 790.2737 & 395.6405 & 773.2472 & 387.1272 & 772.2632 & 386.6352 & $\mathbf{E}$ & 1044.5724 & 522.7898 & 1027.5459 & 514.2766 & 1026.5619 & 513.7846 & 8 \\
\hline 8 & 903.3578 & 452.1825 & 886.3312 & 443.6693 & 885.3472 & 443.1773 & I & 915.5298 & 458.2686 & 898.5033 & 449.7553 & 897.5193 & 449.2633 & 7 \\
\hline 9 & 1016.4419 & 508.7246 & 999.4153 & 500.2113 & 998.4313 & 499.7193 & $\mathbf{L}$ & 802.4458 & 401.7265 & 785.4192 & 393.2132 & 784.4352 & 392.7212 & 6 \\
\hline 10 & 1153.5008 & 577.2540 & 1136.4742 & 568.7407 & 1135.4902 & 568.2487 & $\mathbf{H}$ & 689.3617 & 345.1845 & 672.3352 & 336.6712 & 671.3511 & 336.1792 & 5 \\
\hline 11 & 1316.5641 & 658.7857 & 1299.5375 & 650.2724 & 1298.5535 & 649.7804 & $\mathbf{Y}$ & 552.3028 & 276.6550 & 535.2762 & 268.1418 & 534.2922 & 267.6498 & 4 \\
\hline 12 & 1429.6482 & 715.3277 & 1412.6216 & 706.8144 & 1411.6376 & 706.3224 & $\mathbf{L}$ & 389.2395 & 195.1234 & 372.2129 & 186.6101 & 371.2289 & 186.1181 & 3 \\
\hline 13 & 1558.6908 & 779.8490 & 1541.6642 & 771.3357 & 1540.6802 & 770.8437 & $\mathbf{E}$ & 276.1554 & 138.5813 & 259.1288 & 130.0681 & 258.1448 & 129.5761 & 2 \\
\hline 14 & & & & & & & $\mathbf{K}$ & 147.1128 & 74.0600 & 130.0863 & 65.5468 & & & 1 \\
\hline
\end{tabular}



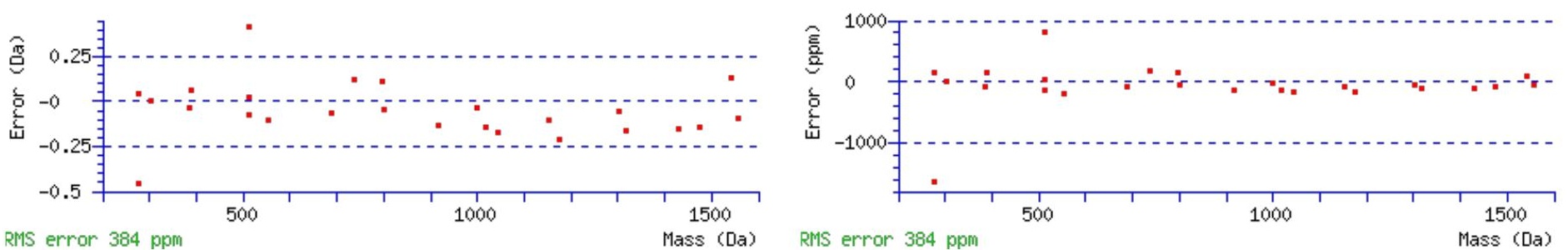

\section{All matches to this query}

\begin{tabular}{|l|l|l|l|}
\hline Score & Mr(calc): & Delta & \multicolumn{1}{c|}{ Sequence } \\
\hline 80.9 & 1703.7890 & 0.0010 & DNATEEEILHYLEK \\
\hline 14.1 & 1703.8011 & -0.0111 & YIVRNTMNCYLEK \\
\hline 14.1 & 1703.8011 & -0.0111 & YIVRNTMNCYLEK \\
\hline 10.1 & 1703.7987 & -0.0088 & KTSVADSMLRMLEK \\
\hline 10.1 & 1703.7987 & -0.0088 & KTSVADSMLRMLEK \\
\hline 5.6 & 1703.7987 & -0.0088 & $\underline{\text { KTSVADSMLRMLEK }}$ \\
\hline 5.6 & 1703.7987 & -0.0088 & KTSVADSMLRMLEK \\
\hline 5.0 & 1703.7896 & 0.0003 & $\underline{\text { SLVKSAEGSTVPLTR }}$ \\
\hline 5.0 & 1703.7896 & 0.0003 & SLVKSAEGSTVPLTR \\
\hline 5.0 & 1703.7896 & 0.0003 & $\underline{\text { SLVKSAEGSTVPLTR }}$ \\
\hline
\end{tabular}

Spectrum No: 224; Query: 2105; Rank: 1

\section{Peptide View}

MS/MS Fragmentation of FVLLMTNCYATPSSNSTDPVK

Found in IPI00198021, Tax_Id=10116 Gene_Symbol=Umod Uromodulin precursor

Match to Query 2105: 2361.094468 from(1181.554510,2+)

Title: 100101RatKid_NS_deglyco_27.2959.2959.2.dta

Data file K:INewmanPaper|Piliangl3SubProteomes\Piliang3SP\mgf5ppm\ERLIC_3SubProteomes5ppm.mgf

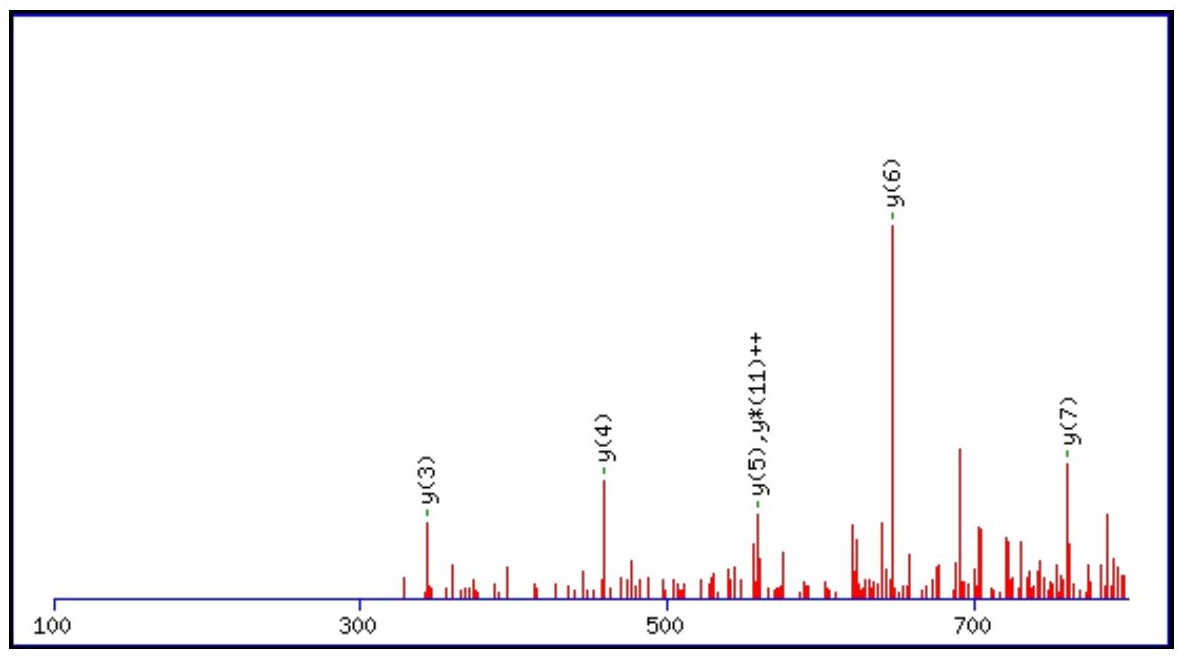



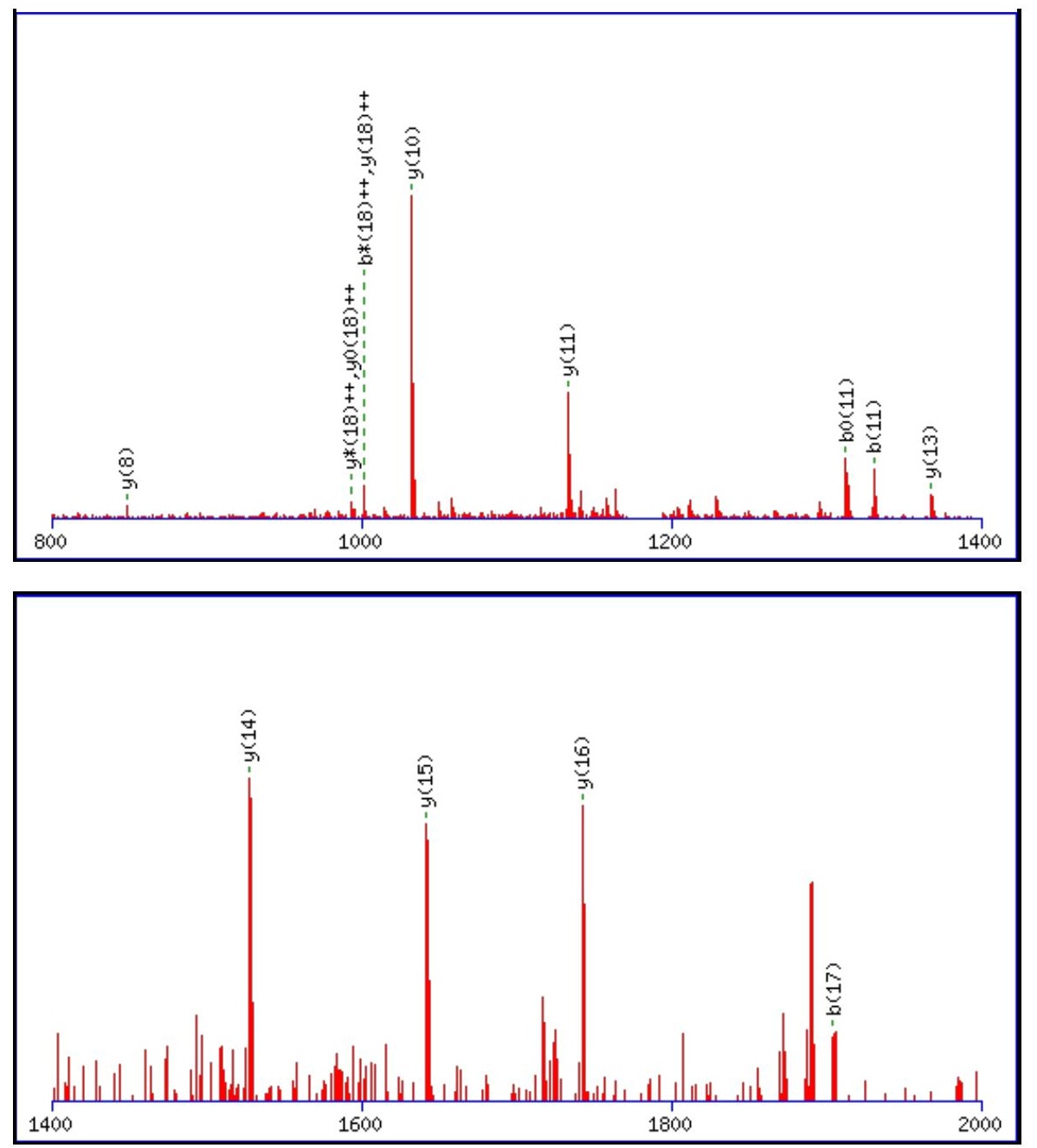

Monoisotopic mass of neutral peptide $\operatorname{Mr}($ calc): 2361.0869

Fixed modifications: Carbamidomethyl (C)

Variable modifications:

M5 : Oxidation (M)

N15 : Deamidated_N (N)

Ions Score: 81 Expect: $2.7 \mathrm{e}-006$

Matches (Bold Red): 20/212 fragment ions using 30 most intense peaks

\begin{tabular}{|c|c|c|c|c|c|c|c|c|c|c|c|c|c|c|}
\hline \# & b & $\mathbf{b}^{++}$ & $\mathbf{b}^{*}$ & $\mathbf{b}^{*^{++}}$ & $\mathbf{b}^{\mathbf{0}}$ & & Seq. & $\mathbf{y}$ & $\mathbf{y}^{++}$ & $\mathbf{y}^{*}$ & $\mathbf{y}^{*^{++}}$ & $\mathbf{y}^{0}$ & $\mathbf{y}^{0}$ & \# \\
\hline 1 & 148.0757 & 74.5415 & & & & & $\mathbf{F}$ & & & & & & & 21 \\
\hline 2 & 247.1441 & 124.0757 & & & & & $\mathbf{V}$ & & & & & 2197.0151 & & 20 \\
\hline 3 & 360.2282 & 180.6177 & & & & & $\mathbf{L}$ & 2115.9573 & 1058.4823 & 2098.9307 & 1049.9690 & 2097.9467 & 1049.4770 & 19 \\
\hline 4 & 473.3122 & 237.1598 & & & & & $\mathbf{L}$ & 2002.8732 & 1001.9403 & 1985.8467 & & 1984.8627 & 992.9350 & 18 \\
\hline 5 & 620.3476 & 310.6775 & & & & & $\mathbf{M}$ & 1889.7892 & 945.3982 & 1872.7626 & 936.8849 & 1871.7786 & 936.3929 & 17 \\
\hline 6 & 721.3953 & 361.2013 & & & & & $\mathbf{T}$ & 1742.7538 & 871.8805 & 1725.7272 & 863.3672 & 1724.7432 & 862.8752 & 16 \\
\hline 7 & 835.4382 & 418.2228 & 818.4117 & 409.7095 & 817.4277 & 409.2175 & $\mathbf{N}$ & 1641.7061 & 821.3567 & 1624.6795 & 812.8434 & 1623.6955 & 812.3514 & 15 \\
\hline 8 & 995.4689 & 498.2381 & 978.4423 & 489.7248 & 977.4583 & 489.2328 & C & 1527.6632 & 764.3352 & 1510.6366 & 755.8219 & 1509.6526 & 755.3299 & 14 \\
\hline 9 & 1158.5322 & 579.7697 & 1141.5057 & 571.2565 & 1140.5217 & 570.7645 & $\mathbf{Y}$ & 1367.6325 & 684.3199 & 1350.6060 & 675.8066 & 1349.6219 & 675.3146 & 13 \\
\hline 10 & 1229.5693 & 615.2883 & 1212.5428 & 606.7750 & 1211.5588 & 606.2830 & A & 1204.5692 & 602.7882 & 1187.5426 & 594.2750 & 1186.5586 & 593.7829 & 12 \\
\hline 11 & 1330.6170 & 665.8121 & 1313.5905 & 657.2989 & 1312.6064 & 656.8069 & $\mathbf{T}$ & 1133.5321 & 567.2697 & 1116.5055 & 558.7564 & 1115.5215 & 558.2644 & 11 \\
\hline 12 & 1427.6698 & 714.3385 & 1410.6432 & 705.8253 & 1409.6592 & 705.3332 & $\mathbf{P}$ & 1032.4844 & 516.7458 & 1015.4578 & 508.2326 & 1014.4738 & 507.7405 & 10 \\
\hline 13 & 1514.7018 & 757.8545 & 1497.6753 & 749.3413 & 1496.6912 & 748.8493 & $\mathrm{~S}$ & 935.4316 & 468.2195 & 918.4051 & 459.7062 & 917.4211 & 459.2142 & 9 \\
\hline 14 & 1601.7338 & 801.3706 & 1584.7073 & 792.8573 & 1583.7233 & 792.3653 & $S$ & 848.3996 & 424.7034 & 831.3730 & 416.1902 & 830.3890 & 415.6982 & 8 \\
\hline 15 & 1716.7608 & 858.8840 & 1699.7342 & 850.3707 & 1698.7502 & 849.8787 & $\mathbf{N}$ & 761.3676 & 381.1874 & 744.3410 & 372.6741 & 743.3570 & 372.1821 & 7 \\
\hline 16 & 1803.7928 & 902.4000 & 1786.7662 & 893.8868 & 1785.7822 & 893.3948 & S & 646.3406 & 323.6740 & 629.3141 & 315.1607 & 628.3301 & 314.6687 & 6 \\
\hline 17 & 1904.8405 & 952.9239 & 1887.8139 & 944.4106 & 1886.8299 & 943.9186 & $\mathbf{T}$ & 559.3086 & 280.1579 & 542.2821 & 271.6447 & 541.2980 & 271.1527 & 5 \\
\hline 18 & 2019.8674 & 1010.4373 & 2002.8409 & 1001.9241 & 2001.8569 & 1001.4321 & D & 458.2609 & 229.6341 & 441.2344 & 221.1208 & 440.2504 & 220.6288 & 4 \\
\hline
\end{tabular}




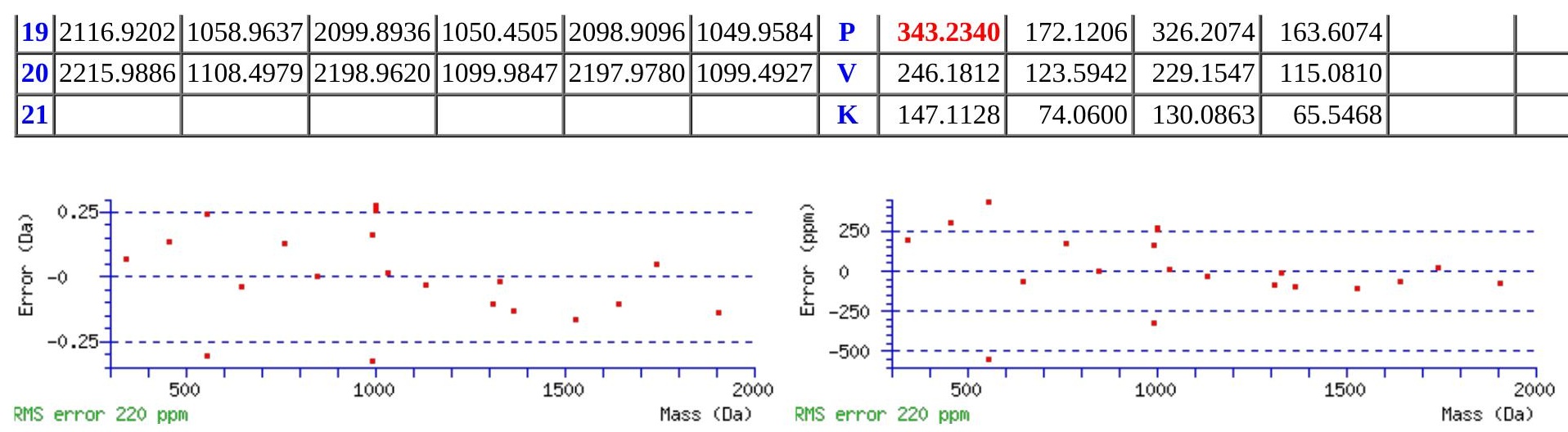

\section{All matches to this query}

\begin{tabular}{|l|l|c|l|}
\hline Score & Mr(calc): & Delta & \multicolumn{1}{|c|}{ Sequence } \\
\hline 80.7 & 2361.0869 & 0.0076 & FVLLMTNCYATPSSNSTDPVK \\
\hline 23.4 & 2361.0869 & 0.0076 & FVLLMTNCYATPSSNSTDPVK \\
\hline 8.2 & 2361.1109 & -0.0165 & NLMSMELTDTDIRPKFNLK \\
\hline 8.0 & 2361.1109 & -0.0165 & NLMSMELTDTDIRPKFNLK \\
\hline 7.0 & 2360.1028 & 0.9916 & FVLLMTNCYATPSSNSTDPVK \\
\hline 3.8 & 2361.1046 & -0.0101 & SAANWNTTGEVVVSMEPEVPVK \\
\hline 2.2 & 2359.0723 & 2.0222 & KNSSAEKSVVSLAGQAPHFSR \\
\hline 0.6 & 2361.0907 & 0.0038 & MNFDHKNETLSISVQPGEGNK \\
\hline 0.6 & 2360.0728 & 1.0217 & VSTDGADNRDSLDPQALSDEVR \\
\hline 0.1 & 2361.1109 & -0.0165 & NLMSMELTDTDIRPKFNLK \\
\hline
\end{tabular}

Spectrum No: 225; Query: 913; Rank: 1

\section{Peptide View}

MS/MS Fragmentation of DMSDGFISNLTIQR

Found in IPI00209863, Tax_Id=10116 Gene_Symbol=P4ha1 Prolyl 4-hydroxylase subunit alpha-1 precursor

Match to Query 913: 1612.745328 from(807.379940,2+)

Title: 100101RatKid_NS_deglyco_19.3844.3844.2.dta

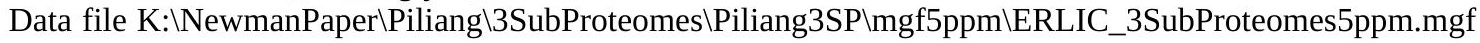

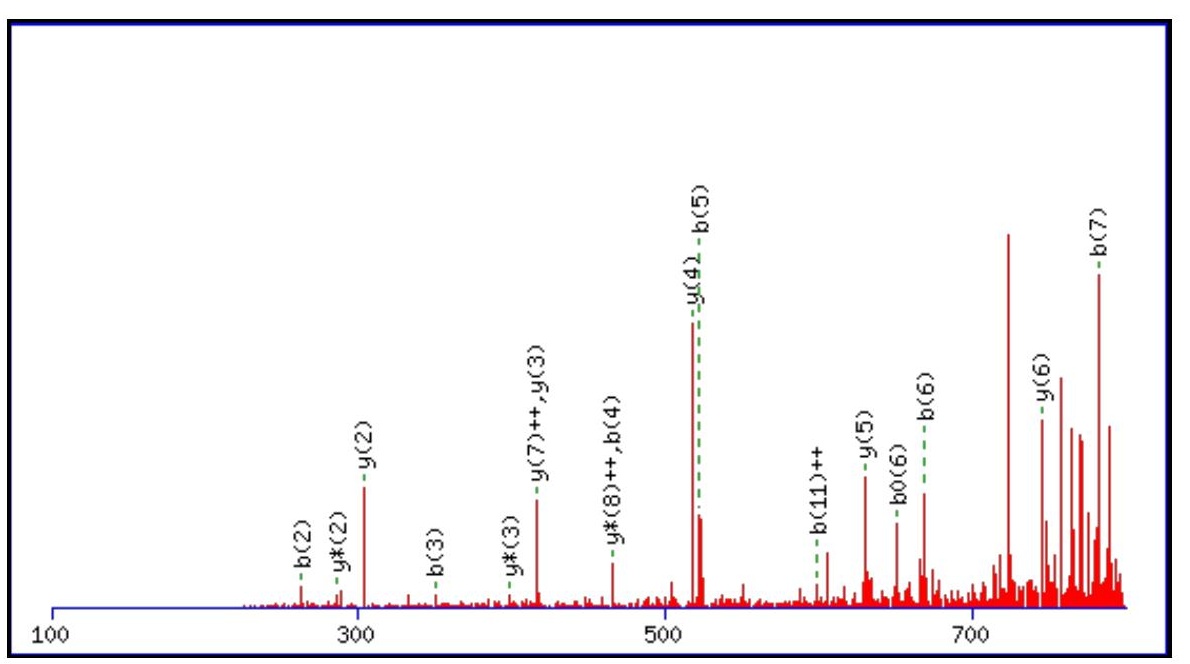



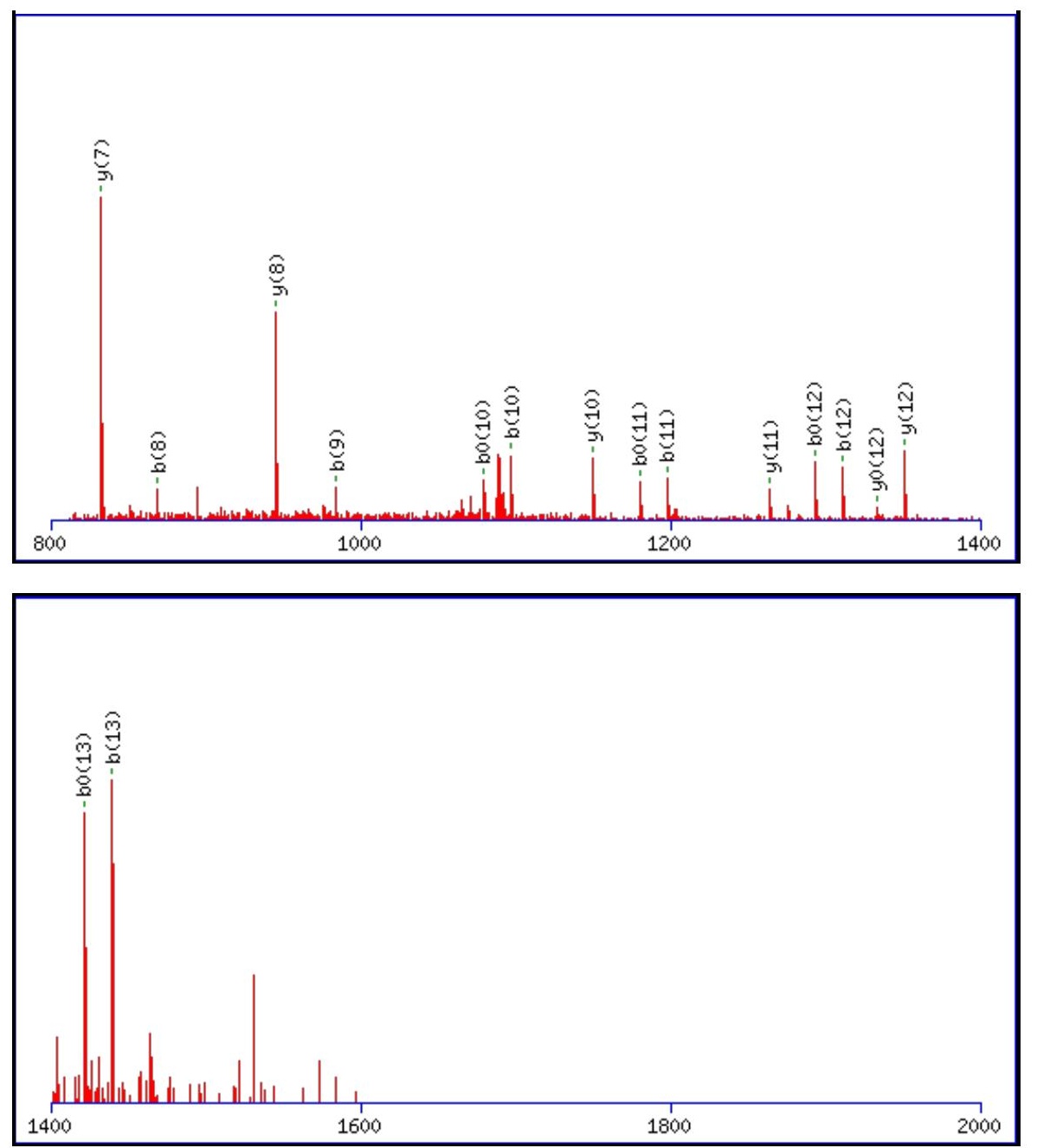

Monoisotopic mass of neutral peptide $\operatorname{Mr}($ calc): 1612.7403

Fixed modifications: Carbamidomethyl (C)

Variable modifications:

M2 : Oxidation (M)

N9 : Deamidated_N (N)

Ions Score: 81 Expect: $1.6 \mathrm{e}-006$

Matches (Bold Red): 33/134 fragment ions using 58 most intense peaks

\begin{tabular}{|r|c|c|c|c|c|c|c|c|c|c|c|c|c|c|}
\hline$\#$ & $\mathbf{b}$ & $\mathbf{b}^{++}$ & $\mathbf{b}^{*}$ & $\mathbf{b}^{\mathbf{*}^{++}}$ & $\mathbf{b}^{\mathbf{0}}$ & $\mathbf{b}^{\mathbf{0 + +}}$ & $\mathbf{S e q}$ & $\mathbf{y}$ & $\mathbf{y}^{++}$ & $\mathbf{y}^{\mathbf{*}}$ & $\mathbf{y}^{\mathbf{*}^{++}}$ & $\mathbf{y}^{\mathbf{0}}$ & $\mathbf{y}^{\mathbf{0 + +}}$ & $\#$ \\
\hline $\mathbf{1}$ & 116.0342 & 58.5207 & & & 98.0237 & 49.5155 & $\mathbf{D}$ & & & & & & & $\mathbf{1 4}$ \\
\hline $\mathbf{2}$ & $\mathbf{2 6 3 . 0 6 9 6}$ & 132.0384 & & & 245.0591 & 123.0332 & $\mathbf{M}$ & 1498.7206 & 749.8639 & 1481.6941 & 741.3507 & 1480.7101 & 740.8587 & $\mathbf{1 3}$ \\
\hline $\mathbf{3}$ & $\mathbf{3 5 0 . 1 0 1 7}$ & 175.5545 & & & 332.0911 & 166.5492 & $\mathbf{S}$ & $\mathbf{1 3 5 1 . 6 8 5 2}$ & 676.3462 & 1334.6587 & 667.8330 & $\mathbf{1 3 3 3 . 6 7 4 6}$ & 667.3410 & $\mathbf{1 2}$ \\
\hline $\mathbf{4}$ & $\mathbf{4 6 5 . 1 2 8 6}$ & 233.0679 & & & 447.1180 & 224.0627 & $\mathbf{D}$ & $\mathbf{1 2 6 4 . 6 5 3 2}$ & 632.8302 & 1247.6266 & 624.3170 & 1246.6426 & 623.8249 & $\mathbf{1 1}$ \\
\hline $\mathbf{5}$ & $\mathbf{5 2 2 . 1 5 0 1}$ & 261.5787 & & & 504.1395 & 252.5734 & $\mathbf{G}$ & $\mathbf{1 1 4 9 . 6 2 6 2}$ & 575.3168 & 1132.5997 & 566.8035 & 1131.6157 & 566.3115 & $\mathbf{1 0}$ \\
\hline $\mathbf{6}$ & $\mathbf{6 6 9 . 2 1 8 5}$ & 335.1129 & & & $\mathbf{6 5 1 . 2 0 7 9}$ & 326.1076 & $\mathbf{F}$ & 1092.6048 & 546.8060 & 1075.5782 & 538.2928 & 1074.5942 & 537.8007 & $\mathbf{9}$ \\
\hline $\mathbf{7}$ & $\mathbf{7 8 2 . 3 0 2 5}$ & 391.6549 & & & 764.2920 & 382.6496 & $\mathbf{I}$ & $\mathbf{9 4 5 . 5 3 6 4}$ & 473.2718 & 928.5098 & $\mathbf{4 6 4 . 7 5 8 5}$ & 927.5258 & 464.2665 & $\mathbf{8}$ \\
\hline $\mathbf{8}$ & $\mathbf{8 6 9 . 3 3 4 6}$ & 435.1709 & & & 851.3240 & 426.1656 & $\mathbf{S}$ & $\mathbf{8 3 2 . 4 5 2 3}$ & $\mathbf{4 1 6 . 7 2 9 8}$ & 815.4258 & 408.2165 & 814.4417 & 407.7245 & $\mathbf{7}$ \\
\hline $\mathbf{9}$ & $\mathbf{9 8 4 . 3 6 1 5}$ & 492.6844 & 967.3350 & 484.1711 & 966.3509 & 483.6791 & $\mathbf{N}$ & $\mathbf{7 4 5 . 4 2 0 3}$ & 373.2138 & 728.3937 & 364.7005 & 727.4097 & 364.2085 & $\mathbf{6}$ \\
\hline $\mathbf{1 0}$ & $\mathbf{1 0 9 7 . 4 4 5 6}$ & 549.2264 & 1080.4190 & 540.7131 & $\mathbf{1 0 7 9 . 4 3 5 0}$ & 540.2211 & $\mathbf{L}$ & $\mathbf{6 3 0 . 3 9 3 3}$ & 315.7003 & 613.3668 & 307.1870 & 612.3828 & 306.6950 & $\mathbf{5}$ \\
\hline $\mathbf{1 1}$ & $\mathbf{1 1 9 8 . 4 9 3 2}$ & $\mathbf{5 9 9 . 7 5 0 3}$ & 1181.4667 & 591.2370 & $\mathbf{1 1 8 0 . 4 8 2 7}$ & 590.7450 & $\mathbf{T}$ & $\mathbf{5 1 7 . 3 0 9 3}$ & 259.1583 & 500.2827 & 250.6450 & 499.2987 & 250.1530 & $\mathbf{4}$ \\
\hline $\mathbf{1 2}$ & $\mathbf{1 3 1 1 . 5 7 7 3}$ & 656.2923 & 1294.5508 & 647.7790 & $\mathbf{1 2 9 3 . 5 6 6 7}$ & 647.2870 & $\mathbf{I}$ & $\mathbf{4 1 6 . 2 6 1 6}$ & 208.6344 & 399.2350 & 200.1212 & & & $\mathbf{3}$ \\
\hline $\mathbf{1 3}$ & $\mathbf{1 4 3 9 . 6 3 5 9}$ & 720.3216 & 1422.6093 & 711.8083 & $\mathbf{1 4 2 1 . 6 2 5 3}$ & 711.3163 & $\mathbf{Q}$ & $\mathbf{3 0 3 . 1 7 7 5}$ & 152.0924 & $\mathbf{2 8 6 . 1 5 1 0}$ & 143.5791 & & & $\mathbf{2}$ \\
\hline $\mathbf{1 4}$ & & & & & & & $\mathbf{R}$ & 175.1190 & 88.0631 & 158.0924 & 79.5498 & & & $\mathbf{1}$ \\
\hline
\end{tabular}



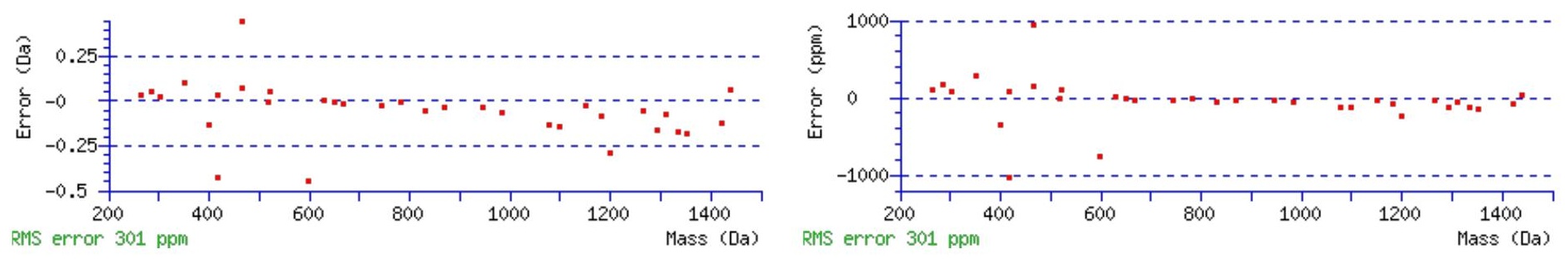

\section{All matches to this query}

\begin{tabular}{|l|l|l|l|}
\hline Score & Mr(calc): & Delta & \multicolumn{1}{|c|}{ Sequence } \\
\hline 80.7 & 1612.7403 & 0.0050 & DMSDGFISNLTIQR \\
\hline 29.8 & 1611.7563 & 0.9891 & DMSDGFISNLTIQR \\
\hline 10.5 & 1612.7457 & -0.0004 & ADAADGSLASSATIRK \\
\hline 7.8 & 1612.7354 & 0.0099 & MMALQTDIVDLQR \\
\hline 7.3 & 1612.7529 & -0.0075 & SFMNQSHLQTHQR \\
\hline 6.7 & 1612.7419 & 0.0034 & SAEPMGITLKLNDK \\
\hline 3.0 & 1612.7403 & 0.0050 & LLDDSEYTCTIQR \\
\hline 2.1 & 1611.7440 & 1.0014 & QEISEINRMIQR \\
\hline 0.4 & 1611.7450 & 1.0003 & MAEGELNIDSFITR \\
\hline 0.3 & 1612.7596 & -0.0143 & SELKEDSVEELKK \\
\hline
\end{tabular}

Spectrum No: 226; Query: 2454; Rank: 1

\section{Peptide View}

MS/MS Fragmentation of DLVVGLYSQWMNNSDNNPIHPNLR

Found in IPI00230862, Tax_Id=10116 Gene_Symbol=Anpep Aminopeptidase N

Match to Query 2454: 2812.319232 from(938.447020,3+)

Title: 091008RatKidney_NoSalt_27.4170.4170.3.dta

Data file K:INewmanPaper|Piliang\3SubProteomes\Piliang3SP\mgf5ppm\ERLIC_3SubProteomes5ppm.mgf

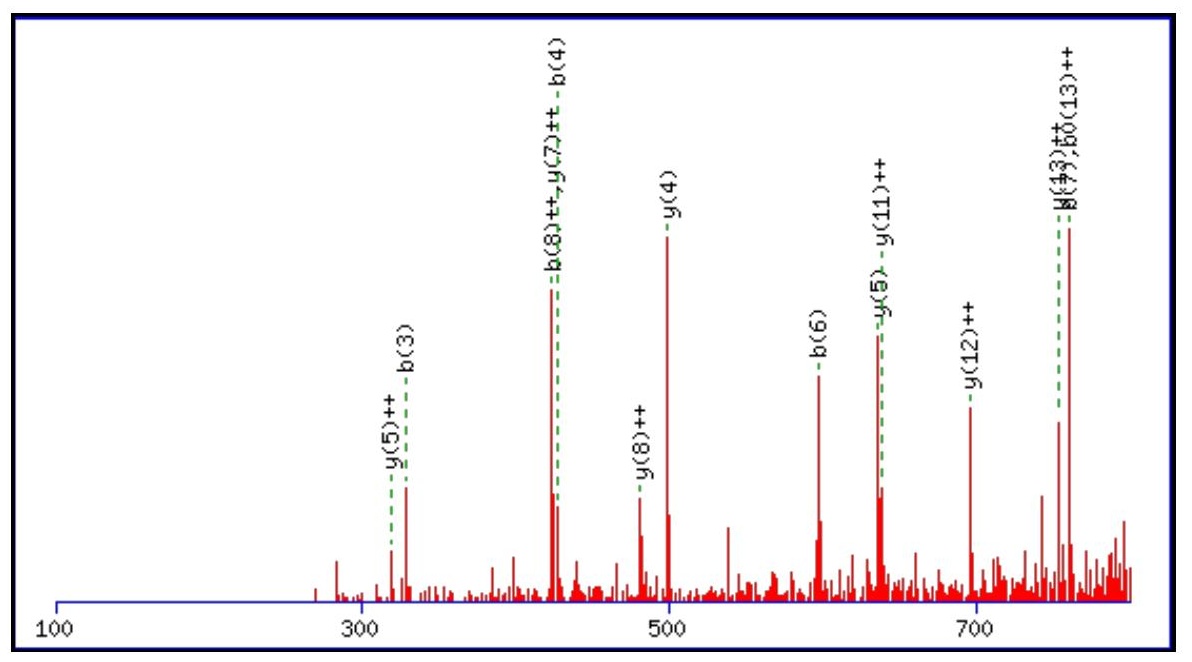



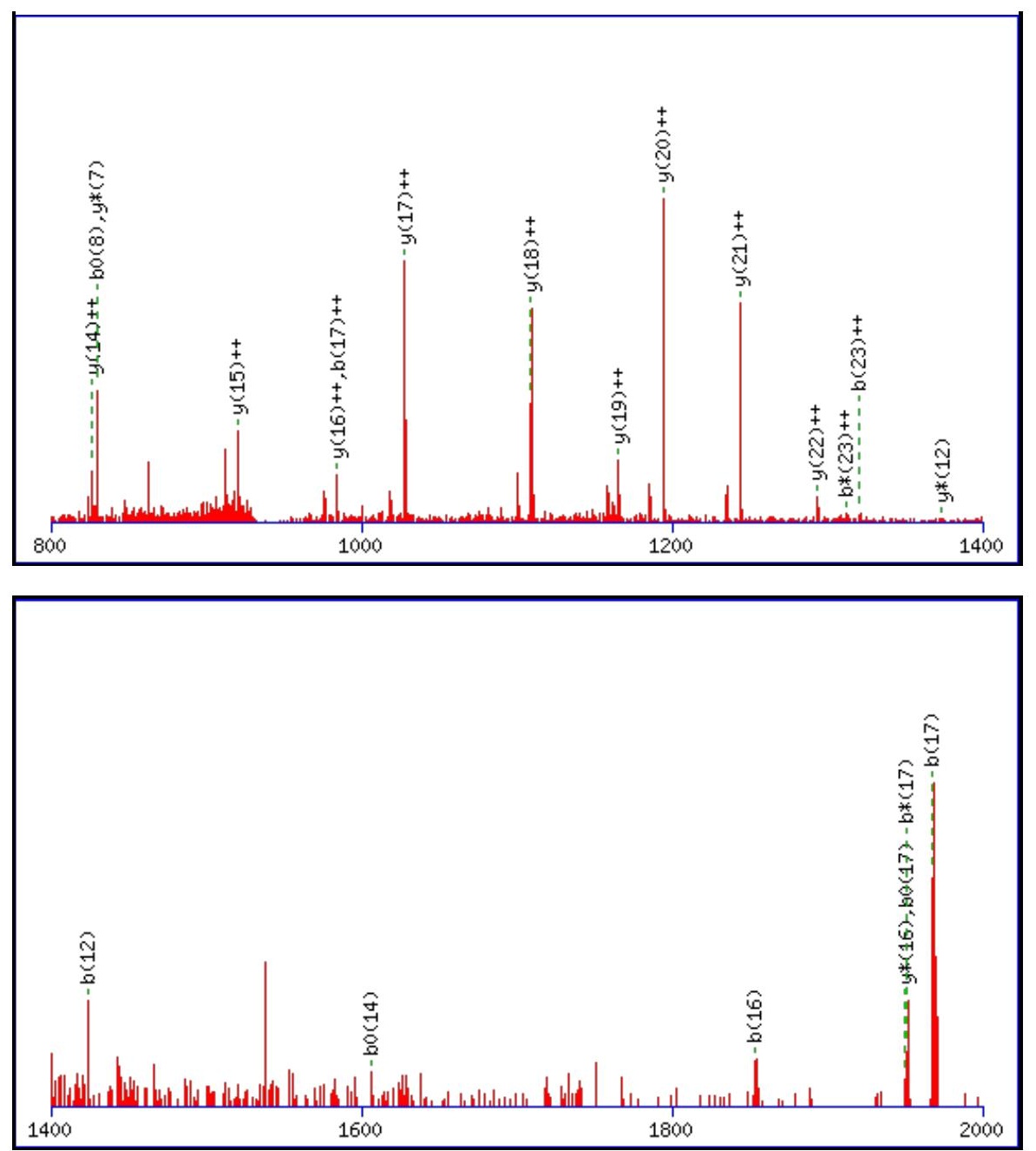

Monoisotopic mass of neutral peptide $\operatorname{Mr}($ calc): 2812.3239

Fixed modifications: Carbamidomethyl (C)

Variable modifications:

M11 : Oxidation (M)

N12 : Deamidated_N (N)

Ions Score: 80 Expect: $3 \mathrm{e}-\odot \odot 6$

Matches (Bold Red): 36/242 fragment ions using 64 most intense peaks

\begin{tabular}{|c|c|c|c|c|c|c|c|c|c|c|c|c|c|c|}
\hline \# & b & $\mathbf{b}^{++}$ & $\mathbf{b}^{*}$ & $\mathbf{b}^{*^{++}}$ & $\mathbf{b}^{0}$ & $\mathbf{b}^{\mathbf{0 + +}}$ & Seq. & $\mathbf{y}$ & $y^{++}$ & $\mathbf{y}^{*}$ & $\mathrm{y}^{*^{++}}$ & $\mathbf{y}^{0}$ & $\mathbf{y}^{0++}$ & \# \\
\hline 1 & 116.0342 & 58.5207 & & & 98.0237 & 49.5155 & D & & & & & & & 24 \\
\hline 2 & 229.1183 & 115.0628 & & & 211.1077 & 106.0575 & $\mathbf{L}$ & 2698.3042 & 1349.6557 & 2681.2777 & & 2680.2936 & & 23 \\
\hline 3 & 328.1867 & 164.5970 & & & 310.1761 & 155.5917 & $\mathbf{V}$ & 2585.2201 & 1293.1137 & 2568.1936 & 1284.6004 & 2567.2096 & 1284.1084 & 22 \\
\hline 4 & 427.2551 & 214.1312 & & & 409.2445 & 205.1259 & $\mathbf{V}$ & 2486.1517 & 1243.5795 & 2469.1252 & 1235.0662 & 2468.1412 & 1234.5742 & 21 \\
\hline 5 & 484.2766 & 242.6419 & & & 466.2660 & 233.6366 & G & 2387.0833 & 1194.0453 & 2370.0568 & 5320 & 2369.0727 & 1185.0400 & 20 \\
\hline 6 & 597.3606 & 299.1840 & & & 579.3501 & 290.1787 & $\mathbf{L}$ & 2330.0619 & 1165.5346 & 2313.0353 & 1157.0213 & 2312.0513 & 1156.5293 & 19 \\
\hline 7 & 760.4240 & 380.7156 & & & 742.4134 & 371.7103 & $\mathbf{Y}$ & 2216.9778 & 1108.9925 & 2199.9512 & 1100.4793 & 2198.9672 & 1099.9872 & 18 \\
\hline 8 & 847.4560 & 424.2316 & & & 829.4454 & 415.2264 & S & 2053.9145 & 1027.4609 & 2036.8879 & 1018.9476 & 2035.9039 & 1018.4556 & 17 \\
\hline 9 & 975.5146 & 488.2609 & 958.4880 & & 957.5040 & 479.2556 & $\mathbf{Q}$ & 1966.8824 & 983.9449 & 1949.8559 & 975.4316 & 1948.8719 & 974.9396 & 16 \\
\hline 10 & 1161.5939 & 581.3006 & 1144.5673 & 572.7873 & 1143.5833 & 572.2953 & $\mathbf{W}$ & 1838.8239 & 919.9156 & 1821.7973 & 911.4023 & 1820.8133 & 910.9103 & 15 \\
\hline 11 & 1308.6293 & 654.8183 & 1291.6027 & 646.3050 & 1290.6187 & 645.8130 & $\mathbf{M}$ & 1652.7445 & 826.8759 & 1635.7180 & 818.3626 & 1634.7340 & 817.8706 & 14 \\
\hline 12 & 1423.6562 & 712.3318 & 1406.6297 & 703.8185 & 1405.6457 & 703.3265 & $\mathbf{N}$ & 1505.7091 & 753.3582 & 1488.6826 & 744.8449 & 1487.6986 & 744.3529 & 13 \\
\hline 13 & 1537.6992 & 769.3532 & 1520.6726 & 760.8399 & 1519.6886 & 760.3479 & $\mathbf{N}$ & 1390.6822 & 695.8447 & 1373.6557 & 687.3315 & 1372.6716 & 686.8395 & 12 \\
\hline 14 & 1624.7312 & 812.8692 & 1607.7046 & 804.3560 & 1606.7206 & 803.8639 & $S$ & 1276.6393 & 638.8233 & 1259.6127 & 630.3100 & 1258.6287 & 629.8180 & 11 \\
\hline 15 & 1739.7581 & 870.3827 & 1722.7316 & 861.8694 & 1721.7476 & 861.3774 & D & 1189.6072 & 595.3073 & 1172.5807 & 586.7940 & 1171.5967 & 586.3020 & 10 \\
\hline 16 & 1853.8011 & 927.4042 & 1836.7745 & 918.8909 & 1835.7905 & 918.3989 & $\mathbf{N}$ & 1074.5803 & 537.7938 & 1057.5538 & 529.2805 & & & 9 \\
\hline 17 & 1967.8440 & 984.4256 & 1950.8174 & 975.9124 & 1949.8334 & 975.4203 & $\mathbf{N}$ & 960.5374 & 480.7723 & 943.5108 & 472.2591 & & & 8 \\
\hline 18 & 2064.8967 & 1032.9520 & 2047.8702 & 1024.4387 & 2046.8862 & 1023.9467 & $\mathbf{P}$ & 846.4944 & 423.7509 & 829.4679 & 415.2376 & & & 7 \\
\hline
\end{tabular}




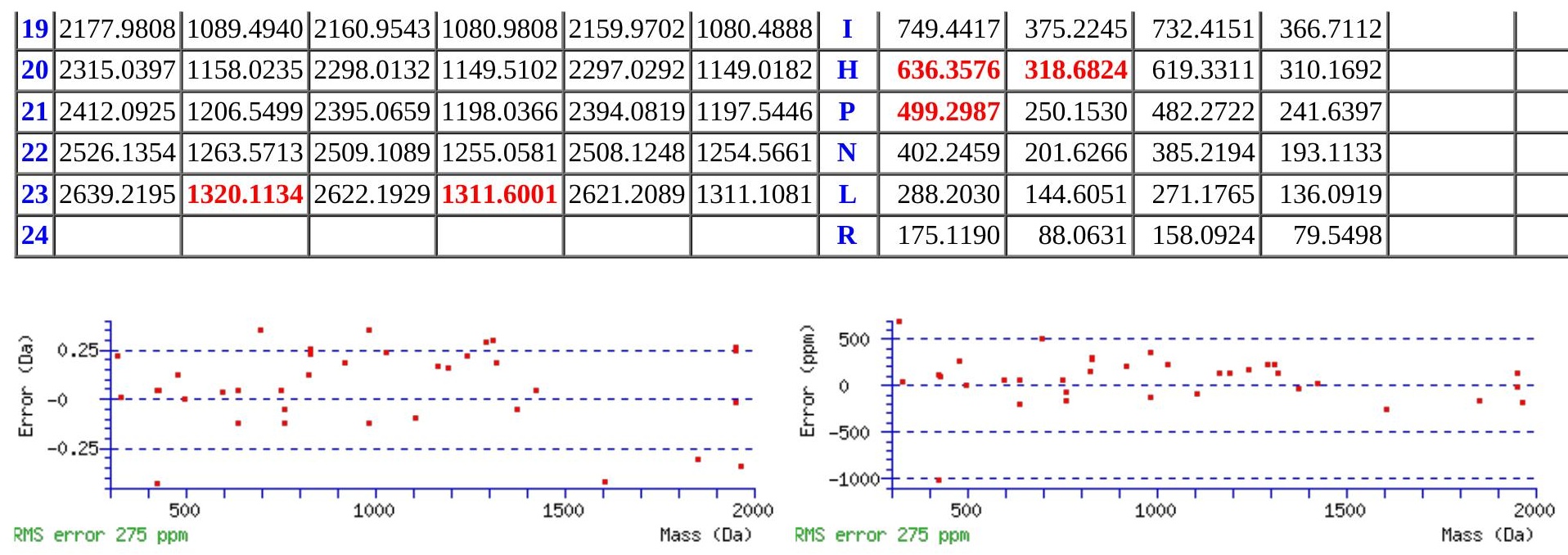

\section{All matches to this query}

\begin{tabular}{|l|l|l|l|}
\hline Score & Mr(calc): & Delta & \multicolumn{1}{|c|}{ Sequence } \\
\hline 80.4 & 2812.3239 & -0.0046 & DLVVGLYSQWMNNSDNNPIHPNLR \\
\hline 78.4 & 2812.3239 & -0.0046 & DLVVGLYSQWMNNSDNNPIHPNLR \\
\hline 67.5 & 2812.3239 & -0.0046 & DLVVGLYSQWMNNSDNNPIHPNLR \\
\hline 65.6 & 2812.3239 & -0.0046 & DLVVGLYSQWMNNSDNNPIHPNLR \\
\hline 65.4 & 2812.3239 & -0.0046 & DLVVGLYSQWMNNSDNNPIHPNLR \\
\hline 9.2 & 2811.3398 & 0.9794 & DLVVGLYSQWMNNSDNNPIHPNLR \\
\hline 3.4 & 2812.3382 & -0.0190 & VPQRTSSIATALNTSGAGGSRPAQAVR \\
\hline 2.2 & 2811.3051 & 1.0141 & LHNGGDVTFHDVLSMLSYRSVDIR \\
\hline 1.2 & 2811.3133 & 1.0059 & LLVVDFSSQALFQDKNSSQVLGEK \\
\hline
\end{tabular}

Spectrum No: 227; Query: 359; Rank: 1

\section{Peptide View}

MS/MS Fragmentation of LVNLTFLDLTR

Found in IPI00360317, Tax_Id=10116 Gene_Symbol=Cd180_predicted CD180 antigen

Match to Query 359: 1304.734928 from(653.374740,2+)

Title: 100101RatKid_NS_deglyco_20.4985.4985.2.dta

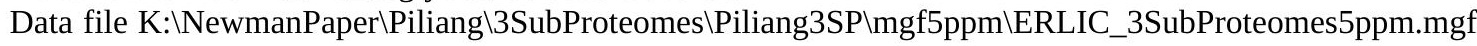

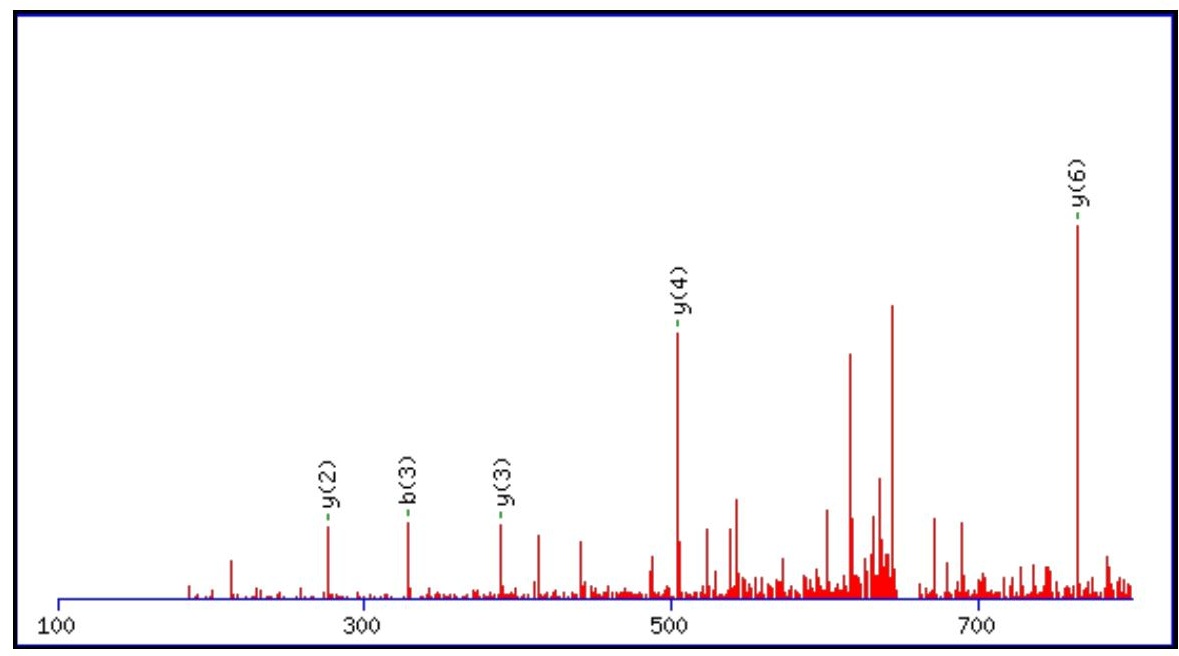



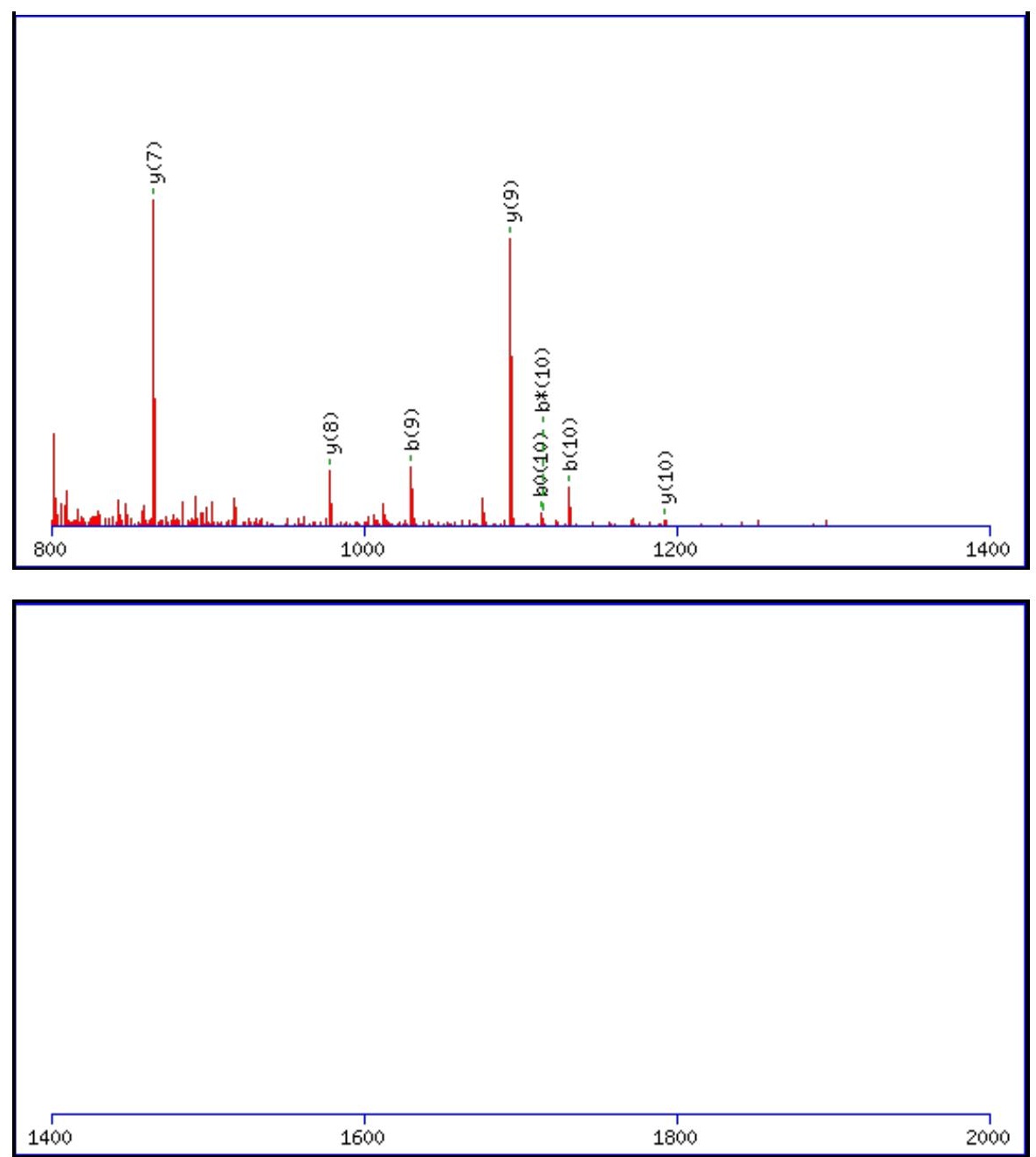

Monoisotopic mass of neutral peptide $\operatorname{Mr}($ calc): 1304.7340

Fixed modifications: Carbamidomethyl (C)

Variable modifications:

N3 : Deamidated $\mathrm{N}(\mathrm{N})$

Ions Score: 80 Expect: $5.2 \mathrm{e}-007$

Matches (Bold Red): 13/106 fragment ions using 21 most intense peaks

\begin{tabular}{|r|c|c|c|c|c|c|c|c|c|c|c|c|c|c|}
\hline$\#$ & $\mathbf{b}$ & $\mathbf{b}^{++}$ & $\mathbf{b}^{*}$ & $\mathbf{b}^{\mathbf{*}^{++}}$ & $\mathbf{b}^{\mathbf{0}}$ & $\mathbf{b}^{\mathbf{0}+}$ & $\mathbf{S e q}$ & $\mathbf{y}$ & $\mathbf{y}^{++}$ & $\mathbf{y}^{\mathbf{*}}$ & $\mathbf{y}^{\mathbf{*}^{++}}$ & $\mathbf{y}^{\mathbf{0}}$ & $\mathbf{y}^{\mathbf{0 + +}}$ & $\#$ \\
\hline $\mathbf{1}$ & 114.0913 & 57.5493 & & & & & $\mathbf{L}$ & & & & & & & $\mathbf{1 1}$ \\
\hline $\mathbf{2}$ & 213.1598 & 107.0835 & & & & & $\mathbf{V}$ & $\mathbf{1 1 9 2 . 6 5 7 2}$ & 596.8322 & 1175.6307 & 588.3190 & 1174.6466 & 587.8270 & $\mathbf{1 0}$ \\
\hline $\mathbf{3}$ & $\mathbf{3 2 8 . 1 8 6 7}$ & 164.5970 & 311.1601 & 156.0837 & & & $\mathbf{N}$ & $\mathbf{1 0 9 3 . 5 8 8 8}$ & 547.2980 & 1076.5622 & 538.7848 & 1075.5782 & 538.2928 & $\mathbf{9}$ \\
\hline $\mathbf{4}$ & 441.2708 & 221.1390 & 424.2442 & 212.6257 & & & $\mathbf{L}$ & $\mathbf{9 7 8 . 5 6 1 9}$ & 489.7846 & 961.5353 & 481.2713 & 960.5513 & 480.7793 & $\mathbf{8}$ \\
\hline $\mathbf{5}$ & 542.3184 & 271.6629 & 525.2919 & 263.1496 & 524.3079 & 262.6576 & $\mathbf{T}$ & $\mathbf{8 6 5 . 4 7 7 8}$ & 433.2425 & 848.4512 & 424.7293 & 847.4672 & 424.2373 & $\mathbf{7}$ \\
\hline $\mathbf{6}$ & 689.3868 & 345.1971 & 672.3603 & 336.6838 & 671.3763 & 336.1918 & $\mathbf{F}$ & $\mathbf{7 6 4 . 4 3 0 1}$ & 382.7187 & 747.4036 & 374.2054 & 746.4196 & 373.7134 & $\mathbf{6}$ \\
\hline $\mathbf{7}$ & 802.4709 & 401.7391 & 785.4444 & 393.2258 & 784.4603 & 392.7338 & $\mathbf{L}$ & 617.3617 & 309.1845 & 600.3352 & 300.6712 & 599.3511 & 300.1792 & $\mathbf{5}$ \\
\hline $\mathbf{8}$ & 917.4979 & 459.2526 & 900.4713 & 450.7393 & 899.4873 & 450.2473 & $\mathbf{D}$ & $\mathbf{5 0 4 . 2 7 7 6}$ & 252.6425 & 487.2511 & 244.1292 & 486.2671 & 243.6372 & $\mathbf{4}$ \\
\hline $\mathbf{9}$ & $\mathbf{1 0 3 0 . 5 8 1 9}$ & 515.7946 & 1013.5554 & 507.2813 & 1012.5714 & 506.7893 & $\mathbf{L}$ & $\mathbf{3 8 9 . 2 5 0 7}$ & 195.1290 & 372.2241 & 186.6157 & 371.2401 & 186.1237 & $\mathbf{3}$ \\
\hline $\mathbf{1 0}$ & $\mathbf{1 1 3 1 . 6 2 9 6}$ & 566.3184 & $\mathbf{1 1 1 4 . 6 0 3 0}$ & 557.8052 & $\mathbf{1 1 1 3 . 6 1 9 0}$ & 557.3132 & $\mathbf{T}$ & $\mathbf{2 7 6 . 1 6 6 6}$ & 138.5870 & 259.1401 & 130.0737 & 258.1561 & 129.5817 & $\mathbf{2}$ \\
\hline $\mathbf{1 1}$ & & & & & & & $\mathbf{R}$ & 175.1190 & 88.0631 & 158.0924 & 79.5498 & & & $\mathbf{1}$ \\
\hline
\end{tabular}
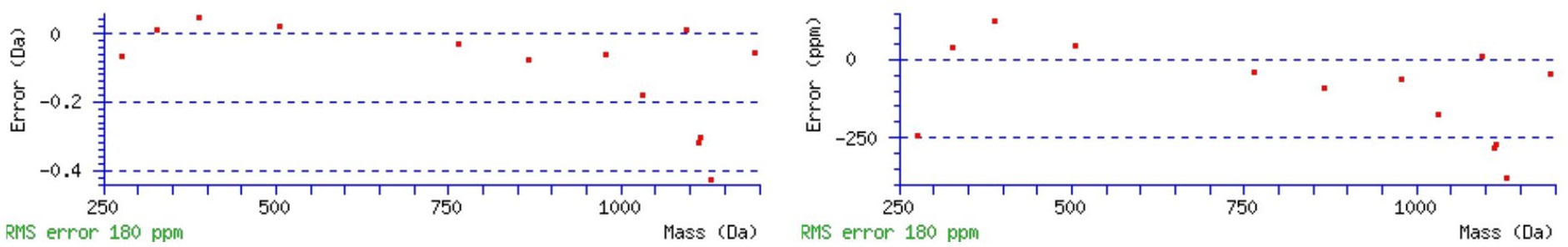


\section{All matches to this query}

\begin{tabular}{|l|l|l|l|}
\hline Score & Mr(calc): & Delta & \multicolumn{1}{c|}{ Sequence } \\
\hline 80.2 & 1304.7340 & 0.0009 & LVNLTFLDLTR \\
\hline 6.1 & 1304.7241 & 0.0109 & QKAIDLLYWR \\
\hline 5.0 & 1304.7374 & -0.0024 & LITLMGQKTSLT \\
\hline 4.7 & 1304.7452 & -0.0103 & IVNFVVNSKKR \\
\hline 2.8 & 1304.7452 & -0.0103 & SLGQIYRVLEK \\
\hline 2.0 & 1304.7313 & 0.0037 & ERKPYRSLTR \\
\hline
\end{tabular}

Spectrum No: 228; Query: 792; Rank: 1

\section{Peptide View}

MS/MS Fragmentation of VVNVSELYGTPCTK

Found in IPI00206668, Tax_Id=10116 Gene_Symbol=Entpd1 Ectonucleoside triphosphate diphosphohydrolase 1

Match to Query 792: 1566.761568 from(784.388060,2+)

Title: 091008RatKidney_NoSalt_30.1984.1984.2.dta

Data file K:INewmanPaper|Piliang|3SubProteomes\Piliang3SP\mgf5ppm\ERLIC_3SubProteomes5ppm.mgf
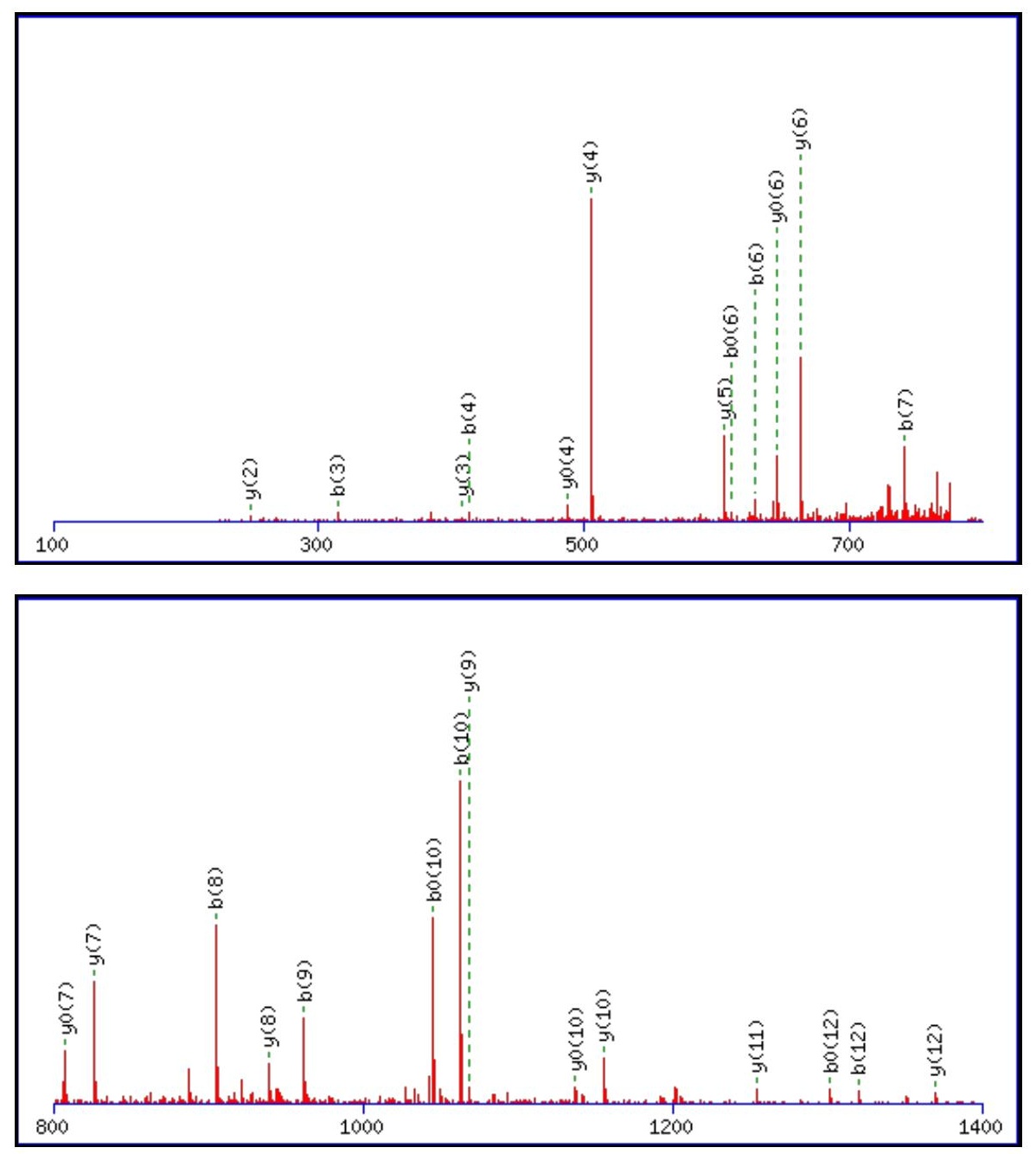


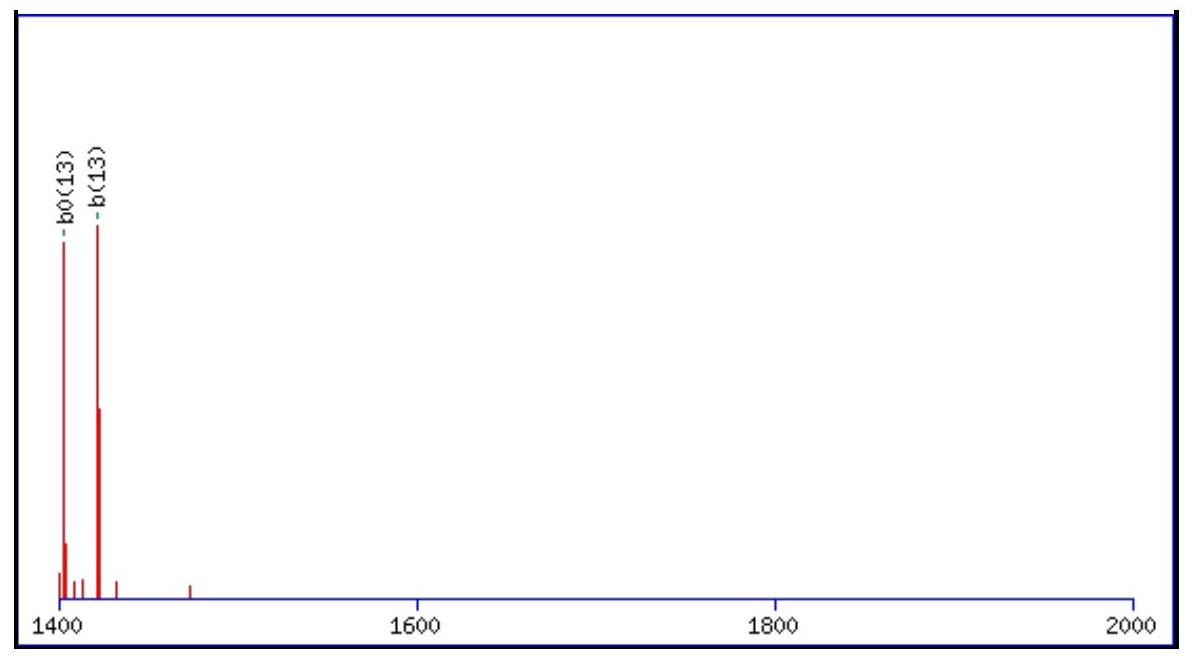

Monoisotopic mass of neutral peptide $\operatorname{Mr}($ calc): 1566.7600

Fixed modifications: Carbamidomethyl (C)

Variable modifications:

N3 : Deamidated $\mathrm{N}(\mathrm{N})$

Ions Score: 80 Expect: $1.7 \mathrm{e}-006$

Matches (Bold Red): 28/142 fragment ions using 42 most intense peaks

\begin{tabular}{|c|c|c|c|c|c|c|c|c|c|c|c|c|c|c|}
\hline \# & b & $\mathbf{b}^{++}$ & b* & $\mathbf{b}^{*^{++}}$ & $\mathbf{b}^{0}$ & $\mathbf{b}^{0++}$ & Seq. & $\mathbf{y}$ & $\mathbf{y}^{++}$ & $\mathbf{y}^{*}$ & $\mathbf{y}^{*^{++}}$ & $\mathbf{y}^{0}$ & $\mathbf{y}^{0++}$ & \# \\
\hline 1 & 100.0757 & 50.5415 & & & & & $\mathbf{V}$ & & & & & & & 14 \\
\hline 2 & 199.1441 & 100.0757 & & & & & V & 1468.6988 & 734.8530 & 1451.6723 & 726.3398 & 1450.6883 & 725.8478 & 13 \\
\hline 3 & 314.1710 & 157.5892 & 297.1445 & 149.0759 & & & $\mathbf{N}$ & 1369.6304 & 685.3188 & 1352.6039 & 676.8056 & 1351.6198 & 676.3136 & 12 \\
\hline 4 & 413.2395 & 207.1234 & 396.2129 & 198.6101 & & & V & 1254.6035 & 627.8054 & 1237.5769 & 619.2921 & 1236.5929 & 618.8001 & 11 \\
\hline 5 & 500.2715 & 250.6394 & 483.2449 & 242.1261 & 482.2609 & 241.6341 & $\mathbf{S}$ & 1155.5351 & 578.2712 & 1138.5085 & 569.7579 & 1137.5245 & 569.2659 & 10 \\
\hline 6 & 629.3141 & 315.1607 & 612.2875 & 306.6474 & 611.3035 & 306.1554 & $\mathbf{E}$ & 1068.5030 & 534.7552 & 1051.4765 & 526.2419 & 1050.4925 & 525.7499 & 9 \\
\hline 7 & 742.3981 & 371.7027 & 725.3716 & 363.1894 & 724.3876 & 362.6974 & $\mathbf{L}$ & 939.4604 & 470.2339 & 922.4339 & 461.7206 & 921.4499 & 461.2286 & 8 \\
\hline 8 & 905.4615 & 453.2344 & 888.4349 & 444.7211 & 887.4509 & 444.2291 & $\mathbf{Y}$ & 826.3764 & 413.6918 & 809.3498 & 405.1785 & 808.3658 & 404.6865 & 7 \\
\hline 9 & 962.4829 & 481.7451 & 945.4564 & 473.2318 & 944.4724 & 472.7398 & G & 663.3130 & 332.1602 & 646.2865 & 323.6469 & 645.3025 & 323.1549 & 6 \\
\hline 10 & 1063.5306 & 532.2689 & 1046.5041 & 523.7557 & 1045.5200 & 523.2637 & $\mathbf{T}$ & 606.2916 & 303.6494 & 589.2650 & 295.1362 & 588.2810 & 294.6441 & 5 \\
\hline 11 & 1160.5834 & 580.7953 & 1143.5568 & 572.2821 & 1142.5728 & 571.7900 & $\mathbf{P}$ & 505.2439 & 253.1256 & 488.2173 & 244.6123 & 487.2333 & 244.1203 & 4 \\
\hline 12 & 1320.6140 & 660.8107 & 1303.5875 & 652.2974 & 1302.6035 & 651.8054 & $\mathrm{C}$ & 408.1911 & 204.5992 & 391.1646 & 196.0859 & 390.1806 & 195.5939 & 3 \\
\hline 13 & 1421.6617 & 711.3345 & 1404.6352 & 702.8212 & 1403.6511 & 702.3292 & $\mathbf{T}$ & 248.1605 & 124.5839 & 231.1339 & 116.0706 & 230.1499 & 115.5786 & 2 \\
\hline 14 & & & & & & & $\mathbf{K}$ & 147.1128 & 74.0600 & 130.0863 & 65.5468 & & & 1 \\
\hline
\end{tabular}
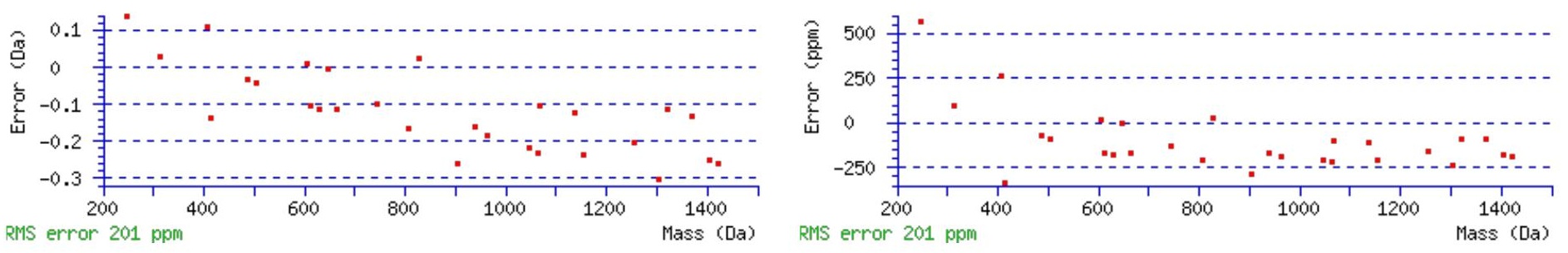

\section{All matches to this query}

\begin{tabular}{|l|l|c|l|}
\hline Score & Mr(calc): & Delta & \multicolumn{1}{c|}{ Sequence } \\
\hline 80.2 & 1566.7600 & 0.0016 & VVNVSELYGTPCTK \\
\hline 7.4 & 1566.7599 & 0.0016 & YVELTEGVECIQK \\
\hline 3.0 & 1566.7654 & -0.0039 & $\underline{\text { KGTEKQITPETQK }}$ \\
\hline 2.9 & 1566.7712 & -0.0096 & ILYNTSQECLAVR \\
\hline 1.1 & 1566.7542 & 0.0074 & DDPNSILLLGSKSK \\
\hline 0.6 & 1566.7668 & -0.0052 & $\underline{\text { RQAFGKPRPTSDK }}$ \\
\hline 0.6 & 1566.7668 & -0.0052 & $\underline{\text { RQAFGKPRPTSDK }}$ \\
\hline
\end{tabular}


Spectrum No: 229; Query: 969; Rank: 1

\section{Peptide View}

MS/MS Fragmentation of NLQVYNATSNSLTVK

Found in IPI00189714, Tax_Id=10116 Gene_Symbol=Col12a1 similar to Collagen alpha-1(XII) chain precursor

Match to Query 969: 1651.840148 from(826.927350,2+)

Title: 091008RatKidney_NH4Format01_23.2126.2126.2.dta

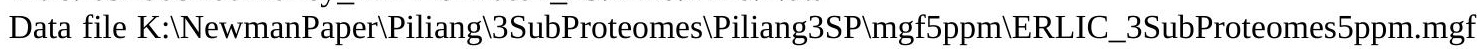
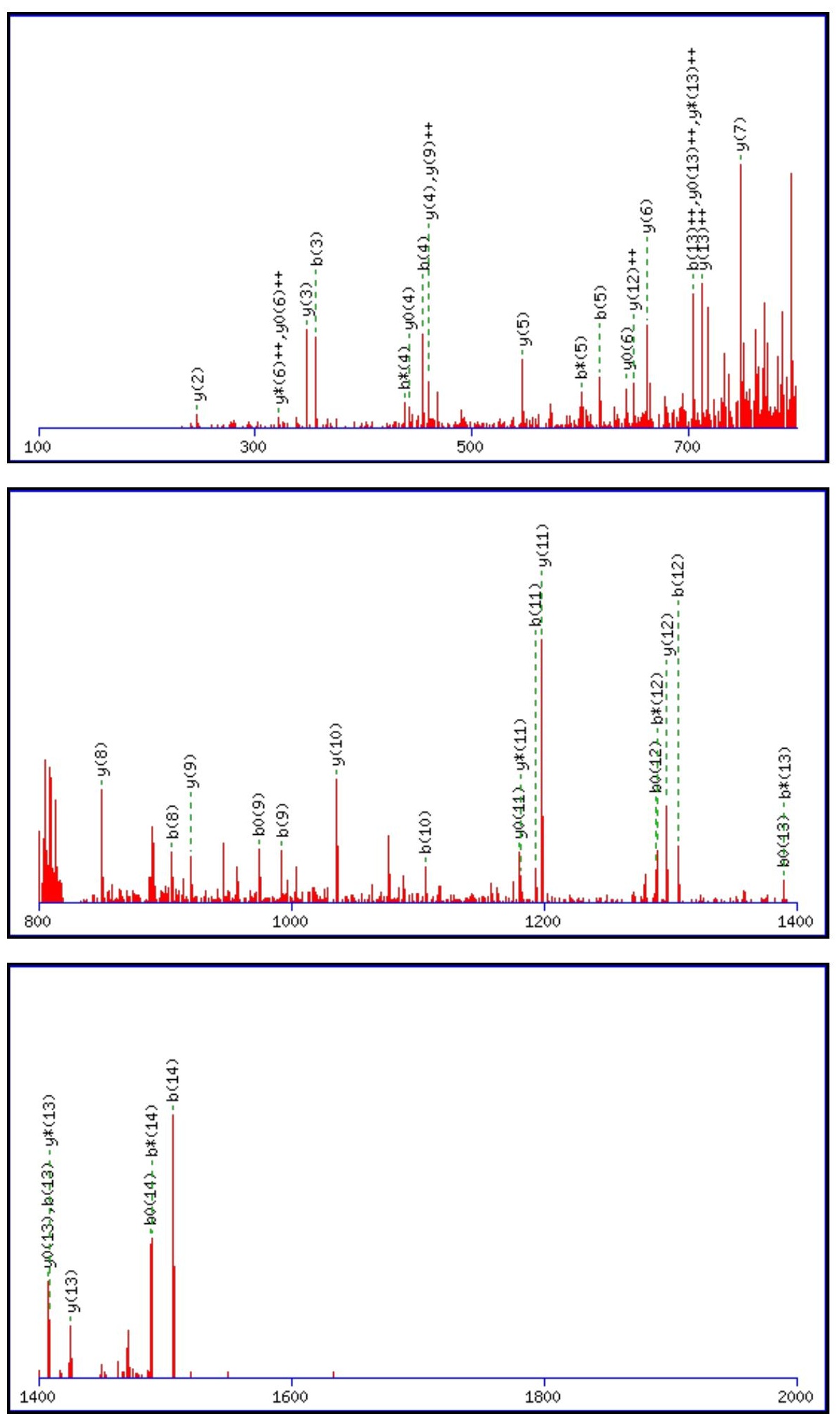
Monoisotopic mass of neutral peptide $\operatorname{Mr}($ calc): 1651.8417

Fixed modifications: Carbamidomethyl (C)

Variable modifications:

N6 : Deamidated_N (N)

Ions Score: 80 Expect: $1.6 \mathrm{e}-006$

Matches (Bold Red): 45/150 fragment ions using 70 most intense peaks

\begin{tabular}{|c|c|c|c|c|c|c|c|c|c|c|c|c|c|c|}
\hline \# & b & $\mathbf{b}^{++}$ & $\mathbf{b}^{*}$ & $\mathbf{b}^{*^{++}}$ & $\mathbf{b}^{0}$ & $\mathbf{b}^{0++}$ & Seq. & $\mathbf{y}$ & $y^{++}$ & $\mathrm{y}^{*}$ & $\mathrm{y}^{*^{++}}$ & $\mathbf{y}^{\mathbf{0}}$ & $y^{0++}$ & $\#$ \\
\hline 1 & 115.0502 & 58.0287 & 98.0237 & 49.5155 & & & $\mathbf{N}$ & & & & & & & 15 \\
\hline 2 & 228.1343 & 114.5708 & 211.1077 & 106.0575 & & & $\mathbf{L}$ & 1538.8061 & 769.9067 & 1521.7795 & 761.3934 & 1520.7955 & 760.9014 & 14 \\
\hline 3 & 356.1928 & 178.6001 & 339.1663 & 170.0868 & & & $\mathbf{Q}$ & 1425.7220 & 713.3646 & \begin{tabular}{|l|}
1408.6954 \\
\end{tabular} & 704.8514 & 1407.7114 & 704.3594 & 13 \\
\hline 4 & 455.2613 & 228.1343 & 438.2347 & 219.6210 & & & V & 1297.6634 & 649.3353 & 1280.6369 & 640.8221 & 1279.6529 & 640.3301 & 12 \\
\hline 5 & 618.3246 & 309.6659 & 601.2980 & 301.1527 & & & $\mathbf{Y}$ & 1198.5950 & 599.8011 & 1181.5685 & 591.2879 & 1180.5844 & 590.7959 & 11 \\
\hline 6 & 733.3515 & 367.1794 & 716.3250 & 358.6661 & & & $\mathbf{N}$ & 1035.5317 & 518.2695 & 1018.5051 & 509.7562 & 1017.5211 & 509.2642 & 10 \\
\hline 7 & 804.3886 & 402.6980 & 787.3621 & 394.1847 & & & A & 920.5047 & 460.7560 & 903.4782 & 452.2427 & 902.4942 & 451.7507 & 9 \\
\hline 8 & 905.4363 & 453.2218 & 888.4098 & 444.7085 & 887.4258 & 444.2165 & $\mathbf{T}$ & 849.4676 & 425.2374 & 832.4411 & 416.7242 & 831.4571 & 416.2322 & 8 \\
\hline 9 & 992.4683 & 496.7378 & 975.4418 & 488.2245 & 974.4578 & 487.7325 & $\mathrm{~S}$ & 748.4199 & 374.7136 & 731.3934 & 366.2003 & 730.4094 & 365.7083 & 7 \\
\hline 10 & 1106.5113 & 553.7593 & 1089.4847 & 545.2460 & 1088.5007 & 544.7540 & $\mathbf{N}$ & 661.3879 & 331.1976 & 644.3614 & 322.6843 & 643.3774: & 322.1923 & 6 \\
\hline 11 & 1193.5433 & 597.2753 & 1176.5168 & 588.7620 & 1175.5327 & 588.2700 & $S$ & 547.3450 & 274.1761 & 530.3184 & 265.6629 & 529.3344 & 265.1709 & 5 \\
\hline 121 & 1306.6274 & 653.8173 & 1289.6008 & 645.3040 & \begin{tabular}{|l|}
1288.6168 \\
\end{tabular} & 644.8120 & $\mathbf{L}$ & 460.3130 & 230.6601 & 443.2864 & 222.1468 & 442.3024 & 221.6548 & 4 \\
\hline 131 & 1407.6750 & 704.3412 & 1390.6485 & 695.8279 & 1389.6645 & 695.3359 & $T$ & 347.2289 & 174.1181 & 330.2023 & 165.6048 & 329.2183 & 165.1128 & 3 \\
\hline 14 & 1506.7435 & 753.8754 & 1489.7169 & 745.3621 & 1488.7329 & 744.8701 & $\mathbf{V}$ & 246.1812 & 123.5942 & 229.1547 & 115.0810 & & & 2 \\
\hline 15 & & & & & & & $\mathbf{K}$ & 147.1128 & \begin{tabular}{|l|}
74.0600 \\
\end{tabular} & 130.0863 & 65.5468 & & & 1 \\
\hline
\end{tabular}
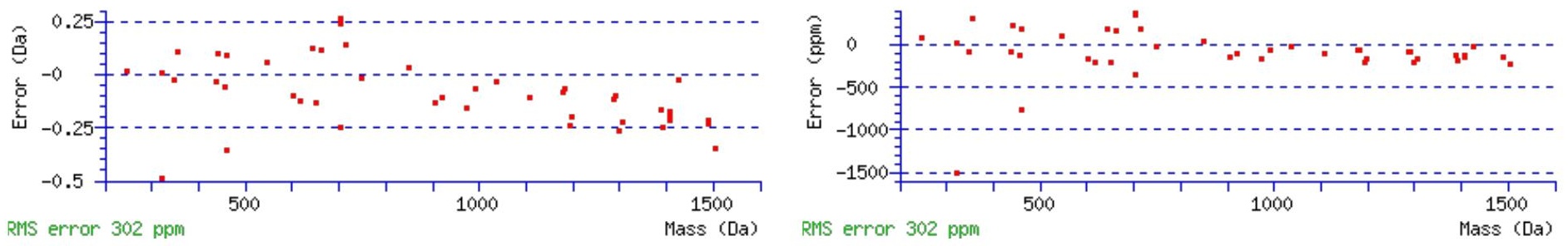

\section{All matches to this query}

\begin{tabular}{|l|c|c|c|}
\hline Score & Mr(calc): & Delta & Sequence \\
\hline 80.0 & 1651.8417 & -0.0015 & NLQVYNATSNSLTVK \\
\hline 42.2 & 1651.8417 & -0.0015 & NLQVYNATSNSLTVK \\
\hline 40.6 & 1651.8417 & -0.0015 & NLQVYNATSNSLTVK \\
\hline 4.7 & 1651.8529 & -0.0127 & EPEKPNIINANREK \\
\hline 3.6 & 1651.8433 & -0.0032 & GITQALNLVDSLSLK \\
\hline 3.2 & 1651.8525 & -0.0123 & LNTLDMDTVKKMVK \\
\hline 0.9 & 1651.8294 & 0.0107 & VKQTKSNVNIGNLR \\
\hline
\end{tabular}

Spectrum No: 230; Query: 298; Rank: 1

\section{Peptide View}

MS/MS Fragmentation of SCINESAIDSR

Found in IPI00231789, Tax_Id=10116 Gene_Symbol=Mme Neprilysin

Match to Query 298: 1251.539228 from(626.776890,2+)

Title: 091008RatKidney_NH4Format01_31.610.610.2.dta

Data file K:INewmanPaper|Piliangl3SubProteomes\Piliang3SP\mgf5ppm\ERLIC_3SubProteomes5ppm.mgf 

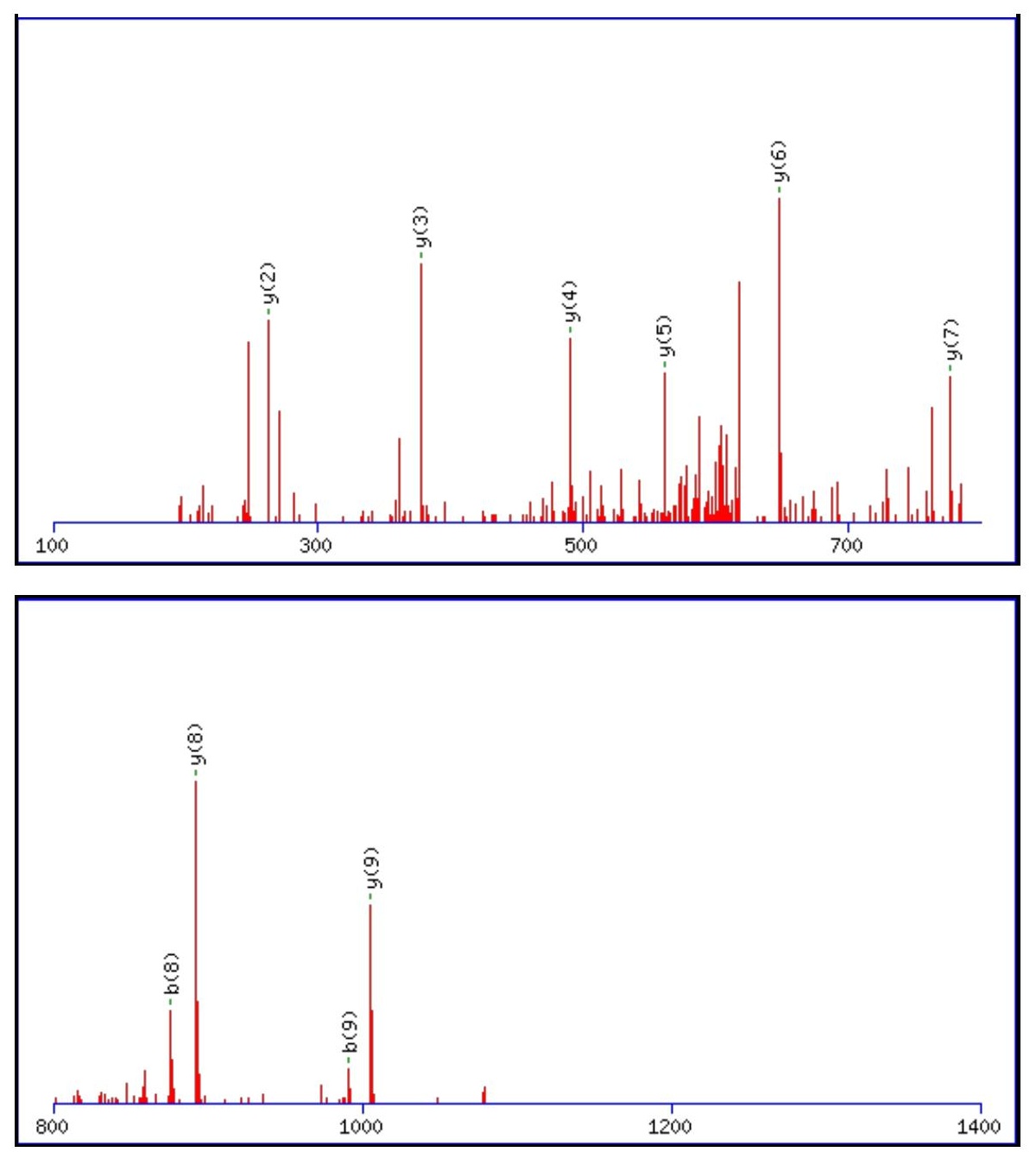

\begin{tabular}{rrrr}
\hline 00 & 1600 & 1800 & 2000 \\
\hline
\end{tabular}

Monoisotopic mass of neutral peptide $\operatorname{Mr}($ calc): 1251.5401

Fixed modifications: Carbamidomethyl (C)

Variable modifications:

N4 : Deamidated $\mathrm{N}(\mathrm{N})$

Ions Score: 80 Expect: $1.1 \mathrm{e}-006$

Matches (Bold Red): 10/112 fragment ions using 12 most intense peaks

\begin{tabular}{|r|c|c|c|c|r|r|r|r|r|r|r|r|r|r|}
\hline$\#$ & $\mathbf{b}$ & $\mathbf{b}^{++}$ & $\mathbf{b}^{*}$ & $\mathbf{b}^{\boldsymbol{*}^{++}}$ & \multicolumn{1}{|c|}{$\mathbf{b}^{\mathbf{0}}$} & \multicolumn{1}{|c|}{$\mathbf{b}^{\mathbf{0 + +}}$} & Seq. & $\mathbf{y}$ & $\mathbf{y}^{++}$ & $\mathbf{y}^{\mathbf{*}}$ & $\mathbf{y}^{\boldsymbol{*}^{++}}$ & $\mathbf{y}^{\mathbf{0}}$ & $\mathbf{y}^{\mathbf{0 + +}}$ & $\#$ \\
\hline $\mathbf{1}$ & 88.0393 & 44.5233 & & & 70.0287 & 35.5180 & $\mathbf{S}$ & & & & & & & $\mathbf{1 1}$ \\
\hline $\mathbf{2}$ & 248.0700 & 124.5386 & & & 230.0594 & 115.5333 & $\mathbf{C}$ & 1165.5154 & 583.2613 & 1148.4888 & 574.7480 & 1147.5048 & 574.2560 & $\mathbf{1 0}$ \\
\hline $\mathbf{3}$ & 361.1540 & 181.0806 & & & 343.1435 & 172.0754 & $\mathbf{I}$ & $\mathbf{1 0 0 5 . 4 8 4 7}$ & 503.2460 & 988.4582 & 494.7327 & 987.4742 & 494.2407 & $\mathbf{9}$ \\
\hline $\mathbf{4}$ & 476.1810 & 238.5941 & 459.1544 & 230.0808 & 458.1704 & 229.5888 & $\mathbf{N}$ & $\mathbf{8 9 2 . 4 0 0 7}$ & 446.7040 & 875.3741 & 438.1907 & 874.3901 & 437.6987 & $\mathbf{8}$ \\
\hline $\mathbf{5}$ & 605.2235 & 303.1154 & 588.1970 & 294.6021 & 587.2130 & 294.1101 & $\mathbf{E}$ & 777.3737 & 389.1905 & 760.3472 & 380.6772 & 759.3632 & 380.1852 & $\mathbf{7}$ \\
\hline
\end{tabular}




\begin{tabular}{|r|r|r|r|r|r|r|r|r|r|r|r|r|r|r|r|}
$\mathbf{6}$ & 692.2556 & 346.6314 & 675.2290 & 338.1182 & 674.2450 & 337.6261 & S & $\mathbf{6 4 8 . 3 3 1 1}$ & 324.6692 & 631.3046 & 316.1559 & 630.3206 & 315.6639 & $\mathbf{6}$ \\
\hline $\mathbf{7}$ & 763.2927 & 382.1500 & 746.2661 & 373.6367 & 745.2821 & 373.1447 & A & $\mathbf{5 6 1 . 2 9 9 1}$ & 281.1532 & 544.2726 & 272.6399 & 543.2885 & 272.1479 & $\mathbf{5}$ \\
\hline $\mathbf{8}$ & $\mathbf{8 7 6 . 3 7 6 8}$ & 438.6920 & 859.3502 & 430.1787 & 858.3662 & 429.6867 & $\mathbf{I}$ & $\mathbf{4 9 0 . 2 6 2 0}$ & 245.6346 & $\mathbf{4 7 3 . 2 3 5 4}$ & 237.1214 & $\mathbf{4 7 2 . 2 5 1 4}$ & 236.6293 & $\mathbf{4}$ \\
\hline $\mathbf{9}$ & $\mathbf{9 9 1 . 4 0 3 7}$ & 496.2055 & 974.3771 & 487.6922 & 973.3931 & 487.2002 & $\mathbf{D}$ & $\mathbf{3 7 7 . 1 7 7 9}$ & 189.0926 & 360.1514 & 180.5793 & 359.1674 & 180.0873 & $\mathbf{3}$ \\
\hline $\mathbf{1 0}$ & 1078.4357 & 539.7215 & 1061.4092 & 531.2082 & 1060.4252 & 530.7162 & S & $\mathbf{2 6 2 . 1 5 1 0}$ & 131.5791 & 245.1244 & 123.0659 & 244.1404 & 122.5738 & $\mathbf{2}$ \\
\hline $\mathbf{1 1}$ & & & & & & & $\mathbf{R}$ & 175.1190 & 88.0631 & 158.0924 & 79.5498 & & & $\mathbf{1}$ \\
\hline
\end{tabular}
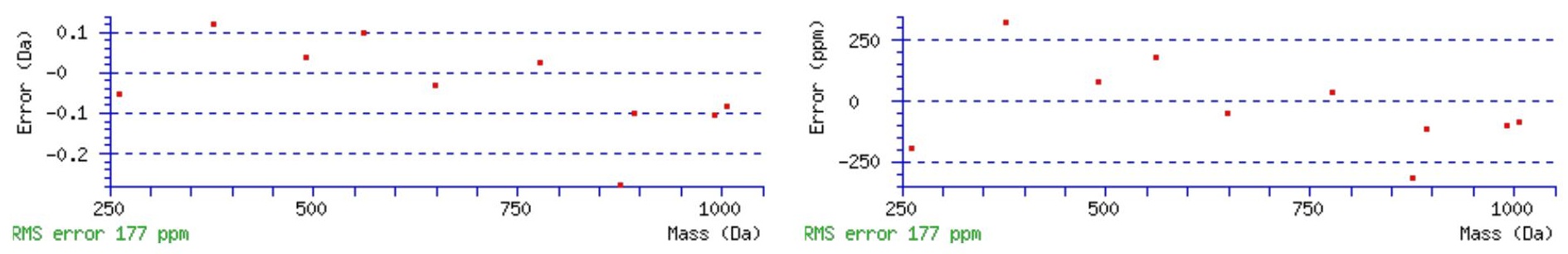

\section{All matches to this query}

\begin{tabular}{|l|l|l|l|}
\hline Score & Mr(calc): & Delta & \multicolumn{1}{c|}{ Sequence } \\
\hline 79.8 & 1251.5401 & -0.0009 & SCINESAIDSR \\
\hline 9.9 & 1250.5449 & 0.9944 & LDLMDEGTDAR \\
\hline 8.8 & 1251.5335 & 0.0057 & MVSTILSVSR \\
\hline 8.8 & 1251.5335 & 0.0057 & MVSTILSVSR \\
\hline 8.1 & 1251.5401 & -0.0009 & KMADENSNVSR \\
\hline 8.0 & 1251.5319 & 0.0074 & HIGSCKDLNK \\
\hline 7.9 & 1251.5335 & 0.0057 & MVSTILSVSR \\
\hline 7.9 & 1251.5280 & 0.0112 & EYQMMLLTK \\
\hline 7.0 & 1249.5439 & 1.9953 & SSSSVTSTSSIK \\
\hline 7.0 & 1249.5439 & 1.9953 & $\underline{\text { SSSSVTSTSSIK }}$ \\
\hline
\end{tabular}

Spectrum No: 231; Query: 135; Rank: 1

\section{Peptide View}

MS/MS Fragmentation of EGNATGHLIGR

Found in IPI00196620, Tax_Id=10116 Gene_Symbol=Cubn Cubilin precursor

Match to Query 135: 1124.558128 from(563.286340,2+)

Title: 100101RatKid_NS_deglyco_20.657.657.2.dta

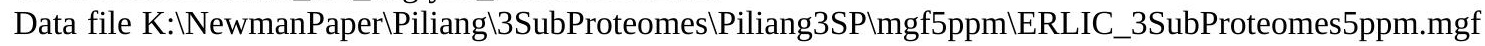

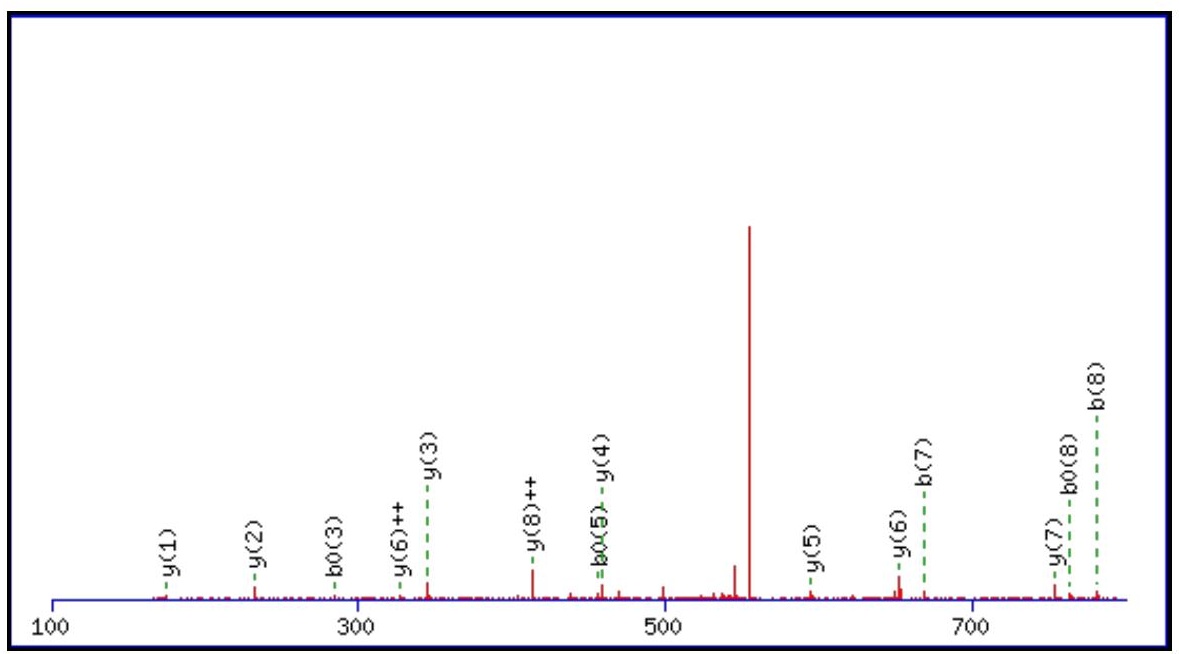



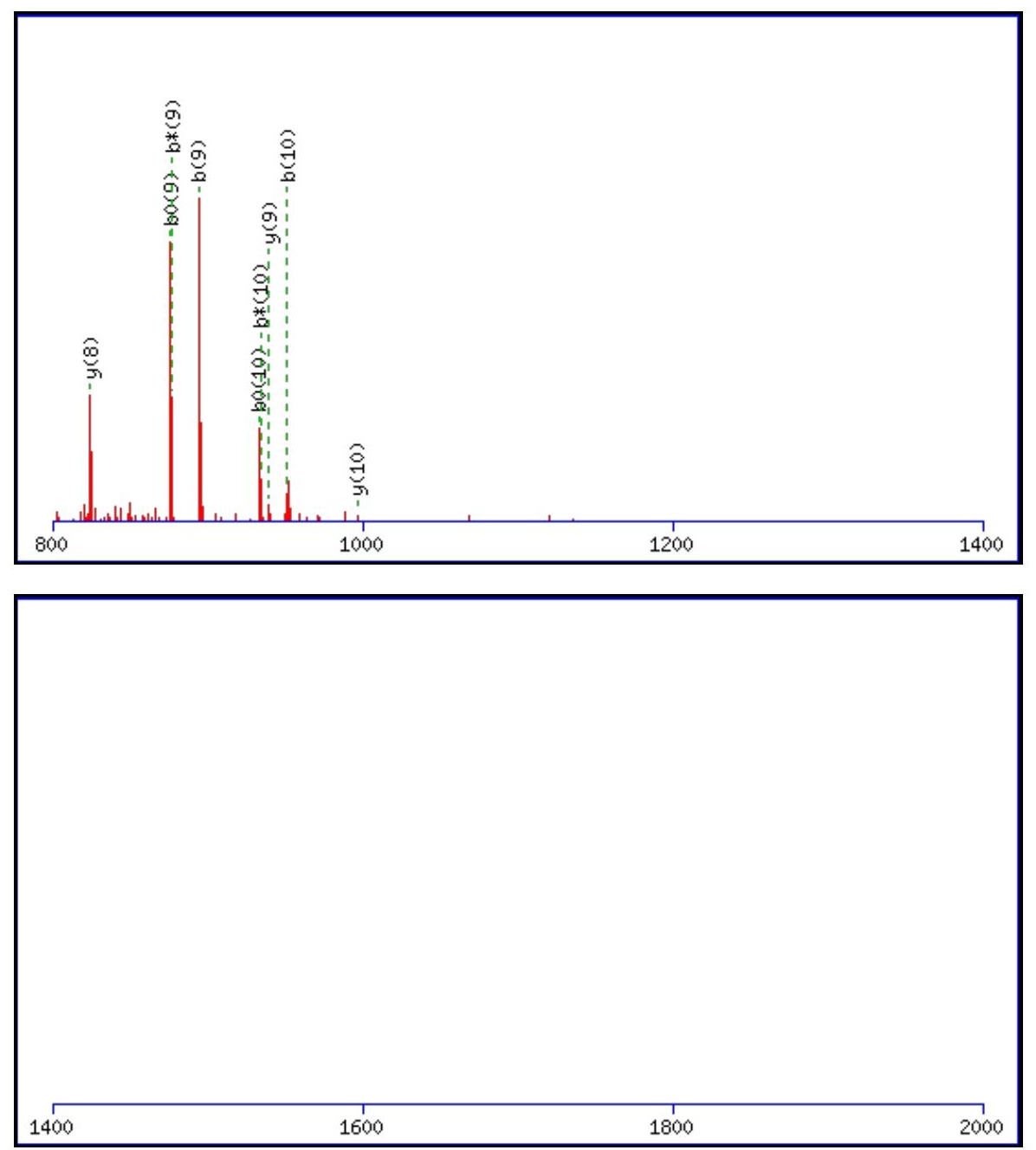

Monoisotopic mass of neutral peptide $\operatorname{Mr}($ calc): 1124.5574

Fixed modifications: Carbamidomethyl (C)

Variable modifications:

N3 : Deamidated_N (N)

Ions Score: 80 Expect: $1.2 \mathrm{e}-006$

Matches (Bold Red): 23/104 fragment ions using 42 most intense peaks

\begin{tabular}{|r|c|c|c|c|c|c|c|c|c|c|c|c|c|c|}
\hline$\#$ & $\mathbf{b}$ & $\mathbf{b}^{++}$ & $\mathbf{b}^{*}$ & $\mathbf{b}^{*_{++}^{+}}$ & $\mathbf{b}^{\mathbf{0}}$ & $\mathbf{b}^{\mathbf{0 + +}}$ & $\mathbf{S e q}$ & $\mathbf{y}$ & $\mathbf{y}^{++}$ & $\mathbf{y}^{*}$ & $\mathbf{y}^{\mathbf{*}^{++}}$ & $\mathbf{y}^{\mathbf{0}}$ & $\mathbf{y}^{\mathbf{0}+}$ & $\#$ \\
\hline $\mathbf{1}$ & 130.0499 & 65.5286 & & & 112.0393 & 56.5233 & $\mathbf{E}$ & & & & & & & $\mathbf{1 1}$ \\
\hline $\mathbf{2}$ & 187.0713 & 94.0393 & & & 169.0608 & 85.0340 & $\mathbf{G}$ & $\mathbf{9 9 6 . 5 2 2 1}$ & 498.7647 & 979.4956 & 490.2514 & 978.5115 & 489.7594 & $\mathbf{1 0}$ \\
\hline $\mathbf{3}$ & 302.0983 & 151.5528 & 285.0717 & 143.0395 & $\mathbf{2 8 4 . 0 8 7 7}$ & 142.5475 & $\mathbf{N}$ & $\mathbf{9 3 9 . 5 0 0 6}$ & 470.2540 & 922.4741 & 461.7407 & 921.4901 & 461.2487 & $\mathbf{9}$ \\
\hline $\mathbf{4}$ & 373.1354 & 187.0713 & 356.1088 & 178.5581 & 355.1248 & 178.0660 & $\mathbf{A}$ & $\mathbf{8 2 4 . 4 7 3 7}$ & $\mathbf{4 1 2 . 7 4 0 5}$ & 807.4472 & 404.2272 & 806.4631 & 403.7352 & $\mathbf{8}$ \\
\hline $\mathbf{5}$ & 474.1831 & 237.5952 & 457.1565 & 229.0819 & $\mathbf{4 5 6 . 1 7 2 5}$ & 228.5899 & $\mathbf{T}$ & 753.4366 & 377.2219 & 736.4101 & 368.7087 & 735.4260 & 368.2167 & $\mathbf{7}$ \\
\hline $\mathbf{6}$ & 531.2045 & 266.1059 & 514.1780 & 257.5926 & 513.1940 & 257.1006 & $\mathbf{G}$ & $\mathbf{6 5 2 . 3 8 8 9}$ & 326.6981 & 635.3624 & 318.1848 & & & $\mathbf{6}$ \\
\hline $\mathbf{7}$ & $\mathbf{6 6 8 . 2 6 3 4}$ & 334.6354 & 651.2369 & 326.1221 & 650.2529 & 325.6301 & $\mathbf{H}$ & $\mathbf{5 9 5 . 3 6 7 5}$ & 298.1874 & 578.3409 & 289.6741 & & & $\mathbf{5}$ \\
\hline $\mathbf{8}$ & $\mathbf{7 8 1 . 3 4 7 5}$ & 391.1774 & 764.3210 & 382.6641 & $\mathbf{7 6 3 . 3 3 6 9}$ & 382.1721 & $\mathbf{L}$ & $\mathbf{4 5 8 . 3 0 8 5}$ & 229.6579 & 441.2820 & 221.1446 & & & $\mathbf{4}$ \\
\hline $\mathbf{9}$ & $\mathbf{8 9 4 . 4 3 1 6}$ & 447.7194 & $\mathbf{8 7 7 . 4 0 5 0}$ & 439.2061 & $\mathbf{8 7 6 . 4 2 1 0}$ & 438.7141 & $\mathbf{I}$ & 345.2245 & 173.1159 & 328.1979 & 164.6026 & & & $\mathbf{3}$ \\
\hline $\mathbf{1 0}$ & $\mathbf{9 5 1 . 4 5 3 0}$ & 476.2302 & $\mathbf{9 3 4 . 4 2 6 5}$ & 467.7169 & $\mathbf{9 3 3 . 4 4 2 5}$ & $\mathbf{4 6 7 . 2 2 4 9}$ & $\mathbf{G}$ & 232.1404 & 116.5738 & 215.1139 & 108.0606 & & & $\mathbf{2}$ \\
\hline $\mathbf{1 1}$ & & & & & & & $\mathbf{R}$ & $\mathbf{1 7 5 . 1 1 9 0}$ & 88.0631 & 158.0924 & 79.5498 & & & $\mathbf{1}$ \\
\hline
\end{tabular}

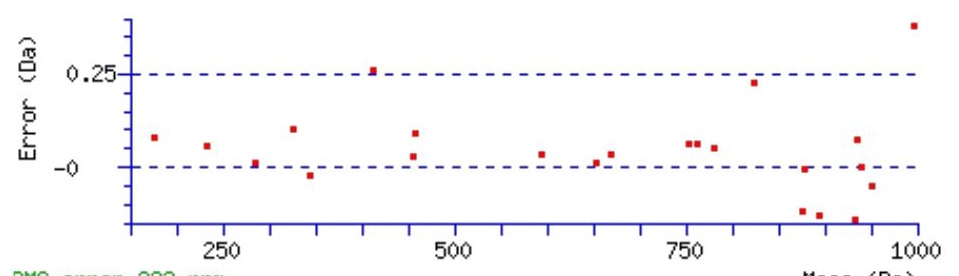

RMS error 222 ppm

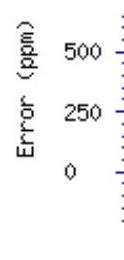

RMS error 222 ppm

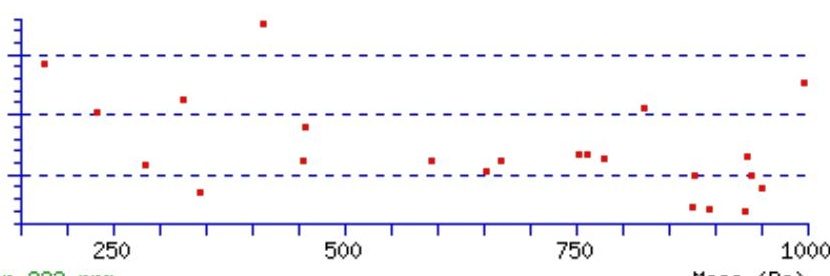

Mass (Da) 


\section{All matches to this query}

\begin{tabular}{|l|l|l|l|}
\hline Score & Mr(calc): & Delta & \multicolumn{1}{c|}{ Sequence } \\
\hline 79.7 & 1124.5574 & 0.0007 & EGNATGHLIGR \\
\hline 24.5 & 1124.5526 & 0.0056 & $\underline{\text { ATCVRLLGR }}$ \\
\hline 19.2 & 1124.5526 & 0.0056 & VAMSGRVIGR \\
\hline 17.7 & 1124.5583 & -0.0002 & $\underline{\text { ACILCVHPR }}$ \\
\hline 14.8 & 1123.5611 & 0.9970 & $\underline{\text { RGSRGSVAVR }}$ \\
\hline 13.4 & 1123.5499 & 1.0082 & $\underline{\text { RGSLGGALTGR }}$ \\
\hline 13.3 & 1123.5638 & 0.9943 & $\underline{\text { ETLGISVGLR }}$ \\
\hline 12.9 & 1124.5591 & -0.0009 & NETIKSVVR \\
\hline 12.9 & 1124.5615 & -0.0033 & $\underline{\text { FTSQPGYIGR }}$ \\
\hline 12.6 & 1123.5638 & 0.9943 & $\underline{\text { QTLDLVLSR }}$ \\
\hline
\end{tabular}

Spectrum No: 232; Query: 206; Rank: 1

\section{Peptide View}

MS/MS Fragmentation of VFGSQNLTTVK

Found in IPI00210975, Tax_Id=10116 Gene_Symbol=Hyou1 Hypoxia up-regulated protein 1 precursor

Match to Query 206: 1193.626748 from(597.820650,2+)

Title: 091008RatKidney_NH4Format01_25.1682.1682.2.dta

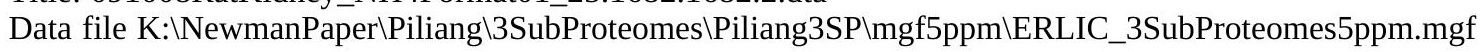

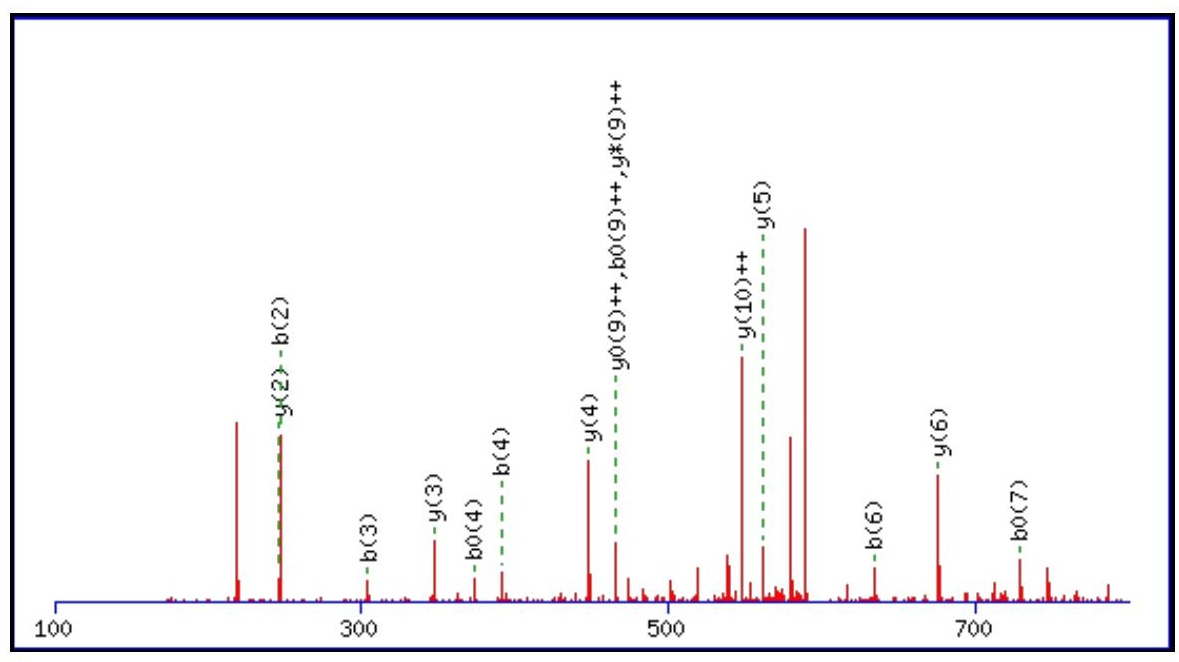




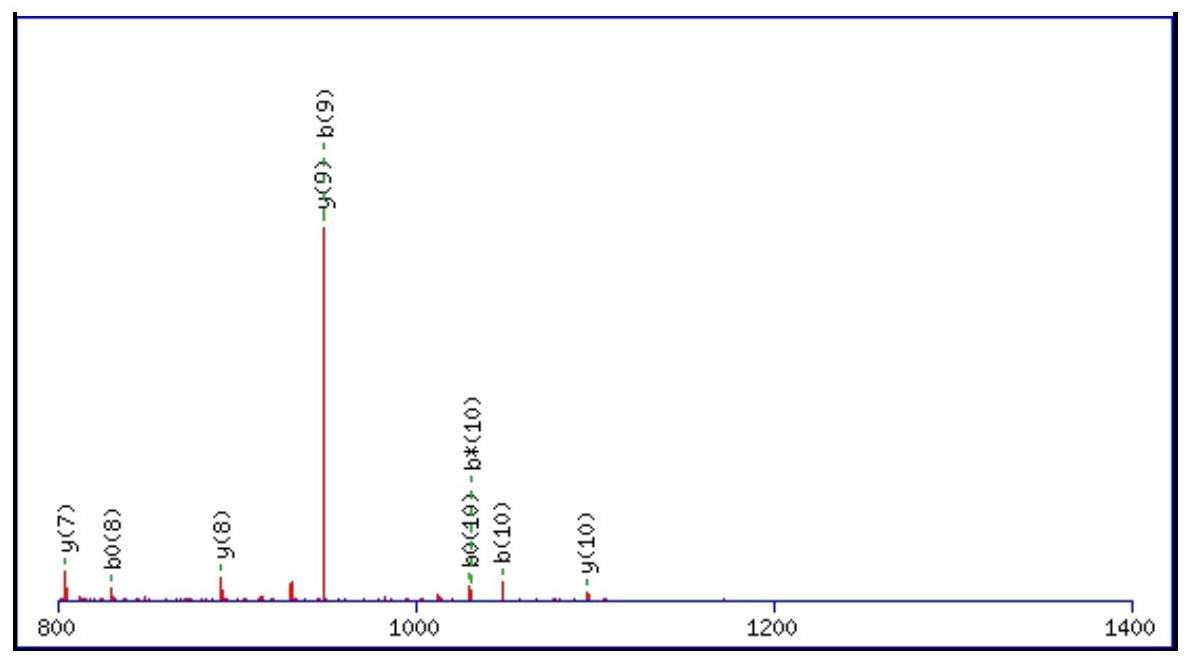

\begin{tabular}{rrrr}
\hline 1400 & 1600 & 1800 & 2000 \\
\hline
\end{tabular}

Monoisotopic mass of neutral peptide $\operatorname{Mr}($ calc): 1193.6292

Fixed modifications: Carbamidomethyl (C)

Variable modifications:

N6: Deamidated $\mathrm{N}(\mathrm{N})$

Ions Score: 80 Expect: $1 \mathrm{e}-006$

Matches (Bold Red): 24/102 fragment ions using 32 most intense peaks

\begin{tabular}{|r|c|c|c|c|c|c|c|c|c|c|c|c|c|c|}
\hline$\#$ & $\mathbf{b}$ & $\mathbf{b}^{++}$ & $\mathbf{b}^{*}$ & $\mathbf{b}^{\mathbf{*}^{++}}$ & $\mathbf{b}^{\mathbf{0}}$ & $\mathbf{b}^{\mathbf{0 + +}}$ & $\mathbf{S e q}$ & $\mathbf{y}$ & $\mathbf{y}^{++}$ & $\mathbf{y}^{\mathbf{*}}$ & $\mathbf{y}^{\mathbf{*}^{++}}$ & $\mathbf{y}^{\mathbf{0}}$ & $\mathbf{y}^{\mathbf{0}++}$ & $\#$ \\
\hline $\mathbf{1}$ & 100.0757 & 50.5415 & & & & & $\mathbf{V}$ & & & & & & & $\mathbf{1 1}$ \\
\hline $\mathbf{2}$ & $\mathbf{2 4 7 . 1 4 4 1}$ & 124.0757 & & & & & $\mathbf{F}$ & $\mathbf{1 0 9 5 . 5 6 8 1}$ & $\mathbf{5 4 8 . 2 8 7 7}$ & 1078.5415 & 539.7744 & 1077.5575 & 539.2824 & $\mathbf{1 0}$ \\
\hline $\mathbf{3}$ & $\mathbf{3 0 4 . 1 6 5 6}$ & 152.5864 & & & & & $\mathbf{G}$ & $\mathbf{9 4 8 . 4 9 9 6}$ & 474.7535 & 931.4731 & $\mathbf{4 6 6 . 2 4 0 2}$ & 930.4891 & $\mathbf{4 6 5 . 7 4 8 2}$ & $\mathbf{9}$ \\
\hline $\mathbf{4}$ & $\mathbf{3 9 1 . 1 9 7 6}$ & 196.1024 & & & 373.1870 & 187.0972 & $\mathbf{S}$ & $\mathbf{8 9 1 . 4 7 8 2}$ & 446.2427 & 874.4516 & 437.7295 & 873.4676 & 437.2374 & $\mathbf{8}$ \\
\hline $\mathbf{5}$ & 519.2562 & 260.1317 & 502.2296 & 251.6185 & 501.2456 & 251.1264 & $\mathbf{Q}$ & $\mathbf{8 0 4 . 4 4 6 2}$ & 402.7267 & 787.4196 & 394.2134 & 786.4356 & 393.7214 & $\mathbf{7}$ \\
\hline $\mathbf{6}$ & $\mathbf{6 3 4 . 2 8 3 1}$ & 317.6452 & 617.2566 & 309.1319 & 616.2725 & 308.6399 & $\mathbf{N}$ & $\mathbf{6 7 6 . 3 8 7 6}$ & 338.6974 & 659.3610 & 330.1842 & 658.3770 & 329.6921 & $\mathbf{6}$ \\
\hline $\mathbf{7}$ & $\mathbf{7 4 7 . 3 6 7 2}$ & 374.1872 & 730.3406 & 365.6740 & $\mathbf{7 2 9 . 3 5 6 6}$ & 365.1819 & $\mathbf{L}$ & $\mathbf{5 6 1 . 3 6 0 6}$ & 281.1840 & 544.3341 & 272.6707 & 543.3501 & 272.1787 & $\mathbf{5}$ \\
\hline $\mathbf{8}$ & 848.4149 & 424.7111 & 831.3883 & 416.1978 & $\mathbf{8 3 0 . 4 0 4 3}$ & 415.7058 & $\mathbf{T}$ & $\mathbf{4 4 8 . 2 7 6 6}$ & 224.6419 & 431.2500 & 216.1287 & 430.2660 & 215.6366 & $\mathbf{4}$ \\
\hline $\mathbf{9}$ & $\mathbf{9 4 9 . 4 6 2 5}$ & 475.2349 & 932.4360 & 466.7216 & 931.4520 & $\mathbf{4 6 6 . 2 2 9 6}$ & $\mathbf{T}$ & $\mathbf{3 4 7 . 2 2 8 9}$ & 174.1181 & 330.2023 & 165.6048 & 329.2183 & 165.1128 & $\mathbf{3}$ \\
\hline $\mathbf{1 0}$ & $\mathbf{1 0 4 8 . 5 3 0 9}$ & 524.7691 & $\mathbf{1 0 3 1 . 5 0 4 4}$ & 516.2558 & $\mathbf{1 0 3 0 . 5 2 0 4}$ & 515.7638 & $\mathbf{V}$ & $\mathbf{2 4 6 . 1 8 1 2}$ & 123.5942 & 229.1547 & 115.0810 & & & $\mathbf{2}$ \\
\hline $\mathbf{1 1}$ & & & & & & & $\mathbf{K}$ & 147.1128 & 74.0600 & 130.0863 & 65.5468 & & & $\mathbf{1}$ \\
\hline
\end{tabular}
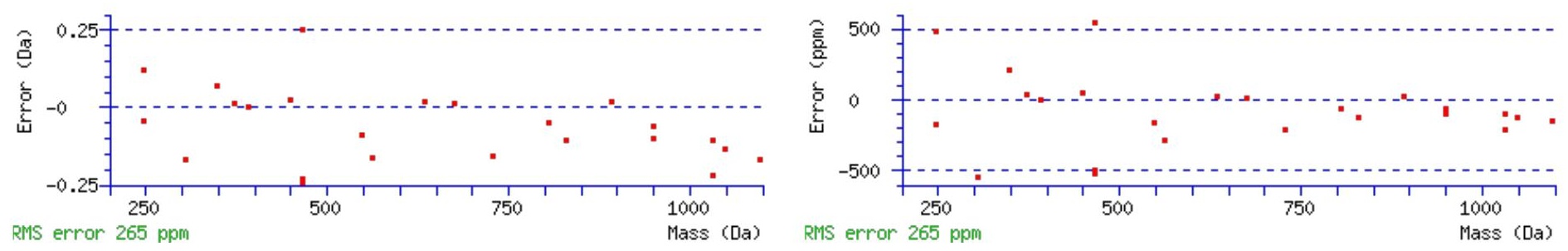


\section{All matches to this query}

\begin{tabular}{|l|l|l|l|}
\hline Score & Mr(calc): & Delta & \multicolumn{1}{|c|}{ Sequence } \\
\hline 79.7 & 1193.6292 & -0.0025 & VFGSQNLTTVK \\
\hline 10.4 & 1193.6326 & -0.0058 & TVMKTGLDSVK \\
\hline 9.3 & 1191.6169 & 2.0099 & MLTNGSLSEIK \\
\hline 7.5 & 1192.6121 & 1.0146 & MELKQISSNK \\
\hline 7.3 & 1193.6292 & -0.0024 & ANKAVDFTTVK \\
\hline 7.3 & 1192.6162 & 1.0105 & MPTSFVAPTVK \\
\hline 6.5 & 1193.6227 & 0.0041 & VFKKSSPNCK \\
\hline 4.7 & 1193.6326 & -0.0058 & KMASTSVDLVK \\
\hline 4.6 & 1193.6153 & 0.0115 & VQTHLENPTR \\
\hline 4.2 & 1193.6301 & -0.0033 & STKIWMAMVK \\
\hline
\end{tabular}

Spectrum No: 233; Query: 1677; Rank: 1

\section{Peptide View}

MS/MS Fragmentation of FSPLLYCQSQELPNGSVK

Found in IPI00200998, Tax_Id=10116 Gene_Symbol=Pdzk1 PDZ domain-containing protein 1

Match to Query 1677: 2067.002508 from(1034.508530,2+)

Title: 100101RatKid_NS_deglyco_05.3936.3936.2.dta

Data file K:INewmanPaper|Piliang|3SubProteomes\Piliang3SP\mgf5ppm\ERLIC_3SubProteomes5ppm.mgf
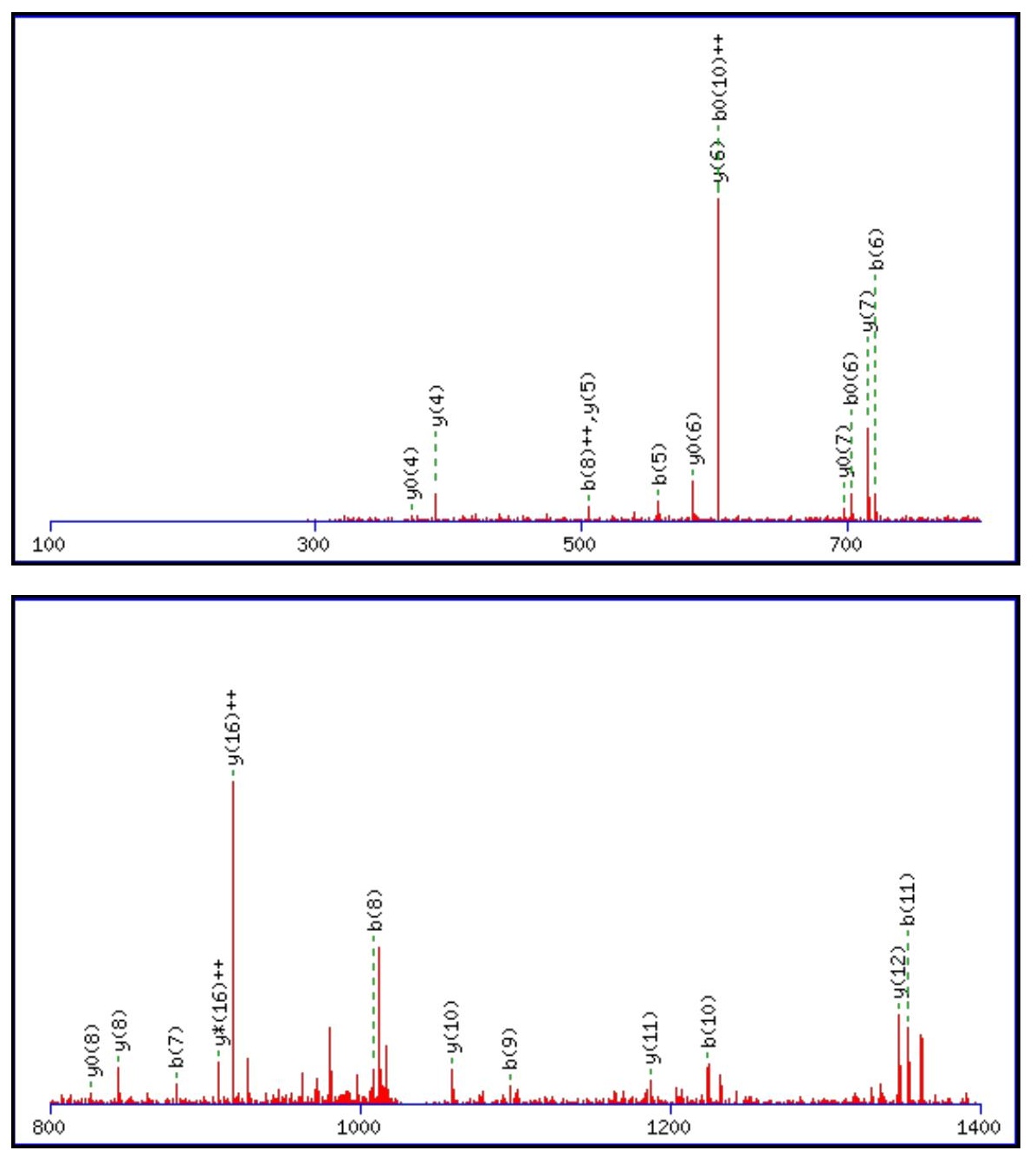


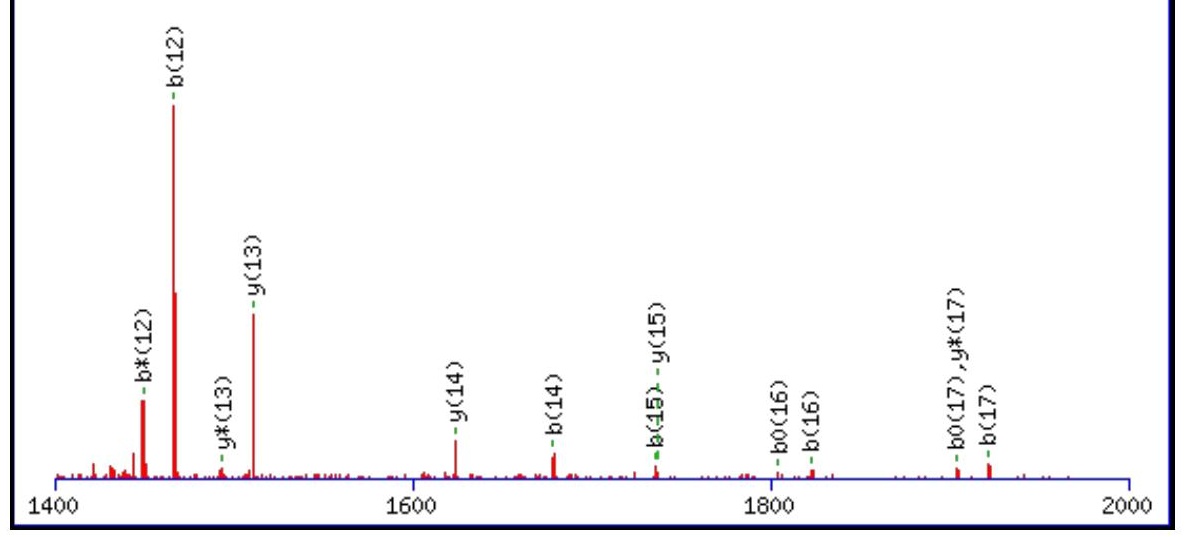

Monoisotopic mass of neutral peptide $\operatorname{Mr}($ calc): 2066.9983

Fixed modifications: Carbamidomethyl (C)

Variable modifications:

N14 : Deamidated_N ( N )

Ions Score: 80 Expect: 2.8e-006

Matches (Bold Red): 37/184 fragment ions using 56 most intense peaks

\begin{tabular}{|c|c|c|c|c|c|c|c|c|c|c|c|c|c|c|}
\hline \# & b & $\mathbf{b}^{++}$ & b* & $\mathbf{b}^{*^{++}}$ & $\mathbf{b}^{\mathbf{0}}$ & $\mathbf{b}^{0++}$ & Seq. & $\mathbf{y}$ & $\mathbf{y}^{++}$ & $\mathbf{y}^{*}$ & $\mathrm{y}^{*^{++}}$ & $\mathbf{y}^{0}$ & $\mathbf{y}^{0++}$ & \# \\
\hline 1 & 148.0757 & 74.5415 & & & & & $\mathbf{F}$ & & & & & & & 18 \\
\hline 2 & 235.1077 & 118.0575 & & & 217.0972 & 109.0522 & $S$ & 1920.9371 & 960.9722 & 1903.9106 & 952.4589 & 1902.9266 & 951.9669 & 17 \\
\hline 3 & 332.1605 & 166.5839 & & & 314.1499 & 157.5786 & $\mathbf{P}$ & 1833.9051 & 917.4562 & 1816.8786 & 908.9429 & 1815.8946 & 908.4509 & 16 \\
\hline 4 & 445.2445 & 223.1259 & & & 427.2340 & 214.1206 & $\mathbf{L}$ & 1736.8524 & 868.9298 & 1719.8258 & 860.4165 & 1718.8418 & 859.9245 & 15 \\
\hline 5 & 558.3286 & 279.6679 & & & 540.3180 & 270.6627 & $\mathbf{L}$ & 1623.7683 & 812.3878 & 1606.7417 & 803.8745 & 1605.7577 & 803.3825 & 14 \\
\hline 6 & 721.3919 & 361.1996 & & & 703.3814 & 352.1943 & $\mathbf{Y}$ & 1510.6842 & 755.8458 & 1493.6577 & 747.3325 & 1492.6737 & 746.8405 & 13 \\
\hline 7 & 881.4226 & 441.2149 & & & 863.4120 & 432.2096 & C & 1347.6209 & 674.3141 & 1330.5944 & 665.8008 & 1329.6103 & 665.3088 & 12 \\
\hline 8 & 1009.4812 & 505.2442 & 992.4546 & 496.7309 & 991.4706 & 496.2389 & $\mathbf{Q}$ & 1187.5903 & 594.2988 & 1170.5637 & 585.7855 & 1169.5797 & 585.2935 & 11 \\
\hline 9 & 1096.5132 & 548.7602 & 1079.4866 & 540.2470 & 1078.5026 & 539.7550 & S & 1059.5317 & 530.2695 & 1042.5051 & 521.7562 & 1041.5211 & 521.2642 & 10 \\
\hline 10 & 1224.5718 & 612.7895 & 1207.5452 & 604.2762 & 1206.5612 & 603.7842 & $\mathbf{Q}$ & 972.4996 & 486.7535 & 955.4731 & 478.2402 & 954.4891 & 477.7482 & 9 \\
\hline 11 & 1353.6144 & 677.3108 & 1336.5878 & 668.7975 & 1335.6038 & 668.3055 & $\mathbf{E}$ & 844.4411 & 422.7242 & 827.4145 & 414.2109 & 826.4305 & 413.7189 & 8 \\
\hline 12 & 1466.6984 & 733.8529 & 1449.6719 & 725.3396 & 1448.6879 & 724.8476 & $\mathbf{L}$ & 715.3985 & 358.2029 & 698.3719 & 349.6896 & 697.3879 & 349.1976 & 7 \\
\hline 13 & 1563.7512 & 782.3792 & 1546.7246 & 773.8660 & 1545.7406 & 773.3740 & $\mathbf{P}$ & 602.3144 & 301.6608 & 585.2879 & 293.1476 & 584.3038 & 292.6556 & 6 \\
\hline 14 & 1678.7781 & 839.8927 & 1661.7516 & 831.3794 & 1660.7676 & 830.8874 & $\mathbf{N}$ & 505.2616 & 253.1345 & 488.2351 & 244.6212 & 487.2511 & 244.1292 & 5 \\
\hline 15 & 1735.7996 & 868.4034 & 1718.7730 & 859.8902 & 1717.7890 & 859.3982 & G & 390.2347 & 195.6210 & 373.2082 & 187.1077 & 372.2241 & 186.6157 & 4 \\
\hline 16 & 1822.8316 & 911.9194 & 1805.8051 & 903.4062 & 1804.8211 & 902.9142 & S & 333.2132 & 167.1103 & 316.1867 & 158.5970 & 315.2027 & 158.1050 & 3 \\
\hline 17 & 1921.9000 & 961.4537 & 1904.8735 & 952.9404 & 1903.8895 & 952.4484 & V & 246.1812 & 123.5942 & 229.1547 & 115.0810 & & & 2 \\
\hline 18 & & & & & & & $\mathbf{K}$ & 147.1128 & 74.0600 & 130.0863 & 65.5468 & & & 1 \\
\hline
\end{tabular}
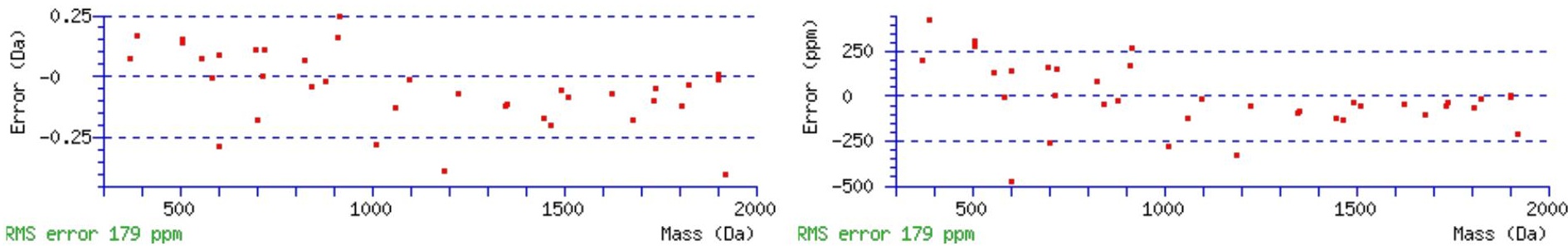

\section{All matches to this query}

\begin{tabular}{|l|c|c|l|}
\hline Score & Mr(calc): & Delta & \multicolumn{1}{c|}{ Sequence } \\
\hline 79.6 & 2066.9983 & 0.0042 & FSPLLYCQSQELPNGSVK \\
\hline 18.9 & 2066.0143 & 0.9882 & FSPLLYCQSQELPNGSVK \\
\hline 10.0 & 2067.0169 & -0.0144 & $\underline{\text { GDWNKLGILDMFLGCIAK }}$ \\
\hline
\end{tabular}




\begin{tabular}{|l|l|l|l|}
4.4 & 2067.0016 & 0.0009 & EAMCIGAEVYDNLKNVIK \\
\hline 4.0 & 2066.9925 & 0.0100 & QVLNFTLEDVLLPQSDR \\
\hline 2.5 & 2065.9905 & 1.0120 & ISAAGNSGNESGVRELLRR \\
\hline 1.3 & 2066.9981 & 0.0044 & EASREAESHPVTDGLRDAK \\
\hline 0.9 & 2066.9972 & 0.0053 & RKILQAFPDMHNSSISK \\
\hline 0.9 & 2066.9972 & 0.0053 & RKILQAFPDMHNSSISK \\
\hline 0.9 & 2067.0181 & -0.0156 & VFQECGTPHPVQSRNRR \\
\hline
\end{tabular}

Spectrum No: 234; Query: 1540; Rank: 1

\section{Peptide View}

MS/MS Fragmentation of TVTNAVVTVPAYFNDSQR

Found in IPI00208205, Tax_Id=10116 Gene_Symbol=Hspa8 Heat shock cognate 71 kDa protein

Match to Query 1540: 1981.967128 from(991.990840,2+)

Title: 091008RatKidney_NH4Format02_22.3468.3468.2.dta

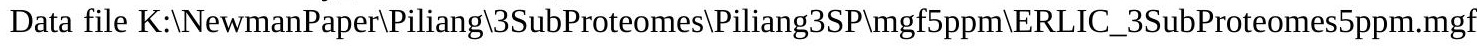
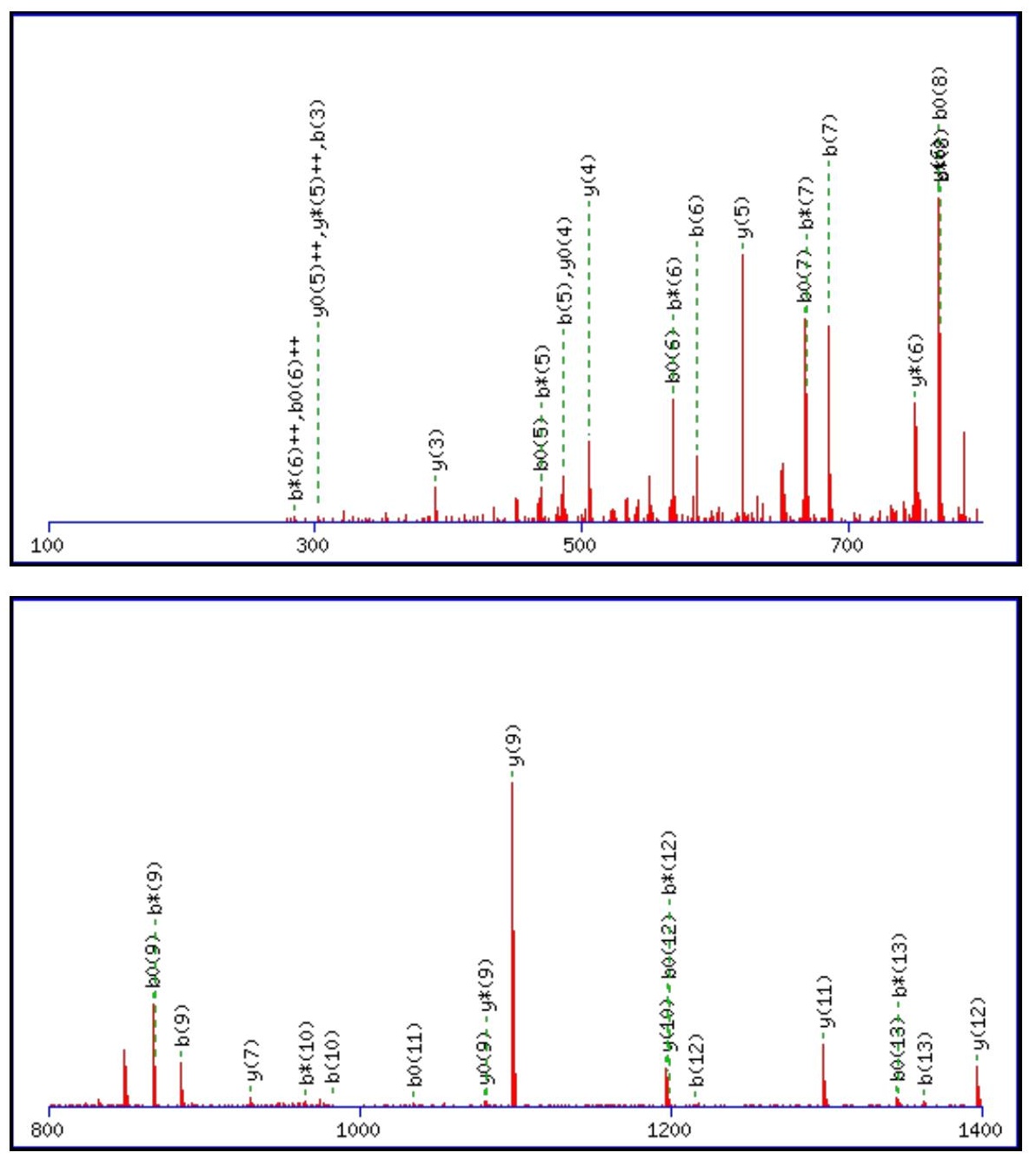


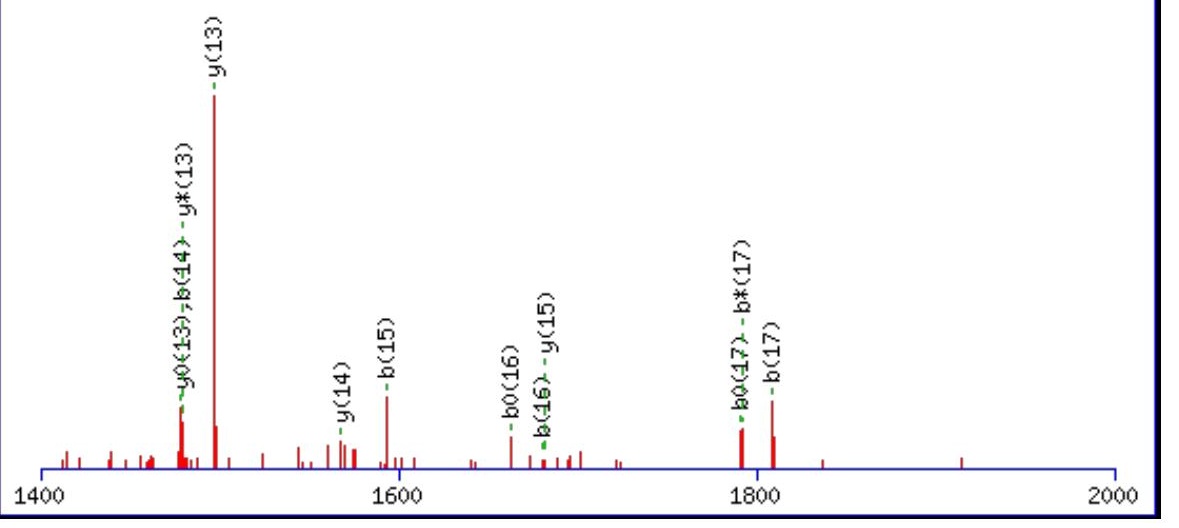

Monoisotopic mass of neutral peptide $\operatorname{Mr}($ calc): 1981.9745

Fixed modifications: Carbamidomethyl (C)

Variable modifications:

N14 : Deamidated_N (N)

Ions Score: 80 Expect: 2.3e- 006

Matches (Bold Red): 53/194 fragment ions using 86 most intense peaks

\begin{tabular}{|c|c|c|c|c|c|c|c|c|c|c|c|c|c|c|}
\hline \# & b & $\mathbf{b}^{++}$ & $\mathbf{b}^{*}$ & $\mathbf{b}^{*^{++}}$ & $\mathbf{b}^{\mathbf{0}}$ & $\mathbf{b}^{\mathbf{0 + +}}$ & Seq. & $\mathbf{y}$ & $\mathbf{y}^{++}$ & $\mathbf{y}^{*}$ & $\mathbf{y}^{*^{++}}$ & $\mathbf{y}^{0}$ & $\mathbf{y}^{0++}$ & \# \\
\hline 1 & 102.0550 & 51.5311 & & & 84.0444 & 42.5258 & $\mathbf{T}$ & & & & & & & 18 \\
\hline 2 & 201.1234 & 101.0653 & & & 183.1128 & 92.0600 & V & & & 1864.9076 & & 1863. & 932.4654 & 17 \\
\hline 3 & 302.1710 & 151.5892 & & & 284.1605 & 142.5839 & $\mathbf{T}$ & 1782.8657 & 891.9365 & 1765.8392 & 883.4232 & 1764.8551 & 882.9312 & 16 \\
\hline 4 & 416.2140 & 208.6106 & 399.1874 & 200.0974 & 398.2034 & 199.6053 & $\mathbf{N}$ & 1681.8180 & 841.4126 & 1664.7915 & 832.8994 & 1663.8075 & 832.4074 & 15 \\
\hline 5 & 487.2511 & 244.1292 & 470.2245 & 235.6159 & 469.2405 & 235.1239 & A & 1567.7751 & 784.3912 & 1550.7485 & 775.8779 & 1549.7645 & 775.3859 & 14 \\
\hline 6 & 586.3195 & 293.6634 & 569.2930 & 285.1501 & 568.3089 & 284.6581 & $\mathbf{V}$ & 1496.7380 & 748.8726 & 1479.7114 & 740.3594 & 1478.7274 & 739.8673 & 13 \\
\hline 7 & 685.3879 & 343.1976 & 668.3614 & 334.6843 & 667.3774 & 334.1923 & $\mathbf{V}$ & 1397.6696 & 699.3384 & 1380.6430 & 690.8251 & 1379.6590 & 690.3331 & 12 \\
\hline 8 & 786.4356 & 393.7214 & 769.4090 & 385.2082 & 768.4250 & 384.7162 & $\mathbf{T}$ & 1298.6012 & 649.8042 & 1281.5746 & 641.2909 & 1280.5906 & 640.7989 & 11 \\
\hline 9 & 885.5040 & 443.2556 & 868.4775 & 434.7424 & 867.4934 & 434.2504 & V & 1197.5535 & 599.2804 & 1180.5269 & 590.7671 & 1179.5429 & 590.2751 & 10 \\
\hline 10 & 982.5568 & 491.7820 & 965.5302 & 483.2688 & 964.5462 & 482.7767 & $\mathbf{P}$ & 1098.4851 & 549.7462 & 1081.4585 & 541.2329 & 1080.4745 & 540.7409 & 9 \\
\hline 11 & 1053.5939 & 527.3006 & 1036.5673 & 518.7873 & 1035.5833 & 518.2953 & A & 1001.4323 & 501.2198 & 984.4057 & 492.7065 & 983.4217 & 492.2145 & 8 \\
\hline 12 & 1216.6572 & 608.8322 & 1199.6307 & 600.3190 & 1198.6467 & 599.8270 & $\mathbf{Y}$ & 930.3952 & 465.7012 & 913.3686 & 457.1880 & 912.3846 & 456.6959 & 7 \\
\hline 13 & 1363.7256 & 682.3665 & 1346.6991 & 673.8532 & 1345.7151 & 673.3612 & $\mathbf{F}$ & 767.3319 & 384.1696 & 750.3053 & 375.6563 & 749.3213 & 375.1643 & 6 \\
\hline 14 & 1478.7526 & 739.8799 & 1461.7260 & 731.3666 & 1460.7420 & 730.8746 & $\mathbf{N}$ & 620.2634 & 310.6354 & 603.2369 & 302.1221 & 602.2529 & 301.6301 & 5 \\
\hline 15 & 1593.7795 & 797.3934 & 1576.7530 & 788.8801 & 1575.7689 & 788.3881 & D & 505.2365 & 253.1219 & 488.2100 & 244.6086 & 487.2259 & 244.1166 & 4 \\
\hline 16 & 1680.8115 & 840.9094 & 1663.7850 & 832.3961 & 1662.8010 & 831.9041 & S & 390.2096 & 195.6084 & 373.1830 & 187.0951 & 372.1990 & 186.6031 & 3 \\
\hline 17 & 1808.8701 & 904.9387 & 1791.8436 & 896.4254 & 1790.8596 & 895.9334 & $\mathbf{Q}$ & 303.1775 & 152.0924 & 286.1510 & 143.5791 & & & 2 \\
\hline 18 & & & & & & & $\mathbf{R}$ & 175.1190 & 88.0631 & 158.0924 & 79.5498 & & & 1 \\
\hline
\end{tabular}
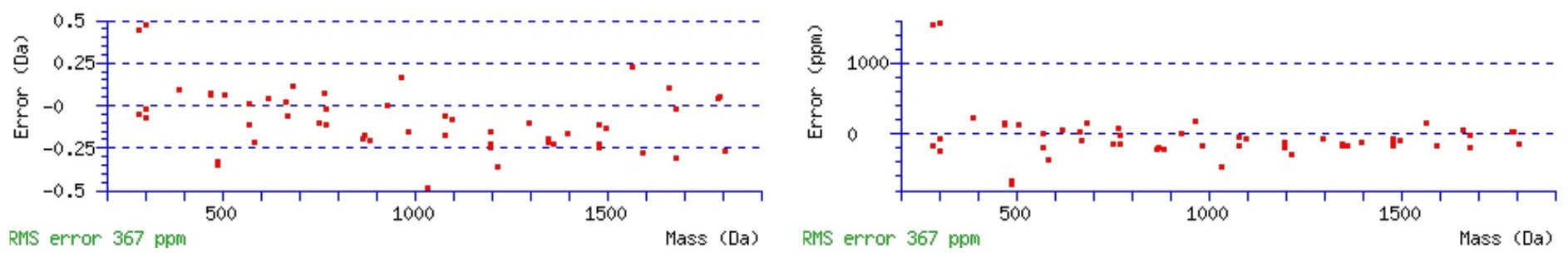

\section{All matches to this query}

\begin{tabular}{|l|l|l|l|}
\hline Score & Mr(calc): & Delta & \multicolumn{1}{c|}{ Sequence } \\
\hline 79.5 & 1981.9745 & -0.0074 & TVTNAVVTVPAYFNDSQR \\
\hline 11.2 & 1981.9745 & -0.0074 & TVTNAVVTVPAYFNDSQR \\
\hline 9.2 & 1981.9795 & -0.0124 & CLIVAQKSVTNTTPLEK \\
\hline & & & \\
\hline
\end{tabular}




\begin{tabular}{|l|l|l|l|}
8.0 & 1979.9717 & 1.9954 & EEQVVQVWSKKNIGEK \\
\hline 5.5 & 1981.9795 & -0.0124 & CLIVAQKSVTNTTPLEK \\
\hline 3.7 & 1979.9791 & 1.9880 & ALMQLLVNPFVGPLTNR \\
\hline 3.5 & 1981.9735 & -0.0063 & QNQDRINKTVLYGTPR \\
\hline 3.4 & 1981.9656 & 0.0016 & RKSMEASLTIVPANETR \\
\hline 2.7 & 1981.9735 & -0.0063 & QNQDRINKTVLYGTPR \\
\hline 2.5 & 1981.9730 & -0.0059 & AMDMRSPVLSTLLPSLR \\
\hline
\end{tabular}

Spectrum No: 235; Query: 1232; Rank: 1

\section{Peptide View}

MS/MS Fragmentation of EEEAIQLDGLNASQIR

Found in IPI00365985, Tax_Id=10116 Gene_Symbol=Tra1_predicted 93 kDa protein

Match to Query 1232: 1785.879948 from(893.947250,2+)

Title: 100101RatKid_NS_deglyco_16.3592.3592.2.dta

Data file K:INewmanPaper|Piliang|3SubProteomes\Piliang3SP\mgf5ppm\ERLIC_3SubProteomes5ppm.mgf
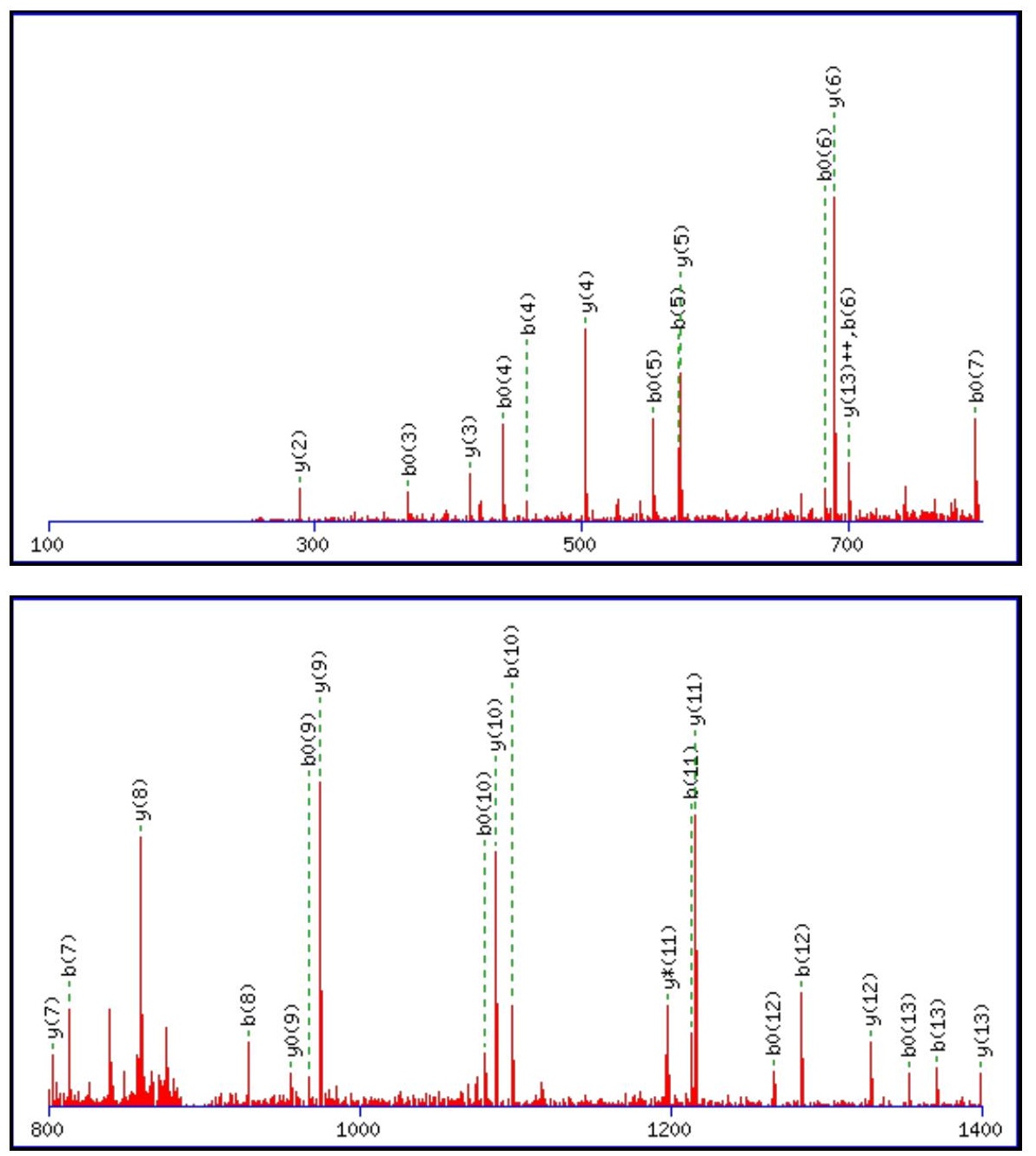


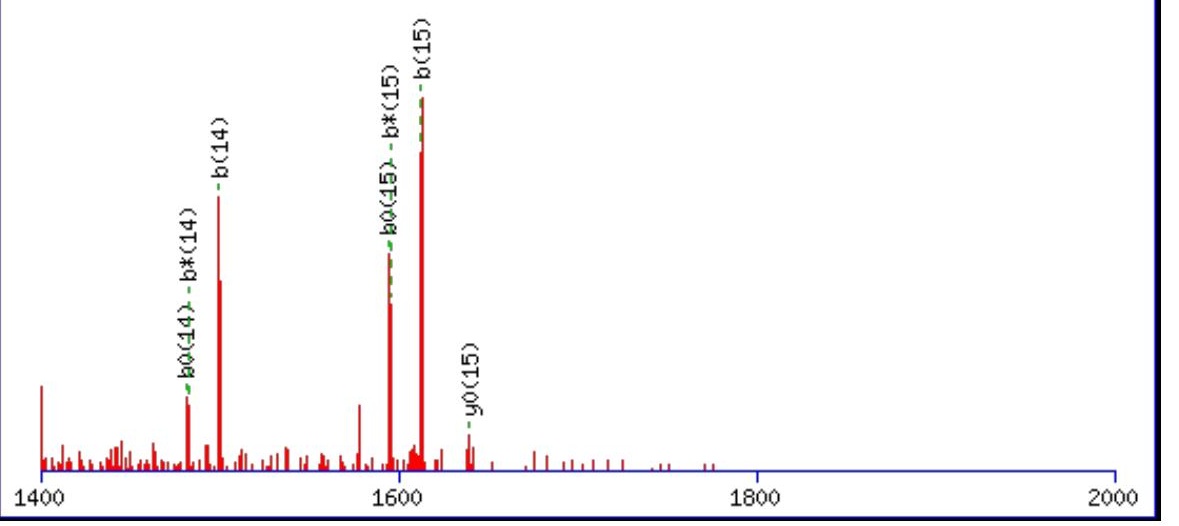

Monoisotopic mass of neutral peptide $\operatorname{Mr}($ calc): 1785.8744

Fixed modifications: Carbamidomethyl (C)

Variable modifications:

N11 : Deamidated_N (N)

Ions Score: 80 Expect: $2.1 \mathrm{e}-006$

Matches (Bold Red): 40/164 fragment ions using 72 most intense peaks

\begin{tabular}{|c|c|c|c|c|c|c|c|c|c|c|c|c|c|c|}
\hline$\#$ & b & $\mathbf{b}^{++}$ & b* & $\mathbf{b}^{*^{++}}$ & $\mathbf{b}^{0}$ & $\mathbf{b}^{\mathbf{0 + +}}$ & Seq. & $\mathbf{y}$ & $\mathbf{y}^{++}$ & $\mathbf{y}^{*}$ & $\mathrm{y}^{*^{++}}$ & $y^{0}$ & $\mathbf{y}^{0++}$ & \# \\
\hline 1 & 130.0499 & 65.5286 & & & 112.0393 & 56.5233 & $\mathbf{E}$ & & & & & & & 16 \\
\hline 2 & 259.0925 & 130.0499 & & & 241.0819 & 121.0446 & $\mathbf{E}$ & 1657.8391 & 829.4232 & 1640.8126 & 820.9099 & 1639.8286 & 820.4179 & 15 \\
\hline 3 & 388.1351 & 194.5712 & & & 370.1245 & 185.5659 & $\mathbf{E}$ & 1528.7966 & 764.9019 & 1511.7700 & 756.3886 & 1510.7860 & 755.8966 & 14 \\
\hline 4 & 459.1722 & 230.0897 & & & 441.1616 & 221.0844 & A & 1399.7540 & 700.3806 & 1382.7274 & 691.8673 & 1381.7434 & 691.3753 & 13 \\
\hline 5 & 572.2562 & 286.6318 & & & 554.2457 & 277.6265 & I & 1328.7168 & 664.8621 & 1311.6903 & 656.3488 & 1310.7063 & 655.8568 & 12 \\
\hline 6 & 700.3148 & 350.6610 & 683.2883 & 342.1478 & 682.3042 & 341.6558 & Q & 1215.6328 & 608.3200 & 1198.6062 & 599.8068 & 1197.6222 & 599.3147 & 11 \\
\hline 7 & 813.3989 & 407.2031 & 796.3723 & 398.6898 & 795.3883 & 398.1978 & $\mathbf{L}$ & 1087.5742 & 544.2907 & 1070.5477 & 535.7775 & 1069.5636 & 535.2855 & 10 \\
\hline 8 & 928.4258 & 464.7165 & 911.3993 & 456.2033 & 910.4153 & 455.7113 & D & 974.4901 & 487.7487 & 957.4636 & 479.2354 & 956.4796 & 478.7434 & 9 \\
\hline 9 & 985.4473 & 493.2273 & 968.4207 & 484.7140 & 967.4367 & 484.2220 & G & 859.4632 & 430.2352 & 842.4367 & 421.7220 & 841.4526 & 421.2300 & 8 \\
\hline 10 & 1098.5313 & 549.7693 & 1081.5048 & 541.2560 & 1080.5208 & 540.7640 & $\mathbf{L}$ & 802.4417 & 401.7245 & 785.4152 & 393.2112 & 784.4312 & 392.7192 & 7 \\
\hline 11 & 1213.5583 & 607.2828 & 1196.5317 & 598.7695 & 1195.5477 & 598.2775 & $\mathbf{N}$ & 689.3577 & 345.1825 & 672.3311 & 336.6692 & 671.3471 & 336.1772 & 6 \\
\hline 12 & 1284.5954 & 642.8013 & 1267.5688 & 634.2881 & 1266.5848 & 633.7961 & A & 574.3307 & 287.6690 & 557.3042 & 279.1557 & 556.3202 & 278.6637 & 5 \\
\hline 13 & 1371.6274 & 686.3174 & 1354.6009 & 677.8041 & 1353.6169 & 677.3121 & $S$ & 503.2936 & 252.1504 & 486.2671 & 243.6372 & 485.2831 & 243.1452 & 4 \\
\hline 14 & 1499.6860 & 750.3466 & 1482.6595 & 741.8334 & 1481.6754 & 741.3414 & $\mathbf{Q}$ & 416.2616 & 208.6344 & 399.2350 & 200.1212 & & & 3 \\
\hline 15 & 1612.7701 & 806.8887 & 1595.7435 & 798.3754 & 1594.7595 & 797.8834 & I & 288.2030 & 144.6051 & 271.1765 & 136.0919 & & & 2 \\
\hline 16 & & & & & & & $\mathbf{R}$ & 175.1190 & 88.0631 & 158.0924 & 79.5498 & & & 1 \\
\hline
\end{tabular}

$$
\text { 㩊 }
$$$$
\text { 営 }
$$$$
3
$$$$
\text { 聚- - }
$$
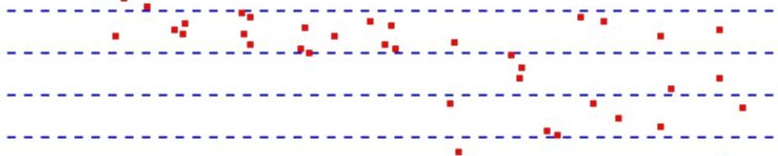

RMS error 137 ppm

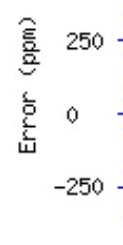

RMS error $137 \mathrm{ppm}$

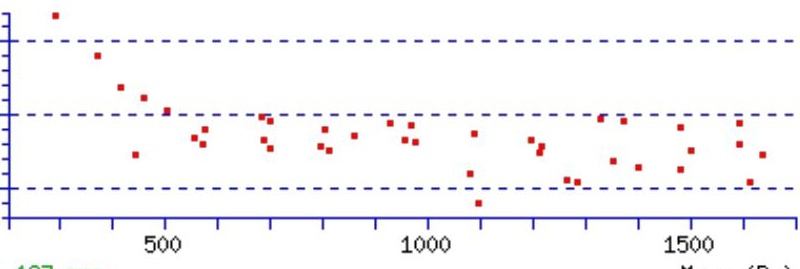

Mass (Da)

\section{All matches to this query}

\begin{tabular}{|l|l|l|l|}
\hline Score & Mr(calc): & Delta & \multicolumn{1}{c|}{ Sequence } \\
\hline 79.5 & 1785.8744 & 0.0055 & EEEAIQLDGLNASQIR \\
\hline 19.1 & 1784.8904 & 0.9895 & EEEAIQLDGLNASQIR \\
\hline 8.3 & 1785.8903 & -0.0104 & ANVTVLDTQIRKLR \\
\hline 0.2 & 1783.8886 & 1.9914 & TQPILDILLKNQTK \\
\hline 0.2 & 1784.8726 & 1.0074 & TQPILDILLKNQTK \\
\hline
\end{tabular}


Spectrum No: 236; Query: 2668; Rank: 1

\section{Peptide View}

MS/MS Fragmentation of RGVDVSQVAWQSQGDTPCSCCIVNNSNGSR

Found in IPI00211989, Tax_Id=10116 Gene_Symbol=Khk Isoform C of Ketohexokinase

Match to Query 2668: 3338.453682 from(1113.825170,3+)

Title: 100101RatKid_NS_deglyco_14.2737.2737.3.dta

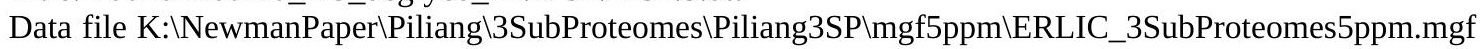
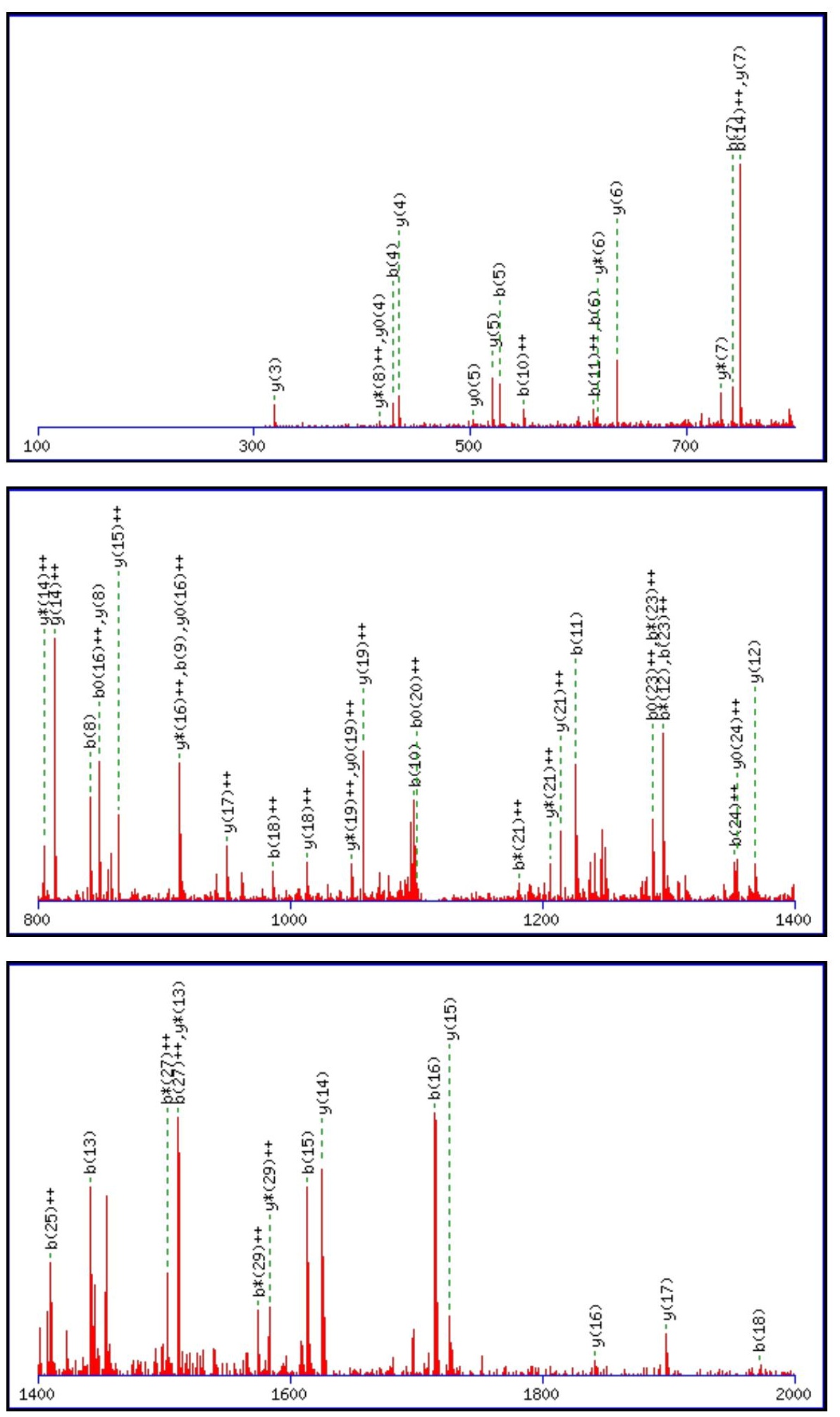
Monoisotopic mass of neutral peptide $\operatorname{Mr}($ calc): 3338.4463

Fixed modifications: Carbamidomethyl (C)

Variable modifications:

N27 : Deamidated_N (N)

Ions Score: 80 Expect: 5 e- 006

Matches (Bold Red): 59/340 fragment ions using 81 most intense peaks

\begin{tabular}{|c|c|c|c|c|c|c|c|c|c|c|c|c|c|c|}
\hline \# & b & $\mathbf{b}^{++}$ & b* & $\mathbf{b}^{*^{++}}$ & $\mathbf{b}^{0}$ & & Seq. & $\mathbf{y}$ & $\mathbf{y}^{++}$ & $\mathbf{y}^{*}$ & $\mathbf{y}^{*^{++}}$ & $\mathbf{y}^{\mathbf{0}}$ & $\mathbf{y}^{\mathbf{0 + +}}$ & \# \\
\hline 1 & 157.1084 & 79.0578 & 140.0818 & 70.5446 & & & $\mathbf{R}$ & & & & & & & 30 \\
\hline 2 & 214.1299 & 107.5686 & 197.1033 & 99.0553 & & & G & 3183.3524 & 1592.1799 & 3166.3259 & 1583.6666 & 3165.3419 & 1583.1746 & 29 \\
\hline 4 & 428.2252 & 214.6162 & 411.1987 & 206.1030 & 410.2146 & 205.6110 & D & 3027.2626 & 1514.1349 & 3010.2360 & 1505.6216 & 3009.2520 & 1505.1296 & 27 \\
\hline 5 & 527.2936 & 264.1504 & 510.2671 & 255.6372 & 509.2831 & 255.1452 & $\mathbf{V}$ & 2912.2356 & 1456.6214 & 2895.2091 & 1448.1082 & 2894.2250 & 1447.6162 & 26 \\
\hline 6 & 614.3257 & 307.6665 & 597.2991 & 299.1532 & 596.3151 & 298.6612 & $S$ & 2813.1672 & 1407.0872 & 2796.1406 & 1398.5740 & 2795.1566 & 1398.0820 & 25 \\
\hline 7 & 742.3842 & 371.6958 & 725.3577 & 363.1825 & 724.3737 & 362.6905 & $\mathbf{Q}$ & 2726.1352 & 1363.5712 & 2709.1086 & 1355.0579 & 2708.1246 & 1354.5659 & 24 \\
\hline 8 & 841.4526 & 421.2300 & 824.4261 & 412.7167 & 823.4421 & 412.2247 & V & 2598.0766 & 1299.5419 & 2581.0500 & 1291.0287 & 2580.0660 & 1290.5367 & 23 \\
\hline 9 & 912.4898 & 456.7485 & 895.4632 & 448.2352 & 894.4792 & 447.7432 & A & 2499.0082 & 1250.0077 & 2481.9816 & 1241.4945 & 2480.9976 & 1241.0024 & 22 \\
\hline 10 & 1098.5691 & 549.7882 & 1081.5425 & 541.2749 & 1080.5585 & 540.7829 & $\mathbf{W}$ & 2427.9711 & 1214.4892 & 2410.9445 & 1205.9759 & 2409.9605 & 1205.4839 & 21 \\
\hline 11 & 1226.6276 & 613.8175 & 1209.6011 & 605.3042 & 1208.6171 & 604.8122 & $\mathbf{Q}$ & 2241.8917 & 1121.4495 & 2224.8652 & 1112.9362 & 2223.8812 & 1112.4442 & 20 \\
\hline 12 & 1313.6597 & 657.3335 & 1296.6331 & 648.8202 & 1295.6491 & 648.3282 & $\mathrm{~S}$ & 2113.8332 & 1057.4202 & 2096.8066 & 1048.9069 & 2095.8226 & 1048.4149 & 19 \\
\hline 14 & 1498.7397 & 749.8735 & 1481.7132 & 741.3602 & 1480.7292 & 740.8682 & G & 1898.7426 & 949.8749 & 1881.7160 & 941.3616 & 1880.7320 & 940.8696 & 17 \\
\hline 15 & 1613.7667 & 807.3870 & 1596.7401 & 798.8737 & 1595.7561 & 798.3817 & D & 1841.7211 & 921.3642 & 1824.6946 & 912.8509 & 1823.7105 & 912.3589 & 16 \\
\hline 16 & 1714.8143 & 857.9108 & 1697.7878 & 849.3975 & 1696.8038 & 848.9055 & $\mathbf{T}$ & 1726.6942 & 863.8507 & 1709.6676 & 855.3374 & 1708.6836 & 854.8454 & 15 \\
\hline 17 & 1811.8671 & 906.4372 & 1794.8406 & 897.9239 & 1793.8565 & 897.4319 & $\mathbf{P}$ & 1625.6465 & 813.3269 & 1608.6199 & 804.8136 & 1607.6359 & 804.3216 & 14 \\
\hline 18 & 1971.8978 & 986.4525 & 1954.8712 & 977.9392 & 1953.8872 & 977.4472 & C & 1528.5937 & 764.8005 & 1511.5672 & 756.2872 & 1510.5831 & 755.7952 & 13 \\
\hline 19 & 2058.9298 & 1029.9685 & 2041.9032 & 1021.4553 & 2040.9192 & 1020.9632 & S & 1368.5631 & 684.7852 & 1351.5365 & 676.2719 & 1350.5525 & 675.7799 & 12 \\
\hline 20 & 2218.9604 & 1109.9839 & 2201.9339 & 1101.4706 & 2200.9499 & 1100.9786 & C & 1281.5310 & 641.2692 & 1264.5045 & 632.7559 & 1263.5205 & 632.2639 & 11 \\
\hline 21 & 2378.9911 & 1189.9992 & 2361.9645 & 1181.4859 & 2360.9805 & 1180.9939 & C & 1121.5004 & 561.2538 & 1104.4738 & 552.7406 & 1103.4898 & 552.2485 & 10 \\
\hline 22 & 2492.0751 & 1246.5412 & 2475.0486 & 1238.0279 & 2474.0646 & 1237.5359 & I & 961.4697 & 481.2385 & 944.4432 & 472.7252 & 943.4592 & 472.2332 & 9 \\
\hline 23 & 2591.1436 & 1296.0754 & 2574.1170 & 1287.5621 & 2573.1330 & 1287.0701 & V & 848.3857 & 424.6965 & 831.3591 & 416.1832 & 830.3751 & 415.6912 & 8 \\
\hline 24 & 2705.1865 & 1353.0969 & 2688.1599 & 1344.5836 & 2687.1759 & 1344.0916 & $\mathbf{N}$ & 749.3173 & 375.1623 & 732.2907 & 366.6490 & 731.3067 & 366.1570 & 7 \\
\hline 25 & 2819.2294 & 1410.1183 & 2802.2029 & 1401.6051 & 2801.2188 & 1401.1131 & $\mathbf{N}$ & 635.2743 & 318.1408 & 618.2478 & 309.6275 & 617.2638 & 309.1355 & 6 \\
\hline 26 & 2906.2614 & 1453.6344 & 2889.2349 & 1445.1211 & 2888.2509 & 1444.6291 & S & 521.2314 & 261.1193 & 504.2049 & 252.6061 & 503.2208 & 252.1141 & 5 \\
\hline 28 & 3078.3098 & 1539.6586 & 3061.2833 & 1531.1453 & 3060.2993 & 1530.6533 & G & 319.1724 & 160.0899 & 302.1459 & 151.5766 & 301.1619 & 151.0846 & 3 \\
\hline 29 & 3165.3419 & 1583.1746 & 3148.3153 & 1574.6613 & 3147.3313 & 1574.1693 & $\mathrm{~S}$ & 262.1510 & 131.5791 & 245.1244 & 123.0659 & 244.1404 & 122.5738 & 2 \\
\hline 30 & & & & & & & $\mathbf{R}$ & 175.1190 & 88.0631 & 158.0924 & 79.5498 & & & 1 \\
\hline
\end{tabular}
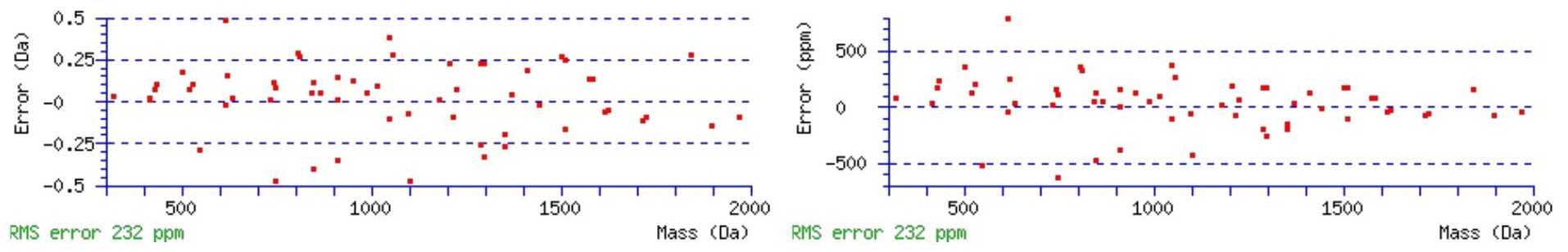

\section{All matches to this query}

\begin{tabular}{|l|c|c|c|}
\hline Score & Mr(calc): & Delta & \multicolumn{1}{c|}{ Sequence } \\
\hline 79.5 & 3338.4463 & 0.0074 & RGVDVSQVAWQSQGDTPCSCCIVNNSNGSR \\
\hline 71.6 & 3338.4463 & 0.0074 & RGVDVSQVAWQSQGDTPCSCCIVNNSNGSR \\
\hline 68.5 & 3338.4463 & 0.0074 & RGVDVSQVAWQSQGDTPCSCCIVNNSNGSR \\
\hline 39.4 & 3337.4623 & 0.9914 & RGVDVSOVAWQSQGDTPCSCCIVNNSNGSR \\
\hline & & &
\end{tabular}




\begin{tabular}{|l|l|l|l|}
29.2 & 3337.4736 & 0.9801 & VYHCNWLMPSQKASVESCIRVLSDVAK \\
\hline 29.2 & 3337.4736 & 0.9801 & VYHCNWLMPSQKASVESCIRVLSDVAK \\
\hline 15.8 & 3337.4736 & 0.9801 & VYHCNWLMPSQKASVESCIRVLSDVAK \\
\hline 15.8 & 3337.4736 & 0.9801 & VYHCNWLMPSQKASVESCIRVLSDVAK \\
\hline 7.0 & 3337.4736 & 0.9801 & VYHCNWLMPSQKASVESCIRVLSDVAK \\
\hline 6.2 & 3338.4646 & -0.0109 & GEGQNRSPIYSFIGGEHFPRITNVSDATK \\
\hline
\end{tabular}

Spectrum No: 237; Query: 152; Rank: 1

\section{Peptide View}

MS/MS Fragmentation of LNASIADLQSK

Found in IPI00190577, Tax_Id=10116 Gene_Symbol=Lama5 similar to Laminin alpha-5 chain precursor

Match to Query 152: 1159.607608 from(580.811080,2+)

Title: 091008RatKidney_NoSalt_27.1695.1695.2.dta

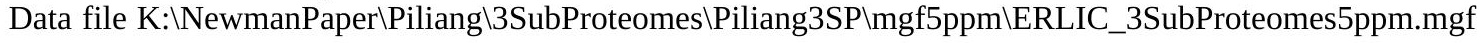
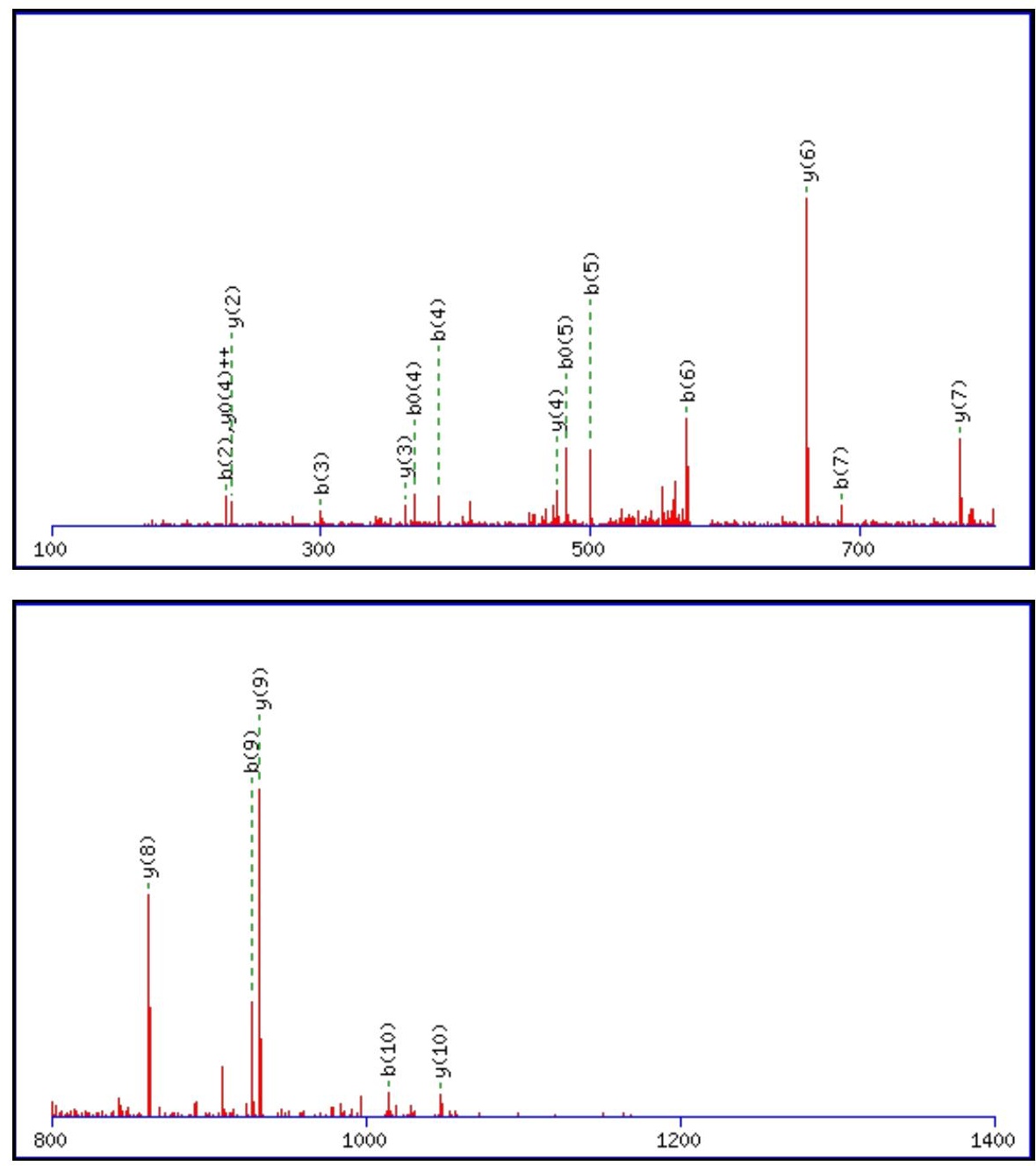


$1400 \quad 1600 \quad 1800 \quad 2000$

Monoisotopic mass of neutral peptide $\operatorname{Mr}($ calc): 1159.6084

Fixed modifications: Carbamidomethyl (C)

Variable modifications:

N2 : Deamidated $\mathrm{N}(\mathrm{N})$

Ions Score: 79 Expect: $1.3 \mathrm{e}-006$

Matches (Bold Red): 19/110 fragment ions using 26 most intense peaks

\begin{tabular}{|r|c|c|c|c|c|c|c|c|c|c|c|c|c|c|}
\hline$\#$ & $\mathbf{b}$ & $\mathbf{b}^{++}$ & $\mathbf{b}^{*}$ & $\mathbf{b}^{*^{++}}$ & $\mathbf{b}^{\mathbf{0}}$ & $\mathbf{b}^{\mathbf{0 + +}}$ & $\mathbf{S e q}$ & $\mathbf{y}$ & $\mathbf{y}^{++}$ & $\mathbf{y}^{\mathbf{*}}$ & $\mathbf{y}^{\mathbf{*}^{++}}$ & $\mathbf{y}^{\mathbf{0}}$ & $\mathbf{y}^{\mathbf{0 + +}}$ & $\#$ \\
\hline $\mathbf{1}$ & 114.0913 & 57.5493 & & & & & $\mathbf{L}$ & & & & & & & $\mathbf{1 1}$ \\
\hline $\mathbf{2}$ & $\mathbf{2 2 9 . 1 1 8 3}$ & 115.0628 & 212.0917 & 106.5495 & & & $\mathbf{N}$ & $\mathbf{1 0 4 7 . 5 3 1 7}$ & 524.2695 & 1030.5051 & 515.7562 & 1029.5211 & 515.2642 & $\mathbf{1 0}$ \\
\hline $\mathbf{3}$ & $\mathbf{3 0 0 . 1 5 5 4}$ & 150.5813 & 283.1288 & 142.0681 & & & $\mathbf{A}$ & $\mathbf{9 3 2 . 5 0 4 7}$ & 466.7560 & 915.4782 & 458.2427 & 914.4942 & 457.7507 & $\mathbf{9}$ \\
\hline $\mathbf{4}$ & $\mathbf{3 8 7 . 1 8 7 4}$ & 194.0973 & 370.1609 & 185.5841 & 369.1769 & 185.0921 & $\mathbf{S}$ & $\mathbf{8 6 1 . 4 6 7 6}$ & 431.2374 & 844.4411 & 422.7242 & 843.4571 & 422.2322 & $\mathbf{8}$ \\
\hline $\mathbf{5}$ & $\mathbf{5 0 0 . 2 7 1 5}$ & 250.6394 & 483.2449 & 242.1261 & $\mathbf{4 8 2 . 2 6 0 9}$ & 241.6341 & $\mathbf{I}$ & 774.4356 & 387.7214 & 757.4090 & 379.2082 & 756.4250 & 378.7162 & $\mathbf{7}$ \\
\hline $\mathbf{6}$ & $\mathbf{5 7 1 . 3 0 8 6}$ & 286.1579 & 554.2820 & 277.6447 & 553.2980 & 277.1527 & $\mathbf{A}$ & $\mathbf{6 6 1 . 3 5 1 5}$ & 331.1794 & 644.3250 & 322.6661 & 643.3410 & 322.1741 & $\mathbf{6}$ \\
\hline $\mathbf{7}$ & $\mathbf{6 8 6 . 3 3 5 5}$ & 343.6714 & 669.3090 & 335.1581 & 668.3250 & 334.6661 & $\mathbf{D}$ & 590.3144 & 295.6608 & 573.2879 & 287.1476 & 572.3039 & 286.6556 & $\mathbf{5}$ \\
\hline $\mathbf{8}$ & 799.4196 & 400.2134 & 782.3931 & 391.7002 & 781.4090 & 391.2082 & $\mathbf{L}$ & 475.2875 & 238.1474 & 458.2609 & 229.6341 & 457.2769 & $\mathbf{2 2 9 . 1 4 2 1}$ & $\mathbf{4}$ \\
\hline $\mathbf{9}$ & $\mathbf{9 2 7 . 4 7 8 2}$ & 464.2427 & 910.4516 & 455.7295 & 909.4676 & 455.2374 & $\mathbf{Q}$ & $\mathbf{3 6 2 . 2 0 3 4}$ & 181.6053 & 345.1769 & 173.0921 & 344.1928 & 172.6001 & $\mathbf{3}$ \\
\hline $\mathbf{1 0}$ & $\mathbf{1 0 1 4 . 5 1 0 2}$ & 507.7587 & 997.4837 & 499.2455 & 996.4996 & 498.7535 & $\mathbf{S}$ & $\mathbf{2 3 4 . 1 4 4 8}$ & 117.5761 & 217.1183 & 109.0628 & 216.1343 & 108.5708 & $\mathbf{2}$ \\
\hline $\mathbf{1 1}$ & & & & & & & $\mathbf{K}$ & 147.1128 & 74.0600 & 130.0863 & 65.5468 & & & $\mathbf{1}$ \\
\hline
\end{tabular}
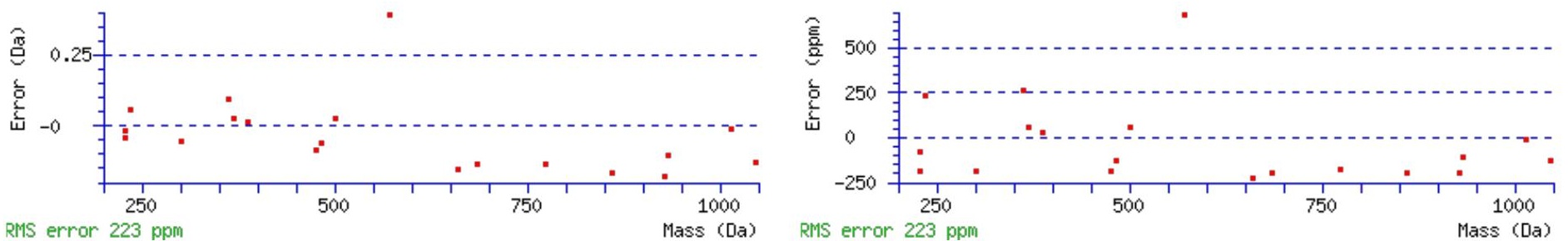

\section{All matches to this query}

\begin{tabular}{|l|l|l|l|}
\hline Score & Mr(calc): & Delta & \multicolumn{1}{|c|}{ Sequence } \\
\hline 79.3 & 1159.6084 & -0.0008 & LNASIADLQSK \\
\hline 22.4 & 1159.6084 & -0.0008 & LEGSLEQEKK \\
\hline 7.3 & 1159.6019 & 0.0057 & GNLTKHMKSK \\
\hline 6.8 & 1159.6115 & -0.0038 & LHVSSGLLVR \\
\hline 5.8 & 1159.6115 & -0.0038 & LHVSSGLLVR \\
\hline 5.4 & 1159.6019 & 0.0057 & LDSAVQMAGLR \\
\hline 5.1 & 1159.6084 & -0.0008 & GESAILVENTK \\
\hline 4.6 & 1159.6084 & -0.0008 & LEQELKDSAK \\
\hline 3.5 & 1157.6015 & 2.0061 & LVPGVSMWNR \\
\hline 3.3 & 1159.6132 & -0.0056 & LDRPTKQMR \\
\hline
\end{tabular}


Spectrum No: 238; Query: 189; Rank: 1

\section{Peptide View}

MS/MS Fragmentation of ISSNETEIMR

Found in IPI00370427, Tax_Id=10116 Gene_Symbol=Tinag Tubulointerstitial nephritis antigen

Match to Query 189: 1179.545028 from(590.779790,2+)

Title: 100101RatKid_NS_deglyco_23.1241.1241.2.dta

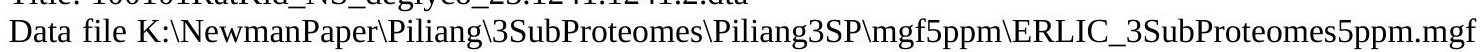
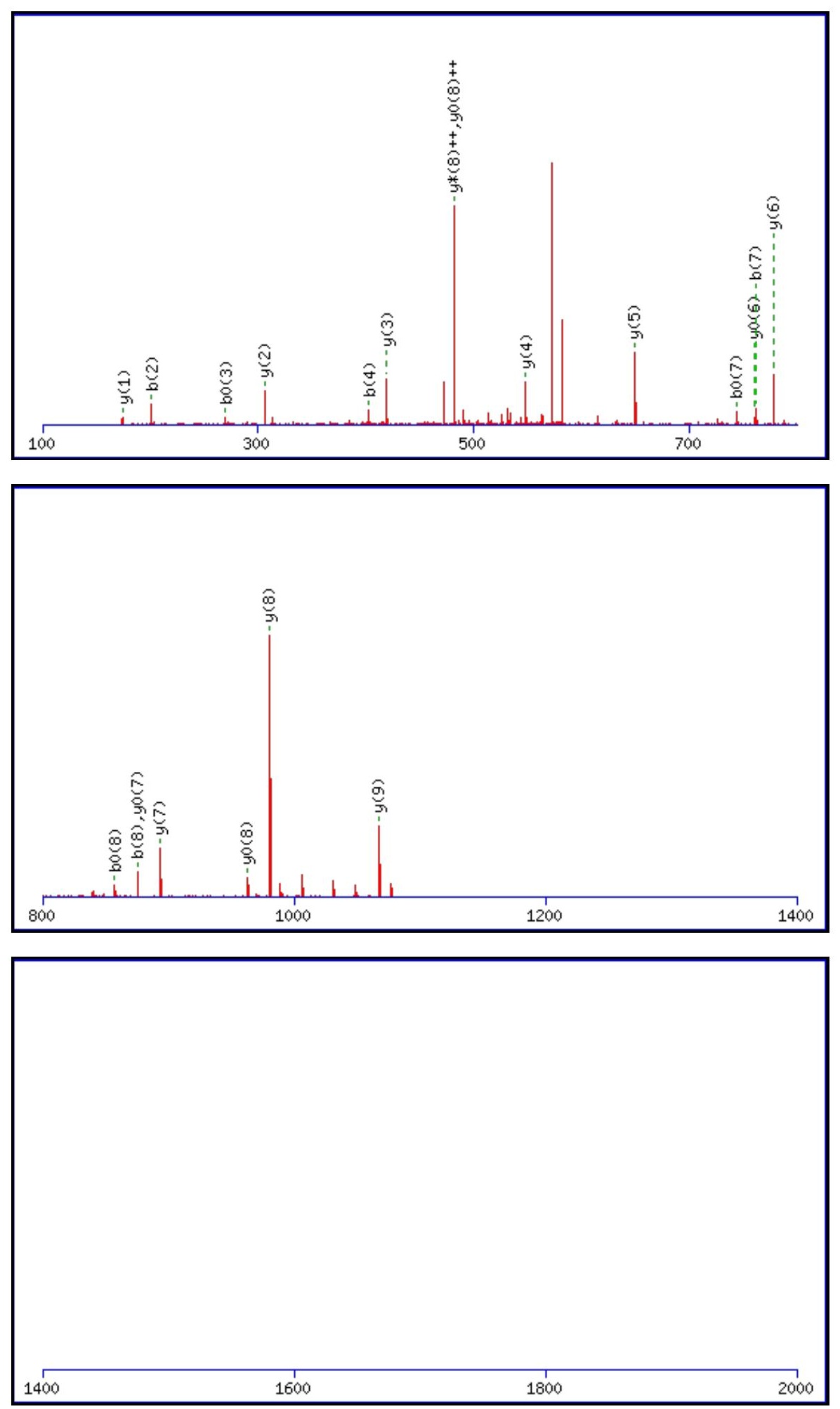
Monoisotopic mass of neutral peptide $\operatorname{Mr}($ calc): 1179.5441

Fixed modifications: Carbamidomethyl (C)

Variable modifications:

N4 : Deamidated_N (N)

Ions Score: 79 Expect: $1.3 e-006$

Matches (Bold Red): 21/94 fragment ions using 30 most intense peaks

\begin{tabular}{|c|c|c|c|c|c|c|c|c|c|c|c|c|c|c|}
\hline \# & b & $\mathbf{b}^{++}$ & b* & $\mathbf{b}^{*^{++}}$ & $\mathbf{b}^{0}$ & $\mathbf{b}^{0++}$ & Seq. & $\mathbf{y}$ & $\mathbf{y}^{++}$ & $\mathbf{y}^{*}$ & $\mathbf{y}^{*^{++}}$ & $\mathbf{y}^{0}$ & $\mathbf{y}^{0++}$ & \# \\
\hline 1 & 114.0913 & 57.5493 & & & & & I & & & & & & & 10 \\
\hline 2 & 201.1234 & 101.0653 & & & 183.1128 & 92.0600 & $\mathrm{~S}$ & 1067.4674 & 534.2373 & 1050.4408 & 525.7240 & 1049.4568 & 525.2320 & 9 \\
\hline 3 & 288.1554 & 144.5813 & & & 270.1448 & 135.5761 & S & 980.4353 & 490.7213 & 963.4088 & 482.2080 & 962.4248 & 481.7160 & 8 \\
\hline 4 & 403.1823 & 202.0948 & 386.1558 & 193.5815 & 385.1718 & 193.0895 & $\mathbf{N}$ & 893.4033 & 447.2053 & 876.3768 & 438.6920 & 875.3927 & 438.2000 & 7 \\
\hline 5 & 532.2249 & 266.6161 & 515.1984 & 258.1028 & 514.2144 & 257.6108 & $\mathbf{E}$ & 778.3764 & 389.6918 & 761.3498 & 381.1785 & 760.3658 & 380.6865 & 6 \\
\hline 6 & 633.2726 & 317.1399 & 616.2461 & 308.6267 & 615.2620 & 308.1347 & $\mathbf{T}$ & 649.3338 & 325.1705 & 632.3072 & 316.6573 & 631.3232 & 316.1652 & 5 \\
\hline 7 & 762.3152 & 381.6612 & 745.2886 & 373.1480 & 744.3046 & 372.6560 & $E$ & 548.2861 & 274.6467 & 531.2595 & 266.1334 & 530.2755 & 265.6414 & 4 \\
\hline 8 & 875.3993 & 438.2033 & 858.3727 & 429.6900 & 857.3887 & 429.1980 & I & 419.2435 & 210.1254 & 402.2170 & 201.6121 & & & 3 \\
\hline 9 & 1006.4397 & 503.7235 & 989.4132 & 495.2102 & 988.4292 & 494.7182 & $\mathbf{M}$ & 306.1594 & 153.5834 & 289.1329 & 145.0701 & & & 2 \\
\hline 10 & & & & & & & $\mathbf{R}$ & 175.1190 & 88.0631 & 158.0924 & 79.5498 & & & 1 \\
\hline
\end{tabular}
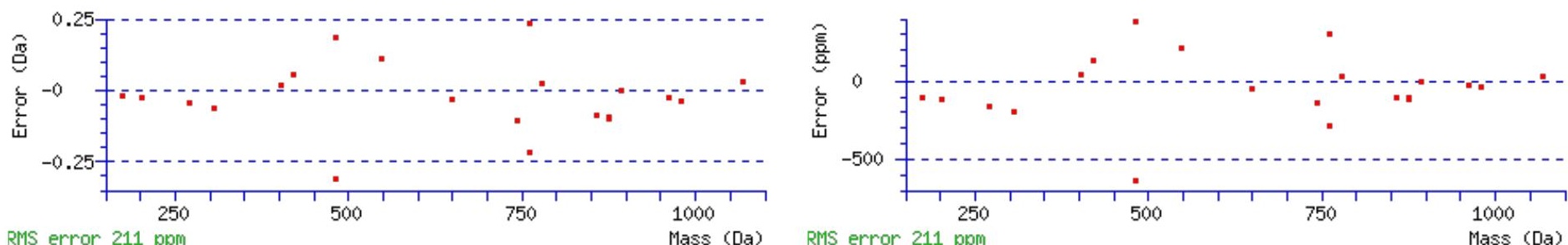

RMS error 211 ppm

All matches to this query

\begin{tabular}{|l|l|l|l|}
\hline Score & Mr(calc): & Delta & \multicolumn{1}{c|}{ Sequence } \\
\hline 79.3 & 1179.5441 & 0.0009 & $\underline{\text { ISSNETEIMR }}$ \\
\hline 12.9 & 1179.5554 & -0.0103 & $\underline{\text { ISSGAKDMAER }}$ \\
\hline 12.8 & 1179.5520 & -0.0070 & $\underline{\text { TVPSYNQTNR }}$ \\
\hline 12.7 & 1179.5536 & -0.0086 & $\underline{\text { LSAIPNDTAAK }}$ \\
\hline 11.5 & 1179.5520 & -0.0070 & $\underline{\text { TVPSYNQTNR }}$ \\
\hline 11.3 & 1179.5560 & -0.0110 & $\underline{\text { LWGLYEEDR }}$ \\
\hline 9.1 & 1179.5536 & -0.0086 & $\underline{\text { LASLSDPELR }}$ \\
\hline 8.8 & 1179.5397 & 0.0053 & $\underline{\text { GDDGKPRSLR }}$ \\
\hline 8.2 & 1178.5332 & 1.0118 & $\underline{\text { KASPDPELSR }}$ \\
\hline 8.2 & 1179.5488 & -0.0038 & $\underline{\text { NREMKQAMR }}$ \\
\hline
\end{tabular}

Spectrum No: 239; Query: 943; Rank: 1

\section{Peptide View}

MS/MS Fragmentation of IAVDWESLGYNITR

Found in IPI00204137, Tax_Id=10116 Gene_Symbol=Ptprk Receptor-like protein tyrosine phosphatase kappa extracellular region

Match to Query 943: 1636.811808 from(819.413180,2+)

Title: 100101RatKid_NS_deglyco_25.4471.4471.2.dta

Data file K:\NewmanPaper\Piliang \3SubProteomes \Piliang3SP $\backslash$ mgf5ppm\ERLIC_3SubProteomes5ppm.mgf 

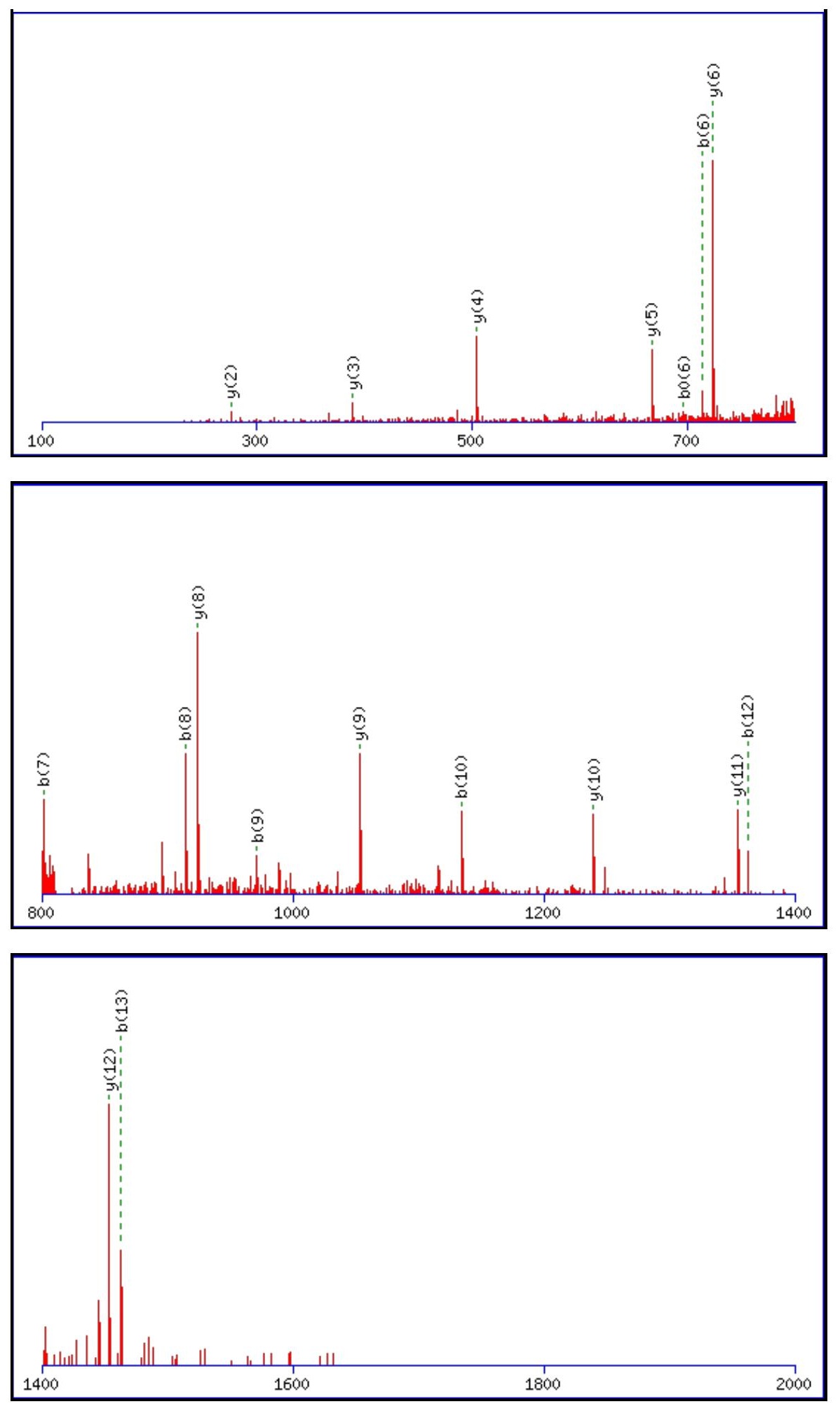

Monoisotopic mass of neutral peptide $\operatorname{Mr}($ calc): 1636.8097

Fixed modifications: Carbamidomethyl (C)

Variable modifications:

N11 : Deamidated $\mathrm{N}(\mathrm{N})$

Ions Score: 79 Expect: $2.2 \mathrm{e}-006$

Matches (Bold Red): 18/128 fragment ions using 34 most intense peaks

\begin{tabular}{|r|c|c|c|c|c|c|c|c|c|c|c|c|c|c|}
\hline$\#$ & $\mathbf{b}$ & $\mathbf{b}^{++}$ & $\mathbf{b}^{*}$ & $\mathbf{b}^{\boldsymbol{*}^{++}}$ & $\mathbf{b}^{\mathbf{0}}$ & $\mathbf{b}^{\mathbf{0 + +}}$ & Seq. & $\mathbf{y}$ & $\mathbf{y}^{++}$ & $\mathbf{y}^{\mathbf{*}}$ & $\mathbf{y}^{\boldsymbol{*}^{++}}$ & $\mathbf{y}^{\mathbf{0}}$ & $\mathbf{y}^{\mathbf{0 + +}}$ & $\#$ \\
\hline $\mathbf{1}$ & 114.0913 & 57.5493 & & & & & $\mathbf{I}$ & & & & & & & $\mathbf{1 4}$ \\
\hline $\mathbf{2}$ & 185.1285 & 93.0679 & & & & & $\mathbf{A}$ & 1524.7329 & 762.8701 & 1507.7063 & 754.3568 & 1506.7223 & $\mathbf{7 5 3 . 8 6 4 8}$ & $\mathbf{1 3}$ \\
\hline 3 & 284.1969 & 142.6021 & & & & & $\mathbf{V}$ & $\mathbf{1 4 5 3 . 6 9 5 8}$ & 727.3515 & 1436.6692 & 718.8383 & 1435.6852 & 718.3462 & $\mathbf{1 2}$ \\
\hline $\mathbf{4}$ & 399.2238 & 200.1155 & & & 381.2132 & 191.1103 & $\mathbf{D}$ & $\mathbf{1 3 5 4 . 6 2 7 4}$ & 677.8173 & 1337.6008 & 669.3040 & 1336.6168 & 668.8120 & $\mathbf{1 1}$ \\
\hline $\mathbf{5}$ & 585.3031 & 293.1552 & & & 567.2926 & 284.1499 & $\mathbf{W}$ & $\mathbf{1 2 3 9 . 6 0 0 4}$ & 620.3038 & 1222.5739 & 611.7906 & 1221.5899 & 611.2986 & $\mathbf{1 0}$ \\
\hline
\end{tabular}




\begin{tabular}{|c|c|c|c|c|c|c|c|c|c|c|c|c|c|c|}
\hline 6 & 714.3457 & |357.6765 & & & 696.3352 & |348.6712| & $\mathbf{E}$ & 1053.5211 & |527.2642| & |1036.4946 & |518.7509| & 1035.5105 & |518.2589 & 9 \\
\hline 7 & 801.3777 & 401.1925 & & & 783.3672 & 392.1872 & S & 924.4785 & 462.7429 & 907.4520 & 454.2296 & 906.4680 & 453.7376 & 8 \\
\hline 8 & 914.4618 & 457.7345 & & & 896.4512 & 448.7293 & $\mathbf{L}$ & 837.4465 & 419.2269 & 820.4199 & 410.7136 & 819.4359 & 410.2216 & 7 \\
\hline 9 & 971.4833 & 486.2453 & & & 953.4727 & 477.2400 & $\mathbf{G}$ & 724.3624 & 362.6849 & 707.3359 & 354.1716 & 706.3519 & 353.6796 & 6 \\
\hline 10 & 1134.5466 & 567.7769 & & & 1116.5360 & 558.7717 & $\mathbf{Y}$ & 667.3410 & 334.1741 & 650.3144 & 325.6608 & 649.3304 & 325.1688 & 5 \\
\hline 11 & 1249.5735 & 625.2904 & 1232.5470 & 616.7771 & 1231.5630 & 616.2851 & $\mathbf{N}$ & 504.2776 & 252.6425 & 487.2511 & 244.1292 & 486.2671 & 243.6372 & 4 \\
\hline 12 & 1362.6576 & 681.8324 & 1345.6311 & 673.3192 & 1344.6470 & 672.8272 & I & 389.2507 & 195.1290 & 372.2241 & 186.6157 & 371.2401 & 186.1237 & 3 \\
\hline 13 & 1463.7053 & 732.3563 & 1446.6787 & 723.8430 & 1445.6947 & 723.3510 & $\mathbf{T}$ & 276.1666 & 138.5870 & 259.1401 & 130.0737 & 258.1561 & 129.5817 & 2 \\
\hline 14 & & & & & & & $\mathbf{R}$ & 175.1190 & 88.0631 & 158.0924 & 79.5498 & & & 1 \\
\hline
\end{tabular}
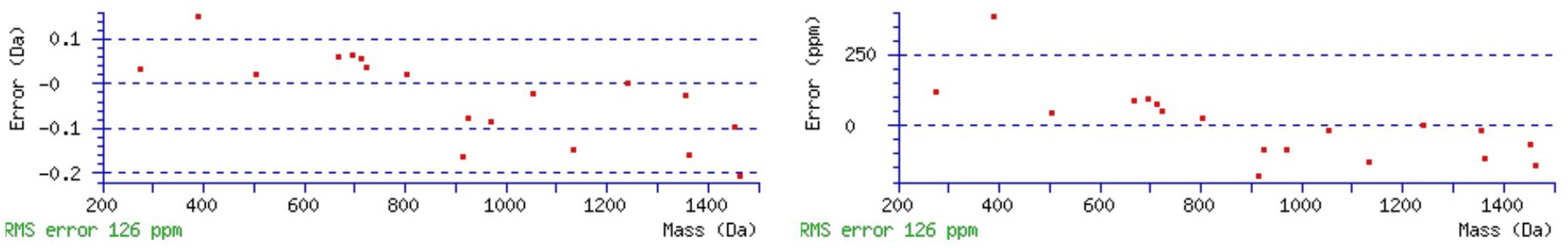

\section{All matches to this query}

\begin{tabular}{|l|l|c|c|}
\hline Score & Mr(calc): & Delta & Sequence \\
\hline 79.1 & 1636.8097 & 0.0021 & IAVDWESLGYNITR \\
\hline 5.1 & 1636.7977 & 0.0141 & TKLLESEAMNESLR \\
\hline 3.8 & 1636.8130 & -0.0012 & $\underline{\text { IEVLEEGSFMNLTR }}$ \\
\hline
\end{tabular}

Spectrum No: 240; Query: 1792; Rank: 1

\section{Peptide View}

MS/MS Fragmentation of TLQEDPSWNVTEFHPTPK

Found in IPI00230862, Tax_Id=10116 Gene_Symbol=Anpep Aminopeptidase N

Match to Query 1792: 2125.997248 from(1064.005900,2+)

Title: 091008RatKidney_NoSalt_23.2698.2698.2.dta

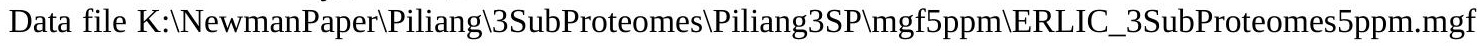

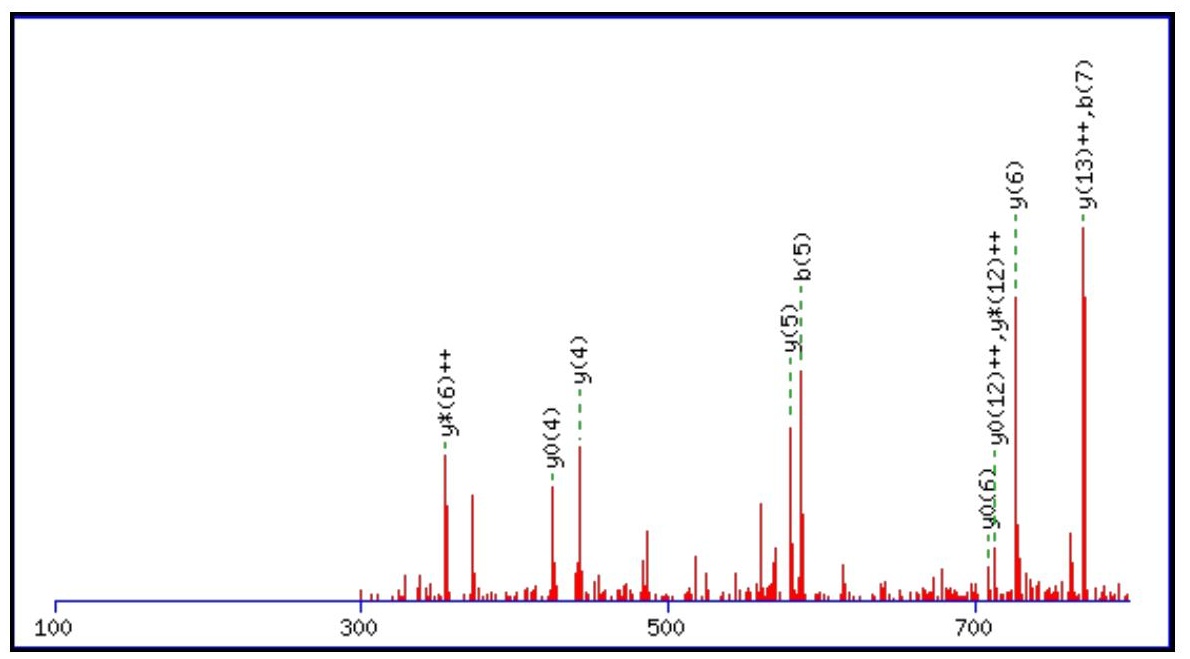



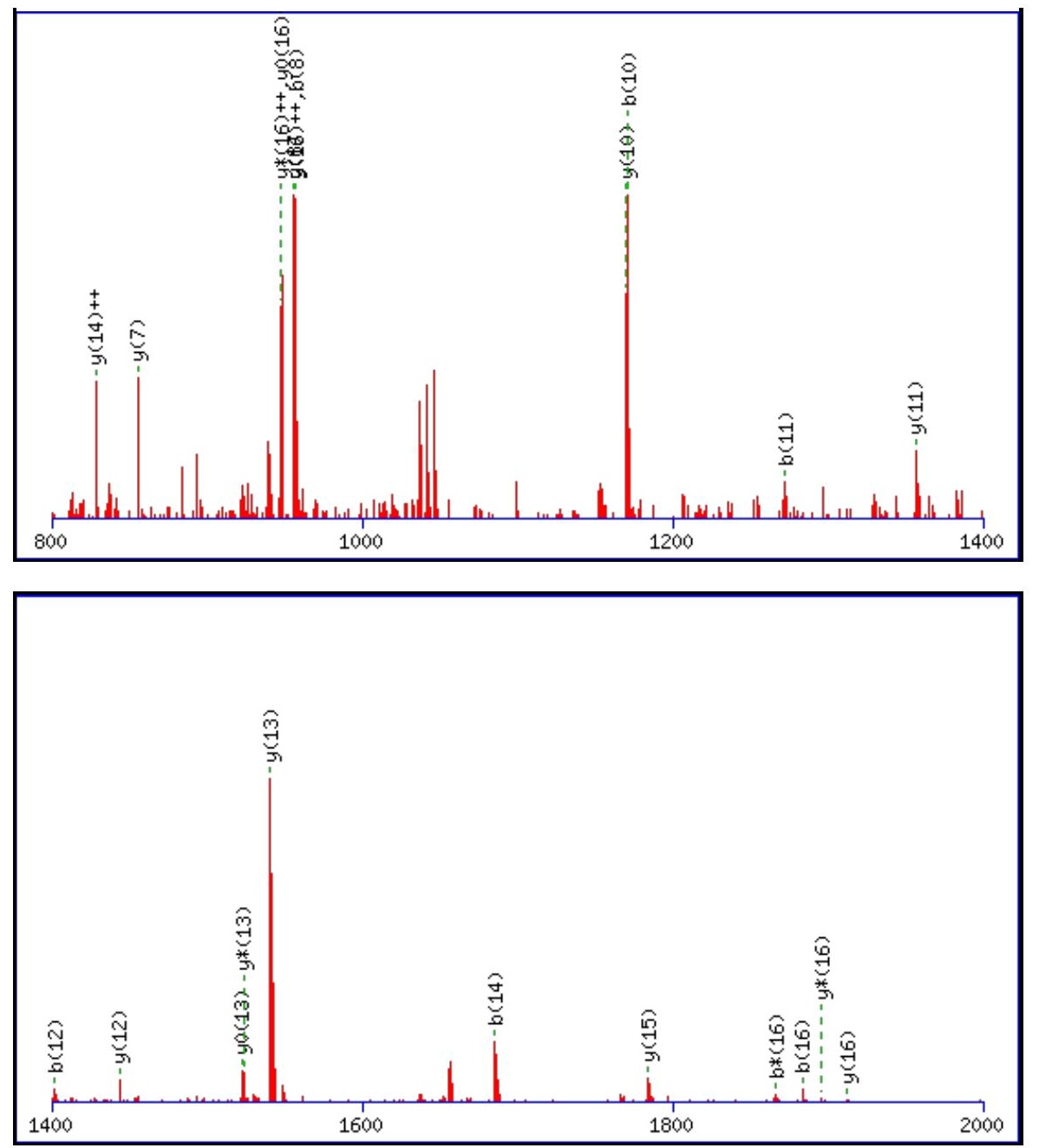

Monoisotopic mass of neutral peptide $\operatorname{Mr}($ calc): 2125.9957

Fixed modifications: Carbamidomethyl (C)

Variable modifications:

N9: Deamidated $\mathrm{N}(\mathrm{N})$

Ions Score: 79 Expect: $3.3 \mathrm{e}-006$

Matches (Bold Red): 33/196 fragment ions using 47 most intense peaks

\begin{tabular}{|c|c|c|c|c|c|c|c|c|c|c|c|c|c|c|}
\hline \# & b & $\mathbf{b}^{++}$ & $\mathbf{b}^{*}$ & & $\mathbf{b}^{\mathbf{0}}$ & $\mathbf{b}^{\mathbf{0 + +}}$ & Seq. & $\mathbf{y}$ & & $\mathbf{y}^{*}$ & & $\mathbf{y}^{\mathbf{0}}$ & & $\#$ \\
\hline 1 & 102.0550 & 51.5311 & & & & & $\mathbf{T}$ & & & & & & & 18 \\
\hline 2 & 215.1390 & 108.0731 & & & 197.1285 & 99.0679 & $\mathbf{L}$ & & & & & & 1004.4760 & 17 \\
\hline 3 & 343.1976 & 72.1024 & & & 325.1870 & 163.0972 & $\mathbf{Q}$ & & & & & & & 16 \\
\hline 4 & 472.2402 & 236.6237 & 455.2136 & 228.1105 & 454.2296 & 227.6185 & $\mathbf{E}$ & 1784.8126 & 892.9099 & 1767.7861 & 884.3967 & 1766.8020 & 883.9047 & 15 \\
\hline 5 & 587.2671 & 294.1372 & 570.2406 & 285.6239 & 569.2566 & 285.1319 & D & 1655.7700 & 828.3886 & 1638.7435 & & 1637.7594 & 819.3834 & 14 \\
\hline 6 & 684.3199 & 342.6636 & 667.2933 & 334.1503 & 666.3093 & 333.6583 & $\mathbf{P}$ & 1540.7431 & 770.8752 & 1523.7165 & 762.3619 & 1522.7325 & 8699 & 13 \\
\hline 7 & 771.3519 & 386.1796 & 754.3254 & 377.6663 & 753.3414 & 377.1743 & $\mathbf{S}$ & 1443.6903 & 722.3488 & 1426.6638 & 713.8355 & 1425.6797 & 435 & 12 \\
\hline 8 & 957.4312 & 479.2193 & 940.4047 & 470.7060 & 939.4207 & 470.2140 & W & 1356.6583 & 678.8328 & 1339.6317 & 670.3195 & 1338.6477 & 669.8275 & 11 \\
\hline 9 & 1072.4582 & 536.7327 & 1055.4316 & 528.2195 & 1054.4476 & 527.7274 & $\mathbf{N}$ & 1170.5790 & 585.7931 & 1153.5524 & 577.2798 & 1152.5684 & 576.7878 & 10 \\
\hline 10 & 171.5266 & 586.2669 & 1154.5000 & 577.7537 & 1153.5160 & 577.2616 & $\mathbf{V}$ & 1055.5520 & 528.2796 & 1038.5255 & 519.7664 & 1037.5415 & 519.2744 & 9 \\
\hline 11 & 272.5743 & 636.7908 & 1255.5477 & 628.2775 & 1254.5637 & 627.7855 & $\mathbf{T}$ & 956.4836 & 478.7454 & 939.4571 & 322 & 938.4730 & 402 & 8 \\
\hline 12 & 401.6169 & 701.3121 & 1384.5903 & 692.7988 & 1383.6063 & 692.3068 & $\mathbf{E}$ & 855.4359 & 428.2216 & 838.4094 & 419.7083 & 837.4254 & 419.2163 & 7 \\
\hline 13 & 548.6853 & 774.8463 & 1531.6587 & 766.3330 & 1530.6747 & 765.8410 & $\mathbf{F}$ & 726.3933 & 363.7003 & 709.3668 & & 708.3828 & 6950 & 6 \\
\hline 14 & 1685.7442 & 843.3757 & 1668.7176 & 834.8625 & 1667.7336 & 834.3704 & $\mathbf{H}$ & 579.3249 & 290.1661 & 562.2984 & 281.6528 & 561.3144 & 281.1608 & 5 \\
\hline 15 & 1782.7970 & 891.9021 & 1765.7704 & 883.3888 & 1764.7864 & 882.8968 & $\mathbf{P}$ & 442.2660 & 221.6366 & 425.2395 & 213.1234 & 424.2554 & 212.6314 & 4 \\
\hline 16 & 1883.8446 & 942.4260 & 1866.8181 & 933.9127 & 1865.8341 & 933.4207 & $\mathbf{T}$ & 345.2132 & 173.1103 & 328.1867 & 164.5970 & 327.2027 & 164.1050 & 3 \\
\hline 17 & 1980.8974 & 990.9523 & 1963.8708 & 982.4391 & 1962.8868 & 981.9471 & $\mathbf{P}$ & 244.1656 & 122.5864 & 227.1390 & 114.0731 & & & 2 \\
\hline 18 & & & & & & & $\mathbf{K}$ & 147.1128 & 74.0600 & 130.0863 & 65.5468 & & & 1 \\
\hline
\end{tabular}



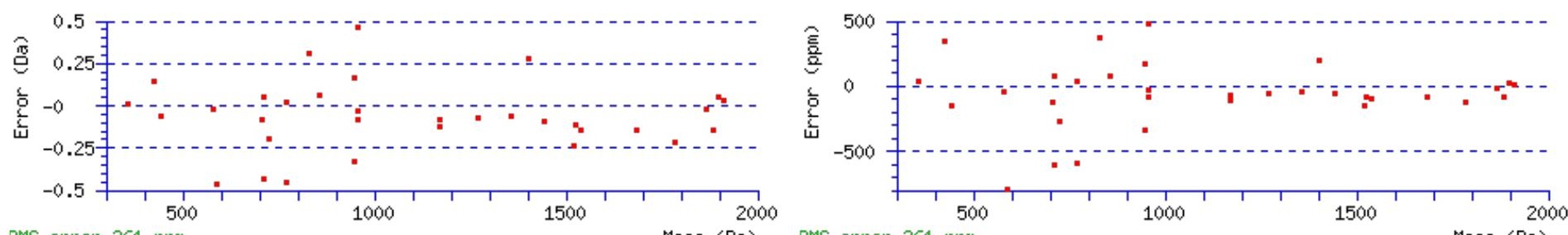

RMS error $261 \mathrm{ppm}$

Mass (Da) RMS error 261 ppm

Mass (Da)

\section{All matches to this query}

\begin{tabular}{|l|l|l|l|}
\hline Score & Mr(calc): & Delta & \multicolumn{1}{|c|}{ Sequence } \\
\hline 78.9 & 2125.9957 & 0.0016 & TLQEDPSWNVTEFHPTPK \\
\hline 11.9 & 2125.0116 & 0.9856 & TLQEDPSWNVTEFHPTPK \\
\hline 7.6 & 2126.0144 & -0.0171 & KDENLVTGEKQEVSVSVGK \\
\hline 5.2 & 2123.9922 & 2.0050 & IKTMVTEQAAQGSSPPDLR \\
\hline 4.1 & 2123.9922 & 2.0050 & IKTMVTEQAAQGSSPPDLR \\
\hline 3.9 & 2123.9863 & 2.0109 & LQENWMDIGFGILSRHK \\
\hline 3.3 & 2125.9854 & 0.0119 & NQEIEELTKICDELIAK \\
\hline 3.0 & 2125.0013 & 0.9959 & NQEIEELTKICDELIAK \\
\hline 2.2 & 2124.0028 & 1.9945 & LVEDVNPQTFTEPSVQLK \\
\hline 1.9 & 2126.0152 & -0.0180 & $\underline{\text { LEMLLDIERNSKMAAAPK }}$ \\
\hline
\end{tabular}

Spectrum No: 241; Query: 2656; Rank: 1

\section{Peptide View}

MS/MS Fragmentation of CIPQYWVCDGDADCSDALDELQNCTMR

Found in IPI00205325, Tax_Id=10116 Gene_Symbol=Lrp2 Low-density lipoprotein receptor-related protein 2 precursor

Match to Query 2656: 3292.299762 from(1098.440530,3+)

Title: 100101RatKid_NS_deglyco_24.4619.4619.3.dta

Data file K:INewmanPaper|Piliangl3SubProteomes\Piliang3SP\mgf5ppm\ERLIC_3SubProteomes5ppm.mgf

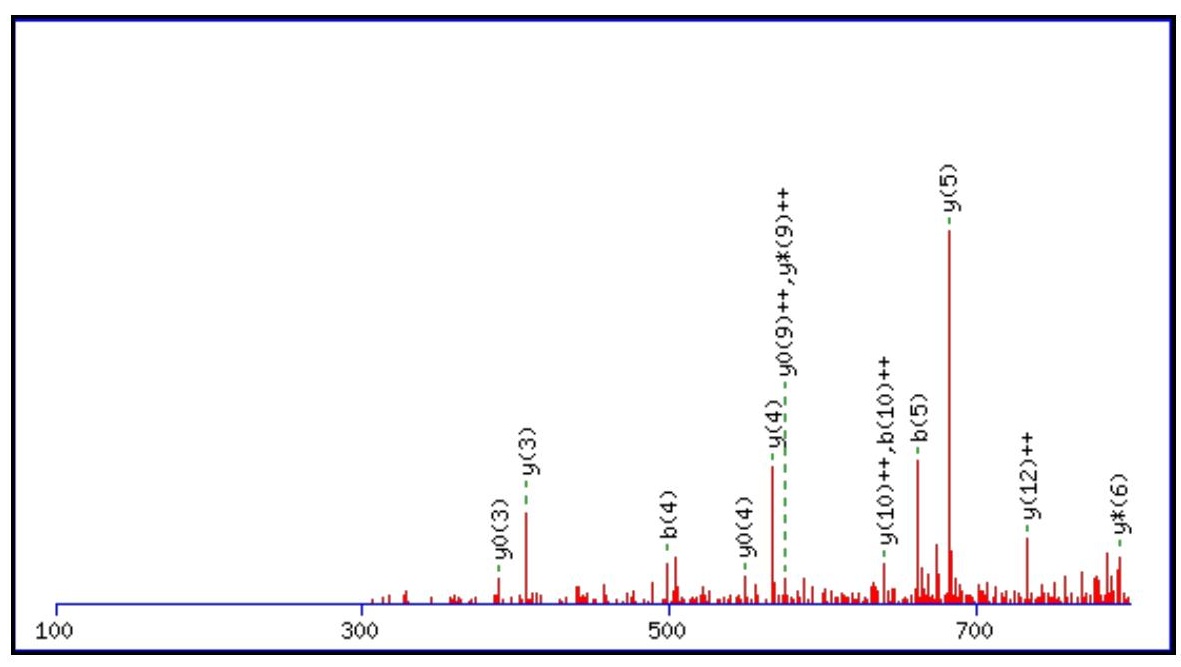



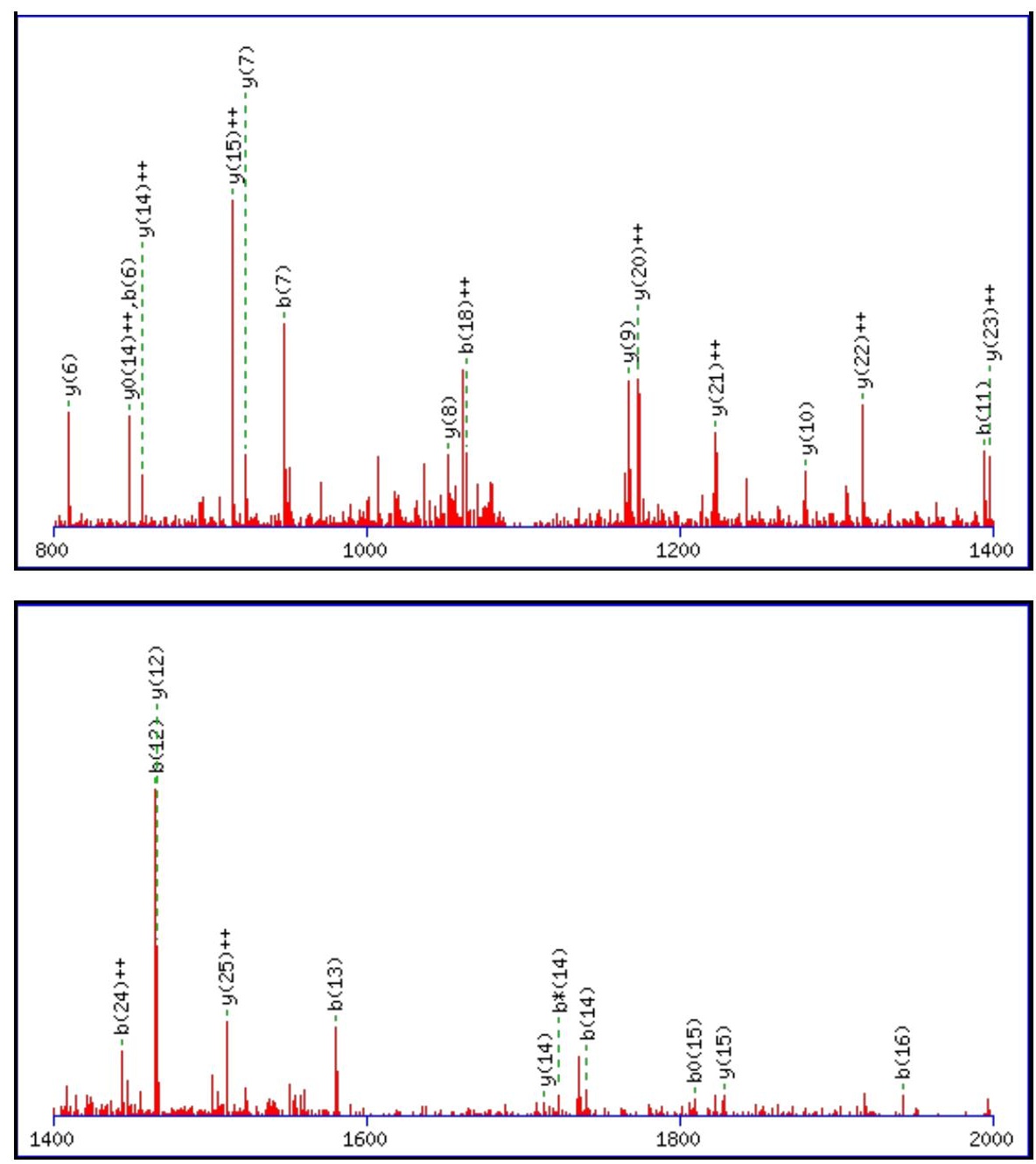

Monoisotopic mass of neutral peptide $\operatorname{Mr}($ calc): 3292.2876

Fixed modifications: Carbamidomethyl (C)

Variable modifications:

N23 : Deamidated N (N)

Ions Score: 79 Expect: $4 \mathrm{e}-006$

Matches (Bold Red): 40/286 fragment ions using 55 most intense peaks

\begin{tabular}{|c|c|c|c|c|c|c|c|c|c|c|c|c|c|c|}
\hline \# & b & $\mathbf{b}^{++}$ & b* & $\mathbf{b}^{*^{++}}$ & $\mathbf{b}^{\mathbf{0}}$ & $\mathbf{b}^{0++}$ & Seq. & $\mathbf{y}$ & $\mathbf{y}^{++}$ & $\mathbf{y}^{*}$ & $\mathrm{y}^{*^{++}}$ & $\mathbf{y}^{\mathbf{0}}$ & $\mathbf{y}^{0++}$ & \# \\
\hline 1 & 161.0379 & 81.0226 & & & & & C & & & & & & & 27 \\
\hline 2 & 274.1220 & 137.5646 & & & & & I & 3133.2642 & 1567.1357 & 3116.2376 & 1558.6225 & 3115.2536 & 1558.1305 & 26 \\
\hline 3 & 371.1748 & 186.0910 & & & & & $\mathbf{P}$ & 3020.1801 & 1510.5937 & 3003.1536 & 1502.0804 & 3002.1696 & 1501.5884 & 425 \\
\hline 4 & 499.2333 & 250.1203 & 482.2068 & 241.6070 & & & $\mathbf{Q}$ & 2923.1274 & 1462.0673 & 2906.1008 & 1453.5540 & 2905.1168 & 1453.0620 & 24 \\
\hline 5 & 662.2967 & 331.6520 & 645.2701 & 323.1387 & & & $\mathbf{Y}$ & 2795.0688 & 1398.0380 & 2778.0422 & 1389.5248 & 2777.0582 & 1389.0327 & 723 \\
\hline 6 & 848.3760 & 424.6916 & 831.3494 & 416.1783 & & & W & 2632.0055 & 1316.5064 & 2614.9789 & 1307.9931 & 2613.9949 & 1307.5011 & 22 \\
\hline 7 & 947.4444 & 474.2258 & 930.4178 & 465.7126 & & & $\mathbf{V}$ & 2445.9261 & 1223.4667 & 2428.8996 & 1214.9534 & 2427.9156 & 1214.4614 & 421 \\
\hline 8 & 1107.4750 & 554.2412 & 1090.4485 & 545.7279 & & & C & 2346.8577 & 1173.9325 & 2329.8312 & 1165.4192 & 2328.8472 & 1164.9272 & 20 \\
\hline 9 & 1222.5020 & 611.7546 & 1205.4754 & 603.2414 & 1204.4914 & 602.7493 & D & 2186.8271 & 1093.9172 & 2169.8005 & 1085.4039 & 2168.8165 & 1084.9119 & 19 \\
\hline 10 & 1279.5234 & 640.2654 & 1262.4969 & 631.7521 & 1261.5129 & 631.2601 & G & 2071.8001 & 1036.4037 & 2054.7736 & 1027.8904 & 2053.7896 & 1027.3984 & 418 \\
\hline 11 & 1394.5504 & 697.7788 & 1377.5238 & 689.2656 & 1376.5398 & 688.7735 & D & 2014.7787 & 1007.8930 & 1997.7521 & 999.3797 & 1996.7681 & 998.8877 & 717 \\
\hline 12 & 1465.5875 & 733.2974 & 1448.5610 & 724.7841 & 1447.5769 & 724.2921 & A & 1899.7517 & 950.3795 & 1882.7252 & 941.8662 & 1881.7412 & 941.3742 & 16 \\
\hline 13 & 1580.6144 & 790.8109 & 1563.5879 & 782.2976 & 1562.6039 & 781.8056 & D & 1828.7146 & 914.8609 & 1811.6881 & 906.3477 & 1810.7041 & 905.8557 & 15 \\
\hline 14 & 1740.6451 & 870.8262 & 1723.6185 & 862.3129 & 1722.6345 & 861.8209 & C & 1713.6877 & 857.3475 & 1696.6611 & 848.8342 & 1695.6771 & 848.3422 & 14 \\
\hline 15 & 1827.6771 & 914.3422 & 1810.6506 & 905.8289 & 1809.6666 & 905.3369 & S & 1553.6570 & 777.3322 & 1536.6305 & 768.8189 & 1535.6465 & 768.3269 & 13 \\
\hline 16 & 1942.7041 & 971.8557 & 1925.6775 & 963.3424 & 1924.6935 & 962.8504 & D & 1466.6250 & 733.8161 & 1449.5985 & 725.3029 & 1448.6144 & 724.8109 & 12 \\
\hline 17 & 2013.7412 & 1007.3742 & 1996.7146 & 998.8610 & 1995.7306 & 998.3689 & A & 1351.5981 & 676.3027 & 1334.5715 & 667.7894 & 1333.5875 & 667.2974 & 11 \\
\hline 18 & 2126.8252 & 1063.9163 & 2109.7987 & 1055.4030 & 2108.8147 & 1054.9110 & $\mathbf{L}$ & 1280.5609 & 640.7841 & 1263.5344 & 632.2708 & 1262.5504 & 631.7788 & 10 \\
\hline
\end{tabular}




\begin{tabular}{|c|c|c|c|c|c|c|c|c|c|c|c|c|c|c|}
\hline 19| & |2241.8522 & |1121.4297 & |2224.8256 & |1112.9165 & |2223.8416 & |1112.4244 & D & 1167.4769 & 584.2421 & |1150.4503 & 575.7288 & |1149.4663 & 575.2368 & 9 \\
\hline 20 & 2370.8948 & 1185.9510 & 2353.8682 & 1177.4378 & 2352.8842 & 1176.9457 & $\mathbf{E}$ & 1052.4499 & 526.7286 & 1035.4234 & 518.2153 & 1034.4394 & 517.7233 & 8 \\
\hline 21 & 2483.9788 & 1242.4931 & 2466.9523 & 1233.9798 & 2465.9683 & 1233.4878 & $\mathbf{L}$ & 923.4073 & 462.2073 & 906.3808 & 453.6940 & 905.3968 & 453.2020 & 7 \\
\hline 22 & 2612.0374 & 1306.5223 & 2595.0109 & 1298.0091 & 2594.0269 & 1297.5171 & $\mathbf{Q}$ & 810.3233 & 405.6653 & 793.2967 & 397.1520 & 792.3127 & 396.6600 & 6 \\
\hline 23 & 2727.0644 & 1364.0358 & 2710.0378 & 1355.5225 & 2709.0538 & 1355.0305 & $\mathbf{N}$ & 682.2647 & 341.6360 & 665.2382 & 333.1227 & 664.2541 & 332.6307 & 5 \\
\hline 24 & 2887.0950 & 1444.0511 & 2870.0685 & 1435.5379 & 2869.0844 & 1435.0459 & C & 567.2378 & 284.1225 & 550.2112 & 275.6092 & 549.2272 & 275.1172 & 4 \\
\hline 25 & 2988.1427 & 1494.5750 & 2971.1161 & 1486.0617 & 2970.1321 & 1485.5697 & $\mathbf{T}$ & 407.2071 & 204.1072 & 390.1806 & 195.5939 & 389.1966 & 195.1019 & 3 \\
\hline 26 & 3119.1832 & 1560.0952 & 3102.1566 & 1551.5819 & 3101.1726 & 1551.0899 & $\mathbf{M}$ & 306.1594 & 153.5834 & 289.1329 & 145.0701 & & & 2 \\
\hline 27 & & & & & & & $\mathbf{R}$ & 175.1190 & 88.0631 & 158.0924 & 79.5498 & & & 1 \\
\hline
\end{tabular}
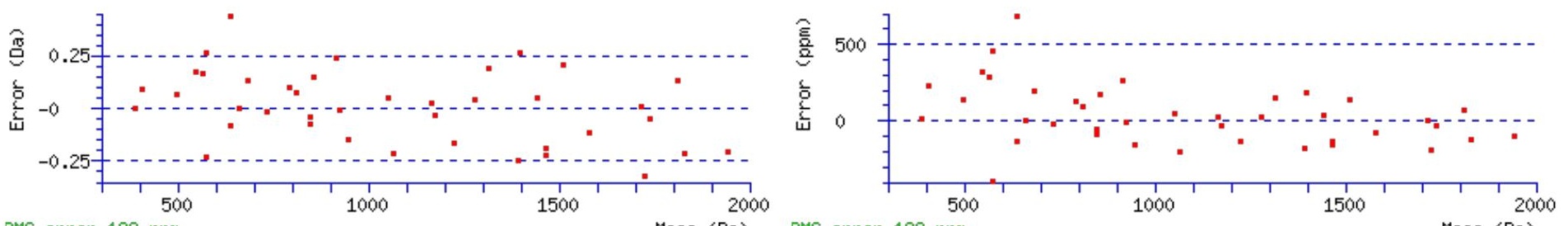

RMS error 198 ppm

Mass (Da) RMS error 198 ppm

Mass (Da)

\section{All matches to this query}

\begin{tabular}{|l|l|l|l|}
\hline Score & Mr(calc): & Delta & \multicolumn{1}{|c|}{ Sequence } \\
\hline 78.9 & 3292.2876 & 0.0122 & CIPQYWVCDGDADCSDALDELQNCTMR \\
\hline 35.0 & 3291.3036 & 0.9962 & CIPQYWVCDGDADCSDALDELQNCTMR \\
\hline 5.5 & 3290.3167 & 1.9831 & MTLSEIYQWICDNFPYYREAGSGWK \\
\hline 4.8 & 3291.2747 & 1.0251 & LLASFFDRNTLANSCGTGIRSSTNDPR \\
\hline 3.5 & 3292.2722 & 0.0276 & LYNWISETLANARHSDAFLTETVNK \\
\hline 2.1 & 3291.3209 & 0.9789 & SVIGDCTQLLTHYYDDARTMYQVFR \\
\hline 1.0 & 3290.3167 & 1.9831 & MTLSEIYQWICDNFPYYREAGSGWK \\
\hline 0.9 & 3290.3167 & 1.9831 & MTLSEIYQWICDNFPYYREAGSGWK \\
\hline 0.3 & 3291.2958 & 1.0040 & PHQWVEGNIPANSQCTVCHESCGSYHR \\
\hline 0.0 & 3292.2722 & 0.0276 & LYNWISETLANARHSDAFLTETVNK \\
\hline
\end{tabular}

Spectrum No: 242; Query: 1819; Rank: 1

\section{Peptide View}

MS/MS Fragmentation of LTNQTMGFSFAVEQDIPVK

Found in IPI00326140, Tax_Id=10116 Gene_Symbol=Pzp Alpha-1-macroglobulin precursor

Match to Query 1819: 2141.035388 from(1071.524970,2+)

Title: 100101RatKid_NS_deglyco_27.3729.3729.2.dta

Data file K:INewmanPaper|Piliang|3SubProteomes\Piliang3SP\mgf5ppm\ERLIC_3SubProteomes5ppm.mgf 

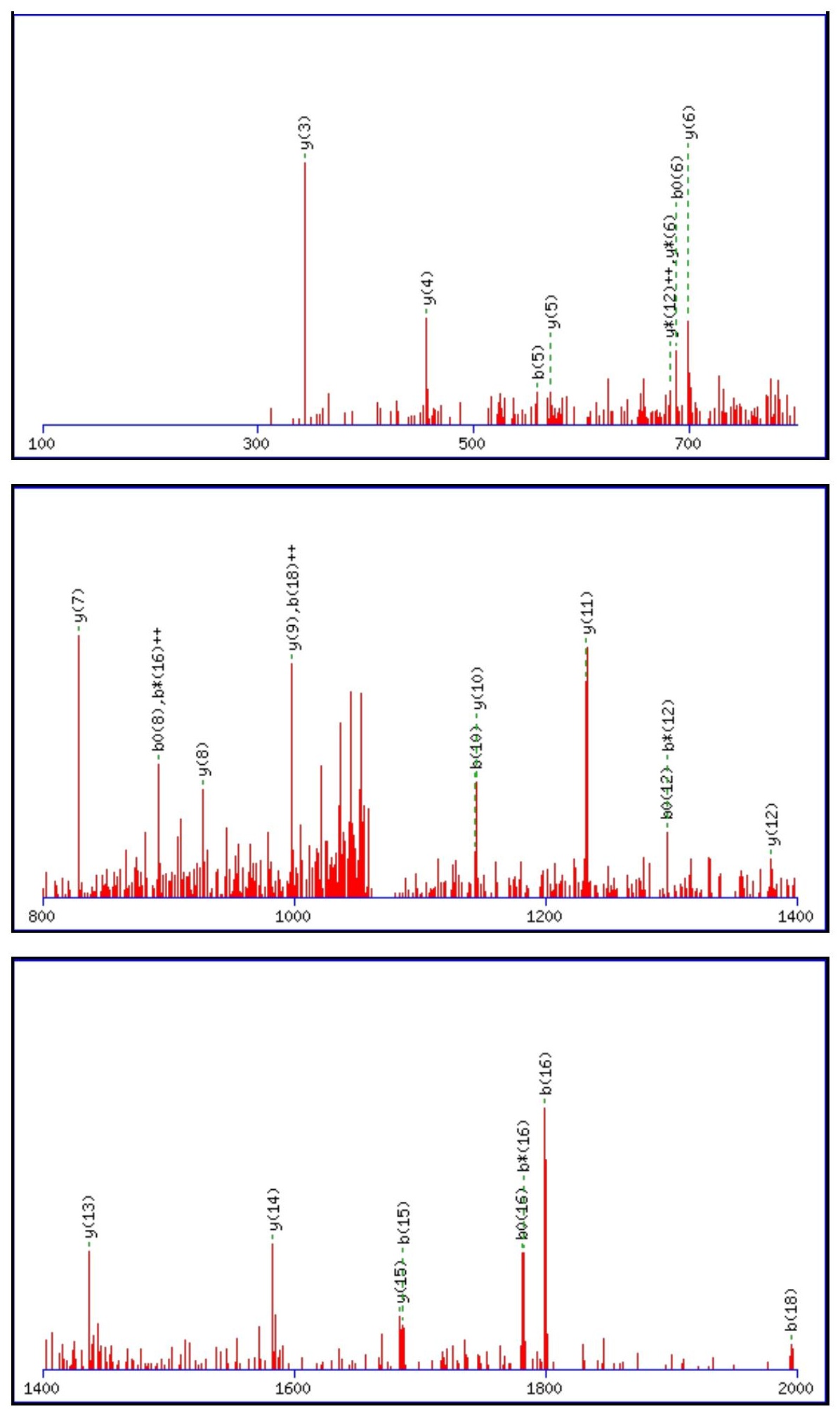

Monoisotopic mass of neutral peptide $\operatorname{Mr}($ calc): 2141.0351

Fixed modifications: Carbamidomethyl (C)

Variable modifications:

N3 : Deamidated_N (N)

M6 : Oxidation (M)

Ions Score: 79 Expect: $3.2 \mathrm{e}-006$

Matches (Bold Red): 28/202 fragment ions using 48 most intense peaks

\begin{tabular}{|c|c|c|c|c|c|r|r|r|r|r|r|r|r|r|}
\hline$\#$ & $\mathbf{b}$ & $\mathbf{b}^{++}$ & $\mathbf{b}^{*}$ & $\mathbf{b}^{\boldsymbol{*}^{++}}$ & $\mathbf{b}^{\mathbf{0}}$ & $\mathbf{b}^{\mathbf{0}++}$ & Seq. & $\mathbf{y}$ & $\mathbf{y}^{++}$ & $\mathbf{y}^{\mathbf{*}}$ & $\mathbf{y}^{\boldsymbol{*}^{++}}$ & $\mathbf{y}^{\mathbf{0}}$ & $\mathbf{y}^{\mathbf{0 + +}}$ & $\#$ \\
\hline $\mathbf{1}$ & 114.0913 & 57.5493 & & & & & $\mathbf{L}$ & & & & & & & $\mathbf{1 9}$ \\
\hline $\mathbf{2}$ & 215.1390 & 108.0731 & & & 197.1285 & 99.0679 & $\mathbf{T}$ & 2028.9583 & 1014.9828 & 2011.9317 & 1006.4695 & 2010.9477 & 1005.9775 & $\mathbf{1 8}$ \\
\hline $\mathbf{3}$ & 330.1660 & 165.5866 & 313.1394 & 157.0733 & 312.1554 & 156.5813 & $\mathbf{N}$ & 1927.9106 & 964.4589 & 1910.8841 & 955.9457 & 1909.9000 & 955.4537 & $\mathbf{1 7}$ \\
\hline $\mathbf{4}$ & 458.2245 & 229.6159 & 441.1980 & 221.1026 & 440.2140 & 220.6106 & $\mathbf{Q}$ & 1812.8837 & 906.9455 & 1795.8571 & 898.4322 & 1794.8731 & 897.9402 & $\mathbf{1 6}$ \\
\hline & & & & & & & & & & & & & &
\end{tabular}




\begin{tabular}{|c|c|c|c|c|c|c|c|c|c|c|c|c|c|c|}
\hline & 559.2722 & |280.1397 & 542.2457 & 271.6265 & 541.2616 & |271.1345 & $T$ & |1684.8251 & 842.9162 & |1667.7985 & 834.4029| & 1666.8145 & 833.9109| & $\mid 15$ \\
\hline 6 & 706.3076 & 353.6574 & 689.2811 & 345.1442 & 688.2971 & 344.6522 & $\mathbf{M}$ & 1583.7774 & 792.3923 & 1566.7509 & 783.8791 & 1565.7668 & 783.3871 & 14 \\
\hline 7 & 763.3291 & 382.1682 & 746.3025 & 373.6549 & 745.3185 & 373.1629 & G & 1436.7420 & 718.8746 & 1419.7155 & 710.3614 & 1418.7314 & 709.8694 & 13 \\
\hline 8 & 910.3975 & 455.7024 & 893.3709 & 447.1891 & 892.3869 & 446.6971 & $\mathbf{F}$ & 1379.7205 & 690.3639 & 1362.6940 & 681.8506 & 1361.7100 & 681.3586 & 12 \\
\hline 9 & 997.4295 & 499.2184 & 980.4030 & 490.7051 & 979.4190 & 490.2131 & S & 1232.6521 & 616.8297 & 1215.6256 & 608.3164 & 1214.6416 & 607.8244 & 11 \\
\hline 10 & 1144.4979 & 572.7526 & 1127.4714 & 564.2393 & 1126.4874 & 563.7473 & $\mathbf{F}$ & 1145.6201 & 573.3137 & 1128.5936 & 564.8004 & 1127.6095 & 564.3084 & 10 \\
\hline 11 & 1215.5351 & 608.2712 & 1198.5085 & 599.7579 & 1197.5245 & 599.2659 & A & 998.5517 & 499.7795 & 981.5251 & 491.2662 & 980.5411 & 490.7742 & 9 \\
\hline 12 & 1314.6035 & 657.8054 & 1297.5769 & 649.2921 & 1296.5929 & 648.8001 & $\bar{V}$ & 927.5146 & 464.2609 & 910.4880 & 455.7477 & 909.5040 & 455.2556 & 8 \\
\hline 13 & 1443.6461 & 722.3267 & 1426.6195 & 713.8134 & 1425.6355 & 713.3214 & $\mathbf{E}$ & 828.4462 & 414.7267 & 811.4196 & 406.2134 & 810.4356 & 405.7214 & 7 \\
\hline 14 & 1571.7046 & 786.3560 & 1554.6781 & 777.8427 & 1553.6941 & 777.3507 & $\mathbf{Q}$ & 699.4036 & 350.2054 & 682.3770 & 341.6921 & 681.3930 & 341.2001 & 6 \\
\hline 15 & 1686.7316 & 843.8694 & 1669.7050 & 835.3562 & 1668.7210 & 834.8641 & D & 571.3450 & 286.1761 & 554.3184 & 277.6629 & 553.3344 & 277.1708 & 5 \\
\hline 16 & 1799.8156 & 900.4115 & 1782.7891 & 891.8982 & 1781.8051 & 891.4062 & I & 456.3180 & 228.6627 & 439.2915 & 220.1494 & & & 4 \\
\hline 17 & 1896.8684 & 948.9378 & 1879.8419 & 940.4246 & 1878.8578 & 939.9326 & $\mathbf{P}$ & 343.2340 & 172.1206 & 326.2074 & 163.6074 & & & 3 \\
\hline 18 & \begin{tabular}{|l|}
1995.9368 \\
\end{tabular} & 998.4720 & 1978.9103 & 989.9588 & 1977.9263 & 989.4668 & $\mathrm{~V}$ & 246.1812 & 123.5942 & 229.1547 & 115.0810 & & & 2 \\
\hline 19 & & & & & & & $\mathbf{K}$ & 147.1128 & 74.0600 & 130.0863 & 65.5468 & & & 1 \\
\hline
\end{tabular}
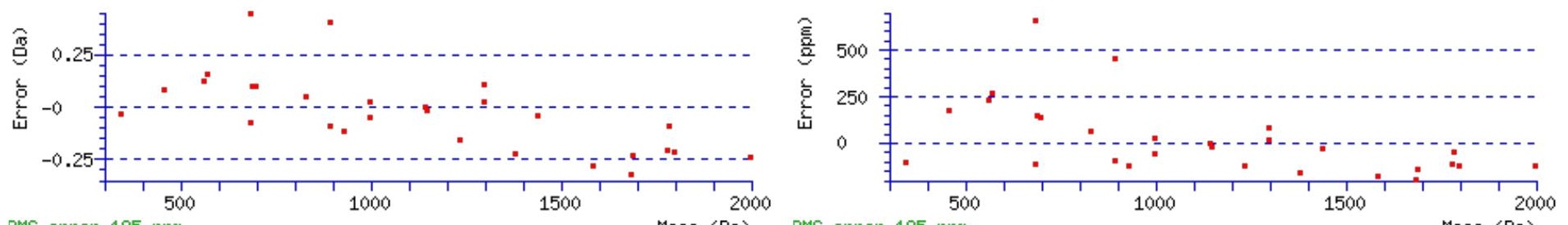

RMS error $195 \mathrm{ppm}$

Mass (Da) RMS error 195 ppm

\section{All matches to this query}

\begin{tabular}{|l|l|l|l|}
\hline Score & Mr(calc): & Delta & \multicolumn{1}{|c|}{ Sequence } \\
\hline 78.9 & 2141.0351 & 0.0003 & LTNQTMGFSFAVEQDIPVK \\
\hline 57.1 & 2140.0511 & 0.9843 & LTNQTMGFSFAVEQDIPVK \\
\hline 10.7 & 2141.0251 & 0.0103 & KLGANLFTIGFAIYEVPK \\
\hline 3.4 & 2141.0397 & -0.0043 & MNPITLTIIYLTTFKGR \\
\hline 1.1 & 2141.0414 & -0.0060 & GGKPLEMAHKIKTVMFDK \\
\hline 0.3 & 2141.0194 & 0.0160 & VPPPPPTPRDNTEEKYPK \\
\hline 0.0 & 2141.0259 & 0.0095 & TKLPCVSHXCRTGESPAR \\
\hline 0.0 & 2141.0251 & 0.0103 & FXPSLSFKLFNVLKTNK \\
\hline 0.0 & 2141.0251 & 0.0103 & FXPSLSFKLFNVLKTNK \\
\hline
\end{tabular}

Spectrum No: 243; Query: 1515; Rank: 1

\section{Peptide View}

MS/MS Fragmentation of FSPNLNISESVIPAIIEK

Found in IPI00195148, Tax_Id=10116 Gene_Symbol=Es2 Liver carboxylesterase 1 precursor

Match to Query 1515: 1971.057248 from(986.535900,2+)

Title: 100101RatKid_NS_deglyco_24.4986.4986.2.dta

Data file K:INewmanPaper|Piliang 3 SSubProteomes\Piliang3SP\mgf5ppm\ERLIC_3SubProteomes5ppm.mgf 

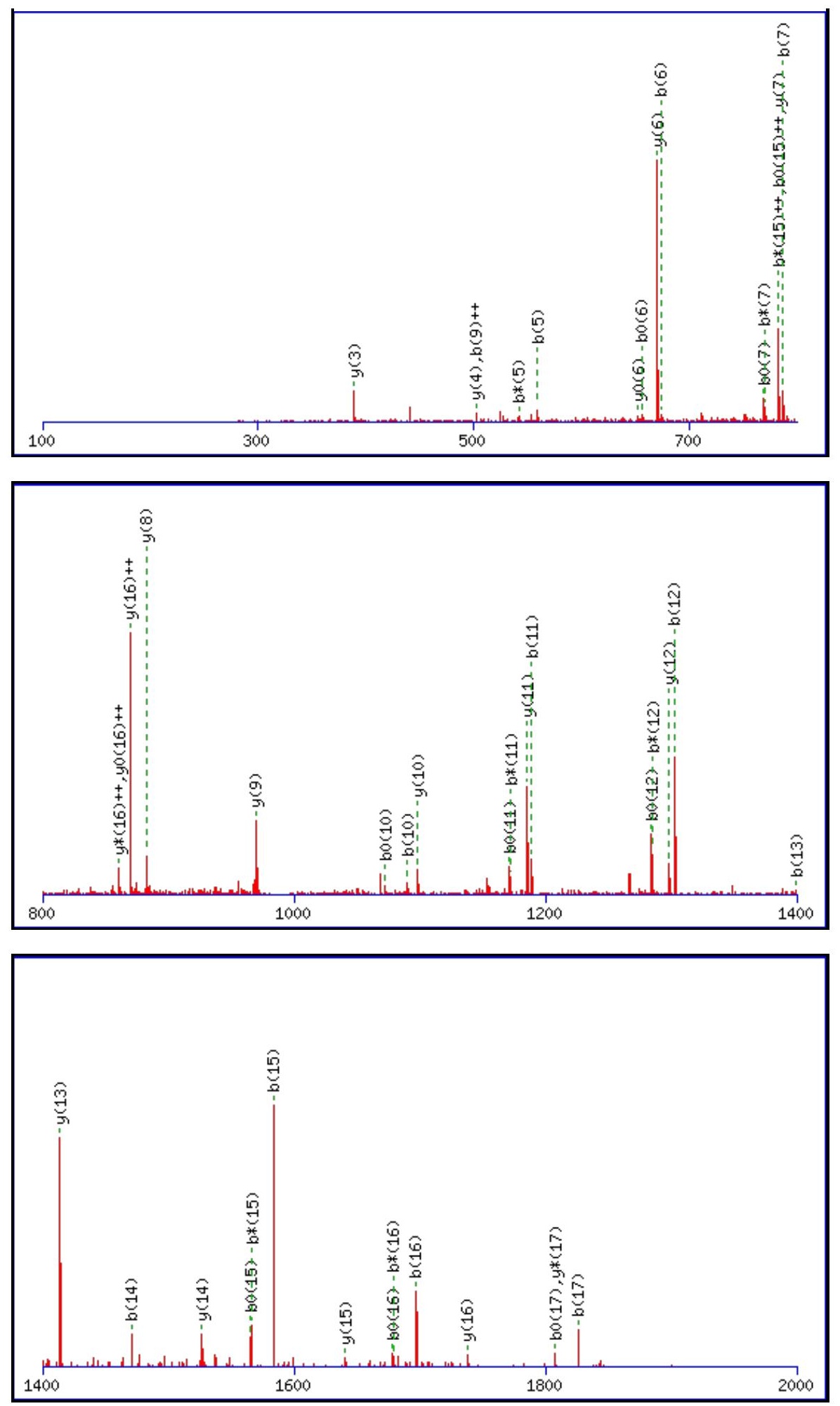

Monoisotopic mass of neutral peptide $\operatorname{Mr}($ calc): 1971.0564

Fixed modifications: Carbamidomethyl (C)

Variable modifications:

N6

Ions Score: 79 Expect: $1.6 \mathrm{e}-006$

Matches (Bold Red): 46/194 fragment ions using 82 most intense peaks

\begin{tabular}{|r|c|c|c|c|c|c|c|c|c|c|c|c|c|c|}
\hline$\#$ & $\mathbf{b}$ & $\mathbf{b}^{++}$ & $\mathbf{b}^{*}$ & $\mathbf{b}^{\boldsymbol{*}^{++}}$ & $\mathbf{b}^{\mathbf{0}}$ & $\mathbf{b}^{\mathbf{0 + +}}$ & Seq. & $\mathbf{y}$ & $\mathbf{y}^{++}$ & $\mathbf{y}^{\mathbf{*}}$ & $\mathbf{y}^{\boldsymbol{*}^{++}}$ & $\mathbf{y}^{\mathbf{0}}$ & $\mathbf{y}^{\mathbf{0 + +}}$ & $\#$ \\
\hline $\mathbf{1}$ & 148.0757 & 74.5415 & & & & & $\mathbf{F}$ & & & & & & & $\mathbf{1 8}$ \\
\hline $\mathbf{2}$ & 235.1077 & 118.0575 & & & 217.0972 & 109.0522 & S & 1824.9953 & 913.0013 & $\mathbf{1 8 0 7 . 9 6 8 8}$ & 904.4880 & 1806.9847 & 903.9960 & $\mathbf{1 7}$ \\
\hline 3 & 332.1605 & 166.5839 & & & 314.1499 & 157.5786 & $\mathbf{P}$ & $\mathbf{1 7 3 7 . 9 6 3 3}$ & $\mathbf{8 6 9 . 4 8 5 3}$ & 1720.9367 & $\mathbf{8 6 0 . 9 7 2 0}$ & 1719.9527 & $\mathbf{8 6 0 . 4 8 0 0}$ & $\mathbf{1 6}$ \\
\hline $\mathbf{4}$ & 446.2034 & 223.6053 & 429.1769 & 215.0921 & 428.1928 & 214.6001 & $\mathbf{N}$ & $\mathbf{1 6 4 0 . 9 1 0 5}$ & 820.9589 & 1623.8840 & 812.4456 & 1622.9000 & 811.9536 & $\mathbf{1 5}$ \\
\hline $\mathbf{5}$ & $\mathbf{5 5 9 . 2 8 7 5}$ & 280.1474 & 542.2609 & 271.6341 & 541.2769 & 271.1421 & $\mathbf{L}$ & $\mathbf{1 5 2 6 . 8 6 7 6}$ & 763.9374 & 1509.8410 & 755.4242 & 1508.8570 & 754.9322 & $\mathbf{1 4}$ \\
\hline
\end{tabular}




\begin{tabular}{|c|c|c|c|c|c|c|c|c|c|c|c|c|c|c|}
\hline 6 & 674.3144 & |337.6608| & 657.2879 & |329.1476 & 656.3038 & |328.6556| & $\mathbf{N}$ & |1413.7835 & |707.3954| & |1396.7570 & |698.8821 & |1395.7730 & |698.3901 & 13 \\
\hline 7 & 787.3985 & 394.2029 & 770.3719 & 385.6896 & 769.3879 & 385.1976 & I & 1298.7566 & 649.8819 & 1281.7300 & 641.3687 & 1280.7460 & 640.8767 & 12 \\
\hline 8 & 874.4305 & 437.7189 & 857.4040 & 429.2056 & 856.4199 & 428.7136 & $S$ & 1185.6725 & 593.3399 & 1168.6460 & 584.8266 & 1167.6620 & 584.3346 & 11 \\
\hline 9 & 1003.4731 & 502.2402 & 986.4465 & 493.7269 & 985.4625 & 493.2349 & $\mathbf{E}$ & 1098.6405 & 549.8239 & 1081.6140 & 541.3106 & 1080.6299 & 540.8186 & 10 \\
\hline 10 & 1090.5051 & 545.7562 & 1073.4786 & 537.2429 & 1072.4946 & 536.7509 & $S$ & 969.5979 & 485.3026 & 952.5714 & 476.7893 & 951.5873 & 476.2973 & 9 \\
\hline 11 & 1189.5735 & 595.2904 & 1172.5470 & 586.7771 & 1171.5630 & 586.2851 & V & 882.5659 & 441.7866 & 865.5393 & 433.2733 & 864.5553 & 432.7813 & 8 \\
\hline 12 & 1302.6576 & 651.8324 & 1285.6311 & 643.3192 & 1284.6470 & 642.8272 & I & 783.4975 & 392.2524 & 766.4709 & 383.7391 & 765.4869 & 383.2471 & 7 \\
\hline 13 & 1399.7104 & 700.3588 & 1382.6838 & 691.8455 & 1381.6998 & 691.3535 & $\mathbf{P}$ & 670.4134 & 335.7103 & 653.3869 & 327.1971 & 652.4028 & 326.7051 & 6 \\
\hline 14 & 1470.7475 & 735.8774 & 1453.7209 & 727.3641 & 1452.7369 & 726.8721 & A & 573.3606 & 287.1840 & 556.3341 & 278.6707 & 555.3501 & 278.1787 & 5 \\
\hline 15 & 1583.8315 & 792.4194 & 1566.8050 & 783.9061 & 1565.8210 & 783.4141 & I & 502.3235 & 251.6654 & 485.2970 & 243.1521 & 484.3130 & 242.6601 & 4 \\
\hline 16 & 1696.9156 & 848.9614 & 1679.8891 & 840.4482 & 1678.9050 & 839.9562 & I & 389.2395 & 195.1234 & 372.2129 & 186.6101 & 371.2289 & 186.1181 & 3 \\
\hline 17 & 1825.9582 & 913.4827 & 1808.9317 & 904.9695 & 1807.9476 & 904.4775 & $\mathbf{E}$ & 276.1554 & 138.5813 & 259.1288 & 130.0681 & 258.1448 & 129.5761 & 2 \\
\hline 18 & & & & & & & K & 147.1128 & 74.0600 & 130.0863 & 65.5468 & & & 1 \\
\hline
\end{tabular}
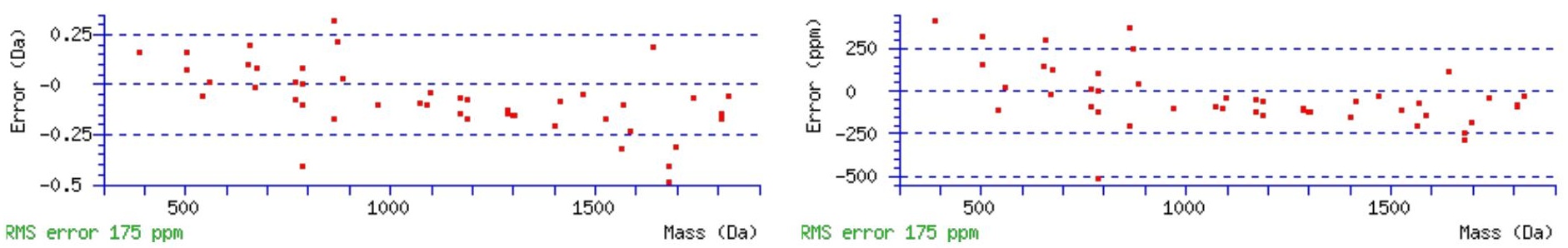

\section{All matches to this query}

\begin{tabular}{|l|c|c|l|}
\hline Score & Mr(calc): & Delta & \multicolumn{1}{|c|}{ Sequence } \\
\hline 78.8 & 1971.0564 & 0.0008 & FSPNLNISESVIPAIIEK \\
\hline 58.4 & 1971.0564 & 0.0008 & FSPNLNISESVIPAIIEK \\
\hline 15.2 & 1970.0724 & 0.9848 & FSPNLNISESVIPAIIEK \\
\hline 14.1 & 1971.0636 & -0.0064 & KEQVETSPQASLKTGLQK \\
\hline 8.2 & 1971.0554 & 0.0019 & LHKILGDKVNNTAVIEK \\
\hline 8.2 & 1971.0710 & -0.0138 & SPADIVKNLKESMAVLEK \\
\hline 7.0 & 1971.0398 & 0.0174 & SELSTHQRLHLGKNSHK \\
\hline 6.2 & 1971.0707 & -0.0134 & TLNVKPLVTHRFPLEK \\
\hline 4.8 & 1971.0465 & 0.0107 & FKWPIIAAENEGQGALVK \\
\hline 2.7 & 1971.0524 & 0.0049 & GSLEEAEAERLLLIATEK \\
\hline
\end{tabular}

Spectrum No: 244; Query: 2769; Rank: 1

\section{Peptide View}

MS/MS Fragmentation of AVATVGPISVAMDASHPSLQFYSSGIYYEPNCSSK

Found in IPI00326070, Tax_Id=10116 Gene_Symbol=Ctsl Cathepsin L1 precursor

Match to Query 2769: 3733.744872 from(1245.588900,3+)

Title: 091008RatKidney_NoSalt_11.5478.5478.3.dta

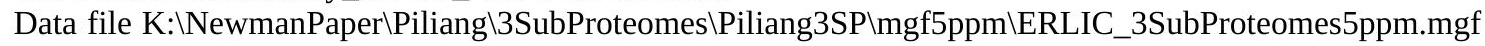



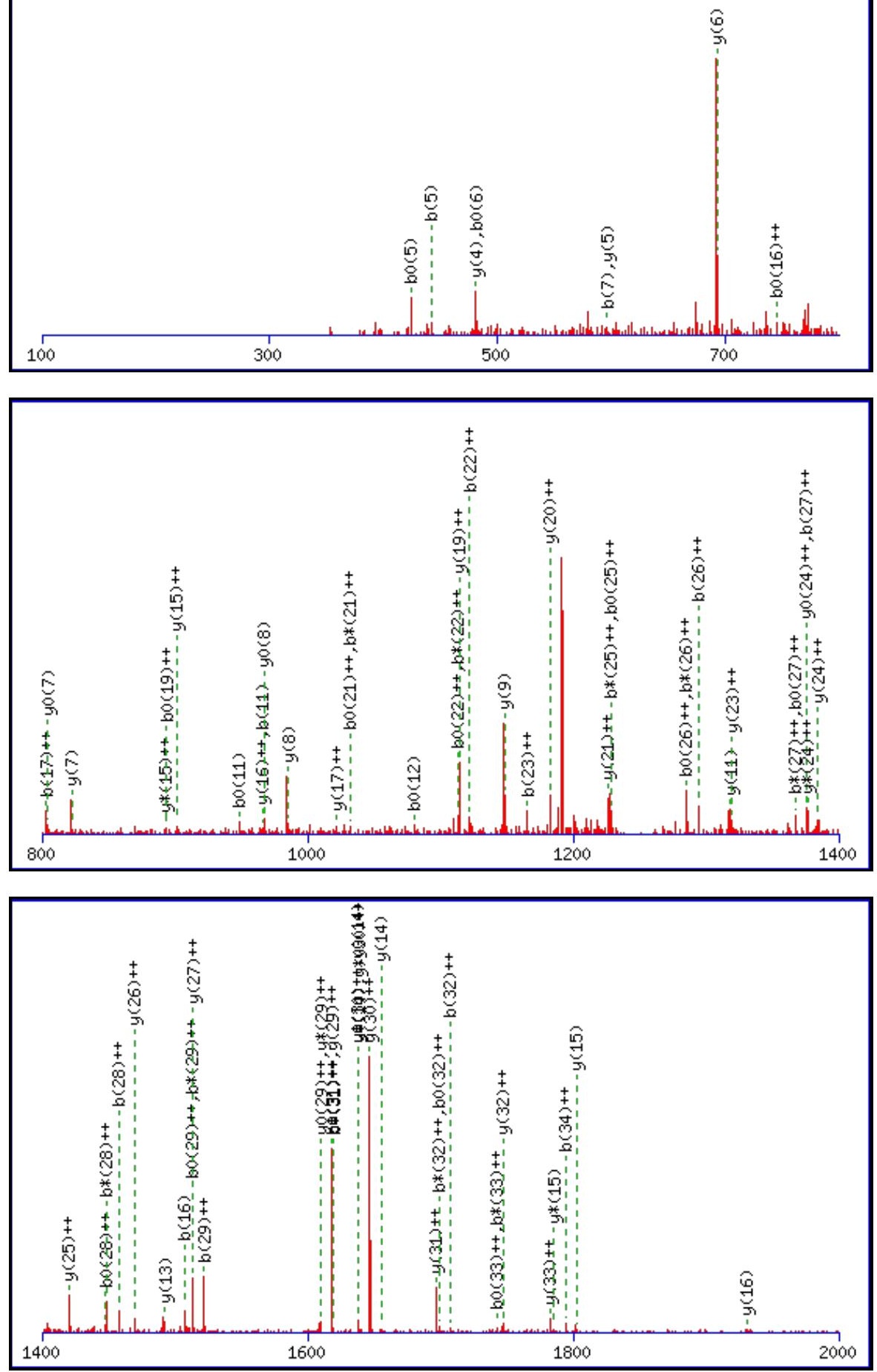

Monoisotopic mass of neutral peptide $\operatorname{Mr}($ calc): 3733.7280

Fixed modifications: Carbamidomethyl (C)

Variable modifications:

N31 : Deamidated $\mathrm{N}(\mathrm{N})$

Ions Score: 79 Expect: $5.6 \mathrm{e}-006$

Matches (Bold Red): 78/362 fragment ions using 144 most intense peaks

\begin{tabular}{|c|c|c|c|c|c|c|c|c|c|c|c|c|c|c|}
\hline$\#$ & b & $\mathbf{b}^{++}$ & $\mathbf{b}^{*}$ & $\mathbf{b}^{*^{++}}$ & $\mathbf{b}^{0}$ & $\mathbf{b}^{0++}$ & Seq. & $\mathbf{y}$ & $\mathbf{y}^{++}$ & $\mathbf{y}^{*}$ & $\mathrm{y}^{\mathrm{*}^{++}}$ & $\mathbf{y}^{0}$ & $y^{0++}$ & \# \\
\hline 1 & 72.0444 & 36.5258 & & & & & A & & & & & & & 35 \\
\hline 2 & 171.1128 & 86.0600 & & & & & V & 3663.6982 & 1832.3527 & 3646.6716 & 1823.8394 & 3645.6876 & 1823.3474 & 34 \\
\hline 3 & 242.1499 & 121.5786 & & & & & A & 3564.6298 & 1782.8185 & 3547.6032 & 1774.3052 & 3546.6192 & 1773.8132 & 33 \\
\hline 4 & 343.1976 & 172.1024 & & & 325.1870 & 163.0972 & $\mathbf{T}$ & 3493.5926 & 1747.3000 & 3476.5661 & 1738.7867 & 3475.5821 & 1738.2947 & 32 \\
\hline 5 & 442.2660 & 221.6366 & & & 424.2554 & 212.6314 & V & 3392.5450 & 1696.7761 & 3375.5184 & 1688.2628 & 3374.5344 & 1687.7708 & 31 \\
\hline
\end{tabular}




\begin{tabular}{|c|c|c|c|c|c|c|c|c|c|c|c|c|c|c|}
\hline 6 & 499.2875 & 250.1474 & & & 481.2769 & & G & & & 3276.4500 & & 4660 & 2366 & 30 \\
\hline 7 & 596.3402 & 298.6738 & & & 578.3297 & 289.6685 & $\mathbf{P}$ & 3236.4551 & 1618.7312 & 3219.4285 & 1610.2179 & 3218.4445 & 1609.7259 & 29 \\
\hline 8 & 709.4243 & 355.2158 & & & 691.4137 & 346.2105 & I & 3139.4023 & 1570.2048 & 3122.3758 & 1561.6915 & 3121.3918 & 1561.1995 & 28 \\
\hline 9 & 796.4563 & 398.7318 & & & 778.4458 & 389.7265 & S & 3026.3183 & 1513.6628 & 3009.2917 & & 008.3077 & 1504.6575 & \\
\hline 10 & 895.5247 & 448.2660 & & & 877.5142 & 439.2607 & $\mathbf{V}$ & 2939.2862 & 1470.1468 & 2922.2597 & 1461.6335 & 2921.2757 & 1461.1415 & \\
\hline 11 & 966.5619 & 483.7846 & & & 948.5513 & 474.7793 & A & 2840.2178 & 1420.6125 & 2823.1913 & 1412.0993 & 2822.2073 & 6073 & \\
\hline 121 & 1097.6023 & 549.3048 & & & 1079.5918 & 540.2995 & $\mathbf{M}$ & 2769.1807 & 1385.0940 & 2752.1542 & 1376.5807 & 1701 & 887 & \\
\hline 13 & 1212.6293 & 606.8183 & & & 1194.6 & 597.8130 & D & 2638.1402 & 1319.5737 & 2621.1137 & 1311.0605 & 1297 & 5685 & \\
\hline 14 & 1283.6664 & 642.3368 & & & 1265.6558 & 633.3316 & A & 2523.1133 & 1262.0603 & 2506.0867 & 1253.5470 & 2505.1027 & 1253.0550 & \\
\hline 15 & 1370.6984 & 685.8529 & & & 1352.6879 & 676.8476 & $S$ & 2452.0762 & 1226.5417 & 2435.0496 & 1218.0284 & 2434.0656 & 5364 & \\
\hline 16 ] & 1507.7573 & 754.3823 & & & 1489.7468 & 745.3770 & $\mathbf{H}$ & 2365.0441 & 1183.0257 & 2348.0176 & 1174.5124 & 2347.0336 & 0204 & \\
\hline 17 & 1604.8101 & 802.9087 & & & 1586.7995 & 793.9034 & $\mathbf{P}$ & 2227.9852 & 1114.4962 & 2210.9587 & 1105.9830 & 2209.9747 & 1105.4910 & 19 \\
\hline 18 & 1691.8421 & 846.4247 & & & 1673.8 & 837.4194 & S & 2130 & 1065.9699 & 2113.9059 & 1057.4566 & 9219 & 1056.9646 & \\
\hline 19 & 1804.9262 & 902.9667 & & & 1786.9156 & 893.9615 & $\mathbf{L}$ & 2043.9004 & 2.4539 & 2026.8739 & 1013.9406 & 5.8899 & 1013.4486 & \\
\hline 201 & 1932.9848 & 966.9960 & 15.9582 & 958.4828 & 1914.9742 & 957.9907 & $\mathbf{Q}$ & 1930.8164 & 965.9118 & 1913.7898 & 957.3985 & 1912.8058 & 956.9065 & 16 \\
\hline 212 & 2080.0532 & 340.5302 & 56 & & 2062.0426 & 1031. & $\mathbf{F}$ & 1802. & 901.8825 & 7312 & 3693 & 1784.7472 & .8772 & \\
\hline 222 & 2243.1165 & 1122.0619 & & & 2225.1060 & 1113.0566 & $\mathbf{Y}$ & 1655.6894 & 828.3483 & 8.6628 & 8351 & 1637.6788 & 3430 & \\
\hline 232 & 2330.1485 & 1165.5779 & 2313.1220 & 1157.0646 & 2312.1380 & 1156.5726 & $S$ & 1492.6260 & 746.8167 & 1475.5995 & 738.3034 & 1474.6155 & 737.8114 & 13 \\
\hline 242 & 2417.1806 & 1209.0939 & 240 & 1 & 2399. & 1200.0886 & $\mathrm{~S}$ & 1405.5940 & 3006 & 5675 & 7874 & 138 & 694.2954 & \\
\hline 252 & 2474.2020 & 1237.6047 & 2457.1755 & 1229 & 2456.1915 & \begin{tabular}{|l|}
1228.5994 \\
\end{tabular} & G & 1318.5620 & 659.7846 & .5354 & 2714 & 5514 & .7793 & \\
\hline 26 & 2587.2861 & 1294.1467 & 2570.2596 & 1285.6334 & 2569.2755 & 1285.1414 & I & 1261.5405 & 631.2739 & 1244.5140 & 622.7606 & 1243.5300 & 622.2686 & 10 \\
\hline 272 & 2750.3494 & \begin{tabular}{|l|}
$\mathbf{1 3 7 5 . 6 7 8 4}$ \\
\end{tabular} & 33.3229 & \begin{tabular}{|l}
1367.1651 \\
\end{tabular} & 2732.3389 & 1366.6731 & $\mathbf{Y}$ & 1148.4565 & 574.7319 & 1131.4299 & 566.2186 & 1130.4459 & 565.7266 & \\
\hline 282 & 2913.4128 & 1457.2100 & 396.3862 & 1448.6967 & 2895.4022 & 1448.2047 & $\mathbf{Y}$ & 985.3931 & 493.2002 & 968.3666 & 484.6869 & 7.3826 & 484.1949 & \\
\hline 293 & 3042.4554 & 1521.7313 & 3025.4288 & 1513.2180 & 3024.4448 & 1512.7260 & $\mathbf{E}$ & 822.3298 & 411.6685 & 805.3033 & 403.1553 & 804.3192 & 402.6633 & 7 \\
\hline 303 & 3139.5081 & 1570.2577 & 3122.4816 & 1561.7444 & 3121.4976 & 1561.2524 & $\mathbf{P}$ & 693.2872 & 347.1472 & 676.2607 & 338.6340 & 675.2766 & 338.1420 & \\
\hline 313 & 3254.5351 & 1627.7712 & 3237.5085 & \begin{tabular}{|l}
1619.2579 \\
\end{tabular} & 3236.5245 & \begin{tabular}{|l|}
1618.7659 \\
\end{tabular} & $\mathbf{N}$ & 596.2344 & 298.6209 & 579.2079 & 290.1076 & 578.2239 & 289.6156 & \\
\hline 323 & 3414.5657 & 1707.7865 & 3397.5392 & 1699.2732 & 3396.5551 & 1698.7812 & $\mathrm{C}$ & 481.2075 & 241.1074 & 464.1810 & 232.5941 & 463.1969 & 232.1021 & 4 \\
\hline 333 & 3501.5977 & 1751.3025 & 3484.5712 & \begin{tabular}{|l}
1742.7892 \\
\end{tabular} & 3483.5872 & \begin{tabular}{|l|}
1742.2972 \\
\end{tabular} & $S$ & 321.1769 & 161.0921 & 304.1503 & 152.5788 & 303.1663 & 152.0868 & 3 \\
\hline 343 & 3588.6298 & 1794.8185 & 3571.6032 & 1786.3052 & 3570.6192 & 1785.8132 & $\mathrm{~S}$ & 234.1448 & 117.5761 & 217.1183 & 109.0628 & 216.1343 & 108.5708 & 2 \\
\hline 35 & & & & & & & $\mathbf{K}$ & 147.1128 & 74.0600 & 130.0863 & 65.5468 & & & \\
\hline
\end{tabular}
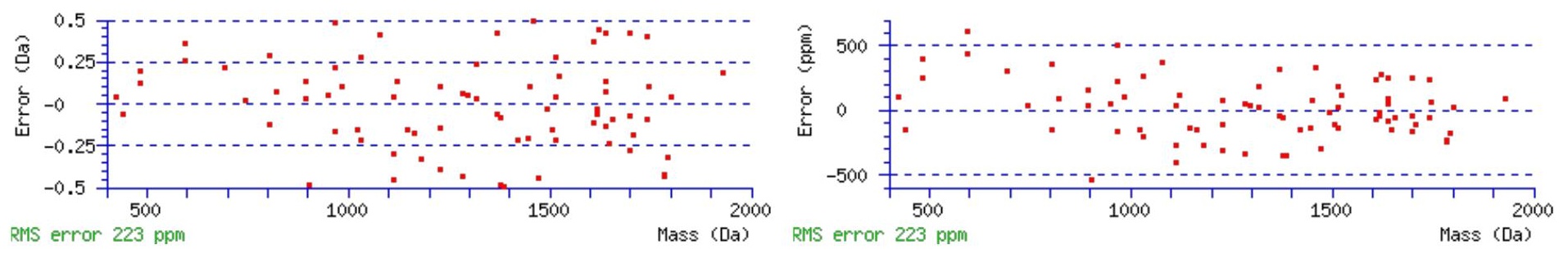

\section{All matches to this query}

\begin{tabular}{|l|l|l|l|}
\hline Score & Mr(calc): & Delta & \multicolumn{1}{|c|}{ Sequence } \\
\hline 78.8 & 3733.7280 & 0.0169 & AVATVGPISVAMDASHPSLQFYSSGIYYEPNCSSK \\
\hline 68.9 & 3732.7440 & 1.0009 & AVATVGPISVAMDASHPSLQFYSSGIYYEPNCSSK \\
\hline 12.4 & 3733.7706 & -0.0257 & LVGATHIVTGHNADDMAETVLMNFLRGDAGRLAR \\
\hline 8.3 & 3731.7549 & 1.9899 & TLRCYHMWIVMARPACSVPCAVPLLTKXSR \\
\hline 8.3 & 3731.7549 & 1.9899 & TLRCYHMWIVMARPACSVPCAVPLLTKXSR \\
\hline 6.2 & 3731.7380 & 2.0069 & VSSVFYTTIVPMLNPLIYSLRNKDVTLALK \\
\hline 6.0 & 3732.7613 & 0.9835 & ALASGFPHVSAYAHEFNPLSPLYSLDVLADASHR \\
\hline 5.8 & 3731.7582 & 1.9866 & WTAHDVENRVECLYNLLSYVNIDIDPVYLK \\
\hline 5.8 & 3732.7422 & 1.0026 & WTAHDVENRVECLYNLLSYVNIDIDPVYLK \\
\hline 5.8 & 3732.7422 & 1.0026 & WTAHDVENRVECLYNLLSYVNIDIDPVYLK \\
\hline
\end{tabular}


Spectrum No: 245; Query: 903; Rank: 1

\section{Peptide View}

MS/MS Fragmentation of LHNQLLPNTTTVEK

Found in IPI00206254, Tax_Id=10116 Gene_Symbol=Ggt1 Gamma-glutamyltranspeptidase 1 precursor

Match to Query 903: 1607.853148 from(804.933850,2+)

Title: 091008RatKidney_NoSalt_28.1420.1420.2.dta

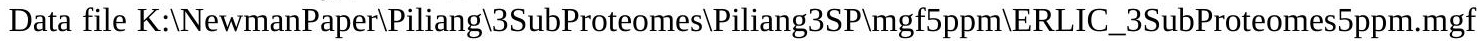
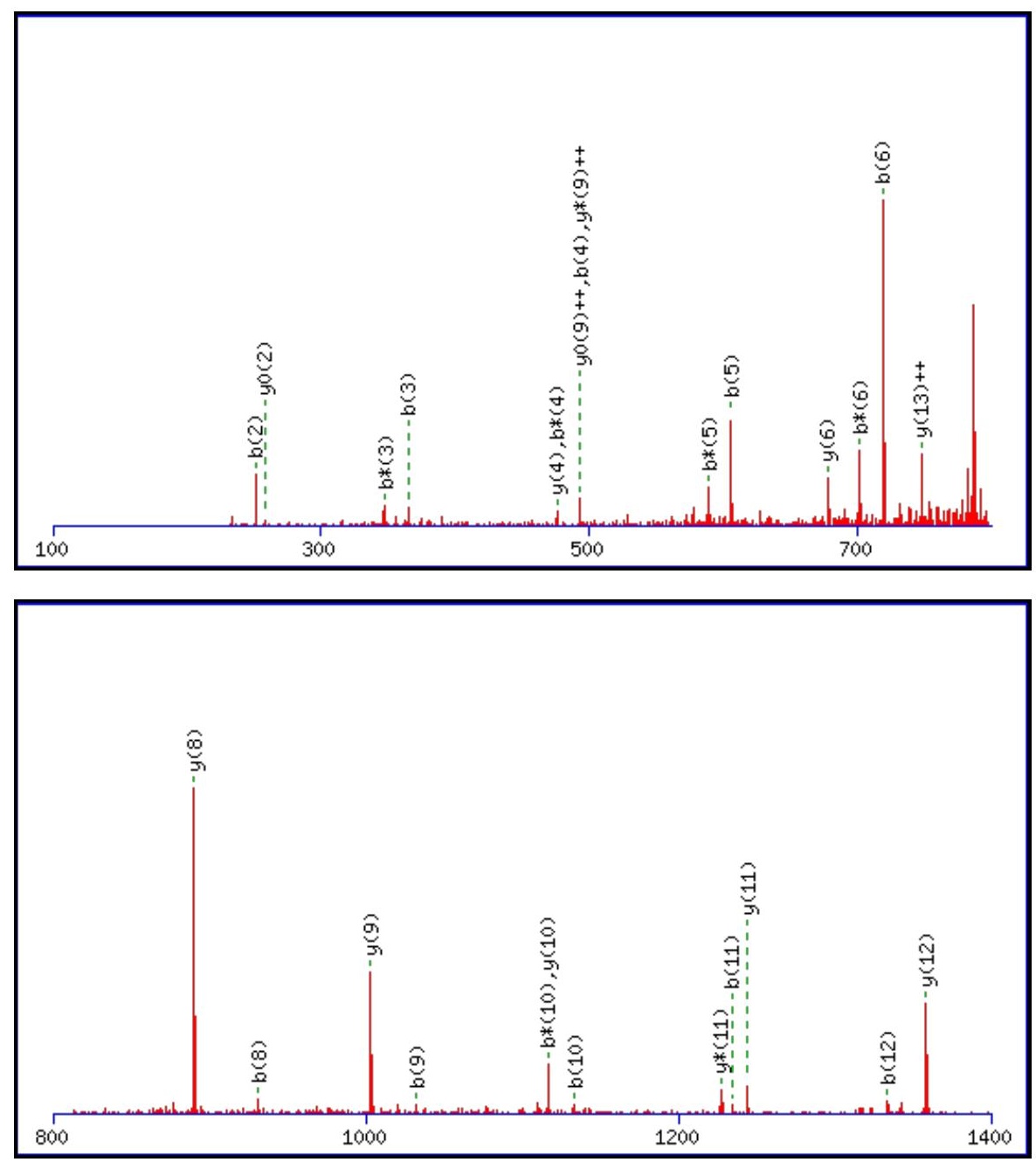


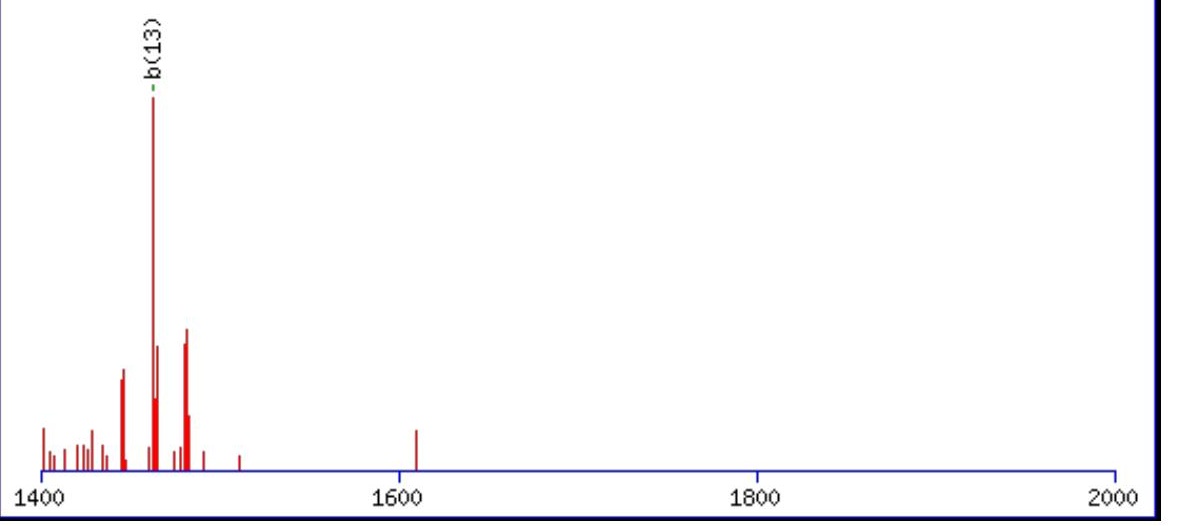

Monoisotopic mass of neutral peptide $\operatorname{Mr}($ calc): 1607.8519

Fixed modifications: Carbamidomethyl (C)

Variable modifications:

N8: Deamidated $\mathrm{N}(\mathrm{N})$

Ions Score: 79 Expect: $1.4 \mathrm{e}-006$

Matches (Bold Red): 28/134 fragment ions using 42 most intense peaks

\begin{tabular}{|c|c|c|c|c|c|c|c|c|c|c|c|c|c|c|}
\hline \# & b & $\mathbf{b}^{++}$ & $\mathbf{b}^{*}$ & $\mathbf{b}^{*^{++}}$ & $\mathbf{b}^{\mathbf{0}}$ & $\mathbf{b}^{0++}$ & Seq. & $\mathbf{y}$ & $\mathbf{y}^{++}$ & $\mathbf{y}^{*}$ & $\mathbf{y}^{*^{++}}$ & $\mathbf{y}^{0}$ & $\mathbf{y}^{0++}$ & \# \\
\hline 1 & 114.0913 & 57.5493 & & & & & $\mathbf{L}$ & & & & & & & 14 \\
\hline 2 & 251.1503 & 126.0788 & & & & & H & 1495.7751 & 748.3912 & 1478.7485 & 739.8779 & 1477.7645 & 739.3859 & 13 \\
\hline 3 & 365.1932 & 183.1002 & 348.1666 & 174.5870 & & & $\mathbf{N}$ & 1358.7162 & 679.8617 & 1341.6896 & 671.3485 & 1340.7056 & 670.8564 & 12 \\
\hline 4 & 493.2518 & 247.1295 & 476.2252 & 238.6162 & & & $\mathbf{Q}$ & 1244.6733 & 622.8403 & 1227.6467 & 614.3270 & 1226.6627 & 613.8350 & 11 \\
\hline 5 & 606.3358 & 303.6715 & 589.3093 & 295.1583 & & & $\mathbf{L}$ & 1116.6147 & 558.8110 & 1099.5881 & 550.2977 & 1098.6041 & 549.8057 & 10 \\
\hline 6 & 719.4199 & 360.2136 & 702.3933 & 351.7003 & & & $\mathbf{L}$ & 1003.5306 & 502.2689 & 986.5041 & 493.7557 & 985.5200 & 493.2637 & 9 \\
\hline 7 & 816.4726 & 408.7400 & 799.4461 & 400.2267 & & & $\mathbf{P}$ & 890.4465 & 445.7269 & 873.4200 & 437.2136 & 872.4360 & 436.7216 & 8 \\
\hline 8 & 931.4996 & 466.2534 & 914.4730 & 457.7402 & & & $\mathbf{N}$ & 793.3938 & 397.2005 & 776.3672 & 388.6873 & 775.3832 & 388.1952 & 7 \\
\hline 9 & 1032.5473 & 516.7773 & 1015.5207 & 508.2640 & 1014.5367 & 507.7720 & $\mathbf{T}$ & 678.3668 & 339.6871 & 661.3403 & 331.1738 & 660.3563 & 330.6818 & 6 \\
\hline 10 & 1133.5949 & 567.3011 & 1116.5684 & 558.7878 & 1115.5844 & 558.2958 & $\mathbf{T}$ & 577.3192 & 289.1632 & 560.2926 & 280.6499 & 559.3086 & 280.1579 & 5 \\
\hline 11 & 1234.6426 & 617.8249 & 1217.6161 & 609.3117 & 1216.6321 & 608.8197 & $\mathbf{T}$ & 476.2715 & 238.6394 & 459.2449 & 230.1261 & 458.2609 & 229.6341 & 4 \\
\hline 12 & 1333.7110 & 667.3592 & 1316.6845 & 658.8459 & 1315.7005 & 658.3539 & $\mathbf{V}$ & 375.2238 & 188.1155 & 358.1973 & 179.6023 & 357.2132 & 179.1103 & 3 \\
\hline 13 & 1462.7536 & 731.8805 & 1445.7271 & 723.3672 & 1444.7431 & 722.8752 & $\mathbf{E}$ & 276.1554 & 138.5813 & 259.1288 & 130.0681 & 258.1448 & 129.5761 & 2 \\
\hline 14 & & & & & & & $K$ & 147.1128 & 74.0600 & 130.0863 & 65.5468 & & & 1 \\
\hline
\end{tabular}

$$
\text { 인 }
$$
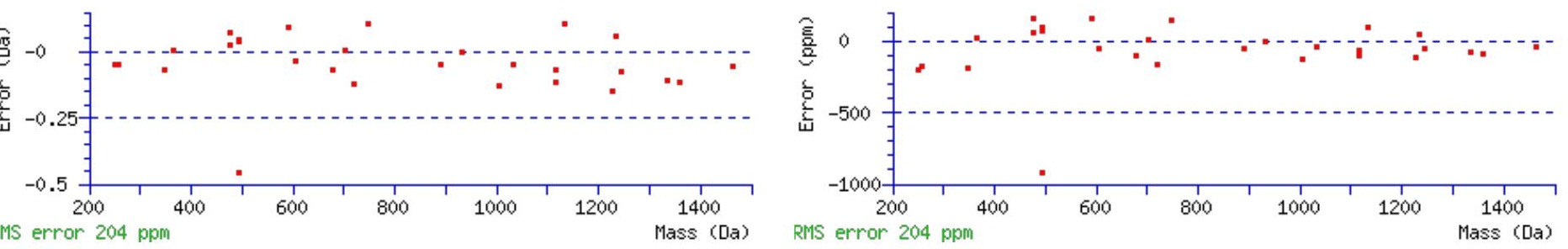

\section{All matches to this query}

\begin{tabular}{|l|l|l|l|}
\hline Score & Mr(calc): & Delta & \multicolumn{1}{|c|}{ Sequence } \\
\hline 78.7 & 1607.8519 & 0.0013 & $\underline{\text { LHNQLLPNTTTVEK }}$ \\
\hline 39.5 & 1607.8519 & 0.0013 & LHNQLLPNTTTVEK \\
\hline 11.1 & 1607.8592 & -0.0061 & $\underline{\text { EISLYIPRMLTEK }}$ \\
\hline 10.9 & 1607.8420 & 0.0112 & $\underline{\text { HLHKVFQENNITK }}$ \\
\hline 10.9 & 1607.8420 & 0.0112 & $\underline{\text { HLHKVFQENNITK }}$ \\
\hline 5.0 & 1607.8519 & 0.0013 & $\underline{\text { FSLGSTINVKVGGNSK }}$ \\
\hline 3.5 & 1607.8572 & -0.0041 & $\underline{\text { LHNNLLTWWGNLK }}$ \\
\hline & & & \\
\hline
\end{tabular}




\begin{tabular}{|l|l|l|l|}
2.5 & 1607.8645 & -0.0113 & VKHAGGHSPQVNHLK \\
\hline 2.0 & 1607.8413 & 0.0118 & MTQRSSVTIKSGGTR \\
\hline 1.8 & 1607.8453 & 0.0078 & EKAAVFRSMNSALGK \\
\hline
\end{tabular}

Spectrum No: 246; Query: 2384; Rank: 1

\section{Peptide View}

MS/MS Fragmentation of FQISPQLQFSPEEVLGMVLNYSR

Found in IPI00210975, Tax_Id=10116 Gene_Symbol=Hyou1 Hypoxia up-regulated protein 1 precursor

Match to Query 2384: 2700.336488 from(1351.175520,2+)

Title: 091008RatKidney_NH4Format01_22.4707.4707.2.dta

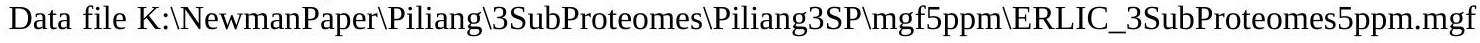
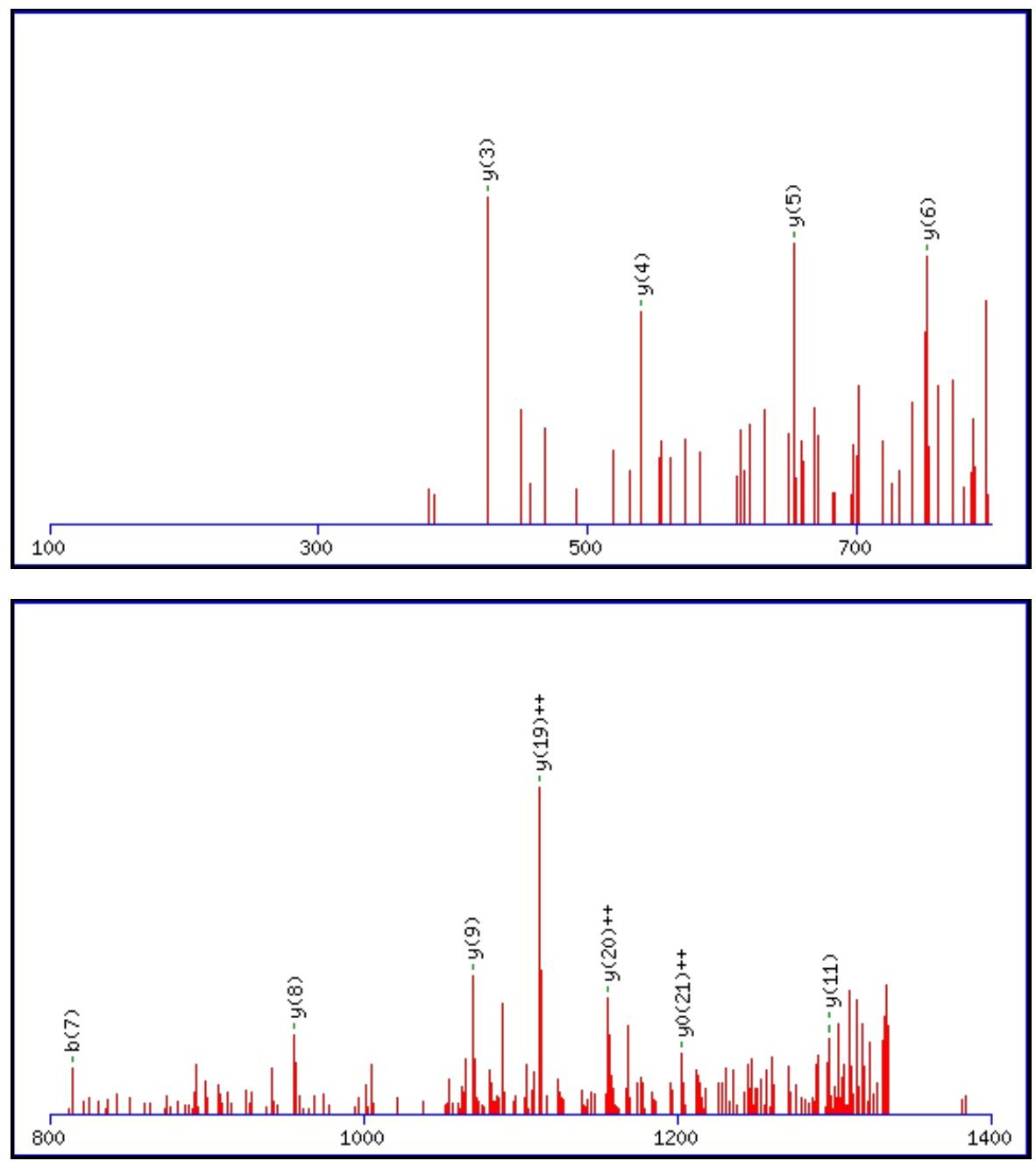


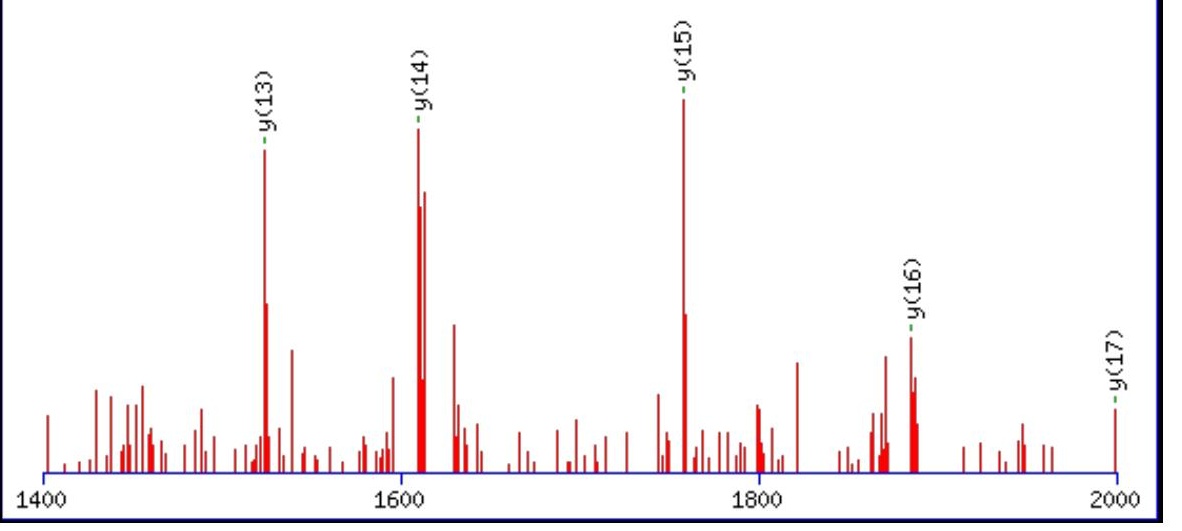

Monoisotopic mass of neutral peptide $\operatorname{Mr}($ calc): 2698.3312

Fixed modifications: Carbamidomethyl (C)

Variable modifications:

M17 : Oxidation (M)

N20 : Deamidated_N (N)

Ions Score: 79 Expect: $3.1 \mathrm{e}-006$

Matches (Bold Red): 16/254 fragment ions using 35 most intense peaks

\begin{tabular}{|c|c|c|c|c|c|c|c|c|c|c|c|c|c|c|}
\hline \# & b & $\mathbf{b}^{++}$ & $\mathbf{b}^{*}$ & $\mathbf{b}^{*^{++}}$ & $\mathbf{b}^{0}$ & & Seq. & $\mathbf{y}$ & $\mathbf{y}^{++}$ & $\mathbf{y}^{*}$ & $\mathbf{y}^{*^{++}}$ & $\mathbf{y}^{\mathbf{0}}$ & & \# \\
\hline 1 & 148.0757 & 74.5415 & & & & & $\mathbf{F}$ & & & & & & & 23 \\
\hline 2 & 276.1343 & 138.5708 & 259.1077 & 130.0575 & & & $\mathbf{Q}$ & 2552.2701 & 6387 & 2535.2436 & 1268.1254 & 2534.2595 & 1267.6334 & 22 \\
\hline 3 & 389.2183 & 195.1128 & 372.1918 & 186.5995 & & & I & 2424.2115 & 1212.6094 & 2407.1850 & 1204.0961 & 2406.2010 & 1203.6041 & 21 \\
\hline 4 & 476.2504 & 238.6288 & 459.2238 & 230.1155 & 458.2398 & 229.6235 & S & 2311.1275 & 1156.0674 & 2294.1009 & 1147.5541 & 2293.1169 & 1147.0621 & 20 \\
\hline 5 & 573.3031 & 287.1552 & 556.2766 & 278.6419 & 555.2926 & 278.1499 & $\mathbf{P}$ & 2224.0954 & 1112.5514 & 2207.0689 & 1104.0381 & 2206.0849 & 1103.5461 & 19 \\
\hline 6 & 701.3617 & 351.1845 & 684.3352 & 342.6712 & 683.3511 & 342.1792 & $\mathbf{Q}$ & 2127.0427 & 1064.0250 & 2110.0161 & 1055.5117 & 2109.0321 & 1055.0197 & 18 \\
\hline 7 & 814.4458 & 407.7265 & 797.4192 & 399.2132 & 796.4352 & 398.7212 & $\mathbf{L}$ & 1998.9841 & 999.9957 & 1981.9576 & 991.4824 & 1980.9735 & 990.9904 & 17 \\
\hline 8 & 942.5043 & 471.7558 & 925.4778 & 463.2425 & 924.4938 & 462.7505 & $\mathbf{Q}$ & 1885.9000 & 943.4537 & 1868.8735 & 934.9404 & 1867.8895 & 934.4484 & 16 \\
\hline 9 & 1089.5728 & 545.2900 & 1072.5462 & 536.7767 & 1071.5622 & 536.2847 & $\mathbf{F}$ & 1757.8415 & 879.4244 & 1740.8149 & 870.9111 & 1739.8309 & 870.4191 & 15 \\
\hline 10 & 1176.6048 & 588.8060 & 1159.5782 & 580.2928 & 1158.5942 & 579.8007 & $\mathbf{S}$ & 1610.7730 & 805.8902 & 1593.7465 & 797.3769 & 1592.7625 & 796.8849 & 14 \\
\hline 11 & 1273.6576 & 637.3324 & 1256.6310 & 628.8191 & 1255.6470 & 628.3271 & $\mathbf{P}$ & 1523.7410 & 762.3741 & 1506.7145 & 753.8609 & 1505.7305 & 753.3689 & 13 \\
\hline 12 & 1402.7001 & 701.8537 & 1385.6736 & 693.3404 & 1384.6896 & 692.8484 & $\mathbf{E}$ & 1426.6883 & 713.8478 & 1409.6617 & 705.3345 & 1408.6777 & 704.8425 & 12 \\
\hline 13 & 1531.7427 & 766.3750 & 1514.7162 & 757.8617 & 1513.7322 & 757.3697 & $\mathbf{E}$ & 1297.6457 & 649.3265 & 1280.6191 & 640.8132 & 1279.6351 & 640.3212 & 11 \\
\hline 14 & 1630.8112 & 815.9092 & 1613.7846 & 807.3959 & 1612.8006 & 806.9039 & $\mathbf{V}$ & 1168.6031 & 584.8052 & 1151.5765 & 576.2919 & 1150.5925 & 575.7999 & 10 \\
\hline 15 & 1743.8952 & 872.4512 & 1726.8687 & 863.9380 & 1725.8846 & 863.4460 & $\mathbf{L}$ & 1069.5347 & 535.2710 & 1052.5081 & 526.7577 & 1051.5241 & 526.2657 & 9 \\
\hline 16 & 1800.9167 & 900.9620 & 1783.8901 & 892.4487 & 1782.9061 & 891.9567 & G & 956.4506 & 478.7289 & 939.4240 & 470.2157 & 938.4400 & 469.7237 & 8 \\
\hline 17 & 1947.9521 & 974.4797 & 1930.9255 & 965.9664 & 1929.9415 & 965.4744 & $\mathbf{M}$ & 899.4291 & 450.2182 & 882.4026 & 441.7049 & 881.4186 & 441.2129 & 7 \\
\hline 18 & 2047.0205 & 1024.0139 & 2029.9939 & 1015.5006 & 2029.0099 & 1015.0086 & $\mathbf{V}$ & 752.3937 & 376.7005 & 735.3672 & 368.1872 & 734.3832 & 367.6952 & 6 \\
\hline 19 & 2160.1046 & 1080.5559 & 2143.0780 & 1072.0426 & 2142.0940 & 1071.5506 & $\mathbf{L}$ & 653.3253 & 327.1663 & 636.2988 & 318.6530 & 635.3147 & 318.1610 & 5 \\
\hline 20 & 2275.1315 & 1138.0694 & 2258.1049 & 1129.5561 & 2257.1209 & 1129.0641 & $\mathbf{N}$ & 540.2412 & 270.6243 & 523.2147 & 262.1110 & 522.2307 & 261.6190 & 4 \\
\hline 21 & 2438.1948 & 1219.6011 & 2421.1683 & 1211.0878 & 2420.1843 & 1210.5958 & $\mathbf{Y}$ & 425.2143 & 213.1108 & 408.1878 & 204.5975 & 407.2037 & 204.1055 & 3 \\
\hline 22 & 2525.2269 & 1263.1171 & 2508.2003 & 1254.6038 & 2507.2163 & 1254.1118 & $\mathrm{~S}$ & 262.1510 & 131.5791 & 245.1244 & 123.0659 & 244.1404 & 122.5738 & 2 \\
\hline 23 & & & & & & & $\mathbf{R}$ & 175.1190 & 88.0631 & 158.0924 & 79.5498 & & & 1 \\
\hline
\end{tabular}
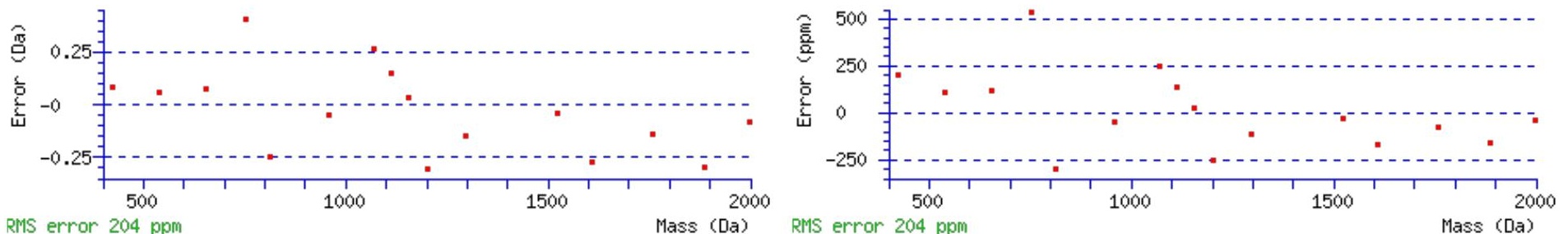
Mascot Search Results: Peptide View

\section{All matches to this query}

\begin{tabular}{|l|l|l|l|}
\hline Score & Mr(calc): & Delta & \multicolumn{1}{c|}{ Sequence } \\
\hline 78.7 & 2698.3312 & 2.0053 & FQISPQLQFSPEEVLGMVLNYSR \\
\hline 2.5 & 2699.3241 & 1.0124 & GSITTMLTLAAPQGDGLYKELADVR \\
\hline 2.5 & 2699.3241 & 1.0124 & GSITTMLTLAAPQGDGLYKELADVR \\
\hline 2.5 & 2699.3241 & 1.0124 & GSITTMLTLAAPQGDGLYKELADVR \\
\hline
\end{tabular}

Spectrum No: 247; Query: 2714; Rank: 1

\section{Peptide View}

MS/MS Fragmentation of SFADENNVFHDPVDGWQNETPSGSLESPAQAR

Found in IPI00199445, Tax_Id=10116 Gene_Symbol=Ank3 ankyrin 3, epithelial isoform 2

Match to Query 2714: 3501.534102 from(1168.185310,3+)

Title: 091008RatKidney_NH4Format02_22.4072.4072.3.dta

Data file K:INewmanPaper|Piliang|3SubProteomes\Piliang3SP\mgf5ppm\ERLIC_3SubProteomes5ppm.mgf
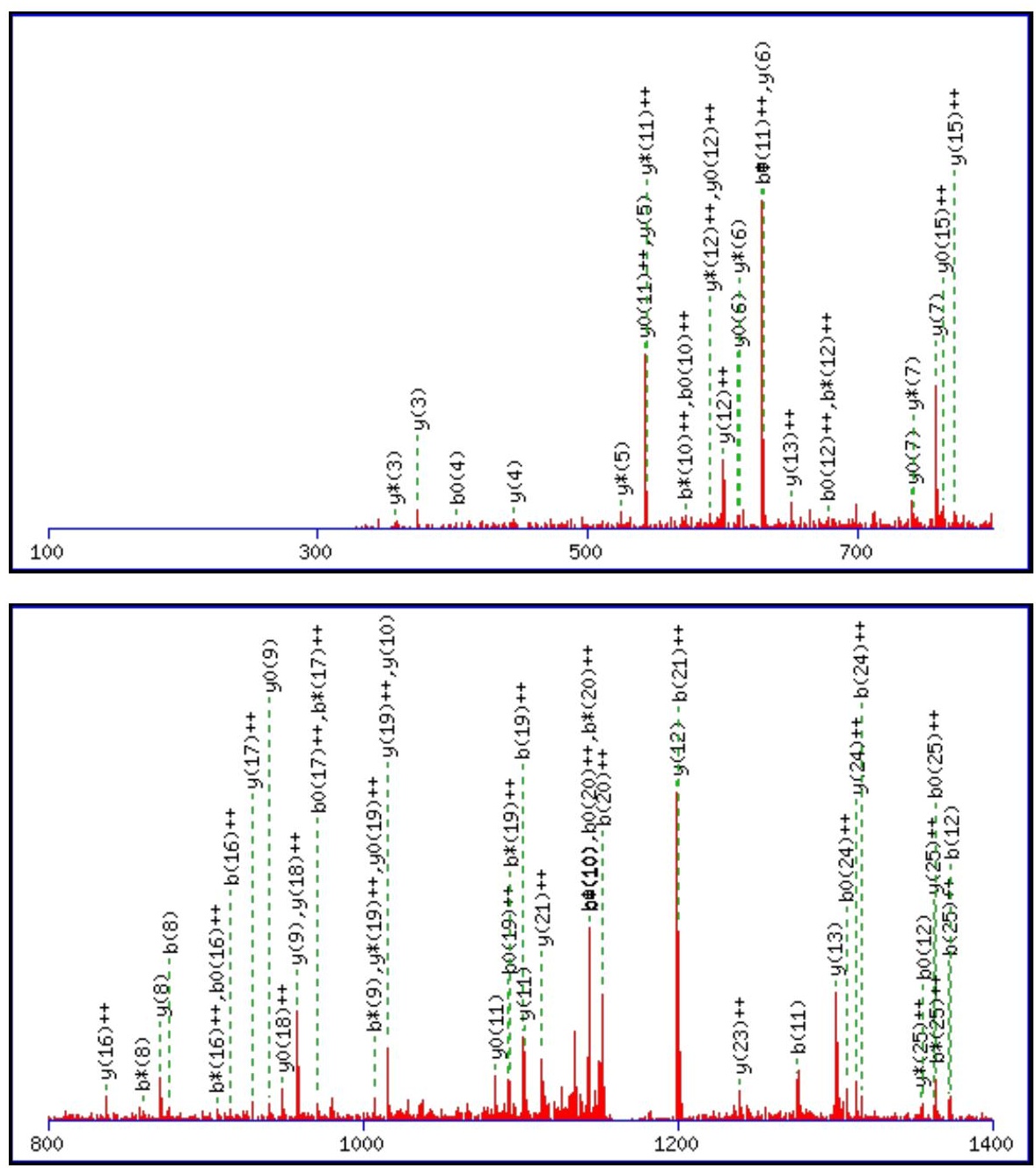


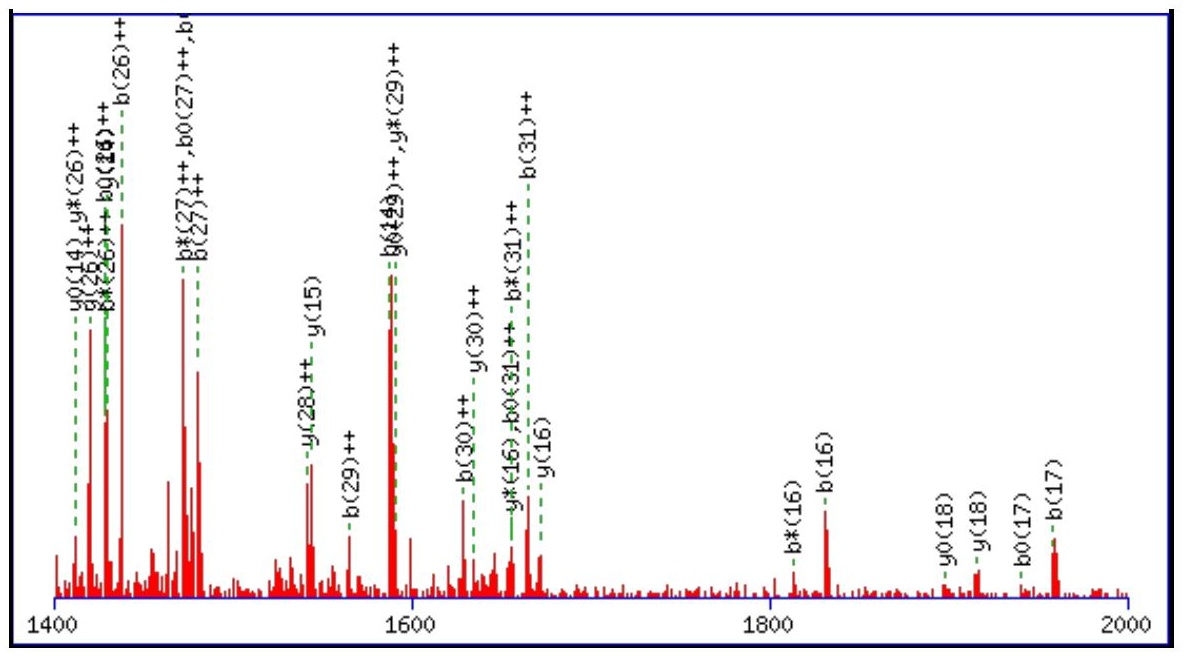

Monoisotopic mass of neutral peptide $\operatorname{Mr}($ calc): 3501.5345

Fixed modifications: Carbamidomethyl (C)

Variable modifications:

N18: Deamidated $\mathrm{N}(\mathrm{N})$

Ions Score: 79 Expect: $6.5 \mathrm{e}-006$

Matches (Bold Red): 101/352 fragment ions using 171 most intense peaks

\begin{tabular}{|c|c|c|c|c|c|c|c|c|c|c|c|c|c|c|}
\hline \# & b & $\mathbf{b}^{++}$ & $\mathbf{b}^{*}$ & $\mathbf{b}^{*^{++}}$ & $\mathbf{b}^{0}$ & $\mathbf{b}^{0++}$ & Seq. & $\mathbf{y}$ & $\mathbf{y}^{++}$ & $\mathbf{y}^{*}$ & $\mathbf{y}^{*}$ & $\mathbf{y}^{0}$ & & \# \\
\hline 1 & 88.0393 & 44.5233 & & & 70.0287 & 35.5180 & $\mathbf{S}$ & & & & & & & 32 \\
\hline 2 & 235.1077 & 118.0575 & & & 217.0972 & 109.0522 & $\mathbf{F}$ & & & & & & & 31 \\
\hline 3 & 306.1448 & 153.5761 & & & 288.1343 & 144.5708 & A & 3268.4414 & 7243 & 3251.4148 & & 4308 & 7190 & 30 \\
\hline 4 & 421.1718 & 211.0895 & & & 403.1612 & 202.0842 & D & 3197.4042 & 1599.2058 & 3180.3777 & 1590.6925 & 3179.3937 & 1590.2005 & 29 \\
\hline 5 & 550.2144 & 275.6108 & & & 532.2038 & 266.6055 & $\mathbf{E}$ & 3082.3773 & 1541.6923 & 3065.3508 & 1533.1790 & 3064.3667 & 1532.6870 & 28 \\
\hline 6 & 664.2573 & 332.6323 & 647.2307 & 324.1190 & 646.2467 & 323.6270 & $\mathbf{N}$ & 2953.3347 & 1477.1710 & 2936.3082 & 1468.6577 & 2935.3241 & 1468.1657 & 27 \\
\hline 7 & 778.3002 & 389.6537 & 761.2 & 381. & 760.2897 & 380.6485 & $\mathbf{N}$ & 2839.2918 & 1420. & 2822.2652 & 1411.6363 & 2821.2812 & 1411.1442 & 26 \\
\hline 8 & 877.3686 & 439.1880 & 860.3421 & 430.6747 & 859.3581 & 430.1827 & V & 2725.2489 & 1363.1281 & 2708.2223 & 1354.6148 & 2707.2383 & 1354.1228 & 25 \\
\hline 9 & 1024.4370 & 512.7222 & 105 & 504. & 1006.4 & 503.7 & $F$ & 2626.1 & & 2609.1539 & & 2608 & & 24 \\
\hline 10 & 1161.4960 & 581.2516 & 1144.4694 & 572.7383 & 1143.4854 & 572.2463 & $\mathbf{H}$ & 2479.1120 & 1240.0597 & 2462.0855 & 1231.5464 & 2461.1015 & 1231.0544 & 23 \\
\hline 11 & 1276.5229 & 638.7651 & 1259.4964 & 630. & 1258.5123 & 629.7598 & D & 2342.0531 & 1171 & 2325.0266 & 1163.0169 & 2324.0426 & .5249 & 22 \\
\hline 12 & 1373.5757 & 687.2915 & 491 & & 1355.5651 & 678.2862 & $\mathbf{P}$ & 2227.0262 & & 996 & & 156 & 114 & 21 \\
\hline 13 & \begin{tabular}{|l|}
1472.6441 \\
\end{tabular} & 736.8257 & 1455.6175 & 728.3124 & 1454.6335 & 727.8204 & V & 2129.9734 & 1065.4903 & 2112.9469 & 1056.9771 & 2111.9628 & 1056.4851 & 20 \\
\hline 14 & 1587.6710 & 794.3392 & 1570.6445 & 785.8259 & 1569.6605 & 785.3339 & D & 2030.9050 & 1015.9561 & 2013.8784 & 1007.4429 & 2012.8944 & 1006.9509 & 19 \\
\hline 5 & 925 & & & & & & G & & & & & & & 18 \\
\hline 16 & \begin{tabular}{|l|}
1830.7718 \\
\end{tabular} & 915.8895 & 1813.7453 & 907.3763 & 1812.7612 & 906.8843 & $\mathbf{W}$ & 1858.8566 & 929.9319 & 1841.8300 & 921.4187 & 1840.8460 & 920.9267 & 17 \\
\hline 17 & 1958.8304 & 979.9188 & 1941.8038 & 971.4056 & 1940.8198 & 970.9135 & $\mathbf{Q}$ & 1672.7773 & 8923 & 1655.7507 & 828.3790 & 1654.7667 & 827.8870 & 16 \\
\hline 18 & 573 & & 2056 & & & 1028 & $\mathbf{N}$ & 1544.7 & & 6922 & 3497 & 1526.7081 & & 15 \\
\hline 19 & 2202.8999 & 1101.9536 & 2185.8734 & 1093.4403 & 2184.8893 & 1092.9483 & $\mathbf{E}$ & 1429.6918 & 715.3495 & 1412.6652 & 706.8362 & 1411.6812 & 706.3442 & 14 \\
\hline 20 & 2303.9476 & 1152.4774 & 2286.9210 & 1143.9642 & 2285.9370 & \begin{tabular}{|l|}
1143.4721 \\
\end{tabular} & $T$ & 1300.6492 & 650.8282 & 1283.6226 & 3149 & 1282.6386 & 641.8229 & 13 \\
\hline 21 & 2401.0004 & \begin{tabular}{|l|}
1201.0038 \\
\end{tabular} & 2383.9738 & 92.4905 & 2382.9898 & 1191.9985 & $\mathbf{P}$ & 1199.6015 & 3044 & 1182.5749 & 591.7911 & 1181.5909 & 591.2991 & 12 \\
\hline 22 & 2488.0324 & 1244.5198 & 2471.0058 & 1236.0066 & 2470.0218 & 1235.5145 & $\mathrm{~S}$ & 1102.5487 & 551.7780 & 1085.5222 & 543.2647 & \begin{tabular}{|l|l|}
1084.5382 \\
\end{tabular} & 542.7727 & 11 \\
\hline 23 & 2545.0538 & 1273.0306 & 2528.0273 & 1264.5173 & 2527.0433 & 1264.0253 & G & 1015.5167 & 508.2620 & 998.4902 & 499.7487 & 997.5061 & 499.2567 & 10 \\
\hline 24 & 2632.0859 & 1316.5466 & 2615.0593 & 1308.0333 & 2614.0753 & 1307.5413 & $\mathrm{~S}$ & 958.4952 & 7513 & 941.4687 & 471.2380 & 940.4847 & 470.7460 & 9 \\
\hline 25 & 2745.1699 & 1373.0886 & 2728.1434 & 1364.5753 & 2727.1594 & 1364.0833 & $\mathbf{L}$ & 871.4632 & 436.2352 & 854.4367 & 427.7220 & 853.4526 & 427.2300 & 8 \\
\hline 26 & 2874.2125 & 1437.6099 & 2857.1860 & 1429.0966 & 2856.2020 & 1428.6046 & $\mathbf{E}$ & 758.3791 & 379.6932 & 741.3526 & 371.1799 & 740.3686 & 370.6879 & 7 \\
\hline 27 & 2961.2446 & \begin{tabular}{|l|}
1481.1259 \\
\end{tabular} & 2944.2180 & \begin{tabular}{|l|}
1472.6126 \\
\end{tabular} & 2943.2340 & \begin{tabular}{|l}
1472.1206 \\
\end{tabular} & $\mathrm{~S}$ & 629.3366 & 315.1719 & 612.3100 & 306.6586 & 611.3260 & 306.1666 & 6 \\
\hline 28 & 3058.2973 & 1529.6523 & 3041.2708 & 1521.1390 & 3040.2868 & 1520.6470 & $\mathbf{P}$ & 542.3045 & 271.6559 & 525.2780 & 263.1426 & & & \\
\hline 29 & 3129.3344 & \begin{tabular}{|l|}
1565.1709 \\
\end{tabular} & 3112.3079 & 1556.6576 & 3111.3239 & 1556.1656 & A & 445.2518 & 223.1295 & 428.2252 & 214.6162 & & & 4 \\
\hline 30 & 3257.3930 & 1629.2001 & 3240.3665 & 1620.6869 & 3239.3824 & 1620.1949 & $\mathbf{Q}$ & 374.2146 & 187.6110 & 357.1881 & 179.0977 & & & \\
\hline 31 & 3328.4301 & 1664.7187 & 3311.4036 & 1656.2054 & 3310.4196 & 1655.7134 & A & 246.1561 & 123.5817 & 229.1295 & 115.0684 & & & 2 \\
\hline 32 & & & & & & & $\mathbf{R}$ & 175.1190 & 88.0631 & 158.0924 & 79.5498 & & & \\
\hline
\end{tabular}



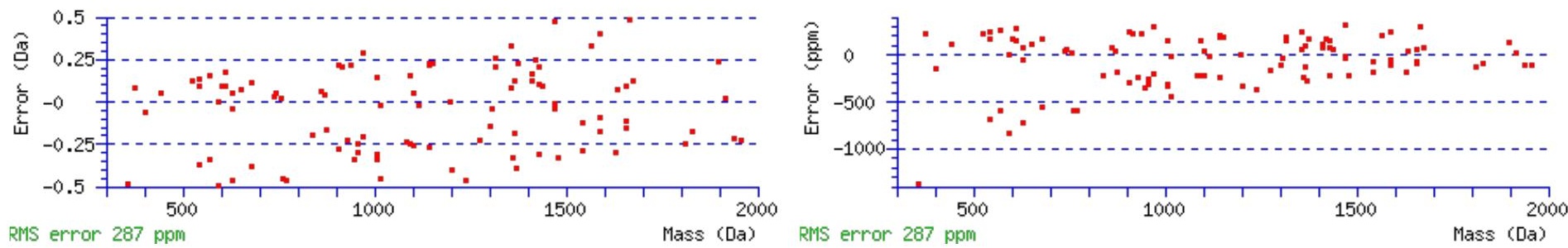

\section{All matches to this query}

\begin{tabular}{|l|l|l|l|}
\hline Score & Mr(calc): & Delta & \multicolumn{1}{c|}{ Sequence } \\
\hline 78.5 & 3501.5345 & -0.0004 & SFADENNVFHDPVDGWQNETPSGSLESPAQAR \\
\hline 76.1 & 3501.5345 & -0.0004 & SFADENNVFHDPVDGWQNETPSGSLESPAQAR \\
\hline 75.5 & 3501.5345 & -0.0004 & SFADENNVFHDPVDGWQNETPSGSLESPAQAR \\
\hline 64.9 & 3500.5505 & 0.9836 & SFADENNVFHDPVDGWQNETPSGSLESPAQAR \\
\hline 16.6 & 3501.5454 & -0.0113 & IMPHTEAQIVNMETGELTKLNMPGELCIR \\
\hline 9.6 & 3501.5451 & -0.0110 & CSTEVPFNSWDRLMRHMGLTDNQIOMVR \\
\hline 9.6 & 3499.5222 & 2.0119 & EIQSTWTQQPTANSSYPYVEISLGDVAAQR \\
\hline 9.1 & 3499.5222 & 2.0119 & EIQSTWTQQPTANSSYPYVEISLGDVAAQR \\
\hline 7.7 & 3499.5222 & 2.0119 & EIQSTWTQQPTANSSYPYVEISLGDVAAQR \\
\hline 7.0 & 3501.5201 & 0.0140 & WTPKSPLDPDSGLLSCTLPNGFGGLSGPEGER \\
\hline
\end{tabular}

Spectrum No: 248; Query: 1858; Rank: 1

\section{Peptide View}

MS/MS Fragmentation of SLTQGSMIVGNLAPVNGTSQGK

Found in IPI00388323, Tax_Id=10116 Gene_Symbol=Hspg2 396 kDa protein

Match to Query 1858: 2175.092008 from(1088.553280,2+)

Title: 100101RatKid_NS_deglyco_21.2701.2701.2.dta

Data file K:INewmanPaper|Piliangl3SubProteomes\Piliang3SP\mgf5ppm\ERLIC_3SubProteomes5ppm.mgf

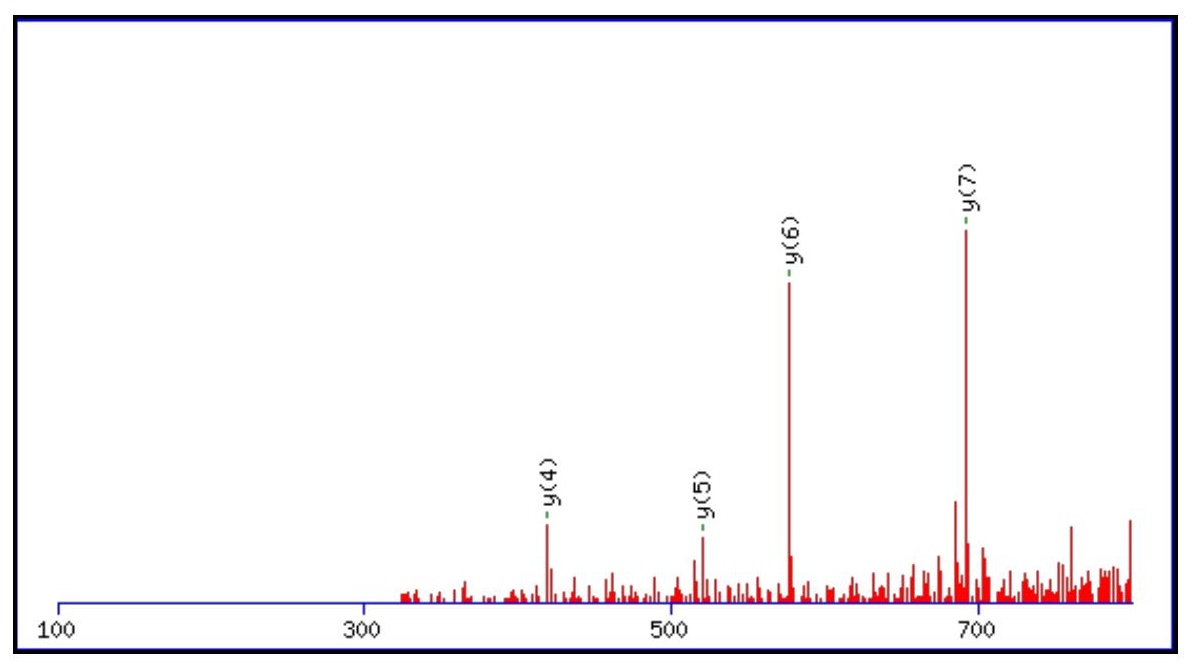



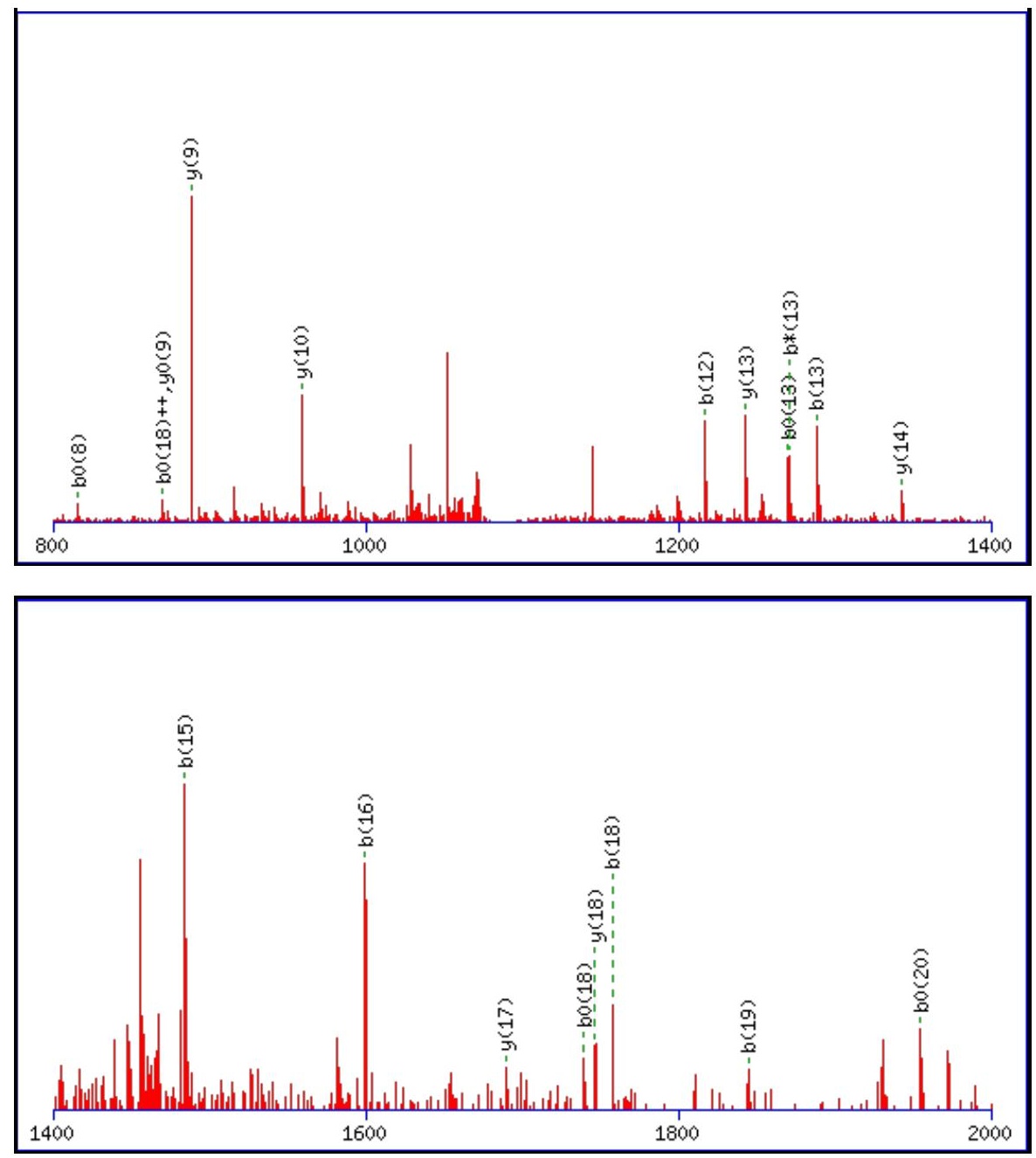

Monoisotopic mass of neutral peptide $\operatorname{Mr}($ calc): 2175.0842

Fixed modifications: Carbamidomethyl (C)

Variable modifications:

M7 : Oxidation (M)

N16 : Deamidated_N (N)

Ions Score: 78 Expect: $2.5 \mathrm{e}-006$

Matches (Bold Red): 23/240 fragment ions using 29 most intense peaks

\begin{tabular}{|c|c|c|c|c|c|c|c|c|c|c|c|c|c|c|}
\hline \# & b & $\mathbf{b}^{++}$ & $\mathbf{b}^{*}$ & $\mathbf{b}^{*^{++}}$ & $\mathbf{b}^{0}$ & & Seq. & $\mathbf{y}$ & & $\mathbf{y}^{*}$ & & $\mathbf{y}^{0}$ & & \# \\
\hline 1 & 88.0393 & 233 & & & 70.0 & 35.5180 & S & & & & & & & 22 \\
\hline 2 & & & & & & & $\mathbf{L}$ & & & & & & & 21 \\
\hline 3 & 302.1710 & & & & 605 & & $\mathbf{T}$ & & & 9488 & 9780 & 1957.9648 & 979.4860 & 20 \\
\hline 4 & 430.2296 & 185 & & & & & $\mathbf{Q}$ & & & & & & & 19 \\
\hline 5 & 487.2511 & 292 & 470. & 59 & 469.2 & 239 & G & 691 & 382 & 425 & 4249 & 8585 & 329 & 18 \\
\hline 6 & 574.2831 & 287.6452 & 557.2 & & 556.2 & 278. & S & 476 & & 1672 & & 1671 & 222 & 17 \\
\hline 7 & 721.3185 & 361.1629 & 704.2 & 496 & 703.3080 & 352.1576 & $\mathbf{M}$ & 156 & 114 & 890 & 982 & 3050 & 061 & 16 \\
\hline 8 & 834.4026 & 417.7049 & & & 816.3 & 408.6 & I & & 3937 & 1438. & 8805 & & 3884 & 15 \\
\hline 9 & 933.4710 & 467.2391 & 916.4444 & 458.7259 & 915.4604 & 458.2339 & $\bar{V}$ & 1342.6961 & 671.8517 & 1325. & 3384 & 1324 & 662.8464 & 14 \\
\hline 10 & 990.4925 & 495.7499 & 659 & 487. & 972.4819 & 486.7446 & G & 1243. & 175 & 1226 & 8042 & 1225.6171 & 613.3122 & 13 \\
\hline 11 & 104.5354 & 552.7713 & 087.5088 & 544.2581 & 1086.5248 & 543.7661 & $\mathbf{N}$ & 1186.6062 & 593.8068 & 1169.5797 & 585.2935 & 1168.5957 & 015 & 12 \\
\hline 12 & 217.6195 & 609.3134 & 200.5929 & 600.8001 & 89 & 600.3 & $\mathbf{L}$ & 1072 & 536. & 1055. & 720 & 1054 & 527.7800 & 11 \\
\hline 13 & 1288.6566 & 644.8319 & 1271.6300 & 636.3186 & 1270.6460 & 635.8266 & A & 959.4792 & 480.2433 & 942.4527 & 471.7300 & 941.4687 & 471.2380 & 10 \\
\hline 14 & 1385.7093 & 693.3583 & 1368.6828 & 684.8450 & 1367.6988 & 684.3530 & $\mathbf{P}$ & & 444. & & 2114 & 870.4 & 194 & 9 \\
\hline 15 & 1484.7777 & 742.8925 & 1467.7512 & 734.3792 & 1466.7672 & 733.8872 & $\mathbf{V}$ & 791.3894 & 396.1983 & 774.3628 & 387.6850 & 773.3788 & 387.1930 & 8 \\
\hline 16 & 1599.8047 & 800.4060 & 1582.7781 & 791.8927 & 1581.7941 & 791.4007 & $\mathbf{N}$ & 692.3210 & 346.6641 & 675.2944 & 338.1508 & 674.3104 & 337.6588 & 7 \\
\hline 17 & 1656.8261 & 828.9167 & 1639.7996 & 820.4034 & 1638.8156 & 819.9114 & G & 577.2940 & 289.1506 & 560.2675 & 280.6374 & 559.2835 & 280.1454 & 6 \\
\hline 18 & 1757.8738 & 879.4406 & 1740.8473 & 870.9273 & 1739.8633 & 870.4353 & $\mathbf{T}$ & 520.2726 & 260.6399 & 503.2460 & 252.1266 & 502.2620 & 251.6346 & 5 \\
\hline
\end{tabular}




\begin{tabular}{|r|r|r|r|r|r|r|r|r|r|r|r|r|r|r|r|}
$\mathbf{1 9}$ & $\mathbf{1 8 4 4 . 9 0 5 9}$ & 922.9566 & 1827.8793 & 914.4433 & 1826.8953 & 913.9513 & S & $\mathbf{4 1 9 . 2 2 4 9}$ & 210.1161 & 402.1983 & 201.6028 & 401.2143 & 201.1108 & $\mathbf{4}$ \\
\hline $\mathbf{2 0}$ & 1972.9644 & 986.9859 & 1955.9379 & 978.4726 & $\mathbf{1 9 5 4 . 9 5 3 9}$ & 977.9806 & $\mathbf{Q}$ & 332.1928 & 166.6001 & 315.1663 & 158.0868 & & & $\mathbf{3}$ \\
\hline $\mathbf{2 1}$ & 2029.9859 & 1015.4966 & 2012.9593 & 1006.9833 & 2011.9753 & 1006.4913 & G & 204.1343 & 102.5708 & 187.1077 & 94.0575 & & & $\mathbf{2}$ \\
\hline $\mathbf{2 2}$ & & & & & & & K & 147.1128 & 74.0600 & 130.0863 & 65.5468 & & & $\mathbf{1}$ \\
\hline
\end{tabular}
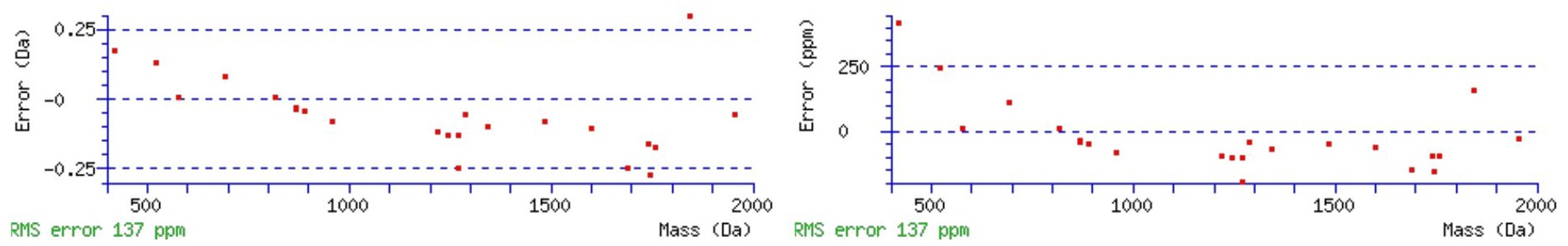

\section{All matches to this query}

\begin{tabular}{|l|l|c|l|}
\hline Score & Mr(calc): & Delta & \multicolumn{1}{c|}{ Sequence } \\
\hline 78.5 & 2175.0842 & 0.0079 & SLTQGSMIVGNLAPVNGTSQGK \\
\hline 31.5 & 2175.0842 & 0.0079 & SLTQGSMIVGNLAPVNGTSQGK \\
\hline 7.9 & 2174.1001 & 0.9919 & SLTQGSMIVGNLAPVNGTSQGK \\
\hline 0.7 & 2175.1072 & -0.0152 & NVAQYGLDPATRYPNLNLR \\
\hline
\end{tabular}

Spectrum No: 249; Query: 1304; Rank: 1

\section{Peptide View}

MS/MS Fragmentation of LANASLYLAEWQNITK

Found in IPI00211616, Tax_Id=10116 Gene_Symbol=Slc3a2 4F2 cell-surface antigen heavy chain

Match to Query 1304: 1835.931408 from(918.972980,2+)

Title: 100101RatKid_NS_deglyco_24.5037.5037.2.dta

Data file K:INewmanPaper|Piliang|3SubProteomes\Piliang3SP\mgf5ppm\ERLIC_3SubProteomes5ppm.mgf

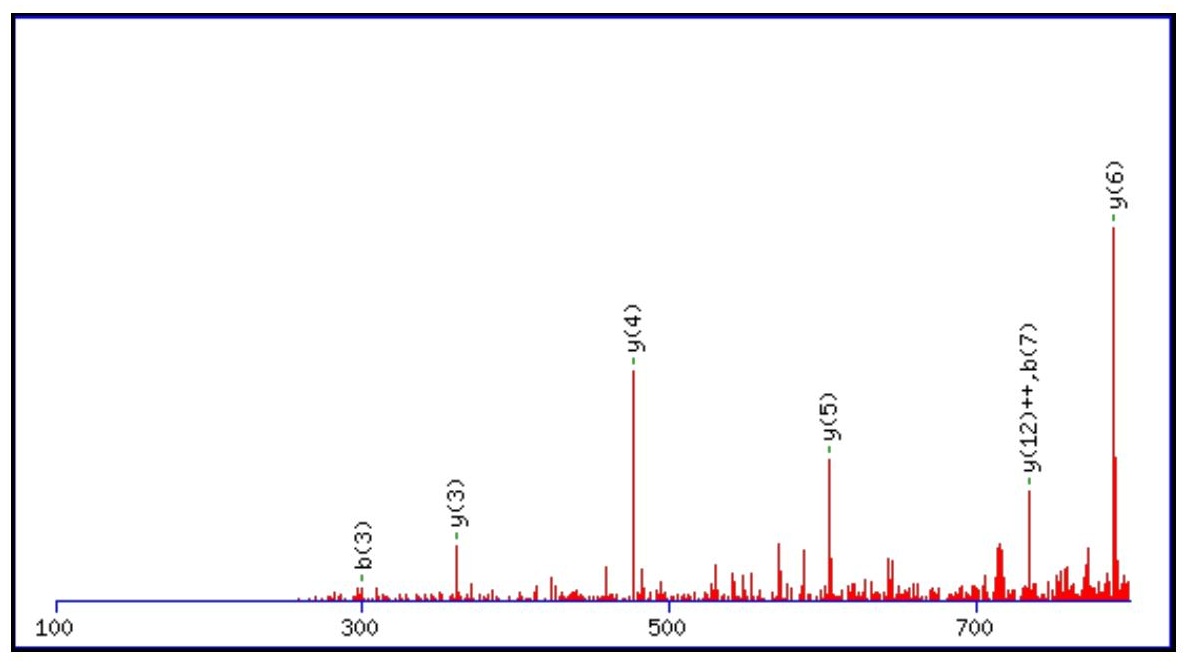



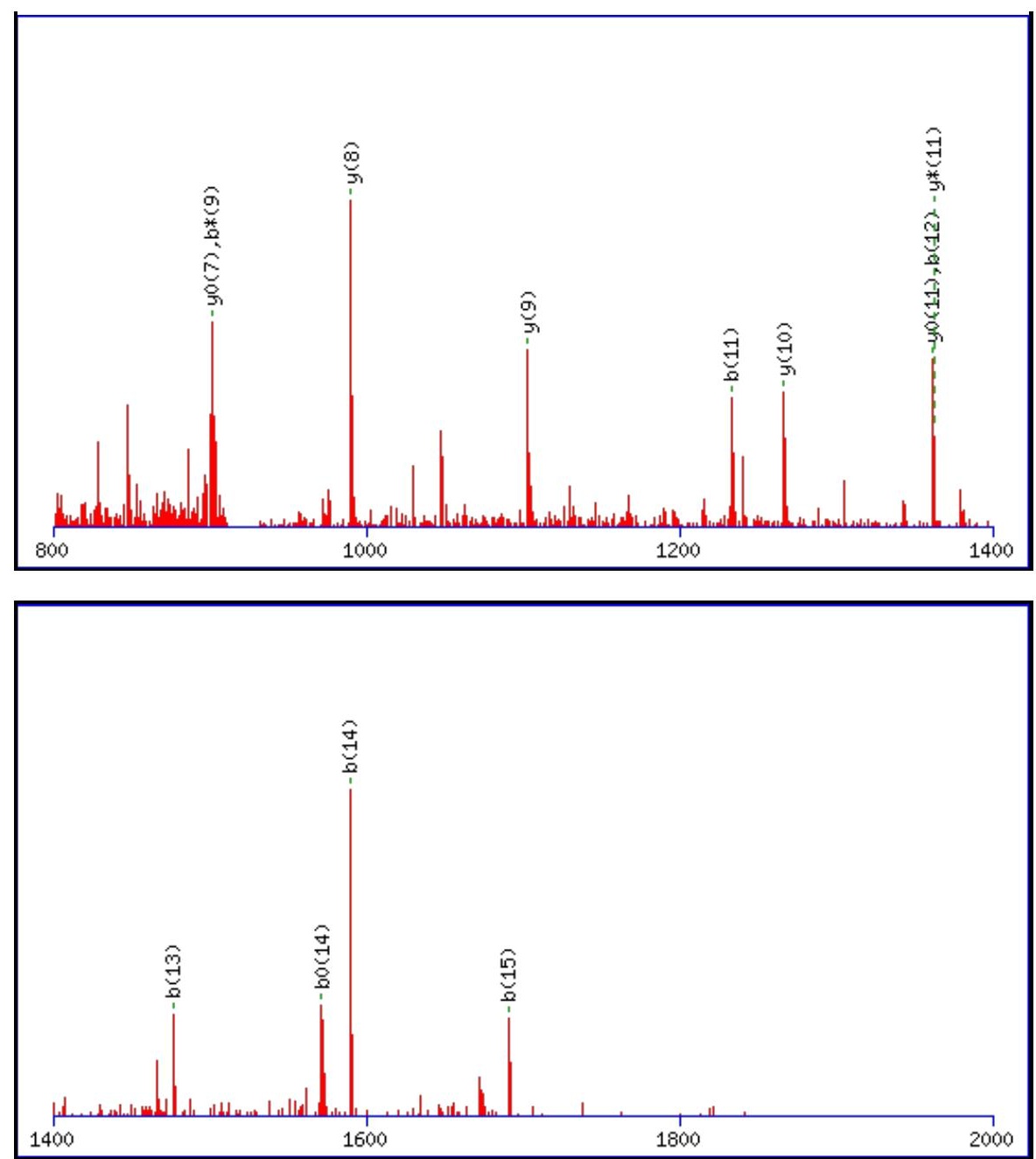

Monoisotopic mass of neutral peptide $\operatorname{Mr}($ calc): 1835.9305

Fixed modifications: Carbamidomethyl (C)

Variable modifications:

N3 : Deamidated_N (N)

N13 : Deamidated_N $(\mathrm{N})$

Ions Score: 78 Expect: $2.4 \mathrm{e}-006$

Matches (Bold Red): 20/166 fragment ions using 22 most intense peaks

\begin{tabular}{|c|c|c|c|c|c|c|c|c|c|c|c|c|c|c|}
\hline \# & b & $\mathbf{b}^{++}$ & $\mathbf{b}^{*}$ & $\mathbf{b}^{*^{++}}$ & $\mathbf{b}^{\mathbf{0}}$ & $\mathbf{b}^{0++}$ & Seq. & $\mathbf{y}$ & $\mathbf{y}^{++}$ & $\mathbf{y}^{*}$ & $\mathbf{y}^{*^{++}}$ & $\mathbf{y}^{0}$ & $y^{0++}$ & \# \\
\hline 1 & 114.0913 & 57.5493 & & & & & $\mathbf{L}$ & & & & & & & 16 \\
\hline 2 & 185.1285 & 93.0679 & & & & & A & 1723.8537 & 862.4305 & 1706.8272 & 853.9172 & 1705.8432 & 853.4252 & 15 \\
\hline 3 & 300.1554 & 150.5813 & 283.1288 & 142.0681 & & & $\mathbf{N}$ & 1652.8166 & 826.9119 & 1635.7901 & 818.3987 & 1634.8061 & 817.9067 & 14 \\
\hline 4 & 371.1925 & 186.0999 & 354.1660 & 177.5866 & & & A & 1537.7897 & 769.3985 & 1520.7631 & 760.8852 & 1519.7791 & 760.3932 & 13 \\
\hline 5 & 458.2245 & 229.6159 & 441.1980 & 221.1026 & 440.2140 & 220.6106 & S & 1466.7526 & 733.8799 & 1449.7260 & 725.3666 & 1448.7420 & 724.8746 & 12 \\
\hline 6 & 571.3086 & 286.1579 & 554.2820 & 277.6447 & 553.2980 & 277.1527 & $\mathbf{L}$ & 1379.7205 & 690.3639 & 1362.6940 & 681.8506 & 1361.7100 & 681.3586 & 11 \\
\hline 7 & 734.3719 & 367.6896 & 717.3454 & 359.1763 & 716.3614 & 358.6843 & $\mathbf{Y}$ & 1266.6365 & 633.8219 & 1249.6099 & 625.3086 & 1248.6259 & 624.8166 & 10 \\
\hline 8 & 847.4560 & 424.2316 & 830.4294 & 415.7184 & 829.4454 & 415.2264 & $\mathbf{L}$ & 1103.5731 & 552.2902 & 1086.5466 & 543.7769 & 1085.5626 & 543.2849 & 9 \\
\hline 9 & 918.4931 & 459.7502 & 901.4666 & 451.2369 & 900.4825 & 450.7449 & A & 990.4891 & 495.7482 & 973.4625 & 487.2349 & 972.4785 & 486.7429 & 8 \\
\hline 10 & 1047.5357 & 524.2715 & 1030.5091 & 515.7582 & 1029.5251 & 515.2662 & $\mathbf{E}$ & 919.4520 & 460.2296 & 902.4254 & 451.7163 & 901.4414 & 451.2243 & 7 \\
\hline 11 & 1233.6150 & 617.3111 & 1216.5885 & 608.7979 & 1215.6044 & 608.3059 & $\mathbf{W}$ & 790.4094 & 395.7083 & 773.3828 & 387.1951 & 772.3988 & 386.7030 & 6 \\
\hline 12 & 1361.6736 & 681.3404 & 1344.6470 & 672.8272 & 1343.6630 & 672.3351 & $\mathbf{Q}$ & 604.3301 & 302.6687 & 587.3035 & 294.1554 & 586.3195 & 293.6634 & 5 \\
\hline 13 & 1476.7005 & 738.8539 & 1459.6740 & 730.3406 & 1458.6900 & 729.8486 & $\mathbf{N}$ & 476.2715 & 238.6394 & 459.2449 & 230.1261 & 458.2609 & 229.6341 & 4 \\
\hline 14 & 1589.7846 & 795.3959 & 1572.7580 & 786.8827 & 1571.7740 & 786.3906 & I & 361.2445 & 181.1259 & 344.2180 & 172.6126 & 343.2340 & 172.1206 & 3 \\
\hline 15 & 1690.8323 & 845.9198 & 1673.8057 & 837.4065 & 1672.8217 & 836.9145 & $\mathbf{T}$ & 248.1605 & 124.5839 & 231.1339 & 116.0706 & 230.1499 & 115.5786 & 2 \\
\hline 16 & & & & & & & $\mathbf{K}$ & 147.1128 & 74.0600 & 130.0863 & 65.5468 & & & 1 \\
\hline
\end{tabular}



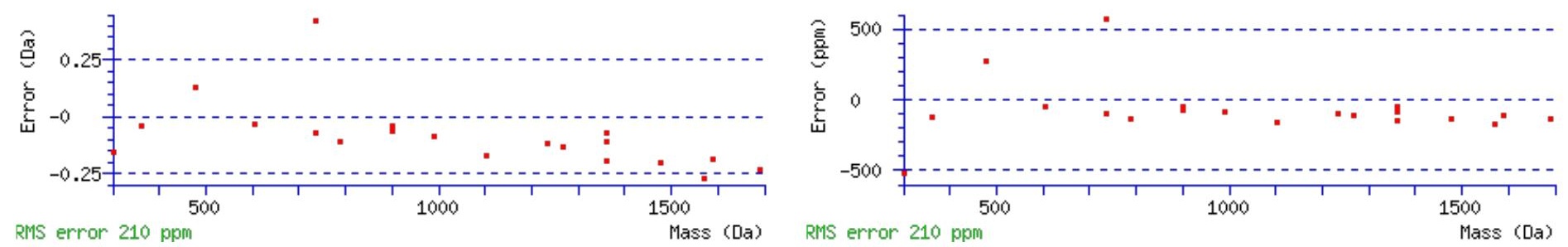

\section{All matches to this query}

\begin{tabular}{|l|l|l|l|}
\hline Score & Mr(calc): & Delta & \multicolumn{1}{c|}{ Sequence } \\
\hline 78.3 & 1835.9305 & 0.0009 & LANASLYLAEWQNITK \\
\hline 8.4 & 1835.9394 & -0.0080 & GSTIRQELVLPSXTLK \\
\hline 8.4 & 1835.9394 & -0.0080 & GSTIRQELVLPSXTLK \\
\hline 1.9 & 1835.9281 & 0.0033 & LKEIINNTPLGSSELK \\
\hline 1.9 & 1835.9281 & 0.0033 & LKEIINNTPLGSSELK \\
\hline 0.2 & 1835.9272 & 0.0042 & MVVTRSGSRIQESSQR \\
\hline 0.0 & 1835.9328 & -0.0014 & GMKAGKPVVAASGAGNLTK \\
\hline
\end{tabular}

Spectrum No: 250; Query: 473; Rank: 1

\section{Peptide View}

MS/MS Fragmentation of SENSSLTLSSSNR

Found in IPI00324585, Tax_Id=10116 Gene_Symbol=Itga1 Integrin alpha-1 precursor

Match to Query 473: 1381.634508 from(691.824530,2+)

Title: 100101RatKid_NS_deglyco_15.1103.1103.2.dta

Data file K:INewmanPaper|Piliangl3SubProteomes\Piliang3SP\mgf5ppm\ERLIC_3SubProteomes5ppm.mgf

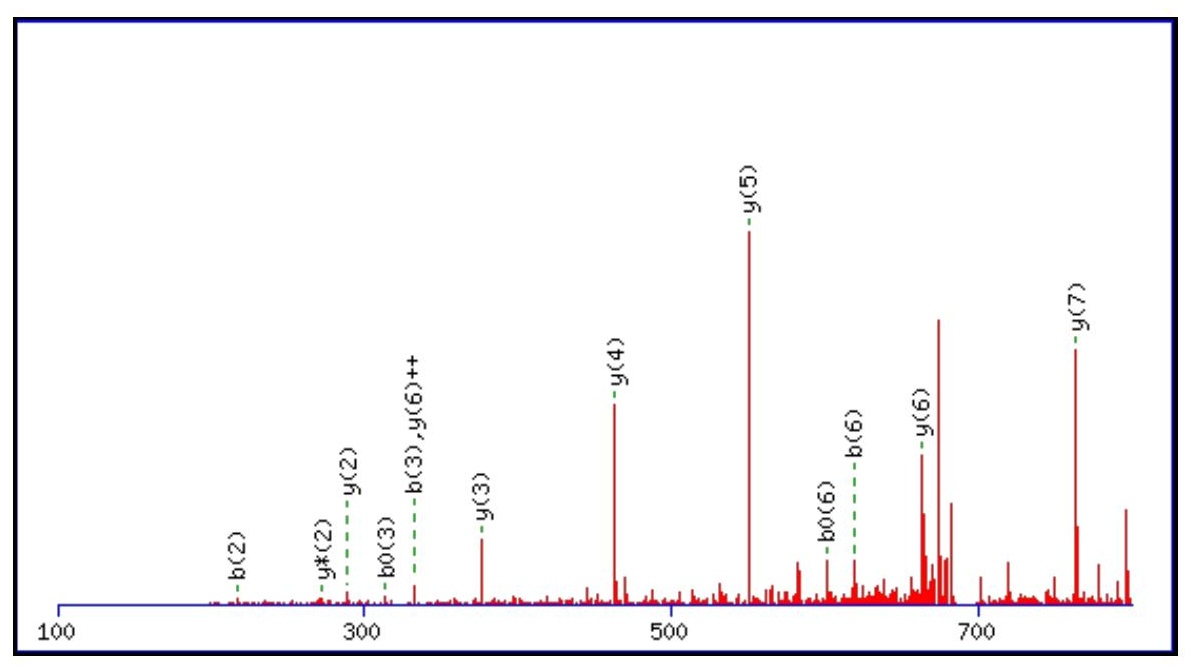



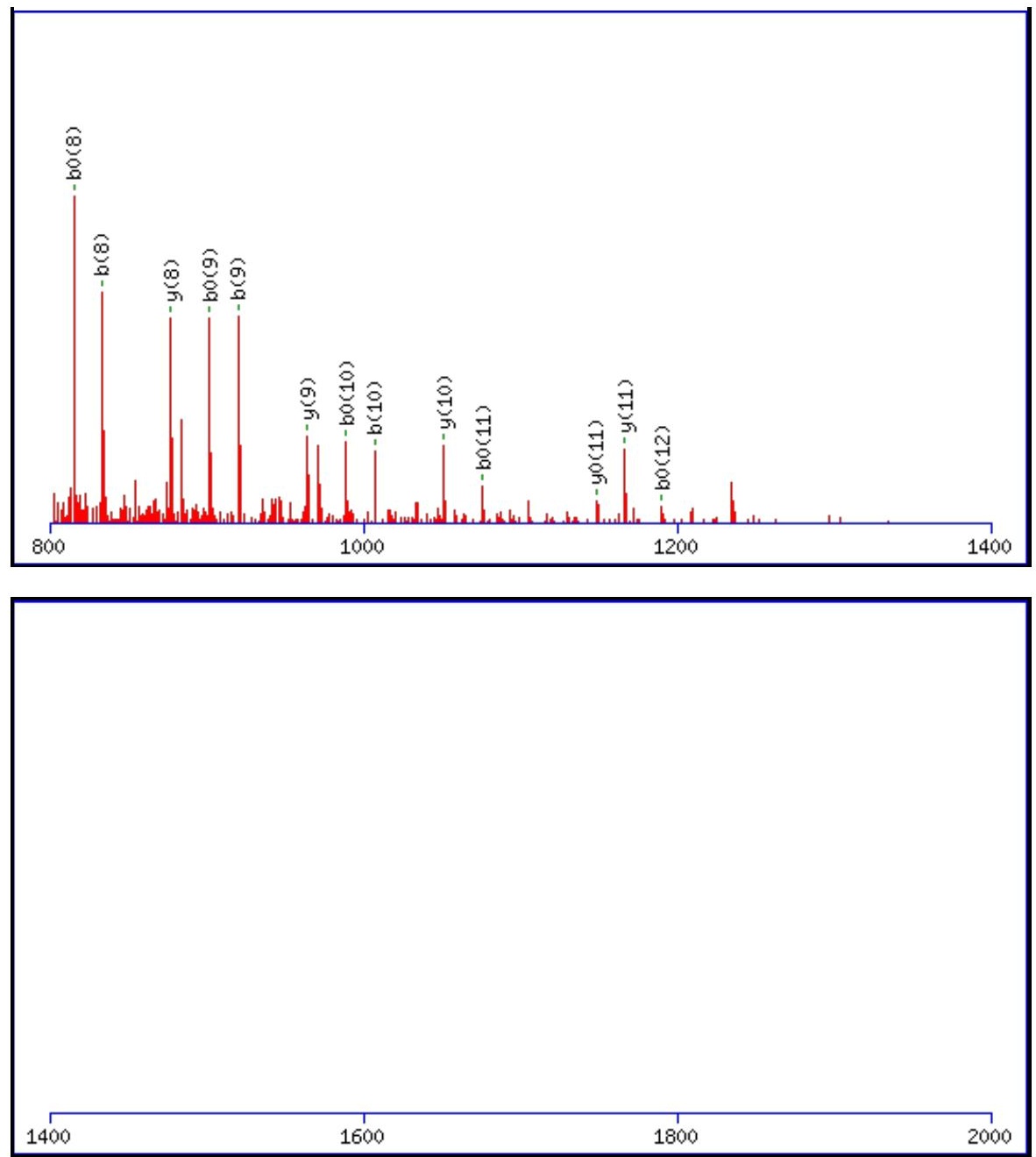

Monoisotopic mass of neutral peptide $\operatorname{Mr}($ calc): 1381.6321

Fixed modifications: Carbamidomethyl (C)

Variable modifications:

N3 : Deamidated $\mathrm{N}(\mathrm{N})$

Ions Score: 78 Expect: $2.1 \mathrm{e}-006$

Matches (Bold Red): 26/136 fragment ions using 45 most intense peaks

\begin{tabular}{|c|c|c|c|c|c|c|c|c|c|c|c|c|c|c|}
\hline \# & b & $\mathbf{b}^{++}$ & b* & $\mathbf{b}^{*^{++}}$ & $\mathbf{b}^{\mathbf{0}}$ & $\mathbf{b}^{\mathbf{0 + +}}$ & Seq. & $\mathbf{y}$ & $\mathrm{y}^{++}$ & $\mathbf{y}^{*}$ & $\mathrm{y}^{*^{++}}$ & $\mathbf{y}^{0}$ & $\mathbf{y}^{0++}$ & \# \\
\hline 1 & 88.0393 & 44.5233 & & & 70.0287 & 35.5180 & S & & & & & & & 13 \\
\hline 2 & 217.0819 & 109.0446 & & & 199.0713 & 100.0393 & $\mathbf{E}$ & 1295.6074 & 648.3073 & 1278.5808 & 639.7940 & 1277.5968 & 639.3020 & 12 \\
\hline 3 & 332.1088 & 166.5581 & 315.0823 & 158.0448 & 314.0983 & 157.5528 & $\mathbf{N}$ & 1166.5648 & 583.7860 & 1149.5382 & 575.2727 & 1148.5542 & 574.7807 & 11 \\
\hline 4 & 419.1409 & 210.0741 & 402.1143 & 201.5608 & 401.1303 & 201.0688 & S & 1051.5378 & 526.2726 & 1034.5113 & 517.7593 & 1033.5273 & 517.2673 & 10 \\
\hline 5 & 506.1729 & 253.5901 & 489.1463 & 245.0768 & 488.1623 & 244.5848 & S & 964.5058 & 482.7565 & 947.4792 & 474.2433 & 946.4952 & 473.7513 & 9 \\
\hline 6 & 619.2570 & 310.1321 & 602.2304 & 301.6188 & 601.2464 & 301.1268 & $\mathbf{L}$ & 877.4738 & 439.2405 & 860.4472 & 430.7272 & 859.4632 & 430.2352 & 8 \\
\hline 7 & 720.3046 & 360.6560 & 703.2781 & 352.1427 & 702.2941 & 351.6507 & $\mathbf{T}$ & 764.3897 & 382.6985 & 747.3632 & 374.1852 & 746.3791 & 373.6932 & 7 \\
\hline 8 & 833.3887 & 417.1980 & 816.3621 & 408.6847 & 815.3781 & 408.1927 & $\mathbf{L}$ & 663.3420 & 332.1747 & 646.3155 & 323.6614 & 645.3315 & 323.1694 & 6 \\
\hline 9 & 920.4207 & 460.7140 & 903.3942 & 452.2007 & 902.4102 & 451.7087 & $\mathrm{~S}$ & 550.2580 & 275.6326 & 533.2314 & 267.1193 & 532.2474 & 266.6273 & 5 \\
\hline 10 & 1007.4528 & 504.2300 & 990.4262 & 495.7167 & 989.4422 & 495.2247 & $\mathrm{~S}$ & 463.2259 & 232.1166 & 446.1994 & 223.6033 & 445.2154 & 223.1113 & 4 \\
\hline 11 & 1094.4848 & 547.7460 & 1077.4582 & 539.2328 & 1076.4742 & 538.7407 & $\mathrm{~S}$ & 376.1939 & 188.6006 & 359.1674 & 180.0873 & 358.1833 & 179.5953 & 3 \\
\hline 12 & 1208.5277 & 604.7675 & 1191.5012 & 596.2542 & 1190.5171 & 595.7622 & $\mathbf{N}$ & 289.1619 & 145.0846 & 272.1353 & 136.5713 & & & 2 \\
\hline 13 & & & & & & & $\mathbf{R}$ & 175.1190 & 88.0631 & 158.0924 & 79.5498 & & & 1 \\
\hline
\end{tabular}



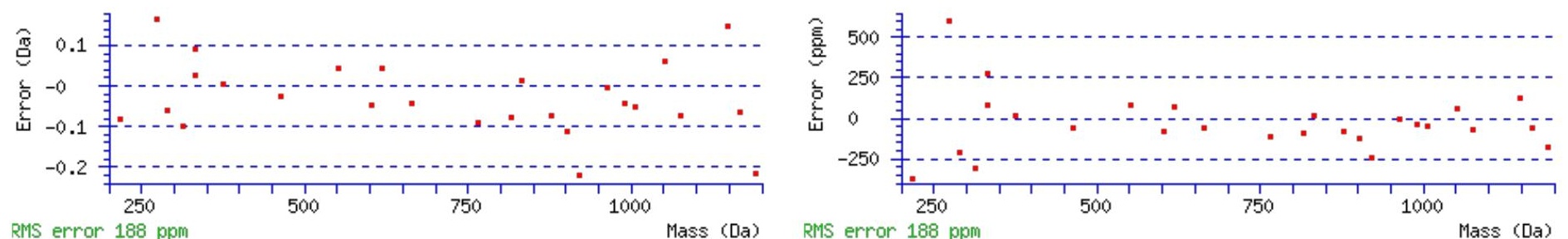

RNS error $188 \mathrm{ppm}$

\section{All matches to this query}

\begin{tabular}{|l|l|l|l|}
\hline Score & Mr(calc): & Delta & Sequence \\
\hline 78.3 & 1381.6321 & 0.0024 & SENSSLTLSSSNR \\
\hline 18.3 & 1381.6321 & 0.0024 & SENSSLTLSSSNR \\
\hline 5.9 & 1380.6269 & 1.0076 & SNSFNADRNIDK \\
\hline 5.8 & 1381.6217 & 0.0128 & MSEDSNMEKAIK \\
\hline 5.3 & 1380.6360 & 0.9985 & SPTPVTMEKSPK \\
\hline 5.3 & 1380.6360 & 0.9985 & SPTPVTMEKSPK \\
\hline 3.3 & 1381.6330 & 0.0015 & MDLSGGMLQDKR \\
\hline
\end{tabular}

Spectrum No: 251; Query: 127; Rank: 1

\section{Peptide View}

\section{MS/MS Fragmentation of LEVLLANVSR}

Found in IPI00199867, Tax_Id=10116 Gene_Symbol=Emilin1_predicted elastin microfibril interfacer 1

Match to Query 127: 1113.640448 from(557.827500,2+)

Title: 100101RatKid_NS_deglyco_27.2689.2689.2.dta

Data file K:INewmanPaper|Piliangl3SubProteomes\Piliang3SP\mgf5ppm\ERLIC_3SubProteomes5ppm.mgf

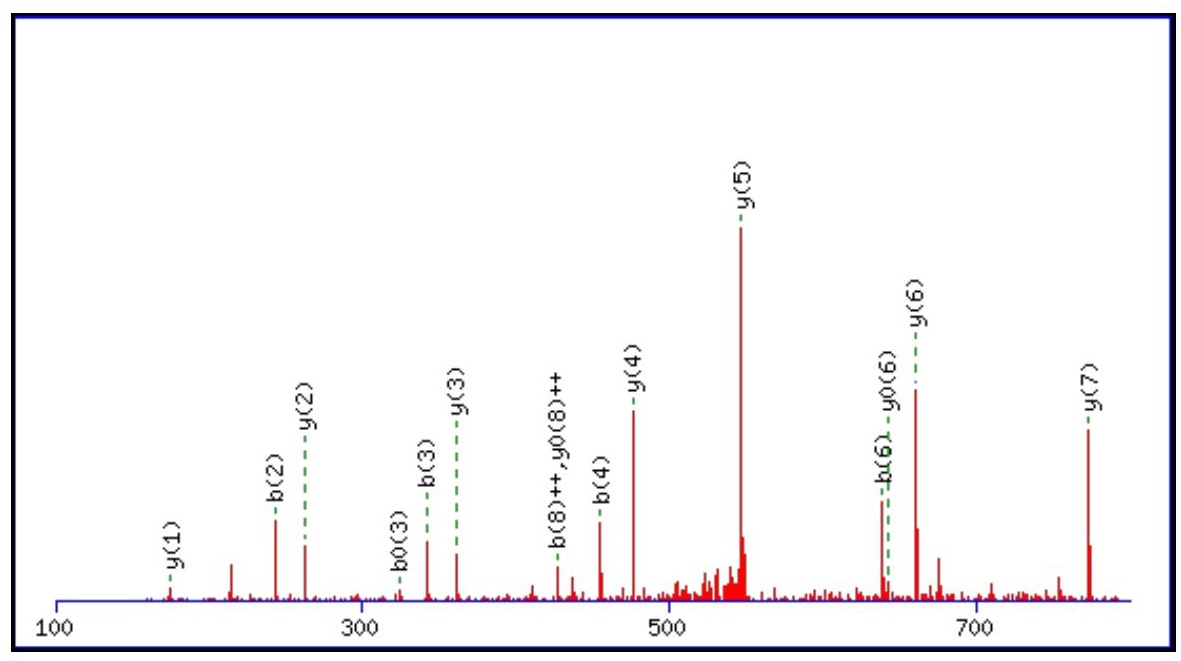



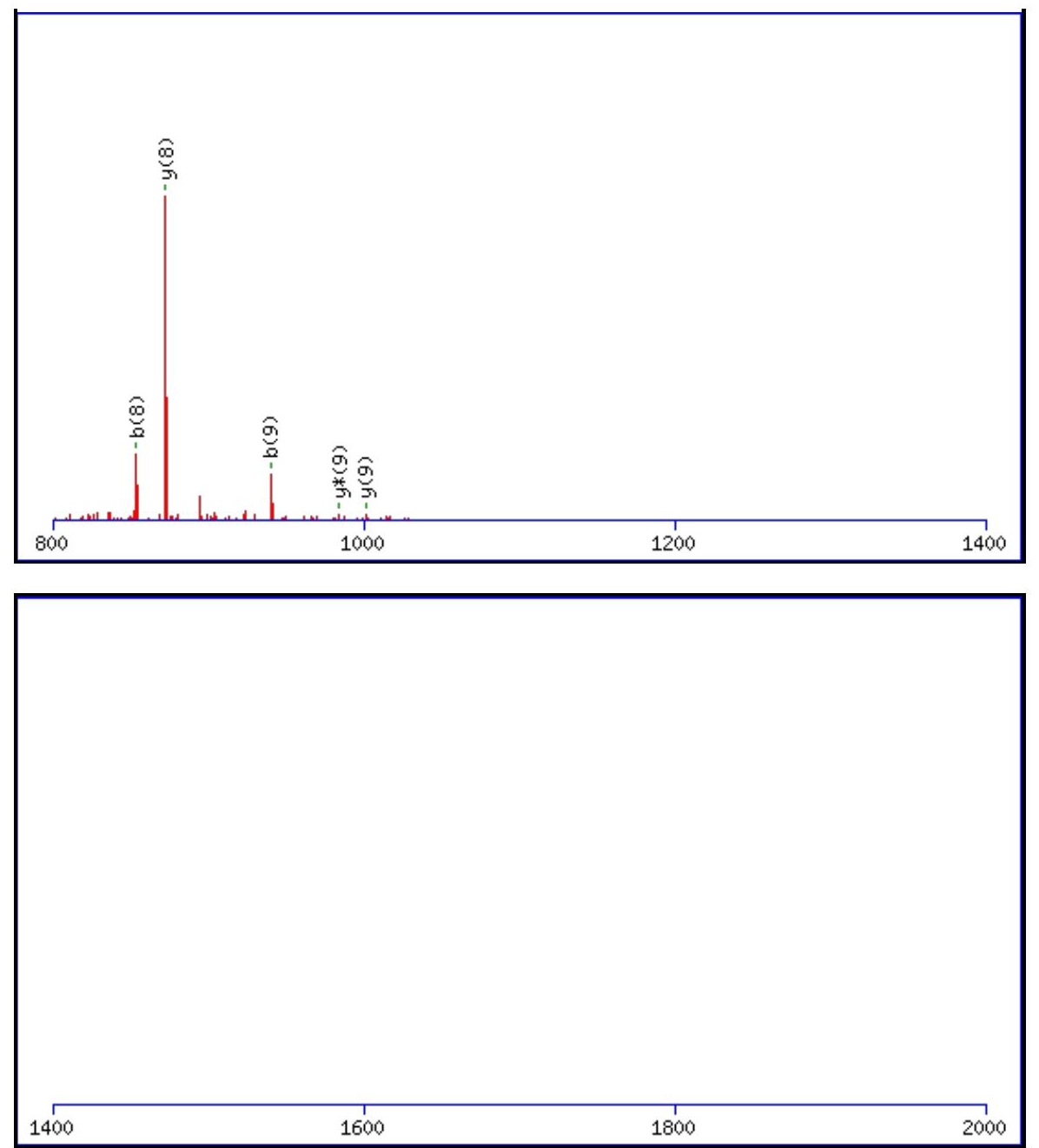

Monoisotopic mass of neutral peptide $\operatorname{Mr}($ calc): 1113.6393

Fixed modifications: Carbamidomethyl (C)

Variable modifications:

N7 : Deamidated_N (N)

Ions Score: 78 Expect: $9.3 \mathrm{e}-007$

Matches (Bold Red): 20/92 fragment ions using 28 most intense peaks

\begin{tabular}{|c|c|c|c|c|c|c|c|c|c|c|c|c|c|c|}
\hline \# & b & $\mathbf{b}^{++}$ & $\mathbf{b}^{*}$ & $\mathbf{b}^{*^{++}}$ & $\mathbf{b}^{\mathbf{0}}$ & $\mathbf{b}^{\mathbf{0 + +}}$ & Seq. & $\mathbf{y}$ & $\mathbf{y}^{++}$ & $\mathbf{y}^{*}$ & $\mathrm{y}^{*^{++}}$ & $\mathbf{y}^{0}$ & $\mathbf{y}^{\mathbf{0}^{++}}$ & $\#$ \\
\hline 1 & 114.0913 & 57.5493 & & & & & $\mathbf{L}$ & & & & & & & 10 \\
\hline 2 & 243.1339 & 122.0706 & & & 225.1234 & 113.0653 & $\mathbf{E}$ & 1001.5626 & 501.2849 & 984.5360 & 492.7717 & 983.5520 & 492.2796 & 9 \\
\hline 3 & 342.2023 & 171.6048 & & & 324.1918 & 162.5995 & V & 872.5200 & 436.7636 & 855.4934 & 428.2504 & 854.5094 & 427.7583 & 8 \\
\hline 4 & 455.2864 & 228.1468 & & & 437.2758 & 219.1416 & L & 773.4516 & 387.2294 & 756.4250 & 378.7161 & 755.4410 & 378.2241 & 7 \\
\hline 5 & 568.3705 & 284.6889 & & & 550.3599 & 275.6836 & $\mathbf{L}$ & 660.3675 & 330.6874 & 643.3410 & 322.1741 & 642.3569 & 321.6821 & 6 \\
\hline 6 & 639.4076 & 320.2074 & & & 621.3970 & |311.2022 & A & 547.2834 & 274.1454 & 530.2569 & 265.6321 & 529.2729 & 265.1401 & 5 \\
\hline 7 & 754.4345 & 377.7209 & 737.4080 & 369.2076 & 736.4240 & 368.7156 & $\mathbf{N}$ & 476.2463 & 238.6268 & 459.2198 & 230.1135 & 458.2358 & 229.6215 & 4 \\
\hline 8 & 853.5029 & 427.2551 & 836.4764 & 418.7418 & 835.4924 & 418.2498 & $\mathbf{V}$ & 361.2194 & 181.1133 & 344.1928 & 172.6001 & 343.2088 & 172.1081 & 3 \\
\hline 9 & 940.5350 & 470.7711 & 923.5084 & 462.2578 & 922.5244 & 461.7658 & $\mathrm{~S}$ & 262.1510 & 131.5791 & 245.1244 & 123.0659 & 244.1404 & 122.5738 & 2 \\
\hline 10 & & & & & & & $\mathbf{R}$ & 175.1190 & 88.0631 & 158.0924 & 79.5498 & & & 1 \\
\hline
\end{tabular}$$
\text { 蒠 }
$$
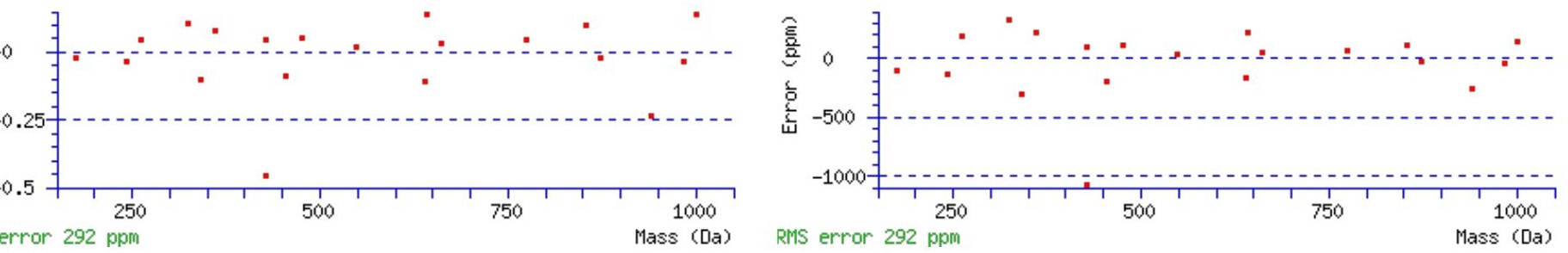

\section{All matches to this query}




\begin{tabular}{|l|l|l|l|}
\hline Score & Mr(calc): & Delta & Sequence \\
\hline 78.2 & 1113.6393 & 0.0011 & LEVLLANVSR \\
\hline 13.4 & 1113.6393 & 0.0011 & DILLISQNAK \\
\hline 11.1 & 1113.6393 & 0.0011 & LINLVGESLR \\
\hline 11.1 & 1113.6393 & 0.0011 & LLNLNLERK \\
\hline 9.6 & 1113.6506 & -0.0101 & NIVKLAEATR \\
\hline 4.5 & 1113.6394 & 0.0011 & EIVSLLLGDR \\
\hline 4.1 & 1113.6294 & 0.0110 & LQLEKAAWR \\
\hline 4.0 & 1113.6506 & -0.0101 & LQVQERLTK \\
\hline 3.5 & 1112.6414 & 0.9990 & NQVIKAERR \\
\hline 3.4 & 1113.6393 & 0.0011 & VIELENRLK \\
\hline
\end{tabular}

Spectrum No: 252; Query: 249; Rank: 1

\section{Peptide View}

MS/MS Fragmentation of ELAVAANETAAR

Found in IPI00363534, Tax_Id=10116 Gene_Symbol=Lama1 laminin, alpha 1

Match to Query 249: 1215.606508 from(608.810530,2+)

Title: 091008RatKidney_NH4Format01_25.921.921.2.dta

Data file K:INewmanPaper|Piliang|3SubProteomes\Piliang3SP\mgf5ppm\ERLIC_3SubProteomes5ppm.mgf
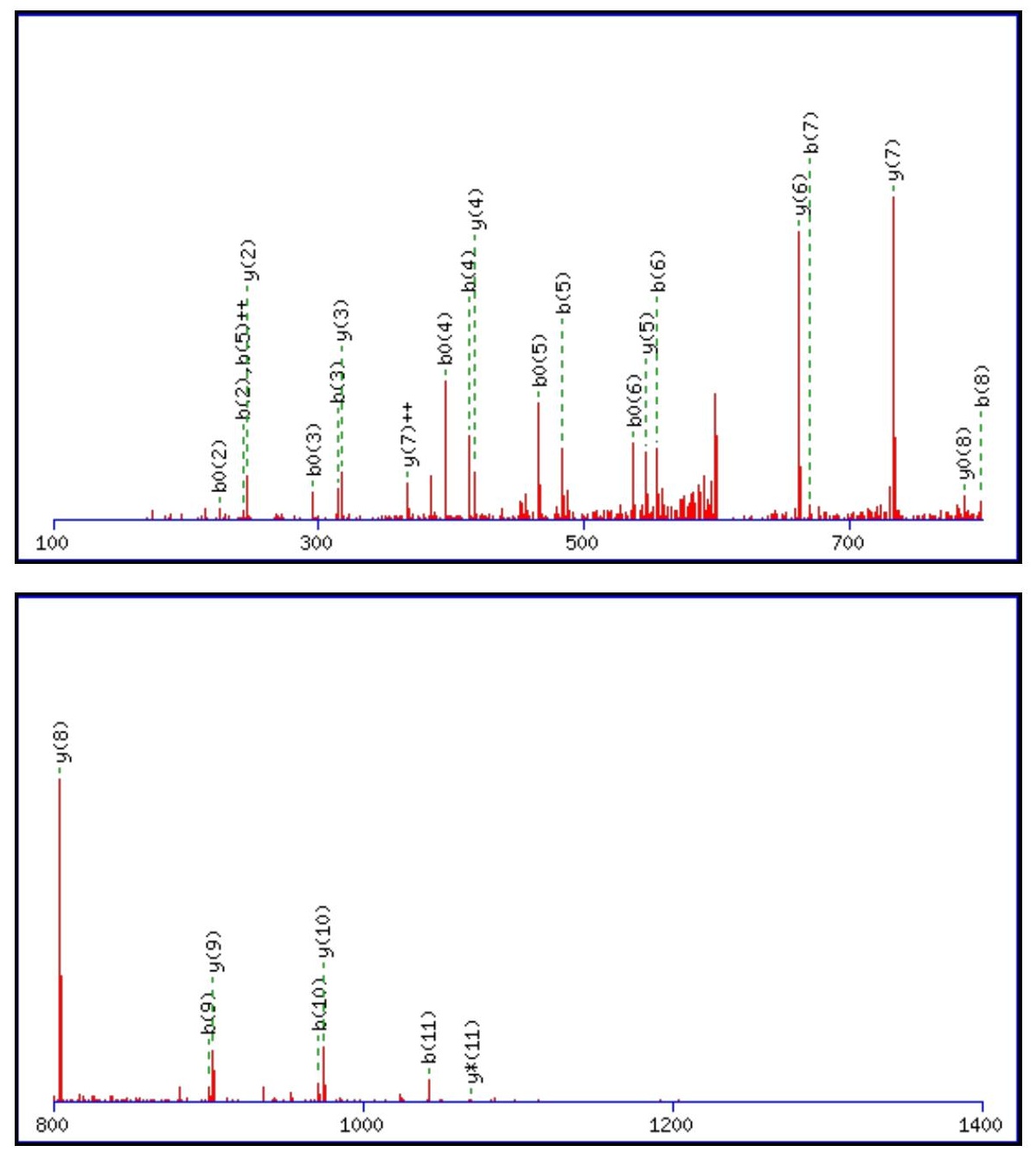


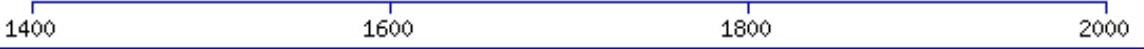

Monoisotopic mass of neutral peptide $\operatorname{Mr}($ calc): 1215.6095

Fixed modifications: Carbamidomethyl (C)

Variable modifications:

N7 : Deamidated $\mathrm{N}(\mathrm{N})$

Ions Score: 78 Expect: $1.8 \mathrm{e}-006$

Matches (Bold Red): 28/114 fragment ions using 44 most intense peaks

\begin{tabular}{|r|c|c|c|c|c|c|c|c|c|c|c|c|c|c|}
\hline$\#$ & $\mathbf{b}$ & $\mathbf{b}^{++}$ & $\mathbf{b}^{*}$ & $\mathbf{b}^{\mathbf{*}^{++}}$ & $\mathbf{b}^{\mathbf{0}}$ & $\mathbf{b}^{\mathbf{0 + +}}$ & $\mathbf{S e q}$ & $\mathbf{y}$ & $\mathbf{y}^{++}$ & $\mathbf{y}^{\mathbf{*}}$ & $\mathbf{y}^{\mathbf{*}^{++}}$ & $\mathbf{y}^{\mathbf{0}}$ & $\mathbf{y}^{\mathbf{0 + +}}$ & $\#$ \\
\hline $\mathbf{1}$ & 130.0499 & 65.5286 & & & 112.0393 & 56.5233 & $\mathbf{E}$ & & & & & & & $\mathbf{1 2}$ \\
\hline $\mathbf{2}$ & $\mathbf{2 4 3 . 1 3 3 9}$ & 122.0706 & & & 225.1234 & 113.0653 & $\mathbf{L}$ & 1087.5742 & 544.2907 & $\mathbf{1 0 7 0 . 5 4 7 7}$ & 535.7775 & 1069.5636 & 535.2855 & $\mathbf{1 1}$ \\
\hline $\mathbf{3}$ & $\mathbf{3 1 4 . 1 7 1 0}$ & 157.5892 & & & $\mathbf{2 9 6 . 1 6 0 5}$ & 148.5839 & $\mathbf{A}$ & $\mathbf{9 7 4 . 4 9 0 1}$ & 487.7487 & 957.4636 & 479.2354 & 956.4796 & 478.7434 & $\mathbf{1 0}$ \\
\hline $\mathbf{4}$ & $\mathbf{4 1 3 . 2 3 9 5}$ & 207.1234 & & & 395.2289 & 198.1181 & $\mathbf{V}$ & $\mathbf{9 0 3 . 4 5 3 0}$ & 452.2302 & 886.4265 & 443.7169 & 885.4425 & 443.2249 & $\mathbf{9}$ \\
\hline $\mathbf{5}$ & $\mathbf{4 8 4 . 2 7 6 6}$ & $\mathbf{2 4 2 . 6 4 1 9}$ & & & $\mathbf{4 6 6 . 2 6 6 0}$ & 233.6366 & $\mathbf{A}$ & $\mathbf{8 0 4 . 3 8 4 6}$ & 402.6959 & 787.3581 & 394.1827 & $\mathbf{7 8 6 . 3 7 4 1}$ & 393.6907 & $\mathbf{8}$ \\
\hline $\mathbf{6}$ & $\mathbf{5 5 5 . 3 1 3 7}$ & 278.1605 & & & 537.3031 & 269.1552 & $\mathbf{A}$ & $\mathbf{7 3 3 . 3 4 7 5}$ & 367.1774 & 716.3210 & 358.6641 & 715.3369 & 358.1721 & $\mathbf{7}$ \\
\hline $\mathbf{7}$ & $\mathbf{6 7 0 . 3 4 0 6}$ & 335.6740 & 653.3141 & 327.1607 & 652.3301 & 326.6687 & $\mathbf{N}$ & $\mathbf{6 6 2 . 3 1 0 4}$ & 331.6588 & 645.2838 & 323.1456 & 644.2998 & 322.6535 & $\mathbf{6}$ \\
\hline $\mathbf{8}$ & $\mathbf{7 9 9 . 3 8 3 2}$ & 400.1952 & 782.3567 & 391.6820 & 781.3727 & 391.1900 & $\mathbf{E}$ & 547.2835 & 274.1454 & 530.2569 & 265.6321 & 529.2729 & 265.1401 & $\mathbf{5}$ \\
\hline $\mathbf{9}$ & $\mathbf{9 0 0 . 4 3 0 9}$ & 450.7191 & 883.4043 & 442.2058 & 882.4203 & 441.7138 & $\mathbf{T}$ & $\mathbf{4 1 8 . 2 4 0 9}$ & 209.6241 & 401.2143 & 201.1108 & 400.2303 & 200.6188 & $\mathbf{4}$ \\
\hline $\mathbf{1 0}$ & $\mathbf{9 7 1 . 4 6 8 0}$ & 486.2376 & 954.4415 & 477.7244 & 953.4574 & 477.2324 & $\mathbf{A}$ & $\mathbf{3 1 7 . 1 9 3 2}$ & 159.1002 & 300.1666 & 150.5870 & & & $\mathbf{3}$ \\
\hline $\mathbf{1 1}$ & $\mathbf{1 0 4 2 . 5 0 5 1}$ & 521.7562 & 1025.4786 & 513.2429 & 1024.4946 & 512.7509 & $\mathbf{A}$ & $\mathbf{2 4 6 . 1 5 6 1}$ & 123.5817 & 229.1295 & 115.0684 & & & $\mathbf{2}$ \\
\hline $\mathbf{1 2}$ & & & & & & & $\mathbf{R}$ & 175.1190 & 88.0631 & 158.0924 & 79.5498 & & & $\mathbf{1}$ \\
\hline
\end{tabular}
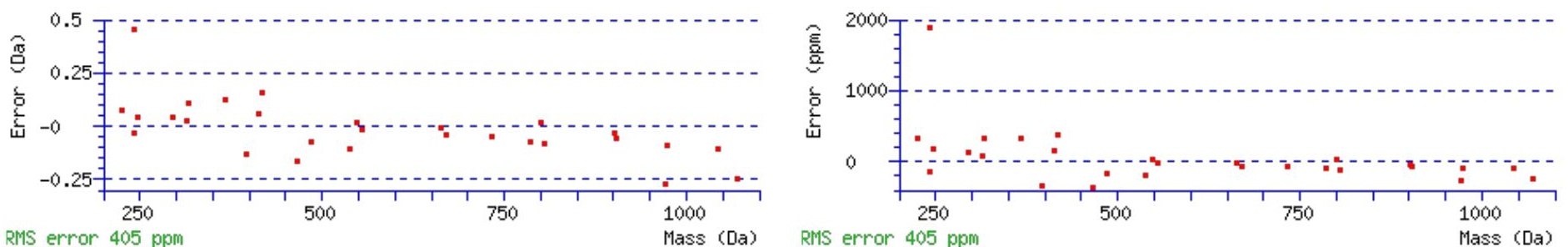

All matches to this query

\begin{tabular}{|l|l|l|l|}
\hline Score & Mr(calc): & Delta & \multicolumn{1}{|c|}{ Sequence } \\
\hline 78.2 & 1215.6095 & -0.0030 & ELAVAANETAAR \\
\hline 7.9 & 1215.6013 & 0.0052 & NGITPLHVASK \\
\hline 7.2 & 1215.6095 & -0.0030 & NQLGKNEDGLK \\
\hline 6.1 & 1214.6077 & 0.9988 & TAPAAAVSPMQR \\
\hline 5.1 & 1215.6046 & 0.0019 & RAKLMSTVSK \\
\hline 4.5 & 1213.5979 & 2.0086 & SSPNPFVGSPPK \\
\hline 4.0 & 1214.5982 & 1.0083 & LTSSLLGMVAK \\
\hline 4.0 & 1214.5982 & 1.0083 & LTSSLLGMVAK \\
\hline 3.3 & 1215.6125 & -0.0060 & VKPRHDSGIK \\
\hline & & & \\
\end{tabular}




\section{|3.0 |1215.6141|-0.0076|SKPLRKTVK}

Spectrum No: 253; Query: 827; Rank: 1

\section{Peptide View}

MS/MS Fragmentation of EAGNITTDGYEILGK

Found in IPI00189714, Tax_Id=10116 Gene_Symbol=Col12a1 similar to Collagen alpha-1(XII) chain precursor

Match to Query 827: 1580.754188 from(791.384370,2+)

Title: 091008RatKidney_NoSalt_27.2733.2733.2.dta

Data file K:\NewmanPaper\Piliang \3SubProteomes \Piliang3SP $\backslash$ mgf5ppm\ERLIC_3SubProteomes5ppm.mgf
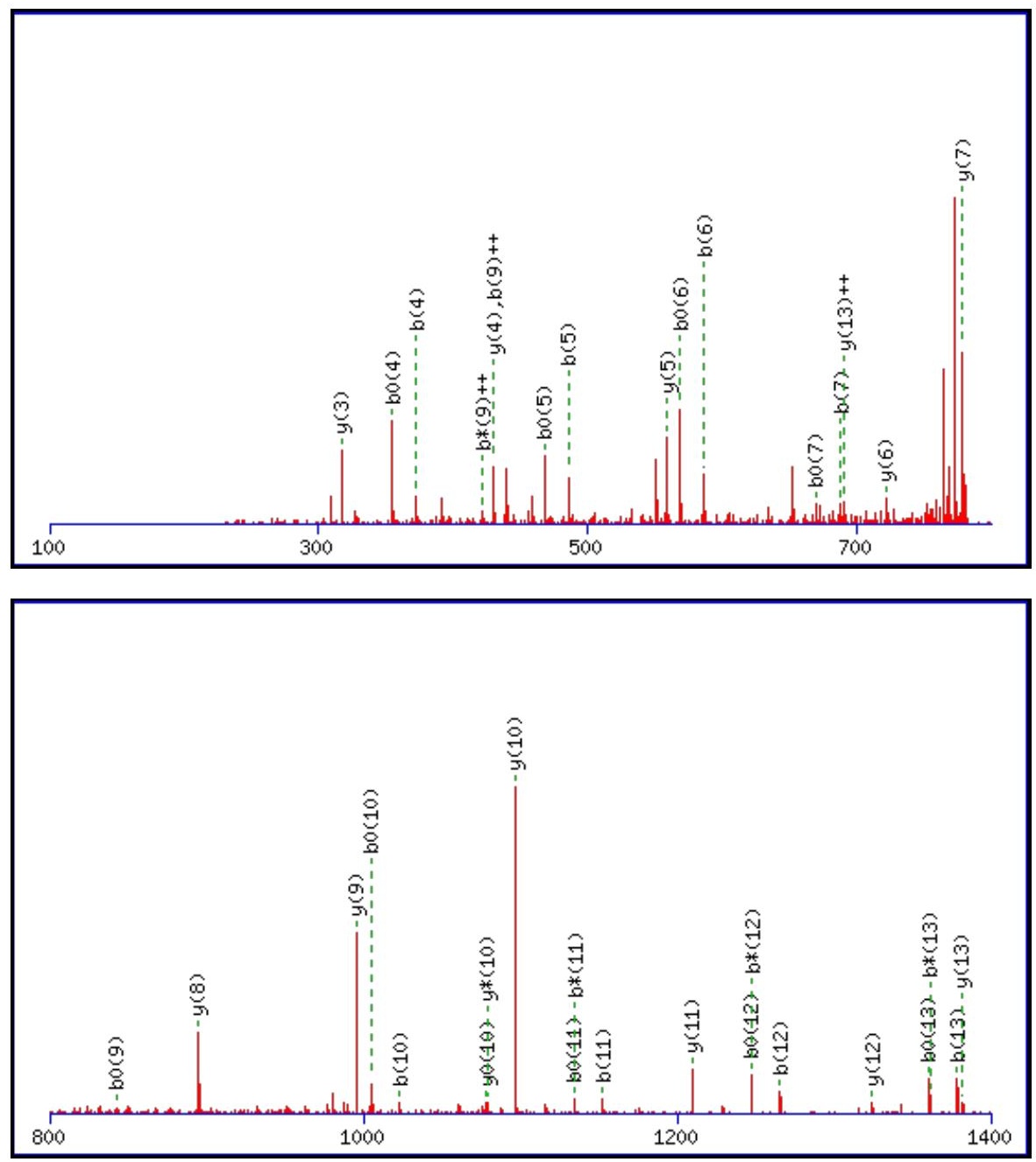


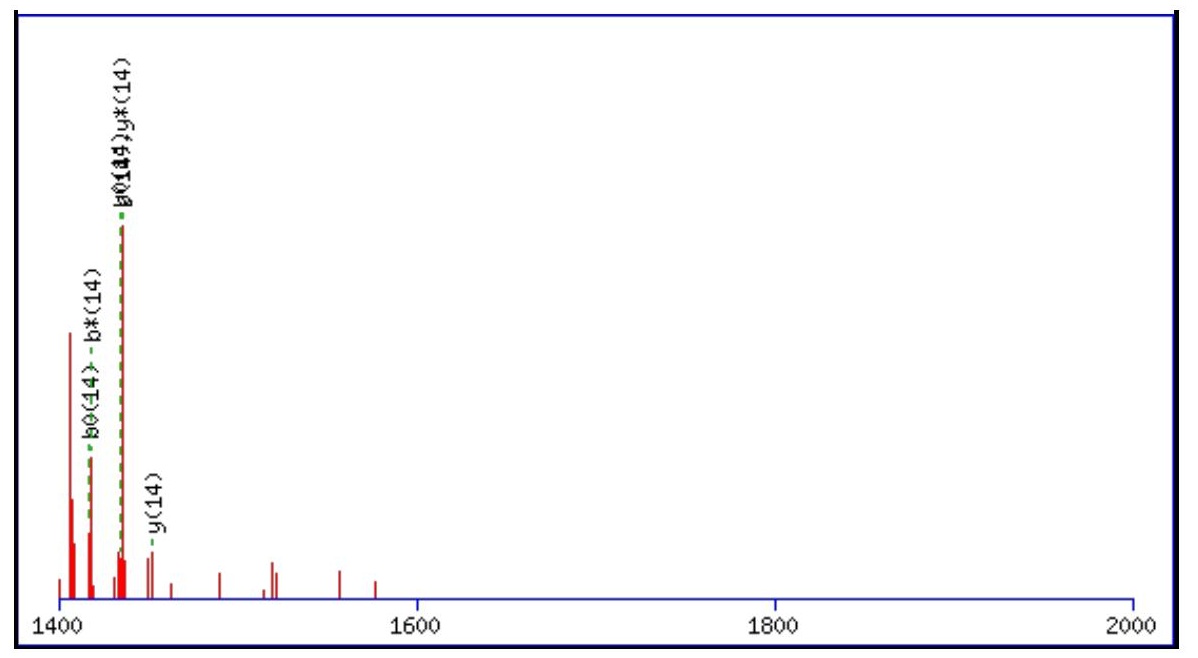

Monoisotopic mass of neutral peptide $\operatorname{Mr}($ calc): 1580.7570

Fixed modifications: Carbamidomethyl (C)

Variable modifications:

N4 : Deamidated $\mathrm{N}(\mathrm{N})$

Ions Score: 78 Expect: $2.7 \mathrm{e}-006$

Matches (Bold Red): 42/154 fragment ions using 77 most intense peaks

\begin{tabular}{|c|c|c|c|c|c|c|c|c|c|c|c|c|c|c|}
\hline \# & b & $\mathbf{b}^{++}$ & b* & $\mathbf{b}^{*^{++}}$ & $\mathbf{b}^{\mathbf{0}}$ & $\mathbf{b}^{\mathbf{0 + +}}$ & Seq. & $\mathbf{y}$ & $\mathbf{y}^{++}$ & $\mathbf{y}^{*}$ & $\mathrm{y}^{\mathrm{*}^{++}}$ & $\mathbf{y}^{\mathbf{0}}$ & $\mathbf{y}^{\mathbf{0 + +}}$ & \# \\
\hline 1 & 130.0499 & 65.5286 & & & 112.0393 & 56.5233 & $\mathbf{E}$ & & & & & & & 15 \\
\hline 2 & 201.0870 & 101.0471 & & & 183.0764 & 92.0418 & A & 1452.7217 & 726.8645 & 1435.6951 & 718.3512 & 1434.7111 & 717.8592 & 14 \\
\hline 3 & 258.1084 & 129.5579 & & & 240.0979 & 120.5526 & G & 1381.6845 & 691.3459 & 1364.6580 & 682.8326 & 1363.6740 & 682.3406 & 13 \\
\hline 4 & 373.1354 & 187.0713 & 356.1088 & 178.5581 & 355.1248 & 178.0660 & $\mathbf{N}$ & 1324.6631 & 662.8352 & 1307.6365 & 654.3219 & 1306.6525 & 653.8299 & 12 \\
\hline 5 & 486.2194 & 243.6134 & 469.1929 & 235.1001 & 468.2089 & 234.6081 & I & 1209.6361 & 605.3217 & 1192.6096 & 596.8084 & 1191.6256 & 596.3164 & 11 \\
\hline 6 & 587.2671 & 294.1372 & 570.2406 & 285.6239 & 569.2566 & 285.1319 & $\mathbf{T}$ & 1096.5521 & 548.7797 & 1079.5255 & 540.2664 & 1078.5415 & 539.7744 & 10 \\
\hline 7 & 688.3148 & 344.6610 & 671.2883 & 336.1478 & 670.3042 & 335.6558 & $\mathbf{T}$ & 995.5044 & 498.2558 & 978.4779 & 489.7426 & 977.4938 & 489.2506 & 9 \\
\hline 8 & 803.3417 & 402.1745 & 786.3152 & 393.6612 & 785.3312 & 393.1692 & D & 894.4567 & 447.7320 & 877.4302 & 439.2187 & 876.4462 & 438.7267 & 8 \\
\hline 9 & 860.3632 & 430.6852 & 843.3367 & 422.1720 & 842.3526 & 421.6800 & G & 779.4298 & 390.2185 & 762.4032 & 381.7053 & 761.4192 & 381.2132 & 7 \\
\hline 10 & 1023.4265 & 512.2169 & 1006.4000 & 503.7036 & 1005.4160 & 503.2116 & $\mathbf{Y}$ & 722.4083 & 361.7078 & 705.3818 & 353.1945 & 704.3978 & 352.7025 & 6 \\
\hline 11 & 1152.4691 & 576.7382 & 1135.4426 & 568.2249 & 1134.4586 & 567.7329 & $\mathbf{E}$ & 559.3450 & 280.1761 & 542.3184 & 271.6629 & 541.3344 & 271.1708 & 5 \\
\hline 12 & 1265.5532 & 633.2802 & 1248.5266 & 624.7670 & 1247.5426 & 624.2750 & I & 430.3024 & 215.6548 & 413.2758 & 207.1416 & & & 4 \\
\hline 13 & 1378.6373 & 689.8223 & 1361.6107 & 681.3090 & 1360.6267 & 680.8170 & $\mathbf{L}$ & 317.2183 & 159.1128 & 300.1918 & 150.5995 & & & 3 \\
\hline 14 & 1435.6587 & 718.3330 & 1418.6322 & 709.8197 & 1417.6482 & 709.3277 & G & 204.1343 & 102.5708 & 187.1077 & 94.0575 & & & 2 \\
\hline 15 & & & & & & & $\mathbf{K}$ & 147.1128 & 74.0600 & 130.0863 & 65.5468 & & & 1 \\
\hline
\end{tabular}
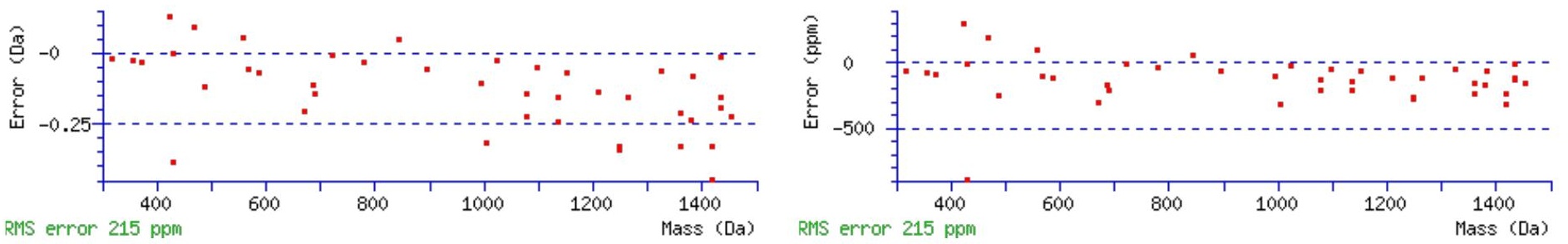

\section{All matches to this query}

\begin{tabular}{|l|l|l|l|}
\hline Score & Mr(calc): & Delta & \multicolumn{1}{c|}{ Sequence } \\
\hline 78.1 & 1580.7570 & -0.0028 & EAGNITTDGYEILGK \\
\hline 11.1 & 1580.7575 & -0.0033 & GHFYMHVGSLLLK \\
\hline 5.9 & 1580.7487 & 0.0055 & TPDLDKWEEIKK \\
\hline 5.5 & 1580.7422 & 0.0120 & MTNGVVHANLFGIK \\
\hline 2.7 & 1580.7682 & -0.0140 & $\underline{\text { ERVPENPSNALPEK }}$ \\
\hline 2.5 & 1578.7403 & 2.0139 & $\underline{\text { AEVAGEEPASKQRK }}$ \\
\hline & & & \\
\hline
\end{tabular}




\begin{tabular}{|l|l|l|l|}
2.3 & 1580.7575 & -0.0033 & GHFYMHVGSLLLK \\
\hline 1.8 & 1578.7331 & 2.0211 & FISQETGNLYISK \\
\hline 1.5 & 1580.7521 & 0.0021 & TDLPCDAAIATILK \\
\hline 1.3 & 1578.7473 & 2.0069 & $\underline{\text { KSTFWRPKTLR }}$ \\
\hline
\end{tabular}

Spectrum No: 254; Query: 487; Rank: 1

\section{Peptide View}

MS/MS Fragmentation of VETGENCTSPAPK

Found in IPI00210027, Tax_Id=10116 Gene_Symbol=Itpr1 Isoform 1 of Inositol 1,4,5-trisphosphate receptor type 1

Match to Query 487: 1389.605288 from(695.809920,2+)

Title: 091008RatKidney_NH4Format01_25.326.326.2.dta

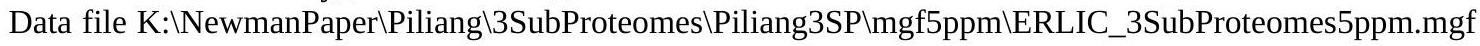
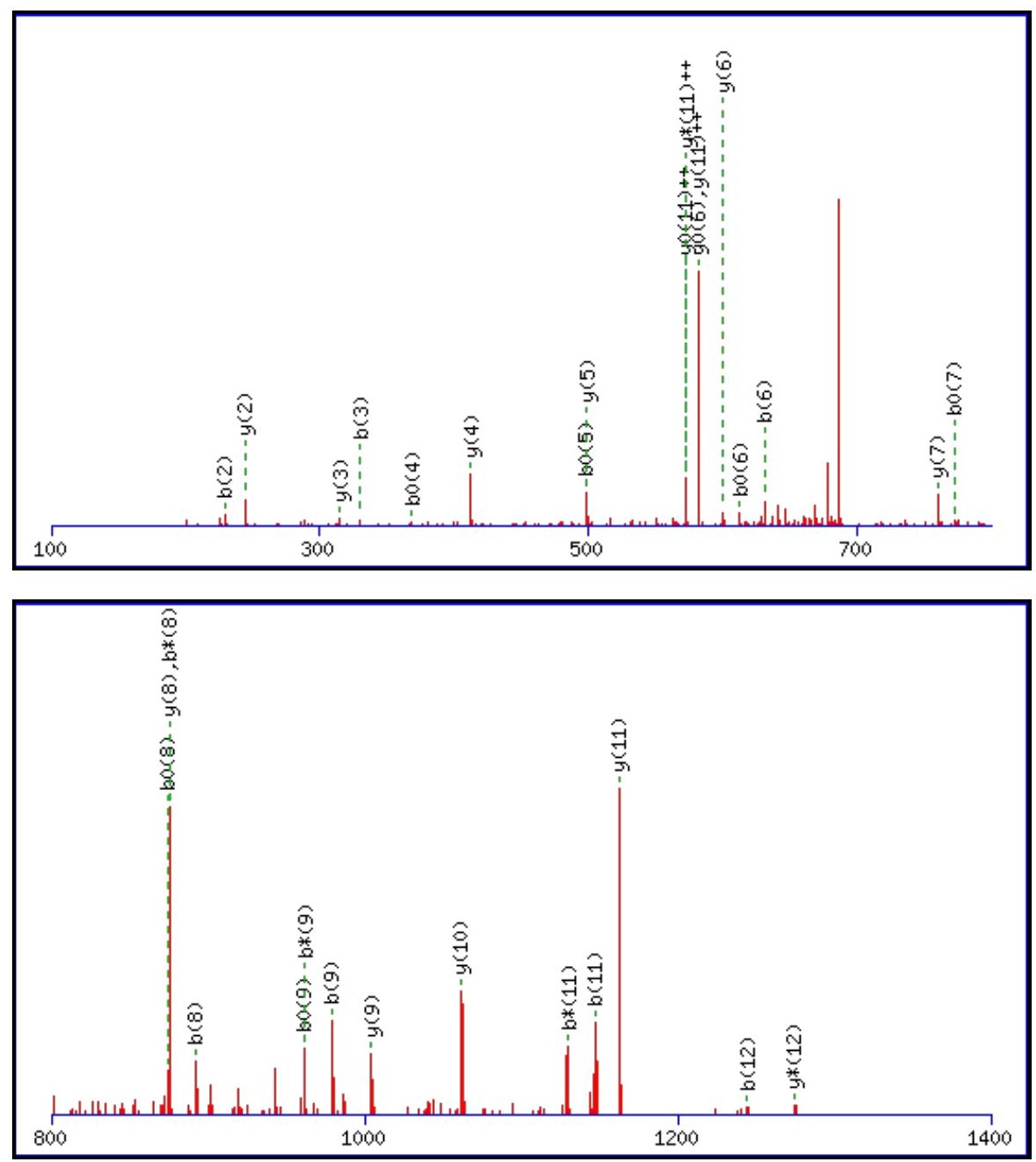
Monoisotopic mass of neutral peptide $\operatorname{Mr}$ (calc): 1389.6082

Fixed modifications: Carbamidomethyl (C)

Variable modifications:

N6 : Deamidated_N (N)

Ions Score: 78 Expect : $2 \mathrm{e}-006$

Matches (Bold Red): 31/124 fragment ions using 46 most intense peaks

\begin{tabular}{|c|c|c|c|c|c|c|c|c|c|c|c|c|c|c|}
\hline \# & b & $\mathbf{b}^{++}$ & b* & $\mathbf{b}^{*^{++}}$ & $\mathbf{b}^{0}$ & $\mathbf{b}^{0++}$ & Seq. & $\mathbf{y}$ & $\mathbf{y}^{++}$ & $\mathbf{y}^{*}$ & $\mathrm{y}^{*^{++}}$ & $\mathbf{y}^{0}$ & $\mathbf{y}^{0++}$ & \# \\
\hline 1 & 100.0757 & 50.5415 & & & & & $\mathbf{V}$ & & & & & & & 13 \\
\hline 2 & 229.1183 & 115.0628 & & & 211.1077 & 106.0575 & $\mathbf{E}$ & 1291.5471 & 646.2772 & 1274.5205 & 637.7639 & 1273.5365 & 637.2719 & 12 \\
\hline 3 & 330.1660 & 165.5866 & & & 312.1554 & 156.5813 & $\mathbf{T}$ & 1162.5045 & 581.7559 & 1145.4779 & 573.2426 & 1144.4939 & 572.7506 & 11 \\
\hline 4 & 387.1874 & 194.0974 & & & 369.1769 & 185.0921 & $\mathbf{G}$ & 1061.4568 & 531.2320 & 1044.4302 & 522.7188 & 1043.4462 & 522.2268 & 10 \\
\hline 5 & 516.2300 & 258.6186 & & & 498.2195 & 249.6134 & $\mathbf{E}$ & 1004.4353 & 502.7213 & 987.4088 & 494.2080 & 986.4248 & 493.7160 & 9 \\
\hline 6 & 631.2570 & 316.1321 & 614.2304 & 307.6188 & 613.2464 & 307.1268 & $\mathbf{N}$ & 875.3927 & 438.2000 & 858.3662 & 429.6867 & 857.3822 & 429.1947 & 8 \\
\hline 7 & 791.2876 & 396.1474 & 774.2611 & 387.6342 & 773.2770 & 387.1422 & C & 760.3658 & 380.6865 & 743.3393 & 372.1733 & 742.3552 & 371.6813 & 7 \\
\hline 8 & 892.3353 & 446.6713 & 875.3087 & 438.1580 & 874.3247 & 437.6660 & $\mathbf{T}$ & 600.3352 & 300.6712 & 583.3086 & 292.1579 & 582.3246 & 291.6659 & 6 \\
\hline 9 & 979.3673 & 490.1873 & 962.3408 & 481.6740 & 961.3567 & 481.1820 & S & 499.2875 & 250.1474 & 482.2609 & 241.6341 & 481.2769 & 241.1421 & 5 \\
\hline 10 & 1076.4201 & 538.7137 & 1059.3935 & 530.2004 & 1058.4095 & 529.7084 & $\mathbf{P}$ & 412.2554 & 206.6314 & 395.2289 & 198.1181 & & & 4 \\
\hline 11 & 1147.4572 & 574.2322 & 1130.4306 & 565.7190 & 1129.4466 & 565.2269 & A & 315.2027 & 158.1050 & 298.1761 & 149.5917 & & & 3 \\
\hline 12 & 1244.5100 & 622.7586 & 1227.4834 & 614.2453 & 1226.4994 & 613.7533 & $\mathbf{P}$ & 244.1656 & 122.5864 & 227.1390 & 114.0731 & & & 2 \\
\hline 13 & & & & & & & $\mathbf{K}$ & 147.1128 & 74.0600 & 130.0863 & 65.5468 & & & 1 \\
\hline
\end{tabular}

$$
\begin{aligned}
& \text { 吾 }
\end{aligned}
$$

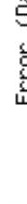

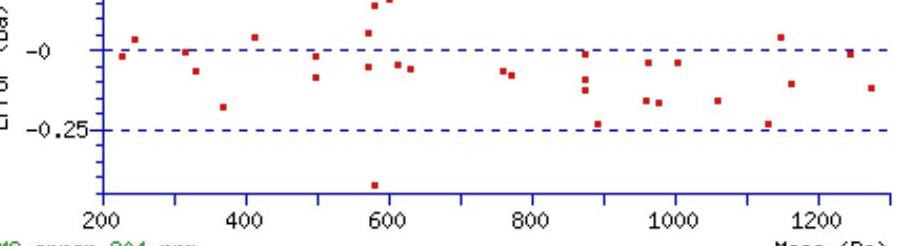

RMS error $204 \mathrm{ppm}$

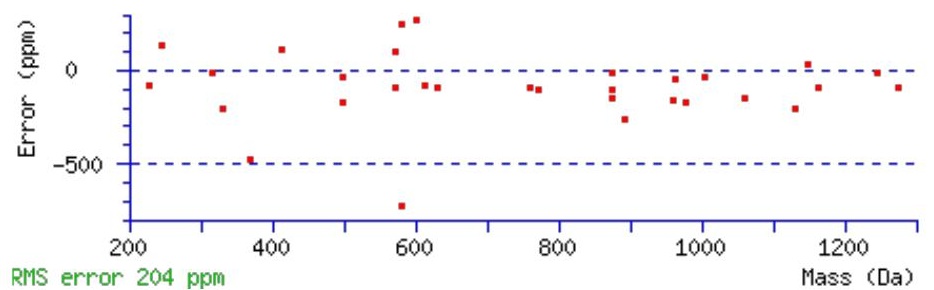

\section{All matches to this query}

\begin{tabular}{|l|l|l|l|}
\hline Score & Mr(calc): & Delta & \multicolumn{1}{c|}{ Sequence } \\
\hline 78.1 & 1389.6082 & -0.0029 & $\underline{\text { VETGENCTSPAPK }}$ \\
\hline 14.0 & 1389.5982 & 0.0071 & $\underline{\text { YAEYSTKKLK }}$ \\
\hline 10.9 & 1389.6038 & 0.0015 & $\underline{\text { RSDLQSTFSGGR }}$ \\
\hline 10.9 & 1389.6038 & 0.0015 & $\underline{\text { RSDLQSTFSGGR }}$ \\
\hline 9.8 & 1387.6101 & 1.9952 & $\underline{\text { KGLKCCASAASR }}$ \\
\hline 8.7 & 1388.6081 & 0.9972 & $\underline{\text { LLASCSADMTIK }}$ \\
\hline 7.8 & 1389.5982 & 0.0071 & $\underline{\text { YAEYSTKKLK }}$ \\
\hline 7.8 & 1389.5982 & 0.0071 & $\underline{\text { YAEYSTKKLK }}$ \\
\hline & & & \\
\hline
\end{tabular}




\begin{tabular}{|l|l|l|l|}
\hline 7.3 & 1388.6102 & 0.9951 & ASRSPLVTSSPK \\
\hline 6.8 & 1388.6102 & 0.9951 & $\underline{\text { IISGDIQTVQR }}$ \\
\hline
\end{tabular}

Spectrum No: 255; Query: 1599; Rank: 1

\section{Peptide View}

\section{MS/MS Fragmentation of VFADGAPHYVAFYSNVTR}

Found in IPI00190577, Tax_Id=10116 Gene_Symbol=Lama5 similar to Laminin alpha-5 chain precursor

Match to Query 1599: 2013.962322 from(672.328050,3+)

Title: 100101RatKid_NS_deglyco_24.3309.3309.3.dta

Data file K:INewmanPaper|Piliangl3SubProteomes\Piliang3SP\mgf5ppm\ERLIC_3SubProteomes5ppm.mgf
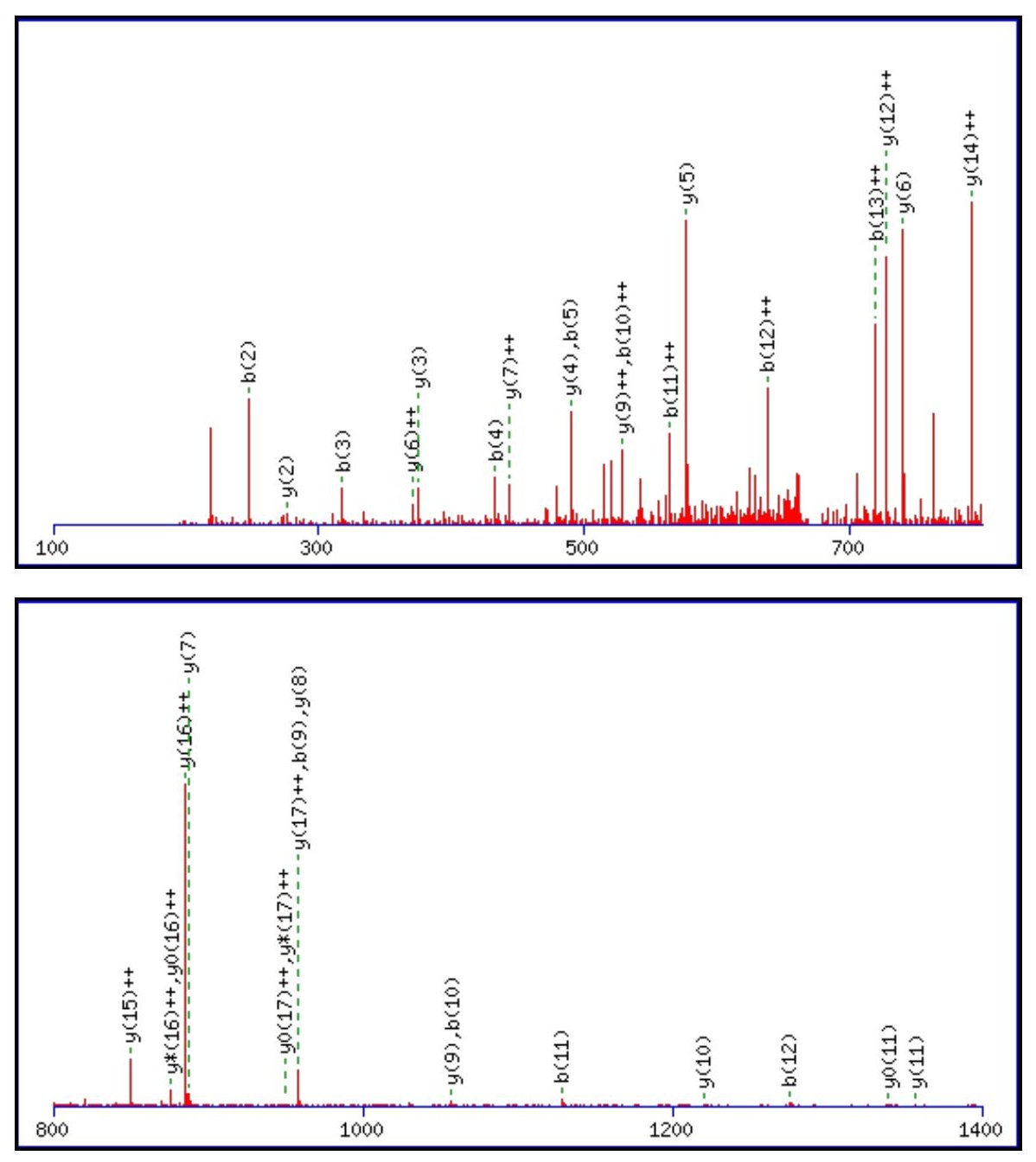


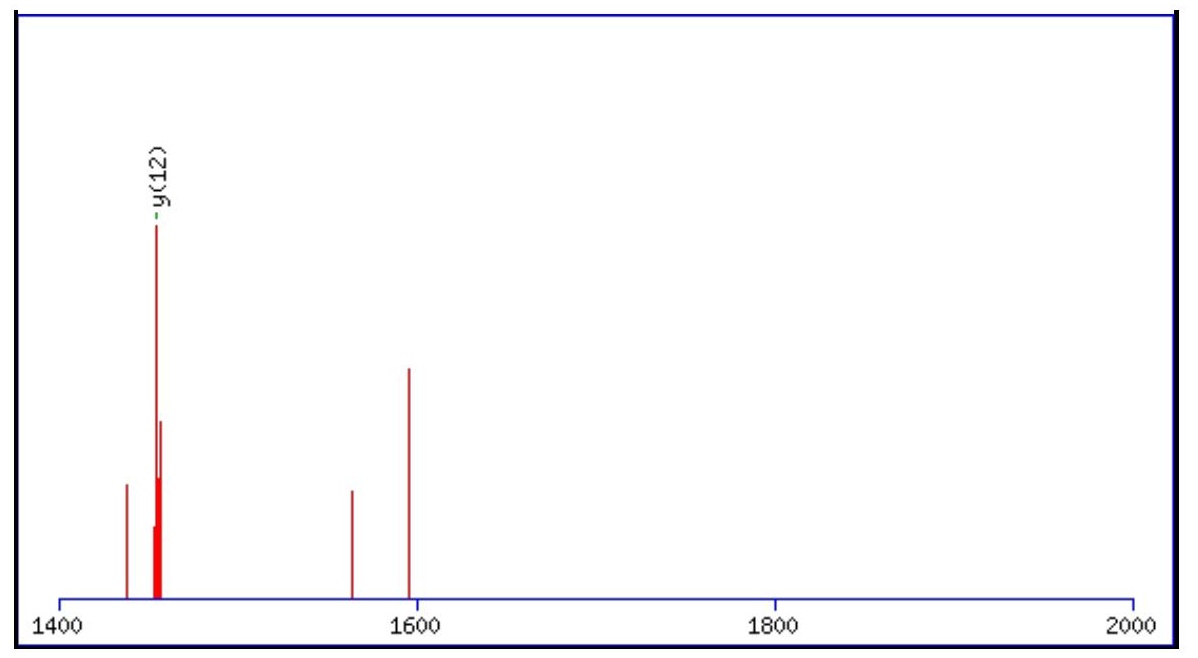

Monoisotopic mass of neutral peptide $\operatorname{Mr}($ calc): 2013.9585

Fixed modifications: Carbamidomethyl (C)

Variable modifications:

N15 : Deamidated_N (N)

Ions Score: 78 Expect: $3.6 \mathrm{e}-006$

Matches (Bold Red): 36/168 fragment ions using 47 most intense peaks

\begin{tabular}{|c|c|c|c|c|c|c|c|c|c|c|c|c|c|c|}
\hline \# & b & $\mathbf{b}^{++}$ & $\mathbf{b}^{*}$ & $\mathbf{b}^{*^{++}}$ & $\mathbf{b}^{0}$ & $\mathbf{b}^{\mathbf{0 + +}}$ & Seq. & $\mathbf{y}$ & $\mathbf{y}^{++}$ & $\mathbf{y}^{*}$ & $\mathrm{y}^{\mathrm{*}^{++}}$ & $\mathbf{y}^{0}$ & $\mathbf{y}^{0++}$ & \# \\
\hline 1 & 100.0757 & 50.5415 & & & & & $\mathbf{V}$ & & & & & & & \\
\hline 2 & 247.1441 & 124.0757 & & & & & $\mathbf{F}$ & 1915.8973 & 958.4523 & 1898.8708 & 949.9390 & 1897.8868 & 949.4470 & 1 \\
\hline 3 & 318.1812 & 159.5942 & & & & & A & 1768.8289 & 884.9181 & 1751.8024 & 876.4048 & 1750.8184 & 875.9128 & \\
\hline 4 & 433.2082 & 217.1077 & & & 415.1976 & 208.1024 & D & 1697.7918 & 849.3995 & 1680.7653 & 840.8863 & 1679.7812 & 840.3943 & \\
\hline 5 & 490.2296 & 245.6185 & & & 472.2191 & 236.6132 & G & 1582.7649 & 791.8861 & 1565.7383 & 783.3728 & 1564.7543 & 782.8808 & \\
\hline 6 & 561.2667 & 281.1370 & & & 543.2562 & 272.1317 & A & 1525.7434 & 763.3753 & 1508.7169 & 754.8621 & 1507.7328 & 754.3701 & \\
\hline 7 & 658.3195 & 329.6634 & & & 640.3089 & 320.6581 & $\mathbf{P}$ & 1454.7063 & 727.8568 & 1437.6797 & 719.3435 & 1436.6957 & 718.8515 & 1 \\
\hline 8 & 795.3784 & 398.1928 & & & 777.3678 & 389.1876 & $\mathbf{H}$ & 1357.6535 & 679.3304 & 1340.6270 & 670.8171 & \begin{tabular}{|l|l|}
1339.6430 \\
\end{tabular} & 670.3251 & 11 \\
\hline 9 & 958.4417 & 479.7245 & & & 940.4312 & 470.7192 & $\mathbf{Y}$ & 1220.5946 & 610.8009 & 1203.5681 & 602.2877 & 1202.5840 & 601.7957 & 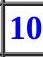 \\
\hline 10 & 1057.5102 & 529.2587 & & & 1039.4996 & 520.2534 & $\mathrm{~V}$ & 1057.5313 & 529.2693 & 1040.5047 & 520.7560 & 1039.5207 & 520.2640 & \\
\hline 11 & 1128.5473 & 564.7773 & & & 1110.5367 & 555.7720 & $\mathbf{A}$ & 958.4629 & 479.7351 & 941.4363 & 471.2218 & 940.4523 & 470.7298 & \\
\hline 12 & 1275.6157 & 638.3115 & & & 1257.6051 & 629.3062 & $\mathbf{F}$ & 887.4258 & 444.2165 & 870.3992 & 435.7032 & 869.4152 & 435.2112 & . \\
\hline 13 & 1438.6790 & 719.8431 & & & 1420.6685 & 710.8379 & $\mathbf{Y}$ & 740.3573 & 370.6823 & 723.3308 & 362.1690 & 722.3468 & 361.6770 & \\
\hline 14 & 1525.7110 & 763.3592 & & & 1507.7005 & 754.3539 & $\mathrm{~S}$ & 577.2940 & 289.1506 & 560.2675 & 280.6374 & 559.2834 & 280.1454 & \\
\hline 15 & 1640.7380 & 820.8726 & 1623.7114 & 812.3594 & 1622.7274 & 811.8673 & $\mathbf{N}$ & 490.2620 & 245.6346 & 473.2354 & 237.1214 & 472.2514 & 236.6293 & 4 \\
\hline 16 & 1739.8064 & 870.4068 & 1722.7798 & 861.8936 & 1721.7958 & 861.4016 & $\mathbf{V}$ & 375.2350 & 188.1212 & 358.2085 & 179.6079 & 357.2245 & 179.1159 & \\
\hline 17 & 1840.8541 & 920.9307 & 1823.8275 & 912.4174 & 1822.8435 & 911.9254 & $\mathbf{T}$ & 276.1666 & 138.5870 & 259.1401 & 130.0737 & 258.1561 & 129.5817 & \\
\hline 18 & & & & & & & $\mathbf{R}$ & 175.1190 & 88.0631 & 158.0924 & 79.5498 & & & \\
\hline
\end{tabular}
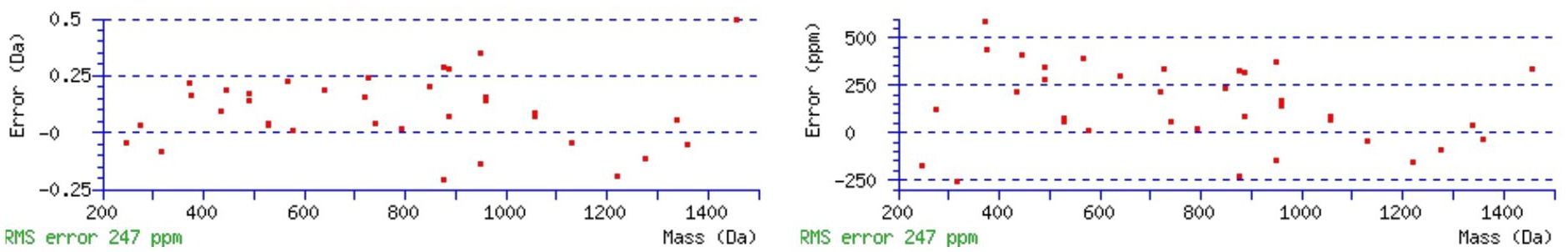

\section{All matches to this query}

\begin{tabular}{|l|l|l|l|}
\hline Score & Mr(calc): & Delta & \multicolumn{1}{c|}{ Sequence } \\
\hline 78.1 & 2013.9585 & 0.0038 & VFADGAPHYVAFYSNVTR \\
\hline 30.4 & 2012.9745 & 0.9879 & VFADGAPHYVAFYSNVTR \\
\hline 9.1 & 2011.9656 & 1.9967 & NDVLTLLSSINKDWWK \\
\hline & & & \\
\hline
\end{tabular}




\begin{tabular}{|l|l|l|l|}
6.0 & 2011.9373 & 2.0250 & GQICVVHYTGMLQNGKK \\
\hline 5.7 & 2013.9449 & 0.0175 & FATVGSDKILFHEVNEK \\
\hline 4.6 & 2012.9754 & 0.9869 & MAKRNYVGISEIPPVSR \\
\hline 4.6 & 2012.9754 & 0.9869 & MAKRNYVGISEIPPVSR \\
\hline 4.5 & 2012.9642 & 0.9981 & WMNGSILNTNLLRITGK \\
\hline 4.5 & 2012.9642 & 0.9981 & WMNGSILNTNLLRITGK \\
\hline 4.3 & 2011.9656 & 1.9967 & NDVLTLLSSINKDWWK \\
\hline
\end{tabular}

Spectrum No: 256; Query: 557; Rank: 1

\section{Peptide View}

MS/MS Fragmentation of YTADPNITSVGPAK

Found in IPI00388914, Tax_Id=10116 Gene_Symbol=Plxnb1_predicted plexin B1

Match to Query 557: 1433.706508 from(717.860530,2+)

Title: 100101RatKid_NS_deglyco_25.1631.1631.2.dta

Data file K:INewmanPaper|Piliang|3SubProteomes\Piliang3SP\mgf5ppm\ERLIC_3SubProteomes5ppm.mgf
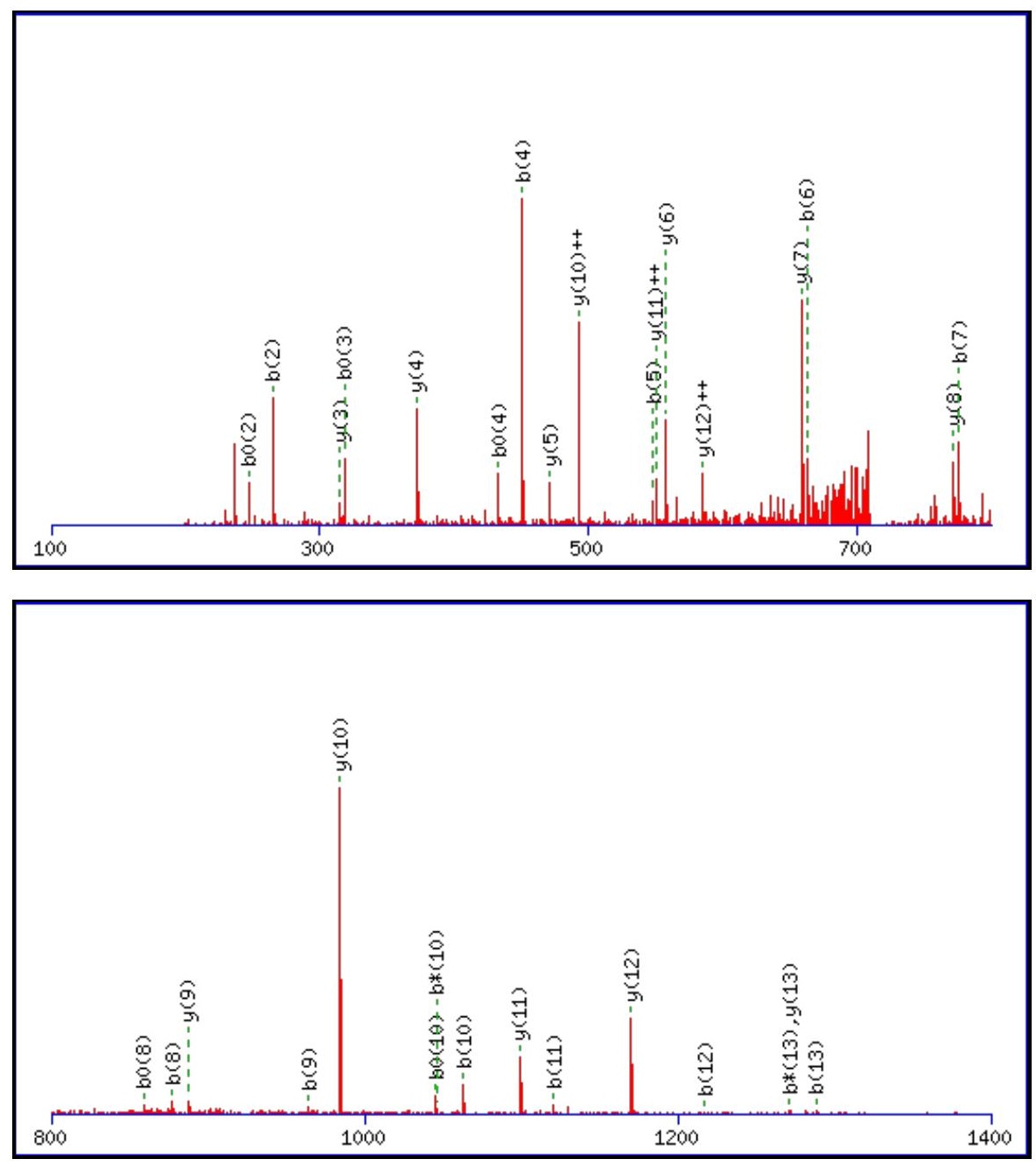


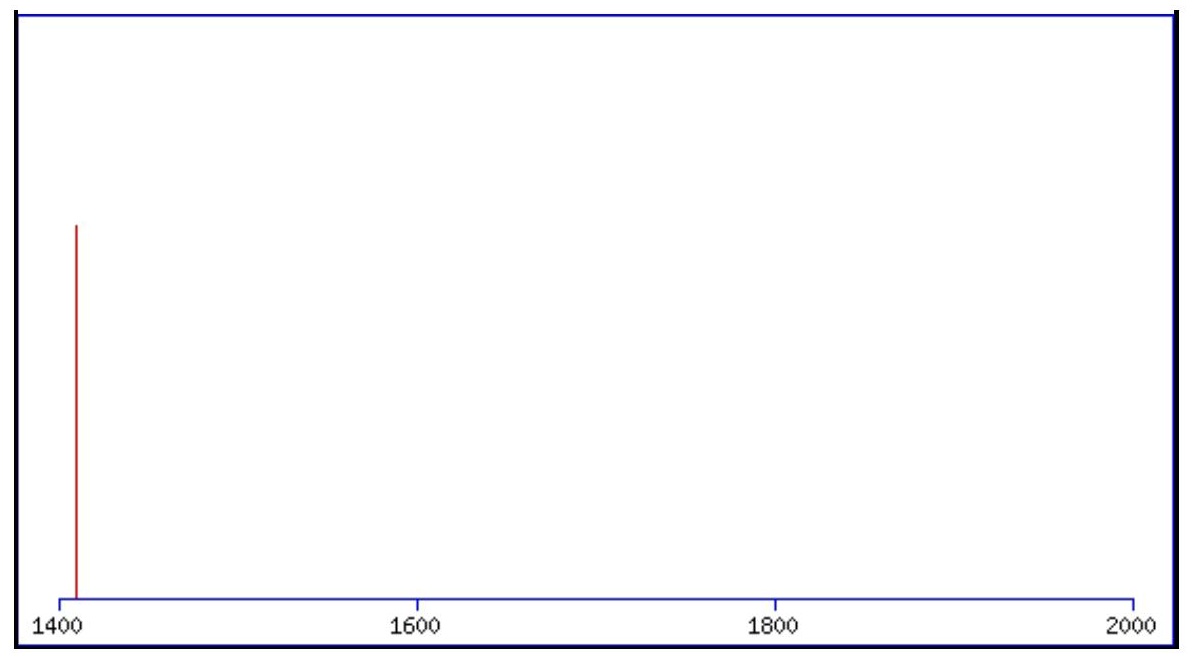

Monoisotopic mass of neutral peptide $\operatorname{Mr}($ calc): 1433.7038

Fixed modifications: Carbamidomethyl (C)

Variable modifications:

N6: Deamidated $\mathrm{N}(\mathrm{N})$

Ions Score: 78 Expect: 2.5e-006

Matches (Bold Red): 32/134 fragment ions using 64 most intense peaks

\begin{tabular}{|c|c|c|c|c|c|c|c|c|c|c|c|c|c|c|}
\hline \# & b & $\mathbf{b}^{++}$ & $\mathbf{b}^{*}$ & $\mathbf{b}^{*^{++}}$ & $\mathbf{b}^{0}$ & $\mathbf{b}^{0++}$ & Seq. & $\mathbf{y}$ & $\mathbf{y}^{++}$ & $\mathbf{y}^{*}$ & $\mathrm{y}^{*^{++}}$ & $\mathbf{y}^{0}$ & $\mathbf{y}^{0++}$ & \# \\
\hline 1 & 164.0706 & 82.5389 & & & & & $\mathbf{Y}$ & & & & & & & 14 \\
\hline 2 & 265.1183 & 133.0628 & & & 247.1077 & 124.0575 & $\mathbf{T}$ & 1271.6478 & 636.3275 & 1254.6212 & 627.8142 & 1253.6372 & 627.3222 & 13 \\
\hline 3 & 336.1554 & 168.5813 & & & 318.1448 & 159.5761 & A & 1170.6001 & 585.8037 & 1153.5735 & 577.2904 & 1152.5895 & 576.7984 & 12 \\
\hline 4 & 451.1823 & 226.0948 & & & 433.1718 & 217.0895 & D & 1099.5630 & 550.2851 & 1082.5364 & 541.7719 & 1081.5524 & 541.2798 & 11 \\
\hline 5 & 548.2351 & 274.6212 & & & 530.2245 & 265.6159 & $\mathbf{P}$ & 984.5360 & 492.7717 & 967.5095 & 484.2584 & 966.5255 & 483.7664 & 10 \\
\hline 6 & 663.2620 & 332.1347 & 646.2355 & 323.6214 & 645.2515 & 323.1294 & $\mathbf{N}$ & 887.4833 & 444.2453 & 870.4567 & 435.7320 & 869.4727 & 435.2400 & 9 \\
\hline 7 & 776.3461 & 388.6767 & 759.3196 & 380.1634 & 758.3355 & 379.6714 & I & 772.4563 & 386.7318 & 755.4298 & 378.2185 & 754.4458 & 377.7265 & 8 \\
\hline 8 & 877.3938 & 439.2005 & 860.3672 & 430.6873 & 859.3832 & 430.1952 & $\mathbf{T}$ & 659.3723 & 330.1898 & 642.3457 & 321.6765 & 641.3617 & 321.1845 & 7 \\
\hline 9 & 964.4258 & 482.7165 & 947.3993 & 474.2033 & 946.4152 & 473.7113 & S & 558.3246 & 279.6659 & 541.2980 & 271.1527 & 540.3140 & 270.6606 & 6 \\
\hline 10 & 1063.4942 & 532.2508 & 1046.4677 & 523.7375 & 1045.4837 & 523.2455 & V & 471.2926 & 236.1499 & 454.2660 & 227.6366 & & & 5 \\
\hline 11 & 1120.5157 & 560.7615 & 1103.4891 & 552.2482 & 1102.5051 & 551.7562 & G & 372.2241 & 186.6157 & 355.1976 & 178.1024 & & & 4 \\
\hline 12 & 1217.5685 & 609.2879 & 1200.5419 & 600.7746 & 1199.5579 & 600.2826 & $\mathbf{P}$ & 315.2027 & 158.1050 & 298.1761 & 149.5917 & & & 3 \\
\hline 13 & 1288.6056 & 644.8064 & 1271.5790 & 636.2931 & 1270.5950 & 635.8011 & A & 218.1499 & 109.5786 & 201.1234 & 101.0653 & & & 2 \\
\hline 14 & & & & & & & $\mathbf{K}$ & 147.1128 & 74.0600 & 130.0863 & 65.5468 & & & 1 \\
\hline
\end{tabular}
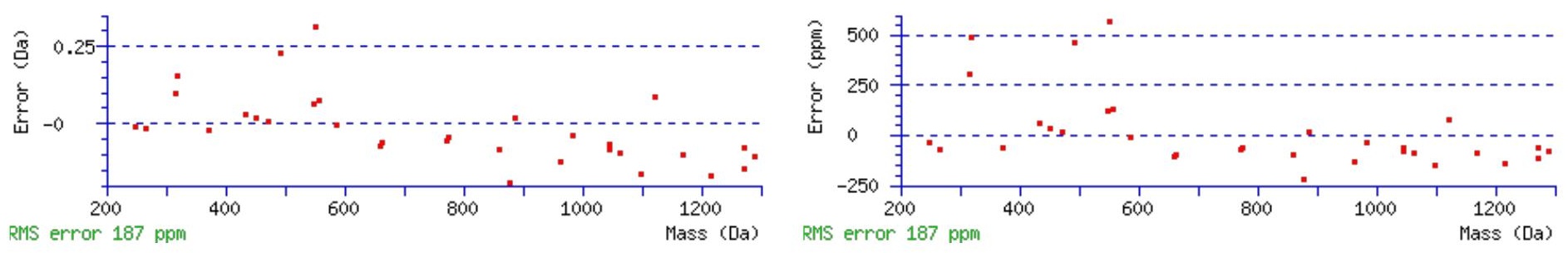

\section{All matches to this query}

\begin{tabular}{|l|l|l|l|}
\hline Score & Mr(calc): & Delta & \multicolumn{1}{c|}{ Sequence } \\
\hline 78.0 & 1433.7038 & 0.0027 & $\underline{\text { YTADPNITSVGPAK }}$ \\
\hline 6.1 & 1433.7150 & -0.0085 & VEEPIHATADPQK \\
\hline 3.9 & 1433.7007 & 0.0058 & VQINDMCSVKPK \\
\hline 0.8 & 1433.7068 & -0.0003 & YTVGLRNXFSVK \\
\hline 0.6 & 1433.7072 & -0.0006 & $\underline{\text { MVNSNLASVEELK }}$ \\
\hline 0.3 & 1433.7068 & -0.0003 & $\underline{\text { SPAPPLPAPPPASR }}$ \\
\hline
\end{tabular}


Spectrum No: 257; Query: 321; Rank: 1

\section{Peptide View}

MS/MS Fragmentation of FNDTNVVHTVK

Found in IPI00327469, Tax_Id=10116 Gene_Symbol=Ahsg Alpha-2-HS-glycoprotein precursor

Match to Query 321: 1273.627448 from(637.821000,2+)

Title: 091008RatKidney_NoSalt_29.945.945.2.dta

Data file K:\NewmanPaper\Piliang \3SubProteomes \Piliang3SP $\backslash$ mgf5ppm\ERLIC_3SubProteomes5ppm.mgf
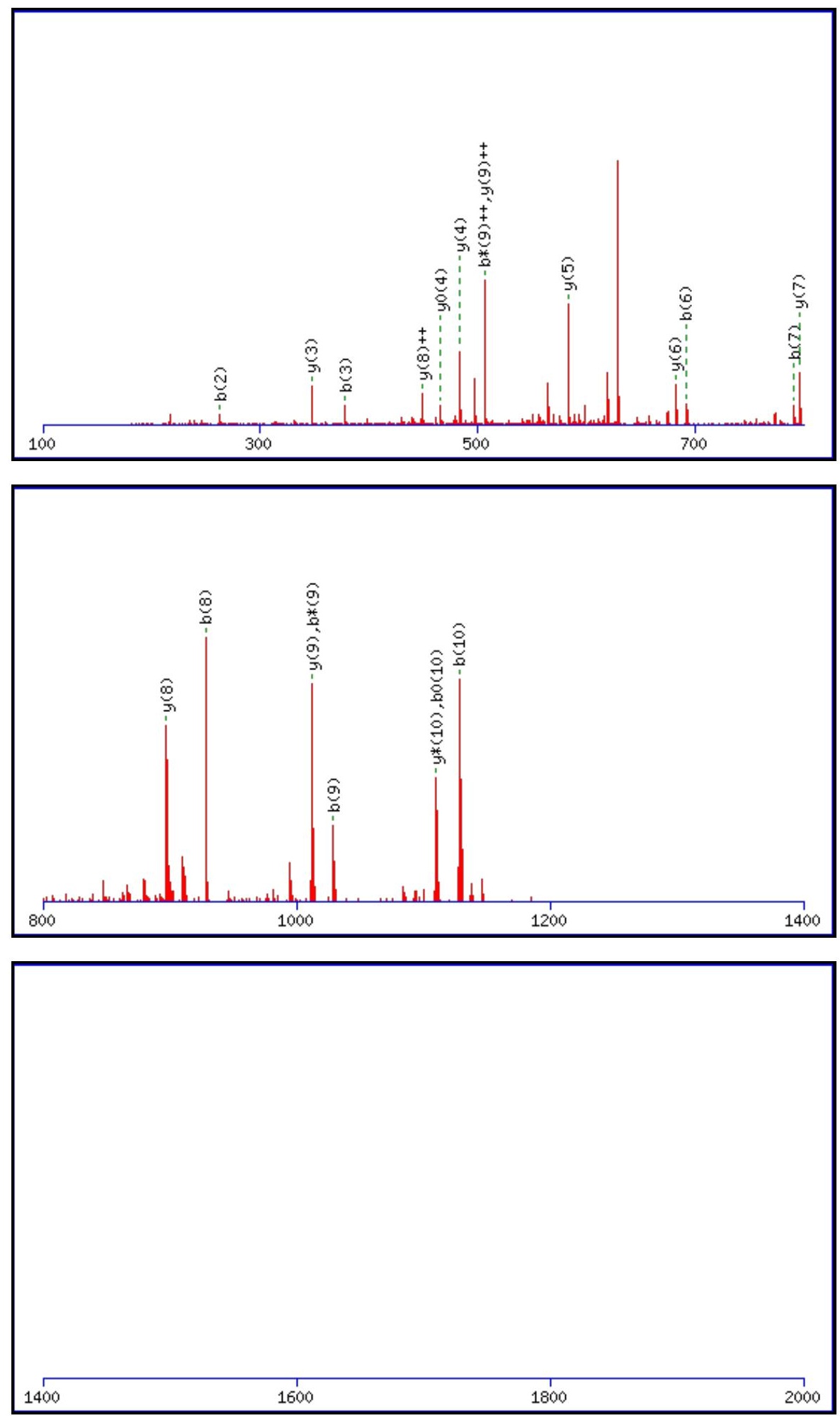

Monoisotopic mass of neutral peptide $\operatorname{Mr}($ calc): 1273.6303 
Fixed modifications: Carbamidomethyl (C)

Variable modifications:

N2 : Deamidated $\mathrm{N}(\mathrm{N})$

Ions Score: 78 Expect: 2.1e-006

Matches (Bold Red): 21/110 fragment ions using 28 most intense peaks

\begin{tabular}{|r|c|c|c|c|c|c|c|c|c|c|c|c|c|c|}
\hline$\#$ & $\mathbf{b}$ & $\mathbf{b}^{++}$ & $\mathbf{b}^{*}$ & $\mathbf{b}^{\mathbf{*}^{++}}$ & $\mathbf{b}^{\mathbf{0}}$ & $\mathbf{b}^{\mathbf{0 + +}}$ & $\mathbf{S e q}$ & $\mathbf{y}$ & $\mathbf{y}^{++}$ & $\mathbf{y}^{\mathbf{*}}$ & $\mathbf{y}^{\boldsymbol{*}^{++}}$ & $\mathbf{y}^{\mathbf{0}}$ & $\mathbf{y}^{\mathbf{0 + +}}$ & $\#$ \\
\hline $\mathbf{1}$ & $\mathbf{1 4 8 . 0 7 5 7}$ & 74.5415 & & & & & $\mathbf{F}$ & & & & & & & $\mathbf{1 1}$ \\
\hline $\mathbf{2}$ & $\mathbf{2 6 3 . 1 0 2 6}$ & 132.0550 & 246.0761 & 123.5417 & & & $\mathbf{N}$ & 1127.5691 & 564.2882 & $\mathbf{1 1 1 0 . 5 4 2 6}$ & 555.7749 & 1109.5586 & 555.2829 & $\mathbf{1 0}$ \\
\hline $\mathbf{3}$ & $\mathbf{3 7 8 . 1 2 9 6}$ & 189.5684 & 361.1030 & 181.0551 & 360.1190 & 180.5631 & $\mathbf{D}$ & $\mathbf{1 0 1 2 . 5 4 2 2}$ & $5 \mathbf{0 6 . 7 7 4 7}$ & 995.5156 & 498.2615 & 994.5316 & 497.7694 & $\mathbf{9}$ \\
\hline $\mathbf{4}$ & 479.1772 & 240.0923 & 462.1507 & 231.5790 & 461.1667 & 231.0870 & $\mathbf{T}$ & $\mathbf{8 9 7 . 5 1 5 2}$ & $\mathbf{4 4 9 . 2 6 1 3}$ & 880.4887 & 440.7480 & 879.5047 & 440.2560 & $\mathbf{8}$ \\
\hline $\mathbf{5}$ & 593.2202 & 297.1137 & 576.1936 & 288.6005 & 575.2096 & 288.1084 & $\mathbf{N}$ & $\mathbf{7 9 6 . 4 6 7 6}$ & 398.7374 & 779.4410 & 390.2241 & 778.4570 & 389.7321 & $\mathbf{7}$ \\
\hline $\mathbf{6}$ & $\mathbf{6 9 2 . 2 8 8 6}$ & 346.6479 & 675.2620 & 338.1347 & 674.2780 & 337.6427 & $\mathbf{V}$ & $\mathbf{6 8 2 . 4 2 4 6}$ & 341.7160 & 665.3981 & 333.2027 & 664.4141 & 332.7107 & $\mathbf{6}$ \\
\hline $\mathbf{7}$ & $\mathbf{7 9 1 . 3 5 7 0}$ & 396.1821 & 774.3305 & 387.6689 & 773.3464 & 387.1769 & $\mathbf{V}$ & $\mathbf{5 8 3 . 3 5 6}$ & 292.1817 & 566.3297 & 283.6685 & 565.3457 & 283.1765 & $\mathbf{5}$ \\
\hline $\mathbf{8}$ & $\mathbf{9 2 8 . 4 1 5 9}$ & 464.7116 & 911.3894 & 456.1983 & 910.4053 & 455.7063 & $\mathbf{H}$ & $\mathbf{4 8 4 . 2 8 7 8}$ & 242.6475 & 467.2613 & 234.1343 & $\mathbf{4 6 6 . 2 7 7 2}$ & 233.6423 & $\mathbf{4}$ \\
\hline $\mathbf{9}$ & $\mathbf{1 0 2 9 . 4 6 3 6}$ & 515.2354 & $\mathbf{1 0 1 2 . 4 3 7 0}$ & $\mathbf{5 0 6 . 7 2 2 2}$ & 1011.4530 & 506.2302 & $\mathbf{T}$ & $\mathbf{3 4 7 . 2 2 8 9}$ & 174.1181 & 330.2023 & 165.6048 & 329.2183 & 165.1128 & $\mathbf{3}$ \\
\hline $\mathbf{1 0}$ & $\mathbf{1 1 2 8 . 5 3 2 0}$ & 564.7696 & 1111.5055 & 556.2564 & $\mathbf{1 1 1 0 . 5 2 1 4}$ & 555.7644 & $\mathbf{V}$ & 246.1812 & 123.5942 & 229.1547 & 115.0810 & & & $\mathbf{2}$ \\
\hline $\mathbf{1 1}$ & & & & & & & $\mathbf{K}$ & 147.1128 & 74.0600 & 130.0863 & 65.5468 & & & $\mathbf{1}$ \\
\hline
\end{tabular}
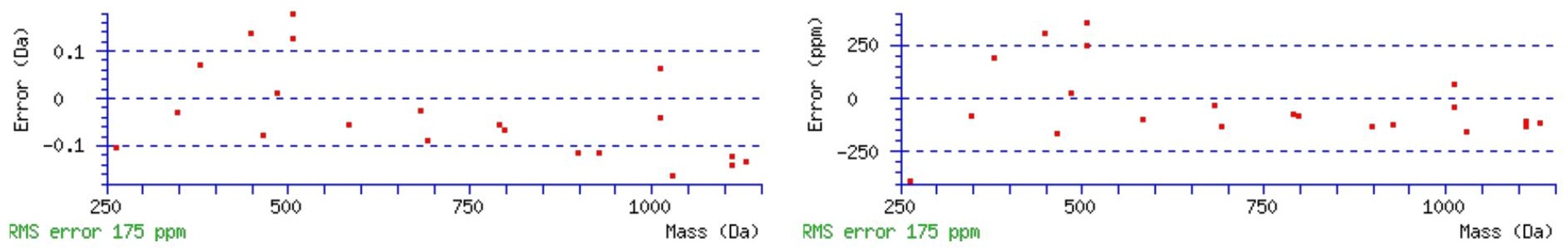

\section{All matches to this query}

\begin{tabular}{|l|l|l|l|}
\hline Score & Mr(calc): & Delta & \multicolumn{1}{|c|}{ Sequence } \\
\hline 78.0 & 1273.6303 & -0.0028 & FNDTNVVHTVK \\
\hline 59.8 & 1273.6303 & -0.0028 & FNDTNVVHTVK \\
\hline 9.7 & 1273.6150 & 0.0125 & QEAENGALDISK \\
\hline 9.1 & 1273.6263 & 0.0012 & VNLGVDSQGGTAR \\
\hline 7.9 & 1273.6197 & 0.0078 & RNQDRPCLSK \\
\hline 7.2 & 1273.6336 & -0.0062 & TALPNMDREVK \\
\hline 6.2 & 1272.6115 & 1.0159 & ANKAVDFTTVK \\
\hline 6.2 & 1273.6319 & -0.0044 & SLTSLVLYGNK \\
\hline 5.6 & 1273.6401 & -0.0127 & ELISEGADVNVK \\
\hline 5.4 & 1272.6115 & 1.0159 & ANKAVDFTTVK \\
\hline
\end{tabular}

Spectrum No: 258; Query: 1251; Rank: 1

\section{Peptide View}

MS/MS Fragmentation of ELYESIWQNFTDQK

Found in IPI00324300, Tax_Id=10116 Gene_Symbol=Ace Angiotensin-converting enzyme, somatic isoform precursor

Match to Query 1251: 1800.820408 from(901.417480,2+)

Title: 100101RatKid_NS_deglyco_25.4889.4889.2.dta

Data file K:INewmanPaper|Piliangl3SubProteomes\Piliang3SP\mgf5ppm\ERLIC_3SubProteomes5ppm.mgf 

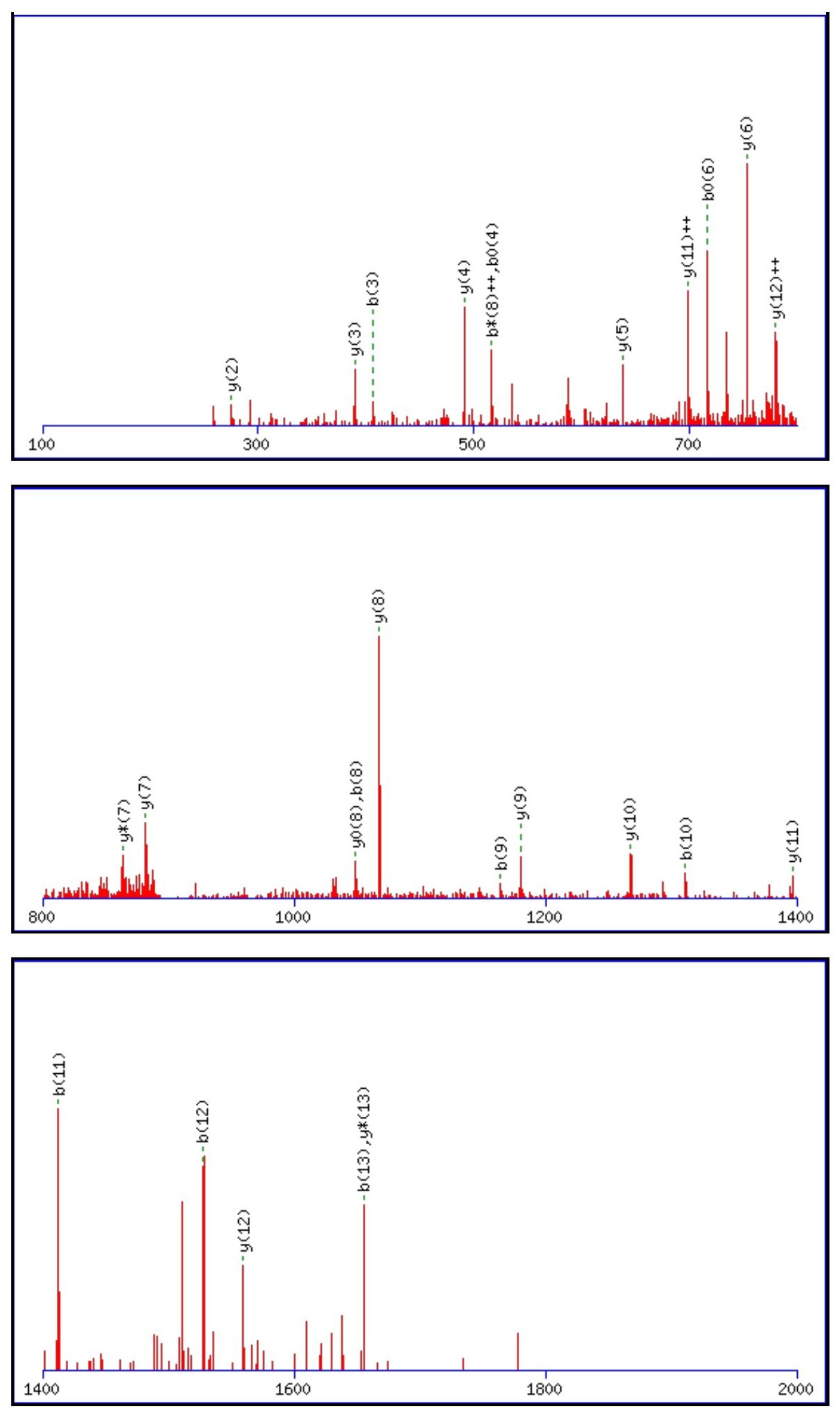

Monoisotopic mass of neutral peptide $\operatorname{Mr}($ calc): 1800.8206

Fixed modifications: Carbamidomethyl (C)

Variable modifications:

N9 : Deamidated $\mathrm{N}(\mathrm{N})$

Ions Score: 78 Expect: $3.9 \mathrm{e}-006$

Matches (Bold Red): 26/138 fragment ions using 38 most intense peaks

\begin{tabular}{|r|c|c|c|c|c|c|c|c|c|c|c|c|c|c|}
\hline$\#$ & $\mathbf{b}$ & $\mathbf{b}^{++}$ & $\mathbf{b}^{*}$ & $\mathbf{b}^{\boldsymbol{*}^{++}}$ & $\mathbf{b}^{\mathbf{0}}$ & $\mathbf{b}^{\mathbf{0 + +}}$ & Seq. & $\mathbf{y}$ & $\mathbf{y}^{++}$ & $\mathbf{y}^{\mathbf{*}}$ & $\mathbf{y}^{\boldsymbol{*}^{++}}$ & $\mathbf{y}^{\mathbf{0}}$ & $\mathbf{y}^{\mathbf{0 + +}}$ & $\#$ \\
\hline $\mathbf{1}$ & 130.0499 & 65.5286 & & & 112.0393 & 56.5233 & $\mathbf{E}$ & & & & & & & $\mathbf{1 4}$ \\
\hline $\mathbf{2}$ & 243.1339 & 122.0706 & & & 225.1234 & 113.0653 & $\mathbf{L}$ & 1672.7853 & 836.8963 & $\mathbf{1 6 5 5 . 7 5 8 8}$ & 828.3830 & 1654.7748 & 827.8910 & $\mathbf{1 3}$ \\
\hline $\mathbf{3}$ & $\mathbf{4 0 6 . 1 9 7 3}$ & 203.6023 & & & 388.1867 & 194.5970 & $\mathbf{Y}$ & $\mathbf{1 5 5 9 . 7 0 1 3}$ & $\mathbf{7 8 0 . 3 5 4 3}$ & 1542.6747 & 771.8410 & 1541.6907 & 771.3490 & $\mathbf{1 2}$ \\
\hline $\mathbf{4}$ & 535.2399 & 268.1236 & & & 517.2293 & 259.1183 & $\mathbf{E}$ & $\mathbf{1 3 9 6 . 6 3 7 9}$ & $\mathbf{6 9 8 . 8 2 2 6}$ & 1379.6114 & 690.3093 & 1378.6274 & 689.8173 & $\mathbf{1 1}$ \\
\hline $\mathbf{5}$ & 622.2719 & 311.6396 & & & 604.2613 & 302.6343 & S & $\mathbf{1 2 6 7 . 5 9 5 3}$ & 634.3013 & 1250.5688 & 625.7880 & 1249.5848 & 625.2960 & $\mathbf{1 0}$ \\
\hline
\end{tabular}




\begin{tabular}{|c|c|c|c|c|c|c|c|c|c|c|c|c|c|c|}
\hline 6 & 735.3559 & 368.1816 & & & 717.3454 & |359.1763 & I & |1180.5633| & 590.7853 & 1163.5368 & 582.2720 & 1162.5527 & 581.7800 & 9 \\
\hline 7 & 921.4353 & 461.2213 & & & 903.4247 & 452.2160 & W & 1067.4792 & 534.2433 & 1050.4527 & 525.7300 & 1049.4687 & 525.2380 & 8 \\
\hline 8 & 1049.4938 & 525.2506 & 1032.4673 & 516.7373 & 1031.4833 & 516.2453 & $\mathbf{Q}$ & 881.3999 & 441.2036 & 864.3734 & 432.6903 & 863.3894 & 432.1983 & 7 \\
\hline 9 & 1164.5208 & 582.7640 & 1147.4942 & 574.2508 & 1146.5102 & 573.7587 & $\mathbf{N}$ & 753.3414 & 377.1743 & 736.3148 & 368.6610 & 735.3308 & 368.1690 & 6 \\
\hline 10 & 1311.5892 & 656.2982 & 1294.5626 & 647.7850 & 1293.5786 & 647.2929 & $\mathbf{F}$ & 638.3144 & 319.6608 & 621.2879 & 311.1476 & 620.3039 & 310.6556 & 5 \\
\hline 11 & 1412.6369 & |706.8221 & 1395.6103 & 698.3088 & 1394.6263 & 697.8168 & $\mathbf{T}$ & 491.2460 & 246.1266 & 474.2195 & 237.6134 & 473.2354 & 237.1214 & 4 \\
\hline 12 & 1527.6638 & 764.3355 & 1510.6373 & 755.8223 & 1509.6532 & 755.3303 & D & 390.1983 & 195.6028 & 373.1718 & 187.0895 & 372.1878 & 186.5975 & 3 \\
\hline 13 & 1655.7224 & 828.3648 & 1638.6958 & 819.8516 & 1637.7118 & 819.3595 & $\mathbf{Q}$ & 275.1714 & 138.0893 & 258.1448 & 129.5761 & & & 2 \\
\hline 14 & & & & & & & $\mathbf{K}$ & 147.1128 & 74.0600 & 130.0863 & 65.5468 & & & 1 \\
\hline
\end{tabular}
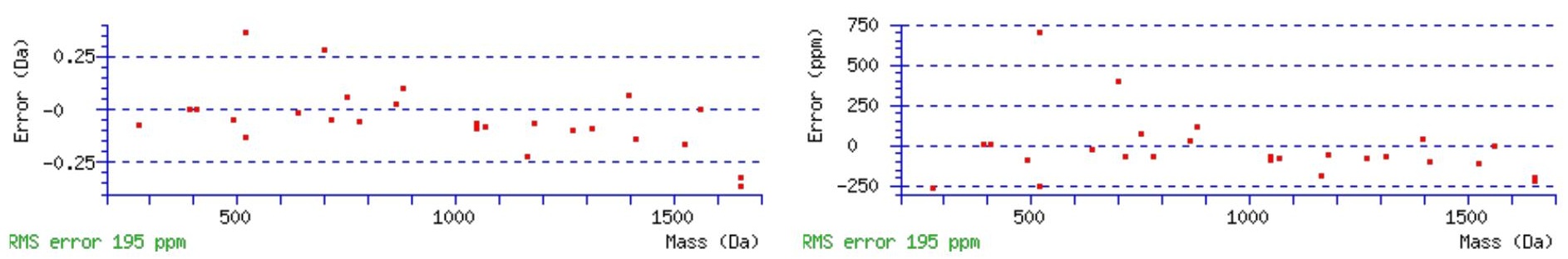

\section{All matches to this query}

\begin{tabular}{|l|l|l|l|}
\hline Score & Mr(calc): & Delta & \multicolumn{1}{c|}{ Sequence } \\
\hline 77.9 & 1800.8206 & -0.0002 & ELYESIWQNFTDQK \\
\hline 8.9 & 1798.8284 & 1.9920 & $\underline{\text { RNAVSELQGELMSIR }}$ \\
\hline 8.5 & 1800.8084 & 0.0121 & $\underline{\text { KHPDASVNFSEFSKK }}$ \\
\hline 6.3 & 1800.8035 & 0.0169 & LCNFAFKTKGNLTK \\
\hline 6.2 & 1800.8382 & -0.0178 & PQHAMGDKHFLSLPK \\
\hline 3.0 & 1800.8124 & 0.0080 & VKFSASYFGPEFQSK \\
\hline 1.5 & 1798.8284 & 1.9920 & $\underline{\text { AARELETLSDMTGGLR }}$ \\
\hline 1.3 & 1800.8304 & -0.0100 & CASKSPNPLLTFCVK \\
\hline 0.3 & 1799.8239 & 0.9965 & VVGFMSIPPSENALMK \\
\hline
\end{tabular}

Spectrum No: 259; Query: 1383; Rank: 1

\section{Peptide View}

MS/MS Fragmentation of GTAGNALMEGASQLVGENR

Found in IPI00205389, Tax_Id=10116 Gene_Symbol=Fgb Isoform 1 of Fibrinogen beta chain precursor

Match to Query 1383: 1874.884648 from(938.449600,2+)

Title: 100101RatKid_NS_deglyco_25.3641.3641.2.dta

Data file K:INewmanPaper|Piliangl3SubProteomes\Piliang3SP\mgf5ppm\ERLIC_3SubProteomes5ppm.mgf 

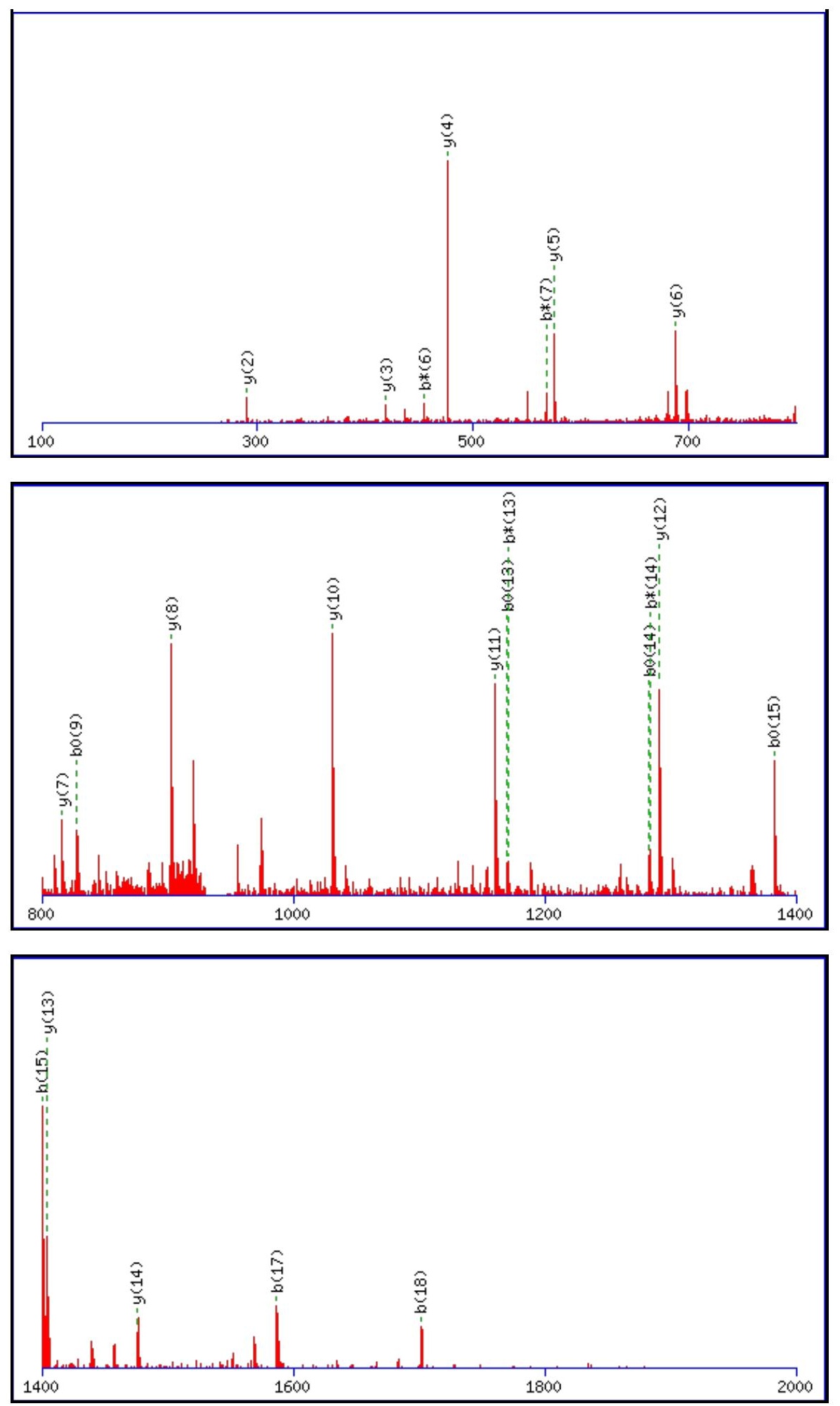

Monoisotopic mass of neutral peptide $\operatorname{Mr}($ calc): 1874.8792

Fixed modifications: Carbamidomethyl (C)

Variable modifications:

N18 : Deamidated $\mathrm{N}(\mathrm{N})$

Ions Score: 78 Expect: $4.1 \mathrm{e}-006$

Matches (Bold Red): 23/202 fragment ions using 41 most intense peaks

\begin{tabular}{|r|r|r|c|c|c|c|c|c|c|c|c|c|c|c|}
\hline$\#$ & $\mathbf{b}$ & $\mathbf{b}^{++}$ & $\mathbf{b}^{*}$ & $\mathbf{b}^{\boldsymbol{*}^{++}}$ & $\mathbf{b}^{\mathbf{0}}$ & $\mathbf{b}^{\mathbf{0 + +}}$ & Seq. & $\mathbf{y}$ & $\mathbf{y}^{++}$ & $\mathbf{y}^{\mathbf{*}}$ & $\mathbf{y}^{\boldsymbol{*}^{++}}$ & $\mathbf{y}^{\mathbf{0}}$ & $\mathbf{y}^{\mathbf{0 + +}}$ & $\#$ \\
\hline $\mathbf{1}$ & 58.0287 & 29.5180 & & & & & $\mathbf{G}$ & & & & & & & $\mathbf{1 9}$ \\
\hline $\mathbf{2}$ & 159.0764 & 80.0418 & & & 141.0659 & 71.0366 & $\mathbf{T}$ & 1818.8650 & 909.9362 & 1801.8385 & 901.4229 & 1800.8545 & 900.9309 & $\mathbf{1 8}$ \\
\hline 3 & 230.1135 & 115.5604 & & & 212.1030 & 106.5551 & $\mathbf{A}$ & 1717.8174 & 859.4123 & 1700.7908 & 850.8990 & 1699.8068 & 850.4070 & $\mathbf{1 7}$ \\
\hline $\mathbf{4}$ & 287.1350 & 144.0711 & & & 269.1244 & 135.0659 & $\mathbf{G}$ & 1646.7803 & 823.8938 & 1629.7537 & 815.3805 & 1628.7697 & 814.8885 & $\mathbf{1 6}$ \\
\hline $\mathbf{5}$ & 401.1779 & 201.0926 & 384.1514 & 192.5793 & 383.1674 & 192.0873 & $\mathbf{N}$ & 1589.7588 & 795.3830 & 1572.7322 & 786.8698 & 1571.7482 & 786.3778 & $\mathbf{1 5}$ \\
\hline
\end{tabular}




\begin{tabular}{|c|c|c|c|c|c|c|c|c|c|c|c|c|c|c|}
\hline 6 & 472.2150 & |236.6112| & 455.1885 & |228.0979 & 454.2045 & |227.6059 & A & |1475.7159 & 738.3616 & |1458.6893 & |729.8483 & |1457.7053 & |729.3563| & 14 \\
\hline 7 & 585.2991 & 293.1532 & 568.2726 & 284.6399 & 567.2885 & 284.1479 & $\mathbf{L}$ & 1404.6787 & 702.8430 & 1387.6522 & 694.3297 & 1386.6682 & 693.8377 & 13 \\
\hline 8 & 716.3396 & 358.6734 & 699.3130 & 350.1602 & 698.3290 & 349.6681 & $\mathbf{M}$ & 1291.5947 & 646.3010 & 1274.5681 & 637.7877 & 1273.5841 & 637.2957 & 12 \\
\hline 9 & 845.3822 & 423.1947 & 828.3556 & 414.6815 & 827.3716 & 414.1894 & $\mathbf{E}$ & 1160.5542 & 580.7807 & 1143.5277 & 572.2675 & 1142.5436 & 571.7755 & 11 \\
\hline 10 & 902.4036 & 451.7055 & 885.3771 & 443.1922 & 884.3931 & 442.7002 & G & 1031.5116 & 516.2594 & 1014.4851 & 507.7462 & 1013.5010 & 507.2542 & 10 \\
\hline 11 & 973.4408 & 487.2240 & 956.4142 & 478.7107 & 955.4302 & 478.2187 & A & 974.4901 & 487.7487 & 957.4636 & 479.2354 & 956.4796 & 478.7434 & 9 \\
\hline 12 & 1060.4728 & 530.7400 & 1043.4462 & 522.2268 & 1042.4622 & 521.7347 & $\mathbf{S}$ & 903.4530 & 452.2302 & 886.4265 & 443.7169 & 885.4425 & 443.2249 & 8 \\
\hline 13 & 1188.5314 & 594.7693 & 1171.5048 & 586.2560 & 1170.5208 & 585.7640 & $\mathbf{Q}$ & 816.4210 & 408.7141 & 799.3945 & 400.2009 & 798.4104 & 399.7089 & 7 \\
\hline 14 & 1301.6154 & 651.3114 & 1284.5889 & 642.7981 & 1283.6049 & 642.3061 & $\mathbf{L}$ & 688.3624 & 344.6848 & 671.3359 & 336.1716 & 670.3519 & 335.6796 & 6 \\
\hline 15 & 1400.6838 & 700.8456 & 1383.6573 & 692.3323 & 1382.6733 & 691.8403 & $\mathbf{V}$ & 575.2784 & 288.1428 & 558.2518 & 279.6295 & 557.2678 & 279.1375 & 5 \\
\hline 16 & 1457.7053 & 729.3563 & 1440.6788 & 720.8430 & 1439.6947 & 720.3510 & G & 476.2099 & 238.6086 & 459.1834 & 230.0953 & 458.1994 & 229.6033 & 4 \\
\hline 17 & 1586.7479 & 793.8776 & 1569.7214 & 785.3643 & 1568.7373 & 784.8723 & $\mathbf{E}$ & 419.1885 & 210.0979 & 402.1619 & 201.5846 & 401.1779 & 201.0926 & 3 \\
\hline 18 & 1701.7748 & 851.3911 & 1684.7483 & 842.8778 & 1683.7643 & 842.3858 & $\mathbf{N}$ & 290.1459 & 145.5766 & 273.1193 & 137.0633 & & & 2 \\
\hline 19 & & & & & & & $\mathbf{R}$ & 175.1190 & 88.0631 & 158.0924 & 79.5498 & & & 1 \\
\hline
\end{tabular}
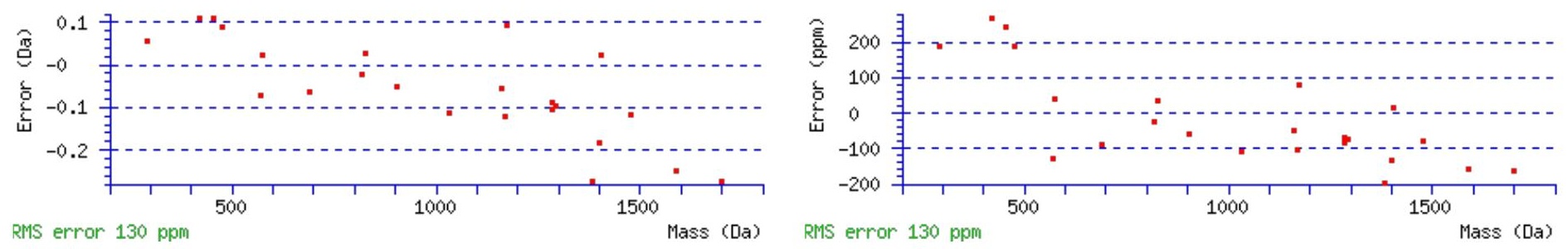

\section{All matches to this query}

\begin{tabular}{|l|l|l|l|}
\hline Score & Mr(calc): & Delta & \multicolumn{1}{|c|}{ Sequence } \\
\hline 77.7 & 1874.8792 & 0.0054 & GTAGNALMEGASQLVGENR \\
\hline 14.0 & 1873.8952 & 0.9894 & GTAGNALMEGASQLVGENR \\
\hline 3.8 & 1874.8792 & 0.0054 & GTAGNALMEGASQLVGENR \\
\hline 2.1 & 1874.8806 & 0.0041 & MALRPSEGDGSAGRWNR \\
\hline 0.8 & 1873.8645 & 1.0202 & VRTMFSEQQLGELEK \\
\hline 0.6 & 1874.8945 & -0.0098 & VAGAELSVCGNVKDWNR \\
\hline 0.5 & 1874.8999 & -0.0153 & VQAESQKHSLRLEDR \\
\hline 0.0 & 1874.8961 & -0.0115 & ARKVLNDIISTMFNR \\
\hline 0.0 & 1874.8961 & -0.0115 & ARKVLNDIISTMFNR \\
\hline
\end{tabular}

Spectrum No: 260; Query: 1453; Rank: 1

\section{Peptide View}

MS/MS Fragmentation of KSEANLSALAETHTNIPK

Found in IPI00327697, Tax_Id=10116 Gene_Symbol=Dpep1 Dipeptidase 1 precursor

Match to Query 1453: 1923.995588 from(963.005070,2+)

Title: 091008RatKidney_NH4Format01_23.1733.1733.2.dta

Data file K:INewmanPaper|Piliang|3SubProteomes\Piliang3SP\mgf5ppm\ERLIC_3SubProteomes5ppm.mgf 

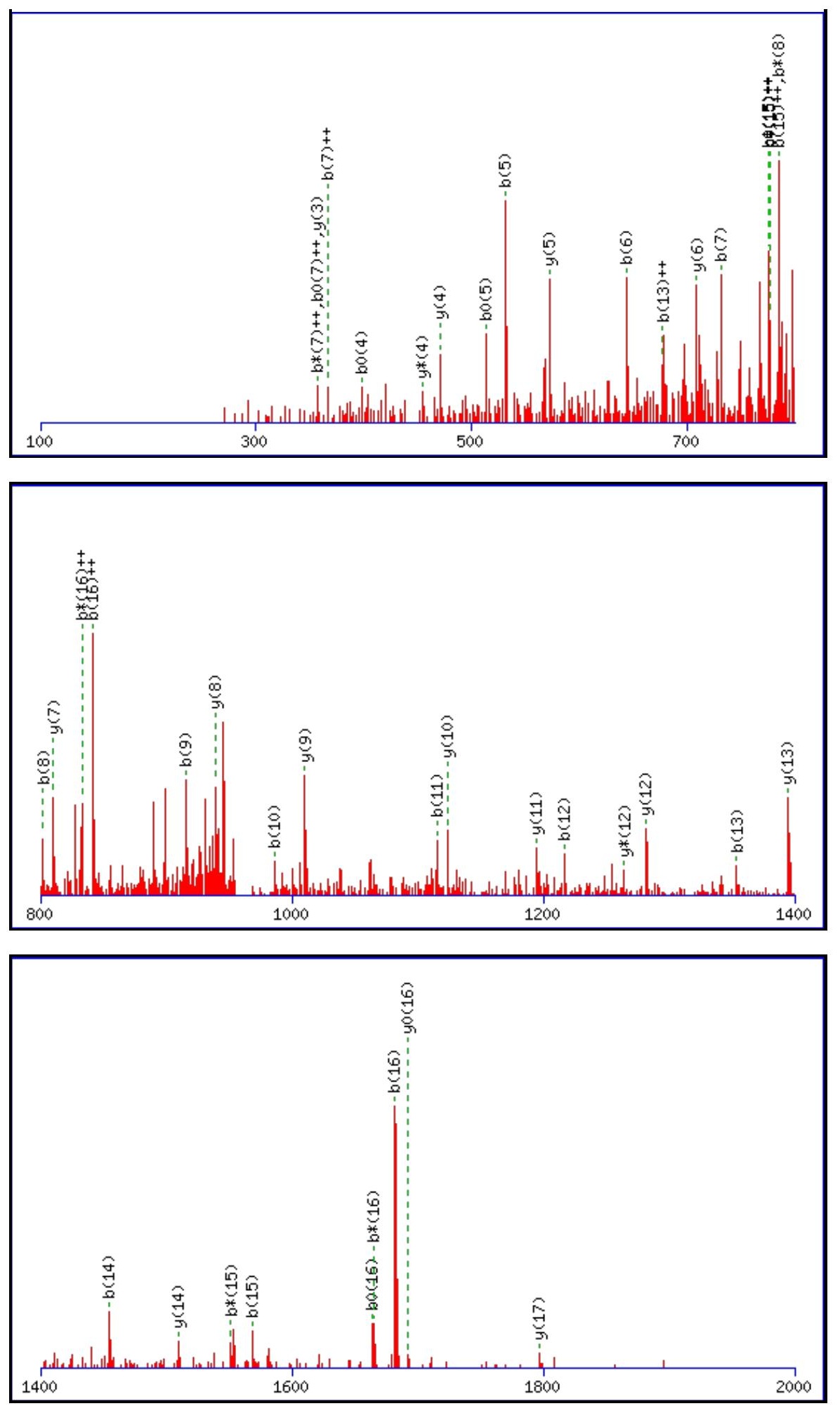

Monoisotopic mass of neutral peptide $\operatorname{Mr}($ calc): 1923.9901

Fixed modifications: Carbamidomethyl (C)

Variable modifications:

N5 : Deamidated $\mathrm{N}(\mathrm{N})$

Ions Score: 78 Expect: $2.4 \mathrm{e}-006$

Matches (Bold Red): 43/194 fragment ions using 76 most intense peaks

\begin{tabular}{|r|c|c|c|c|c|c|c|c|c|c|c|c|c|c|}
\hline$\#$ & $\mathbf{b}$ & $\mathbf{b}^{++}$ & $\mathbf{b}^{*}$ & $\mathbf{b}^{\boldsymbol{*}_{++}}$ & $\mathbf{b}^{\mathbf{0}}$ & $\mathbf{b}^{\mathbf{0 + +}}$ & $\mathbf{S e q}$ & $\mathbf{y}$ & $\mathbf{y}^{++}$ & $\mathbf{y}^{\mathbf{*}}$ & $\mathbf{y}^{\boldsymbol{*}^{++}}$ & $\mathbf{y}^{\mathbf{0}}$ & $\mathbf{y}^{\mathbf{0 + +}}$ & $\#$ \\
\hline $\mathbf{1}$ & 129.1022 & 65.0548 & 112.0757 & 56.5415 & & & $\mathbf{K}$ & & & & & & & $\mathbf{1 8}$ \\
\hline $\mathbf{2}$ & 216.1343 & 108.5708 & 199.1077 & 100.0575 & 198.1237 & 99.5655 & $\mathbf{S}$ & $\mathbf{1 7 9 6 . 9 0 2 5}$ & 898.9549 & 1779.8759 & 890.4416 & 1778.8919 & 889.9496 & $\mathbf{1 7}$ \\
\hline $\mathbf{3}$ & 345.1769 & 173.0921 & 328.1503 & 164.5788 & 327.1663 & 164.0868 & $\mathbf{E}$ & 1709.8704 & 855.4389 & 1692.8439 & 846.9256 & $\mathbf{1 6 9 1 . 8 5 9 9}$ & 846.4336 & $\mathbf{1 6}$ \\
\hline $\mathbf{4}$ & 416.2140 & 208.6106 & 399.1874 & 200.0974 & 398.2034 & 199.6053 & $\mathbf{A}$ & 1580.8279 & 790.9176 & 1563.8013 & 782.4043 & 1562.8173 & 781.9123 & $\mathbf{1 5}$ \\
\hline $\mathbf{5}$ & 531.2409 & 266.1241 & 514.2144 & 257.6108 & 513.2303 & 257.1188 & $\mathbf{N}$ & $\mathbf{1 5 0 9 . 7 9 0 7}$ & 755.3990 & 1492.7642 & 746.8857 & 1491.7802 & 746.3937 & $\mathbf{1 4}$ \\
\hline
\end{tabular}




\begin{tabular}{|c|c|c|c|c|c|c|c|c|c|c|c|c|c|c|}
\hline 6 & 644.3250 & |322.6661| & 627.2984 & |314.1529 & 626.3144 & |313.6608 | & $\mathbf{L}$ & |1394.7638 & |697.8855 & |1377.7373 & |689.3723 & |1376.7532 & |688.8803| & 13 \\
\hline 7 & 731.3570 & 366.1821 & 714.3305 & 357.6689 & 713.3464 & 357.1769 & S & 1281.6797 & 641.3435 & 1264.6532 & 632.8302 & 1263.6692 & 632.3382 & 12 \\
\hline 8 & 802.3941 & 401.7007 & 785.3676 & 393.1874 & 784.3836 & 392.6954 & A & 1194.6477 & 597.8275 & 1177.6212 & 589.3142 & 1176.6371 & 588.8222 & 11 \\
\hline 9 & 915.4782 & 458.2427 & 898.4516 & 449.7295 & 897.4676 & 449.2374 & $\mathbf{L}$ & 1123.6106 & 562.3089 & 1106.5841 & 553.7957 & 1105.6000 & 553.3037 & 10 \\
\hline 10 & 986.5153 & 493.7613 & 969.4887 & 485.2480 & 968.5047 & 484.7560 & A & 1010.5265 & 505.7669 & 993.5000 & 497.2536 & 992.5160 & 496.7616 & 9 \\
\hline 11 & 1115.5579 & 558.2826 & 1098.5313 & 549.7693 & 1097.5473 & 549.2773 & $\mathbf{E}$ & 939.4894 & 470.2483 & 922.4629 & 461.7351 & 921.4789 & 461.2431 & 8 \\
\hline 12 & 1216.6056 & 608.8064 & 1199.5790 & 600.2931 & 1198.5950 & 599.8011 & $\mathbf{T}$ & 810.4468 & 405.7271 & 793.4203 & 397.2138 & 792.4363 & 396.7218 & 7 \\
\hline 13 & 1353.6645 & 677.3359 & 1336.6379 & 668.8226 & 1335.6539 & 668.3306 & $\mathbf{H}$ & 709.3991 & 355.2032 & 692.3726 & 346.6899 & 691.3886 & 346.1979 & 6 \\
\hline 14 & 1454.7122 & 727.8597 & 1437.6856 & 719.3464 & 1436.7016 & 718.8544 & $\mathbf{T}$ & 572.3402 & 286.6738 & 555.3137 & 278.1605 & 554.3297 & 277.6685 & 5 \\
\hline 15 & 1568.7551 & 784.8812 & 1551.7285 & 776.3679 & 1550.7445 & 775.8759 & $\mathbf{N}$ & 471.2926 & 236.1499 & 454.2660 & 227.6366 & & & 4 \\
\hline 16 & 1681.8391 & 841.4232 & 1664.8126 & 832.9099 & 1663.8286 & 832.4179 & I & 357.2496 & 179.1285 & 340.2231 & 170.6152 & & & 3 \\
\hline 17 & 1778.8919 & 889.9496 & 1761.8654 & 881.4363 & 1760.8813 & 880.9443 & $\mathbf{P}$ & 244.1656 & 122.5864 & 227.1390 & 114.0731 & & & 2 \\
\hline 18 & & & & & & & $\mathbf{K}$ & 147.1128 & 74.0600 & 130.0863 & 65.5468 & & & 1 \\
\hline
\end{tabular}
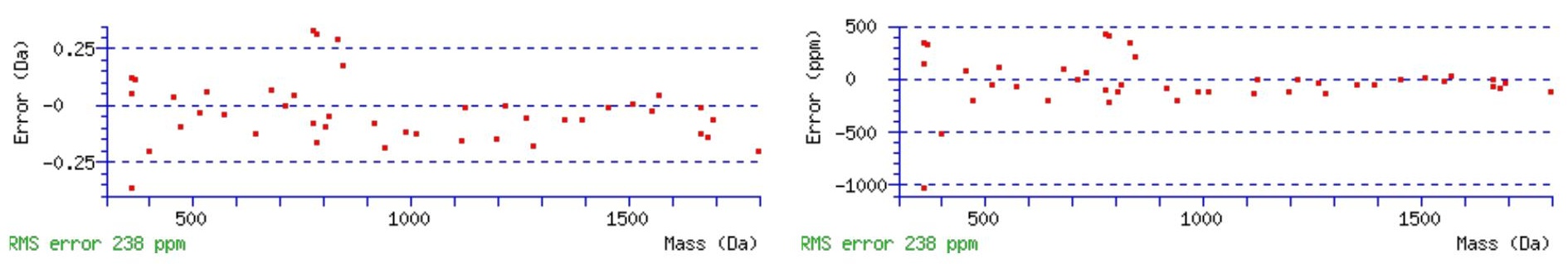

\section{All matches to this query}

\begin{tabular}{|l|r|r|l|}
\hline Score & Mr(calc): & Delta & \multicolumn{1}{|c|}{ Sequence } \\
\hline 77.6 & 1923.9901 & 0.0055 & KSEANLSALAETHTNIPK \\
\hline 26.4 & 1923.0061 & 0.9895 & KSEANLSALAETHTNIPK \\
\hline 22.6 & 1923.9901 & 0.0055 & KSEANLSALAETHTNIPK \\
\hline 10.2 & 1923.9779 & 0.0177 & TRKLLENSTASIQQQK \\
\hline 10.2 & 1923.9779 & 0.0177 & TRKLLENSTASIQQQK \\
\hline 10.2 & 1923.9779 & 0.0177 & TRKLLENSTASIQQQK \\
\hline 3.3 & 1924.0013 & -0.0058 & LEEDARALINAGSIPAQR \\
\hline
\end{tabular}

Spectrum No: 261; Query: 1292; Rank: 1

\section{Peptide View}

\section{MS/MS Fragmentation of TSDTGDQTISNGTEANSK}

Found in IPI00193425, Tax_Id=10116 Gene_Symbol=Bsg Isoform 2 of Basigin precursor

Match to Query 1292: 1825.789648 from(913.902100,2+)

Title: 100101RatKid_NS_deglyco_23.463.463.2.dta

Data file K:INewmanPaper|Piliangl3SubProteomes\Piliang3SP\mgf5ppm\ERLIC_3SubProteomes5ppm.mgf 

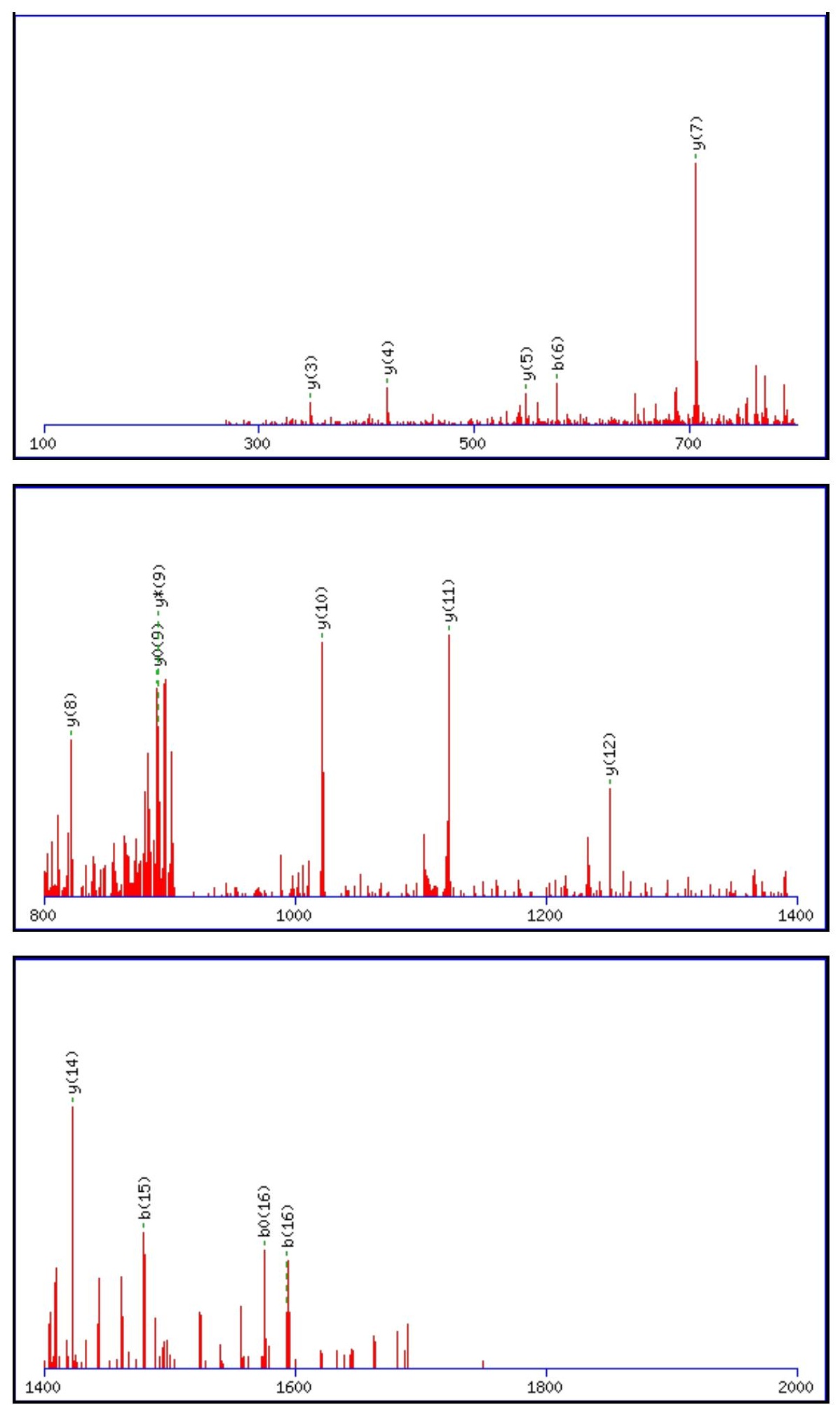

Monoisotopic mass of neutral peptide $\operatorname{Mr}($ calc): 1825.7814

Fixed modifications: Carbamidomethyl (C)

Variable modifications:

N11 : Deamidated $\mathrm{N}(\mathrm{N})$

Ions Score: 78 Expect: $4.3 \mathrm{e}-006$

Matches (Bold Red): 15/190 fragment ions using 23 most intense peaks

\begin{tabular}{|r|c|c|c|c|r|r|r|r|c|c|c|c|c|c|}
\hline$\#$ & $\mathbf{b}$ & $\mathbf{b}^{++}$ & $\mathbf{b}^{*}$ & $\mathbf{b}^{\boldsymbol{*}^{++}}$ & $\mathbf{b}^{\mathbf{0}}$ & $\mathbf{b}^{\mathbf{0 + +}}$ & Seq. & $\mathbf{y}$ & $\mathbf{y}^{++}$ & $\mathbf{y}^{\mathbf{*}}$ & $\mathbf{y}^{\boldsymbol{*}^{++}}$ & $\mathbf{y}^{\mathbf{0}}$ & $\mathbf{y}^{\mathbf{0 + +}}$ & $\#$ \\
\hline $\mathbf{1}$ & 102.0550 & 51.5311 & & & 84.0444 & 42.5258 & $\mathbf{T}$ & & & & & & & $\mathbf{1 8}$ \\
\hline $\mathbf{2}$ & 189.0870 & 95.0471 & & & 171.0764 & 86.0418 & S & 1725.7410 & 863.3741 & 1708.7144 & 854.8608 & 1707.7304 & 854.3688 & $\mathbf{1 7}$ \\
\hline 3 & 304.1139 & 152.5606 & & & 286.1034 & 143.5553 & $\mathbf{D}$ & 1638.7089 & 819.8581 & 1621.6824 & 811.3448 & 1620.6984 & 810.8528 & $\mathbf{1 6}$ \\
\hline $\mathbf{4}$ & 405.1616 & 203.0844 & & & 387.1510 & 194.0792 & $\mathbf{T}$ & 1523.6820 & 762.3446 & 1506.6554 & 753.8314 & 1505.6714 & 753.3393 & $\mathbf{1 5}$ \\
\hline $\mathbf{5}$ & 462.1831 & 231.5952 & & & 444.1725 & 222.5899 & $\mathbf{G}$ & $\mathbf{1 4 2 2 . 6 3 4 3}$ & 711.8208 & 1405.6078 & 703.3075 & 1404.6237 & 702.8155 & $\mathbf{1 4}$ \\
\hline
\end{tabular}




\begin{tabular}{|r|r|r|r|r|r|r|r|r|r|r|r|r|r|r|r|r|}
$\mathbf{6}$ & $\mathbf{5 7 7 . 2 1 0 0}$ & 289.1086 & & & 559.1994 & 280.1034 & $\mathbf{D}$ & 1365.6128 & 683.3101 & 1348.5863 & 674.7968 & 1347.6023 & 674.3048 & $\mathbf{1 3}$ \\
\hline $\mathbf{7}$ & 705.2686 & 353.1379 & 688.2420 & 344.6247 & 687.2580 & 344.1327 & $\mathbf{Q}$ & $\mathbf{1 2 5 0 . 5 8 5 9}$ & 625.7966 & 1233.5593 & 617.2833 & 1232.5753 & 616.7913 & $\mathbf{1 2}$ \\
\hline $\mathbf{8}$ & 806.3163 & 403.6618 & 789.2897 & 395.1485 & 788.3057 & 394.6565 & $\mathbf{T}$ & $\mathbf{1 1 2 2 . 5 2 7 3}$ & 561.7673 & 1105.5008 & 553.2540 & 1104.5168 & 552.7620 & $\mathbf{1 1}$ \\
\hline $\mathbf{9}$ & 919.4003 & 460.2038 & 902.3738 & 451.6905 & 901.3898 & 451.1985 & $\mathbf{I}$ & $\mathbf{1 0 2 1 . 4 7 9 6}$ & 511.2435 & 1004.4531 & 502.7302 & 1003.4691 & 502.2382 & $\mathbf{1 0}$ \\
\hline $\mathbf{1 0}$ & 1006.4324 & 503.7198 & 989.4058 & 495.2065 & 988.4218 & 494.7145 & S & 908.3956 & 454.7014 & $\mathbf{8 9 1 . 3 6 9 0}$ & 446.1882 & $\mathbf{8 9 0 . 3 8 5 0}$ & 445.6961 & $\mathbf{9}$ \\
\hline $\mathbf{1 1}$ & 1121.4593 & 561.2333 & 1104.4327 & 552.7200 & 1103.4487 & 552.2280 & $\mathbf{N}$ & $\mathbf{8 2 1 . 3 6 3 5}$ & 411.1854 & 804.3370 & 402.6721 & 803.3530 & 402.1801 & $\mathbf{8}$ \\
\hline $\mathbf{1 2}$ & 1178.4808 & 589.7440 & 1161.4542 & 581.2307 & 1160.4702 & 580.7387 & $\mathbf{G}$ & $\mathbf{7 0 6 . 3 3 6 6}$ & 353.6719 & 689.3101 & 345.1587 & 688.3260 & 344.6667 & $\mathbf{7}$ \\
\hline $\mathbf{1 3}$ & 1279.5284 & 640.2679 & 1262.5019 & 631.7546 & 1261.5179 & 631.2626 & $\mathbf{T}$ & 649.3151 & 325.1612 & 632.2886 & 316.6479 & 631.3046 & 316.1559 & $\mathbf{6}$ \\
\hline $\mathbf{1 4}$ & 1408.5710 & 704.7892 & 1391.5445 & 696.2759 & 1390.5605 & 695.7839 & $\mathbf{E}$ & $\mathbf{5 4 8 . 2 6 7 5}$ & 274.6374 & 531.2409 & 266.1241 & 530.2569 & 265.6321 & $\mathbf{5}$ \\
\hline $\mathbf{1 5}$ & $\mathbf{1 4 7 9 . 6 0 8 1}$ & 740.3077 & 1462.5816 & 731.7944 & 1461.5976 & 731.3024 & $\mathbf{A}$ & $\mathbf{4 1 9 . 2 2 4 9}$ & 210.1161 & 402.1983 & 201.6028 & 401.2143 & 201.1108 & $\mathbf{4}$ \\
\hline $\mathbf{1 6}$ & $\mathbf{1 5 9 3 . 6 5 1 1}$ & 797.3292 & 1576.6245 & 788.8159 & $\mathbf{1 5 7 5 . 6 4 0 5}$ & 788.3239 & $\mathbf{N}$ & 348.1878 & 174.5975 & 331.1612 & 166.0842 & 330.1772 & 165.5922 & $\mathbf{3}$ \\
\hline $\mathbf{1 7}$ & 1680.6831 & 840.8452 & 1663.6566 & 832.3319 & 1662.6725 & 831.8399 & S & 234.1448 & 117.5761 & 217.1183 & 109.0628 & 216.1343 & 108.5708 & $\mathbf{2}$ \\
\hline $\mathbf{1 8}$ & & & & & & & K & 147.1128 & 74.0600 & 130.0863 & 65.5468 & & & $\mathbf{1}$ \\
\hline
\end{tabular}
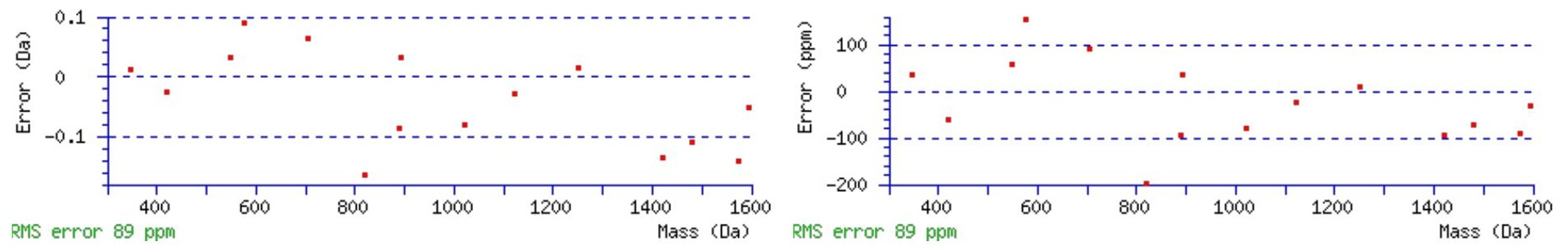

\section{All matches to this query}

\begin{tabular}{|l|l|c|l|}
\hline Score & Mr(calc): & Delta & \multicolumn{1}{c|}{ Sequence } \\
\hline 77.6 & 1825.7814 & 0.0083 & TSDTGDQTISNGTEANSK \\
\hline 46.3 & 1825.7814 & 0.0083 & TSDTGDQTISNGTEANSK \\
\hline 23.8 & 1824.7973 & 0.9923 & TSDTGDQTISNGTEANSK \\
\hline 3.5 & 1825.8039 & -0.0143 & $\underline{\text { MRLEDFNNNINNSLK }}$ \\
\hline
\end{tabular}

Spectrum No: 262; Query: 2148; Rank: 1

\section{Peptide View}

\section{MS/MS Fragmentation of DNLTLWTSDTQGDEAEAGEGGEN}

Found in IPI00324893, Tax_Id=10116 Gene_Symbol=Ywhaz 14-3-3 protein zeta/delta

Match to Query 2148: 2408.982568 from(1205.498560,2+)

Title: 091008RatKidney_NoSalt_30.2811.2811.2.dta

Data file K:INewmanPaper|Piliangl3SubProteomes\Piliang3SP\mgf5ppm\ERLIC_3SubProteomes5ppm.mgf 

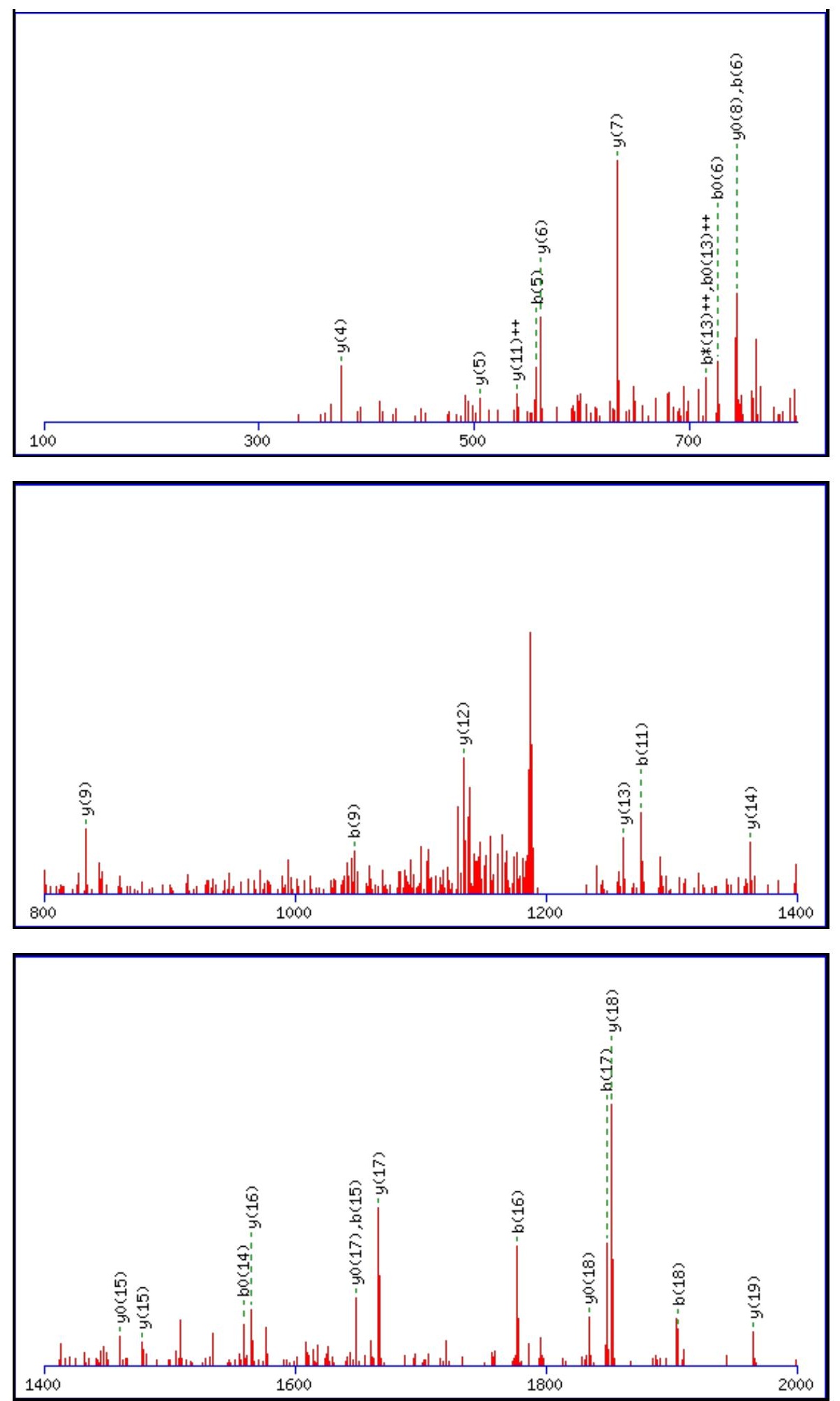

Monoisotopic mass of neutral peptide $\operatorname{Mr}($ calc): 2408.9728

Fixed modifications: Carbamidomethyl (C)

Variable modifications:

N2 : Deamidated $\mathrm{N}(\mathrm{N})$

Ions Score: 78 Expect: $4.6 \mathrm{e}-006$

Matches (Bold Red): 30/260 fragment ions using 49 most intense peaks

\begin{tabular}{|r|c|c|c|c|r|r|r|r|r|r|r|r|r|r|}
\hline$\#$ & $\mathbf{b}$ & \multicolumn{1}{|c|}{$\mathbf{b}^{++}$} & $\mathbf{b}^{*}$ & $\mathbf{b}^{\boldsymbol{*}^{++}}$ & \multicolumn{1}{|c|}{$\mathbf{b}^{\mathbf{0}}$} & \multicolumn{1}{|c|}{$\mathbf{b}^{\mathbf{0 + +}}$} & Seq. & $\mathbf{y}$ & $\mathbf{y}^{++}$ & $\mathbf{y}^{\mathbf{*}}$ & $\mathbf{y}^{\mathbf{*}^{++}}$ & $\mathbf{y}^{\mathbf{0}}$ & $\mathbf{y}^{\mathbf{0 + +}}$ & $\#$ \\
\hline $\mathbf{1}$ & 116.0342 & 58.5207 & & & 98.0237 & 49.5155 & $\mathbf{D}$ & & & & & & & $\mathbf{2 3}$ \\
\hline $\mathbf{2}$ & 231.0612 & 116.0342 & 214.0346 & 107.5209 & 213.0506 & 107.0289 & $\mathbf{N}$ & 2294.9531 & 1147.9802 & 2277.9266 & 1139.4669 & 2276.9426 & 1138.9749 & $\mathbf{2 2}$ \\
\hline $\mathbf{3}$ & 344.1452 & 172.5762 & 327.1187 & 164.0630 & 326.1347 & 163.5710 & $\mathbf{L}$ & 2179.9262 & 1090.4667 & 2162.8996 & 1081.9535 & 2161.9156 & 1081.4615 & $\mathbf{2 1}$ \\
\hline $\mathbf{4}$ & 445.1929 & 223.1001 & 428.1663 & 214.5868 & 427.1823 & 214.0948 & $\mathbf{T}$ & 2066.8421 & 1033.9247 & 2049.8156 & 1025.4114 & 2048.8316 & 1024.9194 & $\mathbf{2 0}$ \\
\hline $\mathbf{5}$ & $\mathbf{5 5 8 . 2 7 7 0}$ & 279.6421 & 541.2504 & 271.1288 & 540.2664 & 270.6368 & $\mathbf{L}$ & $\mathbf{1 9 6 5 . 7 9 4 5}$ & 983.4009 & 1948.7679 & 974.8876 & 1947.7839 & 974.3956 & $\mathbf{1 9}$ \\
\hline
\end{tabular}




\begin{tabular}{|c|c|c|c|c|c|c|c|c|c|c|c|c|c|c|}
\hline 6 & 744.3563 & 372.6818 & 727.3297 & 364.1685 & 726.3457 & 363.6765 & W & 1852.7104 & 926.8588 & |1835.6838 & 3456 & 1834.6998 & 535 & 18 \\
\hline 7 & 845.4040 & 423.2056 & 828.3774 & 414.6923 & 827.3934 & 414.2003 & $\mathbf{T}$ & 1666.6311 & 833.8192 & 1649.6045 & 825.3059 & 1648.6205 & 824.8139 & 17 \\
\hline 8 & 932.4360 & 466.7216 & 915.4094 & 458.2084 & 914.4254 & 457.7163 & $S$ & 1565.5834 & 783.2953 & 1548.5568 & 774.7821 & 1547.5728 & 774.2901 & 16 \\
\hline 9 & 1047.4629 & 524.2351 & 1030.4364 & 515.7218 & 1029.4524 & 515.2298 & D & 1478.5514 & 739.7793 & 1461.5248 & 731.2660 & 1460.5408 & 730.7740 & 15 \\
\hline 10 & 1148.5106 & 574.7589 & 1131.4841 & 566.2457 & 1130.5000 & 565.7537 & $\mathbf{T}$ & 1363.5244 & 682.2658 & 1346.4979 & 673.7526 & 1345.5139 & 673.2606 & 14 \\
\hline 11 & 1276.5692 & 638.7882 & 1259.5426 & 630.2750 & 1258.5586 & 629.7829 & $\mathbf{Q}$ & 1262.4767 & 631.7420 & 1245.4502 & 623.2287 & 1244.4662 & 622.7367 & 3 \\
\hline 12 & 1333.5906 & 667.2990 & 1316.5641 & 658.7857 & 1315.5801 & 658.2937 & G & 1134.4182 & 567.7127 & 1117.3916 & 559.1994 & 1116.4076 & 558.7074 & 12 \\
\hline 13 & 1448.6176 & 724.8124 & 1431.5910 & 716.2992 & 1430.6070 & 715.8071 & D & 1077.3967 & 539.2020 & 1060.3702 & 530.6887 & 1059.3861 & 530.1967 & 11 \\
\hline 14 & 1577.6602 & 789.3337 & 1560.6336 & 780.8205 & 1559.6496 & 780.3284 & $\mathbf{E}$ & 962.3698 & 481.6885 & 945.3432 & 473.1752 & 944.3592 & 472.6832 & 10 \\
\hline 15 & 1648.6973 & 824.8523 & 1631.6707 & 816.3390 & 1630.6867 & 815.8470 & A & 833.3272 & 417.1672 & 816.3006 & 408.6539 & 815.3166 & 408.1619 & 9 \\
\hline 16 & 1777.7399 & 889.3736 & 1760.7133 & 880.8603 & 1759.7293 & 880.3683 & $\mathbf{E}$ & 762.2901 & 381.6487 & 745.2635 & 373.1354 & 744.2795 & 372.6434 & 8 \\
\hline 17 & 1848.7770 & 924.8921 & 1831.7505 & 916.3789 & 1830.7664 & 915.8869 & A & 633.2475 & 317.1274 & 616.2209 & 308.6141 & 615.2369 & 308.1221 & 7 \\
\hline 18 & 1905.7985 & 953.4029 & 1888.7719 & 944.8896 & 1887.7879 & 944.3976 & G & 562.2103 & 281.6088 & 545.1838 & 273.0955 & 544.1998 & 272.6035 & 6 \\
\hline 19 & 2034.8411 & 1017.9242 & 2017.8145 & 1009.4109 & 2016.8305 & 1008.9189 & $\mathbf{E}$ & 505.1889 & 253.0981 & 488.1623 & 244.5848 & 487.1783 & 244.0928 & 5 \\
\hline 21 & 2148.8840 & 1074.9456 & 2131.8574 & 1066.4324 & 2130.8734 & 1065.9403 & G & 319.1248 & 160.0661 & 302.0983 & 151.5528 & 301.1143 & 151.0608 & 3 \\
\hline 22 & 2277.9266 & 1139.4669 & 2260.9000 & 1130.9537 & 2259.9160 & 1130.4616 & $\mathbf{E}$ & 262.1034 & 131.5553 & 245.0768 & 123.0420 & 244.0928 & 122.5500 & 2 \\
\hline 23 & & & & & & & $\mathbf{N}$ & 133.0608 & 67.0340 & 116.0342 & 58.5207 & & & 1 \\
\hline
\end{tabular}
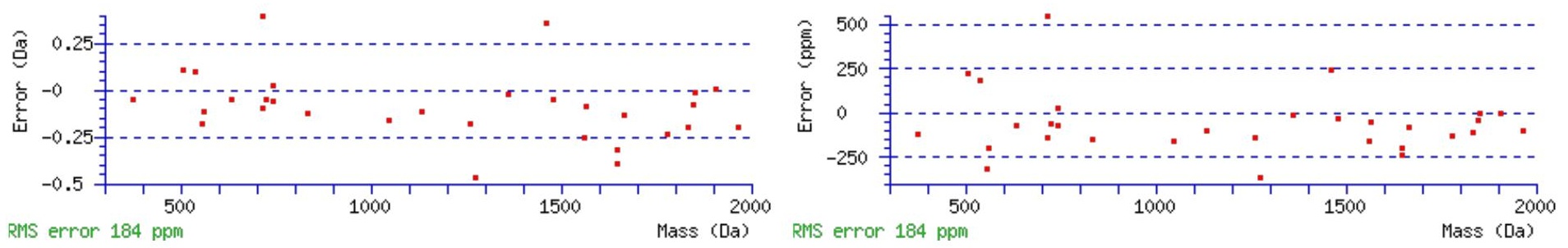

\section{All matches to this query}

\begin{tabular}{|l|l|l|l|}
\hline Score & Mr(calc): & Delta & \multicolumn{1}{c|}{ Sequence } \\
\hline 77.5 & 2408.9728 & 0.0098 & DNLTLWTSDTQGDEAEAGEGGEN \\
\hline 68.2 & 2407.9888 & 0.9938 & DNLTLWTSDTQGDEAEAGEGGEN \\
\hline 8.8 & 2408.9728 & 0.0098 & DNLTLWTSDTQGDEAEAGEGGEN \\
\hline 0.6 & 2408.9709 & 0.0117 & VMSSIGNELANSVWEEGSQGR \\
\hline
\end{tabular}

Spectrum No: 263; Query: 2546; Rank: 1

\section{Peptide View}

MS/MS Fragmentation of CISIDKVCDGVPDCPEGDDENNVTSGR

Found in IPI00205325, Tax_Id=10116 Gene_Symbol=Lrp2 Low-density lipoprotein receptor-related protein 2 precursor

Match to Query 2546: 3008.257362 from(1003.759730,3+)

Title: 100101RatKid_NS_deglyco_23.2435.2435.3.dta

Data file K:\NewmanPaper\Piliang \3SubProteomes\Piliang3SP \mgf5ppm\ERLIC_3SubProteomes5ppm.mgf 

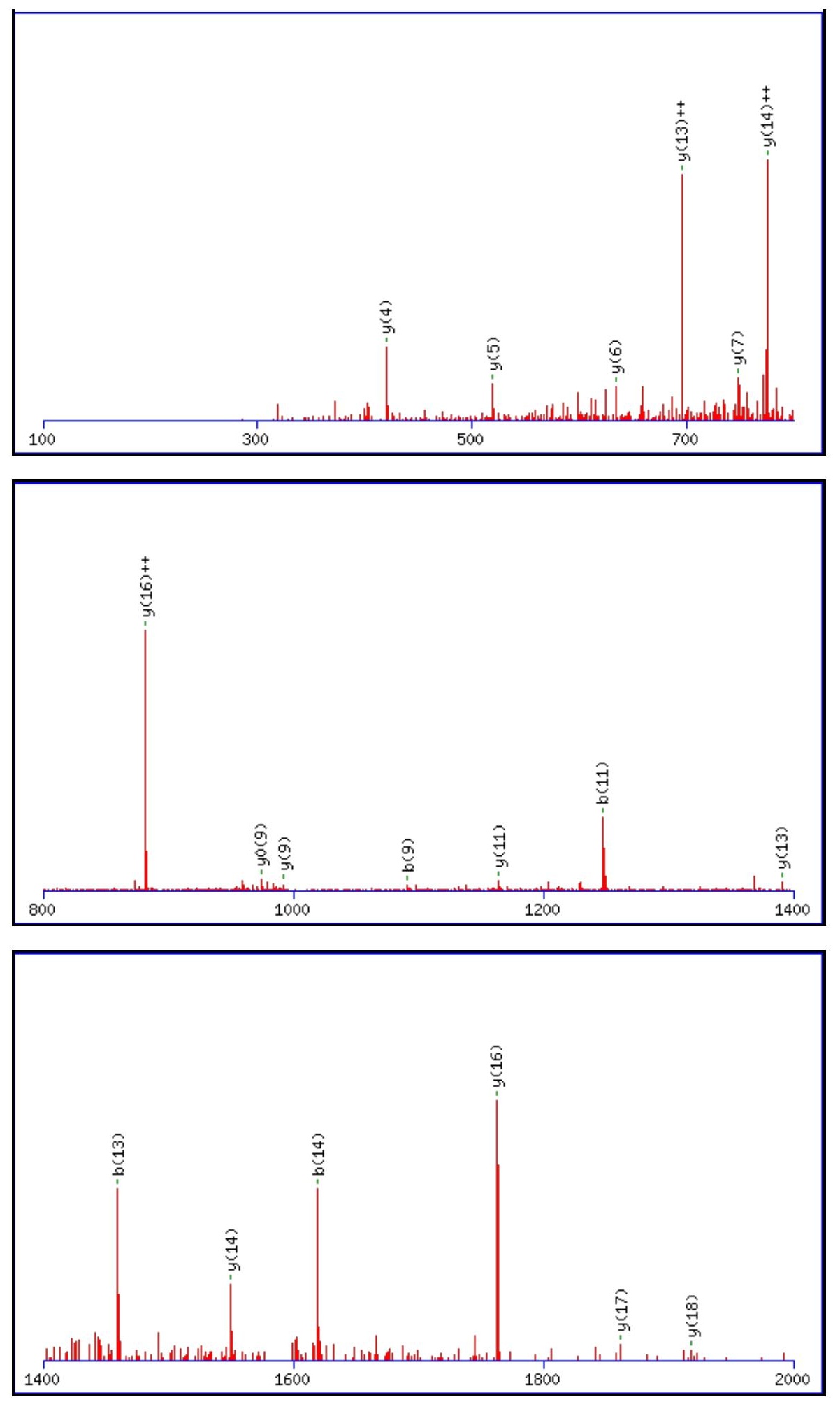

Monoisotopic mass of neutral peptide $\operatorname{Mr}($ calc): 3008.2434

Fixed modifications: Carbamidomethyl (C)

Variable modifications:

N22 : Deamidated_N (N)

Ions Score: 77 Expect: $7 \mathrm{e}-006$

Matches (Bold Red): 19/294 fragment ions using 34 most intense peaks

\begin{tabular}{|r|c|c|c|c|c|c|c|c|c|c|c|c|c|c|}
\hline$\#$ & $\mathbf{b}$ & $\mathbf{b}^{++}$ & $\mathbf{b}^{*}$ & $\mathbf{b}^{\boldsymbol{*}^{++}}$ & $\mathbf{b}^{\mathbf{0}}$ & $\mathbf{b}^{\mathbf{0 + +}}$ & Seq. & $\mathbf{y}$ & $\mathbf{y}^{++}$ & $\mathbf{y}^{\mathbf{*}}$ & $\mathbf{y}^{\mathbf{*}^{++}}$ & $\mathbf{y}^{\mathbf{0}}$ & $\mathbf{y}^{\mathbf{0 + +}}$ & $\#$ \\
\hline $\mathbf{1}$ & 161.0379 & 81.0226 & & & & & $\mathbf{C}$ & & & & & & & $\mathbf{2 7}$ \\
\hline $\mathbf{2}$ & 274.1220 & 137.5646 & & & & & $\mathbf{I}$ & 2849.2200 & 1425.1136 & 2832.1935 & 1416.6004 & 2831.2094 & 1416.1084 & $\mathbf{2 6}$ \\
\hline 3 & 361.1540 & 181.0806 & & & 343.1435 & 172.0754 & S & 2736.1359 & 1368.5716 & 2719.1094 & 1360.0583 & 2718.1254 & 1359.5663 & 25 \\
\hline $\mathbf{4}$ & 474.2381 & 237.6227 & & & 456.2275 & 228.6174 & $\mathbf{I}$ & 2649.1039 & 1325.0556 & 2632.0774 & 1316.5423 & 2631.0933 & 1316.0503 & 24 \\
\hline $\mathbf{5}$ & 589.2650 & 295.1362 & & & 571.2545 & 286.1309 & $\mathbf{D}$ & 2536.0198 & 1268.5136 & 2518.9933 & 1260.0003 & 2518.0093 & 1259.5083 & $2 \mathbf{2 3}$ \\
\hline
\end{tabular}




\begin{tabular}{|c|c|c|c|c|c|c|c|c|c|c|c|c|c|c|}
\hline 6 & 717.3600 & 359.1836 & 700.3334 & 350.6704 & 699.3494 & 350.1783 & K & |2420.9929 & |1211.0001 | & 2403.9664 & |1202.4868 & |2402.9823 & $\mid$ & 22 \\
\hline 7 & 816.4284 & 408.7178 & 799.4019 & 400.2046 & 798.4178 & 399.7126 & V & 2292.8979 & 1146.9526 & 2275.8714 & 1138.4393 & 2274.8874 & 1137.9473 & 21 \\
\hline 8 & 976.4591 & 488.7332 & 959.4325 & 480.2199 & 958.4485 & 479.7279 & C & 2193.8295 & 1097.4184 & 2176.8030 & 1088.9051 & 2175.8190 & 1088.4131 & 20 \\
\hline 9 & 1091.4860 & 546.2466 & 1074.4594 & 537.7334 & 1073.4754 & 537.2414 & D & 2033.7989 & 1017.4031 & 2016.7723 & 1008.8898 & 2015.7883 & 1008.3978 & 19 \\
\hline 10 & 1148.5075 & 574.7574 & 1131.4809 & 566.2441 & 1130.4969 & 565.7521 & G & 1918.7719 & 959.8896 & 1901.7454 & 951.3763 & 1900.7614 & 950.8843 & 18 \\
\hline 11 & 1247.5759 & 624.2916 & 1230.5493 & 615.7783 & 1229.5653 & 615.2863 & V & 1861.7505 & 931.3789 & 1844.7239 & 922.8656 & 1843.7399 & 922.3736 & 17 \\
\hline 12 & 1344.6286 & 672.8180 & 1327.6021 & 664.3047 & 1326.6181 & 663.8127 & $\mathbf{P}$ & 1762.6821 & 881.8447 & 1745.6555 & 873.3314 & 1744.6715 & 872.8394 & 6 \\
\hline 13 & 1459.6556 & 730.3314 & 1442.6290 & 721.8182 & 1441.6450 & 721.3261 & D & 1665.6293 & 833.3183 & 1648.6027 & 824.8050 & 1647.6187 & 824.3130 & 15 \\
\hline 14 & 1619.6862 & 810.3468 & 1602.6597 & 801.8335 & 1601.6757 & 801.3415 & C & 1550.6023 & 775.8048 & 1533.5758 & 767.2915 & 1532.5918 & 766.7995 & 14 \\
\hline 15 & 1716.7390 & 858.8731 & 1699.7124 & 850.3599 & 1698.7284 & 849.8679 & $\mathbf{P}$ & 1390.5717 & 695.7895 & 1373.5452 & 687.2762 & 1372.5611 & 686.7842 & 13 \\
\hline 16 & 1845.7816 & 923.3944 & 1828.7550 & 914.8812 & 1827.7710 & 914.3891 & $\mathbf{E}$ & 1293.5189 & 647.2631 & 1276.4924 & 638.7498 & 1275.5084 & 638.2578 & 12 \\
\hline 17 & 1902.8030 & 951.9052 & 1885.7765 & 943.3919 & 1884.7925 & 942.8999 & G & 1164.4763 & 582.7418 & 1147.4498 & 574.2285 & 1146.4658 & 573.7365 & 11 \\
\hline 18 & 2017.8300 & 1009.4186 & 2000.8034 & 1000.9054 & 1999.8194 & 1000.4134 & D & 1107.4549 & 554.2311 & 1090.4283 & 545.7178 & 1089.4443 & 545.2258 & 10 \\
\hline 19 & 2132.8569 & 1066.9321 & 2115.8304 & 1058.4188 & 2114.8464 & 1057.9268 & D & 992.4279 & 496.7176 & 975.4014 & 488.2043 & 974.4174 & 487.7123 & 9 \\
\hline 21 & 2375.9425 & 1188.4749 & 2358.9159 & 1179.9616 & 2357.9319 & 1179.4696 & $\mathbf{N}$ & 748.3584 & 374.6828 & 731.3319 & 366.1696 & 730.3478 & 365.6776 & 7 \\
\hline 22 & 2490.9694 & 1245.9883 & 2473.9428 & 1237.4751 & 2472.9588 & 1236.9831 & $\mathbf{N}$ & 634.3155 & 317.6614 & 617.2889 & 309.1481 & 616.3049 & 308.6561 & 6 \\
\hline 23 & 2590.0378 & 1295.5225 & 2573.0113 & 1287.0093 & 2572.0272 & 1286.5173 & $\mathbf{V}$ & 519.2885 & 260.1479 & 502.2620 & 251.6346 & 501.2780 & 251.1426 & 5 \\
\hline 24 & 2691.0855 & 1346.0464 & 2674.0589 & 1337.5331 & 2673.0749 & 1337.0411 & $\mathbf{T}$ & 420.2201 & 210.6137 & 403.1936 & 202.1004 & 402.2096 & 201.6084 & 4 \\
\hline 25 & 2778.1175 & 1389.5624 & 2761.0910 & 1381.0491 & 2760.1069 & 1380.5571 & $\mathrm{~S}$ & 319.1724 & 160.0899 & 302.1459 & 151.5766 & 301.1619 & 151.0846 & 3 \\
\hline 26 & 2835.1390 & 1418.0731 & 2818.1124 & 1409.5599 & 2817.1284 & 1409.0678 & G & 232.1404 & 116.5738 & 215.1139 & 108.0606 & & & 2 \\
\hline 27 & & & & & & & $\mathbf{R}$ & 175.1190 & 88.0631 & 158.0924 & 79.5498 & & & 1 \\
\hline
\end{tabular}
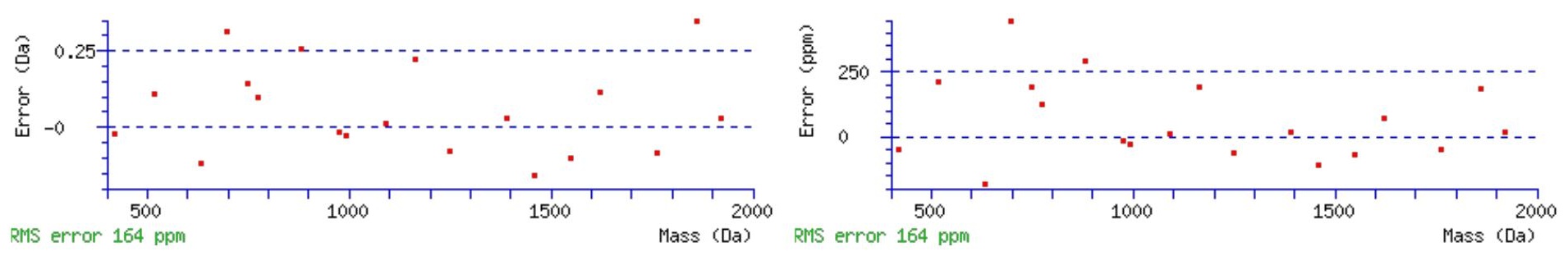

\section{All matches to this query}

\begin{tabular}{|l|c|c|l|}
\hline Score & Mr(calc): & Delta & \multicolumn{1}{|c|}{ Sequence } \\
\hline 77.4 & 3008.2434 & 0.0140 & CISIDKVCDGVPDCPEGDDENNVTSGR \\
\hline 63.3 & 3008.2434 & 0.0140 & CISIDKVCDGVPDCPEGDDENNVTSGR \\
\hline 9.8 & 3007.2759 & 0.9815 & VHTGEKPYHCEACGKSFSQSANLIK \\
\hline 9.8 & 3007.2759 & 0.9815 & VHTGEKPYHCEACGKSFSOSANLIK \\
\hline 8.3 & 3007.2759 & 0.9815 & VHTGEKPYHCEACGKSFSQSANLIK \\
\hline 7.5 & 3008.2602 & -0.0029 & EEMELSVLMSORWDSDTEEQCAKK \\
\hline 5.2 & 3008.2316 & 0.0257 & EQINALTSFLDASLVYSPEPSMANR \\
\hline 5.2 & 3008.2316 & 0.0257 & EQINALTSFLDASLVYSPEPSMANR \\
\hline 5.2 & 3008.2316 & 0.0257 & EQINALTSFLDASLVYSPEPSMANR \\
\hline 5.0 & 3007.2828 & 0.9746 & KSSDTVTGTPAPVQDENNTMAFMQTPK \\
\hline
\end{tabular}

Spectrum No: 264; Query: 679; Rank: 1

\section{Peptide View}

MS/MS Fragmentation of NRTDVEYELDEK

Found in IPI00327143, Tax_Id=10116 Gene_Symbol=Alpl Alkaline phosphatase, tissue-nonspecific isozyme precursor 
Match to Query 679: 1510.680008 from(756.347280,2+)

Title: 091008RatKidney_NH4Format01_27.1105.1105.2.dta

Data file K:INewmanPaper|Piliang|3SubProteomes\Piliang3SP\mgf5ppm\ERLIC_3SubProteomes5ppm.mgf
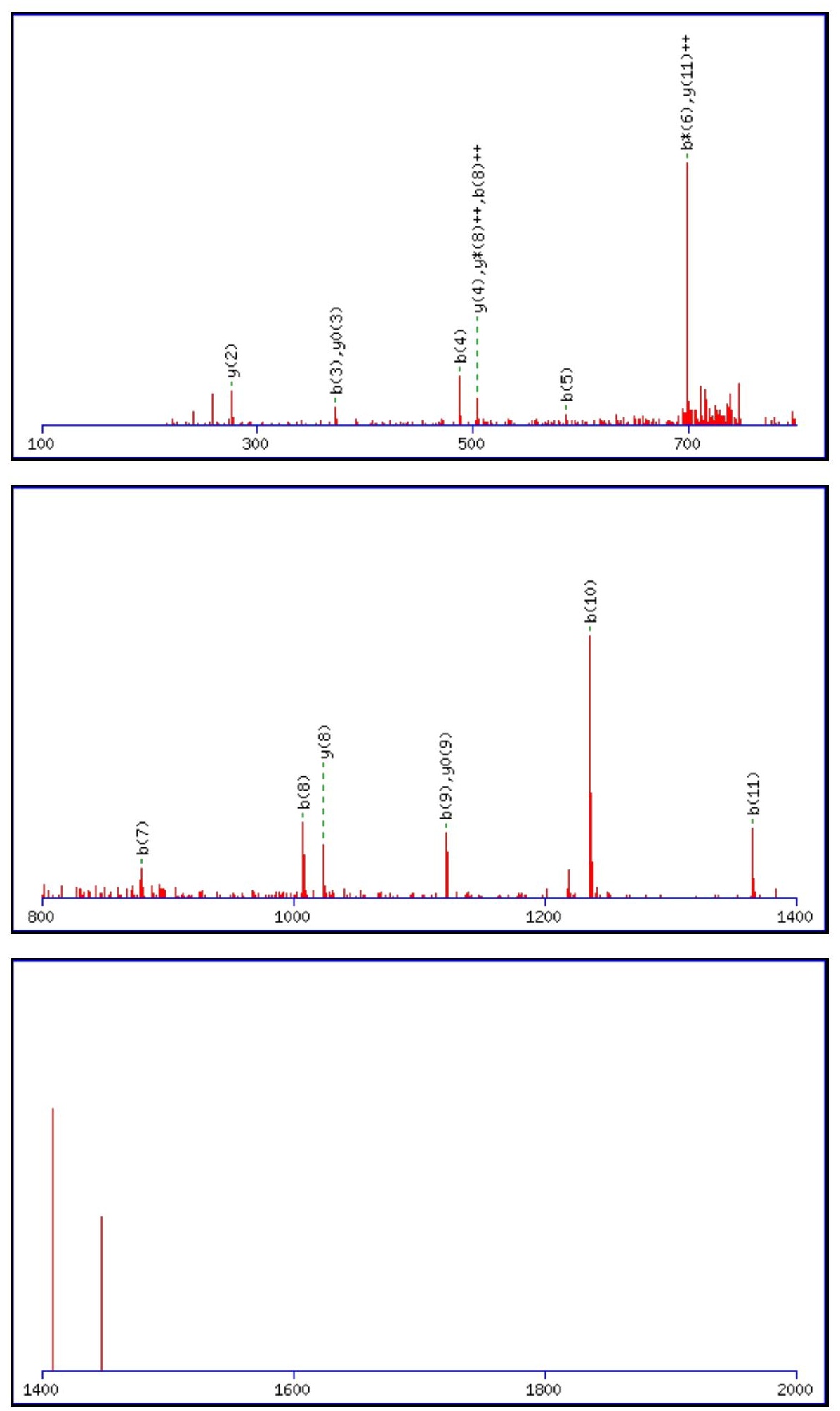

Monoisotopic mass of neutral peptide $\operatorname{Mr}($ calc): 1510.6787

Fixed modifications: Carbamidomethyl (C)

Variable modifications:

N1 : Deamidated_N (N)

Ions Score: 77 Expect: $2.7 \mathrm{e}-006$

Matches (Bold Red): 17/126 fragment ions using 24 most intense peaks

\begin{tabular}{|r|c|c|c|c|c|c|c|c|c|c|c|c|c|c|}
\hline$\#$ & $\mathbf{b}$ & $\mathbf{b}^{++}$ & $\mathbf{b}^{*}$ & $\mathbf{b}^{\mathbf{*}^{++}}$ & $\mathbf{b}^{\mathbf{0}}$ & $\mathbf{b}^{\mathbf{0}++}$ & Seq. & $\mathbf{y}$ & $\mathbf{y}^{++}$ & $\mathbf{y}^{\mathbf{*}}$ & $\mathbf{y}^{\boldsymbol{*}^{++}}$ & $\mathbf{y}^{\mathbf{0}}$ & $\mathbf{y}^{\mathbf{0 + +}}$ & $\#$ \\
\hline $\mathbf{1}$ & 116.0342 & 58.5207 & 99.0077 & 50.0075 & & & $\mathbf{N}$ & & & & & & & $\mathbf{1 2}$ \\
\hline $\mathbf{2}$ & 272.1353 & 136.5713 & 255.1088 & 128.0580 & & & $\mathbf{R}$ & 1396.6591 & $\mathbf{6 9 8 . 8 3 3 2}$ & 1379.6325 & 690.3199 & 1378.6485 & 689.8279 & $\mathbf{1 1}$ \\
\hline
\end{tabular}




\begin{tabular}{|c|c|c|c|c|c|c|c|c|c|c|c|c|c|c|}
\hline 3 & 373.1830 & 187.0951 & 356.1565 & |178.5819 & 355.1724 & |178.0899 || & $\mathbf{T}$ & 1240.5580 & |620.7826 & 1223.53 & 612.2693 & & 773 & 10 \\
\hline 4 & 488.2099 & 244.6086 & 471.1834 & 236.0953 & 470.1994 & 235.6033 & D & 1139.5103 & 570.2588 & 1122.4837 & 561.7455 & 1121.4997 & 561.2535 & 9 \\
\hline 5 & 587.2784 & 294.1428 & 570.2518 & 285.6295 & 569.2678 & 285.1375 & V & 1024.4833 & 512.7453 & 1007.4568 & 504.2320 & 1006.4728 & 503.7400 & 8 \\
\hline 6 & 716.3210 & 358.6641 & 699.2944 & 350.1508 & 698.3104 & 349.6588 & $\mathbf{E}$ & 925.4149 & 463.2111 & 908.3884 & 454.6978 & 907.4044 & 454.2058 & 7 \\
\hline 7 & 879.3843 & 440.1958 & 862.3577 & 431.6825 & 861.3737 & 431.1905 & $\mathbf{Y}$ & 796.3723 & 398.6898 & 779.3458 & 390.1765 & 778.3618 & 389.6845 & 6 \\
\hline 8 & 1008.4269 & 504.7171 & 991.4003 & 496.2038 & 990.4163 & 495.7118 & $\mathbf{E}$ & 633.3090 & 317.1581 & 616.2824 & 308.6449 & 615.2984 & 308.1529 & 5 \\
\hline 9 & 1121.5109 & 561.2591 & 1104.4844 & 552.7458 & 1103.5004 & 552.2538 & $\mathbf{L}$ & 504.2664 & 252.6368 & 487.2399 & 244.1236 & 486.2558 & 243.6316 & 4 \\
\hline 10 & 1236.5379 & 618.7726 & 1219.5113 & 610.2593 & 1218.5273 & 609.7673 & D & 391.1823 & 196.0948 & 374.1558 & 187.5815 & 373.1718 & 187.0895 & 3 \\
\hline 11 & 1365.5805 & 683.2939 & 1348.5539 & 674.7806 & 1347.5699 & 674.2886 & $\mathbf{E}$ & 276.1554 & 138.5813 & 259.1288 & 130.0681 & 258.1448 & 129.5761 & 2 \\
\hline 12 & & & & & & & $\mathbf{K}$ & 147.1128 & 74.0600 & 130.0863 & 65.5468 & & & 1 \\
\hline
\end{tabular}
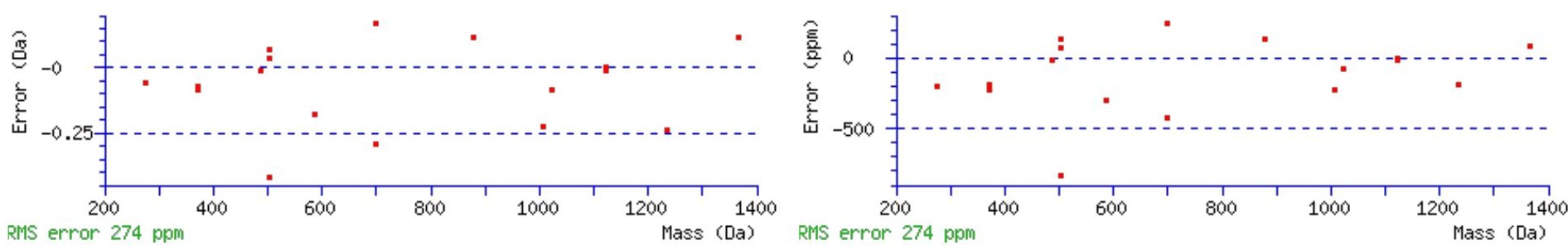

\section{All matches to this query}

\begin{tabular}{|l|l|l|l|}
\hline Score & Mr(calc): & Delta & \multicolumn{1}{|c|}{ Sequence } \\
\hline 77.4 & 1510.6787 & 0.0013 & NRTDVEYELDEK \\
\hline 17.4 & 1510.6680 & 0.0121 & IYNYNFLMPTR \\
\hline 17.2 & 1510.6778 & 0.0022 & DKMYNLYIVEK \\
\hline 16.8 & 1510.6778 & 0.0022 & DKMYNLYIVEK \\
\hline 16.5 & 1510.6778 & 0.0022 & DYEMKFTKIEK \\
\hline 14.9 & 1510.6777 & 0.0023 & KTGGSGERNATPEK \\
\hline 14.7 & 1509.6657 & 1.0143 & GSEDEDKFDPLMK \\
\hline 12.9 & 1510.6745 & 0.0055 & FFSVFKGNINEK \\
\hline 12.9 & 1510.6916 & -0.0116 & TIETQAVNALDEK \\
\hline 12.9 & 1510.6916 & -0.0116 & TIETQAVNALDEK \\
\hline
\end{tabular}

Spectrum No: 265; Query: 712; Rank: 1

\section{Peptide View}

MS/MS Fragmentation of CCGASNYTDWER

Found in IPI00231686, Tax_Id=10116 Gene_Symbol=Cd63 CD63 antigen

Match to Query 712: 1518.552548 from(760.283550,2+)

Title: 100101RatKid_NS_deglyco_26.1908.1908.2.dta

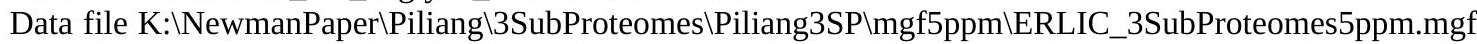



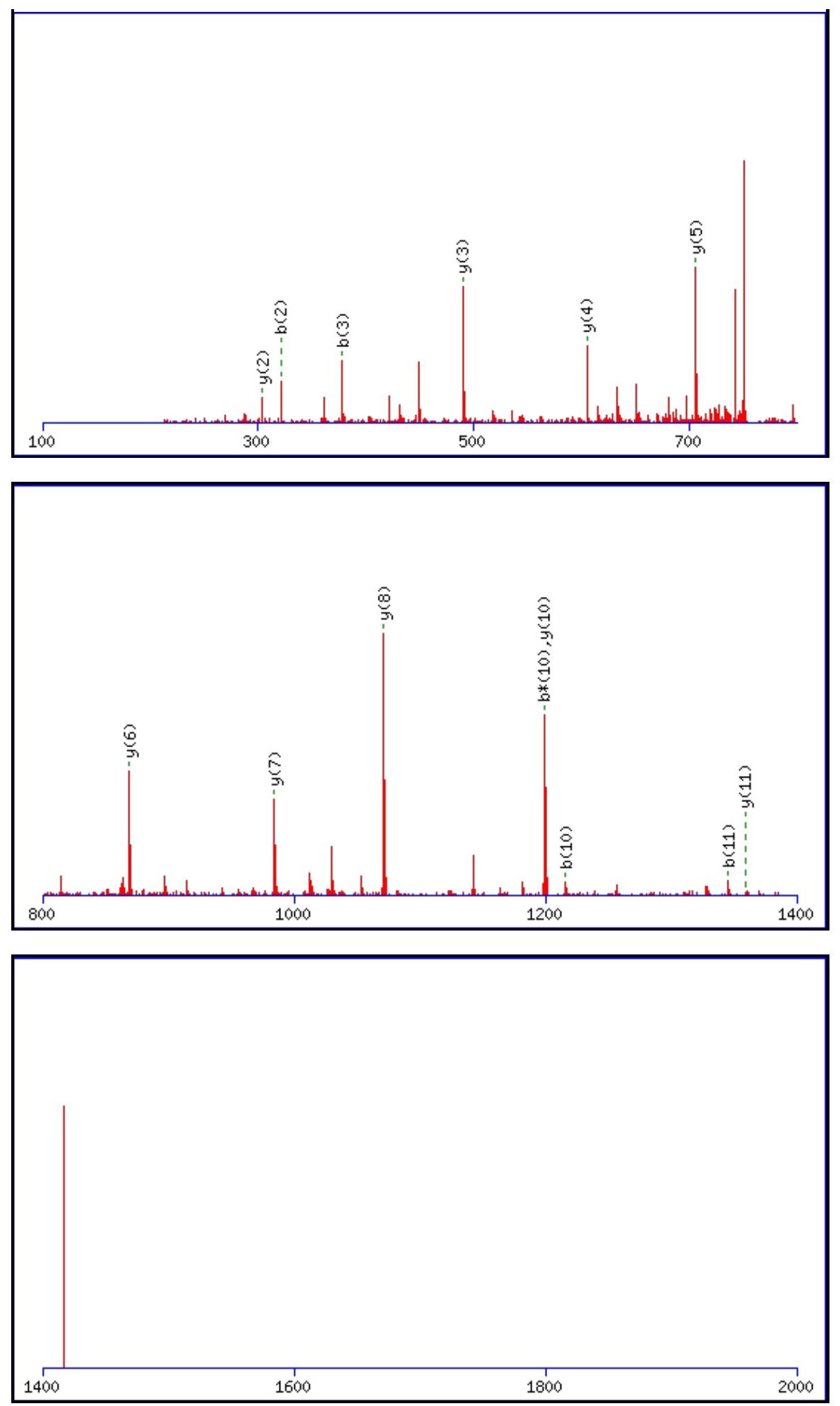

Monoisotopic mass of neutral peptide $\operatorname{Mr}($ calc): 1518.5504

Fixed modifications: Carbamidomethyl (C)

Variable modifications:

N6 : Deamidated $\mathrm{N}(\mathrm{N})$

Ions Score: 77 Expect: $1.3 \mathrm{e}-006$

Matches (Bold Red): 14/112 fragment ions using 28 most intense peaks

\begin{tabular}{|r|c|c|c|c|c|c|c|c|c|c|c|c|c|c|}
\hline$\#$ & $\mathbf{b}$ & $\mathbf{b}^{++}$ & $\mathbf{b}^{*}$ & $\mathbf{b}^{\boldsymbol{*}^{++}}$ & $\mathbf{b}^{\mathbf{0}}$ & $\mathbf{b}^{\mathbf{0 + +}}$ & Seq. & $\mathbf{y}$ & $\mathbf{y}^{++}$ & $\mathbf{y}^{\mathbf{*}}$ & $\mathbf{y}^{\boldsymbol{*}^{++}}$ & $\mathbf{y}^{\mathbf{0}}$ & $\mathbf{y}^{\mathbf{0 + +}}$ & $\#$ \\
\hline $\mathbf{1}$ & 161.0379 & 81.0226 & & & & & $\mathbf{C}$ & & & & & & & $\mathbf{1 2}$ \\
\hline $\mathbf{2}$ & $\mathbf{3 2 1 . 0 6 8 6}$ & 161.0379 & & & & & $\mathbf{C}$ & $\mathbf{1 3 5 9 . 5 2 7 0}$ & 680.2671 & 1342.5005 & 671.7539 & 1341.5164 & 671.2619 & $\mathbf{1 1}$ \\
\hline $\mathbf{3}$ & $\mathbf{3 7 8 . 0 9 0 0}$ & 189.5487 & & & & & $\mathbf{G}$ & $\mathbf{1 1 9 9 . 4 9 6 4}$ & 600.2518 & 1182.4698 & 591.7385 & 1181.4858 & 591.2465 & $\mathbf{1 0}$ \\
\hline $\mathbf{4}$ & 449.1272 & 225.0672 & & & & & A & 1142.4749 & 571.7411 & 1125.4483 & 563.2278 & 1124.4643 & 562.7358 & $\mathbf{9}$ \\
\hline $\mathbf{5}$ & 536.1592 & 268.5832 & & & 518.1486 & 259.5779 & S & $\mathbf{1 0 7 1 . 4 3 7 8}$ & 536.2225 & 1054.4112 & 527.7093 & 1053.4272 & 527.2172 & $\mathbf{8}$ \\
\hline
\end{tabular}




\begin{tabular}{|r|r|r|r|r|r|r|r|r|r|r|r|r|r|r|r|r|r|}
$\mathbf{6}$ & 651.1861 & 326.0967 & 634.1596 & 317.5834 & 633.1756 & 317.0914 & $\mathbf{N}$ & $\mathbf{9 8 4 . 4 0 5 7}$ & 492.7065 & 967.3792 & 484.1932 & 966.3952 & 483.7012 & $\mathbf{7}$ \\
\hline $\mathbf{7}$ & 814.2494 & 407.6284 & 797.2229 & 399.1151 & 796.2389 & 398.6231 & $\mathbf{Y}$ & $\mathbf{8 6 9 . 3 7 8 8}$ & 435.1930 & 852.3523 & 426.6798 & 851.3682 & 426.1878 & $\mathbf{6}$ \\
\hline $\mathbf{8}$ & 915.2971 & 458.1522 & 898.2706 & 449.6389 & 897.2866 & 449.1469 & $\mathbf{T}$ & $\mathbf{7 0 6 . 3 1 5 5}$ & 353.6614 & 689.2889 & 345.1481 & 688.3049 & 344.6561 & $\mathbf{5}$ \\
\hline $\mathbf{9}$ & 1030.3241 & 515.6657 & 1013.2975 & 507.1524 & 1012.3135 & 506.6604 & $\mathbf{D}$ & $\mathbf{6 0 5 . 2 6 7 8}$ & 303.1375 & 588.2413 & 294.6243 & 587.2572 & 294.1323 & $\mathbf{4}$ \\
\hline $\mathbf{1 0}$ & $\mathbf{1 2 1 6 . 4 0 3 4}$ & 608.7053 & $\mathbf{1 1 9 9 . 3 7 6 8}$ & 600.1921 & 1198.3928 & 599.7000 & $\mathbf{W}$ & $\mathbf{4 9 0 . 2 4 0 9}$ & 245.6241 & 473.2143 & 237.1108 & 472.2303 & 236.6188 & $\mathbf{3}$ \\
\hline $\mathbf{1 1}$ & $\mathbf{1 3 4 5 . 4 4 6 0}$ & 673.2266 & 1328.4194 & 664.7134 & 1327.4354 & 664.2213 & $\mathbf{E}$ & $\mathbf{3 0 4 . 1 6 1 5}$ & 152.5844 & 287.1350 & 144.0711 & 286.1510 & 143.5791 & $\mathbf{2}$ \\
\hline $\mathbf{1 2}$ & & & & & & & $\mathbf{R}$ & 175.1190 & 88.0631 & 158.0924 & 79.5498 & & & $\mathbf{1}$ \\
\hline
\end{tabular}
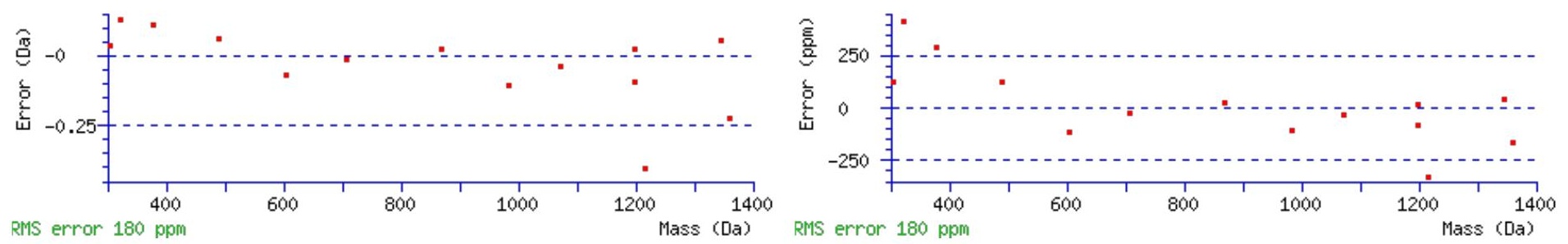

\section{All matches to this query}

\begin{tabular}{|l|c|c|l|}
\hline Score & Mr(calc): & Delta & \multicolumn{1}{c|}{ Sequence } \\
\hline 77.3 & 1518.5504 & 0.0022 & CCGASNYTDWER \\
\hline 5.3 & 1518.5541 & -0.0016 & DVPHSNRYPTSS \\
\hline 4.2 & 1518.5479 & 0.0046 & TASSVIELTCAK \\
\hline 1.5 & 1518.5450 & 0.0075 & ESTSDMMDGTEER \\
\hline 1.1 & 1518.5388 & 0.0137 & ETSEQHESKER \\
\hline 1.0 & 1518.5462 & 0.0063 & TKSSVNNMSSFR \\
\hline
\end{tabular}

Spectrum No: 266; Query: 2289; Rank: 1

\section{Peptide View}

MS/MS Fragmentation of ADECALPYLGATCYCDLFCNR

Found in IPI00190428, Tax_Id=10116 Gene_Symbol=Lcn7 Tubulointerstitial nephritis antigen-like precursor

Match to Query 2289: 2569.049608 from(1285.532080,2+)

Title: 100101RatKid_NS_deglyco_23.5020.5020.2.dta

Data file K:INewmanPaper|Piliangl3SubProteomes\Piliang3SP\mgf5ppm\ERLIC_3SubProteomes5ppm.mgf

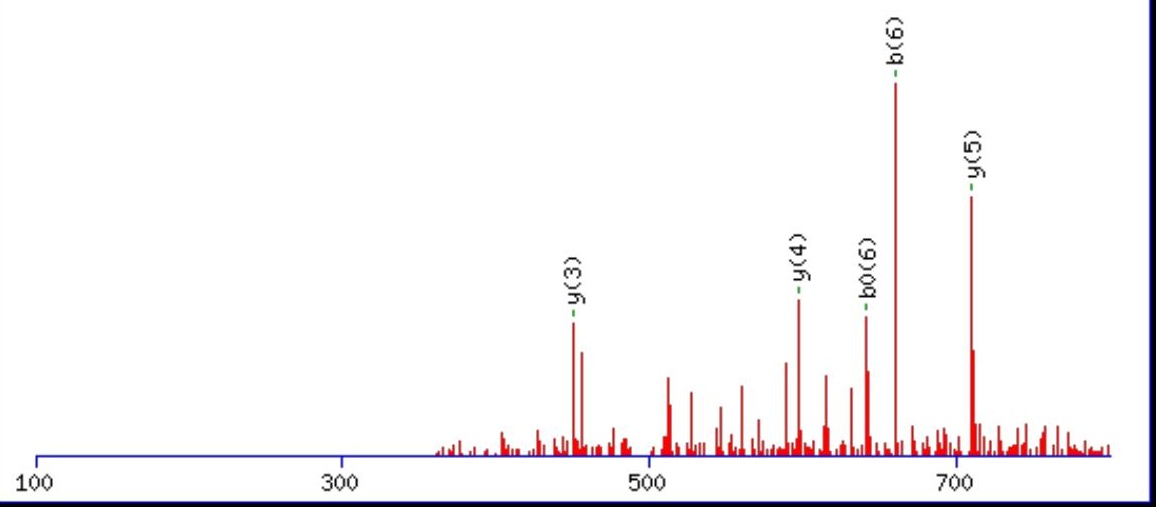



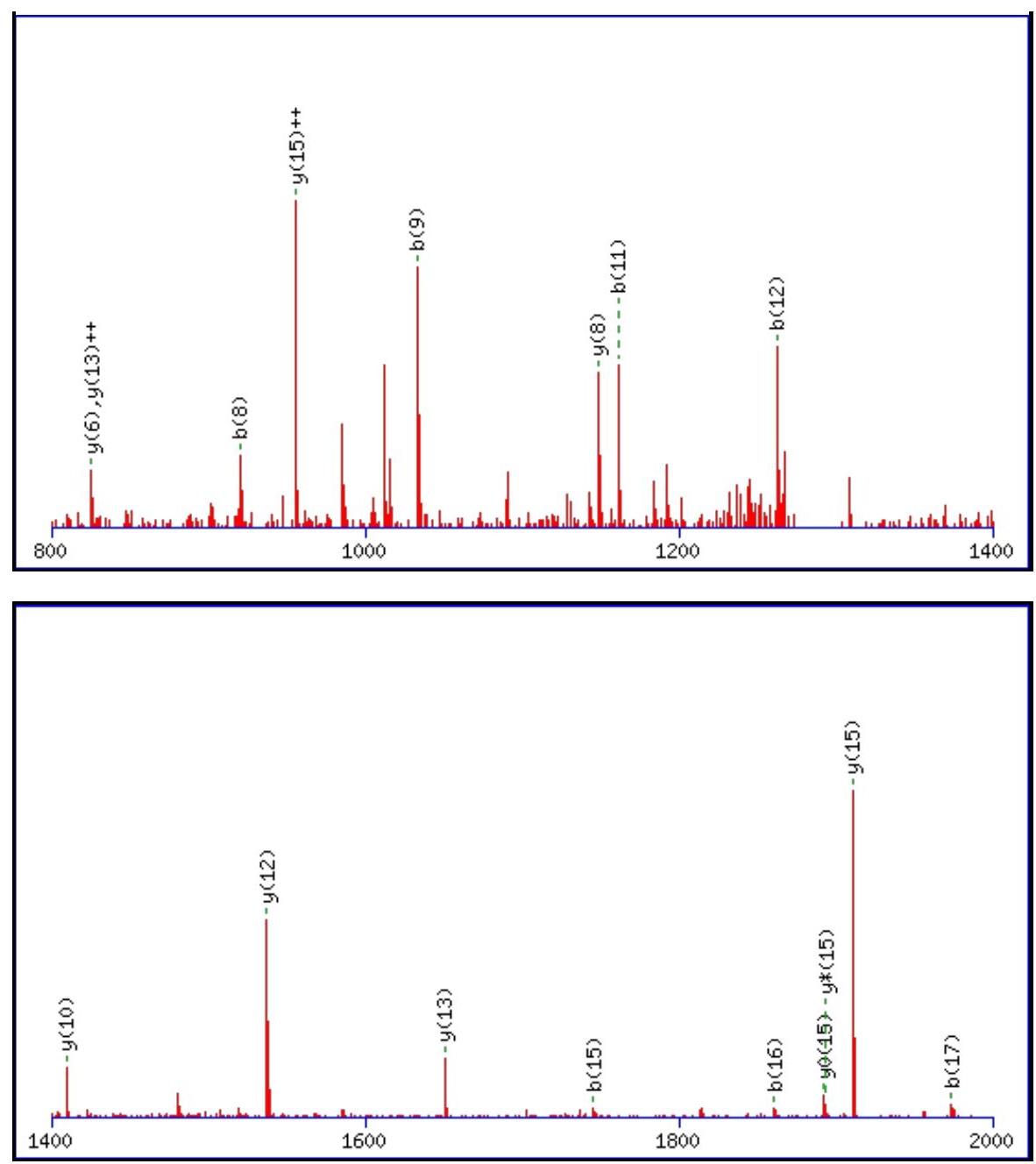

Monoisotopic mass of neutral peptide $\operatorname{Mr}($ calc): 2569.0382

Fixed modifications: Carbamidomethyl (C)

Variable modifications:

N20 : Deamidated $\mathrm{N}(\mathrm{N})$

Ions Score: 77 Expect: $4.9 \mathrm{e}-006$

Matches (Bold Red): 22/190 fragment ions using 30 most intense peaks

\begin{tabular}{|c|c|c|c|c|c|c|c|c|c|c|c|c|c|c|}
\hline \# & b & $\mathbf{b}^{++}$ & b* & $\mathbf{b}^{*^{++}}$ & $\mathbf{b}^{0}$ & $\mathbf{b}^{0++}$ & Seq. & $\mathbf{y}$ & $\mathrm{y}^{++}$ & $\mathbf{y}^{*}$ & $\mathbf{y}^{*^{++}}$ & $\mathbf{y}^{0}$ & $y^{0++}$ & \# \\
\hline 1 & 72.0444 & 36.5258 & & & & & A & & & & & & & 21 \\
\hline 2 & 187.0713 & 94.0393 & & & 169.0608 & 85.0340 & D & 2499.0084 & 1250.0078 & 2481.9818 & 1241.4945 & 2480.9978 & 1241.0025 & 20 \\
\hline 3 & 316.1139 & 158.5606 & & & 298.1034 & 149.5553 & $\mathbf{E}$ & 2383.9814 & 1192.4943 & 2366.9549 & 1183.9811 & 2365.9709 & 1183.4891 & 19 \\
\hline 4 & 476.1446 & 238.5759 & & & 458.1340 & 229.5706 & $\mathrm{C}$ & 2254.9388 & 1127.9731 & 2237.9123 & 1119.4598 & 2236.9283 & 1118.9678 & 18 \\
\hline 5 & 547.1817 & 274.0945 & & & 529.1711 & 265.0892 & $\mathbf{A}$ & 2094.9082 & 1047.9577 & 2077.8816 & 1039.4445 & 2076.8976 & 1038.9524 & 17 \\
\hline 6 & 660.2658 & 330.6365 & & & 642.2552 & 321.6312 & $\mathbf{L}$ & 2023.8711 & 1012.4392 & 2006.8445 & 1003.9259 & 2005.8605 & 1003.4339 & 16 \\
\hline 7 & 757.3185 & 379.1629 & & & 739.3080 & 370.1576 & $\mathbf{P}$ & 1910.7870 & 955.8971 & 1893.7604 & 947.3839 & 1892.7764 & 946.8919 & 15 \\
\hline 8 & 920.3818 & 460.6946 & & & 902.3713 & 451.6893 & $\mathbf{Y}$ & 1813.7342 & 907.3708 & 1796.7077 & 898.8575 & 1795.7237 & 898.3655 & 14 \\
\hline 9 & 1033.4659 & 517.2366 & & & 1015.4553 & 508.2313 & $\mathbf{L}$ & 1650.6709 & 825.8391 & 1633.6444 & 817.3258 & 1632.6603 & 816.8338 & 13 \\
\hline 10 & 1090.4874 & 545.7473 & & & 1072.4768 & 536.7420 & G & 1537.5868 & 769.2971 & 1520.5603 & 760.7838 & 1519.5763 & 760.2918 & 12 \\
\hline 11 & 1161.5245 & 581.2659 & & & 1143.5139 & 572.2606 & A & 1480.5654 & 740.7863 & 1463.5388 & 732.2731 & 1462.5548 & 731.7810 & 11 \\
\hline 12 & 1262.5722 & 631.7897 & & & 1244.5616 & 622.7844 & $\mathbf{T}$ & 1409.5283 & 705.2678 & 1392.5017 & 696.7545 & 1391.5177 & 696.2625 & 10 \\
\hline 13 & 1422.6028 & 711.8050 & & & 1404.5923 & 702.7998 & C & 1308.4806 & 654.7439 & 1291.4540 & 646.2307 & 1290.4700 & 645.7386 & 9 \\
\hline 14 & 1585.6661 & 793.3367 & & & 1567.6556 & 784.3314 & $\mathbf{Y}$ & 1148.4499 & 574.7286 & 1131.4234 & 566.2153 & 1130.4394 & 565.7233 & 8 \\
\hline 15 & 1745.6968 & 873.3520 & & & 1727.6862 & 864.3468 & C & 985.3866 & 493.1969 & 968.3601 & 484.6837 & 967.3760 & 484.1917 & 7 \\
\hline 16 & 1860.7237 & 930.8655 & & & 1842.7132 & 921.8602 & D & 825.3560 & 413.1816 & 808.3294 & 404.6683 & 807.3454 & 404.1763 & 6 \\
\hline 17 & 1973.8078 & 987.4075 & & & 1955.7972 & 978.4023 & $\mathbf{L}$ & 710.3290 & 355.6681 & 693.3025 & 347.1549 & & & 5 \\
\hline 18 & 2120.8762 & 1060.9417 & & & 2102.8656 & 1051.9365 & $\mathbf{F}$ & 597.2450 & 299.1261 & 580.2184 & 290.6128 & & & 4 \\
\hline
\end{tabular}




\begin{tabular}{|l|r|r|r|r|r|r|r|r|r|r|r|r|r|r|r|}
$\mathbf{1 9}$ & 2280.9069 & 1140.9571 & & & 2262.8963 & 1131.9518 & $\mathbf{C}$ & $\mathbf{4 5 0 . 1 7 6 5}$ & 225.5919 & 433.1500 & 217.0786 & & & $\mathbf{3}$ \\
\hline $\mathbf{2 0}$ & 2395.9338 & 1198.4705 & 2378.9073 & 1189.9573 & 2377.9232 & 1189.4653 & $\mathbf{N}$ & 290.1459 & 145.5766 & 273.1193 & 137.0633 & & & $\mathbf{2}$ \\
\hline $\mathbf{2 1}$ & & & & & & & $\mathbf{R}$ & 175.1190 & 88.0631 & 158.0924 & 79.5498 & & & $\mathbf{1}$ \\
\hline
\end{tabular}
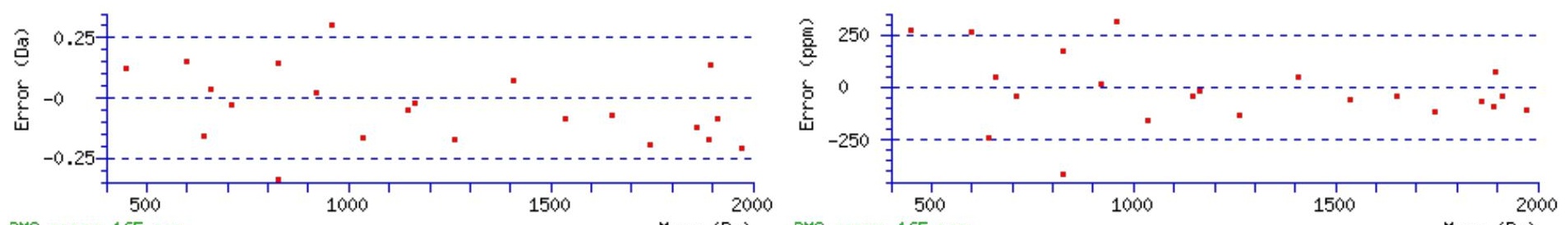

RMS error 165 ppm

Mass (Da) RMS error 165 ppm

Mass (Da)

\section{All matches to this query}

\begin{tabular}{|l|l|c|l|}
\hline Score & Mr(calc): & Delta & \multicolumn{1}{|c|}{ Sequence } \\
\hline 77.3 & 2569.0382 & 0.0114 & ADECALPYLGATCYCDLFCNR \\
\hline 23.1 & 2568.0542 & 0.9954 & ADECALPYLGATCYCDLFCNR \\
\hline 5.0 & 2569.0286 & 0.0210 & VTILIYGLHHNPSYWPNPK \\
\hline 3.5 & 2569.0726 & -0.0230 & ATNDSVVADKSCEKIHFIAPK \\
\hline 3.4 & 2569.0379 & 0.0117 & LEEACGMGEAPTKSSRTWQQK \\
\hline 3.4 & 2569.0379 & 0.0117 & LEEACGMGEAPTKSSRTWQQK \\
\hline 3.4 & 2569.0379 & 0.0117 & LEEACGMGEAPTKSSRTWQQK \\
\hline 2.5 & 2569.0358 & 0.0138 & EDGEDQDDDQMSRGTKSPTEGEK \\
\hline 1.7 & 2569.0493 & 0.0003 & ESLQNGNLNDPSSVESSNGQWPK \\
\hline 1.6 & 2569.0286 & 0.0210 & VTILIYGLHHNPSYWPNPK \\
\hline
\end{tabular}

Spectrum No: 267; Query: 710; Rank: 1

\section{Peptide View}

MS/MS Fragmentation of LWNSTFLEEYSK

Found in IPI00205166, Tax_Id=10116 Gene_Symbol=Itga6 similar to integrin alpha 6 isoform 2

Match to Query 710: 1516.714388 from(759.364470,2+)

Title: 100101RatKid_NS_deglyco_14.4548.4548.2.dta

Data file K:\NewmanPaper|Piliangl3SubProteomes\Piliang3SP\mgf5ppm\ERLIC_3SubProteomes5ppm.mgf

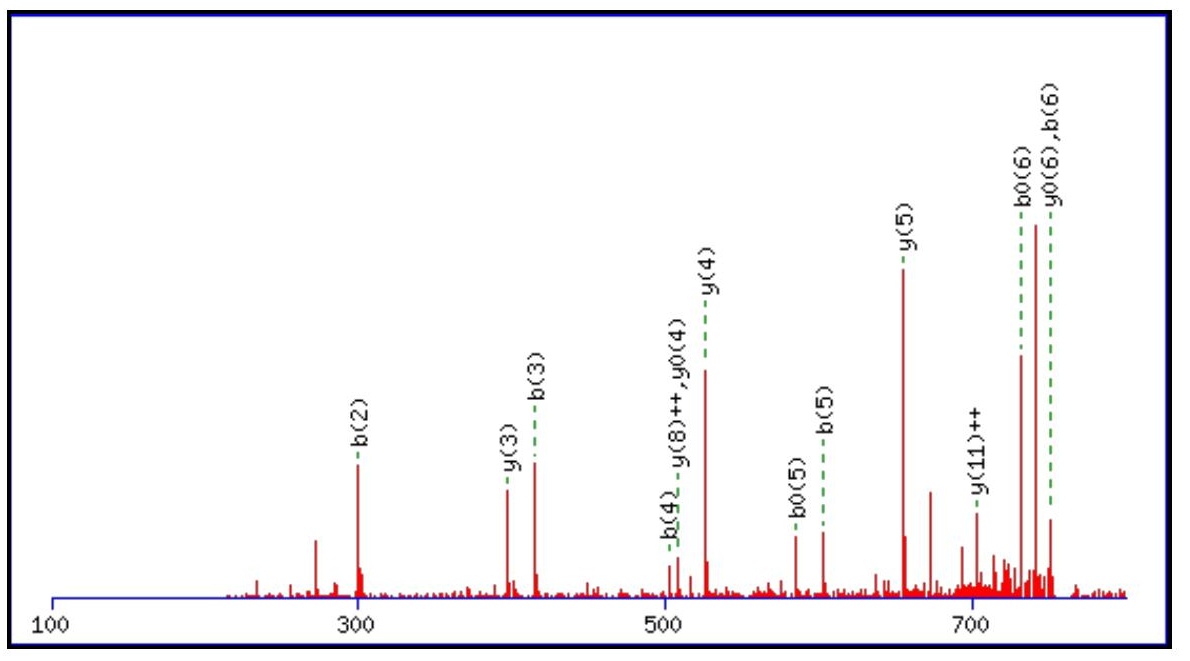



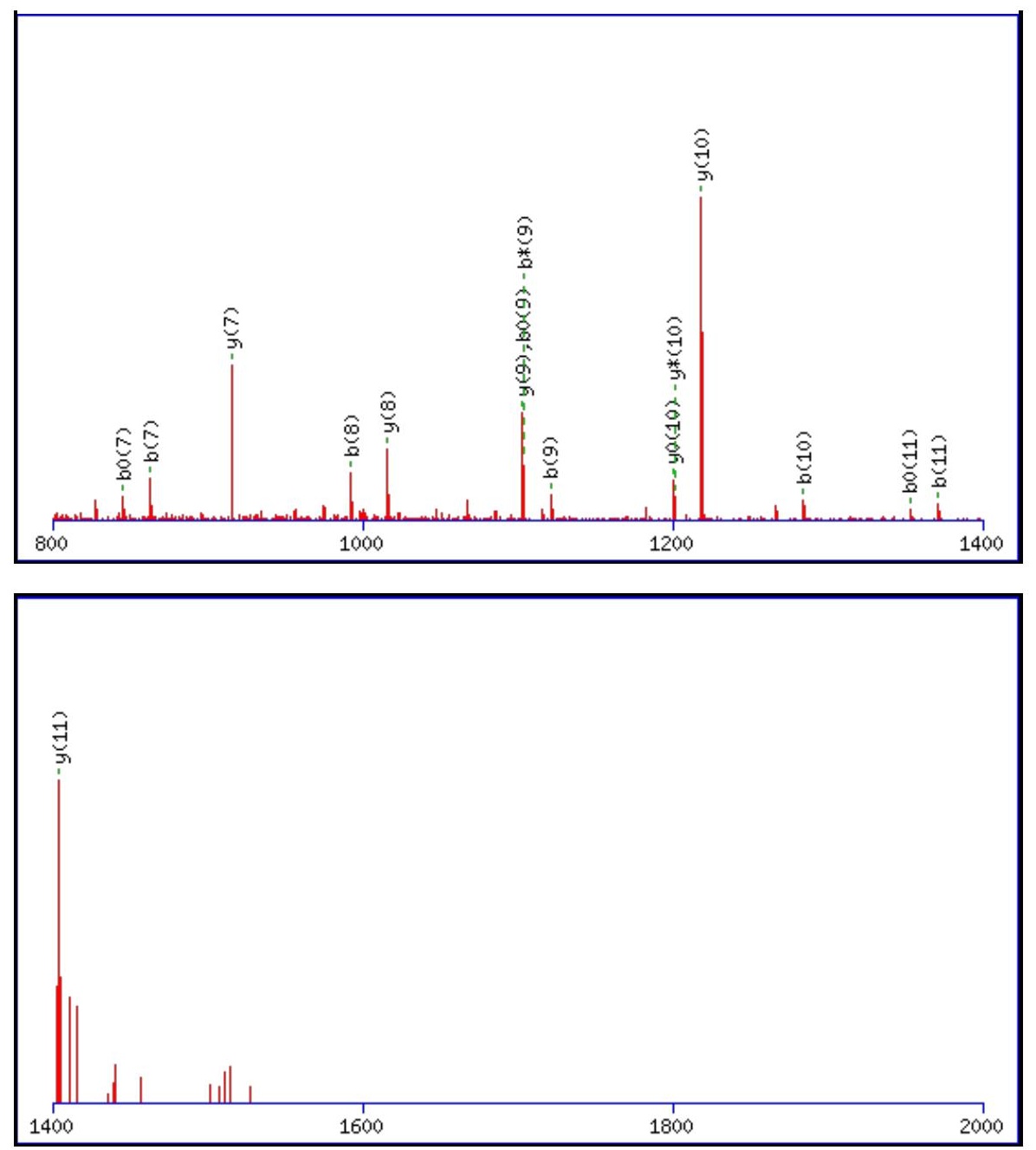

Monoisotopic mass of neutral peptide $\operatorname{Mr}($ calc): 1516.7085

Fixed modifications: Carbamidomethyl (C)

Variable modifications:

N3 : Deamidated $\mathrm{N}(\mathrm{N})$

Ions Score: 77 Expect: $3 \mathrm{e}-006$

Matches (Bold Red): 30/120 fragment ions using 44 most intense peaks

\begin{tabular}{|c|c|c|c|c|c|c|c|c|c|c|c|c|c|c|}
\hline \# & b & $\mathbf{b}^{++}$ & $\mathbf{b}^{*}$ & $\mathbf{b}^{*^{++}}$ & $\mathbf{b}^{\mathbf{0}}$ & $\mathbf{b}^{\mathbf{0 + +}}$ & Seq. & $\mathbf{y}$ & $\mathbf{y}^{++}$ & $\mathbf{y}^{*}$ & $\mathrm{y}^{*^{++}}$ & $\mathbf{y}^{\mathbf{0}}$ & $\mathbf{y}^{0++}$ & \# \\
\hline 1 & 114.0913 & 57.5493 & & & & & $\mathbf{L}$ & & & & & & & 12 \\
\hline 2 & 300.1707 & 150.5890 & & & & & $\mathbf{W}$ & 1404.6318 & 702.8195 & 1387.6052 & 694.3063 & 1386.6212 & 693.8142 & 11 \\
\hline 3 & 415.1976 & 208.1024 & 398.1710 & 199.5892 & & & $\mathbf{N}$ & 1218.5525 & 609.7799 & 1201.5259 & 601.2666 & 1200.5419 & 600.7746 & 10 \\
\hline 4 & 502.2296 & 251.6184 & 485.2031 & 243.1052 & 484.2191 & 242.6132 & $\mathbf{S}$ & 1103.5255 & 552.2664 & 1086.4990 & 543.7531 & 1085.5150 & 543.2611 & 9 \\
\hline 5 & 603.2773 & 302.1423 & 586.2507 & 293.6290 & 585.2667 & 293.1370 & $\mathbf{T}$ & 1016.4935 & 508.7504 & 999.4670 & 500.2371 & 998.4829 & 499.7451 & 8 \\
\hline 6 & 750.3457 & 375.6765 & 733.3192 & 367.1632 & 732.3351 & 366.6712 & $\mathbf{F}$ & 915.4458 & 458.2266 & 898.4193 & 449.7133 & 897.4353 & 449.2213 & 7 \\
\hline 7 & 863.4298 & 432.2185 & 846.4032 & 423.7053 & 845.4192 & 423.2132 & $\mathbf{L}$ & 768.3774 & 384.6923 & 751.3509 & 376.1791 & 750.3668 & 375.6871 & 6 \\
\hline 8 & 992.4724 & 496.7398 & 975.4458 & 488.2265 & 974.4618 & 487.7345 & $\mathbf{E}$ & 655.2933 & 328.1503 & 638.2668 & 319.6370 & 637.2828 & 319.1450 & 5 \\
\hline 9 & 1121.5150 & 561.2611 & 1104.4884 & 552.7478 & 1103.5044 & 552.2558 & $\mathbf{E}$ & 526.2508 & 263.6290 & 509.2242 & 255.1157 & 508.2402 & 254.6237 & 4 \\
\hline 10 & 1284.5783 & 642.7928 & 1267.5517 & 634.2795 & 1266.5677 & 633.7875 & $\mathbf{Y}$ & 397.2082 & 199.1077 & 380.1816 & 190.5944 & 379.1976 & 190.1024 & 3 \\
\hline 11 & 1371.6103 & 686.3088 & 1354.5838 & 677.7955 & 1353.5998 & 677.3035 & S & 234.1448 & 117.5761 & 217.1183 & 109.0628 & 216.1343 & 108.5708 & 2 \\
\hline 12 & & & & & & & $\mathbf{K}$ & 147.1128 & 74.0600 & 130.0863 & 65.5468 & & & 1 \\
\hline
\end{tabular}



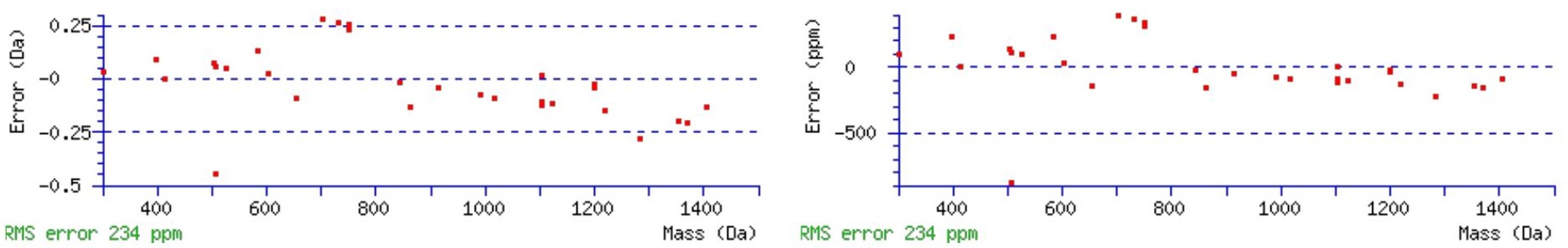

\section{All matches to this query}

\begin{tabular}{|l|l|l|l|}
\hline Score & Mr(calc): & Delta & \multicolumn{1}{c|}{ Sequence } \\
\hline 77.3 & 1516.7085 & 0.0058 & LWNSTFLEEYSK \\
\hline 21.9 & 1515.7245 & 0.9899 & LWNSTFLEEYSK \\
\hline 5.1 & 1516.7035 & 0.0109 & IGEERNSALSPHK \\
\hline 3.2 & 1516.7158 & -0.0014 & TANKVDPEHTDYK \\
\hline 3.0 & 1516.7232 & -0.0088 & VAEVIYCSFGGTSK \\
\hline 2.2 & 1514.6977 & 2.0167 & AELSDSETTARKK \\
\hline 1.2 & 1516.7109 & 0.0035 & SLVQEMVGSFGKR \\
\hline 1.0 & 1514.6977 & 2.0167 & $\underline{\text { AELSDSETTARKK }}$ \\
\hline
\end{tabular}

Spectrum No: 268; Query: 1401; Rank: 1

\section{Peptide View}

MS/MS Fragmentation of NNLTDPSLSEMVEVALR

Found in IPI00327143, Tax_Id=10116 Gene_Symbol=Alpl Alkaline phosphatase, tissue-nonspecific isozyme precursor

Match to Query 1401: 1887.933428 from(944.973990,2+)

Title: 100101RatKid_NS_deglyco_23.5490.5490.2.dta

Data file K:INewmanPaper|Piliang|3SubProteomes\Piliang3SP\mgf5ppm\ERLIC_3SubProteomes5ppm.mgf

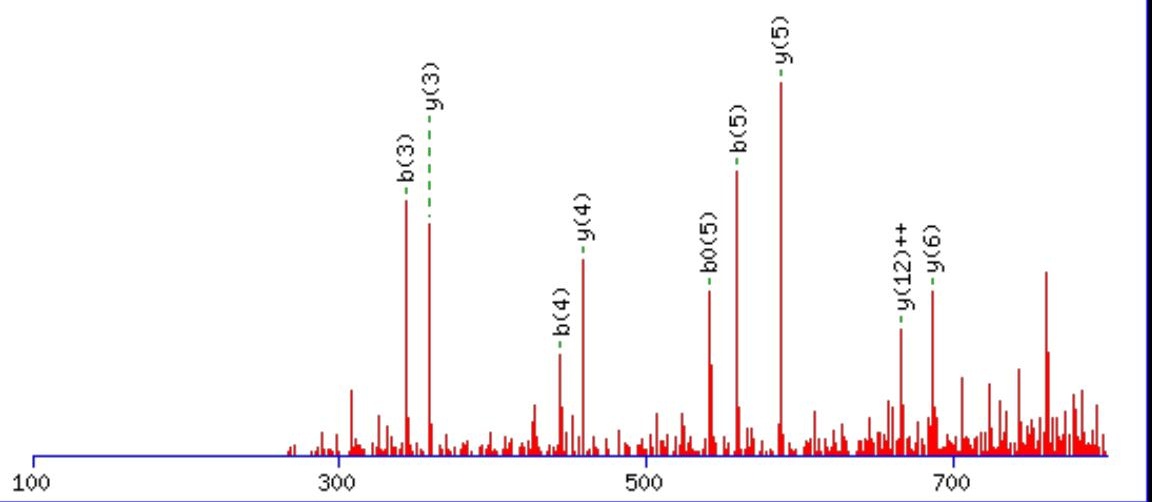



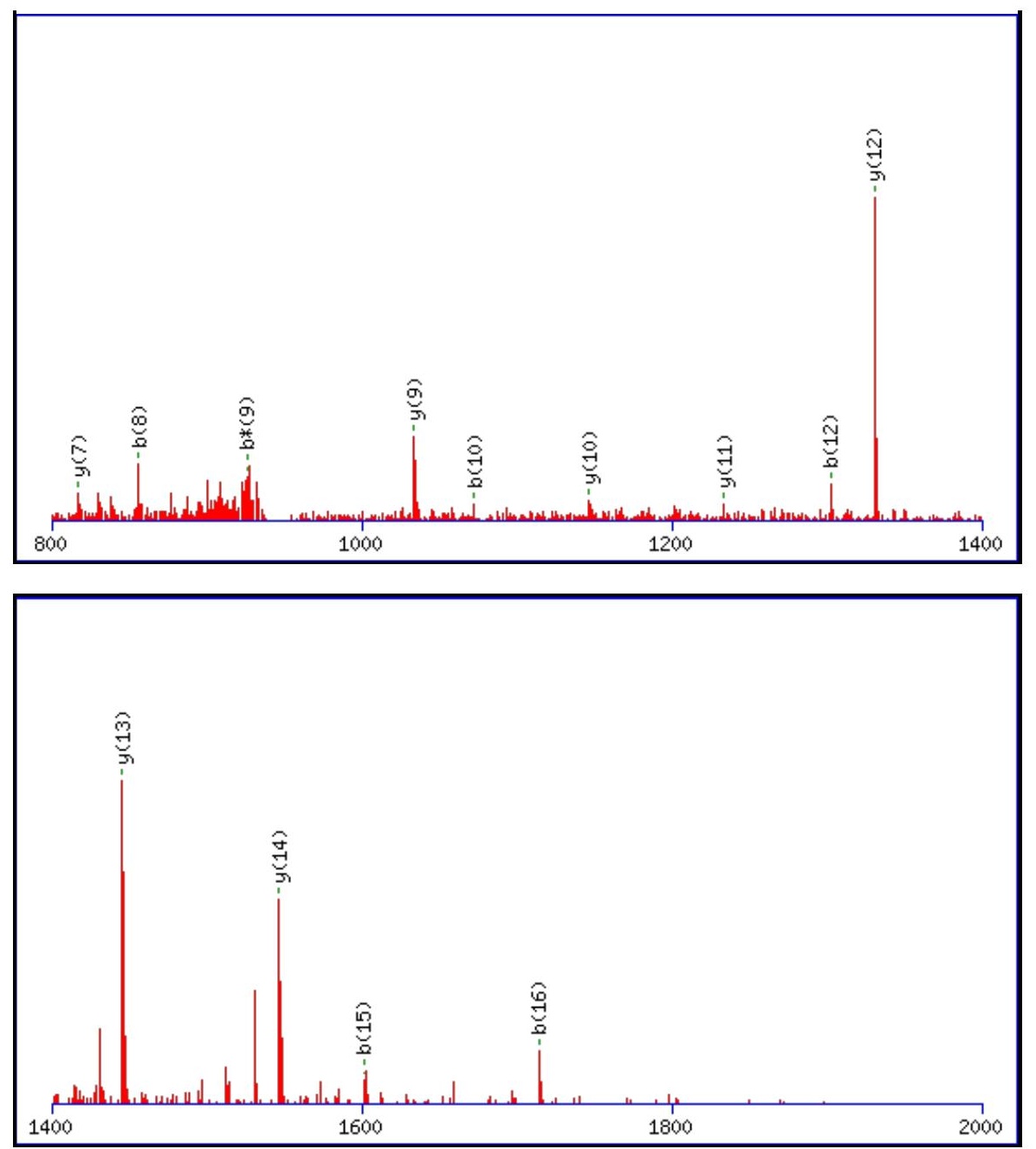

Monoisotopic mass of neutral peptide $\operatorname{Mr}($ calc): 1887.9248

Fixed modifications: Carbamidomethyl (C)

Variable modifications:

N2 : Deamidated_N (N)

Ions Score: 77 Expect: $4 \mathrm{e}-006$

Matches (Bold Red): 22/178 fragment ions using 40 most intense peaks

\begin{tabular}{|c|c|c|c|c|c|c|c|c|c|c|c|c|c|c|}
\hline \# & b & $\mathbf{b}^{++}$ & $\mathbf{b}^{*}$ & $\mathbf{b}^{*^{++}}$ & $\mathbf{b}^{\mathbf{0}}$ & $\mathbf{b}^{\mathbf{0 + +}}$ & Seq. & $\mathbf{y}$ & $\mathbf{y}^{++}$ & $\mathbf{y}^{*}$ & $\mathrm{y}^{*^{++}}$ & $\mathbf{y}^{0}$ & $\mathbf{y}^{\mathbf{0}^{++}}$ & \# \\
\hline 1 & 115.0502 & 58.0287 & 98.0237 & 49.5155 & & & $\mathbf{N}$ & & & & & & & 17 \\
\hline 2 & 230.0771 & 115.5422 & 213.0506 & 107.0289 & & & $\mathbf{N}$ & |1774.8891 & 887.9482 & $\mid 1757.8626$ & 879.4349 & 1756.8786 & 878.9429 & 16 \\
\hline 3 & 343.1612 & 172.0842 & 326.1347 & 163.5710 & & & $\mathbf{L}$ & 1659.8622 & 830.4347 & 1642.8357 & 821.9215 & 1641.8516 & 821.4295 & 15 \\
\hline 4 & 444.2089 & 222.6081 & 427.1823 & 214.0948 & 426.1983 & 213.6028 & $\mathbf{T}$ & 1546.7781 & 773.8927 & |1529.7516 & 765.3794 & 1528.7676 & 764.8874 & 14 \\
\hline 5 & 559.2358 & 280.1216 & 542.2093 & 271.6083 & 541.2253 & 271.1163 & D & 1445.7305 & 723.3689 & 1428.7039 & 714.8556 & 1427.7199 & 714.3636 & 13 \\
\hline 6 & 656.2886 & 328.6479 & 639.2620 & 320.1347 & 638.2780 & 319.6427 & $\mathbf{P}$ & 1330.7035 & 665.8554 & 1313.6770 & 657.3421 & 1312.6929 & 656.8501 & 12 \\
\hline 7 & 743.3206 & 372.1639 & 726.2941 & 363.6507 & 725.3101 & 363.1587 & $S$ & 1233.6507 & 617.3290 & 1216.6242 & 608.8157 & 1215.6402 & 608.3237 & 11 \\
\hline 8 & 856.4047 & 428.7060 & 839.3781 & 420.1927 & 838.3941 & 419.7007 & $\mathbf{L}$ & \begin{tabular}{|l|}
1146.6187 \\
\end{tabular} & 573.8130 & 1129.5922 & 565.2997 & 1128.6082 & 564.8077 & 10 \\
\hline 9 & 943.4367 & 472.2220 & 926.4102 & 463.7087 & 925.4261 & 463.2167 & $S$ & \begin{tabular}{|l}
1033.5347 \\
\end{tabular} & 517.2710 & 1016.5081 & 508.7577 & 1015.5241 & 508.2657 & 9 \\
\hline 10 & 1072.4793 & 536.7433 & 1055.4528 & 528.2300 & 1054.4687 & 527.7380 & $\mathbf{E}$ & 946.5026 & 473.7550 & 929.4761 & 465.2417 & 928.4921 & 464.7497 & 8 \\
\hline 11 & 1203.5198 & 602.2635 & 1186.4932 & 593.7503 & 1185.5092 & 593.2582 & $\mathbf{M}$ & 817.4600 & 409.2337 & 800.4335 & 400.7204 & 799.4495 & 400.2284 & 7 \\
\hline 12 & 1302.5882 & 651.7977 & 1285.5617 & 643.2845 & 1284.5776 & 642.7925 & $\mathrm{~V}$ & 686.4196 & 343.7134 & 669.3930 & 335.2001 & 668.4090 & 334.7081 & 6 \\
\hline 13 & 1431.6308 & 716.3190 & 1414.6042 & 707.8058 & 1413.6202 & 707.3138 & $\mathbf{E}$ & 587.3511 & 294.1792 & 570.3246 & 285.6659 & 569.3406 & 285.1739 & 5 \\
\hline 14 & 1530.6992 & 765.8532 & 1513.6727 & 757.3400 & 1512.6886 & 756.8480 & $\mathbf{V}$ & 458.3085 & 229.6579 & 441.2820 & 221.1446 & & & 4 \\
\hline 15 & 1601.7363 & 801.3718 & 1584.7098 & 792.8585 & 1583.7258 & 792.3665 & A & 359.2401 & 180.1237 & 342.2136 & 171.6104 & & & 3 \\
\hline 16 & 1714.8204 & 857.9138 & 1697.7938 & 849.4006 & 1696.8098 & 848.9085 & $\mathbf{L}$ & 288.2030 & 144.6051 & 271.1765 & 136.0919 & & & 2 \\
\hline 17 & & & & & & & $\mathbf{R}$ & 175.1190 & 88.0631 & 158.0924 & 79.5498 & & & 1 \\
\hline
\end{tabular}



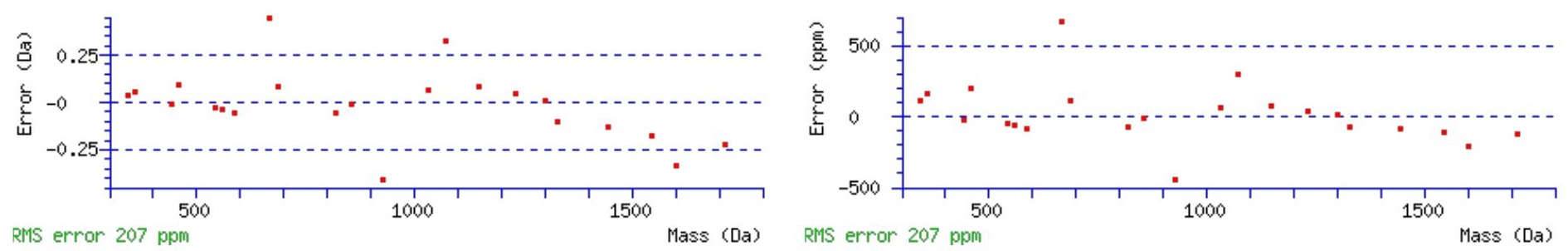

\section{All matches to this query}

\begin{tabular}{|l|c|c|l|}
\hline Score & Mr(calc): & Delta & \multicolumn{1}{c|}{ Sequence } \\
\hline 77.2 & 1887.9248 & 0.0087 & NNLTDPSLSEMVEVALR \\
\hline 77.2 & 1887.9248 & 0.0087 & NNLTDPSLSEMVEVALR \\
\hline 52.7 & 1886.9408 & 0.9927 & NNLTDPSLSEMVEVALR \\
\hline 3.4 & 1887.9286 & 0.0049 & LSRSNEKEQLQGLNDR \\
\hline 3.3 & 1887.9149 & 0.0185 & EWDKPSCTVPEVSAKR \\
\hline 1.0 & 1887.9343 & -0.0008 & LTKNIATDSYISVNLR \\
\hline 0.6 & 1887.9455 & -0.0121 & DQTLSPTIISGLHSIAR \\
\hline 0.6 & 1887.9455 & -0.0121 & DQTLSPTIISGLHSIAR \\
\hline 0.3 & 1887.9343 & -0.0008 & SKEVNLHEKANTPVLK \\
\hline
\end{tabular}

Spectrum No: 269; Query: 1374; Rank: 1

\section{Peptide View}

MS/MS Fragmentation of GQPIPNKNLTVQVNSVR

Found in IPI00326140, Tax_Id=10116 Gene_Symbol=Pzp Alpha-1-macroglobulin precursor

Match to Query 1374: 1864.019788 from(933.017170,2+)

Title: 100101RatKid_NS_deglyco_23.1952.1952.2.dta

Data file K:INewmanPaper|Piliangl3SubProteomes\Piliang3SP\mgf5ppm\ERLIC_3SubProteomes5ppm.mgf

\begin{tabular}{|c|c|c|c|c|c|c|}
\hline & (⿳⺈⿴囗十心 & 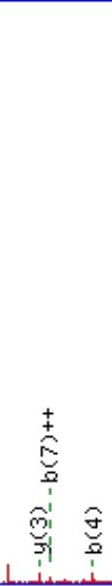 & 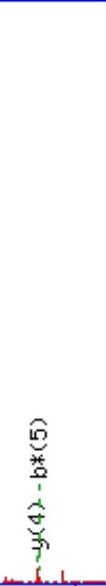 & 仓ิ & 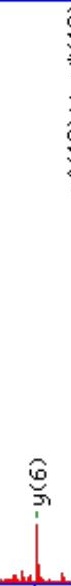 & 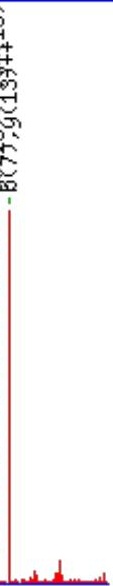 \\
\hline 100 & 30 & & 500 & & 700 & \\
\hline
\end{tabular}



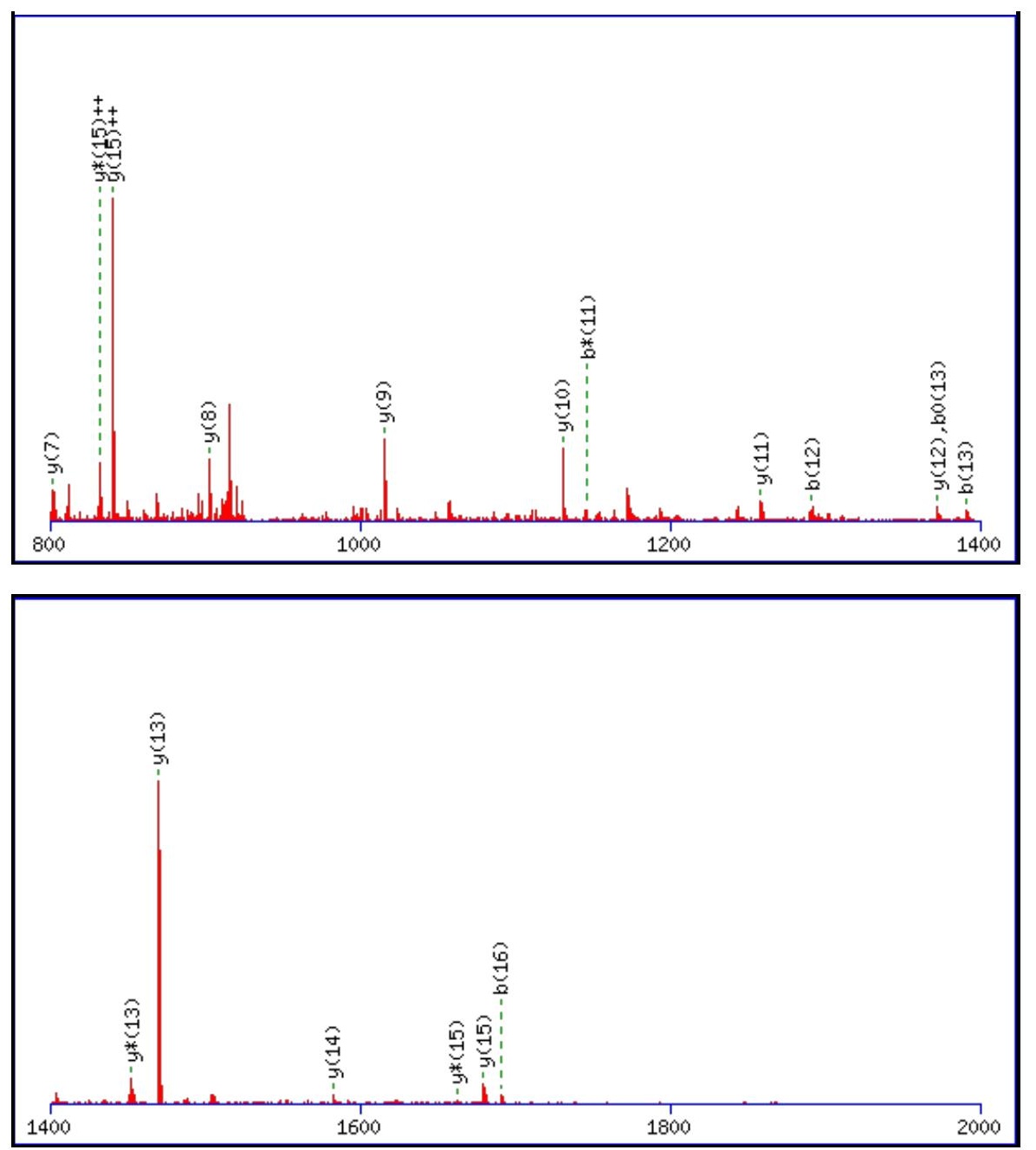

Monoisotopic mass of neutral peptide $\operatorname{Mr}($ calc): 1864.0166

Fixed modifications: Carbamidomethyl (C)

Variable modifications:

N8: Deamidated $\mathrm{N}(\mathrm{N})$

Ions Score: 77 Expect: $1.2 \mathrm{e}-006$

Matches (Bold Red): 33/168 fragment ions using 86 most intense peaks

\begin{tabular}{|c|c|c|c|c|c|c|c|c|c|c|c|c|c|c|}
\hline \# & b & $\mathbf{b}^{++}$ & $\mathbf{b}^{*}$ & $\mathbf{b}^{*^{++}}$ & $\mathbf{b}^{\mathbf{0}}$ & $\mathbf{b}^{\mathbf{0 + +}}$ & Seq. & $\mathbf{y}$ & $\mathbf{y}^{++}$ & $\mathbf{y}^{*}$ & $\mathrm{y}^{*^{++}}$ & $\mathbf{y}^{0}$ & $\mathbf{y}^{\mathbf{0}^{++}}$ & \# \\
\hline 1 & 58.0287 & 29.5180 & & & & & G & & & & & & & 17 \\
\hline 2 & 186.0873 & 93.5473 & 169.0608 & 85.0340 & & & $\mathbf{Q}$ & 1808.0025 & 904.5049 & $\mid 1790.9759$ & 895.9916 & 1789.9919 & 895.4996 & 16 \\
\hline 3 & 283.1401 & 142.0737 & 266.1135 & 133.5604 & & & $\mathbf{P}$ & \begin{tabular}{|l|}
1679.9439 \\
\end{tabular} & 840.4756 & 1662.9173 & 831.9623 & 1661.9333 & 831.4703 & 15 \\
\hline 4 & 396.2241 & 198.6157 & 379.1976 & 190.1024 & & & I & 1582.8911 & 791.9492 & 1565.8646 & 783.4359 & 1564.8806 & 782.9439 & 14 \\
\hline 5 & 493.2769 & 247.1421 & 476.2504 & 238.6288 & & & $\mathbf{P}$ & \begin{tabular}{|l|}
1469.8071 \\
\end{tabular} & 735.4072 & 1452.7805 & \begin{tabular}{|l|}
726.8939 \\
\end{tabular} & 1451.7965 & 726.4019 & 13 \\
\hline 6 & 607.3198 & 304.1636 & 590.2933 & 295.6503 & & & $\mathbf{N}$ & 1372.7543 & 686.8808 & 1355.7277 & 678.3675 & 1354.7437 & 677.8755 & 12 \\
\hline 7 & 735.4148 & 368.2110 & 718.3883 & 359.6978 & & & $\mathbf{K}$ & 1258.7114 & 629.8593 & 1241.6848 & 621.3460 & 1240.7008 & 620.8540 & 11 \\
\hline 8 & 850.4417 & 425.7245 & 833.4152 & 417.2112 & & & $\mathbf{N}$ & 1130.6164 & 565.8118 & 1113.5899 & 557.2986 & 1112.6058 & 556.8066 & 10 \\
\hline 9 & \begin{tabular}{|l|}
963.5258 \\
\end{tabular} & 482.2665 & 946.4993 & 473.7533 & & & $\mathbf{L}$ & 1015.5895 & 508.2984 & 998.5629 & 499.7851 & 997.5789 & 499.2931 & 9 \\
\hline 10 & 1064.5735 & 532.7904 & 1047.5469 & 524.2771 & 1046.5629 & 523.7851 & $\mathbf{T}$ & 902.5054 & 451.7563 & 885.4789 & 443.2431 & 884.4948 & 442.7511 & 8 \\
\hline 11 & 1163.6419 & 582.3246 & 1146.6153 & 573.8113 & 1145.6313 & 573.3193 & V & 801.4577 & 401.2325 & 784.4312 & 392.7192 & 783.4472 & 392.2272 & 7 \\
\hline 12 & 1291.7005 & 646.3539 & 1274.6739 & 637.8406 & 1273.6899 & 637.3486 & $\mathbf{Q}$ & 702.3893 & 351.6983 & 685.3628 & 343.1850 & 684.3787 & 342.6930 & 6 \\
\hline 13 & \begin{tabular}{|l|}
1390.7689 \\
\end{tabular} & 695.8881 & 1373.7423 & 687.3748 & \begin{tabular}{|l}
1372.7583 \\
\end{tabular} & 686.8828 & $\mathbf{V}$ & 574.3307 & 287.6690 & 557.3042 & 279.1557 & 556.3202 & 278.6637 & 5 \\
\hline 14 & 1504.8118 & 752.9095 & \begin{tabular}{|l}
1487.7853 \\
\end{tabular} & 744.3963 & 1486.8012 & 743.9043 & $\mathbf{N}$ & 475.2623 & 238.1348 & 458.2358 & 229.6215 & 457.2518 & 229.1295 & 4 \\
\hline 15 & 1591.8438 & 796.4256 & 1574.8173 & 787.9123 & 1573.8333 & 787.4203 & $\mathrm{~S}$ & 361.2194 & 181.1133 & 344.1928 & 172.6001 & 343.2088 & 172.1081 & 3 \\
\hline 16 & 1690.9123 & 845.9598 & 1673.8857 & 837.4465 & 1672.9017 & 836.9545 & V & 274.1874 & 137.5973 & 257.1608 & 129.0840 & & & 2 \\
\hline 17 & & & & & & & $\mathbf{R}$ & 175.1190 & 88.0631 & 158.0924 & 79.5498 & & & 1 \\
\hline
\end{tabular}



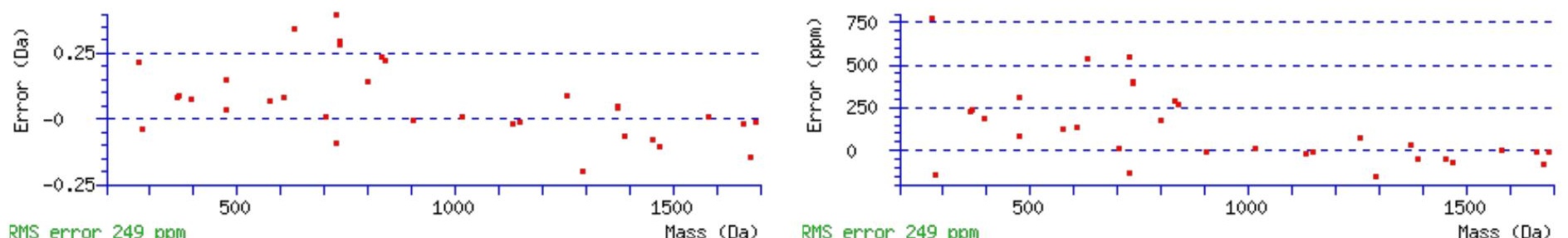

All matches to this query

\begin{tabular}{|l|l|c|l|}
\hline Score & Mr(calc): & Delta & \multicolumn{1}{c|}{ Sequence } \\
\hline 77.0 & 1864.0166 & 0.0031 & GQPIPNKNLTVQVNSVR \\
\hline 61.2 & 1864.0166 & 0.0031 & GQPIPNKNLTVQVNSVR \\
\hline 58.2 & 1864.0166 & 0.0031 & GQPIPNKNLTVQVNSVR \\
\hline 19.9 & 1863.0326 & 0.9871 & GQPIPNKNLTVQVNSVR \\
\hline 2.0 & 1864.0305 & -0.0107 & APLLPSQVNLEDKGALAK \\
\hline 1.5 & 1864.0213 & -0.0015 & AIASHPSAALMALRRGSR \\
\hline
\end{tabular}

Spectrum No: 270; Query: 2432; Rank: 1

\section{Peptide View}

MS/MS Fragmentation of HTGPGILSMANAGPNTNGSQFFICTAK

Found in IPI00364439, Tax_Id=10116 Gene_Symbol=RGD1559682_predicted similar to Peptidyl-prolyl cis-trans isomerase A

Match to Query 2432: 2791.304982 from(931.442270,3+)

Title: 091008RatKidney_NoSalt_12.4393.4393.3.dta

Data file K:INewmanPaper|Piliangl3SubProteomes\Piliang3SP\mgf5ppm\ERLIC_3SubProteomes5ppm.mgf

\begin{tabular}{|c|c|c|c|c|c|c|}
\hline & 俧 & 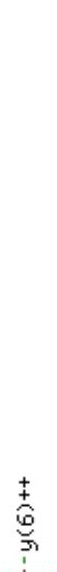 & 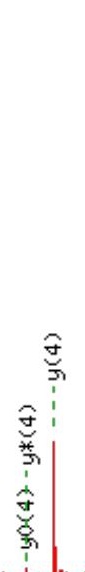 & 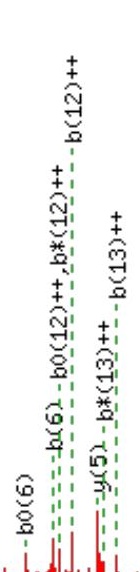 & (⿳亠丷厂亍亍 & 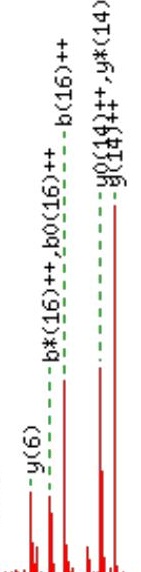 \\
\hline 100 & 300 & & 50 & & 700 & \\
\hline
\end{tabular}



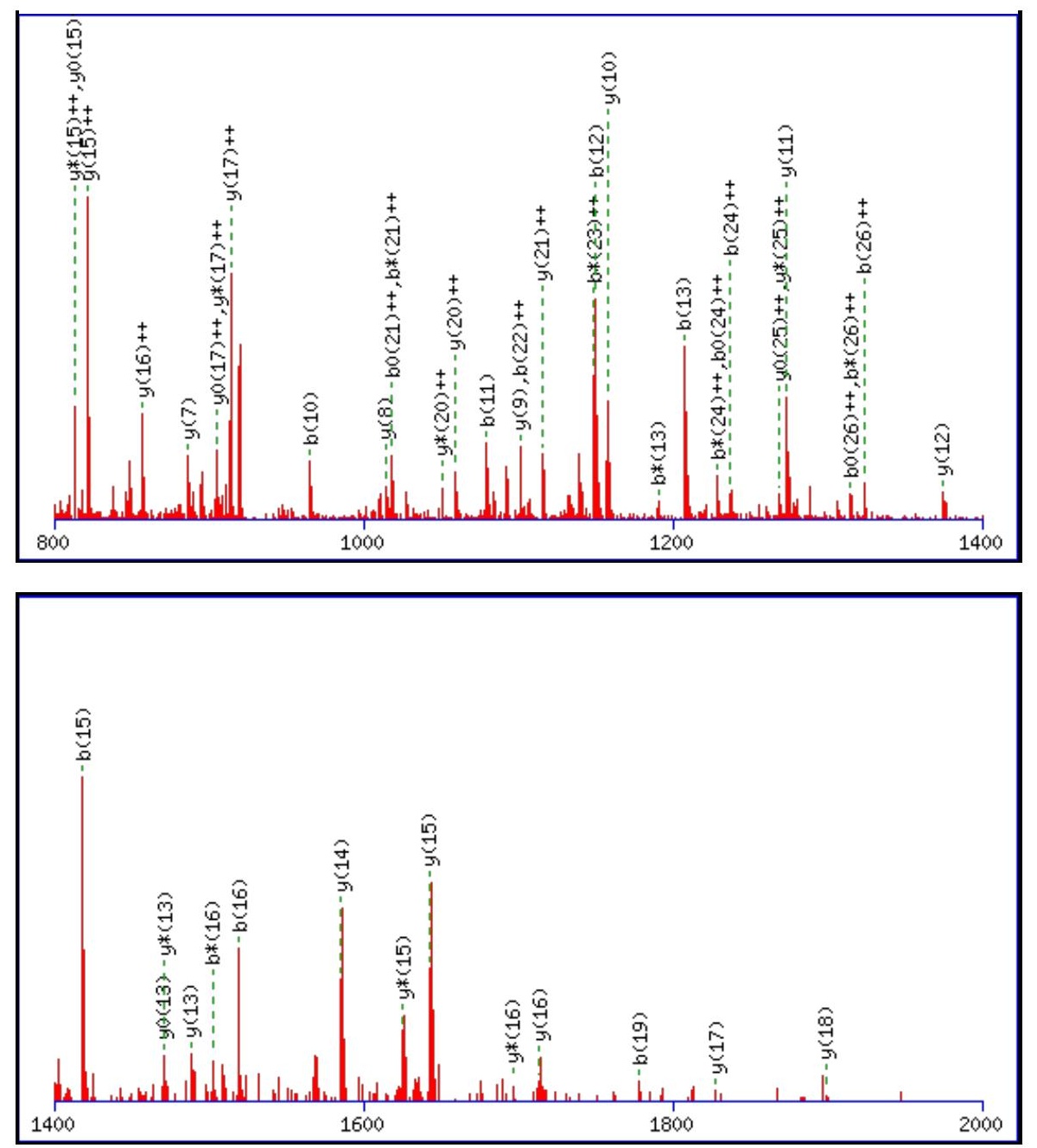

Monoisotopic mass of neutral peptide $\operatorname{Mr}($ calc): 2791.3058

Fixed modifications: Carbamidomethyl (C)

Variable modifications:

N17 : Deamidated_N (N)

Ions Score: 77 Expect: $7.1 \mathrm{e}-006$

Matches (Bold Red): 73/286 fragment ions using 126 most intense peaks

\begin{tabular}{|c|c|c|c|c|c|c|c|c|c|c|c|c|c|c|}
\hline \# & b & $\mathbf{b}^{++}$ & $\mathbf{b}^{*}$ & $\mathbf{b}^{*^{++}}$ & $\mathbf{b}^{\mathbf{0}}$ & $\mathbf{b}^{\mathbf{0 + +}}$ & Seq. & $\mathbf{y}$ & $\mathbf{y}^{++}$ & $\mathbf{y}^{*}$ & $\mathbf{y}^{*^{++}}$ & $\mathbf{y}^{0}$ & $\mathbf{y}^{0++}$ & \# \\
\hline 1 & 138.0662 & 69.5367 & & & & & $\mathbf{H}$ & & & & & & & 27 \\
\hline 2 & 239.1139 & 120.0606 & & & 221.1033 & 111.0553 & $\mathbf{T}$ & 2655.2541 & 1328.1307 & 2638.2276 & 1319.6174 & 2637.2436 & 1319.1254 & 26 \\
\hline 3 & 296.1353 & 148.5713 & & & 278.1248 & 139.5660 & G & 2554.2065 & 1277.6069 & 2537.1799 & 1269.0936 & 2536.1959 & 1268.6016 & 25 \\
\hline 4 & 393.1881 & 197.0977 & & & 375.1775 & 188.0924 & $\mathbf{P}$ & 2497.1850 & 1249.0961 & 2480.1585 & 1240.5829 & 2479.1744 & 1240.0909 & 24 \\
\hline 5 & 450.2096 & 225.6084 & & & 432.1990 & 216.6031 & G & 2400.1322 & 1200.5698 & 2383.1057 & 1192.0565 & 2382.1217 & 1191.5645 & 23 \\
\hline 6 & 563.2936 & 282.1504 & & & 545.2831 & 273.1452 & $\mathbf{I}$ & 2343.1108 & 1172.0590 & 2326.0842 & 1163.5458 & 2325.1002 & 1163.0537 & 22 \\
\hline 7 & 676.3777 & 338.6925 & & & 658.3671 & 329.6872 & $\mathbf{L}$ & 2230.0267 & 1115.5170 & 2213.0002 & 1107.0037 & 2212.0161 & 1106.5117 & 21 \\
\hline 8 & 763.4097 & 382.2085 & & & 745.3991 & 373.2032 & $\mathrm{~S}$ & 2116.9426 & 1058.9750 & 2099.9161 & 1050.4617 & 2098.9321 & 1049.9697 & 20 \\
\hline 9 & 894.4502 & 447.7287 & & & 876.4396 & 438.7235 & $\mathbf{M}$ & 2029.9106 & 1015.4589 & 2012.8841 & 1006.9457 & 2011.9001 & 1006.4537 & 19 \\
\hline 10 & 965.4873 & 483.2473 & & & 947.4767 & 474.2420 & A & 1898.8701 & 949.9387 & 1881.8436 & 941.4254 & 1880.8596 & 940.9334 & 18 \\
\hline 11 & 1079.5302 & 540.2688 & 1062.5037 & 531.7555 & 1061.5197 & 531.2635 & $\mathbf{N}$ & 1827.8330 & 914.4201 & 1810.8065 & 905.9069 & 1809.8225 & 905.4149 & 17 \\
\hline 12 & 1150.5674 & 575.7873 & 1133.5408 & 567.2740 & 1132.5568 & 566.7820 & A & 1713.7901 & 857.3987 & 1696.7635 & 848.8854 & 1695.7795 & 848.3934 & 16 \\
\hline 13 & \begin{tabular}{|l|}
1207.5888 \\
\end{tabular} & 604.2980 & \begin{tabular}{|l}
1190.5623 \\
\end{tabular} & 595.7848 & 1189.5783 & 595.2928 & G & 1642.7530 & 821.8801 & \begin{tabular}{|l}
1625.7264 \\
\end{tabular} & 813.3669 & 1624.7424 & 812.8748 & 15 \\
\hline 14 & 1304.6416 & 652.8244 & 1287.6150 & 644.3112 & 1286.6310 & 643.8191 & $\mathbf{P}$ & \begin{tabular}{|l}
1585.7315 \\
\end{tabular} & 793.3694 & 1568.7050 & 784.8561 & 1567.7209 & 784.3641 & 14 \\
\hline 15 & 1418.6845 & 709.8459 & 1401.6580 & 701.3326 & 1400.6739 & 700.8406 & $\mathbf{N}$ & 1488.6787 & 744.8430 & 1471.6522 & 736.3297 & 1470.6682 & 735.8377 & 13 \\
\hline 16 & \begin{tabular}{|l|}
1519.7322 \\
\end{tabular} & 760.3697 & 1502.7056 & 751.8565 & 1501.7216 & 751.3644 & $T$ & 1374.6358 & 687.8215 & 1357.6093 & 679.3083 & 1356.6253 & 678.8163 & 12 \\
\hline 17 & 1634.7591 & 817.8832 & 1617.7326 & 809.3699 & 1616.7486 & 808.8779 & $\mathbf{N}$ & 1273.5881 & 637.2977 & 1256.5616 & 628.7844 & 1255.5776 & 628.2924 & 11 \\
\hline 18 & 1691.7806 & 846.3939 & 1674.7540 & 837.8807 & 1673.7700 & 837.3886 & $\mathbf{G}$ & 1158.5612 & 579.7842 & 1141.5347 & 571.2710 & 1140.5506 & 570.7790 & 10 \\
\hline
\end{tabular}




\begin{tabular}{|c|c|c|c|c|c|c|c|c|c|c|c|c|c|c|}
\hline 19 & |1778.8126 & 889.9099 & |1761.7861 & 881.3967 & 1760.8021 & 880.9047 & S & |1101.5397 & 551.2735 & 1084.5132 & 542.7602 & |1083.5292 & 542.2682 & 9 \\
\hline 20 & 1906.8712 & 953.9392 & 1889.8446 & 945.4260 & 1888.8606 & 944.9340 & $\mathbf{Q}$ & 1014.5077 & 507.7575 & 997.4812 & 499.2442 & 996.4972 & 498.7522 & 8 \\
\hline 21 & 2053.9396 & 1027.4734 & 2036.9131 & 1018.9602 & 2035.9290 & 1018.4682 & $\mathbf{F}$ & 886.4491 & 443.7282 & 869.4226 & 435.2149 & 868.4386 & 434.7229 & 7 \\
\hline 22 & 2201.0080 & 1101.0076 & 2183.9815 & 1092.4944 & 2182.9975 & 1092.0024 & $\mathbf{F}$ & 739.3807 & 370.1940 & 722.3542 & 361.6807 & 721.3702 & 361.1887 & 6 \\
\hline 23 & 2314.0921 & 1157.5497 & 2297.0655 & 1149.0364 & 2296.0815 & 1148.5444 & I & 592.3123 & 296.6598 & 575.2858 & 288.1465 & 574.3017 & 287.6545 & 5 \\
\hline 24 & 2474.1227 & 1237.5650 & 2457.0962 & 1229.0517 & 2456.1122 & 1228.5597 & C & 479.2282 & 240.1178 & 462.2017 & 231.6045 & 461.2177 & 231.1125 & 4 \\
\hline 25 & 2575.1704 & 1288.0888 & 2558.1439 & 1279.5756 & 2557.1598 & 1279.0836 & $\mathbf{T}$ & 319.1976 & 160.1024 & 302.1710 & 151.5892 & 301.1870 & 151.0972 & 3 \\
\hline 26 & 2646.2075 & 1323.6074 & 2629.1810 & 1315.0941 & 2628.1970 & 1314.6021 & A & 218.1499 & 109.5786 & 201.1234 & 101.0653 & & & 2 \\
\hline 27 & & & & & & & $\mathbf{K}$ & 147.1128 & 74.0600 & 130.0863 & 65.5468 & & & 1 \\
\hline
\end{tabular}
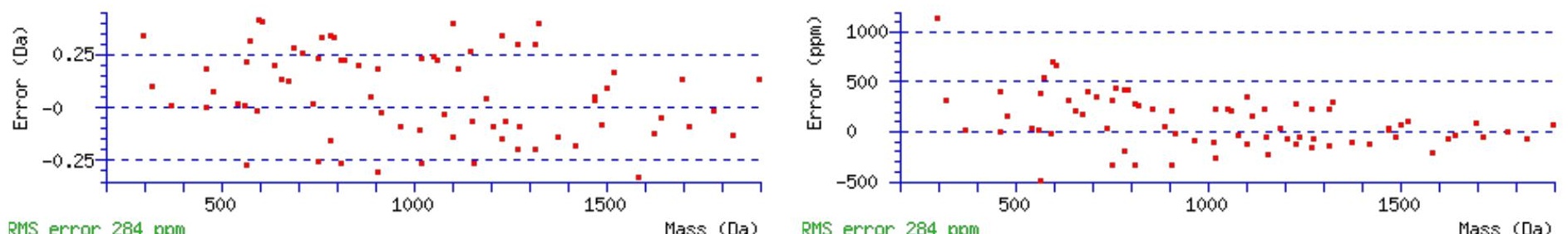

RMS error 284 ppm

\section{All matches to this query}

\begin{tabular}{|l|l|l|l|}
\hline Score & Mr(calc): & Delta & \multicolumn{1}{|c|}{ Sequence } \\
\hline 76.9 & 2791.3058 & -0.0008 & HTGPGILSMANAGPNTNGSQFFICTAK \\
\hline 57.4 & 2791.3058 & -0.0008 & HTGPGILSMANAGPNTNGSOFFICTAK \\
\hline 29.3 & 2791.3058 & -0.0008 & HTGPGILSMANAGPNTNGSQFFICTAK \\
\hline 24.7 & 2790.3218 & 0.9832 & HTGPGILSMANAGPNTNGSQFFICTAK \\
\hline 4.2 & 2791.3225 & -0.0175 & QREQQAYMVGLPQDALGQRPSAPR \\
\hline 3.5 & 2790.2894 & 1.0155 & SPAAGGADTAVVEIVSKIKPEVTSPK \\
\hline 3.0 & 2790.3143 & 0.9907 & YLRSLPSPFSPQPNSLSTKALGDR \\
\hline 1.8 & 2789.3017 & 2.0033 & CVSSSAPANTPLAPCPQSEVIIPSVK \\
\hline 1.7 & 2789.3129 & 1.9921 & AGSVELPASSPMPQLPPDTLEMRVR \\
\hline 1.5 & 2789.2721 & 2.0329 & RSSVLVTHAELMPDKTATHEVHR \\
\hline
\end{tabular}

Spectrum No: 271; Query: 2747; Rank: 1

\section{Peptide View}

MS/MS Fragmentation of LADDVDLEQVANETHGHVGADLAALCSEAALQAIR

Found in IPI00212014, Tax_Id=10116 Gene_Symbol=Vcp Transitional endoplasmic reticulum ATPase

Match to Query 2747: 3672.771372 from(1225.264400,3+)

Title: 091008RatKidney_NoSalt_14.5976.5976.3.dta

Data file K:INewmanPaper|Piliang $\mid 3 S$ SubProteomes\Piliang3SP\mgf5ppm\ERLIC_3SubProteomes5ppm.mgf 

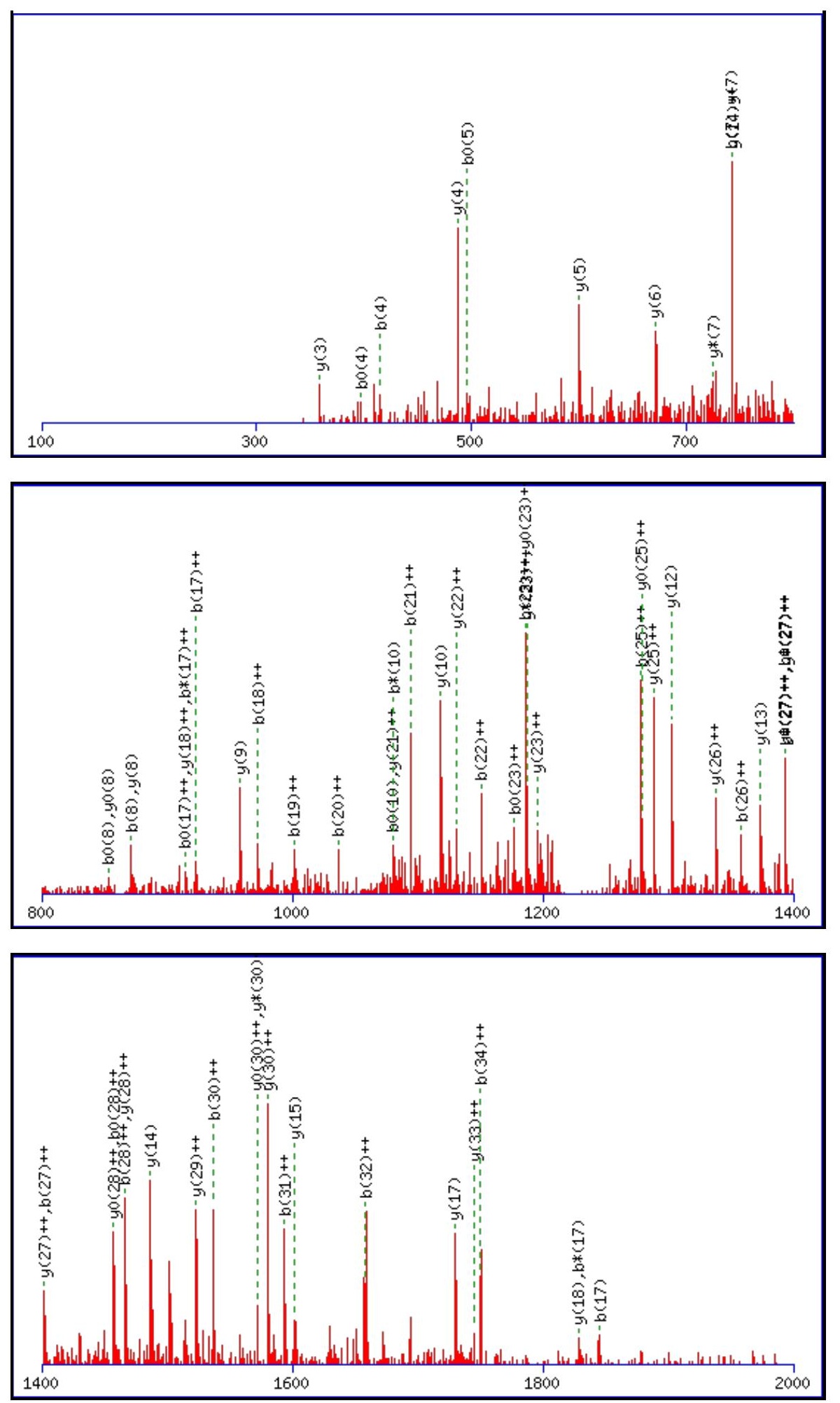

Monoisotopic mass of neutral peptide $\operatorname{Mr}($ calc): 3672.7689

Fixed modifications: Carbamidomethyl (C)

Variable modifications:

N12 : Deamidated_N (N)

Ions Score: 77 Expect: $6.5 \mathrm{e}-006$

Matches (Bold Red): 67/374 fragment ions using 110 most intense peaks

\begin{tabular}{|c|c|c|c|c|c|c|c|c|c|c|c|c|c|c|}
\hline \# & b & $\mathbf{b}^{++}$ & $\mathbf{b}^{*}$ & $\mathbf{b}^{*^{++}}$ & $\mathbf{b}^{0}$ & $\mathbf{b}^{\mathbf{0 + +}}$ & Seq. & $\mathbf{y}$ & $\mathbf{y}^{++}$ & $\mathbf{y}^{*}$ & $\mathbf{y}^{*^{++}}$ & $\mathbf{y}^{\mathbf{0}}$ & $\mathbf{y}^{\mathbf{0 + +}}$ & \# \\
\hline 1 & 114.0913 & 57.5493 & & & & & L & & & & & & & 35 \\
\hline 2 & 185.1285 & 93.0679 & & & & & A & 3560.6922 & 1780.8497 & 3543.6656 & 1772.3365 & 3542.6816 & 1771.8444 & 34 \\
\hline 3 & 300.1554 & 150.5813 & & & 282.1448 & 141.5761 & D & 3489.6551 & 1745.3312 & 3472.6285 & 1736.8179 & 3471.6445 & 1736.3259 & 33 \\
\hline 4 & 415.1823 & 208.0948 & & & 397.1718 & 199.0895 & D & 3374.6281 & 1687.8177 & 3357.6016 & 1679.3044 & 3356.6176 & 1678.8124 & 32 \\
\hline 5 & 514.2508 & 257.6290 & & & 496.2402 & 248.6237 & $\mathbf{V}$ & 3259.6012 & 1630.3042 & 3242.5746 & 1621.7910 & 3241.5906 & 1621.2989 & 31 \\
\hline
\end{tabular}




\begin{tabular}{|c|c|c|c|c|c|c|c|c|c|c|c|c|c|c|}
\hline 61 & 629.2777 & 315.1425 & & & 611.2671 & 306.1372 & D & 3160.5328| & 1580.7700 & |3143.5062| & |1572.2567 & 3142.5222 & |1571.7647 & 30 \\
\hline 7 & 742.3618 & 371.6845 & & & 724.3512 & 362.6792 & $\mathbf{L}$ & 3045.5058 & 1523.2565 & 3028.4793 & 1514.7433 & 3027.4953 & 1514.2513 & 29 \\
\hline 8 & 871.4044 & 436.2058 & & & 853.3938 & 427.2005 & $\mathbf{E}$ & 2932.4218 & 1466.7145 & 2915.3952 & 1458.2012 & 2914.4112 & 1457.7092 & 28 \\
\hline 9 & 999.4629 & 500.2351 & 982.4364 & 491.7218 & 981.4524 & 491.2298 & $\mathbf{Q}$ & 2803.3792 & 1402.1932 & 2786.3526 & 1393.6799 & 2785.3686 & 1393.1879 & 27 \\
\hline 10 & 1098.5313 & 549.7693 & 1081.5048 & 541.2560 & 1080.5208 & 540.7640 & V & 2675.3206 & 1338.1639 & 2658.2940 & 1329.6507 & 2657.3100 & 1329.1586 & 26 \\
\hline 11 & 1169.5685 & 585.2879 & 1152.5419 & 576.7746 & 1151.5579 & 576.2826 & A & 2576.2522 & 1288.6297 & 2559.2256 & 1280.1164 & 2558.2416 & 1279.6244 & 25 \\
\hline 12 & 1284.5954 & 642.8013 & 1267.5688 & 634.2881 & 1266.5848 & 633.7961 & $\mathbf{N}$ & 2505.2151 & 1253.1112 & 2488.1885 & 1244.5979 & 2487.2045 & 1244.1059 & 24 \\
\hline 13 & 1413.6380 & 707.3226 & 1396.6114 & 698.8094 & 1395.6274 & 698.3174 & $\mathbf{E}$ & 2390.1881 & 1195.5977 & 2373.1616 & 1187.0844 & 2372.1776 & 1186.5924 & 23 \\
\hline 14 & 1514.6857 & 757.8465 & 1497.6591 & 749.3332 & 1496.6751 & 748.8412 & $T$ & 2261.1455 & 1131.0764 & 2244.1190 & 1122.5631 & 2243.1350 & 1122.0711 & 22 \\
\hline 15 & 1651.7446 & 826.3759 & 1634.7180 & 817.8627 & 1633.7340 & 817.3706 & $\mathbf{H}$ & 2160.0978 & 1080.5526 & 2143.0713 & 1072.0393 & 2142.0873 & 1071.5473 & 21 \\
\hline 16 & 1708.7660 & 854.8867 & 1691.7395 & 846.3734 & 1690.7555 & 845.8814 & G & 2023.0389 & 1012.0231 & 2006.0124 & 1003.5098 & 2005.0284 & 1003.0178 & 20 \\
\hline 17 & 1845.8250 & 923.4161 & 1828.7984 & 914.9028 & 1827.8144 & 914.4108 & H & 1966.0175 & 983.5124 & 1948.9909 & 974.9991 & 1948.0069 & 974.5071 & 19 \\
\hline 18 & 1944.8934 & 972.9503 & 1927.8668 & 964.4370 & 1926.8828 & 963.9450 & $\mathbf{V}$ & 1828.9586 & 914.9829 & 1811.9320 & 906.4696 & 1810.9480 & 905.9776 & 8 \\
\hline 19 & 2001.9148 & 1001.4611 & 1984.8883 & 992.9478 & 1983.9043 & 992.4558 & G & 1729.8901 & 865.4487 & 1712.8636 & 856.9354 & 1711.8796 & 856.4434 & 17 \\
\hline 20 & 2072.9519 & 1036.9796 & 2055.9254 & 1028.4663 & 2054.9414 & 1027.9743 & A & 1672.8687 & 836.9380 & 1655.8421 & 828.4247 & 1654.8581 & 827.9327 & 16 \\
\hline 21 & 2187.9789 & 1094.4931 & 2170.9523 & 1085.9798 & 2169.9683 & 1085.4878 & D & 1601.8316 & 801.4194 & 1584.8050 & 792.9061 & 1583.8210 & 792.4141 & 15 \\
\hline 22 & 2301.0630 & 1151.0351 & 2284.0364 & 1142.5218 & 2283.0524 & 1142.0298 & $\mathbf{L}$ & 1486.8046 & 743.9060 & 1469.7781 & 735.3927 & 1468.7941 & 734.9007 & 14 \\
\hline 23 & 2372.1001 & 1186.5537 & 2355.0735 & 1178.0404 & 2354.0895 & 1177.5484 & A & 1373.7206 & 687.3639 & 1356.6940 & 678.8506 & 1355.7100 & 678.3586 & 13 \\
\hline 24 & 2443.1372 & 1222.0722 & 2426.1106 & 1213.5590 & 2425.1266 & 1213.0669 & A & 1302.6834 & 651.8454 & 1285.6569 & 643.3321 & 1284.6729 & 642.8401 & 12 \\
\hline 25 & 2556.2212 & 1278.6143 & 2539.1947 & 1270.1010 & 2538.2107 & 1269.6090 & $\mathbf{L}$ & 1231.6463 & 616.3268 & 1214.6198 & 607.8135 & 1213.6358 & 607.3215 & 11 \\
\hline 26 & 2716.2519 & 1358.6296 & 2699.2253 & 1350.1163 & 2698.2413 & 1349.6243 & C & 1118.5623 & 559.7848 & 1101.5357 & 551.2715 & 1100.5517 & 550.7795 & 10 \\
\hline 27 & 2803.2839 & \begin{tabular}{|l|l|}
1402.1456 \\
\end{tabular} & 2786.2574 & 1393.6323 & 2785.2734 & 1393.1403 & $S$ & 958.5316 & 479.7694 & 941.5051 & 471.2562 & 940.5211 & 470.7642 & 9 \\
\hline 28 & 2932.3265 & 1466.6669 & 2915.3000 & 1458.1536 & 2914.3160 & 1457.6616 & $\mathbf{E}$ & 871.4996 & 436.2534 & 854.4730 & 427.7402 & 853.4890 & 427.2482 & 8 \\
\hline 29 & 3003.3636 & 1502.1855 & 2986.3371 & 1493.6722 & 2985.3531 & 1493.1802 & A & 742.4570 & 371.7321 & 725.4305 & 363.2189 & & & 7 \\
\hline 30 & 3074.4007 & 1537.7040 & 3057.3742 & 1529.1907 & 3056.3902 & 1528.6987 & $\mathbf{A}$ & 671.4199 & 336.2136 & 654.3933 & 327.7003 & & & 6 \\
\hline 31 & 3187.4848 & 1594.2460 & 3170.4583 & 1585.7328 & 3169.4742 & 1585.2408 & $\mathbf{L}$ & 600.3828 & 300.6950 & 583.3562 & 292.1817 & & & 5 \\
\hline 32 & 3315.5434 & 1658.2753 & 3298.5168 & 1649.7621 & 3297.5328 & 1649.2700 & $\mathbf{Q}$ & 487.2987 & 244.1530 & 470.2722 & 235.6397 & & & 4 \\
\hline 33 & 3386.5805 & 1693.7939 & 3369.5540 & 1685.2806 & 3368.5699 & 1684.7886 & A & 359.2401 & 180.1237 & 342.2136 & 171.6104 & & & 3 \\
\hline 34 & 3499.6646 & 1750.3359 & 3482.6380 & 1741.8226 & 3481.6540 & 1741.3306 & I & 288.2030 & 144.6051 & 271.1765 & 136.0919 & & & 2 \\
\hline 35 & & & & & & & $\mathbf{R}$ & 175.1190 & 88.0631 & 158.0924 & 79.5498 & & & 1 \\
\hline
\end{tabular}
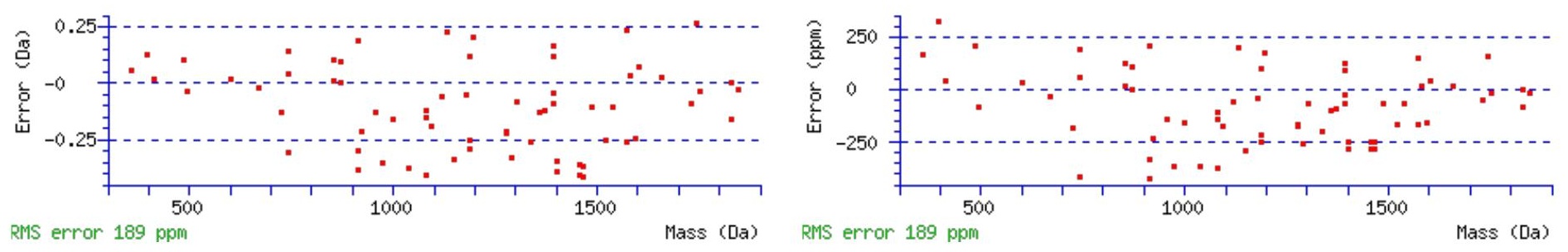

\section{All matches to this query}

\begin{tabular}{|l|l|l|l|}
\hline Score & Mr(calc): & Delta & \multicolumn{1}{c|}{ Sequence } \\
\hline 76.9 & 3672.7689 & 0.0024 & LADDVDLEQVANETHGHVGADLAALCSEAALQAIR \\
\hline 67.6 & 3671.7849 & 0.9864 & LADDVDLEQVANETHGHVGADLAALCSEAALQAIR \\
\hline 4.5 & 3671.7758 & 0.9956 & MLLASYSLIVRPATPAPAPQAPATPGAQLAHDMR \\
\hline 4.5 & 3671.7758 & 0.9956 & MLLASYSLIVRPATPAPAPQAPATPGAQLAHDMR \\
\hline 3.8 & 3670.7345 & 2.0369 & AVHTVDFTADKYHVVSGADDYTVKLWDIPNSK \\
\hline 3.6 & 3672.8028 & -0.0314 & TQFVLLNQHNTALLSMLSSAIASEGPRMANFHR \\
\hline 3.6 & 3672.7997 & -0.0284 & TYKLENILPELGIMDVFNTQADLSGIAGAKDVR \\
\hline 2.6 & 3672.7627 & 0.0087 & EEPMTLLGDPSASLTLLSLSSISECNSTRSLLR \\
\hline 2.4 & 3670.7994 & 1.9720 & GTNQLDGMDLLVLLDLIGAANPTFPNFFPKTTR \\
\hline 2.3 & 3670.8005 & 1.9709 & TFPQDHDVYVGKPSLNRPIHASELQSKNRTR \\
\hline
\end{tabular}


Spectrum No: 272; Query: 2710; Rank: 1

\section{Peptide View}

MS/MS Fragmentation of SLGNVNFSVSVEAQQSPELCGSQVATVPETGRK

Found in IPI00212666, Tax_Id=10116 Gene_Symbol=Mug1 Isoform 1 of Murinoglobulin-1 precursor

Match to Query 2710: 3476.700642 from(1159.907490,3+)

Title: 100101RatKid_NS_deglyco_23.4529.4529.3.dta

Data file K:INewmanPaper|Piliang|3SubProteomes\Piliang3SP\mgf5ppm\ERLIC_3SubProteomes5ppm.mgf
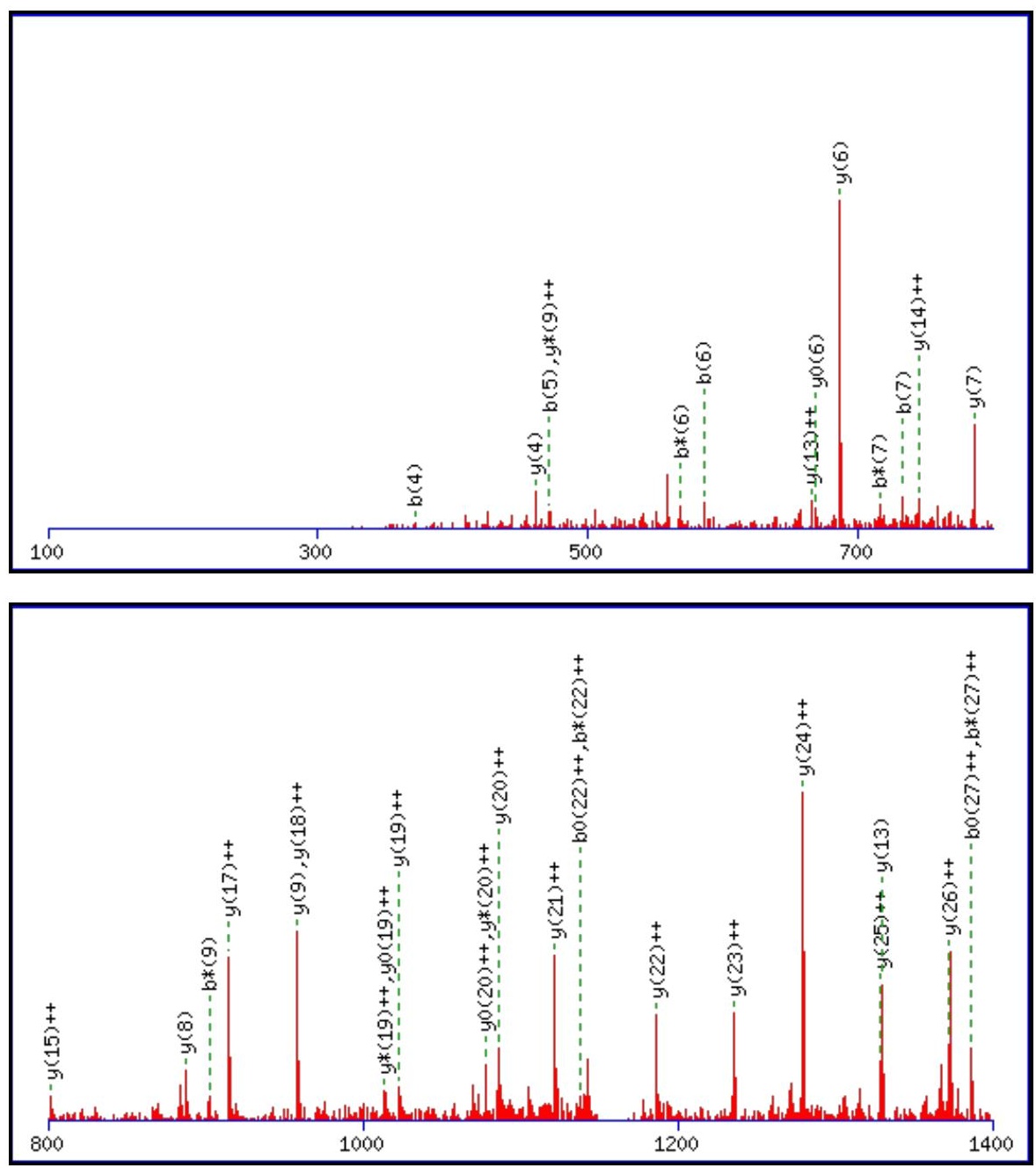


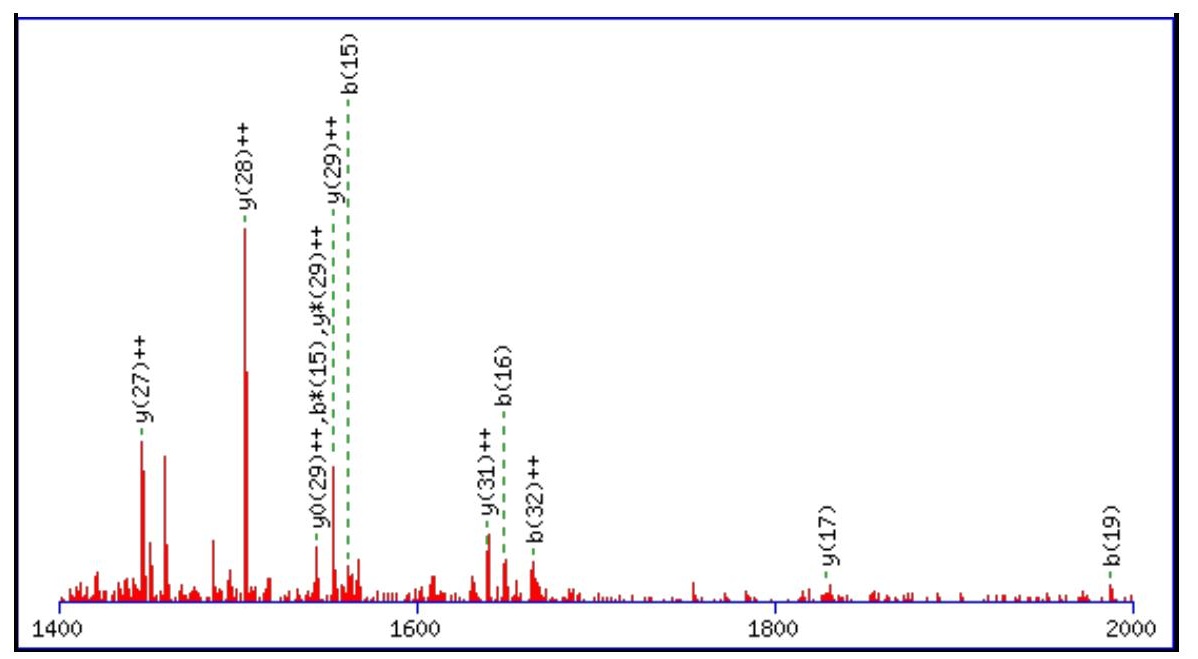

Monoisotopic mass of neutral peptide $\operatorname{Mr}($ calc): 3475.6889

Fixed modifications: Carbamidomethyl (C)

Variable modifications:

N6 : Deamidated_N (N)

Ions Score: 77 Expect: $5.9 \mathrm{e}-006$

Matches (Bold Red): 48/372 fragment ions using 91 most intense peaks

\begin{tabular}{|c|c|c|c|c|c|c|c|c|c|c|c|c|c|c|}
\hline \# & b & $\mathbf{b}^{++}$ & $\mathbf{b}^{*}$ & $\mathbf{b}^{*^{++}}$ & $\mathbf{b}^{0}$ & $\mathbf{b}^{\mathbf{0}^{++}}$ & Seq. & $\mathbf{y}$ & $\mathbf{y}^{++}$ & $\mathbf{y}^{*}$ & $\mathrm{y}^{\mathrm{*}^{++}}$ & $\mathbf{y}^{0}$ & $\mathbf{y}^{\mathbf{0}^{++}}$ & \# \\
\hline 1 & 88.0393 & 44.5233 & & & 70.0287 & 35.5180 & $S$ & & & & & & & 33 \\
\hline 2 & 201.1234 & 101.0653 & & & 183.1128 & 92.0600 & $\mathbf{L}$ & 3389.6642 & 1695.3357 & 3372.6376 & 1686.8224 & 3371.6536 & 1686.3304 & 32 \\
\hline 3 & 258.1448 & 129.5761 & & & 240.1343 & 120.5708 & G & 3276.5801 & 1638.7937 & 3259.5536 & 1630.2804 & 3258.5695 & 1629.7884 & 31 \\
\hline 4 & 372.1878 & 186.5975 & 355.1612 & 178.0842 & 354.1772 & 177.5922 & $\mathbf{N}$ & 3219.5586 & 1610.2830 & 3202.5321 & 1601.7697 & 3201.5481 & 1601.2777 & 30 \\
\hline 5 & 471.2562 & 236.1317 & 454.2296 & 227.6185 & 453.2456 & 227.1264 & $\mathbf{V}$ & 3105.5157 & 1553.2615 & 3088.4892 & 1544.7482 & 3087.5051 & 1544.2562 & 29 \\
\hline 6 & 586.2831 & 293.6452 & 569.2566 & 285.1319 & 568.2725 & 284.6399 & $\mathbf{N}$ & 3006.4473 & 1503.7273 & 2989.4208 & 1495.2140 & |2988.4367 & 1494.7220 & 28 \\
\hline 7 & 733.3515 & 367.1794 & 716.3250 & 358.6661 & 715.3410 & 358.1741 & $\mathbf{F}$ & 2891.4204 & 1446.2138 & 2874.3938 & 1437.7005 & 2873.4098 & 1437.2085 & 27 \\
\hline 8 & 820.3836 & 410.6954 & 803.3570 & 402.1821 & 802.3730 & 401.6901 & $\mathrm{~S}$ & 2744.3520 & 1372.6796 & 2727.3254 & 1364.1663 & 2726.3414 & 1363.6743 & 26 \\
\hline 9 & 919.4520 & 460.2296 & 902.4254 & 451.7163 & 901.4414 & 451.2243 & $\mathbf{V}$ & 2657.3199 & 1329.1636 & 2640.2934 & 1320.6503 & |2639.3094 & 1320.1583 & 25 \\
\hline 10 & 1006.4840 & 503.7456 & 989.4574 & 495.2324 & 988.4734 & 494.7404 & $\mathrm{~S}$ & 2558.2515 & 1279.6294 & 2541.2250 & 1271.1161 & 2540.2409 & 1270.6241 & 24 \\
\hline 11 & 1105.5524 & 553.2798 & 1088.5259 & 544.7666 & 1087.5418 & 544.2746 & $\mathbf{V}$ & 2471.2195 & 1236.1134 & 2454.1929 & 1227.6001 & 2453.2089 & 1227.1081 & 23 \\
\hline 12 & 1234.5950 & 617. & 1217 & 609.2879 & 1216.5844 & 608.7959 & $\mathbf{E}$ & & & 1245 & 1178.0659 & 1405 & 1177.5739 & 22 \\
\hline 13 & 1305.6321 & 653.3197 & 1288.6056 & 644.8064 & 1287.6215 & 644.3144 & A & 2243.1085 & 1122.0579 & 2226.0819 & 1113.5446 & 2225.0979 & 1113.0526 & 21 \\
\hline 14 & 1433.6907 & 717.3490 & 1416.6641 & 708.8357 & 1415.6801 & 708.3437 & $\mathbf{Q}$ & 2172.0714 & 1086.5393 & 2155.0448 & 1078.0260 & 2154.0608 & 1077.5340 & 20 \\
\hline 15 & 1561.7493 & 783 & 1544 & & 1543. & & $\mathbf{Q}$ & & & 2026 & & & 5047 & 19 \\
\hline 16 & 1648.7813 & 824.8943 & 1631.7547 & 816.3810 & 1630.7707 & 815.8890 & $\mathrm{~S}$ & 1915.9542 & 958.4807 & 1898.9277 & 949.9675 & 1897.9436 & 949.4755 & 18 \\
\hline 17 & 1745.8341 & 873.4207 & 1728.8075 & 864.9074 & 1727.8235 & 864.4154 & $\mathbf{P}$ & 1828.9222 & 914.9647 & 1811.8956 & 906.4515 & 1810.9116 & 905.9594 & 17 \\
\hline 18 & 1874.8767 & 420 & 1857.8501 & 1287 & 1856.8661 & 928.9367 & $\mathbf{E}$ & 1731.8694 & 866.4383 & 1714.8429 & 857.9251 & 1713.8588 & 857.4331 & 16 \\
\hline 19 & 1987.9607 & 994.4840 & 1970.9342 & 985.9707 & 1969.9502 & 985.4787 & $\mathbf{L}$ & 1602.8268 & 801.9170 & 1585.8003 & 793.4038 & 1584.8163 & 792.9118 & 15 \\
\hline 20 & 2147.9914 & 1074.4993 & 2130.9648 & 1065.9860 & 2129.9808 & 1065.4940 & $\mathrm{C}$ & 1489.7428 & 745.3750 & 1472.7162 & 736.8617 & 1471.7322 & 736.3697 & 14 \\
\hline 21 & 2205.0128 & 1103.0101 & 2187.9863 & 1094.4968 & 2187.0023 & 1094.0048 & $\mathbf{G}$ & 1329.7121 & 665.3597 & 1312.6856 & 656.8464 & 1311.7015 & 656.3544 & 13 \\
\hline 22 & 2292.0449 & 1146.5261 & 2275.0183 & 1138.0128 & 2274.0343 & 1137.5208 & $\mathrm{~S}$ & 1272.6906 & 636.8490 & 1255.6641 & 628.3357 & 1254.6801 & 627.8437 & 12 \\
\hline 23 & 2420.1034 & 1210.5554 & 2403.0769 & 1202.0421 & 2402.0929 & 1201.5501 & $\mathbf{Q}$ & 1185.6586 & 593.3329 & 1168.6321 & 584.8197 & 1167.6480 & 584.3277 & 11 \\
\hline 24 & 2519.1719 & 1260.0896 & 2502.1453 & 1251.5763 & 2501.1613 & 1251.0843 & $\mathbf{V}$ & 1057.6000 & 529.3037 & 1040.5735 & 520.7904 & 1039.5895 & 520.2984 & 10 \\
\hline 25 & 2590.2090 & 1295.6081 & 2573.1824 & 1287.0948 & 2572.1984 & 1286.6028 & A & 958.5316 & 479.7694 & 941.5051 & 471.2562 & 940.5211 & 470.7642 & 9 \\
\hline 26 & 2691.2566 & 1346.1320 & 2674.2301 & 1337.6187 & 2673.2461 & 1337.1267 & $T$ & 887.4945 & 444.2509 & 870.4680 & 435.7376 & 869.4839 & 435.2456 & 8 \\
\hline 27 & 2790.3251 & 1395.6662 & 2773.2985 & 1387.1529 & 2772.3145 & \begin{tabular}{|l|}
1386.6609 \\
\end{tabular} & $\mathrm{~V}$ & 786.4468 & 393.7271 & 769.4203 & 385.2138 & 768.4363 & 384.7218 & 7 \\
\hline 28 & 2887.3778 & 1444.1925 & 2870.3513 & 1435.6793 & 2869.3673 & 1435.1873 & $\mathbf{P}$ & 687.3784 & 344.1928 & 670.3519 & 335.6796 & 669.3678 & 335.1876 & 6 \\
\hline 29 & 3016.4204 & 1508.7138 & 2999.3939 & 1500.2006 & 2998.4099 & 1499.7086 & $E$ & 590.3257 & 295.6665 & 573.2991 & 287.1532 & 572.3151 & 286.6612 & 5 \\
\hline 30 & 3117.4681 & 1559.2377 & 3100.4415 & 1550.7244 & 3099.4575 & 1550.2324 & $T$ & 461.2831 & 231.1452 & 444.2565 & 222.6319 & 443.2725 & 222.1399 & 4 \\
\hline 31 & 3174.4896 & 1587.7484 & 3157.4630 & 1579.2351 & 3156.4790 & 1578.7431 & G & 360.2354 & 180.6213 & 343.2088 & 172.1081 & & & 3 \\
\hline 32 & 3330.5907 & 1665.7990 & 3313.5641 & 1657.2857 & 3312.5801 & $\mid 1656.7937$ & $\mathbf{R}$ & 303.2139 & 152.1106 & 286.1874 & 143.5973 & & & 2 \\
\hline
\end{tabular}




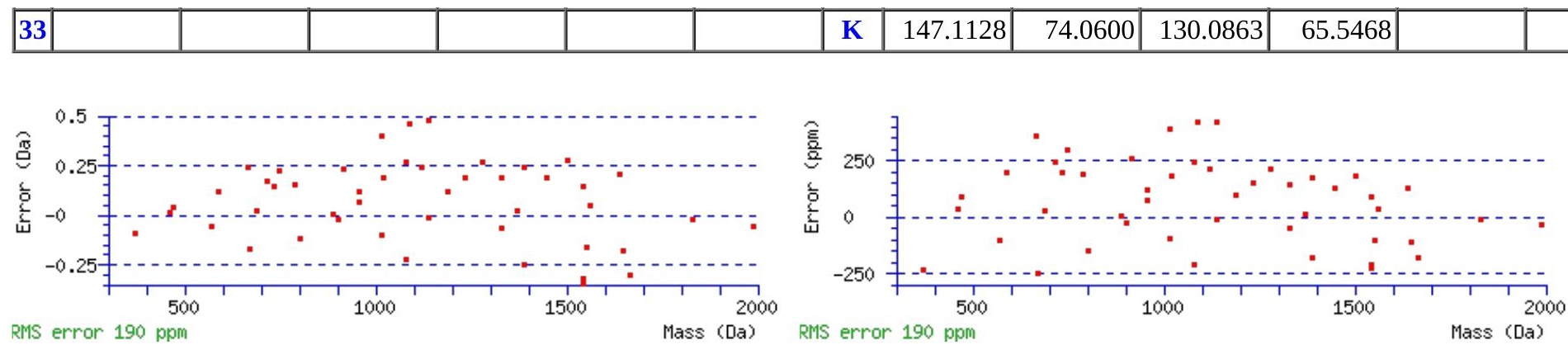

\section{All matches to this query}

\begin{tabular}{|l|l|l|l|}
\hline Score & Mr(calc): & Delta & \multicolumn{1}{|c|}{ Sequence } \\
\hline 76.9 & 3475.6889 & 1.0117 & SLGNVNFSVSVEAQQSPELCGSQVATVPETGRK \\
\hline 73.9 & 3476.6729 & 0.0277 & SLGNVNFSVSVEAQQSPELCGSQVATVPETGRK \\
\hline 67.0 & 3475.6889 & 1.0117 & SLGNVNFSVSVEAQOSPELCGSOVATVPETGRK \\
\hline 43.9 & 3474.7049 & 1.9957 & SLGNVNFSVSVEAQQSPELCGSQVATVPETGRK \\
\hline 2.9 & 3474.6663 & 2.0344 & ELEVKYASLTDLVNNQSVMITVLEEQCLR \\
\hline 2.9 & 3474.6663 & 2.0344 & ELEVKYASLTDLVNNQSVMITVLEEQCLR \\
\hline 2.8 & 3476.7179 & -0.0172 & RKAFSTCASHFTFVVIGYGTCLFLYVKPK \\
\hline 1.6 & 3474.7105 & 1.9901 & VTAGKDSYLALVDKNIMGYIASLHELATTER \\
\hline 0.8 & 3475.7113 & 0.9894 & QQQASQQASTAADPTLKINPSLTELKALVHK \\
\hline 0.8 & 3475.6711 & 1.0295 & QPCCSNIEKPVVSNIQTRSATVSWALNSAEK \\
\hline
\end{tabular}

Spectrum No: 273; Query: 1533; Rank: 1

\section{Peptide View}

MS/MS Fragmentation of RQDPVSWNETFEDISR

Found in IPI00765413, Tax_Id=10116 Gene_Symbol=- 58 kDa protein

Match to Query 1533: 1978.906182 from(660.642670,3+)

Title: 100101RatKid_NS_deglyco_21.3546.3546.3.dta

Data file K:INewmanPaper|Piliangl3SubProteomes\Piliang3SP\mgf5ppm\ERLIC_3SubProteomes5ppm.mgf

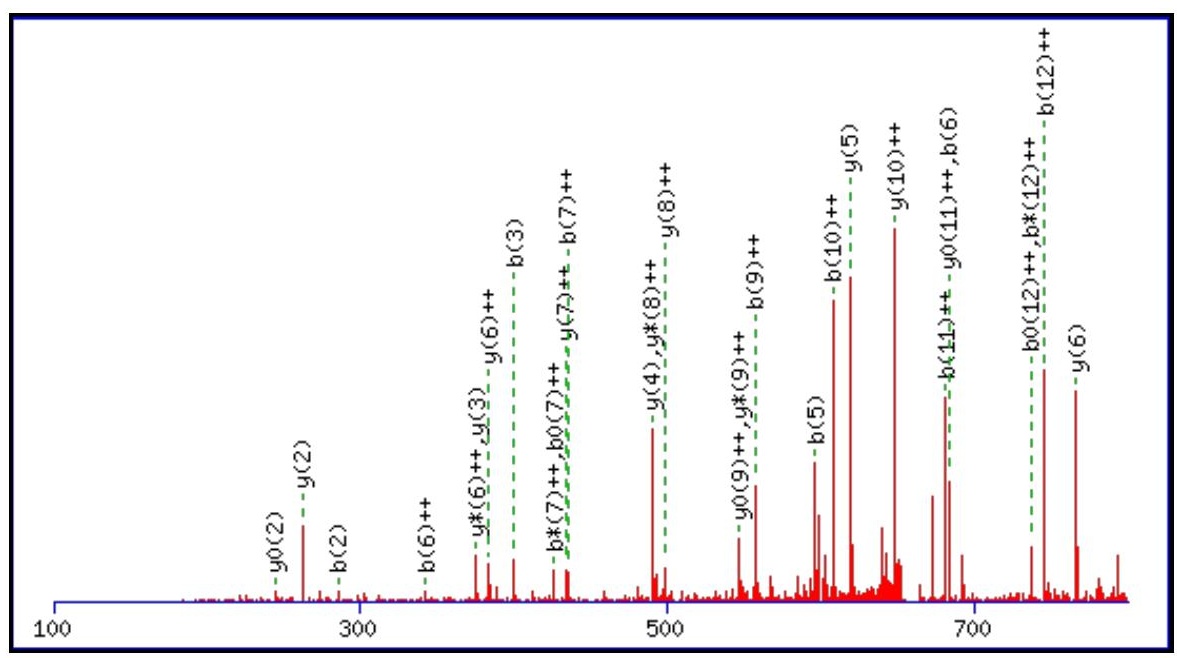



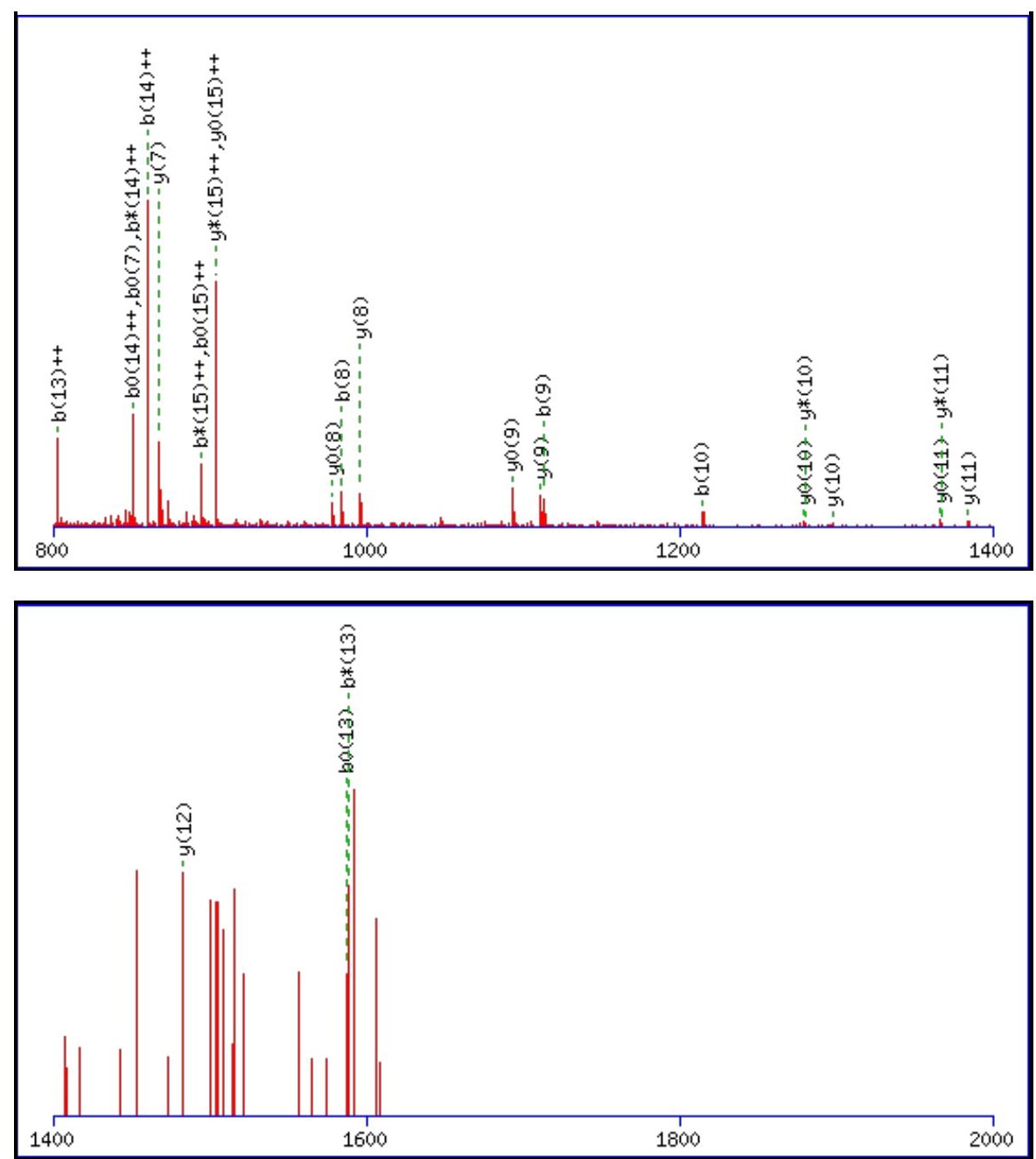

Monoisotopic mass of neutral peptide $\operatorname{Mr}($ calc): 1978.9021

Fixed modifications: Carbamidomethyl (C)

Variable modifications:

N8 : Deamidated_N (N)

Ions Score: 77 Expect: $5 \mathrm{e}-006$

Matches (Bold Red): 56/174 fragment ions using 64 most intense peaks

\begin{tabular}{|c|c|c|c|c|c|c|c|c|c|c|c|c|c|c|}
\hline \# & b & $\mathbf{b}^{++}$ & b* & $\mathbf{b}^{*^{++}}$ & $\mathbf{b}^{0}$ & & Seq. & $\mathbf{y}$ & $\mathbf{y}^{++}$ & $\mathbf{y}^{*}$ & $\mathrm{y}^{*^{++}}$ & $\mathbf{y}^{\mathbf{0}}$ & $y^{0++}$ & \# \\
\hline 1 & 157.1084 & 79.0578 & 140.0818 & 70.5446 & & & $\mathbf{R}$ & & & & & & & 16 \\
\hline 2 & 285.1670 & 143.0871 & 268.1404 & 134.5738 & & & $\mathbf{Q}$ & & 078 & 1806.7817 & & 7977 & & 15 \\
\hline 3 & 400.1939 & 200.6006 & 383.1674 & 192.0873 & 382.1833 & 191.5953 & D & 1695.7497 & 785 & 1678.7231 & 839.8652 & 1677.7391 & 839.3732 & 14 \\
\hline 4 & 497.2467 & 249.1270 & 480.2201 & 240.6137 & 479.2361 & 240.1217 & $\mathbf{P}$ & 1580.7227 & 790.8650 & 1563.6962 & 782.3517 & 1562.7122 & 781.8597 & 13 \\
\hline 5 & 596.3151 & 298.6612 & 579.2885 & 290.1479 & 578.3045 & 289.6559 & V & 1483.6700 & 742.3386 & 1466.6434 & 733.8253 & 1465.6594 & 733.3333 & 12 \\
\hline 6 & 683.3471 & 342.1772 & 666.3206 & 333.6639 & 665.3365 & 333.1719 & S & 1384.6015 & 692.8044 & 1367.5750 & 684.2911 & 1366.5910 & 683.7991 & 11 \\
\hline 7 & 869.4264 & 435.2169 & 852.3999 & 426.7036 & 851.4159 & 426.2116 & $\mathbf{W}$ & 1297.5695 & 649.2884 & 1280.5430 & 640.7751 & 1279.5590 & 640.2831 & 10 \\
\hline 8 & 984.4534 & 492.7303 & 967.4268 & 484.2170 & 966.4428 & 483.7250 & $\mathbf{N}$ & 1111.4902 & 556.2487 & 1094.4637 & 547.7355 & 1093.4796 & 547.2435 & 9 \\
\hline 9 & 1113.4960 & 557.2516 & 1096.4694 & 548.7383 & 1095.4854 & 548.2463 & $\mathbf{E}$ & 996.4633 & 498.7353 & 979.4367 & 490.2220 & 978.4527 & 489.7300 & 8 \\
\hline 10 & 1214.5436 & 607.7755 & 1197.5171 & 599.2622 & 1196.5331 & 598.7702 & $\mathbf{T}$ & 867.4207 & 434.2140 & 850.3941 & 425.7007 & 849.4101 & 425.2087 & 7 \\
\hline 11 & 1361.6120 & 681.3097 & 1344.5855 & 672.7964 & 1343.6015 & 672.3044 & $\mathbf{F}$ & 766.3730 & 383.6901 & 749.3464 & 375.1769 & 748.3624 & 374.6849 & 6 \\
\hline 12 & 1490.6546 & 745.8310 & 1473.6281 & 737.3177 & 1472.6441 & 736.8257 & $\mathbf{E}$ & 619.3046 & 310.1559 & 602.2780 & 301.6427 & 601.2940 & 301.1506 & 5 \\
\hline 13 & 1605.6816 & 803.3444 & 1588.6550 & 794.8312 & 1587.6710 & 794.3391 & D & 490.2620 & 245.6346 & 473.2354 & 237.1214 & 472.2514 & 236.6293 & 4 \\
\hline 14 & 1718.7656 & 859.8865 & 1701.7391 & 851.3732 & 1700.7551 & 850.8812 & I & 375.2350 & 188.1212 & 358.2085 & 179.6079 & 357.2245 & 179.1159 & 3 \\
\hline 15 & 1805.7977 & 903.4025 & 1788.7711 & 894.8892 & 1787.7871 & 894.3972 & S & 262.1510 & 131.5791 & 245.1244 & 123.0659 & 244.1404 & 122.5738 & 2 \\
\hline 16 & & & & & & & $\mathbf{R}$ & 175.1190 & 88.0631 & 158.0924 & 79.5498 & & & 1 \\
\hline
\end{tabular}



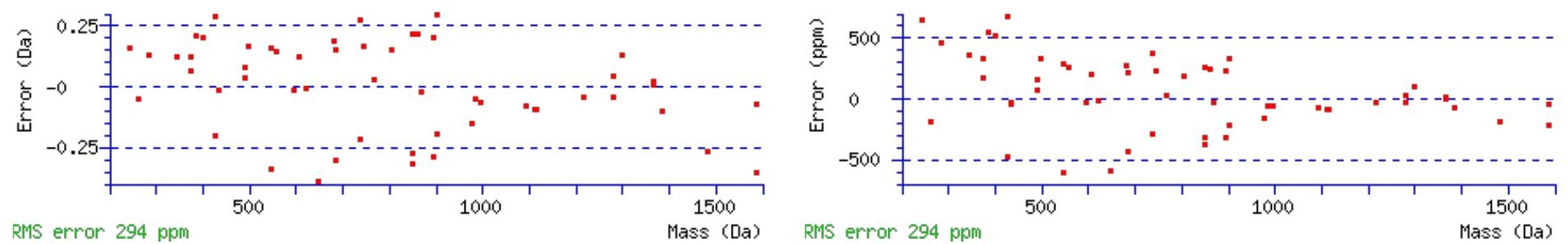

\section{All matches to this query}

\begin{tabular}{|l|l|l|l|}
\hline Score & Mr(calc): & Delta & \multicolumn{1}{|c|}{ Sequence } \\
\hline 76.8 & 1978.9021 & 0.0041 & RQDPVSWNETFEDISR \\
\hline 29.1 & 1977.9181 & 0.9881 & RQDPVSWNETFEDISR \\
\hline 18.9 & 1978.9046 & 0.0016 & CTLPMFVNLTFREVR \\
\hline 9.4 & 1978.9010 & 0.0052 & THAAAAQDTLTQLEHHR \\
\hline 7.1 & 1978.8938 & 0.0124 & AVKYFWLAASNGDSQSR \\
\hline 4.3 & 1978.9006 & 0.0056 & MPSSIVRMDTSNVPPPR \\
\hline 4.3 & 1978.9006 & 0.0056 & MPSSIVRMDTSNVPPPR \\
\hline 4.3 & 1976.8969 & 2.0093 & SQLDINNKKSVTDSIR \\
\hline 4.0 & 1978.9006 & 0.0056 & MPSSIVRMDTSNVPPPR \\
\hline 4.0 & 1978.9006 & 0.0056 & MPSSIVRMDTSNVPPPR \\
\hline
\end{tabular}

Spectrum No: 274; Query: 131; Rank: 1

\section{Peptide View}

MS/MS Fragmentation of FNDTEVLQR

Found in IPI00358014, Tax_Id=10116 Gene_Symbol=Btd Biotinidase precursor

Match to Query 131: 1121.534128 from(561.774340,2+)

Title: 091008RatKidney_NH4Format01_24.1462.1462.2.dta

Data file K:INewmanPaper\Piliang\3SubProteomes\Piliang3SP\mgf5ppm\ERLIC_3SubProteomes5ppm.mgf

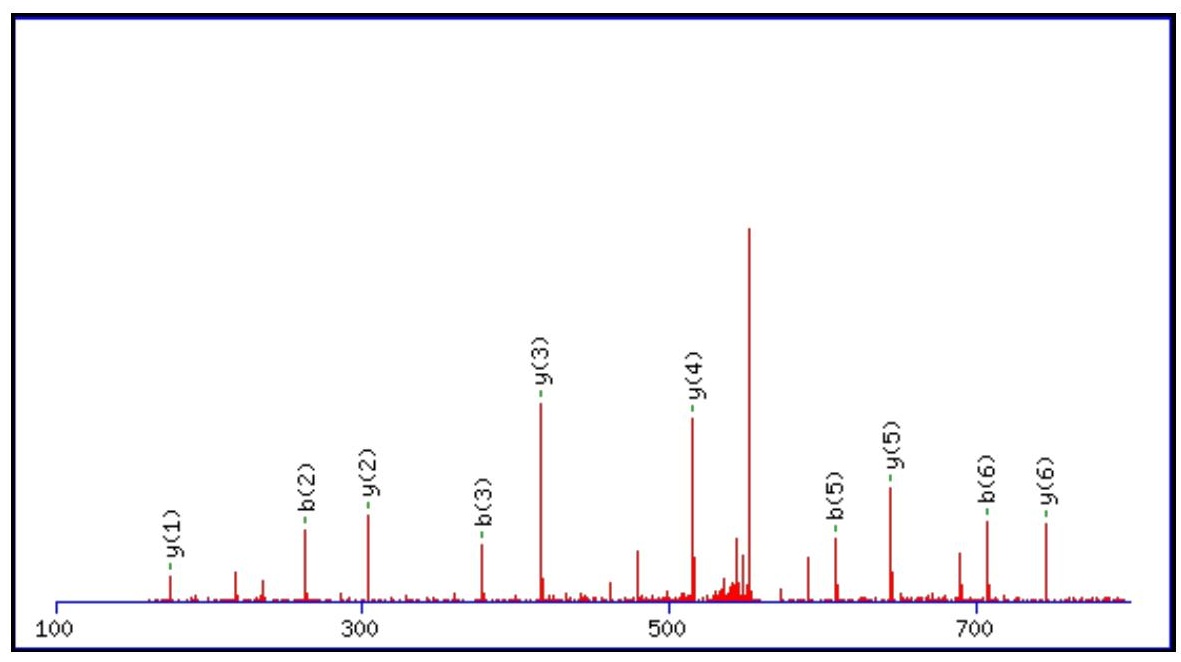



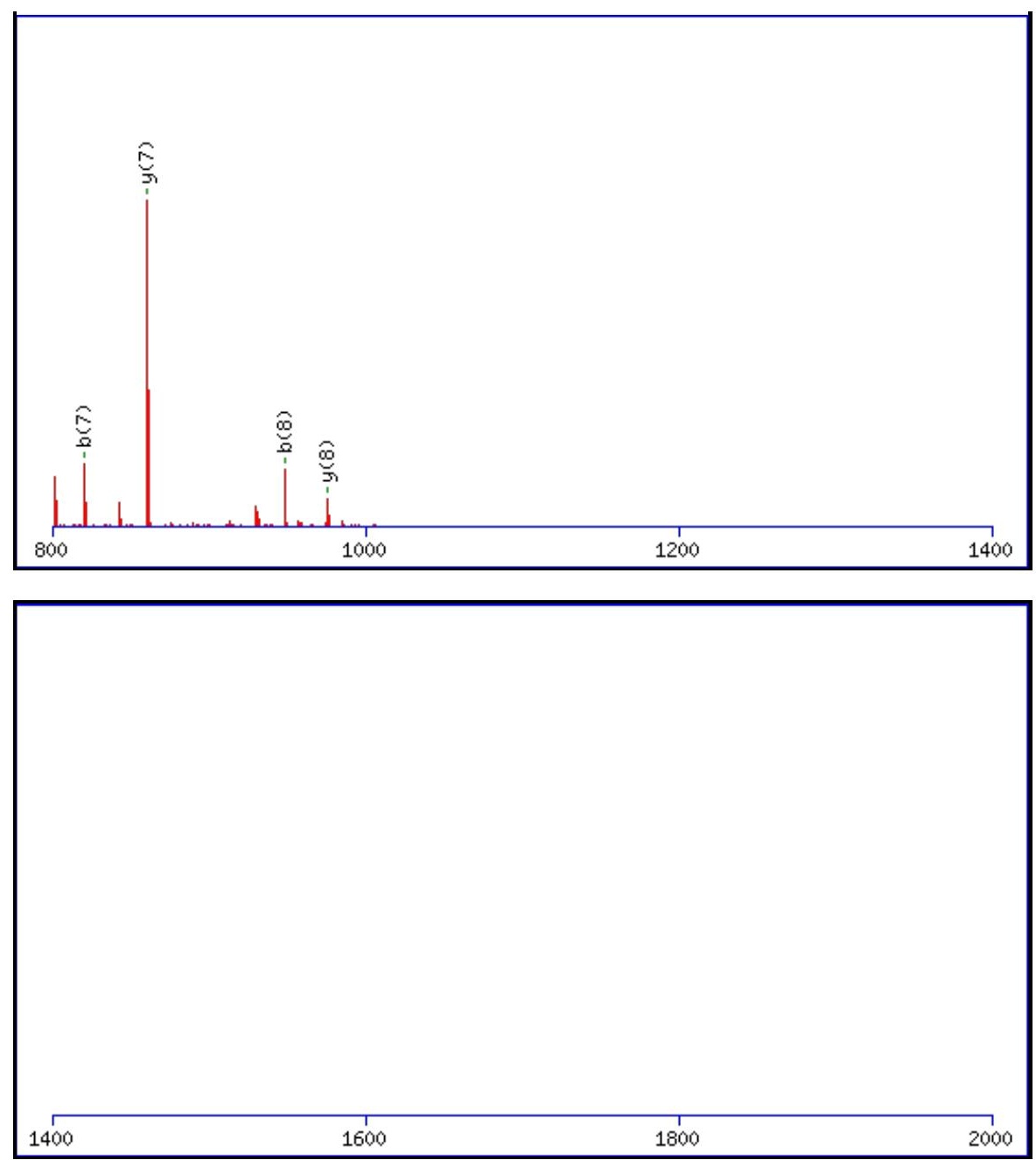

Monoisotopic mass of neutral peptide $\operatorname{Mr}($ calc): 1121.5353

Fixed modifications: Carbamidomethyl (C)

Variable modifications:

N2 : Deamidated $\mathrm{N}(\mathrm{N})$

Ions Score: 77 Expect: $2.3 \mathrm{e}-006$

Matches (Bold Red): 14/82 fragment ions using 19 most intense peaks

\begin{tabular}{|c|c|c|c|c|c|c|c|c|c|c|c|c|c|c|}
\hline \# & b & $\mathbf{b}^{++}$ & b* & $\mathbf{b}^{*^{++}}$ & $\mathbf{b}^{\mathbf{0}}$ & $\mathbf{b}^{0++}$ & Seq. & $\mathbf{y}$ & $y^{++}$ & $\mathbf{y}^{*}$ & $\mathrm{y}^{*^{++}}$ & $\mathbf{y}^{0}$ & $\mathbf{y}^{0++}$ & $\#$ \\
\hline 1 & 148.0757 & 74.5415 & & & & & $\mathbf{F}$ & & & & & & & 9 \\
\hline 2 & 263.1026 & 132.0550 & 246.0761 & 123.5417 & & & $\mathbf{N}$ & 975.4742 & 488.2407 & 958.4476 & 479.7274 & 957.4636 & 479.2354 & 8 \\
\hline 4 & 479.1772 & 240.0923 & 462.1507 & 231.5790 & 461.1667 & 231.0870 & $\mathbf{T}$ & 745.4203 & 373.2138 & 728.3937 & 364.7005 & 727.4097 & 364.2085 & 6 \\
\hline 5 & 608.2198 & 304.6136 & 591.1933 & 296.1003 & 590.2093 & 295.6083 & $\mathbf{E}$ & 644.3726 & 322.6899 & 627.3461 & 314.1767 & 626.3620 & 313.6847 & 5 \\
\hline 7 & 820.3723 & 410.6898 & 803.3458 & 402.1765 & 802.3618 & 401.6845 & $\mathbf{L}$ & 416.2616 & 208.6344 & 399.2350 & 200.1212 & & & 3 \\
\hline 8 & 948.4309 & 474.7191 & 931.4043 & 466.2058 & 930.4203 & 465.7138 & $\mathbf{Q}$ & 303.1775 & 152.0924 & 286.1510 & 143.5791 & & & 2 \\
\hline 9 & & & & & & & $\mathbf{R}$ & 175.1190 & 88.0631 & 158.0924 & 79.5498 & & & 1 \\
\hline
\end{tabular}

.
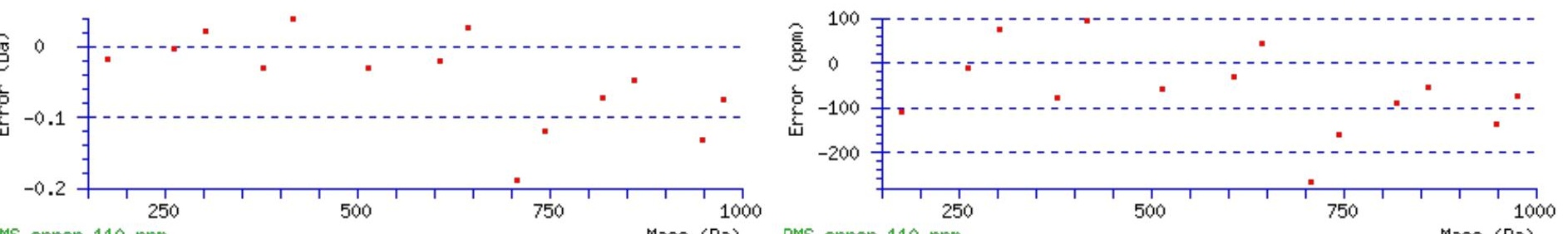

RMS error $110 \mathrm{ppm}$ Mass (Da) RMS error $110 \mathrm{ppm}$

\section{All matches to this query}




\begin{tabular}{|l|l|l|l|} 
Score & Mr(calc): & Delta & \multicolumn{1}{|c|}{ Sequence } \\
\hline 76.8 & 1121.5353 & -0.0012 & FNDTEVLQR \\
\hline 21.4 & 1121.5386 & -0.0045 & EMTEAELKR \\
\hline 20.9 & 1121.5386 & -0.0045 & MNDLLTNRK \\
\hline 13.7 & 1121.5230 & 0.0111 & STTLSHLQR \\
\hline 13.7 & 1121.5230 & 0.0111 & STTLSHLQR \\
\hline 13.7 & 1121.5230 & 0.0111 & STTLSHLQR \\
\hline 13.7 & 1121.5230 & 0.0111 & STTLSHLQR \\
\hline 11.5 & 1121.5353 & -0.0012 & GELDEYIQR \\
\hline 11.1 & 1120.5400 & 0.9941 & LFPGKDNSNK \\
\hline 9.7 & 1121.5326 & 0.0015 & DTHVHRDSR \\
\hline
\end{tabular}

Spectrum No: 275; Query: 2606; Rank: 1

\section{Peptide View}

MS/MS Fragmentation of GVDVSQVAWQSQGDTPCSCCIVNNSNGSR

Found in IPI00211989, Tax_Id=10116 Gene_Symbol=Khk Isoform C of Ketohexokinase

Match to Query 2606: 3183.333162 from(1062.118330,3+)

Title: 100101RatKid_NS_deglyco_19.3211.3211.3.dta

Data file K:INewmanPaper|Piliangl3SubProteomes\Piliang3SP\mgf5ppm\ERLIC_3SubProteomes5ppm.mgf
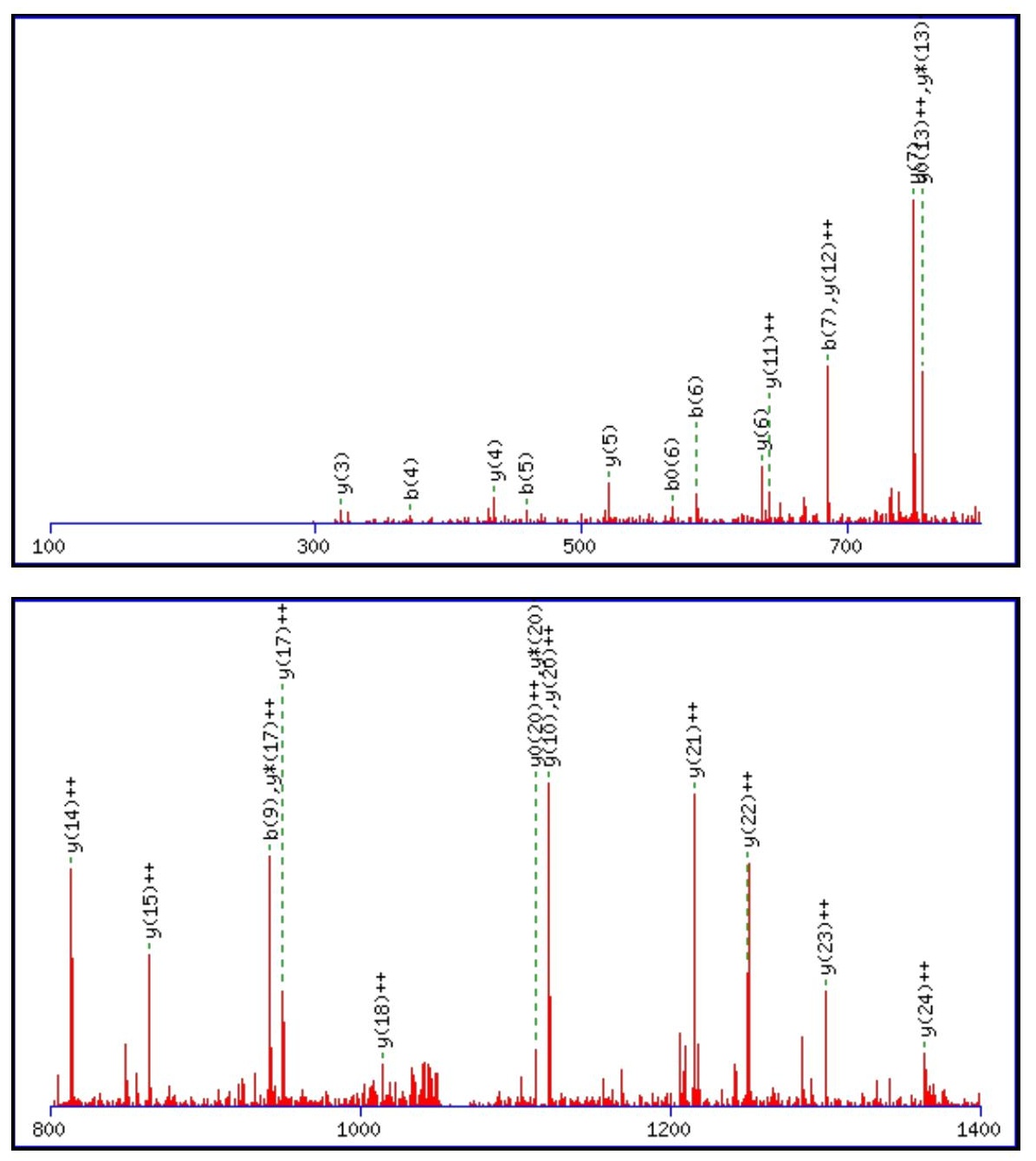

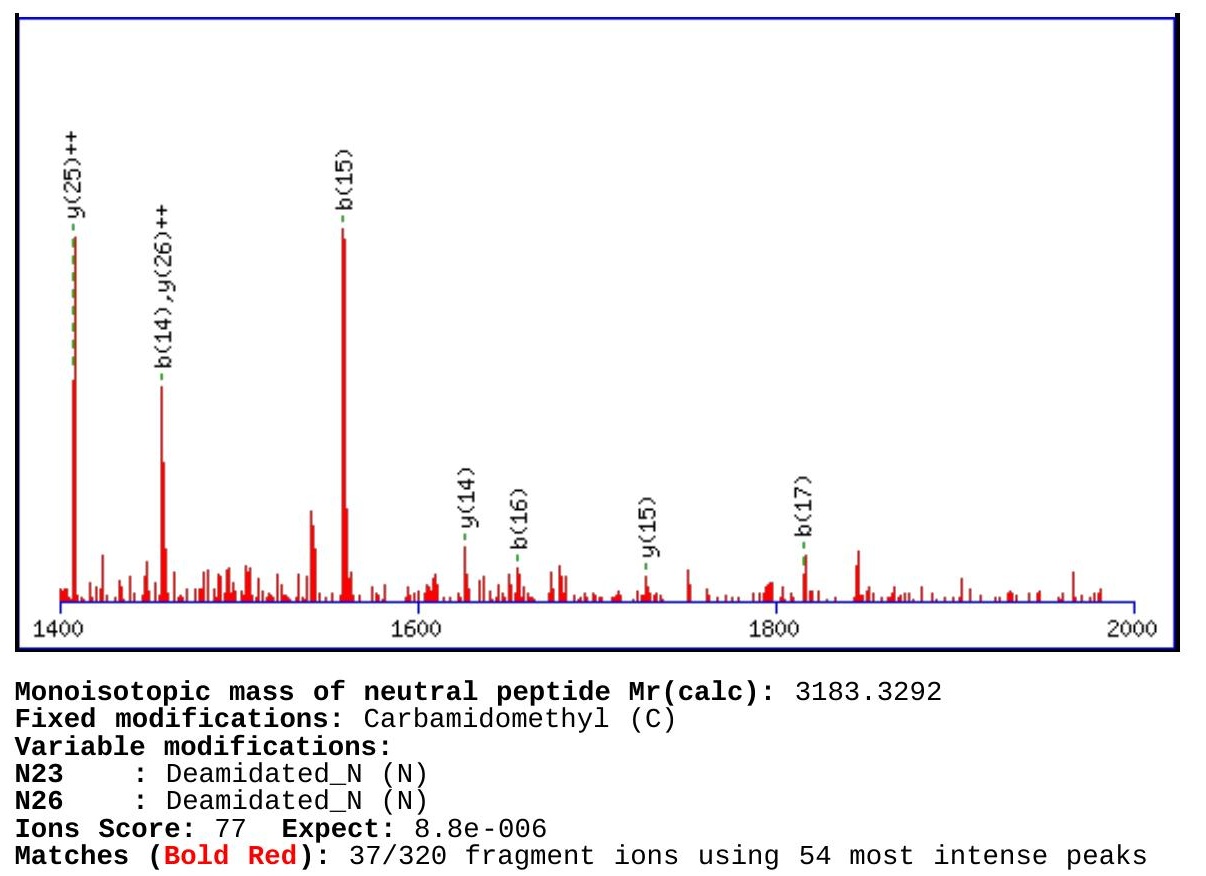

\begin{tabular}{|c|c|c|c|c|c|c|c|c|c|c|c|c|c|c|}
\hline \# & b & $\mathbf{b}^{++}$ & $\mathbf{b}^{*}$ & $\mathbf{b}^{*^{++}}$ & $\mathbf{b}^{0}$ & $\mathbf{b}^{\mathbf{0 + +}}$ & Seq. & $\mathbf{y}$ & $\mathbf{y}^{++}$ & $\mathbf{y}^{*}$ & $\mathrm{y}^{*^{++}}$ & $\mathbf{y}^{0}$ & $\mathbf{y}^{0++}$ & \# \\
\hline 1 & 58.0287 & 29.5180 & & & & & G & & & & & & & 29 \\
\hline 2 & 157.0972 & 79.0522 & & & & & $\mathbf{V}$ & 3127.3150 & 1564.1611 & 3110.2884 & & 3109.3044 & 1558 & 28 \\
\hline 3 & 272.1241 & 136.5657 & & & 254.1135 & 127.5604 & D & 3028.2466 & 1514.6269 & 3011.2200 & 1506.1136 & 3010.2360 & 1505.6216 & 27 \\
\hline 4 & 371.1925 & 186.0999 & & & 353.1819 & 177.0946 & V & 2913.2196 & 1457.1134 & 2896.1931 & 1448.6002 & 2895.2091 & 1448.1082 & 26 \\
\hline 5 & 458.2245 & 229.6159 & & & 440.2140 & 220.6106 & S & 2814.1512 & 1407.5792 & 2797.1247 & 1399.0660 & 2796.1406 & 1398.5740 & 25 \\
\hline 6 & 586.2831 & 293.6452 & 569.2566 & 285.1319 & 568.2726 & 284.6399 & $\mathbf{Q}$ & 2727.1192 & 1364.0632 & 2710.0926 & 1355.5500 & 2709.1086 & 1355.0579 & 24 \\
\hline 7 & 685.3515 & 343.1794 & 668.3250 & 334.6661 & 667.3410 & 334.1741 & V & 2599.0606 & 1300 & 2582.0340 & 1291.5207 & 2581.0500 & 1291.0287 & 23 \\
\hline 8 & 756.3886 & 378.6980 & 739.3621 & 370.1847 & 738.3781 & 369.6927 & A & 2499.9922 & 1250.4997 & 2482.9656 & 1241.9865 & 2481.9816 & 1241.4944 & 22 \\
\hline 9 & 942.4680 & 471.7376 & 925.4414 & 463.2243 & 924.4574 & 462.7323 & $\mathbf{W}$ & 2428.9551 & 1214.9812 & 2411.9285 & 1206.4679 & 2410.9445 & 1205.9759 & 21 \\
\hline 10 & 1070.5265 & 535.7669 & 1053.5000 & 527.2536 & 1052.5160 & 526.7616 & $\mathbf{Q}$ & 2242.8758 & 1121.9415 & 2225.8492 & 1113.4282 & 2224.8652 & 1112.9362 & 20 \\
\hline 11 & 1157.5586 & 579.2829 & 1140.5320 & 570.7696 & 1139.5480 & 570.2776 & S & 2114.8172 & 1057.9122 & 2097.7906 & 1049.3990 & 2096.8066 & 1048.9069 & 19 \\
\hline 12 & 1285.6171 & 643.3122 & 1268.5906 & 634.7989 & 1267.6066 & 634.3069 & $\mathbf{Q}$ & 2027.7852 & 1014.3962 & 2010.7586 & 1005.8829 & 2009.7746 & 1005.3909 & 18 \\
\hline 13 & 1342.6386 & 671.8229 & 1325.6121 & 663.3097 & 1324.6280 & 662.8177 & G & 1899.7266 & 950.3669 & 1882.7000 & 941.8537 & 1881.7160 & 941.3616 & 17 \\
\hline 14 & 1457.6655 & 729.3364 & 1440.6390 & 720.8231 & 1439.6550 & 720.3311 & D & 1842.7051 & 921.8562 & 1825.6786 & 913.3429 & 1824.6945 & 912.8509 & 16 \\
\hline 15 & 1558.7132 & 779.8603 & 1541.6867 & 771.3470 & 1540.7027 & 770.8550 & $\mathbf{T}$ & 1727.6782 & 864.3427 & 1710.6516 & 855.8294 & 1709.6676 & 855.3374 & 15 \\
\hline 16 & 1655.7660 & 828.3866 & 1638.7394 & 819.8734 & 1637.7554 & 819.3814 & $\mathbf{P}$ & 1626.6305 & 813.8189 & 1609.6039 & 805.3056 & 1608.6199 & 804.8136 & 14 \\
\hline 17 & 1815.7966 & 908.4020 & 1798.7701 & 899.8887 & 1797.7861 & 899.3967 & C & 1529.5777 & 765.2925 & 1512.5512 & 756.7792 & 1511.5672 & 756.2872 & 13 \\
\hline 18 & 1902.8287 & 951.9180 & 1885.8021 & 943.4047 & 1884.8181 & 942.9127 & S & 1369.5471 & 685.2772 & 1352.5205 & 676.7639 & 1351.5365 & 676.2719 & 12 \\
\hline 19 & 2062.8593 & 1031.9333 & 2045.8328 & 1023.4200 & 2044.8488 & 1022.9280 & C & 1282.5150 & 641.7612 & 1265.4885 & 633.2479 & 1264.5045 & 632.7559 & 11 \\
\hline 20 & 2222.8900 & 1111.9486 & 2205.8634 & 1103.4353 & 2204.8794 & 1102.9433 & C & 1122.4844 & 561.7458 & 1105.4578 & 553.2326 & 1104.4738 & 552.7406 & 10 \\
\hline 21 & 2335.9740 & 1168.4907 & 2318.9475 & 1159.9774 & 2317.9635 & 1159.4854 & I & 962.4537 & 481.7305 & 945.4272 & 473.2172 & 944.4432 & 472.7252 & 9 \\
\hline 22 & 2435.0424 & 1218.0249 & 2418.0159 & 1209.5116 & 2417.0319 & 1209.0196 & V & 849.3697 & 425.1885 & 832.3431 & 416.6752 & 831.3591 & 416.1832 & 8 \\
\hline 23 & 2550.0694 & 1275.5383 & 2533.0428 & 1267.0251 & 2532.0588 & 1266.5330 & $\mathbf{N}$ & 750.3013 & 375.6543 & 733.2747 & 367.1410 & 732.2907 & 366.6490 & 7 \\
\hline 24 & 2664.1123 & 1332.5598 & 2647.0858 & 1324.0465 & 2646.1017 & 1323.5545 & $\mathbf{N}$ & 635.2743 & 318.1408 & 618.2478 & 309.6275 & 617.2638 & 309.1355 & 6 \\
\hline 25 & 2751.1443 & 1376.0758 & 2734.1178 & 1367.5625 & 2733.1338 & 1367.0705 & S & 521.2314 & 261.1193 & 504.2049 & 252.6061 & 503.2208 & 252.1141 & 5 \\
\hline 26 & 2866.1713 & 1433.5893 & 2849.1447 & 1425.0760 & 2848.1607 & 1424.5840 & $\mathbf{N}$ & 434.1994 & 217.6033 & 417.1728 & 209.0901 & 416.1888 & 208.5980 & 4 \\
\hline 27 & 2923.1927 & 1462.1000 & 2906.1662 & 1453.5867 & 2905.1822 & 1453.0947 & G & 319.1724 & 160.0899 & 302.1459 & 151.5766 & 301.1619 & 151.0846 & 3 \\
\hline 28 & 3010.2248 & 1505.6160 & 2993.1982 & 1497.1027 & 2992.2142 & 1496.6107 & S & 262.1510 & 131.5791 & 245.1244 & 123.0659 & 244.1404 & 122.5738 & 2 \\
\hline 29 & & & & & & & $\mathbf{R}$ & 175.1190 & 88.0631 & 158.0924 & 79.5498 & & & 1 \\
\hline
\end{tabular}



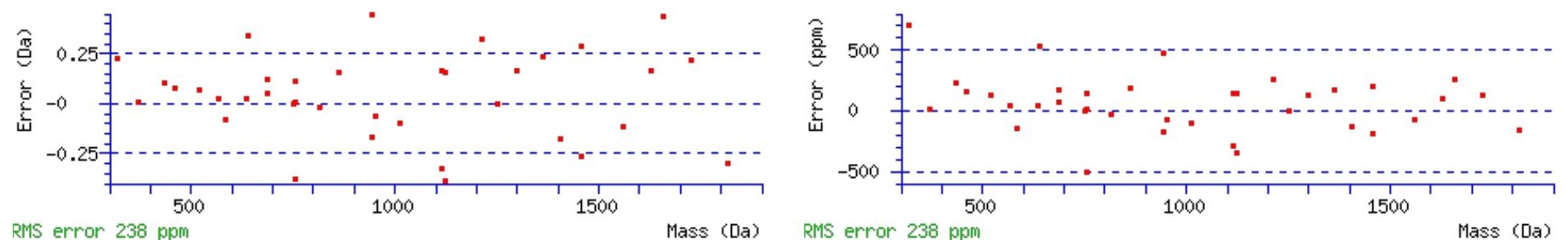

\section{All matches to this query}

\begin{tabular}{|l|l|l|l|}
\hline Score & Mr(calc): & Delta & \multicolumn{1}{c|}{ Sequence } \\
\hline 76.7 & 3183.3292 & 0.0040 & GVDVSQVAWQSQGDTPCSCCIVNNSNGSR \\
\hline 68.5 & 3183.3292 & 0.0040 & GVDVSQVAWQSQGDTPCSCCIVNNSNGSR \\
\hline 66.1 & 3183.3292 & 0.0040 & GVDVSQVAWQSQGDTPCSCCIVNNSNGSR \\
\hline 32.4 & 3182.3452 & 0.9880 & GVDVSQVAWQSQGDTPCSCCIVNNSNGSR \\
\hline 21.1 & 3182.3452 & 0.9880 & GVDVSQVAWQSQGDTPCSCCIVNNSNGSR \\
\hline 20.1 & 3182.3452 & 0.9880 & GVDVSQVAWQSQGDTPCSCCIVNNSNGSR \\
\hline 7.5 & 3181.3199 & 2.0133 & ESLSSYEHHVSAEMKSAASEEKSLEEK \\
\hline 7.5 & 3181.3199 & 2.0133 & ESLSSYEHHVSAEMKSAASEEKSLEEK \\
\hline 5.6 & 3183.3015 & 0.0317 & ESNVNYTICQCNHLTHFGVLMDLSR \\
\hline 4.5 & 3183.3600 & -0.0269 & HFKGSLQDEHWTLNDHQIYNKQGAR \\
\hline
\end{tabular}

Spectrum No: 276; Query: 960; Rank: 1

\section{Peptide View}

MS/MS Fragmentation of DSSGVINVMLNGSEPK

Found in IPI00204984, Tax_Id=10116 Gene_Symbol=Bst1 ADP-ribosyl cyclase 2 precursor

Match to Query 960: 1646.789628 from(824.402090,2+)

Title: 100101RatKid_NS_deglyco_08.3781.3781.2.dta

Data file K:INewmanPaper|Piliang|3SubProteomes\Piliang3SP\mgf5ppm\ERLIC_3SubProteomes5ppm.mgf

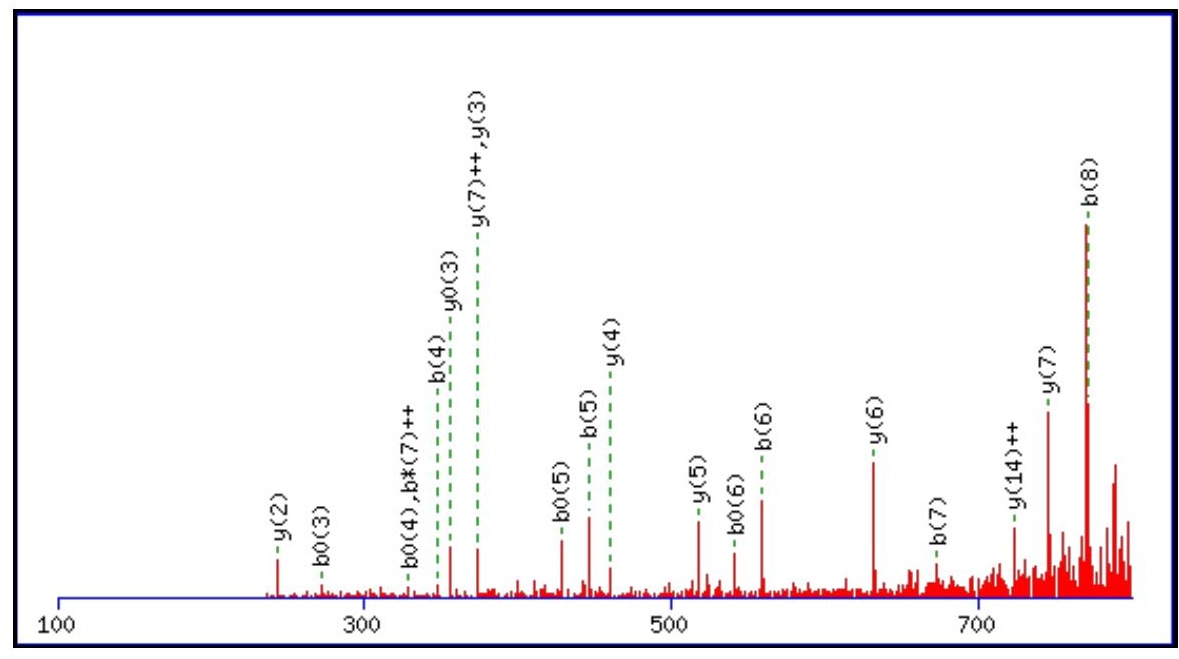



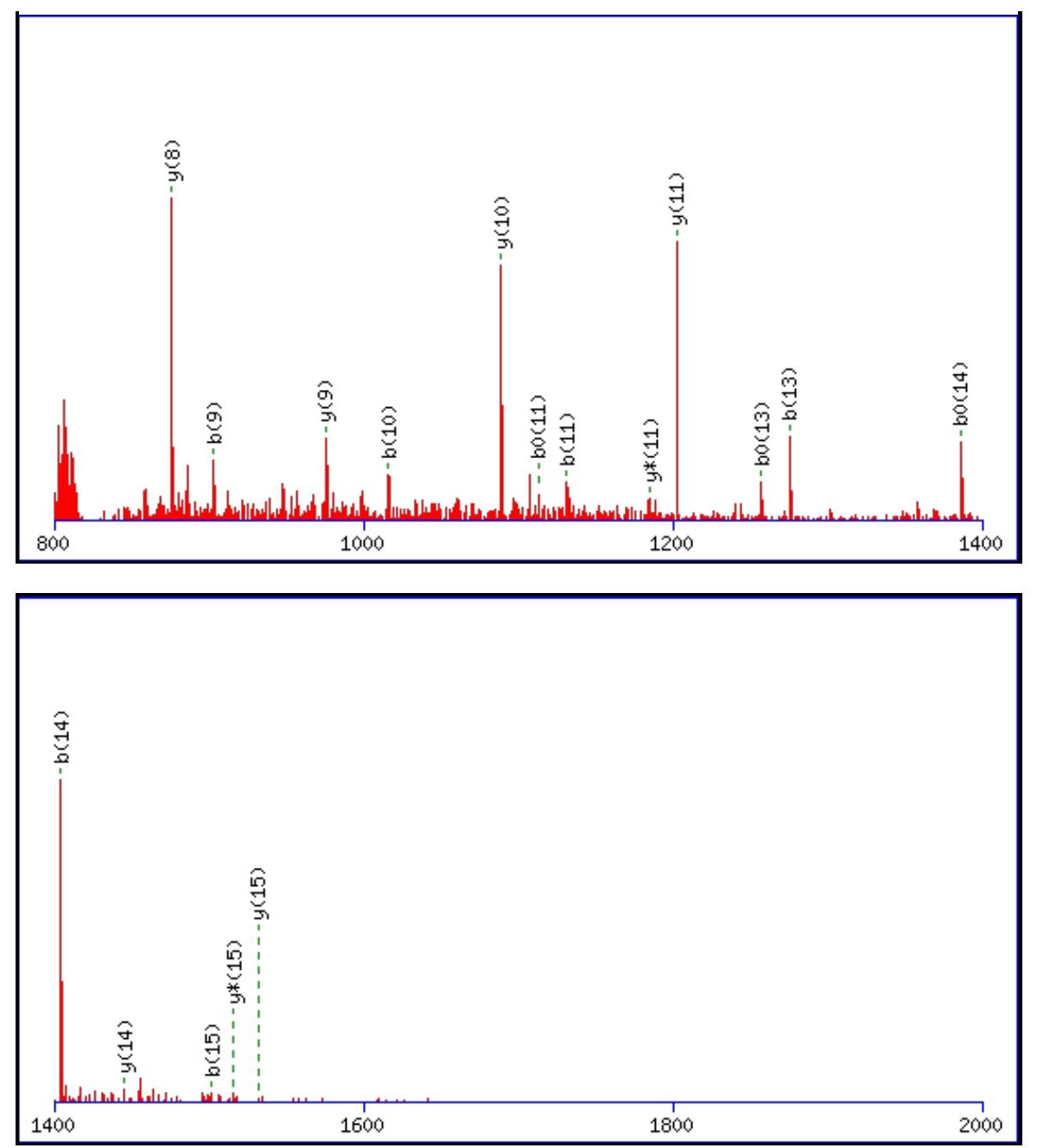

Monoisotopic mass of neutral peptide $\operatorname{Mr}($ calc): 1646.7821

Fixed modifications: Carbamidomethyl (C)

Variable modifications:

N11 : Deamidated $\mathrm{N}(\mathrm{N})$

Ions Score: 77 Expect: $3.9 \mathrm{e}-006$

Matches (Bold Red): 36/164 fragment ions using 65 most intense peaks

\begin{tabular}{|c|c|c|c|c|c|c|c|c|c|c|c|c|c|c|}
\hline \# & b & $\mathbf{b}^{++}$ & b* & $\mathbf{b}^{*^{++}}$ & $\mathbf{b}^{0}$ & $\mathbf{b}^{0++}$ & Seq. & $\mathbf{y}$ & $y^{++}$ & $\mathbf{y}^{*}$ & $\mathrm{y}^{*^{++}}$ & $\mathbf{y}^{\mathbf{0}}$ & $\mathbf{y}^{0++}$ & \# \\
\hline 1 & 116.0342 & 58.5207 & & & 98.0237 & 49.5155 & D & & & & & & & 16 \\
\hline 2 & 203.0662 & 102.0368 & & & 185.0557 & 93.0315 & S & 1532.7625 & 766.8849 & 1515.7359 & 758.3716 & 1514.7519 & 757.8796 & 15 \\
\hline 3 & 290.0983 & 145.5528 & & & 272.0877 & 136.5475 & S & 1445.7304 & 723.3689 & 1428.7039 & 714.8556 & 1427.7199 & 714.3636 & 14 \\
\hline 4 & 347.1197 & 174.0635 & & & 329.1092 & 165.0582 & G & 1358.6984 & 679.8528 & 1341.6719 & 671.3396 & 1340.6879 & 670.8476 & 13 \\
\hline 5 & 446.1882 & 223.5977 & & & 428.1776 & 214.5924 & $\mathbf{V}$ & 1301.6770 & 651.3421 & 1284.6504 & 642.8288 & 1283.6664 & 642.3368 & 12 \\
\hline 6 & 559.2722 & 280.1397 & & & 541.2617 & 271.1345 & I & 1202.6085 & 601.8079 & 1185.5820 & 593.2946 & 1184.5980 & 592.8026 & 11 \\
\hline 7 & 673.3151 & 337.1612 & 656.2886 & 328.6479 & 655.3046 & 328.1559 & $\mathbf{N}$ & 1089.5245 & 545.2659 & 1072.4979 & 536.7526 & 1071.5139 & 536.2606 & 10 \\
\hline 8 & 772.3836 & 386.6954 & 755.3570 & 378.1821 & 754.3730 & 377.6901 & V & 975.4816 & 488.2444 & 958.4550 & 479.7311 & 957.4710 & 479.2391 & 9 \\
\hline 9 & 903.4240 & 452.2157 & 886.3975 & 443.7024 & 885.4135 & 443.2104 & $\mathbf{M}$ & 876.4131 & 438.7102 & 859.3866 & 430.1969 & 858.4026 & 429.7049 & 8 \\
\hline 10 & 1016.5081 & 508.7577 & 999.4816 & 500.2444 & 998.4975 & 499.7524 & $\mathbf{L}$ & 745.3727 & 373.1900 & 728.3461 & 364.6767 & 727.3621 & 364.1847 & 7 \\
\hline 11 & 1131.5350 & 566.2712 & 1114.5085 & 557.7579 & 1113.5245 & 557.2659 & $\mathbf{N}$ & 632.2886 & 316.6479 & 615.2620 & 308.1347 & 614.2780 & 307.6427 & 6 \\
\hline 12 & 1188.5565 & 594.7819 & 1171.5300 & 586.2686 & 1170.5459 & 585.7766 & G & 517.2617 & 259.1345 & 500.2351 & 250.6212 & 499.2511 & 250.1292 & 5 \\
\hline 13 & 1275.5885 & 638.2979 & 1258.5620 & 629.7846 & 1257.5780 & 629.2926 & S & 460.2402 & 230.6237 & 443.2136 & 222.1105 & 442.2296 & 221.6185 & 4 \\
\hline 14 & 1404.6311 & 702.8192 & 1387.6046 & 694.3059 & 1386.6206 & 693.8139 & $\mathbf{E}$ & 373.2082 & 187.1077 & 356.1816 & 178.5944 & 355.1976 & 178.1024 & 3 \\
\hline 15 & 1501.6839 & 751.3456 & 1484.6573 & 742.8323 & 1483.6733 & 742.3403 & $\mathbf{P}$ & 244.1656 & 122.5864 & 227.1390 & 114.0731 & & & 2 \\
\hline 16 & & & & & & & $\mathbf{K}$ & 147.1128 & 74.0600 & 130.0863 & 65.5468 & & & 1 \\
\hline
\end{tabular}



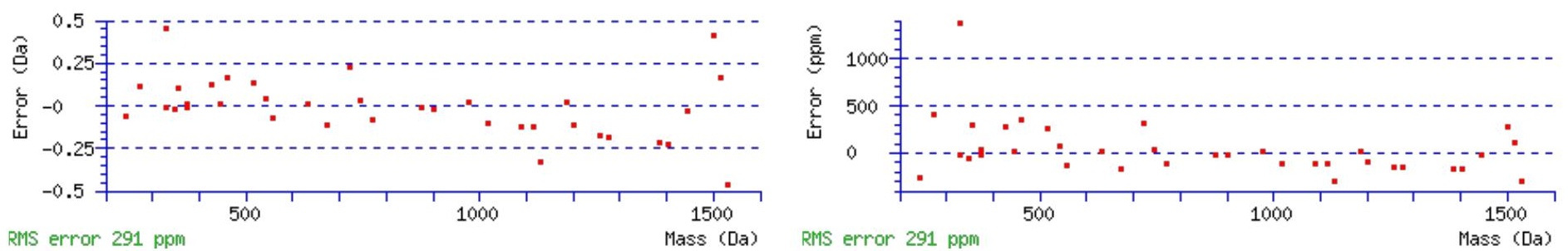

\section{All matches to this query}

\begin{tabular}{|l|c|c|c|}
\hline Score & Mr(calc): & Delta & \multicolumn{1}{c|}{ Sequence } \\
\hline 76.7 & 1646.7821 & 0.0075 & DSSGVINVMLNGSEPK \\
\hline 35.2 & 1646.7821 & 0.0075 & DSSGVINVMLNGSEPK \\
\hline 23.4 & 1645.7981 & 0.9915 & DSSGVINVMLNGSEPK \\
\hline 8.5 & 1646.7748 & 0.0148 & STPSSGDVQVTEDAVR \\
\hline 3.9 & 1645.7921 & 0.9975 & RDGVRSGFEEGGAGPR \\
\hline 2.3 & 1646.8004 & -0.0107 & KATGFQRPYKMPK \\
\hline
\end{tabular}

Spectrum No: 277; Query: 254; Rank: 1

\section{Peptide View}

MS/MS Fragmentation of IEVEANNTVTK

Found in IPI00200640, Tax_Id=10116 Gene_Symbol=Mucdhl Isoform 1 of Mucin and cadherin-like protein precursor

Match to Query 254: 1217.610948 from(609.812750,2+)

Title: 091008RatKidney_NoSalt_31.610.610.2.dta

Data file K:INewmanPaper|Piliang \3SubProteomes\Piliang3SP\mgf5ppm\ERLIC_3SubProteomes5ppm.mgf

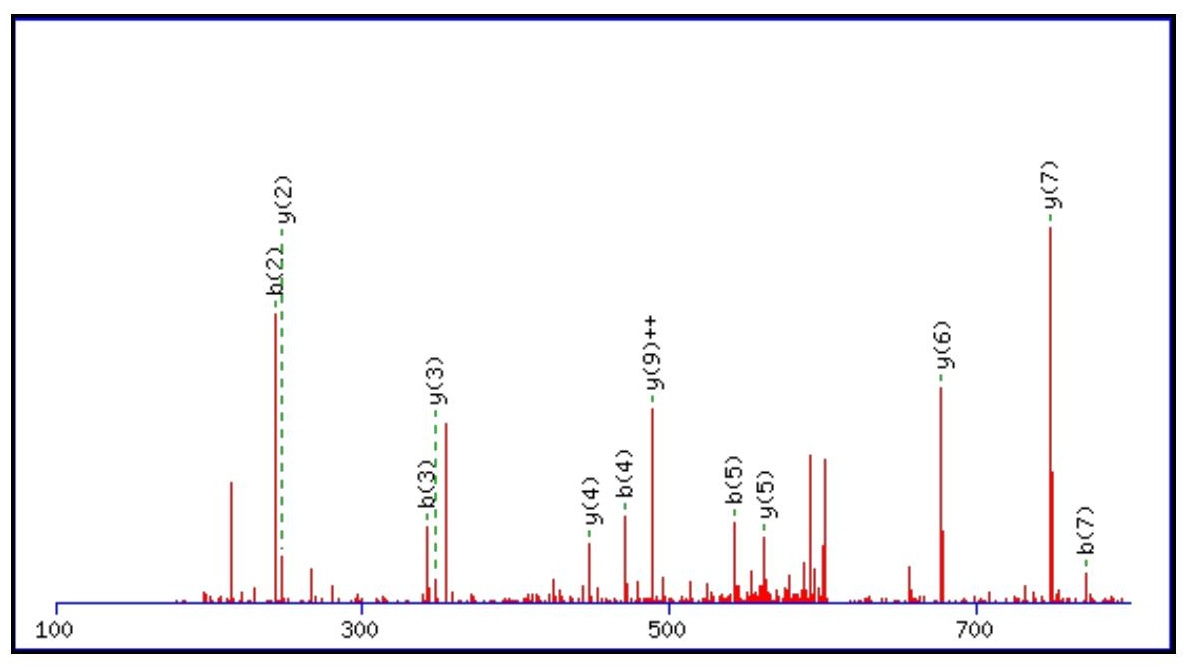



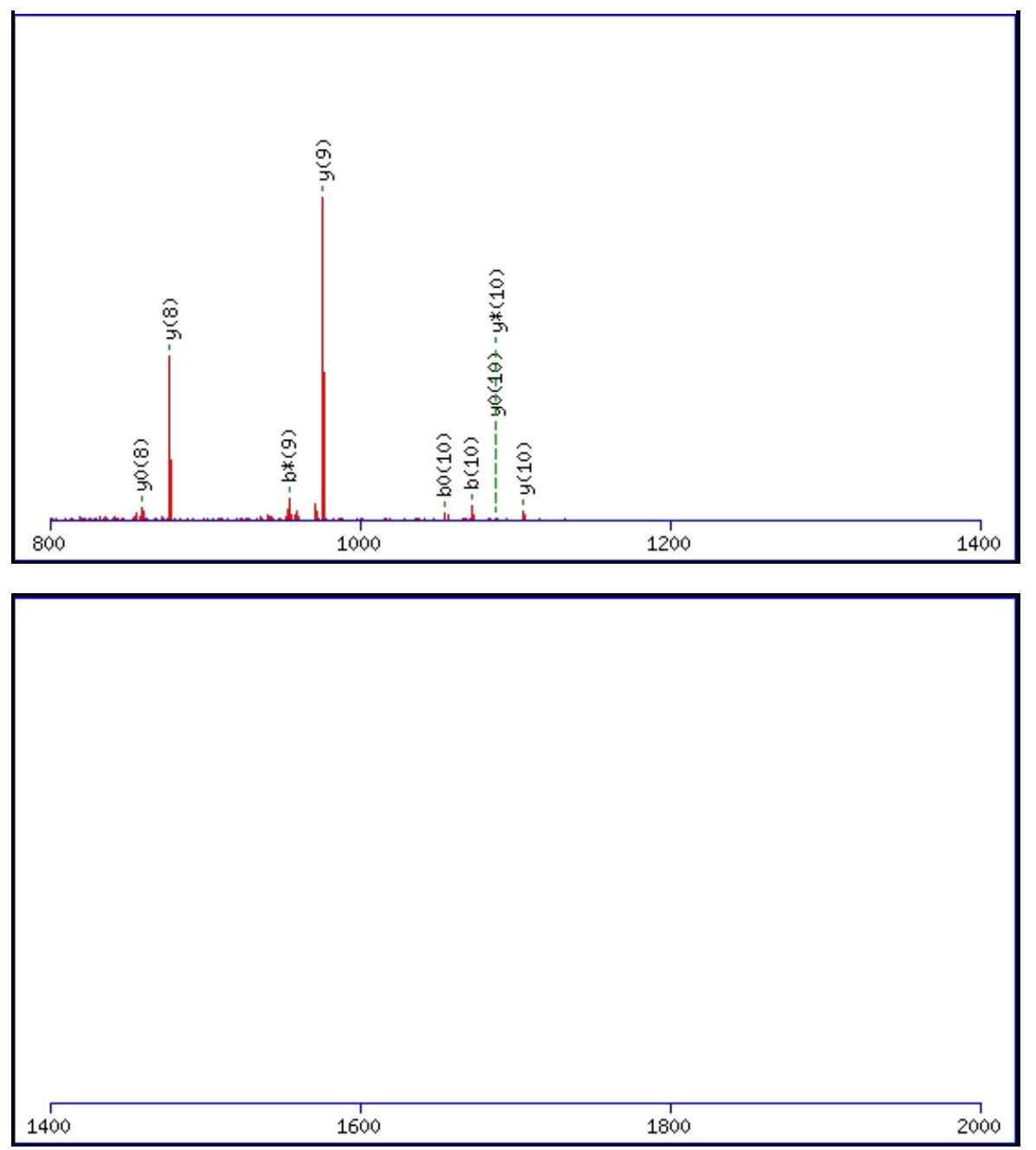

Monoisotopic mass of neutral peptide $\operatorname{Mr}($ calc): 1217.6139

Fixed modifications: Carbamidomethyl (C)

Variable modifications:

N6: Deamidated $\mathrm{N}(\mathrm{N})$

Ions Score: 77 Expect: $2.9 \mathrm{e}-006$

Matches (Bold Red): 21/106 fragment ions using 32 most intense peaks

\begin{tabular}{|r|c|c|c|c|c|c|c|c|c|c|c|c|c|c|}
\hline$\#$ & $\mathbf{b}$ & $\mathbf{b}^{++}$ & $\mathbf{b}^{*}$ & $\mathbf{b}^{\mathbf{*}^{++}}$ & $\mathbf{b}^{\mathbf{0}}$ & $\mathbf{b}^{\mathbf{0}+}$ & $\mathbf{S e q}$ & $\mathbf{y}$ & $\mathbf{y}^{++}$ & $\mathbf{y}^{\mathbf{*}}$ & $\mathbf{y}^{\mathbf{*}^{++}}$ & $\mathbf{y}^{\mathbf{0}}$ & $\mathbf{y}^{\mathbf{0 + +}}$ & $\#$ \\
\hline $\mathbf{1}$ & 114.0913 & 57.5493 & & & & & $\mathbf{I}$ & & & & & & & $\mathbf{1 1}$ \\
\hline $\mathbf{2}$ & $\mathbf{2 4 3 . 1 3 3 9}$ & 122.0706 & & & 225.1234 & 113.0653 & $\mathbf{E}$ & $\mathbf{1 1 0 5 . 5 3 7 2}$ & 553.2722 & $\mathbf{1 0 8 8 . 5 1 0 6}$ & 544.7589 & $\mathbf{1 0 8 7 . 5 2 6 6}$ & $\mathbf{5 4 4 . 2 6 6 9}$ & $\mathbf{1 0}$ \\
\hline $\mathbf{3}$ & $\mathbf{3 4 2 . 2 0 2 3}$ & 171.6048 & & & 324.1918 & 162.5995 & $\mathbf{V}$ & $\mathbf{9 7 6 . 4 9 4 6}$ & $\mathbf{4 8 8 . 7 5 0 9}$ & 959.4680 & 480.2376 & 958.4840 & 479.7456 & $\mathbf{9}$ \\
\hline $\mathbf{4}$ & $\mathbf{4 7 1 . 2 4 4 9}$ & 236.1261 & & & 453.2344 & 227.1208 & $\mathbf{E}$ & $\mathbf{8 7 7 . 4 2 6 1}$ & 439.2167 & 860.3996 & 430.7034 & $\mathbf{8 5 9 . 4 1 5 6}$ & 430.2114 & $\mathbf{8}$ \\
\hline $\mathbf{5}$ & $\mathbf{5 4 2 . 2 8 2 1}$ & 271.6447 & & & 524.2715 & 262.6394 & $\mathbf{A}$ & $\mathbf{7 4 8 . 3 8 3 6}$ & 374.6954 & 731.3570 & 366.1821 & 730.3730 & 365.6901 & $\mathbf{7}$ \\
\hline $\mathbf{6}$ & 657.3090 & 329.1581 & 640.2824 & 320.6449 & 639.2984 & 320.1529 & $\mathbf{N}$ & $\mathbf{6 7 7 . 3 4 6 4}$ & 339.1769 & 660.3199 & 330.6636 & 659.3359 & 330.1716 & $\mathbf{6}$ \\
\hline $\mathbf{7}$ & $\mathbf{7 7 1 . 3 5 1 9}$ & 386.1796 & 754.3254 & 377.6663 & 753.3414 & 377.1743 & $\mathbf{N}$ & $\mathbf{5 6 2 . 3 1 9 5}$ & 281.6634 & 545.2930 & 273.1501 & 544.3089 & 272.6581 & $\mathbf{5}$ \\
\hline $\mathbf{8}$ & $\mathbf{8 7 2 . 3 9 9 6}$ & 436.7034 & 855.3730 & 428.1902 & 854.3890 & 427.6982 & $\mathbf{T}$ & $\mathbf{4 4 8 . 2 7 6 6}$ & 224.6419 & 431.2500 & 216.1287 & 430.2660 & 215.6366 & $\mathbf{4}$ \\
\hline $\mathbf{9}$ & 971.4680 & 486.2376 & $\mathbf{9 5 4 . 4 4 1 5}$ & 477.7244 & 953.4574 & 477.2324 & $\mathbf{V}$ & 347.2289 & 174.1181 & 330.2023 & 165.6048 & 329.2183 & 165.1128 & $\mathbf{3}$ \\
\hline $\mathbf{1 0}$ & $\mathbf{1 0 7 2 . 5 1 5 7}$ & 536.7615 & 1055.4891 & 528.2482 & $\mathbf{1 0 5 4 . 5 0 5 1}$ & 527.7562 & $\mathbf{T}$ & $\mathbf{2 4 8 . 1 6 0 5}$ & 124.5839 & 231.1339 & 116.0706 & 230.1499 & 115.5786 & $\mathbf{2}$ \\
\hline $\mathbf{1 1}$ & & & & & & & $\mathbf{K}$ & 147.1128 & 74.0600 & 130.0863 & 65.5468 & & & $\mathbf{1}$ \\
\hline
\end{tabular}
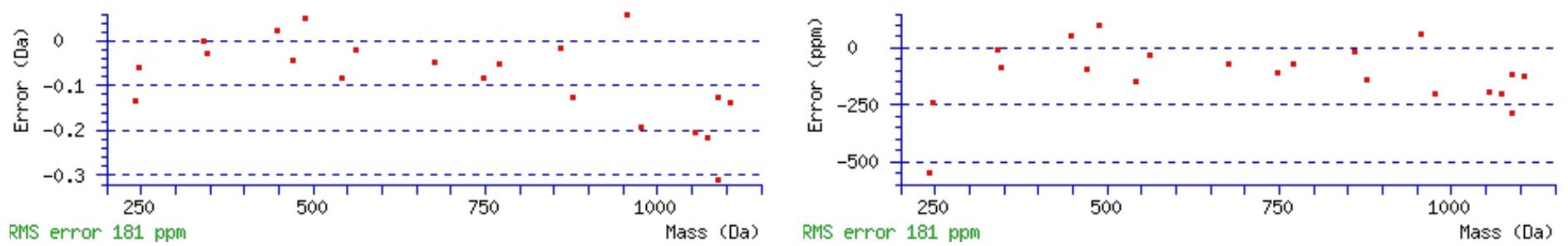


\section{All matches to this query}

\begin{tabular}{|l|c|c|l|}
\hline Score & Mr(calc): & Delta & \multicolumn{1}{c|}{ Sequence } \\
\hline 76.6 & 1217.6139 & -0.0030 & IEVEANNTVTK \\
\hline 61.6 & 1217.6139 & -0.0030 & IEVEANNTVTK \\
\hline 18.4 & 1217.6179 & -0.0070 & LEDLNFPEIK \\
\hline 17.4 & 1217.6169 & -0.0060 & NKLDHSAIIK \\
\hline 12.3 & 1217.6193 & -0.0083 & IEDFWGPAKR \\
\hline 9.3 & 1217.6139 & -0.0030 & IDEIASNLQSK \\
\hline 7.8 & 1217.6097 & 0.0012 & DILFYQVIK \\
\hline 7.5 & 1217.6000 & 0.0109 & XNVQTSESGLR \\
\hline 7.1 & 1217.6000 & 0.0109 & LEQTGETERR \\
\hline 5.9 & 1217.6114 & -0.0005 & LEFPSLPQCK \\
\hline
\end{tabular}

Spectrum No: 278; Query: 927; Rank: 1

\section{Peptide View}

MS/MS Fragmentation of GANLTEDQIFFFPK

Found in IPI00373395, Tax_Id=10116 Gene_Symbol=C8g_predicted 22 kDa protein

Match to Query 927: 1626.793448 from(814.404000,2+)

Title: 091008RatKidney_NoSalt_28.3885.3885.2.dta

Data file K:INewmanPaper|Piliangl3SubProteomes\Piliang3SP\mgf5ppm\ERLIC_3SubProteomes5ppm.mgf
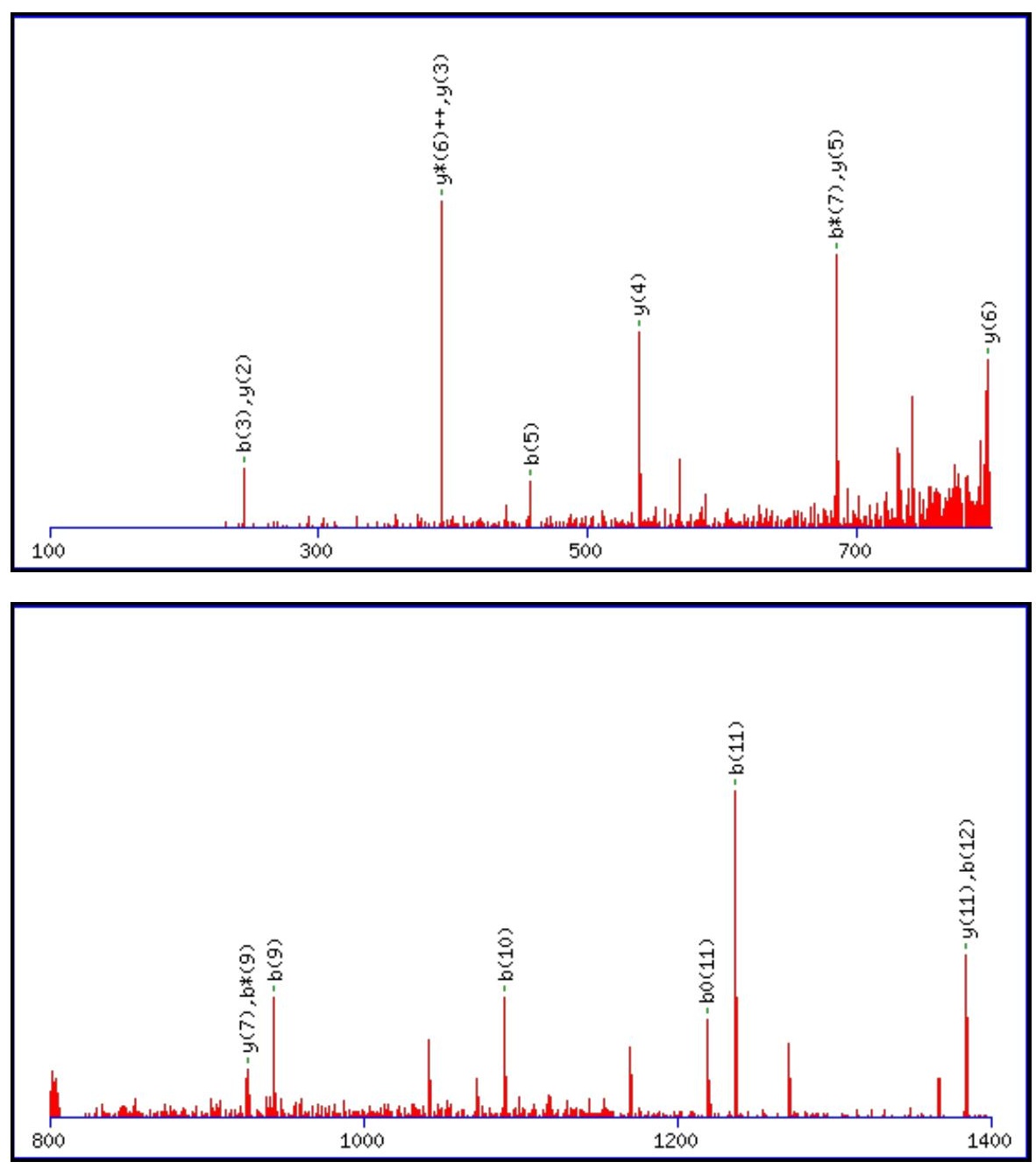


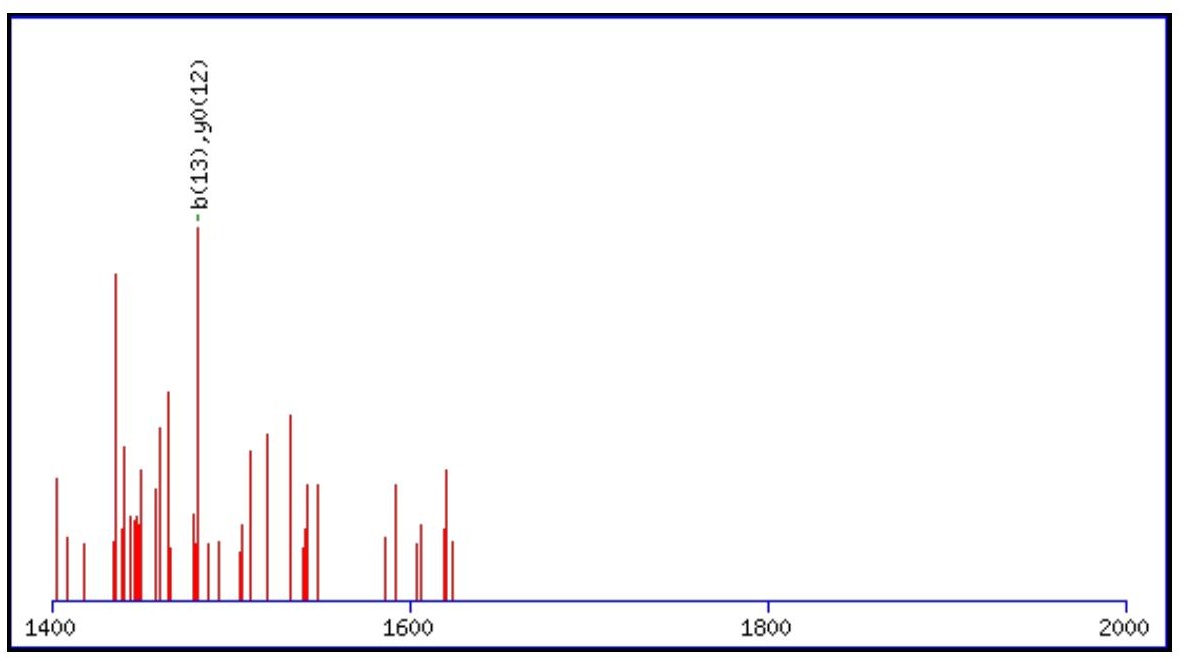

Monoisotopic mass of neutral peptide $\operatorname{Mr}($ calc): 1626.7930

Fixed modifications: Carbamidomethyl (C)

Variable modifications:

N3 : Deamidated_N (N)

Ions Score: 77 Expect: $4 \mathrm{e}-006$

Matches (Bold Red): 19/130 fragment ions using 15 most intense peaks

\begin{tabular}{|c|c|c|c|c|c|c|c|c|c|c|c|c|c|c|}
\hline \# & b & $\mathbf{b}^{++}$ & $\mathbf{b}^{*}$ & $\mathbf{b}^{*++}$ & $\mathbf{b}^{0}$ & $\mathbf{b}^{0++}$ & Seq. & $\mathbf{y}$ & $y^{++}$ & $\mathrm{y}^{*}$ & $\mathrm{y}^{*^{++}}$ & $\mathbf{y}^{0}$ & $y^{0++}$ & $\#$ \\
\hline 1 & 58.0287 & 29.5180 & & & & & G & & & & & & & 14 \\
\hline 2 & 129.0659 & 65.0366 & & & & & A & 1570.7788 & 785.8930 & 1553.7522 & 777.3798 & 1552.7682 & 776.8877 & 13 \\
\hline 3 & 244.0928 & 122.5500 & 227.0662 & 114.0368 & & & $\mathbf{N}$ & 1499.7417 & 750.3745 & 1482.7151 & 741.8612 & 1481.7311 & 741.3692 & 12 \\
\hline 4 & 357.1769 & 179.0921 & 340.1503 & 170.5788 & & & $\mathbf{L}$ & 1384.7147 & 692.8610 & 1367.6882 & 684.3477 & 1366.7042 & 683.8557 & 11 \\
\hline 5 & 458.2245 & 229.6159 & 441.1980 & 221.1026 & 440.2140 & 220.6106 & $T$ & 1271.6307 & 636.3190 & 1254.6041 & 627.8057 & 1253.6201 & 627.3137 & 10 \\
\hline 6 & 587.2671 & 294.1372 & 570.2406 & 285.6239 & 569.2566 & 285.1319 & $\mathbf{E}$ & 1170.5830 & 585.7951 & 1153.5564 & 577.2819 & 1152.5724 & 576.7898 & 9 \\
\hline 7 & 702.2941 & 351.6507 & 685.2675 & 343.1374 & 684.2835 & 342.6454 & D & 1041.5404 & 521.2738 & 1024.5138 & 512.7606 & 1023.5298 & 512.2686 & 8 \\
\hline 8 & 830.3526 & 415.6800 & 813.3261 & 407.1667 & 812.3421 & 406.6747 & $\mathbf{Q}$ & 926.5135 & 463.7604 & 909.4869 & 455.2471 & & & 7 \\
\hline 9 & 943.4367 & 472.2220 & 926.4102 & 463.7087 & 925.4261 & 463.2167 & I & 798.4549 & 399.7311 & 781.4283 & 391.2178 & & & 6 \\
\hline 10 & 1090.5051 & 545.7562 & 1073.4786 & 537.2429 & 1072.4946 & 536.7509 & F & 685.3708 & 343.1890 & 668.3443 & 334.6758 & & & 5 \\
\hline 11 & 1237.5735 & 619.2904 & 1220.5470 & 610.7771 & 1219.5630 & 610.2851 & $\mathbf{F}$ & 538.3024 & 269.6548 & 521.2758 & 261.1416 & & & 4 \\
\hline 121 & 1384.6420 & 692.8246 & 1367.6154 & 684.3113 & 1366.6314 & 683.8193 & $\mathbf{F}$ & 391.2340 & 196.1206 & 374.2074 & 187.6074 & & & 3 \\
\hline 13 & 1481.6947 & 741.3510 & 1464.6682 & 732.8377 & 1463.6842 & 732.3457 & $\mathbf{P}$ & 244.1656 & 122.5864 & 227.1390 & 114.0731 & & & 2 \\
\hline 14 & & & & & & & $\mathbf{K}$ & 147.1128 & 74.0600 & 130.0863 & 65.5468 & & & 1 \\
\hline
\end{tabular}

$$
\text { 照 }
$$
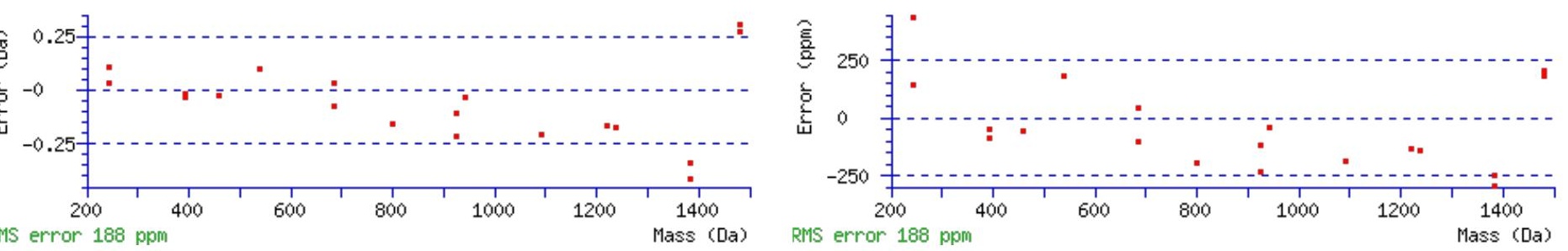

\section{All matches to this query}

\begin{tabular}{|l|l|l|l|}
\hline Score & Mr(calc): & Delta & \multicolumn{1}{c|}{ Sequence } \\
\hline 76.5 & 1626.7930 & 0.0005 & GANLTEDQIFFFPK \\
\hline 12.4 & 1626.8018 & -0.0084 & LVVQDKNKYNTPK \\
\hline 12.1 & 1626.8018 & -0.0084 & YLTGLGSSGLGIGPOK \\
\hline 11.5 & 1626.8018 & -0.0084 & LVVQDKNKYNTPK \\
\hline 11.4 & 1626.7930 & 0.0005 & QDPYLVNFFLENK \\
\hline 9.0 & 1626.8018 & -0.0083 & YEILAQKNLQEAK \\
\hline 5.8 & 1626.7807 & 0.0128 & HPAPPPSNYEVLVK \\
\hline
\end{tabular}




\begin{tabular}{|l|l|l|l|}
5.7 & 1625.7814 & 1.0121 & IAGSLIENYPGERK \\
\hline 5.2 & 1626.7841 & 0.0094 & LYTLLNTCIPPSR \\
\hline 4.6 & 1626.7906 & 0.0029 & IGSTVEDNPLYVLK \\
\hline
\end{tabular}

Spectrum No: 279; Query: 610; Rank: 1

\section{Peptide View}

MS/MS Fragmentation of VWVNSSDGNALIGK

Found in IPI00193894, Tax_Id=10116 Gene_Symbol=Mgam_predicted 198 kDa protein

Match to Query 610: 1459.731028 from(730.872790,2+)

Title: 091008RatKidney_NH4Format01_23.2168.2168.2.dta

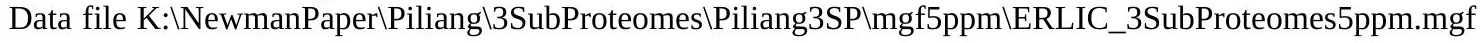
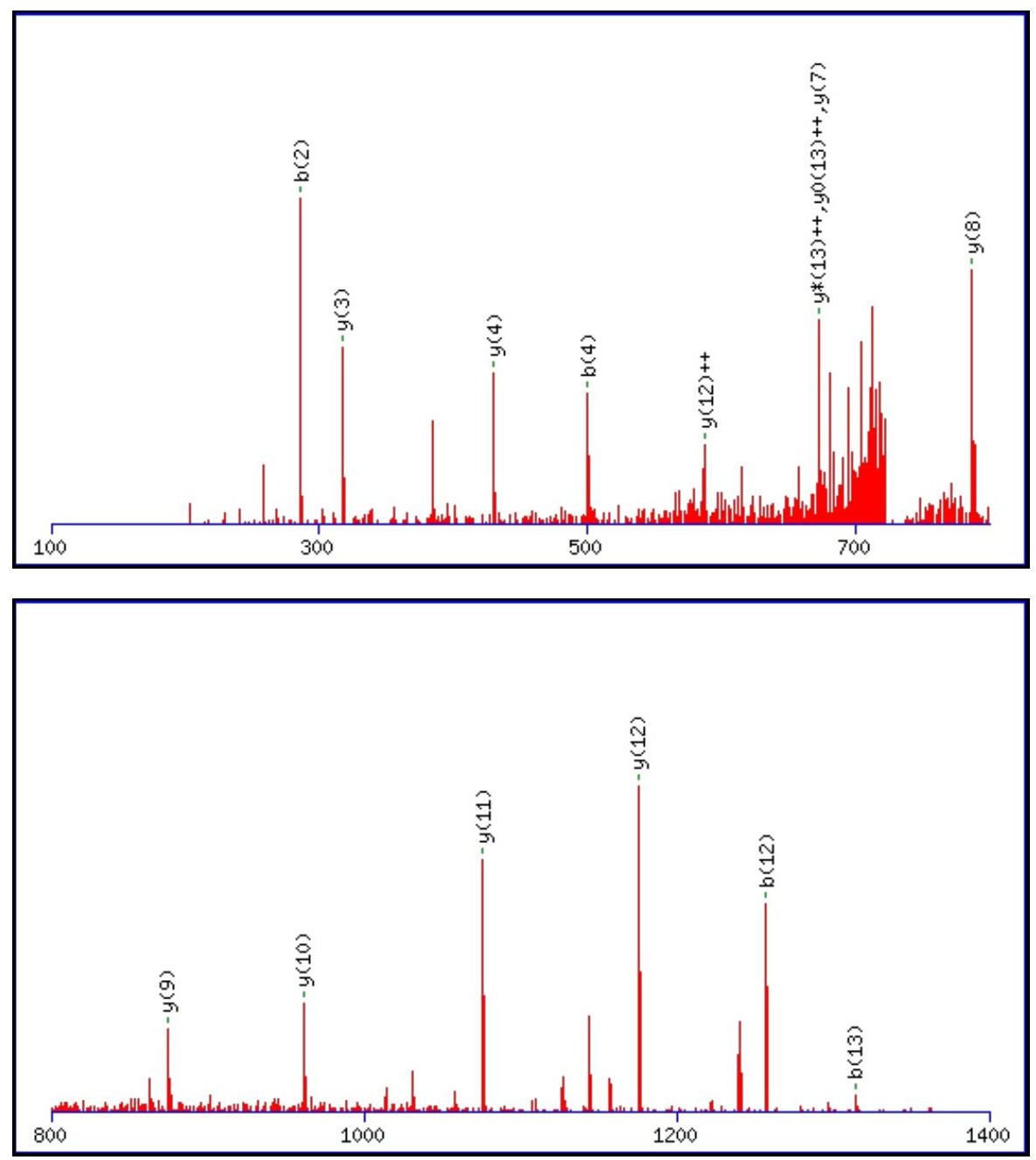


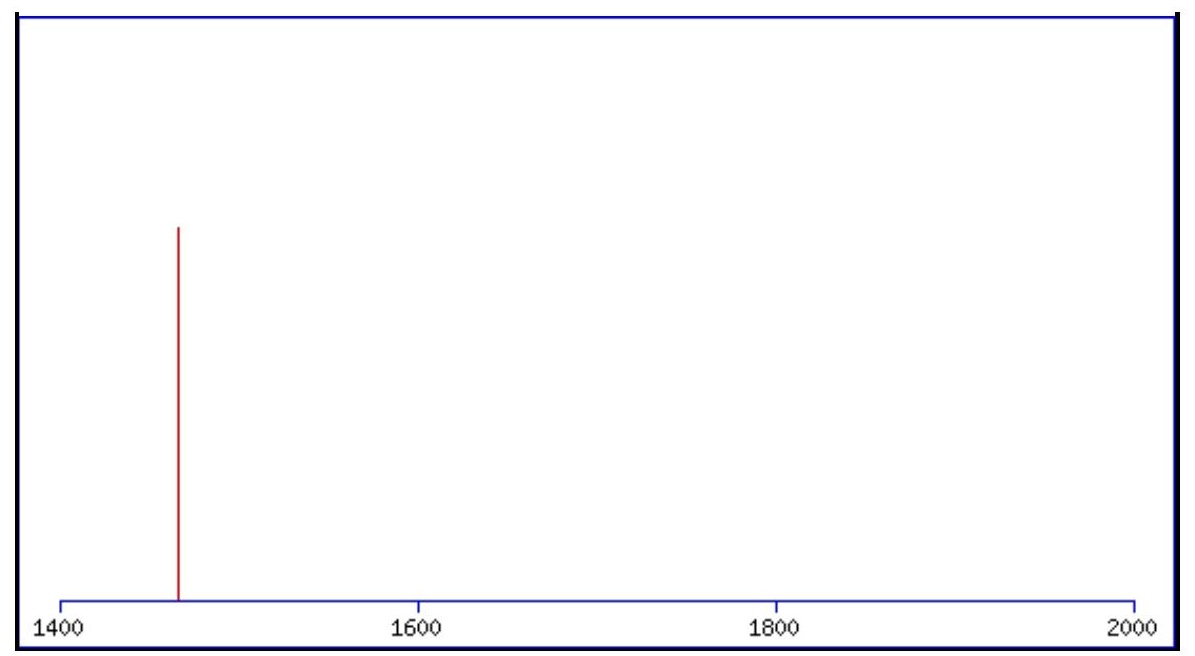

Monoisotopic mass of neutral peptide $\operatorname{Mr}($ calc): 1459.7307

Fixed modifications: Carbamidomethyl (C)

Variable modifications:

N4 : Deamidated_N (N)

Ions Score: 76 Expect : $3.3 \mathrm{e}-006$

Matches (Bold Red): 15/128 fragment ions using 15 most intense peaks

\begin{tabular}{|c|c|c|c|c|c|c|c|c|c|c|c|c|c|c|}
\hline \# & b & $\mathbf{b}^{++}$ & $\mathbf{b}^{*}$ & $\mathbf{b}^{*^{++}}$ & $\mathbf{b}^{0}$ & $\mathbf{b}^{0++}$ & Seq. & $\mathbf{y}$ & $y^{++}$ & $\mathbf{y}^{*}$ & $\mathbf{y}^{*^{++}}$ & $\mathbf{y}^{0}$ & $\mathbf{y}^{\mathbf{0 + +}}$ & \# \\
\hline 1 & 100.0757 & 50.5415 & & & & & $\mathbf{V}$ & & & & & & & 14 \\
\hline 2 & 286.1550 & 143.5811 & & & & & $\mathbf{W}$ & 1361.6696 & 681.3384 & 1344.6430 & 672.8251 & 1343.6590 & 672.3331 & 13 \\
\hline 3 & 385.2234 & 193.1153 & & & & & V & 1175.5902 & 588.2988 & 1158.5637 & 579.7855 & 1157.5797 & 579.2935 & 12 \\
\hline 4 & 500.2504 & 250.6288 & 483.2238 & 242.1155 & & & $\mathbf{N}$ & 1076.5218 & 538.7646 & 1059.4953 & 530.2513 & 1058.5113 & 529.7593 & 11 \\
\hline 5 & 587.2824 & 294.1448 & 570.2558 & 285.6316 & 569.2718 & 285.1395 & $S$ & 961.4949 & 481.2511 & 944.4684 & 472.7378 & 943.4843 & 472.2458 & 10 \\
\hline 6 & 674.3144 & 337.6608 & 657.2879 & 329.1476 & 656.3038 & 328.6556 & S & 874.4629 & 437.7351 & 857.4363 & 429.2218 & 856.4523 & 428.7298 & 9 \\
\hline 7 & 789.3414 & 395.1743 & 772.3148 & 386.6610 & 771.3308 & 386.1690 & D & 787.4308 & 394.2191 & 770.4043 & 385.7058 & 769.4203 & 385.2138 & 8 \\
\hline 8 & 846.3628 & 423.6850 & 829.3363 & 415.1718 & 828.3523 & 414.6798 & G & 672.4039 & 336.7056 & 655.3774 & 328.1923 & & & 7 \\
\hline 9 & 960.4057 & 480.7065 & 943.3792 & 472.1932 & 942.3952 & 471.7012 & $\mathbf{N}$ & 615.3824 & 308.1949 & 598.3559 & 299.6816 & & & 6 \\
\hline 10 & 1031.4429 & 516.2251 & 1014.4163 & 507.7118 & 1013.4323 & 507.2198 & A & 501.3395 & 251.1734 & 484.3130 & 242.6601 & & & 5 \\
\hline 11 & 1144.5269 & 572.7671 & 1127.5004 & 564.2538 & 1126.5164 & 563.7618 & $\mathbf{L}$ & 430.3024 & 215.6548 & 413.2758 & 207.1416 & & & 4 \\
\hline 12 & 1257.6110 & 629.3091 & 1240.5844 & 620.7959 & 1239.6004 & 620.3038 & I & 317.2183 & 159.1128 & 300.1918 & 150.5995 & & & 3 \\
\hline 13 & 1314.6324 & 657.8199 & 1297.6059 & 649.3066 & 1296.6219 & 648.8146 & G & 204.1343 & 102.5708 & 187.1077 & 94.0575 & & & 2 \\
\hline 14 & & & & & & & $\mathbf{K}$ & 147.1128 & 74.0600 & 130.0863 & 65.5468 & & & 1 \\
\hline
\end{tabular}
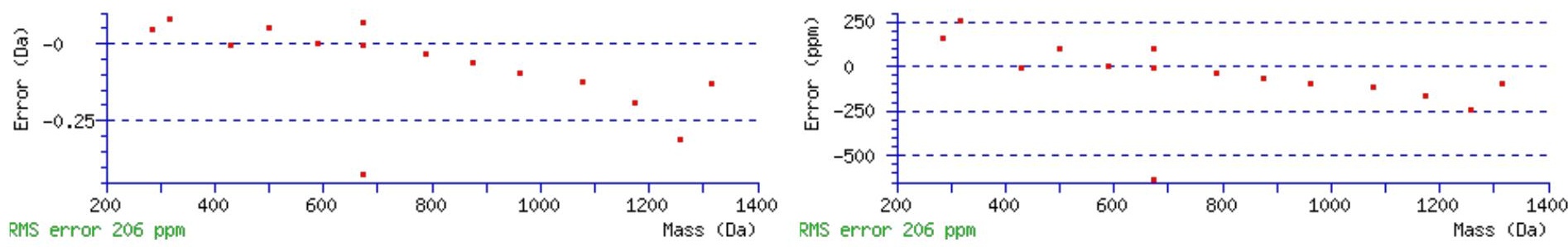

\section{All matches to this query}

\begin{tabular}{|l|l|l|l|}
\hline Score & Mr(calc): & Delta & \multicolumn{1}{c|}{ Sequence } \\
\hline 76.4 & 1459.7307 & 0.0003 & VWVNSSDGNALIGK \\
\hline 57.3 & 1459.7307 & 0.0003 & VWVNSSDGNALIGK \\
\hline 11.4 & 1459.7201 & 0.0110 & IASLSRSVVALGK \\
\hline 4.9 & 1459.7225 & 0.0086 & AITAFTAGFVNIR \\
\hline 4.4 & 1459.7200 & 0.0110 & KNLAKSQTLLGK \\
\hline 3.6 & 1457.7110 & 2.0200 & LSTVPSQDAGAQER \\
\hline 3.4 & 1459.7184 & 0.0126 & ANNLSSLSKKYR \\
\hline & & & \\
\hline
\end{tabular}




\begin{tabular}{|l|l|l|l|}
2.1 & 1459.7201 & 0.0110 & IASLSRSVVALGK \\
\hline 1.6 & 1459.7224 & 0.0086 & NGLWGHALLLASK \\
\hline 1.2 & 1459.7225 & 0.0086 & KRDSFLPFSVGK \\
\hline
\end{tabular}

Spectrum No: 280; Query: 989; Rank: 1

\section{Peptide View}

MS/MS Fragmentation of VGVEAFFPNGTQFSR

Found in IPI00204808, Tax_Id=10116 Gene_Symbol=Mep1b Meprin A subunit beta precursor

Match to Query 989: 1655.793008 from(828.903780,2+)

Title: 091008RatKidney_NH4Format01_24.3507.3507.2.dta

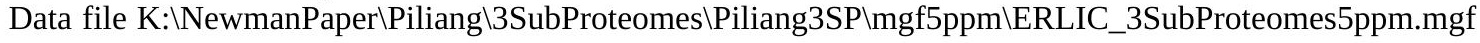
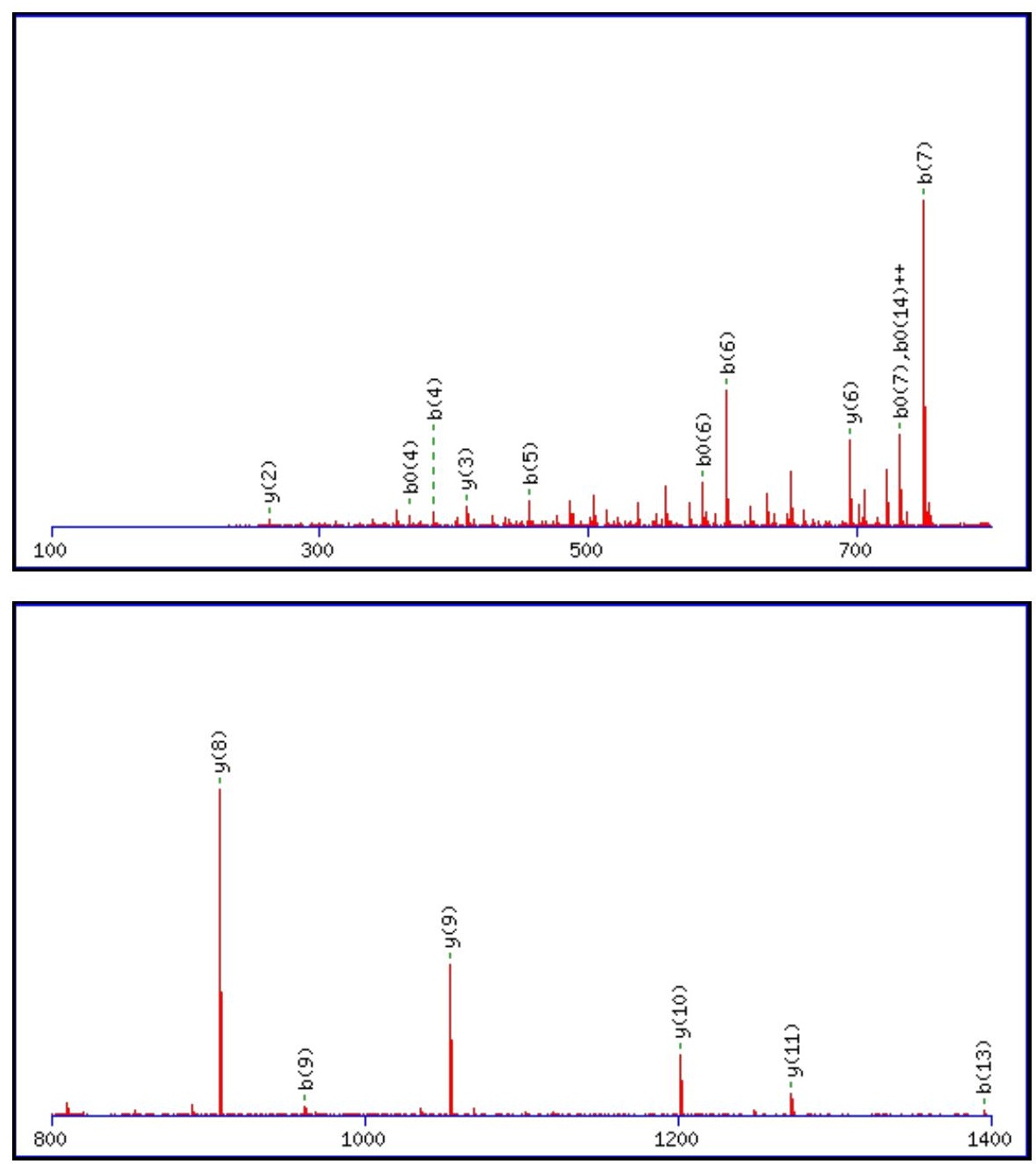


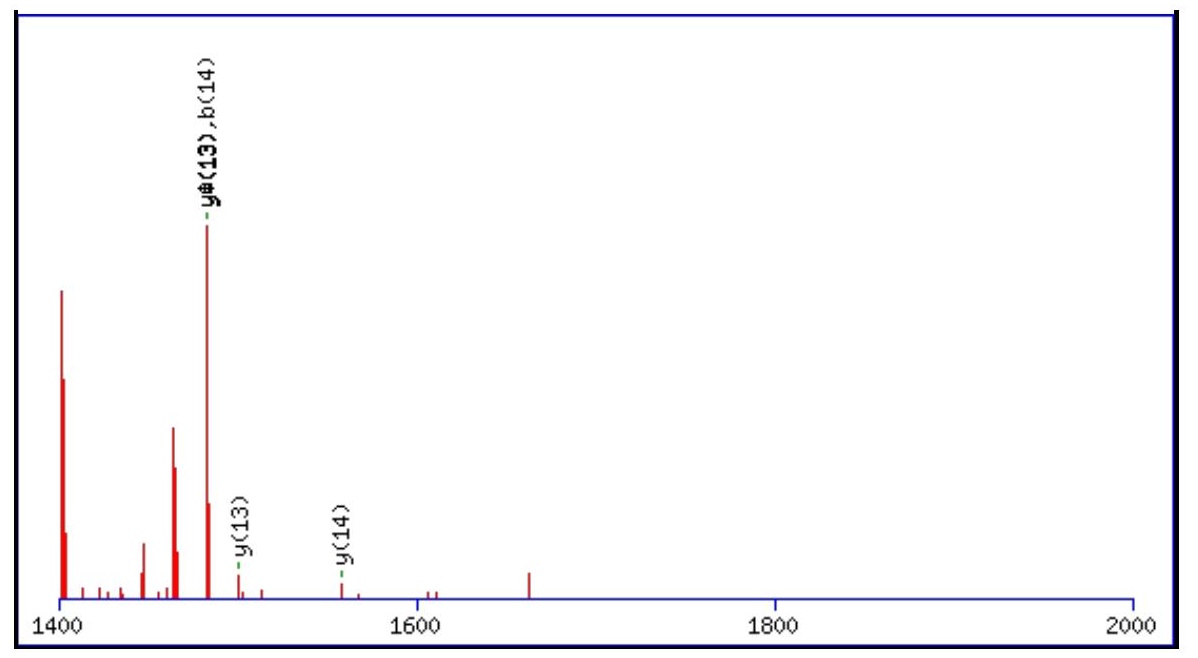

Monoisotopic mass of neutral peptide $\operatorname{Mr}($ calc): 1655.7944

Fixed modifications: Carbamidomethyl (C)

Variable modifications:

N9 : Deamidated_N (N)

Ions Score: 76 Expect : $4.2 \mathrm{e}-006$

Matches (Bold Red): 22/144 fragment ions using 32 most intense peaks

\begin{tabular}{|c|c|c|c|c|c|c|c|c|c|c|c|c|c|c|}
\hline$\#$ & b & $\mathbf{b}^{++}$ & $\mathbf{b}^{*}$ & $\mathbf{b}^{*^{++}}$ & $\mathbf{b}^{0}$ & $\mathbf{b}^{\mathbf{0 + +}}$ & Seq. & $\mathbf{y}$ & $\mathbf{y}^{++}$ & $\mathbf{y}^{*}$ & $\mathrm{y}^{\mathrm{*}^{++}}$ & $\mathbf{y}^{0}$ & $\mathbf{y}^{\mathbf{0}^{++}}$ & \# \\
\hline 1 & 100.0757 & 50.5415 & & & & & V & & & & & & & \\
\hline 2 & 157.0972 & 79.0522 & & & & & $\mathbf{G}$ & 1557.7332 & 779.3703 & 1540.7067 & 770.8570 & 1539.7227 & 770.3650 & \\
\hline 3 & 256.1656 & 128.5864 & & & & & $\mathbf{V}$ & 1500.7118 & 750.8595 & 1483.6852 & 742.3462 & 1482.7012 & 741.8542 & \\
\hline 4 & 385.2082 & 193.1077 & & & 367.1976 & 184.1024 & $\mathbf{E}$ & 1401.6434 & 701.3253 & 1384.6168 & 692.8120 & 1383.6328 & 692.3200 & \\
\hline 5 & 456.2453 & 228.6263 & & & 438.2347 & 219.6210 & A & 1272.6008 & 636.8040 & 1255.5742 & 628.2907 & 1254.5902 & 627.7987 & 11 \\
\hline 6 & 603.3137 & 302.1605 & & & 585.3031 & 293.1552 & $\mathbf{F}$ & 1201.5636 & 601.2855 & 1184.5371 & 592.7722 & 1183.5531 & 592.2802 & \\
\hline 7 & 750.3821 & 375.6947 & & & 732.3715 & 366.6894 & $\mathbf{F}$ & 1054.4952 & 527.7513 & 1037.4687 & 519.2380 & 1036.4847 & 518.7460 & \\
\hline 8 & 847.4349 & 424.2211 & & & 829.4243 & 415.2158 & $\mathbf{P}$ & 907.4268 & 454.2170 & 890.4003 & 445.7038 & 889.4163 & 445.2118 & \\
\hline 9 & 962.4618 & 481.7345 & 945.4353 & 473.2213 & 944.4512 & 472.7293 & $\mathbf{N}$ & 810.3741 & 405.6907 & 793.3475 & 397.1774 & 792.3635 & 396.6854 & \\
\hline 10 & 1019.4833 & 510.2453 & 1002.4567 & 501.7320 & 1001.4727 & 501.2400 & $\mathbf{G}$ & 695.3471 & 348.1772 & 678.3206 & 339.6639 & 677.3366 & 339.1719 & \\
\hline 11 & 1120.5309 & 560.7691 & 1103.5044 & 552.2558 & 1102.5204 & 551.7638 & $T$ & 638.3257 & 319.6665 & 621.2991 & 311.1532 & 620.3151 & 310.6612 & \\
\hline 12 & 1248.5895 & 624.7984 & 1231.5630 & 616.2851 & 1230.5790 & 615.7931 & $\mathbf{Q}$ & 537.2780 & 269.1426 & 520.2514 & 260.6293 & 519.2674 & 260.1373 & \\
\hline 13 & 1395.6579 & 698.3326 & 1378.6314 & 689.8193 & 1377.6474 & 689.3273 & $\mathbf{F}$ & 409.2194 & 205.1133 & 392.1928 & 196.6001 & 391.2088 & 196.1081 & \\
\hline 14 & 1482.6900 & 741.8486 & 1465.6634 & 733.3353 & 1464.6794 & 732.8433 & $\mathrm{~S}$ & 262.1510 & 131.5791 & 245.1244 & 123.0659 & 244.1404 & 122.5738 & \\
\hline 15 & & & & & & & $\mathbf{R}$ & 175.1190 & 88.0631 & 158.0924 & 79.5498 & & & \\
\hline
\end{tabular}
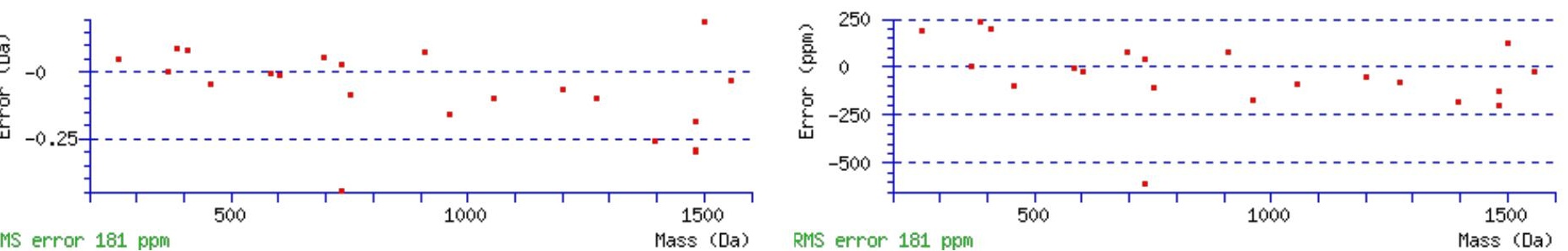

\section{All matches to this query}

\begin{tabular}{|l|l|l|l|}
\hline Score & Mr(calc): & Delta & \multicolumn{1}{c|}{ Sequence } \\
\hline 76.4 & 1655.7944 & -0.0014 & VGVEAFFPNGTQFSR \\
\hline 12.3 & 1654.8001 & 0.9929 & RDTMLGVSIVLENK \\
\hline 7.4 & 1654.8001 & 0.9929 & RDTMLGVSIVLENK \\
\hline 6.8 & 1654.7941 & 0.9990 & ATSPFRSPVRGASSR \\
\hline 5.9 & 1654.8039 & 0.9891 & ATLAEVATVSSGSARR \\
\hline 5.5 & 1654.8039 & 0.9891 & ATLAEVATVSSGSARR \\
\hline & & & \\
\hline
\end{tabular}




\begin{tabular}{|l|l|l|l|}
\hline 5.5 & 1655.7821 & 0.0109 & SSFASLFSFRRSGK \\
\hline 5.5 & 1655.7821 & 0.0109 & SSFASLFSFRRSGK \\
\hline 4.0 & 1655.7976 & -0.0046 & ATIAAGTSQAGQGSGHSR \\
\hline 2.8 & 1655.8011 & -0.0081 & ANIMRMSDGLFLQK \\
\hline
\end{tabular}

Spectrum No: 281; Query: 2729; Rank: 1

\section{Peptide View}

MS/MS Fragmentation of GFDTYFGYLLGSEDYYTHEACAPIECLNGTR

Found in IPI00198405, Tax_Id=10116 Gene_Symbol=Arsb Arylsulfatase B

Match to Query 2729: 3620.566542 from(1207.862790,3+)

Title: 100101RatKid_NS_deglyco_09.7047.7047.3.dta

Data file K:\NewmanPaper\Piliang \3SubProteomes \Piliang3SP $\backslash$ mgf5ppm\ERLIC_3SubProteomes5ppm.mgf
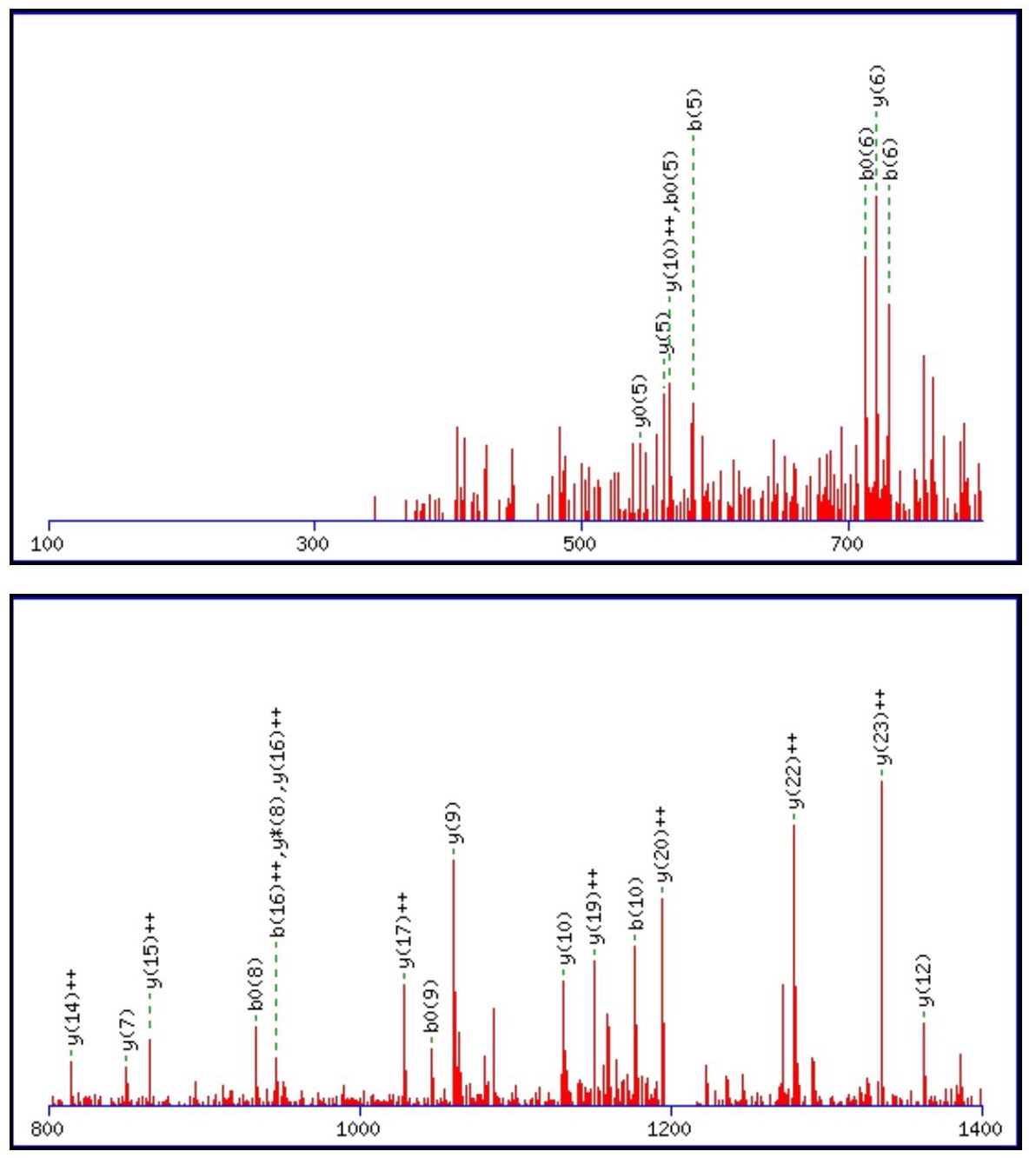


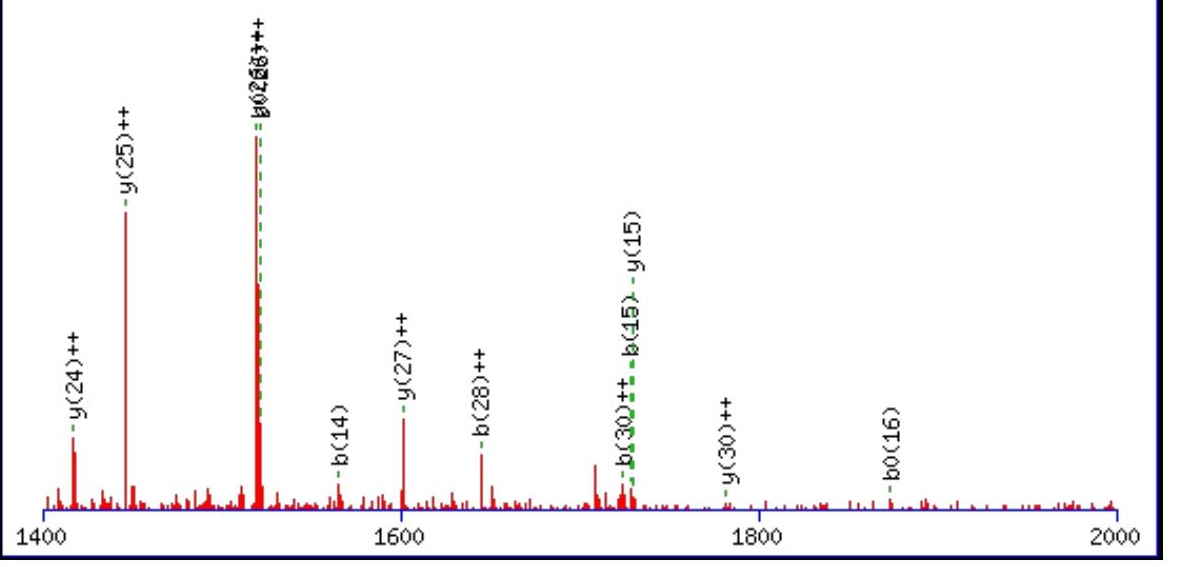

Monoisotopic mass of neutral peptide $\operatorname{Mr}($ calc): 3619.5548

Fixed modifications: Carbamidomethyl (C)

Variable modifications:

N28 : Deamidated_N (N)

Ions Score: 76 Expect: $1.1 \mathrm{e}-005$

Matches (Bold Red): 37/300 fragment ions using 61 most intense peaks

\begin{tabular}{|c|c|c|c|c|c|c|c|c|c|c|c|c|c|c|}
\hline \# & b & $\mathbf{b}^{++}$ & $\mathbf{b}^{*}$ & $\mathbf{b}^{*^{++}}$ & $\mathbf{b}^{\mathbf{0}}$ & $\mathbf{b}^{\mathbf{0 + +}}$ & Seq. & $\mathbf{y}$ & $\mathbf{y}^{++}$ & $\mathbf{y}^{*}$ & $\mathbf{y}^{*^{++}}$ & $\mathbf{y}^{0}$ & $\mathbf{y}^{\mathbf{0 + +}}$ & \# \\
\hline 1 & 58.0287 & 29.5180 & & & & & G & & & & & & & 31 \\
\hline 2 & 205.0972 & 103.0522 & & & & & F & 3563.5406 & 1782.2739 & 3546.5141 & 1773.7607 & 3545.5300 & 1773.2687 & 30 \\
\hline 3 & 320.1241 & 160.5657 & & & 302.1135 & 151.5604 & D & 3416.4722 & 1708.7397 & 3399.4456 & 1700.2265 & 3398.4616 & 1699.7345 & 29 \\
\hline 4 & 421.1718 & 211.0895 & & & 403.1612 & 202.0842 & $\mathbf{T}$ & 3301.4453 & 1651.2263 & 3284.4187 & 1642.7130 & 3283.4347 & 1642.2210 & 28 \\
\hline 5 & 584.2351 & 292.6212 & & & 566.2245 & 283.6159 & $\mathbf{Y}$ & 3200.3976 & 1600.7024 & 3183.3710 & 1592.1892 & 3182.3870 & 1591.6971 & 27 \\
\hline 6 & 731.3035 & 366.1554 & & & 713.2930 & 357.1501 & $\mathbf{F}$ & 3037.3342 & $\mid 1519.1708$ & |3020.3077 & 1510.6575 & 3019.3237 & 1510.1655 & 26 \\
\hline 7 & 788.3250 & 394.6661 & & & 770.3144 & 385.6608 & G & 2890.2658 & 1445.6366 & 2873.2393 & 1437.1233 & 2872.2553 & 1436.6313 & 25 \\
\hline 8 & 951.3883 & 476.1978 & & & 933.3777 & 467.1925 & $\mathbf{Y}$ & 2833.2444 & 1417.1258 & 2816.2178 & 1408.6125 & 2815.2338 & 1408.1205 & 24 \\
\hline 9 & 1064.4724 & 532.7398 & & & 1046.4618 & 523.7345 & $\mathbf{L}$ & 2670.1810 & 1335.5942 & 2653.1545 & 1327.0809 & 2652.1705 & 1326.5889 & 23 \\
\hline 10 & 1177.5564 & 589.2819 & & & 1159.5459 & 580.2766 & $\mathbf{L}$ & 2557.0970 & 1279.0521 & 2540.0704 & 1270.5389 & 2539.0864 & 1270.0468 & 22 \\
\hline 11 & 1234.5779 & 617.7926 & & & 1216.5673 & 608.7873 & G & 2444.0129 & 1222.5101 & 2426.9864 & 1213.9968 & 2426.0023 & 1213.5048 & 21 \\
\hline 12 & 1321.6099 & 661.3086 & & & 1303.5994 & 652.3 & $\mathrm{~S}$ & 2386.9914 & 1193.9994 & 2369 & 861 & 2368.9809 & 1184.9941 & 20 \\
\hline 13 & 1450.6525 & 725.8299 & & & 1432.6420 & 716.8246 & $\mathbf{E}$ & 2299.9594 & \begin{tabular}{|l|l|}
1150.483 \\
\end{tabular} & 2282.9329 & 1141.9701 & 2281.9489 & 1141.4781 & 19 \\
\hline 14 & 1565.6795 & 783.3434 & & & 1547.6689 & 774.3381 & D & 2170.9168 & 1085.9621 & 2153.8903 & 1077.4488 & 2152.9063 & 1076.9568 & 18 \\
\hline 15 & 1728.7428 & 864.8750 & & & & & $\mathbf{Y}$ & 899 & 1028 & 2038 & & 793 & & 17 \\
\hline 16 & 1891.8061 & 946.4067 & & & 1873.7956 & 937.4014 & $\mathbf{Y}$ & 1892.8266 & \begin{tabular}{|l}
946.9169 \\
\end{tabular} & 1875.8000 & 938.4036 & 1874.8160 & 937.9116 & 16 \\
\hline 17 & 1992.8538 & 996.9305 & & & 1974.8432 & 987.9253 & $T$ & 1729.7632 & 865.3853 & 1712.7367 & 856.8720 & 1711.7527 & 856.3800 & 15 \\
\hline 18 & 2129.9127 & 1065.4600 & & & & 1056 & $\mathbf{H}$ & 1628.7155 & 3614 & 1611 & 481 & 1610.7050 & 8561 & 14 \\
\hline 19 & 2258.9553 & 1129.9813 & & & 2240.9447 & 1120.9760 & $\mathbf{E}$ & 1491.6566 & 746.3320 & 1474.6301 & 737.8187 & 1473.6461 & 737.3267 & 13 \\
\hline 20 & 2329.9924 & 1165.4998 & & & 2311.9819 & 1156.4946 & $\mathbf{A}$ & 1362.6140 & 681.8107 & 1345.5875 & 673.2974 & 1344.6035 & 672.8054 & 12 \\
\hline 21 & 2490.0231 & 1245.5152 & & & 2472.0125 & 1236.5099 & $\mathrm{C}$ & 1291.5769 & 646.2921 & 1274.5504 & 637.7788 & 1273.5664 & 637.2868 & 11 \\
\hline 22 & 2561.0602 & 1281.0337 & & & 2543.0496 & 1272.0284 & $\mathbf{A}$ & 1131.5463 & 566.2768 & 1114.5197 & 557.7635 & 1113.5357 & 557.2715 & 10 \\
\hline 23 & 2658.1130 & 1329.5601 & & & 2640.1024 & 1320.5548 & $\mathbf{P}$ & 1060.5092 & 530.7582 & 1043.4826 & 522.2449 & 1042.4986 & 521.7529 & 9 \\
\hline 24 & 2771.1970 & 1386.1021 & & & 2753.1865 & 1377.0969 & I & 963.4564 & 482.2318 & 946.4299 & 473.7186 & 945.4458 & 473.2266 & 8 \\
\hline 25 & 2900.2396 & 1450.6234 & & & 2882.2290 & 1441.6182 & $\mathbf{E}$ & 850.3723 & 425.6898 & 833.3458 & 417.1765 & 832.3618 & 416.6845 & 7 \\
\hline 26 & 3060.2703 & 1530.6388 & & & 3042.2597 & 1521.6335 & $\mathbf{C}$ & 721.3297 & 361.1685 & 704.3032 & 352.6552 & 703.3192 & 352.1632 & 6 \\
\hline 27 & 3173.3543 & 1587.1808 & & & 3155.3438 & 1578.1755 & $\mathbf{L}$ & 561.2991 & 281.1532 & 544.2725 & 272.6399 & 543.2885 & 272.1479 & 5 \\
\hline 28 & 3288.3813 & \begin{tabular}{|l|l|}
1644.6943 \\
\end{tabular} & 3271.3547 & 1636.1810 & 3270.3707 & 1635.6890 & $\mathbf{N}$ & 448.2150 & 224.6112 & 431.1885 & 216.0979 & 430.2045 & 215.6059 & 4 \\
\hline 29 & 3345.4027 & 1673.2050 & 3328.3762 & 1664.6917 & 3327.3922 & 1664.1997 & G & 333.1881 & 167.0977 & 316.1615 & 158.5844 & 315.1775 & 158.0924 & 3 \\
\hline 30 & 3446.4504 & 1723.7288 & 3429.4239 & 1715.2156 & 3428.4398 & 1714.7236 & $T$ & 276.1666 & 138.5870 & 259.1401 & 130.0737 & 258.1561 & 129.5817 & 2 \\
\hline 31 & & & & & & & $\mathbf{R}$ & 175.1190 & 88.0631 & 158.0924 & 79.5498 & & & \\
\hline
\end{tabular}



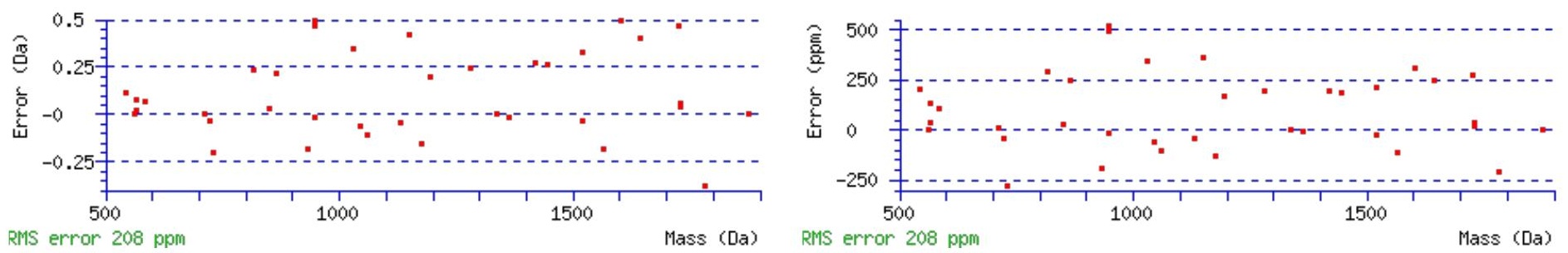

\section{All matches to this query}

\begin{tabular}{|l|l|l|l|}
\hline Score & Mr(calc): & Delta & \multicolumn{1}{|c|}{ Sequence } \\
\hline 76.4 & 3619.5548 & 1.0117 & GFDTYFGYLLGSEDYYTHEACAPIECLNGTR \\
\hline 6.1 & 3618.5708 & 1.9958 & GFDTYFGYLLGSEDYYTHEACAPIECLNGTR \\
\hline 3.9 & 3620.5604 & 0.0061 & MNNNATDLTTGYLSSAVAILLFGSNFVPLKK \\
\hline 3.9 & 3620.5604 & 0.0061 & MNNNATDLTTGYLSSAVAILLFGSNFVPLKK \\
\hline 2.2 & 3618.5789 & 1.9876 & AEIQCLQERLQAVSEATVQPSLSMDSVVFK \\
\hline 1.3 & 3620.5540 & 0.0125 & TSVVVHCSDGWDRTAQLTSLAMLMLDGYYR \\
\hline 1.3 & 3620.5540 & 0.0125 & TSVVVHCSDGWDRTAQLTSLAMLMLDGYYR \\
\hline 1.3 & 3620.5540 & 0.0125 & TSVVVHCSDGWDRTAQLTSLAMLMLDGYYR \\
\hline 1.3 & 3620.5540 & 0.0125 & TSVVVHCSDGWDRTAQLTSLAMLMLDGYYR \\
\hline 0.6 & 3620.5627 & 0.0039 & LTNAAATSGDGYRGQTSPHTPNEKFYGVTVLK \\
\hline
\end{tabular}

Spectrum No: 282; Query: 2193; Rank: 1

\section{Peptide View}

MS/MS Fragmentation of KQILEGLEFNLTQIPEADIHK

Found in IPI00324019, Tax_Id=10116 Gene_Symbol=Serpina1 Alpha-1-antiproteinase precursor

Match to Query 2193: 2437.294988 from(1219.654770,2+)

Title: 100101RatKid_NS_deglyco_20.4569.4569.2.dta

Data file K:INewmanPaper|Piliangl3SubProteomes\Piliang3SP\mgf5ppm\ERLIC_3SubProteomes5ppm.mgf

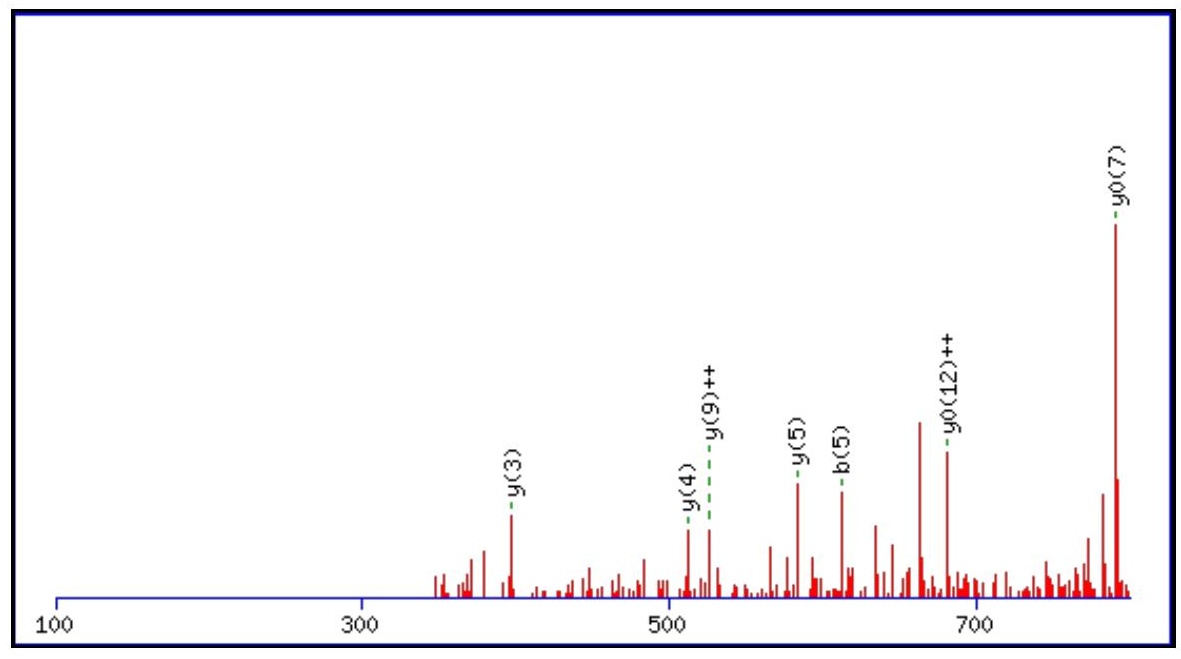



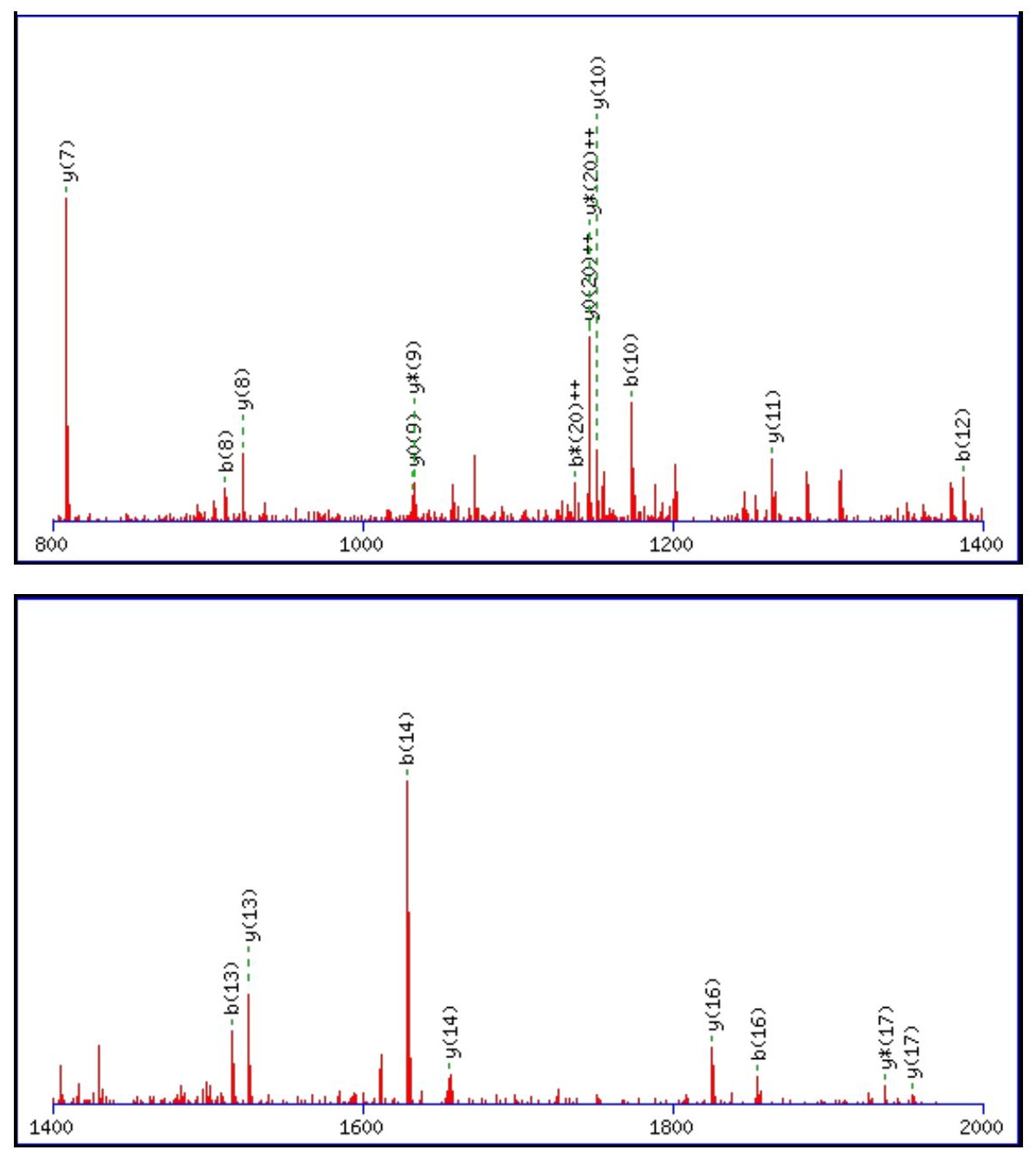

Monoisotopic mass of neutral peptide $\operatorname{Mr}($ calc): 2436.2900

Fixed modifications: Carbamidomethyl (C)

Variable modifications:

N10 : Deamidated_N (N)

Ions Score: 76 Expect: $1.9 \mathrm{e}-006$

Matches (Bold Red): 28/226 fragment ions using 38 most intense peaks

\begin{tabular}{|c|c|c|c|c|c|c|c|c|c|c|c|c|c|c|}
\hline \# & b & $\mathbf{b}^{++}$ & $\mathbf{b}^{*}$ & $\mathbf{b}^{*^{++}}$ & $\mathbf{b}^{\mathbf{0}}$ & $\mathbf{b}^{\mathbf{0 + +}}$ & Seq. & $\mathbf{y}$ & $y^{++}$ & $\mathbf{y}^{*}$ & $\mathbf{y}^{*^{++}}$ & $\mathbf{y}^{0}$ & $\mathbf{y}^{0++}$ & \# \\
\hline 1 & 129.1022 & 65.0548 & 112.0757 & 56.5415 & & & $\mathbf{K}$ & & & & & & & 21 \\
\hline 2 & 257.1608 & 129.0840 & 240.1343 & 120.5708 & & & $\mathbf{Q}$ & 2309.2023 & .1048 & 2292.1758 & 5915 & 2291.1918 & 1146.0995 & 20 \\
\hline 3 & 370.2449 & 185.6261 & 353.2183 & 177.1128 & & & I & 2181.1438 & 1091.0755 & 2164.1172 & 1082.5622 & 2163.1332 & 1082.0702 & 19 \\
\hline 4 & 483.3289 & 242.1681 & 466.3024 & 233.6548 & & & $\mathbf{L}$ & 2068.0597 & 1034. & 2051.0332 & 1026.0202 & 2050.0491 & 1025.5282 & 18 \\
\hline 5 & 612.3715 & 306.6894 & 595.3450 & 298.1761 & 594.3610 & 297.6841 & $\mathbf{E}$ & 1954.9756 & 977.9915 & 1937.9491 & 969.4782 & 1936.9651 & 968.9862 & 17 \\
\hline 6 & 669.3930 & 335.2001 & 652.3665 & 326.6869 & 651.3824 & 326.1949 & G & 1825.9330 & 913.4702 & 1808.9065 & 904.9569 & 1807.9225 & 904.4649 & 16 \\
\hline 7 & 782.4771 & 391.7422 & 765.4505 & 383.2289 & 764.4665 & 382.7369 & $\mathbf{L}$ & 1768.9116 & 884.9594 & 1751.8850 & 876.4462 & 1750.9010 & 875.9541 & 15 \\
\hline 8 & 911.5197 & 456.2635 & 894.4931 & 447.7502 & 893.5091 & 447.2582 & $\mathbf{E}$ & 1655.8275 & 828.4174 & 1638.8010 & 819.9041 & 1637.8170 & 819.4121 & 14 \\
\hline 9 & 1058.5881 & 529.7977 & 1041.5615 & 521.2844 & 1040.5775 & 520.7924 & $\mathbf{F}$ & 1526.7849 & 763.8961 & 1509.7584 & 755.3828 & 1508.7744 & 754.8908 & 13 \\
\hline 10 & 1173.6150 & 587.3111 & 1156.5885 & 578.7979 & 1155.6044 & 578.3059 & $\mathbf{N}$ & 1379.7165 & 690.3619 & 1362.6900 & 681.8486 & 1361.7059 & 681.3566 & 12 \\
\hline 11 & 1286.6991 & 643.8532 & 1269.6725 & 635.3399 & 1268.6885 & 634.8479 & $\mathbf{L}$ & 1264.6896 & 632.8484 & 1247.6630 & 624.3352 & 1246.6790 & 623.8431 & 11 \\
\hline 12 & 1387.7468 & 694.3770 & 1370.7202 & 685.8637 & 1369.7362 & 685.3717 & $\mathbf{T}$ & 1151.6055 & 576.3064 & 1134.5790 & 567.7931 & 1133.5949 & 567.3011 & 10 \\
\hline 13 & 1515.8053 & 758.4063 & 1498.7788 & 749.8930 & 1497.7948 & 749.4010 & $\mathbf{Q}$ & 1050.5578 & 525.7826 & 1033.5313 & 517.2693 & 1032.5473 & 516.7773 & 9 \\
\hline 14 & 1628.8894 & 814.9483 & 1611.8628 & 806.4351 & 1610.8788 & 805.9431 & I & 922.4993 & 461.7533 & 905.4727 & 453.2400 & 904.4887 & 452.7480 & 8 \\
\hline 15 & 1725.9422 & 863.4747 & 1708.9156 & 854.9614 & 1707.9316 & 854.4694 & $\mathbf{P}$ & 809.4152 & 405.2112 & 792.3886 & 396.6980 & 791.4046 & 396.2060 & 7 \\
\hline 16 & 1854.9848 & 927.9960 & 1837.9582 & 919.4827 & 1836.9742 & 918.9907 & $\mathbf{E}$ & 712.3624 & 356.6849 & 695.3359 & 348.1716 & 694.3519 & 347.6796 & 6 \\
\hline 17 & 1926.0219 & 963.5146 & 1908.9953 & 955.0013 & 1908.0113 & 954.5093 & A & 583.3198 & 292.1636 & 566.2933 & 283.6503 & 565.3093 & 283.1583 & 5 \\
\hline 18 & 2041.0488 & 1021.0280 & 2024.0223 & 1012.5148 & 2023.0382 & 1012.0228 & D & 512.2827 & 256.6450 & 495.2562 & 248.1317 & 494.2722 & 247.6397 & 4 \\
\hline
\end{tabular}




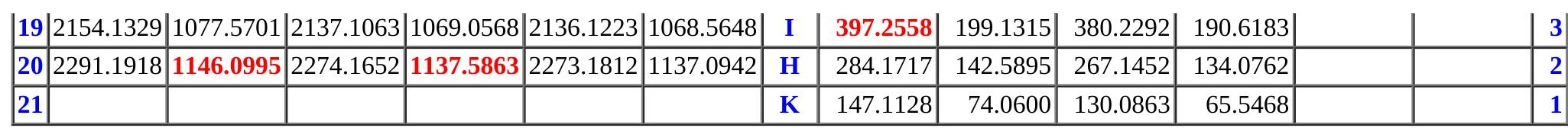
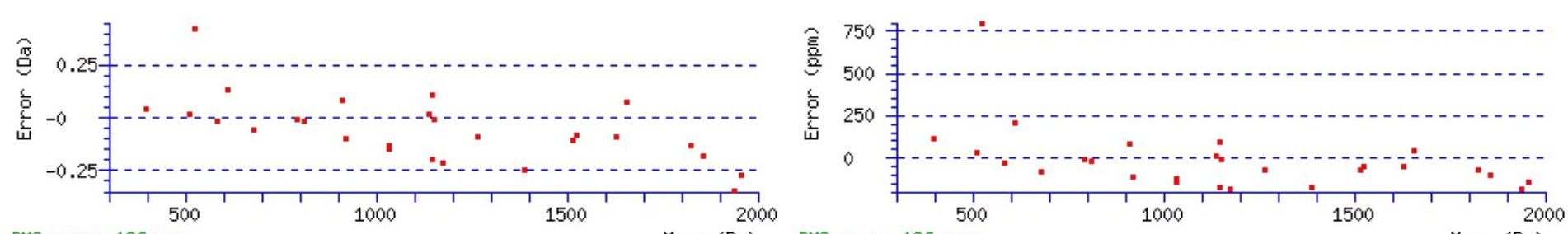

RMS error 186 ppm Mass (Da) RMS error 186 ppm

\section{All matches to this query}

\begin{tabular}{|l|l|l|l|}
\hline Score & Mr(calc): & Delta & Sequence \\
\hline 76.1 & 2436.2900 & 1.0050 & KQILEGLEFNLTOIPEADIHK \\
\hline 25.8 & 2435.3060 & 1.9890 & KQILEGLEFNLTQIPEADIHK \\
\hline
\end{tabular}

Spectrum No: 283; Query: 1802; Rank: 1

\section{Peptide View}

MS/MS Fragmentation of EGNCSVQSGLTWQDCDFK

Found in IPI00327182, Tax_Id=10116 Gene_Symbol=Kng1 T-kininogen 1 precursor

Match to Query 1802: 2130.869628 from(1066.442090,2+)

Title: 100101RatKid_NS_deglyco_25.2944.2944.2.dta

Data file K:INewmanPaper|Piliangl3SubProteomes\Piliang3SP\mgf5ppm\ERLIC_3SubProteomes5ppm.mgf

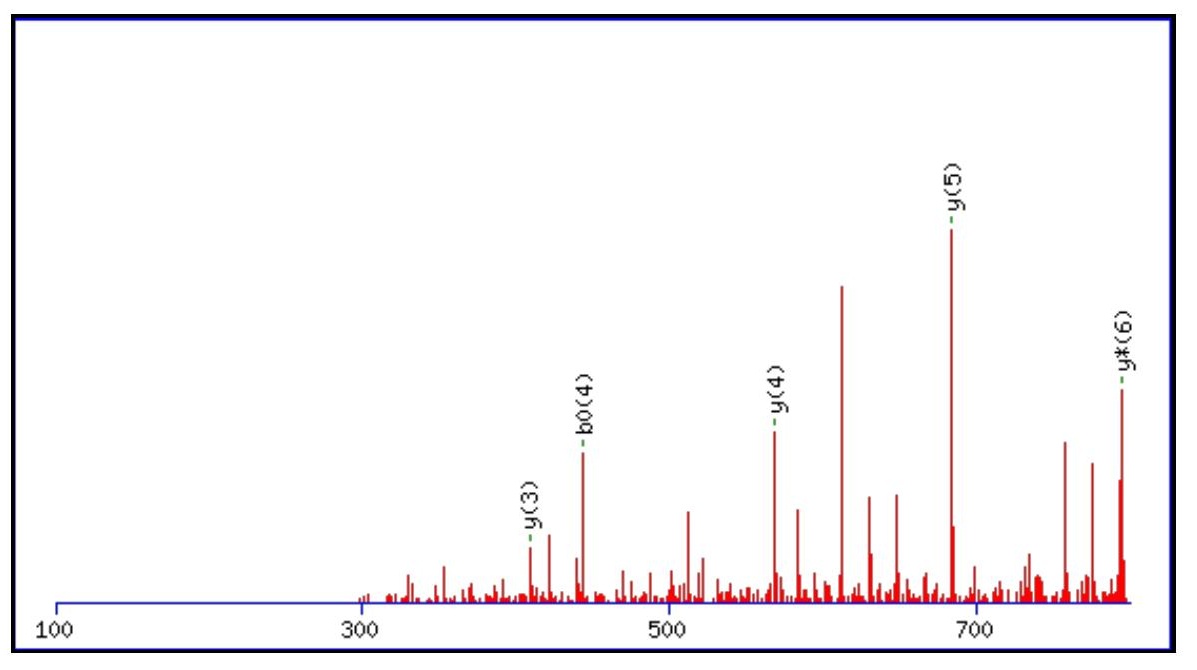



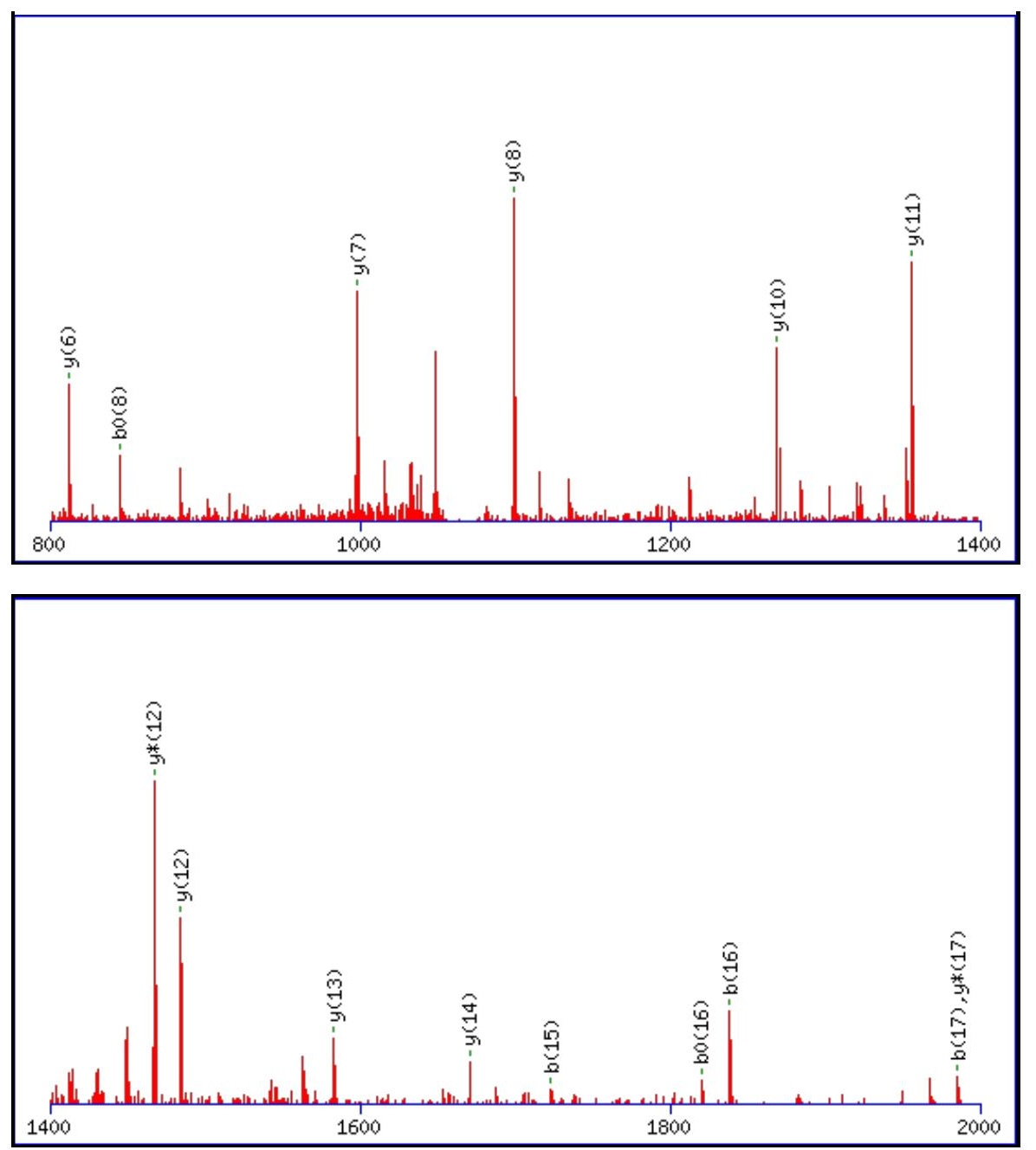

Monoisotopic mass of neutral peptide $\operatorname{Mr}($ calc): 2130.8623

Fixed modifications: Carbamidomethyl (C)

Variable modifications:

N3: Deamidated $\mathrm{N}(\mathrm{N})$

Ions Score: 76 Expect: $5.1 \mathrm{e}-006$

Matches (Bold Red): 20/196 fragment ions using 30 most intense peaks

\begin{tabular}{|c|c|c|c|c|c|c|c|c|c|c|c|c|c|c|}
\hline \# & b & $\mathbf{b}^{++}$ & $\mathbf{b}^{*}$ & $\mathbf{b}^{*^{++}}$ & $\mathbf{b}^{0}$ & $\mathbf{b}^{0++}$ & Seq. & $\mathbf{y}$ & $\mathbf{y}^{++}$ & $\mathbf{y}^{*}$ & $\mathbf{y}^{*^{++}}$ & $\mathbf{y}^{0}$ & $\mathbf{y}^{0++}$ & \# \\
\hline 1 & 130.0499 & 65.5286 & & & 112.0393 & 56.5233 & $\mathbf{E}$ & & & & & & & 18 \\
\hline 2 & 187.0713 & 94.0393 & & & & 85.0340 & $\mathbf{G}$ & & & & & & & 17 \\
\hline 3 & 302.0983 & 151.5528 & 285.0717 & & 284.0877 & 142.5475 & $\mathbf{N}$ & & 4064 & 1928.7789 & 931 & 1927.7949 & 964.4011 & 16 \\
\hline 4 & 462.1289 & 231.5681 & 445.1024 & 223.0548 & 444.1184 & 222.5628 & C & 1830.7785 & & 1813.7520 & & 1812.7680 & 906.8876 & 15 \\
\hline 5 & 549.1609 & 275.0841 & 532.1 & 266.5708 & 531.1 & 266.0788 & S & 479 & & 214 & 643 & .7373 & 826.8723 & 14 \\
\hline 6 & 648.2294 & 324.6183 & 631.2028 & 316.1050 & 630.2188 & 315.6130 & V & 1583.7159 & 3616 & 1566.6893 & 8483 & 1565.7053 & 783.3563 & 13 \\
\hline 7 & 776.2879 & 388.6476 & 759.2614 & 380.1343 & 758.2774 & 379.6423 & $\mathbf{Q}$ & 1484.6475 & 3274 & 209 & 141 & 1466.6369 & 733.8221 & 12 \\
\hline 8 & 863.3200 & 432.1636 & 846.2934 & 423.6503 & 845.3094 & 423.1583 & S & 1356.5889 & 7981 & 1339.5623 & 2848 & 1338.5783 & 669.7928 & 11 \\
\hline 9 & 920.3414 & 460.6744 & 903.3149 & 452.1611 & 902.3309 & 451.6691 & $\mathbf{G}$ & 1269.5569 & 2821 & 1252.5303 & 626.7688 & 1251.5463 & 626.2768 & 10 \\
\hline 10 & 1033.4255 & 517.2164 & 1016.3989 & 508.7031 & 1015.4149 & 508.2111 & $\mathbf{L}$ & 1212.5354 & 713 & 1195.5088 & 581 & 1194.5248 & 597.7660 & 9 \\
\hline 11 & 1134.4732 & 567.7402 & 1117.4466 & 559.2270 & 1116.4626 & 558.7349 & $\mathbf{T}$ & 1099.4513 & 550.2293 & 1082.4248 & 541.7160 & 1081.4408 & 541.2240 & 8 \\
\hline 12 & 1320.5525 & 660.7799 & 1303.5259 & 652.2666 & 1302.5419 & 651.7746 & $\mathbf{W}$ & 998.4036 & 7055 & 981.3771 & 491. & 980.3931 & 490.7002 & 7 \\
\hline 13 & 1448.6111 & 724.8092 & 1431.5845 & 716.2959 & 1430.6005 & 715.8039 & $\mathbf{Q}$ & 812.3243 & 406.6658 & 795.2978 & 398.1525 & 794.3138 & 397.6605 & 6 \\
\hline 14 & 1563.6380 & 782.3226 & 1546.6115 & 773.8094 & 1545.6274 & 773.3174 & D & 684.2658 & 342.6365 & 667.2392 & 334.1232 & 666.2552 & 333.6312 & 5 \\
\hline 15 & 1723.6687 & 862.3380 & 1706.6421 & 853.8247 & 1705.6581 & 853.3327 & C & 569.2388 & 285.1230 & 552.2123 & 276.6098 & 551.2282 & 276.1178 & 4 \\
\hline 16 & 1838.6956 & 919.8514 & 1821.6691 & 911.3382 & 1820.6850 & 910.8462 & D & 409.2082 & 205.1077 & 392.1816 & 196.5944 & 391.1976 & 196.1024 & 3 \\
\hline 17 & 1985.7640 & 993.3856 & 1968.7375 & 984.8724 & 1967.7534 & 984.3804 & $\mathbf{F}$ & 294.1812 & 147.5942 & 277.1547 & 139.0810 & & & 2 \\
\hline 18 & & & & & & & $\mathbf{K}$ & 147.1128 & 74.0600 & 130.0863 & 65.5468 & & & 1 \\
\hline
\end{tabular}



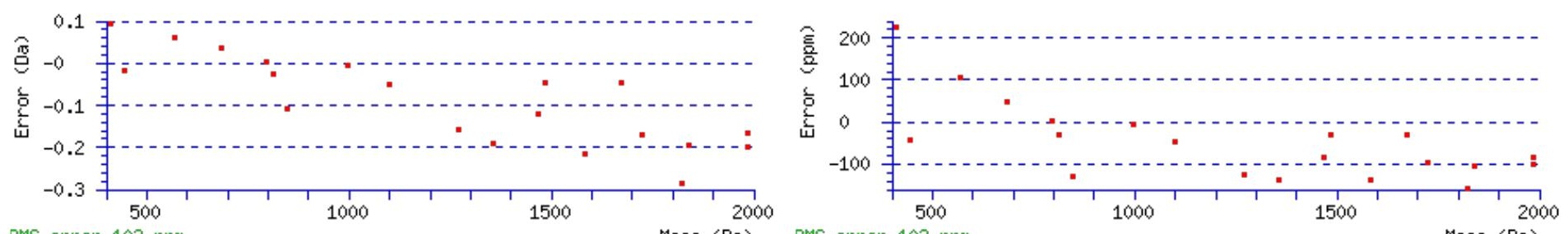

RMS error 102 ppm

Mass (Da) RMS error 102 ppm

Mass (Da)

\section{All matches to this query}

\begin{tabular}{|l|l|l|l|}
\hline Score & Mr(calc): & Delta & \multicolumn{1}{|c|}{ Sequence } \\
\hline 76.1 & 2130.8623 & 0.0073 & EGNCSVQSGLTWODCDFK \\
\hline 60.9 & 2129.8783 & 0.9913 & EGNCSVQSGLTWQDCDFK \\
\hline 8.2 & 2130.8882 & -0.0185 & SYLIMMLEYFKKDMK \\
\hline 8.2 & 2130.8882 & -0.0185 & SYLIMMLEYFKKDMK \\
\hline 8.2 & 2130.8882 & -0.0185 & SYLIMMLEYFKKDMK \\
\hline 8.2 & 2130.8882 & -0.0185 & SYLIMMLEYFKKDMK \\
\hline 3.6 & 2130.8792 & -0.0096 & YNMVGPCVSTTFQGTNFK \\
\hline 1.3 & 2130.8589 & 0.0107 & KTNNNNNNTTDNLQSINK \\
\hline 0.6 & 2129.8481 & 1.0215 & YSKAYGRMGLALTAMNK \\
\hline
\end{tabular}

Spectrum No: 284; Query: 1427; Rank: 1

\section{Peptide View}

MS/MS Fragmentation of LHINYNNLTESVGPLPK

Found in IPI00206403, Tax_Id=10116 Gene_Symbol=Lum Lumican precursor

Match to Query 1427: 1908.995448 from(955.505000,2+)

Title: 091008RatKidney_NH4Format01_25.2924.2924.2.dta

Data file K:INewmanPaper|Piliangl3SubProteomes\Piliang3SP\mgf5ppm\ERLIC_3SubProteomes5ppm.mgf

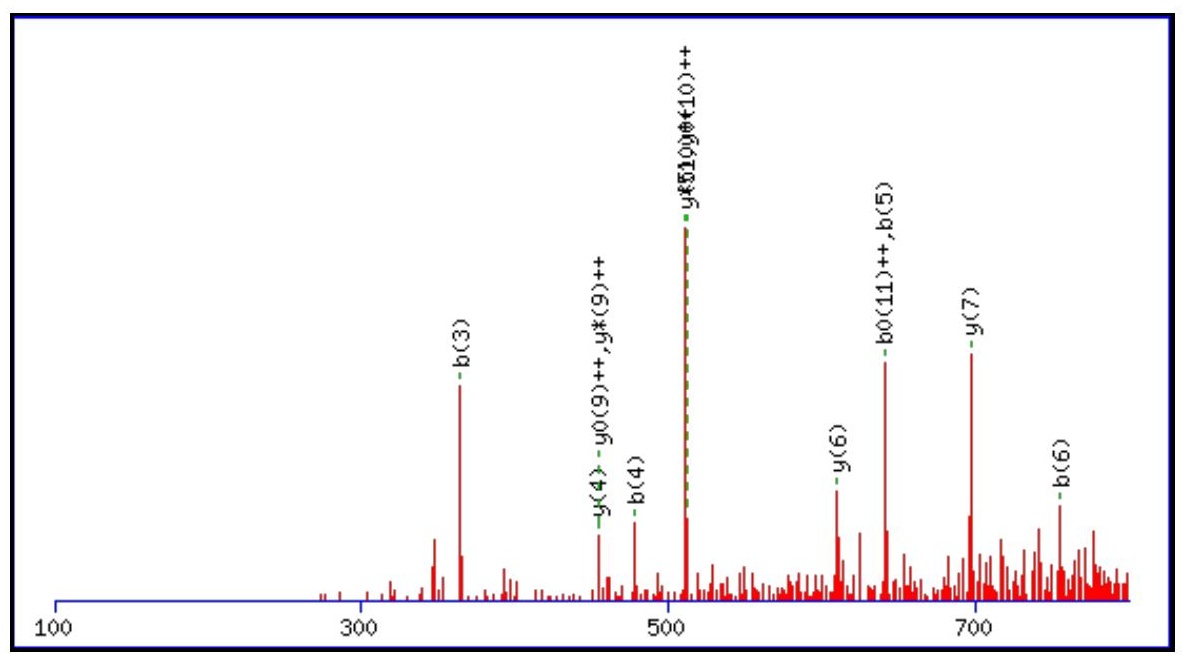



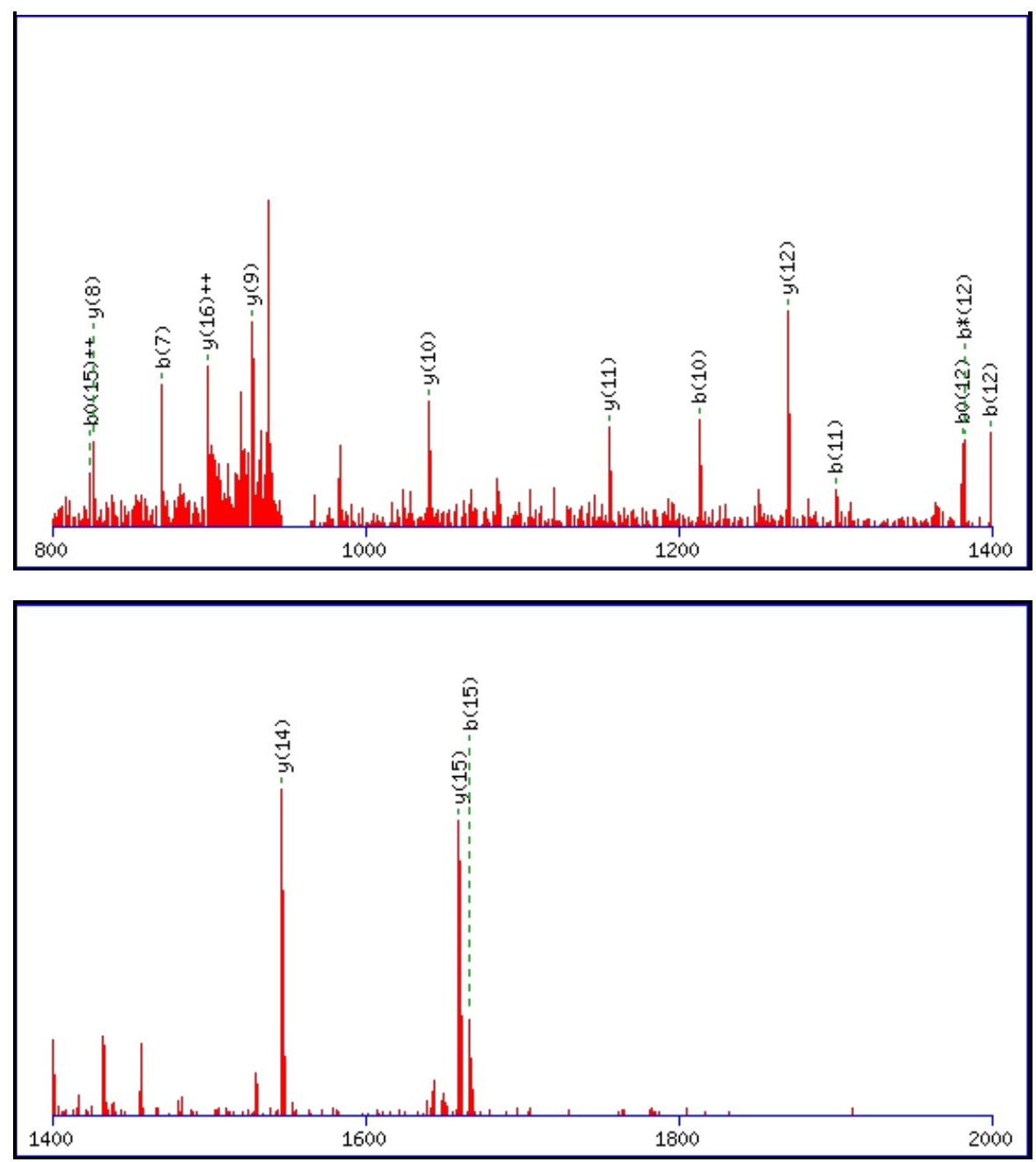

Monoisotopic mass of neutral peptide $\operatorname{Mr}($ calc): 1908.9945

Fixed modifications: Carbamidomethyl (C)

Variable modifications:

N7 : Deamidated $\mathrm{N}(\mathrm{N})$

Ions Score: 76 Expect: $3 \mathrm{e}-006$

Matches (Bold Red): 29/158 fragment ions using 46 most intense peaks

\begin{tabular}{|c|c|c|c|c|c|c|c|c|c|c|c|c|c|c|}
\hline \# & b & $\mathbf{b}^{++}$ & $\mathbf{b}^{*}$ & $\mathbf{b}^{*++}$ & $\mathbf{b}^{0}$ & $\mathbf{b}^{\mathbf{0 + +}}$ & Seq. & $\mathbf{y}$ & $y^{++}$ & $\mathbf{y}^{*}$ & $\mathrm{y}^{*^{++}}$ & $\mathbf{y}^{\mathbf{0}}$ & $\mathbf{y}^{0++}$ & \# \\
\hline 1 & 114.0913 & 57.5493 & & & & & $\mathbf{L}$ & & & & & & & 17 \\
\hline 2 & 251.1503 & 126.0788 & & & & & $\mathbf{H}$ & 1796.9177 & 898.9625 & 1779.8912 & 890.4492 & 1778.9072 & 889.9572 & 16 \\
\hline 3 & 364.2343 & 182.6208 & & & & & I & 1659.8588 & 830.4330 & 1642.8323 & 821.9198 & 1641.8483 & 821.4278 & 15 \\
\hline 4 & 478.2772 & 239.6423 & 461.2507 & 231.1290 & & & $\mathbf{N}$ & 1546.7748 & 773.8910 & 1529.7482 & 765.3777 & 1528.7642 & 764.8857 & 14 \\
\hline 5 & 641.3406 & 321.1739 & 624.3140 & 312.6606 & & & $\mathbf{Y}$ & 1432.7318 & 716.8696 & 1415.7053 & 708.3563 & 1414.7213 & 707.8643 & 13 \\
\hline 6 & 755.3835 & 378.1954 & 738.3569 & 369.6821 & & & $\mathbf{N}$ & 1269.6685 & 635.3379 & 1252.6420 & 626.8246 & 1251.6579 & 626.3326 & 12 \\
\hline 7 & 870.4104 & 435.7089 & 853.3839 & 427.1956 & & & $\mathbf{N}$ & 1155.6256 & 578.3164 & 1138.5990 & 569.8032 & 1137.6150 & 569.3111 & 11 \\
\hline 8 & 983.4945 & 492.2509 & 966.4679 & 483.7376 & & & $\mathbf{L}$ & 1040.5986 & 520.8030 & 1023.5721 & 512.2897 & 1022.5881 & 511.7977 & 10 \\
\hline 9 & 1084.5422 & 542.7747 & 1067.5156 & 534.2615 & 1066.5316 & 533.7694 & $\mathbf{T}$ & 927.5146 & 464.2609 & 910.4880 & 455.7477 & 909.5040 & 455.2556 & 9 \\
\hline 10 & 1213.5848 & 607.2960 & 1196.5582 & 598.7827 & 1195.5742 & 598.2907 & $\mathbf{E}$ & 826.4669 & 413.7371 & 809.4403 & 405.2238 & 808.4563 & 404.7318 & 8 \\
\hline 11 & 1300.6168 & 650.8120 & 1283.5903 & 642.2988 & 1282.6062 & 641.8068 & S & 697.4243 & 349.2158 & 680.3978 & 340.7025 & 679.4137 & 340.2105 & 7 \\
\hline 12 & 1399.6852 & 700.3462 & 1382.6587 & 691.8330 & 1381.6746 & 691.3410 & V & 610.3923 & 305.6998 & 593.3657 & 297.1865 & & & 6 \\
\hline 13 & 1456.7067 & 728.8570 & 1439.6801 & 720.3437 & 1438.6961 & 719.8517 & G & 511.3239 & 256.1656 & 494.2973 & 247.6523 & & & 5 \\
\hline 14 & 1553.7594 & 777.3834 & 1536.7329 & 768.8701 & 1535.7489 & 768.3781 & $\mathbf{P}$ & 454.3024 & 227.6548 & 437.2758 & 219.1416 & & & 4 \\
\hline 15 & 1666.8435 & 833.9254 & 1649.8170 & 825.4121 & 1648.8329 & 824.9201 & $\mathbf{L}$ & 357.2496 & 179.1285 & 340.2231 & 170.6152 & & & 3 \\
\hline 16 & 1763.8963 & 882.4518 & 1746.8697 & 873.9385 & 1745.8857 & 873.4465 & $\mathbf{P}$ & 244.1656 & 122.5864 & 227.1390 & 114.0731 & & & 2 \\
\hline 17 & & & & & & & $\mathbf{K}$ & 147.1128 & 74.0600 & 130.0863 & 65.5468 & & & 1 \\
\hline
\end{tabular}



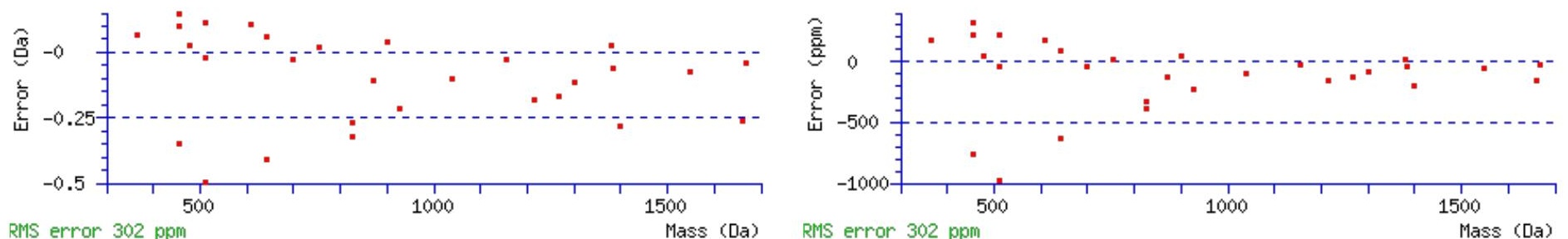

RMS error 302 ppm

\section{All matches to this query}

\begin{tabular}{|l|c|c|c|}
\hline Score & Mr(calc): & Delta & Sequence \\
\hline 76.1 & 1908.9945 & 0.0010 & LHINYNNLTESVGPLPK \\
\hline 62.9 & 1908.9945 & 0.0010 & LHINYNNLTESVGPLPK \\
\hline 50.6 & 1908.9945 & 0.0010 & LHINYNNLTESVGPLPK \\
\hline 21.2 & 1908.0105 & 0.9850 & LHINYNNLTESVGPLPK \\
\hline
\end{tabular}

Spectrum No: 285; Query: 1259; Rank: 1

\section{Peptide View}

MS/MS Fragmentation of DLQNFLENVTSSVDVK

Found in IPI00565109, Tax_Id=10116 Gene_Symbol=RGD1307220_predicted similar to alpha 3 type VI collagen isoform 1 precursor

Match to Query 1259: 1807.886168 from(904.950360,2+)

Title: 091008RatKidney_NoSalt_27.5028.5028.2.dta

Data file K:\NewmanPaper|Piliang|3SubProteomes\Piliang3SP\mgf5ppm\ERLIC_3SubProteomes5ppm.mgf

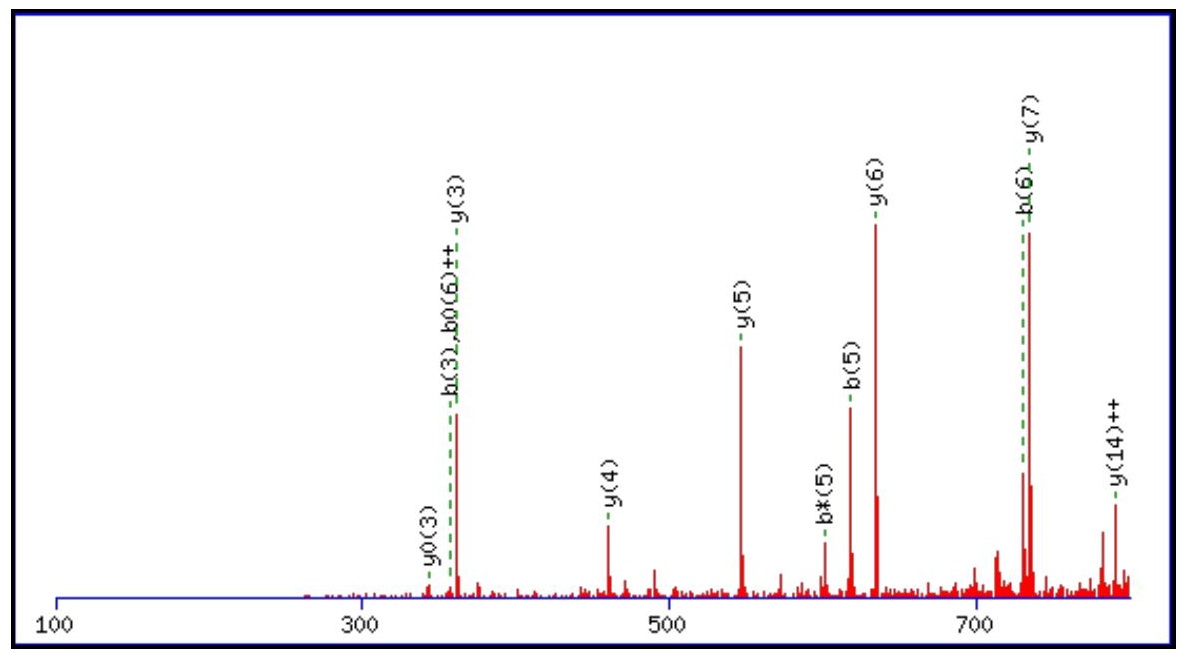



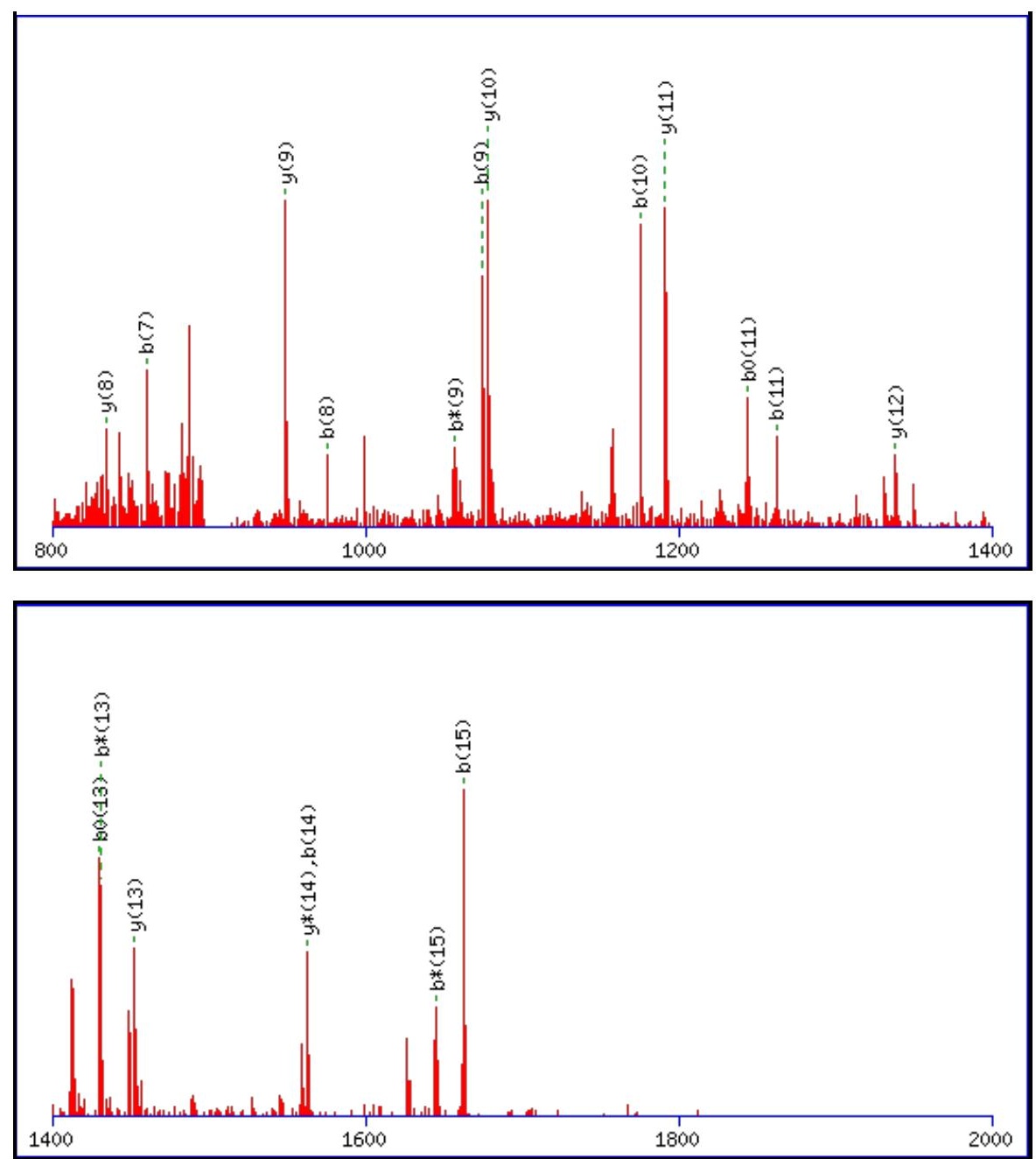

Monoisotopic mass of neutral peptide $\operatorname{Mr}($ calc): 1807.8840

Fixed modifications: Carbamidomethyl (C)

Variable modifications:

N8 : Deamidated_N (N)

Ions Score: 76 Expect: $4.6 \mathrm{e}-006$

Matches (Bold Red): 31/172 fragment ions using 55 most intense peaks

\begin{tabular}{|c|c|c|c|c|c|c|c|c|c|c|c|c|c|c|}
\hline \# & b & $\mathbf{b}^{++}$ & $\mathbf{b}^{*}$ & $\mathbf{b}^{*^{++}}$ & $\mathbf{b}^{0}$ & $\mathbf{b}^{0++}$ & Seq. & $\mathbf{y}$ & $\mathbf{y}^{++}$ & $\mathbf{y}^{*}$ & $\mathbf{y}^{*^{++}}$ & $\mathbf{y}^{\mathbf{0}}$ & $\mathbf{y}^{\mathbf{0 + +}}$ & \# \\
\hline 1 & 116.0342 & 58.5207 & & & 98.0237 & 49.5155 & D & & & & & & & 16 \\
\hline 2 & 229.1183 & 115.0628 & & & 211.1077 & 106.0575 & $\mathbf{L}$ & & 847.4358 & 1676.8378 & & 1675.8537 & 838.4305 & 15 \\
\hline 3 & 357.1769 & 179.0921 & 340.1503 & & 339.1663 & 170.0868 & $\mathbf{Q}$ & 1580.7802 & 790.8938 & 1563.7537 & & 1562.7697 & 781.8885 & 14 \\
\hline 4 & 471.2198 & 236.1135 & 454.1932 & 227.6003 & 453.2092 & 227.1082 & $\mathbf{N}$ & 1452.7217 & 726.8645 & 1435.6951 & 718.3512 & 1434.7111 & 717.8592 & 13 \\
\hline 5 & 618.2882 & 309.6477 & 601.2617 & 301.1345 & 600.2776 & 300.6425 & $\mathbf{F}$ & 1338.6787 & 669.8430 & 1321.6522 & 661.3297 & 1320.6682 & 660.8377 & 12 \\
\hline 6 & 731.3723 & 366.1898 & 714.3457 & 357.6765 & 713.3617 & 357.1845 & $\mathbf{L}$ & 1191.6103 & 596.3088 & 1174.5838 & 587.7955 & 1173.5998 & 587.3035 & 11 \\
\hline 7 & 860.4149 & 430.7111 & 843.3883 & 422.1978 & 842.4043 & 421.7058 & $\mathbf{E}$ & 1078.5263 & 539.7668 & 1061.4997 & 531.2535 & 1060.5157 & 530.7615 & 10 \\
\hline 8 & 975.4418 & 488.2245 & 958.4152 & 479.7113 & 957.4312 & 479.2193 & $\mathbf{N}$ & 949.4837 & 475.2455 & 932.4571 & 466.7322 & 931.4731 & 466.2402 & 9 \\
\hline 9 & 1074.5102 & 537.7587 & 1057.4837 & 529.2455 & 1056.4996 & 528.7535 & $\mathbf{V}$ & 834.4567 & 417.7320 & 817.4302 & 409.2187 & 816.4462 & 408.7267 & 8 \\
\hline 10 & 1175.5579 & 588.2826 & 1158.5313 & 579.7693 & 1157.5473 & 579.2773 & $\mathbf{T}$ & 735.3883 & 368.1978 & 718.3618 & 359.6845 & 717.3777 & 359.1925 & 7 \\
\hline 11 & 1262.5899 & 631.7986 & 1245.5634 & 623.2853 & 1244.5794 & 622.7933 & S & 634.3406 & 317.6740 & 617.3141 & 309.1607 & 616.3301 & 308.6687 & 6 \\
\hline 12 & 1349.6219 & 675.3146 & 1332.5954 & 666.8013 & 1331.6114 & 666.3093 & $\mathrm{~S}$ & 547.3086 & 274.1579 & 530.2821 & 265.6447 & 529.2980 & 265.1527 & 5 \\
\hline 13 & 1448.6904 & 724.8488 & 1431.6638 & 716.3355 & 1430.6798 & 715.8435 & V & 460.2766 & 230.6419 & 443.2500 & 222.1287 & 442.2660 & 221.6366 & 4 \\
\hline 14 & 1563.7173 & 782.3623 & 1546.6908 & 773.8490 & 1545.7067 & 773.3570 & D & 361.2082 & 181.1077 & 344.1816 & 172.5944 & 343.1976 & 172.1024 & 3 \\
\hline 15 & 1662.7857 & 831.8965 & 1645.7592 & 823.3832 & 1644.7752 & 822.8912 & $\mathbf{V}$ & 246.1812 & 123.5942 & 229.1547 & 115.0810 & & & 2 \\
\hline 16 & & & & & & & $\mathbf{K}$ & 147.1128 & 74.0600 & 130.0863 & 65.5468 & & & 1 \\
\hline
\end{tabular}



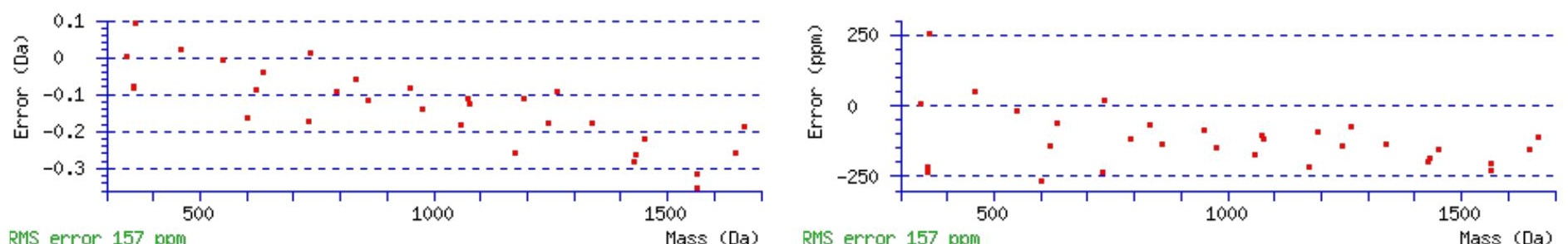

RMS error 157 ppm

\section{All matches to this query}

\begin{tabular}{|l|l|l|l|}
\hline Score & Mr(calc): & Delta & \multicolumn{1}{|c|}{ Sequence } \\
\hline 76.1 & 1807.8840 & 0.0022 & DLQNFLENVTSSVDVK \\
\hline 56.5 & 1807.8840 & 0.0022 & DLQNFLENVTSSVDVK \\
\hline 20.2 & 1806.9000 & 0.9862 & DLQNFLENVTSSVDVK \\
\hline 8.5 & 1807.8838 & 0.0024 & LCCLLARASNLPNVK \\
\hline 2.0 & 1807.8869 & -0.0008 & QDQTLELLGPPPPPAR \\
\hline 1.1 & 1806.8933 & 0.9928 & YLIQDSSILQKVLK \\
\hline
\end{tabular}

Spectrum No: 286; Query: 2009; Rank: 1

\section{Peptide View}

MS/MS Fragmentation of VCDGVPDCPEGDDENNVTSGR

Found in IPI00205325, Tax_Id=10116 Gene_Symbol=Lrp2 Low-density lipoprotein receptor-related protein 2 precursor

Match to Query 2009: 2291.898228 from(1146.956390,2+)

Title: 100101RatKid_NS_deglyco_24.1502.1502.2.dta

Data file K:INewmanPaper|Piliangl3SubProteomes\Piliang3SP\mgf5ppm\ERLIC_3SubProteomes5ppm.mgf

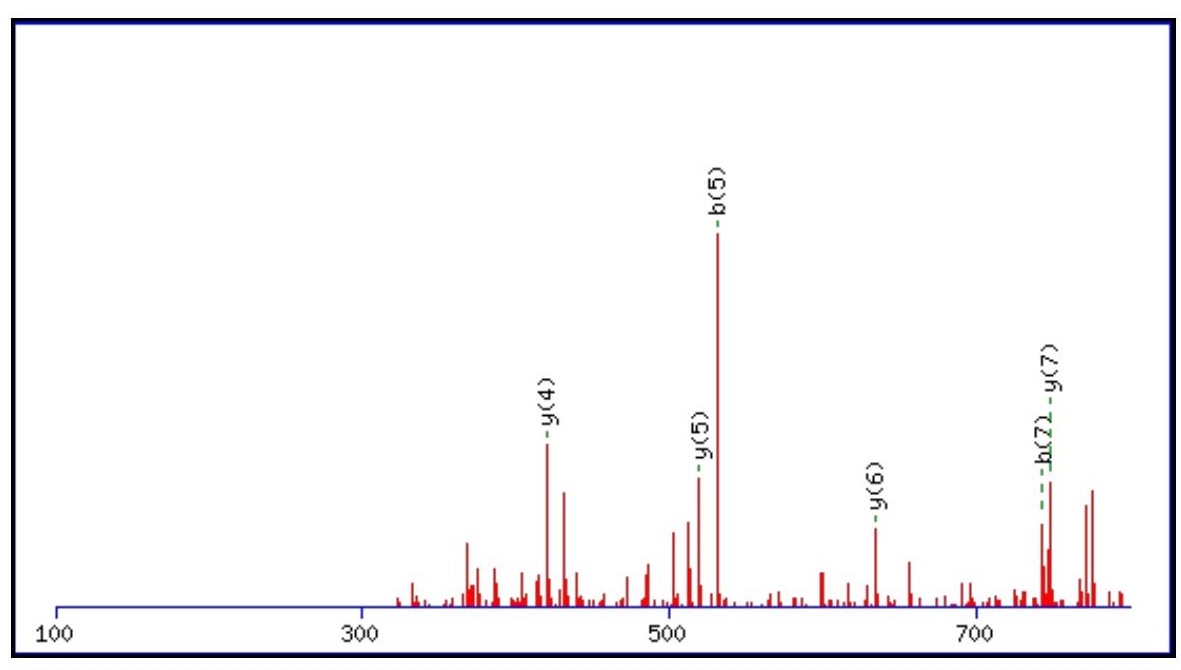



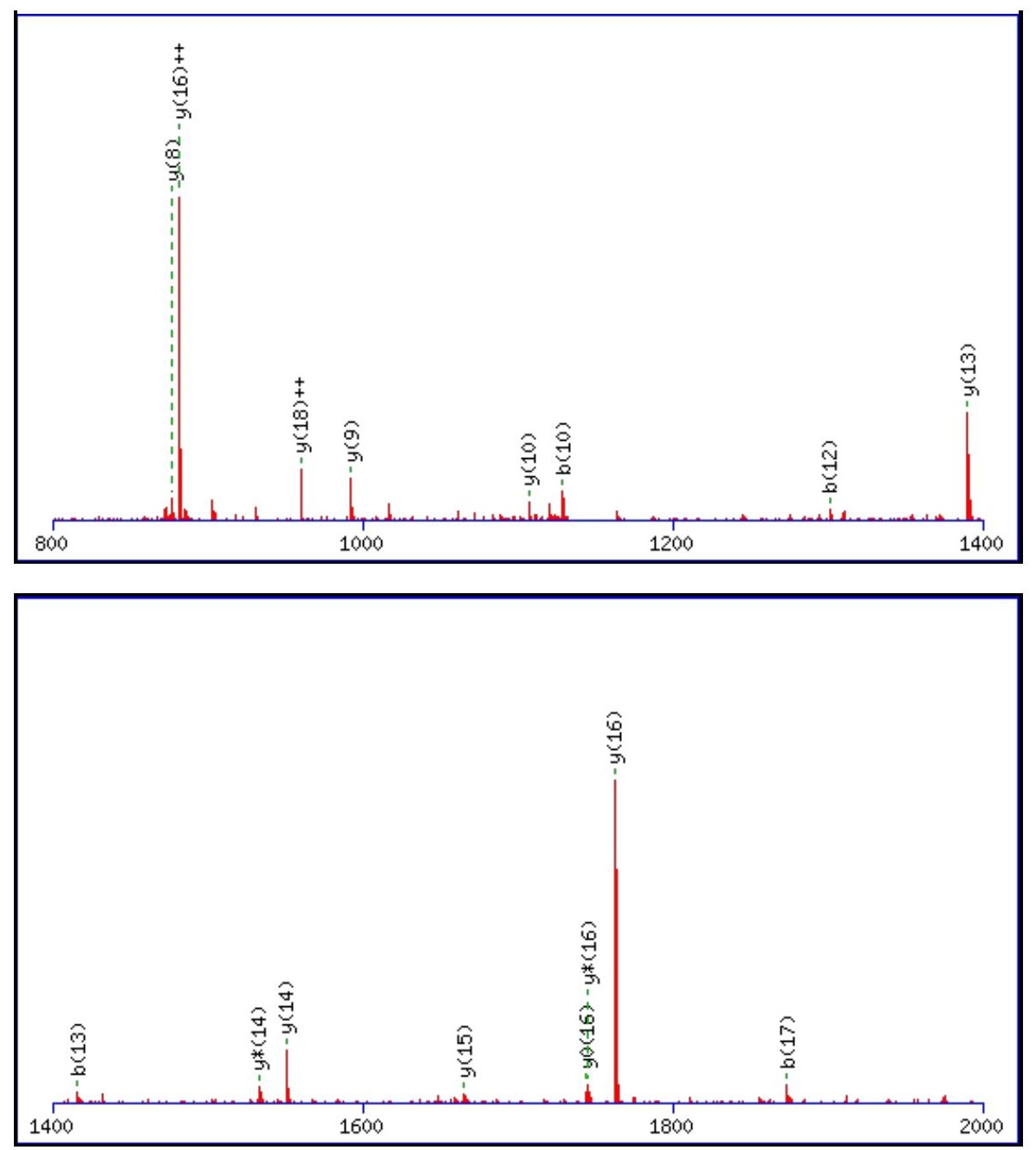

Monoisotopic mass of neutral peptide $\operatorname{Mr}($ calc): 2291.8907

Fixed modifications: Carbamidomethyl (C)

Variable modifications:

N16 : Deamidated_N (N)

Ions Score: 76 Expect: $4.6 \mathrm{e}-006$

Matches (Bold Red): 22/204 fragment ions using 36 most intense peaks

\begin{tabular}{|c|c|c|c|c|c|c|c|c|c|c|c|c|c|c|}
\hline \# & b & $\mathbf{b}^{++}$ & $\mathbf{b}^{*}$ & $\mathbf{b}^{*^{++}}$ & $\mathbf{b}^{0}$ & $\mathbf{b}^{0++}$ & Seq. & $\mathbf{y}$ & $\mathbf{y}^{++}$ & $\mathbf{y}^{*}$ & $\mathbf{y}^{*^{++}}$ & $\mathbf{y}^{0}$ & $\mathbf{y}^{\mathbf{0 + +}}$ & \# \\
\hline 1 & 100.0757 & 50.5415 & & & & & V & & & & & & & 21 \\
\hline 2 & 260.1063 & 130.5568 & & & & & C & 2193.8295 & 1097.4184 & 2176.8030 & 1088.9051 & 2175.8190 & 1088.4131 & 20 \\
\hline 3 & 375.1333 & 188.0703 & & & 357.1227 & 179.0650 & D & 2033.7989 & 1017.4031 & 2016.7723 & 1008.8898 & 2015.7883 & 1008.3978 & 19 \\
\hline 4 & 432.1547 & 216.5810 & & & 414.1442 & 207.5757 & $\mathbf{G}$ & 1918.7719 & 959.8896 & 1901.7454 & 951.3763 & 1900.7614 & 950.8843 & 18 \\
\hline 5 & 531.2232 & 266.1152 & & & 513.2126 & 257.1099 & $\mathbf{V}$ & 1861.7505 & 931.3789 & 1844.7239 & 922.8656 & 1843.7399 & 922.3736 & 17 \\
\hline 6 & 628.2759 & 314.6416 & & & 610.2654 & 305.6363 & $\mathbf{P}$ & 1762.6821 & 881.8447 & 1745.6555 & 873.3314 & 1744.6715 & 872.8394 & 16 \\
\hline 7 & 743.3029 & 372.1551 & & & 725.2923 & 363.1498 & D & 1665.6293 & 833.3183 & 1648.6027 & 824.8050 & 1647.6187 & 824.3130 & 15 \\
\hline 8 & 903.3335 & 452.1704 & & & 885.3230 & 443.1651 & C & 1550.6023 & 775.8048 & 1533.5758 & 767.2915 & 1532.5918 & 766.7995 & 14 \\
\hline 9 & 1000.3863 & 500.6968 & & & 982.3757 & 491.6915 & $\mathbf{P}$ & 1390.5717 & 695.7895 & 1373.5452 & 687.2762 & 1372.5611 & 686.7842 & 13 \\
\hline 10 & 1129.4289 & 565.2181 & & & 1111.4183 & 556.2128 & $\mathbf{E}$ & 1293.5189 & 647.2631 & 1276.4924 & 638.7498 & 1275.5084 & 638.2578 & 12 \\
\hline 11 & 1186.4503 & 593.7288 & & & 1168.4398 & 584.7235 & $\mathbf{G}$ & 1164.4763 & 582.7418 & 1147.4498 & 574.2285 & 1146.4658 & 573.7365 & 11 \\
\hline 12 & 1301.4773 & 651.2423 & & & 1283.4667 & 642.2370 & D & 1107.4549 & 554.2311 & 1090.4283 & 545.7178 & 1089.4443 & 545.2258 & 10 \\
\hline 13 & 1416.5042 & 708.7557 & & & 1398.4937 & 699.7505 & D & 992.4279 & 496.7176 & 975.4014 & 488.2043 & 974.4174 & 487.7123 & 9 \\
\hline 14 & 1545.5468 & 773.2770 & & & 1527.5363 & 764.2718 & $\mathbf{E}$ & 877.4010 & 439.2041 & 860.3744 & 430.6909 & 859.3904 & 430.1989 & 8 \\
\hline 15 & 1659.5897 & 830.2985 & 1642.5632 & 821.7852 & 1641.5792 & 821.2932 & $\mathbf{N}$ & 748.3584 & 374.6828 & 731.3319 & 366.1696 & 730.3478 & 365.6776 & 7 \\
\hline 16 & 1774.6167 & 887.8120 & 1757.5901 & 879.2987 & 1756.6061 & 878.8067 & $\mathbf{N}$ & 634.3155 & 317.6614 & 617.2889 & 309.1481 & 616.3049 & 308.6561 & 6 \\
\hline 17 & 1873.6851 & 937.3462 & 1856.6585 & 928.8329 & 1855.6745 & 928.3409 & $\mathbf{V}$ & 519.2885 & 260.1479 & 502.2620 & 251.6346 & 501.2780 & 251.1426 & 5 \\
\hline 18 & 1974.7328 & 987.8700 & 1957.7062 & 979.3567 & 1956.7222 & 978.8647 & $\mathbf{T}$ & 420.2201 & 210.6137 & 403.1936 & 202.1004 & 402.2096 & 201.6084 & 4 \\
\hline
\end{tabular}




\begin{tabular}{|r|r|r|r|r|r|r|r|r|r|r|r|r|r|r|r|r|r|r|r|r|r|r|r|}
$\mathbf{1 9}$ & 2061.7648 & 1031.3860 & 2044.7383 & 1022.8728 & 2043.7542 & 1022.3808 & $\mathbf{S}$ & 319.1724 & 160.0899 & 302.1459 & 151.5766 & 301.1619 & 151.0846 & $\mathbf{3}$ \\
\hline $\mathbf{2 0}$ & 2118.7863 & 1059.8968 & 2101.7597 & 1051.3835 & 2100.7757 & 1050.8915 & $\mathbf{G}$ & 232.1404 & 116.5738 & 215.1139 & 108.0606 & \\
\hline $\mathbf{2 1}$ & & & & & & & $\mathbf{R}$ & 175.1190 & 88.0631 & 158.0924 & 79.5498 & & \\
\hline
\end{tabular}
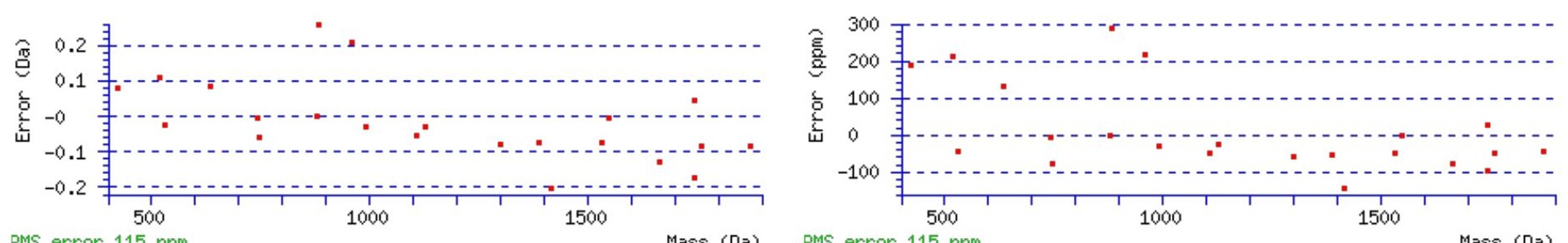

RMS error 115 ppm

Mass (Da) RMS error 115 ppm

Mass (Da)

\section{All matches to this query}

\begin{tabular}{|l|l|l|l|}
\hline Score & Mr(calc): & Delta & \multicolumn{1}{|c|}{ Sequence } \\
\hline 76.1 & 2291.8907 & 0.0075 & VCDGVPDCPEGDDENNVTSGR \\
\hline 61.0 & 2291.8907 & 0.0075 & VCDGVPDCPEGDDENNVTSGR \\
\hline 6.3 & 2290.9067 & 0.9915 & VCDGVPDCPEGDDENNVTSGR \\
\hline 3.9 & 2290.9082 & 0.9900 & MKPAMETAAEENTEQSOER \\
\hline 2.6 & 2289.9085 & 1.9897 & SPSSDLQSLIQSKAEKPR \\
\hline 2.6 & 2289.9085 & 1.9897 & SPSSDLQSLIOSKAEKPR \\
\hline 2.6 & 2289.9085 & 1.9897 & SPSSDLQSLIQSKAEKPR \\
\hline 2.4 & 2291.8923 & 0.0060 & MKPAMETAAEENTEQSOER \\
\hline 2.1 & 2290.9123 & 0.9859 & WQMMKQXDQELNNPTSDK \\
\hline 2.1 & 2290.9123 & 0.9859 & WQMMKQXDQELNNPTSDK \\
\hline
\end{tabular}

Spectrum No: 287; Query: 1224; Rank: 1

\section{Peptide View}

MS/MS Fragmentation of LSALDNLLNHSSIFLK

Found in IPI00210975, Tax_Id=10116 Gene_Symbol=Hyou1 Hypoxia up-regulated protein 1 precursor

Match to Query 1224: 1784.962148 from(893.488350,2+)

Title: 091008RatKidney_NH4Format02_24.5410.5410.2.dta

Data file K:\NewmanPaper|Piliang|3SubProteomes\Piliang3SP\mgf5ppm\ERLIC_3SubProteomes5ppm.mgf

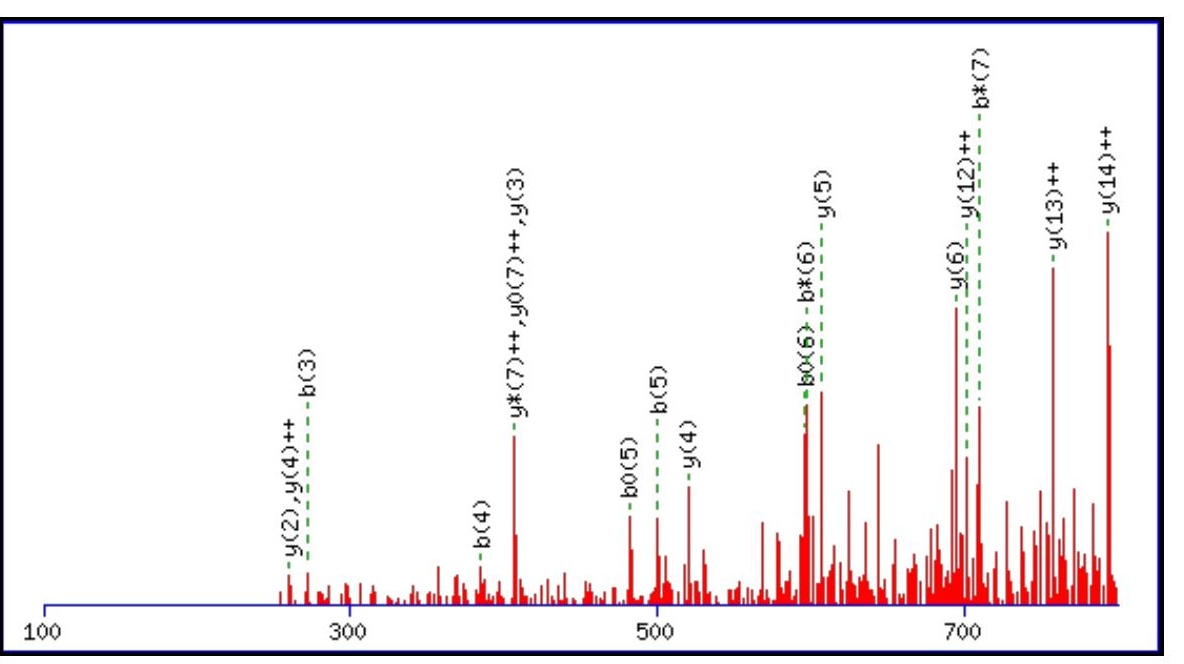



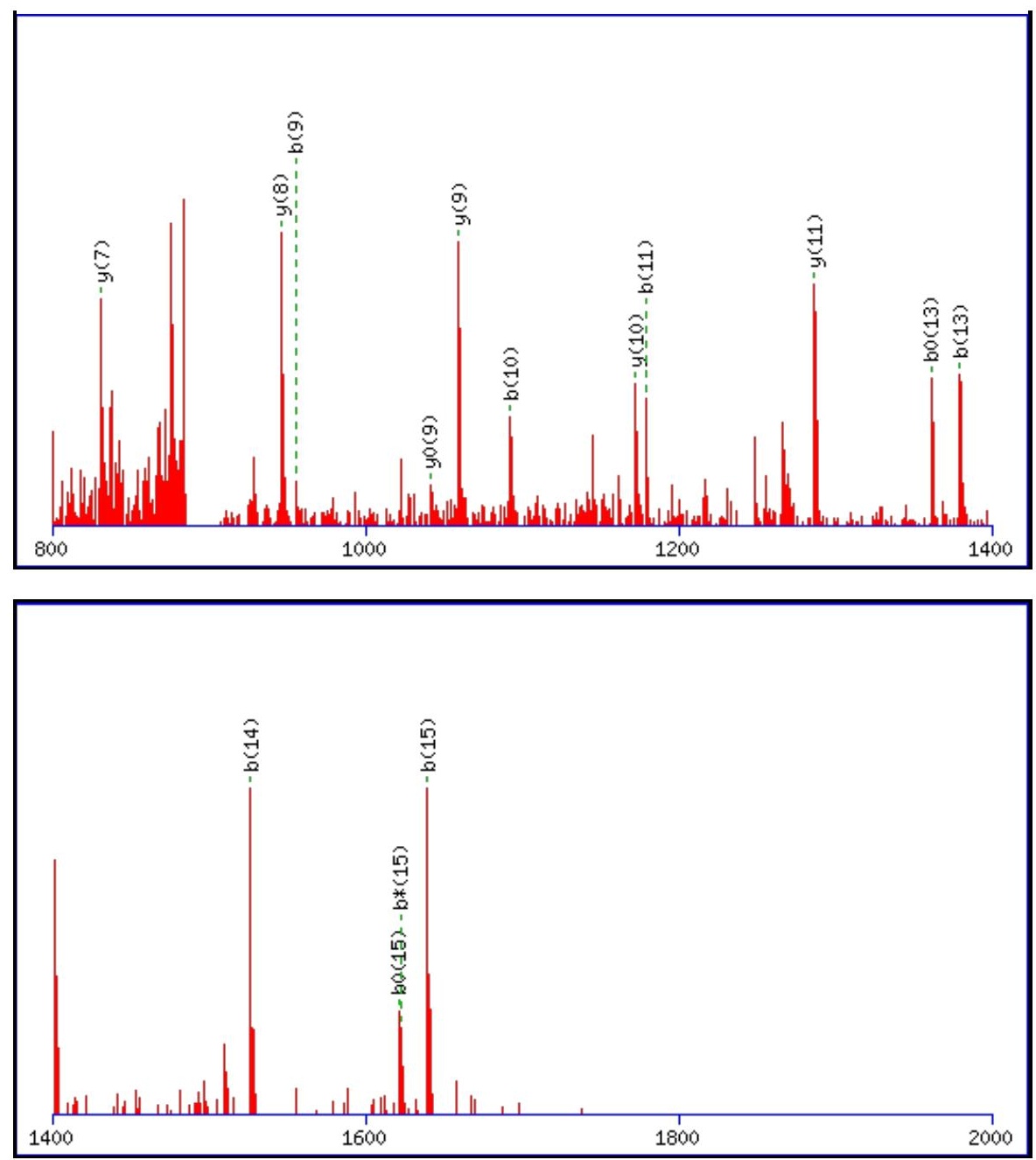

Monoisotopic mass of neutral peptide $\operatorname{Mr}($ calc): 1784.9672

Fixed modifications: Carbamidomethyl (C)

Variable modifications:

N9: Deamidated $\mathrm{N}(\mathrm{N})$

Ions Score: 76 Expect: $2.3 \mathrm{e}-006$

Matches (Bold Red): 33/160 fragment ions using 52 most intense peaks

\begin{tabular}{|c|c|c|c|c|c|c|c|c|c|c|c|c|c|c|}
\hline \# & b & $\mathbf{b}^{++}$ & $\mathbf{b}^{*}$ & $\mathbf{b}^{*^{++}}$ & $\mathbf{b}^{0}$ & $\mathbf{b}^{0++}$ & Seq. & $\mathbf{y}$ & $\mathbf{y}^{++}$ & $\mathbf{y}^{*}$ & $\mathbf{y}^{*^{++}}$ & $\mathbf{y}^{0}$ & $\mathbf{y}^{0++}$ & \# \\
\hline 1 & 114.0913 & 57.5493 & & & & & $\mathbf{L}$ & & & & & & & 16 \\
\hline 2 & 201.1234 & 101.0653 & & & 183.1128 & 92.0600 & S & 1672.8905 & 836.9489 & 1655.8639 & 828.4356 & 1654.8799 & 827.9436 & 15 \\
\hline 3 & 272.1605 & 136.5839 & & & 254.1499 & 127.5786 & A & 1585.8584 & 793.4329 & 1568.8319 & 784.9196 & 1567.8479 & 784.4276 & 14 \\
\hline 4 & 385.2445 & 193.1259 & & & 367.2340 & 184.1206 & $\mathbf{L}$ & 1514.8213 & 757.9143 & 1497.7948 & 749.4010 & 1496.8107 & 748.9090 & 13 \\
\hline 5 & 500.2715 & 250.6394 & & & 482.2609 & 241.6341 & D & 1401.7372 & 701.3723 & 1384.7107 & 692.8590 & 1383.7267 & 692.3670 & 12 \\
\hline 6 & 614.3144 & 307.6608 & 597.2879 & 299.1476 & 596.3039 & 298.6556 & $\mathbf{N}$ & 1286.7103 & 643.8588 & 1269.6838 & 635.3455 & 1268.6997 & 634.8535 & 11 \\
\hline 7 & 727.3985 & 364.2029 & 710.3719 & 355.6896 & 709.3879 & 355.1976 & $\mathbf{L}$ & 1172.6674 & 586.8373 & 1155.6408 & 578.3241 & 1154.6568 & 577.8320 & 10 \\
\hline 8 & 840.4825 & 420.7449 & 823.4560 & 412.2316 & 822.4720 & 411.7396 & $\mathbf{L}$ & 1059.5833 & 530.2953 & 1042.5568 & 521.7820 & 1041.5727 & 521.2900 & 9 \\
\hline 9 & 955.5095 & 478.2584 & 938.4829 & 469.7451 & 937.4989 & 469.2531 & $\mathbf{N}$ & 946.4993 & 473.7533 & 929.4727 & 465.2400 & 928.4887 & 464.7480 & 8 \\
\hline 10 & 1092.5684 & 546.7878 & 1075.5418 & 538.2746 & 1074.5578 & 537.7826 & $\mathbf{H}$ & 831.4723 & 416.2398 & 814.4458 & 407.7265 & 813.4617 & 407.2345 & 7 \\
\hline 11 & 1179.6004 & 590.3038 & 1162.5739 & 581.7906 & 1161.5899 & 581.2986 & S & 694.4134 & 347.7103 & 677.3869 & 339.1971 & 676.4028 & 338.7051 & 6 \\
\hline 12 & 1266.6324 & 633.8199 & 1249.6059 & 625.3066 & 1248.6219 & 624.8146 & $\mathrm{~S}$ & 607.3814 & 304.1943 & 590.3548 & 295.6811 & 589.3708 & 295.1890 & 5 \\
\hline 13 & 1379.7165 & 690.3619 & 1362.6900 & 681.8486 & 1361.7059 & 681.3566 & I & 520.3493 & 260.6783 & 503.3228 & 252.1650 & & & 4 \\
\hline 14 & 1526.7849 & 763.8961 & 1509.7584 & 755.3828 & 1508.7744 & 754.8908 & $\mathbf{F}$ & 407.2653 & 204.1363 & 390.2387 & 195.6230 & & & 3 \\
\hline 15 & 1639.8690 & 820.4381 & 1622.8424 & 811.9249 & 1621.8584 & 811.4329 & $\mathbf{L}$ & 260.1969 & 130.6021 & 243.1703 & 122.0888 & & & 2 \\
\hline 16 & & & & & & & $\mathbf{K}$ & 147.1128 & 74.0600 & 130.0863 & 65.5468 & & & 1 \\
\hline
\end{tabular}



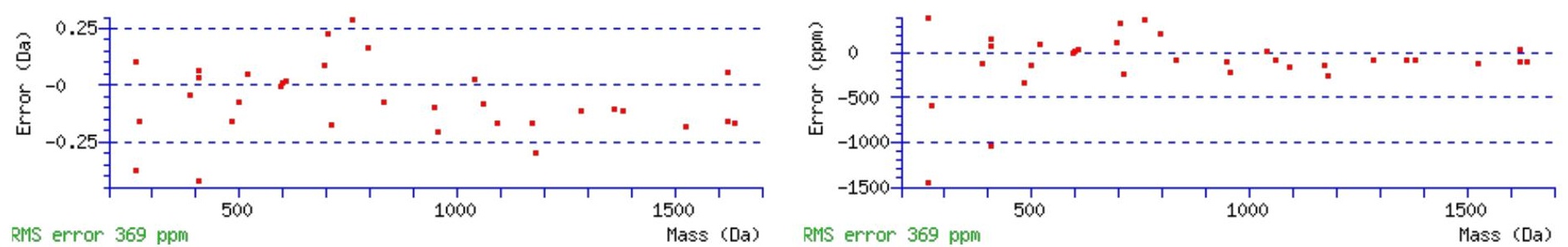

\section{All matches to this query}

\begin{tabular}{|l|l|l|l|}
\hline Score & Mr(calc): & Delta & \multicolumn{1}{|c|}{ Sequence } \\
\hline 76.0 & 1784.9672 & -0.0051 & LSALDNLLNHSSIFLK \\
\hline 53.9 & 1784.9672 & -0.0051 & LSALDNLLNHSSIFLK \\
\hline 5.6 & 1784.9632 & -0.0010 & EELNKELVQASERLK \\
\hline 4.9 & 1784.9519 & 0.0102 & DINRLLEETQAELLK \\
\hline 4.4 & 1784.9771 & -0.0149 & KVELKENLIAELEEK \\
\hline 3.6 & 1784.9585 & 0.0037 & YPLMVKLNLLLXLK \\
\hline 2.5 & 1784.9454 & 0.0167 & LSSPECLNASLLGGVLR \\
\hline 2.3 & 1784.9785 & -0.0163 & VRELSTSEKHVPYIK \\
\hline 2.3 & 1784.9477 & 0.0144 & VAALLAANSFDIIFLK \\
\hline 1.6 & 1782.9393 & 2.0228 & ASAVSPANLPAVLLQPR \\
\hline
\end{tabular}

Spectrum No: 288; Query: 920; Rank: 1

\section{Peptide View}

MS/MS Fragmentation of ECLGNCSEPDDPTK

Found in IPI00393267, Tax_Id=10116 Gene_Symbol=Insr Tyrosine-protein kinase receptor

Match to Query 920: 1621.625928 from(811.820240,2+)

Title: 100101RatKid_NS_deglyco_20.879.879.2.dta

Data file K:INewmanPaper|Piliangl3SubProteomes\Piliang3SP\mgf5ppm\ERLIC_3SubProteomes5ppm.mgf

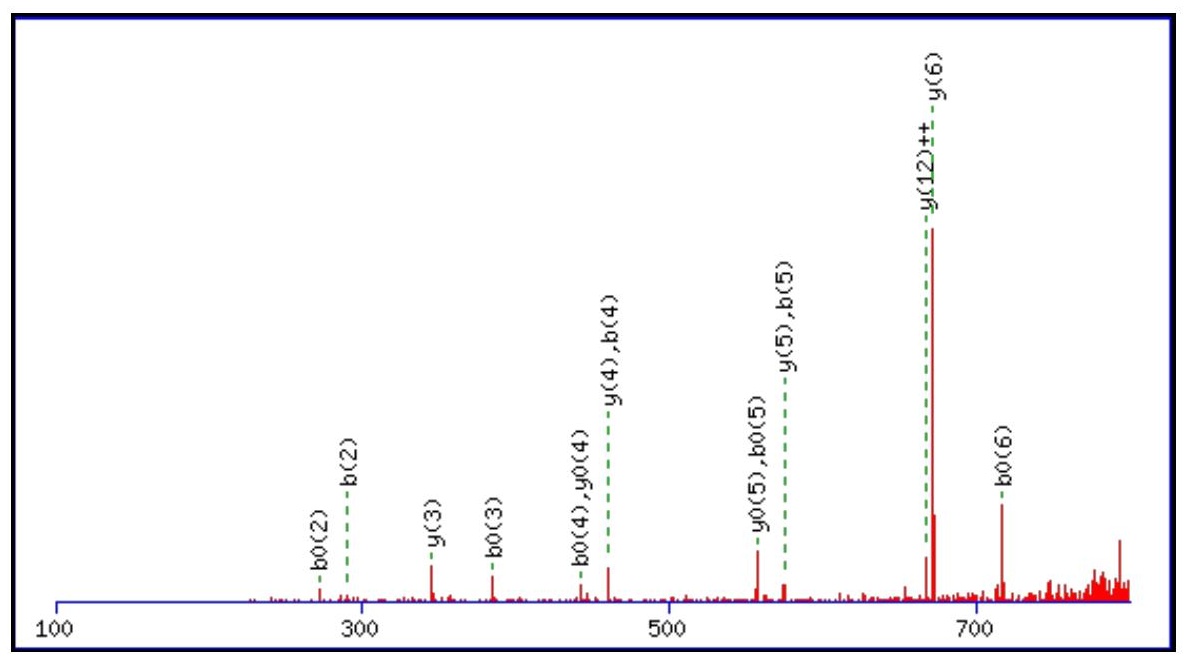



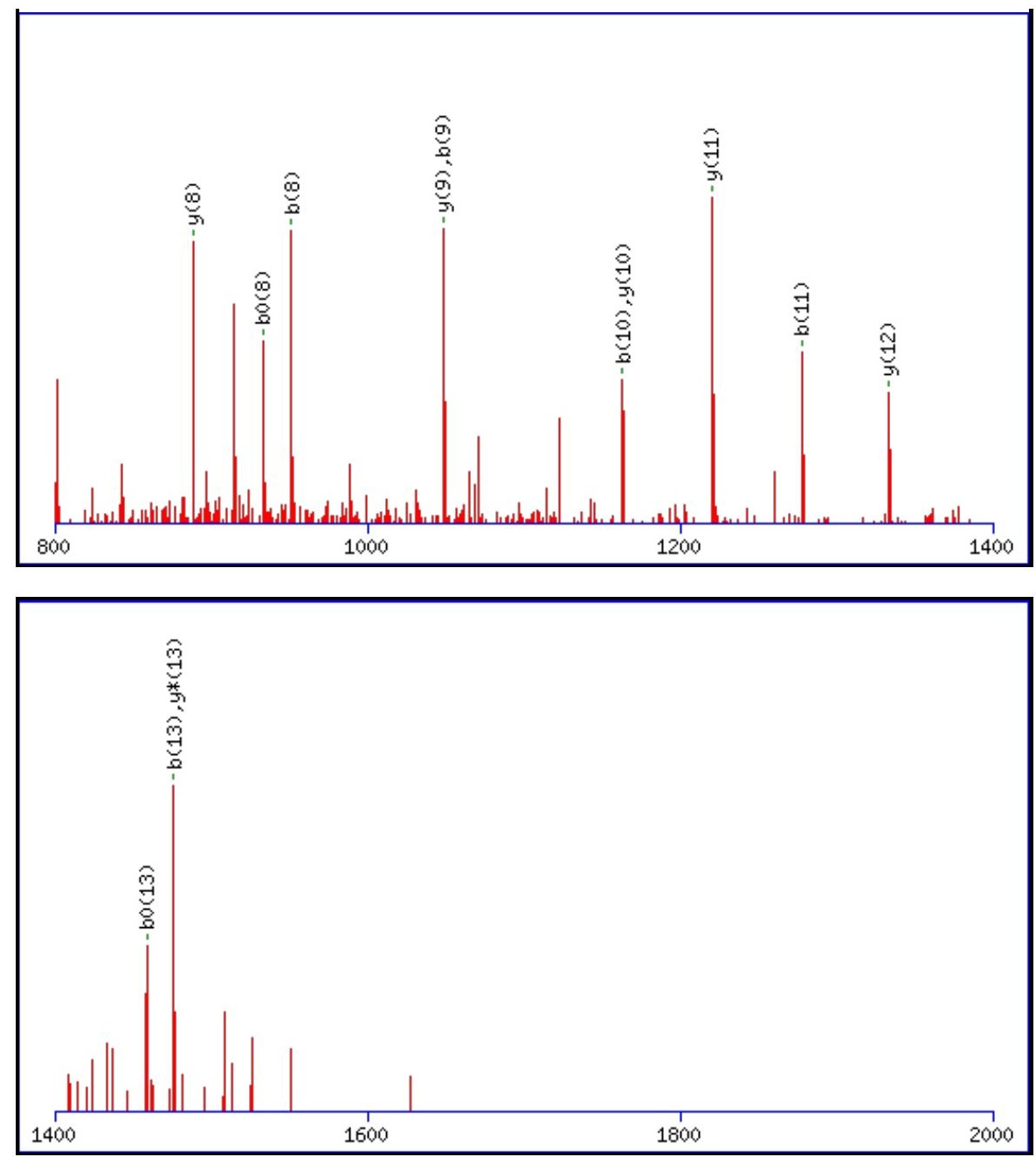

Monoisotopic mass of neutral peptide $\operatorname{Mr}($ calc): 1621.6236

Fixed modifications: Carbamidomethyl (C)

Variable modifications:

N5 : Deamidated $\mathrm{N}(\mathrm{N})$

Ions Score: 76 Expect: $2.7 \mathrm{e}-006$

Matches (Bold Red): 28/146 fragment ions using 32 most intense peaks

\begin{tabular}{|c|c|c|c|c|c|c|c|c|c|c|c|c|c|c|}
\hline \# & b & $\mathbf{b}^{++}$ & b* & $\mathbf{b}^{*^{++}}$ & $\mathbf{b}^{\mathbf{0}}$ & $\mathbf{b}^{\mathbf{0 + +}}$ & Seq. & $\mathbf{y}$ & $\mathbf{y}^{++}$ & $\mathbf{y}^{*}$ & $\mathbf{y}^{*^{++}}$ & $\mathbf{y}^{\mathbf{0}}$ & $\mathbf{y}^{0++}$ & \# \\
\hline 1 & 130.0499 & 65.5286 & & & 112.0393 & 56.5233 & $\mathbf{E}$ & & & & & & & 14 \\
\hline 2 & 290.0805 & 145.5439 & & & 272.0700 & 136.5386 & C & 1493.5883 & 747.2978 & 1476.5617 & 738.7845 & 1475.5777 & 738.2925 & 13 \\
\hline 3 & 403.1646 & 202.0859 & & & 385.1540 & 193.0806 & $\mathbf{L}$ & 1333.5576 & 667.2825 & 1316.5311 & 658.7692 & 1315.5471 & 658.2772 & 12 \\
\hline 4 & 460.1860 & 230.5967 & & & 442.1755 & 221.5914 & $\mathbf{G}$ & 1220.4736 & 610.7404 & 1203.4470 & 602.2271 & 1202.4630 & 601.7351 & 11 \\
\hline 5 & 575.2130 & 288.1101 & 558.1864 & 279.5969 & 557.2024 & 279.1048 & $\mathbf{N}$ & 1163.4521 & 582.2297 & 1146.4256 & 573.7164 & 1145.4415 & 573.2244 & 10 \\
\hline 6 & 735.2436 & 368.1255 & 718.2171 & 359.6122 & 717.2331 & 359.1202 & C & 1048.4252 & 524.7162 & 1031.3986 & 516.2029 & 1030.4146 & 515.7109 & 9 \\
\hline 7 & 822.2757 & 411.6415 & 805.2491 & 403.1282 & 804.2651 & 402.6362 & $\mathbf{S}$ & 888.3945 & 444.7009 & 871.3680 & 436.1876 & 870.3840 & 435.6956 & 8 \\
\hline 8 & 951.3183 & 476.1628 & 934.2917 & 467.6495 & 933.3077 & 467.1575 & $\mathbf{E}$ & 801.3625 & 401.1849 & 784.3359 & 392.6716 & 783.3519 & 392.1796 & 7 \\
\hline 9 & 1048.3710 & 524.6891 & 1031.3445 & 516.1759 & 1030.3605 & 515.6839 & $\mathbf{P}$ & 672.3199 & 336.6636 & 655.2933 & 328.1503 & 654.3093 & 327.6583 & 6 \\
\hline 10 & 1163.3980 & 582.2026 & 1146.3714 & 573.6893 & 1145.3874 & 573.1973 & D & 575.2671 & 288.1372 & 558.2406 & 279.6239 & 557.2566 & 279.1319 & 5 \\
\hline 11 & 1278.4249 & 639.7161 & 1261.3984 & 631.2028 & 1260.4143 & 630.7108 & D & 460.2402 & 230.6237 & 443.2136 & 222.1105 & 442.2296 & 221.6185 & 4 \\
\hline 12 & 1375.4777 & 688.2425 & 1358.4511 & 679.7292 & 1357.4671 & 679.2372 & $\mathbf{P}$ & 345.2132 & 173.1103 & 328.1867 & 164.5970 & 327.2027 & 164.1050 & 3 \\
\hline 13 & 1476.5253 & 738.7663 & 1459.4988 & 730.2530 & 1458.5148 & 729.7610 & $\mathbf{T}$ & 248.1605 & 124.5839 & 231.1339 & 116.0706 & 230.1499 & 115.5786 & 2 \\
\hline 14 & & & & & & & $\mathbf{K}$ & 147.1128 & 74.0600 & 130.0863 & 65.5468 & & & 1 \\
\hline
\end{tabular}



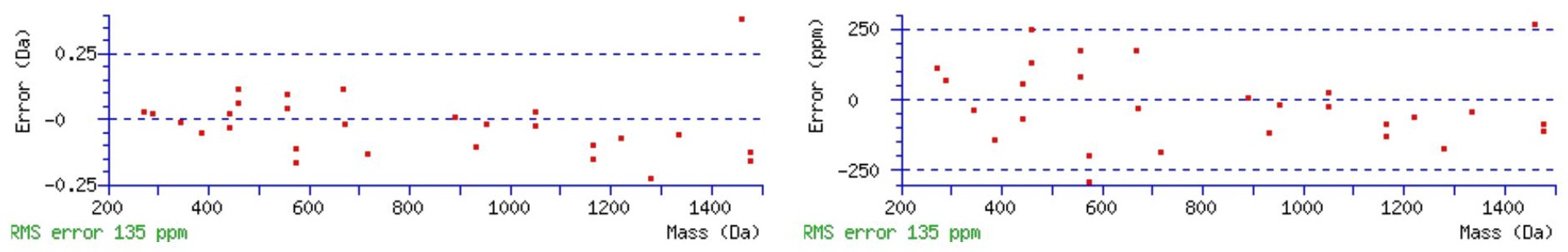

\section{All matches to this query}

\begin{tabular}{|l|l|l|l|}
\hline Score & Mr(calc): & Delta & Sequence \\
\hline 75.9 & 1621.6236 & 0.0023 & ECLGNCSEPDDPTK \\
\hline 8.0 & 1621.6274 & -0.0014 & ASSVGNVADSTEPTK \\
\hline 7.5 & 1621.6274 & -0.0014 & $\underline{\text { ASSVGNVADSTEPTK }}$ \\
\hline 5.7 & 1621.6274 & -0.0014 & ASSVGNVADSTEPTK \\
\hline 5.7 & 1621.6274 & -0.0014 & $\underline{\text { ASSVGNVADSTEPTK }}$ \\
\hline 5.3 & 1621.6274 & -0.0014 & $\underline{\text { ASSVGNVADSTEPTK }}$ \\
\hline 5.3 & 1621.6227 & 0.0032 & DGDEESGNKNENSPK \\
\hline 1.2 & 1621.6274 & -0.0014 & ASSVGNVADSTEPTK \\
\hline 0.7 & 1621.6287 & -0.0027 & EKSHGYQRENSK \\
\hline 0.4 & 1620.6387 & 0.9873 & DGDEESGNKNENSPK \\
\hline
\end{tabular}

Spectrum No: 289; Query: 1909; Rank: 1

\section{Peptide View}

MS/MS Fragmentation of YHYNGTLLDGTLFDSSYSR

Found in IPI00215190, Tax_Id=10116 Gene_Symbol=Fkbp9 FK506-binding protein 9 precursor

Match to Query 1909: 2209.001208 from(1105.507880,2+)

Title: 100101RatKid_NS_deglyco_13.4624.4624.2.dta

Data file K:INewmanPaper|Piliangl3SubProteomes\Piliang3SP\mgf5ppm\ERLIC_3SubProteomes5ppm.mgf

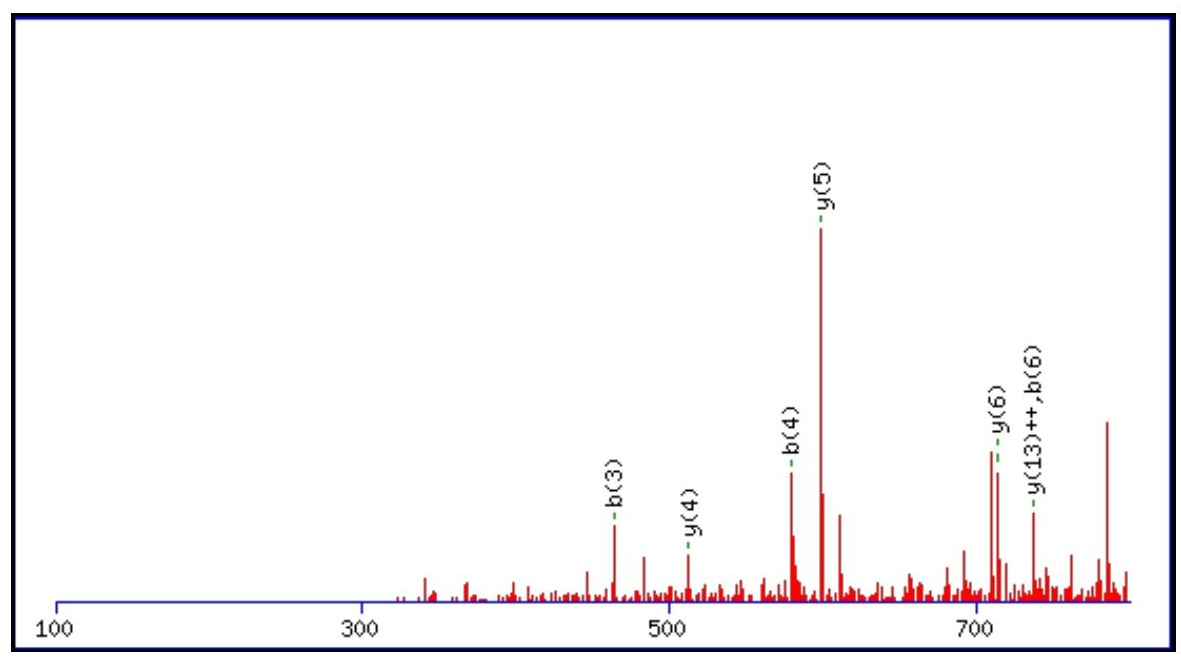



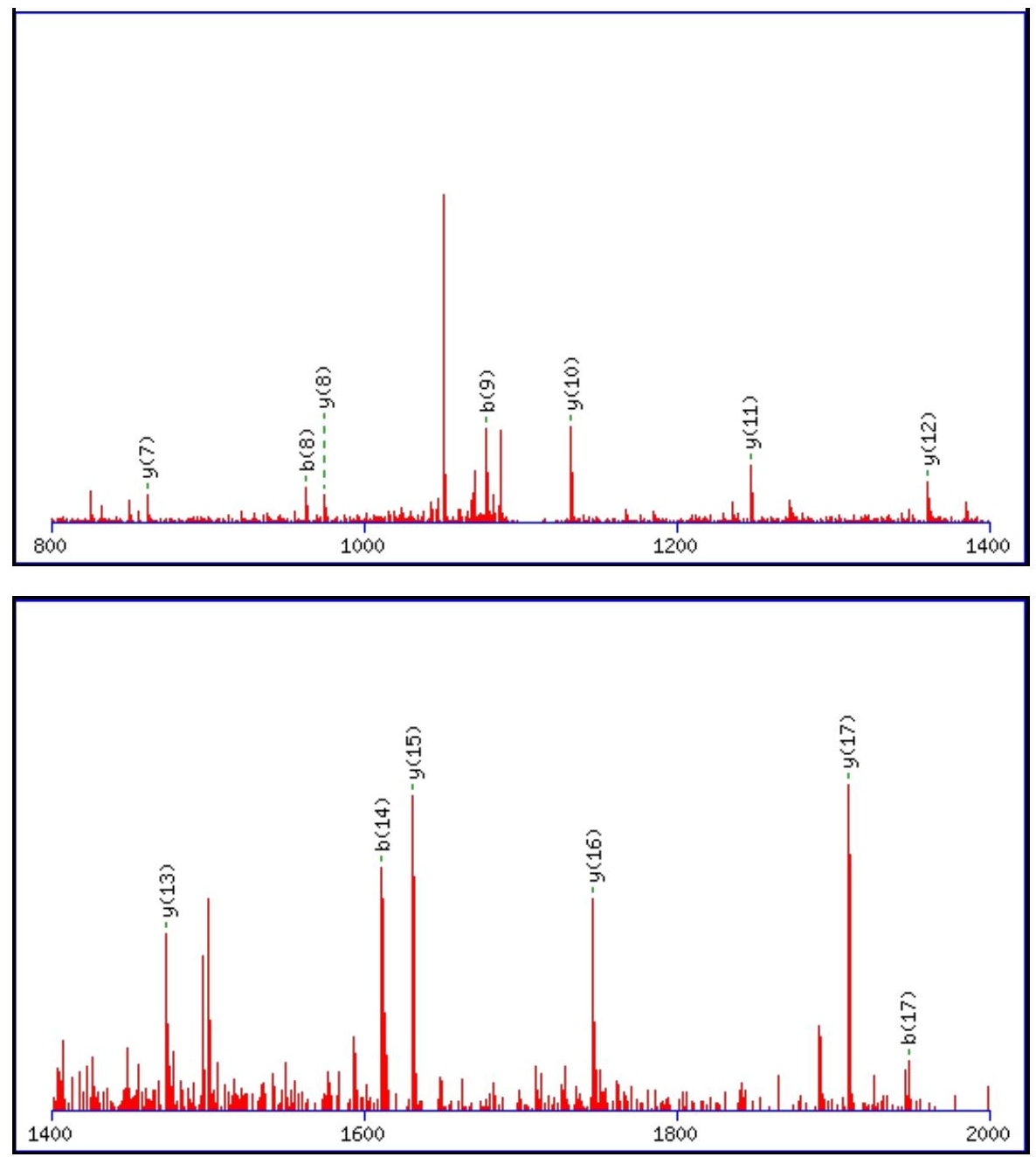

Monoisotopic mass of neutral peptide $\operatorname{Mr}($ calc): 2208.9964

Fixed modifications: Carbamidomethyl (C)

Variable modifications:

N4 : Deamidated N (N)

Ions Score: 76 Expect: $6.9 \mathrm{e}-006$

Matches (Bold Red): 20/198 fragment ions using 38 most intense peaks

\begin{tabular}{|c|c|c|c|c|c|c|c|c|c|c|c|c|c|c|}
\hline \# & b & $\mathbf{b}^{++}$ & $\mathbf{b}^{*}$ & $\mathbf{b}^{*^{++}}$ & $\mathbf{b}^{\mathbf{0}}$ & $\mathbf{b}^{0++}$ & Seq. & $\mathbf{y}$ & $y^{++}$ & $\mathbf{y}^{*}$ & $\mathbf{y}^{*^{++}}$ & $\mathbf{y}^{0}$ & $\mathbf{y}^{\mathbf{0 + +}}$ & \# \\
\hline 1 & 164.0706 & 82.5389 & & & & & $\mathbf{Y}$ & & & & & & & 19 \\
\hline 2 & 301.1295 & 151.0684 & & & & & H & 2046.9403 & 1023.9738 & 2029.9138 & 1015.4605 & 2028.9298 & 1014.9685 & 18 \\
\hline 3 & 464.1928 & 232.6001 & & & & & $\mathbf{Y}$ & 1909.8814 & 955.4443 & 1892.8549 & 946.9311 & 1891.8708 & 946.4391 & 17 \\
\hline 4 & 579.2198 & 290.1135 & 562.1932 & 281.6003 & & & $\mathbf{N}$ & 1746.8181 & 873.9127 & 1729.7915 & 865.3994 & 1728.8075 & 864.9074 & 16 \\
\hline 5 & 636.2412 & 318.6243 & 619.2147 & 310.1110 & & & $\mathbf{G}$ & 1631.7911 & 816.3992 & 1614.7646 & 807.8859 & 1613.7806 & 807.3939 & 15 \\
\hline 6 & 737.2889 & 369.1481 & 720.2624 & 360.6348 & 719.2784 & 360.1428 & $\mathbf{T}$ & 1574.7697 & 787.8885 & 1557.7431 & 779.3752 & 1556.7591 & 778.8832 & 14 \\
\hline 7 & 850.3730 & 425.6901 & 833.3464 & 417.1769 & 832.3624 & 416.6849 & $\mathbf{L}$ & 1473.7220 & 737.3646 & 1456.6955 & 728.8514 & 1455.7114 & 728.3594 & 13 \\
\hline 8 & 963.4571 & 482.2322 & 946.4305 & 473.7189 & 945.4465 & 473.2269 & $\mathbf{L}$ & 1360.6379 & 680.8226 & 1343.6114 & 672.3093 & 1342.6274 & 671.8173 & 12 \\
\hline 9 & 1078.4840 & 539.7456 & 1061.4574 & 531.2324 & 1060.4734 & 530.7404 & D & 1247.5539 & 624.2806 & 1230.5273 & 615.7673 & 1229.5433 & 615.2753 & 11 \\
\hline 10 & 1135.5055 & 568.2564 & 1118.4789 & 559.7431 & 1117.4949 & 559.2511 & G & 1132.5269 & 566.7671 & 1115.5004 & 558.2538 & 1114.5164 & 557.7618 & 10 \\
\hline 11 & 1236.5531 & 618.7802 & 1219.5266 & 610.2669 & 1218.5426 & 609.7749 & $\mathbf{T}$ & 1075.5055 & 538.2564 & 1058.4789 & 529.7431 & 1057.4949 & 529.2511 & 9 \\
\hline 12 & 1349.6372 & 675.3222 & 1332.6107 & 666.8090 & 1331.6266 & 666.3170 & $\mathbf{L}$ & 974.4578 & 487.7325 & 957.4312 & 479.2193 & 956.4472 & 478.7272 & 8 \\
\hline 13 & 1496.7056 & 748.8564 & 1479.6791 & 740.3432 & 1478.6951 & 739.8512 & $\mathbf{F}$ & 861.3737 & 431.1905 & 844.3472 & 422.6772 & 843.3632 & 422.1852 & 7 \\
\hline 14 & 1611.7326 & 806.3699 & 1594.7060 & 797.8566 & 1593.7220 & 797.3646 & D & 714.3053 & 357.6563 & 697.2788 & 349.1430 & 696.2947 & 348.6510 & 6 \\
\hline 15 & 1698.7646 & 849.8859 & 1681.7380 & 841.3727 & 1680.7540 & 840.8806 & S & 599.2784 & 300.1428 & 582.2518 & 291.6295 & 581.2678 & 291.1375 & 5 \\
\hline 16 & 1785.7966 & 893.4019 & 1768.7701 & 884.8887 & 1767.7861 & 884.3967 & $\mathrm{~S}$ & 512.2463 & 256.6268 & 495.2198 & 248.1135 & 494.2358 & 247.6215 & 4 \\
\hline 17 & 1948.8599 & 974.9336 & 1931.8334 & 966.4203 & 1930.8494 & 965.9283 & $\mathbf{Y}$ & 425.2143 & 213.1108 & 408.1878 & 204.5975 & 407.2037 & 204.1055 & 3 \\
\hline 18 & 2035.8920 & 1018.4496 & 2018.8654 & 1009.9364 & 2017.8814 & 1009.4443 & $S$ & 262.1510 & 131.5791 & 245.1244 & 123.0659 & 244.1404 & 122.5738 & 2 \\
\hline
\end{tabular}




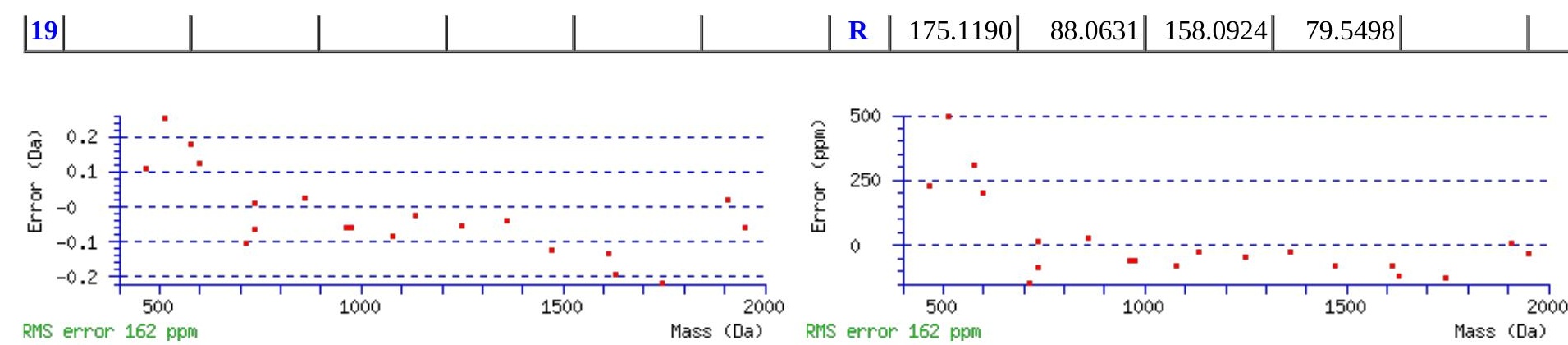

\section{All matches to this query}

\begin{tabular}{|l|l|c|l|}
\hline Score & Mr(calc): & Delta & \multicolumn{1}{|c|}{ Sequence } \\
\hline 75.9 & 2208.9964 & 0.0048 & YHYNGTLLDGTLFDSSYSR \\
\hline 37.3 & 2208.0124 & 0.9888 & YHYNGTLLDGTLFDSSYSR \\
\hline 2.6 & 2208.9946 & 0.0066 & KRALSISPLSDASLDLQR \\
\hline 1.2 & 2207.0147 & 1.9865 & LSLKGHEDWVTDVAISNDK \\
\hline 0.4 & 2207.9832 & 1.0180 & TIMLLGVSSQKISVRLK \\
\hline
\end{tabular}

Spectrum No: 290; Query: 231; Rank: 1

\section{Peptide View}

MS/MS Fragmentation of QTAEYAANITK

Found in IPI00327398, Tax_Id=10116 Gene_Symbol=Enpep Isoform 1 of Glutamyl aminopeptidase

Match to Query 231: 1209.588308 from(605.801430,2+)

Title: 091008RatKidney_NoSalt_26.810.810.2.dta

Data file K:\NewmanPaper|Piliang|3SubProteomes\Piliang3SP\mgf5ppm\ERLIC_3SubProteomes5ppm.mgf

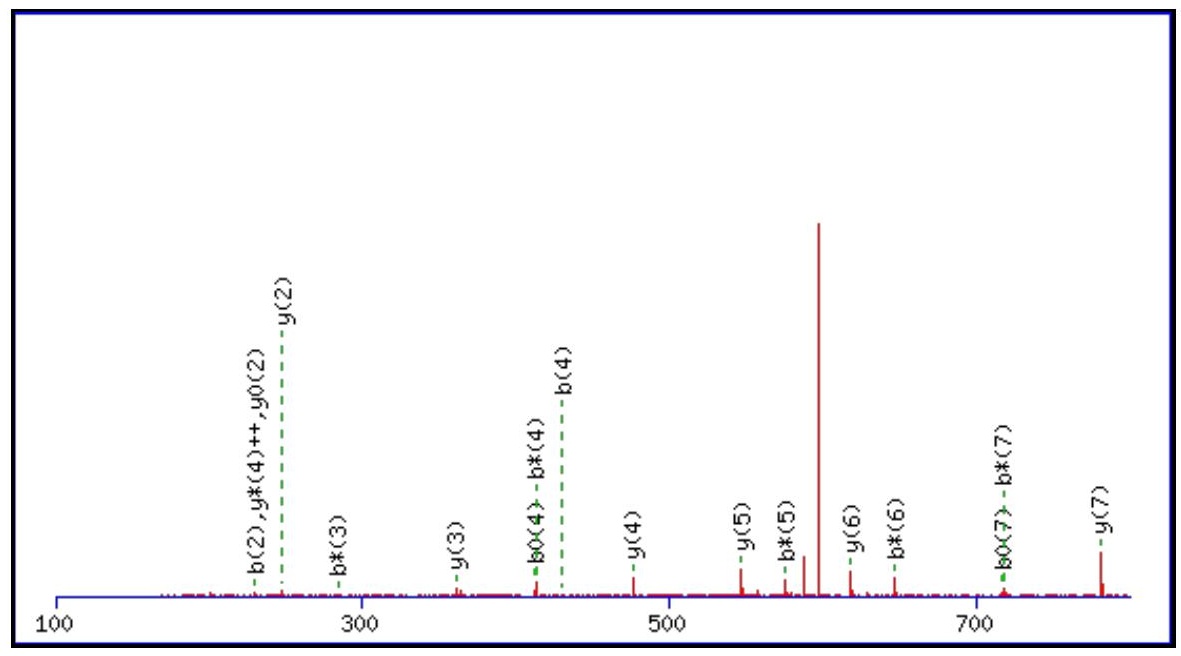




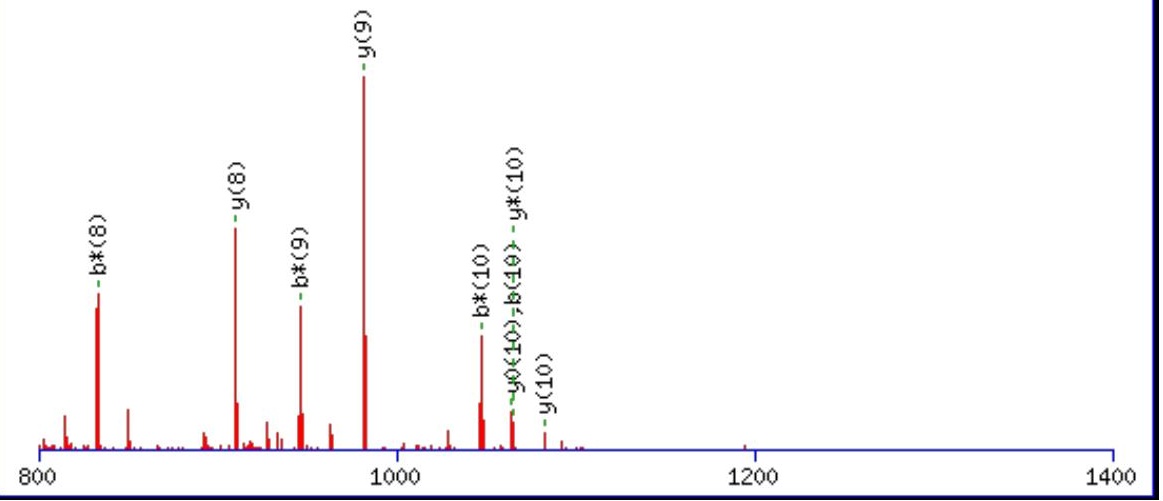

\begin{tabular}{|c|c|c|c|}
\hline 1400 & 1600 & 1800 & 2000 \\
\hline
\end{tabular}

Monoisotopic mass of neutral peptide $\operatorname{Mr}($ calc): 1209.5877

Fixed modifications: Carbamidomethyl (C)

Variable modifications:

N8: : Deamidated $\mathrm{N}(\mathrm{N})$

Ions Score: 76 Expect: $3.7 \mathrm{e}-006$

Matches (Bold Red): 26/116 fragment ions using 40 most intense peaks

\begin{tabular}{|c|c|c|c|c|c|c|c|c|c|c|c|c|c|c|}
\hline \# & b & $\mathbf{b}^{++}$ & b* & $\mathbf{b}^{*^{++}}$ & $\mathbf{b}^{0}$ & $\mathbf{b}^{\mathbf{0 + +}}$ & Seq. & $\mathbf{y}$ & $\mathbf{y}^{++}$ & $\mathbf{y}^{*}$ & $\mathrm{y}^{*^{++}}$ & $\mathbf{y}^{\mathbf{0}}$ & $\mathbf{y}^{0++}$ & \# \\
\hline 1 & 129.0659 & 65.0366 & 112.0393 & 56.5233 & & & $\mathbf{Q}$ & & & & & & & 11 \\
\hline 2 & 230.1135 & 115.5604 & 213.0870 & 107.0471 & 212.1030 & 106.5551 & $\mathbf{T}$ & 1082.5364 & 541.7719 & 1065.5099 & 533.2586 & 1064.5259 & 532.7666 & 10 \\
\hline 3 & 301.1506 & 151.0790 & 284.1241 & 142.5657 & 283.1401 & 142.0737 & A & 981.4887 & 491.2480 & 964.4622 & 482.7347 & 963.4782 & 482.2427 & 9 \\
\hline 4 & 430.1932 & 215.6003 & 413.1667 & 207.0870 & 412.1827 & 206.5950 & $\mathbf{E}$ & 910.4516 & 455.7295 & 893.4251 & 447.2162 & 892.4411 & 446.7242 & 8 \\
\hline 5 & 593.2566 & 297.1319 & 576.2300 & 288.6186 & 575.2460 & 288.1266 & $\mathbf{Y}$ & 781.4090 & 391.2082 & 764.3825 & 382.6949 & 763.3985 & 382.2029 & 7 \\
\hline 6 & 664.2937 & 332.6505 & 647.2671 & 324.1372 & 646.2831 & 323.6452 & A & 618.3457 & 309.6765 & 601.3192 & 301.1632 & 600.3351 & 300.6712 & 6 \\
\hline 7 & 735.3308 & 368.1690 & 718.3042 & 359.6558 & 717.3202 & 359.1638 & A & 547.3086 & 274.1579 & 530.2820 & 265.6447 & 529.2980 & 265.1527 & 5 \\
\hline 8 & 850.3577 & 425.6825 & 833.3312 & 417.1692 & 832.3472 & 416.6772 & $\mathbf{N}$ & 476.2715 & 238.6394 & 459.2449 & 230.1261 & 458.2609 & 229.6341 & 4 \\
\hline 9 & 963.4418 & 482.2245 & 946.4152 & 473.7113 & 945.4312 & 473.2193 & I & 361.2445 & 181.1259 & 344.2180 & 172.6126 & 343.2340 & 172.1206 & 3 \\
\hline 10 & 1064.4895 & 532.7484 & 1047.4629 & 524.2351 & 1046.4789 & 523.7431 & $\mathbf{T}$ & 248.1605 & 124.5839 & 231.1339 & 116.0706 & 230.1499 & 115.5786 & 2 \\
\hline 11 & & & & & & & $\mathbf{K}$ & 147.1128 & 74.0600 & 130.0863 & 65.5468 & & & 1 \\
\hline
\end{tabular}
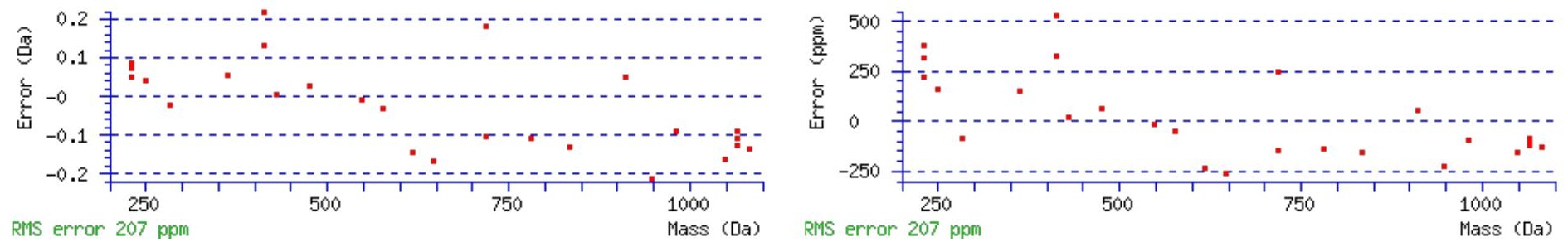


\section{All matches to this query}

\begin{tabular}{|l|l|l|l|}
\hline Score & Mr(calc): & Delta & \multicolumn{1}{|c|}{ Sequence } \\
\hline 75.9 & 1209.5877 & 0.0006 & QTAEYAANITK \\
\hline 12.0 & 1208.5826 & 1.0057 & DTVLAYWADR \\
\hline 5.9 & 1208.5781 & 1.0102 & MVEVCVESIK \\
\hline 5.0 & 1207.5710 & 2.0173 & EAEATAKRPR \\
\hline 3.5 & 1208.5955 & 0.9928 & DILSVQIXR \\
\hline 3.4 & 1208.5914 & 0.9969 & SETPVTIARR \\
\hline 3.4 & 1208.5914 & 0.9969 & SETPVTIARR \\
\hline 3.4 & 1208.5914 & 0.9969 & SETPVTIARR \\
\hline 3.3 & 1208.5802 & 1.0081 & QVLSGSGGPVTK \\
\hline 3.2 & 1208.5785 & 1.0098 & EVARNQNYSK \\
\hline
\end{tabular}

Spectrum No: 291; Query: 635; Rank: 1

\section{Peptide View}

MS/MS Fragmentation of VLTNQENPYQNR

Found in IPI00215296, Tax_Id=10116 Gene_Symbol=C1qa Complement C1q subcomponent subunit A precursor

Match to Query 635: 1475.704308 from(738.859430,2+)

Title: 100101RatKid_NS_deglyco_23.986.986.2.dta

Data file K:INewmanPaper|Piliang|3SubProteomes\Piliang3SP\mgf5ppm\ERLIC_3SubProteomes5ppm.mgf
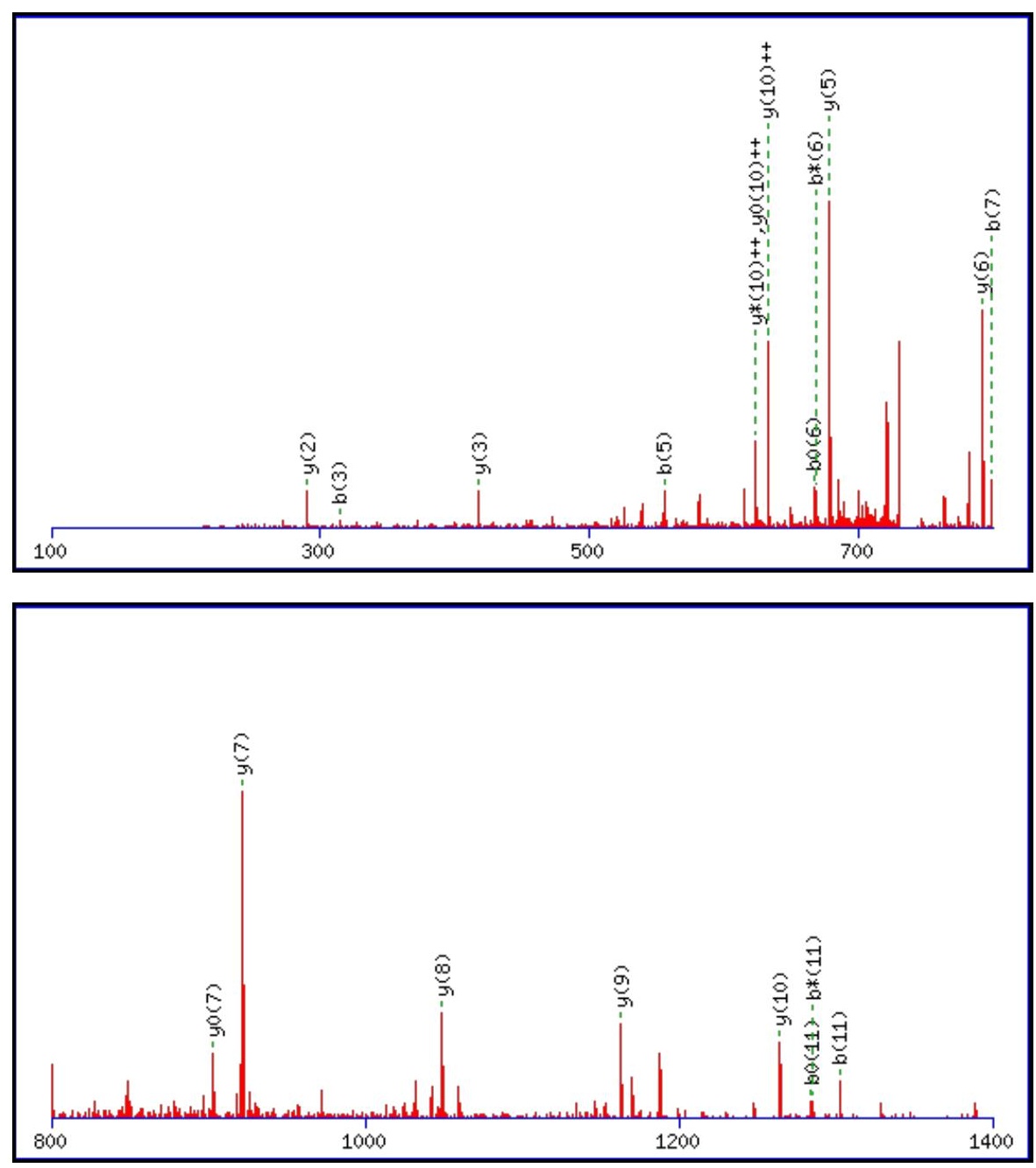


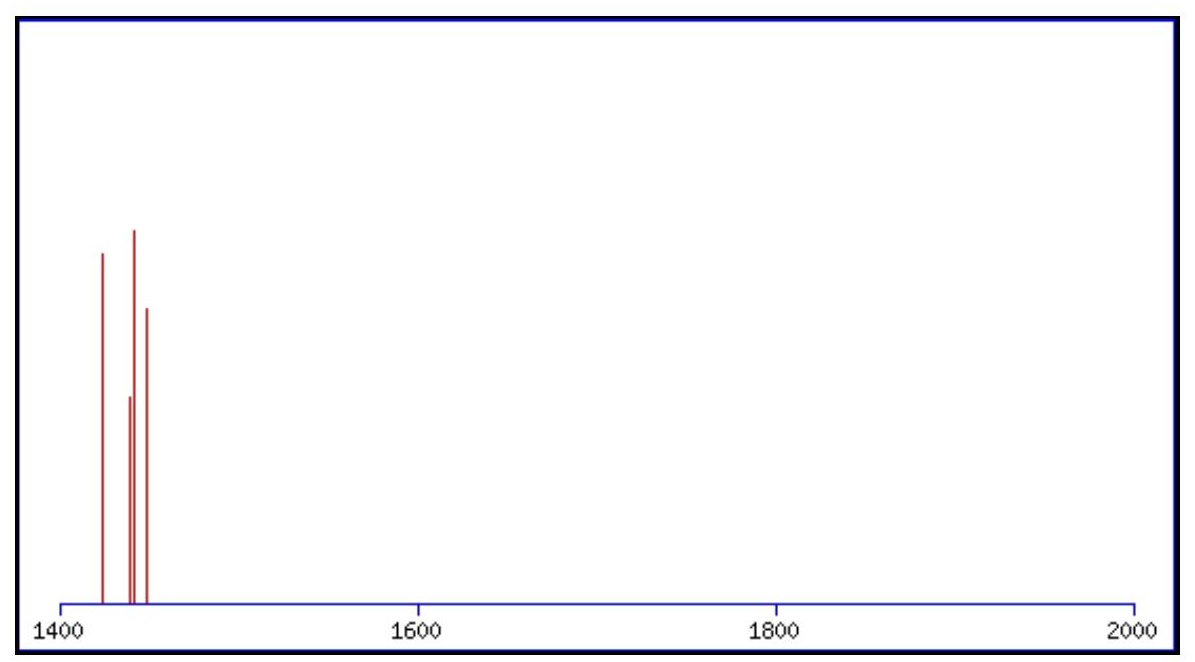

Monoisotopic mass of neutral peptide $\operatorname{Mr}($ calc): 1475.7004

Fixed modifications: Carbamidomethyl (C)

Variable modifications:

N11 : Deamidated_N (N)

Ions Score: 76 Expect: $4 \mathrm{e}-006$

Matches (Bold Red): 20/110 fragment ions using 33 most intense peaks

\begin{tabular}{|r|c|c|c|c|c|c|c|c|c|c|c|c|c|c|}
\hline$\#$ & $\mathbf{b}$ & $\mathbf{b}^{++}$ & $\mathbf{b}^{*}$ & $\mathbf{b}^{\boldsymbol{*}^{++}}$ & $\mathbf{b}^{\mathbf{0}}$ & $\mathbf{b}^{\mathbf{0 + +}}$ & $\mathbf{S e q}$ & $\mathbf{y}$ & $\mathbf{y}^{++}$ & $\mathbf{y}^{\mathbf{*}}$ & $\mathbf{y}^{\boldsymbol{*}^{++}}$ & $\mathbf{y}^{\mathbf{0}}$ & $\mathbf{y}^{\mathbf{0 + +}}$ & $\#$ \\
\hline $\mathbf{1}$ & 100.0757 & 50.5415 & & & & & $\mathbf{V}$ & & & & & & & $\mathbf{1 2}$ \\
\hline $\mathbf{2}$ & 213.1598 & 107.0835 & & & & & $\mathbf{L}$ & 1377.6393 & 689.3233 & 1360.6128 & 680.8100 & 1359.6288 & 680.3180 & $\mathbf{1 1}$ \\
\hline $\mathbf{3}$ & $\mathbf{3 1 4 . 2 0 7 4}$ & 157.6074 & & & 296.1969 & 148.6021 & $\mathbf{T}$ & $\mathbf{1 2 6 4 . 5 5 5 3}$ & $\mathbf{6 3 2 . 7 8 1 3}$ & 1247.5287 & $\mathbf{6 2 4 . 2 6 8 0}$ & 1246.5447 & $\mathbf{6 2 3 . 7 7 6 0}$ & $\mathbf{1 0}$ \\
\hline $\mathbf{4}$ & 428.2504 & 214.6288 & 411.2238 & 206.1155 & 410.2398 & 205.6235 & $\mathbf{N}$ & $\mathbf{1 1 6 3 . 5 0 7 6}$ & 582.2574 & 1146.4810 & 573.7442 & 1145.4970 & 573.2521 & $\mathbf{9}$ \\
\hline $\mathbf{5}$ & $\mathbf{5 5 6 . 3 0 8 9}$ & 278.6581 & 539.2824 & 270.1448 & 538.2984 & 269.6528 & $\mathbf{Q}$ & $\mathbf{1 0 4 9 . 4 6 4 7}$ & 525.2360 & 1032.4381 & 516.7227 & 1031.4541 & 516.2307 & $\mathbf{8}$ \\
\hline $\mathbf{6}$ & 685.3515 & 343.1794 & $\mathbf{6 6 8 . 3 2 5 0}$ & 334.6661 & $\mathbf{6 6 7 . 3 4 1 0}$ & 334.1741 & $\mathbf{E}$ & $\mathbf{9 2 1 . 4 0 6 1}$ & 461.2067 & 904.3795 & 452.6934 & $\mathbf{9 0 3 . 3 9 5 5}$ & 452.2014 & $\mathbf{7}$ \\
\hline $\mathbf{7}$ & $\mathbf{7 9 9 . 3 9 4 5}$ & 400.2009 & 782.3679 & 391.6876 & 781.3839 & 391.1956 & $\mathbf{N}$ & $\mathbf{7 9 2 . 3 6 3 5}$ & 396.6854 & 775.3369 & 388.1721 & & & $\mathbf{6}$ \\
\hline $\mathbf{8}$ & 896.4472 & 448.7272 & 879.4207 & 440.2140 & 878.4367 & 439.7220 & $\mathbf{P}$ & $\mathbf{6 7 8 . 3 2 0 6}$ & 339.6639 & 661.2940 & 331.1506 & & & \\
\hline $\mathbf{9}$ & 1059.5106 & 530.2589 & 1042.4840 & 521.7456 & 1041.5000 & 521.2536 & $\mathbf{Y}$ & 581.2678 & 291.1375 & 564.2412 & 282.6243 & & & \\
\hline $\mathbf{1 0}$ & 1187.5691 & 594.2882 & 1170.5426 & 585.7749 & 1169.5586 & 585.2829 & $\mathbf{Q}$ & $\mathbf{4 1 8 . 2 0 4 5}$ & 209.6059 & 401.1779 & 201.0926 & & $\mathbf{4}$ \\
\hline $\mathbf{1 1}$ & $\mathbf{1 3 0 2 . 5 9 6 1}$ & 651.8017 & $\mathbf{1 2 8 5 . 5 6 9 5}$ & 643.2884 & $\mathbf{1 2 8 4 . 5 8 5 5}$ & 642.7964 & $\mathbf{N}$ & $\mathbf{2 9 0 . 1 4 5 9}$ & 145.5766 & 273.1193 & 137.0633 & & & $\mathbf{3}$ \\
\hline $\mathbf{1 2}$ & & & & & & & $\mathbf{R}$ & 175.1190 & 88.0631 & 158.0924 & 79.5498 & & & $\mathbf{2}$ \\
\hline
\end{tabular}
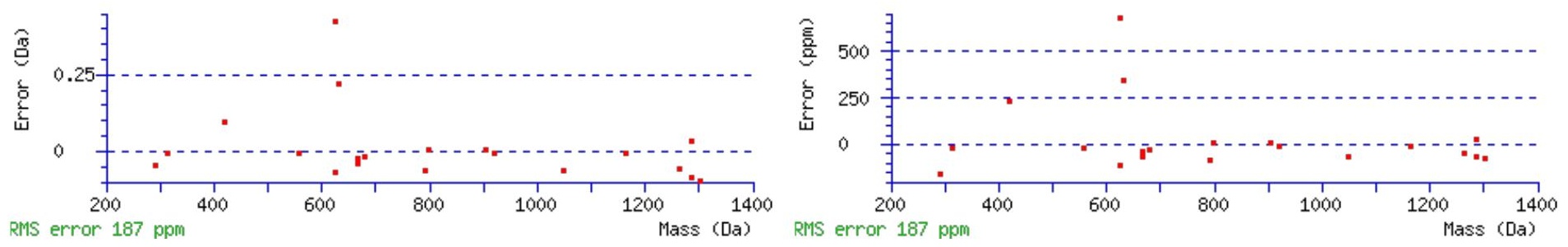

\section{All matches to this query}

\begin{tabular}{|l|l|l|l|}
\hline Score & Mr(calc): & Delta & \multicolumn{1}{|c|}{ Sequence } \\
\hline 75.8 & 1475.7004 & 0.0039 & VLTNQENPYQNR \\
\hline 28.1 & 1475.7004 & 0.0039 & VLTNQENPYQNR \\
\hline 7.5 & 1475.7133 & -0.0090 & LVSRTASKYTDR \\
\hline 7.4 & 1475.7150 & -0.0107 & VAKVTGGAASKLSK \\
\hline 7.0 & 1475.7133 & -0.0090 & LVSRTASKYTDR \\
\hline 6.5 & 1475.7133 & -0.0090 & LVSRTASKYTDR \\
\hline 5.1 & 1475.7004 & 0.0039 & VLTNQENPYQNR \\
\hline 3.6 & 1475.7150 & -0.0107 & VAKVTGGAASKLSK \\
\hline 1.7 & 1474.6891 & 1.0152 & KMPQYQKELSK \\
\hline
\end{tabular}




\section{|1.2 $1473.7106 \mid 1.9938$ |RAPTTALTLRSK}

Spectrum No: 292; Query: 863; Rank: 1

\section{Peptide View}

\section{MS/MS Fragmentation of CNTTQGNEVTSILR}

Found in IPI00327143, Tax_Id=10116 Gene_Symbol=Alpl Alkaline phosphatase, tissue-nonspecific isozyme precursor

Match to Query 863: 1592.749768 from(797.382160,2+)

Title: 100101RatKid_NS_deglyco_23.2388.2388.2.dta

Data file K:\NewmanPaper\Piliang $\backslash 3 S$ ubProteomes $\backslash$ Piliang3SP $\backslash$ mgf5ppm\ERLIC_3SubProteomes5ppm.mgf
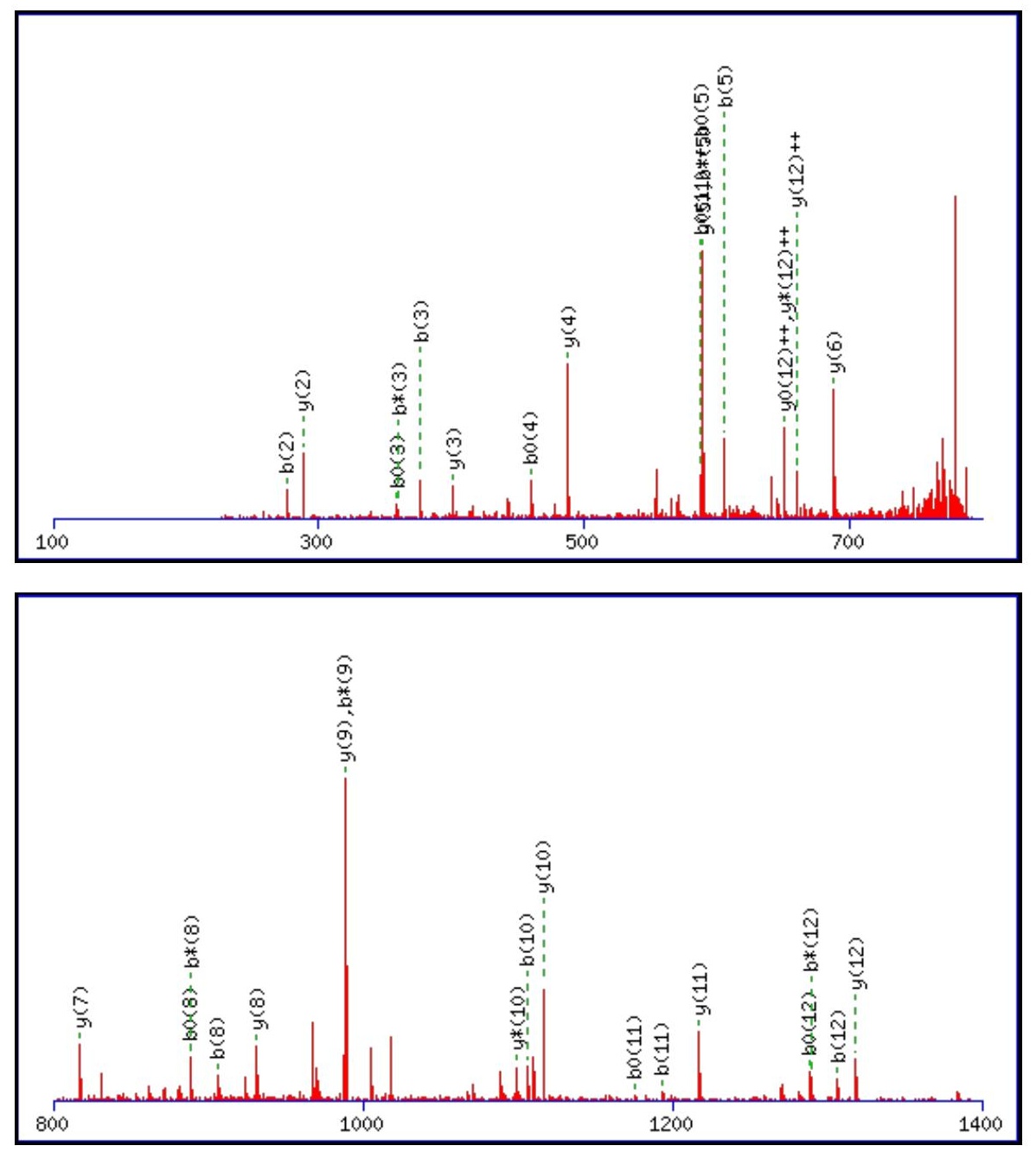


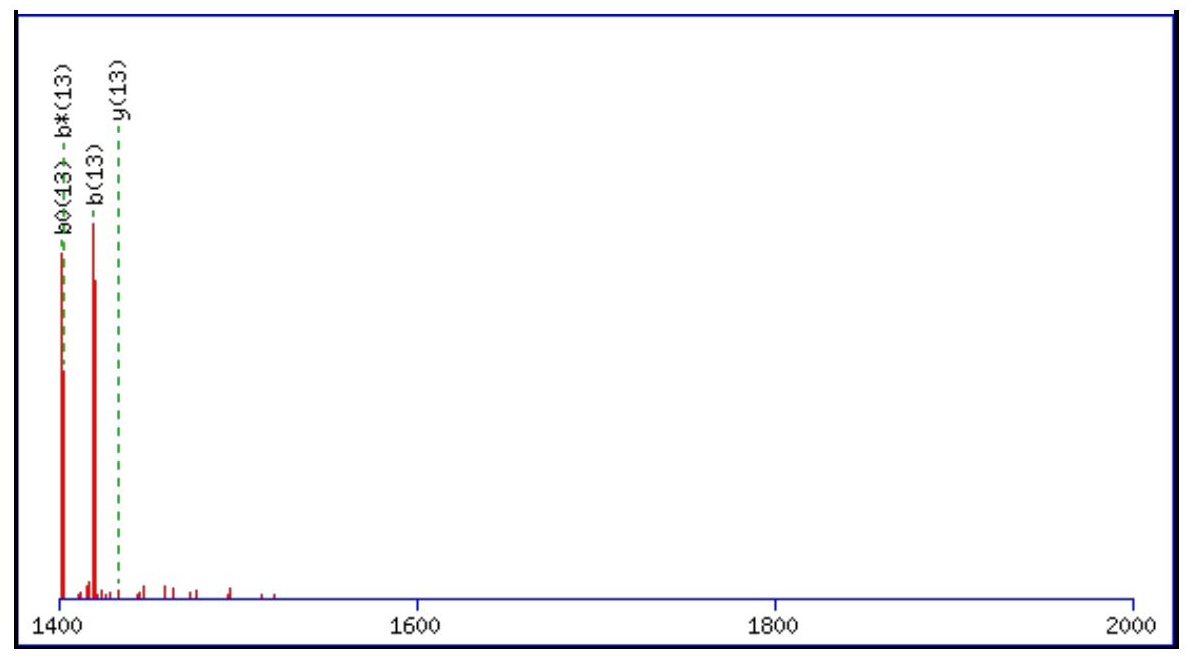

Monoisotopic mass of neutral peptide $\operatorname{Mr}($ calc): 1592.7464

Fixed modifications: Carbamidomethyl (C)

Variable modifications:

N2 : Deamidated_N (N)

Ions Score: 76 Expect: $4.9 \mathrm{e}-006$

Matches (Bold Red): 38/144 fragment ions using 65 most intense peaks

\begin{tabular}{|c|c|c|c|c|c|c|c|c|c|c|c|c|c|c|}
\hline \# & b & $\mathbf{b}^{++}$ & $\mathbf{b}^{*}$ & $\mathbf{b}^{*^{++}}$ & $\mathbf{b}^{0}$ & $\mathbf{b}^{0++}$ & Seq. & $\mathbf{y}$ & $y^{++}$ & $\mathbf{y}^{*}$ & $\mathrm{y}^{\mathrm{*}^{++}}$ & $\mathbf{y}^{0}$ & $y^{0++}$ & \# \\
\hline 1 & 161.0379 & 81.0226 & & & & & $\mathrm{C}$ & & & & & & & 14 \\
\hline 2 & 276.0649 & 138.5361 & 259.0383 & 130.0228 & & & $\mathbf{N}$ & 1433.7231 & 717.3652 & 1416.6965 & 708.8519 & 1415.7125 & 708.3599 & 13 \\
\hline 3 & 377.1125 & 189.0599 & 360.0860 & 180.5466 & 359.1020 & 180.0546 & $\mathbf{T}$ & 1318.6961 & 659.8517 & 1301.6696 & 651.3384 & 1300.6856 & 650.8464 & 12 \\
\hline 4 & 478.1602 & 239.5837 & 461.1337 & 231.0705 & 460.1497 & 230.5785 & $\mathbf{T}$ & 1217.6484 & 609.3279 & 1200.6219 & 600.8146 & 1199.6379 & 600.3226 & 11 \\
\hline 5 & 606.2188 & 303.6130 & 589.1922 & 295.0998 & 588.2082 & 294.6078 & $\mathbf{Q}$ & 1116.6008 & 558.8040 & 1099.5742 & 550.2907 & 1098.5902 & 549.7987 & 10 \\
\hline 6 & 663.2403 & 332.1238 & 646.2137 & 323.6105 & 645.2297 & 323.1185 & G & 988.5422 & 494.7747 & 971.5156 & 486.2615 & 970.5316 & 485.7694 & 9 \\
\hline 7 & 777.2832 & 389.1452 & 760.2566 & 380.6320 & 759.2726 & 380.1399 & $\mathbf{N}$ & 931.5207 & 466.2640 & 914.4942 & 457.7507 & 913.5102 & 457.2587 & 8 \\
\hline 8 & 906.3258 & 453.6665 & 889.2992 & 445.1533 & 888.3152 & 444.6612 & $\mathbf{E}$ & 817.4778 & 409.2425 & 800.4512 & 400.7293 & 799.4672 & 400.2373 & 7 \\
\hline 9 & 1005.3942 & 503.2007 & 988.3676 & 494.6875 & 987.3836 & 494.1955 & $\bar{V}$ & 688.4352 & 344.7212 & 671.4087 & 336.2080 & 670.4246 & 335.7160 & 6 \\
\hline 10 & 1106.4419 & 553.7246 & 1089.4153 & 545.2113 & 1088.4313 & 544.7193 & $\mathbf{T}$ & 589.3668 & 295.1870 & 572.3402 & 286.6738 & 571.3562 & 286.1817 & 5 \\
\hline 11 & 1193.4739 & 597.2406 & 1176.4474 & 588.7273 & 1175.4633 & 588.2353 & $\mathrm{~S}$ & 488.3191 & 244.6632 & 471.2926 & 236.1499 & 470.3085 & 235.6579 & 4 \\
\hline 12 & 1306.5580 & 653.7826 & 1289.5314 & 645.2693 & 1288.5474 & 644.7773 & I & 401.2871 & 201.1472 & 384.2605 & 192.6339 & & & 3 \\
\hline 13 & 1419.6420 & 710.3247 & 1402.6155 & 701.8114 & 1401.6315 & 701.3194 & $\mathbf{L}$ & 288.2030 & 144.6051 & 271.1765 & 136.0919 & & & 2 \\
\hline 14 & & & & & & & $\mathbf{R}$ & 175.1190 & 88.0631 & 158.0924 & 79.5498 & & & 1 \\
\hline
\end{tabular}
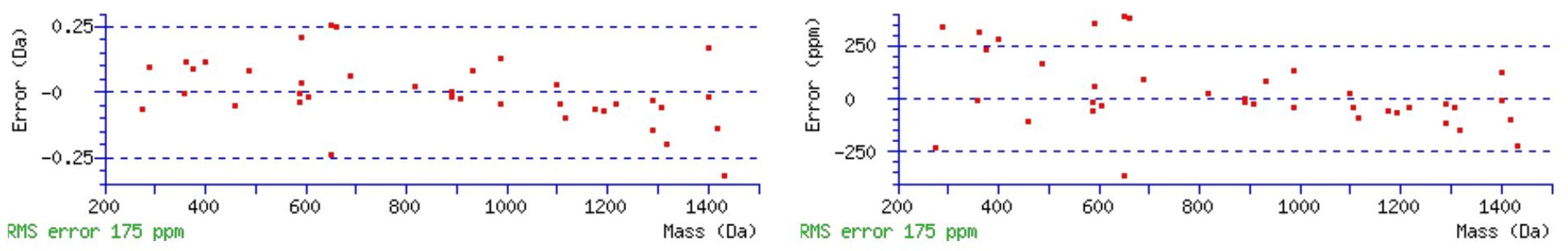

\section{All matches to this query}

\begin{tabular}{|l|l|l|l|}
\hline Score & Mr(calc): & Delta & \multicolumn{1}{c|}{ Sequence } \\
\hline 75.7 & 1592.7464 & 0.0033 & $\underline{\text { CNTTQGNEVTSILR }}$ \\
\hline 64.5 & 1592.7464 & 0.0033 & CNTTQGNEVTSILR \\
\hline 12.5 & 1592.7529 & -0.0031 & TATNGISANGVNKSEK \\
\hline 11.8 & 1591.7607 & 0.9891 & $\underline{\text { ESHVINQVKNSKK }}$ \\
\hline 8.7 & 1592.7577 & -0.0079 & TLGESQRTDCSLAR \\
\hline 8.0 & 1592.7633 & -0.0136 & DCTKELLPPTALR \\
\hline 7.7 & 1592.7365 & 0.0132 & NRYGDVPCLDQTR \\
\hline & & & \\
\hline
\end{tabular}




\begin{tabular}{|l|l|l|l|}
7.2 & 1592.7390 & 0.0108 & NRERVESSEETTR \\
\hline 6.7 & 1592.7487 & 0.0011 & IENFTTDYKIIR \\
\hline 6.5 & 1592.7633 & -0.0136 & DCTKELLPPTALR \\
\hline
\end{tabular}

Spectrum No: 293; Query: 1140; Rank: 1

\section{Peptide View}

MS/MS Fragmentation of LTSCATNASMCGDEAR

Found in IPI00369995, Tax_Id=10116 Gene_Symbol=Lrp1 similar to low density lipoprotein receptor-related protein 1

Match to Query 1140: 1743.691808 from(872.853180,2+)

Title: 100101RatKid_NS_deglyco_23.915.915.2.dta

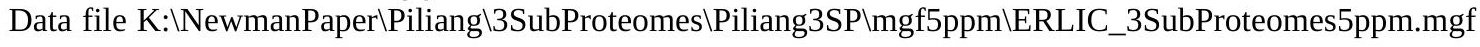
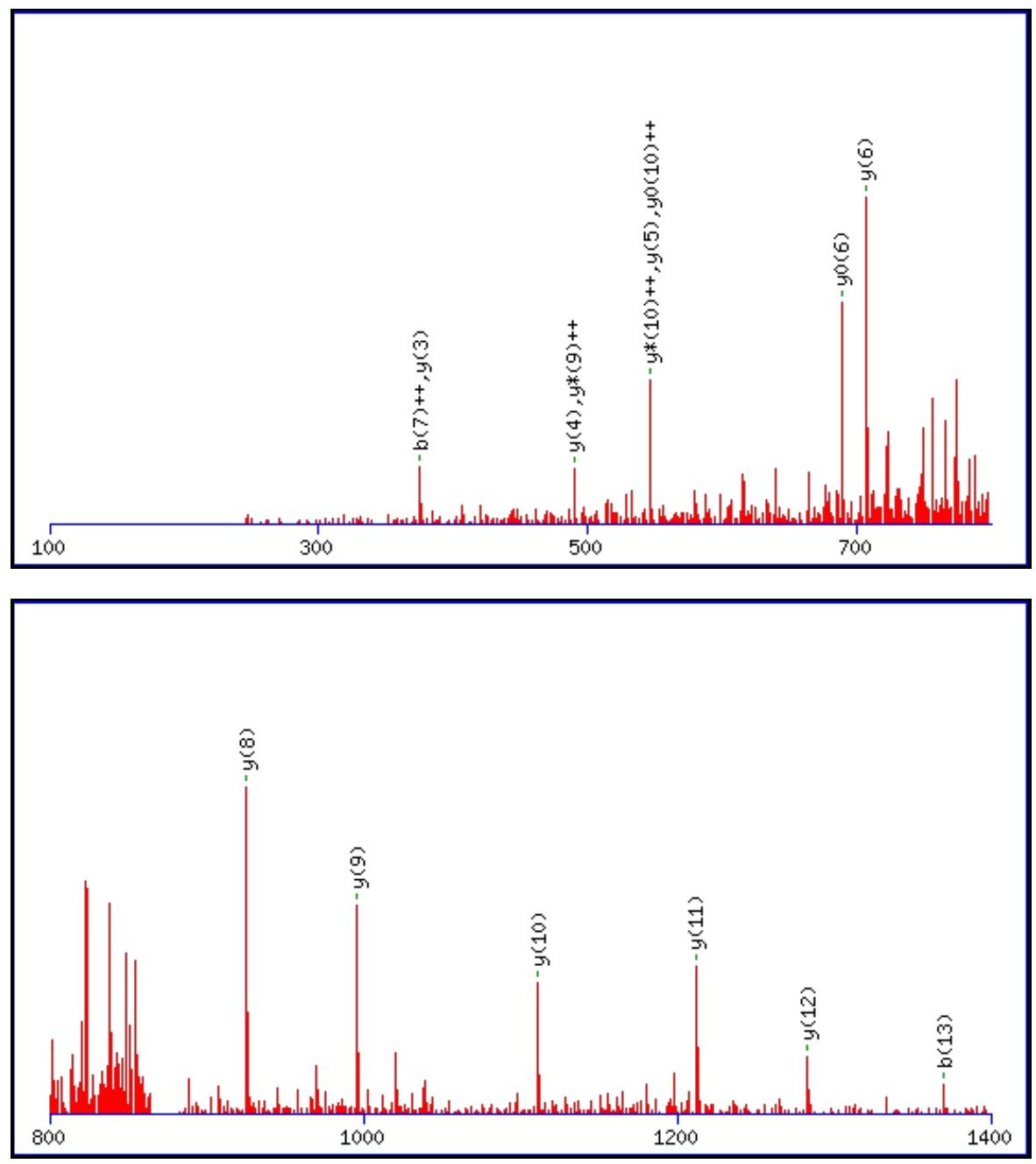


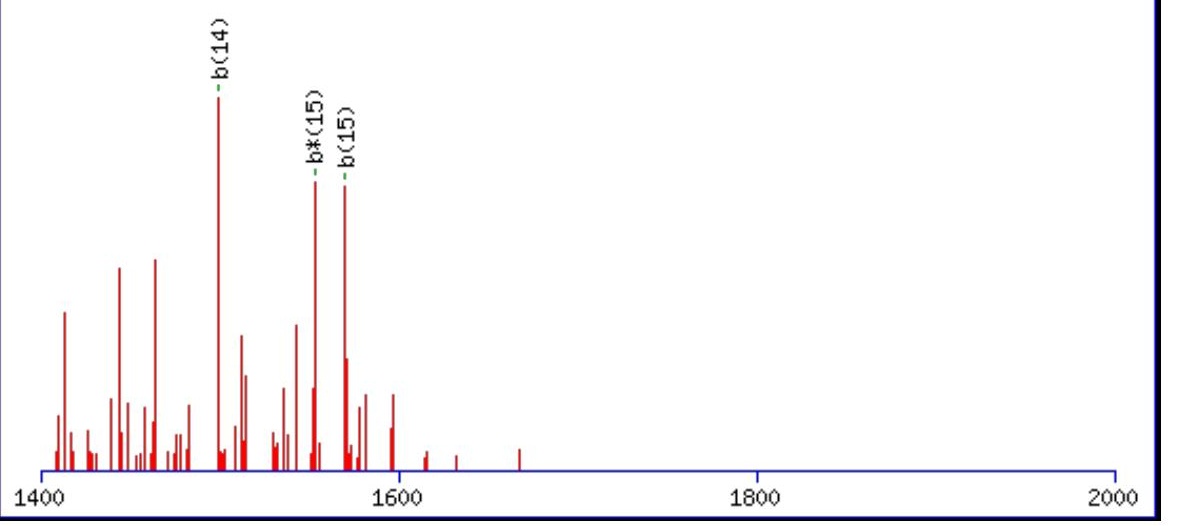

Monoisotopic mass of neutral peptide $\operatorname{Mr}($ calc): 1743.6862

Fixed modifications: Carbamidomethyl (C)

Variable modifications:

N7 : Deamidated $\mathrm{N}(\mathrm{N})$

Ions Score: 75 Expect: $3.7 \mathrm{e}-006$

Matches (Bold Red): 18/162 fragment ions using 27 most intense peaks

\begin{tabular}{|c|c|c|c|c|c|c|c|c|c|c|c|c|c|c|}
\hline \# & b & $\mathbf{b}^{++}$ & $\mathbf{b}^{*}$ & $\mathbf{b}^{*^{++}}$ & $\mathbf{b}^{0}$ & $\mathbf{b}^{\mathbf{0 + +}}$ & Seq. & $\mathbf{y}$ & $\mathbf{y}^{++}$ & $\mathbf{y}^{*}$ & $\mathbf{y}^{*^{++}}$ & $\mathbf{y}^{0}$ & $\mathbf{y}^{0++}$ & \# \\
\hline 1 & 114.0913 & 57.5493 & & & & & $\mathbf{L}$ & & & & & & & 16 \\
\hline 2 & 215.1390 & 108.0731 & & & 197.1285 & 99.0679 & $\mathbf{T}$ & 1631.6094 & 816.3084 & 1614.5829 & 807.7951 & 1613.5989 & 807.3031 & 15 \\
\hline 3 & 302.1710 & 151.5892 & & & 284.1605 & 142.5839 & S & 1530.5617 & 765.7845 & 1513.5352 & 757.2712 & 1512.5512 & 756.7792 & 14 \\
\hline 4 & 462.2017 & 231.6045 & & & 444.1911 & 222.5992 & $\mathrm{C}$ & 1443.5297 & 722.2685 & 1426.5032 & 713.7552 & 1425.5192 & 713.2632 & 13 \\
\hline 5 & 533.2388 & 267.1230 & & & 515.2282 & 258.1178 & A & 1283.4991 & 642.2532 & 1266.4725 & 633.7399 & 1265.4885 & 633.2479 & 12 \\
\hline 6 & 634.2865 & 317.6469 & & & 616.2759 & 308.6416 & $\mathbf{T}$ & 1212.4620 & 606.7346 & 1195.4354 & 598.2213 & 1194.4514 & 597.7293 & 11 \\
\hline 7 & 749.3134 & 375.1604 & 732.2869 & 366.6471 & 731.3029 & 366.1551 & $\mathbf{N}$ & 1111.4143 & 556.2108 & 1094.3877 & 547.6975 & 1093.4037 & 547.2055 & 10 \\
\hline 8 & 820.3505 & 410.6789 & 803.3240 & 402.1656 & 802.3400 & 401.6736 & A & 996.3873 & 498.6973 & 979.3608 & 490.1840 & 978.3768 & 489.6920 & 9 \\
\hline 9 & 907.3826 & 454.1949 & 890.3560 & 445.6816 & 889.3720 & 445.1896 & S & 925.3502 & 463.1788 & 908.3237 & 454.6655 & 907.3397 & 454.1735 & 8 \\
\hline 10 & 1038.4231 & 519.7152 & 1021.3965 & 511.2019 & 1020.4125 & 510.7099 & $\mathbf{M}$ & 838.3182 & 419.6627 & 821.2917 & 411.1495 & 820.3076 & 410.6575 & 7 \\
\hline 11 & 1198.4537 & 599.7305 & 1181.4272 & 591.2172 & 1180.4431 & 590.7252 & C & 707.2777 & 354.1425 & 690.2512 & 345.6292 & 689.2672 & 345.1372 & 6 \\
\hline 12 & 1255.4752 & 628.2412 & 1238.4486 & 619.7279 & 1237.4646 & 619.2359 & G & 547.2471 & 274.1272 & 530.2205 & 265.6139 & 529.2365 & 265.1219 & 5 \\
\hline 13 & 1370.5021 & 685.7547 & 1353.4756 & 677.2414 & 1352.4915 & 676.7494 & D & 490.2256 & 245.6164 & 473.1991 & 237.1032 & 472.2150 & 236.6112 & 4 \\
\hline 14 & 1499.5447 & 750.2760 & 1482.5182 & 741.7627 & 1481.5341 & 741.2707 & $\mathbf{E}$ & 375.1987 & 188.1030 & 358.1721 & 179.5897 & 357.1881 & 179.0977 & 3 \\
\hline 15 & 1570.5818 & 785.7945 & 1553.5553 & 777.2813 & 1552.5713 & 776.7893 & A & 246.1561 & 123.5817 & 229.1295 & 115.0684 & & & 2 \\
\hline 16 & & & & & & & $\mathbf{R}$ & 175.1190 & 88.0631 & 158.0924 & 79.5498 & & & 1 \\
\hline
\end{tabular}
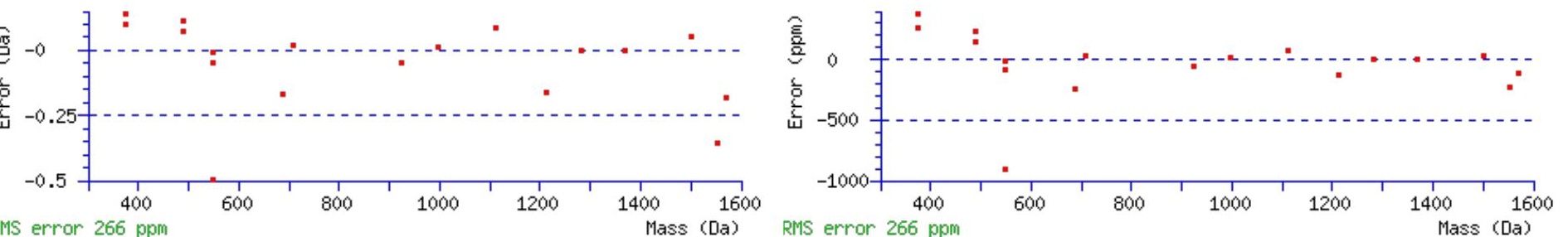

\section{All matches to this query}

\begin{tabular}{|l|l|l|l|}
\hline Score & Mr(calc): & Delta & \multicolumn{1}{c|}{ Sequence } \\
\hline 75.5 & 1743.6862 & 0.0056 & LTSCATNASMCGDEAR \\
\hline 24.0 & 1742.7022 & 0.9896 & LTSCATNASMCGDEAR \\
\hline 12.0 & 1743.6828 & 0.0091 & ISTATPYMNGETATK \\
\hline 8.5 & 1742.6801 & 1.0117 & KSAENGIYSVSGDEK \\
\hline 7.5 & 1742.6971 & 0.9947 & HFNSDLMAGDNSGAVK \\
\hline & & & \\
\hline
\end{tabular}




\begin{tabular}{|l|l|l|l|}
0.5 & 1743.7042 & -0.0124 & FFGNSWSETYRNR \\
\hline 0.4 & 1742.6801 & 1.0117 & KSAENGIYSVSGDEK \\
\hline 0.3 & 1741.6783 & 2.0135 & LKSDMXSWTENLR \\
\hline
\end{tabular}

Spectrum No: 294; Query: 141; Rank: 1

\section{Peptide View}

MS/MS Fragmentation of NSSGVILAALGK

Found in IPI00363534, Tax_Id=10116 Gene_Symbol=Lama1 laminin, alpha 1

Match to Query 141: 1129.633408 from(565.823980,2+)

Title: 091008RatKidney_NH4Format01_24.2942.2942.2.dta

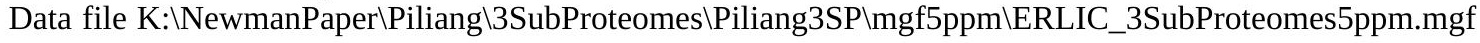
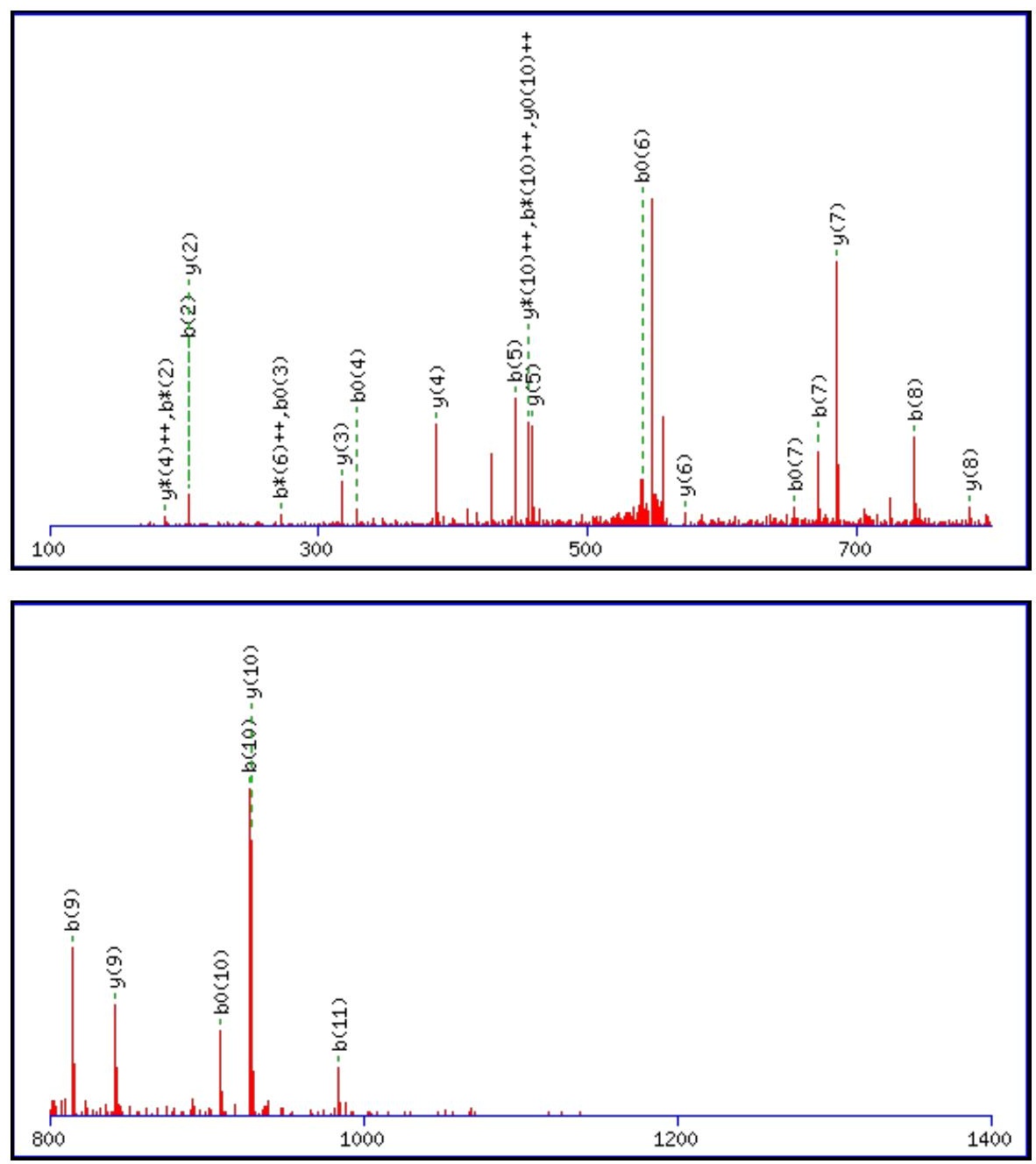


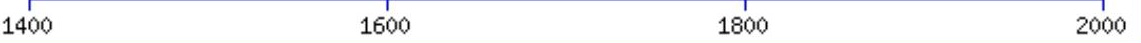

Monoisotopic mass of neutral peptide $\operatorname{Mr}($ calc): 1129.6343

Fixed modifications: Carbamidomethyl (C)

Variable modifications:

N1 : Deamidated_N (N)

Ions Score: 75 Expect: $2 \mathrm{e}-006$

Matches (Bold Red): 27/112 fragment ions using 34 most intense peaks

\begin{tabular}{|r|c|c|c|c|c|c|c|c|c|c|c|c|c|c|}
\hline$\#$ & $\mathbf{b}$ & $\mathbf{b}^{++}$ & $\mathbf{b}^{*}$ & $\mathbf{b}^{*^{++}}$ & $\mathbf{b}^{\mathbf{0}}$ & $\mathbf{b}^{\mathbf{0 + +}}$ & $\mathbf{S e q}$ & $\mathbf{y}$ & $\mathbf{y}^{++}$ & $\mathbf{y}^{*}$ & $\mathbf{y}^{\mathbf{*}^{++}}$ & $\mathbf{y}^{\mathbf{0}}$ & $\mathbf{y}^{\mathbf{0 + +}}$ & $\#$ \\
\hline $\mathbf{1}$ & 116.0342 & 58.5207 & 99.0077 & 50.0075 & & & $\mathbf{N}$ & & & & & & & $\mathbf{1 2}$ \\
\hline $\mathbf{2}$ & $\mathbf{2 0 3 . 0 6 6 2}$ & 102.0368 & $\mathbf{1 8 6 . 0 3 9 7}$ & 93.5235 & 185.0557 & 93.0315 & $\mathbf{S}$ & 1015.6146 & 508.3109 & 998.5881 & 499.7977 & 997.6041 & 499.3057 & $\mathbf{1 1}$ \\
\hline $\mathbf{3}$ & 290.0983 & 145.5528 & 273.0717 & 137.0395 & $\mathbf{2 7 2 . 0 8 7 7}$ & 136.5475 & $\mathbf{S}$ & $\mathbf{9 2 8 . 5 8 2 6}$ & 464.7949 & 911.5560 & $\mathbf{4 5 6 . 2 8 1 7}$ & 910.5720 & $\mathbf{4 5 5 . 7 8 9 7}$ & $\mathbf{1 0}$ \\
\hline $\mathbf{4}$ & 347.1197 & 174.0635 & 330.0932 & 165.5502 & 329.1092 & 165.0582 & $\mathbf{G}$ & $\mathbf{8 4 1 . 5 5 0 6}$ & 421.2789 & 824.5240 & 412.7656 & & & $\mathbf{9}$ \\
\hline $\mathbf{5}$ & 446.1881 & 223.5977 & 429.1616 & 215.0844 & 428.1776 & 214.5924 & $\mathbf{V}$ & $\mathbf{7 8 4 . 5 2 9 1}$ & 392.7682 & 767.5026 & 384.2549 & & & $\mathbf{8}$ \\
\hline $\mathbf{6}$ & 559.2722 & 280.1397 & 542.2457 & 271.6265 & 541.2616 & 271.1345 & $\mathbf{I}$ & $\mathbf{6 8 5 . 4 6 0 7}$ & 343.2340 & 668.4341 & 334.7207 & & & $\mathbf{7}$ \\
\hline $\mathbf{7}$ & $\mathbf{6 7 2 . 3 5 6 3}$ & 336.6818 & 655.3297 & 328.1685 & $\mathbf{6 5 4 . 3 4 5 7}$ & 327.6765 & $\mathbf{L}$ & $\mathbf{5 7 2 . 3 7 6 6}$ & 286.6920 & 555.3501 & 278.1787 & & & $\mathbf{6}$ \\
\hline $\mathbf{8}$ & $\mathbf{7 4 3 . 3 9 3 4}$ & 372.2003 & 726.3668 & 363.6871 & 725.3828 & 363.1950 & $\mathbf{A}$ & $\mathbf{4 5 9 . 2 9 2 6}$ & 230.1499 & 442.2660 & 221.6366 & & & $\mathbf{5}$ \\
\hline $\mathbf{9}$ & $\mathbf{8 1 4 . 4 3 0 5}$ & 407.7189 & 797.4040 & 399.2056 & 796.4199 & 398.7136 & $\mathbf{A}$ & $\mathbf{3 8 8 . 2 5 5 4}$ & 194.6314 & 371.2289 & $\mathbf{1 8 6 . 1 1 8 1}$ & & & $\mathbf{4}$ \\
\hline $\mathbf{1 0}$ & $\mathbf{9 2 7 . 5 1 4 6}$ & 464.2609 & 910.4880 & $\mathbf{4 5 5 . 7 4 7 6}$ & $\mathbf{9 0 9 . 5 0 4 0}$ & 455.2556 & $\mathbf{L}$ & $\mathbf{3 1 7 . 2 1 8 3}$ & 159.1128 & 300.1918 & 150.5995 & & & 3 \\
\hline $\mathbf{1 1}$ & $\mathbf{9 8 4 . 5 3 6 0}$ & 492.7717 & 967.5095 & 484.2584 & 966.5255 & 483.7664 & $\mathbf{G}$ & $\mathbf{2 0 4 . 1 3 4 3}$ & 102.5708 & 187.1077 & 94.0575 & & & $\mathbf{2}$ \\
\hline $\mathbf{1 2}$ & & & & & & & $\mathbf{K}$ & 147.1128 & 74.0600 & 130.0863 & 65.5468 & & & $\mathbf{1}$ \\
\hline
\end{tabular}
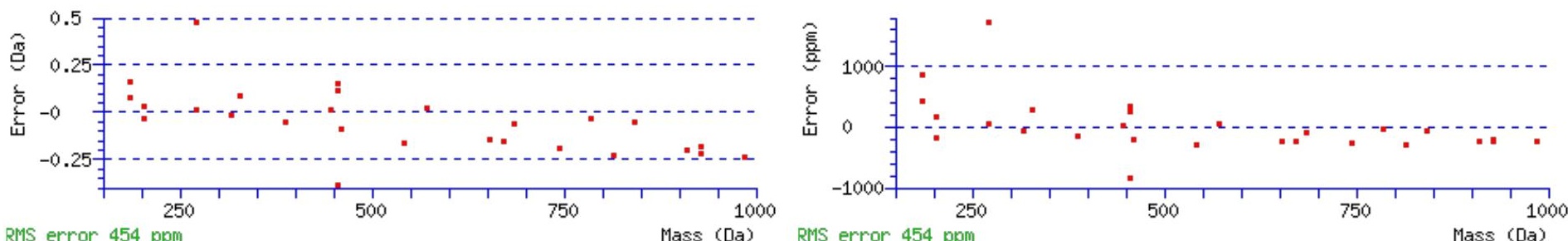

RMS error 454 ppm

Mass (Da) RMS error 454 ppm

\section{All matches to this query}

\begin{tabular}{|l|c|c|l|}
\hline Score & Mr(calc): & Delta & \multicolumn{1}{c|}{ Sequence } \\
\hline 75.5 & 1129.6343 & -0.0009 & NSSGVILAALGK \\
\hline 5.4 & 1129.6356 & -0.0022 & WATVTIQRR \\
\hline 4.9 & 1129.6277 & 0.0057 & MAVANAGQKIK \\
\hline 3.1 & 1128.6291 & 1.0043 & LSGWLXEIGR \\
\hline 2.9 & 1129.6244 & 0.0090 & LKLFGHSGSGK \\
\hline 2.8 & 1128.6390 & 0.9944 & NSGVVILADLK \\
\hline 2.7 & 1128.6390 & 0.9944 & INELLQLIGK \\
\hline 2.5 & 1128.6251 & 1.0083 & LSQQVTQLGR \\
\hline 2.3 & 1129.6343 & -0.0009 & ENTQVISVLK \\
\hline & & & \\
\hline
\end{tabular}




\begin{tabular}{|l|l|l|l|}
1.9 & 1127.6339 & 1.9995 & AGKWEIPLSK \\
\hline
\end{tabular}

Spectrum No: 295; Query: 1728; Rank: 1

\section{Peptide View}

MS/MS Fragmentation of IDCYPDEHGVSEANCTAR

Found in IPI00193894, Tax_Id=10116 Gene_Symbol=Mgam_predicted 198 kDa protein

Match to Query 1728: 2093.849508 from(1047.932030,2+)

Title: 100101RatKid_NS_deglyco_21.1319.1319.2.dta

Data file K:INewmanPaper|Piliangl3SubProteomes\Piliang3SP\mgf5ppm\ERLIC_3SubProteomes5ppm.mgf
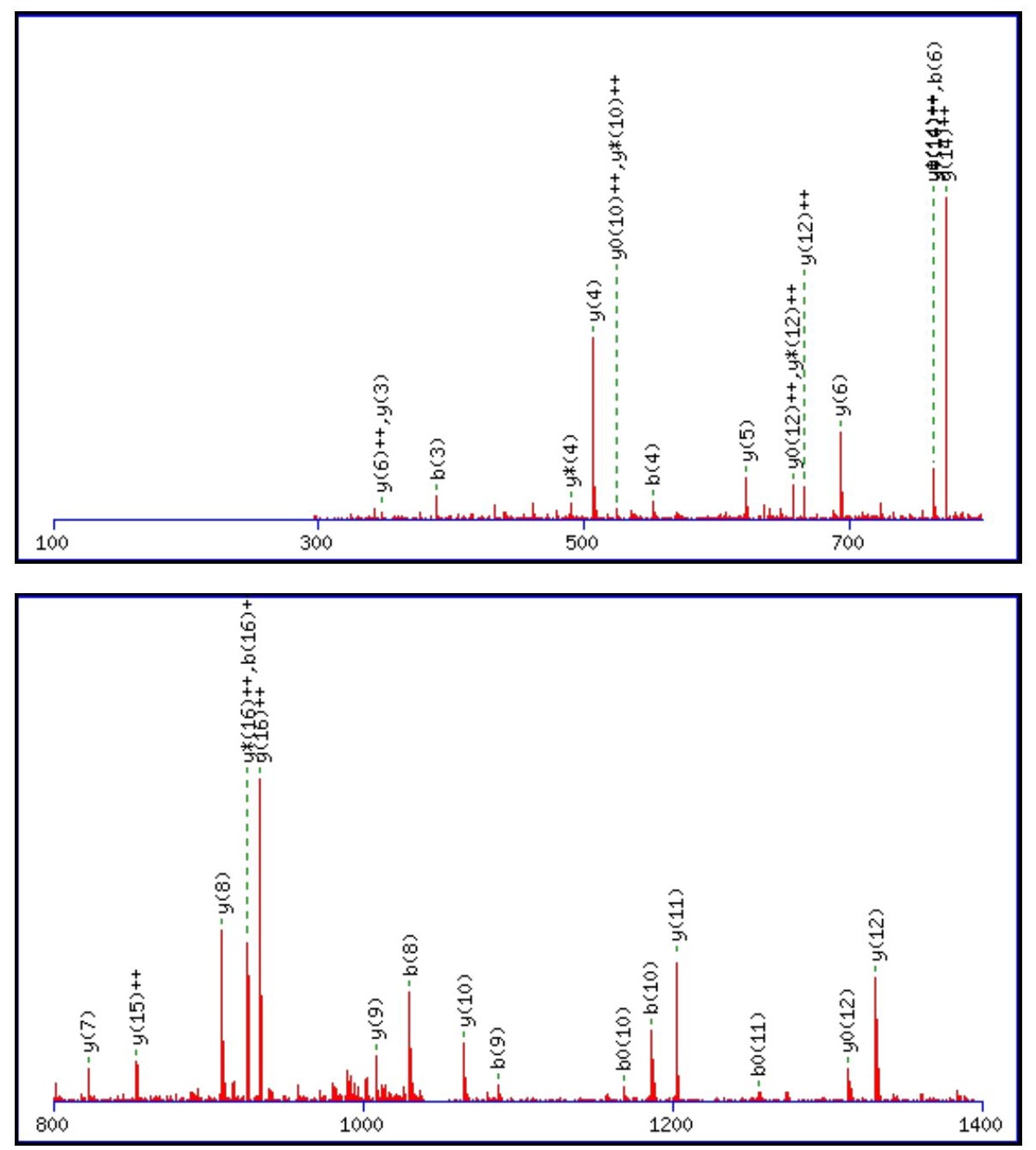


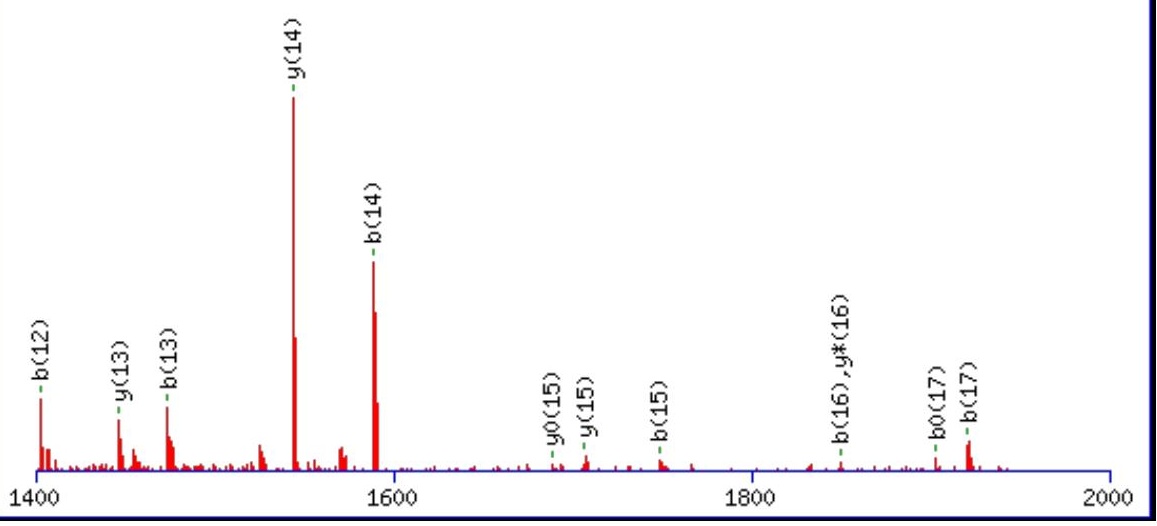

Monoisotopic mass of neutral peptide $\operatorname{Mr}($ calc): 2093.8419

Fixed modifications: Carbamidomethyl (C)

Variable modifications:

N14 : Deamidated_N (N)

Ions Score: 75 Expect: $5.7 \mathrm{e}-006$

Matches (Bold Red): 46/172 fragment ions using 74 most intense peaks

\begin{tabular}{|c|c|c|c|c|c|c|c|c|c|c|c|c|c|c|}
\hline \# & b & $\mathbf{b}^{++}$ & $\mathbf{b}^{*}$ & $\mathbf{b}^{*^{++}}$ & $\mathbf{b}^{0}$ & $\mathbf{b}^{0++}$ & Seq. & $\mathbf{y}$ & $\mathbf{y}^{++}$ & $\mathrm{y}^{*}$ & $\mathrm{y}^{*^{++}}$ & $\mathbf{y}^{0}$ & $\mathbf{y}^{\mathbf{0 + +}^{++}}$ & \# \\
\hline 1 & 114.0913 & 57.5493 & & & & & I & & & & & & & 18 \\
\hline 2 & 229.1183 & 115.0628 & & & 211.1077 & 106.0575 & D & 1981.7651 & 991.3862 & 1964.7385 & 982.8729 & 1963.7545 & 982.3809 & 17 \\
\hline 3 & 389.1489 & 195.0781 & & & 371.1384 & 186.0728 & C & 1866.7381 & 933.8727 & 1849.7116 & 925.3594 & 1848.7276 & 924.8674 & 16 \\
\hline 4 & 552.2123 & 276.6098 & & & 534.2017 & 267.6045 & $\mathbf{Y}$ & 1706.7075 & \begin{tabular}{|l|}
853.8574 \\
\end{tabular} & 1689.6809 & 845.3441 & \begin{tabular}{|l}
1688.6969 \\
\end{tabular} & 844.8521 & 15 \\
\hline 5 & 649.2650 & 325.1362 & & & 631.2545 & 316.1309 & $\mathbf{P}$ & 1543.6442 & 772.3257 & 1526.6176 & 763.8124 & 1525.6336 & 763.3204 & 14 \\
\hline 6 & 764.2920 & 382.6496 & & & 746.2814 & 373.6443 & D & 1446.5914 & 723.7993 & 1429.5648 & 715.2861 & 1428.5808 & 714.7941 & 13 \\
\hline 7 & 893.3346 & 447.1709 & & & 875.3240 & 438.1656 & $\mathbf{E}$ & 1331.5644 & 666.2859 & 1314.5379 & 657.7726 & \begin{tabular}{|l}
1313.5539 \\
\end{tabular} & 657.2806 & 12 \\
\hline 8 & 1030.3935 & 515.7004 & & & 1012.3829 & 506.6951 & H & 1202.5219 & 601.7646 & 1185.4953 & 593.2513 & 1184.5113 & 592.7593 & 11 \\
\hline 9 & 1087.4149 & 544.2111 & & & 1069.4044 & 535.2058 & G & 1065.4629 & 533.2351 & 1048.4364 & 524.7218 & 1047.4524 & 524.2298 & 10 \\
\hline 101 & 1186.4834 & 593.7453 & & & 1168.4728 & 584.7400 & V & 1008.4415 & 504.7244 & 991.4149 & 496.2111 & 990.4309 & 495.7191 & 9 \\
\hline 111 & 1273.5154 & 637.2613 & & & 1255.5048 & 628.2560 & $S$ & 909.3731 & 455.1902 & 892.3465 & 446.6769 & 891.3625 & 446.1849 & 8 \\
\hline 121 & 1402.5580 & 701.7826 & & & 1384.5474 & 692.7773 & $\mathbf{E}$ & 822.3410 & 411.6742 & 805.3145 & 403.1609 & 804.3305 & 402.6689 & 7 \\
\hline 13 & \begin{tabular}{|l|}
1473.5951 \\
\end{tabular} & \begin{tabular}{|l|l|}
737.3012 \\
\end{tabular} & & & 1455.5845 & 728.2959 & A & 693.2984 & 347.1529 & 676.2719 & 338.6396 & 675.2879 & 338.1476 & 6 \\
\hline 14 & 1588.6220 & 794.8146 & 1571.5955 & 786.3014 & 1570.6115 & 785.8094 & $\mathbf{N}$ & 622.2613 & 311.6343 & 605.2348 & 303.1210 & 604.2508 & 302.6290 & 5 \\
\hline 151 & 1748.6527 & 874.8300 & 1731.6261 & 866.3167 & 1730.6421 & 865.8247 & C & 507.2344 & 254.1208 & 490.2078 & 245.6076 & 489.2238 & 245.1156 & 4 \\
\hline 16 & 1849.7004 & 925.3538 & 1832.6738 & 916.8405 & 1831.6898 & 916.3485 & $\mathbf{T}$ & 347.2037 & 174.1055 & 330.1772 & 165.5922 & 329.1932 & 165.1002 & 3 \\
\hline 17 & 1920.7375 & 960.8724 & 1903.7109 & 952.3591 & \begin{tabular}{|l|}
1902.7269 \\
\end{tabular} & 951.8671 & $\mathbf{A}$ & 246.1561 & 123.5817 & 229.1295 & 115.0684 & & & 2 \\
\hline 18 & & & & & & & $\mathbf{R}$ & 175.1190 & 88.0631 & 158.0924 & 79.5498 & & & 1 \\
\hline
\end{tabular}
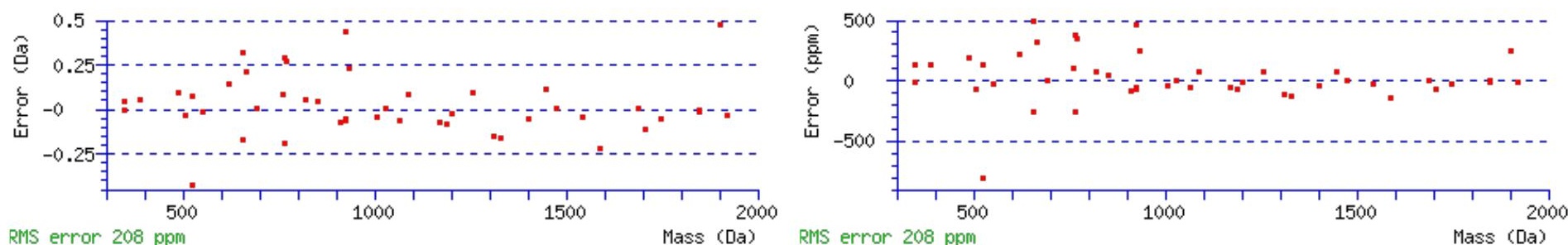

RMS error $208 \mathrm{ppm}$ Mass (Da) RMS error 208 ppm

\section{All matches to this query}

\begin{tabular}{|l|c|c|l|}
\hline Score & Mr(calc): & Delta & \multicolumn{1}{c|}{ Sequence } \\
\hline 75.4 & 2093.8419 & 0.0076 & IDCYPDEHGVSEANCTAR \\
\hline 7.0 & 2092.8578 & 0.9917 & IDCYPDEHGVSEANCTAR \\
\hline 5.9 & 2092.8373 & 1.0122 & NIRSNYRDMHTHMVK \\
\hline & & & \\
\hline
\end{tabular}




\begin{tabular}{|l|l|l|l|}
5.2 & 2093.8490 & 0.0005 & STSACNRVLNGAVPTDGSK \\
\hline 4.7 & 2093.8548 & -0.0052 & APGAPLDCKETDCGSPSPR \\
\hline 4.1 & 2093.8486 & 0.0009 & TAEPPSPPASRAAYRQR \\
\hline 4.0 & 2091.8407 & 2.0088 & GPAGSQLASXQSNVCLPSK \\
\hline 3.0 & 2093.8490 & 0.0005 & STSACNRVLNGAVPTDGSK \\
\hline 1.8 & 2091.8577 & 1.9918 & KDHSVNKMCASSCDFVK \\
\hline 0.6 & 2092.8669 & 0.9827 & SPDEATAADQESEDDLSASR \\
\hline
\end{tabular}

Spectrum No: 296; Query: 1864; Rank: 1

\section{Peptide View}

MS/MS Fragmentation of FNEVYDALANAHPNLTVYK

Found in IPI00454558, Tax_Id=10116 Gene_Symbol=Enpp5 Ectonucleotide pyrophosphatase/phosphodiesterase family member 5 precursor

Match to Query 1864: 2180.059448 from(1091.037000,2+)

Title: 091008RatKidney_NH4Format01_18.4100.4100.2.dta

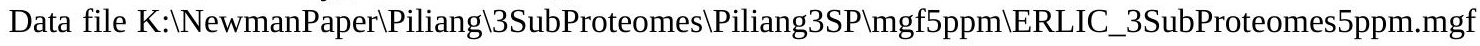
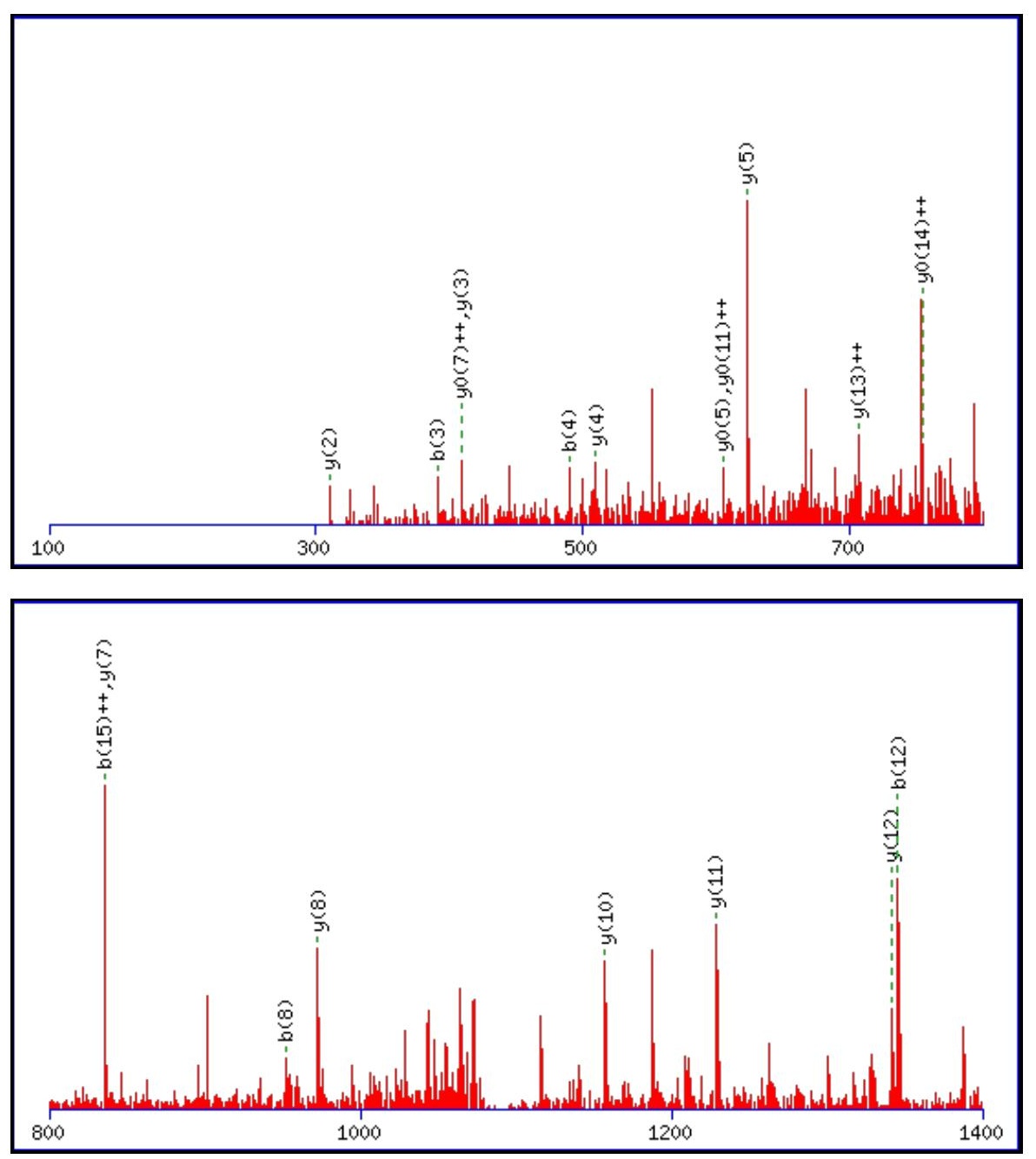


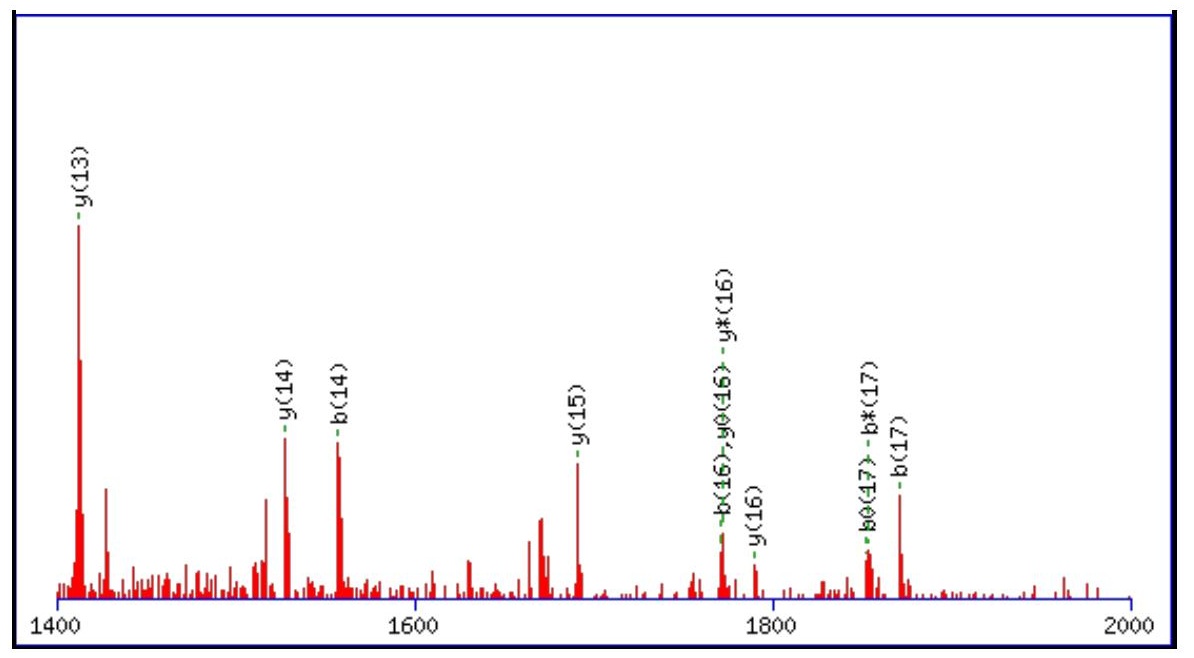

Monoisotopic mass of neutral peptide $\operatorname{Mr}($ calc): 2179.0585

Fixed modifications: Carbamidomethyl (C)

Variable modifications:

N14 : Deamidated $\mathrm{N}(\mathrm{N})$

Ions Score: 75 Expect: $6.7 \mathrm{e}-006$

Matches (Bold Red): 30/204 fragment ions using 59 most intense peaks

\begin{tabular}{|c|c|c|c|c|c|c|c|c|c|c|c|c|c|c|}
\hline \# & b & $\mathbf{b}^{++}$ & $\mathbf{b}^{*}$ & & $\mathbf{b}^{0}$ & & Seq. & $\mathbf{y}$ & & $\mathbf{y}^{*}$ & & $\mathbf{y}^{0}$ & & \# \\
\hline 1 & 148.0757 & 74.5415 & & & & & $\mathbf{F}$ & & & & & & & 19 \\
\hline 2 & 262.1186 & 131.5629 & 245.0921 & & & & $\mathbf{N}$ & & 1017.0024 & & & 9869 & & 18 \\
\hline 3 & 391.1612 & 196.0842 & 374.1347 & & 506 & & $\mathbf{E}$ & & 9809 & 9280 & 4676 & & 950.9756 & 17 \\
\hline 4 & 490.2296 & 245.6185 & 473.2031 & 237.1052 & 472.2191 & 236.6132 & $\mathbf{V}$ & 1789.9119 & 895.4596 & $\mathbf{1 7 7 2 . 8 8 5 4}$ & 886.9463 & & 886.4543 & 16 \\
\hline 5 & 653.2930 & 327.1501 & 636.2 & 318. & 635. & 148 & $\mathbf{Y}$ & 435 & 254 & 170 & 121 & 3329 & 836.9201 & 15 \\
\hline 6 & 768.3199 & 384.6636 & 751.2933 & 376.1503 & 750.3093 & 375.6583 & D & & 764.3937 & 1510.7536 & 755.8805 & & & 14 \\
\hline 7 & 839.3570 & 420.1 & 822. & 411. & 821. & 769 & A & 532 & 803 & 267 & 3670 & 427 & 697.8750 & 13 \\
\hline 8 & 952.4411 & 476.7242 & 935.4145 & 468.2109 & 934.4305 & 467.7189 & $\mathbf{L}$ & 1341.7161 & 671.3617 & 1324.6896 & 662.8484 & 1323.7056 & 662.3564 & 12 \\
\hline 9 & 1023.4782 & 512.2427 & 1006.4516 & 503.7295 & 1005.4676 & 503.2374 & A & 1228.6321 & 614.8197 & 1211.6055 & 606.3064 & 1210.6215 & 605.8144 & 11 \\
\hline 10 & 1137.5211 & 569.2642 & 120.4946 & 560.7509 & 119.5106 & 560.2589 & $\mathbf{N}$ & 1157.5949 & 579.3011 & 1140.5684 & 570.7878 & 1139.5844 & 570.2958 & 10 \\
\hline 11 & 1208.5582 & 604.7828 & 1191.5317 & 596.2695 & 1190.5477 & 595.7775 & A & 1043.5520 & 522.2796 & 1026.5255 & 513.7664 & 1025.5415 & 513.2744 & 9 \\
\hline 12 & 1345.6171 & 673.3122 & 1328.5906 & 664.7989 & 1327.6066 & 664.3069 & $\mathbf{H}$ & & 486.7611 & 955.4884 & & 954.5043 & 477.7558 & 8 \\
\hline 13 & 1442.6699 & 721.8386 & 1425.6434 & 713.3253 & 1424.6593 & 712.8333 & $\mathbf{P}$ & 835.4560 & 418.2316 & 818.4294 & 409.7184 & 817.4454 & 409.2264 & 7 \\
\hline 14 & 1557.6968 & 779.3521 & 1540.6703 & 770.8388 & 1539.6863 & 770.3468 & $\mathbf{N}$ & 738.4032 & 369.7053 & 721.3767 & 361.1920 & 720.3927 & 360.7000 & 6 \\
\hline 15 & 1670.7809 & 835.8941 & 1653.7544 & 827.3808 & 1652.7703 & 826.8888 & $\mathbf{L}$ & 623.3763 & 312.1918 & 606.3497 & 303.6785 & 605.3657 & 303.1865 & 5 \\
\hline 16 & 1771.8286 & 886.4179 & 1754.8020 & 877.9047 & 1753.8180 & 877.4126 & $\mathbf{T}$ & 510.2922 & 255.6498 & 493.2657 & 247.1365 & 492.2817 & 246.6445 & 4 \\
\hline 17 . & \begin{tabular}{|l|}
1870.8970 \\
\end{tabular} & 935.9521 & 1853.8704 & 927.4389 & 1852.8864 & 926.9469 & $\mathbf{V}$ & 409.2445 & 205.1259 & 392.2180 & 196.6126 & & & 3 \\
\hline 18 & 2033.9603 & 1017.4838 & 2016.9338 & 1008.9705 & 2015.9498 & 1008.4785 & $\mathbf{Y}$ & 310.1761 & 155.5917 & 293.1496 & 147.0784 & & & 2 \\
\hline 19 & & & & & & & $\mathbf{K}$ & 147.1128 & 74.0600 & 130.0863 & 65.5468 & & & 1 \\
\hline
\end{tabular}
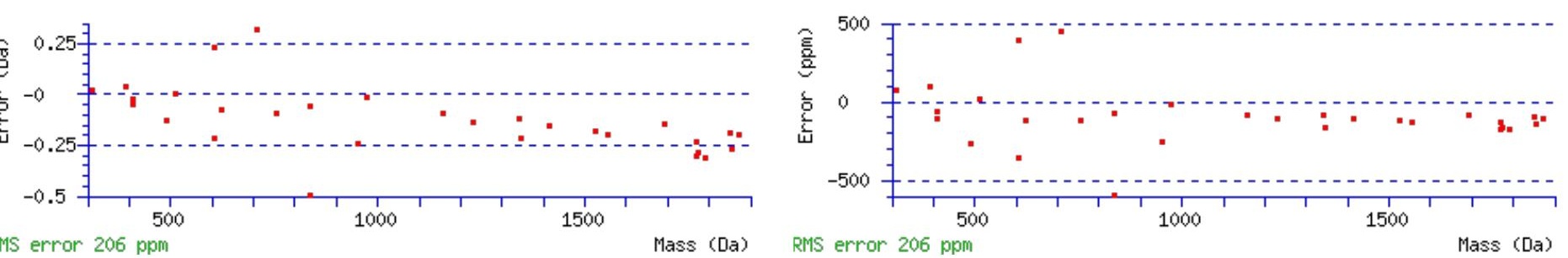

\section{All matches to this query}

\begin{tabular}{|l|l|l|l|}
\hline Score & Mr(calc): & Delta & \multicolumn{1}{c|}{ Sequence } \\
\hline 75.4 & 2179.0585 & 1.0009 & FNEVYDALANAHPNLTVYK \\
\hline 71.8 & 2180.0426 & 0.0169 & FNEVYDALANAHPNLTVYK \\
\hline & & & \\
\hline
\end{tabular}




\begin{tabular}{|l|l|l|l|}
61.0 & 2180.0426 & 0.0169 & FNEVYDALANAHPNLTVYK \\
\hline 52.5 & 2179.0585 & 1.0009 & FNEVYDALANAHPNLTVYK \\
\hline 48.4 & 2180.0426 & 0.0169 & FNEVYDALANAHPNLTVYK \\
\hline 13.7 & 2179.0585 & 1.0009 & FNEVYDALANAHPNLTVYK \\
\hline 5.8 & 2178.0745 & 1.9849 & FNEVYDALANAHPNLTVYK \\
\hline 0.9 & 2179.0390 & 1.0204 & VLPIMVRSTAINASRALK \\
\hline 0.4 & 2179.0773 & 0.9822 & SKLEDIANAALAANAVTQVAK \\
\hline
\end{tabular}

Spectrum No: 297; Query: 630; Rank: 1

\section{Peptide View}

MS/MS Fragmentation of SFSGLLDCGNCSR

Found in IPI00391769, Tax_Id=10116 Gene_Symbol=Cacna2d1 Voltage-gated calcium channel alpha2/delta-1 subunit

Match to Query 630: 1472.606448 from(737.310500,2+)

Title: 100101RatKid_NS_deglyco_23.2554.2554.2.dta

Data file K:INewmanPaper|Piliang|3SubProteomes\Piliang3SP\mgf5ppm\ERLIC_3SubProteomes5ppm.mgf
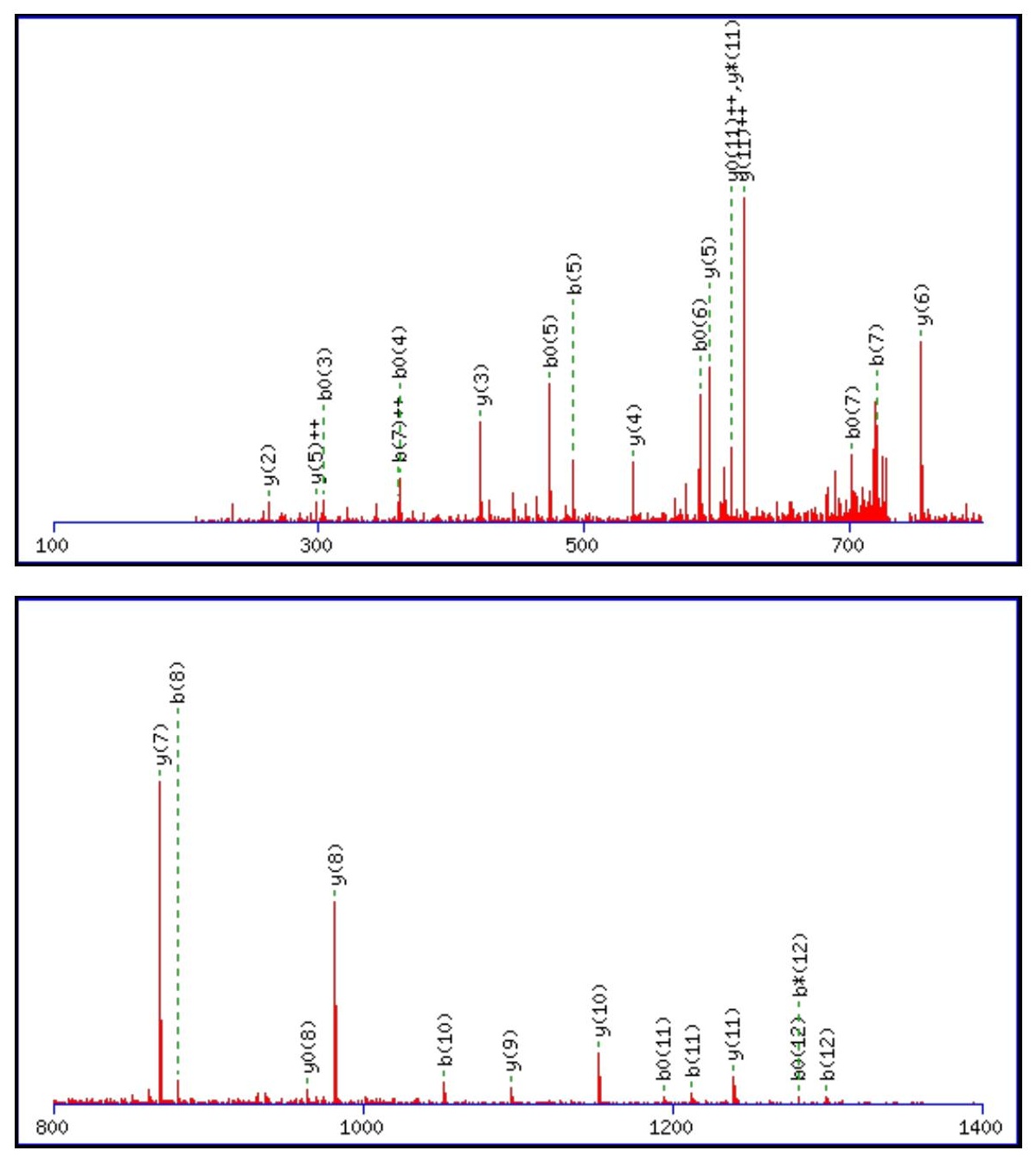


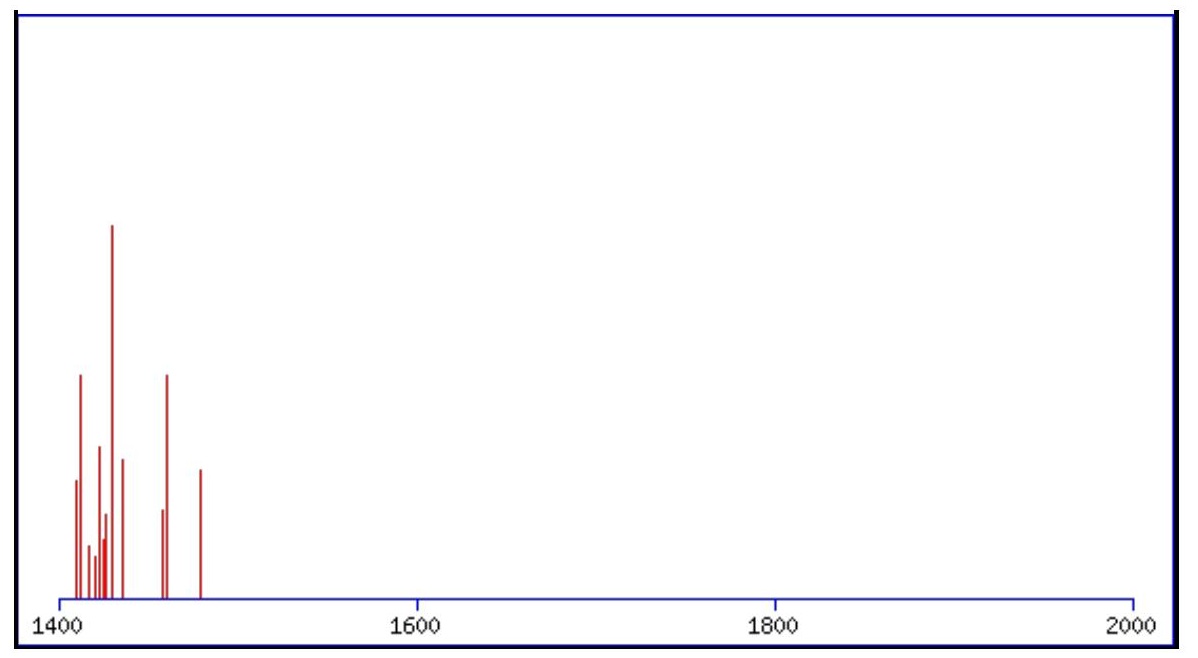

Monoisotopic mass of neutral peptide $\operatorname{Mr}($ calc): 1472.6024

Fixed modifications: Carbamidomethyl (C)

Variable modifications:

N10 : Deamidated_N (N)

Ions Score: 75 Expect: $3.8 \mathrm{e}-006$

Matches (Bold Red): 30/124 fragment ions using 47 most intense peaks

\begin{tabular}{|c|c|c|c|c|c|c|c|c|c|c|c|c|c|c|}
\hline \# & b & $\mathbf{b}^{++}$ & b* & $\mathbf{b}^{*^{++}}$ & $\mathbf{b}^{\mathbf{0}}$ & $\mathbf{b}^{\mathbf{0 + +}}$ & Seq. & $\mathbf{y}$ & $y^{++}$ & $\mathbf{y}^{*}$ & $\mathrm{y}^{*^{++}}$ & $\mathbf{y}^{\mathbf{0}}$ & $\mathbf{y}^{0++}$ & \# \\
\hline 1 & 88.0393 & 44.5233 & & & 70.0287 & 35.5180 & S & & & & & & & 13 \\
\hline 2 & 235.1077 & 118.0575 & & & 217.0972 & 109.0522 & $\mathbf{F}$ & 1386.5777 & 693.7925 & 1369.5511 & 685.2792 & 1368.5671 & 684.7872 & 12 \\
\hline 3 & 322.1397 & 161.5735 & & & 304.1292 & 152.5682 & S & 1239.5092 & 620.2583 & 1222.4827 & 611.7450 & 1221.4987 & 611.2530 & 11 \\
\hline 4 & 379.1612 & 190.0842 & & & 361.1506 & 181.0790 & G & 1152.4772 & 576.7422 & 1135.4507 & 568.2290 & 1134.4666 & 567.7370 & 10 \\
\hline 5 & 492.2453 & 246.6263 & & & 474.2347 & 237.6210 & $\mathbf{L}$ & 1095.4557 & 548.2315 & 1078.4292 & 539.7182 & 1077.4452 & 539.2262 & 9 \\
\hline 6 & 605.3293 & 303.1683 & & & 587.3188 & 294.1630 & $\mathbf{L}$ & 982.3717 & 491.6895 & 965.3451 & 483.1762 & 964.3611 & 482.6842 & 8 \\
\hline 7 & 720.3563 & 360.6818 & & & 702.3457 & 351.6765 & D & 869.2876 & 435.1474 & 852.2611 & 426.6342 & 851.2771 & 426.1422 & 7 \\
\hline 8 & 880.3869 & 440.6971 & & & 862.3764 & 431.6918 & C & 754.2607 & 377.6340 & 737.2341 & 369.1207 & 736.2501 & 368.6287 & 6 \\
\hline 9 & 937.4084 & 469.2078 & & & 919.3978 & 460.2026 & G & 594.2300 & 297.6187 & 577.2035 & 289.1054 & 576.2195 & 288.6134 & 5 \\
\hline 10 & 1052.4353 & 526.7213 & 1035.4088 & 518.2080 & 1034.4248 & 517.7160 & $\mathbf{N}$ & 537.2086 & 269.1079 & 520.1820 & 260.5946 & 519.1980 & 260.1026 & 4 \\
\hline 11 & 1212.4660 & 606.7366 & 1195.4394 & 598.2234 & 1194.4554 & 597.7313 & C & 422.1816 & 211.5945 & 405.1551 & 203.0812 & 404.1711 & 202.5892 & 3 \\
\hline 12 & 1299.4980 & 650.2526 & 1282.4715 & 641.7394 & 1281.4874 & 641.2474 & $\mathrm{~S}$ & 262.1510 & 131.5791 & 245.1244 & 123.0659 & 244.1404 & 122.5738 & 2 \\
\hline 13 & & & & & & & $\mathbf{R}$ & 175.1190 & 88.0631 & 158.0924 & 79.5498 & & & 1 \\
\hline
\end{tabular}
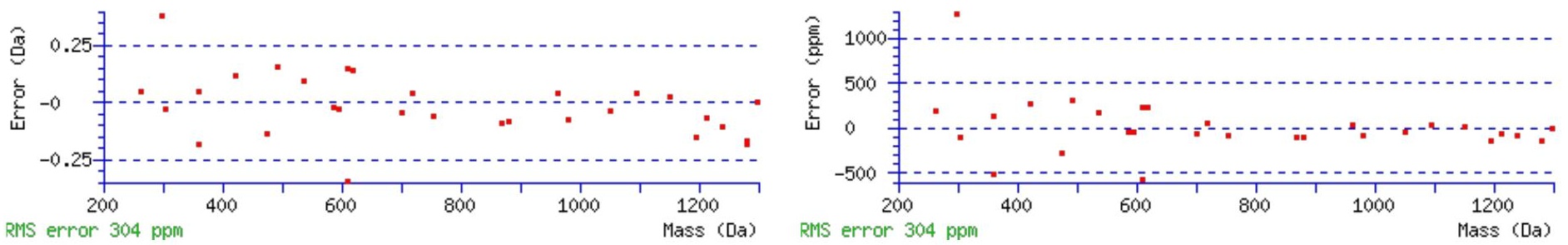

\section{All matches to this query}

\begin{tabular}{|l|c|c|l|}
\hline Score & $\mathbf{M r}(\mathbf{c a l c}):$ & Delta & \multicolumn{1}{c|}{ Sequence } \\
\hline 75.3 & 1472.6024 & 0.0040 & $\underline{\text { SFSGLLDCGNCSR }}$ \\
\hline 12.3 & 1472.6119 & -0.0055 & $\underline{\text { NSPLPNCTYATR }}$ \\
\hline 3.2 & 1471.5914 & 1.0150 & $\underline{\text { ASTAINWLTKK }}$ \\
\hline 2.5 & 1471.6093 & 0.9972 & $\underline{\text { SFSQSSGLNNHSK }}$ \\
\hline 2.1 & 1472.6003 & 0.0062 & $\underline{\text { RSSIWQFFSR }}$ \\
\hline 1.5 & 1472.5933 & 0.0132 & $\underline{\text { SFSQSSGLNNHSK }}$ \\
\hline 1.5 & 1472.5933 & 0.0132 & SFSQSSGLNNHSK \\
\hline 1.0 & 1470.5867 & 2.0198 & $\underline{\text { FQTVESVNLMK }}$ \\
\hline & & & \\
\hline
\end{tabular}




\begin{tabular}{|l|l|l|l|}
0.9 & 1470.5859 & 2.0206 & ADEAEANGYSSGSGR \\
\hline 0.6 & 1472.5966 & 0.0098 & ASESPDSAARQMK \\
\hline
\end{tabular}

Spectrum No: 298; Query: 1832; Rank: 1

\section{Peptide View}

MS/MS Fragmentation of SLTFNESYQDVSEIVYGAK

Found in IPI00372372, Tax_Id=10116 Gene_Symbol=Serpinc1 Serine (Or cysteine) peptidase inhibitor, clade C (Antithrombin), member 1

Match to Query 1832: 2150.012528 from(1076.013540,2+)

Title: 100101RatKid_NS_deglyco_24.5051.5051.2.dta

Data file K:INewmanPaper|Piliangl3SubProteomes\Piliang3SP\mgf5ppm\ERLIC_3SubProteomes5ppm.mgf
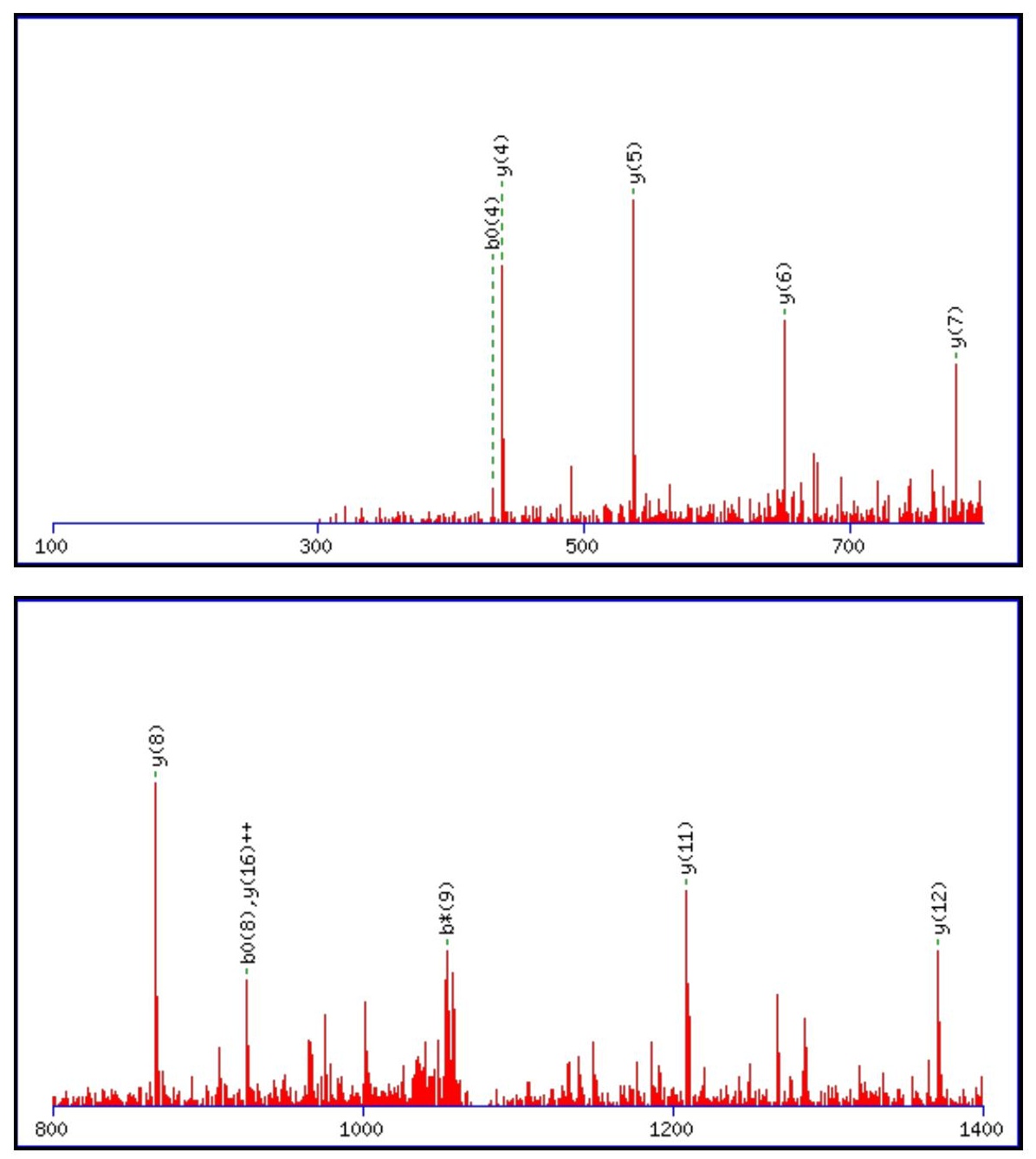


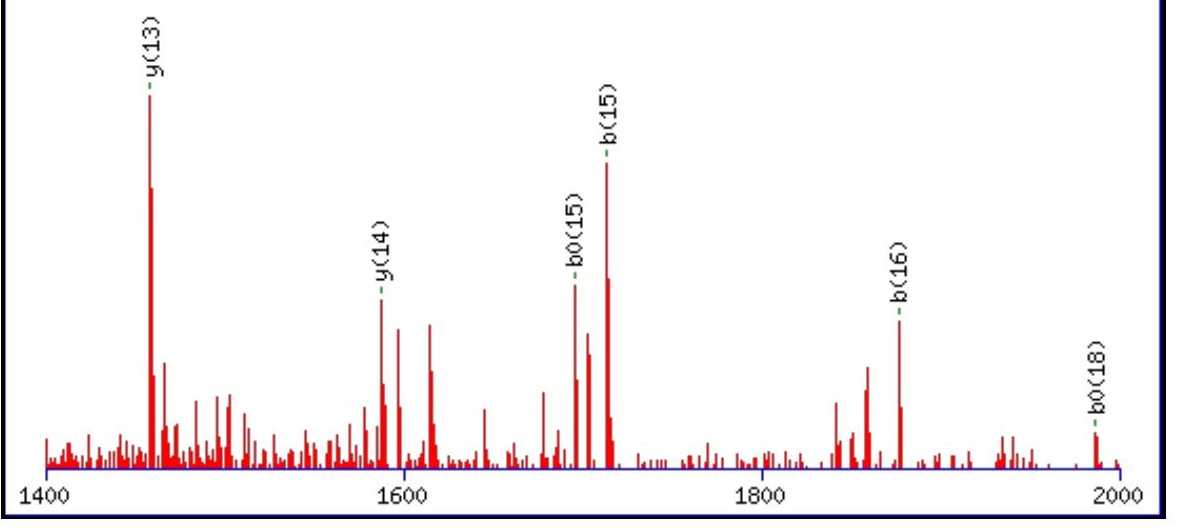

Monoisotopic mass of neutral peptide $\operatorname{Mr}($ calc): 2150.0055

Fixed modifications: Carbamidomethyl (C)

Variable modifications:

N5 : Deamidated_N (N)

Ions Score: 75 Expect: $7.8 \mathrm{e}-006$

Matches (Bold Red): 17/196 fragment ions using 25 most intense peaks

\begin{tabular}{|c|c|c|c|c|c|c|c|c|c|c|c|c|c|c|}
\hline \# & b & $\mathbf{b}^{++}$ & $\mathbf{b}^{*}$ & $\mathbf{b}^{*^{++}}$ & $\mathbf{b}^{0}$ & $\mathbf{b}^{0++}$ & Seq. & $\mathbf{y}$ & $\mathbf{y}^{++}$ & $\mathbf{y}^{*}$ & $\mathbf{y}^{*^{++}}$ & $\mathbf{y}^{\mathbf{0}}$ & $\mathbf{y}^{0++}$ & \# \\
\hline 1 & 88.0393 & 44.5233 & & & 70.0287 & 35.5180 & S & & & & & & & 19 \\
\hline 2 & 201.1234 & 101.0653 & & & 183.1128 & 92.0600 & $\mathbf{L}$ & 2063.9808 & 1032.4940 & 2046.9542 & 1023.9808 & 2045.9702 & 1023.4887 & 18 \\
\hline 3 & 302.1710 & 151.5892 & & & 284.1605 & 142.5839 & $\mathbf{T}$ & 1950.8967 & 975.9520 & 1933.8702 & 967.4387 & 1932.8862 & 966.9467 & 17 \\
\hline 4 & 449.2395 & 225.1234 & & & 431.2289 & 216.1181 & $\mathbf{F}$ & 1849.8490 & 925.4282 & 1832.8225 & 916.9149 & 1831.8385 & 916.4229 & 16 \\
\hline 5 & 564.2664 & 282.6368 & 547.2398 & 274.1236 & 546.2558 & 273.6316 & $\mathbf{N}$ & 1702.7806 & 851.8940 & 1685.7541 & 843.3807 & 1684.7701 & 842.8887 & 15 \\
\hline 6 & 693.3090 & 347.1581 & 676.2824 & 338.6449 & 675.2984 & 338.1529 & $\mathbf{E}$ & 1587.7537 & 794.3805 & 1570.7271 & 785.8672 & 1569.7431 & 785.3752 & 14 \\
\hline 7 & 780.3410 & 390.6741 & 763.3145 & 382.1609 & 762.3305 & 381.6689 & S & 1458.7111 & 729.8592 & 1441.6846 & 721.3459 & 1440.7005 & 720.8539 & 13 \\
\hline 8 & 943.4043 & 472.2058 & 926.3778 & 463.6925 & 925.3938 & 463.2005 & $\mathbf{Y}$ & 1371.6791 & 686.3432 & 1354.6525 & 677.8299 & 1353.6685 & 677.3379 & 12 \\
\hline 9 & 1071.4629 & 536.2351 & 1054.4364 & 527.7218 & 1053.4524 & 527.2298 & $\mathbf{Q}$ & 1208.6157 & 604.8115 & 1191.5892 & 596.2982 & 1190.6052 & 595.8062 & 11 \\
\hline 10 & 1186.4899 & 593.7486 & 1169.4633 & 585.2353 & 1168.4793 & 584.7433 & D & 1080.5572 & 540.7822 & 1063.5306 & 532.2689 & 1062.5466 & 531.7769 & 10 \\
\hline 11 & 1285.5583 & 643.2828 & 1268.5317 & 634.7695 & 1267.5477 & 634.2775 & $\mathbf{V}$ & 965.5302 & 483.2688 & 948.5037 & 474.7555 & 947.5197 & 474.2635 & 9 \\
\hline 12 & 1372.5903 & 686.7988 & 1355.5638 & 678.2855 & 1354.5797 & 677.7935 & S & 866.4618 & 433.7345 & 849.4353 & 425.2213 & 848.4512 & 424.7293 & 8 \\
\hline 13 & 1501.6329 & 751.3201 & 1484.6064 & 742.8068 & 1483.6223 & 742.3148 & $\mathbf{E}$ & 779.4298 & 390.2185 & 762.4032 & 381.7053 & 761.4192 & 381.2132 & 7 \\
\hline 14 & 1614.7170 & 807.8621 & 1597.6904 & 799.3488 & 1596.7064 & 798.8568 & I & 650.3872 & 325.6972 & 633.3606 & 317.1840 & & & 6 \\
\hline 15 & 1713.7854 & 857.3963 & 1696.7588 & 848.8831 & 1695.7748 & 848.3910 & V & 537.3031 & 269.1552 & 520.2766 & 260.6419 & & & 5 \\
\hline 16 & 1876.8487 & 938.9280 & 1859.8222 & 930.4147 & 1858.8381 & 929.9227 & $\mathbf{Y}$ & 438.2347 & 219.6210 & 421.2082 & 211.1077 & & & 4 \\
\hline 17 & 1933.8702 & 967.4387 & 1916.8436 & 958.9255 & 1915.8596 & 958.4334 & G & 275.1714 & 138.0893 & 258.1448 & 129.5761 & & & 3 \\
\hline 18 & 2004.9073 & 1002.9573 & 1987.8807 & 994.4440 & 1986.8967 & 993.9520 & A & 218.1499 & 109.5786 & 201.1234 & 101.0653 & & & 2 \\
\hline 19 & & & & & & & $\mathbf{K}$ & 147.1128 & 74.0600 & 130.0863 & 65.5468 & & & 1 \\
\hline
\end{tabular}

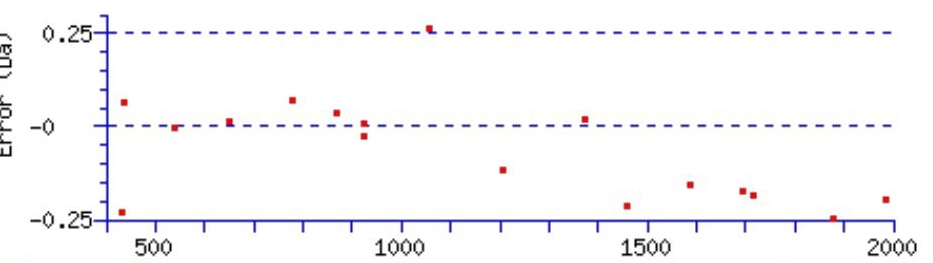

RMS error 165 ppm

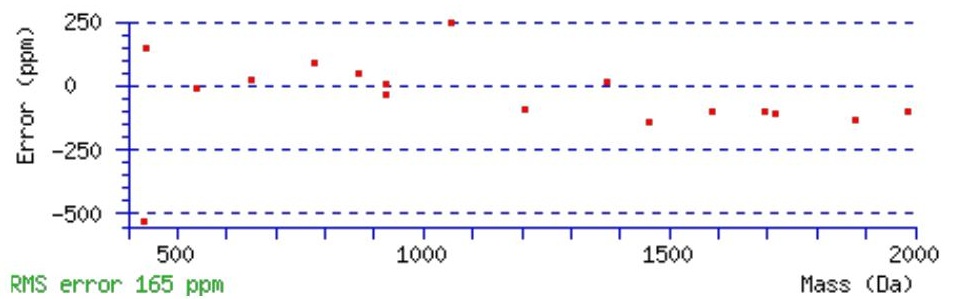

All matches to this query

\begin{tabular}{|l|c|c|c|}
\hline Score & Mr(calc): & Delta & Sequence \\
\hline 75.2 & 2150.0055 & 0.0070 & SLTFNESYQDVSEIVYGAK \\
\hline 62.9 & 2149.0215 & 0.9910 & SLTFNESYQDVSEIVYGAK \\
\hline
\end{tabular}


Spectrum No: 299; Query: 1067; Rank: 1

\section{Peptide View}

MS/MS Fragmentation of DGWTGEYCNCTTSR

Found in IPI00372094, Tax_Id=10116 Gene_Symbol=Itgb6 Integrin beta-6 precursor

Match to Query 1067: 1706.637188 from(854.325870,2+)

Title: 100101RatKid_NS_deglyco_20.1723.1723.2.dta

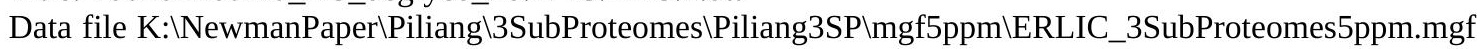
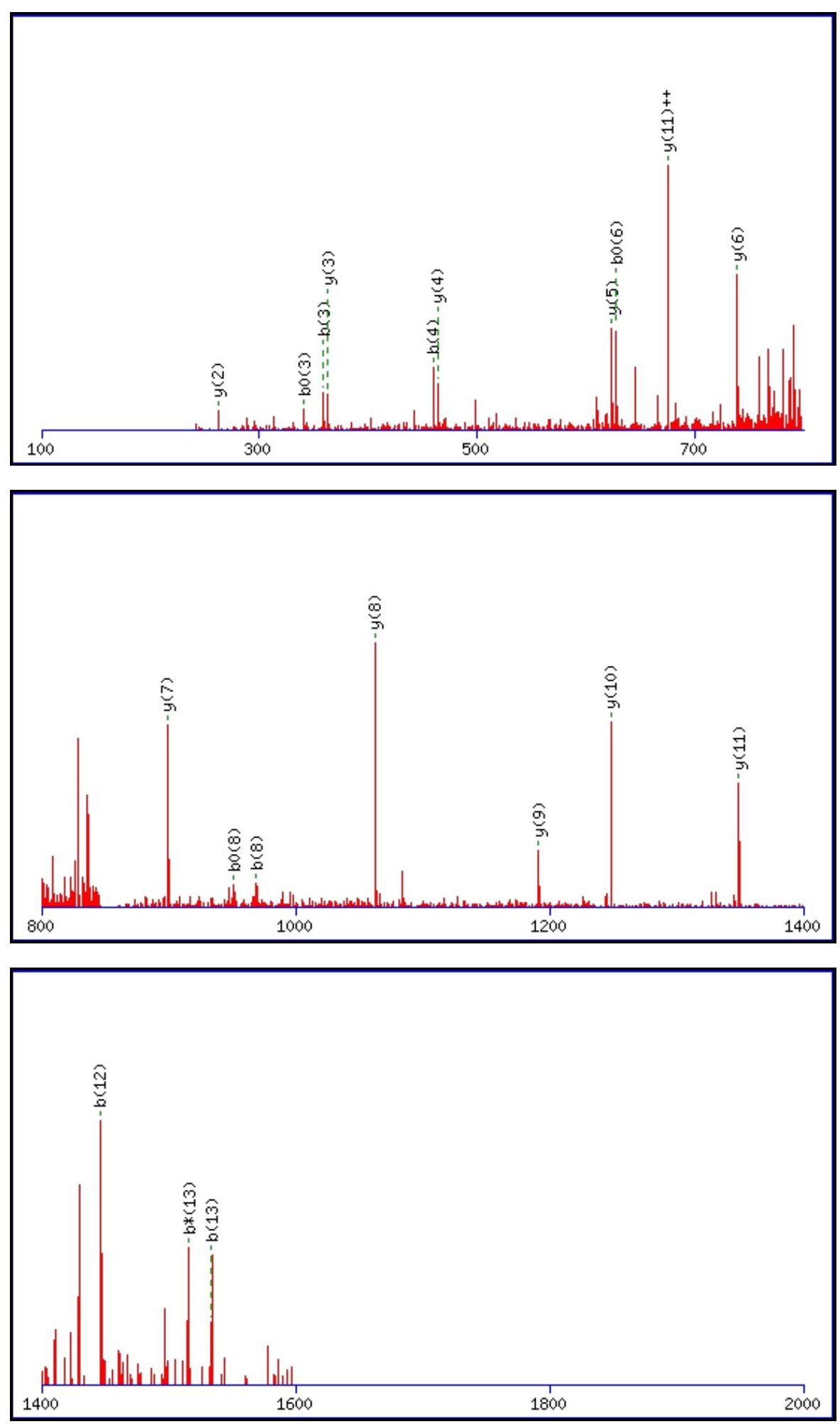
Monoisotopic mass of neutral peptide $\operatorname{Mr}(\mathrm{calc}): 1706.6301$

Fixed modifications: Carbamidomethyl (C)

Variable modifications:

N9 : Deamidated_N (N)

Ions Score: 75 Expect: 2.9e- -006

Matches (Bold Red): 20/138 fragment ions using 30 most intense peaks

\begin{tabular}{|c|c|c|c|c|c|c|c|c|c|c|c|c|c|c|}
\hline \# & b & $\mathbf{b}^{++}$ & $\mathbf{b}^{*}$ & $\mathbf{b}^{*^{++}}$ & $\mathbf{b}^{0}$ & $\mathbf{b}^{0++}$ & Seq. & $\mathbf{y}$ & $\mathbf{y}^{++}$ & $\mathrm{y}^{*}$ & $\mathrm{y}^{\mathrm{*}^{++}}$ & $\mathbf{y}^{\mathbf{0}}$ & $y^{0++}$ & $\#$ \\
\hline 1 & 116.0342 & 58.5207 & & & 98.0237 & 49.5155 & D & & & & & & & 14 \\
\hline 2 & 173.0557 & 87.0315 & & & 155.0451 & 78.0262 & G & 1592.6104 & 796.8088 & 1575.5839 & 788.2956 & 1574.5998 & 787.8036 & 13 \\
\hline 3 & 359.1350 & 180.0711 & & & 341.1244 & 171.0659 & $\mathbf{W}$ & 1535.5890 & 768.2981 & 1518.5624 & 759.7848 & \begin{tabular}{|l}
1517.5784 \\
\end{tabular} & 759.2928 & 12 \\
\hline 4 & 460.1827 & 230.5950 & & & 442.1721 & 221.5897 & $T$ & 1349.5096 & 675.2585 & 1332.4831 & 666.7452 & 1331.4991 & 666.2532 & 11 \\
\hline 5 & 517.2041 & 259.1057 & & & 499.1936 & 250.1004 & G & 1248.4620 & 624.7346 & 1231.4354 & 616.2213 & 1230.4514 & 615.7293 & 10 \\
\hline 6 & 646.2467 & 323.6270 & & & 628.2362 & 314.6217 & $\mathbf{E}$ & 1191.4405 & 596.2239 & 1174.4139 & 587.7106 & 1173.4299 & 587.2186 & 9 \\
\hline 7 & 809.3101 & 405.1587 & & & 791.2995 & 396.1534 & $\mathbf{Y}$ & 1062.3979 & 531.7026 & 1045.3714 & 523.1893 & 1044.3873 & 522.6973 & 8 \\
\hline 8 & 969.3407 & 485.1740 & & & 951.3301 & 476.1687 & C & 899.3346 & 450.1709 & 882.3080 & 441.6576 & 881.3240 & 441.1656 & 7 \\
\hline 9 & 1084.3676 & 542.6875 & 1067.3411 & 534.1742 & 1066.3571 & 533.6822 & $\mathbf{N}$ & 739.3039 & 370.1556 & 722.2774 & 361.6423 & 721.2934 & 361.1503 & 6 \\
\hline 101 & 1244.3983 & 622.7028 & 1227.3717 & 614.1895 & 1226.3877 & 613.6975 & $\mathrm{C}$ & 624.2770 & 312.6421 & 607.2504 & 304.1289 & 606.2664 & 303.6368 & 5 \\
\hline 111 & 1345.4460 & 673.2266 & 1328.4194 & 664.7134 & 1327.4354 & 664.2213 & $T$ & 464.2463 & 232.6268 & 447.2198 & 224.1135 & 446.2358 & 223.6215 & 4 \\
\hline 121 & 1446.4937 & 723.7505 & 1429.4671 & 715.2372 & 1428.4831 & 714.7452 & $\mathbf{T}$ & 363.1987 & 182.1030 & 346.1721 & 173.5897 & 345.1881 & 173.0977 & 3 \\
\hline 131 & 1533.5257 & 767.2665 & 1516.4991 & 758.7532 & 1515.5151 & 758.2612 & $\mathrm{~S}$ & 262.1510 & 131.5791 & 245.1244 & 123.0659 & 244.1404 & 122.5738 & 2 \\
\hline 14 & & & & & & & $\mathbf{R}$ & 175.1190 & 88.0631 & 158.0924 & 79.5498 & & & 1 \\
\hline
\end{tabular}

.
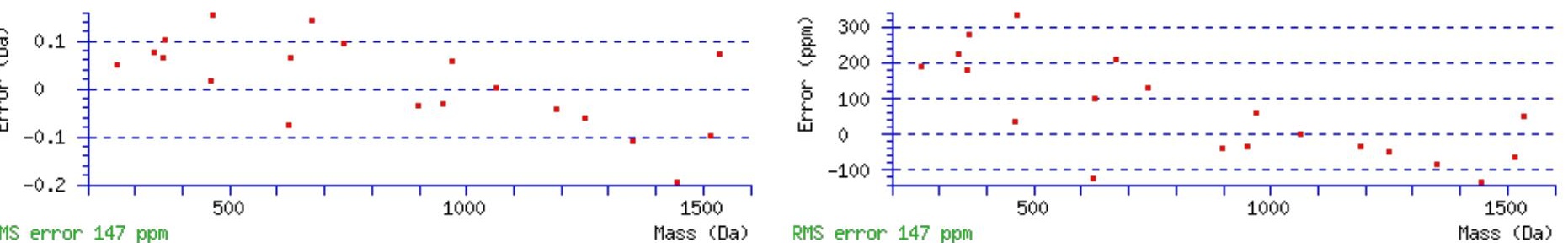

\section{All matches to this query}

\begin{tabular}{|l|l|c|c|}
\hline Score & Mr(calc): & Delta & \multicolumn{1}{c|}{ Sequence } \\
\hline 75.2 & 1706.6301 & 0.0071 & DGWTGEYCNCTTSR \\
\hline 21.6 & 1705.6461 & 0.9911 & DGWTGEYCNCTTSR \\
\hline 1.2 & 1706.6355 & 0.0017 & EPNLLSGTAHSVNK \\
\hline 0.2 & 1706.6511 & -0.0139 & $\underline{\text { NQDTLEPMGPSTIK }}$ \\
\hline
\end{tabular}

Spectrum No: 300; Query: 1203; Rank: 1

\section{Peptide View}

MS/MS Fragmentation of FTCNETTNVIIIHSK

Found in IPI00230862, Tax_Id=10116 Gene_Symbol=Anpep Aminopeptidase N

Match to Query 1203: 1776.874408 from(889.444480,2+)

Title: 100101RatKid_NS_deglyco_23.2169.2169.2.dta

Data file K:INewmanPaper|Piliang|3SubProteomes\Piliang3SP\mgf5ppm\ERLIC_3SubProteomes5ppm.mgf 

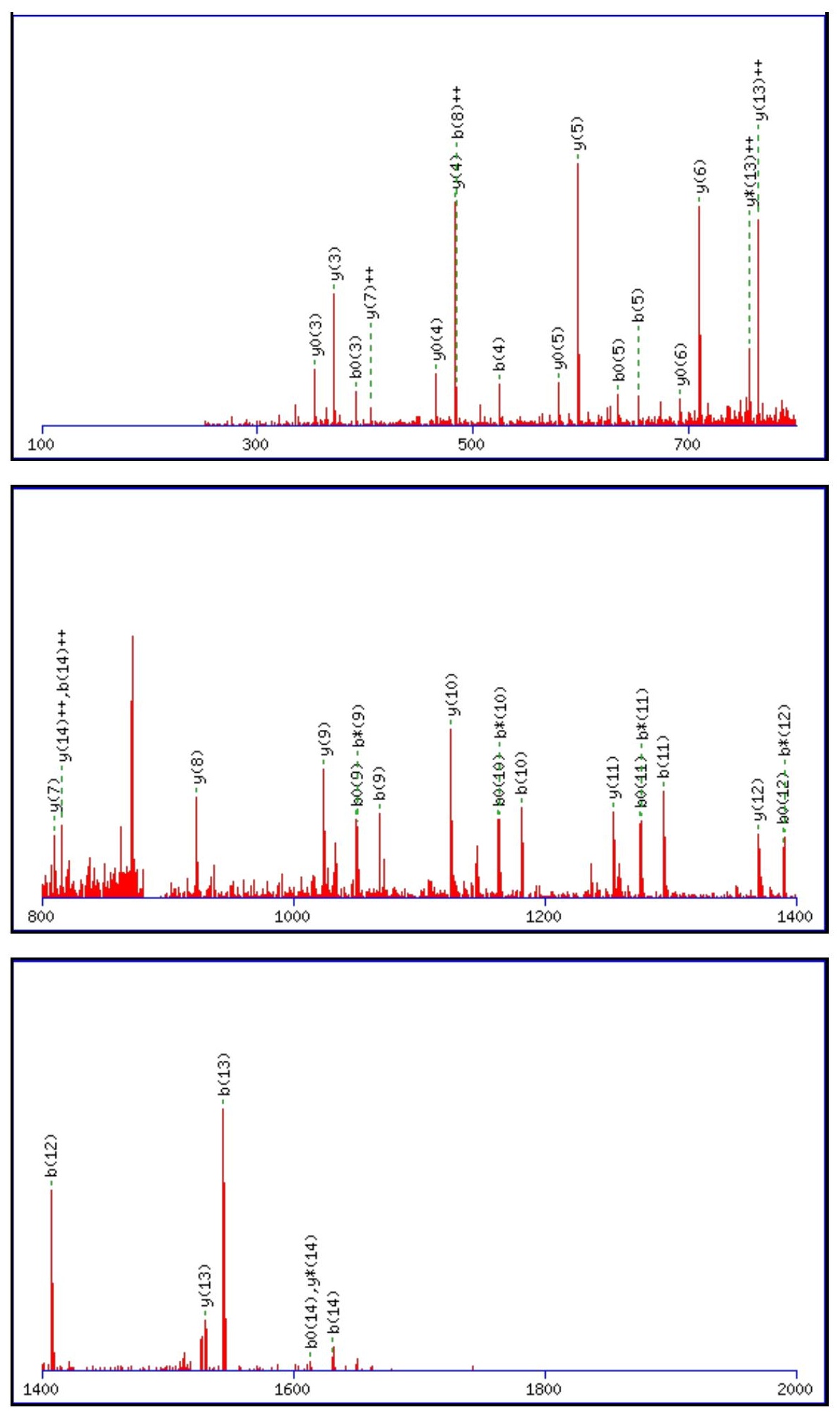

Monoisotopic mass of neutral peptide $\operatorname{Mr}($ calc): 1776.8716

Fixed modifications: Carbamidomethyl (C)

Variable modifications:

N4 : Deamidated $\mathrm{N}(\mathrm{N})$

Ions Score: 75 Expect: $5.4 \mathrm{e}-006$

Matches (Bold Red): 41/158 fragment ions using 61 most intense peaks

\begin{tabular}{|r|c|c|c|c|c|c|c|c|c|c|c|c|c|c|}
\hline$\#$ & $\mathbf{b}$ & $\mathbf{b}^{++}$ & $\mathbf{b}^{*}$ & $\mathbf{b}^{\mathbf{*}^{++}}$ & $\mathbf{b}^{\mathbf{0}}$ & $\mathbf{b}^{\mathbf{0 + +}}$ & Seq. & $\mathbf{y}$ & $\mathbf{y}^{++}$ & $\mathbf{y}^{\mathbf{*}}$ & $\mathbf{y}^{\boldsymbol{*}^{++}}$ & $\mathbf{y}^{\mathbf{0}}$ & $\mathbf{y}^{\mathbf{0 + +}}$ & $\#$ \\
\hline $\mathbf{1}$ & 148.0757 & 74.5415 & & & & & $\mathbf{F}$ & & & & & & & $\mathbf{1 5}$ \\
\hline $\mathbf{2}$ & 249.1234 & 125.0653 & & & 231.1128 & 116.0600 & $\mathbf{T}$ & 1630.8105 & $\mathbf{8 1 5 . 9 0 8 9}$ & $\mathbf{1 6 1 3 . 7 8 3 9}$ & 807.3956 & 1612.7999 & 806.9036 & $\mathbf{1 4}$ \\
\hline $\mathbf{3}$ & 409.1540 & 205.0806 & & & 391.1435 & 196.0754 & $\mathbf{C}$ & $\mathbf{1 5 2 9 . 7 6 2 8}$ & $\mathbf{7 6 5 . 3 8 5 0}$ & 1512.7363 & $\mathbf{7 5 6 . 8 7 1 8}$ & 1511.7522 & 756.3798 & $\mathbf{1 3}$ \\
\hline $\mathbf{4}$ & $\mathbf{5 2 4 . 1 8 1 0}$ & 262.5941 & 507.1544 & 254.0808 & 506.1704 & 253.5888 & $\mathbf{N}$ & $\mathbf{1 3 6 9 . 7 3 2 2}$ & 685.3697 & 1352.7056 & 676.8564 & 1351.7216 & 676.3644 & $\mathbf{1 2}$ \\
\hline $\mathbf{5}$ & $\mathbf{6 5 3 . 2 2 3 5}$ & 327.1154 & 636.1970 & 318.6021 & $\mathbf{6 3 5 . 2 1 3 0}$ & 318.1101 & $\mathbf{E}$ & $\mathbf{1 2 5 4 . 7 0 5 2}$ & 627.8563 & 1237.6787 & 619.3430 & 1236.6947 & 618.8510 & $\mathbf{1 1}$ \\
\hline
\end{tabular}




\begin{tabular}{|c|c|c|c|c|c|c|c|c|c|c|c|c|c|c|}
\hline 6 & 754.2712 & |377.6393 & 737.2447 & 369.1260 & 736.2607 & 368.6340 & $\mathbf{T}$ & 1125.6626 & 563.3350 & 1108.6361 & 554.8217 & 1107.6521 & 554.3297 & 10 \\
\hline 7 & 855.3189 & 428.1631 & 838.2924 & 419.6498 & 837.3083 & 419.1578 & $\mathbf{T}$ & 1024.6150 & 512.8111 & 1007.5884 & 504.2978 & 1006.6044 & 503.8058 & 9 \\
\hline 8 & 969.3618 & 485.1846 & 952.3353 & 476.6713 & 951.3513 & 476.1793 & $\mathbf{N}$ & 923.5673 & 462.2873 & 906.5407 & 453.7740 & 905.5567 & 453.2820 & 8 \\
\hline 9 & 1068.4302 & 534.7188 & 1051.4037 & 526.2055 & 1050.4197 & 525.7135 & V & 809.5243 & 405.2658 & 792.4978 & 396.7525 & 791.5138 & 396.2605 & 7 \\
\hline 10 & 1181.5143 & 591.2608 & 1164.4878 & 582.7475 & 1163.5037 & 582.2555 & I & 710.4559 & 355.7316 & 693.4294 & 347.2183 & 692.4454 & 346.7263 & 6 \\
\hline 11 & 1294.5984 & 647.8028 & 1277.5718 & 639.2896 & 1276.5878 & 638.7975 & I & 597.3719 & 299.1896 & 580.3453 & 290.6763 & 579.3613 & 290.1843 & 5 \\
\hline 12 & 1407.6824 & 704.3449 & 1390.6559 & 695.8316 & 1389.6719 & 695.3396 & I & 484.2878 & 242.6475 & 467.2613 & 234.1343 & 466.2772 & 233.6423 & 4 \\
\hline 13 & 1544.7413 & 772.8743 & 1527.7148 & 764.3610 & 1526.7308 & 763.8690 & $\mathbf{H}$ & 371.2037 & 186.1055 & 354.1772 & 177.5922 & 353.1932 & 177.1002 & 3 \\
\hline 14 & 1631.7734 & 816.3903 & 1614.7468 & 807.8771 & 1613.7628 & 807.3850 & S & 234.1448 & 117.5761 & 217.1183 & 109.0628 & 216.1343 & 108.5708 & 2 \\
\hline 15 & & & & & & & $\mathbf{K}$ & 147.1128 & 74.0600 & 130.0863 & 65.5468 & & & 1 \\
\hline
\end{tabular}
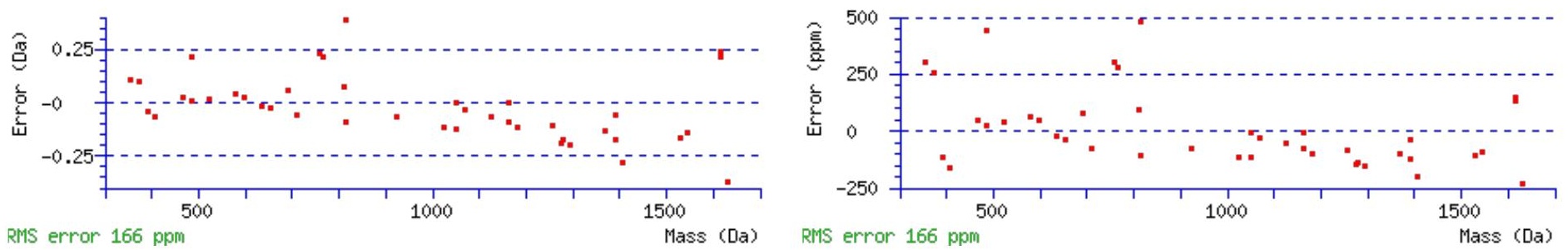

All matches to this query

\begin{tabular}{|l|l|c|l|}
\hline Score & Mr(calc): & Delta & \multicolumn{1}{|c|}{ Sequence } \\
\hline 75.1 & 1776.8716 & 0.0028 & FTCNETTNVIIIHSK \\
\hline 55.1 & 1776.8716 & 0.0028 & FTCNETTNVIIIHSK \\
\hline 23.7 & 1775.8876 & 0.9868 & FTCNETTNVIIIHSK \\
\hline 8.8 & 1776.8712 & 0.0032 & FPSGIKHLANYVHSK \\
\hline 8.3 & 1776.8675 & 0.0069 & QIKNLEMERNAESSK \\
\hline 4.7 & 1776.8593 & 0.0151 & NHREDGILTKLICK \\
\hline 4.0 & 1776.8811 & -0.0067 & KIELEDALALGPSWR \\
\hline 4.0 & 1776.8576 & 0.0168 & VGDSNTLIFIRILR \\
\hline 3.5 & 1774.8771 & 1.9973 & LVRDDIPSTQENLMK \\
\hline 3.4 & 1774.8767 & 1.9977 & TKATLIRTTFNWSR \\
\hline
\end{tabular}

Spectrum No: 301; Query: 1756; Rank: 1

\section{Peptide View}

MS/MS Fragmentation of EGETCGAEDNDTCGVSLYK

Found in IPI00365178, Tax_Id=10116 Gene_Symbol=Fbln2 similar to Fibulin-2 precursor isoform 1

Match to Query 1756: 2104.824708 from(1053.419630,2+)

Title: 100101RatKid_NS_deglyco_26.1813.1813.2.dta

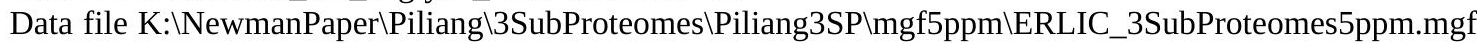



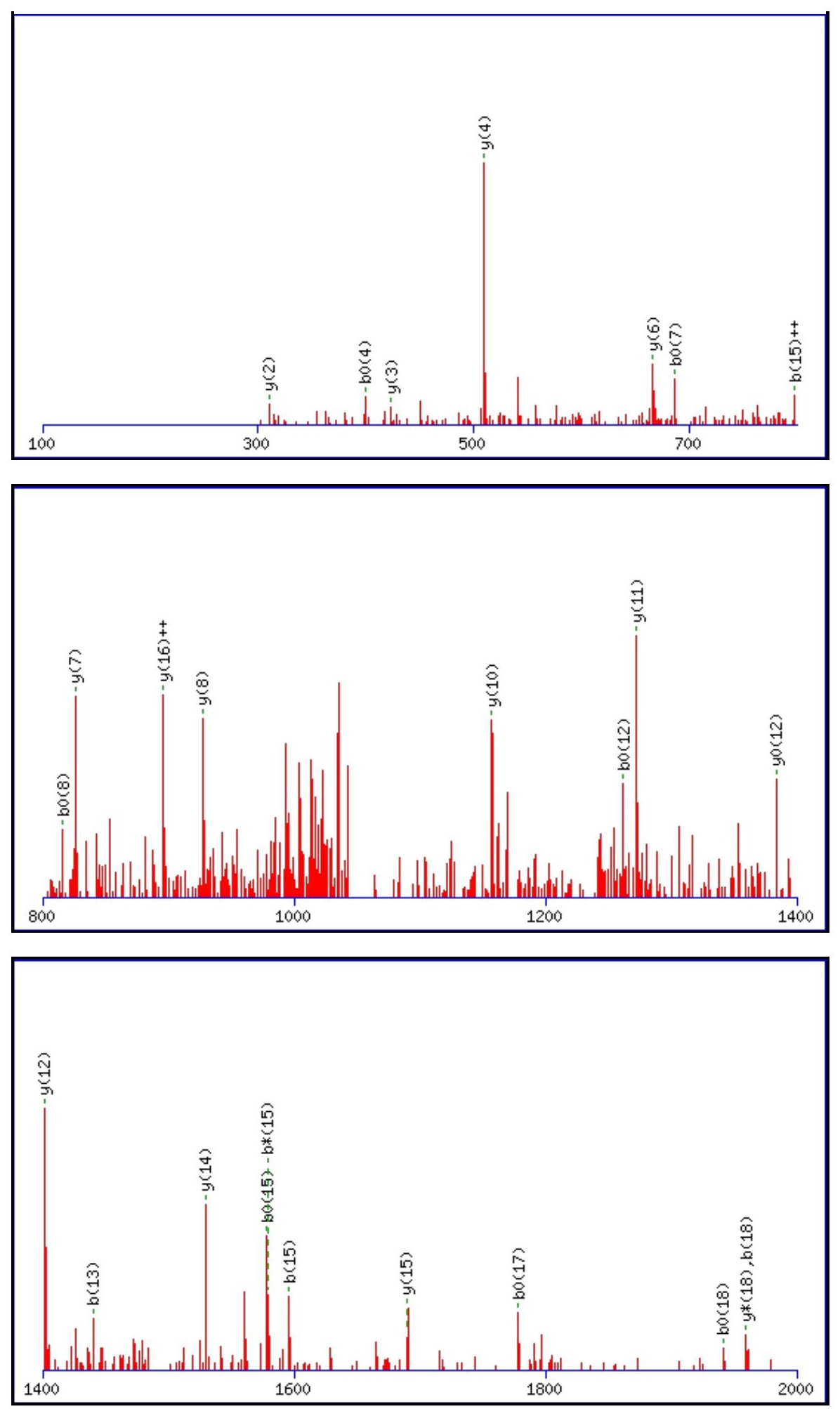

Monoisotopic mass of neutral peptide $\operatorname{Mr}($ calc): 2104.8201

Fixed modifications: Carbamidomethyl (C)

Variable modifications:

N10 : Deamidated $\mathrm{N}(\mathrm{N})$

Ions Score: 75 Expect: $5.3 \mathrm{e}-006$

Matches (Bold Red): 26/192 fragment ions using 41 most intense peaks

\begin{tabular}{|r|c|c|c|c|c|c|c|c|c|c|c|c|c|c|}
\hline$\#$ & $\mathbf{b}$ & $\mathbf{b}^{++}$ & $\mathbf{b}^{*}$ & $\mathbf{b}^{\boldsymbol{*}^{++}}$ & $\mathbf{b}^{\mathbf{0}}$ & $\mathbf{b}^{\mathbf{0 + +}}$ & Seq. & $\mathbf{y}$ & $\mathbf{y}^{++}$ & $\mathbf{y}^{\mathbf{*}}$ & $\mathbf{y}^{\boldsymbol{*}^{++}}$ & $\mathbf{y}^{\mathbf{0}}$ & $\mathbf{y}^{\mathbf{0 + +}}$ & $\#$ \\
\hline $\mathbf{1}$ & 130.0499 & 65.5286 & & & 112.0393 & 56.5233 & $\mathbf{E}$ & & & & & & & $\mathbf{1 9}$ \\
\hline $\mathbf{2}$ & 187.0713 & 94.0393 & & & 169.0608 & 85.0340 & $\mathbf{G}$ & 1976.7848 & 988.8960 & $\mathbf{1 9 5 9 . 7 5 8 3}$ & 980.3828 & 1958.7742 & 979.8908 & $\mathbf{1 8}$ \\
\hline 3 & 316.1139 & 158.5606 & & & 298.1034 & 149.5553 & $\mathbf{E}$ & 1919.7633 & 960.3853 & 1902.7368 & 951.8720 & 1901.7528 & 951.3800 & $\mathbf{1 7}$ \\
\hline $\mathbf{4}$ & 417.1616 & 209.0844 & & & 399.1510 & 200.0792 & $\mathbf{T}$ & 1790.7208 & $\mathbf{8 9 5 . 8 6 4 0}$ & 1773.6942 & 887.3507 & 1772.7102 & 886.8587 & $\mathbf{1 6}$ \\
\hline $\mathbf{5}$ & 577.1923 & 289.0998 & & & 559.1817 & 280.0945 & $\mathbf{C}$ & $\mathbf{1 6 8 9 . 6 7 3 1}$ & 845.3402 & 1672.6465 & 836.8269 & 1671.6625 & 836.3349 & $\mathbf{1 5}$ \\
\hline
\end{tabular}




\begin{tabular}{|c|c|c|c|c|c|c|c|c|c|c|c|c|c|c|}
\hline 6 & 634.2137 & 317.6105 & & & 616.2032 & 308.6052 & G & |1529.6424| & 765.3248 & 1512.6159 & 756.8116 & 1511.6319 & |756.3196 & 14 \\
\hline 7 & 705.2508 & 353.1291 & & & 687.2403 & 344.1238 & $\mathbf{A}$ & 1472.6210 & 736.8141 & 1455.5944 & 728.3008 & 1454.6104 & 727.8088 & 13 \\
\hline 8 & 834.2934 & 417.6504 & & & 816.2829 & 408.6451 & E & 1401.5838 & 701.2956 & 1384.5573 & 692.7823 & 1383.5733 & 692.2903 & 12 \\
\hline 9 & 949.3204 & 475.1638 & & & 931.3098 & 466.1585 & D & 1272.5413 & 636.7743 & 1255.5147 & 628.2610 & 1254.5307 & 627.7690 & 11 \\
\hline 10 & 1064.3473 & 532.6773 & 1047.3208 & 524.1640 & 1046.3367 & 523.6720 & $\mathbf{N}$ & 1157.5143 & 579.2608 & 1140.4878 & 570.7475 & 1139.5037 & 570.2555 & 10 \\
\hline 11 & 1179.3742 & 590.1908 & 1162.3477 & 581.6775 & 1161.3637 & 581.1855 & D & 1042.4874 & 521.7473 & 1025.4608 & 513.2341 & 1024.4768 & 512.7420 & 9 \\
\hline 12 & 1280.4219 & 640.7146 & 1263.3954 & 632.2013 & 1262.4114 & 631.7093 & $T$ & 927.4604 & 464.2339 & 910.4339 & 455.7206 & 909.4499 & 455.2286 & 8 \\
\hline 13 & 1440.4526 & 720.7299 & 1423.4260 & 712.2167 & 1422.4420 & 711.7246 & $\mathrm{C}$ & 826.4128 & 413.7100 & 809.3862 & 405.1967 & 808.4022 & 404.7047 & 7 \\
\hline 14 & 1497.4740 & 749.2407 & 1480.4475 & 740.7274 & 1479.4635 & 740.2354 & $\mathbf{G}$ & 666.3821 & 333.6947 & 649.3556 & 325.1814 & 648.3715 & 324.6894 & 6 \\
\hline 15 & 1596.5425 & \begin{tabular}{|l|}
798.7749 \\
\end{tabular} & 1579.5159 & 790.2616 & 1578.5319 & 789.7696 & $\mathbf{V}$ & 609.3606 & 305.1840 & 592.3341 & 296.6707 & 591.3501 & 296.1787 & 5 \\
\hline 16 & 1683.5745 & 842.2909 & 1666.5479 & 833.7776 & 1665.5639 & 833.2856 & $S$ & 510.2922 & 255.6498 & 493.2657 & 247.1365 & 492.2817 & 246.6445 & 4 \\
\hline 17 & 1796.6585 & 898.8329 & 1779.6320 & 890.3196 & 1778.6480 & 889.8276 & $\mathbf{L}$ & 423.2602 & 212.1337 & 406.2336 & 203.6205 & & & 3 \\
\hline 18 & 1959.7219 & 980.3646 & 1942.6953 & 971.8513 & 1941.7113 & 971.3593 & $\mathbf{Y}$ & 310.1761 & 155.5917 & 293.1496 & 147.0784 & & & 2 \\
\hline 19 & & & & & & & $\mathbf{K}$ & 147.1128 & 74.0600 & 130.0863 & 65.5468 & & & 1 \\
\hline
\end{tabular}
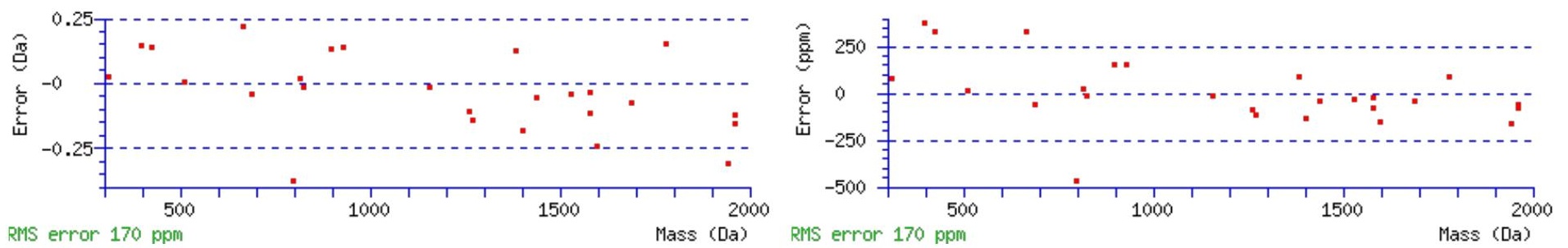

\section{All matches to this query}

\begin{tabular}{|l|c|c|l|}
\hline Score & Mr(calc): & Delta & \multicolumn{1}{|c|}{ Sequence } \\
\hline 75.1 & 2104.8201 & 0.0046 & EGETCGAEDNDTCGVSLYK \\
\hline 8.7 & 2103.8361 & 0.9886 & EGETCGAEDNDTCGVSLYK \\
\hline 6.2 & 2104.8302 & -0.0055 & KLTSSGCIDDATRGSAVK \\
\hline 6.2 & 2104.8302 & -0.0055 & KLTSSGCIDDATRGSAVK \\
\hline 3.2 & 2104.8073 & 0.0174 & SSSSSQSRSHSGSREHSR \\
\hline 0.3 & 2104.8302 & -0.0055 & KLTSSGCIDDATRGSAVK \\
\hline
\end{tabular}

Spectrum No: 302; Query: 844; Rank: 1

\section{Peptide View}

\section{MS/MS Fragmentation of FNLTETPETEIHR}

Found in IPI00211075, Tax_Id=10116 Gene_Symbol=Serpina3n Serine protease inhibitor A3N precursor

Match to Query 844: 1586.756048 from(794.385300,2+)

Title: 091008RatKidney_NH4Format01_27.1843.1843.2.dta

Data file K:\NewmanPaper\Piliang\3SubProteomes\Piliang3SP\mgf5ppm\ERLIC_3SubProteomes5ppm.mgf 

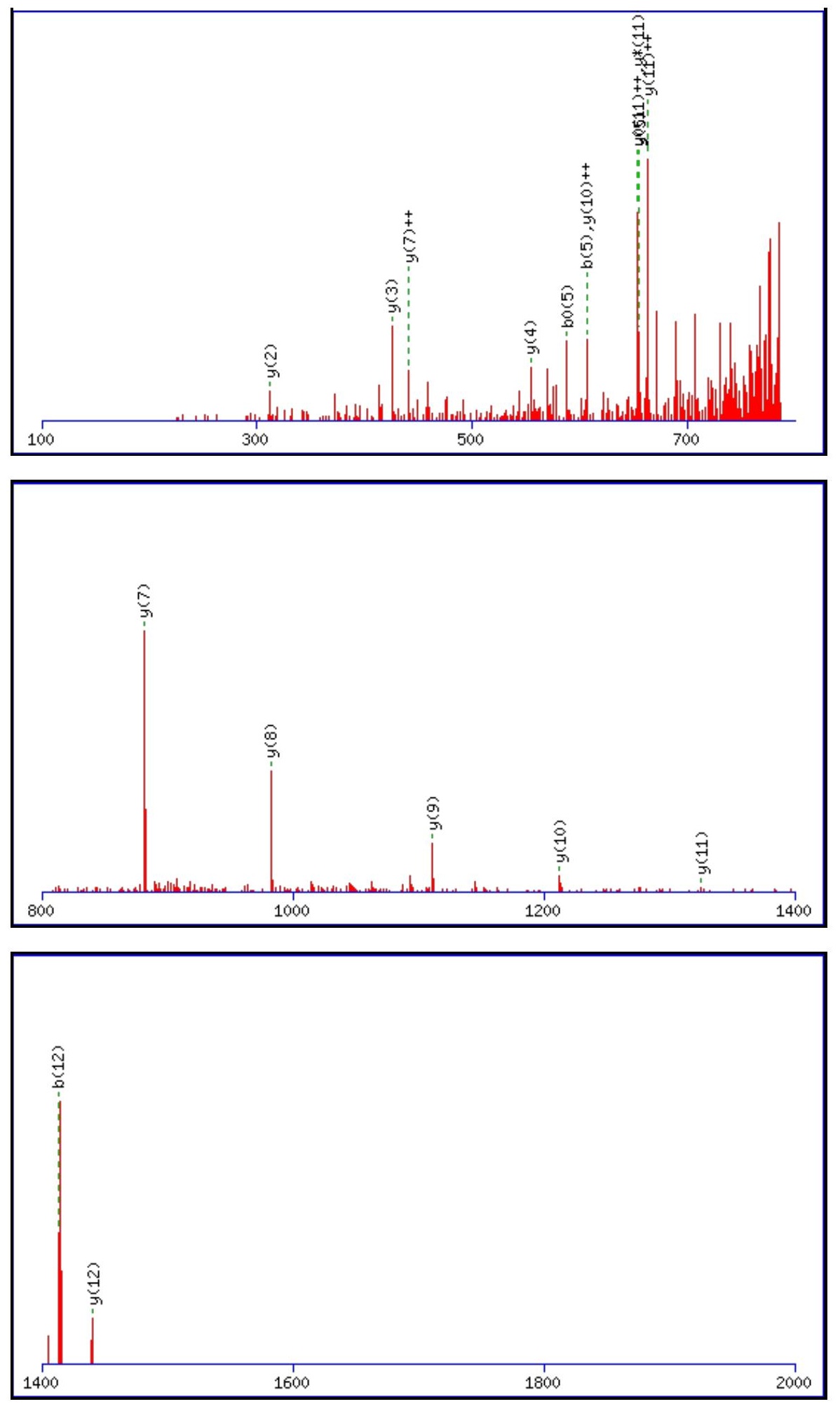

Monoisotopic mass of neutral peptide $\operatorname{Mr}($ calc): 1586.7576

Fixed modifications: Carbamidomethyl (C)

Variable modifications:

N2 : Deamidated $\mathrm{N}(\mathrm{N})$

Ions Score: 75 Expect: $5.2 \mathrm{e}-006$

Matches (Bold Red): 18/130 fragment ions using 24 most intense peaks

\begin{tabular}{|r|c|c|c|c|c|c|c|c|c|c|c|c|c|c|}
\hline$\#$ & $\mathbf{b}$ & $\mathbf{b}^{++}$ & $\mathbf{b}^{*}$ & $\mathbf{b}^{\boldsymbol{*}^{++}}$ & $\mathbf{b}^{\mathbf{0}}$ & $\mathbf{b}^{\mathbf{0}+}$ & Seq. & $\mathbf{y}$ & $\mathbf{y}^{++}$ & $\mathbf{y}^{*}$ & $\mathbf{y}^{\boldsymbol{*}^{++}}$ & $\mathbf{y}^{\mathbf{0}}$ & $\mathbf{y}^{\mathbf{0 + +}}$ & $\#$ \\
\hline $\mathbf{1}$ & 148.0757 & 74.5415 & & & & & $\mathbf{F}$ & & & & & & & $\mathbf{1 3}$ \\
\hline $\mathbf{2}$ & 263.1026 & 132.0550 & 246.0761 & 123.5417 & & & $\mathbf{N}$ & $\mathbf{1 4 4 0 . 6 9 6 5}$ & 720.8519 & 1423.6700 & 712.3386 & 1422.6859 & 711.8466 & $\mathbf{1 2}$ \\
\hline $\mathbf{3}$ & 376.1867 & 188.5970 & 359.1601 & 180.0837 & & & $\mathbf{L}$ & $\mathbf{1 3 2 5 . 6 6 9 6}$ & $\mathbf{6 6 3 . 3 3 8 4}$ & 1308.6430 & $\mathbf{6 5 4 . 8 2 5 1}$ & 1307.6590 & $\mathbf{6 5 4 . 3 3 3 1}$ & $\mathbf{1 1}$ \\
\hline $\mathbf{4}$ & 477.2344 & 239.1208 & 460.2078 & 230.6075 & 459.2238 & 230.1155 & $\mathbf{T}$ & $\mathbf{1 2 1 2 . 5 8 5 5}$ & $\mathbf{6 0 6 . 7 9 6 4}$ & 1195.5590 & 598.2831 & 1194.5749 & 597.7911 & $\mathbf{1 0}$ \\
\hline $\mathbf{5}$ & $\mathbf{6 0 6 . 2 7 7 0}$ & 303.6421 & 589.2504 & 295.1288 & $\mathbf{5 8 8 . 2 6 6 4}$ & 294.6368 & $\mathbf{E}$ & $\mathbf{1 1 1 1 . 5 3 7 8}$ & 556.2726 & 1094.5113 & 547.7593 & 1093.5273 & 547.2673 & $\mathbf{9}$ \\
\hline
\end{tabular}




\begin{tabular}{|c|c|c|c|c|c|c|c|c|c|c|c|c|c|c|}
\hline 6 & 707.3246 & |354.1660| & 690.2981 & |345.6527 & 689.3141 & |345.1607 & $\mathbf{T}$ & 982.4952 & |491.7513 & 965.4687 & |483.2380 & 964.4847 & |482.7460| & 8 \\
\hline 7 & 804.3774 & 402.6923 & 787.3509 & 394.1791 & 786.3668 & 393.6871 & $\mathbf{P}$ & 881.4476 & 441.2274 & 864.4210 & 432.7141 & 863.4370 & 432.2221 & 7 \\
\hline 8 & 933.4200 & 467.2136 & 916.3934 & 458.7004 & 915.4094 & 458.2084 & $\mathbf{E}$ & 784.3948 & 392.7010 & 767.3682 & 384.1878 & 766.3842 & 383.6958 & 6 \\
\hline 9 & 1034.4677 & 517.7375 & 1017.4411 & 509.2242 & 1016.4571 & 508.7322 & $\mathbf{T}$ & 655.3522 & 328.1797 & 638.3257 & 319.6665 & 637.3416 & 319.1745 & 5 \\
\hline 10 & 1163.5103 & 582.2588 & 1146.4837 & 573.7455 & 1145.4997 & 573.2535 & $\mathbf{E}$ & 554.3045 & 277.6559 & 537.2780 & 269.1426 & 536.2940 & 268.6506 & 4 \\
\hline 11 & 1276.5943 & 638.8008 & 1259.5678 & 630.2875 & 1258.5838 & 629.7955 & I & 425.2619 & 213.1346 & 408.2354 & 204.6213 & & & 3 \\
\hline 12 & 1413.6532 & 707.3303 & 1396.6267 & 698.8170 & 1395.6427 & 698.3250 & H & 312.1779 & 156.5926 & 295.1513 & 148.0793 & & & 2 \\
\hline 13 & & & & & & & $\mathbf{R}$ & 175.1190 & 88.0631 & 158.0924 & 79.5498 & & & 1 \\
\hline
\end{tabular}
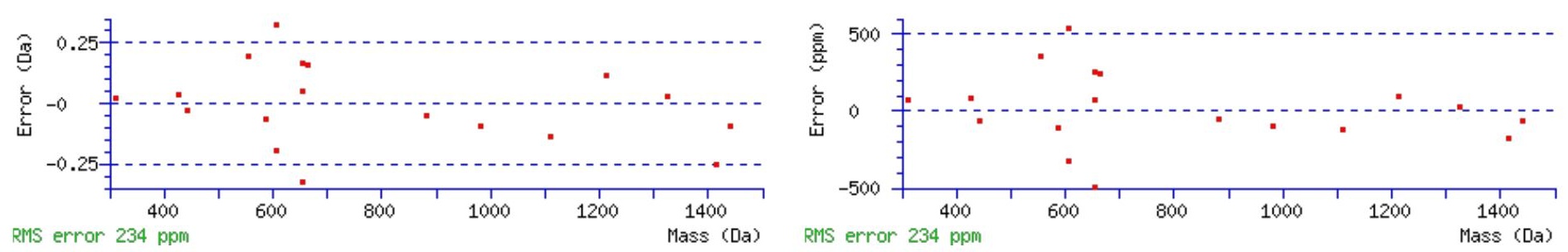

\section{All matches to this query}

\begin{tabular}{|l|l|l|l|}
\hline Score & Mr(calc): & Delta & \multicolumn{1}{c|}{ Sequence } \\
\hline 75.0 & 1586.7576 & -0.0016 & FNLTETPETEIHR \\
\hline 17.4 & 1585.7541 & 1.0019 & SVFPLEQAFINNK \\
\hline 17.3 & 1585.7535 & 1.0025 & MVKVSIGLGEGTGSR \\
\hline 14.9 & 1585.7670 & 0.9890 & YLTVKVKASYVR \\
\hline 11.3 & 1586.7471 & 0.0090 & MDPVERRSEAEPR \\
\hline 11.0 & 1584.7548 & 2.0012 & GKSPAQAELSYLNK \\
\hline 11.0 & 1584.7548 & 2.0012 & GKSPAQAELSYLNK \\
\hline 10.9 & 1585.7388 & 1.0172 & GKSPAQAELSYLNK \\
\hline 10.9 & 1585.7388 & 1.0172 & GKSPAQAELSYLNK \\
\hline 9.0 & 1586.7464 & 0.0096 & DINDNPPVFPTTQK \\
\hline
\end{tabular}

Spectrum No: 303; Query: 728; Rank: 1

\section{Peptide View}

MS/MS Fragmentation of YTGNSSALFILPDK

Found in IPI00210091, Tax_Id=10116 Gene_Symbol=Serpina3m similar to Serine protease inhibitor A3M precursor

Match to Query 728: 1525.768468 from(763.891510,2+)

Title: 091008RatKidney_NoSalt_30.2807.2807.2.dta

Data file K:INewmanPaper|Piliangl3SubProteomes\Piliang3SP\mgf5ppm\ERLIC_3SubProteomes5ppm.mgf 

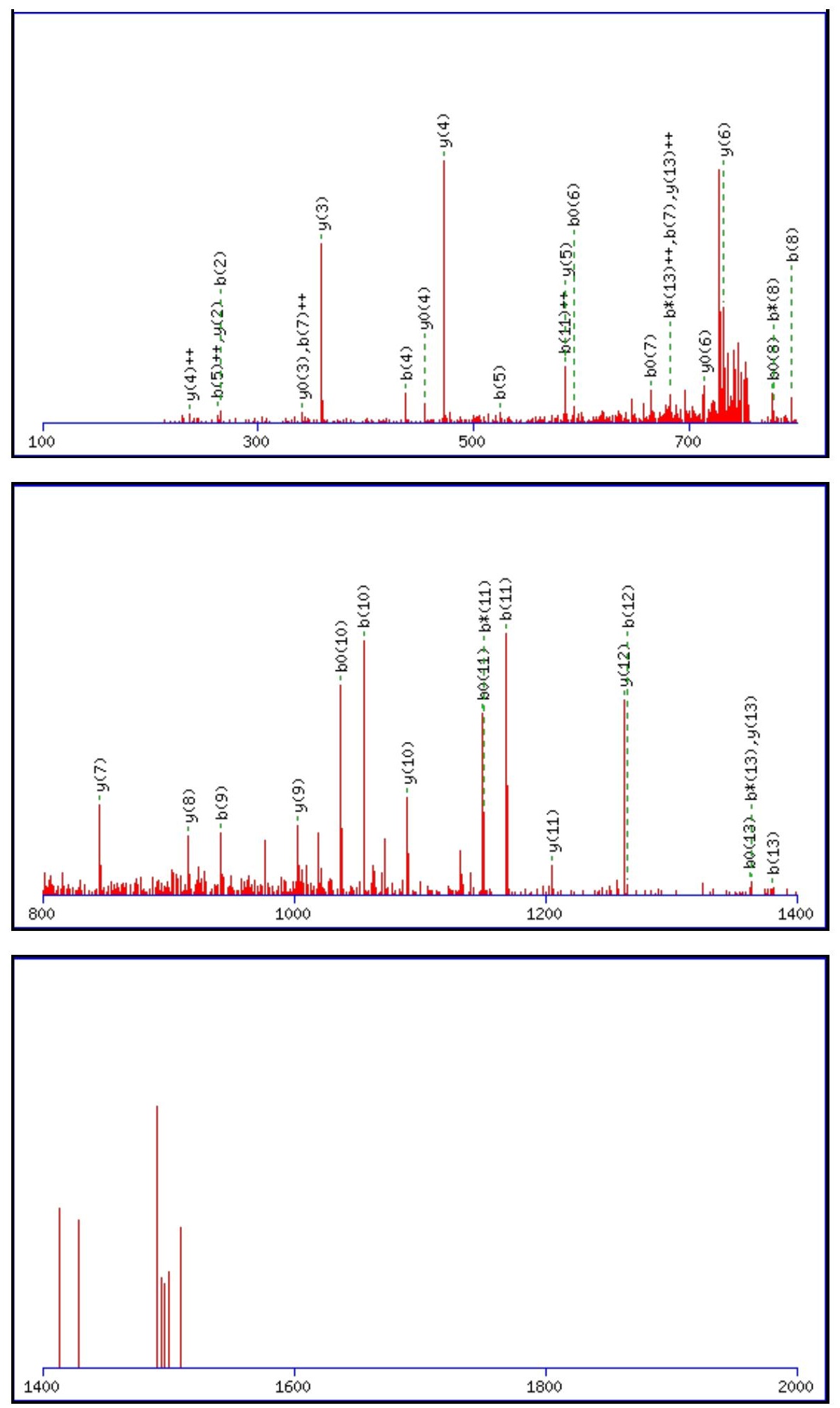

Monoisotopic mass of neutral peptide $\operatorname{Mr}($ calc): 1525.7664

Fixed modifications: Carbamidomethyl (C)

Variable modifications:

N4 : Deamidated_N (N)

Ions Score: 75 Expect: $4.6 \mathrm{e}-006$

Matches (Bold Red): 4๑/146 fragment ions using 78 most intense peaks

\begin{tabular}{|r|c|c|c|c|c|c|c|c|c|c|c|c|c|c|}
\hline$\#$ & $\mathbf{b}$ & $\mathbf{b}^{++}$ & $\mathbf{b}^{*}$ & $\mathbf{b}^{\boldsymbol{*}^{++}}$ & $\mathbf{b}^{\mathbf{0}}$ & $\mathbf{b}^{\mathbf{0 + +}}$ & $\mathbf{S e q}$ & $\mathbf{y}$ & $\mathbf{y}^{++}$ & $\mathbf{y}^{\mathbf{*}}$ & $\mathbf{y}^{\boldsymbol{*}^{++}}$ & $\mathbf{y}^{\mathbf{0}}$ & $\mathbf{y}^{\mathbf{0 + +}}$ & $\#$ \\
\hline $\mathbf{1}$ & 164.0706 & 82.5389 & & & & & $\mathbf{Y}$ & & & & & & & $\mathbf{1 4}$ \\
\hline $\mathbf{2}$ & $\mathbf{2 6 5 . 1 1 8 3}$ & 133.0628 & & & 247.1077 & 124.0575 & $\mathbf{T}$ & $\mathbf{1 3 6 3 . 7 1 0 4}$ & $\mathbf{6 8 2 . 3 5 8 8}$ & 1346.6838 & 673.8455 & 1345.6998 & 673.3535 & $\mathbf{1 3}$ \\
\hline $\mathbf{3}$ & 322.1397 & 161.5735 & & & 304.1292 & 152.5682 & $\mathbf{G}$ & $\mathbf{1 2 6 2 . 6 6 2 7}$ & 631.8350 & 1245.6361 & 623.3217 & 1244.6521 & 622.8297 & $\mathbf{1 2}$ \\
\hline $\mathbf{4}$ & $\mathbf{4 3 7 . 1 6 6 7}$ & 219.0870 & 420.1401 & 210.5737 & 419.1561 & 210.0817 & $\mathbf{N}$ & $\mathbf{1 2 0 5 . 6 4 1 2}$ & 603.3242 & 1188.6147 & 594.8110 & 1187.6307 & 594.3190 & $\mathbf{1 1}$ \\
\hline $\mathbf{5}$ & $\mathbf{5 2 4 . 1 9 8 7}$ & $\mathbf{2 6 2 . 6 0 3 0}$ & 507.1722 & 254.0897 & 506.1881 & 253.5977 & $\mathbf{S}$ & $\mathbf{1 0 9 0 . 6 1 4 3}$ & 545.8108 & 1073.5877 & 537.2975 & 1072.6037 & 536.8055 & $\mathbf{1 0}$ \\
\hline
\end{tabular}




\begin{tabular}{|c|c|c|c|c|c|c|c|c|c|c|c|c|c|c|}
\hline 6 & 611.2307 & 306.1190 & 594.2042 & |297.6057| & 593.2202 & |297.1137| & S & 1003.5823 & |502.2948| & 986.5557| & 493.7815 & 985.5717 & 493.2895| & 9 \\
\hline 7 & 682.2679 & 341.6376 & 665.2413 & 333.1243 & 664.2573 & 332.6323 & A & 916.5502 & 458.7788 & 899.5237 & 450.2655 & 898.5397 & 449.7735 & 8 \\
\hline 8 & 795.3519 & 398.1796 & 778.3254 & 389.6663 & 777.3414 & 389.1743 & $\mathbf{L}$ & 845.5131 & 423.2602 & 828.4866 & 414.7469 & 827.5026 & 414.2549 & 7 \\
\hline 9 & 942.4203 & 471.7138 & 925.3938 & 463.2005 & 924.4098 & 462.7085 & $\mathbf{F}$ & 732.4291 & 366.7182 & 715.4025 & 358.2049 & 714.4185 & 357.7129 & 6 \\
\hline 10 & 1055.5044 & 528.2558 & 1038.4778 & 519.7426 & 1037.4938 & 519.2506 & I & 585.3606 & 293.1840 & 568.3341 & 284.6707 & 567.3501 & 284.1787 & 5 \\
\hline 11 & 1168.5885 & 584.7979 & 1151.5619 & 576.2846 & 1150.5779 & 575.7926 & $\mathbf{L}$ & 472.2766 & 236.6419 & 455.2500 & 228.1287 & 454.2660 & 227.6366 & 4 \\
\hline 12 & 1265.6412 & 633.3243 & 1248.6147 & 624.8110 & 1247.6307 & 624.3190 & $\mathbf{P}$ & 359.1925 & 180.0999 & 342.1660 & 171.5866 & 341.1819 & 171.0946 & 3 \\
\hline 13 & 1380.6682 & 690.8377 & 1363.6416 & 682.3244 & 1362.6576 & 681.8324 & D & 262.1397 & 131.5735 & 245.1132 & 123.0602 & 244.1292 & 122.5682 & 2 \\
\hline 14 & & & & & & & $\mathbf{K}$ & 147.1128 & 74.0600 & 130.0863 & 65.5468 & & & 1 \\
\hline
\end{tabular}
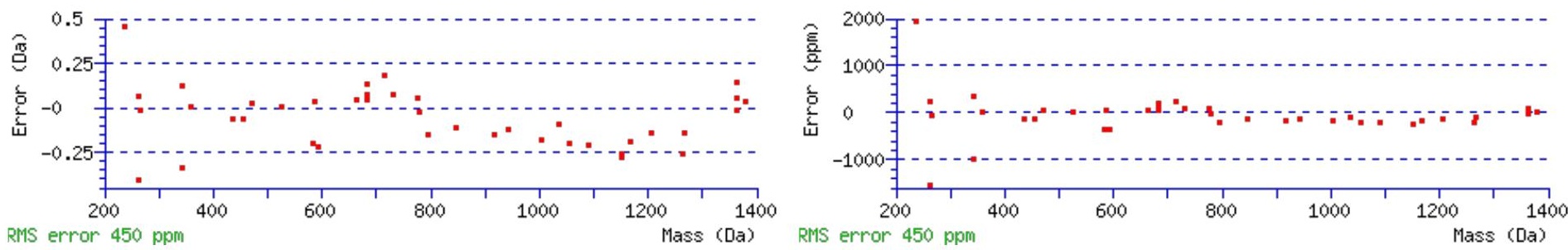

\section{All matches to this query}

\begin{tabular}{|l|l|l|l|}
\hline Score & Mr(calc): & Delta & \multicolumn{1}{|c|}{ Sequence } \\
\hline 75.0 & 1525.7664 & 0.0021 & YTGNSSALFILPDK \\
\hline 9.7 & 1525.7694 & -0.0009 & KYNWTPALIALR \\
\hline 7.7 & 1525.7694 & -0.0009 & KYNWTPALIALR \\
\hline 7.7 & 1525.7687 & -0.0003 & TKELIGSMKINGR \\
\hline 5.6 & 1523.7596 & 2.0089 & NNELIAIGKGSSLK \\
\hline 5.6 & 1523.7596 & 2.0089 & NNELIAIGKGSSLK \\
\hline 5.6 & 1523.7596 & 2.0089 & NNELIAIGKGSSLK \\
\hline 5.6 & 1523.7596 & 2.0089 & NNELIAIGKGSSLK \\
\hline 3.8 & 1524.7532 & 1.0153 & AATNLENQLHSAQK \\
\hline 3.4 & 1525.7810 & -0.0126 & SSVLVTHAELMPDK \\
\hline
\end{tabular}

Spectrum No: 304; Query: 2003; Rank: 1

\section{Peptide View}

MS/MS Fragmentation of NVIIWGNHSSTQYPDVNHAK

Found in IPI00198717, Tax_Id=10116 Gene_Symbol=Mdh1 Malate dehydrogenase, cytoplasmic

Match to Query 2003: 2280.099848 from(1141.057200,2+)

Title: 091008RatKidney_NoSalt_06.1125.1125.2.dta

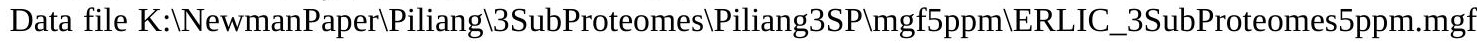



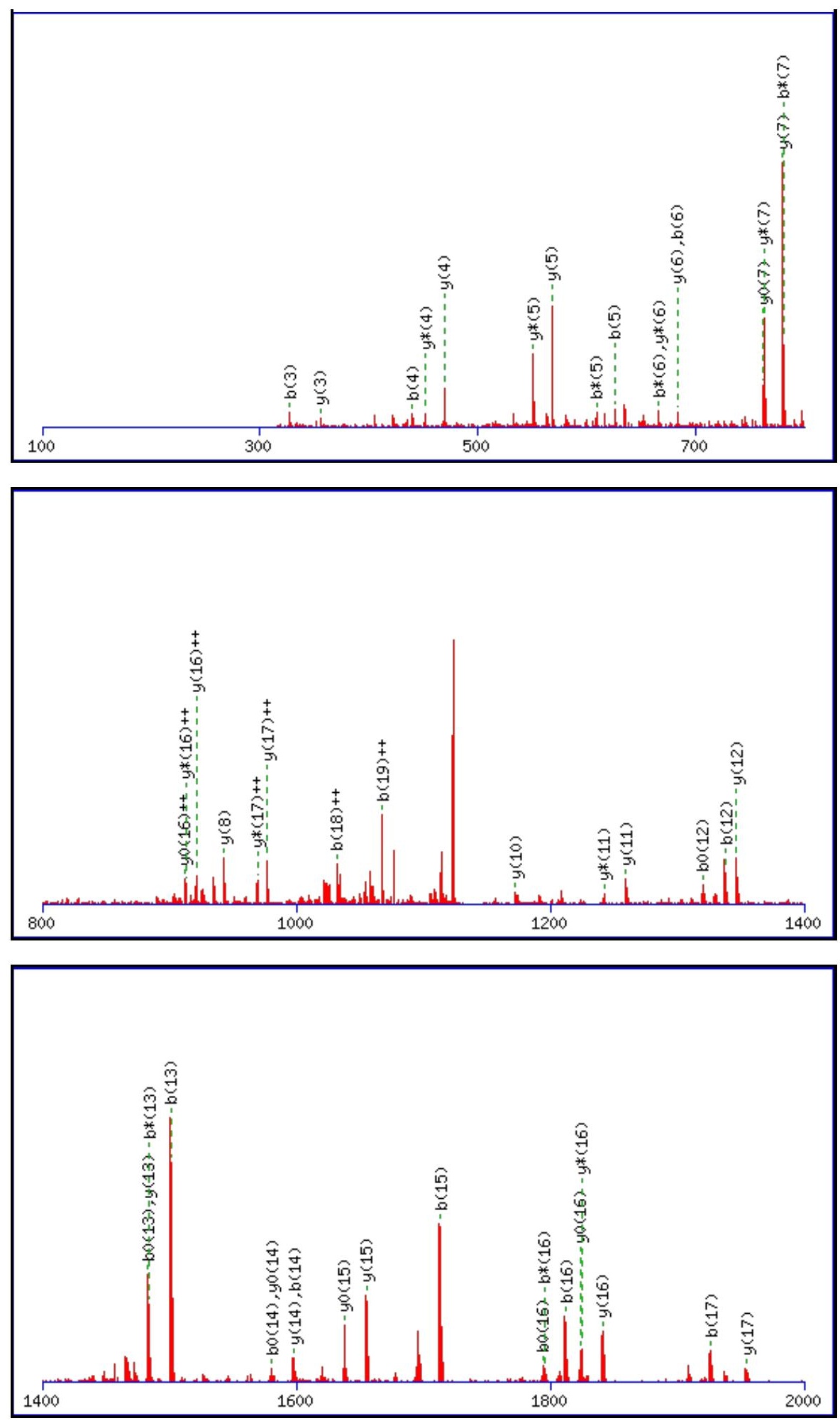

Monoisotopic mass of neutral peptide $\operatorname{Mr}($ calc): 2280.0923

Fixed modifications: Carbamidomethyl (C)

Variable modifications:

N7 : Deamidated $\mathrm{N}(\mathrm{N})$

Ions Score: 75 Expect: $8.8 \mathrm{e}-006$

Matches (Bold Red): 50/202 fragment ions using 93 most intense peaks

\begin{tabular}{|r|c|r|r|r|r|r|r|r|r|r|r|r|r|r|}
\hline$\#$ & $\mathbf{b}$ & \multicolumn{1}{|c|}{$\mathbf{b}^{++}$} & $\mathbf{b}^{*}$ & $\mathbf{b}^{\boldsymbol{*}^{++}}$ & $\mathbf{b}^{\mathbf{0}}$ & $\mathbf{b}^{\mathbf{0 + +}}$ & $\mathbf{S e q}$ & $\mathbf{y}$ & $\mathbf{y}^{++}$ & $\mathbf{y}^{\mathbf{*}}$ & $\mathbf{y}^{\mathbf{*}^{++}}$ & $\mathbf{y}^{\mathbf{0}}$ & $\mathbf{y}^{\mathbf{0 + +}}$ & $\#$ \\
\hline $\mathbf{1}$ & 115.0502 & 58.0287 & 98.0237 & 49.5155 & & & $\mathbf{N}$ & & & & & & & $\mathbf{2 0}$ \\
\hline $\mathbf{2}$ & 214.1186 & 107.5629 & 197.0921 & 99.0497 & & & $\mathbf{V}$ & 2167.0567 & 1084.0320 & 2150.0301 & 1075.5187 & 2149.0461 & 1075.0267 & $\mathbf{1 9}$ \\
\hline $\mathbf{3}$ & $\mathbf{3 2 7 . 2 0 2 7}$ & 164.1050 & 310.1761 & 155.5917 & & & $\mathbf{I}$ & 2067.9883 & 1034.4978 & 2050.9617 & 1025.9845 & 2049.9777 & 1025.4925 & $\mathbf{1 8}$ \\
\hline $\mathbf{4}$ & $\mathbf{4 4 0 . 2 8 6 7}$ & 220.6470 & 423.2602 & 212.1337 & & & $\mathbf{I}$ & $\mathbf{1 9 5 4 . 9 0 4 2}$ & $\mathbf{9 7 7 7 . 9 5 5 7}$ & 1937.8777 & $\mathbf{9 6 9 . 4 4 2 5}$ & 1936.8936 & 968.9505 & $\mathbf{1 7}$ \\
\hline $\mathbf{5}$ & $\mathbf{6 2 6 . 3 6 6 1}$ & 313.6867 & $\mathbf{6 0 9 . 3 3 9 5}$ & 305.1734 & & & $\mathbf{W}$ & $\mathbf{1 8 4 1 . 8 2 0 1}$ & $\mathbf{9 2 1 . 4 1 3 7}$ & $\mathbf{1 8 2 4 . 7 9 3 6}$ & $\mathbf{9 1 2 . 9 0 0 4}$ & $\mathbf{1 8 2 3 . 8 0 9 6}$ & $\mathbf{9 1 2 . 4 0 8 4}$ & $\mathbf{1 6}$ \\
\hline
\end{tabular}




\begin{tabular}{|c|c|c|c|c|c|c|c|c|c|c|c|c|c|c|}
\hline 6 & 683.3875 & 342.1974 & 666.3610 & 333.6841 & & & $\mathbf{G}$ & |1655.7408 & 828.3741 & |1638.7143 & 819.8608 & |1637.7303| & 819.3688 & 15 \\
\hline 7 & 798.4145 & 399.7109 & 781.3879 & 391.1976 & & & $\mathbf{N}$ & 1598.7194 & 799.8633 & 1581.6928 & 791.3500 & 1580.7088 & 790.8580 & 14 \\
\hline 8 & 935.4734 & 468.2403 & 918.4468 & 459.7270 & & & $\mathbf{H}$ & 1483.6924 & 742.3499 & 1466.6659 & 733.8366 & 1465.6819 & 733.3446 & 13 \\
\hline 9 & 1022.5054 & 511.7563 & 1005.4788 & 503.2431 & 1004.4948 & 502.7511 & S & 1346.6335 & 673.8204 & 1329.6070 & 665.3071 & 1328.6230 & 664.8151 & 12 \\
\hline 10 & 1109.5374 & 555.2724 & 1092.5109 & 546.7591 & 1091.5269 & 546.2671 & S & 1259.6015 & 630.3044 & 1242.5749 & 621.7911 & 1241.5909 & 621.2991 & 11 \\
\hline 11 & 1210.5851 & 605.7962 & 1193.5586 & 597.2829 & 1192.5745 & 596.7909 & $\mathbf{T}$ & 1172.5695 & 586.7884 & 1155.5429 & 578.2751 & 1154.5589 & 577.7831 & 10 \\
\hline 12 & 1338.6437 & 669.8255 & 1321.6171 & 661.3122 & 1320.6331 & 660.8202 & $\mathbf{Q}$ & 1071.5218 & 536.2645 & 1054.4952 & 527.7513 & 1053.5112 & 527.2592 & 9 \\
\hline 13 & 1501.7070 & 751.3571 & 1484.6805 & 742.8439 & 1483.6964 & 742.3519 & $\mathbf{Y}$ & 943.4632 & 472.2352 & 926.4367 & 463.7220 & 925.4526 & 463.2300 & 8 \\
\hline 14 & 1598.7598 & 799.8835 & 1581.7332 & 791.3703 & 1580.7492 & 790.8782 & $\mathbf{P}$ & 780.3999 & 390.7036 & 763.3733 & 382.1903 & 762.3893 & 381.6983 & 7 \\
\hline 15 & 1713.7867 & 857.3970 & 1696.7602 & 848.8837 & 1695.7762 & 848.3917 & D & 683.3471 & 342.1772 & 666.3206 & 333.6639 & 665.3365 & 333.1719 & 6 \\
\hline 16 & 1812.8551 & 906.9312 & 1795.8286 & 898.4179 & 1794.8446 & 897.9259 & V & 568.3202 & 284.6637 & 551.2936 & 276.1504 & & & 5 \\
\hline 17 & 1926.8981 & 963.9527 & 1909.8715 & 955.4394 & 1908.8875 & 954.9474 & $\mathbf{N}$ & 469.2518 & 235.1295 & 452.2252 & 226.6162 & & & 4 \\
\hline 18 & 2063.9570 & 1032.4821 & 2046.9304 & 1023.9688 & 2045.9464 & 1023.4768 & $\mathbf{H}$ & 355.2088 & 178.1081 & 338.1823 & 169.5948 & & & 3 \\
\hline 19 & 2134.9941 & 1068.0007 & 2117.9675 & 1059.4874 & 2116.9835 & 1058.9954 & A & 218.1499 & 109.5786 & 201.1234 & 101.0653 & & & 2 \\
\hline
\end{tabular}
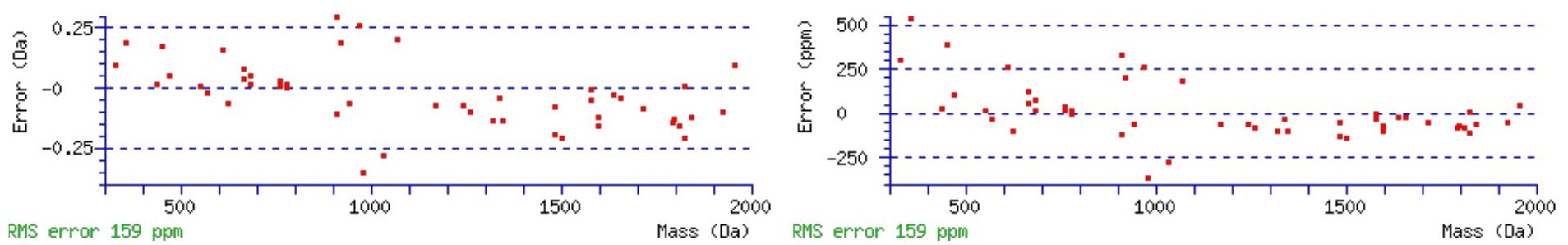

\section{All matches to this query}

\begin{tabular}{|l|l|l|l|}
\hline Score & Mr(calc): & Delta & \multicolumn{1}{|c|}{ Sequence } \\
\hline 74.9 & 2280.0923 & 0.0075 & NVIIWGNHSSTQYPDVNHAK \\
\hline 68.8 & 2280.0923 & 0.0075 & $\underline{\text { NVIIWGNHSSTQYPDVNHAK }}$ \\
\hline 67.8 & 2279.1083 & 0.9915 & NVIIWGNHSSTQYPDVNHAK \\
\hline 62.2 & 2280.0923 & 0.0075 & NVIIWGNHSSTQYPDVNHAK \\
\hline 4.2 & 2280.0916 & 0.0083 & KLLYERSASLAATEAQLTR \\
\hline 2.6 & 2280.1134 & -0.0136 & NELVESRREAPTQSDVFFR \\
\hline 2.5 & 2280.1102 & -0.0104 & TMANGILKVPAIHVNNSITK \\
\hline 2.1 & 2280.1031 & -0.0032 & GVASGLCVLVSWLTAFVLTK \\
\hline 0.1 & 2280.1030 & -0.0032 & ASKYLHVSLMEPPVVLPLQ \\
\hline 0.1 & 2280.1030 & -0.0032 & ASKYLHVSLMEPPVVLPLQ \\
\hline
\end{tabular}

Spectrum No: 305; Query: 475; Rank: 1

\section{Peptide View}

MS/MS Fragmentation of NMTLFSDLVAEK

Found in IPI00372839, Tax_Id=10116 Gene_Symbol=Col6a2 similar to procollagen, type VI, alpha 2

Match to Query 475: 1383.657148 from(692.835850,2+)

Title: 091008RatKidney_NH4Format01_26.3262.3262.2.dta

Data file K:\NewmanPaper \Piliang \3SubProteomes\Piliang3SP \mgf5ppm\ERLIC_3SubProteomes5ppm.mgf 

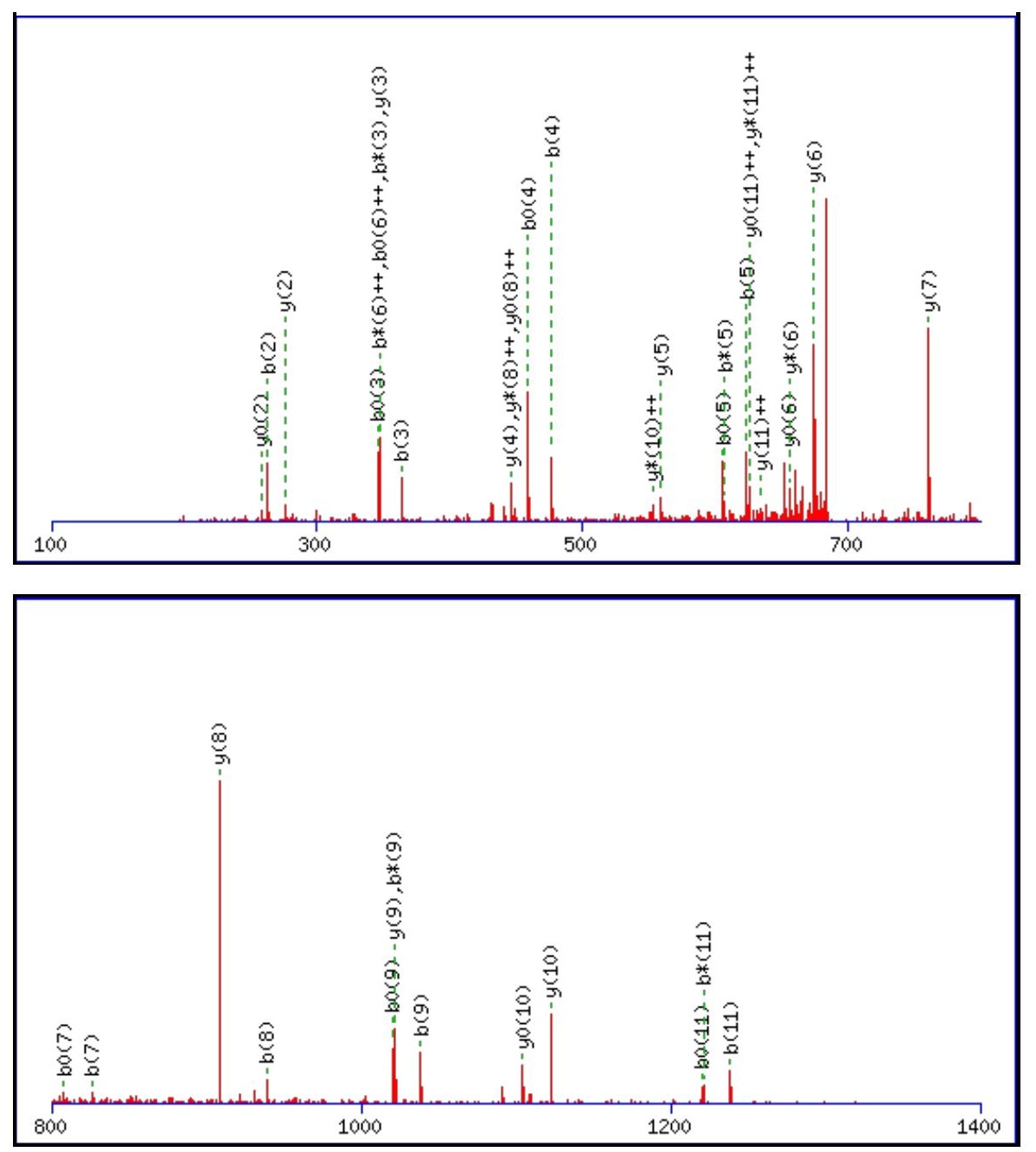

\begin{tabular}{rrrr}
\hline 00 & 1600 & 1800 & 2000 \\
\hline
\end{tabular}

Monoisotopic mass of neutral peptide $\operatorname{Mr}($ calc): 1383.6592

Fixed modifications: Carbamidomethyl (C)

Variable modifications:

N1 : Deamidated_N (N)

M2 : Oxidation (M)

Ions Score: 75 Expect : $5.8 \mathrm{e}-006$

Matches (Bold Red): 39/126 fragment ions using 57 most intense peaks

\begin{tabular}{|r|c|c|c|c|c|c|c|c|c|c|c|c|c|c|}
\hline$\#$ & $\mathbf{b}$ & $\mathbf{b}^{++}$ & $\mathbf{b}^{*}$ & $\mathbf{b}^{\boldsymbol{*}^{++}}$ & $\mathbf{b}^{\mathbf{0}}$ & $\mathbf{b}^{\mathbf{0}+}$ & $\mathbf{S e q}$ & $\mathbf{y}$ & $\mathbf{y}^{++}$ & $\mathbf{y}^{\mathbf{*}}$ & $\mathbf{y}^{\mathbf{*}^{++}}$ & $\mathbf{y}^{\mathbf{0}}$ & $\mathbf{y}^{\mathbf{0}++}$ & $\#$ \\
\hline $\mathbf{1}$ & 116.0342 & 58.5207 & 99.0077 & 50.0075 & & & $\mathbf{N}$ & & & & & & & $\mathbf{1 2}$ \\
\hline $\mathbf{2}$ & $\mathbf{2 6 3 . 0 6 9 6}$ & 132.0384 & 246.0431 & 123.5252 & & & $\mathbf{M}$ & 1269.6395 & $\mathbf{6 3 5 . 3 2 3 4}$ & 1252.6130 & $\mathbf{6 2 6 . 8 1 0 1}$ & 1251.6290 & $\mathbf{6 2 6 . 3 1 8 1}$ & $\mathbf{1 1}$ \\
\hline $\mathbf{3}$ & $\mathbf{3 6 4 . 1 1 7 3}$ & 182.5623 & 347.0907 & 174.0490 & 346.1067 & 173.5570 & $\mathbf{T}$ & $\mathbf{1 1 2 2 . 6 0 4 1}$ & 561.8057 & 1105.5776 & 553.2924 & $\mathbf{1 1 0 4 . 5 9 3 6}$ & 552.8004 & $\mathbf{1 0}$ \\
\hline $\mathbf{4}$ & $\mathbf{4 7 7 . 2 0 1 4}$ & 239.1043 & 460.1748 & 230.5910 & $\mathbf{4 5 9 . 1 9 0 8}$ & 230.0990 & $\mathbf{L}$ & $\mathbf{1 0 2 1 . 5 5 6 4}$ & 511.2819 & 1004.5299 & 502.7686 & 1003.5459 & 502.2766 & $\mathbf{9}$ \\
\hline & & & & & & & & & & & & & &
\end{tabular}




\begin{tabular}{|c|c|c|c|c|c|c|c|c|c|c|c|c|c|c|}
\hline 5 & 624.2698 & |312.6385 & 607.2432 & 304.1253 & 606.2592 & |303.6332 & $\mathbf{F}$ & 908.4724 & |454.7398 & 891.4458 & |446.2266 & 890.4618 & 345 & 8 \\
\hline 6 & 711.3018 & 356.1545 & 694.2753 & 347.6413 & 693.2912 & 347.1493 & S & 761.4040 & 381.2056 & 744.3774 & 372.6923 & 743.3934 & 372.2003 & 7 \\
\hline 7 & 826.3287 & 413.6680 & 809.3022 & 405.1547 & 808.3182 & 404.6627 & D & 674.3719 & 337.6896 & 657.3454 & 329.1763 & 656.3614 & 328.6843 & 6 \\
\hline 8 & 939.4128 & 470.2100 & 922.3863 & 461.6968 & 921.4022 & 461.2048 & $\mathbf{L}$ & 559.3450 & 280.1761 & 542.3184 & 271.6629 & 541.3344 & 271.1709 & 5 \\
\hline 9 & 1038.4812 & 519.7442 & 1021.4547 & 511.2310 & 1020.4707 & 510.7390 & V & 446.2609 & 223.6341 & 429.2344 & 215.1208 & 428.2504 & 214.6288 & 4 \\
\hline 10 & 1109.5183 & 555.2628 & 1092.4918 & 546.7495 & 1091.5078 & 546.2575 & A & 347.1925 & 174.0999 & 330.1660 & 165.5866 & 329.1819 & 165.0946 & 3 \\
\hline 11 & 1238.5609 & 619.7841 & 1221.5344 & 611.2708 & 1220.5504 & 610.7788 & $\mathbf{E}$ & 276.1554 & 138.5813 & 259.1288 & 130.0681 & 258.1448 & 129.5761 & 2 \\
\hline 12 & & & & & & & K & 147.1128 & 74.0600 & 130.0863 & 65.5468 & & & 1 \\
\hline
\end{tabular}
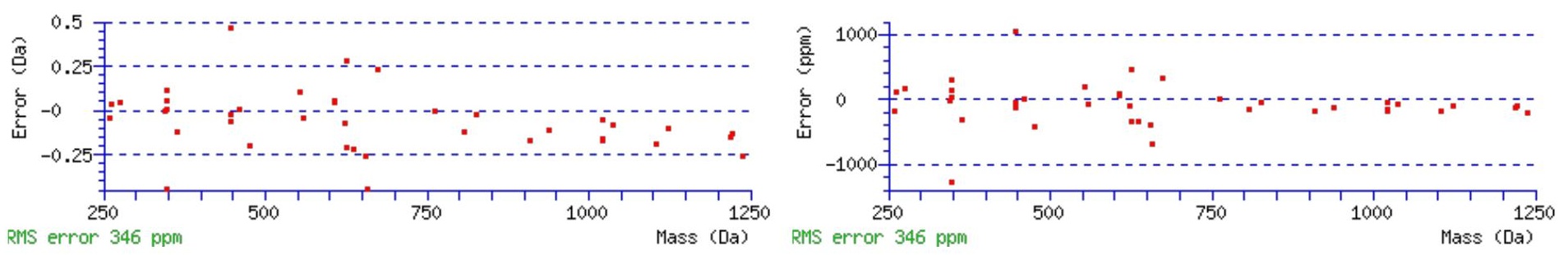

\section{All matches to this query}

\begin{tabular}{|l|l|l|l|}
\hline Score & Mr(calc): & Delta & \multicolumn{1}{c|}{ Sequence } \\
\hline 74.9 & 1383.6592 & -0.0020 & NMTLFSDLVAEK \\
\hline 20.7 & 1383.6646 & -0.0075 & SAANKSVSIVAEK \\
\hline 14.8 & 1383.6646 & -0.0075 & SAANKSVSIVAEK \\
\hline 14.8 & 1383.6646 & -0.0075 & SAANKSVSIVAEK \\
\hline 10.7 & 1383.6435 & 0.0136 & SFEGLSKPSPQK \\
\hline 8.1 & 1383.6646 & -0.0075 & KELQSNKESIK \\
\hline 7.9 & 1382.6574 & 0.9997 & $\underline{\text { IMNTFSVMPSPK }}$ \\
\hline 6.4 & 1383.6639 & -0.0067 & VYRGCSCILEK \\
\hline 6.1 & 1382.6534 & 1.0038 & EMAKMVADTISR \\
\hline 5.7 & 1383.6630 & -0.0059 & $\underline{\text { ANTKGHTDIDSPK }}$ \\
\hline
\end{tabular}

Spectrum No: 306; Query: 559; Rank: 1

\section{Peptide View}

MS/MS Fragmentation of DITNLINNTFIR

Found in IPI00373408, Tax_Id=10116 Gene_Symbol=Sort1 Sortilin precursor

Match to Query 559: 1433.751608 from(717.883080,2+)

Title: 091008RatKidney_NoSalt_27.4439.4439.2.dta

Data file K:INewmanPaper|Piliangl3SubProteomes\Piliang3SP\mgf5ppm\ERLIC_3SubProteomes5ppm.mgf 

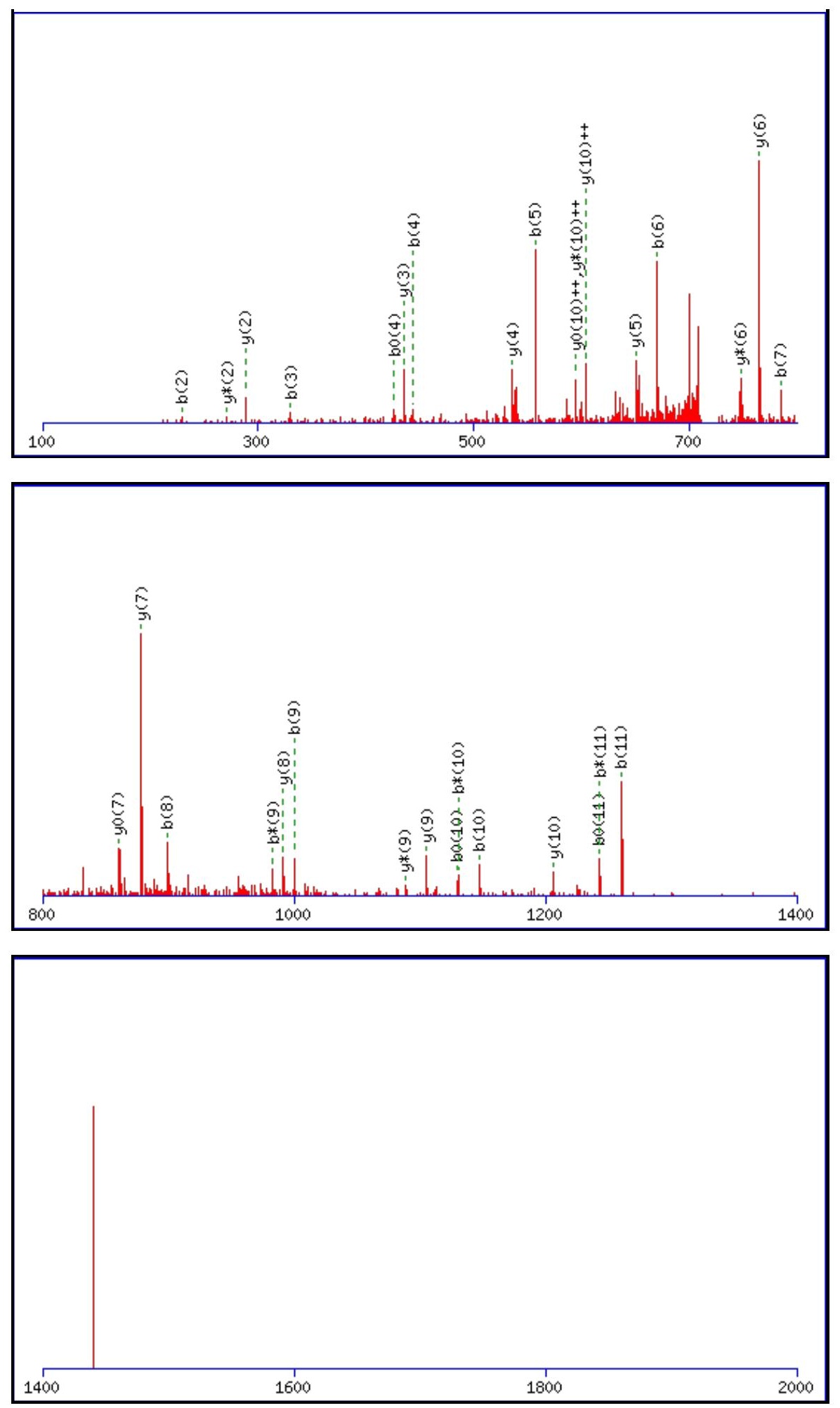

Monoisotopic mass of neutral peptide $\operatorname{Mr}($ calc): 1433.7514

Fixed modifications: Carbamidomethyl (C)

Variable modifications:

N7 : Deamidated $\mathrm{N}(\mathrm{N})$

Ions Score: 75 Expect: $4.1 \mathrm{e}-006$

Matches (Bold Red): 32/120 fragment ions using 60 most intense peaks

\begin{tabular}{|r|c|c|c|c|r|r|r|r|r|r|r|r|r|r|}
\hline$\#$ & $\mathbf{b}$ & $\mathbf{b}^{++}$ & $\mathbf{b}^{*}$ & $\mathbf{b}^{\boldsymbol{*}^{++}}$ & $\mathbf{b}^{\mathbf{0}}$ & \multicolumn{1}{|c|}{$\mathbf{b}^{\mathbf{0 + +}}$} & Seq. & $\mathbf{y}$ & $\mathbf{y}^{++}$ & $\mathbf{y}^{\mathbf{*}}$ & $\mathbf{y}^{\boldsymbol{*}^{++}}$ & $\mathbf{y}^{\mathbf{0}}$ & $\mathbf{y}^{\mathbf{0 + +}}$ & $\#$ \\
\hline $\mathbf{1}$ & 116.0342 & 58.5207 & & & 98.0237 & 49.5155 & $\mathbf{D}$ & & & & & & & $\mathbf{1 2}$ \\
\hline $\mathbf{2}$ & $\mathbf{2 2 9 . 1 1 8 3}$ & 115.0628 & & & 211.1077 & 106.0575 & $\mathbf{I}$ & 1319.7318 & 660.3695 & 1302.7052 & 651.8562 & 1301.7212 & 651.3642 & $\mathbf{1 1}$ \\
\hline $\mathbf{3}$ & $\mathbf{3 3 0 . 1 6 6 0}$ & 165.5866 & & & 312.1554 & 156.5813 & $\mathbf{T}$ & $\mathbf{1 2 0 6 . 6 4 7 7}$ & $\mathbf{6 0 3 . 8 2 7 5}$ & 1189.6212 & $\mathbf{5 9 5 . 3 1 4 2}$ & 1188.6371 & $\mathbf{5 9 4 . 8 2 2 2}$ & $\mathbf{1 0}$ \\
\hline $\mathbf{4}$ & $\mathbf{4 4 4 . 2 0 8 9}$ & 222.6081 & 427.1823 & 214.0948 & $\mathbf{4 2 6 . 1 9 8 3}$ & 213.6028 & $\mathbf{N}$ & $\mathbf{1 1 0 5 . 6 0 0 0}$ & 553.3037 & $\mathbf{1 0 8 8 . 5 7 3 5}$ & 544.7904 & 1087.5895 & 544.2984 & $\mathbf{9}$ \\
\hline $\mathbf{5}$ & $\mathbf{5 5 7 . 2 9 3 0}$ & 279.1501 & 540.2664 & 270.6368 & 539.2824 & 270.1448 & $\mathbf{L}$ & $\mathbf{9 9 1 . 5 5 7 1}$ & 496.2822 & 974.5306 & 487.7689 & 973.5465 & 487.2769 & $\mathbf{8}$ \\
\hline
\end{tabular}




\begin{tabular}{|c|c|c|c|c|c|c|c|c|c|c|c|c|c|c|}
\hline 6 & 670.3770 & |335.6921 & 653.3505 & 327.1789 & 652.3665 & |326.6869 | & I & 878.4730 & |439.7402 & 861.4465 & 431.2269 & 860.4625 & |430.7349 & 7 \\
\hline 7 & 785.4040 & 393.2056 & 768.3774 & 384.6923 & 767.3934 & 384.2003 & $\mathbf{N}$ & 765.3890 & |383.1981 & 748.3624 & 374.6848 & 747.3784 & |374.1928 & 6 \\
\hline 8 & 899.4469 & 450.2271 & 882.4203 & 441.7138 & 881.4363 & |441.2218 & $\mathbf{N}$ & 650.3620 & 325.6847 & 633.3355 & 317.1714 & 632.3515 & 316.6794 & 5 \\
\hline 9 & 1000.4946 & 500.7509 & 983.4680 & 492.2376 & 982.4840 & 491.7456 & $\mathbf{T}$ & 536.3191 & 268.6632 & 519.2926 & 260.1499 & 518.3085 & |259.6579 & 4 \\
\hline 10 & 1147.5630 & 574.2851 & 1130.5364 & 565.7719 & 1129.5524 & 565.2798 & $\mathbf{F}$ & 435.2714 & 218.1394 & 418.2449 & 209.6261 & & & 3 \\
\hline 11 & 1260.6470 & 630.8272 & 1243.6205 & 622.3139 & 1242.6365 & 621.8219 & I & 288.2030 & 144.6051 & 271.1765 & 136.0919 & & & 2 \\
\hline 12 & & & & & & & $\mathbf{R}$ & 175.1190 & 88.0631 & 158.0924 & 79.5498 & & & 1 \\
\hline
\end{tabular}
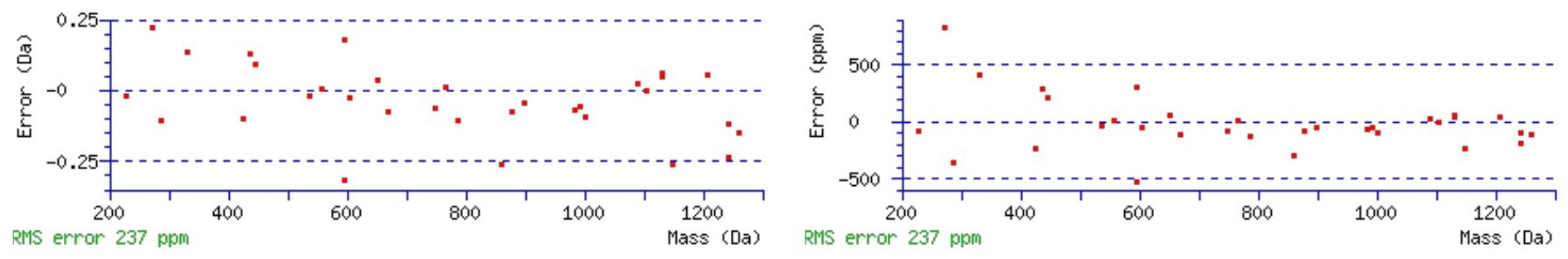

\section{All matches to this query}

\begin{tabular}{|l|l|l|l|}
\hline Score & Mr(calc): & Delta & \multicolumn{1}{|c|}{ Sequence } \\
\hline 74.7 & 1433.7514 & 0.0002 & DITNLINNTFIR \\
\hline 72.4 & 1433.7514 & 0.0002 & DITNLINNTFIR \\
\hline 33.1 & 1433.7514 & 0.0002 & DITNLINNTFIR \\
\hline 14.2 & 1433.7626 & -0.0110 & GEKELLFNKNSR \\
\hline 12.8 & 1433.7626 & -0.0110 & DLFIDGRSKNIR \\
\hline 7.9 & 1433.7627 & -0.0111 & SISDVASPIRSFR \\
\hline 6.2 & 1433.7402 & 0.0114 & LKDLSLNDPQYK \\
\hline 5.8 & 1433.7392 & 0.0124 & 1 TIAPDGGVSRLR \\
\hline 4.5 & 1432.7609 & 0.9907 & RGMNIPQPPIGPR \\
\hline 4.0 & 1431.7470 & 2.0046 & GDDGIPGPPGPKGIR \\
\hline
\end{tabular}

Spectrum No: 307; Query: 780; Rank: 1

\section{Peptide View}

MS/MS Fragmentation of DGNCTSPQALCNAR

Found in IPI00205325, Tax_Id=10116 Gene_Symbol=Lrp2 Low-density lipoprotein receptor-related protein 2 precursor

Match to Query 780: 1563.636468 from(782.825510,2+)

Title: 100101RatKid_NS_deglyco_19.1055.1055.2.dta

Data file K:INewmanPaper|Piliangl3SubProteomes\Piliang3SP\mgf5ppm\ERLIC_3SubProteomes5ppm.mgf 

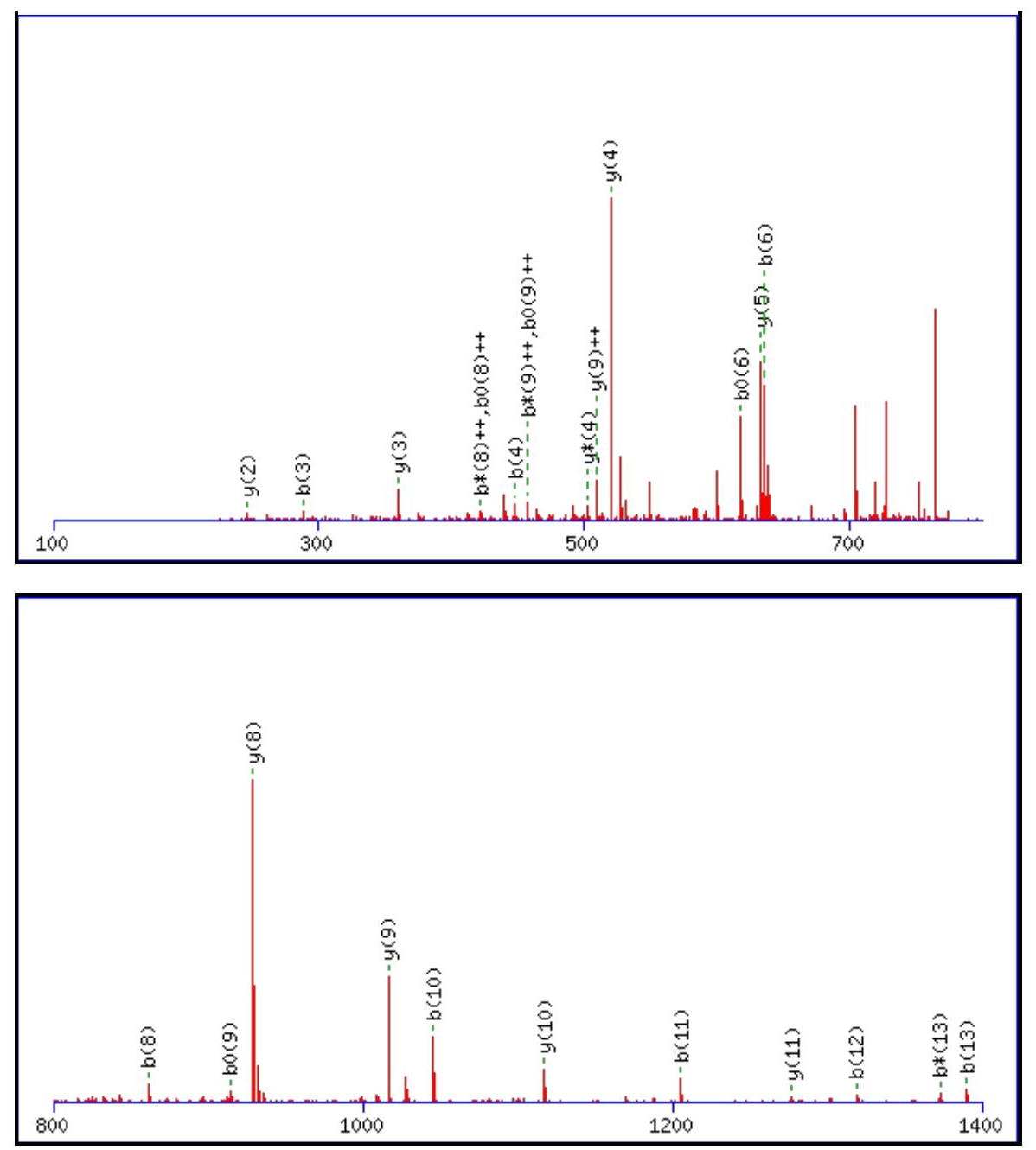

\begin{tabular}{rrrr}
\hline 00 & 1600 & 1800 & 2000 \\
\hline
\end{tabular}

Monoisotopic mass of neutral peptide $\operatorname{Mr}($ calc): 1563.6406

Fixed modifications: Carbamidomethyl (C)

Variable modifications:

N3 : Deamidated $\mathrm{N}(\mathrm{N})$

Ions Score: 75 Expect: $4.2 \mathrm{e}-006$

Matches (Bold Red): 25/136 fragment ions using 33 most intense peaks

\begin{tabular}{|r|c|c|c|c|r|r|r|c|c|c|c|c|c|c|}
\hline$\#$ & $\mathbf{b}$ & $\mathbf{b}^{++}$ & $\mathbf{b}^{*}$ & $\mathbf{b}^{\boldsymbol{*}^{++}}$ & $\mathbf{b}^{\mathbf{0}}$ & $\mathbf{b}^{\mathbf{0 + +}}$ & Seq. & $\mathbf{y}$ & $\mathbf{y}^{++}$ & $\mathbf{y}^{\mathbf{*}}$ & $\mathbf{y}^{\boldsymbol{*}^{++}}$ & $\mathbf{y}^{\mathbf{0}}$ & $\mathbf{y}^{\mathbf{0 + +}}$ & $\#$ \\
\hline $\mathbf{1}$ & 116.0342 & 58.5207 & & & 98.0237 & 49.5155 & $\mathbf{D}$ & & & & & & & $\mathbf{1 4}$ \\
\hline $\mathbf{2}$ & 173.0557 & 87.0315 & & & 155.0451 & 78.0262 & $\mathbf{G}$ & 1449.6209 & 725.3141 & 1432.5944 & 716.8008 & 1431.6104 & 716.3088 & $\mathbf{1 3}$ \\
\hline $\mathbf{3}$ & $\mathbf{2 8 8 . 0 8 2 6}$ & 144.5449 & 271.0561 & 136.0317 & 270.0721 & 135.5397 & $\mathbf{N}$ & 1392.5995 & 696.8034 & 1375.5729 & 688.2901 & 1374.5889 & 687.7981 & $\mathbf{1 2}$ \\
\hline $\mathbf{4}$ & $\mathbf{4 4 8 . 1 1 3 3}$ & 224.5603 & 431.0867 & 216.0470 & 430.1027 & 215.5550 & $\mathbf{C}$ & $\mathbf{1 2 7 7 . 5 7 2 5}$ & 639.2899 & 1260.5460 & 630.7766 & 1259.5620 & 630.2846 & $\mathbf{1 1}$ \\
\hline $\mathbf{5}$ & 549.1609 & 275.0841 & 532.1344 & 266.5708 & 531.1504 & 266.0788 & $\mathbf{T}$ & $\mathbf{1 1 1 7 . 5 4 1 9}$ & 559.2746 & 1100.5153 & 550.7613 & 1099.5313 & 550.2693 & $\mathbf{1 0}$ \\
\hline
\end{tabular}




\begin{tabular}{|c|c|c|c|c|c|c|c|c|c|c|c|c|c|c|}
\hline 6 & 636.1930 & |318.6001 & 619.1664 & |310.0869 & 618.1824 & |309.5948 | & S & |1016.4942 & |508.7507| & 999.4676 & 500.2375 & 998.4836 & | 499.7455 & 9 \\
\hline 7 & 733.2457 & 367.1265 & 716.2192 & 358.6132 & 715.2352 & 358.1212 & $\mathbf{P}$ & 929.4622 & 465.2347 & 912.4356 & 456.7214 & & & 8 \\
\hline 8 & \begin{tabular}{|l|}
861.3043 \\
\end{tabular} & 431.1558 & 844.2778 & 422.6425 & 843.2938 & 422.1505 & $\mathbf{Q}$ & 832.4094 & 416.7083 & 815.3828 & 408.1951 & & & 7 \\
\hline 9 & 932.3414 & 466.6744 & 915.3149 & 458.1611 & 914.3309 & 457.6691 & A & 704.3508 & 352.6790 & 687.3243 & 344.1658 & & & 6 \\
\hline 10 & 1045.4255 & 523.2164 & 1028.3989 & 514.7031 & 1027.4149 & 514.2111 & $\mathbf{L}$ & 633.3137 & 317.1605 & 616.2872 & 308.6472 & & & 5 \\
\hline 11 & 1205.4561 & 603.2317 & 1188.4296 & 594.7184 & 1187.4456 & 594.2264 & $\mathrm{C}$ & 520.2296 & 260.6185 & 503.2031 & 252.1052 & & & 4 \\
\hline 12 & 1319.4991 & 660.2532 & 1302.4725 & 651.7399 & 1301.4885 & 651.2479 & $\mathbf{N}$ & 360.1990 & 180.6031 & 343.1724 & 172.0899 & & & 3 \\
\hline 13 & 1390.5362 & 695.7717 & 1373.5096 & 687.2585 & 1372.5256 & 686.7664 & A & 246.1561 & 123.5817 & 229.1295 & 115.0684 & & & 2 \\
\hline 14 & & & & & & & $\mathbf{R}$ & 175.1190 & 88.0631 & 158.0924 & 79.5498 & & & 1 \\
\hline
\end{tabular}
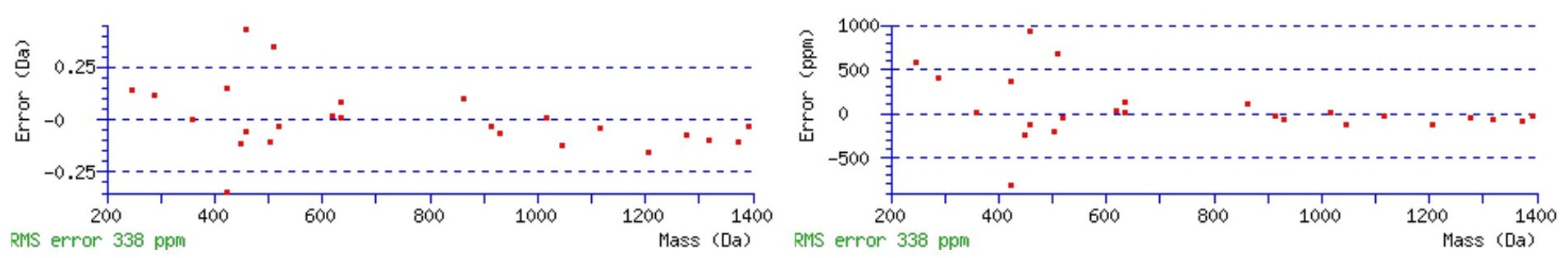

\section{All matches to this query}

\begin{tabular}{|l|l|l|l|}
\hline Score & Mr(calc): & Delta & \multicolumn{1}{|c|}{ Sequence } \\
\hline 74.7 & 1563.6406 & -0.0041 & DGNCTSPQALCNAR \\
\hline 20.9 & 1563.6283 & 0.0082 & MRSESQRMSNAR \\
\hline 16.5 & 1563.6283 & 0.0082 & MRSESQRMSNAR \\
\hline 13.2 & 1563.6517 & -0.0153 & SPGSESKVLGCKR \\
\hline 11.3 & 1561.6418 & 1.9946 & DKPYACDICGKR \\
\hline 11.2 & 1563.6406 & -0.0041 & DGNCTSPQALCNAR \\
\hline 10.3 & 1563.6355 & 0.0010 & ASSFYGSASSNHLR \\
\hline 4.1 & 1563.6357 & 0.0008 & RDSIQMSMKGCR \\
\hline 3.7 & 1563.6517 & -0.0153 & SPGSESKVLGCKR \\
\hline 3.7 & 1563.6517 & -0.0153 & SPGSESKVLGCKR \\
\hline
\end{tabular}

Spectrum No: 308; Query: 2316; Rank: 1

\section{Peptide View}

MS/MS Fragmentation of VIDLWDLAQSANFTEKELESFR

Found in IPI00364124, Tax_Id=10116 Gene_Symbol=Lrpap1 Alpha-2-macroglobulin receptor-associated protein precursor

Match to Query 2316: 2611.287612 from(871.436480,3+)

Title: 091008RatKidney_NoSalt_21.6217.6217.3.dta

Data file K:INewmanPaper|Piliang $\mid 3 S$ SubProteomes\Piliang3SP\mgf5ppm\ERLIC_3SubProteomes5ppm.mgf 

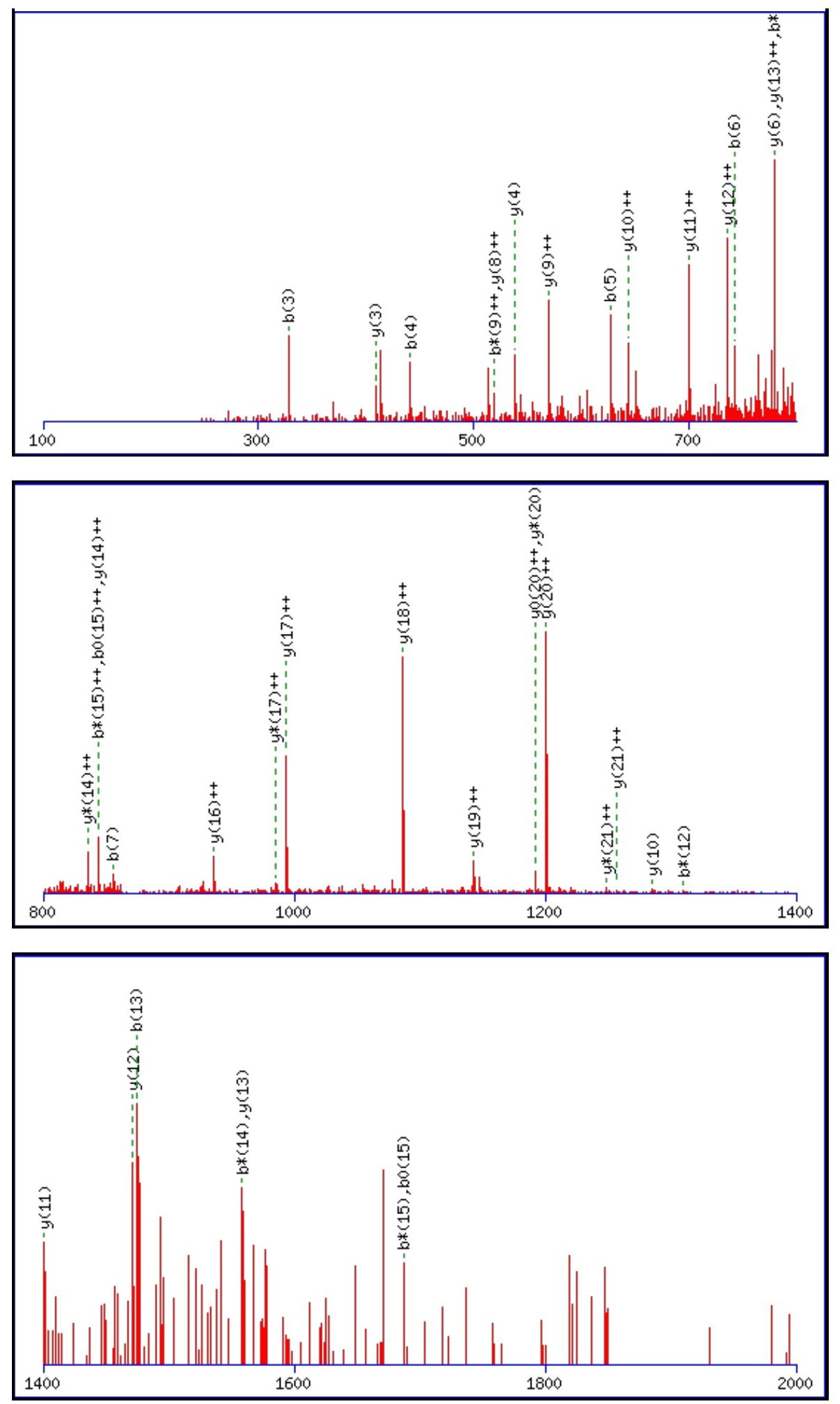

Monoisotopic mass of neutral peptide $\operatorname{Mr}($ calc): 2611.2806

Fixed modifications: Carbamidomethyl (C)

Variable modifications:

N12 : Deamidated_N (N)

Ions Score: 75 Expect: $9.3 \mathrm{e}-006$

Matches (Bold Red): 39/228 fragment ions using 63 most intense peaks

\begin{tabular}{|r|c|c|c|c|c|c|c|c|c|c|c|c|c|c|}
\hline$\#$ & $\mathbf{b}$ & $\mathbf{b}^{++}$ & $\mathbf{b}^{*}$ & $\mathbf{b}^{\boldsymbol{*}^{++}}$ & $\mathbf{b}^{\mathbf{0}}$ & $\mathbf{b}^{\mathbf{0 + +}}$ & Seq. & $\mathbf{y}$ & $\mathbf{y}^{++}$ & $\mathbf{y}^{\mathbf{*}}$ & $\mathbf{y}^{\mathbf{*}^{++}}$ & $\mathbf{y}^{\mathbf{0}}$ & $\mathbf{y}^{\mathbf{0 + +}}$ & $\#$ \\
\hline $\mathbf{1}$ & 100.0757 & 50.5415 & & & & & $\mathbf{V}$ & & & & & & & $\mathbf{2 2}$ \\
\hline $\mathbf{2}$ & 213.1598 & 107.0835 & & & & & $\mathbf{I}$ & 2513.2195 & $\mathbf{1 2 5 7 . 1 1 3 4}$ & 2496.1929 & $\mathbf{1 2 4 8 . 6 0 0 1}$ & 2495.2089 & 1248.1081 & $\mathbf{2 1}$ \\
\hline $\mathbf{3}$ & $\mathbf{3 2 8 . 1 8 6 7}$ & 164.5970 & & & 310.1761 & 155.5917 & $\mathbf{D}$ & 2400.1354 & $\mathbf{1 2 0 0 . 5 7 1 3}$ & 2383.1088 & $\mathbf{1 1 9 2 . 0 5 8 1}$ & 2382.1248 & $\mathbf{1 1 9 1 . 5 6 6 1}$ & $\mathbf{2 0}$ \\
\hline $\mathbf{4}$ & $\mathbf{4 4 1 . 2 7 0 8}$ & 221.1390 & & & 423.2602 & 212.1337 & $\mathbf{L}$ & 2285.1084 & $\mathbf{1 1 4 3 . 0 5 7 9}$ & 2268.0819 & 1134.5446 & 2267.0979 & 1134.0526 & $\mathbf{1 9}$ \\
\hline $\mathbf{5}$ & $\mathbf{6 2 7 . 3 5 0 1}$ & 314.1787 & & & 609.3395 & 305.1734 & $\mathbf{W}$ & 2172.0244 & $\mathbf{1 0 8 6 . 5 1 5 8}$ & 2154.9978 & 1078.0026 & 2154.0138 & 1077.5105 & $\mathbf{1 8}$ \\
\hline
\end{tabular}




\begin{tabular}{|c|c|c|c|c|c|c|c|c|c|c|c|c|c|c|}
\hline & 742.3770 & 371.6921 & & & 724.3665 & 362.6869 & D & 1985.9451 & 993.4762 & |1968.9185 & 984.9629 & 1967.9345 & 984.4709 & 17 \\
\hline 7 & 855.4611 & 428.2342 & & & 837.4505 & 419.2289 & L & 1870.9181 & 935.9627 & 1853.8916 & 927.4494 & 1852.9076 & 926.9574 & 16 \\
\hline 8 & 926.4982 & 463.7527 & & & 908.4876 & 454.7475 & A & 1757.8341 & 879.4207 & 1740.8075 & 870.9074 & 1739.8235 & 870.4154 & 15 \\
\hline 9 & 1054.5568 & 527.7820 & 1037.5302 & 519.2688 & 1036.5462 & 518.7767 & $\mathbf{Q}$ & 1686.7969 & 843.9021 & 1669.7704 & 835.3888 & 1668.7864 & 834.8968 & 4 \\
\hline 10 & 1141.5888 & 571.2980 & 1124.5623 & 562.7848 & 1123.5782 & 562.2928 & S & 1558.7384 & 779.8728 & 1541.7118 & 771.3595 & 1540.7278 & 770.8675 & 13 \\
\hline 11 & 1212.6259 & 606.8166 & 1195.5994 & 598.3033 & 1194.6154 & 597.8113 & A & 1471.7063 & 736.3568 & 1454.6798 & 727.8435 & 1453.6958 & 727.3515 & 12 \\
\hline 12 & 1327.6529 & 664.3301 & 1310.6263 & 655.8168 & 1309.6423 & 655.3248 & $\mathbf{N}$ & 1400.6692 & 700.8383 & 1383.6427 & 692.3250 & 1382.6587 & 691.8330 & 11 \\
\hline 13 & 1474.7213 & 737.8643 & 1457.6947 & 729.3510 & 1456.7107 & 728.8590 & $\mathbf{F}$ & 1285.6423 & 643.3248 & 1268.6157 & 634.8115 & 1267.6317 & 3195 & 10 \\
\hline 14 & 1575.7689 & 788.3881 & 1558.7424 & 779.8748 & 1557.7584 & 779.3828 & $\mathrm{~T}$ & 1138.5739 & 569.7906 & 1121.5473 & 561.2773 & 1120.5633 & 560.7853 & 9 \\
\hline 15 & 1704.8115 & 852.9094 & 1687.7850 & 844.3961 & 1686.8010 & 843.9041 & $\mathbf{E}$ & 1037.5262 & 519.2667 & 1020.4997 & 510.7535 & 1019.5156 & 510.2615 & 8 \\
\hline 16 & 1832.9065 & 916.9569 & 1815.8800 & 908.4436 & 1814.8959 & 907.9516 & $\mathbf{K}$ & 908.4836 & 454.7454 & 891.4571 & 446.2322 & 890.4730 & 445.7402 & 7 \\
\hline 17 & 1961.9491 & 981.4782 & 1944.9225 & 972.9649 & 1943.9385 & 972.4729 & $\mathbf{E}$ & 780.3886 & 390.6980 & 763.3621 & 382.1847 & 762.3781 & 381.6927 & 6 \\
\hline 18 & 2075.0332 & 1038.0202 & 2058.0066 & 1029.5069 & 2057.0226 & 1029.0149 & $\mathbf{L}$ & 651.3461 & 326.1767 & 634.3195 & 317.6634 & 633.3355 & 317.1714 & 5 \\
\hline 19 & 2204.0758 & 1102.5415 & 2187.0492 & 1094.0282 & 2186.0652 & 1093.5362 & $E$ & 538.2620 & 269.6346 & 521.2354 & 261.1214 & 520.2514 & 260.6293 & 4 \\
\hline 20 & 2291.1078 & 1146.0575 & 2274.0812 & 1137.5443 & 2273.0972 & 1137.0522 & $S$ & 409.2194 & 205.1133 & 392.1928 & 196.6001 & 391.2088 & 196.1081 & 3 \\
\hline 21 & 2438.1762 & 1219.5917 & 2421.1496 & 1211.0785 & 2420.1656 & 1210.5865 & $\mathbf{F}$ & 322.1874 & 161.5973 & 305.1608 & 153.0840 & & & 2 \\
\hline 22 & & & & & & & $\mathbf{R}$ & 175.1190 & 88.0631 & 158.0924 & 79.5498 & & & 1 \\
\hline
\end{tabular}
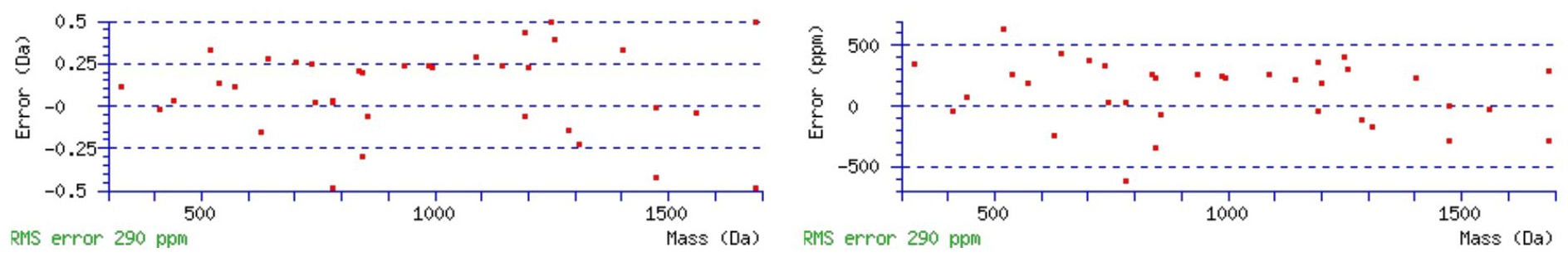

\section{All matches to this query}

\begin{tabular}{|l|l|l|l|}
\hline Score & Mr(calc): & Delta & \multicolumn{1}{|c|}{ Sequence } \\
\hline 74.6 & 2611.2806 & 0.0070 & VIDLWDLAQSANFTEKELESFR \\
\hline 5.9 & 2610.2966 & 0.9911 & VIDLWDLAQSANFTEKELESFR \\
\hline 5.7 & 2609.2954 & 1.9922 & IPVEANFLYVYSTKPNYYSWR \\
\hline 4.9 & 2611.3002 & -0.0126 & IEGLDSEMIMKPLVTEINAKLR \\
\hline 2.2 & 2611.2975 & -0.0099 & NIDGEALVAIESFYPVFTPPPKK \\
\hline 2.2 & 2611.2975 & -0.0099 & NIDGEALVAIESFYPVFTPPPKK \\
\hline 2.2 & 2610.2794 & 1.0082 & IPVEANFLYVYSTKPNYYSWR \\
\hline 1.7 & 2610.2764 & 1.0112 & VLIEFSLLRLENPDEACAVSQK \\
\hline 1.6 & 2609.2880 & 1.9996 & TLLRAAGPLSPPGAGHTVPGPGVATR \\
\hline 1.5 & 2611.2993 & -0.0117 & KIQELQSEKETLSTQLDLAETK \\
\hline
\end{tabular}

Spectrum No: 309; Query: 2653; Rank: 1

\section{Peptide View}

MS/MS Fragmentation of KKPTPIQLNPAPDGSAVNGTSSAETNLEALQK

Found in IPI00231247, Tax_Id=10116 Gene_Symbol=Map2k1 Dual specificity mitogen-activated protein kinase kinase 1

Match to Query 2653: 3276.688212 from(1093.236680,3+)

Title: 091008RatKidney_NH4Format01_13.2839.2839.3.dta

Data file K:INewmanPaper|Piliang \3SubProteomes\Piliang3SP\mgf5ppm\ERLIC_3SubProteomes5ppm.mgf 

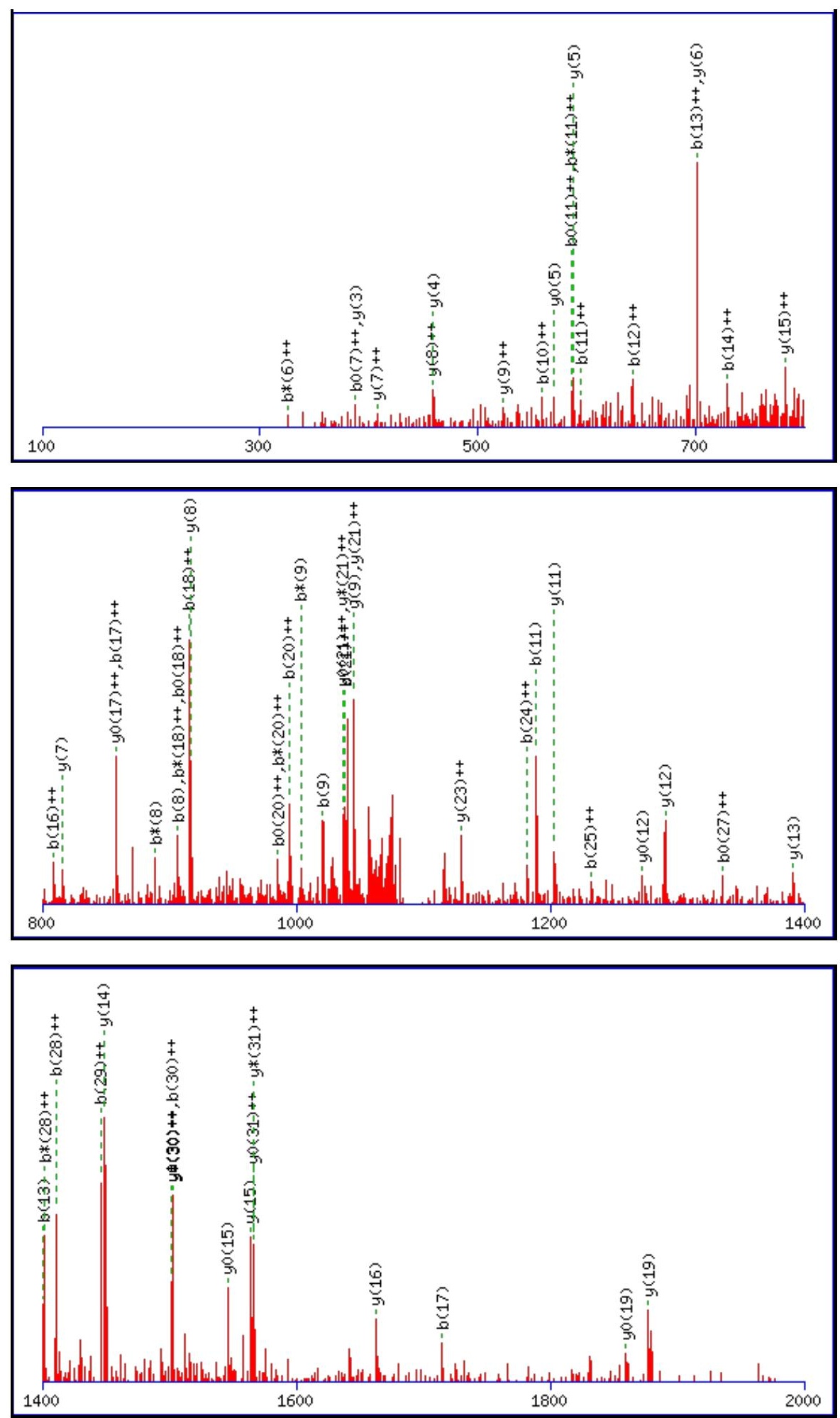

Monoisotopic mass of neutral peptide $\operatorname{Mr}($ calc): 3276.6837

Fixed modifications: Carbamidomethyl (C)

Variable modifications:

N18 : Deamidated $\mathrm{N}(\mathrm{N})$

Ions Score: 74 Expect: $5.2 \mathrm{e}-006$

Matches (Bold Red): 64/358 fragment ions using 108 most intense peaks

\begin{tabular}{|c|c|c|c|c|c|c|c|c|c|c|c|c|c|c|}
\hline$\#$ & $\mathbf{b}$ & $\mathbf{b}^{++}$ & $\mathbf{b}^{*}$ & $\mathbf{b}^{\boldsymbol{*}_{++}}$ & $\mathbf{b}^{\mathbf{0}}$ & $\mathbf{b}^{\mathbf{0 + +}}$ & $\mathbf{S e q}$ & $\mathbf{y}$ & $\mathbf{y}^{++}$ & $\mathbf{y}^{\mathbf{*}}$ & $\mathbf{y}^{\mathbf{*}^{++}}$ & $\mathbf{y}^{\mathbf{0}}$ & $\mathbf{y}^{\mathbf{0 + +}}$ & $\#$ \\
\hline $\mathbf{1}$ & 129.1022 & 65.0548 & 112.0757 & 56.5415 & & & $\mathbf{K}$ & & & & & & & 32 \\
\hline $\mathbf{2}$ & 257.1972 & 129.1022 & 240.1707 & 120.5890 & & & $\mathbf{K}$ & 3149.5961 & 1575.3017 & 3132.5695 & $\mathbf{1 5 6 6 . 7 8 8 4}$ & 3131.5855 & $\mathbf{1 5 6 6 . 2 9 6 4}$ & $\mathbf{3 1}$ \\
\hline $\mathbf{3}$ & 354.2500 & 177.6286 & 337.2234 & 169.1153 & & & $\mathbf{P}$ & 3021.5011 & 1511.2542 & 3004.4746 & $\mathbf{1 5 0 2 . 7 4 0 9}$ & 3003.4905 & $\mathbf{1 5 0 2 . 2 4 8 9}$ & $\mathbf{3 0}$ \\
\hline $\mathbf{4}$ & 455.2976 & 228.1525 & 438.2711 & 219.6392 & 437.2871 & 219.1472 & $\mathbf{T}$ & 2924.4483 & 1462.7278 & 2907.4218 & 1454.2145 & 2906.4378 & 1453.7225 & $\mathbf{2 9}$ \\
\hline $\mathbf{5}$ & 552.3504 & 276.6788 & 535.3239 & 268.1656 & 534.3398 & 267.6736 & $\mathbf{P}$ & 2823.4007 & 1412.2040 & 2806.3741 & 1403.6907 & 2805.3901 & 1403.1987 & $\mathbf{2 8}$ \\
\hline
\end{tabular}




\begin{tabular}{|c|c|c|c|c|c|c|c|c|c|c|c|c|c|c|}
\hline 6 & 665.4345 & 333.2209 & 648.4079 & 324.7076 & 647.4239| & 324.2156 & I & 2726.3479 & |1363.6776 & |2709.3214 & |1355.1643 & |2708.3373 & |1354.6723 & 27 \\
\hline 7 & 793.4931 & 397.2502 & 776.4665 & 388.7369 & 775.4825 & 388.2449 & $\mathbf{Q}$ & 2613.2638 & 1307.1356 & 2596.2373 & 1298.6223 & 2595.2533 & 1298.1303 & 26 \\
\hline 8 & 906.5771 & 453.7922 & 889.5506 & 445.2789 & 888.5666 & 444.7869 & $\mathbf{L}$ & 2485.2053 & 1243.1063 & 2468.1787 & 1234.5930 & 2467.1947 & 1234.1010 & 25 \\
\hline 9 & 1020.6200 & 510.8137 & 1003.5935 & 502.3004 & 1002.6095 & 501.8084 & $\mathbf{N}$ & 2372.1212 & 1186.5642 & 2355.0946 & 1178.0510 & 2354.1106 & 1177.5590 & 24 \\
\hline 10 & 1117.6728 & 559.3400 & 1100.6463 & 550.8268 & 1099.6622 & 550.3348 & $\mathbf{P}$ & 2258.0783 & 1129.5428 & 2241.0517 & 1121.0295 & 2240.0677 & 1120.5375 & 23 \\
\hline 11 & 1188.7099 & 594.8586 & 1171.6834 & 586.3453 & 1170.6994 & 585.8533 & A & 2161.0255 & 1081.0164 & 2143.9990 & 1072.5031 & 2143.0149 & 1072.0111 & 22 \\
\hline 12 & 1285.7627 & 643.3850 & 1268.7361 & 634.8717 & 1267.7521 & 634.3797 & $\mathbf{P}$ & 2089.9884 & 1045.4978 & 2072.9618 & 1036.9846 & 2071.9778 & 1036.4926 & 21 \\
\hline 13 & 1400.7896 & 700.8985 & 1383.7631 & 692.3852 & 1382.7791 & 691.8932 & D & 1992.9356 & 996.9715 & 1975.9091 & 988.4582 & 1974.9251 & 987.9662 & 20 \\
\hline 14 & 1457.8111 & 729.4092 & 1440.7845 & 720.8959 & 1439.8005 & 720.4039 & $\mathbf{G}$ & 1877.9087 & 939.4580 & 1860.8821 & 930.9447 & 1859.8981 & 930.4527 & 19 \\
\hline 15 & 1544.8431 & 772.9252 & 1527.8166 & 764.4119 & 1526.8326 & 763.9199 & S & 1820.8872 & 910.9472 & 1803.8607 & 902.4340 & 1802.8767 & 901.9420 & 18 \\
\hline 16 & 1615.8802 & 808.4438 & 1598.8537 & 799.9305 & 1597.8697 & 799.4385 & A & 1733.8552 & 867.4312 & 1716.8286 & 858.9180 & 1715.8446 & 858.4260 & 17 \\
\hline 17 & 1714.9486 & 857.9780 & 1697.9221 & 849.4647 & 1696.9381 & 848.9727 & V & 1662.8181 & 831.9127 & 1645.7915 & 823.3994 & 1644.8075 & 822.9074 & 16 \\
\hline 18 & 1829.9756 & 915.4914 & 1812.9490 & 906.9782 & 1811.9650 & 906.4861 & $\mathbf{N}$ & 1563.7497 & 782.3785 & 1546.7231 & 773.8652 & 1545.7391 & 773.3732 & 15 \\
\hline 19 & 1886.9970 & 944.0022 & 1869.9705 & 935.4889 & 1868.9865 & 934.9969 & $\mathbf{G}$ & 1448.7227 & 724.8650 & 1431.6962 & 716.3517 & 1430.7122 & 715.8597 & 14 \\
\hline 21 & 2075.0768 & 1038.0420 & 2058.0502 & 1029.5287 & 2057.0662 & 1029.0367 & S & 1290.6536 & 645.8304 & 1273.6270 & 637.3172 & 1272.6430 & 636.8251 & 12 \\
\hline 22 & 2162.1088 & 1081.5580 & 2145.0822 & 1073.0448 & 2144.0982 & 1072.5527 & $\mathrm{~S}$ & 1203.6216 & 602.3144 & 1186.5950 & 593.8011 & 1185.6110 & 593.3091 & 11 \\
\hline 23 & 2233.1459 & 1117.0766 & 2216.1193 & 1108.5633 & 2215.1353 & 1108.0713 & A & 1116.5895 & 558.7984 & 1099.5630 & 550.2851 & 1098.5790 & 549.7931 & 10 \\
\hline 24 & 2362.1885 & 1181.5979 & 2345.1619 & 1173.0846 & 2344.1779 & 1172.5926 & $\mathbf{E}$ & 1045.5524 & 523.2798 & 1028.5259 & 514.7666 & 1027.5419 & 514.2746 & 9 \\
\hline 25 & 2463.2362 & 1232.1217 & 2446.2096 & 1223.6084 & 2445.2256 & 1223.1164 & $\mathbf{T}$ & 916.5098 & 458.7585 & 899.4833 & 450.2453 & 898.4993 & 449.7533 & 8 \\
\hline 26 & 2577.2791 & 1289.1432 & 2560.2525 & 1280.6299 & 2559.2685 & 1280.1379 & $\mathbf{N}$ & 815.4621 & 408.2347 & 798.4356 & 399.7214 & 797.4516 & 399.2294 & 7 \\
\hline 27 & 2690.3632 & 1345.6852 & 2673.3366 & 1337.1719 & 2672.3526 & 1336.6799 & $\mathbf{L}$ & 701.4192 & 351.2132 & 684.3927 & 342.7000 & 683.4087 & 342.2080 & 6 \\
\hline 28 & 2819.4058 & 1410.2065 & 2802.3792 & 1401.6932 & 2801.3952 & 1401.2012 & $\mathbf{E}$ & 588.3352 & 294.6712 & 571.3086 & 286.1579 & 570.3246 & 285.6659 & 5 \\
\hline 29 & 2890.4429 & 1445.7251 & 2873.4163 & 1437.2118 & 2872.4323 & 1436.7198 & A & 459.2926 & 230.1499 & 442.2660 & 221.6366 & & & 4 \\
\hline 30 & 3003.5269 & 1502.2671 & 2986.5004 & 1493.7538 & 2985.5164 & 1493.2618 & $\mathbf{L}$ & 388.2554 & 194.6314 & 371.2289 & 186.1181 & & & 3 \\
\hline 31 & 3131.5855 & 1566.2964 & 3114.5590 & 1557.7831 & 3113.5749 & 1557.2911 & $\mathbf{Q}$ & 275.1714 & 138.0893 & 258.1448 & 129.5761 & & & 2 \\
\hline 32 & & & & & & & $\mathbf{K}$ & 147.1128 & 74.0600 & 130.0863 & 65.5468 & & & 1 \\
\hline
\end{tabular}
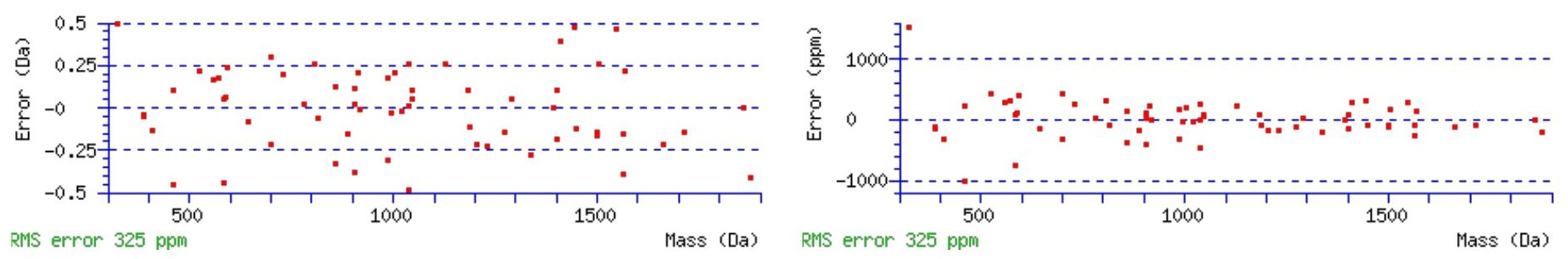

\section{All matches to this query}

\begin{tabular}{|l|l|l|l|}
\hline Score & Mr(calc): & Delta & \multicolumn{1}{c|}{ Sequence } \\
\hline 74.4 & 3276.6837 & 0.0045 & KKPTPIQLNPAPDGSAVNGTSSAETNLEALQK \\
\hline 62.9 & 3276.6837 & 0.0045 & KKPTPIQLNPAPDGSAVNGTSSAETNLEALQK \\
\hline 54.6 & 3275.6997 & 0.9885 & KKPTPIQLNPAPDGSAVNGTSSAETNLEALQK \\
\hline 50.4 & 3276.6837 & 0.0045 & KKPTPIQLNPAPDGSAVNGTSSAETNLEALQK \\
\hline 0.9 & 3274.6673 & 2.0210 & MPSLEVPVPKISGPDANVHLKTPNVGISGPK \\
\hline
\end{tabular}

Spectrum No: 310; Query: 585; Rank: 1

\section{Peptide View}

MS/MS Fragmentation of DEELNCTVVELK

Found in IPI00211075, Tax_Id=10116 Gene_Symbol=Serpina3n Serine protease inhibitor A3N precursor 
Match to Query 585: 1448.668568 from(725.341560,2+)

Title: 091008RatKidney_NoSalt_42.1491.1491.2.dta

Data file K:INewmanPaper|Piliang|3SubProteomes\Piliang3SP\mgf5ppm\ERLIC_3SubProteomes5ppm.mgf
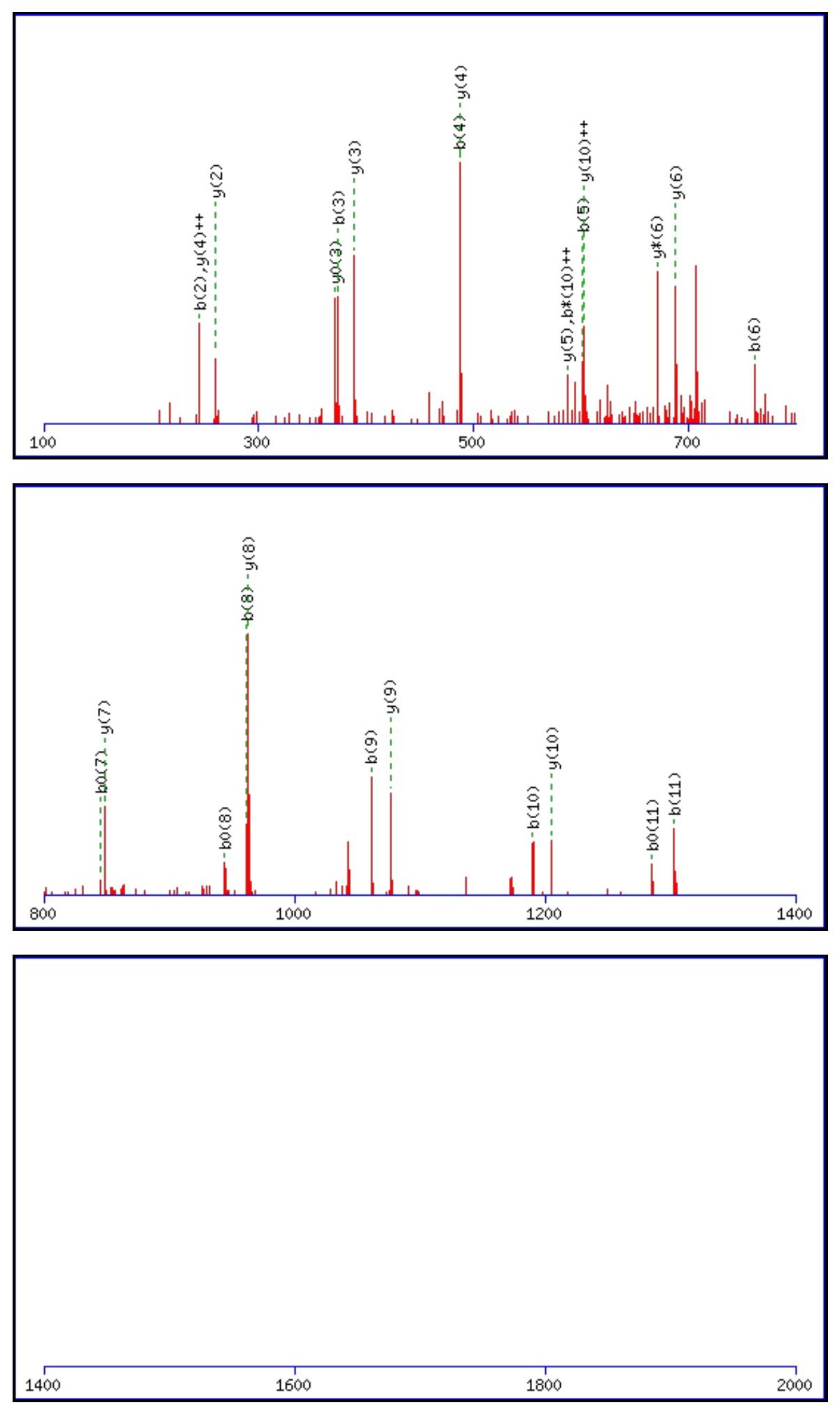

Monoisotopic mass of neutral peptide $\operatorname{Mr}($ calc): 1448.6705

Fixed modifications: Carbamidomethyl (C)

Variable modifications:

N5 : Deamidated_N (N)

Ions Score: 74 Expect: $5.5 \mathrm{e}-006$

Matches (Bold Red): 26/120 fragment ions using 36 most intense peaks

\begin{tabular}{|r|c|c|c|c|c|c|c|c|c|c|c|c|c|c|}
\hline$\#$ & $\mathbf{b}$ & $\mathbf{b}^{++}$ & $\mathbf{b}^{*}$ & $\mathbf{b}^{*^{++}}$ & $\mathbf{b}^{\mathbf{0}}$ & $\mathbf{b}^{\mathbf{0}++}$ & Seq. & $\mathbf{y}$ & $\mathbf{y}^{++}$ & $\mathbf{y}^{\mathbf{*}}$ & $\mathbf{y}^{\mathbf{*}^{++}}$ & $\mathbf{y}^{\mathbf{0}}$ & $\mathbf{y}^{\mathbf{0}++}$ & $\#$ \\
\hline $\mathbf{1}$ & 116.0342 & 58.5207 & & & 98.0237 & 49.5155 & $\mathbf{D}$ & & & & & & & $\mathbf{1 2}$ \\
\hline $\mathbf{2}$ & $\mathbf{2 4 5 . 0 7 6 8}$ & 123.0420 & & & 227.0662 & 114.0368 & $\mathbf{E}$ & 1334.6508 & 667.8290 & 1317.6243 & 659.3158 & 1316.6402 & 658.8238 & $\mathbf{1 1}$ \\
\hline
\end{tabular}




\begin{tabular}{|c|c|c|c|c|c|c|c|c|c|c|c|c|c|c|}
\hline & 374.1194 & |187.5633 & & & 356.1088 & 178.5581 & $\mathbf{E}$ & 1205.6082 & 603.3077 & 1188.5817 & 594.7945 & 1187.5976 & 594.3025 & 10 \\
\hline 4 & 487.2035 & 244.1054 & & & 469.1929 & 235.1001 & L & 1076.5656 & 538.7864 & 1059.5391 & 530.2732 & 1058.5551 & 529.7812 & 9 \\
\hline 5 & 602.2304 & 301.6188 & 585.2039 & 293.1056 & 584.2198 & 292.6136 & $\mathbf{N}$ & 963.4816 & 482.2444 & 946.4550 & 473.7311 & 945.4710 & 473.2391 & 8 \\
\hline 6 & 762.2611 & |381.6342 & 745.2345 & 373.1209 & 744.2505 & 372.6289 & C & 848.4546 & 424.7309 & 831.4281 & 416.2177 & 830.4441 & 415.7257 & 7 \\
\hline 7 & 863.3087 & 432.1580 & 846.2822 & 423.6447 & 845.2982 & 423.1527 & $\mathbf{T}$ & 688.4240 & 344.7156 & 671.3974 & 336.2023 & 670.4134 & 335.7103 & 6 \\
\hline 8 & 962.3771 & 481.6922 & 945.3506 & 473.1789 & 944.3666 & 472.6869 & V & 587.3763 & 294.1918 & 570.3497 & 285.6785 & 569.3657 & 285.1865 & 5 \\
\hline 9 & 1061.4456 & 531.2264 & 1044.4190 & 522.7131 & 1043.4350 & 522.2211 & V & 488.3079 & 244.6576 & 471.2813 & 236.1443 & 470.2973 & 235.6523 & 4 \\
\hline 10 & 1190.4882 & 595.7477 & 1173.4616 & 587.2344 & 1172.4776 & 586.7424 & E & 389.2395 & 195.1234 & 372.2129 & 186.6101 & 371.2289 & 186.1181 & 3 \\
\hline 11 & 1303.5722 & 652.2897 & 1286.5457 & 643.7765 & 1285.5617 & 643.2845 & L & 260.1969 & 130.6021 & 243.1703 & 122.0888 & & & 2 \\
\hline 12 & & & & & & & $\mathbf{K}$ & 147.1128 & 74.0600 & 130.0863 & 65.5468 & & & 1 \\
\hline
\end{tabular}
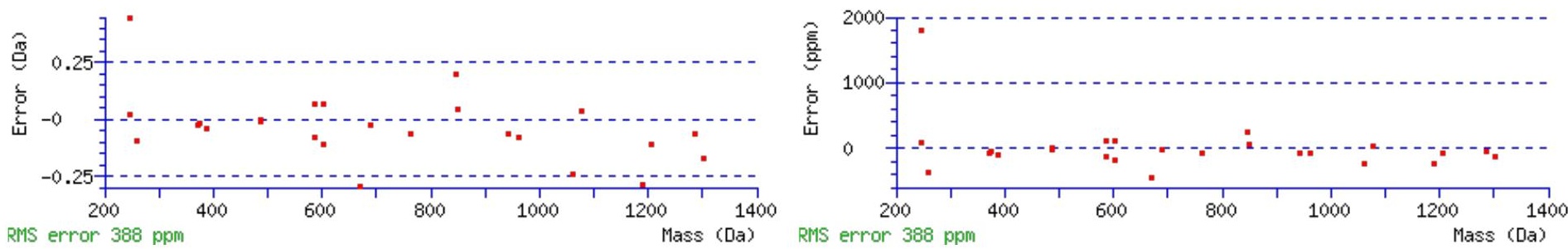

All matches to this query

\begin{tabular}{|l|l|l|l|}
\hline Score & Mr(calc): & Delta & \multicolumn{1}{|c|}{ Sequence } \\
\hline 74.4 & 1448.6705 & -0.0019 & DEELNCTVVELK \\
\hline 17.0 & 1448.6783 & -0.0097 & DAGENNFINVDLK \\
\hline 16.8 & 1448.6783 & -0.0097 & DAGENNFINVDLK \\
\hline 15.2 & 1448.6606 & 0.0080 & NEAATAEVWGCLK \\
\hline 15.0 & 1448.6565 & 0.0120 & KMNDSNSSGAGGPVK \\
\hline 12.1 & 1448.6701 & -0.0015 & TYQFLNDIRAK \\
\hline 12.1 & 1448.6701 & -0.0015 & TYQFLNDIRAK \\
\hline 10.8 & 1448.6639 & 0.0046 & QDGAVAMEMQPLK \\
\hline 9.7 & 1448.6588 & 0.0097 & DYFNQTLNILK \\
\hline 8.9 & 1448.6734 & -0.0049 & ILEMEHTQQLK \\
\hline
\end{tabular}

Spectrum No: 311; Query: 529; Rank: 1

\section{Peptide View}

MS/MS Fragmentation of VIPVCNASLPAQR

Found in IPI00192504, Tax_Id=10116 Gene_Symbol=Mrc2 Macrophage mannose receptor 2 precursor

Match to Query 529: 1424.747568 from(713.381060,2+)

Title: 100101RatKid_NS_deglyco_21.1950.1950.2.dta

Data file K:INewmanPaper|Piliangl3SubProteomes\Piliang3SP\mgf5ppm\ERLIC_3SubProteomes5ppm.mgf 

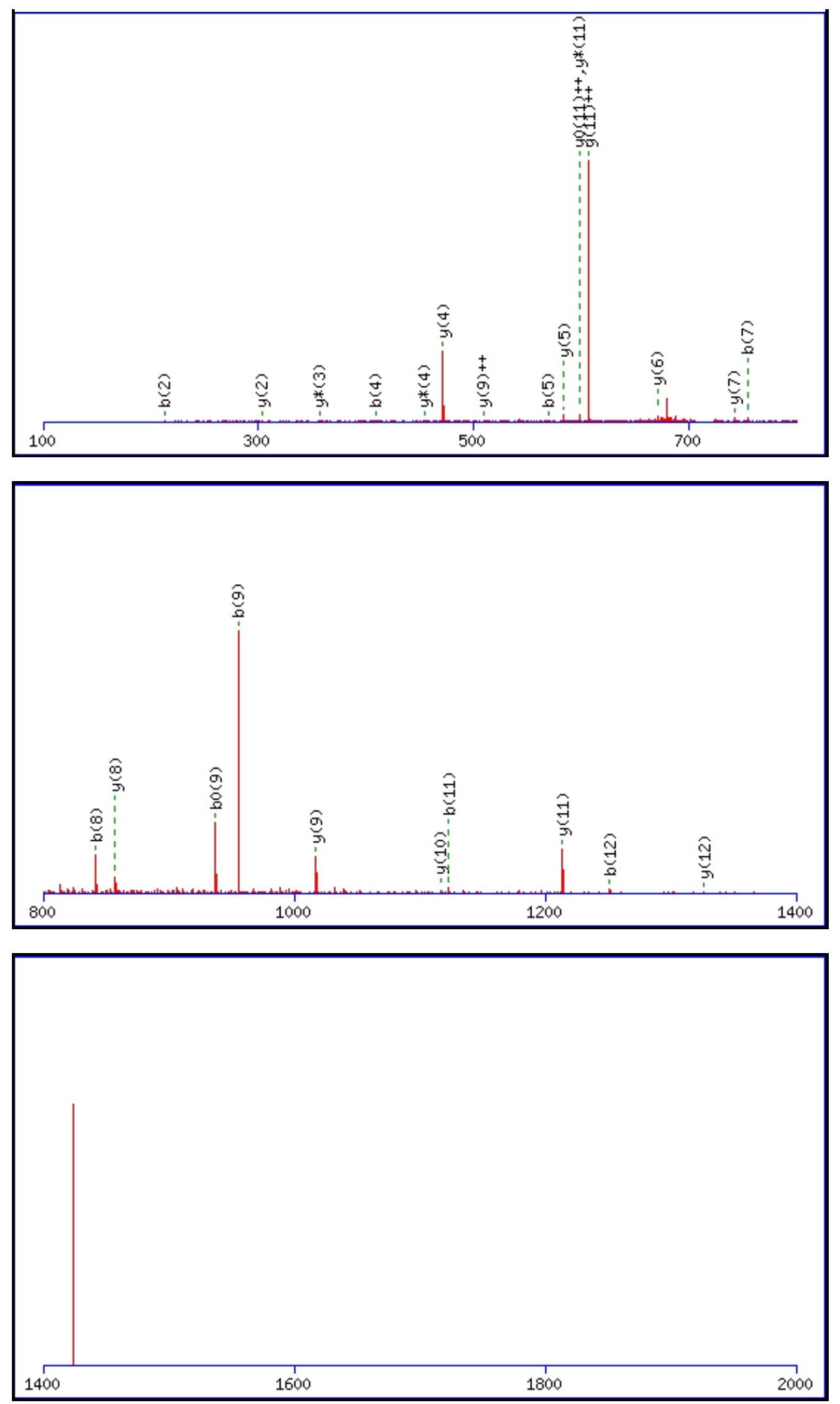

Monoisotopic mass of neutral peptide $\operatorname{Mr}($ calc): 1424.7446

Fixed modifications: Carbamidomethyl (C)

Variable modifications:

N6 : Deamidated $\mathrm{N}(\mathrm{N})$

Ions Score: 74 Expect: $3.5 \mathrm{e}-006$

Matches (Bold Red): 25/110 fragment ions using 46 most intense peaks

\begin{tabular}{|r|c|c|c|c|c|c|c|c|c|c|c|c|c|c|}
\hline$\#$ & $\mathbf{b}$ & $\mathbf{b}^{++}$ & $\mathbf{b}^{*}$ & $\mathbf{b}^{\boldsymbol{*}^{++}}$ & $\mathbf{b}^{\mathbf{0}}$ & $\mathbf{b}^{\mathbf{0 + +}}$ & Seq. & $\mathbf{y}$ & $\mathbf{y}^{++}$ & $\mathbf{y}^{\mathbf{*}}$ & $\mathbf{y}^{\boldsymbol{*}^{++}}$ & $\mathbf{y}^{\mathbf{0}}$ & $\mathbf{y}^{\mathbf{0 + +}}$ & $\#$ \\
\hline $\mathbf{1}$ & 100.0757 & 50.5415 & & & & & $\mathbf{V}$ & & & & & & & $\mathbf{1 3}$ \\
\hline $\mathbf{2}$ & $\mathbf{2 1 3 . 1 5 9 8}$ & 107.0835 & & & & & $\mathbf{I}$ & $\mathbf{1 3 2 6 . 6 8 3 4}$ & 663.8454 & 1309.6569 & 655.3321 & 1308.6729 & 654.8401 & $\mathbf{1 2}$ \\
\hline 3 & 310.2125 & 155.6099 & & & & & $\mathbf{P}$ & $\mathbf{1 2 1 3 . 5 9 9 4}$ & $\mathbf{6 0 7 . 3 0 3 3}$ & 1196.5728 & $\mathbf{5 9 8 . 7 9 0 1}$ & 1195.5888 & 598.2980 & $\mathbf{1 1}$ \\
\hline $\mathbf{4}$ & $\mathbf{4 0 9 . 2 8 0 9}$ & 205.1441 & & & & & $\mathbf{V}$ & $\mathbf{1 1 1 6 . 5 4 6 6}$ & 558.7769 & 1099.5201 & 550.2637 & 1098.5360 & 549.7717 & $\mathbf{1 0}$ \\
\hline $\mathbf{5}$ & $\mathbf{5 6 9 . 3 1 1 6}$ & 285.1594 & & & & & $\mathbf{C}$ & $\mathbf{1 0 1 7 . 4 7 8 2}$ & $\mathbf{5 0 9 . 2 4 2 7}$ & 1000.4516 & 500.7295 & 999.4676 & 500.2375 & $\mathbf{9}$ \\
\hline
\end{tabular}




\begin{tabular}{|c|c|c|c|c|c|c|c|c|c|c|c|c|c|c|}
\hline 6 & 684.3385 & |342.6729 & 667.3120 & |334.1596 & & & $\mathbf{N}$ & 857.4475 & |429.2274| & 840.4210 & 420.7141 & 839.4370 & |420.2221 & 8 \\
\hline 7 & 755.3756 & 378.1915 & 738.3491 & |369.6782 & & & A & 742.4206 & |371.7139 & 725.3941 & 363.2007 & 724.4100 & 362.7087 & 7 \\
\hline 8 & 842.4077 & |421.7075 & 825.3811 & 413.1942 & 824.3971 & 412.7022 & S & 671.3835 & 336.1954 & 654.3570 & 327.6821 & 653.3729 & 327.1901 & 6 \\
\hline 9 & 955.4917 & 478.2495 & 938.4652 & 469.7362 & 937.4812 & |469.2442 & $\mathbf{L}$ & 584.3515 & 292.6794 & 567.3249 & 284.1661 & & & 5 \\
\hline 10 & 1052.5445 & 526.7759 & 1035.5179 & 518.2626 & 1034.5339 & 517.7706 & $\mathbf{P}$ & 471.2674 & 236.1373 & 454.2409 & 227.6241 & & & 4 \\
\hline 11 & 1123.5816 & 562.2944 & 1106.5551 & 553.7812 & 1105.5710 & 553.2892 & A & 374.2146 & 187.6110 & 357.1881 & 179.0977 & & & 3 \\
\hline 12 & 1251.6402 & 626.3237 & 1234.6136 & 617.8105 & 1233.6296 & 617.3184 & $\mathbf{Q}$ & 303.1775 & 152.0924 & 286.1510 & 143.5791 & & & 2 \\
\hline 13 & & & & & & & $\mathbf{R}$ & 175.1190 & 88.0631 & 158.0924 & 79.5498 & & & 1 \\
\hline
\end{tabular}
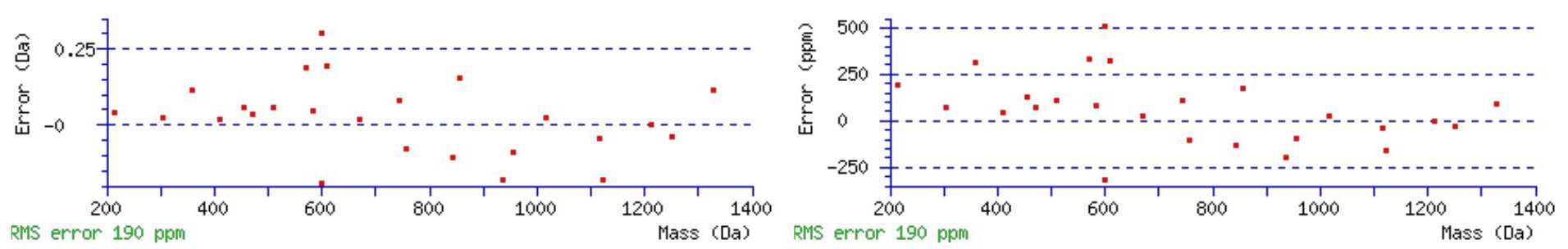

\section{All matches to this query}

\begin{tabular}{|l|l|l|l|}
\hline Score & Mr(calc): & Delta & \multicolumn{1}{|c|}{ Sequence } \\
\hline 74.4 & 1424.7446 & 0.0030 & VIPVCNASLPAQR \\
\hline 13.3 & 1424.7558 & -0.0082 & NSRLLMSSFSKR \\
\hline 7.9 & 1423.7323 & 1.0152 & LGSKVITDPSLSK \\
\hline 7.9 & 1423.7323 & 1.0152 & LGSKVITDPSLSK \\
\hline 7.9 & 1423.7323 & 1.0152 & LGSKVITDPSLSK \\
\hline 3.6 & 1423.7320 & 1.0156 & NPPVLYHNSRAR \\
\hline 2.8 & 1424.7524 & -0.0049 & IVRDYDVYARR \\
\hline 2.4 & 1423.7436 & 1.0040 & LGLSTAEILSGKR \\
\hline 2.4 & 1423.7337 & 1.0139 & LIASRFKHSGTK \\
\hline 2.4 & 1423.7337 & 1.0139 & LIASRFKHSGTK \\
\hline
\end{tabular}

Spectrum No: 312; Query: 2024; Rank: 1

\section{Peptide View}

MS/MS Fragmentation of QILEGLEFNLTQIPEADIHK

Found in IPI00324019, Tax_Id=10116 Gene_Symbol=Serpina1 Alpha-1-antiproteinase precursor

Match to Query 2024: 2308.200788 from(1155.107670,2+)

Title: 091008RatKidney_NoSalt_26.4790.4790.2.dta

Data file K:INewmanPaper|Piliangl3SubProteomes\Piliang3SP\mgf5ppm\ERLIC_3SubProteomes5ppm.mgf 

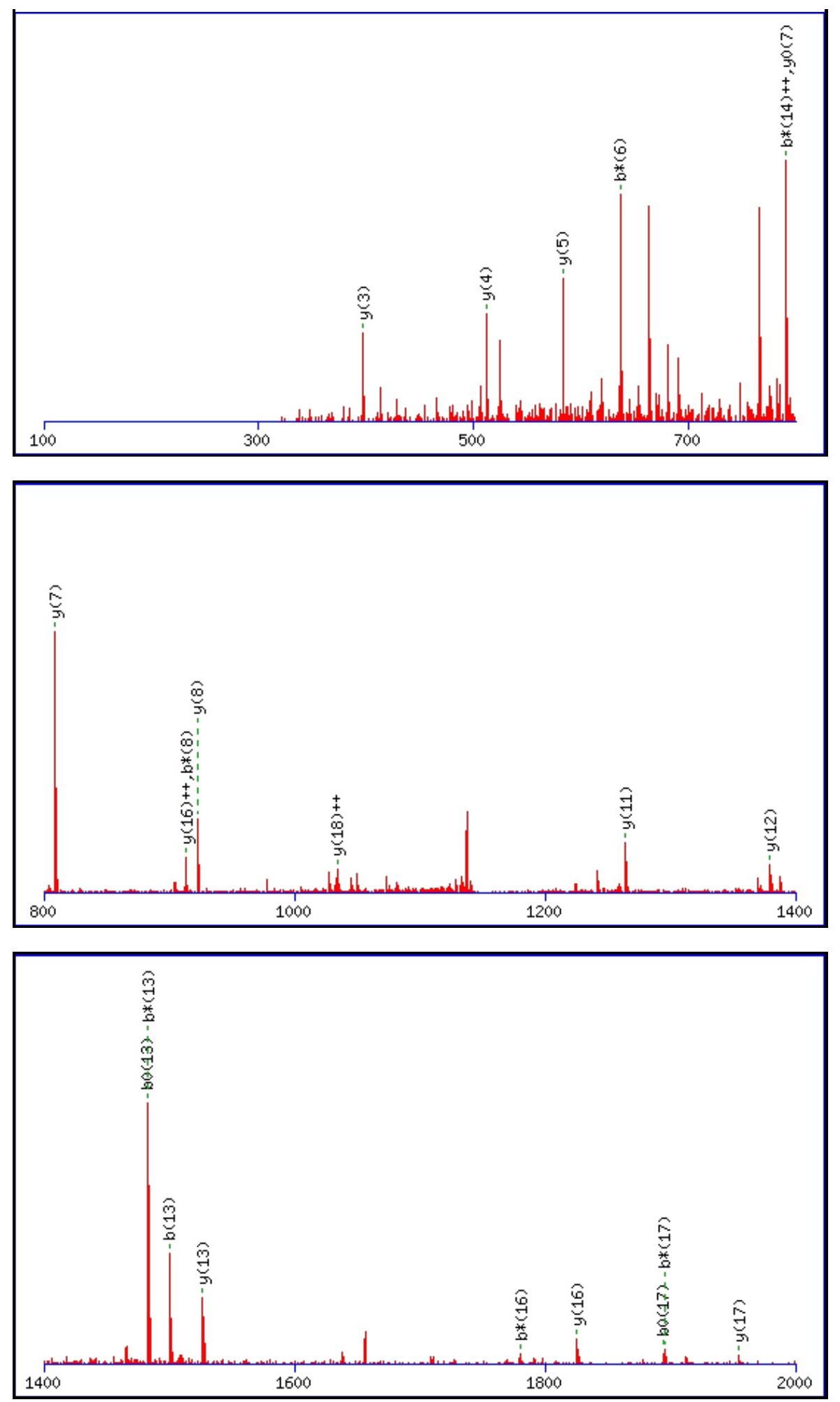

Monoisotopic mass of neutral peptide $\operatorname{Mr}($ calc): 2308.1950

Fixed modifications: Carbamidomethyl (C)

Variable modifications:

N9 : Deamidated_N (N)

Ions Score: 74 Expect: $4.4 \mathrm{e}-006$

Matches (Bold Red): 22/216 fragment ions using 31 most intense peaks

\begin{tabular}{|r|c|c|c|c|c|c|c|c|c|c|c|c|c|c|}
\hline$\#$ & $\mathbf{b}$ & $\mathbf{b}^{++}$ & $\mathbf{b}^{*}$ & $\mathbf{b}^{*^{++}}$ & $\mathbf{b}^{\mathbf{0}}$ & $\mathbf{b}^{\mathbf{0 + +}}$ & Seq. & $\mathbf{y}$ & $\mathbf{y}^{++}$ & $\mathbf{y}^{\mathbf{*}}$ & $\mathbf{y}^{\boldsymbol{*}^{++}}$ & $\mathbf{y}^{\mathbf{0}}$ & $\mathbf{y}^{\mathbf{0 + +}}$ & $\#$ \\
\hline $\mathbf{1}$ & 129.0659 & 65.0366 & 112.0393 & 56.5233 & & & $\mathbf{Q}$ & & & & & & & $\mathbf{2 0}$ \\
\hline $\mathbf{2}$ & 242.1499 & 121.5786 & 225.1234 & 113.0653 & & & $\mathbf{I}$ & 2181.1438 & 1091.0755 & 2164.1172 & 1082.5622 & 2163.1332 & 1082.0702 & $\mathbf{1 9}$ \\
\hline 3 & 355.2340 & 178.1206 & 338.2074 & 169.6074 & & & $\mathbf{L}$ & 2068.0597 & $\mathbf{1 0 3 4 . 5 3 3 5}$ & 2051.0332 & 1026.0202 & 2050.0491 & 1025.5282 & $\mathbf{1 8}$ \\
\hline $\mathbf{4}$ & 484.2766 & 242.6419 & 467.2500 & 234.1287 & 466.2660 & 233.6366 & $\mathbf{E}$ & $\mathbf{1 9 5 4 . 9 7 5 6}$ & 977.9915 & 1937.9491 & 969.4782 & 1936.9651 & 968.9862 & $\mathbf{1 7}$ \\
\hline $\mathbf{5}$ & 541.2980 & 271.1527 & 524.2715 & 262.6394 & 523.2875 & 262.1474 & $\mathbf{G}$ & $\mathbf{1 8 2 5 . 9 3 3 0}$ & $\mathbf{9 1 3 . 4 7 0 2}$ & 1808.9065 & 904.9569 & 1807.9225 & 904.4649 & $\mathbf{1 6}$ \\
\hline
\end{tabular}




\begin{tabular}{|c|c|c|c|c|c|c|c|c|c|c|c|c|c|c|}
\hline 6 & 654.3821 & 327.6947 & 637.3556 & 319.1814 & 636.3715 & 318.6894 & $\mathbf{L}$ & |1768.9116 & 884.9594 & |1751.8850| & 876.4462 & $|1750.9010|$ & 875.9541 & $|15|$ \\
\hline 7 & 783.4247 & 392.2160 & 766.3981 & 383.7027 & 765.4141 & 383.2107 & $\mathbf{E}$ & 1655.8275 & 828.4174 & 1638.8010 & 819.9041 & 1637.8170 & 819.4121 & 14 \\
\hline 8 & 930.4931 & 465.7502 & 913.4666 & 457.2369 & 912.4825 & 456.7449 & $\mathbf{F}$ & 1526.7849 & 763.8961 & 1509.7584 & 755.3828 & 1508.7744 & 754.8908 & 13 \\
\hline 9 & 1045.5200 & 523.2637 & 1028.4935 & 514.7504 & 1027.5095 & 514.2584 & $\mathbf{N}$ & 1379.7165 & 690.3619 & 1362.6900 & 681.8486 & 1361.7059 & 681.3566 & 12 \\
\hline 10 & 1158.6041 & 579.8057 & 1141.5776 & 571.2924 & 1140.5935 & 570.8004 & $\mathbf{L}$ & 1264.6896 & 632.8484 & 1247.6630 & 624.3352 & 1246.6790 & 623.8431 & 11 \\
\hline 11 & 1259.6518 & 630.3295 & 1242.6252 & 621.8163 & 1241.6412 & 621.3243 & $\mathbf{T}$ & 1151.6055 & 576.3064 & 1134.5790 & 567.7931 & 1133.5949 & 567.3011 & 10 \\
\hline 12 & 1387.7104 & 694.3588 & 1370.6838 & 685.8455 & 1369.6998 & 685.3535 & $\mathbf{Q}$ & 1050.5578 & 525.7826 & 1033.5313 & 517.2693 & 1032.5473 & 516.7773 & 9 \\
\hline 13 & 1500.7944 & 750.9009 & 1483.7679 & 742.3876 & 1482.7839 & 741.8956 & I & 922.4993 & 461.7533 & 905.4727 & 453.2400 & 904.4887 & 452.7480 & 8 \\
\hline 14 & 1597.8472 & 799.4272 & 1580.8206 & 790.9140 & 1579.8366 & 790.4220 & $\mathbf{P}$ & 809.4152 & 405.2112 & 792.3886 & 396.6980 & 791.4046 & 396.2060 & 7 \\
\hline 15 & 1726.8898 & 863.9485 & 1709.8632 & 855.4353 & 1708.8792 & 854.9432 & $\mathbf{E}$ & 712.3624 & 356.6849 & 695.3359 & 348.1716 & 694.3519 & 347.6796 & 6 \\
\hline 16 & 1797.9269 & 899.4671 & 1780.9004 & 890.9538 & 1779.9163 & 890.4618 & A & 583.3198 & 292.1636 & 566.2933 & 283.6503 & 565.3093 & 283.1583 & 5 \\
\hline 17 & 1912.9538 & 956.9806 & 1895.9273 & 948.4673 & 1894.9433 & 947.9753 & D & 512.2827 & 256.6450 & 495.2562 & 248.1317 & 494.2722 & 247.6397 & 4 \\
\hline 18 & 2026.0379 & 1013.5226 & 2009.0114 & 1005.0093 & 2008.0273 & 1004.5173 & I & 397.2558 & 199.1315 & 380.2292 & 190.6183 & & & 3 \\
\hline 19 & 2163.0968 & 1082.0520 & 2146.0703 & 1073.5388 & 2145.0863 & 1073.0468 & $\mathbf{H}$ & 284.1717 & 142.5895 & 267.1452 & 134.0762 & & & 2 \\
\hline
\end{tabular}
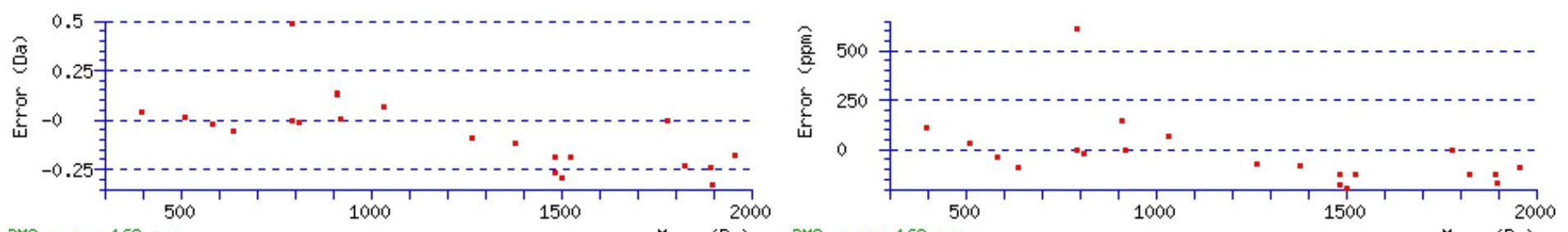

RMS error 169 ppm Mass (Da) RMS error 169 ppm

\section{All matches to this query}

\begin{tabular}{|l|l|c|c|}
\hline Score & Mr(calc): & Delta & Sequence \\
\hline 74.3 & 2308.1950 & 0.0057 & QILEGLEFNLTQIPEADIHK \\
\hline 19.4 & 2307.2110 & 0.9898 & QILEGLEFNLTQIPEADIHK \\
\hline
\end{tabular}

Spectrum No: 313; Query: 293; Rank: 1

\section{Peptide View}

MS/MS Fragmentation of QELSQDNATLK

Found in IPI00190577, Tax_Id=10116 Gene_Symbol=Lama5 similar to Laminin alpha-5 chain precursor

Match to Query 293: 1246.606048 from(624.310300,2+)

Title: 100101RatKid_NS_deglyco_23.839.839.2.dta

Data file K:INewmanPaper|Piliangl3SubProteomes\Piliang3SP\mgf5ppm\ERLIC_3SubProteomes5ppm.mgf 

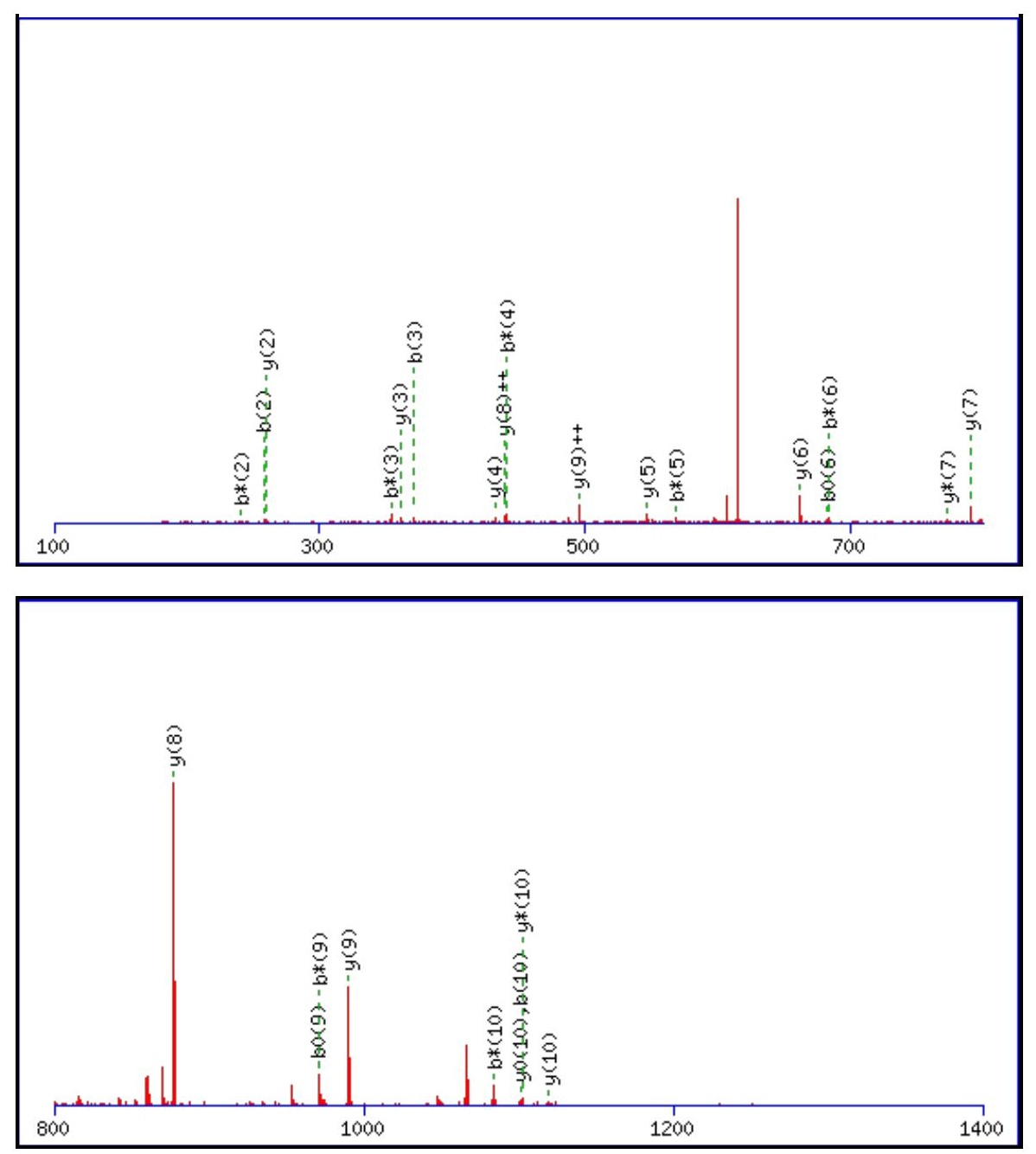

\begin{tabular}{rrrr}
\hline 00 & 1600 & 1800 & 2000 \\
\hline
\end{tabular}

Monoisotopic mass of neutral peptide $\operatorname{Mr}($ calc): 1246.6041

Fixed modifications: Carbamidomethyl (C)

Variable modifications:

N7 : Deamidated $\mathrm{N}(\mathrm{N})$

Ions Score: 74 Expect : $4.9 \mathrm{e}-006$

Matches (Bold Red): 26/114 fragment ions using 41 most intense peaks

\begin{tabular}{|r|c|c|c|c|c|c|c|c|c|c|c|c|c|c|}
\hline$\#$ & $\mathbf{b}$ & $\mathbf{b}^{++}$ & $\mathbf{b}^{*}$ & $\mathbf{b}^{\boldsymbol{*}_{++}}$ & $\mathbf{b}^{\mathbf{0}}$ & $\mathbf{b}^{\mathbf{0 + +}}$ & Seq. & $\mathbf{y}$ & $\mathbf{y}^{++}$ & $\mathbf{y}^{\mathbf{*}}$ & $\mathbf{y}^{\boldsymbol{*}^{++}}$ & $\mathbf{y}^{\mathbf{0}}$ & $\mathbf{y}^{\mathbf{0 + +}}$ & $\#$ \\
\hline $\mathbf{1}$ & 129.0659 & 65.0366 & 112.0393 & 56.5233 & & & $\mathbf{Q}$ & & & & & & & $\mathbf{1 1}$ \\
\hline $\mathbf{2}$ & $\mathbf{2 5 8 . 1 0 8 4}$ & 129.5579 & 241.0819 & 121.0446 & 240.0979 & 120.5526 & $\mathbf{E}$ & $\mathbf{1 1 1 9 . 5 5 2 8}$ & 560.2800 & $\mathbf{1 1 0 2 . 5 2 6 3}$ & 551.7668 & $\mathbf{1 1 0 1 . 5 4 2 2}$ & 551.2748 & $\mathbf{1 0}$ \\
\hline $\mathbf{3}$ & $\mathbf{3 7 1 . 1 9 2 5}$ & 186.0999 & 354.1660 & 177.5866 & 353.1819 & 177.0946 & $\mathbf{L}$ & $\mathbf{9 9 0 . 5 1 0 2}$ & $\mathbf{4 9 5 . 7 5 8 7}$ & 973.4837 & 487.2455 & 972.4996 & 486.7535 & $\mathbf{9}$ \\
\hline $\mathbf{4}$ & 458.2245 & 229.6159 & 441.1980 & 221.1026 & 440.2140 & 220.6106 & S & $\mathbf{8 7 7 . 4 2 6 1}$ & $\mathbf{4 3 9 . 2 1 6 7}$ & 860.3996 & 430.7034 & 859.4156 & 430.2114 & $\mathbf{8}$ \\
\hline $\mathbf{5}$ & 586.2831 & 293.6452 & $\mathbf{5 6 9 . 2 5 6 6}$ & 285.1319 & 568.2726 & 284.6399 & $\mathbf{Q}$ & $\mathbf{7 9 0 . 3 9 4 1}$ & 395.7007 & $\mathbf{7 7 3 . 3 6 7 6}$ & 387.1874 & 772.3836 & 386.6954 & $\mathbf{7}$ \\
\hline
\end{tabular}




\begin{tabular}{|c|c|c|c|c|c|c|c|c|c|c|c|c|c|c|}
\hline 6 & 701.3101 & |351.1587 & 684.2835 & |342.6454 & 683.2995 & |342.1534 & D & 662.3355 & |331.6714| & 645.3090 & |323.1581 & 644.3250 & |322.6661| & 6 \\
\hline 7 & 816.3370 & 408.6721 & 799.3104 & 400.1589 & 798.3264 & 399.6669 & $\mathbf{N}$ & 547.3086 & 274.1579 & 530.2820 & 265.6447 & 529.2980 & 265.1527 & 5 \\
\hline 8 & 887.3741 & 444.1907 & 870.3476 & 435.6774 & 869.3635 & 435.1854 & A & 432.2817 & 216.6445 & 415.2551 & 208.1312 & 414.2711 & 207.6392 & 4 \\
\hline 9 & 988.4218 & 494.7145 & 971.3952 & 486.2013 & 970.4112 & 485.7093 & $\mathbf{T}$ & 361.2445 & |181.1259 & 344.2180 & 172.6126 & 343.2340 & $\mid 172.1206$ & 3 \\
\hline 10 & 1101.5059 & 551.2566 & 1084.4793 & 542.7433 & 1083.4953 & 542.2513 & L & 260.1969 & |130.6021 & 243.1703 & 122.0888 & & & 2 \\
\hline 11 & & & & & & & $\mathbf{K}$ & 147.1128 & 74.0600 & 130.0863 & 65.5468 & & & 1 \\
\hline
\end{tabular}
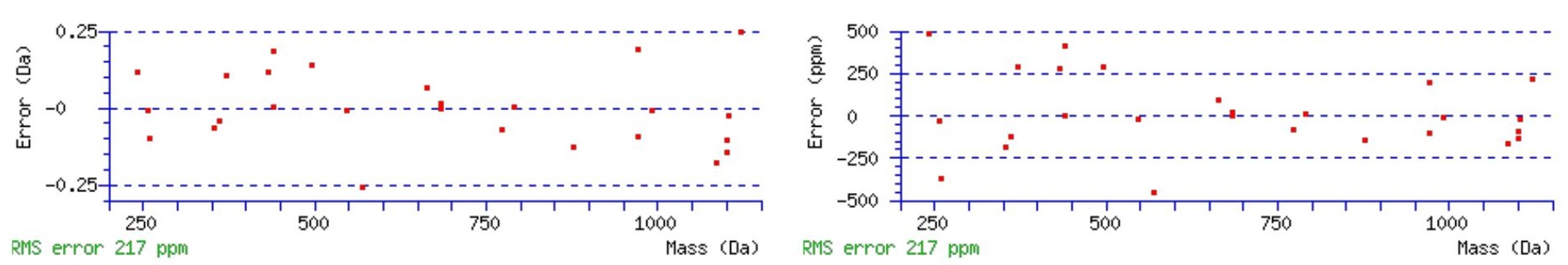

\section{All matches to this query}

\begin{tabular}{|l|l|c|l|}
\hline Score & Mr(calc): & Delta & \multicolumn{1}{|c|}{ Sequence } \\
\hline 74.2 & 1246.6041 & 0.0020 & QELSODNATLK \\
\hline 23.3 & 1246.6041 & 0.0020 & AGKLSENPSESK \\
\hline 17.5 & 1246.5958 & 0.0102 & KIESFGSKSGK \\
\hline 12.3 & 1246.6128 & -0.0068 & WALGVMAADASR \\
\hline 12.1 & 1246.6032 & 0.0028 & EKSMATFILK \\
\hline 10.6 & 1246.5958 & 0.0102 & KIESFGSKSGK \\
\hline 7.4 & 1246.6111 & -0.0051 & FKTLFVAGER \\
\hline 6.8 & 1246.6153 & -0.0093 & KQKEQSGGEEK \\
\hline 6.5 & 1246.6111 & -0.0050 & KQNVKYYPK \\
\hline 6.0 & 1246.6153 & -0.0093 & ESSQSSAQPAKK \\
\hline
\end{tabular}

Spectrum No: 314; Query: 1769; Rank: 1

\section{Peptide View}

MS/MS Fragmentation of AWGTPCELCPPVNTSEYK

Found in IPI00204006, Tax_Id=10116 Gene_Symbol=Fbn1 Fibrillin-1

Match to Query 1769: 2108.927488 from(1055.471020,2+)

Title: 100101RatKid_NS_deglyco_23.3372.3372.2.dta

Data file K:INewmanPaper|Piliangl3SubProteomes\Piliang3SP\mgf5ppm\ERLIC_3SubProteomes5ppm.mgf

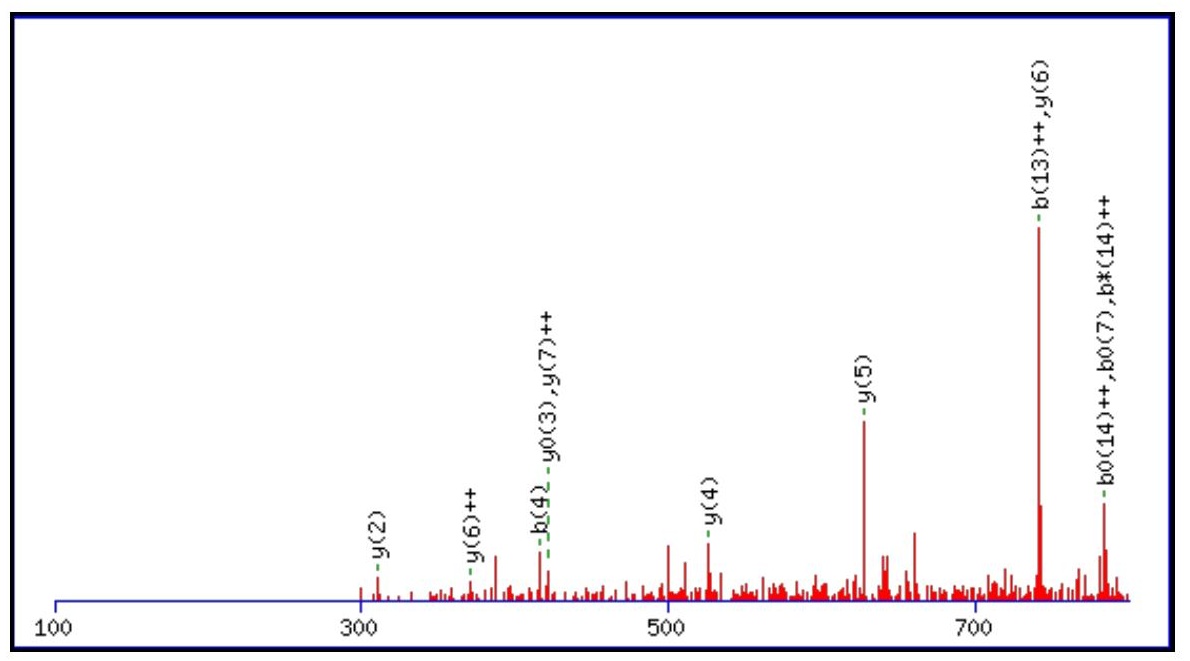



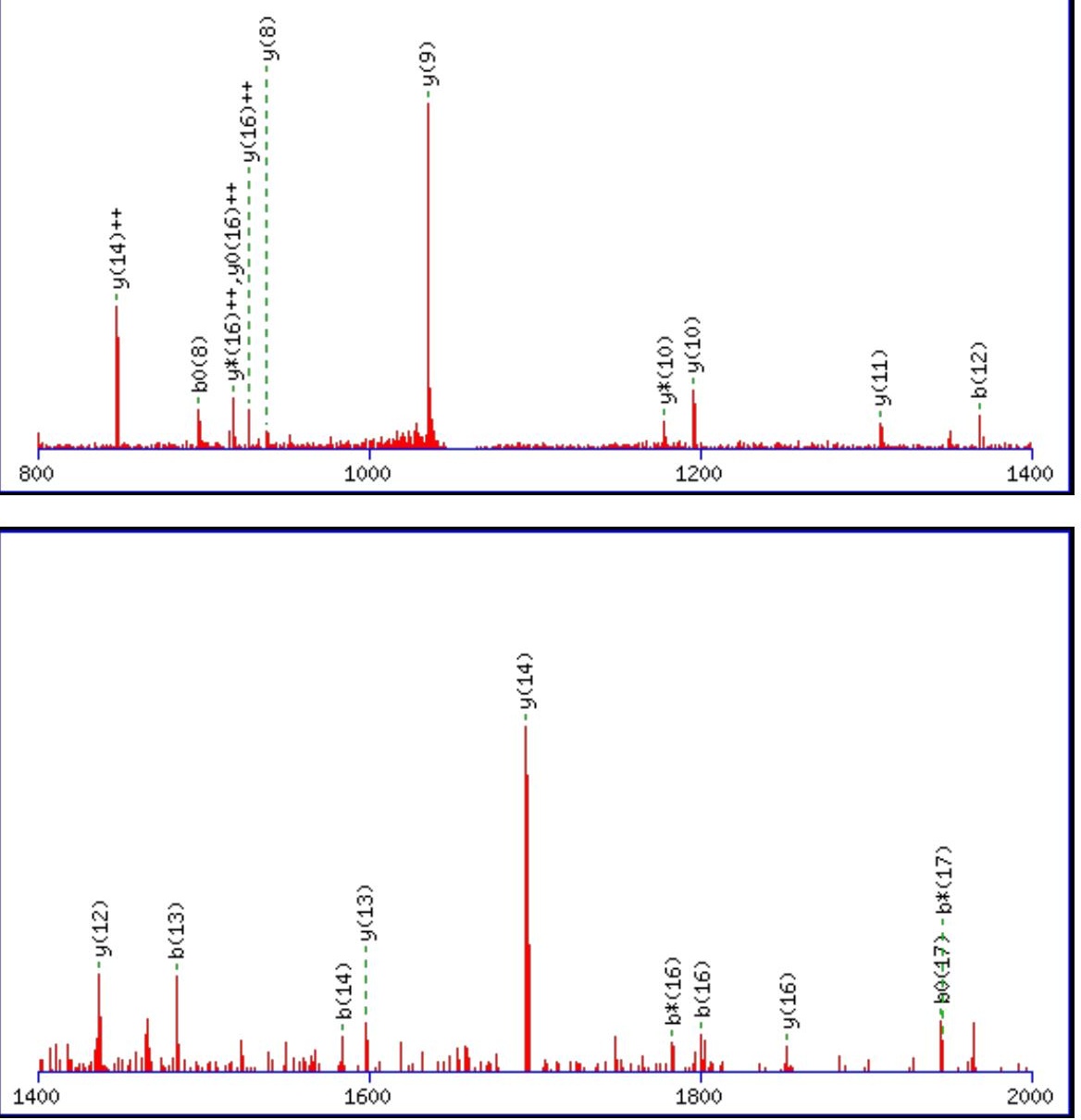

Monoisotopic mass of neutral peptide $\operatorname{Mr}$ (calc): 2108.9183

Fixed modifications: Carbamidomethyl (C)

Variable modifications:

N13 : Deamidated_N (N)

Ions Score: 74 Expect: $1 \mathrm{e}-005$

Matches (Bold Red): 33/170 fragment ions using 57 most intense peaks

\begin{tabular}{|c|c|c|c|c|c|c|c|c|c|c|c|c|c|c|}
\hline \# & b & $\mathbf{b}^{++}$ & b* & $\mathbf{b}^{*^{++}}$ & $\mathbf{b}^{0}$ & $\mathbf{b}^{\mathbf{0 + +}}$ & Seq. & $\mathbf{y}$ & $\mathbf{y}^{++}$ & $\mathbf{y}^{*}$ & $\mathrm{y}^{*^{++}}$ & $\mathbf{y}^{\mathbf{0}}$ & $\mathbf{y}^{0++}$ & \# \\
\hline 1 & 72.0444 & 36.5258 & & & & & A & & & & & & & 18 \\
\hline 2 & 258.1237 & 129.5655 & & & & & $\mathbf{W}$ & 2038.8885 & 1019.9479 & 2021.8619 & 1011.4346 & 2020.8779 & 1010.9426 & 17 \\
\hline 3 & 315.1452 & 158.0762 & & & & & G & 1852.8092 & 926.9082 & 1835.7826 & 918.3950 & 1834.7986 & 917.9029 & 16 \\
\hline 4 & 416.1928 & 208.6001 & & & 398.1823 & 199.5948 & $\mathbf{T}$ & 1795.7877 & 898.3975 & 1778.7612 & 889.8842 & 1777.7771 & 889.3922 & 15 \\
\hline 5 & 513.2456 & 257.1264 & & & 495.2350 & 248.1212 & $\mathbf{P}$ & 1694.7400 & 847.8737 & 1677.7135 & 839.3604 & 1676.7295 & 838.8684 & 14 \\
\hline 6 & 673.2763 & 337.1418 & & & 655.2657 & 328.1365 & C & 1597.6873 & 799.3473 & 1580.6607 & 790.8340 & 1579.6767 & 790.3420 & 13 \\
\hline 7 & 802.3189 & 401.6631 & & & 784.3083 & 392.6578 & $\mathbf{E}$ & 1437.6566 & 719.3319 & 1420.6301 & 710.8187 & 1419.6461 & 710.3267 & 12 \\
\hline 8 & 915.4029 & 458.2051 & & & 897.3924 & 449.1998 & $\mathbf{L}$ & 1308.6140 & 654.8107 & 1291.5875 & 646.2974 & 1290.6035 & 645.8054 & 11 \\
\hline 9 & 1075.4336 & 538.2204 & & & 1057.4230 & 529.2151 & C & 1195.5300 & 598.2686 & 1178.5034 & 589.7553 & 1177.5194 & 589.2633 & 10 \\
\hline 10 & 1172.4863 & 586.7468 & & & 1154.4758 & 577.7415 & $\mathbf{P}$ & 1035.4993 & 518.2533 & 1018.4728 & 509.7400 & 1017.4887 & 509.2480 & 9 \\
\hline 11 & 1269.5391 & 635.2732 & & & 1251.5285 & 626.2679 & $\mathbf{P}$ & 938.4465 & 469.7269 & 921.4200 & 461.2136 & 920.4360 & 460.7216 & 8 \\
\hline 12 & 1368.6075 & 684.8074 & & & 1350.5969 & 675.8021 & V & 841.3938 & 421.2005 & 824.3672 & 412.6873 & 823.3832 & 412.1952 & 7 \\
\hline 13 & 1483.6344 & 742.3209 & 1466.6079 & 733.8076 & 1465.6239 & 733.3156 & $\mathbf{N}$ & 742.3254 & 371.6663 & 725.2988 & 363.1530 & 724.3148 & 362.6610 & 6 \\
\hline 14 & 1584.6821 & 792.8447 & 1567.6556 & 784.3314 & 1566.6716 & 783.8394 & $\mathbf{T}$ & 627.2984 & 314.1529 & 610.2719 & 305.6396 & 609.2879 & 305.1476 & 5 \\
\hline 15 & 1671.7142 & 836.3607 & 1654.6876 & 827.8474 & 1653.7036 & 827.3554 & S & 526.2508 & 263.6290 & 509.2242 & 255.1157 & 508.2402 & 254.6237 & 4 \\
\hline 16 & 1800.7567 & 900.8820 & 1783.7302 & 892.3687 & 1782.7462 & 891.8767 & $\mathbf{E}$ & 439.2187 & 220.1130 & 422.1922 & 211.5997 & 421.2082 & 211.1077 & 3 \\
\hline 17 & 1963.8201 & 982.4137 & 1946.7935 & 973.9004 & 1945.8095 & 973.4084 & $\mathbf{Y}$ & 310.1761 & 155.5917 & 293.1496 & 147.0784 & & & 2 \\
\hline 18 & & & & & & & $\mathbf{K}$ & 147.1128 & 74.0600 & 130.0863 & 65.5468 & & & 1 \\
\hline
\end{tabular}



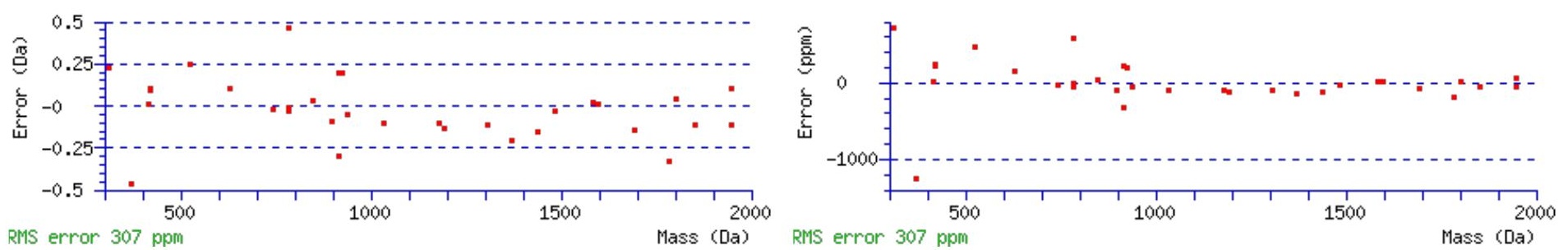

\section{All matches to this query}

\begin{tabular}{|l|l|l|l|}
\hline Score & Mr(calc): & Delta & \multicolumn{1}{|c|}{ Sequence } \\
\hline 74.1 & 2108.9183 & 0.0092 & AWGTPCELCPPVNTSEYK \\
\hline 3.3 & 2107.9197 & 1.0078 & MSVCTLSVPVSSISPGRR \\
\hline 2.4 & 2107.9197 & 1.0078 & MSVCTLSVPVSSISPGRR \\
\hline 2.4 & 2107.9197 & 1.0078 & MSVCTLSVPVSSISPGRR \\
\hline 2.0 & 2106.9388 & 1.9887 & ERKPNNLSDTVKELFR \\
\hline 1.4 & 2106.9115 & 2.0160 & ALMNTQYGLERIMSDER \\
\hline 1.3 & 2106.9228 & 2.0047 & MSVPCSEYRKTSGLGSPR \\
\hline 0.3 & 2108.9126 & 0.0149 & QFSQLPANVAVCSSETYK \\
\hline
\end{tabular}

Spectrum No: 315; Query: 1809; Rank: 1

\section{Peptide View}

MS/MS Fragmentation of CDPAIKPHEELLILNLTR

Found in IPI00325860, Tax_Id=10116 Gene_Symbol=Podxl Podocalyxin precursor

Match to Query 1809: 2132.137122 from(711.719650,3+)

Title: 100101RatKid_NS_deglyco_14.4023.4023.3.dta

Data file K:INewmanPaper|Piliangl3SubProteomes\Piliang3SP\mgf5ppm\ERLIC_3SubProteomes5ppm.mgf

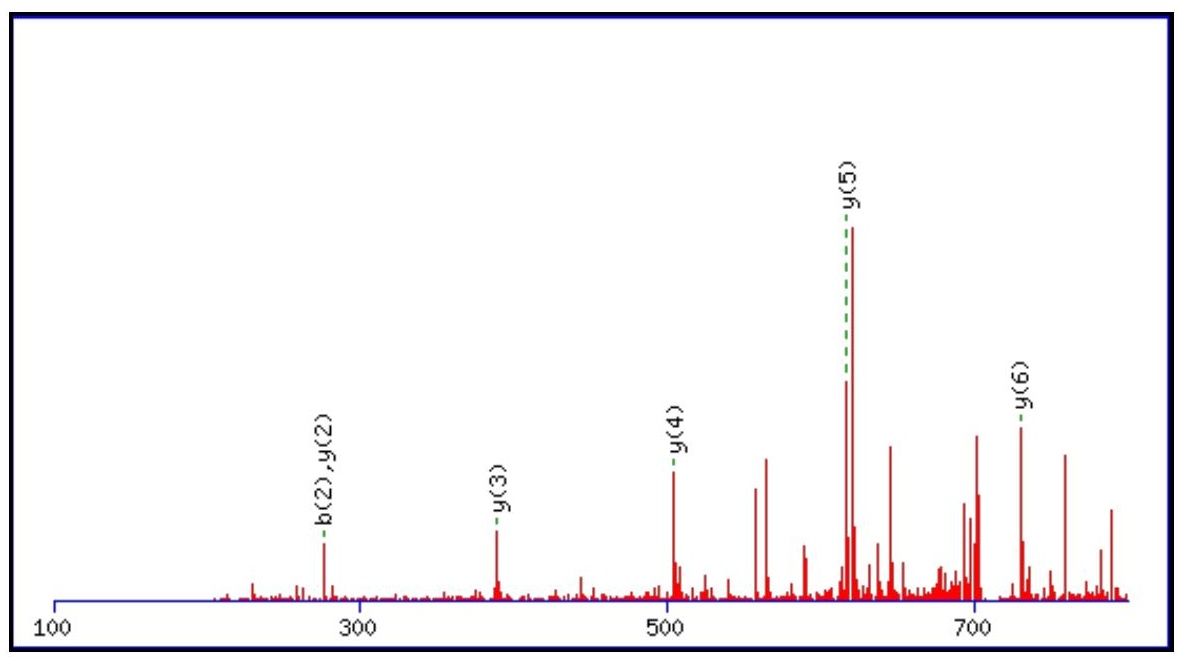



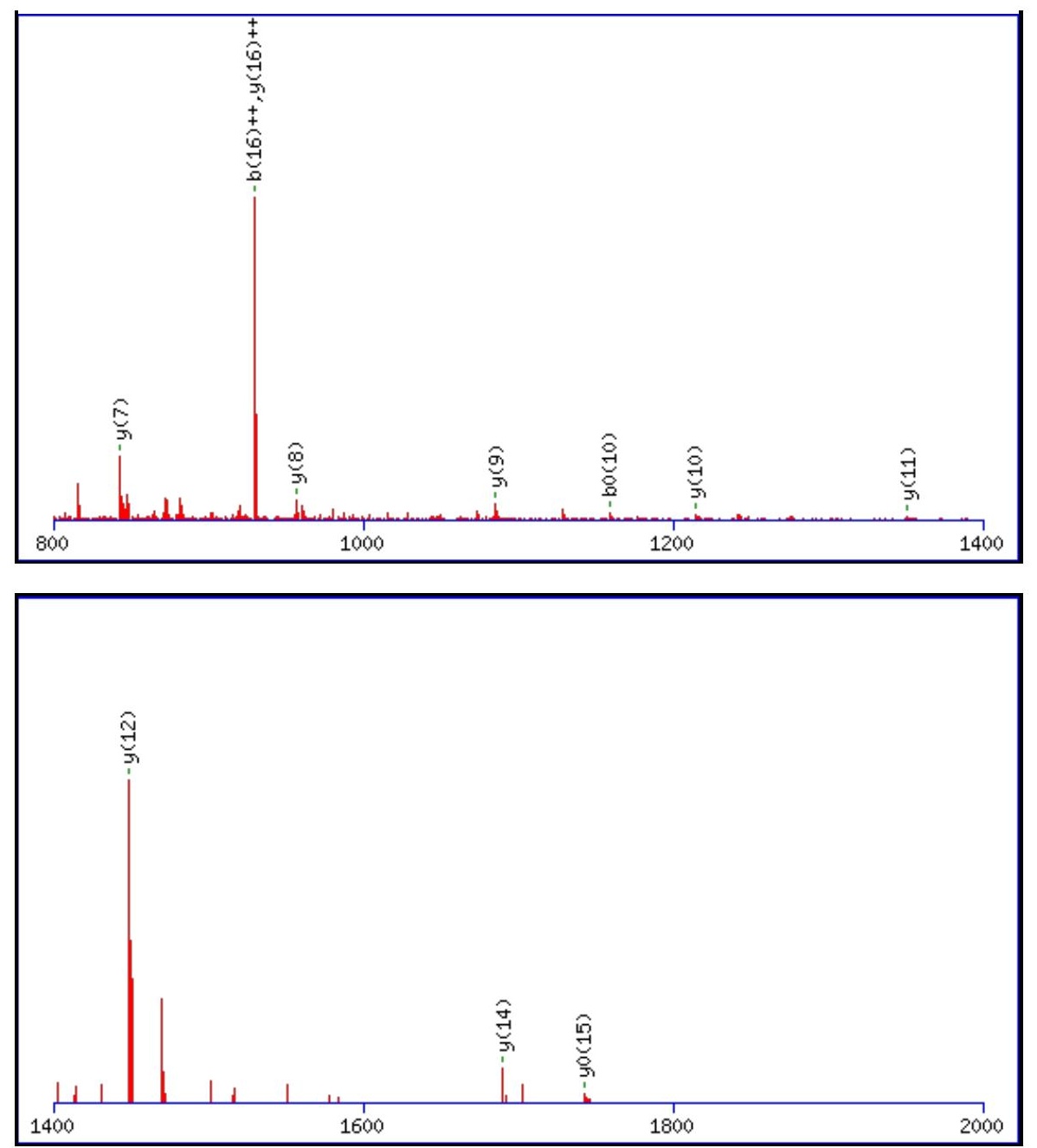

Monoisotopic mass of neutral peptide $\operatorname{Mr}$ (calc): 2132.1299

Fixed modifications: Carbamidomethyl (C)

Variable modifications:

N15 : Deamidated_N (N)

Ions Score: 74 Expect: $3.9 \mathrm{e}-006$

Matches (Bold Red): 17/190 fragment ions using 31 most intense peaks

\begin{tabular}{|c|c|c|c|c|c|c|c|c|c|c|c|c|c|c|}
\hline \# & b & $\mathbf{b}^{++}$ & $\mathbf{b}^{*}$ & $\mathbf{b}^{*^{++}}$ & $\mathbf{b}^{0}$ & & Seq. & $\mathbf{y}$ & $\mathbf{y}^{++}$ & $\mathbf{y}^{*}$ & $\mathbf{y}^{*^{++}}$ & $\mathbf{y}^{0}$ & $y^{0++}$ & \# \\
\hline 1 & 161.0379 & 81.0226 & & & & & C & & & & & & & 18 \\
\hline 2 & 276.0649 & 138.5361 & & & 258.0543 & 129.5308 & D & 1973.1066 & 987.0569 & 1956.0800 & & 1955 & & 17 \\
\hline 3 & 373.1176 & 187.0625 & & & 355.1071 & 178.0572 & $\mathbf{P}$ & 1858.0797 & 929.5435 & 1841.0531 & 921.0302 & 1840.0691 & 920.5382 & 16 \\
\hline 4 & 444.1547 & 222.5810 & & & 426.1442 & 213.5757 & A & 1761.0269 & 881.0171 & 1744.0003 & 872.5038 & 1743.0163 & 872.0118 & 15 \\
\hline 5 & 557.2388 & 279.1230 & & & 539.2282 & 270.1178 & I & 1689.9898 & 845.4985 & 1672.9632 & 836.9853 & 1671.9792 & 836.4932 & 14 \\
\hline 6 & 685.3338 & 343.1705 & 668.3072 & 334.6573 & 667.3232 & 334.1652 & $\mathbf{K}$ & 1576.9057 & 788.9565 & 1559.8792 & 780.4432 & 1558.8951 & 779.9512 & 13 \\
\hline 7 & 782.3865 & 391.6969 & 765.3600 & 383.1836 & 764.3760 & 382.6916 & $\mathbf{P}$ & 1448.8107 & 724.9090 & 1431.7842 & 716.3957 & 1430.8002 & 715.9037 & 12 \\
\hline 8 & 919.4454 & 460.2264 & 902.4189 & 451.7131 & 901.4349 & 451.2211 & $\mathbf{H}$ & 1351.7580 & 676.3826 & 1334.7314 & 667.8694 & 1333.7474 & 667.3773 & 11 \\
\hline 9 & 1048.4880 & 524.7477 & 1031.4615 & 516.2344 & 1030.4775 & 515.7424 & $\mathbf{E}$ & 1214.6991 & 607.8532 & 1197.6725 & 599.3399 & 1196.6885 & 598.8479 & 10 \\
\hline 10 & 1177.5306 & 589.2690 & 1160.5041 & 580.7557 & 1159.5201 & 580.2637 & $\mathbf{E}$ & 1085.6565 & 543.3319 & 1068.6299 & 534.8186 & 1067.6459 & 534.3266 & 9 \\
\hline 11 & 1290.6147 & 645.8110 & 1273.5882 & 637.2977 & 1272.6041 & 636.8057 & $\mathbf{L}$ & 956.6139 & 478.8106 & 939.5873 & 470.2973 & 938.6033 & 469.8053 & 8 \\
\hline 12 & 1403.6988 & 702.3530 & 1386.6722 & 693.8397 & 1385.6882 & 693.3477 & $\mathbf{L}$ & 843.5298 & 422.2685 & 826.5033 & 413.7553 & 825.5193 & 413.2633 & 7 \\
\hline 13 & 1516.7828 & 758.8951 & 1499.7563 & 750.3818 & 1498.7723 & 749.8898 & I & 730.4458 & 365.7265 & 713.4192 & 357.2132 & 712.4352 & 356.7212 & 6 \\
\hline 14 & 1629.8669 & 815.4371 & 1612.8403 & 806.9238 & 1611.8563 & 806.4318 & $\mathbf{L}$ & 617.3617 & 309.1845 & 600.3351 & 300.6712 & 599.3511 & 300.1792 & 5 \\
\hline 15 & 1744.8938 & 872.9506 & 1727.8673 & 864.4373 & 1726.8833 & 863.9453 & $\mathbf{N}$ & 504.2776 & 252.6425 & 487.2511 & 244.1292 & 486.2671 & 243.6372 & 4 \\
\hline 16 & 1857.9779 & 929.4926 & 1840.9513 & 920.9793 & 1839.9673 & 920.4873 & $\mathbf{L}$ & 389.2507 & 195.1290 & 372.2241 & 186.6157 & 371.2401 & 186.1237 & 3 \\
\hline 17 & 1959.0256 & 980.0164 & 1941.9990 & 971.5031 & 1941.0150 & 971.0111 & $\mathbf{T}$ & 276.1666 & 138.5870 & 259.1401 & 130.0737 & 258.1561 & 129.5817 & 2 \\
\hline 18 & & & & & & & $\mathbf{R}$ & 175.1190 & 88.0631 & 158.0924 & 79.5498 & & & 1 \\
\hline
\end{tabular}



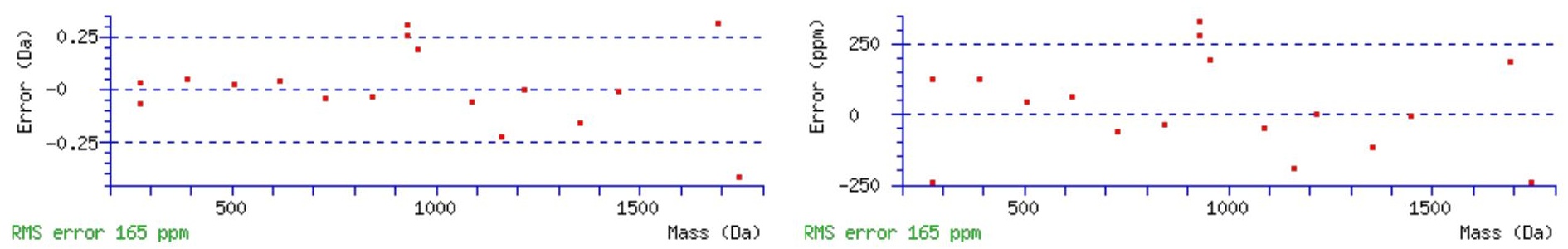

\section{All matches to this query}

\begin{tabular}{|l|l|l|l|}
\hline Score & Mr(calc): & Delta & \multicolumn{1}{|c|}{ Sequence } \\
\hline 74.1 & 2132.1299 & 0.0072 & CDPAIKPHEELLILNLTR \\
\hline 9.8 & 2131.1459 & 0.9912 & CDPAIKPHEELLILNLTR \\
\hline 5.6 & 2130.1256 & 2.0116 & IPQGTVGQVMLDDRAYLVR \\
\hline 4.5 & 2132.1306 & 0.0065 & VFPVLWTLVSPFINENTR \\
\hline 2.5 & 2132.1378 & -0.0007 & LFNHQEHKVIIVGLDNAGK \\
\hline 1.8 & 2132.1239 & 0.0132 & EFLRARRPPGPDLSPDGPR \\
\hline 0.9 & 2131.1241 & 1.0130 & LKMQIVSLKAGSLVVTLR \\
\hline 0.8 & 2132.1428 & -0.0057 & SVILHLLSQLKLGMDLTR \\
\hline 0.6 & 2132.1306 & 0.0065 & VFPVLWTLVSPFINENTR \\
\hline 0.0 & 2132.1428 & -0.0057 & SVILHLLSQLKLGMDLTR \\
\hline
\end{tabular}

Spectrum No: 316; Query: 970; Rank: 1

\section{Peptide View}

\section{MS/MS Fragmentation of KIENLEQNISLPVR}

Found in IPI00400747, Tax_Id=10116 Gene_Symbol=Man2b1 Mannosidase 2, alpha B1

Match to Query 970: 1652.908908 from(827.461730,2+)

Title: 091008RatKidney_NH4Format01_18.3090.3090.2.dta

Data file K:INewmanPaper|Piliang|3SubProteomes\Piliang3SP\mgf5ppm\ERLIC_3SubProteomes5ppm.mgf

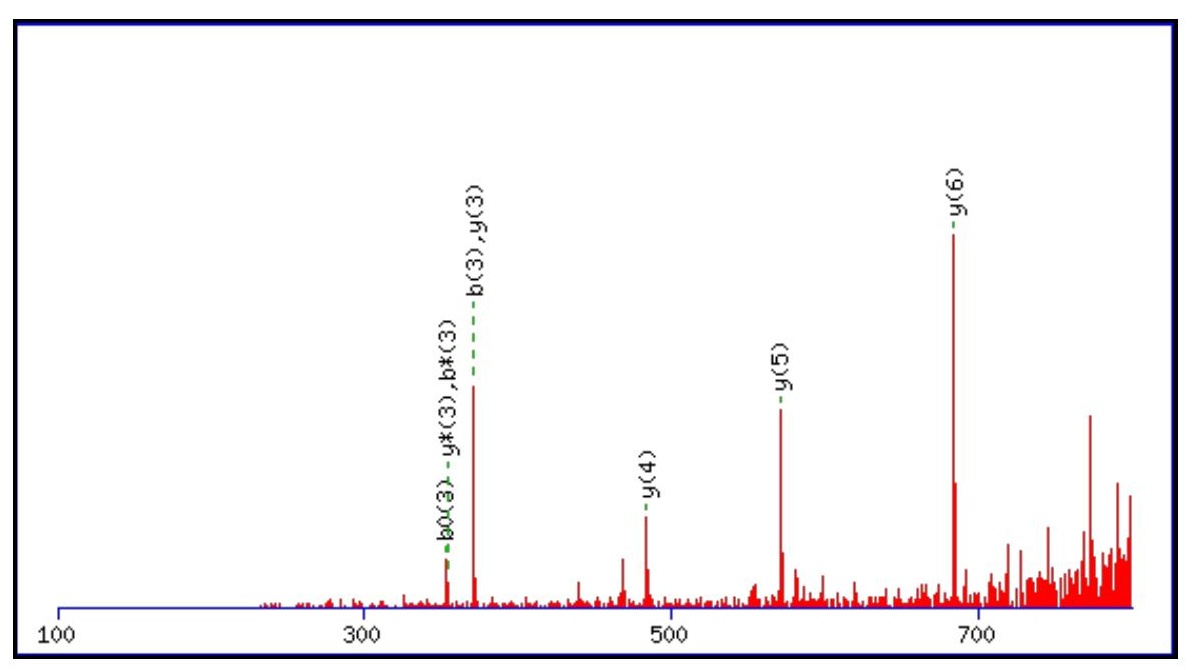



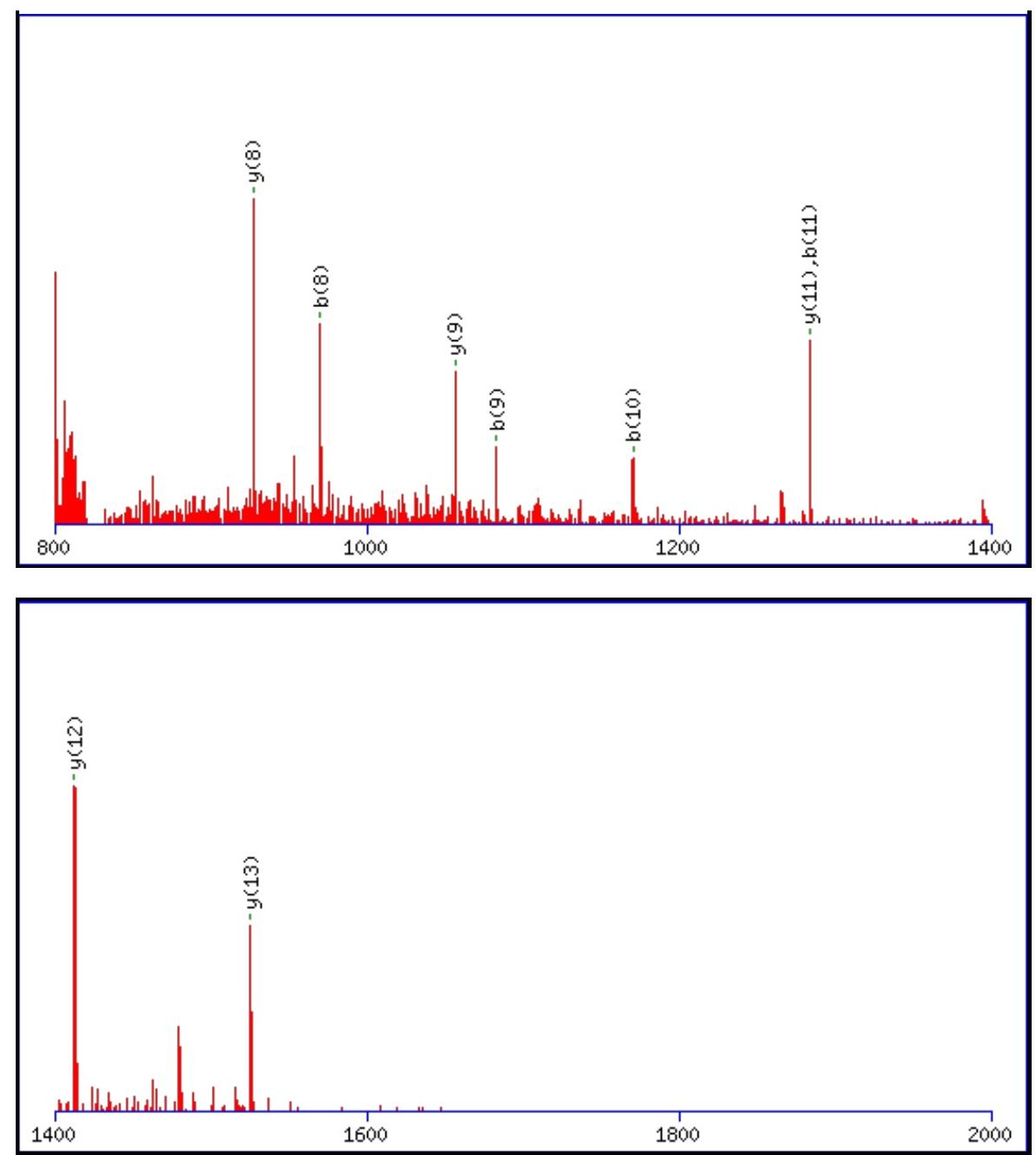

Monoisotopic mass of neutral peptide $\operatorname{Mr}($ calc): 1652.9097

Fixed modifications: Carbamidomethyl (C)

Variable modifications:

N8: : Deamidated $\mathrm{N}(\mathrm{N})$

Ions Score: 74 Expect: $2.5 \mathrm{e}-006$

Matches (Bold Red): 17/144 fragment ions using 26 most intense peaks

\begin{tabular}{|c|c|c|c|c|c|c|c|c|c|c|c|c|c|c|}
\hline \# & b & $\mathbf{b}^{++}$ & $\mathbf{b}^{*}$ & $\mathbf{b}^{*^{++}}$ & $\mathbf{b}^{\mathbf{0}}$ & $\mathbf{b}^{0++}$ & Seq. & $\mathbf{y}$ & $\mathbf{y}^{++}$ & $\mathbf{y}^{*}$ & $\mathrm{y}^{*^{++}}$ & $\mathbf{y}^{\mathbf{0}}$ & $\mathbf{y}^{\mathbf{0 + +}}$ & \# \\
\hline 1 & 129.1022 & 65.0548 & 112.0757 & 56.5415 & & & $\mathbf{K}$ & & & & & & & 14 \\
\hline 2 & 242.1863 & 121.5968 & 225.1598 & 113.0835 & & & I & 1525.8220 & 763.4147 & 1508.7955 & 754.9014 & 1507.8115 & 754.4094 & 13 \\
\hline 3 & 371.2289 & 186.1181 & 354.2023 & 177.6048 & 353.2183 & 177.1128 & $\mathbf{E}$ & 1412.7380 & 706.8726 & 1395.7114 & 698.3594 & 1394.7274 & 697.8673 & 12 \\
\hline 4 & 485.2718 & 243.1395 & 468.2453 & 234.6263 & 467.2613 & 234.1343 & $\mathbf{N}$ & 1283.6954 & 642.3513 & 1266.6688 & 633.8381 & 1265.6848 & 633.3460 & 11 \\
\hline 5 & 598.3559 & 299.6816 & 581.3293 & 291.1683 & 580.3453 & 290.6763 & $\mathbf{L}$ & 1169.6525 & 585.3299 & 1152.6259 & 576.8166 & 1151.6419 & 576.3246 & 10 \\
\hline 6 & 727.3985 & 364.2029 & 710.3719 & 355.6896 & 709.3879 & 355.1976 & $\mathbf{E}$ & 1056.5684 & 528.7878 & 1039.5418 & 520.2746 & 1038.5578 & 519.7826 & 9 \\
\hline 7 & 855.4571 & 428.2322 & 838.4305 & 419.7189 & 837.4465 & 419.2269 & $\mathbf{Q}$ & 927.5258 & 464.2665 & 910.4993 & 455.7533 & 909.5152 & 455.2613 & 8 \\
\hline 8 & 970.4840 & 485.7456 & 953.4574 & 477.2324 & 952.4734 & 476.7404 & $\mathbf{N}$ & 799.4672 & 400.2372 & 782.4407 & 391.7240 & 781.4567 & 391.2320 & 7 \\
\hline 9 & 1083.5681 & 542.2877 & 1066.5415 & 533.7744 & 1065.5575 & 533.2824 & I & 684.4403 & 342.7238 & 667.4137 & 334.2105 & 666.4297 & 333.7185 & 6 \\
\hline 10 & 1170.6001 & 585.8037 & 1153.5735 & 577.2904 & 1152.5895 & 576.7984 & $\mathrm{~S}$ & 571.3562 & 286.1817 & 554.3297 & 277.6685 & 553.3457 & 277.1765 & 5 \\
\hline 11 & 1283.6842 & 642.3457 & 1266.6576 & 633.8324 & 1265.6736 & 633.3404 & $\mathbf{L}$ & 484.3242 & 242.6657 & 467.2976 & 234.1525 & & & 4 \\
\hline 12 & 1380.7369 & 690.8721 & 1363.7104 & 682.3588 & 1362.7263 & 681.8668 & $\mathbf{P}$ & 371.2401 & 186.1237 & 354.2136 & 177.6104 & & & 3 \\
\hline 13 & 1479.8053 & 740.4063 & 1462.7788 & 731.8930 & 1461.7948 & 731.4010 & $\mathbf{V}$ & 274.1874 & 137.5973 & 257.1608 & 129.0840 & & & 2 \\
\hline 14 & & & & & & & $\mathbf{R}$ & 175.1190 & 88.0631 & 158.0924 & 79.5498 & & & 1 \\
\hline
\end{tabular}



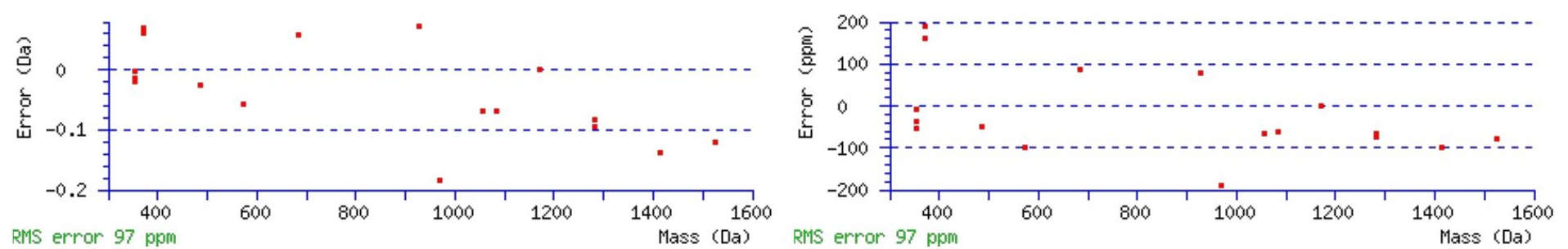

All matches to this query

\begin{tabular}{|l|l|l|l|}
\hline Score & Mr(calc): & Delta & \multicolumn{1}{|c|}{ Sequence } \\
\hline 74.1 & 1652.9097 & -0.0008 & KIENLEQNISLPVR \\
\hline 43.4 & 1652.9097 & -0.0008 & KIENLEQNISLPVR \\
\hline 6.1 & 1652.9209 & -0.0120 & KQNLPQNIKIESNK \\
\hline 1.9 & 1652.8958 & 0.0132 & $\underline{\text { LQLNQSKNNIPRAR }}$ \\
\hline 1.8 & 1651.9174 & 0.9915 & LYATVVRKGSLLPR \\
\hline 1.8 & 1652.9209 & -0.0120 & RSAANALEGPAVKIEK \\
\hline 1.8 & 1652.8985 & 0.0104 & KSAPETLTLPDPEKK \\
\hline 1.6 & 1652.8958 & 0.0132 & LQLNQSKNNIPRAR \\
\hline 0.3 & 1652.9097 & -0.0008 & $\underline{\text { ADLTGISKSPSLHLSK }}$ \\
\hline
\end{tabular}

Spectrum No: 317; Query: 2119; Rank: 1

\section{Peptide View}

MS/MS Fragmentation of VLTLANFTTKDEGDYMCELR

Found in IPI00188956, Tax_Id=10116 Gene_Symbol=Thy1 Thy-1 membrane glycoprotein precursor

Match to Query 2119: 2376.105942 from(793.042590,3+)

Title: 100101RatKid_NS_deglyco_13.4855.4855.3.dta

Data file K:INewmanPaper|Piliangl3SubProteomes\Piliang3SP\mgf5ppm|ERLIC_3SubProteomes5ppm.mgf

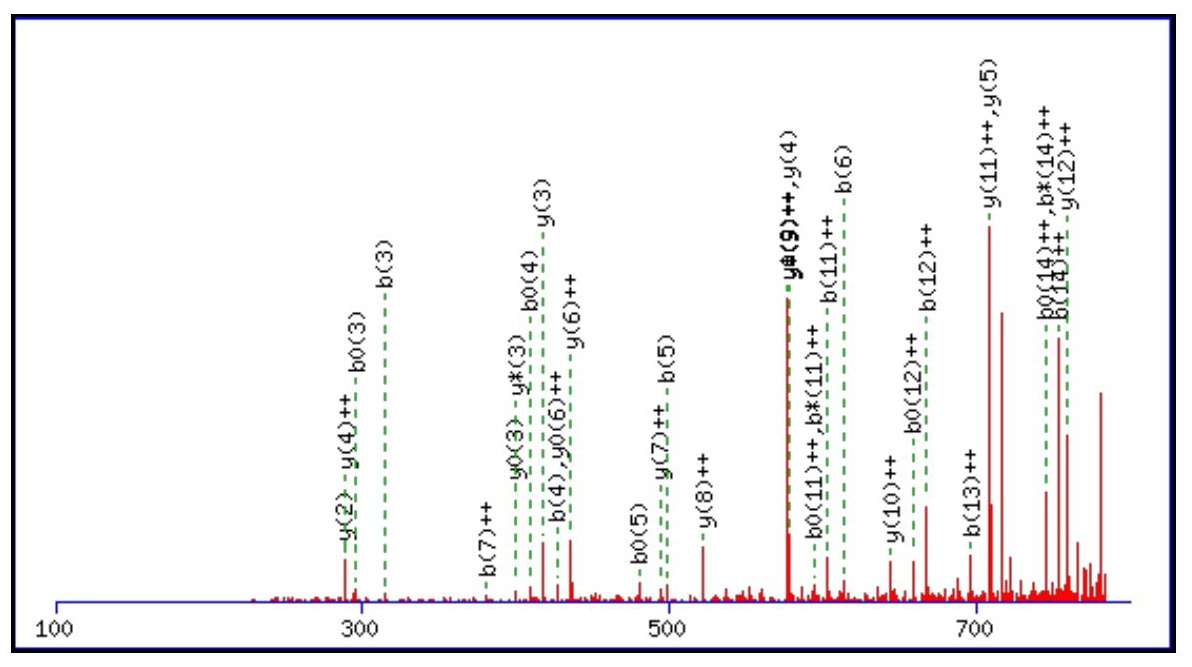



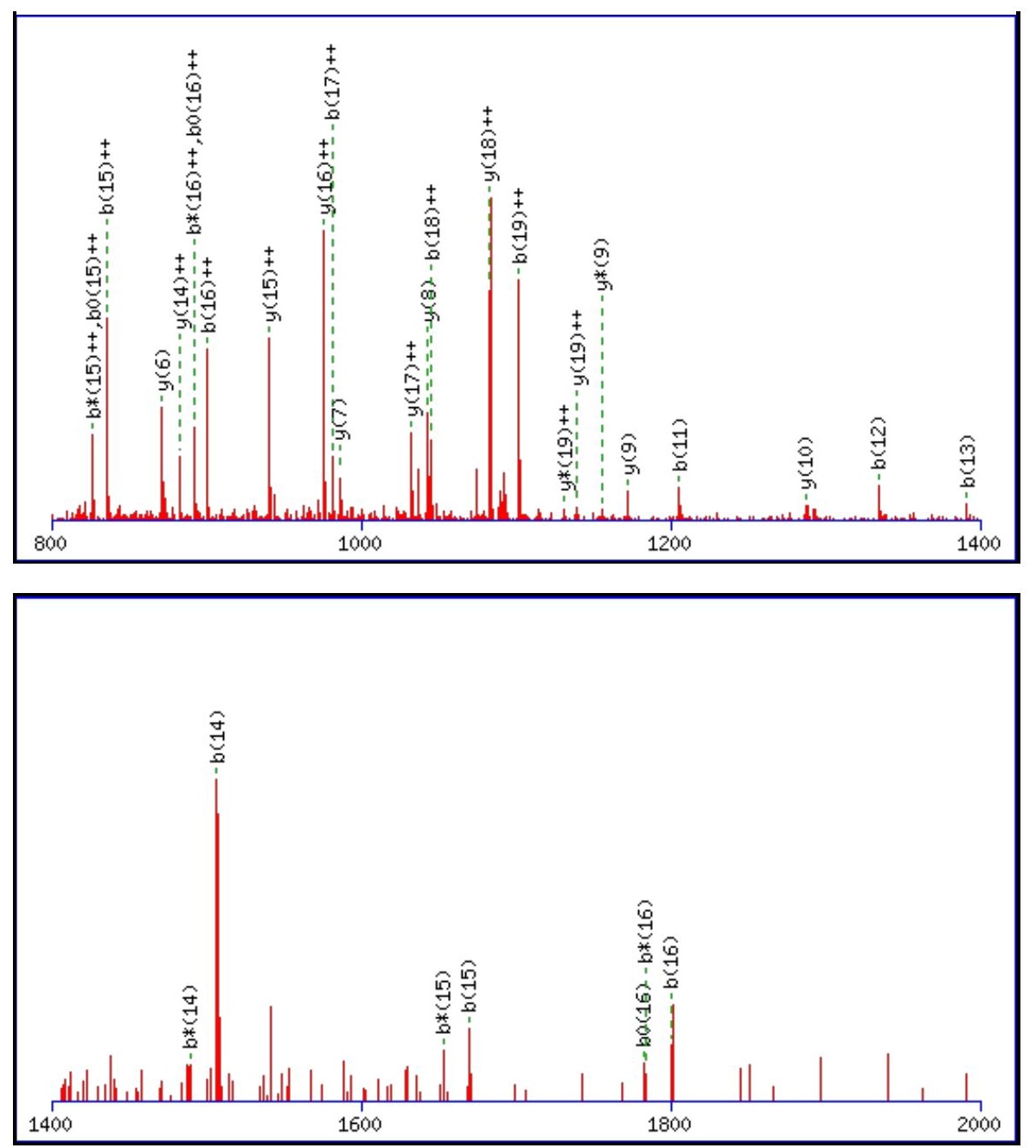

Monoisotopic mass of neutral peptide $\operatorname{Mr}($ calc): 2376.0977

Fixed modifications: Carbamidomethyl (C)

Variable modifications:

N6 : Deamidated $\mathrm{N}(\mathrm{N})$

Ions Score: 74 Expect: $1.1 \mathrm{e}-005$

Matches (Bold Red): 65/210 fragment ions using 107 most intense peaks

\begin{tabular}{|c|c|c|c|c|c|c|c|c|c|c|c|c|c|c|}
\hline \# & b & $\mathbf{b}^{++}$ & b* & $\mathbf{b}^{*^{++}}$ & $\mathbf{b}^{0}$ & $\mathbf{b}^{\mathbf{0 + +}}$ & Seq. & $\mathbf{y}$ & $\mathbf{y}^{++}$ & $\mathbf{y}^{*}$ & $\mathbf{y}^{*^{++}}$ & $\mathbf{y}^{0}$ & $\mathbf{y}^{\mathbf{0 + +}}$ & \# \\
\hline 1 & 100.0757 & 50.5415 & & & & & $\mathbf{V}$ & & & & & & & 20 \\
\hline 2 & 213.1598 & 107.0835 & & & & & $\mathbf{L}$ & 2278.0366 & 1139.5219 & 2261.0101 & 1131.0087 & 2260.0260 & 1130.5167 & 19 \\
\hline 3 & 314.2074 & 157.6074 & & & 296.1969 & 148.6021 & $\mathbf{T}$ & 2164.9525 & 1082.9799 & 2147.9260 & 1074.4666 & 2146.9420 & 1073.9746 & 18 \\
\hline 4 & 427.2915 & 214.1494 & & & 409.2809 & 205.1441 & $\mathbf{L}$ & 2063.9049 & 1032.4561 & 2046.8783 & 1023.9428 & 2045.8943 & 1023.4508 & 17 \\
\hline 5 & 498.3286 & 249.6679 & & & 480.3180 & 240.6627 & A & 1950.8208 & 975.9140 & 1933.7943 & 967.4008 & 1932.8102 & 966.9088 & 16 \\
\hline 6 & 613.3555 & 307.1814 & 596.3290 & 298.6681 & 595.3450 & 298.1761 & $\mathbf{N}$ & 1879.7837 & 940.3955 & 1862.7571 & 931.8822 & 1861.7731 & 931.3902 & 15 \\
\hline 7 & 760.4240 & 380.7156 & 743.3974 & 372.2023 & 742.4134 & 371.7103 & $\mathbf{F}$ & 1764.7568 & 882.8820 & 1747.7302 & 874.3687 & 1746.7462 & 873.8767 & 14 \\
\hline 8 & 861.4716 & 431.2395 & 844.4451 & 422.7262 & 843.4611 & 422.2342 & $\mathbf{T}$ & 1617.6883 & 809.3478 & 1600.6618 & 800.8345 & 1599.6778 & 800.3425 & 13 \\
\hline 9 & 962.5193 & 481.7633 & 945.4928 & 473.2500 & 944.5088 & 472.7580 & $T$ & 1516.6407 & 758.8240 & 1499.6141 & 750.3107 & 1498.6301 & 749.8187 & 12 \\
\hline 10 & 1090.6143 & 545.8108 & 1073.5877 & 537.2975 & 1072.6037 & 536.8055 & $\mathbf{K}$ & 1415.5930 & 708.3001 & 1398.5664 & 699.7869 & 1397.5824 & 699.2948 & 11 \\
\hline 11 & 1205.6412 & 603.3243 & 1188.6147 & 594.8110 & 1187.6307 & 594.3190 & D & 1287.4980 & 644.2526 & 1270.4715 & 635.7394 & 1269.4875 & 635.2474 & 10 \\
\hline 12 & 1334.6838 & 667.8455 & 1317.6573 & 659.3323 & 1316.6733 & 658.8403 & $\mathbf{E}$ & 1172.4711 & 586.7392 & 1155.4445 & 578.2259 & 1154.4605 & 577.7339 & 9 \\
\hline 13 & 1391.7053 & 696.3563 & 1374.6787 & 687.8430 & 1373.6947 & 687.3510 & G & 1043.4285 & 522.2179 & 1026.4019 & 513.7046 & 1025.4179 & 513.2126 & 8 \\
\hline 14 & 1506.7322 & 753.8698 & 1489.7057 & 745.3565 & 1488.7217 & 744.8645 & D & 986.4070 & 493.7071 & 969.3805 & 485.1939 & 968.3965 & 484.7019 & 7 \\
\hline 15 & 1669.7956 & 835.4014 & 1652.7690 & 826.8881 & 1651.7850 & 826.3961 & $\mathbf{Y}$ & 871.3801 & 436.1937 & 854.3535 & 427.6804 & 853.3695 & 427.1884 & 6 \\
\hline 16 & 1800.8360 & 900.9217 & 1783.8095 & 892.4084 & 1782.8255 & 891.9164 & $\mathbf{M}$ & 708.3167 & 354.6620 & 691.2902 & 346.1487 & 690.3062 & 345.6567 & 5 \\
\hline 17 & 1960.8667 & 980.9370 & 1943.8401 & 972.4237 & 1942.8561 & 971.9317 & $\mathrm{C}$ & 577.2763 & 289.1418 & 560.2497 & 280.6285 & 559.2657 & 280.1365 & 4 \\
\hline 18 & 2089.9093 & 1045.4583 & 2072.8827 & 1036.9450 & 2071.8987 & 1036.4530 & $\mathbf{E}$ & 417.2456 & 209.1264 & 400.2191 & 200.6132 & 399.2350 & 200.1212 & 3 \\
\hline
\end{tabular}




\begin{tabular}{|l|l|r|r|r|r|r|r|r|r|r|r|r|r|r|r|r|}
$\mathbf{1 9}$ & 2202.9933 & $\mathbf{1 1 0 2 . 0 0 0 3}$ & 2185.9668 & 1093.4870 & 2184.9828 & 1092.9950 & $\mathbf{L}$ & $\mathbf{2 8 8 . 2 0 3 0}$ & 144.6051 & 271.1765 & 136.0919 & & & $\mathbf{2}$ \\
\hline $\mathbf{2 0}$ & & & & & & & $\mathbf{R}$ & 175.1190 & 88.0631 & 158.0924 & 79.5498 & & & $\mathbf{1}$ \\
\hline
\end{tabular}
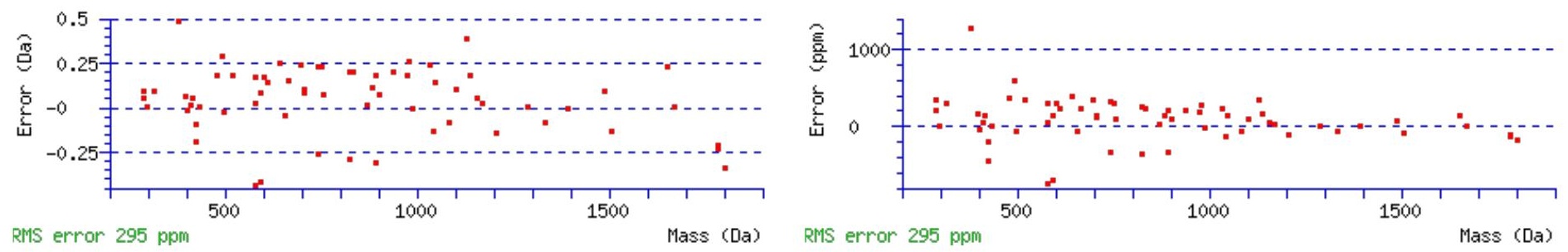

\section{All matches to this query}

\begin{tabular}{|l|l|l|l|}
\hline Score & Mr(calc): & Delta & \multicolumn{1}{|c|}{ Sequence } \\
\hline 74.0 & 2376.0977 & 0.0082 & VLTLANFTTKDEGDYMCELR \\
\hline 23.8 & 2375.1137 & 0.9922 & VLTLANFTTKDEGDYMCELR \\
\hline 5.9 & 2376.1049 & 0.0010 & XLLDMDTGVTEGGLNVTLTIR \\
\hline 5.1 & 2376.1056 & 0.0003 & GEVYQLDILDTSGNHPFPAMR \\
\hline 4.3 & 2376.0876 & 0.0184 & SSKNHASASSAFNKTALPLTGK \\
\hline 0.1 & 2375.1175 & 0.9885 & SYDYRVSLKTAISTTLELR \\
\hline 0.1 & 2375.1175 & 0.9885 & SYDYRVSLKTAISTTLELR \\
\hline 0.1 & 2375.1175 & 0.9885 & SYDYRVSLKTAISTTLELR \\
\hline 0.1 & 2375.1175 & 0.9885 & SYDYRVSLKTAISTTLELR \\
\hline 0.1 & 2375.1175 & 0.9885 & SYDYRVSLKTAISTTLELR \\
\hline
\end{tabular}

Spectrum No: 318; Query: 2660; Rank: 1

\section{Peptide View}

MS/MS Fragmentation of CIPQYWVCDGDADCSDALDELQNCTMR

Found in IPI00205325, Tax_Id=10116 Gene_Symbol=Lrp2 Low-density lipoprotein receptor-related protein 2 precursor

Match to Query 2660: 3309.291102 from(1104.104310,3+)

Title: 100101RatKid_NS_deglyco_24.4321.4321.3.dta

Data file K:INewmanPaper|Piliangl3SubProteomes\Piliang3SP\mgf5ppm\ERLIC_3SubProteomes5ppm.mgf

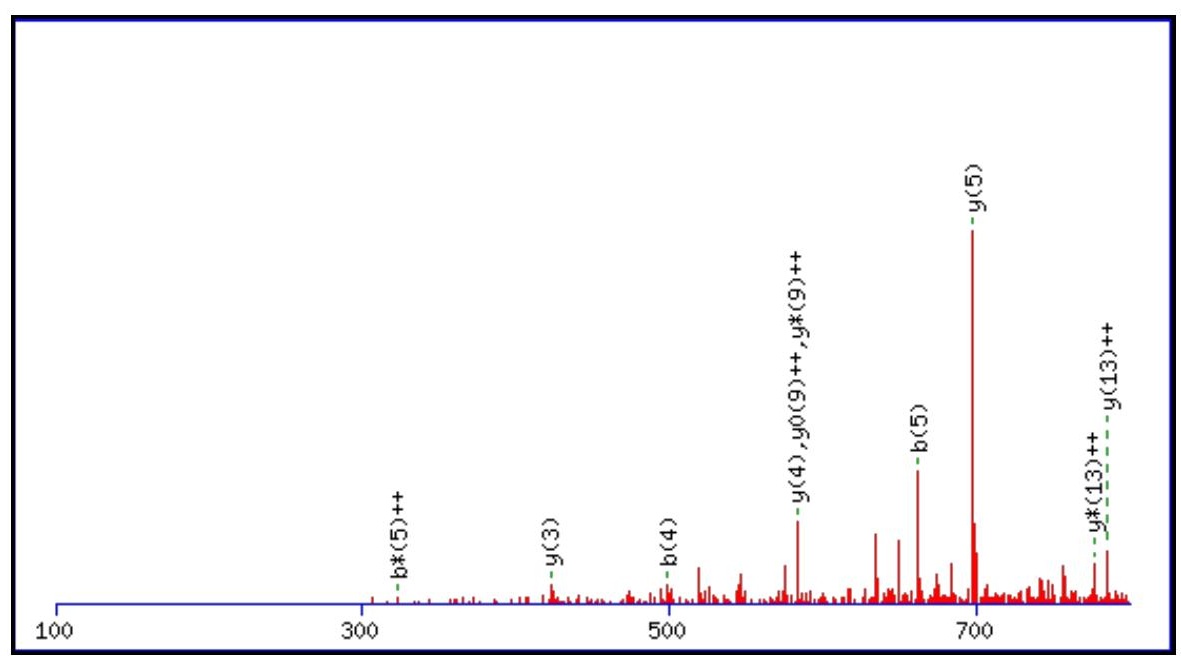



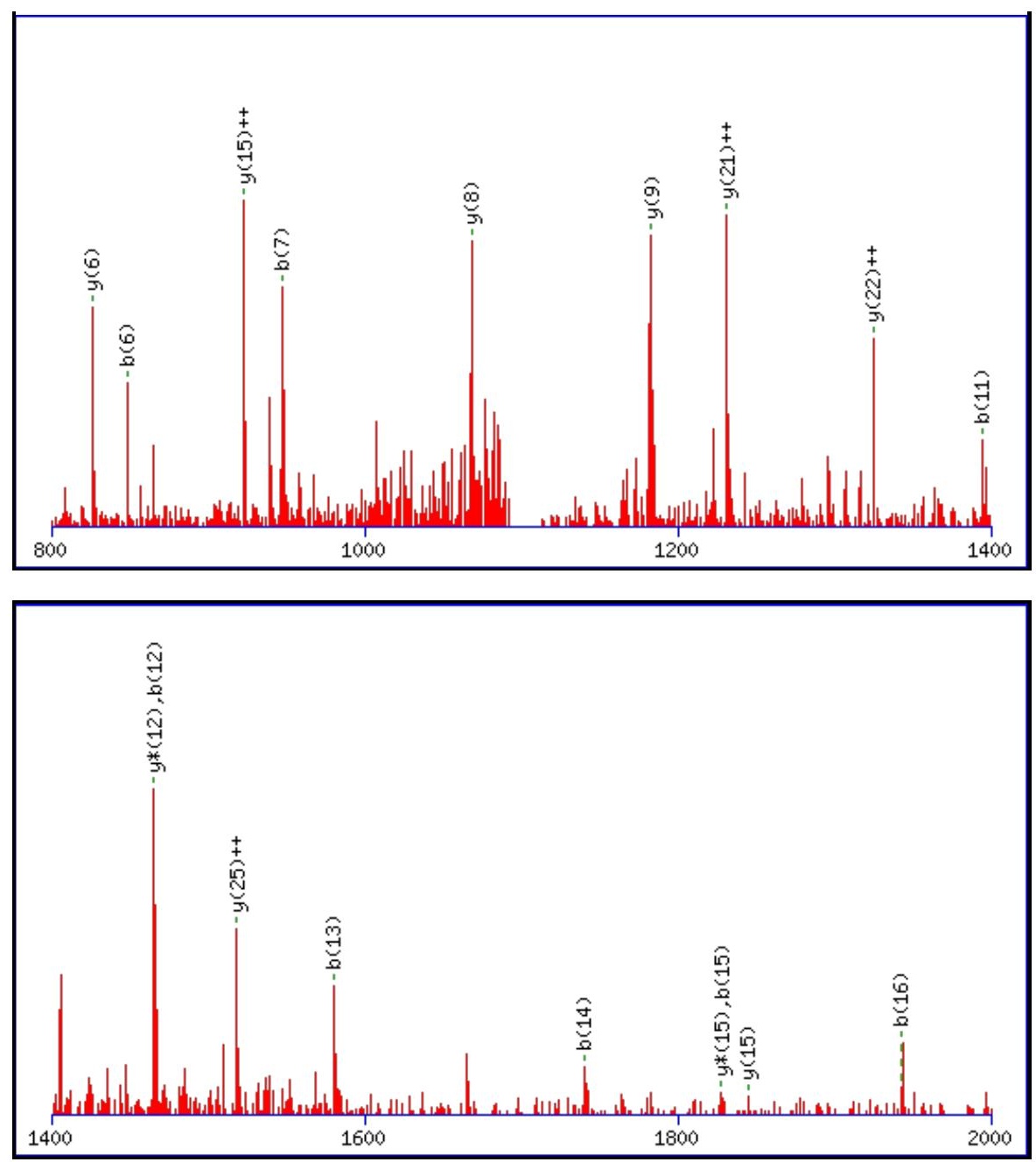

Monoisotopic mass of neutral peptide $\operatorname{Mr}($ calc): 3308.2825

Fixed modifications: Carbamidomethyl (C)

Variable modifications:

N23 : Deamidated_N (N)

M26 : Oxidation $(M)$

Ions Score: 74 Expect : $1.3 \mathrm{e}-005$

Matches (Bold Red): 28/286 fragment ions using 43 most intense peaks

\begin{tabular}{|c|c|c|c|c|c|c|c|c|c|c|c|c|c|c|}
\hline \# & b & $\mathbf{b}^{++}$ & $\mathbf{b}^{*}$ & $\mathbf{b}^{*^{++}}$ & $\mathbf{b}^{\mathbf{0}}$ & & Seq. & $\mathbf{y}$ & $\mathbf{y}^{++}$ & $\mathbf{y}^{*}$ & $\mathbf{y}^{*^{++}}$ & $\mathbf{y}^{0}$ & & \# \\
\hline 1 & 161.0379 & 81.0226 & & & & & C & & & & & & & 27 \\
\hline 2 & 274.1220 & 137.5646 & & & & & I & & 1575.1332 & 3132.2326 & 1566.6199 & 3131.2485 & 1566.1279 & 26 \\
\hline 3 & 371.1748 & 186.0910 & & & & & $\mathbf{P}$ & 3036.1750 & 1518.5912 & 3019.1485 & 1510.0779 & 3018.1645 & 1509.5859 & 25 \\
\hline 4 & 499.2333 & 250.1203 & 482.2068 & & & & $\mathbf{Q}$ & 2939.1223 & 1470.0648 & 2922.0957 & 5515 & 2921.1117 & 595 & 24 \\
\hline 5 & 662.2967 & 331.6520 & 645.2701 & 323.1387 & & & $\mathbf{Y}$ & 2811.0637 & 1406.0355 & 2794.0372 & 1397.5222 & 2793.0531 & 1397.0302 & 23 \\
\hline 6 & 848.3760 & 424.6916 & 831.3494 & 416.1783 & & & $\mathbf{W}$ & 2648.0004 & 1324.5038 & 2630.9738 & 1315.9906 & 2629.9898 & 1315.4985 & 22 \\
\hline 7 & 947.4444 & 474.2258 & 930.4178 & 465.7126 & & & $\mathbf{V}$ & 2461.9211 & 1231. & 2444 & 9509 & 2443.9105 & 1222.4589 & 21 \\
\hline 8 & 1107.4750 & 554.2412 & 1090.4485 & 545.7279 & & & C & 2362.8527 & 1181.9300 & 2345.8261 & 1173.4167 & 2344.8421 & 1172.9247 & 20 \\
\hline 9 & 1222.5020 & 611.7546 & 205.4754 & 603.2414 & 1204 & & D & 2202.8220 & 1101 & 2185. & 1093.4014 & 2184.8114 & 1092.9094 & 19 \\
\hline 10 & 1279.5234 & 640.2654 & 1262.4969 & 631.7521 & 1261.5129 & 631.2601 & G & 2087.7951 & 1044.4012 & 2070.7685 & 1035.8879 & 2069.7845 & 1035.3959 & 18 \\
\hline 11 & 1394.5504 & 697.7788 & 1377.5238 & 689.2656 & 1376.5398 & 688.7735 & D & 2030.7736 & 1015.8904 & 2013.7470 & 1007.3772 & 2012.7630 & 1006.8852 & 17 \\
\hline 12 & 1465.5875 & 733.2974 & 1448.5610 & 724.7841 & 1447.5769 & 724.2921 & A & 1915.7467 & 958.3770 & 1898.7201 & 949.8637 & 1897.7361 & 949.3717 & 16 \\
\hline 13 & 1580.6144 & 790.8109 & 1563.5879 & 782.2976 & 1562.6039 & 781.8056 & D & 1844.7095 & 922.8584 & 1827.6830 & 914.3451 & 1826.6990 & 913.8531 & 15 \\
\hline 14 & 1740.6451 & 870.8262 & 1723.6185 & 862.3129 & 1722.6345 & 861.8209 & $\mathrm{C}$ & 1729.6826 & 865.3449 & 1712.6560 & 856.8317 & 1711.6720 & 856.3397 & 14 \\
\hline 15 & 1827.6771 & 914.3422 & 1810.6506 & 905.8289 & 1809.6666 & 905.3369 & $\mathrm{~S}$ & 1569.6519 & 785.3296 & 1552.6254 & 776.8163 & 1551.6414 & 776.3243 & 13 \\
\hline 16 & 1942.7041 & 971.8557 & 1925.6775 & 963.3424 & 1924.6935 & 962.8504 & D & 1482.6199 & 741.8136 & 1465.5934 & 733.3003 & 1464.6094 & 732.8083 & 12 \\
\hline 17 & 2013.7412 & 1007.3742 & 1996.7146 & 998.8610 & 1995.7306 & 998.3689 & A & 1367.5930 & 684.3001 & 1350.5664 & 675.7869 & 1349.5824 & 675.2948 & 11 \\
\hline 18 & 2126.8252 & 1063.9163 & 2109.7987 & 1055.4030 & 2108.8147 & 1054.9110 & $\mathbf{L}$ & 1296.5559 & 648.7816 & 1279.5293 & 640.2683 & 1278.5453 & 639.7763 & 10 \\
\hline
\end{tabular}




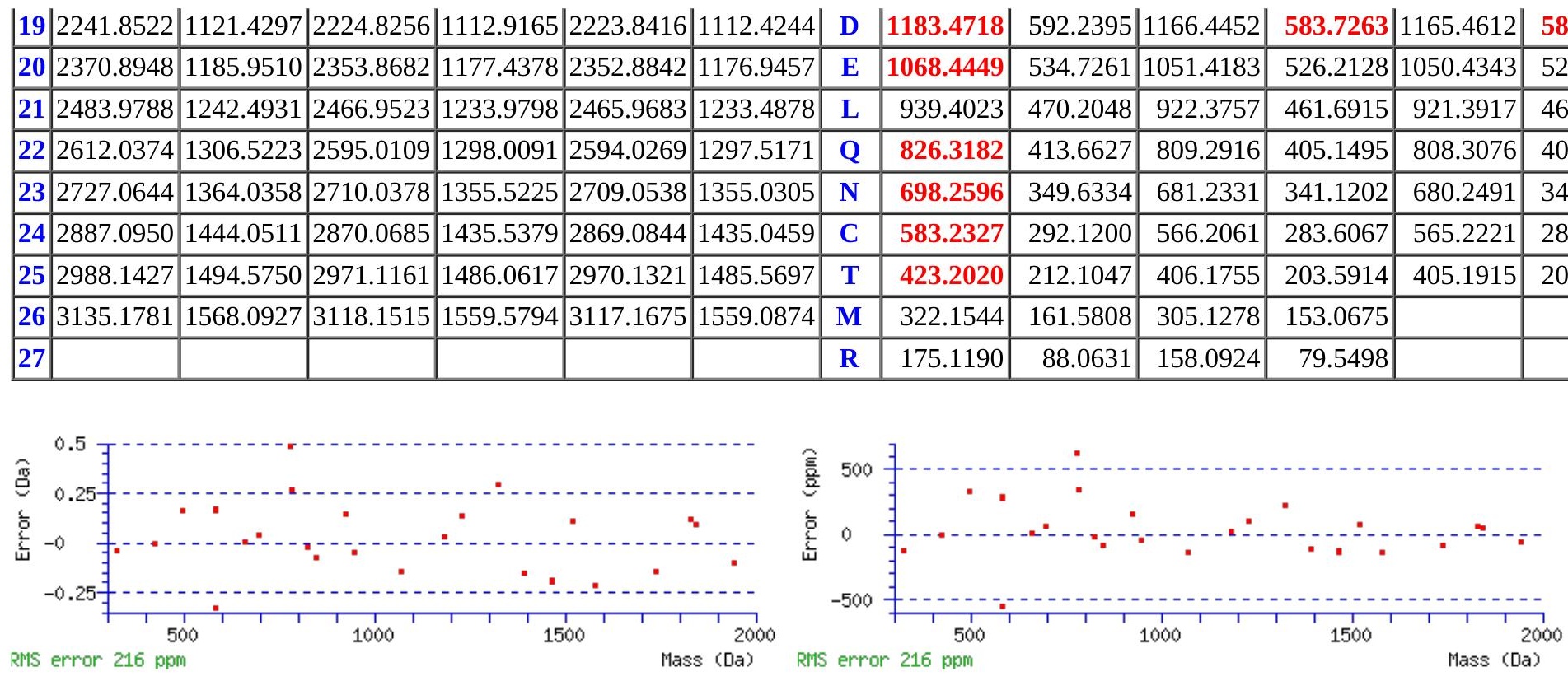

\section{All matches to this query}

\begin{tabular}{|l|l|l|l|}
\hline Score & Mr(calc): & Delta & \multicolumn{1}{|c|}{ Sequence } \\
\hline 73.6 & 3308.2825 & 1.0086 & CIPQYWVCDGDADCSDALDELQNCTMR \\
\hline 35.0 & 3307.2985 & 1.9926 & CIPQYWVCDGDADCSDALDELQNCTMR \\
\hline 6.2 & 3307.2760 & 2.0151 & VLFSFPGNQVTSVAGVYFTDVYGGINR \\
\hline 5.4 & 3307.2650 & 2.0261 & NSTTPNAPYQQGMGMPDMLGRMPYAPNK \\
\hline 5.4 & 3307.2650 & 2.0261 & NSTTPNAPYQQGMGMPDMLGRMPYAPNK \\
\hline 2.9 & 3307.2760 & 2.0151 & VLFSFPGNQVTSVAGVYFTDVYGGINR \\
\hline 1.0 & 3308.2600 & 1.0311 & DSYFWIALQDQNNTGEYTWKTMGQR \\
\hline 1.0 & 3307.2650 & 2.0261 & NSTTPNAPYQQGMGMPDMLGRMPYAPNK \\
\hline 1.0 & 3307.2650 & 2.0261 & NSTTPNAPYQQGMGMPDMLGRMPYAPNK \\
\hline 0.9 & 3307.3140 & 1.9771 & ITAHCLAYSNSSVNPIIYAFLSENFR \\
\hline
\end{tabular}

Spectrum No: 319; Query: 615; Rank: 1

\section{Peptide View}

MS/MS Fragmentation of VSNMTLPASAEVLK

Found in IPI00206336, Tax_Id=10116 Gene_Symbol=Lamp1 Lysosome-associated membrane glycoprotein 1 precursor

Match to Query 615: 1459.760148 from(730.887350,2+)

Title: 100101RatKid_NS_deglyco_25.2839.2839.2.dta

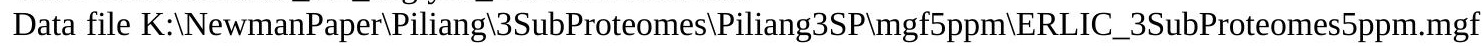



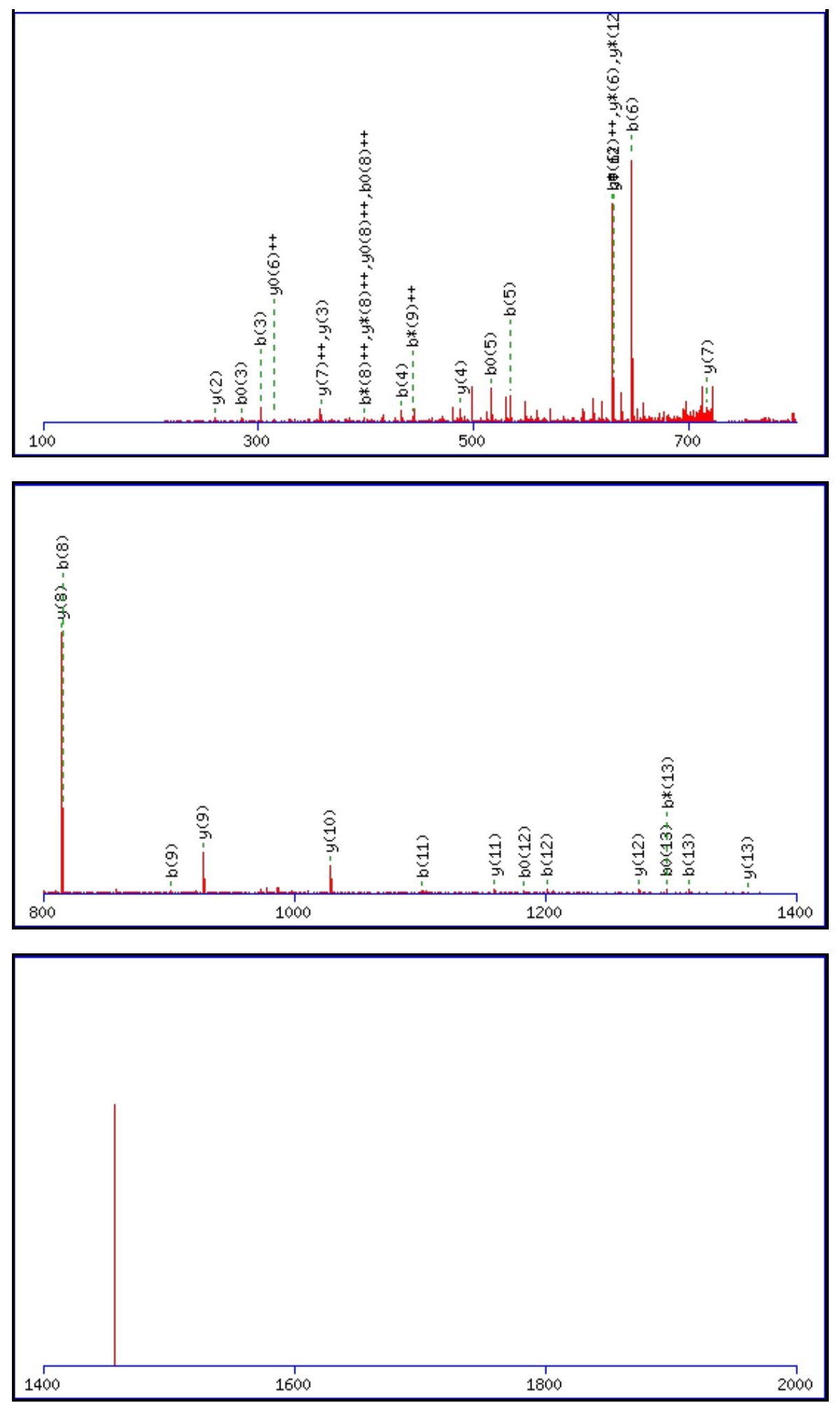

Monoisotopic mass of neutral peptide $\operatorname{Mr}($ calc): 1459.7592

Fixed modifications: Carbamidomethyl (C)

Variable modifications:

N3

Ions Score: 74 Expect: $5.3 \mathrm{e}-006$

Matches (Bold Red): 36/144 fragment ions using 60 most intense peaks

\begin{tabular}{|r|c|c|c|c|c|c|c|c|c|c|c|c|c|c|}
\hline$\#$ & $\mathbf{b}$ & $\mathbf{b}^{++}$ & $\mathbf{b}^{*}$ & $\mathbf{b}^{\boldsymbol{*}^{++}}$ & $\mathbf{b}^{\mathbf{0}}$ & $\mathbf{b}^{\mathbf{0}+}$ & Seq. & $\mathbf{y}$ & $\mathbf{y}^{++}$ & $\mathbf{y}^{*}$ & $\mathbf{y}^{\boldsymbol{*}^{++}}$ & $\mathbf{y}^{\mathbf{0}}$ & $\mathbf{y}^{\mathbf{0 + +}}$ & $\#$ \\
\hline $\mathbf{1}$ & 100.0757 & 50.5415 & & & & & $\mathbf{V}$ & & & & & & & $\mathbf{1 4}$ \\
\hline $\mathbf{2}$ & 187.1077 & 94.0575 & & & 169.0972 & 85.0522 & $\mathbf{S}$ & $\mathbf{1 3 6 1 . 6 9 8 1}$ & 681.3527 & 1344.6715 & 672.8394 & 1343.6875 & 672.3474 & $\mathbf{1 3}$ \\
\hline $\mathbf{3}$ & $\mathbf{3 0 2 . 1 3 4 7}$ & 151.5710 & 285.1081 & 143.0577 & $\mathbf{2 8 4 . 1 2 4 1}$ & 142.5657 & $\mathbf{N}$ & $\mathbf{1 2 7 4 . 6 6 6 1}$ & 637.8367 & 1257.6395 & $\mathbf{6 2 9 . 3 2 3 4}$ & 1256.6555 & $\mathbf{6 2 8 . 8 3 1 4}$ & $\mathbf{1 2}$ \\
\hline $\mathbf{4}$ & $\mathbf{4 3 3 . 1 7 5 1}$ & 217.0912 & 416.1486 & 208.5779 & 415.1646 & 208.0859 & $\mathbf{M}$ & $\mathbf{1 1 5 9 . 6 3 9 1}$ & 580.3232 & 1142.6126 & 571.8099 & 1141.6286 & 571.3179 & $\mathbf{1 1}$ \\
\hline $\mathbf{5}$ & 534.2228 & 267.6150 & 517.1963 & 259.1018 & 516.2123 & 258.6098 & $\mathbf{T}$ & $\mathbf{1 0 2 8 . 5 9 8 6}$ & 514.8030 & 1011.5721 & 506.2897 & 1010.5881 & 505.7977 & $\mathbf{1 0}$ \\
\hline
\end{tabular}




\begin{tabular}{|c|c|c|c|c|c|c|c|c|c|c|c|c|c|c|}
\hline 6 & 647.3069 & |324.1571| & 630.2803 & |315.6438 & 629.2963 & |315.1518| & $\mathbf{L}$ & 927.5510 & |464.2791 & 910.5244 & |455.7658 & 909.5404 & |455.2738| & 9 \\
\hline 7 & 744.3596 & 372.6835 & 727.3331 & 364.1702 & 726.3491 & 363.6782 & $\mathbf{P}$ & 814.4669 & 407.7371 & 797.4403 & 399.2238 & 796.4563 & 398.7318 & 8 \\
\hline 8 & 815.3968 & 408.2020 & 798.3702 & 399.6887 & 797.3862 & 399.1967 & A & 717.4141 & 359.2107 & 700.3876 & 350.6974 & 699.4036 & 350.2054 & 7 \\
\hline 9 & 902.4288 & 451.7180 & 885.4022 & 443.2048 & 884.4182 & 442.7127 & S & 646.3770 & 323.6921 & 629.3505 & 315.1789 & 628.3665 & 314.6869 & 6 \\
\hline 10 & 973.4659 & 487.2366 & 956.4394 & 478.7233 & 955.4553 & 478.2313 & A & 559.3450 & 280.1761 & 542.3184 & 271.6629 & 541.3344 & 271.1709 & 5 \\
\hline 11 & 1102.5085 & 551.7579 & 1085.4819 & 543.2446 & 1084.4979 & 542.7526 & $\mathbf{E}$ & 488.3079 & 244.6576 & 471.2813 & 236.1443 & 470.2973 & 235.6523 & 4 \\
\hline 12 & 1201.5769 & 601.2921 & 1184.5504 & 592.7788 & 1183.5663 & 592.2868 & V & 359.2653 & 180.1363 & 342.2387 & 171.6230 & & & 3 \\
\hline 13 & 1314.6610 & 657.8341 & 1297.6344 & 649.3209 & 1296.6504 & 648.8288 & $\mathbf{L}$ & 260.1969 & 130.6021 & 243.1703 & 122.0888 & & & 2 \\
\hline 14 & & & & & & & K & 147.1128 & 74.0600 & 130.0863 & 65.5468 & & & 1 \\
\hline
\end{tabular}
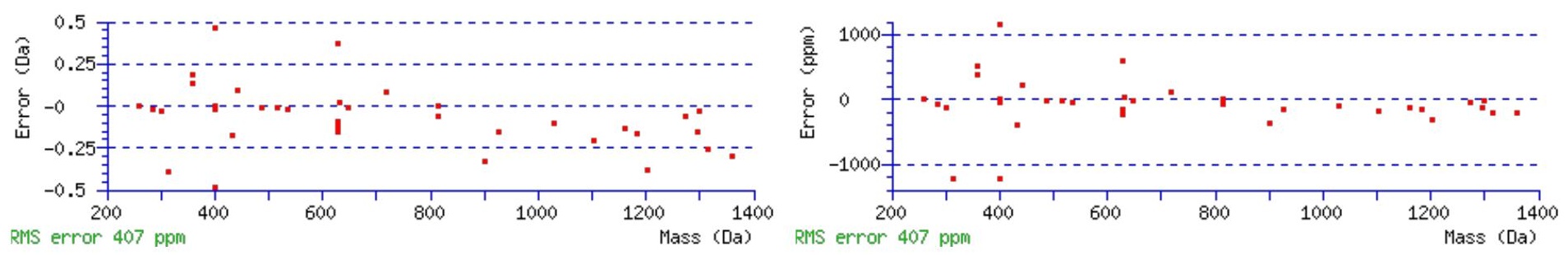

\section{All matches to this query}

\begin{tabular}{|l|l|c|l|}
\hline Score & Mr(calc): & Delta & \multicolumn{1}{|c|}{ Sequence } \\
\hline 73.6 & 1459.7592 & 0.0009 & VSNMTLPASAEVLK \\
\hline 16.2 & 1459.7671 & -0.0069 & WLGTGNKGSNALLK \\
\hline 14.5 & 1459.7606 & -0.0004 & KSCGXIPGKQAWK \\
\hline 8.9 & 1459.7518 & 0.0084 & KLNESLKQQNEK \\
\hline 8.4 & 1459.7466 & 0.0135 & GFRQASTLCRHK \\
\hline 5.9 & 1458.7467 & 1.0135 & $\underline{\text { IKDDVGDNWRIK }}$ \\
\hline 4.7 & 1459.7671 & -0.0069 & AVLQPHENIVPNK \\
\hline 4.6 & 1459.7558 & 0.0043 & SSYKYDSLLLGSK \\
\hline 4.2 & 1459.7476 & 0.0125 & FTLGKVWITTSK \\
\hline 3.4 & 1459.7476 & 0.0125 & FTLGKVWITTSK \\
\hline
\end{tabular}

Spectrum No: 320; Query: 352; Rank: 1

\section{Peptide View}

MS/MS Fragmentation of VYNGSVPFEER

Found in IPI00339118, Tax_Id=10116 Gene_Symbol=Enpp1 Isoform 2 of Ectonucleotide pyrophosphatase/phosphodiesterase family member 1

Match to Query 352: 1296.599948 from(649.307250,2+)

Title: 100101RatKid_NS_deglyco_23.2021.2021.2.dta

Data file K:INewmanPaper|Piliang|3SubProteomes\Piliang3SP\mgf5ppm\ERLIC_3SubProteomes5ppm.mgf 

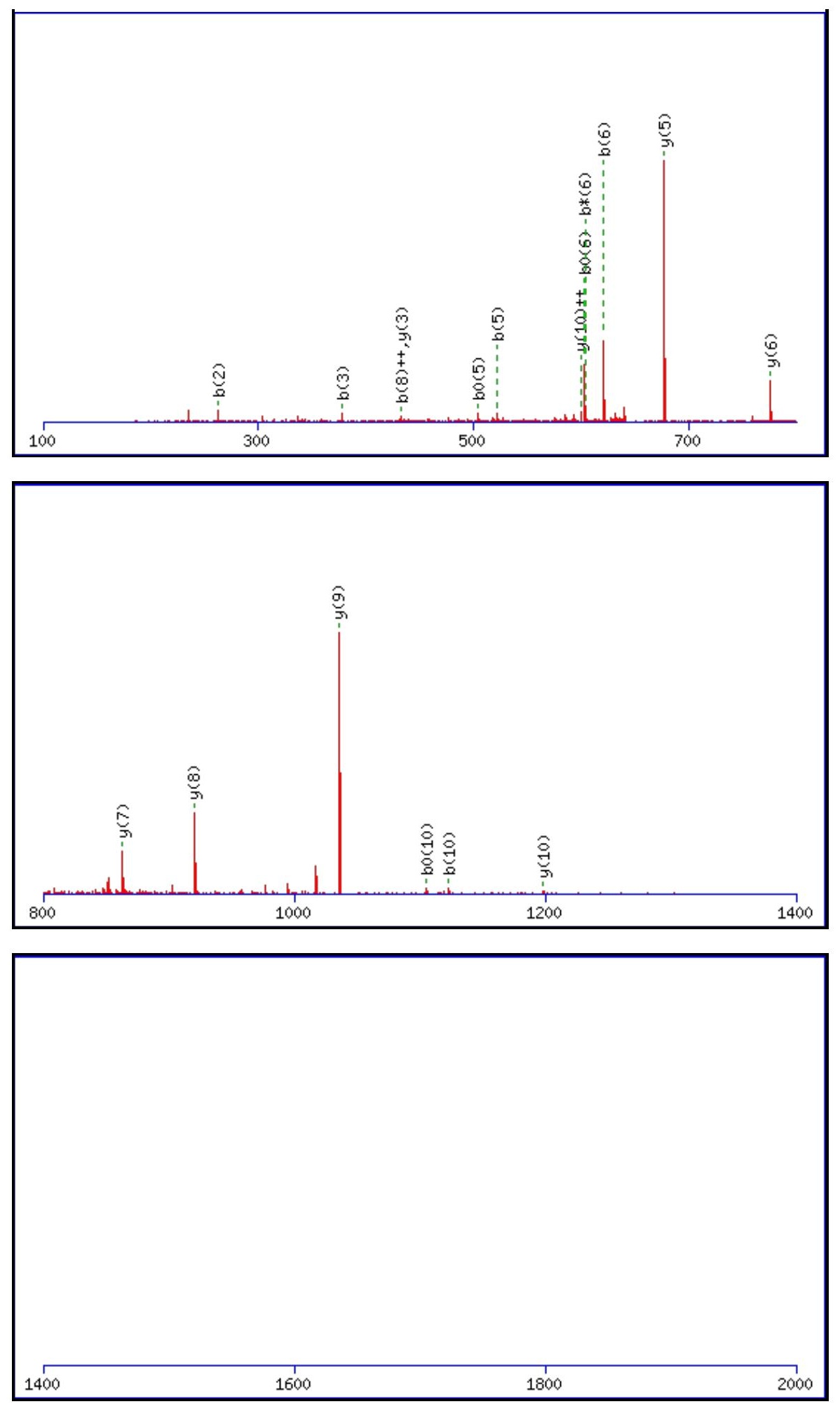

Monoisotopic mass of neutral peptide $\operatorname{Mr}($ calc): 1296.5986

Fixed modifications: Carbamidomethyl (C)

Variable modifications:

N3 : Deamidated $\mathrm{N}(\mathrm{N})$

Ions Score: 73 Expect: $5.9 \mathrm{e}-006$

Matches (Bold Red): 18/106 fragment ions using 22 most intense peaks

\begin{tabular}{|r|c|c|c|c|c|c|c|c|c|c|c|c|c|c|}
\hline$\#$ & $\mathbf{b}$ & $\mathbf{b}^{++}$ & $\mathbf{b}^{*}$ & $\mathbf{b}^{\boldsymbol{*}^{++}}$ & $\mathbf{b}^{\mathbf{0}}$ & $\mathbf{b}^{\mathbf{0 + +}}$ & $\mathbf{S e q}$ & $\mathbf{y}$ & $\mathbf{y}^{++}$ & $\mathbf{y}^{\mathbf{*}}$ & $\mathbf{y}^{\boldsymbol{*}^{++}}$ & $\mathbf{y}^{\mathbf{0}}$ & $\mathbf{y}^{\mathbf{0 + +}}$ & $\#$ \\
\hline $\mathbf{1}$ & 100.0757 & 50.5415 & & & & & $\mathbf{V}$ & & & & & & & $\mathbf{1 1}$ \\
\hline $\mathbf{2}$ & $\mathbf{2 6 3 . 1 3 9 0}$ & 132.0731 & & & & & $\mathbf{Y}$ & $\mathbf{1 1 9 8 . 5 3 7 5}$ & $5 \mathbf{9 9 . 7 7 2 4}$ & 1181.5109 & 591.2591 & 1180.5269 & 590.7671 & $\mathbf{1 0}$ \\
\hline $\mathbf{3}$ & $\mathbf{3 7 8 . 1 6 6 0}$ & 189.5866 & 361.1394 & 181.0733 & & & $\mathbf{N}$ & $\mathbf{1 0 3 5 . 4 7 4 2}$ & 518.2407 & 1018.4476 & 509.7274 & 1017.4636 & 509.2354 & $\mathbf{9}$ \\
\hline $\mathbf{4}$ & 435.1874 & 218.0973 & 418.1609 & 209.5841 & & & $\mathbf{G}$ & $\mathbf{9 2 0 . 4 4 7 2}$ & 460.7272 & 903.4207 & 452.2140 & 902.4367 & 451.7220 & $\mathbf{8}$ \\
\hline $\mathbf{5}$ & $\mathbf{5 2 2 . 2 1 9 4}$ & 261.6134 & 505.1929 & 253.1001 & $\mathbf{5 0 4 . 2 0 8 9}$ & 252.6081 & $\mathbf{S}$ & $\mathbf{8 6 3 . 4 2 5 8}$ & 432.2165 & 846.3992 & 423.7032 & 845.4152 & 423.2112 & $\mathbf{7}$ \\
\hline
\end{tabular}




\begin{tabular}{|r|r|r|r|r|r|r|r|r|r|r|r|r|r|r|r|}
$\mathbf{6}$ & $\mathbf{6 2 1 . 2 8 7 9}$ & 311.1476 & $\mathbf{6 0 4 . 2 6 1 3}$ & 302.6343 & $\mathbf{6 0 3 . 2 7 7 3}$ & 302.1423 & $\mathbf{V}$ & $\mathbf{7 7 6 . 3 9 3 7}$ & 388.7005 & $\mathbf{7 5 9 . 3 6 7 2}$ & 380.1872 & $\mathbf{7 5 8 . 3 8 3 2}$ & 379.6952 & $\mathbf{6}$ \\
\hline $\mathbf{7}$ & 718.3406 & 359.6740 & 701.3141 & 351.1607 & 700.3301 & 350.6687 & $\mathbf{P}$ & $\mathbf{6 7 7 . 3 2 5 3}$ & 339.1663 & 660.2988 & 330.6530 & 659.3148 & 330.1610 & $\mathbf{5}$ \\
\hline $\mathbf{8}$ & 865.4090 & $\mathbf{4 3 3 . 2 0 8 2}$ & 848.3825 & 424.6949 & 847.3985 & 424.2029 & $\mathbf{F}$ & 580.2726 & 290.6399 & 563.2460 & 282.1266 & 562.2620 & $\mathbf{2 8 1 . 6 3 4 6}$ & $\mathbf{4}$ \\
\hline $\mathbf{9}$ & 994.4516 & 497.7295 & 977.4251 & 489.2162 & 976.4411 & 488.7242 & $\mathbf{E}$ & $\mathbf{4 3 3 . 2 0 4 1}$ & 217.1057 & 416.1776 & 208.5924 & 415.1936 & 208.1004 & $\mathbf{3}$ \\
\hline $\mathbf{1 0}$ & $\mathbf{1 1 2 3 . 4 9 4 2}$ & 562.2508 & 1106.4677 & 553.7375 & $\mathbf{1 1 0 5 . 4 8 3 7}$ & 553.2455 & $\mathbf{E}$ & 304.1615 & 152.5844 & 287.1350 & 144.0711 & 286.1510 & $\mathbf{1 4 3 . 5 7 9 1}$ & $\mathbf{2}$ \\
\hline $\mathbf{1 1}$ & & & & & & & $\mathbf{R}$ & 175.1190 & 88.0631 & 158.0924 & 79.5498 & & & $\mathbf{1}$ \\
\hline
\end{tabular}
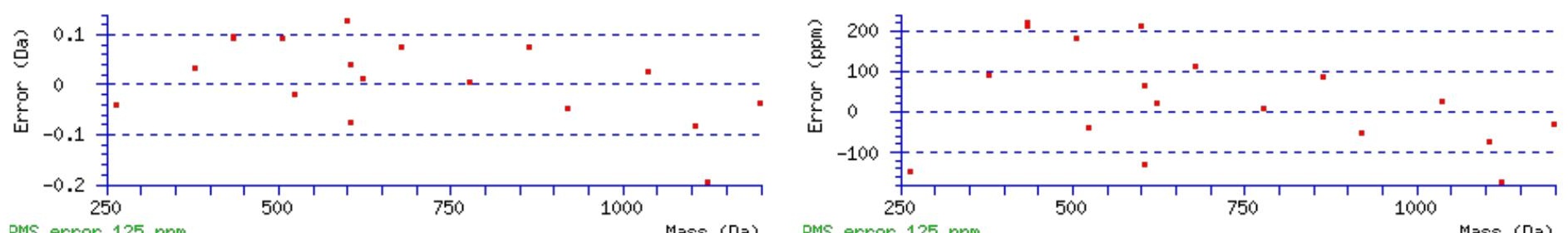

RMS error 125 ppm

\section{All matches to this query}

\begin{tabular}{|l|l|l|l|}
\hline Score & Mr(calc): & Delta & \multicolumn{1}{|c|}{ Sequence } \\
\hline 73.5 & 1296.5986 & 0.0013 & VYNGSVPFEER \\
\hline 11.1 & 1296.6020 & -0.0020 & MDVAFLLDNSR \\
\hline 5.0 & 1294.5863 & 2.0136 & WTLSNMLGDNK \\
\hline 4.0 & 1295.5890 & 1.0110 & NMADLMWSTVK \\
\hline 2.7 & 1296.5914 & 0.0085 & SLMGSRAAMDSR \\
\hline 1.9 & 1296.6067 & -0.0067 & WRMMSLSQSR \\
\hline 1.9 & 1296.6067 & -0.0067 & WRMMSLSQSR \\
\hline 1.3 & 1295.5849 & 1.0150 & TMERCNLIDK \\
\hline 1.0 & 1296.5976 & 0.0024 & RGPTANGFLER \\
\hline 0.9 & 1296.5992 & 0.0007 & VLSIQSHVVR \\
\hline
\end{tabular}

Spectrum No: 321; Query: 1825; Rank: 1

\section{Peptide View}

MS/MS Fragmentation of DCSTNPPRLPVTAVNTTMR

Found in IPI00197684, Tax_Id=10116 Gene_Symbol=Xpnpep2 Membrane-bound aminopeptidase P

Match to Query 1825: 2146.017312 from(716.346380,3+)

Title: 091008RatKidney_NoSalt_25.1872.1872.3.dta

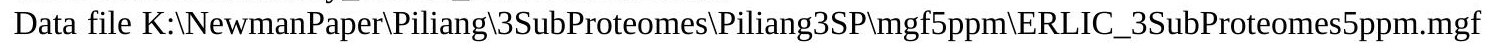

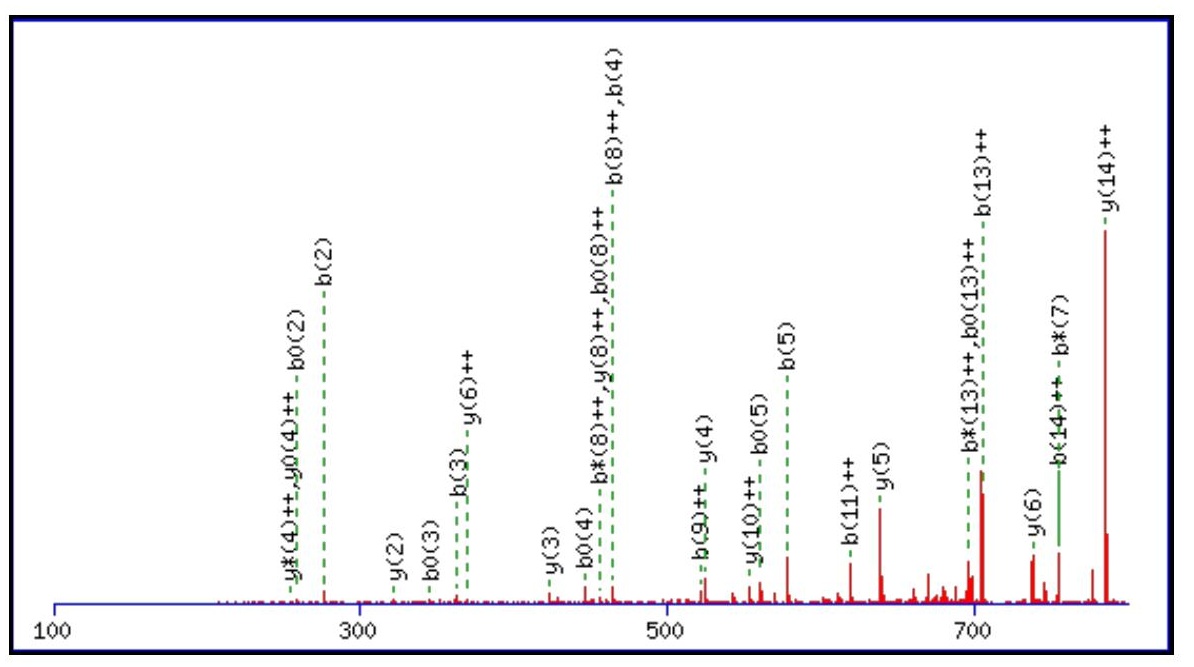



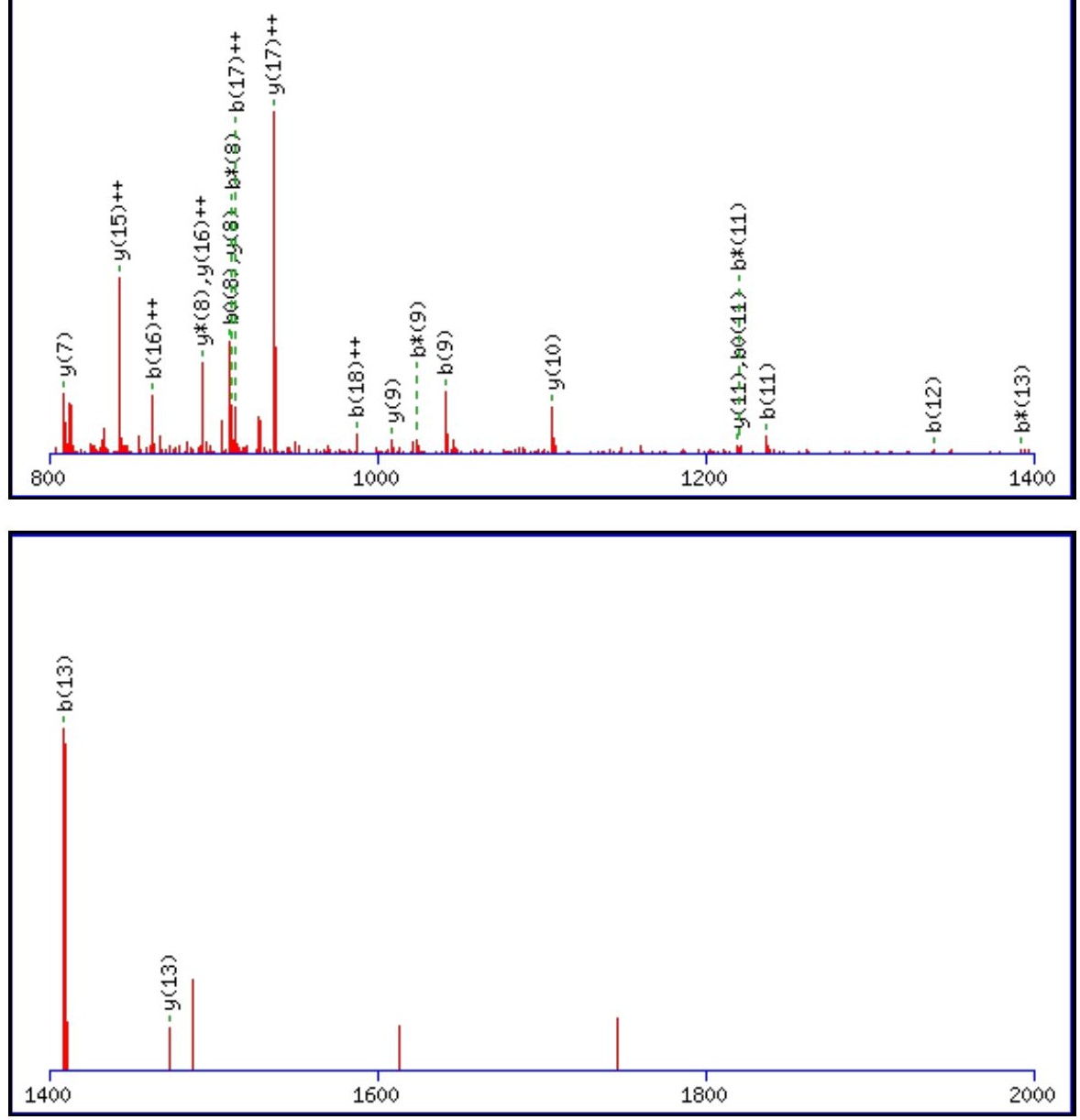

Monoisotopic mass of neutral peptide $\operatorname{Mr}($ calc): 2146.0147

Fixed modifications: Carbamidomethyl (C)

Variable modifications:

N15 : Deamidated_N (N)

M18 : Oxidation (M)

Ions Score: 73 Expect: $1.2 \mathrm{e}-005$

Matches (Bold Red): 52/204 fragment ions using 68 most intense peaks

\begin{tabular}{|c|c|c|c|c|c|c|c|c|c|c|c|c|c|c|}
\hline \# & b & $\mathbf{b}^{++}$ & $\mathbf{b}^{*}$ & $\mathbf{b}^{*++}$ & $\mathbf{b}^{0}$ & $\mathbf{b}^{0++}$ & Seq. & $\mathbf{y}$ & $y^{++}$ & $\mathbf{y}^{*}$ & $\mathbf{y}^{*^{++}}$ & $\mathbf{y}^{\mathbf{0}}$ & $y^{0++}$ & \# \\
\hline 1 & 116.0342 & 58.5207 & & & 98.0237 & 49.5155 & D & & & & & & & 19 \\
\hline 2 & 276.0649 & 138.5361 & & & 258.0543 & 129.5308 & $\mathrm{C}$ & 2031.9950 & 1016.5011 & 2014.9685 & 1007.9879 & 2013.9845 & 1007.4959 & 18 \\
\hline 3 & 363.0969 & 182.0521 & & & 345.0863 & 173.0468 & $\mathbf{S}$ & 1871.9644 & 936.4858 & 1854.9378 & 927.9725 & 1853.9538 & 927.4805 & 17 \\
\hline 4 & 464.1446 & 232.5759 & & & 446.1340 & 223.5706 & $\mathbf{T}$ & 1784.9323 & 892.9698 & 1767.9058 & 884.4565 & 1766.9218 & 883.9645 & 16 \\
\hline 5 & 578.1875 & 289.5974 & 561.1610 & 281.0841 & 560.1769 & 280.5921 & $\mathbf{N}$ & 1683.8847 & 842.4460 & 1666.8581 & 833.9327 & 1665.8741 & 833.4407 & 15 \\
\hline 6 & 675.2403 & 338.1238 & 658.2137 & 329.6105 & 657.2297 & 329.1185 & $\mathbf{P}$ & 1569.8417 & 785.4245 & 1552.8152 & 776.9112 & 1551.8312 & 776.4192 & 14 \\
\hline 7 & 772.2930 & 386.6502 & 755.2665 & 378.1369 & 754.2825 & 377.6449 & $\mathbf{P}$ & 1472.7890 & 736.8981 & 1455.7624 & 728.3849 & 1454.7784 & 727.8928 & 13 \\
\hline 8 & 928.3941 & 464.7007 & 911.3676 & \begin{tabular}{|l|l|}
456.1874 \\
\end{tabular} & 910.3836 & 455.6954 & $\mathbf{R}$ & 1375.7362 & 688.3717 & 1358.7097 & 679.8585 & 1357.7256 & 679.3665 & 12 \\
\hline 9 & 1041.4782 & 521.2427 & 1024.4517 & 512.7295 & 1023.4676 & 512.2375 & $\mathbf{L}$ & 1219.6351 & 610.3212 & 1202.6086 & 601.8079 & 1201.6245 & 601.3159 & 11 \\
\hline 10 & 1138.5310 & 569.7691 & 1121.5044 & 561.2558 & 1120.5204 & 560.7638 & $\mathbf{P}$ & 1106.5510 & 553.7792 & 1089.5245 & 545.2659 & 1088.5405 & 544.7739 & 10 \\
\hline 11 & 1237.5994 & 619.3033 & 1220.5728 & 610.7901 & 1219.5888 & 610.2980 & $\mathbf{V}$ & 1009.4983 & 505.2528 & 992.4717 & 496.7395 & 991.4877 & 496.2475 & 9 \\
\hline 12 & 1338.6471 & 669.8272 & 1321.6205 & 661.3139 & 1320.6365 & 660.8219 & $\mathbf{T}$ & 910.4299 & 455.7186 & 893.4033 & 447.2053 & 892.4193 & 446.7133 & 8 \\
\hline 13 & 1409.6842 & 705.3457 & 1392.6576 & 696.8325 & 1391.6736 & 696.3404 & A & 809.3822 & 405.1947 & 792.3556 & 396.6815 & 791.3716 & 396.1894 & 7 \\
\hline 14 & 1508.7526 & 754.8799 & 1491.7260 & 746.3667 & 1490.7420 & 745.8747 & V & 738.3451 & 369.6762 & 721.3185 & 361.1629 & 720.3345 & 360.6709 & 6 \\
\hline 15 & 1623.7795 & 812.3934 & 1606.7530 & 803.8801 & 1605.7690 & 803.3881 & $\mathbf{N}$ & 639.2767 & 320.1420 & 622.2501 & 311.6287 & 621.2661 & 311.1367 & 5 \\
\hline 16 & 1724.8272 & 862.9172 & 1707.8007 & 854.4040 & 1706.8166 & 853.9120 & $\mathbf{T}$ & 524.2497 & 262.6285 & 507.2232 & 254.1152 & 506.2391 & 253.6232 & 4 \\
\hline 17 & 1825.8749 & 913.4411 & 1808.8483 & 904.9278 & 1807.8643 & 904.4358 & $\mathbf{T}$ & 423.2020 & 212.1047 & 406.1755 & 203.5914 & 405.1915 & 203.0994 & 3 \\
\hline
\end{tabular}




\begin{tabular}{|r|r|r|r|r|r|r|r|r|r|r|r|r|r|}
$\mathbf{1 8}$ & 1972.9103 & $\mathbf{9 8 6 . 9 5 8 8}$ & 1955.8837 & 978.4455 & 1954.8997 & 977.9535 & $\mathbf{M}$ & $\mathbf{3 2 2 . 1 5 4 4}$ & 161.5808 & 305.1278 & 153.0675 & \\
\hline $\mathbf{1 9}$ & & & & & & & $\mathbf{R}$ & 175.1190 & 88.0631 & 158.0924 & $\mathbf{7 9 . 5 4 9 8}$ & \\
\hline
\end{tabular}
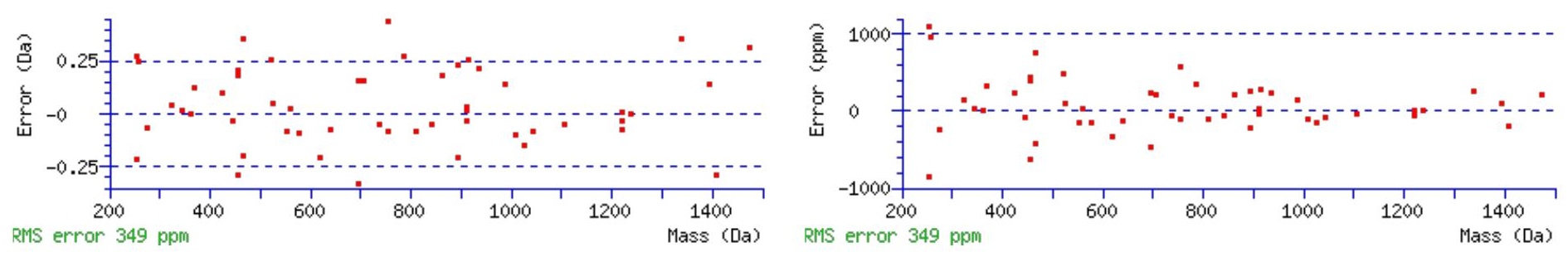

\section{All matches to this query}

\begin{tabular}{|l|l|l|l|}
\hline Score & Mr(calc): & Delta & \multicolumn{1}{|c|}{ Sequence } \\
\hline 73.2 & 2146.0147 & 0.0026 & DCSTNPPRLPVTAVNTTMR \\
\hline 41.9 & 2146.0147 & 0.0026 & DCSTNPPRLPVTAVNTTMR \\
\hline 33.1 & 2145.0307 & 0.9866 & DCSTNPPRLPVTAVNTTMR \\
\hline 4.2 & 2145.0078 & 1.0095 & VNCSDKYGTTPLVWAARK \\
\hline 3.9 & 2144.0263 & 1.9910 & DIALEITVTNGPSDPRNPR \\
\hline 3.4 & 2145.0338 & 0.9835 & DAEDGNANFLGKASGVDLVPR \\
\hline 1.8 & 2145.0303 & 0.9870 & HWVQMGGPRVTLGADGRTK \\
\hline 1.7 & 2143.9990 & 2.0183 & LSDSGNYTCMAANIVAKRR \\
\hline 1.6 & 2145.9983 & 0.0190 & SLSLFSNVSQSHGIYIGEK \\
\hline 1.6 & 2144.0276 & 1.9897 & RTRHVHSFSVSLHASSEK \\
\hline
\end{tabular}

Spectrum No: 322; Query: 1440; Rank: 1

\section{Peptide View}

MS/MS Fragmentation of DAFTNVSSPDAGATLPNDK

Found in IPI00369140, Tax_Id=10116 Gene_Symbol=RGD1309019_predicted 38 kDa protein

Match to Query 1440: 1919.875788 from(960.945170,2+)

Title: 091008RatKidney_NoSalt_32.1881.1881.2.dta

Data file K:INewmanPaper|Piliang \3SubProteomes\Piliang3SP\mgf5ppm\ERLIC_3SubProteomes5ppm.mgf

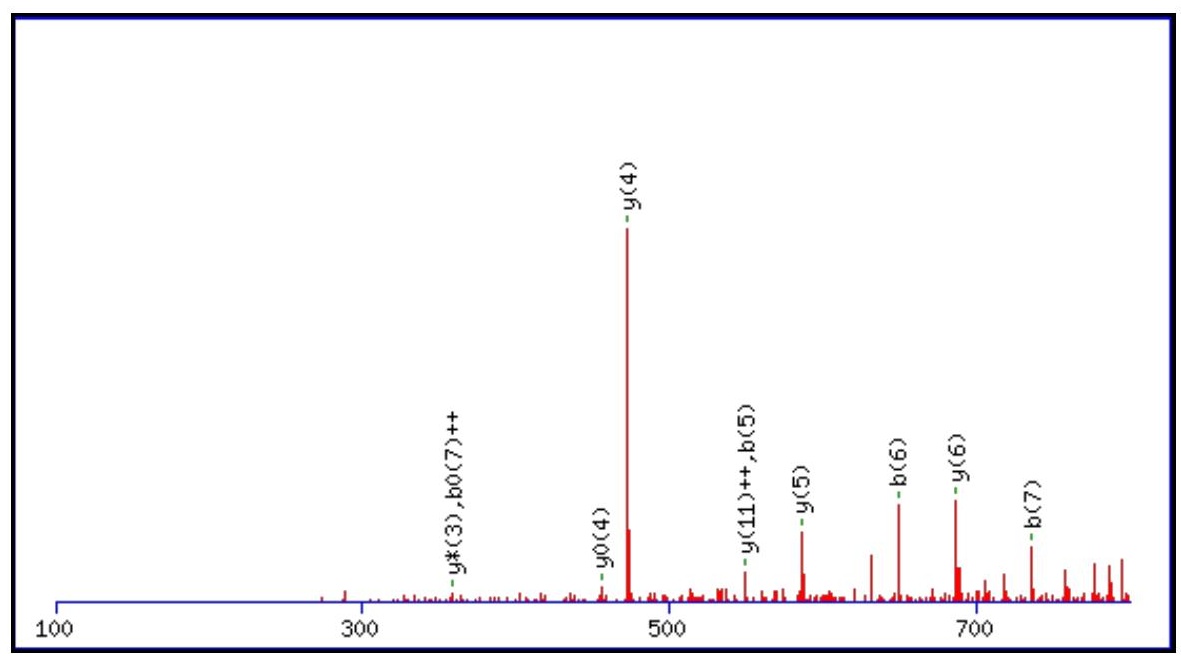



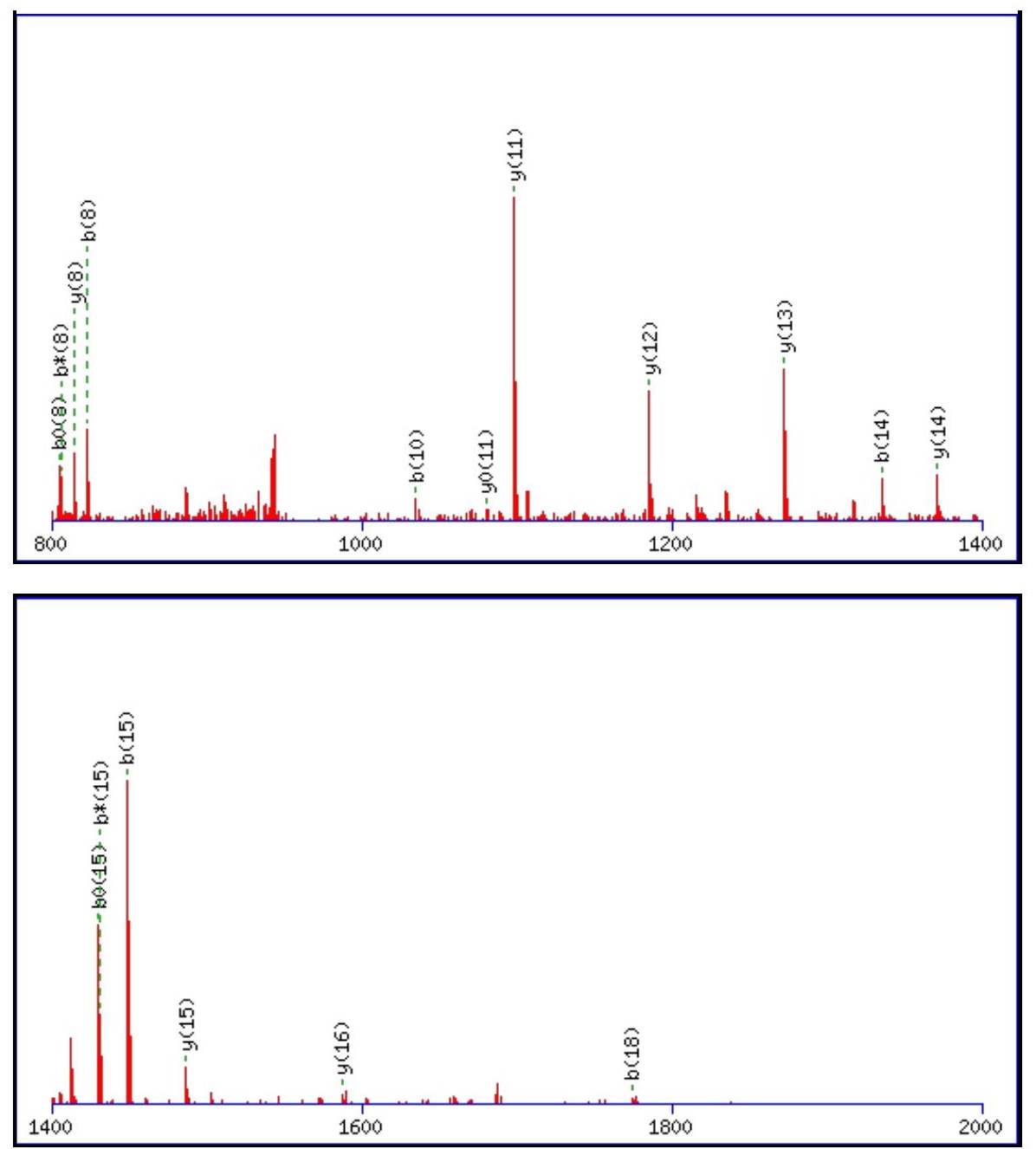

Monoisotopic mass of neutral peptide $\operatorname{Mr}($ calc): 1919.8749

Fixed modifications: Carbamidomethyl (C)

Variable modifications:

N5: Deamidated_N (N)

Ions Score: 73 Expect: $1.1 \mathrm{e}-005$

Matches (Bold Red): 27/206 fragment ions using 43 most intense peaks

\begin{tabular}{|c|c|c|c|c|c|c|c|c|c|c|c|c|c|c|}
\hline \# & b & $\mathbf{b}^{++}$ & $\mathbf{b}^{*}$ & $\mathbf{b}^{*^{++}}$ & $\mathbf{b}^{0}$ & $\mathbf{b}^{\mathbf{0 + +}}$ & Seq. & $\mathbf{y}$ & $\mathbf{y}^{++}$ & $\mathbf{y}^{*}$ & $\mathrm{y}^{*^{++}}$ & $\mathbf{y}^{0}$ & $\mathbf{y}^{0++}$ & \# \\
\hline $\mathbf{1}$ & 116.0342 & 58.5207 & & & 98.0237 & 49.5155 & D & & & & & & & 19 \\
\hline 2 & 187.0713 & 94.0393 & & & 169.0608 & 85.0340 & A & 1805.8552 & 903.4312 & 1788.8286 & & 1787.8446 & 894.4260 & 18 \\
\hline 3 & 334.1397 & 167.5735 & & & 316.1292 & 158.5682 & $\mathbf{F}$ & 1734.8181 & 867.9127 & 1717.7915 & 859.3994 & 1716.8075 & 858.9074 & 17 \\
\hline 4 & 435.1874 & 218.0974 & & & 417.1769 & 209.0921 & $\mathbf{T}$ & 1587.7497 & 794.3785 & 1570.7231 & 785.8652 & 1569.7391 & 785.3732 & 16 \\
\hline 5 & 550.2144 & 275.6108 & 533.1878 & 267.0975 & 532.2038 & 266.6055 & $\mathbf{N}$ & 1486.7020 & 743.8546 & 1469.6754 & 735.3414 & 1468.6914 & 734.8493 & 15 \\
\hline 6 & 649.2828 & 325.1450 & 632.2562 & 316.6318 & 631.2722 & 316.1397 & $\mathbf{V}$ & 1371.6751 & 686.3412 & 1354.6485 & 677.8279 & 1353.6645 & 677.3359 & 14 \\
\hline 7 & 736.3148 & 368.6610 & 719.2883 & 360.1478 & 718.3042 & 359.6558 & S & 1272.6066 & 636.8070 & 1255.5801 & 628.2937 & 1254.5961 & 627.8017 & 13 \\
\hline 8 & 823.3468 & 412.1771 & 806.3203 & 403.6638 & 805.3363 & 403.1718 & $\mathrm{~S}$ & 1185.5746 & 593.2909 & 1168.5481 & 584.7777 & 1167.5640 & 584.2857 & 12 \\
\hline 9 & 920.3996 & 460.7034 & 903.3730 & 452.1902 & 902.3890 & 451.6982 & $\mathbf{P}$ & 1098.5426 & 549.7749 & 1081.5160 & 541.2617 & 1080.5320 & 540.7696 & 11 \\
\hline 10 & 1035.4265 & 518.2169 & 1018.4000 & 509.7036 & 1017.4160 & 509.2116 & D & 1001.4898 & 501.2485 & 984.4633 & 492.7353 & 983.4793 & 492.2433 & 10 \\
\hline 11 & 1106.4637 & 553.7355 & 1089.4371 & 545.2222 & 1088.4531 & 544.7302 & A & 886.4629 & 443.7351 & 869.4363 & 435.2218 & 868.4523 & 434.7298 & 9 \\
\hline 12 & 1163.4851 & 582.2462 & 1146.4586 & 573.7329 & 1145.4746 & 573.2409 & G & 815.4258 & 408.2165 & 798.3992 & 399.7032 & 797.4152 & 399.2112 & 8 \\
\hline 13 & 1234.5222 & 617.7648 & 1217.4957 & 609.2515 & 1216.5117 & 608.7595 & A & 758.4043 & 379.7058 & 741.3777 & 371.1925 & 740.3937 & 370.7005 & 7 \\
\hline 14 & 1335.5699 & 668.2886 & 1318.5434 & 659.7753 & 1317.5593 & 659.2833 & $\mathbf{T}$ & 687.3672 & 344.1872 & 670.3406 & 335.6740 & 669.3566 & 335.1819 & 6 \\
\hline 15 & 1448.6540 & 724.8306 & 1431.6274 & 716.3174 & 1430.6434 & 715.8253 & $\mathbf{L}$ & 586.3195 & 293.6634 & 569.2930 & 285.1501 & 568.3089 & 284.6581 & 5 \\
\hline 16 & 1545.7067 & 773.3570 & 1528.6802 & 764.8437 & 1527.6962 & 764.3517 & $\mathbf{P}$ & 473.2354 & 237.1214 & 456.2089 & 228.6081 & 455.2249 & 228.1161 & 4 \\
\hline 17 & 1659.7497 & 830.3785 & 1642.7231 & 821.8652 & 1641.7391 & 821.3732 & $\mathbf{N}$ & 376.1827 & 188.5950 & 359.1561 & 180.0817 & 358.1721 & 179.5897 & 3 \\
\hline 18 & 1774.7766 & 887.8919 & 1757.7501 & 879.3787 & 1756.7660 & 878.8867 & D & 262.1397 & 131.5735 & 245.1132 & 123.0602 & 244.1292 & 122.5682 & 2 \\
\hline
\end{tabular}



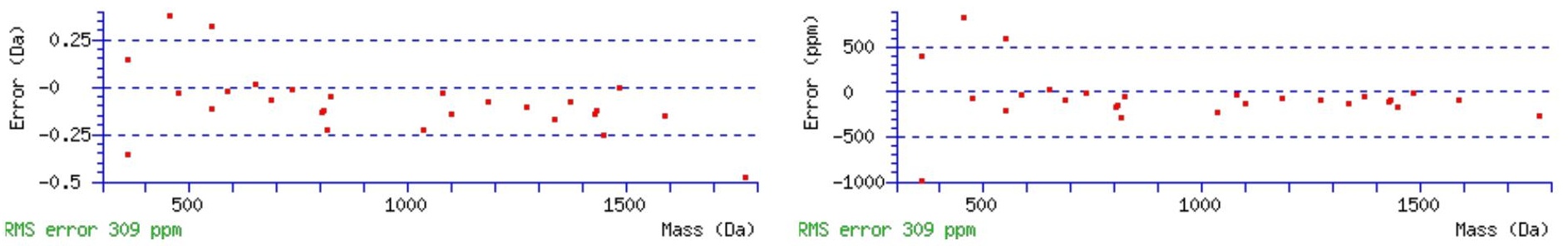

\section{All matches to this query}

\begin{tabular}{|l|l|l|l|}
\hline Score & Mr(calc): & Delta & \multicolumn{1}{|c|}{ Sequence } \\
\hline 73.0 & 1919.8749 & 0.0009 & DAFTNVSSPDAGATLPNDK \\
\hline 35.7 & 1919.8749 & 0.0009 & DAFTNVSSPDAGATLPNDK \\
\hline 26.3 & 1918.8909 & 0.9849 & DAFTNVSSPDAGATLPNDK \\
\hline 4.9 & 1919.8599 & 0.0159 & RNGSKLSNDHQQNQSK \\
\hline 0.6 & 1919.8740 & 0.0018 & YNNLLLLENQFICGK \\
\hline 0.5 & 1918.8703 & 1.0055 & HETYRSTLKTLPGTR \\
\hline 0.5 & 1918.8703 & 1.0055 & HETYRSTLKTLPGTR \\
\hline 0.2 & 1919.8852 & -0.0094 & MKGGVIYIYPDEAAASR \\
\hline
\end{tabular}

Spectrum No: 323; Query: 2484; Rank: 1

\section{Peptide View}

MS/MS Fragmentation of GNSMEEILEGLKFNLTETPETEIHR

Found in IPI00211075, Tax_Id=10116 Gene_Symbol=Serpina3n Serine protease inhibitor A3N precursor

Match to Query 2484: 2903.387622 from(968.803150,3+)

Title: 091008RatKidney_NH4Format01_25.3668.3668.3.dta

Data file K:INewmanPaper|Piliang|3SubProteomes\Piliang3SP\mgf5ppm\ERLIC_3SubProteomes5ppm.mgf

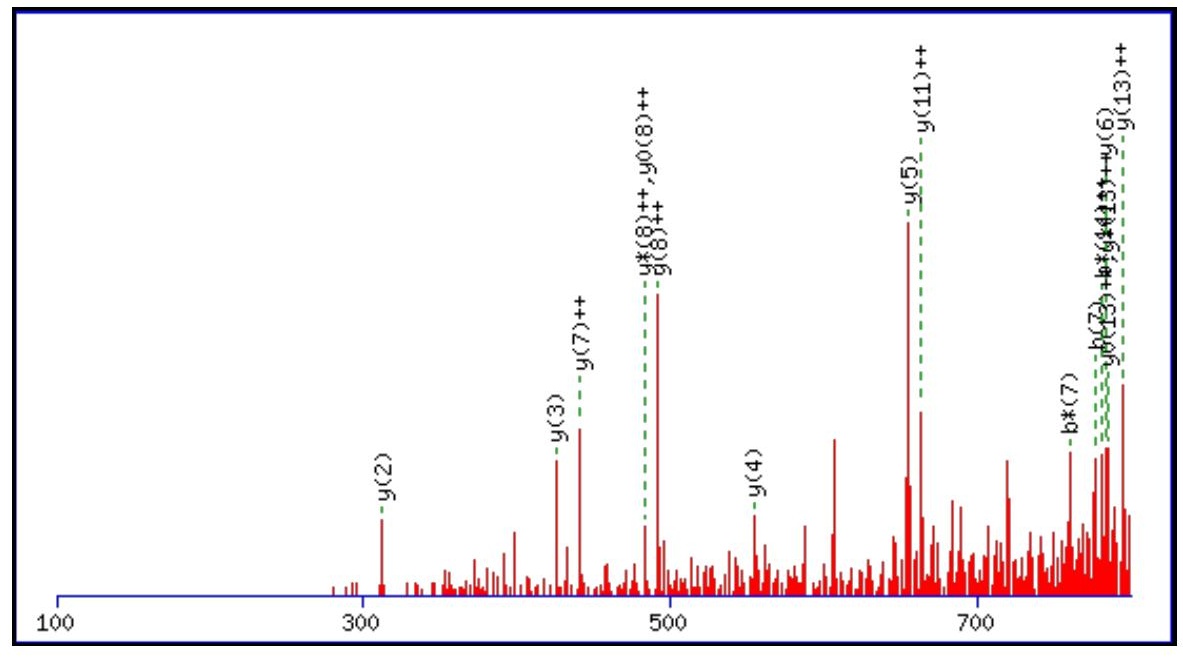



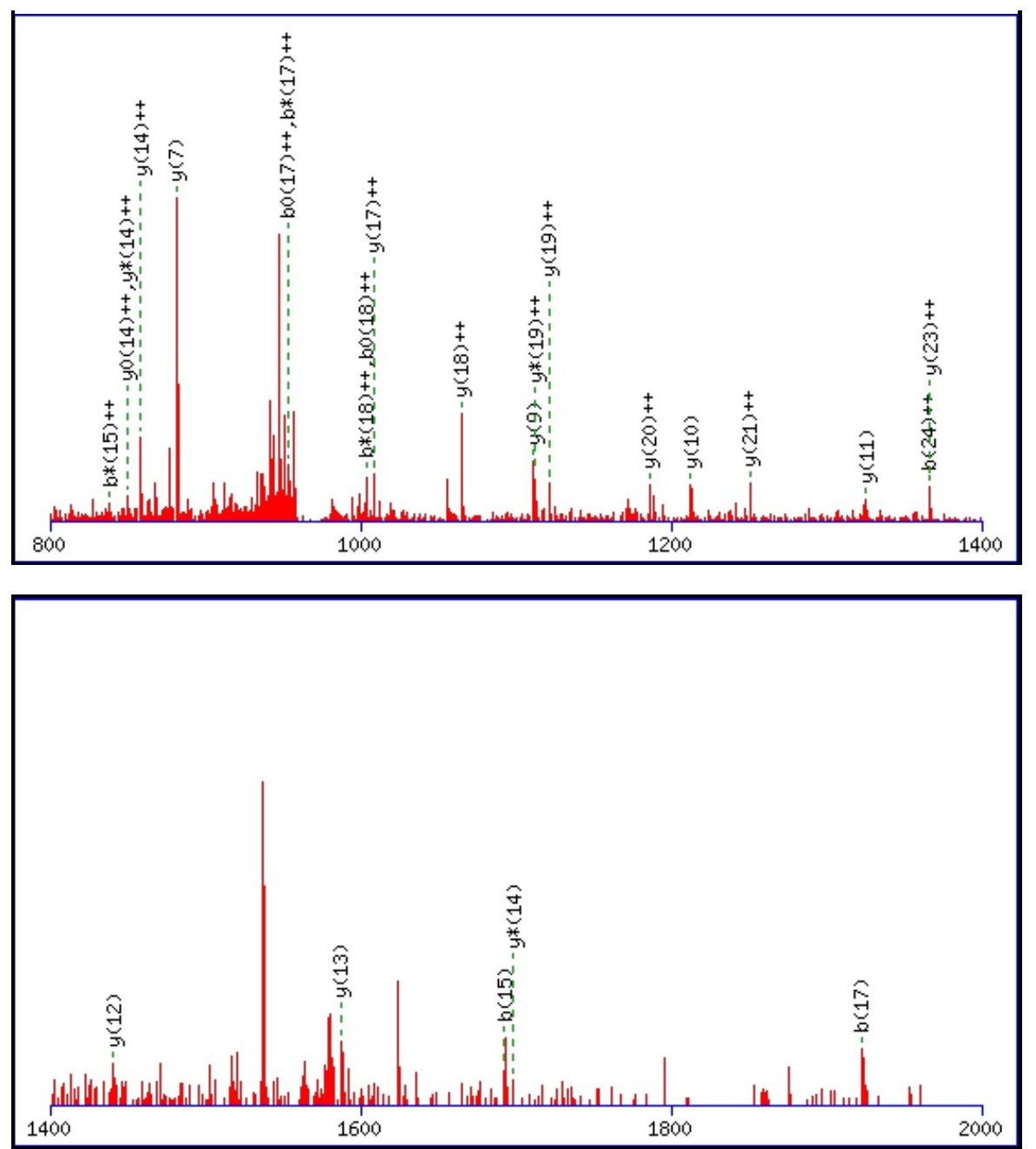

Monoisotopic mass of neutral peptide $\operatorname{Mr}($ calc): 2903.3858

Fixed modifications: Carbamidomethyl (C)

Variable modifications:

M4 : Oxidation (M)

N14 : Deamidated_N (N)

Ions Score: 73 Expect: $1.7 \mathrm{e}-005$

Matches (Bold Red): 41/276 fragment ions using 69 most intense peaks

\begin{tabular}{|c|c|c|c|c|c|c|c|c|c|c|c|c|c|c|}
\hline \# & b & & & & $\mathbf{b}^{0}$ & & eq. & $\mathbf{y}$ & & & & $\mathbf{y}^{0}$ & & \# \\
\hline 1 & & & & & & & $\mathbf{G}$ & & & & & & & 25 \\
\hline 2 & 172.0717 & & & & & & $\mathbf{N}$ & & & & & & & 24 \\
\hline 3 & 259.1037 & & & & & & S & & & & & 3182 & & 23 \\
\hline 4 & 406.1391 & 203.5732 & 389.1 & & & & $\mathbf{M}$ & & & & & & & 22 \\
\hline 5 & 535. & 945 & 518. & & & & $\mathbf{E}$ & & & & & 508 & 290 & 21 \\
\hline 6 & 664.2243 & 332.6158 & 647.1 & & & & $\mathbf{E}$ & & & & & & & 20 \\
\hline 7 & 777.3 & 78 & 760. & 380 & 78 & & I & & & 2224 & & 656 & 0864 & 19 \\
\hline 8 & 890.3924 & 445.6998 & 873.3659 & & & & $\mathbf{L}$ & & & & & & & 18 \\
\hline 9 & 1019.4350 & 510.2211 & 02.4085 & 501. & 244 & 159 & $\mathbf{E}$ & 0080 & 1008.0076 & 1997. & 944 & 1996.9974 & 999.0024 & 17 \\
\hline 10 & 076.4565 & 538.7319 & 299 & 530.2 & 59 & 529 & G & 654 & 943 & 1868 & & 186 & 811 & 16 \\
\hline 11 & 1189.5405 & 595.2739 & 172.5140 & 586.7606 & 300 & 586.2686 & L & 1828.9439 & 9756 & 1811. & 4623 & 1810.9334 & 905.9703 & 15 \\
\hline 12 & 1317.6355 & 659.3214 & 300.6089 & 650.8081 & & & $\mathbf{K}$ & & & & & & 1283 & 14 \\
\hline 13 & 1464.7039 & 732.8556 & 447.6774 & 724.3423 & & 723.8 & $\mathbf{F}$ & 1587.7649 & 3861 & 1570.7384 & 8728 & 1569.7544 & 785.3808 & 13 \\
\hline 14 & 1579.7308 & 790.3691 & 1562.7043 & 781.8558 & 1561.7203 & 781. & $\mathbf{N}$ & 1440.6965 & 720 & 1423.6700 & 3386 & 1422 & 8466 & 12 \\
\hline 15 & 1692.8149 & 846.9111 & 1675.7884 & 838.3978 & 1674.8043 & 837.9058 & L & 1325.6696 & 663.3384 & 1308.6430 & 654.8251 & 1307.6590 & 654.3331 & 11 \\
\hline 16 & 1793.8626 & 897.4349 & 1776.8360 & 888.9217 & 1775.8520 & 888.4297 & $\mathbf{T}$ & 5855 & 606.7964 & 1195.5590 & 598.2831 & 1194.5749 & 597.7911 & 10 \\
\hline 17 & 1922.9052 & 961.9562 & 1905.8786 & 953.4430 & 1904.8946 & 952.9509 & $\mathbf{E}$ & 1111.5378 & 556.2726 & 1094.5113 & 547.7593 & 1093.5273 & 547.2673 & 9 \\
\hline 18 & 2023.9529 & 1012.4801 & 2006.9263 & 1003.9668 & 2005.9423 & 1003.4748 & $\mathbf{T}$ & 982.4952 & 491.7513 & 965.4687 & 483.2380 & 964.4847 & 482.7460 & 8 \\
\hline
\end{tabular}




\begin{tabular}{|l|l|r|r|r|r|r|r|r|r|r|r|r|r|r|r|}
$\mathbf{1 9}$ & 2121.0056 & 1061.0065 & 2103.9791 & 1052.4932 & 2102.9951 & 1052.0012 & $\mathbf{P}$ & $\mathbf{8 8 1 . 4 4 7 6}$ & $\mathbf{4 4 1 . 2 2 7 4}$ & 864.4210 & 432.7141 & 863.4370 & 432.2221 & $\mathbf{7}$ \\
\hline $\mathbf{2 0}$ & 2250.0482 & 1125.5277 & 2233.0217 & 1117.0145 & 2232.0377 & 1116.5225 & $\mathbf{E}$ & $\mathbf{7 8 4 . 3 9 4 8}$ & 392.7010 & 767.3682 & 384.1878 & 766.3842 & 383.6958 & $\mathbf{6}$ \\
\hline $\mathbf{2 1}$ & 2351.0959 & 1176.0516 & 2334.0693 & 1167.5383 & 2333.0853 & 1167.0463 & $\mathbf{T}$ & $\mathbf{6 5 5 . 3 5 2 2}$ & 328.1797 & 638.3257 & 319.6665 & 637.3416 & 319.1745 & $\mathbf{5}$ \\
\hline $\mathbf{2 2}$ & 2480.1385 & 1240.5729 & 2463.1119 & 1232.0596 & 2462.1279 & 1231.5676 & $\mathbf{E}$ & $\mathbf{5 5 4 . 3 0 4 5}$ & 277.6559 & 537.2780 & 269.1426 & 536.2940 & 268.6506 & $\mathbf{4}$ \\
\hline $\mathbf{2 3}$ & 2593.2226 & 1297.1149 & 2576.1960 & 1288.6016 & 2575.2120 & 1288.1096 & $\mathbf{I}$ & $\mathbf{4 2 5 . 2 6 1 9}$ & 213.1346 & 408.2354 & 204.6213 & & & & $\mathbf{3}$ \\
\hline $\mathbf{2 4}$ & 2730.2815 & $\mathbf{1 3 6 5 . 6 4 4 4}$ & $\mathbf{2 7 1 3 . 2 5 4 9}$ & 1357.1311 & 2712.2709 & 1356.6391 & $\mathbf{H}$ & $\mathbf{3 1 2 . 1 7 7 9}$ & 156.5926 & 295.1513 & 148.0793 & & & $\mathbf{2}$ \\
\hline $\mathbf{2 5}$ & & & & & & & $\mathbf{R}$ & 175.1190 & 88.0631 & 158.0924 & 79.5498 & & & $\mathbf{1}$ \\
\hline
\end{tabular}
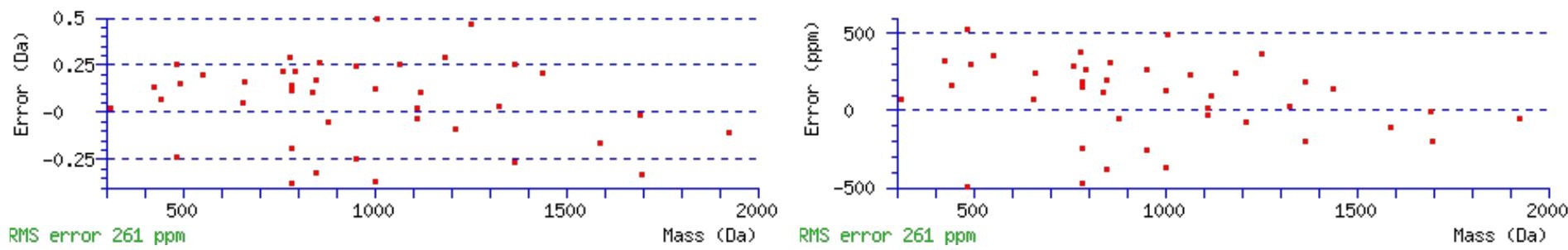

\section{All matches to this query}

\begin{tabular}{|l|l|l|l|}
\hline Score & Mr(calc): & Delta & \multicolumn{1}{|c|}{ Sequence } \\
\hline 72.9 & 2903.3858 & 0.0018 & GNSMEEILEGLKFNLTETPETEIHR \\
\hline 14.7 & 2903.3858 & 0.0018 & GNSMEEILEGLKFNLTETPETEIHR \\
\hline 12.5 & 2902.4018 & 0.9858 & GNSMEEILEGLKFNLTETPETEIHR \\
\hline 5.2 & 2901.3787 & 2.0089 & GDTADARPPTPLLPGHPTASSTSIKVR \\
\hline
\end{tabular}

Spectrum No: 324; Query: 2603; Rank: 1

\section{Peptide View}

MS/MS Fragmentation of GVDVSQVAWQSQGDTPCSCCIVNNSNGSR

Found in IPI00211989, Tax_Id=10116 Gene_Symbol=Khk Isoform C of Ketohexokinase

Match to Query 2603: 3182.360862 from(1061.794230,3+)

Title: 100101RatKid_NS_deglyco_08.3131.3131.3.dta

Data file K:INewmanPaper|Piliangl3SubProteomes\Piliang3SP\mgf5ppm\ERLIC_3SubProteomes5ppm.mgf

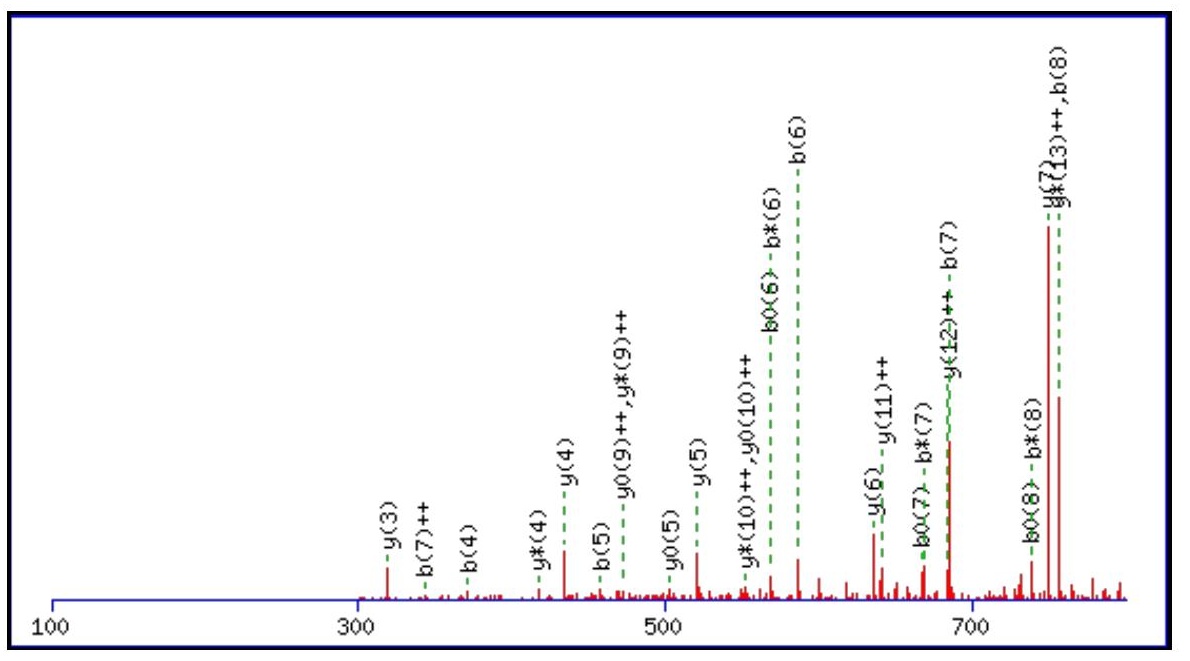



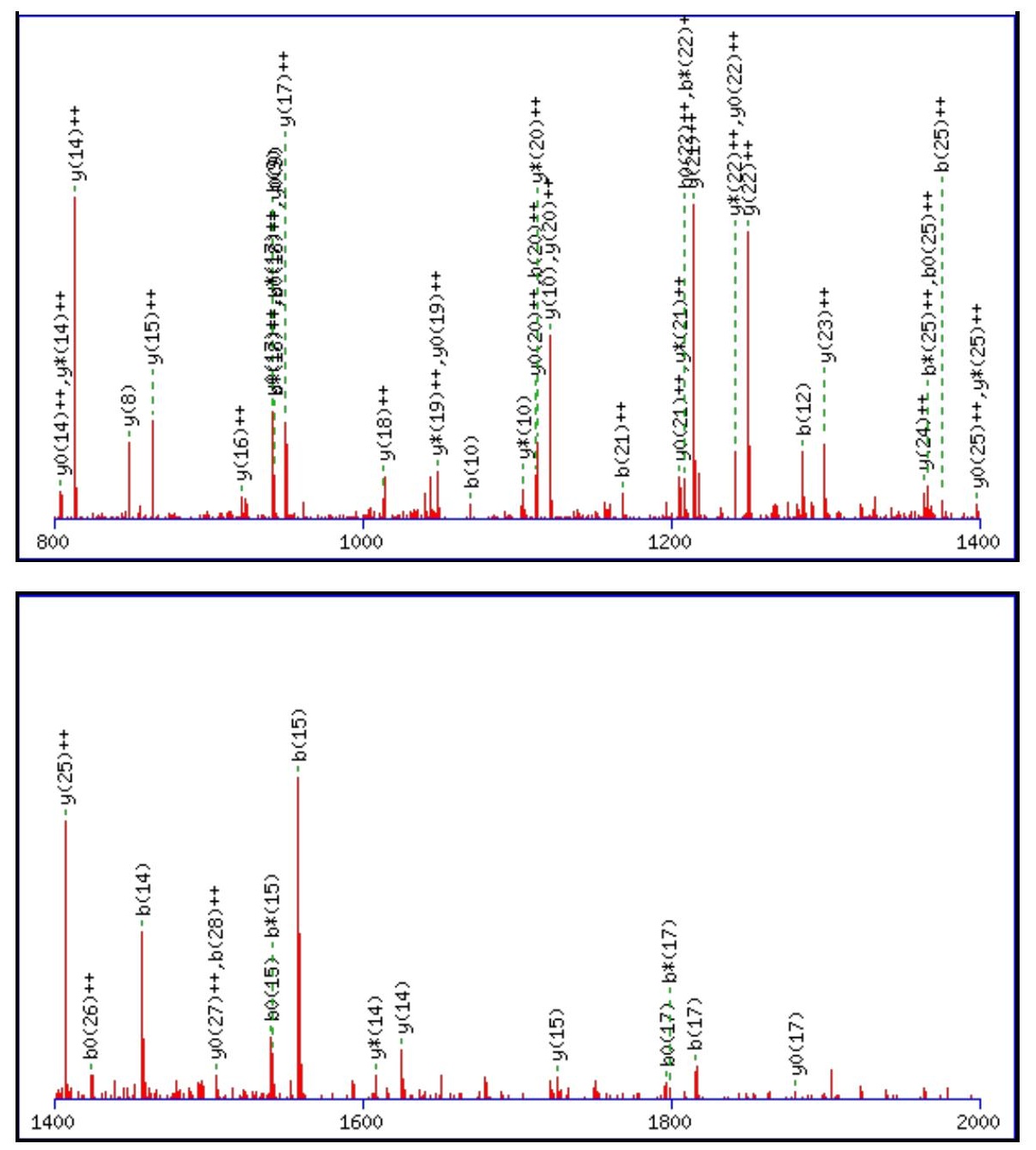

Monoisotopic mass of neutral peptide $\operatorname{Mr}($ calc): 3182.3452

Fixed modifications: Carbamidomethyl (C)

Variable modifications:

N26: Deamidated $\mathrm{N}(\mathrm{N})$

Ions Score: 73 Expect: $2.3 \mathrm{e}-005$

Matches (Bold Red): 81/320 fragment ions using 107 most intense peaks

\begin{tabular}{|c|c|c|c|c|c|c|c|c|c|c|c|c|c|c|}
\hline \# & b & $\mathbf{b}^{++}$ & b* & $\mathbf{b}^{*^{++}}$ & $\mathbf{b}^{0}$ & $\mathbf{b}^{0++}$ & Seq. & $\mathbf{y}$ & $y^{++}$ & $\mathbf{y}^{*}$ & $\mathrm{y}^{*^{++}}$ & $\mathbf{y}^{\mathbf{0}}$ & $\mathbf{y}^{0++}$ & \# \\
\hline 1 & 58.0287 & 29.5180 & & & & & G & & & & & & & 29 \\
\hline 2 & 157.0972 & 79.0522 & & & & & $\mathbf{V}$ & 3126.3310 & 1563.6691 & 3109.3044 & 1555.1558 & 3108.3204 & 1554.6638 & 28 \\
\hline 3 & 272.1241 & 136.5657 & & & 254.1135 & 127.5604 & D & 3027.2626 & 1514.1349 & 3010.2360 & 1505.6216 & 3009.2520 & 1505.1296 & 27 \\
\hline 4 & 371.1925 & 186.0999 & & & 353.1819 & 177.0946 & $\mathbf{V}$ & 2912.2356 & 1456.6214 & 2895.2091 & 1448.1082 & 2894.2250 & 1447.6162 & 26 \\
\hline 5 & 458.2245 & 229.6159 & & & 440.2140 & 220.6106 & $\mathrm{~S}$ & 2813.1672 & 1407.0872 & 2796.1406 & 1398.5740 & 2795.1566 & 1398.0820 & 25 \\
\hline 6 & 586.2831 & 293.6452 & 569.2566 & 285.1319 & 568.2726 & 284.6399 & $\mathbf{Q}$ & 2726.1352 & 1363.5712 & 2709.1086 & 1355.0579 & 2708.1246 & 1354.5659 & 24 \\
\hline 7 & 685.3515 & 343.1794 & 668.3250 & 334.6661 & 667.3410 & 334.1741 & $\bar{V}$ & 2598.0766 & 1299.5419 & 2581.0500 & 1291.0287 & 2580.0660 & 1290.5367 & 23 \\
\hline 8 & 756.3886 & 378.6980 & 739.3621 & 370.1847 & 738.3781 & 369.6927 & A & 2499.0082 & 1250.0077 & 2481.9816 & 1241.4945 & 2480.9976 & 1241.0024 & 22 \\
\hline 9 & 942.4680 & 471.7376 & 925.4414 & 463.2243 & 924.4574 & 462.7323 & $\mathbf{W}$ & 2427.9711 & 1214.4892 & 2410.9445 & 1205.9759 & 2409.9605 & 1205.4839 & 21 \\
\hline 10 & 1070.5265 & 535.7669 & 1053.5000 & 527.2536 & 1052.5160 & 526.7616 & $\mathbf{Q}$ & 2241.8917 & 1121.4495 & 2224.8652 & 1112.9362 & 2223.8812 & 1112.4442 & 20 \\
\hline 11 & 1157.5586 & 579.2829 & 1140.5320 & 570.7696 & 1139.5480 & 570.2776 & $\mathrm{~S}$ & 2113.8332 & 1057.4202 & 2096.8066 & 1048.9069 & 2095.8226 & 1048.4149 & 19 \\
\hline 12 & 1285.6171 & 643.3122 & 1268.5906 & 634.7989 & 1267.6066 & 634.3069 & $\mathbf{Q}$ & 2026.8011 & 1013.9042 & 2009.7746 & 1005.3909 & 2008.7906 & 1004.8989 & 18 \\
\hline 13 & 1342.6386 & 671.8229 & 1325.6121 & 663.3097 & 1324.6280 & 662.8177 & G & 1898.7426 & 949.8749 & 1881.7160 & 941.3616 & 1880.7320 & 940.8696 & 17 \\
\hline 14 & 1457.6655 & 729.3364 & 1440.6390 & 720.8231 & 1439.6550 & 720.3311 & D & 1841.7211 & 921.3642 & 1824.6946 & 912.8509 & 1823.7105 & 912.3589 & 16 \\
\hline 15 & 1558.7132 & 779.8603 & 1541.6867 & 771.3470 & 1540.7027 & 770.8550 & $\mathbf{T}$ & 1726.6942 & 863.8507 & 1709.6676 & 855.3374 & 1708.6836 & 854.8454 & 15 \\
\hline 16 & 1655.7660 & 828.3866 & 1638.7394 & 819.8734 & 1637.7554 & 819.3814 & $\mathbf{P}$ & 1625.6465 & 813.3269 & 1608.6199 & 804.8136 & 1607.6359 & 804.3216 & 14 \\
\hline 17 & 1815.7966 & 908.4020 & 1798.7701 & 899.8887 & 1797.7861 & 899.3967 & $\mathrm{C}$ & 1528.5937 & 764.8005 & 1511.5672 & 756.2872 & 1510.5831 & 755.7952 & 13 \\
\hline 18 & 1902.8287 & 951.9180 & 1885.8021 & 943.4047 & 1884.8181 & 942.9127 & $\mathrm{~S}$ & 1368.5631 & 684.7852 & 1351.5365 & 676.2719 & 1350.5525 & 675.7799 & 12 \\
\hline
\end{tabular}




\begin{tabular}{|c|c|c|c|c|c|c|c|c|c|c|c|c|c|c|}
\hline 19 & 2062.8593 & |1031.9333 & |2045.8328 & |1023.4200 & |2044.8488 & |1022.9280 & C & 1281.5310 & 641.2692 & |1264.5045 & 632.7559 & 1263.5205 & 632.2639 & |11 \\
\hline 20 & 2222.8900 & 1111.9486 & 2205.8634 & 1103.4353 & 2204.8794 & 1102.9433 & C & 1121.5004 & 561.2538 & 1104.4738 & 552.7406 & 1103.4898 & 552.2485 & 10 \\
\hline 21 & 2335.9740 & 1168.4907 & 2318.9475 & 1159.9774 & 2317.9635 & 1159.4854 & I & 961.4697 & 481.2385 & 944.4432 & 472.7252 & 943.4592 & 472.2332 & 9 \\
\hline 22 & 2435.0424 & 1218.0249 & 2418.0159 & 1209.5116 & 2417.0319 & 1209.0196 & V & 848.3857 & 424.6965 & 831.3591 & 416.1832 & 830.3751 & 415.6912 & 8 \\
\hline 23 & 2549.0854 & 1275.0463 & 2532.0588 & 1266.5330 & 2531.0748 & 1266.0410 & $\mathbf{N}$ & 749.3173 & 375.1623 & 732.2907 & 366.6490 & 731.3067 & 366.1570 & 7 \\
\hline 24 & 2663.1283 & 1332.0678 & 2646.1017 & 1323.5545 & 2645.1177 & 1323.0625 & $\mathbf{N}$ & 635.2743 & 318.1408 & 618.2478 & 309.6275 & 617.2638 & 309.1355 & 6 \\
\hline 25 & 2750.1603 & 1375.5838 & 2733.1338 & 1367.0705 & 2732.1498 & 1366.5785 & $\mathrm{~S}$ & 521.2314 & 261.1193 & 504.2049 & 252.6061 & 503.2208 & 252.1141 & 5 \\
\hline 26 & 2865.1873 & 1433.0973 & 2848.1607 & 1424.5840 & 2847.1767 & 1424.0920 & $\mathbf{N}$ & 434.1994 & 217.6033 & 417.1728 & 209.0901 & 416.1888 & 208.5980 & 4 \\
\hline 27 & 2922.2087 & 1461.6080 & 2905.1822 & 1453.0947 & 2904.1982 & 1452.6027 & G & 319.1724 & 160.0899 & 302.1459 & 151.5766 & 301.1619 & 151.0846 & 3 \\
\hline 28 & 3009.2408 & 1505.1240 & 2992.2142 & 1496.6107 & 2991.2302 & 1496.1187 & $S$ & 262.1510 & 131.5791 & 245.1244 & 123.0659 & 244.1404 & 122.5738 & 2 \\
\hline 29 & & & & & & & $\mathbf{R}$ & 175.1190 & 88.0631 & 158.0924 & 79.5498 & & & 1 \\
\hline
\end{tabular}
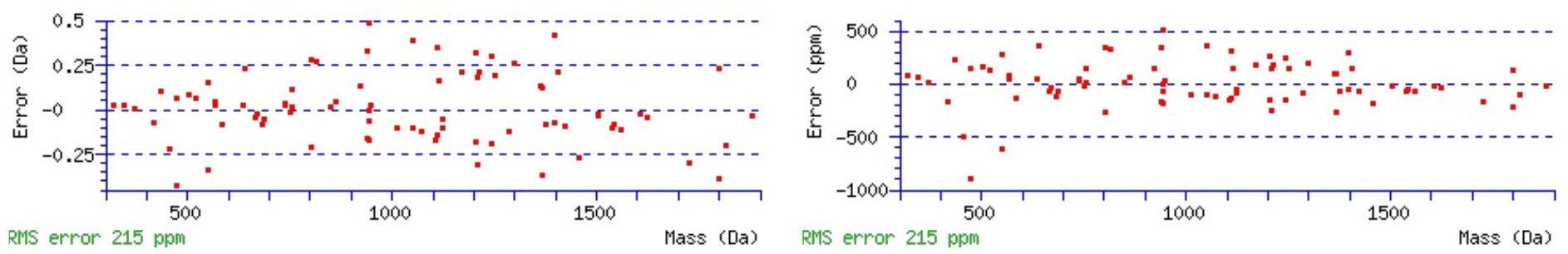

\section{All matches to this query}

\begin{tabular}{|l|l|l|l|}
\hline Score & Mr(calc): & Delta & \multicolumn{1}{|c|}{ Sequence } \\
\hline 72.7 & 3182.3452 & 0.0157 & GVDVSQVAWQSQGDTPCSCCIVNNSNGSR \\
\hline 61.7 & 3182.3452 & 0.0157 & GVDVSQVAWQSQGDTPCSCCIVNNSNGSR \\
\hline 60.8 & 3182.3452 & 0.0157 & GVDVSQVAWQSQGDTPCSCCIVNNSNGSR \\
\hline 20.0 & 3181.3612 & 0.9997 & GVDVSQVAWQSQGDTPCSCCIVNNSNGSR \\
\hline 5.2 & 3182.3562 & 0.0047 & SLKEFQENTNRQTVLLQATCLELK \\
\hline 2.3 & 3181.3811 & 0.9798 & QKTNQQIEYLIFVSTGYNQRPLDGK \\
\hline 2.3 & 3181.3811 & 0.9798 & QKTNQQIEYLIFVSTGYNQRPLDGK \\
\hline 2.3 & 3181.3801 & 0.9808 & DEVAHTVTESRVLQNTRHPFLTALK \\
\hline 2.1 & 3182.3742 & -0.0133 & YLVENNANISIANKYDNTCLMIAAYK \\
\hline 2.1 & 3182.3742 & -0.0133 & YLVENNANISIANKYDNTCLMIAAYK \\
\hline
\end{tabular}

Spectrum No: 325; Query: 2539; Rank: 1

\section{Peptide View}

MS/MS Fragmentation of AFINGTGVETVVSADLPNAHGLAVDWVSR

Found in IPI00369995, Tax_Id=10116 Gene_Symbol=Lrp1 similar to low density lipoprotein receptor-related protein 1

Match to Query 2539: 2996.510232 from(999.844020,3+)

Title: 100101RatKid_NS_deglyco_16.5694.5694.3.dta

Data file K:INewmanPaper|Piliangl3SubProteomes\Piliang3SP\mgf5ppm\ERLIC_3SubProteomes5ppm.mgf 

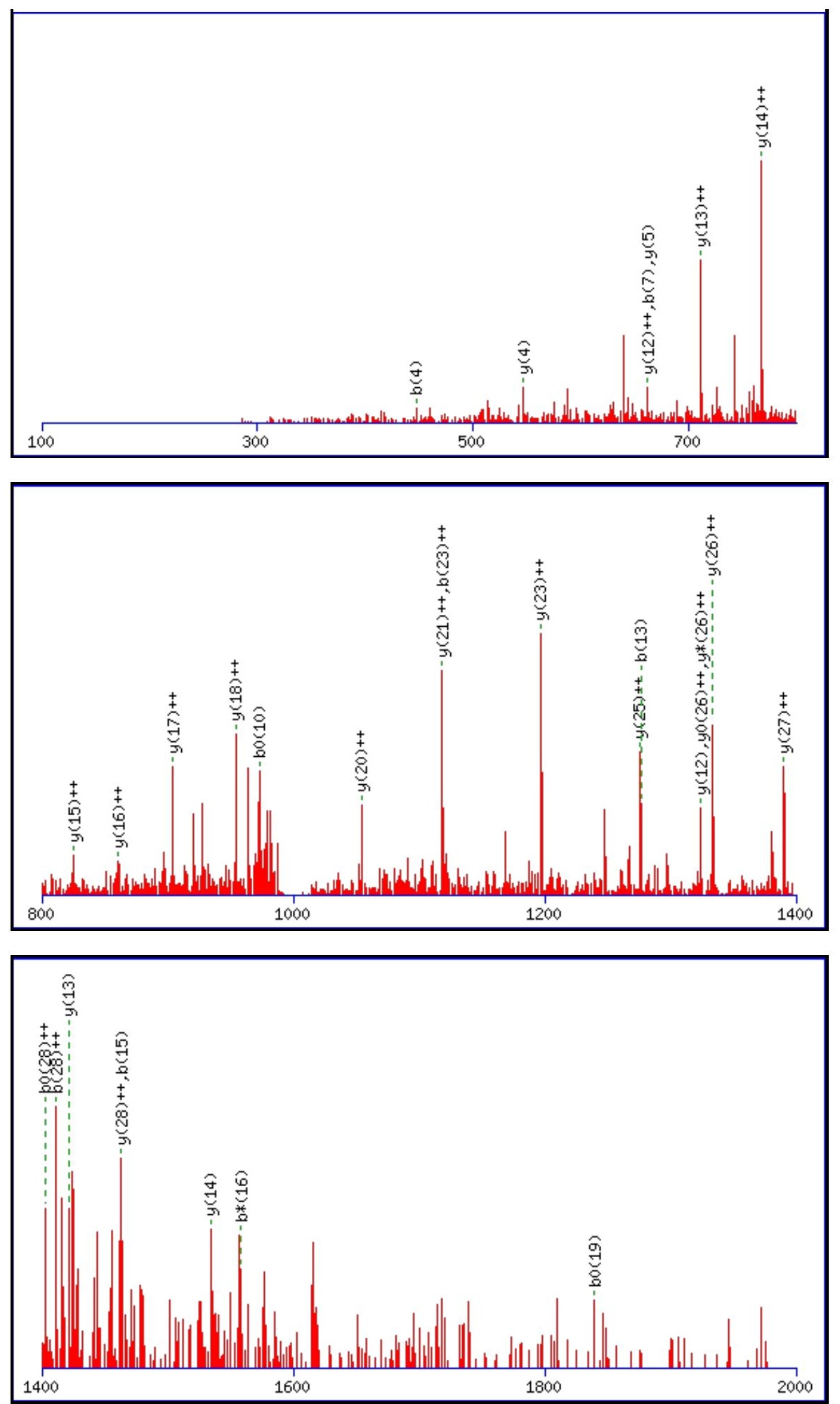

Monoisotopic mass of neutral peptide $\operatorname{Mr}($ calc): 2995.5040

Fixed modifications: Carbamidomethyl (C)

Variable modifications:

N4 : Deamidated_N (N)

Ions Score: 73 Expect: $1.1 \mathrm{e}-005$

Matches (Bold Red): 31/318 fragment ions using 60 most intense peaks

\begin{tabular}{|r|c|c|c|c|c|c|c|c|c|c|c|c|c|c|}
\hline$\#$ & $\mathbf{b}$ & $\mathbf{b}^{++}$ & $\mathbf{b}^{*}$ & $\mathbf{b}^{\boldsymbol{*}^{++}}$ & $\mathbf{b}^{\mathbf{0}}$ & $\mathbf{b}^{\mathbf{0 + +}}$ & Seq. & $\mathbf{y}$ & $\mathbf{y}^{++}$ & $\mathbf{y}^{*}$ & $\mathbf{y}^{\boldsymbol{*}^{++}}$ & $\mathbf{y}^{\mathbf{0}}$ & $\mathbf{y}^{\mathbf{0 + +}}$ & $\#$ \\
\hline $\mathbf{1}$ & 72.0444 & 36.5258 & & & & & $\mathbf{A}$ & & & & & & & $\mathbf{2 9}$ \\
\hline $\mathbf{2}$ & 219.1128 & 110.0600 & & & & & $\mathbf{F}$ & 2925.4741 & $\mathbf{1 4 6 3 . 2 4 0 7}$ & 2908.4476 & 1454.7274 & 2907.4635 & 1454.2354 & $\mathbf{2 8}$ \\
\hline $\mathbf{3}$ & 332.1969 & 166.6021 & & & & & $\mathbf{I}$ & 2778.4057 & $\mathbf{1 3 8 9 . 7 0 6 5}$ & 2761.3791 & 1381.1932 & 2760.3951 & 1380.7012 & $\mathbf{2 7}$ \\
\hline $\mathbf{4}$ & 447.2238 & 224.1155 & 430.1973 & 215.6023 & & & $\mathbf{N}$ & 2665.3216 & $\mathbf{1 3 3 3 . 1 6 4 5}$ & 2648.2951 & $\mathbf{1 3 2 4 . 6 5 1 2}$ & 2647.3111 & $\mathbf{1 3 2 4 . 1 5 9 2}$ & $\mathbf{2 6}$ \\
\hline $\mathbf{5}$ & 504.2453 & 252.6263 & 487.2187 & 244.1130 & & & $\mathbf{G}$ & 2550.2947 & $\mathbf{1 2 7 5 . 6 5 1 0}$ & 2533.2681 & 1267.1377 & 2532.2841 & 1266.6457 & $\mathbf{2 5}$ \\
\hline
\end{tabular}




\begin{tabular}{|c|c|c|c|c|c|c|c|c|c|c|c|c|c|c|}
\hline 6 & 605.2929 & 303.1501 & 588.2664 & 294.6368 & 587.2824 & 294.1448 & $\mathbf{T}$ & 2493.2732 & |1247.1403 & |2476.2467| & |1238.6270 & |2475.2627 & |1238.1350 & 24 \\
\hline 7 & 662.3144 & 331.6608 & 645.2879 & 323.1476 & 644.3038 & 322.6556 & G & 2392.2255 & 1196.6164 & 2375.1990 & 1188.1031 & 2374.2150 & 1187.6111 & 23 \\
\hline 8 & 761.3828 & 381.1951 & 744.3563 & 372.6818 & 743.3723 & 372.1898 & V & 2335.2041 & 1168.1057 & 2318.1775 & 1159.5924 & 2317.1935 & 1159.1004 & 2 \\
\hline 9 & 890.4254 & 445.7163 & 873.3989 & 437.2031 & 872.4149 & 436.7111 & $\mathbf{E}$ & 2236.1357 & 1118.5715 & 2219.1091 & 1110.0582 & 2218.1251 & 1109.5662 & 21 \\
\hline 10 & 991.4731 & 496.2402 & 974.4465 & 487.7269 & 973.4625 & 487.2349 & $\mathbf{T}$ & 2107.0931 & 1054.0502 & 2090.0665 & 1045.5369 & 2089.0825 & 1045.0449 & 20 \\
\hline 11 & 1090.5415 & 545.7744 & 1073.5150 & 537.2611 & 1072.5309 & 536.7691 & $\mathbf{V}$ & 2006.0454 & 1003.5263 & 1989.0189 & 995.0131 & 1988.0348 & 994.5211 & 19 \\
\hline 12 & 1189.6099 & 595.3086 & 1172.5834 & 586.7953 & 1171.5994 & 586.3033 & $\mathbf{V}$ & 1906.9770 & 953.9921 & 1889.9504 & 945.4789 & 1888.9664 & 944.9868 & 18 \\
\hline 13 & 1276.6420 & 638.8246 & 1259.6154 & 630.3113 & 1258.6314 & 629.8193 & $\mathbf{S}$ & 1807.9086 & 904.4579 & 1790.8820 & 895.9446 & 1789.8980 & 895.4526 & 17 \\
\hline 14 & 1347.6791 & 674.3432 & 1330.6525 & 665.8299 & 1329.6685 & 665.3379 & A & 1720.8765 & 860.9419 & 1703.8500 & 852.4286 & 1702.8660 & 851.9366 & 16 \\
\hline 15 & 1462.7060 & 731.8566 & 1445.6795 & 723.3434 & 1444.6954 & 722.8514 & D & 1649.8394 & 825.4234 & 1632.8129 & 816.9101 & 1631.8289 & 816.4181 & 15 \\
\hline 16 & 1575.7901 & 788.3987 & 1558.7635 & 779.8854 & 1557.7795 & 779.3934 & $\mathbf{L}$ & 1534.8125 & 767.9099 & 1517.7859 & 759.3966 & 1516.8019 & 758.9046 & 14 \\
\hline 17 & 1672.8428 & 836.9251 & 1655.8163 & 828.4118 & 1654.8323 & 827.9198 & $\mathbf{P}$ & 1421.7284 & 711.3678 & 1404.7019 & 702.8546 & 1403.7179 & 702.3626 & 13 \\
\hline 18 & 1786.8858 & 893.9465 & 1769.8592 & 885.4332 & 1768.8752 & 884.9412 & $\mathbf{N}$ & 1324.6757 & 662.8415 & 1307.6491 & 654.3282 & 1306.6651 & 653.8362 & 12 \\
\hline 19 & 1857.9229 & 929.4651 & 1840.8963 & 920.9518 & 1839.9123 & 920.4598 & A & 1210.6327 & 605.8200 & 1193.6062 & 597.3067 & 1192.6222 & 596.8147 & 11 \\
\hline 21 & 2052.0033 & 1026.5053 & 2034.9767 & 1017.9920 & 2033.9927 & 1017.5000 & $\mathbf{G}$ & 1002.5367 & 501.7720 & 985.5102 & 493.2587 & 984.5261 & 492.7667 & 9 \\
\hline 22 & 2165.0873 & 1083.0473 & 2148.0608 & 1074.5340 & 2147.0768 & 1074.0420 & $\mathbf{L}$ & 945.5152 & 473.2613 & 928.4887 & 464.7480 & 927.5047 & 464.2560 & 8 \\
\hline 23 & 2236.1244 & 1118.5659 & 2219.0979 & 1110.0526 & 2218.1139 & 1109.5606 & A & 832.4312 & 416.7192 & 815.4046 & 408.2060 & 814.4206 & 407.7139 & 7 \\
\hline 24 & 2335.1928 & 1168.1001 & 2318.1663 & 1159.5868 & 2317.1823 & 1159.0948 & $\mathbf{V}$ & 761.3941 & 381.2007 & 744.3675 & 372.6874 & 743.3835 & 372.1954 & 6 \\
\hline 25 & 2450.2198 & 1225.6135 & 2433.1932 & 1217.1003 & 2432.2092 & 1216.6083 & D & 662.3257 & 331.6665 & 645.2991 & 323.1532 & 644.3151 & 322.6612 & 5 \\
\hline 26 & 2636.2991 & 1318.6532 & 2619.2726 & 1310.1399 & 2618.2885 & 1309.6479 & $\mathbf{W}$ & 547.2987 & 274.1530 & 530.2722 & 265.6397 & 529.2881 & 265.1477 & 4 \\
\hline 27 & 2735.3675 & 1368.1874 & 2718.3410 & 1359.6741 & 2717.3570 & 1359.1821 & $\mathbf{V}$ & 361.2194 & 181.1133 & 344.1928 & 172.6001 & 343.2088 & 172.1081 & 3 \\
\hline 28 & 2822.3995 & 1411.7034 & 2805.3730 & 1403.1901 & 2804.3890 & 1402.6981 & S & 262.1510 & 131.5791 & 245.1244 & 123.0659 & 244.1404 & 122.5738 & 2 \\
\hline 29 & & & & & & & $\mathbf{R}$ & 175.1190 & 88.0631 & 158.0924 & 79.5498 & & & 1 \\
\hline
\end{tabular}
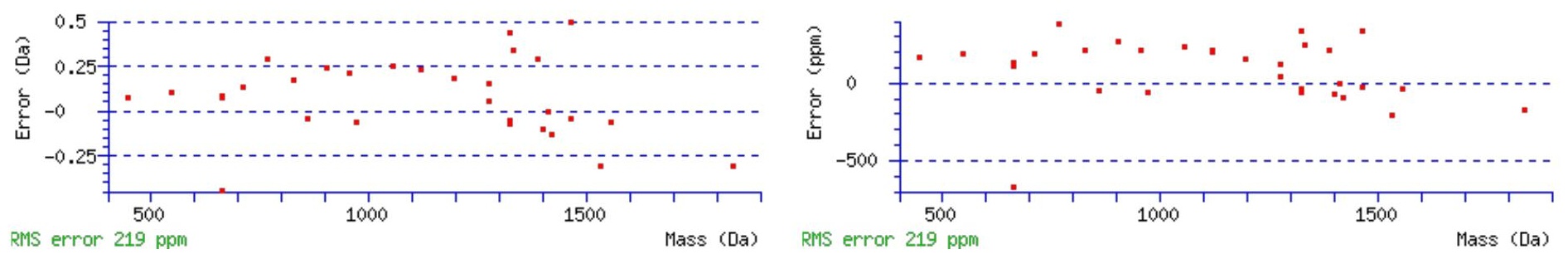

\section{All matches to this query}

\begin{tabular}{|l|l|l|l|}
\hline Score & Mr(calc): & Delta & \multicolumn{1}{c|}{ Sequence } \\
\hline 72.7 & 2995.5040 & 1.0063 & AFINGTGVETVVSADLPNAHGLAVDWVSR \\
\hline 49.5 & 2996.4880 & 0.0223 & AFINGTGVETVVSADLPNAHGLAVDWVSR \\
\hline 46.7 & 2995.5040 & 1.0063 & AFINGTGVETVVSADLPNAHGLAVDWVSR \\
\hline 45.8 & 2994.5200 & 1.9903 & AFINGTGVETVVSADLPNAHGLAVDWVSR \\
\hline 6.0 & 2996.5154 & -0.0052 & CIIVGNGGVLANKSLGSRIDDYDIVIR \\
\hline 2.6 & 2994.5144 & 1.9958 & RQVTRATVPTSVQTLGMTPATMTSKPR \\
\hline 1.9 & 2994.5144 & 1.9958 & RQVTRATVPTSVQTLGMTPATMTSKPR \\
\hline 0.1 & 2995.5211 & 0.9891 & AFIVVLGIITAVICPAALCCRDGTLGR \\
\hline
\end{tabular}

Spectrum No: 326; Query: 1339; Rank: 1

\section{Peptide View}

MS/MS Fragmentation of VGLIGSCTNSSYEDMGR

Found in IPI00421539, Tax_Id=10116 Gene_Symbol=Aco2 Aconitate hydratase, mitochondrial precursor 
Match to Query 1339: 1845.790328 from(923.902440,2+)

Title: 091008RatKidney_NoSalt_18.2370.2370.2.dta

Data file K:INewmanPaper|Piliang|3SubProteomes\Piliang3SP\mgf5ppm\ERLIC_3SubProteomes5ppm.mgf
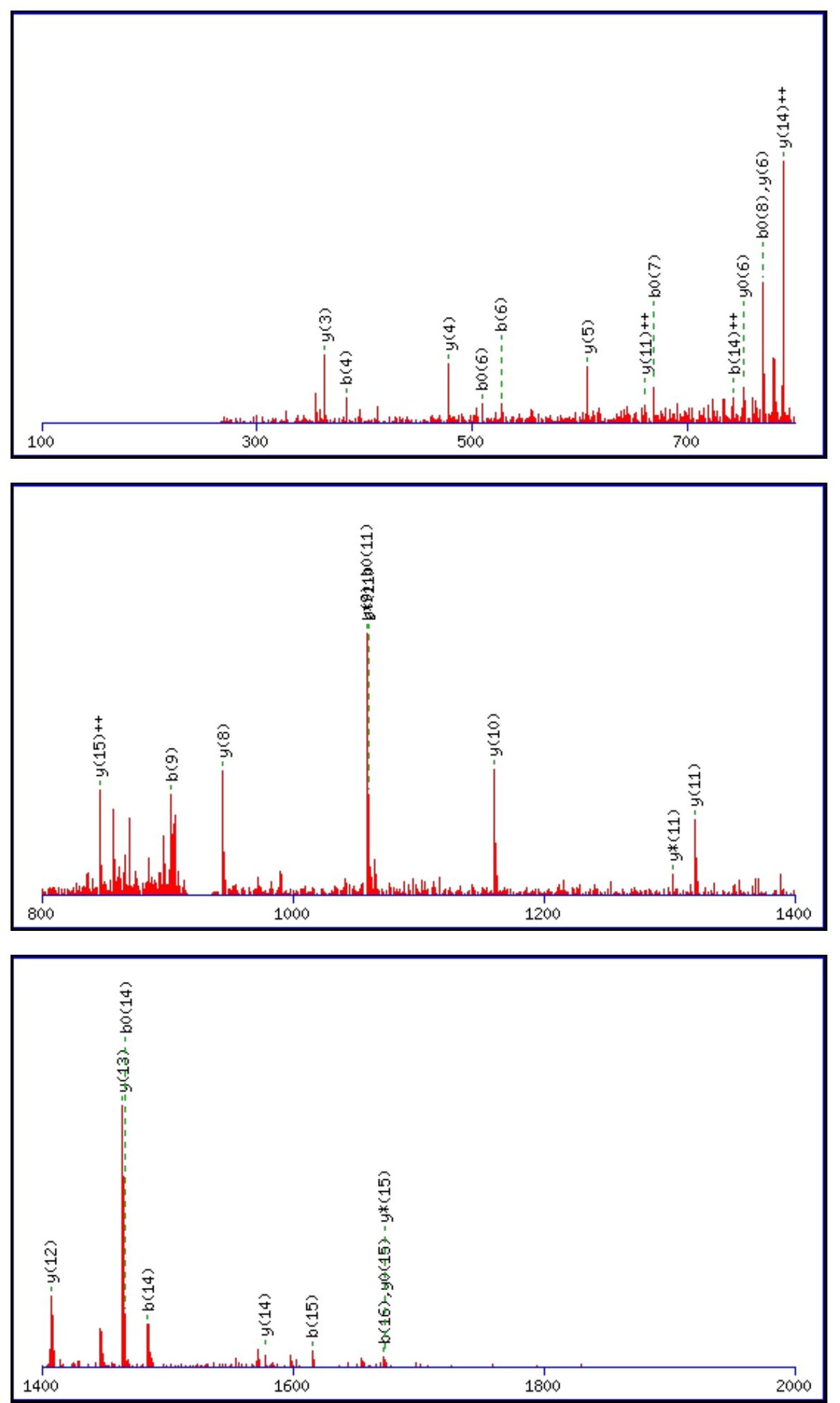

Monoisotopic mass of neutral peptide $\operatorname{Mr}($ calc): 1845.7873

Fixed modifications: Carbamidomethyl (C)

Variable modifications:

N9 : Deamidated_N (N)

Ions Score: 73 Expect: $1.1 \mathrm{e}-005$

Matches (Bold Red): 31/160 fragment ions using 51 most intense peaks

\begin{tabular}{|c|c|c|c|c|c|c|c|c|c|c|c|c|c|c|}
\hline$\#$ & $\mathbf{b}$ & $\mathbf{b}^{++}$ & $\mathbf{b}^{*}$ & $\mathbf{b}^{\mathbf{*}^{++}}$ & $\mathbf{b}^{\mathbf{0}}$ & $\mathbf{b}^{\mathbf{0}+}$ & Seq. & $\mathbf{y}$ & $\mathbf{y}^{++}$ & $\mathbf{y}^{\mathbf{*}}$ & $\mathbf{y}^{\mathbf{*}^{++}}$ & $\mathbf{y}^{\mathbf{0}}$ & $\mathbf{y}^{\mathbf{0 + +}}$ & $\#$ \\
\hline $\mathbf{1}$ & 100.0757 & 50.5415 & & & & & $\mathbf{V}$ & & & & & & & $\mathbf{1 7}$ \\
\hline $\mathbf{2}$ & 157.0972 & 79.0522 & & & & & $\mathbf{G}$ & 1747.7262 & 874.3667 & 1730.6996 & 865.8534 & 1729.7156 & 865.3614 & $1 \mathbf{1 6}$ \\
\hline
\end{tabular}




\begin{tabular}{|c|c|c|c|c|c|c|c|c|c|c|c|c|c|c|}
\hline & 270.1812 & |135.5942 & & & & & L & |1690.7047| & 845.8560 & 1673.6782 & 837.3427 & 1672.6941 & 836.8507| & 15 \\
\hline 4 & 383.2653 & 192.1363 & & & & & I & 1577.6206 & 789.3140 & 1560.5941 & 780.8007 & 1559.6101 & 780.3087 & 14 \\
\hline 5 & 440.2867 & 220.6470 & & & & & G & 1464.5366 & 732.7719 & 1447.5100 & 724.2587 & 1446.5260 & 723.7666 & 13 \\
\hline 6 & 527.3188 & 264.1630 & & & 509.3082 & 255.1577 & $S$ & 1407.5151 & 704.2612 & 1390.4886 & 695.7479 & 1389.5045 & 695.2559 & 12 \\
\hline 7 & 687.3494 & 344.1783 & & & 669.3389 & 335.1731 & C & 1320.4831 & 660.7452 & 1303.4565 & 652.2319 & 1302.4725 & 651.7399 & 11 \\
\hline 8 & 788.3971 & 394.7022 & & & 770.3865 & 385.6969 & $\mathbf{T}$ & 1160.4524 & 580.7299 & 1143.4259 & 572.2166 & 1142.4419 & 571.7246 & 10 \\
\hline 9 & 903.4240 & 452.2157 & 886.3975 & 443.7024 & 885.4135 & 443.2104 & $\mathbf{N}$ & 1059.4048 & 530.2060 & 1042.3782 & 521.6927 & 1041.3942 & 521.2007 & 9 \\
\hline 10 & 990.4561 & 495.7317 & 973.4295 & 487.2184 & 972.4455 & 486.7264 & $S$ & 944.3778 & 472.6925 & 927.3513 & 464.1793 & 926.3673 & 463.6873 & 8 \\
\hline 11 & 1077.4881 & 539.2477 & 1060.4615 & 530.7344 & 1059.4775 & 530.2424 & $\mathrm{~S}$ & 857.3458 & 429.1765 & 840.3192 & 420.6633 & 839.3352 & 420.1713 & 7 \\
\hline 12 & 1240.5514 & 620.7793 & 1223.5249 & 612.2661 & 1222.5409 & 611.7741 & $\mathbf{Y}$ & 770.3138 & 385.6605 & 753.2872 & 377.1472 & 752.3032 & 376.6552 & 6 \\
\hline 13 & 1369.5940 & 685.3006 & 1352.5675 & 676.7874 & 1351.5835 & 676.2954 & $\mathbf{E}$ & 607.2504 & 304.1289 & 590.2239 & 295.6156 & 589.2399 & 295.1236 & 5 \\
\hline 14 & 1484.6210 & 742.8141 & 1467.5944 & 734.3008 & 1466.6104 & 733.8088 & D & 478.2078 & 239.6076 & 461.1813 & 231.0943 & 460.1973 & 230.6023 & 4 \\
\hline 15 & 1615.6614 & 808.3344 & 1598.6349 & 799.8211 & 1597.6509 & 799.3291 & $\mathbf{M}$ & 363.1809 & 182.0941 & 346.1544 & 173.5808 & & & 3 \\
\hline 16 & 1672.6829 & 836.8451 & 1655.6564 & 828.3318 & 1654.6723 & 827.8398 & G & 232.1404 & 116.5738 & 215.1139 & 108.0606 & & & 2 \\
\hline 17 & & & & & & & $\mathbf{R}$ & 175.1190 & 88.0631 & 158.0924 & 79.5498 & & & 1 \\
\hline
\end{tabular}
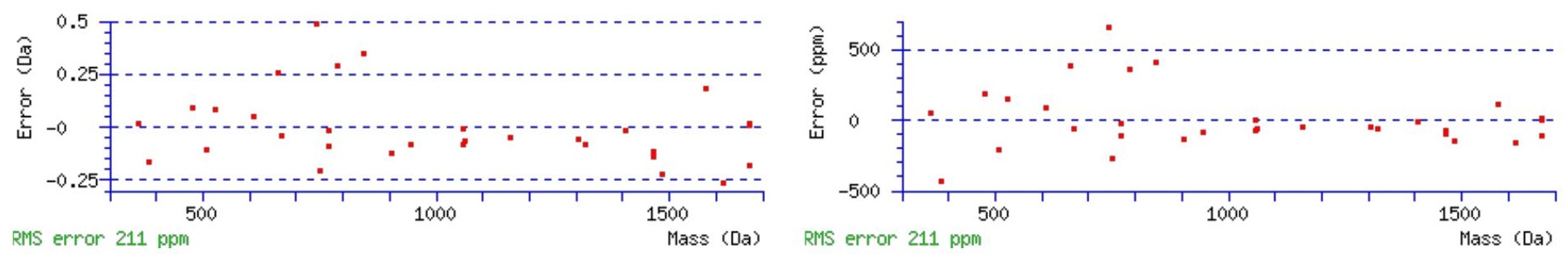

\section{All matches to this query}

\begin{tabular}{|l|l|l|l|}
\hline Score & Mr(calc): & Delta & \multicolumn{1}{|c|}{ Sequence } \\
\hline 72.7 & 1845.7873 & 0.0030 & VGLIGSCTNSSYEDMGR \\
\hline 7.2 & 1844.8033 & 0.9870 & VGLIGSCTNSSYEDMGR \\
\hline 2.0 & 1844.7859 & 1.0044 & ALLEAGASPNAPNTFGR \\
\hline 1.4 & 1844.7706 & 1.0197 & ANGKPEGKSQEPATSGK \\
\hline 0.2 & 1845.7856 & 0.0048 & QAYTNTPMVDNELLR \\
\hline
\end{tabular}

Spectrum No: 327; Query: 2652; Rank: 1

\section{Peptide View}

MS/MS Fragmentation of SPLGPHNQTAEQLQTLEQQSISLQQDTER

Found in IPI00190577, Tax_Id=10116 Gene_Symbol=Lama5 similar to Laminin alpha-5 chain precursor

Match to Query 2652: 3276.596262 from(1093.206030,3+)

Title: 100101RatKid_NS_deglyco_19.3885.3885.3.dta

Data file K:INewmanPaper|Piliangl3SubProteomes\Piliang3SP\mgf5ppm\ERLIC_3SubProteomes5ppm.mgf 

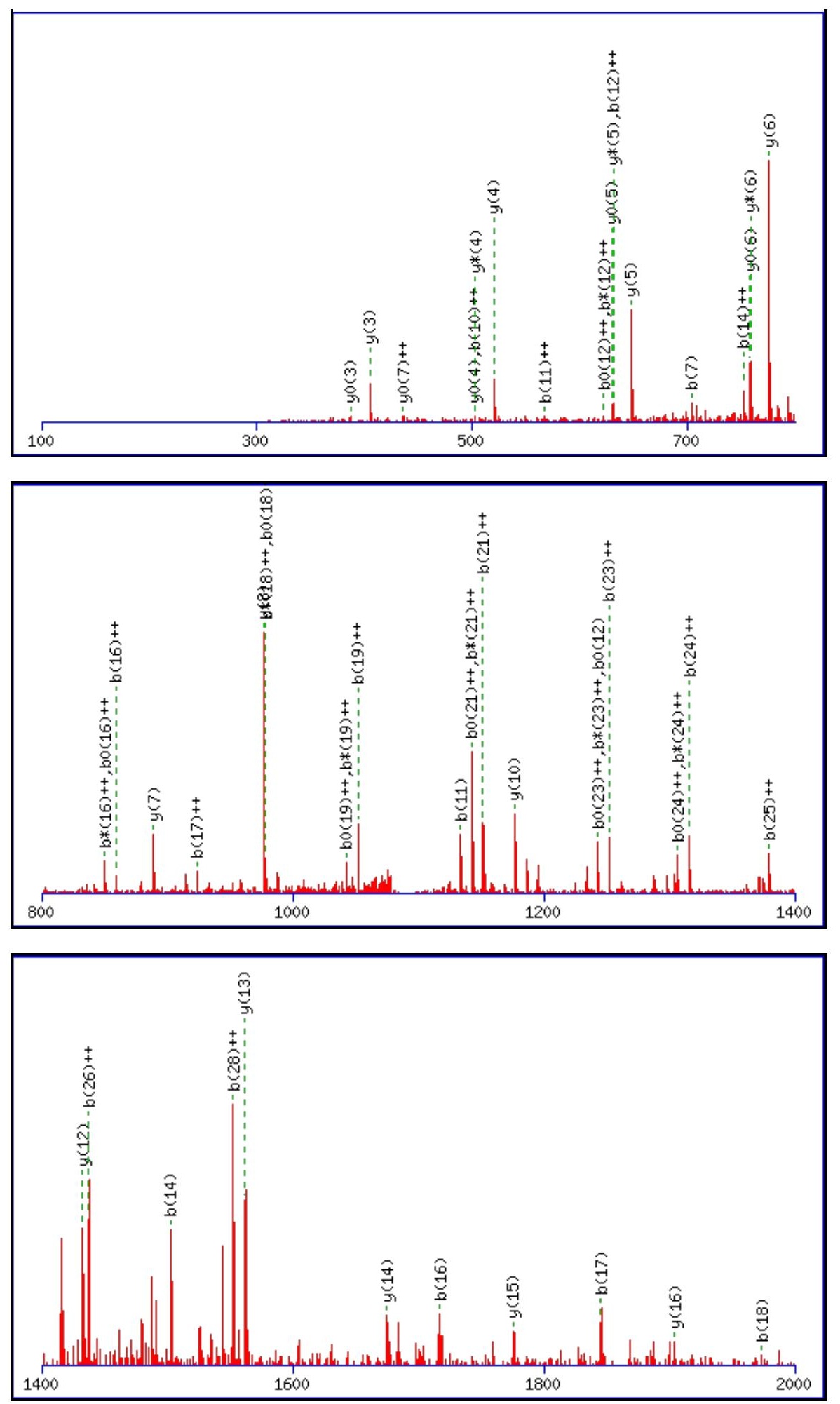

Monoisotopic mass of neutral peptide $\operatorname{Mr}($ calc): 3276.5858

Fixed modifications: Carbamidomethyl (C)

Variable modifications:

N7 : Deamidated $\mathrm{N}(\mathrm{N})$

Ions Score: 73 Expect: $1.7 \mathrm{e}-005$

Matches (Bold Red): 54/322 fragment ions using 73 most intense peaks

\begin{tabular}{|c|c|c|c|c|c|c|c|c|c|c|c|c|c|c|}
\hline \# & b & $\mathbf{b}^{++}$ & b* & $\mathbf{b}^{*^{++}}$ & $\mathbf{b}^{0}$ & $\mathbf{b}^{\mathbf{0}^{++}}$ & Seq. & $\mathbf{y}$ & $\mathbf{y}^{++}$ & $\mathbf{y}^{*}$ & $\mathbf{y}^{*^{++}}$ & $\mathbf{y}^{0}$ & $\mathbf{y}^{\mathbf{0}^{++}}$ & \# \\
\hline 1 & 88.0393 & 44.5233 & & & 70.0287 & 35.5180 & $\mathrm{~S}$ & & & & & & & 29 \\
\hline 2 & 185.0921 & 93.0497 & & & 167.0815 & 84.0444 & $\mathbf{P}$ & 3190.5611 & 1595.7842 & 3173.5345 & 1587.2709 & 3172.5505 & 1586.7789 & 28 \\
\hline 3 & 298.1761 & 149.5917 & & & 280.1656 & 140.5864 & $\mathbf{L}$ & 3093.5083 & 1547.2578 & 3076.4818 & 1538.7445 & 3075.4978 & 1538.2525 & 27 \\
\hline 4 & 355.1976 & 178.1024 & & & 337.1870 & 169.0972 & G & 2980.4243 & 1490.7158 & 2963.3977 & 1482.2025 & 2962.4137 & 1481.7105 & 26 \\
\hline 5 & 452.2504 & 226.6288 & & & 434.2398 & 217.6235 & $\mathbf{P}$ & 2923.4028 & 1462.2050 & 2906.3762 & 1453.6918 & 2905.3922 & 1453.1998 & 25 \\
\hline
\end{tabular}




\begin{tabular}{|c|c|c|c|c|c|c|c|c|c|c|c|c|c|c|}
\hline 6 & 589.3093 & 295.1583 & & & & & H & 500 & |1413.6787 & & & |2808.3395 & |1404.6734| & \\
\hline 7 & 704.3362 & 352.6717 & 687.3097 & 344.1585 & 686.3256 & 343.6665 & $\mathbf{N}$ & 2689.2911 & 1345.1492 & 2672.2646 & 1336.6359 & 2671.2806 & 1336.1439 & 23 \\
\hline 8 & 832.3948 & 416.7010 & 815.3682 & 408.1878 & 814.3842 & 407.6957 & $\mathbf{Q}$ & 2574.2642 & 1287.6357 & 2557.2376 & 1279.1225 & 2556.2536 & 1278.6304 & 2 \\
\hline 9 & 933.4425 & 467.2249 & 916.4159 & 458.7116 & 915.4319 & 458.2196 & $\mathbf{T}$ & & & 2429.1791 & & 2428.1950 & 1214.6012 & \\
\hline 10 & 1004.4796 & 502.7434 & 987.4530 & 494.2302 & 986.4690 & 493.7381 & A & 2345.1579 & 1173.0826 & 2328.1314 & 1164.5693 & 2327.1474 & 1164.0773 & \\
\hline 11 & 1133.5222 & 567.2647 & 1116.4956 & 558.7514 & 1115.5116 & 558.2594 & $\mathbf{E}$ & 2274.1208 & 1137.5640 & 2257.0943 & 1129.0508 & 2256.1102 & 1128.5588 & 9 \\
\hline 121 & 1261.5807 & 631.2940 & 244.5542 & 622.7807 & 1243.5702 & 622.2887 & $\mathbf{Q}$ & 2145.0782 & 1073.0427 & 2128.0517 & 1064.5295 & 2127.0677 & .0375 & \\
\hline 13 & 1374.6648 & 687.8360 & 1357.6383 & 679.3228 & 1356.6542 & 678.8308 & L & 2017.0196 & 1009.0135 & 1999.9931 & 1000.5002 & 1999.0091 & 1000.0082 & 17 \\
\hline 14 & 1502.7234 & 751.8653 & 1485.6968 & 743.3521 & 1484.7128 & 742.8601 & $\mathbf{Q}$ & 1903.9356 & 952.4714 & 1886.9090 & 943.9582 & 1885.9250 & 943.4661 & 16 \\
\hline 151 & 1603.7711 & 802.3892 & 1586.7445 & 793.8759 & 1585.7605 & 793.3839 & $T$ & 1775.8770 & 888.4421 & 1758.8505 & 879.9289 & 1757.8664 & 879.4369 & \\
\hline 16 & 1716.8551 & 858.9312 & 1699.8286 & 850.4179 & 1698.8446 & 849.9259 & $\mathbf{L}$ & 1674.8293 & .9183 & 1657.8028 & 829.4050 & 1656.8188 & 9130 & \\
\hline 17 & 1845.8977 & 923.4525 & 1828.8712 & 914.9392 & 1827.8872 & 914.4472 & $E$ & 1561.7453 & 781.3763 & 1544.7187 & 772.8630 & 1543.7347 & 772.3710 & 13 \\
\hline 18 & 1973.9563 & 987.4818 & 1956.9298 & 978.9685 & 1955.9457 & 978.4765 & $\mathbf{Q}$ & 1432.7027 & 716.8550 & 1415.6761 & 708.3417 & 1414.6921 & 707.8497 & \\
\hline 192 & 2102.0149 & 1051.5111 & 2084.9883 & 1042.9978 & 2084.0043 & 1042.5058 & $\mathbf{Q}$ & 1304.6441 & 652.8257 & 1287.6175 & 644.3124 & 1286.6335 & 8204 & \\
\hline 20 & 2189.0469 & 1095.0271 & 2172.0204 & 1086.5138 & 2171.0363 & 1086.0218 & $S$ & 1176.5855 & 588.7964 & 1159.5590 & 580.2831 & 1158.5749 & 579.7911 & 10 \\
\hline 21 & 2302.1310 & 1151.5691 & 2285.1044 & 1143.0559 & 2284.1204 & 1142.5638 & I & 1089.5535 & 545.2804 & 1072.5269 & 536.7671 & 1071.5429 & 536.2751 & 9 \\
\hline 22 & 2389.1630 & 1195.0851 & 2372.1365 & 1186.5719 & 2371.1524 & 1186.0799 & $S$ & 976.4694 & 488.7383 & 959.4429 & 480.2251 & 958.4589 & 479.7331 & \\
\hline 232 & 2502.2471 & \begin{tabular}{|l|}
1251.6272 \\
\end{tabular} & 2485.2205 & 1243.1139 & 2484.2365 & 1242.6219 & $\mathbf{L}$ & 889.4374 & 445.2223 & 872.4108 & 436.7091 & 871.4268 & 436.2170 & 7 \\
\hline 24 & 2630.3056 & 1315.6565 & 2613.2791 & \begin{tabular}{|l|l|}
1307.1432 \\
\end{tabular} & 2612.2951 & \begin{tabular}{|l|}
1306.6512 \\
\end{tabular} & $\mathbf{Q}$ & 776.3533 & 388.6803 & 759.3268 & 380.1670 & 758.3428 & 379.6750 & 6 \\
\hline 252 & 2758.3642 & \begin{tabular}{|l|}
1379.6858 \\
\end{tabular} & 2741.3377 & 1371.1725 & 2740.3537 & 1370.6805 & $\mathbf{Q}$ & 648.2947 & 324.6510 & 631.2682 & 316.1377 & 630.2842 & 315.6457 & \\
\hline 26 & 2873.3912 & 1437.1992 & 2856.3646 & 1428.6859 & 2855.3806 & 1428.1939 & D & 520.2362 & 260.6217 & 503.2096 & 252.1084 & 502.2256 & 251.6164 & 4 \\
\hline 272 & 2974.4388 & 1487.7231 & 2957.4123 & 1479.2098 & 2956.4283 & 1478.7178 & $\mathbf{T}$ & 405.2092 & 203.1082 & 388.1827 & 194.5950 & 387.1987 & 194.1030 & 3 \\
\hline 283 & 3103.4814 & 1552.2444 & 3086.4549 & 1543.7311 & 3085.4709 & 1543.2391 & $\mathbf{E}$ & 304.1615 & 152.5844 & 287.1350 & 144.0711 & 286.1510 & 143.5791 & 2 \\
\hline 29 & & & & & & & $\mathbf{R}$ & 175.1190 & 88.0631 & 158.0924 & 79.5498 & & & \\
\hline
\end{tabular}
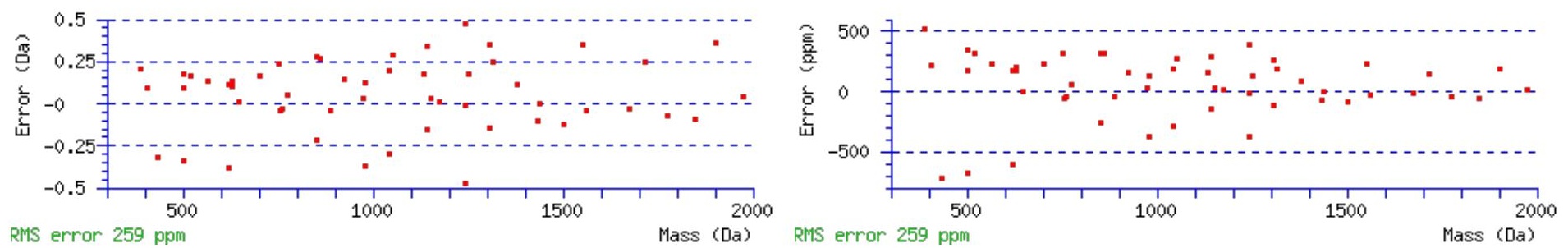

\section{All matches to this query}

\begin{tabular}{|l|l|c|l|}
\hline Score & Mr(calc): & Delta & \multicolumn{1}{c|}{ Sequence } \\
\hline 72.6 & 3276.5858 & 0.0104 & SPLGPHNQTAEQLQTLEQQSISLQQDTER \\
\hline 28.8 & 3275.6018 & 0.9945 & SPLGPHNQTAEQLQTLEQQSISLQQDTER \\
\hline 4.0 & 3276.5730 & 0.0233 & MRVCTKWVLLSHWLVLAYMLMVCCK \\
\hline 3.0 & 3276.6059 & -0.0096 & GALGATPQLMAVFGSLSLYALGNSLLLPWR \\
\hline 2.5 & 3276.5944 & 0.0019 & FHIHLYNPDSLIACVLPYHETRVFVR \\
\hline 1.6 & 3274.5883 & 2.0080 & AELQDMCDNPELTALQIALAECQEKLKK \\
\hline 1.4 & 3276.5808 & 0.0155 & YLQKSSISLAAVLPGWLPPPPTPGSPCAGR \\
\hline 1.4 & 3276.5808 & 0.0155 & YLQKSSISLAAVLPGWLPPPPTPGSPCAGR \\
\hline 1.4 & 3276.5808 & 0.0155 & YLQKSSISLAAVLPGWLPPPPTPGSPCAGR \\
\hline 1.0 & 3275.5797 & 1.0166 & GSPTPSGTRPTAGQAAPPSQQPPKPQEQSRR \\
\hline
\end{tabular}

Spectrum No: 328; Query: 608; Rank: 1 


\section{MS/MS Fragmentation of FVNSTGYLTEAEK}

Found in IPI00358387, Tax_Id=10116 Gene_Symbol=Sumf1_predicted sulfatase modifying factor 1

Match to Query 608: 1458.685708 from(730.350130,2+)

Title: 091008RatKidney_NH4Format01_24.2004.2004.2.dta

Data file K:INewmanPaper|Piliang|3SubProteomes\Piliang3SP\mgf5ppm\ERLIC_3SubProteomes5ppm.mgf
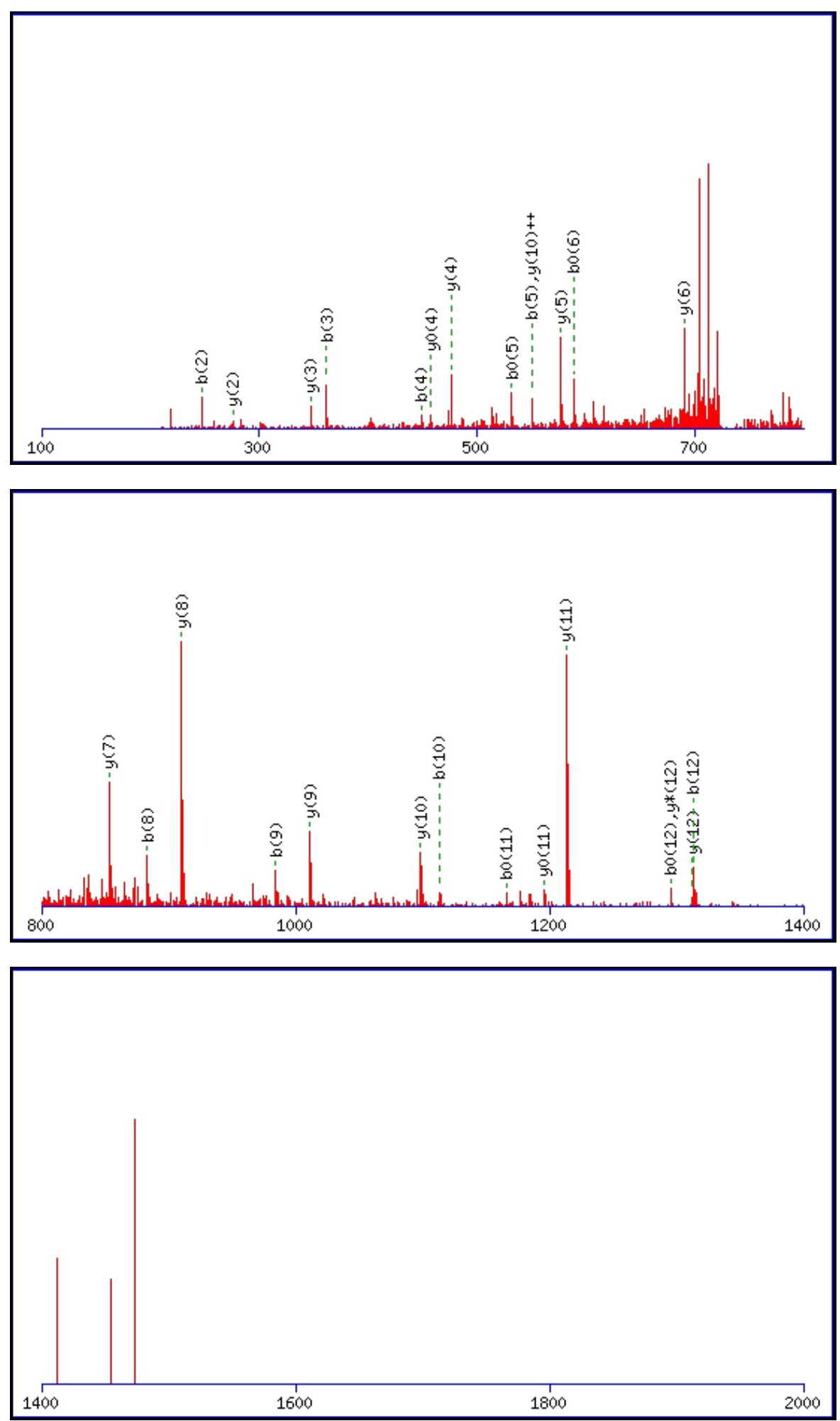

Monoisotopic mass of neutral peptide $\operatorname{Mr}($ calc): 1458.6878

Fixed modifications: Carbamidomethyl (C)

Variable modifications:

N3 : Deamidated N (N)

Ions Score: 72 Expect: $9.3 \mathrm{e}-006$

Matches (Bold Red): 27/132 fragment ions using 66 most intense peaks

\begin{tabular}{|l|l|l|l|l|l|l|l|l|l|l|l|l|l|l|l|}
\hline$\#$ & $\mathbf{b}$ & $\mathbf{b}^{++}$ & $\mathbf{b}^{*}$ & $\mathbf{b}^{*^{++}}$ & $\mathbf{b}^{\mathbf{0}}$ & $\mathbf{b}^{\mathbf{0 + +}}$ & Seq. & $\mathbf{y}$ & $\mathbf{y}^{++}$ & $\mathbf{y}^{*}$ & $\mathbf{y}^{*^{++}}$ & $\mathbf{y}^{\mathbf{0}}$ & $\mathbf{y}^{\mathbf{0 + +}}$ & $\#$ \\
\hline
\end{tabular}




\begin{tabular}{|c|c|c|c|c|c|c|c|c|c|c|c|c|c|c|}
\hline 1 & 148.0757 & 74.5415 & & & & & $\mathbf{F}$ & & & & & & & |13| \\
\hline 2 & 247.1441 & 124.0757 & & & & & V & 1312.6267 & 656.8170 & 1295.6001 & 648.3037 & 1294.6161 & 647.8117 & 12 \\
\hline 3 & 362.1710 & |181.5892 & 345.1445 & 173.0759 & & & $\mathbf{N}$ & 1213.5583 & 607.2828 & 1196.5317 & 598.7695 & 1195.5477 & 598.2775 & 11 \\
\hline 4 & 449.2031 & 225.1052 & 432.1765 & 216.5919 & 431.1925 & 216.0999 & S & 1098.5313 & 549.7693 & 1081.5048 & 541.2560 & 1080.5208 & 540.7640 & 10 \\
\hline 5 & 550.2507 & 275.6290 & 533.2242 & 267.1157 & 532.2402 & 266.6237 & $\mathbf{T}$ & 1011.4993 & 506.2533 & 994.4728 & 497.7400 & 993.4888 & 497.2480 & 9 \\
\hline 6 & 607.2722 & 304.1397 & 590.2457 & 295.6265 & 589.2616 & 295.1345 & G & 910.4516 & 455.7295 & 893.4251 & 447.2162 & 892.4411 & 446.7242 & 8 \\
\hline 7 & 770.3355 & 385.6714 & 753.3090 & 377.1581 & 752.3250 & 376.6661 & $\mathbf{Y}$ & 853.4302 & 427.2187 & 836.4036 & 418.7055 & 835.4196 & 418.2134 & 7 \\
\hline 8 & 883.4196 & 442.2134 & 866.3931 & 433.7002 & 865.4090 & 433.2082 & $\mathbf{L}$ & 690.3668 & 345.6871 & 673.3403 & 337.1738 & 672.3563 & 336.6818 & 6 \\
\hline 9 & 984.4673 & 492.7373 & 967.4407 & 484.2240 & 966.4567 & 483.7320 & $\mathbf{T}$ & 577.2828 & 289.1450 & 560.2562 & 280.6318 & 559.2722 & 280.1397 & 5 \\
\hline 10 & 1113.5099 & 557.2586 & 1096.4833 & 548.7453 & 1095.4993 & 548.2533 & $\mathbf{E}$ & 476.2351 & 238.6212 & 459.2086 & 230.1079 & 458.2245 & 229.6159 & 4 \\
\hline 11 & 1184.5470 & 592.7771 & 1167.5204 & 584.2639 & 1166.5364 & 583.7719 & A & 347.1925 & 174.0999 & 330.1660 & 165.5866 & 329.1819 & 165.0946 & 3 \\
\hline 12 & \begin{tabular}{|l|}
1313.5896 \\
\end{tabular} & 657.2984 & 1296.5630 & 648.7852 & 1295.5790 & 648.2931 & $\mathbf{E}$ & 276.1554 & 138.5813 & 259.1288 & 130.0681 & 258.1448 & 129.5761 & 2 \\
\hline 13 & & & & & & & K & 147.1128 & 74.0600 & 130.0863 & 65.5468 & & & 1 \\
\hline
\end{tabular}
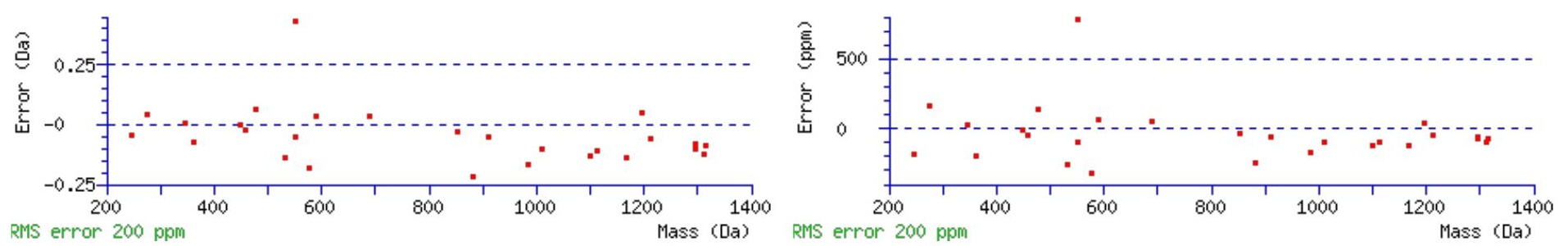

\section{All matches to this query}

\begin{tabular}{|l|l|l|l|}
\hline Score & Mr(calc): & Delta & \multicolumn{1}{|c|}{ Sequence } \\
\hline 72.5 & 1458.6878 & -0.0021 & FVNSTGYLTEAEK \\
\hline 7.1 & 1458.6990 & -0.0133 & EYRKDYEESIK \\
\hline 6.1 & 1458.6891 & -0.0034 & FNVRNGYGFINR \\
\hline 5.9 & 1456.6671 & 2.0186 & RKQQDGGTTGTTK \\
\hline 5.7 & 1458.6878 & -0.0021 & FEAEYVTEKSEK \\
\hline 3.6 & 1457.6915 & 0.9942 & KYAPSANVTTAQK \\
\hline 3.3 & 1458.6812 & 0.0046 & REGGGGGGGGEGGLQSK \\
\hline 2.7 & 1456.6851 & 2.0006 & FINLQPSTLTDK \\
\hline 0.5 & 1457.6803 & 1.0054 & FDEAALSIQKEK \\
\hline
\end{tabular}

Spectrum No: 329; Query: 2536; Rank: 1

\section{Peptide View}

MS/MS Fragmentation of LQAALDNEAGGRPAMEPGNGSLDLGGDAAGR

Found in IPI00210090, Tax_Id=10116 Gene_Symbol=Hnrpu SP120

Match to Query 2536: 2996.383962 from(999.801930,3+)

Title: 091008RatKidney_NH4Format02_24.2597.2597.3.dta

Data file K:INewmanPaper|Piliang|3SubProteomes\Piliang3SP\mgf5ppm\ERLIC_3SubProteomes5ppm.mgf 

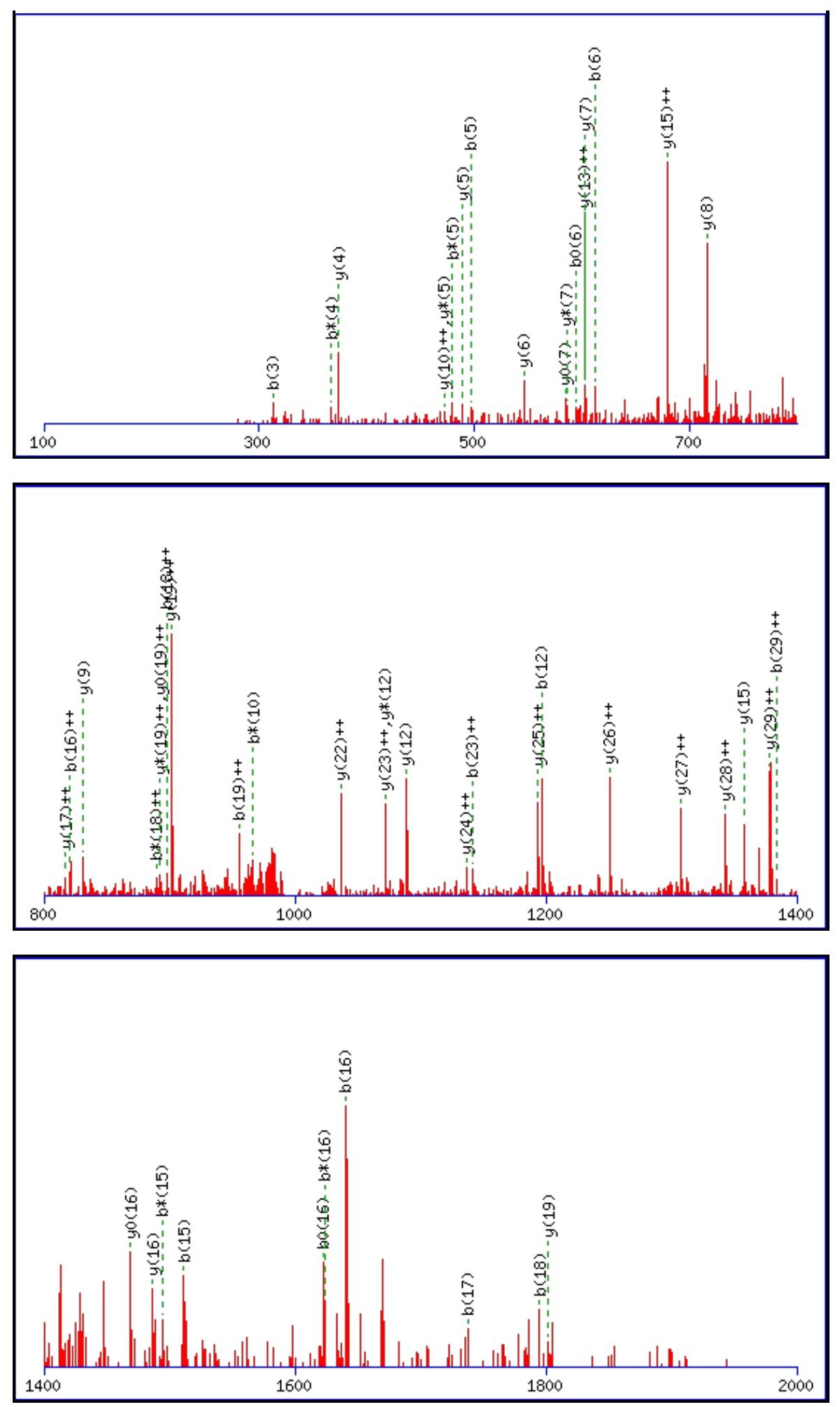

Monoisotopic mass of neutral peptide $\operatorname{Mr}($ calc): 2996.3894

Fixed modifications: Carbamidomethyl (C)

Variable modifications:

M15: Oxidation (M)

N19: Deamidated_N (N)

Ions Score: 72 Expect: 2.1e-005

Matches (Bold Red): 51/340 fragment ions using 97 most intense peaks

\begin{tabular}{|c|c|c|c|c|c|c|c|c|c|c|c|c|c|c|}
\hline$\#$ & $\mathbf{b}$ & $\mathbf{b}^{++}$ & $\mathbf{b}^{*}$ & $\mathbf{b}^{*^{++}}$ & $\mathbf{b}^{\mathbf{0}}$ & $\mathbf{b}^{\mathbf{0}+}$ & Seq. & $\mathbf{y}$ & $\mathbf{y}^{++}$ & $\mathbf{y}^{\mathbf{*}}$ & $\mathbf{y}^{\mathbf{*}^{++}}$ & $\mathbf{y}^{\mathbf{0}}$ & $\mathbf{y}^{\mathbf{0}+}$ & $\#$ \\
\hline $\mathbf{1}$ & 114.0913 & 57.5493 & & & & & $\mathbf{L}$ & & & & & & & $\mathbf{3 1}$ \\
\hline $\mathbf{2}$ & 242.1499 & 121.5786 & 225.1234 & 113.0653 & & & $\mathbf{Q}$ & 2884.3126 & 1442.6599 & 2867.2861 & 1434.1467 & 2866.3020 & 1433.6547 & $\mathbf{3 0}$ \\
\hline $\mathbf{3}$ & $\mathbf{3 1 3 . 1 8 7 0}$ & 157.0972 & 296.1605 & 148.5839 & & & $\mathbf{A}$ & 2756.2540 & $\mathbf{1 3 7 8 . 6 3 0 7}$ & 2739.2275 & 1370.1174 & 2738.2435 & 1369.6254 & $\mathbf{2 9}$ \\
\hline $\mathbf{4}$ & 384.2241 & 192.6157 & 367.1976 & 184.1024 & & & $\mathbf{A}$ & 2685.2169 & $\mathbf{1 3 4 3 . 1 1 2 1}$ & 2668.1904 & 1334.5988 & 2667.2063 & 1334.1068 & $\mathbf{2 8}$ \\
\hline & & & & & & & & & & & & & &
\end{tabular}




\begin{tabular}{|c|c|c|c|c|c|c|c|c|c|c|c|c|c|c|}
\hline & 497.3082 & 249.1577| & 480.2817 & 240.6445 & & & L & & & & & & 1298.5883 & |27| \\
\hline 6 & 612.3352 & 306.6712 & 595.3086 & 298.1579 & 594.3246 & 297.6659 & D & 2501.0957 & 1251.0515 & 2484.0692 & 1242.5382 & 2483.0852 & 1242.0462 & 26 \\
\hline 7 & 726.3781 & 363.6927 & 709.3515 & 355.1794 & 708.3675 & 354.6874 & $\mathbf{N}$ & 2386.0688 & 1193.5380 & 2369.0422 & 1185.0248 & 2368.0582 & 1184.5328 & 25 \\
\hline 8 & 855.4207 & 428.2140 & 838.3941 & 419.7007 & 837.4101 & 419.2087 & $\mathbf{E}$ & 2272.0259 & 1136.5166 & 2254.9993 & 1128.0033 & 2254.0153 & 1127.5113 & 24 \\
\hline 9 & 926.4578 & 463.7325 & 909.4312 & 455.2193 & 908.4472 & 454.7272 & A & 2142.9833 & 1071.9953 & 2125.9567 & $\mid 1063.4820$ & 2124.9727 & 1062.9900 & 2 \\
\hline 10 & 983.4793 & 492.2433 & 966.4527 & 483.7300 & 965.4687 & 483.2380 & G & 2071.9462 & 1036.4767 & 2054.9196 & 1027.9634 & 2053.9356 & 1027.4714 & 22 \\
\hline 11 & 1040.5007 & 520.7540 & 1023.4742 & 512.2407 & 1022.4901 & 511.7487 & G & 2014.9247 & 1007.9660 & 1997.8981 & 999.4527 & 1996.9141 & 998.9607 & 1 \\
\hline 12 & 1196.6018 & 598.8046 & 1179.5753 & 590.2913 & 1178.5913 & 589.7993 & $\mathbf{R}$ & 1957.9032 & 979.4553 & 1940.8767 & 970.9420 & 1939.8927 & 970.4500 & 20 \\
\hline 131 & 1293.6546 & 647.3309 & 1276.6280 & 638.8177 & 1275.6440 & 638.3257 & $\mathbf{P}$ & 1801.8021 & 901.4047 & 1784.7756 & 892.8914 & 1783.7916 & 892.3994 & 19 \\
\hline 141 & 1364.6917 & 682.8495 & 1347.6652 & 674.3362 & 1346.6811 & 673.8442 & $\mathbf{A}$ & 1704.7494 & 852.8783 & 1687.7228 & 844.3650 & 1686.7388 & 843.8730 & 18 \\
\hline 151 & 1511.7271 & 756.3672 & 1494.7006 & 747.8539 & 1493.7165 & 747.3619 & M & 1633.7122 & 817.3598 & 1616.6857 & 808.8465 & 1615.7017 & 808.3545 & 17 \\
\hline 161 & 1640.7697 & 820.8885 & 1623.7432 & 812.3752 & \begin{tabular}{|l|}
1622.7591 \\
\end{tabular} & 811.8832 & $\mathbf{E}$ & 1486.6768 & 743.8421 & 1469.6503 & 735.3288 & 1468.6663 & 734.8368 & 16 \\
\hline 17 & 1737.8225 & 869.4149 & 1720.7959 & 860.9016 & 1719.8119 & 860.4096 & $\mathbf{P}$ & 1357.6342 & 679.3208 & 1340.6077 & 670.8075 & 1339.6237 & 670.3155 & 15 \\
\hline 181 & 1794.8439 & 897.9256 & 1777.8174 & 889.4123 & 1776. & 888. & G & 815 & 944 & 1243 & 2811 & 5709 & 621.7891 & 4 \\
\hline 191 & 1909.8709 & 955.4391 & 1892.8443 & 946.9258 & 1891.8603 & 946.4338 & $\mathbf{N}$ & 1203.5600 & 602.2836 & 1186.5335 & 593.7704 & 1185.5494 & 593.2784 & 3 \\
\hline 201 & 1966.8923 & 983.9498 & 1949.8658 & 975.4365 & 1948.8818 & 974.9445 & G & 1088.5331 & 544.7702 & 1071.5065 & 536.2569 & 1070.5225 & 535.7649 & 12 \\
\hline 212 & 2053.9244 & 1027.4658 & 2036.8978 & 1018.9525: & 2035.9138 & 1018.4605 & $S$ & 1031.5116 & 516.2594 & 1014.4851 & 507.7462 & 1013.5010 & 507.2542 & 11 \\
\hline 222 & 2167.0084 & 1084.0078 & 2149.9819 & 1075.4946 & 2148.9979 & 1075.0026 & $\mathbf{L}$ & 944.4796 & 472.7434 & 927.4530 & 464.2302 & 926.4690 & 463.7381 & 10 \\
\hline 232 & 2282.0354 & 1141.5213 & 2265.0088 & 1133.0080 & 2264.0248 & 1132.5160 & $\mathbf{D}$ & 831.3955 & 416.2014 & 814.3690 & 407.6881 & 813.3850 & 407.1961 & 9 \\
\hline $24 \sqrt{2}$ & 2395.1194 & 1198.0634 & 2378.0929 & 1189.5501 & 2377.1089 & 1189.0581 & $\mathbf{L}$ & 716.3686 & 358.6879 & 699.3420 & 350.1747 & 698.3580 & 349.6826 & 8 \\
\hline 252 & 2452.1409 & 1226.5741 & 2435.1143 & 1218.0608 & 2434.1303 & 1217.5688 & G & 603.2845 & 302.1459 & 586.2580 & 293.6326 & 585.2740 & 293.1406 & 7 \\
\hline 26 & 2509.1624 & 1255.0848 & 2492.1358 & 1246.5715 & 2491.1518 & 1246.0795 & G & 546.2631 & 273.6352 & 529.2365 & 265.1219 & 528.2525 & 264.6299 & 6 \\
\hline 272 & 2624.1893 & 1312.5983 & 2607.1627 & 1304.0850 & 2606.1787 & 1303.5930 & D & 489.2416 & 245.1244 & 472.2150 & 236.6112 & 471.2310 & 236.1191 & 5 \\
\hline 282 & 2695.2264 & 1348.1168 & 2678.1999 & 1339.6036 & 2677.2158 & 1339.1116 & $\mathbf{A}$ & 374.2146 & 187.6110 & 357.1881 & 179.0977 & & & 4 \\
\hline 292 & 2766.2635 & 1383.6354 & 2749.2370 & 1375.1221 & 2748.2530 & 1374.6301 & A & 303.1775 & 152.0924 & 286.1510 & 143.5791 & & & 3 \\
\hline 302 & 2823.2850 & 1412.1461 & 2806.2584 & 1403.6329 & 2805.2744 & 1403.1409 & G & 232.1404 & 116.5738 & 215.1139 & 108.0606 & & & 2 \\
\hline 31 & & & & & & & $\mathbf{R}$ & 175.1190 & 88.0631 & 158.0924 & 79.5498 & & & 1 \\
\hline
\end{tabular}
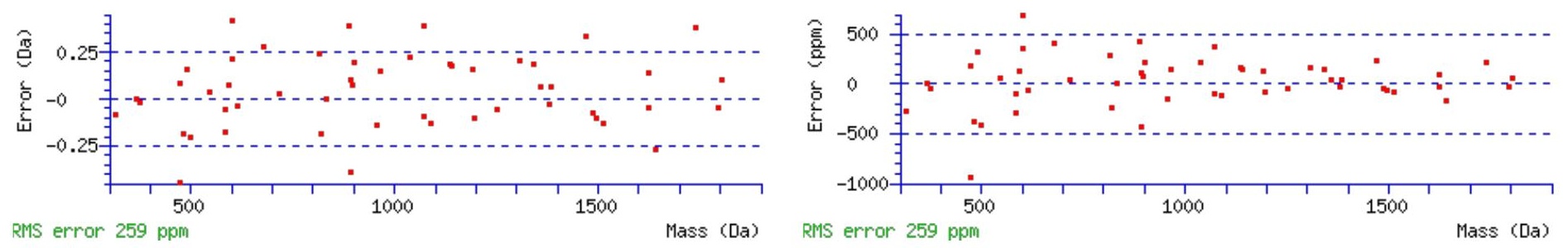

\section{All matches to this query}

\begin{tabular}{|l|l|l|l|}
\hline Score & Mr(calc): & Delta & \multicolumn{1}{c|}{ Sequence } \\
\hline 72.4 & 2996.3894 & -0.0054 & LQAALDNEAGGRPAMEPGNGSLDLGGDAAGR \\
\hline 31.1 & 2996.3894 & -0.0054 & LQAALDNEAGGRPAMEPGNGSLDLGGDAAGR \\
\hline 27.9 & 2995.4054 & 0.9786 & LQAALDNEAGGRPAMEPGNGSLDLGGDAAGR \\
\hline 2.4 & 2996.4042 & -0.0203 & SFNQSSHLSHVQAHERIHTGEKPYK \\
\hline
\end{tabular}

Spectrum No: 330; Query: 115; Rank: 1

\section{Peptide View}

MS/MS Fragmentation of VLTLANFTTK

Found in IPI00188956, Tax_Id=10116 Gene_Symbol=Thy1 Thy-1 membrane glycoprotein precursor

Match to Query 115: 1107.619228 from(554.816890,2+) 
Title: 100101RatKid_NS_deglyco_20.3455.3455.2.dta

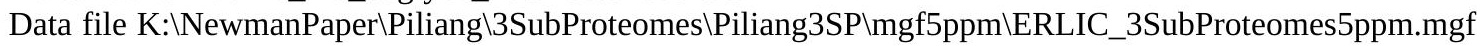
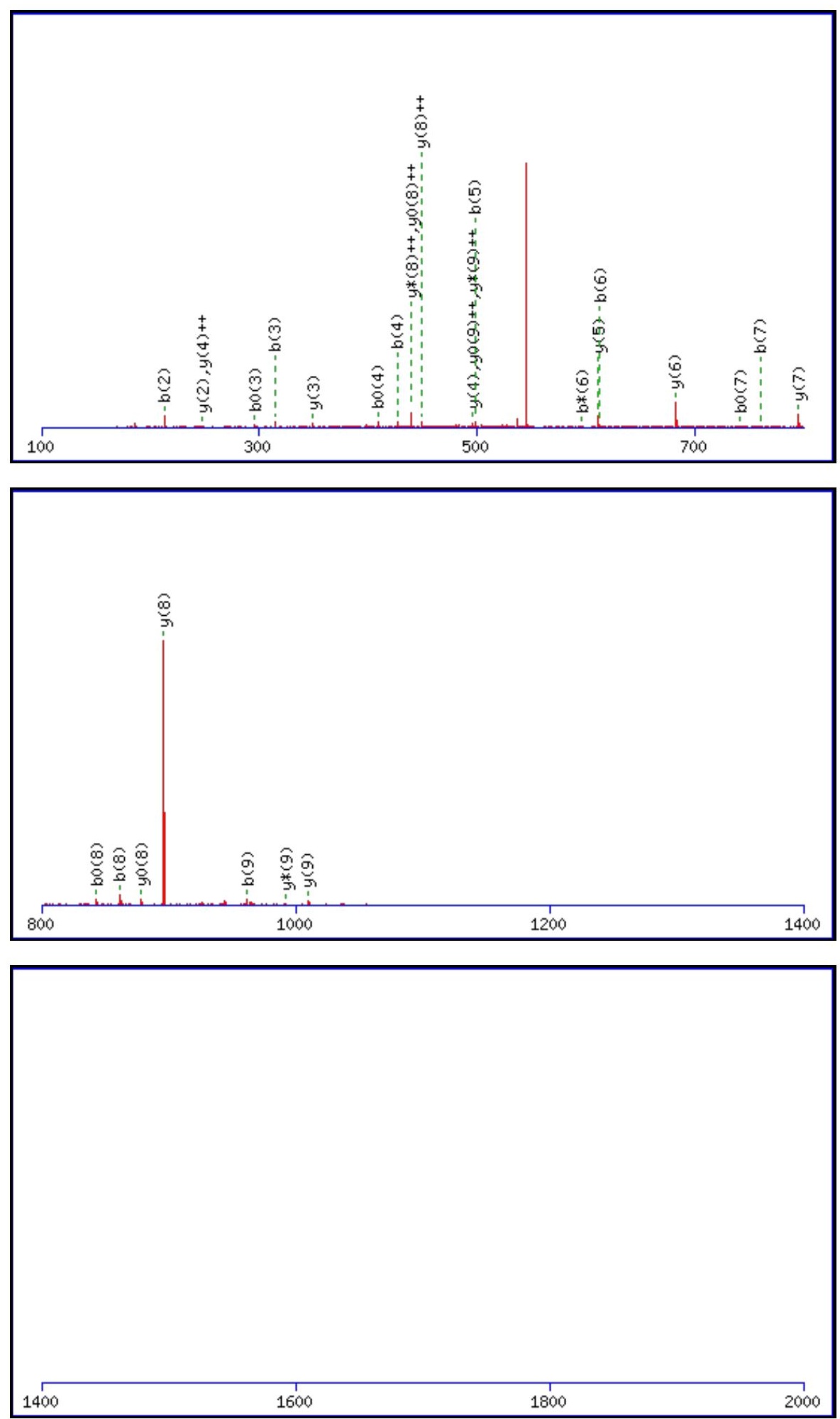

Monoisotopic mass of neutral peptide $\operatorname{Mr}($ calc): 1107.6176

Fixed modifications: Carbamidomethyl (C)

Variable modifications:

N6 : Deamidated $\mathrm{N}(\mathrm{N})$

Ions Score: 72 Expect: $2.9 \mathrm{e}-006$

Matches (Bold Red): 29/92 fragment ions using 40 most intense peaks

\begin{tabular}{|r|c|c|c|c|c|c|c|c|c|c|c|c|c|c|}
\hline$\#$ & $\mathbf{b}$ & $\mathbf{b}^{++}$ & $\mathbf{b}^{*}$ & $\mathbf{b}^{\boldsymbol{*}^{++}}$ & $\mathbf{b}^{\mathbf{0}}$ & $\mathbf{b}^{\mathbf{0 + +}}$ & Seq. & $\mathbf{y}$ & $\mathbf{y}^{++}$ & $\mathbf{y}^{*}$ & $\mathbf{y}^{\mathbf{*}^{++}}$ & $\mathbf{y}^{\mathbf{0}}$ & $\mathbf{y}^{\mathbf{0 + +}}$ & $\#$ \\
\hline $\mathbf{1}$ & 100.0757 & 50.5415 & & & & & $\mathbf{V}$ & & & & & & & $\mathbf{1 0}$ \\
\hline $\mathbf{2}$ & 213.1598 & 107.0835 & & & & & $\mathbf{L}$ & $\mathbf{1 0 0 9 . 5 5 6 4}$ & 505.2819 & $\mathbf{9 9 2 . 5 2 9 9}$ & $\mathbf{4 9 6 . 7 6 8 6}$ & 991.5459 & $\mathbf{4 9 6 . 2 7 6 6}$ & $\mathbf{9}$ \\
\hline 3 & 314.2074 & 157.6074 & & & 296.1969 & 148.6021 & $\mathbf{T}$ & $\mathbf{8 9 6 . 4 7 2 4}$ & $\mathbf{4 4 8 . 7 3 9 8}$ & 879.4458 & $\mathbf{4 4 0 . 2 2 6 5}$ & $\mathbf{8 7 8 . 4 6 1 8}$ & $\mathbf{4 3 9 . 7 3 4 5}$ & $\mathbf{8}$ \\
\hline
\end{tabular}




\begin{tabular}{|c|c|c|c|c|c|c|c|c|c|c|c|c|c|c|}
\hline & |427.2915 & |214.1494 & & & |409.2809| & 5.1441 & $\mathbf{L}$ & 795.4247 & & |778.3981 & |389.7027 & |777.4141 & 107| & \\
\hline 5 & 498.3286 & 249.6679 & & & 480.3180 & 240.6627 & A & 682.3406 & 341.6740 & 665.3141 & 333.1607 & 664.3301 & 332.6687 & \\
\hline 6 & 613.3555 & 307.1814 & 596.3290 & 298.6681 & 595.3450 & 298.1761 & $\mathbf{N}$ & 611.3035 & 306.1554 & 594.2770 & 297.6421 & 593.2929 & 297.1501 & \\
\hline 7 & 760.4240 & 380.7156 & 743.3974 & 372.2023 & 742.4134 & 371.7103 & $\mathbf{F}$ & 496.2766 & 248.6419 & 479.2500 & 240.1287 & 478.2660 & 239.6366 & \\
\hline 8 & 861.4716 & 431.2395 & 844.4451 & 422.7262 & 843.4611 & 422.2342 & $T$ & 349.2082 & 175.1077 & 332.1816 & 166.5944 & 331.1976 & 166.1024 & \\
\hline 9 & 962.5193 & 481.7633 & 945.4928 & 473.2500 & 944.5088 & 472.7580 & $\mathbf{T}$ & 248.1605 & 124.5839 & 231.1339 & 116.0706 & 230.1499 & 115.5786 & 2 \\
\hline 10 & & & & & & & $\mathbf{K}$ & 147.1128 & 74.0600 & 130.0863 & 65.5468 & & & \\
\hline
\end{tabular}
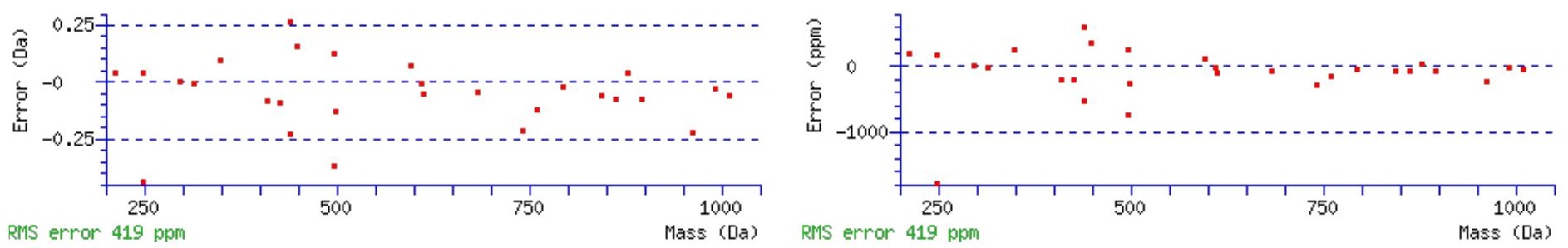

\section{All matches to this query}

\begin{tabular}{|l|l|l|l|}
\hline Score & Mr(calc): & Delta & \multicolumn{1}{|c|}{ Sequence } \\
\hline 72.3 & 1107.6176 & 0.0017 & VLTLANFTTK \\
\hline 12.3 & 1107.6288 & -0.0096 & LVTFSASOKK \\
\hline 9.3 & 1107.6165 & 0.0027 & NLLGKRISK \\
\hline 8.3 & 1107.6165 & 0.0027 & AKARAILTGK \\
\hline 5.0 & 1107.6189 & 0.0003 & VIHWNSPKK \\
\hline 5.0 & 1107.6111 & 0.0082 & VLIFSQMVR \\
\hline 5.0 & 1107.6111 & 0.0082 & VLIFTQMTR \\
\hline 4.0 & 1106.6158 & 1.0035 & IAQLYMIQK \\
\hline 4.0 & 1106.6084 & 1.0109 & KAILTNQYR \\
\hline 4.0 & 1106.6073 & 1.0119 & VGRLSRLAR \\
\hline
\end{tabular}

Spectrum No: 331; Query: 2466; Rank: 1

\section{Peptide View}

MS/MS Fragmentation of AFMTVDGQESPSVTVVGNATTLDVERK

Found in IPI00363534, Tax_Id=10116 Gene_Symbol=Lama1 laminin, alpha 1

Match to Query 2466: 2851.398822 from(951.473550,3+)

Title: 100101RatKid_NS_deglyco_19.3739.3739.3.dta

Data file K:INewmanPaper|Piliangl3SubProteomes\Piliang3SP\mgf5ppm\ERLIC_3SubProteomes5ppm.mgf 

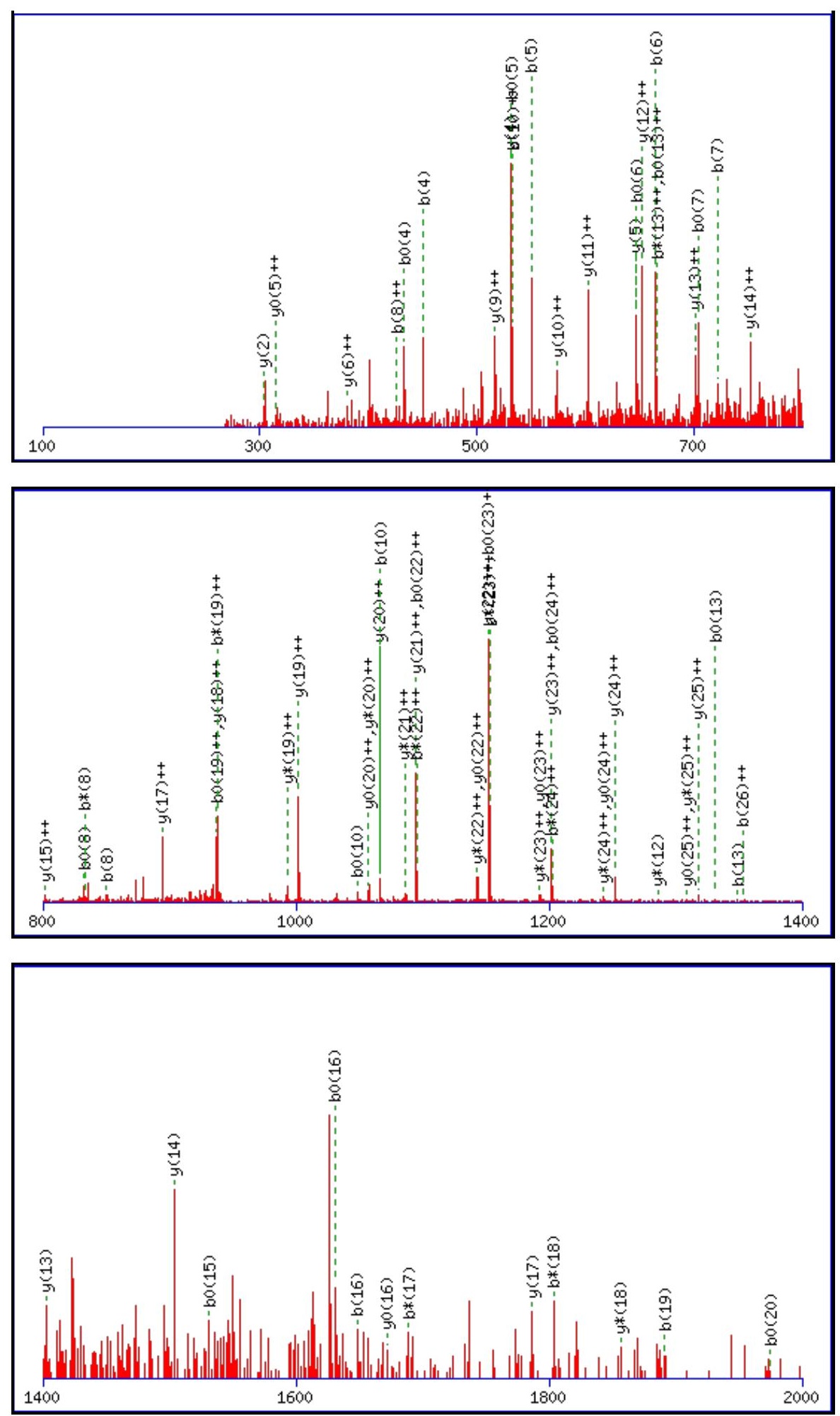

Monoisotopic mass of neutral peptide $\operatorname{Mr}($ calc): 2851.3910

Fixed modifications: Carbamidomethyl (C)

Variable modifications:

N18 : Deamidated_N (N)

Ions Score: 72 Expect: $1.5 \mathrm{e}-005$

Matches (Bold Red): 74/288 fragment ions using 151 most intense peaks

\begin{tabular}{|r|c|c|c|c|c|c|c|c|c|c|c|c|c|c|}
\hline$\#$ & $\mathbf{b}$ & $\mathbf{b}^{++}$ & $\mathbf{b}^{*}$ & $\mathbf{b}^{\boldsymbol{*}^{++}}$ & $\mathbf{b}^{\mathbf{0}}$ & $\mathbf{b}^{\mathbf{0 + +}}$ & Seq. & $\mathbf{y}$ & $\mathbf{y}^{++}$ & $\mathbf{y}^{\mathbf{*}}$ & $\mathbf{y}^{\mathbf{*}^{++}}$ & $\mathbf{y}^{\mathbf{0}}$ & $\mathbf{y}^{\mathbf{0}+}$ & $\#$ \\
\hline $\mathbf{1}$ & $\mathbf{7 2 . 0 4 4 4}$ & 36.5258 & & & & & $\mathbf{A}$ & & & & & & & 27 \\
\hline $\mathbf{2}$ & 219.1128 & 110.0600 & & & & & $\mathbf{F}$ & 2781.3611 & 1391.1842 & 2764.3346 & 1382.6709 & 2763.3505 & 1382.1789 & $\mathbf{2 6}$ \\
\hline 3 & 350.1533 & 175.5803 & & & & & $\mathbf{M}$ & 2634.2927 & $\mathbf{1 3 1 7 . 6 5 0 0}$ & 2617.2661 & $\mathbf{1 3 0 9 . 1 3 6 7}$ & 2616.2821 & $\mathbf{1 3 0 8 . 6 4 4 7}$ & $\mathbf{2 5}$ \\
\hline $\mathbf{4}$ & $\mathbf{4 5 1 . 2 0 1 0}$ & 226.1041 & & & $\mathbf{4 3 3 . 1 9 0 4}$ & 217.0988 & $\mathbf{T}$ & 2503.2522 & $\mathbf{1 2 5 2 . 1 2 9 7}$ & 2486.2257 & $\mathbf{1 2 4 3 . 6 1 6 5}$ & 2485.2416 & $\mathbf{1 2 4 3 . 1 2 4 5}$ & $\mathbf{2 4}$ \\
\hline $\mathbf{5}$ & $\mathbf{5 5 0 . 2 6 9 4}$ & 275.6383 & & & 532.2588 & 266.6330 & $\mathbf{V}$ & 2402.2045 & $\mathbf{1 2 0 1 . 6 0 5 9}$ & 2385.1780 & $\mathbf{1 1 9 3 . 0 9 2 6}$ & 2384.1940 & $\mathbf{1 1 9 2 . 6 0 0 6}$ & $\mathbf{2 3}$ \\
\hline
\end{tabular}




\begin{tabular}{|c|c|c|c|c|c|c|c|c|c|c|c|c|c|c|}
\hline 6 & 665.2963 & 333.1518 & & & 647.2858 & 324.1465 & D & 2303.1361 & $|1152.0717|$ & |2286.1096| & 1143.5584 & |2285.1256 & $1143.0664 \mid 2$ & |22| \\
\hline 7 & 722.3178 & 361.6625 & & & 704.3072 & 352.6573 & G & 2188.1092 & 1094.5582 & 2171.0826 & 1086.0450 & 2170.0986 & 1085.5529 & 21 \\
\hline 8 & 850.3764 & 425.6918 & 833.3498 & 417.1785 & 832.3658 & 416.6865 & $\mathbf{Q}$ & 2131.0877 & $\mid 1066.0475$ & 2114.0612 & 1057.5342 & 2113.0771 & 1057.0422 & 0 \\
\hline 9 & 979.4190 & 490.2131 & 962.3924 & 481.6998 & 961.4084 & 481.2078 & $\mathbf{E}$ & 2003.0291 & 1002.0182 & 1986.0026 & 993.5049 & 1985.0186 & 993.0129 & \\
\hline 10 & 1066.4510 & 533.7291 & 1049.4244 & 525.2159 & $\mid 1048.4404$ & 524.7238 & $\mathbf{S}$ & 1873.9865 & 937.4969 & 1856.9600 & 928.9836 & 1855.9760 & 928.4916 & 18 \\
\hline 11 & 1163.5038 & 582.2555 & 1146.4772 & 573.7422 & 1145.4932 & 573.2502 & $\mathbf{P}$ & 1786.9545 & 893.9809 & 1769.9280 & 885.4676 & 1768.9439 & 884.9756 & 17 \\
\hline 12 & 1250.5358 & 625.7715 & 1233.5092 & 617.2583 & 1232.5252 & 616.7662 & S & 1689.9018 & 845.4545 & 1672.8752 & 836.9412 & 1671.8912 & 836.4492 & 6 \\
\hline 13 & 1349.6042 & 675.3057 & 1332.5776 & 666.7925 & 1331.5936 & 666.3005 & V & 1602.8697 & 801.9385 & 1585.8432 & 793.4252 & 1584.8592 & 792.9332 & 15 \\
\hline 14 & 1450.6519 & 725.8296 & 1433.6253 & 717.3163 & 1432.6413 & 716.8243 & $\mathbf{T}$ & 1503.8013 & 752.4043 & 1486.7748 & 743.8910 & 1485.7907 & 743.3990 & 14 \\
\hline 15 & 1549.7203 & 775.3638 & 1532.6937 & 766.8505 & 1531.7097 & 766.3585 & $\mathbf{V}$ & 1402.7536 & 701.8805 & 1385.7271 & 693.3672 & 1384.7431 & 692.8752 & 13 \\
\hline 16 & \begin{tabular}{|l|}
1648.7887 \\
\end{tabular} & 824.8980 & 1631.7622 & 816.3847 & 1630.7781 & 815.8927 & $\mathbf{V}$ & 1303.6852 & 652.3462 & 1286.6587 & 643.8330 & 1285.6746 & 643.3410 & 12 \\
\hline 17 & 1705.8102 & 853.4087 & \begin{tabular}{|l|}
1688.7836 \\
\end{tabular} & 844.8954 & 1687.7996 & 844.4034 & G & 1204.6168 & 602.8120 & 1187.5903 & 594.2988 & 1186.6062 & 593.8068 & 11 \\
\hline 18 & 1820.8371 & 910.9222 & 1803.8106 & 902.4089 & 1802.8265 & 901.9169 & $\mathbf{N}$ & 1147.5953 & 574.3013 & 1130.5688 & 565.7880 & 1129.5848 & 565.2960 & 10 \\
\hline 19 & \begin{tabular}{|l|}
1891.8742 \\
\end{tabular} & 946.4407 & 1874.8477 & 937.9275 & 1873.8637 & 937.4355 & A & 1032.5684 & 516.7878 & 1015.5419 & 508.2746 & 1014.5578 & 507.7826 & 9 \\
\hline 20 & 1992.9219 & 996.9646 & 1975.8953 & 988.4513 & 1974.9113 & 987.9593 & $\mathbf{T}$ & 961.5313 & 481.2693 & 944.5047 & 472.7560 & 943.5207 & 472.2640 & 8 \\
\hline 21 & 2093.9696 & 1047.4884 & 2076.9430 & 1038.9751 & 2075.9590 & 1038.4831 & $\mathbf{T}$ & 860.4836 & 430.7454 & 843.4571 & 422.2322 & 842.4730 & 421.7402 & 7 \\
\hline 22 & 2207.0536 & 1104.0305 & 2190.0271 & 1095.5172 & 2189.0431 & \begin{tabular}{|l|}
1095.0252 \\
\end{tabular} & $\mathbf{L}$ & 759.4359 & 380.2216 & 742.4094 & 371.7083 & 741.4254 & 371.2163 & 6 \\
\hline 23 & 2322.0806 & 1161.5439 & 2305.0540 & 1153.0307 & 2304.0700 & 1152.5386 & D & 646.3519 & 323.6796 & 629.3253 & 315.1663 & 628.3413 & 314.6743 & 5 \\
\hline 24 & 2421.1490 & 1211.0781 & 2404.1224 & 1202.5649 & 2403.1384 & \begin{tabular}{|l|}
1202.0729 \\
\end{tabular} & $\mathbf{V}$ & 531.3249 & 266.1661 & 514.2984 & 257.6528 & 513.3144 & 257.1608 & 4 \\
\hline 25 & 2550.1916 & 1275.5994 & 2533.1650 & 1267.0862 & 2532.1810 & 1266.5941 & $\mathbf{E}$ & 432.2565 & 216.6319 & 415.2300 & 208.1186 & 414.2459 & 207.6266 & 3 \\
\hline 26 & 2706.2927 & 1353.6500 & 2689.2661 & 1345.1367 & 2688.2821 & 1344.6447 & $\mathbf{R}$ & 303.2139 & 152.1106 & 286.1874 & 143.5973 & & & 2 \\
\hline 27 & & & & & & & $\mathbf{K}$ & 147.1128 & 74.0600 & 130.0863 & 65.5468 & & & 1 \\
\hline
\end{tabular}
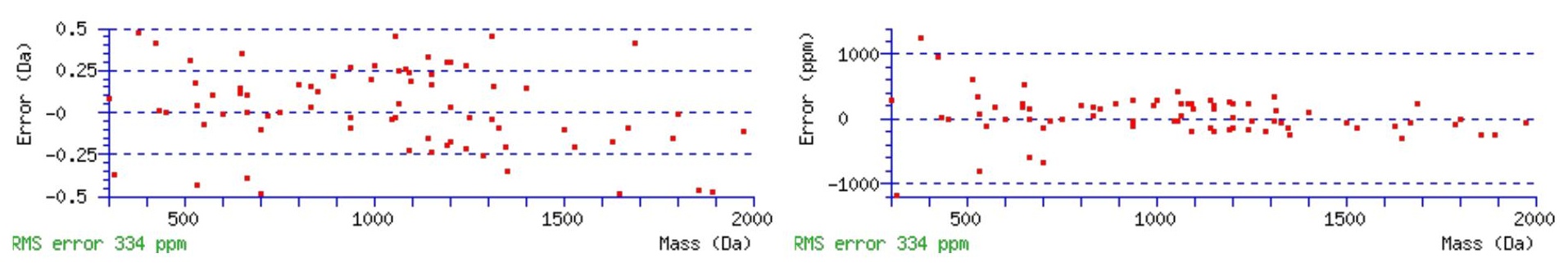

\section{All matches to this query}

\begin{tabular}{|l|l|l|l|}
\hline Score & Mr(calc): & Delta & \multicolumn{1}{|c|}{ Sequence } \\
\hline 72.3 & 2851.3910 & 0.0078 & AFMTVDGQESPSVTVVGNATTLDVERK \\
\hline 17.2 & 2850.4070 & 0.9919 & AFMTVDGQESPSVTVVGNATTLDVERK \\
\hline 5.1 & 2851.3844 & 0.0144 & VHTIVISVQHDEEVCLDEMRDALK \\
\hline 1.0 & 2849.3922 & 2.0066 & FVTAKKNGQLAATVETAGDSELFLMK \\
\hline 1.0 & 2849.3922 & 2.0066 & FVTAKKNGQLAATVETAGDSELFLMK \\
\hline 1.0 & 2849.3922 & 2.0066 & FVTAKKNGQLAATVETAGDSELFLMK \\
\hline 0.8 & 2850.3874 & 1.0114 & MITQTSLTPYIASPVSAYQVAKETR \\
\hline 0.4 & 2850.3874 & 1.0114 & MITQTSLTPYIASPVSAYQVAKETR \\
\hline 0.3 & 2851.3762 & 0.0227 & RVMKLSTLSSPSAILWAPDSPPCSR \\
\hline 0.3 & 2851.3762 & 0.0227 & RVMKLSTLSSPSAILWAPDSPPCSR \\
\hline
\end{tabular}

Spectrum No: 332; Query: 218; Rank: 1

\section{Peptide View}


Match to Query 218: 1202.633188 from(602.323870,2+)

Title: 100101RatKid_NS_deglyco_19.1959.1959.2.dta

Data file K:INewmanPaper|Piliang|3SubProteomes\Piliang3SP\mgf5ppm\ERLIC_3SubProteomes5ppm.mgf
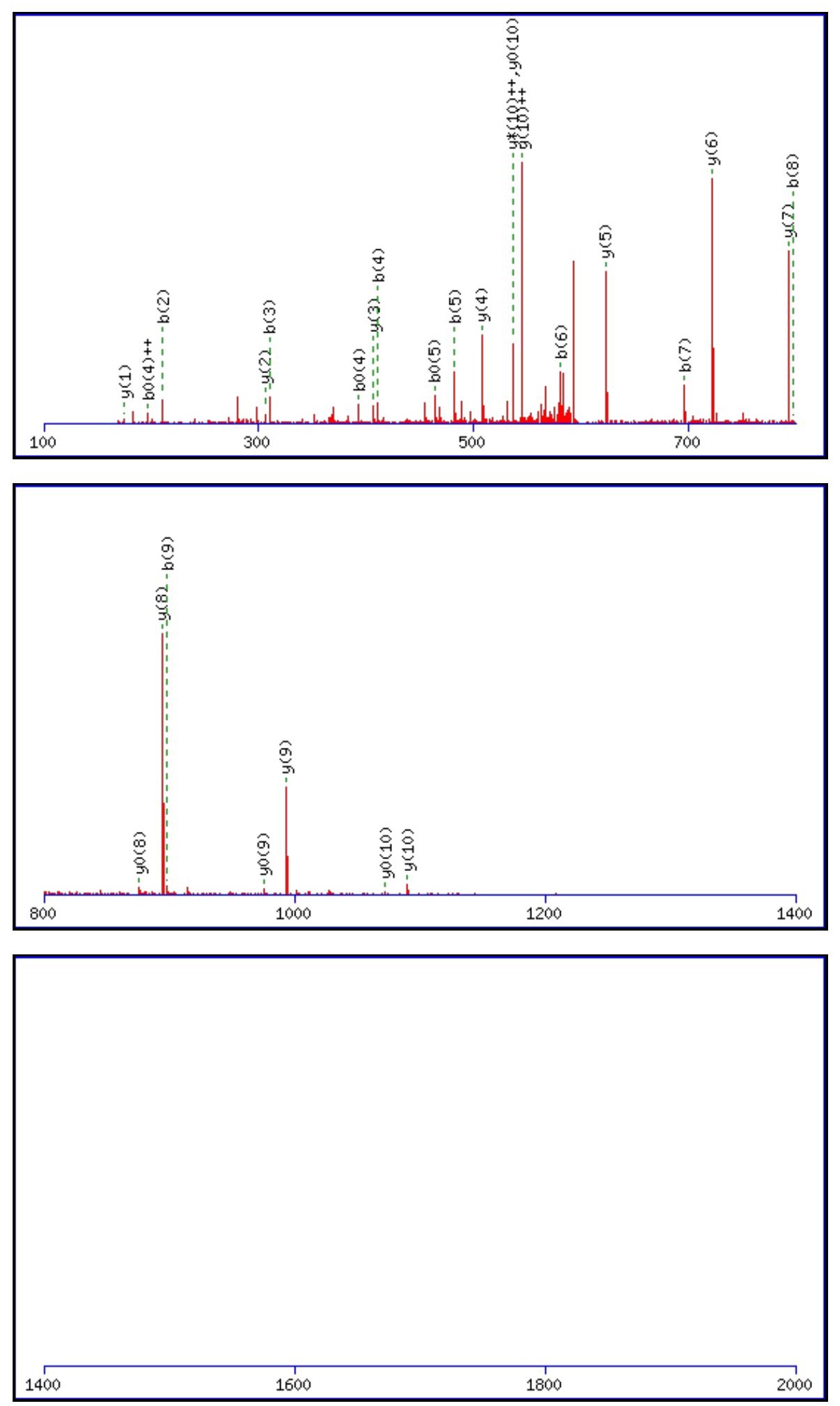

Monoisotopic mass of neutral peptide $\operatorname{Mr}$ (calc): 1202.6329

Fixed modifications: Carbamidomethyl (C)

Variable modifications:

N7 : Deamidated_N (N)

Ions Score: 72 Expect: $7.2 \mathrm{e}-006$

Matches (Bold Red): 27/98 fragment ions using 51 most intense peaks

\begin{tabular}{|c|c|c|c|c|c|c|c|c|c|c|c|c|c|c|}
\hline$\#$ & $\mathbf{b}$ & $\mathbf{b}^{++}$ & $\mathbf{b}^{*}$ & $\mathbf{b}^{*^{++}}$ & $\mathbf{b}^{\mathbf{0}}$ & $\mathbf{b}^{\mathbf{0}+}$ & Seq. & $\mathbf{y}$ & $\mathbf{y}^{++}$ & $\mathbf{y}^{*}$ & $\mathbf{y}^{*^{++}}$ & $\mathbf{y}^{\mathbf{0}}$ & $\mathbf{y}^{\mathbf{0 + +}}$ & $\#$ \\
\hline $\mathbf{1}$ & 114.0913 & 57.5493 & & & & & $\mathbf{L}$ & & & & & & & $\mathbf{1 1}$ \\
\hline $\mathbf{2}$ & 211.1441 & 106.0757 & & & & & $\mathbf{P}$ & $\mathbf{1 0 9 0 . 5 5 6 1}$ & $\mathbf{5 4 5 . 7 8 1 7}$ & 1073.5296 & $\mathbf{5 3 7 . 2 6 8 4}$ & $\mathbf{1 0 7 2 . 5 4 5 6}$ & $\mathbf{5 3 6 . 7 7 6 4}$ & $\mathbf{1 0}$ \\
\hline
\end{tabular}




\begin{tabular}{|c|c|c|c|c|c|c|c|c|c|c|c|c|c|c|}
\hline 3 & 310.2125 & |155.6099 & & & & & V & 993.5034 & |497.2553| & 976.4768 & 488.7420 & 975.4928 & |488.2500| & 9 \\
\hline 4 & 411.2602 & 206.1337 & & & 393.2496 & 197.1285 & $\mathbf{T}$ & 894.4349 & 447.7211 & 877.4084 & 439.2078 & 876.4244 & 438.7158 & 8 \\
\hline 5 & 482.2973 & 241.6523 & & & 464.2867 & 232.6470 & A & 793.3873 & 397.1973 & 776.3607 & 388.6840 & 775.3767 & 388.1920 & 7 \\
\hline 6 & 581.3657 & 291.1865 & & & 563.3552 & 282.1812 & V & 722.3501 & |361.6787 & 705.3236 & 353.1654 & 704.3396 & |352.6734 & 6 \\
\hline 7 & 696.3927 & 348.7000 & 679.3661 & 340.1867 & 678.3821 & 339.6947 & $\mathbf{N}$ & 623.2817 & 312.1445 & 606.2552 & 303.6312 & 605.2712 & 303.1392 & 5 \\
\hline 8 & 797.4403 & 399.2238 & 780.4138 & 390.7105 & 779.4298 & 390.2185 & $\mathbf{T}$ & 508.2548 & 254.6310 & 491.2282 & 246.1178 & 490.2442 & 245.6258 & 4 \\
\hline 9 & 898.4880 & 449.7476 & 881.4615 & 441.2344 & 880.4775 & 440.7424 & $T$ & 407.2071 & 204.1072 & 390.1806 & 195.5939 & 389.1966 & 195.1019 & 3 \\
\hline 10 & 1029.5285 & 515.2679 & 1012.5020 & 506.7546 & 1011.5179 & 506.2626 & M & 306.1594 & 153.5834 & 289.1329 & 145.0701 & & & 2 \\
\hline 11 & & & & & & & $\mathbf{R}$ & 175.1190 & 88.0631 & 158.0924 & 79.5498 & & & 1 \\
\hline
\end{tabular}
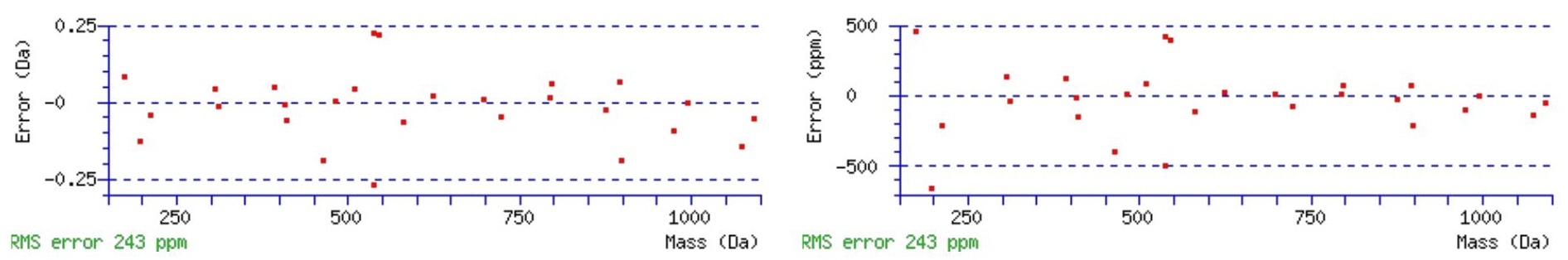

\section{All matches to this query}

\begin{tabular}{|l|l|c|c|}
\hline Score & Mr(calc): & Delta & Sequence \\
\hline 72.2 & 1202.6329 & 0.0003 & LPVTAVNTTMR \\
\hline 4.6 & 1200.6211 & 2.0121 & EAPRKETTGGR \\
\hline
\end{tabular}

Spectrum No: 333; Query: 923; Rank: 1

\section{Peptide View}

\section{MS/MS Fragmentation of ANTTQPGIIEGGQVLK}

Found in IPI00764984, Tax_Id=10116 Gene_Symbol=Itgav_predicted 106 kDa protein

Match to Query 923: 1625.867088 from(813.940820,2+)

Title: 100101RatKid_NS_deglyco_21.2210.2210.2.dta

Data file K:INewmanPaper|Piliangl3SubProteomes\Piliang3SP\mgf5ppm\ERLIC_3SubProteomes5ppm.mgf

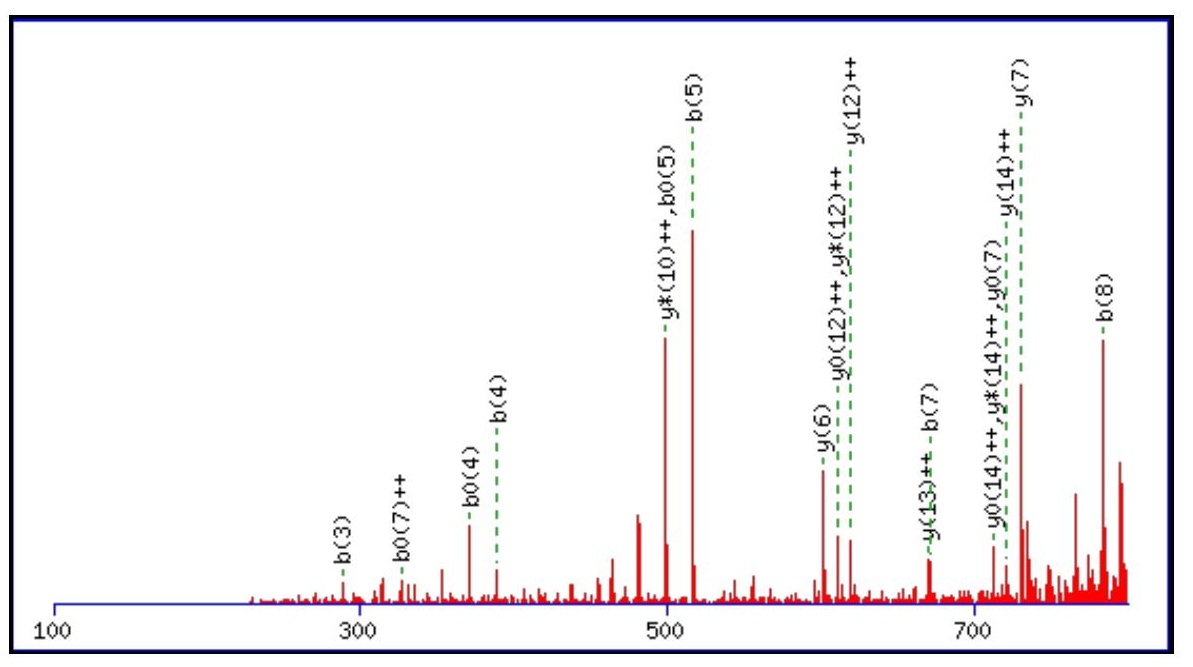



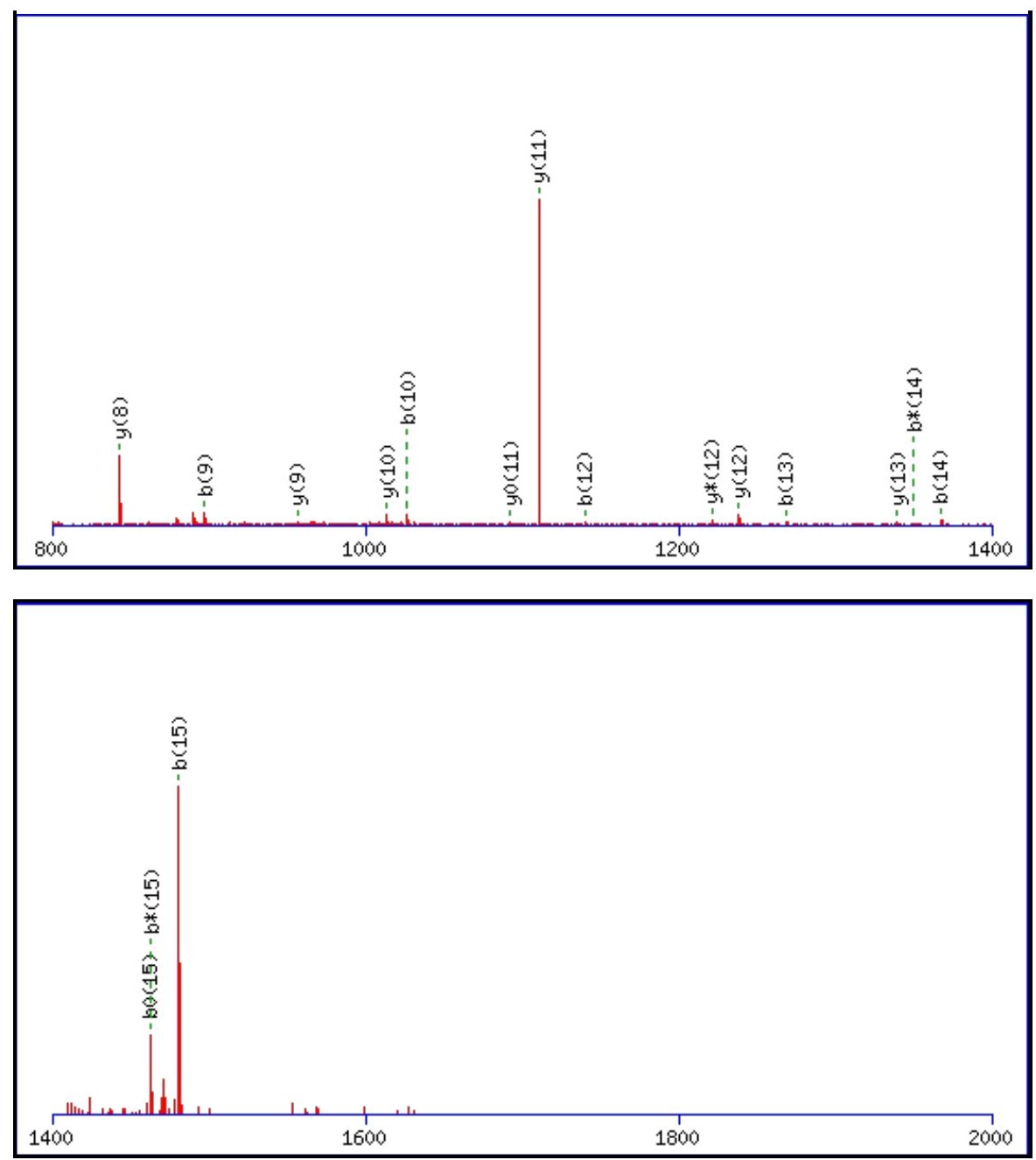

Monoisotopic mass of neutral peptide $\operatorname{Mr}($ calc): 1625.8624

Fixed modifications: Carbamidomethyl (C)

Variable modifications:

N2 : Deamidated $\mathrm{N}(\mathrm{N})$

Ions Score: 72 Expect: $6 \mathrm{e}-006$

Matches (Bold Red): 36/162 fragment ions using 58 most intense peaks

\begin{tabular}{|c|c|c|c|c|c|c|c|c|c|c|c|c|c|c|}
\hline \# & b & $\mathbf{b}^{++}$ & $\mathbf{b}^{*}$ & $\mathbf{b}^{*^{++}}$ & $\mathbf{b}^{\mathbf{0}}$ & $\mathbf{b}^{0++}$ & Seq. & $\mathbf{y}$ & $\mathbf{y}^{++}$ & $\mathbf{y}^{*}$ & $\mathrm{y}^{*^{++}}$ & $\mathbf{y}^{\mathbf{0}}$ & $\mathbf{y}^{\mathbf{0 + +}}$ & \# \\
\hline 1 & 72.0444 & 36.5258 & & & & & A & & & & & & & 16 \\
\hline 2 & 187.0713 & 94.0393 & 170.0448 & 85.5260 & & & $\mathbf{N}$ & 1555.8326 & 778.4199 & 1538.8061 & 769.9067 & 1537.8220 & 769.4147 & 15 \\
\hline 3 & 288.1190 & 144.5631 & 271.0925 & 136.0499 & 270.1084 & 135.5579 & $\mathbf{T}$ & 1440.8057 & 720.9065 & 1423.7791 & 712.3932 & 1422.7951 & 711.9012 & 14 \\
\hline 4 & 389.1667 & 195.0870 & 372.1401 & 186.5737 & 371.1561 & 186.0817 & $\mathbf{T}$ & 1339.7580 & 670.3826 & 1322.7314 & 661.8694 & 1321.7474 & 661.3774 & 13 \\
\hline 5 & 517.2253 & 259.1163 & 500.1987 & 250.6030 & 499.2147 & 250.1110 & $\mathbf{Q}$ & 1238.7103 & 619.8588 & 1221.6838 & 611.3455 & 1220.6998 & 610.8535 & 12 \\
\hline 6 & 614.2780 & 307.6427 & 597.2515 & 299.1294 & 596.2675 & 298.6374 & $\mathbf{P}$ & 1110.6517 & 555.8295 & 1093.6252 & 547.3162 & 1092.6412 & 546.8242 & 11 \\
\hline 7 & 671.2995 & 336.1534 & 654.2729 & 327.6401 & 653.2889 & 327.1481 & G & 1013.5990 & 507.3031 & 996.5724 & 498.7899 & 995.5884 & 498.2978 & 10 \\
\hline 8 & 784.3836 & 392.6954 & 767.3570 & 384.1821 & 766.3730 & 383.6901 & I & 956.5775 & 478.7924 & 939.5510 & 470.2791 & 938.5669 & 469.7871 & 9 \\
\hline 9 & 897.4676 & 449.2374 & 880.4411 & 440.7242 & 879.4571 & 440.2322 & I & 843.4934 & 422.2504 & 826.4669 & 413.7371 & 825.4829 & 413.2451 & 8 \\
\hline 10 & 1026.5102 & 513.7587 & 1009.4837 & 505.2455 & 1008.4996 & 504.7535 & $\mathbf{E}$ & 730.4094 & 365.7083 & 713.3828 & 357.1951 & 712.3988 & 356.7030 & 7 \\
\hline 11 & 1083.5317 & 542.2695 & 1066.5051 & 533.7562 & 1065.5211 & 533.2642 & G & 601.3668 & 301.1870 & 584.3402 & 292.6738 & & & 6 \\
\hline 12 & 1140.5531 & 570.7802 & 1123.5266 & 562.2669 & 1122.5426 & 561.7749 & G & 544.3453 & 272.6763 & 527.3188 & 264.1630 & & & 5 \\
\hline 13 & 1268.6117 & 634.8095 & 1251.5852 & 626.2962 & 1250.6012 & 625.8042 & $\mathbf{Q}$ & 487.3239 & 244.1656 & 470.2973 & 235.6523 & & & 4 \\
\hline 14 & 1367.6801 & 684.3437 & 1350.6536 & 675.8304 & 1349.6696 & 675.3384 & $\mathbf{V}$ & 359.2653 & 180.1363 & 342.2387 & 171.6230 & & & 3 \\
\hline 15 & 1480.7642 & 740.8857 & 1463.7376 & 732.3725 & 1462.7536 & 731.8805 & $\mathbf{L}$ & 260.1969 & 130.6021 & 243.1703 & 122.0888 & & & 2 \\
\hline 16 & & & & & & & $\mathbf{K}$ & 147.1128 & 74.0600 & 130.0863 & 65.5468 & & & 1 \\
\hline
\end{tabular}



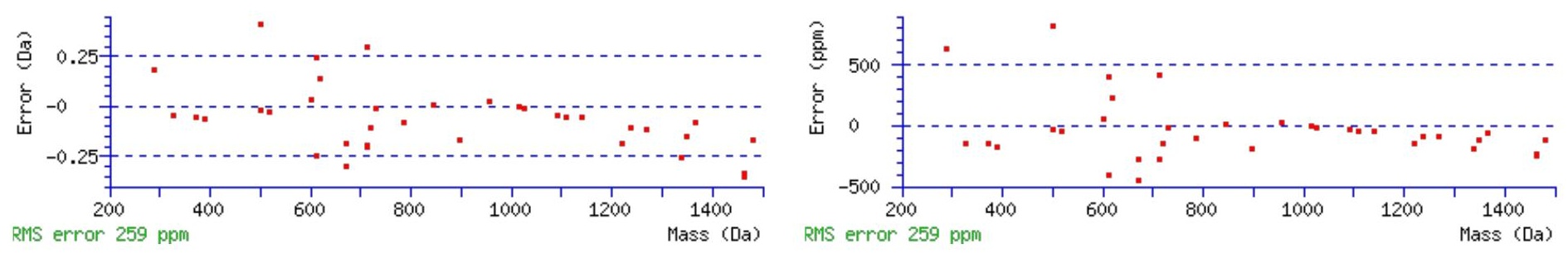

\section{All matches to this query}

\begin{tabular}{|l|l|l|l|}
\hline Score & Mr(calc): & Delta & \multicolumn{1}{|c|}{ Sequence } \\
\hline 72.2 & 1625.8624 & 0.0046 & ANTTQPGIIEGGQVLK \\
\hline 23.5 & 1624.8784 & 0.9887 & ANTTQPGIIEGGQVLK \\
\hline 13.0 & 1625.8624 & 0.0047 & DINTKLPQAQQELK \\
\hline 12.9 & 1625.8575 & 0.0095 & TRILVTSKMEQLK \\
\hline 12.9 & 1625.8575 & 0.0095 & TRILVTSKMEQLK \\
\hline 5.8 & 1625.8575 & 0.0095 & TRILVTSKMEQLK \\
\hline 5.5 & 1625.8624 & 0.0047 & EGTPSGAAPSSALAAVLK \\
\hline 4.7 & 1625.8624 & 0.0047 & SLDEGAPQDTPKLKK \\
\hline 4.5 & 1624.8798 & 0.9873 & LVHNFTDAVIQGRR \\
\hline 3.6 & 1625.8616 & 0.0055 & IYVVQPKAMKIEK \\
\hline
\end{tabular}

Spectrum No: 334; Query: 619; Rank: 1

\section{Peptide View}

MS/MS Fragmentation of VEAIGSLANTTMEK

Found in IPI00193397, Tax_Id=10116 Gene_Symbol=Slc34a1 Sodium-dependent phosphate transport protein 2A

Match to Query 619: 1463.719888 from(732.867220,2+)

Title: 100101RatKid_NS_deglyco_27.2072.2072.2.dta

Data file K:INewmanPaper|Piliangl3SubProteomes\Piliang3SP\mgf5ppm\ERLIC_3SubProteomes5ppm.mgf

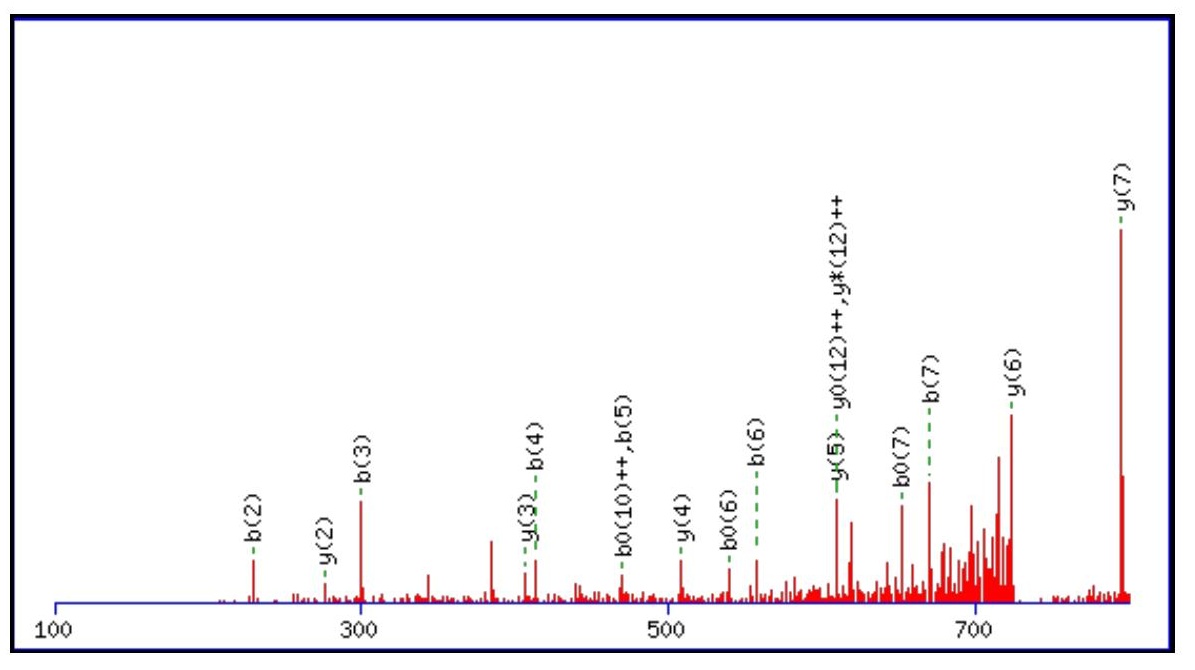



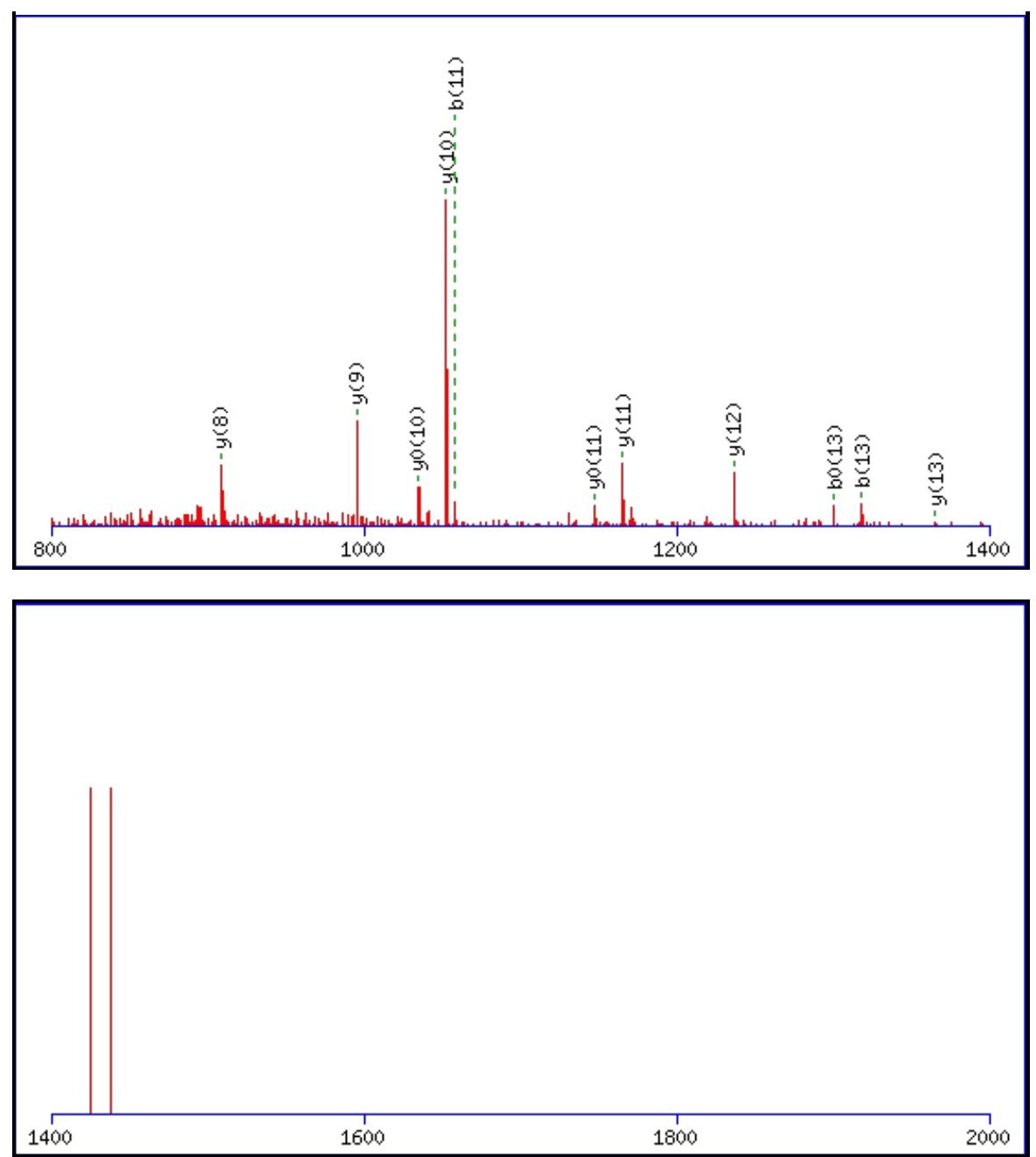

Monoisotopic mass of neutral peptide $\operatorname{Mr}($ calc): 1463.7177

Fixed modifications: Carbamidomethyl (C)

Variable modifications:

N9: Deamidated $\mathrm{N}(\mathrm{N})$

Ions Score: 72 Expect: $9 \mathrm{e}-006$

Matches (Bold Red): 28/136 fragment ions using 47 most intense peaks

\begin{tabular}{|c|c|c|c|c|c|c|c|c|c|c|c|c|c|c|}
\hline \# & b & $\mathbf{b}^{++}$ & b* & $\mathbf{b}^{*^{++}}$ & $\mathbf{b}^{0}$ & $\mathbf{b}^{0++}$ & Seq. & $\mathbf{y}$ & $\mathrm{y}^{++}$ & $\mathbf{y}^{*}$ & $\mathrm{y}^{*^{++}}$ & $\mathbf{y}^{0}$ & $\mathbf{y}^{\mathbf{0 + +}}$ & \# \\
\hline 1 & 100.0757 & 50.5415 & & & & & V & & & & & & & 14 \\
\hline 2 & 229.1183 & 115.0628 & & & 211.1077 & 106.0575 & $\mathbf{E}$ & 1365.6566 & 683.3319 & 1348.6301 & 674.8187 & 1347.6461 & 674.3267 & 13 \\
\hline 3 & 300.1554 & 150.5813 & & & 282.1448 & 141.5761 & A & 1236.6140 & 618.8107 & 1219.5875 & 610.2974 & 1218.6035 & 609.8054 & 12 \\
\hline 4 & 413.2395 & 207.1234 & & & 395.2289 & 198.1181 & I & 1165.5769 & 583.2921 & 1148.5504 & 574.7788 & 1147.5663 & 574.2868 & 11 \\
\hline 5 & 470.2609 & 235.6341 & & & 452.2504 & 226.6288 & G & 1052.4928 & 526.7501 & 1035.4663 & 518.2368 & 1034.4823 & 517.7448 & 10 \\
\hline 6 & 557.2930 & 279.1501 & & & 539.2824 & 270.1448 & S & 995.4714 & 498.2393 & 978.4448 & 489.7261 & 977.4608 & 489.2340 & 9 \\
\hline 7 & 670.3770 & 335.6921 & & & 652.3665 & 326.6869 & $\mathbf{L}$ & 908.4394 & 454.7233 & 891.4128 & 446.2100 & 890.4288 & 445.7180 & 8 \\
\hline 8 & 741.4141 & 371.2107 & & & 723.4036 & 362.2054 & A & 795.3553 & 398.1813 & 778.3287 & 389.6680 & 777.3447 & 389.1760 & 7 \\
\hline 9 & 856.4411 & 428.7242 & 839.4145 & 420.2109 & 838.4305 & 419.7189 & $\mathbf{N}$ & 724.3182 & 362.6627 & 707.2916 & 354.1495 & 706.3076 & 353.6574 & 6 \\
\hline 10 & 957.4887 & 479.2480 & 940.4622 & 470.7347 & 939.4782 & 470.2427 & $\mathbf{T}$ & 609.2912 & 305.1493 & 592.2647 & 296.6360 & 591.2807 & 296.1440 & 5 \\
\hline 11 & 1058.5364 & 529.7719 & 1041.5099 & 521.2586 & 1040.5259 & 520.7666 & $\mathbf{T}$ & 508.2436 & 254.6254 & 491.2170 & 246.1121 & 490.2330 & 245.6201 & 4 \\
\hline 12 & 1189.5769 & 595.2921 & 1172.5504 & 586.7788 & 1171.5663 & 586.2868 & $\mathbf{M}$ & 407.1959 & 204.1016 & 390.1693 & 195.5883 & 389.1853 & 195.0963 & 3 \\
\hline 13 & 1318.6195 & 659.8134 & 1301.5930 & 651.3001 & 1300.6089 & 650.8081 & $\mathbf{E}$ & 276.1554 & 138.5813 & 259.1288 & 130.0681 & 258.1448 & 129.5761 & 2 \\
\hline 14 & & & & & & & $\mathbf{K}$ & 147.1128 & 74.0600 & 130.0863 & 65.5468 & & & 1 \\
\hline
\end{tabular}



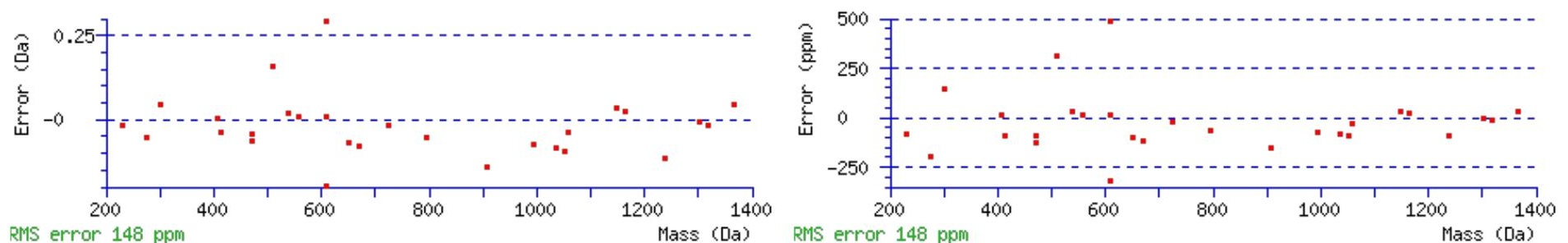

\section{All matches to this query}

\begin{tabular}{|l|l|l|l|}
\hline Score & Mr(calc): & Delta & \multicolumn{1}{|c|}{ Sequence } \\
\hline 72.2 & 1463.7177 & 0.0022 & VEAIGSLANTTMEK \\
\hline 17.6 & 1463.7133 & 0.0066 & RVSTISALSHEGK \\
\hline 16.2 & 1463.7256 & -0.0057 & NNKLSLQFEEDK \\
\hline 12.7 & 1463.7272 & -0.0074 & IDPVEKLQESVK \\
\hline 11.5 & 1463.7133 & 0.0066 & RVSTISALSHEGK \\
\hline 11.5 & 1463.7133 & 0.0066 & RVSTISALSHEGK \\
\hline 6.3 & 1463.7273 & -0.0074 & EEGPVLVADTVKK \\
\hline 4.7 & 1463.7061 & 0.0138 & DAILKYNVAYSK \\
\hline 4.7 & 1463.7061 & 0.0138 & DAILKYNVAYSK \\
\hline 4.7 & 1463.7061 & 0.0138 & DAILKYNVAYSK \\
\hline
\end{tabular}

Spectrum No: 335; Query: 2459; Rank: 1

\section{Peptide View}

MS/MS Fragmentation of MCQLYPETFECVTNSSDILQAFR

Found in IPI00327697, Tax_Id=10116 Gene_Symbol=Dpep1 Dipeptidase 1 precursor

Match to Query 2459: 2825.247928 from(1413.631240,2+)

Title: 100101RatKid_NS_deglyco_20.5460.5460.2.dta

Data file K:INewmanPaper|Piliangl3SubProteomes\Piliang3SP\mgf5ppm\ERLIC_3SubProteomes5ppm.mgf

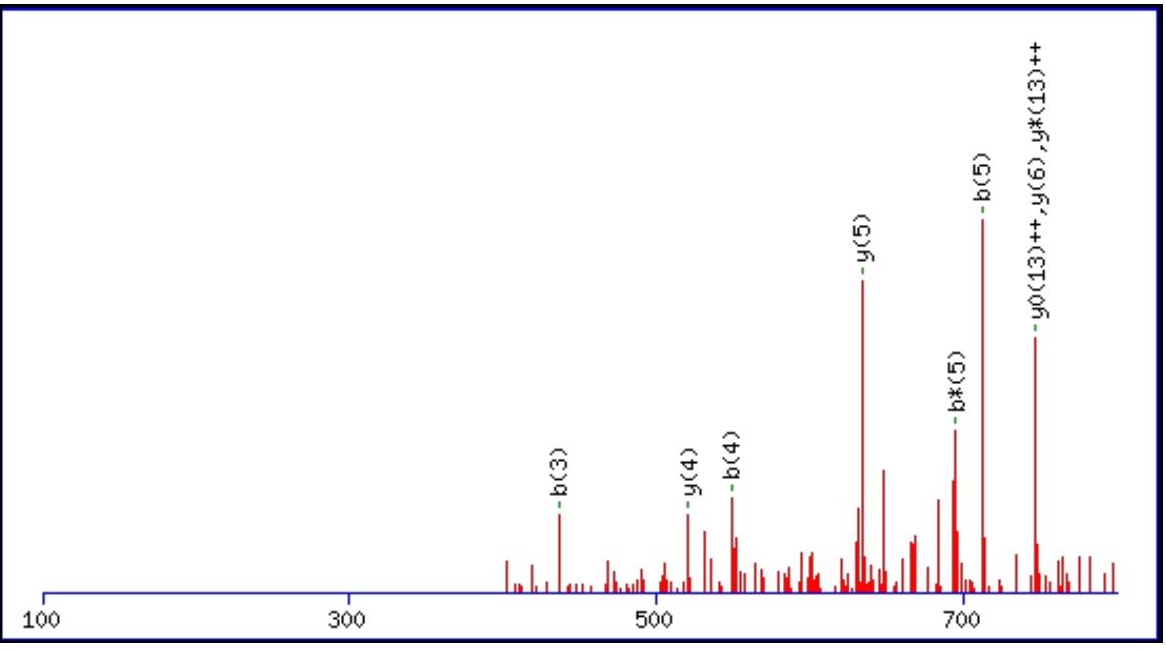



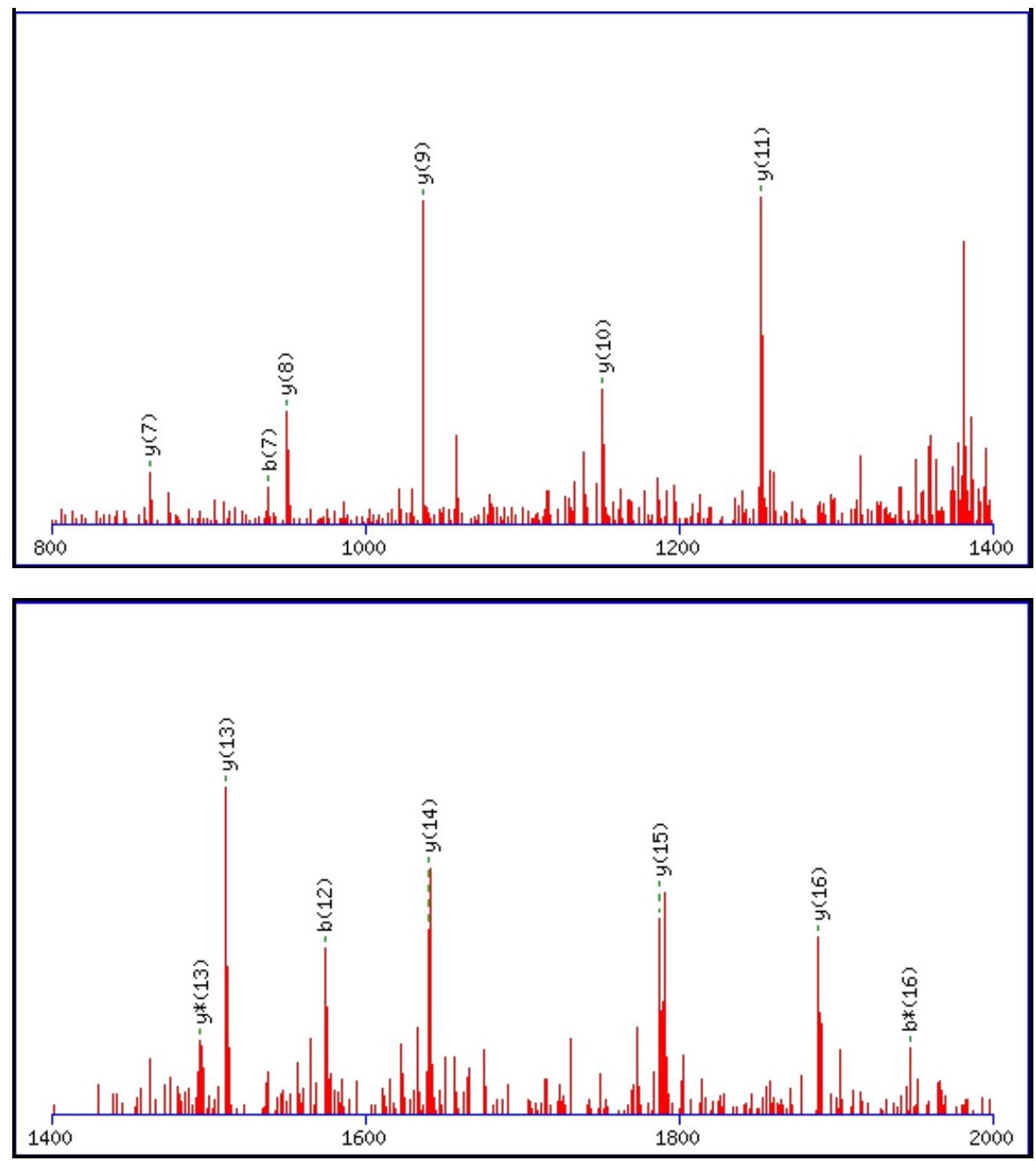

Monoisotopic mass of neutral peptide $\operatorname{Mr}($ calc): 2825.2346

Fixed modifications: Carbamidomethyl (C)

Variable modifications:

M1 : Oxidation (M)

N14 : Deamidated_N (N)

Ions Score: 72 Expect: $2.3 e-005$

Matches (Bold Red): 22/236 fragment ions using 35 most intense peaks

\begin{tabular}{|c|c|c|c|c|c|c|c|c|c|c|c|c|c|c|}
\hline \# & b & $\mathbf{b}^{++}$ & $\mathbf{b}^{*}$ & $\mathbf{b}^{*^{++}}$ & $\mathbf{b}^{\mathbf{0}}$ & & Seq. & $\mathbf{y}$ & $\mathbf{y}^{++}$ & $\mathbf{y}^{*}$ & $\mathbf{y}^{*^{++}}$ & $\mathbf{y}^{0}$ & & \# \\
\hline 1 & 148.0427 & 74.5250 & & & & & $\mathbf{M}$ & & & & & & & 23 \\
\hline 2 & 308.0733 & 154.5403 & & & & & C & & & 2662.1800 & 1331.5936 & 2661.1960 & 1331.1016 & 22 \\
\hline 3 & 436.1319 & 218.5696 & 419.1054 & 210.0563 & & & $\mathbf{Q}$ & 2519.1759 & 1260.0916 & 2502.1493 & 1251.5783 & 2501.1653 & 1251.0863 & 21 \\
\hline 4 & 549.2160 & 275.1116 & 532.1894 & 266.5 & & & $\mathbf{L}$ & 2391.1173 & 623 & 907 & .5490 & 2373.1067 & 1187.0570 & 20 \\
\hline 5 & 712.2793 & 356.6433 & 695.2528 & 348.1300 & & & $\mathbf{Y}$ & 2278.0332 & 5203 & 0067 & .0070 & 2260.0227 & 1130.5150 & 19 \\
\hline 6 & 809.3321 & 405.1697 & 792.3055 & 396.6564 & & & $\mathbf{P}$ & 2114.9699 & 1057.9886 & 2097.9434 & 1049.4753 & 2096.9593 & 1048.9833 & 18 \\
\hline 7 & 938.3747 & 469.6910 & 921.3 & 461. & 1 & & $\mathbf{E}$ & 2017. & $100 s$ & 2000 & 489 & 1999 & 1000.4569 & 17 \\
\hline 8 & 1039.4223 & 520.2148 & 1022.3958 & 511.7015 & 1021.4118 & 511.2095 & $\mathbf{T}$ & 1888.8745 & 944.9409 & 1871.8480 & 936.4276 & 1870.8640 & 935.9356 & 16 \\
\hline 9 & 1186.4908 & 593.7490 & 169.4642 & 585.2357 & 168.4802 & 584.7437 & $\mathbf{F}$ & 1787.8269 & 4171 & 1770.8003 & 038 & 1769.8163 & 885.4118 & 15 \\
\hline 10 & 1315.5333 & 658.2703 & 1298.5068 & 649.7570 & 1297.5228 & 649.2650 & $\mathbf{E}$ & 1640.7585 & 820.8829 & 1623.7319 & 812.3696 & 1622.7479 & 811.8776 & 14 \\
\hline 11 & 1475.5640 & 738.2856 & 1458.5374 & 729.7724 & 1457.5534 & 729.2804 & $\mathrm{C}$ & 1511.7159 & 756.3616 & 1494.6893 & 747.8483 & 1493.7053 & 747.3563 & 13 \\
\hline 12 & 1574.6324 & 787.8198 & 1557.6059 & 779.3066 & 556.6218 & 778.8146 & $\mathbf{V}$ & 1351.6852 & 676.3462 & 1334.6587 & 667.8330 & 1333.6746 & 667.3410 & 12 \\
\hline 13 & 1675.6801 & 838.3437 & 1658.6535 & 829.8304 & 1657.6695 & 829.3384 & $T$ & 1252.6168 & 626.8120 & 1235.5903 & 618.2988 & 1234.6062 & 617.8068 & 11 \\
\hline 14 & 1790.7070 & 895.8571 & 1773.6805 & 887.3439 & 1772.6965 & 886.8519 & $\mathbf{N}$ & 1151.5691 & 576.2882 & 1134.5426 & 567.7749 & 1133.5586 & 567.2829 & 10 \\
\hline 15 & 1877.7390 & 939.3732 & 1860.7125 & 930.8599 & 1859.7285 & 930.3679 & $\mathrm{~S}$ & 1036.5422 & 518.7747 & 1019.5156 & 510.2615 & 1018.5316 & 509.7694 & 9 \\
\hline 16 & 1964.7711 & 982.8892 & 1947.7445 & 974.3759 & 1946.7605 & 973.8839 & $S$ & 949.5102 & 475.2587 & 932.4836 & 466.7454 & 931.4996 & 466.2534 & 8 \\
\hline 17 & 2079.7980 & 1040.4026 & 2062.7715 & 1031.8894 & 2061.7875 & 1031.3974 & D & 862.4781 & 431.7427 & 845.4516 & 423.2294 & 844.4676 & 422.7374 & 7 \\
\hline 18 & 2192.8821 & 1096.9447 & 2175.8555 & 1088.4314 & 2174.8715 & 1087.9394 & I & 747.4512 & 374.2292 & 730.4246 & 365.7160 & & & 6 \\
\hline
\end{tabular}




\begin{tabular}{|l|r|r|r|r|r|r|r|r|r|r|r|r|r|r|r|}
$\mathbf{1 9}$ & 2305.9661 & 1153.4867 & 2288.9396 & 1144.9734 & 2287.9556 & 1144.4814 & L & $\mathbf{6 3 4 . 3 6 7 1}$ & 317.6872 & 617.3406 & 309.1739 & & & $\mathbf{5}$ \\
\hline $\mathbf{2 0}$ & 2434.0247 & 1217.5160 & 2416.9982 & 1209.0027 & 2416.0142 & 1208.5107 & Q & $\mathbf{5 2 1 . 2 8 3 1}$ & 261.1452 & 504.2565 & 252.6319 & & & $\mathbf{4}$ \\
\hline $\mathbf{2 1}$ & 2505.0618 & 1253.0346 & 2488.0353 & 1244.5213 & 2487.0513 & 1244.0293 & A & 393.2245 & 197.1159 & 376.1979 & 188.6026 & & & $\mathbf{3}$ \\
\hline $\mathbf{2 2}$ & 2652.1303 & 1326.5688 & 2635.1037 & 1318.0555 & 2634.1197 & 1317.5635 & F & 322.1874 & 161.5973 & 305.1608 & 153.0840 & & & $\mathbf{2}$ \\
\hline $\mathbf{2 3}$ & & & & & & & R & 175.1190 & 88.0631 & 158.0924 & 79.5498 & & & $\mathbf{1}$ \\
\hline
\end{tabular}
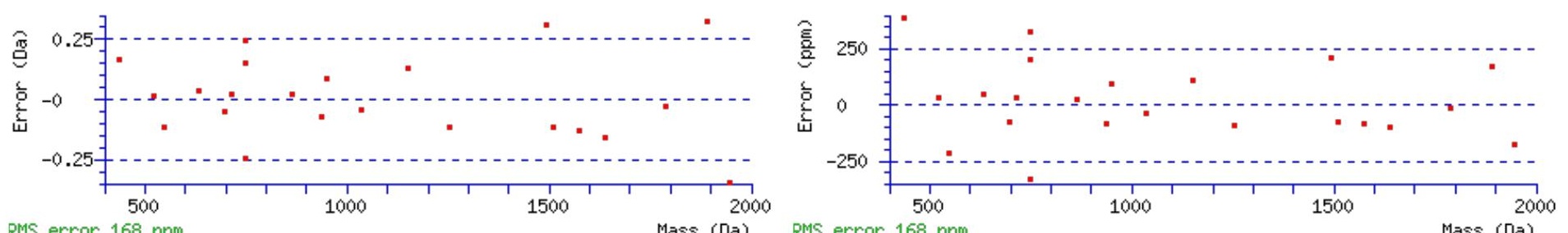

RMS error $168 \mathrm{ppm}$ Mass (Da) RMS error 168 ppm

\section{All matches to this query}

\begin{tabular}{|l|l|l|l|}
\hline Score & Mr(calc): & Delta & \multicolumn{1}{|c|}{ Sequence } \\
\hline 72.1 & 2825.2346 & 0.0133 & MCQLYPETFECVTNSSDILQAFR \\
\hline 19.9 & 2824.2506 & 0.9973 & MCQLYPETFECVTNSSDILQAFR \\
\hline 2.4 & 2823.2171 & 2.0309 & AQGNNYGQTSNGVAEESPNMLVYRK \\
\hline 2.4 & 2823.2171 & 2.0309 & AQGNNYGQTSNGVAEESPNMLVYRK \\
\hline 1.3 & 2825.2595 & -0.0116 & DSTMNVSQNLYELLPRTSPLKNK \\
\hline 1.3 & 2824.2660 & 0.9819 & QINDIOLSRDMTMFVTASKDNTAK \\
\hline 0.6 & 2825.2595 & -0.0116 & DSTMNVSQNLYELLPRTSPLKNK \\
\hline
\end{tabular}

Spectrum No: 336; Query: 2167; Rank: 1

\section{Peptide View}

MS/MS Fragmentation of GANSSSQNPLVYLDVGADGQPLGR

Found in IPI00201489, Tax_Id=10116 Gene_Symbol=Ppif Peptidyl-prolyl cis-trans isomerase, mitochondrial precursor

Match to Query 2167: 2415.164248 from(1208.589400,2+)

Title: 091008RatKidney_NH4Format01_12.4414.4414.2.dta

Data file K:INewmanPaper|Piliang\3SubProteomes\Piliang3SP\mgf5ppm\ERLIC_3SubProteomes5ppm.mgf

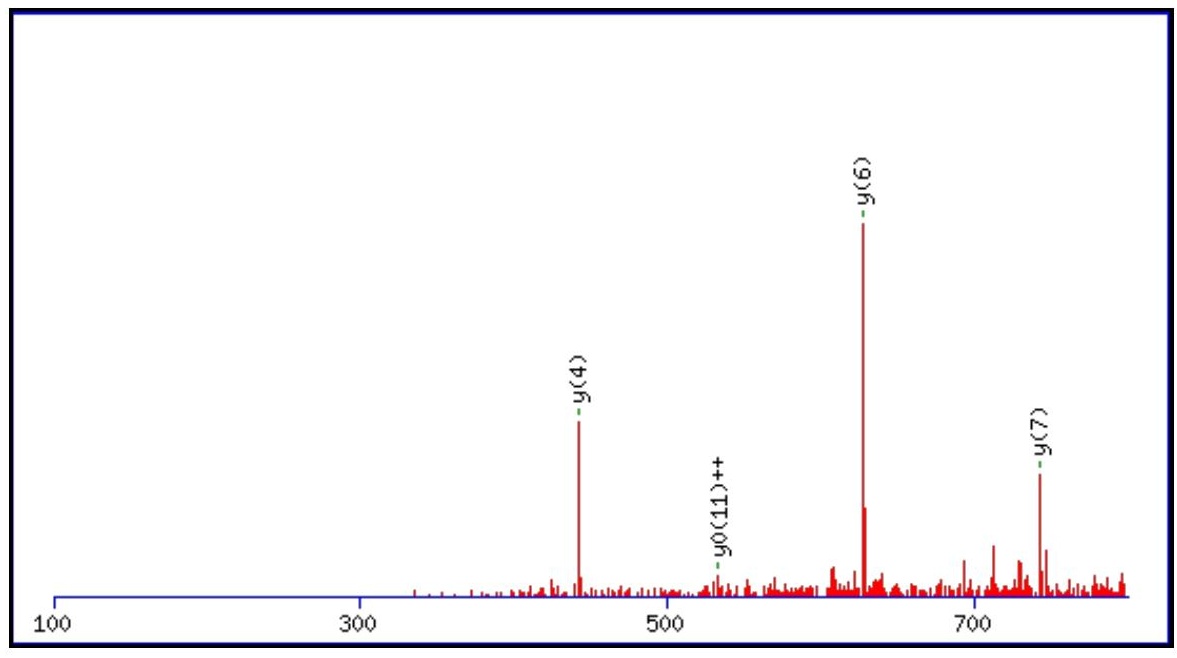



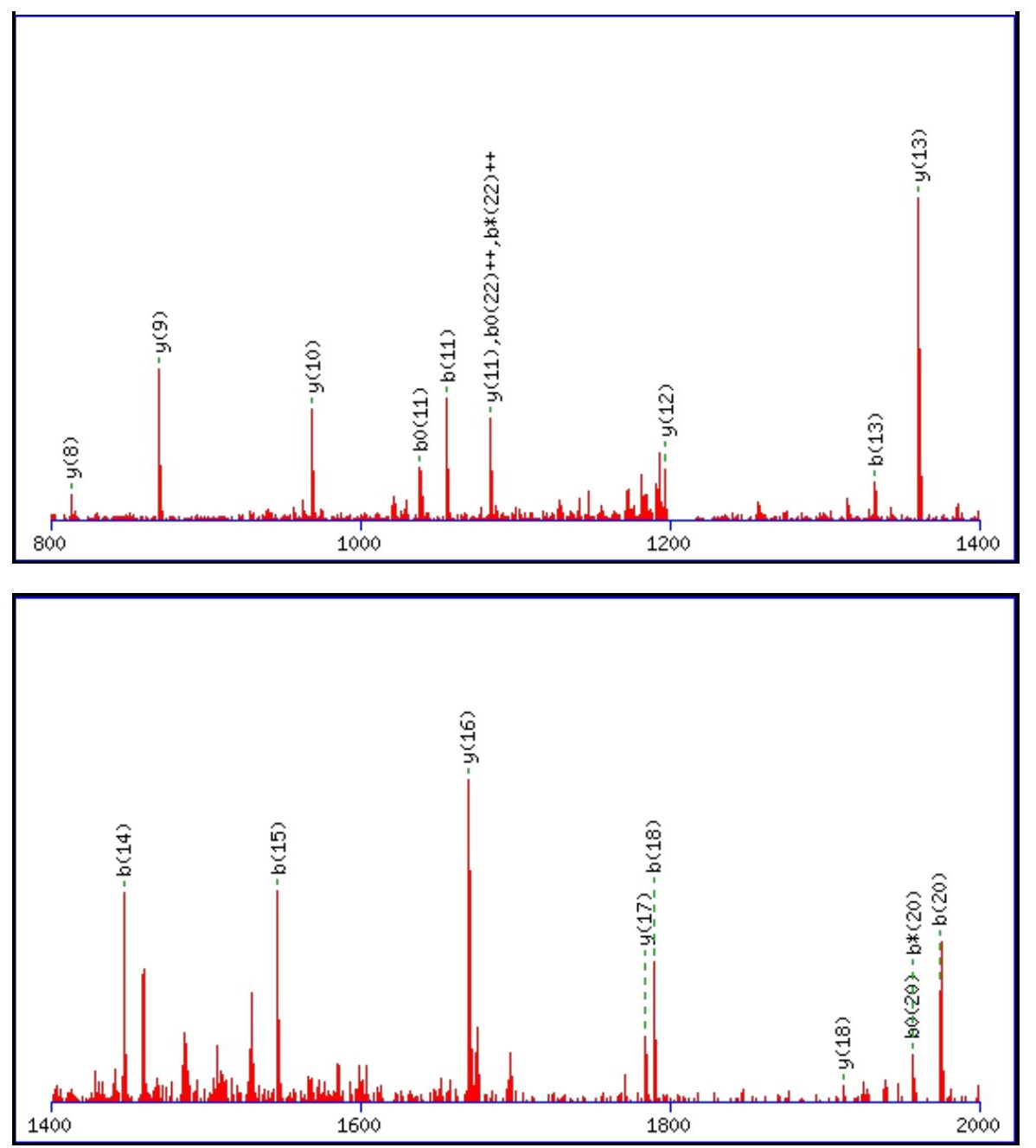

Monoisotopic mass of neutral peptide $\operatorname{Mr}($ calc): 2415.1666

Fixed modifications: Carbamidomethyl (C)

Variable modifications:

N3: Deamidated $\mathrm{N}(\mathrm{N})$

Ions Score: 72 Expect: $1.7 \mathrm{e}-005$

Matches (Bold Red): 24/254 fragment ions using 46 most intense peaks

\begin{tabular}{|c|c|c|c|c|c|c|c|c|c|c|c|c|c|c|}
\hline \# & b & $\mathbf{b}^{++}$ & $\mathbf{b}^{*}$ & $\mathbf{b}^{*^{++}}$ & $\mathbf{b}^{0}$ & $\mathbf{b}^{0++}$ & Seq. & $\mathbf{y}$ & $\mathrm{y}^{++}$ & $\mathbf{y}^{*}$ & $\mathbf{y}^{*^{++}}$ & $\mathbf{y}^{0}$ & $\mathbf{y}^{\mathbf{0 + +}}$ & \# \\
\hline 1 & 58.0287 & 29.5180 & & & & & G & & & & & & & 24 \\
\hline 2 & 129.0659 & 65.0366 & & & & & A & 2359.1524 & 1180.0799 & 2342.1259 & 1171.5666 & 2341.1419 & 1171.0746 & 23 \\
\hline 4 & 331.1248 & 166.0660 & 314.0983 & 157.5528 & 313.1143 & 157.0608 & S & 2173.0884 & 1087.0478 & 2156.0618 & 1078.5346 & 2155.0778 & 1078.0425 & 21 \\
\hline 5 & 418.1568 & 209.5821 & 401.1303 & 201.0688 & 400.1463 & 200.5768 & $\mathrm{~S}$ & 2086.0564 & 1043.5318 & 2069.0298 & 1035.0185 & 2068.0458 & 1034.5265 & 20 \\
\hline 6 & 505.1889 & 253.0981 & 488.1623 & 244.5848 & 487.1783 & 244.0928 & S & 1999.0243 & 1000.0158 & 1981.9978 & 991.5025 & 1981.0138 & 991.0105 & 19 \\
\hline 7 & 633.2475 & 317.1274 & 616.2209 & 308.6141 & 615.2369 & 308.1221 & $\mathbf{Q}$ & 1911.9923 & 956.4998 & 1894.9658 & 947.9865 & 1893.9817 & 947.4945 & 18 \\
\hline 8 & 747.2904 & 374.1488 & 730.2638 & 365.6356 & 729.2798 & 365.1435 & $\mathbf{N}$ & 1783.9337 & 892.4705 & 1766.9072 & 883.9572 & 1765.9232 & 883.4652 & 17 \\
\hline 9 & 844.3431 & 422.6752 & 827.3166 & 414.1619 & 826.3326 & 413.6699 & $\mathbf{P}$ & 1669.8908 & 835.4490 & 1652.8643 & 826.9358 & 1651.8802 & 826.4438 & 16 \\
\hline 10 & 957.4272 & 479.2172 & 940.4007 & 470.7040 & 939.4166 & 470.2120 & $\mathbf{L}$ & 1572.8380 & 786.9227 & 1555.8115 & 778.4094 & 1554.8275 & 777.9174 & 15 \\
\hline 11 & 1056.4956 & 528.7514 & 1039.4691 & 520.2382 & 1038.4851 & 519.7462 & V & 1459.7540 & 730.3806 & 1442.7274 & 721.8673 & 1441.7434 & 721.3753 & 14 \\
\hline 12 & 1219.5590 & 610.2831 & 1202.5324 & 601.7698 & 1201.5484 & 601.2778 & $\mathbf{Y}$ & 1360.6856 & 680.8464 & 1343.6590 & 672.3331 & 1342.6750 & 671.8411 & 13 \\
\hline 14 & 1447.6700 & 724.3386 & 1430.6434 & 715.8253 & 1429.6594 & 715.3333 & D & 1084.5382 & 542.7727 & 1067.5116 & 534.2594 & 1066.5276 & 533.7674 & 11 \\
\hline 15 & 1546.7384 & 773.8728 & 1529.7118 & 765.3595 & 1528.7278 & 764.8675 & V & 969.5112 & 485.2592 & 952.4847 & 476.7460 & 951.5007 & 476.2540 & 10 \\
\hline 16 & 1603.7598 & 802.3836 & 1586.7333 & 793.8703 & 1585.7493 & 793.3783 & $\mathbf{G}$ & 870.4428 & 435.7250 & 853.4163 & 427.2118 & 852.4322 & 426.7198 & 9 \\
\hline 17 & 1674.7969 & 837.9021 & 1657.7704 & 829.3888 & 1656.7864 & 828.8968 & A & 813.4213 & 407.2143 & 796.3948 & 398.7010 & 795.4108 & 398.2090 & 8 \\
\hline 18 & 1789.8239 & 895.4156 & 1772.7973 & 886.9023 & 1771.8133 & 886.4103 & D & 742.3842 & 371.6958 & 725.3577 & 363.1825 & 724.3737 & 362.6905 & 7 \\
\hline
\end{tabular}




\begin{tabular}{|l|r|r|r|r|r|r|r|r|r|r|r|r|r|r|r|}
$\mathbf{1 9}$ & $\mathbf{1 8 4 6 . 8 4 5 4}$ & 923.9263 & 1829.8188 & 915.4130 & 1828.8348 & 914.9210 & G & $\mathbf{6 2 7 . 3 5 7 3}$ & 314.1823 & 610.3307 & 305.6690 & & & $\mathbf{6}$ \\
\hline $\mathbf{2 0}$ & $\mathbf{1 9 7 4 . 9 0 3 9}$ & 987.9556 & $\mathbf{1 9 5 7 . 8 7 7 4}$ & 979.4423 & $\mathbf{1 9 5 6 . 8 9 3 4}$ & $\mathbf{9 7 8 . 9 5 0 3}$ & $\mathbf{Q}$ & 570.3358 & 285.6715 & 553.3093 & 277.1583 & & & $\mathbf{5}$ \\
\hline $\mathbf{2 1}$ & 2071.9567 & 1036.4820 & 2054.9301 & 1027.9687 & 2053.9461 & 1027.4767 & $\mathbf{P}$ & $\mathbf{4 4 2 . 2 7 7 2}$ & 221.6423 & 425.2507 & 213.1290 & & & $\mathbf{4}$ \\
\hline $\mathbf{2 2}$ & 2185.0408 & 1093.0240 & 2168.0142 & $\mathbf{1 0 8 4 . 5 1 0 7}$ & 2167.0302 & $\mathbf{1 0 8 4 . 0 1 8 7}$ & L & 345.2245 & 173.1159 & 328.1979 & 164.6026 & & & $\mathbf{3}$ \\
\hline $\mathbf{2 3}$ & 2242.0622 & 1121.5348 & 2225.0357 & 1113.0215 & 2224.0517 & 1112.5295 & G & 232.1404 & 116.5738 & 215.1139 & 108.0606 & & & $\mathbf{2}$ \\
\hline $\mathbf{2 4}$ & & & & & & & R & 175.1190 & 88.0631 & 158.0924 & $\mathbf{7 9 . 5 4 9 8}$ & & & $\mathbf{1}$ \\
\hline
\end{tabular}
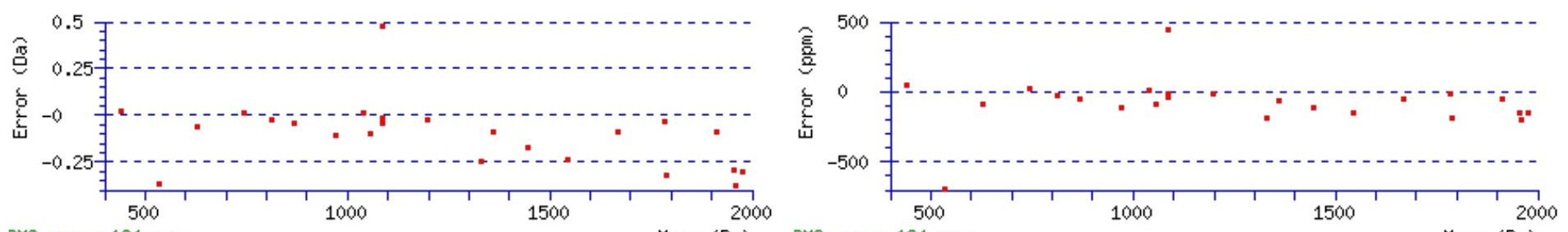

RMS error 194 ppm

Mass (Da) RMS error 194 ppm

Mass (Da)

\section{All matches to this query}

\begin{tabular}{|l|c|c|l|}
\hline Score & Mr(calc): & Delta & \multicolumn{1}{c|}{ Sequence } \\
\hline 71.9 & 2415.1666 & -0.0024 & GANSSSONPLVYLDVGADGQPLGR \\
\hline 57.1 & 2415.1666 & -0.0024 & GANSSSQNPLVYLDVGADGQPLGR \\
\hline 56.1 & 2414.1826 & 0.9816 & GANSSSONPLVYLDVGADGQPLGR \\
\hline 1.8 & 2413.1752 & 1.9890 & NWWASLEVSSLNSLVQMLKK \\
\hline 1.6 & 2415.1570 & 0.0073 & IAEQVASFQEEKSKLDAEVSK \\
\hline 1.4 & 2414.1592 & 1.0050 & NWWASLEVSSLNSLVQMLKK \\
\hline 0.3 & 2415.1471 & 0.0171 & ETPNPWKETLGPNKIPSGQNK \\
\hline 0.0 & 2415.1835 & -0.0193 & YLTSNVAYGSTGIRDVHLELK \\
\hline
\end{tabular}

Spectrum No: 337; Query: 236; Rank: 1

\section{Peptide View}

MS/MS Fragmentation of STNSSLTLLFK

Found in IPI00196620, Tax_Id=10116 Gene_Symbol=Cubn Cubilin precursor

Match to Query 236: 1210.645608 from(606.330080,2+)

Title: 091008RatKidney_NoSalt_16.3521.3521.2.dta

Data file K:INewmanPaper|Piliang\3SubProteomes\Piliang3SP\mgf5ppm\ERLIC_3SubProteomes5ppm.mgf

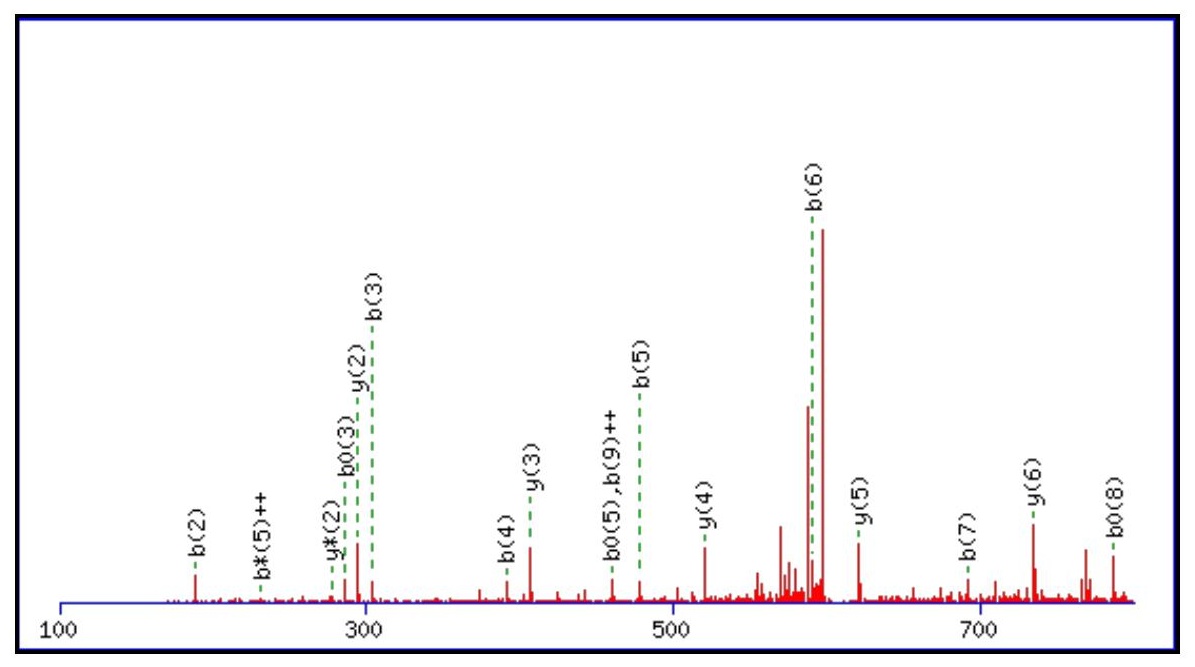



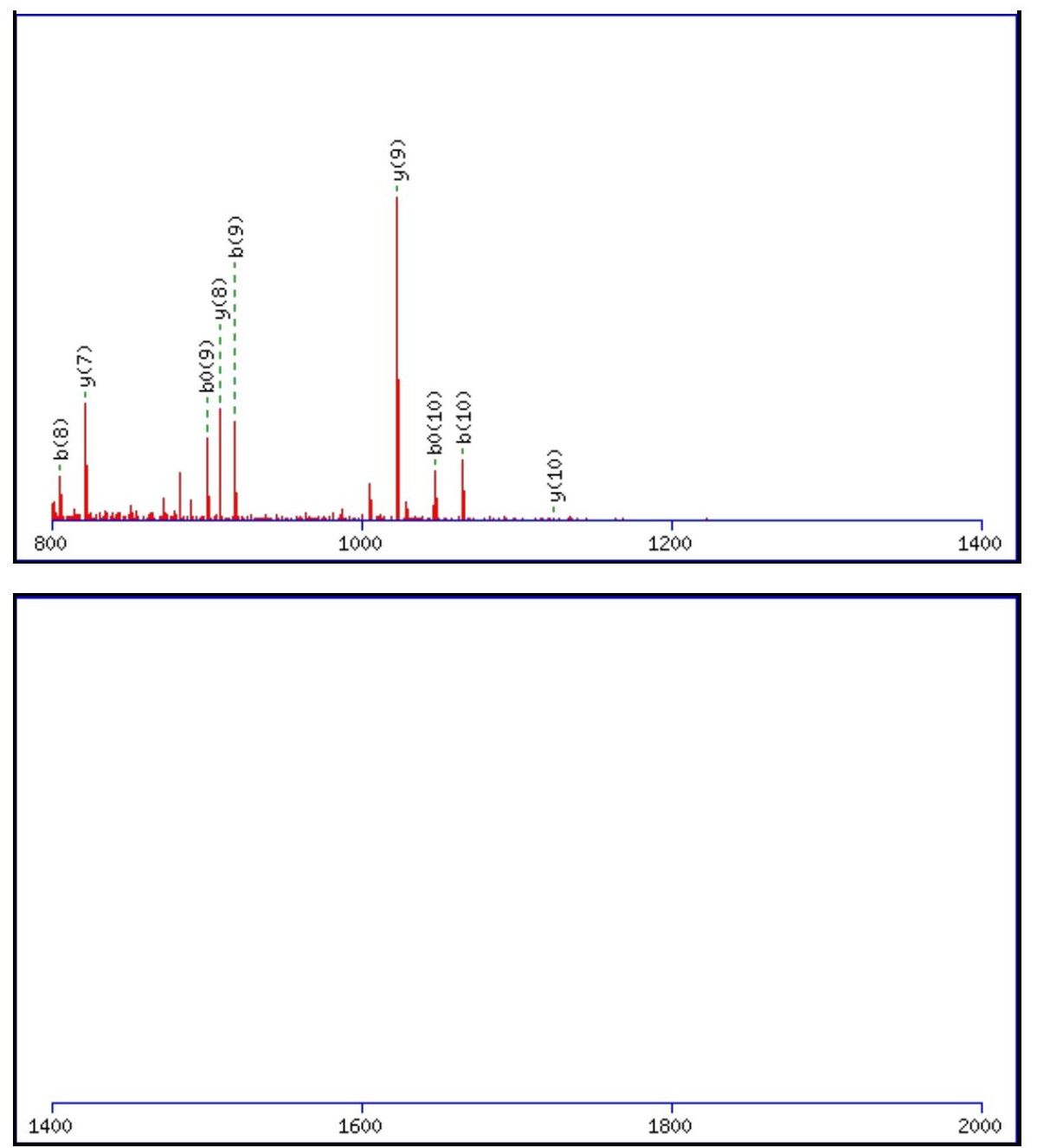

Monoisotopic mass of neutral peptide $\operatorname{Mr}($ calc): 1210.6445

Fixed modifications: Carbamidomethyl (C)

Variable modifications:

N3 : Deamidated N $(\mathrm{N})$

Ions Score: 72 Expect: $5.5 \mathrm{e}-006$

Matches (Bold Red): 26/108 fragment ions using 43 most intense peaks

\begin{tabular}{|c|c|c|c|c|c|c|c|c|c|c|c|c|c|c|}
\hline \# & b & $\mathbf{b}^{++}$ & $\mathbf{b}^{*}$ & $\mathbf{b}^{*^{++}}$ & $\mathbf{b}^{\mathbf{0}}$ & $\mathbf{b}^{0++}$ & Seq. & $\mathbf{y}$ & $\mathbf{y}^{++}$ & $\mathbf{y}^{*}$ & $\mathbf{y}^{*^{++}}$ & $\mathbf{y}^{\mathbf{0}}$ & $\mathbf{y}^{\mathbf{0}^{++}}$ & \# \\
\hline 1 & 88.0393 & 44.5233 & & & 70.0287 & 35.5180 & S & & & & & & & 11 \\
\hline 2 & 189.0870 & 95.0471 & & & 171.0764 & 86.0418 & $T$ & 1124.6198 & 562.8135 & 1107.5932 & 554.3002 & 1106.6092 & 553.8082 & 10 \\
\hline 3 & 304.1139 & 152.5606 & 287.0874 & 144.0473 & 286.1034 & 143.5553 & $\mathbf{N}$ & 1023.5721 & 512.2897 & 1006.5455 & 503.7764 & 1005.5615 & 503.2844 & 9 \\
\hline 4 & 391.1459 & 196.0766 & 374.1194 & 187.5633 & 373.1354 & 187.0713 & S & 908.5451 & 454.7762 & 891.5186 & 446.2629 & 890.5346 & 445.7709 & 8 \\
\hline 5 & 478.1780 & 239.5926 & 461.1514 & 231.0794 & 460.1674 & 230.5873 & S & 821.5131 & 411.2602 & 804.4866 & 402.7469 & 803.5026 & 402.2549 & 7 \\
\hline 6 & 591.2620 & 296.1347 & 574.2355 & 287.6214 & 573.2515 & 287.1294 & $\mathbf{L}$ & 734.4811 & 367.7442 & 717.4545 & 359.2309 & 716.4705 & 358.7389 & 6 \\
\hline 7 & 692.3097 & 346.6585 & 675.2832 & 338.1452 & 674.2992 & 337.6532 & $\mathbf{T}$ & 621.3970 & 311.2022 & 604.3705 & 302.6889 & 603.3865 & 302.1969 & 5 \\
\hline 8 & 805.3938 & 403.2005 & 788.3672 & 394.6873 & 787.3832 & 394.1952 & $\mathbf{L}$ & 520.3493 & 260.6783 & 503.3228 & 252.1650 & & & 4 \\
\hline 9 & 918.4778 & 459.7426 & 901.4513 & 451.2293 & 900.4673 & 450.7373 & L & 407.2653 & 204.1363 & 390.2387 & 195.6230 & & & 3 \\
\hline 10 & \begin{tabular}{|l|}
1065.5463 \\
\end{tabular} & 533.2768 & 1048.5197 & 524.7635 & 1047.5357 & 524.2715 & $\mathbf{F}$ & 294.1812 & 147.5942 & 277.1547 & 139.0810 & & & 2 \\
\hline 11 & & & & & & & $\mathbf{K}$ & 147.1128 & 74.0600 & 130.0863 & 65.5468 & & & 1 \\
\hline
\end{tabular}
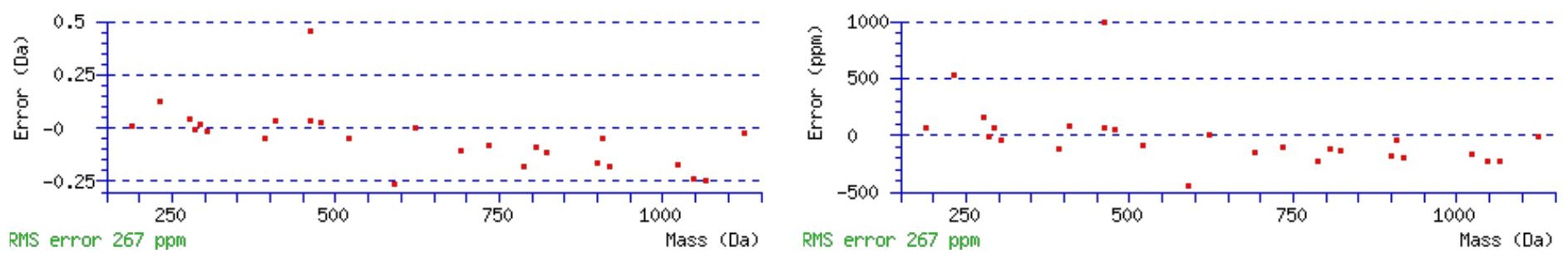


\section{All matches to this query}

\begin{tabular}{|l|l|l|l|}
\hline Score & Mr(calc): & Delta & \multicolumn{1}{|c|}{ Sequence } \\
\hline 71.8 & 1210.6445 & 0.0011 & STNSSLTLLFK \\
\hline 10.6 & 1210.6492 & -0.0036 & FNSRTKAIMK \\
\hline 8.9 & 1210.6532 & -0.0076 & QCVAFKALFK \\
\hline 7.5 & 1210.6346 & 0.0110 & RFSELGFEVK \\
\hline 7.3 & 1210.6363 & 0.0093 & ITGAPAIIFTK \\
\hline 6.3 & 1209.6370 & 1.0086 & SLLKTKPSEK \\
\hline 5.8 & 1210.6557 & -0.0101 & TSVVLNHLAEK \\
\hline 5.8 & 1209.6394 & 1.0062 & KEPSIFSAGFK \\
\hline 4.3 & 1210.6458 & -0.0002 & EHRVGIPEFK \\
\hline 3.8 & 1210.6454 & 0.0003 & YLVLANMLMK \\
\hline
\end{tabular}

Spectrum No: 338; Query: 1695; Rank: 1

\section{Peptide View}

MS/MS Fragmentation of EYQLNDSASYYLNDLDR

Found in IPI00231726, Tax_Id=10116 Gene_Symbol=Gnai3 Guanine nucleotide-binding protein G

Match to Query 1695: 2079.903188 from(1040.958870,2+)

Title: 091008RatKidney_NoSalt_21.4116.4116.2.dta

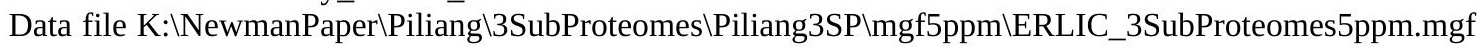
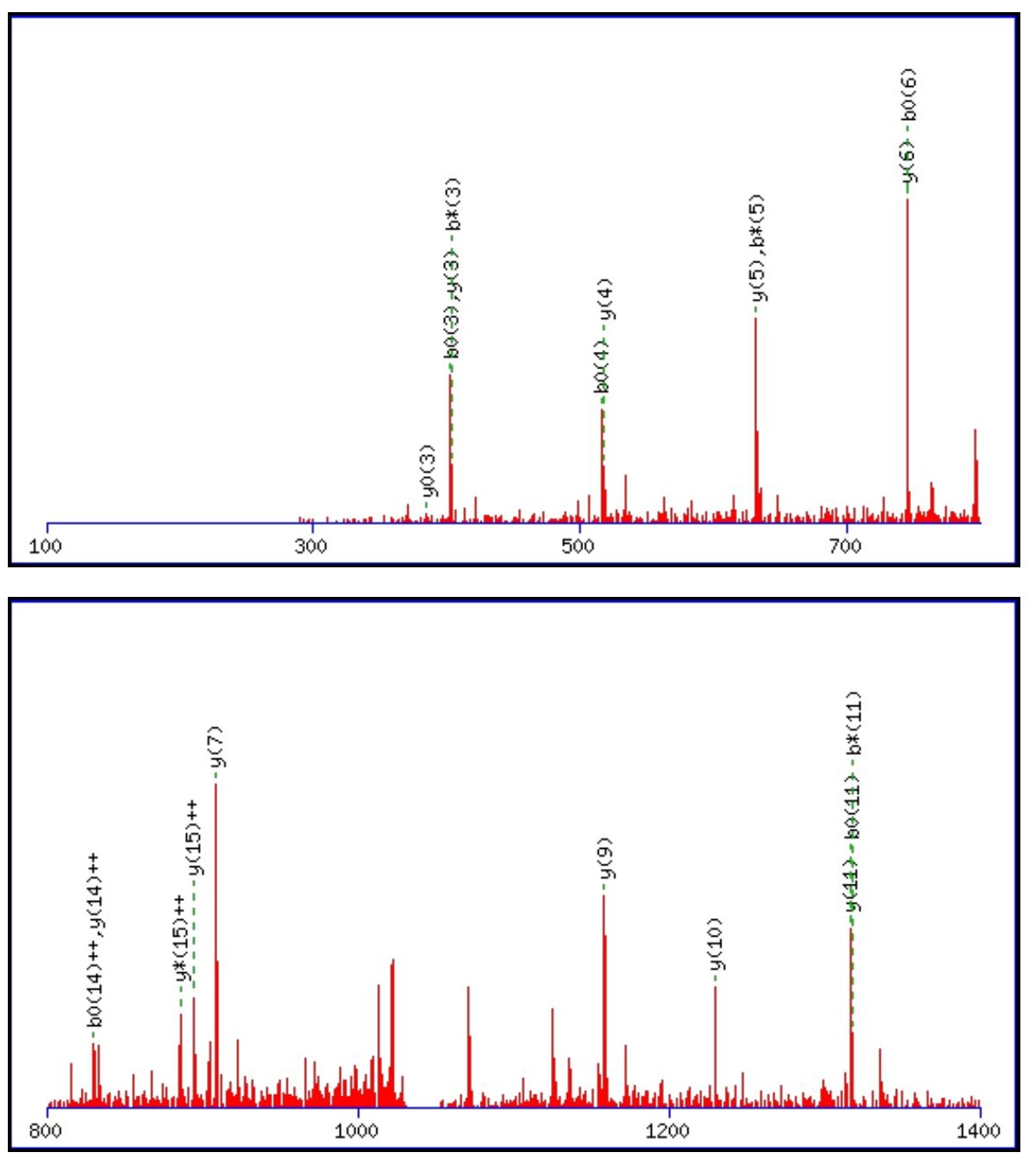


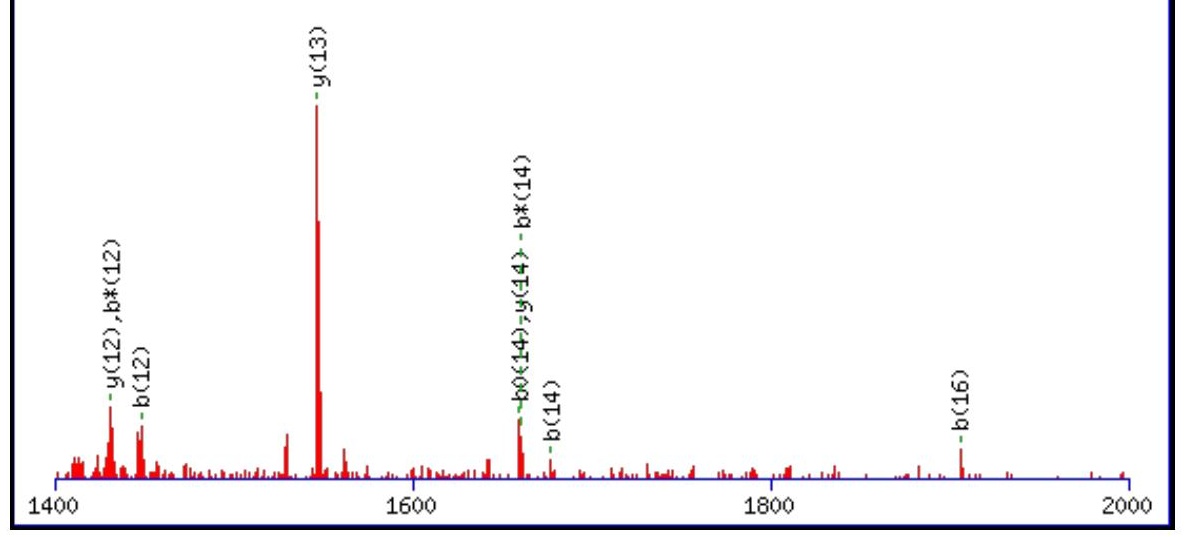

Monoisotopic mass of neutral peptide $\operatorname{Mr}($ calc): 2078.9068

Fixed modifications: Carbamidomethyl (C)

Variable modifications:

N5 : Deamidated_N (N)

Ions Score: 72 Expect: $1.6 \mathrm{e}-005$

Matches (Bold Red): 29/186 fragment ions using 42 most intense peaks

\begin{tabular}{|c|c|c|c|c|c|c|c|c|c|c|c|c|c|c|}
\hline \# & b & $\mathbf{b}^{++}$ & $\mathbf{b}^{*}$ & $\mathbf{b}^{*++}$ & $\mathbf{b}^{0}$ & $\mathbf{b}^{0++}$ & Seq. & $\mathbf{y}$ & $y^{++}$ & $\mathbf{y}^{*}$ & $\mathrm{y}^{*++}$ & $\mathbf{y}^{\mathbf{0}}$ & $y^{0++}$ & $\#$ \\
\hline 1 & 130.0499 & 65.5286 & & & 112.0393 & 56.5233 & $\mathbf{E}$ & & & & & & & 17 \\
\hline 2 & 293.1132 & 147.0602 & & & 275.1026 & 138.0550 & $\mathbf{Y}$ & 1950.8716 & 975.9394 & 1933.8450 & 967.4261 & 1932.8610 & 966.9341 & 16 \\
\hline 3 & 421.1718 & 211.0895 & 404.1452 & 202.5763 & 403.1612 & 202.0842 & $\mathbf{Q}$ & 1787.8082 & 894.4078 & 1770.7817 & 885.8945 & 1769.7977 & 885.4025 & 15 \\
\hline 4 & 534.2558 & 267.6316 & 517.2293 & 259.1183 & 516.2453 & 258.6263 & $\mathbf{L}$ & 1659.7497 & 830.3785 & 1642.7231 & 821.8652 & 1641.7391 & 821.3732 & 14 \\
\hline 5 & 649.2828 & 325.1450 & 632.2562 & 316.6318 & 631.2722 & 316.1397 & $\mathbf{N}$ & 1546.6656 & 773.8364 & 1529.6391 & 765.3232 & 1528.6550 & 764.8312 & 13 \\
\hline 6 & 764.3097 & 382.6585 & 747.2832 & 374.1452 & 746.2992 & 373.6532 & D & \begin{tabular}{|l|}
1431.6387 \\
\end{tabular} & 716.3230 & 1414.6121 & \begin{tabular}{|l|}
707.8097 \\
\end{tabular} & 1413.6281 & 707.3177 & 12 \\
\hline 7 & 851.3417 & 426.1745 & 834.3152 & 417.6612 & 833.3312 & 417.1692 & $\mathrm{~S}$ & 1316.6117 & 658.8095 & 1299.5852 & 650.2962 & 1298.6012 & 649.8042 & 11 \\
\hline 8 & 922 & & & & & & A & & & & 606.7802 & 691 & & 10 \\
\hline 9 & 1009.4109 & 505.2091 & 992.3843 & 496.6958 & 991.4003 & 496.2038 & $S$ & 1158.5426 & 579.7749 & 1141.5160 & \begin{tabular}{|l|}
571.2617 \\
\end{tabular} & 1140.5320 & 570.7696 & 9 \\
\hline 10 & 1172.4742 & 586.7407 & 1155.4477 & 578.2275 & 1154.4637 & 577.7355 & $\mathbf{Y}$ & 1071.5106 & 536.2589 & 1054.4840 & 527.7456 & 1053.5000 & 527.2536 & 8 \\
\hline 11 & 1335.5375 & 668.2724 & 1318.5110 & 659.7591 & 1317.5270 & 659.2671 & $\mathbf{Y}$ & 908.4472 & 454.7272 & 891.4207 & 446.2140 & 890.4367 & 445.7220 & 7 \\
\hline 12 & 1448.6216 & 724.8144 & 1431.5951 & 716.3012 & 1430.6110 & 715.8092 & $\mathbf{L}$ & 745.3839 & 373.1956 & 728.3573 & 364.6823 & 727.3733 & 364.1903 & 6 \\
\hline 131 & 1562.6645 & 781.8359 & 1545.6380 & 773.3226 & 1544.6540 & 772.8306 & $\mathbf{N}$ & 632.2998 & 316.6536 & 615.2733 & 308.1403 & 614.2893 & 307.6483 & 5 \\
\hline 141 & 1677.6915 & 839.3494 & 1660.6649 & 830.8361 & \begin{tabular}{|l|}
1659.6809 \\
\end{tabular} & 830.3441 & D & 518.2569 & 259.6321 & 501.2304 & 251.1188 & 500.2463 & 250.6268 & 4 \\
\hline 151 & 1790.7755 & 895.8914 & 1773.7490 & 887.3781 & 1772.7650 & 886.8861 & $\mathbf{L}$ & 403.2300 & 202.1186 & 386.2034 & 193.6053 & 385.2194 & 193.1133 & 3 \\
\hline 16 & 1905.8025 & 953.4049 & 1888.7759 & 944.8916 & 1887.7919 & 944.3996 & D & 290.1459 & 145.5766 & 273.1193 & 137.0633 & 272.1353 & 136.5713 & 2 \\
\hline 17 & & & & & & & $\mathbf{R}$ & 175.1190 & 88.0631 & 158.0924 & 79.5498 & & & 1 \\
\hline
\end{tabular}

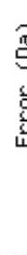
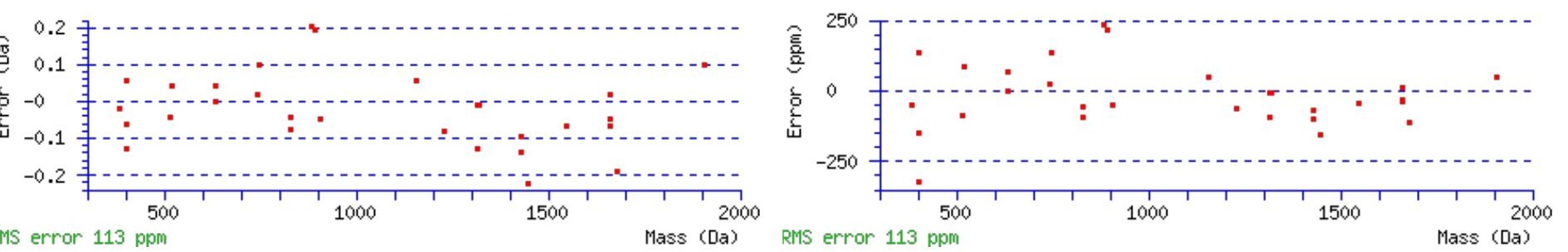

\section{All matches to this query}

\begin{tabular}{|l|l|l|l|}
\hline Score & Mr(calc): & Delta & \multicolumn{1}{c|}{ Sequence } \\
\hline 71.7 & 2078.9068 & 0.9963 & EYQLNDSASYYLNDLDR \\
\hline 56.5 & 2079.8909 & 0.0123 & EYQLNDSASYYLNDLDR \\
\hline 50.3 & 2078.9068 & 0.9963 & EYQLNDSASYYLNDLDR \\
\hline 3.6 & 2077.9116 & 1.9916 & EYQLNDSAAYYLNDLER \\
\hline
\end{tabular}




\begin{tabular}{|l|l|l|l|}
3.6 & 2078.9204 & 0.9828 & MEEQNNSAGKELQHKTR \\
\hline 2.6 & 2077.8782 & 2.0250 & KYNISWELGNEPNNYR \\
\hline 1.6 & 2079.8915 & 0.0117 & DSPNQSLLTGYIQTDLR \\
\hline 1.5 & 2079.8915 & 0.0117 & DSPNQSLLTGYIQTDLR \\
\hline 1.5 & 2079.8915 & 0.0117 & DSPNQSLLTGYIQTDLR \\
\hline
\end{tabular}

Spectrum No: 339; Query: 684; Rank: 1

\section{Peptide View}

MS/MS Fragmentation of TIVYLNGSAQECR

Found in IPI00204348, Tax_Id=10116 Gene_Symbol=Nt5e 5'-nucleotidase precursor

Match to Query 684: 1510.711828 from(756.363190,2+)

Title: 100101RatKid_NS_deglyco_15.2067.2067.2.dta

Data file K:INewmanPaper|Piliangl3SubProteomes\Piliang3SP\mgf5ppm\ERLIC_3SubProteomes5ppm.mgf
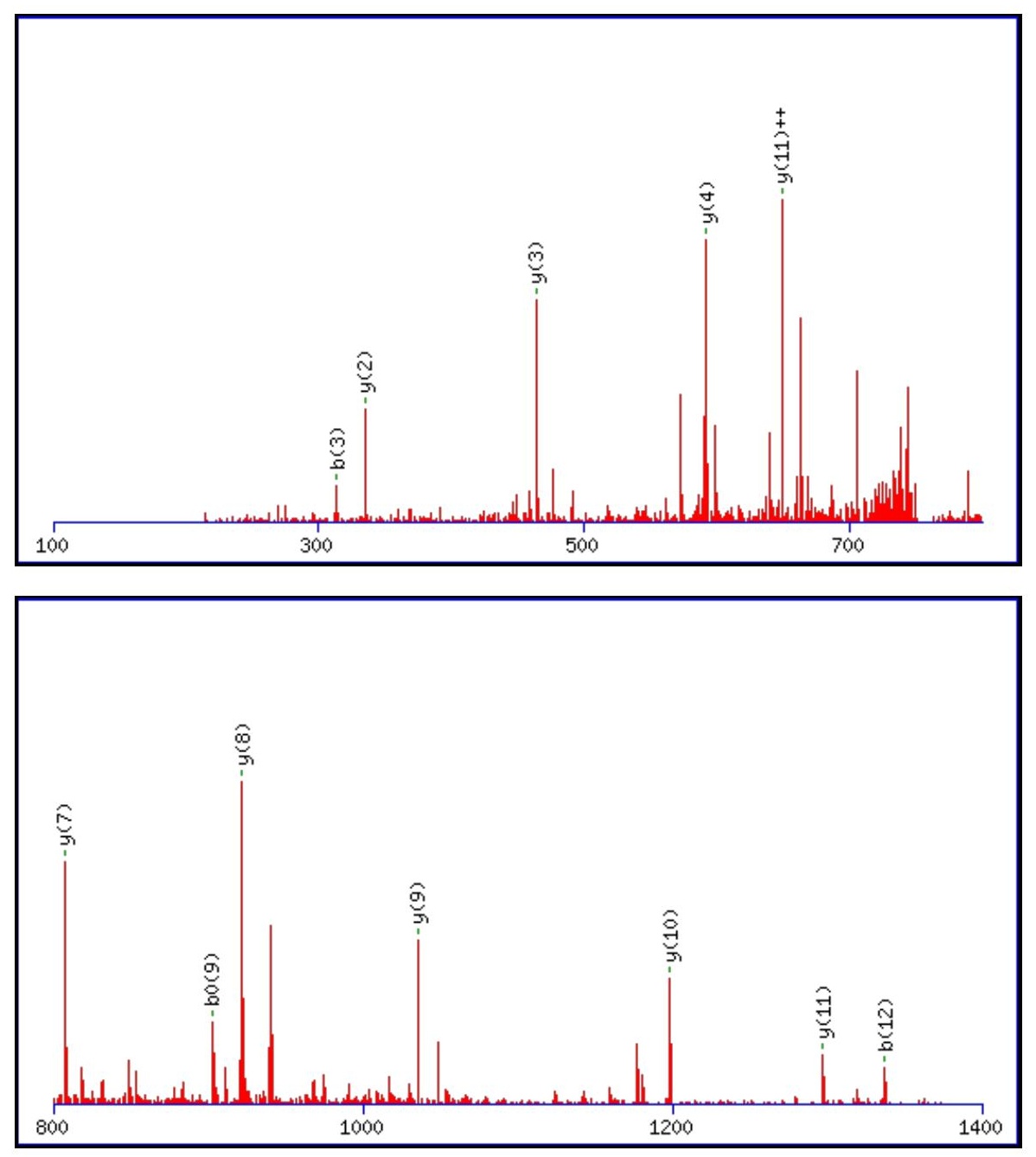


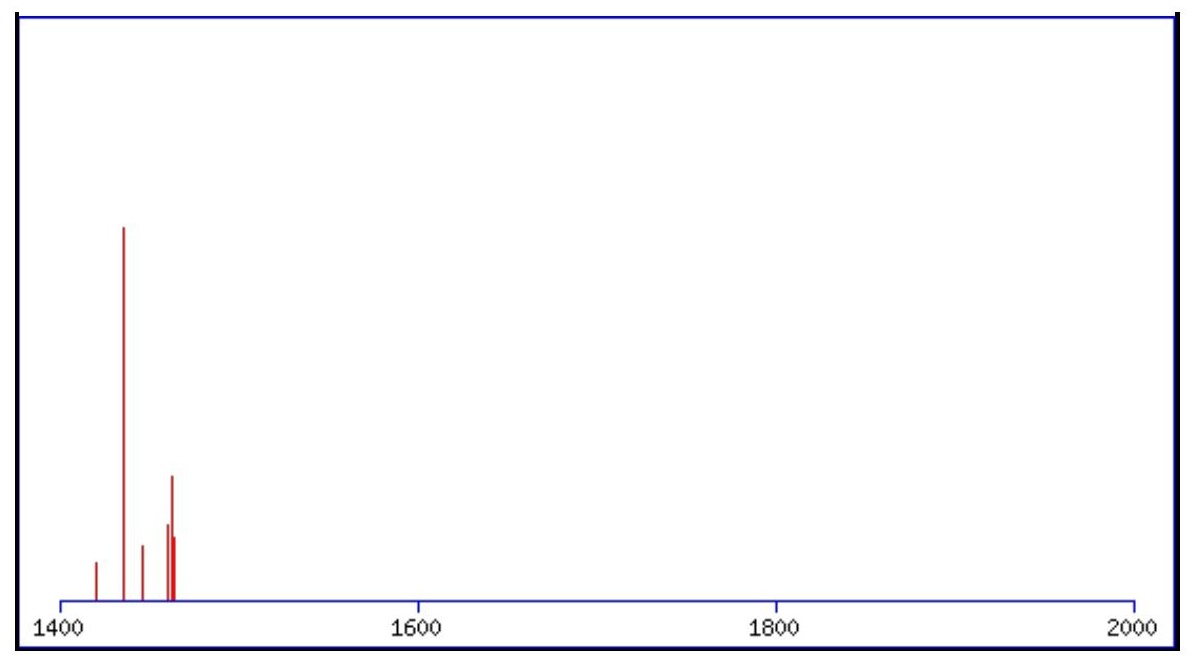

Monoisotopic mass of neutral peptide $\operatorname{Mr}($ calc): 1510.7086

Fixed modifications: Carbamidomethyl (C)

Variable modifications:

N6 : Deamidated $\mathrm{N}(\mathrm{N})$

Ions Score: 72 Expect: $1.2 \mathrm{e}-005$

Matches (Bold Red): 12/130 fragment ions using 17 most intense peaks

\begin{tabular}{|c|c|c|c|c|c|c|c|c|c|c|c|c|c|c|}
\hline \# & b & $\mathbf{b}^{++}$ & $\mathbf{b}^{*}$ & $\mathbf{b}^{*++}$ & $\mathbf{b}^{0}$ & $\mathbf{b}^{0++}$ & Seq. & $\mathbf{y}$ & $y^{++}$ & $\mathbf{y}^{*}$ & $\mathbf{y}^{*^{++}}$ & $\mathbf{y}^{\mathbf{0}}$ & $\mathbf{y}^{0++}$ & \# \\
\hline 1 & 102.0550 & 51.5311 & & & 84.0444 & 42.5258 & $\mathbf{T}$ & & & & & & & 13 \\
\hline 2 & 215.1390 & 108.0731 & & & 197.1285 & 99.0679 & I & 1410.6682 & 705.8377 & 1393.6416 & 697.3245 & 1392.6576 & 696.8324 & 12 \\
\hline 3 & 314.2074 & 157.6074 & & & 296.1969 & 148.6021 & V & 1297.5841 & 649.2957 & 1280.5576 & 640.7824 & 1279.5736 & 640.2904 & 11 \\
\hline 4 & 477.2708 & 239.1390 & & & 459.2602 & 230.1337 & $\mathbf{Y}$ & 1198.5157 & 599.7615 & 1181.4892 & 591.2482 & 1180.5051 & 590.7562 & 10 \\
\hline 5 & 590.3548 & 295.6811 & & & 572.3443 & 286.6758 & $\mathbf{L}$ & 1035.4524 & 518.2298 & 1018.4258 & 509.7166 & 1017.4418 & 509.2245 & 9 \\
\hline 6 & 705.3818 & 353.1945 & 688.3552 & 344.6812 & 687.3712 & 344.1892 & $\mathbf{N}$ & 922.3683 & 461.6878 & 905.3418 & 453.1745 & 904.3577 & 452.6825 & 8 \\
\hline 7 & 762.4032 & 381.7053 & 745.3767 & 373.1920 & 744.3927 & 372.7000 & G & 807.3414 & 404.1743 & 790.3148 & 395.6611 & 789.3308 & 395.1690 & 7 \\
\hline 8 & 849.4353 & 425.2213 & 832.4087 & 416.7080 & 831.4247 & 416.2160 & S & 750.3199 & 375.6636 & 733.2934 & 367.1503 & 732.3093 & 366.6583 & 6 \\
\hline 9 & 920.4724 & 460.7398 & 903.4458 & 452.2265 & 902.4618 & 451.7345 & A & 663.2879 & 332.1476 & 646.2613 & 323.6343 & 645.2773 & 323.1423 & 5 \\
\hline 10 & 1048.5309 & 524.7691 & 1031.5044 & 516.2558 & 1030.5204 & 515.7638 & $\mathbf{Q}$ & 592.2508 & 296.6290 & 575.2242 & 288.1157 & 574.2402 & 287.6237 & 4 \\
\hline 11 & 1177.5735 & 589.2904 & 1160.5470 & 580.7771 & 1159.5630 & 580.2851 & $\mathbf{E}$ & 464.1922 & 232.5997 & 447.1656 & 224.0865 & 446.1816 & 223.5945 & 3 \\
\hline 12 & 1337.6042 & 669.3057 & 1320.5776 & 660.7925 & 1319.5936 & 660.3004 & C & 335.1496 & 168.0784 & 318.1231 & 159.5652 & & & 2 \\
\hline 13 & & & & & & & $\mathbf{R}$ & 175.1190 & 88.0631 & 158.0924 & 79.5498 & & & 1 \\
\hline
\end{tabular}

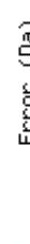
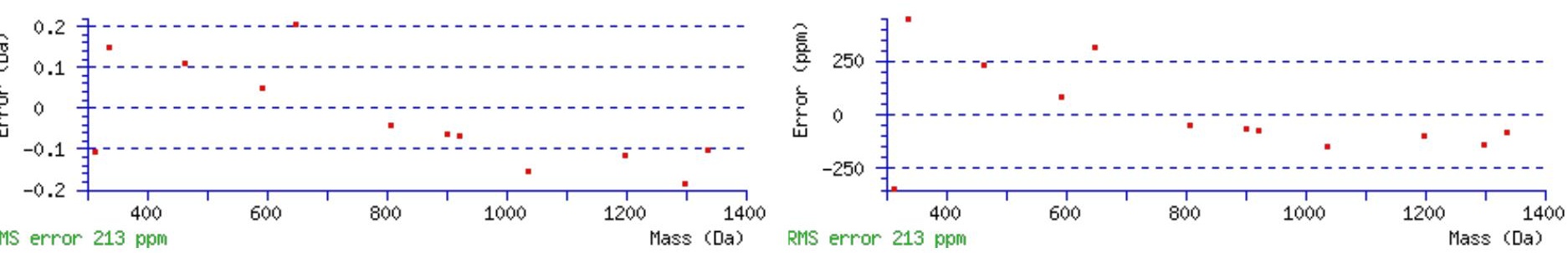

\section{All matches to this query}

\begin{tabular}{|l|l|c|c|}
\hline Score & Mr(calc): & Delta & Sequence \\
\hline 71.6 & 1510.7086 & 0.0033 & TIVYLNGSAQECR \\
\hline 1.6 & 1510.7001 & 0.0117 & ASRLQTDSQGRGR \\
\hline
\end{tabular}

Spectrum No: 340; Query: 2095; Rank: 1

\section{Peptide View}




\section{MS/MS Fragmentation of EIDRDQLMTVLANVTHLLIR}

Found in IPI00363534, Tax_Id=10116 Gene_Symbol=Lama1 laminin, alpha 1

Match to Query 2095: 2350.270992 from(784.430940,3+)

Title: 100101RatKid_NS_deglyco_10.7536.7536.3.dta

Data file K:\NewmanPaper\Piliang \3SubProteomes \Piliang3SP \mgf5ppm\ERLIC_3SubProteomes5ppm.mgf
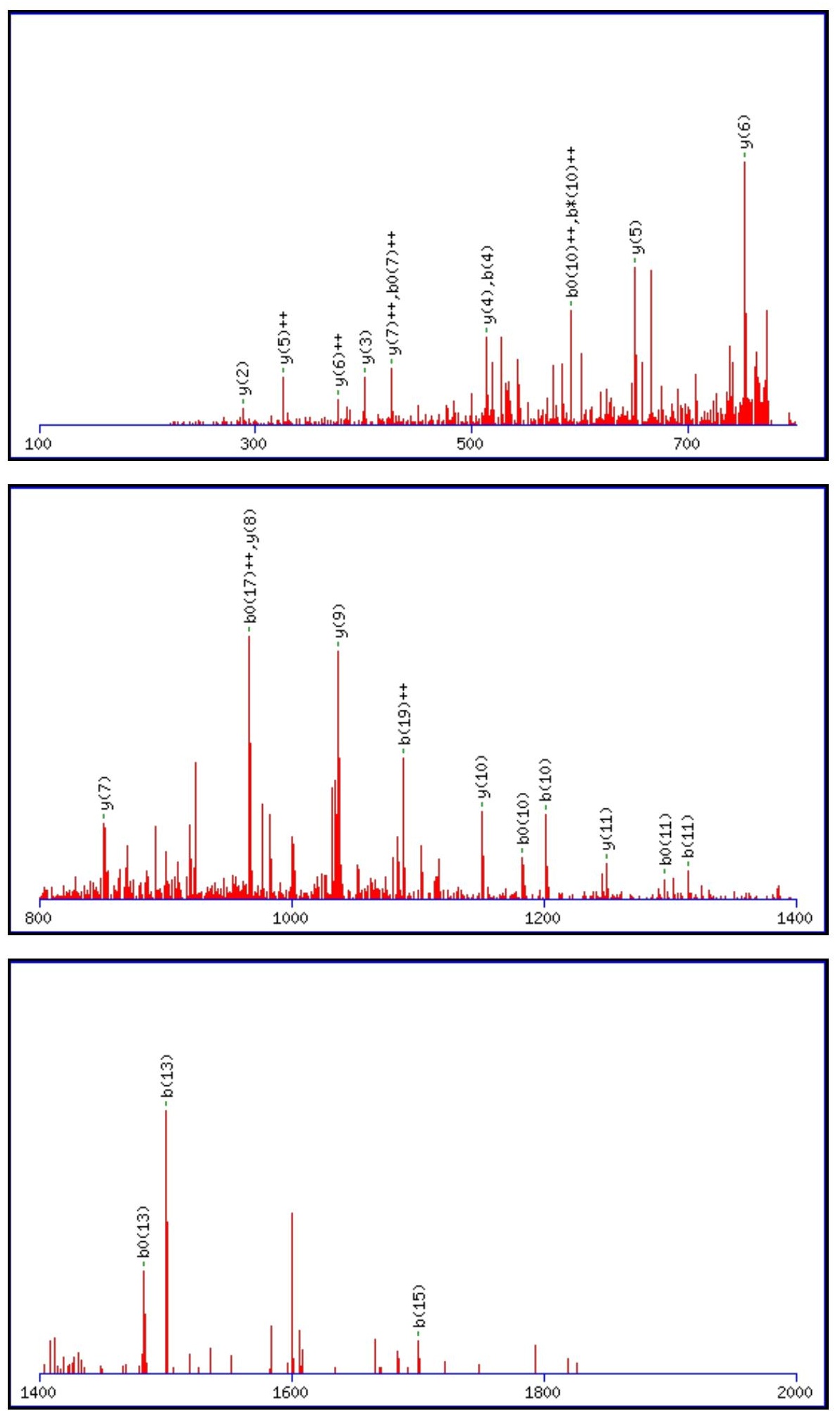

Monoisotopic mass of neutral peptide $\operatorname{Mr}($ calc): 2350.2678

Fixed modifications: Carbamidomethyl (C)

Variable modifications:

N13 : Deamidated_N (N)

Ions Score: 72 Expect: $4.2 \mathrm{e}-006$

Matches (Bold Red): 26/212 fragment ions using 37 most intense peaks

\begin{tabular}{|c|c|c|c|c|c|c|c|c|c|c|c|c|c|c|}
\hline \# & $\mathbf{b}$ & $\mathbf{b}^{++}$ & $\mathbf{b}^{*}$ & $\mathbf{b}^{*++}$ & $\mathbf{b}^{0}$ & $\mathbf{b}^{0++}$ & Seq. & $\mathbf{y}$ & $\mathbf{y}^{++}$ & $\mathbf{y}^{*}$ & $\mathbf{y}^{*^{++}}$ & $\mathbf{y}^{\mathbf{0}}$ & $\mathbf{y}^{\mathbf{0 + +}}$ & \# \\
\hline
\end{tabular}




\begin{tabular}{|c|c|c|c|c|c|c|c|c|c|c|c|c|c|c|}
\hline & 130.0499 & 65.5286 & & & 112.0393 & 56.5233 & E & & & & & & & 20 \\
\hline 2 & 243.1339 & 122.0706 & & & 225.1234 & 113.0653 & I & 2222.2325 & 1111.6199 & 2205.2060 & 1103.1066 & 2204.2220 & 1102.6146 & 19 \\
\hline 3 & 358.1609 & 179.5841 & & & 340.1503 & 170.5788 & D & 2109.1485 & 1055.0779 & 2092.1219 & 1046.5646 & 2091.1379 & 1046.0726 & 18 \\
\hline 4 & 514.2620 & 257.6346 & 497.2354 & 249.1214 & 496.2514 & 248.6293 & $\mathbf{R}$ & 1994.1215 & 997.5644 & 1977.0950 & 989.0511 & 1976.1110 & 988.5591 & 17 \\
\hline 5 & 629.2889 & 315.1481 & 612.2624 & 306.6348 & 611.2784 & 306.1428 & D & 1838.0204 & 919.5139 & 1820.9939 & 911.0006 & 1820.0099 & 910.5086 & 16 \\
\hline 6 & 757.3475 & 379.1774 & 740.3210 & 370.6641 & 739.3369 & 370.1721 & $\mathbf{Q}$ & 1722.9935 & 862.0004 & 1705.9669 & 853.4871 & 1704.9829 & 852.9951 & 15 \\
\hline 7 & 870.4316 & 435.7194 & 853.4050 & 427.2061 & 852.4210 & 426.7141 & L & 1594.9349 & 797.9711 & 1577.9084 & 789.4578 & 1576.9243 & 788.9658 & 14 \\
\hline 81 & 1001.4721 & 501.2397 & 984.4455 & 492.7264 & 983.4615 & 492.2344 & $\mathbf{M}$ & 1481.8508 & 741.4291 & 1464.8243 & 732.9158 & 1463.8403 & 732.4238 & 13 \\
\hline 91 & 1102.5197 & 551.7635 & 1085.4932 & 543.2502 & 1084.5092 & 542.7582 & $T$ & 1350.8104 & 675.9088 & 1333.7838 & 667.3955 & 1332.7998 & 666.9035 & 12 \\
\hline 101 & 1201.5882 & 601.2977 & 1184.5616 & 592.7844 & 1183.5776 & 592.2924 & $\mathbf{V}$ & 1249.7627 & 625.3850 & 1232.7361 & 616.8717 & 1231.7521 & 616.3797 & 11 \\
\hline 111 & \begin{tabular}{|l|}
1314.6722 \\
\end{tabular} & 657.8397 & 1297.6457 & 649.3265 & 1296.6616 & 648.8345 & $\mathbf{L}$ & 1150.6943 & 575.8508 & 1133.6677 & 567.3375 & 1132.6837 & 566.8455 & 10 \\
\hline 121 & 1385.7093 & 693.3583 & 1368.6828 & 684.8450 & 1367.6988 & 684.3530 & A & 1037.6102 & 519.3087 & 1020.5836 & 510.7955 & 1019.5996 & 510.3035 & 9 \\
\hline 131 & 1500.7363 & 750.8718 & 1483.7097 & 742.3585 & 1482.7257 & 741.8665 & $\mathbf{N}$ & 966.5731 & 483.7902 & 949.5465 & 475.2769 & 948.5625 & 474.7849 & 8 \\
\hline 141 & 1599.8047 & 800.4060 & 1582.7781 & 791.8927 & 1581.7941 & 791.4007 & $\mathbf{V}$ & 851.5461 & 426.2767 & 834.5196 & 417.7634 & 833.5356 & 417.2714 & 7 \\
\hline 151 & 1700.8524 & 850.9298 & 1683.8258 & 842.4165 & 1682.8418 & 841.9245 & $\mathbf{T}$ & 752.4777 & 376.7425 & 735.4512 & 368.2292 & 734.4672 & 367.7372 & 6 \\
\hline 161 & 1837.9113 & 919.4593 & 1820.8847 & 910.9460 & 1819.9007 & 910.4540 & $\mathbf{H}$ & 651.4301 & 326.2187 & 634.4035 & 317.7054 & & & 5 \\
\hline 171 & 1950.9953 & 976.0013 & 1933.9688 & 967.4880 & 1932.9848 & 966.9960 & $\mathbf{L}$ & 514.3711 & 257.6892 & 497.3446 & 249.1759 & & & 4 \\
\hline 182 & 2064.0794 & 1032.5433 & 2047.0528 & 1024.0301 & 2046.0688 & 1023.5381 & $\mathbf{L}$ & 401.2871 & 201.1472 & 384.2605 & 192.6339 & & & 3 \\
\hline 192 & 2177.1635 & 1089.0854 & 2160.1369 & 1080.5721 & 2159.1529 & 1080.0801 & I & 288.2030 & 144.6051 & 271.1765 & 136.0919 & & & 2 \\
\hline 20 & & & & & & & $\mathbf{R}$ & 175.1190 & 88.0631 & 158.0924 & 79.5498 & & & 1 \\
\hline
\end{tabular}
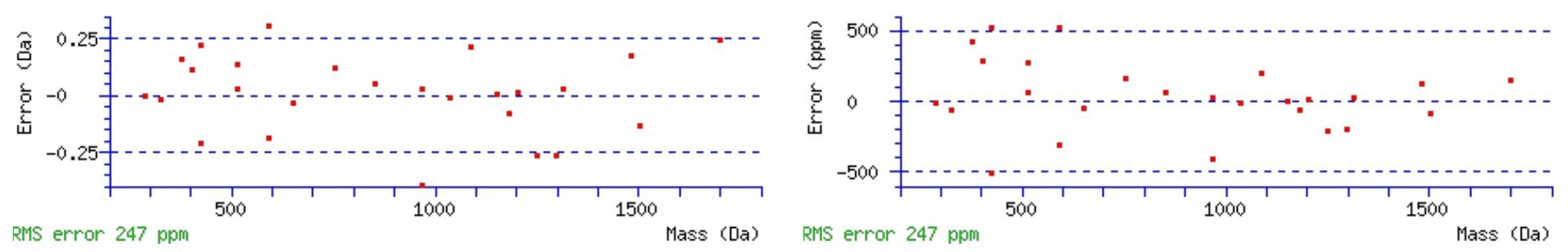

\section{All matches to this query}

\begin{tabular}{|l|l|l|l|}
\hline Score & Mr(calc): & Delta & \multicolumn{1}{c|}{ Sequence } \\
\hline 71.6 & 2350.2678 & 0.0032 & EIDRDQLMTVLANVTHLLIR \\
\hline 19.7 & 2349.2838 & 0.9872 & EIDRDQLMTVLANVTHLLIR \\
\hline 2.8 & 2350.2733 & -0.0023 & STNLLLTRTLSNSLQNVIKR \\
\hline 0.2 & 2350.2930 & -0.0220 & NVVAECLGKLTLIDPETLLPR \\
\hline
\end{tabular}

Spectrum No: 341; Query: 2832; Rank: 1

\section{Peptide View}

MS/MS Fragmentation of DYGPRPDADSESDNSDNNTIFVQGLGEGVSTDQVGEFFK

Found in IPI00364132, Tax_Id=10116 Gene_Symbol=Taf15_predicted 59 kDa protein

Match to Query 2832: 4206.857172 from(1403.293000,3+)

Title: 091008RatKidney_NoSalt_22.5159.5159.3.dta

Data file K:INewmanPaper|Piliangl3SubProteomes\Piliang3SP\mgf5ppm\ERLIC_3SubProteomes5ppm.mgf 

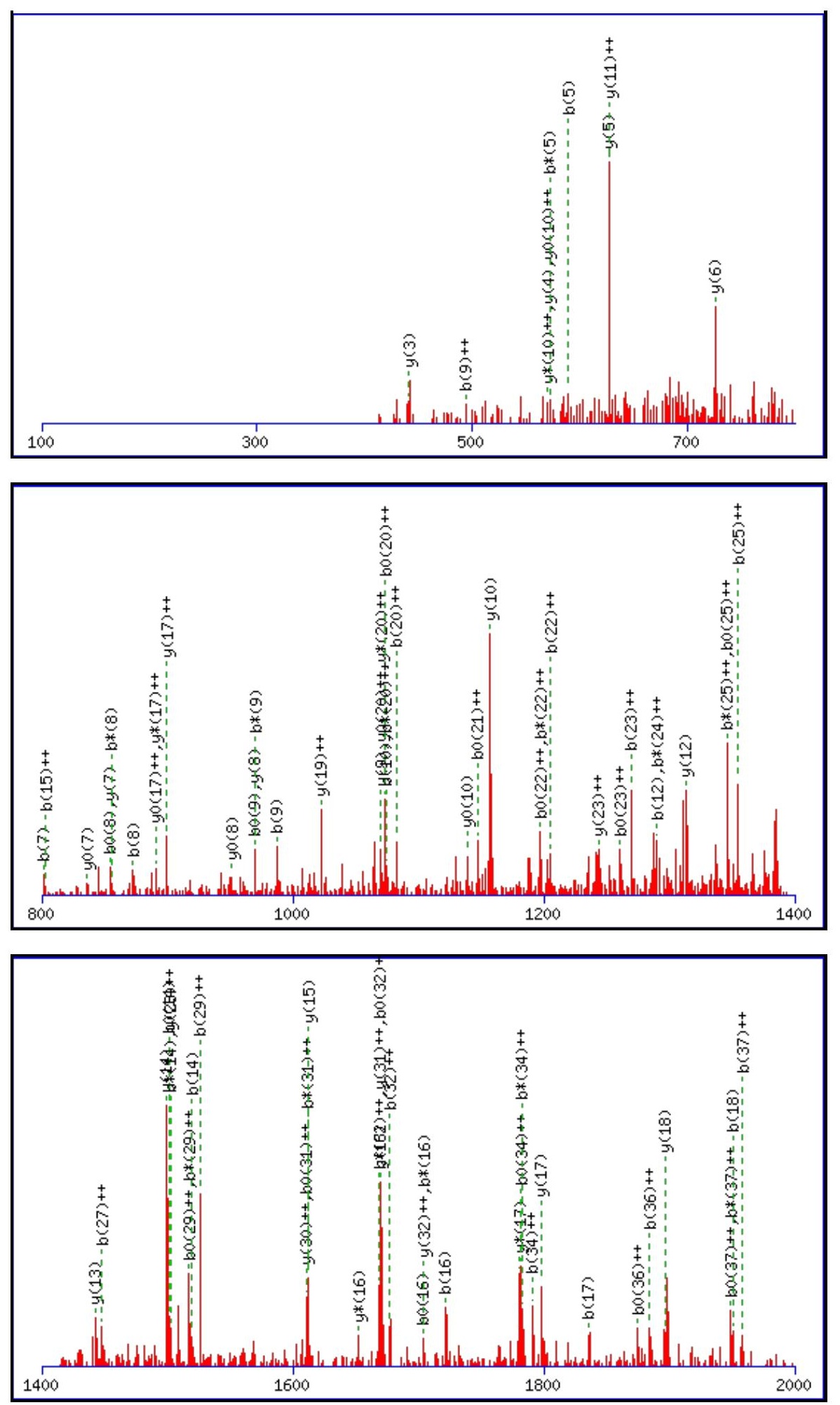

Monoisotopic mass of neutral peptide $\operatorname{Mr}($ calc): 4206.8414

Fixed modifications: Carbamidomethyl (C)

Variable modifications:

N18 : Deamidated_N (N)

Ions Score: 72 Expect: $3.6 \mathrm{e}-005$

Matches (Bold Red): 85/442 fragment ions using 150 most intense peaks

\begin{tabular}{|c|c|c|c|c|c|c|c|c|c|c|c|c|c|c|}
\hline \# & b & $\mathbf{b}^{++}$ & $\mathbf{b}^{*}$ & $\mathbf{b}^{*++}$ & $\mathbf{b}^{0}$ & $\mathbf{b}^{\mathbf{0}^{++}}$ & Seq. & $\mathbf{y}$ & $\mathbf{y}^{++}$ & $\mathbf{y}^{*}$ & $\mathbf{y}^{*^{++}}$ & $\mathbf{y}^{\mathbf{0}}$ & $\mathbf{y}^{\mathbf{0}^{++}}$ & \# \\
\hline 1 & 116.0342 & 58.5207 & & & 98.0237 & 49.5155 & D & & & & & & & 39 \\
\hline 2 & 279.0975 & 140.0524 & & & 261.0870 & 131.0471 & $\mathbf{Y}$ & 4092.8217 & 2046.9145 & 4075.7952 & 2038.4012 & 4074.8112 & 2037.9092 & 38 \\
\hline 3 & 336.1190 & 168.5631 & & & 318.1084 & 159.5579 & G & 3929.7584 & 1965.3828 & 3912.7318 & 1956.8696 & 3911.7478 & 1956.3776 & 37 \\
\hline 4 & 433.1718 & 217.0895 & & & 415.1612 & 208.0842 & $\mathbf{P}$ & 3872.7369 & 1936.8721 & 3855.7104 & 1928.3588 & 3854.7264 & 1927.8668 & 36 \\
\hline 5 & 589.2729 & 295.1401 & 572.2463 & 286.6268 & 571.2623 & 286.1348 & $\mathbf{R}$ & 3775.6842 & 1888.3457 & 3758.6576 & 1879.8324 & 3757.6736 & 1879.3404 & 35 \\
\hline
\end{tabular}




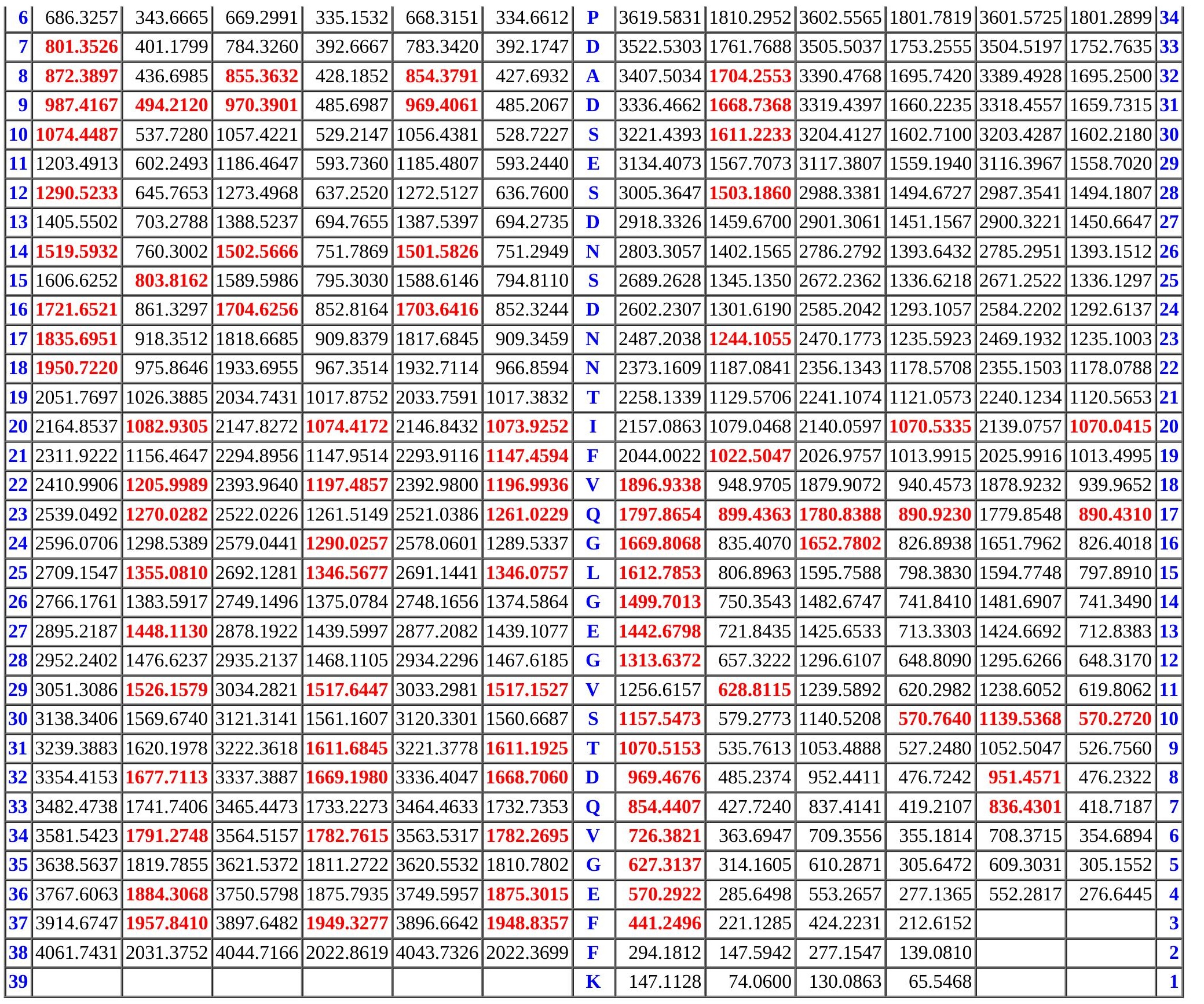
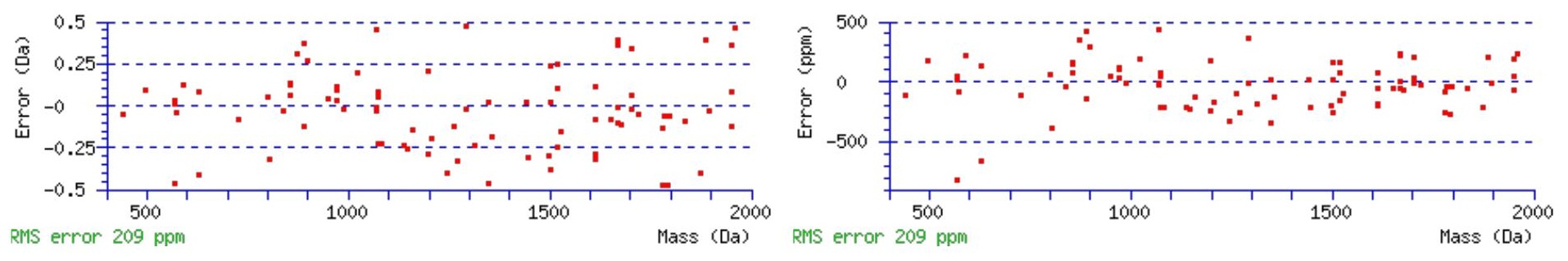

\section{All matches to this query}

\begin{tabular}{|l|l|l|l|}
\hline Score & Mr(calc): & Delta & \multicolumn{1}{c|}{ Sequence } \\
\hline 71.6 & 4206.8414 & 0.0157 & DYGPRPDADSESDNSDNNTIFVQGLGEGVSTDQVGEFFK \\
\hline 71.6 & 4206.8414 & 0.0157 & DYGPRPDADSESDNSDNNTIFVQGLGEGVSTDQVGEFFK \\
\hline 66.7 & 4205.8574 & 0.9998 & DYGPRPDADSESDNSDNNTIFVQGLGEGVSTDQVGEFFK \\
\hline 66.2 & 4206.8414 & 0.0157 & DYGPRPDADSESDNSDNNTIFVQGLGEGVSTDQVGEFFK \\
\hline 9.5 & 4205.8209 & 1.0362 & VEIDAINDPFIDLNYMVYMLQYVSTHGKFNGTVK \\
\hline 7.5 & 4206.8718 & -0.0147 & LHTPMYFFLGNFAFLEIWYVSSTAPNMLANILSK \\
\hline
\end{tabular}




\begin{tabular}{|l|l|l|l|}
6.5 & 4205.8209 & 1.0362 & VEIDAINDPFIDLNYMVYMLQYVSTHGKFNGTVK \\
\hline 5.6 & 4206.8718 & -0.0147 & LHTPMYFFLGNFAFLEIWYVSSTAPNMLANILSK \\
\hline 4.9 & 4204.8675 & 1.9897 & EMLPTNPIRLGLALNFSVFHYEIANSPEEAISLAK \\
\hline 4.9 & 4204.8311 & 2.0261 & EMPPTNPIRLGLALNFSVFHYEIANSPEEAISLAK \\
\hline
\end{tabular}

Spectrum No: 342; Query: 1866; Rank: 1

\section{Peptide View}

MS/MS Fragmentation of RSGPSDEGEEEMEDGTVTNGS

Found in IPI00192279, Tax_Id=10116 Gene_Symbol=- 28 kDa protein

Match to Query 1866: 2182.847308 from(1092.430930,2+)

Title: 100101RatKid_NS_deglyco_24.1138.1138.2.dta

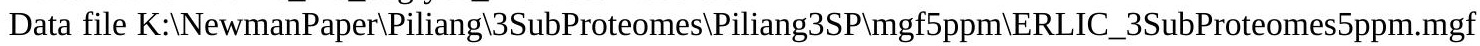
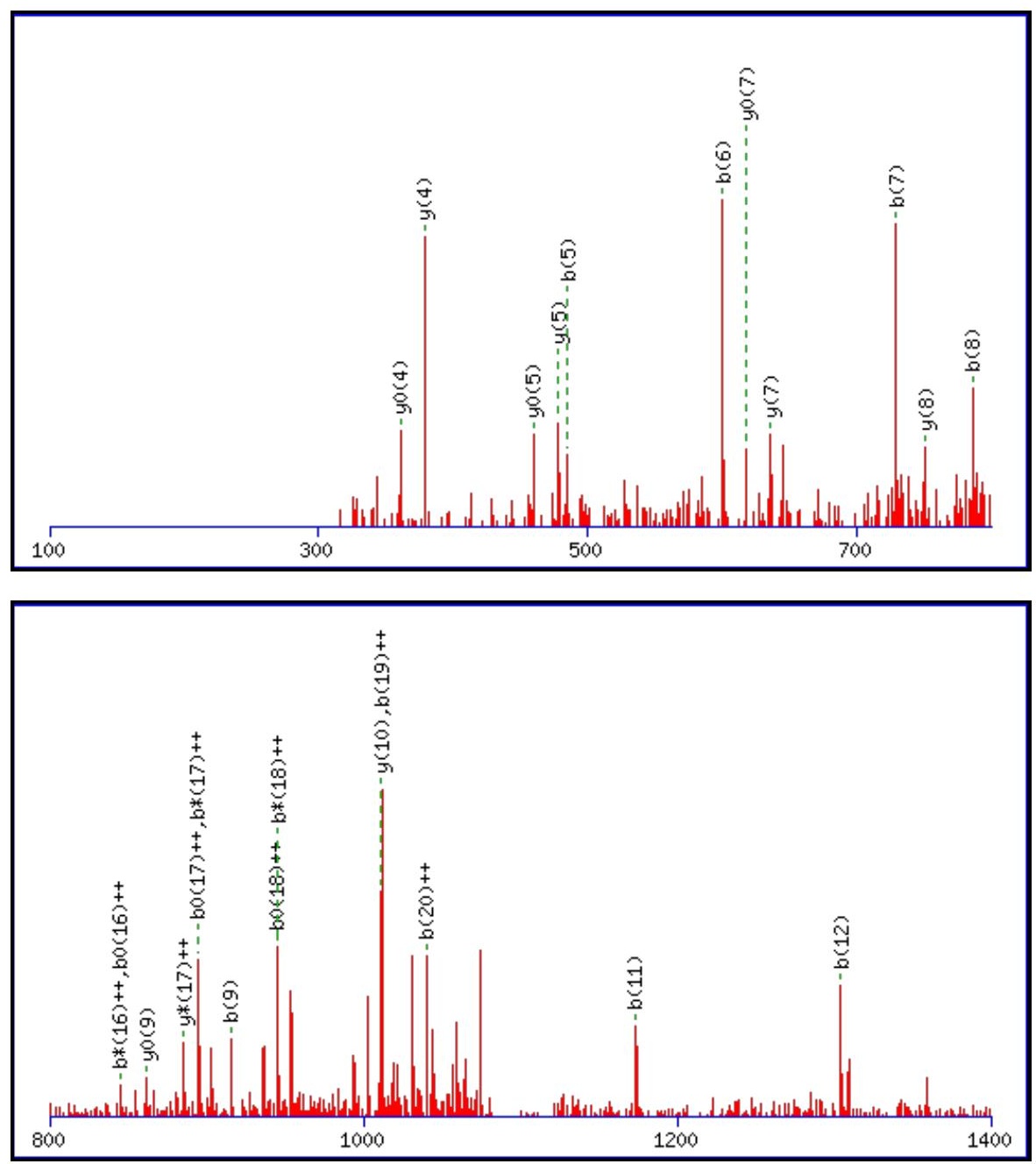


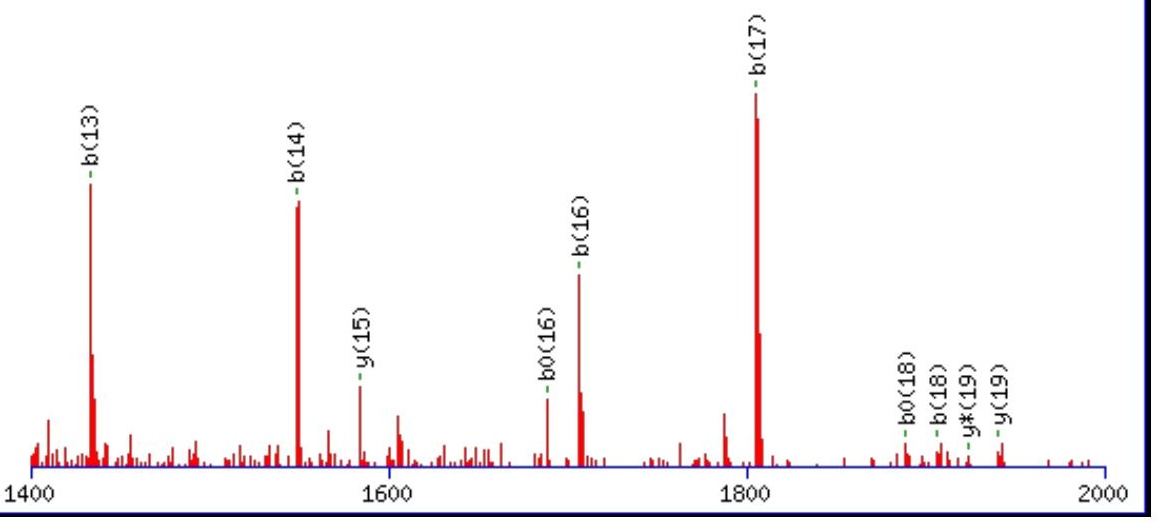

Monoisotopic mass of neutral peptide $\operatorname{Mr}($ calc): 2182.8444

Fixed modifications: Carbamidomethyl (C)

Variable modifications:

N19: Deamidated N ( N)

Ions Score: 72 Expect: $1.3 \mathrm{e}-005$

Matches (Bold Red): 35/234 fragment ions using 56 most intense peaks

\begin{tabular}{|c|c|c|c|c|c|c|c|c|c|c|c|c|c|c|}
\hline \# & b & $\mathbf{b}^{++}$ & b* & $\mathbf{b}^{*^{++}}$ & $\mathbf{b}^{0}$ & $\mathbf{b}^{0++}$ & Seq. & $\mathbf{y}$ & $\mathbf{y}^{++}$ & $\mathbf{y}^{*}$ & $\mathbf{y}^{*^{++}}$ & $\mathbf{y}^{0}$ & $\mathbf{y}^{\mathbf{0 + +}}$ & \# \\
\hline 1 & 157.1084 & 79.0578 & 140.0818 & 70.5446 & & & $\mathbf{R}$ & & & & & & & 21 \\
\hline 2 & 244.1404 & 122.5738 & 227.1139 & 114.0606 & 226.1298 & 113.5686 & S & 2027.7506 & 1014.3789 & 2010.7240 & 1005.8657 & 2009.7400 & 1005.3737 & 20 \\
\hline 3 & 301.1619 & 151.0846 & 284.1353 & 142.5713 & 283.1513 & 142.0793 & G & 1940.7186 & 970.8629 & 1923.6920 & 962.3496 & 1922.7080 & 961.8576 & 19 \\
\hline 4 & 398.2146 & 199.6110 & 381.1881 & 191.0977 & 380.2041 & 190.6057 & $\mathbf{P}$ & 1883.6971 & 942.3522 & 1866.6705 & 933.8389 & 1865.6865 & 933.3469 & 18 \\
\hline 5 & 485.2467 & 243.1270 & 468.2201 & 234.6137 & 467.2361 & 234.1217 & S & 1786.6443 & 893.8258 & 1769.6178 & 885.3125 & 1768.6338 & 884.8205 & 17 \\
\hline 6 & 600.2736 & 300.6404 & 583.2471 & 292.1272 & 582.2630 & 291.6352 & D & 1699.6123 & 850.3098 & 1682.5858 & 841.7965 & 1681.6017 & 841.3045 & 16 \\
\hline 7 & 729.3162 & 365.1617 & 712.2897 & 356.6485 & 711.3056 & 356.1565 & $\mathbf{E}$ & 1584.5854 & 792.7963 & 1567.5588 & 784.2830 & 1566.5748 & 783.7910 & 15 \\
\hline 8 & 786.3377 & 393.6725 & 769.3111 & 385.1592 & 768.3271 & 384.6672 & G & 1455.5428 & 728.2750 & 1438.5162 & 719.7617 & 1437.5322 & 719.2697 & 14 \\
\hline 9 & 915.3803 & 458.1938 & 898.3537 & 449.6805 & 897.3697 & 449.1885 & $\mathbf{E}$ & 1398.5213 & 699.7643 & 1381.4948 & 691.2510 & 1380.5107 & 690.7590 & 13 \\
\hline 10 & 1044.4229 & 522.7151 & 1027.3963 & 514.2018 & 1026.4123 & 513.7098 & $\bar{E}$ & 1269.4787 & 635.2430 & 1252.4522 & 626.7297 & 1251.4681 & 626.2377 & 12 \\
\hline 11 & 1173.4654 & 587.2364 & 1156.4389 & 578.7231 & 1155.4549 & 578.2311 & $\mathbf{E}$ & 1140.4361 & 570.7217 & 1123.4096 & 562.2084 & 1122.4256 & 561.7164 & 11 \\
\hline 12 & 1304.5059 & 652.7566 & 1287.4794 & 644.2433 & 1286.4954 & 643.7513 & $\mathbf{M}$ & 1011.3935 & 506.2004 & 994.3670 & 497.6871 & 993.3830 & 497.1951 & 10 \\
\hline 13 & 1433.5485 & 717.2779 & 1416.5220 & 708.7646 & 1415.5380 & 708.2726 & $\mathbf{E}$ & 880.3530 & 440.6802 & 863.3265 & 432.1669 & 862.3425 & 431.6749 & 9 \\
\hline 14 & 1548.5755 & 774.7914 & 1531.5489 & 766.2781 & 1530.5649 & 765.7861 & D & 751.3104 & 376.1589 & 734.2839 & 367.6456 & 733.2999 & 367.1536 & 8 \\
\hline 15 & 1605.5969 & 803.3021 & 1588.5704 & 794.7888 & 1587.5864 & 794.2968 & G & 636.2835 & 318.6454 & 619.2570 & 310.1321 & 618.2729 & 309.6401 & 7 \\
\hline 16 & 1706.6446 & 853.8259 & 1689.6181 & 845.3127 & 1688.6340 & 844.8207 & $\mathbf{T}$ & 579.2620 & 290.1347 & 562.2355 & 281.6214 & 561.2515 & 281.1294 & 6 \\
\hline 17 & 1805.7130 & 903.3602 & 1788.6865 & 894.8469 & 1787.7025 & 894.3549 & $\mathbf{V}$ & 478.2144 & 239.6108 & 461.1878 & 231.0975 & 460.2038 & 230.6055 & 5 \\
\hline 18 & 1906.7607 & 953.8840 & 1889.7342 & 945.3707 & 1888.7501 & 944.8787 & $\mathbf{T}$ & 379.1459 & 190.0766 & 362.1194 & 181.5633 & 361.1354 & 181.0713 & 4 \\
\hline 19 & 2021.7876 & 1011.3975 & 2004.7611 & 1002.8842 & 2003.7771 & 1002.3922 & $\mathbf{N}$ & 278.0983 & 139.5528 & 261.0717 & 131.0395 & 260.0877 & 130.5475 & 3 \\
\hline 20 & 2078.8091 & 1039.9082 & 2061.7826 & 1031.3949 & 2060.7985 & 1030.9029 & G & 163.0713 & 82.0393 & & & 145.0608 & 73.0340 & 2 \\
\hline 21 & & & & & & & $\mathbf{S}$ & 106.0499 & 53.5286 & & & 88.0393 & 44.5233 & 1 \\
\hline
\end{tabular}

$$
\text { 要 }
$$$$
\text { 舜 }
$$

\section{All matches to this query}

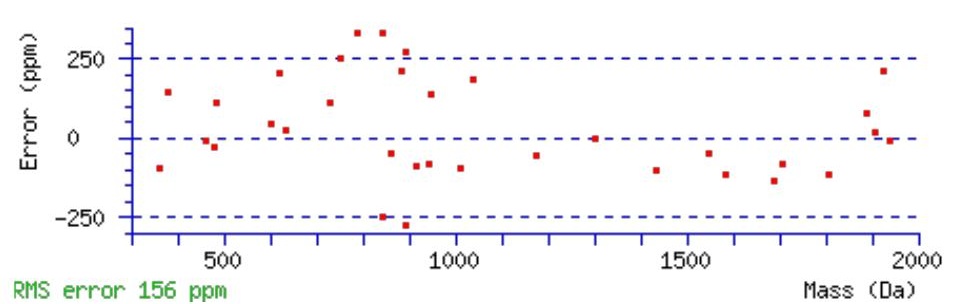

Mass (Da) RMS error 156 ppm
Sequence 


\section{|71.5 $2182.8444|0.0029|$ RSGPSDEGEEEMEDGTVTNGS \\ \begin{tabular}{|l|l|l|l|}
\hline 48.2 & 2181.8604 & 0.9869 & RSGPSDEGEEEMEDGTVTNGS \\
\hline
\end{tabular}}

Spectrum No: 343; Query: 280; Rank: 1

\section{Peptide View}

MS/MS Fragmentation of FVHVNTSAILK

Found in IPI00201298, Tax_Id=10116 Gene_Symbol=Pomt1 Protein O-mannosyl-transferase 1

Match to Query 280: 1228.679892 from(410.567240,3+)

Title: 091008RatKidney_NH4Format01_23.2091.2091.3.dta

Data file K:INewmanPaper|Piliang|3SubProteomes\Piliang3SP\mgf5ppm\ERLIC_3SubProteomes5ppm.mgf
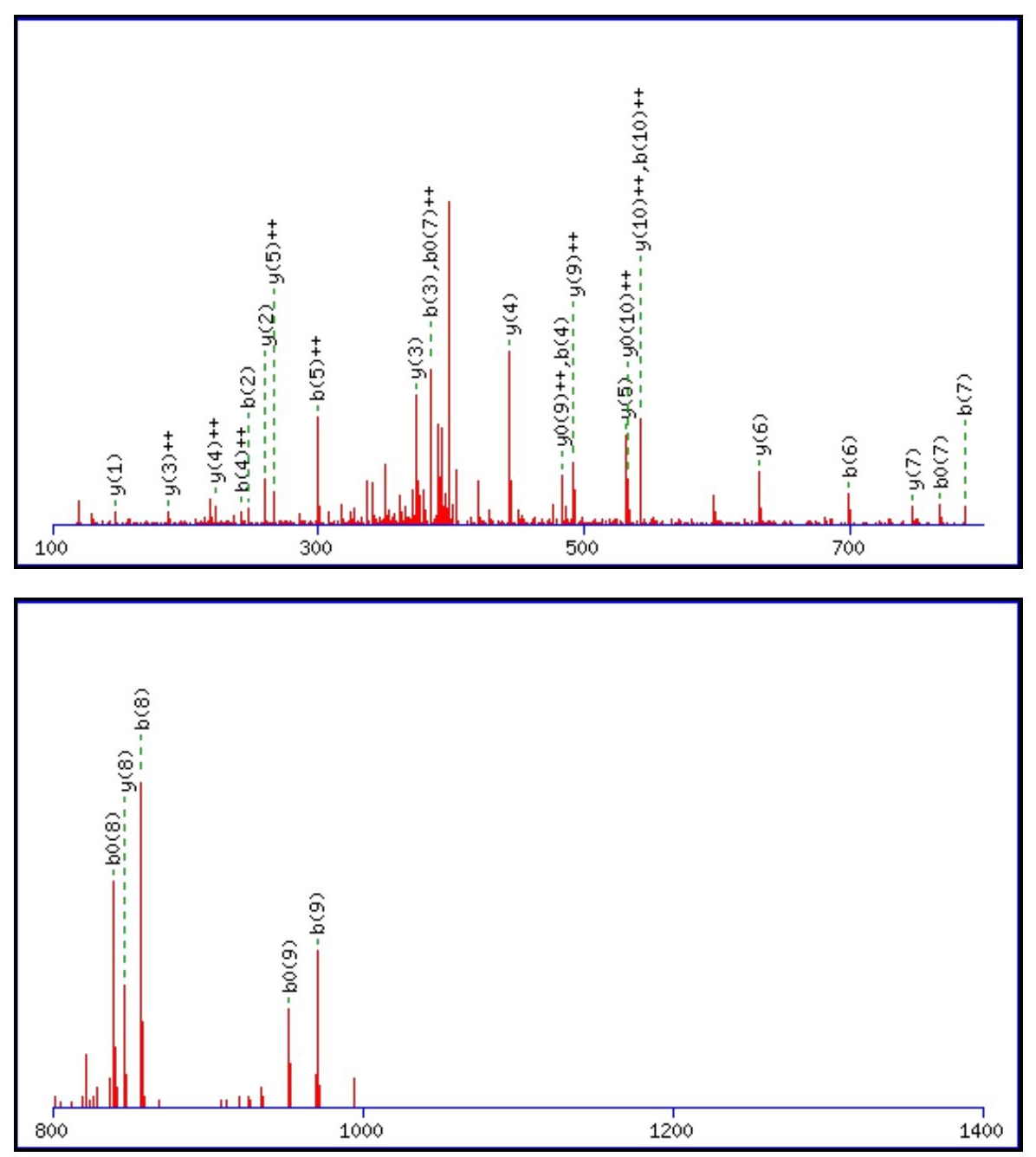


$1400 \quad 1600 \quad 1800 \quad 2000$

Monoisotopic mass of neutral peptide $\operatorname{Mr}($ calc): 1228.6816

Fixed modifications: Carbamidomethyl (C)

Variable modifications:

N5 : Deamidated_N (N)

Ions Score: 71 Expect: $5.3 \mathrm{e}-006$

Matches (Bold Red): 29/94 fragment ions using 31 most intense peaks

\begin{tabular}{|c|c|c|c|c|c|c|c|c|c|c|c|c|c|c|}
\hline \# & b & $\mathbf{b}^{++}$ & $\mathbf{b}^{*}$ & $\mathbf{b}^{*^{++}}$ & $\mathbf{b}^{\mathbf{0}}$ & $\mathbf{b}^{0++}$ & Seq. & $\mathbf{y}$ & $\mathbf{y}^{++}$ & $\mathbf{y}^{*}$ & $\mathrm{y}^{\mathrm{*}^{++}}$ & $\mathbf{y}^{\mathbf{0}}$ & $y^{0++}$ & \# \\
\hline 1 & 148.0757 & 74.5415 & & & & & $\mathbf{F}$ & & & & & & & 11 \\
\hline 2 & 247.1441 & 124.0757 & & & & & V & 1082.6204 & 541.8139 & 1065.5939 & 533.3006 & 1064.6099 & 532.8086 & 10 \\
\hline 3 & 384.2030 & 192.6051 & & & & & $\mathbf{H}$ & 983.5520 & 492.2796 & 966.5255 & 483.7664 & 965.5415 & 483.2744 & 9 \\
\hline 4 & 483.2714 & 242.1394 & & & & & V & 846.4931 & 423.7502 & 829.4666 & 415.2369 & 828.4825 & 414.7449 & 8 \\
\hline 5 & 598.2984 & 299.6528 & 581.2718 & 291.1395 & & & $\mathbf{N}$ & 747.4247 & 374.2160 & 730.3981 & 365.7027 & 729.4141 & 365.2107 & 7 \\
\hline 6 & 699.3460 & 350.1767 & 682.3195 & 341.6634 & 681.3355 & 341.1714 & $\mathbf{T}$ & 632.3978 & 316.7025 & 615.3712 & 308.1892 & 614.3872 & 307.6972 & 6 \\
\hline 7 & 786.3781 & 393.6927 & 769.3515 & 385.1794 & 768.3675 & 384.6874 & $\mathrm{~S}$ & 531.3501 & 266.1787 & 514.3235 & 257.6654 & 513.3395 & 257.1734 & 5 \\
\hline 8 & 857.4152 & 429.2112 & 840.3886 & 420.6980 & 839.4046 & 420.2059 & A & 444.3180 & 222.6627 & 427.2915 & 214.1494 & & & 4 \\
\hline 9 & 970.4993 & 485.7533 & 953.4727 & 477.2400 & 952.4887 & 476.7480 & I & 373.2809 & \begin{tabular}{|l|}
187.1441 \\
\end{tabular} & 356.2544 & 178.6308 & & & 3 \\
\hline 10 & 1083.5833 & 542.2953 & 1066.5568 & 533.7820 & 1065.5728 & 533.2900 & $\mathbf{L}$ & 260.1969 & 130.6021 & 243.1703 & 122.0888 & & & 2 \\
\hline 11 & & & & & & & $\mathbf{K}$ & 147.1128 & 74.0600 & 130.0863 & 65.5468 & & & 1 \\
\hline
\end{tabular}
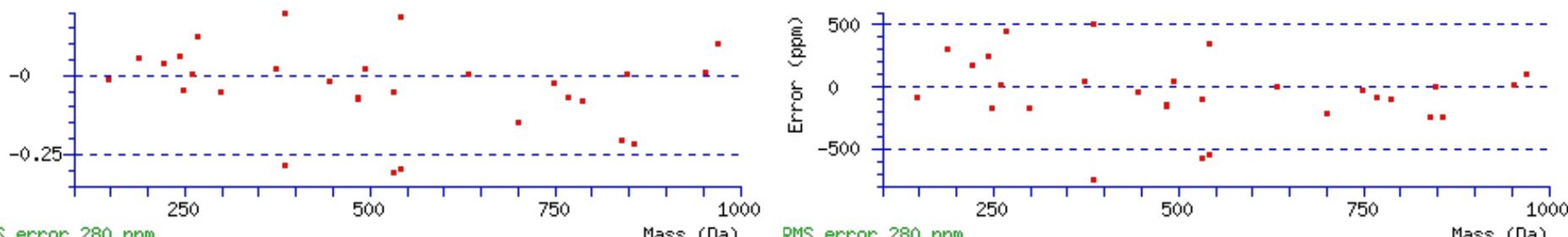

RIS error 280 ppm

Mass (Da) RMS error 280 ppm

\section{All matches to this query}

\begin{tabular}{|l|l|l|l|}
\hline Score & Mr(calc): & Delta & \multicolumn{1}{|c|}{ Sequence } \\
\hline 71.5 & 1228.6816 & -0.0017 & FVHVNTSAILK \\
\hline 9.8 & 1227.6823 & 0.9976 & KLGNLAVAADEK \\
\hline 8.3 & 1227.6710 & 1.0089 & EEIKVEKPEK \\
\hline 7.8 & 1227.6757 & 1.0042 & EKSRMSHLIK \\
\hline 7.0 & 1227.6823 & 0.9976 & NATQEAVAALLK \\
\hline 6.8 & 1226.6843 & 1.9956 & QELRQELRR \\
\hline 6.0 & 1228.6887 & -0.0088 & QRSIEAERLK \\
\hline 6.0 & 1228.6888 & -0.0089 & NRLVNQSALSK \\
\hline 4.1 & 1228.6888 & -0.0089 & EVVRQRSDLK \\
\hline 3.3 & 1228.6676 & 0.0123 & NQWLRRNIK \\
\hline
\end{tabular}


Spectrum No: 344; Query: 1212; Rank: 1

\section{Peptide View}

MS/MS Fragmentation of TDYNASVSVPDSSGPER

Found in IPI00194974, Tax_Id=10116 Gene_Symbol=Hnrpk Hnrpk protein

Match to Query 1212: 1780.771268 from(891.392910,2+)

Title: 091008RatKidney_NH4Format01_18.1423.1423.2.dta

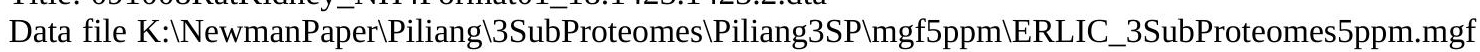
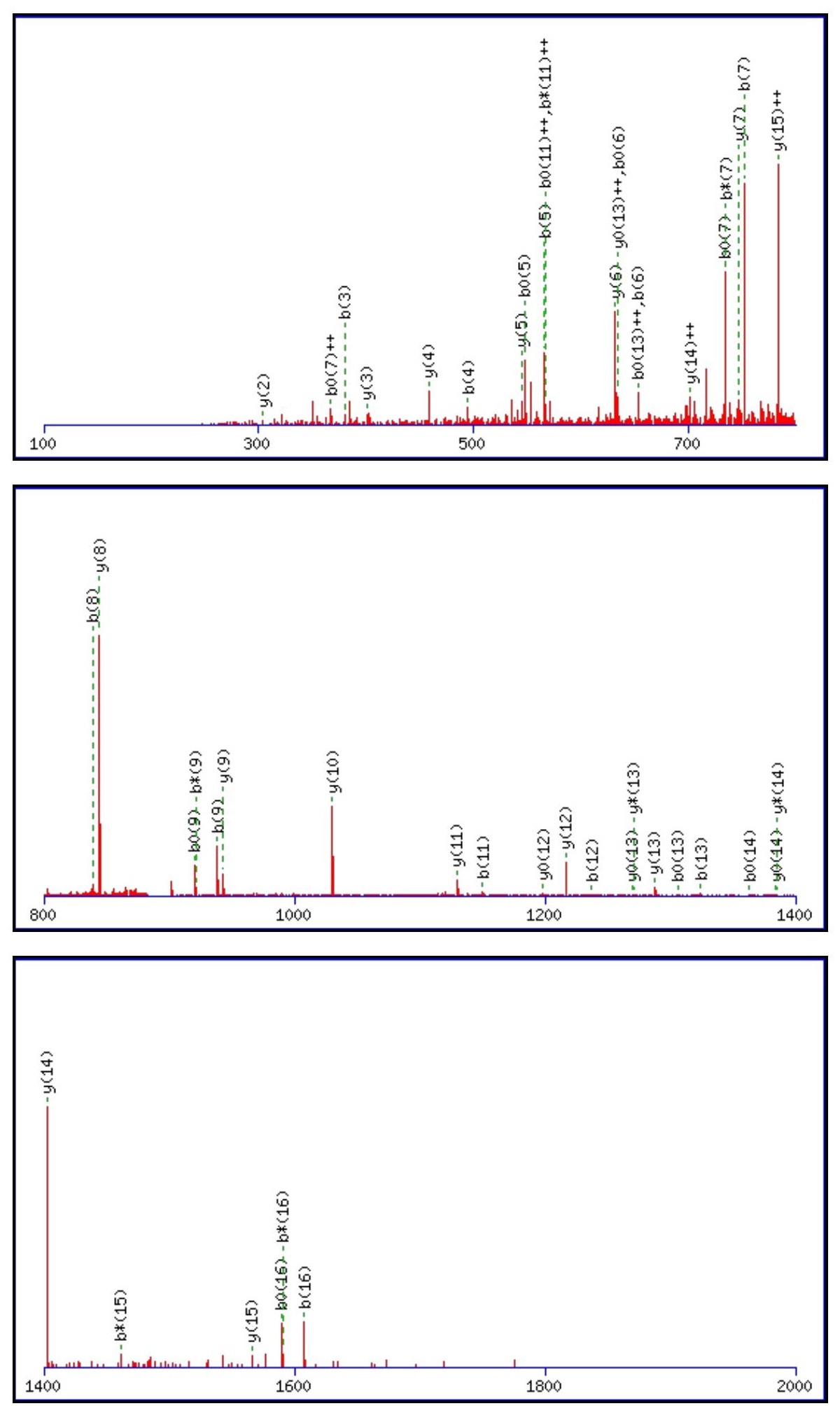
Monoisotopic mass of neutral peptide $\operatorname{Mr}(\mathrm{calc}): 1780.7751$

Fixed modifications: Carbamidomethyl (C)

Variable modifications:

N4 : Deamidated_N (N)

Ions Score: 71 Expect: $1.5 \mathrm{e}-005$

Matches (Bold Red): 48/184 fragment ions using 109 most intense peaks

\begin{tabular}{|c|c|c|c|c|c|c|c|c|c|c|c|c|c|c|}
\hline \# & b & $\mathbf{b}^{++}$ & b* & & $\mathbf{b}^{\mathbf{0}}$ & & Seq. & $\mathbf{y}$ & & $\mathbf{y}^{*}$ & & $\mathbf{y}^{0}$ & & \# \\
\hline 1 & 102.0550 & 51.5311 & & & 84.0444 & 42.5258 & $\mathbf{T}$ & & & & & & & 17 \\
\hline 2 & 217.0819 & 109.0446 & & & 199.0713 & 100.0393 & D & & 710 & 1663.7082 & & 1662.7242 & 831.8657 & 16 \\
\hline 3 & 380.1452 & 190.5763 & & & 362.1347 & 181.5710 & $\mathbf{Y}$ & 1565.7078 & 783.3575 & 1548.6813 & 774.8443 & 1547.6972 & 3523 & 15 \\
\hline 4 & 495.1722 & 248.0897 & 478.1456 & 239.5764 & 477.1616 & 239.0844 & $\mathbf{N}$ & 1402.6445 & 701.8259 & 1385.6179 & 693.3126 & 1384.6339 & 692.8206 & 14 \\
\hline 5 & 566.2093 & 283.6083 & 549.1827 & 275.0950 & 548.1987 & 274.6030 & A & 1287.6175 & 644.3124 & 1270.5910 & 635.7991 & 1269.6070 & 635.3071 & 13 \\
\hline 6 & 653.2413 & 327.1243 & 636.2148 & 318.6110 & 635.2307 & 318.1190 & S & 1216.5804 & 608.7938 & 1199.5539 & 600.2806 & 1198.5699 & 599.7886 & 12 \\
\hline 7 & 752.3097 & 376.6585 & 735.2832 & 368.1452 & 734.2992 & 367.6532 & $\mathbf{V}$ & 1129.5484 & 565.2778 & 1112.5218 & 556.7646 & 1111.5378 & 556.2726 & 11 \\
\hline 8 & 839.3417 & 420.1745 & 822.3152 & 411.6612 & 821.3312 & 411.1692 & S & 1030.4800 & 515.7436 & 1013.4534 & 507.2304 & 1012.4694 & 506.7383 & 10 \\
\hline 9 & 938.4102 & 469.7087 & 921.3836 & 461.1954 & 920.3996 & 460.7034 & V & 943.4480 & 472.2276 & 926.4214 & 463.7143 & 925.4374 & 463.2223 & 9 \\
\hline 10 & 1035.4629 & 518.2351 & 1018.4364 & 509.7218 & 1017.4524 & 509.2298 & $\mathbf{P}$ & 844.3795 & 422.6934 & 827.3530 & 414.1801 & 826.3690 & 413.6881 & 8 \\
\hline 11 & 1150.4899 & 575.7486 & 1133.4633 & 567.2353 & 1132.4793 & 566.7433 & D & 747.3268 & 374.1670 & 730.3002 & 365.6537 & 729.3162 & 365.1617 & 7 \\
\hline 12 & 1237.5219 & 619.2646 & 1220.4953 & 610.7513 & 1219.5113 & 610.2593 & S & 632.2998 & 316.6536 & 615.2733 & 308.1403 & 614.2893 & 307.6483 & 6 \\
\hline 13 & 1324.5539 & 662.7806 & 1307.5274 & 654.2673 & 1306.5434 & 653.7753 & S & 545.2678 & 273.1375 & 528.2413 & 264.6243 & 527.2572 & 264.1323 & 5 \\
\hline 14 & 1381.5754 & 691.2913 & 1364.5488 & 682.7781 & 1363.5648 & 682.2860 & G & 458.2358 & 229.6215 & 441.2092 & 221.1082 & 440.2252 & 220.6162 & 4 \\
\hline 15 & 1478.6282 & 739.8177 & 1461.6016 & 731.3044 & 1460.6176 & 730.8124 & $\mathbf{P}$ & 401.2143 & 201.1108 & 384.1878 & 192.5975 & 383.2037 & 192.1055 & 3 \\
\hline 16 & 1607.6707 & 804.3390 & 1590.6442 & 795.8257 & 1589.6602 & 795.3337 & $\mathbf{E}$ & 304.1615 & 152.5844 & 287.1350 & 144.0711 & 286.1510 & 143.5791 & 2 \\
\hline 17 & & & & & & & $\mathbf{R}$ & 175.1190 & 88.0631 & 158.0924 & 79.5498 & & & 1 \\
\hline
\end{tabular}
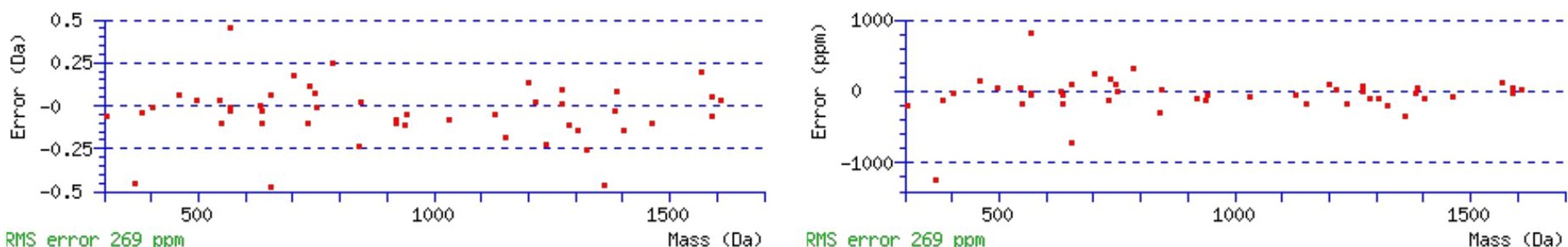

All matches to this query

\begin{tabular}{|l|l|l|l|}
\hline Score & Mr(calc): & Delta & \multicolumn{1}{c|}{ Sequence } \\
\hline 71.5 & 1780.7751 & -0.0039 & TDYNASVSVPDSSGPER \\
\hline 2.7 & 1780.7855 & -0.0142 & IFMQESDASNFLKR \\
\hline 1.6 & 1780.7546 & 0.0167 & RSELYEAEERALR \\
\hline 0.6 & 1779.7553 & 1.0159 & DVTRTNSSNARLTGK \\
\hline
\end{tabular}

Spectrum No: 345; Query: 655; Rank: 1

\section{Peptide View}

MS/MS Fragmentation of GVVDSDDLPLNVSR

Found in IPI00365985, Tax_Id=10116 Gene_Symbol=Tra1_predicted 93 kDa protein

Match to Query 655: 1485.734668 from(743.874610,2+)

Title: 100101RatKid_NS_deglyco_19.3030.3030.2.dta

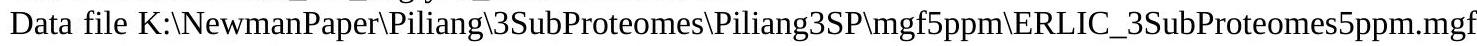



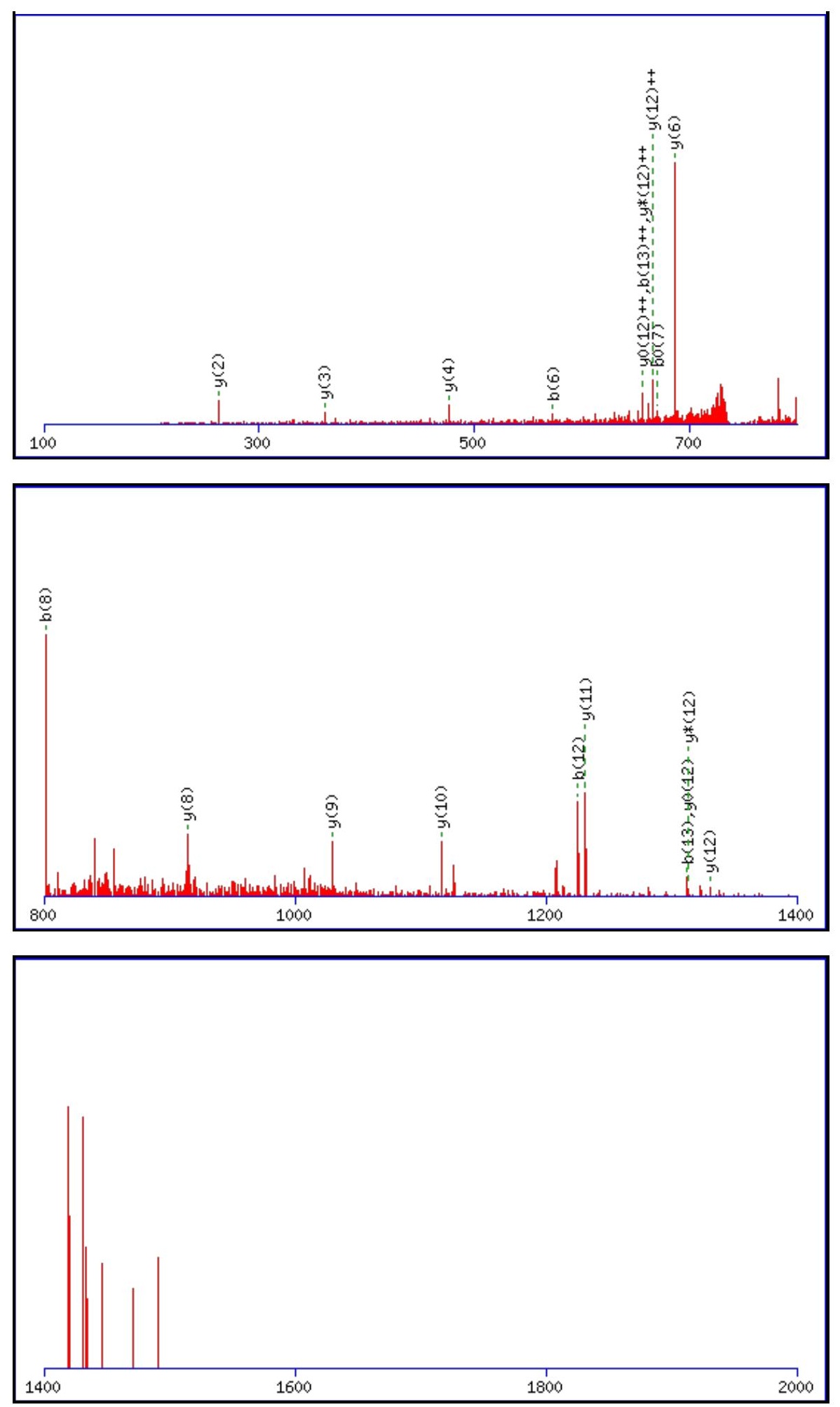

Monoisotopic mass of neutral peptide $\operatorname{Mr}($ calc): 1485.7311

Fixed modifications: Carbamidomethyl (C)

Variable modifications:

N11 : Deamidated N (N)

Ions Score: 71 Expect: $1 \mathrm{e}-005$

Matches (Bold Red): 20/128 fragment ions using 33 most intense peaks

\begin{tabular}{|r|r|r|c|c|c|c|c|c|c|c|c|c|c|c|}
\hline$\#$ & $\mathbf{b}$ & $\mathbf{b}^{++}$ & $\mathbf{b}^{*}$ & $\mathbf{b}^{\boldsymbol{*}^{++}}$ & $\mathbf{b}^{\mathbf{0}}$ & $\mathbf{b}^{\mathbf{0 + +}}$ & Seq. & $\mathbf{y}$ & $\mathbf{y}^{++}$ & $\mathbf{y}^{\mathbf{*}}$ & $\mathbf{y}^{\boldsymbol{*}^{++}}$ & $\mathbf{y}^{\mathbf{0}}$ & $\mathbf{y}^{\mathbf{0 + +}}$ & $\#$ \\
\hline $\mathbf{1}$ & 58.0287 & 29.5180 & & & & & $\mathbf{G}$ & & & & & & & $\mathbf{1 4}$ \\
\hline $\mathbf{2}$ & 157.0972 & 79.0522 & & & & & $\mathbf{V}$ & 1429.7169 & 715.3621 & 1412.6904 & 706.8488 & 1411.7063 & 706.3568 & $\mathbf{1 3}$ \\
\hline 3 & 256.1656 & 128.5864 & & & & & $\mathbf{V}$ & $\mathbf{1 3 3 0 . 6 4 8 5}$ & $\mathbf{6 6 5 . 8 2 7 9}$ & $\mathbf{1 3 1 3 . 6 2 1 9}$ & $\mathbf{6 5 7 . 3 1 4 6}$ & $\mathbf{1 3 1 2 . 6 3 7 9}$ & $\mathbf{6 5 6 . 8 2 2 6}$ & $\mathbf{1 2}$ \\
\hline $\mathbf{4}$ & 371.1925 & 186.0999 & & & 353.1819 & 177.0946 & $\mathbf{D}$ & $\mathbf{1 2 3 1 . 5 8 0 1}$ & 616.2937 & 1214.5535 & 607.7804 & 1213.5695 & 607.2884 & $\mathbf{1 1}$ \\
\hline $\mathbf{5}$ & 458.2245 & 229.6159 & & & 440.2140 & 220.6106 & $\mathbf{S}$ & $\mathbf{1 1 1 6 . 5 5 3 1}$ & 558.7802 & 1099.5266 & 550.2669 & 1098.5426 & 549.7749 & $\mathbf{1 0}$ \\
\hline
\end{tabular}




\begin{tabular}{|r|r|r|r|r|r|r|r|r|r|r|r|r|r|r|r|r|r|}
$\mathbf{6}$ & $\mathbf{5 7 3 . 2 5 1 5}$ & 287.1294 & & & 555.2409 & 278.1241 & $\mathbf{D}$ & $\mathbf{1 0 2 9 . 5 2 1 1}$ & 515.2642 & 1012.4946 & 506.7509 & 1011.5105 & 506.2589 & $\mathbf{9}$ \\
\hline $\mathbf{7}$ & 688.2784 & 344.6429 & & & $\mathbf{6 7 0 . 2 6 7 9}$ & 335.6376 & $\mathbf{D}$ & $\mathbf{9 1 4 . 4 9 4 2}$ & 457.7507 & 897.4676 & 449.2374 & 896.4836 & 448.7454 & $\mathbf{8}$ \\
\hline $\mathbf{8}$ & $\mathbf{8 0 1 . 3 6 2 5}$ & 401.1849 & & & 783.3519 & 392.1796 & $\mathbf{L}$ & 799.4672 & 400.2372 & 782.4407 & 391.7240 & 781.4567 & 391.2320 & $\mathbf{7}$ \\
\hline $\mathbf{9}$ & 898.4153 & 449.7113 & & & 880.4047 & 440.7060 & $\mathbf{P}$ & $\mathbf{6 8 6 . 3 8 3 2}$ & 343.6952 & 669.3566 & 335.1819 & 668.3726 & 334.6899 & $\mathbf{6}$ \\
\hline $\mathbf{1 0}$ & 1011.4993 & 506.2533 & & & 993.4888 & 497.2480 & L & 589.3304 & 295.1688 & 572.3038 & 286.6556 & 571.3198 & 286.1636 & $\mathbf{5}$ \\
\hline $\mathbf{1 1}$ & 1126.5263 & 563.7668 & 1109.4997 & 555.2535 & 1108.5157 & 554.7615 & $\mathbf{N}$ & $\mathbf{4 7 6 . 2 4 6 3}$ & 238.6268 & 459.2198 & 230.1135 & 458.2358 & 229.6215 & $\mathbf{4}$ \\
\hline $\mathbf{1 2}$ & $\mathbf{1 2 2 5 . 5 9 4 7}$ & 613.3010 & 1208.5681 & 604.7877 & 1207.5841 & 604.2957 & $\mathbf{V}$ & $\mathbf{3 6 1 . 2 1 9 4}$ & 181.1133 & 344.1928 & 172.6001 & 343.2088 & 172.1081 & $\mathbf{3}$ \\
\hline $\mathbf{1 3}$ & $\mathbf{1 3 1 2 . 6 2 6 7}$ & $\mathbf{6 5 6 . 8 1 7 0}$ & 1295.6001 & 648.3037 & 1294.6161 & 647.8117 & S & $\mathbf{2 6 2 . 1 5 1 0}$ & 131.5791 & 245.1244 & 123.0659 & 244.1404 & 122.5738 & $\mathbf{2}$ \\
\hline $\mathbf{1 4}$ & & & & & & & $\mathbf{R}$ & 175.1190 & 88.0631 & 158.0924 & 79.5498 & & & $\mathbf{1}$ \\
\hline
\end{tabular}
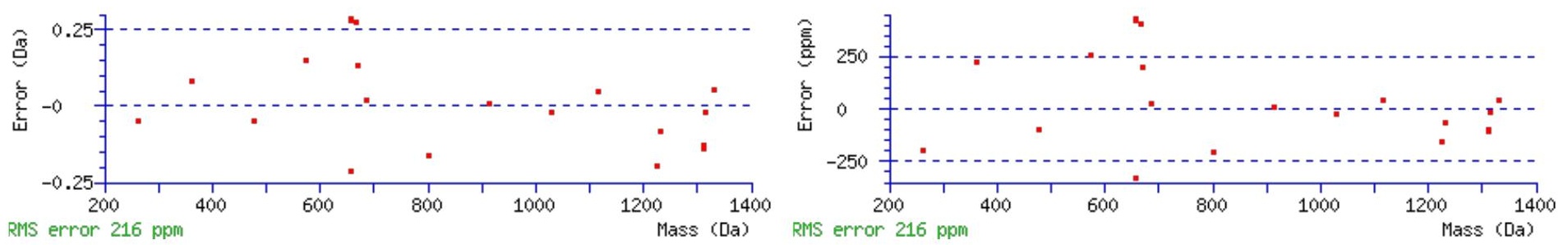

\section{All matches to this query}

\begin{tabular}{|l|l|l|l|}
\hline Score & Mr(calc): & Delta & \multicolumn{1}{|c|}{ Sequence } \\
\hline 71.4 & 1485.7311 & 0.0036 & GVVDSDDLPLNVSR \\
\hline 7.8 & 1485.7385 & -0.0038 & $\underline{\text { LDECGLNLPDVK }}$ \\
\hline 7.4 & 1484.7245 & 1.0102 & CTVVCTLVRLGK \\
\hline 6.3 & 1483.7168 & 2.0179 & NGWPQGNXDAVLR \\
\hline 4.2 & 1485.7228 & 0.0118 & NFSLSVNSVAVLR \\
\hline 4.2 & 1485.7228 & 0.0118 & NFSLSVNSVAVLR \\
\hline 4.1 & 1485.7341 & 0.0006 & VKNFAQLTVSGSR \\
\hline 4.1 & 1485.7341 & 0.0006 & VKNFAQLTVSGSR \\
\hline 4.0 & 1484.7300 & 1.0047 & FITEVWHPDVDK \\
\hline 3.3 & 1485.7212 & 0.0135 & EWEERLPADVSR \\
\hline
\end{tabular}

Spectrum No: 346; Query: 2591; Rank: 1

\section{Peptide View}

MS/MS Fragmentation of DSSDEANCTTLCSQKEFECGSGECILR

Found in IPI00205325, Tax_Id=10116 Gene_Symbol=Lrp2 Low-density lipoprotein receptor-related protein 2 precursor

Match to Query 2591: 3154.275852 from(1052.432560,3+)

Title: 100101RatKid_NS_deglyco_24.2834.2834.3.dta

Data file K:INewmanPaper|Piliang|3SubProteomes\Piliang3SP\mgf5ppm\ERLIC_3SubProteomes5ppm.mgf 

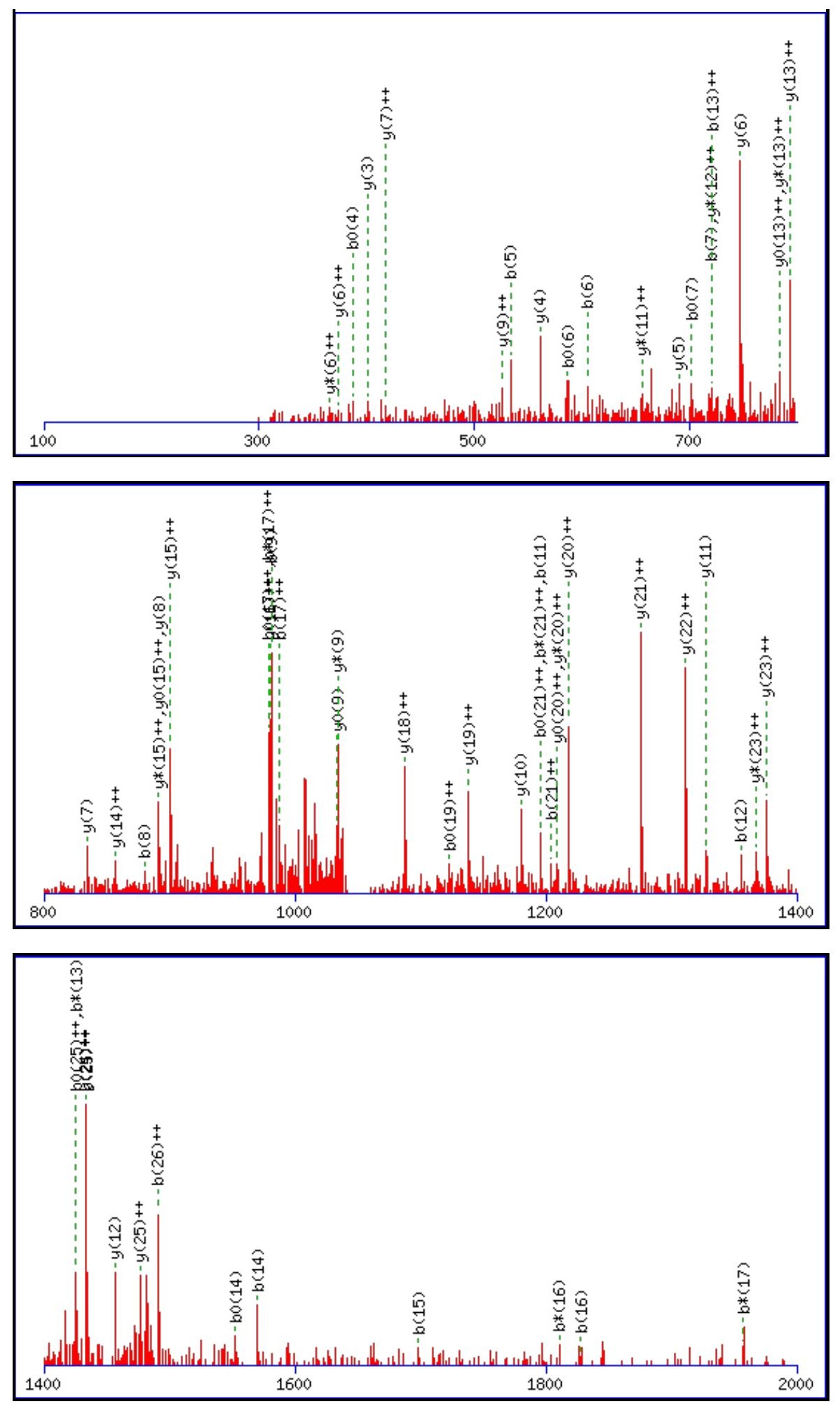

Monoisotopic mass of neutral peptide $\operatorname{Mr}($ calc): 3153.2631

Fixed modifications: Carbamidomethyl (C)

Variable modifications:

N7 : Deamidated $\mathrm{N}(\mathrm{N})$

Ions Score: 71 Expect: 2.6e-005

Matches (Bold Red): 65/292 fragment ions using 128 most intense peaks

\begin{tabular}{|c|c|c|c|c|c|c|c|c|c|c|c|c|c|c|}
\hline \# & b & $\mathbf{b}^{++}$ & $\mathbf{b}^{*}$ & $\mathbf{b}^{*^{++}}$ & $\mathbf{b}^{\mathbf{0}}$ & $\mathbf{b}^{\mathbf{0 + +}}$ & Seq. & $\mathbf{y}$ & $y^{++}$ & $\mathbf{y}^{*}$ & $\mathrm{y}^{*^{++}}$ & $\mathbf{y}^{\mathbf{0}}$ & $y^{0++}$ & \# \\
\hline 1 & 116.0342 & 58.5207 & & & 98.0237 & 49.5155 & D & & & & & & & 27 \\
\hline 2 & 203.0662 & 102.0368 & & & 185.0557 & 93.0315 & S & 3039.2435 & 1520.1254 & 3022.2169 & 1511.6121 & 3021.2329 & 1511.1201 & 26 \\
\hline 3 & 290.0983 & 145.5528 & & & 272.0877 & 136.5475 & S & 2952.2114 & 1476.6094 & 2935.1849 & 1468.0961 & 2934.2009 & 1467.6041 & 25 \\
\hline 4 & 405.1252 & 203.0662 & & & 387.1147 & 194.0610 & D & 2865.1794 & 1433.0933 & 2848.1529 & 1424.5801 & 2847.1688 & 1424.0881 & 24 \\
\hline 5 & 534.1678 & 267.5875 & & & 516.1572 & 258.5823 & $\mathbf{E}$ & 2750.1525 & 1375.5799 & 2733.1259 & 1367.0666 & 2732.1419 & 1366.5746 & 23 \\
\hline
\end{tabular}




\begin{tabular}{|c|c|c|c|c|c|c|c|c|c|c|c|c|c|c|}
\hline 6] & 605.2049 & 303.1061 & & & 587.1944 & 294.1008 & A & |2621.1099| & 1311.0586 & |2604.0833| & 1302.5453 & 2603.0993 & |1302.0533 & $3 \mid 22$ \\
\hline 7 & 720.2319 & 360.6196 & 703.2053 & 352.1063 & 702.2213 & 351.6143 & $\mathbf{N}$ & 2550.0728 & 1275.5400 & 2533.0462 & 1267.0267 & 2532.0622 & 1266.5347 & 21 \\
\hline 8 & 880.2625 & 440.6349 & 863.2360 & 432.1216 & 862.2519 & 431.6296 & C & 2435.0458 & 1218.0265 & 2418.0193 & 1209.5133 & 2417.0353 & 1209.0213 & 20 \\
\hline 9 & 981.3102 & 491.1587 & 964.2836 & 482.6455 & 963.2996 & 482.1535 & $T$ & 2275.0152 & 1138.0112 & 2257.9886 & 1129.4979 & 2257.0046 & 1129.0059 & 19 \\
\hline 10 & 1082.3579 & 541.6826 & 1065.3313 & 533.1693 & 1064.3473 & 532.6773 & $\mathbf{T}$ & 2173.9675 & 1087.4874 & 2156.9409 & 1078.9741 & 2155.9569 & 1078.4821 & 18 \\
\hline 11 & 1195.4419 & 598.2246 & 1178.4154 & 589.7113 & 1177.4314 & 589.2193 & $\mathbf{L}$ & 2072.9198 & 1036.9635 & 2055.8933 & 1028.4503 & 2054.9092 & 1027.9583 & 17 \\
\hline 12 & 1355.4726 & 678.2399 & 1338.4460 & 669.7267 & 1337.4620 & 669.2346 & $\mathrm{C}$ & 1959.8357 & 980.4215 & 1942.8092 & 971.9082 & 1941.8252 & 971.4162 & 16 \\
\hline 13 & 1442.5046 & 721.7559 & 1425.4781 & 713.2427 & 1424.4940 & 712.7507 & $S$ & 1799.8051 & 900.4062 & 1782.7785 & 891.8929 & 1781.7945 & 891.4009 & 15 \\
\hline 14 & 1570.5632 & 785.7852 & 1553.5366 & 777.2720 & 1552.5526 & 776.7799 & $\mathbf{Q}$ & 1712.7731 & 856.8902 & 1695.7465 & 848.3769 & 1694.7625 & 847.8849 & 14 \\
\hline 15 & 1698.6582 & 849.8327 & 1681.6316 & 841.3194 & 1680.6476 & 840.8274 & $\mathbf{K}$ & 1584.7145 & 792.8609 & 1567.6879 & 784.3476 & 1566.7039 & 783.8556 & 13 \\
\hline 16 & 1827.7007 & 914.3540 & 1810.6742 & 905.8407 & 1809.6902 & 905.3487 & $\mathbf{E}$ & 1456.6195 & 728.8134 & 1439.5930 & 720.3001 & 1438.6090 & 719.8081 & 12 \\
\hline 17 & 1974.7692 & 987.8882 & 1957.7426 & 979.3749 & 1956.7586 & 978.8829 & $\mathbf{F}$ & 1327.5769 & 664.2921 & 1310.5504 & 655.7788 & 1309.5664 & 655.2868 & 11 \\
\hline 18 & 2103.8117 & 1052.4095 & 2086.7852 & 1043.8962 & 2085.8012 & 1043.4042 & $\mathbf{E}$ & 1180.5085 & 590.7579 & 1163.4820 & 582.2446 & 1162.4980 & 581.7526 & 10 \\
\hline 19 & 2263.8424 & 1132.4248 & 2246.8158 & 1123.9116 & 2245.8318 & 1123.4196 & $\mathrm{C}$ & 1051.4659 & 526.2366 & 1034.4394 & 517.7233 & 1033.4554 & 517.2313 & 9 \\
\hline 20 & 2320.8639 & 1160.9356 & 2303.8373 & 1152.4223 & 2302.8533 & 1151.9303 & G & 891.4353 & 446.2213 & 874.4087 & 437.7080 & 873.4247 & 437.2160 & 8 \\
\hline 21 & 2407.8959 & 1204.4516 & 2390.8693 & 1195.9383 & 2389.8853 & 1195.4463 & S & 834.4138 & 417.7105 & 817.3873 & 409.1973 & 816.4032 & 408.7053 & 7 \\
\hline 22 & 2464.9174 & 1232.9623 & 2447.8908 & 1224.4490 & 2446.9068 & 1223.9570 & G & 747.3818 & 374.1945 & 730.3552 & 365.6813 & 729.3712 & 365.1892 & 6 \\
\hline 23 & 2593.9599 & 1297.4836 & 2576.9334 & 1288.9703 & 2575.9494 & 1288.4783 & E & 690.3603 & 345.6838 & 673.3338 & 337.1705 & 672.3498 & 336.6785 & 5 \\
\hline 24 & 2753.9906 & 1377.4989 & 2736.9640 & 1368.9857 & 2735.9800 & 1368.4937 & C & 561.3177 & 281.1625 & 544.2912 & 272.6492 & & & 4 \\
\hline 25 & 2867.0747 & 1434.0410 & 2850.0481 & 1425.5277 & 2849.0641 & 1425.0357 & I & 401.2871 & 201.1472 & 384.2605 & 192.6339 & & & 3 \\
\hline 26 & 2980.1587 & 1490.5830 & 2963.1322 & 1482.0697 & 2962.1482 & 1481.5777 & $\mathbf{L}$ & 288.2030 & 144.6051 & 271.1765 & 136.0919 & & & 2 \\
\hline 27 & & & & & & & $\mathbf{R}$ & 175.1190 & 88.0631 & 158.0924 & 79.5498 & & & 1 \\
\hline
\end{tabular}
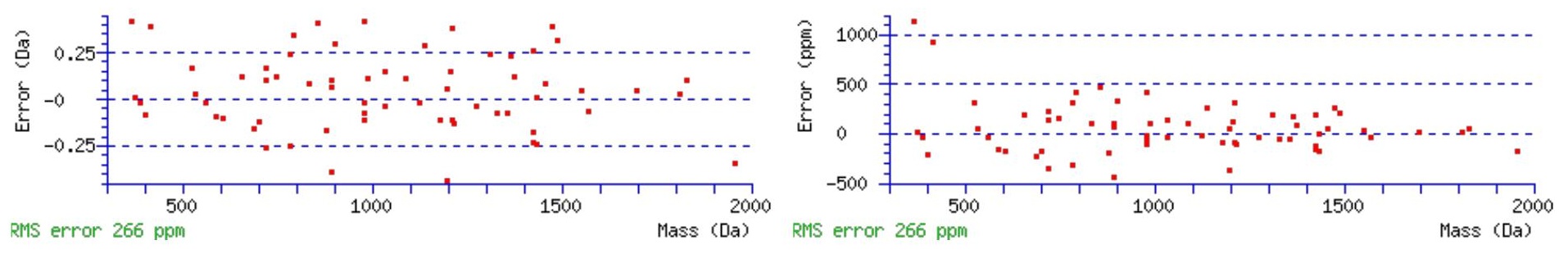

\section{All matches to this query}

\begin{tabular}{|l|l|l|l|}
\hline Score & Mr(calc): & Delta & \multicolumn{1}{c|}{ Sequence } \\
\hline 71.4 & 3153.2631 & 1.0127 & DSSDEANCTTLCSQKEFECGSGECILR \\
\hline 46.1 & 3152.2791 & 1.9967 & DSSDEANCTTLCSQKEFECGSGECILR \\
\hline 1.7 & 3153.2986 & 0.9772 & AFSRYSQLISHQSIHIGVKPYDCK \\
\hline 0.8 & 3153.2986 & 0.9772 & AFSRYSQLISHQSIHIGVKPYDCK \\
\hline 0.8 & 3153.2986 & 0.9772 & AFSRYSQLISHQSIHIGVKPYDCK \\
\hline 0.6 & 3152.2543 & 2.0216 & EQKLHQDTATVGNFISTKSIQSDER \\
\hline 0.5 & 3152.2814 & 1.9944 & GFGFVTFENIDDAKDAMMAMNGKSVDGR \\
\hline
\end{tabular}

Spectrum No: 347; Query: 427; Rank: 1

\section{Peptide View}

MS/MS Fragmentation of LFIVGSNSSSSTR

Found in IPI00191738, Tax_Id=10116 Gene_Symbol=Gclm Glutamate--cysteine ligase regulatory subunit

Match to Query 427: 1354.674808 from(678.344680,2+)

Title: 100101RatKid_NS_deglyco_04.2134.2134.2.dta

Data file K:INewmanPaper|Piliang|3SubProteomes\Piliang3SP\mgf5ppm\ERLIC_3SubProteomes5ppm.mgf 

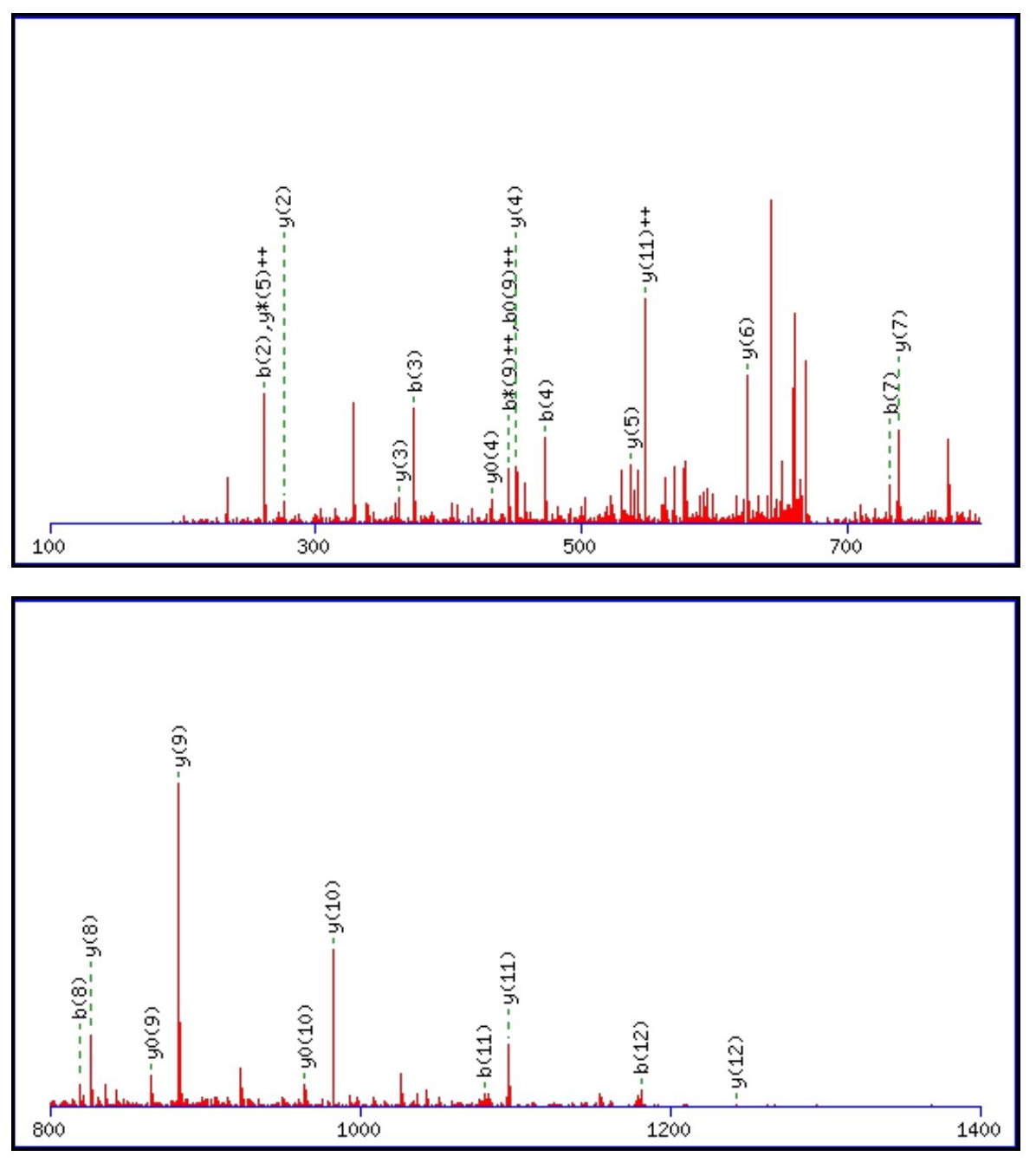

\begin{tabular}{|c|c|c|c|}
\hline & & & \\
\hline 1400 & 1600 & 1800 & 2000 \\
\hline
\end{tabular}

Monoisotopic mass of neutral peptide $\operatorname{Mr}($ calc): 1354.6729

Fixed modifications: Carbamidomethyl (C)

Variable modifications:

N7 : Deamidated_N (N)

Ions Score: 71 Expect: 1 e- 005

Matches (Bold Red): 25/120 fragment ions using 51 most intense peaks

\begin{tabular}{|c|c|c|c|c|c|c|c|c|c|c|c|c|c|c|}
\hline$\#$ & $\mathbf{b}$ & $\mathbf{b}^{++}$ & $\mathbf{b}^{*}$ & $\mathbf{b}^{\mathbf{*}^{++}}$ & $\mathbf{b}^{\mathbf{0}}$ & $\mathbf{b}^{\mathbf{0}+}$ & Seq. & $\mathbf{y}$ & $\mathbf{y}^{++}$ & $\mathbf{y}^{\mathbf{*}}$ & $\mathbf{y}^{\boldsymbol{*}^{++}}$ & $\mathbf{y}^{\mathbf{0}}$ & $\mathbf{y}^{\mathbf{0}+}$ & $\#$ \\
\hline $\mathbf{1}$ & 114.0913 & 57.5493 & & & & & $\mathbf{L}$ & & & & & & & $\mathbf{1 3}$ \\
\hline $\mathbf{2}$ & $\mathbf{2 6 1 . 1 5 9 8}$ & 131.0835 & & & & & $\mathbf{F}$ & $\mathbf{1 2 4 2 . 5 9 6 1}$ & 621.8017 & 1225.5695 & 613.2884 & 1224.5855 & 612.7964 & $\mathbf{1 2}$ \\
\hline $\mathbf{3}$ & $\mathbf{3 7 4 . 2 4 3 8}$ & 187.6255 & & & & & $\mathbf{I}$ & $\mathbf{1 0 9 5 . 5 2 7 6}$ & 548.2675 & 1078.5011 & 539.7542 & 1077.5171 & 539.2622 & $\mathbf{1 1}$ \\
\hline $\mathbf{4}$ & $\mathbf{4 7 3 . 3 1 2 2}$ & 237.1598 & & & & & $\mathbf{V}$ & $\mathbf{9 8 2 . 4 4 3 6}$ & 491.7254 & 965.4170 & 483.2122 & $\mathbf{9 6 4 . 4 3 3 0}$ & 482.7201 & $\mathbf{1 0}$ \\
\hline $\mathbf{5}$ & 530.3337 & 265.6705 & & & & & $\mathbf{G}$ & $\mathbf{8 8 3 . 3 7 5 2}$ & 442.1912 & 866.3486 & 433.6779 & $\mathbf{8 6 5 . 3 6 4 6}$ & 433.1859 & $\mathbf{9}$ \\
\hline
\end{tabular}




\begin{tabular}{|r|r|r|r|r|r|r|r|r|r|r|r|r|r|r|r|r|}
$\mathbf{6}$ & 617.3657 & 309.1865 & & & 599.3552 & 300.1812 & S & $\mathbf{8 2 6 . 3 5 3 7}$ & 413.6805 & 809.3272 & 405.1672 & 808.3431 & 404.6752 & $\mathbf{8}$ \\
\hline $\mathbf{7}$ & 732.3927 & 366.7000 & 715.3661 & 358.1867 & 714.3821 & 357.6947 & $\mathbf{N}$ & 739.3217 & 370.1645 & 722.2951 & 361.6512 & 721.3111 & 361.1592 & $\mathbf{7}$ \\
\hline $\mathbf{8}$ & $\mathbf{8 1 9 . 4 2 4 7}$ & 410.2160 & 802.3981 & 401.7027 & 801.4141 & 401.2107 & S & $\mathbf{6 2 4 . 2 9 4 7}$ & 312.6510 & 607.2682 & 304.1377 & 606.2842 & 303.6457 & $\mathbf{6}$ \\
\hline $\mathbf{9}$ & 906.4567 & 453.7320 & 889.4302 & 445.2187 & 888.4462 & $\mathbf{4 4 4 . 7 2 6 7}$ & S & 537.2627 & 269.1350 & 520.2362 & 260.6217 & 519.2521 & 260.1297 & $\mathbf{5}$ \\
\hline $\mathbf{1 0}$ & 993.4887 & 497.2480 & 976.4622 & 488.7347 & 975.4782 & 488.2427 & S & $\mathbf{4 5 0 . 2 3 0 7}$ & 225.6190 & 433.2041 & 217.1057 & 432.2201 & 216.6137 & $\mathbf{4}$ \\
\hline $\mathbf{1 1}$ & $\mathbf{1 0 8 0 . 5 2 0 8}$ & 540.7640 & 1063.4942 & 532.2507 & 1062.5102 & 531.7587 & S & 363.1987 & 182.1030 & 346.1721 & 173.5897 & 345.1881 & 173.0977 & $\mathbf{3}$ \\
\hline $\mathbf{1 2}$ & $\mathbf{1 1 8 1 . 5 6 8 5}$ & 591.2879 & 1164.5419 & 582.7746 & 1163.5579 & 582.2826 & T & $\mathbf{2 7 6 . 1 6 6 6}$ & 138.5870 & 259.1401 & 130.0737 & 258.1561 & 129.5817 & $\mathbf{2}$ \\
\hline $\mathbf{1 3}$ & & & & & & & R & 175.1190 & 88.0631 & 158.0924 & 79.5498 & & & $\mathbf{1}$ \\
\hline
\end{tabular}
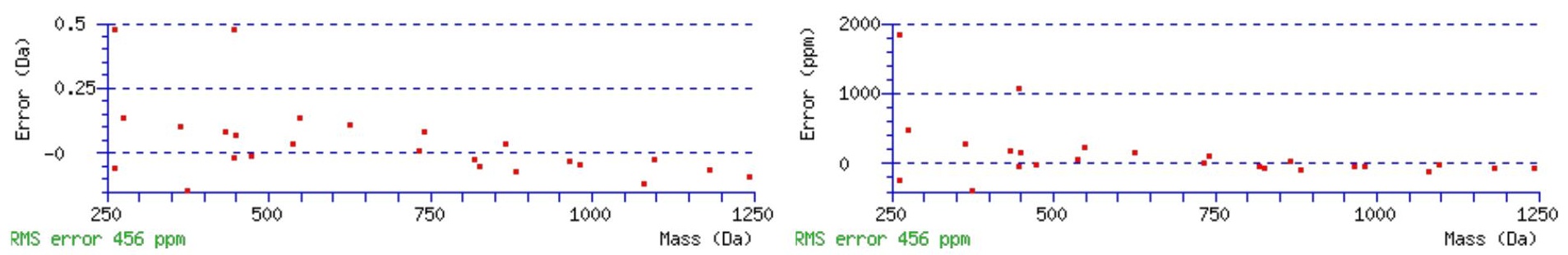

\section{All matches to this query}

\begin{tabular}{|l|l|c|l|}
\hline Score & Mr(calc): & Delta & \multicolumn{1}{c|}{ Sequence } \\
\hline 71.4 & 1354.6729 & 0.0020 & LFIVGSNSSSSTR \\
\hline 8.0 & 1354.6663 & 0.0085 & SKFQGATGMLGSR \\
\hline 6.3 & 1354.6679 & 0.0069 & MLIRVANNISK \\
\hline 5.8 & 1353.6653 & 1.0095 & QSQDLSRSLLK \\
\hline 5.0 & 1353.6823 & 0.9925 & MKRNNFSTVNK \\
\hline 4.7 & 1354.6625 & 0.0123 & MILSTISWMGGK \\
\hline 4.6 & 1354.6663 & 0.0085 & MKRNNFSTVNK \\
\hline 4.6 & 1354.6663 & 0.0085 & MKRNNFSTVNK \\
\hline 4.1 & 1354.6728 & 0.0020 & EAKEAPAQPASEK \\
\hline 3.9 & 1353.6806 & 0.9942 & HFISSSRSLLK \\
\hline
\end{tabular}

Spectrum No: 348; Query: 2212; Rank: 1

\section{Peptide View}

MS/MS Fragmentation of IISANGCKVDNSSLTGESEPQTR

Found in IPI00326305, Tax_Id=10116 Gene_Symbol=Atp1a1 Sodium/potassium-transporting ATPase subunit alpha-1 precursor

Match to Query 2212: 2463.148872 from(822.056900,3+)

Title: 091008RatKidney_NH4Format02_24.1628.1628.3.dta

Data file K:INewmanPaper|Piliangl3SubProteomes\Piliang3SP\mgf5ppm\ERLIC_3SubProteomes5ppm.mgf 

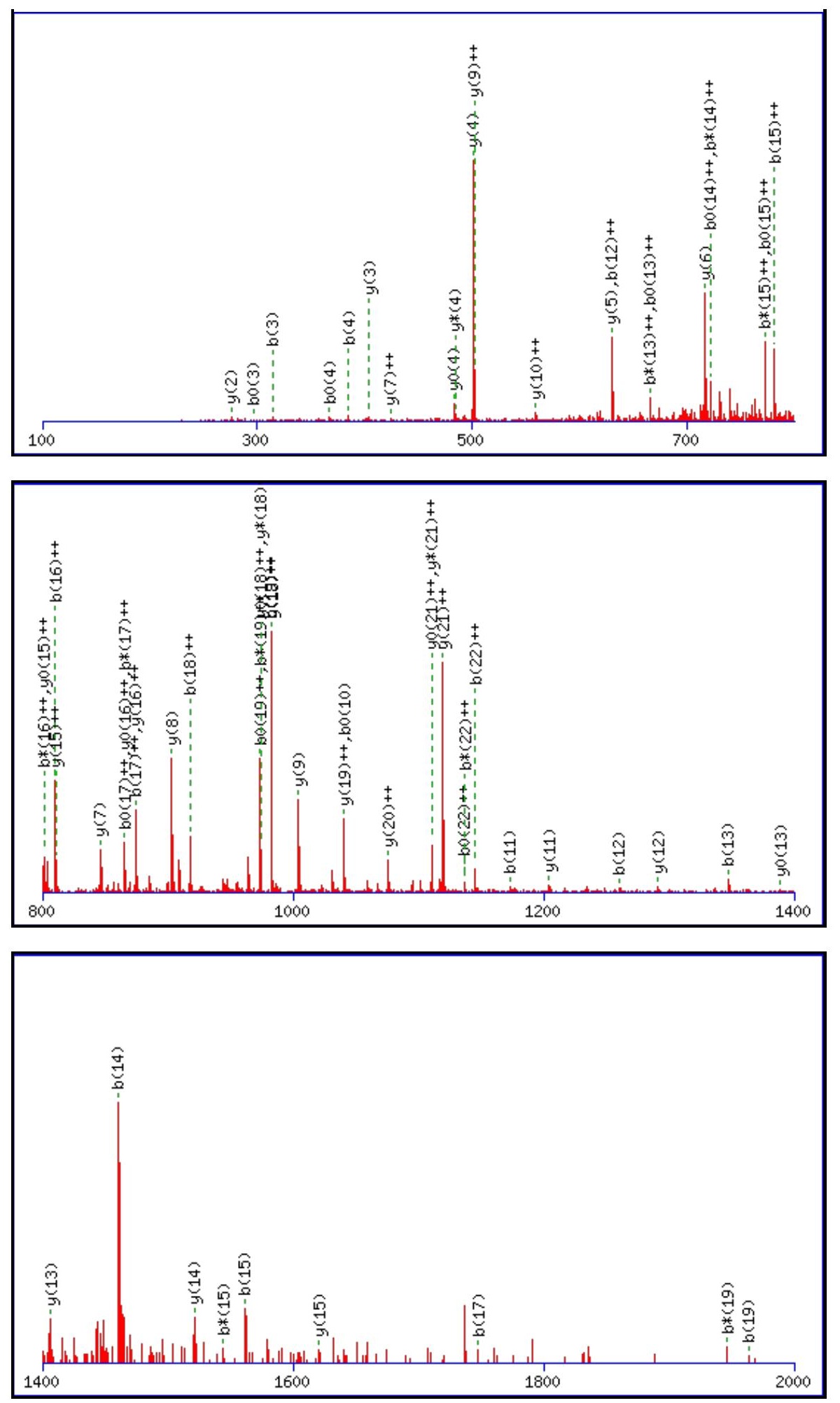

Monoisotopic mass of neutral peptide $\operatorname{Mr}($ calc): 2463.1547

Fixed modifications: Carbamidomethyl (C)

Variable modifications:

N11 : Deamidated $\mathrm{N}(\mathrm{N})$

Ions Score: 71 Expect: $2.3 \mathrm{e}-005$

Matches (Bold Red): 65/250 fragment ions using 113 most intense peaks

\begin{tabular}{|r|c|c|c|c|c|c|c|c|c|c|c|c|c|c|}
\hline$\#$ & $\mathbf{b}$ & $\mathbf{b}^{++}$ & $\mathbf{b}^{*}$ & $\mathbf{b}^{\boldsymbol{*}^{++}}$ & $\mathbf{b}^{\mathbf{0}}$ & $\mathbf{b}^{\mathbf{0 + +}}$ & Seq. & $\mathbf{y}$ & $\mathbf{y}^{++}$ & $\mathbf{y}^{\mathbf{*}}$ & $\mathbf{y}^{\mathbf{*}^{++}}$ & $\mathbf{y}^{\mathbf{0}}$ & $\mathbf{y}^{\mathbf{0 + +}}$ & $\#$ \\
\hline $\mathbf{1}$ & 114.0913 & 57.5493 & & & & & $\mathbf{I}$ & & & & & & & $\mathbf{2 3}$ \\
\hline $\mathbf{2}$ & 227.1754 & 114.0913 & & & & & $\mathbf{I}$ & 2351.0780 & 1176.0426 & 2334.0514 & 1167.5293 & 2333.0674 & 1167.0373 & $\mathbf{2 2}$ \\
\hline $\mathbf{3}$ & $\mathbf{3 1 4 . 2 0 7 4}$ & 157.6074 & & & $\mathbf{2 9 6 . 1 9 6 9}$ & 148.6021 & $\mathbf{S}$ & 2237.9939 & $\mathbf{1 1 1 9 . 5 0 0 6}$ & 2220.9673 & $\mathbf{1 1 1 0 . 9 8 7 3}$ & 2219.9833 & $\mathbf{1 1 1 0 . 4 9 5 3}$ & $\mathbf{2 1}$ \\
\hline $\mathbf{4}$ & $\mathbf{3 8 5 . 2 4 4 5}$ & 193.1259 & & & 367.2340 & 184.1206 & $\mathbf{A}$ & 2150.9619 & $\mathbf{1 0 7 5 . 9 8 4 6}$ & 2133.9353 & 1067.4713 & 2132.9513 & 1066.9793 & $\mathbf{2 0}$ \\
\hline $\mathbf{5}$ & 499.2875 & 250.1474 & 482.2609 & 241.6341 & 481.2769 & 241.1421 & $\mathbf{N}$ & 2079.9247 & $\mathbf{1 0 4 0 . 4 6 6 0}$ & 2062.8982 & 1031.9527 & 2061.9142 & 1031.4607 & $\mathbf{1 9}$ \\
\hline
\end{tabular}




\begin{tabular}{|c|c|c|c|c|c|c|c|c|c|c|c|c|c|c|}
\hline 6 & 556.3089 & 278.6581 & 539.2824 & 270.1448 & 538.2984 & 269.6528 & G & 1965.8818 & 983.4445 & 1948.8553 & 974.9313 & |1947.8713 & 974.4393 & 18 \\
\hline 7 & 716.3396 & 358.6734 & 699.3130 & 350.1602 & 698.3290 & 349.6681 & C & 1908.8604 & 954.9338 & 1891.8338 & 946.4205 & 1890.8498 & 945.9285 & 17 \\
\hline 8 & 844.4345 & 422.7209 & 827.4080 & 414.2076 & 826.4240 & 413.7156 & $\mathbf{K}$ & 1748.8297 & 874.9185 & 1731.8032 & 866.4052 & 1730.8191 & 865.9132 & 16 \\
\hline 9 & 943.5030 & 472.2551 & 926.4764 & 463.7418 & 925.4924 & 463.2498 & V & 1620.7347 & 810.8710 & 1603.7082 & 802.3577 & 1602.7242 & 801.8657 & 15 \\
\hline 10 & 1058.5299 & 529.7686 & 1041.5034 & 521.2553 & 1040.5193 & 520.7633 & D & 1521.6663 & 761.3368 & 1504.6398 & 752.8235 & 1503.6558 & 752.3315 & 14 \\
\hline 11 & 1173.5568 & 587.2821 & 1156.5303 & 578.7688 & 1155.5463 & 578.2768 & $\mathbf{N}$ & 1406.6394 & 703.8233 & 1389.6128 & 695.3101 & 1388.6288 & 694.8180 & 13 \\
\hline 12 & 1260.5889 & 630.7981 & 1243.5623 & 622.2848 & 1242.5783 & 621.7928 & $\mathbf{S}$ & 1291.6125 & 646.3099 & 1274.5859 & 637.7966 & 1273.6019 & 637.3046 & 12 \\
\hline 13 & 1347.6209 & 674.3141 & 1330.5943 & 665.8008 & 1329.6103 & 665.3088 & $\mathbf{S}$ & 1204.5804 & 602.7938 & 1187.5539 & 594.2806 & 1186.5699 & 593.7886 & 11 \\
\hline 14 & 1460.7050 & 730.8561 & 1443.6784 & 722.3428 & 1442.6944 & 721.8508 & $\mathbf{L}$ & 1117.5484 & 559.2778 & 1100.5218 & 550.7646 & 1099.5378 & 550.2726 & 10 \\
\hline 15 & 1561.7526 & 781.3800 & 1544.7261 & 772.8667 & 1543.7421 & 772.3747 & $\mathbf{T}$ & 1004.4643 & 502.7358 & 987.4378 & 494.2225 & 986.4538 & 493.7305 & 9 \\
\hline 16 & 1618.7741 & 809.8907 & 1601.7476 & 801.3774 & 1600.7635 & 800.8854 & G & 903.4167 & 452.2120 & 886.3901 & 443.6987 & 885.4061 & 443.2067 & 8 \\
\hline 17 & 1747.8167 & 874.4120 & 1730.7901 & 865.8987 & 1729.8061 & 865.4067 & $\mathbf{E}$ & 846.3952 & 423.7012 & 829.3686 & 415.1880 & 828.3846 & 414.6959 & 7 \\
\hline 18 & 1834.8487 & 917.9280 & 1817.8222 & 909.4147 & 1816.8382 & 908.9227 & S & 717.3526 & 359.1799 & 700.3260 & 350.6667 & 699.3420 & 350.1747 & 6 \\
\hline 19 & 1963.8913 & 982.4493 & 1946.8648 & 973.9360 & 1945.8808 & 973.4440 & E & 630.3206 & 315.6639 & 613.2940 & 307.1506 & 612.3100 & 306.6586 & 5 \\
\hline 21 & 2189.0027 & 1095.0050 & 2171.9761 & 1086.4917 & 2170.9921 & 1085.9997 & $\mathbf{Q}$ & 404.2252 & 202.6162 & 387.1987 & 194.1030 & 386.2146 & 193.6110 & 3 \\
\hline 22 & 2290.0503 & 1145.5288 & 2273.0238 & 1137.0155 & 2272.0398 & 1136.5235 & $\mathbf{T}$ & 276.1666 & 138.5870 & 259.1401 & 130.0737 & 258.1561 & 129.5817 & 2 \\
\hline 23 & & & & & & & $\mathbf{R}$ & 175.1190 & 88.0631 & 158.0924 & 79.5498 & & & 1 \\
\hline
\end{tabular}
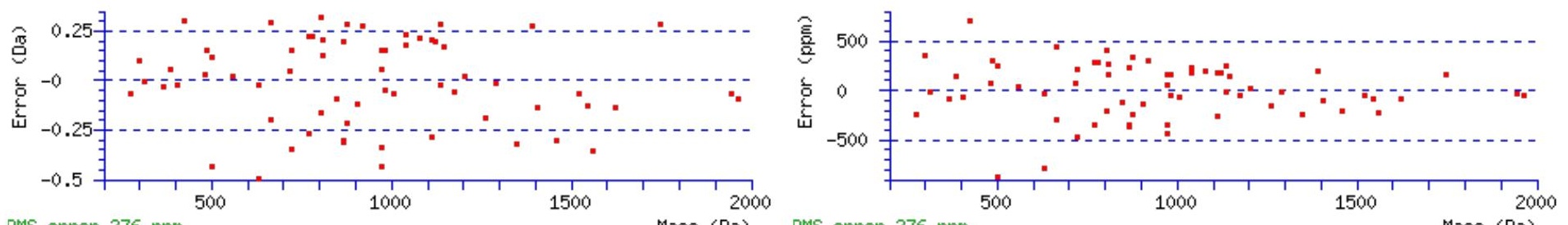

RNS error 276 ppm

Mass (Da) RMS error 276 ppm

Mass (Da)

\section{All matches to this query}

\begin{tabular}{|l|l|l|l|}
\hline Score & Mr(calc): & Delta & \multicolumn{1}{|c|}{ Sequence } \\
\hline 71.2 & 2463.1547 & -0.0059 & IISANGCKVDNSSLTGESEPQTR \\
\hline 54.7 & 2463.1547 & -0.0059 & $\underline{\text { ISANGCKVDNSSLTGESEPQTR }}$ \\
\hline 6.3 & 2461.1325 & 2.0164 & GGAKSIITTSSVNAPMGVNQEK \\
\hline 1.7 & 2462.1389 & 1.0099 & KNSSRPAVTQGTAPSTAXMRTK \\
\hline 1.7 & 2462.1389 & 1.0099 & KNSSRPAVTQGTAPSTAXMRTK \\
\hline 1.6 & 2461.1186 & 2.0303 & KNSSRPAVTQGTAPSTAXMRTK \\
\hline 1.4 & 2461.1325 & 2.0164 & GGAKSIITTSSVNAPMGVNQEK \\
\hline 0.8 & 2463.1672 & -0.0183 & LOYLAAQNKGLQTOLSESRR \\
\hline 0.5 & 2461.1501 & 1.9988 & WMSYEERHLLDELYITKR \\
\hline 0.5 & 2461.1501 & 1.9988 & WMSYEERHLLDELYITKR \\
\hline
\end{tabular}

Spectrum No: 349; Query: 1020; Rank: 1

\section{Peptide View}

MS/MS Fragmentation of TYCSSELVSNCTQK

Found in IPI00212725, Tax_Id=10116 Gene_Symbol=Cd164 Endolyn precursor

Match to Query 1020: 1676.706548 from(839.360550,2+)

Title: 100101RatKid_NS_deglyco_25.1264.1264.2.dta

Data file K:INewmanPaper|Piliangl3SubProteomes\Piliang3SP\mgf5ppm\ERLIC_3SubProteomes5ppm.mgf 

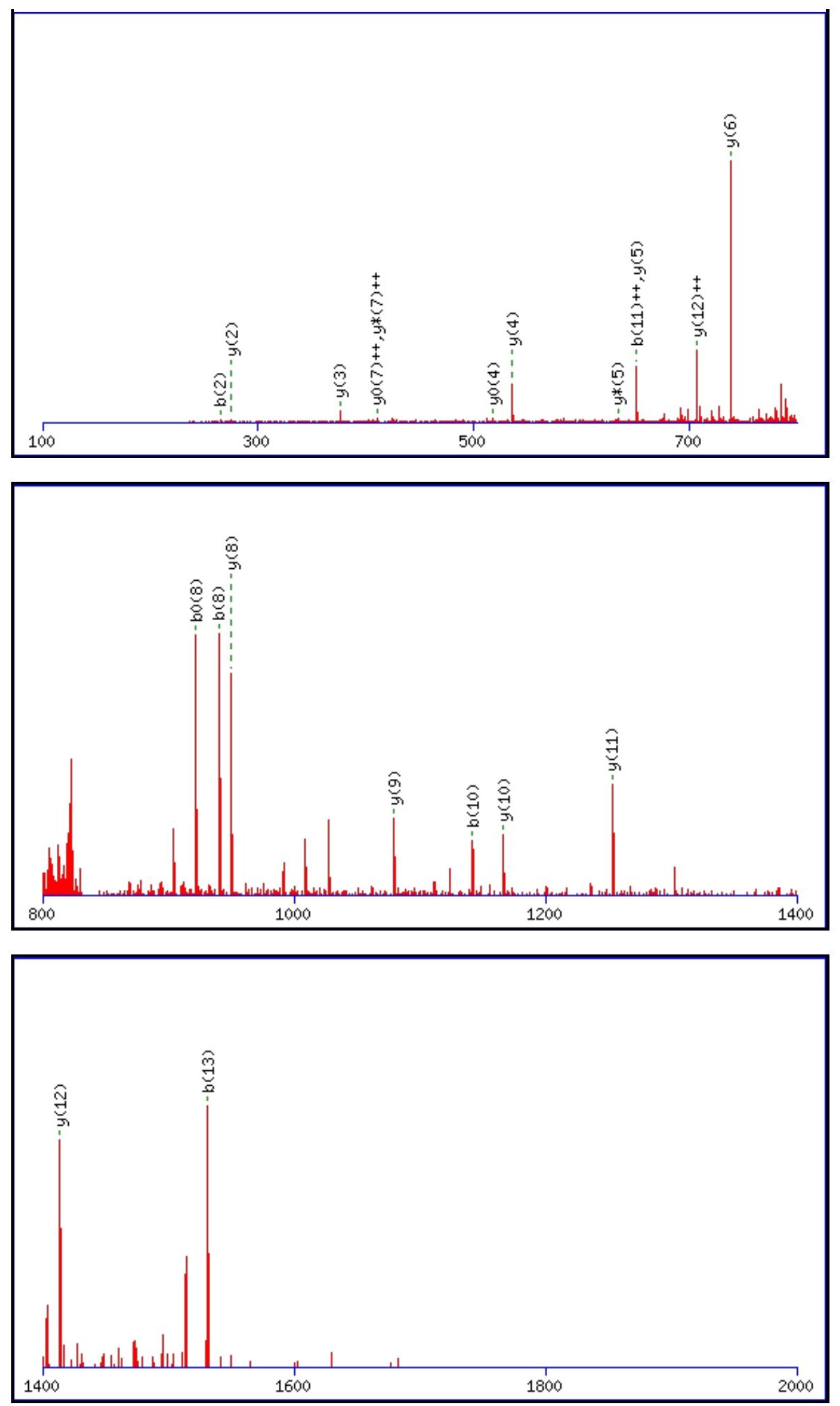

Monoisotopic mass of neutral peptide $\operatorname{Mr}($ calc): 1676.7022

Fixed modifications: Carbamidomethyl (C)

Variable modifications:

N10 : Deamidated $\mathrm{N}(\mathrm{N})$

Ions Score: 71 Expect: $1.4 \mathrm{e}-005$

Matches (Bold Red): 21/134 fragment ions using 30 most intense peaks

\begin{tabular}{|r|c|c|c|c|c|c|c|c|c|c|c|c|c|c|}
\hline$\#$ & $\mathbf{b}$ & $\mathbf{b}^{++}$ & $\mathbf{b}^{*}$ & $\mathbf{b}^{\boldsymbol{*}^{++}}$ & $\mathbf{b}^{\mathbf{0}}$ & $\mathbf{b}^{\mathbf{0 + +}}$ & Seq. & $\mathbf{y}$ & $\mathbf{y}^{++}$ & $\mathbf{y}^{\mathbf{*}}$ & $\mathbf{y}^{\boldsymbol{*}^{++}}$ & $\mathbf{y}^{\mathbf{0}}$ & $\mathbf{y}^{\mathbf{0 + +}}$ & $\#$ \\
\hline $\mathbf{1}$ & 102.0550 & 51.5311 & & & 84.0444 & 42.5258 & $\mathbf{T}$ & & & & & & & $\mathbf{1 4}$ \\
\hline $\mathbf{2}$ & $\mathbf{2 6 5 . 1 1 8 3}$ & 133.0628 & & & 247.1077 & 124.0575 & $\mathbf{Y}$ & 1576.6618 & 788.8345 & 1559.6352 & 780.3213 & 1558.6512 & 779.8292 & $\mathbf{1 3}$ \\
\hline $\mathbf{3}$ & 425.1489 & 213.0781 & & & 407.1384 & 204.0728 & $\mathbf{C}$ & $\mathbf{1 4 1 3 . 5 9 8 5}$ & $\mathbf{7 0 7 . 3 0 2 9}$ & 1396.5719 & 698.7896 & 1395.5879 & 698.2976 & $\mathbf{1 2}$ \\
\hline $\mathbf{4}$ & 512.1810 & 256.5941 & & & 494.1704 & 247.5888 & S & $\mathbf{1 2 5 3 . 5 6 7 8}$ & 627.2875 & 1236.5413 & 618.7743 & 1235.5572 & 618.2823 & $\mathbf{1 1}$ \\
\hline $\mathbf{5}$ & 599.2130 & 300.1101 & & & 581.2024 & 291.1049 & S & $\mathbf{1 1 6 6 . 5 3 5 8}$ & 583.7715 & 1149.5092 & 575.2583 & 1148.5252 & 574.7662 & $\mathbf{1 0}$ \\
\hline
\end{tabular}




\begin{tabular}{|r|r|r|r|r|r|r|r|r|r|r|r|r|r|r|r|r|r|}
$\mathbf{6}$ & 728.2556 & 364.6314 & & & 710.2450 & 355.6261 & $\mathbf{E}$ & $\mathbf{1 0 7 9 . 5 0 3 7}$ & 540.2555 & 1062.4772 & 531.7422 & 1061.4932 & 531.2502 & $\mathbf{9}$ \\
\hline $\mathbf{7}$ & 841.3396 & 421.1735 & & & 823.3291 & 412.1682 & L & $\mathbf{9 5 0 . 4 6 1 2}$ & 475.7342 & 933.4346 & 467.2209 & 932.4506 & 466.7289 & $\mathbf{8}$ \\
\hline $\mathbf{8}$ & $\mathbf{9 4 0 . 4 0 8 1}$ & 470.7077 & & & $\mathbf{9 2 2 . 3 9 7 5}$ & 461.7024 & V & 837.3771 & 419.1922 & 820.3505 & $\mathbf{4 1 0 . 6 7 8 9}$ & 819.3665 & $\mathbf{4 1 0 . 1 8 6 9}$ & $\mathbf{7}$ \\
\hline $\mathbf{9}$ & 1027.4401 & 514.2237 & & & 1009.4295 & 505.2184 & S & 738.3087 & 369.6580 & 721.2821 & 361.1447 & 720.2981 & 360.6527 & $\mathbf{6}$ \\
\hline $\mathbf{1 0}$ & $\mathbf{1 1 4 2 . 4 6 7 0}$ & 571.7372 & 1125.4405 & 563.2239 & 1124.4565 & 562.7319 & $\mathbf{N}$ & $\mathbf{6 5 1 . 2 7 6 6}$ & 326.1420 & $\mathbf{6 3 4 . 2 5 0 1}$ & 317.6287 & 633.2661 & 317.1367 & $\mathbf{5}$ \\
\hline $\mathbf{1 1}$ & 1302.4977 & $\mathbf{6 5 1 . 7 5 2 5}$ & 1285.4711 & 643.2392 & 1284.4871 & 642.7472 & $\mathbf{C}$ & $\mathbf{5 3 6 . 2 4 9 7}$ & 268.6285 & 519.2232 & 260.1152 & $\mathbf{5 1 8 . 2 3 9 1}$ & 259.6232 & $\mathbf{4}$ \\
\hline $\mathbf{1 2}$ & 1403.5454 & 702.2763 & 1386.5188 & 693.7630 & 1385.5348 & 693.2710 & T & 376.2191 & 188.6132 & 359.1925 & 180.0999 & 358.2085 & 179.6079 & $\mathbf{3}$ \\
\hline $\mathbf{1 3}$ & $\mathbf{1 5 3 1 . 6 0 3 9}$ & 766.3056 & 1514.5774 & 757.7923 & 1513.5934 & 757.3003 & $\mathbf{Q}$ & $\mathbf{2 7 5 . 1 7 1 4}$ & 138.0893 & 258.1448 & 129.5761 & & & \\
\hline $\mathbf{1 4}$ & & & & & & & $\mathbf{K}$ & 147.1128 & 74.0600 & 130.0863 & 65.5468 & & & $\mathbf{1}$ \\
\hline
\end{tabular}
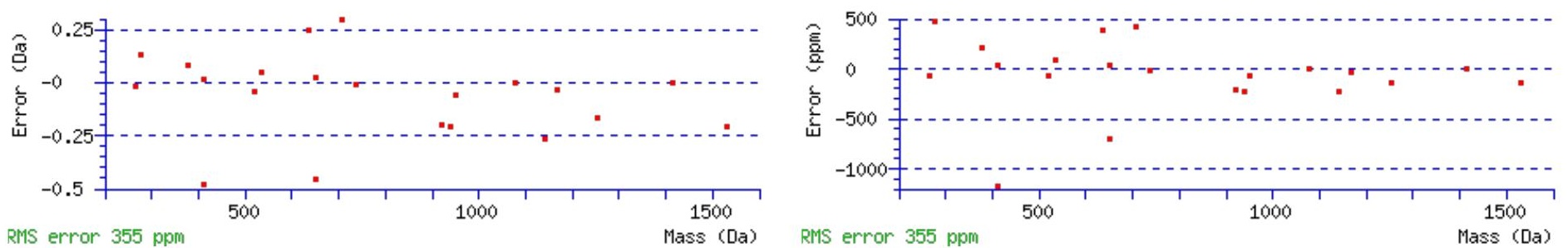

\section{All matches to this query}

\begin{tabular}{|l|l|l|l|}
\hline Score & Mr(calc): & Delta & \multicolumn{1}{c|}{ Sequence } \\
\hline 71.2 & 1676.7022 & 0.0044 & TYCSSELVSNCTQK \\
\hline 16.7 & 1675.7182 & 0.9884 & TYCSSELVSNCTQK \\
\hline 2.9 & 1675.6967 & 1.0098 & VKAGNANSKTGEPSR \\
\hline 2.5 & 1676.7117 & -0.0051 & MLNFGASLQQASEGK \\
\hline 1.3 & 1676.6906 & 0.0160 & QMTAQHYVTEFNK \\
\hline 1.0 & 1675.7164 & 0.9901 & SGNSLPLSNSTMFIQ \\
\hline 1.0 & 1675.7164 & 0.9901 & SGNSLPLSNSTMFIQ \\
\hline 1.0 & 1676.7108 & -0.0043 & IMSFLKXVCTQK \\
\hline 0.4 & 1676.7229 & -0.0164 & KIDERMSDAQGGYK \\
\hline 0.1 & 1674.7032 & 2.0033 & STKGPREESTSCTR \\
\hline
\end{tabular}

Spectrum No: 350; Query: 312; Rank: 1

\section{Peptide View}

MS/MS Fragmentation of MTLSQDNSTLR

Found in IPI00199607, Tax_Id=10116 Gene_Symbol=Ceacam1_v4;Ceacam1_v1;Ceacam1;Ceacam1_v3 carcinoembryonic antigen-related cell adhesion molecule isoform 2

Match to Query 312: 1265.596608 from(633.805580,2+)

Title: 100101RatKid_NS_deglyco_23.1366.1366.2.dta

Data file K:INewmanPaper|Piliang|3SubProteomes\Piliang3SP\mgf5ppm\ERLIC_3SubProteomes5ppm.mgf 

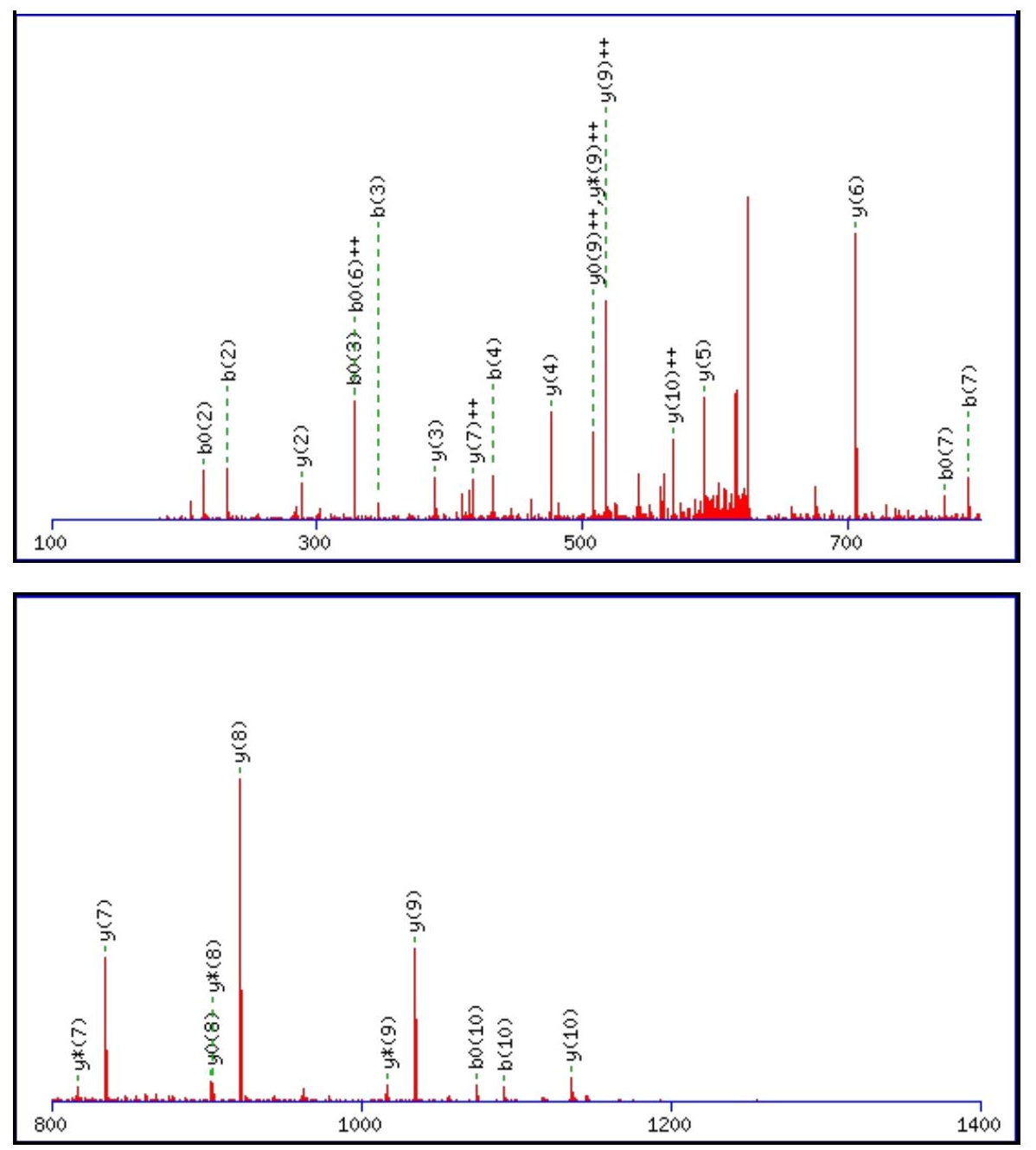

\begin{tabular}{rrrr}
\hline 00 & 1600 & 1800 & 2000 \\
\hline
\end{tabular}

Monoisotopic mass of neutral peptide $\operatorname{Mr}($ calc): 1265.5921

Fixed modifications: Carbamidomethyl (C)

Variable modifications:

N7 : Deamidated $\mathrm{N}(\mathrm{N})$

Ions Score: 71 Expect: $1.1 \mathrm{e}-005$

Matches (Bold Red): 28/106 fragment ions using 44 most intense peaks

\begin{tabular}{|r|c|c|c|c|c|c|c|c|c|c|c|c|c|c|}
\hline$\#$ & $\mathbf{b}$ & $\mathbf{b}^{++}$ & $\mathbf{b}^{*}$ & $\mathbf{b}^{\boldsymbol{*}^{++}}$ & $\mathbf{b}^{\mathbf{0}}$ & $\mathbf{b}^{\mathbf{0 + +}}$ & Seq. & $\mathbf{y}$ & $\mathbf{y}^{++}$ & $\mathbf{y}^{\mathbf{*}}$ & $\mathbf{y}^{\boldsymbol{*}^{++}}$ & $\mathbf{y}^{\mathbf{0}}$ & $\mathbf{y}^{\mathbf{0 + +}}$ & $\#$ \\
\hline $\mathbf{1}$ & 132.0478 & 66.5275 & & & & & $\mathbf{M}$ & & & & & & & $\mathbf{1 1}$ \\
\hline $\mathbf{2}$ & 233.0954 & 117.0514 & & & $\mathbf{2 1 5 . 0 8 4 9}$ & 108.0461 & $\mathbf{T}$ & $\mathbf{1 1 3 5 . 5 5 9 0}$ & $5 \mathbf{6 8 . 2 8 3 1}$ & 1118.5324 & 559.7698 & 1117.5484 & 559.2778 & $\mathbf{1 0}$ \\
\hline 3 & 346.1795 & 173.5934 & & & 328.1689 & 164.5881 & $\mathbf{L}$ & $\mathbf{1 0 3 4 . 5 1 1 3}$ & 517.7593 & $\mathbf{1 0 1 7 . 4 8 4 7}$ & $\mathbf{5 0 9 . 2 4 6 0}$ & 1016.5007 & $\mathbf{5 0 8 . 7 5 4 0}$ & $\mathbf{9}$ \\
\hline $\mathbf{4}$ & $\mathbf{4 3 3 . 2 1 1 5}$ & 217.1094 & & & 415.2010 & 208.1041 & S & $\mathbf{9 2 1 . 4 2 7 2}$ & 461.2172 & $\mathbf{9 0 4 . 4 0 0 7}$ & 452.7040 & $\mathbf{9 0 3 . 4 1 6 6}$ & 452.2120 & $\mathbf{8}$ \\
\hline $\mathbf{5}$ & 561.2701 & 281.1387 & 544.2436 & 272.6254 & 543.2595 & 272.1334 & $\mathbf{Q}$ & $\mathbf{8 3 4 . 3 9 5 2}$ & $\mathbf{4 1 7 . 7 0 1 2}$ & $\mathbf{8 1 7 . 3 6 8 6}$ & 409.1880 & 816.3846 & 408.6959 & $\mathbf{7}$ \\
\hline
\end{tabular}




\begin{tabular}{|c|c|c|c|c|c|c|c|c|c|c|c|c|c|c|}
\hline 6 & 676.2971 & |338.6522| & 659.2705 & |330.1389 & 658.2865 & |329.6469| & D & 706.3366 & |353.6719 & 689.3101 & 345.1587 & 688.3260 & |344.6667| & 6 \\
\hline 7 & 791.3240 & 396.1656 & 774.2974 & 387.6524 & 773.3134 & 387.1603 & $\mathbf{N}$ & 591.3097 & 296.1585 & 574.2831 & 287.6452 & 573.2991 & 287.1532 & 5 \\
\hline 8 & 878.3560 & 439.6816 & 861.3295 & 431.1684 & 860.3455 & 430.6764 & $S$ & 476.2827 & 238.6450 & 459.2562 & 230.1317 & 458.2722 & 229.6397 & 4 \\
\hline 9 & 979.4037 & 490.2055 & 962.3771 & 481.6922 & 961.3931 & 481.2002 & $\mathbf{T}$ & 389.2507 & 195.1290 & 372.2241 & 186.6157 & 371.2401 & 186.1237 & 3 \\
\hline 10 & 1092.4878 & 546.7475 & 1075.4612 & 538.2342 & 1074.4772 & 537.7422 & $\mathbf{L}$ & 288.2030 & 144.6051 & 271.1765 & 136.0919 & & & 2 \\
\hline 11 & & & & & & & $\mathbf{R}$ & 175.1190 & 88.0631 & 158.0924 & 79.5498 & & & 1 \\
\hline
\end{tabular}
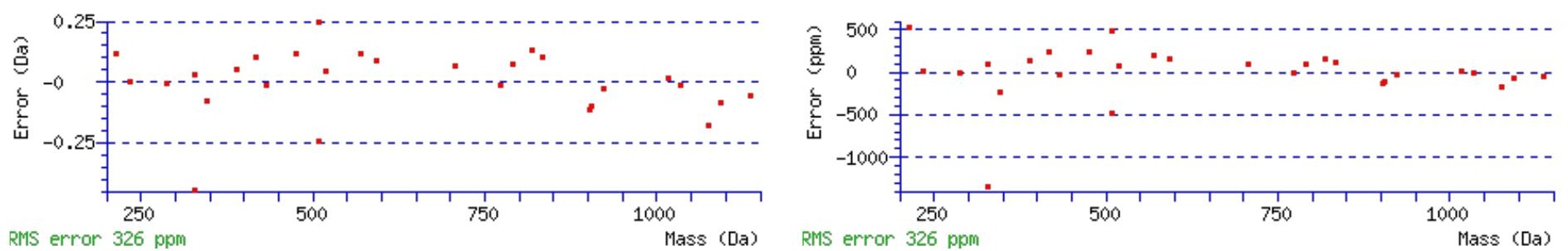

\section{All matches to this query}

\begin{tabular}{|l|l|l|l|}
\hline Score & Mr(calc): & Delta & \multicolumn{1}{|c|}{ Sequence } \\
\hline 71.1 & 1265.5921 & 0.0045 & MTLSODNSTLR \\
\hline 13.0 & 1265.5856 & 0.0110 & TGMREEMSLGR \\
\hline 8.4 & 1265.5922 & 0.0044 & VKDDMTEVTGR \\
\hline 5.5 & 1263.5876 & 2.0090 & VNSLSKLKSK \\
\hline 4.0 & 1265.6017 & -0.0050 & QEQLELTLGR \\
\hline 3.5 & 1265.6043 & -0.0077 & MMPGRMVGVTR \\
\hline 3.2 & 1265.5888 & 0.0078 & DGNGYISAAELR \\
\hline 3.0 & 1265.5921 & 0.0045 & TSLMSAESPTSR \\
\hline 2.7 & 1265.5921 & 0.0045 & STLSESMQLDR \\
\hline 2.7 & 1264.5982 & 0.9984 & QAFENGVRMGR \\
\hline
\end{tabular}

Spectrum No: 351; Query: 1896; Rank: 1

\section{Peptide View}

MS/MS Fragmentation of DLRNEIQVNTSQIPDTVTR

Found in IPI00211648, Tax_Id=10116 Gene_Symbol=Slc3a1 Neutral and basic amino acid transport protein rBAT

Match to Query 1896: 2199.120868 from(1100.567710,2+)

Title: 100101RatKid_NS_deglyco_16.3817.3817.2.dta

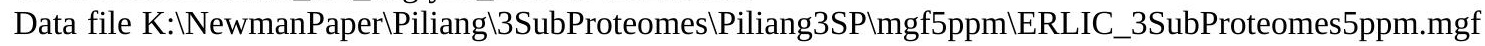

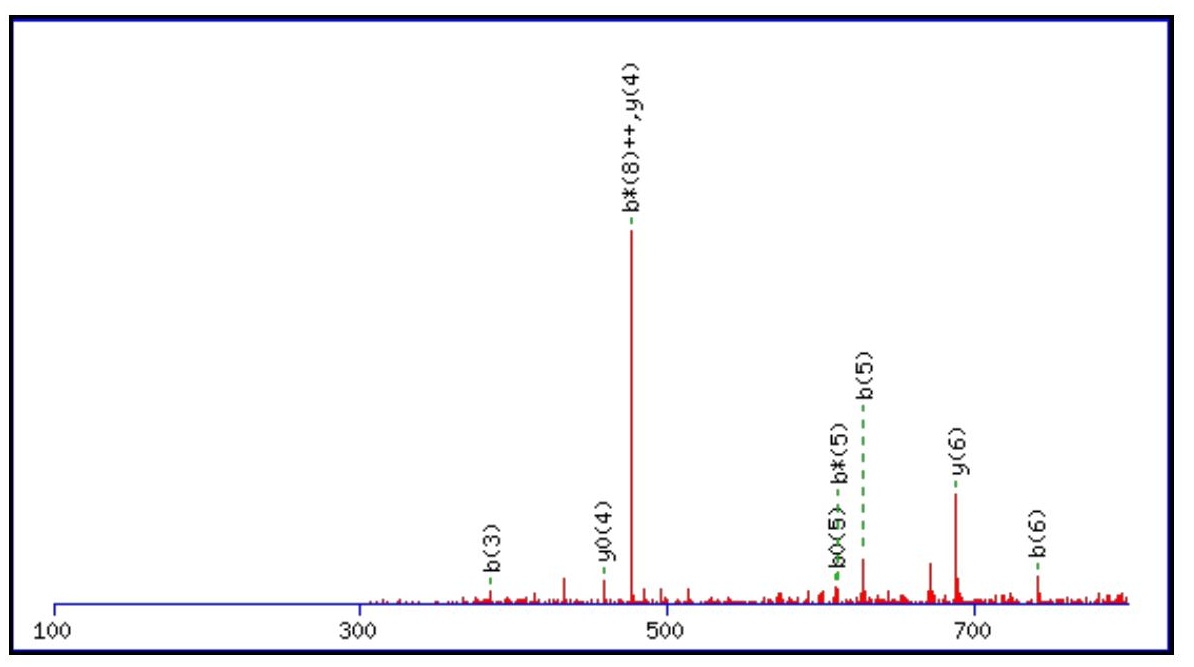



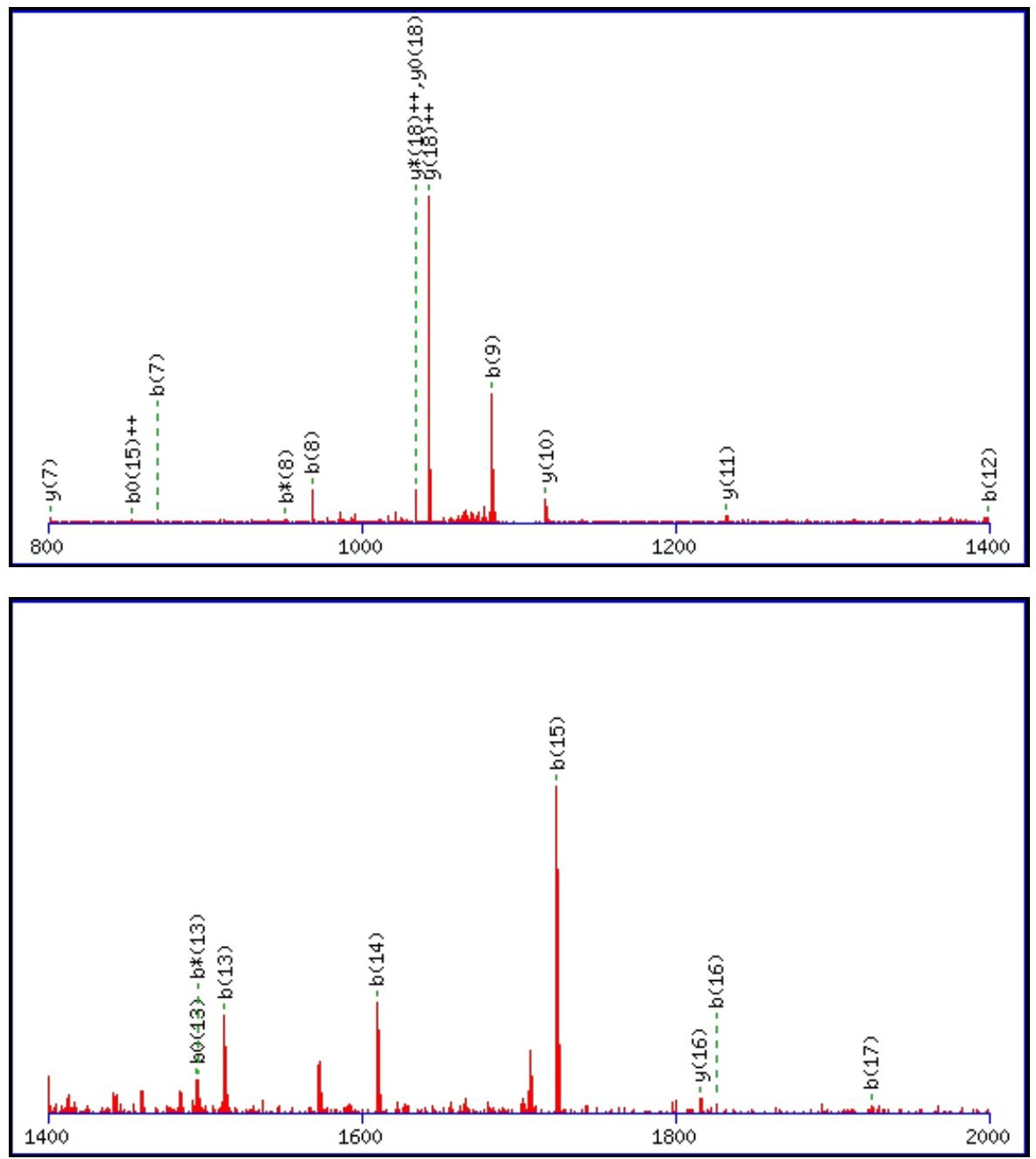

Monoisotopic mass of neutral peptide $\operatorname{Mr}$ (calc): 2199.1131

Fixed modifications: Carbamidomethyl (C)

Variable modifications:

N9 : Deamidated_N (N)

Ions Score: 71 Expect: $1.2 \mathrm{e}-005$

Matches (Bold Red): 29/210 fragment ions using 46 most intense peaks

\begin{tabular}{|c|c|c|c|c|c|c|c|c|c|c|c|c|c|c|}
\hline \# & b & & & & $\mathbf{b}^{0}$ & & Seq. & $\mathbf{y}$ & & & & $\mathbf{y}^{0}$ & & \# \\
\hline 1 & 116.0342 & & & & & & D & & & & & & & 19 \\
\hline 2 & 229.1183 & & & & & & $\mathbf{L}$ & & & & & & & 18 \\
\hline 3 & 385.2194 & & & & 088 & & $\mathrm{n}$ & & & & & & & 17 \\
\hline 4 & 499.2623 & 250.1348 & & & 481.2 & & $\mathbf{N}$ & & & & & & & 16 \\
\hline 5 & 628. & 61 & & & & 508 & $\mathbf{E}$ & & & & & & 310 & 15 \\
\hline 6 & 741.3890 & 371.1981 & & & 723.' & & I & & & & & & & 14 \\
\hline 7 & 869.4 & 435. & 10 & & 70 & & $\mathbf{Q}$ & & & & & 281 & 677 & 13 \\
\hline 8 & 968.5160 & 484.7616 & & & 950. & & V & & & & & & & 12 \\
\hline 9 & 1083.5429 & 542.2751 & 164 & 533. & 323 & 533.2698 & $\mathbf{N}$ & & 095 & 852 & 962 & 6012 & 3042 & 11 \\
\hline 10 & 184.5906 & 592.7989 & 40 & 584 & 00 & 583.7 & $\mathbf{T}$ & & 559 & 582 & & 1099 & 907 & 10 \\
\hline 11 & 1271.6226 & 636.3149 & 961 & 627. & 253.6120 & 627.3097 & S & 371 & 722 & 106 & 589 & 998.5265 & 499.7669 & 9 \\
\hline 12 & 1399.6812 & 700.3442 & & & 706 & 691.3 & $\mathbf{Q}$ & & & & & & & 8 \\
\hline 13 & 1512.7653 & 756.8863 & 495.7387 & 748.3730 & 494.7547 & 747.8810 & I & & 401 & & 392.7136 & 783.4359 & 392.2216 & 7 \\
\hline 14 & 1609.8180 & 805.4126 & 1592.7915 & 796.8994 & 1591.8075 & 796.4074 & $\mathbf{P}$ & 688.3624 & 344.6849 & 671.3359 & 336.1716 & 3519 & 6796 & 6 \\
\hline 15 & 1724.8450 & 862.9261 & 1707.8184 & 854.4128 & 1706.8344 & 853.9208 & D & 591.3097 & 296.1585 & 574.2831 & & 573.2991 & 287.1532 & 5 \\
\hline 16 & 1825.8926 & 913.4500 & 1808.8661 & 904.9367 & 1807.8821 & 904.4447 & $\mathbf{T}$ & 476.2827 & 238.6450 & 459.2562 & 230.1317 & 458.2722 & 229.6397 & 4 \\
\hline 17 & 1924.9611 & 962.9842 & 1907.9345 & 954.4709 & 1906.9505 & 953.9789 & $\mathbf{V}$ & & 188.1212 & 358.2085 & 179.6079 & 357.2245 & 179.1159 & 3 \\
\hline 18 & 2026.0087 & 1013.5080 & 2008.9822 & 1004.9947 & 2007.9982 & 1004.5027 & $\mathbf{T}$ & 276.1666 & 138.5870 & 259.1401 & 130.0737 & 258.1561 & 129.5817 & 2 \\
\hline
\end{tabular}




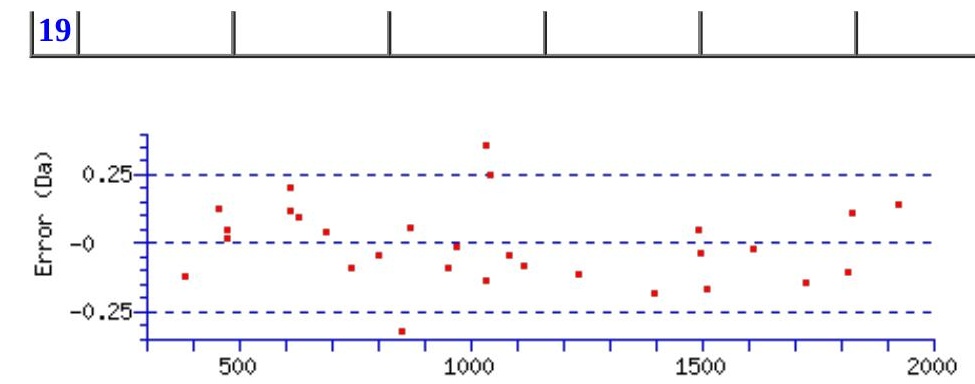

RMS error 165 ppm

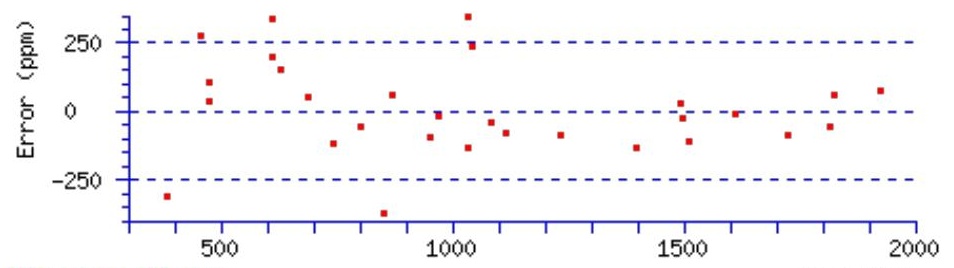

Mass (Da) RMS error 165 ppm

Mass (Da)

\section{All matches to this query}

\begin{tabular}{|l|l|c|l|}
\hline Score & Mr(calc): & Delta & \multicolumn{1}{|c|}{ Sequence } \\
\hline 71.0 & 2199.1131 & 0.0077 & DLRNEIQVNTSQIPDTVTR \\
\hline 39.7 & 2199.1131 & 0.0077 & DLRNEIQVNTSQIPDTVTR \\
\hline 11.4 & 2198.1291 & 0.9917 & DLRNEIQVNTSQIPDTVTR \\
\hline 3.9 & 2198.1378 & 0.9831 & LSAKHPHSQTSNSHPLKAMK \\
\hline 2.2 & 2199.1032 & 0.0177 & NDPTYPRPSEPSLERARSK \\
\hline 1.9 & 2199.1405 & -0.0196 & FKTPGQGMVVHLSNPIMRR \\
\hline 1.6 & 2198.1282 & 0.9926 & NLRREEGIYSVLVALMAGK \\
\hline 1.6 & 2198.1282 & 0.9926 & NLRREEGIYSVLVALMAGK \\
\hline 1.4 & 2199.1089 & 0.0120 & NLLPKSLVNGVPWPQEATR \\
\hline 1.3 & 2197.0983 & 2.0226 & LQELLKPSTGIIRTLPQM \\
\hline
\end{tabular}

Spectrum No: 352; Query: 1357; Rank: 1

\section{Peptide View}

\section{MS/MS Fragmentation of MAATFIGNSTAIQELFK}

Found in IPI00765366, Tax_Id=10116 Gene_Symbol=Tubb4 tubulin, beta 4

Match to Query 1357: 1858.928848 from(930.471700,2+)

Title: 091008RatKidney_NoSalt_04.4989.4989.2.dta

Data file K:INewmanPaper|Piliangl3SubProteomes\Piliang3SP\mgf5ppm\ERLIC_3SubProteomes5ppm.mgf

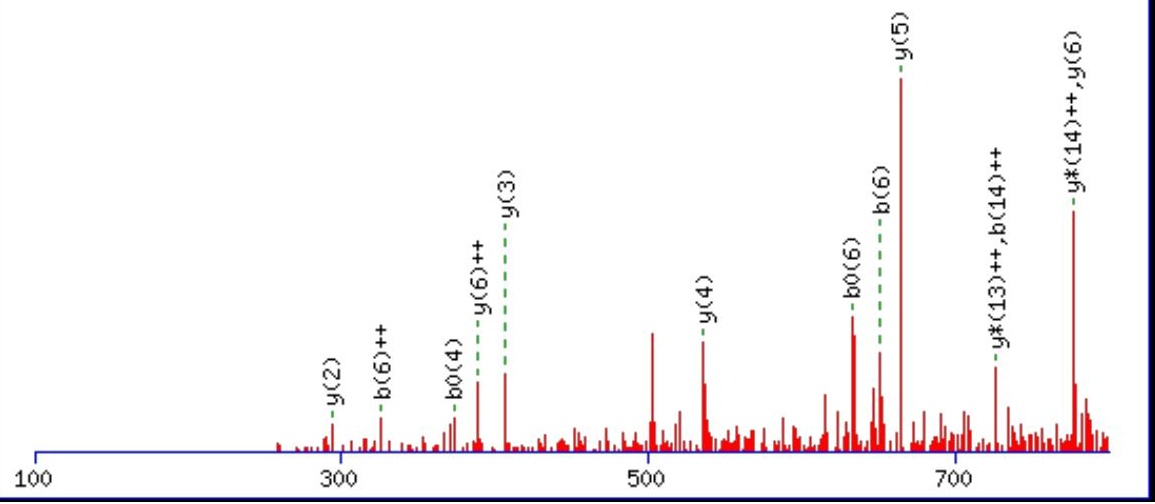



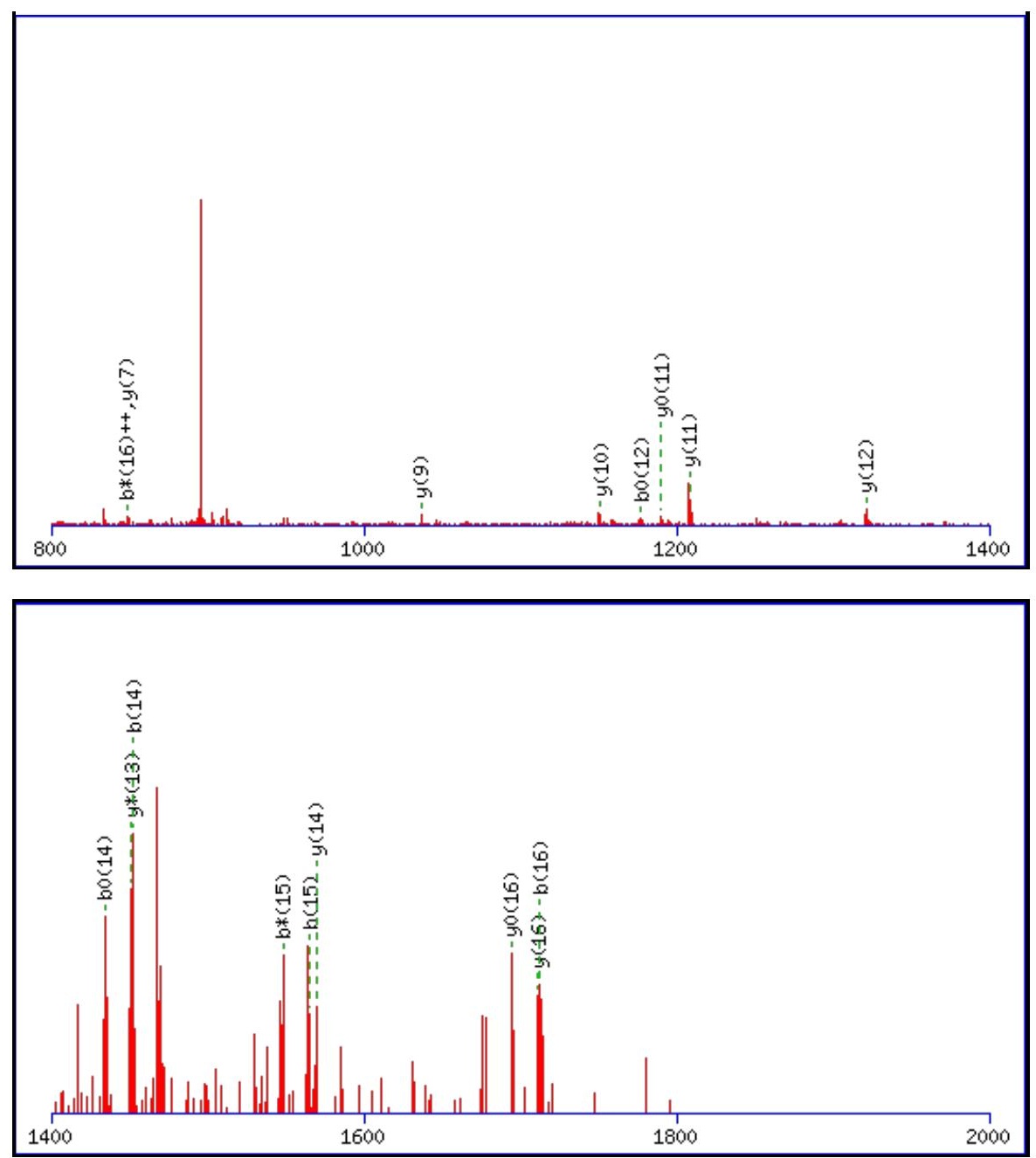

Monoisotopic mass of neutral peptide $\operatorname{Mr}($ calc): 1857.9182

Fixed modifications: Carbamidomethyl (C)

Variable modifications:

M1 : Oxidation (M)

N8 : Deamidated_N (N)

Ions Score: 71 Expect: $1.5 \mathrm{e}-005$

Matches (Bold Red): 30/166 fragment ions using 56 most intense peaks

\begin{tabular}{|c|c|c|c|c|c|c|c|c|c|c|c|c|c|c|}
\hline \# & b & $\mathbf{b}^{++}$ & $\mathbf{b}^{*}$ & $\mathbf{b}^{*^{++}}$ & $\mathbf{b}^{0}$ & $\mathbf{b}^{0++}$ & Seq. & $\mathbf{y}$ & $\mathbf{y}^{++}$ & $\mathbf{y}^{*}$ & $\mathbf{y}^{*^{++}}$ & $\mathbf{y}^{0}$ & $\mathbf{y}^{0++}$ & \# \\
\hline 1 & 148.0427 & 74.5250 & & & & & $\mathbf{M}$ & & & & & & & 17 \\
\hline 2 & 219.0798 & 110.0435 & & & & & A & 1711.8901 & 856.4487 & 1694.8636 & 847.9354 & 1693.8796 & 847.4434 & 16 \\
\hline 3 & 290.1169 & 145.5621 & & & & & A & 1640.8530 & 820.9301 & 1623.8265 & 812.4169 & 1622.8424 & 811.9249 & 15 \\
\hline 4 & 391.1646 & 196.0859 & & & 373.1540 & 187.0806 & $\mathbf{T}$ & 1569.8159 & 785.4116 & 1552.7893 & 776.8983 & 1551.8053 & 776.4063 & 14 \\
\hline 5 & 538.2330 & 269.6201 & & & 520.2224 & 260.6149 & $\mathbf{F}$ & 1468.7682 & 734.8877 & 1451.7417 & 726.3745 & 1450.7577 & 725.8825 & 13 \\
\hline 6 & 651.3171 & 326.1622 & & & 633.3065 & 317.1569 & I & 1321.6998 & 661.3535 & 1304.6733 & 652.8403 & 1303.6892 & 652.3483 & 12 \\
\hline 7 & 708.3385 & 354.6729 & & & 690.3280 & 345.6676 & G & 1208.6157 & 604.8115 & 1191.5892 & 596.2982 & 1190.6052 & 595.8062 & 11 \\
\hline 8 & 823.3655 & 412.1864 & 806.3389 & 403.6731 & 805.3549 & 403.1811 & $\mathbf{N}$ & 1151.5943 & 576.3008 & 1134.5677 & 567.7875 & 1133.5837 & 567.2955 & 10 \\
\hline 9 & 910.3975 & 455.7024 & 893.3709 & 447.1891 & 892.3869 & 446.6971 & $\mathrm{~S}$ & 1036.5673 & 518.7873 & 1019.5408 & 510.2740 & 1018.5568 & 509.7820 & 9 \\
\hline 10 & 1011.4452 & 506.2262 & 994.4186 & 497.7129 & \begin{tabular}{|l|}
993.4346 \\
\end{tabular} & 497.2209 & $\mathbf{T}$ & 949.5353 & 475.2713 & 932.5088 & 466.7580 & 931.5247 & 466.2660 & 8 \\
\hline 11 & 1082.4823 & 541.7448 & 1065.4557 & 533.2315 & 1064.4717 & 532.7395 & A & 848.4876 & 424.7475 & 831.4611 & 416.2342 & 830.4771 & 415.7422 & 7 \\
\hline 12 & 1195.5664 & 598.2868 & 1178.5398 & 589.7735 & 1177.5558 & 589.2815 & $\mathbf{I}$ & 777.4505 & \begin{tabular}{|l|}
389.2289 \\
\end{tabular} & 760.4240 & 380.7156 & 759.4400 & 380.2236 & 6 \\
\hline 13 & 1323.6249 & 662.3161 & 1306.5984 & 653.8028 & 1305.6144 & 653.3108 & $\mathbf{Q}$ & 664.3665 & 332.6869 & 647.3399 & 324.1736 & 646.3559 & 323.6816 & 5 \\
\hline 14 & 1452.6675 & 726.8374 & 1435.6410 & 718.3241 & 1434.6570 & 717.8321 & $\mathbf{E}$ & 536.3079 & 268.6576 & 519.2813 & 260.1443 & 518.2973 & 259.6523 & 4 \\
\hline 15 & 1565.7516 & 783.3794 & 1548.7250 & 774.8662 & 1547.7410 & 774.3741 & $\mathbf{L}$ & 407.2653 & 204.1363 & 390.2387 & 195.6230 & & & 3 \\
\hline 16 & 1712.8200 & 856.9136 & 1695.7935 & 848.4004 & 1694.8094 & 847.9084 & $\mathbf{F}$ & 294.1812 & 147.5942 & 277.1547 & 139.0810 & & & 2 \\
\hline 17 & & & & & & & $\mathbf{K}$ & 147.1128 & 74.0600 & 130.0863 & 65.5468 & & & 1 \\
\hline
\end{tabular}



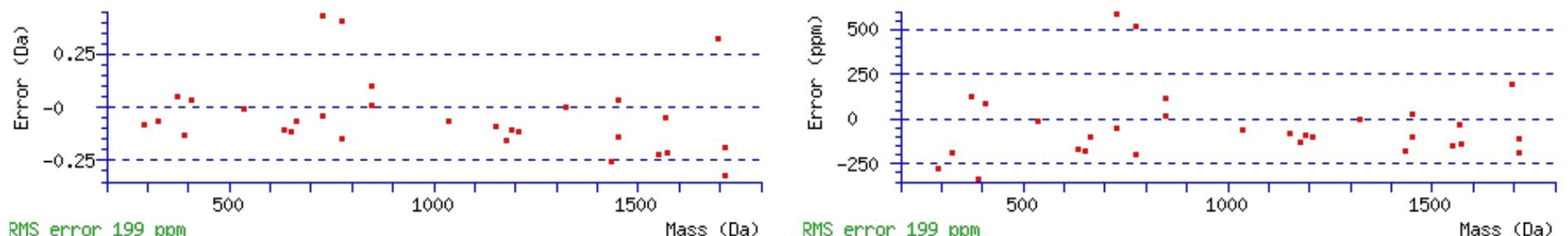

\section{All matches to this query}

\begin{tabular}{|l|l|l|l|}
\hline Score & Mr(calc): & Delta & \multicolumn{1}{|c|}{ Sequence } \\
\hline 70.8 & 1857.9182 & 1.0106 & MAATFIGNSTAIQELFK \\
\hline 57.1 & 1856.9342 & 1.9946 & MAATFIGNSTAIQELFK \\
\hline 57.1 & 1856.9342 & 1.9946 & MASTFIGNSTAIQELFK \\
\hline 57.1 & 1856.9342 & 1.9946 & MSATFIGNSTAIQELFK \\
\hline 56.9 & 1857.9182 & 1.0106 & MASTFIGNSTAIQELFK \\
\hline 56.9 & 1857.9182 & 1.0106 & MSATFIGNSTAIQELFK \\
\hline 8.8 & 1856.9113 & 2.0175 & FKKNNFIIISNYFK \\
\hline 7.8 & 1856.9205 & 2.0083 & SNKNLLLKSEIEIMK \\
\hline 4.0 & 1856.9258 & 2.0031 & TKNISAISYINQRNR \\
\hline 3.2 & 1856.9206 & 2.0083 & SDEKLIKSIINMEIK \\
\hline
\end{tabular}

Spectrum No: 353; Query: 264; Rank: 1

\section{Peptide View}

MS/MS Fragmentation of TGNWSALPSCK

Found in IPI00195241, Tax_Id=10116 Gene_Symbol=Apoh Beta-2-glycoprotein 1 precursor

Match to Query 264: 1220.547368 from(611.280960,2+)

Title: 091008RatKidney_NoSalt_28.1494.1494.2.dta

Data file K:INewmanPaper|Piliang|3SubProteomes\Piliang3SP\mgf5ppm\ERLIC_3SubProteomes5ppm.mgf

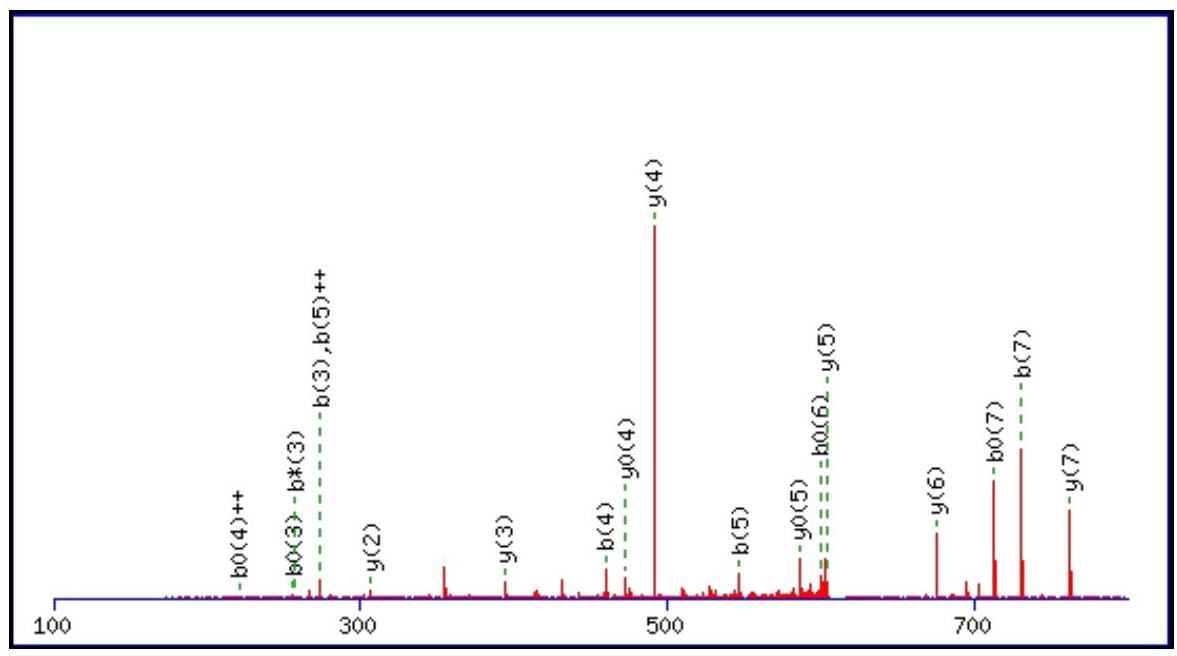



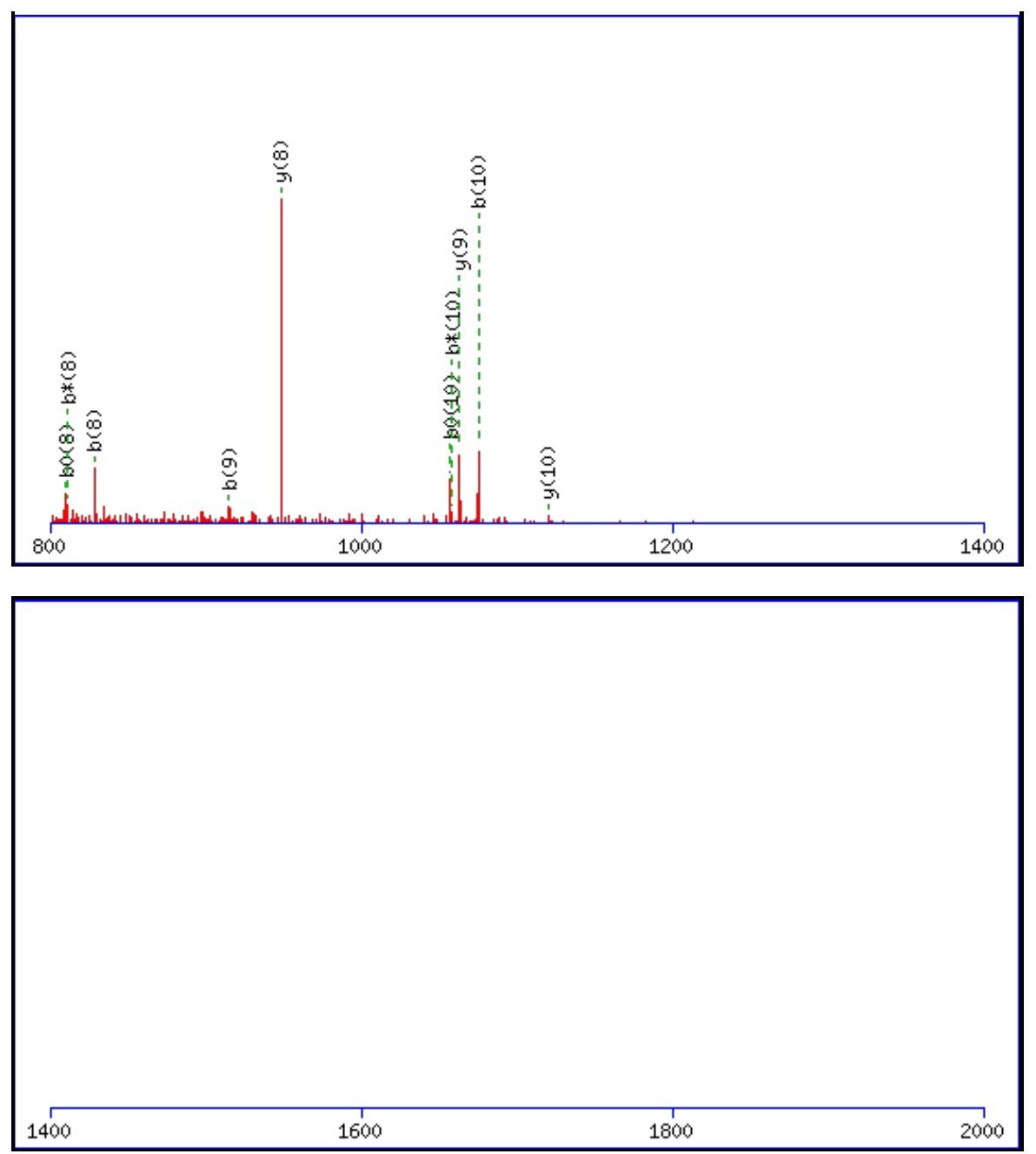

Monoisotopic mass of neutral peptide $\operatorname{Mr}($ calc): 1220.5496

Fixed modifications: Carbamidomethyl (C)

Variable modifications:

N3: Deamidated $\mathrm{N}(\mathrm{N})$

Ions Score: 71 Expect: $9.4 \mathrm{e}-006$

Matches (Bold Red): 28/112 fragment ions using 43 most intense peaks

\begin{tabular}{|c|c|c|c|c|c|c|c|c|c|c|c|c|c|c|}
\hline \# & b & $\mathbf{b}^{++}$ & $\mathbf{b}^{*}$ & $\mathbf{b}^{*^{++}}$ & $\mathbf{b}^{0}$ & $\mathbf{b}^{\mathbf{0 + +}}$ & Seq. & $\mathbf{y}$ & $\mathbf{y}^{++}$ & $\mathrm{y}^{*}$ & $\mathbf{y}^{*^{++}}$ & $\mathbf{y}^{\mathbf{0}}$ & $\mathbf{y}^{0++}$ & \# \\
\hline 1 & 102.0550 & 51.5311 & & & 84.0444 & 42.5258 & $\mathbf{T}$ & & & & & & & 11 \\
\hline 2 & 159.0764 & 80.0418 & & & 141.0659 & 71.0366 & G & 1120.5092 & 560.7582 & 1103.4826 & 552.2449 & 1102.4986 & 551.7529 & 10 \\
\hline 3 & 274.1034 & 137.5553 & 257.0768 & 129.0420 & 256.0928 & 128.5500 & $\mathbf{N}$ & 1063.4877 & 532.2475 & 1046.4612 & 523.7342 & 1045.4771 & 523.2422 & 9 \\
\hline 4 & 460.1827 & 230.5950 & 443.1561 & 222.0817 & 442.1721 & 221.5897 & $\mathbf{W}$ & 948.4608 & 474.7340 & 931.4342 & 466.2207 & 930.4502 & 465.7287 & 8 \\
\hline 5 & 547.2147 & 274.1110 & 530.1881 & 265.5977 & 529.2041 & 265.1057 & $\mathrm{~S}$ & 762.3815 & 381.6944 & 745.3549 & 373.1811 & 744.3709 & 372.6891 & 7 \\
\hline 6 & 618.2518 & 309.6295 & 601.2253 & 301.1163 & 600.2412 & 300.6243 & A & 675.3494 & 338.1783 & 658.3229 & 329.6651 & 657.3389 & 329.1731 & 6 \\
\hline 7 & 731.3359 & 366.1716 & 714.3093 & 357.6583 & 713.3253 & 357.1663 & $\mathbf{L}$ & 604.3123 & 302.6598 & 587.2858 & 294.1465 & 586.3017 & 293.6545 & 5 \\
\hline 8 & 828.3886 & 414.6980 & 811.3621 & 406.1847 & 810.3781 & 405.6927 & $\mathbf{P}$ & 491.2282 & 246.1178 & 474.2017 & 237.6045 & 473.2177 & 237.1125 & 4 \\
\hline 9 & 915.4207 & 458.2140 & 898.3941 & 449.7007 & 897.4101 & 449.2087 & $\mathrm{~S}$ & 394.1755 & 197.5914 & 377.1489 & 189.0781 & 376.1649 & 188.5861 & 3 \\
\hline 10 & 1075.4513 & 538.2293 & 1058.4248 & 529.7160 & 1057.4408 & 529.2240 & C & 307.1435 & 154.0754 & 290.1169 & 145.5621 & & & 2 \\
\hline 11 & & & & & & & $\mathbf{K}$ & 147.1128 & 74.0600 & 130.0863 & 65.5468 & & & 1 \\
\hline
\end{tabular}
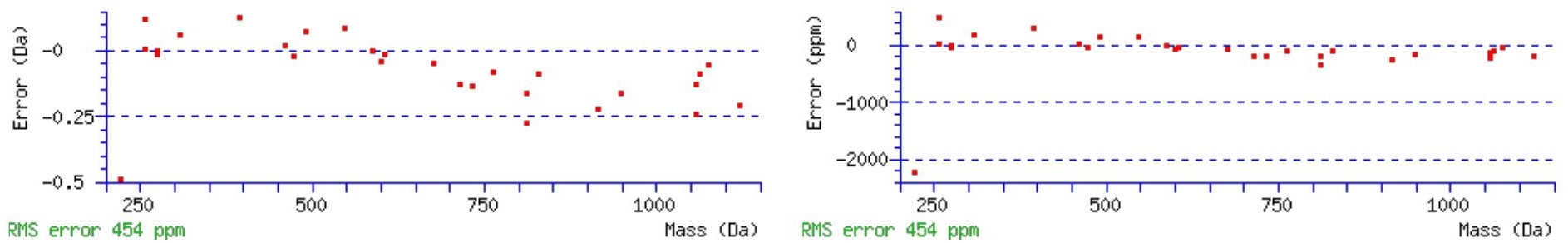


\section{All matches to this query}

\begin{tabular}{|l|l|l|l|}
\hline Score & Mr(calc): & Delta & \multicolumn{1}{|c|}{ Sequence } \\
\hline 70.6 & 1220.5496 & -0.0022 & TGNWSALPSCK \\
\hline 15.1 & 1220.5594 & -0.0121 & LEELNLNSCK \\
\hline 2.1 & 1220.5455 & 0.0018 & NSADPGTSMGKR \\
\hline 1.8 & 1218.5452 & 2.0022 & DCVNSPSVWR \\
\hline 1.6 & 1220.5455 & 0.0019 & MEGERKNNNK \\
\hline 1.6 & 1220.5455 & 0.0019 & MEGERKNNNK \\
\hline 1.6 & 1220.5455 & 0.0019 & MEGERKNNNK \\
\hline 0.5 & 1220.5520 & -0.0047 & ADLSNDDEKSK \\
\hline 0.5 & 1220.5438 & 0.0036 & KFSSSNKTDK \\
\hline 0.5 & 1220.5438 & 0.0036 & KFSSSNKTDK \\
\hline
\end{tabular}

Spectrum No: 354; Query: 981; Rank: 1

\section{Peptide View}

MS/MS Fragmentation of SVVTEEFNGSDWER

Found in IPI00197723, Tax_Id=10116 Gene_Symbol=RGD1565681_predicted similar to proline rich 6

Match to Query 981: 1654.719008 from(828.366780,2+)

Title: 100101RatKid_NS_deglyco_15.3315.3315.2.dta

Data file K:INewmanPaper|Piliang|3SubProteomes\Piliang3SP\mgf5ppm\ERLIC_3SubProteomes5ppm.mgf
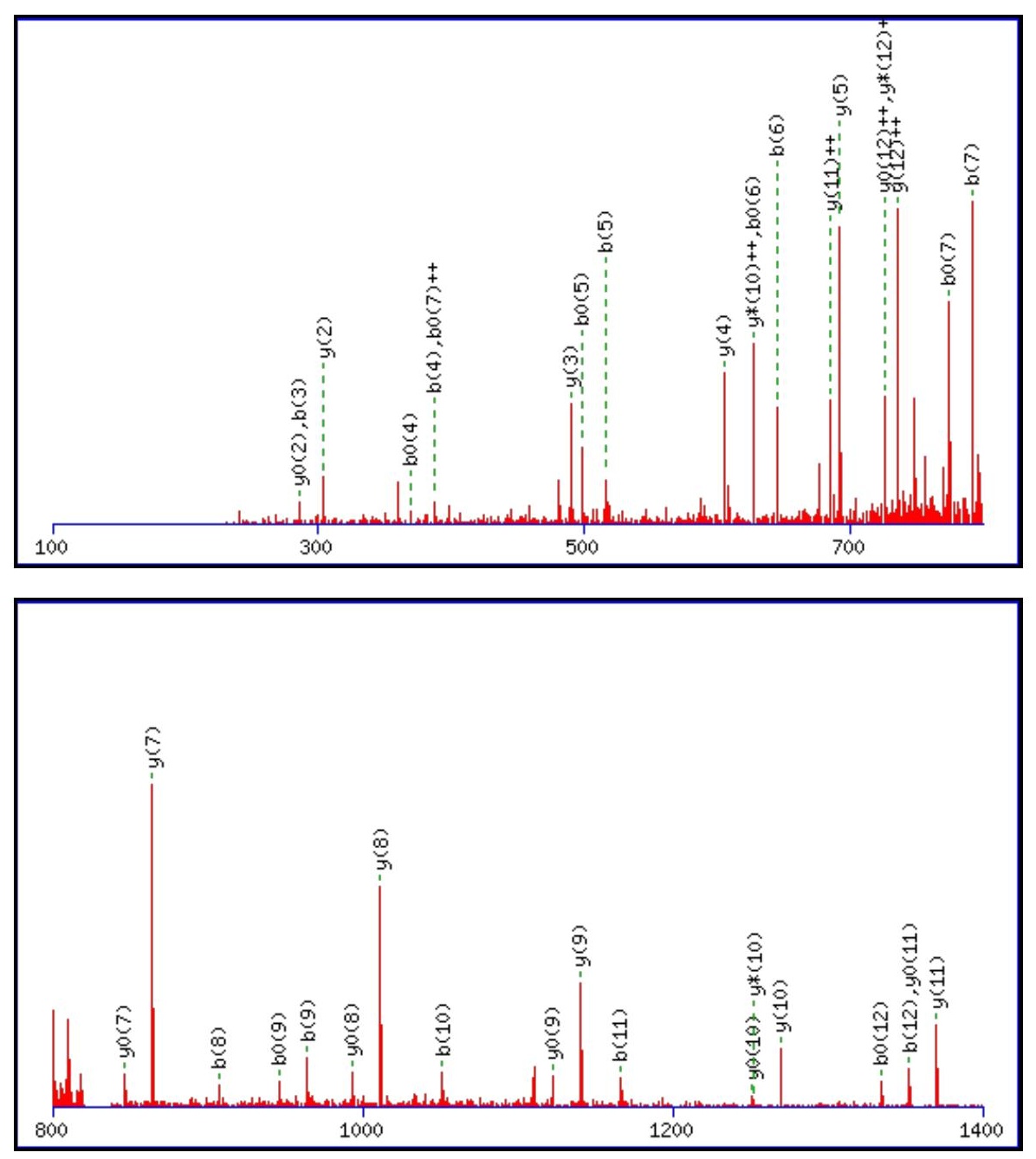


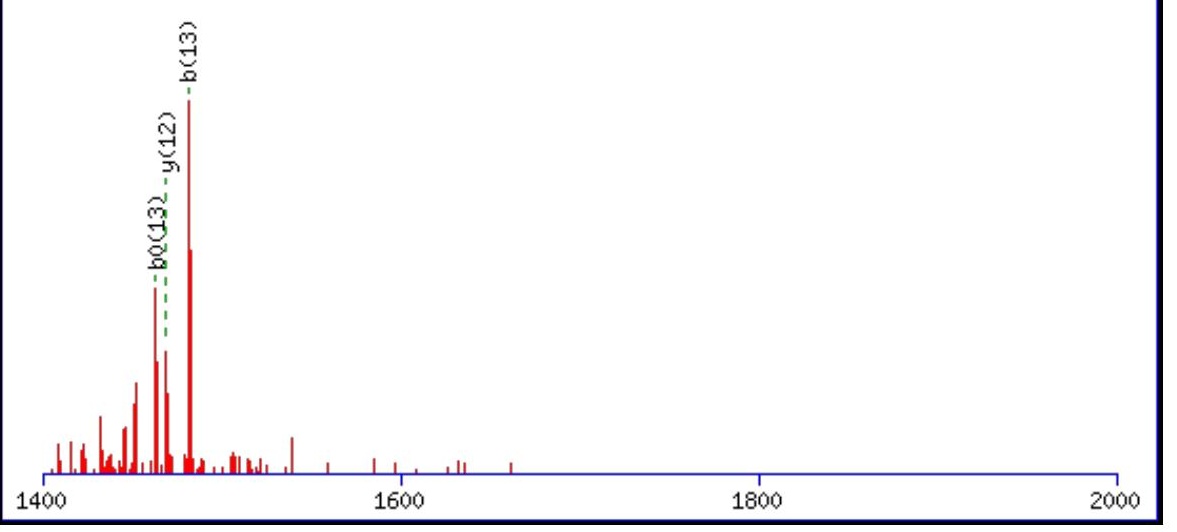

Monoisotopic mass of neutral peptide $\operatorname{Mr}($ calc): 1654.7111

Fixed modifications: Carbamidomethyl (C)

Variable modifications:

N8 : Deamidated_N (N)

Ions Score: 71 Expect: $1.5 \mathrm{e}-005$

Matches (Bold Red): 41/140 fragment ions using 71 most intense peaks

\begin{tabular}{|c|c|c|c|c|c|c|c|c|c|c|c|c|c|c|}
\hline$\#$ & b & $\mathbf{b}^{++}$ & $\mathbf{b}^{*}$ & $\mathbf{b}^{*^{++}}$ & $\mathbf{b}^{0}$ & $\mathbf{b}^{0++}$ & Seq. & $\mathbf{y}$ & $y^{++}$ & $\mathbf{y}^{*}$ & $\mathrm{y}^{*^{++}}$ & $\mathbf{y}^{\mathbf{0}}$ & $\mathbf{y}^{\mathbf{0}^{++}}$ & $\#$ \\
\hline 1 & 88.0393 & 44.5233 & & & 70.0287 & 35.5180 & S & & & & & & & 14 \\
\hline 2 & 187.1077 & 94.0575 & & & 169.0972 & 85.0522 & $\mathbf{V}$ & 1568.6863 & 784.8468 & 1551.6598 & 776.3335 & 1550.6758 & 775.8415 & 13 \\
\hline 3 & 286.1761 & 143.5917 & & & 268.1656 & 134.5864 & $\mathrm{~V}$ & 1469.6179 & 735.3126 & 1452.5914 & 726.7993 & 1451.6074 & 726.3073 & 12 \\
\hline 4 & 387.2238 & 194.1155 & & & 369.2132 & 185.1103 & $\mathbf{T}$ & 1370.5495 & 685.7784 & 1353.5230 & 677.2651 & 1352.5389 & 676.7731 & 11 \\
\hline 5 & 516.2664 & 258.6368 & & & 498.2558 & 249.6316 & $\mathbf{E}$ & 1269.5018 & 635.2546 & 1252.4753 & 626.7413 & 1251.4913 & 626.2493 & 10 \\
\hline 6 & 645.3090 & 323.1581 & & & 627.2984 & 314.1529 & $\mathbf{E}$ & 1140.4592 & 570.7333 & 1123.4327 & 562.2200 & 1122.4487 & 561.7280 & 9 \\
\hline 7 & 792.3774 & 396.6923 & & & 774.3668 & 387.6871 & $\mathbf{F}$ & 1011.4166 & 506.2120 & 994.3901 & 497.6987 & 993.4061 & 497.2067 & 8 \\
\hline 8 & 907.4043 & 454.2058 & 890.3778 & 445.6925 & 889.3938 & 445.2005 & $\mathbf{N}$ & 864.3482 & 432.6778 & 847.3217 & 424.1645 & 846.3377 & 423.6725 & 7 \\
\hline 9 & 964.4258 & 482.7165 & 947.3993 & 474.2033 & 946.4152 & 473.7113 & G & 749.3213 & 375.1643 & 732.2947 & 366.6510 & 731.3107: & 366.1590 & 6 \\
\hline 10 & 1051.4578 & 526.2326 & 1034.4313 & 517.7193 & 1033.4473 & 517.2273 & $S$ & 692.2998 & 346.6536 & 675.2733 & 338.1403 & 674.2893 & 337.6483 & 5 \\
\hline 11 & 1166.4848 & 583.7460 & 1149.4582 & 575.2328 & 1148.4742 & 574.7407 & D & 605.2678 & 303.1375 & 588.2413 & 294.6243 & 587.2572 & 294.1323 & 4 \\
\hline 12 & 1352.5641 & 676.7857 & 1335.5375 & 668.2724 & 1334.5535 & 667.7804 & $\mathbf{W}$ & 490.2409 & 245.6241 & 473.2143 & 237.1108 & 472.2303 & 236.6188 & 3 \\
\hline 13 & 1481.6067 & 741.3070 & 1464.5801 & 732.7937 & 1463.5961 & 732.3017 & $\mathbf{E}$ & 304.1615 & 152.5844 & 287.1350 & 144.0711 & 286.1510 & 143.5791 & 2 \\
\hline 14 & & & & & & & $\mathbf{R}$ & 175.1190 & 88.0631 & 158.0924 & 79.5498 & & & 1 \\
\hline
\end{tabular}
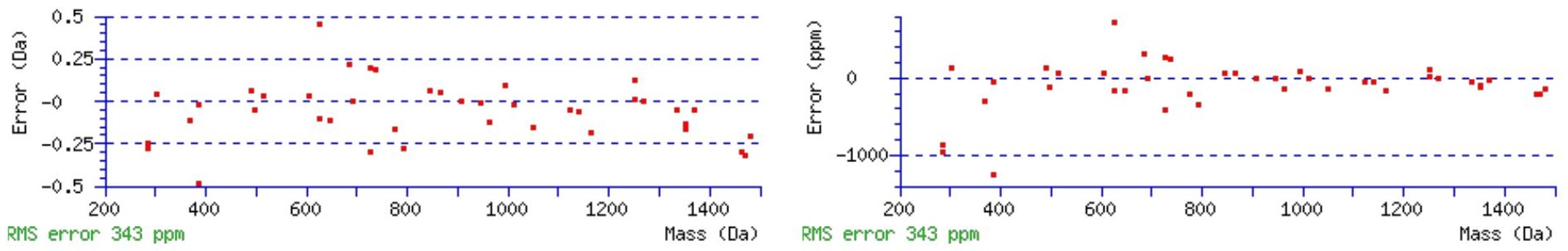

\section{All matches to this query}

\begin{tabular}{|l|l|l|l|}
\hline Score & Mr(calc): & Delta & \multicolumn{1}{c|}{ Sequence } \\
\hline 70.6 & 1654.7111 & 0.0079 & SVVTEEFNGSDWER \\
\hline 16.6 & 1653.7271 & 0.9919 & SVVTEEFNGSDWER \\
\hline 6.7 & 1654.7213 & -0.0023 & GRTTHSPYEERSR \\
\hline 6.7 & 1654.7213 & -0.0023 & GRTTHSPYEERSR \\
\hline 6.7 & 1654.7213 & -0.0023 & GRTTHSPYEERSR \\
\hline 3.9 & 1652.7078 & 2.0112 & IATMETMNIFLEK \\
\hline 3.6 & 1652.7253 & 1.9937 & $\underline{\text { SPISNGTFGFEGHMR }}$ \\
\hline
\end{tabular}




\begin{tabular}{|l|l|l|l|}
2.2 & 1652.7078 & 2.0112 & IATMETMNIFLEK \\
\hline 1.6 & 1654.7352 & -0.0162 & SIWEEQINSRDAK \\
\hline 1.4 & 1653.7108 & 1.0083 & SVDSRNRGPDSLNR \\
\hline
\end{tabular}

Spectrum No: 355; Query: 1907; Rank: 1

\section{Peptide View}

MS/MS Fragmentation of VAANFSTPVISTSGSSDPGQER

Found in IPI00391338, Tax_Id=10116 Gene_Symbol=RGD1562791_predicted similar to ICOS ligand precursor

Match to Query 1907: 2207.040808 from(1104.527680,2+)

Title: 100101RatKid_NS_deglyco_24.2430.2430.2.dta

Data file K:INewmanPaper|Piliangl3SubProteomes\Piliang3SP\mgf5ppm|ERLIC_3SubProteomes5ppm.mgf
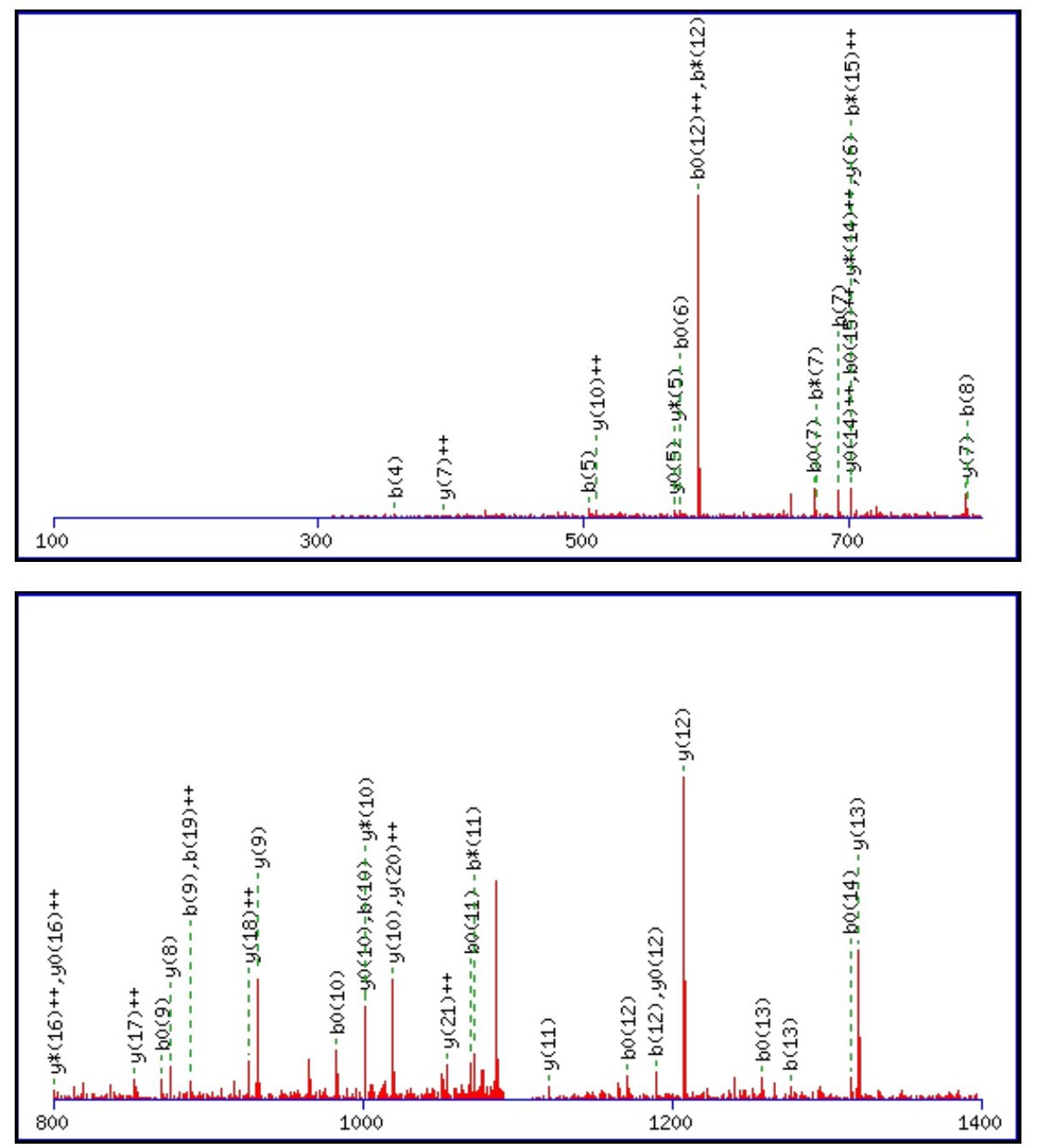


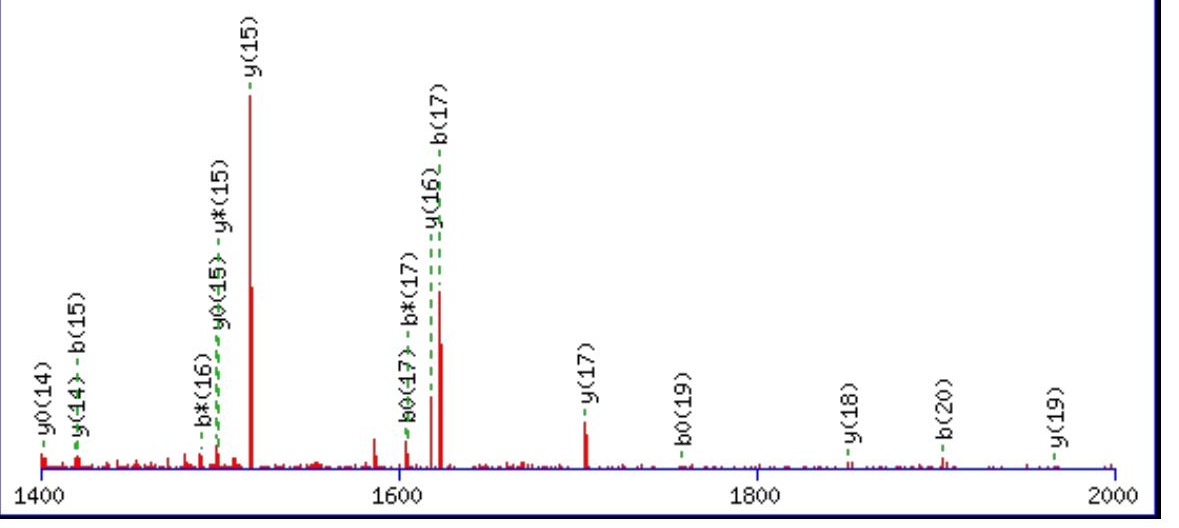

Monoisotopic mass of neutral peptide $\operatorname{Mr}($ calc): 2207.0342

Fixed modifications: Carbamidomethyl (C)

Variable modifications:

N4 : Deamidated_N (N)

Ions Score: 70 Expect: $2.5 \mathrm{e}-005$

Matches (Bold Red): 63/234 fragment ions using 107 most intense peaks

\begin{tabular}{|c|c|c|c|c|c|c|c|c|c|c|c|c|c|c|}
\hline \# & b & $\mathbf{b}^{++}$ & b* & $\mathbf{b}^{*^{++}}$ & $\mathbf{b}^{\mathbf{0}}$ & $\mathbf{b}^{\mathbf{0 + +}}$ & Seq. & $\mathbf{y}$ & $y^{++}$ & $\mathbf{y}^{*}$ & $\mathrm{y}^{\mathrm{*}^{++}}$ & $\mathbf{y}^{0}$ & $y^{0++}$ & \# \\
\hline 1 & 100.0757 & 50.5415 & & & & & $\mathbf{V}$ & & & & & & & 22 \\
\hline 2 & 171.1128 & 86.0600 & & & & & A & 2108.9731 & 1054.9902 & 2091.9465 & 1046.4769 & 2090.9625 & 1045.9849 & 21 \\
\hline 3 & 242.1499 & 121.5786 & & & & & A & 2037.9360 & 1019.4716 & 2020.9094 & 1010.9583 & 2019.9254 & 1010.4663 & 20 \\
\hline 4 & 357.1769 & 179.0921 & 340.1503 & 170.5788 & & & $\mathbf{N}$ & 1966.8988 & 983.9531 & 1949.8723 & 975.4398 & 1948.8883 & 974.9478 & 19 \\
\hline 5 & 504.2453 & 252.6263 & 487.2187 & 244.1130 & & & $\mathbf{F}$ & 1851.8719 & 926.4396 & 1834.8454 & 917.9263 & 1833.8613 & 917.4343 & 18 \\
\hline 6 & 591.2773 & 296.1423 & 574.2507 & 287.6290 & 573.2667 & 287.1370 & S & 1704.8035 & 852.9054 & 1687.7769 & 844.3921 & 1686.7929 & 843.9001 & 17 \\
\hline 7 & 692.3250 & 346.6661 & 675.2984 & 338.1529 & 674.3144 & 337.6608 & $\mathbf{T}$ & 1617.7715 & 809.3894 & 1600.7449 & 800.8761 & 1599.7609 & 800.3841 & 16 \\
\hline 8 & 789.3777 & 395.1925 & 772.3512 & 386.6792 & 771.3672 & 386.1872 & $\mathbf{P}$ & 1516.7238 & 758.8655 & 1499.6972 & 750.3523 & 1498.7132 & 749.8603 & 15 \\
\hline 9 & 888.4462 & 444.7267 & 871.4196 & 436.2134 & 870.4356 & 435.7214 & V & 1419.6710 & 710.3392 & 1402.6445 & 701.8259 & 1401.6605 & 701.3339 & 14 \\
\hline 10 & 1001.5302 & 501.2687 & 984.5037 & 492.7555 & 983.5197 & 492.2635 & I & 1320.6026 & 660.8049 & 1303.5761 & 652.2917 & 1302.5920 & 651.7997 & 13 \\
\hline 11 & 1088.5622 & 544.7848 & 1071.5357 & 536.2715 & 1070.5517 & 535.7795 & $S$ & 1207.5185 & 604.2629 & 1190.4920 & 595.7496 & 1189.5080 & 595.2576 & 12 \\
\hline 12 & 1189.6099 & 595.3086 & 1172.5834 & 586.7953 & 1171.5994 & 586.3033 & $\mathbf{T}$ & 1120.4865 & 560.7469 & 1103.4600 & 552.2336 & 1102.4760 & 551.7416 & 11 \\
\hline 13 & 1276.6420 & 638.8246 & 1259.6154 & 630.3113 & 1258.6314 & 629.8193 & $S$ & 1019.4388 & 510.2231 & 1002.4123 & 501.7098 & 1001.4283 & 501.2178 & 10 \\
\hline 14 & 1333.6634 & 667.3353 & 1316.6369 & 658.8221 & 1315.6529 & 658.3301 & $\mathbf{G}$ & 932.4068 & 466.7070 & 915.3803 & 458.1938 & 914.3962 & 457.7018 & 9 \\
\hline 15 & 1420.6954 & 710.8514 & 1403.6689 & 702.3381 & 1402.6849 & 701.8461 & $S$ & 875.3854 & 438.1963 & 858.3588 & 429.6830 & 857.3748 & 429.1910 & 8 \\
\hline 16 & 1507.7275 & 754.3674 & 1490.7009 & 745.8541 & 1489.7169 & 745.3621 & $S$ & 788.3533 & 394.6803 & 771.3268 & 386.1670 & 770.3428 & 385.6750 & 7 \\
\hline 17 & 1622.7544 & 811.8808 & 1605.7279 & 803.3676 & 1604.7439 & 802.8756 & D & 701.3213 & 351.1643 & 684.2947 & 342.6510 & 683.3107 & 342.1590 & 6 \\
\hline 18 & 1719.8072 & 860.4072 & 1702.7806 & 851.8940 & 1701.7966 & 851.4019 & $\mathbf{P}$ & 586.2944 & 293.6508 & 569.2678 & 285.1375 & 568.2838 & 284.6455 & 5 \\
\hline 19 & 1776.8286 & 888.9180 & 1759.8021 & 880.4047 & 1758.8181 & 879.9127 & G & 489.2416 & 245.1244 & 472.2150 & 236.6112 & 471.2310 & 236.1191 & 4 \\
\hline 20 & 1904.8872 & 952.9472 & 1887.8607 & 944.4340 & 1886.8767 & 943.9420 & $\mathbf{Q}$ & 432.2201 & 216.6137 & 415.1936 & 208.1004 & 414.2096 & 207.6084 & 3 \\
\hline 21 & 2033.9298 & 1017.4685 & 2016.9033 & 1008.9553 & 2015.9192 & 1008.4633 & $\mathbf{E}$ & 304.1615 & 152.5844 & 287.1350 & 144.0711 & 286.1510 & 143.5791 & 2 \\
\hline 22 & & & & & & & $\mathbf{R}$ & 175.1190 & 88.0631 & 158.0924 & 79.5498 & & & 1 \\
\hline
\end{tabular}

$$
\text { RHS }
$$
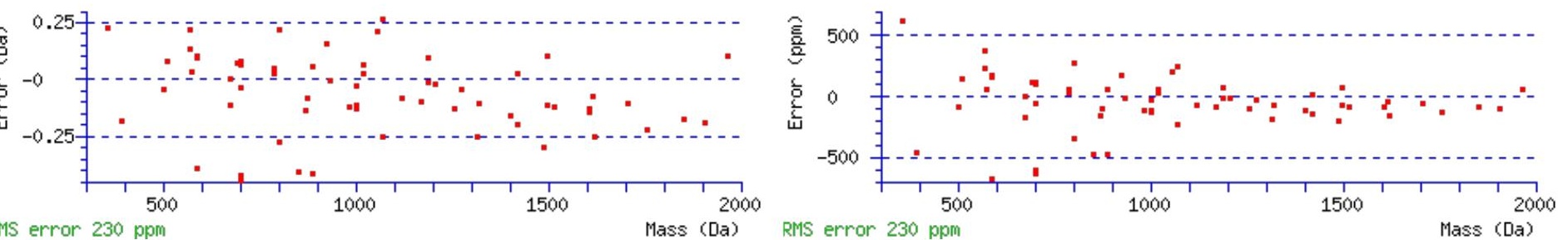

All matches to this query 


\begin{tabular}{|l|l|l|l|} 
Score & Mr(calc): & Delta & \multicolumn{1}{|c|}{ Sequence } \\
\hline 70.4 & 2207.0342 & 0.0066 & VAANFSTPVISTSGSSDPGQER \\
\hline 43.7 & 2206.0502 & 0.9906 & VAANFSTPVISTSGSSDPGQER \\
\hline 7.1 & 2205.0500 & 1.9908 & MRSTNQSSVSEFLLLGLSR \\
\hline 5.0 & 2206.0469 & 0.9939 & KDIQTISNILISIGRCSK \\
\hline 5.0 & 2206.0469 & 0.9939 & KDIQTISNILISIGRCSK \\
\hline 3.4 & 2205.0483 & 1.9925 & KATSFQLITSLHKESINK \\
\hline 3.4 & 2205.0483 & 1.9925 & KATSFQLITSLHKESINK \\
\hline 3.3 & 2207.0228 & 0.0180 & MARGPGQLSGPRPDTVTMPK \\
\hline 3.3 & 2205.0426 & 1.9982 & QTSSYNIPASASISRTSISR \\
\hline 1.0 & 2205.0219 & 2.0189 & ELDEATESNEAMGREVNALK \\
\hline
\end{tabular}

Spectrum No: 356; Query: 2597; Rank: 1

\section{Peptide View}

MS/MS Fragmentation of GEDDSWDFGTGAGFFVNATEDPWNTNYR

Found in IPI00362395, Tax_Id=10116 Gene_Symbol=Esd esterase D/formylglutathione hydrolase

Match to Query 2597: 3168.316482 from(1057.112770,3+)

Title: 091008RatKidney_NoSalt_19.6861.6861.3.dta

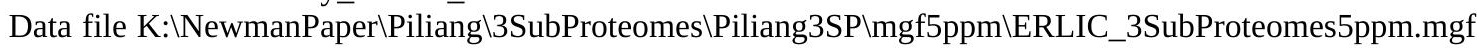
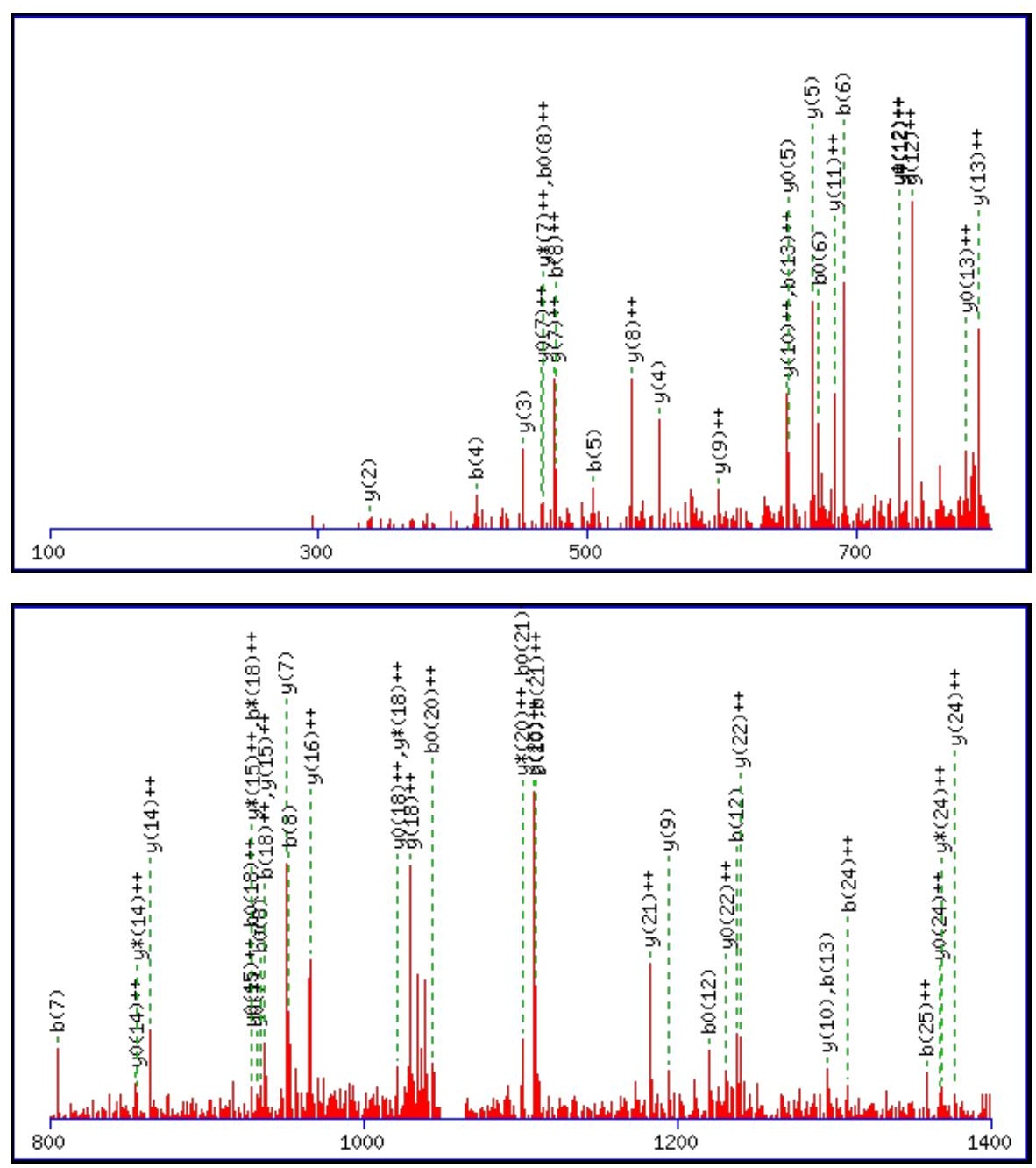


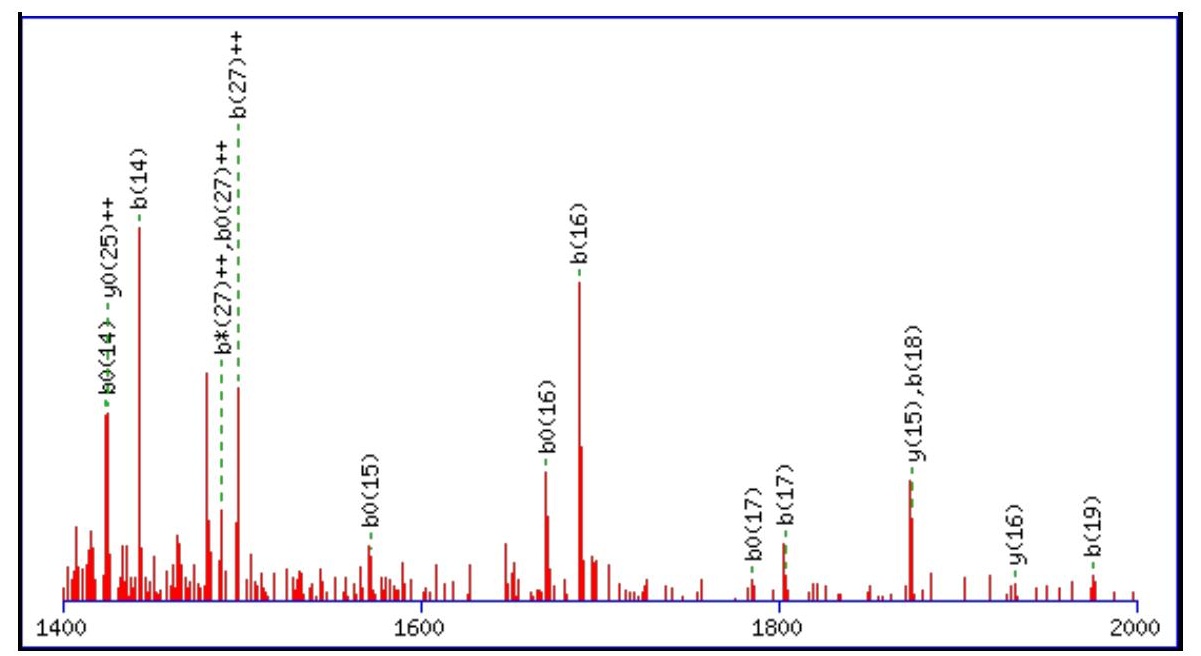

Monoisotopic mass of neutral peptide $\operatorname{Mr}($ calc): 3168.3010

Fixed modifications: Carbamidomethyl (C)

Variable modifications:

N17 : Deamidated_N (N)

Ions Score: 70 Expect: $3.5 \mathrm{e}-005$

Matches (Bold Red): 76/284 fragment ions using 114 most intense peaks

\begin{tabular}{|c|c|c|c|c|c|c|c|c|c|c|c|c|c|c|}
\hline \# & b & $\mathbf{b}^{++}$ & $\mathbf{b}^{*}$ & $\mathbf{b}^{*^{++}}$ & $\mathbf{b}^{0}$ & $\mathbf{b}^{0++}$ & Seq. & $\mathbf{y}$ & $\mathbf{y}^{++}$ & $\mathbf{y}^{*}$ & $\mathrm{y}^{\mathrm{*}^{++}}$ & $\mathbf{y}^{\mathbf{0}}$ & & \# \\
\hline 1 & 58.0287 & 29.5180 & & & & & G & & & & & & & 28 \\
\hline 2 & 187.0713 & 94.0393 & & & 169.0608 & 85.0340 & $\mathbf{E}$ & 3112.2868 & 1556.6470 & 3095.2602 & & 3094.2762 & & 27 \\
\hline 3 & 302.0983 & 151.5528 & & & 284.0877 & 142.5475 & D & 2983.2442 & 1492.1257 & 2966.2176 & 5124 & 2336 & 1204 & 26 \\
\hline 4 & 417.1252 & 209.0662 & & & 399.1147 & 200.0610 & D & 2868.2172 & 1434.6122 & 2851.1907 & 1426.0990 & 2850.2067 & 1425.6070 & 25 \\
\hline 5 & 504.1572 & 252.5823 & & & 486.1467 & 243.5770 & S & 2753.1903 & 1377.0988 & 2736.1637 & 1368.5855 & 2735.1797 & 1368.0935 & 24 \\
\hline 6 & 690.2366 & 345.6219 & & & 672.2260 & 336.6166 & W & 2666.1583 & 1333 & 2649.1317 & 695 & 2648.1477 & 5775 & 23 \\
\hline 7 & 805.2635 & 403.1354 & & & 787.2529 & 394.1301 & D & 2480.0789 & 1240.5431 & 2463.0524 & 1232.0298 & 2462.0684 & 1231.5378 & 22 \\
\hline 8 & 952.3319 & 476.6696 & & & 934.3214 & 467.6643 & $\mathbf{F}$ & 2365.0520 & 1183.0296 & 2348.0254 & 1174.5164 & 2347.0414 & 1174.0244 & 21 \\
\hline 91 & 1009.3534 & 505.1803 & & & 991.3428 & 750 & G & 2217.9836 & 954 & 2200.9570 & 822 & 2199.9730 & 901 & 20 \\
\hline 101 & 1110.4011 & 555.7042 & & & 1092.3905 & 546.6989 & $T$ & 2160.9621 & 1080.9847 & 2143.9356 & 1072.4714 & 2142.9516 & 1071.9794 & 19 \\
\hline 11 & 1167.4225 & 584.2149 & & & 1149.4120 & 575.2096 & G & 2059.9144 & 1030.4609 & 2042.8879 & 1021.9476 & 2041.9039 & 1021.4556 & 18 \\
\hline 121 & 1238.4596 & 619. & & & 1220.4491 & 610.7282 & A & 2002.8930 & 1001.9 & & & 824 & & 17 \\
\hline 13 & \begin{tabular}{|l|}
1295.4811 \\
\end{tabular} & 648.2442 & & & 1277.4705 & 639.2389 & G & 1931.8559 & 966.4316 & 1914.8293 & 957.9183 & 1913.8453 & 957.4263 & 16 \\
\hline 14 & 1442.5495 & 721.7784 & & & 1424.5390 & 712.7731 & $\mathbf{F}$ & 1874.8344 & 937.9208 & 1857.8078 & 929.4076 & 1856.8238 & 928.9156 & 15 \\
\hline 151 & 179 & 795. & & & & & $\mathbf{F}$ & & & & & 1709 & & 14 \\
\hline 16 & \begin{tabular}{|l|}
1688.6863 \\
\end{tabular} & 844.8468 & & & 1670.6758 & 835.8415 & $\mathrm{~V}$ & 1580.6976 & 790.8524 & 1563.6710 & 782.3391 & 1562.6870 & 781.8471 & 13 \\
\hline 17 & 1803.7133 & 902.3603 & 86.6867 & & 1785.7027 & 893.3550 & $\mathbf{N}$ & 1481.6292 & 741.3182 & 1464.6026 & 732.8049 & 1463.6186 & 732.3129 & 12 \\
\hline 18 & \begin{tabular}{|l|}
1874.7504 \\
\end{tabular} & 937.8788 & 57.7238 & 929.3656 & 1856.7398 & 928.8736 & A & 1366.6022 & 683.8047 & 1349.5757 & 675.2915 & 1348.5917 & 674.7995 & 11 \\
\hline 191 & \begin{tabular}{|l|}
1975.7981 \\
\end{tabular} & \begin{tabular}{|l|}
988.4027 \\
\end{tabular} & 1958.7715 & 979.8894 & 1957.7875 & 979.3974 & $\mathbf{T}$ & 1295.5651 & 648.2862 & 1278.5386 & 639.7729 & 1277.5545 & 639.2809 & 10 \\
\hline 202 & 2104.8407 & 1052.9240 & 2087.8141 & 1044.4107 & 2086.8301 & \begin{tabular}{|l|}
1043.9187 \\
\end{tabular} & $\mathbf{E}$ & 1194.5174 & 597.7624 & 1177.4909 & 589.2491 & 1176.5069 & 588.7571 & 9 \\
\hline 212 & 2219.8676 & \begin{tabular}{|l|}
1110.4374 \\
\end{tabular} & 2202.8411 & 242 & 2201.8570 & \begin{tabular}{|l|}
1101.4322 \\
\end{tabular} & D & 1065.4748 & 533.2411 & 1048.4483 & 524.7278 & 1047.4643 & 524.2358 & 8 \\
\hline \begin{tabular}{l|l}
22 & 2 \\
2
\end{tabular} & 2316.9204 & 1158.9638 & 2299.8938 & 1150.4505 & 2298.9098 & 1149.9585 & $\mathbf{P}$ & 950.4479 & 475.7276 & 933.4213 & 467.2143 & 932.4373 & 466.7223 & 7 \\
\hline 23 & 2502.9997 & 1252.0035 & 2485.9731 & 1243.4902 & 2484.9891 & 1242.9982 & W & 853.3951 & 427.2012 & 836.3686 & 418.6879 & 835.3846 & 418.1959 & 6 \\
\hline $24:$ & 2617.0426 & 1309.0249 & 2600.0161 & 1300.5117 & 2599.0320 & 1300.0197 & $\mathbf{N}$ & 667.3158 & 334.1615 & 650.2893 & 325.6483 & 649.3052 & 325.1563 & 5 \\
\hline 25 & 2718.0903 & 1359.5488 & 2701.0637 & 1351.0355 & 2700.0797 & 1350.5435 & $T$ & 553.2729 & 277.1401 & 536.2463 & 268.6268 & 535.2623 & 268.1348 & 4 \\
\hline 26 & 2832.1332 & 1416.5702 & 2815.1067 & 1408.0570 & 2814.1227 & 1407.5650 & $\mathbf{N}$ & 452.2252 & 226.6162 & 435.1987 & 218.1030 & & & 3 \\
\hline 27 & 2995.1965 & 1498.1019 & 2978.1700 & 1489.5886 & 2977.1860 & 1489.0966 & $\mathbf{Y}$ & 338.1823 & 169.5948 & 321.1557 & 161.0815 & & & 2 \\
\hline 28 & & & & & & & $\mathbf{R}$ & 175.1190 & 88.0631 & 158.0924 & 79.5498 & & & \\
\hline
\end{tabular}



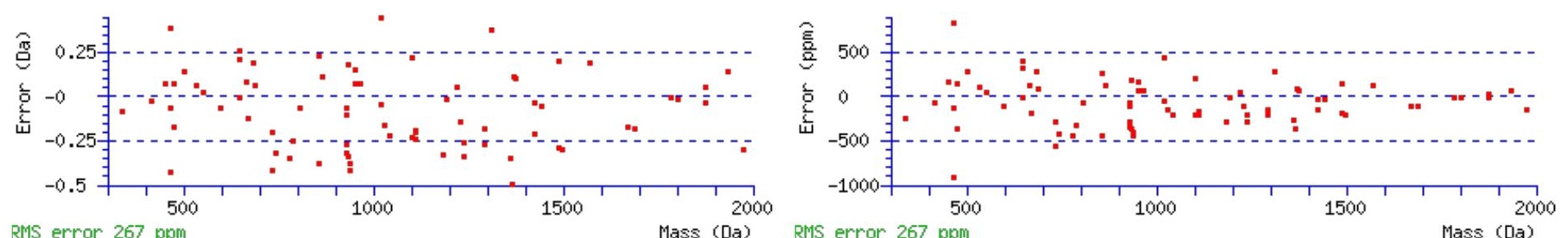

RMS error 267 ppm Mass (Da) RMS error 267 ppm

\section{All matches to this query}

\begin{tabular}{|l|l|l|l|}
\hline Score & Mr(calc): & Delta & \multicolumn{1}{|c|}{ Sequence } \\
\hline 70.3 & 3168.3010 & 0.0155 & GEDDSWDFGTGAGFFVNATEDPWNTNYR \\
\hline 67.8 & 3167.3170 & 0.9995 & GEDDSWDFGTGAGFFVNATEDPWNTNYR \\
\hline 63.5 & 3168.3010 & 0.0155 & GEDDSWDFGTGAGFFVNATEDPWNTNYR \\
\hline 61.2 & 3168.3010 & 0.0155 & GEDDSWDFGTGAGFFVNATEDPWNTNYR \\
\hline 3.6 & 3166.3169 & 1.9996 & SLSSAEDIHTPEQDRPFFSANSVSEQK \\
\hline 2.2 & 3166.3186 & 1.9978 & QNSSSSQGSSSNSGGSSGIPQPPPGMSFYAAK \\
\hline 0.7 & 3167.2931 & 1.0234 & SDNFNTGNMTVLSPYLNTTVLPSSSSSR \\
\hline 0.2 & 3167.2848 & 1.0317 & MAEGERQPPPDSSEETPSATPTFIIPK \\
\hline
\end{tabular}

Spectrum No: 357; Query: 2731; Rank: 1

\section{Peptide View}

MS/MS Fragmentation of VVNLNGELGDSWIVPLDNLTKEDLDEEEDTHL

Found in IPI00325860, Tax_Id=10116 Gene_Symbol=Podxl Podocalyxin precursor

Match to Query 2731: 3621.724002 from(1208.248610,3+)

Title: 091008RatKidney_NoSalt_19.6408.6408.3.dta

Data file K:INewmanPaper|Piliangl3SubProteomes\Piliang3SP\mgf5ppm\ERLIC_3SubProteomes5ppm.mgf

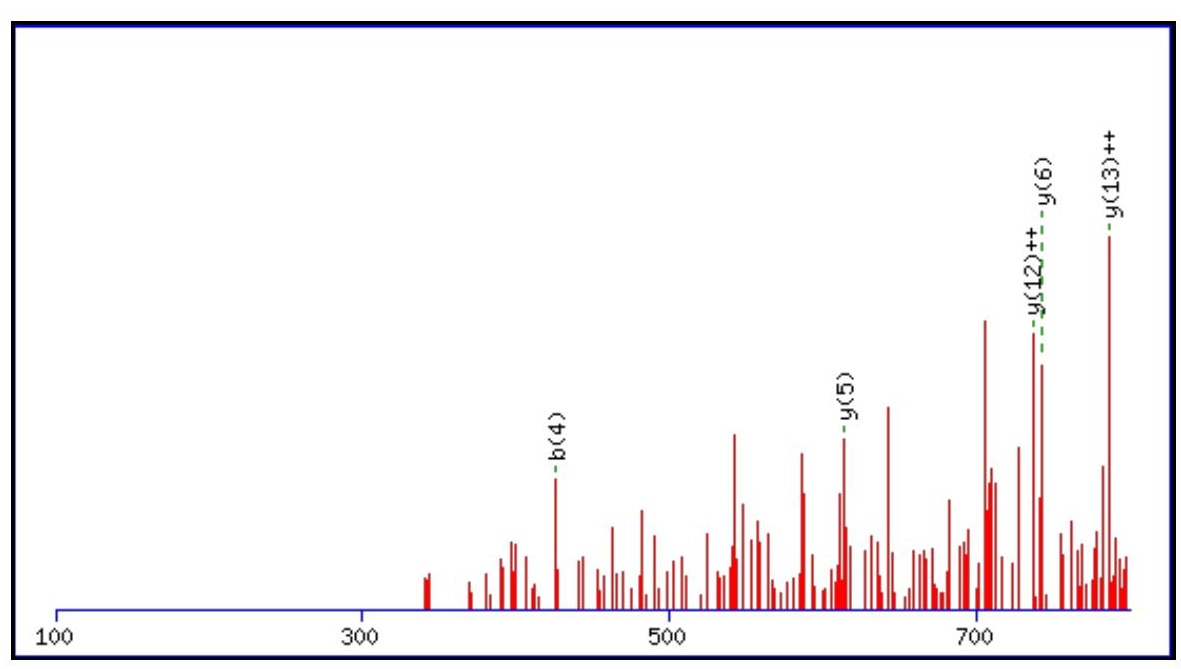




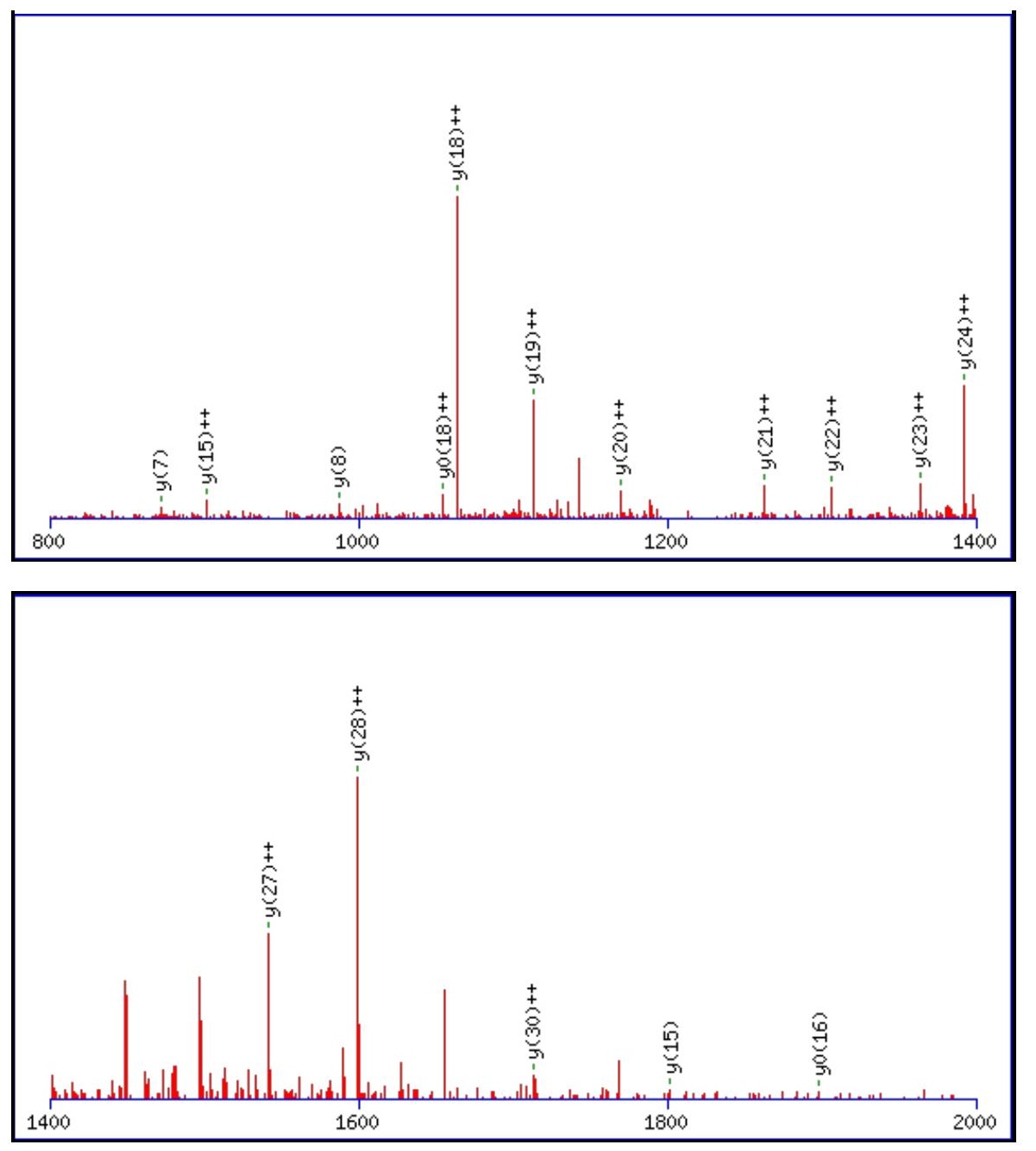

Monoisotopic mass of neutral peptide $\operatorname{Mr}($ calc): 3621.7210

Fixed modifications: Carbamidomethyl (C)

Variable modifications:

N18 : Deamidated $\mathrm{N}(\mathrm{N})$

Ions Score: 70 Expect: $3.6 \mathrm{e}-005$

Matches (Bold Red): 21/330 fragment ions using 43 most intense peaks

\begin{tabular}{|c|c|c|c|c|c|c|c|c|c|c|c|c|c|c|}
\hline \# & b & $\mathbf{b}^{++}$ & b* & $\mathbf{b}^{*^{++}}$ & $\mathbf{b}^{\mathbf{0}}$ & $\mathbf{b}^{0++}$ & Seq. & $\mathbf{y}$ & $\mathbf{y}^{++}$ & $\mathbf{y}^{*}$ & $\mathrm{y}^{\mathrm{*}^{++}}$ & $\mathbf{y}^{0}$ & $y^{0++}$ & \# \\
\hline 1 & 100.0757 & 50.5415 & & & & & $\mathbf{V}$ & & & & & & & 32 \\
\hline 2 & 199.1441 & 100.0757 & & & & & $\mathbf{V}$ & 3523.6599 & 1762.3336 & 3506.6333 & 1753.8203 & 3505.6493 & 1753.3283 & 31 \\
\hline 3 & 313.1870 & 157.0972 & 296.1605 & 148.5839 & & & $\mathbf{N}$ & 3424.5914 & 1712.7994 & 3407.5649 & 1704.2861 & 3406.5809 & 1703.7941 & 30 \\
\hline 4 & 426.2711 & 213.6392 & 409.2445 & 205.1259 & & & $\mathbf{L}$ & 3310.5485 & 1655.7779 & 3293.5220 & 1647.2646 & 3292.5379 & 1646.7726 & 29 \\
\hline 5 & 540.3140 & 270.6606 & 523.2875 & 262.1474 & & & $\mathbf{N}$ & 3197.4644 & 1599.2359 & 3180.4379 & 1590.7226 & 3179.4539 & 1590.2306 & 28 \\
\hline 6 & 597.3355 & 299.1714 & 580.3089 & 290.6581 & & & G & 3083.4215 & 1542.2144 & 3066.3950 & 1533.7011 & 3065.4110 & 1533.2091 & 27 \\
\hline 7 & 726.3781 & 363.6927 & 709.3515 & 355.1794 & 708.3675 & 354.6874 & $\mathbf{E}$ & 3026.4001 & 1513.7037 & 3009.3735 & 1505.1904 & 3008.3895 & 1504.6984 & 426 \\
\hline 8 & 839.4621 & 420.2347 & 822.4356 & 411.7214 & 821.4516 & 411.2294 & $\mathbf{L}$ & 2897.3575 & 1449.1824 & 2880.3309 & 1440.6691 & 2879.3469 & 1440.1771 & 25 \\
\hline 9 & 896.4836 & 448.7454 & 879.4571 & 440.2322 & 878.4730 & 439.7402 & G & 2784.2734 & 1392.6403 & 2767.2469 & 1384.1271 & 2766.2628 & 1383.6351 & 24 \\
\hline 10 & 1011.5106 & 506.2589 & 994.4840 & 497.7456 & 993.5000 & 497.2536 & D & 2727.2519 & 1364.1296 & 2710.2254 & 1355.6163 & 2709.2414 & 1355.1243 & 23 \\
\hline 11 & 1098.5426 & 549.7749 & 1081.5160 & 541.2617 & 1080.5320 & 540.7696 & S & 2612.2250 & 1306.6161 & 2595.1984 & 1298.1029 & 2594.2144 & 1297.6109 & 22 \\
\hline 12 & 1284.6219 & 642.8146 & 1267.5953 & 634.3013 & 1266.6113 & 633.8093 & W & 2525.1930 & 1263.1001 & 2508.1664 & 1254.5868 & 2507.1824 & 1254.0948 & 21 \\
\hline 13 & 1397.7060 & 699.3566 & 1380.6794 & 690.8433 & 1379.6954 & 690.3513 & I & 2339.1137 & 1170.0605 & 2322.0871 & 1161.5472 & 2321.1031 & 1161.0552 & 20 \\
\hline 14 & 1496.7744 & 748.8908 & 1479.7478 & 740.3775 & 1478.7638 & 739.8855 & $\mathbf{V}$ & 2226.0296 & 1113.5184 & 2209.0030 & 1105.0052 & 2208.0190 & 1104.5131 & 19 \\
\hline 15 & 1593.8271 & 797.4172 & 1576.8006 & 788.9039 & 1575.8166 & 788.4119 & $\mathbf{P}$ & 2126.9612 & 1063.9842 & 2109.9346 & 1055.4710 & 2108.9506 & 1054.9789 & 18 \\
\hline 16 & 1706.9112 & 853.9592 & 1689.8846 & 845.4460 & 1688.9006 & 844.9540 & $\mathbf{L}$ & 2029.9084 & 1015.4578 & 2012.8819 & 1006.9446 & 2011.8978 & 1006.4526 & 17 \\
\hline 17 & 1821.9381 & 911.4727 & 1804.9116 & 902.9594 & 1803.9276 & 902.4674 & D & 1916.8243 & 958.9158 & 1899.7978 & 950.4025 & 1898.8138 & 949.9105 & 16 \\
\hline 18 & 1936.9651 & 968.9862 & 1919.9385 & 960.4729 & 1918.9545 & 959.9809 & $\mathbf{N}$ & 1801.7974 & 901.4023 & 1784.7709 & 892.8891 & 1783.7868 & 892.3971 & 15 \\
\hline
\end{tabular}




\begin{tabular}{|l|l|l|l|l|l|l|l|l|l|l|l|l|l|l|l|l|l|l|}
$\mathbf{1 9}$ & 2050.0491 & 1025.5282 & 2033.0226 & 1017.0149 & 2032.0386 & 1016.5229 & L & 1686.7705 & 843.8889 & 1669.7439 & 835.3756 & 1668.7599 & 834.8836 & $\mathbf{1 4}$ \\
\hline $\mathbf{2 0}$ & 2151.0968 & 1076.0520 & 2134.0703 & 1067.5388 & 2133.0863 & 1067.0468 & T & 1573.6864 & $\mathbf{7 8 7 . 3 4 6 8}$ & 1556.6599 & 778.8336 & 1555.6758 & $\mathbf{7 7 8 . 3 4 1 6}$ & $\mathbf{1 3}$ \\
\hline $\mathbf{2 1}$ & 2279.1918 & 1140.0995 & 2262.1652 & 1131.5863 & 2261.1812 & 1131.0942 & K & 1472.6387 & 736.8230 & 1455.6122 & 728.3097 & 1454.6282 & 727.8177 & $\mathbf{1 2}$ \\
\hline $\mathbf{2 2}$ & 2408.2344 & 1204.6208 & 2391.2078 & 1196.1076 & 2390.2238 & 1195.6155 & E & 1344.5438 & 672.7755 & & & & 1326.5332 & 663.7702 & $\mathbf{1 1}$ \\
\hline $\mathbf{2 3}$ & 2523.2613 & 1262.1343 & 2506.2348 & 1253.6210 & 2505.2508 & 1253.1290 & D & 1215.5012 & 608.2542 & & & & 1197.4906 & 599.2489 & $\mathbf{1 0}$ \\
\hline $\mathbf{2 4}$ & 2636.3454 & 1318.6763 & 2619.3188 & 1310.1631 & 2618.3348 & 1309.6710 & L & 1100.4742 & 550.7408 & & & 1082.4637 & 541.7355 & $\mathbf{9}$ \\
\hline $\mathbf{2 5}$ & 2751.3723 & 1376.1898 & 2734.3458 & 1367.6765 & 2733.3618 & 1367.1845 & D & $\mathbf{9 8 7 . 3 9 0 2}$ & 494.1987 & & & 969.3796 & 485.1934 & $\mathbf{8}$ \\
\hline $\mathbf{2 6}$ & 2880.4149 & 1440.7111 & 2863.3884 & 1432.1978 & 2862.4044 & 1431.7058 & E & $\mathbf{8 7 2 . 3 6 3 2}$ & 436.6852 & & & & 854.3527 & 427.6800 & $\mathbf{7}$ \\
\hline $\mathbf{2 7}$ & 3009.4575 & 1505.2324 & 2992.4310 & 1496.7191 & 2991.4469 & 1496.2271 & E & 743.3206 & 372.1640 & & & & 725.3101 & 363.1587 & $\mathbf{6}$ \\
\hline $\mathbf{2 8}$ & 3138.5001 & 1569.7537 & 3121.4736 & 1561.2404 & 3120.4895 & 1560.7484 & E & $\mathbf{6 1 4 . 2 7 8 0}$ & 307.6427 & & & & 596.2675 & 298.6374 & $\mathbf{5}$ \\
\hline $\mathbf{2 9}$ & 3253.5270 & 1627.2672 & 3236.5005 & 1618.7539 & 3235.5165 & 1618.2619 & D & 485.2354 & 243.1214 & & & & 467.2249 & 234.1161 & $\mathbf{4}$ \\
\hline $\mathbf{3 0}$ & 3354.5747 & 1677.7910 & 3337.5482 & 1669.2777 & 3336.5642 & 1668.7857 & T & 370.2085 & 185.6079 & & & & 352.1979 & 176.6026 & $\mathbf{3}$ \\
\hline $\mathbf{3 1}$ & 3491.6336 & 1746.3205 & 3474.6071 & 1737.8072 & 3473.6231 & 1737.3152 & H & 269.1608 & 135.0840 & & & & & & $\mathbf{2}$ \\
\hline $\mathbf{3 2}$ & & & & & & & L & 132.1019 & 66.5546 & & & & & & $\mathbf{1}$ \\
\hline
\end{tabular}
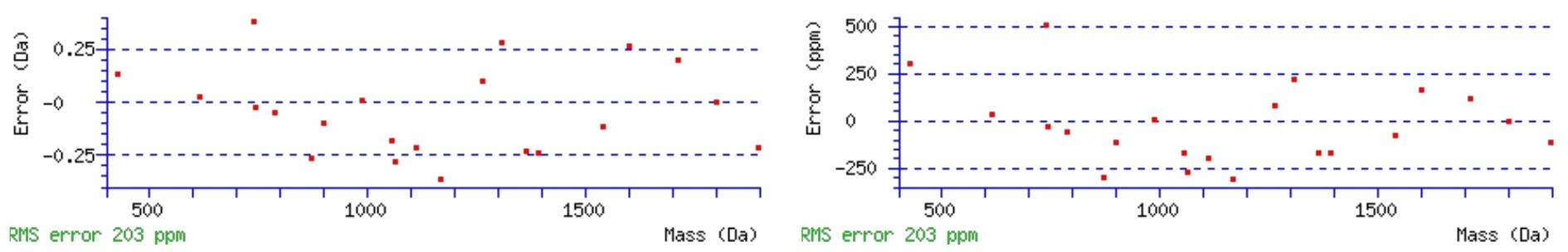

\section{All matches to this query}

\begin{tabular}{|l|l|l|l|}
\hline Score & Mr(calc): & Delta & \multicolumn{1}{|c|}{ Sequence } \\
\hline 70.2 & 3621.7210 & 0.0030 & VVNLNGELGDSWIVPLDNLTKEDLDEEEDTHL \\
\hline 66.3 & 3621.7210 & 0.0030 & VVNLNGELGDSWIVPLDNLTKEDLDEEEDTHL \\
\hline 53.0 & 3621.7210 & 0.0030 & VVNLNGELGDSWIVPLDNLTKEDLDEEEDTHL \\
\hline 39.5 & 3620.7370 & 0.9870 & VVNLNGELGDSWIVPLDNLTKEDLDEEEDTHL \\
\hline 6.4 & 3619.7455 & 1.9785 & QDLVDKLISLFYGVLTPMLNPIIYSLRNK \\
\hline 5.3 & 3620.6956 & 1.0284 & DGIKINDSNFLGKILLQVANLNATTLSYTLK \\
\hline 4.6 & 3621.6897 & 0.0343 & SIFLWLGYSNSFFNPLIYTAFNRSYSSAFK \\
\hline 4.2 & 3619.7455 & 1.9785 & QDLVDKLISLFYGVLTPMLNPIIYSLRNK \\
\hline 4.0 & 3620.6956 & 1.0284 & DGIKINDSNFLGKILLQVANLNATTLSYTLK \\
\hline 4.0 & 3620.6956 & 1.0284 & DGIKINDSNFLGKILLQVANLNATTLSYTLK \\
\hline
\end{tabular}

Spectrum No: 358; Query: 1800; Rank: 1

\section{Peptide View}

MS/MS Fragmentation of DCSTNPPRLPVTAVNTTMR

Found in IPI00197684, Tax_Id=10116 Gene_Symbol=Xpnpep2 Membrane-bound aminopeptidase P

Match to Query 1800: 2130.025962 from(711.015930,3+)

Title: 100101RatKid_NS_deglyco_20.2694.2694.3.dta

Data file K:INewmanPaper|Piliang 3 SubProteomes\Piliang3SP\mgf5ppm\ERLIC_3SubProteomes5ppm.mgf 

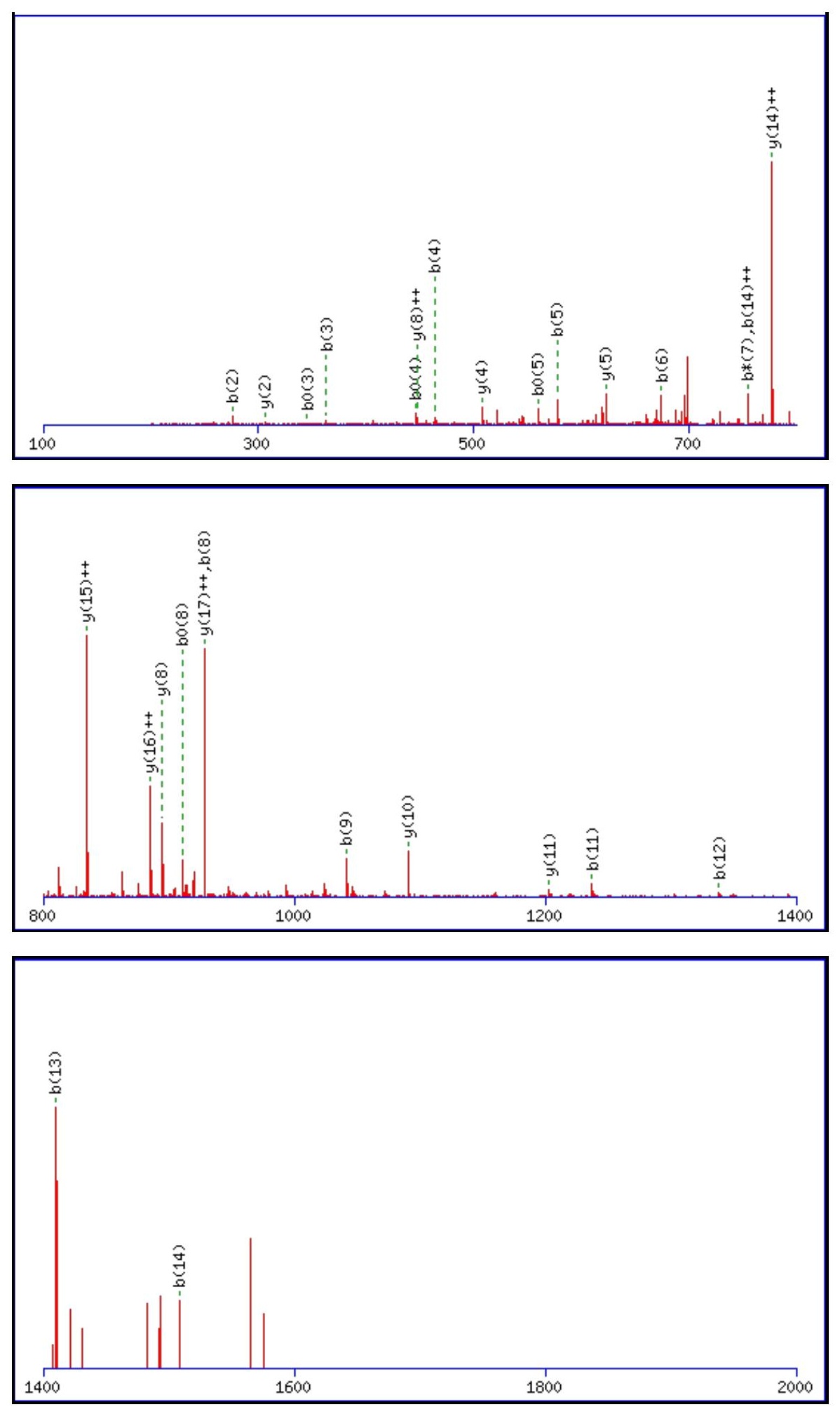

Monoisotopic mass of neutral peptide $\operatorname{Mr}($ calc): 2130.0198

Fixed modifications: Carbamidomethyl (C)

Variable modifications:

N15 : Deamidated $\mathrm{N}(\mathrm{N})$

Ions Score: 70 Expect: $2.3 \mathrm{e}-005$

Matches (Bold Red): 28/204 fragment ions using 45 most intense peaks

\begin{tabular}{|c|c|c|c|c|c|c|c|c|c|c|c|c|c|c|}
\hline \# & b & $\mathbf{b}^{++}$ & $\mathbf{b}^{*}$ & $\mathbf{b}^{*^{++}}$ & $\mathbf{b}^{0}$ & $\mathbf{b}^{\mathbf{0 + +}}$ & Seq. & $\mathbf{y}$ & $y^{++}$ & $\mathrm{y}^{*}$ & $y^{*^{++}}$ & $\mathbf{y}^{\mathbf{0}}$ & $y^{0++}$ & \# \\
\hline 1 & 116.0342 & 58.5207 & & & 98.0237 & 49.5155 & D & & & & & & & 19 \\
\hline 2 & 276.0649 & 138.5361 & & & 258.0543 & 129.5308 & C & 2016.0001 & 1008.5037 & 1998.9736 & 999.9904 & 1997.9895 & 999.4984 & 18 \\
\hline 3 & 363.0969 & 182.0521 & & & 345.0863 & 173.0468 & S & 1855.9695 & 928.4884 & 1838.9429 & 919.9751 & 1837.9589 & 919.4831 & 17 \\
\hline 4 & 464.1446 & 232.5759 & & & 446.1340 & 223.5706 & $\mathbf{T}$ & 1768.9374 & 884.9724 & 1751.9109 & 876.4591 & 1750.9269 & 875.9671 & 16 \\
\hline 5 & 578.1875 & 289.5974 & 561.1610 & 281.0841 & 560.1769 & 280.5921 & $\mathbf{N}$ & 1667.8897 & 834.4485 & 1650.8632 & 825.9352 & 1649.8792 & 825.4432 & 15 \\
\hline
\end{tabular}




\begin{tabular}{|c|c|c|c|c|c|c|c|c|c|c|c|c|c|c|}
\hline 6 & 675.2403 & 338.1238 & 658.2137 & |329.6105 | & 657.2297 & 329.1185 & $\mathbf{P}$ & |1553.8468| & 777.4270 & |1536.8203| & 768.9138| & |1535.8363| & 768.4218 & 14 \\
\hline 7 & 772.2930 & 386.6502 & 755.2665 & 378.1369 & 754.2825 & 377.6449 & $\mathbf{P}$ & 1456.7941 & 728.9007 & 1439.7675 & 720.3874 & 1438.7835 & 719.8954 & 13 \\
\hline 8 & 928.3941 & 464.7007 & 911.3676 & 456.1874 & 910.3836 & 455.6954 & $\mathbf{R}$ & 1359.7413 & 680.3743 & 1342.7147 & 671.8610 & 1341.7307 & 671.3690 & 12 \\
\hline 9 & 1041.4782 & 521.2427 & 1024.4517 & 512.7295 & 1023.4676 & 512.2375 & $\mathbf{L}$ & 1203.6402 & 602.3237 & 1186.6136 & 593.8105 & 1185.6296 & 593.3184 & 11 \\
\hline 10 & 1138.5310 & 569.7691 & 1121.5044 & 561.2558 & 1120.5204 & 560.7638 & $\mathbf{P}$ & 1090.5561 & 545.7817 & 1073.5296 & 537.2684 & 1072.5456 & 536.7764 & 10 \\
\hline 11 & 1237.5994 & 619.3033 & 1220.5728 & 610.7901 & 1219.5888 & 610.2980 & $\mathbf{V}$ & 993.5034 & 497.2553 & 976.4768 & 488.7420 & 975.4928 & 488.2500 & 9 \\
\hline 12 & 1338.6471 & 669.8272 & 1321.6205 & 661.3139 & 1320.6365 & 660.8219 & $\mathbf{T}$ & 894.4349 & 447.7211 & 877.4084 & 439.2078 & 876.4244 & 438.7158 & 8 \\
\hline 13 & 1409.6842 & 705.3457 & 1392.6576 & 696.8325 & 1391.6736 & 696.3404 & A & 793.3873 & 397.1973 & 776.3607 & 388.6840 & 775.3767 & 388.1920 & 7 \\
\hline 14 & 1508.7526 & 754.8799 & 1491.7260 & 746.3667 & 1490.7420 & 745.8747 & $\mathbf{V}$ & 722.3501 & 361.6787 & 705.3236 & 353.1654 & 704.3396 & 352.6734 & 6 \\
\hline 15 & 1623.7795 & 812.3934 & 1606.7530 & 803.8801 & 1605.7690 & 803.3881 & $\mathbf{N}$ & 623.2817 & 312.1445 & 606.2552 & 303.6312 & 605.2712 & 303.1392 & 5 \\
\hline 16 & 1724.8272 & 862.9172 & 1707.8007 & 854.4040 & 1706.8166 & 853.9120 & $\mathbf{T}$ & 508.2548 & 254.6310 & 491.2282 & 246.1178 & 490.2442 & 245.6258 & 4 \\
\hline 17 & 1825.8749 & 913.4411 & 1808.8483 & 904.9278 & 1807.8643 & 904.4358 & $\mathbf{T}$ & 407.2071 & 204.1072 & 390.1806 & 195.5939 & 389.1966 & 195.1019 & 3 \\
\hline 18 & 1956.9154 & 978.9613 & 1939.8888 & 970.4480 & 1938.9048 & 969.9560 & $\mathbf{M}$ & 306.1594 & 153.5834 & 289.1329 & 145.0701 & & & 2 \\
\hline 19 & & & & & & & $\mathbf{R}$ & 175.1190 & 88.0631 & 158.0924 & 79.5498 & & & 1 \\
\hline
\end{tabular}
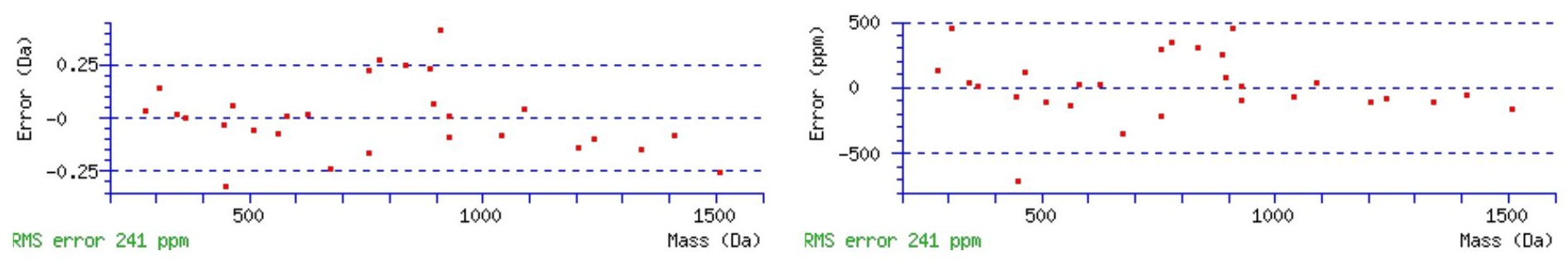

\section{All matches to this query}

\begin{tabular}{|l|l|l|l|}
\hline Score & Mr(calc): & Delta & \multicolumn{1}{c|}{ Sequence } \\
\hline 70.1 & 2130.0198 & 0.0062 & DCSTNPPRLPVTAVNTTMR \\
\hline 52.8 & 2129.0358 & 0.9902 & $\underline{\text { DCSTNPPRLPVTAVNTTMR }}$ \\
\hline 30.8 & 2130.0198 & 0.0062 & DCSTNPPRLPVTAVNTTMR \\
\hline 7.0 & 2130.0064 & 0.0195 & DKVSLAALGGRVFFFSXR \\
\hline 6.8 & 2128.0388 & 1.9872 & $\underline{\text { HQELLVNLNPMVTPLASR }}$ \\
\hline 5.6 & 2129.0201 & 1.0059 & $\underline{\text { LSYNGRSSVVQCRPLTGR }}$ \\
\hline 4.4 & 2128.0388 & 1.9872 & $\underline{\text { HQELLVNLNPMVTPLASR }}$ \\
\hline 3.5 & 2130.0309 & -0.0050 & $\underline{\text { IRVNSVNPTVVLTDMGKK }}$ \\
\hline 3.4 & 2130.0309 & -0.0050 & $\underline{\text { IRVNSVNPTVVLTDMGKK }}$ \\
\hline 3.4 & 2130.0309 & -0.0050 & $\underline{\text { IRVNSVNPTVVLTDMGKK }}$ \\
\hline
\end{tabular}

Spectrum No: 359; Query: 2621; Rank: 1

\section{Peptide View}

MS/MS Fragmentation of SFSGNASILSPLLQVPDIDGDGAPDLLMLSR

Found in IPI00372350, Tax_Id=10116 Gene_Symbol=Itfg3 Protein ITFG3

Match to Query 2621: 3198.621792 from(1067.214540,3+)

Title: 100101RatKid_NS_deglyco_22.6316.6316.3.dta

Data file K:INewmanPaper|Piliang 3 SSubProteomes\Piliang3SP\mgf5ppm\ERLIC_3SubProteomes5ppm.mgf 

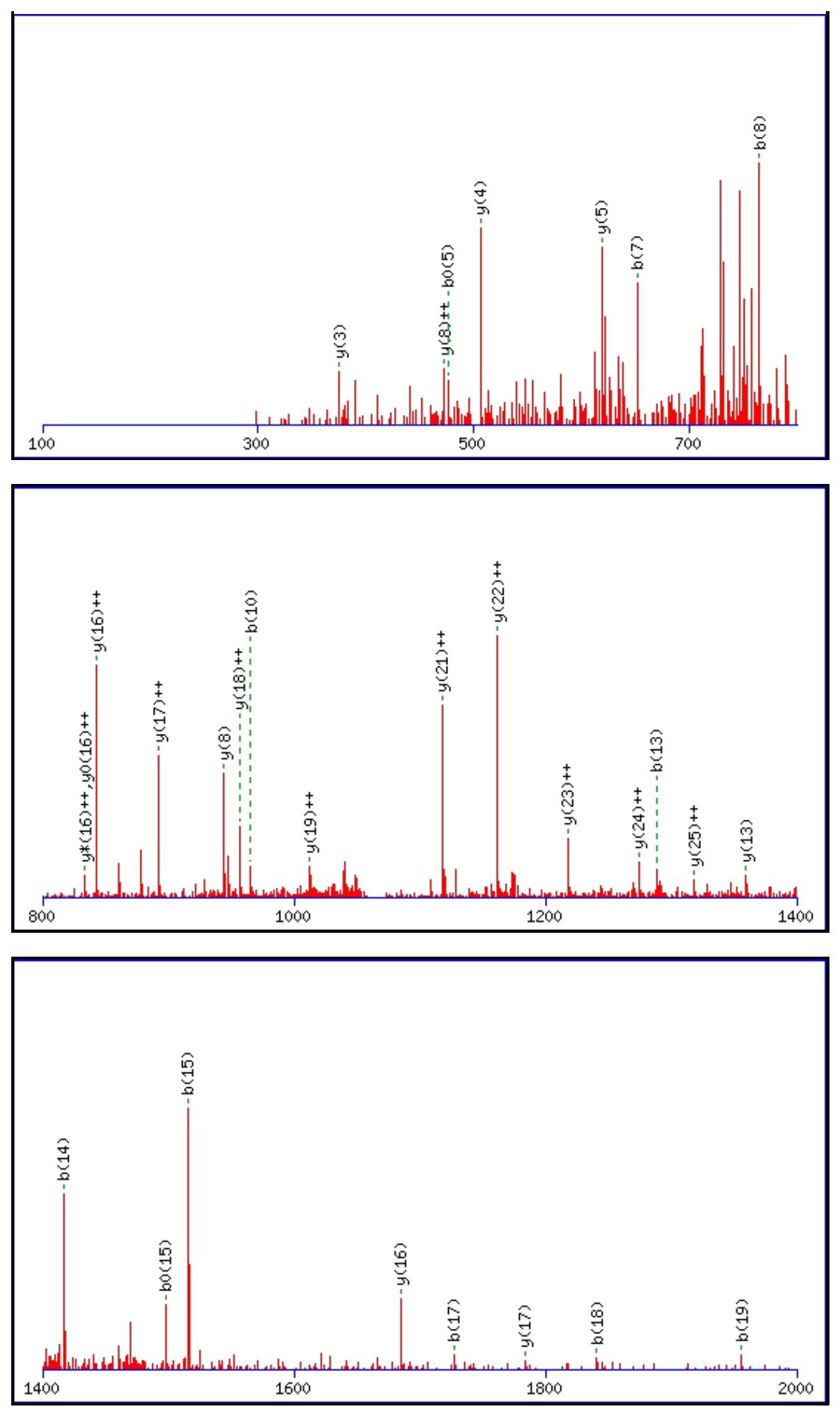

Monoisotopic mass of neutral peptide $\operatorname{Mr}($ calc): 3198.6118

Fixed modifications: Carbamidomethyl (C)

Variable modifications:

N5: Deamidated $\mathrm{N}(\mathrm{N})$

Ions Score: 70 Expect: $2 \mathrm{e}-005$

Matches (Bold Red): $30 / 350$ fragment ions using 42 most intense peaks

\begin{tabular}{|c|c|c|c|c|c|c|c|c|c|c|c|c|c|c|}
\hline \# & b & $\mathbf{b}^{++}$ & $\mathbf{b}^{*}$ & $\mathbf{b}^{*^{++}}$ & $\mathbf{b}^{\mathbf{0}}$ & $\mathbf{b}^{0++}$ & Seq. & $\mathbf{y}$ & $\mathbf{y}^{++}$ & $\mathrm{y}^{*}$ & $\mathbf{y}^{*^{++}}$ & $y^{0}$ & $\mathbf{y}^{\mathbf{0 + +}}$ & \# \\
\hline 1 & 88.0393 & 44.5233 & & & 70.0287 & 35.5180 & $S$ & & & & & & & 31 \\
\hline 2 & 235.1077 & 118.0575 & & & 217.0972 & 109.0522 & $\mathbf{F}$ & 3112.5871 & 1556.7972 & 3095.5605 & |1548.2839 & 3094.5765 & 1547.7919 & 30 \\
\hline 3 & 322.1397 & 161.5735 & & & 304.1292 & 152.5682 & $S$ & 2965.5187 & 1483.2630 & 2948.4921 & 1474.7497 & 2947.5081 & 1474.2577 & 29 \\
\hline 4 & 379.1612 & 190.0842 & & & 361.1506 & 181.0790 & G & 2878.4866 & 1439.7470 & 2861.4601 & 1431.2337 & 2860.4761 & 1430.7417 & 28 \\
\hline 5 & 494.1881 & 247.5977 & 477.1616 & 239.0844 & 476.1776 & 238.5924 & $\mathbf{N}$ & 2821.4652 & 1411.2362 & 2804.4386 & 1402.7230 & 2803.4546 & 1402.2309 & 27 \\
\hline
\end{tabular}




\begin{tabular}{|c|c|c|c|c|c|c|c|c|c|c|c|c|c|c|}
\hline 6 & 565.2253 & 283.1163 & 548.1987 & 274.6030 & 547.2147| & 274.1110 & A & 2706.4382 & |1353.7228 & 2689.4117| & .2095 & 2688.4277 & 7175 & 26 \\
\hline 7 & 652.2573 & 326.6323 & 635.2307 & 318.1190 & 634.2467 & 317.6270 & $\mathbf{S}$ & 2635.4011 & 1318.2042 & 2618.3746 & 1309.6909 & 2617.3906 & |1309.1989| & 25 \\
\hline 8 & 765.3414 & 383.1743 & 748.3148 & 374.6610 & 747.3308 & 374.1690 & I & 2548.3691 & 1274.6882 & 2531.3426 & 1266.1749 & 2530.3585 & 1265.6829 & 24 \\
\hline 9 & 878.4254 & 439.7163 & 861.3989 & 431.2031 & 860.4149 & 430.7111 & $\mathbf{L}$ & 2435.2850 & 1218.1462 & 2418.2585 & 1209.6329 & 2417.2745 & 1209.1409 & \\
\hline 10 & 965.4574 & 483.2324 & 948.4309 & 474.7191 & 947.4469 & 474.2271 & S & 2322.2010 & 1161.6041 & 2305.1744 & 1153.0909 & 2304.1904 & 1152.5988 & 22 \\
\hline 11 & 1062.5102 & 531.7587 & 1045.4837 & 523.2455 & 1044.4996 & 522.7535 & $\mathbf{P}$ & 2235.1689 & 1118.0881 & 2218.1424 & 1109.5748 & 2217.1584 & 1109.0828 & \\
\hline 12 & 1175.5943 & 588.3008 & 1158.5677 & 579.7875 & 1157.5837 & 579.2955 & $\mathbf{L}$ & 2138.1162 & 1069.5617 & 2121.0896 & 1061.0485 & 2120.1056 & 1060.5564 & 0 \\
\hline 13 & 1288.6783 & 644.8428 & 1271.6518 & 636.3295 & 1270.6678 & 635.8375 & $\mathbf{L}$ & 2025.0321 & 1013.0197 & 2008.0056 & 1004.5064 & 2007.0216 & 1004.0144 & \\
\hline 14 & 1416.7369 & 708.8721 & 1399.7104 & 700.3588 & 1398.7263 & 699.8668 & $\mathbf{Q}$ & 1911.9481 & 956.4777 & 1894.9215 & 947.9644 & 1893.9375 & 947.4724 & 18 \\
\hline 15 & 1515.8053 & 758.4063 & 1498.7788 & 749.8930 & 1497.7948 & 749.4010 & V & 1783.8895 & 892.4484 & 1766.8629 & 883.9351 & 1765.8789 & 883.4431 & \\
\hline 16 & 1612.8581 & 806.9327 & 1595.8315 & 798.4194 & 1594.8475 & 797.9274 & $\mathbf{P}$ & 1684.8211 & 842.9142 & 1667.7945 & 834.4009 & 1666.8105 & 833.9089 & 16 \\
\hline 17 & 1727.8850 & 864.4462 & 1710.8585 & 855.9329 & 1709.8745 & 855.4409 & D & 1587.7683 & 794.3878 & 1570.7418 & 785.8745 & 1569.7577 & 785.3825 & 15 \\
\hline 18 & 1840.9691 & 920.9882 & 1823.9426 & 912.4749 & 1822.9585 & 911.9829 & I & 1472.7414 & 736.8743 & 1455.7148 & 728.3610 & 1454.7308 & 727.8690 & 14 \\
\hline 19 & 1955.9960 & 978.5017 & 1938.9695 & 969.9884 & 1937.9855 & 969.4964 & D & 1359.6573 & 680.3323 & 1342.6307 & 671.8190 & 1341.6467 & 671.3270 & 13 \\
\hline 21 & 2128.0444 & 1064.5259 & 2111.0179 & 1056.0126 & 2110.0339 & 1055.5206 & D & 1187.6089 & 594.3081 & 1170.5823 & 585.7948 & 1169.5983 & 585.3028 & 11 \\
\hline 22 & 2185.0659 & 1093.0366 & 2168.0394 & 1084.5233 & 2167.0553 & 1084.0313 & $\mathbf{G}$ & 1072.5819 & 536.7946 & 1055.5554 & 528.2813 & 1054.5714 & 527.7893 & 10 \\
\hline 23 & 2256.1030 & 1128.5552 & 2239.0765 & 1120.0419 & 2238.0925 & 1119.5499 & A & 1015.5605 & 508.2839 & 998.5339 & 499.7706 & 997.5499 & 499.2786 & 9 \\
\hline 242 & 2353.1558 & 1177.0815 & 2336.1292 & 1168.5683 & 2335.1452 & 1168.0763 & $\mathbf{P}$ & 944.5234 & 472.7653 & 927.4968 & 464.2520 & 926.5128 & 463.7600 & 8 \\
\hline 25 & 2468.1827 & 1234.5950 & 2451.1562 & 1226.0817 & 2450.1722 & 1225.5897 & D & 847.4706 & 424.2389 & 830.4441 & 415.7257 & 829.4600 & 415.2337 & 7 \\
\hline 26 & 2581.2668 & 1291.1370 & 2564.2402 & 1282.6238 & 2563.2562 & 1282.1318 & $\mathbf{L}$ & 732.4437 & 366.7255 & 715.4171 & 358.2122 & 714.4331 & 357.7202 & 6 \\
\hline 272 & 2694.3509 & 1347.6791 & 2677.3243 & 1339.1658 & 2676.3403 & 1338.6738 & $\mathbf{L}$ & 619.3596 & 310.1834 & 602.3330 & 301.6702 & 601.3490 & 301.1782 & 5 \\
\hline 28 & 2825.3913 & 1413.1993 & 2808.3648 & 1404.6860 & 2807.3808 & 1404.1940 & $\mathbf{M}$ & 506.2755 & 253.6414 & 489.2490 & 245.1281 & 488.2650 & 244.6361 & 4 \\
\hline 29 & 2938.4754 & 1469.7413 & 2921.4489 & 1461.2281 & 2920.4648 & 1460.7361 & $\mathbf{L}$ & 375.2350 & 188.1212 & 358.2085 & 179.6079 & 357.2245 & 179.1159 & 3 \\
\hline 30 & 3025.5074 & 1513.2574 & 3008.4809 & 1504.7441 & 3007.4969 & 1504.2521 & $\mathrm{~S}$ & 262.1510 & 131.5791 & 245.1244 & 123.0659 & 244.1404 & 122.5738 & 2 \\
\hline 31 & & & & & & & $\mathbf{R}$ & 175.1190 & 88.0631 & 158.0924 & 79.5498 & & & 1 \\
\hline
\end{tabular}
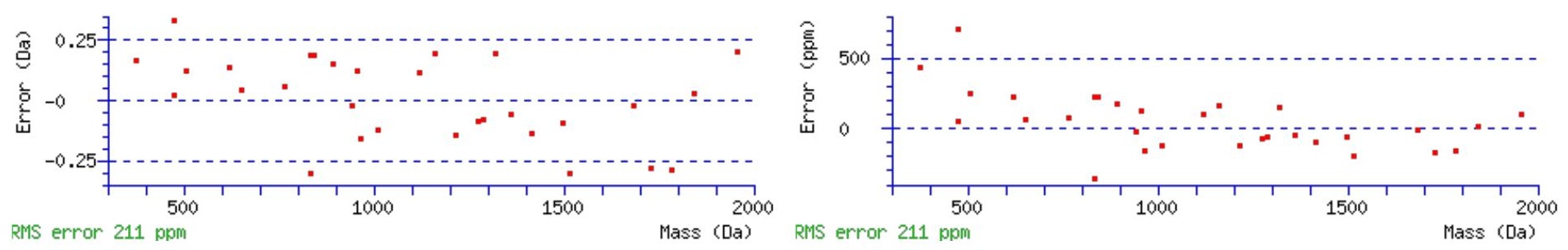

\section{All matches to this query}

\begin{tabular}{|l|l|l|l|}
\hline Score & Mr(calc): & Delta & \multicolumn{1}{c|}{ Sequence } \\
\hline 70.0 & 3198.6118 & 0.0100 & SFSGNASILSPLLQVPDIDGDGAPDLLMLSR \\
\hline 46.1 & 3197.6278 & 0.9940 & SFSGNASILSPLLQVPDIDGDGAPDLLMLSR \\
\hline 12.2 & 3196.6404 & 1.9814 & DRDDVNVEIVFLHNISPNLELEALFKR \\
\hline 7.1 & 3196.6404 & 1.9814 & DRDDVNVEIVFLHNISPNLELEALFKR \\
\hline 6.5 & 3197.6244 & 0.9974 & DRDDVNVEIVFLHNISPNLELEALFKR \\
\hline 4.6 & 3197.6140 & 1.0078 & IVAVFYTLIIPMLNPLIYSLRNTEVK \\
\hline 4.0 & 3198.6400 & -0.0182 & VMKTIPLYNLTGLSVSNGKDQLVVFHTK \\
\hline 3.0 & 3197.6447 & 0.9770 & GVSPEPIHLKVFSPNVVNLTLVDLPGMTK \\
\hline 2.8 & 3196.6049 & 2.0169 & DPLGMGLTIISLGFSALTGLIIGVFVNYR \\
\hline 2.8 & 3196.6049 & 2.0169 & DPLGMGLTIISLGFSALTGLIIGVFVNYR \\
\hline
\end{tabular}

Spectrum No: 360; Query: 304; Rank: 1 


\section{Peptide View}

MS/MS Fragmentation of ATYNISLIHPK

Found in IPI00327398, Tax_Id=10116 Gene_Symbol=Enpep Isoform 1 of Glutamyl aminopeptidase

Match to Query 304: 1256.673788 from(629.344170,2+)

Title: 091008RatKidney_NH4Format02_26.2031.2031.2.dta

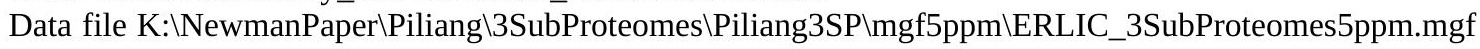
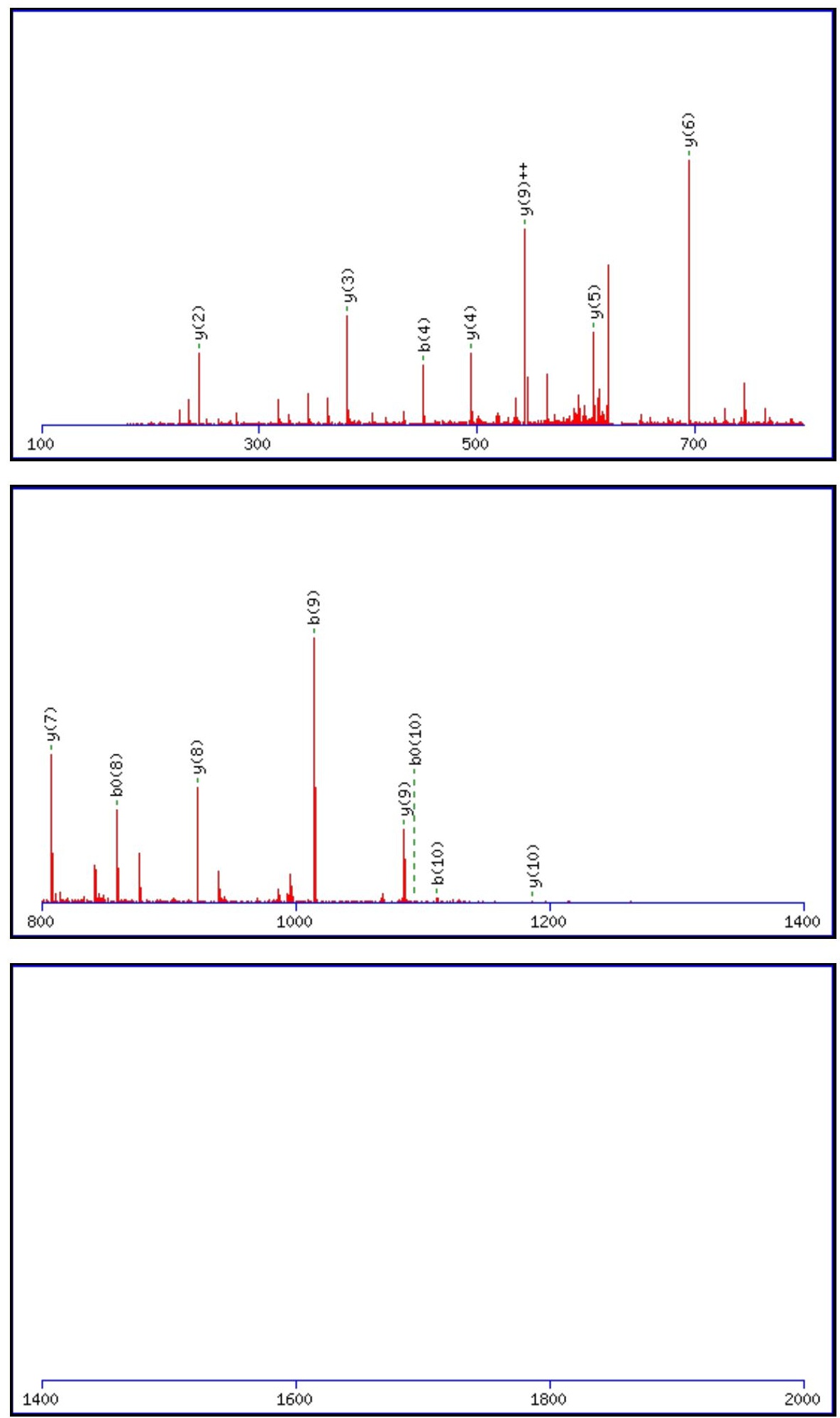

Monoisotopic mass of neutral peptide $\operatorname{Mr}($ calc): 1256.6765

Fixed modifications: Carbamidomethyl (C)

Variable modifications:

N4 : Deamidated_N (N)

Ions Score: 70 Expect: $8.5 \mathrm{e}-006$ 
Matches (Bold Red): 15/102 fragment ions using 29 most intense peaks

\begin{tabular}{|c|c|c|c|c|c|c|c|c|c|c|c|c|c|c|}
\hline \# & b & $\mathbf{b}^{++}$ & $\mathbf{b}^{*}$ & $\mathbf{b}^{*^{++}}$ & $\mathbf{b}^{0}$ & $\mathbf{b}^{\mathbf{0 + +}}$ & Seq. & $\mathbf{y}$ & $\mathbf{y}^{++}$ & $\mathbf{y}^{*}$ & $\mathrm{y}^{\mathrm{*}^{++}}$ & $\mathbf{y}^{\mathbf{0}}$ & $\mathbf{y}^{\mathbf{0 + +}}$ & $\#$ \\
\hline 1 & 72.0444 & 36.5258 & & & & & A & & & & & & & 11 \\
\hline 2 & 173.0921 & 87.0497 & & & 155.0815 & 78.0444 & $\mathbf{T}$ & 1186.6466 & 593.8270 & 1169.6201 & 585.3137 & 1168.6361 & 584.8217 & 10 \\
\hline 3 & 336.1554 & 168.5813 & & & 318.1448 & 159.5761 & $\mathbf{Y}$ & 1085.5990 & 543.3031 & 1068.5724 & 534.7898 & 1067.5884 & 534.2978 & 9 \\
\hline 4 & 451.1823 & 226.0948 & 434.1558 & 217.5815 & 433.1718 & 217.0895 & $\mathbf{N}$ & 922.5356 & 461.7715 & 905.5091 & 453.2582 & 904.5251 & 452.7662 & 8 \\
\hline 5 & 564.2664 & 282.6368 & 547.2398 & 274.1236 & 546.2558 & 273.6316 & I & 807.5087 & 404.2580 & 790.4822 & 395.7447 & 789.4981 & 395.2527 & 7 \\
\hline 6 & 651.2984 & 326.1529 & 634.2719 & 317.6396 & 633.2879 & 317.1476 & $\mathrm{~S}$ & 694.4246 & 347.7160 & 677.3981 & 339.2027 & 676.4141 & 338.7107 & 6 \\
\hline 7 & 764.3825 & 382.6949 & 747.3559 & 374.1816 & 746.3719 & 373.6896 & $\mathbf{L}$ & 607.3926 & 304.1999 & 590.3661 & 295.6867 & & & , \\
\hline 8 & 877.4666 & 439.2369 & 860.4400 & 430.7236 & 859.4560 & 430.2316 & I & 494.3085 & 247.6579 & 477.2820 & 239.1446 & & & 4 \\
\hline 9 & 1014.5255 & 507.7664 & 997.4989 & 499.2531 & 996.5149 & 498.7611 & $\mathbf{H}$ & 381.2245 & 191.1159 & 364.1979 & 182.6026 & & & 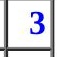 \\
\hline 10 & 1111.5782 & 556.2928 & 1094.5517 & 547.7795 & 1093.5677 & 547.2875 & $\mathbf{P}$ & 244.1656 & 122.5864 & 227.1390 & 114.0731 & & & 2 \\
\hline 11 & & & & & & & $\mathbf{K}$ & 147.1128 & 74.0600 & 130.0863 & 65.5468 & & & ( \\
\hline
\end{tabular}
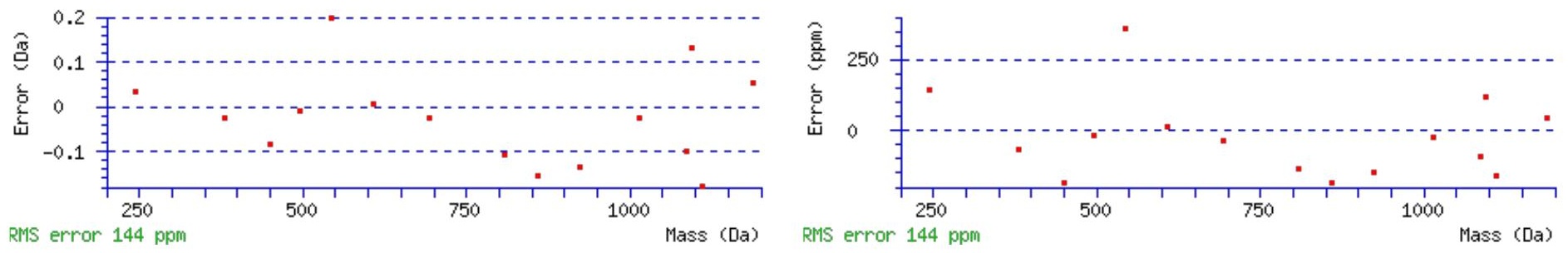

\section{All matches to this query}

\begin{tabular}{|l|l|l|l|}
\hline Score & Mr(calc): & Delta & \multicolumn{1}{|c|}{ Sequence } \\
\hline 70.0 & 1256.6765 & -0.0027 & ATYNISLIHPK \\
\hline 5.2 & 1256.6765 & -0.0027 & SLIHLDLGFNK \\
\hline 4.7 & 1256.6613 & 0.0125 & TDDTPVVLVGNK \\
\hline 4.4 & 1254.6721 & 2.0017 & YAVDTVLRYR \\
\hline 3.8 & 1256.6837 & -0.0099 & AGDTIRLEAGVR \\
\hline 0.4 & 1256.6837 & -0.0099 & RSSDPALRLDK \\
\hline
\end{tabular}

Spectrum No: 361; Query: 97; Rank: 1

\section{Peptide View}

MS/MS Fragmentation of IANISMEVGR

Found in IPI00363534, Tax_Id=10116 Gene_Symbol=Lama1 laminin, alpha 1

Match to Query 97: 1089.547568 from(545.781060,2+)

Title: 091008RatKidney_NoSalt_26.1658.1658.2.dta

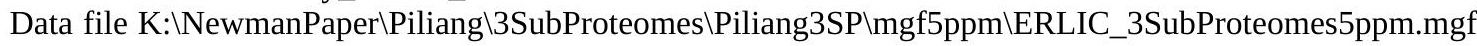



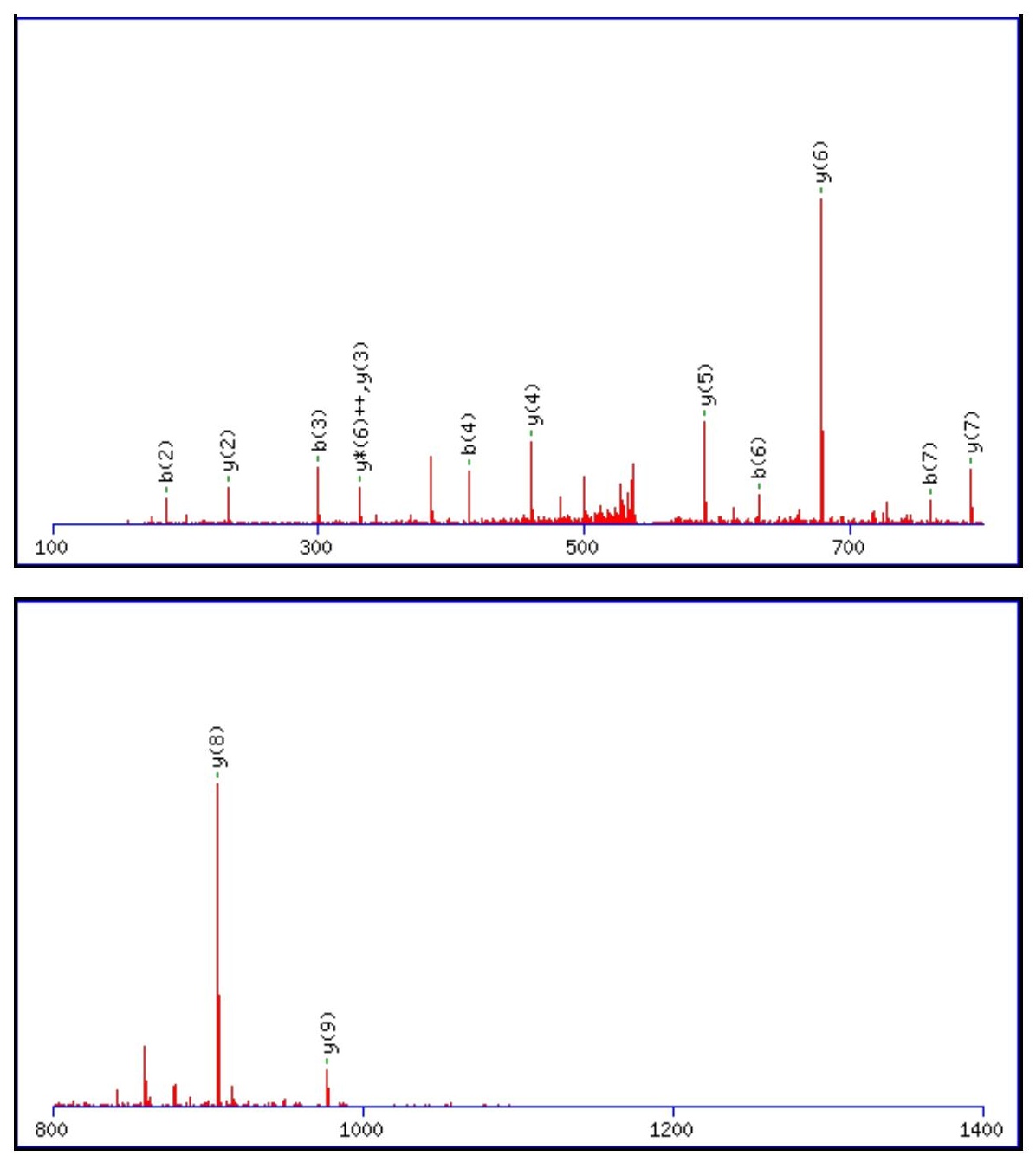

\begin{tabular}{rrrr}
\hline 00 & 1600 & 1800 & 2000 \\
\hline
\end{tabular}

Monoisotopic mass of neutral peptide $\operatorname{Mr}($ calc): 1089.5488 Fixed modifications: Carbamidomethyl (C)

Variable modifications:

N3 : Deamidated_N (N)

Ions Score: 70 Expect: $1.3 \mathrm{e}-005$

Matches (Bold Red): 14/90 fragment ions using 20 most intense peaks

\begin{tabular}{|c|c|c|c|c|c|c|c|c|c|c|c|c|c|c|}
\hline$\#$ & $\mathbf{b}$ & $\mathbf{b}^{++}$ & $\mathbf{b}^{*}$ & $\mathbf{b}^{\boldsymbol{*}^{++}}$ & $\mathbf{b}^{\mathbf{0}}$ & $\mathbf{b}^{\mathbf{0}++}$ & $\mathbf{S e q}$ & $\mathbf{y}$ & $\mathbf{y}^{++}$ & $\mathbf{y}^{*}$ & $\mathbf{y}^{\boldsymbol{*}^{++}}$ & $\mathbf{y}^{\mathbf{0}}$ & $\mathbf{y}^{\mathbf{0 + +}}$ & $\#$ \\
\hline $\mathbf{1}$ & 114.0913 & 57.5493 & & & & & $\mathbf{I}$ & & & & & & & $\mathbf{1 0}$ \\
\hline $\mathbf{2}$ & $\mathbf{1 8 5 . 1 2 8 5}$ & 93.0679 & & & & & $\mathbf{A}$ & $\mathbf{9 7 7 . 4 7 2 1}$ & 489.2397 & 960.4455 & 480.7264 & 959.4615 & 480.2344 & $\mathbf{9}$ \\
\hline $\mathbf{3}$ & $\mathbf{3 0 0 . 1 5 5 4}$ & 150.5813 & 283.1288 & 142.0681 & & & $\mathbf{N}$ & $\mathbf{9 0 6 . 4 3 4 9}$ & 453.7211 & 889.4084 & 445.2078 & 888.4244 & 444.7158 & $\mathbf{8}$ \\
\hline $\mathbf{4}$ & $\mathbf{4 1 3 . 2 3 9 5}$ & 207.1234 & 396.2129 & 198.6101 & & & $\mathbf{I}$ & $\mathbf{7 9 1 . 4 0 8 0}$ & 396.2076 & 774.3815 & 387.6944 & 773.3974 & 387.2024 & $\mathbf{7}$ \\
\hline $\mathbf{5}$ & 500.2715 & 250.6394 & 483.2449 & 242.1261 & 482.2609 & 241.6341 & $\mathbf{S}$ & $\mathbf{6 7 8 . 3 2 3 9}$ & 339.6656 & 661.2974 & $\mathbf{3 3 1 . 1 5 2 3}$ & 660.3134 & 330.6603 & $\mathbf{6}$ \\
\hline
\end{tabular}




\begin{tabular}{|r|c|c|c|c|c|c|c|c|c|c|c|c|c|c|c|c|}
$\mathbf{6}$ & $\mathbf{6 3 1 . 3 1 2 0}$ & 316.1596 & 614.2854 & 307.6463 & 613.3014 & 307.1543 & $\mathbf{M}$ & $\mathbf{5 9 1 . 2 9 1 9}$ & 296.1496 & 574.2654 & 287.6363 & 573.2813 & 287.1443 & $\mathbf{5}$ \\
\hline $\mathbf{7}$ & $\mathbf{7 6 0 . 3 5 4 6}$ & 380.6809 & 743.3280 & 372.1676 & 742.3440 & 371.6756 & $\mathbf{E}$ & $\mathbf{4 6 0 . 2 5 1 4}$ & 230.6293 & 443.2249 & 222.1161 & 442.2409 & 221.6241 & $\mathbf{4}$ \\
\hline $\mathbf{8}$ & 859.4230 & 430.2151 & 842.3964 & 421.7019 & 841.4124 & 421.2098 & $\mathbf{V}$ & 331.2088 & 166.1081 & 314.1823 & 157.5948 & & & $\mathbf{3}$ \\
\hline $\mathbf{9}$ & 916.4444 & 458.7259 & 899.4179 & 450.2126 & 898.4339 & 449.7206 & $\mathbf{G}$ & $\mathbf{2 3 2 . 1 4 0 4}$ & 116.5738 & 215.1139 & 108.0606 & & & $\mathbf{2}$ \\
\hline $\mathbf{1 0}$ & & & & & & & $\mathbf{R}$ & 175.1190 & 88.0631 & 158.0924 & 79.5498 & & & $\mathbf{1}$ \\
\hline
\end{tabular}
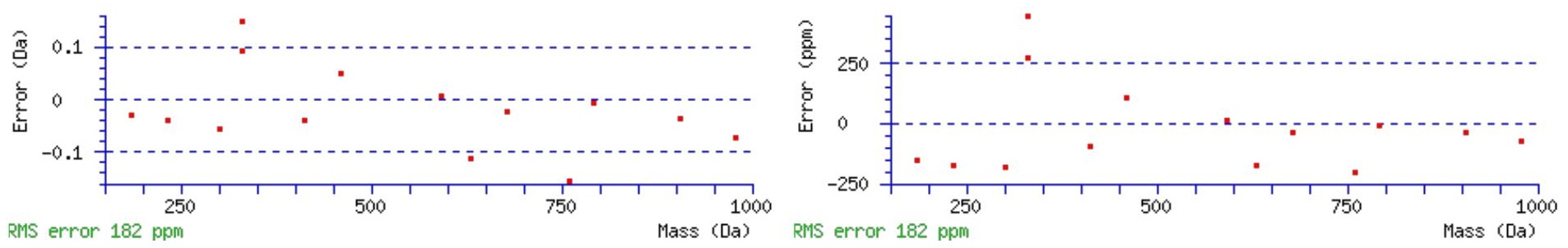

\section{All matches to this query}

\begin{tabular}{|l|l|l|l|}
\hline Score & Mr(calc): & Delta & \multicolumn{1}{|c|}{ Sequence } \\
\hline 70.0 & 1089.5488 & -0.0012 & IANISMEVGR \\
\hline 10.8 & 1089.5567 & -0.0091 & IGKYESHTR \\
\hline 9.7 & 1089.5488 & -0.0012 & AIDLENIMR \\
\hline 9.4 & 1089.5389 & 0.0086 & LAOYMAHTR \\
\hline 8.4 & 1089.5488 & -0.0013 & EALVAMGIDR \\
\hline 8.0 & 1089.5528 & -0.0053 & IALNMDWVK \\
\hline 7.1 & 1089.5529 & -0.0053 & ILWAMPSLGS \\
\hline 4.7 & 1089.5567 & -0.0091 & LTSGLHSYGR \\
\hline 4.7 & 1089.5389 & 0.0086 & LAWGMLNGGR \\
\hline 3.0 & 1089.5461 & 0.0014 & RGNTMAERR \\
\hline
\end{tabular}

Spectrum No: 362; Query: 94; Rank: 1

\section{Peptide View}

MS/MS Fragmentation of DGLALNLTLR

Found in IPI00212868, Tax_Id=10116 Gene_Symbol=Lamb2 Laminin subunit beta-2 precursor

Match to Query 94: 1085.606308 from(543.810430,2+)

Title: 091008RatKidney_NoSalt_18.3269.3269.2.dta

Data file K:INewmanPaper|Piliangl3SubProteomes\Piliang3SP\mgf5ppm\ERLIC_3SubProteomes5ppm.mgf

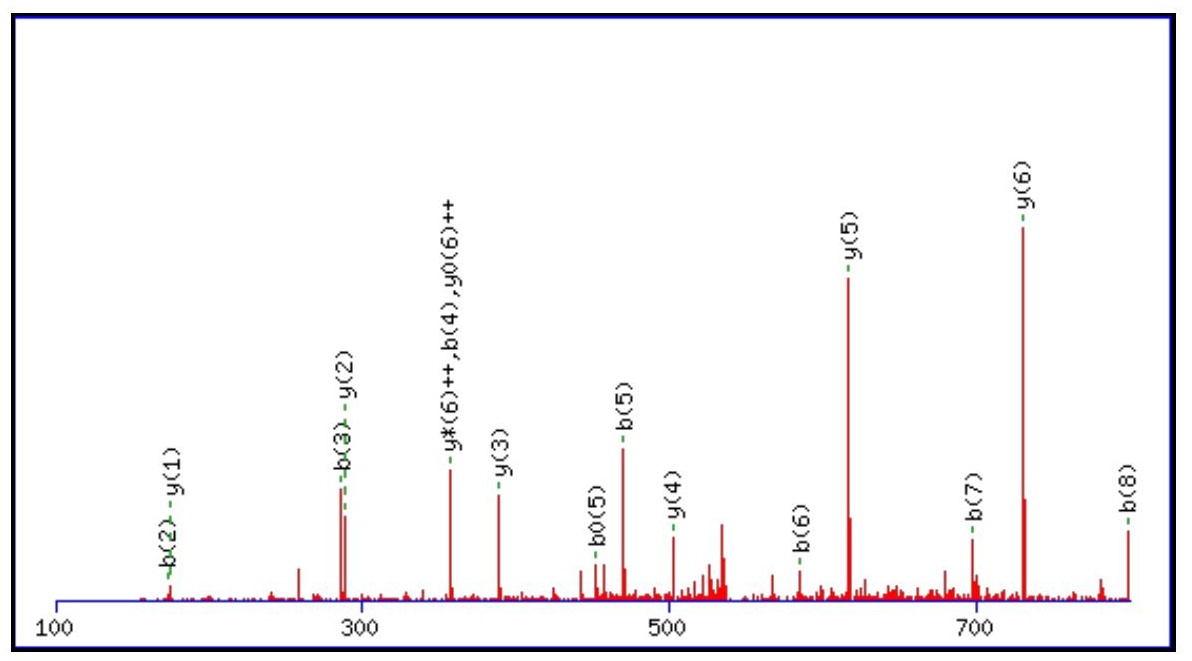




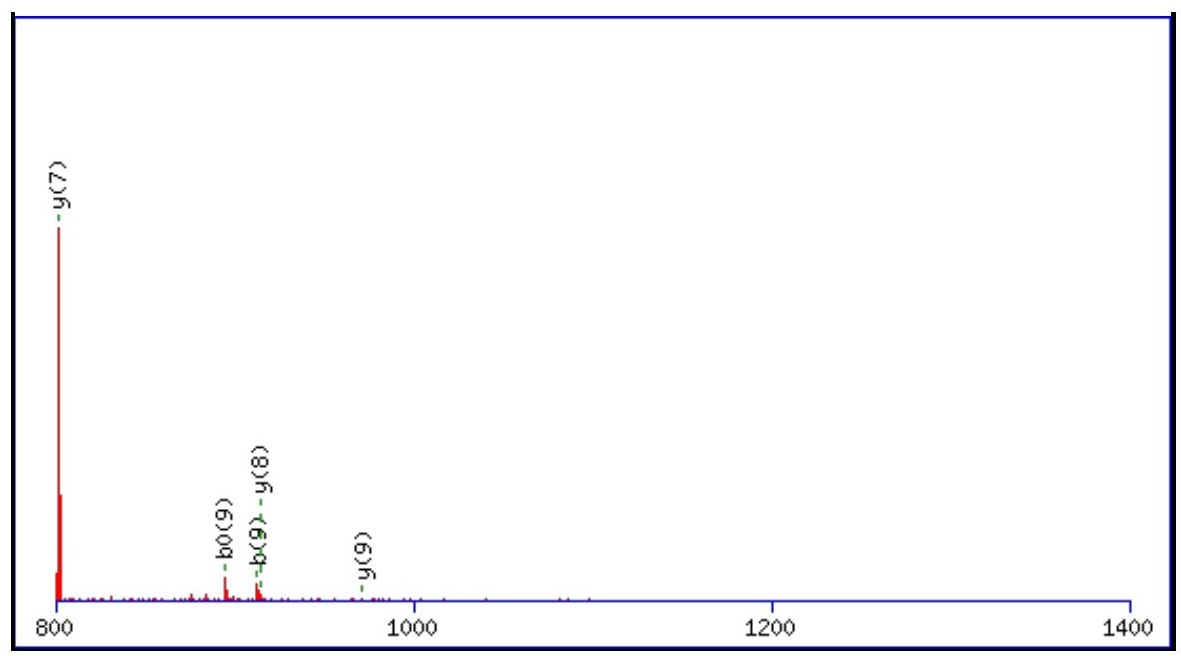

\begin{tabular}{rrr}
\hline 1600 & 1800 & 2000 \\
1400 & 1600
\end{tabular}

Monoisotopic mass of neutral peptide $\operatorname{Mr}($ calc): 1085.6080 Fixed modifications: Carbamidomethyl (C)

Variable modifications:

N6: Deamidated N (N)

Ions Score: 70 Expect: $7.5 \mathrm{e}-006$

Matches (Bold Red): 21/94 fragment ions using 32 most intense peaks

\begin{tabular}{|c|c|c|c|c|c|c|c|c|c|c|c|c|c|c|}
\hline \# & b & $\mathbf{b}^{++}$ & b* & $\mathbf{b}^{*^{++}}$ & $\mathbf{b}^{0}$ & $\mathbf{b}^{0++}$ & Seq. & $\mathbf{y}$ & $y^{++}$ & $\mathbf{y}^{*}$ & $\mathrm{y}^{*^{++}}$ & $\mathbf{y}^{0}$ & $\mathbf{y}^{0++}$ & \# \\
\hline 1 & 116.0342 & 58.5207 & & & 98.0237 & 49.5155 & D & & & & & & & 10 \\
\hline 2 & 173.0557 & 87.0315 & & & 155.0451 & 78.0262 & G & 971.5884 & 486.2978 & 954.5619 & 477.7846 & 953.5778 & 477.2926 & 9 \\
\hline 3 & 286.1397 & 143.5735 & & & 268.1292 & 134.5682 & $\mathbf{L}$ & 914.5669 & 457.7871 & 897.5404 & 449.2738 & 896.5564 & 448.7818 & 8 \\
\hline 4 & 357.1769 & 179.0921 & & & 339.1663 & 170.0868 & A & 801.4829 & 401.2451 & 784.4563 & 392.7318 & 783.4723 & 392.2398 & 7 \\
\hline 5 & 470.2609 & 235.6341 & & & 452.2504 & 226.6288 & $\mathbf{L}$ & 730.4458 & 365.7265 & 713.4192 & 357.2132 & 712.4352 & 356.7212 & 6 \\
\hline 6 & 585.2879 & 293.1476 & 568.2613 & 284.6343 & 567.2773 & 284.1423 & $\mathbf{N}$ & 617.3617 & 309.1845 & 600.3351 & 300.6712 & 599.3511 & 300.1792 & 5 \\
\hline 7 & 698.3719 & 349.6896 & 681.3454 & 341.1763 & 680.3614 & 340.6843 & $\mathbf{L}$ & 502.3348 & 251.6710 & 485.3082 & 243.1577 & 484.3242 & 242.6657 & 4 \\
\hline 8 & 799.4196 & 400.2134 & 782.3931 & 391.7002 & 781.4090 & 391.2082 & $\mathbf{T}$ & 389.2507 & 195.1290 & 372.2241 & 186.6157 & 371.2401 & 186.1237 & 3 \\
\hline 9 & 912.5037 & 456.7555 & 895.4771 & 448.2422 & 894.4931 & 447.7502 & $\mathbf{L}$ & 288.2030 & 144.6051 & 271.1765 & 136.0919 & & & 2 \\
\hline 10 & & & & & & & $\mathbf{R}$ & 175.1190 & 88.0631 & 158.0924 & 79.5498 & & & 1 \\
\hline
\end{tabular}

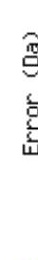$$
\text { 要 }
$$

RMS error 304 ppm

All matches to this query
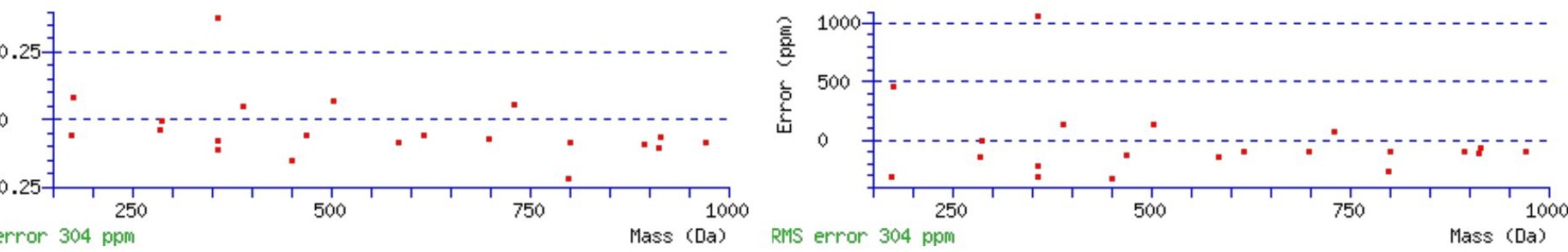

file:///R//wwwroot/SupportingData/3SubProteomes/ERLIC_3SubProteo_Glyco/ERLIC_3SubProteo_Glyco_002.html[4/27/2010 12:05:29 PM] 


\begin{tabular}{|l|l|l|l|}
\hline Score & Mr(calc): & Delta & \multicolumn{1}{|c|}{ Sequence } \\
\hline 69.9 & 1085.6080 & -0.0017 & DGLALNLTLR \\
\hline 19.0 & 1085.6081 & -0.0018 & LQDELVTLR \\
\hline 16.6 & 1085.6080 & -0.0017 & NLEISILER \\
\hline 14.1 & 1085.5968 & 0.0095 & DQLIEVIEK \\
\hline 14.1 & 1084.5989 & 1.0074 & ARQLEEALR \\
\hline 12.1 & 1084.5989 & 1.0074 & IQNISNLQR \\
\hline 11.0 & 1084.6128 & 0.9935 & ELLITSOPGK \\
\hline 10.7 & 1085.6080 & -0.0017 & $\underline{\text { ILKNNIDVR }}$ \\
\hline 9.3 & 1085.5968 & 0.0095 & QEVDILELK \\
\hline 8.3 & 1083.6036 & 2.0027 & $\underline{\text { NNASLPLSLR }}$ \\
\hline
\end{tabular}

Spectrum No: 363; Query: 2713; Rank: 1

\section{Peptide View}

MS/MS Fragmentation of SNSTAGELDALQAEAGSLDKTVNELAEQLEFIK

Found in IPI00365542, Tax_Id=10116 Gene_Symbol=Lamb1_predicted laminin, beta 1

Match to Query 2713: 3492.732372 from(1165.251400,3+)

Title: 100101RatKid_NS_deglyco_12.7567.7567.3.dta

Data file K:INewmanPaper|Piliangl3SubProteomes\Piliang3SP\mgf5ppm\ERLIC_3SubProteomes5ppm.mgf
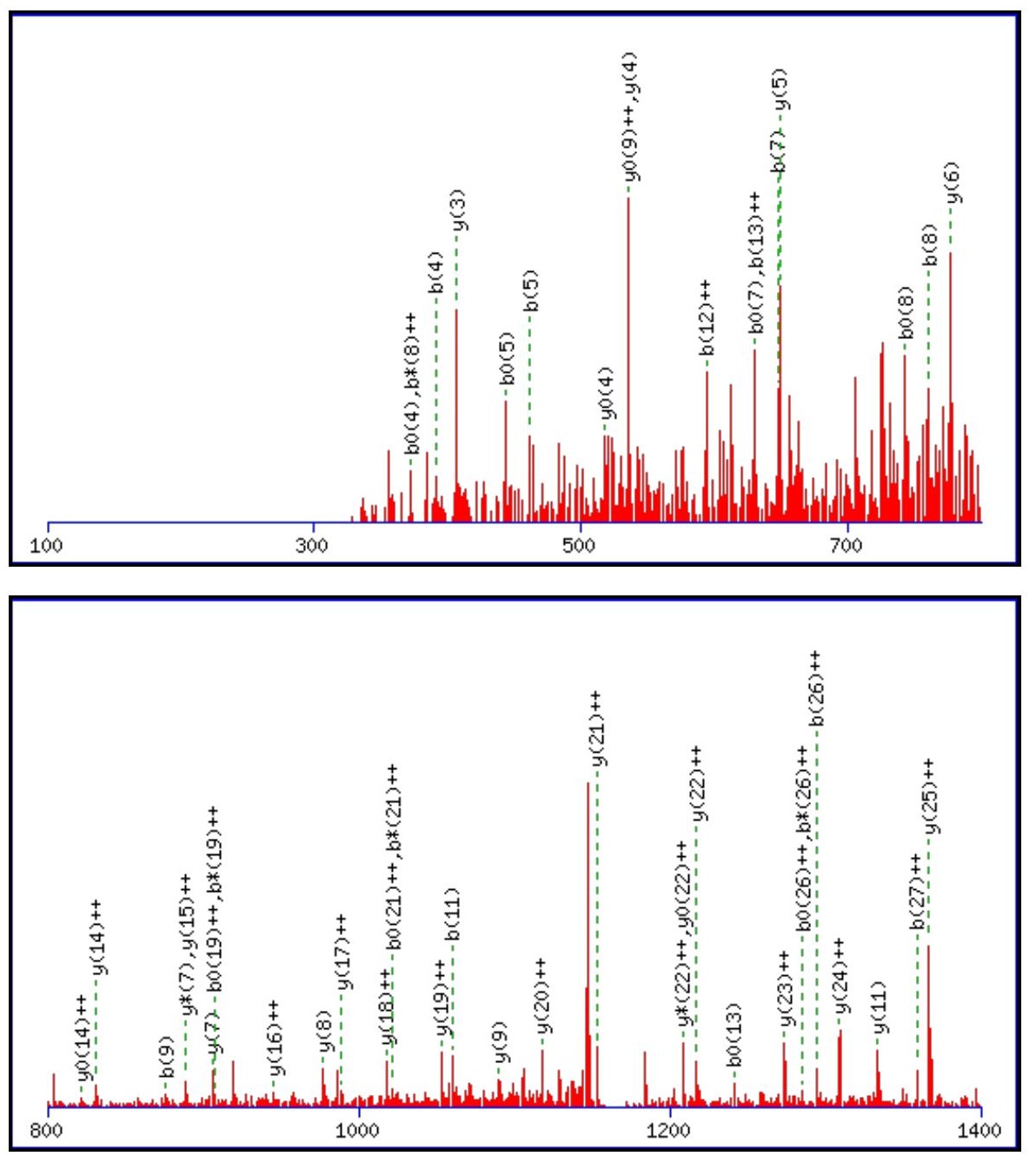


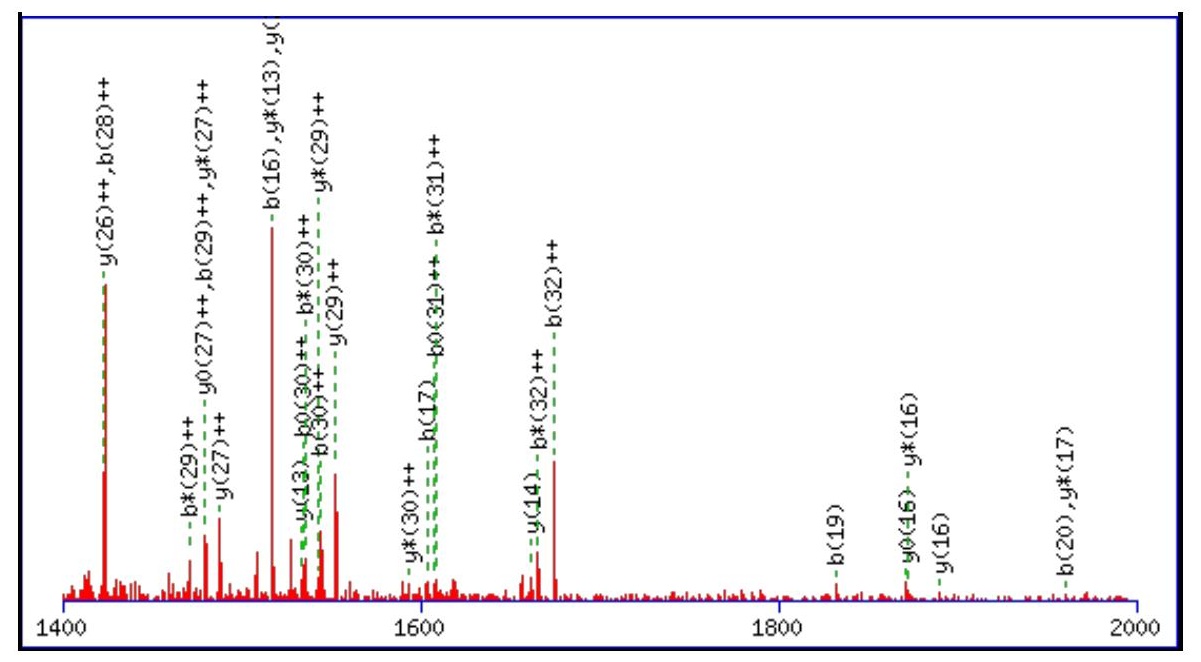

Monoisotopic mass of neutral peptide $\operatorname{Mr}($ calc): 3491.7155

Fixed modifications: Carbamidomethyl (C)

Variable modifications:

N2 : Deamidated_N (N)

Ions Score: 70 Expect: $2.4 \mathrm{e}-005$

Matches (Bold Red): 77/376 fragment ions using 146 most intense peaks

\begin{tabular}{|c|c|c|c|c|c|c|c|c|c|c|c|c|c|c|}
\hline \# & b & $\mathbf{b}^{++}$ & $\mathbf{b}^{*}$ & $\mathbf{b}^{*^{++}}$ & $\mathbf{b}^{0}$ & $\mathbf{b}^{\mathbf{0 + +}}$ & Seq. & $\mathbf{y}$ & $\mathbf{y}^{++}$ & $\mathbf{y}^{*}$ & $\mathrm{y}^{\mathrm{*}^{++}}$ & $\mathbf{y}^{0}$ & $\mathbf{y}^{\mathbf{0 + +}}$ & \# \\
\hline 1 & 88.0393 & 44.5233 & & & 70.0287 & 35.5180 & $\mathrm{~S}$ & & & & & & & 33 \\
\hline 2 & 203.0662 & 102.0368 & 186.0397 & 3.5235 & 185.0557 & 93.0315 & $\mathbf{N}$ & 3405.6908 & 1703.3490 & 3388.6642 & 1694.8357 & 3387.6802 & 1694.3437 & 32 \\
\hline 3 & 290.0983 & 145.5528 & 273.0717 & 137.0395 & 272.0877 & 136.5475 & S & 3290.6638 & 1645.8356 & 3273.6373 & 1637.3223 & 3272.6533 & 1636.8303 & 31 \\
\hline 4 & 391.1459 & 196.0766 & 374.1194 & 187.5633 & 373.1354 & 187.0713 & $\mathbf{T}$ & 3203.6318 & 1602.3195 & 3186.6052 & 1593.8063 & 3185.6212 & 1593.3143 & 30 \\
\hline 5 & 462.1831 & 231.5952 & 445.1565 & 223.0819 & 444.1725 & 222.5899 & A & 3102.5841 & 1551.7957 & 3085.5576 & 1543.2824 & 3084.5736 & 1542.7904 & 29 \\
\hline 6 & 519.2045 & 260.1059 & 502.1780 & 251.5926 & 501.1940 & 251.1006 & G & 3031.5470 & 1516.2771 & 3014.5205 & 1507.7639 & 3013.5364 & 1507.2719 & 28 \\
\hline 7 & 648.2471 & 324.6272 & 631.2206 & 316.1139 & 630.2366 & 315.6219 & $E$ & 2974.5255 & 1487.7664 & 2957.4990 & 1479.2531 & |2956.5150 | & 1478.7611 & 27 \\
\hline 8 & 761.3312 & 381.1692 & 744.3046 & 372.6560 & 743.3206 & 372.1639 & $\mathbf{L}$ & 2845.4829 & 1423.2451 & 2828.4564 & 1414.7318 & 2827.4724 & 1414.2398 & 26 \\
\hline 9 & 876.3581 & 438.6827 & 859.3316 & 430.1694 & 858.3476 & 429.6774 & D & 2732.3989 & 1366.7031 & 2715.3723 & 1358.1898 & 2714.3883 & 1357.6978 & 25 \\
\hline 10 & 947.3952 & 474.2013 & 930.3687 & 465.6880 & 929.3847 & 465.1960 & A & 2617.3719 & 1309.1896 & 2600.3454 & 1300.6763 & 2599.3614 & 1300.1843 & 24 \\
\hline 11 & 1060.4793 & 530.7433 & 1043.4528 & 522.2300 & 1042.4687 & 521.7380 & $\mathbf{L}$ & 2546.3348 & 1273.6711 & 2529.3083 & 1265.1578 & 2528.3243 & 1264.6658 & 23 \\
\hline 12 & 1188.5379 & 594.7726 & 1171.5113 & 586.2593 & 1170.5273 & 585.7673 & $\mathbf{Q}$ & 2433.2508 & 1217.1290 & 2416.2242 & 1208.6157 & 2415.2402 & 1208.1237 & 22 \\
\hline 13 & 1259.5750 & 630. & 1242. & & 1241.5644 & 621.2859 & A & 2305.1922 & 1153 & 2288.1656 & .5865 & 1816 & 0944 & 21 \\
\hline 14 & 1388.6176 & 694.8124 & 1371.5910 & 686.2992 & 1370.6070 & 685.8071 & $\mathbf{E}$ & 2234.1551 & 1117.5812 & 2217.1285 & 1109.0679 & 2216.1445 & 1108.5759 & 20 \\
\hline 15 & 1459.6547 & 730.3310 & 1442.6282 & 721.8177 & 1441.6441 & 721.3257 & A & 2105.1125 & 1053.0599 & 2088.0859 & 1044.5466 & 2087.1019 & 1044.0546 & 19 \\
\hline 16 & \begin{tabular}{|l|}
1516.6762 \\
\end{tabular} & 758.8417 & 1499.6496 & 750.3284 & 1498.6656 & 749.8364 & $\mathbf{G}$ & 2034.0754 & 1017.5413 & 2017.0488 & 1009.0280 & 2016.0648 & 1008.5360 & 18 \\
\hline 17 & 1603.7082 & 802.3577 & 1586.6816 & 793.8445 & 1585.6976 & 793.3525 & $\mathrm{~S}$ & 1977.0539 & \begin{tabular}{|l}
889.0306 \\
\end{tabular} & 1960.0274 & 980.5173 & 1959.0433 & 980.0253 & 17 \\
\hline 18 & 1716.7923 & 858.8998 & 1699.7657 & 850.3865 & 1698.7817 & 849.8945 & $\mathbf{L}$ & 1890.0219 & 945.5146 & \begin{tabular}{|l|l|}
1872.9953 \\
\end{tabular} & 937.0013 & 1872.0113 & 936.5093 & 16 \\
\hline 19 & \begin{tabular}{|l|}
1831.8192 \\
\end{tabular} & 916.4132 & 1814.7927 & 907.9000 & 1813.8086 & 907.4080 & D & 1776.9378 & 888.9725 & 1759.9113 & 880.4593 & 1758.9272 & 879.9673 & 15 \\
\hline 20 & 1959.9142 & 980.4607 & 1942.8876 & 971.9474 & 1941.9036 & 971.4554 & $\mathbf{K}$ & 1661.9109 & 831.4591 & 1644.8843 & 822.9458 & 1643.9003 & 822.4538 & 14 \\
\hline 21 & 2060.9618 & 1030.9846 & 2043.9353 & 1022.4713 & 2042.9513 & 1021.9793 & $\mathbf{T}$ & \begin{tabular}{|l}
1533.8159 \\
\end{tabular} & 767.4116 & \begin{tabular}{|l|l|}
1516.7894 \\
\end{tabular} & 758.8983 & 1515.8053 & 758.4063 & 13 \\
\hline 22 & 2160.0303 & 1080.5188 & 2143.0037 & 1072.0055 & 2142.0197 & 1071.5135 & $\mathbf{V}$ & 1432.7682 & 716.8877 & 1415.7417 & 708.3745 & 1414.7577 & 707.8825 & 12 \\
\hline 23 & 2274.0732 & 1137.5402 & 2257.0466 & 1129.0270 & 2256.0626 & 1128.5349 & $\mathbf{N}$ & \begin{tabular}{|l|l|}
1333.6998 \\
\end{tabular} & 667.3535 & 1316.6733 & 658.8403 & 1315.6892 & 658.3483 & 11 \\
\hline 24 & 2403.1158 & 1202.0615 & 2386.0892 & 1193.5483 & 2385.1052 & 1193.0562 & $\mathbf{E}$ & 1219.6569 & 610.3321 & 1202.6303 & 601.8188 & 1201.6463 & 601.3268 & 10 \\
\hline 25 & 2516.1998 & 1258.6036 & 2499.1733 & 1250.0903 & 2498.1893 & 1249.5983 & $\mathbf{L}$ & 1090.6143 & 545.8108 & \begin{tabular}{|l|l|}
1073.5877 \\
\end{tabular} & 537.2975 & 1072.6037 & 536.8055 & 9 \\
\hline 26 & 2587.2370 & \begin{tabular}{|l|}
1294.1221 \\
\end{tabular} & 2570.2104 & 1285.6088 & 2569.2264 & \begin{tabular}{|l|}
1285.1168 \\
\end{tabular} & A & 977.5302 & 489.2688 & \begin{tabular}{|l|}
960.5037 \\
\end{tabular} & 480.7555 & 959.5197 & 480.2635 & 8 \\
\hline 27 & 2716.2795 & 1358.6434 & 2699.2530 & 1350.1301 & 2698.2690 & 1349.6381 & $\mathbf{E}$ & 906.4931 & 453.7502 & 889.4666 & 445.2369 & 888.4825 & 444.7449 & 7 \\
\hline 28 & 2844.3381 & 1422.6727 & 2827.3116 & 1414.1594 & 2826.3276 & 1413.6674 & $\mathbf{Q}$ & 777.4505 & 389.2289 & 760.4240 & 380.7156 & 759.4400 & 380.2236 & 6 \\
\hline 29 & 2957.4222 & 1479.2147 & 2940.3956 & 1470.7015 & 2939.4116 & 1470.2095 & $\mathbf{L}$ & 649.3919 & 325.1996 & 632.3654 & 316.6863 & 631.3814 & 316.1943 & 5 \\
\hline 30 & 3086.4648 & 1543.7360 & 3069.4382 & 1535.2228 & 3068.4542 & 1534.7307 & $\mathbf{E}$ & 536.3079 & 268.6576 & 519.2813 & 260.1443 & 518.2973 & 259.6523 & 4 \\
\hline 31 & 3233.5332 & 1617.2702 & 3216.5066 & 1608.7570 & 3215.5226 & \begin{tabular}{|l|}
1608.2650 \\
\end{tabular} & $\mathbf{F}$ & 407.2653 & 204.1363 & 390.2387 & 195.6230 & & & 3 \\
\hline 32 & 3346.6173 & 1673.8123 & 3329.5907 & |1665.2990 & $\mid$ & $|1664.8070|$ & I & 260.1969 & 130.6021 & 243.1703 & 122.0888 & & & 2 \\
\hline
\end{tabular}




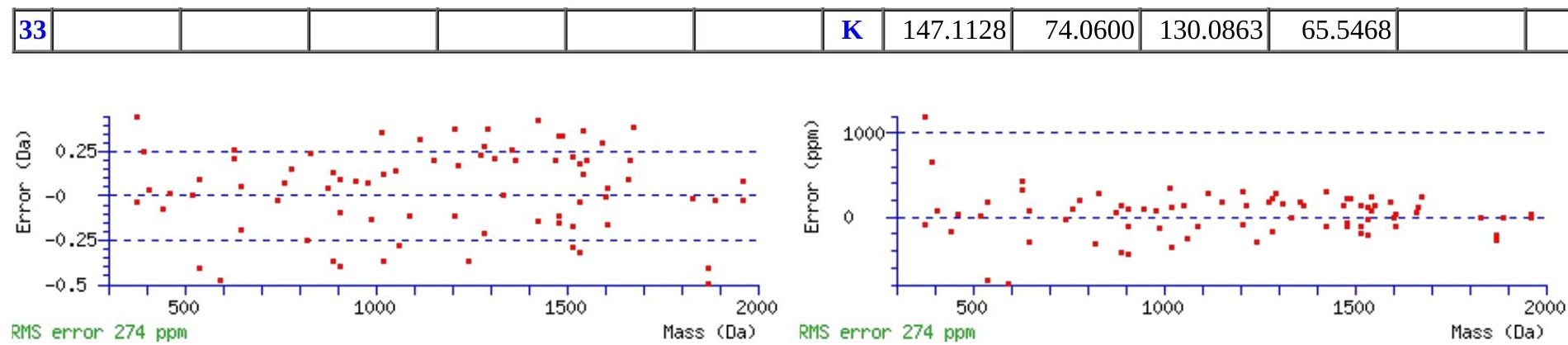

\section{All matches to this query}

\begin{tabular}{|l|l|r|l|}
\hline Score & Mr(calc): & Delta & \multicolumn{1}{c|}{ Sequence } \\
\hline 69.9 & 3491.7155 & 1.0169 & SNSTAGELDALQAEAGSLDKTVNELAEQLEFIK \\
\hline 59.8 & 3492.6995 & 0.0329 & SNSTAGELDALQAEAGSLDKTVNELAEQLEFIK \\
\hline 48.3 & 3490.7314 & 2.0009 & SNSTAGELDALQAEAGSLDKTVNELAEQLEFIK \\
\hline 48.1 & 3491.7155 & 1.0169 & SNSTAGELDALQAEAGSLDKTVNELAEQLEFIK \\
\hline 4.1 & 3490.7311 & 2.0013 & GLNISYLGIQRQVQLLMSFFYEAIRLMK \\
\hline 2.7 & 3491.7631 & 0.9692 & LVAMNMPLNSDGTVMFNATLFALVRTALKIK \\
\hline 2.7 & 3491.7631 & 0.9692 & LVAMNMPLNSDGTVMFNATLFALVRTALKIK \\
\hline 2.4 & 3492.7471 & -0.0148 & LVAMNMPLNSDGTVMFNATLFALVRTALKIK \\
\hline 2.4 & 3492.7471 & -0.0148 & LVAMNMPLNSDGTVMFNATLFALVRTALKIK \\
\hline 2.4 & 3492.7471 & -0.0148 & LVAMNMPLNSDGTVMFNATLFALVRTALKIK \\
\hline
\end{tabular}

Spectrum No: 364; Query: 1904; Rank: 1

\section{Peptide View}

MS/MS Fragmentation of VNCSTSCAAPEIGGLETPTNK

Found in IPI00372952, Tax_Id=10116 Gene_Symbol=Icam2 Intercellular adhesion molecule 2

Match to Query 1904: 2205.996688 from(1104.005620,2+)

Title: 100101RatKid_NS_deglyco_25.2138.2138.2.dta

Data file K:INewmanPaper|Piliangl3SubProteomes\Piliang3SP\mgf5ppm\ERLIC_3SubProteomes5ppm.mgf

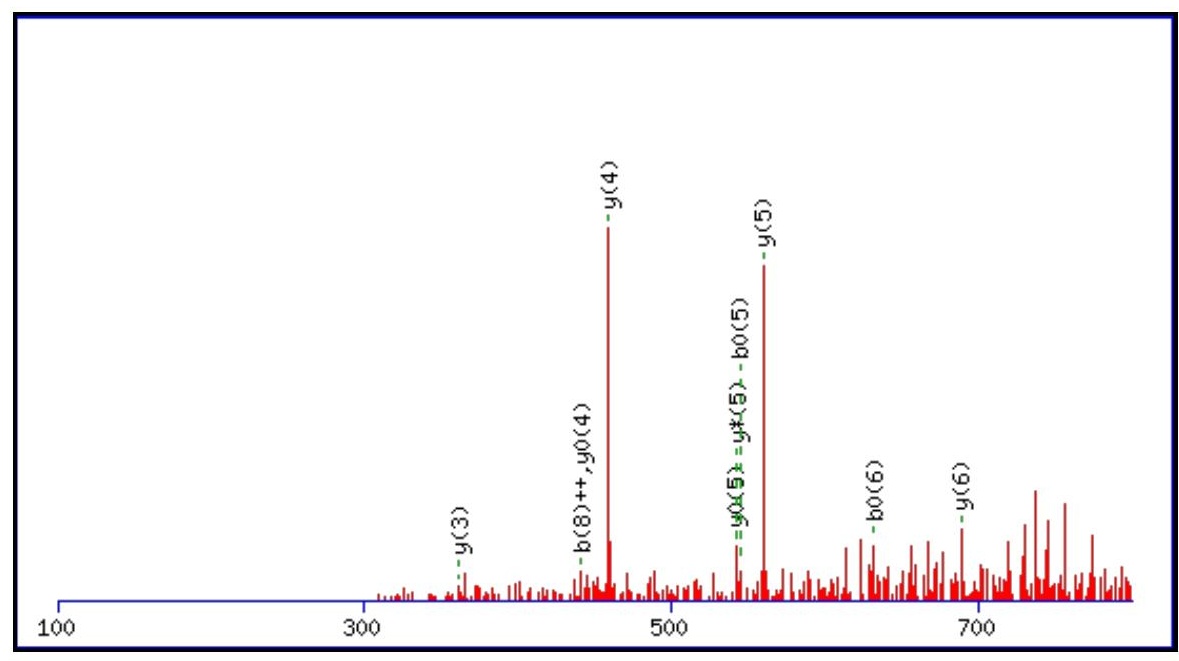




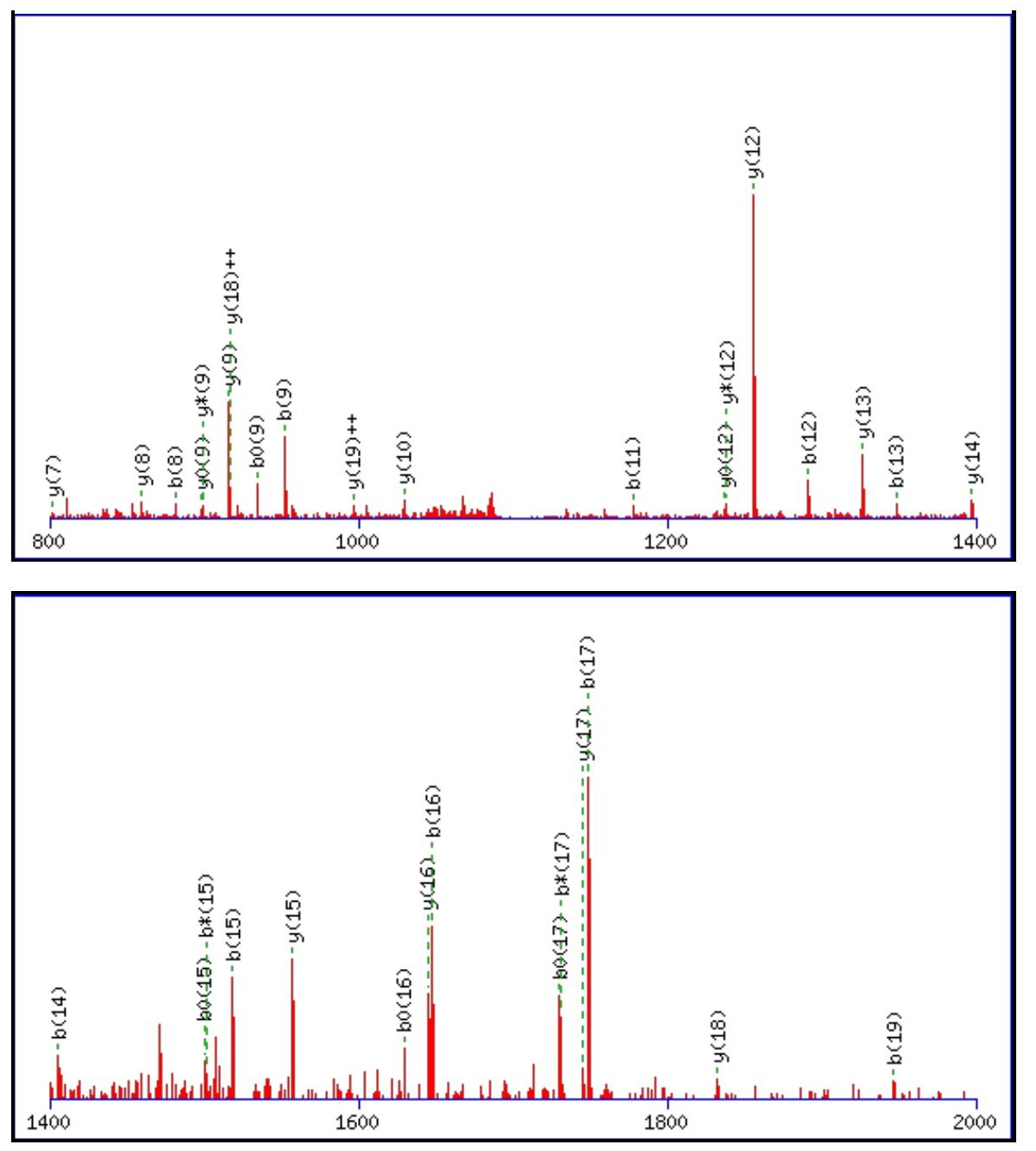

Monoisotopic mass of neutral peptide $\operatorname{Mr}($ calc): 2205.9882

Fixed modifications: Carbamidomethyl (C)

Variable modifications:

N2: Deamidated $\mathrm{N}(\mathrm{N})$

Ions Score: 70 Expect: $2.9 \mathrm{e}-005$

Matches (Bold Red): 43/228 fragment ions using 109 most intense peaks

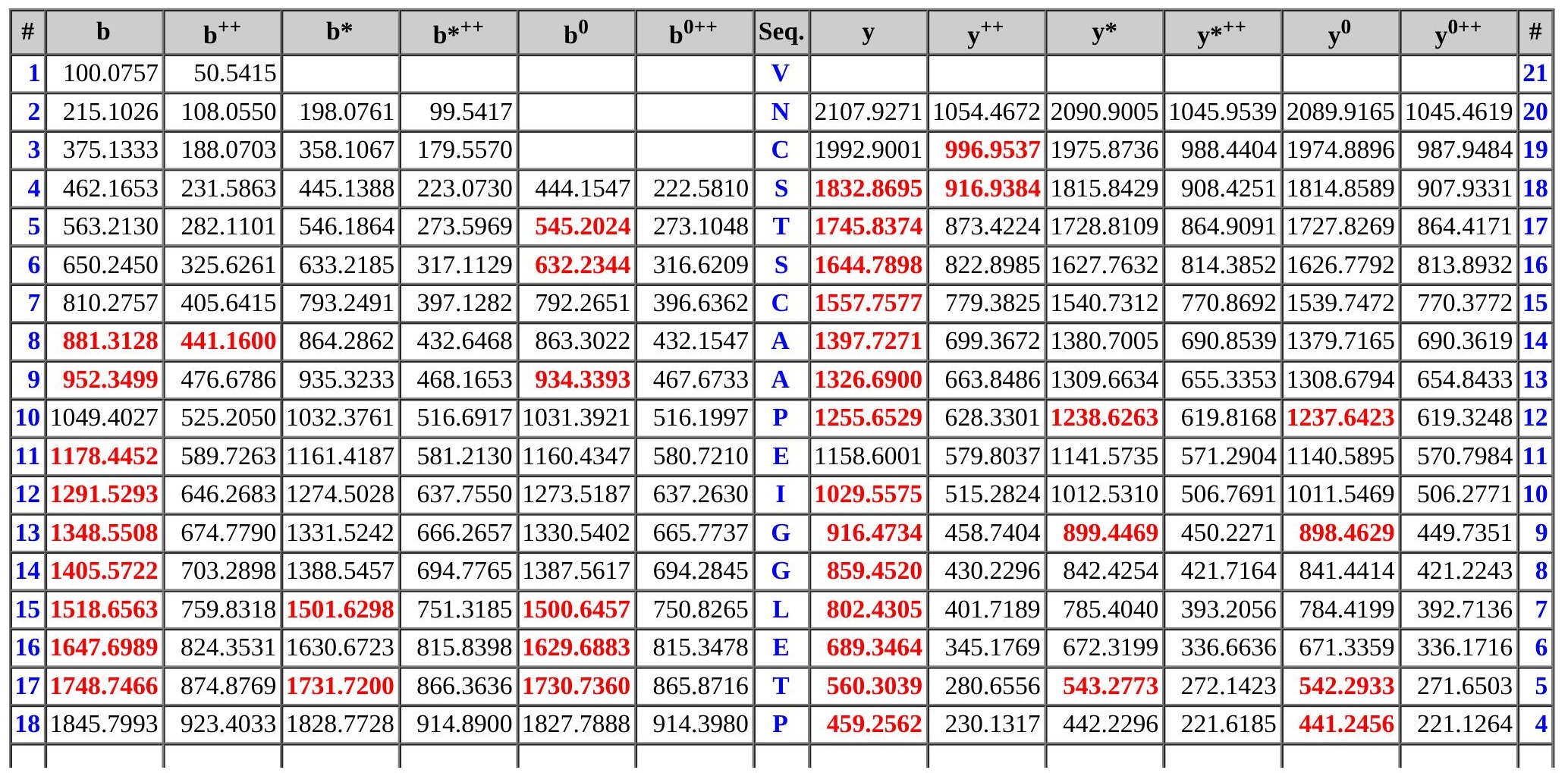




\begin{tabular}{|l|r|r|r|r|r|r|r|r|r|r|r|r|r|r|r|}
$\mathbf{1 9}$ & $\mathbf{1 9 4 6 . 8 4 7 0}$ & 973.9271 & 1929.8205 & 965.4139 & 1928.8364 & 964.9219 & $\mathbf{T}$ & 362.2034 & 181.6053 & 345.1769 & 173.0921 & 344.1928 & 172.6001 & $\mathbf{3}$ \\
\hline $\mathbf{2 0}$ & 2060.8899 & 1030.9486 & 2043.8634 & 1022.4353 & 2042.8794 & 1021.9433 & $\mathbf{N}$ & 261.1557 & 131.0815 & 244.1292 & 122.5682 & & & $\mathbf{2}$ \\
\hline $\mathbf{2 1}$ & & & & & & & $\mathbf{K}$ & 147.1128 & 74.0600 & 130.0863 & 65.5468 & & & $\mathbf{1}$ \\
\hline
\end{tabular}
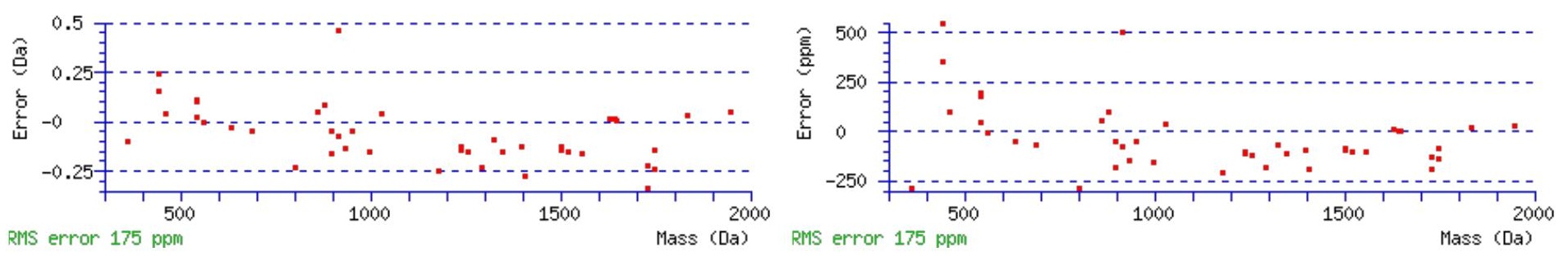

\section{All matches to this query}

\begin{tabular}{|l|c|c|l|}
\hline Score & Mr(calc): & Delta & \multicolumn{1}{|c|}{ Sequence } \\
\hline 69.8 & 2205.9882 & 0.0085 & VNCSTSCAAPEIGGLETPTNK \\
\hline 44.0 & 2205.0042 & 0.9925 & VNCSTSCAAPEIGGLETPTNK \\
\hline 10.5 & 2205.9882 & 0.0085 & VNCSTSCAAPEIGGLETPTNK \\
\hline 2.0 & 2206.0089 & -0.0122 & MVGANNOKNLTSFKTTAER \\
\hline 1.5 & 2206.0089 & -0.0122 & MVGANNQKNLTSFKTTAER \\
\hline 1.5 & 2206.0089 & -0.0122 & MVGANNOKNLTSFKTTAER \\
\hline 1.3 & 2205.9782 & 0.0185 & VFTTLVLCDKPAVSENPR \\
\hline 1.3 & 2205.9782 & 0.0185 & VFTTLVLCDKPAVSENPR \\
\hline 1.1 & 2206.0042 & -0.0075 & GPSENGELSAPLELGSLVNGVS \\
\hline 0.9 & 2206.0042 & -0.0075 & GPSENGELSAPLELGSLVNGVS \\
\hline
\end{tabular}

Spectrum No: 365; Query: 2704; Rank: 1

\section{Peptide View}

MS/MS Fragmentation of LLYISPENFQIDNSPNSTAGPSCSLLLEAFR

Found in IPI00464518, Tax_Id=10116 Gene_Symbol=Hexb Beta-hexosaminidase beta chain precursor

Match to Query 2704: 3453.684522 from(1152.235450,3+)

Title: 100101RatKid_NS_deglyco_11.7176.7176.3.dta

Data file K:INewmanPaper|Piliangl3SubProteomes\Piliang3SP\mgf5ppm\ERLIC_3SubProteomes5ppm.mgf

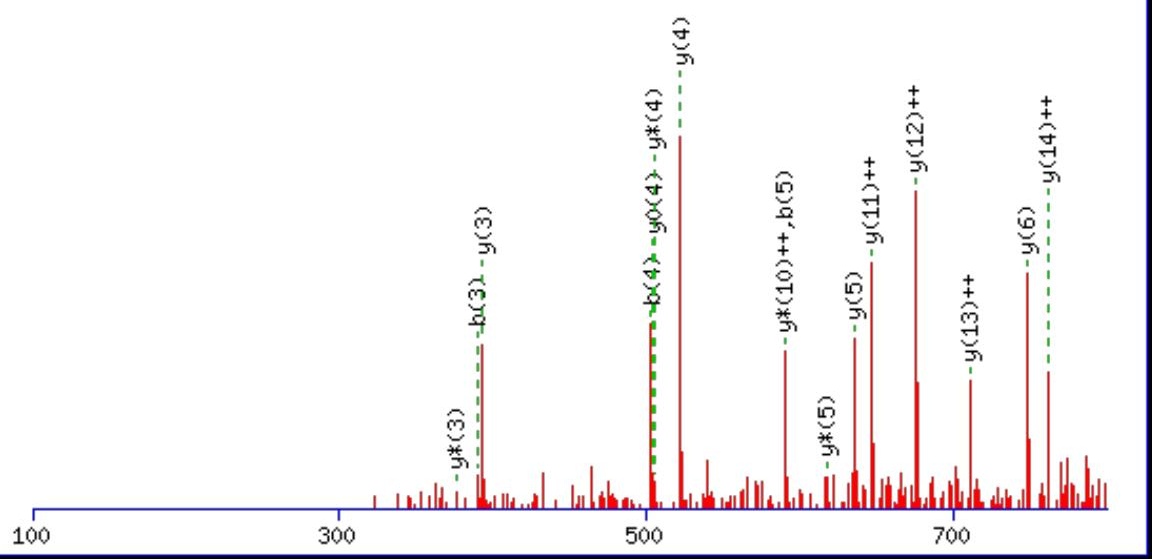



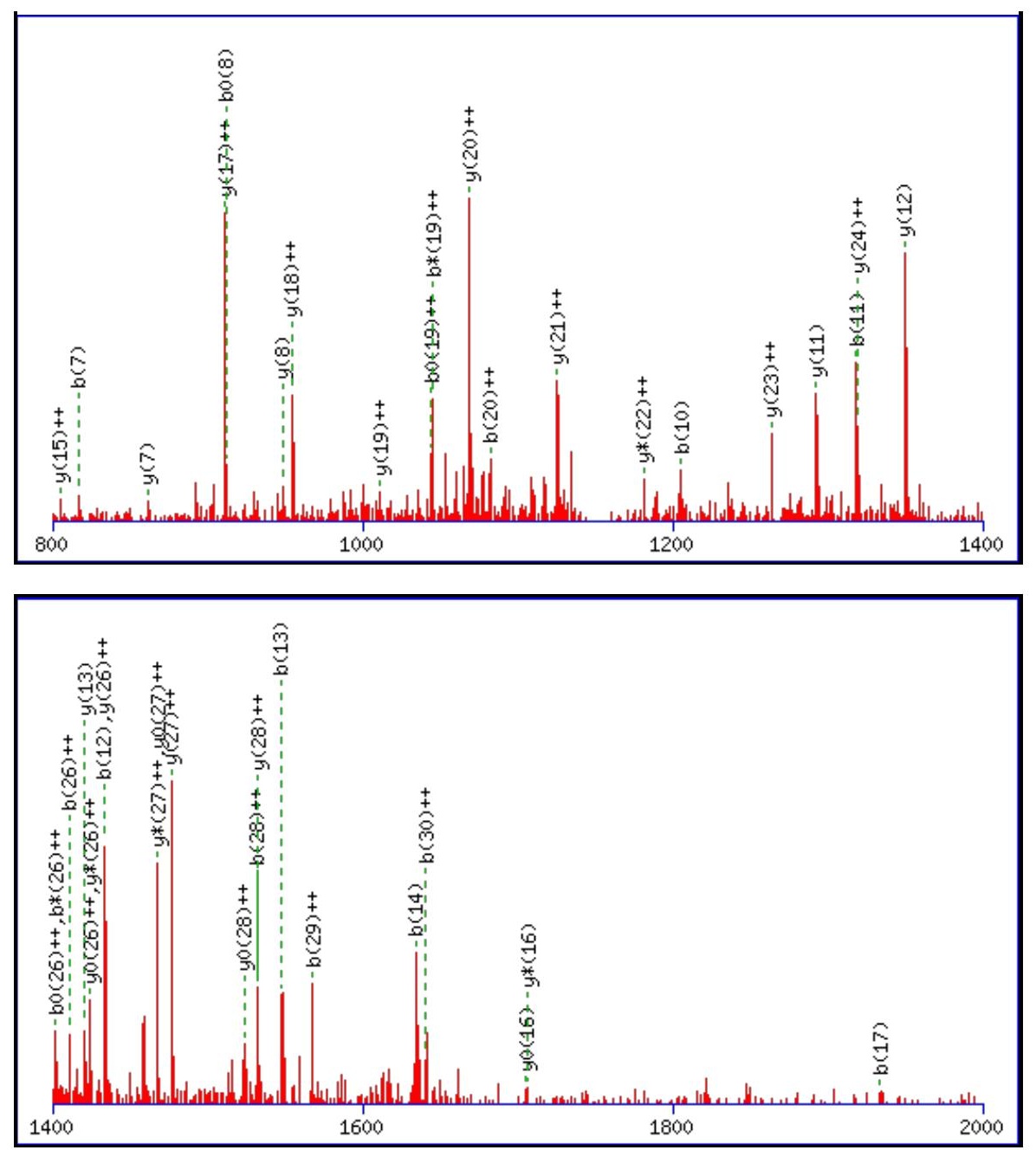

Monoisotopic mass of neutral peptide $\operatorname{Mr}($ calc): 3453.6762

Fixed modifications: Carbamidomethyl (C)

Variable modifications:

N16 : Deamidated N (N)

Ions Score: 70 Expect: $3 \mathrm{e}-005$

Matches (Bold Red): 57/332 fragment ions using 113 most intense peaks

\begin{tabular}{|c|c|c|c|c|c|c|c|c|c|c|c|c|c|c|}
\hline$\#$ & b & $\mathbf{b}^{++}$ & $\mathbf{b}^{*}$ & $\mathbf{b}^{*++}$ & $\mathbf{b}^{0}$ & $\mathbf{b}^{\mathbf{0 + +}}$ & Seq. & $\mathbf{y}$ & $y^{++}$ & $\mathbf{y}^{*}$ & $\mathrm{y}^{*^{++}}$ & $\mathbf{y}^{0}$ & $y^{0++}$ & \# \\
\hline 1 & \begin{tabular}{|l|}
114.0913 \\
\end{tabular} & 57.5493 & & & & & $\mathbf{L}$ & & & & & & & 31 \\
\hline 2 & 227.1754 & 114.0913 & & & & & $\mathbf{L}$ & 3341.5994 & 1671.3034 & 3324.5729 & 1662.7901 & 3323.5889 & 1662.2981 & 30 \\
\hline 3 & 390.2387 & 195.6230 & & & & & $\mathbf{Y}$ & 3228.5154 & 1614.7613 & 3211.4888 & 1606.2481 & 3210.5048 & 1605.7560 & 29 \\
\hline 4 & 503.3228 & 252.1650 & & & & & I & 3065.4520 & |1533.2297 & 3048.4255 & 1524.7164 & 3047.4415 & 1524.2244 & 28 \\
\hline 5 & 590.3548 & 295.6811 & & & 572.3443 & 286.6758 & $S$ & 2952.3680 & 1476.6876 & 2935.3414 & 1468.1744 & 2934.3574 & 1467.6823 & 27 \\
\hline 6 & 687.4076 & 344.2074 & & & 669.3970 & 335.2022 & $\mathbf{P}$ & 2865.3360 & 1433.1716 & 2848.3094 & 1424.6583 & 2847.3254 & 1424.1663 & 26 \\
\hline 7 & 816.4502 & 408.7287 & & & 798.4396 & 399.7234 & $\mathbf{E}$ & 2768.2832 & 1384.6452 & 2751.2566 & 1376.1320 & 2750.2726 & 1375.6400 & 25 \\
\hline 8 & 930.4931 & 465.7502 & 913.4666 & 457.2369 & 912.4825 & 456.7449 & $\mathbf{N}$ & 2639.2406 & 1320.1239 & 2622.2141 & 1311.6107 & 2621.2300 & 1311.1187 & 24 \\
\hline 9 & 1077.5615 & 539.2844 & 1060.5350 & 530.7711 & 1059.5510 & 530.2791 & $\mathbf{F}$ & 2525.1977 & 1263.1025 & 2508.1711 & 1254.5892 & 2507.1871 & 1254.0972 & 23 \\
\hline 10 & 1205.6201 & 603.3137 & 1188.5936 & 594.8004 & 1187.6095 & 594.3084 & $\mathbf{Q}$ & 2378.1293 & 1189.5683 & 2361.1027 & 1181.0550 & 2360.1187 & 1180.5630 & 22 \\
\hline 11 & 1318.7042 & 659.8557 & 1301.6776 & 651.3424 & 1300.6936 & 650.8504 & I & 2250.0707 & 1125.5390 & 2233.0441 & 1117.0257 & 2232.0601 & 1116.5337 & 21 \\
\hline 12 & 1433.7311 & 717.3692 & 1416.7046 & 708.8559 & 1415.7205 & 708.3639 & D & 2136.9866 & 1068.9969 & 2119.9601 & 1060.4837 & 2118.9761 & 1059.9917 & 20 \\
\hline 13 & 1547.7740 & 774.3907 & 1530.7475 & 765.8774 & 1529.7635 & 765.3854 & $\mathbf{N}$ & 2021.9597 & 1011.4835 & 2004.9331 & 1002.9702 & 2003.9491 & 1002.4782 & 19 \\
\hline 14 & 1634.8061 & 817.9067 & 1617.7795 & 809.3934 & 1616.7955 & 808.9014 & $\mathrm{~S}$ & 1907.9167 & 954.4620 & 1890.8902 & 945.9487 & 1889.9062 & 945.4567 & 18 \\
\hline 15 & 1731.8588 & 866.4331 & 1714.8323 & 857.9198 & 1713.8483 & 857.4278 & $\mathbf{P}$ & 1820.8847 & 910.9460 & 1803.8582 & 902.4327 & 1802.8742 & 901.9407 & 17 \\
\hline 16 & 1846.8858 & 923.9465 & 1829.8592 & 915.4332 & 1828.8752 & 914.9412 & $\mathbf{N}$ & 1723.8320 & 862.4196 & 1706.8054 & 853.9063 & 1705.8214 & 853.4143 & 16 \\
\hline 17 & 1933.9178 & 967.4625 & 1916.8912 & 958.9493 & 1915.9072 & 958.4573 & $\mathrm{~S}$ & 1608.8050 & 804.9061 & 1591.7785 & 796.3929 & 1590.7945 & 795.9009 & 15 \\
\hline 18 & 2034.9655 & 1017.9864 & 2017.9389 & 1009.4731 & 2016.9549 & 1008.9811 & $T$ & 1521.7730 & 761.3901 & 1504.7464 & 752.8769 & 1503.7624 & 752.3849 & 14 \\
\hline
\end{tabular}




\begin{tabular}{|c|c|c|c|c|c|c|c|c|c|c|c|c|c|c|}
\hline 19 & 2106.0026 & |1053.5049 & |2088.9760 & 1044.9917 & |2087.9920 & $\mid 1044.4996$ & A & 1420.7253 & 710.8663 & |1403.6988 & 702.3530 & 1402.7147 & 701.8610 & 13 \\
\hline 20 & 2163.0240 & 1082.0157 & 2145.9975 & 1073.5024 & 2145.0135 & 1073.0104 & G & 1349.6882 & 675.3477 & 1332.6616 & 666.8345 & 1331.6776 & 666.3425 & 12 \\
\hline 21 & 2260.0768 & 1130.5420 & 2243.0503 & 1122.0288 & 2242.0662 & 1121.5368 & $\mathbf{P}$ & 1292.6667 & 646.8370 & 1275.6402 & 638.3237 & 1274.6562 & 637.8317 & 11 \\
\hline 22 & 2347.1088 & 1174.0581 & 2330.0823 & 1165.5448 & 2329.0983 & 1165.0528 & $\mathrm{~S}$ & 1195.6140 & 598.3106 & 1178.5874 & 589.7973 & 1177.6034 & 589.3053 & 10 \\
\hline 23 & 2507.1395 & 1254.0734 & 2490.1129 & 1245.5601 & 2489.1289 & 1245.0681 & C & 1108.5819 & 554.7946 & 1091.5554 & 546.2813 & 1090.5714 & 545.7893 & 9 \\
\hline 24 & 2594.1715 & 1297.5894 & 2577.1450 & 1289.0761 & 2576.1610 & 1288.5841 & $S$ & 948.5513 & 474.7793 & 931.5247 & 466.2660 & 930.5407 & 465.7740 & 8 \\
\hline 25 & 2707.2556 & 1354.1314 & 2690.2290 & 1345.6182 & 2689.2450 & 1345.1261 & L & 861.5193 & 431.2633 & 844.4927 & 422.7500 & 843.5087 & 422.2580 & 7 \\
\hline 26 & 2820.3396 & 1410.6735 & 2803.3131 & 1402.1602 & 2802.3291 & 1401.6682 & $\mathbf{L}$ & 748.4352 & 374.7212 & 731.4087 & 366.2080 & 730.4246 & 365.7160 & 6 \\
\hline 27 & 2933.4237 & 1467.2155 & 2916.3972 & 1458.7022 & 2915.4131 & 1458.2102 & $\mathbf{L}$ & 635.3511 & 318.1792 & 618.3246 & 309.6659 & 617.3406 & 309.1739 & 5 \\
\hline 28 & 3062.4663 & 1531.7368 & 3045.4398 & 1523.2235 & 3044.4557 & 1522.7315 & E & 522.2671 & 261.6372 & 505.2405 & 253.1239 & 504.2565 & 252.6319 & 4 \\
\hline 29 & 3133.5034 & \begin{tabular}{|l|l|}
1567.2553 \\
\end{tabular} & 3116.4769 & 1558.7421 & 3115.4929 & 1558.2501 & A & 393.2245 & 197.1159 & 376.1979 & 188.6026 & & & 3 \\
\hline 30 & 3280.5718 & 1640.7896 & 3263.5453 & 1632.2763 & 3262.5613 & 1631.7843 & $\mathbf{F}$ & 322.1874 & 161.5973 & 305.1608 & 153.0840 & & & 2 \\
\hline 31 & & & & & & & $\mathbf{R}$ & 175.1190 & 88.0631 & 158.0924 & 79.5498 & & & 1 \\
\hline
\end{tabular}
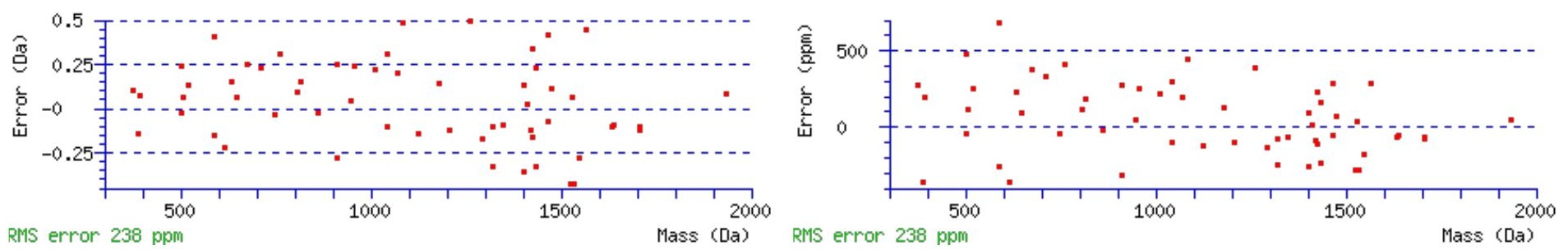

\section{All matches to this query}

\begin{tabular}{|l|l|l|l|}
\hline Score & Mr(calc): & Delta & \multicolumn{1}{|c|}{ Sequence } \\
\hline 69.8 & 3453.6762 & 0.0083 & LLYISPENFQIDNSPNSTAGPSCSLLLEAFR \\
\hline 57.6 & 3453.6762 & 0.0083 & LLYISPENFOIDNSPNSTAGPSCSLLLEAFR \\
\hline 44.3 & 3453.6762 & 0.0083 & LLYISPENFQIDNSPNSTAGPSCSLLLEAFR \\
\hline 37.2 & 3452.6922 & 0.9923 & LLYISPENFQIDNSPNSTAGPSCSLLLEAFR \\
\hline 7.2 & 3453.7084 & -0.0239 & LDGHNTFSYISIFTPLWLSLLTLMATTFR \\
\hline 2.8 & 3452.7051 & 0.9794 & DRIEHLTFANCLSIISNGIPQTLSDLGFK \\
\hline 2.5 & 3453.7133 & -0.0288 & FFFEPVPEFPLNPNFTIVDNKDNTAGIITR \\
\hline 2.5 & 3453.7133 & -0.0288 & FFFEPVPEFPLNPNFTIVDNKDNTAGIITR \\
\hline 2.5 & 3453.7133 & -0.0288 & FFFEPVPEFPLNPNFTIVDNKDNTAGIITR \\
\hline 2.5 & 3453.7133 & -0.0288 & FFFEPVPEFPLNPNFTIVDNKDNTAGIITR \\
\hline
\end{tabular}

Spectrum No: 366; Query: 1042; Rank: 1

\section{Peptide View}

MS/MS Fragmentation of NNSNDIVNAIMELTM

Found in IPI00371266, Tax_Id=10116 Gene_Symbol=Naca_predicted nascent-polypeptide-associated complex alpha polypeptide

Match to Query 1042: 1694.754408 from(848.384480,2+)

Title: 100101RatKid_NS_deglyco_19.6755.6755.2.dta

Data file K:INewmanPaper|Piliang|3SubProteomes\Piliang3SP\mgf5ppm\ERLIC_3SubProteomes5ppm.mgf 

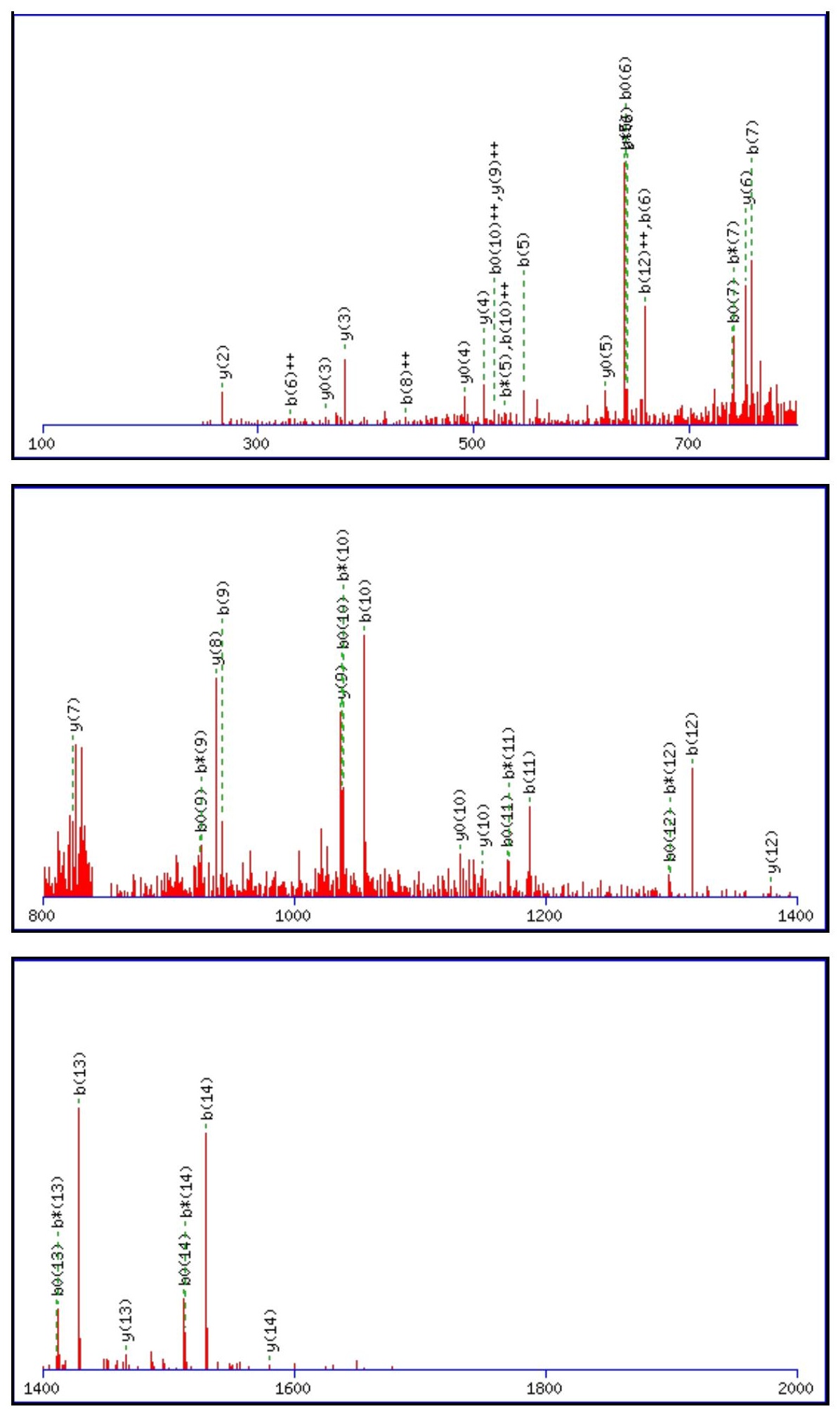

Monoisotopic mass of neutral peptide $\operatorname{Mr}($ calc): 1694.7491

Fixed modifications: Carbamidomethyl (C)

Variable modifications:

N1 : Deamidated_N (N)

M15 : Oxidation (M)

Ions Score: 70 Expect : $2.1 \mathrm{e}-005$

Matches (Bold Red): 48/148 fragment ions using 109 most intense peaks

\begin{tabular}{|c|c|c|c|c|c|c|c|c|c|c|c|c|c|c|}
\hline$\#$ & $\mathbf{b}$ & $\mathbf{b}^{++}$ & $\mathbf{b}^{*}$ & $\mathbf{b}^{\boldsymbol{*}^{++}}$ & $\mathbf{b}^{\mathbf{0}}$ & $\mathbf{b}^{\mathbf{0}+}$ & Seq. & $\mathbf{y}$ & $\mathbf{y}^{++}$ & $\mathbf{y}^{\mathbf{*}}$ & $\mathbf{y}^{\boldsymbol{*}^{++}}$ & $\mathbf{y}^{\mathbf{0}}$ & $\mathbf{y}^{\mathbf{0 + +}}$ & $\#$ \\
\hline $\mathbf{1}$ & 116.0342 & 58.5207 & 99.0077 & 50.0075 & & & $\mathbf{N}$ & & & & & & & $\mathbf{1 5}$ \\
\hline $\mathbf{2}$ & 230.0771 & 115.5422 & 213.0506 & 107.0289 & & & $\mathbf{N}$ & $\mathbf{1 5 8 0 . 7 2 9 5}$ & 790.8684 & 1563.7029 & 782.3551 & 1562.7189 & $\mathbf{7 8 1 . 8 6 3 1}$ & $\mathbf{1 4}$ \\
\hline $\mathbf{3}$ & 317.1092 & 159.0582 & 300.0826 & 150.5449 & 299.0986 & 150.0529 & $\mathbf{S}$ & $\mathbf{1 4 6 6 . 6 8 6 5}$ & 733.8469 & 1449.6600 & 725.3336 & 1448.6760 & 724.8416 & $\mathbf{1 3}$ \\
\hline $\mathbf{4}$ & 431.1521 & 216.0797 & 414.1255 & 207.5664 & 413.1415 & 207.0744 & $\mathbf{N}$ & $\mathbf{1 3 7 9 . 6 5 4 5}$ & 690.3309 & 1362.6280 & 681.8176 & 1361.6440 & 681.3256 & $\mathbf{1 2}$ \\
\hline & & & & & & & & & & & & & &
\end{tabular}




\begin{tabular}{|c|c|c|c|c|c|c|c|c|c|c|c|c|c|c|}
\hline 5 & 546.1790 & |273.5932 & 529.1525 & |265.0799 & 528.1685 & 264.5879 & D & 1265.6116 & 633.3094 & 1248.5850 & 624.7962 & |1247.6010 & 624.3042 & |11 \\
\hline 6 & 659.2631 & 330.1352 & 642.2366 & 321.6219 & 641.2525 & 321.1299 & I & 1150.5846 & 575.7960 & 1133.5581 & 567.2827 & 1132.5741 & 566.7907 & 10 \\
\hline 7 & 758.3315 & 379.6694 & 741.3050 & 371.1561 & 740.3210 & 370.6641 & $\mathbf{V}$ & 1037.5006 & 519.2539 & 1020.4740 & 510.7407 & 1019.4900 & 510.2486 & 9 \\
\hline 8 & 872.3744 & 436.6909 & 855.3479 & 428.1776 & 854.3639 & 427.6856 & $\mathbf{N}$ & 938.4322 & 469.7197 & 921.4056 & 461.2064 & 920.4216 & 460.7144 & 8 \\
\hline 9 & 943.4116 & 472.2094 & 926.3850 & 463.6961 & 925.4010 & 463.2041 & A & 824.3892 & 412.6983 & & & 806.3787 & 403.6930 & 7 \\
\hline 10 & 1056.4956 & 528.7514 & 1039.4691 & 520.2382 & 1038.4851 & 519.7462 & I & 753.3521 & 377.1797 & & & 735.3416 & 368.1744 & 6 \\
\hline 11 & 1187.5361 & 594.2717 & 1170.5096 & 585.7584 & 1169.5255 & 585.2664 & $\mathbf{M}$ & 640.2681 & 320.6377 & & & 622.2575 & 311.6324 & 5 \\
\hline 12 & 1316.5787 & 658.7930 & 1299.5521 & 650.2797 & 1298.5681 & 649.7877 & $E$ & 509.2276 & 255.1174 & & & 491.2170 & 246.1121 & 4 \\
\hline 13 & 1429.6628 & 715.3350 & 1412.6362 & 706.8217 & 1411.6522 & 706.3297 & $\mathbf{L}$ & 380.1850 & 190.5961 & & & 362.1744 & 181.5908 & 3 \\
\hline 14 & 1530.7104 & 765.8589 & 1513.6839 & 757.3456 & 1512.6999 & 756.8536 & $\mathbf{T}$ & 267.1009 & 134.0541 & & & 249.0904 & 125.0488 & 2 \\
\hline 15 & & & & & & & M & 166.0532 & 83.5303 & & & & & 1 \\
\hline
\end{tabular}
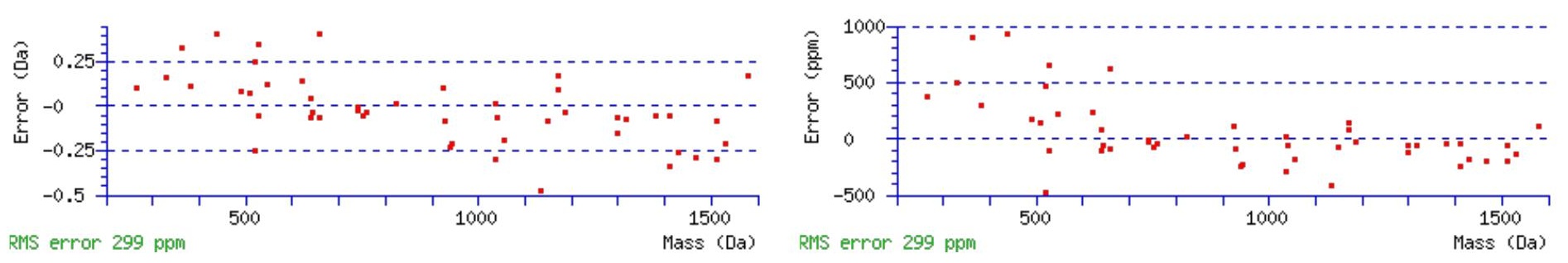

\section{All matches to this query}

\begin{tabular}{|l|l|l|l|}
\hline Score & Mr(calc): & Delta & Sequence \\
\hline 69.7 & 1694.7491 & 0.0053 & NNSNDIVNAIMELTM \\
\hline 66.7 & 1694.7491 & 0.0053 & NNSNDIVNAIMELTM \\
\hline 66.7 & 1694.7491 & 0.0053 & NNSNDIVNAIMELTM \\
\hline 31.1 & 1693.7651 & 0.9893 & NNSNDIVNAIMELTM \\
\hline 28.6 & 1694.7491 & 0.0053 & NNSNDIVNAIMELTM \\
\hline 24.4 & 1694.7491 & 0.0053 & NNSNDIVNAIMELTM \\
\hline 21.6 & 1694.7491 & 0.0053 & NNSNDIVNAIMELTM \\
\hline 13.8 & 1694.7491 & 0.0053 & NNSNDIVNAIMELTM \\
\hline 9.8 & 1694.7487 & 0.0057 & TCGNLLNPWTPSQK \\
\hline 8.1 & 1693.7651 & 0.9893 & NNSNDIVNAIMELTM \\
\hline
\end{tabular}

Spectrum No: 367; Query: 1257; Rank: 1

\section{Peptide View}

MS/MS Fragmentation of WNDSPCNQSLPSICK

Found in IPI00192504, Tax_Id=10116 Gene_Symbol=Mrc2 Macrophage mannose receptor 2 precursor

Match to Query 1257: 1805.772588 from(903.893570,2+)

Title: 100101RatKid_NS_deglyco_23.2514.2514.2.dta

Data file K:INewmanPaper|Piliangl3SubProteomes\Piliang3SP\mgf5ppm\ERLIC_3SubProteomes5ppm.mgf 

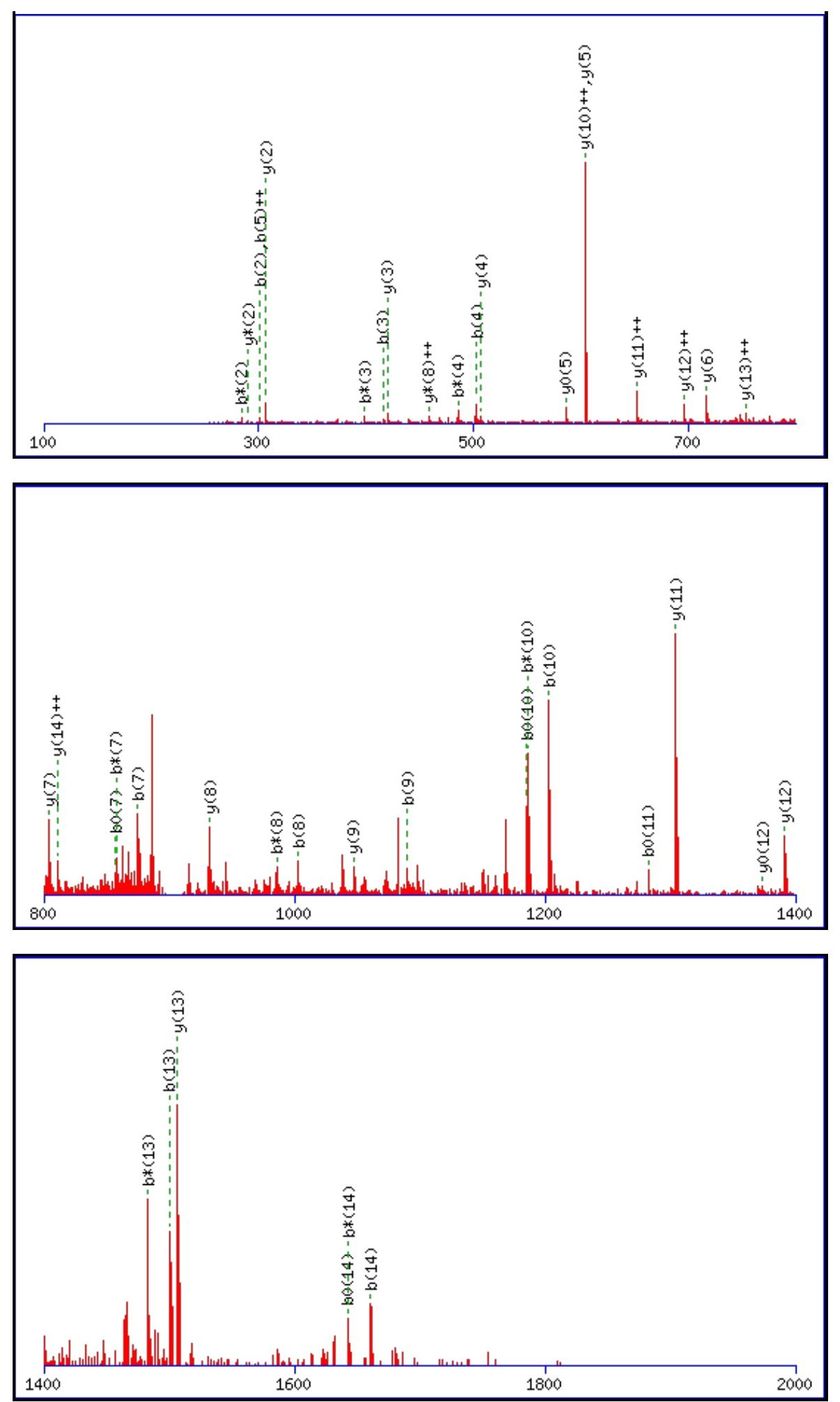

Monoisotopic mass of neutral peptide $\operatorname{Mr}($ calc): 1805.7713

Fixed modifications: Carbamidomethyl (C)

Variable modifications:

N7 : Deamidated $\mathrm{N}(\mathrm{N})$

Ions Score: 70 Expect: $2.2 \mathrm{e}-005$

Matches (Bold Red): 42/156 fragment ions using 75 most intense peaks

\begin{tabular}{|r|c|c|c|c|c|c|c|c|c|c|c|c|c|c|}
\hline$\#$ & $\mathbf{b}$ & $\mathbf{b}^{++}$ & $\mathbf{b}^{*}$ & $\mathbf{b}^{\mathbf{*}^{++}}$ & $\mathbf{b}^{\mathbf{0}}$ & $\mathbf{b}^{\mathbf{0 + +}}$ & Seq. & $\mathbf{y}$ & $\mathbf{y}^{++}$ & $\mathbf{y}^{\mathbf{*}}$ & $\mathbf{y}^{\boldsymbol{*}^{++}}$ & $\mathbf{y}^{\mathbf{0}}$ & $\mathbf{y}^{\mathbf{0 + +}}$ & $\#$ \\
\hline $\mathbf{1}$ & 187.0866 & 94.0469 & & & & & $\mathbf{W}$ & & & & & & & $\mathbf{1 5}$ \\
\hline $\mathbf{2}$ & $\mathbf{3 0 1 . 1 2 9 5}$ & 151.0684 & $\mathbf{2 8 4 . 1 0 3 0}$ & 142.5551 & & & $\mathbf{N}$ & 1620.6992 & $\mathbf{8 1 0 . 8 5 3 3}$ & 1603.6727 & 802.3400 & 1602.6887 & 801.8480 & $\mathbf{1 4}$ \\
\hline $\mathbf{3}$ & $\mathbf{4 1 6 . 1 5 6 5}$ & 208.5819 & 399.1299 & 200.0686 & 398.1459 & 199.5766 & $\mathbf{D}$ & $\mathbf{1 5 0 6 . 6 5 6 3}$ & 753.8318 & 1489.6298 & 745.3185 & 1488.6457 & 744.8265 & $\mathbf{1 3}$ \\
\hline $\mathbf{4}$ & $\mathbf{5 0 3 . 1 8 8 5}$ & 252.0979 & $\mathbf{4 8 6 . 1 6 1 9}$ & 243.5846 & 485.1779 & 243.0926 & S & $\mathbf{1 3 9 1 . 6 2 9 4}$ & $\mathbf{6 9 6 . 3 1 8 3}$ & 1374.6028 & 687.8050 & $\mathbf{1 3 7 3 . 6 1 8 8}$ & 687.3130 & $\mathbf{1 2}$ \\
\hline $\mathbf{5}$ & 600.2413 & $\mathbf{3 0 0 . 6 2 4 3}$ & 583.2147 & 292.1110 & 582.2307 & 291.6190 & $\mathbf{P}$ & $\mathbf{1 3 0 4 . 5 9 7 3}$ & $\mathbf{6 5 2 . 8 0 2 3}$ & 1287.5708 & 644.2890 & 1286.5868 & 643.7970 & $\mathbf{1 1}$ \\
\hline
\end{tabular}




\begin{tabular}{|c|c|c|c|c|c|c|c|c|c|c|c|c|c|c|}
\hline 6 & 760.2719 & |380.6396 & 743.2454 & |372.1263 & 742.2613 & |371.6343 & C & |1207.5446 & 604.2759 & |1190.5180 & $|595.7626|$ & |1189.5340 & |595.2706 | & 10 \\
\hline 7 & 875.2988 & 438.1531 & 858.2723 & 429.6398 & 857.2883 & 429.1478 & $\mathbf{N}$ & 1047.5139 & 524.2606 & 1030.4874 & 515.7473 & 1029.5034 & 515.2553 & 9 \\
\hline 8 & 1003.3574 & 502.1823 & 986.3309 & 493.6691 & 985.3468 & 493.1771 & $\mathbf{Q}$ & 932.4870 & 466.7471 & 915.4604 & 458.2339 & 914.4764 & 457.7418 & 8 \\
\hline 9 & 1090.3894 & 545.6984 & 1073.3629 & 537.1851 & 1072.3789 & 536.6931 & $S$ & 804.4284 & 402.7178 & 787.4019 & 394.2046 & 786.4178 & 393.7126 & 7 \\
\hline 10 & 1203.4735 & 602.2404 & 1186.4470 & 593.7271 & 1185.4629 & 593.2351 & $\mathbf{L}$ & 717.3964 & 359.2018 & 700.3698 & 350.6886 & 699.3858 & 350.1965 & 6 \\
\hline 11 & 1300.5263 & 650.7668 & 1283.4997 & 642.2535 & 1282.5157 & 641.7615 & $\mathbf{P}$ & 604.3123 & 302.6598 & 587.2858 & 294.1465 & 586.3017 & 293.6545 & 5 \\
\hline 12 & 1387.5583 & 694.2828 & 1370.5317 & 685.7695 & 1369.5477 & 685.2775 & $S$ & 507.2595 & 254.1334 & 490.2330 & 245.6201 & 489.2490 & 245.1281 & 4 \\
\hline 13 & 1500.6424 & 750.8248 & 1483.6158 & 742.3115 & 1482.6318 & 741.8195 & I & 420.2275 & 210.6174 & 403.2010 & 202.1041 & & & 3 \\
\hline 14 & 1660.6730 & 830.8401 & 1643.6465 & 822.3269 & 1642.6624 & 821.8349 & C & 307.1435 & 154.0754 & 290.1169 & 145.5621 & & & 2 \\
\hline 15 & & & & & & & $\mathbf{K}$ & 147.1128 & 74.0600 & 130.0863 & 65.5468 & & & 1 \\
\hline
\end{tabular}
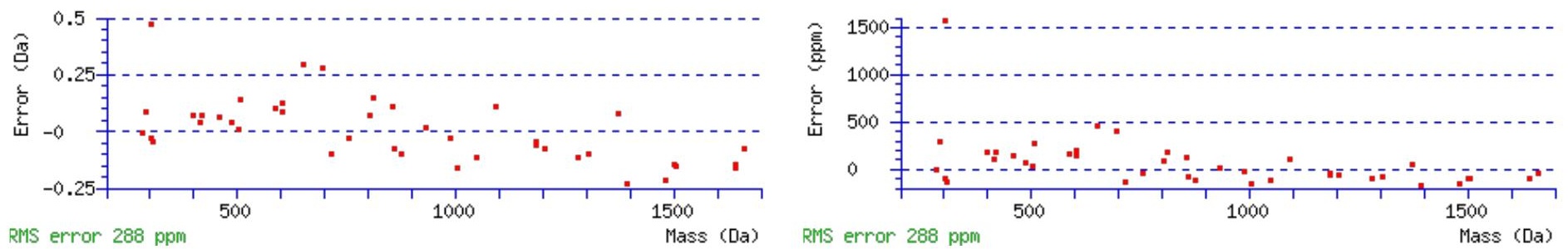

\section{All matches to this query}

\begin{tabular}{|l|l|l|l|}
\hline Score & Mr(calc): & Delta & \multicolumn{1}{c|}{ Sequence } \\
\hline 69.6 & 1805.7713 & 0.0013 & WNDSPCNQSLPSICK \\
\hline 23.0 & 1805.7713 & 0.0013 & WNDSPCNQSLPSICK \\
\hline 15.9 & 1804.7872 & 0.9853 & WNDSPCNQSLPSICK \\
\hline 11.0 & 1805.7737 & -0.0011 & LESEGSPETLTNLKK \\
\hline 11.0 & 1805.7737 & -0.0011 & LESEGSPETLTNLKK \\
\hline 11.0 & 1805.7737 & -0.0011 & LESEGSPETLTNLKK \\
\hline 7.9 & 1805.7824 & -0.0098 & SLTSKFMTTFSQLR \\
\hline 6.8 & 1805.7672 & 0.0054 & SMEETRPVPTVKEK \\
\hline 6.8 & 1805.7824 & -0.0098 & SLTSKFMTTFSQLR \\
\hline 6.2 & 1805.7824 & -0.0098 & SLTSKFMTTFSQLR \\
\hline
\end{tabular}

Spectrum No: 368; Query: 997; Rank: 1

\section{Peptide View}

MS/MS Fragmentation of ANEQVIQNLNQTYK

Found in IPI00205333, Tax_Id=10116 Gene_Symbol=Gpr116 Probable G-protein coupled receptor 116 precursor

Match to Query 997: 1662.824828 from(832.419690,2+)

Title: 100101RatKid_NS_deglyco_25.2260.2260.2.dta

Data file K:INewmanPaper|Piliang|3SubProteomes\Piliang3SP\mgf5ppm\ERLIC_3SubProteomes5ppm.mgf 

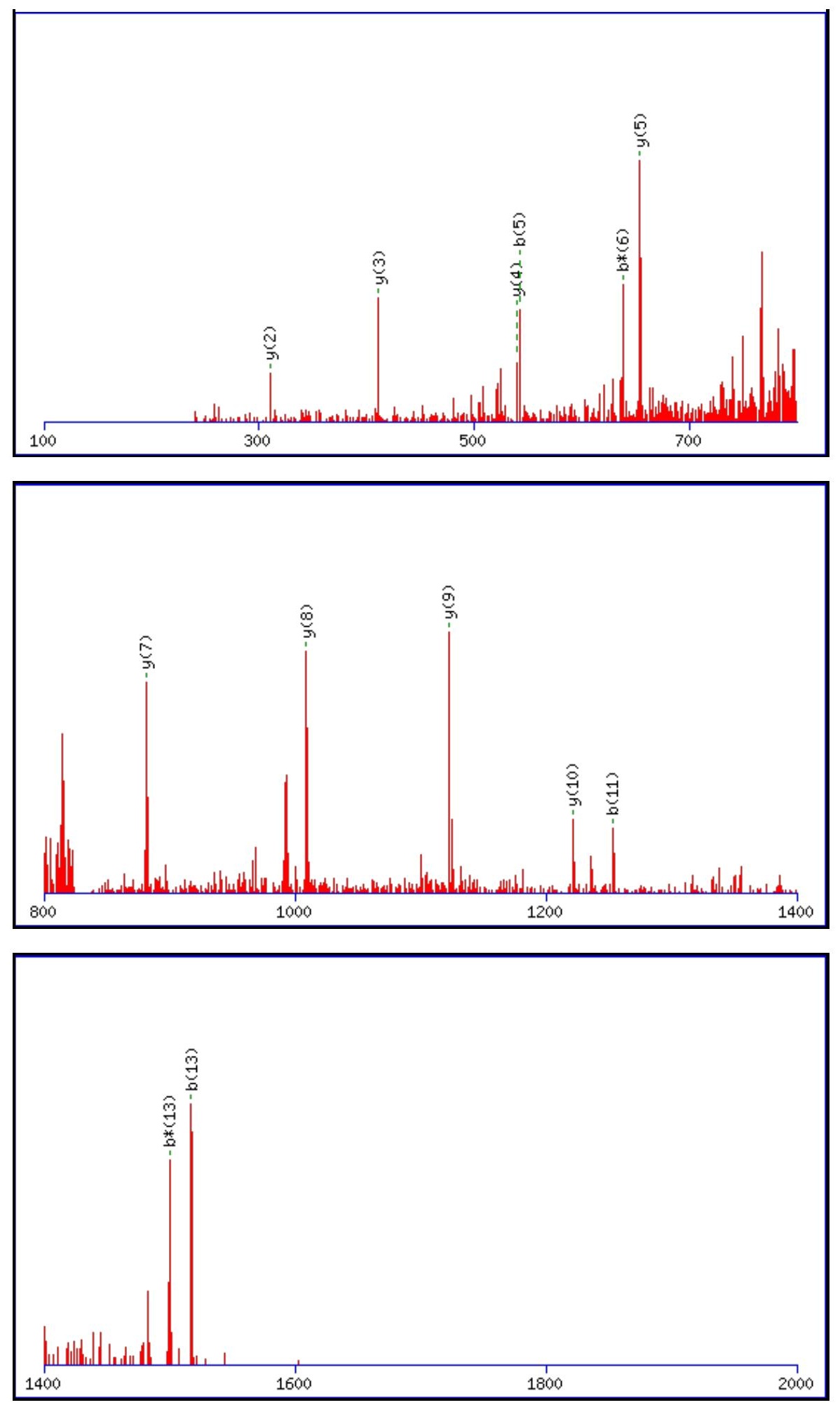

Monoisotopic mass of neutral peptide $\operatorname{Mr}($ calc): 1662.8213

Fixed modifications: Carbamidomethyl (C)

Variable modifications:

N10 : Deamidated $\mathrm{N}(\mathrm{N})$

Ions Score: 70 Expect: $1.9 \mathrm{e}-005$

Matches (Bold Red): 13/146 fragment ions using 19 most intense peaks

\begin{tabular}{|r|c|c|c|c|c|c|c|c|c|c|c|c|c|c|}
\hline$\#$ & $\mathbf{b}$ & $\mathbf{b}^{++}$ & $\mathbf{b}^{*}$ & $\mathbf{b}^{\boldsymbol{*}^{++}}$ & $\mathbf{b}^{\mathbf{0}}$ & $\mathbf{b}^{\mathbf{0}+}$ & Seq. & $\mathbf{y}$ & $\mathbf{y}^{++}$ & $\mathbf{y}^{*}$ & $\mathbf{y}^{\boldsymbol{*}^{++}}$ & $\mathbf{y}^{\mathbf{0}}$ & $\mathbf{y}^{\mathbf{0 + +}}$ & $\#^{\#}$ \\
\hline $\mathbf{1}$ & 72.0444 & 36.5258 & & & & & $\mathbf{A}$ & & & & & & & $\mathbf{1 4}$ \\
\hline $\mathbf{2}$ & 186.0873 & 93.5473 & 169.0608 & 85.0340 & & & $\mathbf{N}$ & 1592.7915 & 796.8994 & 1575.7649 & 788.3861 & 1574.7809 & 787.8941 & $\mathbf{1 3}$ \\
\hline $\mathbf{3}$ & 315.1299 & 158.0686 & 298.1034 & 149.5553 & 297.1193 & 149.0633 & $\mathbf{E}$ & 1478.7485 & 739.8779 & 1461.7220 & 731.3646 & 1460.7380 & 730.8726 & $\mathbf{1 2}$ \\
\hline $\mathbf{4}$ & 443.1885 & 222.0979 & 426.1619 & 213.5846 & 425.1779 & 213.0926 & $\mathbf{Q}$ & 1349.7060 & 675.3566 & 1332.6794 & 666.8433 & 1331.6954 & 666.3513 & $\mathbf{1 1}$ \\
\hline $\mathbf{5}$ & $\mathbf{5 4 2 . 2 5 6 9}$ & 271.6321 & 525.2304 & 263.1188 & 524.2463 & 262.6268 & $\mathbf{V}$ & $\mathbf{1 2 2 1 . 6 4 7 4}$ & 611.3273 & 1204.6208 & 602.8141 & 1203.6368 & 602.3220 & $\mathbf{1 0}$ \\
\hline
\end{tabular}




\begin{tabular}{|c|c|c|c|c|c|c|c|c|c|c|c|c|c|c|}
\hline 6 & 655.3410 & 328.1741 & 638.3144 & |319.6608 & 637.3304 & |319.1688| & I & 1122.5790 & 561.7931 & 1105.5524 & 553.2798 & 1104.5684 & 552.7878 & 9 \\
\hline 7 & 783.3995 & 392.2034 & 766.3730 & 383.6901 & 765.3890 & 383.1981 & $\mathbf{Q}$ & 1009.4949 & 505.2511 & 992.4683 & 496.7378 & 991.4843 & 496.2458 & 8 \\
\hline 8 & 897.4425 & 449.2249 & 880.4159 & 440.7116 & 879.4319 & 440.2196 & $\mathbf{N}$ & 881.4363 & 441.2218 & 864.4098 & 432.7085 & 863.4258 & 432.2165 & 7 \\
\hline 9 & 1010.5265 & 505.7669 & 993.5000 & 497.2536 & 992.5160 & 496.7616 & $\mathbf{L}$ & 767.3934 & 384.2003 & 750.3668 & 375.6871 & 749.3828 & 375.1951 & 6 \\
\hline 10 & 1125.5535 & 563.2804 & 1108.5269 & 554.7671 & 1107.5429 & 554.2751 & $\mathbf{N}$ & 654.3093 & 327.6583 & 637.2828 & 319.1450 & 636.2988 & 318.6530 & 5 \\
\hline 11 & 1253.6120 & 627.3097 & 1236.5855 & 618.7964 & 1235.6015 & 618.3044 & $\mathbf{Q}$ & 539.2824 & 270.1448 & 522.2558 & 261.6316 & 521.2718 & 261.1396 & 4 \\
\hline 12 & 1354.6597 & 677.8335 & 1337.6332 & 669.3202 & 1336.6492 & 668.8282 & $\mathbf{T}$ & 411.2238 & 206.1155 & 394.1973 & 197.6023 & 393.2132 & 197.1103 & 3 \\
\hline 13 & 1517.7231 & 759.3652 & 1500.6965 & 750.8519 & 1499.7125 & 750.3599 & $\mathbf{Y}$ & 310.1761 & 155.5917 & 293.1496 & 147.0784 & & & 2 \\
\hline 14 & & & & & & & $\mathbf{K}$ & 147.1128 & 74.0600 & 130.0863 & 65.5468 & & & 1 \\
\hline
\end{tabular}
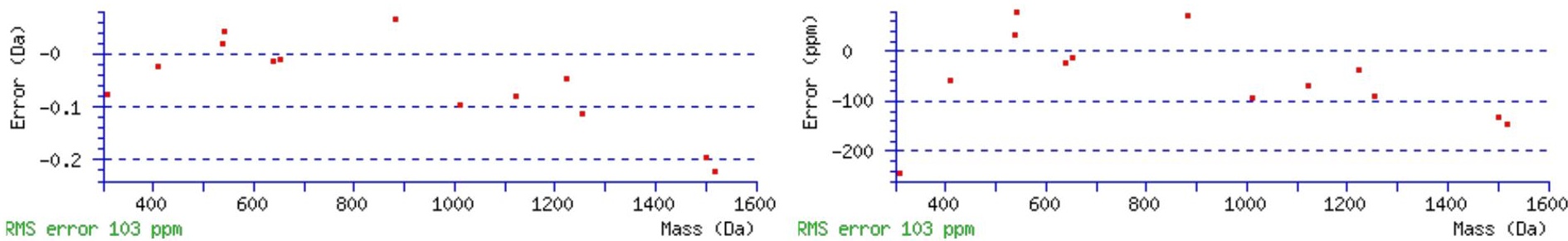

RMS error 103 ppm

\section{All matches to this query}

\begin{tabular}{|l|l|c|l|}
\hline Score & Mr(calc): & Delta & \multicolumn{1}{c|}{ Sequence } \\
\hline 69.6 & 1662.8213 & 0.0036 & ANEQVIQNLNQTYK \\
\hline 53.3 & 1662.8213 & 0.0036 & ANEQVIQNLNQTYK \\
\hline 19.9 & 1661.8373 & 0.9876 & ANEQVIQNLNQTYK \\
\hline 11.6 & 1662.8213 & 0.0036 & ANEQVIQNLNQTYK \\
\hline 8.3 & 1662.8270 & -0.0022 & SPFVVNVAPPLDLSK \\
\hline 6.9 & 1660.8225 & 2.0023 & ALKKVLEDYEAFR \\
\hline 6.2 & 1662.8382 & -0.0134 & $\underline{\text { HFVTGIIDSLAVSPK }}$ \\
\hline 5.5 & 1662.8259 & -0.0011 & VYISELKNIRLR \\
\hline 5.3 & 1662.8101 & 0.0148 & LLVNVDEEDYELGR \\
\hline 3.4 & 1662.8342 & -0.0093 & KTEPAAGKETGAAKPK \\
\hline
\end{tabular}

Spectrum No: 369; Query: 665; Rank: 1

\section{Peptide View}

MS/MS Fragmentation of ILYGLENATQELK

Found in IPI00361301, Tax_Id=10116 Gene_Symbol=Lama2_predicted similar to Laminin alpha-2 chain precursor

Match to Query 665: 1491.782848 from(746.898700,2+)

Title: 100101RatKid_NS_deglyco_21.3449.3449.2.dta

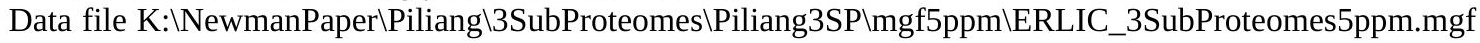



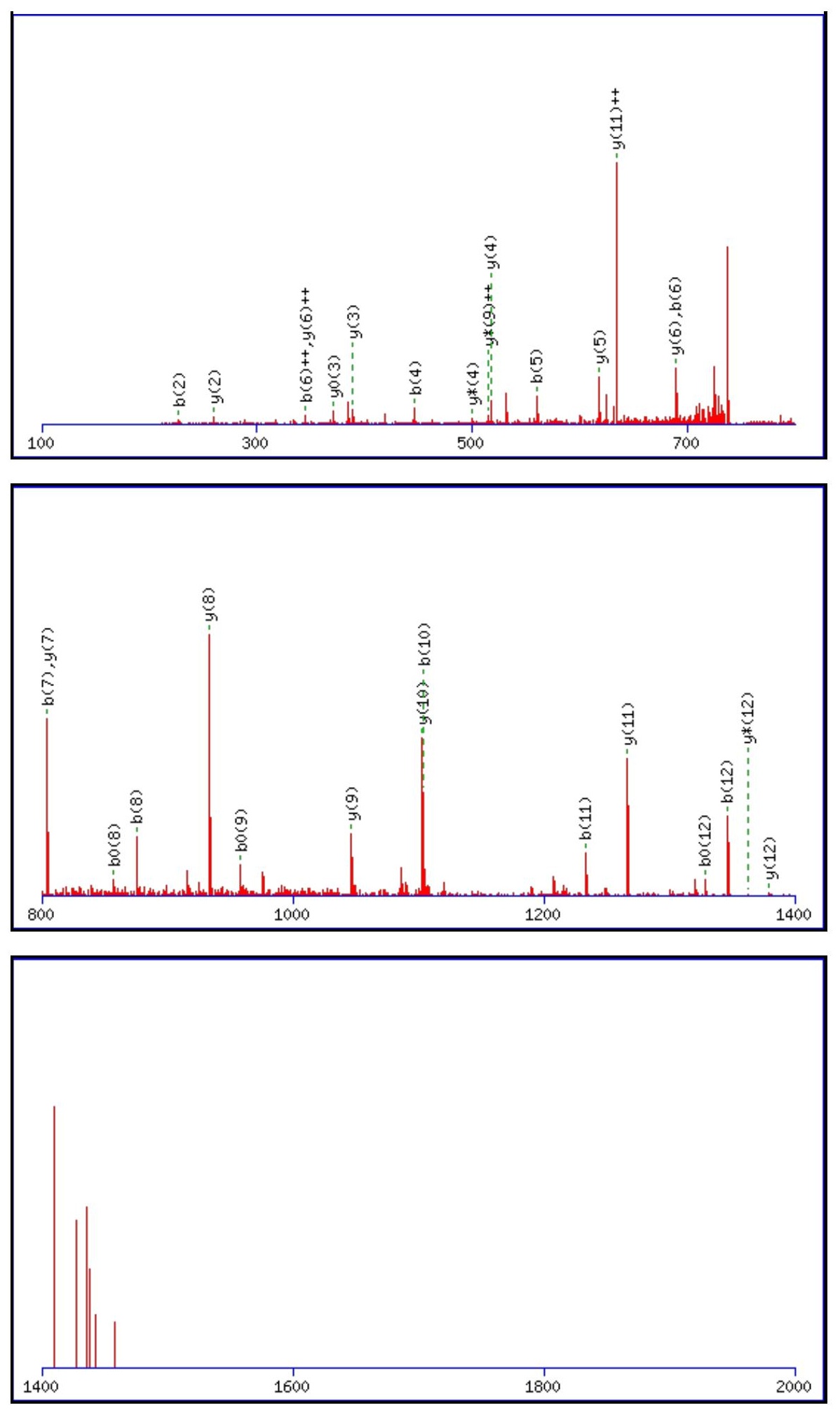

Monoisotopic mass of neutral peptide $\operatorname{Mr}($ calc): 1491.7820

Fixed modifications: Carbamidomethyl (C)

Variable modifications:

N7 : Deamidated $\mathrm{N}(\mathrm{N})$

Ions Score: 70 Expect: $1.3 \mathrm{e}-005$

Matches (Bold Red): 30/118 fragment ions using 48 most intense peaks

\begin{tabular}{|r|c|c|c|c|c|c|c|c|c|c|c|c|c|c|}
\hline$\#$ & $\mathbf{b}$ & $\mathbf{b}^{++}$ & $\mathbf{b}^{*}$ & $\mathbf{b}^{\boldsymbol{*}^{++}}$ & $\mathbf{b}^{\mathbf{0}}$ & $\mathbf{b}^{\mathbf{0 + +}}$ & Seq. & $\mathbf{y}$ & $\mathbf{y}^{++}$ & $\mathbf{y}^{\mathbf{*}}$ & $\mathbf{y}^{\boldsymbol{*}^{++}}$ & $\mathbf{y}^{\mathbf{0}}$ & $\mathbf{y}^{\mathbf{0 + +}}$ & $\#$ \\
\hline $\mathbf{1}$ & 114.0913 & 57.5493 & & & & & $\mathbf{I}$ & & & & & & & $\mathbf{1 3}$ \\
\hline $\mathbf{2}$ & $\mathbf{2 2 7 . 1 7 5 4}$ & 114.0913 & & & & & L & $\mathbf{1 3 7 9 . 7 0 5 3}$ & 690.3563 & $\mathbf{1 3 6 2 . 6 7 8 7}$ & 681.8430 & 1361.6947 & 681.3510 & $\mathbf{1 2}$ \\
\hline 3 & 390.2387 & 195.6230 & & & & & Y & $\mathbf{1 2 6 6 . 6 2 1 2}$ & $\mathbf{6 3 3 . 8 1 4 2}$ & 1249.5947 & 625.3010 & 1248.6107 & 624.8090 & $\mathbf{1 1}$ \\
\hline $\mathbf{4}$ & $\mathbf{4 4 7 . 2 6 0 2}$ & 224.1337 & & & & & G & $\mathbf{1 1 0 3 . 5 5 7 9}$ & 552.2826 & 1086.5313 & 543.7693 & 1085.5473 & 543.2773 & $\mathbf{1 0}$ \\
\hline $\mathbf{5}$ & $\mathbf{5 6 0 . 3 4 4 3}$ & 280.6758 & & & & & L & $\mathbf{1 0 4 6 . 5 3 6 4}$ & 523.7719 & 1029.5099 & $\mathbf{5 1 5 . 2 5 8 6}$ & 1028.5259 & 514.7666 & $\mathbf{9}$ \\
\hline
\end{tabular}




\begin{tabular}{|c|c|c|c|c|c|c|c|c|c|c|c|c|c|c|}
\hline 6 & 689.3869 & |345.1971 & & & 671.3763 & |336.1918 & $\mathbf{E}$ & 933.4524 & 467.2298 & 916.4258 & 458.7165 & 915.4418 & |458.2245 & 8 \\
\hline 7 & 804.4138 & 402.7105 & 787.3872 & |394.1973 & 786.4032 & 393.7053 & $\mathbf{N}$ & 804.4098 & 402.7085 & 787.3832 & 394.1952 & 786.3992 & 393.7032 & 7 \\
\hline 8 & 875.4509 & 438.2291 & 858.4244 & |429.7158 & 857.4403 & 429.2238 & A & 689.3828 & 345.1951 & 672.3563 & 336.6818 & 671.3723 & |336.1898 & 6 \\
\hline 9 & 976.4986 & 488.7529 & 959.4720 & 480.2397 & 958.4880 & 479.7476 & $\mathbf{T}$ & 618.3457 & 309.6765 & 601.3192 & 301.1632 & 600.3352 & 300.6712 & 5 \\
\hline 10 & 1104.5572 & 552.7822 & 1087.5306 & 544.2689 & 1086.5466 & 543.7769 & $\mathbf{Q}$ & 517.2980 & 259.1527 & 500.2715 & 250.6394 & 499.2875 & 250.1474 & 4 \\
\hline 11 & 1233.5998 & 617.3035 & 1216.5732 & 608.7902 & 1215.5892 & 608.2982 & $\mathbf{E}$ & 389.2395 & 195.1234 & 372.2129 & 186.6101 & 371.2289 & |186.1181 & 3 \\
\hline 12 & 1346.6838 & 673.8455 & 1329.6573 & 665.3323 & 1328.6733 & 664.8403 & $\mathbf{L}$ & 260.1969 & 130.6021 & 243.1703 & 122.0888 & & & 2 \\
\hline 13 & & & & & & & $\mathbf{K}$ & 147.1128 & 74.0600 & 130.0863 & 65.5468 & & & 1 \\
\hline
\end{tabular}
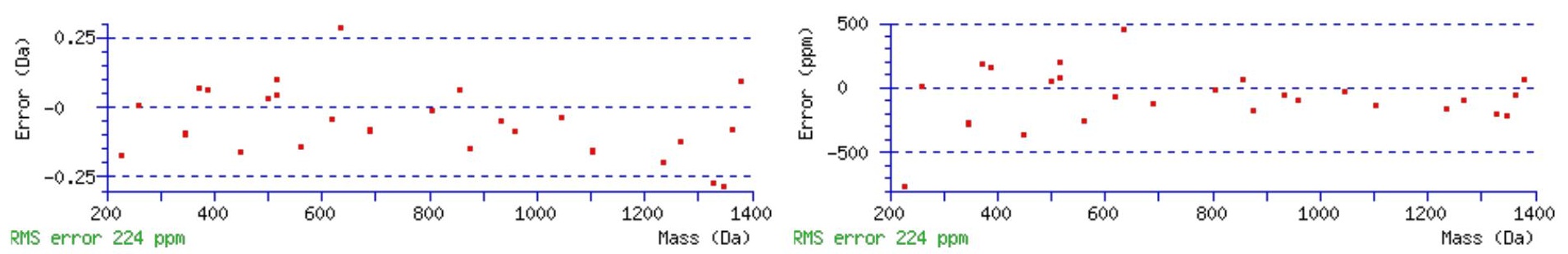

\section{All matches to this query}

\begin{tabular}{|l|l|l|l|}
\hline Score & Mr(calc): & Delta & \multicolumn{1}{|c|}{ Sequence } \\
\hline 69.5 & 1491.7820 & 0.0008 & $\underline{\text { LYGLENATOELK }}$ \\
\hline 8.2 & 1491.7722 & 0.0107 & DNSWGKVFGILEK \\
\hline 7.5 & 1491.7821 & 0.0008 & GDNVLINTFSGLLK \\
\hline 5.6 & 1491.7698 & 0.0131 & LLPGLETESKLGR \\
\hline 5.2 & 1491.7868 & -0.0039 & ATSDWKGLRMTVK \\
\hline 4.6 & 1491.7755 & 0.0073 & QCNNVLVYELLK \\
\hline 4.2 & 1491.7933 & -0.0105 & LFKGSQGEDGTLIK \\
\hline 4.1 & 1491.7810 & 0.0018 & LLTRSSTLSGHIK \\
\hline 4.1 & 1491.7810 & 0.0018 & LLTRSSTLSGHIK \\
\hline 4.1 & 1491.7810 & 0.0018 & LLTRSSTLSGHIK \\
\hline
\end{tabular}

Spectrum No: 370; Query: 2565; Rank: 1

\section{Peptide View}

MS/MS Fragmentation of MGAGEALGNASQLFEAWLKGNESIPVNLR

Found in IPI00327398, Tax_Id=10116 Gene_Symbol=Enpep Isoform 1 of Glutamyl aminopeptidase

Match to Query 2565: 3090.522132 from(1031.181320,3+)

Title: 100101RatKid_NS_deglyco_19.6450.6450.3.dta

Data file K:INewmanPaper|Piliangl3SubProteomes\Piliang3SP\mgf5ppm\ERLIC_3SubProteomes5ppm.mgf 

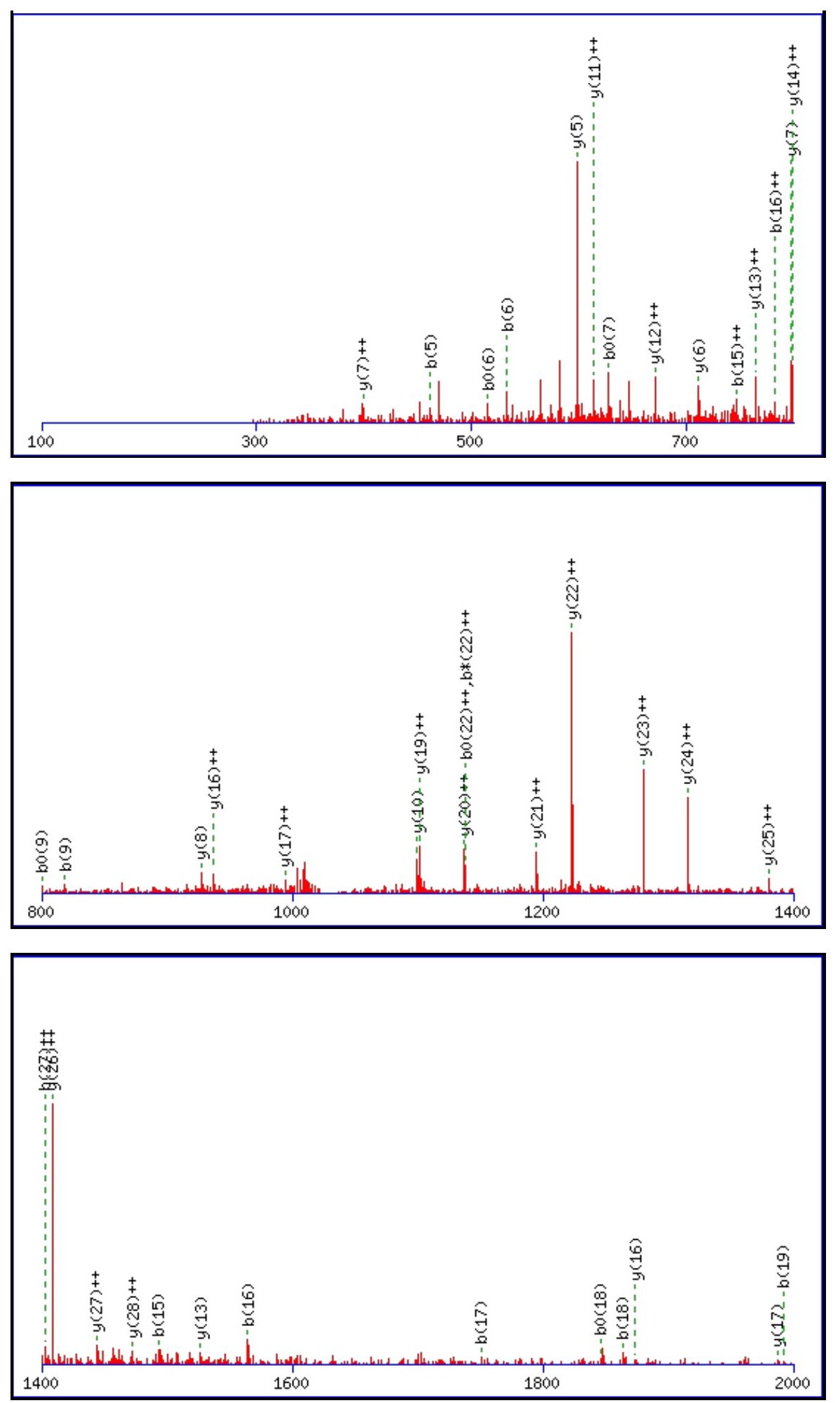

Monoisotopic mass of neutral peptide $\operatorname{Mr}($ calc): 3089.5127

Fixed modifications: Carbamidomethyl (C)

Variable modifications:

M1 : Oxidation (M)

N9 $\quad$ Deamidated_N (N)

N21 : Deamidated_N (N)

Ions Score: 70 Expect: $2.9 \mathrm{e}-005$

Matches (Bold Red): 42/300 fragment ions using 100 most intense peaks

\begin{tabular}{|c|c|c|c|c|c|c|c|c|c|c|c|c|c|c|}
\hline \# & b & $\mathbf{b}^{++}$ & $\mathbf{b}^{*}$ & $\mathbf{b}^{*^{++}}$ & $\mathbf{b}^{\mathbf{0}}$ & $\mathbf{b}^{0++}$ & Seq. & $\mathbf{y}$ & $y^{++}$ & $\mathbf{y}^{*}$ & $y^{*^{++}}$ & $\mathbf{y}^{\mathbf{0}}$ & $y^{0++}$ & $\#$ \\
\hline 1 & 148.0427 & 74.5250 & & & & & M & & & & & & & 29 \\
\hline 2 & 205.0641 & 103.0357 & & & & & G & 2943.4847 & 1472.2460 & 2926.4581 & 1463.7327 & 2925.4741 & 1463.2407 & \begin{tabular}{|l|l|}
7728 \\
\end{tabular} \\
\hline 3 & 276.1013 & 138.5543 & & & & & A & 2886.4632 & 1443.7352 & 2869.4367 & 1435.2220 & 2868.4526 & 1434.7300 & 27 \\
\hline 4 & 333.1227 & 167.0650 & & & & & G & 2815.4261 & 1408.2167 & 2798.3995 & 1399.7034 & 2797.4155 & 1399.2114 & 426 \\
\hline
\end{tabular}




\begin{tabular}{|c|c|c|c|c|c|c|c|c|c|c|c|c|c|c|}
\hline & 462.1653 & 231.5863 & & & 444.1547 & 222.5810 & $\mathbf{E}$ & 2758.4046 & $\mid 1379.7059$ & |2741.3781| & |1371.1927 & |2740.3941 & | 1370.7007 & $\mid 25$ \\
\hline 6 & 533.2024 & 267.1049 & & & 515.1919 & 258.0996 & A & 2629.3620 & 1315.1847 & 2612.3355 & 1306.6714 & 2611.3515 & 1306.1794 & 24 \\
\hline 7 & 646.2865 & 323.6469 & & & 628.2759 & 314.6416 & $\mathbf{L}$ & 2558.3249 & 1279.6661 & 2541.2984 & 1271.1528 & 2540.3143 & 1270.6608 & 23 \\
\hline 8 & 703.3080 & 352.1576 & & & 685.2974 & 343.1523 & G & 2445.2409 & 1223.1241 & 2428.2143 & 1214.6108 & 2427.2303 & 1214.1188 & 22 \\
\hline 9 & 818.3349 & 409.6711 & 801.3083 & 401.1578 & 800.3243 & 400.6658 & $\mathbf{N}$ & 2388.2194 & 1194.6133 & 2371.1928 & 1186.1001 & 2370.2088 & 1185.6080 & 21 \\
\hline 10 & 889.3720 & 445.1896 & 872.3455 & 436.6764 & 871.3614 & 436.1844 & A & 2273.1925 & 1137.0999 & 2256.1659 & 1128.5866 & 2255.1819 & 1128.0946 & 20 \\
\hline 11 & 976.4040 & 488.7057 & 959.3775 & 480.1924 & 958.3935 & 479.7004 & $\mathrm{~S}$ & 2202.1553 & 1101.5813 & 2185.1288 & 1093.0680 & 2184.1448 & 1092.5760 & 19 \\
\hline 12 & 1104.4626 & 552.7349 & 1087.4361 & 544.2217 & 1086.4520 & 543.7297 & $\mathbf{Q}$ & 2115.1233 & 1058.0653 & 2098.0968 & 1049.5520 & 2097.1127 & 1049.0600 & 18 \\
\hline 13 & 1217.5467 & 609.2770 & 1200.5201 & 600.7637 & 1199.5361 & 600.2717 & L & \begin{tabular}{|l|l|}
1987.0647 \\
\end{tabular} & 994.0360 & 1970.0382 & 985.5227 & 1969.0542 & 985.0307 & 17 \\
\hline 14 & 1364.6151 & 682.8112 & 1347.5885 & 674.2979 & 1346.6045 & 673.8059 & $\mathbf{F}$ & 1873.9807 & 937.4940 & 1856.9541 & 928.9807 & 1855.9701 & 928.4887 & $\overline{16}$ \\
\hline 15 & 1493.6577 & 747.3325 & 1476.6311 & 738.8192 & 1475.6471 & 738.3272 & E & 1726.9123 & 863.9598 & 1709.8857 & 855.4465 & 1708.9017 & 854.9545 & 15 \\
\hline 16 & 1564.6948 & 782.8510 & 1547.6682 & 774.3378 & 1546.6842 & 773.8458 & A & 1597.8697 & 799.4385 & 1580.8431 & 790.9252 & 1579.8591 & 790.4332 & 14 \\
\hline 17 & 1750.7741 & 875.8907 & 1733.7476 & 867.3774 & 1732.7635 & 866.8854 & W & 1526.8325 & 763.9199 & 1509.8060 & 755.4066 & 1508.8220 & 754.9146 & 13 \\
\hline 18 & 1863.8 & 932 & 1846. & & 1845.8476 & 923.4274 & $\mathbf{L}$ & 1340.7532 & 670.8803 & 1323.7267 & 662.3670 & 1322.7427 & 661.8750 & 12 \\
\hline 19 & 1991.9531 & 996.4802 & 1974.9266 & 987.9669 & 1973.9426 & 987.4749 & $\mathbf{K}$ & 1227.6692 & 614.3382 & 1210.6426 & 605.8249 & 1209.6586 & 605.3329 & 11 \\
\hline 20 & 2048.9746 & 1024.9909 & 2031.9481 & 1016.4777 & 2030.9640 & 1015.9857 & G & 1099.5742 & 550.2907 & 1082.5477 & 541.7775 & 1081.5636 & 541.2855 & 10 \\
\hline 21 & 2164.0015 & 1082.5044 & 2146.9750 & 1073.9911 & 2145.9910 & 1073.4991 & $\mathbf{N}$ & 1042.5527 & 521.7800 & 1025.5262 & 513.2667 & 1024.5422 & 512.7747 & 9 \\
\hline 22 & 2293.0441 & 1147.0257 & 2276.0176 & 1138.5124 & 2275.0336 & 1138.0204 & $\mathbf{E}$ & 927.5258 & 464.2665 & 910.4993 & 455.7533 & 909.5152 & 455.2613 & 8 \\
\hline 23 & 2380.0762 & 1190.5417 & 2363.0496 & 1182.0284 & 2362.0656 & 1181.5364 & S & 798.4832 & 399.7452 & 781.4567 & 391.2320 & 780.4726 & 390.7400 & 7 \\
\hline 24 & 2493.1602 & 1247.0837 & 2476.1337 & 1238.5705 & 2475.1497 & 1238.0785 & I & 711.4512 & 356.2292 & 694.4246 & 347.7160 & & & 6 \\
\hline 25 & 2590.2130 & 1295.6101 & 2573.1864 & 1287.0969 & 2572.2024 & 1286.6048 & $\mathbf{P}$ & 598.3671 & 299.6872 & 581.3406 & 291.1739 & & & 5 \\
\hline 26 & 2689.2814 & 1345.1443 & 2672.2549 & 1336.6311 & 2671.2708 & 1336.1391 & V & 501.3144 & 251.1608 & 484.2878 & 242.6475 & & & 4 \\
\hline 27 & 2803.3243 & \begin{tabular}{|l|}
1402.1658 \\
\end{tabular} & 2786.2978 & 1393.6525 & 2785.3138 & 1393.1605 & $\mathbf{N}$ & 402.2459 & 201.6266 & 385.2194 & 193.1133 & & & 3 \\
\hline 28 & 2916.4084 & 1458.7078 & 2899.3818 & 1450.1946 & 2898.3978 & 1449.7026 & $\mathbf{L}$ & 288.2030 & 144.6051 & 271.1765 & 136.0919 & & & 2 \\
\hline 29 & & & & & & & $\mathbf{R}$ & 175.1190 & 88.0631 & 158.0924 & 79.5498 & & & 1 \\
\hline
\end{tabular}
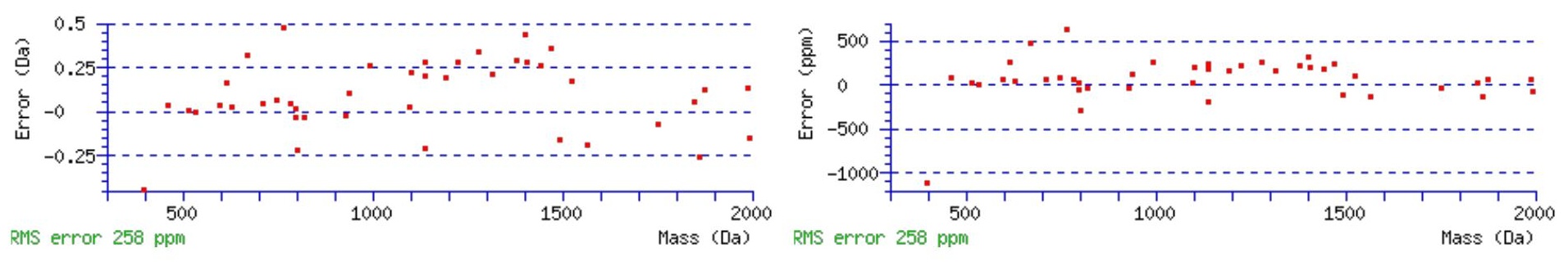

\section{All matches to this query}

\begin{tabular}{|l|l|l|l|}
\hline Score & Mr(calc): & Delta & \multicolumn{1}{|c|}{ Sequence } \\
\hline 69.5 & 3089.5127 & 1.0094 & MGAGEALGNASOLFEAWLKGNESIPVNLR \\
\hline 67.8 & 3090.4967 & 0.0254 & MGAGEALGNASQLFEAWLKGNESIPVNLR \\
\hline 58.7 & 3089.5127 & 1.0094 & MGAGEALGNASOLFEAWLKGNESIPVNLR \\
\hline 50.0 & 3089.5127 & 1.0094 & MGAGEALGNASQLFEAWLKGNESIPVNLR \\
\hline 9.8 & 3088.5287 & 1.9934 & MGAGEALGNASQLFEAWLKGNESIPVNLR \\
\hline 8.3 & 3088.5036 & 2.0185 & CLSVSPGGQWLASGSDDGTLRLWEVATAR \\
\hline 5.9 & 3088.5287 & 1.9934 & MGAGEALGNASQLFEAWLKGNESIPVNLR \\
\hline 4.5 & 3088.5320 & 1.9901 & ELLILEILQMGLKQISWTEVKNASR \\
\hline 2.5 & 3089.5290 & 0.9931 & IILLSHAKSMSLIEIGNPSQSLENVCR \\
\hline 2.4 & 3089.5290 & 0.9931 & IILLSHAKSMSLIEIGNPSQSLENVCR \\
\hline
\end{tabular}

Spectrum No: 371; Query: 1988; Rank: 1 


\section{Peptide View}

MS/MS Fragmentation of LLNTTDVYLLPSLNPDGFER

Found in IPI00213182, Tax_Id=10116 Gene_Symbol=Cpd Isoform 1 of Carboxypeptidase D precursor

Match to Query 1988: 2277.156328 from(1139.585440,2+)

Title: 091008RatKidney_NoSalt_25.5204.5204.2.dta

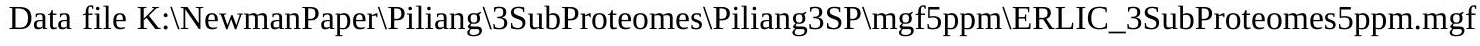
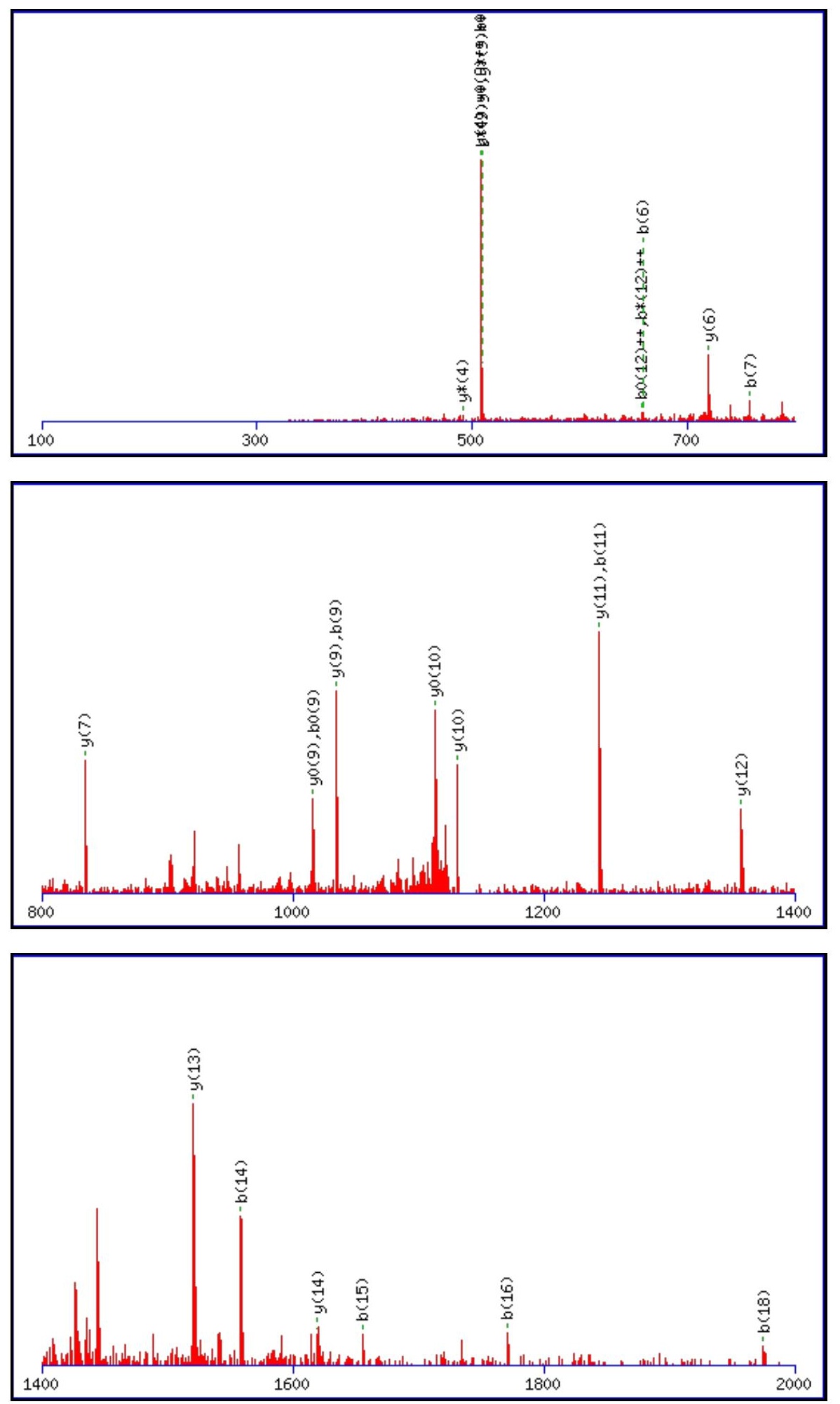

Monoisotopic mass of neutral peptide $\operatorname{Mr}($ calc): 2277.1529

Fixed modifications: Carbamidomethyl (C)

Variable modifications:

N3 : Deamidated_N (N)

Ions Score: 69 Expect: 2e-005

Matches (Bold Red): 27/216 fragment ions using 40 most intense peaks 


\begin{tabular}{|c|c|c|c|c|c|c|c|c|c|c|c|c|c|c|}
\hline \# & b & $\mathbf{b}^{++}$ & $\mathbf{b}^{*}$ & $\mathbf{b}^{*^{++}}$ & $\mathbf{b}^{0}$ & $\mathbf{b}^{\mathbf{0 + +}}$ & Seq. & $\mathbf{y}$ & $\mathbf{y}^{++}$ & $\mathbf{y}^{*}$ & $\mathrm{y}^{\mathrm{*}^{++}}$ & $\mathbf{y}^{0}$ & $\mathbf{y}^{0++}$ & \# \\
\hline 1 & 114.0913 & 57.5493 & & & & & $\mathbf{L}$ & & & & & & & 20 \\
\hline 2 & 227.1754 & 114.0913 & & & & & $\mathbf{L}$ & 2165.0761 & 1083.0417 & 2148.0495 & 1074.5284 & 2147.0655 & 1074.0364 & 19 \\
\hline 3 & 342.2023 & 171.6048 & 325.1758 & 163.0915 & & & $\mathbf{N}$ & 2051.9920 & 1026.4996 & 2034.9655 & 1017.9864 & 2033.9815 & 1017.4944 & 18 \\
\hline 4 & 443.2500 & 222.1286 & 426.2235 & 213.6154 & 425.2395 & 213.1234 & $T$ & 1936.9651 & 968.9862 & 1919.9385 & 960.4729 & 1918.9545 & 959.9809 & 17 \\
\hline 5 & 544.2977 & 272.6525 & 527.2711 & 264.1392 & 526.2871 & 263.6472 & $T$ & 1835.9174 & 918.4623 & 1818.8909 & 909.9491 & 1817.9068 & 909.4571 & 16 \\
\hline 6 & 659.3246 & 330.1660 & 642.2981 & 321.6527 & 641.3141 & 321.1607 & D & 1734.8697 & 867.9385 & 1717.8432 & 859.4252 & 1716.8592 & 858.9332 & 15 \\
\hline 7 & 758.3931 & 379.7002 & 741.3665 & 371.1869 & 740.3825 & 370.6949 & $\mathbf{V}$ & 1619.8428 & 810.4250 & 1602.8162 & 801.9118 & 1601.8322 & 801.4197 & 14 \\
\hline 8 & 921.4564 & 461.2318 & \begin{tabular}{|l}
904.4298 \\
\end{tabular} & 452.7186 & 903.4458 & 452.2265 & $\mathbf{Y}$ & 1520.7744 & 760.8908 & 1503.7478 & 752.3775 & 1502.7638 & 751.8855 & 13 \\
\hline 9 & 1034.5404 & 517.7739 & 1017.5139 & 509.2606 & 1016.5299 & 508.7686 & L & 1357.7110 & 679.3592 & 1340.6845 & 670.8459 & 1339.7005 & 670.3539 & 12 \\
\hline 10 & 1147.6245 & 574.3159 & 1130.5980 & 565.8026 & 1129.6139 & 565.3106 & $\mathbf{L}$ & 1244.6270 & 622.8171 & 1227.6004 & 614.3039 & 1226.6164 & 613.8118 & 11 \\
\hline 11 & 1244.6773 & 622.8423 & 1227.6507 & 614.3290 & 1226.6667 & 613.8370 & $\mathbf{P}$ & 1131.5429 & 566.2751 & 1114.5164 & 557.7618 & 1113.5323 & 557.2698 & 10 \\
\hline 12 & 1331.7093 & 666.3583 & 1314.6828 & 657.8450 & 1313.6987 & 657.3530 & $\mathrm{~S}$ & 1034.4901 & 517.7487 & 1017.4636 & 509.2354 & 1016.4796 & 508.7434 & 9 \\
\hline 13 & 1444.7934 & 722.9003 & 1427.7668 & 714.3870 & 1426.7828 & 713.8950 & $\mathbf{L}$ & 947.4581 & 474.2327 & 930.4316 & 465.7194 & 929.4476 & 465.2274 & 8 \\
\hline 14 & \begin{tabular}{|l|}
1558.8363 \\
\end{tabular} & 779.9218 & 1541.8097 & 771.4085 & 1540.8257 & 770.9165 & $\mathbf{N}$ & 834.3741 & 417.6907 & 817.3475 & 409.1774 & 816.3635 & 408.6854 & 7 \\
\hline 15 & 1655.8891 & 828.4482 & 1638.8625 & 819.9349 & 1637.8785 & 819.4429 & $\mathbf{P}$ & 720.3311 & 360.6692 & 703.3046 & 352.1559 & 702.3206 & 351.6639 & 6 \\
\hline 16 & 1770.9160 & 885.9616 & 1753.8895 & 877.4484 & 1752.9054 & 876.9564 & D & 623.2784 & 312.1428 & 606.2518 & 303.6295 & 605.2678 & 303.1375 & 5 \\
\hline 17 & 1827.9375 & 914.4724 & 1810.9109 & 905.9591 & 1809.9269 & 905.4671 & G & 508.2514 & 254.6293 & 491.2249 & 246.1161 & 490.2409 & 245.6241 & 4 \\
\hline 18 & 1975.0059 & 988.0066 & 1957.9793 & 979.4933 & 1956.9953 & 979.0013 & $\mathbf{F}$ & 451.2300 & 226.1186 & 434.2034 & 217.6053 & 433.2194 & 217.1133 & 3 \\
\hline 19 & 2104.0485 & 1052.5279 & 2087.0219 & 1044.0146 & 2086.0379 & 1043.5226 & E & 304.1615 & 152.5844 & 287.1350 & 144.0711 & 286.1510 & 143.5791 & 2 \\
\hline 20 & & & & & & & $\mathbf{R}$ & 175.1190 & 88.0631 & 158.0924 & 79.5498 & & & 1 \\
\hline
\end{tabular}
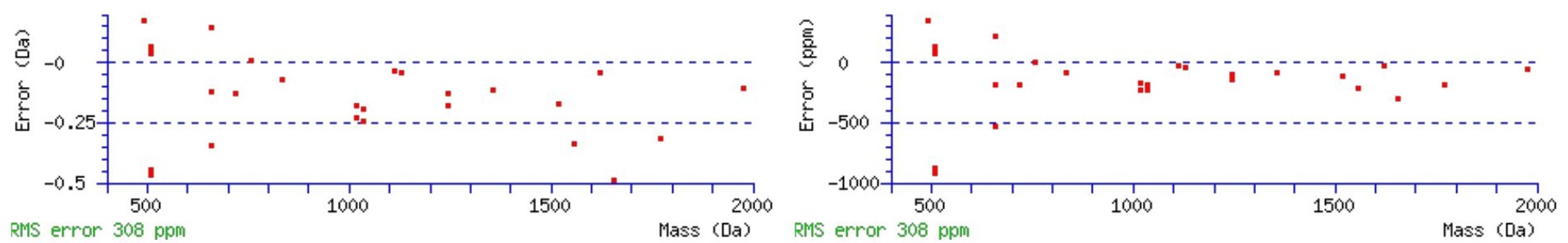

\section{All matches to this query}

\begin{tabular}{|l|l|l|l|}
\hline Score & Mr(calc): & Delta & \multicolumn{1}{|c|}{ Sequence } \\
\hline 69.4 & 2277.1529 & 0.0035 & LLNTTDVYLLPSLNPDGFER \\
\hline 46.8 & 2276.1689 & 0.9875 & LLNTTDVYLLPSLNPDGFER \\
\hline 40.9 & 2277.1529 & 0.0035 & LLNTTDVYLLPSLNPDGFER \\
\hline 4.8 & 2277.1497 & 0.0066 & MDKSELGCLRAIVLFNPDAK \\
\hline 3.9 & 2275.1660 & 1.9903 & SSIFQRLLHCLLGKAHSTK \\
\hline 3.9 & 2275.1660 & 1.9903 & SSIFQRLLHCLLGKAHSTK \\
\hline 3.8 & 2276.1752 & 0.9811 & VMHRDIKPANVFITATGVVK \\
\hline 1.2 & 2277.1437 & 0.0127 & MVRYPGQSVSTVSPAHYARR \\
\hline 1.0 & 2276.1566 & 0.9998 & LQLGPETLQQDNPKLIYAR \\
\hline 0.9 & 2277.1383 & 0.0181 & LKRASSEDMLNKPGSASSGVAR \\
\hline
\end{tabular}

Spectrum No: 372; Query: 2104; Rank: 1

\section{Peptide View}

MS/MS Fragmentation of ALNELASQGFPGNATTPSGEQLR

Found in IPI00190577, Tax_Id=10116 Gene_Symbol=Lama5 similar to Laminin alpha-5 chain precursor 
Match to Query 2104: 2358.155508 from(1180.085030,2+)

Title: 100101RatKid_NS_deglyco_23.3257.3257.2.dta

Data file K:\NewmanPaper\Piliang \3SubProteomes $\backslash$ Piliang3SP $\backslash$ mgf5ppm\ERLIC_3SubProteomes5ppm.mgf
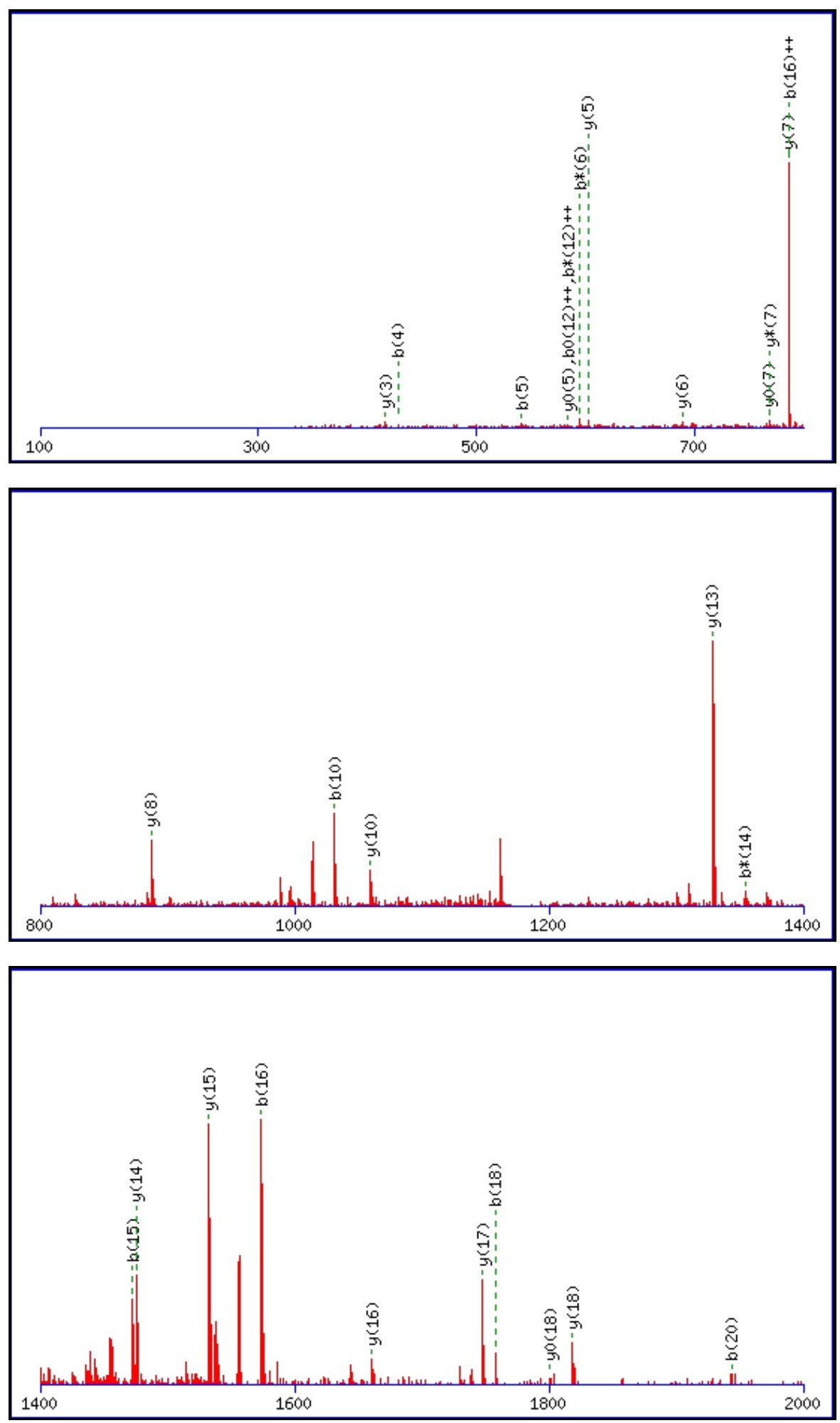

Monoisotopic mass of neutral peptide $\operatorname{Mr}($ calc): 2358.1451

Fixed modifications: Carbamidomethyl (C)

Variable modifications:

N13 : Deamidated_N (N)

Ions Score: 69 Expect: $2.6 \mathrm{e}-005$

Matches (Bold Red): 28/248 fragment ions using 46 most intense peaks

\begin{tabular}{|c|c|c|c|c|c|c|c|c|c|c|c|c|c|c|}
\hline \# & b & $\mathbf{b}^{++}$ & $\mathbf{b}^{*}$ & $\mathbf{b}^{*++}$ & $\mathbf{b}^{0}$ & $\mathbf{b}^{\mathbf{0 + +}}$ & Seq. & $\mathbf{y}$ & $y^{++}$ & $\mathbf{y}^{*}$ & $\mathrm{y}^{\mathrm{*}^{++}}$ & $\mathbf{y}^{\mathbf{0}}$ & $\mathbf{y}^{0++}$ & \# \\
\hline 1 & 72.0444 & 36.5258 & & & & & A & & & & & & & 23 \\
\hline 2 & 185.1285 & 93.0679 & & & & & $\mathbf{L}$ & 2288.1153 & 1144.5613 & 2271.0888 & 1136.0480 & 2270.1048 & 1135.5560 & 22 \\
\hline
\end{tabular}




\begin{tabular}{|c|c|c|c|c|c|c|c|c|c|c|c|c|c|c|}
\hline & 299.1714 & 150.0893 & 282.1448 & 141.5761 & & & $\mathbf{N}$ & |2175.0313 & & |2158.0047 & |1079.5060 & 2157.0207 & 1079.0140 & 21 \\
\hline 4 & 428.2140 & 214.6106 & 411.1874 & 206.0974 & 410.2034 & 205.6053 & $\mathbf{E}$ & 2060.9883 & 1030.9978 & 2043.9618 & 1022.4845 & 2042.9778 & 1021.9925 & 20 \\
\hline 5 & 541.2980 & 271.1527 & 524.2715 & 262.6394 & 523.2875 & 262.1474 & $\mathbf{L}$ & 1931.9457 & 966.4765 & 1914.9192 & 957.9632 & 1913.9352 & 957.4712 & 19 \\
\hline 6 & 612.3352 & 306.6712 & 595.3086 & 298.1579 & 594.3246 & 297.6659 & A & 1818.8617 & 909.9345 & 1801.8351 & 901.4212 & 1800.8511 & 900.9292 & 18 \\
\hline 7 & 699.3672 & 350.1872 & 682.3406 & 341.6740 & 681.3566 & 341.1819 & $\mathbf{S}$ & 1747.8246 & 874.4159 & 1730.7980 & 865.9026 & 1729.8140 & 865.4106 & 17 \\
\hline 8 & 827.4258 & 414.2165 & 810.3992 & 405.7032 & 809.4152 & 405.2112 & $\mathbf{Q}$ & 1660.7925 & 830.8999 & 1643.7660 & 822.3866 & 1642.7820 & 821.8946 & 16 \\
\hline 9 & 884.4472 & 442.7272 & 867.4207 & 434.2140 & 866.4367 & 433.7220 & G & 1532.7340 & 766.8706 & 1515.7074 & 758.3573 & 1514.7234 & 757.8653 & 15 \\
\hline 10 & 1031.5156 & 516.2615 & 1014.4891 & 507.7482 & 1013.5051 & 507.2562 & $\mathbf{F}$ & 1475.7125 & 738.3599 & 1458.6859 & 729.8466 & 1457.7019 & 729.3546 & 14 \\
\hline 11 & 1128.5684 & 564.7878 & 1111.5419 & 556.2746 & 1110.5578 & 555.7826 & $\mathbf{P}$ & 1328.6441 & 664.8257 & 1311.6175 & 656.3124 & 1310.6335 & 655.8204 & 13 \\
\hline 12 & 1185.5899 & 593.2986 & 1168.5633 & 584.7853 & 1167.5793 & 584.2933 & $\mathbf{G}$ & 1231.5913 & 616.2993 & 1214.5648 & 607.7860 & 1213.5808 & 607.2940 & 12 \\
\hline 13 & 1300.6168 & 650.8120 & 1283.5903 & 642.2988 & 1282.6062 & 641.8068 & $\mathbf{N}$ & 1174.5699 & 587.7886 & 1157.5433 & 579.2753 & 1156.5593 & 578.7833 & 11 \\
\hline 14 & 1371.6539 & 686.3306 & 1354.6274 & 677.8173 & 1353.6433 & 677.3253 & A & 1059.5429 & 530.2751 & 1042.5164 & 521.7618 & 1041.5324 & 521.2698 & 10 \\
\hline 15 & 1472.7016 & 736.8544 & 1455.6750 & 728.3412 & 1454.6910 & 727.8492 & $T$ & 988.5058 & 494.7565 & 971.4793 & 486.2433 & 970.4952 & 485.7513 & 9 \\
\hline 16 & \begin{tabular}{|l|}
1573.7493 \\
\end{tabular} & 787.3783 & 1556.7227 & 778.8650 & 1555.7387 & 778.3730 & $\mathbf{T}$ & 887.4581 & 444.2327 & 870.4316 & 435.7194 & 869.4476 & 435.2274 & 8 \\
\hline 17 & 1670.8020 & 835.9047 & 1653.7755 & 827.3914 & 1652.7915 & 826.8994 & $\mathbf{P}$ & 786.4104 & 393.7089 & 769.3839 & 385.1956 & 768.3999 & 384.7036 & 7 \\
\hline 18 & 1757.8341 & 879.4207 & 1740.8075 & 870.9074 & 1739.8235 & 870.4154 & S & 689.3577 & 345.1825 & 672.3311 & 336.6692 & 671.3471 & 336.1772 & 6 \\
\hline 19 & 1814.8555 & 907.9314 & 1797.8290 & 899.4181 & 1796.8450 & 898.9261 & G & 602.3257 & 301.6665 & 585.2991 & 293.1532 & 584.3151 & 292.6612 & 5 \\
\hline 20 & \begin{tabular}{|l|l|}
1943.8981 \\
\end{tabular} & 972.4527 & 1926.8716 & 963.9394 & 1925.8876 & 963.4474 & $\mathbf{E}$ & 545.3042 & 273.1557 & 528.2776 & 264.6425 & 527.2936 & 264.1504 & 4 \\
\hline 21 & 2071.9567 & 1036.4820 & 2054.9301 & 1027.9687 & 2053.9461 & 1027.4767 & $\mathbf{Q}$ & 416.2616 & 208.6344 & 399.2350 & 200.1212 & & & 3 \\
\hline 22 & 2185.0408 & 1093.0240 & 2168.0142 & 1084.5107 & 2167.0302 & 1084.0187 & $\mathbf{L}$ & 288.2030 & 144.6051 & 271.1765 & 136.0919 & & & 2 \\
\hline 23 & & & & & & & $\mathbf{R}$ & 175.1190 & 88.0631 & 158.0924 & 79.5498 & & & 1 \\
\hline
\end{tabular}
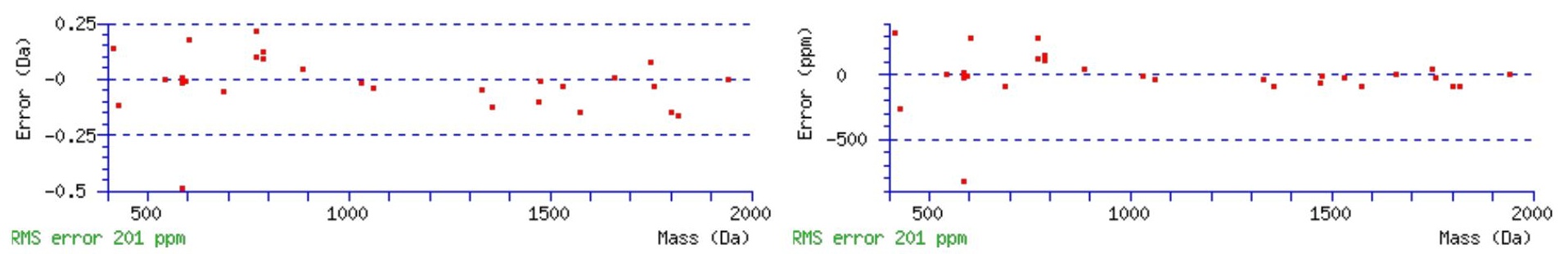

\section{All matches to this query}

\begin{tabular}{|l|l|l|l|}
\hline Score & Mr(calc): & Delta & \multicolumn{1}{|c|}{ Sequence } \\
\hline 69.3 & 2358.1451 & 0.0104 & ALNELASQGFPGNATTPSGEQLR \\
\hline 25.9 & 2357.1611 & 0.9944 & ALNELASQGFPGNATTPSGEQLR \\
\hline 23.6 & 2358.1451 & 0.0104 & ALNELASQGFPGNATTPSGEQLR \\
\hline 3.9 & 2358.1402 & 0.0153 & LLGTHPKKEPMEALNTAQGAR \\
\hline 2.0 & 2358.1515 & 0.0040 & LECPIRTYLVTARSAASSGAR \\
\hline 2.0 & 2358.1515 & 0.0040 & LECPIRTYLVTARSAASSGAR \\
\hline 1.4 & 2358.1402 & 0.0153 & SQLSLSMANHDIPPDAVRAKK \\
\hline 0.9 & 2358.1409 & 0.0146 & HEFLIFEDRKFADIGNTVK \\
\hline 0.8 & 2358.1597 & -0.0041 & ELRSTLDSIPSLSLPSSIKR \\
\hline 0.7 & 2358.1354 & 0.0201 & KLVQLLASSPCESVMKRPR \\
\hline
\end{tabular}

Spectrum No: 373; Query: 169; Rank: 1

\section{Peptide View}

MS/MS Fragmentation of VNETEEGYAR

Found in IPI00371511, Tax_Id=10116 Gene_Symbol=Creg_predicted cellular repressor of E1A-stimulated genes

Match to Query 169: 1167.503068 from(584.758810,2+) 
Title: 091008RatKidney_NH4Format01_23.433.433.2.dta

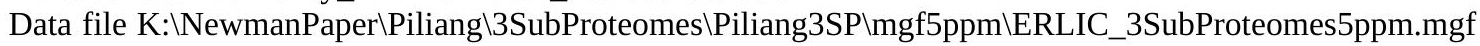
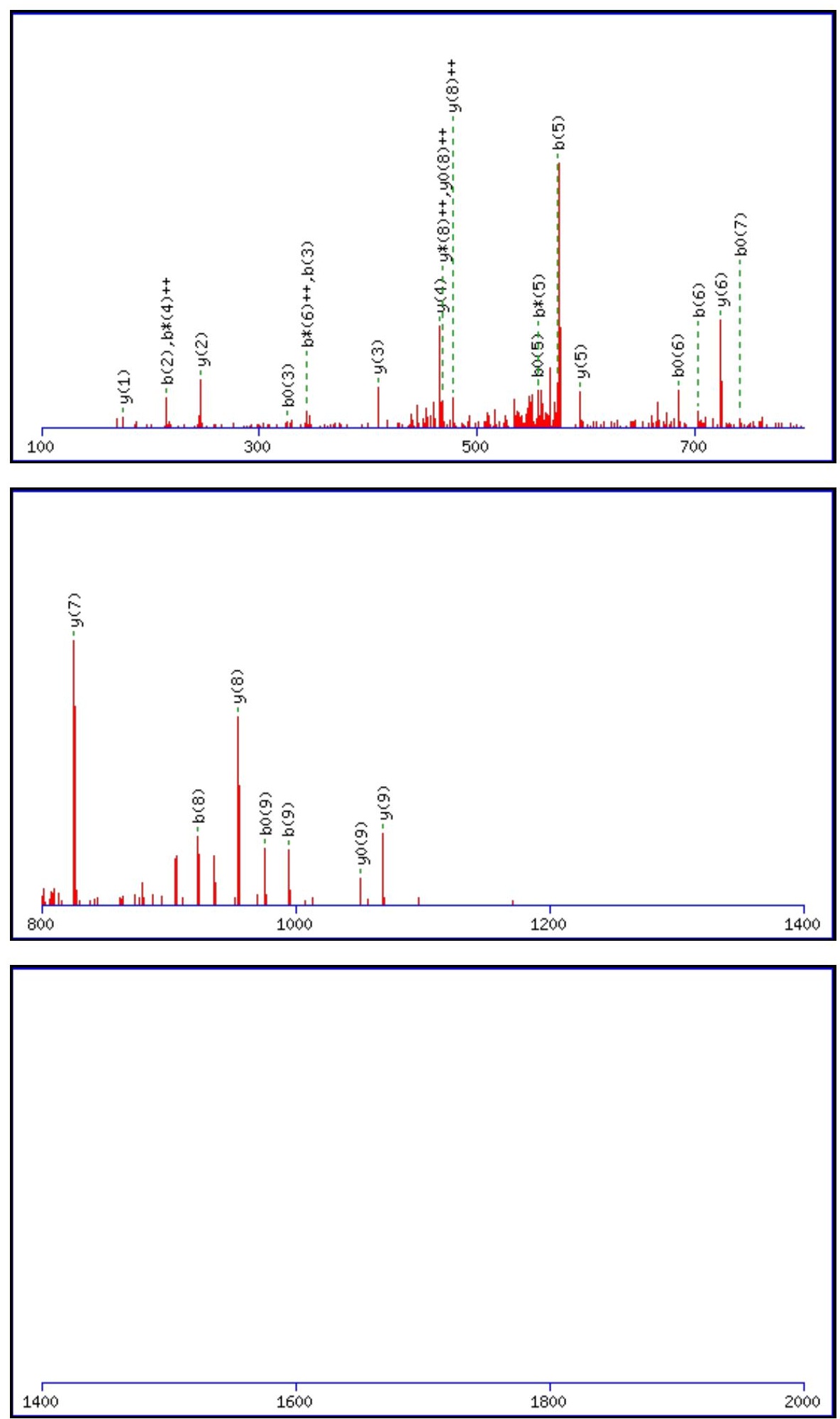

Monoisotopic mass of neutral peptide $\operatorname{Mr}($ calc): 1167.5044

Fixed modifications: Carbamidomethyl (C)

Variable modifications:

N2 : Deamidated N (N)

Ions Score: 69 Expect: $1.1 \mathrm{e}-005$

Matches (Bold Red): 27/94 fragment ions using 45 most intense peaks

\begin{tabular}{|c|c|c|c|c|c|c|c|c|c|c|c|c|c|c|}
\hline$\#$ & $\mathbf{b}$ & $\mathbf{b}^{++}$ & $\mathbf{b}^{*}$ & $\mathbf{b}^{\mathbf{*}^{++}}$ & $\mathbf{b}^{\mathbf{0}}$ & $\mathbf{b}^{\mathbf{0}+}$ & Seq. & $\mathbf{y}$ & $\mathbf{y}^{++}$ & $\mathbf{y}^{\mathbf{*}}$ & $\mathbf{y}^{\mathbf{*}^{++}}$ & $\mathbf{y}^{\mathbf{0}}$ & $\mathbf{y}^{\mathbf{0}+}$ & $\#$ \\
\hline $\mathbf{1}$ & 100.0757 & 50.5415 & & & & & $\mathbf{V}$ & & & & & & & $\mathbf{1 0}$ \\
\hline $\mathbf{2}$ & 215.1026 & 108.0550 & 198.0761 & 99.5417 & & & $\mathbf{N}$ & $\mathbf{1 0 6 9 . 4 4 3 3}$ & 535.2253 & 1052.4167 & 526.7120 & $\mathbf{1 0 5 1 . 4 3 2 7}$ & 526.2200 & $\mathbf{9}$ \\
\hline $\mathbf{3}$ & 344.1452 & 172.5762 & 327.1187 & 164.0630 & 326.1347 & 163.5710 & $\mathbf{E}$ & $\mathbf{9 5 4 . 4 1 6 3}$ & $\mathbf{4 7 7 . 7 1 1 8}$ & 937.3898 & $\mathbf{4 6 9 . 1 9 8 5}$ & 936.4058 & $\mathbf{4 6 8 . 7 0 6 5}$ & $\mathbf{8}$ \\
\hline
\end{tabular}




\begin{tabular}{|c|c|c|c|c|c|c|c|c|c|c|c|c|c|c|}
\hline 4 & 445.1929 & 223.1001 & |428.1663 & 214.5868 & |427.1823 & |214.0948 & $\mathbf{T}$ & 825.3737 & |413.1905| & 808.3472 & |404.6772 & 807.3632 & |404.1852 | & 7 \\
\hline 5 & 574.2355 & 287.6214 & 557.2089 & 279.1081 & 556.2249 & 278.6161 & $\mathbf{E}$ & 724.3260 & 362.6667 & 707.2995 & 354.1534 & 706.3155 & 353.6614 & 6 \\
\hline 6 & 703.2781 & 352.1427 & 686.2515 & 343.6294 & 685.2675 & |343.1374 & E & 595.2835 & 298.1454 & 578.2569 & 289.6321 & 577.2729 & 289.1401 & 5 \\
\hline 7 & 760.2995 & 380.6534 & 743.2730 & 372.1401 & 742.2890 & 371.6481 & G & 466.2409 & 233.6241 & 449.2143 & 225.1108 & & & 4 \\
\hline 8 & 923.3629 & 462.1851 & 906.3363 & 453.6718 & 905.3523 & 453.1798 & $\mathbf{Y}$ & 409.2194 & 205.1133 & 392.1928 & 196.6001 & & & 3 \\
\hline 9 & 994.4000 & 497.7036 & 977.3734 & 489.1904 & 976.3894 & 488.6984 & A & 246.1561 & 123.5817 & 229.1295 & 115.0684 & & & 2 \\
\hline 10 & & & & & & & $\mathbf{R}$ & 175.1190 & 88.0631 & 158.0924 & 79.5498 & & & 1 \\
\hline
\end{tabular}
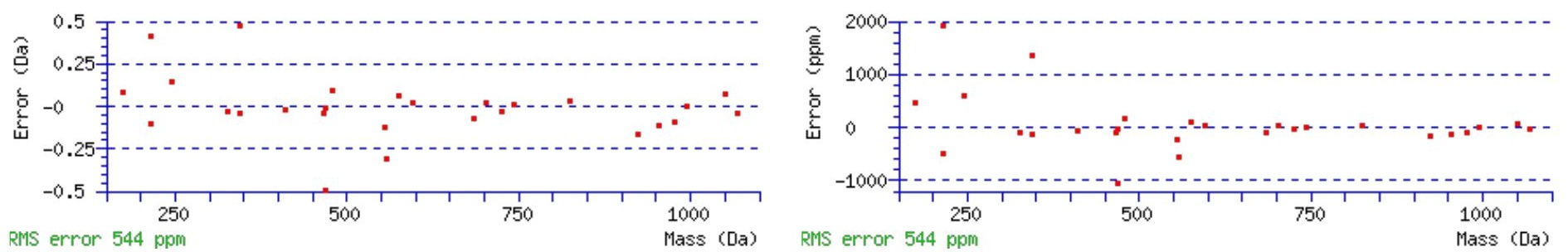

\section{All matches to this query}

\begin{tabular}{|l|l|l|l|}
\hline Score & Mr(calc): & Delta & \multicolumn{1}{|c|}{ Sequence } \\
\hline 69.2 & 1167.5044 & -0.0013 & VNETEEGYAR \\
\hline 11.1 & 1166.5043 & 0.9988 & DMTEVVHIK \\
\hline 10.1 & 1167.4921 & 0.0109 & AEAGVSEVGGGR \\
\hline 8.0 & 1166.4968 & 1.0062 & NVPDSAKEAR \\
\hline 6.0 & 1167.4921 & 0.0110 & QDVDNASLAR \\
\hline 5.9 & 1167.5033 & -0.0003 & ADGNQLRSAR \\
\hline 5.0 & 1166.4926 & 1.0104 & FLSHKFTK \\
\hline 5.0 & 1167.5107 & -0.0077 & QSIPATMNAR \\
\hline 4.7 & 1167.4951 & 0.0080 & ARTXTRTR \\
\hline 4.6 & 1167.4951 & 0.0080 & LTGRSRYR \\
\hline
\end{tabular}

Spectrum No: 374; Query: 419; Rank: 1

\section{Peptide View}

\section{MS/MS Fragmentation of DELADEIANSSGK}

Found in IPI00209113, Tax_Id=10116 Gene_Symbol=Myh9 Myosin-9

Match to Query 419: 1348.599388 from(675.306970,2+)

Title: 100101RatKid_NS_deglyco_21.1559.1559.2.dta

Data file K:INewmanPaper|Piliangl3SubProteomes\Piliang3SP\mgf5ppm\ERLIC_3SubProteomes5ppm.mgf 

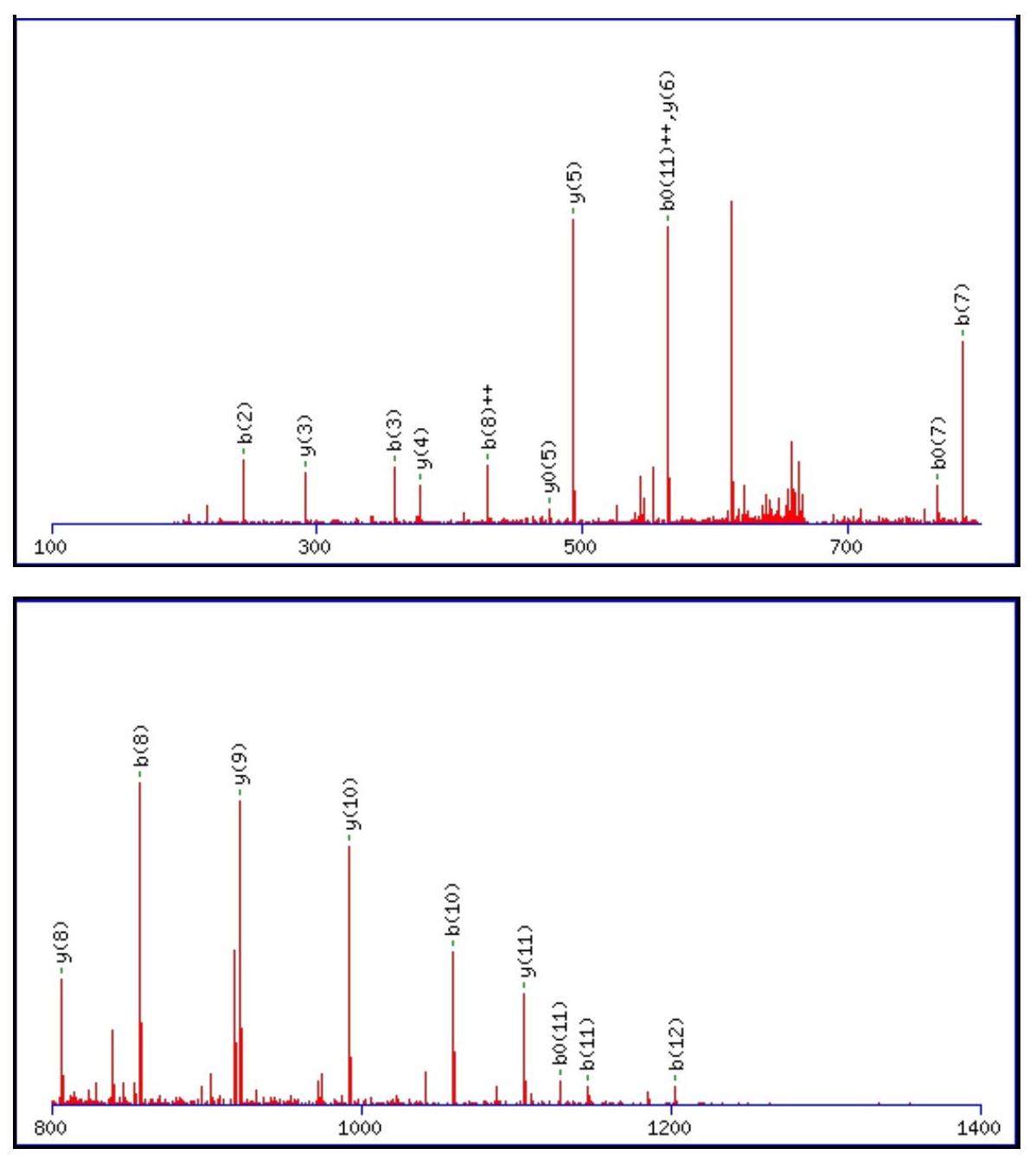

\begin{tabular}{rrrr}
\hline 1600 & 1600 & 1800 & 2000 \\
\hline
\end{tabular}

Monoisotopic mass of neutral peptide $\operatorname{Mr}($ calc): 1348.5994

Fixed modifications: Carbamidomethyl (C)

Variable modifications:

N9 : Deamidated $\mathrm{N}(\mathrm{N})$

Ions Score: 69 Expect: $1.6 \mathrm{e}-005$

Matches (Bold Red): 21/124 fragment ions using 31 most intense peaks

\begin{tabular}{|r|c|c|c|c|r|r|r|r|r|r|r|r|r|r|}
\hline$\#$ & $\mathbf{b}$ & $\mathbf{b}^{++}$ & $\mathbf{b}^{*}$ & $\mathbf{b}^{\boldsymbol{*}^{++}}$ & \multicolumn{1}{|c|}{$\mathbf{b}^{\mathbf{0}}$} & \multicolumn{1}{|c|}{$\mathbf{b}^{\mathbf{0 + +}}$} & Seq. & $\mathbf{y}$ & $\mathbf{y}^{++}$ & $\mathbf{y}^{\mathbf{*}}$ & $\mathbf{y}^{\boldsymbol{*}^{++}}$ & $\mathbf{y}^{\mathbf{0}}$ & $\mathbf{y}^{\mathbf{0 + +}}$ & $\#$ \\
\hline $\mathbf{1}$ & 116.0342 & 58.5207 & & & 98.0237 & 49.5155 & $\mathbf{D}$ & & & & & & & $\mathbf{1 3}$ \\
\hline $\mathbf{2}$ & $\mathbf{2 4 5 . 0 7 6 8}$ & 123.0420 & & & 227.0662 & 114.0368 & $\mathbf{E}$ & 1234.5797 & 617.7935 & 1217.5532 & 609.2802 & 1216.5692 & 608.7882 & $\mathbf{1 2}$ \\
\hline $\mathbf{3}$ & $\mathbf{3 5 8 . 1 6 0 9}$ & 179.5841 & & & 340.1503 & 170.5788 & $\mathbf{L}$ & $\mathbf{1 1 0 5 . 5 3 7 2}$ & 553.2722 & 1088.5106 & 544.7589 & 1087.5266 & 544.2669 & $\mathbf{1 1}$ \\
\hline $\mathbf{4}$ & $\mathbf{4 2 9 . 1 9 8 0}$ & 215.1026 & & & 411.1874 & 206.0974 & $\mathbf{A}$ & $\mathbf{9 9 2 . 4 5 3 1}$ & 496.7302 & 975.4265 & 488.2169 & 974.4425 & 487.7249 & $\mathbf{1 0}$ \\
\hline $\mathbf{5}$ & 544.2249 & 272.6161 & & & 526.2144 & 263.6108 & $\mathbf{D}$ & $\mathbf{9 2 1 . 4 1 6 0}$ & 461.2116 & 904.3894 & 452.6984 & 903.4054 & 452.2063 & $\mathbf{9}$ \\
\hline
\end{tabular}




\begin{tabular}{|c|c|c|c|c|c|c|c|c|c|c|c|c|c|c|}
\hline 6 & 673.2675 & |337.1374 & & & 655.2570 & |328.1321 & $\mathbf{E}$ & 806.3890 & |403.6982 & 789.3625 & |395.1849 & 788.3785 & | 394.6929 & 8 \\
\hline 7 & 786.3516 & 393.6794 & & & 768.3410 & 384.6742 & I & 677.3464 & 339.1769 & 660.3199 & 330.6636 & 659.3359 & 330.1716 & 7 \\
\hline 8 & 857.3887 & 429.1980 & & & 839.3781 & 420.1927 & A & 564.2624 & 282.6348 & 547.2358 & 274.1216 & 546.2518 & 273.6295 & 6 \\
\hline 9 & 972.4156 & 486.7115 & 955.3891 & 478.1982 & 954.4051 & 477.7062 & $\mathbf{N}$ & 493.2253 & 247.1163 & 476.1987 & 238.6030 & 475.2147 & 238.1110 & 5 \\
\hline 10 & 1059.4477 & 530.2275 & 1042.4211 & 521.7142 & 1041.4371 & 521.2222 & S & 378.1983 & 189.6028 & 361.1718 & 181.0895 & 360.1878 & 180.5975 & 4 \\
\hline 11 & 1146.4797 & 573.7435 & 1129.4531 & 565.2302 & 1128.4691 & 564.7382 & $\mathbf{S}$ & 291.1663 & 146.0868 & 274.1397 & 137.5735 & 273.1557 & 137.0815 & 3 \\
\hline 12 & 1203.5012 & 602.2542 & 1186.4746 & 593.7409 & 1185.4906 & 593.2489 & $\mathbf{G}$ & 204.1343 & 102.5708 & 187.1077 & 94.0575 & & & 2 \\
\hline 13 & & & & & & & $\mathbf{K}$ & 147.1128 & 74.0600 & 130.0863 & 65.5468 & & & 1 \\
\hline
\end{tabular}
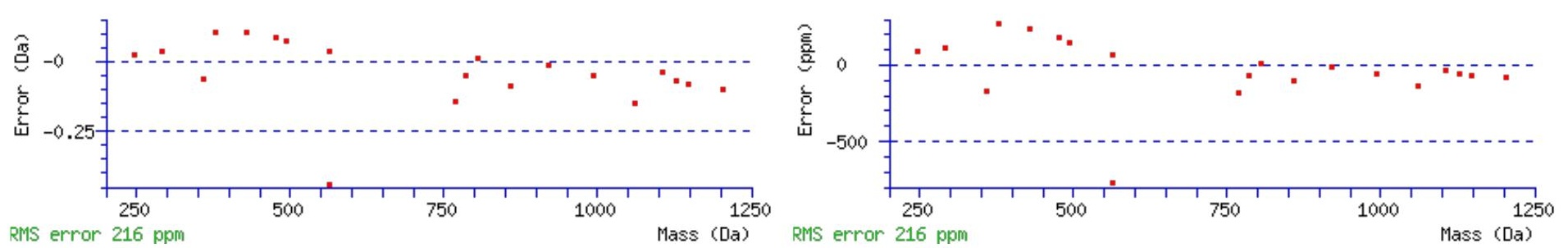

\section{All matches to this query}

\begin{tabular}{|l|l|l|l|}
\hline Score & Mr(calc): & Delta & \multicolumn{1}{|c|}{ Sequence } \\
\hline 69.2 & 1348.5994 & 0.0000 & DELADEIANSSGK \\
\hline 5.8 & 1347.6071 & 0.9923 & NELTEHAKTPK \\
\hline 5.0 & 1348.6064 & -0.0070 & $\underline{\text { LEDALYFDRK }}$ \\
\hline 4.5 & 1347.5894 & 1.0100 & LSFDKDAMVAR \\
\hline 3.3 & 1348.6097 & -0.0104 & MIYANSKNAIK \\
\hline 3.3 & 1348.6097 & -0.0104 & MIYANSKNAIK \\
\hline 2.4 & 1348.6024 & -0.0030 & ELVSDTNQHVK \\
\hline 2.2 & 1348.5958 & 0.0035 & VSMENRSYRK \\
\hline 1.7 & 1348.5920 & 0.0074 & LYKNCPKAMK \\
\hline 1.7 & 1348.6098 & -0.0104 & TASLFEQKSMK \\
\hline
\end{tabular}

Spectrum No: 375; Query: 941; Rank: 1

\section{Peptide View}

MS/MS Fragmentation of LATIANFSALGLEPGR

Found in IPI00199585, Tax_Id=10116 Gene_Symbol=Slc22a5 Organic cation/carnitine transporter 2

Match to Query 941: 1629.876588 from(815.945570,2+)

Title: 100101RatKid_NS_deglyco_23.4633.4633.2.dta

Data file K:INewmanPaper|Piliang|3SubProteomes\Piliang3SP\mgf5ppm\ERLIC_3SubProteomes5ppm.mgf 

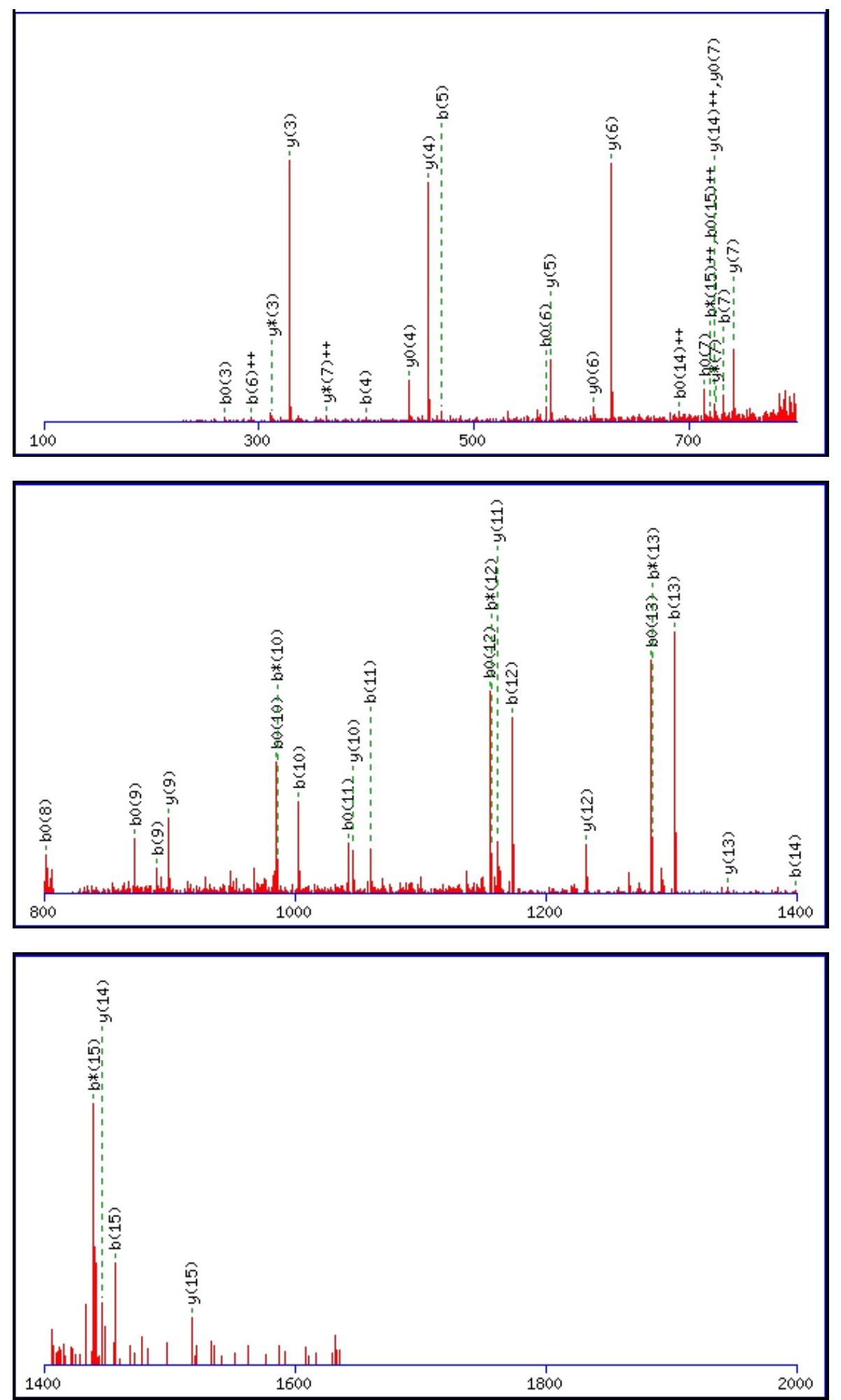

Monoisotopic mass of neutral peptide $\operatorname{Mr}($ calc): 1629.8726

Fixed modifications: Carbamidomethyl (C)

Variable modifications:

N6 Deamidated $\mathrm{N}(\mathrm{N})$

Ions Score: 69 Expect: $1.2 \mathrm{e}-005$

Matches (Bold Red): 46/160 fragment ions using 81 most intense peaks

\begin{tabular}{|c|c|c|c|c|c|c|c|c|c|c|c|c|c|c|}
\hline$\#$ & $\mathbf{b}$ & $\mathbf{b}^{++}$ & $\mathbf{b}^{*}$ & $\mathbf{b}^{\mathbf{*}^{++}}$ & $\mathbf{b}^{\mathbf{0}}$ & $\mathbf{b}^{\mathbf{0}+}$ & Seq. & $\mathbf{y}$ & $\mathbf{y}^{++}$ & $\mathbf{y}^{\mathbf{*}}$ & $\mathbf{y}^{\mathbf{*}^{++}}$ & $\mathbf{y}^{\mathbf{0}}$ & $\mathbf{y}^{\mathbf{0}++}$ & $\#$ \\
\hline $\mathbf{1}$ & 114.0913 & 57.5493 & & & & & $\mathbf{L}$ & & & & & & & $\mathbf{1 6}$ \\
\hline $\mathbf{2}$ & 185.1285 & 93.0679 & & & & & A & $\mathbf{1 5 1 7 . 7 9 5 8}$ & 759.4016 & 1500.7693 & $\mathbf{7 5 0 . 8 8 8 3}$ & 1499.7853 & 750.3963 & $\mathbf{1 5}$ \\
\hline $\mathbf{3}$ & 286.1761 & 143.5917 & & & $\mathbf{2 6 8 . 1 6 5 6}$ & 134.5864 & $\mathbf{T}$ & $\mathbf{1 4 4 6 . 7 5 8 7}$ & $\mathbf{7 2 3 . 8 8 3 0}$ & 1429.7322 & 715.3697 & 1428.7481 & 714.8777 & $\mathbf{1 4}$ \\
\hline $\mathbf{4}$ & $\mathbf{3 9 9 . 2 6 0 2}$ & 200.1337 & & & 381.2496 & 191.1285 & $\mathbf{I}$ & $\mathbf{1 3 4 5 . 7 1 1 0}$ & 673.3592 & 1328.6845 & 664.8459 & 1327.7005 & 664.3539 & $\mathbf{1 3}$ \\
\hline $\mathbf{5}$ & $\mathbf{4 7 0 . 2 9 7 3}$ & 235.6523 & & & 452.2867 & 226.6470 & $\mathbf{A}$ & $\mathbf{1 2 3 2 . 6 2 7 0}$ & 616.8171 & 1215.6004 & 608.3038 & 1214.6164 & 607.8118 & $\mathbf{1 2}$ \\
\hline
\end{tabular}




\begin{tabular}{|c|c|c|c|c|c|c|c|c|c|c|c|c|c|c|}
\hline 6 & 585.3242 & 293.1658 & 568.2977 & |284.6525 & 567.3137 & |284.1605 & $\mathbf{N}$ & 1161.5899 & |581.2986 & |1144.5633 & 572.7853 & 1143.5793 & |572.2933 & 11 \\
\hline 7 & 732.3927 & 366.7000 & 715.3661 & |358.1867 & 714.3821 & |357.6947 & $\mathbf{F}$ & 1046.5629 & 523.7851 & 1029.5364 & 515.2718 & 1028.5524 & 514.7798 & 10 \\
\hline 8 & 819.4247 & 410.2160 & 802.3981 & 401.7027 & 801.4141 & 401.2107 & $\mathrm{~S}$ & 899.4945 & 450.2509 & 882.4680 & 441.7376 & 881.4839 & 441.2456 & 9 \\
\hline 9 & 890.4618 & 445.7345 & 873.4353 & 437.2213 & 872.4512 & 436.7293 & A & 812.4625 & 406.7349 & 795.4359 & 398.2216 & 794.4519 & 397.7296 & 8 \\
\hline 10 & 1003.5459 & 502.2766 & 986.5193 & 493.7633 & 985.5353 & 493.2713 & L & 741.4254 & 371.2163 & 724.3988 & 362.7030 & 723.4148 & 362.2110 & 7 \\
\hline 11 & 1060.5673 & 530.7873 & 1043.5408 & 522.2740 & 1042.5568 & 521.7820 & $\mathbf{G}$ & 628.3413 & 314.6743 & 611.3148 & 306.1610 & 610.3307 & 305.6690 & 6 \\
\hline 12 & 1173.6514 & 587.3293 & 1156.6248 & 578.8161 & 1155.6408 & 578.3241 & $\mathbf{L}$ & 571.3198 & 286.1636 & 554.2933 & 277.6503 & 553.3093 & |277.1583 & 5 \\
\hline 13 & 1302.6940 & 651.8506 & 1285.6674 & 643.3374 & 1284.6834 & 642.8453 & $\mathbf{E}$ & 458.2358 & 229.6215 & 441.2092 & 221.1082 & 440.2252 & 220.6162 & 4 \\
\hline 14 & 1399.7468 & 700.3770 & 1382.7202 & 691.8637 & 1381.7362 & 691.3717 & $\mathbf{P}$ & 329.1932 & 165.1002 & 312.1666 & 156.5870 & & & 3 \\
\hline 15 & 1456.7682 & 728.8877 & 1439.7417 & 720.3745 & 1438.7577 & 719.8825 & G & 232.1404 & 116.5738 & 215.1139 & 108.0606 & & & 2 \\
\hline 16 & & & & & & & $\mathbf{R}$ & 175.1190 & 88.0631 & 158.0924 & 79.5498 & & & 1 \\
\hline
\end{tabular}
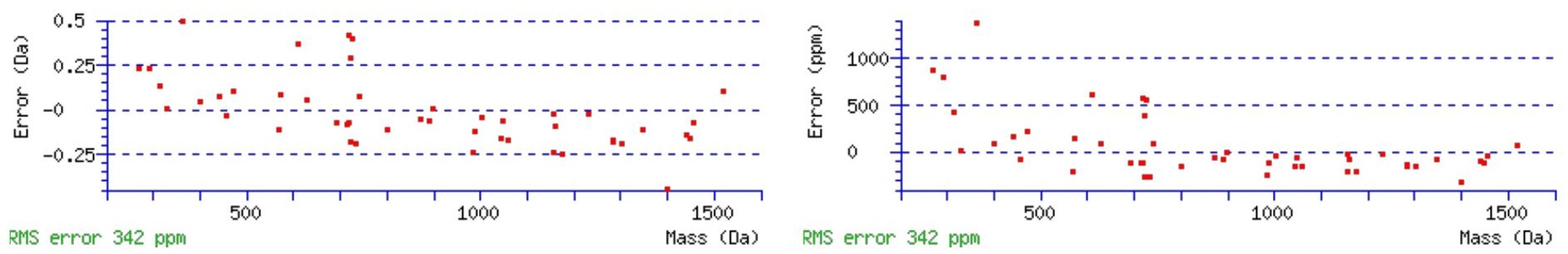

\section{All matches to this query}

\begin{tabular}{|l|l|l|l|}
\hline Score & Mr(calc): & Delta & \multicolumn{1}{|c|}{ Sequence } \\
\hline 69.1 & 1629.8726 & 0.0040 & LATIANFSALGLEPGR \\
\hline 20.0 & 1628.8886 & 0.9880 & LATIANFSALGLEPGR \\
\hline 7.1 & 1628.8733 & 1.0033 & ETEDLRKVALEAQK \\
\hline 5.5 & 1629.8620 & 0.0146 & EEAGALLSLCKRQR \\
\hline 5.1 & 1629.8798 & -0.0032 & ERNGTLISISQQKR \\
\hline 3.7 & 1629.8686 & 0.0080 & GKANSTGTLVITNPTR \\
\hline 1.7 & 1627.8828 & 1.9938 & TQASKQIMKHLTSR \\
\hline 1.1 & 1628.8725 & 1.0041 & YNVLTLVRMLSLK \\
\hline 0.7 & 1628.8872 & 0.9894 & NLTKDLLNELEVVK \\
\hline 0.7 & 1629.8630 & 0.0136 & YLTLLSDVLVVSTK \\
\hline
\end{tabular}

Spectrum No: 376; Query: 944; Rank: 1

\section{Peptide View}

MS/MS Fragmentation of CLVPHTVNLSSAWR

Found in IPI00199585, Tax_Id=10116 Gene_Symbol=Slc22a5 Organic cation/carnitine transporter 2

Match to Query 944: 1639.814982 from(547.612270,3+)

Title: 100101RatKid_NS_deglyco_25.3179.3179.3.dta

Data file K:INewmanPaper|Piliang|3SubProteomes\Piliang3SP\mgf5ppm\ERLIC_3SubProteomes5ppm.mgf 

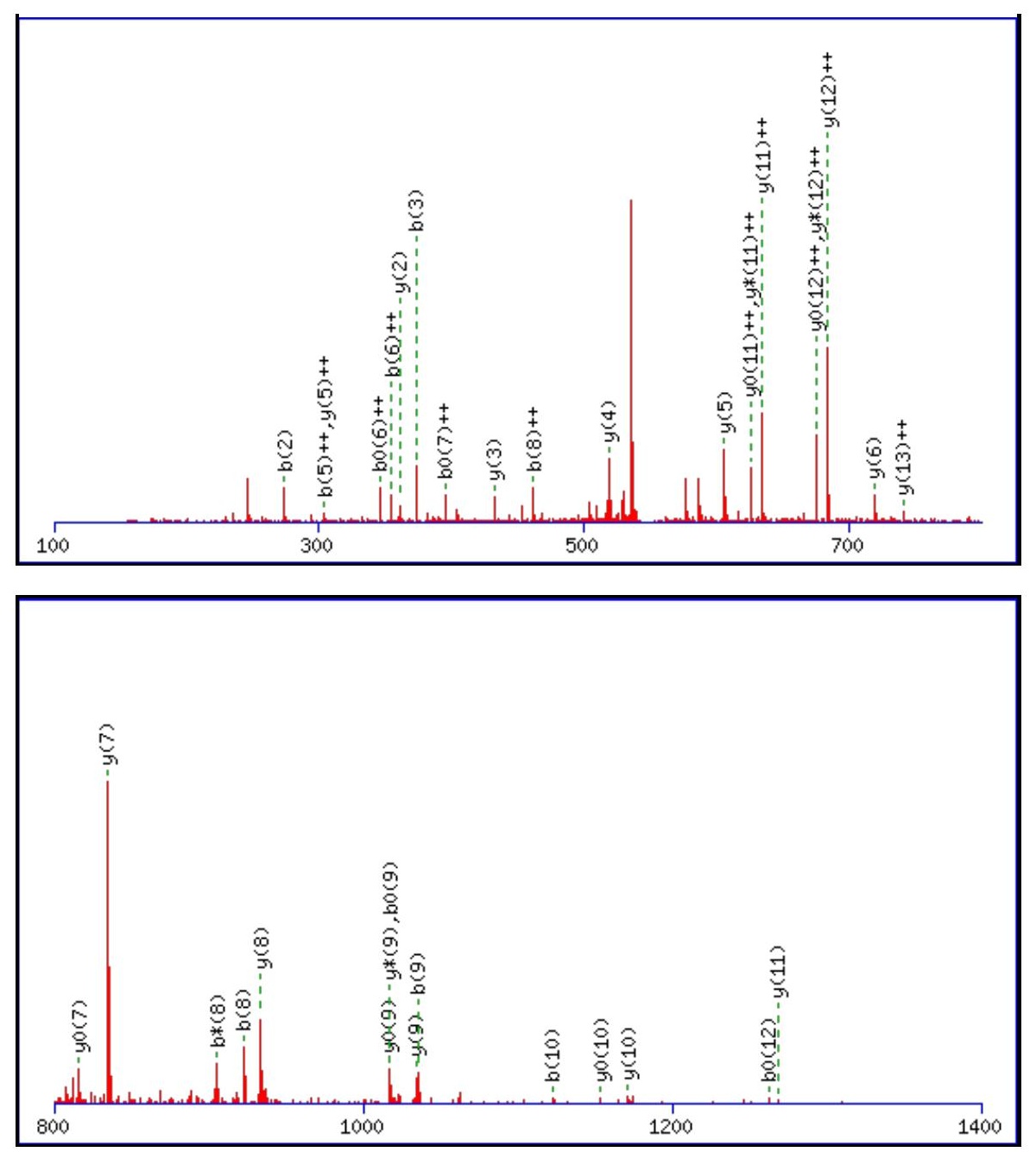

\begin{tabular}{rrrr}
\hline 1600 & 1600 & 1800 & 2000 \\
\hline
\end{tabular}

Monoisotopic mass of neutral peptide $\operatorname{Mr}($ calc): 1639.8141

Fixed modifications: Carbamidomethyl (C)

Variable modifications:

N8 : Deamidated_N (N)

Ions Score: 69 Expect: $2.1 \mathrm{e}-005$

Matches (Bold Red): 35/126 fragment ions using 50 most intense peaks

\begin{tabular}{|r|c|c|c|c|c|c|c|c|c|c|c|c|c|c|}
\hline$\#$ & $\mathbf{b}$ & $\mathbf{b}^{++}$ & $\mathbf{b}^{*}$ & $\mathbf{b}^{\mathbf{*}^{++}}$ & $\mathbf{b}^{\mathbf{0}}$ & $\mathbf{b}^{\mathbf{0 + +}}$ & Seq. & $\mathbf{y}$ & $\mathbf{y}^{++}$ & $\mathbf{y}^{\mathbf{*}}$ & $\mathbf{y}^{\boldsymbol{*}^{++}}$ & $\mathbf{y}^{\mathbf{0}}$ & $\mathbf{y}^{\mathbf{0 + +}}$ & $\#$ \\
\hline $\mathbf{1}$ & 161.0379 & 81.0226 & & & & & $\mathbf{C}$ & & & & & & & $\mathbf{1 4}$ \\
\hline $\mathbf{2}$ & $\mathbf{2 7 4 . 1 2 2 0}$ & 137.5646 & & & & & $\mathbf{L}$ & 1480.7907 & 740.8990 & 1463.7641 & 732.3857 & 1462.7801 & 731.8937 & $\mathbf{1 3}$ \\
\hline 3 & 373.1904 & 187.0988 & & & & & $\mathbf{V}$ & 1367.7066 & $\mathbf{6 8 4 . 3 5 6 9}$ & 1350.6801 & $\mathbf{6 7 5 . 8 4 3 7}$ & 1349.6961 & $\mathbf{6 7 5 . 3 5 1 7}$ & $\mathbf{1 2}$ \\
\hline $\mathbf{4}$ & 470.2432 & 235.6252 & & & & & $\mathbf{P}$ & $\mathbf{1 2 6 8 . 6 3 8 2}$ & $\mathbf{6 3 4 . 8 2 2 7}$ & 1251.6117 & $\mathbf{6 2 6 . 3 0 9 5}$ & 1250.6276 & $\mathbf{6 2 5 . 8 1 7 5}$ & $\mathbf{1 1}$ \\
\hline $\mathbf{5}$ & 607.3021 & $\mathbf{3 0 4 . 1 5 4 7}$ & & & & & $\mathbf{H}$ & $\mathbf{1 1 7 1 . 5 8 5 4}$ & 586.2964 & 1154.5589 & 577.7831 & $\mathbf{1 1 5 3 . 5 7 4 9}$ & 577.2911 & $\mathbf{1 0}$ \\
\hline
\end{tabular}




\begin{tabular}{|c|c|c|c|c|c|c|c|c|c|c|c|c|c|c|}
\hline 6 & 708.3498 & 354.6785 & & & 690.3392 & |345.6732 & $\mathbf{T}$ & |1034.5265 & |517.7669 & 1017.5000 & 509.2536 & 1016.5160 & 508.7616 & 9 \\
\hline 7 & 807.4182 & 404.2127 & & & 789.4076 & 395.2074 & V & 933.4788 & 467.2431 & 916.4523 & 458.7298 & 915.4683 & 458.2378 & 8 \\
\hline 8 & 922.4451 & 461.7262 & 905.4186 & 453.2129 & 904.4345 & 452.7209 & $\mathbf{N}$ & 834.4104 & 417.7089 & 817.3839 & 409.1956 & 816.3999 & 408.7036 & 7 \\
\hline 9 & 1035.5292 & 518.2682 & 1018.5026 & 509.7549 & 1017.5186 & 509.2629 & $\mathbf{L}$ & 719.3835 & 360.1954 & 702.3570 & 351.6821 & 701.3729 & 351.1901 & 6 \\
\hline 10 & 1122.5612 & 561.7842 & 1105.5347 & 553.2710 & 1104.5506 & 552.7790 & S & 606.2994 & 303.6534 & 589.2729 & 295.1401 & 588.2889 & 294.6481 & 5 \\
\hline 11 & 1209.5932 & 605.3003 & 1192.5667 & 596.7870 & 1191.5827 & 596.2950 & $S$ & 519.2674 & 260.1373 & 502.2409 & 251.6241 & 501.2568 & 251.1321 & 4 \\
\hline 12 & 1280.6303 & 640.8188 & 1263.6038 & 632.3055 & 1262.6198 & 631.8135 & A & 432.2354 & 216.6213 & 415.2088 & 208.1081 & & & 3 \\
\hline 13 & 1466.7097 & 733.8585 & 1449.6831 & 725.3452 & 1448.6991 & 724.8532 & W & 361.1983 & 181.1028 & 344.1717 & 172.5895 & & & 2 \\
\hline 14 & & & & & & & $\mathbf{R}$ & 175.1190 & 88.0631 & 158.0924 & 79.5498 & & & 1 \\
\hline
\end{tabular}
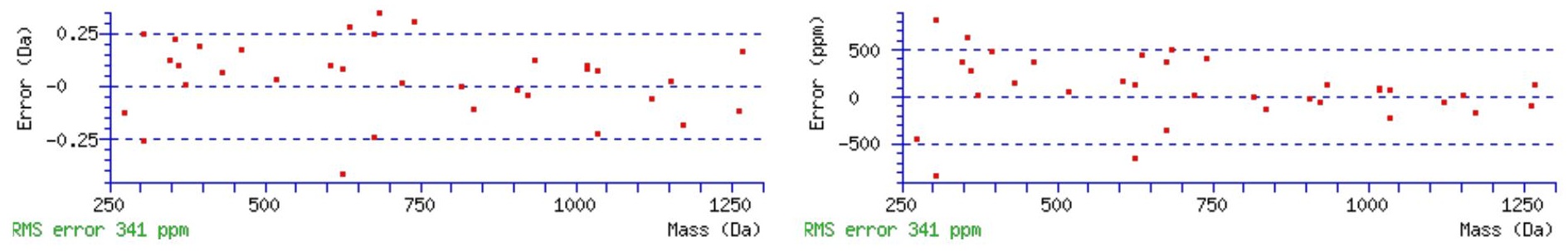

\section{All matches to this query}

\begin{tabular}{|l|l|l|l|}
\hline Score & Mr(calc): & Delta & \multicolumn{1}{|c|}{ Sequence } \\
\hline 69.0 & 1639.8141 & 0.0009 & $\underline{\text { CLVPHTVNLSSAWR }}$ \\
\hline 5.7 & 1637.8039 & 2.0111 & $\underline{\text { SFSOSSILIQHRR }}$ \\
\hline 5.7 & 1638.7978 & 1.0172 & DQINIETKNKTVR \\
\hline 5.7 & 1639.8294 & -0.0145 & GTDLSLASSVVLRSR \\
\hline 5.7 & 1639.8294 & -0.0145 & GTDLSLASSVVLRSR \\
\hline 4.8 & 1639.8004 & 0.0146 & TCKDGATELLLLAR \\
\hline 3.5 & 1638.8277 & 0.9873 & MTGRGKTKPLNTVR \\
\hline 3.3 & 1639.7988 & 0.0162 & AQDCISQGPLPTPTR \\
\hline 2.5 & 1638.8076 & 1.0074 & CNPYVKTYLLPDR \\
\hline 2.4 & 1639.8239 & -0.0090 & VKGVAYTNTTMLGER \\
\hline
\end{tabular}

Spectrum No: 377; Query: 2165; Rank: 1

\section{Peptide View}

MS/MS Fragmentation of YRAEYAVANDTGFVDIPQQEK

Found in IPI00326070, Tax_Id=10116 Gene_Symbol=Ctsl Cathepsin L1 precursor

Match to Query 2165: 2414.146062 from(805.722630,3+)

Title: 100101RatKid_NS_deglyco_14.3110.3110.3.dta

Data file K:\NewmanPaper|Piliangl3SubProteomes\Piliang3SP\mgf5ppm\ERLIC_3SubProteomes5ppm.mgf 

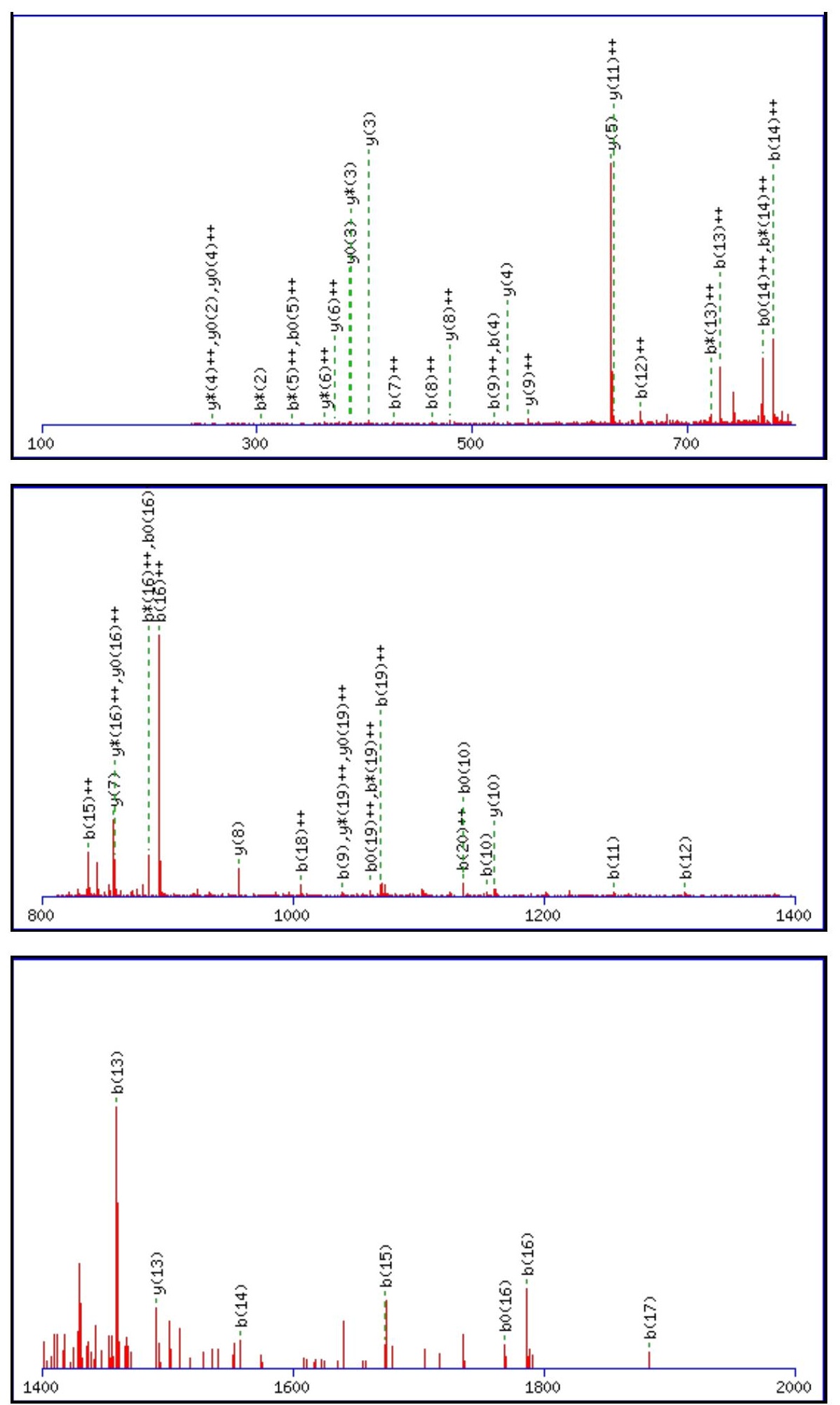

Monoisotopic mass of neutral peptide $\operatorname{Mr}($ calc): 2414.1390

Fixed modifications: Carbamidomethyl (C)

Variable modifications:

N9 : Deamidated $\mathrm{N}(\mathrm{N})$

Ions Score: 69 Expect: $3.7 \mathrm{e}-005$

Matches (Bold Red): 54/230 fragment ions using 75 most intense peaks

\begin{tabular}{|r|c|c|c|c|c|c|c|c|c|c|c|c|c|c|}
\hline$\#$ & $\mathbf{b}$ & $\mathbf{b}^{++}$ & $\mathbf{b}^{*}$ & $\mathbf{b}^{\boldsymbol{*}^{++}}$ & $\mathbf{b}^{\mathbf{0}}$ & $\mathbf{b}^{\mathbf{0 + +}}$ & $\mathbf{S e q}$ & $\mathbf{y}$ & $\mathbf{y}^{++}$ & $\mathbf{y}^{\mathbf{*}}$ & $\mathbf{y}^{\mathbf{*}^{++}}$ & $\mathbf{y}^{\mathbf{0}}$ & $\mathbf{y}^{\mathbf{0 + +}}$ & $\#$ \\
\hline $\mathbf{1}$ & 164.0706 & 82.5389 & & & & & $\mathbf{Y}$ & & & & & & & $\mathbf{2 1}$ \\
\hline $\mathbf{2}$ & 320.1717 & 160.5895 & 303.1452 & 152.0762 & & & $\mathbf{R}$ & 2252.0830 & 1126.5451 & 2235.0564 & 1118.0318 & 2234.0724 & 1117.5398 & $\mathbf{2 0}$ \\
\hline $\mathbf{3}$ & 391.2088 & 196.1081 & 374.1823 & 187.5948 & & & $\mathbf{A}$ & 2095.9819 & 1048.4946 & 2078.9553 & $\mathbf{1 0 3 9 . 9 8 1 3}$ & 2077.9713 & $\mathbf{1 0 3 9 . 4 8 9 3}$ & $\mathbf{1 9}$ \\
\hline $\mathbf{4}$ & $\mathbf{5 2 0 . 2 5 1 4}$ & 260.6293 & 503.2249 & 252.1161 & 502.2409 & 251.6241 & $\mathbf{E}$ & 2024.9447 & 1012.9760 & 2007.9182 & 1004.4627 & 2006.9342 & 1003.9707 & $\mathbf{1 8}$ \\
\hline $\mathbf{5}$ & 683.3148 & 342.1610 & 666.2882 & 333.6477 & 665.3042 & 333.1557 & $\mathbf{Y}$ & 1895.9021 & 948.4547 & 1878.8756 & 939.9414 & 1877.8916 & 939.4494 & $\mathbf{1 7}$ \\
\hline
\end{tabular}




\begin{tabular}{|c|c|c|c|c|c|c|c|c|c|c|c|c|c|c|}
\hline 6 & 754.3519 & 377.6796 & 737.3253 & 369.1663 & 736.3413 & 368.6743 & $\mathbf{A}$ & 1732.8388 & 866.9230 & |1715.8123 & 858.4098 & |1714.8283 & 857.9178 & $\mid 16$ \\
\hline 7 & 853.4203 & 427.2138 & 836.3937 & 418.7005 & 835.4097 & 418.2085 & $\mathbf{V}$ & 1661.8017 & 831.4045 & 1644.7752 & 822.8912 & 1643.7911 & 822.3992 & 15 \\
\hline 8 & 924.4574 & 462.7323 & 907.4308 & 454.2191 & 906.4468 & 453.7271 & A & 1562.7333 & 781.8703 & 1545.7067 & 773.3570 & 1544.7227 & 772.8650 & 4 \\
\hline 9 & 1039.4843 & 520.2458 & 1022.4578 & 511.7325 & 1021.4738 & 511.2405 & $\mathbf{N}$ & 1491.6962 & 746.3517 & 1474.6696 & 737.8385 & 1473.6856 & 737.3464 & 13 \\
\hline 10 & 1154.5113 & 577.7593 & 1137.4847 & 569.2460 & 1136.5007 & 568.7540 & D & 1376.6692 & 688.8383 & 1359.6427 & 680.3250 & 1358.6587 & 679.8330 & 12 \\
\hline 11 & 1255.5590 & 628.2831 & 1238.5324 & 619.7698 & 1237.5484 & 619.2778 & $\mathbf{T}$ & 1261.6423 & 631.3248 & 1244.6157 & 622.8115 & 1243.6317 & 622.3195 & 11 \\
\hline 12 & 1312.5804 & 656.7938 & 1295.5539 & 648.2806 & 1294.5699 & 647.7886 & G & 1160.5946 & 580.8009 & 1143.5681 & 572.2877 & 1142.5841 & 571.7957 & 10 \\
\hline 13 & 1459.6488 & 730.3281 & 1442.6223 & 721.8148 & 1441.6383 & 721.3228 & $\mathbf{F}$ & 1103.5732 & 552.2902 & 1086.5466 & 543.7769 & 1085.5626 & 543.2849 & 9 \\
\hline 14 & 1558.7172 & 779.8623 & 1541.6907 & 771.3490 & 1540.7067 & 770.8570 & V & 956.5047 & 478.7560 & 939.4782 & 470.2427 & 938.4942 & 469.7507 & 8 \\
\hline 15 & 1673.7442 & 837.3757 & 1656.7176 & 828.8625 & 1655.7336 & 828.3704 & D & 857.4363 & 429.2218 & 840.4098 & 420.7085 & 839.4258 & 420.2165 & 7 \\
\hline 16 & 1786.8283 & 893.9178 & 1769.8017 & 885.4045 & 1768.8177 & 884.9125 & I & 742.4094 & 371.7083 & 725.3828 & 363.1951 & 724.3988 & 362.7030 & 6 \\
\hline 17 & 1883.8810 & 942.4441 & 1866.8545 & 933.9309 & 1865.8705 & 933.4389 & $\mathbf{P}$ & 629.3253 & 315.1663 & 612.2988 & 306.6530 & 611.3148 & 306.1610 & 5 \\
\hline 18 & 2011.9396 & 1006.4734 & 1994.9130 & 997.9602 & 1993.9290 & 997.4682 & $\mathbf{Q}$ & 532.2726 & 266.6399 & 515.2460 & 258.1266 & 514.2620 & 257.6346 & 4 \\
\hline 19 & 2139.9982 & 1070.5027 & 2122.9716 & 1061.9894 & 2121.9876 & 1061.4974 & $\mathbf{Q}$ & 404.2140 & 202.6106 & 387.1874 & 194.0974 & 386.2034 & 193.6053 & 3 \\
\hline 21 & & & & & & & $\mathbf{K}$ & 147.1128 & 74.0600 & 130.0863 & 65.5468 & & & 1 \\
\hline
\end{tabular}
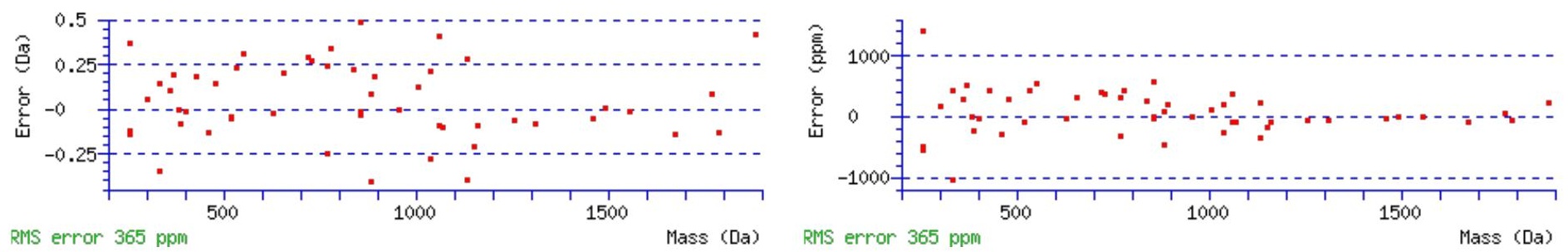

\section{All matches to this query}

\begin{tabular}{|l|l|l|l|}
\hline Score & Mr(calc): & Delta & \multicolumn{1}{|c|}{ Sequence } \\
\hline 68.9 & 2414.1390 & 0.0071 & YRAEYAVANDTGFVDIPQQEK \\
\hline 12.3 & 2413.1550 & 0.9911 & YRAEYAVANDTGFVDIPQQEK \\
\hline 5.8 & 2414.1631 & -0.0170 & HPGSNSLSGPVKALIAQEWSEK \\
\hline 5.1 & 2414.1284 & 0.0177 & YQANLEITGPKVASPGPQEKK \\
\hline 5.1 & 2414.1284 & 0.0177 & YQANLEITGPKVASPGPQEKK \\
\hline 4.1 & 2412.1488 & 1.9973 & TAQPRHHQDPYKAGLSHYVK \\
\hline 3.9 & 2414.1454 & 0.0007 & GVTILSGQPACGLSLQPEGHWK \\
\hline 3.4 & 2414.1375 & 0.0086 & CQPRLMYPAKLSINIDGETK \\
\hline 3.2 & 2414.1495 & -0.0034 & LNSPTDSTPALLSSAVTPRKAK \\
\hline 3.2 & 2414.1375 & 0.0086 & COPRLLYPAKLSMNIDGETK \\
\hline
\end{tabular}

Spectrum No: 378; Query: 767; Rank: 1

\section{Peptide View}

MS/MS Fragmentation of QIDYILGQYNTTK

Found in IPI00325312, Tax_Id=10116 Gene_Symbol=Prom1 prominin 1 isoform 1

Match to Query 767: 1556.775188 from(779.394870,2+)

Title: 100101RatKid_NS_deglyco_25.2958.2958.2.dta

Data file K:INewmanPaper|Piliangl3SubProteomes\Piliang3SP\mgf5ppm|ERLIC_3SubProteomes5ppm.mgf 

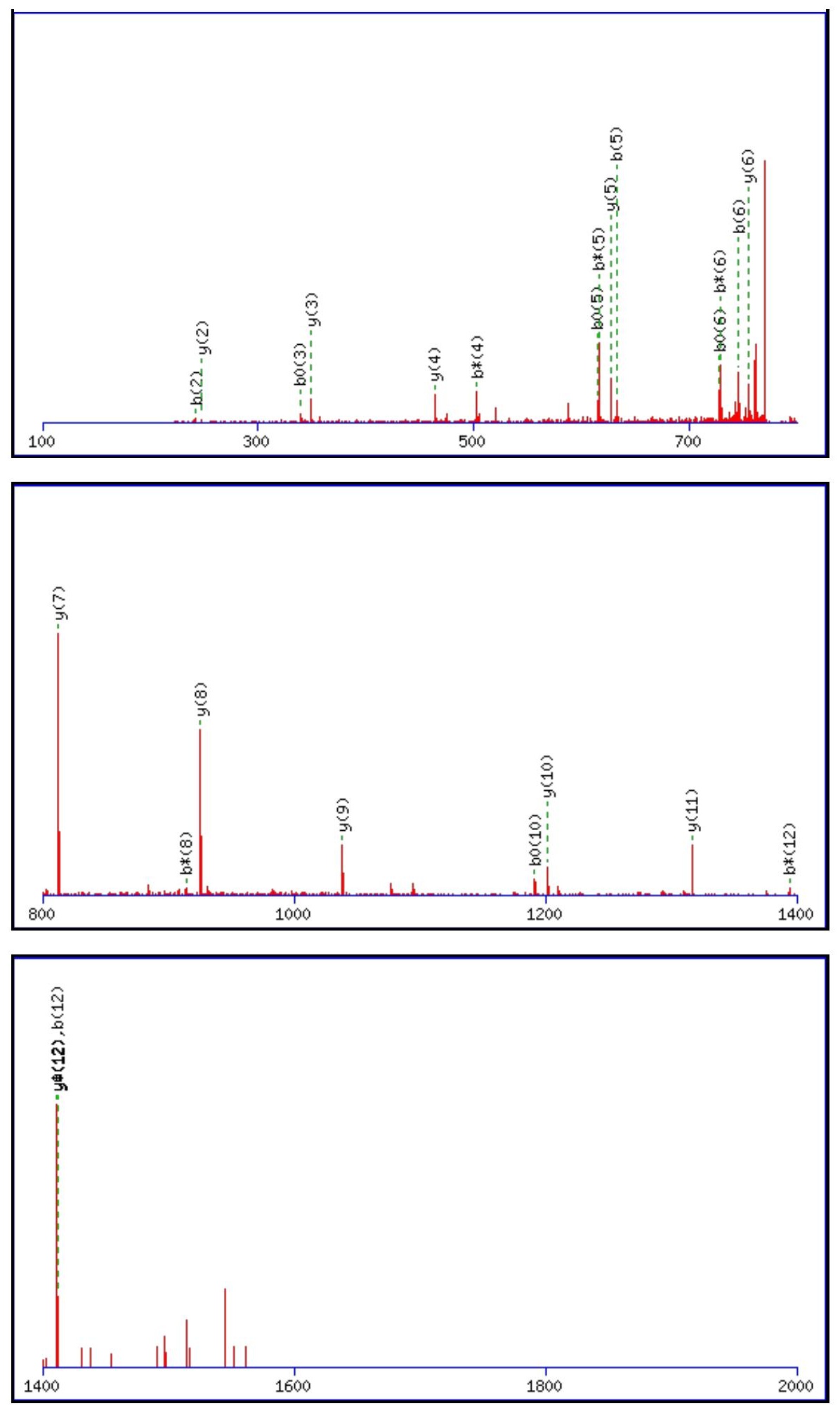

Monoisotopic mass of neutral peptide $\operatorname{Mr}($ calc): 1556.7722

Fixed modifications: Carbamidomethyl (C)

Variable modifications:

N10 : Deamidated $\mathrm{N}(\mathrm{N})$

Ions Score: 69 Expect: $2.2 \mathrm{e}-005$

Matches (Bold Red): 25/138 fragment ions using 37 most intense peaks

\begin{tabular}{|r|c|c|c|c|c|c|c|c|c|c|c|c|c|c|}
\hline$\#$ & $\mathbf{b}$ & $\mathbf{b}^{++}$ & $\mathbf{b}^{*}$ & $\mathbf{b}^{\boldsymbol{*}_{++}}$ & $\mathbf{b}^{\mathbf{0}}$ & $\mathbf{b}^{\mathbf{0 + +}}$ & $\mathbf{S e q}$ & $\mathbf{y}$ & $\mathbf{y}^{++}$ & $\mathbf{y}^{\mathbf{*}}$ & $\mathbf{y}^{\boldsymbol{*}^{++}}$ & $\mathbf{y}^{\mathbf{0}}$ & $\mathbf{y}^{\mathbf{0 + +}}$ & $\#$ \\
\hline $\mathbf{1}$ & 129.0659 & 65.0366 & 112.0393 & 56.5233 & & & $\mathbf{Q}$ & & & & & & & $\mathbf{1 3}$ \\
\hline $\mathbf{2}$ & $\mathbf{2 4 2 . 1 4 9 9}$ & 121.5786 & 225.1234 & 113.0653 & & & $\mathbf{I}$ & 1429.7209 & 715.3641 & $\mathbf{1 4 1 2 . 6 9 4 4}$ & 706.8508 & $\mathbf{1 4 1 1 . 7 1 0 4}$ & $\mathbf{7 0 6 . 3 5 8 8}$ & $\mathbf{1 2}$ \\
\hline $\mathbf{3}$ & 357.1769 & 179.0921 & 340.1503 & 170.5788 & 339.1663 & 170.0868 & $\mathbf{D}$ & $\mathbf{1 3 1 6 . 6 3 6 9}$ & 658.8221 & 1299.6103 & 650.3088 & 1298.6263 & 649.8168 & $\mathbf{1 1}$ \\
\hline $\mathbf{4}$ & 520.2402 & 260.6237 & 503.2136 & 252.1105 & 502.2296 & 251.6185 & $\mathbf{Y}$ & $\mathbf{1 2 0 1 . 6 0 9 9}$ & 601.3086 & 1184.5834 & 592.7953 & 1183.5994 & 592.3033 & $\mathbf{1 0}$ \\
\hline $\mathbf{5}$ & $\mathbf{6 3 3 . 3 2 4 3}$ & 317.1658 & $\mathbf{6 1 6 . 2 9 7 7}$ & 308.6525 & $\mathbf{6 1 5 . 3 1 3 7}$ & 308.1605 & $\mathbf{I}$ & $\mathbf{1 0 3 8 . 5 4 6 6}$ & 519.7769 & 1021.5200 & 511.2637 & 1020.5360 & 510.7717 & $\mathbf{9}$ \\
\hline
\end{tabular}




\begin{tabular}{|c|c|c|c|c|c|c|c|c|c|c|c|c|c|c|}
\hline 6 & 746.4083 & |373.7078| & 729.3818 & |365.1945 & 728.3978 & |364.7025| & $\mathbf{L}$ & 925.4625 & |463.2349| & 908.4360 & |454.7216 & 907.4520 & |454.2296| & 8 \\
\hline 7 & 803.4298 & 402.2185 & 786.4032 & 393.7053 & 785.4192 & 393.2132 & $\mathbf{G}$ & 812.3785 & 406.6929 & 795.3519 & 398.1796 & 794.3679 & 397.6876 & 7 \\
\hline 8 & 931.4884 & 466.2478 & 914.4618 & 457.7345 & 913.4778 & 457.2425 & $\mathbf{Q}$ & 755.3570 & 378.1821 & 738.3305 & 369.6689 & 737.3464 & 369.1769 & 6 \\
\hline 9 & 1094.5517 & 547.7795 & 1077.5251 & 539.2662 & 1076.5411 & 538.7742 & $\mathbf{Y}$ & 627.2984 & 314.1529 & 610.2719 & 305.6396 & 609.2879 & 305.1476 & 5 \\
\hline 10 & 1209.5786 & 605.2930 & 1192.5521 & 596.7797 & 1191.5681 & 596.2877 & $\mathbf{N}$ & 464.2351 & 232.6212 & 447.2085 & 224.1079 & 446.2245 & 223.6159 & 4 \\
\hline 11 & 1310.6263 & 655.8168 & 1293.5998 & 647.3035 & 1292.6157 & 646.8115 & $\mathbf{T}$ & 349.2082 & 175.1077 & 332.1816 & 166.5944 & 331.1976 & 166.1024 & 3 \\
\hline 12 & 1411.6740 & 706.3406 & 1394.6474 & 697.8274 & 1393.6634 & 697.3353 & $\mathbf{T}$ & 248.1605 & 124.5839 & 231.1339 & 116.0706 & 230.1499 & 115.5786 & 2 \\
\hline 13 & & & & & & & $\mathbf{K}$ & 147.1128 & 74.0600 & 130.0863 & 65.5468 & & & 1 \\
\hline
\end{tabular}
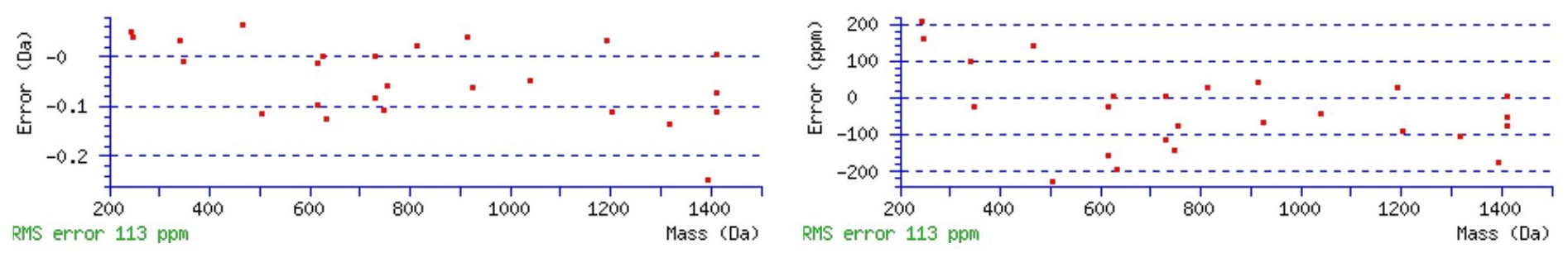

\section{All matches to this query}

\begin{tabular}{|l|l|c|l|}
\hline Score & Mr(calc): & Delta & \multicolumn{1}{|c|}{ Sequence } \\
\hline 68.8 & 1556.7722 & 0.0030 & QIDYILGOYNTTK \\
\hline 6.5 & 1556.7712 & 0.0040 & DSRGHLGLVANPLK \\
\hline 4.6 & 1556.7712 & 0.0040 & IKYDAIARQGQSK \\
\hline 3.8 & 1554.7712 & 2.0040 & MAPPSEETPLISQR \\
\hline 2.9 & 1556.7786 & -0.0034 & GPLAVLTCIATHPK \\
\hline 2.8 & 1556.7699 & 0.0053 & TDLTSVLKGNLSTK \\
\hline 2.3 & 1554.7663 & 2.0089 & RMETKPVITCLK \\
\hline 1.8 & 1555.7647 & 1.0105 & ADSFLLERTLPSK \\
\hline 1.8 & 1555.7647 & 1.0105 & ADSFLLERTLPSK \\
\hline 1.0 & 1556.7738 & 0.0014 & ELLNIDLIKDYK \\
\hline
\end{tabular}

Spectrum No: 379; Query: 422; Rank: 1

\section{Peptide View}

MS/MS Fragmentation of GVAVTNTSQLGFR

Found in IPI00192845, Tax_Id=10116 Gene_Symbol=P2rx4 40 kDa protein

Match to Query 422: 1349.694888 from(675.854720,2+)

Title: 100101RatKid_NS_deglyco_21.2369.2369.2.dta

Data file K:INewmanPaper|Piliang|3SubProteomes\Piliang3SP\mgf5ppm\ERLIC_3SubProteomes5ppm.mgf 

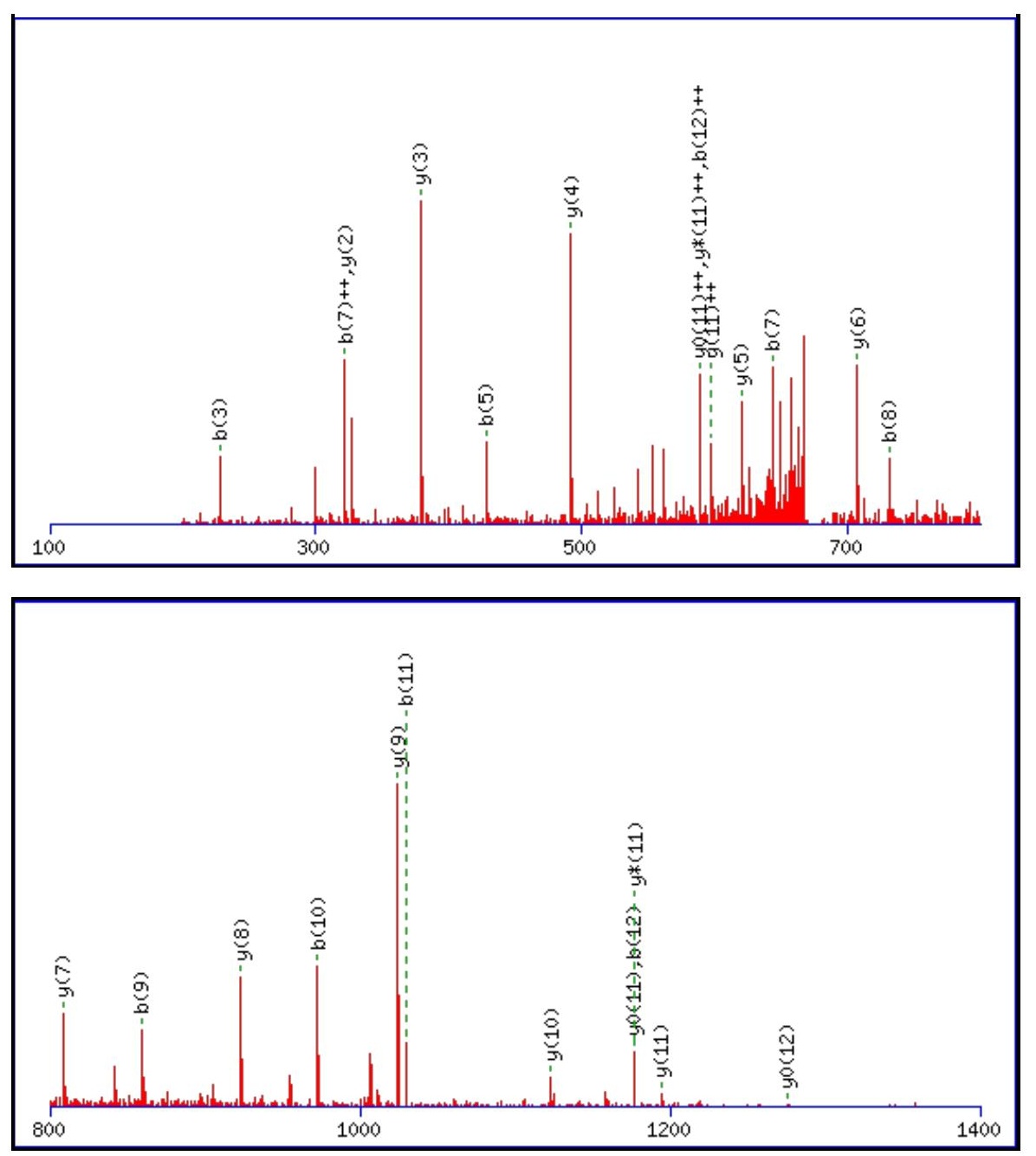

\begin{tabular}{rrrr}
1400 & 1600 & 1800 & 2000 \\
\hline
\end{tabular}

Monoisotopic mass of neutral peptide $\operatorname{Mr}($ calc): 1349.6939

Fixed modifications: Carbamidomethyl (C)

Variable modifications:

N6 : Deamidated $\mathrm{N}(\mathrm{N})$

Ions Score: 69 Expect: $1.7 \mathrm{e}-005$

Matches (Bold Red): 26/116 fragment ions using 38 most intense peaks

\begin{tabular}{|r|r|r|c|c|c|c|c|c|c|c|c|c|c|c|}
\hline$\#$ & $\mathbf{b}$ & $\mathbf{b}^{++}$ & $\mathbf{b}^{*}$ & $\mathbf{b}^{\boldsymbol{*}^{++}}$ & $\mathbf{b}^{\mathbf{0}}$ & $\mathbf{b}^{\mathbf{0 + +}}$ & Seq. & $\mathbf{y}$ & $\mathbf{y}^{++}$ & $\mathbf{y}^{\mathbf{*}}$ & $\mathbf{y}^{\boldsymbol{*}^{++}}$ & $\mathbf{y}^{\mathbf{0}}$ & $\mathbf{y}^{\mathbf{0 + +}}$ & $\#$ \\
\hline $\mathbf{1}$ & 58.0287 & 29.5180 & & & & & $\mathbf{G}$ & & & & & & & $\mathbf{1 3}$ \\
\hline $\mathbf{2}$ & 157.0972 & 79.0522 & & & & & $\mathbf{V}$ & 1293.6797 & 647.3435 & 1276.6532 & 638.8302 & $\mathbf{1 2 7 5 . 6 6 9 2}$ & 638.3382 & $\mathbf{1 2}$ \\
\hline 3 & 228.1343 & 114.5708 & & & & & A & $\mathbf{1 1 9 4 . 6 1 1 3}$ & 597.8093 & $\mathbf{1 1 7 7 . 5 8 4 8}$ & $\mathbf{5 8 9 . 2 9 6 0}$ & $\mathbf{1 1 7 6 . 6 0 0 8}$ & $\mathbf{5 8 8 . 8 0 4 0}$ & $\mathbf{1 1}$ \\
\hline $\mathbf{4}$ & 327.2027 & 164.1050 & & & & & $\mathbf{V}$ & $\mathbf{1 1 2 3 . 5 7 4 2}$ & 562.2907 & 1106.5477 & 553.7775 & 1105.5636 & 553.2855 & $\mathbf{1 0}$ \\
\hline $\mathbf{5}$ & $\mathbf{4 2 8 . 2 5 0 4}$ & 214.6288 & & & 410.2398 & 205.6235 & $\mathbf{T}$ & $\mathbf{1 0 2 4 . 5 0 5 8}$ & 512.7565 & 1007.4792 & 504.2433 & 1006.4952 & 503.7513 & $\mathbf{9}$ \\
\hline
\end{tabular}




\begin{tabular}{|c|c|c|c|c|c|c|c|c|c|c|c|c|c|c|}
\hline 6 & 543.2773 & |272.1423 & 526.2507 & |263.6290 & 525.2667 & |263.1370 & $\mathbf{N}$ & 923.4581 & |462.2327 & 906.4316 & |453.7194 & 905.4476 & $\mid$ & 8 \\
\hline 7 & 644.3250 & 322.6661 & 627.2984 & 314.1529 & 626.3144 & 313.6608 & $\mathbf{T}$ & 808.4312 & 404.7192 & 791.4046 & 396.2060 & 790.4206 & 395.7139 & 7 \\
\hline 8 & 731.3570 & 366.1821 & 714.3305 & 357.6689 & 713.3464 & 357.1769 & $\mathrm{~S}$ & 707.3835 & 354.1954 & 690.3570 & 345.6821 & 689.3729 & 345.1901 & 6 \\
\hline 9 & 859.4156 & 430.2114 & 842.3890 & 421.6982 & 841.4050 & 421.2061 & $\mathbf{Q}$ & 620.3515 & 310.6794 & 603.3249 & 302.1661 & & & 5 \\
\hline 10 & 972.4996 & |486.7535 & 955.4731 & 478.2402 & 954.4891 & 477.7482 & $\mathbf{L}$ & 492.2929 & 246.6501 & 475.2663 & 238.1368 & & & 4 \\
\hline 11 & 1029.5211 & 515.2642 & 1012.4946 & 506.7509 & 1011.5105 & 506.2589 & G & 379.2088 & 190.1081 & 362.1823 & 181.5948 & & & 3 \\
\hline 12 & 1176.5895 & 588.7984 & 1159.5630 & 580.2851 & 1158.5790 & 579.7931 & $\mathbf{F}$ & 322.1874 & 161.5973 & 305.1608 & 153.0840 & & & 2 \\
\hline 13 & & & & & & & $\mathbf{R}$ & 175.1190 & 88.0631 & 158.0924 & 79.5498 & & & 1 \\
\hline
\end{tabular}
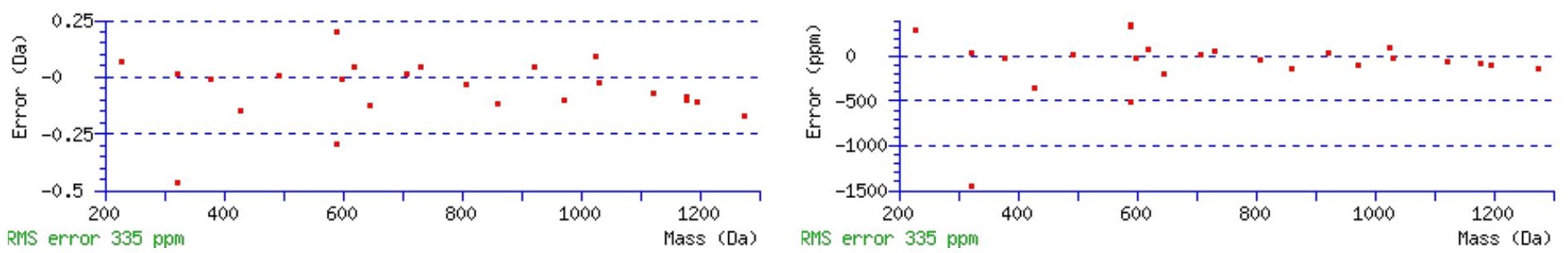

\section{All matches to this query}

\begin{tabular}{|l|c|c|l|}
\hline Score & Mr(calc): & Delta & \multicolumn{1}{|c|}{ Sequence } \\
\hline 68.6 & 1349.6939 & 0.0009 & GVAVTNTSOLGFR \\
\hline 8.4 & 1349.6874 & 0.0074 & VKVGVNGFGCTGR \\
\hline 6.8 & 1349.6912 & 0.0037 & RAHQLRPNDSR \\
\hline 4.8 & 1349.6907 & 0.0042 & ARALQMEKTMR \\
\hline 1.6 & 1349.6816 & 0.0132 & NLSRVPSQITR \\
\hline 1.6 & 1349.6816 & 0.0132 & NLSRVPSQITR \\
\hline 1.5 & 1349.6939 & 0.0010 & ENVYLTAERVR \\
\hline 1.3 & 1349.7068 & -0.0119 & NLSISVSGLRPK \\
\hline
\end{tabular}

Spectrum No: 380; Query: 2701; Rank: 1

\section{Peptide View}

MS/MS Fragmentation of ADCLDASDESACPTRFPNGTYCPAAMFECK

Found in IPI00205325, Tax_Id=10116 Gene_Symbol=Lrp2 Low-density lipoprotein receptor-related protein 2 precursor

Match to Query 2701: 3441.379662 from(1148.133830,3+)

Title: 100101RatKid_NS_deglyco_16.4202.4202.3.dta

Data file K:INewmanPaper|Piliang|3SubProteomes\Piliang3SP\mgf5ppm\ERLIC_3SubProteomes5ppm.mgf

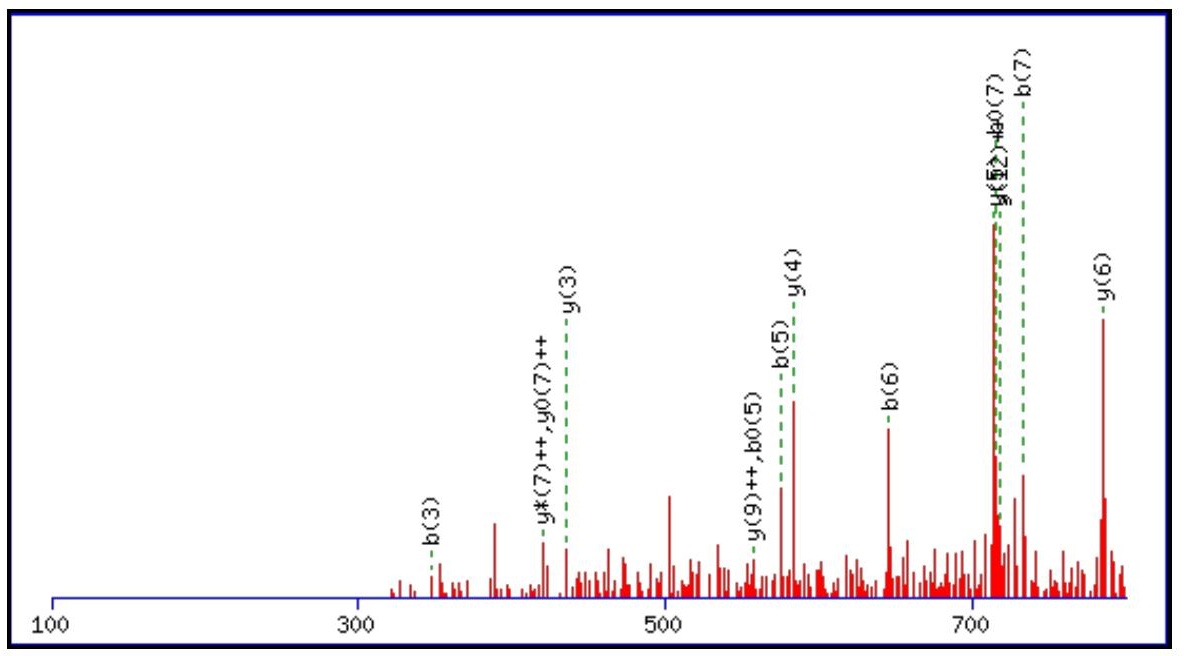



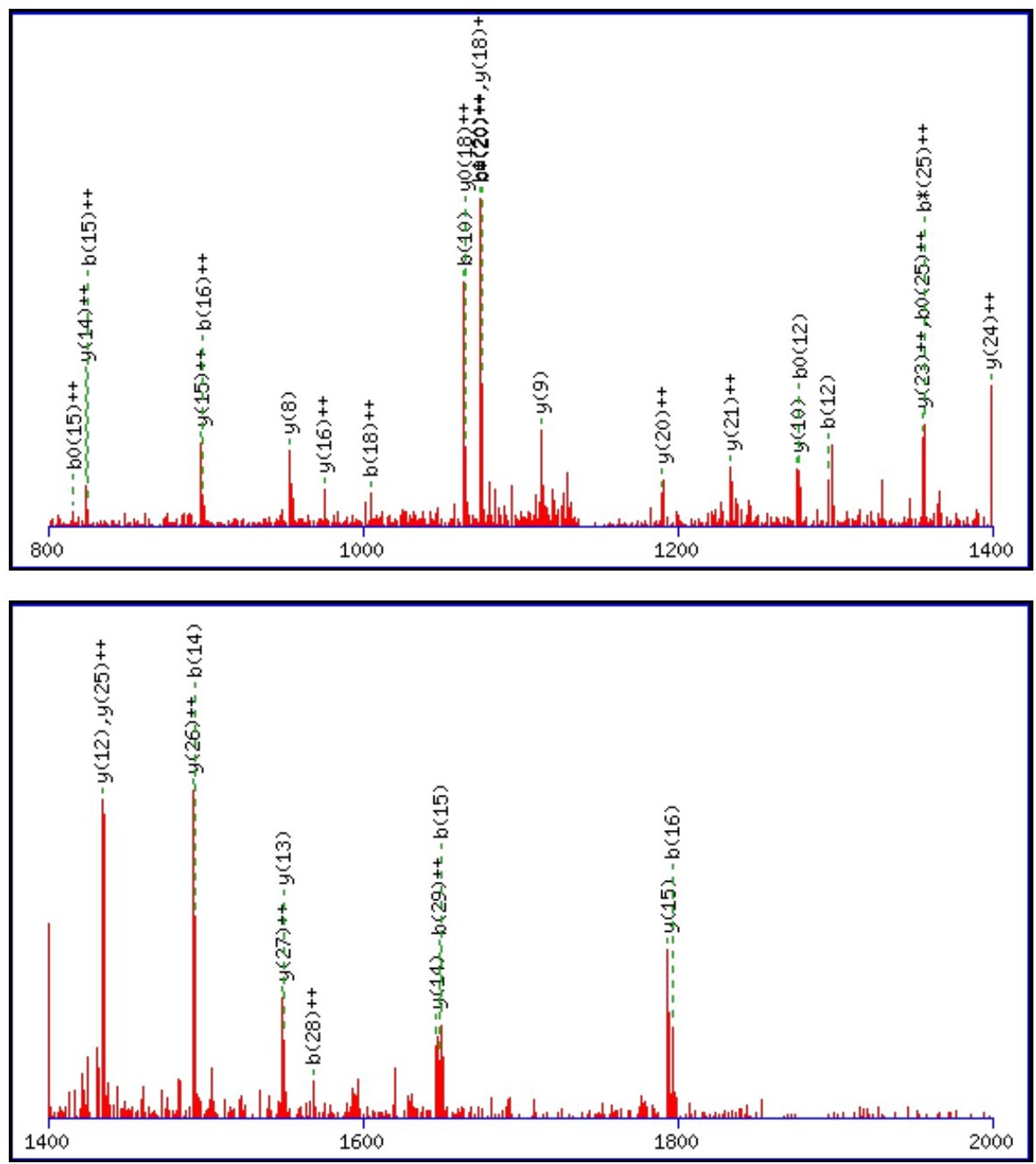

Monoisotopic mass of neutral peptide $\operatorname{Mr}$ (calc): 3441.3716

Fixed modifications: Carbamidomethyl (C)

Variable modifications:

N18 : Deamidated_N (N)

Ions Score: 68 Expect: $5 \mathrm{e}-005$

Matches (Bold Red): 49/314 fragment ions using 89 most intense peaks

\begin{tabular}{|c|c|c|c|c|c|c|c|c|c|c|c|c|c|c|}
\hline \# & b & $\mathbf{b}^{++}$ & $\mathbf{b}^{*}$ & $\mathbf{b}^{*^{++}}$ & $\mathbf{b}^{0}$ & $\mathbf{b}^{0++}$ & Seq. & $\mathbf{y}$ & $y^{++}$ & $\mathbf{y}^{*}$ & $\mathbf{y}^{*^{++}}$ & $\mathbf{y}^{0}$ & $\mathbf{y}^{\mathbf{0 + +}}$ & \# \\
\hline 1 & 72.0444 & 36.5258 & & & & & A & & & & & & & 30 \\
\hline 2 & 187.0713 & 94.0393 & & & 169.0608 & 85.0340 & D & 3371.3418 & 1686.1745 & 3354.3152 & 1677.6613 & 3353.3312 & 1677.1693 & 29 \\
\hline 3 & 347.1020 & 174.0546 & & & 329.0914 & 165.0493 & C & 3256.3149 & 1628.6611 & 3239.2883 & 1620.1478 & 3238.3043 & 1619.6558 & 28 \\
\hline 4 & 460.1860 & 230.5967 & & & 442.1755 & 221.5914 & $\mathbf{L}$ & 3096.2842 & 1548.6457 & 3079.2577 & 1540.1325 & 3078.2736 & 1539.6405 & 27 \\
\hline 5 & 575.2130 & 288.1101 & & & 557.2024 & 279.1049 & D & 2983.2001 & 1492.1037 & 2966.1736 & 1483.5904 & 2965.1896 & 1483.0984 & 26 \\
\hline 6 & 646.2501 & 323.6287 & & & 628.2395 & 314.6234 & A & 2868.1732 & 1434.5902 & 2851.1466 & 1426.0770 & 2850.1626 & 1425.5850 & 25 \\
\hline 7 & 733.2821 & 367.1447 & & & 715.2716 & 358.1394 & $\mathrm{~S}$ & 2797.1361 & 1399.0717 & 2780.1095 & 1390.5584 & 2779.1255 & 1390.0664 & 24 \\
\hline 8 & 848.3091 & 424.6582 & & & 830.2985 & 415.6529 & D & 2710.1041 & 1355.5557 & 2693.0775 & 1347.0424 & 2692.0935 & 1346.5504 & 23 \\
\hline 9 & 977.3517 & 489.1795 & & & 959.3411 & 480.1742 & $\mathbf{E}$ & 2595.0771 & 1298.0422 & 2578.0506 & 1289.5289 & 2577.0665 & 1289.0369 & 22 \\
\hline 10 & 1064.3837 & 532.6955 & & & 1046.3731 & 523.6902 & $\mathrm{~S}$ & 2466.0345 & 1233.5209 & 2449.0080 & 1225.0076 & 2448.0240 & 1224.5156 & 21 \\
\hline 11 & 1135.4208 & 568.2140 & & & 1117.4102 & 559.2088 & $\mathbf{A}$ & 2379.0025 & 1190.0049 & 2361.9759 & 1181.4916 & 2360.9919 & 1180.9996 & 20 \\
\hline 12 & 1295.4515 & 648.2294 & & & 1277.4409 & 639.2241 & $\mathrm{C}$ & 2307.9654 & 1154.4863 & 2290.9388 & 1145.9731 & 2289.9548 & 1145.4810 & 19 \\
\hline 13 & 1392.5042 & 696.7557 & & & 1374.4937 & 687.7505 & $\mathbf{P}$ & 2147.9347 & 1074.4710 & 2130.9082 & 1065.9577 & 2129.9242 & 1065.4657 & 18 \\
\hline 14 & 1493.5519 & 747.2796 & & & 1475.5413 & 738.2743 & $\mathbf{T}$ & 2050.8820 & 1025.9446 & 2033.8554 & 1017.4313 & 2032.8714 & 1016.9393 & 17 \\
\hline 15 & 1649.6530 & 825.3301 & 1632.6265 & 816.8169 & 1631.6424 & 816.3249 & $\mathbf{R}$ & 1949.8343 & 975.4208 & 1932.8077 & 966.9075 & 1931.8237 & 966.4155 & 16 \\
\hline 16 & 1796.7214 & 898.8644 & 1779.6949 & 890.3511 & 1778.7109 & 889.8591 & $\mathbf{F}$ & 1793.7332 & 897.3702 & 1776.7066 & 888.8569 & 1775.7226 & 888.3649 & 15 \\
\hline 17 & 1893.7742 & 947.3907 & 1876.7476 & 938.8775 & 1875.7636 & 938.3855 & $\mathbf{P}$ & 1646.6648 & 823.8360 & 1629.6382 & 815.3227 & 1628.6542 & 814.8307 & 14 \\
\hline 18 & 2008.8011 & 1004.9042 & 1991.7746 & 996.3909 & 1990.7906 & 995.8989 & $\mathbf{N}$ & 1549.6120 & 775.3096 & 1532.5854 & 766.7964 & 1531.6014 & 766.3044 & 13 \\
\hline
\end{tabular}




\begin{tabular}{|c|c|c|c|c|c|c|c|c|c|c|c|c|c|c|}
\hline 19 & 2065.8226 & |1033.4149 & 2048.7960 & |1024.9017| & |2047.8120 & |1024.4097 & G & 1434.5851 & 717.7962 & |1417.5585 & 709.2829 & 1416.5745 & 708.7909 & 12 \\
\hline 20 & 2166.8703 & 1083.9388 & 2149.8437 & 1075.4255 & 2148.8597 & 1074.9335 & $\mathbf{T}$ & 1377.5636 & 689.2854 & 1360.5370 & 680.7722 & 1359.5530 & 680.2802 & 11 \\
\hline 21 & 2329.9336 & 1165.4704 & 2312.9070 & 1156.9572 & 2311.9230 & 1156.4652 & $\mathbf{Y}$ & 1276.5159 & 638.7616 & 1259.4894 & 630.2483 & 1258.5054 & 629.7563 & 10 \\
\hline 22 & 2489.9642 & 1245.4858 & 2472.9377 & 1236.9725 & 2471.9537 & 1236.4805 & C & 1113.4526 & 557.2299 & 1096.4260 & 548.7167 & 1095.4420 & 548.2246 & 9 \\
\hline 23 & 2587.0170 & 1294.0121 & 2569.9905 & 1285.4989 & 2569.0064 & 1285.0069 & $\mathbf{P}$ & 953.4219 & 477.2146 & 936.3954 & 468.7013 & 935.4114 & 468.2093 & 8 \\
\hline 24 & 2658.0541 & 1329.5307 & 2641.0276 & |1321.0174 & 2640.0436 & 1320.5254 & A & 856.3692 & 428.6882 & 839.3426 & 420.1750 & 838.3586 & 419.6829 & 7 \\
\hline 25 & 2729.0912 & 1365.0493 & 2712.0647 & 1356.5360 & 2711.0807 & 1356.0440 & A & 785.3321 & 393.1697 & 768.3055 & 384.6564 & 767.3215 & 384.1644 & 6 \\
\hline 26 & 2860.1317 & 1430.5695 & 2843.1052 & 1422.0562 & 2842.1212 & 1421.5642 & M & 714.2949 & 357.6511 & 697.2684 & 349.1378 & 696.2844 & 348.6458 & 5 \\
\hline 27 & 3007.2001 & 1504.1037 & 2990.1736 & 1495.5904 & 2989.1896 & 1495.0984 & $\mathbf{F}$ & 583.2545 & 292.1309 & 566.2279 & 283.6176 & 565.2439 & 283.1256 & 4 \\
\hline 28 & 3136.2427 & 1568.6250 & 3119.2162 & 1560.1117 & 3118.2322 & 1559.6197 & E & 436.1860 & 218.5967 & 419.1595 & 210.0834 & 418.1755 & 209.5914 & 3 \\
\hline 29 & 3296.2734 & 1648.6403 & 3279.2468 & 1640.1271 & 3278.2628 & 1639.6350 & C & 307.1435 & 154.0754 & 290.1169 & 145.5621 & & & 2 \\
\hline 30 & & & & & & & $\mathbf{K}$ & 147.1128 & 74.0600 & 130.0863 & 65.5468 & & & 1 \\
\hline
\end{tabular}
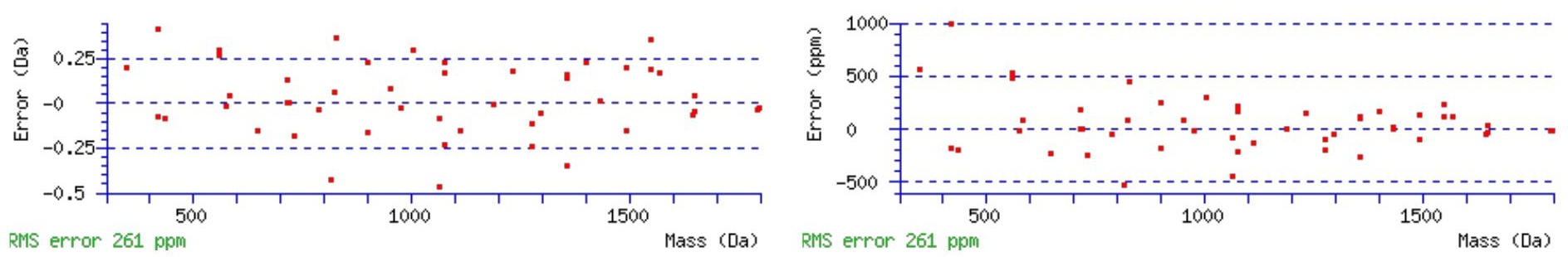

\section{All matches to this query}

\begin{tabular}{|l|l|l|l|}
\hline Score & Mr(calc): & Delta & \multicolumn{1}{|c|}{ Sequence } \\
\hline 68.4 & 3441.3716 & 0.0080 & ADCLDASDESACPTRFPNGTYCPAAMFECK \\
\hline 39.9 & 3440.3876 & 0.9920 & ADCLDASDESACPTRFPNGTYCPAAMFECK \\
\hline 6.2 & 3439.3487 & 2.0310 & TNISEDREECCTTSKNATQENSGDNIPMR \\
\hline 6.2 & 3439.3487 & 2.0310 & TNISEDREECCTTSKNATQENSGDNIPMR \\
\hline 5.7 & 3439.3487 & 2.0310 & TNISEDREECCTTSKNATQENSGDNIPMR \\
\hline 2.4 & 3440.3443 & 1.0353 & FMKDDISAPPTEGVYVYGLYLEGAGWDK \\
\hline 2.1 & 3439.3486 & 2.0310 & GSDELLSGSVLSSPNSNMSGMVVTANGNDSKK \\
\hline 1.9 & 3441.3965 & -0.0168 & TTMNNYLPSNHMTMISQQPNNLGTNSLNK \\
\hline 1.8 & 3441.3965 & -0.0168 & TTMNNYLPSNHMTMISQQPNNLGTNSLNK \\
\hline 1.8 & 3441.4008 & -0.0212 & RWFILTDNCLYYFEFTTVSVWGGGNR \\
\hline
\end{tabular}

Spectrum No: 381; Query: 898; Rank: 1

\section{Peptide View}

MS/MS Fragmentation of EQLFFNLSDPELR

Found in IPI00191766, Tax_Id=10116 Gene_Symbol=L1cam Isoform 1 of Neural cell adhesion molecule L1 precursor

Match to Query 898: 1607.788048 from(804.901300,2+)

Title: 100101RatKid_NS_deglyco_25.4429.4429.2.dta

Data file K:INewmanPaper|Piliang|3SubProteomes\Piliang3SP\mgf5ppm\ERLIC_3SubProteomes5ppm.mgf 

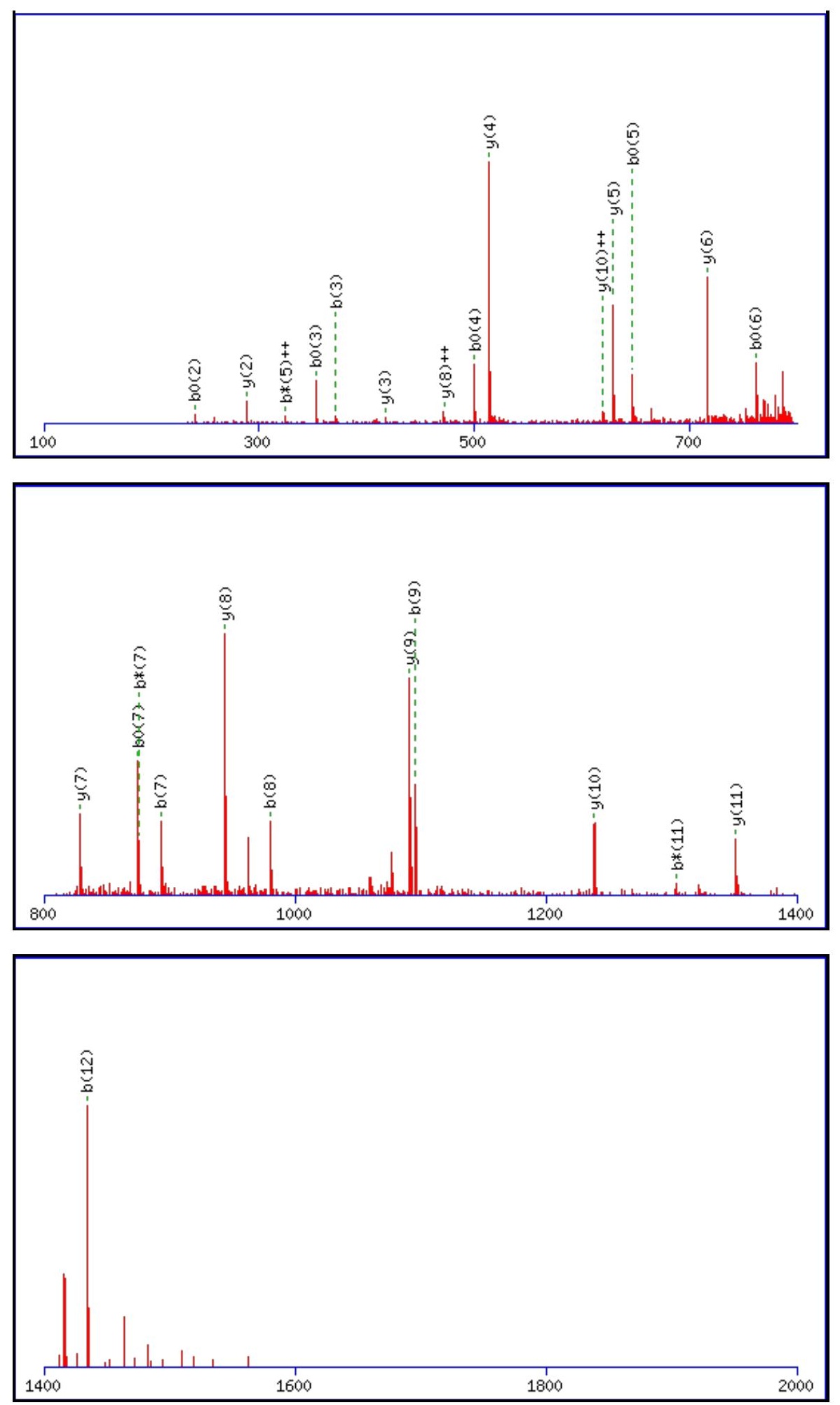

Monoisotopic mass of neutral peptide $\operatorname{Mr}($ calc): 1607.7831

Fixed modifications: Carbamidomethyl (C)

Variable modifications:

N6 : Deamidated $\mathrm{N}(\mathrm{N})$

Ions Score: 68 Expect: $2.5 \mathrm{e}-005$

Matches (Bold Red): 26/138 fragment ions using 53 most intense peaks

\begin{tabular}{|r|c|c|c|c|c|c|c|c|c|c|c|c|c|c|}
\hline$\#$ & $\mathbf{b}$ & $\mathbf{b}^{++}$ & $\mathbf{b}^{*}$ & $\mathbf{b}^{\boldsymbol{*}^{++}}$ & $\mathbf{b}^{\mathbf{0}}$ & $\mathbf{b}^{\mathbf{0 + +}}$ & Seq. & $\mathbf{y}$ & $\mathbf{y}^{++}$ & $\mathbf{y}^{\mathbf{*}}$ & $\mathbf{y}^{\boldsymbol{*}^{++}}$ & $\mathbf{y}^{\mathbf{0}}$ & $\mathbf{y}^{\mathbf{0 + +}}$ & $\#$ \\
\hline $\mathbf{1}$ & 130.0499 & 65.5286 & & & 112.0393 & 56.5233 & $\mathbf{E}$ & & & & & & & $\mathbf{1 3}$ \\
\hline $\mathbf{2}$ & 258.1084 & 129.5579 & 241.0819 & 121.0446 & $\mathbf{2 4 0 . 0 9 7 9}$ & 120.5526 & $\mathbf{Q}$ & 1479.7478 & 740.3775 & 1462.7213 & 731.8643 & 1461.7372 & 731.3723 & $\mathbf{1 2}$ \\
\hline 3 & 371.1925 & 186.0999 & 354.1660 & 177.5866 & 353.1819 & 177.0946 & $\mathbf{L}$ & $\mathbf{1 3 5 1 . 6 8 9 2}$ & 676.3483 & 1334.6627 & 667.8350 & 1333.6787 & 667.3430 & $\mathbf{1 1}$ \\
\hline $\mathbf{4}$ & 518.2609 & 259.6341 & 501.2344 & 251.1208 & $\mathbf{5 0 0 . 2 5 0 4}$ & 250.6288 & $\mathbf{F}$ & $\mathbf{1 2 3 8 . 6 0 5 2}$ & $\mathbf{6 1 9 . 8 0 6 2}$ & 1221.5786 & 611.2929 & 1220.5946 & 610.8009 & $\mathbf{1 0}$ \\
\hline $\mathbf{5}$ & 665.3293 & 333.1683 & 648.3028 & 324.6550 & $\mathbf{6 4 7 . 3 1 8 8}$ & 324.1630 & $\mathbf{F}$ & $\mathbf{1 0 9 1 . 5 3 6 8}$ & 546.2720 & 1074.5102 & 537.7587 & 1073.5262 & 537.2667 & $\mathbf{9}$ \\
\hline
\end{tabular}




\begin{tabular}{|c|c|c|c|c|c|c|c|c|c|c|c|c|c|c|}
\hline 6 & 780.3563 & |390.6818| & 763.3297 & |382.1685| & 762.3457 & |381.6765| & $\mathbf{N}$ & 944.4683 & |472.7378 & 927.4418 & |464.2245| & 926.4578 & |463.7325| & 8 \\
\hline 7 & 893.4403 & 447.2238 & 876.4138 & 438.7105 & 875.4298 & 438.2185 & $\mathbf{L}$ & 829.4414 & 415.2243 & 812.4149 & 406.7111 & 811.4308 & 406.2191 & 7 \\
\hline 8 & 980.4724 & 490.7398 & 963.4458 & 482.2265 & 962.4618 & 481.7345 & S & 716.3573 & 358.6823 & 699.3308 & 350.1690 & 698.3468 & 349.6770 & 6 \\
\hline 9 & 1095.4993 & 548.2533 & 1078.4728 & 539.7400 & 1077.4887 & 539.2480 & D & 629.3253 & 315.1663 & 612.2988 & 306.6530 & 611.3148 & 306.1610 & 5 \\
\hline 10 & 1192.5521 & 596.7797 & 1175.5255 & 588.2664 & 1174.5415 & 587.7744 & $\mathbf{P}$ & 514.2984 & 257.6528 & 497.2718 & 249.1396 & 496.2878 & 248.6475 & 4 \\
\hline 11 & 1321.5947 & 661.3010 & 1304.5681 & 652.7877 & 1303.5841 & 652.2957 & $\mathbf{E}$ & 417.2456 & 209.1264 & 400.2191 & 200.6132 & 399.2350 & 200.1212 & 3 \\
\hline 12 & 1434.6787 & 717.8430 & 1417.6522 & 709.3297 & 1416.6682 & 708.8377 & $\mathbf{L}$ & 288.2030 & 144.6051 & 271.1765 & |136.0919 & & & 2 \\
\hline 13 & & & & & & & $\mathbf{R}$ & 175.1190 & 88.0631 & 158.0924 & 79.5498 & & & 1 \\
\hline
\end{tabular}
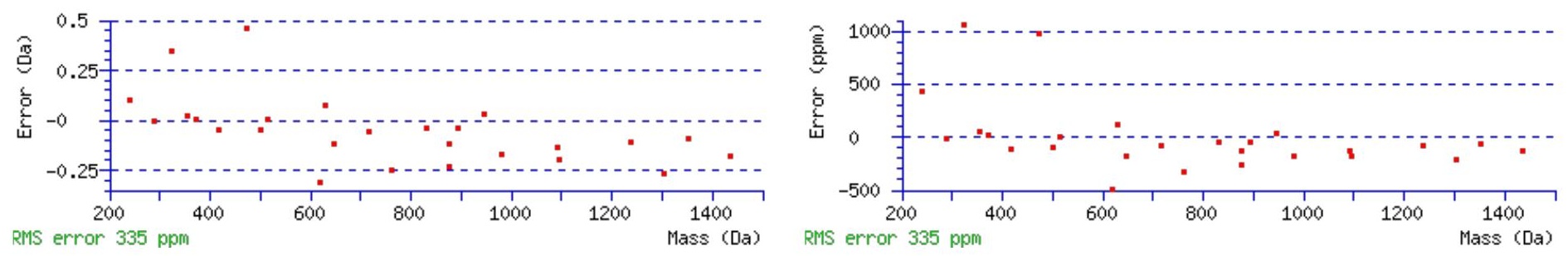

\section{All matches to this query}

\begin{tabular}{|l|c|c|l|}
\hline Score & Mr(calc): & Delta & \multicolumn{1}{c|}{ Sequence } \\
\hline 68.3 & 1607.7831 & 0.0049 & EQLFFNLSDPELR \\
\hline 14.6 & 1606.7991 & 0.9889 & EQLFFNLSDPELR \\
\hline 9.7 & 1607.8032 & -0.0151 & EKNRTSSIIPVER \\
\hline 4.6 & 1607.7920 & -0.0039 & GKLAAATSLPEDTVR \\
\hline 4.6 & 1607.7920 & -0.0039 & GKLAAATSLPEDTVR \\
\hline 2.9 & 1606.7773 & 1.0108 & TGVFITLSIVLER \\
\hline 2.0 & 1607.8032 & -0.0151 & EKNRTSSIIPVER \\
\hline 1.9 & 1605.7780 & 2.0100 & QEAVSMAKKNSEQR \\
\hline 0.6 & 1606.7718 & 1.0163 & ISLFADDMIVYLK \\
\hline 0.3 & 1606.7760 & 1.0121 & ETEATNAILMEQIK \\
\hline
\end{tabular}

Spectrum No: 382; Query: 671; Rank: 1

\section{Peptide View}

MS/MS Fragmentation of EVMSLLQPLNITK

Found in IPI00388265, Tax_Id=10116 Gene_Symbol=Pgcp 48 kDa protein

Match to Query 671: 1501.803448 from(751.909000,2+)

Title: 091008RatKidney_NH4Format01_18.3736.3736.2.dta

Data file K:INewmanPaper|Piliang|3SubProteomes\Piliang3SP\mgf5ppm\ERLIC_3SubProteomes5ppm.mgf 

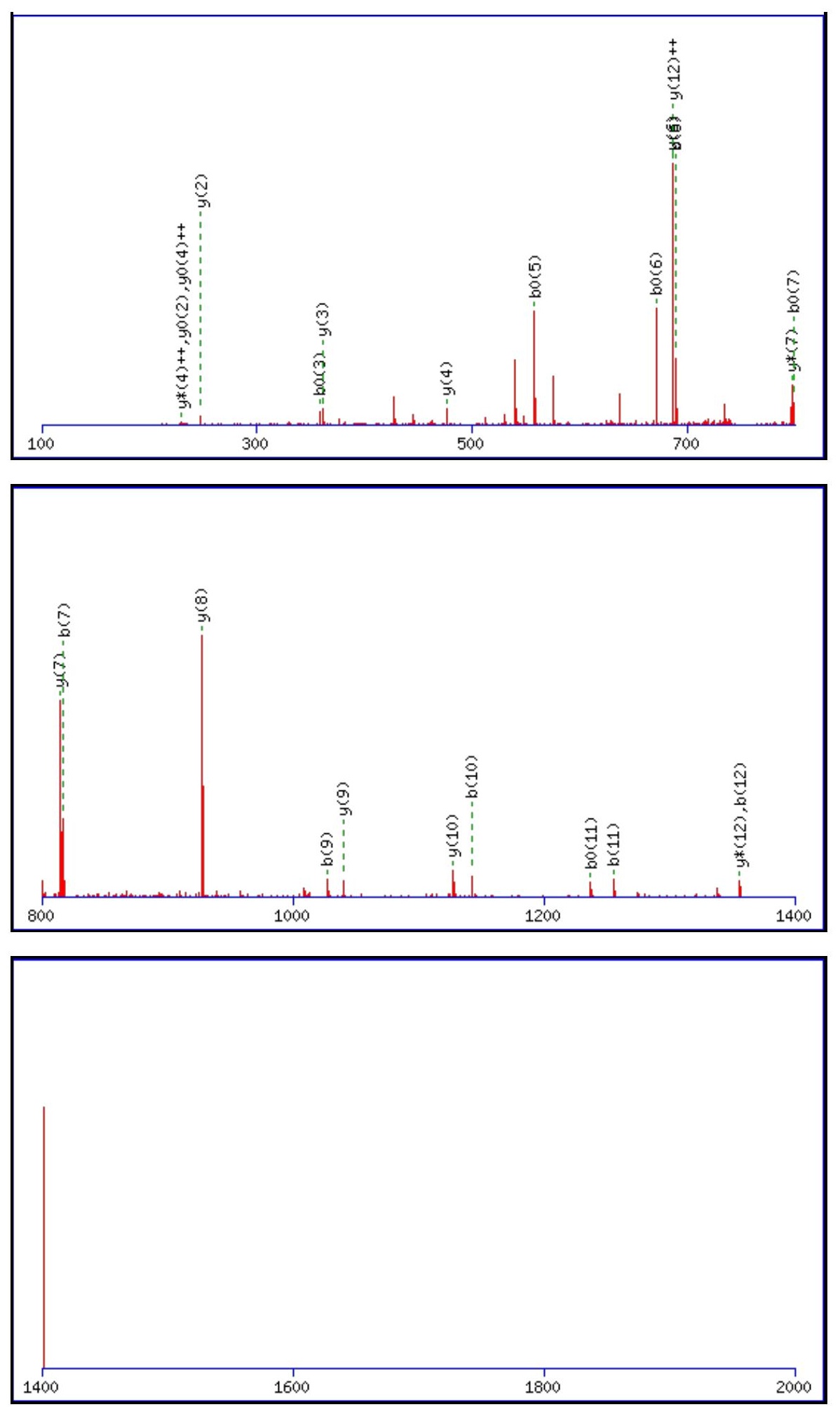

Monoisotopic mass of neutral peptide $\operatorname{Mr}($ calc): 1501.8062

Fixed modifications: Carbamidomethyl (C)

Variable modifications:

M3 : Oxidation (M)

N10 : Deamidated_N (N)

Ions Score: 68 Expect: $1.4 \mathrm{e}-005$

Matches (Bold Red): 25/130 fragment ions using 26 most intense peaks

\begin{tabular}{|c|c|c|c|c|c|c|c|c|c|c|c|c|c|c|}
\hline$\#$ & $\mathbf{b}$ & $\mathbf{b}^{++}$ & $\mathbf{b}^{*}$ & $\mathbf{b}^{\boldsymbol{*}_{+}^{++}}$ & $\mathbf{b}^{\mathbf{0}}$ & $\mathbf{b}^{\mathbf{0 + +}}$ & $\mathbf{S e q}$ & $\mathbf{y}$ & $\mathbf{y}^{++}$ & $\mathbf{y}^{\mathbf{*}}$ & $\mathbf{y}^{\mathbf{*}^{++}}$ & $\mathbf{y}^{\mathbf{0}}$ & $\mathbf{y}^{\mathbf{0}+}$ & $\#$ \\
\hline $\mathbf{1}$ & 130.0499 & 65.5286 & & & 112.0393 & 56.5233 & $\mathbf{E}$ & & & & & & & $\mathbf{1 3}$ \\
\hline $\mathbf{2}$ & 229.1183 & 115.0628 & & & 211.1077 & 106.0575 & $\mathbf{V}$ & 1373.7709 & $\mathbf{6 8 7 . 3 8 9 1}$ & $\mathbf{1 3 5 6 . 7 4 4 3}$ & 678.8758 & 1355.7603 & 678.3838 & $\mathbf{1 2}$ \\
\hline $\mathbf{3}$ & 376.1537 & 188.5805 & & & 358.1431 & 179.5752 & $\mathbf{M}$ & 1274.7024 & 637.8549 & 1257.6759 & 629.3416 & 1256.6919 & 628.8496 & $\mathbf{1 1}$ \\
\hline $\mathbf{4}$ & 463.1857 & 232.0965 & & & 445.1752 & 223.0912 & $\mathbf{S}$ & $\mathbf{1 1 2 7 . 6 6 7 0}$ & 564.3372 & 1110.6405 & 555.8239 & 1109.6565 & 555.3319 & $\mathbf{1 0}$ \\
\hline & & & & & & & & & & & & & &
\end{tabular}




\begin{tabular}{|r|r|r|r|r|r|r|r|r|r|r|r|r|r|r|r|r|r|}
$\mathbf{5}$ & 576.2698 & 288.6385 & & & 558.2592 & 279.6332 & L & $\mathbf{1 0 4 0 . 6 3 5 0}$ & 520.8211 & 1023.6085 & 512.3079 & 1022.6245 & 511.8159 & $\mathbf{9}$ \\
\hline $\mathbf{6}$ & $\mathbf{6 8 9 . 3 5 3 8}$ & 345.1806 & & & $\mathbf{6 7 1 . 3 4 3 3}$ & 336.1753 & L & $\mathbf{9 2 7 . 5 5 1 0}$ & 464.2791 & 910.5244 & 455.7658 & 909.5404 & 455.2738 & $\mathbf{8}$ \\
\hline $\mathbf{7}$ & $\mathbf{8 1 7 . 4 1 2 4}$ & 409.2098 & 800.3859 & 400.6966 & $\mathbf{7 9 9 . 4 0 1 9}$ & 400.2046 & $\mathbf{Q}$ & $\mathbf{8 1 4 . 4 6 6 9}$ & 407.7371 & $\mathbf{7 9 7 . 4 4 0 3}$ & 399.2238 & 796.4563 & 398.7318 & $\mathbf{7}$ \\
\hline $\mathbf{8}$ & 914.4652 & 457.7362 & 897.4386 & 449.2230 & 896.4546 & 448.7309 & $\mathbf{P}$ & $\mathbf{6 8 6 . 4 0 8 3}$ & 343.7078 & 669.3818 & 335.1945 & 668.3977 & 334.7025 & $\mathbf{6}$ \\
\hline $\mathbf{9}$ & $\mathbf{1 0 2 7 . 5 4 9 2}$ & 514.2783 & 1010.5227 & 505.7650 & 1009.5387 & 505.2730 & $\mathbf{L}$ & 589.3555 & 295.1814 & 572.3290 & 286.6681 & 571.3450 & 286.1761 & $\mathbf{5}$ \\
\hline $\mathbf{1 0}$ & $\mathbf{1 1 4 2 . 5 7 6 2}$ & 571.7917 & 1125.5496 & 563.2785 & 1124.5656 & 562.7864 & $\mathbf{N}$ & $\mathbf{4 7 6 . 2 7 1 5}$ & 238.6394 & 459.2449 & $\mathbf{2 3 0 . 1 2 6 1}$ & 458.2609 & $\mathbf{2 2 9 . 6 3 4 1}$ & $\mathbf{4}$ \\
\hline $\mathbf{1 1}$ & $\mathbf{1 2 5 5 . 6 6 0 2}$ & 628.3338 & 1238.6337 & 619.8205 & $\mathbf{1 2 3 7 . 6 4 9 7}$ & 619.3285 & $\mathbf{I}$ & $\mathbf{3 6 1 . 2 4 4 5}$ & 181.1259 & 344.2180 & 172.6126 & 343.2340 & 172.1206 & $\mathbf{3}$ \\
\hline $\mathbf{1 2}$ & $\mathbf{1 3 5 6 . 7 0 7 9}$ & 678.8576 & 1339.6814 & 670.3443 & 1338.6974 & 669.8523 & T & $\mathbf{2 4 8 . 1 6 0 5}$ & 124.5839 & 231.1339 & 116.0706 & $\mathbf{2 3 0 . 1 4 9 9}$ & 115.5786 & $\mathbf{2}$ \\
\hline $\mathbf{1 3}$ & & & & & & & $\mathbf{K}$ & 147.1128 & 74.0600 & 130.0863 & 65.5468 & & & $\mathbf{1}$ \\
\hline
\end{tabular}
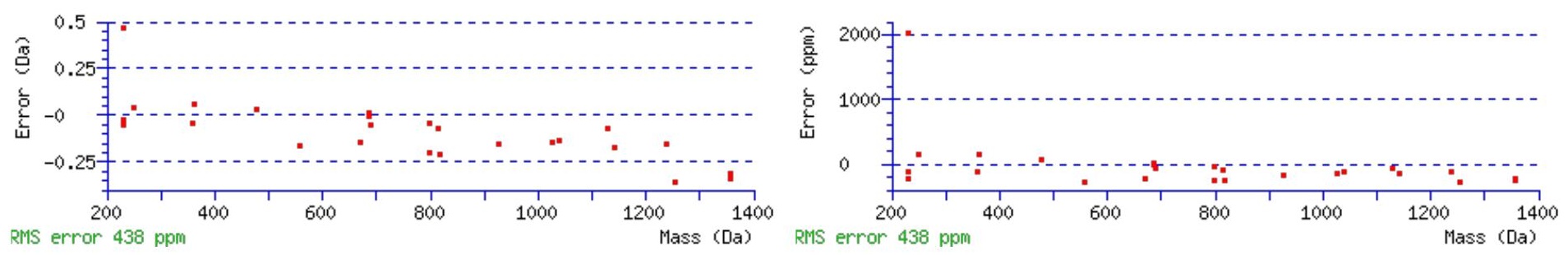

\section{All matches to this query}

\begin{tabular}{|l|l|l|l|}
\hline Score & Mr(calc): & Delta & \multicolumn{1}{c|}{ Sequence } \\
\hline 68.3 & 1501.8062 & -0.0027 & EVMSLLQPLNITK \\
\hline 11.6 & 1501.8174 & -0.0139 & MPILSKLSQDINK \\
\hline 7.1 & 1499.8056 & 1.9979 & ELSVEEQIKRNR \\
\hline 6.2 & 1501.7889 & 0.0146 & AFQELLNQVGGLGR \\
\hline 6.1 & 1499.7943 & 2.0091 & QESEPDKLTIGKR \\
\hline 4.8 & 1500.7896 & 1.0138 & GSLLADGRQKDVDK \\
\hline 4.8 & 1501.7988 & 0.0047 & LKIDTDKENAEVK \\
\hline 4.8 & 1501.8062 & -0.0027 & DLLSKMLVIDPDK \\
\hline 4.0 & 1501.8174 & -0.0139 & VEMALEALRNVIK \\
\hline 3.9 & 1501.7889 & 0.0146 & NAGLAFIELVNEGR \\
\hline
\end{tabular}

Spectrum No: 383; Query: 1697; Rank: 1

\section{Peptide View}

MS/MS Fragmentation of GALLANFLTQGQVCCNGTR

Found in IPI00203690, Tax_Id=10116 Gene_Symbol=Aldh9a1 4-trimethylaminobutyraldehyde dehydrogenase

Match to Query 1697: 2079.986742 from(694.336190,3+)

Title: 091008RatKidney_NH4Format02_21.4235.4235.3.dta

Data file K:INewmanPaper\Piliang $\mid 3 S$ SubProteomes\Piliang3SP\mgf5ppm\ERLIC_3SubProteomes5ppm.mgf 

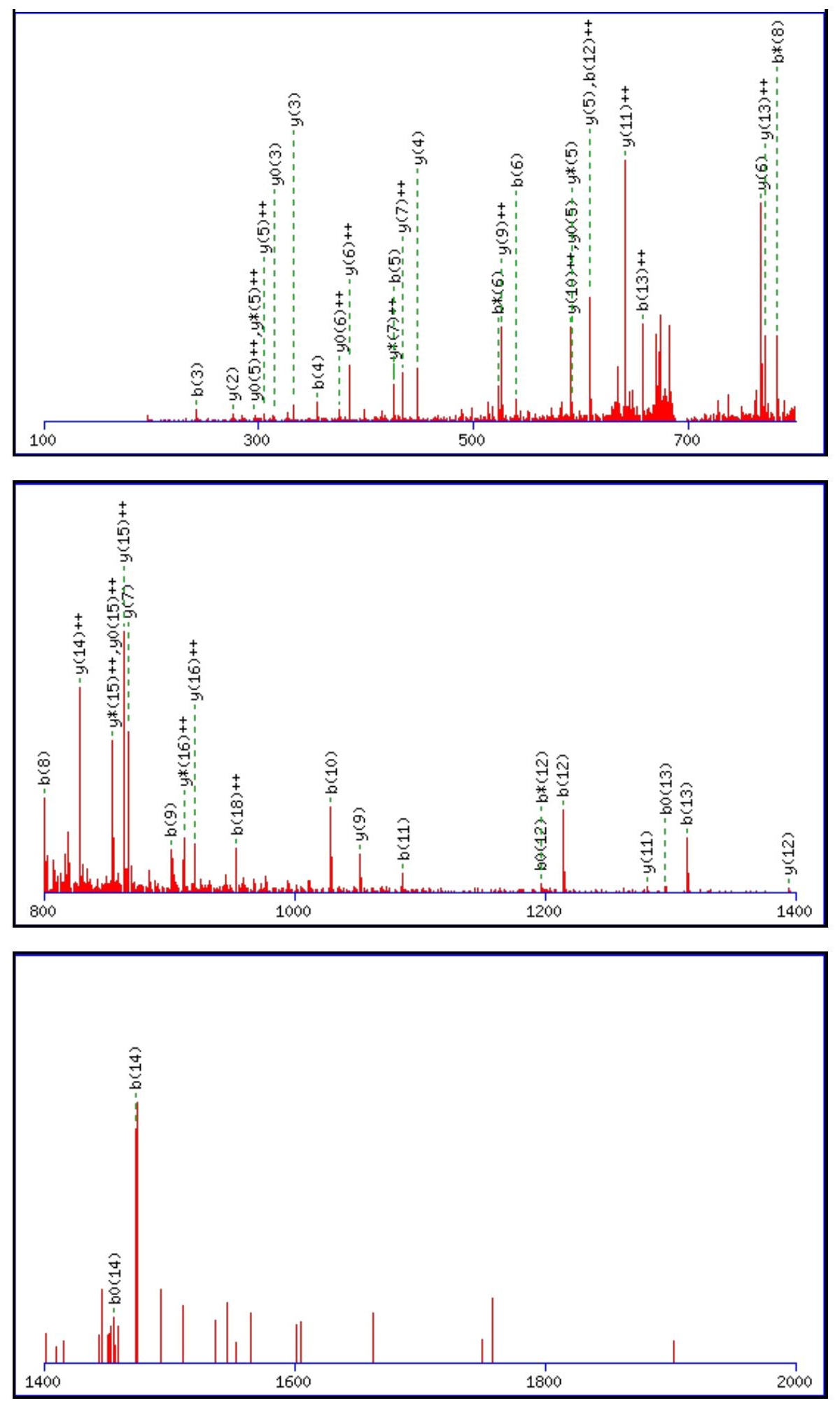

Monoisotopic mass of neutral peptide $\operatorname{Mr}($ calc): 2079.9830

Fixed modifications: Carbamidomethyl (C)

Variable modifications:

N16 : Deamidated_N (N)

Ions Score: 68 Expect: $3.8 \mathrm{e}-005$

Matches (Bold Red): 49/188 fragment ions using 82 most intense peaks

\begin{tabular}{|c|c|c|c|c|c|c|c|c|c|c|c|c|c|c|}
\hline \# & b & $\mathbf{b}^{++}$ & $\mathbf{b}^{*}$ & $\mathbf{b}^{*^{++}}$ & $\mathbf{b}^{\mathbf{0}}$ & $\mathbf{b}^{0++}$ & Seq. & $\mathbf{y}$ & $y^{++}$ & $\mathbf{y}^{*}$ & $\mathrm{y}^{*^{++}}$ & $\mathbf{y}^{\mathbf{0}}$ & $y^{0++}$ & \# \\
\hline 1 & 58.0287 & 29.5180 & & & & & G & & & & & & & 19 \\
\hline 2 & 129.0659 & 65.0366 & & & & & A & 2023.9688 & 1012.4880 & 2006.9423 & 1003.9748 & 2005.9582 & 1003.4828 & 18 \\
\hline 3 & 242.1499 & 121.5786 & & & & & $\mathbf{L}$ & 1952.9317 & 976.9695 & 1935.9051 & 968.4562 & 1934.9211 & 967.9642 & 17 \\
\hline 4 & 355.2340 & 178.1206 & & & & & $\mathbf{L}$ & 1839.8476 & 920.4275 & 1822.8211 & 911.9142 & 1821.8371 & 911.4222 & 16 \\
\hline 5 & 426.2711 & 213.6392 & & & & & A & 1726.7636 & 863.8854 & 1709.7370 & 855.3721 & 1708.7530 & 854.8801 & 15 \\
\hline
\end{tabular}




\begin{tabular}{|c|c|c|c|c|c|c|c|c|c|c|c|c|c|c|}
\hline & 540.3140 & |270.6606 & 523.2875 & 262.1474 & & & $\mathbf{N}$ & |1655.7264| & 828.3669 & & 819.8536 & 1637.7159 & 819.3616 & $\mid 14$ \\
\hline 7 & 687.3824 & 344.1949 & 670.3559 & 335.6816 & & & $\mathbf{F}$ & 1541.6835 & 771.3454 & 1524.6570 & 762.8321 & 1523.6730 & 762.3401 & 13 \\
\hline 8 & 800.4665 & 400.7369 & 783.4400 & 392.2236 & & & $\mathbf{L}$ & 1394.6151 & 697.8112 & 1377.5886 & 689.2979 & 1376.6045 & 688.8059 & 12 \\
\hline 9 & 901.5142 & 451.2607 & 884.4876 & 442.7475 & 883.5036 & 442.2554 & $\mathbf{T}$ & 1281.5310 & 641.2692 & 1264.5045 & 632.7559 & 1263.5205 & 632.2639 & 11 \\
\hline 10 & 029.5728 & 515.2900 & 1012.5462 & 506.7767 & 1011.5622 & 506.2847 & $\mathbf{Q}$ & 1180.4834 & 590.7453 & 1163.4568 & 582.2320 & 1162.4728 & 581.7400 & 10 \\
\hline 11 & 1086.5942 & 543.8007 & 1069.5677 & 535.2875 & 1068.5837 & 534.7955 & G & 1052.4248 & 526.7160 & 1035.3982 & 518.2028 & 1034.4142 & 517.7107 & 9 \\
\hline 12 & 1214.6528 & 607.8300 & 1197.6263 & 599.3168 & 1196.6422 & 598.8248 & $\mathbf{Q}$ & 995.4033 & 498.2053 & 978.3768 & 489.6920 & 977.3928 & 489.2000 & 8 \\
\hline 13 & 1313.7212 & 657.3642 & 1296.6947 & 648.8510 & 1295.7106 & 648.3590 & V & 867.3447 & 434.1760 & 850.3182 & 425.6627 & 849.3342 & 425.1707 & 7 \\
\hline 14 & 1473.7519 & 737.3796 & 1456.7253 & 728.8663 & 1455.7413 & 728.3743 & C & 768.2763 & 384.6418 & 751.2498 & 376.1285 & 750.2658 & 375.6365 & 6 \\
\hline 15 & 1633.7825 & 817.3949 & 1616.7560 & 808.8816 & 1615.7719 & 808.3896 & C & 608.2457 & 304.6265 & 591.2191 & 296.1132 & 590.2351 & 295.6212 & 5 \\
\hline 16 & 1748.8094 & 874.9084 & 1731.7829 & 866.3951 & 1730.7989 & 865.9031 & $\mathbf{N}$ & 448.2150 & 224.6112 & 431.1885 & 216.0979 & 430.2045 & 215.6059 & 4 \\
\hline 17 & 1805.8309 & 903.4191 & 1788.8044 & 894.9058 & 1787.8203 & 894.4138 & G & 333.1881 & 167.0977 & 316.1615 & 158.5844 & 315.1775 & 158.0924 & 3 \\
\hline 18 & 1906.8786 & 953.9429 & 1889.8520 & 945.4297 & 1888.8680 & 944.9377 & $\mathbf{T}$ & 276.1666 & 138.5870 & 259.1401 & 130.0737 & 258.1561 & 129.5817 & 2 \\
\hline 19 & & & & & & & $\mathbf{R}$ & 175.1190 & 88.0631 & 158.0924 & 79.5498 & & & 1 \\
\hline
\end{tabular}
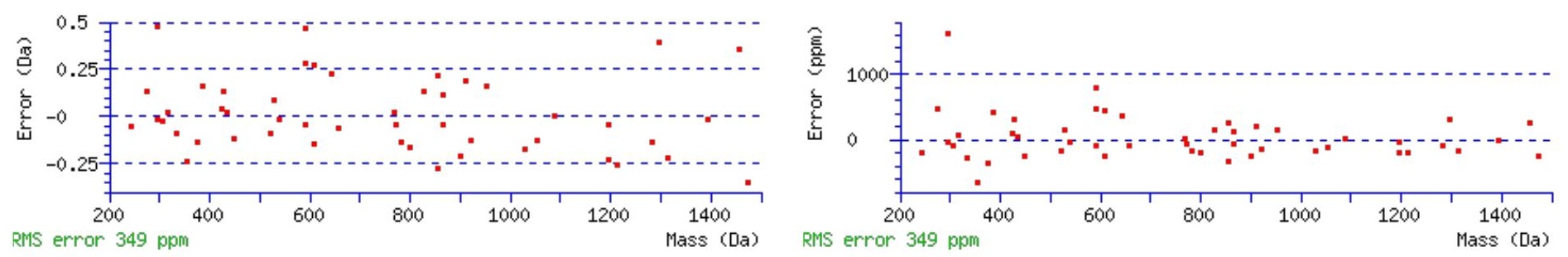

\section{All matches to this query}

\begin{tabular}{|l|l|l|l|}
\hline Score & Mr(calc): & Delta & Sequence \\
\hline 68.3 & 2079.9830 & 0.0037 & GALLANFLTQGQVCCNGTR \\
\hline 30.4 & 2078.9990 & 0.9877 & GALLANFLTQGQVCCNGTR \\
\hline 15.4 & 2079.9830 & 0.0037 & GALLANFLTQGQVCCNGTR \\
\hline
\end{tabular}

Spectrum No: 384; Query: 1636; Rank: 1

\section{Peptide View}

MS/MS Fragmentation of DEYPSVLMYLNATQPER

Found in IPI00207180, Tax_Id=10116 Gene_Symbol=Slc6a18 Sodium- and chloride-dependent transporter XTRP2

Match to Query 1636: 2041.928328 from(1021.971440,2+)

Title: 091008RatKidney_NoSalt_31.3582.3582.2.dta

Data file K:INewmanPaper|Piliang\3SubProteomes\Piliang3SP\mgf5ppm\ERLIC_3SubProteomes5ppm.mgf 

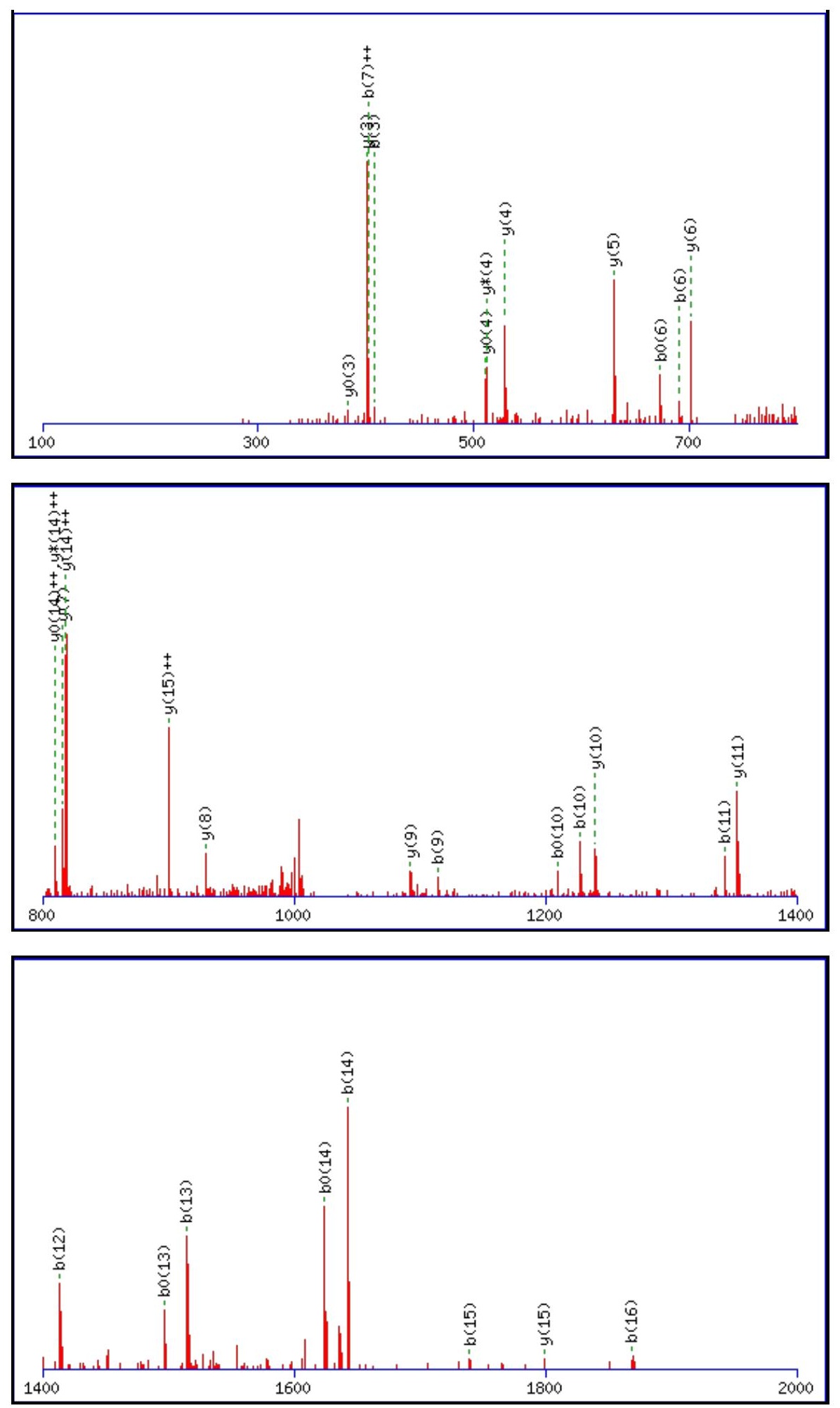

Monoisotopic mass of neutral peptide $\operatorname{Mr}($ calc): 2041.9302

Fixed modifications: Carbamidomethyl (C)

Variable modifications:

M8 : Oxidation $(\mathrm{M})$

N11 : Deamidated_N (N)

Ions Score: 68 Expect: $3.7 \mathrm{e}-005$

Matches (Bold Red): 32/170 fragment ions using 52 most intense peaks

\begin{tabular}{|c|c|c|c|c|r|r|c|c|c|c|c|c|c|c|}
\hline$\#$ & $\mathbf{b}$ & $\mathbf{b}^{++}$ & $\mathbf{b}^{*}$ & $\mathbf{b}^{\boldsymbol{*}^{++}}$ & \multicolumn{1}{|c|}{$\mathbf{b}^{\mathbf{0}}$} & $\mathbf{b}^{\mathbf{0}+}$ & Seq. & $\mathbf{y}$ & $\mathbf{y}^{++}$ & $\mathbf{y}^{\mathbf{*}}$ & $\mathbf{y}^{\mathbf{*}^{++}}$ & $\mathbf{y}^{\mathbf{0}}$ & $\mathbf{y}^{\mathbf{0}+}$ & $\#$ \\
\hline $\mathbf{1}$ & 116.0342 & 58.5207 & & & 98.0237 & 49.5155 & $\mathbf{D}$ & & & & & & & $\mathbf{1 7}$ \\
\hline $\mathbf{2}$ & 245.0768 & 123.0420 & & & 227.0662 & 114.0368 & $\mathbf{E}$ & 1927.9106 & 964.4589 & 1910.8841 & 955.9457 & 1909.9000 & 955.4537 & $\mathbf{1 6}$ \\
\hline $\mathbf{3}$ & $\mathbf{4 0 8 . 1 4 0 1}$ & 204.5737 & & & 390.1296 & 195.5684 & $\mathbf{Y}$ & $\mathbf{1 7 9 8 . 8 6 8 0}$ & $\mathbf{8 9 9 . 9 3 7 6}$ & 1781.8415 & 891.4244 & 1780.8574 & 890.9324 & $\mathbf{1 5}$ \\
\hline $\mathbf{4}$ & 505.1929 & 253.1001 & & & 487.1823 & 244.0948 & $\mathbf{P}$ & 1635.8047 & $\mathbf{8 1 8 . 4 0 6 0}$ & $\mathbf{1 6 1 8 . 7 7 8 1}$ & $\mathbf{8 0 9 . 8 9 2 7}$ & 1617.7941 & $\mathbf{8 0 9 . 4 0 0 7}$ & $\mathbf{1 4}$ \\
\hline & & & & & & & & & & & & & &
\end{tabular}




\begin{tabular}{|r|r|r|r|r|r|r|r|r|r|r|r|r|r|r|r|r|}
$\mathbf{5}$ & 592.2249 & 296.6161 & & & 574.2144 & 287.6108 & S & 1538.7519 & 769.8796 & 1521.7254 & 761.3663 & 1520.7414 & 760.8743 & $\mathbf{1 3}$ \\
\hline $\mathbf{6}$ & $\mathbf{6 9 1 . 2 9 3 3}$ & 346.1503 & & & $\mathbf{6 7 3 . 2 8 2 8}$ & 337.1450 & V & 1451.7199 & 726.3636 & 1434.6933 & 717.8503 & 1433.7093 & 717.3583 & $\mathbf{1 2}$ \\
\hline $\mathbf{7}$ & 804.3774 & $\mathbf{4 0 2 . 6 9 2 3}$ & & & 786.3668 & 393.6871 & L & $\mathbf{1 3 5 2 . 6 5 1 5}$ & 676.8294 & 1335.6249 & 668.3161 & 1334.6409 & 667.8241 & $\mathbf{1 1}$ \\
\hline $\mathbf{8}$ & 951.4128 & 476.2100 & & & 933.4022 & 467.2048 & $\mathbf{M}$ & $\mathbf{1 2 3 9 . 5 6 7 4}$ & 620.2873 & 1222.5409 & 611.7741 & 1221.5568 & 611.2821 & $\mathbf{1 0}$ \\
\hline $\mathbf{9}$ & $\mathbf{1 1 1 4 . 4 7 6 1}$ & 557.7417 & & & 1096.4656 & 548.7364 & $\mathbf{Y}$ & $\mathbf{1 0 9 2 . 5 3 2 0}$ & 546.7696 & 1075.5055 & 538.2564 & 1074.5214 & 537.7644 & $\mathbf{9}$ \\
\hline $\mathbf{1 0}$ & $\mathbf{1 2 2 7 . 5 6 0 2}$ & 614.2837 & & & $\mathbf{1 2 0 9 . 5 4 9 6}$ & 605.2785 & $\mathbf{L}$ & $\mathbf{9 2 9 . 4 6 8 7}$ & 465.2380 & 912.4421 & 456.7247 & 911.4581 & 456.2327 & $\mathbf{8}$ \\
\hline $\mathbf{1 1}$ & $\mathbf{1 3 4 2 . 5 8 7 1}$ & 671.7972 & 1325.5606 & 663.2839 & 1324.5766 & 662.7919 & $\mathbf{N}$ & $\mathbf{8 1 6 . 3 8 4 6}$ & 408.6959 & 799.3581 & 400.1827 & 798.3741 & 399.6907 & $\mathbf{7}$ \\
\hline $\mathbf{1 2}$ & $\mathbf{1 4 1 3 . 6 2 4 3}$ & 707.3158 & 1396.5977 & 698.8025 & 1395.6137 & 698.3105 & $\mathbf{A}$ & $\mathbf{7 0 1 . 3 5 7 7}$ & 351.1825 & 684.3311 & 342.6692 & 683.3471 & 342.1772 & $\mathbf{6}$ \\
\hline $\mathbf{1 3}$ & $\mathbf{1 5 1 4 . 6 7 1 9}$ & 757.8396 & 1497.6454 & 749.3263 & $\mathbf{1 4 9 6 . 6 6 1 4}$ & $\mathbf{7 4 8 . 8 3 4 3}$ & $\mathbf{T}$ & $\mathbf{6 3 0 . 3 2 0 6}$ & 315.6639 & 613.2940 & 307.1506 & 612.3100 & 306.6586 & $\mathbf{5}$ \\
\hline $\mathbf{1 4}$ & $\mathbf{1 6 4 2 . 7 3 0 5}$ & 821.8689 & 1625.7040 & 813.3556 & $\mathbf{1 6 2 4 . 7 1 9 9}$ & 812.8636 & $\mathbf{Q}$ & $\mathbf{5 2 9 . 2 7 2 9}$ & 265.1401 & $\mathbf{5 1 2 . 2 4 6 3}$ & 256.6268 & $\mathbf{5 1 1 . 2 6 2 3}$ & 256.1348 & $\mathbf{4}$ \\
\hline $\mathbf{1 5}$ & $\mathbf{1 7 3 9 . 7 8 3 3}$ & 870.3953 & 1722.7567 & 861.8820 & 1721.7727 & 861.3900 & $\mathbf{P}$ & $\mathbf{4 0 1 . 2 1 4 3}$ & 201.1108 & 384.1878 & 192.5975 & $\mathbf{3 8 3 . 2 0 3 7}$ & 192.1055 & $\mathbf{3}$ \\
\hline $\mathbf{1 6}$ & $\mathbf{1 8 6 8 . 8 2 5 9}$ & 934.9166 & 1851.7993 & 926.4033 & 1850.8153 & 925.9113 & $\mathbf{E}$ & 304.1615 & 152.5844 & 287.1350 & 144.0711 & 286.1510 & 143.5791 & $\mathbf{2}$ \\
\hline $\mathbf{1 7}$ & & & & & & & $\mathbf{R}$ & 175.1190 & 88.0631 & 158.0924 & 79.5498 & & & $\mathbf{1}$ \\
\hline
\end{tabular}
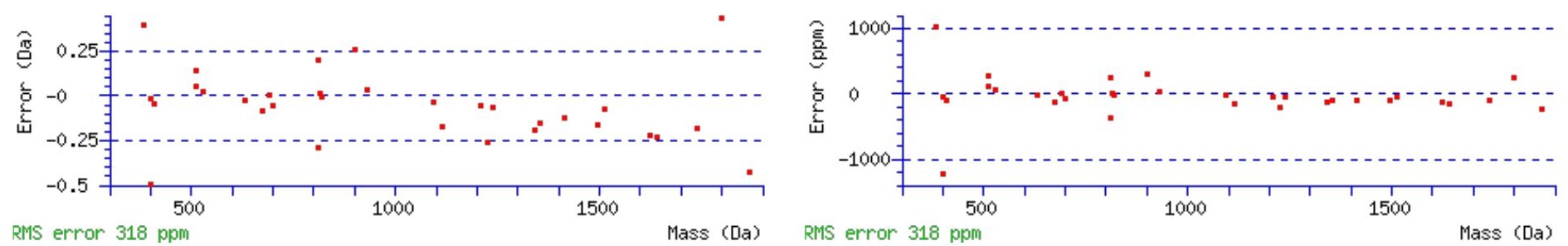

\section{All matches to this query}

\begin{tabular}{|l|c|c|c|}
\hline Score & Mr(calc): & Delta & Sequence \\
\hline 68.3 & 2041.9302 & -0.0019 & DEYPSVLMYLNATQPER \\
\hline 5.1 & 2041.9357 & -0.0074 & NTPYVNEVSNDEALLKR \\
\hline 2.9 & 2041.9308 & -0.0025 & YMRDTGLTALTNLKLR \\
\hline 2.4 & 2041.9308 & -0.0025 & $\underline{\text { YMRDTGLTALTNLKLR }}$ \\
\hline 2.2 & 2041.9218 & 0.0065 & $\underline{\text { ATSRLTTDFHNAEKTNR }}$ \\
\hline 1.4 & 2041.9317 & -0.0034 & $\underline{\text { ASLKPSDSPRSVSSENSSK }}$ \\
\hline 1.4 & 2041.9317 & -0.0034 & $\underline{\text { ASLKPSDSPRSVSSENSSK }}$ \\
\hline 1.0 & 2039.9109 & 2.0174 & $\underline{\text { ARPQPPDGGQCRPPGSQR }}$ \\
\hline 0.0 & 2041.9375 & -0.0091 & $\underline{\text { KRENTFPSSSSCSSSPGVK }}$ \\
\hline
\end{tabular}

Spectrum No: 385; Query: 2075; Rank: 1

\section{Peptide View}

MS/MS Fragmentation of VPFNPNDTFESEFYLDEKR

Found in IPI00200591, Tax_Id=10116 Gene_Symbol=LOC299282 Serine protease inhibitor A3L precursor

Match to Query 2075: 2347.067142 from(783.362990,3+)

Title: 091008RatKidney_NoSalt_26.3801.3801.3.dta

Data file K:INewmanPaper|Piliangl3SubProteomes\Piliang3SP\mgf5ppm\ERLIC_3SubProteomes5ppm.mgf 

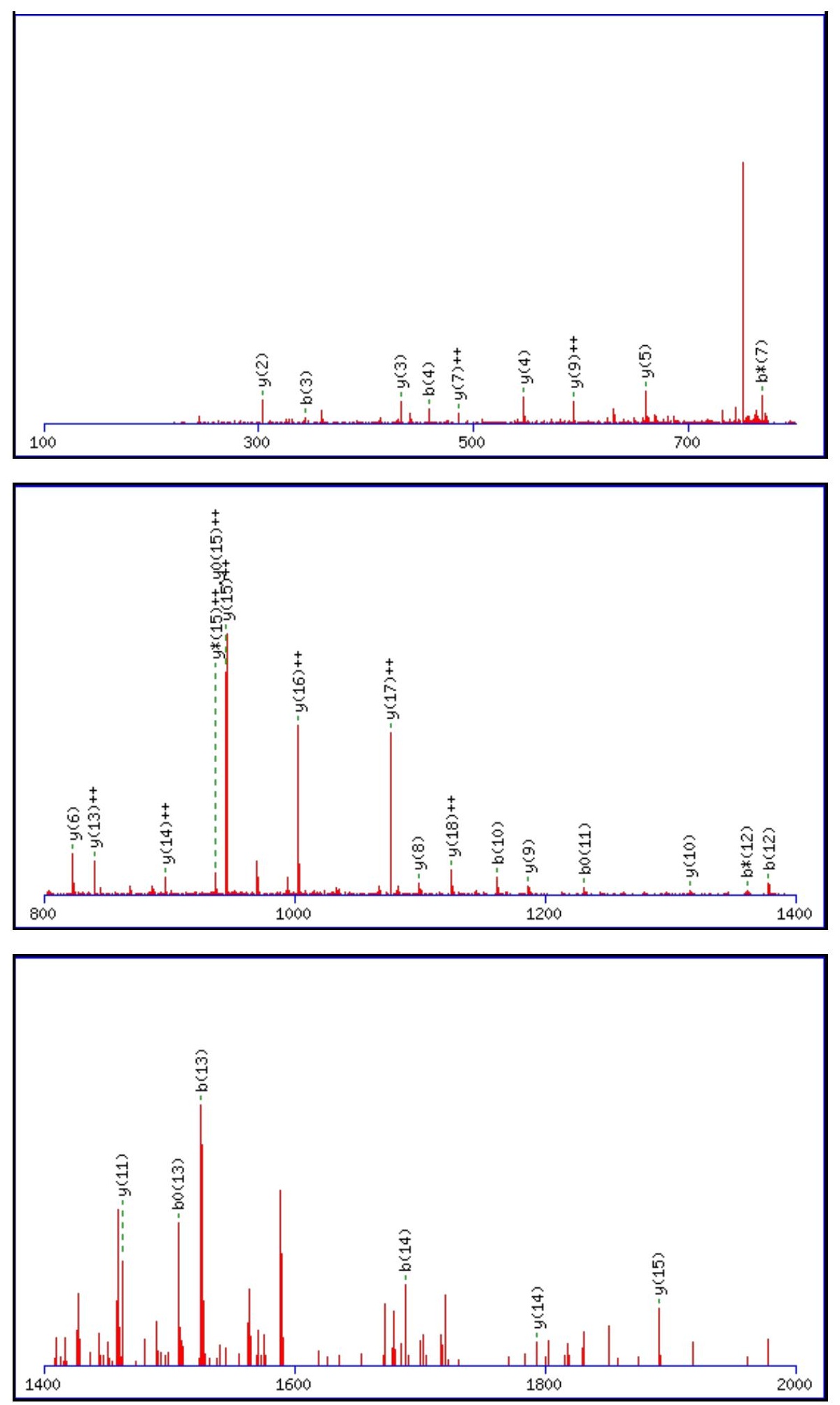

Monoisotopic mass of neutral peptide $\operatorname{Mr}($ calc): 2347.0644

Fixed modifications: Carbamidomethyl (C)

Variable modifications:

N6 : Deamidated $\mathrm{N}(\mathrm{N})$

Ions Score: 68 Expect: $5.4 \mathrm{e}-005$

Matches (Bold Red): 31/194 fragment ions using 61 most intense peaks

\begin{tabular}{|c|c|c|c|c|c|c|c|c|c|c|c|c|c|c|}
\hline$\#$ & b & $\mathbf{b}^{++}$ & $\mathbf{b}^{*}$ & $\mathbf{b}^{*^{++}}$ & $\mathbf{b}^{\mathbf{0}}$ & $\mathbf{b}^{0++}$ & Seq. & $\mathbf{y}$ & $\mathbf{y}^{++}$ & $\mathrm{y}^{*}$ & $\mathrm{y}^{*^{++}}$ & $\mathbf{y}^{0}$ & $y^{0++}$ & \# \\
\hline 1 & 100.0757 & 50.5415 & & & & & V & & & & & & & 19 \\
\hline 2 & 197.1285 & 99.0679 & & & & & $\mathbf{P}$ & 2249.0033 & 1125.0053 & 2231.9768 & 1116.4920 & 2230.9927 & 1116.0000 & 18 \\
\hline 3 & 344.1969 & 172.6021 & & & & & $\mathbf{F}$ & 2151.9505 & 1076.4789 & 2134.9240 & 1067.9656 & 2133.9400 & 1067.4736 & 17 \\
\hline 4 & 458.2398 & 229.6235 & 441.2132 & 221.1103 & & & $\mathbf{N}$ & 2004.8821 & 1002.9447 & 1987.8556 & 994.4314 & 1986.8716 & 993.9394 & 16 \\
\hline 5 & 555.2926 & 278.1499 & 538.2660 & 269.6366 & & & $\mathbf{P}$ & 1890.8392 & 945.9232 & 1873.8127 & 937.4100 & 1872.8286 & 936.9180 & 15 \\
\hline
\end{tabular}




\begin{tabular}{|c|c|c|c|c|c|c|c|c|c|c|c|c|c|c|}
\hline 6 & 670.3195 & 335.6634 & 653.2929 & 327.1501 & & & $\mathbf{N}$ & |1793.7864 & 897.3969 & |1776.7599 & 888.8836 & |1775.7759| & 888.3916 & 14 \\
\hline 7 & 785.3464 & 393.1769 & 768.3199 & 384.6636 & 767.3359 & 384.1716 & D & 1678.7595 & 839.8834 & 1661.7330 & 831.3701 & 1660.7489 & 830.8781 & 13 \\
\hline 8 & 886.3941 & 443.7007 & 869.3676 & 435.1874 & 868.3836 & 434.6954 & $\mathbf{T}$ & 1563.7326 & 782.3699 & 1546.7060 & 773.8566 & 1545.7220 & 773.3646 & 12 \\
\hline 9 & 1033.4625 & 517.2349 & 1016.4360 & 508.7216 & 1015.4520 & 508.2296 & $\mathbf{F}$ & 1462.6849 & 731.8461 & 1445.6583 & 723.3328 & 1444.6743 & 722.8408 & 11 \\
\hline 10 & 1162.5051 & 581.7562 & 1145.4786 & 573.2429 & 1144.4946 & 572.7509 & $\mathbf{E}$ & 1315.6165 & 658.3119 & 1298.5899 & 649.7986 & 1297.6059 & 649.3066 & 10 \\
\hline 11 & 1249.5372 & 625.2722 & 1232.5106 & 616.7589 & 1231.5266 & 616.2669 & S & 1186.5739 & 593.7906 & 1169.5473 & 585.2773 & 1168.5633 & 584.7853 & 9 \\
\hline 12 & 1378.5797 & 689.7935 & 1361.5532 & 681.2802 & 1360.5692 & 680.7882 & $\mathbf{E}$ & 1099.5419 & 550.2746 & 1082.5153 & 541.7613 & 1081.5313 & 541.2693 & 8 \\
\hline 13 & 1525.6482 & 763.3277 & 1508.6216 & 754.8144 & 1507.6376 & 754.3224 & $\mathbf{F}$ & 970.4993 & 485.7533 & 953.4727 & 477.2400 & 952.4887 & 476.7480 & 7 \\
\hline 14 & 1688.7115 & 844.8594 & 1671.6849 & 836.3461 & 1670.7009 & 835.8541 & $\mathbf{Y}$ & 823.4308 & 412.2191 & 806.4043 & 403.7058 & 805.4203 & 403.2138 & 6 \\
\hline 15 & 1801.7956 & 901.4014 & 1784.7690 & 892.8881 & 1783.7850 & 892.3961 & $\mathbf{L}$ & 660.3675 & 330.6874 & 643.3410 & 322.1741 & 642.3569 & 321.6821 & 5 \\
\hline 16 & 1916.8225 & 958.9149 & 1899.7959 & 950.4016 & 1898.8119 & 949.9096 & D & 547.2835 & 274.1454 & 530.2569 & 265.6321 & 529.2729 & 265.1401 & 4 \\
\hline 17 & 2045.8651 & 1023.4362 & 2028.8385 & 1014.9229 & 2027.8545 & 1014.4309 & $\mathbf{E}$ & 432.2565 & 216.6319 & 415.2300 & 208.1186 & 414.2459 & 207.6266 & 3 \\
\hline 18 & 2173.9601 & 1087.4837 & 2156.9335 & 1078.9704 & 2155.9495 & 1078.4784 & $\mathbf{K}$ & 303.2139 & 152.1106 & 286.1874 & 143.5973 & & & 2 \\
\hline 19 & & & & & & & $\mathbf{R}$ & 175.1190 & 88.0631 & 158.0924 & 79.5498 & & & 1 \\
\hline
\end{tabular}
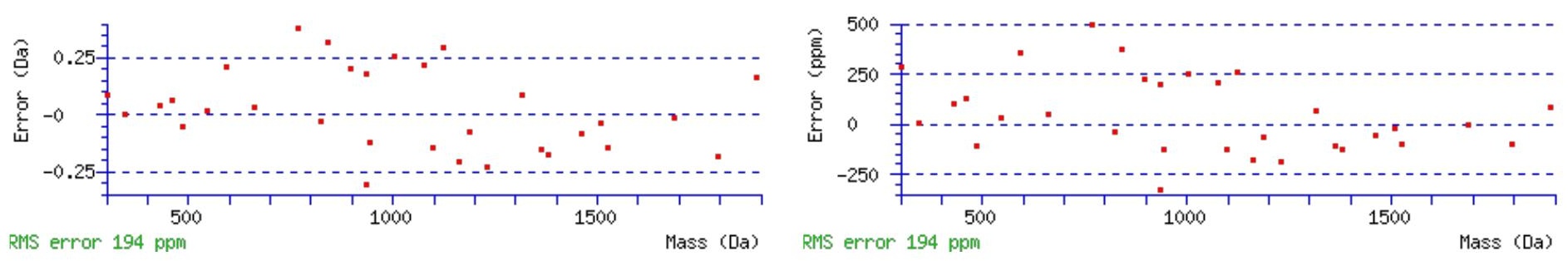

\section{All matches to this query}

\begin{tabular}{|l|l|l|l|}
\hline Score & Mr(calc): & Delta & \multicolumn{1}{|c|}{ Sequence } \\
\hline 68.3 & 2347.0644 & 0.0027 & VPFNPNDTFESEFYLDEKR \\
\hline 50.9 & 2347.0644 & 0.0027 & VPFNPNDTFESEFYLDEKR \\
\hline 28.8 & 2346.0804 & 0.9867 & VPFNPNDTFESEFYLDEKR \\
\hline 9.4 & 2346.0748 & 0.9923 & SSPLRASSLTIMATFLGLLI \\
\hline 5.4 & 2347.0862 & -0.0191 & VDENNPEYLRERNMAPDLR \\
\hline 4.3 & 2346.0748 & 0.9923 & SSPLRASSLTIMATFLGLLI \\
\hline 4.3 & 2347.0861 & -0.0190 & VEARTPNPNLLQSEKSLYK \\
\hline 4.3 & 2347.0861 & -0.0190 & VEARTPNPNLLQSEKSLYK \\
\hline 4.3 & 2347.0861 & -0.0190 & VEARTPNPNLLQSEKSLYK \\
\hline 4.3 & 2347.0861 & -0.0190 & VEARTPNPNLLQSEKSLYK \\
\hline
\end{tabular}

Spectrum No: 386; Query: 636; Rank: 1

\section{Peptide View}

MS/MS Fragmentation of VSNMTLPASAEVLK

Found in IPI00206336, Tax_Id=10116 Gene_Symbol=Lamp1 Lysosome-associated membrane glycoprotein 1 precursor

Match to Query 636: 1475.750968 from(738.882760,2+)

Title: 091008RatKidney_NH4Format01_27.1684.1684.2.dta

Data file K:INewmanPaper|Piliangl3SubProteomes\Piliang3SP\mgf5ppm\ERLIC_3SubProteomes5ppm.mgf 

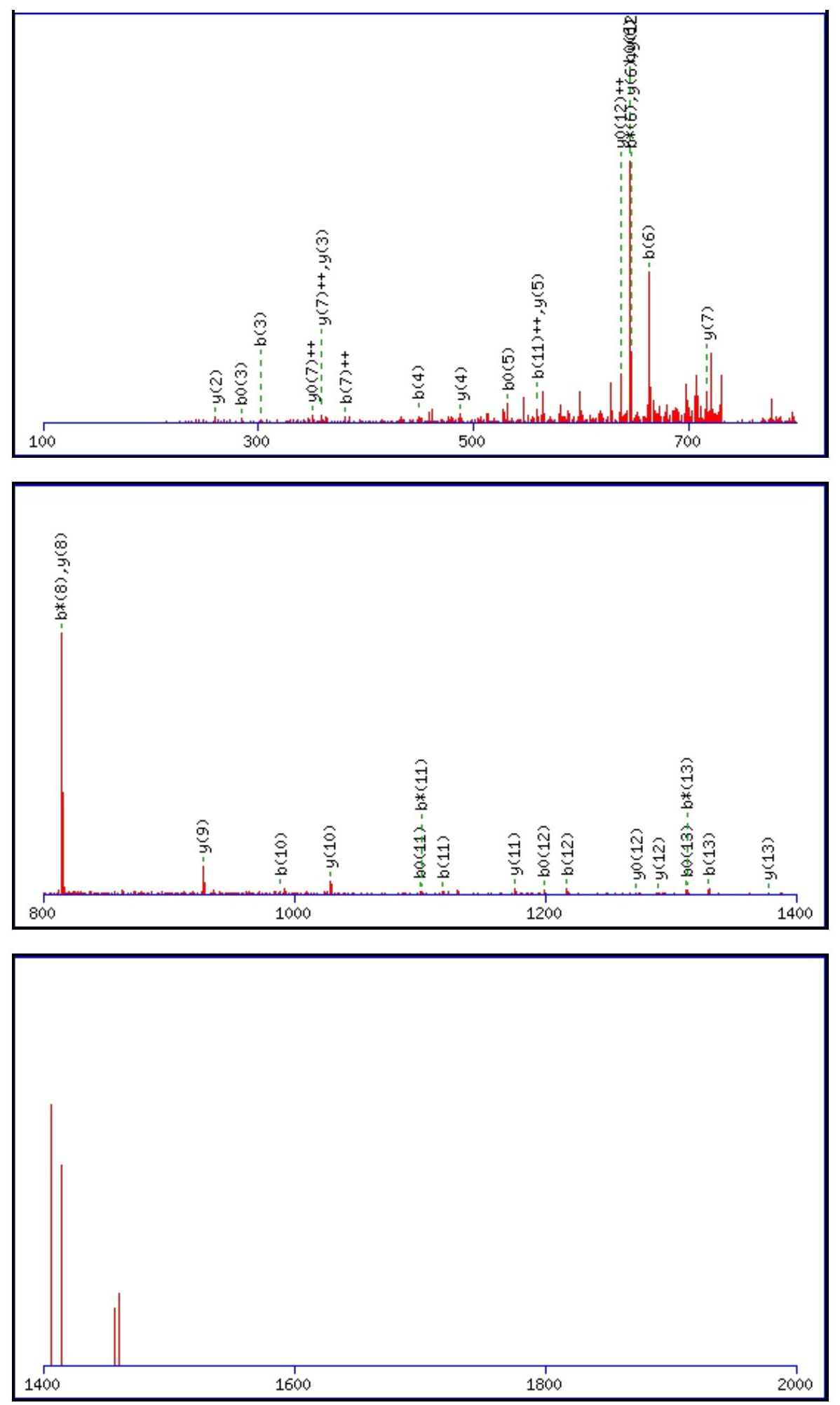

Monoisotopic mass of neutral peptide $\operatorname{Mr}($ calc): 1475.7541

Fixed modifications: Carbamidomethyl (C)

Variable modifications:

N3 : Deamidated_N (N)

M4 : Oxidation (M)

Ions Score: 68 Expect: $2.1 \mathrm{e}-005$

Matches (Bold Red): 36/144 fragment ions using 79 most intense peaks

\begin{tabular}{|c|c|c|c|c|c|c|c|c|c|c|c|c|c|c|}
\hline$\#$ & $\mathbf{b}$ & $\mathbf{b}^{++}$ & $\mathbf{b}^{*}$ & $\mathbf{b}^{\boldsymbol{*}_{++}}$ & $\mathbf{b}^{\mathbf{0}}$ & $\mathbf{b}^{\mathbf{0 + +}}$ & $\mathbf{S e q}$ & $\mathbf{y}$ & $\mathbf{y}^{++}$ & $\mathbf{y}^{\mathbf{*}}$ & $\mathbf{y}^{\mathbf{*}^{++}}$ & $\mathbf{y}^{\mathbf{0}}$ & $\mathbf{y}^{\mathbf{0}+}$ & $\#$ \\
\hline $\mathbf{1}$ & 100.0757 & 50.5415 & & & & & $\mathbf{V}$ & & & & & & & $\mathbf{1 4}$ \\
\hline $\mathbf{2}$ & 187.1077 & 94.0575 & & & 169.0972 & 85.0522 & $\mathbf{S}$ & $\mathbf{1 3 7 7 . 6 9 3 0}$ & 689.3501 & 1360.6665 & 680.8369 & 1359.6824 & 680.3449 & $\mathbf{1 3}$ \\
\hline $\mathbf{3}$ & $\mathbf{3 0 2 . 1 3 4 7}$ & 151.5710 & 285.1081 & 143.0577 & $\mathbf{2 8 4 . 1 2 4 1}$ & 142.5657 & $\mathbf{N}$ & $\mathbf{1 2 9 0 . 6 6 1 0}$ & $\mathbf{6 4 5 . 8 3 4 1}$ & 1273.6344 & 637.3209 & $\mathbf{1 2 7 2 . 6 5 0 4}$ & $\mathbf{6 3 6 . 8 2 8 8}$ & $\mathbf{1 2}$ \\
\hline $\mathbf{4}$ & $\mathbf{4 4 9 . 1 7 0 1}$ & 225.0887 & 432.1435 & 216.5754 & 431.1595 & 216.0834 & $\mathbf{M}$ & $\mathbf{1 1 7 5 . 6 3 4 0}$ & 588.3207 & 1158.6075 & 579.8074 & 1157.6235 & 579.3154 & $\mathbf{1 1}$ \\
\hline & & & & & & & & & & & & & &
\end{tabular}




\begin{tabular}{|c|c|c|c|c|c|c|c|c|c|c|c|c|c|c|}
\hline & 550.2177 & |275.6125 & 533.1912 & 267.0992 & 532.2072 & |266.6072 & $\mathbf{T}$ & |1028.5986 & |514.8030 & 1011.5721 & |506.2897 & 1010.5881 & 505.7977| & 10 \\
\hline 6 & 663.3018 & 332.1545 & 646.2753 & 323.6413 & 645.2912 & 323.1493 & $\mathbf{L}$ & 927.5510 & |464.2791 & 910.5244 & 455.7658 & 909.5404 & 455.2738 & 9 \\
\hline 7 & 760.3546 & 380.6809 & 743.3280 & 372.1676 & 742.3440 & 371.6756 & $\mathbf{P}$ & 814.4669 & 407.7371 & 797.4403 & 399.2238 & 796.4563 & 398.7318 & 8 \\
\hline 8 & 831.3917 & 416.1995 & 814.3651 & 407.6862 & 813.3811 & 407.1942 & A & 717.4141 & 359.2107 & 700.3876 & 350.6974 & 699.4036 & 350.2054 & 7 \\
\hline 9 & 918.4237 & 459.7155 & 901.3972 & 451.2022 & 900.4131 & 450.7102 & S & 646.3770 & 323.6921 & 629.3505 & 315.1789 & 628.3665 & 314.6869 & 6 \\
\hline 10 & 989.4608 & 495.2340 & 972.4343 & 486.7208 & 971.4503 & |486.2288 & A & 559.3450 & |280.1761 & 542.3184 & 271.6629 & 541.3344 & 271.1709 & 5 \\
\hline 11 & 1118.5034 & 559.7553 & 1101.4769 & 551.2421 & 1100.4928 & 550.7501 & E & 488.3079 & 244.6576 & 471.2813 & 236.1443 & 470.2973 & 235.6523 & 4 \\
\hline 12 & 1217.5718 & 609.2896 & 1200.5453 & 600.7763 & 1199.5613 & 600.2843 & V & 359.2653 & |180.1363 & 342.2387 & 171.6230 & & & 3 \\
\hline 13 & 1330.6559 & 665.8316 & 1313.6293 & 657.3183 & 1312.6453 & 656.8263 & L & 260.1969 & 130.6021 & 243.1703 & 122.0888 & & & 2 \\
\hline 14 & & & & & & & $\mathbf{K}$ & 147.1128 & 74.0600 & 130.0863 & 65.5468 & & & 1 \\
\hline
\end{tabular}
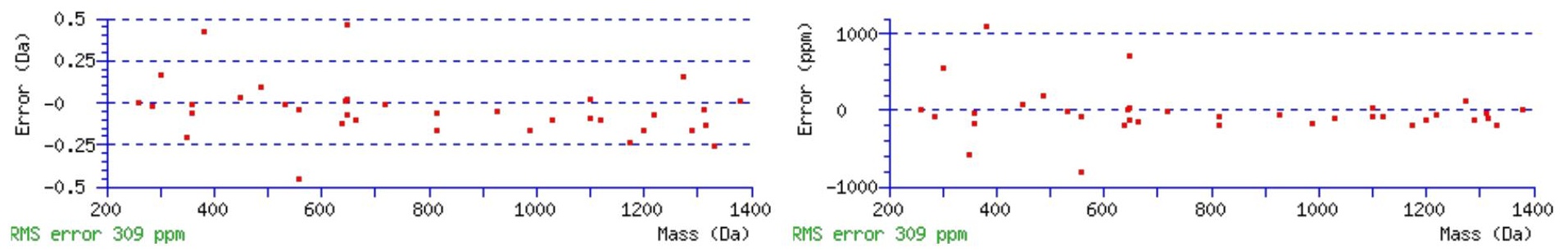

\section{All matches to this query}

\begin{tabular}{|l|l|l|l|}
\hline Score & Mr(calc): & Delta & \multicolumn{1}{c|}{ Sequence } \\
\hline 68.3 & 1475.7541 & -0.0032 & VSNMTLPASAEVLK \\
\hline 14.1 & 1475.7468 & 0.0042 & GDSGDTLAATQSLLK \\
\hline 13.7 & 1475.7620 & -0.0110 & GDFDNLSGKGKPLK \\
\hline 4.3 & 1475.7653 & -0.0144 & MAKTQSQPILNTK \\
\hline 3.7 & 1475.7442 & 0.0067 & TSITALAWSPNGMK \\
\hline 2.2 & 1475.7409 & 0.0101 & SVYWENTLHSLK \\
\hline 2.1 & 1475.7620 & -0.0110 & LLSSYVSVHAESGK \\
\hline 2.0 & 1475.7467 & 0.0042 & SVDSRNTLNDLLK \\
\hline 1.5 & 1475.7497 & 0.0012 & GTTLTNLTGLHR \\
\hline 0.8 & 1475.7541 & -0.0031 & MDLVLELKNNASK \\
\hline
\end{tabular}

Spectrum No: 387; Query: 1630; Rank: 1

\section{Peptide View}

MS/MS Fragmentation of KLHINYNNLTESVGPLPK

Found in IPI00206403, Tax_Id=10116 Gene_Symbol=Lum Lumican precursor

Match to Query 1630: 2037.090908 from(1019.552730,2+)

Title: 091008RatKidney_NH4Format01_25.2595.2595.2.dta

Data file K:INewmanPaper|Piliangl3SubProteomes\Piliang3SP\mgf5ppm\ERLIC_3SubProteomes5ppm.mgf 

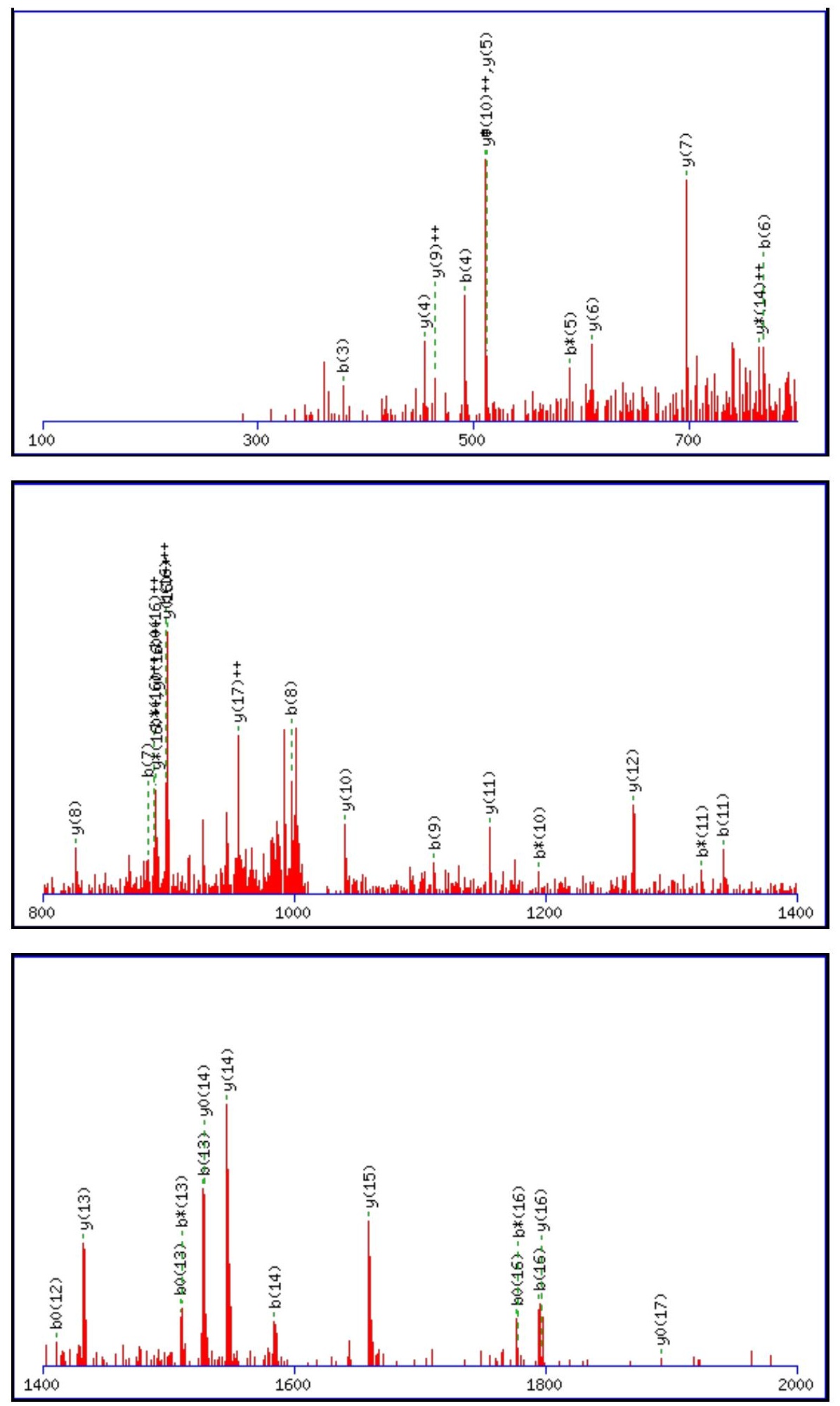

Monoisotopic mass of neutral peptide $\operatorname{Mr}($ calc): 2037.0894

Fixed modifications: Carbamidomethyl (C)

Variable modifications:

N8: Deamidated $\mathrm{N}(\mathrm{N})$

Ions Score: 68 Expect: $1.2 \mathrm{e}-005$

Matches (Bold Red): 44/174 fragment ions using 84 most intense peaks

\begin{tabular}{|r|c|c|c|c|c|c|c|c|c|c|c|c|c|c|}
\hline$\#$ & $\mathbf{b}$ & $\mathbf{b}^{++}$ & $\mathbf{b}^{*}$ & $\mathbf{b}^{\boldsymbol{*}_{++}}$ & $\mathbf{b}^{\mathbf{0}}$ & $\mathbf{b}^{\mathbf{0 + +}}$ & Seq. & $\mathbf{y}$ & $\mathbf{y}^{++}$ & $\mathbf{y}^{\mathbf{*}}$ & $\mathbf{y}^{\boldsymbol{*}^{++}}$ & $\mathbf{y}^{\mathbf{0}}$ & $\mathbf{y}^{\mathbf{0 + +}}$ & $\#$ \\
\hline $\mathbf{1}$ & 129.1022 & 65.0548 & 112.0757 & 56.5415 & & & $\mathbf{K}$ & & & & & & & $\mathbf{1 8}$ \\
\hline $\mathbf{2}$ & 242.1863 & 121.5968 & 225.1598 & 113.0835 & & & $\mathbf{L}$ & 1910.0018 & $\mathbf{9 5 5 . 5 0 4 5}$ & 1892.9752 & 946.9913 & $\mathbf{1 8 9 1 . 9 9 1 2}$ & 946.4993 & $\mathbf{1 7}$ \\
\hline $\mathbf{3}$ & 379.2452 & 190.1262 & 362.2187 & 181.6130 & & & $\mathbf{H}$ & $\mathbf{1 7 9 6 . 9 1 7 7}$ & $\mathbf{8 9 8 . 9 6 2 5}$ & 1779.8912 & $\mathbf{8 9 0 . 4 4 9 2}$ & 1778.9072 & $\mathbf{8 8 9 . 9 5 7 2}$ & $\mathbf{1 6}$ \\
\hline $\mathbf{4}$ & $\mathbf{4 9 2 . 3 2 9 3}$ & 246.6683 & 475.3027 & 238.1550 & & & $\mathbf{I}$ & $\mathbf{1 6 5 9 . 8 5 8 8}$ & 830.4330 & 1642.8323 & 821.9198 & 1641.8483 & 821.4278 & $\mathbf{1 5}$ \\
\hline $\mathbf{5}$ & 606.3722 & 303.6897 & 589.3457 & 295.1765 & & & $\mathbf{N}$ & $\mathbf{1 5 4 6 . 7 7 4 8}$ & 773.8910 & 1529.7482 & $\mathbf{7 6 5 . 3 7 7 7}$ & $\mathbf{1 5 2 8 . 7 6 4 2}$ & $\mathbf{7 6 4 . 8 8 5 7}$ & $\mathbf{1 4}$ \\
\hline
\end{tabular}




\begin{tabular}{|c|c|c|c|c|c|c|c|c|c|c|c|c|c|c|}
\hline 6 & 769.4355 & |385.2214 & 752.4090 & 376.7081 & & & $\mathbf{Y}$ & 1432.7318 & 716.8696 & 1415.7053 & 708.3563 & 1414.7213 & 707.8643 & 13 \\
\hline 7 & 883.4785 & 442.2429 & 866.4519 & 433.7296 & & & $\mathbf{N}$ & 1269.6685 & 635.3379 & 1252.6420 & 626.8246 & 1251.6579 & 626.3326 & 12 \\
\hline 8 & 998.5054 & 499.7563 & 981.4788 & 491.2431 & & & $\mathbf{N}$ & 1155.6256 & 578.3164 & 1138.5990 & 569.8032 & 1137.6150 & 569.3111 & 11 \\
\hline 9 & 1111.5895 & 556.2984 & 1094.5629 & 547.7851 & & & $\mathbf{L}$ & 1040.5986 & 520.8030 & 1023.5721 & 512.2897 & 1022.5881 & 511.7977 & 10 \\
\hline 10 & 1212.6371 & 606.8222 & $\mid 1195.6106$ & 598.3089 & 1194.6266 & 597.8169 & $\mathbf{T}$ & 927.5146 & 464.2609 & 910.4880 & 455.7477 & 909.5040 & 455.2556 & 9 \\
\hline 11 & 1341.6797 & 671.3435 & 1324.6532 & 662.8302 & 1323.6692 & 662.3382 & $\mathbf{E}$ & 826.4669 & 413.7371 & 809.4403 & 405.2238 & 808.4563 & 404.7318 & 8 \\
\hline 12 & 1428.7118 & 714.8595 & 1411.6852 & 706.3462 & 1410.7012 & 705.8542 & S & 697.4243 & 349.2158 & 680.3978 & 340.7025 & 679.4137 & 340.2105 & 7 \\
\hline 13 & 1527.7802 & $\mid 764.3937$ & 1510.7536 & 755.8805 & \begin{tabular}{|l|}
1509.7696 \\
\end{tabular} & 755.3884 & V & 610.3923 & 305.6998 & 593.3657 & 297.1865 & & & 6 \\
\hline 14 & 1584.8016 & 792.9045 & 1567.7751 & 784.3912 & 1566.7911 & 783.8992 & G & 511.3239 & 256.1656 & 494.2973 & 247.6523 & & & 5 \\
\hline 15 & 1681.8544 & 841.4308 & 1664.8279 & 832.9176 & 1663.8438 & 832.4256 & $\mathbf{P}$ & 454.3024 & 227.6548 & 437.2758 & 219.1416 & & & 4 \\
\hline 16 & 1794.9385 & \begin{tabular}{|l|}
897.9729 \\
\end{tabular} & 1777.9119 & 889.4596 & \begin{tabular}{|l|}
1776.9279 \\
\end{tabular} & \begin{tabular}{|l|}
888.9676 \\
\end{tabular} & $\mathbf{L}$ & 357.2496 & 179.1285 & 340.2231 & 170.6152 & & & 3 \\
\hline 17 & 1891.9912 & 946.4993 & 1874.9647 & 937.9860 & 1873.9807 & 937.4940 & $\mathbf{P}$ & 244.1656 & 122.5864 & 227.1390 & 114.0731 & & & 2 \\
\hline 18 & & & & & & & $\mathbf{K}$ & 147.1128 & 74.0600 & 130.0863 & 65.5468 & & & 1 \\
\hline
\end{tabular}
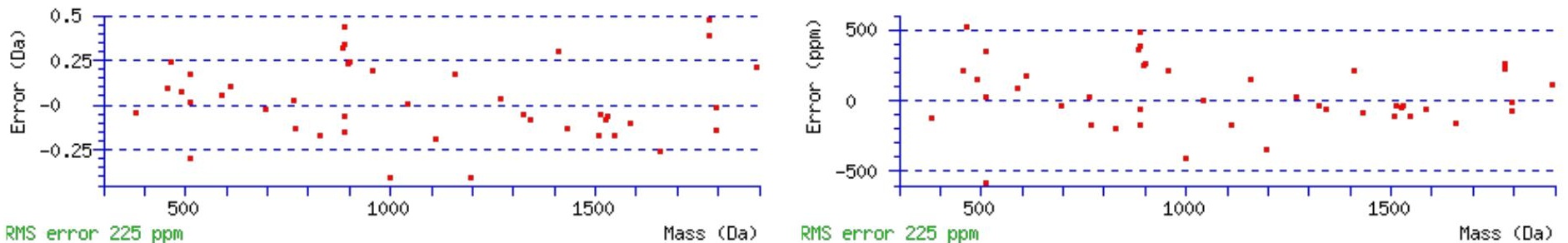

All matches to this query

\begin{tabular}{|l|c|c|c|}
\hline Score & Mr(calc): & Delta & \multicolumn{1}{|c|}{ Sequence } \\
\hline 68.3 & 2037.0894 & 0.0015 & KLHINYNNLTESVGPLPK \\
\hline 55.2 & 2037.0894 & 0.0015 & KLHINYNNLTESVGPLPK \\
\hline 43.3 & 2037.0894 & 0.0015 & KLHINYNNLTESVGPLPK \\
\hline 14.9 & 2036.1054 & 0.9855 & KLHINYNNLTESVGPLPK \\
\hline
\end{tabular}

Spectrum No: 388; Query: 654; Rank: 1

\section{Peptide View}

MS/MS Fragmentation of KYQTLNCSVNVR

Found in IPI00205166, Tax_Id=10116 Gene_Symbol=Itga6 similar to integrin alpha 6 isoform 2

Match to Query 654: 1481.727668 from(741.871110,2+)

Title: 091008RatKidney_NH4Format01_23.978.978.2.dta

Data file K:INewmanPaper|Piliang|3SubProteomes\Piliang3SP\mgf5ppm\ERLIC_3SubProteomes5ppm.mgf 

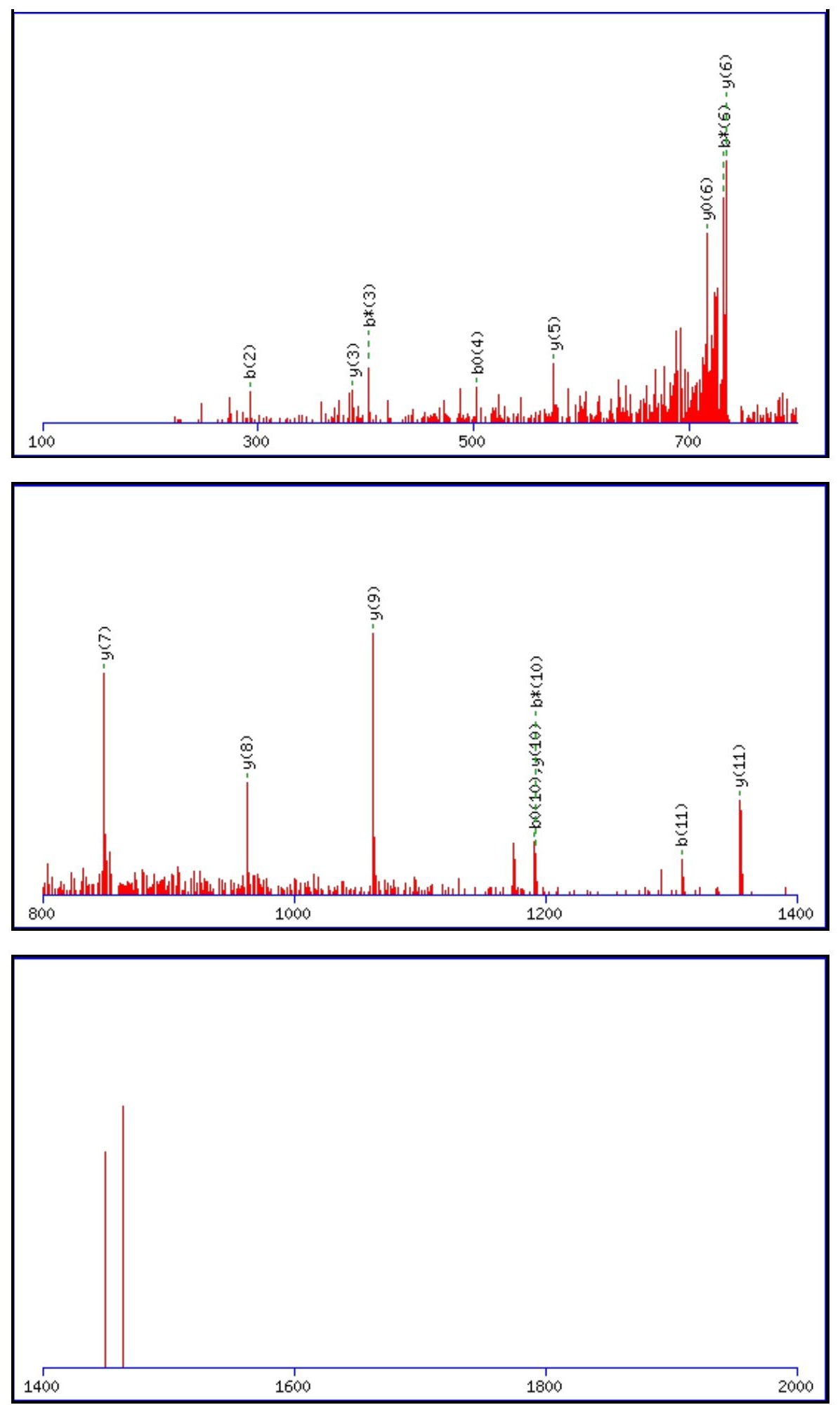

Monoisotopic mass of neutral peptide $\operatorname{Mr}($ calc): 1481.7296

Fixed modifications: Carbamidomethyl (C)

Variable modifications:

N6 : Deamidated $\mathrm{N}(\mathrm{N})$

Ions Score: 68 Expect: $2.3 \mathrm{e}-005$

Matches (Bold Red): 16/118 fragment ions using 26 most intense peaks

\begin{tabular}{|r|c|c|c|c|c|c|c|c|c|c|c|c|c|c|}
\hline$\#$ & $\mathbf{b}$ & $\mathbf{b}^{++}$ & $\mathbf{b}^{*}$ & $\mathbf{b}^{\boldsymbol{*}_{++}}$ & $\mathbf{b}^{\mathbf{0}}$ & $\mathbf{b}^{\mathbf{0 + +}}$ & Seq. & $\mathbf{y}$ & $\mathbf{y}^{++}$ & $\mathbf{y}^{\mathbf{*}}$ & $\mathbf{y}^{\boldsymbol{*}^{++}}$ & $\mathbf{y}^{\mathbf{0}}$ & $\mathbf{y}^{\mathbf{0 + +}}$ & $\#$ \\
\hline $\mathbf{1}$ & 129.1022 & 65.0548 & 112.0757 & 56.5415 & & & $\mathbf{K}$ & & & & & & & $\mathbf{1 2}$ \\
\hline $\mathbf{2}$ & $\mathbf{2 9 2 . 1 6 5 6}$ & 146.5864 & 275.1390 & 138.0731 & & & $\mathbf{Y}$ & $\mathbf{1 3 5 4 . 6 4 2 0}$ & 677.8246 & 1337.6154 & 669.3113 & 1336.6314 & 668.8193 & $\mathbf{1 1}$ \\
\hline $\mathbf{3}$ & 420.2241 & 210.6157 & $\mathbf{4 0 3 . 1 9 7 6}$ & 202.1024 & & & $\mathbf{Q}$ & $\mathbf{1 1 9 1 . 5 7 8 6}$ & 596.2930 & 1174.5521 & 587.7797 & 1173.5681 & 587.2877 & $\mathbf{1 0}$ \\
\hline $\mathbf{4}$ & 521.2718 & 261.1396 & 504.2453 & 252.6263 & $5 \mathbf{5 0 3 . 2 6 1 3}$ & 252.1343 & $\mathbf{T}$ & $\mathbf{1 0 6 3 . 5 2 0 1}$ & 532.2637 & 1046.4935 & 523.7504 & 1045.5095 & 523.2584 & $\mathbf{9}$ \\
\hline $\mathbf{5}$ & 634.3559 & 317.6816 & 617.3293 & 309.1683 & 616.3453 & 308.6763 & $\mathbf{L}$ & $\mathbf{9 6 2 . 4 7 2 4}$ & 481.7398 & 945.4458 & 473.2266 & 944.4618 & 472.7345 & $\mathbf{8}$ \\
\hline
\end{tabular}




\begin{tabular}{|c|c|c|c|c|c|c|c|c|c|c|c|c|c|c|}
\hline 6 & 749.3828 & |375.1951 & 732.3563 & |366.6818 & 731.3723 & |366.1898 & $\mathbf{N}$ & 849.3883 & |425.1978 & 832.3618 & 416.6845 & 831.3778 & |416.1925 & 7 \\
\hline 7 & 909.4135 & 455.2104 & 892.3869 & |446.6971 & 891.4029 & 446.2051 & C & 734.3614 & 367.6843 & 717.3348 & 359.1711 & 716.3508 & 358.6790 & 6 \\
\hline 8 & 996.4455 & |498.7264 & 979.4190 & 490.2131 & 978.4349 & 489.7211 & S & 574.3307 & 287.6690 & 557.3042 & 279.1557 & 556.3202 & 278.6637 & 5 \\
\hline 9 & 1095.5139 & 548.2606 & 1078.4874 & 539.7473 & 1077.5034 & 539.2553 & V & 487.2987 & 244.1530 & 470.2722 & 235.6397 & & & 4 \\
\hline 10 & 1209.5568 & 605.2821 & 1192.5303 & 596.7688 & 1191.5463 & 596.2768 & $\mathbf{N}$ & 388.2303 & |194.6188 & 371.2037 & 186.1055 & & & 3 \\
\hline 11 & 1308.6253 & 654.8163 & 1291.5987 & 646.3030 & 1290.6147 & 645.8110 & V & 274.1874 & 137.5973 & 257.1608 & 129.0840 & & & 2 \\
\hline 12 & & & & & & & $\mathbf{R}$ & 175.1190 & 88.0631 & 158.0924 & 79.5498 & & & 1 \\
\hline
\end{tabular}
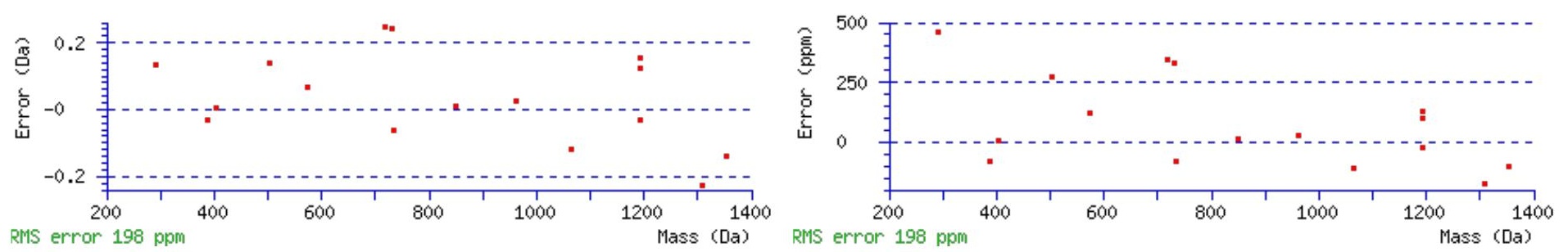

\section{All matches to this query}

\begin{tabular}{|l|l|l|l|}
\hline Score & Mr(calc): & Delta & \multicolumn{1}{c|}{ Sequence } \\
\hline 68.3 & 1481.7296 & -0.0020 & KYQTLNCSVNVR \\
\hline 43.2 & 1481.7296 & -0.0020 & KYOTLNCSVNVR \\
\hline 9.0 & 1481.7140 & 0.0137 & GFSAGSARLPGLNR \\
\hline 3.8 & 1480.7305 & 0.9971 & CFVLMDNILNLK \\
\hline
\end{tabular}

Spectrum No: 389; Query: 603; Rank: 1

\section{Peptide View}

MS/MS Fragmentation of DTNGSQFFITTVK

Found in IPI00213644, Tax_Id=10116 Gene_Symbol=Ppib Peptidyl-prolyl cis-trans isomerase B precursor

Match to Query 603: 1457.696728 from(729.855640,2+)

Title: 091008RatKidney_NH4Format02_21.3308.3308.2.dta

Data file K:INewmanPaper|Piliangl3SubProteomes\Piliang3SP\mgf5ppm\ERLIC_3SubProteomes5ppm.mgf

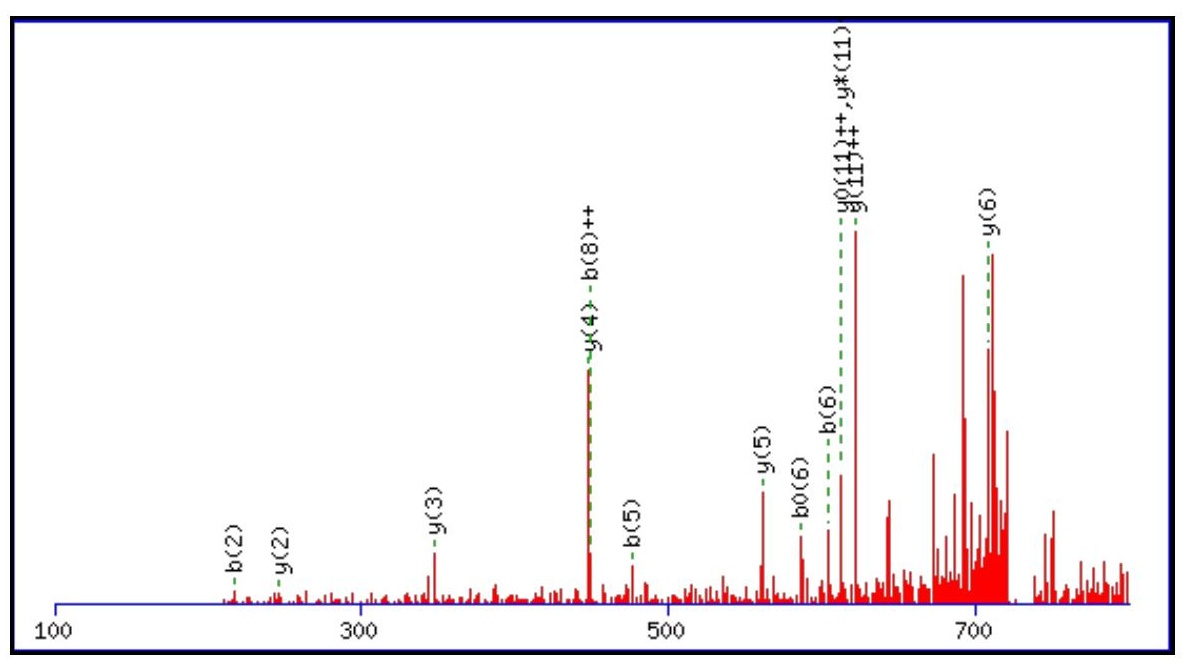



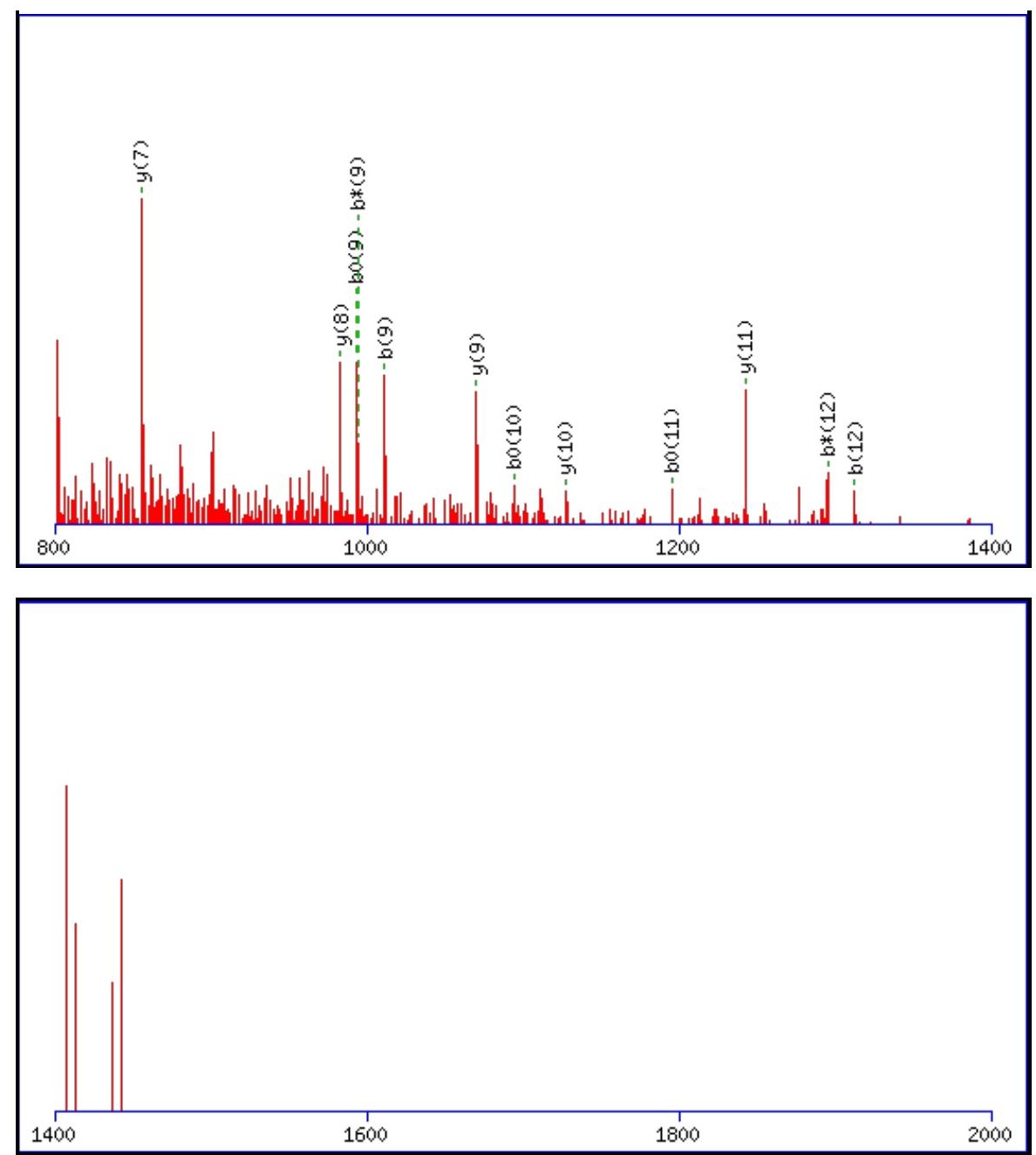

Monoisotopic mass of neutral peptide $\operatorname{Mr}($ calc): 1457.7038

Fixed modifications: Carbamidomethyl (C)

Variable modifications:

N3 : Deamidated $\mathrm{N}(\mathrm{N})$

Ions Score: 68 Expect: $2.5 \mathrm{e}-005$

Matches (Bold Red): 25/136 fragment ions using 41 most intense peaks

\begin{tabular}{|c|c|c|c|c|c|c|c|c|c|c|c|c|c|c|}
\hline \# & b & $\mathbf{b}^{++}$ & $\mathbf{b}^{*}$ & $\mathbf{b}^{*^{++}}$ & $\mathbf{b}^{0}$ & $\mathbf{b}^{0++}$ & Seq. & $\mathbf{y}$ & $\mathbf{y}^{++}$ & $\mathbf{y}^{*}$ & $\mathrm{y}^{*^{++}}$ & $\mathbf{y}^{\mathbf{0}}$ & $\mathbf{y}^{0++}$ & \# \\
\hline 1 & 116.0342 & 58.5207 & & & 98.0237 & 49.5155 & D & & & & & & & 13 \\
\hline 2 & 217.0819 & 109.0446 & & & 199.0713 & 100.0393 & $\mathbf{T}$ & 1343.6842 & 672.3457 & 1326.6576 & 663.8324 & 1325.6736 & 663.3404 & 12 \\
\hline 3 & 332.1088 & 166.5581 & 315.0823 & 158.0448 & 314.0983 & 157.5528 & $\mathbf{N}$ & 1242.6365 & 621.8219 & 1225.6099 & 613.3086 & 1224.6259 & 612.8166 & 11 \\
\hline 4 & 389.1303 & 195.0688 & 372.1037 & 186.5555 & 371.1197 & 186.0635 & $\mathbf{G}$ & 1127.6095 & 564.3084 & 1110.5830 & 555.7951 & 1109.5990 & 555.3031 & 10 \\
\hline 5 & 476.1623 & 238.5848 & 459.1358 & 230.0715 & 458.1518 & 229.5795 & S & 1070.5881 & 535.7977 & 1053.5615 & 527.2844 & 1052.5775 & 526.7924 & 9 \\
\hline 6 & 604.2209 & 302.6141 & 587.1944 & 294.1008 & 586.2103 & 293.6088 & $\mathbf{Q}$ & 983.5560 & 492.2817 & 966.5295 & 483.7684 & 965.5455 & 483.2764 & 8 \\
\hline 7 & 751.2893 & 376.1483 & 734.2628 & 367.6350 & 733.2788 & 367.1430 & $\mathbf{F}$ & 855.4975 & 428.2524 & 838.4709 & 419.7391 & 837.4869 & 419.2471 & 7 \\
\hline 8 & 898.3577 & 449.6825 & 881.3312 & 441.1692 & 880.3472 & 440.6772 & $\mathbf{F}$ & 708.4291 & 354.7182 & 691.4025 & 346.2049 & 690.4185 & 345.7129 & 6 \\
\hline 9 & 1011.4418 & 506.2245 & 994.4152 & 497.7113 & 993.4312 & 497.2193 & I & 561.3606 & 281.1840 & 544.3341 & 272.6707 & 543.3501 & 272.1787 & 5 \\
\hline 10 & 1112.4895 & 556.7484 & 1095.4629 & 548.2351 & 1094.4789 & 547.7431 & $\mathbf{T}$ & 448.2766 & 224.6419 & 431.2500 & 216.1287 & 430.2660 & 215.6366 & 4 \\
\hline 11 & 1213.5372 & 607.2722 & 1196.5106 & 598.7589 & 1195.5266 & 598.2669 & $\mathbf{T}$ & 347.2289 & 174.1181 & 330.2023 & 165.6048 & 329.2183 & 165.1128 & 3 \\
\hline 12 & 1312.6056 & 656.8064 & 1295.5790 & 648.2931 & 1294.5950 & 647.8011 & V & 246.1812 & 123.5942 & 229.1547 & 115.0810 & & & 2 \\
\hline 13 & & & & & & & $\mathbf{K}$ & 147.1128 & 74.0600 & 130.0863 & 65.5468 & & & 1 \\
\hline
\end{tabular}



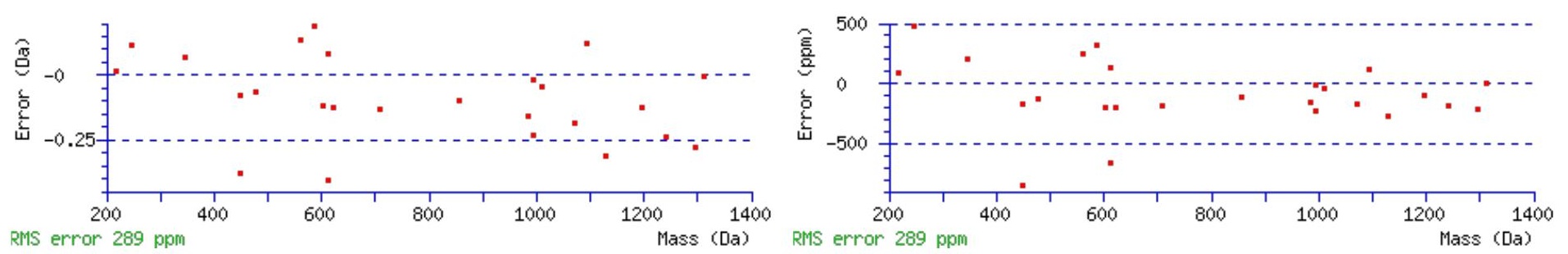

\section{All matches to this query}

\begin{tabular}{|l|l|l|l|}
\hline Score & Mr(calc): & Delta & \multicolumn{1}{|c|}{ Sequence } \\
\hline 68.3 & 1457.7038 & -0.0071 & DTNGSQFFITTVK \\
\hline 8.7 & 1457.6837 & 0.0131 & KMSEQINTSLVK \\
\hline 8.7 & 1457.6837 & 0.0131 & KMSEQINTSLVK \\
\hline 7.6 & 1457.7038 & -0.0071 & FVNSTGYLTEAEK \\
\hline 7.2 & 1457.7071 & -0.0104 & SEYKKMESEISK \\
\hline 7.0 & 1456.6963 & 1.0004 & GNFLLXLTLTDGR \\
\hline 4.0 & 1457.6972 & -0.0005 & SSILPFTPPIVK \\
\hline 3.0 & 1456.6997 & 0.9971 & ETVQKSVCVLSK \\
\hline 3.0 & 1456.6996 & 0.9971 & KMSEQINTSLVK \\
\hline 0.8 & 1457.7085 & -0.0118 & $\underline{\text { HLKNSMNGLDWK }}$ \\
\hline
\end{tabular}

Spectrum No: 390; Query: 518; Rank: 1

\section{Peptide View}

MS/MS Fragmentation of FQLLNFSSSELK

Found in IPI00464765, Tax_Id=10116 Gene_Symbol=Igsf4a Immunoglobulin superfamily, member 4A

Match to Query 518: 1412.720528 from(707.367540,2+)

Title: 100101RatKid_NS_deglyco_25.4270.4270.2.dta

Data file K:INewmanPaper|Piliangl3SubProteomes\Piliang3SP\mgf5ppm\ERLIC_3SubProteomes5ppm.mgf

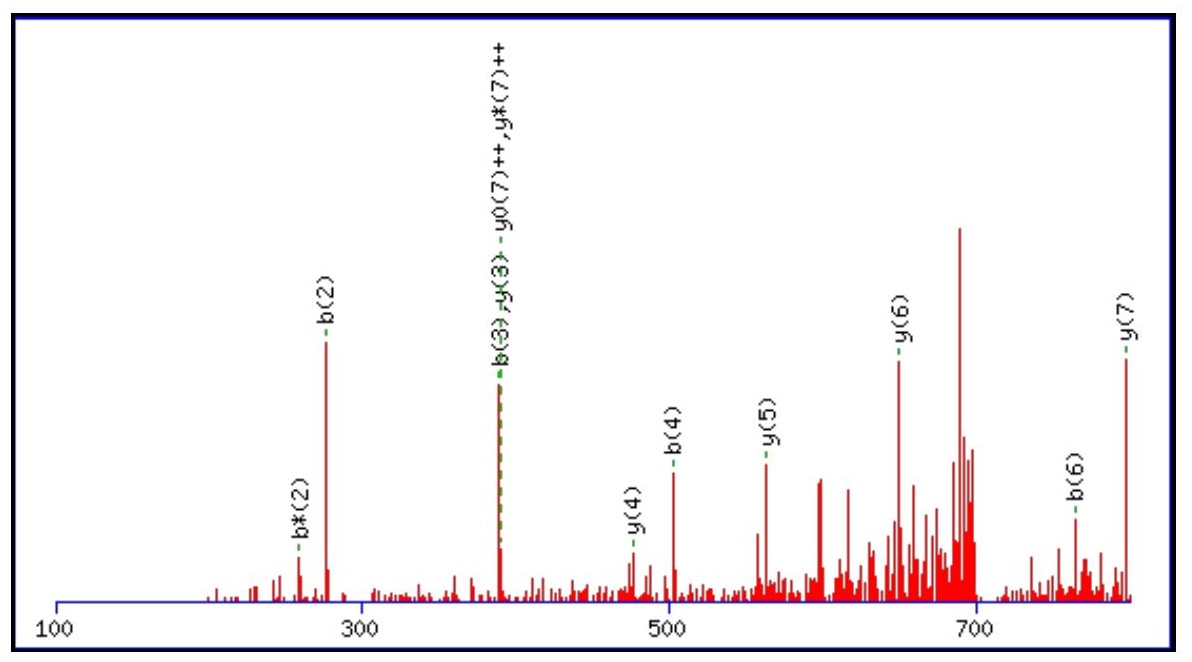



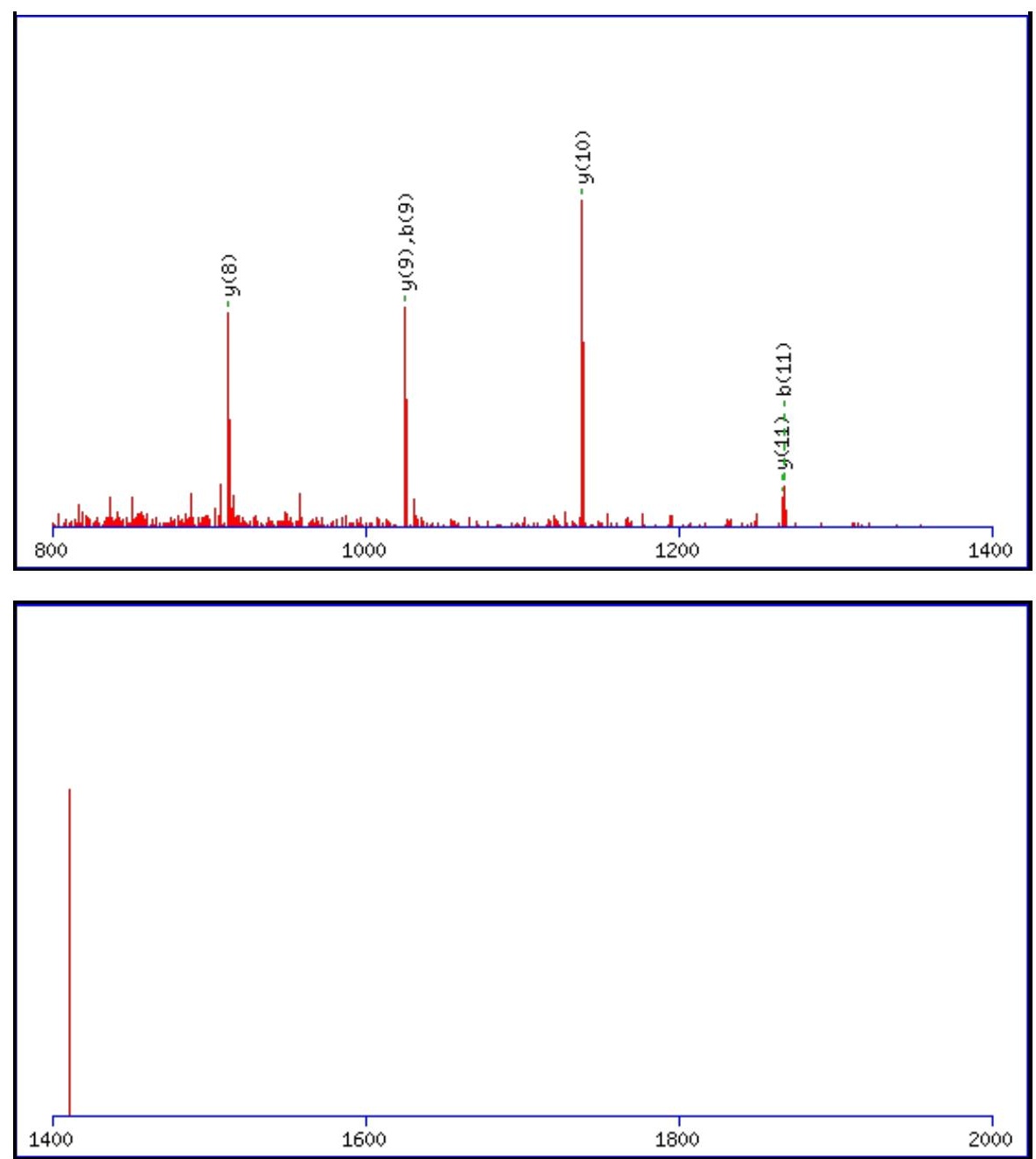

Monoisotopic mass of neutral peptide $\operatorname{Mr}($ calc): 1412.7187

Fixed modifications: Carbamidomethyl (C)

Variable modifications:

N5: Deamidated $\mathrm{N}(\mathrm{N})$

Ions Score: 68 Expect: $1.8 \mathrm{e}-005$

Matches (Bold Red): 18/114 fragment ions using 31 most intense peaks

\begin{tabular}{|c|c|c|c|c|c|c|c|c|c|c|c|c|c|c|}
\hline \# & b & $\mathbf{b}^{++}$ & $\mathbf{b}^{*}$ & $\mathbf{b}^{*^{++}}$ & $\mathbf{b}^{0}$ & $\mathbf{b}^{0++}$ & Seq. & $\mathbf{y}$ & $y^{++}$ & $\mathbf{y}^{*}$ & $\mathrm{y}^{*^{++}}$ & $\mathbf{y}^{0}$ & $\mathbf{y}^{0++}$ & \# \\
\hline 1 & 148.0757 & 74.5415 & & & & & $\mathbf{F}$ & & & & & & & 12 \\
\hline 2 & 276.1343 & 138.5708 & 259.1077 & 130.0575 & & & $\mathbf{Q}$ & 1266.6576 & 633.8324 & 1249.6311 & 625.3192 & 1248.6470 & 624.8272 & 11 \\
\hline 3 & 389.2183 & 195.1128 & 372.1918 & 186.5995 & & & $\mathbf{L}$ & 1138.5990 & 569.8031 & 1121.5725 & 561.2899 & 1120.5885 & 560.7979 & 10 \\
\hline 4 & 502.3024 & 251.6548 & 485.2758 & 243.1416 & & & $\mathbf{L}$ & 1025.5150 & 513.2611 & 1008.4884 & 504.7478 & 1007.5044 & 504.2558 & 9 \\
\hline 5 & 617.3293 & 309.1683 & 600.3028 & 300.6550 & & & $\mathbf{N}$ & 912.4309 & 456.7191 & 895.4043 & 448.2058 & 894.4203 & 447.7138 & 8 \\
\hline 6 & 764.3977 & 382.7025 & 747.3712 & 374.1892 & & & $\mathbf{F}$ & 797.4040 & 399.2056 & 780.3774 & 390.6923 & 779.3934 & 390.2003 & 7 \\
\hline 7 & 851.4298 & 426.2185 & 834.4032 & 417.7053 & 833.4192 & 417.2132 & S & 650.3355 & 325.6714 & 633.3090 & 317.1581 & 632.3250 & 316.6661 & 6 \\
\hline 8 & 938.4618 & 469.7345 & 921.4353 & 461.2213 & 920.4512 & 460.7293 & S & 563.3035 & 282.1554 & 546.2770 & 273.6421 & 545.2930 & 273.1501 & 5 \\
\hline 9 & 1025.4938 & 513.2506 & 1008.4673 & 504.7373 & 1007.4833 & 504.2453 & S & 476.2715 & 238.6394 & 459.2449 & 230.1261 & 458.2609 & 229.6341 & 4 \\
\hline 10 & 1154.5364 & 577.7718 & 1137.5099 & 569.2586 & 1136.5259 & 568.7666 & $E$ & 389.2395 & 195.1234 & 372.2129 & 186.6101 & 371.2289 & 186.1181 & 3 \\
\hline 11 & 1267.6205 & 634.3139 & 1250.5939 & 625.8006 & 1249.6099 & 625.3086 & $\mathbf{L}$ & 260.1969 & 130.6021 & 243.1703 & 122.0888 & & & 2 \\
\hline 12 & & & & & & & $\mathbf{K}$ & 147.1128 & 74.0600 & 130.0863 & 65.5468 & & & 1 \\
\hline
\end{tabular}



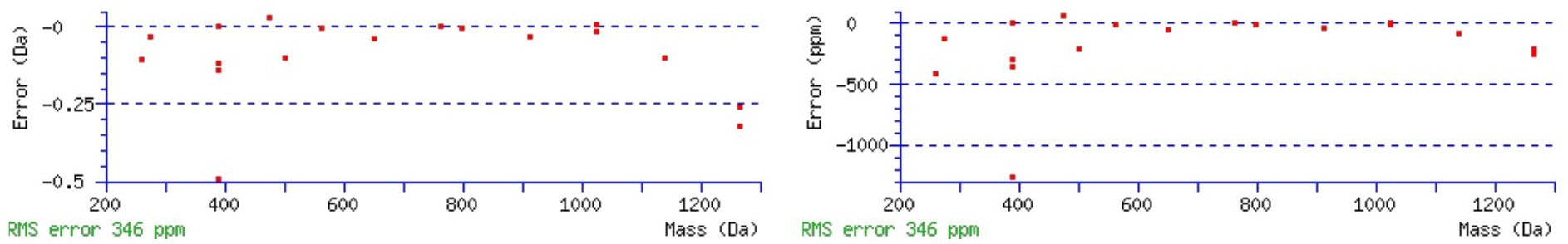

\section{All matches to this query}

\begin{tabular}{|l|l|c|l|}
\hline Score & Mr(calc): & Delta & \multicolumn{1}{|c|}{ Sequence } \\
\hline 68.2 & 1412.7187 & 0.0018 & FQLLNFSSSELK \\
\hline 29.4 & 1412.7156 & 0.0050 & CMLLAHADLIEK \\
\hline 17.4 & 1412.7334 & -0.0128 & VSIVDLTCHLEK \\
\hline 16.2 & 1412.7300 & -0.0094 & VRVESGYFSLEK \\
\hline 13.7 & 1411.7307 & 0.9899 & NDPNQLNNLIKK \\
\hline 12.6 & 1412.7177 & 0.0028 & KFNATERVLQK \\
\hline 12.1 & 1411.7146 & 1.0060 & KMKLVESQLEK \\
\hline 11.4 & 1411.7307 & 0.9899 & NDPNQLNNLIKK \\
\hline 11.4 & 1411.7307 & 0.9899 & NDPNQLNNLIKK \\
\hline 11.4 & 1411.7152 & 1.0053 & YYYVKNIIKK \\
\hline
\end{tabular}

Spectrum No: 391; Query: 887; Rank: 1

\section{Peptide View}

MS/MS Fragmentation of DMSDGFISNLTIQR

Found in IPI00209863, Tax_Id=10116 Gene_Symbol=P4ha1 Prolyl 4-hydroxylase subunit alpha-1 precursor

Match to Query 887: 1596.750428 from(799.382490,2+)

Title: 100101RatKid_NS_deglyco_19.4358.4358.2.dta

Data file K:INewmanPaper|Piliangl3SubProteomes\Piliang3SP\mgf5ppm\ERLIC_3SubProteomes5ppm.mgf

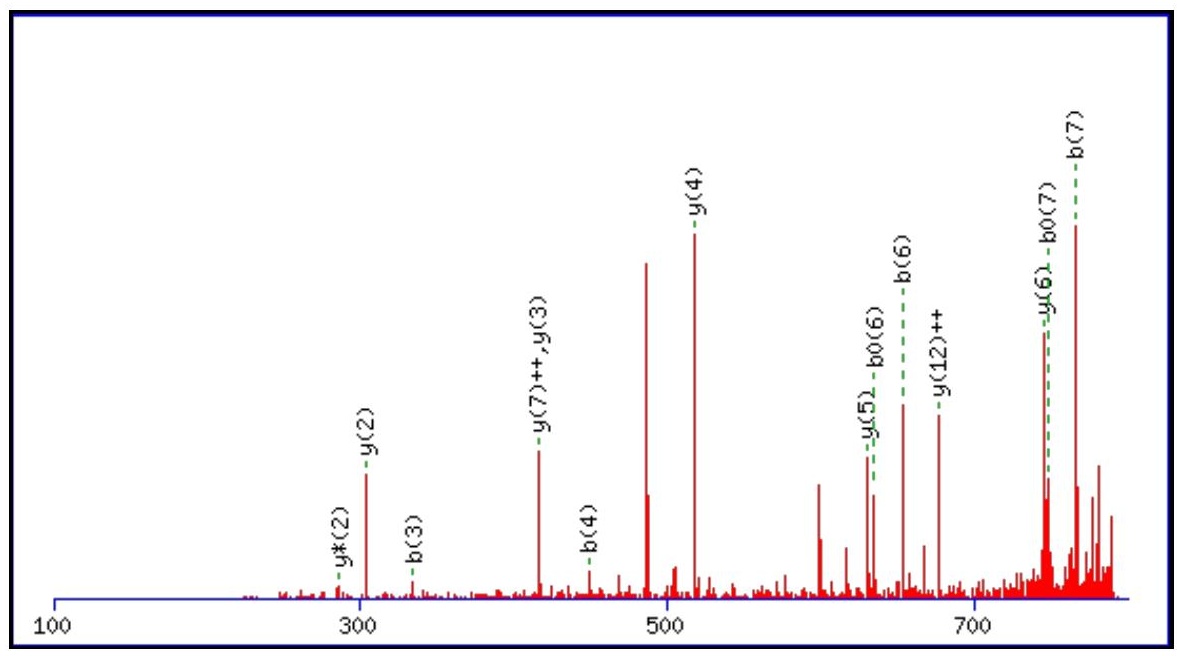



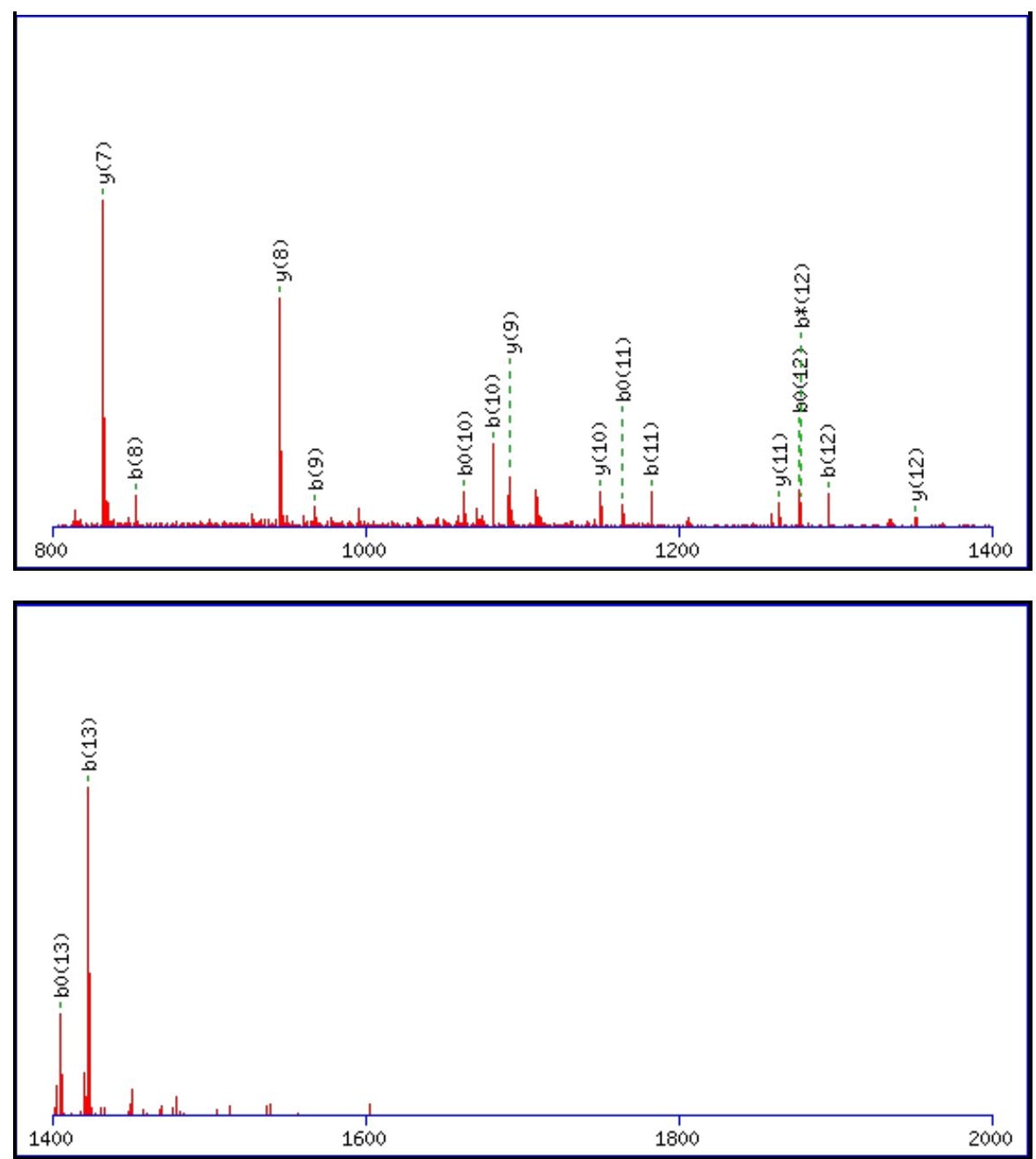

Monoisotopic mass of neutral peptide $\operatorname{Mr}($ calc): 1596.7454

Fixed modifications: Carbamidomethyl (C)

Variable modifications:

N9: Deamidated $\mathrm{N}(\mathrm{N})$

Ions Score: 68 Expect: $2.7 \mathrm{e}-005$

Matches (Bold Red): 31/134 fragment ions using 60 most intense peaks

\begin{tabular}{|c|c|c|c|c|c|c|c|c|c|c|c|c|c|c|}
\hline \# & b & $\mathbf{b}^{++}$ & b* & $\mathbf{b}^{*^{++}}$ & $\mathbf{b}^{0}$ & $\mathbf{b}^{0++}$ & Seq. & $\mathbf{y}$ & $\mathbf{y}^{++}$ & $\mathbf{y}^{*}$ & $\mathrm{y}^{*^{++}}$ & $\mathbf{y}^{0}$ & $\mathbf{y}^{0++}$ & \# \\
\hline 1 & 116.0342 & 58.5207 & & & 98.0237 & 49.5155 & D & & & & & & & 14 \\
\hline 2 & 247.0747 & 124.0410 & & & 229.0641 & 115.0357 & $\mathbf{M}$ & 1482.7257 & 741.8665 & 1465.6991 & 733.3532 & 1464.7151 & 732.8612 & 13 \\
\hline 3 & 334.1067 & 167.5570 & & & 316.0962 & 158.5517 & S & 1351.6852 & 676.3462 & 1334.6587 & 667.8330 & 1333.6746 & 667.3410 & 12 \\
\hline 4 & 449.1337 & 225.0705 & & & 431.1231 & 216.0652 & D & 1264.6532 & 632.8302 & 1247.6266 & 624.3170 & 1246.6426 & 623.8249 & 11 \\
\hline 5 & 506.1551 & 253.5812 & & & 488.1446 & 244.5759 & G & 1149.6262 & 575.3168 & 1132.5997 & 566.8035 & 1131.6157 & 566.3115 & 10 \\
\hline 6 & 653.2236 & 327.1154 & & & 635.2130 & 318.1101 & $\mathbf{F}$ & 1092.6048 & 546.8060 & 1075.5782 & 538.2928 & 1074.5942 & 537.8007 & 9 \\
\hline 7 & 766.3076 & 383.6574 & & & 748.2971 & 374.6522 & I & 945.5364 & 473.2718 & 928.5098 & 464.7585 & 927.5258 & 464.2665 & 8 \\
\hline 8 & 853.3396 & 427.1735 & & & 835.3291 & 418.1682 & $\mathrm{~S}$ & 832.4523 & 416.7298 & 815.4258 & 408.2165 & 814.4417 & 407.7245 & 7 \\
\hline 9 & 968.3666 & 484.6869 & 951.3400 & 476.1737 & 950.3560 & 475.6816 & $\mathbf{N}$ & 745.4203 & 373.2138 & 728.3937 & 364.7005 & 727.4097 & 364.2085 & 6 \\
\hline 10 & 1081.4506 & 541.2290 & 1064.4241 & 532.7157 & 1063.4401 & 532.2237 & $\mathbf{L}$ & 630.3933 & 315.7003 & 613.3668 & 307.1870 & 612.3828 & 306.6950 & 5 \\
\hline 11 & 1182.4983 & 591.7528 & 1165.4718 & 583.2395 & 1164.4878 & 582.7475 & $\mathbf{T}$ & 517.3093 & 259.1583 & 500.2827 & 250.6450 & 499.2987 & 250.1530 & 4 \\
\hline 12 & 1295.5824 & 648.2948 & 1278.5558 & 639.7816 & 1277.5718 & 639.2895 & I & 416.2616 & 208.6344 & 399.2350 & 200.1212 & & & 3 \\
\hline 13 & 1423.6410 & 712.3241 & 1406.6144 & 703.8108 & 1405.6304 & 703.3188 & $\mathbf{Q}$ & 303.1775 & 152.0924 & 286.1510 & 143.5791 & & & 2 \\
\hline 14 & & & & & & & $\mathbf{R}$ & 175.1190 & 88.0631 & 158.0924 & 79.5498 & & & 1 \\
\hline
\end{tabular}



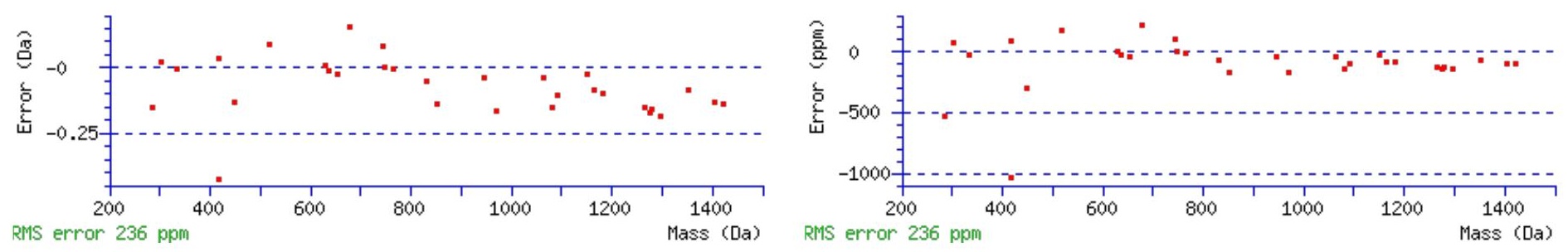

\section{All matches to this query}

\begin{tabular}{|l|l|l|l|}
\hline Score & Mr(calc): & Delta & \multicolumn{1}{|c|}{ Sequence } \\
\hline 68.2 & 1596.7454 & 0.0051 & DMSDGFISNLTIQR \\
\hline 16.1 & 1595.7614 & 0.9891 & DMSDGFISNLTIQR \\
\hline 8.5 & 1594.7409 & 2.0096 & ITYEPITTTLKR \\
\hline 8.2 & 1596.7443 & 0.0062 & MNLQAQSKAQNKR \\
\hline 7.4 & 1596.7466 & 0.0038 & HGLPFVTLTLPSR \\
\hline 7.4 & 1596.7466 & 0.0038 & HGLPFVTLTLPSR \\
\hline 7.0 & 1594.7521 & 1.9983 & VFVATSSGSIAIRK \\
\hline 6.3 & 1595.7629 & 0.9876 & IQMPLSFMWNXK \\
\hline 6.3 & 1595.7461 & 1.0044 & TDSISIQSMIESNR \\
\hline 6.3 & 1594.7331 & 2.0174 & RSECVSEMLDLEK \\
\hline
\end{tabular}

Spectrum No: 392; Query: 1294; Rank: 1

\section{Peptide View}

MS/MS Fragmentation of GTFTDCALANMTQQIR

Found in IPI00372839, Tax_Id=10116 Gene_Symbol=Col6a2 similar to procollagen, type VI, alpha 2

Match to Query 1294: 1826.837468 from(914.426010,2+)

Title: 100101RatKid_NS_deglyco_12.4502.4502.2.dta

Data file K:INewmanPaper|Piliangl3SubProteomes\Piliang3SP\mgf5ppm\ERLIC_3SubProteomes5ppm.mgf

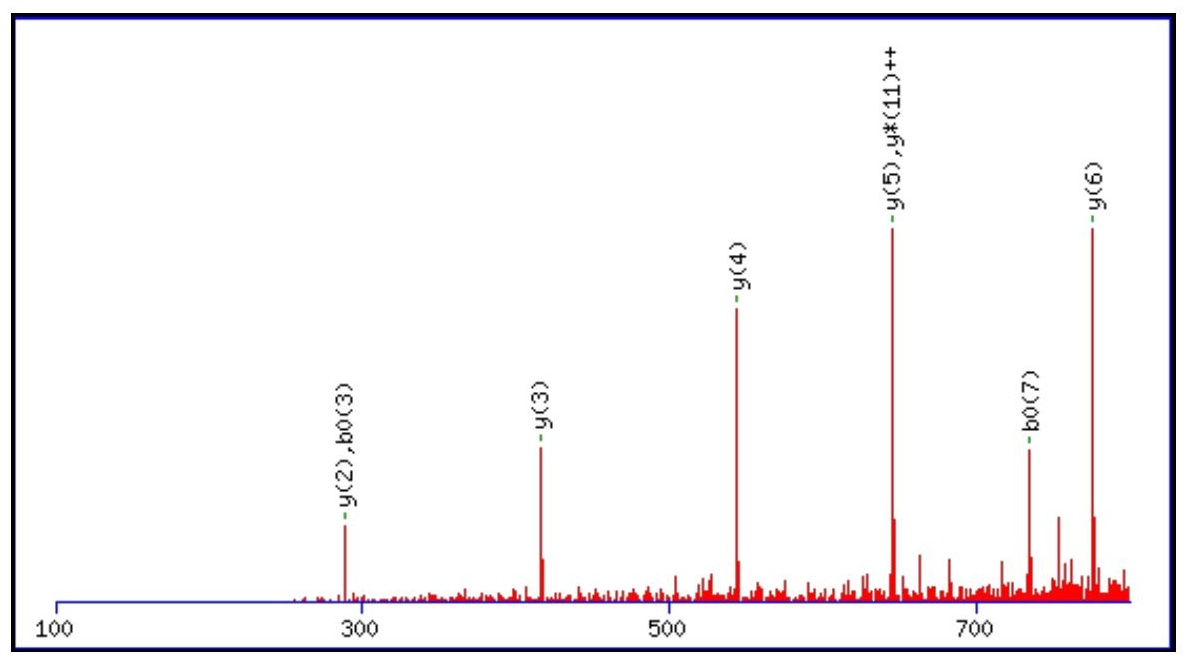



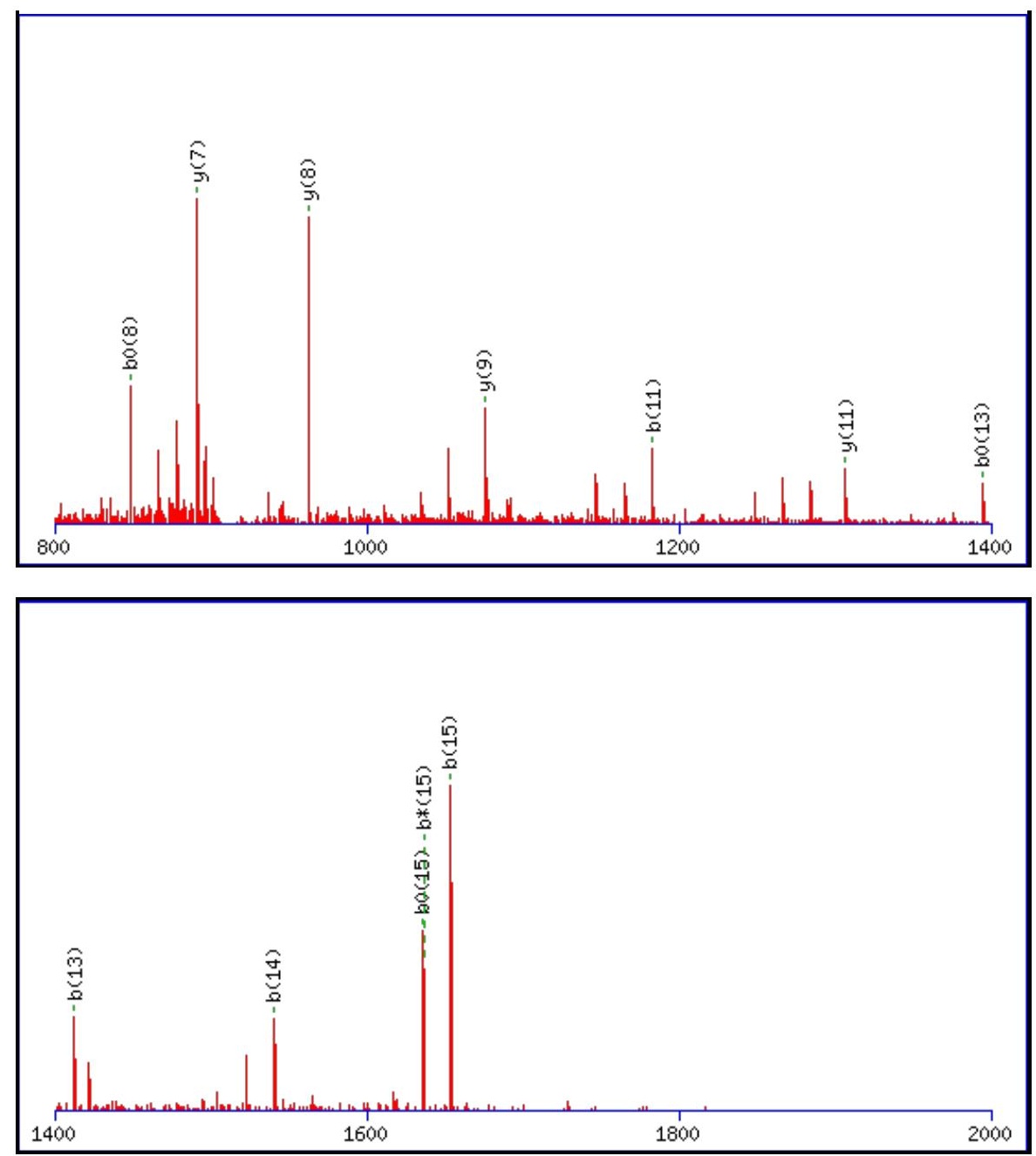

Monoisotopic mass of neutral peptide $\operatorname{Mr}($ calc): 1826.8291

Fixed modifications: Carbamidomethyl (C)

Variable modifications:

N10 : Deamidated N (N)

Ions Score: 68 Expect: $3.4 \mathrm{e}-005$

Matches (Bold Red): 20/152 fragment ions using 21 most intense peaks

\begin{tabular}{|c|c|c|c|c|c|c|c|c|c|c|c|c|c|c|}
\hline \# & b & $\mathbf{b}^{++}$ & b* & $\mathbf{b}^{*^{++}}$ & $\mathbf{b}^{0}$ & $\mathbf{b}^{0++}$ & Seq. & $\mathbf{y}$ & $\mathrm{y}^{++}$ & $\mathbf{y}^{*}$ & $\mathbf{y}^{*^{++}}$ & $\mathbf{y}^{0}$ & $y^{0++}$ & \# \\
\hline 1 & 58.0287 & 29.5180 & & & & & G & & & & & & & 16 \\
\hline 2 & 159.0764 & 80.0418 & & & 141.0659 & 71.0366 & $\mathbf{T}$ & 1770.8149 & 885.9111 & 1753.7884 & 877.3978 & 1752.8044 & 876.9058 & 15 \\
\hline 3 & 306.1448 & 153.5761 & & & 288.1343 & 144.5708 & $\mathbf{F}$ & 1669.7672 & 835.3873 & 1652.7407 & 826.8740 & 1651.7567 & 826.3820 & 14 \\
\hline 4 & 407.1925 & 204.0999 & & & 389.1819 & 195.0946 & $\mathbf{T}$ & 1522.6988 & 761.8531 & 1505.6723 & 753.3398 & 1504.6883 & 752.8478 & 13 \\
\hline 5 & 522.2195 & 261.6134 & & & 504.2089 & 252.6081 & D & 1421.6512 & 711.3292 & 1404.6246 & 702.8159 & 1403.6406 & 702.3239 & 12 \\
\hline 6 & 682.2501 & 341.6287 & & & 664.2395 & 332.6234 & C & 1306.6242 & 653.8157 & 1289.5977 & 645.3025 & 1288.6136 & 644.8105 & 11 \\
\hline 7 & 753.2872 & 377.1472 & & & 735.2767 & 368.1420 & A & 1146.5936 & 573.8004 & 1129.5670 & 565.2871 & 1128.5830 & 564.7951 & 10 \\
\hline 8 & 866.3713 & 433.6893 & & & 848.3607 & 424.6840 & $\mathbf{L}$ & 1075.5565 & 538.2819 & 1058.5299 & 529.7686 & 1057.5459 & 529.2766 & 9 \\
\hline 9 & 937.4084 & 469.2078 & & & 919.3978 & 460.2026 & A & 962.4724 & 481.7398 & 945.4458 & 473.2266 & 944.4618 & 472.7345 & 8 \\
\hline 10 & 1052.4353 & 526.7213 & 1035.4088 & 518.2080 & 1034.4248 & 517.7160 & $\mathbf{N}$ & 891.4353 & 446.2213 & 874.4087 & 437.7080 & 873.4247 & 437.2160 & 7 \\
\hline 11 & 1183.4758 & 592.2415 & 1166.4493 & 583.7283 & 1165.4653 & 583.2363 & $\mathbf{M}$ & 776.4083 & 388.7078 & 759.3818 & 380.1945 & 758.3978 & 379.7025 & 6 \\
\hline 12 & 1284.5235 & 642.7654 & 1267.4969 & 634.2521 & 1266.5129 & 633.7601 & $\mathbf{T}$ & 645.3679 & 323.1876 & 628.3413 & 314.6743 & 627.3573 & 314.1823 & 5 \\
\hline 13 & 1412.5821 & 706.7947 & 1395.5555 & 698.2814 & 1394.5715 & 697.7894 & $\mathbf{Q}$ & 544.3202 & 272.6637 & 527.2936 & 264.1504 & & & 4 \\
\hline 14 & 1540.6407 & 770.8240 & 1523.6141 & 762.3107 & 1522.6301 & 761.8187 & $\mathbf{Q}$ & 416.2616 & 208.6344 & 399.2350 & 200.1212 & & & 3 \\
\hline 15 & 1653.7247 & 827.3660 & 1636.6982 & 818.8527 & 1635.7142 & 818.3607 & I & 288.2030 & 144.6051 & 271.1765 & 136.0919 & & & 2 \\
\hline 16 & & & & & & & $\mathbf{R}$ & 175.1190 & 88.0631 & 158.0924 & 79.5498 & & & 1 \\
\hline
\end{tabular}



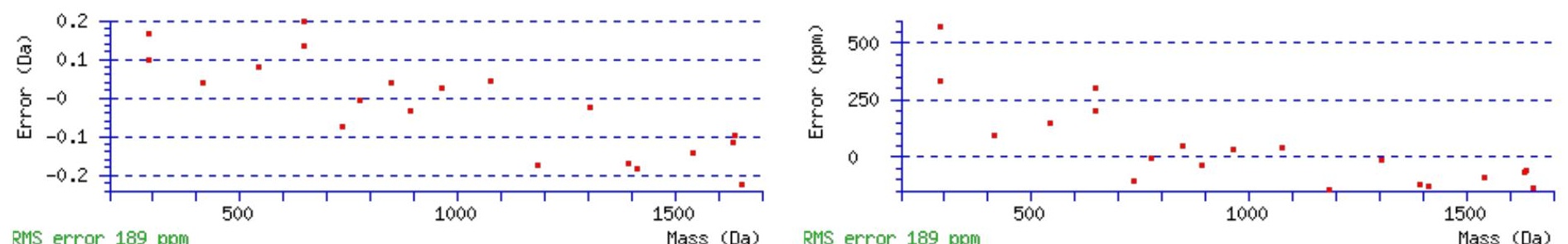

\section{All matches to this query}

\begin{tabular}{|l|l|c|l|}
\hline Score & Mr(calc): & Delta & \multicolumn{1}{c|}{ Sequence } \\
\hline 68.1 & 1826.8291 & 0.0084 & GTFTDCALANMTQQIR \\
\hline 14.0 & 1825.8451 & 0.9924 & GTFTDCALANMTQQIR \\
\hline 3.7 & 1826.8556 & -0.0181 & TIFNWTCSCNGAKLR \\
\hline 3.0 & 1826.8291 & 0.0084 & MNERMNTTEIFDGIR \\
\hline 2.5 & 1826.8291 & 0.0084 & MNERMNTTEIFDGIR \\
\hline 1.1 & 1826.8331 & 0.0043 & TLWMPSSTSXWSMLR \\
\hline 0.9 & 1824.8126 & 2.0249 & EGGNFLDTTDTGNSVAAR \\
\hline 0.6 & 1826.8460 & -0.0085 & HYLLVGAGAIGCEMLK \\
\hline 0.3 & 1826.8240 & 0.0135 & NYGKFLEEYTSQLR \\
\hline 0.3 & 1826.8240 & 0.0135 & NYGKFLEEYTSQLR \\
\hline
\end{tabular}

Spectrum No: 393; Query: 1926; Rank: 1

\section{Peptide View}

MS/MS Fragmentation of KNTTGLPQNILSQVHQLNSK

Found in IPI00196620, Tax_Id=10116 Gene_Symbol=Cubn Cubilin precursor

Match to Query 1926: 2220.184242 from(741.068690,3+)

Title: 091008RatKidney_NH4Format01_23.2589.2589.3.dta

Data file K:INewmanPaper|Piliang|3SubProteomes\Piliang3SP\mgf5ppm\ERLIC_3SubProteomes5ppm.mgf

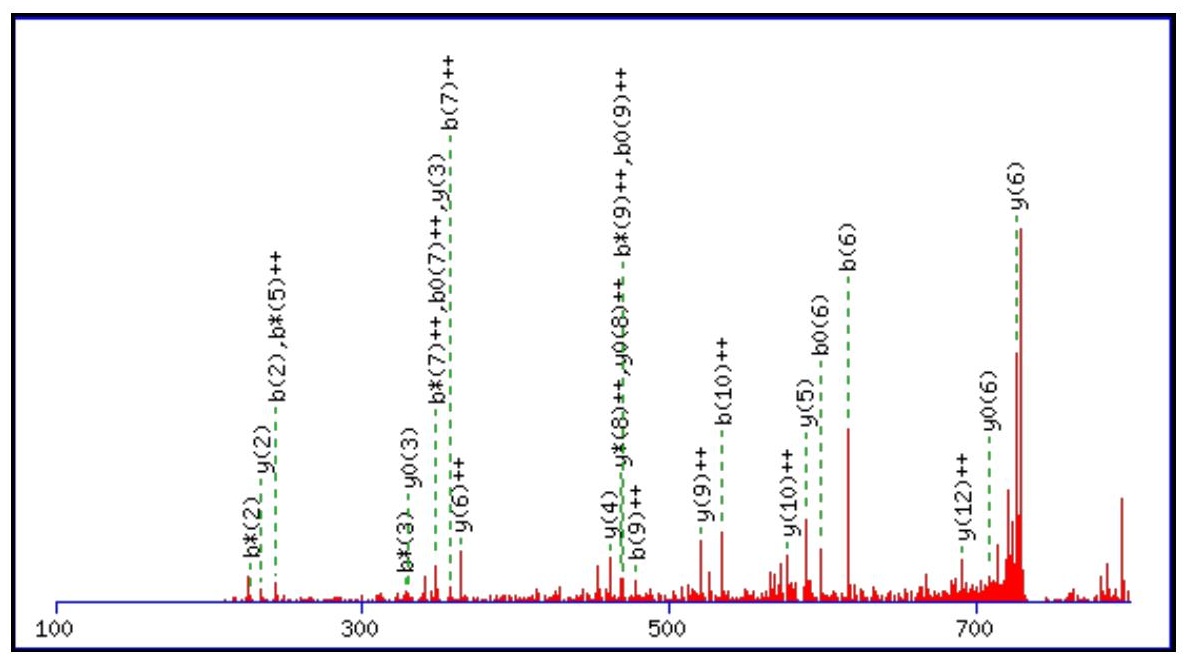



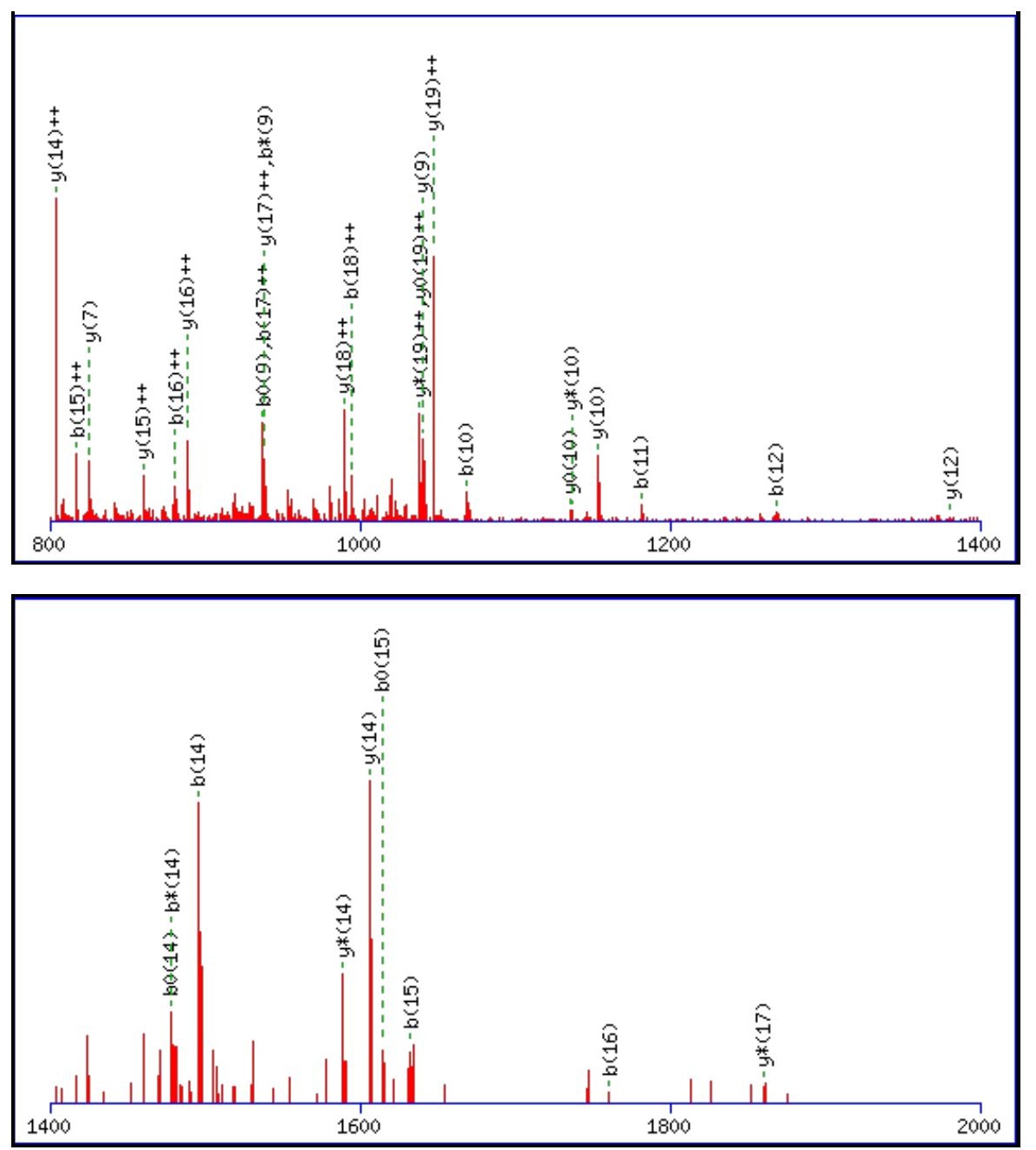

Monoisotopic mass of neutral peptide $\operatorname{Mr}($ calc): 2220.1862

Fixed modifications: Carbamidomethyl (C)

Variable modifications:

N2 : Deamidated $\mathrm{N}(\mathrm{N})$

Ions Score: 68 Expect: $1.4 \mathrm{e}-005$

Matches (Bold Red): 59/222 fragment ions using 100 most intense peaks

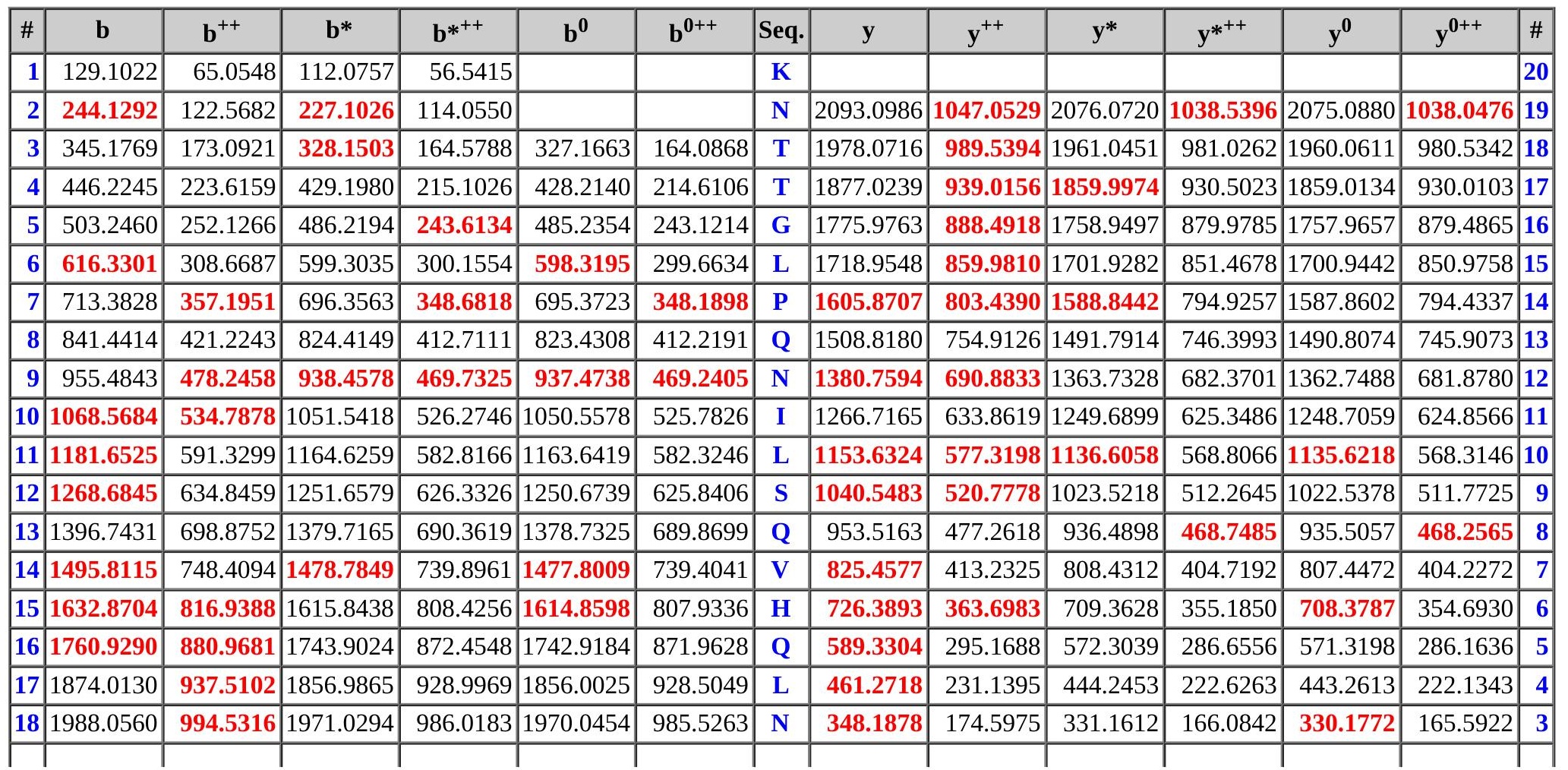




\begin{tabular}{|r|r|r|r|r|r|r|r|r|r|r|r|r|r|r|r|}
$\mathbf{1 9}$ & 2075.0880 & $\mathbf{1 0 3 8 . 0 4 7 6}$ & 2058.0614 & 1029.5344 & 2057.0774 & 1029.0423 & $\mathrm{~S}$ & $\mathbf{2 3 4 . 1 4 4 8}$ & 117.5761 & 217.1183 & 109.0628 & 216.1343 & 108.5708 & $\mathbf{2}$ \\
\hline $\mathbf{2 0}$ & & & & & & & $\mathrm{K}$ & 147.1128 & 74.0600 & 130.0863 & 65.5468 & & & $\mathbf{1}$ \\
\hline
\end{tabular}
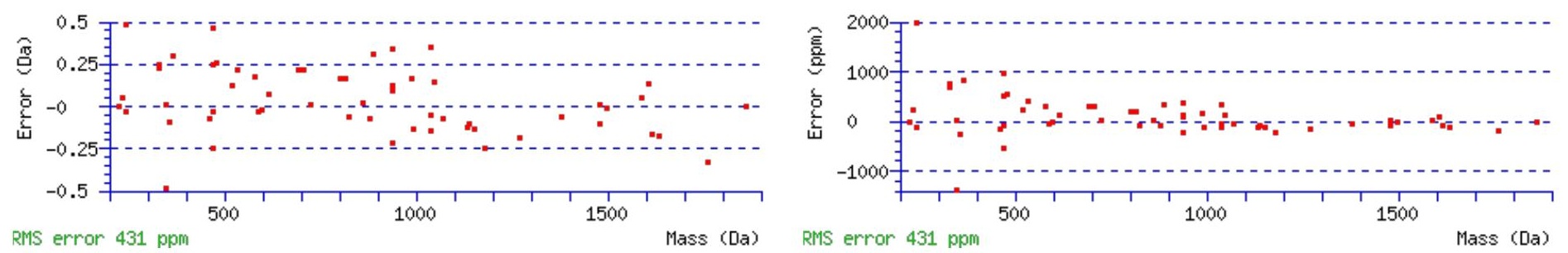

\section{All matches to this query}

\begin{tabular}{|l|l|l|l|}
\hline Score & Mr(calc): & Delta & \multicolumn{1}{c|}{ Sequence } \\
\hline 68.1 & 2220.1862 & -0.0020 & KNTTGLPQNILSQVHQLNSK \\
\hline 57.9 & 2220.1862 & -0.0020 & KNTTGLPQNILSQVHQLNSK \\
\hline 37.6 & 2219.2022 & 0.9820 & KNTTGLPQNILSQVHQLNSK \\
\hline 22.7 & 2220.1862 & -0.0020 & KNTTGLPQNILSQVHQLNSK \\
\hline 1.8 & 2218.1786 & 2.0056 & FTVPIPALNNSPOLKEFFR \\
\hline
\end{tabular}

Spectrum No: 394; Query: 2491; Rank: 1

\section{Peptide View}

MS/MS Fragmentation of ACIENGTSCIDICGEPQFLELMHVK

Found in IPI00372804, Tax_Id=10116 Gene_Symbol=Sccpdh Probable saccharopine dehydrogenase

Match to Query 2491: 2921.307222 from(974.776350,3+)

Title: 091008RatKidney_NH4Format01_09.5044.5044.3.dta

Data file K:INewmanPaper|Piliang|3SubProteomes\Piliang3SP\mgf5ppm|ERLIC_3SubProteomes5ppm.mgf

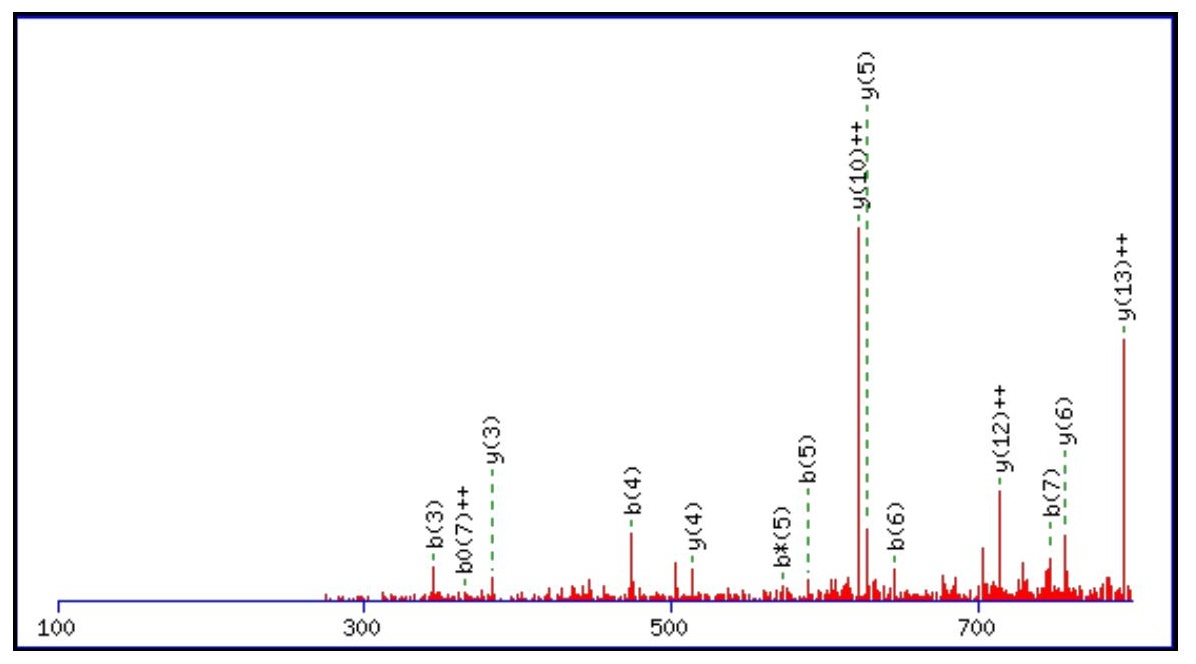



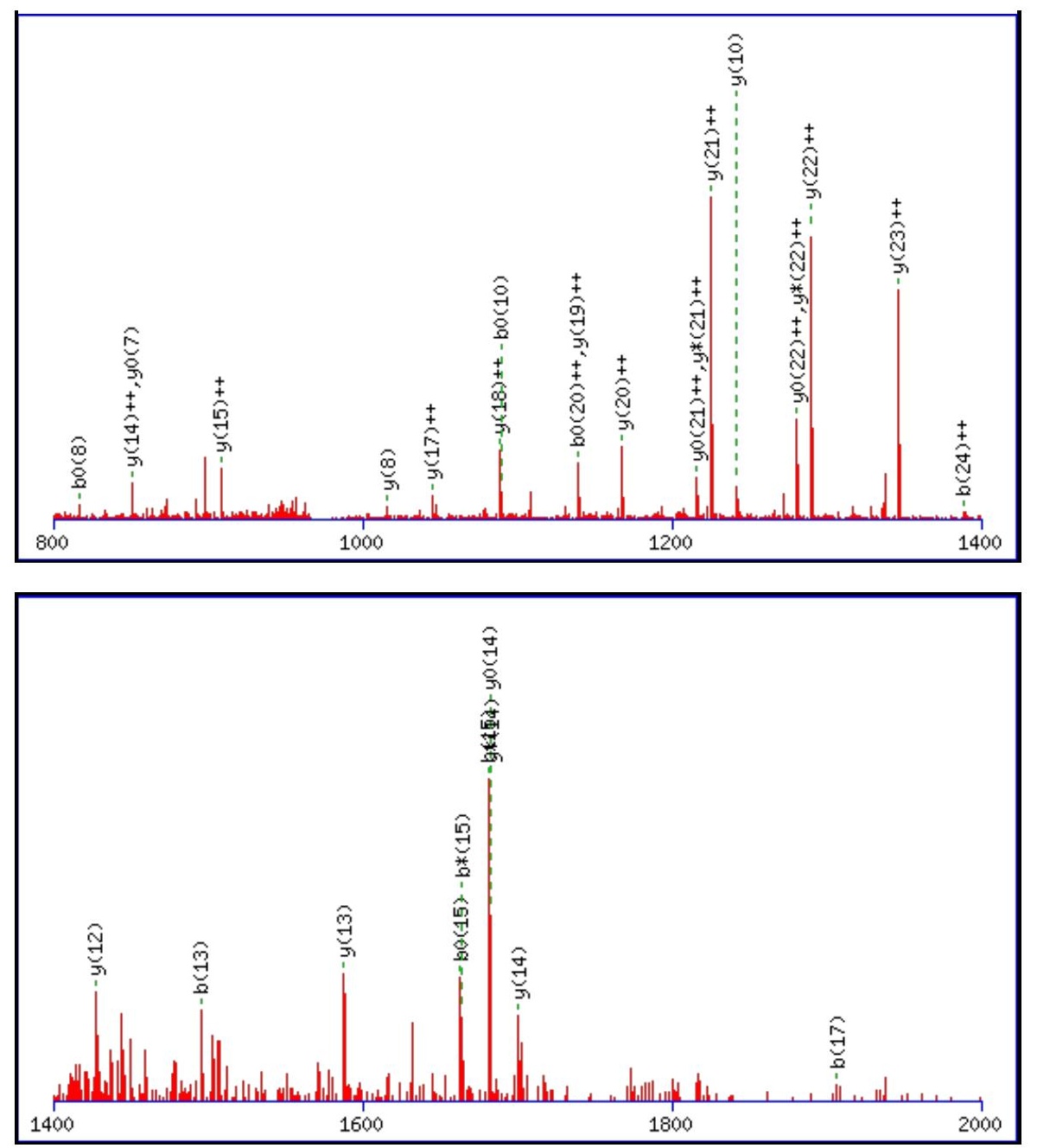

Monoisotopic mass of neutral peptide $\operatorname{Mr}($ calc): 2921.3068

Fixed modifications: Carbamidomethyl (C)

Variable modifications:

N5: Deamidated $\mathrm{N}(\mathrm{N})$

Ions Score: 68 Expect: $6.1 \mathrm{e}-005$

Matches (Bold Red): 44/264 fragment ions using 87 most intense peaks

\begin{tabular}{|c|c|c|c|c|c|c|c|c|c|c|c|c|c|c|}
\hline \# & b & $\mathbf{b}^{++}$ & $\mathbf{b}^{*}$ & $\mathbf{b}^{*+}$ & $\mathbf{b}^{0}$ & $\mathbf{b}^{\mathbf{0 + +}}$ & Seq. & $\mathbf{y}$ & $\mathbf{y}^{++}$ & $\mathbf{y}^{*}$ & $\mathbf{y}^{*^{++}}$ & $\mathbf{y}^{0}$ & $\mathbf{y}^{\mathbf{0 + +}}$ & \# \\
\hline 1 & 72.0444 & 36.5258 & & & & & A & & & & & & & 25 \\
\hline 2 & 232.0750 & 116.5412 & & & & & C & 2851.2769 & 1426.1421 & 2834.2504 & 1417.6288 & 2833.2664 & 1417.1368 & 24 \\
\hline 3 & 345.1591 & 173.0832 & & & & & I & 2691.2463 & 1346.1268 & 2674.2197 & 1337.6135 & 2673.2357 & 1337.1215 & 23 \\
\hline 4 & 474.2017 & 237.6045 & & & 456.1911 & & $\mathbf{E}$ & 2578.1622 & 1289.5847 & 2561.1357 & 1281.0715 & 2560.1517 & 1280.5795 & 22 \\
\hline 5 & 589.2286 & 295.1180 & 572.2021 & 286.6047 & 571.2181 & 286.1127 & $\mathbf{N}$ & 2449.1196 & 1225.0635 & 2432.0931 & 1216.5502 & 2431.1091 & 1216.0582 & 21 \\
\hline 6 & 646.2501 & 323.6287 & 629.2235 & 315.1154 & 628.2395 & 314.6234 & $\mathbf{G}$ & 2334.0927 & 1167.5500 & 2317.0661 & 1159.0367 & 2316.0821 & 1158.5447 & 20 \\
\hline 7 & 747.2978 & 374.1525 & 730.2712 & 365.6393 & 729.2872 & 365.1472 & $\mathbf{T}$ & 2277.0712 & 1139.0393 & 2260.0447 & 1130.5260 & 2259.0607 & 1130.0340 & 19 \\
\hline 8 & 834.3298 & 417.6685 & 817.3033 & 409.1553 & 816.3192 & 408.6633 & S & 2176.0235 & 1088.5154 & 2158.9970 & 1080.0021 & 2158.0130 & 1079.5101 & 18 \\
\hline 9 & 994.3605 & 497.6839 & 977.3339 & 489.1706 & 976.3499 & 488.6786 & C & 2088.9915 & 1044.9994 & 2071.9650 & 1036.4861 & 2070.9810 & 1035.9941 & 17 \\
\hline 10 & 1107.4445 & 554.2259 & 1090.4180 & 545.7126 & 1089.4340 & 545.2206 & I & 1928.9609 & 964.9841 & 1911.9343 & 956.4708 & 1910.9503 & 955.9788 & 16 \\
\hline 11 & 1222.4715 & 611.7394 & 1205.4449 & 603.2261 & 1204.4609 & 602.7341 & D & 1815.8768 & 908.4420 & 1798.8503 & 899.9288 & 1797.8662 & 899.4368 & 15 \\
\hline 12 & 1335.5555 & 668.2814 & 1318.5290 & 659.7681 & 1317.5450 & 659.2761 & I & 1700.8499 & 850.9286 & 1683.8233 & 842.4153 & 1682.8393 & 841.9233 & 14 \\
\hline 13 & 1495.5862 & 748.2967 & 1478.5596 & 739.7834 & 1477.5756 & 739.2914 & C & 1587.7658 & 794.3865 & 1570.7392 & 785.8733 & 1569.7552 & 785.3813 & 13 \\
\hline 14 & 1552.6076 & 776.8075 & 1535.5811 & 768.2942 & 1534.5971 & 767.8022 & G & 1427.7351 & 714.3712 & 1410.7086 & 705.8579 & 1409.7246 & 705.3659 & 12 \\
\hline 15 & 1681.6502 & 841.3288 & 1664.6237 & 832.8155 & 1663.6397 & 832.3235 & $\mathbf{E}$ & 1370.7137 & 685.8605 & 1353.6871 & 677.3472 & 1352.7031 & 676.8552 & 11 \\
\hline 16 & 1778.7030 & 889.8551 & 1761.6764 & 881.3419 & 1760.6924 & 880.8499 & $\mathbf{P}$ & 1241.6711 & 621.3392 & 1224.6445 & 612.8259 & 1223.6605 & 612.3339 & 10 \\
\hline 17 & 1906.7616 & 953.8844 & 1889.7350 & 945.3711 & 1888.7510 & 944.8791 & $\mathbf{Q}$ & 1144.6183 & 572.8128 & 1127.5918 & 564.2995 & 1126.6078 & 563.8075 & 9 \\
\hline 18 & 2053.8300 & 1027.4186 & 2036.8034 & 1018.9054 & 2035.8194 & 1018.4133 & $\mathbf{F}$ & 1016.5598 & 508.7835 & 999.5332 & 500.2702 & 998.5492 & 499.7782 & 8 \\
\hline
\end{tabular}




\begin{tabular}{|c|c|c|c|c|c|c|c|c|c|c|c|c|c|c|}
\hline 19 & 2166.9140 & |1083.9607 & |2149.8875 & 1075.4474 & |2148.9035 & |1074.9554 & L & 869.4913 & 435.2493 & 852.4648 & 426.7360 & 851.4808 & 426.2440 & 7 \\
\hline 20 & 2295.9566 & 1148.4820 & 2278.9301 & 1139.9687 & 2277.9461 & 1139.4767 & $\mathbf{E}$ & 756.4073 & 378.7073 & 739.3807 & 370.1940 & 738.3967 & 369.7020 & 6 \\
\hline 21 & 2409.0407 & 1205.0240 & 2392.0142 & 1196.5107 & 2391.0301 & 1196.0187 & L & 627.3647 & 314.1860 & 610.3381 & 305.6727 & & & 5 \\
\hline 22 & 2540.0812 & 1270.5442 & 2523.0546 & 1262.0310 & 2522.0706 & 1261.5390 & $\mathbf{M}$ & 514.2806 & 257.6439 & 497.2541 & 249.1307 & & & 4 \\
\hline 23 & 2677.1401 & 1339.0737 & 2660.1136 & 1330.5604 & 2659.1295 & 1330.0684 & $\mathbf{H}$ & 383.2401 & 192.1237 & 366.2136 & 183.6104 & & & 3 \\
\hline 24 & 2776.2085 & $\mathbf{1 3 8 8 . 6 0 7 9}$ & 2759.1820 & 1380.0946 & 2758.1980 & 1379.6026 & V & 246.1812 & 123.5942 & 229.1547 & 115.0810 & & & 2 \\
\hline 25 & & & & & & & K & 147.1128 & 74.0600 & 130.0863 & 65.5468 & & & 1 \\
\hline
\end{tabular}
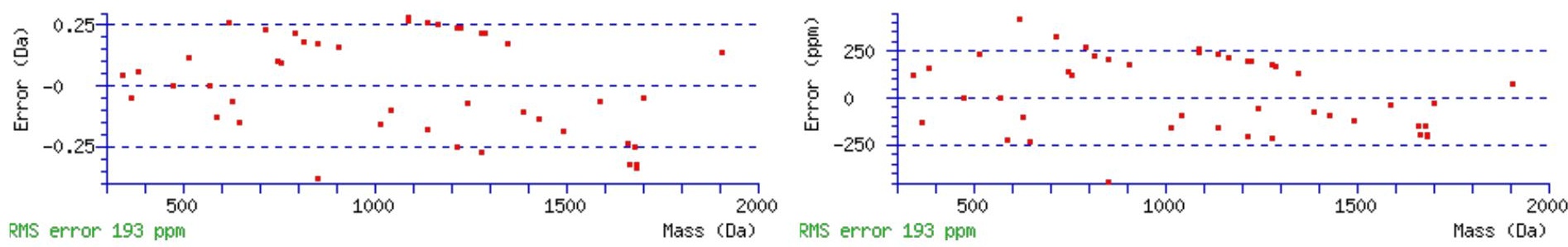

\section{All matches to this query}

\begin{tabular}{|l|l|l|l|}
\hline Score & Mr(calc): & Delta & \multicolumn{1}{c|}{ Sequence } \\
\hline 68.1 & 2921.3068 & 0.0005 & ACIENGTSCIDICGEPQFLELMHVK \\
\hline 31.6 & 2920.3228 & 0.9845 & ACIENGTSCIDICGEPOFLELMHVK \\
\hline 12.2 & 2919.3265 & 1.9807 & MYTDKLETVPPLNELTEIPGEDKK \\
\hline 12.1 & 2919.3232 & 1.9840 & DYDVSFGDGVLTIKLGGDLGTYVINK \\
\hline 5.8 & 2920.3330 & 0.9742 & LMHAGVTDSGETVNIGDVSYKLKTPK \\
\hline 5.8 & 2921.3122 & -0.0050 & CPSDPEMLKSSVSSPLHPTMRINLN \\
\hline 5.7 & 2919.3265 & 1.9807 & MYTDKLETVPPLNELTEIPGEDKK \\
\hline 4.4 & 2920.3330 & 0.9742 & LMHAGVTDSGETVNIGDVSYKLKTPK \\
\hline 4.4 & 2919.2949 & 2.0124 & SHWSMDLLQSGPLVKDMNTKSLEK \\
\hline 4.3 & 2919.3160 & 1.9912 & MPNILTSNLKIINEDTNCISPTQK \\
\hline
\end{tabular}

Spectrum No: 395; Query: 230; Rank: 1

\section{Peptide View}

MS/MS Fragmentation of DALQNMSSSLK

Found in IPI00325312, Tax_Id=10116 Gene_Symbol=Prom1 prominin 1 isoform 1

Match to Query 230: 1209.553488 from(605.784020,2+)

Title: 091008RatKidney_NoSalt_32.646.646.2.dta

Data file K:INewmanPaper|Piliangl3SubProteomes\Piliang3SP\mgf5ppm\ERLIC_3SubProteomes5ppm.mgf 

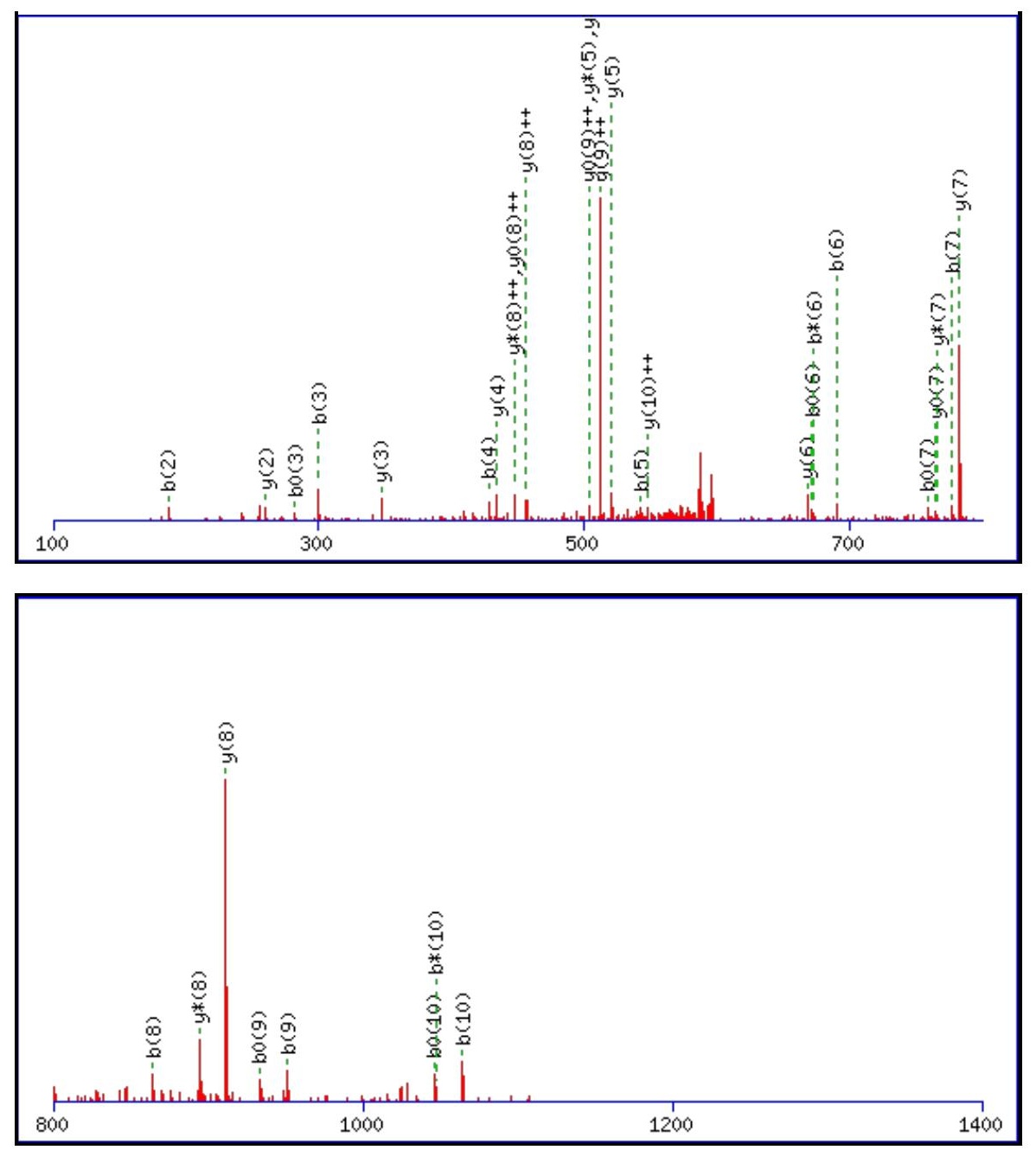

\begin{tabular}{rrrr}
\hline 00 & 1600 & 1800 & 2000 \\
\hline
\end{tabular}

Monoisotopic mass of neutral peptide $\operatorname{Mr}($ calc): 1209.5547

Fixed modifications: Carbamidomethyl (C)

Variable modifications:

N5 : Deamidated_N (N)

M6 : Oxidation (M)

Ions Score: 68 Expect : $2.1 \mathrm{e}-005$

Matches (Bold Red): 34/110 fragment ions using 55 most intense peaks

\begin{tabular}{|c|c|c|c|c|c|c|c|c|c|c|c|c|c|c|}
\hline \# & b & $\mathbf{b}^{++}$ & $\mathbf{b}^{*}$ & $\mathbf{b}^{*^{++}}$ & $\mathbf{b}^{\mathbf{0}}$ & $\mathbf{b}^{0++}$ & Seq. & $\mathbf{y}$ & $\mathbf{y}^{++}$ & $\mathbf{y}^{*}$ & $\mathbf{y}^{*^{++}}$ & $\mathbf{y}^{0}$ & $y^{0++}$ & \# \\
\hline 1 & 116.0342 & 58.5207 & & & 98.0237 & 49.5155 & D & & & & & & & 11 \\
\hline 2 & 187.0713 & 94.0393 & & & 169.0608 & 85.0340 & $\mathbf{A}$ & 1095.5350 & 548.2712 & 1078.5085 & 539.7579 & 1077.5245 & 539.2659 & 10 \\
\hline 3 & 300.1554 & 150.5813 & & & 282.1448 & 141.5761 & $\mathbf{L}$ & 1024.4979 & 512.7526 & 1007.4714 & 504.2393 & 1006.4874 & 503.7473 & 9 \\
\hline 4 & 428.2140 & 214.6106 & 411.1874 & 206.0974 & 410.2034 & 205.6053 & $\mathbf{Q}$ & 911.4139 & 456.2106 & 894.3873 & 447.6973 & 893.4033 & 447.2053 & 8 \\
\hline
\end{tabular}




\begin{tabular}{|c|c|c|c|c|c|c|c|c|c|c|c|c|c|c|}
\hline & 543.2409 & |272.1241 & 526.2144 & |263.6108 & 525.2303 & |263.1188 & $\mathbf{N}$ & 783.3553 & 392.1813 & 766.3287 & |383.6680 | & 765.3447 & |383.1760| & 7 \\
\hline 6 & 690.2763 & 345.6418 & 673.2498 & |337.1285 & 672.2658 & 336.6365 & M & 668.3284 & 334.6678 & 651.3018 & 326.1545 & 650.3178 & 325.6625 & 6 \\
\hline 7 & 777.3083 & 389.1578 & 760.2818 & 380.6445 & 759.2978 & 380.1525 & S & 521.2930 & 261.1501 & 504.2664 & 252.6368 & 503.2824 & 252.1448 & 5 \\
\hline 8 & 864.3404 & 432.6738 & 847.3138 & 424.1605 & 846.3298 & 423.6685 & $\mathbf{S}$ & 434.2609 & 217.6341 & 417.2344 & 209.1208 & 416.2504 & 208.6288 & 4 \\
\hline 9 & 951.3724 & 476.1898 & 934.3458 & 467.6766 & 933.3618 & 467.1846 & S & 347.2289 & 174.1181 & 330.2023 & 165.6048 & 329.2183 & 165.1128 & 3 \\
\hline 10 & 1064.4565 & 532.7319 & 1047.4299 & 524.2186 & 1046.4459 & 523.7266 & $\mathbf{L}$ & 260.1969 & 130.6021 & 243.1703 & 122.0888 & & & 2 \\
\hline 11 & & & & & & & $\mathbf{K}$ & 147.1128 & 74.0600 & 130.0863 & 65.5468 & & & 1 \\
\hline
\end{tabular}
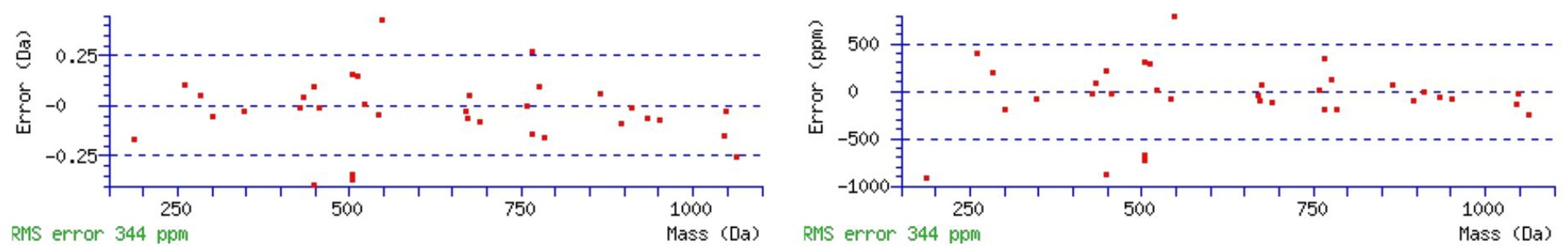

\section{All matches to this query}

\begin{tabular}{|l|l|l|l|}
\hline Score & Mr(calc): & Delta & \multicolumn{1}{|c|}{ Sequence } \\
\hline 68.0 & 1209.5547 & -0.0012 & DALQNMSSSLK \\
\hline 14.2 & 1209.5522 & 0.0013 & EGIQTCMFPK \\
\hline 7.9 & 1208.5607 & 0.9928 & QLLKYGKIS \\
\hline 6.8 & 1209.5448 & 0.0087 & SVITSIFGVK \\
\hline 6.0 & 1209.5481 & 0.0054 & NAEMKNAMRK \\
\hline 5.9 & 1209.5431 & 0.0104 & SYDYRVSLK \\
\hline 5.2 & 1209.5577 & -0.0042 & QPSKMPNGKK \\
\hline 5.0 & 1209.5431 & 0.0104 & ANLWPAQTTK \\
\hline 5.0 & 1209.5431 & 0.0104 & ANLWPAQTTK \\
\hline 4.5 & 1209.5547 & -0.0012 & GDSSQLMLDTK \\
\hline
\end{tabular}

Spectrum No: 396; Query: 2028; Rank: 1

\section{Peptide View}

MS/MS Fragmentation of NVTYGTYLDDPDPDDGFNYK

Found in IPI00554194, Tax_Id=10116 Gene_Symbol=Calu calumenin isoform b

Match to Query 2028: 2308.970948 from(1155.492750,2+)

Title: 100101RatKid_NS_deglyco_20.3666.3666.2.dta

Data file K:INewmanPaper|Piliangl3SubProteomes\Piliang3SP\mgf5ppm\ERLIC_3SubProteomes5ppm.mgf 

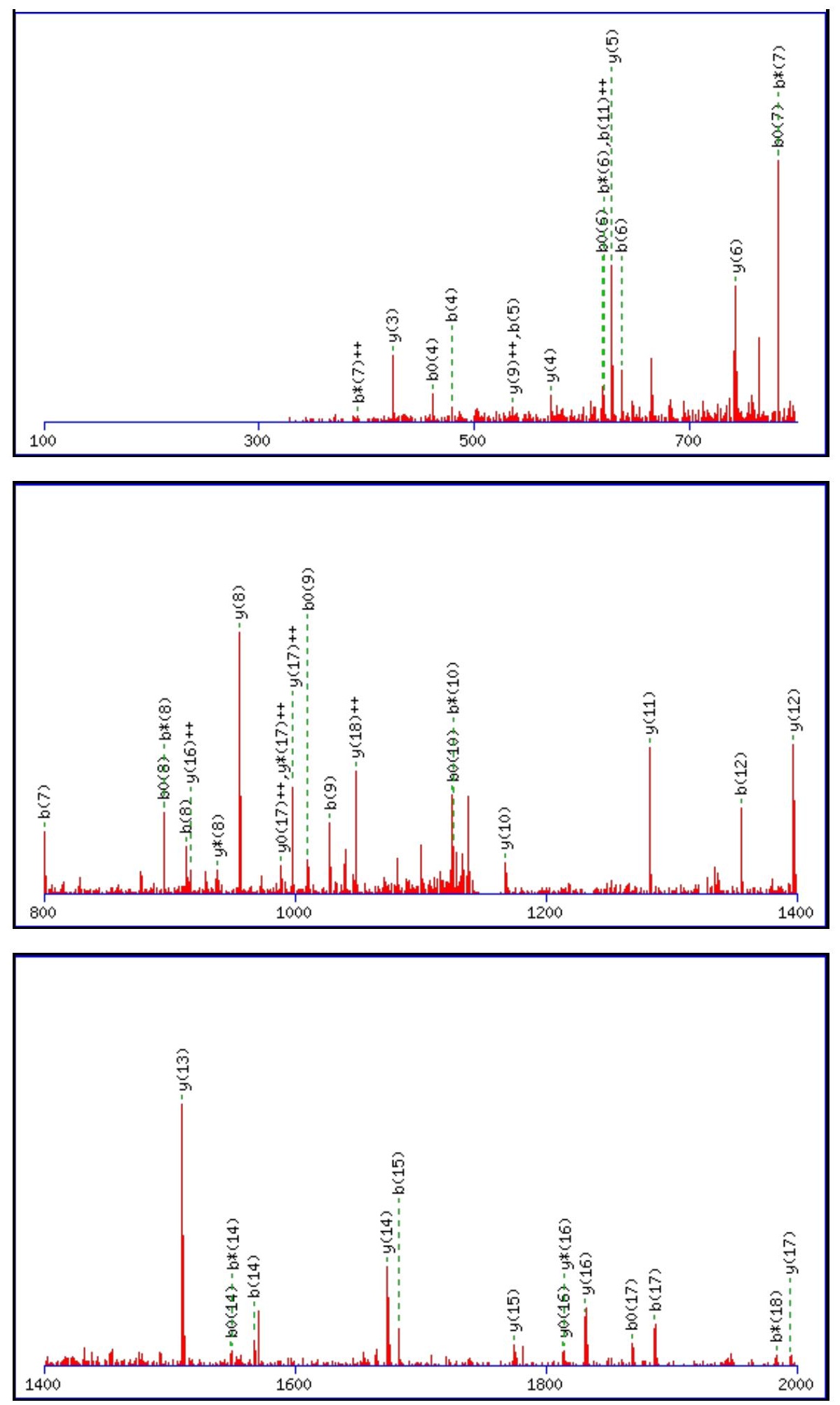

Monoisotopic mass of neutral peptide $\operatorname{Mr}($ calc): 2308.9648

Fixed modifications: Carbamidomethyl (C)

Variable modifications:

N1 : Deamidated $\mathrm{N}(\mathrm{N})$

Ions Score: 68 Expect: $4.5 \mathrm{e}-005$

Matches (Bold Red): 48/214 fragment ions using 91 most intense peaks

\begin{tabular}{|c|c|c|c|c|c|c|c|c|c|c|c|c|c|c|}
\hline \# & b & $\mathbf{b}^{++}$ & $\mathbf{b}^{*}$ & $\mathbf{b}^{*^{++}}$ & $\mathbf{b}^{0}$ & $\mathbf{b}^{\mathbf{0 + +}}$ & Seq. & $\mathbf{y}$ & $y^{++}$ & $\mathbf{y}^{*}$ & $\mathrm{y}^{*^{++}}$ & $\mathbf{y}^{\mathbf{0}}$ & $y^{0++}$ & \# \\
\hline 1 & 116.0342 & 58.5207 & 99.0077 & 50.0075 & & & $\mathbf{N}$ & & & & & & & 20 \\
\hline 2 & 215.1026 & 108.0550 & 198.0761 & 99.5417 & & & V & 2194.9451 & 1097.9762 & 2177.9186 & 1089.4629 & 2176.9346 & 1088.9709 & 19 \\
\hline 3 & 316.1503 & 158.5788 & 299.1238 & 150.0655 & 298.1397 & 149.5735 & $\mathbf{T}$ & 2095.8767 & 1048.4420 & |2078.8502 & 1039.9287 & 2077.8662 & |1039.4367 & 18 \\
\hline 4 & 479.2136 & 240.1105 & 462.1871 & 231.5972 & 461.2031 & 231.1052 & $\mathbf{Y}$ & 1994.8290 & 997.9182 & 1977.8025 & 989.4049 & 1976.8185 & 988.9129 & 17 \\
\hline 5 & 536.2351 & 268.6212 & 519.2085 & 260.1079 & 518.2245 & 259.6159 & G & 1831.7657 & 916.3865 & \begin{tabular}{|l|l|}
1814.7392 \\
\end{tabular} & 907.8732 & 1813.7552 & 907.3812 & 16 \\
\hline
\end{tabular}




\begin{tabular}{|c|c|c|c|c|c|c|c|c|c|c|c|c|c|c|}
\hline 6 & 637.2828 & 319.1450 & 620.2562 & 310.6318 & 619.2722 & 310.1397| & $\mathbf{T}$ & |1774.7443| & 887.8758 & |1757.7177 & 879.3625 & |1756.7337| & 878.8705 & 15 \\
\hline 7 & 800.3461 & 400.6767 & 783.3196 & 392.1634 & 782.3355 & 391.6714 & $\mathbf{Y}$ & 1673.6966 & 837.3519 & 1656.6700 & 828.8387 & 1655.6860 & 828.3466 & 14 \\
\hline 8 & 913.4302 & 457.2187 & 896.4036 & 448.7054 & 895.4196 & 448.2134 & $\mathbf{L}$ & 1510.6332 & 755.8203 & 1493.6067 & 747.3070 & 1492.6227 & 746.8150 & 13 \\
\hline 9 & 1028.4571 & 514.7322 & 1011.4306 & 506.2189 & 1010.4465 & 505.7269 & D & 1397.5492 & 699.2782 & 1380.5226 & 690.7650 & 1379.5386 & 690.2729 & 12 \\
\hline 10 & 1143.4841 & 572.2457 & 1126.4575 & 563.7324 & 1125.4735 & 563.2404 & D & 1282.5222 & 641.7648 & 1265.4957 & 633.2515 & 1264.5117 & 632.7595 & 11 \\
\hline 11 & 1240.5368 & 620.7720 & 1223.5103 & 612.2588 & 1222.5263 & 611.7668 & $\mathbf{P}$ & 1167.4953 & 584.2513 & 1150.4687 & 575.7380 & 1149.4847 & 575.2460 & 10 \\
\hline 12 & 1355.5638 & 678.2855 & 1338.5372 & 669.7722 & 1337.5532 & 669.2802 & D & 1070.4425 & 535.7249 & 1053.4160 & 527.2116 & 1052.4320 & 526.7196 & 9 \\
\hline 13 & 1452.6165 & 726.8119 & 1435.5900 & 718.2986 & 1434.6060 & 717.8066 & $\mathbf{P}$ & 955.4156 & 478.2114 & 938.3890 & 469.6982 & 937.4050 & 469.2061 & 8 \\
\hline 14 & 1567.6435 & 784.3254 & 1550.6169 & 775.8121 & 1549.6329 & 775.3201 & D & 858.3628 & 429.6851 & 841.3363 & 421.1718 & 840.3523 & 420.6798 & 7 \\
\hline 15 & 1682.6704 & 841.8388 & 1665.6439 & 833.3256 & 1664.6598 & 832.8336 & D & 743.3359 & 372.1716 & 726.3093 & 363.6583 & 725.3253 & 363.1663 & 6 \\
\hline 16 & 1739.6919 & 870.3496 & 1722.6653 & 861.8363 & 1721.6813 & 861.3443 & G & 628.3089 & 314.6581 & 611.2824 & 306.1448 & & & 5 \\
\hline 17 & 1886.7603 & 943.8838 & 1869.7337 & 935.3705 & 1868.7497 & 934.8785 & $\mathbf{F}$ & 571.2875 & 286.1474 & 554.2609 & 277.6341 & & & 4 \\
\hline 18 & 2000.8032 & 1000.9052 & 1983.7767 & 992.3920 & 1982.7927 & 991.9000 & $\mathbf{N}$ & 424.2191 & 212.6132 & 407.1925 & 204.0999 & & & 3 \\
\hline 19 & 2163.8665 & 1082.4369 & 2146.8400 & 1073.9236 & 2145.8560 & 1073.4316 & $\mathbf{Y}$ & 310.1761 & 155.5917 & 293.1496 & 147.0784 & & & 2 \\
\hline
\end{tabular}
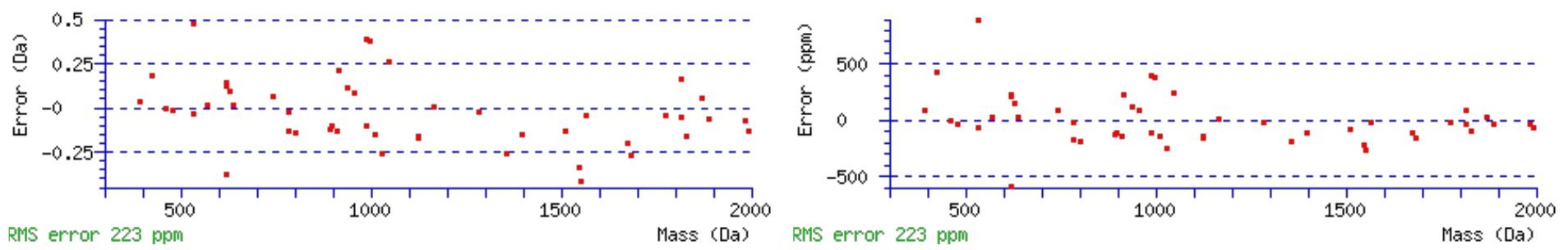

\section{All matches to this query}

\begin{tabular}{|l|l|l|l|}
\hline Score & Mr(calc): & Delta & \multicolumn{1}{|c|}{ Sequence } \\
\hline 67.8 & 2308.9648 & 0.0061 & NVTYGTYLDDPDPDDGFNYK \\
\hline 40.6 & 2307.9808 & 0.9901 & NVTYGTYLDDPDPDDGFNYK \\
\hline 26.2 & 2308.9648 & 0.0062 & NATYSYVLDDPDPDDGFNYK \\
\hline 19.2 & 2308.9648 & 0.0061 & NVTYGTYLDDPDPDDGFNYK \\
\hline 19.1 & 2307.9808 & 0.9902 & NATYSYVLDDPDPDDGFNYK \\
\hline 5.5 & 2308.9783 & -0.0073 & SSGVKTLFNPAPAIADLDPR \\
\hline 2.2 & 2308.9646 & 0.0063 & QYWCTFKDTSISCYKSR \\
\hline 2.2 & 2308.9646 & 0.0063 & QYWCTFKDTSISCYKSR \\
\hline 1.1 & 2308.9825 & -0.0115 & SGELRKEINSNTEQLQSSK \\
\hline 1.1 & 2308.9825 & -0.0115 & SGELRKEINSNTEQLQSSK \\
\hline
\end{tabular}

Spectrum No: 397; Query: 1343; Rank: 1

\section{Peptide View}

MS/MS Fragmentation of TRCNTTQGNEVTSILR

Found in IPI00327143, Tax_Id=10116 Gene_Symbol=Alpl Alkaline phosphatase, tissue-nonspecific isozyme precursor

Match to Query 1343: 1849.899972 from(617.640600,3+)

Title: 100101RatKid_NS_deglyco_19.1947.1947.3.dta

Data file K:\NewmanPaper\Piliang \3SubProteomes\Piliang3SP \mgf5ppm\ERLIC_3SubProteomes5ppm.mgf 

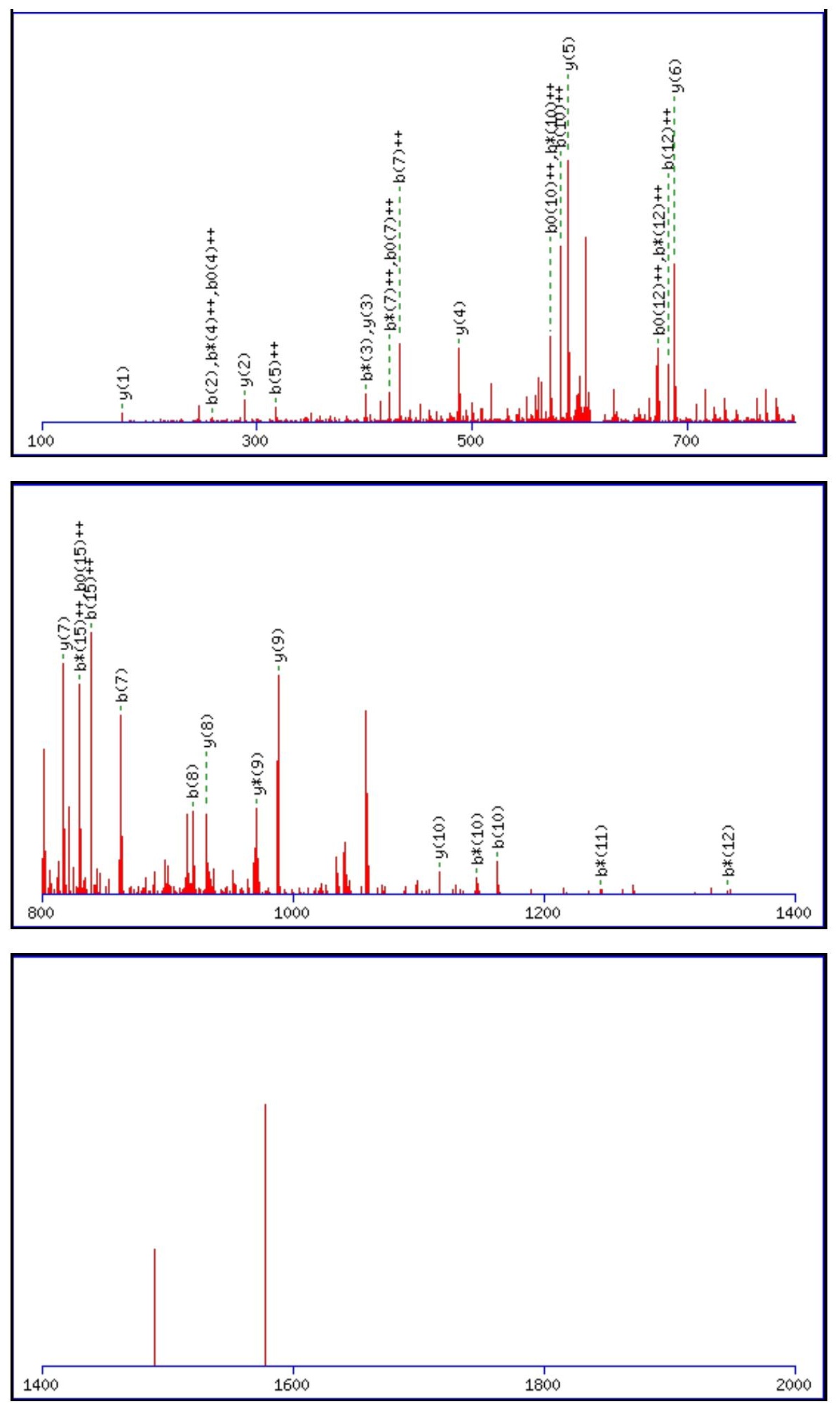

Monoisotopic mass of neutral peptide $\operatorname{Mr}($ calc): 1849.8952

Fixed modifications: Carbamidomethyl (C)

Variable modifications:

N4 : Deamidated $\mathrm{N}(\mathrm{N})$

Ions Score: 68 Expect: $4.2 \mathrm{e}-005$

Matches (Bold Red): 34/172 fragment ions using 43 most intense peaks

\begin{tabular}{|r|c|c|c|c|c|c|c|c|c|c|c|c|c|c|}
\hline$\#$ & $\mathbf{b}$ & $\mathbf{b}^{++}$ & $\mathbf{b}^{*}$ & $\mathbf{b}^{\boldsymbol{*}^{++}}$ & $\mathbf{b}^{\mathbf{0}}$ & $\mathbf{b}^{\mathbf{0 + +}}$ & Seq. & $\mathbf{y}$ & $\mathbf{y}^{++}$ & $\mathbf{y}^{\mathbf{*}}$ & $\mathbf{y}^{\boldsymbol{*}^{++}}$ & $\mathbf{y}^{\mathbf{0}}$ & $\mathbf{y}^{\mathbf{0 + +}}$ & $\#$ \\
\hline $\mathbf{1}$ & 102.0550 & 51.5311 & & & 84.0444 & 42.5258 & $\mathbf{T}$ & & & & & & & $\mathbf{1 6}$ \\
\hline $\mathbf{2}$ & $\mathbf{2 5 8 . 1 5 6 1}$ & 129.5817 & 241.1295 & 121.0684 & 240.1455 & 120.5764 & $\mathbf{R}$ & 1749.8548 & 875.4310 & 1732.8283 & 866.9178 & 1731.8443 & 866.4258 & $\mathbf{1 5}$ \\
\hline $\mathbf{3}$ & 418.1867 & 209.5970 & $\mathbf{4 0 1 . 1 6 0 2}$ & 201.0837 & 400.1762 & 200.5917 & $\mathbf{C}$ & 1593.7537 & 797.3805 & 1576.7272 & 788.8672 & 1575.7431 & 788.3752 & $\mathbf{1 4}$ \\
\hline $\mathbf{4}$ & 533.2137 & 267.1105 & 516.1871 & 258.5972 & 515.2031 & 258.1052 & $\mathbf{N}$ & 1433.7231 & 717.3652 & 1416.6965 & 708.8519 & 1415.7125 & 708.3599 & $\mathbf{1 3}$ \\
\hline $\mathbf{5}$ & 634.2613 & $\mathbf{3 1 7 . 6 3 4 3}$ & 617.2348 & 309.1210 & 616.2508 & 308.6290 & $\mathbf{T}$ & 1318.6961 & 659.8517 & 1301.6696 & 651.3384 & 1300.6856 & 650.8464 & $\mathbf{1 2}$ \\
\hline
\end{tabular}




\begin{tabular}{|c|c|c|c|c|c|c|c|c|c|c|c|c|c|c|}
\hline 6 & 735.3090 & |368.1581| & 718.2825 & |359.6449 & 717.2984 & |359.1529| & & |1217.6484| & 609.3279| & |1200.6219 & 600.8146 & 1199.6379 & |600.3226 & \\
\hline 7 & 863.3676 & 432.1874 & 846.3410 & 423.6742 & 845.3570 & 423.1821 & $\mathbf{Q}$ & 1116.6008 & 558.8040 & 1099.5742 & 550.2907 & 1098.5902 & 549.7987 & 10 \\
\hline 8 & 920.3891 & 460.6982 & 903.3625 & 452.1849 & 902.3785 & 451.6929 & G & 988.5422 & 494.7747 & 971.5156 & 486.2615 & 0.5316 & 485.7694 & 9 \\
\hline 9 & 1034.4320 & 517.7196 & 1017.4054 & 509.2064 & 1016.4214 & 508.7143 & $\mathbf{N}$ & 931.5207 & 466.2640 & 914.4942 & 457.7507 & 913.5102 & 457.2587 & 8 \\
\hline 10 & 1163.4746 & 582.2409 & 1146.4480 & 573.7276 & 1145.4640 & 573.2356 & $\mathbf{E}$ & 817.4778 & 409.2425 & 800.4512 & 400.7293 & 799.4672 & 400.2373 & 7 \\
\hline 11 & 1262.5430 & 631.7751 & 1245.5164 & 623.2619 & 1244.5324 & 622.7698 & V & 688.4352 & 344.7212 & 671.4087 & 336.2080 & 670.4246 & 335.7160 & 6 \\
\hline 12 & 1363.5907 & 682.2990 & 1346.5641 & 673.7857 & 1345.5801 & 673.2937 & $\mathbf{T}$ & 589.3668 & 295.1870 & 572.3402 & 286.6738 & 571.3562 & 286.1817 & 5 \\
\hline 13 & 1450.6227 & 725.8150 & 1433.5961 & 717.3017 & 1432.6121 & 716.8097 & $S$ & 488.3191 & 244.6632 & 471.2926 & 236.1499 & 470.3085 & 235.6579 & 4 \\
\hline 14 & 1563.7068 & 782.3570 & 1546.6802 & 773.8437 & 1545.6962 & 773.3517 & 1 & 401.2871 & 201.1472 & 384.2605 & 192.6339 & & & 3 \\
\hline 15 & 1676.7908 & 838.8990 & 1659.7643 & 830.3858 & 1658.7803 & 829.8938 & $\mathbf{L}$ & 288.2030 & 144.6051 & 271.1765 & 136.0919 & & & 2 \\
\hline 16 & & & & & & & $\mathbf{R}$ & 175.1190 & 88.0631 & 158.0924 & 79.5498 & & & 1 \\
\hline
\end{tabular}
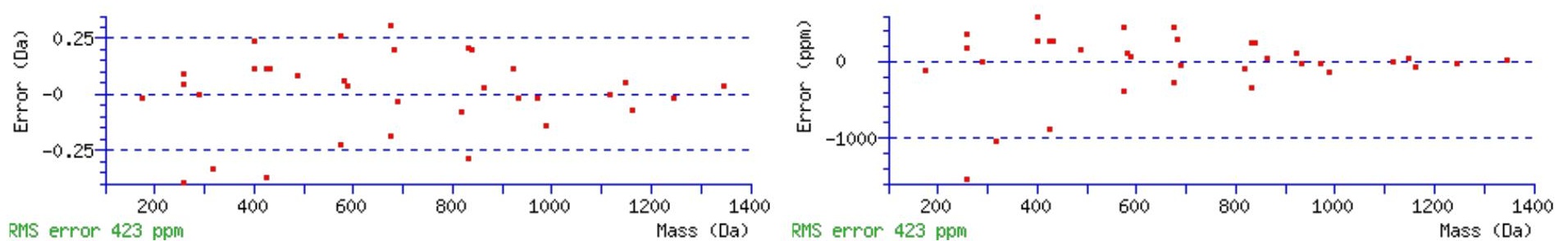

\section{All matches to this query}

\begin{tabular}{|l|l|c|l|}
\hline Score & Mr(calc): & Delta & \multicolumn{1}{|c|}{ Sequence } \\
\hline 67.8 & 1849.8952 & 0.0048 & TRCNTTQGNEVTSILR \\
\hline 38.4 & 1848.9112 & 0.9888 & TRCNTTQGNEVTSILR \\
\hline 32.4 & 1849.8952 & 0.0048 & TRCNTTQGNEVTSILR \\
\hline 8.2 & 1849.8840 & 0.0160 & DQQNMANGSTVVVTLTR \\
\hline 6.5 & 1848.8931 & 1.0069 & HGRSSVCRFGPLQIR \\
\hline 6.5 & 1848.8931 & 1.0069 & HGRSSVCRFGPLQIR \\
\hline 6.4 & 1849.9118 & -0.0119 & TRIFGCQNPFRQGGGR \\
\hline 4.8 & 1848.8813 & 1.0187 & TGNNSTKAETRKPDNSK \\
\hline 4.6 & 1848.8827 & 1.0173 & DRSTIPGQHGDREEPR \\
\hline 4.6 & 1847.8931 & 2.0069 & IYSNAGEKSFDRLLR \\
\hline
\end{tabular}

Spectrum No: 398; Query: 725; Rank: 1

\section{Peptide View}

MS/MS Fragmentation of ENGVFEEISNSSGR

Found in IPI00326260, Tax_Id=10116 Gene_Symbol=Sdfr1 Isoform 2 of Neuroplastin precursor

Match to Query 725: 1524.672228 from(763.343390,2+)

Title: 100101RatKid_NS_deglyco_26.2476.2476.2.dta

Data file K:INewmanPaper|Piliangl3SubProteomes\Piliang3SP\mgf5ppm\ERLIC_3SubProteomes5ppm.mgf 

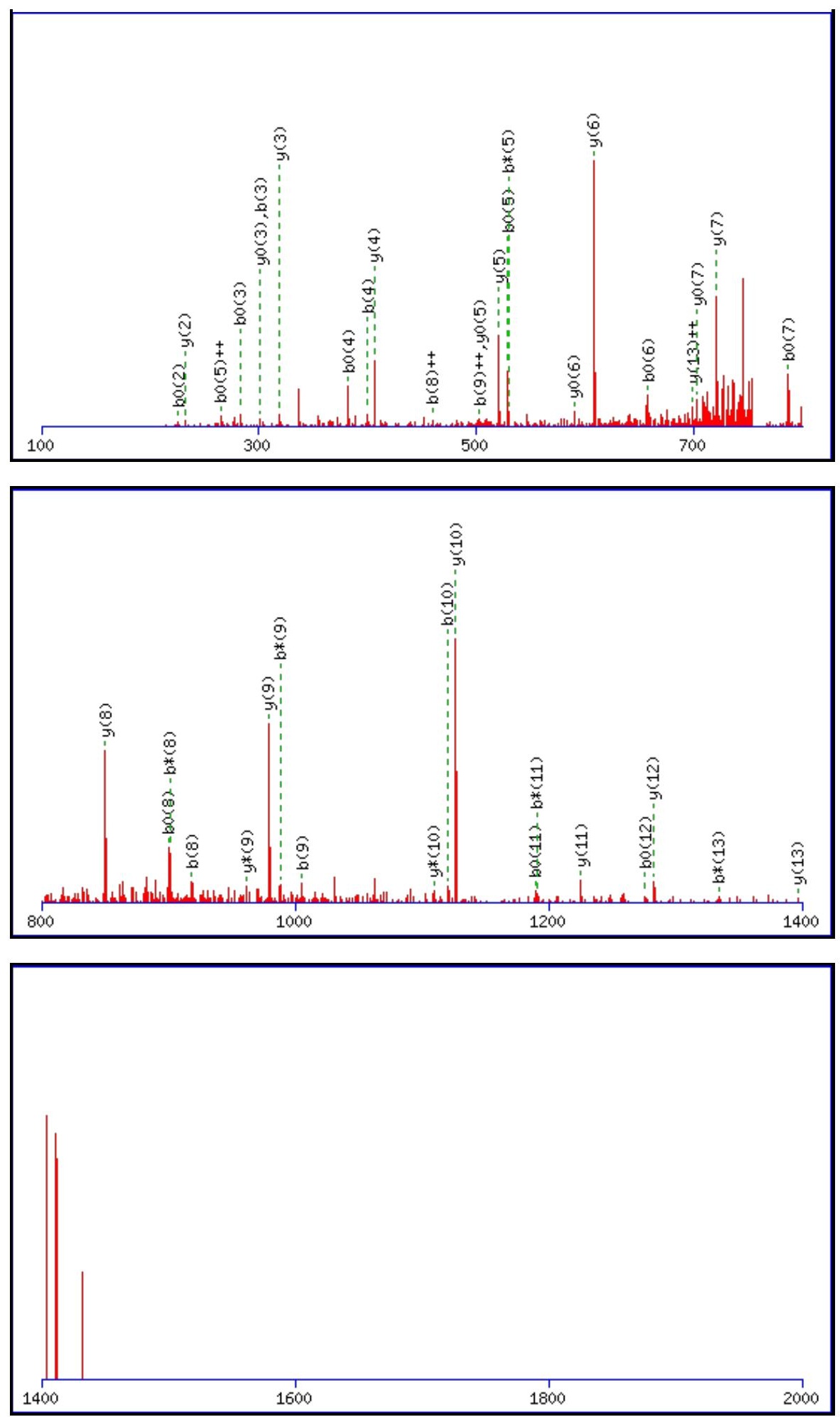

Monoisotopic mass of neutral peptide $\operatorname{Mr}($ calc): 1524.6692

Fixed modifications: Carbamidomethyl (C)

Variable modifications:

N10 : Deamidated $\mathrm{N}(\mathrm{N})$

Ions Score: 68 Expect: $2.5 \mathrm{e}-005$

Matches (Bold Red): 41/150 fragment ions using 89 most intense peaks

\begin{tabular}{|r|c|c|c|c|c|c|c|c|c|c|c|c|c|c|}
\hline$\#$ & $\mathbf{b}$ & $\mathbf{b}^{++}$ & $\mathbf{b}^{*}$ & $\mathbf{b}^{\boldsymbol{*}^{++}}$ & $\mathbf{b}^{\mathbf{0}}$ & $\mathbf{b}^{\mathbf{0 + +}}$ & Seq. & $\mathbf{y}$ & $\mathbf{y}^{++}$ & $\mathbf{y}^{\mathbf{*}}$ & $\mathbf{y}^{\boldsymbol{*}^{++}}$ & $\mathbf{y}^{\mathbf{0}}$ & $\mathbf{y}^{\mathbf{0 + +}}$ & $\#$ \\
\hline $\mathbf{1}$ & 130.0499 & 65.5286 & & & 112.0393 & 56.5233 & $\mathbf{E}$ & & & & & & & $\mathbf{1 4}$ \\
\hline $\mathbf{2}$ & 244.0928 & 122.5500 & 227.0662 & 114.0368 & $\mathbf{2 2 6 . 0 8 2 2}$ & 113.5448 & $\mathbf{N}$ & $\mathbf{1 3 9 6 . 6 3 3 9}$ & $\mathbf{6 9 8 . 8 2 0 6}$ & 1379.6074 & 690.3073 & 1378.6233 & 689.8153 & $\mathbf{1 3}$ \\
\hline 3 & $\mathbf{3 0 1 . 1 1 4 3}$ & 151.0608 & 284.0877 & 142.5475 & $\mathbf{2 8 3 . 1 0 3 7}$ & 142.0555 & $\mathbf{G}$ & $\mathbf{1 2 8 2 . 5 9 1 0}$ & 641.7991 & 1265.5644 & 633.2859 & 1264.5804 & 632.7938 & $\mathbf{1 2}$ \\
\hline $\mathbf{4}$ & $\mathbf{4 0 0 . 1 8 2 7}$ & 200.5950 & 383.1561 & 192.0817 & $\mathbf{3 8 2 . 1 7 2 1}$ & 191.5897 & $\mathbf{V}$ & $\mathbf{1 2 2 5 . 5 6 9 5}$ & 613.2884 & 1208.5430 & 604.7751 & 1207.5589 & 604.2831 & $\mathbf{1 1}$ \\
\hline $\mathbf{5}$ & 547.2511 & 274.1292 & 530.2245 & 265.6159 & 529.2405 & $\mathbf{2 6 5 . 1 2 3 9}$ & $\mathbf{F}$ & $\mathbf{1 1 2 6 . 5 0 1 1}$ & 563.7542 & $\mathbf{1 1 0 9 . 4 7 4 6}$ & 555.2409 & 1108.4905 & 554.7489 & $\mathbf{1 0}$ \\
\hline
\end{tabular}




\begin{tabular}{|c|c|c|c|c|c|c|c|c|c|c|c|c|c|c|}
\hline 6 & 676.2937 & |338.6505 & 659.2671 & 330.1372 & 658.2831 & |329.6452| & $\mathbf{E}$ & 979.4327| & |490.2200| & 962.4061 & |481.7067 & 961.4221 & \begin{tabular}{||c|c|}
$481.2147 \mid$ \\
\end{tabular} & 9 \\
\hline 7 & 805.3363 & 403.1718 & 788.3097 & 394.6585 & 787.3257 & 394.1665 & $\mathbf{E}$ & 850.3901 & 425.6987 & 833.3635 & 417.1854 & 832.3795 & 416.6934 & 8 \\
\hline 8 & 918.4203 & 459.7138 & 901.3938 & 451.2005 & 900.4098 & 450.7085 & I & 721.3475 & 361.1774 & 704.3210 & 352.6641 & 703.3369 & 352.1721 & 7 \\
\hline 9 & 1005.4524 & 503.2298 & 988.4258 & 494.7165 & 987.4418 & 494.2245 & S & 608.2634 & 304.6354 & 591.2369 & 296.1221 & 590.2529 & 295.6301 & 6 \\
\hline 10 & 1120.4793 & 560.7433 & 1103.4528 & 552.2300 & 1102.4687 & 551.7380 & $\mathbf{N}$ & 521.2314 & 261.1193 & 504.2049 & 252.6061 & 503.2208 & 252.1141 & 5 \\
\hline 11 & 1207.5113 & 604.2593 & 1190.4848 & 595.7460 & 1189.5008 & 595.2540 & S & 406.2045 & 203.6059 & 389.1779 & 195.0926 & 388.1939 & 194.6006 & 4 \\
\hline 12 & 1294.5434 & 647.7753 & 1277.5168 & 639.2620 & 1276.5328 & 638.7700 & S & 319.1724 & 160.0899 & 302.1459 & 151.5766 & 301.1619 & 151.0846 & 3 \\
\hline 13 & 1351.5648 & 676.2860 & 1334.5383 & 667.7728 & 1333.5543 & 667.2808 & G & 232.1404 & 116.5738 & 215.1139 & 108.0606 & & & 2 \\
\hline 14 & & & & & & & $\mathbf{R}$ & 175.1190 & 88.0631 & 158.0924 & 79.5498 & & & 1 \\
\hline
\end{tabular}
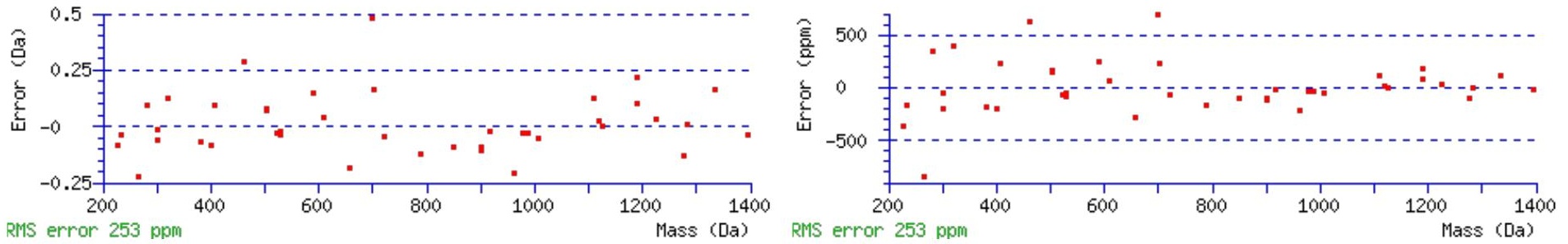

\section{All matches to this query}

\begin{tabular}{|l|l|l|l|}
\hline Score & Mr(calc): & Delta & Sequence \\
\hline 67.8 & 1524.6692 & 0.0030 & ENGVFEEISNSSGR \\
\hline 3.8 & 1523.6664 & 1.0058 & DPSPQTRRTCAR \\
\hline 3.8 & 1523.6664 & 1.0058 & $\underline{\text { DPSPQTRRTCAR }}$ \\
\hline
\end{tabular}

Spectrum No: 399; Query: 2493; Rank: 1

\section{Peptide View}

MS/MS Fragmentation of VWPDYPNITVDPSLDWDSQVQQYR

Found in IPI00191437, Tax_Id=10116 Gene_Symbol=- 64 kDa protein

Match to Query 2493: 2921.365708 from(1461.690130,2+)

Title: 100101RatKid_NS_deglyco_19.5292.5292.2.dta

Data file K:\NewmanPaper $\backslash$ Piliang $\backslash 3$ SubProteomes $\backslash$ Piliang3SP $\backslash$ mgf5ppm\ERLIC_3SubProteomes5ppm.mgf

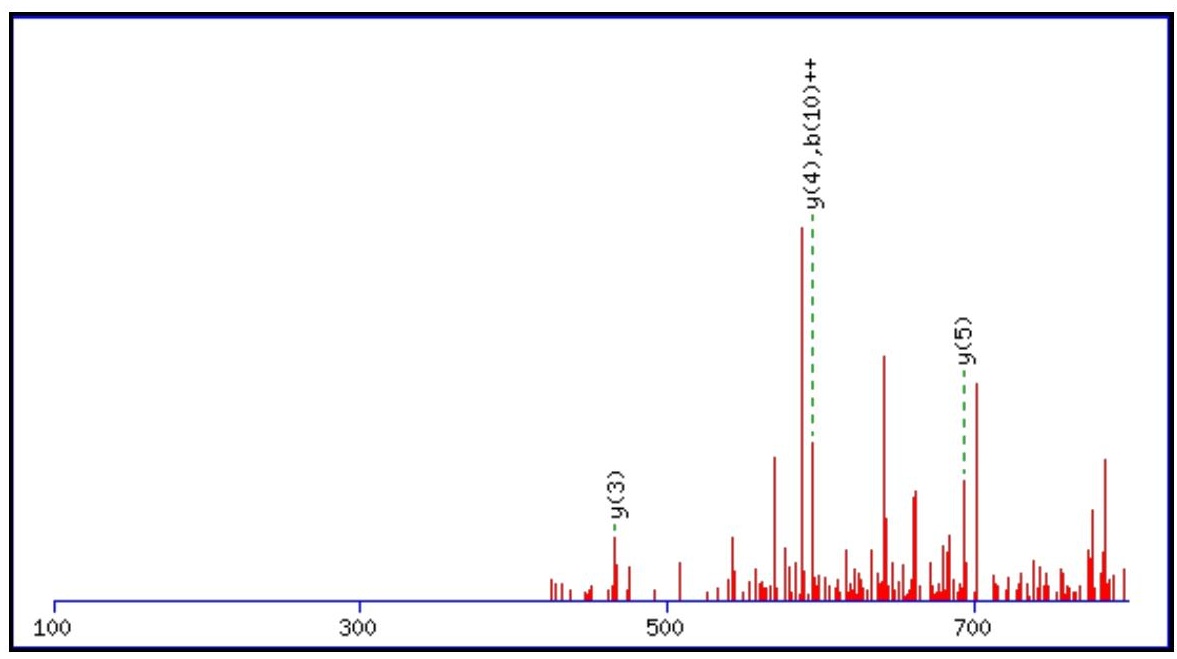



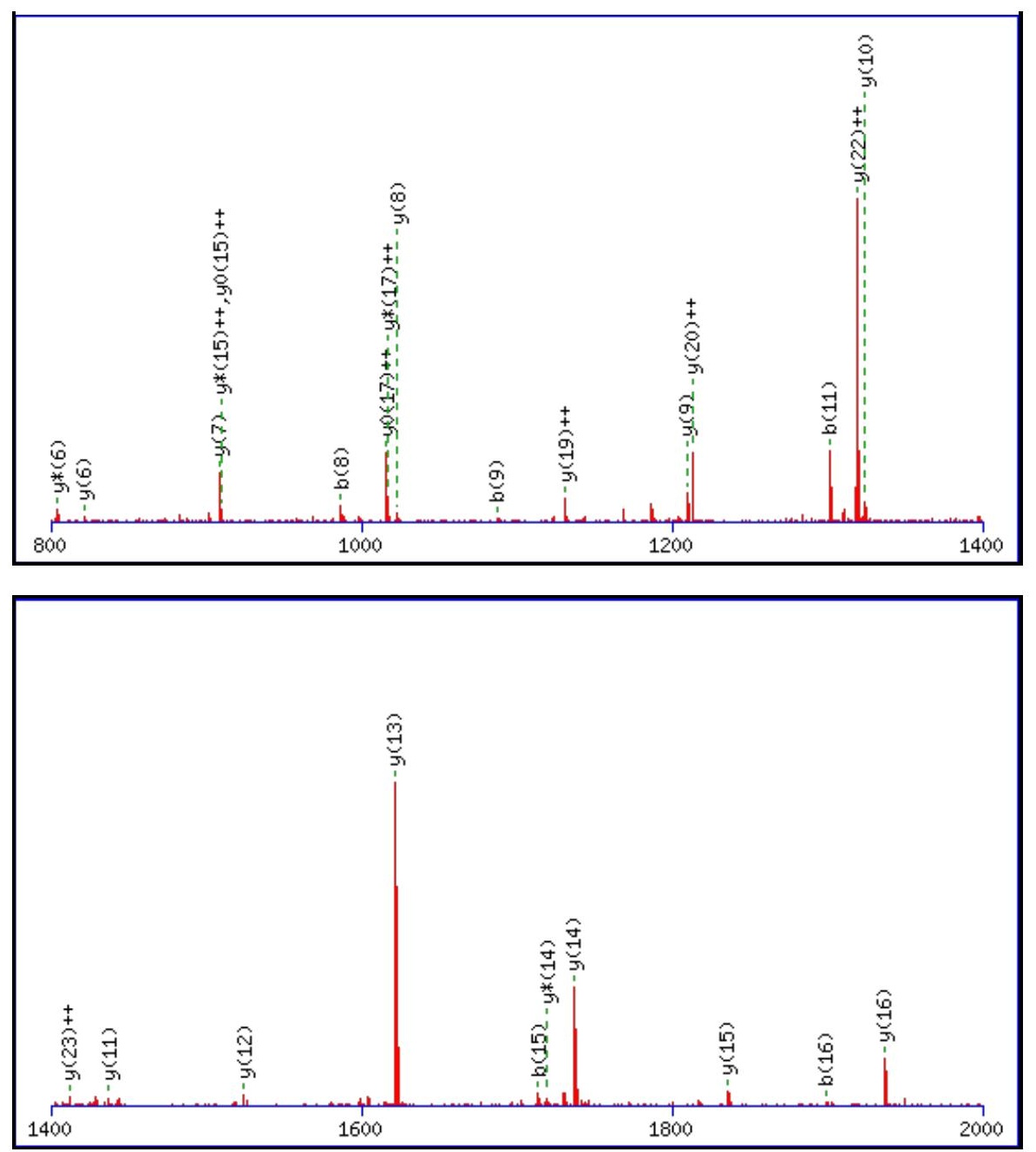

Monoisotopic mass of neutral peptide $\operatorname{Mr}($ calc): 2921.3508

Fixed modifications: Carbamidomethyl (C)

Variable modifications:

N7 : Deamidated_N (N)

Ions Score: 68 Expect: $6.2 \mathrm{e}-005$

Matches (Bold Red): 30/246 fragment ions using 65 most intense peaks

\begin{tabular}{|c|c|c|c|c|c|c|c|c|c|c|c|c|c|c|}
\hline \# & b & $\mathbf{b}^{++}$ & b* & $\mathbf{b}^{*++}$ & $\mathbf{b}^{0}$ & $\mathbf{b}^{0++}$ & Seq. & $\mathbf{y}$ & $y^{++}$ & $\mathbf{y}^{*}$ & $\mathrm{y}^{*^{++}}$ & $\mathbf{y}^{\mathbf{0}}$ & $\mathbf{y}^{0++}$ & \# \\
\hline 1 & 100.0757 & 50.5415 & & & & & $\mathbf{V}$ & & & & & & & 24 \\
\hline 2 & 286.1550 & 143.5811 & & & & & $\mathbf{W}$ & 2823.2897 & 1412.1485 & 2806.2631 & 1403.6352 & 2805.2791 & 1403.1432 & 23 \\
\hline 3 & 383.2078 & 192.1075 & & & & & $\mathbf{P}$ & 2637.2103 & 1319.1088 & 2620.1838 & 1310.5955 & 2619.1998 & 1310.1035 & 22 \\
\hline 4 & 498.2347 & 249.6210 & & & 480.2241 & 240.6157 & D & 2540.1576 & 1270.5824 & 2523.1310 & 1262.0692 & 2522.1470 & 1261.5771 & 21 \\
\hline 5 & 661.2980 & 331.1527 & & & 643.2875 & 322.1474 & $\mathbf{Y}$ & 2425.1306 & 1213.0690 & 2408.1041 & 1204.5557 & 2407.1201 & 1204.0637 & 20 \\
\hline 6 & 758.3508 & 379.6790 & & & 740.3402 & 370.6738 & $\mathbf{P}$ & 2262.0673 & 1131.5373 & 2245.0408 & 1123.0240 & 2244.0567 & 1122.5320 & 19 \\
\hline 7 & 873.3777 & 437.1925 & 856.3512 & 428.6792 & 855.3672 & 428.1872 & $\mathbf{N}$ & 2165.0145 & 1083.0109 & 2147.9880 & 1074.4976 & 2147.0040 & 1074.0056 & 18 \\
\hline 8 & 986.4618 & 493.7345 & 969.4353 & 485.2213 & 968.4512 & 484.7293 & I & 2049.9876 & 1025.4974 & 2032.9611 & 1016.9842 & 2031.9770 & 1016.4922 & 17 \\
\hline 9 & 1087.5095 & 544.2584 & 1070.4829 & 535.7451 & 1069.4989 & 535.2531 & $T$ & 1936.9035 & 968.9554 & 1919.8770 & 960.4421 & 1918.8930 & 959.9501 & 16 \\
\hline 10 & 1186.5779 & 593.7926 & 1169.5513 & 585.2793 & 1168.5673 & 584.7873 & $\mathbf{V}$ & 1835.8559 & 918.4316 & 1818.8293 & 909.9183 & 1817.8453 & 909.4263 & 15 \\
\hline 11 & 1301.6048 & 651.3061 & 1284.5783 & 642.7928 & 1283.5943 & 642.3008 & D & 1736.7875 & 868.8974 & 1719.7609 & 860.3841 & 1718.7769 & 859.8921 & 14 \\
\hline 12 & 1398.6576 & 699.8324 & 1381.6311 & 691.3192 & 1380.6470 & 690.8272 & $\mathbf{P}$ & 1621.7605 & 811.3839 & 1604.7340 & 802.8706 & 1603.7499 & 802.3786 & 13 \\
\hline 13 & 1485.6896 & 743.3485 & 1468.6631 & 734.8352 & 1467.6791 & 734.3432 & $\mathrm{~S}$ & 1524.7077 & 762.8575 & 1507.6812 & 754.3442 & 1506.6972 & 753.8522 & 12 \\
\hline 14 & 1598.7737 & 799.8905 & 1581.7471 & 791.3772 & 1580.7631 & 790.8852 & $\mathbf{L}$ & 1437.6757 & 719.3415 & 1420.6492 & 710.8282 & 1419.6652 & 710.3362 & 11 \\
\hline 15 & 1713.8006 & 857.4040 & 1696.7741 & 848.8907 & 1695.7901 & 848.3987 & D & 1324.5917 & 662.7995 & 1307.5651 & 654.2862 & 1306.5811 & 653.7942 & 10 \\
\hline 16 & 1899.8800 & 950.4436 & 1882.8534 & 941.9303 & 1881.8694 & 941.4383 & $\mathbf{W}$ & 1209.5647 & 605.2860 & 1192.5382 & 596.7727 & 1191.5541 & 596.2807 & 9 \\
\hline 17 & 2014.9069 & 1007.9571 & 1997.8803 & 999.4438 & 1996.8963 & 998.9518 & D & 1023.4854 & 512.2463 & 1006.4589 & 503.7331 & 1005.4748 & 503.2411 & 8 \\
\hline 18 & 2101.9389 & 1051.4731 & 2084.9124 & 1042.9598 & 2083.9284 & 1042.4678 & $\mathrm{~S}$ & 908.4585 & 454.7329 & 891.4319 & 446.2196 & 890.4479 & 445.7276 & 7 \\
\hline
\end{tabular}




\begin{tabular}{|l|l|l|l|l|l|l|l|l|l|l|l|l|l|l|l|l|l|l|l|}
$\mathbf{1 9}$ & 2229.9975 & 1115.5024 & 2212.9710 & 1106.9891 & 2211.9869 & 1106.4971 & $\mathbf{Q}$ & $\mathbf{8 2 1 . 4 2 6 4}$ & $\mathbf{4 1 1 . 2 1 6 9}$ & $\mathbf{8 0 4 . 3 9 9 9}$ & $\mathbf{4 0 2 . 7 0 3 6}$ & & & $\mathbf{6}$ \\
\hline $\mathbf{2 0}$ & 2329.0659 & 1165.0366 & 2312.0394 & 1156.5233 & 2311.0553 & 1156.0313 & $\mathbf{V}$ & $\mathbf{6 9 3 . 3 6 7 9}$ & 347.1876 & 676.3413 & 338.6743 & & & $\mathbf{5}$ \\
\hline $\mathbf{2 1}$ & 2457.1245 & 1229.0659 & 2440.0979 & 1220.5526 & 2439.1139 & 1220.0606 & $\mathbf{Q}$ & $\mathbf{5 9 4 . 2 9 9 4}$ & 297.6534 & 577.2729 & 289.1401 & & & $\mathbf{4}$ \\
\hline $\mathbf{2 2}$ & 2585.1831 & 1293.0952 & 2568.1565 & 1284.5819 & 2567.1725 & 1284.0899 & $\mathbf{Q}$ & $\mathbf{4 6 6 . 2 4 0 9}$ & 233.6241 & 449.2143 & 225.1108 & & & $\mathbf{3}$ \\
\hline $\mathbf{2 3}$ & 2748.2464 & 1374.6268 & 2731.2199 & 1366.1136 & 2730.2358 & 1365.6216 & $\mathbf{Y}$ & 338.1823 & 169.5948 & 321.1557 & 161.0815 & & & $\mathbf{2}$ \\
\hline $\mathbf{2 4}$ & & & & & & & $\mathbf{R}$ & 175.1190 & 88.0631 & 158.0924 & 79.5498 & & & $\mathbf{1}$ \\
\hline
\end{tabular}
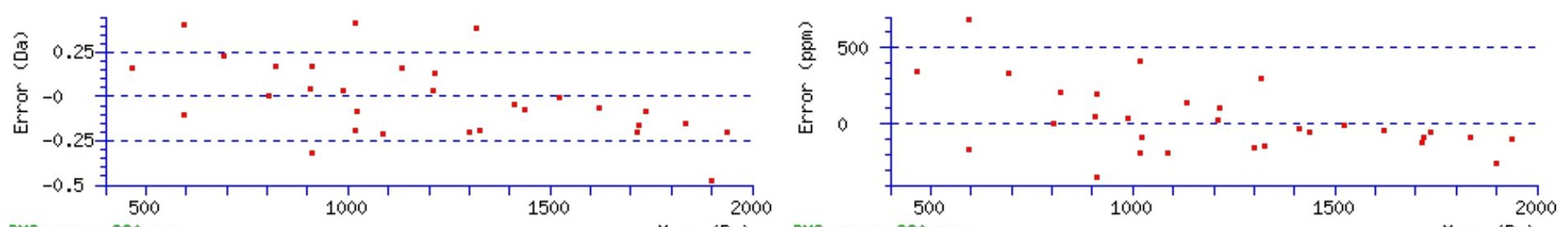

RMS error 220 ppm

Mass (Da) RMS error 220 ppm

Mass (Da)

\section{All matches to this query}

\begin{tabular}{|l|l|l|l|}
\hline Score & Mr(calc): & Delta & \multicolumn{1}{|c|}{ Sequence } \\
\hline 67.8 & 2921.3508 & 0.0149 & VWPDYPNITVDPSLDWDSQVQQYR \\
\hline 47.4 & 2920.3668 & 0.9989 & VWPDYPNITVDPSLDWDSQVQQYR \\
\hline 10.6 & 2921.3766 & -0.0108 & EFPFPTTITAVRTNVANLSDAALWK \\
\hline 4.0 & 2921.3766 & -0.0108 & EFPFPTTITAVRTNVANLSDAALWK \\
\hline 4.0 & 2921.3766 & -0.0108 & EFPFPTTITAVRTNVANLSDAALWK \\
\hline 3.8 & 2921.3766 & -0.0108 & EFPFPTTITAVRTNVANLSDAALWK \\
\hline 3.8 & 2921.3766 & -0.0108 & EFPFPTTITAVRTNVANLSDAALWK \\
\hline 3.7 & 2920.3449 & 1.0208 & LAYLLQQTDEYVANLTNLVWEHK \\
\hline 3.6 & 2921.3525 & 0.0132 & GTAEGAVDAMLVHGQDAITVSNGGRIMR \\
\hline 2.9 & 2921.3907 & -0.0250 & SLGTTTATISFPHMAMPMLLVLIIR \\
\hline
\end{tabular}

Spectrum No: 400; Query: 2244; Rank: 1

\section{Peptide View}

MS/MS Fragmentation of WKVPFNPNDTFESEFYLDEK

Found in IPI00200591, Tax_Id=10116 Gene_Symbol=LOC299282 Serine protease inhibitor A3L precursor

Match to Query 2244: 2505.143682 from(836.055170,3+)

Title: 100101RatKid_NS_deglyco_18.5085.5085.3.dta

Data file K:INewmanPaper|Piliangl3SubProteomes\Piliang3SP\mgf5ppm|ERLIC_3SubProteomes5ppm.mgf

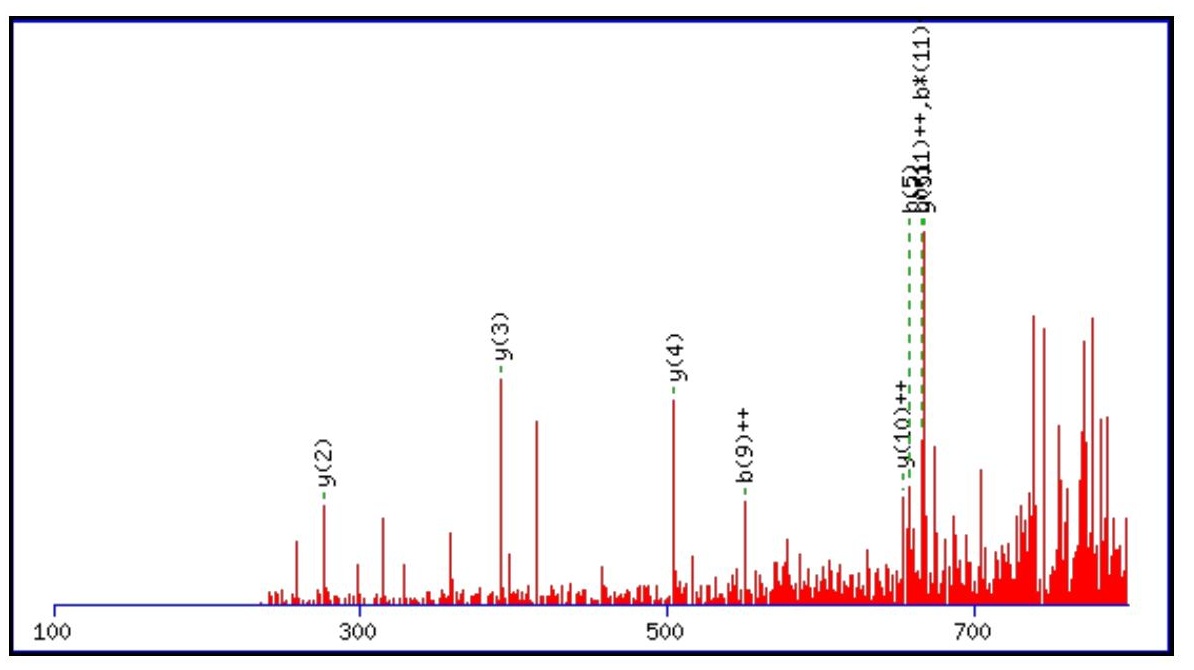



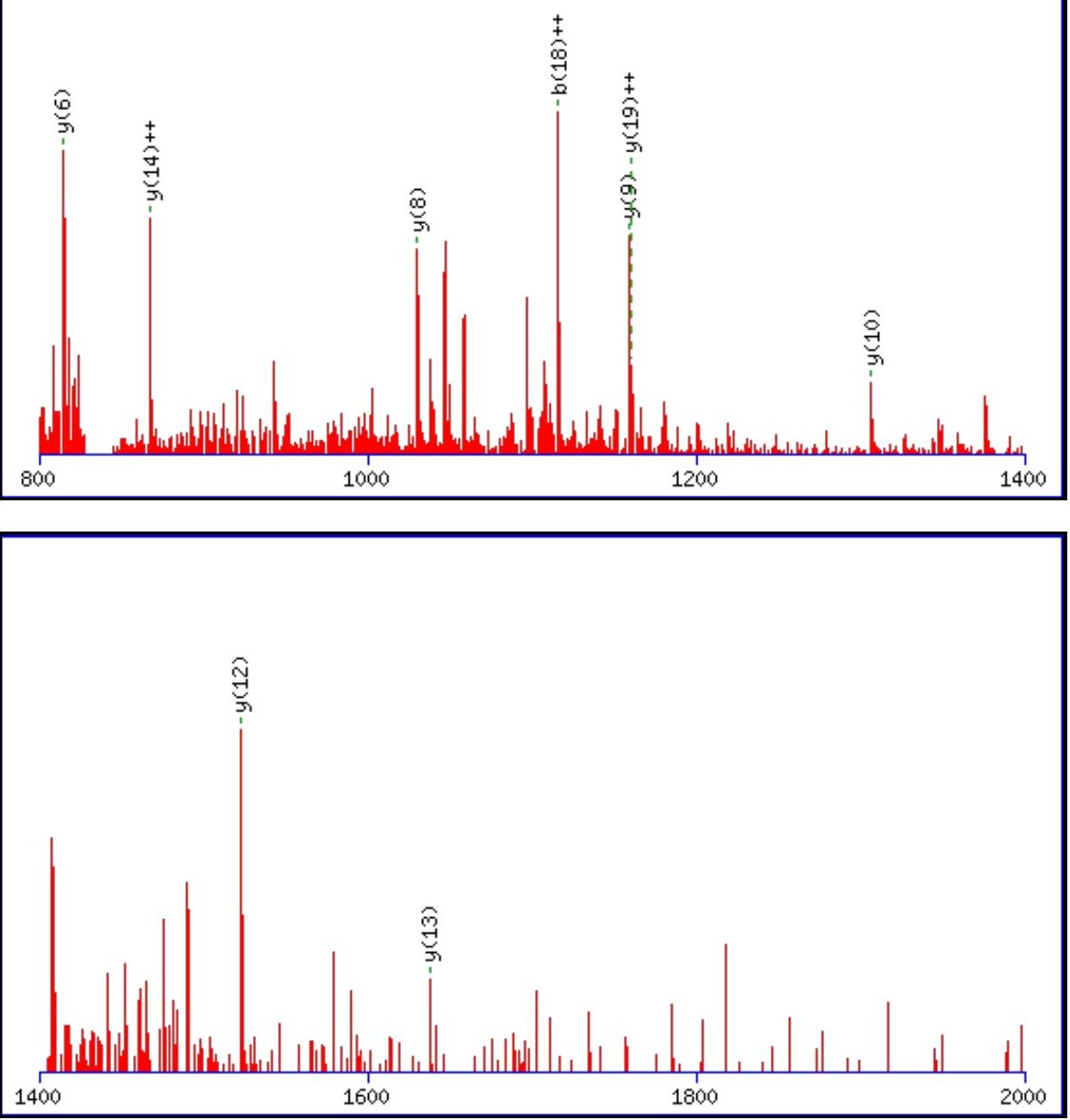

Monoisotopic mass of neutral peptide $\operatorname{Mr}$ (calc): 2505.1376

Fixed modifications: Carbamidomethyl (C)

Variable modifications:

N8 : Deamidated_N (N)

Ions Score: 68 Expect: $5.6 \mathrm{e}-005$

Matches (Bold Red): 18/208 fragment ions using 26 most intense peaks

\begin{tabular}{|c|c|c|c|c|c|c|c|c|c|c|c|c|c|c|}
\hline \# & b & $\mathbf{b}^{++}$ & $\mathbf{b}^{*}$ & $\mathbf{b}^{*^{++}}$ & $\mathbf{b}^{\mathbf{0}}$ & & Seq. & $\mathbf{y}$ & $\mathbf{y}^{++}$ & $\mathbf{y}^{*}$ & $\mathrm{y}^{*^{++}}$ & $\mathbf{y}^{0}$ & & \# \\
\hline 1 & 187.0866 & 94.0469 & & & & & $\mathbf{W}$ & & & & & & & 20 \\
\hline 2 & 315.1816 & 158.0944 & & & & & $\mathbf{K}$ & & & & & 2302.0550 & 1151.5311 & 19 \\
\hline 3 & 414.2500 & 207.6286 & 397.2234 & 199.1153 & & & $\mathbf{V}$ & 2191.9706 & 1096.4889 & 2174.9441 & 1087.9757 & 2173.9601 & 1087.4837 & 18 \\
\hline 4 & 511.3027 & 256.1550 & 494.2762 & 247.6417 & & & $\mathbf{P}$ & & & 2075.8757 & & 2074.8916 & 1037.9495 & 17 \\
\hline 5 & 658.3711 & 329.6892 & 641.3446 & 321.1759 & & & $\mathbf{F}$ & 1995.8494 & 998.4284 & 1978.8229 & 989.9151 & 1977.8389 & 989.4231 & 16 \\
\hline 6 & 772.4141 & 386.7107 & 755.3875 & 378.1974 & & & $\mathbf{N}$ & 1848.7810 & 924.8942 & 1831.7545 & 916.3809 & 1830.7705 & 915.8889 & 15 \\
\hline 7 & 869.4668 & 435.2371 & 852.4403 & 426.7238 & & & $\mathbf{P}$ & 1734.7381 & $\mathbf{8 6 7 . 8 7 2 7}$ & 1717.7115 & 859.3594 & 1716.7275 & 858.8674 & 14 \\
\hline 8 & 984.4938 & 492.7505 & 967.4672 & 484.2372 & & & $\mathbf{N}$ & 1637.6853 & 819.3463 & 1620.6588 & 810.8330 & 1619.6748 & 810.3410 & 13 \\
\hline 9 & 1099.5207 & 550.2640 & 1082.4942 & 541.7507 & 081.5101 & & D & 1522.6584 & 761.8328 & 1505.6318 & 753.3196 & 1504.6478 & 752.8276 & 12 \\
\hline 10 & 1200.5684 & 600.7878 & 1183.5418 & 592.2746 & 1182.5578 & 591.7826 & $\mathbf{T}$ & 1407.6315 & 704.3194 & 1390.6049 & 695.8061 & 1389.6209 & 695.3141 & 11 \\
\hline 11 & 1347.6368 & 674.3220 & 1330.6103 & 665.8088 & 1329.6262 & 665.3168 & $\mathbf{F}$ & 1306.5838 & 653.7955 & 1289.5572 & 645.2823 & 1288.5732 & 644.7902 & 10 \\
\hline 12 & 1476.6794 & 738.8433 & 1459.6529 & 730.3301 & 1458.6688 & 729.8381 & $\mathbf{E}$ & 1159.5154 & 580.2613 & 1142.4888 & 571.7480 & 1141.5048 & 571.2560 & 9 \\
\hline 13 & 1563.7114 & 782.3594 & 1546.6849 & 773.8461 & 1545.7009 & 773.3541 & $S$ & 1030.4728 & 515.7400 & 1013.4462 & 507.2267 & 1012.4622 & 506.7347 & 8 \\
\hline 14 & 1692.7540 & 846.8806 & 1675.7275 & 838.3674 & 1674.7435 & 837.8754 & $E$ & 943.4407 & 472.2240 & 926.4142 & 463.7107 & 925.4302 & 463.2187 & 7 \\
\hline 15 & 1839.8224 & 920.4149 & 1822.7959 & 911.9016 & 1821.8119 & 911.4096 & $\mathbf{F}$ & 814.3981 & 407.7027 & 797.3716 & 399.1894 & 796.3876 & 398.6974 & 6 \\
\hline 16 & 2002.8858 & 1001.9465 & 1985.8592 & 993.4332 & 1984.8752 & 992.9412 & $\mathbf{Y}$ & 667.3297 & 334.1685 & 650.3032 & 325.6552 & 649.3192 & 325.1632 & 5 \\
\hline 17 & 2115.9698 & 1058.4886 & 2098.9433 & 1049.9753 & 2097.9593 & 1049.4833 & $\mathbf{L}$ & 504.2664 & 252.6368 & 487.2399 & 244.1236 & 486.2558 & 243.6316 & 4 \\
\hline 18 & 2230.9968 & 1116.0020 & 2213.9702 & 1107.4887 & 2212.9862 & 1106.9967 & D & 391.1823 & 196.0948 & 374.1558 & 187.5815 & 373.1718 & 187.0895 & 3 \\
\hline
\end{tabular}




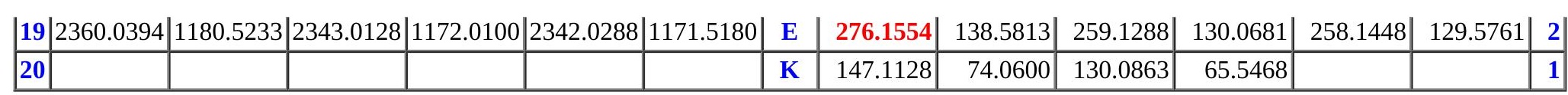
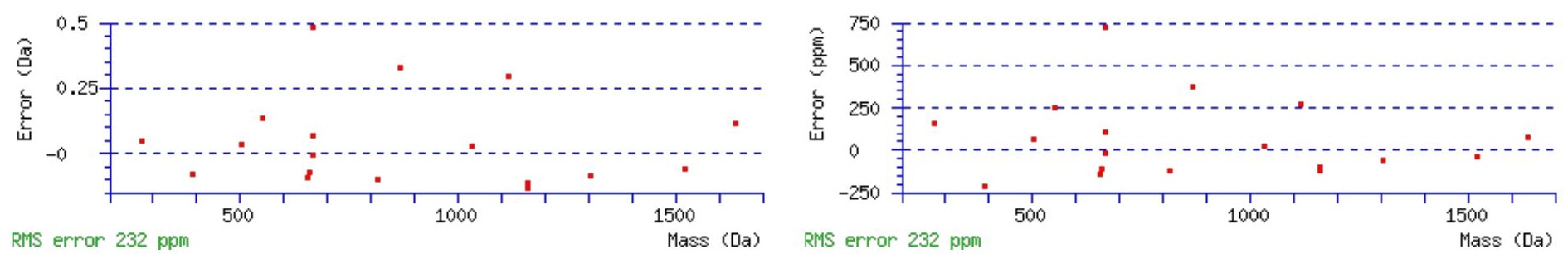

\section{All matches to this query}

\begin{tabular}{|l|l|l|l|}
\hline Score & Mr(calc): & Delta & \multicolumn{1}{|c|}{ Sequence } \\
\hline 67.7 & 2505.1376 & 0.0061 & WKVPFNPNDTFESEFYLDEK \\
\hline 53.8 & 2505.1376 & 0.0061 & WKVPFNPNDTFESEFYLDEK \\
\hline 46.3 & 2504.1536 & 0.9901 & WKVPFNPNDTFESEFYLDEK \\
\hline 14.8 & 2503.1127 & 2.0309 & TLARKNITMEMESNDTIENMK \\
\hline 1.6 & 2505.1285 & 0.0152 & SSSSGSDDYAYTQALLLHQRAR \\
\hline 0.1 & 2503.1454 & 1.9983 & MAHITINQYLQQVYEAIDTR \\
\hline
\end{tabular}

Mascot: http://www.matrixscience.com/ 


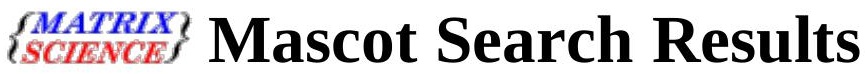

Results Generated by a Script Modified from Mascot Pepitde View

by Newman Sze, School of Biological Sciences, Nanyang Technological University

Spectrum No: 401; Query: 2613; Rank: 1

\section{Peptide View}

MS/MS Fragmentation of VNYEAETWDWIAETLSSNHMNFSSADR

Found in IPI00327398, Tax_Id=10116 Gene_Symbol=Enpep Isoform 1 of Glutamyl aminopeptidase

Match to Query 2613: 3189.371262 from(1064.131030,3+)

Title: 100101RatKid_NS_deglyco_18.6398.6398.3.dta

Data file K:INewmanPaper|Piliang|3SubProteomes\Piliang3SP\mgf5ppm\ERLIC_3SubProteomes5ppm.mgf
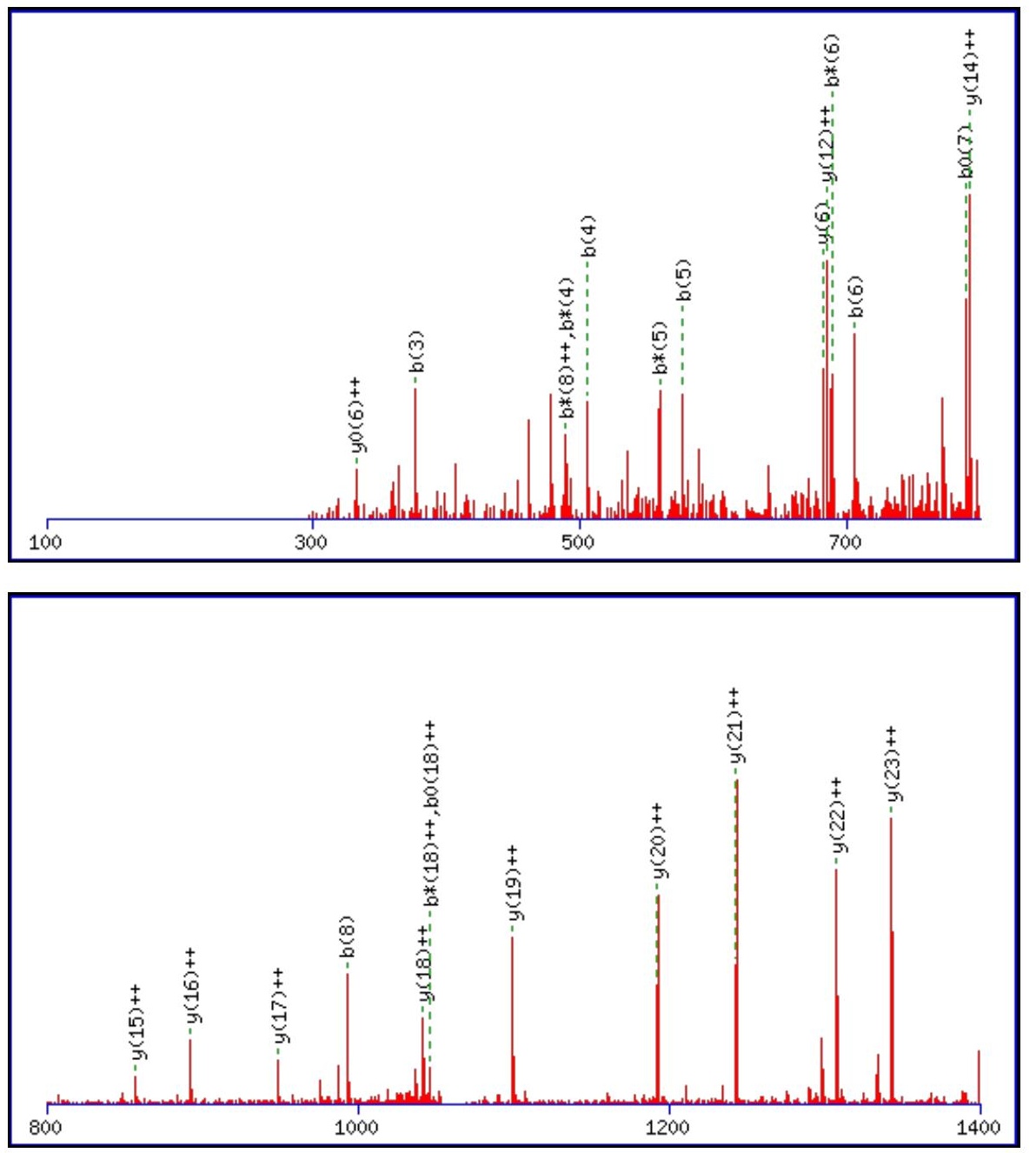


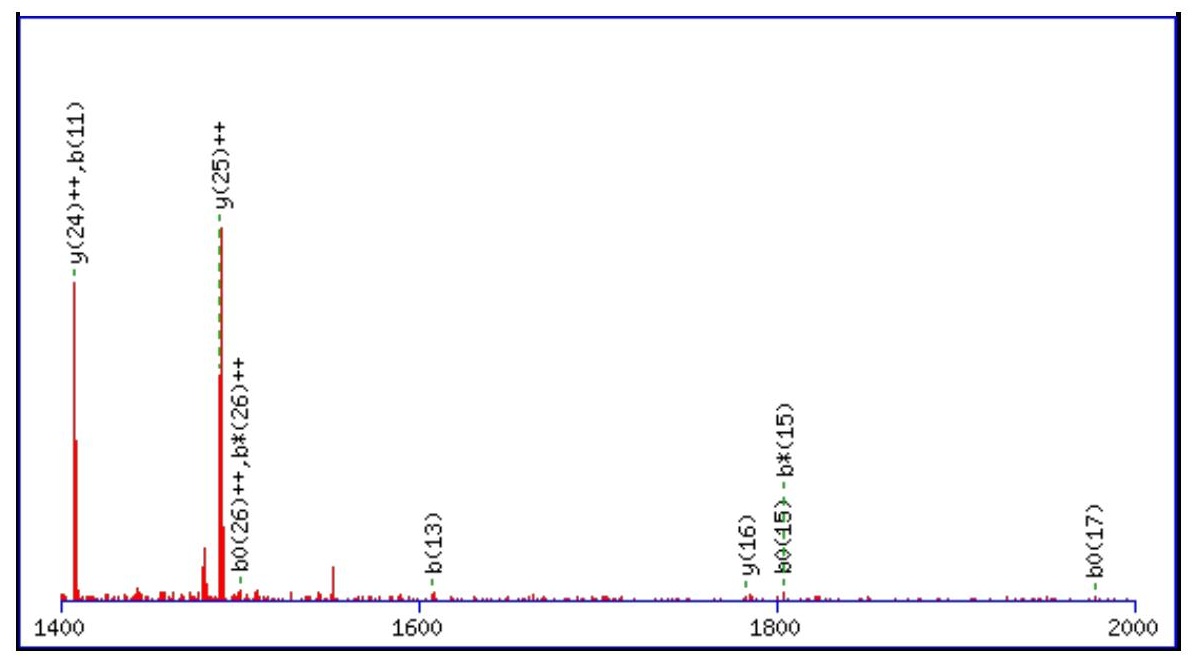

Monoisotopic mass of neutral peptide $\operatorname{Mr}($ calc): 3189.3621

Fixed modifications: Carbamidomethyl (C)

Variable modifications:

M20 : Oxidation (M)

N21 : Deamidated_N (N)

Ions Score: 68 Expect: $7.5 \mathrm{e}-005$

Matches (Bold Red): 35/302 fragment ions using 56 most intense peaks

\begin{tabular}{|c|c|c|c|c|c|c|c|c|c|c|c|c|c|c|}
\hline \# & b & $\mathbf{b}^{++}$ & b* & $\mathbf{b}^{*^{++}}$ & $\mathbf{b}^{\mathbf{0}}$ & $\mathbf{b}^{0++}$ & Seq. & $\mathbf{y}$ & $\mathbf{y}^{++}$ & $\mathbf{y}^{*}$ & $\mathrm{y}^{*^{++}}$ & $\mathbf{y}^{0}$ & $y^{0++}$ & \# \\
\hline 1 & 100.0757 & 50.5415 & & & & & $\mathbf{V}$ & & & & & & & 27 \\
\hline 2 & 214.1186 & 107.5629 & 197.0921 & 99.0497 & & & $\mathbf{N}$ & 3091.3010 & 1546.1542 & 3074.2745 & 1537.6409 & 3073.2905 & 1537.1489 & 26 \\
\hline 3 & 377.1819 & 189.0946 & 360.1554 & 180.5813 & & & $\mathbf{Y}$ & 2977.2581 & 1489.1327 & 2960.2316 & 1480.6194 & 2959.2475 & 1480.1274 & 25 \\
\hline 4 & 506.2245 & 253.6159 & 489.1980 & 245.1026 & 488.2140 & & $\mathbf{E}$ & 2814.1948 & 1407.6010 & 2797.1682 & 1399.0878 & 2796.1842 & 1398.5957 & 24 \\
\hline 5 & 577.2617 & 289.1345 & 560.2351 & 280.6212 & 559.2511 & 280.1292 & A & 2685.1522 & 1343.0797 & 2668.1256 & 1334.5665 & 2667.1416 & 1334.0744 & 23 \\
\hline 6 & 706.3042 & 353.6558 & 689.2777 & 345.1425 & 688.2937 & 344.6505 & $\mathbf{E}$ & 2614.1151 & 1307.5612 & 2597.0885 & 1299.0479 & 2596.1045 & 1298.5559 & 22 \\
\hline 7 & 807.3519 & 404.1796 & 790.3254 & 395.6663 & 789.3414 & 395.1743 & $\mathbf{T}$ & 2485.0725 & 1243.0399 & 2468.0459 & 1234.5266 & 2467.0619 & 1234.0346 & 21 \\
\hline 8 & 993.4312 & 497.2193 & 976.4047 & 488.7060 & 975.4207 & 488.2140 & $\mathbf{W}$ & 2384.0248 & 1192.5160 & 2366.9982 & 1184.0028 & 2366.0142 & 1183.5108 & 20 \\
\hline 9 & 1108.4582 & 554.7327 & 1091.4316 & 546.2195 & 1090.4476 & 545.7274 & D & 2197.9455 & 1099.4764 & 2180.9189 & 1090.9631 & 2179.9349 & 1090.4711 & 19 \\
\hline 10 & 1294.5375 & 647.7724 & 1277.5109 & 639.2591 & 1276.5269 & 638.7671 & $\mathbf{W}$ & 2082.9185 & 1041.9629 & 2065.8920 & 1033.4496 & 2064.9080 & 1032.9576 & 18 \\
\hline 11 & 1407.6216 & 704.3144 & 1390.5950 & 695.8011 & 1389.6110 & 695.3091 & I & 1896.8392 & 948.9233 & 1879.8127 & 940.4100 & 1878.8287 & 939.9180 & 17 \\
\hline 12 & 1478.6587 & 739.8330 & 1461.6321 & 731.3197 & 1460.6481 & 730.8277 & A & 1783.7552 & 892.3812 & 1766.7286 & 883.8679 & 1765.7446 & 883.3759 & 16 \\
\hline 13 & 1607.7013 & 804.3543 & 1590.6747 & 795.8410 & 1589.6907 & 795.3490 & $E$ & 1712.7180 & 856.8627 & 1695.6915 & 848.3494 & 1694.7075 & 847.8574 & 15 \\
\hline 14 & 1708.7489 & 854.8781 & 1691.7224 & 846.3648 & 1690.7384 & 845.8728 & $\mathbf{T}$ & 1583.6755 & 792.3414 & 1566.6489 & 783.8281 & 1565.6649 & 783.3361 & 14 \\
\hline 15 & 1821.8330 & 911.4201 & 1804.8065 & 902.9069 & 1803.8224 & 902.4149 & $\mathbf{L}$ & 1482.6278 & 741.8175 & 1465.6012 & 733.3043 & 1464.6172 & 732.8122 & 13 \\
\hline 16 & 1908.8650 & 954.9362 & 1891.8385 & 946.4229 & 1890.8545 & 945.9309 & $\mathrm{~S}$ & 1369.5437 & 685.2755 & 1352.5172 & 676.7622 & 1351.5331 & 676.2702 & 12 \\
\hline 17 & 1995.8971 & 998.4522 & 1978.8705 & 989.9389 & 1977.8865 & 989.4469 & S & 1282.5117 & 641.7595 & 1265.4851 & 633.2462 & 1264.5011 & 632.7542 & 11 \\
\hline 18 & 2109.9400 & 1055.4736 & 2092.9134 & 1046.9604 & 2091.9294 & 1046.4684 & $\mathbf{N}$ & 1195.4797 & 598.2435 & 1178.4531 & 589.7302 & 1177.4691 & 589.2382 & 10 \\
\hline 19 & 2246.9989 & 1124.0031 & 2229.9724 & 1115.4898 & 2228.9883 & 1114.9978 & $\mathbf{H}$ & 1081.4367 & 541.2220 & 1064.4102 & 532.7087 & 1063.4262 & 532.2167 & 9 \\
\hline 20 & 2394.0343 & 1197.5208 & 2377.0078 & 1189.0075 & 2376.0237 & 1188.5155 & $\mathbf{M}$ & 944.3778 & 472.6925 & 927.3513 & 464.1793 & 926.3673 & 463.6873 & 8 \\
\hline 21 & 2509.0612 & 1255.0343 & 2492.0347 & 1246.5210 & 2491.0507 & 1246.0290 & $\mathbf{N}$ & 797.3424 & 399.1748 & 780.3159 & 390.6616 & 779.3318 & 390.1696 & 7 \\
\hline 22 & 2656.1297 & 1328.5685 & 2639.1031 & 1320.0552 & 2638.1191 & 1319.5632 & $\mathbf{F}$ & 682.3155 & 341.6614 & 665.2889 & 333.1481 & 664.3049 & 332.6561 & 6 \\
\hline 23 & 2743.1617 & 1372.0845 & 2726.1351 & 1363.5712 & 2725.1511 & 1363.0792 & $S$ & 535.2471 & 268.1272 & 518.2205 & 259.6139 & 517.2365 & 259.1219 & 5 \\
\hline 24 & 2830.1937 & 1415.6005 & 2813.1672 & 1407.0872 & 2812.1831 & 1406.5952 & $\mathrm{~S}$ & 448.2150 & 224.6112 & 431.1885 & 216.0979 & 430.2045 & 215.6059 & 4 \\
\hline 25 & 2901.2308 & 1451.1191 & 2884.2043 & 1442.6058 & 2883.2203 & 1442.1138 & A & 361.1830 & 181.0951 & 344.1565 & 172.5819 & 343.1724 & 172.0899 & 3 \\
\hline 26 & 3016.2578 & 1508.6325 & 2999.2312 & 1500.1192 & 2998.2472 & 1499.6272 & D & 290.1459 & 145.5766 & 273.1193 & 137.0633 & 272.1353 & 136.5713 & 2 \\
\hline 27 & & & & & & & $\mathbf{R}$ & 175.1190 & 88.0631 & 158.0924 & 79.5498 & & & 1 \\
\hline
\end{tabular}



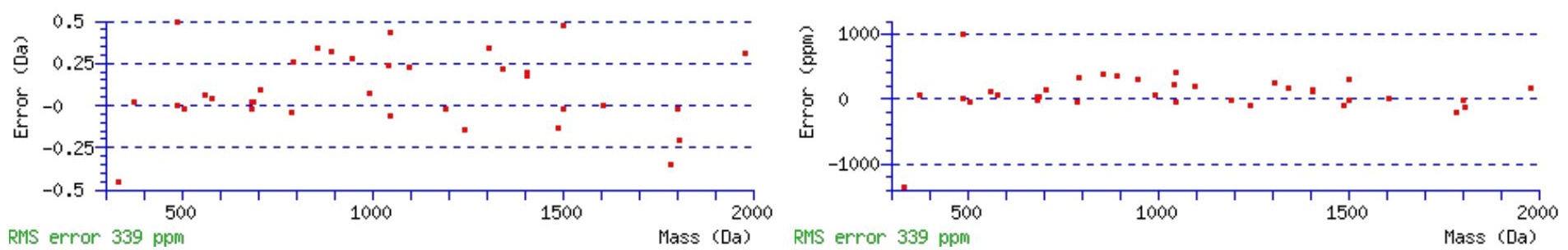

\section{All matches to this query}

\begin{tabular}{|l|l|l|l|}
\hline Score & Mr(calc): & Delta & \multicolumn{1}{c|}{ Sequence } \\
\hline 67.7 & 3189.3621 & 0.0091 & VNYEAETWDWIAETLSSNHMNFSSADR \\
\hline 67.7 & 3189.3621 & 0.0091 & VNYEAETWDWIAETLSSNHMNFSSADR \\
\hline 13.5 & 3188.3781 & 0.9931 & VNYEAETWDWIAETLSSNHMNFSSADR \\
\hline 4.8 & 3188.3613 & 1.0100 & MEEAVMSILHNLEMENAEIHENSHKR \\
\hline 4.0 & 3188.3876 & 0.9836 & GPGKPSVASTSGPQSTRTPSHQELKMQR \\
\hline 2.6 & 3187.3758 & 1.9955 & AALIPAGLSDGQFYSPPESEAGSEEEAEEK \\
\hline 2.6 & 3187.3758 & 1.9955 & AALIPAGLSDGQFYSPPESEAGSEEEAEEK \\
\hline 2.6 & 3187.3758 & 1.9955 & AALIPAGLSDGQFYSPPESEAGSEEEAEEK \\
\hline 2.6 & 3187.3475 & 2.0238 & SALLYGTLSSEAPQDGESNQSGYCAMISR \\
\hline 2.6 & 3187.3475 & 2.0238 & SALLYGTLSSEAPQDGESNQSGYCAMISR \\
\hline
\end{tabular}

Spectrum No: 402; Query: 2382; Rank: 1

\section{Peptide View}

MS/MS Fragmentation of IHVSDQELQSANASVDDSRLEELK

Found in IPI00368347, Tax_Id=10116 Gene_Symbol=Ube1x Ubiquitin-activating enzyme E1, Chr X

Match to Query 2382: 2685.305382 from(896.109070,3+)

Title: 091008RatKidney_NH4Format02_21.2731.2731.3.dta

Data file K:INewmanPaper|Piliang|3SubProteomes\Piliang3SP\mgf5ppm\ERLIC_3SubProteomes5ppm.mgf

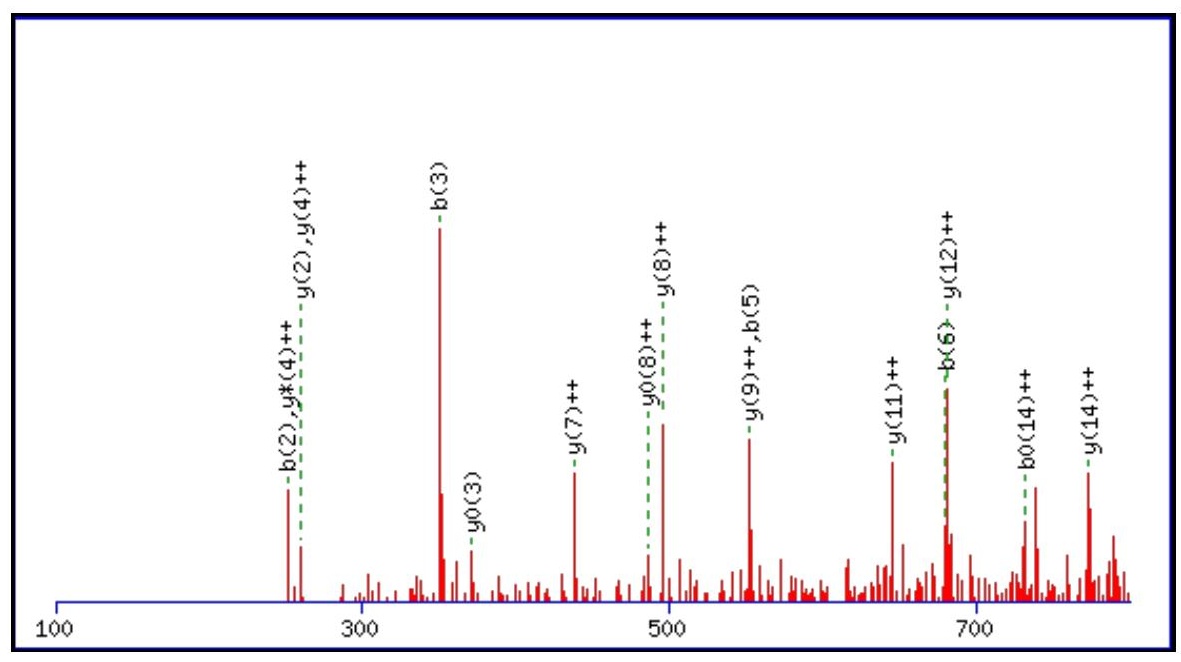



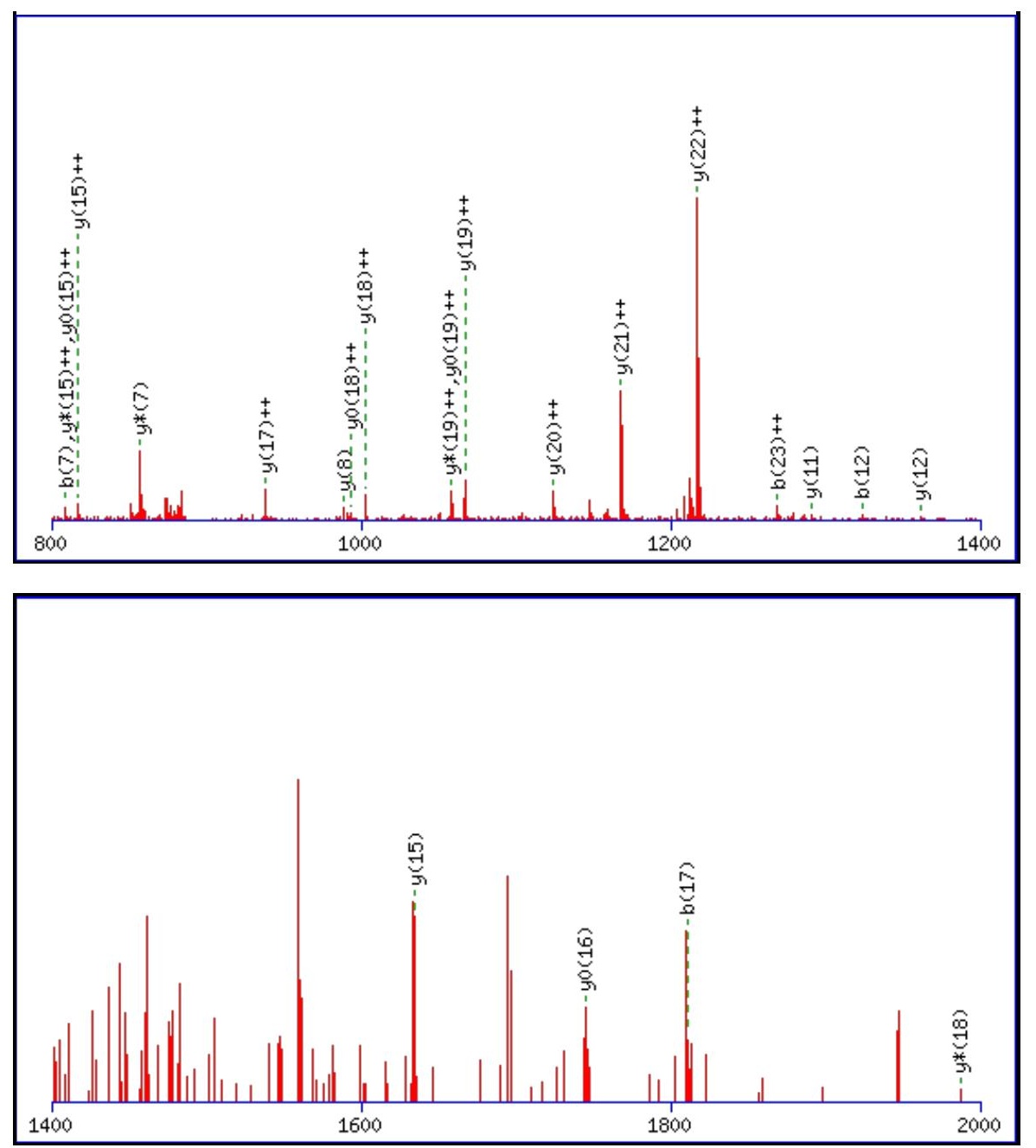

Monoisotopic mass of neutral peptide $\operatorname{Mr}($ calc): 2683.2936

Fixed modifications: Carbamidomethyl (C)

Variable modifications:

N12 : Deamidated_N (N)

Ions Score: 68 Expect: $4.7 \mathrm{e}-005$

Matches (Bold Red): 39/256 fragment ions using 75 most intense peaks

\begin{tabular}{|c|c|c|c|c|c|c|c|c|c|c|c|c|c|c|}
\hline \# & b & $\mathbf{b}^{++}$ & $\mathbf{b}^{*}$ & $\mathbf{b}^{*^{++}}$ & $\mathbf{b}^{\mathbf{0}}$ & $\mathbf{b}^{\mathbf{0 + +}}$ & Seq. & $\mathbf{y}$ & $y^{++}$ & $\mathbf{y}^{*}$ & $\mathbf{y}^{*^{++}}$ & $\mathbf{y}^{0}$ & $\mathbf{y}^{0++}$ & \# \\
\hline 1 & 114.0913 & 57.5493 & & & & & I & & & & & & & 24 \\
\hline 2 & 251.1503 & 126.0788 & & & & & $\mathbf{H}$ & 2571.2169 & 1286.1121 & 2554.1903 & 1277.5988 & 2553.2063 & 1277.1068 & 23 \\
\hline 3 & 350.2187 & 175.6130 & & & & & V & 2434.1580 & 1217.5826 & 2417.1314 & 1209.0694 & 2416.1474 & 1208.5773 & 22 \\
\hline 4 & 437.2507 & 219.1290 & & & 419.2401 & & S & 2335.0896 & 1168.0484 & 2318.0630 & 1159.5351 & 2317.0790 & 1159.0431 & 21 \\
\hline 5 & 552.2776 & 276.6425 & & & 534.2671 & 267.6372 & D & 2248.0575 & 1124.5324 & 2231.0310 & 1116.0191 & 2230.0470 & 1115.5271 & 20 \\
\hline 6 & 680.3362 & 340.6717 & 663.3097 & 332.1585 & 662.3257 & 331.6665 & $\mathbf{Q}$ & 2133.0306 & 1067.0189 & 2116.0040 & 1058.5057 & 2115.0200 & 1058.0137 & 19 \\
\hline 7 & 809.3788 & 405.1930 & 792.3523 & 396.6798 & 791.3682 & 396.1878 & $\mathbf{E}$ & 2004.9720 & 1002.9896 & 1987.9455 & 994.4764 & 1986.9614 & 993.9844 & 18 \\
\hline 8 & 922.4629 & 461.7351 & 905.4363 & 453.2218 & 904.4523 & 452.7298 & $\mathbf{L}$ & 1875.9294 & 938.4683 & 1858.9029 & 929.9551 & 1857.9189 & 929.4631 & 17 \\
\hline 9 & 1050.5215 & 525.7644 & 1033.4949 & 517.2511 & 1032.5109 & 516.7591 & $\mathbf{Q}$ & 1762.8454 & 881.9263 & 1745.8188 & 873.4130 & 1744.8348 & 872.9210 & 16 \\
\hline 10 & 1137.5535 & 569.2804 & 1120.5269 & 560.7671 & 1119.5429 & 560.2751 & S & 1634.7868 & 817.8970 & 1617.7602 & 809.3838 & 1616.7762 & 808.8917 & 15 \\
\hline 11 & 1208.5906 & 604.7989 & 1191.5640 & 596.2857 & 1190.5800 & 595.7937 & A & 1547.7547 & 774.3810 & 1530.7282 & 765.8677 & 1529.7442 & 765.3757 & 14 \\
\hline 12 & 1323.6175 & 662.3124 & 1306.5910 & 653.7991 & 1305.6070 & 653.3071 & $\mathbf{N}$ & 1476.7176 & 738.8625 & 1459.6911 & 730.3492 & 1458.7071 & 729.8572 & 13 \\
\hline 13 & 1394.6546 & 697.8310 & 1377.6281 & 689.3177 & 1376.6441 & 688.8257 & A & 1361.6907 & 681.3490 & 1344.6642 & 672.8357 & 1343.6801 & 672.3437 & 12 \\
\hline 14 & 1481.6867 & 741.3470 & 1464.6601 & 732.8337 & 1463.6761 & 732.3417 & $S$ & 1290.6536 & 645.8304 & 1273.6270 & 637.3172 & 1272.6430 & 636.8251 & 11 \\
\hline 15 & 1580.7551 & 790.8812 & 1563.7285 & 782.3679 & 1562.7445 & 781.8759 & V & 1203.6216 & 602.3144 & 1186.5950 & 593.8011 & 1185.6110 & 593.3091 & 10 \\
\hline 16 & 1695.7820 & 848.3947 & 1678.7555 & 839.8814 & 1677.7715 & 839.3894 & D & 1104.5531 & 552.7802 & 1087.5266 & 544.2669 & 1086.5426 & 543.7749 & 9 \\
\hline 17 & 1810.8090 & 905.9081 & 1793.7824 & 897.3948 & 1792.7984 & 896.9028 & D & 989.5262 & 495.2667 & 972.4997 & 486.7535 & 971.5156 & 486.2615 & 8 \\
\hline 18 & 1897.8410 & 949.4241 & 1880.8144 & 940.9109 & 1879.8304 & 940.4189 & S & 874.4993 & 437.7533 & 857.4727 & 429.2400 & 856.4887 & 428.7480 & 7 \\
\hline
\end{tabular}




\begin{tabular}{|l|r|r|r|r|r|r|r|r|r|r|r|r|r|r|r|r|}
$\mathbf{1 9}$ & 2053.9421 & 1027.4747 & 2036.9156 & 1018.9614 & 2035.9315 & 1018.4694 & $\mathbf{R}$ & 787.4672 & 394.2373 & 770.4407 & 385.7240 & 769.4567 & 385.2320 & $\mathbf{6}$ \\
\hline $\mathbf{2 0}$ & 2167.0262 & 1084.0167 & 2149.9996 & 1075.5034 & 2149.0156 & 1075.0114 & $\mathbf{L}$ & 631.3661 & 316.1867 & 614.3396 & 307.6734 & 613.3556 & 307.1814 & $\mathbf{5}$ \\
\hline $\mathbf{2 1}$ & 2296.0688 & 1148.5380 & 2279.0422 & 1140.0247 & 2278.0582 & 1139.5327 & $\mathbf{E}$ & 518.2821 & $\mathbf{2 5 9 . 6 4 4 7}$ & 501.2555 & $\mathbf{2 5 1 . 1 3 1 4}$ & 500.2715 & 250.6394 & $\mathbf{4}$ \\
\hline $\mathbf{2 2}$ & 2425.1114 & 1213.0593 & 2408.0848 & 1204.5460 & 2407.1008 & 1204.0540 & E & 389.2395 & 195.1234 & 372.2129 & 186.6101 & 371.2289 & 186.1181 & $\mathbf{3}$ \\
\hline $\mathbf{2 3}$ & 2538.1954 & $\mathbf{1 2 6 9 . 6 0 1 3}$ & 2521.1689 & 1261.0881 & 2520.1849 & 1260.5961 & $\mathbf{L}$ & $\mathbf{2 6 0 . 1 9 6 9}$ & 130.6021 & 243.1703 & 122.0888 & & & $\mathbf{2}$ \\
\hline $\mathbf{2 4}$ & & & & & & & $\mathbf{K}$ & 147.1128 & 74.0600 & 130.0863 & 65.5468 & & & $\mathbf{1}$ \\
\hline
\end{tabular}
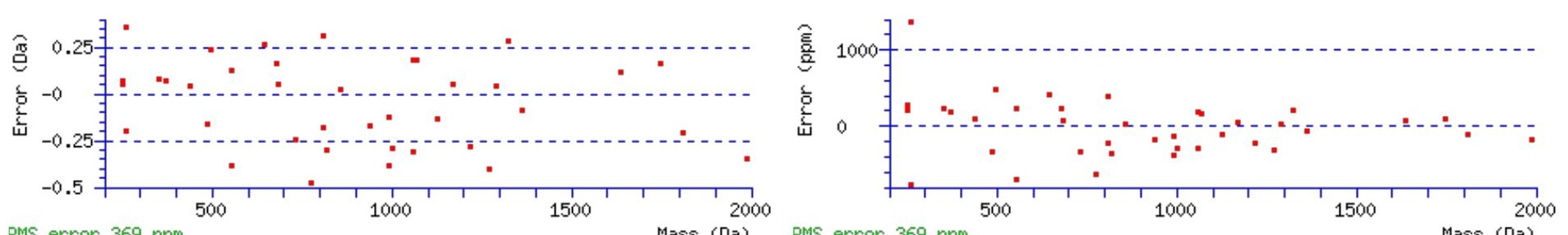

RMS error 369 ppm

Mass (Da) RMS error 369 ppm

Mass (Da)

\section{All matches to this query}

\begin{tabular}{|l|c|c|c|}
\hline Score & Mr(calc): & Delta & Sequence \\
\hline 67.6 & 2683.2936 & 2.0117 & IHVSDQELQSANASVDDSRLEELK \\
\hline 8.1 & 2685.2890 & 0.0164 & GANIFLTSSGLIKLGDFGCSVKLK \\
\hline 6.7 & 2685.2890 & 0.0164 & GANIFLTSSGLIKLGDFGCSVKLK \\
\hline 6.7 & 2685.2890 & 0.0164 & GANIFLTSSGLIKLGDFGCSVKLK \\
\hline 5.8 & 2685.3027 & 0.0027 & ITLSQVGDVLRALGTNPTNAEVKK \\
\hline 5.8 & 2685.3027 & 0.0027 & ITLSQVGDVLRALGTNPTNAEVKK \\
\hline 4.4 & 2685.2890 & 0.0164 & GANIFLTSSGLIKLGDFGCSVKLK \\
\hline 4.4 & 2685.2890 & 0.0164 & GANIFLTSSGLIKLGDFGCSVKLK \\
\hline 4.2 & 2685.2890 & 0.0164 & GANIFLTSSGLIKLGDFGCSVKLK \\
\hline 1.2 & 2683.3136 & 1.9918 & TKIDLQHPEGAVEPKSIVGHLQK \\
\hline
\end{tabular}

Spectrum No: 403; Query: 2358; Rank: 1

\section{Peptide View}

MS/MS Fragmentation of EVLNYTTVPVYLPEITIGAHQGSR

Found in IPI00189759, Tax_Id=10116 Gene_Symbol=LOC316632 NADH dehydrogenase 1 alpha subcomplex 10-like protein

Match to Query 2358: 2657.383008 from(1329.698780,2+)

Title: 091008RatKidney_NoSalt_09.4827.4827.2.dta

Data file K:INewmanPaper|Piliangl3SubProteomes\Piliang3SP\mgf5ppm\ERLIC_3SubProteomes5ppm.mgf 

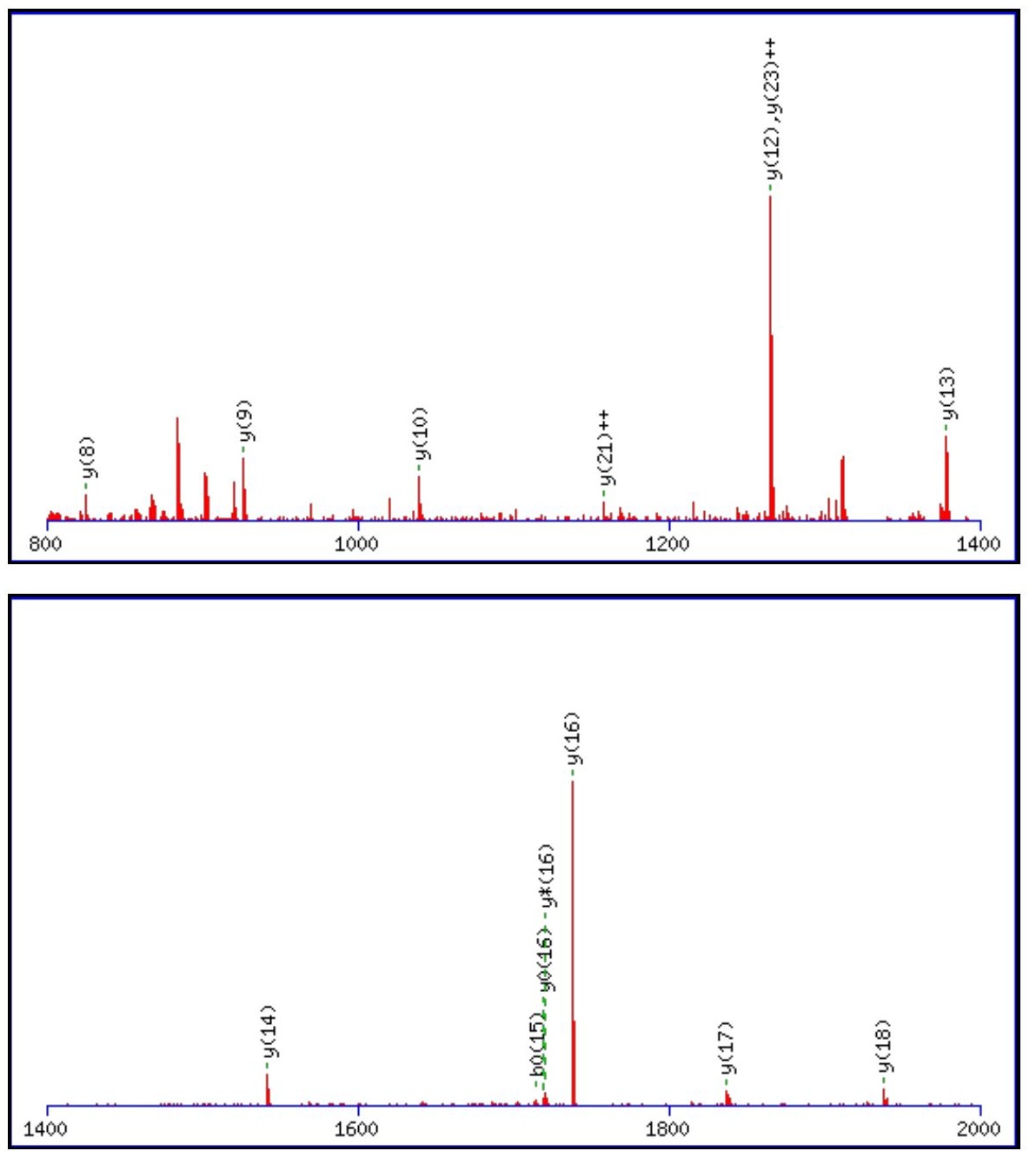

Monoisotopic mass of neutral peptide $\operatorname{Mr}$ (calc): 2657.3701

Fixed modifications: Carbamidomethyl (C)

Variable modifications:

N4 : Deamidated_N (N)

Ions Score: 68 Expect: $2.1 \mathrm{e}-005$

Matches (Bold Red): 18/268 fragment ions using 37 most intense peaks

\begin{tabular}{|c|c|c|c|c|c|c|c|c|c|c|c|c|c|c|}
\hline \# & b & $\mathbf{b}^{++}$ & $\mathbf{b}^{*}$ & & $\mathbf{b}^{0}$ & & eq. & $\mathbf{y}$ & & & & $\mathbf{y}^{0}$ & & \# \\
\hline 1 & 130.0499 & 65.5286 & & & 117 & 56.5233 & $F$ & & & & & & & 24 \\
\hline 2 & 229.1 & & & & & & V & & & & & & & 23 \\
\hline 3 & 342.2023 & 171.6048 & & & & & $\mathbf{L}$ & & & & & & & 22 \\
\hline 4 & 457.2 & 229. & & & & & $\mathbf{N}$ & & & & & & & 21 \\
\hline 5 & 620.2926 & 310.6499 & & & 602.2 & 301. & $\mathbf{Y}$ & & & 288 & & & 5760 & 20 \\
\hline 6 & 721.3403 & 361.1738 & & 352. & 703. & 352. & $\mathbf{T}$ & & & & & 815 & & 19 \\
\hline 7 & 822.3880 & 411.6976 & 14 & 403. & 804.3 & 402.6923 & $\mathbf{T}$ & 443 & 258 & 1921 & 125 & 338 & 5205 & 18 \\
\hline 8 & 921.4564 & 461.2318 & 904.4298 & 452.7186 & 903.4458 & 452.2265 & V & & & 1819 & & 1818.9861 & 967 & 17 \\
\hline 9 & 018.5091 & 509.7582 & 326 & 501.2 & 986 & 500.7 & $\mathbf{P}$ & 282 & & 1720 & & 177 & 625 & 16 \\
\hline 10 & 1117.5776 & 559.2924 & 100.5510 & 550.7791 & 1099.5670 & 550.2871 & $\mathbf{V}$ & 1640.8755 & 414 & 1623 & 4281 & 1622.8649 & 811.9361 & 15 \\
\hline 11 & 1280.6409 & 640.8241 & 143 & 632.3108 & 1262.6303 & 631.8188 & $\bar{Y}$ & 1541.8071 & 771.4072 & 1524 & 3939 & 1523.7965 & 762.4019 & 14 \\
\hline 12 & 1393.7250 & 697.3661 & 376.6984 & 688.8528 & 1375.7144 & 688.3608 & $\mathbf{L}$ & 1378.7437 & 8755 & 1361 & 622 & 1360 & 702 & 13 \\
\hline 13 & 1490.7777 & 745.8925 & 1473.7512 & 737.3792 & 1472.7672 & 736.8872 & $\mathbf{P}$ & 1265.6597 & 335 & 1248.6331 & 8202 & 1247.6491 & 624.3282 & 12 \\
\hline 14 & 1619.8203 & 810.4138 & 1602.7938 & 801.9005 & 1601.8097 & 801.4085 & $\mathbf{E}$ & 1168.6069 & 584.8071 & 1151.5804 & 576.2938 & 1150.5963 & 575.8018 & 11 \\
\hline 15 & 1732.9044 & 866.9558 & 1715.8778 & 858.4426 & 1714.8938 & 857.9505 & I & 1039.5643 & 520.2858 & 1022.5378 & 511.7725 & 1021.5538 & 511.2805 & 10 \\
\hline 16 & 1833.9521 & 917.4797 & 1816.9255 & 908.9664 & 1815.9415 & 908.4744 & $\mathbf{T}$ & 926.4803 & 463.7438 & 909.4537 & 455.2305 & 908.4697 & 454.7385 & 9 \\
\hline 17 & 1947.0361 & 974.0217 & 1930.0096 & 965.5084 & 1929.0256 & 965.0164 & I & 825.4326 & 413.2199 & 808.4060 & 404.7067 & 807.4220 & 404.2146 & 8 \\
\hline 18 & 2004.0576 & 1002.5324 & 1987.0310 & 994.0192 & 1986.0470 & 993.5271 & G & 712.3485 & 356.6779 & 695.3220 & 348.1646 & 694.3379 & 347.6726 & 7 \\
\hline
\end{tabular}




\begin{tabular}{|l|l|r|r|r|r|r|r|r|r|r|r|r|r|r|r|}
$\mathbf{1 9}$ & 2075.0947 & 1038.0510 & 2058.0681 & 1029.5377 & 2057.0841 & 1029.0457 & A & 655.3270 & 328.1672 & 638.3005 & 319.6539 & 637.3165 & 319.1619 & $\mathbf{6}$ \\
\hline $\mathbf{2 0}$ & 2212.1536 & 1106.5804 & 2195.1271 & 1098.0672 & 2194.1430 & 1097.5752 & H & $\mathbf{5 8 4 . 2 8 9 9}$ & 292.6486 & 567.2634 & 284.1353 & 566.2794 & 283.6433 & $\mathbf{5}$ \\
\hline $\mathbf{2 1}$ & 2340.2122 & 1170.6097 & 2323.1856 & 1162.0965 & 2322.2016 & 1161.6044 & $\mathbf{Q}$ & $\mathbf{4 4 7 . 2 3 1 0}$ & 224.1191 & 430.2045 & 215.6059 & 429.2205 & 215.1139 & $\mathbf{4}$ \\
\hline $\mathbf{2 2}$ & 2397.2337 & 1199.1205 & 2380.2071 & 1190.6072 & 2379.2231 & 1190.1152 & G & 319.1724 & 160.0899 & 302.1459 & 151.5766 & 301.1619 & 151.0846 & $\mathbf{3}$ \\
\hline $\mathbf{2 3}$ & 2484.2657 & 1242.6365 & 2467.2391 & 1234.1232 & 2466.2551 & 1233.6312 & S & 262.1510 & 131.5791 & 245.1244 & 123.0659 & 244.1404 & 122.5738 & $\mathbf{2}$ \\
\hline $\mathbf{2 4}$ & & & & & & & R & 175.1190 & 88.0631 & 158.0924 & 79.5498 & & & $\mathbf{1}$ \\
\hline
\end{tabular}
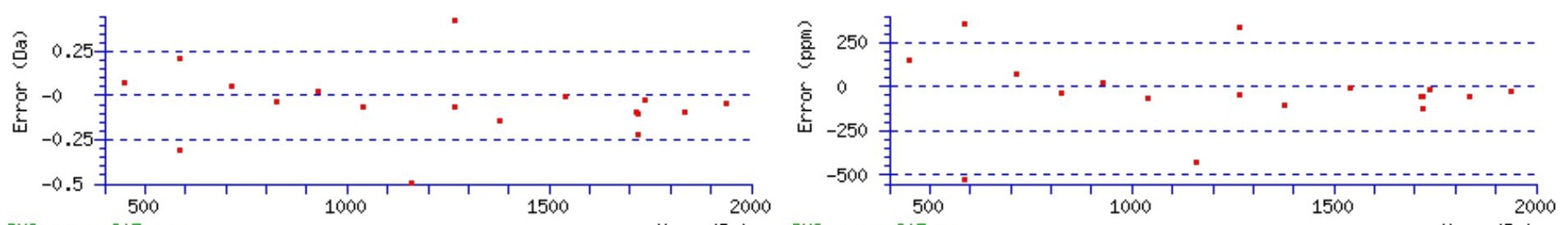

RMS error 207 ppm Mass (Da) RMS error 207 ppm

\section{All matches to this query}

\begin{tabular}{|l|c|c|l|}
\hline Score & Mr(calc): & Delta & \multicolumn{1}{c|}{ Sequence } \\
\hline 67.5 & 2657.3701 & 0.0129 & EVLNYTTVPVYLPEITIGAHQGSR \\
\hline 64.1 & 2656.3861 & 0.9969 & EVLNYTTVPVYLPEITIGAHQGSR \\
\hline 0.4 & 2657.3986 & -0.0156 & NYLDLTVTYTTLMILLSRIEER \\
\hline 0.2 & 2655.3779 & 2.0051 & $\underline{\text { SLGKAVATVSLSVALASVTVRSSGCR }}$ \\
\hline 0.2 & 2655.3779 & 2.0051 & SLGKAVATVSLSVALASVTVRSSGCR \\
\hline 0.2 & 2655.3779 & 2.0051 & SLGKAVATVSLSVALASVTVRSSGCR \\
\hline 0.2 & 2655.3779 & 2.0051 & $\underline{\text { SLGKAVATVSLSVALASVTVRSSGCR }}$ \\
\hline
\end{tabular}

Spectrum No: 404; Query: 2596; Rank: 1

\section{Peptide View}

MS/MS Fragmentation of GYYNQSEGGSHTIQEMFGCDVGSDGSLLR

Found in IPI00855224, Tax_Id=10116 Gene_Symbol=RT1-Aw2 MHC class I RT1.Ab heavy chain precursor

Match to Query 2596: 3164.353422 from(1055.791750,3+)

Title: 100101RatKid_NS_deglyco_20.4320.4320.3.dta

Data file K:INewmanPaper|Piliangl3SubProteomes\Piliang3SP\mgf5ppm|ERLIC_3SubProteomes5ppm.mgf

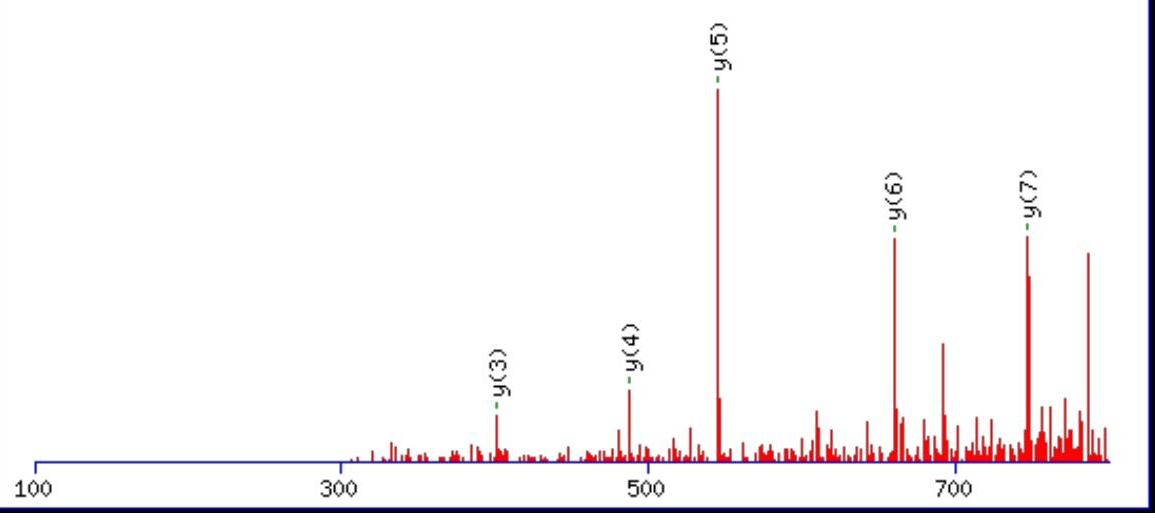



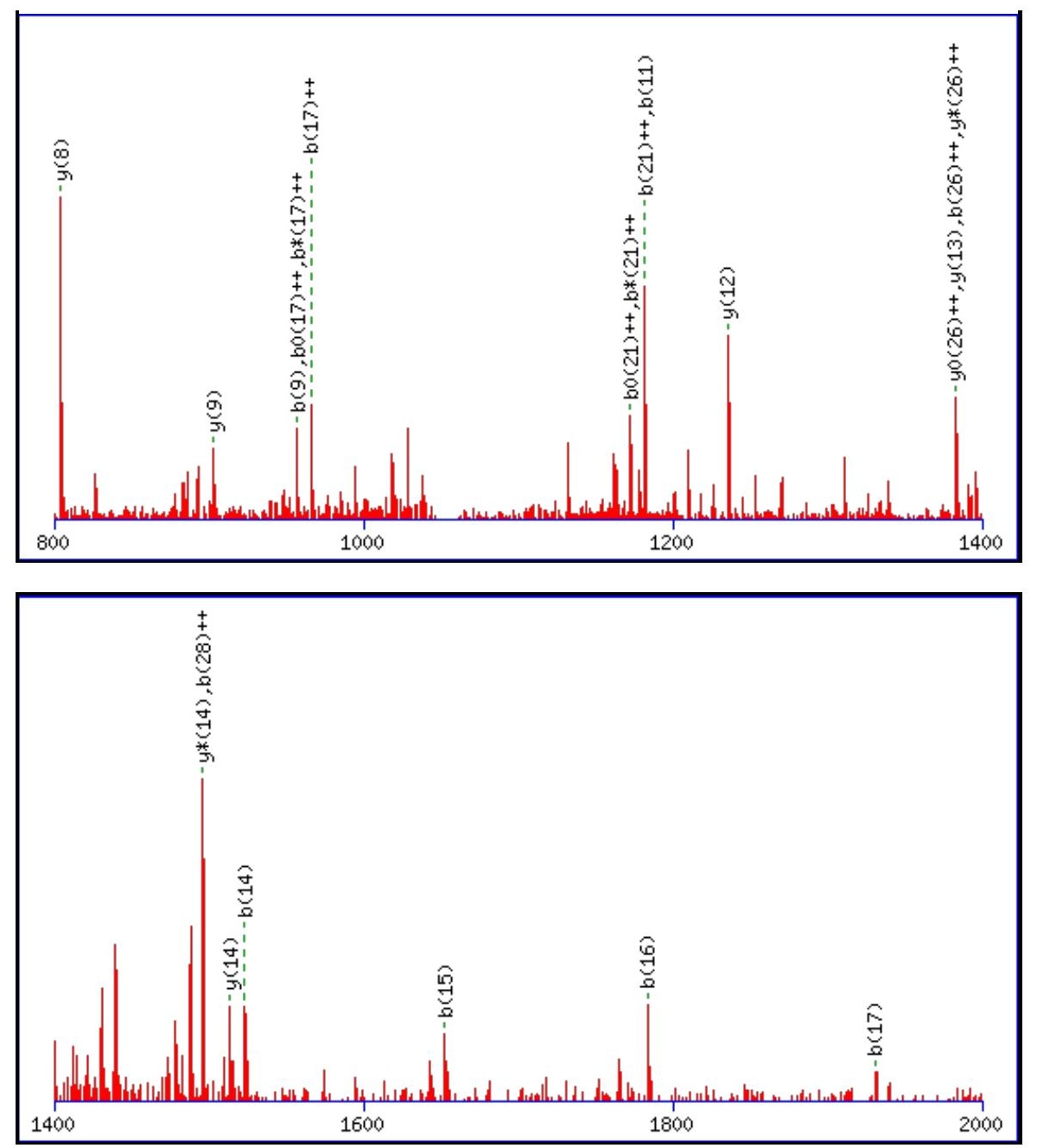

Monoisotopic mass of neutral peptide $\operatorname{Mr}($ calc): 3164.3452

Fixed modifications: Carbamidomethyl (C)

Variable modifications:

N4 : Deamidated $\mathrm{N}(\mathrm{N})$

Ions Score: 68 Expect: $7.2 \mathrm{e}-005$

Matches (Bold Red): 27/314 fragment ions using 28 most intense peaks

\begin{tabular}{|c|c|c|c|c|c|c|c|c|c|c|c|c|c|c|}
\hline \# & b & $\mathbf{b}^{++}$ & $\mathbf{b}^{*}$ & $\mathbf{b}^{*^{++}}$ & $\mathbf{b}^{0}$ & $\mathbf{b}^{\mathbf{0 + +}}$ & Seq. & $\mathbf{y}$ & $\mathbf{y}^{++}$ & $\mathbf{y}^{*}$ & $\mathbf{y}^{*^{++}}$ & $\mathbf{y}^{0}$ & $\mathbf{y}^{0++}$ & \# \\
\hline 1 & 58.0287 & 29.5180 & & & & & G & & & & & & & 29 \\
\hline 2 & 221.0921 & 111.0497 & & & & & $\mathbf{Y}$ & 3108.3310 & 1554.6691 & 3091.3044 & 1546.1558 & 3090.3204 & 1545.6638 & 28 \\
\hline 3 & 384.1554 & 192.5813 & & & & & $\mathbf{Y}$ & 2945.2676 & 1473.1374 & 2928.2411 & 1464.6242 & 2927.2571 & 1464.1322 & 27 \\
\hline 4 & 499.1823 & 250.0948 & 482.1558 & 241.5815 & & & $\mathbf{N}$ & 2782.2043 & 1391.6058 & 2765.1777 & 1383.0925 & 2764.1937 & 1382.6005 & 26 \\
\hline 5 & 627.2409 & 314.1241 & 610.2144 & 305.6108 & & & $\mathbf{Q}$ & 2667.1774 & 1334.0923 & 2650.1508 & 1325.5790 & 2649.1668 & 1325.0870 & 25 \\
\hline 6 & 714.2729 & 357.6401 & 697.2464 & 349.1268 & 696.2624 & 348.6348 & $S$ & 2539.1188 & 1270.0630 & 2522.0922 & 1261.5498 & 2521.1082 & 1261.0577 & 24 \\
\hline 7 & 843.3155 & 422.1614 & 826.2890 & 413.6481 & 825.3050 & 413.1561 & $\mathbf{E}$ & 2452.0868 & 1226.5470 & 2435.0602 & 1218.0337 & 2434.0762 & 1217.5417 & 23 \\
\hline 8 & 900.3370 & 450.6721 & 883.3104 & 442.1589 & 882.3264 & 441.6669 & G & 2323.0442 & 1162.0257 & 2306.0176 & 1153.5124 & 2305.0336 & 1153.0204 & 22 \\
\hline 9 & 957.3585 & 479.1829 & 940.3319 & 470.6696 & 939.3479 & 470.1776 & G & 2266.0227 & 1133.5150 & 2248.9961 & 1125.0017 & 2248.0121 & 1124.5097 & 21 \\
\hline 10 & 1044.3905 & 522.6989 & 1027.3639 & 514.1856 & 1026.3799 & 513.6936 & $S$ & 2209.0012 & 1105.0043 & 2191.9747 & 1096.4910 & 2190.9907 & 1095.9990 & 20 \\
\hline 11 & 1181.4494 & 591.2283 & 1164.4229 & 582.7151 & 1163.4388 & 582.2231 & H & 2121.9692 & 1061.4882 & 2104.9427 & 1052.9750 & 2103.9586 & 1052.4830 & 19 \\
\hline 12 & 1282.4971 & 641.7522 & 1265.4705 & 633.2389 & 1264.4865 & 632.7469 & $T$ & 1984.9103 & 992.9588 & 1967.8837 & 984.4455 & 1966.8997 & 983.9535 & 18 \\
\hline 13 & 1395.5811 & 698.2942 & 1378.5546 & 689.7809 & 1377.5706 & 689.2889 & I & 1883.8626 & 942.4349 & 1866.8361 & 933.9217 & 1865.8520 & 933.4297 & 17 \\
\hline 14 & 1523.6397 & 762.3235 & 1506.6132 & 753.8102 & 1505.6292 & 753.3182 & $\mathbf{Q}$ & 1770.7785 & 885.8929 & 1753.7520 & 877.3796 & 1752.7680 & 876.8876 & 16 \\
\hline 15 & 1652.6823 & 826.8448 & 1635.6558 & 818.3315 & 1634.6718 & 817.8395 & $\mathbf{E}$ & 1642.7200 & 821.8636 & 1625.6934 & 813.3503 & 1624.7094 & 812.8583 & 15 \\
\hline 16 & 1783.7228 & 892.3650 & 1766.6963 & 883.8518 & 1765.7122 & 883.3598 & $\mathbf{M}$ & 1513.6774 & 757.3423 & 1496.6508 & 748.8291 & 1495.6668 & 748.3370 & 14 \\
\hline 17 & 1930.7912 & 965.8992 & 1913.7647 & 957.3860 & 1912.7806 & 956.8940 & $\mathbf{F}$ & 1382.6369 & 691.8221 & 1365.6103 & 683.3088 & 1364.6263 & 682.8168 & 13 \\
\hline 18 & 1987.8127 & 994.4100 & 1970.7861 & 985.8967 & 1969.8021 & 985.4047 & G & 1235.5685 & 618.2879 & 1218.5419 & 609.7746 & 1217.5579 & 609.2826 & 12 \\
\hline
\end{tabular}




\begin{tabular}{|r|r|r|r|r|r|r|r|r|r|r|r|r|r|r|r|r|}
$\mathbf{1 9}$ & 2147.8433 & 1074.4253 & 2130.8168 & 1065.9120 & 2129.8328 & 1065.4200 & C & 1178.5470 & 589.7771 & 1161.5205 & 581.2639 & 1160.5364 & 580.7719 & $\mathbf{1 1}$ \\
\hline $\mathbf{2 0}$ & 2262.8703 & 1131.9388 & 2245.8437 & 1123.4255 & 2244.8597 & 1122.9335 & D & 1018.5164 & 509.7618 & 1001.4898 & 501.2485 & 1000.5058 & 500.7565 & $\mathbf{1 0}$ \\
\hline $\mathbf{2 1}$ & 2361.9387 & $\mathbf{1 1 8 1 . 4 7 3 0}$ & 2344.9121 & $\mathbf{1 1 7 2 . 9 5 9 7}$ & 2343.9281 & $\mathbf{1 1 7 2 . 4 6 7 7}$ & V & $\mathbf{9 0 3 . 4 8 9 4}$ & $\mathbf{4 5 2 . 2 4 8 3}$ & 886.4629 & 443.7351 & 885.4789 & 443.2431 & $\mathbf{9}$ \\
\hline $\mathbf{2 2}$ & 2418.9601 & 1209.9837 & 2401.9336 & 1201.4704 & 2400.9496 & 1200.9784 & G & $\mathbf{8 0 4 . 4 2 1 0}$ & 402.7141 & 787.3945 & 394.2009 & $\mathbf{7 8 6 . 4 1 0 4}$ & 393.7089 & $\mathbf{8}$ \\
\hline $\mathbf{2 3}$ & 2505.9922 & 1253.4997 & 2488.9656 & 1244.9865 & 2487.9816 & 1244.4944 & S & $\mathbf{7 4 7 . 3 9 9 5}$ & 374.2034 & 730.3730 & 365.6901 & 729.3890 & 365.1981 & $\mathbf{7}$ \\
\hline $\mathbf{2 4}$ & 2621.0191 & 1311.0132 & 2603.9926 & 1302.4999 & 2603.0086 & 1302.0079 & D & $\mathbf{6 6 0 . 3 6 7 5}$ & 330.6874 & 643.3410 & 322.1741 & 642.3570 & 321.6821 & $\mathbf{6}$ \\
\hline $\mathbf{2 5}$ & 2678.0406 & 1339.5239 & 2661.0140 & 1331.0107 & 2660.0300 & 1330.5186 & G & $\mathbf{5 4 5 . 3 4 0 6}$ & 273.1739 & 528.3140 & 264.6606 & 527.3300 & 264.1686 & $\mathbf{5}$ \\
\hline $\mathbf{2 6}$ & 2765.0726 & $\mathbf{1 3 8 3 . 0 3 9 9}$ & 2748.0461 & 1374.5267 & 2747.0620 & 1374.0347 & S & $\mathbf{4 8 8 . 3 1 9 1}$ & 244.6632 & 471.2926 & 236.1499 & 470.3085 & 235.6579 & $\mathbf{4}$ \\
\hline $\mathbf{2 7}$ & 2878.1567 & 1439.5820 & 2861.1301 & 1431.0687 & 2860.1461 & 1430.5767 & L & $\mathbf{4 0 1 . 2 8 7 1}$ & 201.1472 & 384.2605 & 192.6339 & & & & $\mathbf{3}$ \\
\hline $\mathbf{2 8}$ & 2991.2407 & $\mathbf{1 4 9 6 . 1 2 4 0}$ & 2974.2142 & 1487.6107 & 2973.2302 & 1487.1187 & L & 288.2030 & 144.6051 & 271.1765 & 136.0919 & & & & $\mathbf{2}$ \\
\hline $\mathbf{2 9}$ & & & & & & & R & 175.1190 & 88.0631 & 158.0924 & 79.5498 & & & $\mathbf{1}$ \\
\hline
\end{tabular}
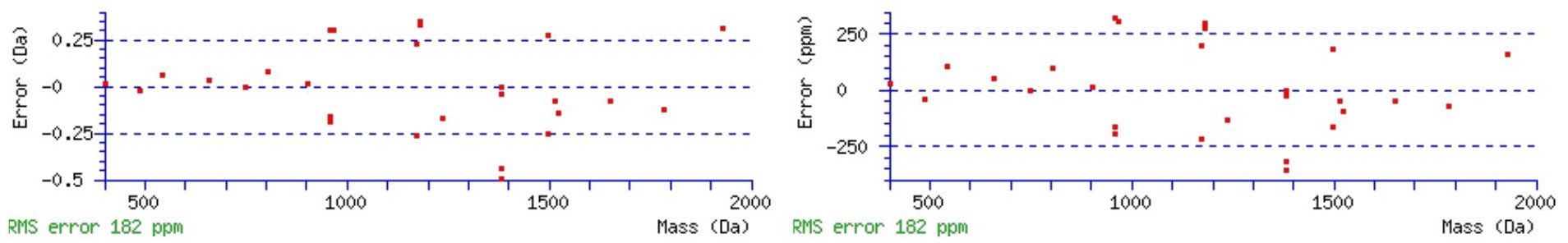

\section{All matches to this query}

\begin{tabular}{|l|l|l|l|}
\hline Score & Mr(calc): & Delta & \multicolumn{1}{|c|}{ Sequence } \\
\hline 67.5 & 3164.3452 & 0.0083 & GYYNQSEGGSHTIQEMFGCDVGSDGSLLR \\
\hline 48.4 & 3163.3611 & 0.9923 & GYYNQSEGGSHTIQEMFGCDVGSDGSLLR \\
\hline 43.0 & 3164.3815 & -0.0281 & GYYNQSEGGSHTLQLMYGCDVGSDGSLLR \\
\hline 4.5 & 3164.3518 & 0.0017 & DGSTALMVALDAGQSEIASMLYSRMNIK \\
\hline 4.2 & 3164.3518 & 0.0017 & DGSTALMVALDAGQSEIASMLYSRMNIK \\
\hline 3.9 & 3164.3518 & 0.0017 & DGSTALMVALDAGQSEIASMLYSRMNIK \\
\hline 3.5 & 3164.3518 & 0.0017 & DGSTALMVALDAGQSEIASMLYSRMNIK \\
\hline 2.3 & 3164.3536 & -0.0002 & LMRYHSVSTETPHERNVDHLPGGESR \\
\hline 1.9 & 3162.3163 & 2.0372 & DTAAAVMYTVVTPMLNPFIYSLRNR \\
\hline 1.7 & 3162.3163 & 2.0372 & DTAAAVMYTVVTPMLNPFIYSLRNR \\
\hline
\end{tabular}

Spectrum No: 405; Query: 355; Rank: 1

\section{Peptide View}

MS/MS Fragmentation of LNAINITSALDR

Found in IPI00454453, Tax_Id=10116 Gene_Symbol=Sgce Epsilon-sarcoglycan

Match to Query 355: 1300.698748 from(651.356650,2+)

Title: 091008RatKidney_NH4Format01_23.2946.2946.2.dta

Data file K:INewmanPaper|Piliang $3 S$ SubProteomes\Piliang3SP\mgf5ppm\ERLIC_3SubProteomes5ppm.mgf 

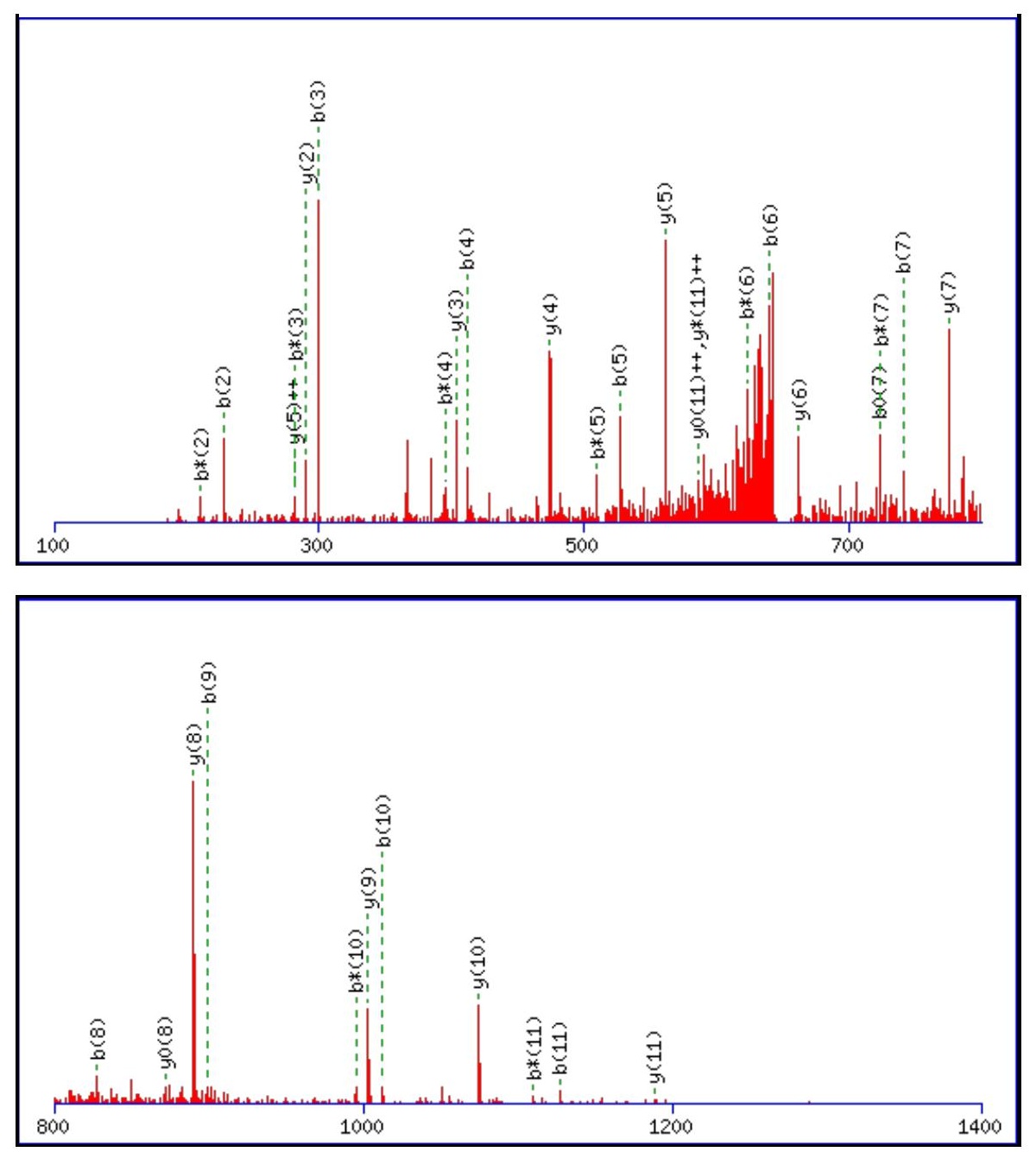

\begin{tabular}{rrrr}
\hline 00 & 1600 & 1800 & 2000 \\
\hline
\end{tabular}

Monoisotopic mass of neutral peptide $\operatorname{Mr}($ calc): 1300.6986

Fixed modifications: Carbamidomethyl (C)

Variable modifications:

N5 : Deamidated $\mathrm{N}(\mathrm{N})$

Ions Score: 67 Expect: $1.8 \mathrm{e}-005$

Matches (Bold Red): 33/116 fragment ions using 76 most intense peaks

\begin{tabular}{|r|c|c|c|c|c|c|c|c|c|c|c|c|c|c|}
\hline$\#$ & $\mathbf{b}$ & $\mathbf{b}^{++}$ & $\mathbf{b}^{*}$ & $\mathbf{b}^{\boldsymbol{*}^{++}}$ & $\mathbf{b}^{\mathbf{0}}$ & $\mathbf{b}^{\mathbf{0 + +}}$ & Seq. & $\mathbf{y}$ & $\mathbf{y}^{++}$ & $\mathbf{y}^{\mathbf{*}}$ & $\mathbf{y}^{\boldsymbol{*}^{++}}$ & $\mathbf{y}^{\mathbf{0}}$ & $\mathbf{y}^{\mathbf{0 + +}}$ & $\#$ \\
\hline $\mathbf{1}$ & 114.0913 & 57.5493 & & & & & $\mathbf{L}$ & & & & & & & $\mathbf{1 2}$ \\
\hline $\mathbf{2}$ & $\mathbf{2 2 8 . 1 3 4 3}$ & 114.5708 & 211.1077 & 106.0575 & & & $\mathbf{N}$ & $\mathbf{1 1 8 8 . 6 2 1 9}$ & 594.8146 & 1171.5953 & $\mathbf{5 8 6 . 3 0 1 3}$ & 1170.6113 & $\mathbf{5 8 5 . 8 0 9 3}$ & $\mathbf{1 1}$ \\
\hline $\mathbf{3}$ & $\mathbf{2 9 9 . 1 7 1 4}$ & 150.0893 & $\mathbf{2 8 2 . 1 4 4 8}$ & 141.5761 & & & $\mathbf{A}$ & $\mathbf{1 0 7 4 . 5 7 9 0}$ & 537.7931 & 1057.5524 & 529.2798 & 1056.5684 & 528.7878 & $\mathbf{1 0}$ \\
\hline $\mathbf{4}$ & $\mathbf{4 1 2 . 2 5 5 4}$ & 206.6314 & 395.2289 & 198.1181 & & & $\mathbf{I}$ & $\mathbf{1 0 0 3 . 5 4 1 8}$ & 502.2746 & 986.5153 & 493.7613 & 985.5313 & 493.2693 & $\mathbf{9}$ \\
\hline $\mathbf{5}$ & $\mathbf{5 2 7 . 2 8 2 4}$ & 264.1448 & $\mathbf{5 1 0 . 2 5 5 8}$ & 255.6316 & & & $\mathbf{N}$ & $\mathbf{8 9 0 . 4 5 7 8}$ & 445.7325 & 873.4312 & 437.2193 & $\mathbf{8 7 2 . 4 4 7 2}$ & 436.7272 & $\mathbf{8}$ \\
\hline
\end{tabular}




\begin{tabular}{|r|c|c|c|c|c|c|c|c|c|c|c|c|c|c|}
$\mathbf{6}$ & $\mathbf{6 4 0 . 3 6 6 4}$ & 320.6869 & $\mathbf{6 2 3 . 3 3 9 9}$ & 312.1736 & & & $\mathbf{I}$ & $\mathbf{7 7 5 . 4 3 0 8}$ & 388.2191 & 758.4043 & 379.7058 & 757.4203 & 379.2138 & $\mathbf{7}$ \\
\hline $\mathbf{7}$ & $\mathbf{7 4 1 . 4 1 4 1}$ & 371.2107 & $\mathbf{7 2 4 . 3 8 7 6}$ & 362.6974 & $\mathbf{7 2 3 . 4 0 3 6}$ & 362.2054 & $\mathbf{T}$ & $\mathbf{6 6 2 . 3 4 6 8}$ & 331.6770 & 645.3202 & 323.1638 & 644.3362 & 322.6717 & $\mathbf{6}$ \\
\hline $\mathbf{8}$ & $\mathbf{8 2 8 . 4 4 6 2}$ & 414.7267 & 811.4196 & 406.2134 & 810.4356 & 405.7214 & S & $\mathbf{5 6 1 . 2 9 9 1}$ & $\mathbf{2 8 1 . 1 5 3 2}$ & 544.2726 & 272.6399 & 543.2885 & 272.1479 & $\mathbf{5}$ \\
\hline $\mathbf{9}$ & $\mathbf{8 9 9 . 4 8 3 3}$ & 450.2453 & 882.4567 & 441.7320 & 881.4727 & 441.2400 & A & $\mathbf{4 7 4 . 2 6 7 1}$ & 237.6372 & 457.2405 & 229.1239 & 456.2565 & 228.6319 & $\mathbf{4}$ \\
\hline $\mathbf{1 0}$ & $\mathbf{1 0 1 2 . 5 6 7 3}$ & 506.7873 & $\mathbf{9 9 5 . 5 4 0 8}$ & $\mathbf{4 9 8 . 2 7 4 0}$ & 994.5568 & 497.7820 & L & $\mathbf{4 0 3 . 2 3 0 0}$ & 202.1186 & 386.2034 & 193.6053 & 385.2194 & 193.1133 & $\mathbf{3}$ \\
\hline $\mathbf{1 1}$ & $\mathbf{1 1 2 7 . 5 9 4 3}$ & 564.3008 & $\mathbf{1 1 1 0 . 5 6 7 7}$ & 555.7875 & 1109.5837 & 555.2955 & $\mathbf{D}$ & $\mathbf{2 9 0 . 1 4 5 9}$ & 145.5766 & 273.1193 & 137.0633 & 272.1353 & 136.5713 & $\mathbf{2}$ \\
\hline $\mathbf{1 2}$ & & & & & & & $\mathbf{R}$ & 175.1190 & 88.0631 & 158.0924 & 79.5498 & & & $\mathbf{1}$ \\
\hline
\end{tabular}
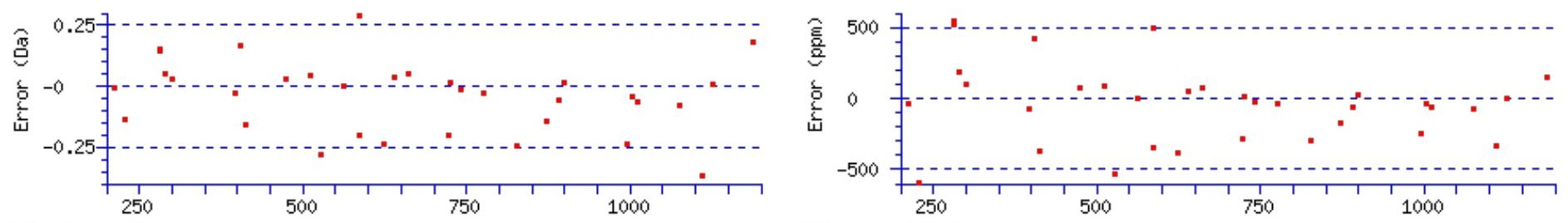

RMS error 276 ppm

Mass (Da) RMS error 276 ppm

\section{All matches to this query}

\begin{tabular}{|l|l|l|l|}
\hline Score & Mr(calc): & Delta & \multicolumn{1}{|c|}{ Sequence } \\
\hline 67.4 & 1300.6986 & 0.0001 & LNAINITSALDR \\
\hline 34.6 & 1300.6986 & 0.0001 & LNAINITSALDR \\
\hline 3.8 & 1300.7074 & -0.0086 & WLCARVLLXR \\
\hline 3.8 & 1300.7074 & -0.0086 & WLCARVLLXR \\
\hline 2.8 & 1300.7034 & -0.0046 & AARLQISVCQR \\
\hline 1.5 & 1300.6987 & 0.0001 & VQDDVTKLDLR \\
\hline 1.3 & 1300.6921 & 0.0066 & $\underline{\text { RLEAGAMVLADR }}$ \\
\hline 0.2 & 1300.6987 & 0.0001 & $\underline{\text { LLLSGTADGADLR }}$ \\
\hline
\end{tabular}

Spectrum No: 406; Query: 242; Rank: 1

\section{Peptide View}

MS/MS Fragmentation of VYVYAVNQTR

Found in IPI00324585, Tax_Id=10116 Gene_Symbol=Itga1 Integrin alpha-1 precursor

Match to Query 242: 1212.615588 from(607.315070,2+)

Title: 100101RatKid_NS_deglyco_20.1583.1583.2.dta

Data file K:INewmanPaper|Piliangl3SubProteomes\Piliang3SP\mgf5ppm\ERLIC_3SubProteomes5ppm.mgf

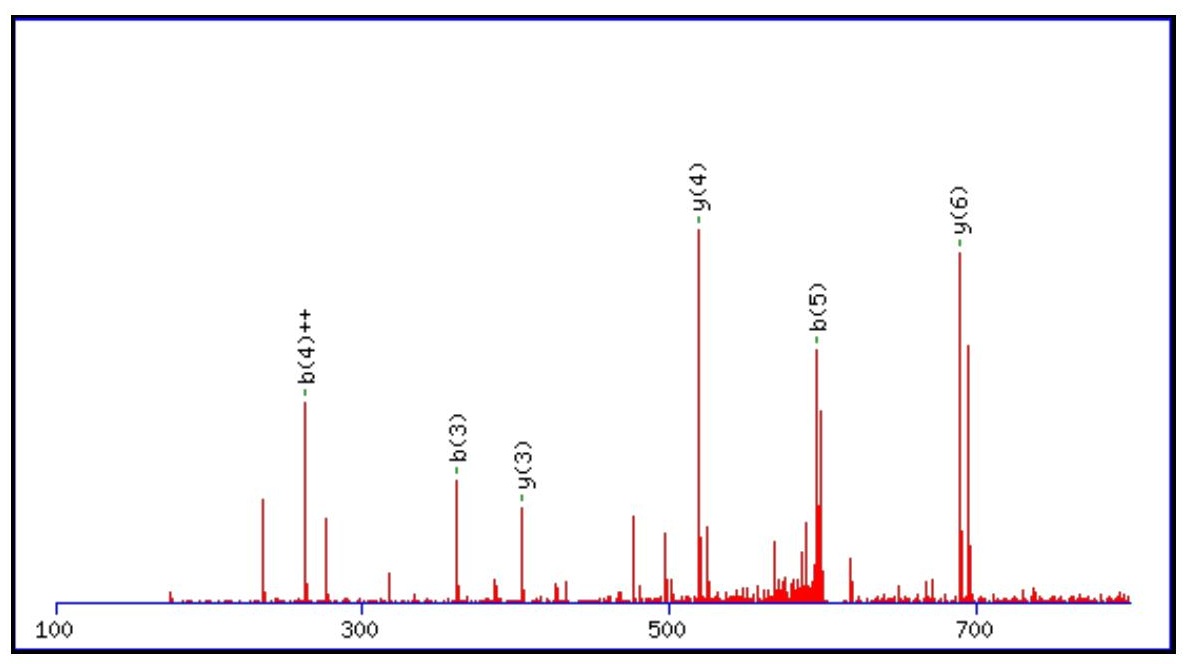



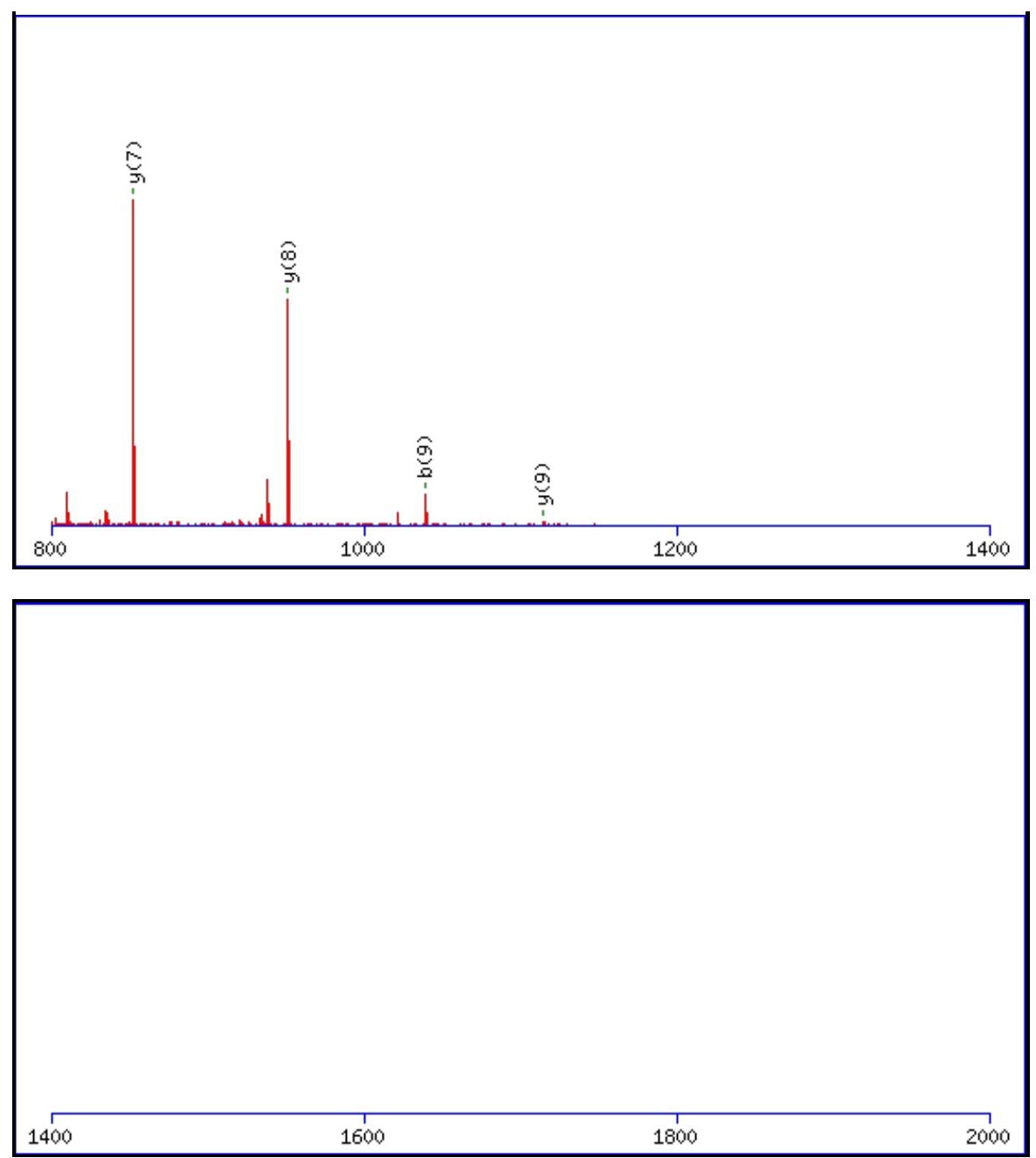

Monoisotopic mass of neutral peptide $\operatorname{Mr}($ calc): 1212.6139

Fixed modifications: Carbamidomethyl (C)

Variable modifications:

N7 : Deamidated $\mathrm{N}(\mathrm{N})$

Ions Score: 67 Expect : $1.8 \mathrm{e}-005$

Matches (Bold Red): 11/78 fragment ions using 12 most intense peaks

\begin{tabular}{|r|c|c|c|c|c|c|c|c|c|c|c|c|c|c|}
\hline$\#$ & $\mathbf{b}$ & $\mathbf{b}^{++}$ & $\mathbf{b}^{*}$ & $\mathbf{b}^{\mathbf{*}^{++}}$ & $\mathbf{b}^{\mathbf{0}}$ & $\mathbf{b}^{\mathbf{0 + +}}$ & $\mathbf{S e q}$ & $\mathbf{y}$ & $\mathbf{y}^{++}$ & $\mathbf{y}^{\mathbf{*}}$ & $\mathbf{y}^{\mathbf{*}^{++}}$ & $\mathbf{y}^{\mathbf{0}}$ & $\mathbf{y}^{\mathbf{0 + +}}$ & $\#$ \\
\hline $\mathbf{1}$ & 100.0757 & 50.5415 & & & & & $\mathbf{V}$ & & & & & & & $\mathbf{1 0}$ \\
\hline $\mathbf{2}$ & $\mathbf{2 6 3 . 1 3 9 0}$ & 132.0731 & & & & & $\mathbf{Y}$ & $\mathbf{1 1 1 4 . 5 5 2 7}$ & 557.7800 & 1097.5262 & 549.2667 & 1096.5422 & 548.7747 & $\mathbf{9}$ \\
\hline $\mathbf{3}$ & $\mathbf{3 6 2 . 2 0 7 4}$ & 181.6074 & & & & & $\mathbf{V}$ & $\mathbf{9 5 1 . 4 8 9 4}$ & 476.2483 & 934.4629 & 467.7351 & 933.4789 & 467.2431 & $\mathbf{8}$ \\
\hline $\mathbf{4}$ & 525.2708 & $\mathbf{2 6 3 . 1 3 9 0}$ & & & & & $\mathbf{Y}$ & $\mathbf{8 5 2 . 4 2 1 0}$ & 426.7141 & 835.3945 & 418.2009 & 834.4104 & 417.7089 & $\mathbf{7}$ \\
\hline $\mathbf{5}$ & $\mathbf{5 9 6 . 3 0 7 9}$ & 298.6576 & & & & & $\mathbf{A}$ & $\mathbf{6 8 9 . 3 5 7 7}$ & 345.1825 & 672.3311 & 336.6692 & 671.3471 & 336.1772 & $\mathbf{6}$ \\
\hline $\mathbf{6}$ & 695.3763 & 348.1918 & & & & & $\mathbf{V}$ & 618.3206 & 309.6639 & 601.2940 & 301.1506 & 600.3100 & 300.6586 & $\mathbf{5}$ \\
\hline $\mathbf{7}$ & 810.4032 & 405.7053 & 793.3767 & 397.1920 & & & $\mathbf{N}$ & $\mathbf{5 1 9 . 2 5 2 1}$ & 260.1297 & 502.2256 & 251.6164 & 501.2416 & 251.1244 & $\mathbf{4}$ \\
\hline $\mathbf{8}$ & 938.4618 & 469.7345 & 921.4353 & 461.2213 & & & $\mathbf{Q}$ & $\mathbf{4 0 4 . 2 2 5 2}$ & 202.6162 & 387.1987 & 194.1030 & 386.2146 & 193.6110 & $\mathbf{3}$ \\
\hline $\mathbf{9}$ & $\mathbf{1 0 3 9 . 5 0 9 5}$ & 520.2584 & 1022.4829 & 511.7451 & 1021.4989 & 511.2531 & $\mathbf{T}$ & 276.1666 & 138.5870 & 259.1401 & 130.0737 & 258.1561 & 129.5817 & $\mathbf{2}$ \\
\hline $\mathbf{1 0}$ & & & & & & & $\mathbf{R}$ & 175.1190 & 88.0631 & 158.0924 & 79.5498 & & & $\mathbf{1}$ \\
\hline
\end{tabular}
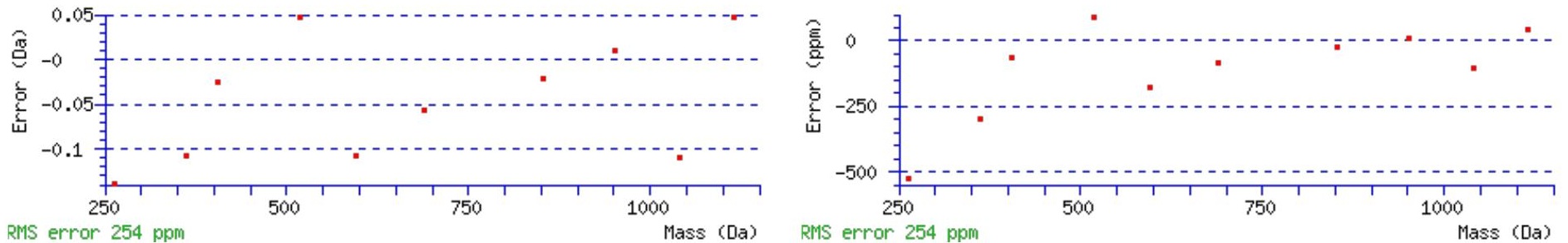

All matches to this query 


\begin{tabular}{|l|l|l|l|}
\hline Score & Mr(calc): & Delta & \multicolumn{1}{c|}{ Sequence } \\
\hline 67.4 & 1212.6139 & 0.0017 & VYVYAVNQTR \\
\hline 17.4 & 1212.6268 & -0.0112 & VYVNGLISLR \\
\hline 7.5 & 1212.6145 & 0.0010 & RCAHXEIKSR \\
\hline 6.7 & 1212.6251 & -0.0095 & VYSGRTFVER \\
\hline 6.2 & 1212.6073 & 0.0082 & KFQYQLACR \\
\hline 5.7 & 1212.6251 & -0.0095 & KFSVEVHDPR \\
\hline 5.3 & 1210.6032 & 2.0124 & MASIVPLKEK \\
\hline 3.6 & 1212.6251 & -0.0095 & VVYSDLHPQR \\
\hline 2.6 & 1210.6054 & 2.0102 & GNSELLRHER \\
\hline 2.2 & 1212.6139 & 0.0017 & FNPVTGEVPPR \\
\hline
\end{tabular}

Spectrum No: 407; Query: 1039; Rank: 1

\section{Peptide View}

MS/MS Fragmentation of SSSHLPPSSYFNASGR

Found in IPI00190499, Tax_Id=10116 Gene_Symbol=Tpp1 Tripeptidyl-peptidase 1 precursor

Match to Query 1039: 1693.764222 from(565.595350,3+)

Title: 091008RatKidney_NH4Format02_24.1718.1718.3.dta

Data file K:INewmanPaper|Piliang|3SubProteomes\Piliang3SP\mgf5ppm\ERLIC_3SubProteomes5ppm.mgf
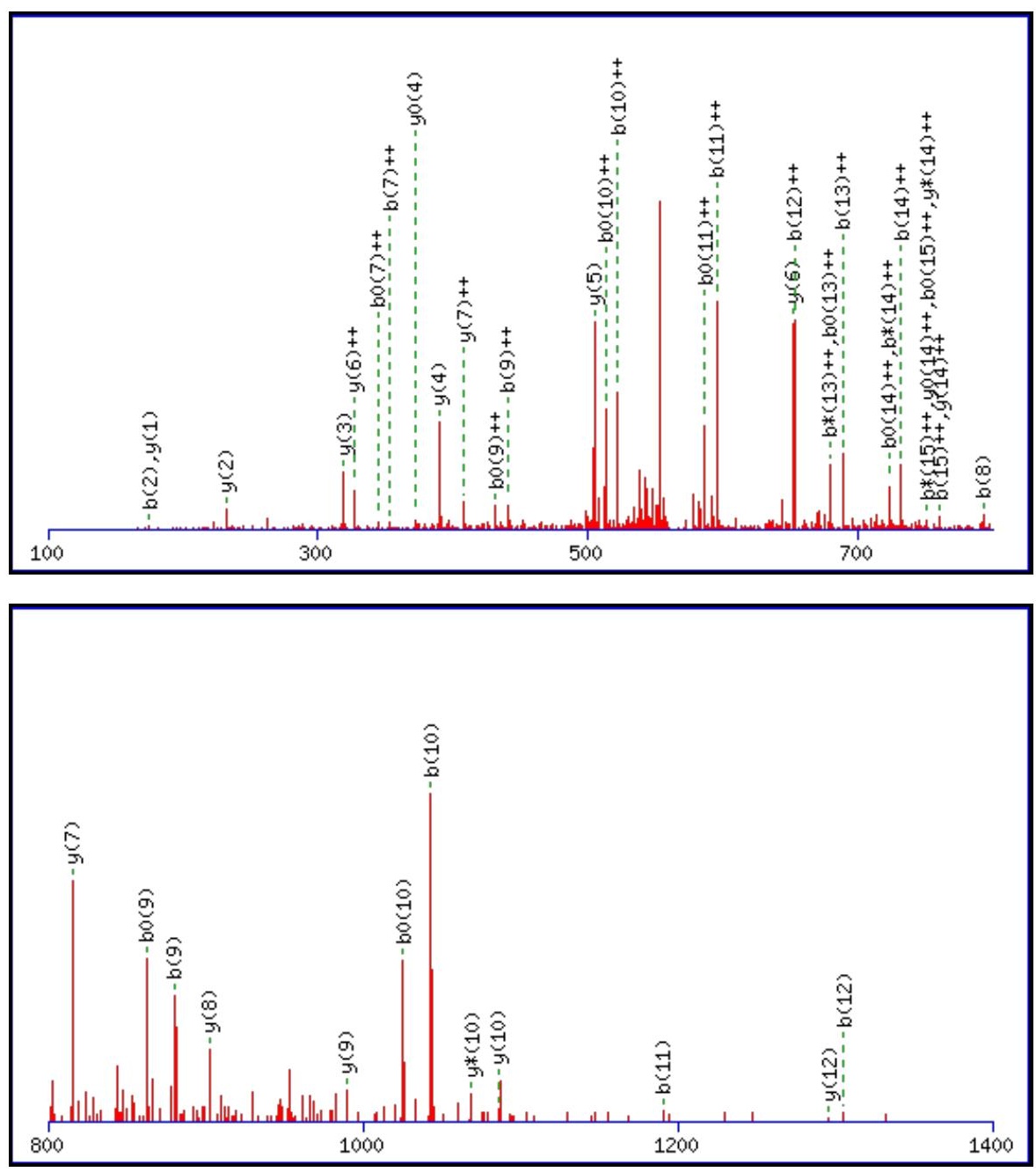
Monoisotopic mass of neutral peptide $\operatorname{Mr}($ calc): 1693.7696

Fixed modifications: Carbamidomethyl (C)

Variable modifications:

N12 : Deamidated_N (N)

Ions Score: 67 Expect: $3.8 \mathrm{e}-005$

Matches (Bold Red): 44/154 fragment ions using 66 most intense peaks

\begin{tabular}{|c|c|c|c|c|c|c|c|c|c|c|c|c|c|c|}
\hline \# & b & $\mathbf{b}^{++}$ & $\mathbf{b}^{*}$ & $\mathbf{b}^{*^{++}}$ & $\mathbf{b}^{\mathbf{0}}$ & $\mathbf{b}^{0++}$ & Seq. & $\mathbf{y}$ & $y^{++}$ & $\mathbf{y}^{*}$ & $\mathbf{y}^{*^{++}}$ & $\mathbf{y}^{0}$ & $\mathbf{y}^{\mathbf{0 + +}}$ & \# \\
\hline 1 & 88.0393 & 44.5233 & & & 70.0287 & 35.5180 & $\mathrm{~S}$ & & & & & & & 16 \\
\hline 2 & 175.0713 & 88.0393 & & & 157.0608 & 79.0340 & $S$ & 1607.7449 & 804.3761 & 1590.7183 & 795.8628 & 1589.7343 & 795.3708 & 15 \\
\hline 3 & 262.1034 & 131.5553 & & & 244.0928 & 122.5500 & S & 1520.7128 & 760.8601 & 1503.6863 & 752.3468 & 1502.7023 & 751.8548 & 14 \\
\hline 4 & 399.1623 & 200.0848 & & & 381.1517 & 191.0795 & H & 1433.6808 & 717.3440 & 1416.6542 & 708.8308 & 1415.6702 & 708.3388 & 13 \\
\hline 5 & 512.2463 & 256.6268 & & & 494.2358 & 247.6215 & $\mathbf{L}$ & 1296.6219 & 648.8146 & 1279.5953 & 640.3013 & 1278.6113 & 639.8093 & 12 \\
\hline 6 & 609.2991 & 305.1532 & & & 591.2885 & 296.1479 & $\mathbf{P}$ & 1183.5378 & 592.2725 & 1166.5113 & 583.7593 & 1165.5273 & 583.2673 & 11 \\
\hline 7 & 706.3519 & 353.6796 & & & 688.3413 & 344.6743 & $\mathbf{P}$ & 1086.4851 & 543.7462 & 1069.4585 & 535.2329 & 1068.4745 & 534.7409 & 10 \\
\hline 8 & 793.3839 & 397.1956 & & & 775.3733 & 388.1903 & S & 989.4323 & 495.2198 & 972.4057 & 486.7065 & 971.4217 & 486.2145 & 9 \\
\hline 9 & 880.4159 & 440.7116 & & & 862.4054 & 431.7063 & $S$ & 902.4003 & 451.7038 & 885.3737 & 443.1905 & 884.3897 & 442.6985 & 8 \\
\hline 10 & 1043.4792 & 522.2433 & & & 1025.4687 & 513.2380 & $\mathbf{Y}$ & 815.3682 & 408.1878 & 798.3417 & 399.6745 & 797.3577 & 399.1825 & 7 \\
\hline 11 & 1190.5477 & 595.7775 & & & 1172.5371 & 586.7722 & $\mathbf{F}$ & 652.3049 & 326.6561 & 635.2784 & 318.1428 & 634.2943 & 317.6508 & 6 \\
\hline 12 & 1305.5746 & 653.2909 & 1288.5481 & 644.7777 & 1287.5640 & 644.2857 & $\mathbf{N}$ & 505.2365 & 253.1219 & 488.2099 & 244.6086 & 487.2259 & 244.1166 & 5 \\
\hline 13 & 1376.6117 & 688.8095 & 1359.5852 & 680.2962 & 1358.6011 & 679.8042 & A & 390.2096 & 195.6084 & 373.1830 & 187.0951 & 372.1990 & 186.6031 & 4 \\
\hline 14 & 1463.6437 & 732.3255 & 1446.6172 & 723.8122 & 1445.6332 & 723.3202 & $\mathbf{S}$ & 319.1724 & 160.0899 & 302.1459 & 151.5766 & 301.1619 & 151.0846 & 3 \\
\hline 15 & 1520.6652 & 760.8362 & 1503.6387 & 752.3230 & 1502.6546 & 751.8310 & G & 232.1404 & 116.5738 & 215.1139 & 108.0606 & & & 2 \\
\hline 16 & & & & & & & $\mathbf{R}$ & 175.1190 & 88.0631 & 158.0924 & 79.5498 & & & 1 \\
\hline
\end{tabular}

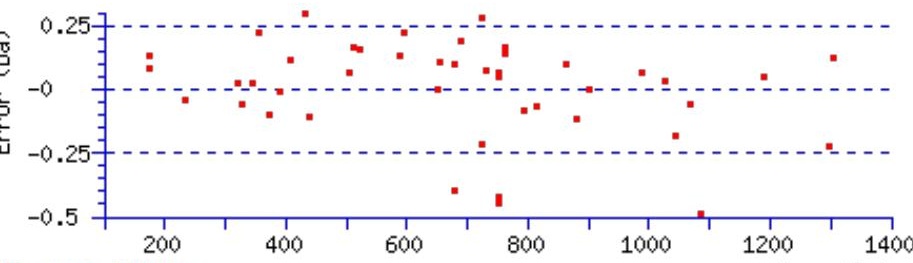

RMS error 304 ppm

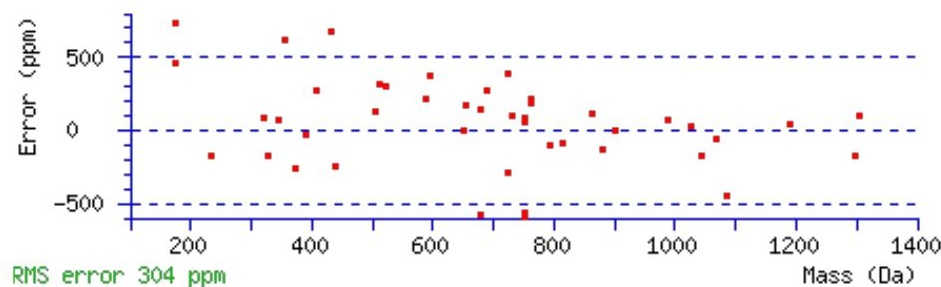

\section{All matches to this query}

\begin{tabular}{|l|l|l|l|}
\hline Score & Mr(calc): & Delta & \multicolumn{1}{c|}{ Sequence } \\
\hline 67.3 & 1693.7696 & -0.0054 & SSSHLPPSSYFNASGR \\
\hline 9.3 & 1693.7560 & 0.0082 & NDSVIVADQTPTPTR \\
\hline 7.6 & 1693.7645 & -0.0003 & TGEHNSSGSRTLRGR \\
\hline 6.6 & 1691.7606 & 2.0036 & ILIANTGMDTDKIK \\
\hline 6.5 & 1693.7560 & 0.0082 & NDSVIVADQTPTPTR \\
\hline & & & \\
\hline
\end{tabular}




\begin{tabular}{|l|l|l|l|}
6.1 & 1692.7581 & 1.0062 & QQGQEPSQLNKGSGR \\
\hline 5.1 & 1691.7718 & 1.9924 & LSGMDLINLSKVSR \\
\hline 4.4 & 1692.7581 & 1.0062 & ENGPRAPASGGSLSGTR \\
\hline 4.4 & 1692.7581 & 1.0062 & ENGPRAPASGGSLSGTR \\
\hline 4.2 & 1692.7720 & 0.9922 & NDSVIVADQTPTPTR \\
\hline
\end{tabular}

Spectrum No: 408; Query: 875; Rank: 1

\section{Peptide View}

MS/MS Fragmentation of DSSKSSNVSLPDCKA

Found in IPI00764367, Tax_Id=10116 Gene_Symbol=LOC686172 similar to RT1 class I, CE1

Match to Query 875: 1594.718548 from(798.366550,2+)

Title: 091008RatKidney_NoSalt_22.1010.1010.2.dta

Data file K:INewmanPaper|Piliangl3SubProteomes\Piliang3SP\mgf5ppm\ERLIC_3SubProteomes5ppm.mgf
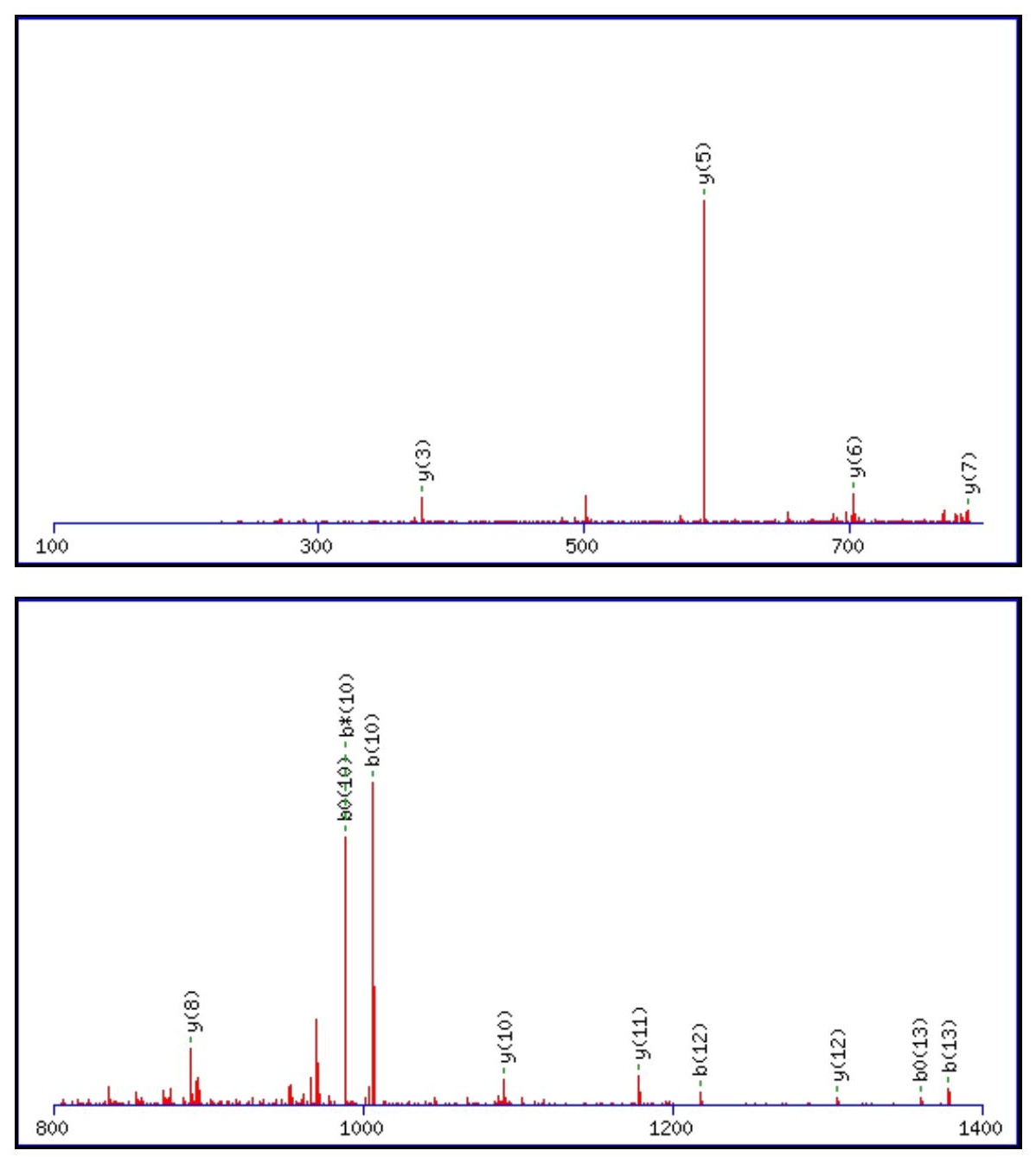


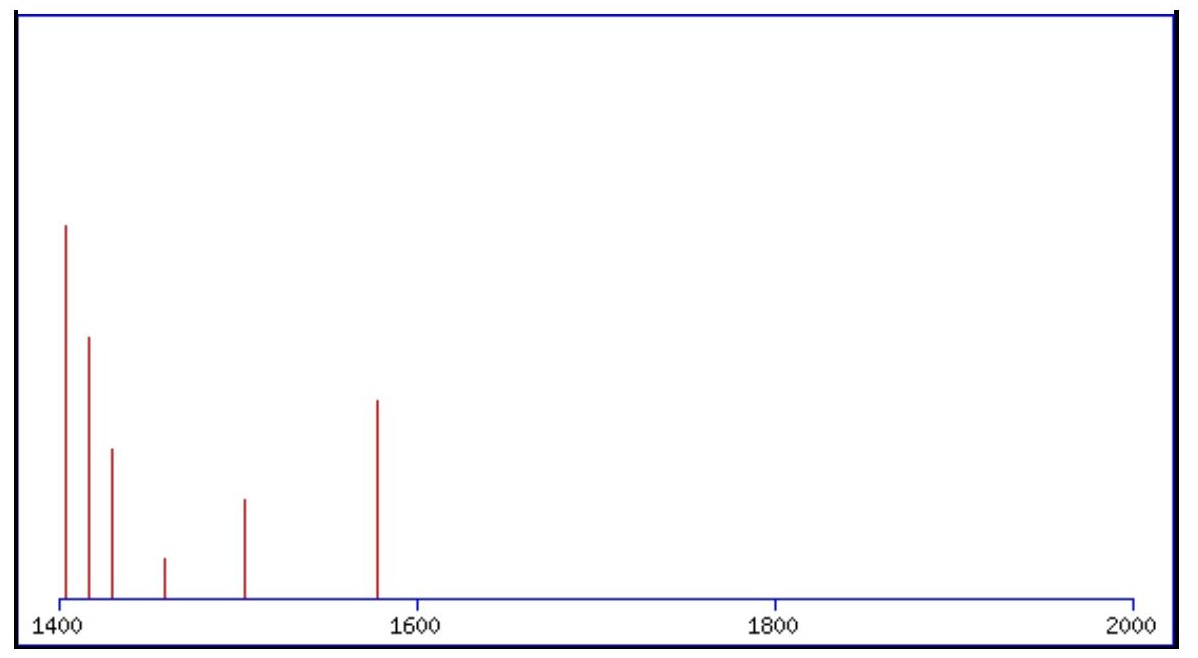

Monoisotopic mass of neutral peptide $\operatorname{Mr}($ calc): 1594.7144

Fixed modifications: Carbamidomethyl (C)

Variable modifications:

N7 : Deamidated_N (N)

Ions Score: 67 Expect: $3.4 \mathrm{e}-005$

Matches (Bold Red): 14/154 fragment ions using 26 most intense peaks

\begin{tabular}{|c|c|c|c|c|c|c|c|c|c|c|c|c|c|c|}
\hline \# & b & $\mathbf{b}^{++}$ & b* & $\mathbf{b}^{*^{++}}$ & $\mathbf{b}^{\mathbf{0}}$ & $\mathbf{b}^{\mathbf{0 + +}}$ & Seq. & $\mathbf{y}$ & $\mathbf{y}^{++}$ & $\mathbf{y}^{*}$ & $\mathbf{y}^{*^{++}}$ & $\mathbf{y}^{0}$ & $\mathbf{y}^{\mathbf{0 + +}}$ & \# \\
\hline 1 & 116.0342 & 58.5207 & & & 98.0237 & 49.5155 & D & & & & & & & 15 \\
\hline 2 & 203.0662 & 102.0368 & & & 185.0557 & 93.0315 & $S$ & 1480.6948 & 740.8510 & 1463.6682 & 732.3378 & 1462.6842 & 731.8458 & 14 \\
\hline 3 & 290.0983 & 145.5528 & & & 272.0877 & 136.5475 & S & 1393.6628 & 697.3350 & 1376.6362 & 688.8217 & 1375.6522 & 688.3297 & 13 \\
\hline 4 & 418.1932 & 209.6003 & 401.1667 & 201.0870 & 400.1827 & 200.5950 & $\mathbf{K}$ & 1306.6307 & 653.8190 & 1289.6042 & 645.3057 & 1288.6202 & 644.8137 & 12 \\
\hline 5 & 505.2253 & 253.1163 & 488.1987 & 244.6030 & 487.2147 & 244.1110 & S & 1178.5358 & 589.7715 & 1161.5092 & 581.2582 & 1160.5252 & 580.7662 & 11 \\
\hline 6 & 592.2573 & 296.6323 & 575.2307 & 288.1190 & 574.2467 & 287.6270 & S & 1091.5037 & 546.2555 & 1074.4772 & 537.7422 & 1073.4932 & 537.2502 & 10 \\
\hline 7 & 707.2842 & 354.1458 & 690.2577 & 345.6325 & 689.2737 & 345.1405 & $\mathbf{N}$ & 1004.4717 & 502.7395 & 987.4452 & 494.2262 & 986.4612 & 493.7342 & 9 \\
\hline 8 & 806.3526 & 403.6800 & 789.3261 & 395.1667 & 788.3421 & 394.6747 & V & 889.4448 & 445.2260 & 872.4182 & 436.7128 & 871.4342 & 436.2207 & 8 \\
\hline 9 & 893.3847 & 447.1960 & 876.3581 & 438.6827 & 875.3741 & 438.1907 & $\mathrm{~S}$ & 790.3764 & 395.6918 & 773.3498 & 387.1785 & 772.3658 & 386.6865 & 7 \\
\hline 10 & 1006.4687 & 503.7380 & 989.4422 & 495.2247 & 988.4582 & 494.7327 & $\mathbf{L}$ & 703.3443 & 352.1758 & 686.3178 & 343.6625 & 685.3338 & 343.1705 & 6 \\
\hline 11 & 1103.5215 & 552.2644 & 1086.4950 & 543.7511 & 1085.5109 & 543.2591 & $\mathbf{P}$ & 590.2603 & 295.6338 & 573.2337 & 287.1205 & 572.2497 & 286.6285 & 5 \\
\hline 12 & 1218.5484 & 609.7779 & 1201.5219 & 601.2646 & 1200.5379 & 600.7726 & D & 493.2075 & 247.1074 & 476.1810 & 238.5941 & 475.1969 & 238.1021 & 4 \\
\hline 13 & 1378.5791 & 689.7932 & 1361.5525 & 681.2799 & 1360.5685 & 680.7879 & C & 378.1806 & 189.5939 & 361.1540 & 181.0806 & & & 3 \\
\hline 14 & 1506.6741 & 753.8407 & 1489.6475 & 745.3274 & 1488.6635 & 744.8354 & $\mathbf{K}$ & 218.1499 & 109.5786 & 201.1234 & 101.0653 & & & 2 \\
\hline 15 & & & & & & & A & 90.0550 & 45.5311 & & & & & 1 \\
\hline
\end{tabular}
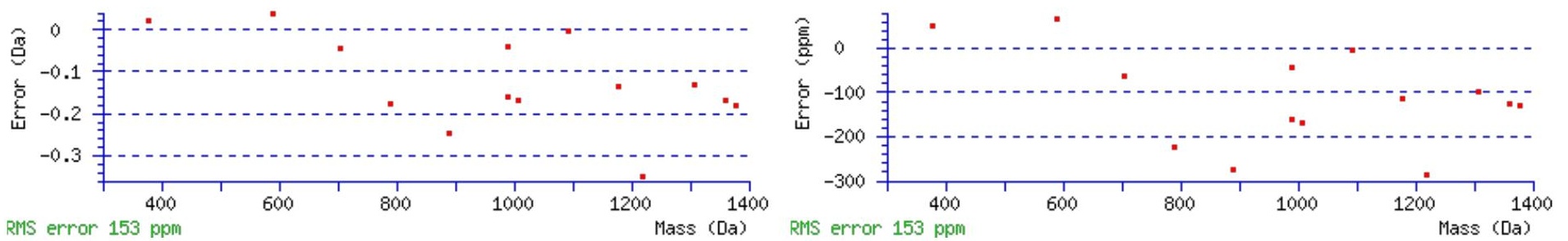

\section{All matches to this query}

\begin{tabular}{|l|l|l|l|}
\hline Score & Mr(calc): & Delta & \multicolumn{1}{c|}{ Sequence } \\
\hline 67.2 & 1594.7144 & 0.0041 & $\underline{\text { DSSKSSNVSLPDCKA }}$ \\
\hline 19.2 & 1593.7304 & 0.9881 & $\underline{\text { DSSKSSNVSLPDCKA }}$ \\
\hline 9.1 & 1594.7062 & 0.0124 & $\underline{\text { FKASRDSTLSEMK }}$ \\
\hline 5.4 & 1592.7092 & 2.0094 & $\underline{\text { NTFREASMSVIMK }}$ \\
\hline 4.9 & 1594.7157 & 0.0029 & QLRTKGELSFEK \\
\hline 3.6 & 1594.7270 & -0.0084 & $\underline{\text { HTLTGHSGKVLSAK }}$ \\
\hline & & & \\
\hline
\end{tabular}




\begin{tabular}{|l|l|l|l|}
3.6 & 1594.7270 & -0.0084 & HTLTGHSGKVLSAK \\
\hline 2.2 & 1593.7222 & 0.9964 & $\underline{\text { IHCSVKAENSVAAK }}$ \\
\hline 1.3 & 1592.7130 & 2.0055 & NHRTSQSPXIMLR \\
\hline 1.0 & 1594.7303 & -0.0118 & $\underline{\text { RAAMSVTLTSVKR }}$ \\
\hline
\end{tabular}

Spectrum No: 409; Query: 2694; Rank: 1

\section{Peptide View}

MS/MS Fragmentation of LTFFNSTLNTSGLSTQGEALPIPGAHRPGVVTK

Found in IPI00196725, Tax_Id=10116 Gene_Symbol=Fthfd 10-formyltetrahydrofolate dehydrogenase

Match to Query 2694: 3410.788932 from(1137.936920,3+)

Title: 091008RatKidney_NoSalt_03.3669.3669.3.dta

Data file K:INewmanPaper|Piliang|3SubProteomes\Piliang3SP\mgf5ppm\ERLIC_3SubProteomes5ppm.mgf
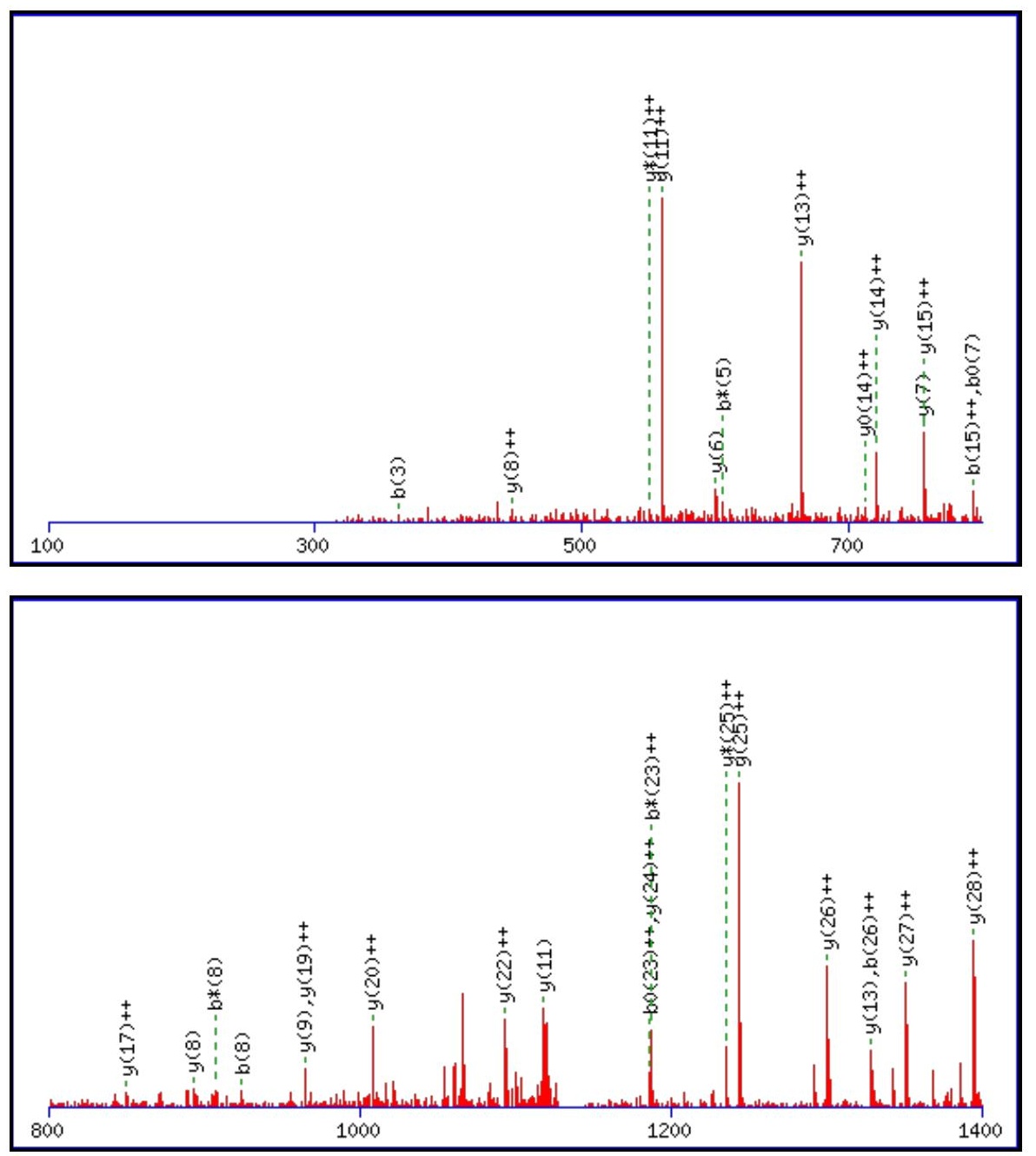


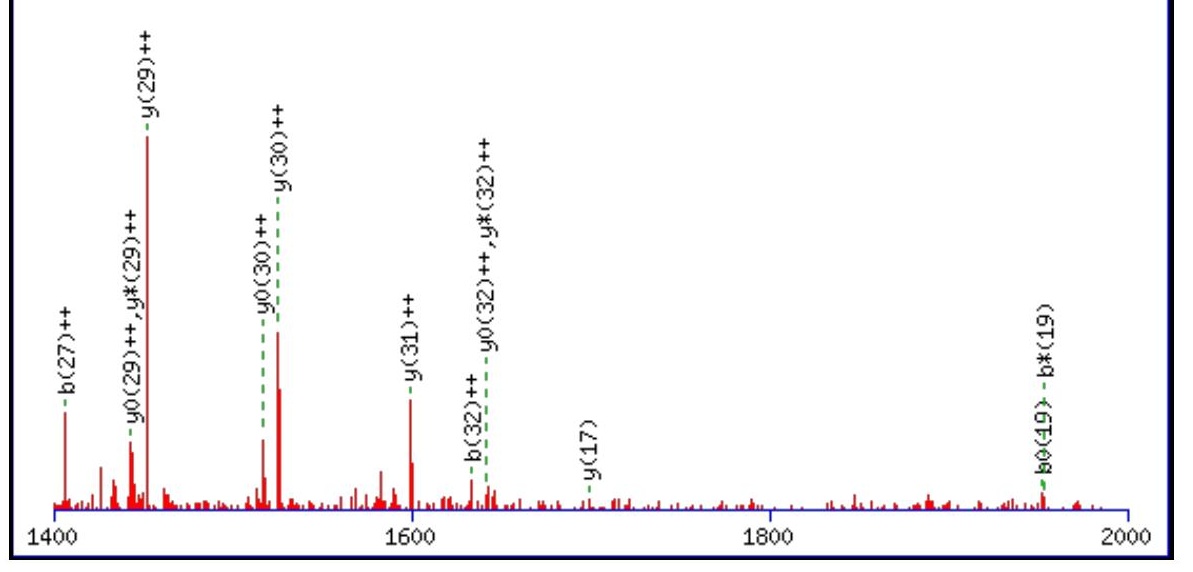

Monoisotopic mass of neutral peptide $\operatorname{Mr}($ calc): 3410.7834

Fixed modifications: Carbamidomethyl (C)

Variable modifications:

N9 : Deamidated_N (N)

Ions Score: 67 Expect : $1.6 \mathrm{e}-005$

Matches (Bold Red): 45/372 fragment ions using 92 most intense peaks

\begin{tabular}{|c|c|c|c|c|c|c|c|c|c|c|c|c|c|c|}
\hline & b & $\mathbf{b}^{++}$ & $\mathbf{b}^{*}$ & $\mathbf{b}^{*^{++}}$ & $\mathbf{b}^{0}$ & & Seq. & $\mathbf{y}$ & & $\mathbf{y}^{*}$ & & $\mathbf{y}^{0}$ & & \\
\hline 1 & 114.0913 & 57.5493 & & & & & $\mathbf{L}$ & & & & & & & 33 \\
\hline 2 & 215.1390 & 108.0731 & & & & & $\mathbf{T}$ & & & & & & & 32 \\
\hline 3 & 362.2074 & 181.6 & & & & 21 & $\mathbf{F}$ & & 331 & & & 484 & & 31 \\
\hline & 509.2758 & 255.1 & & & 653 & 246 & $\mathbf{F}$ & 5905 & 1525 & 3033.5640 & & 5800 & & 30 \\
\hline 5 & 623.3188 & 312.1630 & & & 605.3082 & & $\mathbf{N}$ & 2903.5221 & 1452.2647 & 2886.4956 & & 5116 & & 29 \\
\hline 6 & 710.3508 & 355.6 & 43 & & 02 & 346 & S & 792 & & 277 & & 686 & & 28 \\
\hline 7 & 811.3985 & 406.2029 & 794.3 & 397. & 793.3879 & 397. & $T$ & 2702.4472 & 1351.7272 & 2685.4206 & 2139 & 2684.4366 & 7219 & 27 \\
\hline 8 & 924.4825 & 462.7449 & 907.4 & 454. & 906.4720 & 453. & L & 995 & & 2584 & & & & 26 \\
\hline 9 & 1039.5095 & 520.2 & 29 & & 989 & 31 & $\mathbf{N}$ & 154 & 613 & 889 & & 049 & 561 & 25 \\
\hline 10 & 1140.5572 & 570.7822 & 123.5306 & 562. & 1122.5466 & 561.7 & $\mathbf{T}$ & 2373.2885 & 1479 & 2356.2619 & 6346 & 2355.2779 & 1426 & 24 \\
\hline 1 & 27.5892 & 982 & 326 & & & & $\mathrm{~S}$ & 2272. & & & & & & 23 \\
\hline 2 & 1284.6107 & 642.8 & 41 & 634. & 1266. & 37 & G & 2185. & 109 & 2168 & & 982 & & 22 \\
\hline 13 & 1397.6947 & 699.3510 & 1380.6682 & 690.8 & 1379.6842 & 690.3457 & $\mathbf{L}$ & 2128.1873 & 1064 & 2111.1608 & 0840 & 2110.1768 & 5920 & 21 \\
\hline 4 & 84.7267 & 742.8 & 02 & & 62 & & $\mathrm{~S}$ & 2015 & 1008 & 1998 & & 1997 & & 20 \\
\hline 5 & 1585.7744 & 793.3908 & 79 & 776 & 1567.7639 & $784.3^{3}$ & $\mathbf{T}$ & 1928.0712 & 392 & 191 & 260 & 607 & 5340 & 19 \\
\hline 16 & 1713.8330 & 857.4201 & 696.8065 & 848.9069 & 1695.8224 & 848.4149 & $\mathbf{Q}$ & 1827.0235 & 0154 & 1809. & 905.5021 & 1809.0130 & 0101 & 18 \\
\hline 7 & 70.8545 & 885.9 & 79 & & & & G & 1698. & & & & 168 & 808 & 17 \\
\hline 8 & 1899.8971 & 950.4522 & 382.8 & 941. & 1881.8 & 941. & $\mathbf{E}$ & 1641.9 & & 1624 & & 1623 & 701 & 16 \\
\hline 19 & 1970.9342 & 985.9707 & 1953.9076 & 977.4574 & 1952.9236 & 976.9654 & A & 1512.9009 & 9541 & 1495 & 748.4408 & 1494 & 9488 & 15 \\
\hline 20 & 84.0182 & 128 & 2 & & 77 & 75 & $\mathbf{L}$ & 1441. & & 1424 & & 1423 & 4303 & 14 \\
\hline 1 & 2181.0710 & 91.0391 & 2164.0445 & 259 & 2163.0604 & 1082.0339 & $\mathbf{P}$ & 1328.7797 & 935 & 1311 & & 1310 & 8882 & 13 \\
\hline 22 & 2294.1551 & 147.5812 & 2277.1285 & 139.0679 & 2276.1445 & 1138.5759 & I & 1231.7270 & 616.3671 & 1214.7004 & 607.8538 & 1213.7164 & 607.3618 & 12 \\
\hline 23 & 391.2078 & 076 & 2374 & 943 & 973 & 023 & $\mathbf{P}$ & 1118.6429 & 8251 & 1101.6164 & 3118 & 1100 & 8198 & 11 \\
\hline 24 & 2448.2293 & 24.6183 & 2431.2027 & 050 & 2430.2187 & 1215.6130 & G & 1021.5901 & & 1004 & & 1003 & & 10 \\
\hline 25 & 2519.2664 & 1260.1368 & 2502.2399 & 6236 & 2501.2558 & 1251.1316 & A & 964.5687 & 7880 & 947.5421 & 2747 & 946.5581 & 473.7827 & 9 \\
\hline 26 & 2656.3253 & 1328.6663 & 2639.2988 & 1320.1530 & 2638.3148 & 1319.6610 & $\mathbf{H}$ & 893.5316 & 2694 & 876. & 438.7561 & 875.5210 & 438.2641 & 8 \\
\hline 27 & 2812.4264 & 1406.7169 & 2795.3999 & 1398.2036 & 2794.4159 & 1397.7116 & $\mathbf{R}$ & 756.4726 & 378.7400 & & & 738.4621 & 369.7347 & 7 \\
\hline 28 & 2909.4792 & 1455.2432 & 2892.4526 & 1446.7300 & 2891.4686 & 1446.2380 & $\mathbf{P}$ & 600.3715 & 300.6894 & 583.3450 & 292.1761 & 582.3610 & 6841 & 6 \\
\hline 29 & 2966.5007 & 1483.7540 & 2949.4741 & 1475.2407 & 2948.4901 & 1474.7487 & G & 503.3188 & 252.1630 & 486.2922 & 243.6498 & 485.3082 & 243.1577 & 5 \\
\hline 30 & 3065.5691 & 1533.2882 & 3048.5425 & 1524.7749 & 3047.5585 & 1524.2829 & $\mathbf{V}$ & 446.2973 & 223.6523 & 429.2708 & & 428.2867 & 214.6470 & 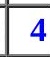 \\
\hline 31 & 3164.6375 & 1582.8224 & 3147.6109 & 1574.3091 & 3146.6269 & \begin{tabular}{|l|}
1573.8171 \\
\end{tabular} & $\mathbf{V}$ & 347.2289 & 174.1181 & 330.2023 & 165.6048 & 329.2183 & 165.1128 & 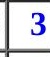 \\
\hline 32 & 3265.6852 & 1633.3462 & 3248.6586 & 1624.8329 & 3247.6746 & 624.3409 & $T$ & 248.1605 & 124.5839 & 231.1339 & 116.0706 & 230.1499 & 115.5786 & \\
\hline
\end{tabular}




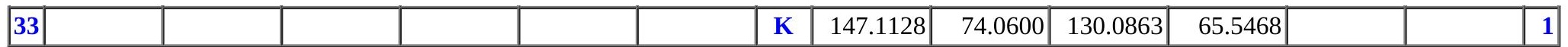
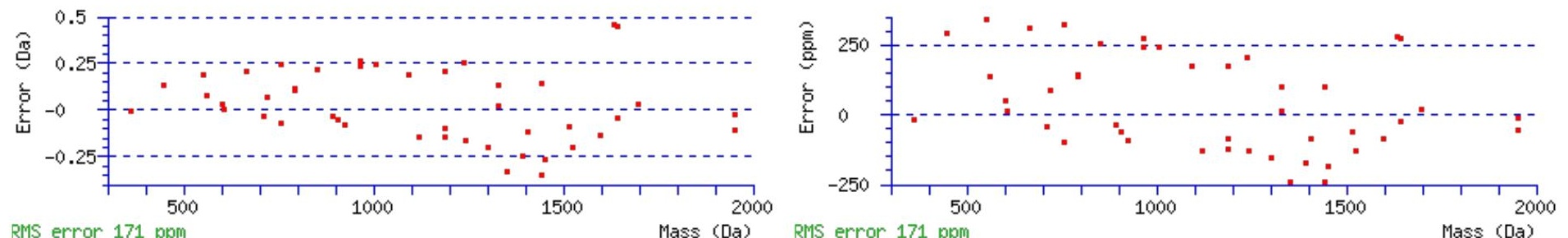

RMS error 171 ppm Mass (Da) RIS error $171 \mathrm{ppm}$

\section{All matches to this query}

\begin{tabular}{|l|l|l|l|}
\hline Score & Mr(calc): & Delta & \multicolumn{1}{c|}{ Sequence } \\
\hline 67.2 & 3410.7834 & 0.0055 & LTFFNSTLNTSGLSTQGEALPIPGAHRPGVVTK \\
\hline 64.8 & 3410.7834 & 0.0055 & LTFFNSTLNTSGLSTQGEALPIPGAHRPGVVTK \\
\hline 64.8 & 3409.7994 & 0.9895 & LTFFNSTLNTSGLSTQGEALPIPGAHRPGVVTK \\
\hline 4.9 & 3408.7500 & 2.0390 & HGLSLLWIWMAELGDGRESNQKLQEEIIK \\
\hline
\end{tabular}

Spectrum No: 410; Query: 2700; Rank: 1

\section{Peptide View}

MS/MS Fragmentation of EHNFNIYNDSVSDSLNVPYDYTSVMHYSK

Found in IPI00204808, Tax_Id=10116 Gene_Symbol=Mep1b Meprin A subunit beta precursor

Match to Query 2700: 3438.512502 from(1147.178110,3+)

Title: 100101RatKid_NS_deglyco_13.4762.4762.3.dta

Data file K:INewmanPaper|Piliang|3SubProteomes\Piliang3SP\mgf5ppm\ERLIC_3SubProteomes5ppm.mgf

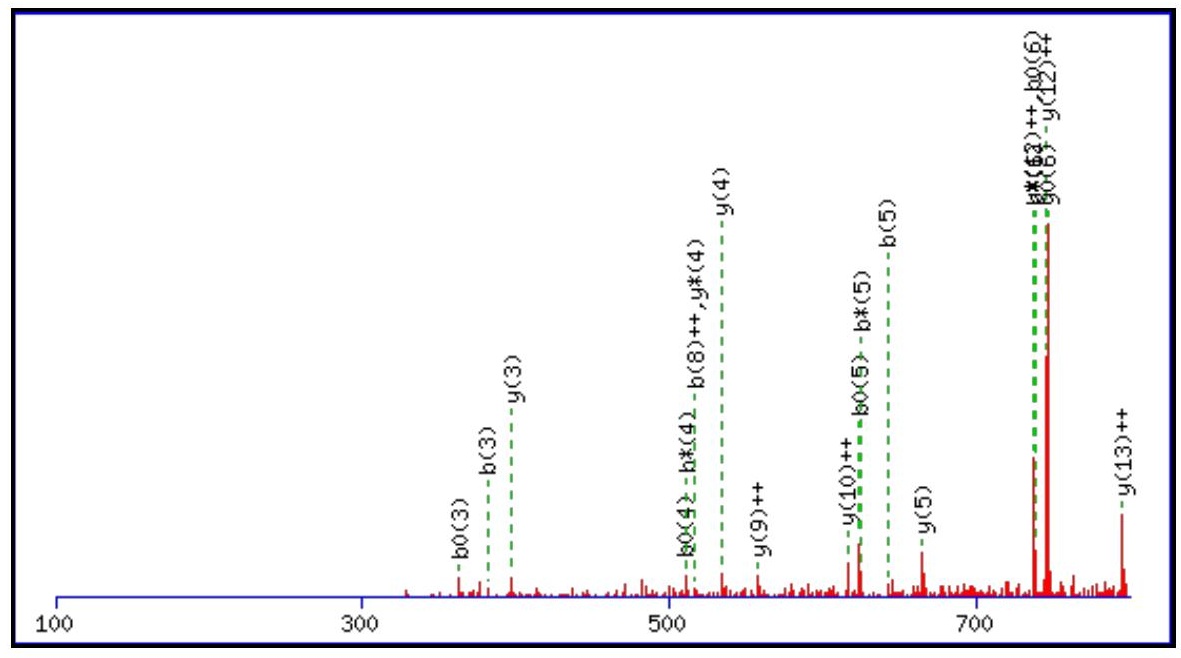



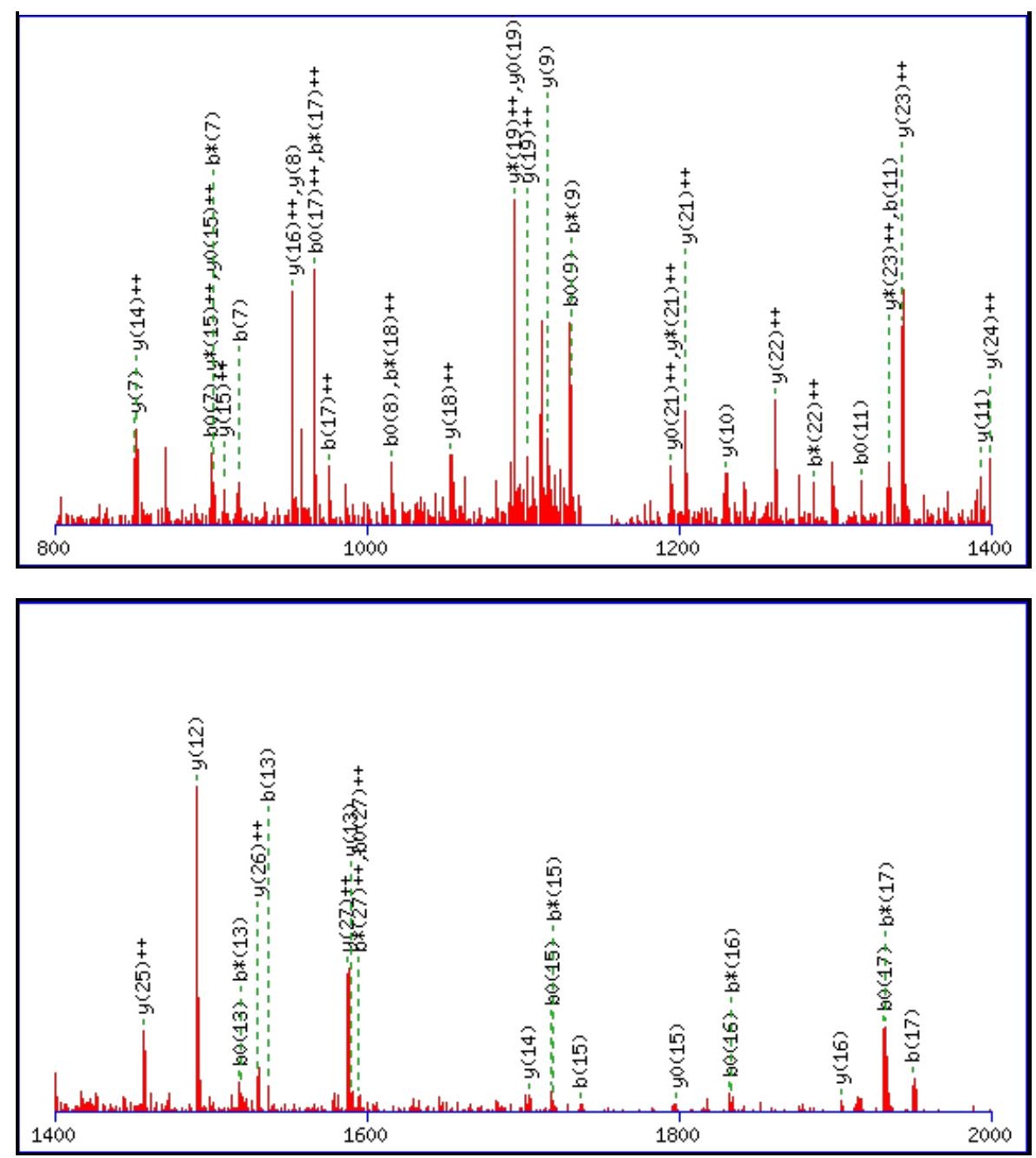

Monoisotopic mass of neutral peptide $\operatorname{Mr}($ calc): 3438.4986

Fixed modifications: Carbamidomethyl (C)

Variable modifications:

N8: Deamidated N (N)

Ions Score: 67 Expect: $9.1 \mathrm{e}-005$

Matches (Bold Red): 75/330 fragment ions using 139 most intense peaks

\begin{tabular}{|c|c|c|c|c|c|c|c|c|c|c|c|c|c|c|}
\hline \# & b & $\mathbf{b}^{++}$ & $\mathbf{b}^{*}$ & $\mathbf{b}^{*^{++}}$ & $\mathbf{b}^{0}$ & $\mathbf{b}^{\mathbf{0 + +}}$ & Seq. & $\mathbf{y}$ & $\mathbf{y}^{++}$ & $\mathbf{y}^{*}$ & $\mathbf{y}^{*^{++}}$ & $\mathbf{y}^{0}$ & $\mathbf{y}^{0++}$ & \# \\
\hline 1 & 130.0499 & 65.5286 & & & 112.0393 & 56.5233 & $\mathbf{E}$ & & & & & & & 29 \\
\hline 2 & 267.1088 & 134.0580 & & & 249.0982 & 125.0527 & $\mathbf{H}$ & 3310.4633 & 1655 & 4368 & 220 & 3292.4528 & 1646.7300 & 28 \\
\hline 3 & 381.1517 & 191.0795 & 364.1252 & 182.5662 & 363.1411 & 182.0742 & $\mathbf{N}$ & 3173.4044 & 1587.2059 & 3156.3779 & 1578.6926 & 3155.3939 & 1578.2006 & 27 \\
\hline 4 & 528.2201 & 264.6137 & 511.1936 & 256.1004 & 510.2096 & 255.6084 & $\mathbf{F}$ & 3059.3615 & 1530.1844 & 3042.3350 & 1521. & 3041.3509 & 1521.1 & 26 \\
\hline 5 & 642.2630 & 321.6352 & 625.2365 & 313.1219 & 624.2525 & 312.6299 & $\mathbf{N}$ & 2912.2931 & 1456.6502 & 2895.2665 & 1448.1369 & 2894.2825 & 1447.6449 & 25 \\
\hline 6 & 755.3471 & 378.1772 & 738.3206 & 369.6639 & 737.3365 & 369.1719 & I & 2798.2502 & 1399.6287 & 2781.2236 & 1391.1154 & 2780.2396 & 1390.6234 & 24 \\
\hline 7 & 918.4104 & 459.7089 & 901.3839 & 451.1956 & 900.3999 & 450.7036 & $\mathbf{Y}$ & 2685.1661 & 1343.0867 & 2668.1395 & 1334.5734 & 2667.1555 & 1334.0814 & 23 \\
\hline 8 & 1033.4374 & 517.2223 & 1016.4108 & 508.7091 & 1015.4268 & 508.2170 & $\mathbf{N}$ & 2522.1028 & 1261.5550 & 2505.0762 & 1253.0417 & 2504.0922 & 1252.5497 & 22 \\
\hline 9 & 1148.4643 & 574.7358 & 1131.4378 & 566.2225 & 1130.4538 & 565.7305 & D & 2407.0758 & 1204.0416 & 2390.0493 & 1195.5283 & 2389.0653 & 1195.0363 & 21 \\
\hline 10 & 1235.4963 & 618.2518 & 1218.4698 & 609.7385 & 1217.4858 & 609.2465 & S & 2292.0489 & 1146.5281 & 2275.0223 & 1138.0148 & 2274.0383 & 1137.5228 & 20 \\
\hline 11 & 1334.5648 & 667.7860 & 1317.5382 & 659.2727 & 1316.5542 & 658.7807 & $\mathbf{V}$ & 2205.0169 & 1103.0121 & 2187.9903 & 1094.4988 & 2187.0063 & 1094.0068 & 19 \\
\hline 12 & 1421.5968 & 711.3020 & 1404.5702 & 702.7888 & 1403.5862 & 702.2968 & $\mathrm{~S}$ & 2105.9484 & 1053.4779 & 2088.9219 & 1044.9646 & 2087.9379 & 1044.4726 & 18 \\
\hline 13 & 1536.6237 & 768.8155 & 1519.5972 & 760.3022 & 1518.6132 & 759.8102 & D & 2018.9164 & 1009.9618 & 2001.8899 & 1001.4486 & 2000.9059 & 1000.9566 & 17 \\
\hline 14 & 1623.6558 & 812.3315 & 1606.6292 & 803.8182 & 1605.6452 & 803.3262 & $S$ & 1903.8895 & 952.4484 & 1886.8629 & 943.9351 & 1885.8789 & 943.4431 & 16 \\
\hline 15 & 1736.7398 & 868.8736 & 1719.7133 & 860.3603 & 1718.7293 & 859.8683 & $\mathbf{L}$ & 1816.8574 & 908.9324 & 1799.8309 & 900.4191 & 1798.8469 & 899.9271 & 15 \\
\hline 16 & 1850.7828 & 925.8950 & 1833.7562 & 917.3817 & 1832.7722 & 916.8897 & $\mathbf{N}$ & 1703.7734 & 852.3903 & 1686.7468 & 843.8771 & 1685.7628 & 843.3850 & 14 \\
\hline 17 & 1949.8512 & 975.4292 & 1932.8246 & 966.9159 & 1931.8406 & 966.4239 & V & 1589.7305 & 795.3689 & 1572.7039 & 786.8556 & 1571.7199 & 786.3636 & 13 \\
\hline 18 & 2046.9039 & 1023.9556 & 2029.8774 & 1015.4423 & 2028.8934 & 1014.9503 & $\mathbf{P}$ & 1490.6620 & 745.8347 & 1473.6355 & 737.3214 & 1472.6515 & 736.8294 & 12 \\
\hline
\end{tabular}




\begin{tabular}{|l|r|r|r|r|r|r|r|r|r|r|r|r|r|r|r|}
$\mathbf{1 9}$ & 2209.9673 & 1105.4873 & 2192.9407 & 1096.9740 & 2191.9567 & 1096.4820 & $\mathbf{Y}$ & $\mathbf{1 3 9 3 . 6 0 9 3}$ & 697.3083 & 1376.5827 & 688.7950 & 1375.5987 & 688.3030 & $\mathbf{1 1}$ \\
\hline $\mathbf{2 0}$ & 2324.9942 & 1163.0007 & 2307.9677 & 1154.4875 & 2306.9836 & 1153.9955 & $\mathbf{D}$ & $\mathbf{1 2 3 0 . 5 4 6 0}$ & $\mathbf{6 1 5 . 7 7 6 6}$ & 1213.5194 & 607.2633 & $\mathbf{1 2 1 2 . 5 3 5 4}$ & 606.7713 & $\mathbf{1 0}$ \\
\hline $\mathbf{2 1}$ & 2488.0575 & 1244.5324 & 2471.0310 & 1236.0191 & 2470.0470 & 1235.5271 & $\mathbf{Y}$ & $\mathbf{1 1 1 5 . 5 1 9 0}$ & $\mathbf{5 5 8 . 2 6 3 1}$ & 1098.4925 & 549.7499 & 1097.5084 & 549.2579 & $\mathbf{9}$ \\
\hline $\mathbf{2 2}$ & 2589.1052 & 1295.0562 & 2572.0787 & $\mathbf{1 2 8 6 . 5 4 3 0}$ & 2571.0946 & 1286.0510 & $\mathbf{T}$ & $\mathbf{9 5 2 . 4 5 5 7}$ & 476.7315 & 935.4291 & 468.2182 & 934.4451 & 467.7262 & $\mathbf{8}$ \\
\hline $\mathbf{2 3}$ & 2676.1372 & 1338.5723 & 2659.1107 & 1330.0590 & 2658.1267 & 1329.5670 & $\mathbf{S}$ & $\mathbf{8 5 1 . 4 0 8 0}$ & 426.2076 & 834.3815 & 417.6944 & 833.3974 & 417.2024 & $\mathbf{7}$ \\
\hline $\mathbf{2 4}$ & 2775.2057 & 1388.1065 & 2758.1791 & 1379.5932 & 2757.1951 & 1379.1012 & $\mathbf{V}$ & 764.3760 & 382.6916 & 747.3494 & 374.1783 & $\mathbf{7 4 6 . 3 6 5 4}$ & 373.6863 & $\mathbf{6}$ \\
\hline $\mathbf{2 5}$ & 2906.2461 & 1453.6267 & 2889.2196 & 1445.1134 & 2888.2356 & 1444.6214 & $\mathbf{M}$ & $\mathbf{6 6 5 . 3 0 7 6}$ & 333.1574 & 648.2810 & 324.6441 & 647.2970 & 324.1521 & $\mathbf{5}$ \\
\hline $\mathbf{2 6}$ & 3043.3050 & 1522.1562 & 3026.2785 & 1513.6429 & 3025.2945 & 1513.1509 & $\mathbf{H}$ & $\mathbf{5 3 4 . 2 6 7 1}$ & 267.6372 & $\mathbf{5 1 7 . 2 4 0 5}$ & 259.1239 & 516.2565 & 258.6319 & $\mathbf{4}$ \\
\hline $\mathbf{2 7}$ & 3206.3684 & 1603.6878 & 3189.3418 & $\mathbf{1 5 9 5 . 1 7 4 6}$ & 3188.3578 & $\mathbf{1 5 9 4 . 6 8 2 5}$ & $\mathbf{Y}$ & $\mathbf{3 9 7 . 2 0 8 2}$ & 199.1077 & 380.1816 & 190.5944 & 379.1976 & 190.1024 & $\mathbf{3}$ \\
\hline $\mathbf{2 8}$ & 3293.4004 & 1647.2038 & 3276.3739 & 1638.6906 & 3275.3898 & 1638.1986 & S & 234.1448 & 117.5761 & 217.1183 & 109.0628 & 216.1343 & 108.5708 & $\mathbf{2}$ \\
\hline $\mathbf{2 9}$ & & & & & & & $\mathbf{K}$ & 147.1128 & 74.0600 & 130.0863 & 65.5468 & & & $\mathbf{1}$ \\
\hline
\end{tabular}
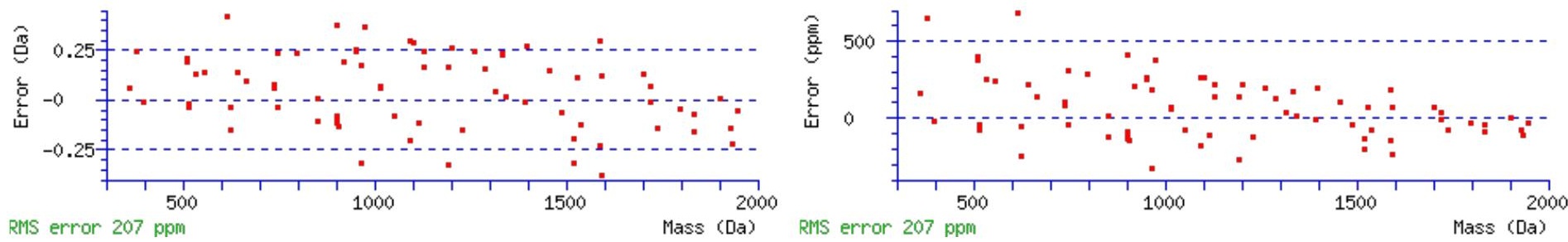

\section{All matches to this query}

\begin{tabular}{|l|l|l|l|}
\hline Score & Mr(calc): & Delta & \multicolumn{1}{|c|}{ Sequence } \\
\hline 67.2 & 3438.4986 & 0.0139 & EHNFNIYNDSVSDSLNVPYDYTSVMHYSK \\
\hline 62.6 & 3438.4986 & 0.0139 & EHNFNIYNDSVSDSLNVPYDYTSVMHYSK \\
\hline 47.4 & 3438.4986 & 0.0139 & EHNFNIYNDSVSDSLNVPYDYTSVMHYSK \\
\hline 35.4 & 3438.4986 & 0.0139 & EHNFNIYNDSVSDSLNVPYDYTSVMHYSK \\
\hline 16.3 & 3437.5146 & 0.9979 & EHNFNIYNDSVSDSLNVPYDYTSVMHYSK \\
\hline 12.6 & 3438.5029 & 0.0096 & NPSVSLDLLNLYMVNQISSKKEISETMK \\
\hline 12.6 & 3438.5029 & 0.0096 & NPSVSLDLLNLYMVNQISSKKEISETMK \\
\hline 11.8 & 3437.5189 & 0.9936 & NPSVSLDLLNLYMVNQISSKKEISETMK \\
\hline 7.2 & 3436.5120 & 2.0005 & YLTVAYACVPKNILTAVDPAVANLNPSLK \\
\hline 5.5 & 3437.5189 & 0.9936 & NPSVSLDLLNLYMVNQISSKKEISETMK \\
\hline
\end{tabular}

Spectrum No: 411; Query: 1839; Rank: 1

\section{Peptide View}

MS/MS Fragmentation of YSNESQDLSVNGYNCTMR

Found in IPI00191789, Tax_Id=10116 Gene_Symbol=Hrg Histidine-rich glycoprotein

Match to Query 1839: 2154.855368 from(1078.434960,2+)

Title: 100101RatKid_NS_deglyco_27.1669.1669.2.dta

Data file K:INewmanPaper|Piliangl3SubProteomes\Piliang3SP\mgf5ppm\ERLIC_3SubProteomes5ppm.mgf 

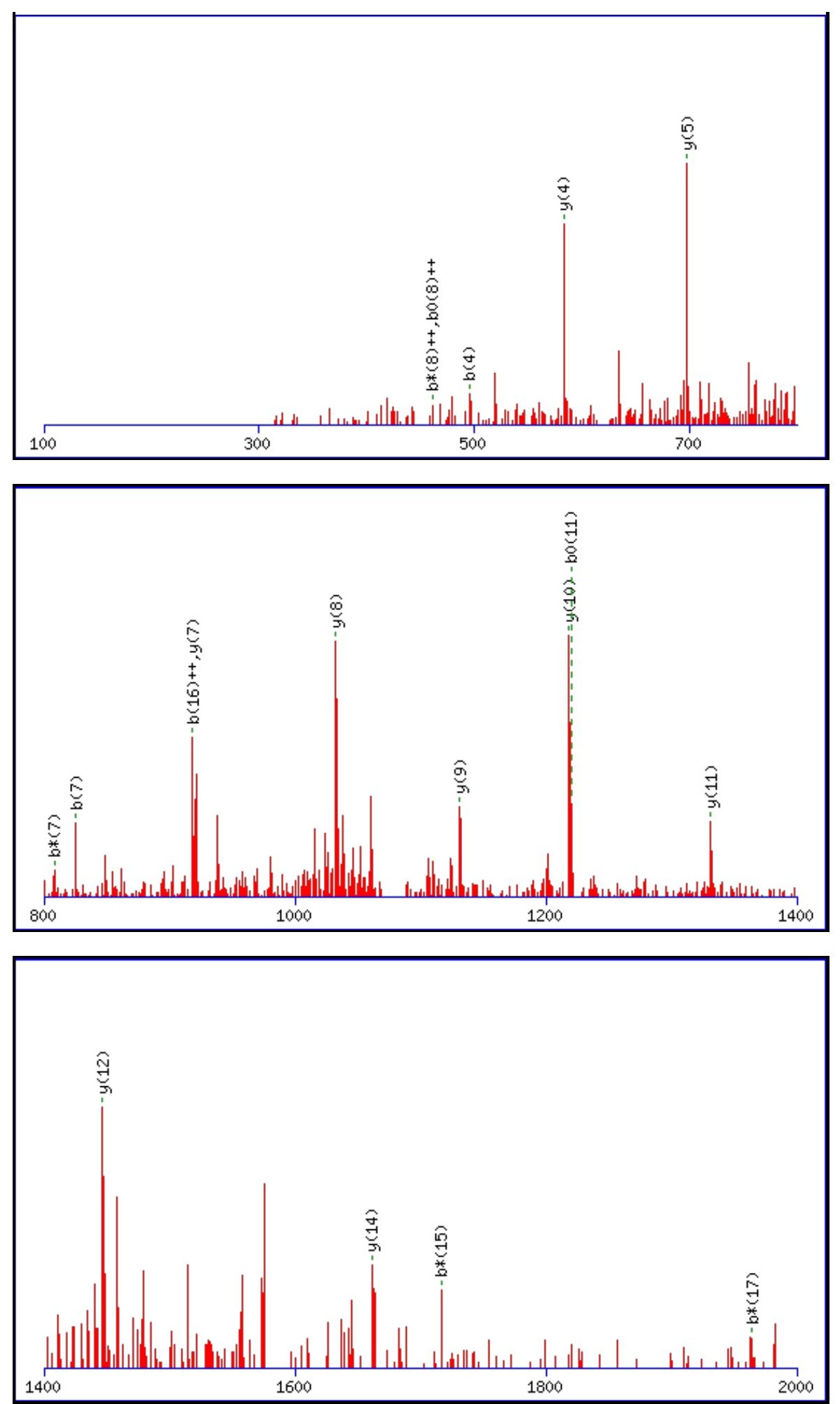

Monoisotopic mass of neutral peptide $\operatorname{Mr}($ calc): 2154.8470

Fixed modifications: Carbamidomethyl (C)

Variable modifications:

N3 : Deamidated_N (N)

N14 : Deamidated_N (N)

M17 : 0xidation (M)

Ions Score: 67 Expect: $4.4 \mathrm{e}-005$

Matches (Bold Red): 18/194 fragment ions using 35 most intense peaks

\begin{tabular}{|c|c|c|c|c|c|c|c|c|c|c|c|c|c|c|}
\hline$\#$ & $\mathbf{b}$ & $\mathbf{b}^{++}$ & $\mathbf{b}^{*}$ & $\mathbf{b}^{\boldsymbol{*}_{++}}$ & $\mathbf{b}^{\mathbf{0}}$ & $\mathbf{b}^{\mathbf{0 + +}}$ & Seq. & $\mathbf{y}$ & $\mathbf{y}^{++}$ & $\mathbf{y}^{\mathbf{*}}$ & $\mathbf{y}^{\boldsymbol{*}^{++}}$ & $\mathbf{y}^{\mathbf{0}}$ & $\mathbf{y}^{\mathbf{0}++}$ & $\#$ \\
\hline $\mathbf{1}$ & 164.0706 & 82.5389 & & & & & $\mathbf{Y}$ & & & & & & & $\mathbf{1 8}$ \\
\hline $\mathbf{2}$ & 251.1026 & 126.0550 & & & 233.0921 & 117.0497 & S & 1992.7910 & 996.8991 & 1975.7644 & 988.3858 & 1974.7804 & 987.8938 & $\mathbf{1 7}$ \\
\hline $\mathbf{3}$ & 366.1296 & 183.5684 & 349.1030 & 175.0551 & 348.1190 & 174.5631 & $\mathbf{N}$ & 1905.7589 & 953.3831 & 1888.7324 & 944.8698 & 1887.7484 & 944.3778 & $\mathbf{1 6}$ \\
\hline $\mathbf{4}$ & $\mathbf{4 9 5 . 1 7 2 2}$ & 248.0897 & 478.1456 & 239.5764 & 477.1616 & 239.0844 & $\mathbf{E}$ & 1790.7320 & 895.8696 & 1773.7054 & 887.3564 & 1772.7214 & 886.8643 & $\mathbf{1 5}$ \\
\hline
\end{tabular}




\begin{tabular}{|c|c|c|c|c|c|c|c|c|c|c|c|c|c|c|}
\hline & 582.2042 & 291.6057 & 565.1776 & & 564.1936 & & S & $6894 \mid$ & 831.3483 & 6628 & 822.8351 & 1643.6788 & 822.3431 & \\
\hline 6 & 710.2628 & 355.6350 & 693.2362 & 347.1217 & 692.2522 & 346.6297 & $\mathbf{Q}$ & 1574.6574 & 787.8323 & 1557.6308 & 779.3190 & 1556.6468 & 778.8270 & 13 \\
\hline 7 & 825.2897 & 413.1485 & 808.2632 & 404.6352 & 807.2791 & 404.1432 & D & 1446.5988 & 723.8030 & 1429.5722 & 715.2898 & 1428.5882 & 714.7978 & 12 \\
\hline 8 & 938.3738 & 469.6905 & 921.3472 & 461.1773 & 920.3632 & 460.6852 & $\mathbf{L}$ & 1331.5718 & 666.2896 & 1314.5453 & 657.7763 & 1313.5613 & 657.2843 & 11 \\
\hline 9 & 025.4058 & 13.2065 & 1008.3793 & 504.6933 & |1007.3952 & 504.2013 & $\mathrm{~S}$ & 1218.4878 & 609.7475 & 1201.4612 & 601.2343 & 1200.4772 & 600.7422 & 10 \\
\hline 10 & 1124.4742 & 562.7407 & 1107.4477 & 554.2275 & 1106.4637 & 553.7355 & $\mathbf{V}$ & 1131.4558 & 566.2315 & 1114.4292 & 557.7182 & 1113.4452 & 557.2262 & 9 \\
\hline 11 & 1238.5171 & 619.7622 & 1221.4906 & 611.2489 & 1220.5066 & 610.7569 & $\mathbf{N}$ & 1032.3873 & 516.6973 & 1015.3608 & 508.1840 & 1014.3768 & 507.6920 & 8 \\
\hline 12 & 1295.5386 & 648.2729 & 1278.5121 & 639.7597 & 1277.5280 & 639.2677 & G & 918.3444 & 459.6758 & 901.3179 & 451.1626 & 900.3338 & 450.6706 & 7 \\
\hline 13 & 1458.6019 & 729.8046 & 1441.5754 & 721.2913 & 1440.5914 & 720.7993 & $\mathbf{Y}$ & 861.3229 & 431.1651 & 844.2964 & 422.6518 & 843.3124 & 422.1598 & 6 \\
\hline 14 & 1573.6289 & 787.3181 & 1556.6023 & 778.8048 & 1555.6183 & 778.3128 & $\mathbf{N}$ & 698.2596 & 349.6334 & 681.2331 & 341.1202 & 680.2491 & 340.6282 & 5 \\
\hline 15 & 1733.6595 & 867.3334 & \begin{tabular}{|l|}
1716.6330 \\
\end{tabular} & 858.8201 & 1715.6490 & 858.3281 & $\mathrm{C}$ & 583.2327 & 292.1200 & 566.2061 & 283.6067 & 565.2221 & 283.1147 & 4 \\
\hline 16 & 1834.7072 & 917.8572 & 1817.6807 & 909.3440 & 1816.6966 & 908.8520 & $\mathbf{T}$ & 423.2020 & 212.1047 & 406.1755 & 203.5914 & 405.1915 & 203.0994 & 3 \\
\hline 17 & 1981.7426 & 991.3749 & 1964.7161 & 982.8617 & 1963.7320 & 982.3697 & $\mathbf{M}$ & 322.1544 & 161.5808 & 305.1278 & 153.0675 & & & 2 \\
\hline 18 & & & & & & & $\mathbf{R}$ & 175.1190 & 88.0631 & 158.0924 & 79.5498 & & & \\
\hline
\end{tabular}
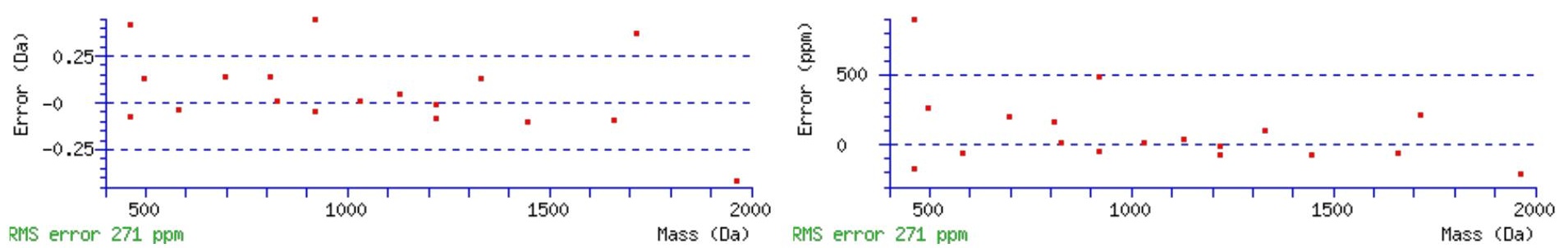

\section{All matches to this query}

\begin{tabular}{|l|l|l|l|}
\hline Score & Mr(calc): & Delta & Sequence \\
\hline 67.2 & 2154.8470 & 0.0084 & YSNESQDLSVNGYNCTMR \\
\hline 53.1 & 2153.8630 & 0.9924 & YSNESQDLSVNGYNCTMR \\
\hline 40.3 & 2154.8470 & 0.0084 & YSNESQDLSVNGYNCTMR \\
\hline 39.0 & 2154.8470 & 0.0084 & YSNESQDLSVNGYNCTMR \\
\hline 28.7 & 2153.8630 & 0.9924 & YSNESQDLSVNGYNCTMR \\
\hline 3.9 & 2153.8630 & 0.9924 & YSNESQDLSVNGYNCTMR \\
\hline 0.9 & 2153.8493 & 1.0061 & SADEFNCLSCPGSMWSYK \\
\hline
\end{tabular}

Spectrum No: 412; Query: 1818; Rank: 1

\section{Peptide View}

MS/MS Fragmentation of IVDNGYGYASNGSVYFDTAK

Found in IPI00371408, Tax_Id=10116 Gene_Symbol=Cars_predicted 95 kDa protein

Match to Query 1818: 2140.969808 from(1071.492180,2+)

Title: 100101RatKid_NS_deglyco_13.3388.3388.2.dta

Data file K:INewmanPaper|Piliang|3SubProteomes\Piliang3SP\mgf5ppm\ERLIC_3SubProteomes5ppm.mgf 

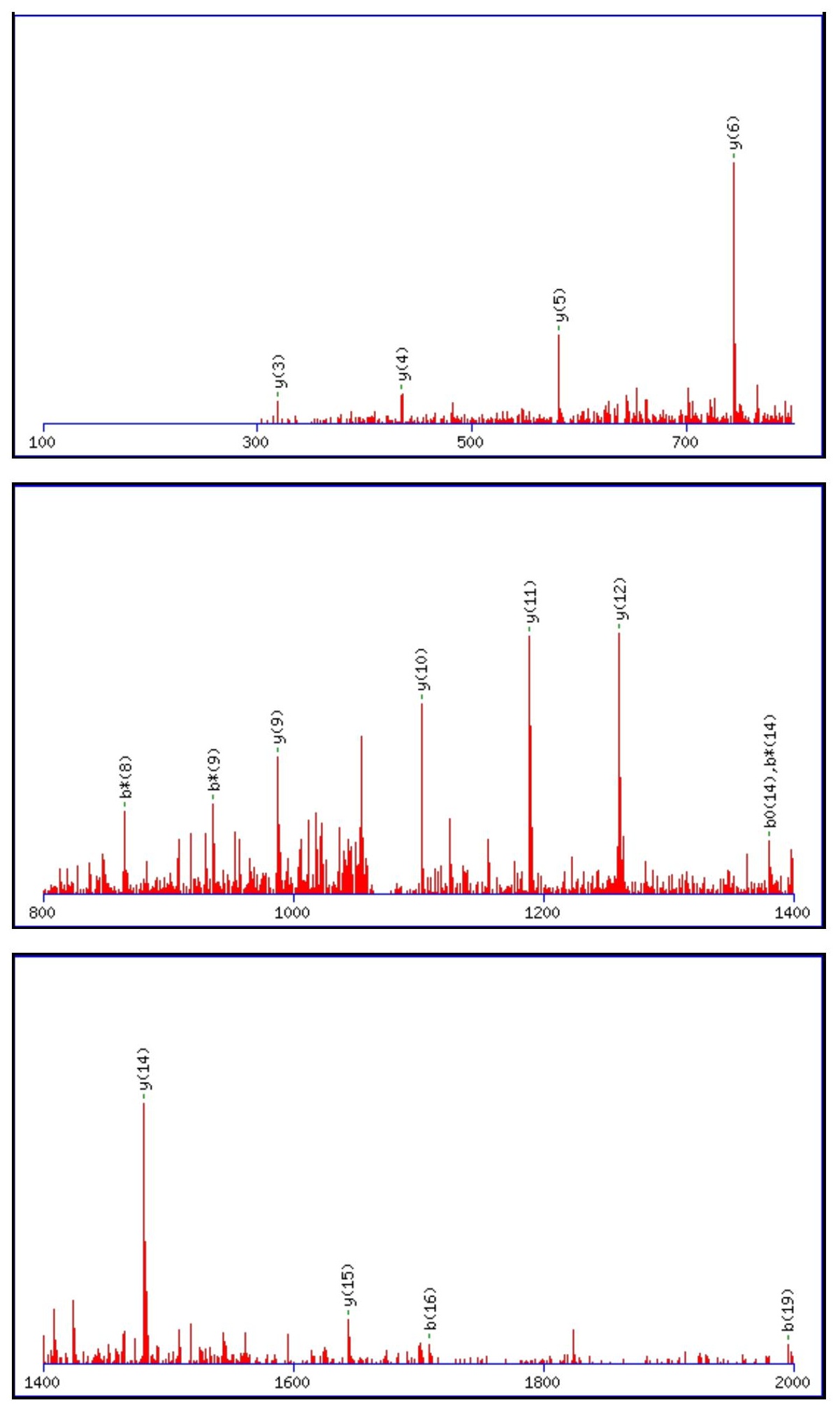

Monoisotopic mass of neutral peptide $\operatorname{Mr}($ calc): 2140.9589

Fixed modifications: Carbamidomethyl (C)

Variable modifications:

N11 : Deamidated $\mathrm{N}(\mathrm{N})$

Ions Score: 67 Expect: $5.1 \mathrm{e}-005$

Matches (Bold Red): 16/214 fragment ions using 27 most intense peaks

\begin{tabular}{|r|c|c|c|c|c|c|c|c|c|c|c|c|c|c|}
\hline$\#$ & $\mathbf{b}$ & $\mathbf{b}^{++}$ & $\mathbf{b}^{*}$ & $\mathbf{b}^{\boldsymbol{*}^{++}}$ & $\mathbf{b}^{\mathbf{0}}$ & $\mathbf{b}^{\mathbf{0 + +}}$ & Seq. & $\mathbf{y}$ & $\mathbf{y}^{++}$ & $\mathbf{y}^{\mathbf{*}}$ & $\mathbf{y}^{\boldsymbol{*}^{++}}$ & $\mathbf{y}^{\mathbf{0}}$ & $\mathbf{y}^{\mathbf{0 + +}}$ & $\#$ \\
\hline $\mathbf{1}$ & 114.0913 & 57.5493 & & & & & $\mathbf{I}$ & & & & & & & $\mathbf{2 0}$ \\
\hline $\mathbf{2}$ & 213.1598 & 107.0835 & & & & & $\mathbf{V}$ & 2028.8821 & 1014.9447 & 2011.8556 & 1006.4314 & 2010.8716 & 1005.9394 & $\mathbf{1 9}$ \\
\hline $\mathbf{3}$ & 328.1867 & 164.5970 & & & 310.1761 & 155.5917 & $\mathbf{D}$ & 1929.8137 & 965.4105 & 1912.7872 & 956.8972 & 1911.8032 & 956.4052 & $\mathbf{1 8}$ \\
\hline $\mathbf{4}$ & 442.2296 & 221.6185 & 425.2031 & 213.1052 & 424.2191 & 212.6132 & $\mathbf{N}$ & 1814.7868 & 907.8970 & 1797.7602 & 899.3838 & 1796.7762 & 898.8917 & $\mathbf{1 7}$ \\
\hline $\mathbf{5}$ & 499.2511 & 250.1292 & 482.2245 & 241.6159 & 481.2405 & 241.1239 & $\mathbf{G}$ & 1700.7439 & 850.8756 & 1683.7173 & 842.3623 & 1682.7333 & 841.8703 & $\mathbf{1 6}$ \\
\hline
\end{tabular}




\begin{tabular}{|c|c|c|c|c|c|c|c|c|c|c|c|c|c|c|}
\hline 6 & 662.3144 & |331.6608 & 645.2879 & |323.1476 & 644.3039 & |322.6556| & $\mathbf{Y}$ & |1643.7224 & 822.3648 & |1626.6958| & 813.8516 & 1625.7118 & 813.3596 & 15 \\
\hline 7 & 719.3359 & 360.1716 & 702.3093 & 351.6583 & 701.3253 & 351.1663 & $\mathbf{G}$ & 1480.6591 & 740.8332 & 1463.6325 & 732.3199 & 1462.6485 & 731.8279 & 14 \\
\hline 8 & 882.3992 & 441.7032 & 865.3727 & 433.1900 & 864.3886 & 432.6980 & $\mathbf{Y}$ & 1423.6376 & 712.3224 & 1406.6110 & 703.8092 & 1405.6270 & 703.3172 & 13 \\
\hline 9 & 953.4363 & 477.2218 & 936.4098 & 468.7085 & 935.4258 & 468.2165 & A & 1260.5743 & 630.7908 & 1243.5477 & 622.2775 & 1242.5637 & 621.7855 & 12 \\
\hline 10 & 1040.4684 & 520.7378 & 1023.4418 & 512.2245 & 1022.4578 & 511.7325 & S & 1189.5372 & 595.2722 & 1172.5106 & 586.7589 & 1171.5266 & 586.2669 & 11 \\
\hline 11 & 1155.4953 & 578.2513 & 1138.4687 & 569.7380 & 1137.4847 & 569.2460 & $\mathbf{N}$ & 1102.5051 & 551.7562 & 1085.4786 & 543.2429 & 1084.4946 & 542.7509 & 10 \\
\hline 12 & 1212.5168 & 606.7620 & 1195.4902 & 598.2487 & 1194.5062 & 597.7567 & G & 987.4782 & 494.2427 & 970.4516 & 485.7295 & 969.4676 & 485.2375 & 9 \\
\hline 13 & 1299.5488 & 650.2780 & 1282.5222 & 641.7648 & 1281.5382 & 641.2727 & S & 930.4567 & 465.7320 & 913.4302 & 457.2187 & 912.4462 & 456.7267 & 8 \\
\hline 14 & 1398.6172 & 699.8122 & 1381.5906 & 691.2990 & 1380.6066 & 690.8070 & V & 843.4247 & 422.2160 & 826.3981 & 413.7027 & 825.4141 & 413.2107 & 7 \\
\hline 15 & 1561.6805 & 781.3439 & 1544.6540 & 772.8306 & 1543.6700 & 772.3386 & $\mathbf{Y}$ & 744.3563 & 372.6818 & 727.3297 & 364.1685 & 726.3457 & 363.6765 & 6 \\
\hline 16 & 1708.7489 & 854.8781 & 1691.7224 & 846.3648 & 1690.7384 & 845.8728 & $\mathbf{F}$ & 581.2930 & 291.1501 & 564.2664 & 282.6368 & 563.2824 & 282.1448 & 5 \\
\hline 17 & 1823.7759 & 912.3916 & 1806.7493 & 903.8783 & 1805.7653 & 903.3863 & D & 434.2245 & 217.6159 & 417.1980 & 209.1026 & 416.2140 & 208.6106 & A \\
\hline 18 & 1924.8236 & 962.9154 & 1907.7970 & 954.4021 & 1906.8130 & 953.9101 & $\mathbf{T}$ & 319.1976 & 160.1024 & 302.1710 & 151.5892 & 301.1870 & 151.0972 & 3 \\
\hline 19 & 1995.8607 & 998.4340 & 1978.8341 & 989.9207 & 1977.8501 & 989.4287 & A & 218.1499 & 109.5786 & 201.1234 & 101.0653 & & & 2 \\
\hline 20 & & & & & & & $\mathbf{K}$ & 147.1128 & 74.0600 & 130.0863 & 65.5468 & & & 1 \\
\hline
\end{tabular}
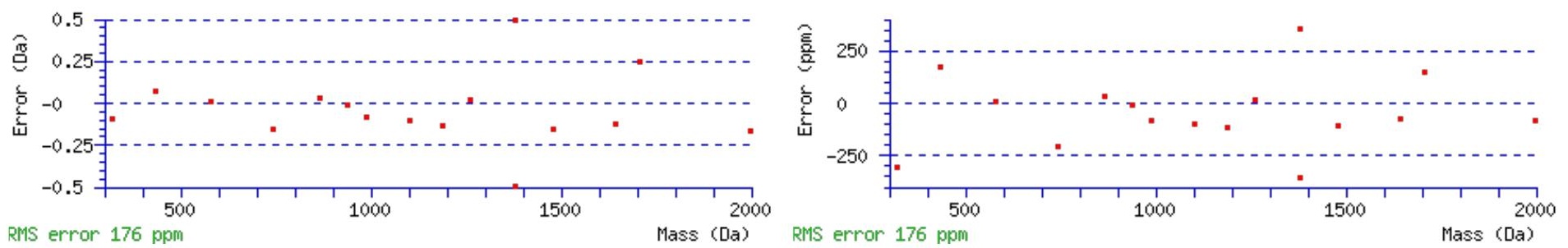

\section{All matches to this query}

\begin{tabular}{|l|c|c|l|}
\hline Score & Mr(calc): & Delta & \multicolumn{1}{|c|}{ Sequence } \\
\hline 67.2 & 2140.9589 & 0.0109 & IVDNGYGYASNGSVYFDTAK \\
\hline 10.5 & 2140.9589 & 0.0109 & $\underline{\text { IVDNGYGYASNGSVYFDTAK }}$ \\
\hline 2.9 & 2139.9749 & 0.9949 & $\underline{\text { IVDNGYGYASNGSVYFDTAK }}$ \\
\hline 0.6 & 2140.9868 & -0.0170 & SEMKMEIDDLASNVETVSK \\
\hline 0.6 & 2140.9868 & -0.0170 & $\underline{\text { SEMKMEIDDLASNVETVSK }}$ \\
\hline
\end{tabular}

Spectrum No: 413; Query: 2200; Rank: 1

\section{Peptide View}

\section{MS/MS Fragmentation of EAGSLEPVTVMGTEYVFYNDTK}

Found in IPI00370450, Tax_Id=10116 Gene_Symbol=Plxnb2 plexin B2

Match to Query 2200: 2450.124568 from(1226.069560,2+)

Title: 100101RatKid_NS_deglyco_23.4748.4748.2.dta

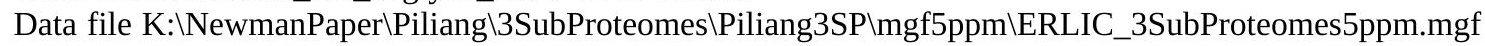



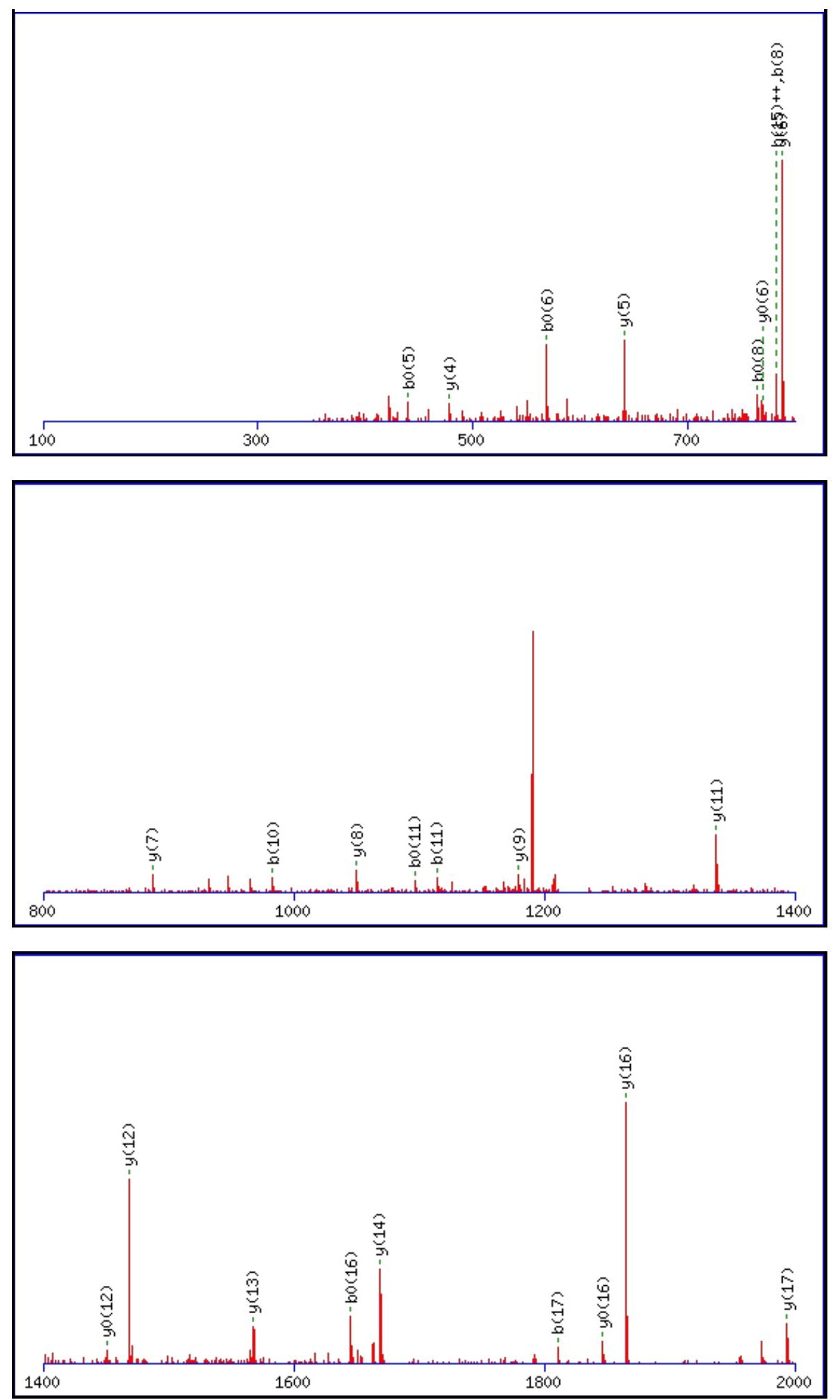

Monoisotopic mass of neutral peptide $\operatorname{Mr}($ calc): 2450.1199

Fixed modifications: Carbamidomethyl (C)

Variable modifications:

N19 : Deamidated_N (N)

Ions Score: 67 Expect : $6.6 \mathrm{e}-005$

Matches (Bold Red): 25/214 fragment ions using 42 most intense peaks

\begin{tabular}{|c|c|c|c|c|c|c|c|c|c|c|c|c|c|c|}
\hline \# & b & $\mathbf{b}^{++}$ & $\mathbf{b}^{*}$ & $\mathbf{b}^{*^{++}}$ & $\mathbf{b}^{\mathbf{0}}$ & $\mathbf{b}^{\mathbf{0 + +}}$ & Seq. & $\mathbf{y}$ & $y^{++}$ & $\mathbf{y}^{*}$ & $\mathrm{y}^{\mathrm{*}^{++}}$ & $\mathbf{y}^{\mathbf{0}}$ & $\mathbf{y}^{\mathbf{0 + +}}$ & \# \\
\hline 1 & 130.0499 & 65.5286 & & & 112.0393 & 56.5233 & $\mathbf{E}$ & & & & & & & 22 \\
\hline 2 & 201.0870 & 101.0471 & & & 183.0764 & 92.0418 & A & 2322.0846 & 1161.5459 & 2305.0581 & 1153.0327 & 2304.0740 & 1152.5407 & 21 \\
\hline 3 & 258.1084 & 129.5579 & & & 240.0979 & 120.5526 & G & 2251.0475 & 1126.0274 & 2234.0209 & 1117.5141 & 2233.0369 & 1117.0221 & 20 \\
\hline 4 & 345.1405 & 173.0739 & & & 327.1299 & 164.0686 & $S$ & 2194.0260 & 1097.5167 & 2176.9995 & 1089.0034 & 2176.0155 & 1088.5114 & 19 \\
\hline 5 & 458.2245 & 229.6159 & & & 440.2140 & 220.6106 & L & 2106.9940 & 1054.0006 & 2089.9674 & 1045.4874 & 2088.9834 & 1044.9954 & 18 \\
\hline
\end{tabular}




\begin{tabular}{|c|c|c|c|c|c|c|c|c|c|c|c|c|c|c|}
\hline 6 & 587.2671 & 294.1372 & & & 569.2566 & 285.1319 & $\mathbf{E}$ & 1993.9099 & 997.4586 & 1976.8834 & 988.9453 & 1975.8994 & 988.4533 & 17 \\
\hline 7 & 684.3199 & 342.6636 & & & 666.3093 & 333.6583 & $\mathbf{P}$ & 1864.8673 & 932.9373 & 1847.8408 & 924.4240 & 1846.8568 & 923.9320 & 16 \\
\hline 8 & 783.3883 & 392.1978 & & & 765.3777 & 383.1925 & V & 1767.8146 & 884.4109 & 1750.7880 & 875.8977 & 1749.8040 & 875.4056 & 15 \\
\hline 9 & 884.4360 & 442.7216 & & & 866.4254 & 433.7164 & $\mathbf{T}$ & 1668.7462 & 834.8767 & 1651.7196 & 826.3634 & 1650.7356 & 825.8714 & 4 \\
\hline 10 & 983.5044 & 492.2558 & & & 965.4938 & 483.2506 & V & 1567.6985 & 784.3529 & 1550.6719 & 775.8396 & 1549.6879 & 775.3476 & 13 \\
\hline 11 & 1114.5449 & 557.7761 & & & 1096.5343 & 548.7708 & M & 1468.6301 & 734.8187 & 1451.6035 & 726.3054 & 1450.6195 & 725.8134 & 12 \\
\hline 12 & 1171.5664 & 586.2868 & & & 1153.5558 & 577.2815 & G & 1337.5896 & 669.2984 & 1320.5630 & 660.7852 & 1319.5790 & 660.2931 & 11 \\
\hline 13 & 1272.6140 & 636.8107 & & & 1254.6035 & 627.8054 & $\mathbf{T}$ & 1280.5681 & 640.7877 & 1263.5416 & 632.2744 & 1262.5576 & 631.7824 & 10 \\
\hline 14 & 1401.6566 & 701.3320 & & & 1383.6461 & 692.3267 & $\mathbf{E}$ & 1179.5204 & 590.2639 & |1162.4939| & 581.7506 & 1161.5099 & 581.2586 & 9 \\
\hline 15 & 1564.7200 & 782.8636 & & & 1546.7094 & 773.8583 & $\mathbf{Y}$ & 1050.4778 & 525.7426 & 1033.4513 & 517.2293 & 1032.4673 & 516.7373 & 8 \\
\hline 16 & 1663.7884 & 832.3978 & & & 1645.7778 & 823.3925 & V & 887.4145 & 444.2109 & 870.3880 & 435.6976 & 869.4040 & 435.2056 & 7 \\
\hline 17 & \begin{tabular}{|l|}
1810.8568 \\
\end{tabular} & 905.9320 & & & 1792.8462 & 896.9267 & $\mathbf{F}$ & 788.3461 & 394.6767 & 771.3196 & 386.1634 & 770.3355 & 385.6714 & 6 \\
\hline 18 & 1973.9201 & 987.4637 & & & 1955.9095 & 978.4584 & $\mathbf{Y}$ & 641.2777 & 321.1425 & 624.2511 & 312.6292 & 623.2671 & 312.1372 & 5 \\
\hline 19 & 2088.9470 & 1044.9772 & 2071.9205 & 1036.4639 & 2070.9365 & 1035.9719 & $\mathbf{N}$ & 478.2144 & 239.6108 & 461.1878 & 231.0975 & 460.2038 & 230.6055 & 4 \\
\hline 20 & 2203.9740 & 1102.4906 & 2186.9474 & 1093.9774 & 2185.9634 & 1093.4853 & D & 363.1874 & 182.0974 & 346.1609 & 173.5841 & 345.1769 & 173.0921 & 3 \\
\hline 21 & 2305.0217 & 1153.0145 & 2287.9951 & 1144.5012 & 2287.0111 & 1144.0092 & $\mathbf{T}$ & 248.1605 & 124.5839 & 231.1339 & 116.0706 & 230.1499 & 115.5786 & 2 \\
\hline 22 & & & & & & & K & 147.1128 & 74.0600 & 130.0863 & 65.5468 & & & 1 \\
\hline
\end{tabular}
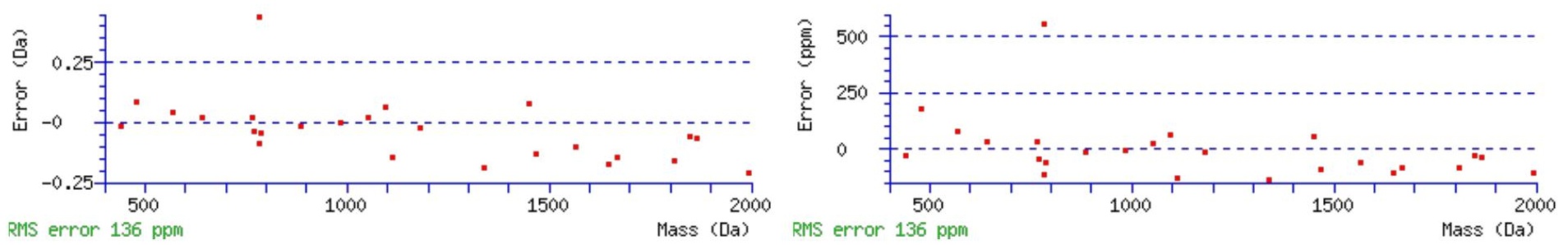

\section{All matches to this query}

\begin{tabular}{|l|l|l|l|}
\hline Score & Mr(calc): & Delta & \multicolumn{1}{|c|}{ Sequence } \\
\hline 67.1 & 2450.1199 & 0.0046 & EAGSLEPVTVMGTEYVFYNDTK \\
\hline 14.9 & 2449.1359 & 0.9887 & EAGSLEPVTVMGTEYVFYNDTK \\
\hline 7.5 & 2449.1058 & 1.0187 & VWLLEMNSNPALHTNCEVLK \\
\hline 1.6 & 2449.1058 & 1.0187 & VWLLEMNSNPALHTNCEVLK \\
\hline 1.2 & 2449.1058 & 1.0187 & VWLLEMNSNPALHTNCEVLK \\
\hline 1.2 & 2449.1058 & 1.0187 & VWLLEMNSNPALHTNCEVLK \\
\hline 0.8 & 2448.0969 & 2.0277 & QTMFELSKMFLLCLNYWK \\
\hline 0.7 & 2449.1348 & 0.9897 & INNLFGNTRNLLTTDFVQMR \\
\hline 0.7 & 2449.1348 & 0.9897 & INNLFGNTRNLLTTDFVQMR \\
\hline 0.7 & 2450.1049 & 0.0196 & VWIMQNERGISHDDIAQQSK \\
\hline
\end{tabular}

Spectrum No: 414; Query: 284; Rank: 1

\section{Peptide View}

MS/MS Fragmentation of TDDIINSSGYR

Found in IPI00198641, Tax_Id=10116 Gene_Symbol=LOC246263 Acyl-coenzyme A synthetase ACSM2, mitochondrial precursor

Match to Query 284: 1240.553208 from(621.283880,2+)

Title: 091008RatKidney_NH4Format01_13.1514.1514.2.dta

Data file K:INewmanPaper|Piliang|3SubProteomes\Piliang3SP\mgf5ppm\ERLIC_3SubProteomes5ppm.mgf 

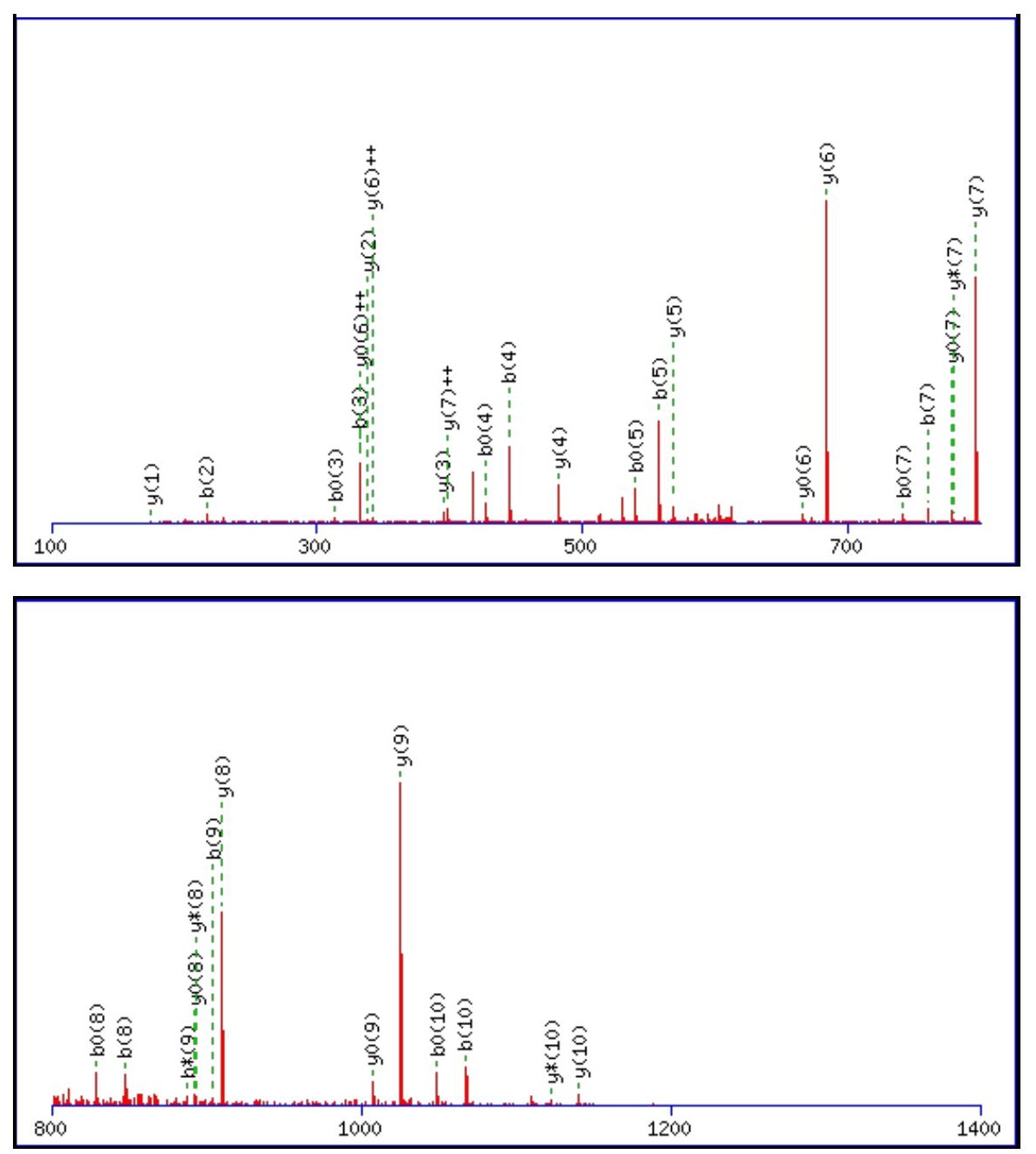

\begin{tabular}{rrrr}
\hline 00 & 1600 & 1800 & 2000 \\
\hline
\end{tabular}

Monoisotopic mass of neutral peptide $\operatorname{Mr}($ calc): 1240.5571

Fixed modifications: Carbamidomethyl (C)

Variable modifications:

N6 : Deamidated $\mathrm{N}(\mathrm{N})$

Ions Score: 67 Expect: $2.6 \mathrm{e}-005$

Matches (Bold Red): 35/104 fragment ions using 71 most intense peaks

\begin{tabular}{|r|c|c|c|c|r|r|r|r|r|r|r|r|r|r|}
\hline$\#$ & $\mathbf{b}$ & $\mathbf{b}^{++}$ & $\mathbf{b}^{*}$ & $\mathbf{b}^{\boldsymbol{*}^{++}}$ & $\mathbf{b}^{\mathbf{0}}$ & \multicolumn{1}{|c|}{$\mathbf{b}^{\mathbf{0 + +}}$} & Seq. & $\mathbf{y}$ & $\mathbf{y}^{++}$ & $\mathbf{y}^{\mathbf{*}}$ & $\mathbf{y}^{\boldsymbol{*}^{++}}$ & $\mathbf{y}^{\mathbf{0}}$ & $\mathbf{y}^{\mathbf{0 + +}}$ & $\#$ \\
\hline $\mathbf{1}$ & 102.0550 & 51.5311 & & & 84.0444 & 42.5258 & $\mathbf{T}$ & & & & & & & $\mathbf{1 1}$ \\
\hline $\mathbf{2}$ & $\mathbf{2 1 7 . 0 8 1 9}$ & 109.0446 & & & 199.0713 & 100.0393 & $\mathbf{D}$ & $\mathbf{1 1 4 0 . 5 1 6 8}$ & 570.7620 & $\mathbf{1 1 2 3 . 4 9 0 2}$ & 562.2487 & 1122.5062 & 561.7567 & $\mathbf{1 0}$ \\
\hline 3 & 332.1088 & 166.5581 & & & 314.0983 & 157.5528 & $\mathbf{D}$ & $\mathbf{1 0 2 5 . 4 8 9 8}$ & 513.2485 & 1008.4633 & 504.7353 & $\mathbf{1 0 0 7 . 4 7 9 2}$ & 504.2433 & $\mathbf{9}$ \\
\hline $\mathbf{4}$ & $\mathbf{4 4 5 . 1 9 2 9}$ & 223.1001 & & & $\mathbf{4 2 7 . 1 8 2 3}$ & 214.0948 & $\mathbf{I}$ & $\mathbf{9 1 0 . 4 6 2 9}$ & 455.7351 & $\mathbf{8 9 3 . 4 3 6 3}$ & 447.2218 & $\mathbf{8 9 2 . 4 5 2 3}$ & 446.7298 & $\mathbf{8}$ \\
\hline $\mathbf{5}$ & $\mathbf{5 5 8 . 2 7 7 0}$ & 279.6421 & & & 540.2664 & 270.6368 & $\mathbf{I}$ & $\mathbf{7 9 7 . 3 7 8 8}$ & 399.1930 & $\mathbf{7 8 0 . 3 5 2 3}$ & 390.6798 & $\mathbf{7 7 9 . 3 6 8 2}$ & 390.1878 & $\mathbf{7}$ \\
\hline
\end{tabular}




\begin{tabular}{|r|r|r|r|r|r|r|r|r|r|r|r|r|r|r|r|r|r|}
$\mathbf{6}$ & 673.3039 & 337.1556 & 656.2774 & 328.6423 & 655.2933 & 328.1503 & $\mathbf{N}$ & $\mathbf{6 8 4 . 2 9 4 7}$ & $\mathbf{3 4 2 . 6 5 1 0}$ & 667.2682 & 334.1377 & $\mathbf{6 6 6 . 2 8 4 2}$ & $\mathbf{3 3 3 . 6 4 5 7}$ & $\mathbf{6}$ \\
\hline $\mathbf{7}$ & $\mathbf{7 6 0 . 3 3 5 9}$ & 380.6716 & 743.3094 & 372.1583 & $\mathbf{7 4 2 . 3 2 5 4}$ & 371.6663 & S & $\mathbf{5 6 9 . 2 6 7 8}$ & 285.1375 & 552.2413 & 276.6243 & 551.2572 & 276.1323 & $\mathbf{5}$ \\
\hline $\mathbf{8}$ & $\mathbf{8 4 7 . 3 6 8 0}$ & 424.1876 & 830.3414 & 415.6743 & $\mathbf{8 2 9 . 3 5 7 4}$ & 415.1823 & S & $\mathbf{4 8 2 . 2 3 5 8}$ & 241.6215 & 465.2092 & 233.1082 & 464.2252 & 232.6162 & $\mathbf{4}$ \\
\hline $\mathbf{9}$ & $\mathbf{9 0 4 . 3 8 9 4}$ & 452.6984 & $\mathbf{8 8 7 . 3 6 2 9}$ & 444.1851 & 886.3789 & 443.6931 & G & 395.2037 & 198.1055 & 378.1772 & 189.5922 & & & \\
\hline $\mathbf{1 0}$ & $\mathbf{1 0 6 7 . 4 5 2 8}$ & 534.2300 & 1050.4262 & 525.7167 & $\mathbf{1 0 4 9 . 4 4 2 2}$ & 525.2247 & $\mathbf{Y}$ & $\mathbf{3 3 8 . 1 8 2 3}$ & 169.5948 & 321.1557 & 161.0815 & & & & $\mathbf{2}$ \\
\hline $\mathbf{1 1}$ & & & & & & & $\mathbf{R}$ & $\mathbf{1 7 5 . 1 1 9 0}$ & 88.0631 & 158.0924 & 79.5498 & & & $\mathbf{1}$ \\
\hline
\end{tabular}
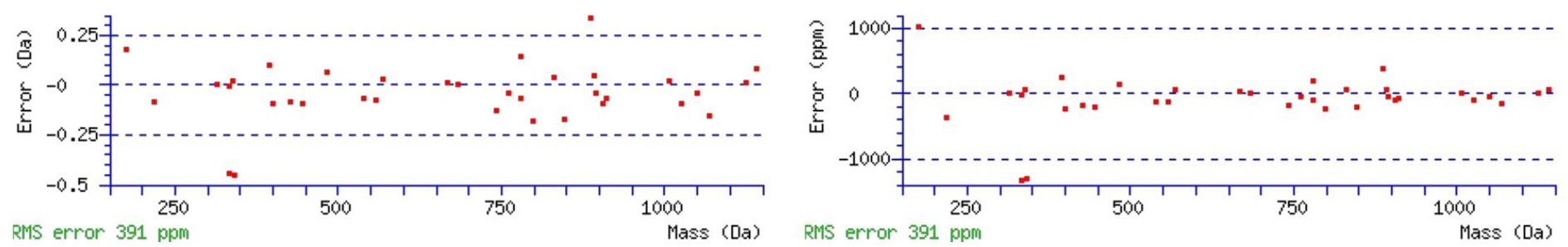

\section{All matches to this query}

\begin{tabular}{|l|l|l|l|}
\hline Score & Mr(calc): & Delta & \multicolumn{1}{c|}{ Sequence } \\
\hline 67.0 & 1240.5571 & -0.0039 & TDDIINSSGYR \\
\hline 14.7 & 1240.5506 & 0.0026 & TDNGGLGYRCK \\
\hline 13.3 & 1240.5449 & 0.0083 & KGESSDLAAQR \\
\hline 8.7 & 1240.5561 & -0.0029 & NKNSLGRSER \\
\hline 8.4 & 1240.5449 & 0.0083 & DSKLNNTNVR \\
\hline 8.4 & 1240.5449 & 0.0083 & DSKLNNTNVR \\
\hline 8.3 & 1240.5561 & -0.0029 & NKNSLGRSER \\
\hline 7.7 & 1240.5449 & 0.0083 & KGESSDLAAQR \\
\hline 7.7 & 1238.5544 & 1.9988 & VDNVNSIIER \\
\hline 6.0 & 1238.5544 & 1.9988 & VDNVNSIIER \\
\hline
\end{tabular}

Spectrum No: 415; Query: 171; Rank: 1

\section{Peptide View}

MS/MS Fragmentation of VFNTSEDLSR

Found in IPI00363534, Tax_Id=10116 Gene_Symbol=Lama1 laminin, alpha 1

Match to Query 171: 1167.541388 from(584.777970,2+)

Title: 100101RatKid_NS_deglyco_26.1465.1465.2.dta

Data file K:INewmanPaper|Piliangl3SubProteomes\Piliang3SP\mgf5ppm\ERLIC_3SubProteomes5ppm.mgf

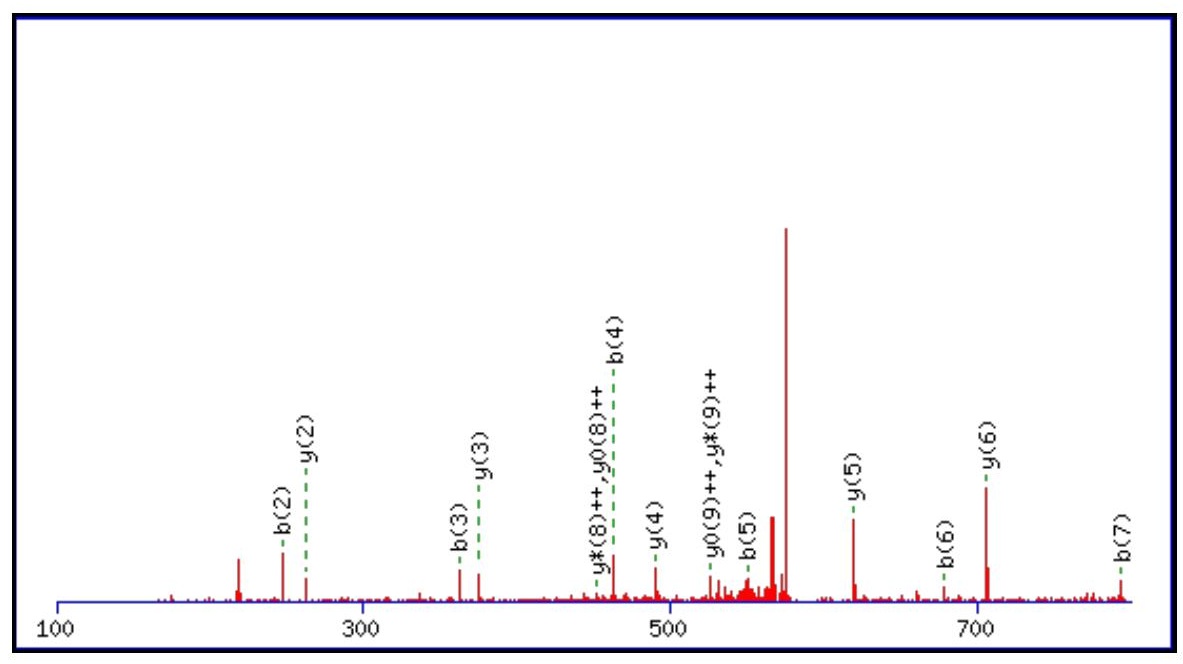




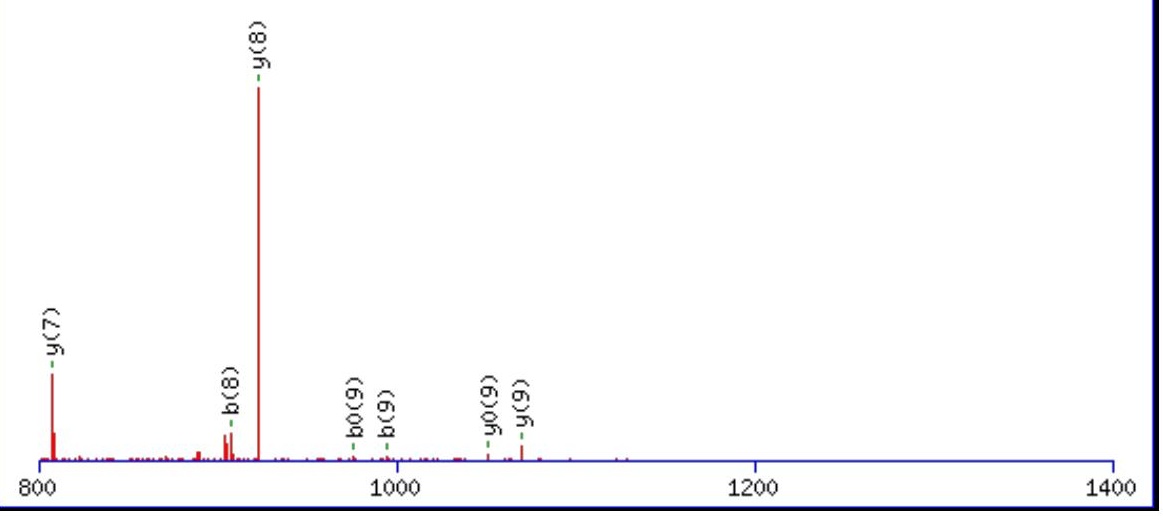

\begin{tabular}{|c|c|c|c|}
\hline 1400 & 1600 & 1800 & 2000 \\
\hline
\end{tabular}

Monoisotopic mass of neutral peptide $\operatorname{Mr}($ calc): 1167.5408

Fixed modifications: Carbamidomethyl (C)

Variable modifications:

N3 : Deamidated_N (N)

Ions Score: 67 Expect: $2.5 \mathrm{e}-005$

Matches (Bold Red): 22/96 fragment ions using 32 most intense peaks

\begin{tabular}{|r|c|c|c|c|c|c|c|c|c|c|c|c|c|c|}
\hline$\#$ & $\mathbf{b}$ & $\mathbf{b}^{++}$ & $\mathbf{b}^{*}$ & $\mathbf{b}^{\mathbf{*}^{++}}$ & $\mathbf{b}^{\mathbf{0}}$ & $\mathbf{b}^{\mathbf{0 + +}}$ & $\mathbf{S e q}$ & $\mathbf{y}$ & $\mathbf{y}^{++}$ & $\mathbf{y}^{*}$ & $\mathbf{y}^{\mathbf{*}^{++}}$ & $\mathbf{y}^{\mathbf{0}}$ & $\mathbf{y}^{\mathbf{0}++}$ & $\#$ \\
\hline $\mathbf{1}$ & 100.0757 & 50.5415 & & & & & $\mathbf{V}$ & & & & & & & $\mathbf{1 0}$ \\
\hline $\mathbf{2}$ & $\mathbf{2 4 7 . 1 4 4 1}$ & 124.0757 & & & & & $\mathbf{F}$ & $\mathbf{1 0 6 9 . 4 7 9 6}$ & 535.2435 & 1052.4531 & $\mathbf{5 2 6 . 7 3 0 2}$ & $\mathbf{1 0 5 1 . 4 6 9 1}$ & $\mathbf{5 2 6 . 2 3 8 2}$ & $\mathbf{9}$ \\
\hline $\mathbf{3}$ & $3 \mathbf{6 2 . 1 7 1 0}$ & 181.5892 & 345.1445 & 173.0759 & & & $\mathbf{N}$ & $\mathbf{9 2 2 . 4 1 1 2}$ & 461.7092 & 905.3847 & $\mathbf{4 5 3 . 1 9 6 0}$ & 904.4007 & $\mathbf{4 5 2 . 7 0 4 0}$ & $\mathbf{8}$ \\
\hline $\mathbf{4}$ & $\mathbf{4 6 3 . 2 1 8 7}$ & 232.1130 & 446.1922 & 223.5997 & 445.2082 & 223.1077 & $\mathbf{T}$ & $\mathbf{8 0 7 . 3 8 4 3}$ & 404.1958 & 790.3577 & 395.6825 & 789.3737 & 395.1905 & $\mathbf{7}$ \\
\hline $\mathbf{5}$ & $\mathbf{5 5 0 . 2 5 0 7}$ & 275.6290 & 533.2242 & 267.1157 & 532.2402 & 266.6237 & $\mathbf{S}$ & $\mathbf{7 0 6 . 3 3 6 6}$ & 353.6719 & 689.3101 & 345.1587 & 688.3260 & 344.6667 & $\mathbf{6}$ \\
\hline $\mathbf{6}$ & $\mathbf{6 7 9 . 2 9 3 3}$ & 340.1503 & 662.2668 & 331.6370 & 661.2828 & 331.1450 & $\mathbf{E}$ & $\mathbf{6 1 9 . 3 0 4 6}$ & 310.1559 & 602.2780 & 301.6427 & 601.2940 & 301.1506 & $\mathbf{5}$ \\
\hline $\mathbf{7}$ & $\mathbf{7 9 4 . 3 2 0 3}$ & 397.6638 & 777.2937 & 389.1505 & 776.3097 & 388.6585 & $\mathbf{D}$ & $\mathbf{4 9 0 . 2 6 2 0}$ & 245.6346 & 473.2354 & 237.1214 & 472.2514 & 236.6293 & $\mathbf{4}$ \\
\hline $\mathbf{8}$ & $\mathbf{9 0 7 . 4 0 4 3}$ & 454.2058 & 890.3778 & 445.6925 & 889.3938 & 445.2005 & $\mathbf{L}$ & $\mathbf{3 7 5 . 2 3 5 0}$ & 188.1212 & 358.2085 & 179.6079 & 357.2245 & 179.1159 & 3 \\
\hline $\mathbf{9}$ & $\mathbf{9 9 4 . 4 3 6 4}$ & 497.7218 & 977.4098 & 489.2086 & $\mathbf{9 7 6 . 4 2 5 8}$ & 488.7165 & $\mathbf{S}$ & $\mathbf{2 6 2 . 1 5 1 0}$ & 131.5791 & 245.1244 & 123.0659 & 244.1404 & 122.5738 & $\mathbf{2}$ \\
\hline $\mathbf{1 0}$ & & & & & & & $\mathbf{R}$ & 175.1190 & 88.0631 & 158.0924 & 79.5498 & & & $\mathbf{1}$ \\
\hline
\end{tabular}
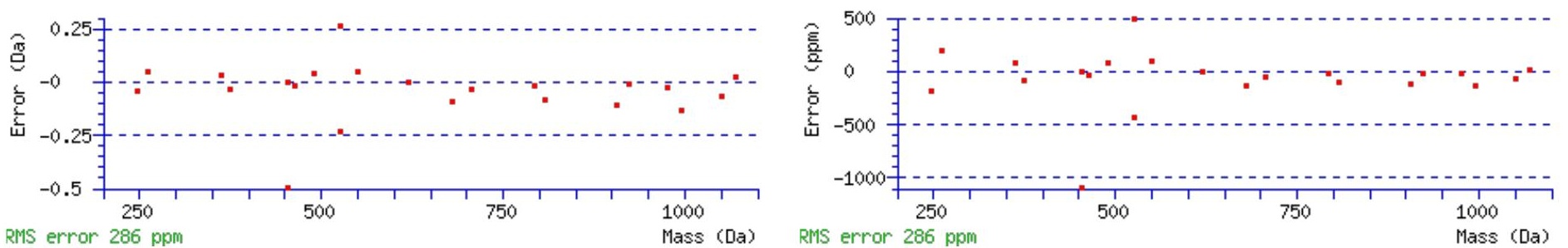

RMS error 286 ppm

Mass (Da) RIS error 286 ppm 


\section{All matches to this query}

\begin{tabular}{|l|l|l|l|}
\hline Score & Mr(calc): & Delta & \multicolumn{1}{c|}{ Sequence } \\
\hline 66.9 & 1167.5408 & 0.0006 & VFNTSEDLSR \\
\hline 12.1 & 1167.5383 & 0.0031 & MVQFHFTNK \\
\hline 7.8 & 1167.5450 & -0.0036 & MALLMAEMSR \\
\hline 7.2 & 1165.5285 & 2.0129 & NEDMKSALNK \\
\hline 6.3 & 1165.5285 & 2.0129 & NKMESDGEKK \\
\hline 6.1 & 1165.5285 & 2.0129 & NSDLCIASGTK \\
\hline 5.5 & 1166.5373 & 1.0041 & FELLPDPTR \\
\hline 5.5 & 1167.5397 & 0.0017 & NLSRKDAER \\
\hline 4.9 & 1167.5488 & -0.0075 & MAMSLQGSRR \\
\hline 4.6 & 1167.5424 & -0.0010 & $\underline{\text { LSVLADEVDK }}$ \\
\hline
\end{tabular}

Spectrum No: 416; Query: 2761; Rank: 1

\section{Peptide View}

MS/MS Fragmentation of RPFGEVYELEIDTLETTCHALDPTPLANCSVR

Found in IPI00327469, Tax_Id=10116 Gene_Symbol=Ahsg Alpha-2-HS-glycoprotein precursor

Match to Query 2761: 3703.759376 from(926.947120,4+)

Title: 100101RatKid_NS_deglyco_20.5565.5565.4.dta

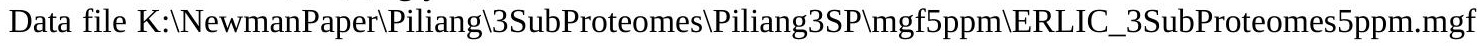
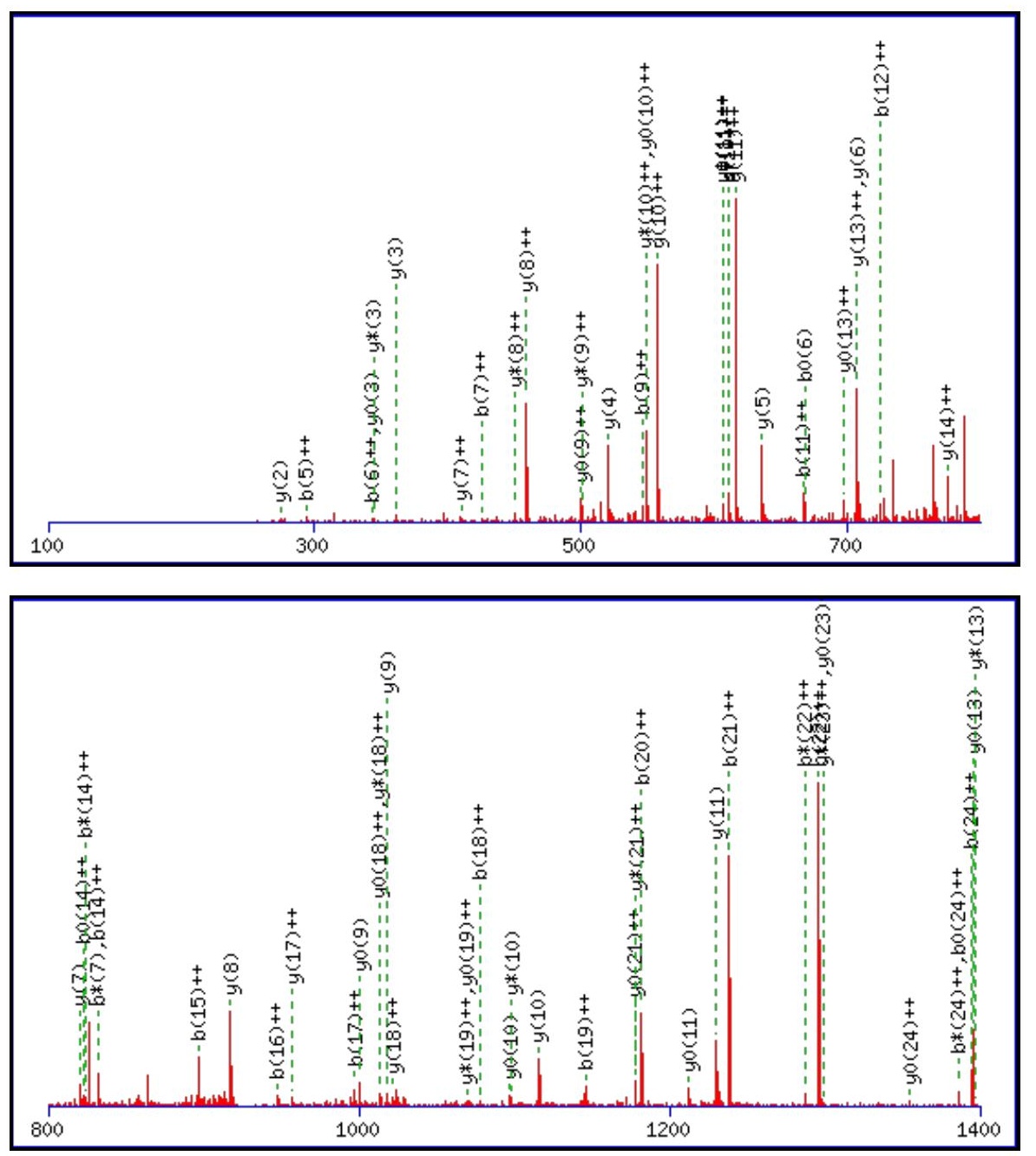


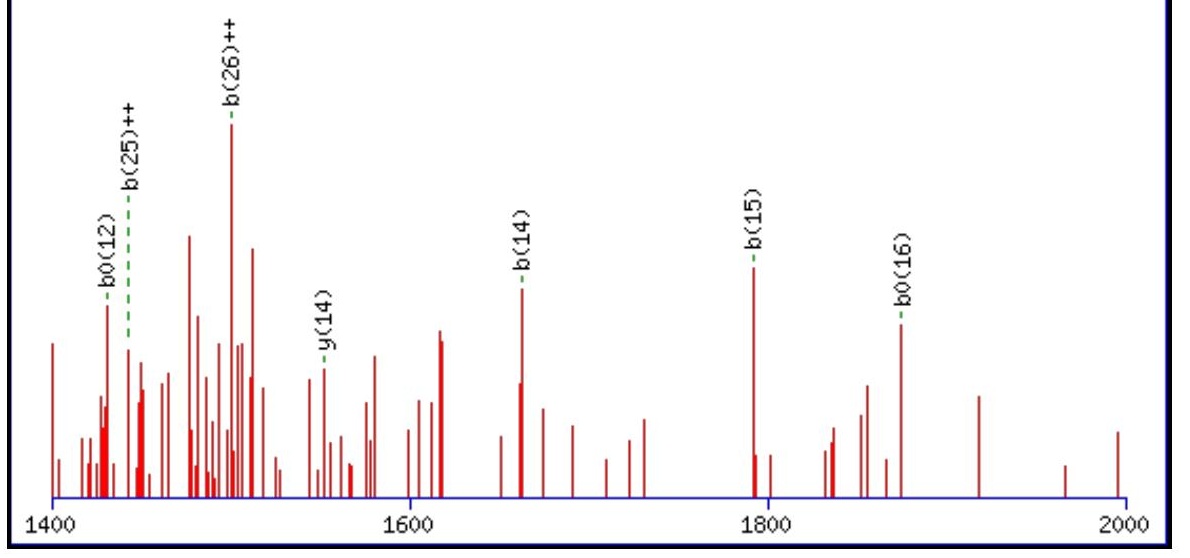

Monoisotopic mass of neutral peptide $\operatorname{Mr}($ calc): 3703.7498

Fixed modifications: Carbamidomethyl (C)

Variable modifications:

N28 : Deamidated_N (N)

Ions Score: 67 Expect : $8.1 \mathrm{e}-005$

Matches (Bold Red): 74/360 fragment ions using 151 most intense peaks

\begin{tabular}{|c|c|c|c|c|c|c|c|c|c|c|c|c|c|c|}
\hline \# & b & $\mathbf{b}^{++}$ & b* & & $\mathbf{b}^{0}$ & & eq. & $\mathbf{y}$ & & & & $\mathbf{y}^{0}$ & & \# \\
\hline 1 & 157.1084 & 79.0578 & 140.0818 & & & & $\mathbf{R}$ & & & & & & & 32 \\
\hline 2 & 254.1612 & 27.5842 & 237.1346 & 709 & & & $\mathbf{P}$ & & & & & & & 31 \\
\hline 3 & 401.2296 & 184 & 384.2030 & 051 & & & $\mathbf{F}$ & 3451.6032 & 3052 & 5767 & 7920 & 3433.5926 & 3000 & 30 \\
\hline 4 & 458.2510 & 229.6292 & 441.2245 & 159 & & & $\mathbf{G}$ & 5348 & 7710 & 3287.5082 & 2578 & 5242 & 7658 & 29 \\
\hline 5 & 587.2936 & 294.1504 & 570.2671 & 372 & 831 & & $\mathbf{E}$ & 5133 & 2603 & 3230.4868 & 7470 & 3229.5028 & 2550 & 28 \\
\hline 6 & 686.3620 & 343.6847 & 669.3355 & 335.1714 & 668.3515 & 334 & V & 3118.4707 & 1559.7390 & 3101.4442 & 2257 & 3100.4602 & 1550.7337 & 27 \\
\hline 7 & 849.4254 & 425.2 & 832.3 & 416. & 148 & & $\mathbf{Y}$ & 023 & 1510 & 3758 & 915 & 3001 & 995 & 26 \\
\hline 8 & 978.4680 & 489.7376 & 961.4414 & 481.2243 & 960.4574 & 480.7 & $\mathbf{E}$ & 2856.3390 & 1428. & 3124 & 1599 & 2838.3284 & 6679 & 25 \\
\hline 9 & 1091.5520 & 546.2796 & 1074.5255 & 537.7664 & 415 & 537. & $\mathbf{L}$ & 2727. & 1364 & 2710.2699 & 1355. & 2709.2858 & 1466 & 24 \\
\hline 10 & 220.5946 & 610.8009 & 1203.5681 & 602.2877 & 1202.5841 & 601. & $\mathbf{E}$ & 2123 & 1307. & 2597.1858 & 9965 & 2596.2018 & 045 & 23 \\
\hline 11 & 333.6787 & 667.3430 & 1316.6521 & 658.8297 & 315.6681 & 658. & I & 2485.1697 & 1243.0885 & 2468.1432 & 1234 & 2467.1592 & 1234.0832 & 22 \\
\hline 12 & 448.7056 & 724.8564 & 431.6791 & 432 & 951 & 715 & D & 0857 & 465 & 0591 & 332 & 2354.0751 & 1177 & 21 \\
\hline 13 & 1549.7533 & 775.3803 & 1532.7268 & 766.8670 & 531.7427 & 766. & $\mathbf{T}$ & 2257.0587 & 1129.0330 & 2240.0322 & 1120. & 2239.0482 & 1120.0277 & 20 \\
\hline 14 & 662.8374 & 831.9223 & 645.8108 & 823.4090 & 268 & 822. & $\mathbf{L}$ & 0111 & 092 & 8445 & 959 & 2138 & 1069 & 19 \\
\hline 15 & 1791.8800 & 896.4436 & 1774.8534 & 887.9303 & 1773.8694 & 887.4383 & $\mathbf{E}$ & 2042.9270 & 1021.9671 & 2025.9004 & 1013.4539 & 2024.9164 & 1012.9619 & 18 \\
\hline 16 & 892.9276 & 946.9675 & 875.9011 & 938.4542 & 171 & 937.9 & $\mathbf{T}$ & 1913. & 957.4458 & 1896 & 326 & 1895 & 406 & 17 \\
\hline 17 & 1993.9753 & 997.4913 & 1976.9488 & 988.9780 & 1975.9648 & 988.4860 & $\mathbf{T}$ & 1812.8367 & 906.9220 & 1795.8102 & 898.4087 & 1794.8262 & 897.9167 & 16 \\
\hline 18 & 154.0060 & 077.5066 & 136.9794 & 068.9933 & 954 & 013 & C & 1711.7890 & 3982 & 7625 & 847.8849 & 1693.7785 & 3929 & 15 \\
\hline 19 & 2291.0649 & 146.0361 & 2274.0383 & 137.5228 & 2273.0543 & 1137.0308 & $\mathbf{H}$ & 1551.7584 & 776.3828 & 1534.7318 & 767.8696 & 1533.7478 & 767.3776 & 14 \\
\hline 20 & 362.1020 & 181.5546 & 345.0754 & 173.0414 & 914 & 1172 & A & 141 & 534 & 7729 & 3401 & 1396 & 481 & 13 \\
\hline 21 & 2475.1861 & 1238.0967 & 2458.1595 & 1229.5834 & 2457.1755 & 1229.0914 & $\mathbf{L}$ & 1343.6624 & 672.3348 & 1326.6358 & 663.8215 & 1325.6518 & 663.3295 & 12 \\
\hline 22 & 2590.2130 & 295.6101 & 2573.1864 & 1287.0969 & 2572.2024 & 1286.6049 & D & 1230.5783 & 615.7928 & 1213.5518 & 2795 & 1212.5677 & 606.7875 & 11 \\
\hline 23 & 2687.2658 & 344.1365 & 2670.2392 & 1335.6232 & 2669.2552 & 1335.1312 & $\mathbf{P}$ & 1115.5514 & 558.2793 & 1098.5248 & 549.7660 & 1097.5408 & 549.2740 & 10 \\
\hline 24 & 2788.3134 & 394.6604 & 2771.2869 & 1386.1471 & 2770.3029 & 1385.6551 & $\mathbf{T}$ & 1018.4986 & 509.7529 & 1001.4721 & 501.2397 & 1000.4880 & 500.7477 & 9 \\
\hline 25 & 2885.3662 & \begin{tabular}{|l}
1443.1867 \\
\end{tabular} & 2868.3397 & 1434.6735 & 2867.3556 & 1434.1815 & $\mathbf{P}$ & 917.4509 & 459.2291 & 900.4244 & 450.7158 & 899.4404 & 450.2238 & 8 \\
\hline 26 & 2998.4503 & 1499.7288 & 2981.4237 & 1491.2155 & 2980.4397 & 1490.7235 & $\mathbf{L}$ & 820.3982 & 410.7027 & 803.3716 & 402.1894 & 802.3876 & 401.6974 & 7 \\
\hline 27 & 3069.4874 & 1535.2473 & 3052.4608 & 1526.7341 & 3051.4768 & 1526.2420 & A & 707.3141 & 354.1607 & 690.2875 & 345.6474 & 689.3035 & 345.1554 & 6 \\
\hline 28 & 3184.5143 & 1592.7608 & 3167.4878 & 1584.2475 & 3166.5038 & 1583.7555 & $\mathbf{N}$ & 636.2770 & 318.6421 & 619.2504 & 310.1289 & 618.2664 & 309.6368 & 5 \\
\hline 29 & 3344.5450 & 1672.7761 & 3327.5184 & 1664.2628 & 3326.5344 & 1663.7708 & C & 521.2500 & 261.1287 & 504.2235 & 252.6154 & 503.2395 & 252.1234 & 4 \\
\hline 30 & 3431.5770 & 1716.2921 & 3414.5504 & 1707.7789 & 3413.5664 & 1707.2869 & $\mathrm{~S}$ & 361.2194 & 181.1133 & 344.1928 & 172.6001 & 343.2088 & 172.1081 & 3 \\
\hline 31 & 3530.6454 & 1765.8263 & 3513.6189 & 1757.3131 & 3512.6348 & 1756.8211 & V & 274.1874 & 137.5973 & 257.1608 & 129.0840 & & & 2 \\
\hline 32 & & & & & & & $\mathbf{R}$ & 175.1190 & 88.0631 & 158.0924 & 79.5498 & & & 1 \\
\hline
\end{tabular}



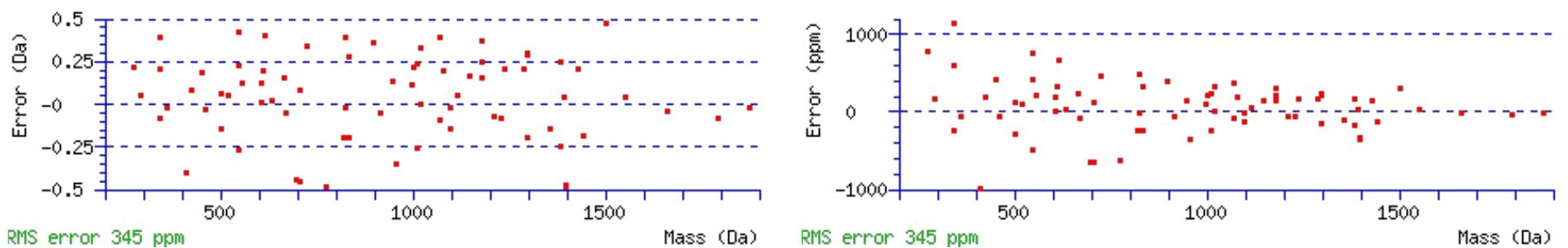

\section{All matches to this query}

\begin{tabular}{|l|c|c|l|}
\hline Score & Mr(calc): & Delta & \multicolumn{1}{c|}{ Sequence } \\
\hline 66.9 & 3703.7498 & 0.0096 & RPFGEVYELEIDTLETTCHALDPTPLANCSVR \\
\hline 25.9 & 3702.7658 & 0.9936 & RPFGEVYELEIDTLETTCHALDPTPLANCSVR \\
\hline 6.2 & 3703.7324 & 0.0269 & EVPPSEPVAEPFPSGDIAPPGLISGGKYSQTHRK \\
\hline 6.2 & 3703.7324 & 0.0269 & EVPPSEPVAEPFPSGDIAPPGLISGGKYSQTHRK \\
\hline 5.8 & 3702.7843 & 0.9751 & SCLIAEMIALGLQSYSLVDNLGFNRLLEYLK \\
\hline 5.4 & 3701.7196 & 2.0398 & AADSDLQITNAQTKEEYTDDNALIPKNSSVIVR \\
\hline 4.8 & 3701.7806 & 1.9787 & VARGVSLSTTSLLSGFQAIKLRPNMSGWLSLR \\
\hline 4.7 & 3701.7806 & 1.9787 & VARGVSLSTTSLLSGFQAIKLRPNMSGWLSLR \\
\hline 4.6 & 3703.7745 & -0.0152 & QVYDIAAKFGGLAAGEDNGQRGYLLTYVIAYMR \\
\hline 3.7 & 3702.7412 & 1.0181 & SDGLIGLYQGFGVSVQGIIVYRASYFGAYDTVK \\
\hline
\end{tabular}

Spectrum No: 417; Query: 2110; Rank: 1

\section{Peptide View}

MS/MS Fragmentation of VYINYYDMNAANVGWNGSTFA

Found in IPI00230907, Tax_Id=10116 Gene_Symbol=Mif Macrophage migration inhibitory factor

Match to Query 2110: 2370.021448 from(1186.018000,2+)

Title: 091008RatKidney_NoSalt_18.6295.6295.2.dta

Data file K:\NewmanPaper|Piliang\3SubProteomes\Piliang3SP\mgf5ppm\ERLIC_3SubProteomes5ppm.mgf

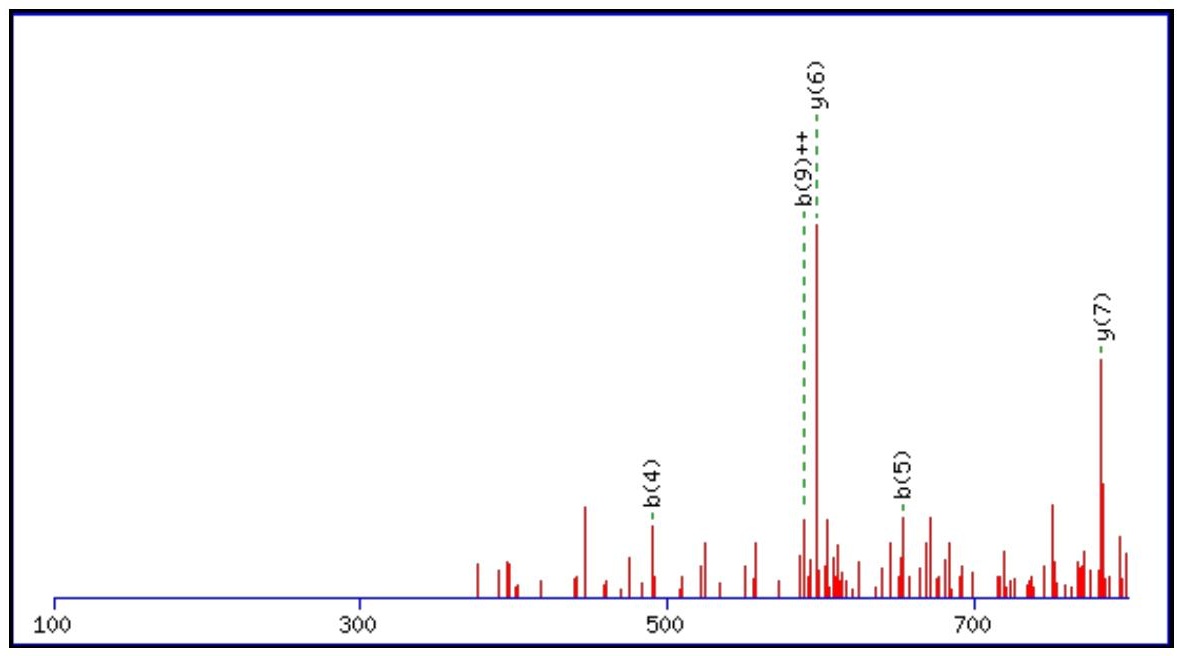



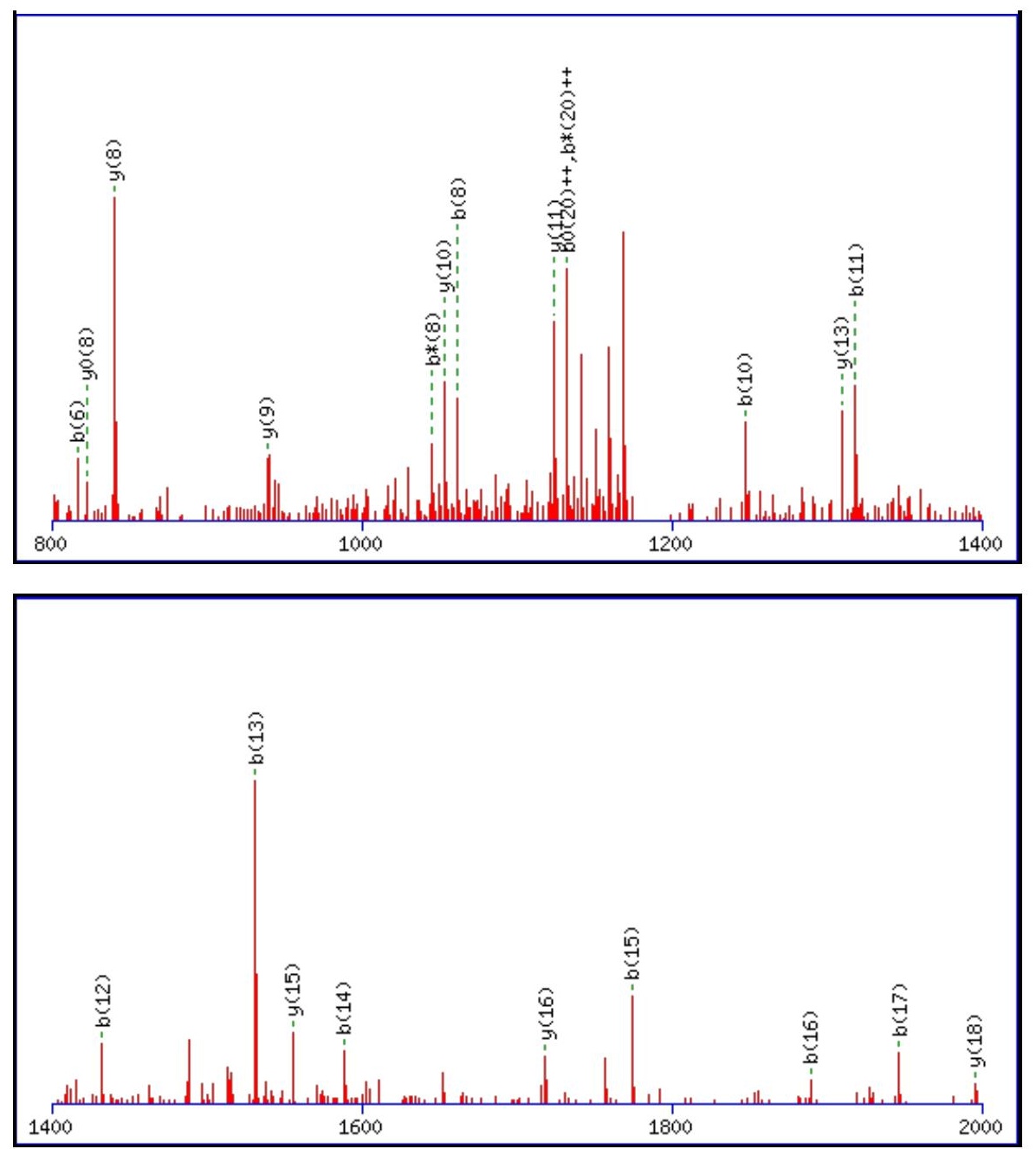

Monoisotopic mass of neutral peptide $\operatorname{Mr}($ calc): 2370.0263

Fixed modifications: Carbamidomethyl (C)

Variable modifications:

N16 : Deamidated_N (N)

Ions Score: 67 Expect: $6.3 \mathrm{e}-005$

Matches (Bold Red): 27/208 fragment ions using 48 most intense peaks

\begin{tabular}{|c|c|c|c|c|c|c|c|c|c|c|c|c|c|c|}
\hline \# & b & $\mathbf{b}^{++}$ & b* & $\mathbf{b}^{*^{++}}$ & $\mathbf{b}^{0}$ & $\mathbf{b}^{0++}$ & Seq. & $\mathbf{y}$ & $y^{++}$ & $\mathbf{y}^{*}$ & $\mathbf{y}^{*^{++}}$ & $\mathbf{y}^{\mathbf{0}}$ & $\mathbf{y}^{0++}$ & \# \\
\hline 1 & 100.0757 & 50.5415 & & & & & $\mathbf{V}$ & & & & & & & 21 \\
\hline 2 & 263.1390 & 132.0731 & & & & & $\mathbf{Y}$ & 2271.9652 & 1136.4862 & 2254.9386 & 1127.9729 & 2253.9546 & 1127.4809 & 20 \\
\hline 4 & 490.2660 & 245.6366 & 473.2395 & 237.1234 & & & $\mathbf{N}$ & 1995.8178 & 998.4125 & 1978.7912 & 989.8992 & 1977.8072 & 989.4072 & 18 \\
\hline 5 & 653.3293 & 327.1683 & 636.3028 & 318.6550 & & & $\mathbf{Y}$ & 1881.7748 & 941.3911 & 1864.7483 & 932.8778 & 1863.7643 & 932.3858 & 17 \\
\hline 6 & 816.3927 & 408.7000 & 799.3661 & 400.1867 & & & $\mathbf{Y}$ & 1718.7115 & 859.8594 & 1701.6850 & 851.3461 & 1700.7009 & 850.8541 & 16 \\
\hline 7 & 931.4196 & 466.2134 & 914.3931 & 457.7002 & 913.4090 & 457.2082 & D & 1555.6482 & 778.3277 & 1538.6216 & 769.8145 & 1537.6376 & 769.3224 & 15 \\
\hline 8 & 1062.4601 & 531.7337 & 1045.4335 & 523.2204 & 1044.4495 & 522.7284 & $\mathbf{M}$ & 1440.6212 & 720.8143 & 1423.5947 & 712.3010 & 1422.6107 & 711.8090 & 14 \\
\hline 9 & 1176.5030 & 588.7551 & 1159.4765 & 580.2419 & 1158.4925 & 579.7499 & $\mathbf{N}$ & 1309.5807 & 655.2940 & 1292.5542 & 646.7807 & 1291.5702 & 646.2887 & 13 \\
\hline 10 & 1247.5401 & 624.2737 & 1230.5136 & 615.7604 & 1229.5296 & 615.2684 & A & 1195.5378 & 598.2725 & 1178.5113 & 589.7593 & 1177.5273 & 589.2673 & 12 \\
\hline 11 & 1318.5773 & 659.7923 & 1301.5507 & 651.2790 & 1300.5667 & 650.7870 & A & 1124.5007 & 562.7540 & 1107.4742 & 554.2407 & 1106.4901 & 553.7487 & 11 \\
\hline 12 & 1432.6202 & 716.8137 & 1415.5936 & 708.3005 & 1414.6096 & 707.8084 & $\mathbf{N}$ & 1053.4636 & 527.2354 & 1036.4370 & 518.7222 & 1035.4530 & 518.2302 & 10 \\
\hline 14 & 1588.7101 & 794.8587 & 1571.6835 & 786.3454 & 1570.6995 & 785.8534 & G & 840.3523 & 420.6798 & 823.3257 & 412.1665 & 822.3417 & 411.6745 & 8 \\
\hline 15 & 1774.7894 & 887.8983 & 1757.7628 & 879.3850 & 1756.7788 & 878.8930 & W & 783.3308 & 392.1690 & 766.3042 & 383.6558 & 765.3202 & 383.1638 & 7 \\
\hline 16 & 1889.8163 & 945.4118 & 1872.7898 & 936.8985 & 1871.8057 & 936.4065 & $\mathbf{N}$ & 597.2515 & 299.1294 & 580.2249 & 290.6161 & 579.2409 & 290.1241 & 6 \\
\hline 17 & 1946.8378 & 973.9225 & 1929.8112 & 965.4092 & 1928.8272 & 964.9172 & G & 482.2245 & 241.6159 & & & 464.2140 & 232.6106 & 5 \\
\hline 18 & 2033.8698 & 1017.4385 & 2016.8432 & 1008.9253 & 2015.8592 & 1008.4333 & $\mathrm{~S}$ & 425.2031 & 213.1052 & & & 407.1925 & 204.0999 & 4 \\
\hline
\end{tabular}




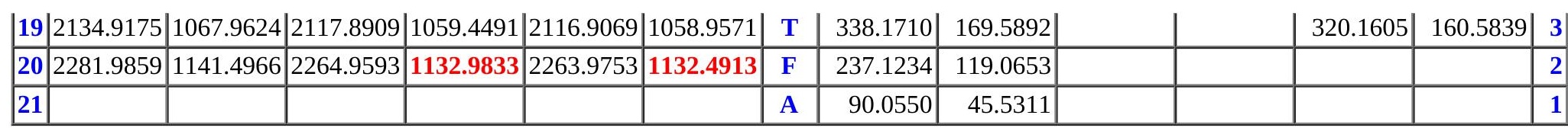
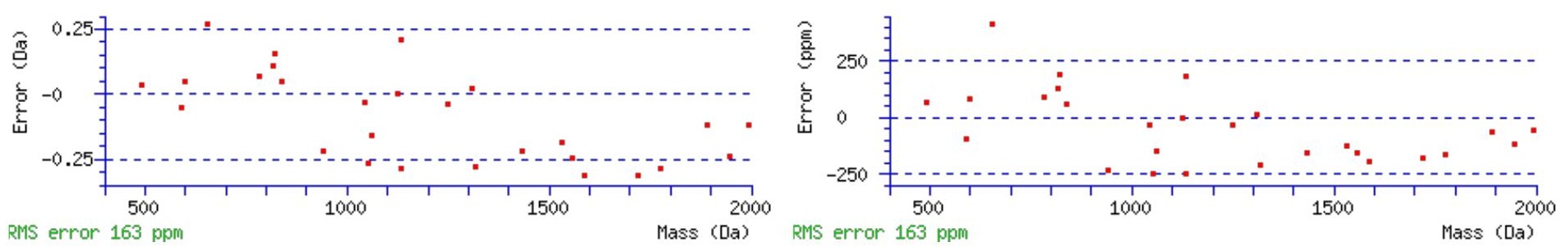

\section{All matches to this query}

\begin{tabular}{|l|l|l|l|}
\hline Score & Mr(calc): & Delta & \multicolumn{1}{|c|}{ Sequence } \\
\hline 66.8 & 2370.0263 & -0.0048 & VYINYYDMNAANVGWNGSTFA \\
\hline 35.9 & 2370.0263 & -0.0048 & VYINYYDMNAANVGWNGSTFA \\
\hline 34.4 & 2370.0263 & -0.0048 & VYINYYDMNAANVGWNGSTFA \\
\hline 8.6 & 2370.0263 & -0.0048 & VYINYYDMNAANVGWNGSTFA \\
\hline 2.1 & 2369.0337 & 0.9878 & DMITGITLPINNTMKSLWK \\
\hline 2.1 & 2369.0337 & 0.9878 & DMITGITLPINNTMKSLWK \\
\hline
\end{tabular}

Spectrum No: 418; Query: 1608; Rank: 1

\section{Peptide View}

MS/MS Fragmentation of YQTSSAINGSVIWDRPTK

Found in IPI00210872, Tax_Id=10116 Gene_Symbol=Mep1a Meprin A subunit alpha precursor

Match to Query 1608: 2023.010768 from(1012.512660,2+)

Title: 100101RatKid_NS_deglyco_19.2812.2812.2.dta

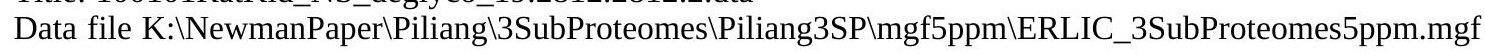

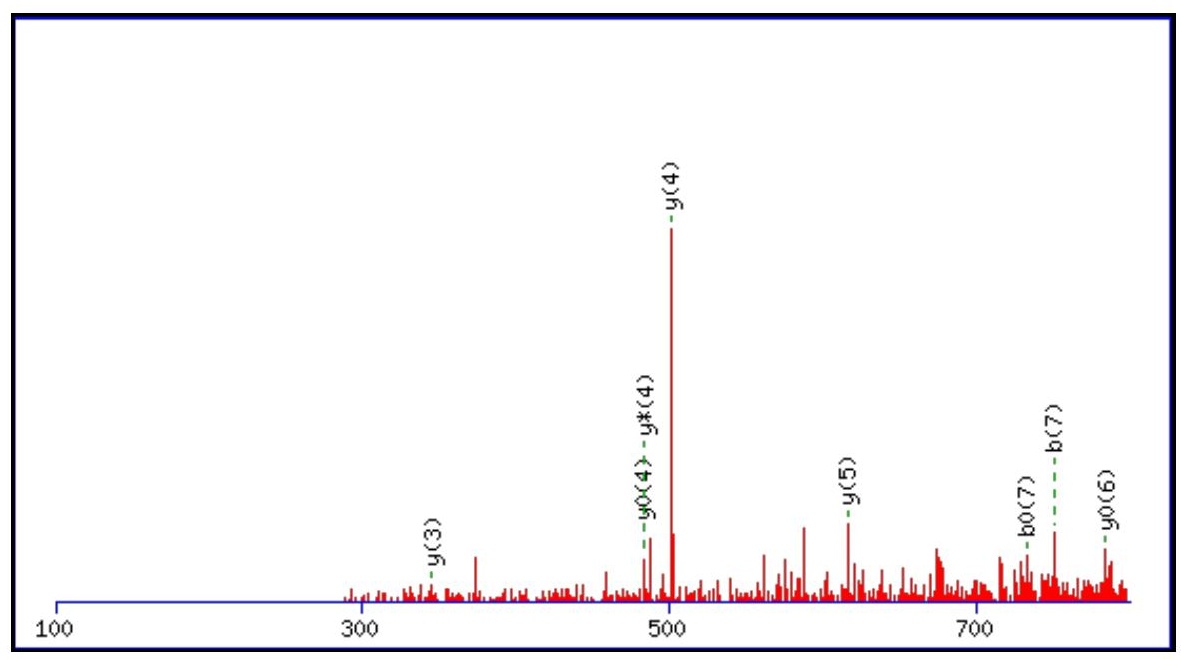




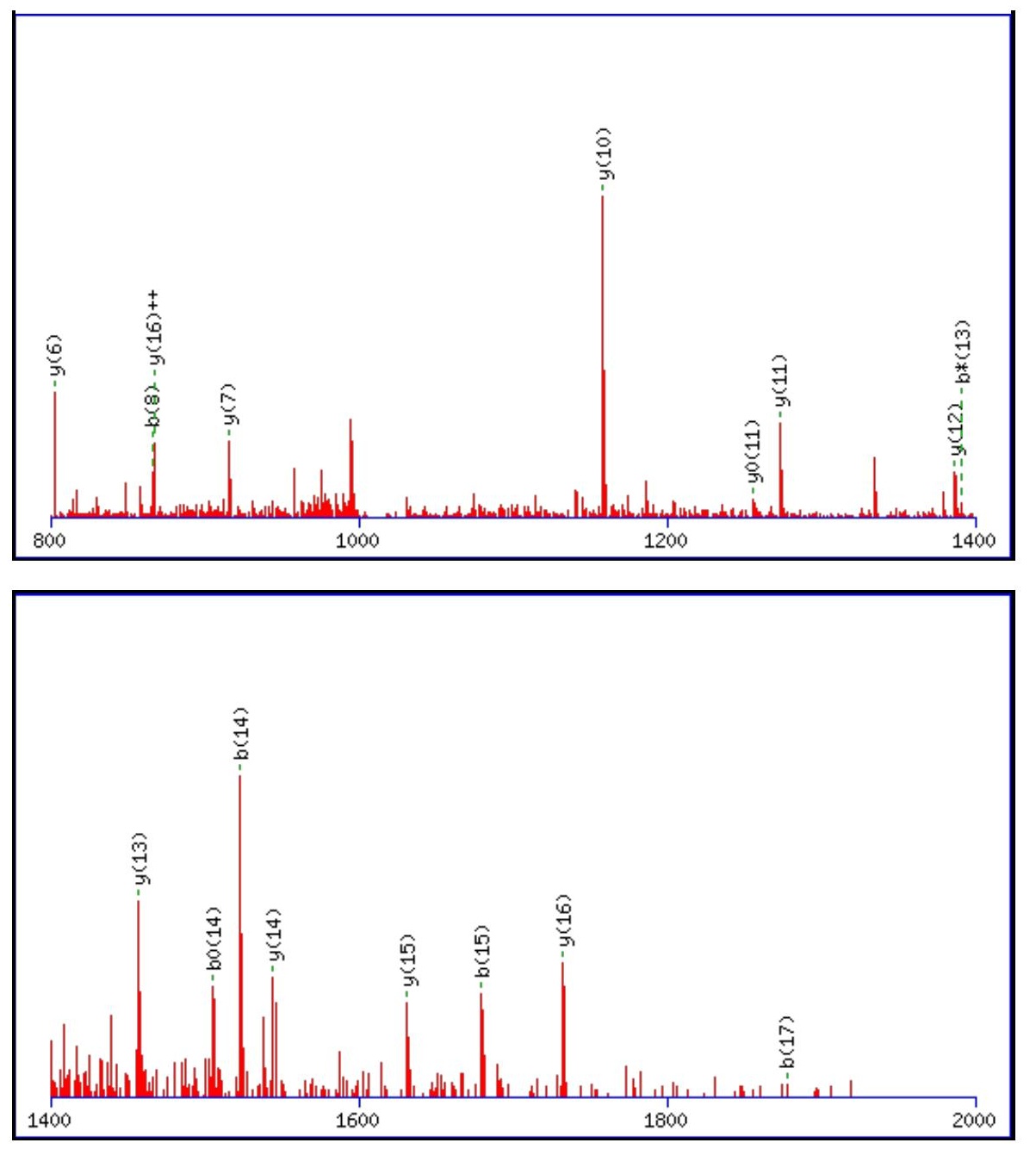

Monoisotopic mass of neutral peptide $\operatorname{Mr}($ calc): 2023.0011

Fixed modifications: Carbamidomethyl (C)

Variable modifications:

N8: Deamidated_N (N)

Ions Score: 67 Expect: $4.4 \mathrm{e}-005$

Matches (Bold Red): 25/196 fragment ions using 58 most intense peaks

\begin{tabular}{|c|c|c|c|c|c|c|c|c|c|c|c|c|c|c|}
\hline \# & b & $\mathbf{b}^{++}$ & $\mathbf{b}^{*}$ & & $\mathbf{b}^{0}$ & & Seq. & $\mathbf{y}$ & & $\mathbf{y}^{*}$ & & $\mathbf{y}^{0}$ & & \# \\
\hline 1 & 164.0706 & 82.5389 & & & & & $\mathbf{Y}$ & & & & & & & 18 \\
\hline 2 & 292.1292 & 46.5682 & 275.1026 & 38.0550 & & & $\mathbf{Q}$ & 450 & & & & & & 17 \\
\hline 3 & 393.1769 & 97.0921 & 376.1 & 788 & 663 & 188. & $\mathbf{T}$ & 864 & 469 & 1715.8599 & 336 & 8759 & 857.9416 & 16 \\
\hline 4 & 480.2089 & 240.6081 & 463.1823 & 232.0948 & 462.1983 & 231.6028 & S & 1631.8388 & 816.4230 & 1614.8122 & 807.9097 & 1613.8282 & 807.4177 & 15 \\
\hline 5 & 567.2409 & 284.1241 & 550.2144 & 275.6 & 549.2 & 275. & S & 1544.8067 & 772.9070 & 7802 & & 1526 & 017 & 14 \\
\hline 6 & 638.2780 & 319.6427 & 621.2515 & 311.1294 & 620.2675 & 310.6374 & A & 1457.7747 & 729.3910 & 1440.7481 & 720.8777 & 1439.7641 & 720.3857 & 13 \\
\hline 7 & 751.3621 & 376.1847 & 734.3355 & 367.6714 & 733.3515 & 367.1794 & I & 1386.7376 & 693.8724 & 7110 & 592 & 1368.7270 & 684.8671 & 12 \\
\hline 8 & 866.3890 & 433.6982 & 849.3625 & 425.1849 & 848.3785 & 424.6929 & $\mathbf{N}$ & 1273.6535 & 637.3304 & 1256.6270 & 628.8171 & 1255.6430 & 628.3251 & 11 \\
\hline 9 & 923.4105 & 462.2089 & 906.3839 & 453.6956 & 905.3999 & 453.2036 & G & 1158.6266 & 579.8169 & 1141.6000 & 571.3037 & 1140.6160 & 570.8116 & 10 \\
\hline 10 & 010.4425 & 505.7249 & 993.4160 & 497.2116 & 992.4320 & 496.7196 & S & 1101.6051 & 551.3062 & 1084.5786 & 542.7929 & 1083.5946 & 542.3009 & 9 \\
\hline 11 & 1109.5109 & 555.2591 & 1092.4844 & 546.7458 & 1091.5004 & 546.2538 & V & 1014.5731 & 507.7902 & 997.5465 & 499.2769 & 996.5625 & 498.7849 & 8 \\
\hline 12 & 1222.5950 & 611.8011 & 1205.5685 & 603.2879 & 1204.5844 & 602.7959 & I & 915.5047 & 458.2560 & 898.4781 & 449.7 & 897.4941 & 449.2507 & 7 \\
\hline 13 & 1408.6743 & 704.8408 & 1391.6478 & 696.3275 & 1390.6637 & 695.8355 & $\mathbf{W}$ & 802.4206 & 401.7139 & 785.3941 & 393.2007 & 784.4100 & 392.7087 & 6 \\
\hline 14 & 1523.7013 & 762.3543 & 1506.6747 & 753.8410 & 1505.6907 & 753.3490 & D & 616.3413 & 308.6743 & 599.3148 & 300.1610 & 598.3307 & 299.6690 & 5 \\
\hline 15 & 1679.8024 & 840.4048 & 1662.7758 & 831.8915 & 1661.7918 & 831.3995 & $\mathbf{R}$ & 501.3144 & 251.1608 & 484.2878 & 242.6475 & 483.3038 & 242.1555 & 4 \\
\hline 16 & 1776.8551 & 888.9312 & 1759.8286 & 880.4179 & 1758.8446 & 879.9259 & $\mathbf{P}$ & 345.2132 & 173.1103 & 328.1867 & 164.5970 & 327.2027 & 164.1050 & 3 \\
\hline 17 & 1877.9028 & 939.4550 & 1860.8763 & 930.9418 & 1859.8922 & 930.4498 & $\mathbf{T}$ & 248.1605 & 124.5839 & 231.1339 & 116.0706 & 230.1499 & 115.5786 & 2 \\
\hline 18 & & & & & & & $\mathbf{K}$ & 147.1128 & 74.0600 & 130.0863 & 65.5468 & & & 1 \\
\hline
\end{tabular}



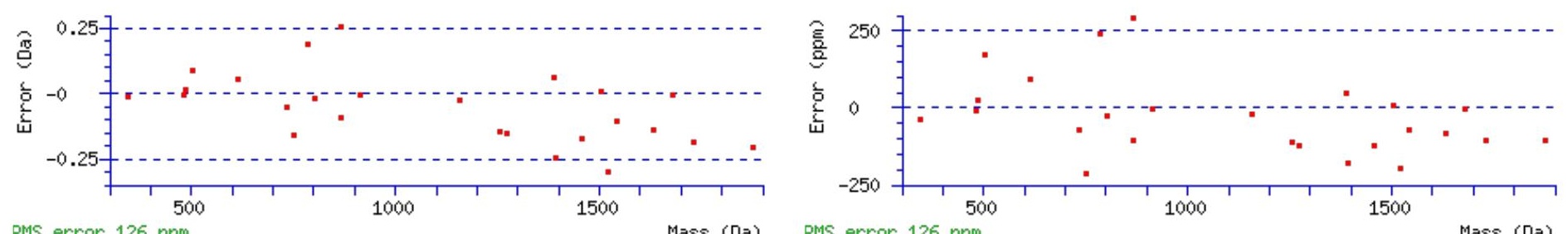

\section{All matches to this query}

\begin{tabular}{|l|l|l|l|}
\hline Score & Mr(calc): & Delta & Sequence \\
\hline 66.7 & 2023.0011 & 0.0097 & YQTSSAINGSVIWDRPTK \\
\hline 12.4 & 2022.0170 & 0.9937 & YQTSSAINGSVIWDRPTK \\
\hline 3.3 & 2022.0048 & 1.0060 & VLKSHGQDYLVGNRLSR \\
\hline
\end{tabular}

Spectrum No: 419; Query: 1693; Rank: 1

\section{Peptide View}

MS/MS Fragmentation of QLLANSSALVETILGHQER

Found in IPI00190577, Tax_Id=10116 Gene_Symbol=Lama5 similar to Laminin alpha-5 chain precursor

Match to Query 1693: 2079.096762 from(694.039530,3+)

Title: 091008RatKidney_NoSalt_22.5095.5095.3.dta

Data file K:INewmanPaper|Piliangl3SubProteomes\Piliang3SP\mgf5ppm\ERLIC_3SubProteomes5ppm.mgf
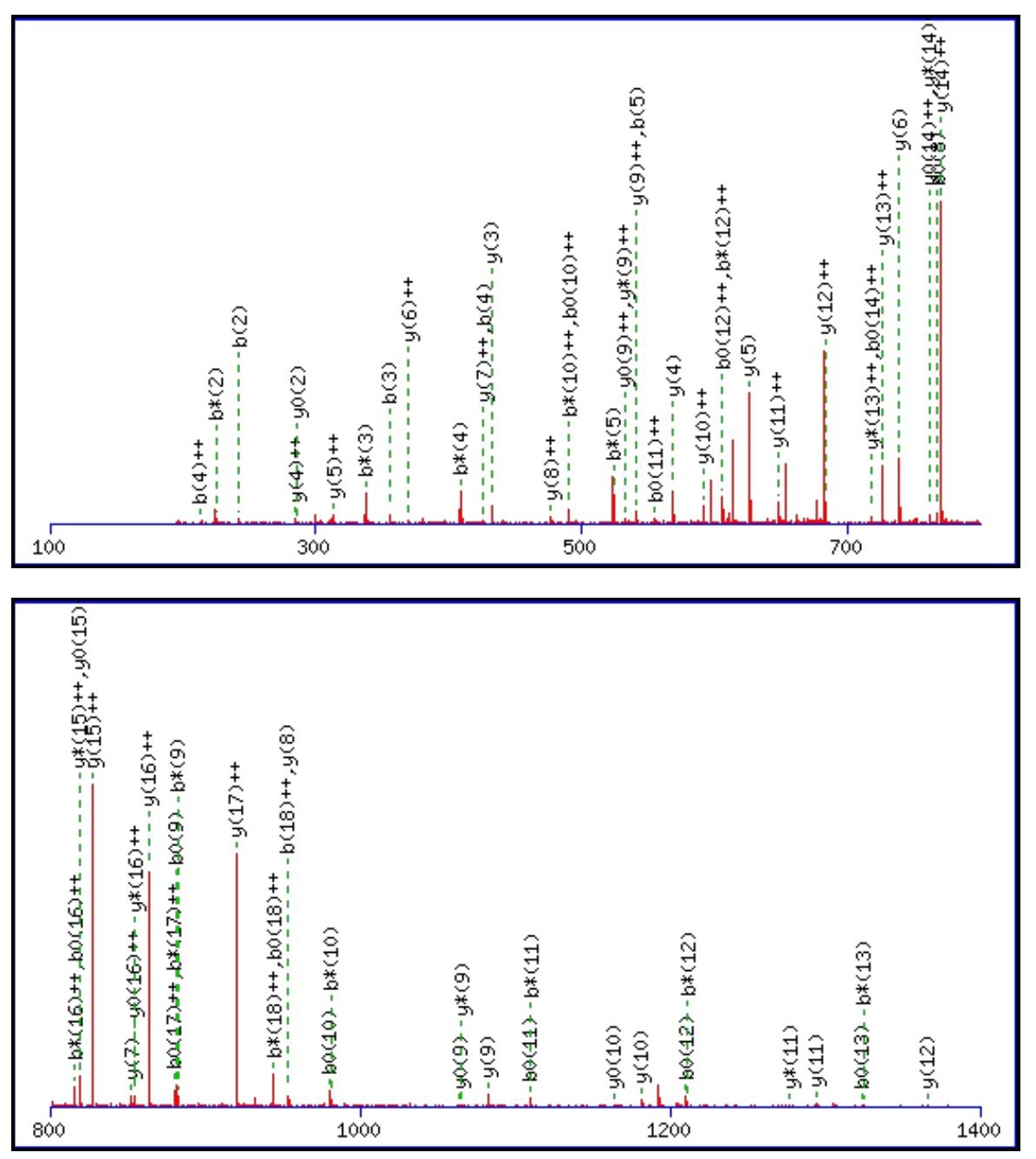


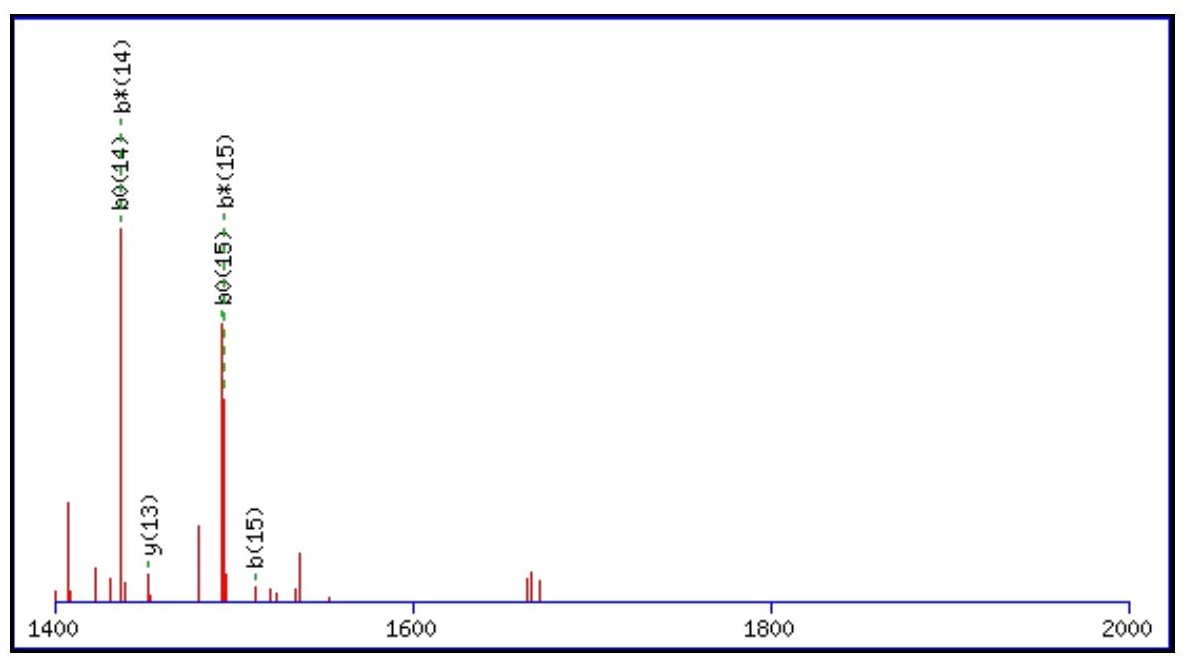

Monoisotopic mass of neutral peptide $\operatorname{Mr}($ calc): 2079.0960

Fixed modifications: Carbamidomethyl (C)

Variable modifications:

N5 : Deamidated_N (N)

Ions Score: 67 Expect: $2.2 \mathrm{e}-005$

Matches (Bold Red): 77/204 fragment ions using 143 most intense peaks

\begin{tabular}{|c|c|c|c|c|c|c|c|c|c|c|c|c|c|c|}
\hline \# & b & $\mathbf{b}^{++}$ & $\mathbf{b}^{*}$ & $\mathbf{b}^{*^{++}}$ & $\mathbf{b}^{0}$ & $\mathbf{b}^{0++}$ & Seq. & $\mathbf{y}$ & $\mathbf{y}^{++}$ & $\mathbf{y}^{*}$ & $\mathrm{y}^{\mathrm{*}^{++}}$ & $\mathbf{y}^{0}$ & $\mathbf{y}^{0++}$ & $\#$ \\
\hline 1 & 129.0659 & 65.0366 & 112.0393 & 56.5233 & & & $\mathbf{Q}$ & & & & & & & 19 \\
\hline 2 & 242.1499 & 121.5786 & 225.1234 & 113.0653 & & & $\mathbf{L}$ & 1952.0447 & 976.5260 & 1935.0182 & 968.0127 & 1934.0342 & 967.5207 & 18 \\
\hline 3 & 355.2340 & 178.1206 & 338.2074 & 169.6074 & & & $\mathbf{L}$ & 1838.9607 & 919.9840 & 1821.9341 & 911.4707 & 1820.9501 & 910.9787 & 17 \\
\hline 4 & 426.2711 & 213.6392 & 409.2445 & 205.1259 & & & $\mathbf{A}$ & 1725.8766 & 863.4419 & 1708.8500 & 854.9287 & 1707.8660 & \begin{tabular}{|l|}
854.4367 \\
\end{tabular} & 16 \\
\hline 5 & 541.2980 & 271.1527 & 524.2715 & 262.6394 & & & $\mathbf{N}$ & 1654.8395 & 827.9234 & 1637.8129 & 819.4101 & 1636.8289 & 818.9181 & 15 \\
\hline 6 & 628.3301 & 314.6687 & 611.3035 & 306.1554 & 610.3195 & 305.6634 & $S$ & 1539.8125 & 770.4099 & 1522.7860 & 761.8966 & 1521.8020 & \begin{tabular}{|l|}
761.4046 \\
\end{tabular} & 14 \\
\hline 7 & 715.3621 & 358.1847 & 698.3355 & 349.6714 & 697.3515 & 349.1794 & S & 1452.7805 & 726.8939 & 1435.7540 & 718.3806 & 1434.7700 & 717.8886 & 13 \\
\hline 8 & 786.3992 & 393.7032 & 769.3727 & 385.1900 & 768.3886 & 384.6980 & $\mathbf{A}$ & 1365.7485 & 683.3779 & 1348.7219 & 674.8646 & 1347.7379 & 674.3726 & 12 \\
\hline 9 & 899.4833 & 450.2453 & 882.4567 & 441.7320 & 881.4727 & 441.2400 & $\mathbf{L}$ & 1294.7114 & 647.8593 & 1277.6848 & 639.3461 & 1276.7008 & 638.8540 & 11 \\
\hline 10 & 998.5517 & 499.7795 & 981.5251 & 491.2662 & 980.5411 & 490.7742 & V & 1181.6273 & 591.3173 & 1164.6008 & 582.8040 & 1163.6167 & 582.3120 & 10 \\
\hline 11 & 1127.5943 & 564.3008 & 1110.5677 & 555.7875 & 1109.5837 & 555.2955 & $\mathbf{E}$ & 1082.5589 & 541.7831 & 1065.5324 & 533.2698 & 1064.5483 & 532.7778 & 9 \\
\hline 12 & 1228.6420 & 614.8246 & 1211.6154 & 606.3113 & 1210.6314 & 605.8193 & $\mathbf{T}$ & 953.5163 & 477.2618 & 936.4898 & 468.7485 & 935.5057 & 468.2565 & 8 \\
\hline 131 & 1341.7260 & 671.3666 & 1324.6995 & 662.8534 & 1323.7155 & 662.3614 & $\mathbf{I}$ & 852.4686 & 426.7380 & 835.4421 & 418.2247 & 834.4581 & 417.7327 & 7 \\
\hline 14 & 1454.8101 & 727.9087 & \begin{tabular}{|l}
1437.7835 \\
\end{tabular} & 719.3954 & 1436.7995 & \begin{tabular}{|l|l|}
718.9034 \\
\end{tabular} & $\mathbf{L}$ & 739.3846 & 370.1959 & 722.3580 & 361.6826 & 721.3740 & 361.1906 & 6 \\
\hline 15 & 1511.8315 & 756.4194 & 1494.8050 & 747.9061 & 1493.8210 & 747.4141 & G & 626.3005 & 313.6539 & 609.2739 & 305.1406 & 608.2899 & 304.6486 & 5 \\
\hline 16 & 1648.8905 & 824.9489 & 1631.8639 & 816.4356 & 1630.8799 & 815.9436 & $\mathbf{H}$ & 569.2790 & 285.1432 & 552.2525 & 276.6299 & 551.2685 & 276.1379 & 4 \\
\hline 17 & 1776.9490 & 888.9782 & 1759.9225 & 880.4649 & 1758.9385 & 879.9729 & $\mathbf{Q}$ & 432.2201 & 216.6137 & 415.1936 & 208.1004 & 414.2096 & 207.6084 & 3 \\
\hline 18 & 1905.9916 & 953.4995 & 1888.9651 & 944.9862 & 1887.9811 & 944.4942 & $\mathbf{E}$ & 304.1615 & 152.5844 & 287.1350 & 144.0711 & 286.1510 & 143.5791 & 2 \\
\hline 19 & & & & & & & $\mathbf{R}$ & 175.1190 & 88.0631 & 158.0924 & 79.5498 & & & 1 \\
\hline
\end{tabular}

$$
\text { 监 }
$$

要

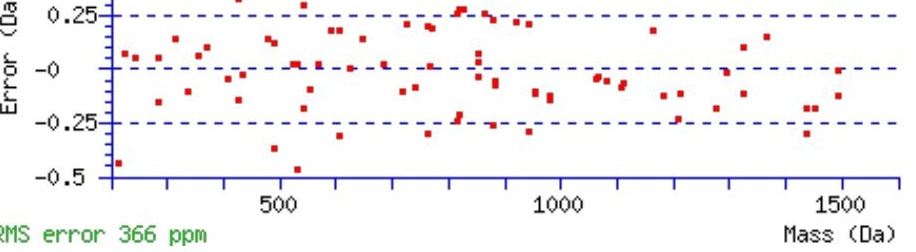

All matches to this query

\begin{tabular}{|l|l|l|l|}
\hline Score & Mr(calc): & Delta & Sequence \\
\hline 66.7 & 2079.0960 & 0.0008 & QLLANSSALVETILGHQER \\
\hline 41.7 & 2078.1120 & 0.9848 & QLLANSSALVETILGHQER \\
\hline
\end{tabular}

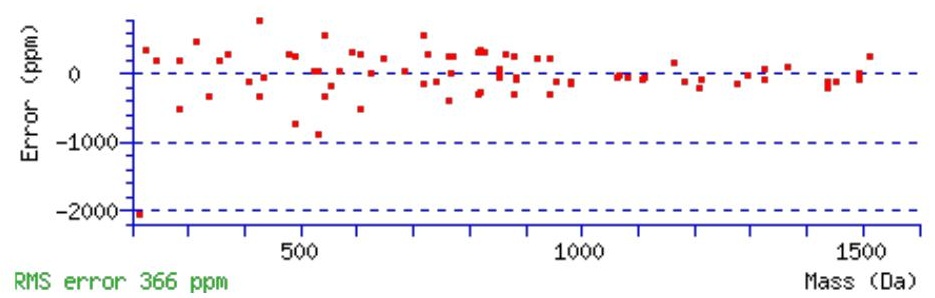




\begin{tabular}{|l|l|l|l|}
\hline 3.0 & 2078.0989 & 0.9979 & RIHIIXVTKPLLTARK \\
\hline 0.3 & 2077.0738 & 2.0229 & GASPNVSNVKVETPLHMAAR \\
\hline
\end{tabular}

Spectrum No: 420; Query: 1263; Rank: 1

\section{Peptide View}

MS/MS Fragmentation of ANEGAIYPDNTTDFQR

Found in IPI00325847, Tax_Id=10116 Gene_Symbol=Cp GPI-anchored ceruloplasmin

Match to Query 1263: 1811.803148 from(906.908850,2+)

Title: 100101RatKid_NS_deglyco_19.2207.2207.2.dta

Data file K:INewmanPaper|Piliangl3SubProteomes\Piliang3SP\mgf5ppm\ERLIC_3SubProteomes5ppm.mgf
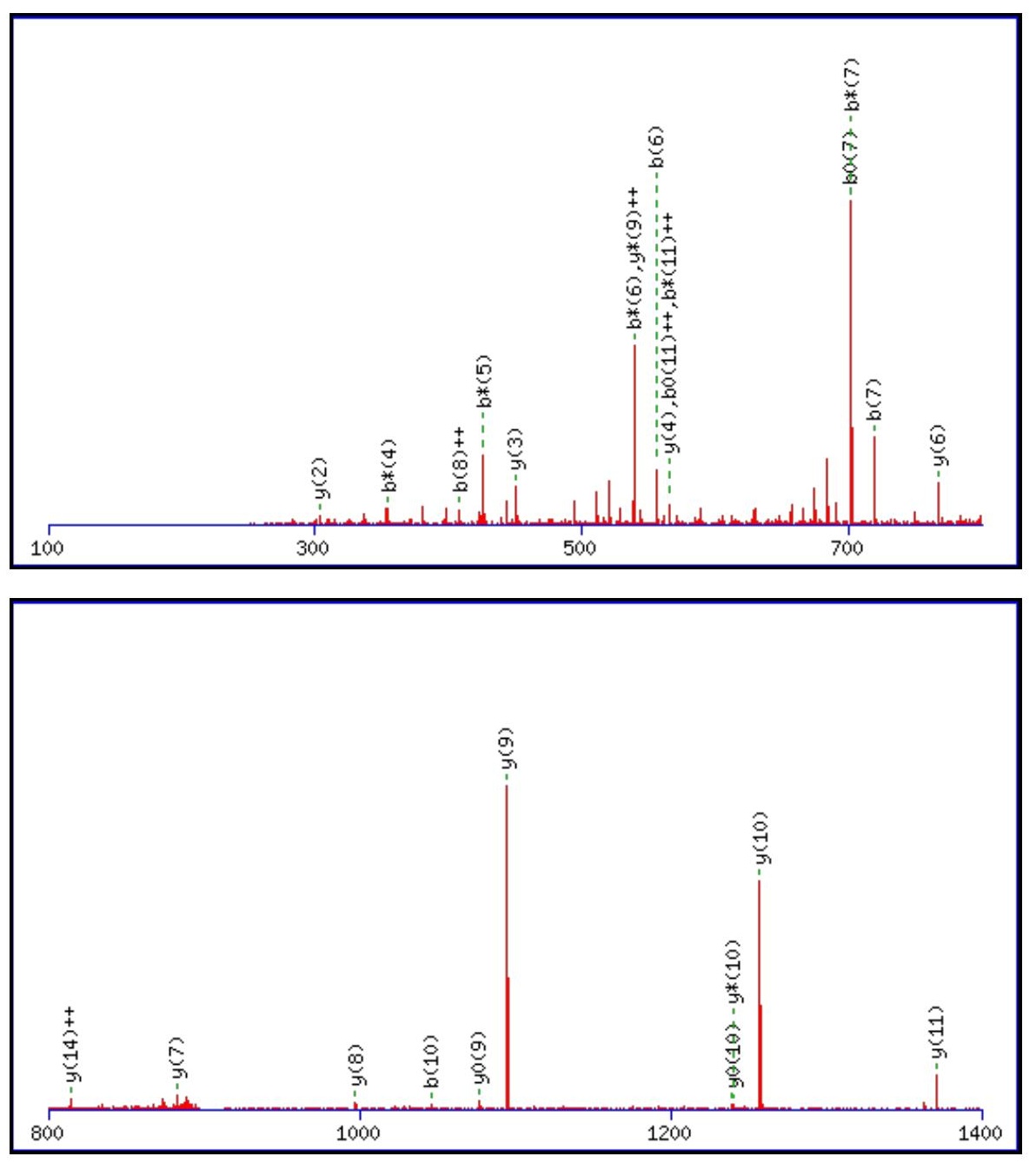


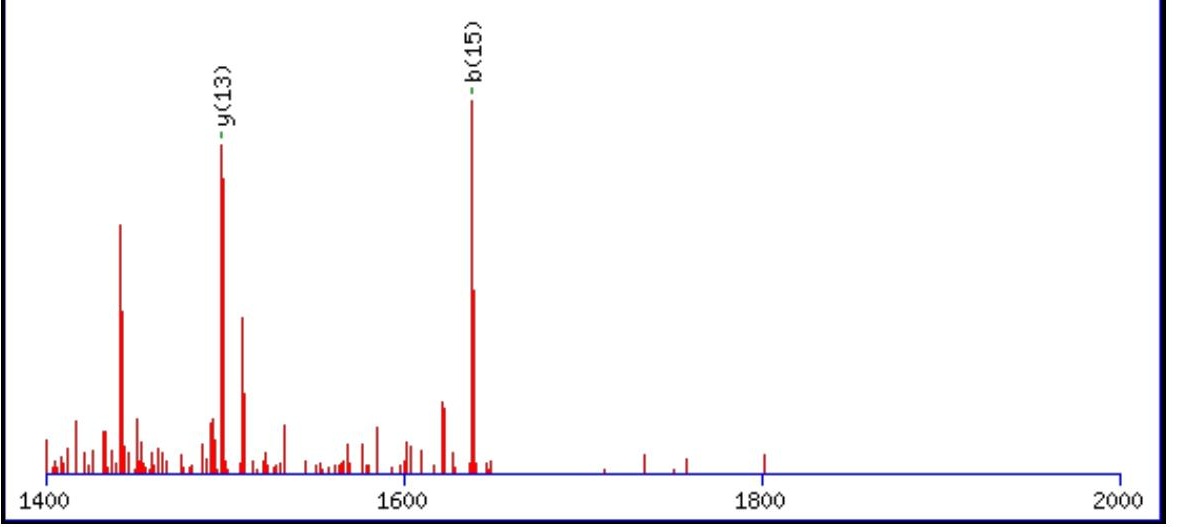

Monoisotopic mass of neutral peptide $\operatorname{Mr}($ calc): 1811.7962

Fixed modifications: Carbamidomethyl (C)

Variable modifications:

N10 : Deamidated_N (N)

Ions Score: 67 Expect: $4.5 \mathrm{e}-005$

Matches (Bold Red): 27/168 fragment ions using 50 most intense peaks

\begin{tabular}{|c|c|c|c|c|c|c|c|c|c|c|c|c|c|c|}
\hline \# & b & $\mathbf{b}^{++}$ & $\mathbf{b}^{*}$ & $\mathbf{b}^{*^{++}}$ & $\mathbf{b}^{0}$ & $\mathbf{b}^{\mathbf{0 + +}}$ & Seq. & $\mathbf{y}$ & $y^{++}$ & $\mathbf{y}^{*}$ & $\mathbf{y}^{*^{++}}$ & $\mathbf{y}^{0}$ & $\mathbf{y}^{0++}$ & \# \\
\hline 1 & 72.0444 & 36.5258 & & & & & A & & & & & & & 16 \\
\hline 2 & 186.0873 & 93.5473 & 169.0608 & 85.0340 & & & $\mathbf{N}$ & 1741.7664 & 871.3868 & 1724.7398 & 862.8736 & 1723.7558 & 862.3815 & 15 \\
\hline 3 & 315.1299 & 158.0686 & 298.1034 & 149.5553 & 297.1193 & 149.0633 & $\mathbf{E}$ & 1627.7235 & 814.3654 & 1610.6969 & 805.8521 & 1609.7129 & 805.3601 & 14 \\
\hline 4 & 372.1514 & 186.5793 & 355.1248 & 178.0661 & 354.1408 & 177.5740 & $\mathbf{G}$ & 1498.6809 & 749.8441 & 1481.6543 & 741.3308 & 1480.6703 & 740.8388 & 13 \\
\hline 5 & 443.1885 & 222.0979 & 426.1619 & 213.5846 & 425.1779 & 213.0926 & A & 1441.6594 & 721.3333 & 1424.6328 & 712.8201 & 1423.6488 & 712.3281 & 12 \\
\hline 6 & 556.2726 & 278.6399 & 539.2460 & 270.1266 & 538.2620 & 269.6346 & I & 1370.6223 & 685.8148 & 1353.5957 & 677.3015 & 1352.6117 & 676.8095 & 11 \\
\hline 7 & 719.3359 & 360.1716 & 702.3093 & 351.6583 & 701.3253 & 351.1663 & $\mathbf{Y}$ & 1257.5382 & 629.2727 & 1240.5117 & 620.7595 & 1239.5277 & 620.2675 & 10 \\
\hline 8 & 816.3886 & 408.6980 & 799.3621 & 400.1847 & 798.3781 & 399.6927 & $\mathbf{P}$ & 1094.4749 & 547.7411 & 1077.4483 & 539.2278 & 1076.4643 & 538.7358 & 9 \\
\hline 9 & 931.4156 & 466.2114 & 914.3890 & 457.6982 & 913.4050 & 457.2061 & D & 997.4221 & 499.2147 & 980.3956 & 490.7014 & 979.4116 & 490.2094 & 8 \\
\hline 10 & 1046.4425 & 523.7249 & 1029.4160 & 515.2116 & 1028.4320 & 514.7196 & $\mathbf{N}$ & 882.3952 & 441.7012 & 865.3686 & 433.1880 & 864.3846 & 432.6959 & 7 \\
\hline 11 & 1147.4902 & 574.2487 & 1130.4637 & 565.7355 & 1129.4796 & 565.2435 & $\mathbf{T}$ & 767.3682 & 384.1878 & 750.3417 & 375.6745 & 749.3577 & 375.1825 & 6 \\
\hline 12 & 1248.5379 & 624.7726 & 1231.5113 & 616.2593 & 1230.5273 & 615.7673 & $\mathbf{T}$ & 666.3206 & 333.6639 & 649.2940 & 325.1506 & 648.3100 & 324.6586 & 5 \\
\hline 13 & 1363.5648 & 682.2861 & 1346.5383 & 673.7728 & 1345.5543 & 673.2808 & D & 565.2729 & 283.1401 & 548.2463 & 274.6268 & 547.2623 & 274.1348 & 4 \\
\hline 14 & 1510.6332 & 755.8203 & 1493.6067 & 747.3070 & 1492.6227 & 746.8150 & $\mathbf{F}$ & 450.2459 & 225.6266 & 433.2194 & 217.1133 & & & 3 \\
\hline 15 & 1638.6918 & 819.8495 & 1621.6653 & 811.3363 & 1620.6813 & 810.8443 & $\mathbf{Q}$ & 303.1775 & 152.0924 & 286.1510 & 143.5791 & & & 2 \\
\hline 16 & & & & & & & $\mathbf{R}$ & 175.1190 & 88.0631 & 158.0924 & 79.5498 & & & 1 \\
\hline
\end{tabular}
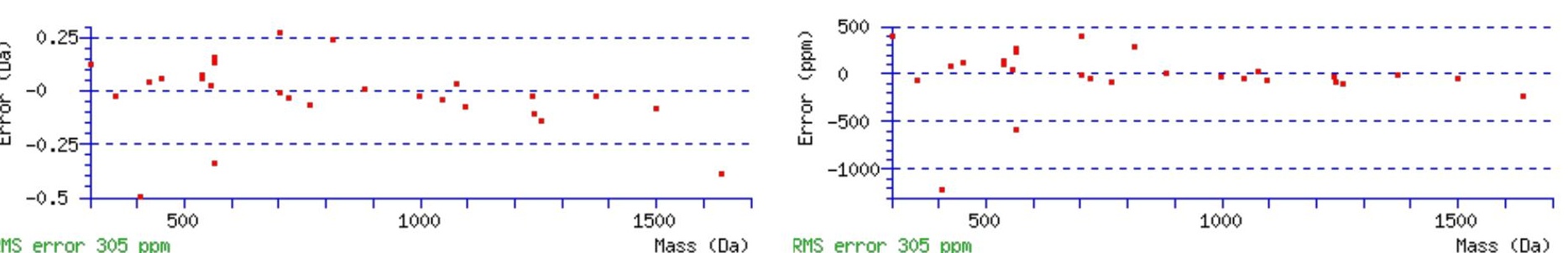

\section{All matches to this query}

\begin{tabular}{|l|l|l|l|}
\hline Score & $\mathbf{M r}$ (calc): & Delta & \multicolumn{1}{c|}{ Sequence } \\
\hline 66.6 & 1811.7962 & 0.0069 & $\underline{\text { ANEGAIYPDNTTDFQR }}$ \\
\hline 10.6 & 1811.8050 & -0.0019 & NLSKITNNNLNDSQR \\
\hline 10.6 & 1811.8050 & -0.0019 & $\underline{\text { NLSKITNNNLNDSQR }}$ \\
\hline 9.9 & 1811.8050 & -0.0019 & $\underline{\text { NLSKITNNNLNDSQR }}$ \\
\hline 8.5 & 1811.8050 & -0.0019 & NLSKITNNNLNDSQR \\
\hline & & & \\
\hline
\end{tabular}




\begin{tabular}{|l|l|l|l|}
7.6 & 1811.8050 & -0.0019 & NLSKITNNNLNDSQR \\
\hline 7.6 & 1811.8050 & -0.0019 & NLSKITNNNLNDSQR \\
\hline 7.5 & 1809.7791 & 2.0241 & CQSYQQSTKQLTMK \\
\hline 4.8 & 1811.7962 & 0.0069 & ANEGAIYPDNTTDFQR \\
\hline 4.4 & 1810.8122 & 0.9910 & ANEGAIYPDNTTDFQR \\
\hline
\end{tabular}

Spectrum No: 421; Query: 995; Rank: 1

\section{Peptide View}

MS/MS Fragmentation of GYLASFEMFNSTFK

Found in IPI00400739, Tax_Id=10116 Gene_Symbol=Entpd5 Ectonucleoside triphosphate diphosphohydrolase 5

Match to Query 995: 1658.732588 from(830.373570,2+)

Title: 091008RatKidney_NH4Format01_24.3877.3877.2.dta

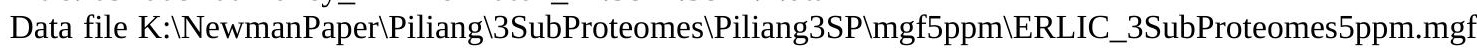
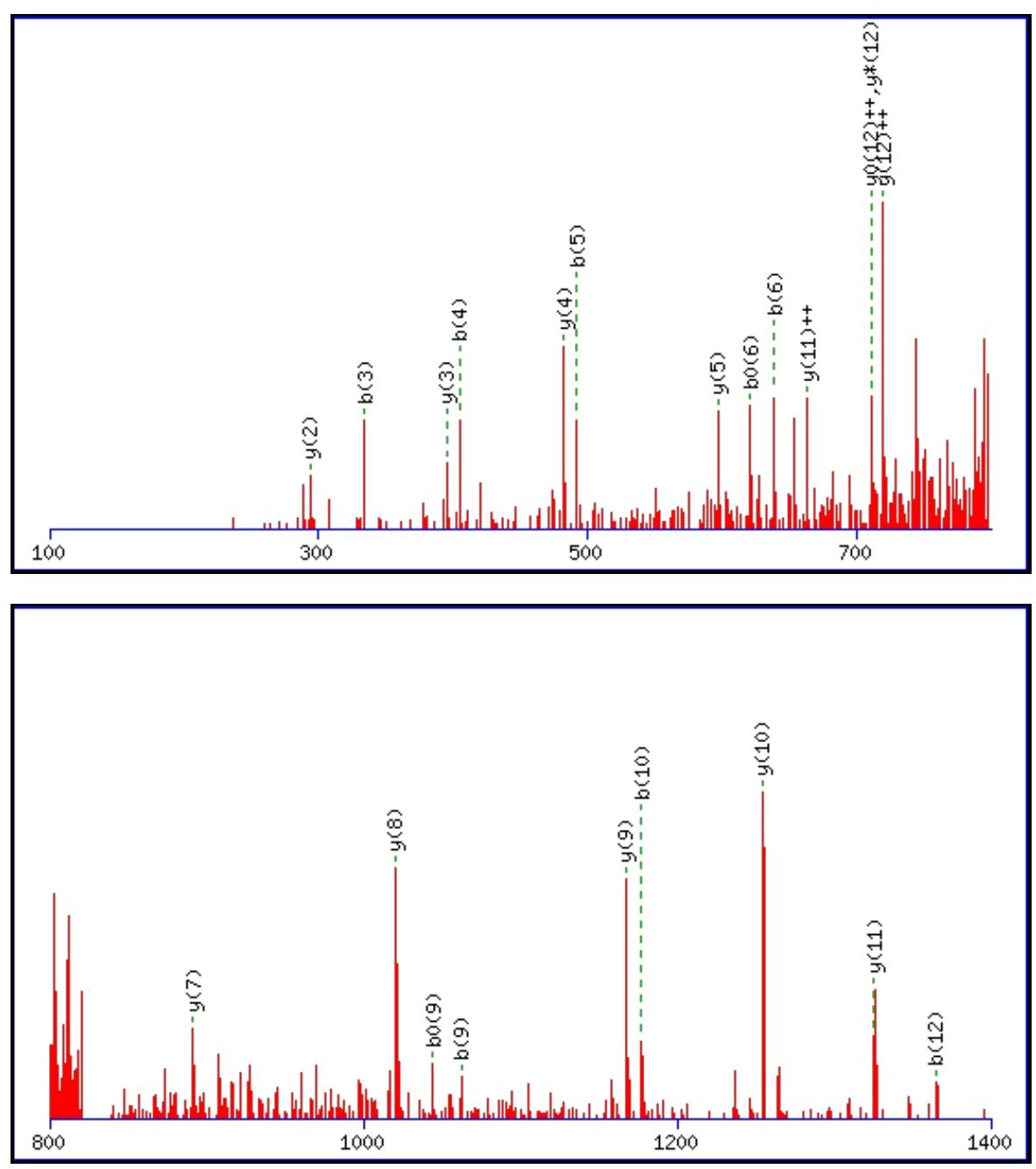


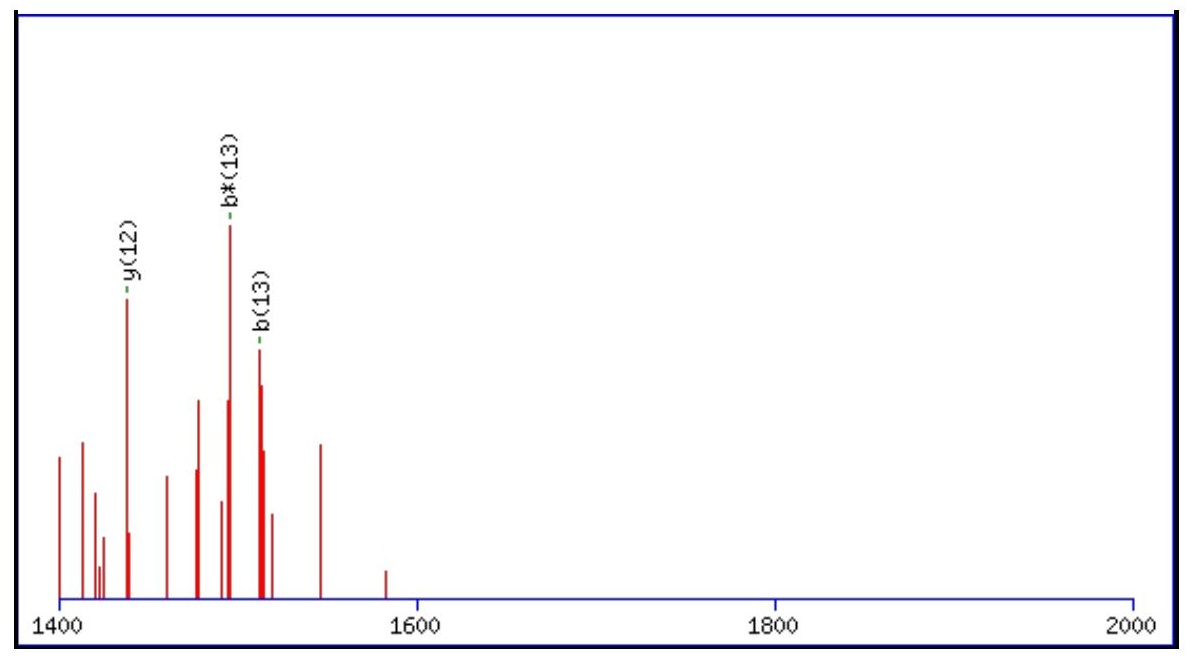

Monoisotopic mass of neutral peptide $\operatorname{Mr}($ calc): 1657.7334

Fixed modifications: Carbamidomethyl (C)

Variable modifications:

M8 : Oxidation (M)

N10 : Deamidated_N (N)

Ions Score: 66 Expect: $4.2 \mathrm{e}-005$

Matches (Bold Red): 25/126 fragment ions using 40 most intense peaks

\begin{tabular}{|c|c|c|c|c|c|c|c|c|c|c|c|c|c|c|}
\hline \# & b & $\mathbf{b}^{++}$ & b* & $\mathbf{b}^{*^{++}}$ & $\mathbf{b}^{\mathbf{0}}$ & $\mathbf{b}^{0++}$ & Seq. & $\mathbf{y}$ & $y^{++}$ & $\mathbf{y}^{*}$ & $\mathrm{y}^{\mathrm{*}^{++}}$ & $\mathbf{y}^{0}$ & $\mathbf{y}^{0++}$ & \# \\
\hline 1 & 58.0287 & 29.5180 & & & & & G & & & & & & & 14 \\
\hline 2 & 221.0921 & 111.0497 & & & & & $\mathbf{Y}$ & 1601.7192 & 801.3632 & 1584.6927 & 792.8500 & 1583.7087 & 792.3580 & 13 \\
\hline 3 & 334.1761 & 167.5917 & & & & & $\mathbf{L}$ & 1438.6559 & 719.8316 & 1421.6293 & 711.3183 & 1420.6453 & 710.8263 & 12 \\
\hline 4 & 405.2132 & 203.1103 & & & & & A & 1325.5718 & 663.2896 & 1308.5453 & 654.7763 & 1307.5613 & 654.2843 & 11 \\
\hline 5 & 492.2453 & 246.6263 & & & 474.2347 & 237.6210 & $\mathrm{~S}$ & 1254.5347 & 627.7710 & 1237.5082 & 619.2577 & 1236.5241 & 618.7657 & 10 \\
\hline 6 & 639.3137 & 320.1605 & & & 621.3031 & 311.1552 & $\mathbf{F}$ & 1167.5027 & 584.2550 & 1150.4761 & 575.7417 & 1149.4921 & 575.2497 & 9 \\
\hline 7 & 768.3563 & 384.6818 & & & 750.3457 & 375.6765 & $\mathbf{E}$ & 1020.4343 & 510.7208 & 1003.4077 & 502.2075 & 1002.4237 & 501.7155 & 8 \\
\hline 8 & 915.3917 & 458.1995 & & & 897.3811 & 449.1942 & $\mathbf{M}$ & 891.3917 & 446.1995 & 874.3651 & 437.6862 & 873.3811 & 437.1942 & 7 \\
\hline 9 & 1062.4601 & 531.7337 & & & 1044.4495 & 522.7284 & $\mathbf{F}$ & 744.3563 & 372.6818 & 727.3297 & 364.1685 & 726.3457 & 363.6765 & 6 \\
\hline 10 & 1177.4870 & 589.2472 & 1160.4605 & 580.7339 & 1159.4765 & 580.2419 & $\mathbf{N}$ & 597.2879 & 299.1476 & 580.2613 & 290.6343 & 579.2773 & 290.1423 & 5 \\
\hline 11 & 1264.5191 & 632.7632 & 1247.4925 & 624.2499 & 1246.5085 & 623.7579 & $\mathrm{~S}$ & 482.2609 & 241.6341 & 465.2344 & 233.1208 & 464.2504 & 232.6288 & 4 \\
\hline 12 & 1365.5667 & 683.2870 & 1348.5402 & 674.7737 & 1347.5562 & 674.2817 & $\mathbf{T}$ & 395.2289 & 198.1181 & 378.2023 & 189.6048 & 377.2183 & 189.1128 & 3 \\
\hline 13 & 1512.6352 & 756.8212 & 1495.6086 & 748.3079 & 1494.6246 & 747.8159 & $\mathbf{F}$ & 294.1812 & 147.5942 & 277.1547 & 139.0810 & & & 2 \\
\hline 14 & & & & & & & $\mathbf{K}$ & 147.1128 & 74.0600 & 130.0863 & 65.5468 & & & 1 \\
\hline
\end{tabular}
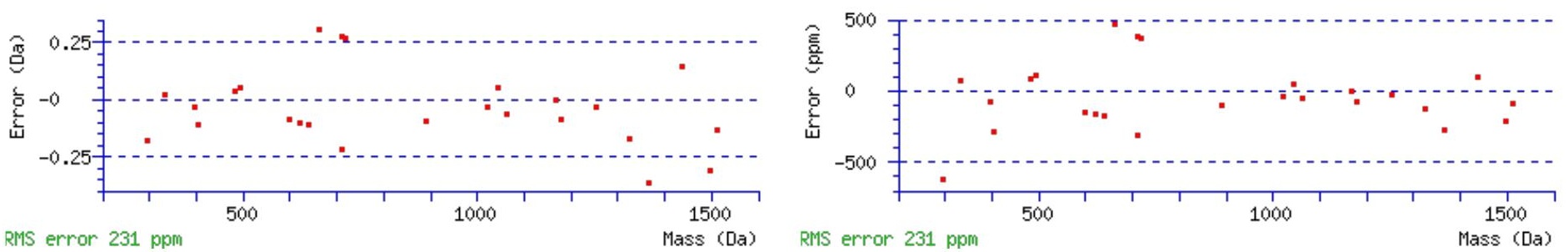

\section{All matches to this query}

\begin{tabular}{|l|l|l|l|}
\hline Score & Mr(calc): & Delta & Sequence \\
\hline 66.4 & 1657.7334 & 0.9992 & GYLASFEMFNSTFK \\
\hline 6.4 & 1657.7211 & 1.0115 & NFAAESFTFLMRK \\
\hline 3.3 & 1657.7382 & 0.9944 & KSTEMRKPESEEK \\
\hline 3.3 & 1657.7382 & 0.9944 & KSTEMRKPESEEK \\
\hline
\end{tabular}


Spectrum No: 422; Query: 1139; Rank: 1

\section{Peptide View}

MS/MS Fragmentation of SCVTITDAFPQNMSR

Found in IPI00370450, Tax_Id=10116 Gene_Symbol=Plxnb2 plexin B2

Match to Query 1139: 1742.763268 from(872.388910,2+)

Title: 100101RatKid_NS_deglyco_25.2413.2413.2.dta

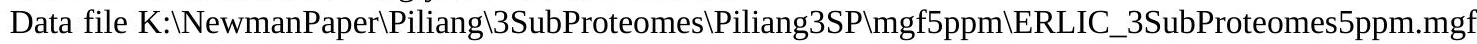
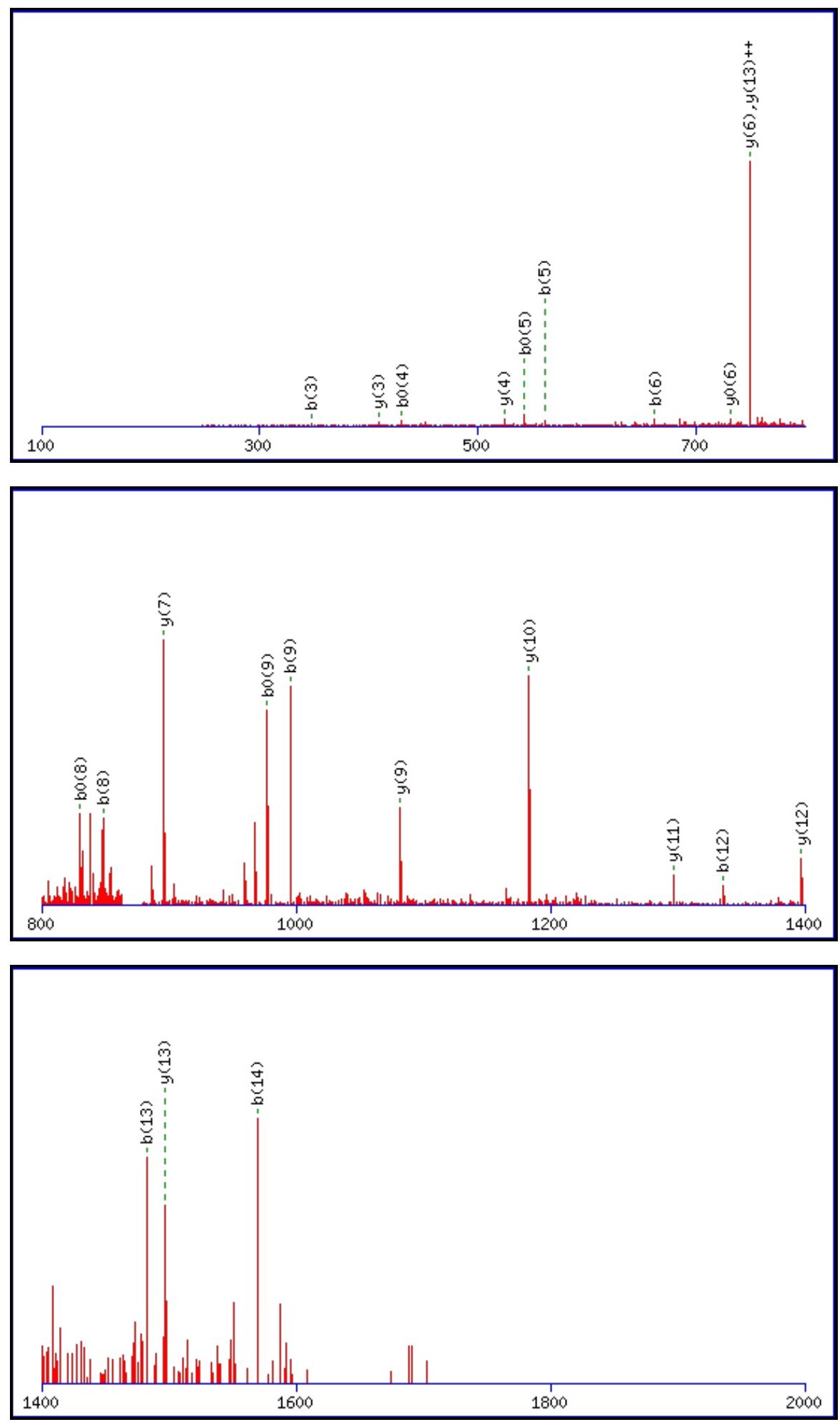

Monoisotopic mass of neutral peptide $\operatorname{Mr}($ calc): 1742.7604

Fixed modifications: Carbamidomethyl (C)

Variable modifications: 
N12 : Deamidated_N (N)

M13 : Oxidation (M)

Ions Score: 66 Expect: $4.7 \mathrm{e}-005$

Matches (Bold Red): 23/146 fragment ions using 35 most intense peaks

\begin{tabular}{|c|c|c|c|c|c|c|c|c|c|c|c|c|c|c|}
\hline \# & b & $\mathbf{b}^{++}$ & $\mathbf{b}^{*}$ & $\mathbf{b}^{*^{++}}$ & $\mathbf{b}^{0}$ & $\mathbf{b}^{0++}$ & Seq. & $\mathbf{y}$ & $y^{++}$ & $\mathbf{y}^{*}$ & $\mathrm{y}^{*^{++}}$ & $\mathbf{y}^{\mathbf{0}}$ & $y^{0++}$ & $\#$ \\
\hline 1 & 88.0393 & 44.5233 & & & 70.0287 & 35.5180 & S & & & & & & & 15 \\
\hline 2 & 248.0700 & 124.5386 & & & 230.0594 & 115.5333 & C & 1656.7356 & 828.8714 & 1639.7091 & 820.3582 & 1638.7251 & 819.8662 & 14 \\
\hline 3 & 347.1384 & 174.0728 & & & 329.1278 & 165.0675 & $\mathrm{~V}$ & 1496.7050 & \begin{tabular}{|l|l|}
748.8561 \\
\end{tabular} & 1479.6784 & 740.3428 & 1478.6944 & 739.8508 & 13 \\
\hline 4 & 448.1860 & 224.5967 & & & 430.1755 & 215.5914 & $T$ & 1397.6366 & 699.3219 & 1380.6100 & 690.8086 & 1379.6260 & 690.3166 & 12 \\
\hline 5 & 561.2701 & 281.1387 & & & 543.2595 & 272.1334 & I & 1296.5889 & 648.7981 & 1279.5623 & 640.2848 & 1278.5783 & 639.7928 & 11 \\
\hline 6 & 662.3178 & 331.6625 & & & 644.3072 & 322.6573 & $T$ & 1183.5048 & 592.2560 & 1166.4783 & 583.7428 & 1165.4942 & 583.2508 & 10 \\
\hline 7 & 777.3447 & 389.1760 & & & 759.3342 & 380.1707 & D & 1082.4571 & 541.7322 & 1065.4306 & 533.2189 & 1064.4466 & 532.7269 & 9 \\
\hline 8 & 848.3818 & 424.6946 & & & 830.3713 & 415.6893 & $\mathbf{A}$ & 967.4302 & 484.2187 & 950.4036 & 475.7055 & 949.4196 & 475.2135 & 8 \\
\hline 9 & 995.4503 & 498.2288 & & & 977.4397 & 489.2235 & $\mathbf{F}$ & 896.3931 & 448.7002 & 879.3665 & 440.1869 & 878.3825 & 439.6949 & 7 \\
\hline 10 & 1092.5030 & 546.7551 & & & 1074.4925 & 537.7499 & $\mathbf{P}$ & 749.3247 & 375.1660 & 732.2981 & 366.6527 & 731.3141 & 366.1607 & 6 \\
\hline 11 & 1220.5616 & 610.7844 & 1203.5351 & 602.2712 & 1202.5510 & 601.7792 & $\mathbf{Q}$ & 652.2719 & 326.6396 & 635.2453 & 318.1263 & 634.2613 & 317.6343 & 5 \\
\hline 12 & 1335.5885 & 668.2979 & 1318.5620 & 659.7846 & 1317.5780 & 659.2926 & $\mathbf{N}$ & 524.2133 & 262.6103 & 507.1868 & 254.0970 & 506.2028 & 253.6050 & 4 \\
\hline 13 & 1482.6239 & 741.8156 & 1465.5974 & 733.3023 & 1464.6134 & 732.8103 & $\mathbf{M}$ & 409.1864 & 205.0968 & 392.1598 & 196.5836 & 391.1758 & 196.0915 & 3 \\
\hline 14 & 1569.6560 & 785.3316 & 1552.6294 & 776.8183 & 1551.6454 & 776.3263 & $S$ & 262.1510 & 131.5791 & 245.1244 & 123.0659 & 244.1404 & 122.5738 & 2 \\
\hline 15 & & & & & & & $\mathbf{R}$ & 175.1190 & 88.0631 & 158.0924 & 79.5498 & & & 1 \\
\hline
\end{tabular}
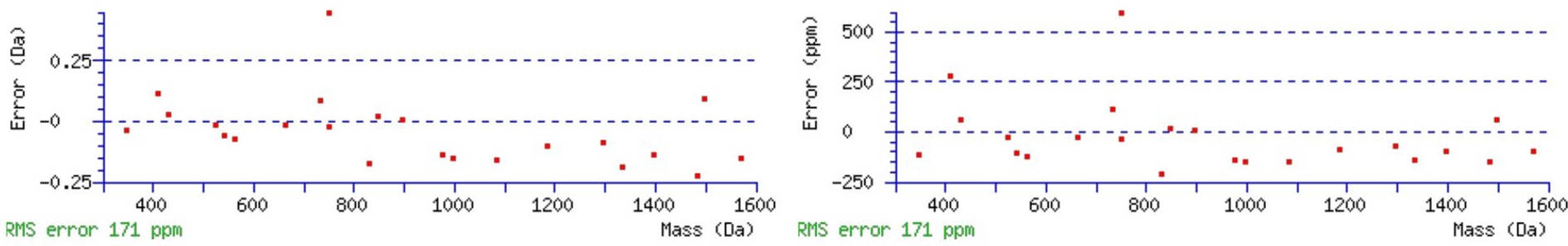

All matches to this query

\begin{tabular}{|l|l|c|l|}
\hline Score & Mr(calc): & Delta & \multicolumn{1}{c|}{ Sequence } \\
\hline 66.3 & 1742.7604 & 0.0029 & SCVTITDAFPQNMSR \\
\hline 8.0 & 1740.7463 & 2.0169 & MKATEEMSDHLPIK \\
\hline 6.5 & 1740.7623 & 2.0010 & MSPARMSPARMSPAR \\
\hline 5.7 & 1740.7623 & 2.0010 & MSPARMSPARMSPAR \\
\hline 4.2 & 1741.7764 & 0.9869 & SCVTITDAFPQNMSR \\
\hline 4.0 & 1740.7623 & 2.0010 & MSPARMSPARMSPAR \\
\hline 3.1 & 1742.7737 & -0.0104 & NSIHFSNSKNSVSSR \\
\hline 2.6 & 1742.7635 & -0.0002 & DVNDWIGPPNNNGVTK \\
\hline 2.4 & 1740.7736 & 1.9896 & DNSLSVSIPQPSPLK \\
\hline 2.4 & 1740.7736 & 1.9896 & DNSLSVSIPQPSPLK \\
\hline
\end{tabular}

Spectrum No: 423; Query: 2151; Rank: 1

\section{Peptide View}

MS/MS Fragmentation of ASFNITLIHPNNLTALSNMLPK

Found in IPI00230862, Tax_Id=10116 Gene_Symbol=Anpep Aminopeptidase N

Match to Query 2151: 2410.261722 from(804.427850,3+)

Title: 100101RatKid_NS_deglyco_13.6464.6464.3.dta

Data file K:INewmanPaper|Piliangl3SubProteomes\Piliang3SP\mgf5ppm\ERLIC_3SubProteomes5ppm.mgf 

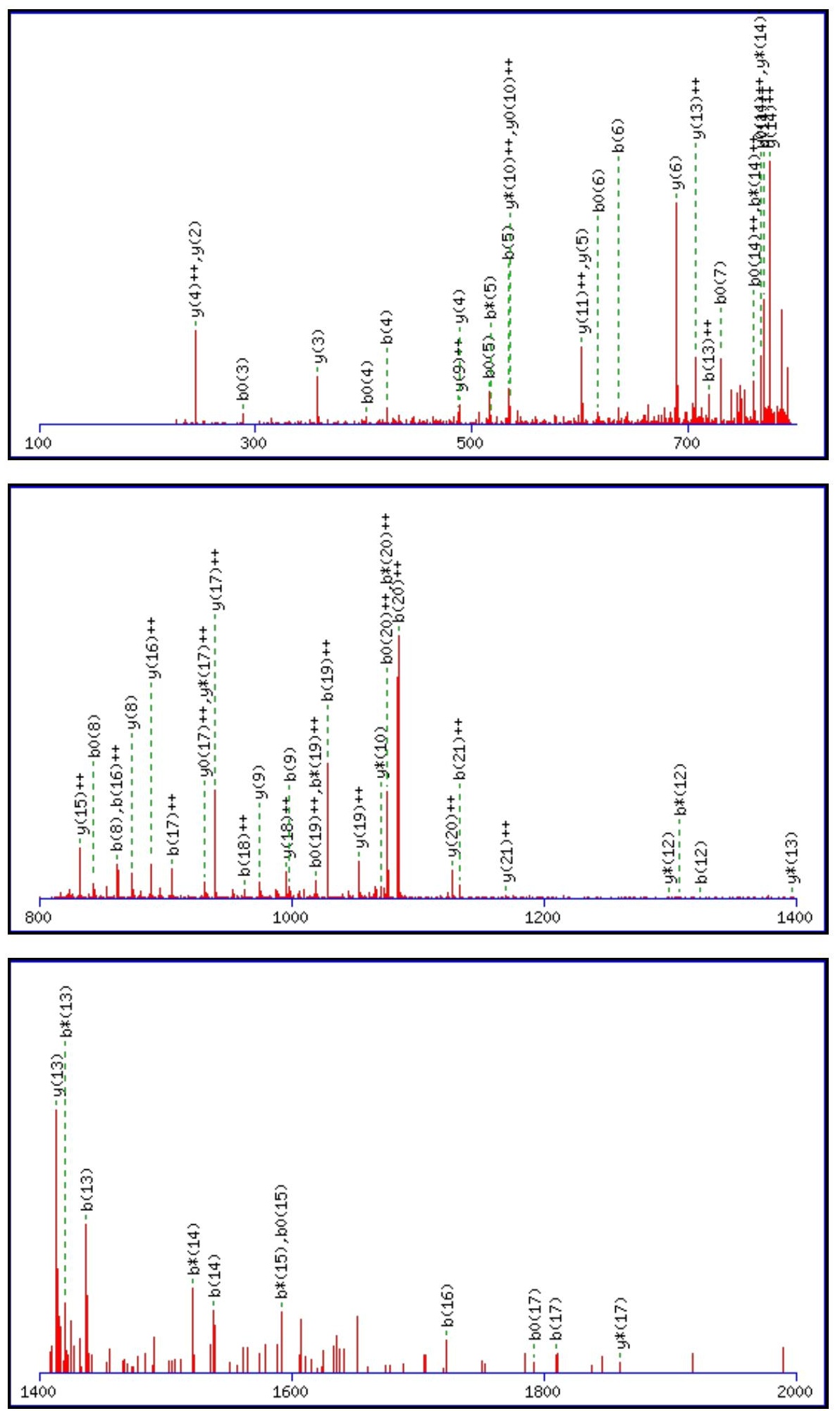

Monoisotopic mass of neutral peptide $\operatorname{Mr}($ calc): 2410.2566

Fixed modifications: Carbamidomethyl (C)

Variable modifications:

N4 : Deamidated_N (N)

N12 : Deamidated_N (N)

Ions Score: 66 Expect: $3 e-005$

Matches (Bold Red): 67/234 fragment ions using 120 most intense peaks

\begin{tabular}{|r|c|c|c|c|c|r|c|c|c|c|c|c|c|c|}
\hline$\#$ & $\mathbf{b}$ & $\mathbf{b}^{++}$ & $\mathbf{b}^{*}$ & $\mathbf{b}^{\boldsymbol{*}^{++}}$ & $\mathbf{b}^{\mathbf{0}}$ & $\mathbf{b}^{\mathbf{0 + +}}$ & Seq. & $\mathbf{y}$ & $\mathbf{y}^{++}$ & $\mathbf{y}^{\mathbf{*}}$ & $\mathbf{y}^{\mathbf{*}^{++}}$ & $\mathbf{y}^{\mathbf{0}}$ & $\mathbf{y}^{\mathbf{0 + +}}$ & $\#$ \\
\hline $\mathbf{1}$ & $\mathbf{7 2 . 0 4 4 4}$ & 36.5258 & & & & & $\mathbf{A}$ & & & & & & & $\mathbf{2 2}$ \\
\hline $\mathbf{2}$ & 159.0764 & 80.0418 & & & 141.0659 & 71.0366 & $\mathbf{S}$ & 2340.2268 & $\mathbf{1 1 7 0 . 6 1 7 0}$ & 2323.2002 & 1162.1038 & 2322.2162 & 1161.6117 & $\mathbf{2 1}$ \\
\hline $\mathbf{3}$ & 306.1448 & 153.5761 & & & $\mathbf{2 8 8 . 1 3 4 3}$ & 144.5708 & $\mathbf{F}$ & 2253.1948 & $\mathbf{1 1 2 7 . 1 0 1 0}$ & 2236.1682 & 1118.5877 & 2235.1842 & 1118.0957 & $\mathbf{2 0}$ \\
\hline $\mathbf{4}$ & $\mathbf{4 2 1 . 1 7 1 8}$ & 211.0895 & 404.1452 & 202.5762 & $\mathbf{4 0 3 . 1 6 1 2}$ & 202.0842 & $\mathbf{N}$ & 2106.1263 & $\mathbf{1 0 5 3 . 5 6 6 8}$ & 2089.0998 & 1045.0535 & 2088.1158 & 1044.5615 & $\mathbf{1 9}$ \\
\hline
\end{tabular}




\begin{tabular}{|c|c|c|c|c|c|c|c|c|c|c|c|c|c|c|}
\hline & 534.2558 & 267.6316 & 517.2293 & 259.1183 & 516.2453 & 258.6263 & I & 1991.0994 & 996.0533 & |1974.0729 & 987.5401 & |1973.0888 & 987.0481 & 18 \\
\hline 6 & 635.3035 & 318.1554 & 618.2770 & 309.6421 & 617.2929 & 309.1501 & $\mathbf{T}$ & 1878.0153 & 939.5113 & \begin{tabular}{|l|}
1860.9888 \\
\end{tabular} & 930.9980 & 1860.0048 & 930.5060 & 17 \\
\hline 7 & 748.3876 & 374.6974 & 731.3610 & 366.1842 & 730.3770 & 365.6921 & $\mathbf{L}$ & 1776.9677 & 888.9875 & 1759.9411 & 880.4742 & 1758.9571 & 879.9822 & 16 \\
\hline 8 & 861.4716 & 431.2395 & 844.4451 & 422.7262 & 843.4611 & 422.2342 & I & 1663.8836 & 832.4454 & 1646.8570 & 823.9322 & 1645.8730 & 823.4402 & 15 \\
\hline 9 & 998.5306 & 499.7689 & 981.5040 & 491.2556 & 980.5200 & 490.7636 & $\mathbf{H}$ & 1550.7995 & 775.9034 & 1533.7730 & 767.3901 & 1532.7890 & 766.8981 & 14 \\
\hline 10 & 1095.5833 & 548.2953 & 1078.5568 & 539.7820 & 1077.5728 & 539.2900 & $\mathbf{P}$ & 1413.7406 & 707.3739 & 1396.7141 & 698.8607 & 1395.7301 & 698.3687 & 13 \\
\hline 11 & 1209.6262 & 605.3168 & 1192.5997 & 596.8035 & 1191.6157 & 596.3115 & $\mathbf{N}$ & 1316.6879 & 658.8476 & 1299.6613 & 650.3343 & 1298.6773 & 649.8423 & 12 \\
\hline 12 & 1324.6532 & 662.8302 & 1307.6266 & 654.3170 & 1306.6426 & 653.8249 & $\mathbf{N}$ & 1202.6449 & 601.8261 & 1185.6184 & 593.3128 & 1184.6344 & 592.8208 & 11 \\
\hline 13 & 1437.7372 & 719.3723 & 1420.7107 & 710.8590 & 1419.7267 & 710.3670 & $\mathbf{L}$ & 1087.6180 & 544.3126 & 1070.5914 & 535.7994 & 1069.6074 & 535.3074 & 10 \\
\hline 14 & 1538.7849 & 769.8961 & 1521.7584 & 761.3828 & 1520.7744 & 760.8908 & $\mathbf{T}$ & 974.5339 & 487.7706 & 957.5074 & 479.2573 & 956.5234 & 478.7653 & 9 \\
\hline 15 & 1609.8220 & 805.4147 & 1592.7955 & 796.9014 & 1591.8115 & 796.4094 & A & 873.4863 & 437.2468 & 856.4597 & 428.7335 & 855.4757 & 428.2415 & 8 \\
\hline 16 & 1722.9061 & 861.9567 & 1705.8795 & 853.4434 & 1704.8955 & 852.9514 & L & 802.4491 & 401.7282 & 785.4226 & 393.2149 & 784.4386 & 392.7229 & 7 \\
\hline 17 & 1809.9381 & 905.4727 & 1792.9116 & 896.9594 & 1791.9276 & 896.4674 & S & 689.3651 & 345.1862 & 672.3385 & 336.6729 & 671.3545 & 336.1809 & 6 \\
\hline 18 & 1923.9811 & 962.4942 & 1906.9545 & 953.9809 & 1905.9705 & 953.4889 & $\mathbf{N}$ & 602.3330 & 301.6702 & 585.3065 & 293.1569 & & & 5 \\
\hline 19 & 2055.0215 & 1028.0144 & 2037.9950 & 1019.5011 & 2037.0110 & 1019.0091 & M & 488.2901 & 244.6487 & 471.2636 & 236.1354 & & & 4 \\
\hline 20 & 2168.1056 & 1084.5564 & 2151.0791 & 1076.0432 & 2150.0950 & $\mathbf{1 0 7 5 . 5 5 1 2}$ & $\mathbf{L}$ & 357.2496 & 179.1285 & 340.2231 & 170.6152 & & & 3 \\
\hline 21 & 2265.1584 & 1133.0828 & 2248.1318 & 1124.5695 & 2247.1478 & 1124.0775 & $\mathbf{P}$ & 244.1656 & 122.5864 & 227.1390 & 114.0731 & & & 2 \\
\hline 22 & & & & & & & $\mathbf{K}$ & 147.1128 & 74.0600 & 130.0863 & 65.5468 & & & 1 \\
\hline
\end{tabular}
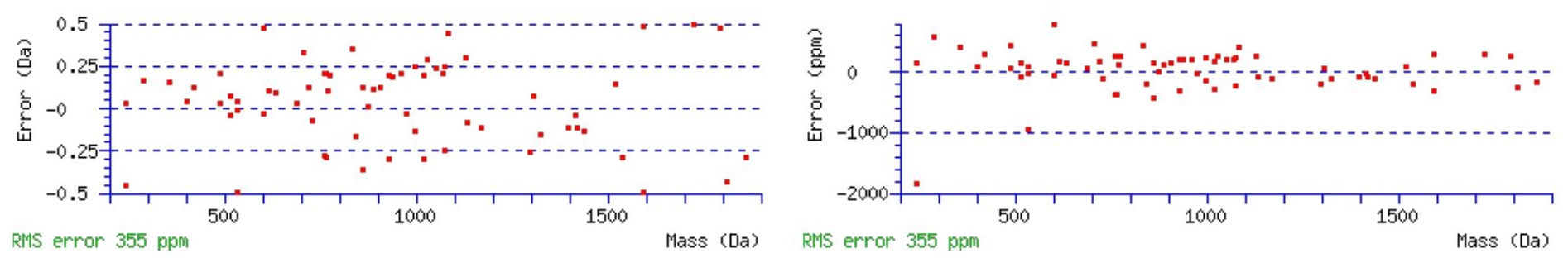

\section{All matches to this query}

\begin{tabular}{|l|l|l|l|}
\hline Score & Mr(calc): & Delta & \multicolumn{1}{c|}{ Sequence } \\
\hline 66.3 & 2410.2566 & 0.0051 & ASFNITLIHPNNLTALSNMLPK \\
\hline 64.8 & 2410.2566 & 0.0051 & ASFNITLIHPNNLTALSNMLPK \\
\hline 59.5 & 2410.2566 & 0.0051 & ASFNITLIHPNNLTALSNMLPK \\
\hline 49.5 & 2410.2566 & 0.0051 & ASFNITLIHPNNLTALSNMLPK \\
\hline 40.3 & 2410.2566 & 0.0051 & ASFNITLIHPNNLTALSNMLPK \\
\hline 40.3 & 2410.2566 & 0.0051 & ASFNITLIHPNNLTALSNMLPK \\
\hline 27.5 & 2409.2726 & 0.9892 & ASFNITLIHPNNLTALSNMLPK \\
\hline 25.5 & 2409.2726 & 0.9892 & ASFNITLIHPNNLTALSNMLPK \\
\hline 25.3 & 2409.2726 & 0.9892 & ASFNITLIHPNNLTALSNMLPK \\
\hline 7.8 & 2409.2726 & 0.9892 & ASFNITLIHPNNLTALSNMLPK \\
\hline
\end{tabular}

Spectrum No: 424; Query: 805; Rank: 1

\section{Peptide View}

MS/MS Fragmentation of GVFITNETGQPLIGK

Found in IPI00400579, Tax_Id=10116 Gene_Symbol=Gaa Lysosomal alpha-glucosidase precursor

Match to Query 805: 1573.833548 from(787.924050,2+)

Title: 091008RatKidney_NH4Format01_23.2641.2641.2.dta

Data file K:INewmanPaper|Piliang|3SubProteomes\Piliang3SP\mgf5ppm\ERLIC_3SubProteomes5ppm.mgf 

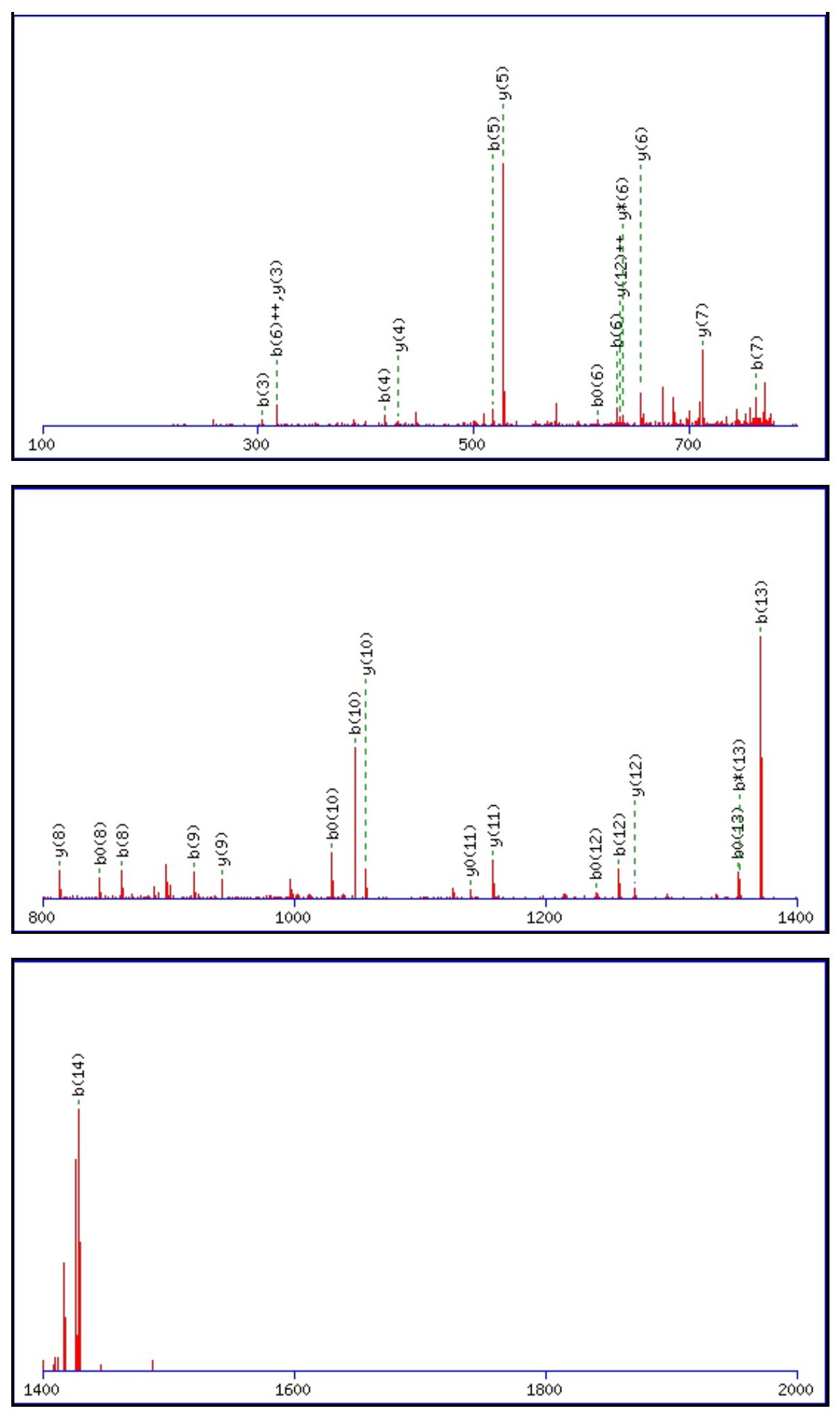

Monoisotopic mass of neutral peptide $\operatorname{Mr}($ calc): 1573.8352

Fixed modifications: Carbamidomethyl (C)

Variable modifications:

N6 : Deamidated $\mathrm{N}(\mathrm{N})$

Ions Score: 66 Expect: $2.7 \mathrm{e}-005$

Matches (Bold Red): 31/136 fragment ions using 57 most intense peaks

\begin{tabular}{|r|r|r|c|c|c|c|c|c|c|c|c|c|c|c|}
\hline$\#$ & $\mathbf{b}$ & $\mathbf{b}^{++}$ & $\mathbf{b}^{*}$ & $\mathbf{b}^{\boldsymbol{*}^{++}}$ & $\mathbf{b}^{\mathbf{0}}$ & $\mathbf{b}^{\mathbf{0 + +}}$ & Seq. & $\mathbf{y}$ & $\mathbf{y}^{++}$ & $\mathbf{y}^{\mathbf{*}}$ & $\mathbf{y}^{\boldsymbol{*}^{++}}$ & $\mathbf{y}^{\mathbf{0}}$ & $\mathbf{y}^{\mathbf{0 + +}}$ & $\#$ \\
\hline $\mathbf{1}$ & 58.0287 & 29.5180 & & & & & $\mathbf{G}$ & & & & & & & $\mathbf{1 5}$ \\
\hline $\mathbf{2}$ & 157.0972 & 79.0522 & & & & & $\mathbf{V}$ & 1517.8210 & 759.4141 & 1500.7944 & 750.9009 & 1499.8104 & 750.4088 & $\mathbf{1 4}$ \\
\hline 3 & $\mathbf{3 0 4 . 1 6 5 6}$ & 152.5864 & & & & & $\mathbf{F}$ & 1418.7526 & 709.8799 & 1401.7260 & 701.3666 & 1400.7420 & 700.8746 & $\mathbf{1 3}$ \\
\hline $\mathbf{4}$ & $\mathbf{4 1 7 . 2 4 9 6}$ & 209.1285 & & & & & $\mathbf{I}$ & $\mathbf{1 2 7 1 . 6 8 4 2}$ & $\mathbf{6 3 6 . 3 4 5 7}$ & 1254.6576 & 627.8324 & 1253.6736 & 627.3404 & $\mathbf{1 2}$ \\
\hline $\mathbf{5}$ & $\mathbf{5 1 8 . 2 9 7 3}$ & 259.6523 & & & 500.2867 & 250.6470 & $\mathbf{T}$ & $\mathbf{1 1 5 8 . 6 0 0 1}$ & 579.8037 & 1141.5735 & 571.2904 & $\mathbf{1 1 4 0 . 5 8 9 5}$ & 570.7984 & $\mathbf{1 1}$ \\
\hline
\end{tabular}




\begin{tabular}{|c|c|c|c|c|c|c|c|c|c|c|c|c|c|c|}
\hline 6 & 633.3242 & |317.1658 & 616.2977 & 308.6525 & 615.3137 & |308.1605 & $\mathbf{N}$ & |1057.5524| & 529.2798 & 1040.5259 & 520.7666 & 1039.5418 & |520.2746 & 10 \\
\hline 7 & 762.3668 & 381.6871 & 745.3403 & 373.1738 & 744.3563 & 372.6818 & $\mathbf{E}$ & 942.5255 & 471.7664 & 925.4989 & 463.2531 & 924.5149 & 462.7611 & 9 \\
\hline 8 & 863.4145 & 432.2109 & 846.3880 & 423.6976 & 845.4040 & 423.2056 & $T$ & 813.4829 & 407.2451 & 796.4563 & 398.7318 & 795.4723 & 398.2398 & 8 \\
\hline 9 & 920.4360 & 460.7216 & 903.4094 & 452.2084 & 902.4254 & 451.7163 & G & 712.4352 & 356.7212 & 695.4087 & 348.2080 & & & 7 \\
\hline 10 & 1048.4946 & 524.7509 & 1031.4680 & 516.2376 & 1030.4840 & 515.7456 & $\mathbf{Q}$ & 655.4137 & 328.2105 & 638.3872 & 319.6972 & & & 6 \\
\hline 11 & 1145.5473 & 573.2773 & 1128.5208 & 564.7640 & 1127.5368 & 564.2720 & $\mathbf{P}$ & 527.3552 & 264.1812 & 510.3286 & 255.6679 & & & 5 \\
\hline 12 & 1258.6314 & 629.8193 & 1241.6048 & 621.3061 & 1240.6208 & 620.8141 & $\mathbf{L}$ & 430.3024 & 215.6548 & 413.2758 & 207.1416 & & & 4 \\
\hline 13 & 1371.7155 & 686.3614 & 1354.6889 & 677.8481 & 1353.7049 & 677.3561 & I & 317.2183 & 159.1128 & 300.1918 & 150.5995 & & & 3 \\
\hline 14 & 1428.7369 & 714.8721 & 1411.7104 & 706.3588 & 1410.7264 & 705.8668 & G & 204.1343 & 102.5708 & 187.1077 & 94.0575 & & & 2 \\
\hline 15 & & & & & & & K & 147.1128 & 74.0600 & 130.0863 & 65.5468 & & & 1 \\
\hline
\end{tabular}
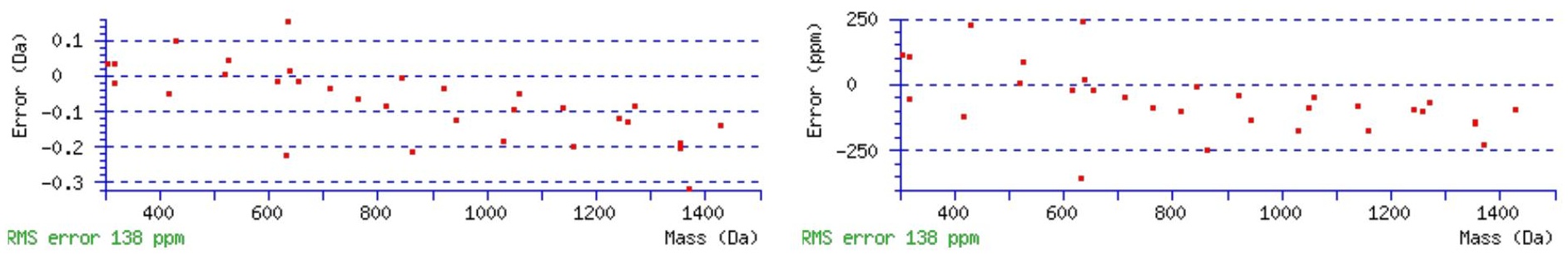

\section{All matches to this query}

\begin{tabular}{|l|l|l|l|}
\hline Score & Mr(calc): & Delta & \multicolumn{1}{c|}{ Sequence } \\
\hline 66.1 & 1573.8352 & -0.0016 & GVFITNETGQPLIGK \\
\hline 7.7 & 1573.8327 & 0.0009 & NMKLQPVPWFIGK \\
\hline 5.1 & 1573.8423 & -0.0088 & SLDESTLRKQIER \\
\hline 4.5 & 1573.8199 & 0.0136 & SDLTDKAEVLDQLK \\
\hline 2.5 & 1571.8229 & 2.0107 & EALMPTLGEIKDQK \\
\hline 2.1 & 1571.8229 & 2.0107 & EGKLKPLPGETMEK \\
\hline 1.4 & 1573.8181 & 0.0155 & VRGGRCAILNCLGK \\
\hline
\end{tabular}

Spectrum No: 425; Query: 1831; Rank: 1

\section{Peptide View}

MS/MS Fragmentation of LWVPNTSFDTASNWNQNR

Found in IPI00870509, Tax_Id=10116 Gene_Symbol=Amn_predicted amnionless

Match to Query 1831: 2149.987028 from(1076.000790,2+)

Title: 100101RatKid_NS_deglyco_14.4243.4243.2.dta

Data file K:INewmanPaper|Piliangl3SubProteomes\Piliang3SP\mgf5ppm\ERLIC_3SubProteomes5ppm.mgf 

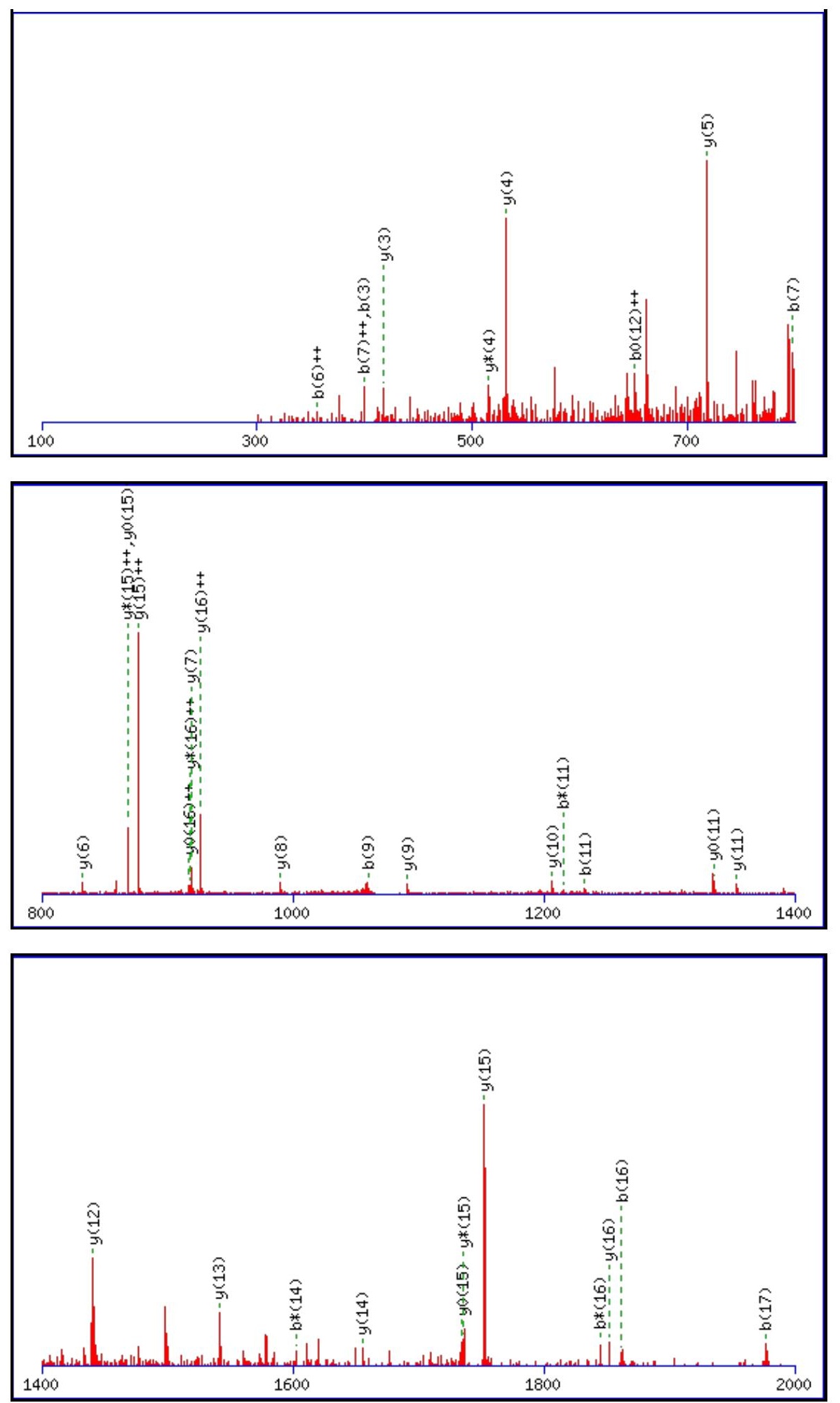

Monoisotopic mass of neutral peptide $\operatorname{Mr}($ calc): 2149.9817

Fixed modifications: Carbamidomethyl (C)

Variable modifications:

N17 : Deamidated N (N)

Ions Score: 66 Expect: $6.6 \mathrm{e}-005$

Matches (Bold Red): 36/174 fragment ions using 88 most intense peaks

\begin{tabular}{|r|c|c|c|c|c|c|c|c|c|c|c|c|c|c|}
\hline$\#$ & $\mathbf{b}$ & $\mathbf{b}^{++}$ & $\mathbf{b}^{*}$ & $\mathbf{b}^{\boldsymbol{*}^{++}}$ & $\mathbf{b}^{\mathbf{0}}$ & $\mathbf{b}^{\mathbf{0 + +}}$ & Seq. & $\mathbf{y}$ & $\mathbf{y}^{++}$ & $\mathbf{y}^{\mathbf{*}}$ & $\mathbf{y}^{\mathbf{*}^{++}}$ & $\mathbf{y}^{\mathbf{0}}$ & $\mathbf{y}^{\mathbf{0 + +}}$ & $\#$ \\
\hline $\mathbf{1}$ & 114.0913 & 57.5493 & & & & & $\mathbf{L}$ & & & & & & & $\mathbf{1 8}$ \\
\hline $\mathbf{2}$ & 300.1707 & 150.5890 & & & & & $\mathbf{W}$ & 2037.9049 & 1019.4561 & 2020.8784 & 1010.9428 & 2019.8944 & 1010.4508 & $\mathbf{1 7}$ \\
\hline $\mathbf{3}$ & 399.2391 & 200.1232 & & & & & $\mathbf{V}$ & $\mathbf{1 8 5 1 . 8 2 5 6}$ & $\mathbf{9 2 6 . 4 1 6 4}$ & 1834.7991 & $\mathbf{9 1 7 . 9 0 3 2}$ & 1833.8151 & $\mathbf{9 1 7 . 4 1 1 2}$ & $\mathbf{1 6}$ \\
\hline $\mathbf{4}$ & 496.2918 & 248.6496 & & & & & $\mathbf{P}$ & $\mathbf{1 7 5 2 . 7 5 7 2}$ & $\mathbf{8 7 6 . 8 8 2 2}$ & $\mathbf{1 7 3 5 . 7 3 0 7}$ & $\mathbf{8 6 8 . 3 6 9 0}$ & $\mathbf{1 7 3 4 . 7 4 6 6}$ & $\mathbf{8 6 7 . 8 7 7 0}$ & $\mathbf{1 5}$ \\
\hline $\mathbf{5}$ & 610.3348 & 305.6710 & 593.3082 & 297.1577 & & & $\mathbf{N}$ & $\mathbf{1 6 5 5 . 7 0 4 4}$ & 828.3559 & 1638.6779 & 819.8426 & 1637.6939 & 819.3506 & $\mathbf{1 4}$ \\
\hline
\end{tabular}




\begin{tabular}{|c|c|c|c|c|c|c|c|c|c|c|c|c|c|c|}
\hline 6 & 711.3824 & |356.1949 & 694.3559 & 347.6816 & 693.3719 & |347.1896 | & $T$ & |1541.6615 & 771.3344 & 1524.6350 & 762.8211| & 1523.6510 & 762.3291| & |13| \\
\hline 7 & 798.4145 & 399.7109 & 781.3879 & 391.1976 & 780.4039 & |390.7056 & S & 1440.6138 & 720.8106 & 1423.5873 & 712.2973 & 1422.6033 & 711.8053 & 12 \\
\hline 8 & 945.4829 & 473.2451 & 928.4563 & 464.7318 & 927.4723 & 464.2398 & $\mathbf{F}$ & 1353.5818 & 677.2945 & 1336.5553 & 668.7813 & 1335.5712 & 668.2893 & 11 \\
\hline 9 & 1060.5098 & 530.7585 & 1043.4833 & 522.2453 & 1042.4993 & 521.7533 & D & 1206.5134 & 603.7603 & 1189.4868 & 595.2471 & 1188.5028 & 594.7551 & 10 \\
\hline 10 & 1161.5575 & 581.2824 & 1144.5310 & 572.7691 & 1143.5469 & 572.2771 & $T$ & 1091.4865 & 546.2469 & 1074.4599 & 537.7336 & 1073.4759 & 537.2416 & 9 \\
\hline 11 & 1232.5946 & 616.8009 & 1215.5681 & 608.2877 & 1214.5840 & 607.7957 & A & 990.4388 & 495.7230 & 973.4122 & 487.2098 & 972.4282 & 486.7177 & 8 \\
\hline 12 & 1319.6266 & 660.3170 & 1302.6001 & 651.8037 & 1301.6161 & 651.3117 & S & 919.4017 & 460.2045 & 902.3751 & 451.6912 & 901.3911 & 451.1992 & 7 \\
\hline 13 & 1433.6696 & 717.3384 & 1416.6430 & 708.8251 & 1415.6590 & 708.3331 & $\mathbf{N}$ & 832.3696 & 416.6885 & 815.3431 & 408.1752 & & & 6 \\
\hline 14 & 1619.7489 & 810.3781 & 1602.7223 & 801.8648 & 1601.7383 & 801.3728 & W & 718.3267 & 359.6670 & 701.3002 & 351.1537 & & & 5 \\
\hline 15 & 1733.7918 & 867.3995 & 1716.7653 & 858.8863 & |1715.7812 & 858.3943 & $\mathbf{N}$ & 532.2474 & 266.6273 & 515.2208 & 258.1141 & & & 4 \\
\hline 16 & 1861.8504 & 931.4288 & 1844.8238 & 922.9156 & 1843.8398 & 922.4235 & $\mathbf{Q}$ & 418.2045 & 209.6059 & 401.1779 & 201.0926 & & & 3 \\
\hline 17 & 1976.8773 & 988.9423 & 1959.8508 & 980.4290 & 1958.8668 & 979.9370 & $\mathbf{N}$ & 290.1459 & 145.5766 & 273.1193 & 137.0633 & & & 2 \\
\hline 18 & & & & & & & $\mathbf{R}$ & 175.1190 & 88.0631 & 158.0924 & 79.5498 & & & 1 \\
\hline
\end{tabular}
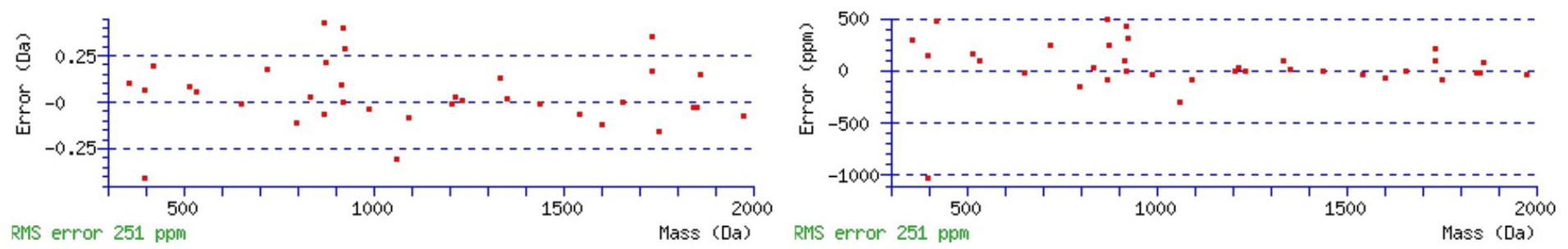

\section{All matches to this query}

\begin{tabular}{|l|l|l|l|}
\hline Score & Mr(calc): & Delta & \multicolumn{1}{|c|}{ Sequence } \\
\hline 66.0 & 2149.9817 & 0.0053 & $\underline{\text { LWVPNTSFDTASNWNQNR }}$ \\
\hline 59.6 & 2149.9817 & 0.0053 & $\underline{\text { LWVPNTSFDTASNWNQNR }}$ \\
\hline 48.5 & 2149.9817 & 0.0053 & $\underline{\text { LWVPNTSFDTASNWNQNR }}$ \\
\hline 14.5 & 2149.9817 & 0.0053 & LWVPNTSFDTASNWNQNR \\
\hline 7.1 & 2150.0004 & -0.0134 & $\underline{\text { RIEELEGQLDQLTQENR }}$ \\
\hline 5.9 & 2149.9753 & 0.0117 & $\underline{\text { RNQKLSSVEEDPGVNQDR }}$ \\
\hline 5.9 & 2149.9753 & 0.0117 & $\underline{\text { RNQKLSSVEEDPGVNQDR }}$ \\
\hline 3.8 & 2150.0062 & -0.0191 & KHDEHNEKELDLMINQR \\
\hline 3.5 & 2149.9785 & 0.0085 & $\underline{\text { WNFLLTAVSKAMTLSHR }}$ \\
\hline 3.0 & 2149.9785 & 0.0085 & $\underline{\text { WNFLLTAVSKAMTLSHR }}$ \\
\hline
\end{tabular}

Spectrum No: 426; Query: 118; Rank: 1

\section{Peptide View}

MS/MS Fragmentation of EYNVSEDTR

Found in IPI00200640, Tax_Id=10116 Gene_Symbol=Mucdhl Isoform 1 of Mucin and cadherin-like protein precursor

Match to Query 118: 1112.462008 from(557.238280,2+)

Title: 091008RatKidney_NoSalt_28.569.569.2.dta

Data file K:INewmanPaper|Piliang|3SubProteomes\Piliang3SP\mgf5ppm\ERLIC_3SubProteomes5ppm.mgf 

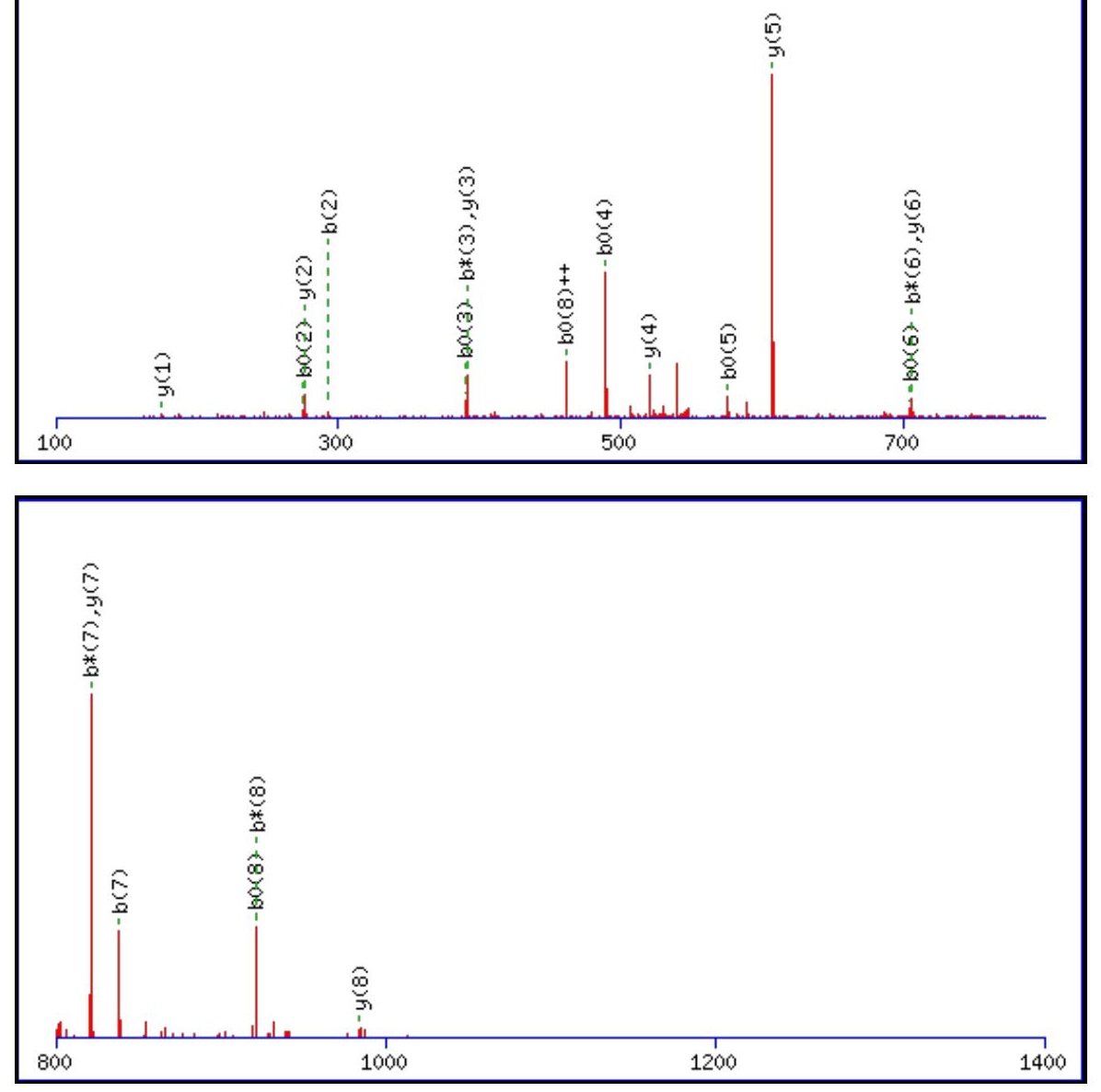

\begin{tabular}{rrrr}
\hline 00 & 1600 & 1800 & 2000 \\
\hline
\end{tabular}

Monoisotopic mass of neutral peptide $\operatorname{Mr}($ calc): 1112.4622

Fixed modifications: Carbamidomethyl (C)

Variable modifications:

N3 : Deamidated $\mathrm{N}(\mathrm{N})$

Ions Score: 66 Expect: $2 \mathrm{e}-005$

Matches (Bold Red): 21/90 fragment ions using 27 most intense peaks

\begin{tabular}{|c|c|c|c|c|c|c|c|c|c|c|c|c|c|c|}
\hline$\#$ & $\mathbf{b}$ & $\mathbf{b}^{++}$ & $\mathbf{b}^{*}$ & $\mathbf{b}^{*_{++}}$ & $\mathbf{b}^{\mathbf{0}}$ & $\mathbf{b}^{\mathbf{0}+}$ & $\mathbf{S e q}$ & $\mathbf{y}$ & $\mathbf{y}^{++}$ & $\mathbf{y}^{*}$ & $\mathbf{y}^{\boldsymbol{*}^{++}}$ & $\mathbf{y}^{\mathbf{0}}$ & $\mathbf{y}^{\mathbf{0}++}$ & $\#$ \\
\hline $\mathbf{1}$ & 130.0499 & 65.5286 & & & 112.0393 & 56.5233 & $\mathbf{E}$ & & & & & & & $\mathbf{9}$ \\
\hline $\mathbf{2}$ & 293.1132 & 147.0602 & & & $\mathbf{2 7 5 . 1 0 2 6}$ & 138.0550 & $\mathbf{Y}$ & $\mathbf{9 8 4 . 4 2 6 9}$ & 492.7171 & 967.4003 & 484.2038 & 966.4163 & 483.7118 & $\mathbf{8}$ \\
\hline $\mathbf{3}$ & 408.1401 & 204.5737 & 391.1136 & 196.0604 & $\mathbf{3 9 0 . 1 2 9 6}$ & 195.5684 & $\mathbf{N}$ & $\mathbf{8 2 1 . 3 6 3 5}$ & 411.1854 & 804.3370 & 402.6721 & 803.3530 & 402.1801 & $\mathbf{7}$ \\
\hline $\mathbf{4}$ & 507.2085 & 254.1079 & 490.1820 & 245.5946 & $\mathbf{4 8 9 . 1 9 8 0}$ & 245.1026 & $\mathbf{V}$ & $\mathbf{7 0 6 . 3 3 6 6}$ & 353.6719 & 689.3101 & 345.1587 & 688.3260 & 344.6667 & $\mathbf{6}$ \\
\hline $\mathbf{5}$ & 594.2406 & 297.6239 & 577.2140 & 289.1107 & $\mathbf{5 7 6 . 2 3 0 0}$ & 288.6186 & $\mathbf{S}$ & $\mathbf{6 0 7 . 2 6 8 2}$ & 304.1377 & 590.2416 & 295.6245 & 589.2576 & 295.1325 & $\mathbf{5}$ \\
\hline
\end{tabular}




\begin{tabular}{|l|l|l|l|l|l|l|l|l|l|l|l|l|l|l|l|l|}
$\mathbf{6}$ & 723.2832 & 362.1452 & $\mathbf{7 0 6 . 2 5 6 6}$ & 353.6319 & $\mathbf{7 0 5 . 2 7 2 6}$ & 353.1399 & $\mathbf{E}$ & $\mathbf{5 2 0 . 2 3 6 2}$ & 260.6217 & 503.2096 & 252.1084 & 502.2256 & 251.6164 & $\mathbf{4}$ \\
\hline $\mathbf{7}$ & $\mathbf{8 3 8 . 3 1 0 1}$ & 419.6587 & $\mathbf{8 2 1 . 2 8 3 6}$ & 411.1454 & 820.2995 & 410.6534 & D & $\mathbf{3 9 1 . 1 9 3 6}$ & 196.1004 & 374.1670 & 187.5872 & 373.1830 & 187.0951 & 3 \\
\hline $\mathbf{8}$ & 939.3578 & 470.1825 & $\mathbf{9 2 2 . 3 3 1 2}$ & 461.6693 & $\mathbf{9 2 1 . 3 4 7 2}$ & $\mathbf{4 6 1 . 1 7 7 3}$ & $\mathbf{T}$ & $\mathbf{2 7 6 . 1 6 6 6}$ & 138.5870 & 259.1401 & 130.0737 & 258.1561 & 129.5817 & $\mathbf{2}$ \\
\hline $\mathbf{9}$ & & & & & & & R & $\mathbf{1 7 5 . 1 1 9 0}$ & 88.0631 & 158.0924 & $\mathbf{7 9 . 5 4 9 8}$ & & & $\mathbf{1}$ \\
\hline
\end{tabular}
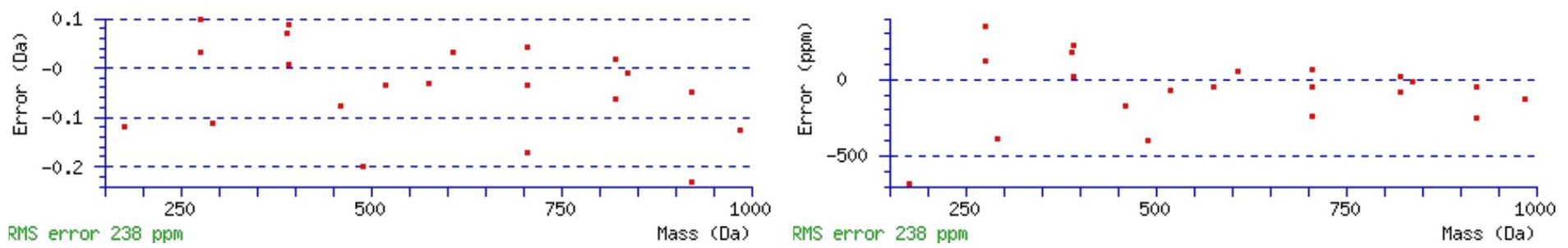

\section{All matches to this query}

\begin{tabular}{|l|l|l|l|}
\hline Score & Mr(calc): & Delta & \multicolumn{1}{|c|}{ Sequence } \\
\hline 65.9 & 1112.4622 & -0.0002 & EYNVSEDTR \\
\hline 7.8 & 1112.4638 & -0.0018 & EPTTVETEK \\
\hline 6.0 & 1111.4669 & 0.9951 & VEDYTNNQK \\
\hline 5.1 & 1110.4495 & 2.0125 & DQFESHLR \\
\hline 3.1 & 1112.4638 & -0.0018 & EPTTVETEK \\
\hline 2.4 & 1110.4495 & 2.0125 & RYSFSESR \\
\hline 1.8 & 1112.4726 & -0.0106 & EVMFPPGTR \\
\hline 1.7 & 1111.4521 & 1.0099 & YYVREMR \\
\hline 1.7 & 1111.4521 & 1.0099 & YYVREMR \\
\hline 1.4 & 1112.4652 & -0.0032 & GESVFHTTR \\
\hline
\end{tabular}

Spectrum No: 427; Query: 2760; Rank: 1

\section{Peptide View}

MS/MS Fragmentation of SYLDDGPLQHYIAVSSPTNTTYVVQYALANLTGK

Found in IPI00192495, Tax_Id=10116 Gene_Symbol=Ncstn Isoform 1 of Nicastrin precursor

Match to Query 2760: 3700.833522 from(1234.618450,3+)

Title: 100101RatKid_NS_deglyco_16.6848.6848.3.dta

Data file K:INewmanPaper|Piliangl3SubProteomes\Piliang3SP\mgf5ppm\ERLIC_3SubProteomes5ppm.mgf

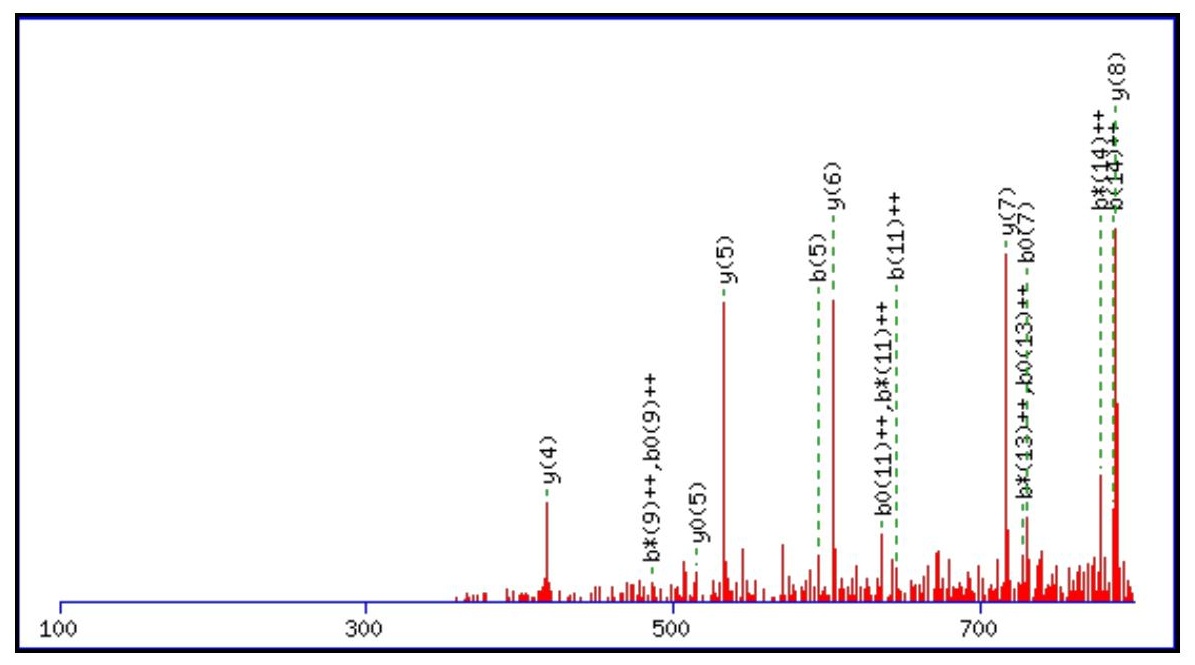



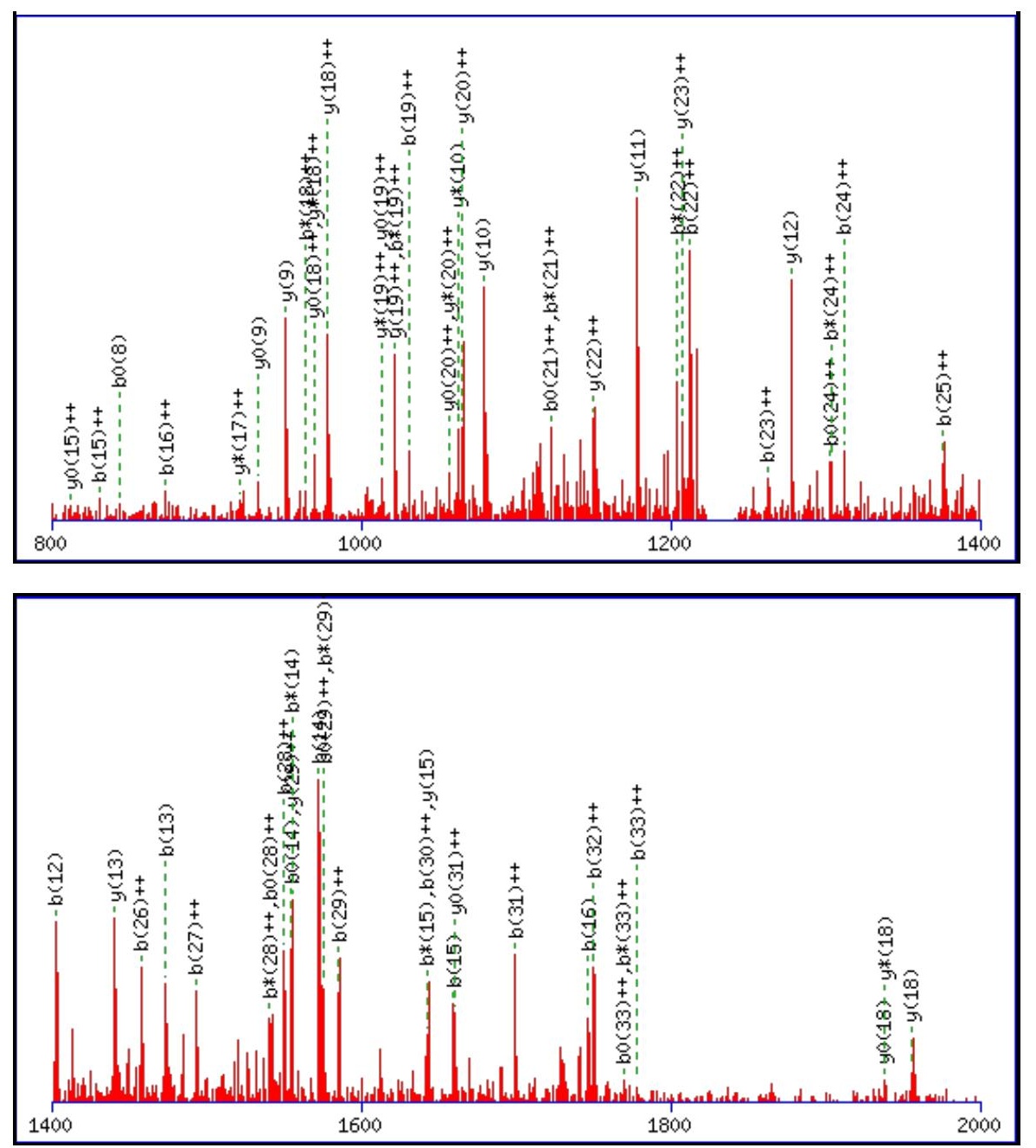

Monoisotopic mass of neutral peptide $\operatorname{Mr}($ calc): 3700.8148

Fixed modifications: Carbamidomethyl (C)

Variable modifications:

N19 : Deamidated_N (N)

N30 : Deamidated_N $(\mathrm{N})$

Ions Score: 66 Expect: $6.1 \mathrm{e}-005$

Matches (Bold Red): 80/376 fragment ions using 145 most intense peaks

\begin{tabular}{|c|c|c|c|c|c|c|c|c|c|c|c|c|c|c|}
\hline \# & b & $\mathbf{b}^{++}$ & $\mathbf{b}^{*}$ & $\mathbf{b}^{*^{++}}$ & $\mathbf{b}^{0}$ & $\mathbf{b}^{0++}$ & Seq. & $\mathbf{y}$ & $\mathbf{y}^{++}$ & $\mathbf{y}^{*}$ & $\mathbf{y}^{*^{++}}$ & $\mathbf{y}^{0}$ & & \# \\
\hline 1 & 88.0393 & & & & 287 & 35.5180 & $\mathrm{~S}$ & & & & & & & 34 \\
\hline 2 & 251.1026 & 26.0550 & & & 233.0921 & & $\mathbf{Y}$ & & & & & & & 433 \\
\hline 3 & 364.1 & 0 & & & & & $\mathbf{L}$ & & & & & & 617 & 32 \\
\hline 4 & 479.2136 & 240.1 & & & 461.2 & 052 & D & 3338.6427 & 1669. & 3321.6161 & 3117 & 3320.6321 & 197 & 31 \\
\hline 5 & 594.2406 & 297.6239 & & & 576.2300 & 288.6186 & D & 3223.6157 & 1612.3115 & 3206.5892 & 1603.7982 & 3205.6052 & 062 & 30 \\
\hline 6 & 651.2 & 26.1 & & & & & G & & & 522 & & & & 29 \\
\hline 7 & 748.3148 & 374.6610 & & & 730.3042 & 365.6558 & $\mathbf{P}$ & 3051.5673 & 1526.2873 & 5408 & 1517.7740 & 3033.5568 & 1517.2820 & 28 \\
\hline 8 & 861.3989 & 431.2031 & & & 843.3883 & 422.1978 & $\mathbf{L}$ & 2954.5146 & 1477.7609 & 2937.4880 & 1469.2476 & 2936.5040 & 1468.7556 & 27 \\
\hline 9 & 989.4575 & 495.23 & & & 69 & & $\mathbf{Q}$ & 2841 & 1421 & 2824 & & 2823 & 136 & 26 \\
\hline 10 & 1126.5164 & 563.7618 & 09.4898 & 555.2 & 1108.5058 & 554.7565 & H & 2713.3719 & 1357.1896 & 2696.3454 & $134 \varepsilon$ & 2695.3614 & 134 & 25 \\
\hline 11 & 1289.5797 & 645.2935 & 72.5531 & 636. & 1271.5691 & 636.2882 & $\mathbf{Y}$ & 2576.3130 & 1288.6601 & 2559.2865 & 1280 & 3025 & 1279.6549 & 24 \\
\hline 12 & 02.6638 & 355 & 2 & 22 & 84.6532 & 692.8302 & I & 2413 & 1207 & 2396. & 5152 & 2395.2391 & 1198.1232 & 23 \\
\hline 13 & 1473.7009 & 737.3541 & 456.6743 & 728.8 & 1455.6903 & 728.3488 & A & 2300.1656 & 1150.5864 & 2283.1391 & 1142 & 2282.1551 & 5812 & 22 \\
\hline 14 & 1572.7693 & 786.8883 & 555.7427 & 778.3750 & 1554.7587 & 777.8830 & $\mathbf{V}$ & 2229.1285 & 1115.0679 & 2212.1020 & 1106.5546 & 2211.1179 & 1106.0626 & 21 \\
\hline 15 & 1659.8013 & 830.4043 & 1642.7748 & 821.8910 & 1641.7907 & 821.3990 & $\mathrm{~S}$ & 2130.0601 & 1065.5337 & 2113.0335 & 1057.0204 & 2112.0495 & 1056.5284 & 20 \\
\hline 16 & 746.8333 & 873.9203 & 1729.8068 & 865.4070 & 1728.8228 & 864.9150 & $\mathrm{~S}$ & 2043.0281 & 1022.0177 & 2026.0015 & 1013.5044 & 2025.0175 & 1013.0124 & 19 \\
\hline 17 & 1843.8861 & 922.4467 & 1826.8596 & 913.9334 & 1825.8755 & 913.4414 & $\mathbf{P}$ & 1955.9960 & 978.5017 & 1938.9695 & 969.9884 & 1937.9855 & 969.4964 & 18 \\
\hline 18 & 1944.9338 & 972.9705 & 1927.9072 & 964.4573 & 1926.9232 & 963.9652 & $T$ & 1858.9433 & 929.9753 & 1841.9167 & 921.4620 & 1840.9327 & 920.9700 & 17 \\
\hline
\end{tabular}




\begin{tabular}{|c|c|c|c|c|c|c|c|c|c|c|c|c|c|c|}
\hline 19 & |2059.9607| & $|1030.4840|$ & 2042. & 1021.9707 & |2041.9502| & 1021.4787 & $\mathbf{N}$ & & 879.4514 & 1740.8690 & 870.9382 & |1739.8850| & 870.4462 & 16 \\
\hline 20 & 2161.0084 & 1081.0078 & |2143.9819 & 1072.4946 & 2142.9978 & 1072.0026 & $T$ & 1642.8687 & 821.9380 & 1625.8421 & 813.4247 & 1624.8581 & 812.9327 & 15 \\
\hline 21 & 2262.0561 & |1131.5317 & 2245.0295 & 1123.0184 & 2244.0455 & 1122.5264 & $\mathbf{T}$ & 1541.8210 & 771.4141 & 1524.7944 & 762.9009 & 1523.8104 & 762.4088 & 14 \\
\hline 22 & 2425.1194 & 1213.0633 & 2408.0929 & 1204.5501 & 2407.1088 & 1204.0581 & $\mathbf{Y}$ & \begin{tabular}{|l|l|}
1440.7733 \\
\end{tabular} & 720.8903 & 1423.7468 & 712.3770 & 1422.7627 & 711.8850 & 13 \\
\hline 23 & 2524.1878 & 1262.5975 & 2507.1613 & 1254.0843 & 2506.1773 & 1253.5923 & V & 1277.7100 & 639.3586 & 1260.6834 & 630.8454 & 1259.6994 & 630.3533 & 12 \\
\hline 24 & 2623.2562 & 1312.1318 & 2606.2297 & 1303.6185 & 2605.2457 & 1303.1265 & V & 1178.6416 & 589.8244 & 1161.6150 & 581.3111 & 1160.6310 & 580.8191 & 11 \\
\hline 25 & 2751.3148 & 1376.1610 & 2734.2883 & 1367.6478 & 2733.3042 & 1367.1558 & $\mathbf{Q}$ & 1079.5731 & 540.2902 & 1062.5466 & 531.7769 & 1061.5626 & 531.2849 & 10 \\
\hline 26 & 2914.3781 & \begin{tabular}{|l|}
1457.6927 \\
\end{tabular} & 2897.3516 & 1449.1794 & 2896.3676 & 1448.6874 & $\mathbf{Y}$ & 951.5146 & 476.2609 & 934.4880 & 467.7476 & 933.5040 & 467.2556 & 9 \\
\hline 27 & 2985.4153 & 1493.2113 & 2968.3887 & 1484.6980 & 2967.4047 & 1484.2060 & A & 788.4512 & 394.7293 & 771.4247 & 386.2160 & 770.4407 & 385.7240 & 8 \\
\hline 28 & 3098.4993 & 1549.7533 & 3081.4728 & 1541.2400 & 3080.4888 & 1540.7480 & $\mathbf{L}$ & 717.4141 & 359.2107 & 700.3876 & 350.6974 & 699.4036 & 350.2054 & 7 \\
\hline 29 & 3169.5364 & 1585.2719 & 3152.5099 & 1576.7586 & 3151.5259 & 1576.2666 & A & 604.3301 & 302.6687 & 587.3035 & 294.1554 & 586.3195 & 293.6634 & 6 \\
\hline 30 & 3284.5634 & 1642.7853 & 3267.5368 & 1634.2720 & 3266.5528 & 1633.7800 & $\mathbf{N}$ & 533.2929 & 267.1501 & 516.2664 & 258.6368 & 515.2824 & 258.1448 & 5 \\
\hline 31 & 3397.6474 & 1699.3274 & 3380.6209 & 1690.8141 & 3379.6369 & 1690.3221 & L & 418.2660 & 209.6366 & 401.2395 & 201.1234 & 400.2554 & 200.6314 & 4 \\
\hline 32 & 3498.6951 & 1749.8512 & 3481.6686 & 1741.3379 & 3480.6845 & 1740.8459 & $\mathbf{T}$ & 305.1819 & 153.0946 & 288.1554 & 144.5813 & 287.1714 & 144.0893 & 3 \\
\hline 33 & 3555.7166 & 1778.3619 & 3538.6900 & 1769.8487 & 3537.7060 & 1769.3566 & G & 204.1343 & 102.5708 & 187.1077 & 94.0575 & & & 2 \\
\hline 34 & & & & & & & $\mathbf{K}$ & 147.1128 & 74.0600 & 130.0863 & 65.5468 & & & 1 \\
\hline
\end{tabular}

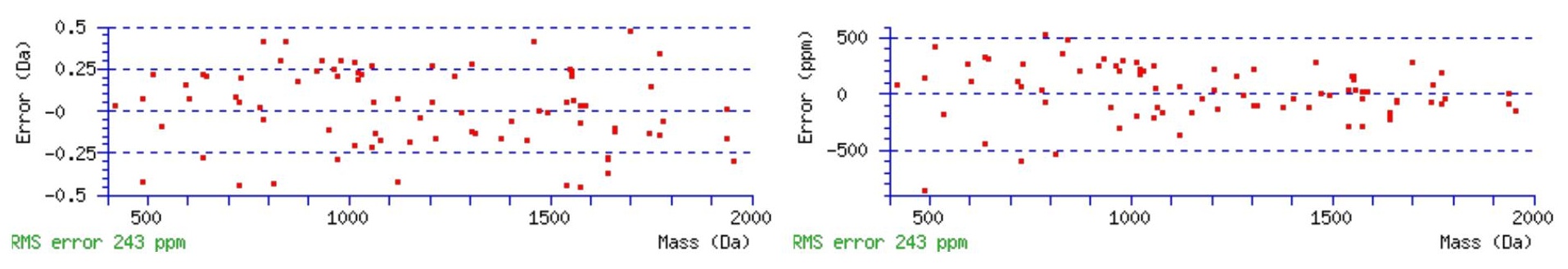

\section{All matches to this query}

\begin{tabular}{|l|l|l|l|}
\hline Score & Mr(calc): & Delta & \multicolumn{1}{c|}{ Sequence } \\
\hline 65.8 & 3700.8148 & 0.0187 & SYLDDGPLQHYIAVSSPTNTTYVVQYALANLTGK \\
\hline 54.4 & 3699.8308 & 1.0027 & SYLDDGPLQHYIAVSSPTNTTYVVQYALANLTGK \\
\hline 27.7 & 3699.8308 & 1.0027 & SYLDDGPLQHYIAVSSPTNTTYVVQYALANLTGK \\
\hline 10.0 & 3699.8493 & 0.9842 & SWCTLGFVRSIALTPQACGALSSPRWITLLMK \\
\hline 3.4 & 3698.8266 & 2.0069 & $\underline{\text { IKTNVINPAYAAELGQIENSLHMGYSALEIKSK }}$ \\
\hline 3.4 & 3698.8266 & 2.0069 & $\underline{\text { IKTNVINPAYAAELGQIENSLHMGYSALEIKSK }}$ \\
\hline 3.0 & 3698.8468 & 1.9867 & SYLDDGPLQHYIAVSSPTNTTYVVQYALANLTGK \\
\hline 2.3 & 3699.8106 & 1.0229 & $\underline{\text { IKTNVINPAYAAELGQIENSLHMGYSALEIKSK }}$ \\
\hline 2.3 & 3699.8106 & 1.0229 & $\underline{\text { IKTNVINPAYAAELGQIENSLHMGYSALEIKSK }}$ \\
\hline 2.0 & 3698.7950 & 2.0385 & $\underline{\text { ISLPLYSILSALTILGMIMASAFLFFNIKNR }}$ \\
\hline
\end{tabular}

Spectrum No: 428; Query: 1844; Rank: 1

\section{Peptide View}

\section{MS/MS Fragmentation of SLTQGSMIVGNLAPVNGTSQGK}

Found in IPI00388323, Tax_Id=10116 Gene_Symbol=Hspg2 396 kDa protein

Match to Query 1844: 2159.095128 from(1080.554840,2+)

Title: 100101RatKid_NS_deglyco_14.3410.3410.2.dta

Data file K:\NewmanPaper|Piliangl3SubProteomes\Piliang3SP\mgf5ppm\ERLIC_3SubProteomes5ppm.mgf 

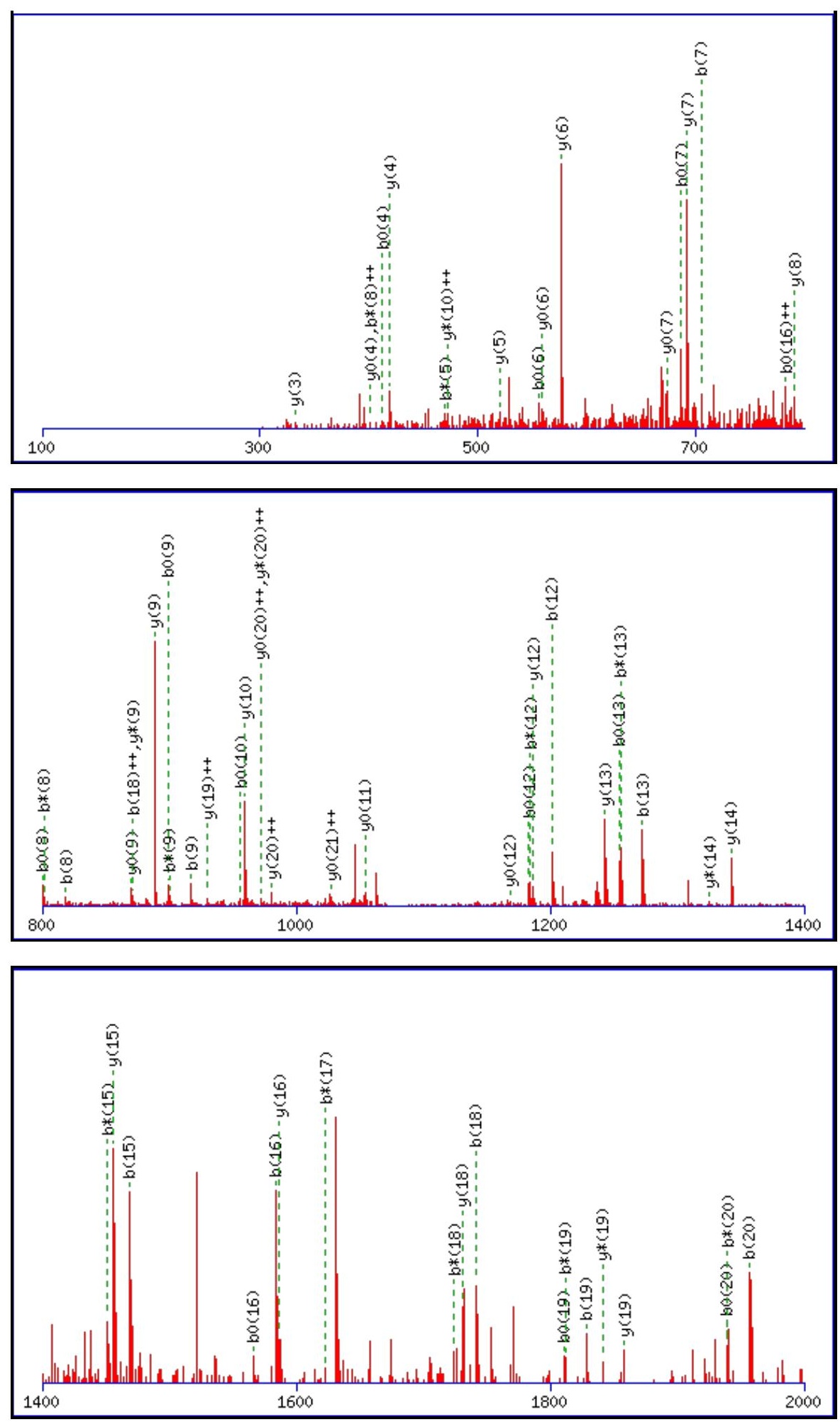

Monoisotopic mass of neutral peptide $\operatorname{Mr}($ calc): 2159.0892

Fixed modifications: Carbamidomethyl (C)

Variable modifications:

N16 : Deamidated N (N)

Ions Score: 66 Expect: $4.4 \mathrm{e}-005$

Matches (Bold Red): 64/240 fragment ions using 143 most intense peaks

\begin{tabular}{|c|c|c|c|c|c|c|c|c|c|c|c|c|c|c|}
\hline \# & b & $\mathbf{b}^{++}$ & $\mathbf{b}^{*}$ & $\mathbf{b}^{*^{++}}$ & $\mathbf{b}^{0}$ & $\mathbf{b}^{0++}$ & Seq. & $\mathbf{y}$ & $y^{++}$ & $\mathrm{y}^{*}$ & $\mathrm{y}^{*^{++}}$ & $\mathbf{y}^{\mathbf{0}}$ & $y^{0++}$ & $\#$ \\
\hline 1 & 88.0393 & 44.5233 & & & 70.0287 & 35.5180 & $\mathbf{S}$ & & & & & & & 22 \\
\hline 2 & 201.1234 & 101.0653 & & & 183.1128 & 92.0600 & $\mathbf{L}$ & 2073.0645 & 1037.0359 & 2056.0379 & 1028.5226 & 2055.0539 & 1028.0306 & 21 \\
\hline 3 & 302.1710 & 151.5892 & & & 284.1605 & 142.5839 & $\mathbf{T}$ & 1959.9804 & 980.4938 & 1942.9539 & 971.9806 & 1941.9698 & 971.4886 & 20 \\
\hline 4 & 430.2296 & 215.6185 & 413.2031 & 207.1052 & 412.2191 & 206.6132 & $\mathbf{Q}$ & 1858.9327 & 929.9700 & 1841.9062 & 921.4567 & 1840.9222 & 920.9647 & 19 \\
\hline 5 & 487.2511 & 244.1292 & 470.2245 & 235.6159 & 469.2405 & 235.1239 & G & 1730.8742 & 865.9407 & 1713.8476 & 857.4274 & 1712.8636 & 856.9354 & 18 \\
\hline
\end{tabular}




\begin{tabular}{|c|c|c|c|c|c|c|c|c|c|c|c|c|c|c|}
\hline 6 & 574.2831 & 287.6452 & 557.2566 & |279.1319| & 556.2726 & |278.6399| & S & 1673.8527 & 837.4300 & |1656.8261 & 828.9167 & |1655.8421 & 828.4247 & $|17|$ \\
\hline 7 & 705.3236 & 353.1654 & 688.2971 & 344.6522 & 687.3130 & 344.1602 & $\mathbf{M}$ & 1586.8207 & 793.9140 & 1569.7941 & 785.4007 & 1568.8101 & 784.9087 & 16 \\
\hline 8 & 818.4077 & 409.7075 & 801.3811 & 401.1942 & 800.3971 & 400.7022 & I & 1455.7802 & 728.3937 & 1438.7536 & 719.8805 & 1437.7696 & 719.3884 & 5 \\
\hline 9 & 917.4761 & 459.2417 & 900.4495 & 450.7284 & 899.4655 & 450.2364 & V & 1342.6961 & 671.8517 & 1325.6696 & 663.3384 & 1324.6855 & 662.8464 & 14 \\
\hline 10 & 974.4975 & 487.7524 & 957.4710 & 479.2391 & 956.4870 & 478.7471 & $\mathbf{G}$ & 1243.6277 & 622.3175 & 1226.6012 & 613.8042 & 1225.6171 & 613.3122 & 13 \\
\hline 11 & 1088.5405 & 544.7739 & 1071.5139 & 536.2606 & 1070.5299 & 535.7686 & $\mathbf{N}$ & 1186.6062 & 593.8068 & 1169.5797 & 585.2935 & 1168.5957 & 584.8015 & 12 \\
\hline 12 & 1201.6245 & 601.3159 & 1184.5980 & 592.8026 & 1183.6140 & 592.3106 & $\mathbf{L}$ & 1072.5633 & 536.7853 & 1055.5368 & 528.2720 & 1054.5527 & 527.7800 & 11 \\
\hline 13 & 1272.6616 & 636.8345 & 1255.6351 & 628.3212 & 1254.6511 & 627.8292 & A & 959.4792 & 480.2433 & 942.4527 & 471.7300 & 941.4687 & 471.2380 & 10 \\
\hline 14 & 1369.7144 & 685.3608 & 1352.6879 & 676.8476 & 1351.7038 & 676.3556 & $\mathbf{P}$ & 888.4421 & 444.7247 & 871.4156 & 436.2114 & 870.4316 & 435.7194 & 9 \\
\hline 15 & 1468.7828 & 734.8951 & 1451.7563 & 726.3818 & 1450.7723 & 725.8898 & $\mathbf{V}$ & 791.3894 & 396.1983 & 774.3628 & 387.6850 & 773.3788 & 387.1930 & 8 \\
\hline 16 & 1583.8098 & 792.4085 & 1566.7832 & 783.8952 & 1565.7992 & 783.4032 & $\mathbf{N}$ & 692.3210 & 346.6641 & 675.2944 & 338.1508 & 674.3104 & 337.6588 & 7 \\
\hline 17 & 1640.8312 & 820.9193 & 1623.8047 & 812.4060 & 1622.8207 & 811.9140 & G & 577.2940 & 289.1506 & 560.2675 & 280.6374 & 559.2835 & 280.1454 & 6 \\
\hline 18 & 1741.8789 & 871.4431 & 1724.8524 & 862.9298 & 1723.8683 & 862.4378 & $\mathbf{T}$ & 520.2726 & 260.6399 & 503.2460 & 252.1266 & 502.2620 & 251.6346 & 5 \\
\hline 19 & 1828.9109 & 914.9591 & 1811.8844 & 906.4458 & 1810.9004 & 905.9538 & S & 419.2249 & 210.1161 & 402.1983 & 201.6028 & 401.2143 & 201.1108 & 4 \\
\hline 20 & 1956.9695 & 978.9884 & 1939.9430 & 970.4751 & 1938.9589 & 969.9831 & $\mathbf{Q}$ & 332.1928 & 166.6001 & 315.1663 & 158.0868 & & & 3 \\
\hline 21 & 2013.9910 & 1007.4991 & 1996.9644 & 998.9859 & 1995.9804 & 998.4938 & G & 204.1343 & 102.5708 & 187.1077 & 94.0575 & & & 2 \\
\hline 22 & & & & & & & $\mathbf{K}$ & 147.1128 & 74.0600 & 130.0863 & 65.5468 & & & 1 \\
\hline
\end{tabular}
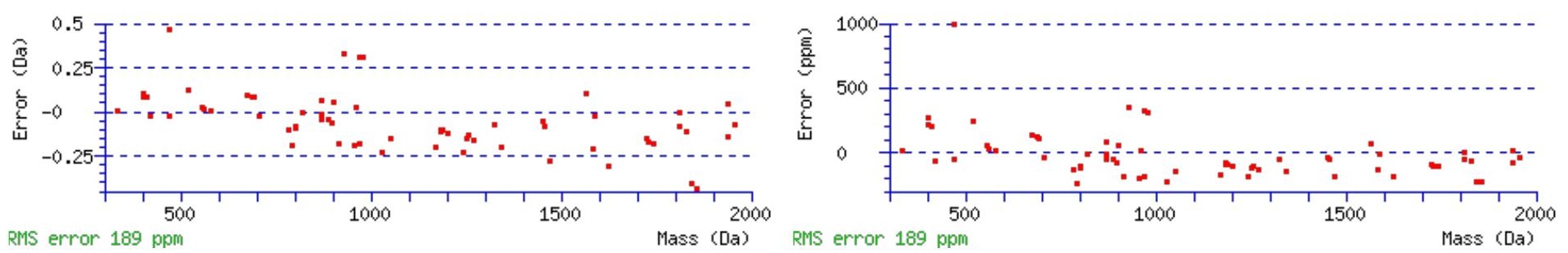

\section{All matches to this query}

\begin{tabular}{|l|l|l|l|}
\hline Score & Mr(calc): & Delta & \multicolumn{1}{c|}{ Sequence } \\
\hline 65.8 & 2159.0892 & 0.0059 & SLTQGSMIVGNLAPVNGTSQGK \\
\hline 38.9 & 2159.0892 & 0.0059 & SLTQGSMIVGNLAPVNGTSQGK \\
\hline 6.2 & 2158.1052 & 0.9899 & SLTQGSMIVGNLAPVNGTSQGK \\
\hline 1.1 & 2159.0736 & 0.0216 & SFISVAGTTGISAKSNVARGR \\
\hline 1.1 & 2159.0736 & 0.0216 & SFISVAGTTGISAKSNVARGR \\
\hline 1.1 & 2159.0736 & 0.0216 & SFISVAGTTGISAKSNVARGR \\
\hline 0.8 & 2159.0736 & 0.0216 & $\underline{\text { SFISVAGTTGISAKSNVARGR }}$ \\
\hline
\end{tabular}

Spectrum No: 429; Query: 2716; Rank: 1

\section{Peptide View}

MS/MS Fragmentation of TVFQKENGTVTAANASTLNDGAAAVVLMTAEAAQR

Found in IPI00324302, Tax_Id=10116 Gene_Symbol=Acat1 Acetyl-CoA acetyltransferase, mitochondrial precursor

Match to Query 2716: 3520.759692 from(1174.593840,3+)

Title: 100101RatKid_NS_deglyco_07.6033.6033.3.dta

Data file K:INewmanPaper|Piliangl3SubProteomes\Piliang3SP\mgf5ppm\ERLIC_3SubProteomes5ppm.mgf 

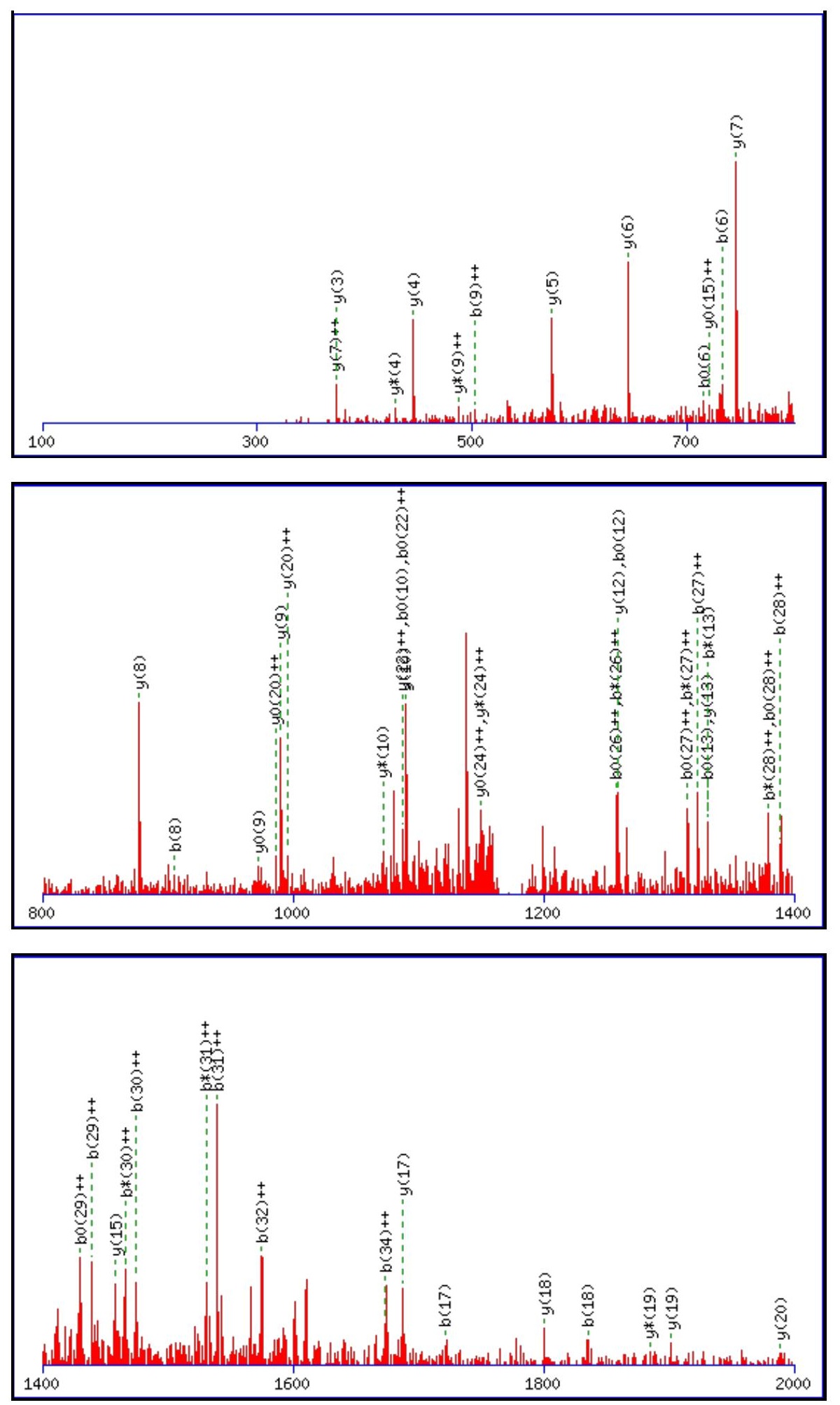

Monoisotopic mass of neutral peptide $\operatorname{Mr}($ calc): 3520.7467

Fixed modifications: Carbamidomethyl (C)

Variable modifications:

N7 : Deamidated $\mathrm{N}(\mathrm{N})$

Ions Score: 66 Expect: $5.7 \mathrm{e}-005$

Matches (Bold Red): 54/394 fragment ions using 92 most intense peaks

\begin{tabular}{|c|c|c|c|c|c|c|c|c|c|c|c|c|c|c|}
\hline \# & b & $\mathbf{b}^{++}$ & $\mathbf{b}^{*}$ & $\mathbf{b}^{*^{++}}$ & $\mathbf{b}^{0}$ & $\mathbf{b}^{0++}$ & Seq. & $\mathbf{y}$ & $y^{++}$ & $\mathbf{y}^{*}$ & $y^{*^{++}}$ & $\mathbf{y}^{0}$ & $y^{0++}$ & \# \\
\hline 1 & 102.0550 & 51.5311 & & & 84.0444 & 42.5258 & $\mathbf{T}$ & & & & & & & 35 \\
\hline 2 & 201.1234 & 101.0653 & & & 183.1128 & 92.0600 & V & 3420.7064 & 1710.8568 & 3403.6798 & 1702.3435 & 3402.6958 & 1701.8515 & 34 \\
\hline 3 & 348.1918 & 174.5995 & & & 330.1812 & 165.5942 & $\mathbf{F}$ & 3321.6380 & 1661.3226 & 3304.6114 & 1652.8093 & 3303.6274 & 1652.3173 & 33 \\
\hline 4 & 476.2504 & 238.6288 & 459.2238 & 230.1155 & 458.2398 & 229.6235 & $\mathbf{Q}$ & 3174.5695 & 1587.7884 & 3157.5430 & 1579.2751 & 3156.5590 & 1578.7831 & 32 \\
\hline 5 & 604.3453 & 302.6763 & 587.3188 & 294.1630 & 586.3348 & 293.6710 & $\bar{K}$ & 3046.5110 & 1523.7591 & 3029.4844 & 1515.2458 & 3028.5004 & 1514.7538 & 31 \\
\hline
\end{tabular}




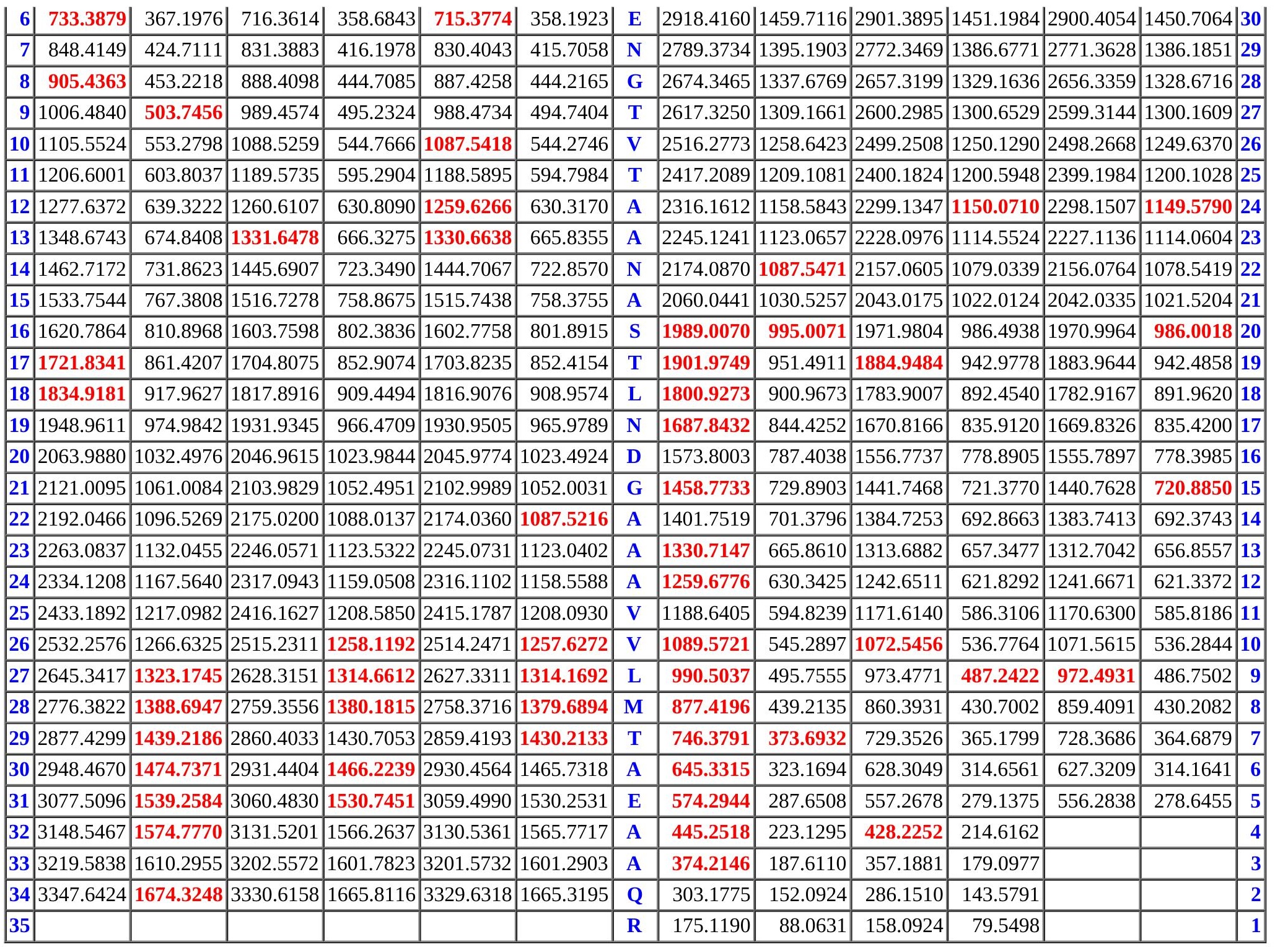
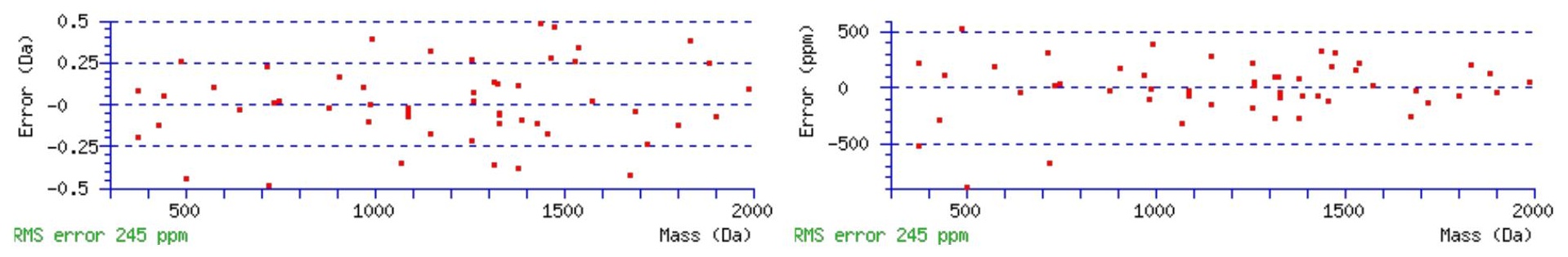

\section{All matches to this query}

\begin{tabular}{|l|c|c|l|}
\hline Score & Mr(calc): & Delta & \multicolumn{1}{c|}{ Sequence } \\
\hline 65.7 & 3520.7467 & 0.0129 & TVFQKENGTVTAANASTLNDGAAAVVLMTAEAAQR \\
\hline 53.8 & 3520.7467 & 0.0129 & TVFQKENGTVTAANASTLNDGAAAVVLMTAEAAQR \\
\hline 53.1 & 3519.7627 & 0.9970 & TVFQKENGTVTAANASTLNDGAAAVVLMTAEAAQR \\
\hline 42.3 & 3520.7467 & 0.0129 & TVFQKENGTVTAANASTLNDGAAAVVLMTAEAAQR \\
\hline 3.0 & 3518.7448 & 2.0149 & DIVHLNSDIVTAPAFALLLEFMYEGKLQFK \\
\hline 1.9 & 3520.7870 & -0.0274 & SIPDEGTLAGKINAIQSLLQQIPEPNFILLK \\
\hline 1.8 & 3520.7729 & -0.0132 & SRQSQPVNVGLVDWISLAYQHYAIAVRNTR \\
\hline 1.8 & 3520.7729 & -0.0132 & SRQSQPVNVGLVDWISLAYQHYAIAVRNTR \\
\hline 0.5 & 3519.7462 & 1.0135 & DSPKHPQLVKNSLCAAPRPTALGLLPNSGPPK \\
\hline
\end{tabular}


Spectrum No: 430; Query: 1026; Rank: 1

\section{Peptide View}

MS/MS Fragmentation of MSGDWELEVNGTEAK

Found in IPI00361686, Tax_Id=10116 Gene_Symbol=C1qbp Complement component 1 Q subcomponent-binding protein, mitochondrial precursor

Match to Query 1026: 1681.717848 from(841.866200,2+)

Title: 091008RatKidney_NH4Format01_13.2482.2482.2.dta

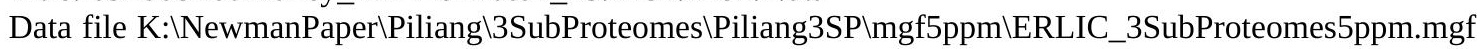
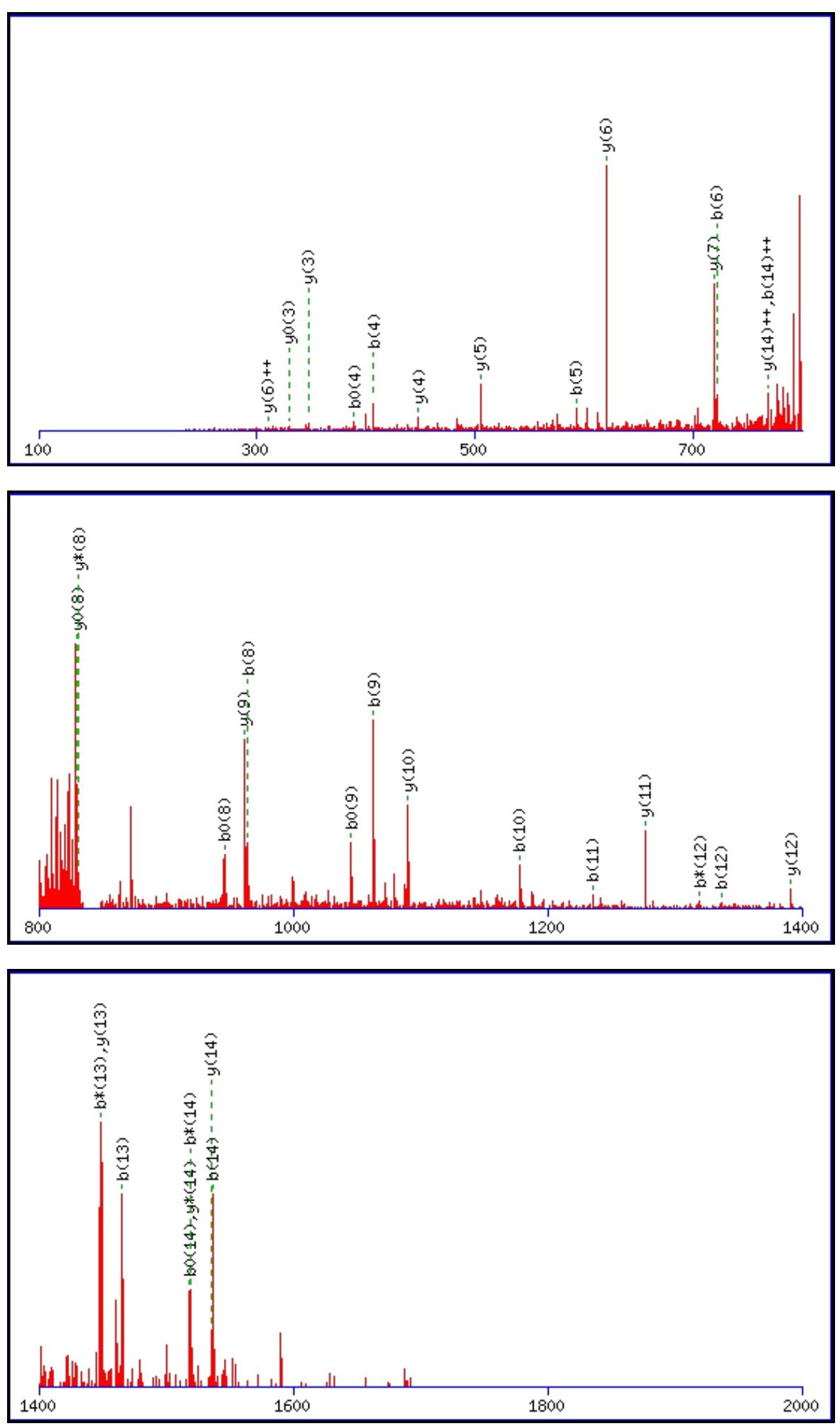
Monoisotopic mass of neutral peptide $\operatorname{Mr}($ calc): 1681.7141

Fixed modifications: Carbamidomethyl (C)

Variable modifications:

M1 : Oxidation (M)

N10 : Deamidated_N (N)

Ions Score: 66 Expect: $4.7 e-005$

Matches (Bold Red): 35/144 fragment ions using 84 most intense peaks

\begin{tabular}{|c|c|c|c|c|c|c|c|c|c|c|c|c|c|c|}
\hline \# & b & $\mathbf{b}^{++}$ & $\mathbf{b}^{*}$ & $\mathbf{b}^{*^{++}}$ & $\mathbf{b}^{0}$ & $\mathbf{b}^{0++}$ & Seq. & $\mathbf{y}$ & $\mathbf{y}^{++}$ & $\mathrm{y}^{*}$ & $\mathbf{y}^{*^{++}}$ & $\mathbf{y}^{0}$ & $y^{0++}$ & \# \\
\hline 1 & 148.0427 & 74.5250 & & & & & $\mathbf{M}$ & & & & & & & 15 \\
\hline 2 & 235.0747 & 118.0410 & & & 217.0641 & 109.0357 & $\mathrm{~S}$ & 1535.6860 & 768.3466 & 1518.6595 & 759.8334 & 1517.6754 & 759.3414 & 14 \\
\hline 3 & 292.0962 & 146.5517 & & & 274.0856 & 137.5464 & G & 1448.6540 & 724.8306 & 1431.6274 & 716.3174 & 1430.6434 & 715.8253 & 13 \\
\hline 4 & 407.1231 & 204.0652 & & & 389.1126 & 195.0599 & D & 1391.6325 & 696.3199 & 1374.6060 & 687.8066 & 1373.6219 & 687.3146 & 12 \\
\hline 5 & 593.2024 & 297.1049 & & & 575.1919 & 288.0996 & $\mathbf{W}$ & 1276.6056 & 638.8064 & 1259.5790 & 630.2931 & 1258.5950 & 629.8011 & 11 \\
\hline 6 & 722.2450 & 361.6261 & & & 704.2345 & 352.6209 & $\mathbf{E}$ & 1090.5263 & 545.7668 & 1073.4997 & 537.2535 & 1072.5157 & 536.7615 & 10 \\
\hline 7 & 835.3291 & 418.1682 & & & 817.3185 & 409.1629 & $\mathbf{L}$ & 961.4837 & 481.2455 & 944.4571 & 472.7322 & 943.4731 & 472.2402 & 9 \\
\hline 8 & 964.3717 & 482.6895 & & & 946.3611 & 473.6842 & $\mathbf{E}$ & 848.3996 & 424.7034 & 831.3730 & 416.1902 & 830.3890 & 415.6982 & 8 \\
\hline 9 & 1063.4401 & 532.2237 & & & 1045.4295 & 523.2184 & $\mathbf{V}$ & 719.3570 & 360.1821 & 702.3305 & 351.6689 & 701.3464 & 351.1769 & 7 \\
\hline 10 & \begin{tabular}{|l|}
1178.4670 \\
\end{tabular} & 589.7372 & 1161.4405 & 581.2239 & 1160.4565 & 580.7319 & $\mathbf{N}$ & 620.2886 & 310.6479 & 603.2620 & 302.1347 & 602.2780 & 301.6427 & 6 \\
\hline 11 & 1235.4885 & 618.2479 & 1218.4619 & 609.7346 & 1217.4779 & 609.2426 & G & 505.2617 & 253.1345 & 488.2351 & 244.6212 & 487.2511 & 244.1292 & 5 \\
\hline 121 & 1336.5362 & 668.7717 & 1319.5096 & 660.2584 & 1318.5256 & 659.7664 & $T$ & 448.2402 & 224.6237 & 431.2136 & 216.1105 & 430.2296 & 215.6185 & 4 \\
\hline 13 & 1465.5788 & 733.2930 & 1448.5522 & 724.7797 & 1447.5682 & 724.2877 & $\mathbf{E}$ & 347.1925 & 174.0999 & 330.1660 & 165.5866 & 329.1819 & 165.0946 & 3 \\
\hline 14 & 1536.6159 & 768.8116 & 1519.5893 & 760.2983 & 1518.6053 & 759.8063 & A & 218.1499 & 109.5786 & 201.1234 & 101.0653 & & & 2 \\
\hline 15 & & & & & & & $\mathbf{K}$ & 147.1128 & 74.0600 & 130.0863 & 65.5468 & & & 1 \\
\hline
\end{tabular}
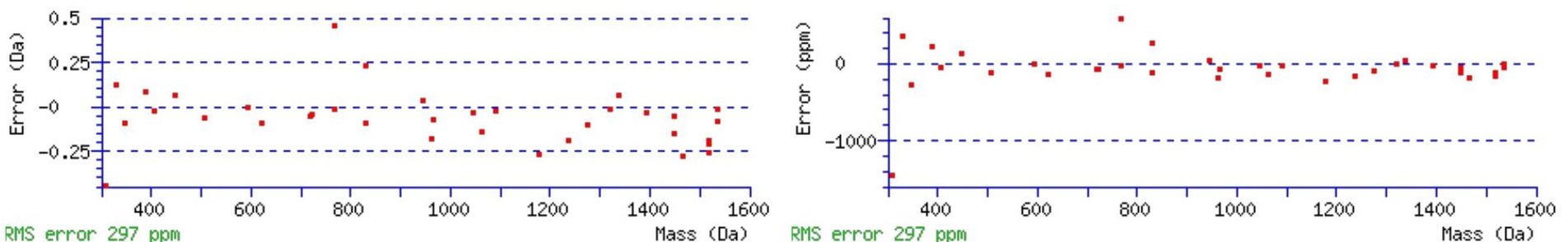

\section{All matches to this query}

\begin{tabular}{|l|l|l|l|}
\hline Score & Mr(calc): & Delta & \multicolumn{1}{c|}{ Sequence } \\
\hline 65.6 & 1681.7141 & 0.0037 & MSGDWELEVNGTEAK \\
\hline 14.5 & 1680.7301 & 0.9877 & MSGDWELEVNGTEAK \\
\hline 2.2 & 1681.7059 & 0.0120 & MSVNYAAGLSPYADK \\
\hline 0.3 & 1681.7076 & 0.0103 & VFEECCXTSKEEK \\
\hline
\end{tabular}

Spectrum No: 431; Query: 271; Rank: 1

\section{Peptide View}

MS/MS Fragmentation of LANTSMFNNSK

Found in IPI00206254, Tax_Id=10116 Gene_Symbol=Ggt1 Gamma-glutamyltranspeptidase 1 precursor

Match to Query 271: 1227.547848 from(614.781200,2+)

Title: 100101RatKid_NS_deglyco_19.1701.1701.2.dta

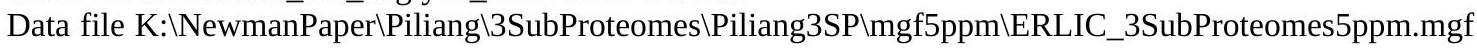



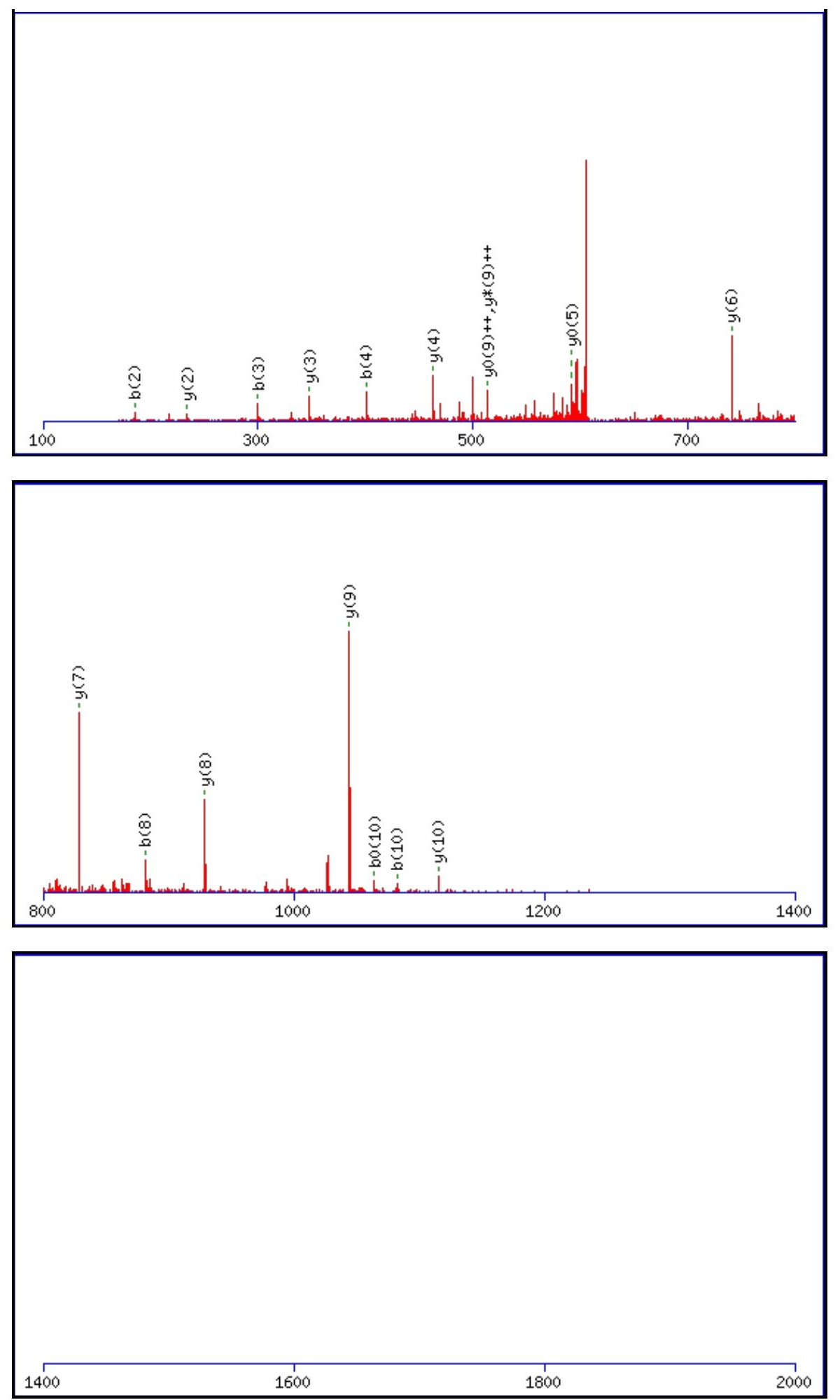

Monoisotopic mass of neutral peptide $\operatorname{Mr}($ calc): 1227.5441

Fixed modifications: Carbamidomethyl (C)

Variable modifications:

N3 : Deamidated_N (N)

N8 : Deamidated_N (N)

Ions Score: 65 Expect: $3.9 \mathrm{e}-005$

Matches (Bold Red): 17/108 fragment ions using 29 most intense peaks

\begin{tabular}{|r|c|c|c|c|c|c|c|c|c|c|c|c|c|c|}
\hline$\#$ & $\mathbf{b}$ & $\mathbf{b}^{++}$ & $\mathbf{b}^{*}$ & $\mathbf{b}^{\boldsymbol{*}^{++}}$ & $\mathbf{b}^{\mathbf{0}}$ & $\mathbf{b}^{\mathbf{0 + +}}$ & $\mathbf{S e q}$ & $\mathbf{y}$ & $\mathbf{y}^{++}$ & $\mathbf{y}^{\mathbf{*}}$ & $\mathbf{y}^{\mathbf{*}^{++}}$ & $\mathbf{y}^{\mathbf{0}}$ & $\mathbf{y}^{\mathbf{0}++}$ & $\#$ \\
\hline $\mathbf{1}$ & 114.0913 & 57.5493 & & & & & $\mathbf{L}$ & & & & & & & $\mathbf{1 1}$ \\
\hline $\mathbf{2}$ & $\mathbf{1 8 5 . 1 2 8 5}$ & 93.0679 & & & & & $\mathbf{A}$ & $\mathbf{1 1 1 5 . 4 6 7 4}$ & 558.2373 & 1098.4408 & 549.7240 & 1097.4568 & 549.2320 & $\mathbf{1 0}$ \\
\hline $\mathbf{3}$ & $\mathbf{3 0 0 . 1 5 5 4}$ & 150.5813 & 283.1288 & 142.0681 & & & $\mathbf{N}$ & $\mathbf{1 0 4 4 . 4 3 0 2}$ & 522.7188 & 1027.4037 & $\mathbf{5 1 4 . 2 0 5 5}$ & 1026.4197 & $\mathbf{5 1 3 . 7 1 3 5}$ & $\mathbf{9}$ \\
\hline $\mathbf{4}$ & $\mathbf{4 0 1 . 2 0 3 1}$ & 201.1052 & 384.1765 & 192.5919 & 383.1925 & 192.0999 & $\mathbf{T}$ & $\mathbf{9 2 9 . 4 0 3 3}$ & 465.2053 & 912.3768 & 456.6920 & 911.3927 & 456.2000 & $\mathbf{8}$ \\
\hline & & & & & & & & & & & & & &
\end{tabular}




\begin{tabular}{|c|c|c|c|c|c|c|c|c|c|c|c|c|c|c|}
\hline & 488.2351 & |244.6212 & 471.2085 & |236.1079 & 470.2245 & |235.6159 & S & 828.3556 & |414.6814 & 811.3291 & 406.1682 & 810.3451 & | 405.6762 & 7 \\
\hline 6 & 619.2756 & 310.1414 & 602.2490 & |301.6282 & 601.2650 & |301.1361 & M & 741.3236 & |371.1654 & 724.2970 & 362.6522 & 723.3130 & 362.1602 & 6 \\
\hline 7 & 766.3440 & 383.6756 & 749.3174 & |375.1624 & 748.3334 & 374.6704 & $\mathbf{F}$ & 610.2831 & 305.6452 & 593.2566 & 297.1319 & 592.2725 & 296.6399 & 5 \\
\hline 8 & 881.3709 & 441.1891 & 864.3444 & |432.6758 & 863.3604 & 432.1838 & $\mathbf{N}$ & 463.2147 & 232.1110 & 446.1881 & 223.5977 & 445.2041 & 223.1057 & 4 \\
\hline 9 & 995.4139 & 498.2106 & 978.3873 & 489.6973 & 977.4033 & 489.2053 & $\mathbf{N}$ & 348.1878 & |174.5975 & 331.1612 & 166.0842 & 330.1772 & 165.5922 & 3 \\
\hline 10 & 1082.4459 & 541.7266 & 1065.4193 & 533.2133 & 1064.4353 & 532.7213 & $\mathbf{S}$ & 234.1448 & 117.5761 & 217.1183 & 109.0628 & 216.1343 & 108.5708 & 2 \\
\hline 11 & & & & & & & $\mathbf{K}$ & 147.1128 & 74.0600 & 130.0863 & 65.5468 & & & 1 \\
\hline
\end{tabular}
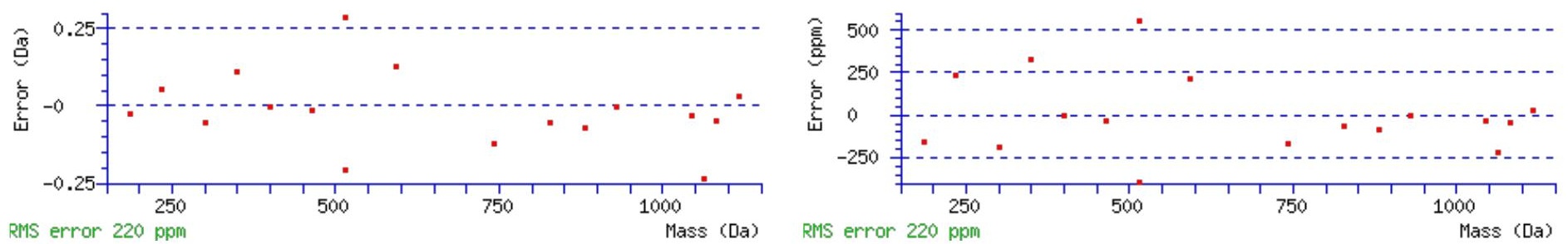

\section{All matches to this query}

\begin{tabular}{|l|l|l|l|}
\hline Score & Mr(calc): & Delta & \multicolumn{1}{|c|}{ Sequence } \\
\hline 65.5 & 1227.5441 & 0.0037 & LANTSMFNNSK \\
\hline 48.8 & 1227.5441 & 0.0037 & LANTSMFNNSK \\
\hline 39.9 & 1227.5441 & 0.0037 & LANTSMFNNSK \\
\hline 17.4 & 1226.5366 & 1.0112 & MSTSIQPLNR \\
\hline 9.8 & 1227.5511 & -0.0033 & LAFLMHSWK \\
\hline 8.3 & 1226.5366 & 1.0112 & MSTSIQPLNR \\
\hline 7.6 & 1227.5495 & -0.0016 & ALYPXVQSXTK \\
\hline 5.8 & 1227.5537 & -0.0058 & ANLSLGTTGWK \\
\hline 5.8 & 1227.5537 & -0.0058 & ANLSLGTTGWK \\
\hline 5.2 & 1227.5384 & 0.0095 & DEKPLTSGSSK \\
\hline
\end{tabular}

Spectrum No: 432; Query: 1560; Rank: 1

\section{Peptide View}

\section{MS/MS Fragmentation of ARDAGYTNATTTDQHGLAK}

Found in IPI00201262, Tax_Id=10116 Gene_Symbol=LOC297568;Mug2;Mug1 Alpha-1-inhibitor 3 precursor

Match to Query 1560: 1990.938312 from(664.653380,3+)

Title: 100101RatKid_NS_deglyco_23.582.582.3.dta

Data file K:INewmanPaper|Piliangl3SubProteomes\Piliang3SP\mgf5ppm\ERLIC_3SubProteomes5ppm.mgf 

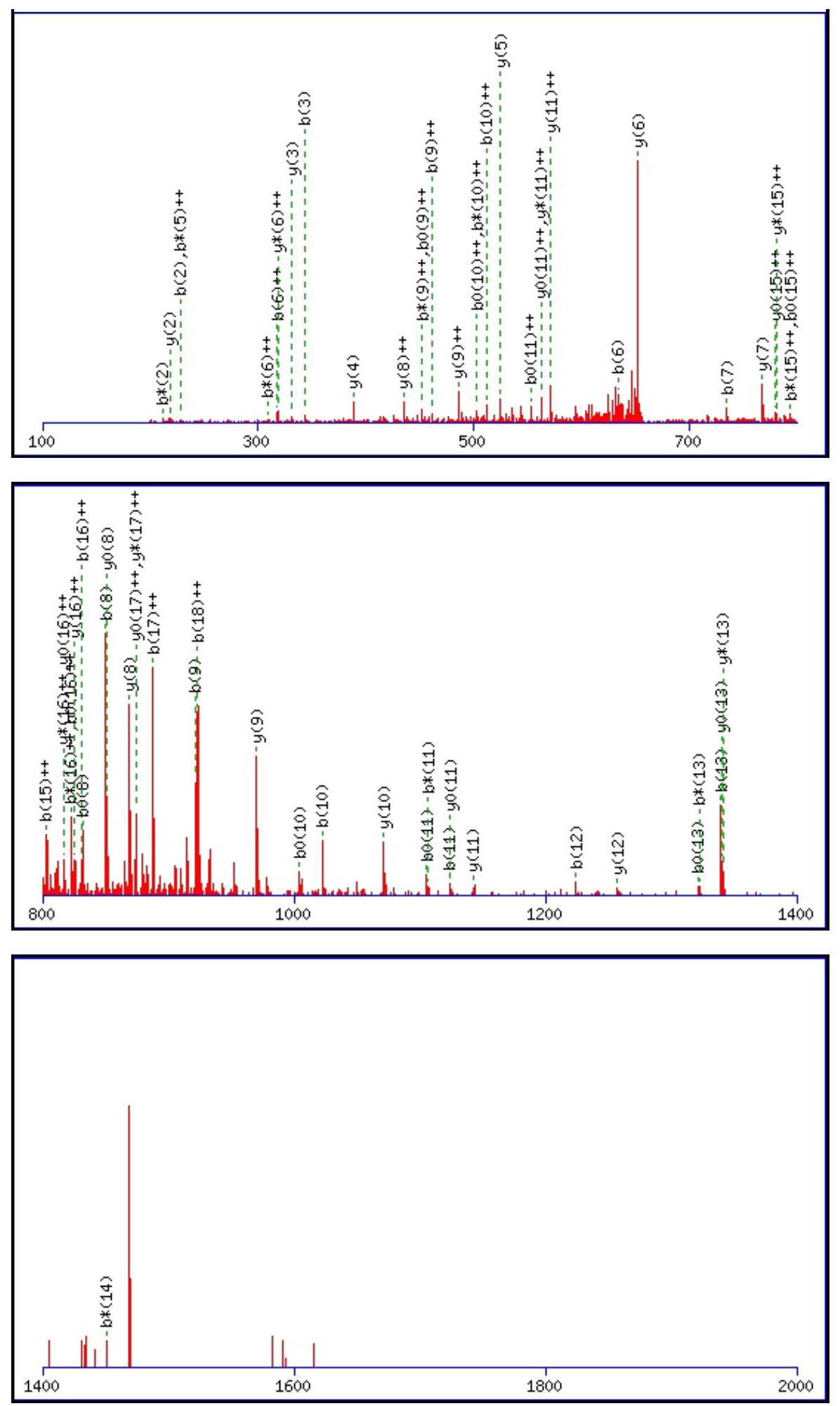

Monoisotopic mass of neutral peptide $\operatorname{Mr}($ calc): 1990.9344

Fixed modifications: Carbamidomethyl (C)

Variable modifications:

N8: Deamidated $\mathrm{N}(\mathrm{N})$

Ions Score: 65 Expect: $6.9 \mathrm{e}-005$

Matches (Bold Red): 64/198 fragment ions using 99 most intense peaks

\begin{tabular}{|r|c|c|c|c|c|c|c|c|c|c|c|c|c|c|}
\hline$\#$ & $\mathbf{b}$ & $\mathbf{b}^{++}$ & $\mathbf{b}^{*}$ & $\mathbf{b}^{\boldsymbol{*}^{++}}$ & $\mathbf{b}^{\mathbf{0}}$ & $\mathbf{b}^{\mathbf{0 + +}}$ & Seq. & $\mathbf{y}$ & $\mathbf{y}^{++}$ & $\mathbf{y}^{\mathbf{*}}$ & $\mathbf{y}^{\boldsymbol{*}^{++}}$ & $\mathbf{y}^{\mathbf{0}}$ & $\mathbf{y}^{\mathbf{0 + +}}$ & $\#$ \\
\hline $\mathbf{1}$ & $\mathbf{7 2 . 0 4 4 4}$ & 36.5258 & & & & & $\mathbf{A}$ & & & & & & & $\mathbf{1 9}$ \\
\hline $\mathbf{2}$ & $\mathbf{2 2 8 . 1 4 5 5}$ & 114.5764 & 211.1190 & 106.0631 & & & $\mathbf{R}$ & 1920.9046 & 960.9559 & 1903.8781 & 952.4427 & 1902.8940 & 951.9507 & $\mathbf{1 8}$ \\
\hline $\mathbf{3}$ & $\mathbf{3 4 3 . 1 7 2 4}$ & 172.0899 & 326.1459 & 163.5766 & 325.1619 & 163.0846 & $\mathbf{D}$ & 1764.8035 & 882.9054 & 1747.7769 & $\mathbf{8 7 4 . 3 9 2 1}$ & 1746.7929 & $\mathbf{8 7 3 . 9 0 0 1}$ & $\mathbf{1 7}$ \\
\hline $\mathbf{4}$ & 414.2096 & 207.6084 & 397.1830 & 199.0951 & 396.1990 & 198.6031 & A & 1649.7766 & $\mathbf{8 2 5 . 3 9 1 9}$ & 1632.7500 & $\mathbf{8 1 6 . 8 7 8 6}$ & 1631.7660 & $\mathbf{8 1 6 . 3 8 6 6}$ & $\mathbf{1 6}$ \\
\hline $\mathbf{5}$ & 471.2310 & 236.1191 & 454.2045 & 227.6059 & 453.2205 & 227.1139 & $\mathbf{G}$ & 1578.7394 & 789.8734 & 1561.7129 & $\mathbf{7 8 1 . 3 6 0 1}$ & 1560.7289 & $\mathbf{7 8 0 . 8 6 8 1}$ & $\mathbf{1 5}$ \\
\hline
\end{tabular}




\begin{tabular}{|c|c|c|c|c|c|c|c|c|c|c|c|c|c|c|}
\hline 6 & 634.2944 & 317.6508 & 617.2678 & |309.1375 & 616.2838 & |308.6455 & $\mathbf{Y}$ & & 761.3626 & |1504.6914 & 752.8494 & 1503.7074 & 752.3573| & 14 \\
\hline 7 & 735.3420 & 368.1747 & 718.3155 & |359.6614 & 717.3315 & 359.1694 & $\mathbf{T}$ & 1358.6546 & 679.8310 & |1341.6281 & 671.3177 & 1340.6441 & 670.8257 & 13 \\
\hline 8 & 850.3690 & 425.6881 & 833.3424 & |417.1748 & 832.3584 & 416.6828 & $\mathbf{N}$ & 1257.6070 & 629.3071 & 1240.5804 & 620.7938 & 1239.5964 & 620.3018 & 12 \\
\hline 9 & 921.4061 & 461.2067 & 904.3795 & 452.6934 & 903.3955 & 452.2014 & A & 1142.5800 & 571.7937 & 1125.5535 & 563.2804 & 1124.5695 & 562.7884 & 11 \\
\hline 10 & 1022.4538 & 511.7305 & 1005.4272 & 503.2172 & 1004.4432 & 502.7252 & $\mathbf{T}$ & 1071.5429 & 536.2751 & 1054.5164 & 527.7618 & 1053.5324 & 527.2698 & 10 \\
\hline 11 & 123.5014 & 562.2544 & \begin{tabular}{|l|}
1106.4749 \\
\end{tabular} & 553.7411 & 1105.4909 & 553.2491 & $\mathbf{T}$ & 970.4952 & 485.7513 & 953.4687 & 477.2380 & 952.4847 & 476.7460 & 9 \\
\hline 12 & 1224.5491 & 612.7782 & 1207.5226 & 604.2649 & 1206.5386 & 603.7729 & $\mathbf{T}$ & 869.4476 & 435.2274 & 852.4210 & 426.7141 & 851.4370 & 426.2221 & 8 \\
\hline 13 & 1339.5761 & 670.2917 & 1322.5495 & 661.7784 & 1321.5655 & 661.2864 & D & 768.3999 & 384.7036 & 751.3733 & 376.1903 & 750.3893 & 375.6983 & 7 \\
\hline 14 & 1467.6346 & 734.3210 & 1450.6081 & 725.8077 & 1449.6241 & 725.3157 & $\mathbf{Q}$ & 653.3729 & 327.1901 & 636.3464 & 318.6768 & & & 6 \\
\hline 15 & 1604.6936 & 802.8504 & 1587.6670 & |794.3371 & 1586.6830 & 793.8451 & H & 525.3144 & 263.1608 & 508.2878 & 254.6475 & & & 5 \\
\hline 16 & 1661.7150 & 831.3611 & 1644.6885 & 822.8479 & 1643.7044 & 822.3559 & G & 388.2554 & 194.6314 & 371.2289 & 186.1181 & & & 4 \\
\hline 17 & 1774.7991 & 887.9032 & 1757.7725 & 879.3899 & 1756.7885 & 878.8979 & $\mathbf{L}$ & 331.2340 & 166.1206 & 314.2074 & 157.6074 & & & 3 \\
\hline 18 & 1845.8362 & 923.4217 & 1828.8096 & 914.9085 & 1827.8256 & 914.4165 & A & 218.1499 & 109.5786 & 201.1234 & 101.0653 & & & 2 \\
\hline 19 & & & & & & & $\mathbf{K}$ & 147.1128 & 74.0600 & 130.0863 & 65.5468 & & & \\
\hline
\end{tabular}
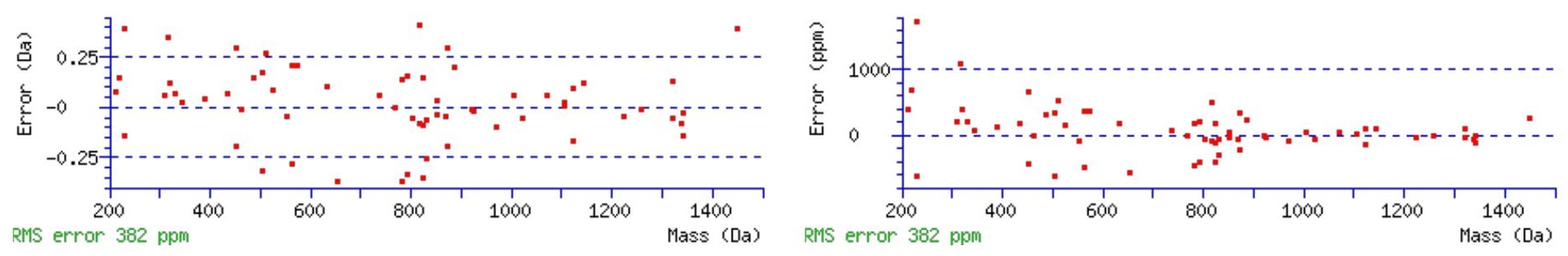

\section{All matches to this query}

\begin{tabular}{|l|l|c|l|}
\hline Score & Mr(calc): & Delta & \multicolumn{1}{|c|}{ Sequence } \\
\hline 65.5 & 1990.9344 & 0.0039 & ARDAGYTNATTTDQHGLAK \\
\hline 41.4 & 1989.9504 & 0.9879 & ARDAGYTNATTTDQHGLAK \\
\hline 6.2 & 1990.9257 & 0.0126 & EKNLFQKSNIGVEMMK \\
\hline 3.2 & 1990.9401 & -0.0018 & AAKEANIHQFIDSLPEK \\
\hline 2.7 & 1990.9554 & -0.0171 & FPQLSLVPFDHILNDR \\
\hline 2.7 & 1990.9531 & -0.0148 & GAVTGHEECVDALLQHGAK \\
\hline 2.0 & 1990.9257 & 0.0126 & EKNLFQKSNIGVEMMK \\
\hline 1.4 & 1990.9297 & 0.0086 & NIKKSAGAPPSMVGMLFY \\
\hline 1.2 & 1990.9248 & 0.0135 & SLSLKLQDSAVYTERDS \\
\hline 1.1 & 1990.9230 & 0.0153 & HVRNRPXASPTMTMGTAK \\
\hline
\end{tabular}

Spectrum No: 433; Query: 2452; Rank: 1

\section{Peptide View}

MS/MS Fragmentation of IYTYADTPNDFQLSNFSLPEEDTK

Found in IPI00764168, Tax_Id=10116 Gene_Symbol=LOC684536 similar to Glucosylceramidase precursor (Beta-glucocerebrosidase) (Acid betaglucosidase) (D-glucosyl-N-acylsphingosine glucohydrolase) isoform 1

Match to Query 2452: 2810.284108 from(1406.149330,2+)

Title: 100101RatKid_NS_deglyco_25.4564.4564.2.dta

Data file K:INewmanPaper|Piliang|3SubProteomes\Piliang3SP\mgf5ppm\ERLIC_3SubProteomes5ppm.mgf 

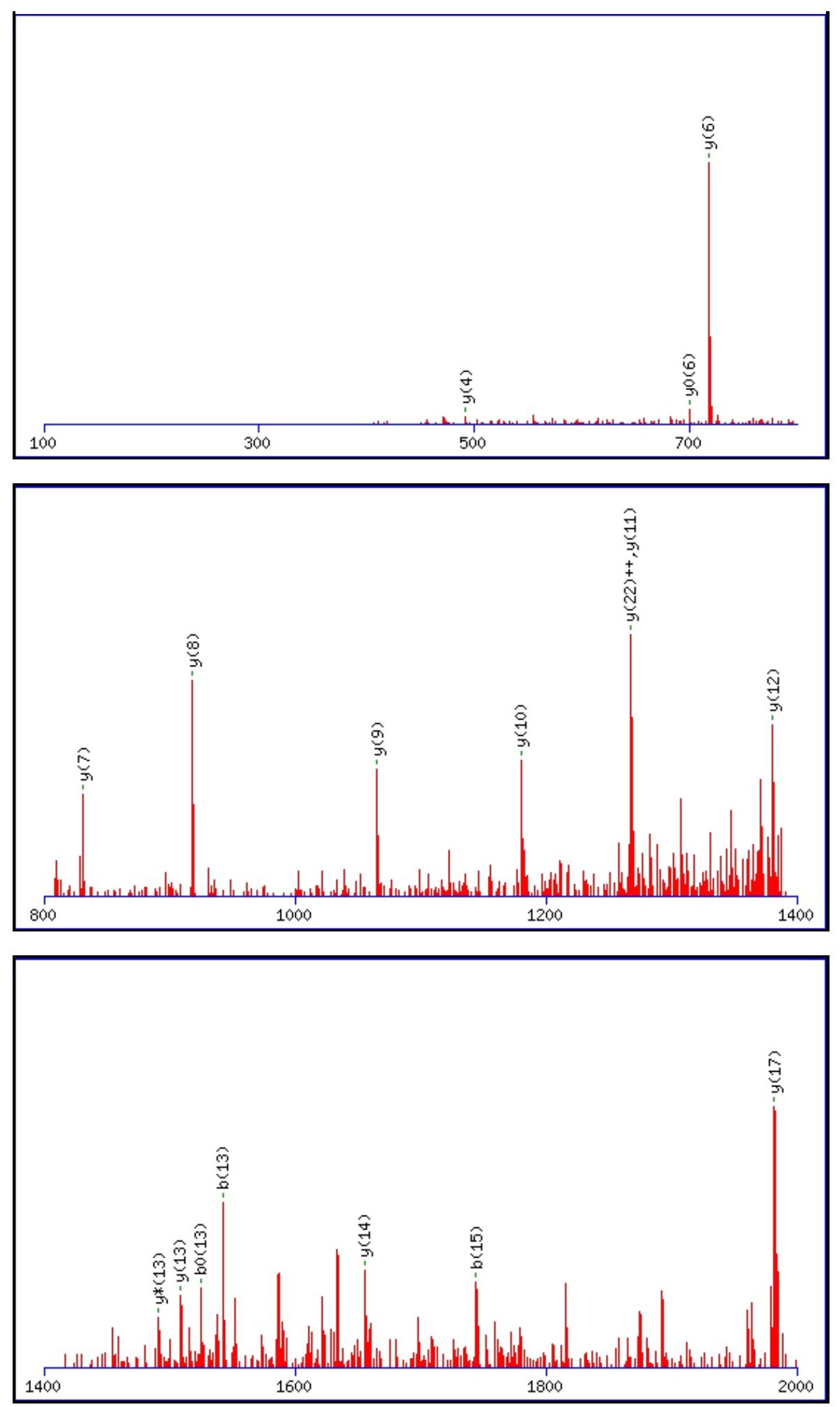

Monoisotopic mass of neutral peptide $\operatorname{Mr}($ calc): 2808.2654

Fixed modifications: Carbamidomethyl (C)

Variable modifications:

N15 : Deamidated N (N)

Ions Score: 66 Expect: $9.9 \mathrm{e}-005$

Matches (Bold Red): 17/254 fragment ions using 27 most intense peaks

\begin{tabular}{|c|c|c|c|c|c|c|c|c|c|c|c|c|c|c|}
\hline$\#$ & $\mathbf{b}$ & $\mathbf{b}^{++}$ & $\mathbf{b}^{*}$ & $\mathbf{b}^{\boldsymbol{*}^{++}}$ & $\mathbf{b}^{\mathbf{0}}$ & $\mathbf{b}^{\mathbf{0 + +}}$ & Seq. & $\mathbf{y}$ & $\mathbf{y}^{++}$ & $\mathbf{y}^{\mathbf{*}}$ & $\mathbf{y}^{\mathbf{*}^{++}}$ & $\mathbf{y}^{\mathbf{0}}$ & $\mathbf{y}^{\mathbf{0 + +}}$ & $\#$ \\
\hline $\mathbf{1}$ & 114.0913 & 57.5493 & & & & & $\mathbf{I}$ & & & & & & & $\mathbf{2 4}$ \\
\hline $\mathbf{2}$ & 277.1547 & 139.0810 & & & & & $\mathbf{Y}$ & 2696.1886 & 1348.5979 & 2679.1621 & 1340.0847 & 2678.1780 & 1339.5927 & $\mathbf{2 3}$ \\
\hline $\mathbf{3}$ & 378.2023 & 189.6048 & & & 360.1918 & 180.5995 & $\mathbf{T}$ & 2533.1253 & $\mathbf{1 2 6 7 . 0 6 6 3}$ & 2516.0987 & 1258.5530 & 2515.1147 & 1258.0610 & 22 \\
\hline $\mathbf{4}$ & 541.2657 & 271.1365 & & & 523.2551 & 262.1312 & $\mathbf{Y}$ & 2432.0776 & 1216.5424 & 2415.0511 & 1208.0292 & 2414.0670 & 1207.5372 & $2 \mathbf{2 1}$ \\
\hline $\mathbf{5}$ & 612.3028 & 306.6550 & & & 594.2922 & 297.6498 & $\mathbf{A}$ & 2269.0143 & 1135.0108 & 2251.9877 & 1126.4975 & 2251.0037 & 1126.0055 & $\mathbf{2 0}$ \\
\hline
\end{tabular}




\begin{tabular}{|c|c|c|c|c|c|c|c|c|c|c|c|c|c|c|}
\hline & 727.3297| & 364.1685 & & & 709.3192 & 355.1632 & D & 2197.9772 & 1099.4922 & |2180.9506 & 1090.9789 & 2179.9666 & 1090.4869 & 19 \\
\hline 7 & 828.3774 & 414.6923 & & & 810.3668 & 405.6871 & $T$ & 2082.9502 & 1041.9787 & 2065.9237 & 1033.4655 & 2064.9396 & 1032.9735 & 18 \\
\hline 8 & 925.4302 & 463.2187 & & & 907.4196 & 454.2134 & $\mathbf{P}$ & 1981.9025 & 991.4549 & 1964.8760 & 982.9416 & 1963.8920 & 982.4496 & 17 \\
\hline 9 & 1039.4731 & 520.2402 & 1022.4466 & 511.7269 & 1021.4625 & 511.2349 & $\mathbf{N}$ & 1884.8498 & 942.9285 & 1867.8232 & 934.4152 & 1866.8392 & 933.9232 & 16 \\
\hline 10 & 1154.5000 & 577.7537 & 1137.4735 & 569.2404 & 1136.4895 & 568.7484 & D & 1770.8068 & 885.9071 & 1753.7803 & 877.3938 & 1752.7963 & 876.9018 & 15 \\
\hline 11 & 1301.5685 & 651.2879 & 1284.5419 & 642.7746 & 1283.5579 & 642.2826 & $\mathbf{F}$ & 1655.7799 & 828.3936 & 1638.7534 & 819.8803 & 1637.7693 & 819.3883 & 4 \\
\hline 12 & 1429.6270 & 715.3172 & 1412.6005 & 706.8039 & 1411.6165 & 706.3119 & $\mathbf{Q}$ & 1508.7115 & 754.8594 & $\mid 1491.6849$ & 746.3461 & 1490.7009 & 745.8541 & 13 \\
\hline 13 & \begin{tabular}{|l|}
1542.7111 \\
\end{tabular} & 771.8592 & 1525.6846 & 763.3459 & 1524.7005 & 762.8539 & $\mathbf{L}$ & 1380.6529 & 690.8301 & 1363.6264 & 682.3168 & 1362.6423 & 681.8248 & 12 \\
\hline 14 & 1629.7431 & 815.3752 & 1612.7166 & 806.8619 & 1611.7326 & 806.3699 & $\mathrm{~S}$ & 1267.5688 & 634.2881 & 1250.5423 & 625.7748 & 1249.5583 & 625.2828 & 11 \\
\hline 15 & 1744.7701 & 872.8887 & 1727.7435 & 864.3754 & 1726.7595 & 863.8834 & $\mathbf{N}$ & 1180.5368 & 590.7720 & 1163.5103 & 582.2588 & 1162.5263 & 581.7668 & 10 \\
\hline 16 & 1891.8385 & 946.4229 & 1874.8119 & 937.9096 & 1873.8279 & 937.4176 & $\mathbf{F}$ & 1065.5099 & 533.2586 & 1048.4833 & 524.7453 & 1047.4993 & 524.2533 & 9 \\
\hline 17 & 1978.8705 & 989.9389 & 1961.8440 & 981.4256 & 1960.8599 & 980.9336 & $\mathrm{~S}$ & 918.4415 & 459.7244 & 901.4149 & 451.2111 & 900.4309 & 450.7191 & 8 \\
\hline 18 & 2091.9546 & 1046.4809 & 2074.9280 & 1037.9676 & 2073.9440 & 1037.4756 & $\mathbf{L}$ & 831.4094 & 416.2084 & 814.3829 & 407.6951 & 813.3989 & 407.2031 & 7 \\
\hline 19 & 2189.0073 & 1095.0073 & 2171.9808 & 1086.4940 & 2170.9968 & 1086.0020 & $\mathbf{P}$ & 718.3254 & 359.6663 & 701.2988 & 351.1531 & 700.3148 & 350.6610 & 6 \\
\hline 20 & 2318.0499 & 1159.5286 & 2301.0234 & 1151.0153 & 2300.0394 & 1150.5233 & $\mathbf{E}$ & 621.2726 & 311.1399 & 604.2461 & 302.6267 & 603.2620 & 302.1347 & 5 \\
\hline 21 & 2447.0925 & 1224.0499 & 2430.0660 & 1215.5366 & 2429.0820 & 1215.0446 & $\mathbf{E}$ & 492.2300 & 246.6186 & 475.2035 & 238.1054 & 474.2195 & 237.6134 & 4 \\
\hline 22 & 2562.1195 & 1281.5634 & 2545.0929 & 1273.0501 & 2544.1089 & 1272.5581 & D & 363.1874 & 182.0974 & 346.1609 & 173.5841 & 345.1769 & 173.0921 & 3 \\
\hline 23 & 2663.1671 & 1332.0872 & 2646.1406 & 1323.5739 & 2645.1566 & 1323.0819 & $\mathbf{T}$ & 248.1605 & 124.5839 & 231.1339 & 116.0706 & 230.1499 & 115.5786 & 2 \\
\hline 24 & & & & & & & $\mathbf{K}$ & 147.1128 & 74.0600 & 130.0863 & 65.5468 & & & 1 \\
\hline
\end{tabular}
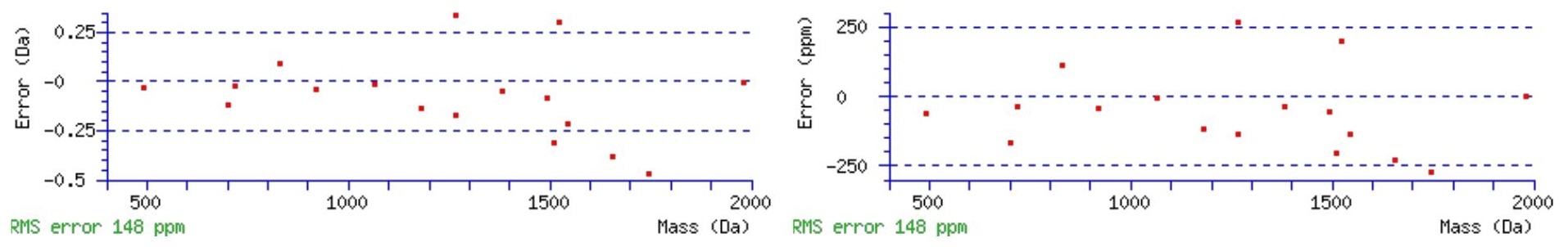

\section{All matches to this query}

\begin{tabular}{|l|l|l|l|}
\hline Score & Mr(calc): & Delta & \multicolumn{1}{|c|}{ Sequence } \\
\hline 65.5 & 2808.2654 & 2.0187 & IYTYADTPNDFQLSNFSLPEEDTK \\
\hline 21.5 & 2808.2654 & 2.0187 & IYTYADTPNDFQLSNFSLPEEDTK \\
\hline 5.6 & 2810.2656 & 0.0185 & GLHACNTYMDSLDIVTSKVSSLFR \\
\hline 5.6 & 2810.2656 & 0.0185 & GLHACNTYMDSLDIVTSKVSSLFR \\
\hline 5.6 & 2810.2656 & 0.0185 & GLHACNTYMDSLDIVTSKVSSLFR \\
\hline 5.6 & 2810.2656 & 0.0185 & GLHACNTYMDSLDIVTSKVSSLFR \\
\hline 5.2 & 2809.2583 & 1.0258 & VASMSLMYTVVTPMLNPFIYSLR \\
\hline 5.2 & 2809.2583 & 1.0258 & VASMSLMYTVVTPMLNPFIYSLR \\
\hline 5.2 & 2809.2583 & 1.0258 & VASMSLMYTVVTPMLNPFIYSLR \\
\hline 5.2 & 2809.2583 & 1.0258 & VASMSLMYTVVTPMLNPFIYSLR \\
\hline
\end{tabular}

Spectrum No: 434; Query: 455; Rank: 1

\section{Peptide View}

MS/MS Fragmentation of NMTLFSDLVAEK

Found in IPI00372839, Tax_Id=10116 Gene_Symbol=Col6a2 similar to procollagen, type VI, alpha 2

Match to Query 455: 1367.668128 from(684.841340,2+)

Title: 100101RatKid_NS_deglyco_21.4679.4679.2.dta

Data file K:INewmanPaper|Piliangl3SubProteomes\Piliang3SP\mgf5ppm\ERLIC_3SubProteomes5ppm.mgf 

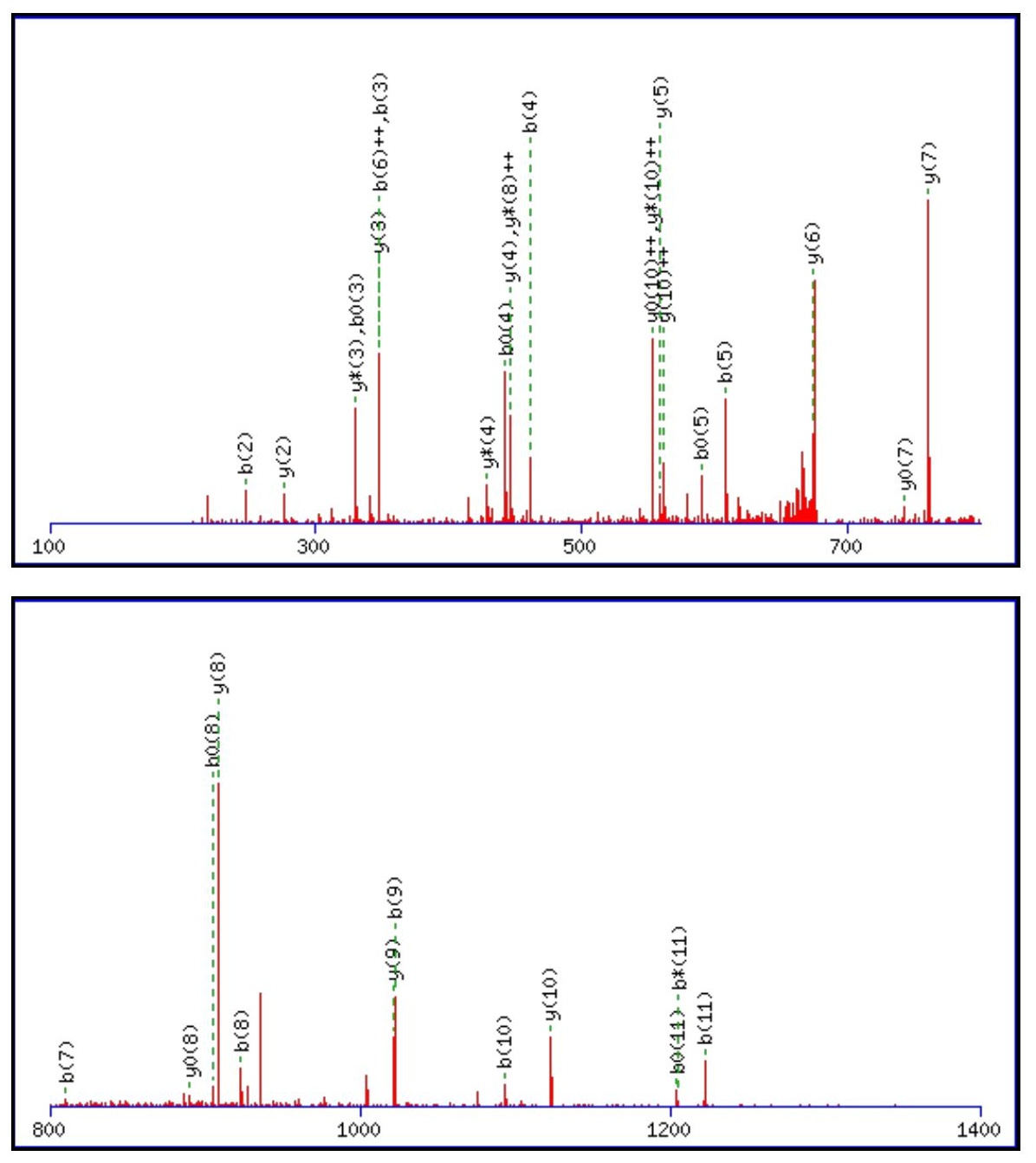

\begin{tabular}{|c|c|c|c|}
\hline 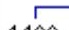 & 1 & 1 & 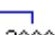 \\
\hline 1400 & 1600 & 1800 & 2000 \\
\hline
\end{tabular}

Monoisotopic mass of neutral peptide $\operatorname{Mr}($ calc): 1367.6642

Fixed modifications: Carbamidomethyl (C)

Variable modifications:

N1 : Deamidated_N (N)

Ions Score: 65 Expect: $4.1 \mathrm{e}-005$

Matches (Bold Red): 33/126 fragment ions using 48 most intense peaks

\begin{tabular}{|c|c|c|c|c|c|c|c|c|c|c|c|c|c|c|}
\hline \# & b & $\mathbf{b}^{++}$ & $\mathbf{b}^{*}$ & $\mathbf{b}^{*^{++}}$ & $\mathbf{b}^{0}$ & $\mathbf{b}^{\mathbf{0}^{++}}$ & Seq. & $\mathbf{y}$ & $\mathbf{y}^{++}$ & $\mathrm{y}^{*}$ & $\mathbf{y}^{*^{++}}$ & $\mathbf{y}^{0}$ & $y^{0++}$ & \# \\
\hline 1 & 116.0342 & 58.5207 & 99.0077 & 50.0075 & & & $\mathbf{N}$ & & & & & & & 12 \\
\hline 2 & 247.0747 & 124.0410 & 230.0481 & 115.5277 & & & $\mathbf{M}$ & 1253.6446 & 627.3259 & 1236.6181 & 618.8127 & 1235.6340 & 618.3207 & 11 \\
\hline 3 & 348.1224 & 174.5648 & 331.0958 & 166.0516 & 330.1118 & 165.5595 & $T$ & 1122.6041 & 561.8057 & 1105.5776 & 553.2924 & 1104.5936 & 552.8004 & 10 \\
\hline 4 & 461.2064 & 231.1069 & 444.1799 & 222.5936 & 443.1959 & 222.1016 & $\mathbf{L}$ & 1021.5564 & 511.2819 & 1004.5299 & 502.7686 & 1003.5459 & 502.2766 & 9 \\
\hline 5 & 608.2749 & 304.6411 & 591.2483 & 296.1278 & 590.2643 & 295.6358 & $\mathbf{F}$ & 908.4724 & 454.7398 & 891.4458 & 446.2266 & 890.4618 & 445.7345 & 8 \\
\hline
\end{tabular}




\begin{tabular}{|c|c|c|c|c|c|c|c|c|c|c|c|c|c|c|}
\hline 6 & 695.3069 & |348.1571| & 678.2803 & |339.6438 & 677.2963 & |339.1518 & S & 761.4040 & |381.2056 & 744.3774 & |372.6923| & 743.3934 & |372.2003| & 7 \\
\hline 7 & 810.3338 & 405.6706 & 793.3073 & 397.1573 & 792.3233 & 396.6653 & D & 674.3719 & 337.6896 & 657.3454 & 329.1763 & 656.3614 & 328.6843 & 6 \\
\hline 8 & 923.4179 & 462.2126 & 906.3913 & 453.6993 & 905.4073 & 453.2073 & $\mathbf{L}$ & 559.3450 & 280.1761 & 542.3184 & 271.6629 & 541.3344 & 271.1708 & 5 \\
\hline 9 & 1022.4863 & 511.7468 & 1005.4598 & 503.2335 & 1004.4757 & 502.7415 & $\mathbf{V}$ & 446.2609 & 223.6341 & 429.2344 & 215.1208 & 428.2504 & 214.6288 & 4 \\
\hline 10 & 1093.5234 & 547.2653 & 1076.4969 & 538.7521 & 1075.5129 & 538.2601 & A & 347.1925 & 174.0999 & 330.1660 & 165.5866 & 329.1819 & 165.0946 & 3 \\
\hline 11 & 1222.5660 & 611.7866 & 1205.5395 & 603.2734 & 1204.5554 & 602.7814 & $\mathbf{E}$ & 276.1554 & 138.5813 & 259.1288 & 130.0681 & 258.1448 & 129.5761 & 2 \\
\hline 12 & & & & & & & $\mathbf{K}$ & 147.1128 & 74.0600 & 130.0863 & 65.5468 & & & 1 \\
\hline
\end{tabular}
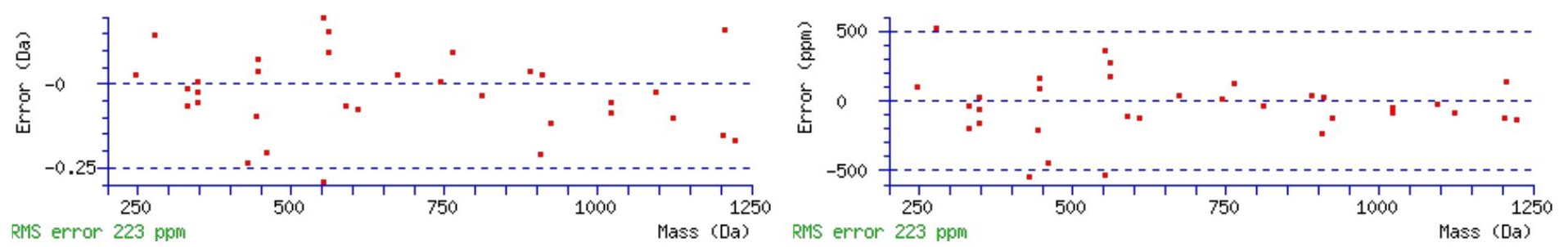

\section{All matches to this query}

\begin{tabular}{|l|l|c|l|}
\hline Score & Mr(calc): & Delta & \multicolumn{1}{|c|}{ Sequence } \\
\hline 65.4 & 1367.6642 & 0.0039 & NMTLFSDLVAEK \\
\hline 9.9 & 1366.6729 & 0.9953 & HINPSODDLTVK \\
\hline 8.9 & 1367.6681 & 0.0001 & YGTVRNSAATAEK \\
\hline 8.4 & 1367.6639 & 0.0042 & FTVFFRKSEK \\
\hline 7.8 & 1367.6681 & 0.0000 & EDSQSPGAHLTVK \\
\hline 7.3 & 1366.6625 & 1.0056 & IMNTFSVMPSPK \\
\hline 6.9 & 1367.6795 & -0.0114 & FAEMYQPLLEK \\
\hline 6.9 & 1365.6516 & 2.0165 & AHLTGWLMTLK \\
\hline 5.4 & 1367.6639 & 0.0042 & FTVFFRKSEK \\
\hline 5.0 & 1367.6789 & -0.0107 & QMVTGLMTKAEK \\
\hline
\end{tabular}

Spectrum No: 435; Query: 73; Rank: 1

\section{Peptide View}

MS/MS Fragmentation of LLNNLTSIK

Found in IPI00363849, Tax_Id=10116 Gene_Symbol=Lamc1 similar to Laminin gamma-1 chain precursor

Match to Query 73: 1015.588508 from(508.801530,2+)

Title: 091008RatKidney_NoSalt_25.2108.2108.2.dta

Data file K:INewmanPaper|Piliang|3SubProteomes\Piliang3SP\mgf5ppm\ERLIC_3SubProteomes5ppm.mgf 

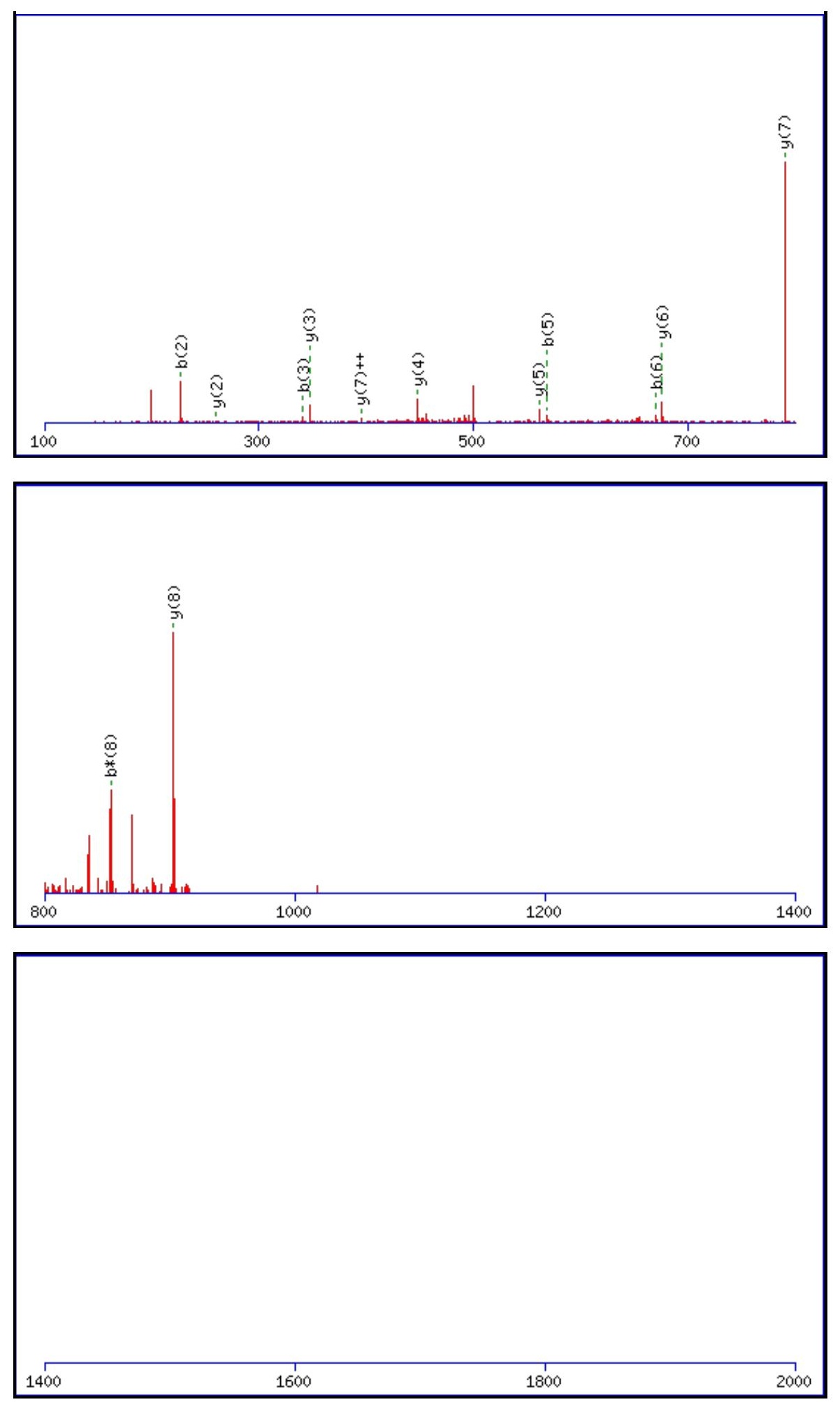

Monoisotopic mass of neutral peptide $\operatorname{Mr}($ calc): 1015.5913

Fixed modifications: Carbamidomethyl (C)

Variable modifications:

N4 : Deamidated_N (N)

Ions Score: 65 Expect: $2 \mathrm{e}-005$

Matches (Bold Red): 13/78 fragment ions using 17 most intense peaks

\begin{tabular}{|c|c|c|c|c|c|c|c|c|c|c|c|c|c|c|}
\hline$\#$ & $\mathbf{b}$ & $\mathbf{b}^{++}$ & $\mathbf{b}^{*}$ & $\mathbf{b}^{*_{+}^{++}}$ & $\mathbf{b}^{\mathbf{0}}$ & $\mathbf{b}^{\mathbf{0}+}$ & Seq. & $\mathbf{y}$ & $\mathbf{y}^{++}$ & $\mathbf{y}^{*}$ & $\mathbf{y}^{\mathbf{*}^{++}}$ & $\mathbf{y}^{\mathbf{0}}$ & $\mathbf{y}^{\mathbf{0}++}$ & $\#$ \\
\hline $\mathbf{1}$ & 114.0913 & 57.5493 & & & & & $\mathbf{L}$ & & & & & & & $\mathbf{9}$ \\
\hline $\mathbf{2}$ & 227.1754 & 114.0913 & & & & & $\mathbf{L}$ & $\mathbf{9 0 3 . 5 1 4 6}$ & 452.2609 & 886.4880 & 443.7476 & 885.5040 & 443.2556 & $\mathbf{8}$ \\
\hline $\mathbf{3}$ & 341.2183 & 171.1128 & 324.1918 & 162.5995 & & & $\mathbf{N}$ & $\mathbf{7 9 0 . 4 3 0 5}$ & $\mathbf{3 9 5 . 7 1 8 9}$ & 773.4040 & 387.2056 & 772.4199 & 386.7136 & $\mathbf{7}$ \\
\hline $\mathbf{4}$ & 456.2453 & 228.6263 & 439.2187 & 220.1130 & & & $\mathbf{N}$ & $\mathbf{6 7 6 . 3 8 7 6}$ & 338.6974 & 659.3610 & 330.1842 & 658.3770 & 329.6921 & $\mathbf{6}$ \\
\hline $\mathbf{5}$ & $\mathbf{5 6 9 . 3 2 9 3}$ & 285.1683 & 552.3028 & 276.6550 & & & $\mathbf{L}$ & $\mathbf{5 6 1 . 3 6 0 6}$ & 281.1840 & 544.3341 & 272.6707 & 543.3501 & 272.1787 & $\mathbf{5}$ \\
\hline
\end{tabular}




\begin{tabular}{|c|c|c|c|c|c|c|c|c|c|c|c|c|c|}
\hline 6 & |670.3770| & |335.6921| & |653.3505 & |327.1789 & |652.3664 & |326.6869| & $\mathbf{T}$ & |448.2766 | & |224.6419 & |431.2500 & |216.1287 & |430.2660| & |215.6366 | \\
\hline 7 & 757.4090 & 379.2082 & 740.3825 & 370.6949 & 739.3985 & 370.2029 & S & 347.2289 & 174.1181 & 330.2023 & 165.6048 & 329.2183 & 165.1128 \\
\hline 8 & 870.4931 & 435.7502 & 853.4666 & 427.2369 & 852.4825 & 426.7449 & I & 260.1969 & 130.6021 & 243.1703 & 122.0888 & & \\
\hline
\end{tabular}
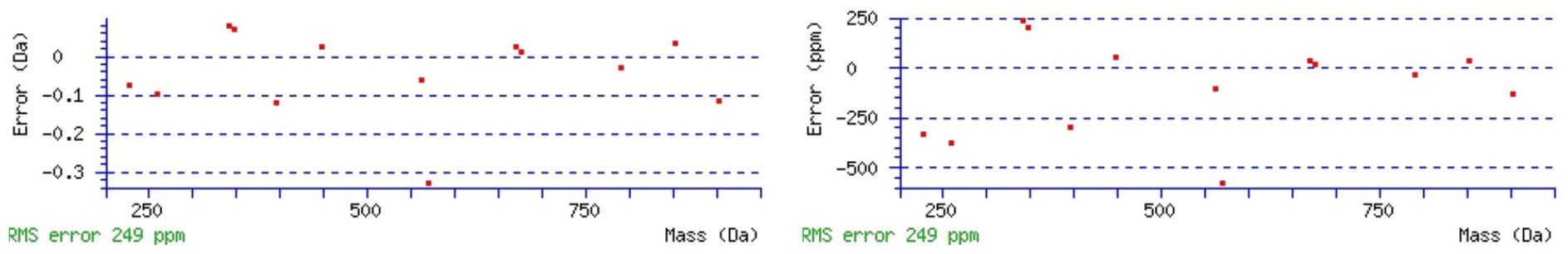

\section{All matches to this query}

\begin{tabular}{|l|l|l|l|}
\hline Score & Mr(calc): & Delta & \multicolumn{1}{|c|}{ Sequence } \\
\hline 65.3 & 1015.5913 & -0.0028 & LLNNLTSIK \\
\hline 46.7 & 1015.5913 & -0.0028 & LLNNLTSIK \\
\hline 17.3 & 1015.5913 & -0.0028 & LNILDTLSK \\
\hline 17.1 & 1015.5913 & -0.0028 & LILDSLSQK \\
\hline 9.7 & 1015.5831 & 0.0054 & LIIHLISK \\
\hline 7.9 & 1015.5927 & -0.0042 & TKVHLPGHK \\
\hline 7.8 & 1015.5913 & -0.0028 & DKLVLATEK \\
\hline 7.2 & 1015.5848 & 0.0037 & ILNMIRQK \\
\hline 5.1 & 1015.5913 & -0.0028 & EVSLLQSLK \\
\hline 5.1 & 1015.5814 & 0.0071 & LIAWERTK \\
\hline
\end{tabular}

Spectrum No: 436; Query: 1081; Rank: 1

\section{Peptide View}

MS/MS Fragmentation of IKTPEKNDTAAEQGGR

Found in IPI00778946, Tax_Id=10116 Gene_Symbol=Itgav_predicted Protein

Match to Query 1081: 1714.848882 from(572.623570,3+)

Title: 100101RatKid_NS_deglyco_23.263.263.3.dta

Data file K:INewmanPaper|Piliangl3SubProteomes\Piliang3SP\mgf5ppm\ERLIC_3SubProteomes5ppm.mgf

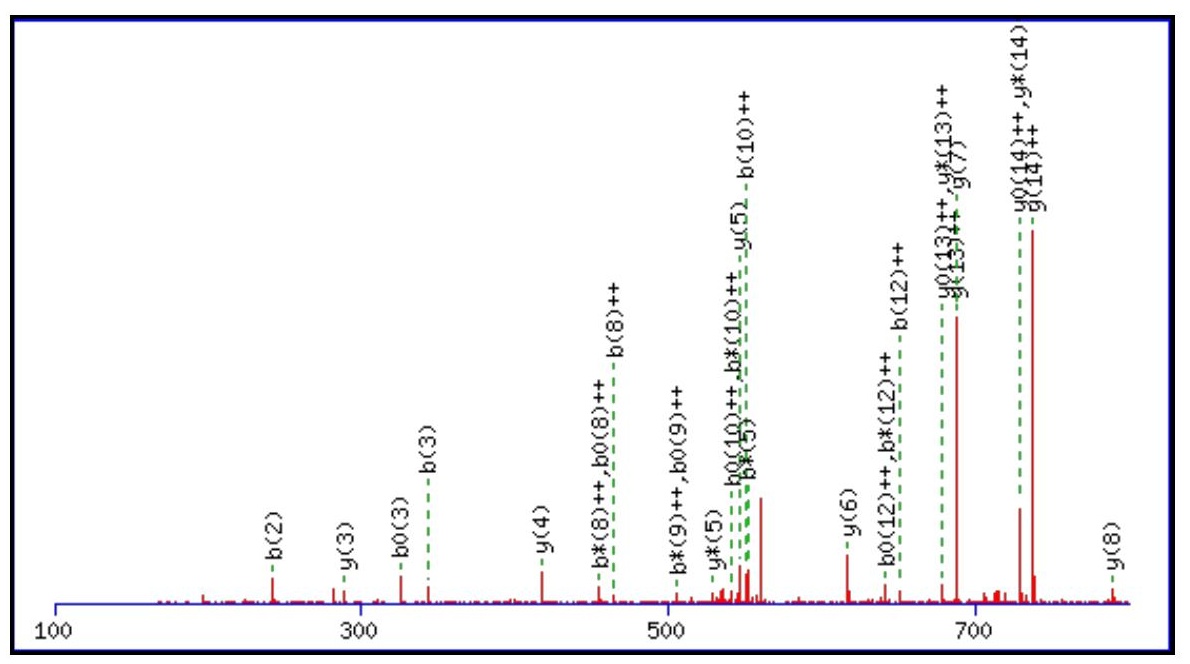



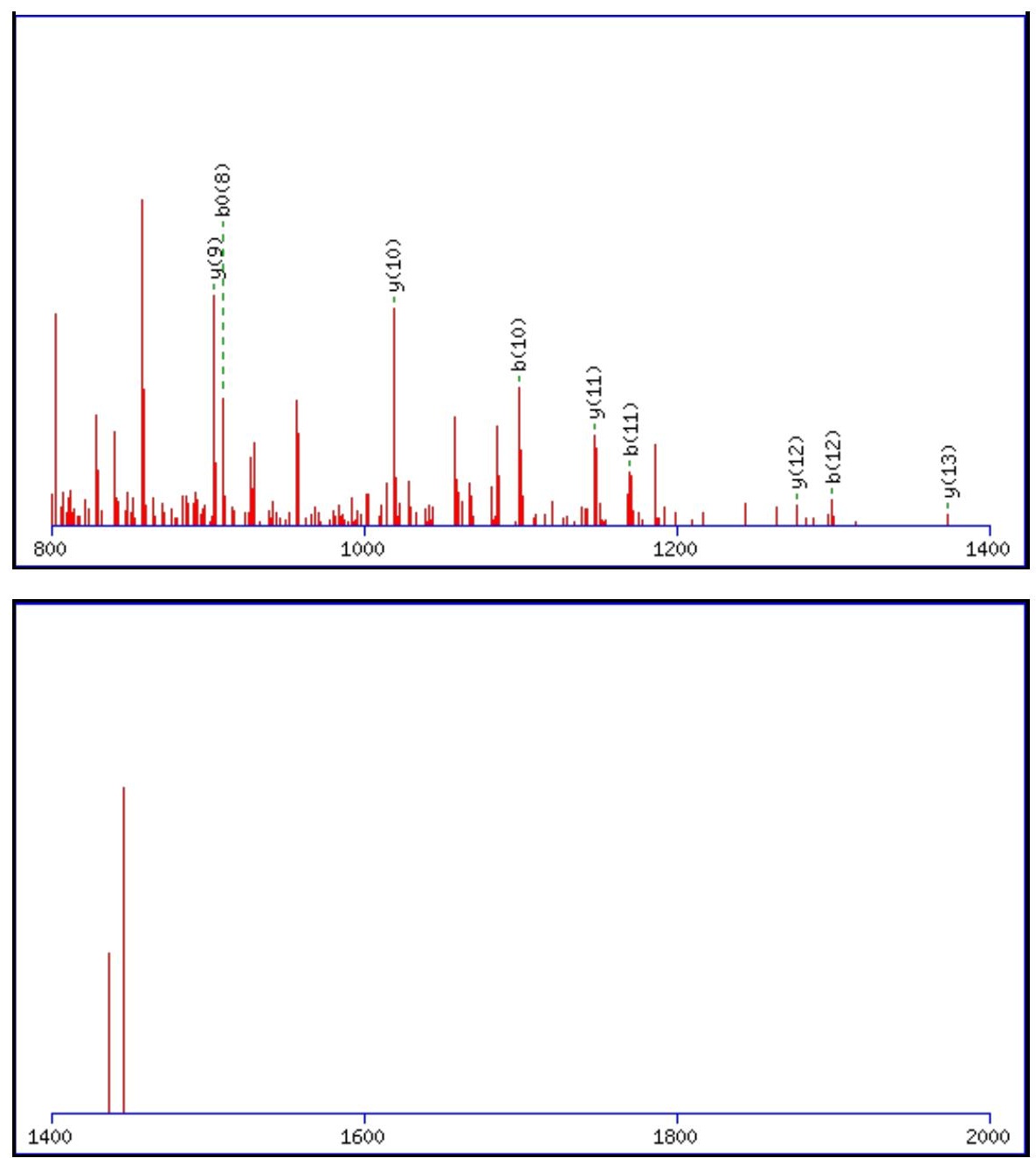

Monoisotopic mass of neutral peptide $\operatorname{Mr}($ calc): 1714.8486

Fixed modifications: Carbamidomethyl (C)

Variable modifications:

N7 : Deamidated $\mathrm{N}(\mathrm{N})$

Ions Score: 65 Expect: $5.2 \mathrm{e}-005$

Matches (Bold Red): 37/166 fragment ions using 61 most intense peaks

\begin{tabular}{|c|c|c|c|c|c|c|c|c|c|c|c|c|c|c|}
\hline \# & b & $\mathbf{b}^{++}$ & $\mathbf{b}^{*}$ & $\mathbf{b}^{*^{++}}$ & $\mathbf{b}^{\mathbf{0}}$ & $\mathbf{b}^{0++}$ & Seq. & $\mathbf{y}$ & $\mathbf{y}^{++}$ & $\mathbf{y}^{*}$ & $\mathrm{y}^{*^{++}}$ & $\mathbf{y}^{\mathbf{0}}$ & $\mathbf{y}^{\mathbf{0 + +}}$ & \# \\
\hline 1 & 114.0913 & 57.5493 & & & & & I & & & & & & & 16 \\
\hline 2 & 242.1863 & 121.5968 & 225.1598 & 113.0835 & & & $\mathbf{K}$ & 1602.7718 & 801.8895 & 1585.7453 & 793.3763 & 1584.7612 & 792.8843 & 15 \\
\hline 3 & 343.2340 & 172.1206 & 326.2074 & 163.6074 & 325.2234 & 163.1153 & $\mathbf{T}$ & 1474.6768 & 737.8421 & 1457.6503 & 729.3288 & 1456.6663 & 728.8368 & 14 \\
\hline 4 & 440.2867 & 220.6470 & 423.2602 & 212.1337 & 422.2762 & 211.6417 & $\mathbf{P}$ & 1373.6292 & 687.3182 & 1356.6026 & 678.8049 & 1355.6186 & 678.3129 & 13 \\
\hline 5 & 569.3293 & 285.1683 & 552.3028 & 276.6550 & 551.3188 & 276.1630 & $\mathbf{E}$ & 1276.5764 & 638.7918 & 1259.5498 & 630.2786 & 1258.5658 & 629.7866 & 12 \\
\hline 6 & 697.4243 & 349.2158 & 680.3978 & 340.7025 & 679.4137 & 340.2105 & $\mathbf{K}$ & 1147.5338 & 574.2705 & 1130.5073 & 565.7573 & 1129.5232 & 565.2653 & 11 \\
\hline 7 & 812.4512 & 406.7293 & 795.4247 & 398.2160 & 794.4407 & 397.7240 & $\mathbf{N}$ & 1019.4388 & 510.2231 & 1002.4123 & 501.7098 & 1001.4283 & 501.2178 & 10 \\
\hline 8 & 927.4782 & 464.2427 & 910.4516 & 455.7295 & 909.4676 & 455.2374 & D & 904.4119 & 452.7096 & 887.3854 & 444.1963 & 886.4013 & 443.7043 & 9 \\
\hline 9 & 1028.5259 & 514.7666 & 1011.4993 & 506.2533 & 1010.5153 & 505.7613 & $\mathbf{T}$ & 789.3850 & 395.1961 & 772.3584 & 386.6828 & 771.3744 & 386.1908 & 8 \\
\hline 10 & 1099.5630 & 550.2851 & 1082.5364 & 541.7719 & 1081.5524 & 541.2798 & A & 688.3373 & 344.6723 & 671.3107 & 336.1590 & 670.3267 & 335.6670 & 7 \\
\hline 11 & 1170.6001 & 585.8037 & 1153.5735 & 577.2904 & 1152.5895 & 576.7984 & A & 617.3002 & 309.1537 & 600.2736 & 300.6404 & 599.2896 & 300.1484 & 6 \\
\hline 12 & 1299.6427 & 650.3250 & 1282.6161 & 641.8117 & 1281.6321 & 641.3197 & $\mathbf{E}$ & 546.2631 & 273.6352 & 529.2365 & 265.1219 & 528.2525 & 264.6299 & 5 \\
\hline 13 & 1427.7013 & 714.3543 & 1410.6747 & 705.8410 & 1409.6907 & 705.3490 & $\mathbf{Q}$ & 417.2205 & 209.1139 & 400.1939 & 200.6006 & & & 4 \\
\hline 14 & 1484.7227 & 742.8650 & 1467.6962 & 734.3517 & 1466.7122 & 733.8597 & G & 289.1619 & 145.0846 & 272.1353 & 136.5713 & & & 3 \\
\hline 15 & 1541.7442 & 771.3757 & 1524.7176 & 762.8625 & 1523.7336 & 762.3704 & G & 232.1404 & 116.5738 & 215.1139 & 108.0606 & & & 2 \\
\hline 16 & & & & & & & $\mathbf{R}$ & 175.1190 & 88.0631 & 158.0924 & 79.5498 & & & 1 \\
\hline
\end{tabular}



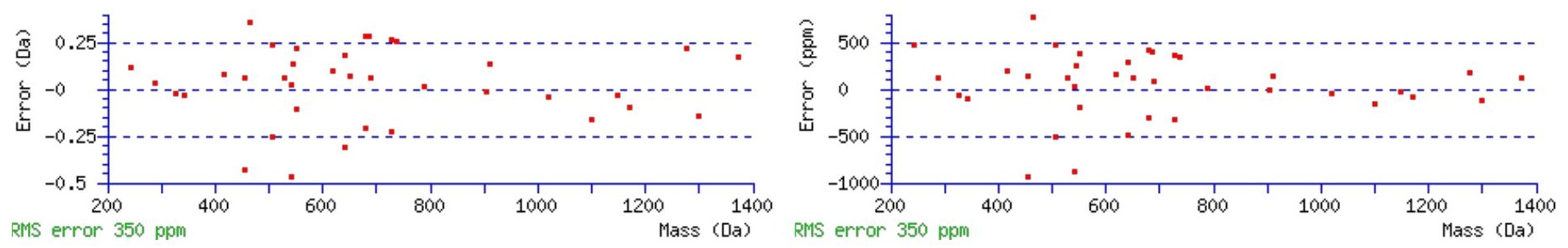

\section{All matches to this query}

\begin{tabular}{|l|l|l|l|}
\hline Score & Mr(calc): & Delta & \multicolumn{1}{|c|}{ Sequence } \\
\hline 65.2 & 1714.8486 & 0.0003 & IKTPEKNDTAAEQGGR \\
\hline 3.8 & 1712.8400 & 2.0089 & THLQLGSVLYHHTK \\
\hline 3.8 & 1712.8400 & 2.0089 & THLQLGSVLYHHTK \\
\hline 3.8 & 1712.8400 & 2.0089 & THLQLGSVLYHHTK \\
\hline 2.5 & 1713.8451 & 1.0038 & YGTGAVSIQAGSALLAR \\
\hline 2.3 & 1714.8348 & 0.0141 & KITCKNGEWEEPPK \\
\hline 0.9 & 1712.8498 & 1.9991 & EIQPKGRSYELVSK \\
\hline 0.8 & 1713.8450 & 1.0038 & NQLSSYPSAALSKLR \\
\hline 0.2 & 1712.8420 & 2.0069 & QTIIGQPMSVTITTK \\
\hline 0.2 & 1712.8420 & 2.0069 & QTIIGQPMSVTITTK \\
\hline
\end{tabular}

Spectrum No: 437; Query: 329; Rank: 1

\section{Peptide View}

MS/MS Fragmentation of ELISNASDALDK

Found in IPI00365985, Tax_Id=10116 Gene_Symbol=Tra1_predicted 93 kDa protein

Match to Query 329: 1275.621628 from(638.818090,2+)

Title: 100101RatKid_NS_deglyco_15.2399.2399.2.dta

Data file K:INewmanPaper|Piliangl3SubProteomes\Piliang3SP\mgf5ppm\ERLIC_3SubProteomes5ppm.mgf

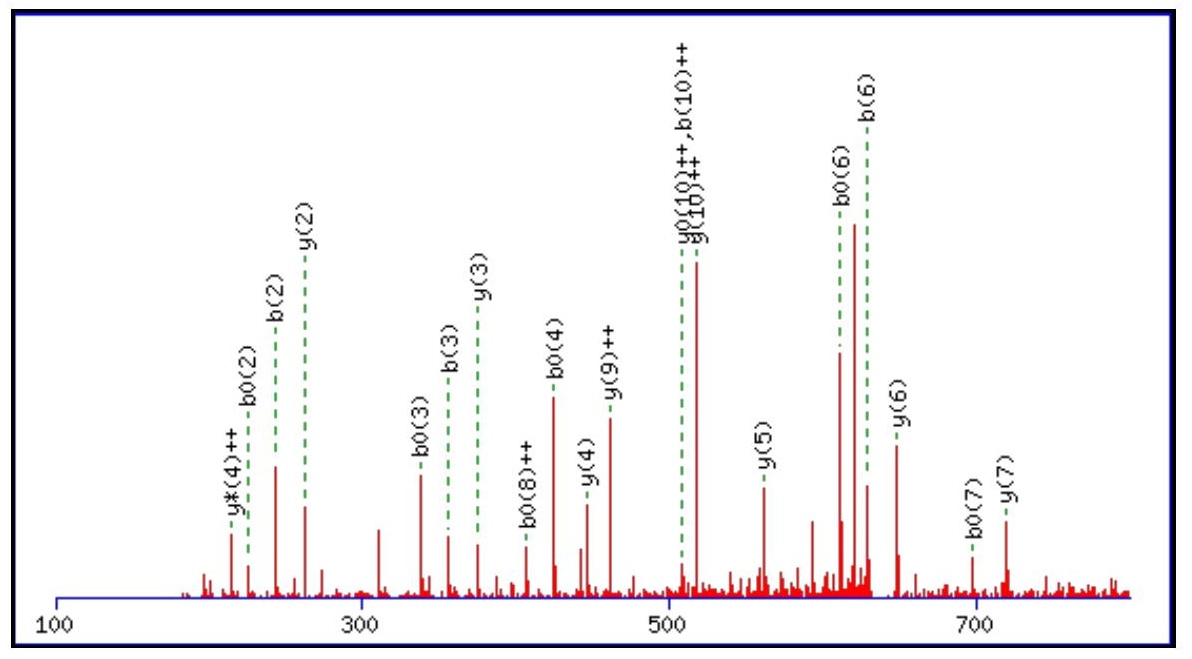




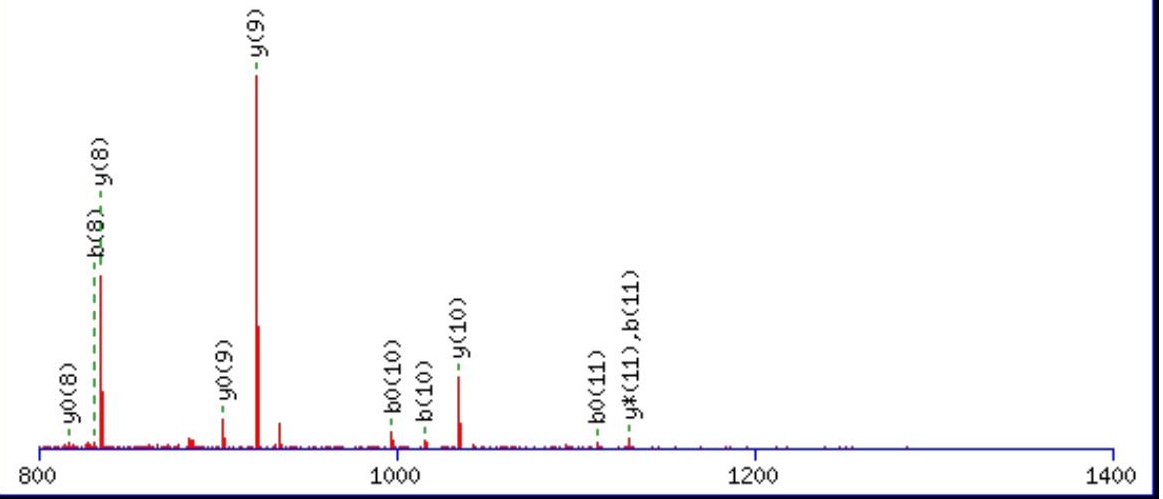

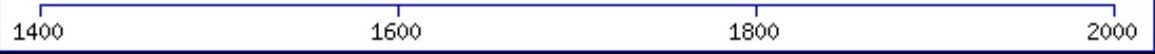

Monoisotopic mass of neutral peptide $\operatorname{Mr}($ calc): 1275.6194

Fixed modifications: Carbamidomethyl (C)

Variable modifications:

N5 : Deamidated $\mathrm{N}(\mathrm{N})$

Ions Score: 65 Expect: $4.2 \mathrm{e}-005$

Matches (Bold Red): 31/122 fragment ions using 46 most intense peaks

\begin{tabular}{|c|c|c|c|c|c|c|c|c|c|c|c|c|c|c|}
\hline \# & b & $\mathbf{b}^{++}$ & $\mathbf{b}^{*}$ & $\mathbf{b}^{*^{++}}$ & $\mathbf{b}^{0}$ & $\mathbf{b}^{0++}$ & Seq. & $\mathbf{y}$ & $\mathbf{y}^{++}$ & $\mathbf{y}^{*}$ & $\mathrm{y}^{*^{++}}$ & $\mathbf{y}^{0}$ & $\mathbf{y}^{\mathbf{0 + +}}$ & \# \\
\hline 1 & 130.0499 & 65.5286 & & & 112.0393 & 56.5233 & $\mathbf{E}$ & & & & & & & 12 \\
\hline 2 & 243.1339 & 122.0706 & & & 225.1234 & 113.0653 & $\mathbf{L}$ & 1147.5841 & 574.2957 & 1130.5576 & 565.7824 & 1129.5735 & 565.2904 & 11 \\
\hline 3 & 356.2180 & 178.6126 & & & 338.2074 & 169.6074 & I & 1034.5000 & 517.7537 & 1017.4735 & 509.2404 & 1016.4895 & 508.7484 & 10 \\
\hline 4 & 443.2500 & 222.1287 & & & 425.2395 & 213.1234 & S & 921.4160 & 461.2116 & 904.3894 & 452.6984 & 903.4054 & 452.2063 & 9 \\
\hline 5 & 558.2770 & 279.6421 & 541.2504 & 271.1288 & 540.2664 & 270.6368 & $\mathbf{N}$ & 834.3839 & 417.6956 & 817.3574 & 409.1823 & 816.3734 & 408.6903 & 8 \\
\hline 6 & 629.3141 & 315.1607 & 612.2875 & 306.6474 & 611.3035 & 306.1554 & A & 719.3570 & 360.1821 & 702.3305 & 351.6689 & 701.3464 & 351.1769 & 7 \\
\hline 7 & 716.3461 & 358.6767 & 699.3196 & 350.1634 & 698.3355 & 349.6714 & S & 648.3199 & 324.6636 & 631.2933 & 316.1503 & 630.3093 & 315.6583 & 6 \\
\hline 8 & 831.3730 & 416.1902 & 814.3465 & 407.6769 & 813.3625 & 407.1849 & D & 561.2879 & 281.1476 & 544.2613 & 272.6343 & 543.2773 & 272.1423 & 5 \\
\hline 9 & 902.4102 & 451.7087 & 885.3836 & 443.1954 & 884.3996 & 442.7034 & A & 446.2609 & 223.6341 & 429.2344 & 215.1208 & 428.2504 & 214.6288 & 4 \\
\hline 10 & 1015.4942 & 508.2507 & 998.4677 & 499.7375 & 997.4837 & 499.2455 & $\mathbf{L}$ & 375.2238 & 188.1155 & 358.1973 & 179.6023 & 357.2132 & 179.1103 & 3 \\
\hline 11 & 1130.5212 & 565.7642 & 1113.4946 & 557.2509 & 1112.5106 & 556.7589 & D & 262.1397 & 131.5735 & 245.1132 & 123.0602 & 244.1292 & 122.5682 & 2 \\
\hline 12 & & & & & & & $\mathbf{K}$ & 147.1128 & 74.0600 & 130.0863 & 65.5468 & & & 1 \\
\hline
\end{tabular}



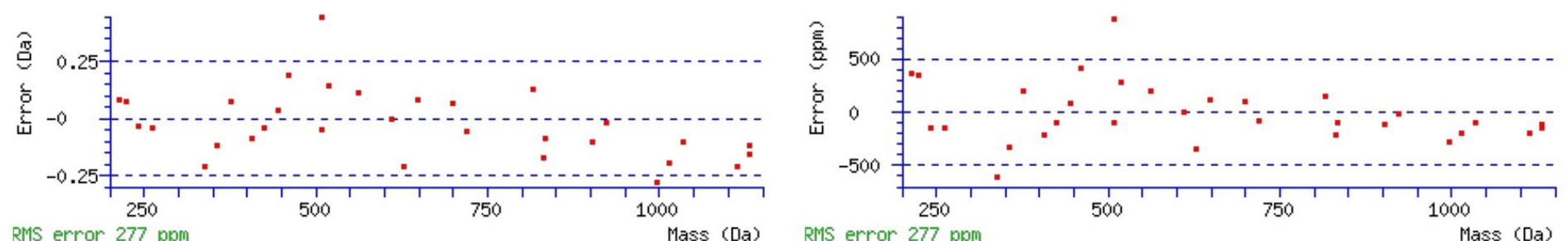

RMS error 277 ppm

Mass (Da) RMS error 277 ppm

\section{All matches to this query}

\begin{tabular}{|l|l|l|l|}
\hline Score & Mr(calc): & Delta & \multicolumn{1}{c|}{ Sequence } \\
\hline 65.2 & 1275.6194 & 0.0022 & ELISNASDALDK \\
\hline 9.0 & 1275.6306 & -0.0090 & $\underline{\text { EISQSDSLER }}$ \\
\hline 8.3 & 1275.6264 & -0.0048 & $\underline{\text { KGGIFSAEFLK }}$ \\
\hline 5.9 & 1275.6112 & 0.0105 & $\underline{\text { FVTLGSETSKK }}$ \\
\hline 5.2 & 1275.6320 & -0.0104 & $\underline{\text { GAVQSEWRTSR }}$ \\
\hline 3.8 & 1275.6159 & 0.0058 & $\underline{\text { HVTSTHMKKK }}$ \\
\hline 3.2 & 1275.6129 & 0.0088 & $\underline{\text { ELALMGETQER }}$ \\
\hline 2.1 & 1274.6271 & 0.9945 & LKNISSKYDK \\
\hline 1.3 & 1275.6111 & 0.0105 & $\underline{\text { LKNISSKYDK }}$ \\
\hline 0.8 & 1275.6125 & 0.0091 & $\underline{\text { KKVHFHGESK }}$ \\
\hline
\end{tabular}

Spectrum No: 438; Query: 507; Rank: 1

\section{Peptide View}

MS/MS Fragmentation of WGEVDLIGNCSR

Found in IPI00204451, Tax_Id=10116 Gene_Symbol=Cfi Complement factor I precursor

Match to Query 507: 1405.632888 from(703.823720,2+)

Title: 100101RatKid_NS_deglyco_28.2758.2758.2.dta

Data file K:\NewmanPaper\Piliang \3SubProteomes\Piliang3SP \mgf5ppm\ERLIC_3SubProteomes5ppm.mgf

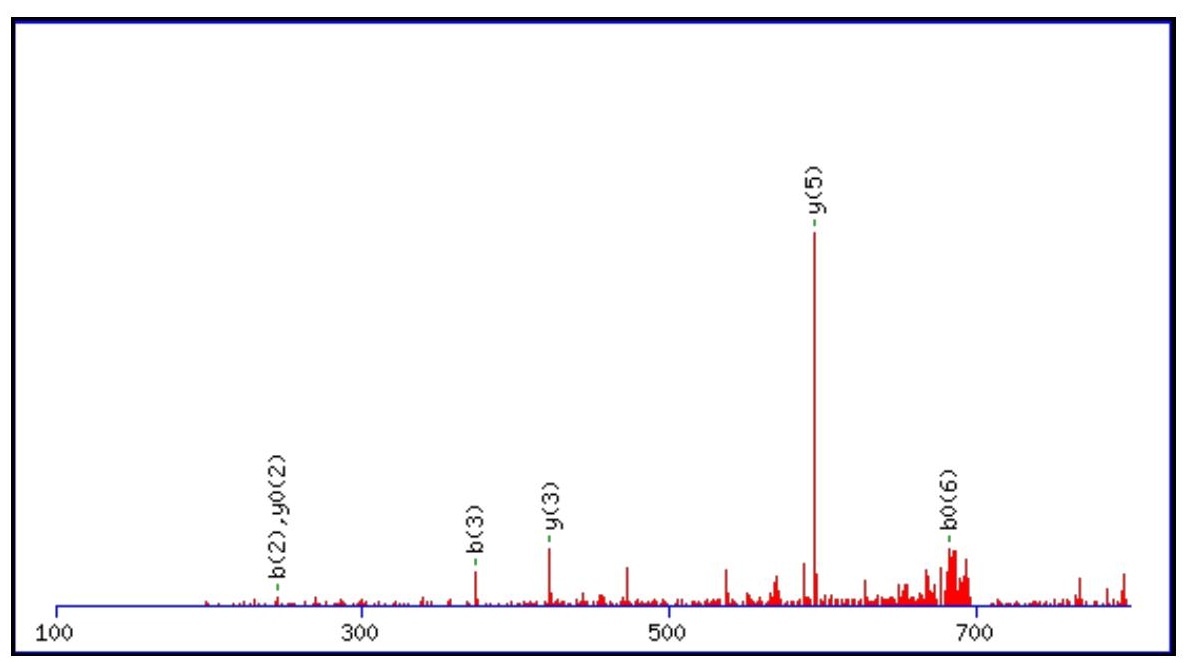



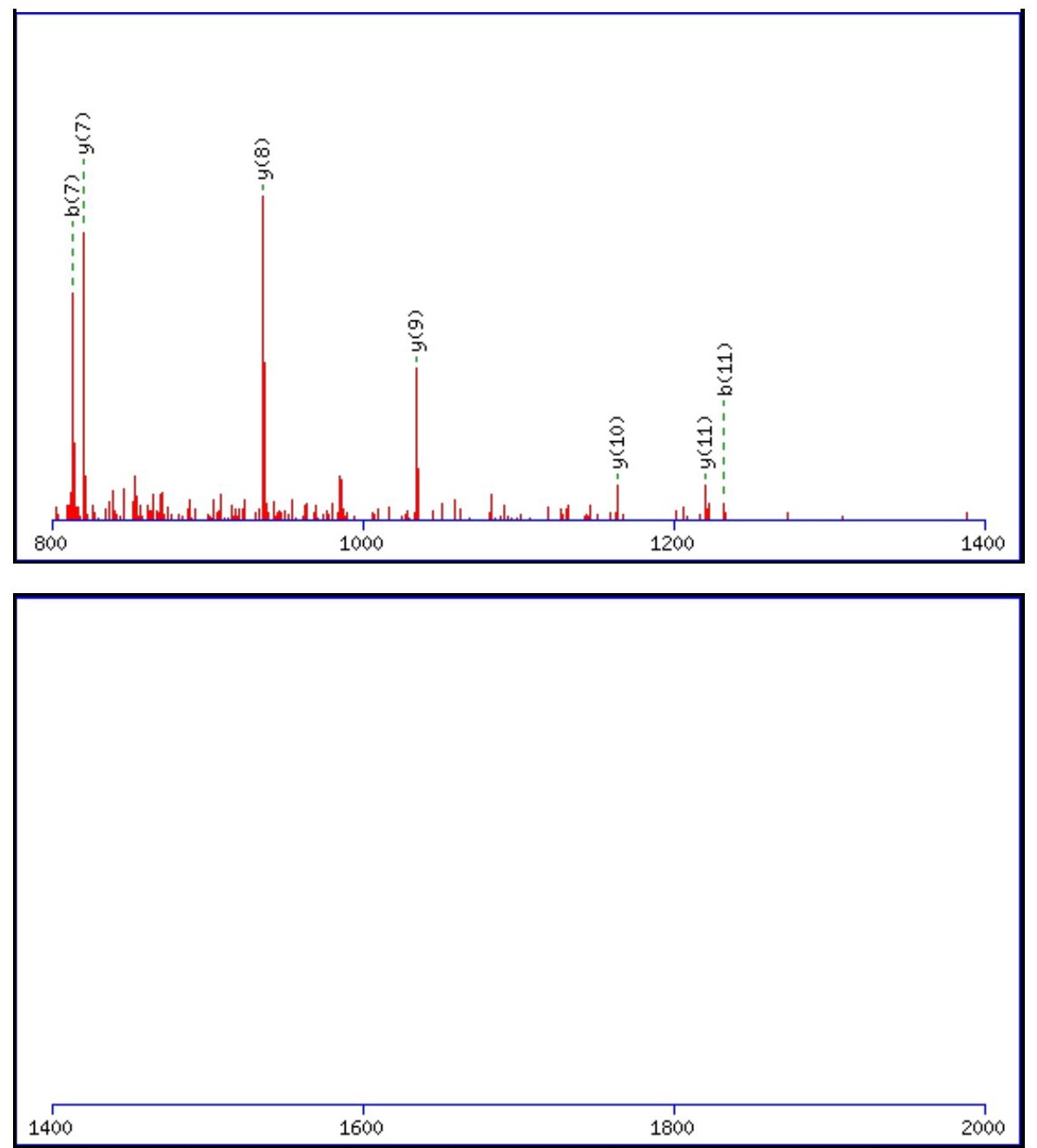

Monoisotopic mass of neutral peptide $\operatorname{Mr}($ calc): 1405.6296

Fixed modifications: Carbamidomethyl (C)

Variable modifications:

N9: Deamidated $\mathrm{N}(\mathrm{N})$

Ions Score: 65 Expect: $4.8 \mathrm{e}-005$

Matches (Bold Red): 13/110 fragment ions using 25 most intense peaks

\begin{tabular}{|r|c|c|c|c|c|c|c|c|c|c|c|c|c|c|}
\hline$\#$ & $\mathbf{b}$ & $\mathbf{b}^{++}$ & $\mathbf{b}^{*}$ & $\mathbf{b}^{\mathbf{*}^{++}}$ & $\mathbf{b}^{\mathbf{0}}$ & $\mathbf{b}^{\mathbf{0 + +}}$ & $\mathbf{S e q}$ & $\mathbf{y}$ & $\mathbf{y}^{++}$ & $\mathbf{y}^{\mathbf{*}}$ & $\mathbf{y}^{\mathbf{*}^{++}}$ & $\mathbf{y}^{\mathbf{0}}$ & $\mathbf{y}^{\mathbf{0 + +}}$ & $\#$ \\
\hline $\mathbf{1}$ & 187.0866 & 94.0469 & & & & & $\mathbf{W}$ & & & & & & & $\mathbf{1 2}$ \\
\hline $\mathbf{2}$ & $\mathbf{2 4 4 . 1 0 8 1}$ & 122.5577 & & & & & $\mathbf{G}$ & $\mathbf{1 2 2 0 . 5 5 7 6}$ & 610.7824 & 1203.5310 & 602.2691 & 1202.5470 & 601.7771 & $\mathbf{1 1}$ \\
\hline $\mathbf{3}$ & $\mathbf{3 7 3 . 1 5 0 6}$ & 187.0790 & & & 355.1401 & 178.0737 & $\mathbf{E}$ & $\mathbf{1 1 6 3 . 5 3 6 1}$ & 582.2717 & 1146.5096 & 573.7584 & 1145.5255 & 573.2664 & $\mathbf{1 0}$ \\
\hline $\mathbf{4}$ & 472.2191 & 236.6132 & & & 454.2085 & 227.6079 & $\mathbf{V}$ & $\mathbf{1 0 3 4 . 4 9 3 5}$ & 517.7504 & 1017.4670 & 509.2371 & 1016.4829 & 508.7451 & $\mathbf{9}$ \\
\hline $\mathbf{5}$ & 587.2460 & 294.1266 & & & 569.2354 & 285.1214 & $\mathbf{D}$ & $\mathbf{9 3 5 . 4 2 5 1}$ & 468.2162 & 918.3986 & 459.7029 & 917.4145 & 459.2109 & $\mathbf{8}$ \\
\hline $\mathbf{6}$ & 700.3301 & 350.6687 & & & $\mathbf{6 8 2 . 3 1 9 5}$ & 341.6634 & $\mathbf{L}$ & $\mathbf{8 2 0 . 3 9 8 2}$ & 410.7027 & 803.3716 & 402.1894 & 802.3876 & 401.6974 & $\mathbf{7}$ \\
\hline $\mathbf{7}$ & $\mathbf{8 1 3 . 4 1 4 1}$ & 407.2107 & & & 795.4036 & 398.2054 & $\mathbf{I}$ & 707.3141 & 354.1607 & 690.2875 & 345.6474 & 689.3035 & 345.1554 & $\mathbf{6}$ \\
\hline $\mathbf{8}$ & 870.4356 & 435.7214 & & & 852.4250 & 426.7162 & $\mathbf{G}$ & 594.2300 & 297.6187 & 577.2035 & 289.1054 & 576.2195 & 288.6134 & $\mathbf{5}$ \\
\hline $\mathbf{9}$ & 985.4625 & 493.2349 & 968.4360 & 484.7216 & 967.4520 & 484.2296 & $\mathbf{N}$ & 537.2086 & 269.1079 & 520.1820 & 260.5946 & 519.1980 & 260.1026 & $\mathbf{4}$ \\
\hline $\mathbf{1 0}$ & 1145.4932 & 573.2502 & 1128.4666 & 564.7370 & 1127.4826 & 564.2449 & $\mathbf{C}$ & $\mathbf{4 2 2 . 1 8 1 6}$ & 211.5945 & 405.1551 & 203.0812 & 404.1711 & 202.5892 & $\mathbf{3}$ \\
\hline $\mathbf{1 1}$ & $\mathbf{1 2 3 2 . 5 2 5 2}$ & 616.7662 & 1215.4987 & 608.2530 & 1214.5146 & 607.7610 & $\mathbf{S}$ & 262.1510 & 131.5791 & 245.1244 & 123.0659 & $\mathbf{2 4 4 . 1 4 0 4}$ & 122.5738 & $\mathbf{2}$ \\
\hline $\mathbf{1 2}$ & & & & & & & $\mathbf{R}$ & 175.1190 & 88.0631 & 158.0924 & 79.5498 & & & $\mathbf{1}$ \\
\hline
\end{tabular}



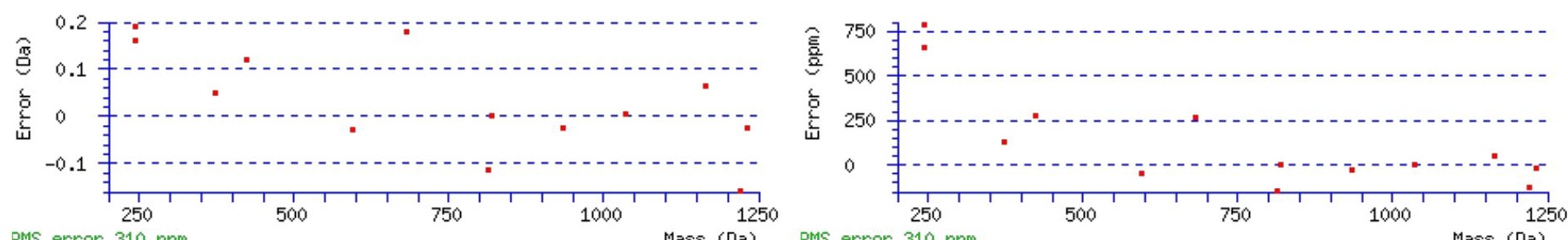

RMS error $310 \mathrm{ppm}$

Mass (Da) RMS error $310 \mathrm{ppm}$

\section{All matches to this query}

\begin{tabular}{|l|l|l|l|}
\hline Score & Mr(calc): & Delta & \multicolumn{1}{c|}{ Sequence } \\
\hline 65.0 & 1405.6296 & 0.0033 & WGEVDLIGNCSR \\
\hline 4.1 & 1403.6323 & 2.0006 & RRLVSELESR \\
\hline 1.6 & 1403.6122 & 2.0207 & YEPNKQFLER \\
\hline
\end{tabular}

Spectrum No: 439; Query: 1090; Rank: 1

\section{Peptide View}

MS/MS Fragmentation of IQNYSVMAICDYDK

Found in IPI00208422, Tax_Id=10116 Gene_Symbol=Dpp4 Dipeptidyl peptidase 4

Match to Query 1090: 1719.752648 from(860.883600,2+)

Title: 100101RatKid_NS_deglyco_23.3364.3364.2.dta

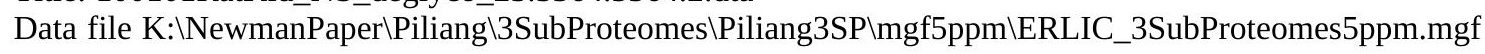
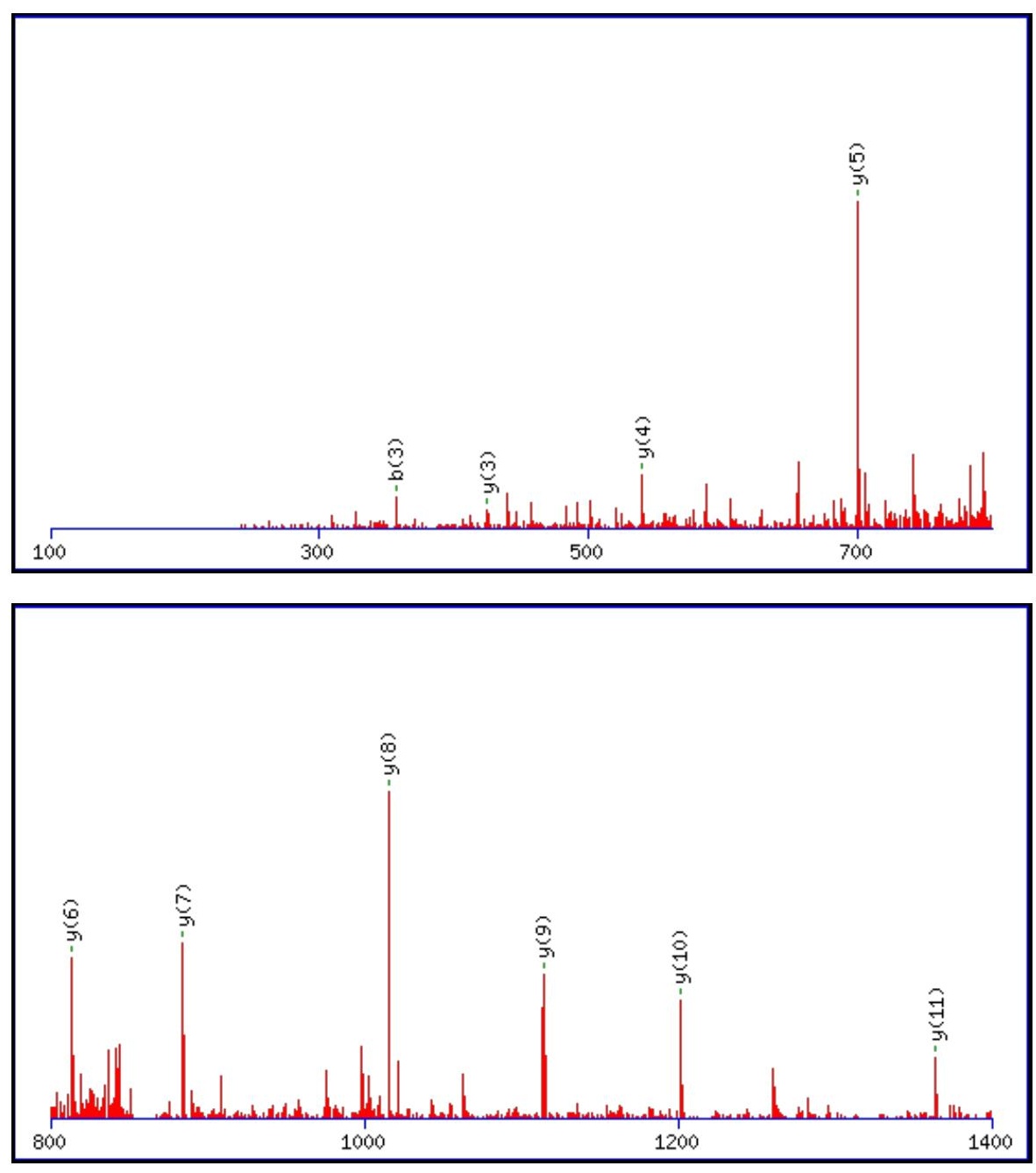


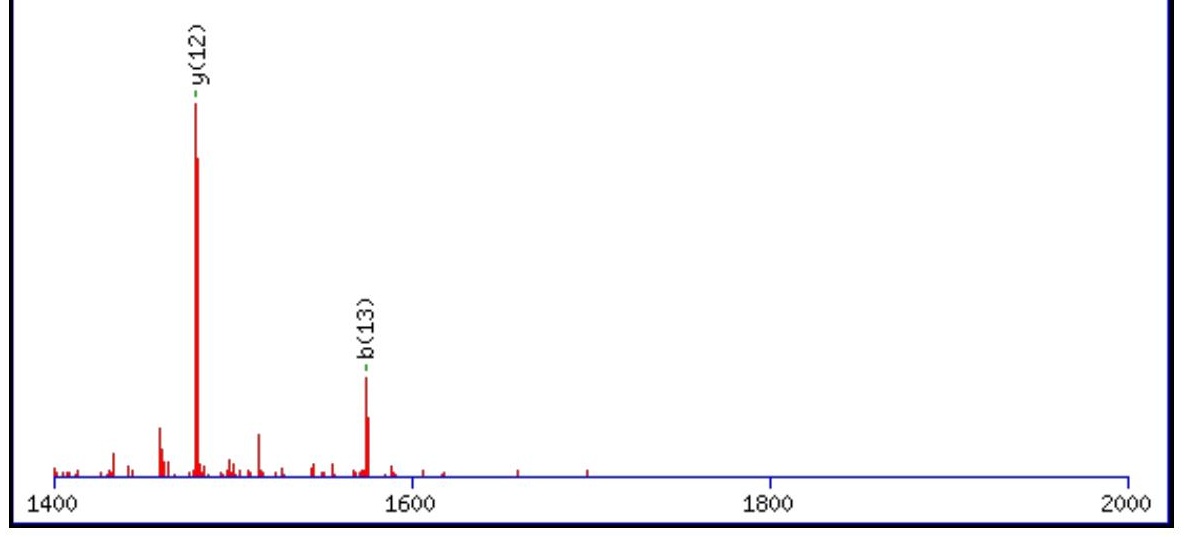

Monoisotopic mass of neutral peptide $\operatorname{Mr}($ calc): 1719.7484

Fixed modifications: Carbamidomethyl (C)

Variable modifications:

N3 : Deamidated_N (N)

Ions Score: 65 Expect: $6 \mathrm{e}-005$

Matches (Bold Red): 12/144 fragment ions using 21 most intense peaks

\begin{tabular}{|c|c|c|c|c|c|c|c|c|c|c|c|c|c|c|}
\hline \# & b & $\mathbf{b}^{++}$ & $\mathbf{b}^{*}$ & $\mathbf{b}^{*^{++}}$ & $\mathbf{b}^{\mathbf{0}}$ & $\mathbf{b}^{0++}$ & Seq. & $\mathbf{y}$ & $y^{++}$ & $\mathbf{y}^{*}$ & $\mathrm{y}^{*^{++}}$ & $\mathbf{y}^{\mathbf{0}}$ & $\mathbf{y}^{\mathbf{0 + +}}$ & \# \\
\hline 1 & 114.0913 & 57.5493 & & & & & I & & & & & & & 14 \\
\hline 2 & 242.1499 & 121.5786 & 225.1234 & 113.0653 & & & $\mathbf{Q}$ & 1607.6716 & 804.3394 & 1590.6451 & 795.8262 & 1589.6611 & 795.3342 & 13 \\
\hline 3 & 357.1769 & 179.0921 & 340.1503 & 170.5788 & & & $\mathbf{N}$ & 1479.6130 & 740.3102 & 1462.5865 & 731.7969 & 1461.6025 & 731.3049 & 12 \\
\hline 4 & 520.2402 & 260.6237 & 503.2136 & 252.1105 & & & $\mathbf{Y}$ & 1364.5861 & 682.7967 & 1347.5596 & 674.2834 & 1346.5755 & 673.7914 & 11 \\
\hline 5 & 607.2722 & 304.1397 & 590.2457 & 295.6265 & 589.2616 & 295.1345 & $S$ & 1201.5228 & 601.2650 & 1184.4962 & 592.7518 & 1183.5122 & 592.2597 & 10 \\
\hline 6 & 706.3406 & 353.6740 & 689.3141 & 345.1607 & 688.3301 & 344.6687 & V & 1114.4907 & 557.7490 & 1097.4642 & 549.2357 & 1096.4802 & 548.7437 & 9 \\
\hline 7 & 837.3811 & 419.1942 & 820.3546 & 410.6809 & 819.3705 & 410.1889 & $\mathbf{M}$ & 1015.4223 & 508.2148 & 998.3958 & 499.7015 & 997.4118 & 499.2095 & 8 \\
\hline 8 & 908.4182 & 454.7128 & 891.3917 & 446.1995 & 890.4077 & 445.7075 & A & 884.3818 & 442.6946 & 867.3553 & 434.1813 & 866.3713 & 433.6893 & 7 \\
\hline 9 & 1021.5023 & 511.2548 & 1004.4757 & 502.7415 & 1003.4917 & 502.2495 & I & 813.3447 & 407.1760 & 796.3182 & 398.6627 & 795.3342 & 398.1707 & 6 \\
\hline 10 & 1181.5329 & 591.2701 & 1164.5064 & 582.7568 & 1163.5224 & 582.2648 & $\mathrm{C}$ & 700.2607 & 350.6340 & 683.2341 & 342.1207 & 682.2501 & 341.6287 & 5 \\
\hline 11 & 1296.5599 & 648.7836 & 1279.5333 & 640.2703 & 1278.5493 & 639.7783 & D & 540.2300 & 270.6186 & 523.2035 & 262.1054 & 522.2195 & 261.6134 & 4 \\
\hline 12 & 1459.6232 & 730.3152 & 1442.5967 & 721.8020 & 1441.6126 & 721.3100 & $\mathbf{Y}$ & 425.2031 & 213.1052 & 408.1765 & 204.5919 & 407.1925 & 204.0999 & 3 \\
\hline 13 & 1574.6502 & 787.8287 & 1557.6236 & 779.3154 & 1556.6396 & 778.8234 & D & 262.1397 & 131.5735 & 245.1132 & 123.0602 & 244.1292 & 122.5682 & 2 \\
\hline 14 & & & & & & & $\mathbf{K}$ & 147.1128 & 74.0600 & 130.0863 & 65.5468 & & & 1 \\
\hline
\end{tabular}
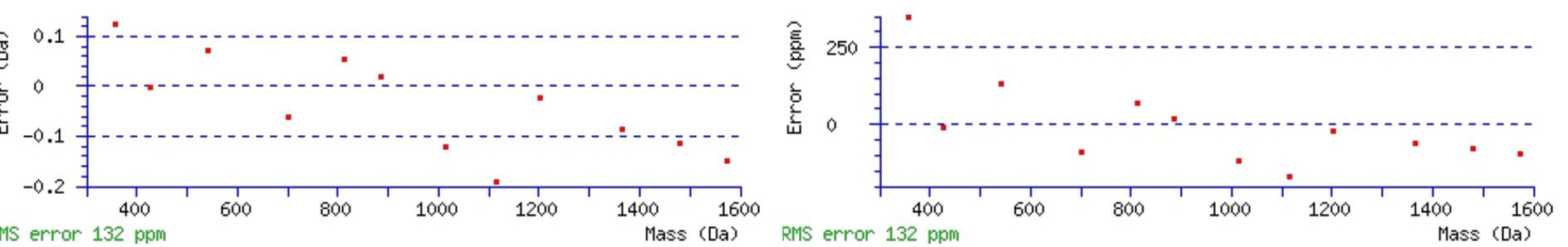

\section{All matches to this query}

\begin{tabular}{|l|l|c|l|}
\hline Score & Mr(calc): & Delta & \multicolumn{1}{|c|}{ Sequence } \\
\hline 65.0 & 1719.7484 & 0.0043 & IQNYSVMAICDYDK \\
\hline 50.3 & 1718.7644 & 0.9883 & IQNYSVMAICDYDK \\
\hline 5.1 & 1719.7621 & -0.0095 & DPSSQVGACIVNTENK \\
\hline 4.0 & 1719.7634 & -0.0107 & SKPVEWTLTRTDK \\
\hline 3.1 & 1718.7365 & 1.0162 & YPMGRVHVGLSSTR \\
\hline 2.7 & 1719.7634 & -0.0107 & SKPVEWTLTRTDK \\
\hline 2.7 & 1719.7634 & -0.0107 & SKPVEWTLTRTDK \\
\hline
\end{tabular}




\begin{tabular}{|l|l|l|l|}
2.1 & 1718.7365 & 1.0162 & YPMGRVHVGLSSTR \\
\hline 1.7 & 1719.7634 & -0.0107 & SKPVEWTLTRTDK \\
\hline 0.1 & 1719.7522 & 0.0005 & LYTLVLTDPDAPSR \\
\hline
\end{tabular}

Spectrum No: 440; Query: 598; Rank: 1

\section{Peptide View}

MS/MS Fragmentation of SVLENSTSYEEAK

Found in IPI00421601, Tax_Id=10116 Gene_Symbol=Asah1 N-acylsphingosine amidohydrolase 1

Match to Query 598: 1456.656948 from(729.335750,2+)

Title: 091008RatKidney_NoSalt_21.1079.1079.2.dta

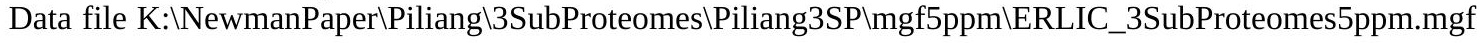
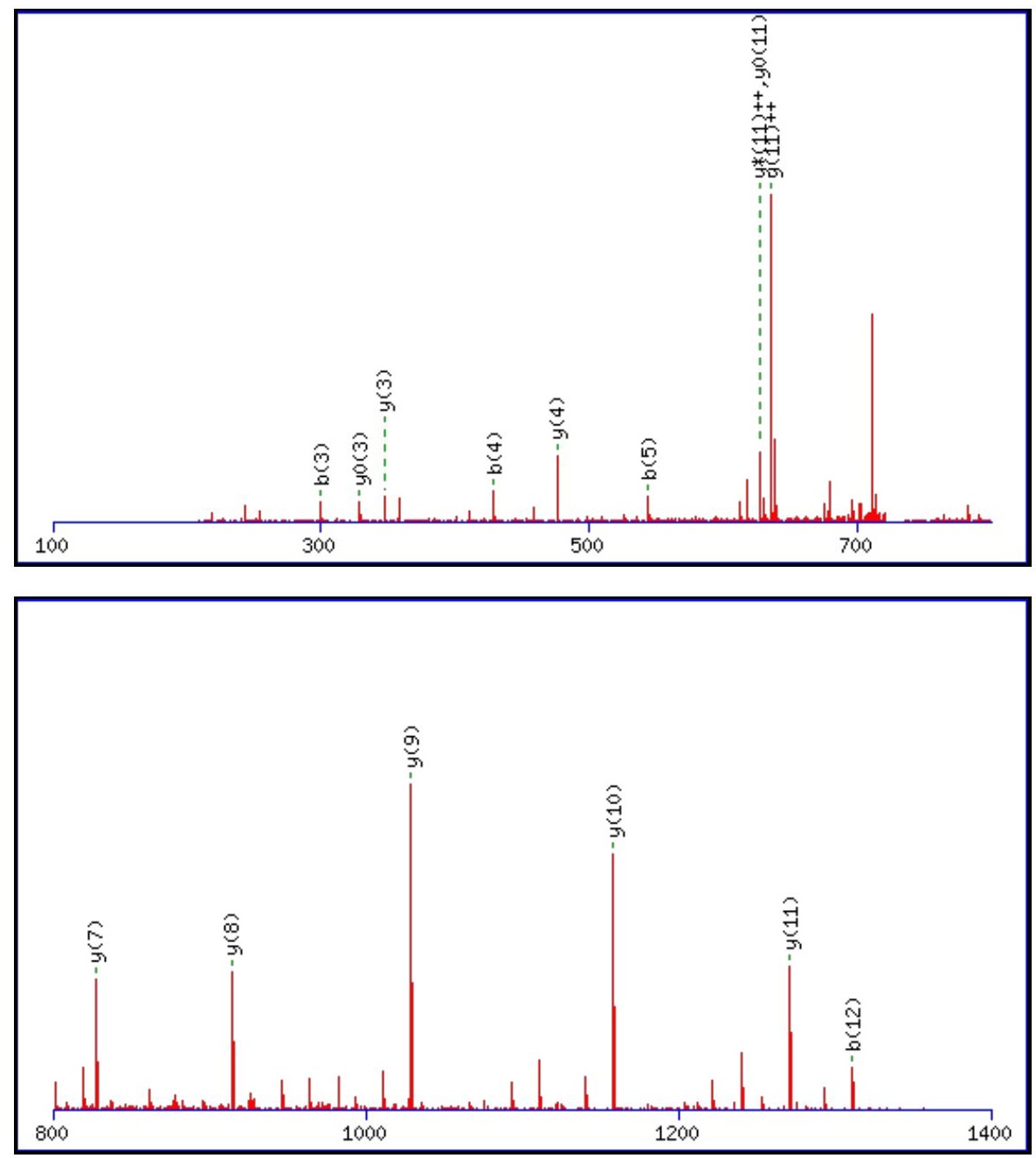
Monoisotopic mass of neutral peptide Mr(calc): 1456.6569

Fixed modifications: Carbamidomethyl (C)

Variable modifications:

N5 : Deamidated $\mathrm{N}(\mathrm{N})$

Ions Score: 65 Expect: $5.2 \mathrm{e}-005$

Matches (Bold Red): 15/132 fragment ions using 17 most intense peaks

\begin{tabular}{|c|c|c|c|c|c|c|c|c|c|c|c|c|c|c|}
\hline \# & b & $\mathbf{b}^{++}$ & $\mathbf{b}^{*}$ & $\mathbf{b}^{*^{++}}$ & $\mathbf{b}^{0}$ & $\mathbf{b}^{0++}$ & Seq. & $\mathbf{y}$ & $y^{++}$ & $\mathbf{y}^{*}$ & $\mathrm{y}^{\mathrm{*}^{++}}$ & $\mathbf{y}^{0}$ & $\mathbf{y}^{0++}$ & \# \\
\hline 1 & 88.0393 & 44.5233 & & & 70.0287 & 35.5180 & S & & & & & & & 13 \\
\hline 2 & 187.1077 & 94.0575 & & & 169.0972 & 85.0522 & $\mathbf{V}$ & 1370.6322 & 685.8197 & 1353.6056 & 677.3065 & 1352.6216 & 676.8144 & 12 \\
\hline 3 & 300.1918 & 150.5995 & & & 282.1812 & 141.5942 & $\mathbf{L}$ & 1271.5638 & 636.2855 & 1254.5372 & 627.7722 & 1253.5532 & 627.2802 & 11 \\
\hline 4 & 429.2344 & 215.1208 & & & 411.2238 & 206.1155 & $\mathbf{E}$ & 1158.4797 & 579.7435 & 1141.4531 & 571.2302 & 1140.4691 & 570.7382 & 10 \\
\hline 5 & 544.2613 & 272.6343 & 527.2348 & 264.1210 & 526.2507 & 263.6290 & $\mathbf{N}$ & 1029.4371 & 515.2222 & 1012.4106 & 506.7089 & 1011.4265 & 506.2169 & 9 \\
\hline 6 & 631.2933 & 316.1503 & 614.2668 & 307.6370 & 613.2828 & 307.1450 & S & 914.4102 & 457.7087 & 897.3836 & 449.1954 & 896.3996 & 448.7034 & 8 \\
\hline 7 & 732.3410 & 366.6741 & 715.3145 & 358.1609 & 714.3305 & 357.6689 & $\mathbf{T}$ & 827.3781 & 414.1927 & 810.3516 & 405.6794 & 809.3676 & 405.1874 & 7 \\
\hline 8 & 819.3730 & 410.1902 & 802.3465 & 401.6769 & 801.3625 & 401.1849 & S & 726.3305 & 363.6689 & 709.3039 & 355.1556 & 708.3199 & 354.6636 & 6 \\
\hline 9 & 982.4364 & 491.7218 & 965.4098 & 483.2086 & 964.4258 & 482.7165 & $\bar{Y}$ & 639.2984 & 320.1529 & 622.2719 & 311.6396 & 621.2879 & 311.1476 & 5 \\
\hline 10 & 1111.4790 & 556.2431 & 1094.4524 & 547.7298 & 1093.4684 & 547.2378 & $\mathbf{E}$ & 476.2351 & 238.6212 & 459.2086 & 230.1079 & 458.2245 & 229.6159 & 4 \\
\hline 11 & 1240.5216 & 620.7644 & 1223.4950 & 612.2511 & 1222.5110 & 611.7591 & $\mathbf{E}$ & 347.1925 & 174.0999 & 330.1660 & 165.5866 & 329.1819 & 165.0946 & 3 \\
\hline 12 & 1311.5587 & 656.2830 & 1294.5321 & 647.7697 & 1293.5481 & 647.2777 & A & 218.1499 & 109.5786 & 201.1234 & 101.0653 & & & 2 \\
\hline 13 & & & & & & & $\mathbf{K}$ & 147.1128 & 74.0600 & 130.0863 & 65.5468 & & & 1 \\
\hline
\end{tabular}
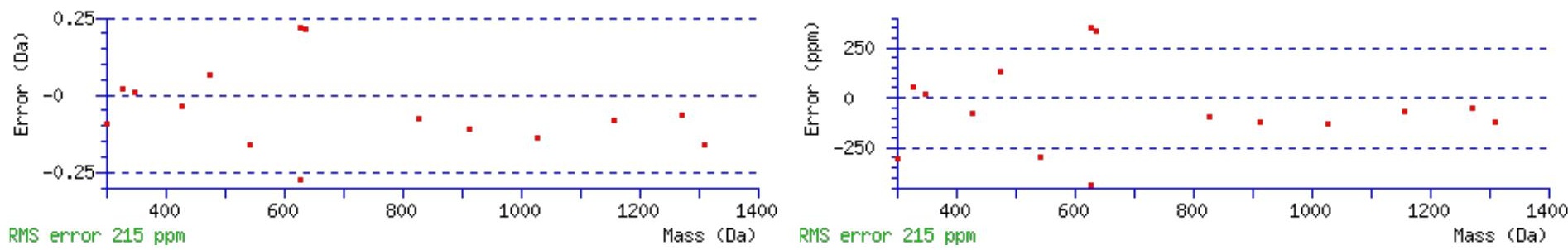

\section{All matches to this query}

\begin{tabular}{|l|l|l|l|}
\hline Score & Mr(calc): & Delta & \multicolumn{1}{c|}{ Sequence } \\
\hline 64.9 & 1456.6569 & 0.0001 & SVLENSTSYEEAK \\
\hline 13.7 & 1455.6408 & 1.0161 & RYSGPWXDPRR \\
\hline 13.7 & 1455.6408 & 1.0161 & RYSGPWXDPRR \\
\hline 11.0 & 1456.6438 & 0.0132 & SLLMVTSGYKAK \\
\hline 10.1 & 1456.6520 & 0.0049 & ADLLDTINTCIK \\
\hline 10.0 & 1456.6599 & -0.0030 & SVFPLSVSDSPSR \\
\hline 9.2 & 1456.6438 & 0.0132 & SLLMVTSGYKAK \\
\hline 8.7 & 1456.6599 & -0.0030 & $\underline{\text { SVFPLSVSDSPSR }}$ \\
\hline & & & \\
\hline
\end{tabular}




\begin{tabular}{|l|l|l|l|}
8.7 & 1456.6599 & -0.0030 & SVFPLSVSDSPSR \\
\hline 6.5 & 1456.6520 & 0.0049 & $\underline{\text { ADLLDTINTCIK }}$ \\
\hline
\end{tabular}

Spectrum No: 441; Query: 1473; Rank: 1

\section{Peptide View}

MS/MS Fragmentation of GHPVDDPTGAFANGSLTFK

Found in IPI00196226, Tax_Id=10116 Gene_Symbol=RGD1303130 Uncharacterized protein C1orf85 homolog precursor

Match to Query 1473: 1930.913982 from(644.645270,3+)

Title: 100101RatKid_NS_deglyco_20.3065.3065.3.dta

Data file K:INewmanPaper|Piliangl3SubProteomes\Piliang3SP\mgf5ppm\ERLIC_3SubProteomes5ppm.mgf
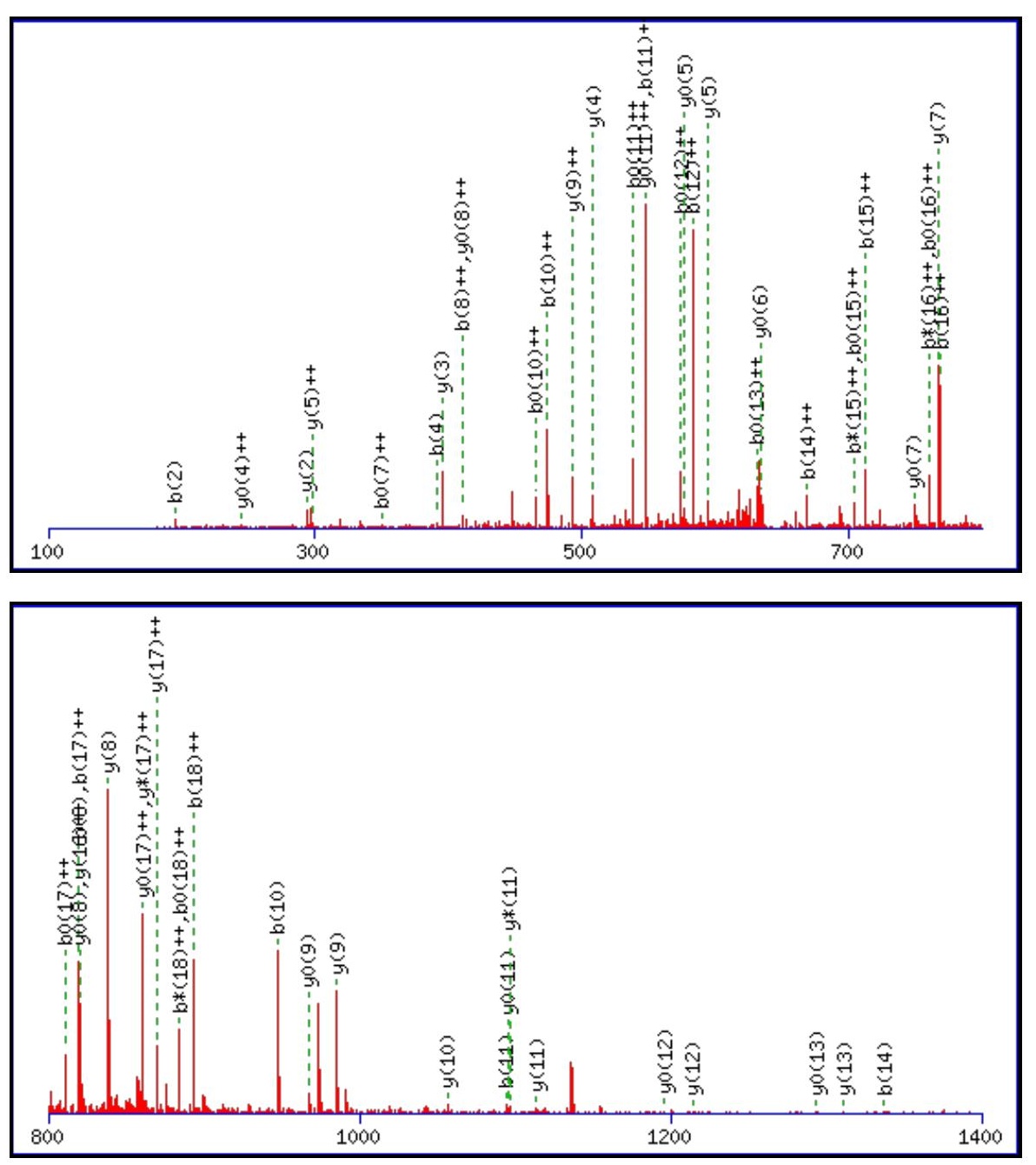


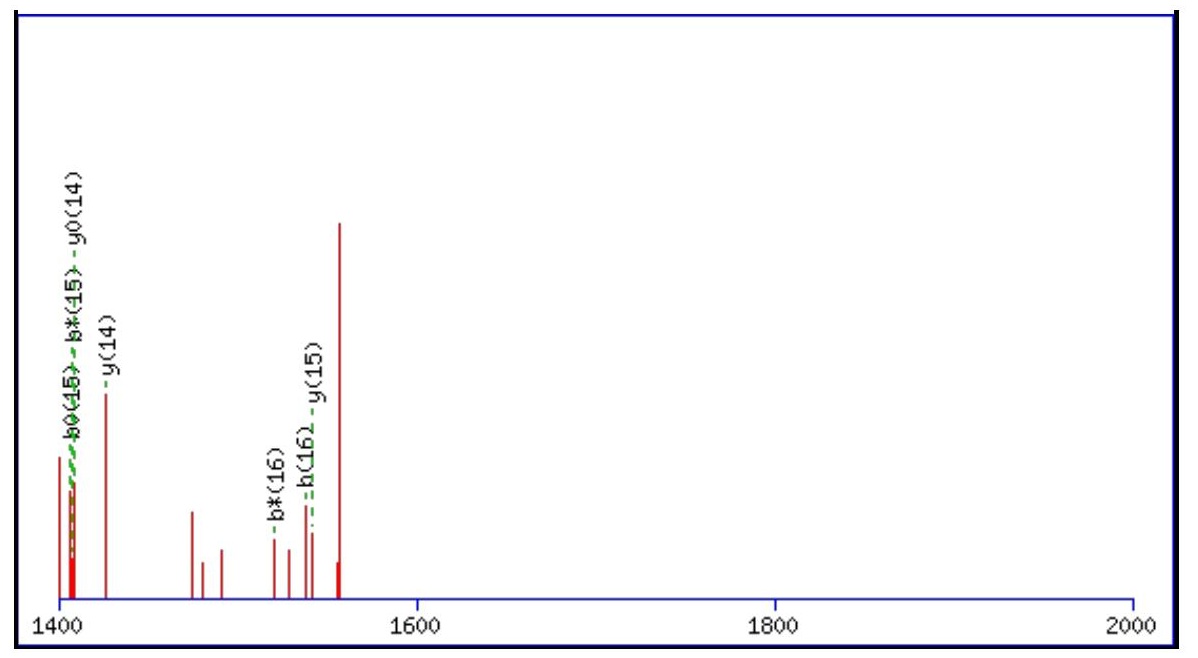

Monoisotopic mass of neutral peptide $\operatorname{Mr}($ calc): 1930.9061

Fixed modifications: Carbamidomethyl (C)

Variable modifications:

N13 : Deamidated_N (N)

Ions Score: 65 Expect: $8 \mathrm{e}-005$

Matches (Bold Red): 63/180 fragment ions using 116 most intense peaks

\begin{tabular}{|c|c|c|c|c|c|c|c|c|c|c|c|c|c|c|}
\hline \# & b & $\mathbf{b}^{++}$ & b* & $\mathbf{b}^{*^{++}}$ & $\mathbf{b}^{\mathbf{0}}$ & $\mathbf{b}^{0++}$ & Seq. & $\mathbf{y}$ & $\mathbf{y}^{++}$ & $\mathbf{y}^{*}$ & $\mathbf{y}^{*^{++}}$ & $\mathbf{y}^{0}$ & $\mathbf{y}^{0++}$ & \# \\
\hline 1 & 58.0287 & 29.5180 & & & & & G & & & & & & & 19 \\
\hline 2 & 195.0877 & 98.0475 & & & & & $\mathbf{H}$ & 1874.8919 & 937.9496 & 1857.8654 & 929.4363 & 1856.8813 & 928.9443 & 18 \\
\hline 3 & 292.1404 & 146.5738 & & & & & $\mathbf{P}$ & 1737.8330 & 869.4201 & 1720.8065 & 860.9069 & 1719.8224 & 860.4149 & 17 \\
\hline 4 & 391.2088 & 196.1081 & & & & & V & 1640.7802 & 820.8938 & 1623.7537 & 812.3805 & 1622.7697 & 811.8885 & 16 \\
\hline 5 & 506.2358 & 253.6215 & & & 488.2252 & 244.6162 & D & 1541.7118 & 771.3596 & 1524.6853 & 762.8463 & 1523.7013 & 762.3543 & 15 \\
\hline 6 & 621.2627 & 311.1350 & & & 603.2522 & 302.1297 & D & 1426.6849 & 713.8461 & 1409.6583 & 705.3328 & 1408.6743 & 704.8408 & 14 \\
\hline 7 & 718.3155 & 359.6614 & & & 700.3049 & 350.6561 & $\mathbf{P}$ & 1311.6579 & 656.3326 & 1294.6314 & 647.8193 & 1293.6474 & 647.3273 & 13 \\
\hline 8 & 819.3632 & 410.1852 & & & 801.3526 & 401.1799 & $\mathbf{T}$ & 1214.6052 & 607.8062 & 1197.5786 & 599.2930 & 1196.5946 & 598.8009 & 12 \\
\hline 9 & 876.3846 & 438.6959 & & & 858.3741 & 429.6907 & G & 1113.5575 & 557.2824 & 1096.5309 & 548.7691 & 1095.5469 & 548.2771 & 11 \\
\hline 10 & 947.4217 & 474.2145 & & & 929.4112 & 465.2092 & A & 1056.5360 & 528.7717 & 1039.5095 & 520.2584 & 1038.5255 & 519.7664 & 10 \\
\hline 11 & 1094.4902 & 547.7487 & & & 1076.4796 & 538.7434 & $\mathbf{F}$ & 985.4989 & 493.2531 & 968.4724 & 484.7398 & 967.4884 & 484.2478 & 9 \\
\hline 12 & 1165.5273 & 583.2673 & & & 1147.5167 & 574.2620 & A & 838.4305 & 419.7189 & 821.4040 & 411.2056 & 820.4199 & 410.7136 & 8 \\
\hline 13 & 1280.5542 & 640.7807 & 1263.5277 & 632.2675 & 1262.5436 & 631.7755 & $\mathbf{N}$ & 767.3934 & 384.2003 & 750.3668 & 375.6871 & 749.3828 & 375.1951 & 7 \\
\hline 14 & 1337.5757 & 669.2915 & 1320.5491 & 660.7782 & 1319.5651 & 660.2862 & G & 652.3665 & 326.6869 & 635.3399 & 318.1736 & 634.3559 & 317.6816 & 6 \\
\hline 15 & 1424.6077 & 712.8075 & 1407.5811 & 704.2942 & 1406.5971 & 703.8022 & S & 595.3450 & 298.1761 & 578.3184 & 289.6629 & 577.3344 & 289.1709 & 5 \\
\hline 16 & 1537.6918 & 769.3495 & 1520.6652 & 760.8362 & 1519.6812 & 760.3442 & $\mathbf{L}$ & 508.3130 & 254.6601 & 491.2864 & 246.1468 & 490.3024 & 245.6548 & 4 \\
\hline 17 & 1638.7394 & 819.8734 & 1621.7129 & 811.3601 & 1620.7289 & 810.8681 & $\mathbf{T}$ & 395.2289 & 198.1181 & 378.2023 & 189.6048 & 377.2183 & 189.1128 & 3 \\
\hline 18 & 1785.8079 & 893.4076 & 1768.7813 & 884.8943 & 1767.7973 & 884.4023 & $\mathbf{F}$ & 294.1812 & 147.5942 & 277.1547 & 139.0810 & & & 2 \\
\hline 19 & & & & & & & $\mathbf{K}$ & 147.1128 & 74.0600 & 130.0863 & 65.5468 & & & 1 \\
\hline
\end{tabular}

$$
\text { 㠃 }
$$
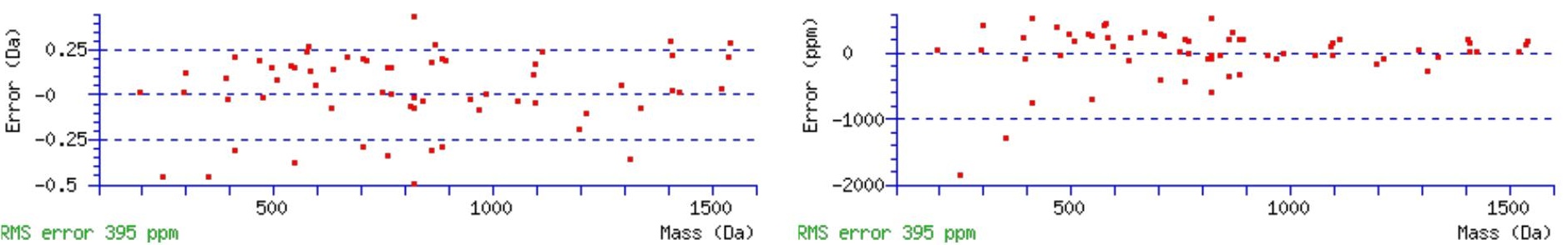

\section{All matches to this query}

\begin{tabular}{|l|c|c|c|}
\hline Score & Mr(calc): & Delta & \multicolumn{1}{|c|}{ Sequence } \\
\hline 64.8 & 1930.9061 & 0.0079 & GHPVDDPTGAFANGSLTFK \\
\hline 11.5 & 1929.9221 & 0.9919 & GHPVDDPTGAFANGSLTFK \\
\hline & & & \\
\hline
\end{tabular}




\begin{tabular}{|l|l|l|l|}
3.2 & 1928.8993 & 2.0147 & LGQNGTAITFINNNSKR \\
\hline 2.6 & 1930.8996 & 0.0144 & YWCERALDAFSEKTR \\
\hline 1.9 & 1930.9173 & -0.0034 & GHQPEGELVFRGDFNKT \\
\hline 1.8 & 1930.9166 & -0.0026 & GTGALLSSAKGVGAPLSSAK \\
\hline 1.8 & 1930.9166 & -0.0026 & GTGALLSSAKGVGAPLSSAK \\
\hline 1.7 & 1930.9196 & -0.0056 & RELSLSARCHQLEPR \\
\hline 1.7 & 1930.9196 & -0.0056 & RELSLSARCHQLEPR \\
\hline 1.5 & 1928.9146 & 1.9994 & RHDFSLFKLSDSEIR \\
\hline
\end{tabular}

Spectrum No: 442; Query: 1828; Rank: 1

\section{Peptide View}

MS/MS Fragmentation of AVGKDNFTLIPEGTNGTEER

Found in IPI00870112, Tax_Id=10116 Gene_Symbol=Dpysl2 Dihydropyrimidinase-related protein 2

Match to Query 1828: 2148.032688 from(1075.023620,2+)

Title: 091008RatKidney_NH4Format02_23.2500.2500.2.dta

Data file K:INewmanPaper|Piliang|3SubProteomes\Piliang3SP\mgf5ppm|ERLIC_3SubProteomes5ppm.mgf
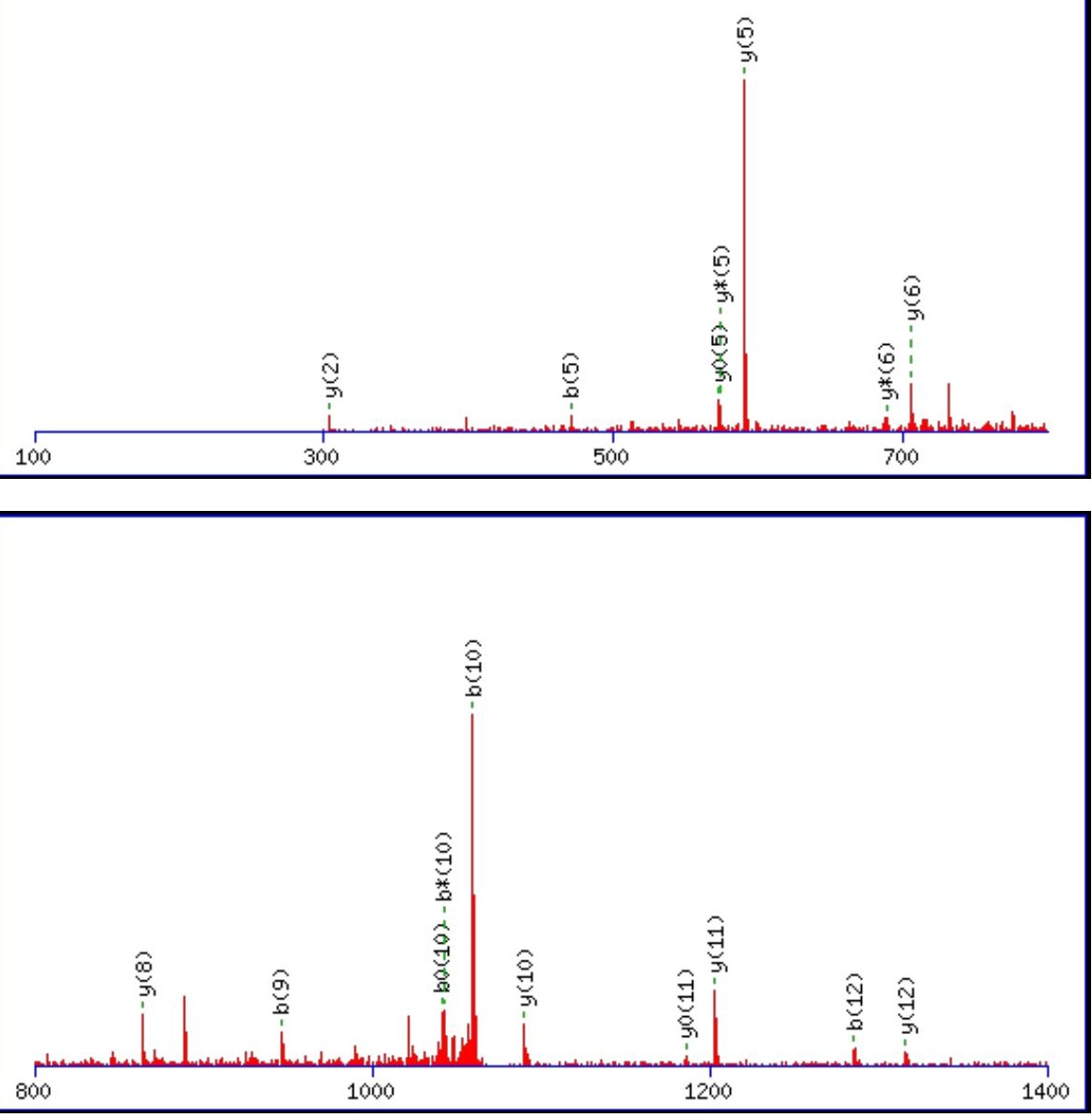


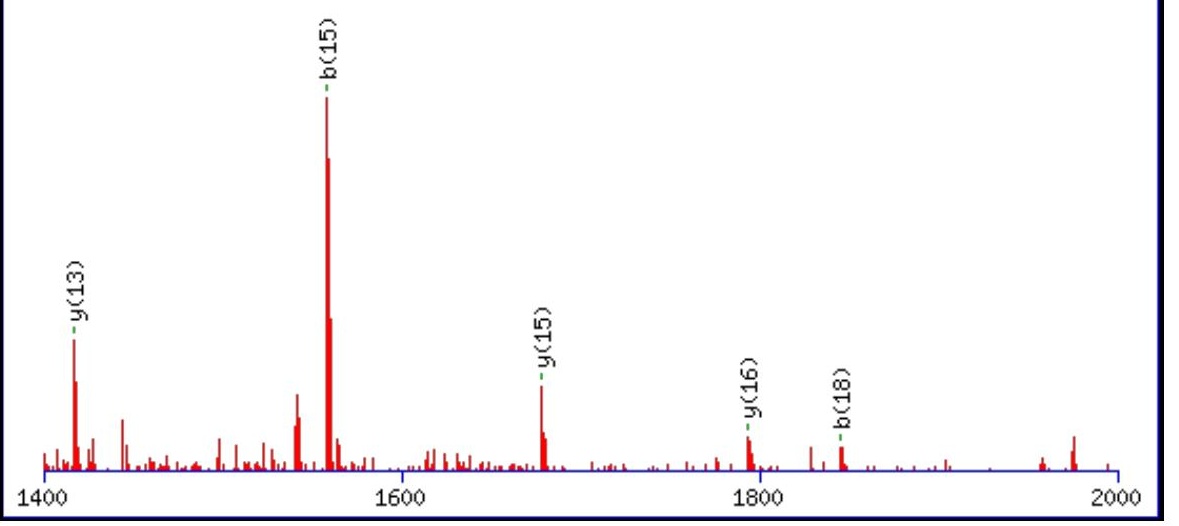

Monoisotopic mass of neutral peptide $\operatorname{Mr}($ calc): 2148.0335

Fixed modifications: Carbamidomethyl (C)

Variable modifications:

N15 : Deamidated_N (N)

Ions Score: 65 Expect: $7.9 \mathrm{e}-005$

Matches (Bold Red): 22/212 fragment ions using 38 most intense peaks

\begin{tabular}{|c|c|c|c|c|c|c|c|c|c|c|c|c|c|c|}
\hline \# & b & $\mathbf{b}^{++}$ & $\mathbf{b}^{*}$ & $\mathbf{b}^{*^{++}}$ & $\mathbf{b}^{0}$ & $\mathbf{b}^{0++}$ & Seq. & $\mathbf{y}$ & $\mathrm{y}^{++}$ & $\mathbf{y}^{*}$ & $\mathbf{y}^{*^{++}}$ & $\mathbf{y}^{0}$ & $\mathbf{y}^{0++}$ & $\#$ \\
\hline 1 & 72.0444 & 36.5258 & & & & & A & & & & & & & 20 \\
\hline 2 & 171.1128 & 86.0600 & & & & & $\mathbf{V}$ & 2078.0036 & 1039.5055 & 2060.9771 & 1030.9922 & 2059.9931 & 1030.5002 & 19 \\
\hline 3 & 228.1343 & 114.5708 & & & & & G & 1978.9352 & 989.9713 & 1961.9087 & 981.4580 & 1960.9247 & 980.9660 & 18 \\
\hline 4 & 356.2292 & 178.6183 & 339.2027 & 170.1050 & & & $\mathbf{K}$ & 1921.9138 & 961.4605 & 1904.8872 & 952.9472 & 1903.9032 & 952.4552 & 17 \\
\hline 5 & 471.2562 & 236.1317 & 454.2296 & 227.6185 & 453.2456 & 227.1264 & D & 1793.8188 & 897.4130 & 1776.7923 & 888.8998 & 1775.8082 & 888.4078 & 16 \\
\hline 6 & 585.2991 & 293.1532 & 568.2726 & 284.6399 & 567.2885 & 284.1479 & $\mathbf{N}$ & 1678.7919 & 839.8996 & 1661.7653 & 831.3863 & 1660.7813 & 830.8943 & 15 \\
\hline 7 & 732.3675 & 366.6874 & 715.3410 & 358.1741 & 714.3569 & 357.6821 & $\mathbf{F}$ & 1564.7489 & 782.8781 & 1547.7224 & 774.3648 & 1546.7384 & 773.8728 & 14 \\
\hline 8 & 833.4152 & 417.2112 & 816.3886 & 408.6980 & 815.4046 & 408.2060 & $\mathbf{T}$ & 1417.6805 & 709.3439 & 1400.6540 & 700.8306 & 1399.6700 & 700.3386 & 13 \\
\hline 9 & 946.4993 & 473.7533 & 929.4727 & 465.2400 & 928.4887 & 464.7480 & $\mathbf{L}$ & 1316.6328 & 658.8201 & 1299.6063 & 650.3068 & 1298.6223 & 649.8148 & 12 \\
\hline 10 & 1059.5833 & 530.2953 & 1042.5568 & 521.7820 & 1041.5728 & 521.2900 & I & 1203.5488 & 602.2780 & 1186.5222 & 593.7648 & 1185.5382 & 593.2727 & 11 \\
\hline 11 & 1156.6361 & 578.8217 & 1139.6095 & 570.3084 & 1138.6255 & 569.8164 & $\mathbf{P}$ & 1090.4647 & 545.7360 & 1073.4382 & 537.2227 & 1072.4542 & 536.7307 & 10 \\
\hline 12 & 1285.6787 & 643.3430 & 1268.6521 & 634.8297 & 1267.6681 & 634.3377 & $\mathbf{E}$ & 993.4120 & 497.2096 & 976.3854 & 488.6963 & 975.4014 & 488.2043 & 9 \\
\hline 13 & 1342.7001 & 671.8537 & 1325.6736 & 663.3404 & 1324.6896 & 662.8484 & G & 864.3694 & 432.6883 & 847.3428 & 424.1750 & 846.3588 & 423.6830 & 8 \\
\hline 14 & 1443.7478 & 722.3775 & 1426.7213 & 713.8643 & 1425.7373 & 713.3723 & $\mathbf{T}$ & 807.3479 & 404.1776 & 790.3213 & 395.6643 & 789.3373 & 395.1723 & 7 \\
\hline 15 & 1558.7748 & 779.8910 & 1541.7482 & 771.3777 & 1540.7642 & 770.8857 & $\mathbf{N}$ & 706.3002 & 353.6537 & 689.2737 & 345.1405 & 688.2897 & 344.6485 & 6 \\
\hline 16 & 1615.7962 & 808.4017 & 1598.7697 & 799.8885 & 1597.7857 & 799.3965 & G & 591.2733 & 296.1403 & 574.2467 & 287.6270 & 573.2627 & 287.1350 & 5 \\
\hline 17 & 1716.8439 & 858.9256 & 1699.8174 & 850.4123 & 1698.8333 & 849.9203 & $\mathbf{T}$ & 534.2518 & 267.6295 & 517.2253 & 259.1163 & 516.2413 & 258.6243 & 4 \\
\hline 18 & 1845.8865 & 923.4469 & 1828.8599 & 914.9336 & 1827.8759 & 914.4416 & $\mathbf{E}$ & 433.2041 & 217.1057 & 416.1776 & 208.5924 & 415.1936 & 208.1004 & 3 \\
\hline 19 & 1974.9291 & 987.9682 & 1957.9025 & 979.4549 & 1956.9185 & 978.9629 & $\mathbf{E}$ & 304.1615 & 152.5844 & 287.1350 & 144.0711 & 286.1510 & 143.5791 & 2 \\
\hline 20 & & & & & & & $\mathbf{R}$ & 175.1190 & 88.0631 & 158.0924 & 79.5498 & & & 1 \\
\hline
\end{tabular}

$$
\text { 䟢 }
$$

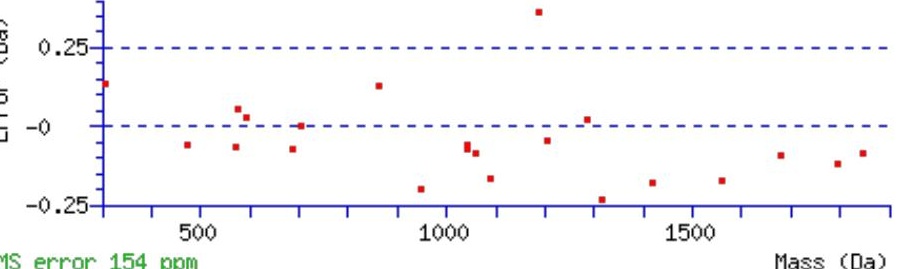

RMS error 154 ppm

\section{All matches to this query}

$$
\begin{array}{|l}
\hline \text { S } \\
\hline 64.7 \\
\hline
\end{array}
$$




\begin{tabular}{|l|l|l|l||}
20.0 & 2148.0335 & -0.0008 & AVGKDNFTLIPEGTNGTEER \\
\hline 12.6 & 2146.0170 & 2.0157 & FPGMSVLLFLQESLGQER \\
\hline 8.7 & 2147.0495 & 0.9832 & AVGKDNFTLIPEGTNGTEER \\
\hline 3.3 & 2146.0170 & 2.0157 & VRYATWSIIMDSVVPSDK \\
\hline 1.1 & 2148.0229 & 0.0098 & KENVAERALAAXSCTEAESR \\
\hline 0.6 & 2148.0197 & 0.0130 & EGKYIIELAHMIKDNGWD \\
\hline 0.5 & 2148.0171 & 0.0156 & SAACLPSDISWPGTHXKER \\
\hline 0.5 & 2148.0366 & -0.0039 & KFISDIPYLKELFNNMP \\
\hline 0.1 & 2147.0289 & 1.0038 & APQSIELPAVAGHTLTARR \\
\hline
\end{tabular}

Spectrum No: 443; Query: 1222; Rank: 1

\section{Peptide View}

MS/MS Fragmentation of NGGLGHMNITLLSDLTK

Found in IPI00208215, Tax_Id=10116 Gene_Symbol=Prdx3 Thioredoxin-dependent peroxide reductase, mitochondrial precursor

Match to Query 1222: 1783.921228 from(892.967890,2+)

Title: 091008RatKidney_NH4Format02_17.4082.4082.2.dta

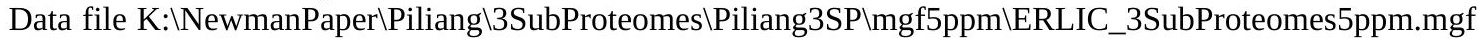
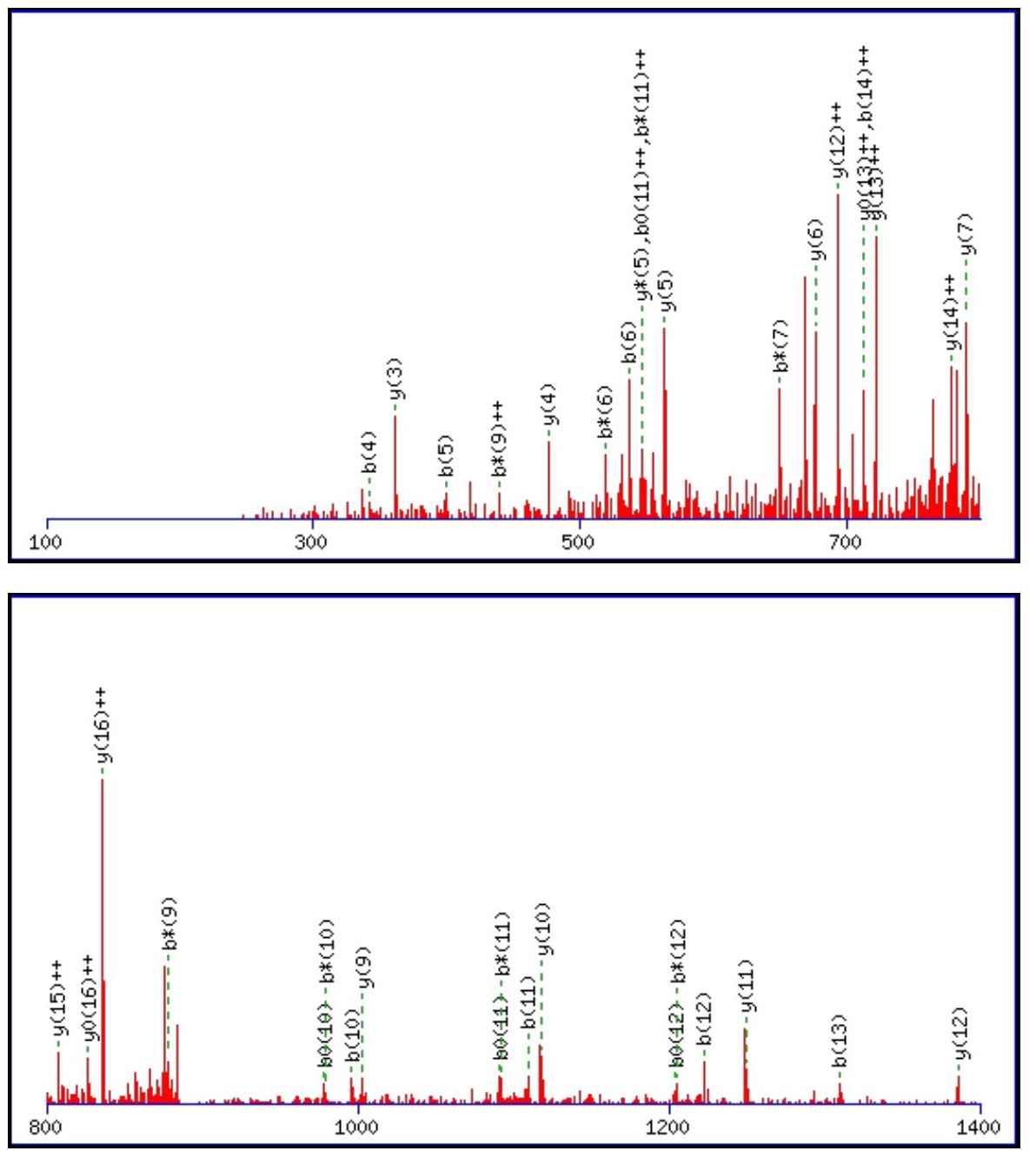


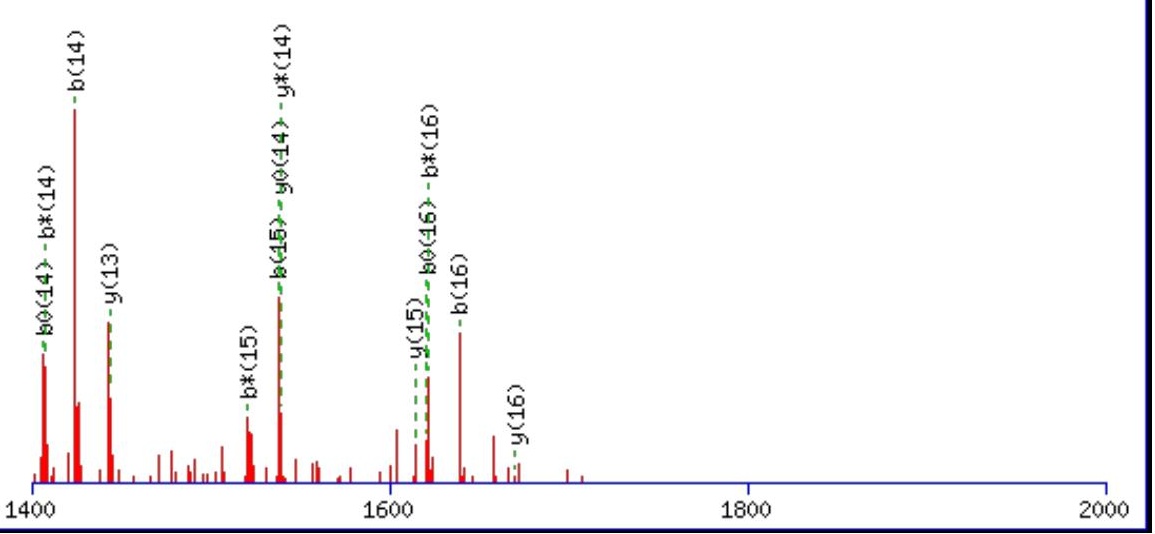

Monoisotopic mass of neutral peptide $\operatorname{Mr}($ calc): 1783.9138

Fixed modifications: Carbamidomethyl (C)

Variable modifications:

N8 : Deamidated_N (N)

Ions Score: 65 Expect: $4.5 \mathrm{e}-005$

Matches (Bold Red): 50/172 fragment ions using 94 most intense peaks

\begin{tabular}{|c|c|c|c|c|c|c|c|c|c|c|c|c|c|c|}
\hline \# & b & $\mathbf{b}^{++}$ & $\mathbf{b}^{*}$ & $\mathbf{b}^{*^{++}}$ & $\mathbf{b}^{\mathbf{0}}$ & $\mathbf{b}^{0++}$ & Seq. & $\mathbf{y}$ & $\mathbf{y}^{++}$ & $\mathbf{y}^{*}$ & $\mathbf{y}^{*^{++}}$ & $\mathbf{y}^{\mathbf{0}}$ & $\mathbf{y}^{0++}$ & \# \\
\hline 1 & 115.0502 & 58.0287 & 98.0237 & 49.5155 & & & $\mathbf{N}$ & & & & & & & 17 \\
\hline 2 & 172.0717 & 86.5395 & 155.0451 & 78.0262 & & & G & 1670.8782 & 835.9427 & 1653.8516 & 827.4295 & 1652.8676 & 826.9374 & 16 \\
\hline 3 & 229.0931 & 115.0502 & 212.0666 & 106.5369 & & & G & 1613.8567 & 807.4320 & 1596.8302 & 798.9187 & 1595.8461 & 798.4267 & 15 \\
\hline 4 & 342.1772 & 171.5922 & 325.1506 & 163.0790 & & & $\mathbf{L}$ & 1556.8352 & 778.9213 & 1539.8087 & 770.4080 & 1538.8247 & 769.9160 & 14 \\
\hline 5 & 399.1987 & 200.1030 & 382.1721 & 191.5897 & & & $\mathbf{G}$ & 1443.7512 & 722.3792 & 1426.7246 & 713.8660 & 1425.7406 & 713.3739 & 13 \\
\hline 6 & 536.2576 & 268.6324 & 519.2310 & 260.1191 & & & $\mathbf{H}$ & 1386.7297 & 693.8685 & 1369.7032 & 685.3552 & 1368.7192 & 684.8632 & 12 \\
\hline 7 & 667.2981 & 334.1527 & 650.2715 & 325.6394 & & & $\mathbf{M}$ & 1249.6708 & 625.3390 & 1232.6443 & 616.8258 & 1231.6602 & 616.3338 & 11 \\
\hline 8 & 782.3250 & 391.6661 & 765.2984 & 383.1529 & & & $\mathbf{N}$ & 1118.6303 & 559.8188 & 1101.6038 & 551.3055 & 1100.6198 & 550.8135 & 10 \\
\hline 9 & 895.4091 & 448.2082 & 878.3825 & 439.6949 & & & I & 1003.6034 & 502.3053 & 986.5768 & 493.7921 & 985.5928 & 493.3001 & 9 \\
\hline 10 & 996.4567 & 498.7320 & 979.4302 & 490.2187 & 978.4462 & 489.7267 & $\mathbf{T}$ & 890.5193 & 445.7633 & 873.4928 & 437.2500 & 872.5088 & 436.7580 & 8 \\
\hline 11 & 1109.5408 & 555.2740 & 1092.5142 & 546.7608 & 1091.5302 & 546.2688 & $\mathbf{L}$ & 789.4716 & 395.2395 & 772.4451 & 386.7262 & 771.4611 & 386.2342 & 7 \\
\hline 12 & 1222.6249 & 611.8161 & 1205.5983 & 603.3028 & 1204.6143 & 602.8108 & $\mathbf{L}$ & 676.3876 & 338.6974 & 659.3610 & 330.1842 & 658.3770 & 329.6921 & 6 \\
\hline 13 & 1309.6569 & 655.3321 & 1292.6303 & 646.8188 & 1291.6463 & 646.3268 & $\mathbf{S}$ & 563.3035 & 282.1554 & 546.2770 & 273.6421 & 545.2930 & 273.1501 & 5 \\
\hline 14 & 1424.6838 & 712.8456 & 1407.6573 & 704.3323 & 1406.6733 & 703.8403 & D & 476.2715 & 238.6394 & 459.2449 & 230.1261 & 458.2609 & 229.6341 & 4 \\
\hline 15 & 1537.7679 & 769.3876 & 1520.7413 & 760.8743 & 1519.7573 & 760.3823 & $\mathbf{L}$ & 361.2445 & 181.1259 & 344.2180 & 172.6126 & 343.2340 & 172.1206 & 3 \\
\hline 16 & 1638.8156 & 819.9114 & 1621.7890 & 811.3982 & 1620.8050 & 810.9061 & $\mathbf{T}$ & 248.1605 & 124.5839 & 231.1339 & 116.0706 & 230.1499 & 115.5786 & 2 \\
\hline 17 & & & & & & & $\mathbf{K}$ & 147.1128 & 74.0600 & 130.0863 & 65.5468 & & & 1 \\
\hline
\end{tabular}
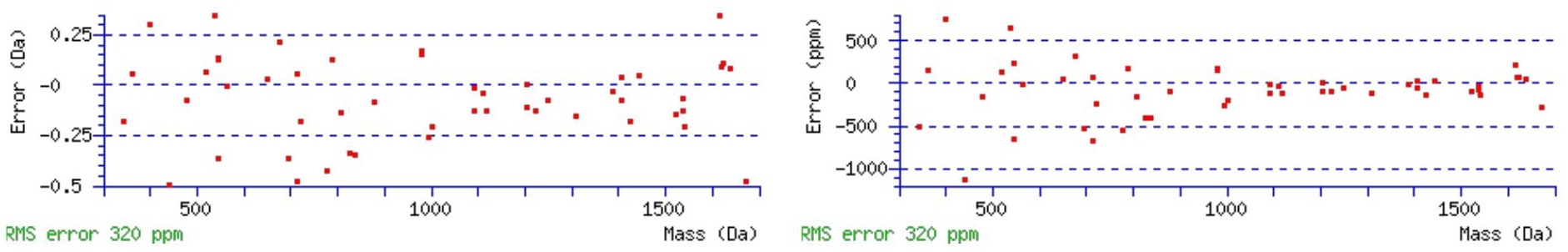

\section{All matches to this query}

\begin{tabular}{|l|l|l|l|}
\hline Score & Mr(calc): & Delta & \multicolumn{1}{c|}{ Sequence } \\
\hline 64.7 & 1783.9138 & 0.0074 & NGGLGHMNITLLSDLTK \\
\hline 58.8 & 1783.9138 & 0.0074 & NGGLGHMNITLLSDLTK \\
\hline 37.4 & 1782.9298 & 0.9914 & NGGLGHMNITLLSDLTK \\
\hline 6.7 & 1783.9039 & 0.0173 & FRGPPGPTPAMGTNASVK \\
\hline & & & \\
\hline
\end{tabular}




\begin{tabular}{|l||l|l|l|}
\hline 2.1 & 1783.9046 & 0.0166 & NSYFFRVLFQPHTK \\
\hline 1.0 & 1783.9039 & 0.0173 & GFXLNSCLVYVDVSR \\
\hline
\end{tabular}

Spectrum No: 444; Query: 367; Rank: 1

\section{Peptide View}

MS/MS Fragmentation of ICGNETLFPIR

Found in IPI00196620, Tax_Id=10116 Gene_Symbol=Cubn Cubilin precursor

Match to Query 367: 1319.651168 from(660.832860,2+)

Title: 091008RatKidney_NH4Format01_25.2714.2714.2.dta

Data file K:INewmanPaper|Piliangl3SubProteomes\Piliang3SP\mgf5ppm\ERLIC_3SubProteomes5ppm.mgf
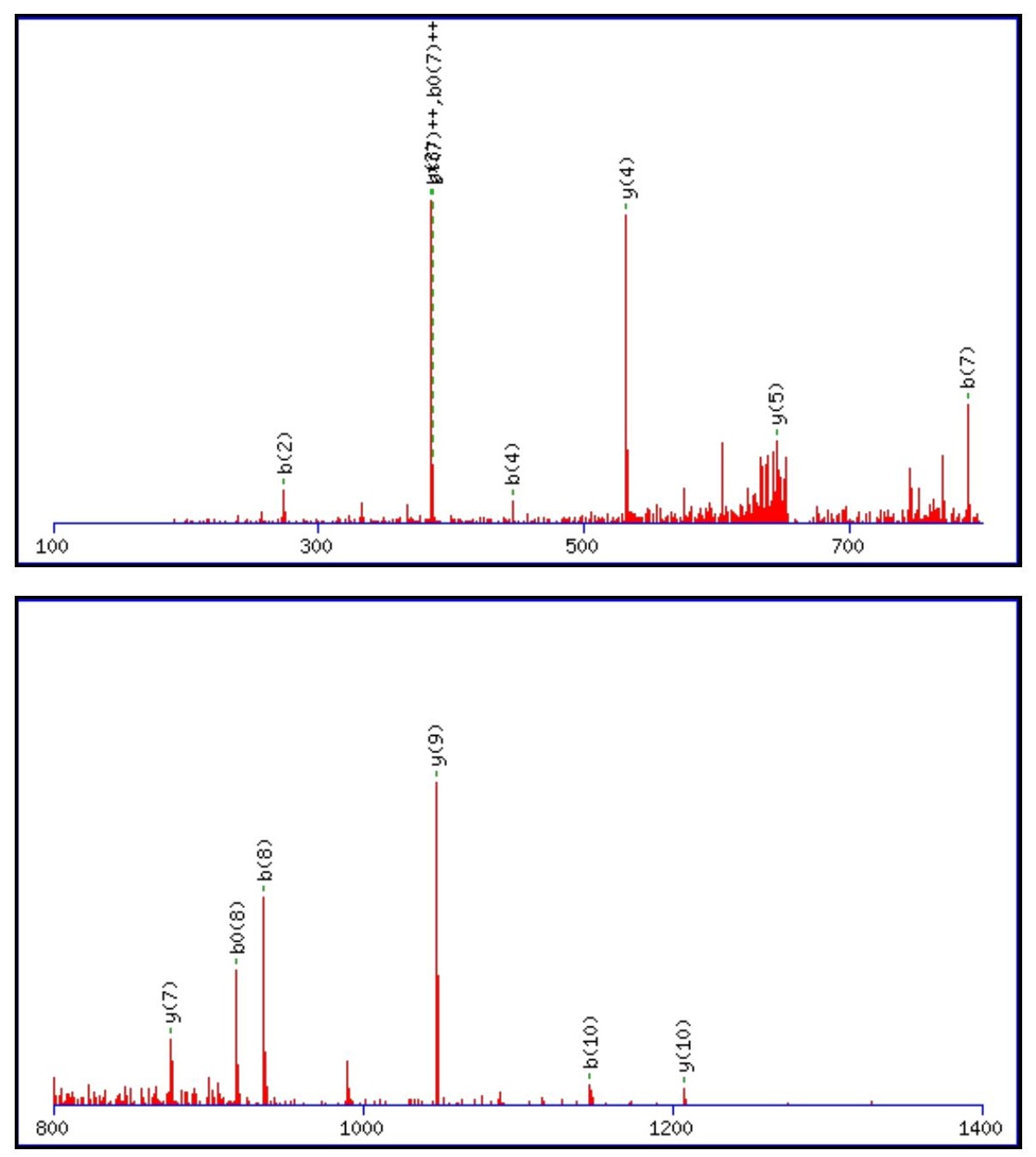


$1400 \quad 1600 \quad 1800 \quad 2000$

Monoisotopic mass of neutral peptide $\operatorname{Mr}($ calc): 1319.6544

Fixed modifications: Carbamidomethyl (C)

Variable modifications:

N4 : Deamidated_N (N)

Ions Score: 65 Expect: $4.5 \mathrm{e}-005$

Matches (Bold Red): 14/96 fragment ions using 15 most intense peaks

\begin{tabular}{|c|c|c|c|c|c|c|c|c|c|c|c|c|c|c|}
\hline \# & b & $\mathbf{b}^{++}$ & $\mathbf{b}^{*}$ & $\mathbf{b}^{*^{++}}$ & $\mathbf{b}^{0}$ & $\mathbf{b}^{0++}$ & Seq. & $\mathbf{y}$ & $\mathbf{y}^{++}$ & $\mathrm{y}^{*}$ & $\mathrm{y}^{*^{++}}$ & $\mathbf{y}^{\mathbf{0}}$ & $y^{0++}$ & \# \\
\hline 1 & 114.0913 & 57.5493 & & & & & I & & & & & & & 11 \\
\hline 2 & 274.1220 & 137.5646 & & & & & C & 1207.5776 & 604.2924 & 1190.5510 & 595.7792 & 1189.5670 & 595.2871 & 10 \\
\hline 3 & 331.1435 & 166.0754 & & & & & G & 1047.5469 & 524.2771 & 1030.5204 & 515.7638 & 1029.5364 & 515.2718 & 9 \\
\hline 4 & 446.1704 & 223.5888 & 429.1438 & 215.0756 & & & $\mathbf{N}$ & 990.5255 & 495.7664 & 973.4989 & 487.2531 & 972.5149 & 486.7611 & 8 \\
\hline 5 & 575.2130 & 288.1101 & 558.1864 & 279.5969 & 557.2024 & 279.1048 & $\mathbf{E}$ & 875.4985 & 438.2529 & 858.4720 & 429.7396 & 857.4880 & 429.2476 & 7 \\
\hline 6 & 676.2607 & 338.6340 & 659.2341 & 330.1207 & 658.2501 & 329.6287 & $\mathbf{T}$ & 746.4559 & 373.7316 & 729.4294 & 365.2183 & 728.4454 & 364.7263 & 6 \\
\hline 7 & 789.3447 & 395.1760 & 772.3182 & 386.6627 & 771.3342 & 386.1707 & $\mathbf{L}$ & 645.4083 & 323.2078 & 628.3817 & 314.6945 & & & 5 \\
\hline 8 & 936.4131 & 468.7102 & 919.3866 & 460.1969 & 918.4026 & 459.7049 & $\mathbf{F}$ & 532.3242 & 266.6657 & 515.2976 & 258.1525 & & & 4 \\
\hline 9 & 1033.4659 & 517.2366 & 1016.4394 & 508.7233 & 1015.4553 & 508.2313 & $\mathbf{P}$ & 385.2558 & 193.1315 & 368.2292 & 184.6183 & & & 3 \\
\hline 10 & 1146.5500 & 573.7786 & 1129.5234 & 565.2653 & 1128.5394 & 564.7733 & I & 288.2030 & 144.6051 & 271.1765 & 136.0919 & & & 2 \\
\hline 11 & & & & & & & $\mathbf{R}$ & 175.1190 & 88.0631 & 158.0924 & 79.5498 & & & 1 \\
\hline
\end{tabular}
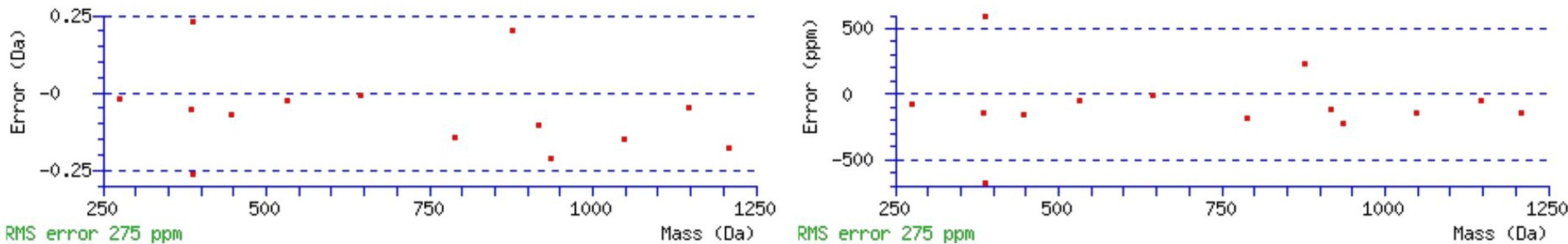

\section{All matches to this query}

\begin{tabular}{|l|l|l|l|}
\hline Score & $\mathbf{M r}$ (calc): & Delta & \multicolumn{1}{|c|}{ Sequence } \\
\hline 64.5 & 1319.6544 & -0.0032 & ICGNETLFPIR \\
\hline 17.0 & 1319.6622 & -0.0110 & GKQAFEGAPYPR \\
\hline 9.8 & 1319.6391 & 0.0121 & GVIDMGNSLIER \\
\hline 8.4 & 1319.6470 & 0.0042 & NSSAGNFIAPSVR \\
\hline 8.4 & 1319.6470 & 0.0042 & NSSAGNFIAPSVR \\
\hline 5.0 & 1317.6329 & 2.0182 & KQKLQYSTDK \\
\hline 4.3 & 1319.6391 & 0.0121 & LMAENSTTALPR \\
\hline 3.1 & 1319.6469 & 0.0042 & RNFSNSIDLPR \\
\hline 2.5 & 1319.6427 & 0.0084 & IYFRFELPR \\
\hline 2.5 & 1319.6542 & -0.0030 & LRDSENTRSSR \\
\hline
\end{tabular}


Spectrum No: 445; Query: 1874; Rank: 1

\section{Peptide View}

MS/MS Fragmentation of LRNPCTSEQNCTSPFSYK

Found in IPI00191681, Tax_Id=10116 Gene_Symbol=Itgb1 Integrin beta-1 precursor

Match to Query 1874: 2188.960752 from(730.660860,3+)

Title: 100101RatKid_NS_deglyco_20.1833.1833.3.dta

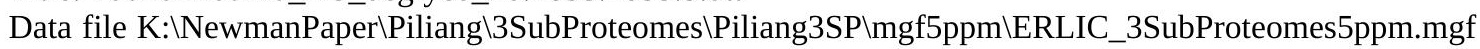
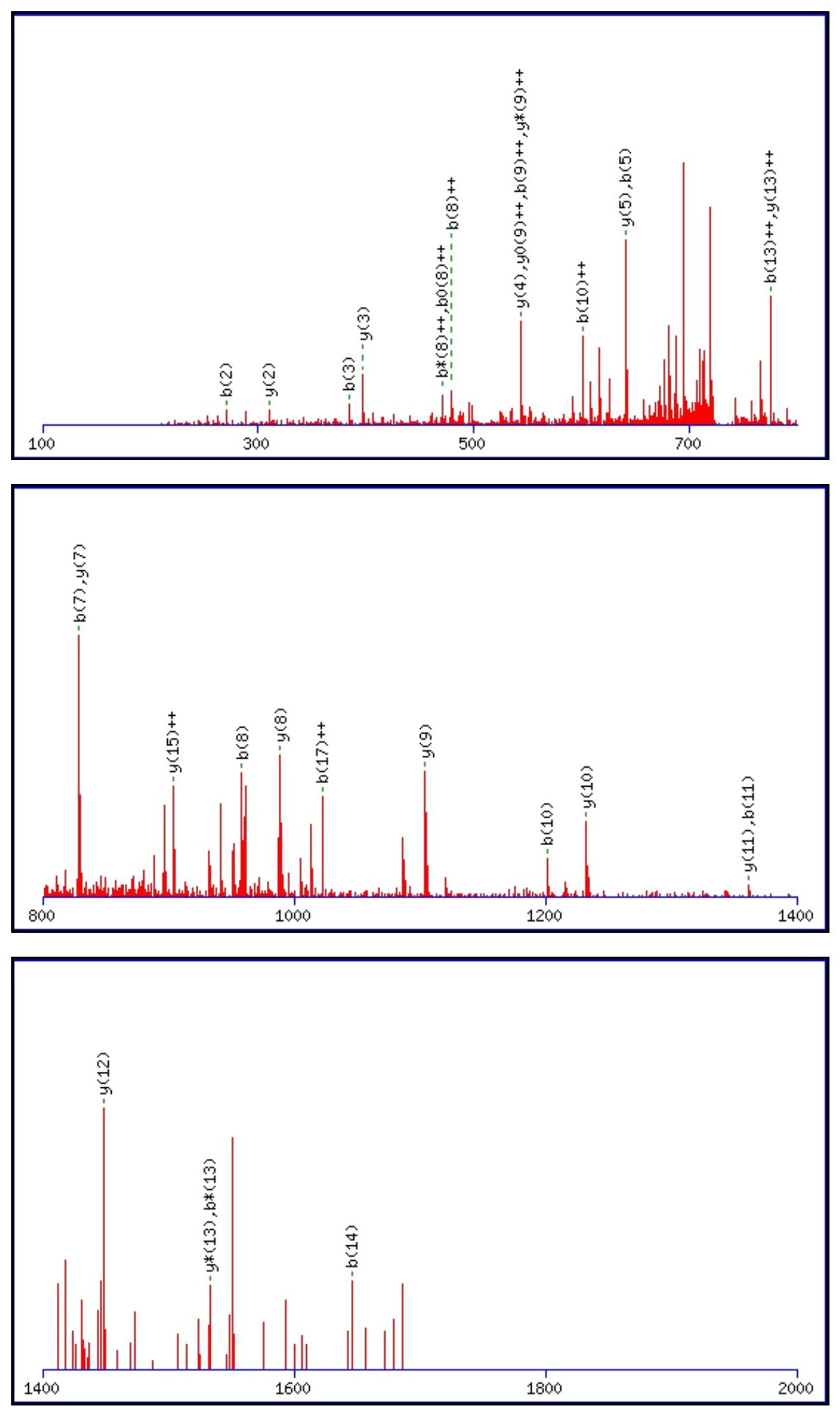
Monoisotopic mass of neutral peptide $\operatorname{Mr}($ calc): 2188.9517

Fixed modifications: Carbamidomethyl (C)

Variable modifications:

N10 : Deamidated_N (N)

Ions Score: 65 Expect: $9.2 \mathrm{e}-005$

Matches (Bold Red): 31/188 fragment ions using 35 most intense peaks

\begin{tabular}{|c|c|c|c|c|c|c|c|c|c|c|c|c|c|c|}
\hline \# & b & $\mathbf{b}^{++}$ & b* & $\mathbf{b}^{*++}$ & $\mathbf{b}^{0}$ & $\mathbf{b}^{0++}$ & Seq. & $\mathbf{y}$ & $\mathbf{y}^{++}$ & $\mathrm{y}^{*}$ & $\mathbf{y}^{*^{++}}$ & $\mathbf{y}^{0}$ & $\mathbf{y}^{\mathbf{0 + +}}$ & \# \\
\hline 1 & 114.0913 & 57.5493 & & & & & $\mathbf{L}$ & & & & & & & 18 \\
\hline 2 & 270.1925 & 135.5999 & 253.1659 & 127.0866 & & & $\mathbf{R}$ & 2076.8750 & 1038.9411 & 2059.8484 & 1030.4278 & 2058.8644 & 1029.9358 & 17 \\
\hline 3 & 384.2354 & 192.6213 & 367.2088 & 184.1081 & & & $\mathbf{N}$ & 1920.7738 & 960.8906 & 1903.7473 & 952.3773 & 1902.7633 & 951.8853 & 16 \\
\hline 4 & 481.2881 & 241.1477 & 464.2616 & 232.6344 & & & $\mathbf{P}$ & 1806.7309 & 903.8691 & 1789.7044 & 895.3558 & 1788.7204 & 894.8638 & 15 \\
\hline 5 & 641.3188 & 321.1630 & 624.2922 & 312.6498 & & & C & 1709.6782 & 855.3427 & 1692.6516 & 846.8294 & 1691.6676 & 846.3374 & 14 \\
\hline 6 & 742.3665 & 371.6869 & 725.3399 & 363.1736 & 724.3559 & 362.6816 & $\mathbf{T}$ & 1549.6475 & 775.3274 & 1532.6210 & 766.8141 & 1531.6369 & 766.3221 & 13 \\
\hline 7 & 829.3985 & 415.2029 & 812.3719 & 406.6896 & 811.3879 & 406.1976 & $\mathbf{S}$ & 1448.5998 & 724.8036 & 1431.5733 & 716.2903 & 1430.5893 & 715.7983 & 12 \\
\hline 8 & 958.4411 & 479.7242 & 941.4145 & 471.2109 & 940.4305 & 470.7189 & $\mathbf{E}$ & 1361.5678 & 681.2875 & 1344.5413 & 672.7743 & 1343.5572 & 672.2823 & 11 \\
\hline 9 & 1086.4997 & 543.7535 & 1069.4731 & 535.2402 & 1068.4891 & 534.7482 & $\mathbf{Q}$ & 1232.5252 & 616.7662 & 1215.4987 & 608.2530 & 1214.5146 & 607.7610 & 10 \\
\hline 10 & 1201.5266 & 601.2669 & 1184.5001 & 592.7537 & 1183.5160 & 592.2617 & $\mathbf{N}$ & 1104.4666 & 552.7370 & 1087.4401 & 544.2237 & 1086.4561 & 543.7317 & 9 \\
\hline 11 & 1361.5573 & 681.2823 & 1344.5307 & 672.7690 & 1343.5467 & 672.2770 & C & 989.4397 & 495.2235 & 972.4131 & 486.7102 & 971.4291 & 486.2182 & 8 \\
\hline 12 & 1462.6049 & 731.8061 & 1445.5784 & 723.2928 & 1444.5944 & 722.8008 & $\mathbf{T}$ & 829.4090 & 415.2082 & 812.3825 & 406.6949 & 811.3985 & 406.2029 & 7 \\
\hline 13 & 1549.6370 & 775.3221 & 1532.6104 & 766.8088 & 1531.6264 & 766.3168 & $S$ & 728.3614 & 364.6843 & 711.3348 & 356.1710 & 710.3508 & 355.6790 & 6 \\
\hline 14 & 1646.6897 & 823.8485 & 1629.6632 & 815.3352 & 1628.6792 & 814.8432 & $\mathbf{P}$ & 641.3293 & 321.1683 & 624.3028 & 312.6550 & 623.3188 & 312.1630 & 5 \\
\hline 15 & 1793.7581 & 897.3827 & 1776.7316 & 888.8694 & 1775.7476 & 888.3774 & $\mathbf{F}$ & 544.2766 & 272.6419 & 527.2500 & 264.1287 & 526.2660 & 263.6366 & 4 \\
\hline 16 & 1880.7902 & 940.8987 & 1863.7636 & 932.3854 & 1862.7796 & 931.8934 & S & 397.2082 & 199.1077 & 380.1816 & 190.5944 & 379.1976 & 190.1024 & 3 \\
\hline 17 & 2043.8535 & 1022.4304 & 2026.8269 & 1013.9171 & 2025.8429 & 1013.4251 & $\mathbf{Y}$ & 310.1761 & 155.5917 & 293.1496 & 147.0784 & & & 2 \\
\hline 18 & & & & & & & $\mathbf{K}$ & 147.1128 & 74.0600 & 130.0863 & 65.5468 & & & 1 \\
\hline
\end{tabular}
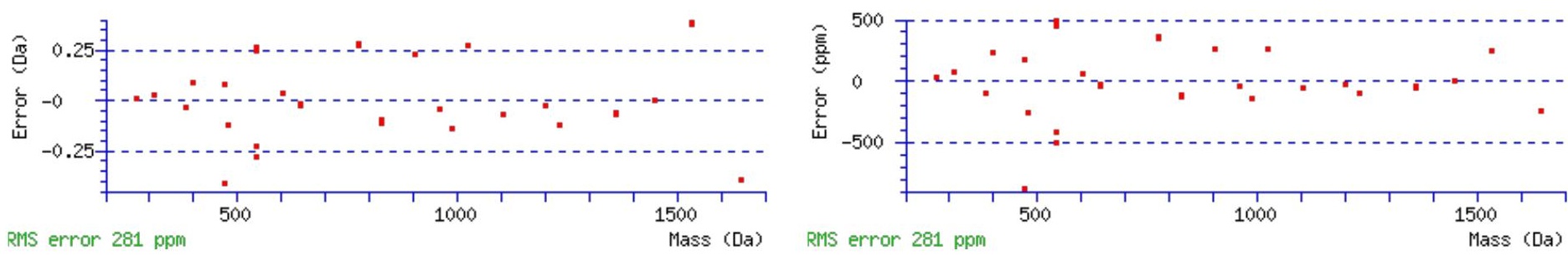

\section{All matches to this query}

\begin{tabular}{|l|l|l|l|}
\hline Score & Mr(calc): & Delta & \multicolumn{1}{c|}{ Sequence } \\
\hline 64.5 & 2188.9517 & 0.0090 & LRNPCTSEQNCTSPFSYK \\
\hline 28.5 & 2188.9517 & 0.0090 & LRNPCTSEQNCTSPFSYK \\
\hline 13.1 & 2187.9677 & 0.9930 & $\underline{\text { LRNPCTSEQNCTSPFSYK }}$ \\
\hline 7.0 & 2188.9629 & -0.0022 & $\underline{\text { TGEMSGPVFTDSGIHIILR }}$ \\
\hline 7.0 & 2188.9629 & -0.0022 & $\underline{\text { TGEMSGPVFTDSGIHIILR }}$ \\
\hline 3.5 & 2188.9493 & 0.0115 & $\underline{\text { SMPVLGSVSSVTKSALNKK }}$ \\
\hline 2.8 & 2187.9685 & 0.9923 & VELGQNGEEKSPSSTSHPPK \\
\hline 2.8 & 2187.9685 & 0.9923 & VELGQNGEEKSPSSTSHPPK \\
\hline 1.8 & 2188.9629 & -0.0022 & TGEMSGPVFTDSGIHIILR \\
\hline 1.8 & 2188.9629 & -0.0022 & $\underline{\text { TGEMSGPVFTDSGIHIILR }}$ \\
\hline
\end{tabular}

Spectrum No: 446; Query: 643; Rank: 1

\section{Peptide View}




\section{MS/MS Fragmentation of EATIVGENETYPR}

Found in IPI00205606, Tax_Id=10116 Gene_Symbol=Sel1h Protein sel-1 homolog 1 precursor

Match to Query 643: 1478.694308 from(740.354430,2+)

Title: 100101RatKid_NS_deglyco_08.1864.1864.2.dta

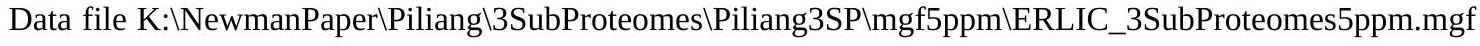
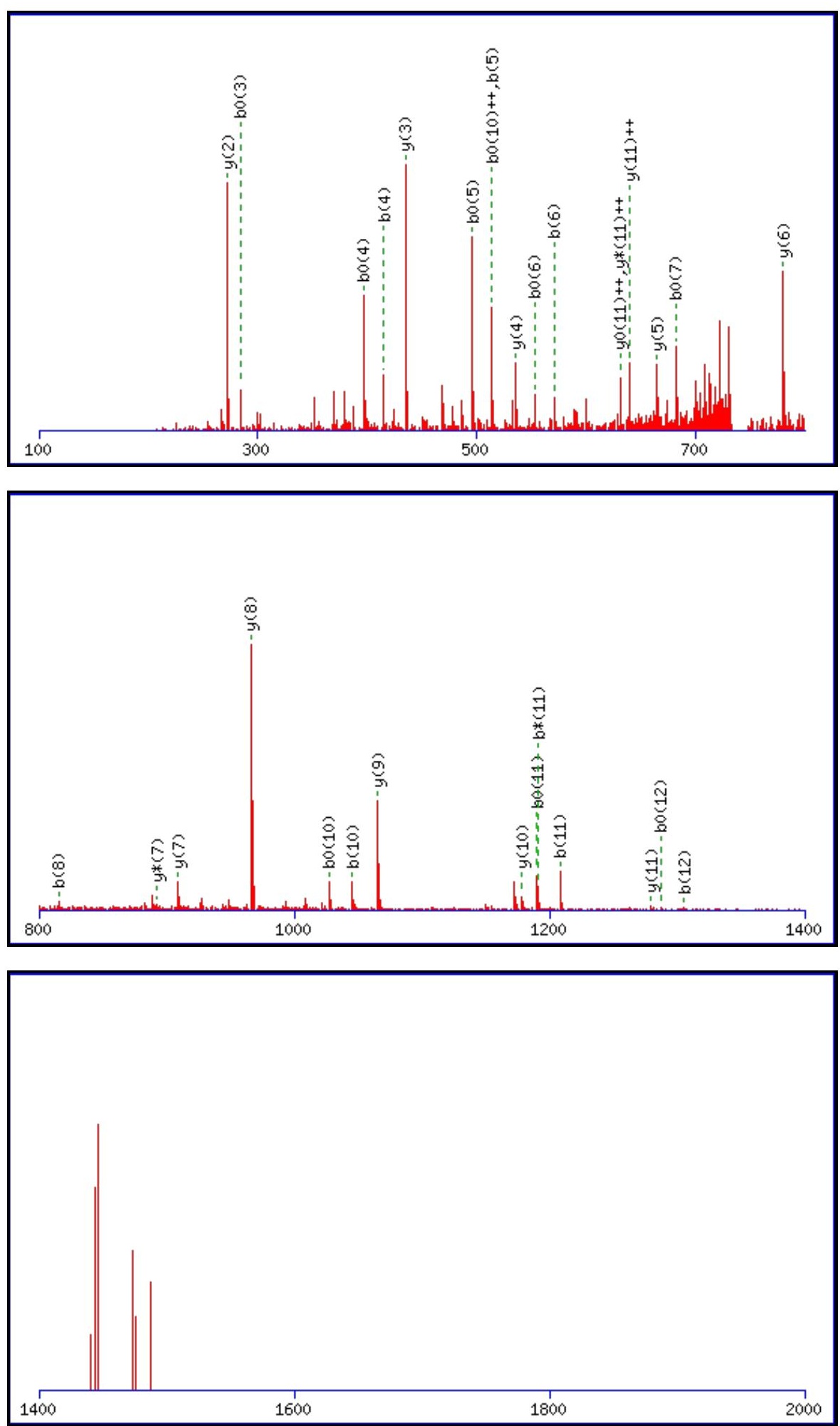

Monoisotopic mass of neutral peptide $\operatorname{Mr}($ calc): 1478.6889

Fixed modifications: Carbamidomethyl (C)

Variable modifications:

N8 : Deamidated_N (N)

Ions Score: 64 Expect: $5.9 \mathrm{e}-005$

Matches (Bold Red): 31/124 fragment ions using 59 most intense peaks

\begin{tabular}{|l|l|l|l|l|l|l|l|l|l|l|l|l|l|l|}
\hline$\#$ & $\mathbf{b}$ & $\mathbf{b}^{++}$ & $\mathbf{b}^{*}$ & $\mathbf{b}^{\boldsymbol{*}^{++}}$ & $\mathbf{b}^{\mathbf{0}}$ & $\mathbf{b}^{\mathbf{0 + +}}$ & Seq. & $\mathbf{y}$ & $\mathbf{y}^{++}$ & $\mathbf{y}^{*}$ & $\mathbf{y}^{*^{++}}$ & $\mathbf{y}^{\mathbf{0}}$ & $\mathbf{y}^{\mathbf{0 + +}}$ & $\#$ \\
\hline
\end{tabular}




\begin{tabular}{|c|c|c|c|c|c|c|c|c|c|c|c|c|c|c|}
\hline 1] & 130.0499 & 65.5286 & & & 112.0393 & 56.5233 & E & & & & & & & 13 \\
\hline 2 & 201.0870 & 101.0471 & & & 183.0764 & 92.0418 & A & 1350.6536 & 675.8304 & 1333.6270 & 667.3172 & 1332.6430 & 666.8251 & 12 \\
\hline 3 & 302.1347 & 151.5710 & & & 284.1241 & 142.5657 & $\mathbf{T}$ & 1279.6165 & 640.3119 & 1262.5899 & 631.7986 & 1261.6059 & 631.3066 & 11 \\
\hline 4 & 415.2187 & 208.1130 & & & 397.2082 & 199.1077 & I & 1178.5688 & 589.7880 & 1161.5422 & 581.2748 & 1160.5582 & 580.7827 & 10 \\
\hline 5 & 514.2871 & 257.6472 & & & 496.2766 & 248.6419 & V & 1065.4847 & 533.2460 & 1048.4582 & 524.7327 & 1047.4742 & 524.2407 & 9 \\
\hline 6 & 571.3086 & 286.1579 & & & 553.2980 & 277.1527 & G & 966.4163 & 483.7118 & 949.3898 & 475.1985 & 948.4057 & 474.7065 & 8 \\
\hline 7 & 700.3512 & 350.6792 & & & 682.3406 & 341.6740 & $\mathbf{E}$ & 909.3948 & 455.2011 & 892.3683 & 446.6878 & 891.3843 & 446.1958 & 7 \\
\hline 8 & 815.3781 & 408.1927 & 798.3516 & 399.6794 & 797.3676 & 399.1874 & $\mathbf{N}$ & 780.3523 & 390.6798 & 763.3257 & 382.1665 & 762.3417 & 381.6745 & 6 \\
\hline 9 & 944.4207 & 472.7140 & 927.3942 & 464.2007 & 926.4102 & 463.7087 & $\mathbf{E}$ & 665.3253 & 333.1663 & 648.2988 & 324.6530 & 647.3148 & 324.1610 & 5 \\
\hline 10 & 1045.4684 & 523.2378 & 1028.4419 & 514.7246 & 1027.4578 & 514.2326 & $\mathbf{T}$ & 536.2827 & 268.6450 & 519.2562 & 260.1317 & 518.2722 & 259.6397 & 4 \\
\hline 11 & 1208.5317 & 604.7695 & 1191.5052 & 596.2562 & 1190.5212 & 595.7642 & $\mathbf{Y}$ & 435.2350 & 218.1212 & 418.2085 & 209.6079 & & & 3 \\
\hline 12 & 1305.5845 & 653.2959 & 1288.5579 & 644.7826 & 1287.5739 & 644.2906 & $\mathbf{P}$ & 272.1717 & 136.5895 & 255.1452 & 128.0762 & & & 2 \\
\hline 13 & & & & & & & $\mathbf{R}$ & 175.1190 & 88.0631 & 158.0924 & 79.5498 & & & 1 \\
\hline
\end{tabular}
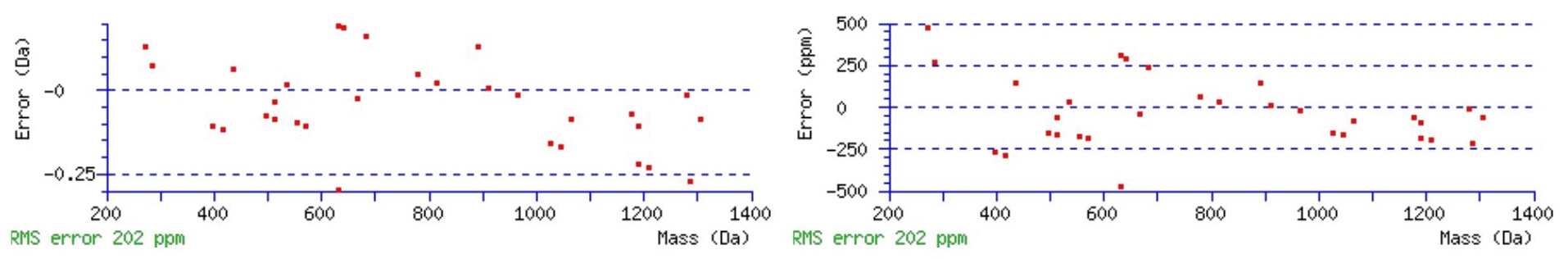

\section{All matches to this query}

\begin{tabular}{|l|l|l|l|}
\hline Score & Mr(calc): & Delta & \multicolumn{1}{c|}{ Sequence } \\
\hline 64.4 & 1478.6889 & 0.0054 & EATIVGENETYPR \\
\hline 10.1 & 1477.7049 & 0.9894 & EATIVGENETYPR \\
\hline 8.9 & 1478.7035 & -0.0092 & SLALESSVSSCSPR \\
\hline 6.2 & 1478.6806 & 0.0137 & STLPNPFHLGTSK \\
\hline 6.2 & 1478.6806 & 0.0137 & STLPNPFHLGTSK \\
\hline 4.1 & 1477.6926 & 1.0017 & TKSDAPGQTQLPR \\
\hline 3.0 & 1476.6779 & 2.0165 & LLASGYPLASGIR \\
\hline 3.0 & 1476.6779 & 2.0165 & LLASGYPLASGIR \\
\hline
\end{tabular}

Spectrum No: 447; Query: 2686; Rank: 1

\section{Peptide View}

MS/MS Fragmentation of SCGECIQAGPNCGWCTNTTFLQEGMPTSAR

Found in IPI00191681, Tax_Id=10116 Gene_Symbol=Itgb1 Integrin beta-1 precursor

Match to Query 2686: 3391.394442 from(1131.472090,3+)

Title: 100101RatKid_NS_deglyco_23.4160.4160.3.dta

Data file K:INewmanPaper|Piliangl3SubProteomes\Piliang3SP\mgf5ppm\ERLIC_3SubProteomes5ppm.mgf 

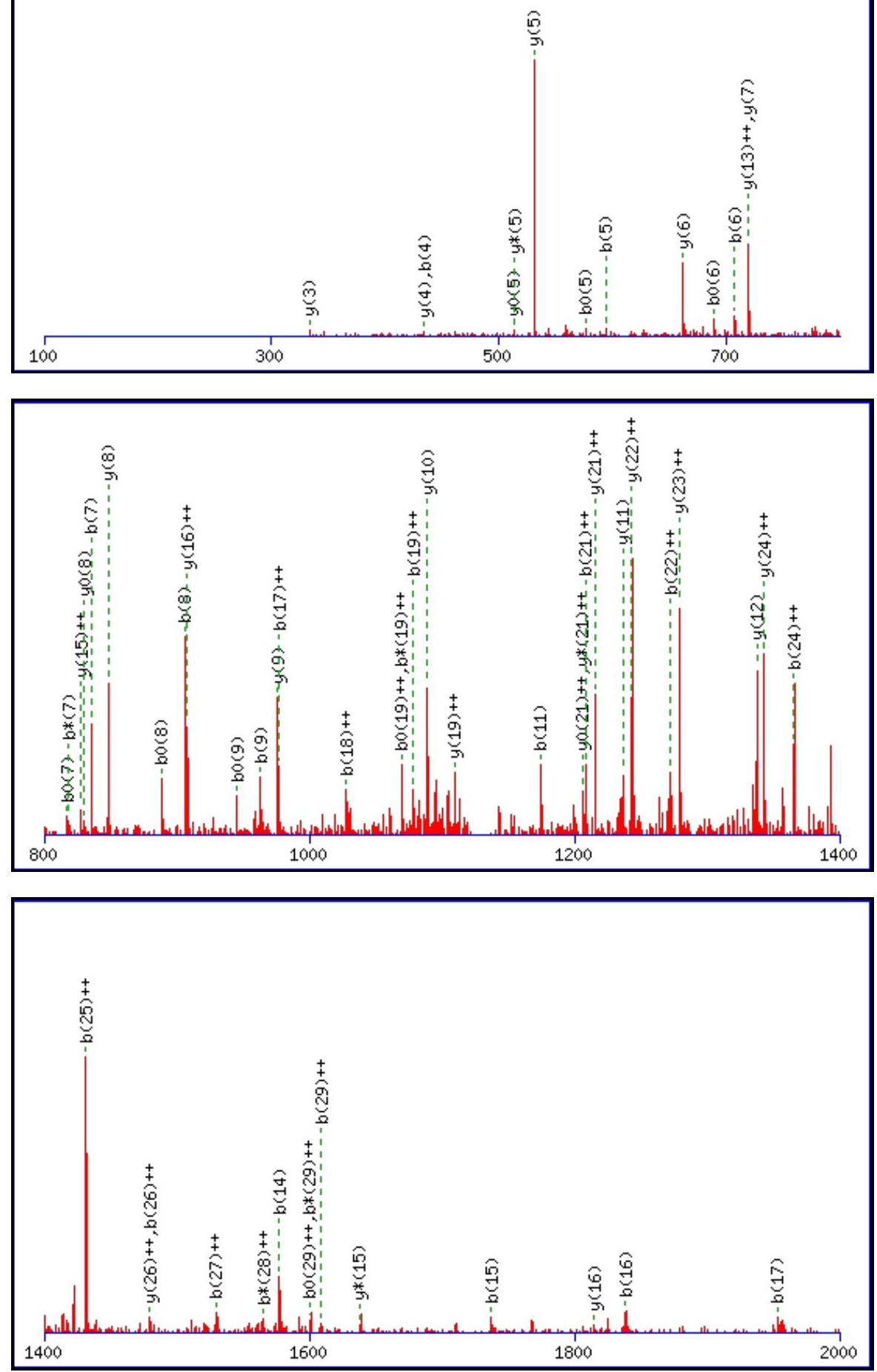

Monoisotopic mass of neutral peptide $\operatorname{Mr}($ calc): 3390.3832

Fixed modifications: Carbamidomethyl (C)

Variable modifications:

N17 : Deamidated_N (N)

Ions Score: 64 Expect: 0.00015

Matches (Bold Red): 58/332 fragment ions using 104 most intense peaks

\begin{tabular}{|c|c|c|c|c|c|c|c|c|c|c|c|c|c|c|}
\hline \# & b & $\mathbf{b}^{++}$ & $\mathbf{b}^{*}$ & $\mathbf{b}^{*^{++}}$ & $\mathbf{b}^{0}$ & $\mathbf{b}^{\mathbf{0 + +}}$ & Seq. & $\mathbf{y}$ & $\mathbf{y}^{++}$ & $\mathbf{y}^{*}$ & $\mathrm{y}^{\mathrm{*}^{++}}$ & $\mathbf{y}^{0}$ & $\mathbf{y}^{\mathbf{0 + +}}$ & \# \\
\hline 1 & 88.0393 & 44.5233 & & & 70.0287 & 35.5180 & $\mathrm{~S}$ & & & & & & & 30 \\
\hline 2 & 248.0700 & 124.5386 & & & 230.0594 & 115.5333 & C & 3304.3584 & 1652.6829 & 3287.3319 & 1644.1696 & 3286.3479 & 1643.6776 & 29 \\
\hline 3 & 305.0914 & 153.0493 & & & 287.0809 & 144.0441 & G & 3144.3278 & |1572.6675 & 3127.3013 & 1564.1543 & |3126.3172 & 1563.6623 & 28 \\
\hline 4 & 434.1340 & 217.5706 & & & 416.1234 & 208.5654 & $\mathbf{E}$ & 3087.3063 & 1544.1568 & 3070.2798 & 1535.6435 & 3069.2958 & 1535.1515 & 27 \\
\hline 5 & 594.1647 & 297.5860 & & & 576.1541 & 288.5807 & C & 2958.2637 & 1479.6355 & 2941.2372 & 1471.1222 & 2940.2532 & 1470.6302 & 26 \\
\hline
\end{tabular}




\begin{tabular}{|c|c|c|c|c|c|c|c|c|c|c|c|c|c|c|}
\hline & 707.2487 & 354.1280 & & & 689.2382 & 345.1227 & I & 331 & 1399.6202 & 2781.2065 & 1391.1069 & 2780.2225 & 149 & 25 \\
\hline 7 & 835.3073 & 418.1573 & 818.2808 & 409.6440 & 817.2967 & 409.1520 & $\mathbf{Q}$ & 2685.1490 & 1343.0782 & 2668.1225 & 1334.5649 & 2667.1385 & 1334.0729 & 24 \\
\hline 8 & 906.3444 & 453.6758 & 889.3179 & 445.1626 & 888.3338 & 444.6706 & A & 2557.0905 & 1279.0489 & 2540.0639 & 1270.5356 & 2539.0799 & 1270.0436 & 5 \\
\hline 9 & 963.3659 & 482.1866 & 946.3393 & 473.6733 & 945.3553 & 473.1813 & G & 2486.0533 & 1243.5303 & $\mid 2469.0268$ & 1235.0170 & & 1234.5250 & \\
\hline 10 - & 1060.4186 & 530.7130 & 1043.3921 & 522.1997 & 1042.4081 & 521.7077 & $\mathbf{P}$ & 2429.0319 & 1215.0196 & 2412.0053 & 1206.5063 & 2411.0213 & 1206.0143 & 3 \\
\hline 11 & 1174.4616 & 587.7344 & 1157.4350 & 579.2211 & 1156.4510 & 578.7291 & $\mathbf{N}$ & 2331.9791 & 1166.4932 & 2314.9526 & 1157.9799 & 2313.9685 & 1157.4879 & 9 \\
\hline 12 & 334.4922 & 667.7497 & 1317.4657 & 659.2365 & 1316.4817 & 658.7445 & C & 2217.9362 & 1109.4717 & 2200.9096 & 1100.9585 & & 1100.4664 & \\
\hline 13 & 391.5137 & 696.2605 & 1374.4871 & 687.7472 & 1373.5031 & 687.2552 & G & 2057.9055 & 1029.4564 & 2040.8790 & 1020.9431 & 2039.8950 & 1020.4511 & 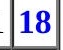 \\
\hline 14 & 1577.5930 & 789.3001 & 1560.5664 & 780.7869 & 1559.5824 & 780.2949 & $\mathbf{W}$ & 2000.8841 & 1000.9457 & 1983.8575 & 992.4324 & 1982.8735 & 991.9404 & 17 \\
\hline 15 & 737.6236 & 869.3155 & 1720.5971 & 860.8022 & 1719.6131 & 860.3102 & $\mathrm{C}$ & 1814.8048 & 907.9060 & 1797.7782 & 899.3927 & 1796.7942 & 9007 & 6 \\
\hline 16 & 1838.6713 & 919.8393 & 1821.6448 & 911.3260 & 1820.6608 & 910.8340 & $T$ & 1654.7741 & 827.8907 & 1637.7476 & 819.3774 & 1636.7635 & 818.8854 & \\
\hline 17 & 1953.6983 & 977.3528 & 1936.6717 & 968.8395 & 1935.6877 & 968.3475 & $\mathbf{N}$ & 1553.7264 & 777.3669 & 1536.6999 & 768.8536 & 1535.7159 & 768.3616 & 14 \\
\hline 18 & 2054.7459 & 1027.8766 & 2037.7194 & 1019.3633 & 2036.7354 & 1018.8713 & $T$ & 1438.6995 & 719.8534 & 1421.6729 & 711.3401 & 1420.6889 & 710.8481 & 3 \\
\hline 19 & 2155.7936 & 078.4004 & 2138.7671 & 1069.8872 & 2137.7831 & 1069.3952 & $\mathbf{T}$ & 1337.6518 & 669.3295 & 1320.6253 & 660.8163 & 1319.6412 & 3243 & 12 \\
\hline 20 & 2302.8620 & 1151.9347 & 2285.8355 & 1143.4214 & 2284.8515 & 1142.9294 & $\mathbf{F}$ & 1236.6041 & 618.8057 & 1219.5776 & 610.2924 & 1218.5936 & 609.8004 & 11 \\
\hline 21 & 2415.9461 & 1208.4767 & 2398.9195 & 1199.9634 & 2397.9355 & 1199.4714 & $\mathbf{L}$ & 1089.5357 & 545.2715 & 1072.5092 & 536.7582 & 1071.5252 & 536.2662 & 0 \\
\hline 22 & 2544.0047 & 1272.5060 & 2526.9781 & 1263.9927 & 2525.9941 & 1263.5007 & $\mathbf{Q}$ & 976.4517 & 488.7295 & 959.4251 & 480.2162 & 958.4411 & 479.7242 & 9 \\
\hline 23 & 2673.0473 & 1337.0273 & 2656.0207 & 1328.5140 & 2655.0367 & 1328.0220 & $\mathbf{E}$ & 848.3931 & 424.7002 & 831.3665 & 416.1869 & 830.3825 & 415.6949 & 8 \\
\hline 24 & 2730.0687 & 1365.5380 & 2713.0422 & 1357.0247 & 2712.0582 & 1356.5327 & G & 719.3505 & 360.1789 & 702.3239 & 351.6656 & 701.3399 & 351.1736 & 7 \\
\hline 25 & 2861.1092 & 1431.0582 & 2844.0827 & 1422.5450 & 2843.0987 & 1422.0530 & M & 662.3290 & 331.6681 & 645.3025 & 323.1549 & 644.3185 & 322.6629 & 6 \\
\hline 26 & 2958.1620 & 1479.5846 & 2941.1354 & 1471.0714 & 2940.1514 & 1470.5793 & $\mathbf{P}$ & 531.2885 & 266.1479 & 514.2620 & 257.6346 & 513.2780 & 257.1426 & 5 \\
\hline 27 & 3059.2097 & 1530.1085 & 3042.1831 & 1521.5952 & 3041.1991 & 1521.1032 & $\mathbf{T}$ & 434.2358 & 217.6215 & 417.2092 & 209.1083 & 416.2252 & 208.6162 & 4 \\
\hline 28 & 3146.2417 & 1573.6245 & 3129.2151 & \begin{tabular}{|l}
1565.1112 \\
\end{tabular} & 3128.2311 & 1564.6192 & $\mathrm{~S}$ & 333.1881 & 167.0977 & 316.1615 & 158.5844 & 315.1775 & 158.0924 & 3 \\
\hline 29 & 3217.2788 & 1609.1430 & 3200.2523 & 1600.6298 & 3199.2682 & 1600.1378 & $\mathbf{A}$ & 246.1561 & 123.5817 & 229.1295 & 115.0684 & & & 2 \\
\hline 30 & & & & & & & $\mathbf{R}$ & 175.1190 & 88.0631 & 158.0924 & 79.5498 & & & 1 \\
\hline
\end{tabular}
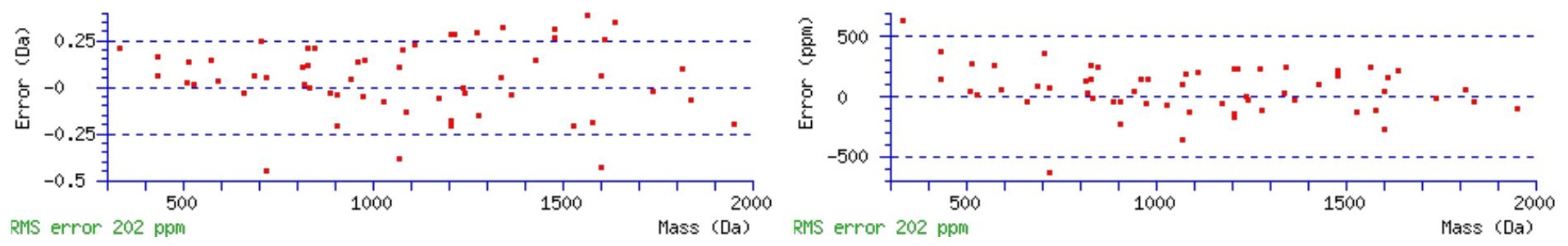

\section{All matches to this query}

\begin{tabular}{|l|l|l|l|}
\hline Score & Mr(calc): & Delta & \multicolumn{1}{|c|}{ Sequence } \\
\hline 64.4 & 3390.3832 & 1.0112 & SCGECIQAGPNCGWCTNTTFLOEGMPTSAR \\
\hline 50.8 & 3391.3672 & 0.0272 & SCGECIQAGPNCGWCTNTTFLQEGMPTSAR \\
\hline 47.9 & 3390.3832 & 1.0112 & SCGECIQAGPNCGWCTNTTFLQEGMPTSAR \\
\hline 45.9 & 3389.3992 & 1.9952 & SCGECIQAGPNCGWCTNTTFLQEGMPTSAR \\
\hline 4.8 & 3390.3586 & 1.0359 & DGDSTVTGARVSNDCSAGTAGVPALSSSEARR \\
\hline 4.4 & 3390.3804 & 1.0141 & QKPSLGTMPVQVTPPRGTFSPNMGMQPR \\
\hline 4.4 & 3391.3644 & 0.0300 & QKPSLGTMPVQVTPPRGTFSPNMGMQPR \\
\hline 2.8 & 3389.3984 & 1.9960 & IDVASIDMVSEVNMDYTLTMYFQQSWK \\
\hline 2.8 & 3389.3608 & 2.0337 & LYVVGGYDGHTYLNTVESYDAQKDEWK \\
\hline 2.0 & 3390.3868 & 1.0076 & TASWLAAELSKEGHQVALLSGEMMVEQR \\
\hline
\end{tabular}

Spectrum No: 448; Query: 110; Rank: 1 


\section{Peptide View}

MS/MS Fragmentation of AAENFTLLVK

Found in IPI00324987, Tax_Id=10116 Gene_Symbol=P2rx4 P2X purinoceptor 4

Match to Query 110: 1105.602708 from(553.808630,2+)

Title: 091008RatKidney_NH4Format01_24.2623.2623.2.dta

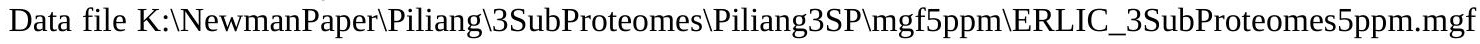
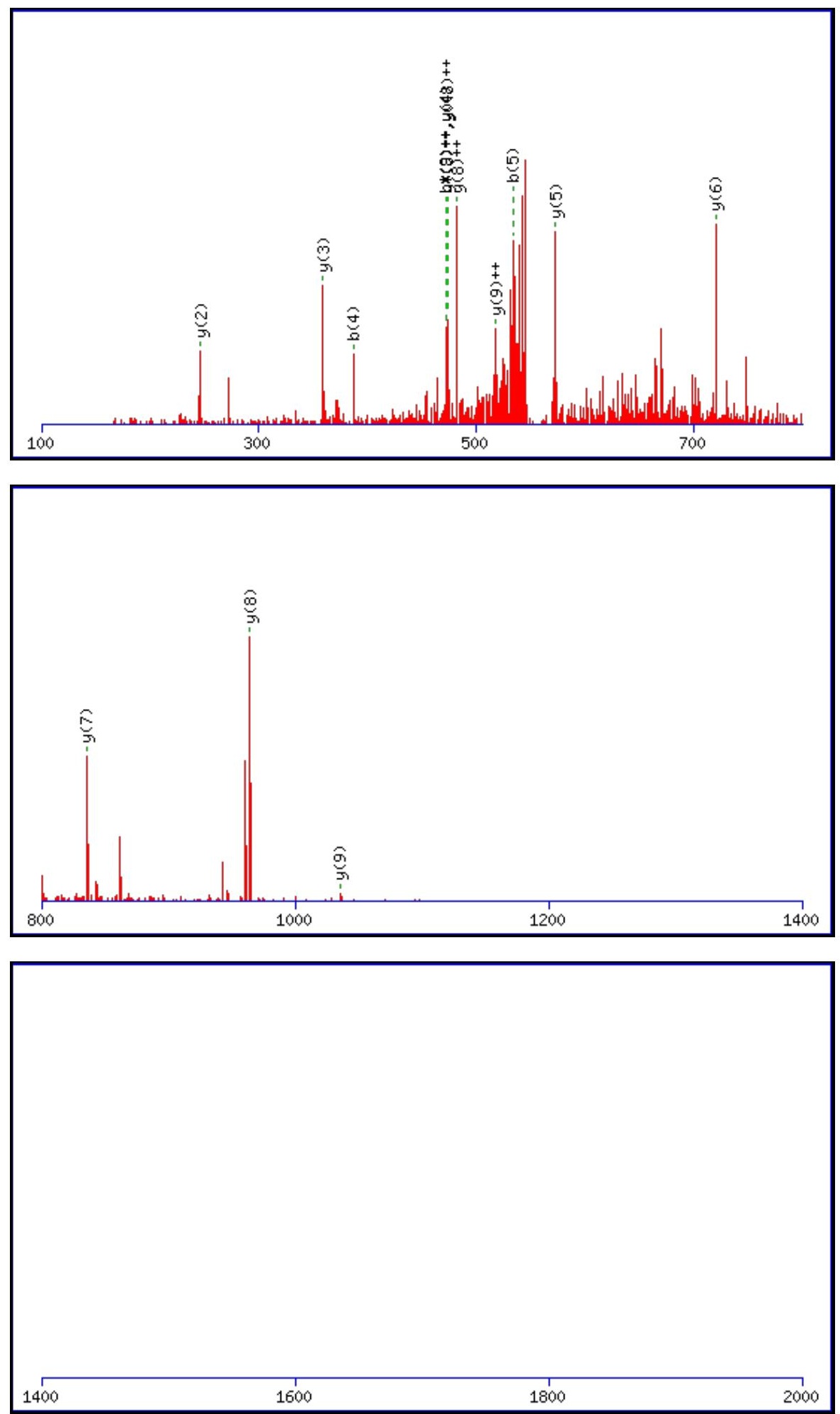

Monoisotopic mass of neutral peptide $\operatorname{Mr}($ calc): 1105.6019

Fixed modifications: Carbamidomethyl (C)

Variable modifications:

N4 : Deamidated_N (N)

Ions Score: 64 Expect: 2.8e-005

Matches (Bold Red): 15/90 fragment ions using 28 most intense peaks 


\begin{tabular}{|c|c|c|c|c|c|c|c|c|c|c|c|c|c|c|}
\hline \# & b & $\mathbf{b}^{++}$ & $\mathbf{b}^{*}$ & $\mathbf{b}^{*++}$ & $\mathbf{b}^{0}$ & $\mathbf{b}^{0++}$ & Seq. & $\mathbf{y}$ & $y^{++}$ & $\mathbf{y}^{*}$ & $\mathrm{y}^{*^{++}}$ & $y^{0}$ & $\mathbf{y}^{\mathbf{0 + +}}$ & $\#$ \\
\hline 1 & 72.0444 & 36.5258 & & & & & A & & & & & & & 10 \\
\hline 2 & 143.0815 & 72.0444 & & & & & $\mathbf{A}$ & 1035.5721 & 518.2897 & 1018.5455 & 509.7764 & 1017.5615 & 509.2844 & 9 \\
\hline $3:$ & 272.1241 & 136.5657 & & & 254.1135 & 127.5604 & $\mathbf{E}$ & 964.5350 & 482.7711 & 947.5084 & 474.2578 & 946.5244 & 473.7658 & 8 \\
\hline $4:$ & 387.1510 & 194.0792 & 370.1245 & 185.5659 & 369.1405 & 185.0739 & $\mathbf{N}$ & 835.4924 & 418.2498 & 818.4658 & 409.7366 & 817.4818 & 409.2445 & 7 \\
\hline 5 & 534.2194 & 267.6134 & 517.1929 & 259.1001 & 516.2089 & 258.6081 & $\mathbf{F}$ & 720.4654 & 360.7364 & 703.4389 & 352.2231 & 702.4549 & 351.7311 & 6 \\
\hline 6 & 635.2671 & 318.1372 & 618.2406 & 309.6239 & 617.2566 & 309.1319 & $\mathbf{T}$ & 573.3970 & 287.2022 & 556.3705 & 278.6889 & 555.3865 & 278.1969 & 5 \\
\hline 7 & 748.3512 & 374.6792 & 731.3246 & 366.1660 & 730.3406 & 365.6740 & $\mathbf{L}$ & 472.3493 & 236.6783 & 455.3228 & 228.1650 & & & 4 \\
\hline 8 & 861.4353 & 431.2213 & 844.4087 & 422.7080 & 843.4247 & 422.2160 & $\mathbf{L}$ & 359.2653 & 180.1363 & 342.2387 & 171.6230 & & & 3 \\
\hline 9 & 960.5037 & 480.7555 & 943.4771 & 472.2422 & 942.4931 & 471.7502 & $\mathbf{V}$ & 246.1812 & 123.5942 & 229.1547 & 115.0810 & & & 2 \\
\hline 10 & & & & & & & $\mathbf{K}$ & 147.1128 & 74.0600 & 130.0863 & 65.5468 & & & 1 \\
\hline
\end{tabular}
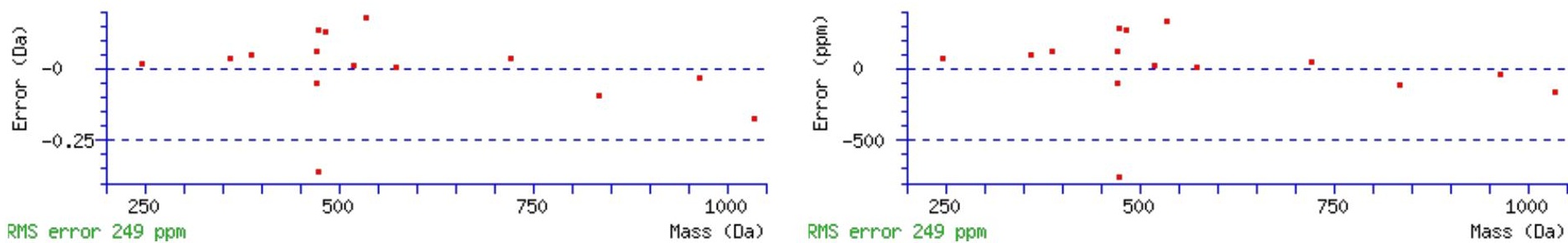

\section{All matches to this query}

\begin{tabular}{|l|l|l|l|}
\hline Score & Mr(calc): & Delta & \multicolumn{1}{c|}{ Sequence } \\
\hline 64.4 & 1105.6019 & 0.0008 & $\underline{\text { AAENFTLLVK }}$ \\
\hline 10.9 & 1105.6131 & -0.0104 & TGISKPYNVK \\
\hline 10.4 & 1105.5992 & 0.0035 & $\underline{\text { APQPGIRNPR }}$ \\
\hline 4.0 & 1103.5988 & 2.0039 & NRNFWVLR \\
\hline 3.5 & 1103.5910 & 2.0117 & $\underline{\text { ARMDVFLPR }}$ \\
\hline 3.0 & 1105.5979 & 0.0048 & KETNSKATVK \\
\hline 3.0 & 1103.5863 & 2.0164 & DPGTFDLLVK \\
\hline 2.3 & 1105.6019 & 0.0008 & $\underline{\text { XSTLPLANVK }}$ \\
\hline 0.9 & 1105.5954 & 0.0073 & $\underline{\text { MFLNLLSPR }}$ \\
\hline 0.1 & 1105.6009 & 0.0018 & $\underline{\text { ARLKTNPVK }}$ \\
\hline
\end{tabular}

Spectrum No: 449; Query: 163; Rank: 1

\section{Peptide View}

MS/MS Fragmentation of VINDTWTWK

Found in IPI00210975, Tax_Id=10116 Gene_Symbol=Hyou1 Hypoxia up-regulated protein 1 precursor

Match to Query 163: 1162.565648 from(582.290100,2+)

Title: 091008RatKidney_NH4Format01_24.2574.2574.2.dta

Data file K:INewmanPaper|Piliang|3SubProteomes\Piliang3SP\mgf5ppm\ERLIC_3SubProteomes5ppm.mgf 

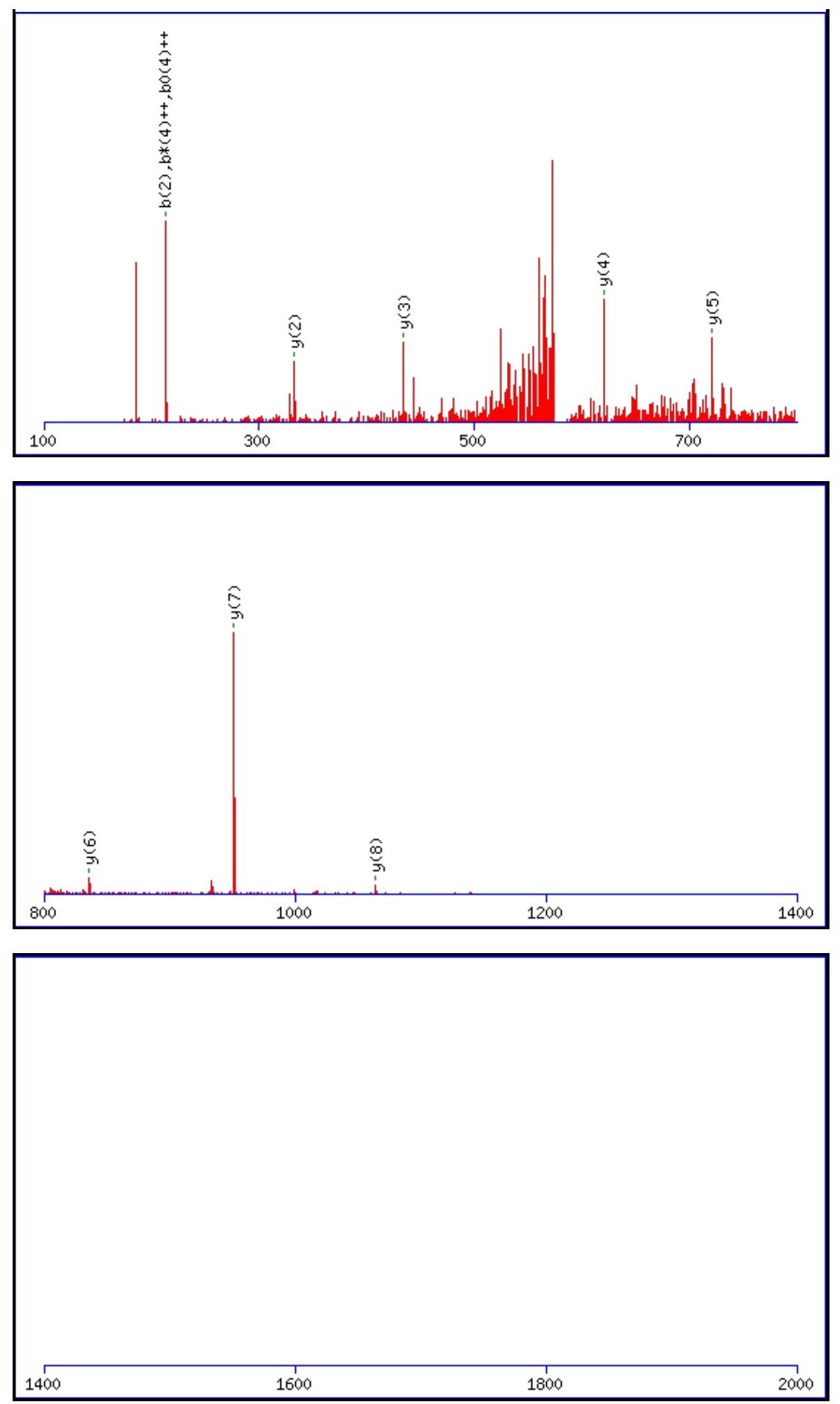

Monoisotopic mass of neutral peptide $\operatorname{Mr}($ calc): 1162.5659

Fixed modifications: Carbamidomethyl (C)

Variable modifications:

N3 : Deamidated N (N)

Ions Score: 64 Expect: $4.6 \mathrm{e}-005$

Matches (Bold Red): 10/82 fragment ions using 18 most intense peaks

\begin{tabular}{|c|c|c|c|c|c|c|c|c|c|c|c|c|c|c|}
\hline$\#$ & $\mathbf{b}$ & $\mathbf{b}^{++}$ & $\mathbf{b}^{*}$ & $\mathbf{b}^{\boldsymbol{*}^{++}}$ & $\mathbf{b}^{\mathbf{0}}$ & $\mathbf{b}^{\mathbf{0}++}$ & Seq. & $\mathbf{y}$ & $\mathbf{y}^{++}$ & $\mathbf{y}^{\mathbf{*}}$ & $\mathbf{y}^{\boldsymbol{*}^{++}}$ & $\mathbf{y}^{\mathbf{0}}$ & $\mathbf{y}^{\mathbf{0}++}$ & $\#$ \\
\hline $\mathbf{1}$ & 100.0757 & 50.5415 & & & & & $\mathbf{V}$ & & & & & & & $\mathbf{9}$ \\
\hline $\mathbf{2}$ & $\mathbf{2 1 3 . 1 5 9 8}$ & 107.0835 & & & & & $\mathbf{I}$ & $\mathbf{1 0 6 4 . 5 0 4 7}$ & 532.7560 & 1047.4782 & 524.2427 & 1046.4942 & 523.7507 & $\mathbf{8}$ \\
\hline $\mathbf{3}$ & 328.1867 & 164.5970 & 311.1601 & 156.0837 & & & $\mathbf{N}$ & $\mathbf{9 5 1 . 4 2 0 7}$ & 476.2140 & 934.3941 & 467.7007 & 933.4101 & 467.2087 & $\mathbf{7}$ \\
\hline $\mathbf{4}$ & 443.2136 & 222.1105 & 426.1871 & 213.5972 & 425.2031 & 213.1052 & $\mathbf{D}$ & $\mathbf{8 3 6 . 3 9 3 7}$ & 418.7005 & 819.3672 & 410.1872 & 818.3832 & 409.6952 & $\mathbf{6}$ \\
\hline $\mathbf{5}$ & 544.2613 & 272.6343 & 527.2348 & 264.1210 & 526.2507 & 263.6290 & $\mathbf{T}$ & $\mathbf{7 2 1 . 3 6 6 8}$ & 361.1870 & 704.3402 & 352.6738 & 703.3562 & 352.1817 & $\mathbf{5}$ \\
\hline
\end{tabular}




\begin{tabular}{|l|r|r|r|r|r|r|r|r|r|r|r|r|r|r|r|r|}
$\mathbf{6}$ & 730.3406 & 365.6740 & 713.3141 & 357.1607 & 712.3301 & 356.6687 & $\mathbf{W}$ & $\mathbf{6 2 0 . 3 1 9 1}$ & 310.6632 & 603.2926 & 302.1499 & 602.3085 & 301.6579 & $\mathbf{4}$ \\
\hline $\mathbf{7}$ & 831.3883 & 416.1978 & 814.3618 & 407.6845 & 813.3777 & 407.1925 & $\mathbf{T}$ & $\mathbf{4 3 4 . 2 3 9 8}$ & 217.6235 & 417.2132 & 209.1103 & 416.2292 & 208.6183 & $\mathbf{3}$ \\
\hline $\mathbf{8}$ & 1017.4676 & 509.2374 & 1000.4411 & 500.7242 & 999.4571 & 500.2322 & $\mathbf{W}$ & 333.1921 & 167.0997 & 316.1656 & 158.5864 & & & 2 \\
\hline $\mathbf{9}$ & & & & & & & K & 147.1128 & 74.0600 & 130.0863 & 65.5468 & & & $\mathbf{1}$ \\
\hline
\end{tabular}
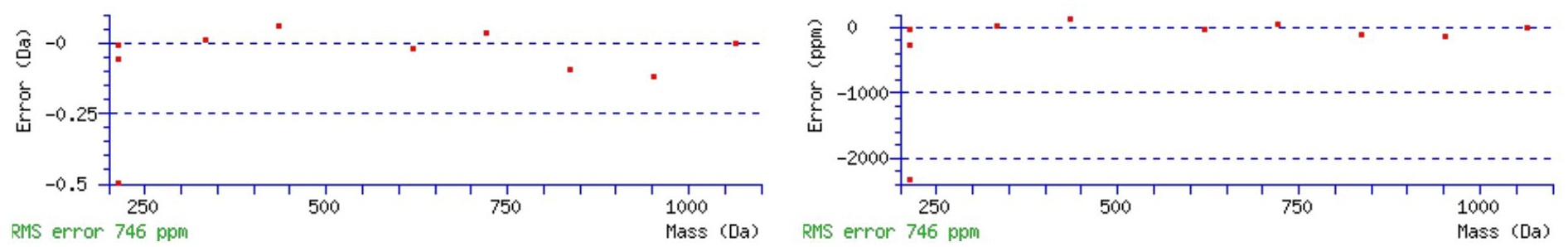

\section{All matches to this query}

\begin{tabular}{|l|l|l|l|}
\hline Score & Mr(calc): & Delta & \multicolumn{1}{|c|}{ Sequence } \\
\hline 64.4 & 1162.5659 & -0.0002 & VINDTWTWK \\
\hline 9.2 & 1162.5675 & -0.0019 & VISELFFTK \\
\hline 3.1 & 1162.5747 & -0.0091 & VLTLPRSNPS \\
\hline 2.6 & 1162.5635 & 0.0021 & VLPGVDALSNV \\
\hline 2.0 & 1162.5731 & -0.0074 & DPDSFRASIR \\
\hline 2.0 & 1162.5747 & -0.0091 & VIKNTDKHK \\
\hline 2.0 & 1162.5675 & -0.0019 & VISELFFTK \\
\hline 2.0 & 1162.5652 & 0.0004 & VISSQECNVK \\
\hline 2.0 & 1162.5747 & -0.0091 & VLTLPRSNPS \\
\hline 2.0 & 1162.5747 & -0.0091 & VLTLPRSNPS \\
\hline
\end{tabular}

Spectrum No: 450; Query: 1047; Rank: 1

\section{Peptide View}

MS/MS Fragmentation of KVVNVSELYGTPCTK

Found in IPI00206668, Tax_Id=10116 Gene_Symbol=Entpd1 Ectonucleoside triphosphate diphosphohydrolase 1

Match to Query 1047: 1694.861588 from(848.438070,2+)

Title: 100101RatKid_NS_deglyco_18.2423.2423.2.dta

Data file K:INewmanPaper|Piliangl3SubProteomes\Piliang3SP\mgf5ppm\ERLIC_3SubProteomes5ppm.mgf

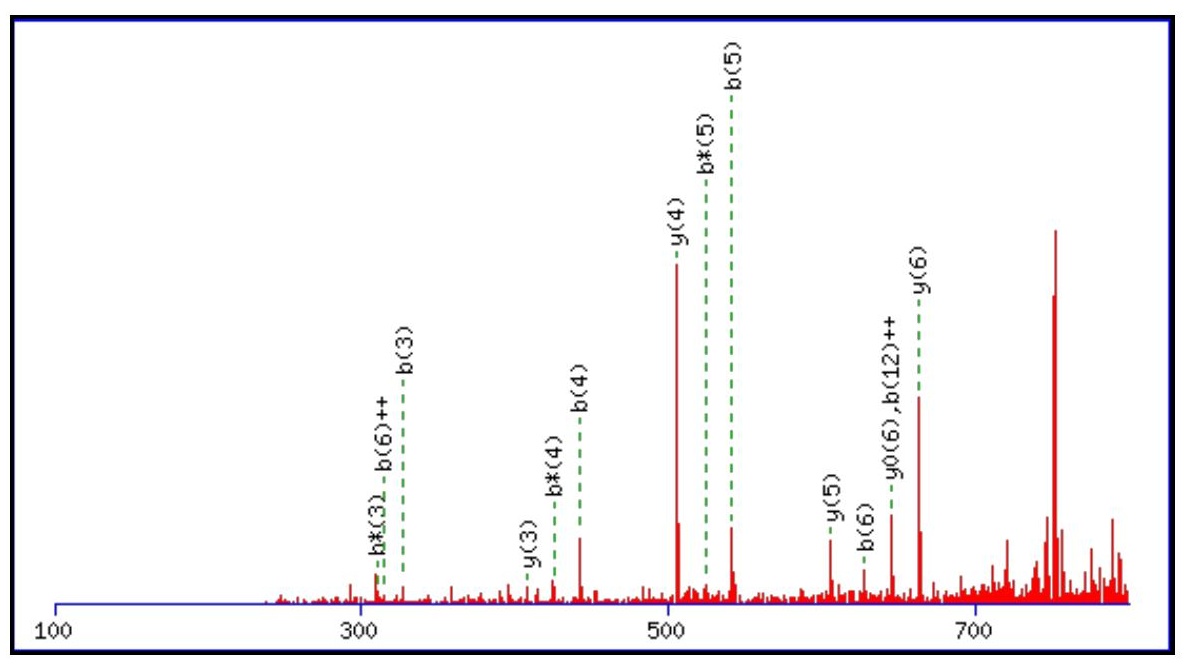



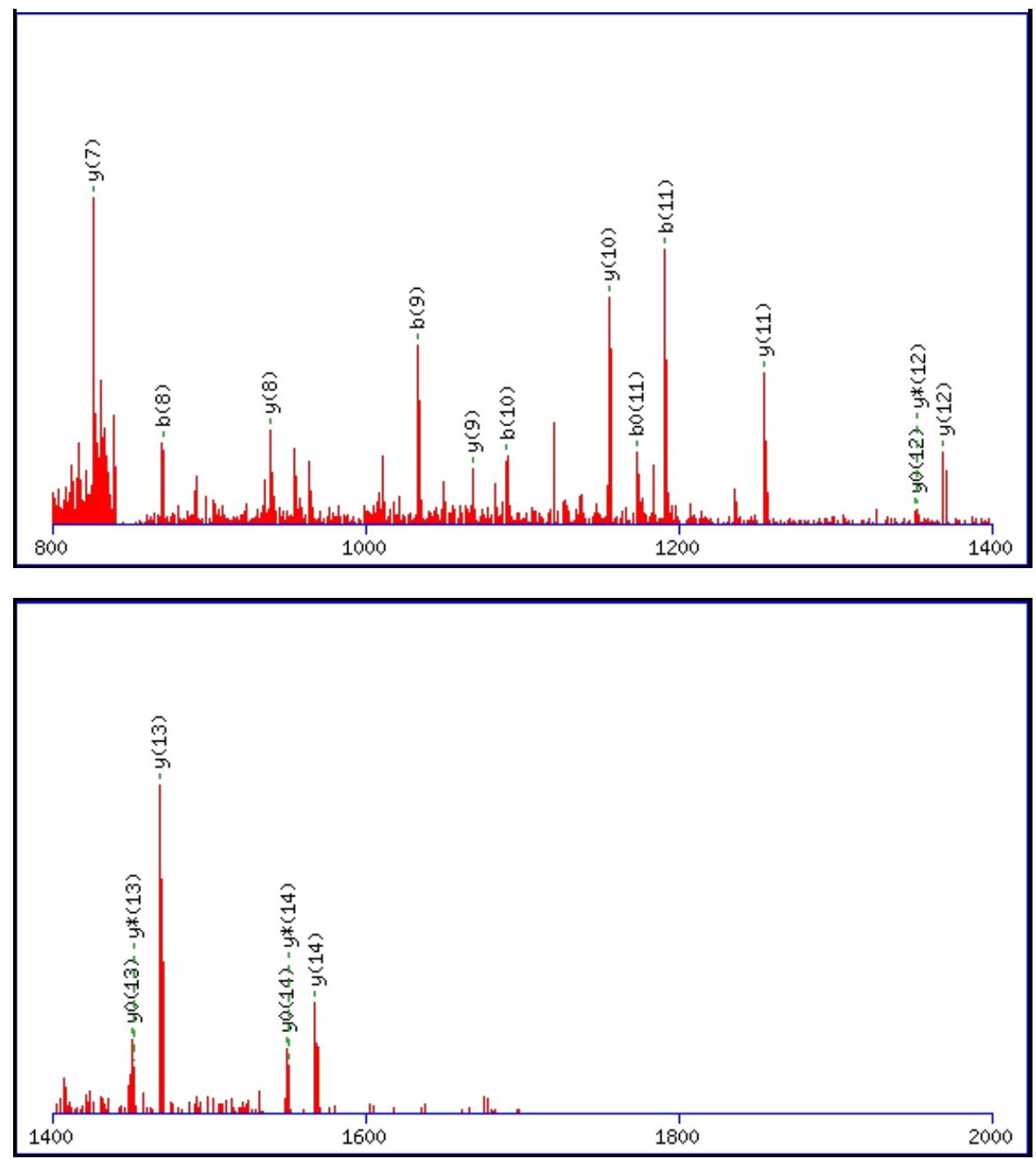

Monoisotopic mass of neutral peptide $\operatorname{Mr}($ calc): 1694.8549

Fixed modifications: Carbamidomethyl (C)

Variable modifications:

N4 : Deamidated $\mathrm{N}(\mathrm{N})$

Ions Score: 64 Expect: $5.7 \mathrm{e}-005$

Matches (Bold Red): 34/156 fragment ions using 87 most intense peaks

\begin{tabular}{|c|c|c|c|c|c|c|c|c|c|c|c|c|c|c|}
\hline \# & b & $\mathbf{b}^{++}$ & b* & $\mathbf{b}^{*^{++}}$ & $\mathbf{b}^{0}$ & $\mathbf{b}^{0++}$ & Seq. & $\mathbf{y}$ & $y^{++}$ & $\mathbf{y}^{*}$ & $\mathbf{y}^{*^{++}}$ & $\mathbf{y}^{0}$ & $\mathbf{y}^{0++}$ & \# \\
\hline 1 & 129.1022 & 65.0548 & 112.0757 & 56.5415 & & & $\mathbf{K}$ & & & & & & & 15 \\
\hline 2 & 228.1707 & 114.5890 & 211.1441 & 106.0757 & & & $\mathbf{V}$ & 1567.7672 & 784.3873 & 1550.7407 & 775.8740 & 1549.7567 & 775.3820 & 14 \\
\hline 3 & 327.2391 & 164.1232 & 310.2125 & 155.6099 & & & $\mathbf{V}$ & 1468.6988 & 734.8530 & 1451.6723 & 726.3398 & 1450.6883 & 725.8478 & 13 \\
\hline 4 & 442.2660 & 221.6366 & 425.2395 & 213.1234 & & & $\mathbf{N}$ & 1369.6304 & 685.3188 & 1352.6039 & 676.8056 & 1351.6198 & 676.3136 & 12 \\
\hline 5 & 541.3344 & 271.1708 & 524.3079 & 262.6576 & & & $\mathbf{V}$ & 1254.6035 & 627.8054 & 1237.5769 & 619.2921 & 1236.5929 & 618.8001 & 11 \\
\hline 6 & 628.3664 & 314.6869 & 611.3399 & 306.1736 & 610.3559 & 305.6816 & S & 1155.5351 & 578.2712 & 1138.5085 & 569.7579 & 1137.5245 & 569.2659 & 10 \\
\hline 7 & 757.4090 & 379.2082 & 740.3825 & 370.6949 & 739.3985 & 370.2029 & $\mathbf{E}$ & 1068.5030 & 534.7552 & 1051.4765 & 526.2419 & 1050.4925 & 525.7499 & 9 \\
\hline 8 & 870.4931 & 435.7502 & 853.4666 & 427.2369 & 852.4825 & 426.7449 & $\mathbf{L}$ & 939.4604 & 470.2339 & 922.4339 & 461.7206 & 921.4499 & 461.2286 & 8 \\
\hline 9 & 1033.5564 & 517.2819 & 1016.5299 & 508.7686 & 1015.5459 & 508.2766 & $\mathbf{Y}$ & 826.3764 & 413.6918 & 809.3498 & 405.1785 & 808.3658 & 404.6865 & 7 \\
\hline 10 & 1090.5779 & 545.7926 & 1073.5513 & 537.2793 & 1072.5673 & 536.7873 & G & 663.3130 & 332.1602 & 646.2865 & 323.6469 & 645.3025 & 323.1549 & 6 \\
\hline 11 & 1191.6256 & 596.3164 & 1174.5990 & 587.8032 & 1173.6150 & 587.3111 & $\mathbf{T}$ & 606.2916 & 303.6494 & 589.2650 & 295.1362 & 588.2810 & 294.6441 & 5 \\
\hline 12 & 1288.6783 & 644.8428 & 1271.6518 & 636.3295 & 1270.6678 & 635.8375 & $\mathbf{P}$ & 505.2439 & 253.1256 & 488.2173 & 244.6123 & 487.2333 & 244.1203 & 4 \\
\hline 13 & 1448.7090 & 724.8581 & 1431.6824 & 716.3449 & 1430.6984 & 715.8528 & C & 408.1911 & 204.5992 & 391.1646 & 196.0859 & 390.1806 & 195.5939 & 3 \\
\hline 14 & 1549.7567 & 775.3820 & 1532.7301 & 766.8687 & 1531.7461 & 766.3767 & $T$ & 248.1605 & 124.5839 & 231.1339 & 116.0706 & 230.1499 & 115.5786 & 2 \\
\hline 15 & & & & & & & $\mathbf{K}$ & 147.1128 & 74.0600 & 130.0863 & 65.5468 & & & 1 \\
\hline
\end{tabular}



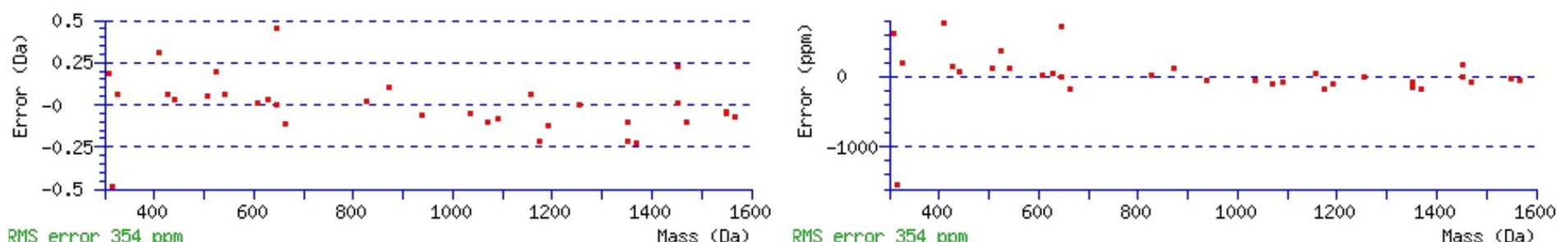

RIS error 354 ppm

\section{All matches to this query}

\begin{tabular}{|l|l|l|l|}
\hline Score & Mr(calc): & Delta & Sequence \\
\hline 64.3 & 1694.8549 & 0.0067 & KVVNVSELYGTPCTK \\
\hline 16.7 & 1693.8709 & 0.9907 & KVVNVSELYGTPCTK \\
\hline 9.9 & 1694.8505 & 0.0111 & $\underline{\text { QVVQSSLLGRWGTGK }}$ \\
\hline
\end{tabular}

Spectrum No: 451; Query: 2807; Rank: 1

\section{Peptide View}

\section{MS/MS Fragmentation of ILTNNSQTPILSPQEVVSCSPYAQGCDGGFPYLIAGK}

Found in IPI00193765, Tax_Id=10116 Gene_Symbol=Ctsc Dipeptidyl-peptidase 1 precursor

Match to Query 2807: 3981.920616 from(996.487430,4+)

Title: 100101RatKid_NS_deglyco_21.5341.5341.4.dta

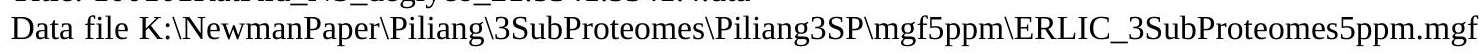
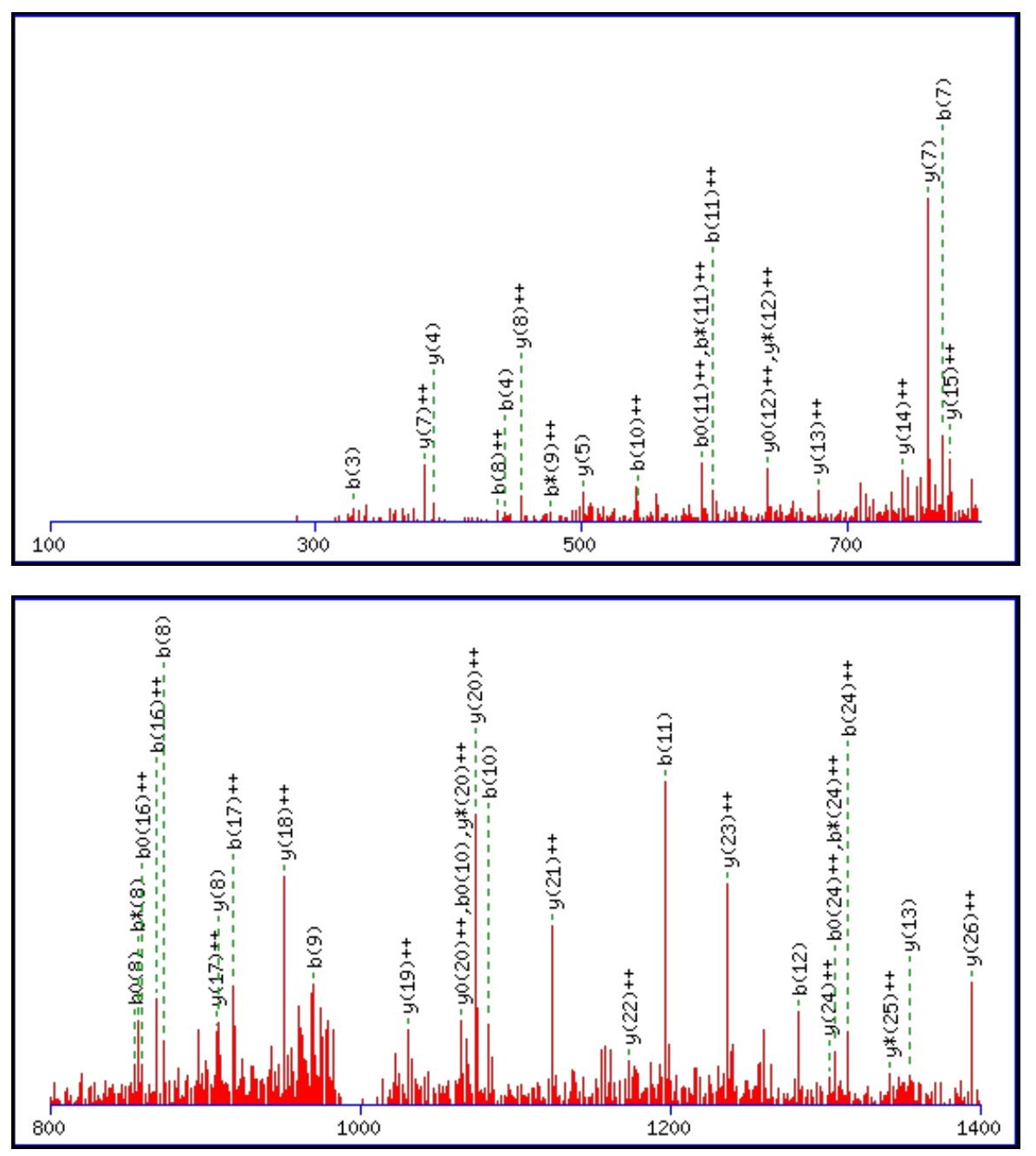


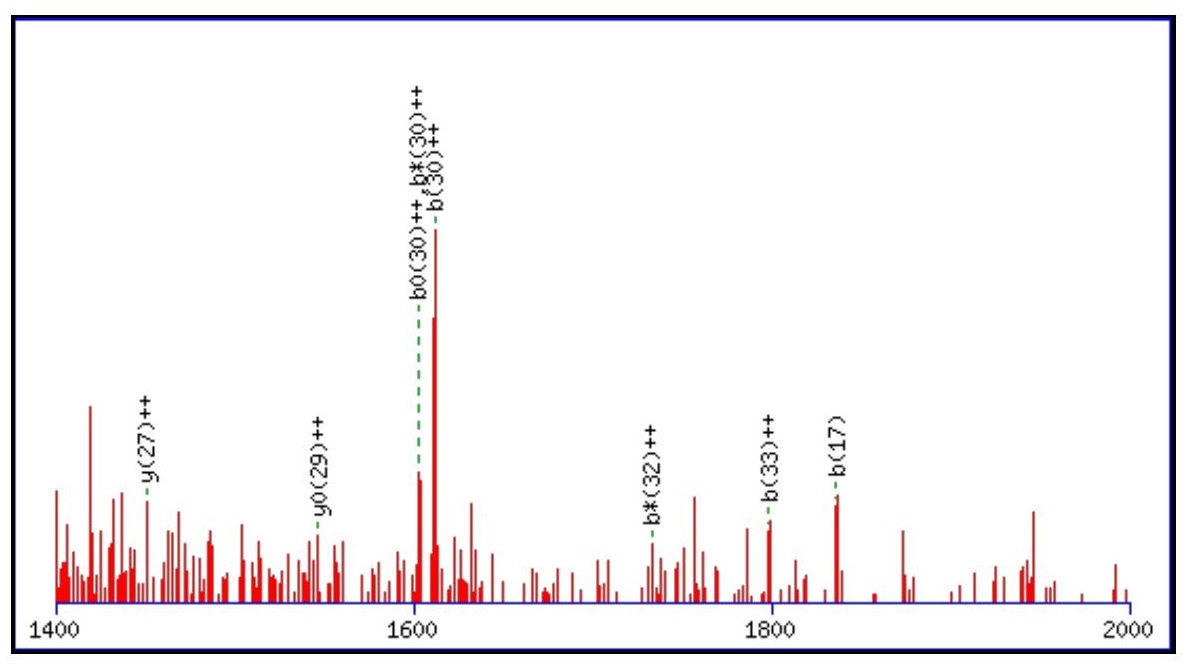

Monoisotopic mass of neutral peptide $\operatorname{Mr}($ calc): 3981.9129

Fixed modifications: Carbamidomethyl (C)

Variable modifications:

N4 : Deamidated_N (N)

Ions Score: 64 Expect: 0.00012

Matches (Bold Red): 55/402 fragment ions using 111 most intense peaks

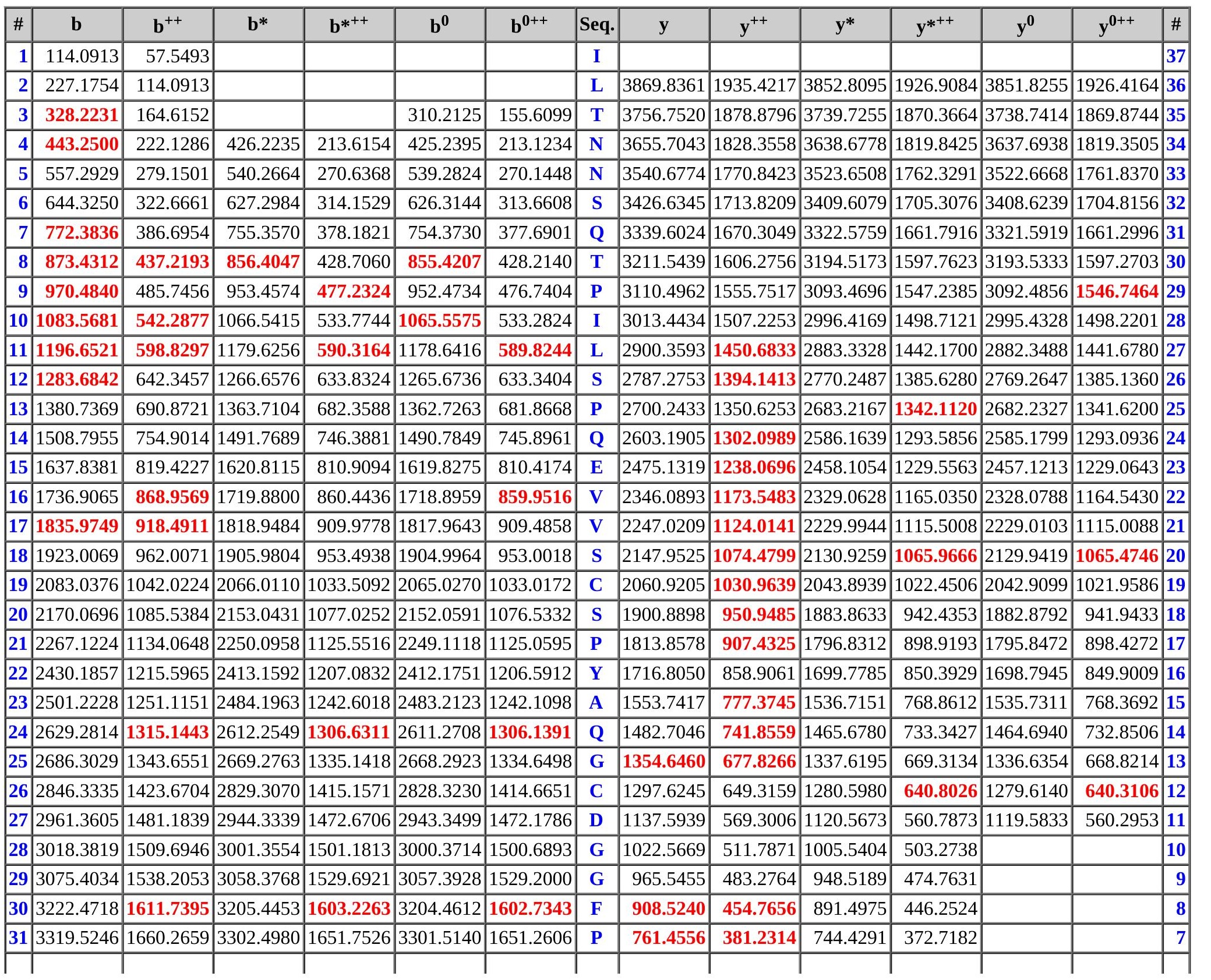




\begin{tabular}{|r|r|r|r|r|r|r|r|r|r|r|r|r|r|r|r|r|r|}
$\mathbf{3 2}$ & 3482.5879 & 1741.7976 & 3465.5613 & $\mathbf{1 7 3 3 . 2 8 4 3}$ & 3464.5773 & 1732.7923 & $\mathbf{Y}$ & 664.4028 & 332.7051 & 647.3763 & 324.1918 & & & $\mathbf{6}$ \\
\hline $\mathbf{3 3}$ & 3595.6720 & $\mathbf{1 7 9 8 . 3 3 9 6}$ & 3578.6454 & 1789.8263 & 3577.6614 & 1789.3343 & L & $\mathbf{5 0 1 . 3 3 9 5}$ & 251.1734 & 484.3130 & 242.6601 & & & $\mathbf{5}$ \\
\hline $\mathbf{3 4}$ & 3708.7560 & 1854.8816 & 3691.7295 & 1846.368 & 3690.745 & 1845.8764 & I & $\mathbf{3 8 8 . 2 5 5 4}$ & 194.6314 & 371.2289 & 186.1181 & & & $\mathbf{4}$ \\
\hline $\mathbf{3 5}$ & 3779.7931 & 1890.4002 & 3762.7666 & 1881.8869 & 3761.7826 & 1881.3949 & $\mathbf{A}$ & 275.1714 & 138.0893 & 258.1448 & 129.5761 & & & $\mathbf{3}$ \\
\hline $\mathbf{3 6}$ & 3836.8146 & 1918.9109 & 3819.7881 & 1910.3977 & 3818.8040 & 1909.9057 & $\mathbf{G}$ & 204.1343 & 102.5708 & 187.1077 & 94.0575 & & & $\mathbf{2}$ \\
\hline $\mathbf{3 7}$ & & & & & & & K & 147.1128 & 74.0600 & 130.0863 & 65.5468 & & & $\mathbf{1}$ \\
\hline
\end{tabular}
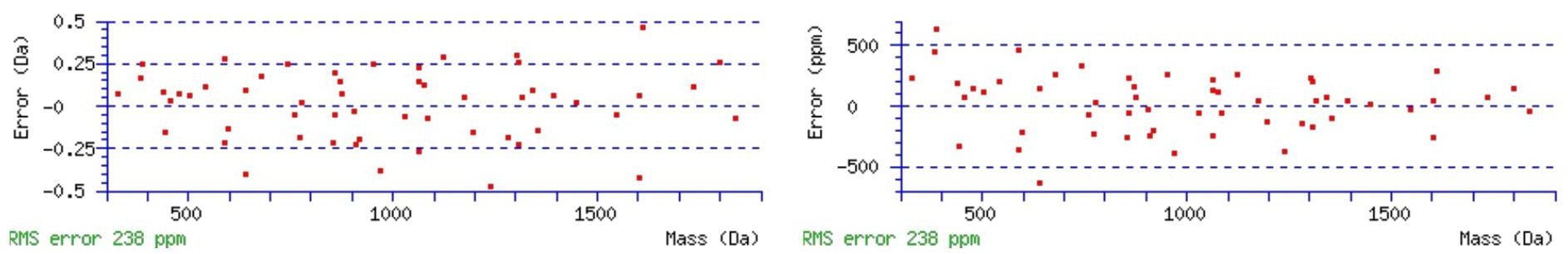

\section{All matches to this query}

\begin{tabular}{|l|l|l|l|}
\hline Score & Mr(calc): & Delta & \multicolumn{1}{|c|}{ Sequence } \\
\hline 64.3 & 3981.9129 & 0.0078 & ILTNNSOTPILSPQEVVSCSPYAQGCDGGFPYLIAGK \\
\hline 58.0 & 3981.9129 & 0.0078 & ILTNNSQTPILSPQEVVSCSPYAQGCDGGFPYLIAGK \\
\hline 35.7 & 3980.9288 & 0.9918 & ILTNNSOTPILSPQEVVSCSPYAQGCDGGFPYLIAGK \\
\hline 4.7 & 3979.9095 & 2.0111 & SVILDDDVLLILTLDEELSAPLTPSNGLGQEDLPSK \\
\hline 3.6 & 3979.9293 & 1.9914 & NAALSTQLREAATQAQQTLGSTIDKATGTLLYGLASR \\
\hline 1.3 & 3979.8758 & 2.0448 & SQIALKLGVTADDVKNVIIWGNHSSTQYPDVNHAK \\
\hline 1.3 & 3979.8758 & 2.0448 & SQIALKLGVTADDVKNVIIWGNHSSTQYPDVNHAK \\
\hline 1.2 & 3980.8935 & 1.0271 & LFSSLGELSTISAQRSPGGPGGGASYSVRPSGRYPVAR \\
\hline 1.1 & 3980.9227 & 0.9979 & LTFNLTSGNHLGHFIVHPFTGLLTTAKQLDRENK \\
\hline 1.1 & 3980.9227 & 0.9979 & LTFNLTSGNHLGHFIVHPFTGLLTTAKQLDRENK \\
\hline
\end{tabular}

Spectrum No: 452; Query: 2541; Rank: 1

\section{Peptide View}

MS/MS Fragmentation of NENFSSGPSFETISASGLNAALAHYSPTK

Found in IPI00197684, Tax_Id=10116 Gene_Symbol=Xpnpep2 Membrane-bound aminopeptidase P

Match to Query 2541: 2997.409332 from(1000.143720,3+)

Title: 100101RatKid_NS_deglyco_23.4786.4786.3.dta

Data file K:INewmanPaper|Piliangl3SubProteomes\Piliang3SP\mgf5ppm\ERLIC_3SubProteomes5ppm.mgf

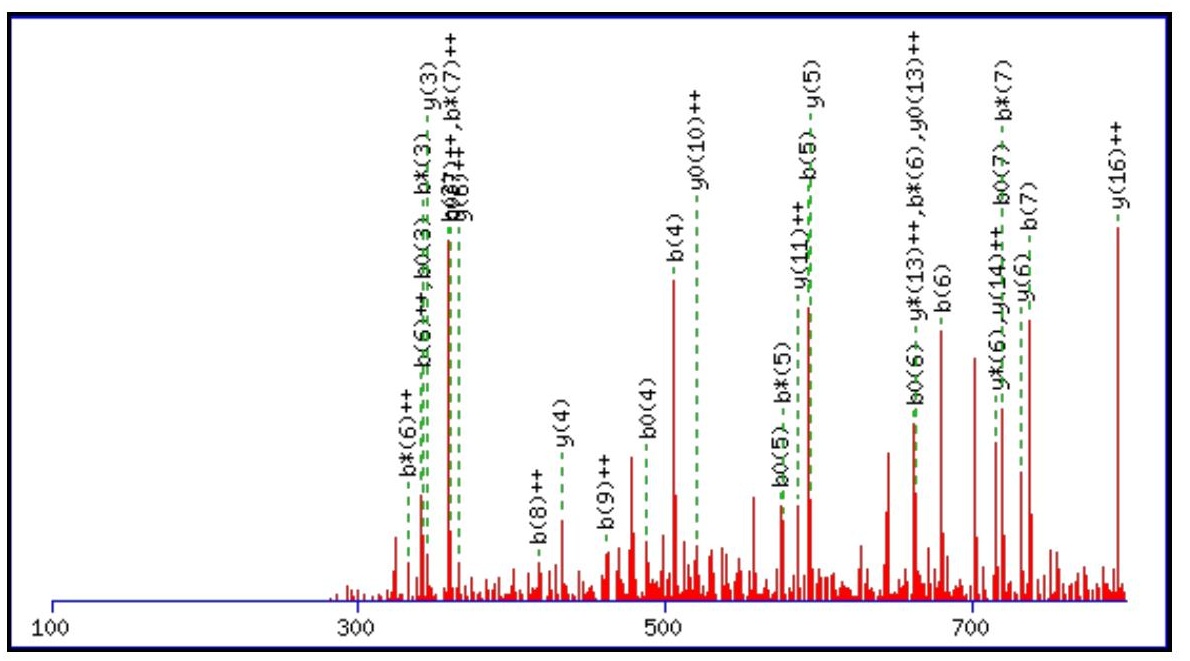



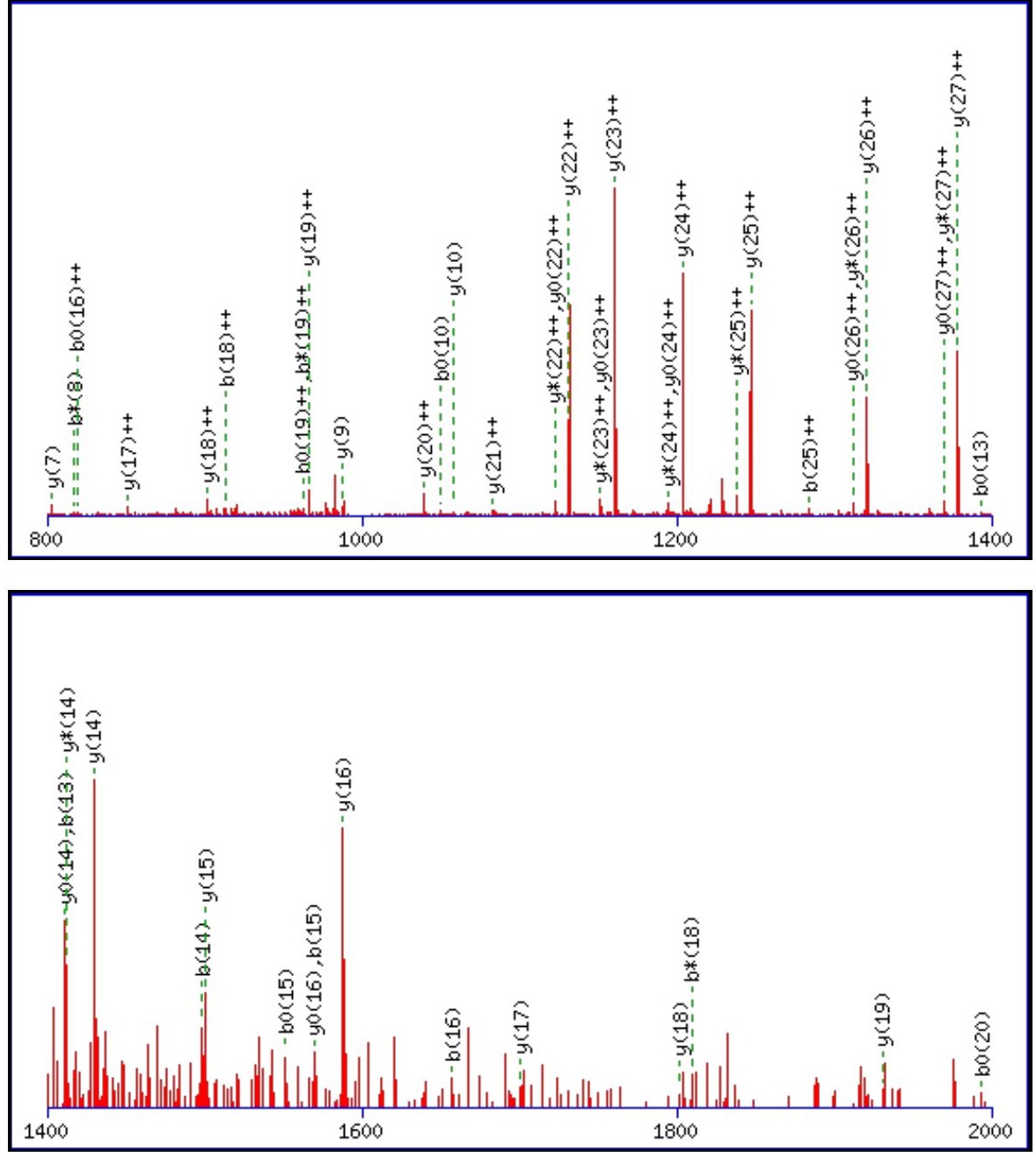

Monoisotopic mass of neutral peptide $\operatorname{Mr}($ calc): 2997.3992

Fixed modifications: Carbamidomethyl (C)

Variable modifications:

N3 : Deamidated_N (N)

Ions Score: 64 Expect: 0.00014

Matches (Bold Red): 81/332 fragment ions using 173 most intense peaks

\begin{tabular}{|c|c|c|c|c|c|c|c|c|c|c|c|c|c|c|}
\hline \# & b & $\mathbf{b}^{++}$ & $\mathbf{b}^{*}$ & $\mathbf{b}^{*^{++}}$ & $\mathbf{b}^{\mathbf{0}}$ & $\mathbf{b}^{\mathbf{0 + +}}$ & Seq. & $\mathbf{y}$ & $\mathbf{y}^{++}$ & $\mathbf{y}^{*}$ & $\mathbf{y}^{*^{++}}$ & $\mathbf{y}^{0}$ & $\mathbf{y}^{0++}$ & \# \\
\hline 1 & 115.0502 & 58.0287 & 98.0237 & 49.5155 & & & $\mathbf{N}$ & & & & & & & 29 \\
\hline 2 & 244.0928 & 122.5500 & 227.0662 & 114.0368 & 226.0822 & 113.5448 & $\mathbf{E}$ & 2884.3636 & 1442.6854 & 2867.3370 & 1434.1721 & 2866.3530 & 1433.6801 & 28 \\
\hline 3 & 359.1197 & 180.0635 & 342.0932 & 171.5502 & 341.1092 & 171.0582 & $\mathbf{N}$ & 2755.3210 & 1378.1641 & 2738.2944 & 1369.6508 & 2737.3104 & 1369.1588 & 27 \\
\hline 4 & 506.1881 & 253.5977 & 489.1616 & 245.0844 & 488.1776 & 244.5924 & $\mathbf{F}$ & 2640.2940 & 1320.6506 & 2623.2675 & 1312.1374 & 2622.2835 & 1311.6454 & 26 \\
\hline 5 & 593.2202 & 297.1137 & 576.1936 & 288.6005 & 575.2096 & 288.1084 & $S$ & 2493.2256 & 1247.1164 & 2476.1991 & 1238.6032 & 2475.2150 & 1238.1112 & 25 \\
\hline 6 & 680.2522 & 340.6297 & 663.2257 & 332.1165 & 662.2416 & 331.6245 & $\mathrm{~S}$ & 2406.1936 & 1203.6004 & 2389.1670 & 1195.0872 & 2388.1830 & 1194.5951 & 24 \\
\hline 7 & 737.2737 & 369.1405 & 720.2471 & 360.6272 & 719.2631 & 360.1352 & G & 2319.1616 & 1160.0844 & 2302.1350 & 1151.5711 & 2301.1510 & 1151.0791 & 23 \\
\hline 8 & 834.3264 & 417.6669 & 817.2999 & 409.1536 & 816.3159 & 408.6616 & $\mathbf{P}$ & 2262.1401 & 1131.5737 & 2245.1135 & 1123.0604 & 2244.1295 & 1122.5684 & 22 \\
\hline 9 & 921.3585 & 461.1829 & 904.3319 & 452.6696 & 903.3479 & 452.1776 & $S$ & 2165.0873 & 1083.0473 & 2148.0608 & 1074.5340 & 2147.0768 & 1074.0420 & 21 \\
\hline 10 & 1068.4269 & 534.7171 & 1051.4003 & 526.2038 & 1050.4163 & 525.7118 & $\mathbf{F}$ & 2078.0553 & 1039.5313 & 2061.0287 & 1031.0180 & 2060.0447 & 1030.5260 & 20 \\
\hline 11 & 1197.4695 & 599.2384 & 1180.4429 & 590.7251 & 1179.4589 & 590.2331 & E & 1930.9869 & 965.9971 & 1913.9603 & 957.4838 & 1912.9763 & 956.9918 & 19 \\
\hline 12 & 1298.5171 & 649.7622 & 1281.4906 & 641.2489 & 1280.5066 & 640.7569 & $\mathbf{T}$ & 1801.9443 & 901.4758 & 1784.9177 & 892.9625 & 1783.9337 & 892.4705 & 18 \\
\hline 13 & 1411.6012 & 706.3042 & 1394.5747 & 697.7910 & 1393.5906 & 697.2990 & I & 1700.8966 & 850.9519 & 1683.8701 & 842.4387 & 1682.8860 & 841.9467 & 17 \\
\hline 14 & 1498.6332 & 749.8203 & \begin{tabular}{|l|}
1481.6067 \\
\end{tabular} & 741.3070 & 1480.6227 & 740.8150 & S & 1587.8125 & 794.4099 & 1570.7860 & 785.8966 & 1569.8020 & 785.4046 & 16 \\
\hline 15 & 1569.6703 & 785.3388 & 1552.6438 & 776.8255 & 1551.6598 & 776.3335 & A & 1500.7805 & 750.8939 & 1483.7540 & 742.3806 & 1482.7700 & 741.8886 & 15 \\
\hline 16 & 1656.7024 & 828.8548 & 1639.6758 & 820.3416 & 1638.6918 & 819.8495 & $\mathrm{~S}$ & 1429.7434 & 715.3753 & 1412.7169 & 706.8621 & 1411.7328 & 706.3701 & 14 \\
\hline 17 & 1713.7238 & 857.3656 & 1696.6973 & 848.8523 & 1695.7133 & 848.3603 & G & 1342.7114 & 671.8593 & 1325.6848 & 663.3461 & 1324.7008 & 662.8540 & 13 \\
\hline 18 & 1826.8079 & 913.9076 & 1809.7814 & 905.3943 & 1808.7973 & 904.9023 & $\mathbf{L}$ & 1285.6899 & 643.3486 & 1268.6634 & 634.8353 & 1267.6793 & 634.3433 & 12 \\
\hline
\end{tabular}




\begin{tabular}{|c|c|c|c|c|c|c|c|c|c|c|c|c|c|c|}
\hline 19 & 1940.8508 & 970.9291 & 1923.8243 & 962.4158 & |1922.8403 & 961.9238 & $\mathbf{N}$ & 1172.6058 & 586.8066 & |1155.5793 & 578.2933 & 1154.5953 & 577.8013 & 11 \\
\hline 20 & 2011.8879 & 1006.4476 & 1994.8614 & 997.9343 & 1993.8774 & 997.4423 & A & 1058.5629 & 529.7851 & 1041.5364 & 521.2718 & 1040.5524 & 520.7798 & 10 \\
\hline 21 & 2082.9251 & 1041.9662 & 2065.8985 & 1033.4529 & 2064.9145 & 1032.9609 & A & 987.5258 & 494.2665 & 970.4993 & 485.7533 & 969.5152 & 485.2613 & 9 \\
\hline 22 & 2196.0091 & 1098.5082 & 2178.9826 & 1089.9949 & 2177.9986 & 1089.5029 & $\mathbf{L}$ & 916.4887 & 458.7480 & 899.4621 & 450.2347 & 898.4781 & 449.7427 & 8 \\
\hline 23 & 2267.0462 & 1134.0268 & 2250.0197 & 1125.5135 & 2249.0357 & 1125.0215 & A & 803.4046 & 402.2060 & 786.3781 & 393.6927 & 785.3941 & 393.2007 & 7 \\
\hline 24 & 2404.1052 & 1202.5562 & 2387.0786 & 1194.0429 & 2386.0946 & 1193.5509 & $\mathbf{H}$ & 732.3675 & 366.6874 & 715.3410 & 358.1741 & 714.3570 & 357.6821 & 6 \\
\hline 25 & 2567.1685 & 1284.0879 & 2550.1419 & 1275.5746 & 2549.1579 & 1275.0826 & $\mathbf{Y}$ & 595.3086 & 298.1579 & 578.2821 & 289.6447 & 577.2980 & 289.1527 & 5 \\
\hline 26 & 2654.2005 & 1327.6039 & 2637.1740 & 1319.0906 & 2636.1899 & 1318.5986 & $\mathrm{~S}$ & 432.2453 & 216.6263 & 415.2187 & 208.1130 & 414.2347 & 207.6210 & 4 \\
\hline 27 & 2751.2533 & 1376.1303 & 2734.2267 & 1367.6170 & 2733.2427 & 1367.1250 & $\mathbf{P}$ & 345.2132 & 173.1103 & 328.1867 & 164.5970 & 327.2027 & 164.1050 & 3 \\
\hline 28 & 2852.3010 & 1426.6541 & 2835.2744 & 1418.1408 & 2834.2904 & 1417.6488 & $T$ & 248.1605 & 124.5839 & 231.1339 & 116.0706 & 230.1499 & 115.5786 & 2 \\
\hline 29 & & & & & & & $\mathbf{K}$ & 147.1128 & 74.0600 & 130.0863 & 65.5468 & & & 1 \\
\hline
\end{tabular}
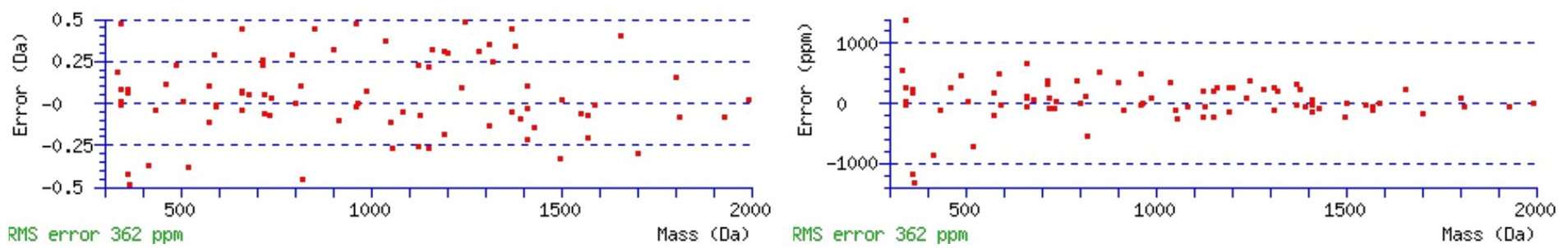

\section{All matches to this query}

\begin{tabular}{|l|l|l|l|}
\hline Score & Mr(calc): & Delta & \multicolumn{1}{|c|}{ Sequence } \\
\hline 64.2 & 2997.3992 & 0.0102 & NENFSSGPSFETISASGLNAALAHYSPTK \\
\hline 57.5 & 2997.3992 & 0.0102 & NENFSSGPSFETISASGLNAALAHYSPTK \\
\hline 41.7 & 2997.3992 & 0.0102 & NENFSSGPSFETISASGLNAALAHYSPTK \\
\hline 37.1 & 2996.4152 & 0.9942 & NENFSSGPSFETISASGLNAALAHYSPTK \\
\hline 7.4 & 2995.3798 & 2.0296 & GGVMGAPSTRAEECTTACKTLDLGTGEAR \\
\hline 4.6 & 2997.3868 & 0.0225 & EFVAQPNCQQLLASRWYDEFPGWR \\
\hline 3.5 & 2995.3782 & 2.0311 & STECLSCLILSPNGSPKPVMASYLIY \\
\hline 3.5 & 2995.3782 & 2.0311 & STECLSCLILSPNGSPKPVMASYLIY \\
\hline 3.5 & 2995.3782 & 2.0311 & STECLSCLILSPNGSPKPVMASYLIY \\
\hline 2.6 & 2997.3919 & 0.0174 & VVATNPIGTGDPSIPSRMIRTNEAVPK \\
\hline
\end{tabular}

Spectrum No: 453; Query: 2548; Rank: 1

\section{Peptide View}

MS/MS Fragmentation of AGVLADDHLIEVNGENVENASHEEVVEK

Found in IPI00200998, Tax_Id=10116 Gene_Symbol=Pdzk1 PDZ domain-containing protein 1

Match to Query 2548: 3016.438332 from(1006.486720,3+)

Title: 100101RatKid_NS_deglyco_12.3427.3427.3.dta

Data file K:INewmanPaper|Piliangl3SubProteomes\Piliang3SP\mgf5ppm\ERLIC_3SubProteomes5ppm.mgf 

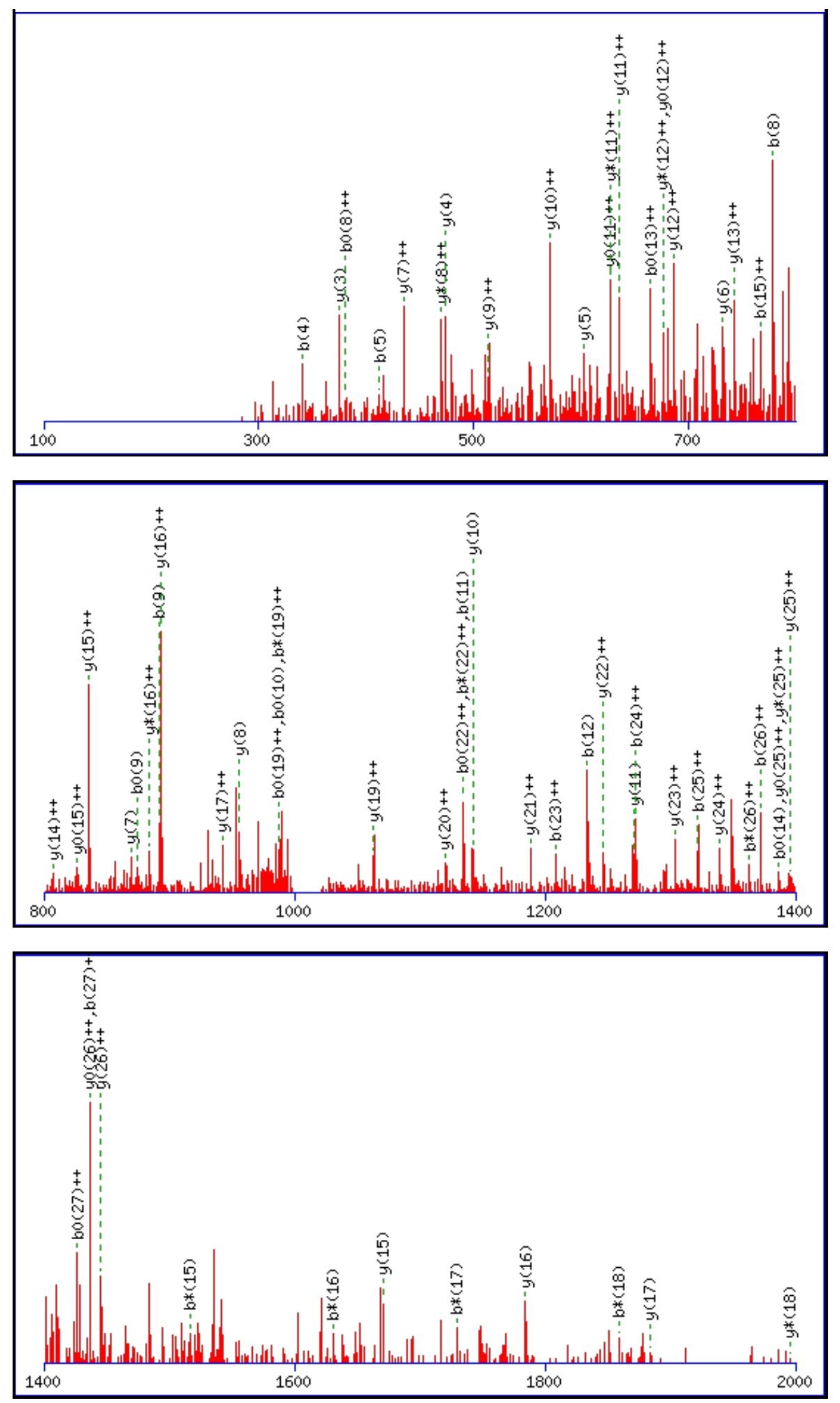

Monoisotopic mass of neutral peptide $\operatorname{Mr}($ calc): 3016.4261

Fixed modifications: Carbamidomethyl (C)

Variable modifications:

N19 : Deamidated_N (N)

Ions Score: 64 Expect: 0.00012

Matches (Bold Red): 68/288 fragment ions using 165 most intense peaks

\begin{tabular}{|c|c|c|c|c|c|c|c|c|c|c|c|c|c|c|}
\hline \# & b & $\mathbf{b}^{++}$ & b* & $\mathbf{b}^{*++}$ & $\mathbf{b}^{0}$ & $\mathbf{b}^{\mathbf{0}^{++}}$ & Seq. & $\mathbf{y}$ & $\mathbf{y}^{++}$ & $\mathbf{y}^{*}$ & $\mathbf{y}^{*^{++}}$ & $\mathbf{y}^{0}$ & $\mathbf{y}^{\mathbf{0}^{++}}$ & \# \\
\hline 1 & 72.0444 & 36.5258 & & & & & A & & & & & & & 28 \\
\hline 2 & 129.0659 & 65.0366 & & & & & G & 2946.3963 & 1473.7018 & 2929.3698 & 1465.1885 & 2928.3857 & 1464.6965 & 27 \\
\hline 3 & 228.1343 & 114.5708 & & & & & $\mathbf{V}$ & 2889.3748 & 1445.1911 & 2872.3483 & 1436.6778 & 2871.3643 & 1436.1858 & 26 \\
\hline 4 & 341.2183 & 171.1128 & & & & & L & 2790.3064 & \begin{tabular}{|l|}
1395.6569 \\
\end{tabular} & 2773.2799 & 1387.1436 & 2772.2959 & 1386.6516 & 25 \\
\hline 5 & 412.2554 & 206.6314 & & & & & $\mathbf{A}$ & 2677.2224 & \begin{tabular}{|l|}
1339.1148 \\
\end{tabular} & 2660.1958 & 1330.6015 & 2659.2118 & 1330.1095 & 24 \\
\hline
\end{tabular}




\begin{tabular}{|c|c|c|c|c|c|c|c|c|c|c|c|c|c|c|}
\hline 6 & 527.2824 & 264.1448 & & & 509.2718 & 255.1396 & D & 2606.1853 & 1303.5963 & |2589.1587| & 1295.0830 & 2588.1747 & 1294.5910 & $23 \mid$ \\
\hline 7 & 642.3093 & 321.6583 & & & 624.2988 & 312.6530 & D & 2491.1583 & 1246.0828 & 2474.1318 & 1237.5695 & 2473.1477 & 1237.0775 & 22 \\
\hline 8 & 779.3682 & 390.1878 & & & 761.3577 & 381.1825 & $\mathbf{H}$ & 2376.1314 & 1188.5693 & 2359.1048 & 1180.0560 & 2358.1208 & 1179.5640 & 21 \\
\hline 9 & 892.4523 & 446.7298 & & & 874.4417 & 437.7245 & L & 2239.0725 & 1120.0399 & 2222.0459 & 1111.5266 & 2221.0619 & 1111.0346 & 20 \\
\hline 10 & 1005.5364 & 503.2718 & & & 987.5258 & 494.2665 & I & 2125.9884 & 1063.4978 & 2108.9618 & 1054.9846 & 2107.9778 & 1054.4926 & 19 \\
\hline 11 & 1134.5790 & 567.7931 & & & 1116.5684 & 558.7878 & $\mathbf{E}$ & 2012.9043 & 1006.9558 & 1995.8778 & 998.4425 & 1994.8938 & 997.9505 & 18 \\
\hline 12 & 1233.6474 & 617.3273 & & & 1215.6368 & 608.3220 & V & 1883.8617 & 942.4345 & 1866.8352 & 933.9212 & 1865.8512 & 933.4292 & 17 \\
\hline 13 & 1347.6903 & 674.3488 & 1330.6638 & 665.8355 & 1329.6797 & 665.3435 & $\mathbf{N}$ & 1784.7933 & 892.9003 & 1767.7668 & 884.3870 & 1766.7828 & 883.8950 & 16 \\
\hline 14 & 1404.7118 & 702.8595 & 1387.6852 & 694.3462 & 1386.7012 & 693.8542 & G & 1670.7504 & 835.8788 & 1653.7238 & 827.3656 & 1652.7398 & 826.8736 & 15 \\
\hline 15 & 1533.7544 & 767.3808 & 1516.7278 & 758.8675 & 1515.7438 & 758.3755 & $\mathbf{E}$ & 1613.7289 & 807.3681 & 1596.7024 & 798.8548 & 1595.7184 & 798.3628 & 14 \\
\hline 16 & 1647.7973 & 824.4023 & 1630.7707 & 815.8890 & 1629.7867 & 815.3970 & $\mathbf{N}$ & 1484.6863 & 742.8468 & 1467.6598 & 734.3335 & 1466.6758 & 733.8415 & 13 \\
\hline 17 & 1746.8657 & 873.9365 & 1729.8392 & 865.4232 & 1728.8551 & 864.9312 & $\mathbf{V}$ & 1370.6434 & 685.8253 & 1353.6169 & 677.3121 & 1352.6328 & 676.8201 & 12 \\
\hline 18 & 1875.9083 & 938.4578 & 1858.8817 & 929.9445 & 1857.8977 & 929.4525 & $E$ & 1271.5750 & 636.2911 & 1254.5484 & 627.7779 & 1253.5644 & 627.2859 & 11 \\
\hline 19 & 1990.9352 & 995.9713 & \begin{tabular}{|l|}
1973.9087 \\
\end{tabular} & 987.4580 & 1972.9247 & 986.9660 & $\mathbf{N}$ & 1142.5324 & 571.7698 & 1125.5059 & 563.2566 & 1124.5218 & 562.7646 & 10 \\
\hline 20 & 2061.9723 & 1031.4898 & 2044.9458 & 1022.9765 & 2043.9618 & 1022.4845 & A & 1027.5055 & 514.2564 & 1010.4789 & 505.7431 & 1009.4949 & 505.2511 & 9 \\
\hline 21 & 2149.0044 & 1075.0058 & 2131.9778 & 1066.4926 & 2130.9938 & 1066.0005 & S & 956.4684 & 478.7378 & 939.4418 & 470.2245 & 938.4578 & 469.7325 & 8 \\
\hline 22 & 2286.0633 & 1143.5353 & 2269.0367 & 1135.0220 & 2268.0527 & 1134.5300 & $\mathbf{H}$ & 869.4363 & 435.2218 & 852.4098 & 426.7085 & 851.4258 & 426.2165 & 7 \\
\hline 23 & 2415.1059 & 1208.0566 & 2398.0793 & 1199.5433 & 2397.0953 & 1199.0513 & $\mathbf{E}$ & 732.3774 & 366.6923 & 715.3509 & 358.1791 & 714.3668 & 357.6871 & 6 \\
\hline 24 & 2544.1485 & \begin{tabular}{|l}
1272.5779 \\
\end{tabular} & 2527.1219 & 1264.0646 & 2526.1379 & 1263.5726 & $\mathbf{E}$ & 603.3348 & 302.1710 & 586.3083 & 293.6578 & 585.3243 & 293.1658 & 5 \\
\hline 25 & 2643.2169 & \begin{tabular}{|l|l|}
1322.1121 \\
\end{tabular} & 2626.1903 & 1313.5988 & 2625.2063 & 1313.1068 & $\mathbf{V}$ & 474.2922 & 237.6498 & 457.2657 & 229.1365 & 456.2817 & 228.6445 & 4 \\
\hline 26 & 2742.2853 & 1371.6463 & 2725.2588 & 1363.1330 & 2724.2747 & 1362.6410 & $\mathbf{V}$ & 375.2238 & 188.1155 & 358.1973 & 179.6023 & 357.2132 & 179.1103 & 3 \\
\hline 27 & 2871.3279 & 1436.1676 & 2854.3013 & 1427.6543 & 2853.3173 & 1427.1623 & $\mathbf{E}$ & 276.1554 & 138.5813 & 259.1288 & 130.0681 & 258.1448 & 129.5761 & 2 \\
\hline 28 & & & & & & & K & 147.1128 & 74.0600 & 130.0863 & 65.5468 & & & 1 \\
\hline
\end{tabular}
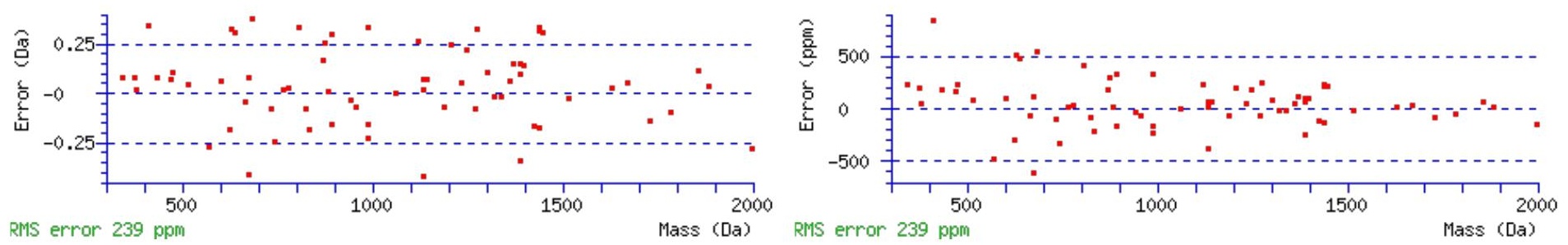

\section{All matches to this query}

\begin{tabular}{|l|l|l|l|}
\hline Score & Mr(calc): & Delta & \multicolumn{1}{|c|}{ Sequence } \\
\hline 64.1 & 3016.4261 & 0.0122 & AGVLADDHLIEVNGENVENASHEEVVEK \\
\hline 61.3 & 3016.4261 & 0.0122 & AGVLADDHLIEVNGENVENASHEEVVEK \\
\hline 58.1 & 3016.4261 & 0.0122 & AGVLADDHLIEVNGENVENASHEEVVEK \\
\hline 22.6 & 3015.4421 & 0.9962 & AGVLADDHLIEVNGENVENASHEEVVEK \\
\hline 4.2 & 3016.4091 & 0.0292 & ISLFGNDVLRKMSIIQIMIDNYOK \\
\hline 4.0 & 3016.4253 & 0.0131 & MTTDTGTIPFAPNLEDINNILESKFR \\
\hline 4.0 & 3016.4253 & 0.0131 & MTTDTGTIPFAPNLEDINNILESKFR \\
\hline 4.0 & 3016.4253 & 0.0131 & MTTDTGTIPFAPNLEDINNILESKFR \\
\hline 4.0 & 3016.4253 & 0.0131 & MTTDTGTIPFAPNLEDINNILESKFR \\
\hline 3.7 & 3016.4091 & 0.0292 & ISLFGNDVLRKMSIIQIMIDNYQK \\
\hline
\end{tabular}

Spectrum No: 454; Query: 319; Rank: 1

\section{Peptide View}

MS/MS Fragmentation of ALSPNSTISSAPK 
Found in IPI00208721, Tax_Id=10116 Gene_Symbol=RGD1563141_predicted 78 kDa protein

Match to Query 319: 1272.652628 from(637.333590,2+)

Title: 091008RatKidney_NH4Format01_24.1085.1085.2.dta

Data file K:INewmanPaper|Piliang|3SubProteomes\Piliang3SP\mgf5ppm\ERLIC_3SubProteomes5ppm.mgf
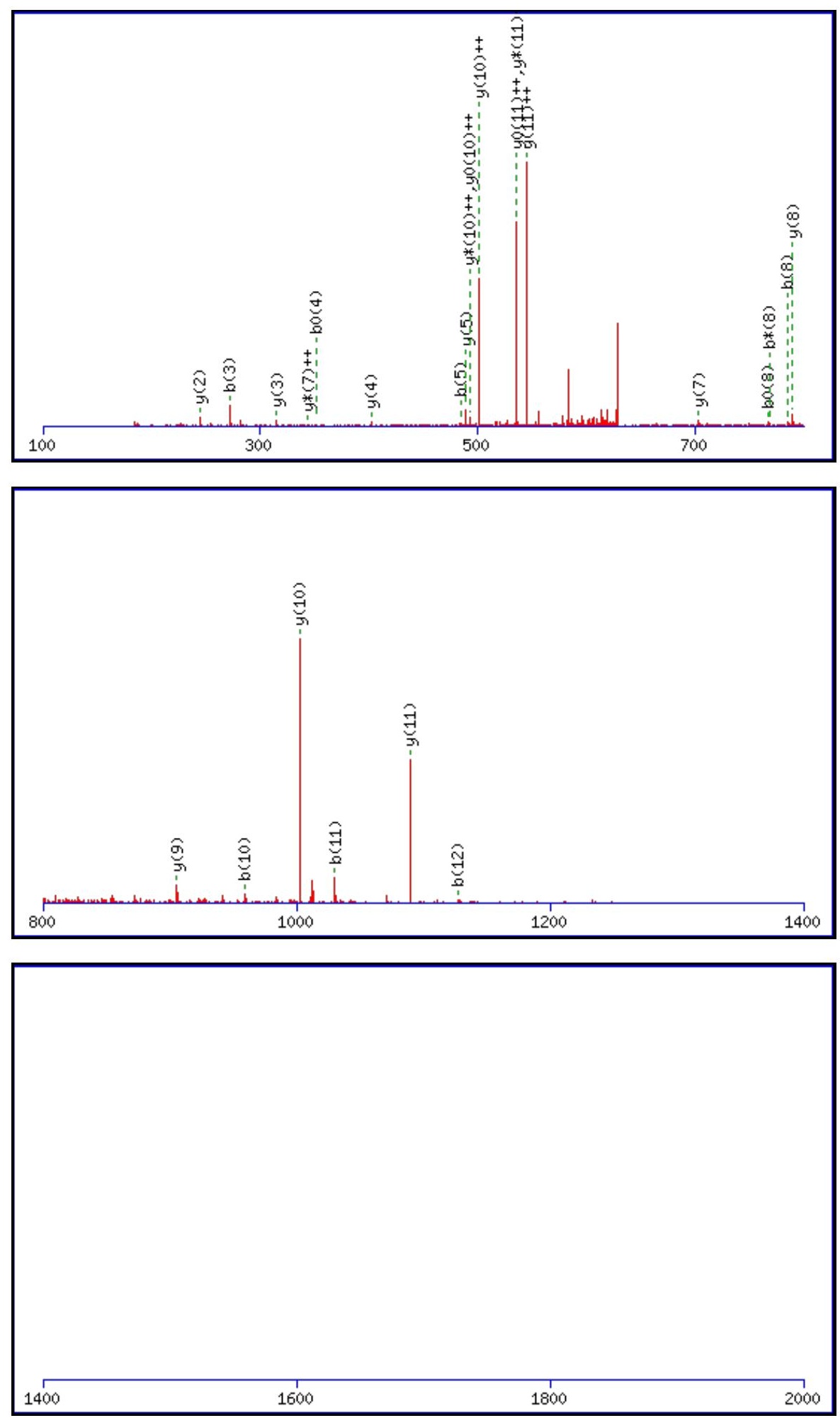

Monoisotopic mass of neutral peptide $\operatorname{Mr}($ calc): 1272.6561

Fixed modifications: Carbamidomethyl (C)

Variable modifications:

N5: Deamidated $\mathrm{N}(\mathrm{N})$

Ions Score: 64 Expect: $4.4 \mathrm{e}-005$

Matches (Bold Red): 25/126 fragment ions using 43 most intense peaks

\begin{tabular}{|c|c|c|c|c|c|c|c|c|c|c|c|c|c|c|}
\hline$\#$ & $\mathbf{b}$ & $\mathbf{b}^{++}$ & $\mathbf{b}^{*}$ & $\mathbf{b}^{*^{++}}$ & $\mathbf{b}^{\mathbf{0}}$ & $\mathbf{b}^{\mathbf{0 + +}}$ & Seq. & $\mathbf{y}$ & $\mathbf{y}^{++}$ & $\mathbf{y}^{\mathbf{*}}$ & $\mathbf{y}^{\mathbf{*}^{++}}$ & $\mathbf{y}^{\mathbf{0}}$ & $\mathbf{y}^{\mathbf{0}}$ & $\#$ \\
\hline $\mathbf{1}$ & $\mathbf{7 2 . 0 4 4 4}$ & 36.5258 & & & & & $\mathbf{A}$ & & & & & & & $\mathbf{1 3}$ \\
\hline
\end{tabular}




\begin{tabular}{|c|c|c|c|c|c|c|c|c|c|c|c|c|c|c|}
\hline 2 & 185.1285 & 93.0679 & & & & & $\mathbf{L}$ & 1202.6263 & 601.8168 & 1185.5998 & 593.3035 & 1184.6157 & 592.8115 & 12 \\
\hline 3 & 272.1605 & 136.5839 & & & 254.1499 & 127.5786 & $\mathbf{S}$ & 1089.5422 & 545.2748 & 1072.5157 & 536.7615 & 1071.5317 & 536.2695 & 11 \\
\hline 4 & 369.2132 & 185.1103 & & & 351.2027 & 176.1050 & $\mathbf{P}$ & 1002.5102 & 501.7587 & 985.4837 & |493.2455 & 984.4996 & 492.7535 & 10 \\
\hline 5 & 484.2402 & 242.6237 & 467.2136 & 234.1105 & 466.2296 & 233.6184 & $\mathbf{N}$ & 905.4574 & 453.2324 & 888.4309 & 444.7191 & 887.4469 & 444.2271 & 9 \\
\hline 6 & 571.2722 & 286.1397 & 554.2457 & 277.6265 & 553.2616 & 277.1345 & $\mathbf{S}$ & 790.4305 & 395.7189 & 773.4040 & 387.2056 & 772.4199 & 386.7136 & 8 \\
\hline 7 & 672.3199 & 336.6636 & 655.2933 & 328.1503 & 654.3093 & 327.6583 & $\mathbf{T}$ & 703.3985 & 352.2029 & 686.3719 & 343.6896 & 685.3879 & 343.1976 & 7 \\
\hline 8 & 785.4040 & 393.2056 & 768.3774 & 384.6923 & 767.3934 & 384.2003 & I & 602.3508 & 301.6790 & 585.3243 & 293.1658 & 584.3402 & 292.6738 & 6 \\
\hline 9 & 872.4360 & 436.7216 & 855.4094 & 428.2084 & 854.4254 & 427.7163 & $\mathrm{~S}$ & 489.2667 & 245.1370 & 472.2402 & 236.6237 & 471.2562 & 236.1317 & 5 \\
\hline 10 & 959.4680 & 480.2376 & 942.4415 & 471.7244 & 941.4574 & 471.2324 & S & 402.2347 & 201.6210 & 385.2082 & 193.1077 & 384.2241 & 192.6157 & 4 \\
\hline 11 & 1030.5051 & 515.7562 & 1013.4786 & 507.2429 & 1012.4946 & 506.7509 & A & 315.2027 & 158.1050 & 298.1761 & 149.5917 & & & 3 \\
\hline 12 & 1127.5579 & 564.2826 & 1110.5313 & 555.7693 & 1109.5473 & 555.2773 & $\mathbf{P}$ & 244.1656 & 122.5864 & 227.1390 & 114.0731 & & & 2 \\
\hline 13 & & & & & & & K & 147.1128 & 74.0600 & 130.0863 & 65.5468 & & & 1 \\
\hline
\end{tabular}
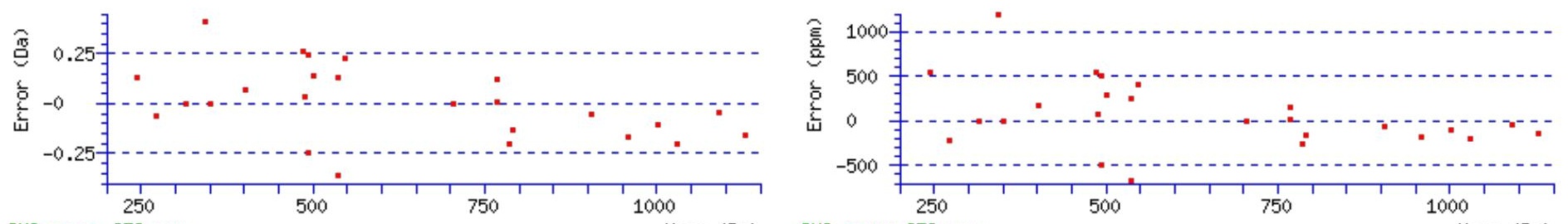

RIS error 378 ppm

Mass (Da) RMS error 378 ppm

\section{All matches to this query}

\begin{tabular}{|l|c|c|l|}
\hline Score & Mr(calc): & Delta & \multicolumn{1}{c|}{ Sequence } \\
\hline 64.1 & 1272.6561 & -0.0035 & ALSPNSTISSAPK \\
\hline 8.9 & 1272.6462 & 0.0064 & LADELSGWVQR \\
\hline 8.8 & 1272.6591 & -0.0065 & GTLKRFSSLGK \\
\hline 6.9 & 1272.6509 & 0.0017 & $\underline{\text { SPGNKCRLXR }}$ \\
\hline 5.9 & 1271.6387 & 1.0139 & NRPNLHILSK \\
\hline 5.5 & 1272.6536 & -0.0010 & LAVTALCWNPK \\
\hline 2.6 & 1272.6575 & -0.0048 & SPKYHSSSKPR \\
\hline 1.0 & 1272.6422 & 0.0104 & LANQKSSNPTGR \\
\hline 0.3 & 1272.6422 & 0.0104 & $\underline{\text { AIERERDTVQG }}$ \\
\hline
\end{tabular}

Spectrum No: 455; Query: 2302; Rank: 1

\section{Peptide View}

MS/MS Fragmentation of LANASLYLAEWQNITKNFSEDR

Found in IPI00211616, Tax_Id=10116 Gene_Symbol=Slc3a2 4F2 cell-surface antigen heavy chain

Match to Query 2302: 2585.232462 from(862.751430,3+)

Title: 100101RatKid_NS_deglyco_22.5849.5849.3.dta

Data file K:INewmanPaper|Piliang|3SubProteomes\Piliang3SP\mgf5ppm\ERLIC_3SubProteomes5ppm.mgf 

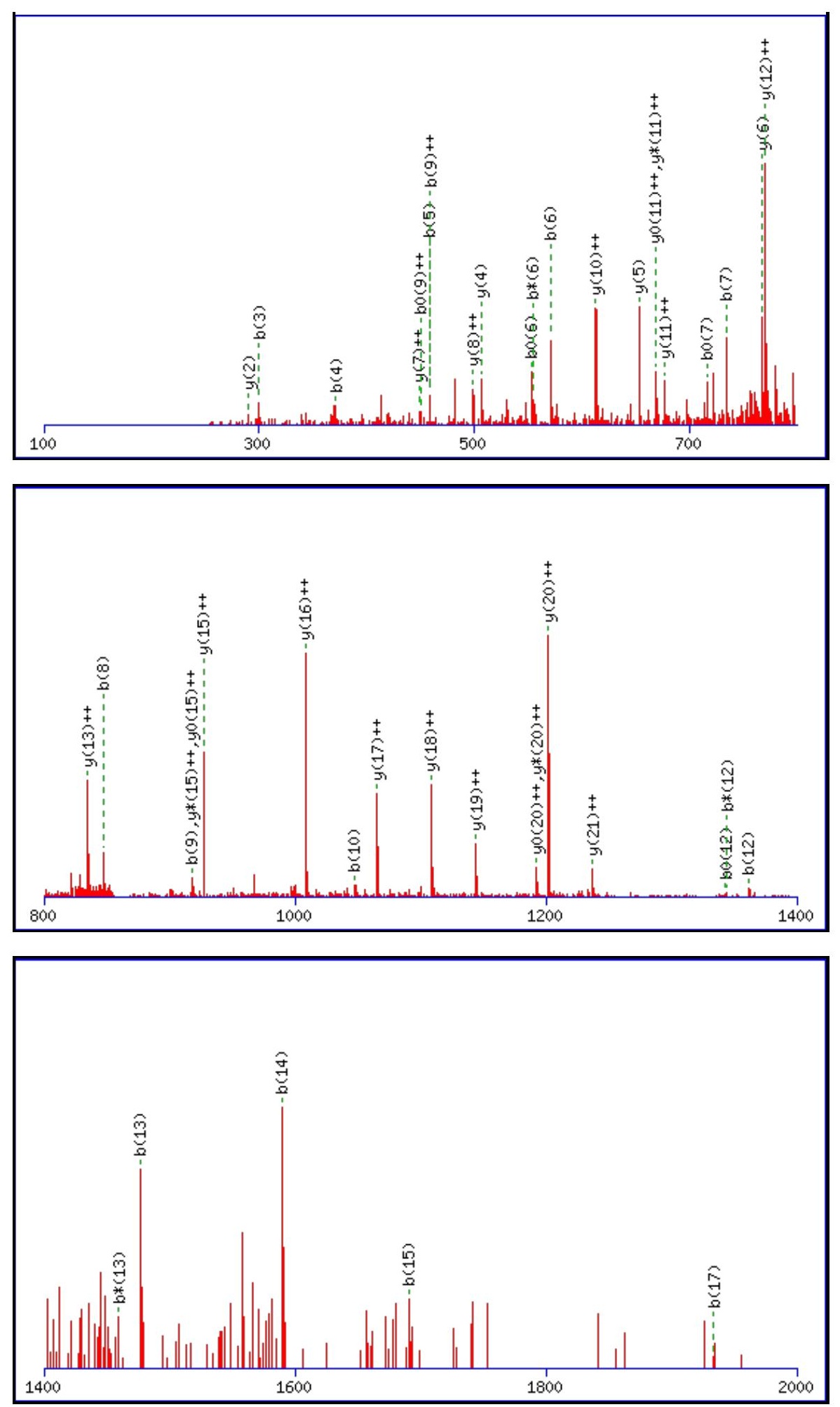

Monoisotopic mass of neutral peptide $\operatorname{Mr}($ calc): 2585.2285

Fixed modifications: Carbamidomethyl (C)

Variable modifications:

N3 : Deamidated_N (N)

N13 : Deamidated_N (N)

N17 : Deamidated_N (N)

Ions Score: 64 Expect: 0.00011

Matches (Bold Red): 44/238 fragment ions using 86 most intense peaks

\begin{tabular}{|c|c|c|c|c|c|c|c|c|c|c|c|c|c|c|}
\hline$\#$ & $\mathbf{b}$ & $\mathbf{b}^{++}$ & $\mathbf{b}^{*}$ & $\mathbf{b}^{\mathbf{*}^{++}}$ & $\mathbf{b}^{\mathbf{0}}$ & $\mathbf{b}^{\mathbf{0}+}$ & Seq. & $\mathbf{y}$ & $\mathbf{y}^{++}$ & $\mathbf{y}^{\mathbf{*}}$ & $\mathbf{y}^{\mathbf{*}^{++}}$ & $\mathbf{y}^{\mathbf{0}}$ & $\mathbf{y}^{\mathbf{0}+}$ & $\#$ \\
\hline $\mathbf{1}$ & 114.0913 & 57.5493 & & & & & $\mathbf{L}$ & & & & & & & $\mathbf{2 2}$ \\
\hline $\mathbf{2}$ & 185.1285 & 93.0679 & & & & & $\mathbf{A}$ & 2473.1518 & $\mathbf{1 2 3 7 . 0 7 9 5}$ & 2456.1252 & 1228.5662 & 2455.1412 & 1228.0742 & $\mathbf{2 1}$ \\
\hline $\mathbf{3}$ & $\mathbf{3 0 0 . 1 5 5 4}$ & 150.5813 & 283.1288 & 142.0681 & & & $\mathbf{N}$ & 2402.1146 & $\mathbf{1 2 0 1 . 5 6 1 0}$ & 2385.0881 & $\mathbf{1 1 9 3 . 0 4 7 7}$ & 2384.1041 & $\mathbf{1 1 9 2 . 5 5 5 7}$ & $\mathbf{2 0}$ \\
\hline $\mathbf{4}$ & $\mathbf{3 7 1 . 1 9 2 5}$ & 186.0999 & 354.1660 & 177.5866 & & & $\mathbf{A}$ & 2287.0877 & $\mathbf{1 1 4 4 . 0 4 7 5}$ & 2270.0612 & 1135.5342 & 2269.0771 & 1135.0422 & $\mathbf{1 9}$ \\
\hline
\end{tabular}




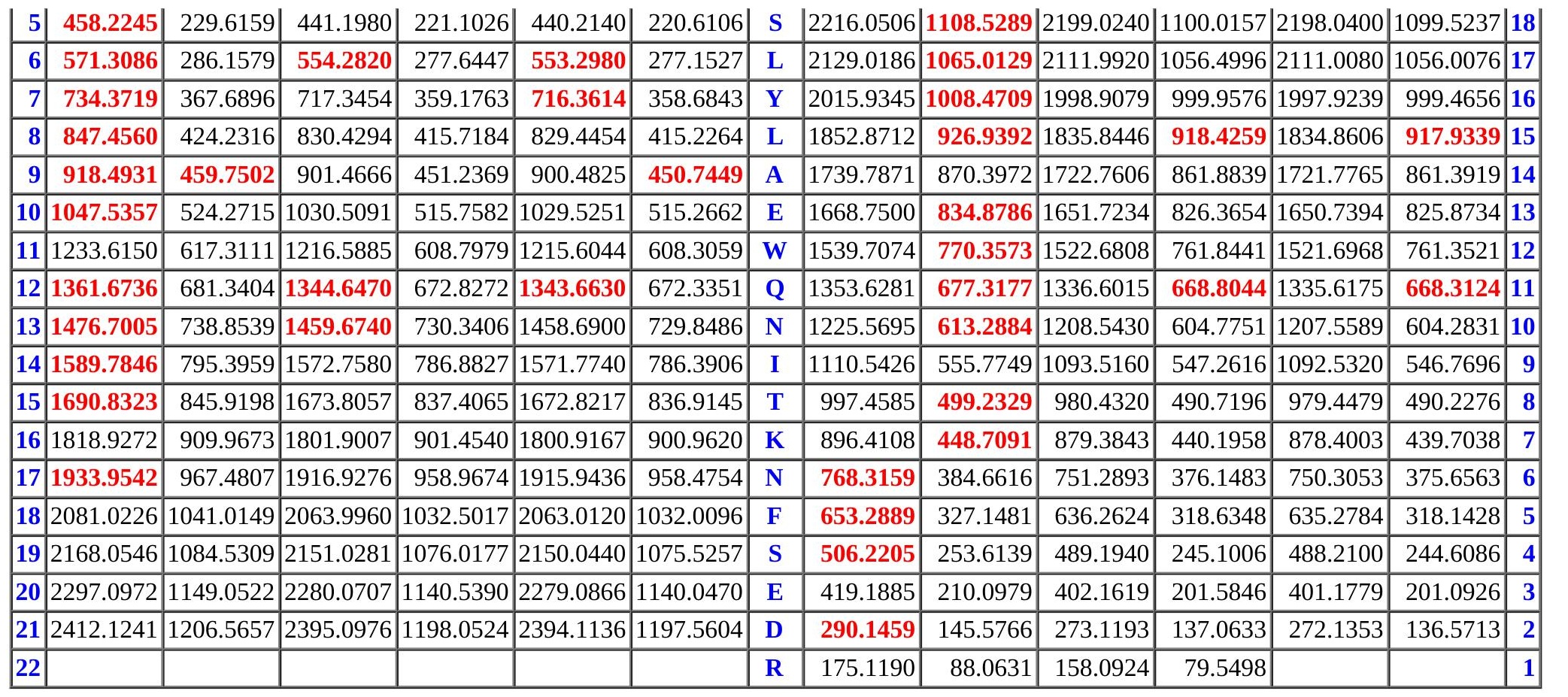
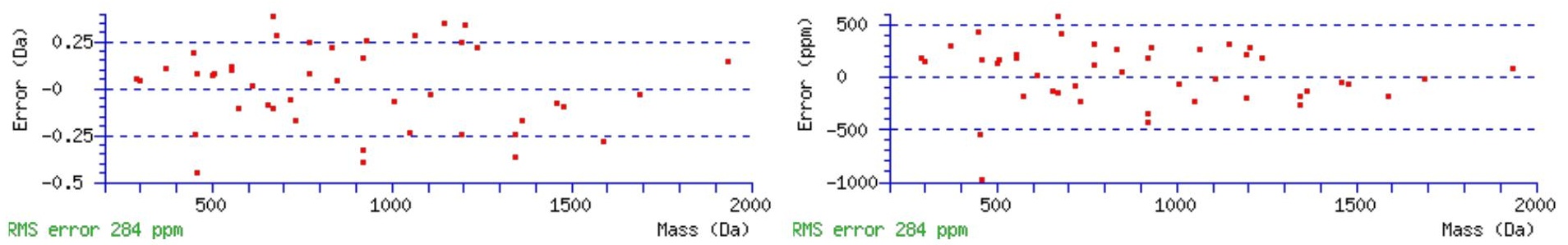

\section{All matches to this query}

\begin{tabular}{|l|l|l|l|}
\hline Score & Mr(calc): & Delta & \multicolumn{1}{|c|}{ Sequence } \\
\hline 64.1 & 2585.2285 & 0.0040 & LANASLYLAEWQNITKNFSEDR \\
\hline 31.3 & 2584.2445 & 0.9880 & LANASLYLAEWQNITKNFSEDR \\
\hline 27.4 & 2584.2445 & 0.9880 & LANASLYLAEWQNITKNFSEDR \\
\hline 27.1 & 2584.2445 & 0.9880 & LANASLYLAEWQNITKNFSEDR \\
\hline 1.8 & 2583.2023 & 2.0302 & KGTELLLGVDALGLHIYDPENR \\
\hline 0.2 & 2583.2459 & 1.9866 & DIKDTTVGTLSQRITNQVHGLK \\
\hline 0.1 & 2584.2329 & 0.9995 & QALQRHVLSCAARSNLQDSPPR \\
\hline
\end{tabular}

Spectrum No: 456; Query: 999; Rank: 1

\section{Peptide View}

MS/MS Fragmentation of WSTGLAHNSSAITYR

Found in IPI00358382, Tax_Id=10116 Gene_Symbol=C8a_predicted complement component 8, alpha polypeptide

Match to Query 999: 1663.800728 from(832.907640,2+)

Title: 100101RatKid_NS_deglyco_23.2136.2136.2.dta

Data file K:INewmanPaper|Piliang|3SubProteomes\Piliang3SP\mgf5ppm\ERLIC_3SubProteomes5ppm.mgf 

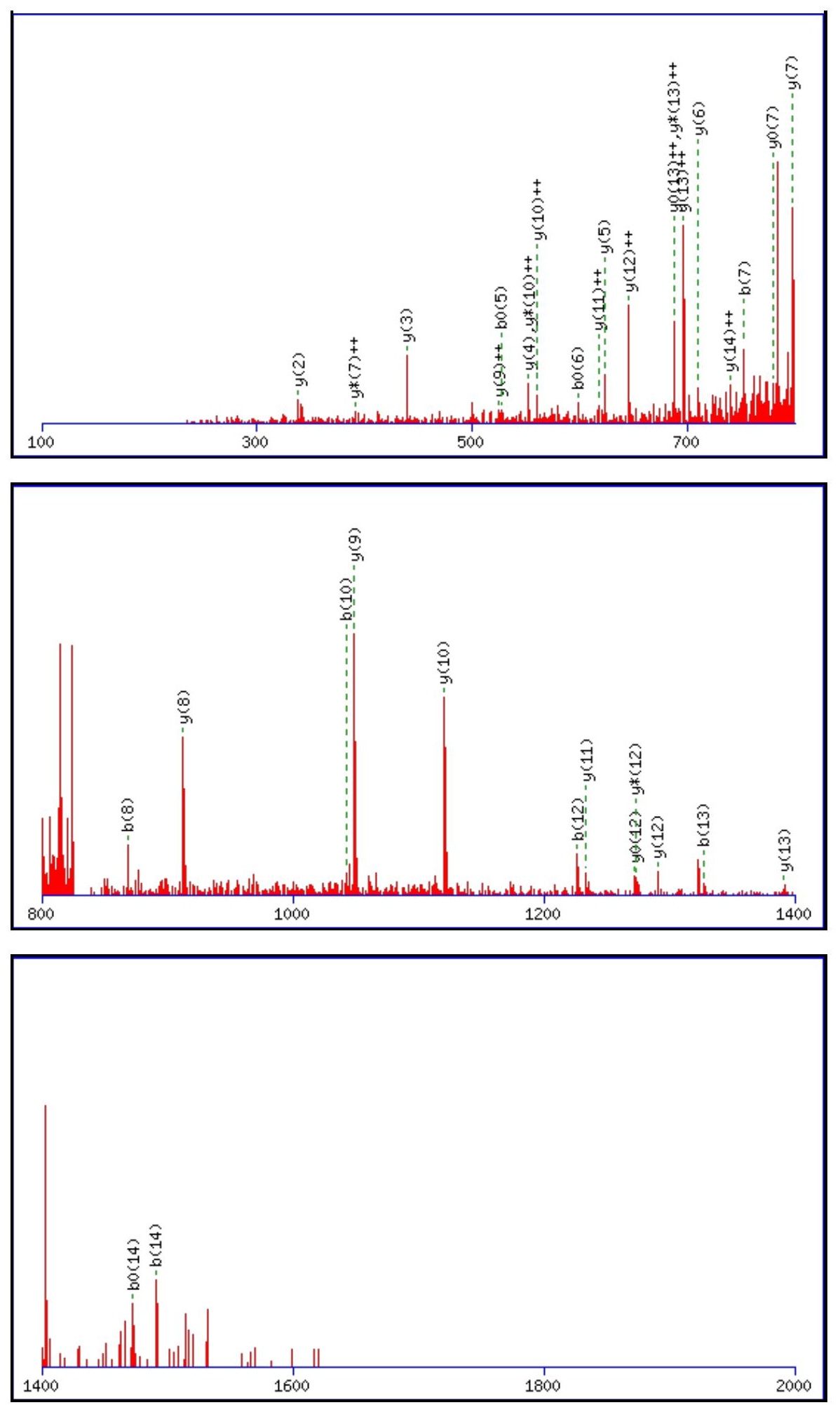

Monoisotopic mass of neutral peptide $\operatorname{Mr}($ calc): 1663.7954

Fixed modifications: Carbamidomethyl (C)

Variable modifications:

N8: Deamidated $\mathrm{N}(\mathrm{N})$

Ions Score: 64 Expect: $7.4 \mathrm{e}-005$

Matches (Bold Red): 34/148 fragment ions using 85 most intense peaks

\begin{tabular}{|r|c|c|c|c|c|c|c|c|c|c|c|c|c|c|}
\hline$\#$ & $\mathbf{b}$ & $\mathbf{b}^{++}$ & $\mathbf{b}^{*}$ & $\mathbf{b}^{\mathbf{*}^{++}}$ & $\mathbf{b}^{\mathbf{0}}$ & $\mathbf{b}^{\mathbf{0 + +}}$ & Seq. & $\mathbf{y}$ & $\mathbf{y}^{++}$ & $\mathbf{y}^{\mathbf{*}}$ & $\mathbf{y}^{\boldsymbol{*}^{++}}$ & $\mathbf{y}^{\mathbf{0}}$ & $\mathbf{y}^{\mathbf{0 + +}}$ & $\#$ \\
\hline $\mathbf{1}$ & 187.0866 & 94.0469 & & & & & $\mathbf{W}$ & & & & & & & $\mathbf{1 5}$ \\
\hline $\mathbf{2}$ & 274.1186 & 137.5629 & & & 256.1081 & 128.5577 & S & 1478.7234 & 739.8653 & 1461.6968 & 731.3521 & 1460.7128 & 730.8601 & $\mathbf{1 4}$ \\
\hline 3 & 375.1663 & 188.0868 & & & 357.1557 & 179.0815 & T & $\mathbf{1 3 9 1 . 6 9 1 4}$ & $\mathbf{6 9 6 . 3 4 9 3}$ & 1374.6648 & $\mathbf{6 8 7 . 8 3 6 0}$ & 1373.6808 & $\mathbf{6 8 7 . 3 4 4 0}$ & $\mathbf{1 3}$ \\
\hline $\mathbf{4}$ & 432.1878 & 216.5975 & & & 414.1772 & 207.5922 & $\mathbf{G}$ & $\mathbf{1 2 9 0 . 6 4 3 7}$ & $\mathbf{6 4 5 . 8 2 5 5}$ & $\mathbf{1 2 7 3 . 6 1 7 1}$ & 637.3122 & $\mathbf{1 2 7 2 . 6 3 3 1}$ & 636.8202 & $\mathbf{1 2}$ \\
\hline $\mathbf{5}$ & 545.2718 & 273.1396 & & & 527.2613 & 264.1343 & L & $\mathbf{1 2 3 3 . 6 2 2 2}$ & $\mathbf{6 1 7 . 3 1 4 7}$ & 1216.5957 & 608.8015 & 1215.6117 & 608.3095 & $\mathbf{1 1}$ \\
\hline
\end{tabular}




\begin{tabular}{|c|c|c|c|c|c|c|c|c|c|c|c|c|c|c|}
\hline & 616.3089| & |308.6581 & & & 598.2984 & |299.6528 & A & |1120.5382 & |560.7727 & |1103.5116 & 552.2594 & 1102.5276 & $|551.7674|$ & 10 \\
\hline 7 & 753.3678 & 377.1876 & & & 735.3573 & 368.1823 & $\mathbf{H}$ & 1049.5010 & 525.2542 & 1032.4745 & 516.7409 & 1031.4905 & 516.2489 & 9 \\
\hline 8 & 868.3948 & 434.7010 & 851.3682 & 426.1878 & 850.3842 & 425.6957 & $\mathbf{N}$ & 912.4421 & 456.7247 & 895.4156 & 448.2114 & 894.4316 & 447.7194 & 8 \\
\hline 9 & 955.4268 & 478.2170 & 938.4003 & 469.7038 & 937.4162 & 469.2118 & $\mathrm{~S}$ & 797.4152 & 399.2112 & 780.3886 & 390.6980 & 779.4046 & 390.2060 & 7 \\
\hline 10 & 1042.4588 & 521.7331 & 1025.4323 & 513.2198 & 1024.4483 & 512.7278 & $\mathrm{~S}$ & 710.3832 & 355.6952 & 693.3566 & 347.1819 & 692.3726 & 346.6899 & 6 \\
\hline 11 & 1113.4960 & 557.2516 & 1096.4694 & 548.7383 & 1095.4854 & 548.2463 & A & 623.3511 & |312.1792 & 606.3246 & 303.6659 & 605.3406 & 303.1739 & 5 \\
\hline 12 & 1226.5800 & 613.7936 & 1209.5535 & 605.2804 & 1208.5695 & 604.7884 & I & 552.3140 & 276.6607 & 535.2875 & 268.1474 & 534.3035 & 267.6554 & 4 \\
\hline 13 & 1327.6277 & 664.3175 & 1310.6012 & 655.8042 & 1309.6171 & 655.3122 & $\mathrm{~T}$ & 439.2300 & 220.1186 & 422.2034 & 211.6053 & 421.2194 & 211.1133 & 3 \\
\hline 14 & 1490.6910 & 745.8492 & 1473.6645 & 737.3359 & 1472.6805 & 736.8439 & $\mathbf{Y}$ & 338.1823 & 169.5948 & 321.1557 & 161.0815 & & & 2 \\
\hline 15 & & & & & & & $\mathbf{R}$ & 175.1190 & 88.0631 & 158.0924 & 79.5498 & & & 1 \\
\hline
\end{tabular}
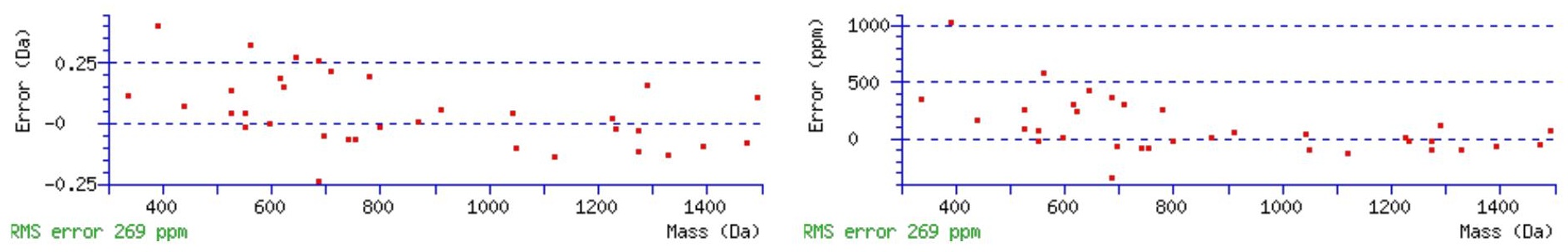

\section{All matches to this query}

\begin{tabular}{|l|l|l|l|}
\hline Score & Mr(calc): & Delta & \multicolumn{1}{|c|}{ Sequence } \\
\hline 64.1 & 1663.7954 & 0.0053 & WSTGLAHNSSAITYR \\
\hline 5.9 & 1663.7963 & 0.0044 & GGFCNFMHLKPISR \\
\hline 5.8 & 1663.7970 & 0.0037 & QYQNILKSGTLYR \\
\hline 5.0 & 1662.7991 & 1.0016 & SPRSPGKSPPPSHPR \\
\hline 4.7 & 1662.8130 & 0.9877 & QYQNILKSGTLYR \\
\hline 4.0 & 1662.7991 & 1.0016 & SPRSPGKSPPPSHPR \\
\hline 2.8 & 1663.8070 & -0.0062 & GDVDISLPKVEGDLK \\
\hline 2.8 & 1663.8070 & -0.0062 & GDVDLSLPKVEGDLK \\
\hline 0.9 & 1663.7978 & 0.0030 & RMXRDGAHVVISSR \\
\hline
\end{tabular}

Spectrum No: 457; Query: 238; Rank: 1

\section{Peptide View}

MS/MS Fragmentation of MLEAYNLTEK

Found in IPI00189714, Tax_Id=10116 Gene_Symbol=Col12a1 similar to Collagen alpha-1(XII) chain precursor

Match to Query 238: 1211.574308 from(606.794430,2+)

Title: 091008RatKidney_NoSalt_25.1910.1910.2.dta

Data file K:INewmanPaper|Piliang $\mid 3 S$ SubProteomes\Piliang3SP\mgf5ppm\ERLIC_3SubProteomes5ppm.mgf 

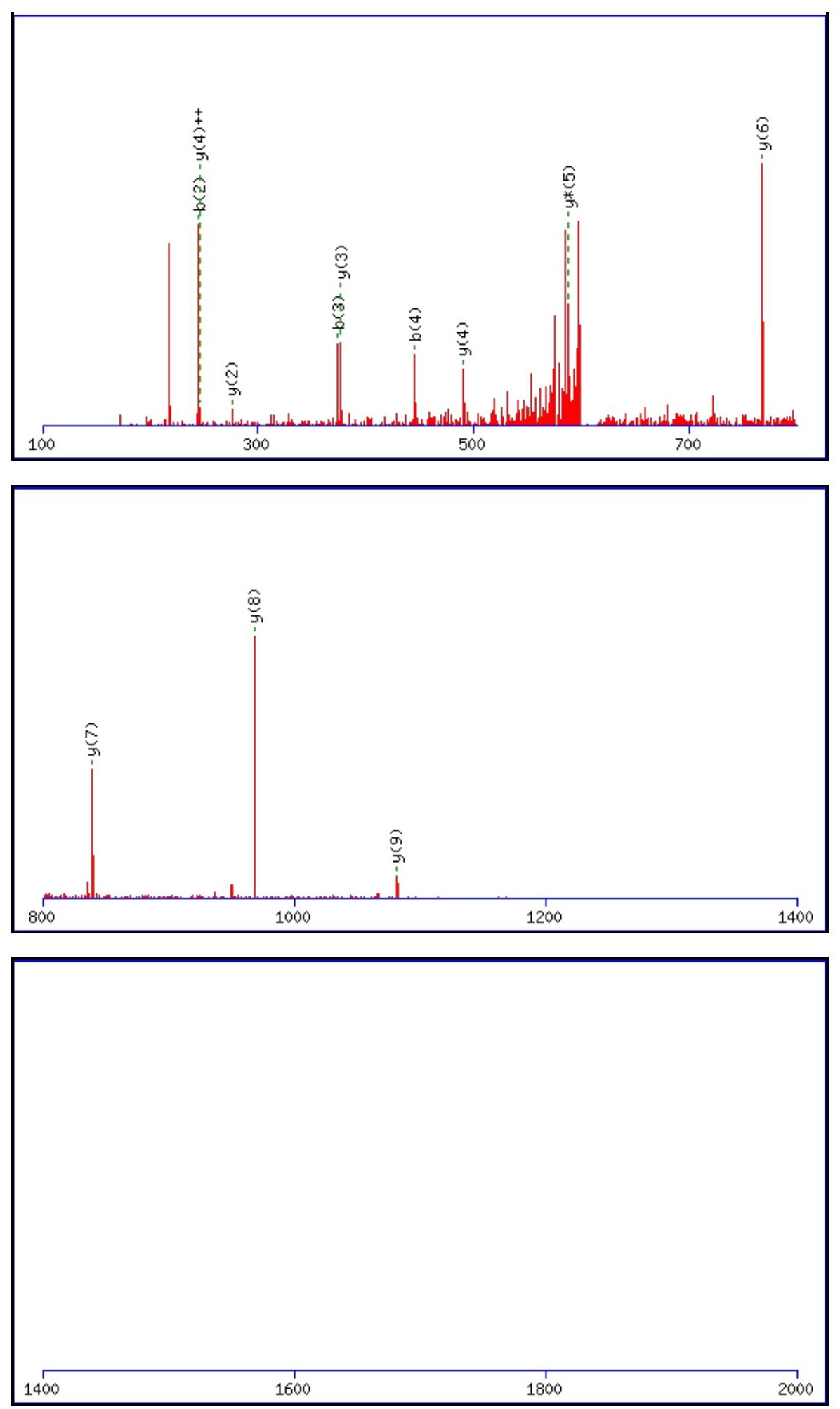

Monoisotopic mass of neutral peptide $\operatorname{Mr}($ calc): 1211.5743

Fixed modifications: Carbamidomethyl (C)

Variable modifications:

N6 : Deamidated_N (N)

Ions Score: 64 Expect: $5.6 \mathrm{e}-005$

Matches (Bold Red): 12/92 fragment ions using 20 most intense peaks

\begin{tabular}{|r|c|c|c|c|c|c|c|c|c|c|c|c|c|c|}
\hline$\#$ & $\mathbf{b}$ & $\mathbf{b}^{++}$ & $\mathbf{b}^{*}$ & $\mathbf{b}^{\boldsymbol{*}^{++}}$ & $\mathbf{b}^{\mathbf{0}}$ & $\mathbf{b}^{\mathbf{0 + +}}$ & Seq. & $\mathbf{y}$ & $\mathbf{y}^{++}$ & $\mathbf{y}^{\mathbf{*}}$ & $\mathbf{y}^{\boldsymbol{*}^{++}}$ & $\mathbf{y}^{\mathbf{0}}$ & $\mathbf{y}^{\mathbf{0 + +}}$ & $\#$ \\
\hline $\mathbf{1}$ & 132.0478 & 66.5275 & & & & & $\mathbf{M}$ & & & & & & & $\mathbf{1 0}$ \\
\hline $\mathbf{2}$ & $\mathbf{2 4 5 . 1 3 1 8}$ & 123.0696 & & & & & $\mathbf{L}$ & $\mathbf{1 0 8 1 . 5 4 1 2}$ & 541.2742 & 1064.5146 & 532.7610 & 1063.5306 & 532.2689 & $\mathbf{9}$ \\
\hline $\mathbf{3}$ & $\mathbf{3 7 4 . 1 7 4 4}$ & 187.5908 & & & 356.1639 & 178.5856 & $\mathbf{E}$ & $\mathbf{9 6 8 . 4 5 7 1}$ & 484.7322 & 951.4306 & 476.2189 & 950.4465 & 475.7269 & $\mathbf{8}$ \\
\hline $\mathbf{4}$ & $\mathbf{4 4 5 . 2 1 1 5}$ & 223.1094 & & & 427.2010 & 214.1041 & $\mathbf{A}$ & $\mathbf{8 3 9 . 4 1 4 5}$ & 420.2109 & 822.3880 & 411.6976 & 821.4040 & 411.2056 & $\mathbf{7}$ \\
\hline $\mathbf{5}$ & 608.2749 & 304.6411 & & & 590.2643 & 295.6358 & $\mathbf{Y}$ & $\mathbf{7 6 8 . 3 7 7 4}$ & 384.6923 & 751.3509 & 376.1791 & 750.3668 & 375.6871 & $\mathbf{6}$ \\
\hline
\end{tabular}




\begin{tabular}{|r|r|r|r|r|r|l|l|l|l|l|l|l|l|l|l|l|}
$\mathbf{6}$ & 723.3018 & 362.1545 & 706.2752 & 353.6413 & 705.2912 & 353.1493 & $\mathbf{N}$ & 605.3141 & 303.1607 & $\mathbf{5 8 8 . 2 8 7 5}$ & 294.6474 & 587.3035 & 294.1554 & $\mathbf{5}$ \\
\hline $\mathbf{7}$ & 836.3859 & 418.6966 & 819.3593 & 410.1833 & 818.3753 & 409.6913 & $\mathbf{L}$ & $\mathbf{4 9 0 . 2 8 7 1}$ & $\mathbf{2 4 5 . 6 4 7 2}$ & 473.2606 & 237.1339 & 472.2766 & 236.6419 & $\mathbf{4}$ \\
\hline $\mathbf{8}$ & 937.4335 & 469.2204 & 920.4070 & 460.7071 & 919.4230 & 460.2151 & $\mathbf{T}$ & $\mathbf{3 7 7 . 2 0 3 1}$ & 189.1052 & 360.1765 & 180.5919 & 359.1925 & 180.0999 & $\mathbf{3}$ \\
\hline $\mathbf{9}$ & 1066.4761 & 533.7417 & 1049.4496 & 525.2284 & 1048.4656 & 524.7364 & $\mathbf{E}$ & $\mathbf{2 7 6 . 1 5 5 4}$ & 138.5813 & 259.1288 & 130.0681 & 258.1448 & 129.5761 & $\mathbf{2}$ \\
\hline $\mathbf{1 0}$ & & & & & & & K & 147.1128 & 74.0600 & 130.0863 & 65.5468 & & & $\mathbf{1}$ \\
\hline
\end{tabular}
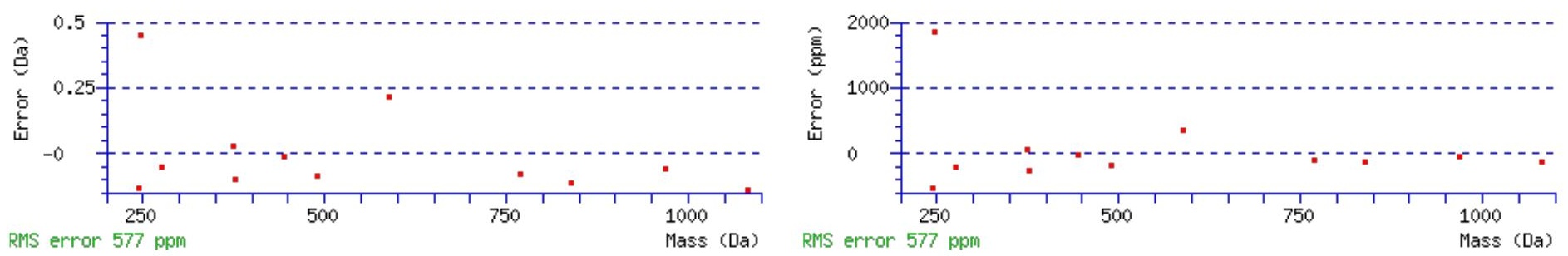

\section{All matches to this query}

\begin{tabular}{|l|l|l|l|}
\hline Score & Mr(calc): & Delta & \multicolumn{1}{|c|}{ Sequence } \\
\hline 63.9 & 1211.5743 & -0.0000 & MLEAYNLTEK \\
\hline 16.8 & 1211.5744 & -0.0001 & EIMFSDITEK \\
\hline 13.1 & 1211.5686 & 0.0057 & NTELETLLAK \\
\hline 10.0 & 1211.5798 & -0.0055 & AKETALAATEK \\
\hline 8.9 & 1209.5754 & 1.9989 & ELQISQREK \\
\hline 8.1 & 1209.5642 & 2.0101 & LEEEIOTLR \\
\hline 6.5 & 1211.5798 & -0.0055 & NKNVTEAVKK \\
\hline 5.3 & 1210.5764 & 0.9979 & ELSPEAICHR \\
\hline 4.9 & 1211.5733 & 0.0010 & QKMTGLQAQK \\
\hline 4.0 & 1209.5642 & 2.0101 & TAKQNPLTEK \\
\hline
\end{tabular}

Spectrum No: 458; Query: 2287; Rank: 1

\section{Peptide View}

MS/MS Fragmentation of RIYYSDFSNQTINSMAEDGSNR

Found in IPI00205325, Tax_Id=10116 Gene_Symbol=Lrp2 Low-density lipoprotein receptor-related protein 2 precursor

Match to Query 2287: 2568.126162 from(857.049330,3+)

Title: 100101RatKid_NS_deglyco_15.3390.3390.3.dta

Data file K:INewmanPaper|Piliangl3SubProteomes\Piliang3SP\mgf5ppm\ERLIC_3SubProteomes5ppm.mgf

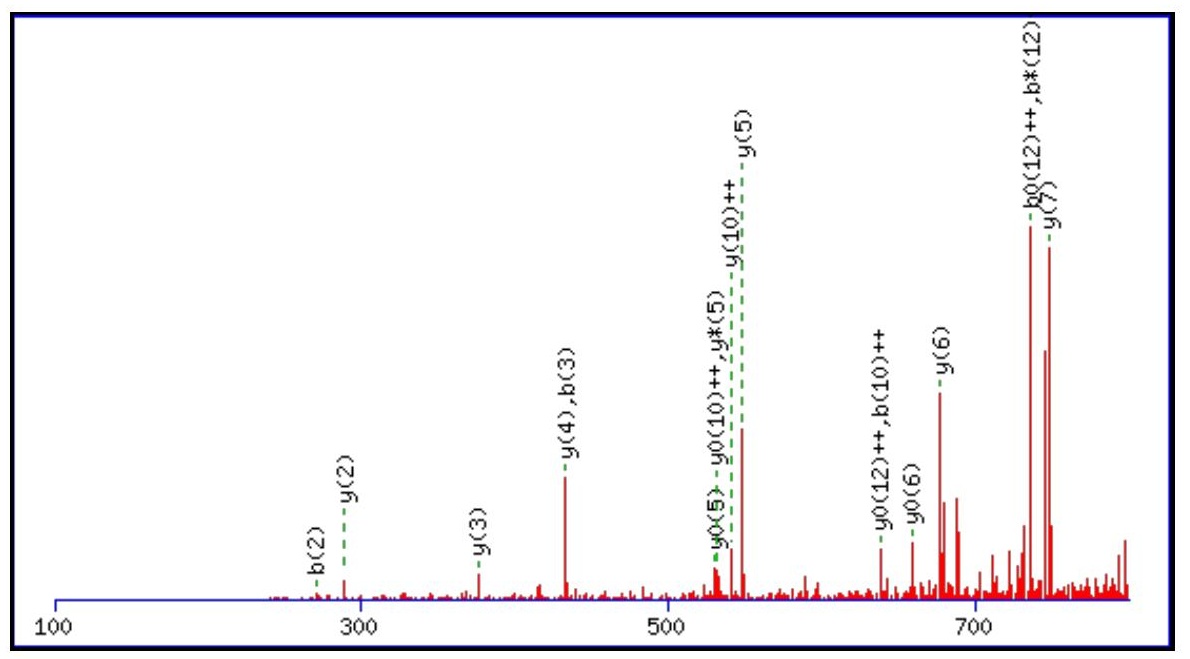



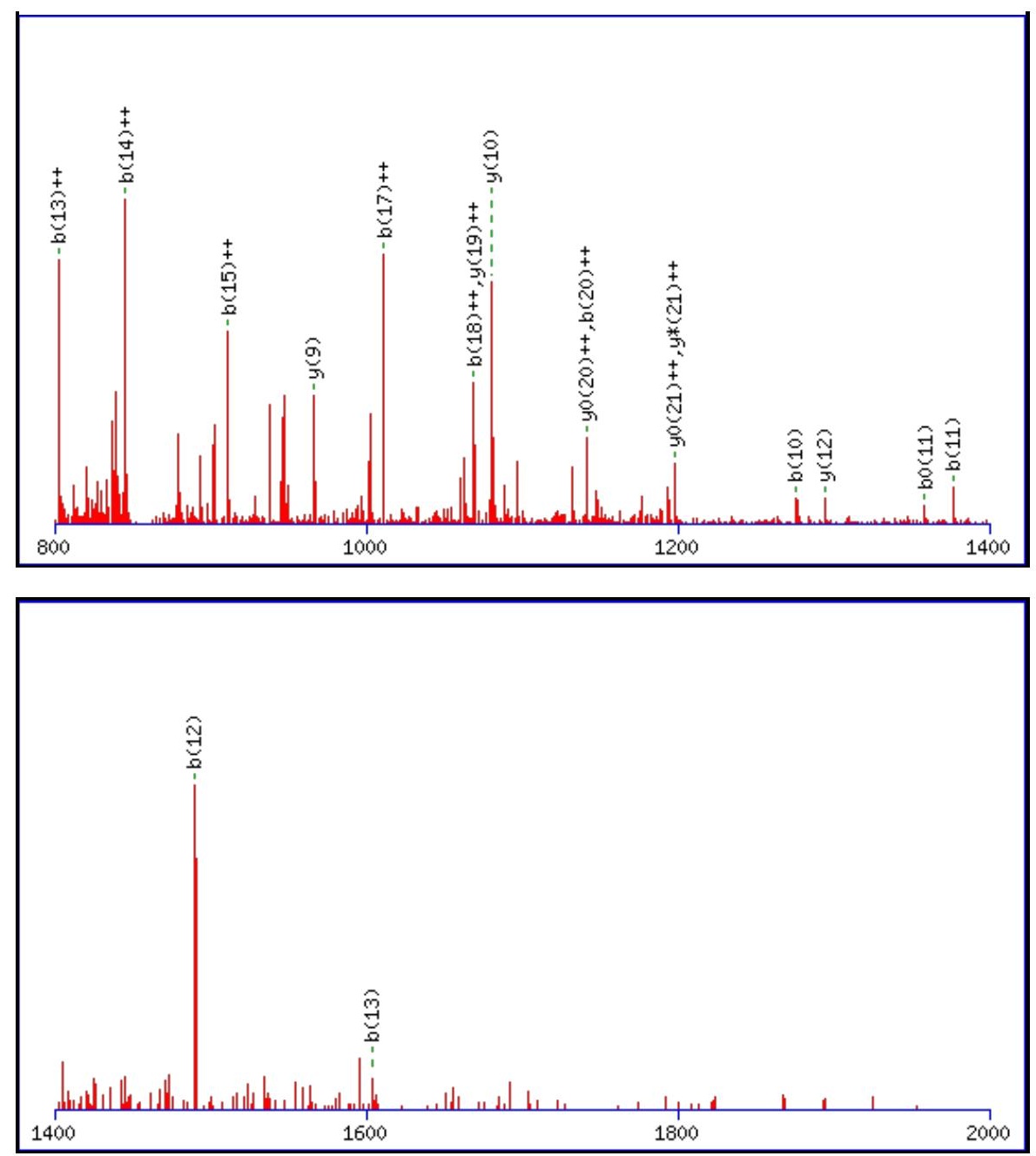

Monoisotopic mass of neutral peptide $\operatorname{Mr}($ calc): 2568.1187

Fixed modifications: Carbamidomethyl (C)

Variable modifications:

N9: Deamidated $\mathrm{N}(\mathrm{N})$

Ions Score: 64 Expect: 0.00015

Matches (Bold Red): 36/240 fragment ions using 38 most intense peaks

\begin{tabular}{|c|c|c|c|c|c|c|c|c|c|c|c|c|c|c|}
\hline \# & b & $\mathbf{b}^{++}$ & $\mathbf{b}^{*}$ & $\mathbf{b}^{*^{++}}$ & $\mathbf{b}^{\mathbf{0}}$ & $\mathbf{b}^{0++}$ & Seq. & $\mathbf{y}$ & $\mathbf{y}^{++}$ & $\mathbf{y}^{*}$ & $\mathrm{y}^{*^{++}}$ & $\mathbf{y}^{\mathbf{0}}$ & $\mathbf{y}^{\mathbf{0 + +}}$ & \# \\
\hline 1 & 157.1084 & 79.0578 & 140.0818 & 70.5446 & & & $\mathbf{R}$ & & & & & & & 22 \\
\hline 2 & 270.1925 & 135.5999 & 253.1659 & 127.0866 & & & I & 2413.0249 & 1207.0161 & 2395.9983 & 1198.5028 & 2395.0143 & 1198.0108 & 21 \\
\hline 3 & 433.2558 & 217.1315 & 416.2292 & 208.6183 & & & $\mathbf{Y}$ & 2299.9408 & 1150.4740 & 2282.9142 & 1141.9608 & 2281.9302 & 1141.4687 & 20 \\
\hline 4 & 596.3191 & 298.6632 & 579.2926 & 290.1499 & & & $\mathbf{Y}$ & 2136.8775 & 1068.9424 & 2119.8509 & 1060.4291 & 2118.8669 & 1059.9371 & 19 \\
\hline 5 & 683.3511 & 342.1792 & 666.3246 & 333.6659 & 665.3406 & 333.1739 & $\mathbf{S}$ & 1973.8141 & 987.4107 & 1956.7876 & 978.8974 & 1955.8036 & 978.4054 & 18 \\
\hline 6 & 798.3781 & 399.6927 & 781.3515 & 391.1794 & 780.3675 & 390.6874 & D & 1886.7821 & 943.8947 & 1869.7556 & 935.3814 & 1868.7715 & 934.8894 & 17 \\
\hline 7 & 945.4465 & 473.2269 & 928.4199 & 464.7136 & 927.4359 & 464.2216 & $\mathbf{F}$ & 1771.7552 & 886.3812 & 1754.7286 & 877.8679 & 1753.7446 & 877.3759 & 16 \\
\hline 8 & 1032.4785 & 516.7429 & 1015.4520 & 508.2296 & 1014.4680 & 507.7376 & $\mathrm{~S}$ & 1624.6867 & 812.8470 & 1607.6602 & 804.3337 & 1606.6762 & 803.8417 & 15 \\
\hline 9 & 1147.5055 & 574.2564 & 1130.4789 & 565.7431 & 1129.4949 & 565.2511 & $\mathbf{N}$ & 1537.6547 & 769.3310 & 1520.6282 & 760.8177 & 1519.6442 & 760.3257 & 14 \\
\hline 10 & 1275.5640 & 638.2857 & 1258.5375 & 629.7724 & 1257.5535 & 629.2804 & $\mathbf{Q}$ & 1422.6278 & 711.8175 & 1405.6012 & 703.3043 & 1404.6172 & 702.8122 & 13 \\
\hline 11 & 1376.6117 & 688.8095 & 1359.5852 & 680.2962 & 1358.6012 & 679.8042 & $\mathbf{T}$ & 1294.5692 & 647.7882 & 1277.5427 & 639.2750 & 1276.5586 & 638.7830 & 12 \\
\hline 12 & 1489.6958 & 745.3515 & 1472.6692 & 736.8383 & 1471.6852 & 736.3462 & I & 1193.5215 & 597.2644 & 1176.4950 & 588.7511 & 1175.5110 & 588.2591 & 11 \\
\hline 13 & 1603.7387 & 802.3730 & 1586.7122 & 793.8597 & 1585.7281 & 793.3677 & $\mathbf{N}$ & 1080.4375 & 540.7224 & 1063.4109 & 532.2091 & 1062.4269 & 531.7171 & 10 \\
\hline 14 & 1690.7707 & 845.8890 & 1673.7442 & 837.3757 & 1672.7602 & 836.8837 & $\mathbf{S}$ & 966.3945 & 483.7009 & 949.3680 & 475.1876 & 948.3840 & 474.6956 & 9 \\
\hline 15 & 1821.8112 & 911.4092 & 1804.7847 & 902.8960 & 1803.8007 & 902.4040 & $\mathbf{M}$ & 879.3625 & 440.1849 & 862.3360 & 431.6716 & 861.3519 & 431.1796 & 8 \\
\hline 16 & 1892.8483 & 946.9278 & 1875.8218 & 938.4145 & 1874.8378 & 937.9225 & A & 748.3220 & 374.6646 & 731.2955 & 366.1514 & 730.3115 & 365.6594 & 7 \\
\hline 17 & 2021.8909 & 1011.4491 & 2004.8644 & 1002.9358 & 2003.8804 & 1002.4438 & $\mathbf{E}$ & 677.2849 & 339.1461 & 660.2584 & 330.6328 & 659.2743 & 330.1408 & 6 \\
\hline 18 & 2136.9179 & 1068.9626 & 2119.8913 & 1060.4493 & 2118.9073 & 1059.9573 & D & 548.2423 & 274.6248 & 531.2158 & 266.1115 & 530.2317 & 265.6195 & 5 \\
\hline
\end{tabular}




\begin{tabular}{|r|r|r|r|r|r|r|r|r|r|r|r|r|r|r|r|}
$\mathbf{1 9}$ & 2193.9393 & 1097.4733 & 2176.9128 & 1088.9600 & 2175.9288 & 1088.4680 & G & $\mathbf{4 3 3 . 2 1 5 4}$ & 217.1113 & 416.1888 & 208.5980 & 415.2048 & 208.1060 & $\mathbf{4}$ \\
\hline $\mathbf{2 0}$ & 2280.9714 & $\mathbf{1 1 4 0 . 9 8 9 3}$ & 2263.9448 & 1132.4760 & 2262.9608 & 1131.9840 & S & $\mathbf{3 7 6 . 1 9 3 9}$ & 188.6006 & 359.1674 & 180.0873 & 358.1833 & 179.5953 & $\mathbf{3}$ \\
\hline $\mathbf{2 1}$ & 2395.0143 & $\mathbf{1 1 9 8 . 0 1 0 8}$ & 2377.9877 & 1189.4975 & 2377.0037 & 1189.0055 & $\mathbf{N}$ & $\mathbf{2 8 9 . 1 6 1 9}$ & 145.0846 & 272.1353 & 136.5713 & & & $\mathbf{2}$ \\
\hline $\mathbf{2 2}$ & & & & & & & R & 175.1190 & 88.0631 & 158.0924 & 79.5498 & & & $\mathbf{1}$ \\
\hline
\end{tabular}
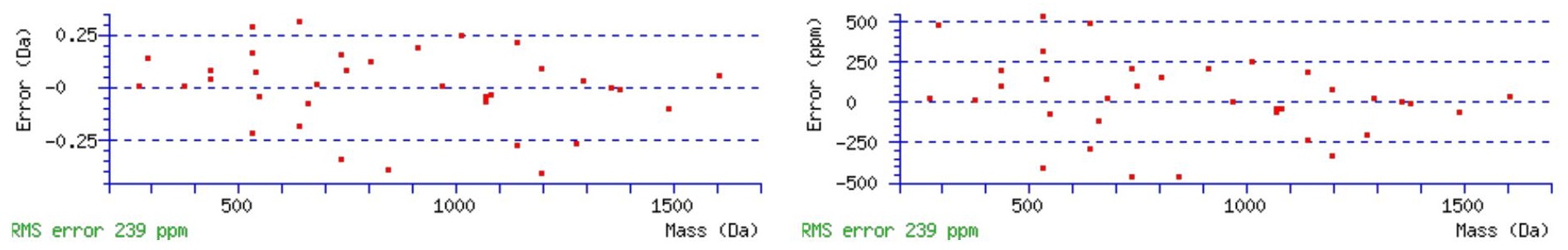

\section{All matches to this query}

\begin{tabular}{|l|l|l|l|}
\hline Score & Mr(calc): & Delta & \multicolumn{1}{|c|}{ Sequence } \\
\hline 63.8 & 2568.1187 & 0.0075 & RIYYSDFSNQTINSMAEDGSNR \\
\hline 44.3 & 2567.1346 & 0.9915 & $\underline{\text { RIYYSDFSNQTINSMAEDGSNR }}$ \\
\hline 39.3 & 2568.1187 & 0.0075 & $\underline{\text { RIYYSDFSNQTINSMAEDGSNR }}$ \\
\hline 16.2 & 2568.1187 & 0.0075 & $\underline{\text { RIYYSDFSNQTINSMAEDGSNR }}$ \\
\hline 6.9 & 2568.1455 & -0.0193 & $\underline{\text { IYNEFISVQATKEVNLDSCTR }}$ \\
\hline 3.6 & 2568.1328 & -0.0067 & $\underline{\text { FLDPGFSSHQAVARTAGSVLLR }}$ \\
\hline 1.1 & 2568.1387 & -0.0125 & KTSVISSPKPPATAARGETSTSR \\
\hline 1.1 & 2568.1387 & -0.0125 & $\underline{\text { KTSVISSPKPPATAARGETSTSR }}$ \\
\hline 0.3 & 2568.1328 & -0.0067 & $\underline{\text { FLDPGFSSHQAVARTAGSVLLR }}$ \\
\hline
\end{tabular}

Spectrum No: 459; Query: 504; Rank: 1

\section{Peptide View}

MS/MS Fragmentation of LNFTGPGEPDSLR

Found in IPI00360541, Tax_Id=10116 Gene_Symbol=Itgb2 integrin beta 2

Match to Query 504: 1402.677308 from(702.345930,2+)

Title: 100101RatKid_NS_deglyco_25.2526.2526.2.dta

Data file K:INewmanPaper|Piliangl3SubProteomes\Piliang3SP\mgf5ppm\ERLIC_3SubProteomes5ppm.mgf

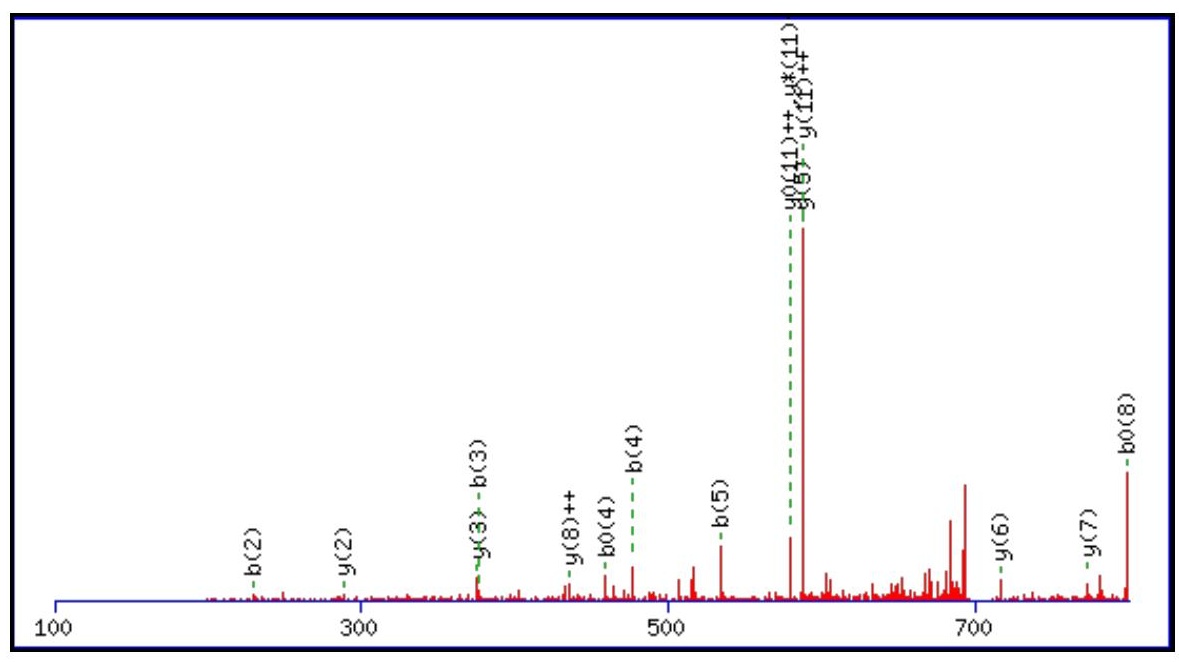



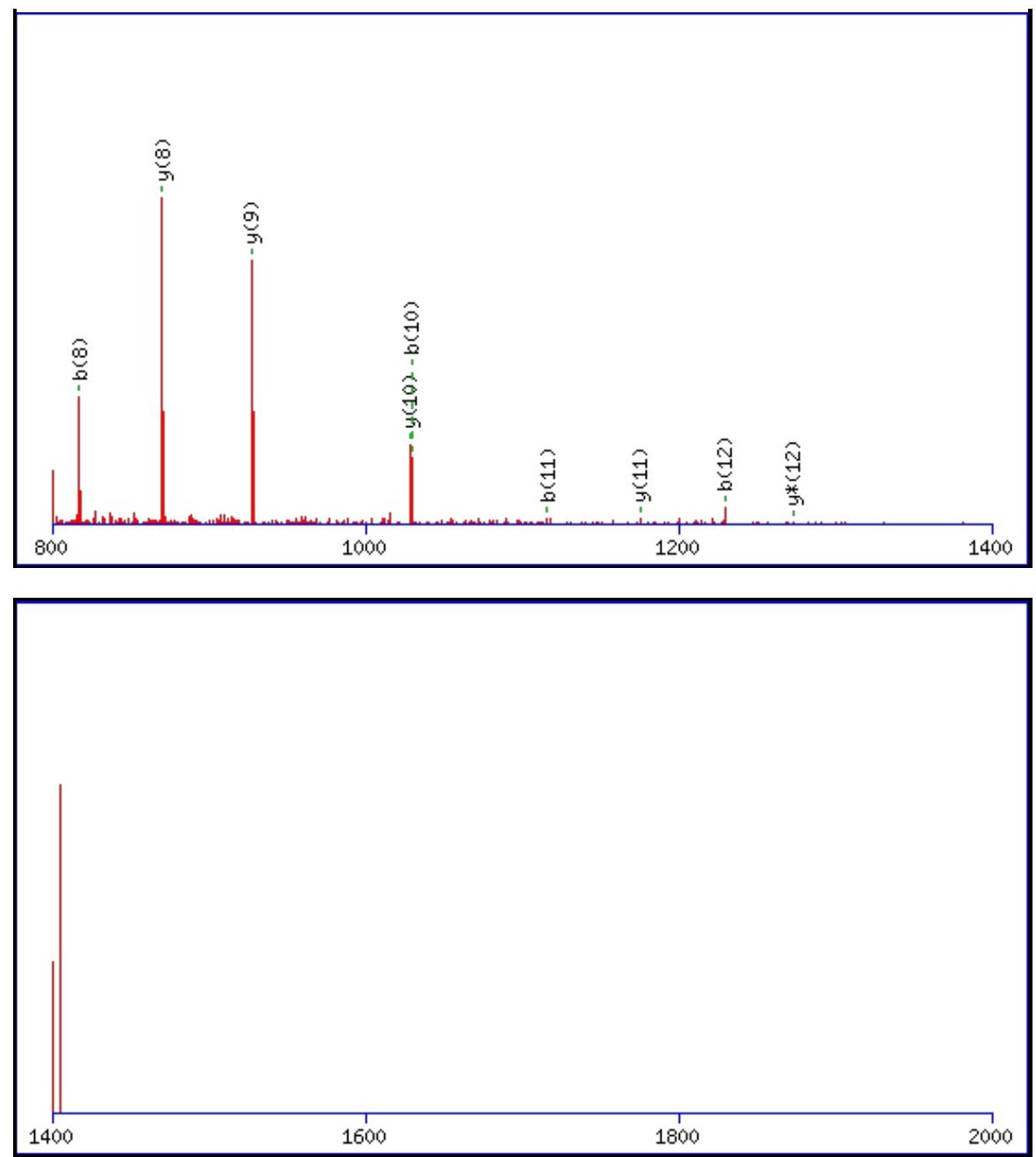

Monoisotopic mass of neutral peptide $\operatorname{Mr}($ calc): 1402.6729

Fixed modifications: Carbamidomethyl (C)

Variable modifications:

N2 : Deamidated $\mathrm{N}(\mathrm{N})$

Ions Score: 64 Expect: $6.1 \mathrm{e}-005$

Matches (Bold Red): 24/132 fragment ions using 47 most intense peaks

\begin{tabular}{|c|c|c|c|c|c|c|c|c|c|c|c|c|c|c|}
\hline \# & b & $\mathbf{b}^{++}$ & $\mathbf{b}^{*}$ & $\mathbf{b}^{*^{++}}$ & $\mathbf{b}^{0}$ & $\mathbf{b}^{0++}$ & Seq. & $\mathbf{y}$ & $\mathbf{y}^{++}$ & $\mathbf{y}^{*}$ & $\mathrm{y}^{*^{++}}$ & $\mathbf{y}^{\mathbf{0}}$ & $\mathbf{y}^{0++}$ & \# \\
\hline 1 & 114.0913 & 57.5493 & & & & & $\mathbf{L}$ & & & & & & & 13 \\
\hline 2 & 229.1183 & 115.0628 & 212.0917 & 106.5495 & & & $\mathbf{N}$ & 1290.5961 & 645.8017 & 1273.5695 & 637.2884 & 1272.5855 & 636.7964 & 12 \\
\hline 3 & 376.1867 & 188.5970 & 359.1601 & 180.0837 & & & $\mathbf{F}$ & 1175.5691 & 588.2882 & 1158.5426 & 579.7749 & 1157.5586 & 579.2829 & 11 \\
\hline 4 & 477.2344 & 239.1208 & 460.2078 & 230.6075 & 459.2238 & 230.1155 & $\mathbf{T}$ & 1028.5007 & 514.7540 & 1011.4742 & 506.2407 & 1010.4902 & 505.7487 & 10 \\
\hline 5 & 534.2558 & 267.6316 & 517.2293 & 259.1183 & 516.2453 & 258.6263 & G & 927.4530 & 464.2302 & 910.4265 & 455.7169 & 909.4425 & 455.2249 & 9 \\
\hline 6 & 631.3086 & 316.1579 & 614.2820 & 307.6447 & 613.2980 & 307.1527 & $\mathbf{P}$ & 870.4316 & 435.7194 & 853.4050 & 427.2061 & 852.4210 & 426.7141 & 8 \\
\hline 7 & 688.3301 & 344.6687 & 671.3035 & 336.1554 & 670.3195 & 335.6634 & $\mathbf{G}$ & 773.3788 & 387.1930 & 756.3523 & 378.6798 & 755.3682 & 378.1878 & 7 \\
\hline 8 & 817.3727 & 409.1900 & 800.3461 & 400.6767 & 799.3621 & 400.1847 & $\mathbf{E}$ & 716.3573 & 358.6823 & 699.3308 & 350.1690 & 698.3468 & 349.6770 & 6 \\
\hline 9 & 914.4254 & 457.7163 & 897.3989 & 449.2031 & 896.4149 & 448.7111 & $\mathbf{P}$ & 587.3148 & 294.1610 & 570.2882 & 285.6477 & 569.3042 & 285.1557 & 5 \\
\hline 10 & 1029.4524 & 515.2298 & 1012.4258 & 506.7165 & 1011.4418 & 506.2245 & D & 490.2620 & 245.6346 & 473.2354 & 237.1214 & 472.2514 & 236.6293 & 4 \\
\hline 11 & 1116.4844 & 558.7458 & 1099.4578 & 550.2326 & 1098.4738 & 549.7406 & S & 375.2350 & 188.1212 & 358.2085 & 179.6079 & 357.2245 & 179.1159 & 3 \\
\hline 12 & 1229.5685 & 615.2879 & 1212.5419 & 606.7746 & 1211.5579 & 606.2826 & $\mathbf{L}$ & 288.2030 & 144.6051 & 271.1765 & 136.0919 & & & 2 \\
\hline 13 & & & & & & & $\mathbf{R}$ & 175.1190 & 88.0631 & 158.0924 & 79.5498 & & & 1 \\
\hline
\end{tabular}



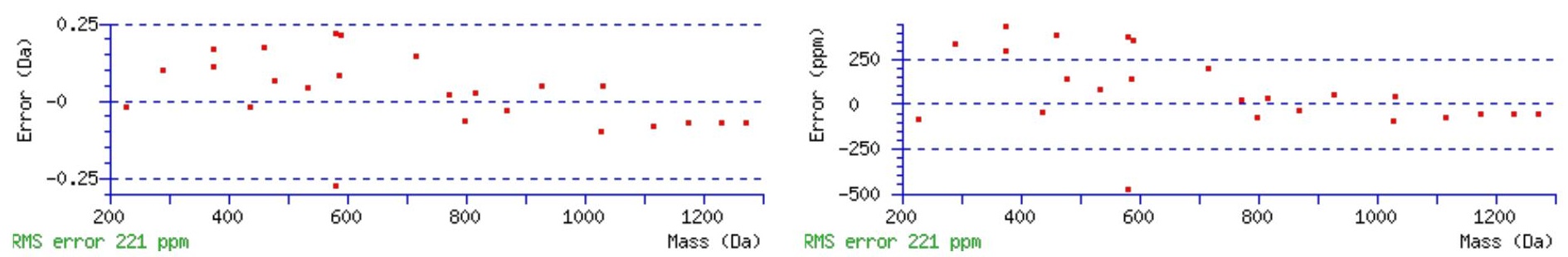

\section{All matches to this query}

\begin{tabular}{|l|l|l|l|}
\hline Score & Mr(calc): & Delta & \multicolumn{1}{|c|}{ Sequence } \\
\hline 63.8 & 1402.6729 & 0.0045 & LNFTGPGEPDSLR \\
\hline 13.5 & 1402.6745 & 0.0028 & LITYLDQTTQK \\
\hline 13.5 & 1402.6745 & 0.0028 & LITYLDQTTQK \\
\hline 8.4 & 1402.6731 & 0.0042 & MTMRMKTTSLR \\
\hline 6.8 & 1402.6841 & -0.0067 & LNSSHEAFAASLR \\
\hline 6.5 & 1402.6874 & -0.0101 & NPVMELNEKRR \\
\hline 6.3 & 1402.6688 & 0.0085 & SSNLNVPSSDDIR \\
\hline 5.4 & 1401.6615 & 1.0158 & VYNLVKETMPK \\
\hline 5.0 & 1402.6792 & -0.0019 & VEARKTYMGLR \\
\hline 4.4 & 1402.6646 & 0.0127 & FETVKLASWSR \\
\hline
\end{tabular}

Spectrum No: 460; Query: 374; Rank: 1

\section{Peptide View}

\section{MS/MS Fragmentation of DVNYTQIVVDR}

Found in IPI00366011, Tax_Id=10116 Gene_Symbol=Sema4d_predicted similar to semaphorin 4D

Match to Query 374: 1321.657108 from(661.835830,2+)

Title: 100101RatKid_NS_deglyco_19.2803.2803.2.dta

Data file K:INewmanPaper|Piliangl3SubProteomes\Piliang3SP\mgf5ppm\ERLIC_3SubProteomes5ppm.mgf

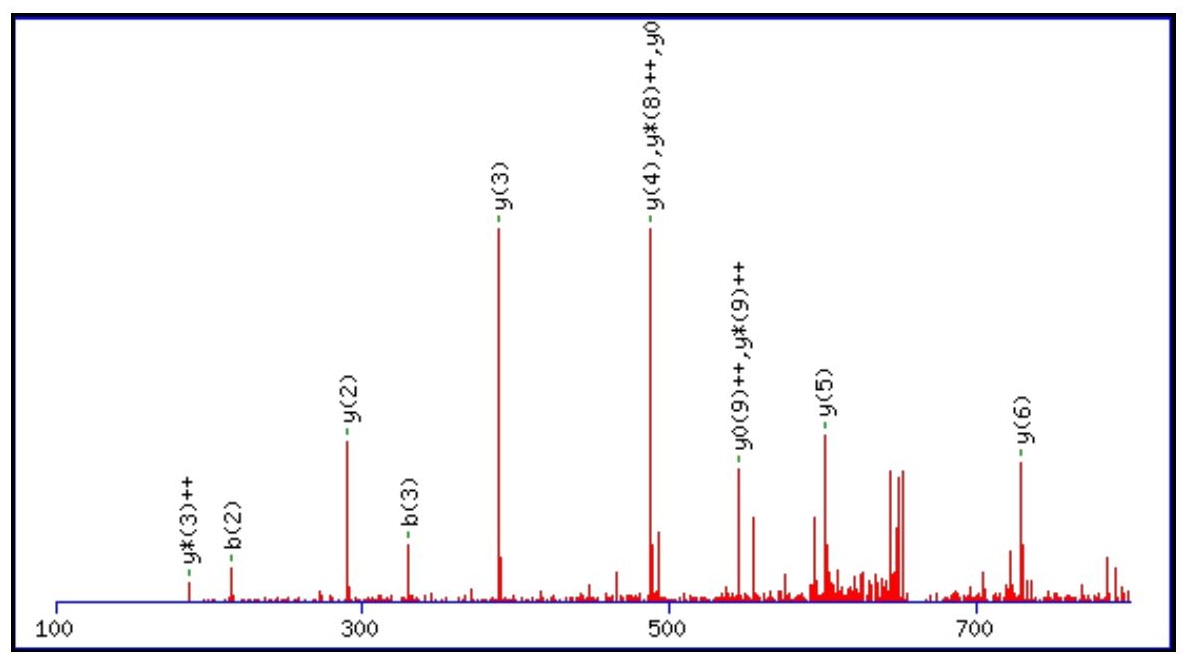



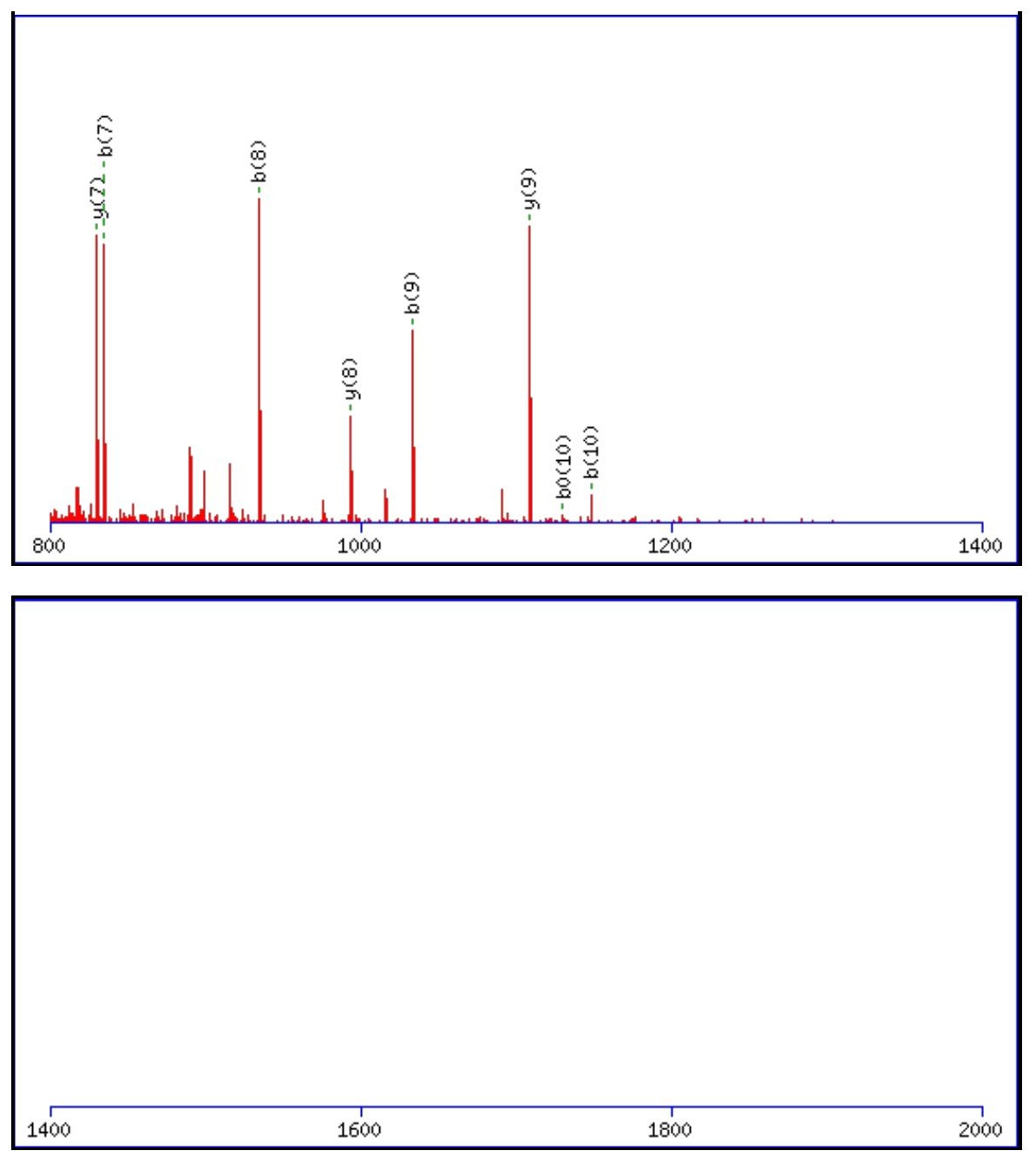

Monoisotopic mass of neutral peptide $\operatorname{Mr}($ calc): 1321.6514

Fixed modifications: Carbamidomethyl (C)

Variable modifications:

N3 : Deamidated $\mathrm{N}(\mathrm{N})$

Ions Score: 64 Expect: $5.3 \mathrm{e}-005$

Matches (Bold Red): 20/114 fragment ions using 31 most intense peaks

\begin{tabular}{|c|c|c|c|c|c|c|c|c|c|c|c|c|c|c|}
\hline$\#$ & b & $\mathbf{b}^{++}$ & $\mathbf{b}^{*}$ & $\mathbf{b}^{*^{++}}$ & $\mathbf{b}^{0}$ & $\mathbf{b}^{0++}$ & Seq. & $\mathbf{y}$ & $\mathbf{y}^{++}$ & $\mathbf{y}^{*}$ & $\mathrm{y}^{*^{++}}$ & $\mathbf{y}^{0}$ & $\mathbf{y}^{0++}$ & \# \\
\hline 1 & 116.0342 & 58.5207 & & & 98.0237 & 49.5155 & D & & & & & & & 11 \\
\hline 2 & 215.1026 & 108.0550 & & & 197.0921 & 99.0497 & V & 1207.6317 & 604.3195 & 1190.6052 & 595.8062 & 1189.6212 & 595.3142 & 10 \\
\hline 3 & 330.1296 & 165.5684 & 313.1030 & 157.0551 & 312.1190 & 156.5631 & $\mathbf{N}$ & 1108.5633 & 554.7853 & 1091.5368 & 546.2720 & 1090.5527 & 545.7800 & 9 \\
\hline 4 & 493.1929 & 247.1001 & 476.1663 & 238.5868 & 475.1823 & 238.0948 & $\mathbf{Y}$ & 993.5364 & 497.2718 & 976.5098 & 488.7586 & 975.5258 & 488.2665 & 8 \\
\hline 5 & 594.2406 & 297.6239 & 577.2140 & 289.1107 & 576.2300 & 288.6186 & $\mathbf{T}$ & 830.4730 & 415.7402 & 813.4465 & 407.2269 & 812.4625 & 406.7349 & 7 \\
\hline 6 & 722.2992 & 361.6532 & 705.2726 & 353.1399 & 704.2886 & 352.6479 & $\mathbf{Q}$ & 729.4254 & 365.2163 & 712.3988 & 356.7030 & 711.4148 & 356.2110 & 6 \\
\hline 7 & 835.3832 & 418.1952 & 818.3567 & 409.6820 & 817.3727 & 409.1900 & I & 601.3668 & 301.1870 & 584.3402 & 292.6738 & 583.3562 & 292.1817 & 5 \\
\hline 8 & 934.4516 & 467.7295 & 917.4251 & 459.2162 & 916.4411 & 458.7242 & $\mathbf{V}$ & 488.2827 & 244.6450 & 471.2562 & 236.1317 & 470.2722 & 235.6397 & 4 \\
\hline 9 & 1033.5200 & 517.2637 & 1016.4935 & 508.7504 & 1015.5095 & 508.2584 & $\mathbf{V}$ & 389.2143 & 195.1108 & 372.1878 & 186.5975 & 371.2037 & 186.1055 & 3 \\
\hline 10 & 1148.5470 & 574.7771 & 1131.5204 & 566.2639 & 1130.5364 & 565.7719 & D & 290.1459 & 145.5766 & 273.1193 & 137.0633 & 272.1353 & 136.5713 & 2 \\
\hline 11 & & & & & & & $\mathbf{R}$ & 175.1190 & 88.0631 & 158.0924 & 79.5498 & & & 1 \\
\hline
\end{tabular}
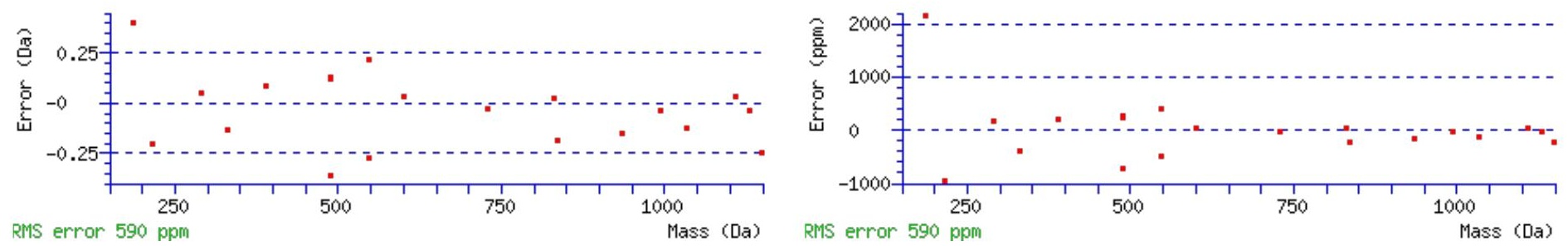


\section{All matches to this query}

\begin{tabular}{|l|l|l|l|}
\hline Score & Mr(calc): & Delta & \multicolumn{1}{|c|}{ Sequence } \\
\hline 63.8 & 1321.6514 & 0.0057 & DVNYTQIVVDR \\
\hline 14.0 & 1320.6551 & 1.0020 & TPRGTKTPINR \\
\hline 4.7 & 1319.6411 & 2.0160 & WTTPNHHIWK \\
\hline 4.5 & 1320.6551 & 1.0020 & TPRGTKTPINR \\
\hline 2.1 & 1319.6374 & 2.0197 & VLDTPGPVSDLK \\
\hline 0.0 & 1321.6473 & 0.0098 & TLSSKNNENTSK \\
\hline
\end{tabular}

Spectrum No: 461; Query: 2268; Rank: 1

\section{Peptide View}

MS/MS Fragmentation of TGDFQLHTNVNNGTEFGGSIYQK

Found in IPI00198327, Tax_Id=10116 Gene_Symbol=Vdac2 Voltage-dependent anion-selective channel protein 2

Match to Query 2268: 2527.157772 from(843.393200,3+)

Title: 091008RatKidney_NH4Format02_20.2989.2989.3.dta

Data file K:\NewmanPaper|Piliang|3SubProteomes\Piliang3SP\mgf5ppm\ERLIC_3SubProteomes5ppm.mgf
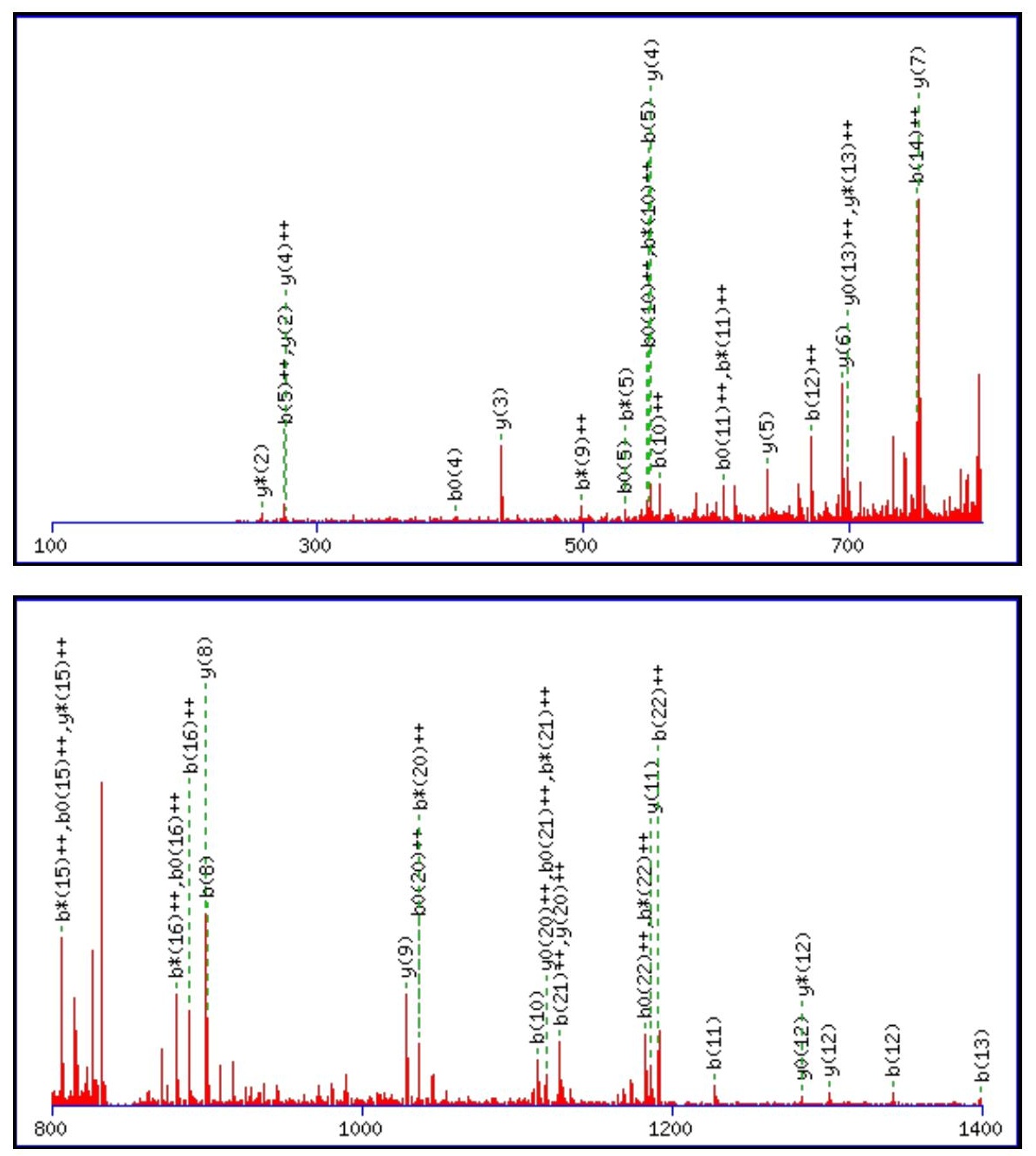


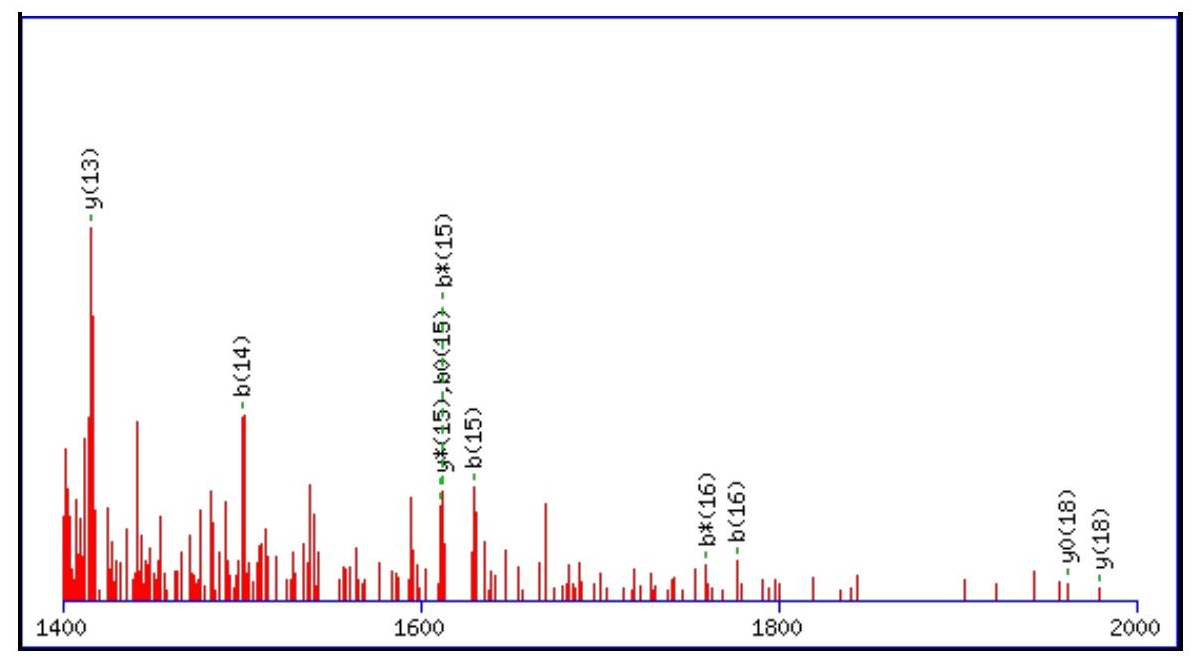

Monoisotopic mass of neutral peptide $\operatorname{Mr}($ calc): 2527.1616

Fixed modifications: Carbamidomethyl (C)

Variable modifications:

N12 : Deamidated $\mathrm{N}(\mathrm{N})$

Ions Score: 64 Expect: 0.00013

Matches (Bold Red): 60/248 fragment ions using 90 most intense peaks

\begin{tabular}{|c|c|c|c|c|c|c|c|c|c|c|c|c|c|c|}
\hline \# & b & $\mathbf{b}^{++}$ & $\mathbf{b}^{*}$ & $\mathbf{b}^{*^{++}}$ & $\mathbf{b}^{0}$ & $\mathbf{b}^{0++}$ & Seq. & $\mathbf{y}$ & $y^{++}$ & $\mathbf{y}^{*}$ & $\mathrm{y}^{\mathrm{*}^{++}}$ & $\mathbf{y}^{0}$ & $y^{0++}$ & \# \\
\hline 1 & 102.0550 & 51.5311 & & & 84.0444 & 42.5258 & $\mathbf{T}$ & & & & & & & 23 \\
\hline 2 & 159.0764 & 80.0418 & & & 141.0659 & 71.0366 & G & 2427.1211 & 1214.0642 & 2410.0946 & 5509 & 2409.1106 & 1205.0589 & 22 \\
\hline 3 & 274.1034 & 137.5553 & & & 256.0928 & 128.5500 & D & 2370.0997 & 1185.5535 & 2353.0731 & 1177.0402 & 2352.0891 & 1176.5482 & 21 \\
\hline 4 & 421.1718 & 211.0895 & & & 403.1612 & 202.0842 & $\mathbf{F}$ & 2255.0727 & 1128.0400 & 2238.0462 & 1119.5267 & 2237.0622 & 1119.0347 & 20 \\
\hline 5 & 549.2304 & 275.1188 & 532.2038 & 266.6055 & 531.2198 & 266.1135 & $\mathbf{Q}$ & 2108.0043 & 1054.5058 & 2090.9778 & 1045.9925 & 2089.9938 & 1045.5005 & 19 \\
\hline 6 & 662.3144 & 331.6608 & 645.2879 & 323.1476 & 644.3039 & 322.6556 & $\mathbf{L}$ & 1979.9457 & 990.4765 & 1962.9192 & 981.9632 & 1961.9352 & 981.4712 & 18 \\
\hline 7 & 799.3733 & 400.1903 & 782.3468 & 391.6770 & 781.3628 & 391.1850 & $\mathbf{H}$ & 1866.8617 & 933.9345 & 1849.8351 & 925.4212 & 1848.8511 & 924.9292 & 17 \\
\hline 8 & 900.4210 & 450.7141 & 883.3945 & 442.2009 & 882.4104 & 441.7089 & $\mathbf{T}$ & 1729.8028 & 865.4050 & 1712.7762 & 856.8917 & 1711.7922 & 856.3997 & 16 \\
\hline 9 & 1014.4639 & 507.7356 & 997.4374 & 499.2223 & 996.4534 & 498.7303 & $\mathbf{N}$ & 1628.7551 & 814.8812 & 1611.7285 & 806.3679 & 1610.7445 & 805.8759 & 15 \\
\hline 10 & 1113.5324 & 557.2698 & 1096.5058 & 548.7565 & 1095.5218 & 548.2645 & $\mathbf{V}$ & 1514.7122 & 757.8597 & 1497.6856 & 749.3464 & 1496.7016 & 748.8544 & 14 \\
\hline 11 & 1227.5753 & 614.2913 & 1210.5487 & 605.7780 & 209.5647 & 605.2860 & $\mathbf{N}$ & 1415.6437 & 708.3255 & 1398.6172 & 699.8122 & 1397.6332 & 699.3202 & 13 \\
\hline 12 & 1342.6022 & 671.8047 & 1325.5757 & 663.2915 & 1324.5916 & 662.7995 & $\mathbf{N}$ & 1301.6008 & 651.3040 & 1284.5743 & 642.7908 & 1283.5903 & 642.2988 & 12 \\
\hline 13 & 1399.6237 & 700.3155 & 1382.5971 & 691.8022 & 1381.6131 & 691.3102 & G & 1186.5739 & 593.7906 & 1169.5473 & 585.2773 & 1168.5633 & 584.7853 & 11 \\
\hline 14 & 1500.6714 & 750.8393 & 1483.6448 & 742.3260 & 1482.6608 & 741.8340 & $\mathbf{T}$ & 1129.5524 & 565.2798 & 1112.5259 & 556.7666 & 1111.5419 & 556.2746 & 10 \\
\hline 15 & 1629.7139 & 815.3606 & 1612.6874 & 806.8473 & 1611.7034 & 806.3553 & $\mathbf{E}$ & 1028.5047 & 514.7560 & 1011.4782 & 506.2427 & 1010.4942 & 505.7507 & 9 \\
\hline 16 & 1776.7824 & 888.8948 & 1759.7558 & 880.3815 & 1758.7718 & 879.8895 & $\mathbf{F}$ & 899.4621 & 450.2347 & 882.4356 & 441.7214 & 881.4516 & 441.2294 & 8 \\
\hline 17 & 1833.8038 & 917.4056 & 1816.7773 & 908.8923 & 1815.7933 & 908.4003 & G & 752.3937 & 376.7005 & 735.3672 & 368.1872 & 734.3832 & 367.6952 & 7 \\
\hline 18 & 1890.8253 & 945.9163 & 1873.7987 & 937.4030 & 1872.8147 & 936.9110 & G & 695.3723 & 348.1898 & 678.3457 & 339.6765 & 677.3617 & 339.1845 & 6 \\
\hline 19 & 1977.8573 & 989.4323 & 1960.8308 & 980.9190 & 1959.8468 & 980.4270 & $\mathrm{~S}$ & 638.3508 & 319.6790 & 621.3243 & 311.1658 & 620.3402 & 310.6738 & 5 \\
\hline 20 & 2090.9414 & 1045.9743 & 2073.9148 & 1037.4611 & 2072.9308 & 1036.9690 & I & 551.3188 & 276.1630 & 534.2922 & 267.6498 & & & 4 \\
\hline 21 & 2254.0047 & 1127.5060 & 2236.9782 & 1118.9927 & 2235.9941 & 1118.5007 & $\mathbf{Y}$ & 438.2347 & 219.6210 & 421.2082 & 211.1077 & & & 3 \\
\hline 22 & 2382.0633 & 1191.5353 & 2365.0367 & 1183.0220 & 2364.0527 & 1182.5300 & $\mathbf{Q}$ & 275.1714 & 138.0893 & 258.1448 & 129.5761 & & & 2 \\
\hline 23 & & & & & & & $\mathbf{K}$ & 147.1128 & 74.0600 & 130.0863 & 65.5468 & & & 1 \\
\hline
\end{tabular}
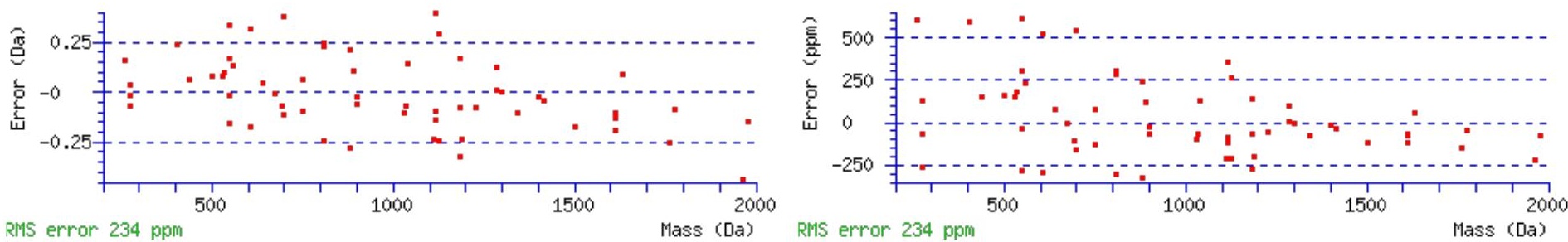

\section{All matches to this query}




\begin{tabular}{|l|c|c|l|}
\hline Score & Mr(calc): & Delta & \multicolumn{1}{c|}{ Sequence } \\
\hline 63.7 & 2527.1616 & -0.0038 & TGDFQLHTNVNNGTEFGGSIYQK \\
\hline 60.4 & 2527.1616 & -0.0038 & TGDFQLHTNVNNGTEFGGSIYQK \\
\hline 54.2 & 2527.1616 & -0.0038 & TGDFQLHTNVNNGTEFGGSIYQK \\
\hline 46.1 & 2526.1776 & 0.9802 & TGDFQLHTNVNNGTEFGGSIYQK \\
\hline 3.5 & 2527.1348 & 0.0230 & NVSVANVCLLSVFQAITISPR \\
\hline 3.1 & 2526.1590 & 0.9987 & SECVPAAAVTHVVEVLSLTAASR \\
\hline 3.1 & 2526.1590 & 0.9987 & SECVPAAAVTHVVEVLSLTAASR \\
\hline 3.1 & 2526.1590 & 0.9987 & SECVPAAAVTHVVEVLSLTAASR \\
\hline 2.0 & 2526.1518 & 1.0059 & ILLLCVGEAGDTVQFAEYIOK \\
\hline 1.6 & 2525.1702 & 1.9876 & CYGYYKNTPQLWSVPSELLK \\
\hline
\end{tabular}

Spectrum No: 462; Query: 247; Rank: 1

\section{Peptide View}

MS/MS Fragmentation of ENISDPTSPVR

Found in IPI00363901, Tax_Id=10116 Gene_Symbol=Igj Immunoglobulin joining chain

Match to Query 247: 1214.573748 from(608.294150,2+)

Title: 091008RatKidney_NH4Format01_31.584.584.2.dta

Data file K:INewmanPaper|Piliang \3SubProteomes\Piliang3SP\mgf5ppm\ERLIC_3SubProteomes5ppm.mgf
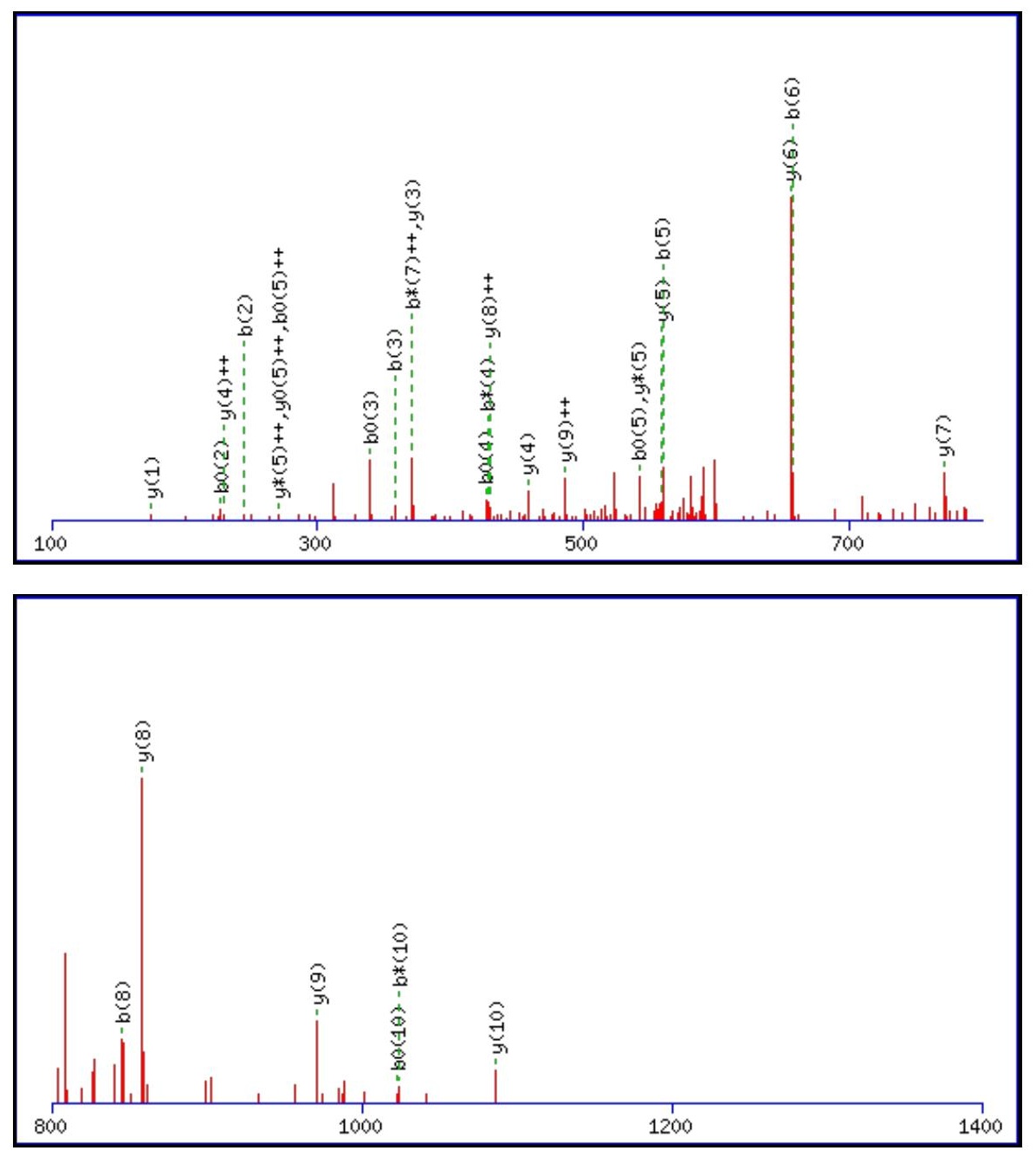


$1400 \quad 1600 \quad 1800 \quad 2000$

Monoisotopic mass of neutral peptide $\operatorname{Mr}($ calc): 1214.5779

Fixed modifications: Carbamidomethyl (C)

Variable modifications:

N2 : Deamidated $\mathrm{N}(\mathrm{N})$

Ions Score: 64 Expect: $5.8 \mathrm{e}-005$

Matches (Bold Red): 29/112 fragment ions using 48 most intense peaks

\begin{tabular}{|c|c|c|c|c|c|c|c|c|c|c|c|c|c|c|}
\hline \# & b & $\mathbf{b}^{++}$ & b* & $\mathbf{b}^{*^{++}}$ & $\mathbf{b}^{0}$ & $\mathbf{b}^{0++}$ & Seq. & $\mathbf{y}$ & $\mathbf{y}^{++}$ & $\mathbf{y}^{*}$ & $\mathbf{y}^{*^{++}}$ & $\mathbf{y}^{0}$ & $\mathbf{y}^{0++}$ & \# \\
\hline 1 & 130.0499 & 65.5286 & & & 112.0393 & 56.5233 & $\mathbf{E}$ & & & & & & & 11 \\
\hline 2 & 245.0768 & 123.0420 & 228.0503 & 114.5288 & 227.0662 & 114.0368 & $\mathbf{N}$ & 1086.5426 & 543.7749 & 1069.5160 & 535.2616 & 1068.5320 & 534.7696 & 10 \\
\hline 3 & 358.1609 & 179.5841 & 341.1343 & 171.0708 & 340.1503 & 170.5788 & I & 971.5156 & 486.2615 & 954.4891 & 477.7482 & 953.5051 & 477.2562 & 9 \\
\hline 4 & 445.1929 & 223.1001 & 428.1663 & 214.5868 & 427.1823 & 214.0948 & S & 858.4316 & 429.7194 & 841.4050 & 421.2061 & 840.4210 & 420.7141 & 8 \\
\hline 5 & 560.2198 & 280.6136 & 543.1933 & 272.1003 & 542.2093 & 271.6083 & D & 771.3995 & 386.2034 & 754.3730 & 377.6901 & 753.3890 & 377.1981 & 7 \\
\hline 6 & 657.2726 & 329.1399 & 640.2461 & 320.6267 & 639.2620 & 320.1347 & $\mathbf{P}$ & 656.3726 & 328.6899 & 639.3461 & 320.1767 & 638.3620 & 319.6847 & 6 \\
\hline 7 & 758.3203 & 379.6638 & 741.2937 & 371.1505 & 740.3097 & 370.6585 & $\mathbf{T}$ & 559.3198 & 280.1636 & 542.2933 & 271.6503 & 541.3093 & 271.1583 & 5 \\
\hline 8 & 845.3523 & 423.1798 & 828.3258 & 414.6665 & 827.3417 & 414.1745 & S & 458.2722 & 229.6397 & 441.2456 & 221.1264 & 440.2616 & 220.6344 & 4 \\
\hline 9 & 942.4051 & 471.7062 & 925.3785 & 463.1929 & 924.3945 & 462.7009 & $\mathbf{P}$ & 371.2401 & 186.1237 & 354.2136 & 177.6104 & & & 3 \\
\hline 10 & 1041.4735 & 521.2404 & 1024.4469 & 512.7271 & 1023.4629 & 512.2351 & V & 274.1874 & 137.5973 & 257.1608 & 129.0840 & & & 2 \\
\hline 11 & & & & & & & $\mathbf{R}$ & 175.1190 & 88.0631 & 158.0924 & 79.5498 & & & 1 \\
\hline
\end{tabular}
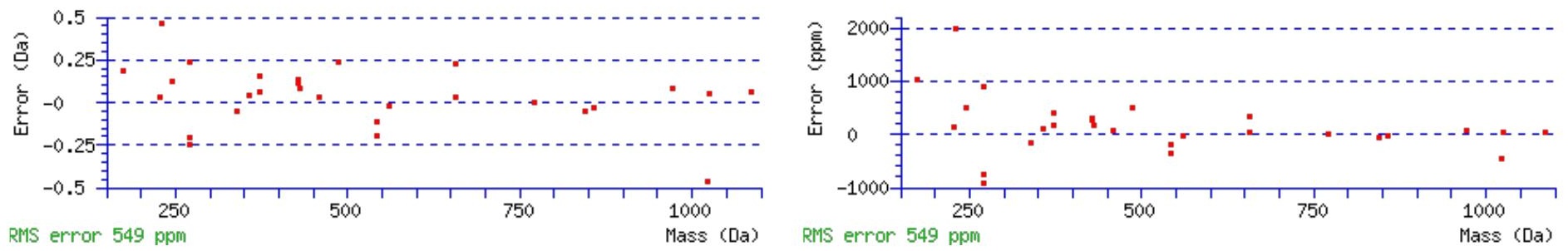

\section{All matches to this query}

\begin{tabular}{|l|l|l|l|}
\hline Score & Mr(calc): & Delta & \multicolumn{1}{|c|}{ Sequence } \\
\hline 63.7 & 1214.5779 & -0.0041 & ENISDPTSPVR \\
\hline 11.7 & 1213.5704 & 1.0034 & KTAEVGSSLSR \\
\hline 8.3 & 1214.5809 & -0.0071 & AFKQNASLTR \\
\hline 8.3 & 1213.5704 & 1.0034 & KTAEVGSSLSR \\
\hline 8.3 & 1214.5779 & -0.0041 & QQTSDSNPPIK \\
\hline 7.8 & 1214.5788 & -0.0050 & MITVNPAHMGK \\
\hline 7.7 & 1212.5751 & 1.9986 & KAKDDATLSGK \\
\hline 7.5 & 1212.5573 & 2.0164 & KNIMALSDGGK \\
\hline 6.7 & 1214.5809 & -0.0071 & NKDVKAAFSR \\
\hline 6.4 & 1213.5704 & 1.0034 & SLETQKTTAR \\
\hline
\end{tabular}


Spectrum No: 463; Query: 214; Rank: 1

\section{Peptide View}

MS/MS Fragmentation of YNQSVSNPCK

Found in IPI00205325, Tax_Id=10116 Gene_Symbol=Lrp2 Low-density lipoprotein receptor-related protein 2 precursor

Match to Query 214: 1196.509028 from(599.261790,2+)

Title: 091008RatKidney_NH4Format01_25.436.436.2.dta

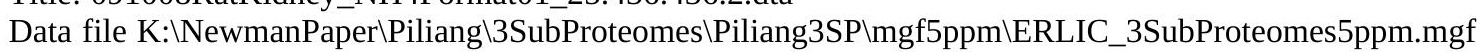
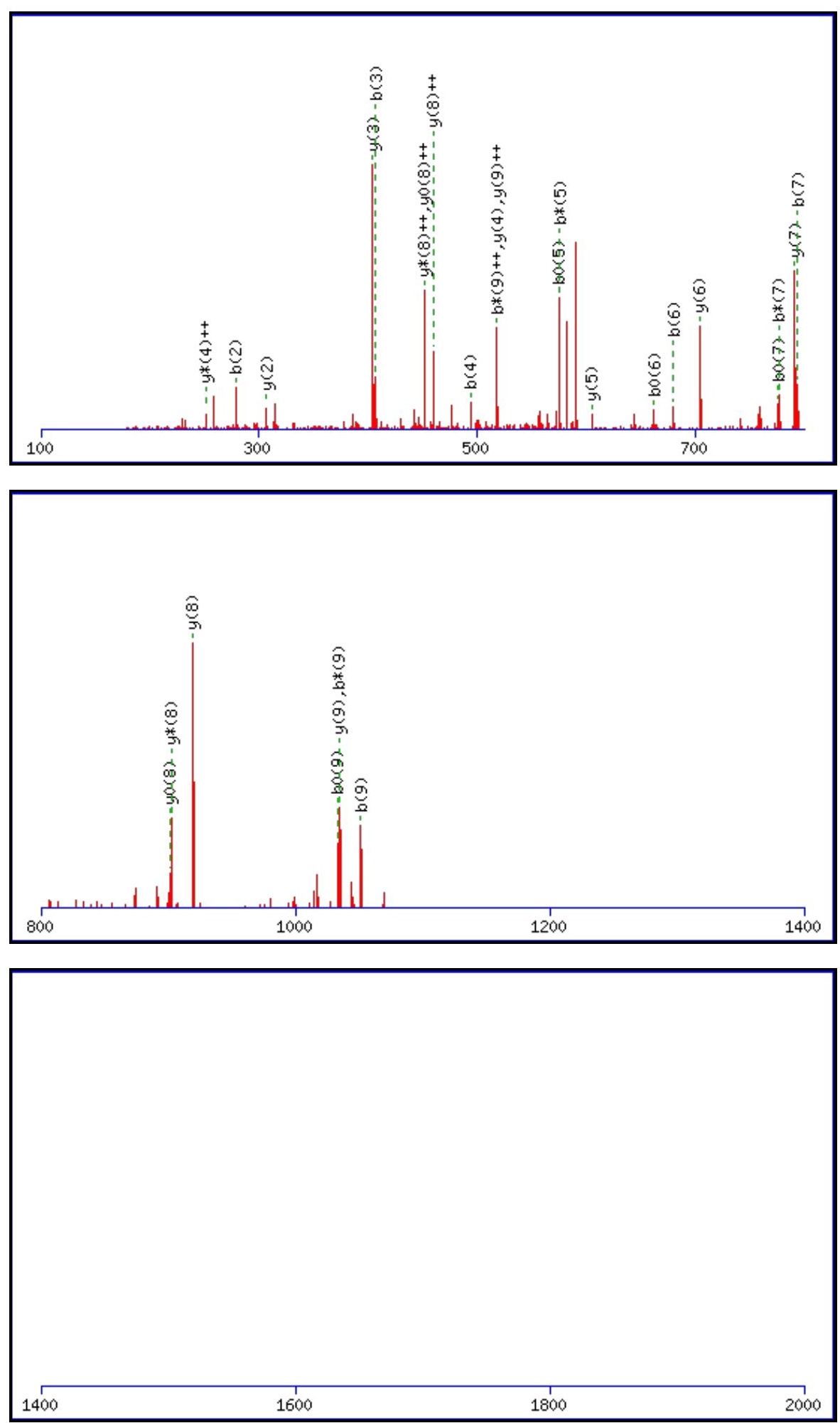
Monoisotopic mass of neutral peptide $\operatorname{Mr}($ calc): 1196.5132

Fixed modifications: Carbamidomethyl (C)

Variable modifications:

N2 : Deamidated_N (N)

Ions Score: 64 Expect: $4.2 \mathrm{e}-005$

Matches (Bold Red): 29/92 fragment ions using 39 most intense peaks

\begin{tabular}{|r|c|c|c|c|c|c|c|c|c|c|c|c|c|c|}
\hline$\#$ & $\mathbf{b}$ & $\mathbf{b}^{++}$ & $\mathbf{b}^{*}$ & $\mathbf{b}^{\mathbf{*}^{++}}$ & $\mathbf{b}^{\mathbf{0}}$ & $\mathbf{b}^{\mathbf{0 + +}}$ & $\mathbf{S e q}$ & $\mathbf{y}$ & $\mathbf{y}^{++}$ & $\mathbf{y}^{\mathbf{*}}$ & $\mathbf{y}^{\mathbf{*}^{++}}$ & $\mathbf{y}^{\mathbf{0}}$ & $\mathbf{y}^{\mathbf{0 + +}}$ & $\#$ \\
\hline $\mathbf{1}$ & 164.0706 & 82.5389 & & & & & $\mathbf{Y}$ & & & & & & & $\mathbf{1 0}$ \\
\hline $\mathbf{2}$ & $\mathbf{2 7 9 . 0 9 7 5}$ & 140.0524 & 262.0710 & 131.5391 & & & $\mathbf{N}$ & $\mathbf{1 0 3 4 . 4 5 7 1}$ & $\mathbf{5 1 7 . 7 3 2 2}$ & 1017.4306 & 509.2189 & 1016.4466 & 508.7269 & $\mathbf{9}$ \\
\hline $\mathbf{3}$ & $\mathbf{4 0 7 . 1 5 6 1}$ & 204.0817 & 390.1296 & 195.5684 & & & $\mathbf{Q}$ & $\mathbf{9 1 9 . 4 3 0 2}$ & $\mathbf{4 6 0 . 2 1 8 7}$ & $\mathbf{9 0 2 . 4 0 3 6}$ & $\mathbf{4 5 1 . 7 0 5 5}$ & $\mathbf{9 0 1 . 4 1 9 6}$ & $\mathbf{4 5 1 . 2 1 3 5}$ & $\mathbf{8}$ \\
\hline $\mathbf{4}$ & $\mathbf{4 9 4 . 1 8 8 1}$ & 247.5977 & 477.1616 & 239.0844 & 476.1776 & 238.5924 & $\mathbf{S}$ & $\mathbf{7 9 1 . 3 7 1 6}$ & 396.1894 & 774.3451 & 387.6762 & 773.3610 & 387.1842 & $\mathbf{7}$ \\
\hline $\mathbf{5}$ & 593.2566 & 297.1319 & 576.2300 & 288.6186 & $\mathbf{5 7 5 . 2 4 6 0}$ & 288.1266 & $\mathbf{V}$ & $\mathbf{7 0 4 . 3 3 9 6}$ & 352.6734 & 687.3130 & 344.1602 & 686.3290 & 343.6681 & $\mathbf{6}$ \\
\hline $\mathbf{6}$ & $\mathbf{6 8 0 . 2 8 8 6}$ & 340.6479 & 663.2620 & 332.1347 & $\mathbf{6 6 2 . 2 7 8 0}$ & 331.6427 & $\mathbf{S}$ & $\mathbf{6 0 5 . 2 7 1 2}$ & 303.1392 & 588.2446 & 294.6259 & 587.2606 & 294.1339 & $\mathbf{5}$ \\
\hline $\mathbf{7}$ & $\mathbf{7 9 4 . 3 3 1 5}$ & 397.6694 & 777.3050 & 389.1561 & $\mathbf{7 7 6 . 3 2 1 0}$ & 388.6641 & $\mathbf{N}$ & $\mathbf{5 1 8 . 2 3 9 1}$ & 259.6232 & 501.2126 & $\mathbf{2 5 1 . 1 0 9 9}$ & & & $\mathbf{4}$ \\
\hline $\mathbf{8}$ & 891.3843 & 446.1958 & 874.3577 & 437.6825 & 873.3737 & 437.1905 & $\mathbf{P}$ & $\mathbf{4 0 4 . 1 9 6 2}$ & 202.6017 & 387.1697 & 194.0885 & & & $\mathbf{3}$ \\
\hline $\mathbf{9}$ & $\mathbf{1 0 5 1 . 4 1 4 9}$ & 526.2111 & $\mathbf{1 0 3 4 . 3 8 8 4}$ & $\mathbf{5 1 7 . 6 9 7 8}$ & $\mathbf{1 0 3 3 . 4 0 4 4}$ & 517.2058 & $\mathbf{C}$ & $\mathbf{3 0 7 . 1 4 3 5}$ & 154.0754 & 290.1169 & 145.5621 & & & $\mathbf{2}$ \\
\hline $\mathbf{1 0}$ & & & & & & & $\mathbf{K}$ & 147.1128 & 74.0600 & 130.0863 & 65.5468 & & & $\mathbf{1}$ \\
\hline
\end{tabular}
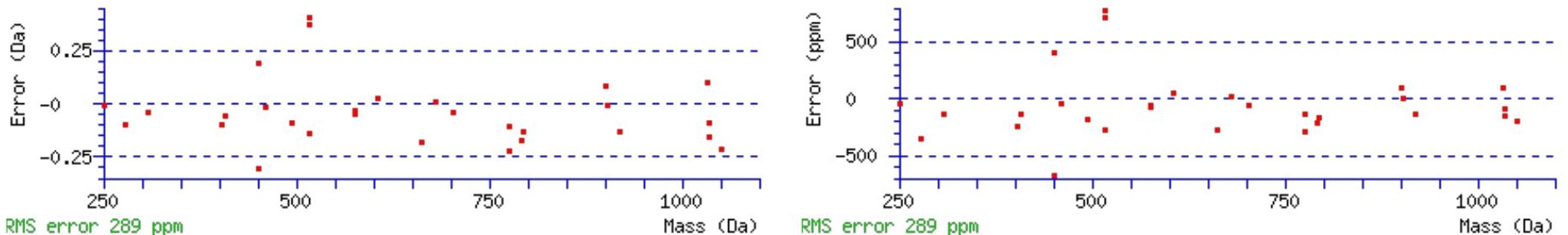

All matches to this query

\begin{tabular}{|l|l|l|l|}
\hline Score & Mr(calc): & Delta & \multicolumn{1}{|c|}{ Sequence } \\
\hline 63.7 & 1196.5132 & -0.0041 & YNQSVSNPCK \\
\hline 36.2 & 1196.5132 & -0.0041 & YNQSVSNPCK \\
\hline 17.7 & 1196.5206 & -0.0115 & MWLEESQMK \\
\hline 13.4 & 1196.5165 & -0.0075 & NSEMKNSMEK \\
\hline 9.2 & 1196.5002 & 0.0088 & YYSLDELSK \\
\hline 9.1 & 1196.5165 & -0.0075 & NAEMKNAMQK \\
\hline 8.3 & 1196.5131 & -0.0041 & STLINTLFK \\
\hline 7.3 & 1196.5131 & -0.0041 & RNSYNPGEMK \\
\hline 7.2 & 1196.5121 & -0.0031 & HSRSRSMEK \\
\hline 7.2 & 1196.5121 & -0.0031 & HSRSRSMEK \\
\hline
\end{tabular}

Spectrum No: 464; Query: 2632; Rank: 1

\section{Peptide View}

MS/MS Fragmentation of ETIGPQQGCGGYLTEDNQSFVSPDSDSNGR

Found in IPI00196620, Tax_Id=10116 Gene_Symbol=Cubn Cubilin precursor

Match to Query 2632: 3215.373402 from(1072.798410,3+)

Title: 100101RatKid_NS_deglyco_20.3067.3067.3.dta

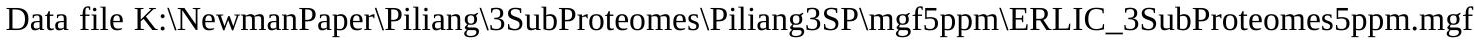



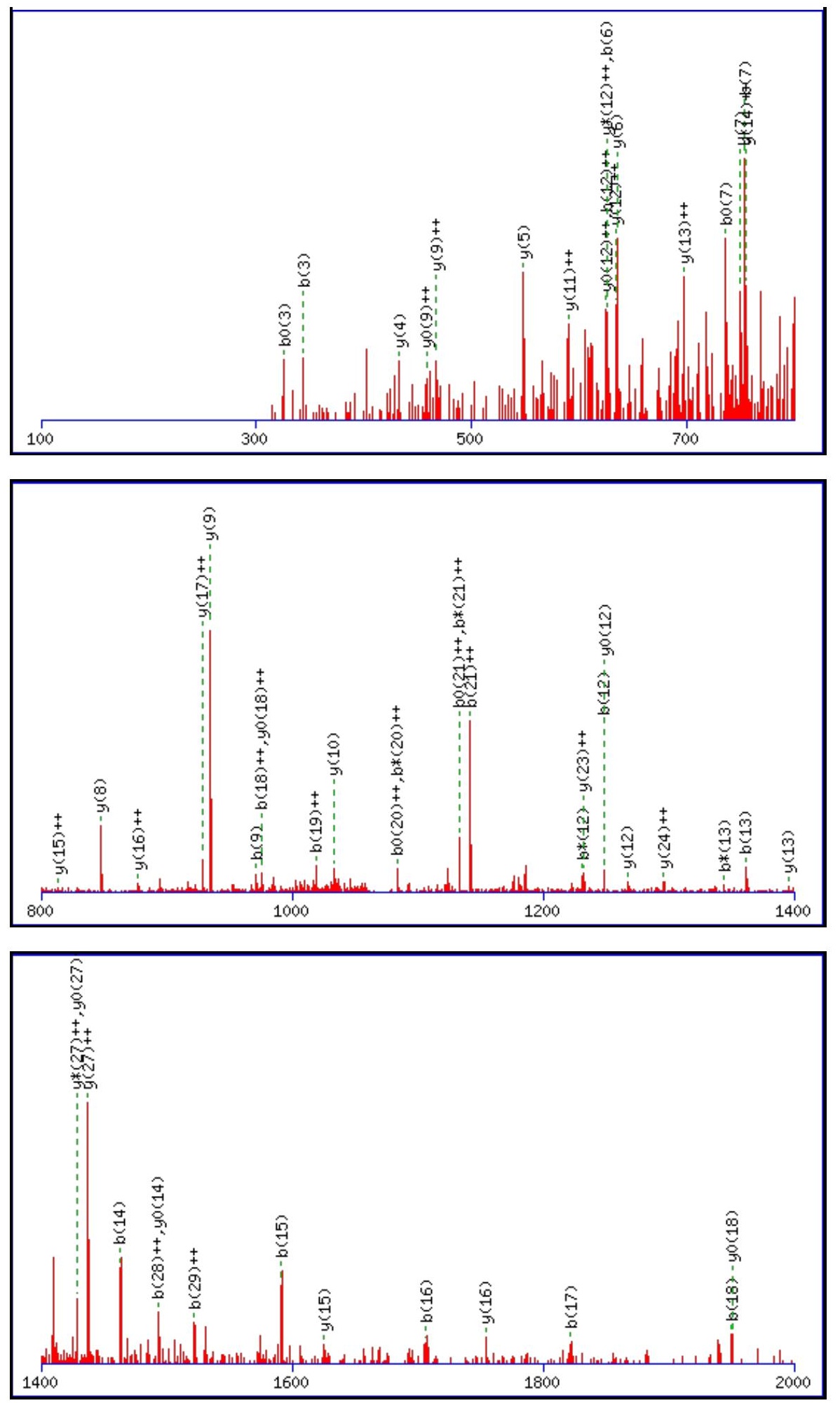

Monoisotopic mass of neutral peptide $\operatorname{Mr}($ calc): 3215.3586

Fixed modifications: Carbamidomethyl (C)

Variable modifications:

N17 : Deamidated_N (N)

Ions Score: 64 Expect: 0.00019

Matches (Bold Red): 56/332 fragment ions using 91 most intense peaks

\begin{tabular}{|c|c|c|c|c|c|c|c|c|c|c|c|c|c|c|}
\hline \# & b & $\mathbf{b}^{++}$ & $\mathbf{b}^{*}$ & $\mathbf{b}^{*^{++}}$ & $\mathbf{b}^{0}$ & $\mathbf{b}^{\mathbf{0 + +}}$ & Seq. & $\mathbf{y}$ & $y^{++}$ & $\mathbf{y}^{*}$ & $\mathrm{y}^{*^{++}}$ & $\mathbf{y}^{\mathbf{0}}$ & $y^{0++}$ & \# \\
\hline 1 & 130.0499 & 65.5286 & & & 112.0393 & 56.5233 & $\mathbf{E}$ & & & & & & & 30 \\
\hline 2 & 231.0975 & 116.0524 & & & 213.0870 & 107.0471 & $\mathbf{T}$ & 3087.3232 & 1544.1652 & 3070.2967 & 1535.6520 & 3069.3127 & 1535.1600 & 29 \\
\hline 3 & 344.1816 & 172.5944 & & & 326.1710 & 163.5892 & I & 2986.2755 & 1493.6414 & 2969.2490 & 1485.1281 & 2968.2650 & 1484.6361 & 28 \\
\hline 4 & 401.2031 & 201.1052 & & & 383.1925 & 192.0999 & G & 2873.1915 & 1437.0994 & 2856.1649 & 1428.5861 & 2855.1809 & 1428.0941 & 27 \\
\hline 5 & 498.2558 & 249.6316 & & & 480.2453 & 240.6263 & $\mathbf{P}$ & 2816.1700 & 1408.5886 & 2799.1435 & 1400.0754 & 2798.1595 & 1399.5834 & 26 \\
\hline
\end{tabular}




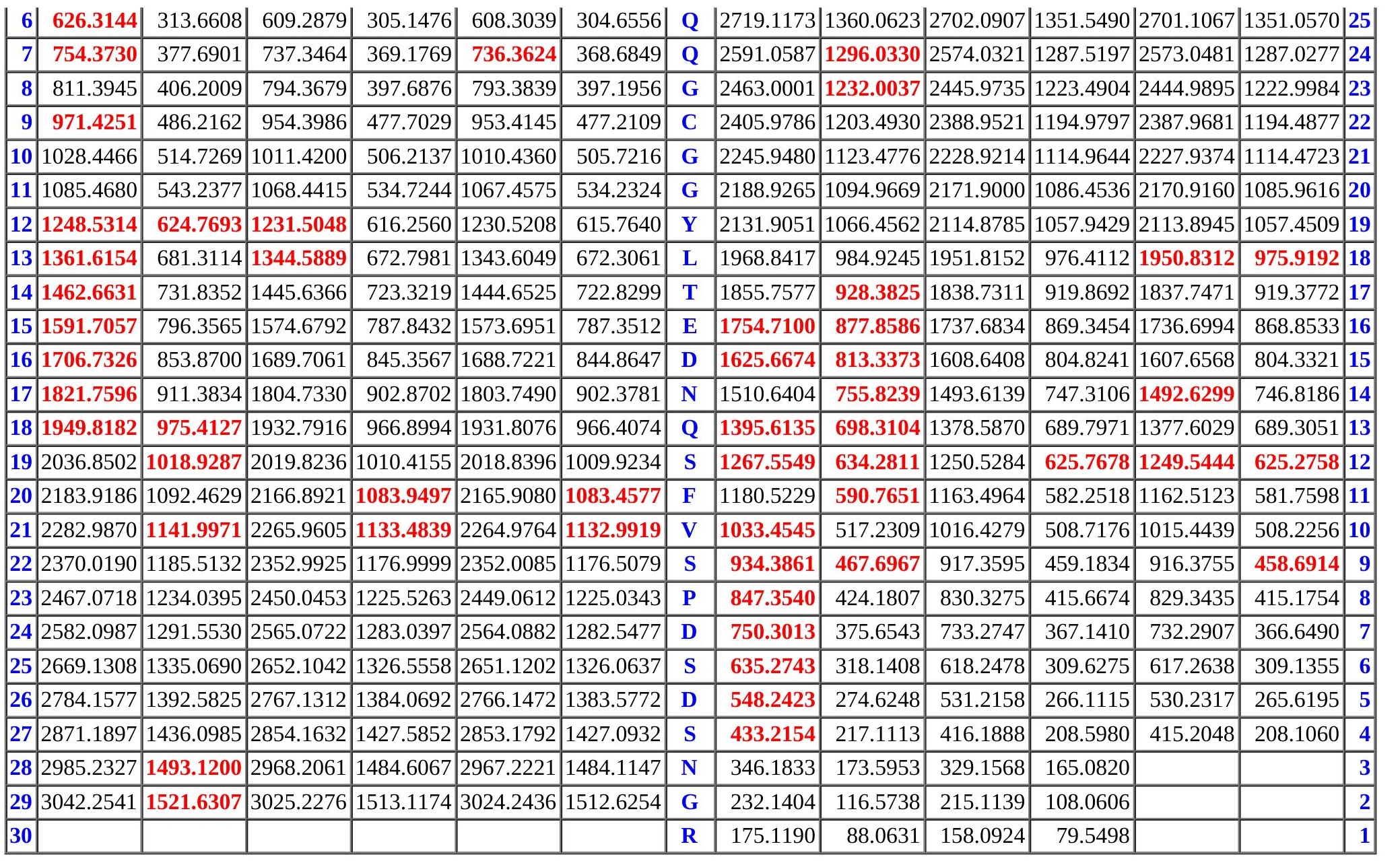
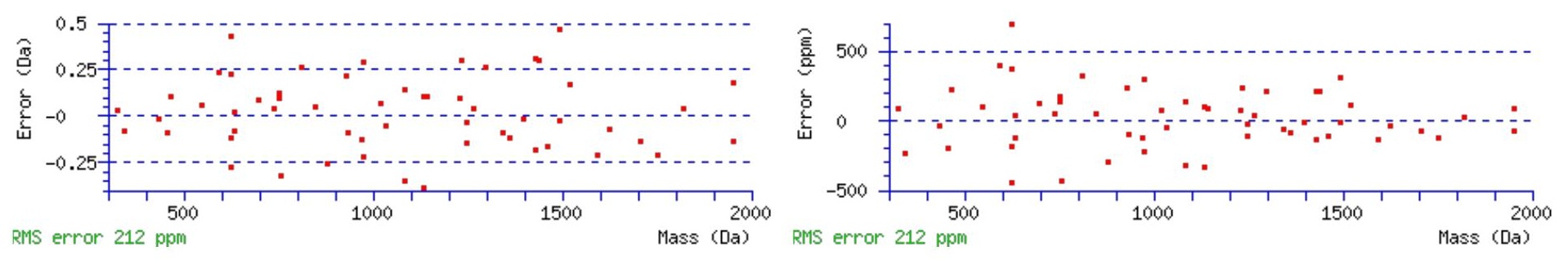

\section{All matches to this query}

\begin{tabular}{|l|l|l|l|}
\hline Score & Mr(calc): & Delta & \multicolumn{1}{|c|}{ Sequence } \\
\hline 63.7 & 3215.3586 & 0.0148 & ETIGPQQGCGGYLTEDNQSFVSPDSDSNGR \\
\hline 42.8 & 3214.3746 & 0.9988 & ETIGPQQGCGGYLTEDNQSFVSPDSDSNGR \\
\hline 28.1 & 3215.3586 & 0.0148 & ETIGPQQGCGGYLTEDNQSFVSPDSDSNGR \\
\hline 12.4 & 3215.4044 & -0.0310 & REALALQGSLEFDDAGGEGVTSHVDDEYR \\
\hline 10.6 & 3215.3491 & 0.0243 & TWRGSSIHQDFLGFYVLDSFRMSAR \\
\hline 10.6 & 3215.3491 & 0.0243 & TWRGSSIHQDFLGFYVLDSFRMSAR \\
\hline 7.5 & 3215.3491 & 0.0243 & TWRGSSIHQDFLGFYVLDSFRMSAR \\
\hline 5.7 & 3214.3573 & 1.0161 & FTEFQTQALQSFFETSAYPKDGEVER \\
\hline 4.8 & 3214.3761 & 0.9973 & YNPSPDQSVSVTVTTNQAAPSSIALVQAK \\
\hline 3.6 & 3215.3705 & 0.0029 & MASHEIEIGTIFTNINATDNHVHSMLK \\
\hline
\end{tabular}

Spectrum No: 465; Query: 1145; Rank: 1 


\section{Peptide View}

MS/MS Fragmentation of SDLNPANGSYPFQALR

Found in IPI00200195, Tax_Id=10116 Gene_Symbol=LOC246120 Putative phospholipase B-like 2 precursor

Match to Query 1145: 1749.833768 from(875.924160,2+)

Title: 091008RatKidney_NH4Format01_24.3086.3086.2.dta

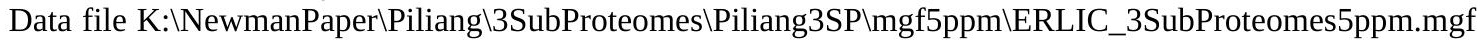
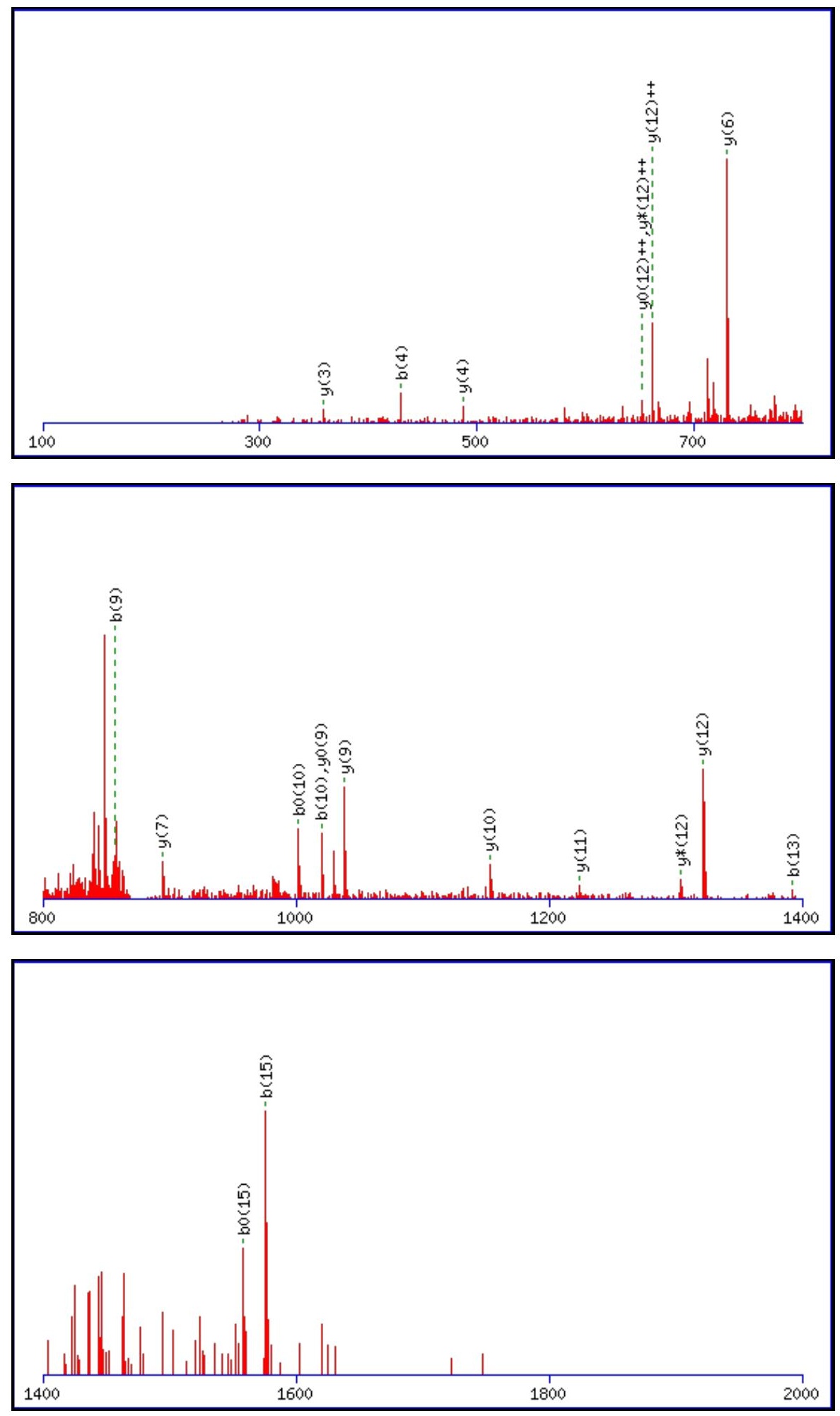

Monoisotopic mass of neutral peptide $\operatorname{Mr}($ calc): 1749.8322

Fixed modifications: Carbamidomethyl (C)

Variable modifications:

N7 : Deamidated_N (N)

Ions Score: 64 Expect: $8.6 \mathrm{e}-005$

Matches (Bold Red): 20/160 fragment ions using 35 most intense peaks 


\begin{tabular}{|c|c|c|c|c|c|c|c|c|c|c|c|c|c|c|}
\hline \# & b & $\mathbf{b}^{++}$ & $\mathbf{b}^{*}$ & $\mathbf{b}^{*^{++}}$ & $\mathbf{b}^{0}$ & $\mathbf{b}^{0++}$ & Seq. & $\mathbf{y}$ & $y^{++}$ & $\mathbf{y}^{*}$ & $\mathrm{y}^{*^{++}}$ & $\mathbf{y}^{0}$ & $\mathbf{y}^{0++}$ & $\#$ \\
\hline 1 & 88.0393 & 44.5233 & & & 70.0287 & 35.5180 & S & & & & & & & 16 \\
\hline 2 & 203.0662 & 102.0368 & & & 185.0557 & 93.0315 & D & 1663.8075 & 832.4074 & 1646.7809 & 823.8941 & 1645.7969 & 823.4021 & 15 \\
\hline 3 & 316.1503 & 158.5788 & & & 298.1397 & 149.5735 & $\mathbf{L}$ & 1548.7805 & 774.8939 & 1531.7540 & 766.3806 & 1530.7699 & 765.8886 & 14 \\
\hline 4 & 430.1932 & 215.6003 & 413.1667 & 207.0870 & 412.1827 & 206.5950 & $\mathbf{N}$ & 1435.6964 & 718.3519 & 1418.6699 & 709.8386 & 1417.6859 & 709.3466 & 13 \\
\hline 5 & 527.2460 & 264.1266 & 510.2195 & 255.6134 & 509.2354 & 255.1214 & $\mathbf{P}$ & 1321.6535 & 661.3304 & \begin{tabular}{|l|}
1304.6270 \\
\end{tabular} & 652.8171 & 1303.6430 & 652.3251 & 12 \\
\hline 6 & 598.2831 & 299.6452 & 581.2566 & 291.1319 & 580.2726 & 290.6399 & A & 1224.6008 & 612.8040 & 1207.5742 & 604.2907 & 1206.5902 & 603.7987 & 11 \\
\hline 7 & 713.3101 & 357.1587 & 696.2835 & 348.6454 & 695.2995 & 348.1534 & $\mathbf{N}$ & 1153.5636 & 577.2855 & 1136.5371 & 568.7722 & 1135.5531 & 568.2802 & 10 \\
\hline 8 & 770.3 & 385.6694 & 753.3050 & 377.1561 & 752.3 & 376.6641 & G & \begin{tabular}{|l|}
1038.5367 \\
\end{tabular} & 519.7720 & 1021.5102 & 511.2587 & 1020.5261 & 510.7667 & 9 \\
\hline 9 & \begin{tabular}{|l|}
857.3635 \\
\end{tabular} & 429.1854 & 840.3370 & 420.6721 & 839.3530 & 420.1801 & $S$ & 981.5152 & 491.2613 & \begin{tabular}{|l|}
964.4887 \\
\end{tabular} & 482.7480 & 963.5047 & 482.2560 & 8 \\
\hline 10 & 1020.4269 & 510.7171 & 1003.4003 & 502.2038 & 1002.4163 & 501.7118 & $\mathbf{Y}$ & 894.4832 & 447.7452 & 877.4567 & 439.2320 & & & 7 \\
\hline 11 & 1117.4796 & 559.2435 & 1100.4531 & 550.7302 & 1099.4691 & 550.2382 & $\mathbf{P}$ & 731.4199 & 366.2136 & 714.3933 & 357.7003 & & & 6 \\
\hline 12 & 1264.5481 & 632.7777 & 1247.5215 & 624.2644 & 1246.5375 & 623.7724 & $\mathbf{F}$ & 634.3671 & 317.6872 & 617.3406 & 309.1739 & & & 5 \\
\hline 13 & 1392.6066 & 696.8070 & 1375.5801 & 688.2937 & 1374.5961 & 687.8017 & $\bar{Q}$ & 487.2987 & 244.1530 & 470.2722 & 235.6397 & & & 4 \\
\hline 14 & 1463.6437 & 732.3255 & 1446.6172 & 723.8122 & 1445.6332 & 723.3202 & A & 359.2401 & 180.1237 & 342.2136 & 171.6104 & & & 3 \\
\hline 15 & 1576.7278 & 788.8675 & 1559.7013 & 780.3543 & 1558.7172 & 779.8623 & $\mathbf{L}$ & 288.2030 & 144.6051 & 271.1765 & 136.0919 & & & 2 \\
\hline 16 & & & & & & & $\mathbf{R}$ & 175.1190 & 88.0631 & 158.0924 & \begin{tabular}{|l|}
79.5498 \\
\end{tabular} & & & 1 \\
\hline
\end{tabular}
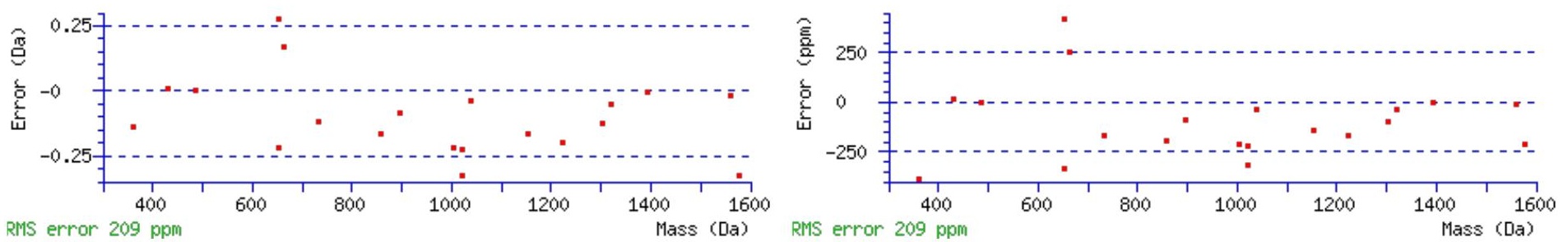

\section{All matches to this query}

\begin{tabular}{|l|l|l|l|}
\hline Score & Mr(calc): & Delta & \multicolumn{1}{c|}{ Sequence } \\
\hline 63.6 & 1749.8322 & 0.0016 & SDLNPANGSYPFQALR \\
\hline 24.6 & 1749.8322 & 0.0016 & SDLNPANGSYPFQALR \\
\hline 12.6 & 1749.8273 & 0.0065 & EKMTILAHDWAALR \\
\hline 9.5 & 1749.8356 & -0.0018 & DSQVGTVNYMAPEAIR \\
\hline 5.2 & 1749.8501 & -0.0163 & ETKTMAKVITTVLR \\
\hline 5.1 & 1749.8506 & -0.0169 & SAEAGAAQAPGEXRGSVR \\
\hline 1.5 & 1749.8355 & -0.0018 & FTVTPSTTQVVGILK \\
\hline 0.9 & 1747.8294 & 2.0043 & KPSDYGYVSLGRAGAK \\
\hline 0.9 & 1747.8294 & 2.0043 & $\underline{\text { KPSDYGYVSLGRAGAK }}$ \\
\hline
\end{tabular}

Spectrum No: 466; Query: 2558; Rank: 1

\section{Peptide View}

MS/MS Fragmentation of MGAGEALGNASQLFEAWLKGNESIPVNLR

Found in IPI00327398, Tax_Id=10116 Gene_Symbol=Enpep Isoform 1 of Glutamyl aminopeptidase

Match to Query 2558: 3073.533102 from(1025.518310,3+)

Title: 100101RatKid_NS_deglyco_14.7143.7143.3.dta

Data file K:INewmanPaper|Piliang|3SubProteomes\Piliang3SP\mgf5ppm\ERLIC_3SubProteomes5ppm.mgf 

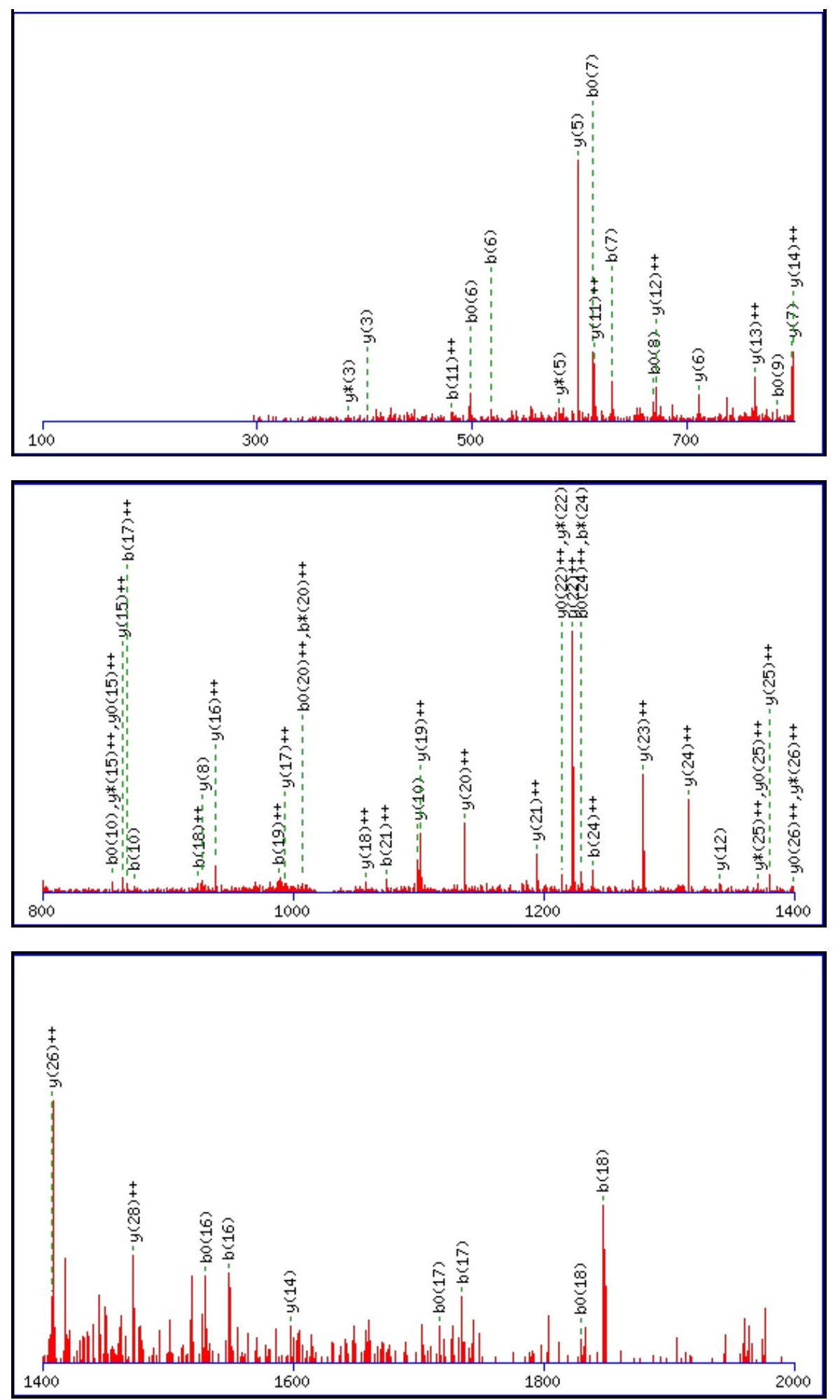

Monoisotopic mass of neutral peptide $\operatorname{Mr}($ calc): 3073.5178

Fixed modifications: Carbamidomethyl (C)

Variable modifications:

N9 : Deamidated_N (N)

N21 : Deamidated_N (N)

Ions Score: 64 Expect : 0.0001

Matches (Bold Red): 59/300 fragment ions using 126 most intense peaks

\begin{tabular}{|c|c|c|c|c|c|c|c|c|c|c|c|c|c|c|}
\hline \# & b & $\mathbf{b}^{++}$ & b* & $\mathbf{b}^{*^{++}}$ & $\mathbf{b}^{\mathbf{0}}$ & $\mathbf{b}^{\mathbf{0}^{++}}$ & Seq. & $\mathbf{y}$ & $\mathbf{y}^{++}$ & $\mathbf{y}^{*}$ & $\mathbf{y}^{*^{++}}$ & $\mathbf{y}^{0}$ & $\mathbf{y}^{0++}$ & \# \\
\hline 1 & 132.0478 & 66.5275 & & & & & M & & & & & & & 29 \\
\hline 2 & 189.0692 & 95.0383 & & & & & G & 2943.4847 & 1472.2460 & 2926.4581 & 1463.7327 & 2925.4741 & 1463.2407 & 28 \\
\hline 3 & 260.1063 & 130.5568 & & & & & A & 2886.4632 & 1443.7352 & 2869.4367 & 1435.2220 & 2868.4526 & 1434.7300 & 27 \\
\hline 4 & 317.1278 & 159.0675 & & & & & G & 2815.4261 & 1408.2167 & 2798.3995 & 1399.7034 & 2797.4155 & 1399.2114 & 26 \\
\hline
\end{tabular}




\begin{tabular}{|c|c|c|c|c|c|c|c|c|c|c|c|c|c|c|}
\hline & 446.1704 & 223.5888 & & & 428.1598 & 214.5836 & $\mathbf{E}$ & 2758.4046 & 1379.7059 & 2741.3781 & |1371.1927 & |2740.3941 & $|1370.7007|$ & 25 \\
\hline 6 & 517.2075 & 259.1074 & & & 499.1969 & 250.1021 & A & 2629.3620 & 1315.1847 & 2612.3355 & 1306.6714 & 2611.3515 & 1306.1794 & \\
\hline 7 & 630.2916 & 315.6494 & & & 612.2810 & 306.6441 & L & 2558.3249 & 1279.6661 & 2541.2984 & 1271.1528 & 2540.3143 & 1270.6608 & \\
\hline 8 & 687.3130 & 344.1602 & & & 669.3025 & 335.1549 & G & 2445.2409 & 1223.1241 & 2428.2143 & 1214.6108 & 2427.2303 & 1214.1188 & 22 \\
\hline 9 & 802.3400 & 401.6736 & 785.3134 & 393.1604 & 784.3294 & 392.6683 & $\mathbf{N}$ & 2388.2194 & 1194.6133 & 2371.1928 & 1186.1001 & 2370.2088 & 1185.6080 & 21 \\
\hline 10 & 873.3771 & 437.1922 & 856.3505 & 428.6789 & 855.3665 & 428.1869 & A & 2273.1925 & 1137.0999 & 2256.1659 & 1128.5866 & 2255.1819 & 1128.0946 & 5 \\
\hline 11 & 960.4091 & 480.7082 & 943.3826 & 472.1949 & 942.3986 & 471.7029 & S & 2202.1553 & 1101.5813 & 2185.1288 & 1093.0680 & 2184.1448 & 1092.5760 & 19 \\
\hline 12 & 1088.4677 & 544.7375 & 1071.4411 & 536.2242 & 1070.4571 & 535.7322 & $\mathbf{Q}$ & 2115.1233 & 1058.0653 & 2098.0968 & 1049.5520 & 2097.1127 & 1049.0600 & 18 \\
\hline 13 & 1201.5518 & 601.2795 & 1184.5252 & 592.7662 & 1183.5412 & 592.2742 & $\mathbf{L}$ & 1987.0647 & 994.0360 & 1970.0382 & 985.5227 & 1969.0542 & 985.0307 & 17 \\
\hline 14 & 348.6202 & 674.8137 & 1331.5936 & 666.30 & 1330.6096 & 665.8084 & $\mathbf{F}$ & 1873.9807 & 937.4940 & 1856.9541 & 807 & 9701 & 4887 & 6 \\
\hline 15 & 1477.6628 & 739.3350 & 1460.6362 & 730.8217 & 1459.6522 & 730.3297 & $\mathbf{E}$ & 1726.9123 & 863.9598 & 1709.8857 & 855.4465 & 1708.9017 & 854.9545 & 15 \\
\hline 16 & 1548.6999 & 774.8536 & 1531.6733 & 766.3403 & 1530.6893 & 765.8483 & A & 1597.8697 & 799.4385 & 1580.8431 & 790.9252 & 1579.8591 & 790.4332 & 4 \\
\hline 17 & 1734.7792 & 867.8932 & 1717.7526 & 859.3800 & 1716.7686 & 858.8880 & W & 1526.8325 & 763.9199 & 1509.8060 & 755.4066 & 1508.8220 & 9146 & 5 \\
\hline 18 & 1847.8633 & 924.4353 & 1830.8367 & 915.9220 & 1829.8527 & 915.4300 & $\mathbf{L}$ & 1340.7532 & 670.8803 & 1323.7267 & 662.3670 & 1322.7427 & 661.8750 & 12 \\
\hline 19 & 1975.9582 & 988.4827 & 1958.9317 & 979.9695 & 1957.9477 & 979.4775 & $\mathbf{K}$ & 1227.6692 & 614.3382 & 1210.6426 & 605.8249 & 1209.6586 & 605.3329 & 11 \\
\hline 20 & 2032.9797 & 1016.9935 & 2015.9531 & \begin{tabular}{|l}
1008.4802 \\
\end{tabular} & 2014.9691 & 1007.9882 & G & 1099.5742 & 550.2907 & 1082.5477 & 541.7775 & 1081.5636 & 541.2855 & 10 \\
\hline 21 & 2148.0066 & 1074.5069 & 2130.9801 & 1065.9937 & 2129.9961 & 1065.5017 & $\mathbf{N}$ & 1042.5527 & 521.7800 & 1025.5262 & 513.2667 & 1024.5422 & 512.7747 & 9 \\
\hline 22 & 2277.0492 & 1139.0282 & 2260.0227 & 1130.5150 & 2259.0386 & 1130.0230 & $\mathbf{E}$ & 927.5258 & 464.2665 & 910.4993 & 455.7533 & 909.5152 & 455.2613 & 8 \\
\hline 23 & 2364.0812 & 1182.5443 & 2347.0547 & 1174.0310 & 2346.0707 & 1173.5390 & $\mathrm{~S}$ & 798.4832 & 399.7452 & 781.4567 & 391.2320 & 780.4726 & 390.7400 & 7 \\
\hline 24 & 2477.1653 & 1239.0863 & 2460.1388 & 1230.5730 & 2459.1547 & 1230.0810 & I & 711.4512 & 356.2292 & 694.4246 & 347.7160 & & & 6 \\
\hline 25 & 2574.2181 & 1287.6127 & 2557.1915 & 1279.0994 & 2556.2075 & 1278.6074 & $\mathbf{P}$ & 598.3671 & 299.6872 & 581.3406 & 291.1739 & & & 5 \\
\hline 26 & 2673.2865 & 1337.1469 & 2656.2599 & 1328.6336 & 2655.2759 & 1328.1416 & V & 501.3144 & 251.1608 & 484.2878 & 242.6475 & & & 4 \\
\hline 27 & 2787.3294 & 1394.1683 & 2770.3029 & 1385.6551 & 2769.3188 & 1385.1631 & $\mathbf{N}$ & 402.2459 & 201.6266 & 385.2194 & 193.1133 & & & 3 \\
\hline 28 & 2900.4135 & 1450.7104 & 2883.3869 & 1442.1971 & 2882.4029 & 1441.7051 & $\mathbf{L}$ & 288.2030 & 144.6051 & 271.1765 & 136.0919 & & & 2 \\
\hline 29 & & & & & & & $\mathbf{R}$ & 175.1190 & 88.0631 & 158.0924 & 79.5498 & & & 1 \\
\hline
\end{tabular}
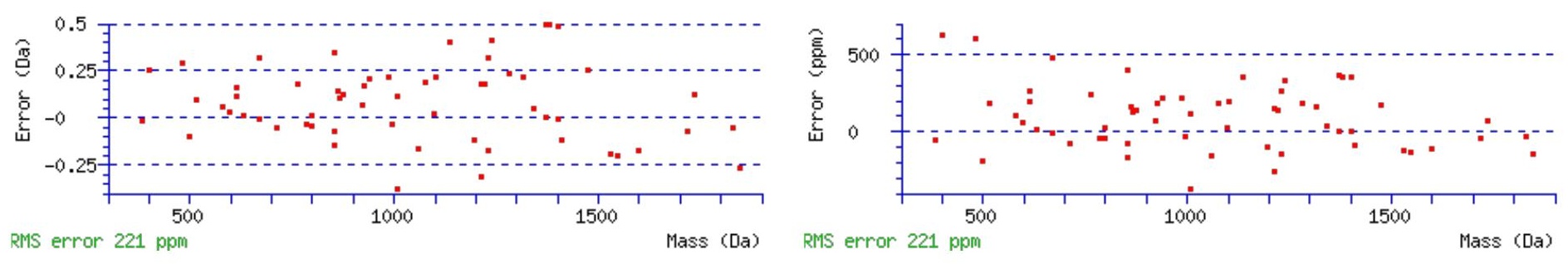

\section{All matches to this query}

\begin{tabular}{|l|l|l|l|}
\hline Score & Mr(calc): & Delta & \multicolumn{1}{c|}{ Sequence } \\
\hline 63.5 & 3073.5178 & 0.0153 & MGAGEALGNASQLFEAWLKGNESIPVNLR \\
\hline 52.2 & 3073.5178 & 0.0153 & MGAGEALGNASQLFEAWLKGNESIPVNLR \\
\hline 44.7 & 3073.5178 & 0.0153 & MGAGEALGNASQLFEAWLKGNESIPVNLR \\
\hline 19.1 & 3072.5338 & 0.9993 & MGAGEALGNASQLFEAWLKGNESIPVNLR \\
\hline 8.9 & 3072.5338 & 0.9993 & MGAGEALGNASQLFEAWLKGNESIPVNLR \\
\hline 3.1 & 3072.5338 & 0.9993 & MGAGEALGNASQLFEAWLKGNESIPVNLR \\
\hline 1.3 & 3071.5539 & 1.9792 & ALGTALIENNQLHVTLTQKQEEIASLR \\
\hline 0.6 & 3073.5219 & 0.0112 & MQVRGREVSGLLISLTAVCLVVTPGSR \\
\hline 0.6 & 3073.5219 & 0.0112 & MQVRGREVSGLLISLTAVCLVVTPGSR \\
\hline 0.5 & 3072.5007 & 1.0324 & LSKNPYLNLASVLPSVCLSTAGKGTPPK \\
\hline
\end{tabular}

Spectrum No: 467; Query: 539; Rank: 1 


\section{Peptide View}

\section{MS/MS Fragmentation of AIPTLVSDGNITVK}

Found in IPI00212933, Tax_Id=10116 Gene_Symbol=Slc5a1 Sodium/glucose cotransporter 1

Match to Query 539: 1427.790028 from(714.902290,2+)

Title: 100101RatKid_NS_deglyco_23.3148.3148.2.dta

Data file K:INewmanPaper|Piliangl3SubProteomes\Piliang3SP\mgf5ppm\ERLIC_3SubProteomes5ppm.mgf
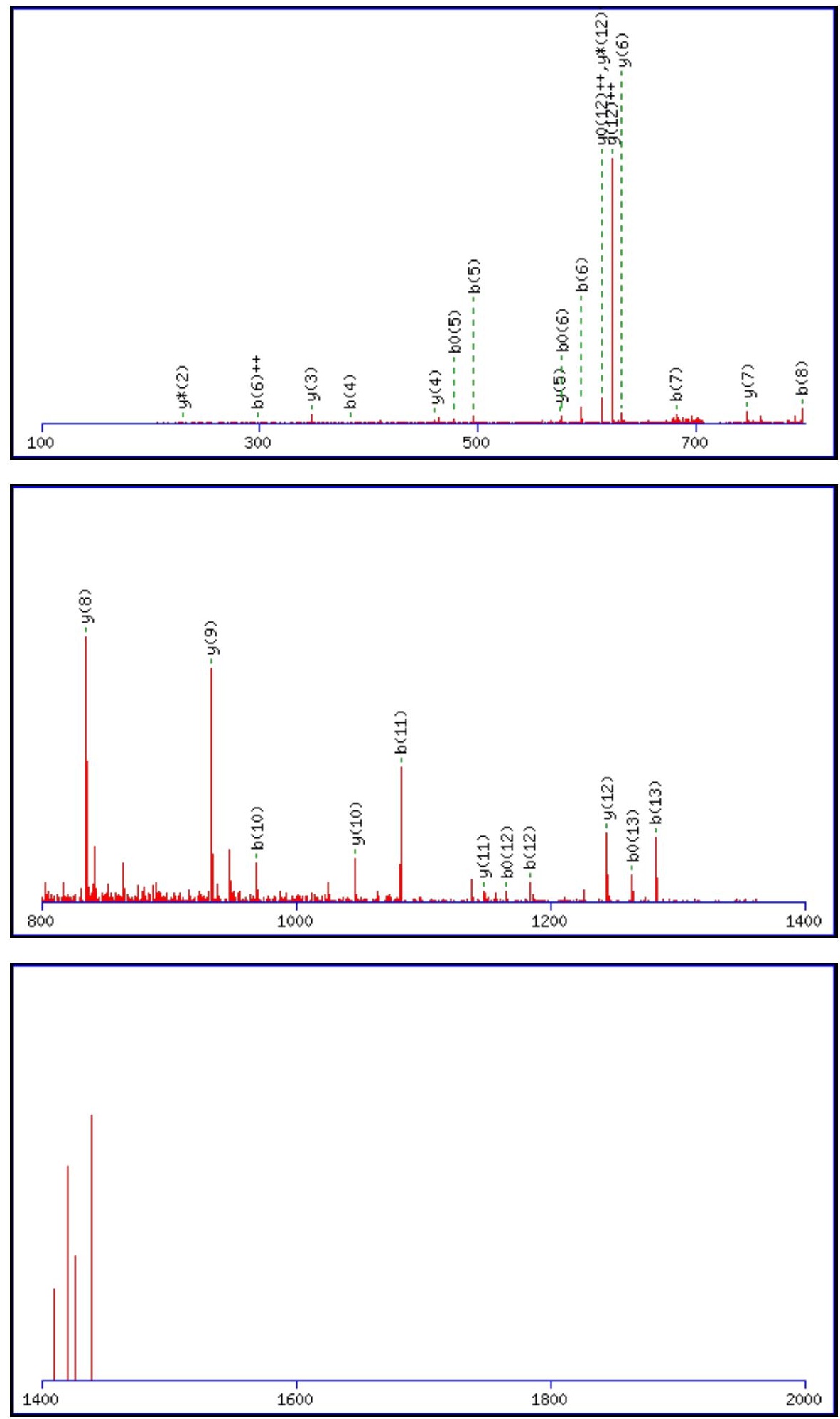

Monoisotopic mass of neutral peptide $\operatorname{Mr}($ calc): 1427.7872

Fixed modifications: Carbamidomethyl (C)

Variable modifications:

N10 : Deamidated_N (N)

Ions Score: 64 Expect: $3.1 \mathrm{e}-005$

Matches (Bold Red): 29/128 fragment ions using 54 most intense peaks 


\begin{tabular}{|c|c|c|c|c|c|c|c|c|c|c|c|c|c|c|}
\hline$\#$ & b & $\mathbf{b}^{++}$ & $\mathbf{b}^{*}$ & $\mathbf{b}^{*^{++}}$ & $\mathbf{b}^{\mathbf{0}}$ & $\mathbf{b}^{0++}$ & Seq. & $\mathbf{y}$ & $\mathbf{y}^{++}$ & $\mathrm{y}^{*}$ & $\mathrm{y}^{*^{++}}$ & $\mathbf{y}^{\mathbf{0}}$ & $y^{0++}$ & \# \\
\hline 1 & 72.0444 & 36.5258 & & & & & A & & & & & & & 14 \\
\hline 2 & 185.1285 & 93.0679 & & & & & I & 1357.7573 & 679.3823 & 1340.7308 & 670.8690 & 1339.7468 & 670.3770 & 13 \\
\hline 3 & 282.1812 & 141.5942 & & & & & $\mathbf{P}$ & 1244.6733 & 622.8403 & 1227.6467 & 614.3270 & 1226.6627 & 613.8350 & 12 \\
\hline 4 & 383.2289 & 192.1181 & & & 365.2183 & 183.1128 & $T$ & 1147.6205 & 574.3139 & 1130.5939 & 565.8006 & 1129.6099 & 565.3086 & 11 \\
\hline 5 & 496.3130 & 248.6601 & & & 478.3024 & 239.6548 & $\mathbf{L}$ & 1046.5728 & 523.7900 & 1029.5463 & 515.2768 & 1028.5622 & 514.7848 & 10 \\
\hline 6 & 595.3814 & 298.1943 & & & 577.3708 & 289.1890 & $\mathrm{~V}$ & 933.4887 & 467.2480 & 916.4622 & 458.7347 & 915.4782 & 458.2427 & 9 \\
\hline 7 & 682.4134 & 341.7103 & & & 664.4028 & 332.7051 & $\mathrm{~S}$ & 834.4203 & 417.7138 & 817.3938 & 409.2005 & 816.4098 & 408.7085 & 8 \\
\hline 8 & 797.4403 & 399.2238 & & & 779.4298 & 390.2185 & D & 747.3883 & 374.1978 & 730.3618 & 365.6845 & 729.3777 & 365.1925 & 7 \\
\hline 9 & 854.4618 & 427.7345 & & & 836.4512 & 418.7293 & G & 632.3614 & 316.6843 & 615.3348 & 308.1710 & 614.3508 & 307.6790 & 6 \\
\hline 10 & 969.4887 & 485.2480 & 952.4622 & 476.7347 & 951.4782 & 476.2427 & $\mathbf{N}$ & 575.3399 & 288.1736 & 558.3133 & 279.6603 & 557.3293 & 279.1683 & 5 \\
\hline 11 & 1082.5728 & 541.7900 & 1065.5463 & 533.2768 & 1064.5622 & 532.7848 & I & 460.3130 & 230.6601 & 443.2864 & 222.1468 & 442.3024 & 221.6548 & 4 \\
\hline 12 & 1183.6205 & 592.3139 & 1166.5939 & 583.8006 & 1165.6099 & 583.3086 & $\mathbf{T}$ & 347.2289 & 174.1181 & 330.2023 & 165.6048 & 329.2183 & 165.1128 & 3 \\
\hline 13 & 1282.6889 & 641.8481 & 1265.6624 & 633.3348 & 1264.6783 & 632.8428 & $\mathrm{~V}$ & 246.1812 & 123.5942 & 229.1547 & 115.0810 & & & 2 \\
\hline 14 & & & & & & & $\mathbf{K}$ & 147.1128 & 74.0600 & 130.0863 & 65.5468 & & & 1 \\
\hline
\end{tabular}
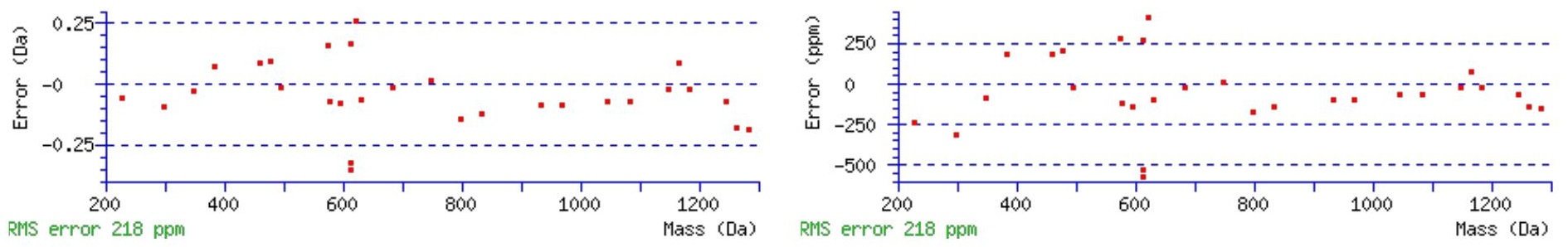

\section{All matches to this query}

\begin{tabular}{|l|l|l|l|}
\hline Score & Mr(calc): & Delta & \multicolumn{1}{c|}{ Sequence } \\
\hline 63.5 & 1427.7872 & 0.0029 & AIPTLVSDGNITVK \\
\hline 5.0 & 1427.7871 & 0.0029 & DLPTLSNVQLISK \\
\hline 4.8 & 1426.7780 & 1.0121 & NVIAPKGQEGSISK \\
\hline 4.1 & 1427.7959 & -0.0059 & VLKELGFCVHVK \\
\hline 3.2 & 1427.8024 & -0.0124 & ALLTVQEPPSFVK \\
\hline 1.5 & 1425.7745 & 2.0156 & LQLGTPPPLLAAR \\
\hline 1.2 & 1427.7901 & -0.0001 & SVLILPPRAGTPK \\
\hline 0.9 & 1427.7844 & 0.0056 & ALRTENRNLLAR \\
\hline 0.9 & 1427.7806 & 0.0094 & LAVLPEDRLQMK \\
\hline 0.8 & 1425.7969 & 1.9931 & $\underline{\text { LARPPRLPVATR }}$ \\
\hline
\end{tabular}

Spectrum No: 468; Query: 1766; Rank: 1

\section{Peptide View}

MS/MS Fragmentation of EGFSANYSVLQSSISEDFK

Found in IPI00212307, Tax_Id=10116 Gene_Symbol=Nrp1 Neuropilin-1 precursor

Match to Query 1766: 2107.966568 from(1054.990560,2+)

Title: 100101RatKid_NS_deglyco_25.4615.4615.2.dta

Data file K:INewmanPaper|Piliangl3SubProteomes\Piliang3SP\mgf5ppm\ERLIC_3SubProteomes5ppm.mgf 

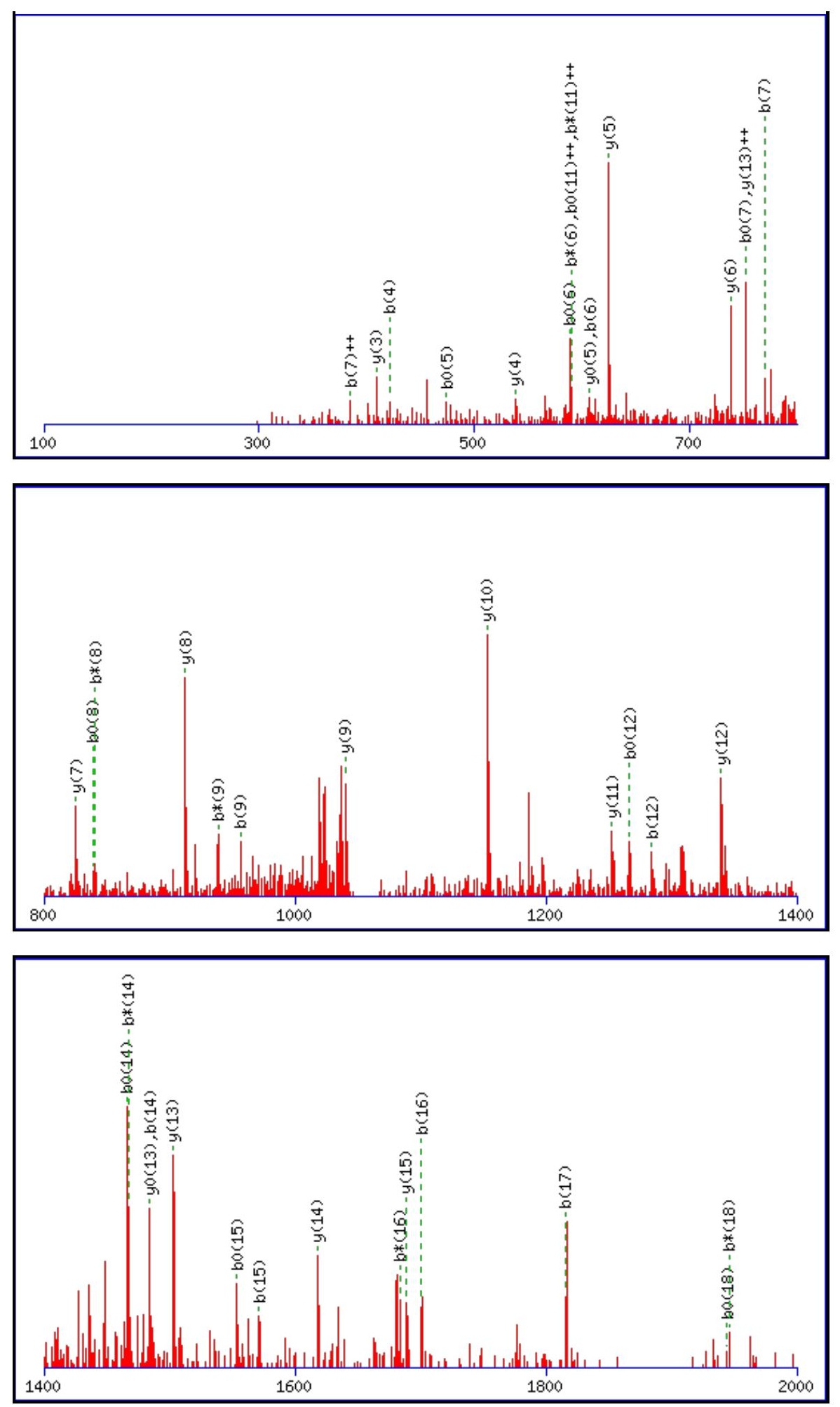

Monoisotopic mass of neutral peptide $\operatorname{Mr}($ calc): 2107.9586

Fixed modifications: Carbamidomethyl (C)

Variable modifications:

N6: Deamidated $\mathrm{N}(\mathrm{N})$

Ions Score: 63 Expect: 0.00012

Matches (Bold Red): 42/202 fragment ions using 71 most intense peaks

\begin{tabular}{|r|c|c|c|c|c|c|c|c|c|c|c|c|c|c|}
\hline$\#$ & $\mathbf{b}$ & $\mathbf{b}^{++}$ & $\mathbf{b}^{*}$ & $\mathbf{b}^{\boldsymbol{*}^{++}}$ & $\mathbf{b}^{\mathbf{0}}$ & $\mathbf{b}^{\mathbf{0 + +}}$ & Seq. & $\mathbf{y}$ & $\mathbf{y}^{++}$ & $\mathbf{y}^{\mathbf{*}}$ & $\mathbf{y}^{\boldsymbol{*}^{++}}$ & $\mathbf{y}^{\mathbf{0}}$ & $\mathbf{y}^{\mathbf{0 + +}}$ & $\#$ \\
\hline $\mathbf{1}$ & 130.0499 & 65.5286 & & & 112.0393 & 56.5233 & $\mathbf{E}$ & & & & & & & $\mathbf{1 9}$ \\
\hline $\mathbf{2}$ & 187.0713 & 94.0393 & & & 169.0608 & 85.0340 & $\mathbf{G}$ & 1979.9233 & 990.4653 & 1962.8967 & 981.9520 & 1961.9127 & 981.4600 & $\mathbf{1 8}$ \\
\hline $\mathbf{3}$ & 334.1397 & 167.5735 & & & 316.1292 & 158.5682 & $\mathbf{F}$ & 1922.9018 & 961.9545 & 1905.8753 & 953.4413 & 1904.8912 & 952.9493 & $\mathbf{1 7}$ \\
\hline $\mathbf{4}$ & $\mathbf{4 2 1 . 1 7 1 8}$ & 211.0895 & & & 403.1612 & 202.0842 & S & 1775.8334 & 888.4203 & 1758.8068 & 879.9071 & 1757.8228 & 879.4151 & $\mathbf{1 6}$ \\
\hline $\mathbf{5}$ & 492.2089 & 246.6081 & & & $\mathbf{4 7 4 . 1 9 8 3}$ & 237.6028 & $\mathbf{A}$ & $\mathbf{1 6 8 8 . 8 0 1 4}$ & 844.9043 & 1671.7748 & 836.3910 & 1670.7908 & 835.8990 & $\mathbf{1 5}$ \\
\hline
\end{tabular}




\begin{tabular}{|c|c|c|c|c|c|c|c|c|c|c|c|c|c|c|}
\hline 6 & 607.2358 & |304.1216 & 590.2093 & 295.6083 & 589.2253 & |295.1163 & $\mathbf{N}$ & |1617.7643 & |809.3858 & |1600.7377 & 800.8725 & |1599.7537 & |800.3805| & 14 \\
\hline 7 & 770.2992 & 385.6532 & 753.2726 & 377.1399 & 752.2886 & 376.6479 & $\mathbf{Y}$ & 1502.7373 & 751.8723 & 1485.7108 & 743.3590 & 1484.7268 & 742.8670 & 13 \\
\hline 8 & 857.3312 & 429.1692 & 840.3046 & 420.6560 & 839.3206 & 420.1639 & $\mathbf{S}$ & 1339.6740 & 670.3406 & 1322.6474 & 661.8274 & 1321.6634 & 661.3353 & 12 \\
\hline 9 & 956.3996 & 478.7034 & 939.3730 & 470.1902 & 938.3890 & 469.6982 & V & 1252.6420 & 626.8246 & 1235.6154 & 618.3113 & 1234.6314 & 617.8193 & 11 \\
\hline 10 & 1069.4837 & 535.2455 & 1052.4571 & 526.7322 & 1051.4731 & 526.2402 & $\mathbf{L}$ & 1153.5735 & 577.2904 & 1136.5470 & 568.7771 & 1135.5630 & 568.2851 & 10 \\
\hline 11 & 1197.5422 & 599.2748 & 1180.5157 & 590.7615 & 1179.5317 & 590.2695 & $\mathbf{Q}$ & 1040.4895 & 520.7484 & 1023.4629 & 512.2351 & 1022.4789 & 511.7431 & 9 \\
\hline 12 & 1284.5743 & 642.7908 & 1267.5477 & 634.2775 & 1266.5637 & 633.7855 & $\mathbf{S}$ & 912.4309 & 456.7191 & 895.4044 & 448.2058 & 894.4203 & 447.7138 & 8 \\
\hline 13 & 1371.6063 & 686.3068 & 1354.5797 & 677.7935 & 1353.5957 & 677.3015 & S & 825.3989 & 413.2031 & 808.3723 & 404.6898 & 807.3883 & 404.1978 & 7 \\
\hline 14 & 1484.6904 & 742.8488 & 1467.6638 & 734.3355 & 1466.6798 & 733.8435 & I & 738.3668 & 369.6871 & 721.3403 & 361.1738 & 720.3563 & 360.6818 & 6 \\
\hline 15 & 1571.7224 & 786.3648 & 1554.6958 & 777.8516 & 1553.7118 & 777.3595 & $\mathrm{S}$ & 625.2828 & 313.1450 & 608.2562 & 304.6318 & 607.2722 & 304.1397 & 5 \\
\hline 16 & 1700.7650 & 850.8861 & 1683.7384 & 842.3729 & 1682.7544 & 841.8808 & $\mathbf{E}$ & 538.2508 & 269.6290 & 521.2242 & 261.1157 & 520.2402 & 260.6237 & 4 \\
\hline 17 & 1815.7919 & 908.3996 & 1798.7654 & 899.8863 & 1797.7814 & 899.3943 & D & 409.2082 & 205.1077 & 392.1816 & 196.5944 & 391.1976 & 196.1024 & 3 \\
\hline 18 & 1962.8603 & 981.9338 & 1945.8338 & 973.4205 & 1944.8498 & 972.9285 & $\mathbf{F}$ & 294.1812 & 147.5942 & 277.1547 & 139.0810 & & & 2 \\
\hline 19 & & & & & & & $\mathbf{K}$ & 147.1128 & 74.0600 & 130.0863 & 65.5468 & & & 1 \\
\hline
\end{tabular}
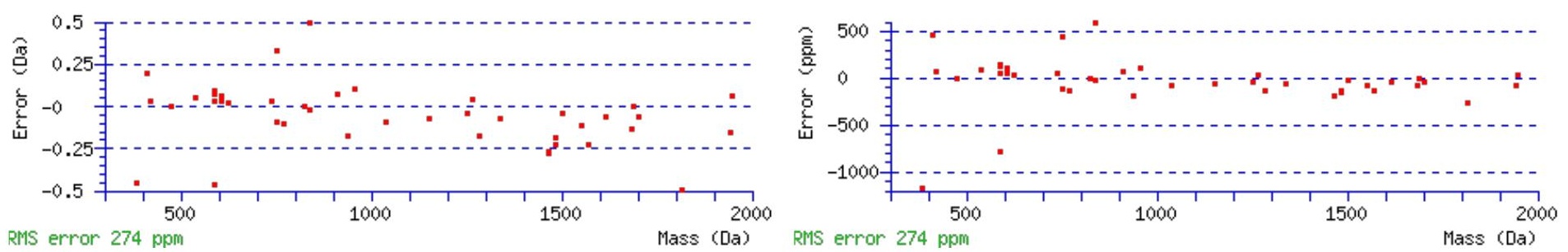

\section{All matches to this query}

\begin{tabular}{|l|l|l|l|}
\hline Score & Mr(calc): & Delta & \multicolumn{1}{c|}{ Sequence } \\
\hline 63.4 & 2107.9586 & 0.0080 & EGFSANYSVLQSSISEDFK \\
\hline 40.9 & 2106.9746 & 0.9920 & EGFSANYSVLQSSISEDFK \\
\hline 3.3 & 2107.9691 & -0.0025 & SIISETSNKIDTEIASLK \\
\hline 3.0 & 2105.9499 & 2.0166 & RPMQQMQPTAPRNSNLR \\
\hline 2.4 & 2107.9691 & -0.0025 & SIISETSNKIDTEIASLK \\
\hline 1.8 & 2105.9656 & 2.0010 & LESQMMAMVERHETQVR \\
\hline 1.8 & 2105.9656 & 2.0010 & LESQMMAMVERHETQVR \\
\hline 1.1 & 2107.9510 & 0.0156 & KSNHLNHCDLSNILPHK \\
\hline
\end{tabular}

Spectrum No: 469; Query: 1742; Rank: 1

\section{Peptide View}

MS/MS Fragmentation of IYHPNINSNGSICLDILR

Found in IPI00192159, Tax_Id=10116 Gene_Symbol=Ube2d3 Ubiquitin-conjugating enzyme E2 D3

Match to Query 1742: 2099.048412 from(700.690080,3+)

Title: 091008RatKidney_NH4Format01_07.3658.3658.3.dta

Data file K:\NewmanPaper\Piliang\3SubProteomes\Piliang3SP\mgf5ppm\ERLIC_3SubProteomes5ppm.mgf 

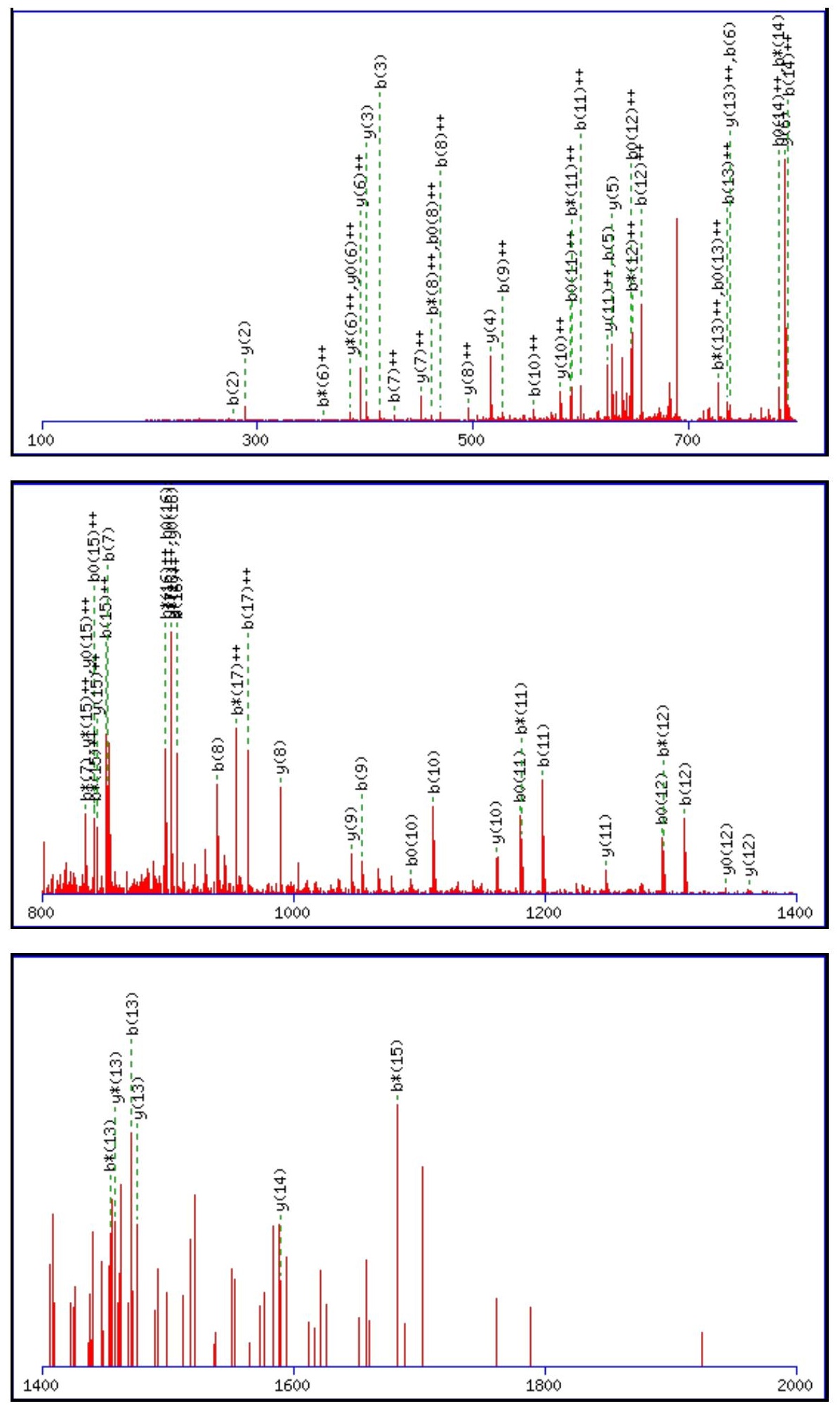

Monoisotopic mass of neutral peptide $\operatorname{Mr}($ calc): 2099.0469

Fixed modifications: Carbamidomethyl (C)

Variable modifications:

N9 : Deamidated_N (N)

Ions Score: 63 Expect : 8.8e- 005

Matches (Bold Red): 74/176 fragment ions using 130 most intense peaks

\begin{tabular}{|r|c|c|c|c|c|c|c|c|c|c|c|c|c|c|}
\hline$\#$ & $\mathbf{b}$ & $\mathbf{b}^{++}$ & $\mathbf{b}^{*}$ & $\mathbf{b}^{\mathbf{*}^{++}}$ & $\mathbf{b}^{\mathbf{0}}$ & $\mathbf{b}^{\mathbf{0 + +}}$ & Seq. & $\mathbf{y}$ & $\mathbf{y}^{++}$ & $\mathbf{y}^{\mathbf{*}}$ & $\mathbf{y}^{\boldsymbol{*}^{++}}$ & $\mathbf{y}^{\mathbf{0}}$ & $\mathbf{y}^{\mathbf{0 + +}}$ & $\#$ \\
\hline $\mathbf{1}$ & 114.0913 & 57.5493 & & & & & $\mathbf{I}$ & & & & & & & $\mathbf{1 8}$ \\
\hline $\mathbf{2}$ & $\mathbf{2 7 7 . 1 5 4 7}$ & 139.0810 & & & & & $\mathbf{Y}$ & 1986.9702 & 993.9887 & 1969.9436 & 985.4755 & 1968.9596 & 984.9834 & $\mathbf{1 7}$ \\
\hline $\mathbf{3}$ & $\mathbf{4 1 4 . 2 1 3 6}$ & 207.6104 & & & & & $\mathbf{H}$ & 1823.9068 & 912.4571 & 1806.8803 & $\mathbf{9 0 3 . 9 4 3 8}$ & 1805.8963 & $\mathbf{9 0 3 . 4 5 1 8}$ & $\mathbf{1 6}$ \\
\hline $\mathbf{4}$ & 511.2663 & 256.1368 & & & & & $\mathbf{P}$ & 1686.8479 & $\mathbf{8 4 3 . 9 2 7 6}$ & 1669.8214 & $\mathbf{8 3 5 . 4 1 4 3}$ & 1668.8374 & $\mathbf{8 3 4 . 9 2 2 3}$ & $\mathbf{1 5}$ \\
\hline $\mathbf{5}$ & $\mathbf{6 2 5 . 3 0 9 3}$ & 313.1583 & 608.2827 & 304.6450 & & & $\mathbf{N}$ & $\mathbf{1 5 8 9 . 7 9 5 2}$ & 795.4012 & 1572.7686 & 786.8880 & 1571.7846 & 786.3959 & $\mathbf{1 4}$ \\
\hline
\end{tabular}




\begin{tabular}{|c|c|c|c|c|c|c|c|c|c|c|c|c|c|c|}
\hline 6 & 738.3933 & 369.7003 & 721.3668 & 361.1870 & & & I & 1475.7522 & |738.3798 & | 1458.7257 & 729.8665 & 1457.7417 & \begin{tabular}{|l|}
729.3745 \\
\end{tabular} & 13 \\
\hline 7 & 852.4363 & 426.7218 & 835.4097 & 418.2085 & & & $\mathbf{N}$ & 1362.6682 & 681.8377 & 1345.6416 & 673.3245 & 1344.6576 & 672.8324 & 12 \\
\hline 8 & 939.4683 & 470.2378 & 922.4417 & 461.7245 & 921.4577 & 461.2325 & S & 1248.6253 & 624.8163 & 1231.5987 & 616.3030 & 1230.6147 & 615.8110 & 11 \\
\hline 9 & 1054.4952 & 527.7513 & 1037.4687 & 519.2380 & 1036.4847 & 518.7460 & $\mathbf{N}$ & 1161.5932 & 581.3003 & |1144.5667 & 572.7870 & 1143.5827 & 572.2950 & 10 \\
\hline 10 & 1111.5167 & 556.2620 & 1094.4901 & 547.7487 & 1093.5061 & 547.2567 & G & 1046.5663 & 523.7868 & 1029.5397 & 515.2735 & 1028.5557 & 514.7815 & 9 \\
\hline 11 & 1198.5487 & 599.7780 & 1181.5222 & 591.2647 & 1180.5382 & 590.7727 & $\mathrm{~S}$ & 989.5448 & |495.2761 & 972.5183 & 486.7628 & 971.5343 & 486.2708 & 8 \\
\hline 12 & 1311.6328 & 656.3200 & 1294.6062 & 647.8068 & 1293.6222 & 647.3147 & I & 902.5128 & 451.7600 & 885.4863 & 443.2468 & 884.5022 & 442.7548 & 7 \\
\hline 13 & 1471.6634 & 736.3354 & 1454.6369 & 727.8221 & 1453.6529 & 727.3301 & $\mathrm{C}$ & 789.4287 & 395.2180 & 772.4022 & 386.7047 & 771.4182 & 386.2127 & 6 \\
\hline 14 & 1584.7475 & 792.8774 & 1567.7209 & 784.3641 & 1566.7369 & 783.8721 & $\mathbf{L}$ & 629.3981 & 315.2027 & 612.3715 & 306.6894 & 611.3875 & 306.1974 & 5 \\
\hline 15 & 1699.7744 & 850.3909 & 1682.7479 & 841.8776 & 1681.7639 & 841.3856 & D & 516.3140 & 258.6606 & 499.2875 & 250.1474 & 498.3035 & 249.6554 & 4 \\
\hline 16 & 1812.8585 & 906.9329 & 1795.8320 & 898.4196 & 1794.8479 & 897.9276 & I & 401.2871 & 201.1472 & 384.2605 & 192.6339 & & & 3 \\
\hline 17 & 1925.9426 & 963.4749 & 1908.9160 & 954.9616 & 1907.9320 & 954.4696 & $\mathbf{L}$ & 288.2030 & 144.6051 & 271.1765 & 136.0919 & & & 2 \\
\hline 18 & & & & & & & $\mathbf{R}$ & 175.1190 & 88.0631 & 158.0924 & 79.5498 & & & 1 \\
\hline
\end{tabular}
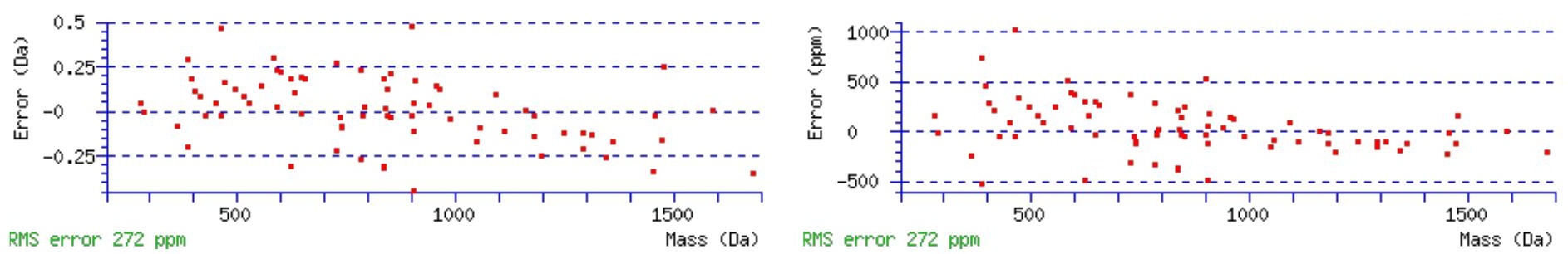

\section{All matches to this query}

\begin{tabular}{|l|l|l|l|}
\hline Score & Mr(calc): & Delta & \multicolumn{1}{|c|}{ Sequence } \\
\hline 63.4 & 2099.0469 & 0.0015 & $\underline{\text { IYHPNINSNGSICLDILR }}$ \\
\hline 48.3 & 2099.0469 & 0.0015 & $\underline{\text { IYHPNINSNGSICLDILR }}$ \\
\hline 41.0 & 2099.0469 & 0.0015 & $\underline{\text { IYHPNINSNGSICLDILR }}$ \\
\hline 39.3 & 2098.0629 & 0.9855 & $\underline{\text { IYHPNINSNGSICLDILR }}$ \\
\hline 14.6 & 2099.0663 & -0.0179 & $\underline{\text { RKINLNISYEINEVSVK }}$ \\
\hline 8.7 & 2098.0360 & 1.0124 & $\underline{\text { FLEELRLSGNHLSHIPR }}$ \\
\hline 8.3 & 2099.0663 & -0.0179 & RKINLNISYEINEVSVK \\
\hline 5.1 & 2099.0485 & -0.0001 & LNKENNELYLELMKLR \\
\hline 4.6 & 2098.0489 & 0.9995 & LSLKFRNGLTLSPVTHR \\
\hline 2.9 & 2099.0486 & -0.0002 & RLMSSILTSIDASKPWSK \\
\hline
\end{tabular}

Spectrum No: 470; Query: 157; Rank: 1

\section{Peptide View}

MS/MS Fragmentation of IYNASELPVR

Found in IPI00369937, Tax_Id=10116 Gene_Symbol=Pigs GPI transamidase component PIG-S

Match to Query 157: 1161.601388 from(581.807970,2+)

Title: 091008RatKidney_NH4Format01_23.1693.1693.2.dta

Data file K:INewmanPaper|Piliangl3SubProteomes\Piliang3SP\mgf5ppm\ERLIC_3SubProteomes5ppm.mgf 

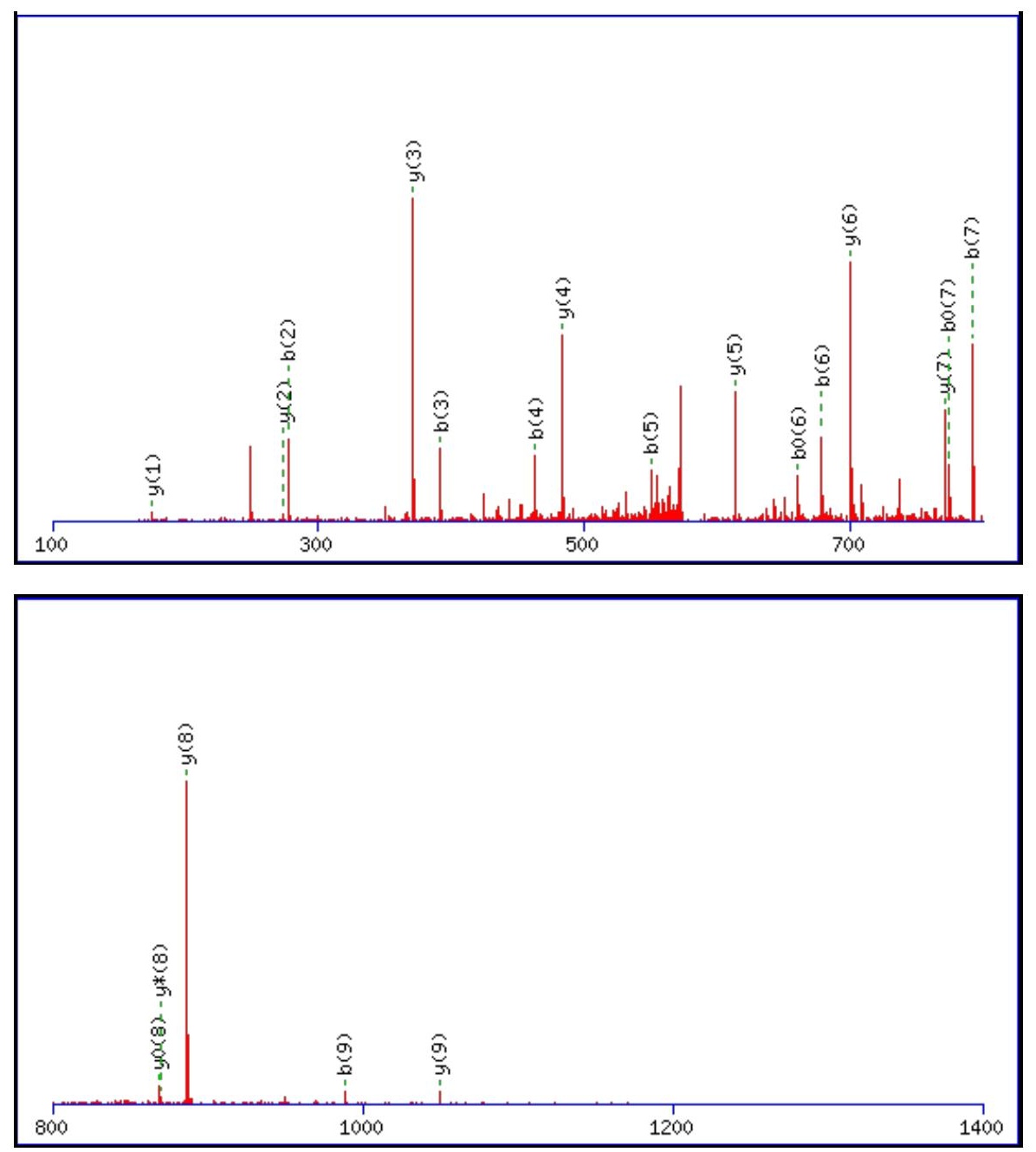

\begin{tabular}{rrrr}
\hline & 1 & 1 & 2000 \\
\hline
\end{tabular}

Monoisotopic mass of neutral peptide $\operatorname{Mr}($ calc): 1161.6030

Fixed modifications: Carbamidomethyl (C)

Variable modifications:

N3 : Deamidated $\mathrm{N}(\mathrm{N})$

Ions Score: 63 Expect : $5 \mathrm{e}-005$

Matches (Bold Red): 20/88 fragment ions using 41 most intense peaks

\begin{tabular}{|c|c|c|c|c|c|c|c|c|c|c|c|c|c|c|}
\hline$\#$ & $\mathbf{b}$ & $\mathbf{b}^{++}$ & $\mathbf{b}^{*}$ & $\mathbf{b}^{\boldsymbol{*}_{+}^{++}}$ & $\mathbf{b}^{\mathbf{0}}$ & $\mathbf{b}^{\mathbf{0 + +}}$ & $\mathbf{S e q}$ & $\mathbf{y}$ & $\mathbf{y}^{++}$ & $\mathbf{y}^{*}$ & $\mathbf{y}^{\mathbf{*}^{++}}$ & $\mathbf{y}^{\mathbf{0}}$ & $\mathbf{y}^{\mathbf{0}++}$ & $\#$ \\
\hline $\mathbf{1}$ & 114.0913 & 57.5493 & & & & & $\mathbf{I}$ & & & & & & & $\mathbf{1 0}$ \\
\hline $\mathbf{2}$ & 277.1547 & 139.0810 & & & & & $\mathbf{Y}$ & $\mathbf{1 0 4 9 . 5 2 6 2}$ & 525.2667 & 1032.4996 & 516.7535 & 1031.5156 & 516.2615 & $\mathbf{9}$ \\
\hline $\mathbf{3}$ & 392.1816 & 196.5944 & 375.1551 & 188.0812 & & & $\mathbf{N}$ & $\mathbf{8 8 6 . 4 6 2 9}$ & 443.7351 & $\mathbf{8 6 9 . 4 3 6 3}$ & 435.2218 & $\mathbf{8 6 8 . 4 5 2 3}$ & 434.7298 & $\mathbf{8}$ \\
\hline $\mathbf{4}$ & $\mathbf{4 6 3 . 2 1 8 7}$ & 232.1130 & 446.1922 & 223.5997 & & & $\mathbf{A}$ & 771.4359 & 386.2216 & 754.4094 & 377.7083 & 753.4254 & 377.2163 & $\mathbf{7}$ \\
\hline $\mathbf{5}$ & $\mathbf{5 5 0 . 2 5 0 7}$ & 275.6290 & 533.2242 & 267.1157 & 532.2402 & 266.6237 & $\mathbf{S}$ & $\mathbf{7 0 0 . 3 9 8 8}$ & 350.7030 & 683.3723 & 342.1898 & 682.3883 & 341.6978 & $\mathbf{6}$ \\
\hline
\end{tabular}




\begin{tabular}{|c|c|c|c|c|c|c|c|c|c|c|c|c|c|c|}
\hline 6 & |679.2933 & |340.1503| & |662.2668 & $|331.6370|$ & $\mid 661.2828$ & |331.1450 & $\mathbf{E}$ & 613.3668 & |307.1870 | & 596.3402 & |298.6738 & 595.3562 & 298.1817 & 5 \\
\hline 7 & 792.3774 & 396.6923 & 775.3509 & 388.1791 & 774.3668 & 387.6871 & $\mathbf{L}$ & 484.3242 & 242.6657 & 467.2976 & 234.1525 & & & 4 \\
\hline 8 & 889.4302 & 445.2187 & 872.4036 & 436.7054 & 871.4196 & 436.2134 & $\mathbf{P}$ & 371.2401 & 186.1237 & 354.2136 & 177.6104 & & & 3 \\
\hline 9 & 988.4986 & 494.7529 & 971.4720 & 486.2397 & 970.4880 & 485.7476 & $\mathrm{~V}$ & 274.1874 & 137.5973 & 257.1608 & 129.0840 & & & 2 \\
\hline 10 & & & & & & & $\mathbf{R}$ & 175.1190 & 88.0631 & 158.0924 & 79.5498 & & & 1 \\
\hline
\end{tabular}
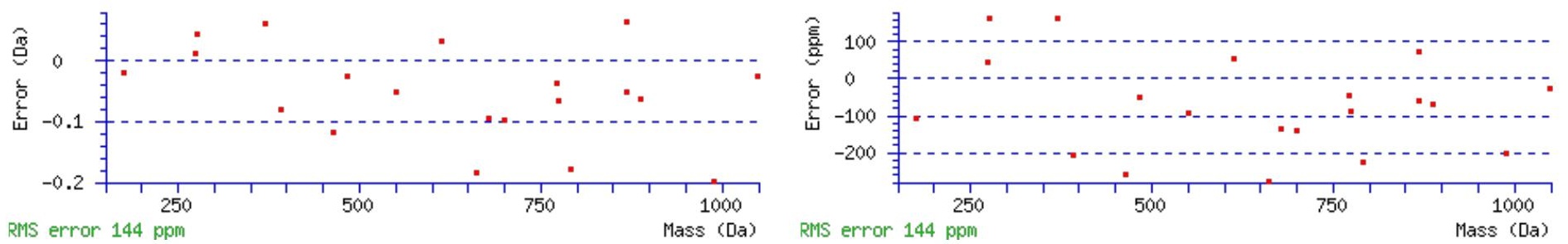

\section{All matches to this query}

\begin{tabular}{|l|c|c|c|}
\hline Score & Mr(calc): & Delta & Sequence \\
\hline 63.4 & 1161.6030 & -0.0016 & $\underline{\text { IYNASELPVR }}$ \\
\hline 14.9 & 1161.5907 & 0.0107 & HLVLSSGTLR \\
\hline 8.6 & 1160.5972 & 1.0042 & NKQIKCNEK \\
\hline 6.4 & 1161.5907 & 0.0107 & $\underline{\text { HLVLSSGTLR }}$ \\
\hline 6.2 & 1161.6030 & -0.0016 & $\underline{\text { LLLNGFDDVR }}$ \\
\hline 5.9 & 1161.5998 & 0.0016 & $\underline{\text { ICXTOKLMR }}$ \\
\hline 5.3 & 1161.5907 & 0.0107 & $\underline{\text { HLVLSSGTLR }}$ \\
\hline 4.6 & 1161.5989 & 0.0024 & TRLDSSNLEK \\
\hline 3.4 & 1161.6030 & -0.0016 & $\underline{\text { SSTLPELPYR }}$ \\
\hline 2.6 & 1159.6019 & 1.9995 & $\underline{\text { EKELCQVVR }}$ \\
\hline
\end{tabular}

Spectrum No: 471; Query: 584; Rank: 1

\section{Peptide View}

MS/MS Fragmentation of NSTLCDLCIGPAK

Found in IPI00196656, Tax_Id=10116 Gene_Symbol=Srprb Ba1-667

Match to Query 584: 1448.667588 from(725.341070,2+)

Title: 100101RatKid_NS_deglyco_25.2621.2621.2.dta

Data file K:INewmanPaper|Piliang|3SubProteomes\Piliang3SP\mgf5ppm\ERLIC_3SubProteomes5ppm.mgf

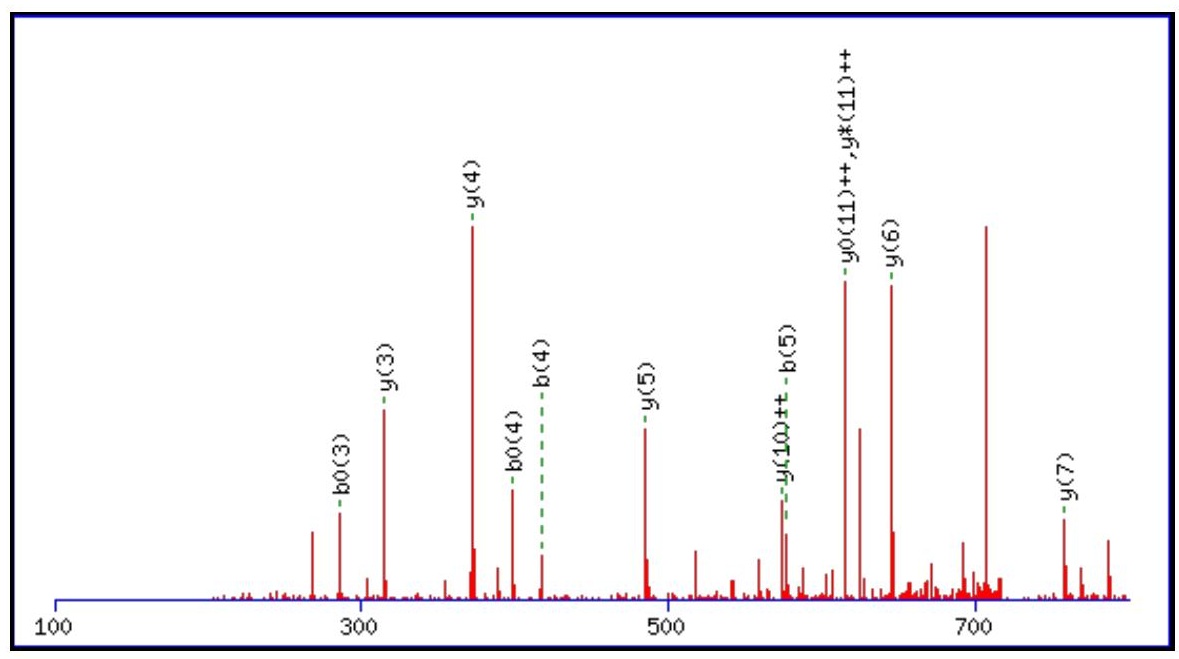



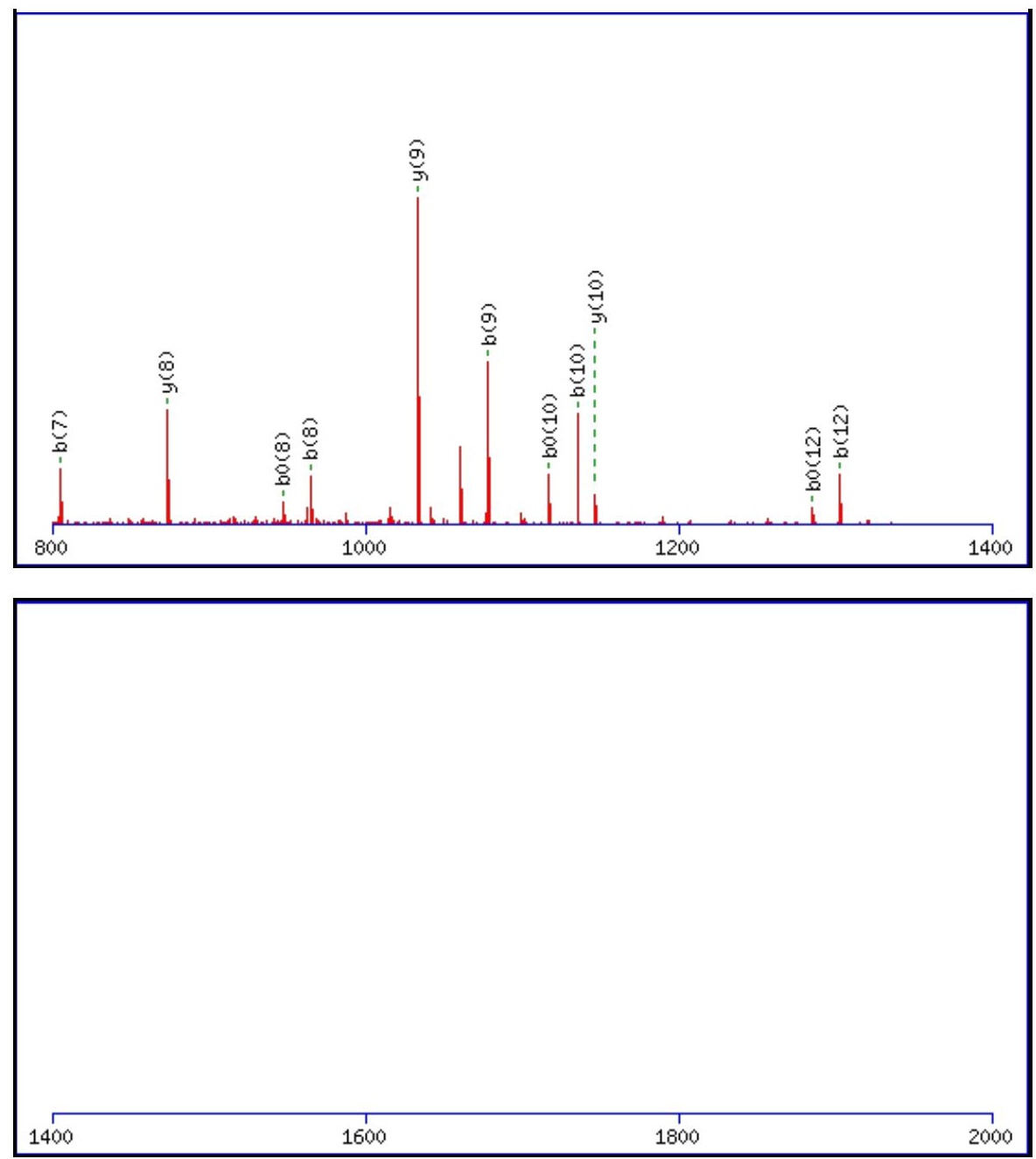

Monoisotopic mass of neutral peptide $\operatorname{Mr}($ calc): 1448.6639

Fixed modifications: Carbamidomethyl (C)

Variable modifications:

N1 : Deamidated $\mathrm{N}(\mathrm{N})$

Ions Score: 63 Expect: $6.9 \mathrm{e}-005$

Matches (Bold Red): 23/128 fragment ions using 31 most intense peaks

\begin{tabular}{|c|c|c|c|c|c|c|c|c|c|c|c|c|c|c|}
\hline \# & b & $\mathbf{b}^{++}$ & $\mathbf{b}^{*}$ & $\mathbf{b}^{*^{++}}$ & $\mathbf{b}^{0}$ & $\mathbf{b}^{0++}$ & Seq. & $\mathbf{y}$ & $\mathbf{y}^{++}$ & $\mathbf{y}^{*}$ & $\mathrm{y}^{*^{++}}$ & $\mathbf{y}^{\mathbf{0}}$ & $\mathbf{y}^{0++}$ & \# \\
\hline 1 & 116.0342 & 58.5207 & 99.0077 & 50.0075 & & & $\mathbf{N}$ & & & & & & & 13 \\
\hline 2 & 203.0662 & 102.0368 & 186.0397 & 93.5235 & 185.0557 & 93.0315 & S & 1334.6443 & 667.8258 & 1317.6177 & 659.3125 & 1316.6337 & 658.8205 & 12 \\
\hline 3 & 304.1139 & 152.5606 & 287.0874 & 144.0473 & 286.1034 & 143.5553 & $\mathbf{T}$ & 1247.6123 & 624.3098 & 1230.5857 & 615.7965 & 1229.6017 & 615.3045 & 11 \\
\hline 4 & 417.1980 & 209.1026 & 400.1714 & 200.5894 & 399.1874 & 200.0973 & $\mathbf{L}$ & 1146.5646 & 573.7859 & 1129.5380 & 565.2727 & 1128.5540 & 564.7806 & 10 \\
\hline 5 & 577.2286 & 289.1180 & 560.2021 & 280.6047 & 559.2181 & 280.1127 & C & 1033.4805 & 517.2439 & 1016.4540 & 508.7306 & 1015.4699 & 508.2386 & 9 \\
\hline 6 & 692.2556 & 346.6314 & 675.2290 & 338.1182 & 674.2450 & 337.6261 & D & 873.4499 & 437.2286 & 856.4233 & 428.7153 & 855.4393 & 428.2233 & 8 \\
\hline 7 & 805.3396 & 403.1735 & 788.3131 & 394.6602 & 787.3291 & 394.1682 & $\mathbf{L}$ & 758.4229 & 379.7151 & 741.3964 & 371.2018 & & & 7 \\
\hline 8 & 965.3703 & 483.1888 & 948.3437 & 474.6755 & 947.3597 & 474.1835 & $\mathrm{C}$ & 645.3389 & 323.1731 & 628.3123 & 314.6598 & & & 6 \\
\hline 9 & 1078.4544 & 539.7308 & 1061.4278 & 531.2175 & 1060.4438 & 530.7255 & I & 485.3082 & 243.1577 & 468.2817 & 234.6445 & & & 5 \\
\hline 10 & 1135.4758 & 568.2415 & 1118.4493 & 559.7283 & 1117.4653 & 559.2363 & G & 372.2241 & 186.6157 & 355.1976 & 178.1024 & & & 4 \\
\hline 11 & 1232.5286 & 616.7679 & 1215.5020 & 608.2547 & 1214.5180 & 607.7626 & $\mathbf{P}$ & 315.2027 & 158.1050 & 298.1761 & 149.5917 & & & 3 \\
\hline 12 & 1303.5657 & 652.2865 & 1286.5391 & 643.7732 & 1285.5551 & 643.2812 & A & 218.1499 & 109.5786 & 201.1234 & 101.0653 & & & 2 \\
\hline 13 & & & & & & & $\mathbf{K}$ & 147.1128 & 74.0600 & 130.0863 & 65.5468 & & & 1 \\
\hline
\end{tabular}



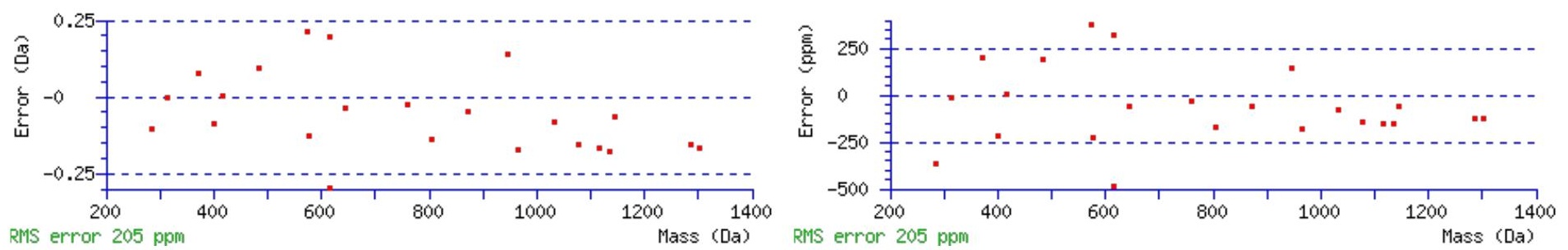

\section{All matches to this query}

\begin{tabular}{|l|l|l|l|}
\hline Score & Mr(calc): & Delta & \multicolumn{1}{|c|}{ Sequence } \\
\hline 63.3 & 1448.6639 & 0.0036 & NSTLCDLCIGPAK \\
\hline 11.6 & 1448.6538 & 0.0138 & ASRTTGGATALXK \\
\hline 10.2 & 1448.6532 & 0.0144 & EGAGGGQDYPQTKAA \\
\hline 8.5 & 1448.6639 & 0.0036 & QDGAVAMEMQPLK \\
\hline 8.2 & 1448.6816 & -0.0141 & ELEEENMALRSK \\
\hline 7.1 & 1448.6701 & -0.0025 & YLGQDFETLRK \\
\hline 5.9 & 1448.6817 & -0.0141 & GKENTDSVVMQPK \\
\hline 5.8 & 1448.6817 & -0.0141 & ERVMEIVDADEK \\
\hline 5.8 & 1448.6622 & 0.0054 & NLGSLDVTIMYK \\
\hline 5.8 & 1448.6557 & 0.0119 & EQMFRLLMASK \\
\hline
\end{tabular}

Spectrum No: 472; Query: 646; Rank: 1

\section{Peptide View}

MS/MS Fragmentation of QSNGSIAIISSMAGK

Found in IPI00211096, Tax_Id=10116 Gene_Symbol=Hsd11b1 Isoform 11-HSD1A of Corticosteroid 11-beta-dehydrogenase isozyme 1

Match to Query 646: 1479.719588 from(740.867070,2+)

Title: 091008RatKidney_NH4Format01_25.1481.1481.2.dta

Data file K:INewmanPaper|Piliang\3SubProteomes|Piliang3SP\mgf5ppm\ERLIC_3SubProteomes5ppm.mgf

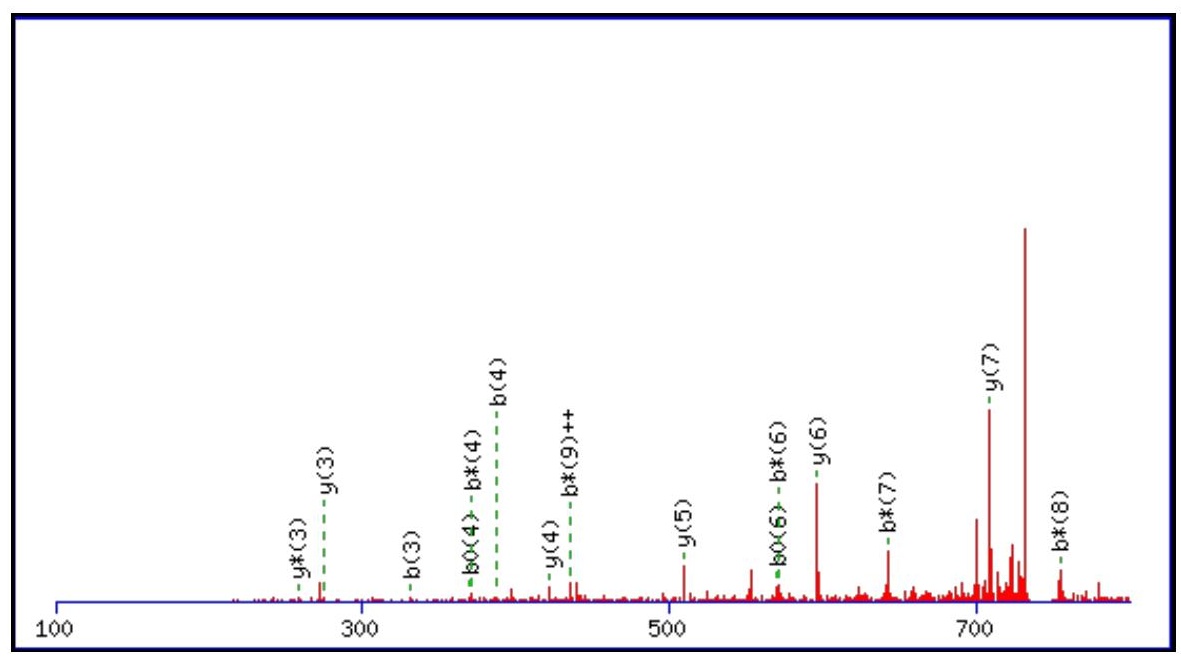



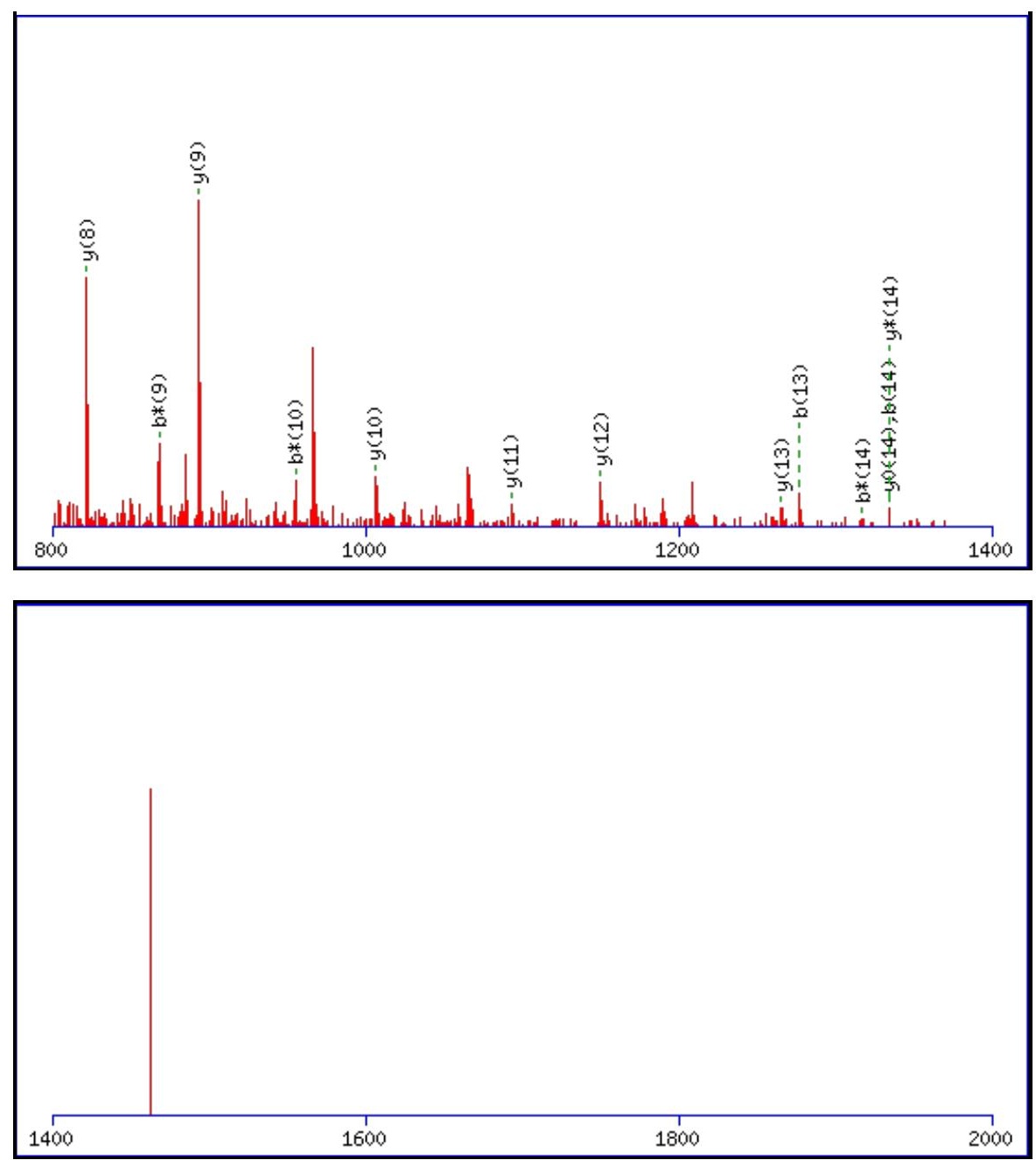

Monoisotopic mass of neutral peptide $\operatorname{Mr}($ calc): 1479.7239

Fixed modifications: Carbamidomethyl (C)

Variable modifications:

N3 : Deamidated_N (N)

M12 : Oxidation $(M)$

Ions Score: 63 Expect : $7.1 \mathrm{e}-005$

Matches (Bold Red): 28/158 fragment ions using 70 most intense peaks

\begin{tabular}{|c|c|c|c|c|c|c|c|c|c|c|c|c|c|c|}
\hline \# & b & $\mathbf{b}^{++}$ & $\mathbf{b}^{*}$ & $\mathbf{b}^{*^{++}}$ & $\mathbf{b}^{\mathbf{0}}$ & $\mathbf{b}^{\mathbf{0 + +}}$ & Seq. & $\mathbf{y}$ & $\mathbf{y}^{++}$ & $\mathrm{y}^{*}$ & $\mathrm{y}^{*^{++}}$ & $\mathbf{y}^{0}$ & $\mathbf{y}^{\mathbf{0 + +}}$ & \# \\
\hline 1 & 129.0659 & 65.0366 & 112.0393 & 56.5233 & & & $\mathbf{Q}$ & & & & & & & 15 \\
\hline 2 & 216.0979 & 108.5526 & 199.0713 & 100.0393 & 198.0873 & 99.5473 & $S$ & 1352.6726 & 676.8399 & 1335.6461 & 668.3267 & 1334.6620 & 667.8347 & 14 \\
\hline 3 & 331.1248 & 166.0660 & 314.0983 & 157.5528 & 313.1143 & 157.0608 & $\mathbf{N}$ & 1265.6406 & 633.3239 & 1248.6140 & 624.8107 & 1247.6300 & 624.3186 & 13 \\
\hline 4 & 388.1463 & 194.5768 & 371.1197 & 186.0635 & 370.1357 & 185.5715 & G & 1150.6136 & 575.8105 & 1133.5871 & 567.2972 & 1132.6031 & 566.8052 & 12 \\
\hline 5 & 475.1783 & 238.0928 & 458.1518 & 229.5795 & 457.1677 & 229.0875 & $S$ & 1093.5922 & 547.2997 & 1076.5656 & 538.7865 & 1075.5816 & 538.2944 & 11 \\
\hline 6 & 588.2624 & 294.6348 & 571.2358 & 286.1216 & 570.2518 & 285.6295 & I & 1006.5601 & 503.7837 & 989.5336 & 495.2704 & 988.5496 & 494.7784 & 10 \\
\hline 7 & 659.2995 & 330.1534 & 642.2729 & 321.6401 & 641.2889 & 321.1481 & A & 893.4761 & 447.2417 & 876.4495 & 438.7284 & 875.4655 & 438.2364 & 9 \\
\hline 8 & 772.3836 & 386.6954 & 755.3570 & 378.1821 & 754.3730 & 377.6901 & I & 822.4390 & 411.7231 & 805.4124 & 403.2098 & 804.4284 & 402.7178 & 8 \\
\hline 9 & 885.4676 & 443.2374 & 868.4411 & 434.7242 & 867.4571 & 434.2322 & I & 709.3549 & 355.1811 & 692.3284 & 346.6678 & 691.3443 & 346.1758 & 7 \\
\hline 10 & 972.4996 & 486.7535 & 955.4731 & 478.2402 & 954.4891 & 477.7482 & S & 596.2708 & 298.6391 & 579.2443 & 290.1258 & 578.2603 & 289.6338 & 6 \\
\hline 11 & 1059.5317 & 530.2695 & 1042.5051 & 521.7562 & 1041.5211 & 521.2642 & S & 509.2388 & 255.1230 & 492.2123 & 246.6098 & 491.2282 & 246.1178 & 5 \\
\hline 12 & 1206.5671 & 603.7872 & 1189.5405 & 595.2739 & 1188.5565 & 594.7819 & $\mathbf{M}$ & 422.2068 & 211.6070 & 405.1802 & 203.0938 & & & 4 \\
\hline 13 & 1277.6042 & 639.3057 & 1260.5776 & 630.7925 & 1259.5936 & 630.3005 & A & 275.1714 & 138.0893 & 258.1448 & 129.5761 & & & 3 \\
\hline 14 & 1334.6257 & 667.8165 & 1317.5991 & 659.3032 & 1316.6151 & 658.8112 & G & 204.1343 & 102.5708 & 187.1077 & 94.0575 & & & 2 \\
\hline 15 & & & & & & & $\mathbf{K}$ & 147.1128 & 74.0600 & 130.0863 & 65.5468 & & & 1 \\
\hline
\end{tabular}



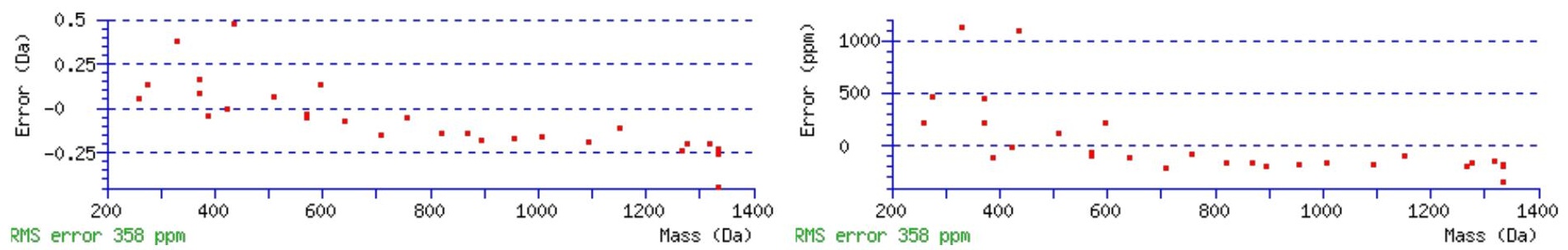

\section{All matches to this query}

\begin{tabular}{|l|l|l|l|}
\hline Score & Mr(calc): & Delta & \multicolumn{1}{|c|}{ Sequence } \\
\hline 63.3 & 1479.7239 & -0.0043 & QSNGSIAIISSMAGK \\
\hline 10.8 & 1479.7139 & 0.0057 & IKYKLSIVGDGK \\
\hline 7.9 & 1478.7221 & 0.9975 & RMEEKPLSSMQK \\
\hline 7.7 & 1479.7195 & 0.0001 & GSSQRLPVARNSK \\
\hline 7.7 & 1479.7195 & 0.0001 & GSSQRLPVARNSK \\
\hline 7.1 & 1479.7205 & -0.0009 & SNSSDTPPLPSPPGK \\
\hline 7.0 & 1479.7334 & -0.0138 & VSLANLKPSLNSR \\
\hline 6.9 & 1479.7334 & -0.0138 & LSSKVAAEVPQGSK \\
\hline 6.5 & 1479.7334 & -0.0138 & SQGPSLDISALKGK \\
\hline 6.4 & 1479.7235 & -0.0040 & KRVDPTVGWTGGK \\
\hline
\end{tabular}

Spectrum No: 473; Query: 2599; Rank: 1

\section{Peptide View}

MS/MS Fragmentation of VNYEAETWDWIAETLSSNHMNFSSADR

Found in IPI00327398, Tax_Id=10116 Gene_Symbol=Enpep Isoform 1 of Glutamyl aminopeptidase

Match to Query 2599: 3173.372922 from(1058.798250,3+)

Title: 100101RatKid_NS_deglyco_18.6588.6588.3.dta

Data file K:INewmanPaper|Piliangl3SubProteomes\Piliang3SP\mgf5ppm\ERLIC_3SubProteomes5ppm.mgf

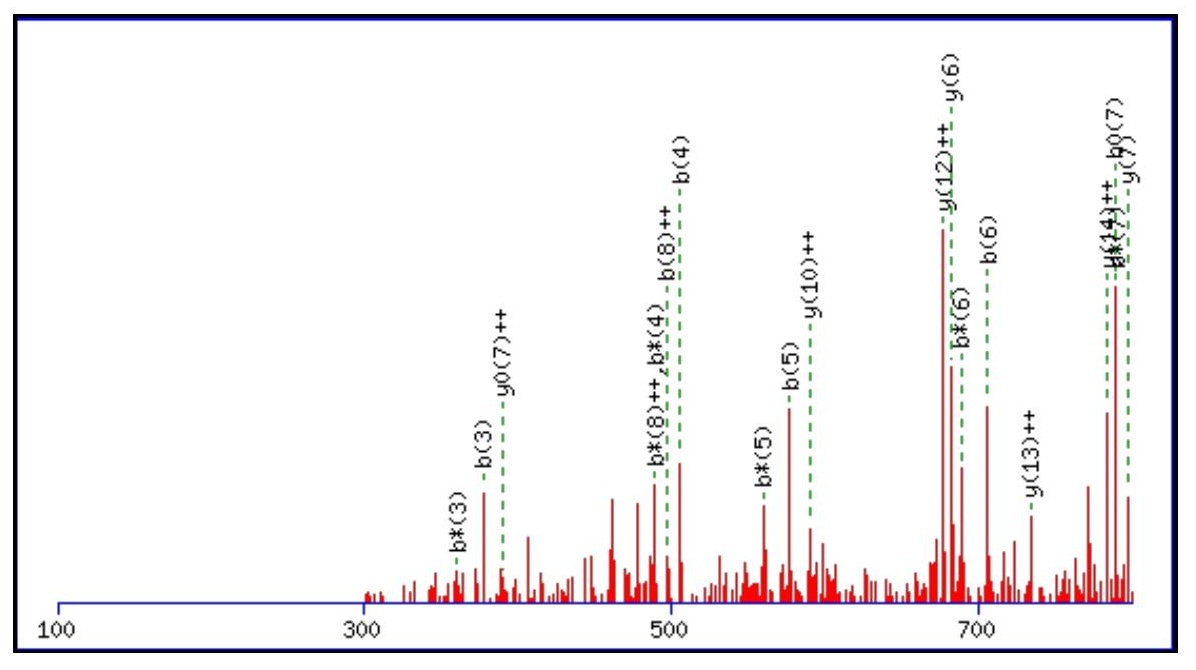



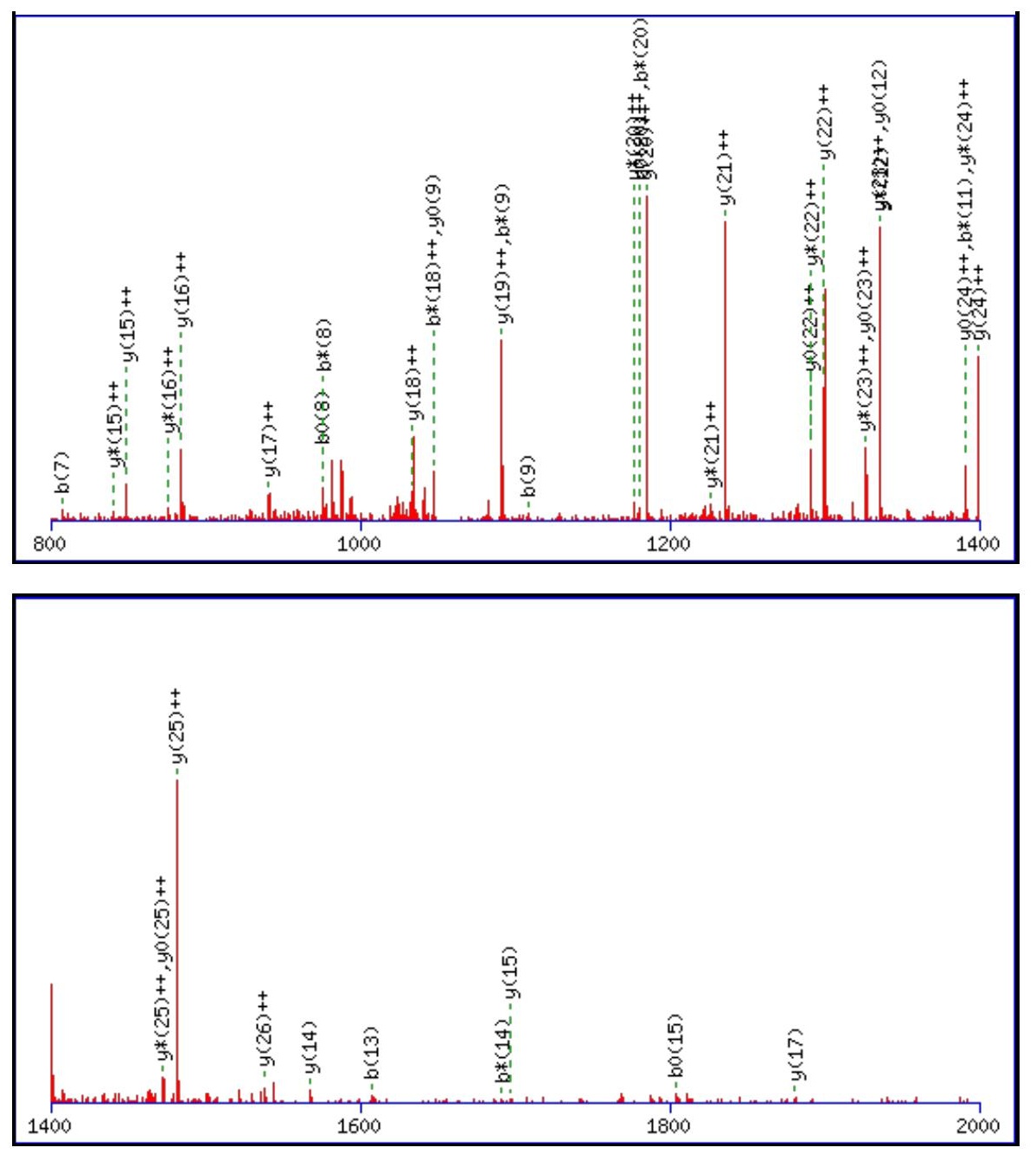

Monoisotopic mass of neutral peptide $\operatorname{Mr}($ calc): 3173.3672

Fixed modifications: Carbamidomethyl (C)

Variable modifications:

N21 : Deamidated_N (N)

Ions Score: 63 Expect: 0.00021

Matches (Bold Red): 61/302 fragment ions using 121 most intense peaks

\begin{tabular}{|c|c|c|c|c|c|c|c|c|c|c|c|c|c|c|}
\hline \# & b & $\mathbf{b}^{++}$ & $\mathbf{b}^{*}$ & $\mathbf{b}^{*^{++}}$ & $\mathbf{b}^{0}$ & $\mathbf{b}^{0++}$ & Seq. & $\mathbf{y}$ & $\mathbf{y}^{++}$ & $\mathbf{y}^{*}$ & $\mathbf{y}^{*^{++}}$ & $\mathbf{y}^{\mathbf{0}}$ & $\mathbf{y}^{\mathbf{0 + +}}$ & \# \\
\hline 1 & 100.0757 & 50.5415 & & & & & V & & & & & & & 27 \\
\hline 2 & 214.1186 & 107.5629 & 197.0921 & & & & $\mathbf{N}$ & 3075.3061 & 1538.1567 & 3058.2796 & & 2955 & 1529.1514 & 26 \\
\hline 3 & 377.1819 & 189.0946 & 360.1554 & 180.5813 & & & $\mathbf{Y}$ & 2961.2632 & 1481.1352 & 2944.2366 & 1472.6220 & 2943.2526 & 1472.1299 & 25 \\
\hline 4 & 506.2245 & 253.6159 & 489.1980 & 245.1026 & 488.2140 & 244.6106 & $\mathbf{E}$ & 2798.1999 & 1399.6036 & 2781.1733 & 1391.0903 & 2780.1893 & 1390.5983 & 24 \\
\hline 5 & 577.2617 & 289.1345 & 560.2351 & 280.6212 & 559.2511 & 280.1292 & A & 2669.1573 & 1335.0823 & 2652.1307 & 1326.5690 & 2651.1467 & 1326.0770 & 23 \\
\hline 6 & 706.3042 & 353.6558 & 689.2777 & 345.1425 & 688.2937 & 344.6505 & $\mathbf{E}$ & 2598.1201 & 1299.5637 & 2581.0936 & 1291.0504 & 2580.1096 & 1290.5584 & 22 \\
\hline 7 & 807.3519 & 404.1796 & 790.3254 & 395.6663 & 789.3414 & 395.1743 & $\mathbf{T}$ & 2469.0776 & 1235.0424 & 2452.0510 & 1226.5291 & 2451.0670 & 1226.0371 & 21 \\
\hline 8 & 993.4312 & 497.2193 & 976.4047 & 488.7060 & 975.4207 & 488.2140 & W & 2368.0299 & 1184.5186 & 2351.0033 & 1176.0053 & 2350.0193 & 1175.5133 & 20 \\
\hline 9 & 1108.4582 & 554.7327 & 1091.4316 & 546.2195 & 1090.4476 & 545.7274 & D & 2181.9506 & 1091.4789 & 2164.9240 & 1082.9656 & 2163.9400 & 1082.4736 & 19 \\
\hline 10 & 1294.5375 & 647.7724 & 1277.5109 & 639.2591 & 1276.5269 & 638.7671 & W & 2066.9236 & 1033.9654 & 2049.8971 & 1025.4522 & 2048.9131 & 1024.9602 & 18 \\
\hline 11 & 1407.6216 & 704.3144 & 1390.5950 & 695.8011 & 1389.6110 & 695.3091 & I & 1880.8443 & 940.9258 & 1863.8178 & 932.4125 & 1862.8337 & 931.9205 & 17 \\
\hline 12 & 1478.6587 & 739.8330 & 1461.6321 & 731.3197 & 1460.6481 & 730.8277 & A & 1767.7602 & 884.3838 & 1750.7337 & 875.8705 & 1749.7497 & 875.3785 & 16 \\
\hline 13 & 1607.7013 & 804.3543 & 1590.6747 & 795.8410 & 1589.6907 & 795.3490 & $\mathbf{E}$ & 1696.7231 & 848.8652 & 1679.6966 & 840.3519 & 1678.7126 & 839.8599 & 15 \\
\hline 14 & 1708.7489 & 854.8781 & 1691.7224 & 846.3648 & 1690.7384 & 845.8728 & $\mathbf{T}$ & 1567.6805 & 784.3439 & 1550.6540 & 775.8306 & 1549.6700 & 775.3386 & 14 \\
\hline 15 & 1821.8330 & 911.4201 & 1804.8065 & 902.9069 & 1803.8224 & 902.4149 & $\mathbf{L}$ & 1466.6329 & 733.8201 & 1449.6063 & 725.3068 & 1448.6223 & 724.8148 & 13 \\
\hline 16 & 1908.8650 & 954.9362 & 1891.8385 & 946.4229 & 1890.8545 & 945.9309 & S & 1353.5488 & 677.2780 & 1336.5222 & 668.7648 & 1335.5382 & 668.2728 & 12 \\
\hline 17 & 1995.8971 & 998.4522 & 1978.8705 & 989.9389 & 1977.8865 & 989.4469 & $\mathrm{~S}$ & 1266.5168 & 633.7620 & 1249.4902 & 625.2487 & 1248.5062 & 624.7567 & 71 \\
\hline 18 & 2109.9400 & 1055.4736 & 2092.9134 & 1046.9604 & 2091.9294 & 1046.4684 & $\mathbf{N}$ & 1179.4847 & 590.2460 & 1162.4582 & 581.7327 & 1161.4742 & 581.2407 & 710 \\
\hline
\end{tabular}




\begin{tabular}{|r|r|r|r|r|r|r|r|r|r|r|r|r|r|r|r|}
$\mathbf{1 9}$ & 2246.9989 & 1124.0031 & 2229.9724 & 1115.4898 & 2228.9883 & 1114.9978 & $\mathbf{H}$ & 1065.4418 & 533.2245 & 1048.4153 & 524.7113 & $\mathbf{1 0 4 7 . 4 3 1 2}$ & 524.2193 & $\mathbf{9}$ \\
\hline $\mathbf{2 0}$ & 2378.0394 & 1189.5233 & 2361.0128 & $\mathbf{1 1 8 1 . 0 1 0 1}$ & 2360.0288 & $\mathbf{1 1 8 0 . 5 1 8 0}$ & $\mathbf{M}$ & $\mathbf{9 2 8 . 3 8 2 9}$ & 464.6951 & 911.3564 & 456.1818 & 910.3723 & 455.6898 & $\mathbf{8}$ \\
\hline $\mathbf{2 1}$ & 2493.0663 & 1247.0368 & 2476.0398 & 1238.5235 & 2475.0558 & 1238.0315 & $\mathbf{N}$ & $\mathbf{7 9 7 . 3 4 2 4}$ & 399.1748 & 780.3159 & 390.6616 & 779.3318 & $\mathbf{3 9 0 . 1 6 9 6}$ & $\mathbf{7}$ \\
\hline $\mathbf{2 2}$ & 2640.1347 & 1320.5710 & 2623.1082 & 1312.0577 & 2622.1242 & 1311.5657 & $\mathbf{F}$ & $\mathbf{6 8 2 . 3 1 5 5}$ & 341.6614 & 665.2889 & 333.1481 & 664.3049 & 332.6561 & $\mathbf{6}$ \\
\hline $\mathbf{2 3}$ & 2727.1668 & 1364.0870 & 2710.1402 & 1355.5737 & 2709.1562 & 1355.0817 & S & 535.2471 & 268.1272 & 518.2205 & 259.6139 & 517.2365 & 259.1219 & $\mathbf{5}$ \\
\hline $\mathbf{2 4}$ & 2814.1988 & 1407.6030 & 2797.1722 & 1399.0898 & 2796.1882 & 1398.5978 & $\mathbf{S}$ & 448.2150 & 224.6112 & 431.1885 & 216.0979 & 430.2045 & 215.6059 & $\mathbf{4}$ \\
\hline $\mathbf{2 5}$ & 2885.2359 & 1443.1216 & 2868.2094 & 1434.6083 & 2867.2253 & 1434.1163 & $\mathbf{A}$ & 361.1830 & 181.0951 & 344.1565 & 172.5819 & 343.1724 & 172.0899 & $\mathbf{3}$ \\
\hline $\mathbf{2 6}$ & 3000.2629 & 1500.6351 & 2983.2363 & 1492.1218 & 2982.2523 & 1491.6298 & $\mathbf{D}$ & 290.1459 & 145.5766 & 273.1193 & 137.0633 & 272.1353 & 136.5713 & $\mathbf{2}$ \\
\hline $\mathbf{2 7}$ & & & & & & & $\mathbf{R}$ & 175.1190 & 88.0631 & 158.0924 & 79.5498 & & & $\mathbf{1}$ \\
\hline
\end{tabular}
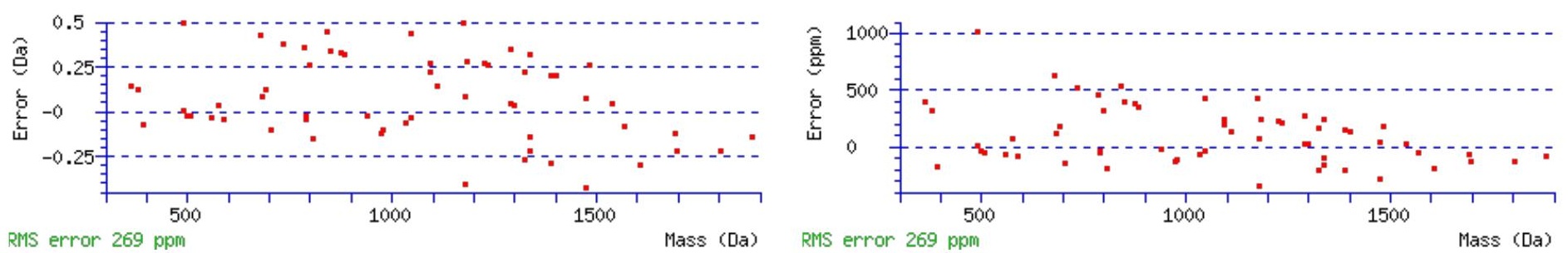

\section{All matches to this query}

\begin{tabular}{|l|l|c|l|}
\hline Score & Mr(calc): & Delta & \multicolumn{1}{|c|}{ Sequence } \\
\hline 63.3 & 3173.3672 & 0.0057 & VNYEAETWDWIAETLSSNHMNFSSADR \\
\hline 62.3 & 3173.3672 & 0.0057 & VNYEAETWDWIAETLSSNHMNFSSADR \\
\hline 9.9 & 3173.3623 & 0.0106 & TKGSMLVSWTPPLDNGGSPITGYWLEK \\
\hline 6.3 & 3173.3623 & 0.0106 & TKGSMLVSWTPPLDNGGSPITGYWLEK \\
\hline 3.8 & 3171.3526 & 2.0204 & SALLYGTLSSEAPQDGESNQSGYCAMISR \\
\hline 2.5 & 3173.3623 & 0.0106 & TKGSMLVSWTPPLDNGGSPITGYWLEK \\
\hline 2.3 & 3171.3420 & 2.0310 & NRLQSVVVVPKNSTLPMEETSPCSSR \\
\hline 1.2 & 3173.3811 & -0.0082 & NFSVGRQTTAPATMSTAASGTTMGLVEQAK \\
\hline 1.0 & 3171.3354 & 2.0375 & EIEMYQGAAQYENPPHIYALADSMYR \\
\hline 0.5 & 3173.3811 & -0.0082 & TLLNTQENMALSLANVSGRLSGMANAFR \\
\hline
\end{tabular}

Spectrum No: 474; Query: 371; Rank: 1

\section{Peptide View}

MS/MS Fragmentation of ILCDNSDNITR

Found in IPI00197864, Tax_Id=10116 Gene_Symbol=LOC314016 Melanoma-associated antigen MG50 (Fragment)

Match to Query 371: 1320.595788 from(661.305170,2+)

Title: 091008RatKidney_NH4Format01_24.997.997.2.dta

Data file K:INewmanPaper\Piliang $\mid 3 S$ ubProteomes\Piliang3SP\mgf5ppm\ERLIC_3SubProteomes5ppm.mgf 

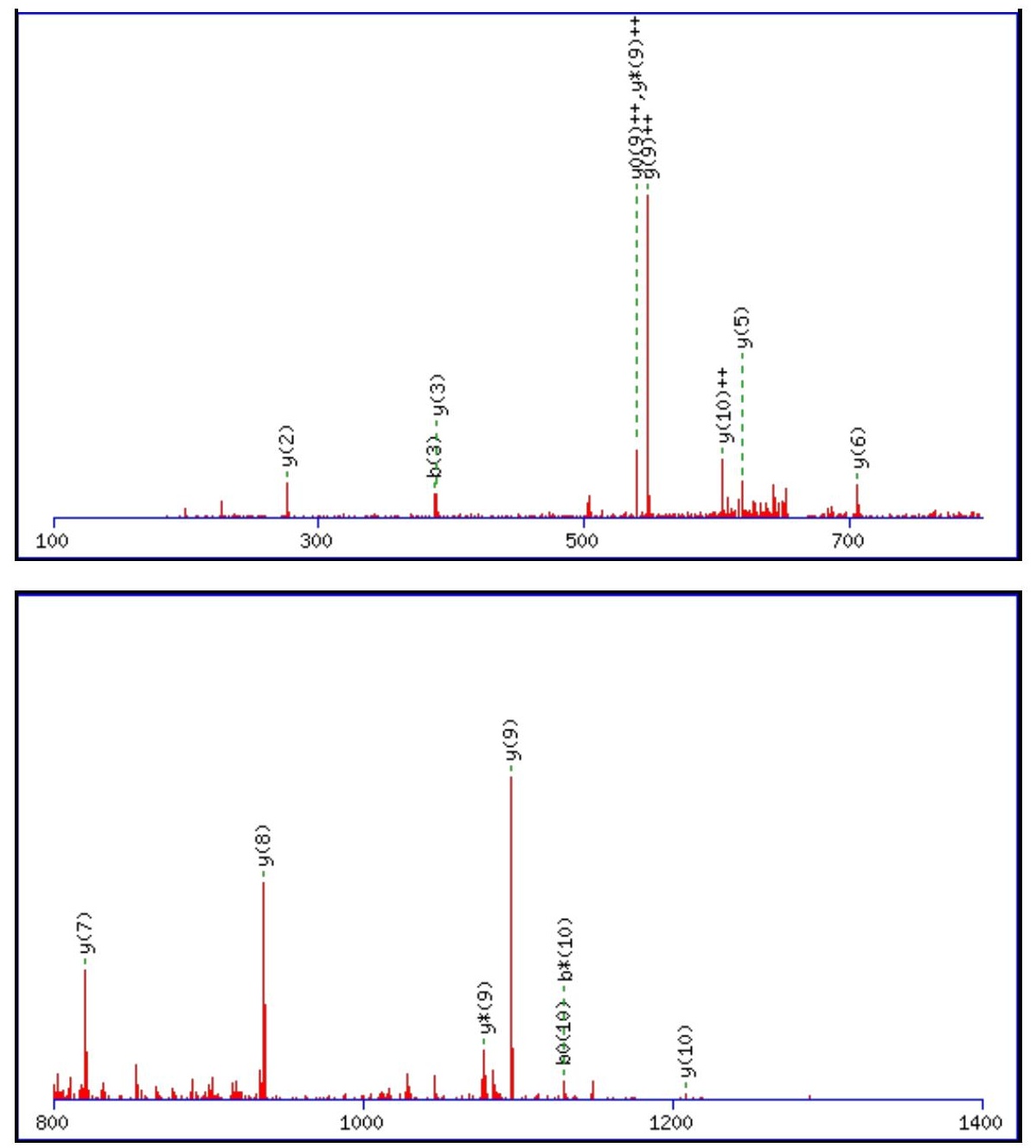

\begin{tabular}{rrrr}
1400 & 1600 & 1800 & 2000 \\
\hline
\end{tabular}

Monoisotopic mass of neutral peptide $\operatorname{Mr}($ calc): 1320.5980

Fixed modifications: Carbamidomethyl (C)

Variable modifications:

N8: Deamidated $\mathrm{N}(\mathrm{N})$

Ions Score: 63 Expect: $6.4 \mathrm{e}-005$

Matches (Bold Red): 16/104 fragment ions using 25 most intense peaks

\begin{tabular}{|r|c|c|c|c|c|c|c|c|c|c|c|c|c|c|}
\hline$\#$ & $\mathbf{b}$ & $\mathbf{b}^{++}$ & $\mathbf{b}^{*}$ & $\mathbf{b}^{\mathbf{*}^{++}}$ & $\mathbf{b}^{\mathbf{0}}$ & $\mathbf{b}^{\mathbf{0 + +}}$ & Seq. & $\mathbf{y}$ & $\mathbf{y}^{++}$ & $\mathbf{y}^{\mathbf{*}}$ & $\mathbf{y}^{\boldsymbol{*}^{++}}$ & $\mathbf{y}^{\mathbf{0}}$ & $\mathbf{y}^{\mathbf{0 + +}}$ & $\#$ \\
\hline $\mathbf{1}$ & 114.0913 & 57.5493 & & & & & $\mathbf{I}$ & & & & & & & $\mathbf{1 1}$ \\
\hline $\mathbf{2}$ & 227.1754 & 114.0913 & & & & & $\mathbf{L}$ & $\mathbf{1 2 0 8 . 5 2 1 2}$ & $\mathbf{6 0 4 . 7 6 4 2}$ & 1191.4946 & 596.2510 & 1190.5106 & 595.7589 & $\mathbf{1 0}$ \\
\hline $\mathbf{3}$ & $\mathbf{3 8 7 . 2 0 6 1}$ & 194.1067 & & & & & $\mathbf{C}$ & $\mathbf{1 0 9 5 . 4 3 7 1}$ & 548.2222 & $\mathbf{1 0 7 8 . 4 1 0 6}$ & $\mathbf{5 3 9 . 7 0 8 9}$ & 1077.4266 & 539.2169 & $\mathbf{9}$ \\
\hline $\mathbf{4}$ & 502.2330 & 251.6201 & & & 484.2224 & 242.6149 & $\mathbf{D}$ & $\mathbf{9 3 5 . 4 0 6 5}$ & 468.2069 & 918.3799 & 459.6936 & 917.3959 & 459.2016 & $\mathbf{8}$ \\
\hline $\mathbf{5}$ & 616.2759 & 308.6416 & 599.2494 & 300.1283 & 598.2654 & 299.6363 & $\mathbf{N}$ & $\mathbf{8 2 0 . 3 7 9 5}$ & 410.6934 & 803.3530 & 402.1801 & 802.3690 & 401.6881 & $\mathbf{7}$ \\
\hline
\end{tabular}




\begin{tabular}{|r|r|r|r|r|r|r|r|r|r|r|r|r|r|r|r|}
$\mathbf{6}$ & 703.3080 & 352.1576 & 686.2814 & 343.6443 & 685.2974 & 343.1523 & S & 706.3366 & 353.6719 & 689.3101 & 345.1587 & 688.3260 & 344.6667 & $\mathbf{6}$ \\
\hline $\mathbf{7}$ & 818.3349 & 409.6711 & 801.3083 & 401.1578 & 800.3243 & 400.6658 & D & $\mathbf{6 1 9 . 3 0 4 6}$ & 310.1559 & 602.2780 & 301.6427 & 601.2940 & 301.1506 & $\mathbf{5}$ \\
\hline $\mathbf{8}$ & 933.3618 & 467.1846 & 916.3353 & 458.6713 & 915.3513 & 458.1793 & $\mathbf{N}$ & 504.2776 & 252.6425 & 487.2511 & 244.1292 & 486.2671 & 243.6372 & $\mathbf{4}$ \\
\hline $\mathbf{9}$ & 1046.4459 & 523.7266 & 1029.4193 & 515.2133 & 1028.4353 & 514.7213 & I & 389.2507 & 195.1290 & 372.2241 & 186.6157 & 371.2401 & 186.1237 & $\mathbf{3}$ \\
\hline $\mathbf{1 0}$ & 1147.4936 & 574.2504 & $\mathbf{1 1 3 0 . 4 6 7 0}$ & 565.7372 & $\mathbf{1 1 2 9 . 4 8 3 0}$ & 565.2451 & $\mathbf{T}$ & $\mathbf{2 7 6 . 1 6 6 6}$ & 138.5870 & 259.1401 & 130.0737 & 258.1561 & 129.5817 & $\mathbf{2}$ \\
\hline $\mathbf{1 1}$ & & & & & & & $\mathbf{R}$ & 175.1190 & 88.0631 & 158.0924 & 79.5498 & & & $\mathbf{1}$ \\
\hline
\end{tabular}
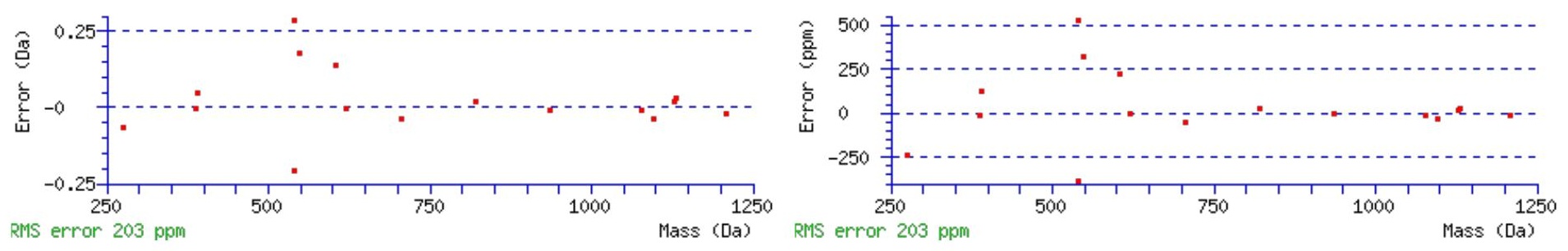

\section{All matches to this query}

\begin{tabular}{|l|l|l|l|}
\hline Score & Mr(calc): & Delta & \multicolumn{1}{|c|}{ Sequence } \\
\hline 63.2 & 1320.5980 & -0.0022 & $\underline{\text { ILCDNSDNITR }}$ \\
\hline 47.2 & 1320.5980 & -0.0022 & $\underline{\text { ILCDNSDNITR }}$ \\
\hline 13.7 & 1319.5983 & 0.9975 & RPAPSGGASGDLR \\
\hline 8.7 & 1319.6027 & 0.9931 & KPCDEDLTSQK \\
\hline 8.4 & 1320.6003 & -0.0045 & KDFINFISEK \\
\hline 5.8 & 1320.5903 & 0.0054 & KPYIWEYSR \\
\hline 5.1 & 1320.5971 & -0.0013 & MKPYSACIKK \\
\hline 3.5 & 1320.6045 & -0.0087 & $\underline{\text { IVSSQSTAEGDVE }}$ \\
\hline 3.1 & 1320.6083 & -0.0125 & $\underline{\text { IYKNKCMR }}$ \\
\hline 3.1 & 1320.5880 & 0.0078 & $\underline{\text { ILPDKVSFSR }}$ \\
\hline
\end{tabular}

Spectrum No: 475; Query: 2059; Rank: 1

\section{Peptide View}

MS/MS Fragmentation of ELINALQINNTAVGHALVLPAR

Found in IPI00565677, Tax_Id=10116 Gene_Symbol=Col6a3_predicted 289 kDa protein

Match to Query 2059: 2327.294952 from(776.772260,3+)

Title: 100101RatKid_NS_deglyco_14.4802.4802.3.dta

Data file K:INewmanPaper|Piliangl3SubProteomes\Piliang3SP\mgf5ppm\ERLIC_3SubProteomes5ppm.mgf

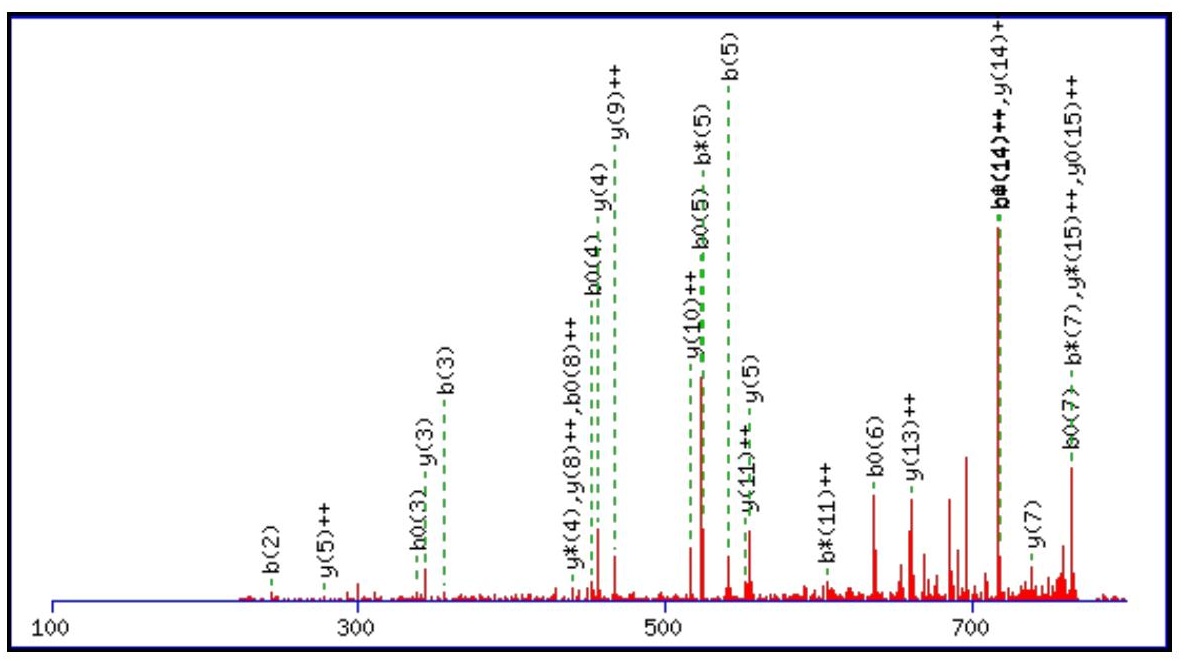



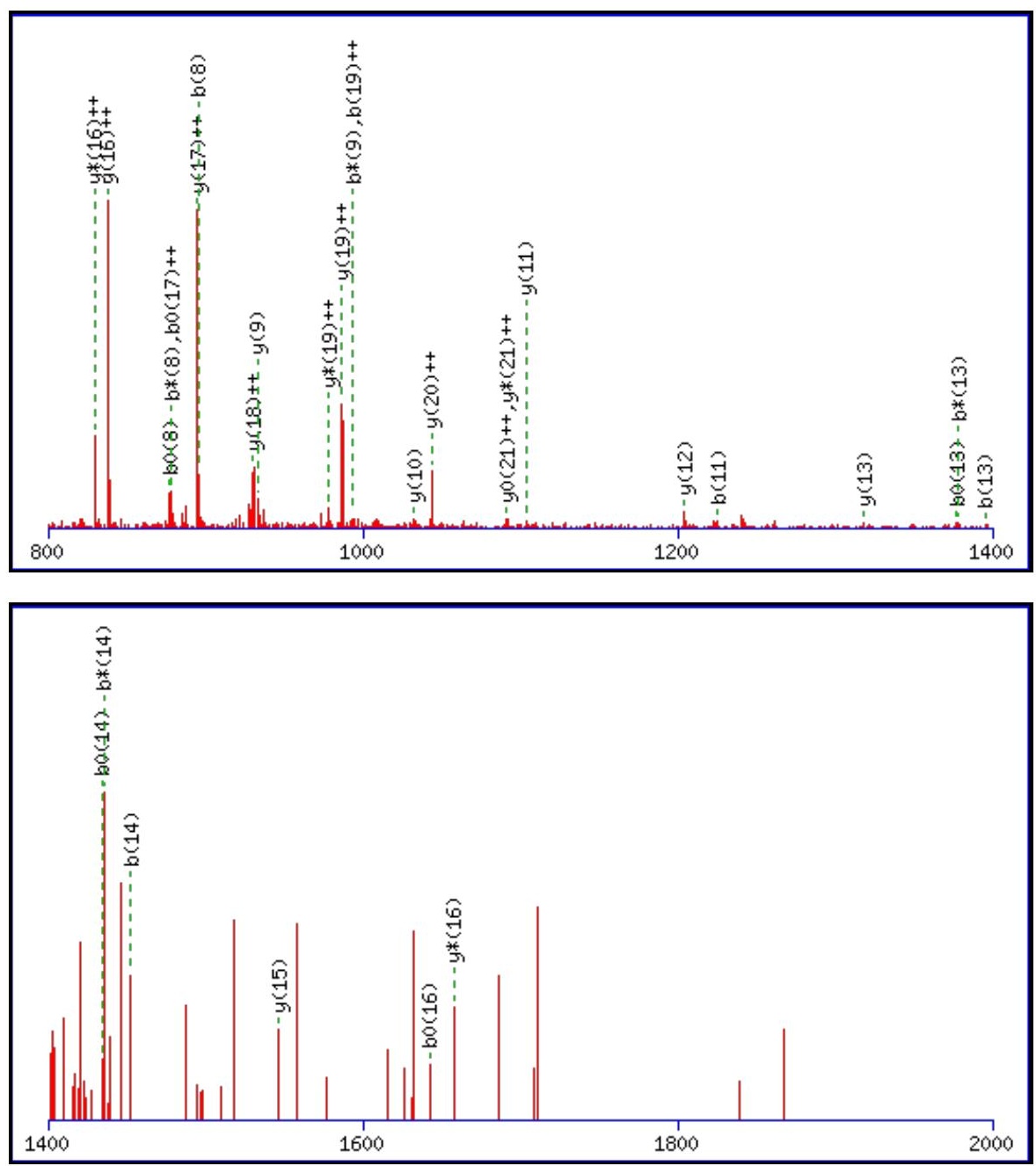

Monoisotopic mass of neutral peptide $\operatorname{Mr}$ (calc): 2327.2961

Fixed modifications: Carbamidomethyl (C)

Variable modifications:

N9 : Deamidated_N (N)

Ions Score: 63 Expect: $1.5 \mathrm{e}-005$

Matches (Bold Red): 58/224 fragment ions using 118 most intense peaks

\begin{tabular}{|c|c|c|c|c|c|c|c|c|c|c|c|c|c|c|}
\hline \# & b & $\mathbf{b}^{++}$ & $\mathbf{b}^{*}$ & $\mathbf{b}^{*^{++}}$ & $\mathbf{b}^{\mathbf{0}}$ & $\mathbf{b}^{\mathbf{0 + +}}$ & Seq. & $\mathbf{y}$ & $\mathbf{y}^{++}$ & $\mathbf{y}^{*}$ & $\mathrm{y}^{\mathrm{*}^{++}}$ & $\mathbf{y}^{\mathbf{0}}$ & $\mathbf{y}^{0++}$ & \# \\
\hline 1 & 130.0499 & 65.5286 & & & 112.0393 & 56.5233 & $\mathbf{E}$ & & & & & & & 22 \\
\hline 2 & 243.1339 & 122.0706 & & & 225.1234 & 113.0653 & $\mathbf{L}$ & & & 2182.2343 & 1091.6208 & 2181.2502 & 1091.1288 & 21 \\
\hline 3 & 356.2180 & 178.6126 & & & 338.2074 & 169.6074 & I & 2086.1767 & 1043.5920 & 2069.1502 & 1035.0787 & 2068.1662 & 1034.5867 & 20 \\
\hline 4 & 470.2609 & 235.6341 & & & 452.2504 & 226.6288 & $\mathbf{N}$ & 1973.0927 & 987.0500 & 1956.0661 & 978.5367 & 1955.0821 & 978.0447 & 19 \\
\hline 5 & 541.2980 & 271.1527 & 524.2715 & 262.6394 & 523.2875 & 262.1474 & A & 1859.0498 & 930.0285 & 1842.0232 & 921.5152 & 1841.0392 & 921.0232 & 18 \\
\hline 6 & 654.3821 & 327.6947 & 637.3556 & 319.1814 & 636.3715 & 318.6894 & $\mathbf{L}$ & 1788.0126 & 894.5100 & 1770.9861 & 885.9967 & 1770.0021 & 885.5047 & 17 \\
\hline 7 & 782.4407 & 391.7240 & 765.4141 & 383.2107 & 764.4301 & 382.7187 & $\mathbf{Q}$ & 1674.9286 & 837.9679 & 1657.9020 & 829.4547 & 1656.9180 & 828.9626 & 16 \\
\hline 8 & 895.5247 & 448.2660 & 878.4982 & 439.7527 & 877.5142 & 439.2607 & I & 1546.8700 & 773.9386 & 1529.8434 & 765.4254 & 1528.8594 & 764.9334 & 15 \\
\hline 9 & 1010.5517 & 505.7795 & 993.5251 & 497.2662 & 992.5411 & 496.7742 & $\mathbf{N}$ & 1433.7859 & 717.3966 & 1416.7594 & 708.8833 & 1415.7754 & 708.3913 & 14 \\
\hline 10 & 1124.5946 & 562.8009 & 1107.5681 & 554.2877 & 1106.5840 & 553.7957 & $\mathbf{N}$ & 1318.7590 & 659.8831 & 1301.7324 & 651.3699 & 1300.7484 & 650.8779 & 13 \\
\hline 11 & 1225.6423 & 613.3248 & 1208.6157 & 604.8115 & 1207.6317 & 604.3195 & $\mathbf{T}$ & 1204.7161 & 602.8617 & 1187.6895 & 594.3484 & 1186.7055 & 593.8564 & 12 \\
\hline 12 & 1296.6794 & 648.8433 & 1279.6529 & 640.3301 & 1278.6688 & 639.8381 & A & 1103.6684 & 552.3378 & 1086.6418 & 543.8246 & & & 11 \\
\hline 13 & 1395.7478 & 698.3775 & 1378.7213 & 689.8643 & 1377.7372 & 689.3723 & $\mathbf{V}$ & 1032.6313 & 516.8193 & 1015.6047 & 508.3060 & & & 10 \\
\hline 14 & 1452.7693 & 726.8883 & 1435.7427 & 718.3750 & 1434.7587 & 717.8830 & G & 933.5629 & 467.2851 & 916.5363 & 458.7718 & & & 9 \\
\hline 15 & 1589.8282 & 795.4177 & 1572.8016 & 786.9045 & 1571.8176 & 786.4125 & H & 876.5414 & 438.7743 & 859.5148 & 430.2611 & & & 8 \\
\hline 16 & 1660.8653 & 830.9363 & 1643.8388 & 822.4230 & 1642.8547 & 821.9310 & A & 739.4825 & 370.2449 & 722.4559 & 361.7316 & & & 7 \\
\hline 17 & 1773.9494 & 887.4783 & 1756.9228 & 878.9650 & 1755.9388 & 878.4730 & $\mathbf{L}$ & 668.4454 & 334.7263 & 651.4188 & 326.2130 & & & 6 \\
\hline 18 & 1873.0178 & 937.0125 & 1855.9912 & 928.4993 & 1855.0072 & 928.0072 & $\mathbf{V}$ & 555.3613 & 278.1843 & 538.3348 & 269.6710 & & & 5 \\
\hline
\end{tabular}




\begin{tabular}{|r|r|r|r|r|r|r|r|r|r|r|r|r|r|r|r|}
$\mathbf{1 9}$ & $\mathbf{1 9 8 6 . 1 0 1 8}$ & $\mathbf{9 9 3 . 5 5 4 6}$ & 1969.0753 & 985.0413 & 1968.0913 & 984.5493 & $\mathbf{L}$ & $\mathbf{4 5 6 . 2 9 2 9}$ & 228.6501 & $\mathbf{4 3 9 . 2 6 6 3}$ & 220.1368 & & & $\mathbf{4}$ \\
\hline $\mathbf{2 0}$ & 2083.1546 & 1042.0809 & 2066.1281 & 1033.5677 & 2065.1440 & 1033.0757 & $\mathbf{P}$ & $\mathbf{3 4 3 . 2 0 8 8}$ & 172.1081 & 326.1823 & 163.5948 & & & $\mathbf{3}$ \\
\hline $\mathbf{2 1}$ & 2154.1917 & 1077.5995 & 2137.1652 & 1069.0862 & 2136.1812 & 1068.5942 & $\mathbf{A}$ & 246.1561 & 123.5817 & 229.1295 & 115.0684 & & & $\mathbf{2}$ \\
\hline $\mathbf{2 2}$ & & & & & & & $\mathbf{R}$ & 175.1190 & 88.0631 & 158.0924 & 79.5498 & & & $\mathbf{1}$ \\
\hline
\end{tabular}
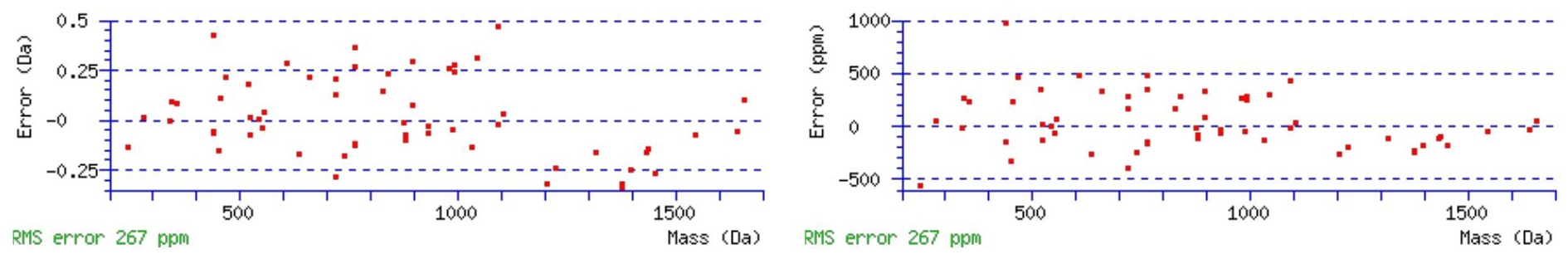

\section{All matches to this query}

\begin{tabular}{|l|l|l|l|}
\hline Score & Mr(calc): & Delta & \multicolumn{1}{c|}{ Sequence } \\
\hline 63.2 & 2327.2961 & -0.0011 & ELINALQINNTAVGHALVLPAR \\
\hline 60.7 & 2327.2961 & -0.0011 & ELINALQINNTAVGHALVLPAR \\
\hline 31.0 & 2326.3121 & 0.9829 & ELINALQINNTAVGHALVLPAR \\
\hline 30.4 & 2327.2961 & -0.0011 & ELINALQINNTAVGHALVLPAR \\
\hline 1.8 & 2327.2974 & -0.0025 & EILQGLAHLHAHOVIHRDIK \\
\hline
\end{tabular}

Spectrum No: 476; Query: 568; Rank: 1

\section{Peptide View}

MS/MS Fragmentation of LQYNSLLSNMSR

Found in IPI00324300, Tax_Id=10116 Gene_Symbol=Ace Angiotensin-converting enzyme, somatic isoform precursor

Match to Query 568: 1442.689388 from(722.351970,2+)

Title: 091008RatKidney_NH4Format01_26.1899.1899.2.dta

Data file K:INewmanPaper|Piliangl3SubProteomes\Piliang3SP\mgf5ppm\ERLIC_3SubProteomes5ppm.mgf

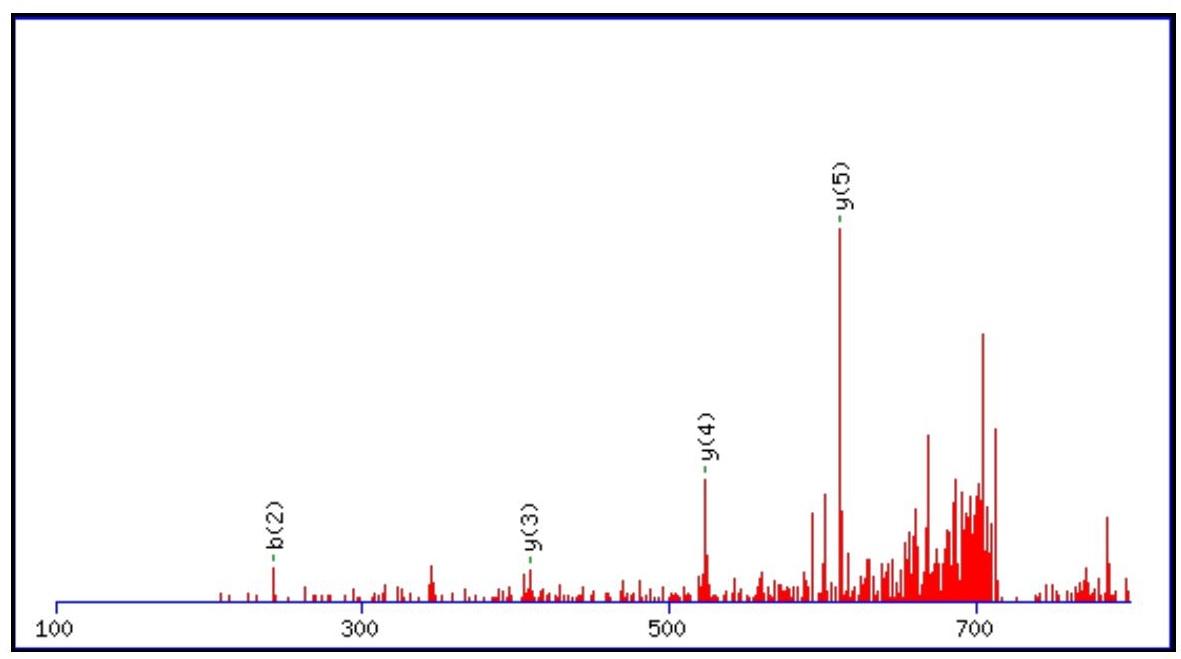



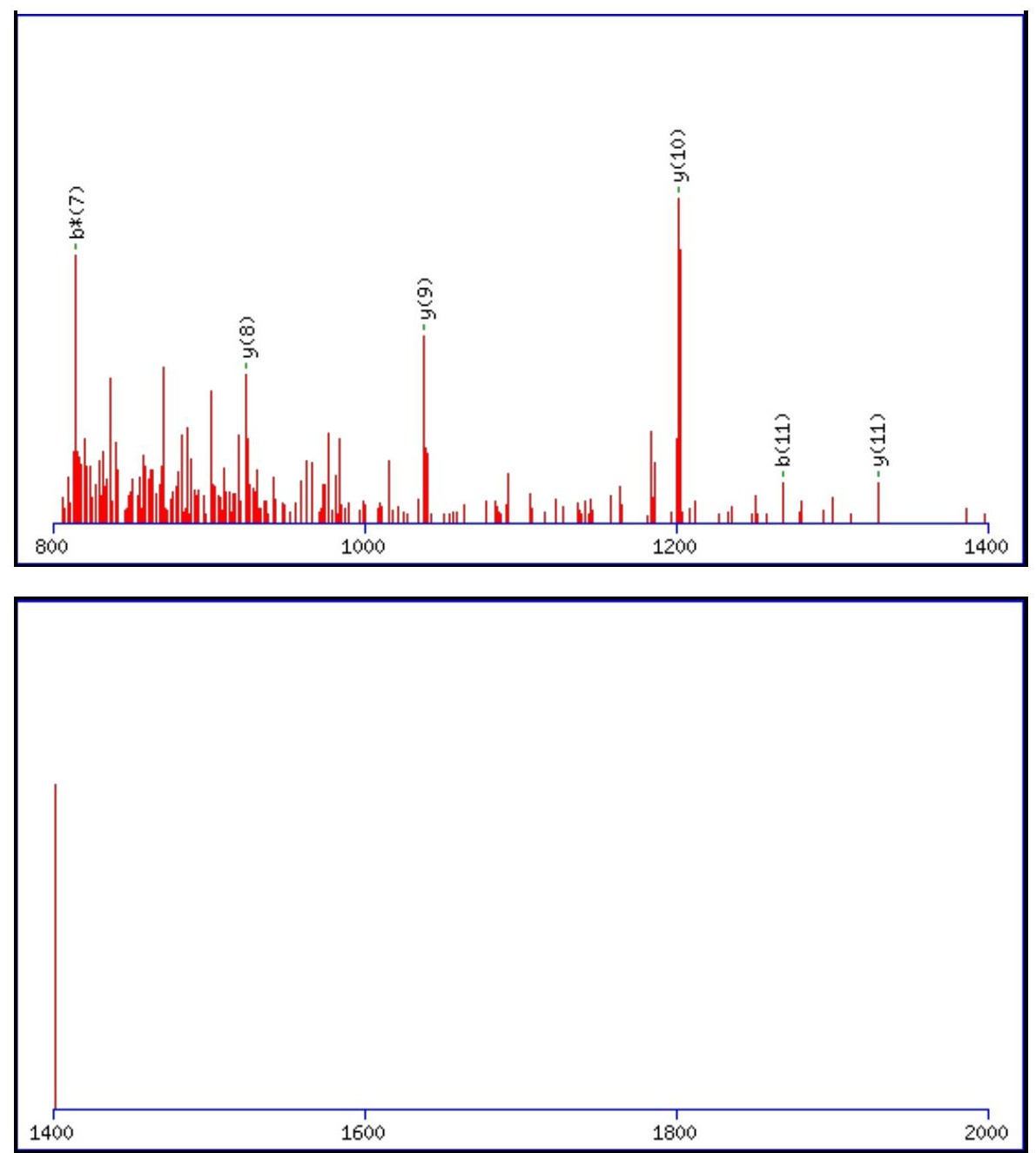

Monoisotopic mass of neutral peptide $\operatorname{Mr}($ calc): 1441.6871

Fixed modifications: Carbamidomethyl (C)

Variable modifications:

N9 : Deamidated_N (N)

M10 : Oxidation $(\mathrm{M})$

Ions Score: 63 Expect : $7.5 \mathrm{e}-005$

Matches (Bold Red): 10/120 fragment ions using 16 most intense peaks

\begin{tabular}{|c|c|c|c|c|c|c|c|c|c|c|c|c|c|c|}
\hline \# & b & $\mathbf{b}^{++}$ & b* & $\mathbf{b}^{*^{++}}$ & $\mathbf{b}^{\mathbf{0}}$ & $\mathbf{b}^{\mathbf{0 + +}}$ & Seq. & $\mathbf{y}$ & $y^{++}$ & $\mathbf{y}^{*}$ & $\mathrm{y}^{*^{++}}$ & $\mathbf{y}^{\mathbf{0}}$ & $\mathbf{y}^{\mathbf{0 + +}}$ & \# \\
\hline 1 & 114.0913 & 57.5493 & & & & & $\mathbf{L}$ & & & & & & & 12 \\
\hline 2 & 242.1499 & 121.5786 & 225.1234 & 113.0653 & & & $\mathbf{Q}$ & 1329.6103 & 665.3088 & 1312.5838 & 656.7955 & 1311.5998 & 656.3035 & 11 \\
\hline 3 & 405.2132 & 203.1103 & 388.1867 & 194.5970 & & & $\mathbf{Y}$ & 1201.5518 & 601.2795 & 1184.5252 & 592.7662 & 1183.5412 & 592.2742 & 10 \\
\hline 4 & 519.2562 & 260.1317 & 502.2296 & 251.6185 & & & $\mathbf{N}$ & 1038.4884 & 519.7479 & 1021.4619 & 511.2346 & 1020.4779 & 510.7426 & 9 \\
\hline 5 & 606.2882 & 303.6477 & 589.2617 & 295.1345 & 588.2776 & 294.6425 & S & 924.4455 & 462.7264 & 907.4190 & 454.2131 & 906.4349 & 453.7211 & 8 \\
\hline 6 & 719.3723 & 360.1898 & 702.3457 & 351.6765 & 701.3617 & 351.1845 & $\mathbf{L}$ & 837.4135 & 419.2104 & 820.3869 & 410.6971 & 819.4029 & 410.2051 & 7 \\
\hline 7 & 832.4563 & 416.7318 & 815.4298 & 408.2185 & 814.4458 & 407.7265 & $\mathbf{L}$ & 724.3294 & 362.6683 & 707.3029 & 354.1551 & 706.3188 & 353.6631 & 6 \\
\hline 8 & 919.4884 & 460.2478 & 902.4618 & 451.7345 & 901.4778 & 451.2425 & $\mathrm{~S}$ & 611.2453 & 306.1263 & 594.2188 & 297.6130 & 593.2348 & 297.1210 & 5 \\
\hline 9 & 1034.5153 & 517.7613 & 1017.4887 & 509.2480 & 1016.5047 & 508.7560 & $\mathbf{N}$ & 524.2133 & 262.6103 & 507.1868 & 254.0970 & 506.2028 & 253.6050 & 4 \\
\hline 10 & 1181.5507 & 591.2790 & 1164.5241 & 582.7657 & 1163.5401 & 582.2737 & $\mathbf{M}$ & 409.1864 & 205.0968 & 392.1598 & 196.5836 & 391.1758 & 196.0915 & 3 \\
\hline 11 & 1268.5827 & 634.7950 & 1251.5562 & 626.2817 & 1250.5722 & 625.7897 & S & 262.1510 & 131.5791 & 245.1244 & 123.0659 & 244.1404 & 122.5738 & 2 \\
\hline 12 & & & & & & & $\mathbf{R}$ & 175.1190 & 88.0631 & 158.0924 & 79.5498 & & & 1 \\
\hline
\end{tabular}



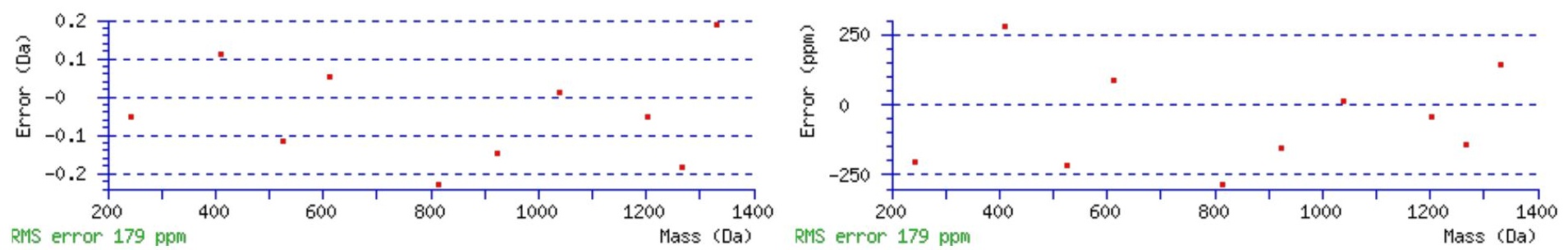

\section{All matches to this query}

\begin{tabular}{|l|l|l|l|}
\hline Score & Mr(calc): & Delta & \multicolumn{1}{|c|}{ Sequence } \\
\hline 63.2 & 1441.6871 & 1.0023 & LQYNSLLSNMSR \\
\hline 22.4 & 1441.6871 & 1.0023 & LQYNSLLSNMSR \\
\hline 9.5 & 1441.6861 & 1.0033 & LRENRTLAMSR \\
\hline 9.0 & 1441.6749 & 1.0145 & \\
\hline 5.3 & 1441.6748 & 1.0146 & LQMSEGERNILSR \\
\hline 4.5 & 1441.6888 & 1.0006 & KIEVIEIMTDR \\
\hline 4.0 & 1441.6888 & 1.0006 & LQSTAMTQELLK \\
\hline 3.5 & 1441.6827 & 1.0067 & HNIATSQSVLHR \\
\hline 3.0 & 1441.7000 & 0.9894 & LKLIASNSTMER \\
\hline 2.8 & 1441.6827 & 1.0067 & HNIATSQSVLHR \\
\hline
\end{tabular}

Spectrum No: 477; Query: 2177; Rank: 1

\section{Peptide View}

MS/MS Fragmentation of ASFNITLIHPNNLTALSNMLPK

Found in IPI00230862, Tax_Id=10116 Gene_Symbol=Anpep Aminopeptidase N

Match to Query 2177: 2426.252022 from(809.757950,3+)

Title: 091008RatKidney_NH4Format01_24.4294.4294.3.dta

Data file K:INewmanPaper|Piliang|3SubProteomes\Piliang3SP\mgf5ppm\ERLIC_3SubProteomes5ppm.mgf

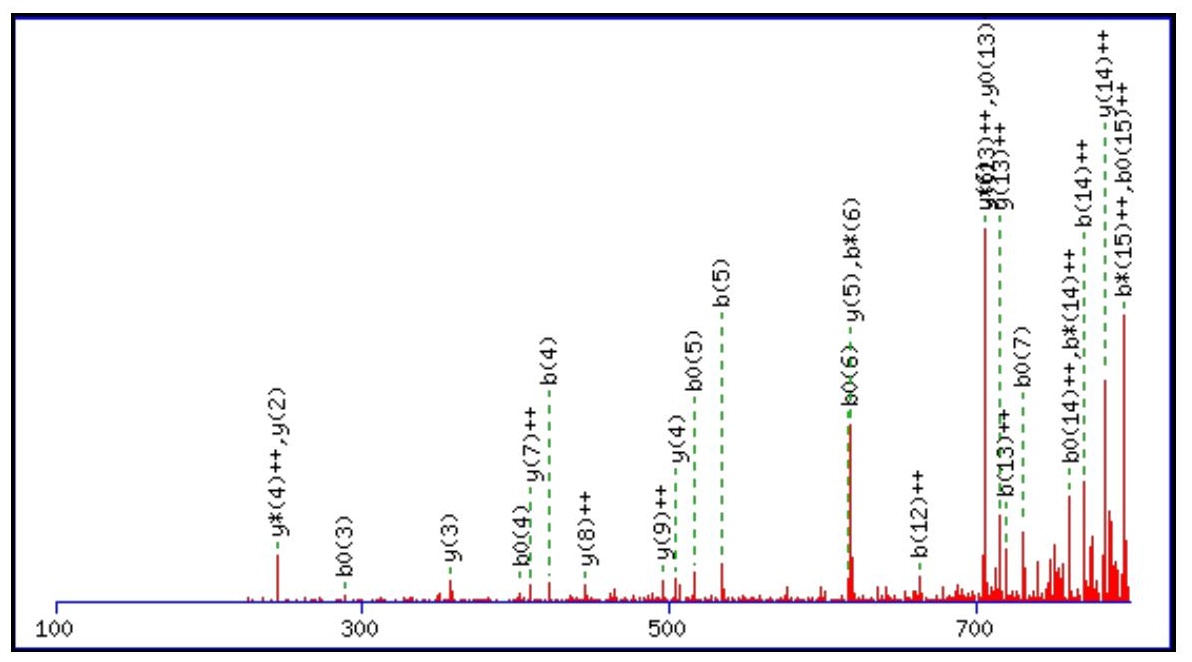



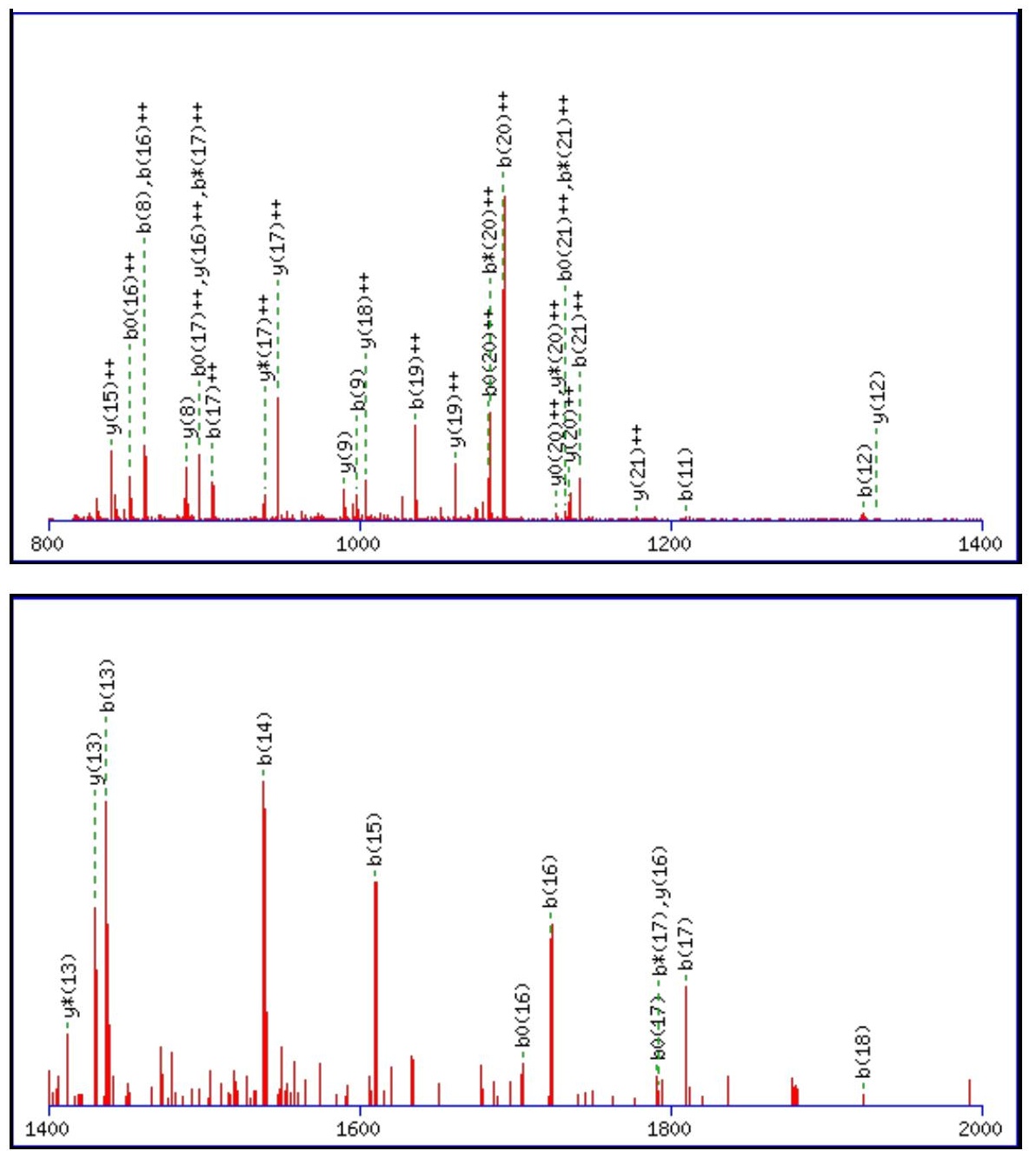

Monoisotopic mass of neutral peptide $\operatorname{Mr}($ calc): 2426.2515

Fixed modifications: Carbamidomethyl (C)

Variable modifications:

N4 : Deamidated_N (N)

N12 : Deamidated_N (N)

M19 : Oxidation (M)

Ions Score: 63 Expect: $6.8 \mathrm{e}-005$

Matches (Bold Red): 69/234 fragment ions using 127 most intense peaks

\begin{tabular}{|c|c|c|c|c|c|c|c|c|c|c|c|c|c|c|}
\hline \# & b & $\mathbf{b}^{++}$ & $\mathbf{b}^{*}$ & $\mathbf{b}^{*^{++}}$ & $\mathbf{b}^{0}$ & & Seq. & $\mathbf{y}$ & $\mathbf{y}^{++}$ & $\mathbf{y}^{*}$ & & $\mathbf{y}^{0}$ & $\mathbf{y}^{\mathbf{0 + +}}$ & \# \\
\hline 1 & 72.0444 & 36.5258 & & & & & A & & & & & & & 22 \\
\hline 2 & 159.0764 & 80.0418 & & & 141.0659 & 71.0366 & $\mathbf{S}$ & 2356.2217 & 1178.6145 & 2339.1952 & 1170.1012 & 2338.2111 & 1169.6092 & 21 \\
\hline 3 & 306.1448 & 153.5761 & & & 288.1343 & 144.5708 & $\mathbf{F}$ & 2269.1897 & 1135.0985 & 2252.1631 & 1126.5852 & 2251.1791 & 1126.0932 & 20 \\
\hline 4 & 421.1718 & 211.0895 & 404.1452 & 202.5762 & 403.1612 & 202.0842 & $\mathbf{N}$ & 2122.1213 & 1061.5643 & 2105.0947 & 1053.0510 & 2104.1107 & 1052.5590 & 19 \\
\hline 5 & 534.2558 & 267.6316 & 517.2293 & 259.1183 & 516.2453 & 258.6263 & I & 2007.0943 & 1004.0508 & 1990.0678 & 995.5375 & 1989.0838 & 995.0455 & 18 \\
\hline 6 & 635.3035 & 318.1554 & 618.2770 & 309.6421 & 617.2929 & 309.1501 & $\mathbf{T}$ & 1894.0103 & 947.5088 & 1876.9837 & 938.9955 & 1875.9997 & 938.5035 & 17 \\
\hline 7 & 748.3876 & 374.6974 & 731.3610 & 366.1842 & 730.3770 & 365.6921 & $\mathbf{L}$ & 1792.9626 & 896.9849 & 1775.9360 & 888.4717 & 1774.9520 & 887.9796 & 16 \\
\hline 8 & 861.4716 & 431.2395 & 844.4451 & 422.7262 & 843.4611 & 422.2342 & I & 1679.8785 & 840.4429 & 1662.8520 & 831.9296 & 1661.8680 & 831.4376 & 15 \\
\hline 9 & 998.5306 & 499.7689 & 981.5040 & 491.2556 & 980.5200 & 490.7636 & $\mathbf{H}$ & 1566.7945 & 783.9009 & 1549.7679 & 775.3876 & 1548.7839 & 774.8956 & 14 \\
\hline 10 & 1095.5833 & 548.2953 & 1078.5568 & 539.7820 & 1077.5728 & 539.2900 & $\mathbf{P}$ & 1429.7355 & 715.3714 & 1412.7090 & 706.8581 & 1411.7250 & 706.3661 & 13 \\
\hline 11 & 1209.6262 & 605.3168 & 1192.5997 & 596.8035 & 1191.6157 & 596.3115 & $\mathbf{N}$ & 1332.6828 & 666.8450 & 1315.6562 & 658.3318 & 1314.6722 & 657.8397 & 12 \\
\hline 12 & 1324.6532 & 662.8302 & 1307.6266 & 654.3170 & 1306.6426 & 653.8249 & $\mathbf{N}$ & 1218.6398 & 609.8236 & 1201.6133 & 601.3103 & 1200.6293 & 600.8183 & 11 \\
\hline 13 & 1437.7372 & 719.3723 & 1420.7107 & 710.8590 & 1419.7267 & 710.3670 & $\mathbf{L}$ & 1103.6129 & 552.3101 & 1086.5864 & 543.7968 & 1085.6023 & 543.3048 & 10 \\
\hline 14 & 1538.7849 & 769.8961 & 1521.7584 & 761.3828 & 1520.7744 & 760.8908 & $\mathbf{T}$ & 990.5288 & 495.7681 & 973.5023 & 487.2548 & 972.5183 & 486.7628 & 9 \\
\hline 15 & 1609.8220 & 805.4147 & 1592.7955 & 796.9014 & 1591.8115 & 796.4094 & A & 889.4812 & 445.2442 & 872.4546 & 436.7309 & 871.4706 & 436.2389 & 8 \\
\hline 16 & 1722.9061 & 861.9567 & 1705.8795 & 853.4434 & 1704.8955 & 852.9514 & $\mathbf{L}$ & 818.4441 & 409.7257 & 801.4175 & 401.2124 & 800.4335 & 400.7204 & 7 \\
\hline 17 & 1809.9381 & 905.4727 & 1792.9116 & 896.9594 & 1791.9276 & 896.4674 & $S$ & 705.3600 & 353.1836 & 688.3334 & 344.6704 & 687.3494 & 344.1784 & 6 \\
\hline
\end{tabular}




\begin{tabular}{|l|l|r|r|r|r|r|r|r|r|r|r|r|r|r|r|}
$\mathbf{1 8}$ & $\mathbf{1 9 2 3 . 9 8 1 1}$ & 962.4942 & 1906.9545 & 953.9809 & 1905.9705 & 953.4889 & $\mathbf{N}$ & $\mathbf{6 1 8 . 3 2 8 0}$ & 309.6676 & 601.3014 & 301.1543 & & & $\mathbf{5}$ \\
\hline $\mathbf{1 9}$ & 2071.0165 & $\mathbf{1 0 3 6 . 0 1 1 9}$ & 2053.9899 & 1027.4986 & 2053.0059 & 1027.0066 & $\mathbf{M}$ & $\mathbf{5 0 4 . 2 8 5 0}$ & 252.6462 & 487.2585 & $\mathbf{2 4 4 . 1 3 2 9}$ & & & $\mathbf{4}$ \\
\hline $\mathbf{2 0}$ & 2184.1005 & $\mathbf{1 0 9 2 . 5 5 3 9}$ & 2167.0740 & $\mathbf{1 0 8 4 . 0 4 0 6}$ & 2166.0900 & $\mathbf{1 0 8 3 . 5 4 8 6}$ & $\mathbf{L}$ & $\mathbf{3 5 7 . 2 4 9 6}$ & 179.1285 & 340.2231 & 170.6152 & & & $\mathbf{3}$ \\
\hline $\mathbf{2 1}$ & 2281.1533 & $\mathbf{1 1 4 1 . 0 8 0 3}$ & 2264.1267 & $\mathbf{1 1 3 2 . 5 6 7 0}$ & 2263.1427 & $\mathbf{1 1 3 2 . 0 7 5 0}$ & $\mathbf{P}$ & $\mathbf{2 4 4 . 1 6 5 6}$ & 122.5864 & 227.1390 & 114.0731 & & & $\mathbf{2}$ \\
\hline $\mathbf{2 2}$ & & & & & & & $\mathbf{K}$ & 147.1128 & 74.0600 & 130.0863 & 65.5468 & & & $\mathbf{1}$ \\
\hline
\end{tabular}
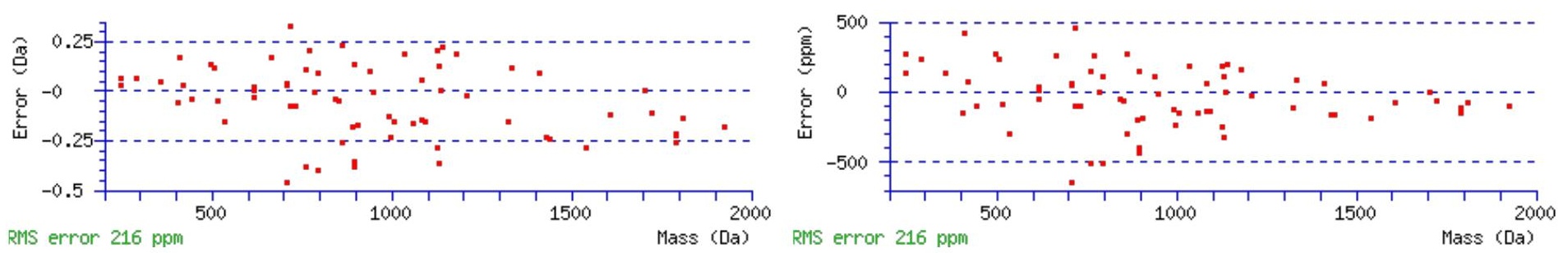

\section{All matches to this query}

\begin{tabular}{|l|l|l|l|}
\hline Score & Mr(calc): & Delta & \multicolumn{1}{|c|}{ Sequence } \\
\hline 63.1 & 2426.2515 & 0.0005 & ASFNITLIHPNNLTALSNMLPK \\
\hline 63.1 & 2426.2515 & 0.0005 & ASFNITLIHPNNLTALSNMLPK \\
\hline 55.2 & 2426.2515 & 0.0005 & ASFNITLIHPNNLTALSNMLPK \\
\hline 48.5 & 2426.2515 & 0.0005 & ASFNITLIHPNNLTALSNMLPK \\
\hline 32.8 & 2425.2675 & 0.9845 & $\underline{\text { ASFNITLIHPNNLTALSNMLPK }}$ \\
\hline 32.8 & 2425.2675 & 0.9845 & $\underline{\text { ASFNITLIHPNNLTALSNMLPK }}$ \\
\hline 31.7 & 2426.2515 & 0.0005 & ASFNITLIHPNNLTALSNMLPK \\
\hline 31.7 & 2426.2515 & 0.0005 & ASFNITLIHPNNLTALSNMLPK \\
\hline 31.3 & 2425.2675 & 0.9845 & ASFNITLIHPNNLTALSNMLPK \\
\hline 23.9 & 2425.2675 & 0.9845 & $\underline{\text { ASFNITLIHPNNLTALSNMLPK }}$ \\
\hline
\end{tabular}

Spectrum No: 478; Query: 51; Rank: 1

\section{Peptide View}

MS/MS Fragmentation of ANLSSQILK

Found in IPI00188338, Tax_Id=10116 Gene_Symbol=Itih1_predicted inter-alpha trypsin inhibitor, heavy chain 1

Match to Query 51: 973.543048 from(487.778800,2+)

Title: 091008RatKidney_NH4Format01_29.1194.1194.2.dta

Data file K:INewmanPaper|Piliangl3SubProteomes\Piliang3SP\mgf5ppm\ERLIC_3SubProteomes5ppm.mgf

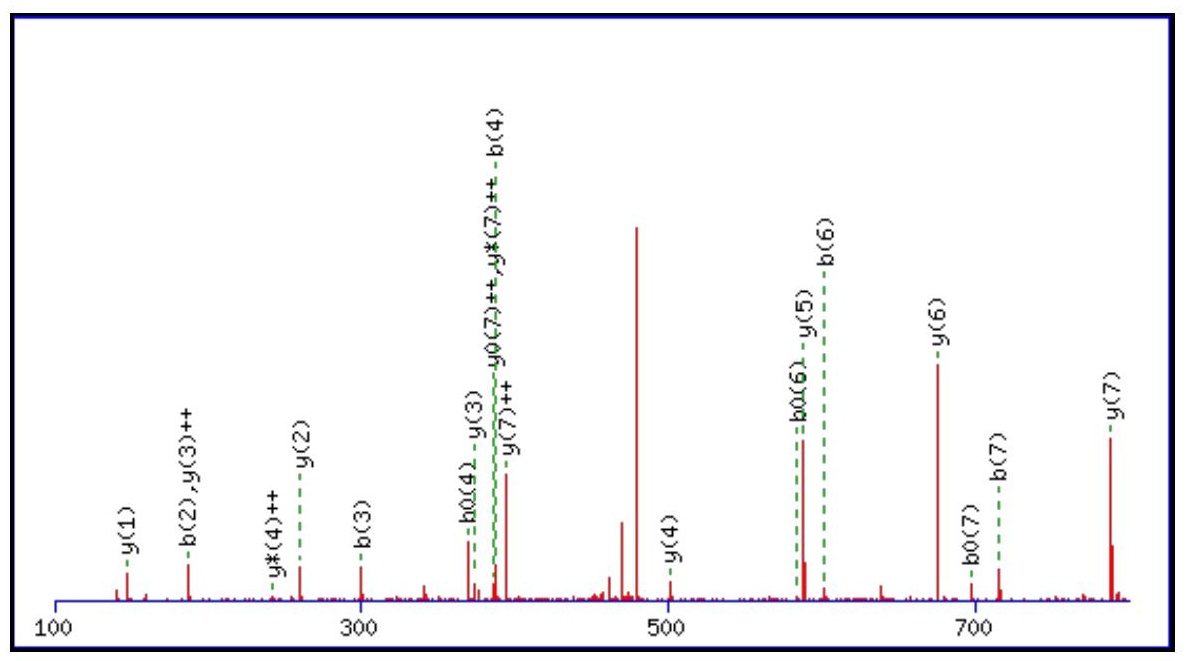



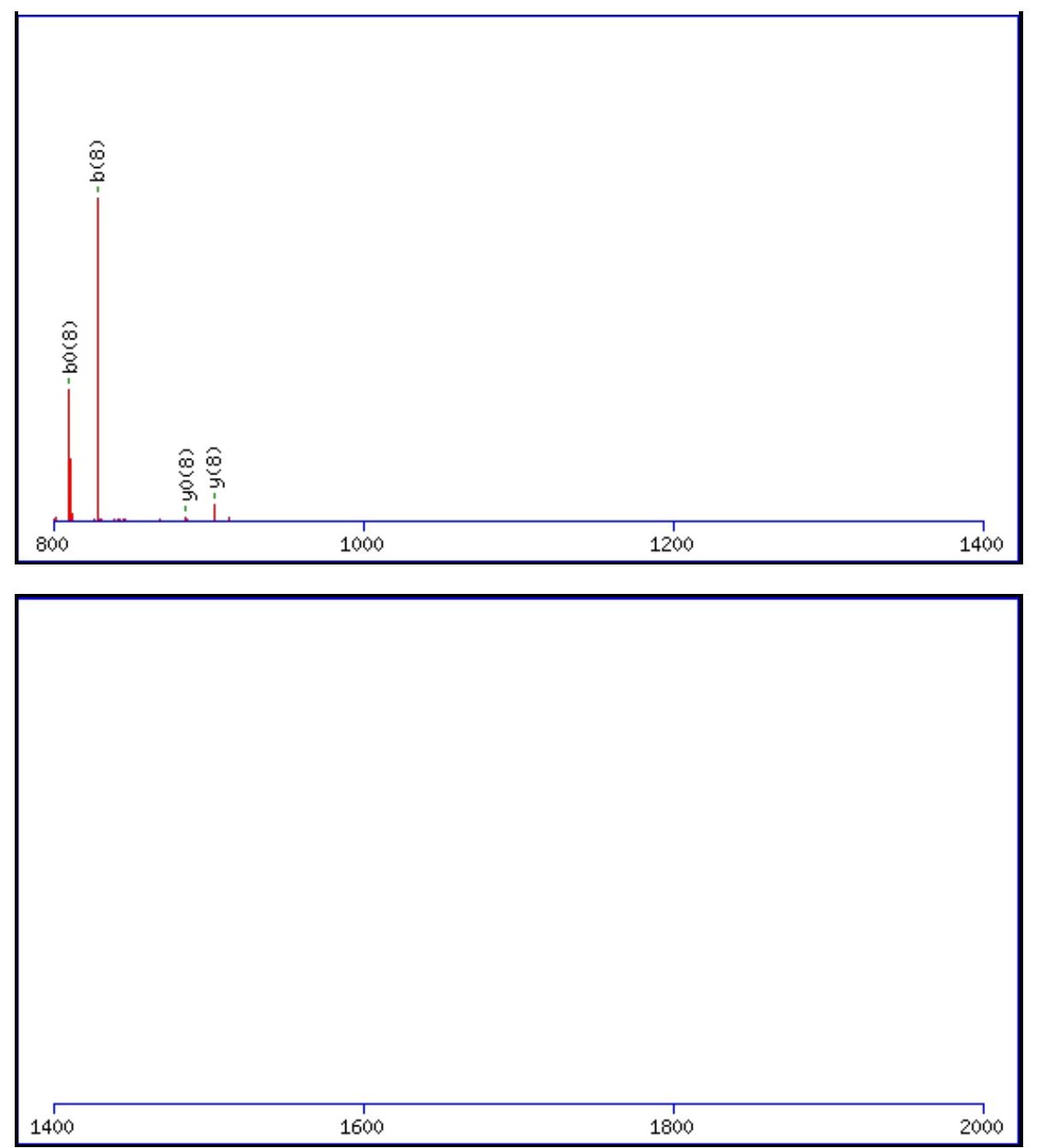

Monoisotopic mass of neutral peptide $\operatorname{Mr}($ calc): 973.5444

Fixed modifications: Carbamidomethyl (C)

Variable modifications:

N2 : Deamidated $\mathrm{N}(\mathrm{N})$

Ions Score: 63 Expect: $4.8 \mathrm{e}-005$

Matches (Bold Red): 24/80 fragment ions using 43 most intense peaks

\begin{tabular}{|c|c|c|c|c|c|c|c|c|c|c|c|c|c|c|}
\hline \# & b & $\mathbf{b}^{++}$ & b* & $\mathbf{b}^{*^{++}}$ & $\mathbf{b}^{\mathbf{0}}$ & $\mathbf{b}^{0++}$ & Seq. & $\mathbf{y}$ & $y^{++}$ & $\mathbf{y}^{*}$ & $\mathrm{y}^{*^{++}}$ & $\mathbf{y}^{\mathbf{0}}$ & $\mathbf{y}^{0++}$ & $\#$ \\
\hline 1 & 72.0444 & 36.5258 & & & & & A & & & & & & & 9 \\
\hline 2 & 187.0713 & 94.0393 & 170.0448 & 85.5260 & & & $\mathbf{N}$ & 903.5146 & 452.2609 & 886.4880 & 443.7476 & 885.5040 & 443.2556 & 8 \\
\hline 4 & 387.1874 & 194.0973 & 370.1609 & 185.5841 & 369.1769 & 185.0921 & S & 675.4036 & 338.2054 & 658.3770 & 329.6921 & 657.3930 & 329.2001 & 6 \\
\hline 5 & 474.2194 & 237.6134 & 457.1929 & 229.1001 & 456.2089 & 228.6081 & S & 588.3715 & 294.6894 & 571.3450 & 286.1761 & 570.3610 & 285.6841 & 5 \\
\hline 7 & 715.3621 & 358.1847 & 698.3355 & 349.6714 & 697.3515 & 349.1794 & I & 373.2809 & 187.1441 & 356.2544 & 178.6308 & & & 3 \\
\hline 8 & 828.4462 & 414.7267 & 811.4196 & 406.2134 & 810.4356 & 405.7214 & $\mathbf{L}$ & 260.1969 & 130.6021 & 243.1703 & 122.0888 & & & 2 \\
\hline 9 & & & & & & & $\mathbf{K}$ & 147.1128 & 74.0600 & 130.0863 & 65.5468 & & & 1 \\
\hline
\end{tabular}
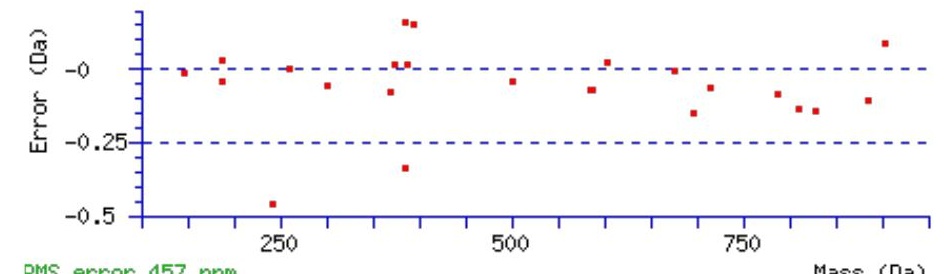

RMS error $457 \mathrm{ppm}$

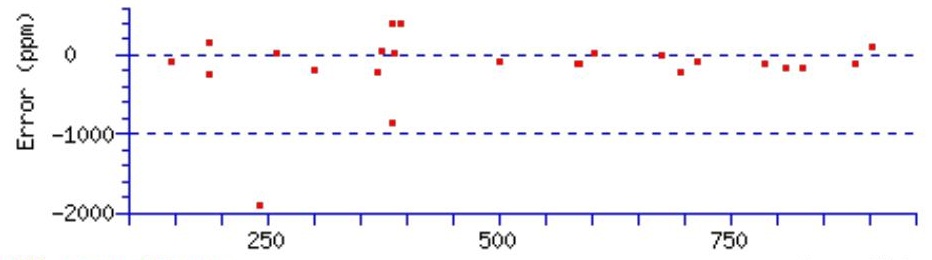

RMS error $457 \mathrm{ppm}$

\section{All matches to this query}


Mascot Search Results: Peptide View

\begin{tabular}{|l|l|l|l|} 
Score & Mr(calc): & Delta & \multicolumn{1}{|c|}{ Sequence } \\
\hline 63.0 & 973.5444 & -0.0013 & ANLSSQILK \\
\hline 22.7 & 973.5444 & -0.0013 & LVSSEQALK \\
\hline 22.2 & 973.5444 & -0.0013 & SVLTAAGNLK \\
\hline 18.1 & 973.5444 & -0.0013 & QENKTLLK \\
\hline 17.0 & 973.5379 & 0.0052 & QGLEMRIK \\
\hline 15.9 & 972.5465 & 0.9966 & QGLSRGLSR \\
\hline 15.0 & 973.5444 & -0.0013 & SASQEVLIK \\
\hline 12.4 & 973.5444 & -0.0013 & KATADNLIK \\
\hline 11.3 & 973.5379 & 0.0052 & EGRKMPIK \\
\hline 11.3 & 973.5345 & 0.0086 & WKDERLK \\
\hline
\end{tabular}

Spectrum No: 479; Query: 148; Rank: 1

\section{Peptide View}

MS/MS Fragmentation of NPNGTVTVISR

Found in IPI00369591, Tax_Id=10116 Gene_Symbol=Pvrl1 similar to poliovirus receptor-related 1

Match to Query 148: 1157.601088 from(579.807820,2+)

Title: 091008RatKidney_NoSalt_26.767.767.2.dta

Data file K:INewmanPaper|Piliangl3SubProteomes\Piliang3SP\mgf5ppm\ERLIC_3SubProteomes5ppm.mgf
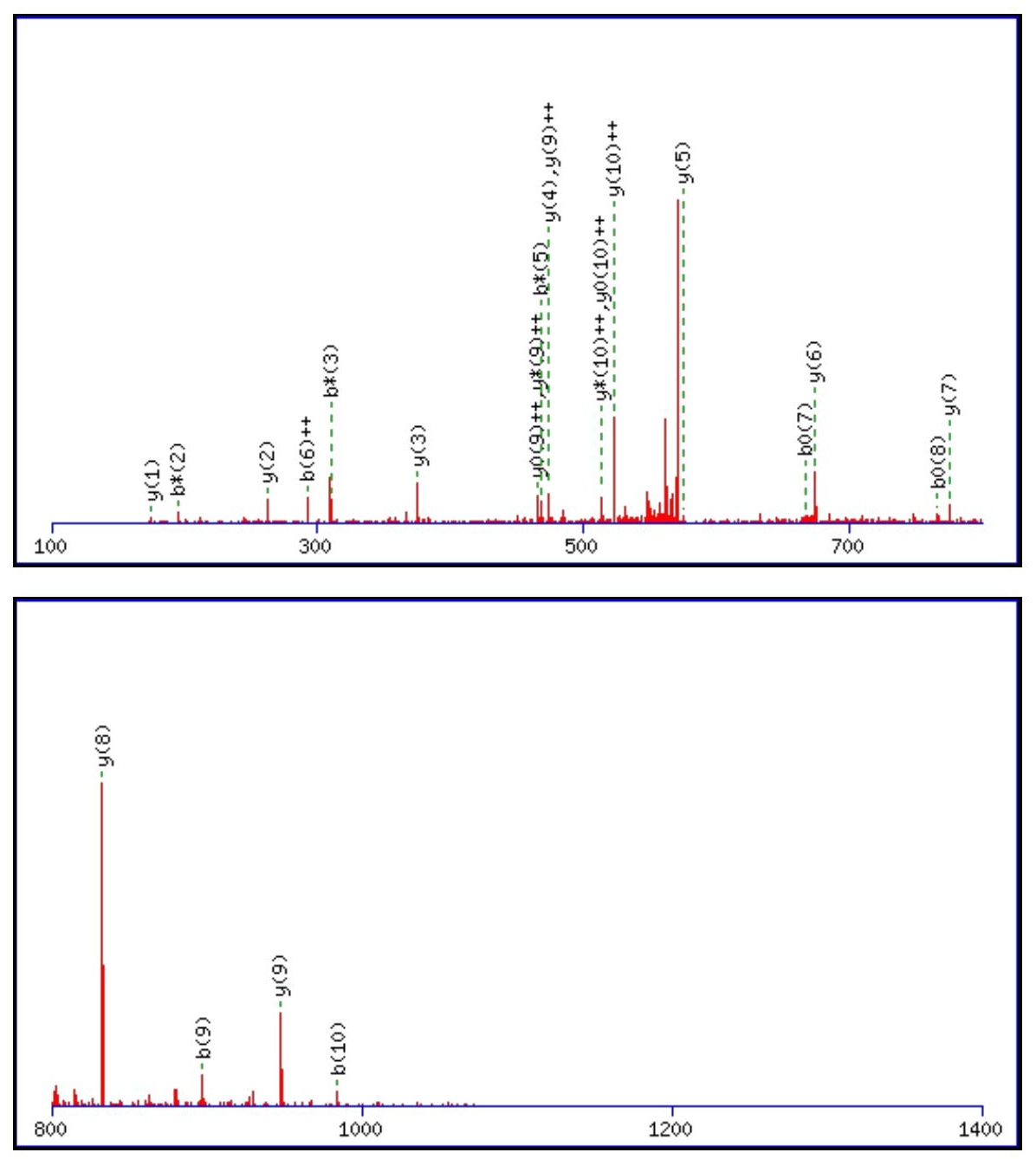


$1400 \quad 1600 \quad 1800 \quad 2000$

Monoisotopic mass of neutral peptide $\operatorname{Mr}($ calc): 1157.6040

Fixed modifications: Carbamidomethyl (C)

Variable modifications:

N3 : Deamidated $\mathrm{N}(\mathrm{N})$

Ions Score: 63 Expect: $5 \mathrm{e}-005$

Matches (Bold Red): 23/110 fragment ions using 29 most intense peaks

\begin{tabular}{|r|c|c|c|c|c|c|c|c|c|c|c|c|c|c|}
\hline$\#$ & $\mathbf{b}$ & $\mathbf{b}^{++}$ & $\mathbf{b}^{*}$ & $\mathbf{b}^{\mathbf{*}^{++}}$ & $\mathbf{b}^{\mathbf{0}}$ & $\mathbf{b}^{\mathbf{0 + +}}$ & $\mathbf{S e q}$ & $\mathbf{y}$ & $\mathbf{y}^{++}$ & $\mathbf{y}^{*}$ & $\mathbf{y}^{\mathbf{*}^{++}}$ & $\mathbf{y}^{\mathbf{0}}$ & $\mathbf{y}^{\mathbf{0 + +}}$ & $\#$ \\
\hline $\mathbf{1}$ & 115.0502 & 58.0287 & 98.0237 & 49.5155 & & & $\mathbf{N}$ & & & & & & & $\mathbf{1 1}$ \\
\hline $\mathbf{2}$ & 212.1030 & 106.5551 & $\mathbf{1 9 5 . 0 7 6 4}$ & 98.0418 & & & $\mathbf{P}$ & 1044.5684 & $\mathbf{5 2 2 . 7 8 7 8}$ & 1027.5418 & $\mathbf{5 1 4 . 2 7 4 6}$ & $\mathbf{1 0 2 6 . 5 5 7 8}$ & $\mathbf{5 1 3 . 7 8 2 6}$ & $\mathbf{1 0}$ \\
\hline $\mathbf{3}$ & 327.1299 & 164.0686 & 310.1034 & 155.5553 & & & $\mathbf{N}$ & $\mathbf{9 4 7 . 5 1 5 6}$ & $\mathbf{4 7 4 . 2 6 1 5}$ & 930.4891 & $\mathbf{4 6 5 . 7 4 8 2}$ & 929.5051 & $\mathbf{4 6 5 . 2 5 6 2}$ & $\mathbf{9}$ \\
\hline $\mathbf{4}$ & 384.1514 & 192.5793 & 367.1248 & 184.0660 & & & $\mathbf{G}$ & $\mathbf{8 3 2 . 4 8 8 7}$ & 416.7480 & 815.4621 & 408.2347 & 814.4781 & 407.7427 & $\mathbf{8}$ \\
\hline $\mathbf{5}$ & 485.1990 & 243.1032 & 468.1725 & 234.5899 & 467.1885 & 234.0979 & $\mathbf{T}$ & 775.4672 & 388.2373 & 758.4407 & 379.7240 & 757.4567 & 379.2320 & $\mathbf{7}$ \\
\hline $\mathbf{6}$ & 584.2675 & 292.6374 & 567.2409 & 284.1241 & 566.2569 & 283.6321 & $\mathbf{V}$ & $\mathbf{6 7 4 . 4 1 9 6}$ & 337.7134 & 657.3930 & 329.2001 & 656.4090 & 328.7081 & $\mathbf{6}$ \\
\hline $\mathbf{7}$ & 685.3151 & 343.1612 & 668.2886 & 334.6479 & $\mathbf{6 6 7 . 3 0 4 6}$ & 334.1559 & $\mathbf{T}$ & $\mathbf{5 7 5 . 3 5 1 1}$ & 288.1792 & 558.3246 & 279.6659 & 557.3406 & 279.1739 & $\mathbf{5}$ \\
\hline $\mathbf{8}$ & 784.3836 & 392.6954 & 767.3570 & 384.1821 & $\mathbf{7 6 6 . 3 7 3 0}$ & 383.6901 & $\mathbf{V}$ & $\mathbf{4 7 4 . 3 0 3 5}$ & 237.6554 & 457.2769 & 229.1421 & 456.2929 & 228.6501 & $\mathbf{4}$ \\
\hline $\mathbf{9}$ & $\mathbf{8 9 7 . 4 6 7 6}$ & 449.2374 & 880.4411 & 440.7242 & 879.4571 & 440.2322 & $\mathbf{I}$ & $\mathbf{3 7 5 . 2 3 5 0}$ & 188.1212 & 358.2085 & 179.6079 & 357.2245 & 179.1159 & $\mathbf{3}$ \\
\hline $\mathbf{1 0}$ & $\mathbf{9 8 4 . 4 9 9 6}$ & 492.7535 & 967.4731 & 484.2402 & 966.4891 & 483.7482 & $\mathbf{S}$ & $\mathbf{2 6 2 . 1 5 1 0}$ & 131.5791 & 245.1244 & 123.0659 & 244.1404 & 122.5738 & $\mathbf{2}$ \\
\hline $\mathbf{1 1}$ & & & & & & & $\mathbf{R}$ & $\mathbf{1 7 5 . 1 1 9 0}$ & 88.0631 & 158.0924 & 79.5498 & & & $\mathbf{1}$ \\
\hline
\end{tabular}
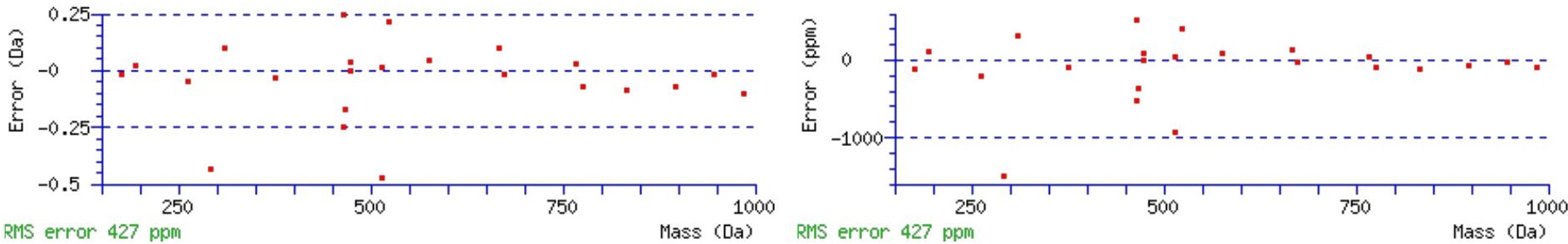

All matches to this query

\begin{tabular}{|l|l|l|l|}
\hline Score & Mr(calc): & Delta & \multicolumn{1}{|c|}{ Sequence } \\
\hline 63.0 & 1157.6040 & -0.0030 & NPNGTVTVISR \\
\hline 46.8 & 1157.6040 & -0.0030 & NPNGTVTVISR \\
\hline 10.3 & 1157.5975 & 0.0036 & QVRPGMDLSR \\
\hline 10.0 & 1156.6023 & 0.9988 & ATHIVQVMSR \\
\hline 9.5 & 1157.6081 & -0.0070 & LVYTVSFSSR \\
\hline 9.0 & 1157.5958 & 0.0053 & RFLTSVISR \\
\hline 9.0 & 1157.5958 & 0.0053 & RFLTSVISR \\
\hline 8.5 & 1157.6040 & -0.0029 & GKPTEASIEAR \\
\hline 7.6 & 1156.5950 & 1.0060 & DILMFYLSR \\
\hline 7.0 & 1156.6088 & 0.9923 & GVAQAALGDLDK \\
\hline
\end{tabular}


Spectrum No: 480; Query: 2784; Rank: 1

\section{Peptide View}

MS/MS Fragmentation of MLVSNIDLGPTILDLAGYDLNKTQMDGTSLLPILK

Found in IPI00366226, Tax_Id=10116 Gene_Symbol=Gns Glucosamine (N-acetyl)-6-sulfatase

Match to Query 2784: 3804.965922 from(1269.329250,3+)

Title: 091008RatKidney_NH4Format01_17.5595.5595.3.dta

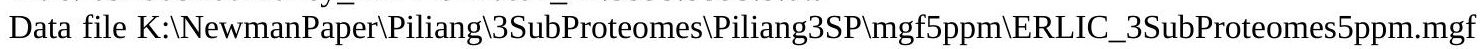
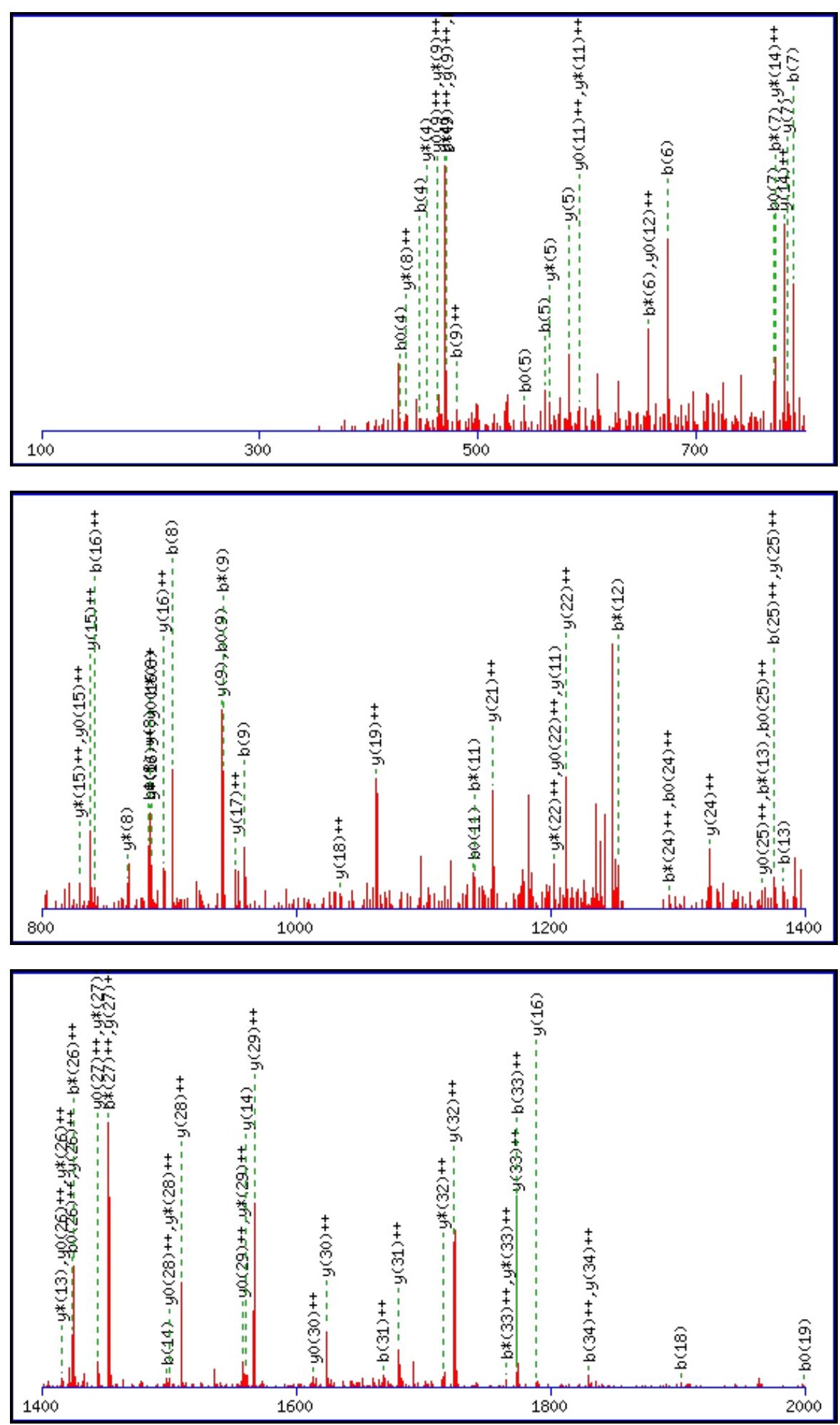
Monoisotopic mass of neutral peptide $\operatorname{Mr}($ calc): 3804.9781

Fixed modifications: Carbamidomethyl (C)

Variable modifications:

M1 : Oxidation (M)

N21 : Deamidated_N (N)

M25 : Oxidation (M)

Ions Score: 63 Expect : $5.7 \mathrm{e}-005$

Matches (Bold Red): 96/382 fragment ions using 184 most intense peaks

\begin{tabular}{|c|c|c|c|c|c|c|c|c|c|c|c|c|c|c|}
\hline \# & b & $\mathbf{b}^{++}$ & $\mathbf{b}^{*}$ & $\mathbf{b}^{*^{++}}$ & $\mathbf{b}^{0}$ & $\mathbf{b}^{\mathbf{0 + +}}$ & Seq. & $\mathbf{y}$ & $y^{++}$ & $\mathbf{y}^{*}$ & $\mathrm{y}^{*^{++}}$ & $\mathbf{y}^{0}$ & $\mathbf{y}^{0++}$ & \# \\
\hline 1 & 148.0427 & 74.5250 & & & & & M & & & & & & & 35 \\
\hline 2 & 261.1267 & 131.0670 & & & & & $\mathbf{L}$ & 3658.9500 & 1829.9786 & 3641.9234 & 1821.4653 & 3640.9394 & 1820.9733 & 34 \\
\hline 3 & 360.1952 & 180.6012 & & & & & V & 3545.8659 & 1773.4366 & 3528.8393 & 1764.9233 & 3527.8553 & 1764.4313 & 33 \\
\hline 4 & 447.2272 & 224.1172 & & & 429.2166 & 215.1119 & $S$ & 3446.7975 & 1723.9024 & $|3429.7709|$ & 1715.3891 & 3428.7869 & 1714.8971 & 32 \\
\hline 5 & 561.2701 & 281.1387 & 544.2436 & 272.6254 & 543.2595 & 272.1334 & $\mathbf{N}$ & 3359.7655 & 1680.3864 & 3342.7389 & 1671.8731 & 3341.7549 & 1671.3811 & 31 \\
\hline 6 & 674.3542 & 337.6807 & 657.3276 & 329.1675 & 656.3436 & 328.6754 & I & 3245.7225 & 1623.3649 & 3228.6960 & 1614.8516 & 3227.7120 & 1614.3596 & 30 \\
\hline 7 & 789.3811 & 395.1942 & 772.3546 & 386.6809 & 771.3706 & 386.1889 & D & 3132.6385 & 1566.8229 & 3115.6119 & 1558.3096 & 3114.6279 & 1557.8176 & 29 \\
\hline 8 & 902.4652 & 451.7362 & 885.4386 & 443.2230 & 884.4546 & 442.7309 & $\mathbf{L}$ & 3017.6115 & 1509.3094 & 3000.5850 & 1500.7961 & 2999.6010 & 1500.3041 & 28 \\
\hline 9 & 959.4866 & 480.2470 & 942.4601 & 471.7337 & 941.4761 & 471.2417 & G & 2904.5275 & 1452.7674 & 2887.5009 & 1444.2541 & 2886.5169 & 1443.7621 & 27 \\
\hline 10 & 1056.5394 & 528.7733 & 1039.5129 & 520.2601 & 1038.5288 & 519.7681 & $\mathbf{P}$ & 2847.5060 & 1424.2566 & 2830.4794 & 1415.7434 & 2829.4954 & 1415.2514 & 26 \\
\hline 11 & 1157.5871 & 579.2972 & 1140.5605 & 570.7839 & 1139.5765 & 570.2919 & $T$ & 2750.4532 & 1375.7303 & 2733.4267 & 1367.2170 & 2732.4427 & 1366.7250 & 25 \\
\hline 12 & 1270.6712 & 635.8392 & 1253.6446 & 627.3259 & 1252.6606 & 626.8339 & I & 2649.4055 & 1325.2064 & 2632.3790 & 1316.6931 & 2631.3950 & 1316.2011 & 24 \\
\hline 13 & 1383.7552 & 692.3812 & \begin{tabular}{|l|}
1366.7287 \\
\end{tabular} & 683.8680 & 1365.7447 & 683.3760 & $\mathbf{L}$ & 2536.3215 & 1268.6644 & 2519.2949 & 1260.1511 & 2518.3109 & 1259.6591 & 23 \\
\hline 14 & 1498.7822 & 749.8947 & 1481.7556 & 741.3814 & 1480.7716 & 740.8894 & D & 2423.2374 & 1212.1223 & 2406.2109 & 1203.6091 & 2405.2269 & 1203.1171 & 22 \\
\hline 15 & 1611.8662 & 806.4368 & 1594.8397 & 797.9235 & 1593.8557 & 797.4315 & $\mathbf{L}$ & 2308.2105 & 1154.6089 & 2291.1839 & 1146.0956 & 2290.1999 & 1145.6036 & 21 \\
\hline 16 & 1682.9033 & 841.9553 & 1665.8768 & 833.4420 & 1664.8928 & 832.9500 & A & 2195.1264 & 1098.0668 & 2178.0999 & 1089.5536 & 2177.1158 & 1089.0616 & 20 \\
\hline 17 & 1739.9248 & 870.4660 & 1722.8983 & 861.9528 & 1721.9142 & 861.4608 & G & 2124.0893 & 1062.5483 & 2107.0627 & 1054.0350 & 2106.0787 & 1053.5430 & 19 \\
\hline 18 & 1902.9881 & 951.9977 & 1885.9616 & 943.4844 & 1884.9776 & 942.9924 & $\mathbf{Y}$ & 2067.0678 & 1034.0376 & 2050.0413 & 1025.5243 & 2049.0573 & 1025.0323 & 18 \\
\hline 19 & 2018.0151 & 1009.5112 & 2000.9885 & 1000.9979 & 2000.0045 & 1000.5059 & D & 1904.0045 & 952.5059 & 1886.9780 & 943.9926 & 1885.9939 & 943.5006 & 17 \\
\hline 20 & 2131.0991 & 1066.0532 & 2114.0726 & 1057.5399 & 2113.0886 & 1057.0479 & $\mathbf{L}$ & 1788.9776 & 894.9924 & 1771.9510 & 886.4791 & 1770.9670 & 885.9871 & 16 \\
\hline 21 & 2246.1261 & 1123 & 2229. & 1115. & 2228.1155 & 1114.5614 & $\mathbf{N}$ & 1675.8935 & 838.4504 & 1658.8669 & 829.9371 & 1657.8829 & 829.4451 & 15 \\
\hline 22 & 2374.2210 & 1187.6142 & 2357.1945 & 1179.1009 & 2356.2105 & 1178.6089 & $\mathbf{K}$ & 1560.8666 & 780.9369 & 1543.8400 & 772.4236 & 1542.8560 & 771.9316 & 14 \\
\hline 23 & 2475.2687 & 1238.1380 & 2458.2422 & 1229.6247 & 2457.2582 & 1229.1327 & $\mathbf{T}$ & 1432.7716 & 716.8894 & 1415.7451 & 708.3762 & 1414.7610 & 707.8842 & 13 \\
\hline 24 & 2603.3273 & 1302.1673 & 2586.3007 & \begin{tabular}{|l|}
1293.6540 \\
\end{tabular} & 2585.3167 & 1293.1620 & $\mathbf{Q}$ & 1331.7239 & 666.3656 & 1314.6974 & 657.8523 & 1313.7134 & 657.3603 & 12 \\
\hline 25 & 2750.3627 & 1375.6850 & 2733.3362 & 1367.1717 & 2732.3521 & 1366.6797 & $\mathbf{M}$ & 1203.6653 & 602.3363 & 1186.6388 & 593.8230 & 1185.6548 & 593.3310 & 11 \\
\hline 26 & 2865.3896 & 1433.1985 & 2848.3631 & 1424.6852 & 2847.3791 & 1424.1932 & D & 1056.6299 & 528.8186 & 1039.6034 & 520.3053 & 1038.6194 & 519.8133 & 10 \\
\hline 27 & 2922.4111 & 1461.7092 & 2905.3846 & 1453.1959 & 2904.4005 & \begin{tabular}{|l|}
1452.7039 \\
\end{tabular} & G & 941.6030 & 471.3051 & 924.5764 & 462.7919 & 923.5924 & 462.2999 & 9 \\
\hline 28 & 3023.4588 & 1512.2330 & 3006.4322 & 1503.7198 & 3005.4482 & 1503.2277 & $T$ & 884.5815 & 442.7944 & 867.5550 & 434.2811 & 866.5710 & 433.7891 & 8 \\
\hline 29 & 3110.4908 & 1555.7490 & 3093.4643 & 1547.2358 & 3092.4802 & 1546.7438 & $S$ & 783.5339 & 392.2706 & 766.5073 & 383.7573 & 765.5233 & 383.2653 & 7 \\
\hline 30 & 3223.5749 & 1612.2911 & 3206.5483 & 1603.7778 & 3205.5643 & 1603.2858 & $\mathbf{L}$ & 696.5018 & 348.7546 & 679.4753 & 340.2413 & & & 6 \\
\hline 31 & 3336.6589 & 1668.8331 & 3319.6324 & 1660.3198 & 3318.6484 & 1659.8278 & $\mathbf{L}$ & 583.4178 & 292.2125 & 566.3912 & 283.6992 & & & 5 \\
\hline 32 & 3433.7117 & \begin{tabular}{|l|l|}
1717.3595 \\
\end{tabular} & 3416.6852 & 1708.8462 & 3415.7011 & 1708.3542 & $\mathbf{P}$ & 470.3337 & 235.6705 & 453.3071 & 227.1572 & & & 4 \\
\hline 33 & 3546.7958 & \begin{tabular}{|l}
1773.9015 \\
\end{tabular} & 3529.7692 & \begin{tabular}{|l|}
1765.3882 \\
\end{tabular} & 3528.7852 & 1764.8962 & $\mathbf{I}$ & 373.2809 & 187.1441 & 356.2544 & 178.6308 & & & 3 \\
\hline 34 & 3659.8798 & 1830.4436 & 3642.8533 & 1821.9303 & 3641.8693 & 1821.4383 & $\mathbf{L}$ & 260.1969 & 130.6021 & 243.1703 & 122.0888 & & & 2 \\
\hline 35 & & & & & & & K & 147.1128 & 74.0600 & 130.0863 & 65.5468 & & & 1 \\
\hline
\end{tabular}
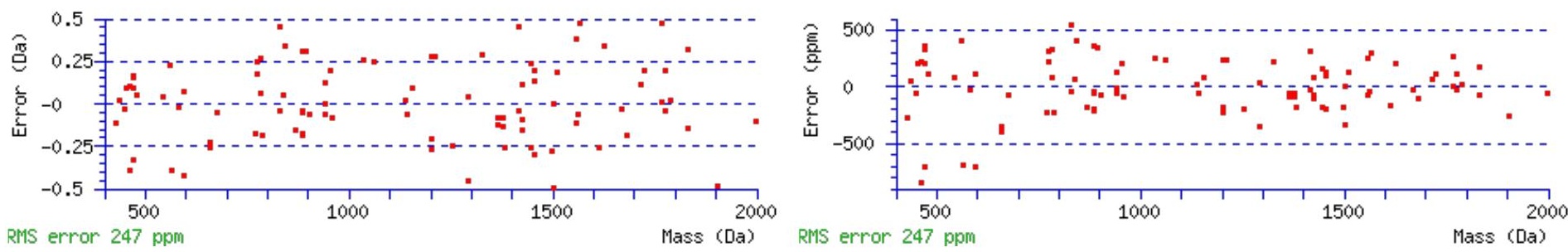

\section{All matches to this query}




\begin{tabular}{|l|l|l|l|}
\hline Score & Mr(calc): & Delta & \multicolumn{1}{|c|}{ Sequence } \\
\hline 62.9 & 3804.9781 & -0.0121 & MLVSNIDLGPTILDLAGYDLNKTQMDGTSLLPILK \\
\hline 13.3 & 3803.9940 & 0.9719 & MLVSNIDLGPTILDLAGYDLNKTQMDGTSLLPILK \\
\hline 6.8 & 3804.9781 & -0.0121 & MLVSNIDLGPTILDLAGYDLNKTQMDGTSLLPILK \\
\hline 5.3 & 3803.9263 & 1.0396 & QNILSLPLGSQIITVPVNSSLSWHINKLRETGK \\
\hline 5.2 & 3803.9263 & 1.0396 & QNILSLPLGSQIITVPVNSSLSWHINKLRETGK \\
\hline 4.3 & 3803.9263 & 1.0396 & QNILSLPLGSQIITVPVNSSLSWHINKLRETGK \\
\hline 3.4 & 3804.9282 & 0.0377 & AATDLERISNAEPEPRSLSLGGHVGFDSLPDQLVSK \\
\hline 3.3 & 3803.9514 & 1.0145 & NLVSDLSPDVKMVLVNYIFFQGLWKKPFPSSR \\
\hline 2.3 & 3803.9514 & 1.0145 & NLVSDLSPDVKMVLVNYIFFQGLWKKPFPSSR \\
\hline 0.7 & 3803.9365 & 1.0294 & DIQYATSESVDLGEIMFSLCYLPTAGRLTLTVIK \\
\hline
\end{tabular}

Spectrum No: 481; Query: 644; Rank: 1

\section{Peptide View}

MS/MS Fragmentation of VEAIGSLANTTMEK

Found in IPI00193397, Tax_Id=10116 Gene_Symbol=Slc34a1 Sodium-dependent phosphate transport protein 2A

Match to Query 644: 1479.713788 from(740.864170,2+)

Title: 100101RatKid_NS_deglyco_26.1825.1825.2.dta

Data file K:INewmanPaper|Piliang \3SubProteomes\Piliang3SP\mgf5ppm\ERLIC_3SubProteomes5ppm.mgf
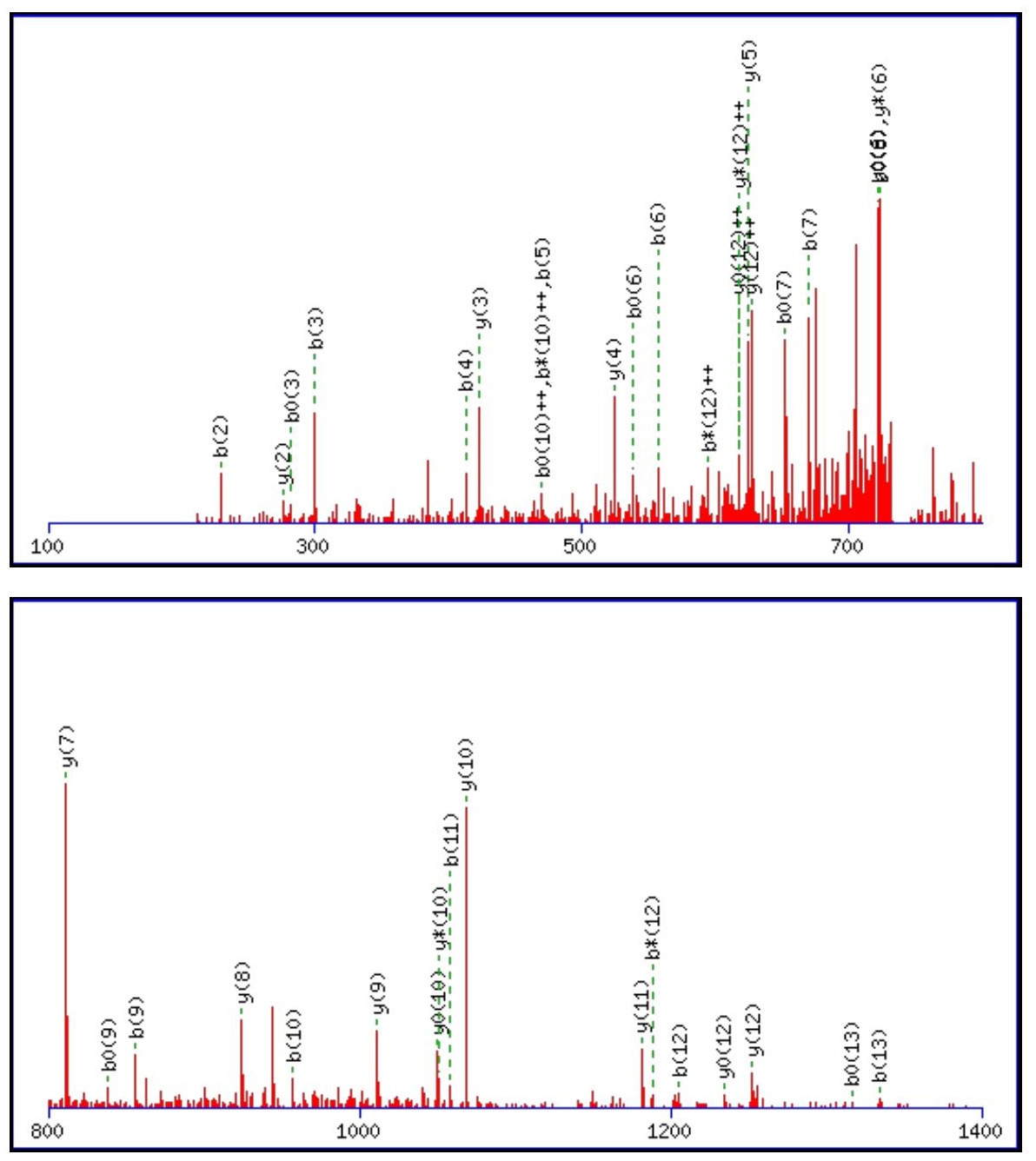
Monoisotopic mass of neutral peptide $\operatorname{Mr}($ calc): 1479.7126

Fixed modifications: Carbamidomethyl (C)

Variable modifications:

N9 : Deamidated_N (N)

M12 : Oxidation (M)

Ions Score: 63 Expect: $7.9 \mathrm{e}-005$

Matches (Bold Red): 39/136 fragment ions using 74 most intense peaks

\begin{tabular}{|c|c|c|c|c|c|c|c|c|c|c|c|c|c|c|}
\hline \# & b & $\mathbf{b}^{++}$ & b* & $\mathbf{b}^{*^{++}}$ & $\mathbf{b}^{\mathbf{0}}$ & $\mathbf{b}^{0++}$ & Seq. & $\mathbf{y}$ & $y^{++}$ & $\mathbf{y}^{*}$ & $\mathrm{y}^{\mathrm{*}^{++}}$ & $\mathbf{y}^{0}$ & $\mathbf{y}^{0++}$ & \# \\
\hline 1 & 100.0757 & 50.5415 & & & & & V & & & & & & & 14 \\
\hline 2 & 229.1183 & 115.0628 & & & 211.1077 & 106.0575 & $\mathbf{E}$ & 1381.6515 & 691.3294 & 1364.6250 & 682.8161 & 1363.6410 & 682.3241 & 13 \\
\hline 3 & 300.1554 & 150.5813 & & & 282.1448 & 141.5761 & A & 1252.6089 & 626.8081 & 1235.5824 & 618.2948 & 1234.5984 & 617.8028 & 12 \\
\hline 4 & 413.2395 & 207.1234 & & & 395.2289 & 198.1181 & I & 1181.5718 & 591.2896 & 1164.5453 & 582.7763 & 1163.5613 & 582.2843 & 11 \\
\hline 5 & 470.2609 & 235.6341 & & & 452.2504 & 226.6288 & G & 1068.4878 & 534.7475 & 1051.4612 & 526.2342 & 1050.4772 & 525.7422 & 10 \\
\hline 6 & 557.2930 & 279.1501 & & & 539.2824 & 270.1448 & $\mathbf{S}$ & 1011.4663 & 506.2368 & 994.4398 & 497.7235 & 993.4557 & 497.2315 & 9 \\
\hline 7 & 670.3770 & 335.6921 & & & 652.3665 & 326.6869 & $\mathbf{L}$ & 924.4343 & 462.7208 & 907.4077 & 454.2075 & 906.4237 & 453.7155 & 8 \\
\hline 8 & 741.4141 & 371.2107 & & & 723.4036 & 362.2054 & A & 811.3502 & 406.1787 & 794.3237 & 397.6655 & 793.3396 & 397.1735 & 7 \\
\hline 9 & 856.4411 & 428.7242 & 839.4145 & 420.2109 & 838.4305 & 419.7189 & $\mathbf{N}$ & 740.3131 & 370.6602 & 723.2865 & 362.1469 & 722.3025 & 361.6549 & 6 \\
\hline 10 & 957.4887 & 479.2480 & 940.4622 & 470.7347 & 939.4782 & 470.2427 & $\mathbf{T}$ & 625.2862 & 313.1467 & 608.2596 & 304.6334 & 607.2756 & 304.1414 & 5 \\
\hline 11 & 1058.5364 & 529.7719 & 1041.5099 & 521.2586 & 1040.5259 & 520.7666 & $\mathbf{T}$ & 524.2385 & 262.6229 & 507.2119 & 254.1096 & 506.2279 & 253.6176 & 4 \\
\hline 12 & 1205.5718 & 603.2896 & 1188.5453 & 594.7763 & 1187.5613 & 594.2843 & $\mathbf{M}$ & 423.1908 & 212.0990 & 406.1643 & 203.5858 & 405.1802 & 203.0938 & 3 \\
\hline 13 & 1334.6144 & 667.8108 & 1317.5879 & 659.2976 & 1316.6039 & 658.8056 & $\mathbf{E}$ & 276.1554 & 138.5813 & 259.1288 & 130.0681 & 258.1448 & 129.5761 & 2 \\
\hline 14 & & & & & & & $\mathbf{K}$ & 147.1128 & 74.0600 & 130.0863 & 65.5468 & & & 1 \\
\hline
\end{tabular}

$$
\text { 甶 }
$$
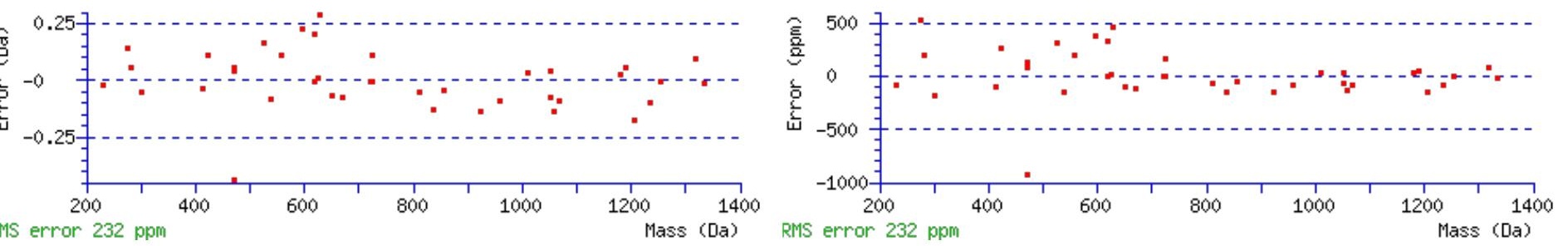

\section{All matches to this query}

\begin{tabular}{|l|l|l|l|}
\hline Score & Mr(calc): & Delta & \multicolumn{1}{c|}{ Sequence } \\
\hline 62.9 & 1479.7126 & 0.0011 & VEAIGSLANTTMEK \\
\hline 2.9 & 1479.7222 & -0.0084 & DKSLADILDPSVK \\
\hline 2.7 & 1479.7017 & 0.0121 & $\underline{\text { QVNQPMIRSATR }}$ \\
\hline 0.9 & 1479.7083 & 0.0055 & $\underline{\text { VAGQNSPSGIQSKK }}$ \\
\hline 0.2 & 1479.7221 & -0.0083 & $\underline{\text { ALNQSNTPLLSLK }}$ \\
\hline
\end{tabular}


Spectrum No: 482; Query: 842; Rank: 1

\section{Peptide View}

MS/MS Fragmentation of NHPFNSSLEDPSSR

Found in IPI00373230, Tax_Id=10116 Gene_Symbol=Muc1 similar to mucin 1, transmembrane

Match to Query 842: 1586.696232 from(529.906020,3+)

Title: 091008RatKidney_NH4Format01_24.1145.1145.3.dta

Data file K:\NewmanPaper\Piliang \3SubProteomes $\backslash$ Piliang3SP $\backslash$ mgf5ppm\ERLIC_3SubProteomes5ppm.mgf
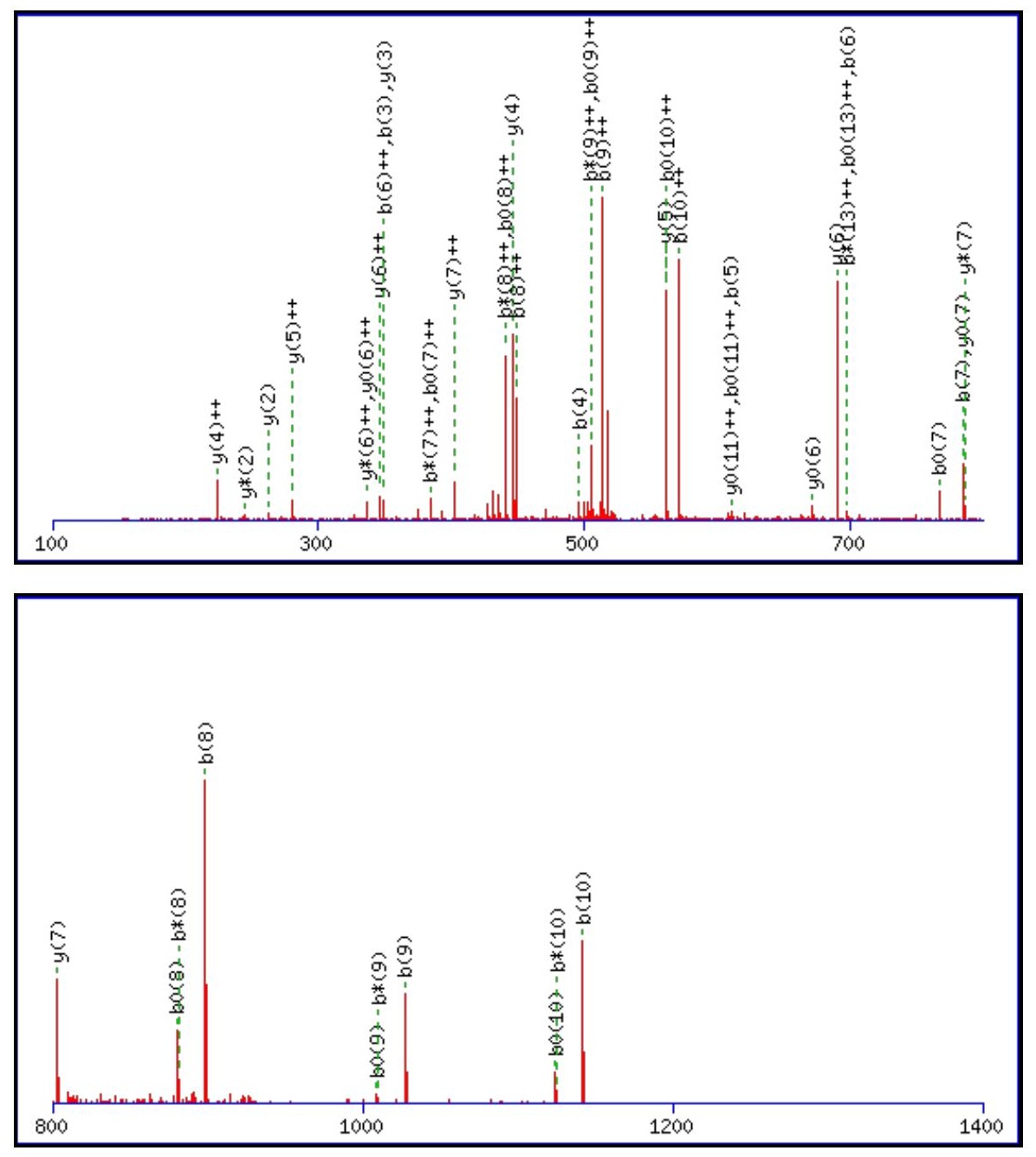

\begin{tabular}{rrrr}
1600 & 1600 & 1800 & 2000 \\
\hline
\end{tabular}

Monoisotopic mass of neutral peptide $\operatorname{Mr}$ (calc): 1586.6961

Fixed modifications: Carbamidomethyl (C) 
Variable modifications:

N5 : Deamidated_N (N)

Ions Score: 63 Expect: $8.3 \mathrm{e}-005$

Matches (Bold Red): 46/144 fragment ions using 43 most intense peaks

\begin{tabular}{|c|c|c|c|c|c|c|c|c|c|c|c|c|c|c|}
\hline \# & b & $\mathbf{b}^{++}$ & $\mathbf{b}^{*}$ & $\mathbf{b}^{*^{++}}$ & $\mathbf{b}^{0}$ & $\mathbf{b}^{0++}$ & Seq. & $\mathbf{y}$ & $\mathbf{y}^{++}$ & $\mathrm{y}^{*}$ & $\mathrm{y}^{\mathrm{*}^{++}}$ & $\mathbf{y}^{0}$ & $\mathbf{y}^{0++}$ & \# \\
\hline 1 & 115.0502 & 58.0287 & 98.0237 & 49.5155 & & & $\mathbf{N}$ & & & & & & & 14 \\
\hline 2 & 252.1091 & 126.5582 & 235.0826 & 118.0449 & & & H & 1473.6605 & 737.3339 & 1456.6339 & 728.8206 & 1455.6499 & 728.3286 & 13 \\
\hline 3 & 349.1619 & 175.0846 & 332.1353 & 166.5713 & & & $\mathbf{P}$ & 1336.6015 & 668.8044 & 1319.5750 & 660.2911 & 1318.5910 & 659.7991 & 12 \\
\hline 4 & 496.2303 & 248.6188 & 479.2037 & 240.1055 & & & $\mathbf{F}$ & 1239.5488 & 620.2780 & 1222.5222 & 611.7648 & 1221.5382 & 611.2727 & 11 \\
\hline 5 & 611.2572 & 306.1323 & 594.2307 & 297.6190 & & & $\mathbf{N}$ & 1092.4804 & 546.7438 & 1075.4538 & 538.2305 & 1074.4698 & 537.7385 & 10 \\
\hline 6 & 698.2893 & 349.6483 & 681.2627 & 341.1350 & 680.2787 & 340.6430 & $\mathrm{~S}$ & 977.4534 & 489.2304 & 960.4269 & 480.7171 & 959.4429 & 480.2251 & 9 \\
\hline 7 & 785.3213 & 393.1643 & 768.2947 & 384.6510 & 767.3107 & 384.1590 & $\mathbf{S}$ & 890.4214 & 445.7143 & 873.3949 & 437.2011 & 872.4108 & 436.7091 & 8 \\
\hline 8 & 898.4053 & 449.7063 & 881.3788 & 441.1930 & 880.3948 & 440.7010 & $\mathbf{L}$ & 803.3894 & 402.1983 & 786.3628 & 393.6850 & 785.3788 & 393.1930 & 7 \\
\hline 9 & 1027.4479 & 514.2276 & 1010.4214 & 505.7143 & 1009.4374 & 505.2223 & $E$ & 690.3053 & 345.6563 & 673.2788 & 337.1430 & 672.2947 & 336.6510 & 6 \\
\hline 10 & 1142.4749 & 571.7411 & 1125.4483 & 563.2278 & 1124.4643 & 562.7358 & D & 561.2627 & 281.1350 & 544.2362 & 272.6217 & 543.2522 & 272.1297 & 5 \\
\hline 11 & 1239.5276 & 620.2675 & 1222.5011 & 611.7542 & 1221.5171 & 611.2622 & $\mathbf{P}$ & 446.2358 & 223.6215 & 429.2092 & 215.1082 & 428.2252 & 214.6162 & 4 \\
\hline 12 & 1326.5597 & 663.7835 & 1309.5331 & 655.2702 & 1308.5491 & 654.7782 & $\mathrm{~S}$ & 349.1830 & 175.0951 & 332.1565 & 166.5819 & 331.1724 & 166.0899 & 3 \\
\hline 13 & 1413.5917 & 707.2995 & 1396.5652 & 698.7862 & 1395.5811 & 698.2942 & $\mathrm{~S}$ & 262.1510 & 131.5791 & 245.1244 & 123.0659 & 244.1404 & 122.5738 & 2 \\
\hline 14 & & & & & & & $\mathbf{R}$ & 175.1190 & 88.0631 & 158.0924 & 79.5498 & & & 1 \\
\hline
\end{tabular}
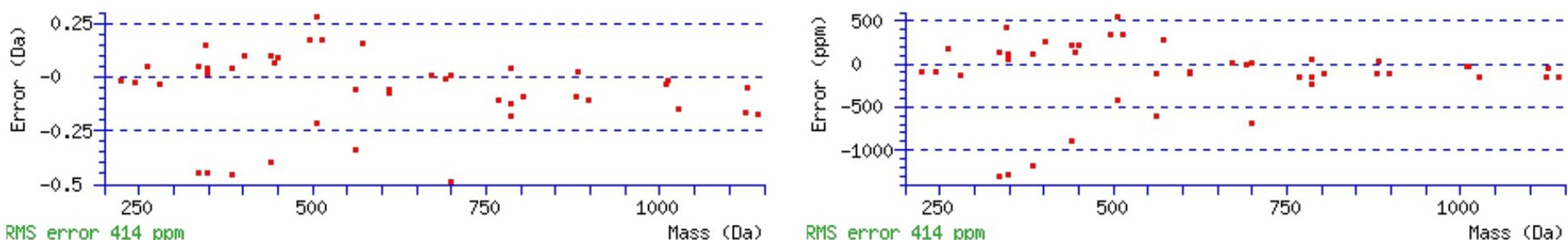

All matches to this query

\begin{tabular}{|l|l|l|l|}
\hline Score & Mr(calc): & Delta & \multicolumn{1}{|c|}{ Sequence } \\
\hline 62.9 & 1586.6961 & 0.0001 & NHPFNSSLEDPSSR \\
\hline 41.7 & 1586.6961 & 0.0001 & NHPFNSSLEDPSSR \\
\hline 23.4 & 1585.7025 & 0.9937 & TPSPPEPEPAGTAQK \\
\hline 23.1 & 1585.7025 & 0.9937 & $\underline{\text { TPSPPEPEPAGTAQK }}$ \\
\hline 14.8 & 1586.6878 & 0.0084 & WTTNXRSLNSNK \\
\hline 11.9 & 1585.6977 & 0.9986 & $\underline{\text { RCKDENYDLAMR }}$ \\
\hline 10.8 & 1585.6798 & 1.0164 & $\underline{\text { QSRGMLLLYSMK }}$ \\
\hline 10.0 & 1586.6912 & 0.0050 & $\underline{\text { SPIMNEFLGGRNR }}$ \\
\hline 8.5 & 1586.6847 & 0.0116 & $\underline{\text { MAPMGWNRKASSR }}$ \\
\hline 8.5 & 1586.6847 & 0.0116 & MAPMGWNRKASSR \\
\hline
\end{tabular}

Spectrum No: 483; Query: 56; Rank: 1

\section{Peptide View}

MS/MS Fragmentation of LGACNNTLK

Found in IPI00372372, Tax_Id=10116 Gene_Symbol=Serpinc1 Serine (Or cysteine) peptidase inhibitor, clade C (Antithrombin), member 1

Match to Query 56: 990.477968 from(496.246260,2+)

Title: 091008RatKidney_NH4Format01_29.350.350.2.dta

Data file K:INewmanPaper|Piliang|3SubProteomes\Piliang3SP\mgf5ppm\ERLIC_3SubProteomes5ppm.mgf 

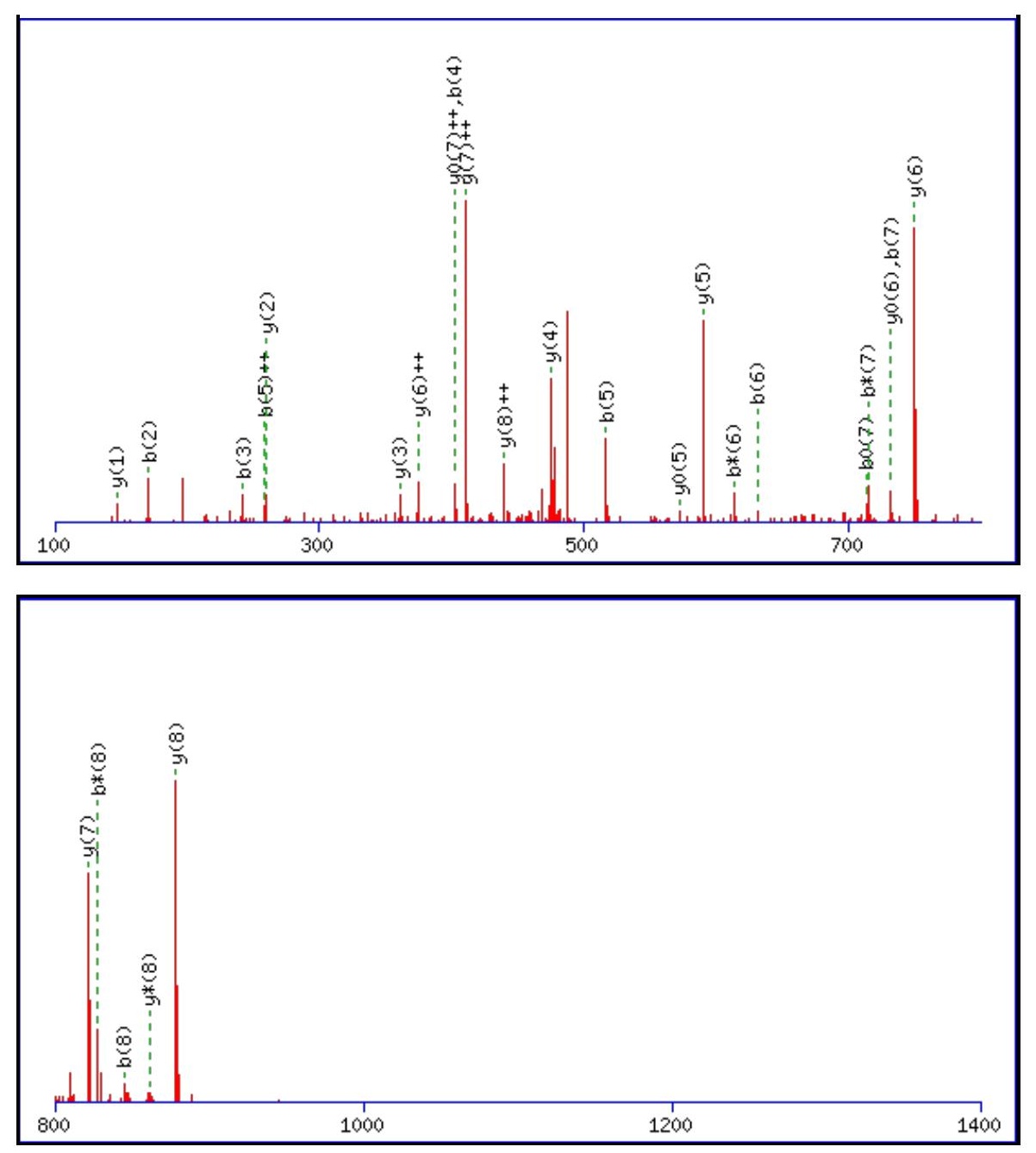

\begin{tabular}{rrrr}
\hline 00 & 1600 & 1800 & 2000 \\
\hline
\end{tabular}

Monoisotopic mass of neutral peptide $\operatorname{Mr}($ calc): 990.4804

Fixed modifications: Carbamidomethyl (C)

Variable modifications:

N5 : Deamidated_N (N)

Ions Score: 63 Expect: $5.4 \mathrm{e}-005$

Matches (Bold Red): 27/72 fragment ions using 45 most intense peaks

\begin{tabular}{|c|c|c|c|c|c|c|c|c|c|c|c|c|c|}
\hline$\#$ & b & $\mathbf{b}^{++}$ & $\mathbf{b}^{*}$ & $\mathbf{b}^{*^{++}}$ & $\mathbf{b}^{0}$ & $\mathbf{b}^{0++}$ & Seq. & $\mathbf{y}$ & $\mathbf{y}^{++}$ & $\mathrm{y}^{*}$ & $\mathrm{y}^{*^{++}}$ & $\mathbf{y}^{0}$ & $y^{0++}$ \\
\hline 1 & 114.0913 & 57.5493 & & & & & $\mathbf{L}$ & & & & & & \\
\hline 2 & 171.1128 & 86.0600 & & & & & G & 878.4036 & 439.7055 & 861.3771 & 431.1922 & 860.3931 & 430.7002 \\
\hline 3 & 242.1499 & 121.5786 & & & & & A & 821.3822 & 411.1947 & 804.3556 & 402.6815 & 803.3716 & 702.1894 \\
\hline 4 & 402.1806 & 201.5939 & & & & & $\mathrm{C}$ & 750.3451 & 375.6762 & 733.3185 & 367.1629 & 732.3345 & 366.6709 \\
\hline 5 & 517.2075 & 259.1074 & 500.1810 & 250.5941 & & & $\mathbf{N}$ & 590.3144 & 295.6608 & 573.2879 & 287.1476 & 572.3038 & 286.6556 \\
\hline
\end{tabular}




\begin{tabular}{|l|l|l|l|l|l|l|l|l|l|l|l|l|l|l|l|l|l|}
$\mathbf{6}$ & $\mathbf{6 3 1 . 2 5 0 4}$ & 316.1289 & $\mathbf{6 1 4 . 2 2 3 9}$ & 307.6156 & & & $\mathbf{N}$ & $\mathbf{4 7 5 . 2 8 7 5}$ & 238.1474 & 458.2609 & 229.6341 & 457.2769 & 229.1421 & $\mathbf{4}$ \\
\hline $\mathbf{7}$ & $\mathbf{7 3 2 . 2 9 8 1}$ & 366.6527 & $\mathbf{7 1 5 . 2 7 1 6}$ & 358.1394 & $\mathbf{7 1 4 . 2 8 7 5}$ & 357.6474 & $\mathbf{T}$ & $\mathbf{3 6 1 . 2 4 4 5}$ & 181.1259 & 344.2180 & 172.6126 & 343.2340 & 172.1206 & 3 \\
\hline $\mathbf{8}$ & $\mathbf{8 4 5 . 3 8 2 2}$ & 423.1947 & $\mathbf{8 2 8 . 3 5 5 6}$ & 414.6815 & 827.3716 & $\mathbf{4 1 4 . 1 8 9 4}$ & $\mathbf{L}$ & $\mathbf{2 6 0 . 1 9 6 9}$ & 130.6021 & 243.1703 & 122.0888 & & & $\mathbf{2}$ \\
\hline $\mathbf{9}$ & & & & & & & $\mathbf{K}$ & $\mathbf{1 4 7 . 1 1 2 8}$ & 74.0600 & 130.0863 & 65.5468 & & & $\mathbf{1}$ \\
\hline
\end{tabular}
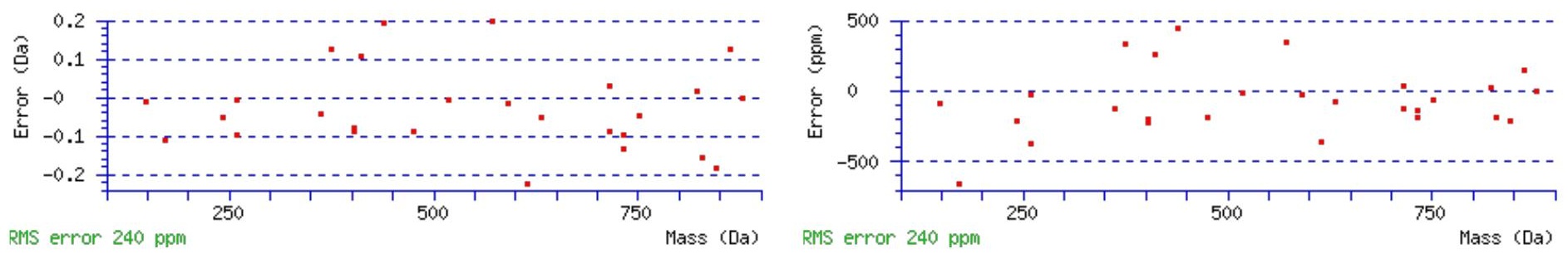

\section{All matches to this query}

\begin{tabular}{|l|l|l|l|}
\hline Score & Mr(calc): & Delta & \multicolumn{1}{|c|}{ Sequence } \\
\hline 62.8 & 990.4804 & -0.0024 & LGACNNTLK \\
\hline 61.0 & 990.4804 & -0.0024 & LGACNNTLK \\
\hline 11.2 & 990.4787 & -0.0007 & LPDSPALAK \\
\hline 10.4 & 990.4787 & -0.0007 & LQETVPPK \\
\hline 8.8 & 990.4730 & 0.0050 & ASRSNNDVK \\
\hline 8.4 & 990.4804 & -0.0025 & $\underline{\text { GETMIDGR }}$ \\
\hline 7.4 & 990.4770 & 0.0009 & THYNSELK \\
\hline 7.2 & 990.4730 & 0.0050 & ASRSNNDVK \\
\hline 6.1 & 990.4688 & 0.0092 & LQLHWSK \\
\hline 5.0 & 990.4722 & 0.0058 & LKMSHPAK \\
\hline
\end{tabular}

Spectrum No: 484; Query: 2299; Rank: 1

\section{Peptide View}

MS/MS Fragmentation of GTDPSLSPSPAAALPAPGTELSYLNR

Found in IPI00192504, Tax_Id=10116 Gene_Symbol=Mrc2 Macrophage mannose receptor 2 precursor

Match to Query 2299: 2582.281568 from(1292.148060,2+)

Title: 091008RatKidney_NH4Format01_23.3513.3513.2.dta

Data file K:INewmanPaper|Piliang|3SubProteomes\Piliang3SP\mgf5ppm\ERLIC_3SubProteomes5ppm.mgf

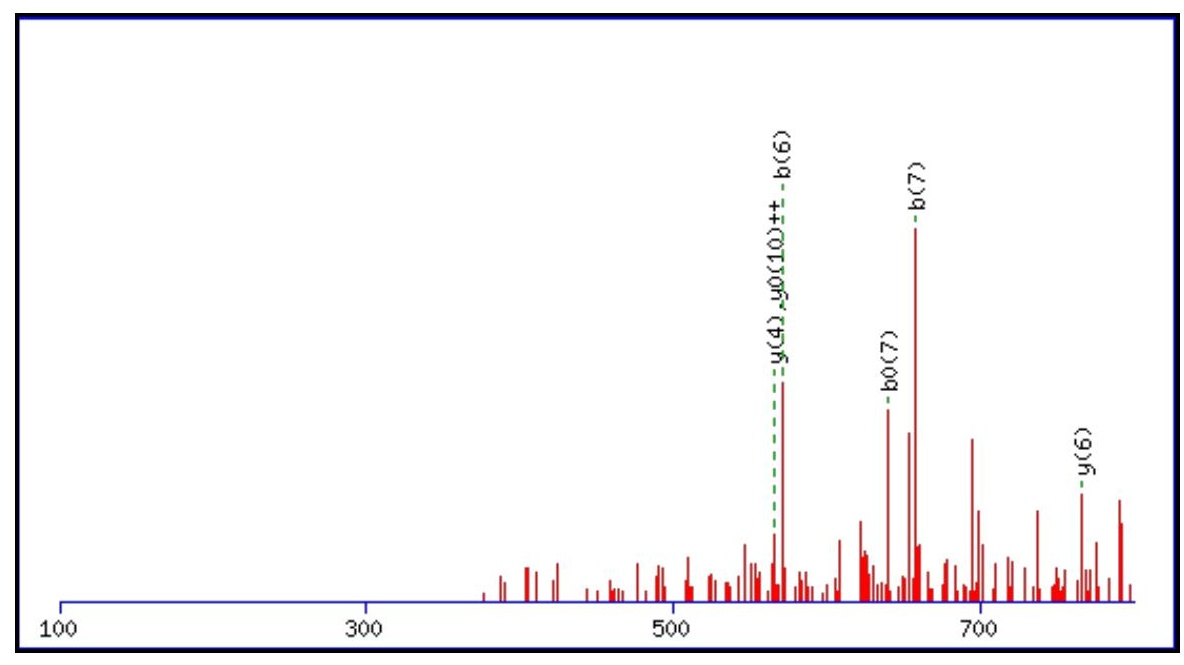



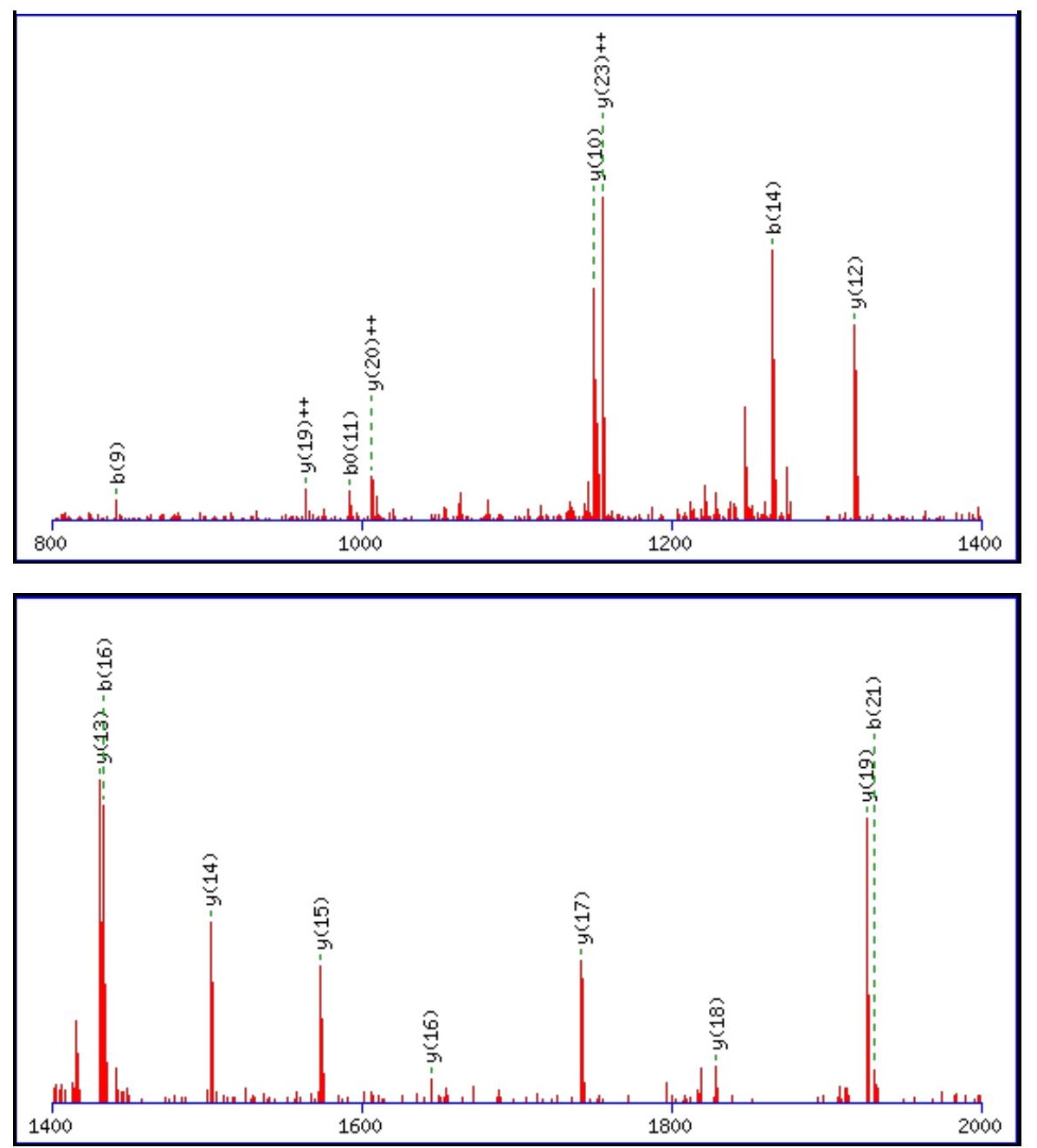

Monoisotopic mass of neutral peptide $\operatorname{Mr}($ calc): 2582.2864

Fixed modifications: Carbamidomethyl (C)

Variable modifications:

N25 : Deamidated_N (N)

Ions Score: 63 Expect: 0.00012

Matches (Bold Red): 23/242 fragment ions using 48 most intense peaks

\begin{tabular}{|c|c|c|c|c|c|c|c|c|c|c|c|c|c|c|}
\hline \# & b & $\mathbf{b}^{++}$ & $\mathbf{b}^{*}$ & $\mathbf{b}^{*+}$ & $\mathbf{b}^{0}$ & $\mathbf{b}^{0++}$ & Seq. & $\mathbf{y}$ & $y^{++}$ & $\mathbf{y}^{*}$ & $\mathbf{y}^{*^{++}}$ & $\mathbf{y}^{\mathbf{0}}$ & $y^{0++}$ & \# \\
\hline 1 & 58.0287 & 29.5180 & & & & & G & & & & & & & 26 \\
\hline 2 & 159.0764 & 80.0418 & & & 141.0659 & 71.0366 & $\mathbf{T}$ & 2526.2722 & 1263.6397 & 2509.2457 & 1255.1265 & 2508.2617 & 1254.6345 & 25 \\
\hline 4 & 371.1561 & 186.0817 & & & 353.1456 & 177.0764 & $\mathbf{P}$ & 2310.1976 & 1155.6024 & 2293.1710 & 1147.0892 & 2292.1870 & 1146.5972 & 23 \\
\hline 5 & 458.1882 & 229.5977 & & & 440.1776 & 220.5924 & $S$ & 2213.1448 & 1107.0761 & 2196.1183 & 1098.5628 & 2195.1343 & 1098.0708 & 22 \\
\hline 6 & 571.2722 & 286.1397 & & & 553.2617 & 277.1345 & $\mathbf{L}$ & 2126.1128 & 1063.5600 & 2109.0863 & 1055.0468 & 2108.1022 & 1054.5548 & 21 \\
\hline 7 & 658.3042 & 329.6558 & & & 640.2937 & 320.6505 & S & 2013.0287 & 1007.0180 & 1996.0022 & 998.5047 & 1995.0182 & 998.0127 & 20 \\
\hline 8 & 755.3570 & 378.1821 & & & 737.3464 & 369.1769 & $\mathbf{P}$ & 1925.9967 & 963.5020 & 1908.9702 & 954.9887 & 1907.9861 & 954.4967 & 19 \\
\hline 9 & 842.3890 & 421.6982 & & & 824.3785 & 412.6929 & $S$ & 1828.9439 & 914.9756 & 1811.9174 & 906.4623 & 1810.9334 & 905.9703 & 18 \\
\hline 10 & 939.4418 & 470.2245 & & & 921.4312 & 461.2193 & $\mathbf{P}$ & 1741.9119 & 871.4596 & 1724.8854 & 862.9463 & 1723.9014 & 862.4543 & 17 \\
\hline 11 & 1010.4789 & 505.7431 & & & 992.4684 & 496.7378 & A & 1644.8592 & 822.9332 & 1627.8326 & 814.4199 & 1626.8486 & 813.9279 & 16 \\
\hline 12 & 1081.5160 & 541.2617 & & & 1063.5055 & 532.2564 & A & 1573.8220 & 787.4147 & 1556.7955 & 778.9014 & 1555.8115 & 778.4094 & 15 \\
\hline 14 & 1265.6372 & 633.3222 & & & 1247.6266 & 624.3170 & $\mathbf{L}$ & 1431.7478 & 716.3775 & 1414.7213 & 707.8643 & 1413.7372 & 707.3723 & 13 \\
\hline 15 & 1362.6900 & 681.8486 & & & 1344.6794 & 672.8433 & $\mathbf{P}$ & 1318.6638 & 659.8355 & 1301.6372 & 651.3222 & 1300.6532 & 650.8302 & 12 \\
\hline 16 & 1433.7271 & 717.3672 & & & 1415.7165 & 708.3619 & A & 1221.6110 & 611.3091 & 1204.5844 & 602.7959 & 1203.6004 & 602.3038 & 11 \\
\hline 17 & 1530.7798 & 765.8936 & & & 1512.7693 & 756.8883 & $\mathbf{P}$ & 1150.5739 & 575.7906 & 1133.5473 & 567.2773 & 1132.5633 & 566.7853 & 10 \\
\hline 18 & 1587.8013 & 794.4043 & & & 1569.7907 & 785.3990 & G & 1053.5211 & 527.2642 & 1036.4946 & 518.7509 & 1035.5105 & 518.2589 & 9 \\
\hline
\end{tabular}




\begin{tabular}{|r|r|r|r|r|r|r|r|r|r|r|r|r|r|r|r|r|}
$\mathbf{1 9}$ & $\mathbf{1 6 8 8 . 8 4 9 0}$ & 844.9281 & & & 1670.8384 & 835.9229 & $\mathbf{T}$ & 996.4996 & 498.7535 & 979.4731 & 490.2402 & 978.4891 & 489.7482 & $\mathbf{8}$ \\
\hline $\mathbf{2 0}$ & $\mathbf{1 8 1 7 . 8 9 1 6}$ & 909.4494 & & & 1799.8810 & 900.4441 & $\mathbf{E}$ & 895.4520 & 448.2296 & 878.4254 & 439.7163 & 877.4414 & 439.2243 & $\mathbf{7}$ \\
\hline $\mathbf{2 1}$ & $\mathbf{1 9 3 0 . 9 7 5 6}$ & 965.9915 & & & 1912.9651 & 956.9862 & L & 766.4094 & 383.7083 & 749.3828 & 375.1950 & 748.3988 & 374.7030 & $\mathbf{6}$ \\
\hline $\mathbf{2 2}$ & 2018.0077 & 1009.5075 & & & 1999.9971 & 1000.5022 & S & 653.3253 & 327.1663 & 636.2988 & 318.6530 & 635.3147 & 318.1610 & $\mathbf{5}$ \\
\hline $\mathbf{2 3}$ & 2181.0710 & 1091.0391 & & & 2163.0604 & 1082.0339 & Y & 566.2933 & 283.6503 & 549.2667 & 275.1370 & & & & $\mathbf{4}$ \\
\hline $\mathbf{2 4}$ & 2294.1551 & 1147.5812 & & & 2276.1445 & 1138.5759 & L & 403.2300 & 202.1186 & 386.2034 & 193.6053 & & & & $\mathbf{3}$ \\
\hline $\mathbf{2 5}$ & 2409.1820 & 1205.0946 & 2392.1555 & 1196.5814 & 2391.1714 & 1196.0894 & N & 290.1459 & 145.5766 & 273.1193 & 137.0633 & & & \\
\hline $\mathbf{2 6}$ & & & & & & & R & 175.1190 & 88.0631 & 158.0924 & 79.5498 & & & $\mathbf{1}$ \\
\hline
\end{tabular}
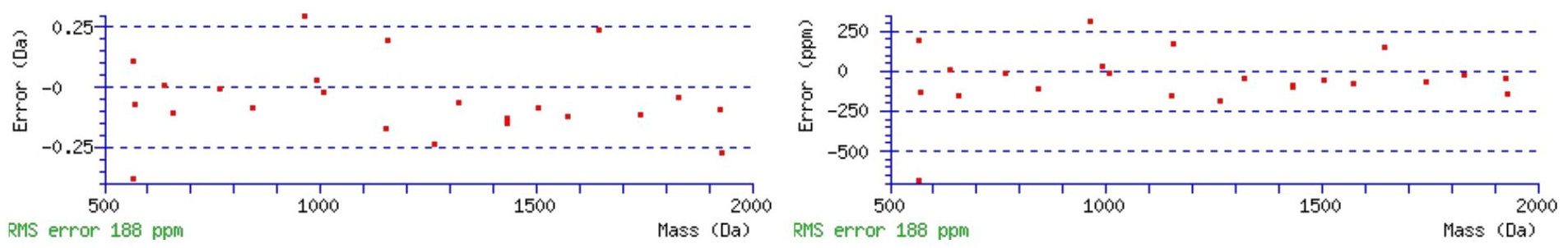

\section{All matches to this query}

\begin{tabular}{|l|l|l|l|}
\hline Score & Mr(calc): & Delta & \multicolumn{1}{|c|}{ Sequence } \\
\hline 62.8 & 2582.2864 & -0.0048 & GTDPSLSPSPAAALPAPGTELSYLNR \\
\hline 9.8 & 2581.3024 & 0.9792 & GTDPSLSPSPAAALPAPGTELSYLNR \\
\hline 0.7 & 2582.2733 & 0.0082 & EPTQPSDARGLIRWMLMVNPDR \\
\hline 0.3 & 2582.2775 & 0.0041 & VVIQEMLKTVAQSQQLETSSQR \\
\hline 0.3 & 2582.2775 & 0.0041 & VVIQEMLKTVAQSQQLETSSQR \\
\hline 0.3 & 2582.2775 & 0.0041 & VVIQEMLKTVAQSQQLETSSQR \\
\hline
\end{tabular}

Spectrum No: 485; Query: 2052; Rank: 1

\section{Peptide View}

MS/MS Fragmentation of GRQQSQEDGDYSPNGSAQPGDK

Found in IPI00364925, Tax_Id=10116 Gene_Symbol=Dhtkd1 Probable 2-oxoglutarate dehydrogenase E1 component DHKTD1, mitochondrial precursor

Match to Query 2052: 2320.982022 from(774.667950,3+)

Title: 091008RatKidney_NH4Format01_23.376.376.3.dta

Data file K:INewmanPaper|Piliangl3SubProteomes\Piliang3SP\mgf5ppm\ERLIC_3SubProteomes5ppm.mgf

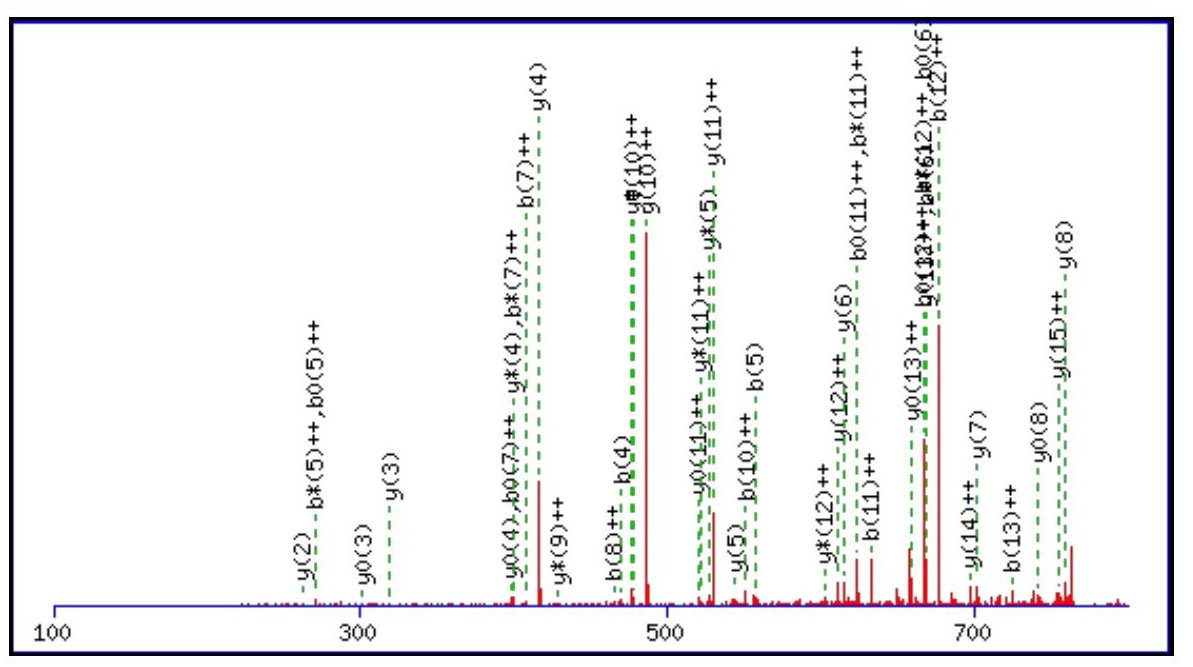



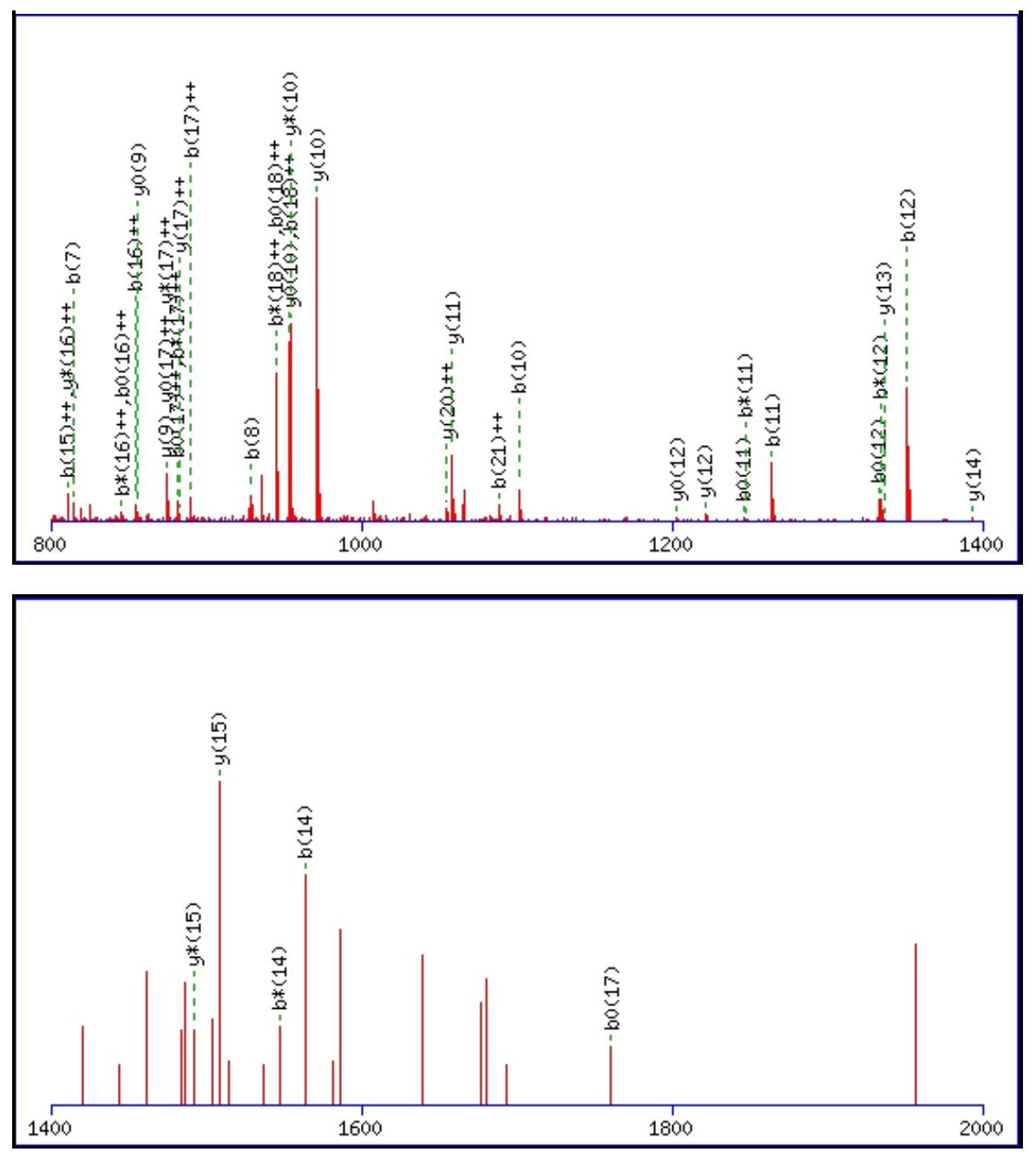

Monoisotopic mass of neutral peptide $\operatorname{Mr}($ calc): 2320.9792

Fixed modifications: Carbamidomethyl (C)

Variable modifications:

N14 : Deamidated_N (N)

Ions Score: 63 Expect: 0.00016

Matches (Bold Red): 83/240 fragment ions using 148 most intense peaks

\begin{tabular}{|c|c|c|c|c|c|c|c|c|c|c|c|c|c|c|}
\hline \# & b & $\mathbf{b}^{++}$ & $\mathbf{b}^{*}$ & $\mathbf{b}^{*^{++}}$ & $\mathbf{b}^{\mathbf{0}}$ & $\mathbf{b}^{\mathbf{0 + +}}$ & Seq. & $\mathbf{y}$ & $y^{++}$ & $\mathbf{y}^{*}$ & $\mathbf{y}^{*^{++}}$ & $\mathbf{y}^{0}$ & $\mathbf{y}^{0++}$ & \# \\
\hline 1 & 58.0287 & 29.5180 & & & & & G & & & & & & & 22 \\
\hline 2 & 214.1299 & 107.5686 & 197.1033 & 99.0553 & & & $\mathbf{R}$ & 2264.9650 & 1132 & 2247.9385 & 1124.4729 & 2246.9545 & 1123.9809 & 21 \\
\hline 3 & 342.1884 & 171.5979 & 325.1619 & 163.0846 & & & $\mathbf{Q}$ & 2108.8639 & 1054.9356 & 2091.8374 & 1046.4223 & 2090.8534 & 1045.9303 & 20 \\
\hline 4 & 470.2470 & 235.6271 & 453.2205 & 227.1139 & & & $\mathbf{Q}$ & 1980.8053 & 990.9063 & 1963.7788 & 982.3930 & 1962.7948 & 981.9010 & 19 \\
\hline 5 & 557.2790 & 279.1432 & 540.2525 & 270.6299 & 539.2685 & 270.1379 & $\mathrm{~S}$ & 1852.7468 & 926.8770 & 1835.7202 & 918.3637 & 1834.7362 & 917.8717 & 18 \\
\hline 6 & 685.3376 & 343.1724 & 668.3111 & 334.6592 & 667.3270 & 334.1672 & $\mathbf{Q}$ & 1765.7147 & 883.3610 & 1748.6882 & 874.8477 & 1747.7042 & 874.3557 & 17 \\
\hline 7 & 814.3802 & 407.6937 & 797.3537 & 399.1805 & 796.3696 & 398.6885 & $\mathbf{E}$ & 1637.6562 & 819.3317 & 1620.6296 & 810.8184 & 1619.6456 & 810.3264 & 16 \\
\hline 8 & 929.4071 & 465.2072 & 912.3806 & 456.6939 & 911.3966 & 456.2019 & D & 1508.6136 & 754.8104 & 1491.5870 & 746.2971 & 1490.6030 & 745.8051 & 15 \\
\hline 9 & 986.4286 & 493.7179 & 969.4021 & 485.2047 & 968.4180 & 484.7127 & G & 1393.5866 & 697.2969 & 1376.5601 & 688.7837 & 1375.5761 & 688.2917 & 14 \\
\hline 10 & 1101.4556 & 551.2314 & 1084.4290 & 542.7181 & 1083.4450 & 542.2261 & D & 1336.5652 & 668.7862 & 1319.5386 & 660.2729 & 1318.5546 & 659.7809 & 13 \\
\hline 11 & 1264.5189 & 632.7631 & 1247.4923 & 624.2498 & 1246.5083 & 623.7578 & $\mathbf{Y}$ & 1221.5382 & 611.2727 & 1204.5117 & 602.7595 & 1203.5277 & 602.2675 & 12 \\
\hline 12 & 1351.5509 & 676.2791 & 1334.5244 & 667.7658 & 1333.5403 & 667.2738 & $\mathrm{~S}$ & 1058.4749 & 529.7411 & 1041.4483 & 521.2278 & 1040.4643 & 520.7358 & 11 \\
\hline 13 & 1448.6037 & 724.8055 & 1431.5771 & 716.2922 & 1430.5931 & 715.8002 & $\mathbf{P}$ & 971.4429 & 486.2251 & 954.4163 & 477.7118 & 953.4323 & 477.2198 & 10 \\
\hline 14 & 1563.6306 & 782.3189 & 1546.6041 & 773.8057 & 1545.6200 & 773.3137 & $\mathbf{N}$ & 874.3901 & 437.6987 & 857.3635 & 429.1854 & 856.3795 & 428.6934 & 9 \\
\hline 15 & 1620.6521 & 810.8297 & 1603.6255 & 802.3164 & 1602.6415 & 801.8244 & G & 759.3632 & 380.1852 & 742.3366 & 371.6719 & 741.3526 & 371.1799 & 8 \\
\hline 16 & 1707.6841 & 854.3457 & 1690.6576 & 845.8324 & 1689.6735 & 845.3404 & $\mathrm{~S}$ & 702.3417 & 351.6745 & 685.3151 & 343.1612 & 684.3311 & 342.6692 & 7 \\
\hline 17 & 1778.7212 & 889.8642 & 1761.6947 & 881.3510 & 1760.7107 & 880.8590 & A & 615.3097 & 308.1585 & 598.2831 & 299.6452 & 597.2991 & 299.1532 & 6 \\
\hline 18 & 1906.7798 & 953.8935 & 1889.7532 & 945.3803 & 1888.7692 & 944.8883 & $\mathbf{Q}$ & 544.2726 & 272.6399 & 527.2460 & 264.1266 & 526.2620 & 263.6346 & 5 \\
\hline
\end{tabular}




\begin{tabular}{|r|r|r|r|r|r|r|r|r|r|r|r|r|r|r|}
$\mathbf{1 9}$ & 2003.8326 & 1002.4199 & 1986.8060 & 993.9066 & 1985.8220 & 993.4146 & $\mathbf{P}$ & $\mathbf{4 1 6 . 2 1 4 0}$ & 208.6106 & $\mathbf{3 9 9 . 1 8 7 4}$ & 200.0974 & $\mathbf{3 9 8 . 2 0 3 4}$ & 199.6053 & $\mathbf{4}$ \\
\hline $\mathbf{2 0}$ & 2060.8540 & 1030.9307 & 2043.8275 & 1022.4174 & 2042.8435 & 1021.9254 & $\mathbf{G}$ & $\mathbf{3 1 9 . 1 6 1 2}$ & 160.0842 & 302.1347 & 151.5710 & $\mathbf{3 0 1 . 1 5 0 6}$ & 151.0790 & $\mathbf{3}$ \\
\hline $\mathbf{2 1}$ & 2175.8810 & $\mathbf{1 0 8 8 . 4 4 4 1}$ & 2158.8544 & 1079.9308 & 2157.8704 & 1079.4388 & $\mathbf{D}$ & $\mathbf{2 6 2 . 1 3 9 7}$ & 131.5735 & 245.1132 & 123.0602 & 244.1292 & 122.5682 & $\mathbf{2}$ \\
\hline $\mathbf{2 2}$ & & & & & & & $\mathbf{K}$ & 147.1128 & 74.0600 & 130.0863 & 65.5468 & & & $\mathbf{1}$ \\
\hline
\end{tabular}
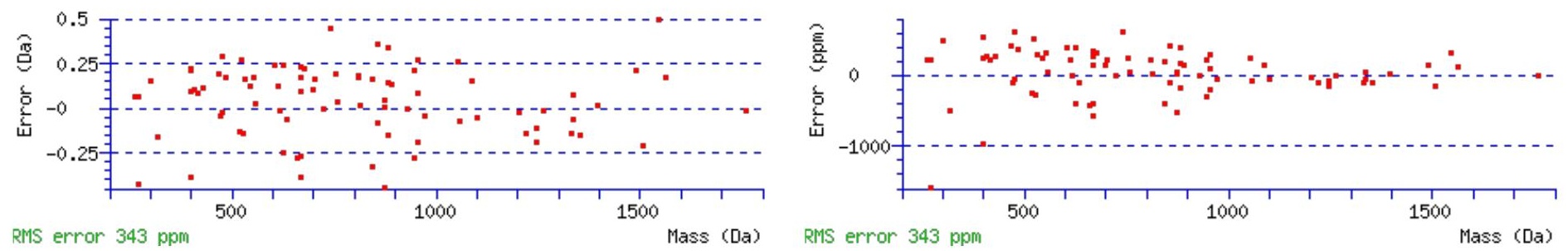

\section{All matches to this query}

\begin{tabular}{|l|l|l|l|}
\hline Score & Mr(calc): & Delta & \multicolumn{1}{|c|}{ Sequence } \\
\hline 62.7 & 2320.9792 & 0.0028 & GRQQSQEDGDYSPNGSAQPGDK \\
\hline 27.7 & 2319.9952 & 0.9868 & GRQQSQEDGDYSPNGSAQPGDK \\
\hline 8.7 & 2321.0046 & -0.0225 & EQMMNSSMSSGSGSLRTSEKR \\
\hline 8.7 & 2321.0046 & -0.0225 & EQMMNSSMSSGSGSLRTSEKR \\
\hline 7.3 & 2318.9816 & 2.0004 & LSSSMCVTSDLLSDIYKPK \\
\hline 5.1 & 2320.9879 & -0.0058 & TWKNNSHSKASETGSTWLK \\
\hline 4.9 & 2320.9661 & 0.0160 & SRGPRGEGGAMSENIWSTLR \\
\hline 4.9 & 2320.9661 & 0.0160 & SRGPRGEGGAMSENIWSTLR \\
\hline 4.9 & 2320.9671 & 0.0150 & VTWTNGTIPPPVTEASSVPK \\
\hline 4.7 & 2320.9743 & 0.0078 & TQAPQVSVSALQPSLPTNSR \\
\hline
\end{tabular}

Spectrum No: 486; Query: 520; Rank: 1

\section{Peptide View}

MS/MS Fragmentation of LSNITNIGPLDMK

Found in IPI00421319, Tax_Id=10116 Gene_Symbol=Tmem106b LRRGT00101

Match to Query 520: 1415.733888 from(708.874220,2+)

Title: 091008RatKidney_NH4Format02_25.3435.3435.2.dta

Data file K:INewmanPaper|Piliang|3SubProteomes\Piliang3SP\mgf5ppm\ERLIC_3SubProteomes5ppm.mgf

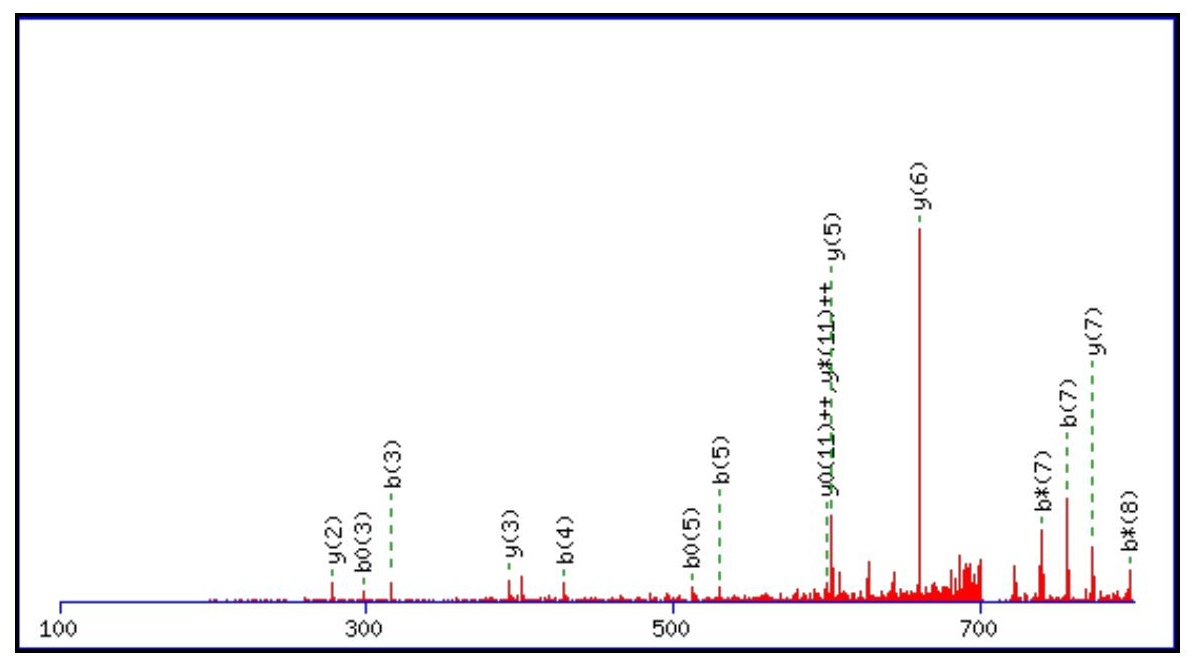



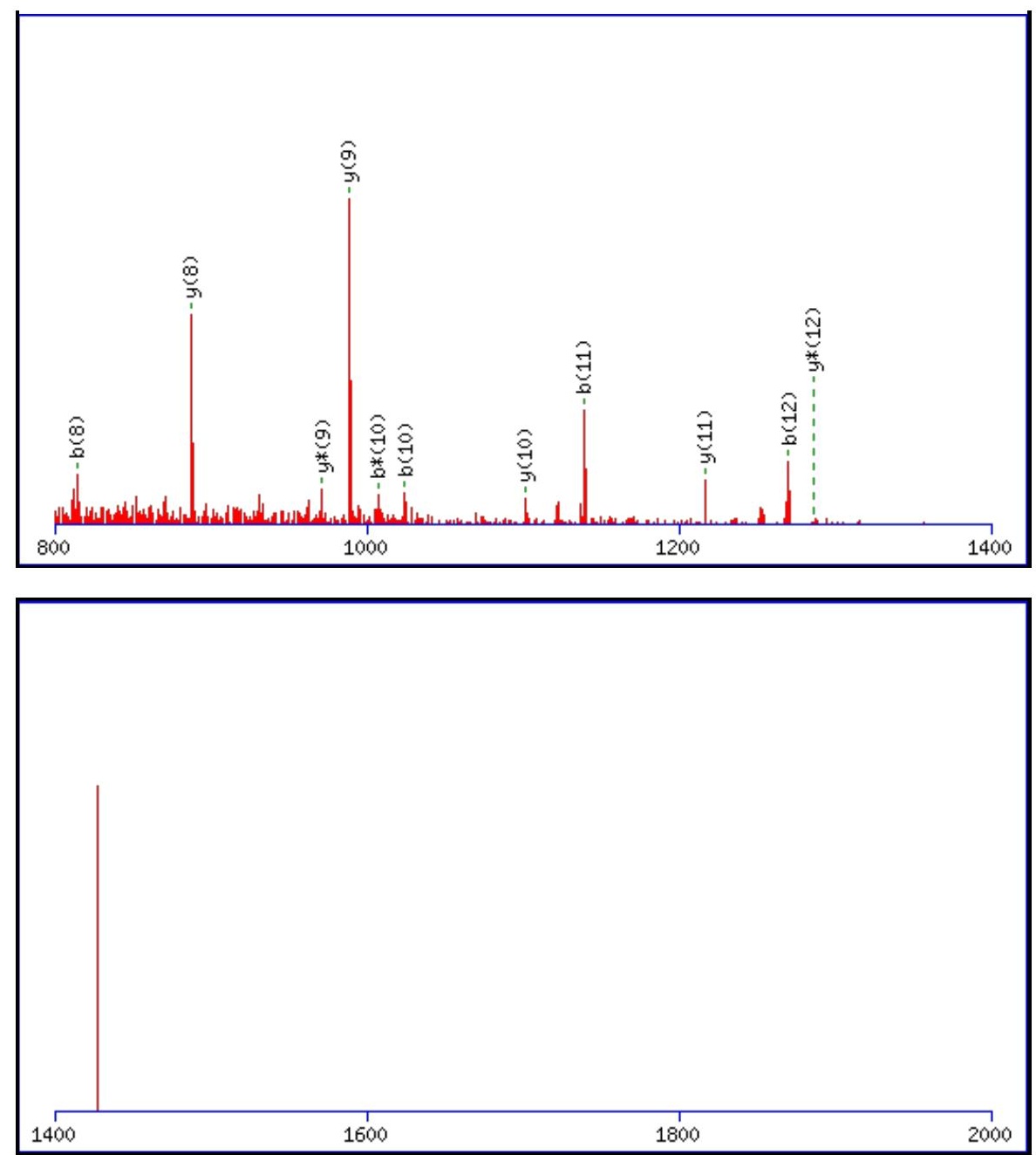

Monoisotopic mass of neutral peptide $\operatorname{Mr}($ calc): 1415.7330

Fixed modifications: Carbamidomethyl (C)

Variable modifications:

N3 : Deamidated N (N)

Ions Score: 63 Expect: $7.2 \mathrm{e}-005$

Matches (Bold Red): 26/134 fragment ions using 43 most intense peaks

\begin{tabular}{|c|c|c|c|c|c|c|c|c|c|c|c|c|c|c|}
\hline \# & b & $\mathbf{b}^{++}$ & $\mathbf{b}^{*}$ & $\mathbf{b}^{*^{++}}$ & $\mathbf{b}^{0}$ & $\mathbf{b}^{\mathbf{0 + +}}$ & Seq. & $\mathbf{y}$ & $y^{++}$ & $\mathbf{y}^{*}$ & $\mathrm{y}^{*^{++}}$ & $\mathbf{y}^{0}$ & $y^{0++}$ & \# \\
\hline 1 & 114.0913 & 57.5493 & & & & & $\mathbf{L}$ & & & & & & & 13 \\
\hline 2 & 201.1234 & 101.0653 & & & 183.1128 & 92.0600 & $S$ & 1303.6562 & 652.3317 & 1286.6297 & 643.8185 & 1285.6457 & 643.3265 & 12 \\
\hline 3 & 316.1503 & 158.5788 & 299.1238 & 150.0655 & 298.1397 & 149.5735 & $\mathbf{N}$ & 1216.6242 & 608.8157 & 1199.5976 & 600.3025 & 1198.6136 & 599.8105 & 11 \\
\hline 4 & 429.2344 & 215.1208 & 412.2078 & 206.6075 & 411.2238 & 206.1155 & I & 1101.5973 & 551.3023 & 1084.5707 & 542.7890 & 1083.5867 & 542.2970 & 10 \\
\hline 5 & 530.2820 & 265.6447 & 513.2555 & 257.1314 & 512.2715 & 256.6394 & $\mathbf{T}$ & 988.5132 & 494.7602 & 971.4866 & 486.2470 & 970.5026 & 485.7550 & 9 \\
\hline 6 & 644.3250 & 322.6661 & 627.2984 & 314.1529 & 626.3144 & 313.6608 & $\mathbf{N}$ & 887.4655 & 444.2364 & 870.4390 & 435.7231 & 869.4550 & 435.2311 & 8 \\
\hline 7 & 757.4090 & 379.2082 & 740.3825 & 370.6949 & 739.3985 & 370.2029 & I & 773.4226 & 387.2149 & 756.3960 & 378.7017 & 755.4120 & 378.2096 & 7 \\
\hline 8 & 814.4305 & 407.7189 & 797.4040 & 399.2056 & 796.4199 & 398.7136 & G & 660.3385 & 330.6729 & 643.3120 & 322.1596 & 642.3280 & 321.6676 & 6 \\
\hline 9 & 911.4833 & 456.2453 & 894.4567 & 447.7320 & 893.4727 & 447.2400 & $\mathbf{P}$ & 603.3171 & 302.1622 & 586.2905 & 293.6489 & 585.3065 & 293.1569 & 5 \\
\hline 10 & 1024.5673 & 512.7873 & 1007.5408 & 504.2740 & 1006.5568 & 503.7820 & $\mathbf{L}$ & 506.2643 & 253.6358 & 489.2377 & 245.1225 & 488.2537 & 244.6305 & 4 \\
\hline 11 & 1139.5943 & 570.3008 & 1122.5677 & 561.7875 & 1121.5837 & 561.2955 & D & 393.1802 & 197.0938 & 376.1537 & 188.5805 & 375.1697 & 188.0885 & 3 \\
\hline 12 & 1270.6348 & 635.8210 & 1253.6082 & 627.3077 & 1252.6242 & 626.8157 & $\mathbf{M}$ & 278.1533 & 139.5803 & 261.1267 & 131.0670 & & & 2 \\
\hline 13 & & & & & & & $\mathbf{K}$ & 147.1128 & 74.0600 & 130.0863 & 65.5468 & & & 1 \\
\hline
\end{tabular}



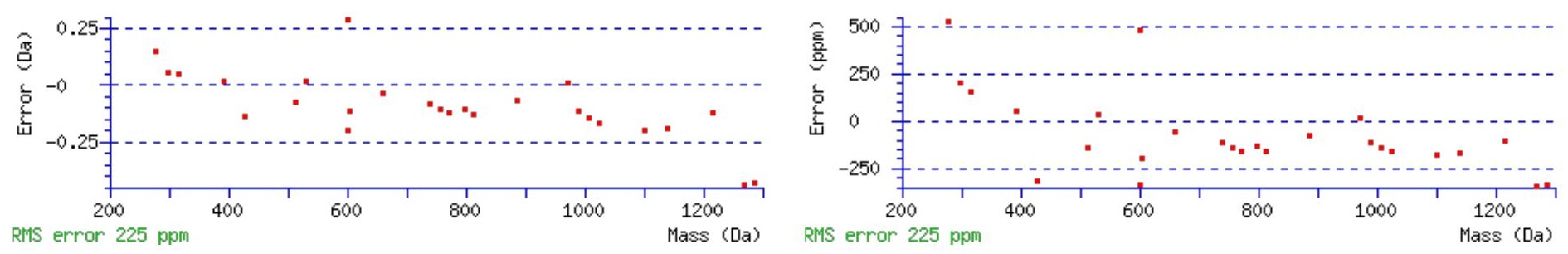

\section{All matches to this query}

\begin{tabular}{|l|l|l|l|}
\hline Score & Mr(calc): & Delta & \multicolumn{1}{|c|}{ Sequence } \\
\hline 62.6 & 1415.7330 & 0.0009 & LSNITNIGPLDMK \\
\hline 44.5 & 1415.7330 & 0.0009 & LSNITNIGPLDMK \\
\hline 9.9 & 1415.7409 & -0.0070 & GLVNLYNLPGDNK \\
\hline 7.2 & 1415.7286 & 0.0053 & TLNVLHNRGALK \\
\hline 4.5 & 1413.7324 & 2.0015 & VTNPNARLRSER \\
\hline 3.3 & 1413.7286 & 2.0053 & SGVLPGMPAVLSNR \\
\hline 1.9 & 1413.7140 & 2.0199 & VWVASDPNLNAVK \\
\hline 0.5 & 1415.7286 & 0.0053 & TLNVLHNRGALK \\
\hline 0.2 & 1415.7256 & 0.0083 & SPESQNLIDGTKK \\
\hline
\end{tabular}

Spectrum No: 487; Query: 1181; Rank: 1

\section{Peptide View}

MS/MS Fragmentation of LTSCATNASMCGDEAR

Found in IPI00369995, Tax_Id=10116 Gene_Symbol=Lrp1 similar to low density lipoprotein receptor-related protein 1

Match to Query 1181: 1759.682968 from(880.848760,2+)

Title: 100101RatKid_NS_deglyco_23.527.527.2.dta

Data file K:INewmanPaper|Piliangl3SubProteomes\Piliang3SP\mgf5ppm\ERLIC_3SubProteomes5ppm.mgf

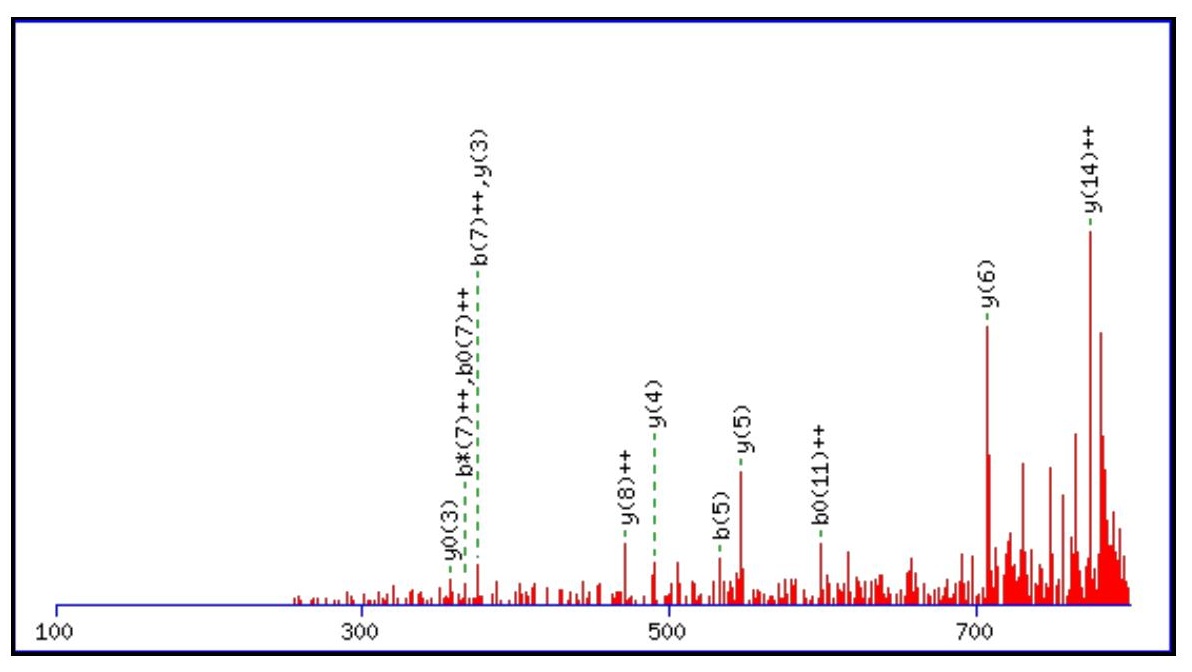



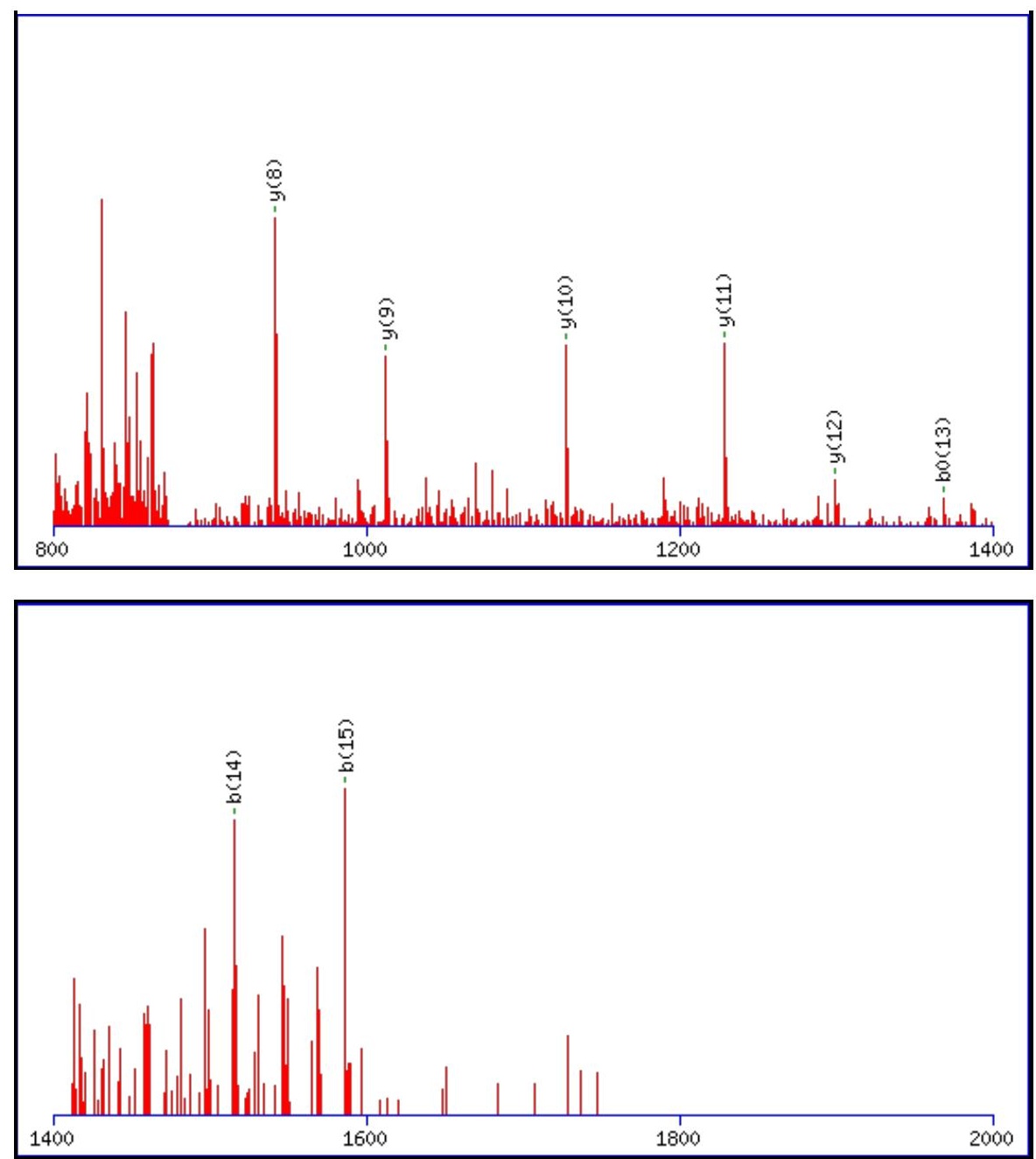

Monoisotopic mass of neutral peptide $\operatorname{Mr}($ calc): 1759.6811

Fixed modifications: Carbamidomethyl (C)

Variable modifications:

N7 : Deamidated_N (N)

M10 : Oxidation $(M)$

Ions Score: 63 Expect: $6.5 \mathrm{e}-005$

Matches (Bold Red): 20/162 fragment ions using 26 most intense peaks

\begin{tabular}{|c|c|c|c|c|c|c|c|c|c|c|c|c|c|c|}
\hline \# & b & $\mathbf{b}^{++}$ & $\mathbf{b}^{*}$ & $\mathbf{b}^{*^{++}}$ & $\mathbf{b}^{0}$ & $\mathbf{b}^{0++}$ & Seq. & $\mathbf{y}$ & $\mathbf{y}^{++}$ & $\mathbf{y}^{*}$ & $\mathbf{y}^{*^{++}}$ & $\mathbf{y}^{\mathbf{0}}$ & $\mathbf{y}^{0++}$ & \# \\
\hline 1 & 114.0913 & 57.5493 & & & & & $\mathbf{L}$ & & & & & & & 16 \\
\hline 2 & 215.1390 & 108.0731 & & & 197.1285 & 99.0679 & $\mathbf{T}$ & 1647.6043 & 824.3058 & 1630.5778 & 815.7925 & 1629.5938 & 815.3005 & 15 \\
\hline 3 & 302.1710 & 151.5892 & & & 284.1605 & 142.5839 & S & 1546.5567 & 773.7820 & 1529.5301 & 765.2687 & 1528.5461 & 764.7767 & 14 \\
\hline 4 & 462.2017 & 231.6045 & & & 444.1911 & 222.5992 & $\mathrm{C}$ & 1459.5246 & 730.2660 & 1442.4981 & 721.7527 & 1441.5141 & 721.2607 & 13 \\
\hline 5 & 533.2388 & 267.1230 & & & 515.2282 & 258.1178 & A & 1299.4940 & 650.2506 & 1282.4674 & 641.7374 & 1281.4834 & 641.2454 & 12 \\
\hline 6 & 634.2865 & 317.6469 & & & 616.2759 & 308.6416 & $\mathbf{T}$ & 1228.4569 & 614.7321 & 1211.4303 & 606.2188 & 1210.4463 & 605.7268 & 11 \\
\hline 7 & 749.3134 & 375.1604 & 732.2869 & 366.6471 & 731.3029 & 366.1551 & $\mathbf{N}$ & 1127.4092 & 564.2082 & 1110.3826 & 555.6950 & 1109.3986 & 555.2030 & 10 \\
\hline 8 & 820.3505 & 410.6789 & 803.3240 & 402.1656 & 802.3400 & 401.6736 & A & 1012.3823 & 506.6948 & 995.3557 & 498.1815 & 994.3717 & 497.6895 & 9 \\
\hline 9 & 907.3826 & 454.1949 & 890.3560 & 445.6816 & 889.3720 & 445.1896 & S & 941.3451 & 471.1762 & 924.3186 & 462.6629 & 923.3346 & 462.1709 & 8 \\
\hline 10 & 1054.4180 & 527.7126 & 1037.3914 & 519.1993 & 1036.4074 & 518.7073 & $\mathbf{M}$ & 854.3131 & 427.6602 & 837.2866 & 419.1469 & 836.3026 & 418.6549 & 7 \\
\hline 11 & 1214.4486 & 607.7279 & 1197.4221 & 599.2147 & 1196.4381 & 598.7227 & C & 707.2777 & 354.1425 & 690.2512 & 345.6292 & 689.2671 & 345.1372 & 6 \\
\hline 12 & 1271.4701 & 636.2387 & 1254.4435 & 627.7254 & 1253.4595 & 627.2334 & G & 547.2471 & 274.1272 & 530.2205 & 265.6139 & 529.2365 & 265.1219 & 5 \\
\hline 13 & 1386.4970 & 693.7522 & 1369.4705 & 685.2389 & 1368.4865 & 684.7469 & D & 490.2256 & 245.6164 & 473.1991 & 237.1032 & 472.2150 & 236.6112 & 4 \\
\hline 14 & 1515.5396 & 758.2734 & 1498.5131 & 749.7602 & 1497.5291 & 749.2682 & $\mathbf{E}$ & 375.1987 & 188.1030 & 358.1721 & 179.5897 & 357.1881 & 179.0977 & 3 \\
\hline 15 & 1586.5767 & 793.7920 & 1569.5502 & 785.2787 & 1568.5662 & 784.7867 & A & 246.1561 & 123.5817 & 229.1295 & 115.0684 & & & 2 \\
\hline 16 & & & & & & & $\mathbf{R}$ & 175.1190 & 88.0631 & 158.0924 & 79.5498 & & & 1 \\
\hline
\end{tabular}



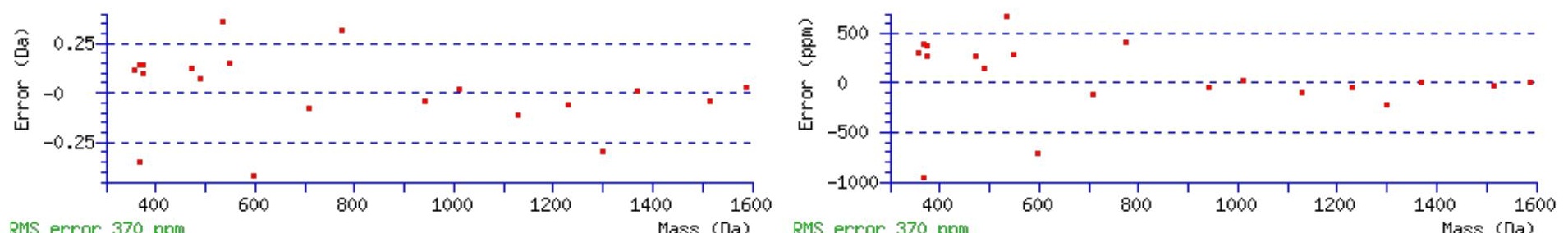

RIS error $370 \mathrm{ppm}$

\section{All matches to this query}

\begin{tabular}{|l|l|l|l|}
\hline Score & Mr(calc): & Delta & \multicolumn{1}{c|}{ Sequence } \\
\hline 62.5 & 1759.6811 & 0.0018 & LTSCATNASMCGDEAR \\
\hline 15.4 & 1758.6824 & 1.0006 & DYLSGELGDNLKMK \\
\hline 12.8 & 1758.6971 & 0.9859 & LTSCATNASMCGDEAR \\
\hline 3.1 & 1759.6832 & -0.0002 & NVATTKVSSTEGVAR \\
\hline 2.3 & 1759.6985 & -0.0155 & CSGEGGAGKGPEGSGXAR \\
\hline 2.3 & 1759.6655 & 0.0175 & CSGEGGAGKGPEGSGXAR \\
\hline 1.5 & 1758.6920 & 0.9910 & HFNSDLMAGDNSGAVK \\
\hline 0.6 & 1759.6687 & 0.0143 & DSFAQDSDIHTDSSR \\
\hline
\end{tabular}

Spectrum No: 488; Query: 890; Rank: 1

\section{Peptide View}

MS/MS Fragmentation of IFNIDSGNGSIFTSK

Found in IPI00215357, Tax_Id=10116 Gene_Symbol=Cdh6 Cadherin-6 precursor

Match to Query 890: 1599.785768 from(800.900160,2+)

Title: 100101RatKid_NS_deglyco_15.4297.4297.2.dta

Data file K:\NewmanPaper\Piliang \3SubProteomes\Piliang3SP \mgf5ppm\ERLIC_3SubProteomes5ppm.mgf

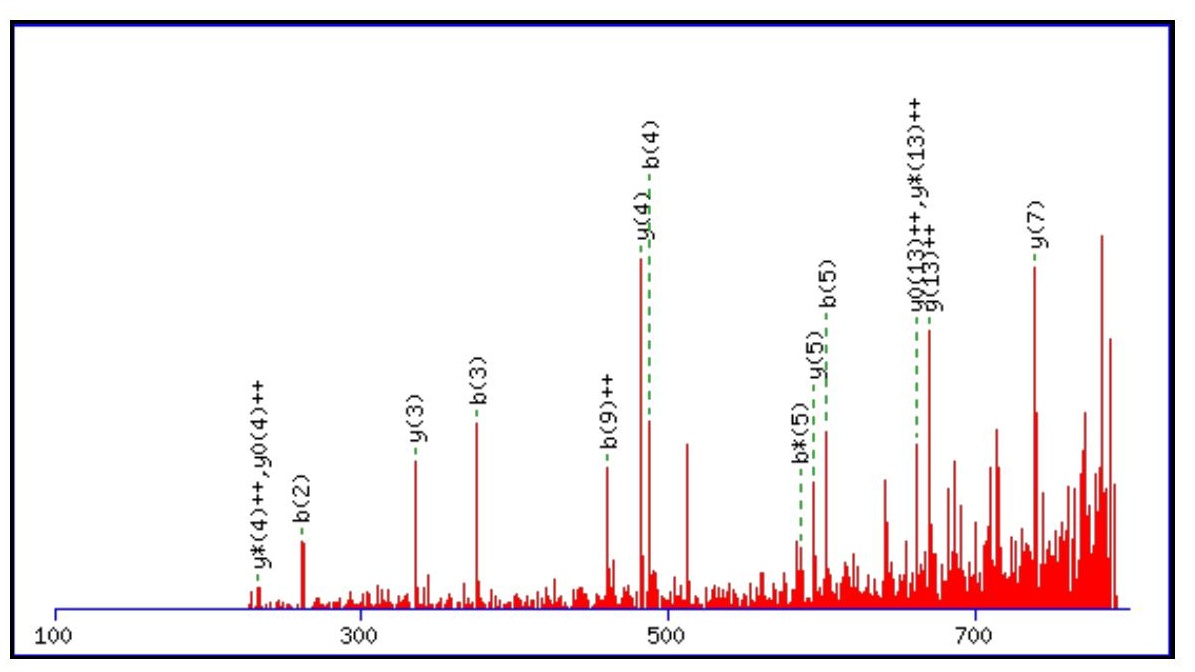



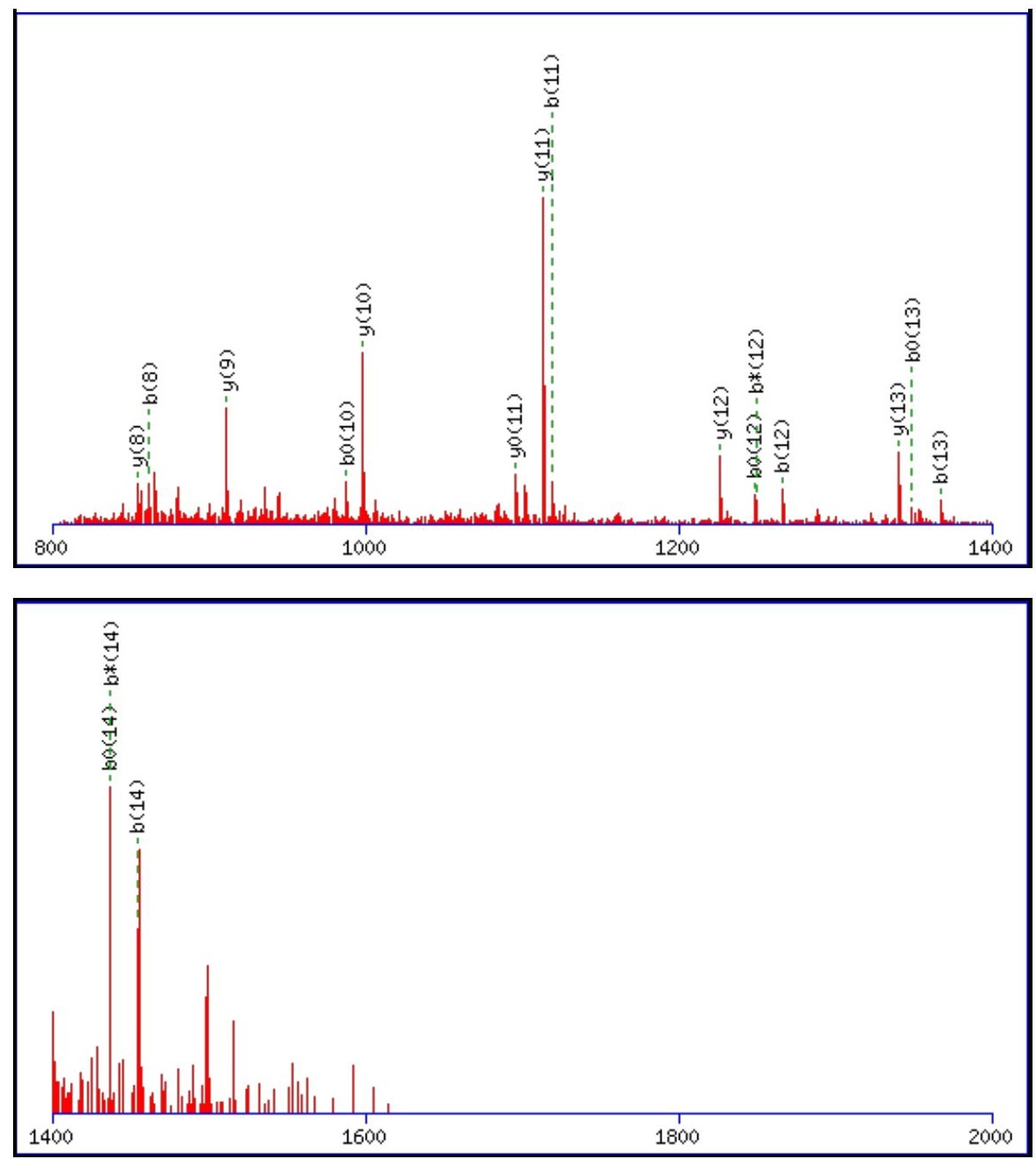

Monoisotopic mass of neutral peptide $\operatorname{Mr}($ calc): 1599.7780

Fixed modifications: Carbamidomethyl (C)

Variable modifications:

N8: : Deamidated $\mathrm{N}(\mathrm{N})$

Ions Score: 62 Expect: $9.6 \mathrm{e}-005$

Matches (Bold Red): 33/154 fragment ions using 61 most intense peaks

\begin{tabular}{|c|c|c|c|c|c|c|c|c|c|c|c|c|c|c|}
\hline \# & b & $\mathbf{b}^{++}$ & $\mathbf{b}^{*}$ & $\mathbf{b}^{*^{++}}$ & $\mathbf{b}^{0}$ & $\mathbf{b}^{\mathbf{0 + +}}$ & Seq. & $\mathbf{y}$ & $\mathbf{y}^{++}$ & $\mathbf{y}^{*}$ & $\mathrm{y}^{*^{++}}$ & $\mathbf{y}^{\mathbf{0}}$ & $\mathbf{y}^{\mathbf{0 + +}}$ & \# \\
\hline 1 & 114.0913 & 57.5493 & & & & & I & & & & & & & 15 \\
\hline 2 & 261.1598 & 131.0835 & & & & & $\mathbf{F}$ & 1487.7013 & 744.3543 & 1470.6747 & 735.8410 & 1469.6907 & 735.3490 & 14 \\
\hline 3 & 375.2027 & 188.1050 & 358.1761 & 179.5917 & & & $\mathbf{N}$ & 1340.6328 & 670.8201 & 1323.6063 & 662.3068 & 1322.6223 & 661.8148 & 13 \\
\hline 4 & 488.2867 & 244.6470 & 471.2602 & 236.1337 & & & I & 1226.5899 & 613.7986 & 1209.5634 & 605.2853 & 1208.5794 & 604.7933 & 12 \\
\hline 5 & 603.3137 & 302.1605 & 586.2871 & 293.6472 & 585.3031 & 293.1552 & D & 1113.5059 & 557.2566 & 1096.4793 & 548.7433 & 1095.4953 & 548.2513 & 11 \\
\hline 6 & 690.3457 & 345.6765 & 673.3192 & 337.1632 & 672.3352 & 336.6712 & $\mathbf{S}$ & 998.4789 & 499.7431 & 981.4524 & 491.2298 & 980.4683 & 490.7378 & 10 \\
\hline 7 & 747.3672 & 374.1872 & 730.3406 & 365.6740 & 729.3566 & 365.1819 & G & 911.4469 & 456.2271 & 894.4203 & 447.7138 & 893.4363 & 447.2218 & 9 \\
\hline 8 & 862.3941 & 431.7007 & 845.3676 & 423.1874 & 844.3836 & 422.6954 & $\mathbf{N}$ & 854.4254 & 427.7163 & 837.3989 & 419.2031 & 836.4149 & 418.7111 & 8 \\
\hline 9 & 919.4156 & 460.2114 & 902.3890 & 451.6982 & 901.4050 & 451.2061 & $\mathbf{G}$ & 739.3985 & 370.2029 & 722.3719 & 361.6896 & 721.3879 & 361.1976 & 7 \\
\hline 10 & 1006.4476 & 503.7274 & 989.4211 & 495.2142 & 988.4370 & 494.7222 & S & 682.3770 & 341.6921 & 665.3505 & 333.1789 & 664.3665 & 332.6869 & 6 \\
\hline 11 & 1119.5317 & 560.2695 & 1102.5051 & 551.7562 & 1101.5211 & 551.2642 & I & 595.3450 & 298.1761 & 578.3184 & 289.6629 & 577.3344 & 289.1709 & 5 \\
\hline 12 & 1266.6001 & 633.8037 & 1249.5735 & 625.2904 & 1248.5895 & 624.7984 & $\mathbf{F}$ & 482.2609 & 241.6341 & 465.2344 & 233.1208 & 464.2504 & 232.6288 & 4 \\
\hline 13 & 1367.6478 & 684.3275 & 1350.6212 & 675.8142 & 1349.6372 & 675.3222 & $\mathbf{T}$ & 335.1925 & 168.0999 & 318.1660 & 159.5866 & 317.1819 & 159.0946 & 3 \\
\hline 14 & 1454.6798 & 727.8435 & 1437.6532 & 719.3303 & 1436.6692 & 718.8383 & S & 234.1448 & 117.5761 & 217.1183 & 109.0628 & 216.1343 & 108.5708 & 2 \\
\hline 15 & & & & & & & $\mathbf{K}$ & 147.1128 & 74.0600 & 130.0863 & 65.5468 & & & 1 \\
\hline
\end{tabular}



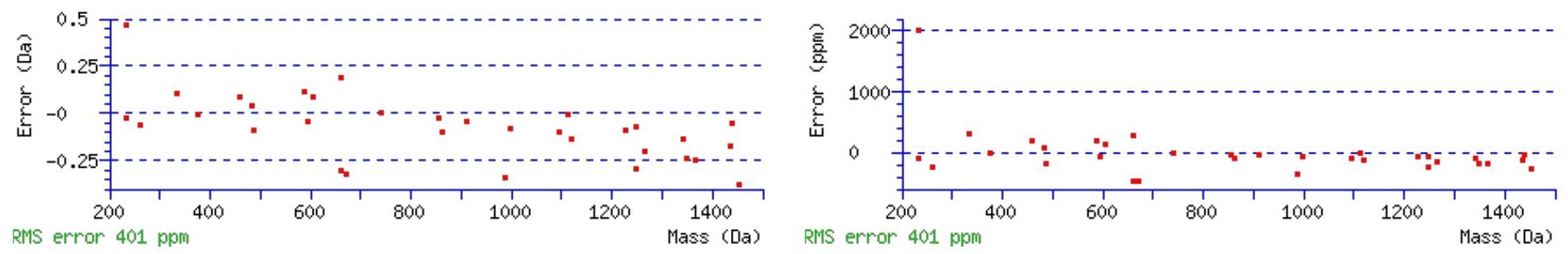

\section{All matches to this query}

\begin{tabular}{|l|l|c|l|}
\hline Score & Mr(calc): & Delta & \multicolumn{1}{|c|}{ Sequence } \\
\hline 62.4 & 1599.7780 & 0.0077 & IFNIDSGNGSIFTSK \\
\hline 25.2 & 1599.7780 & 0.0077 & IFNIDSGNGSIFTSK \\
\hline 13.5 & 1598.7940 & 0.9917 & IFNIDSGNGSIFTSK \\
\hline 5.7 & 1599.7814 & 0.0044 & ELPCLEELENNLK \\
\hline 5.7 & 1599.7786 & 0.0071 & LGRVPSKTLDLNK \\
\hline 4.2 & 1599.7909 & -0.0052 & LFVLDAGASGSKDIK \\
\hline 4.2 & 1599.7867 & -0.0010 & WKNIFYMKNNNK \\
\hline 2.8 & 1599.7909 & -0.0051 & FTGTAESLINLINK \\
\hline 2.8 & 1599.7909 & -0.0051 & FTGTAESLINLINK \\
\hline 2.7 & 1599.7860 & -0.0003 & SSMLIPPGIIRLK \\
\hline
\end{tabular}

Spectrum No: 489; Query: 702; Rank: 1

\section{Peptide View}

MS/MS Fragmentation of LDPTVLDASELANR

Found in IPI00201263, Tax_Id=10116 Gene_Symbol=Cspg4 Chondroitin sulfate proteoglycan 4 precursor

Match to Query 702: 1513.765328 from(757.889940,2+)

Title: 100101RatKid_NS_deglyco_26.3017.3017.2.dta

Data file K:INewmanPaper|Piliangl3SubProteomes\Piliang3SP\mgf5ppm\ERLIC_3SubProteomes5ppm.mgf

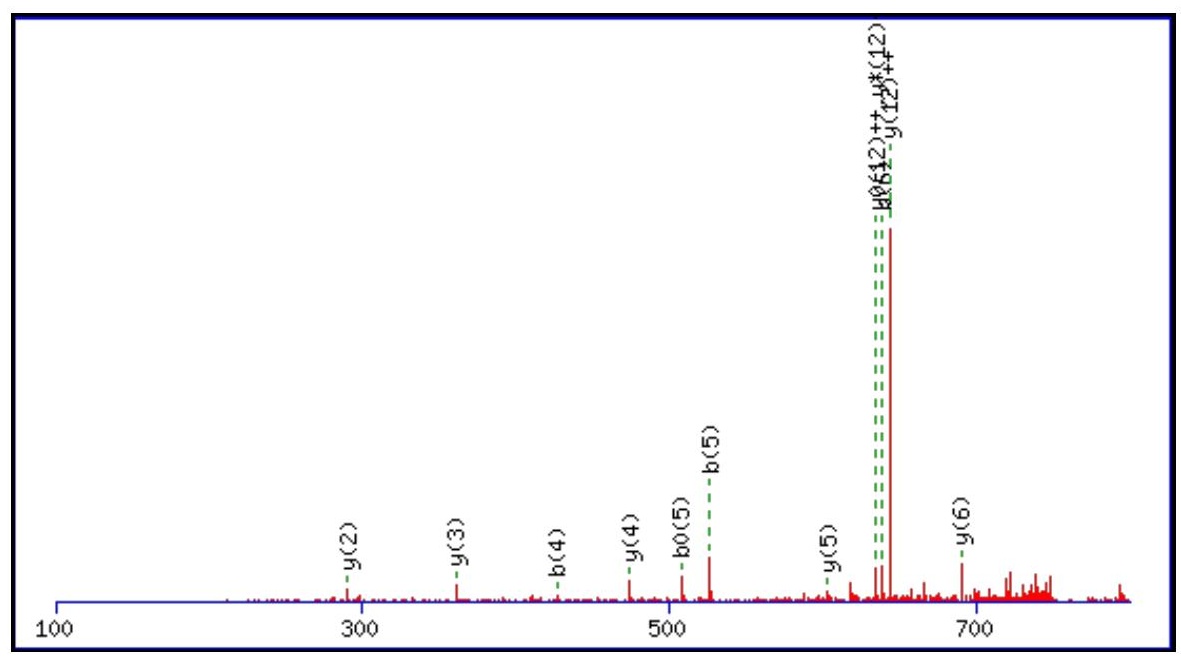



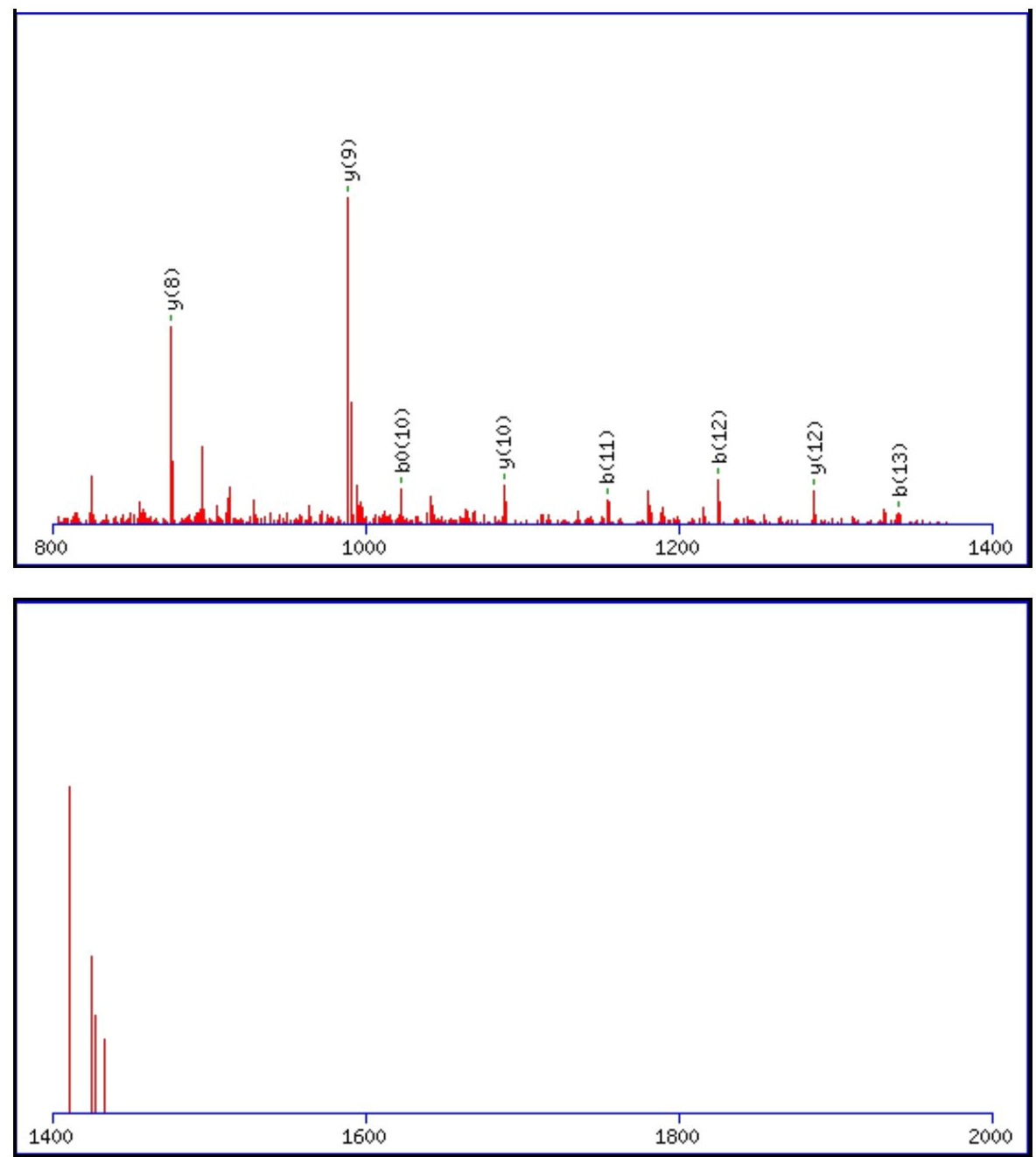

Monoisotopic mass of neutral peptide $\operatorname{Mr}($ calc): 1513.7624

Fixed modifications: Carbamidomethyl (C)

Variable modifications:

N13 : Deamidated $\mathrm{N}(\mathrm{N})$

Ions Score: 62 Expect: $7.6 \mathrm{e}-005$

Matches (Bold Red): 20/122 fragment ions using 40 most intense peaks

\begin{tabular}{|c|c|c|c|c|c|c|c|c|c|c|c|c|c|c|}
\hline \# & b & $\mathbf{b}^{++}$ & $\mathbf{b}^{*}$ & $\mathbf{b}^{*^{++}}$ & $\mathbf{b}^{0}$ & $\mathbf{b}^{0++}$ & Seq. & $\mathbf{y}$ & $\mathrm{y}^{++}$ & $\mathbf{y}^{*}$ & $\mathbf{y}^{*^{++}}$ & $\mathbf{y}^{0}$ & $\mathbf{y}^{\mathbf{0 + +}}$ & \# \\
\hline 1 & 114.0913 & 57.5493 & & & & & $\mathbf{L}$ & & & & & & & 14 \\
\hline 2 & 229.1183 & 115.0628 & & & 211.1077 & 106.0575 & D & 1401.6856 & 701.3464 & 1384.6591 & 692.8332 & 1383.6750 & 692.3412 & 13 \\
\hline 3 & 326.1710 & 163.5892 & & & 308.1605 & 154.5839 & $\mathbf{P}$ & 1286.6587 & 643.8330 & 1269.6321 & 635.3197 & 1268.6481 & 634.8277 & 12 \\
\hline 4 & 427.2187 & 214.1130 & & & 409.2082 & 205.1077 & $\mathbf{T}$ & 1189.6059 & 595.3066 & 1172.5794 & 586.7933 & 1171.5953 & 586.3013 & 11 \\
\hline 5 & 526.2871 & 263.6472 & & & 508.2766 & 254.6419 & $\mathbf{V}$ & 1088.5582 & 544.7827 & 1071.5317 & 536.2695 & 1070.5477 & 535.7775 & 10 \\
\hline 6 & 639.3712 & 320.1892 & & & 621.3606 & 311.1840 & $\mathbf{L}$ & 989.4898 & 495.2485 & 972.4633 & 486.7353 & 971.4792 & 486.2433 & 9 \\
\hline 7 & 754.3981 & 377.7027 & & & 736.3876 & 368.6974 & D & 876.4057 & 438.7065 & 859.3792 & 430.1932 & 858.3952 & 429.7012 & 8 \\
\hline 8 & 825.4353 & 413.2213 & & & 807.4247 & 404.2160 & A & 761.3788 & 381.1930 & 744.3523 & 372.6798 & 743.3682 & 372.1878 & 7 \\
\hline 9 & 912.4673 & 456.7373 & & & 894.4567 & 447.7320 & $\mathbf{S}$ & 690.3417 & 345.6745 & 673.3151 & 337.1612 & 672.3311 & 336.6692 & 6 \\
\hline 10 & 1041.5099 & 521.2586 & & & 1023.4993 & 512.2533 & $\mathbf{E}$ & 603.3097 & 302.1585 & 586.2831 & 293.6452 & 585.2991 & 293.1532 & 5 \\
\hline 11 & 1154.5939 & 577.8006 & & & 1136.5834 & 568.7953 & $\mathbf{L}$ & 474.2671 & 237.6372 & 457.2405 & 229.1239 & & & 4 \\
\hline 12 & 1225.6311 & 613.3192 & & & 1207.6205 & 604.3139 & A & 361.1830 & 181.0951 & 344.1565 & 172.5819 & & & 3 \\
\hline 13 & 1340.6580 & 670.8326 & 1323.6314 & 662.3194 & 1322.6474 & 661.8274 & $\mathbf{N}$ & 290.1459 & 145.5766 & 273.1193 & 137.0633 & & & 2 \\
\hline 14 & & & & & & & $\mathbf{R}$ & 175.1190 & 88.0631 & 158.0924 & 79.5498 & & & 1 \\
\hline
\end{tabular}



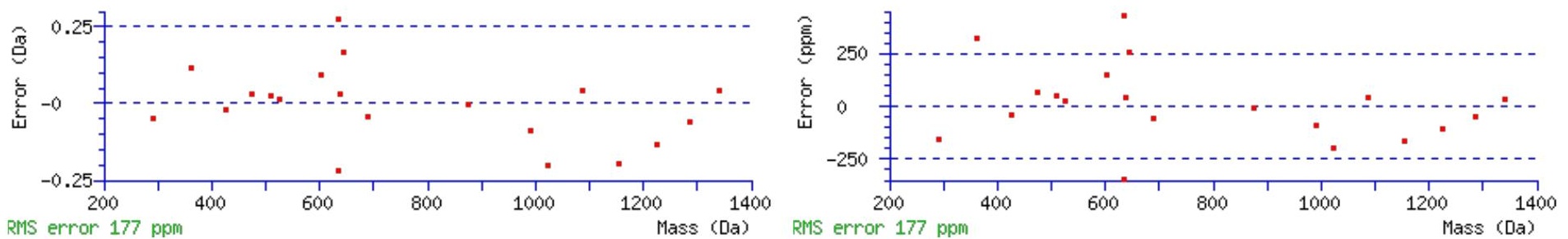

\section{All matches to this query}

\begin{tabular}{|l|l|l|l|}
\hline Score & Mr(calc): & Delta & \multicolumn{1}{c|}{ Sequence } \\
\hline 62.4 & 1513.7624 & 0.0030 & LDPTVLDASELANR \\
\hline 13.0 & 1513.7599 & 0.0055 & INMPSLSPAELWR \\
\hline 9.0 & 1513.7653 & -0.0000 & DKALLATLYRNR \\
\hline 6.2 & 1512.7758 & 0.9895 & INMPSLSPAELWR \\
\hline 4.5 & 1513.7559 & 0.0094 & NLLHVTDTGVGMTR \\
\hline 4.4 & 1513.7541 & 0.0112 & ANLEISYAKGLQK \\
\hline 1.0 & 1513.7558 & 0.0095 & VSVPKADVILVSK \\
\hline 0.5 & 1513.7793 & -0.0139 & LSAGVALASEIYIK \\
\hline 0.4 & 1513.7624 & 0.0029 & DIIEQDIDVLDAR \\
\hline 0.3 & 1511.7442 & 2.0211 & ASYPAFVNIKMDR \\
\hline
\end{tabular}

Spectrum No: 490; Query: 2429; Rank: 1

\section{Peptide View}

MS/MS Fragmentation of NCHYPTCDQLTCANGACYNTSQR

Found in IPI00205325, Tax_Id=10116 Gene_Symbol=Lrp2 Low-density lipoprotein receptor-related protein 2 precursor

Match to Query 2429: 2791.093332 from(931.371720,3+)

Title: 100101RatKid_NS_deglyco_15.1924.1924.3.dta

Data file K:INewmanPaper|Piliangl3SubProteomes\Piliang3SP\mgf5ppm\ERLIC_3SubProteomes5ppm.mgf

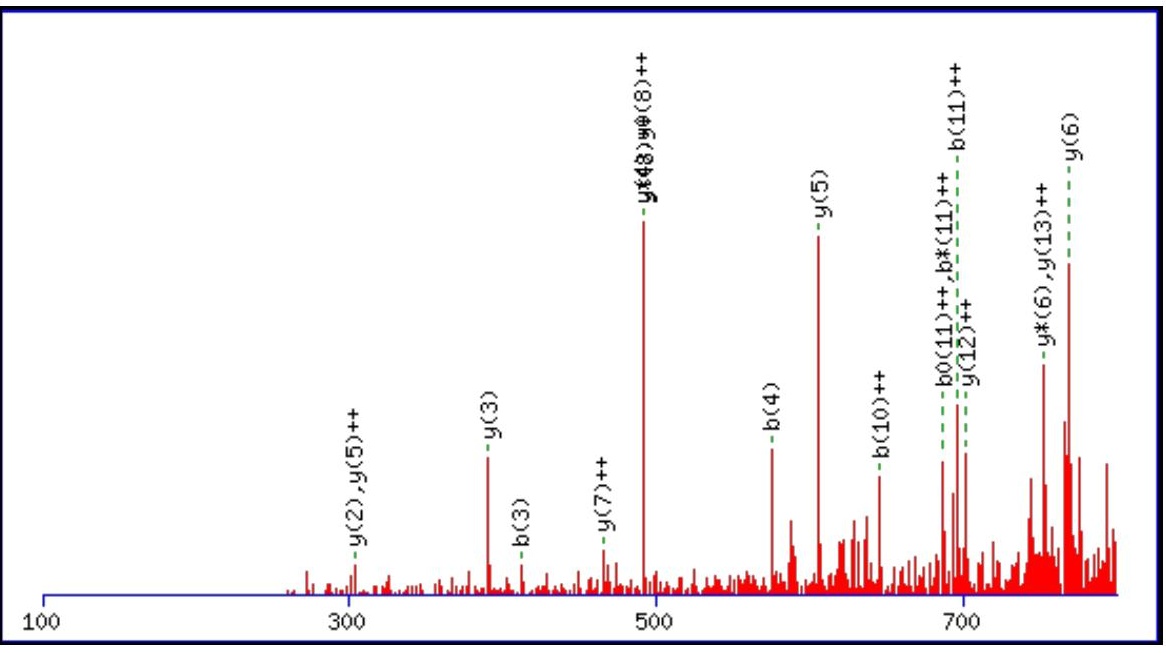



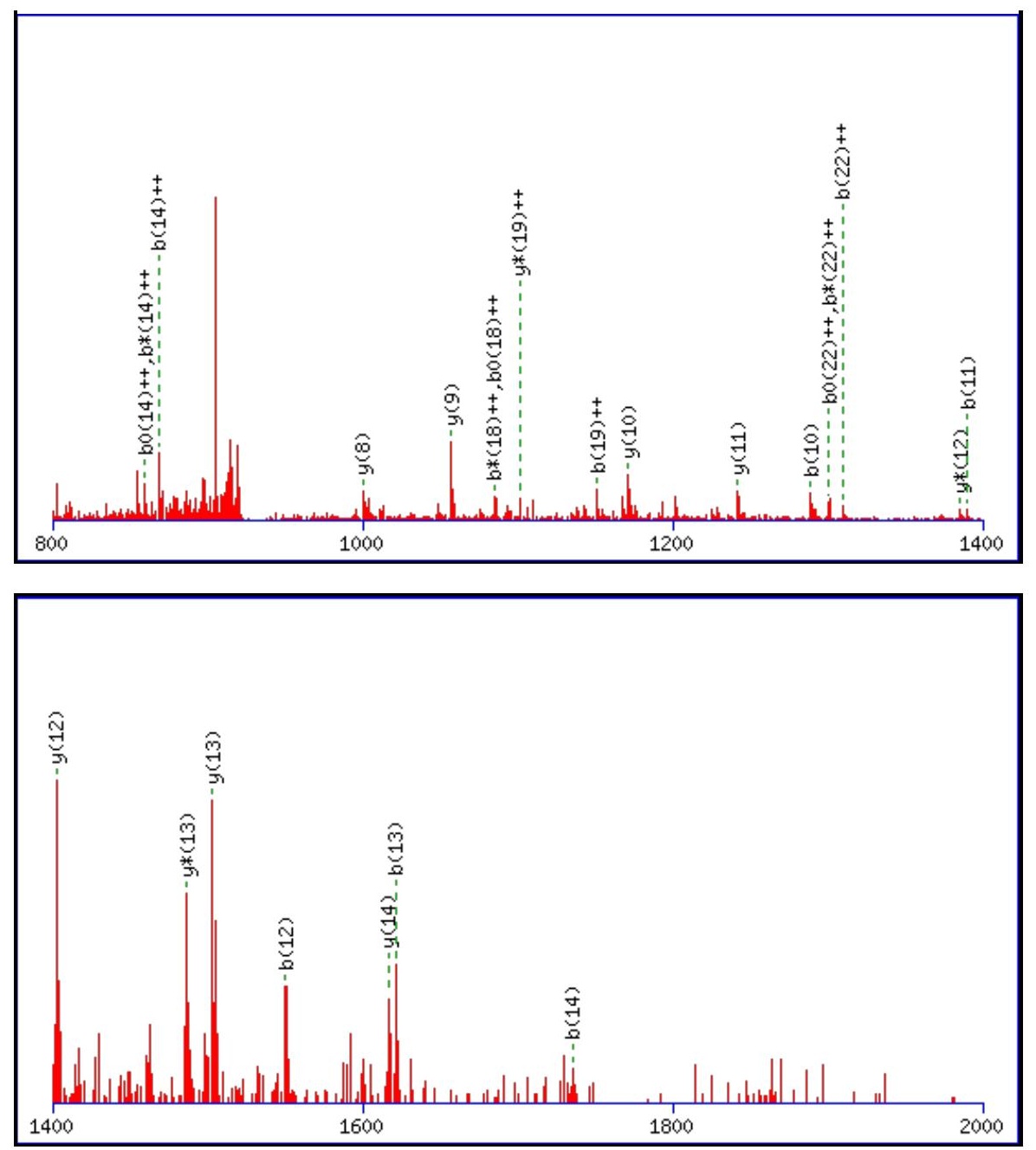

Monoisotopic mass of neutral peptide $\operatorname{Mr}($ calc): 2791.0843

Fixed modifications: Carbamidomethyl (C)

Variable modifications:

N19 : Deamidated_N (N)

Ions Score: 62 Expect: 0.00017

Matches (Bold Red): 42/250 fragment ions using 69 most intense peaks

\begin{tabular}{|c|c|c|c|c|c|c|c|c|c|c|c|c|c|c|}
\hline$\#$ & b & $\mathbf{b}^{++}$ & $\mathbf{b}^{*}$ & $\mathbf{b}^{*++}$ & $\mathbf{b}^{\mathbf{0}}$ & $\mathbf{b}^{\mathbf{0 + +}}$ & Seq. & $\mathbf{y}$ & $\mathbf{y}^{++}$ & $\mathbf{y}^{*}$ & $\mathrm{y}^{*^{++}}$ & $\mathbf{y}^{0}$ & $y^{0++}$ & \# \\
\hline 1 & \begin{tabular}{|l|}
115.0502 \\
\end{tabular} & 58.0287 & 98.0237 & 49.5155 & & & $\mathbf{N}$ & & & & & & & 23 \\
\hline 2 & 275.0809 & 138.0441 & 258.0543 & 129.5308 & & & $\mathrm{C}$ & 2678.0487 & 1339.5280 & 2661.0221 & 1331.0147 & 2660.0381 & 1330.5227 & 22 \\
\hline 3 & 412.1398 & 206.5735 & 395.1132 & 198.0602 & & & H & 2518.0180 & 1259.5126 & 2500.9915 & 1250.9994 & 2500.0075 & 1250.5074 & 21 \\
\hline 4 & \begin{tabular}{|l|}
575.2031 \\
\end{tabular} & 288.1052 & 558.1765 & 279.5919 & & & $\mathbf{Y}$ & 2380.9591 & 1190.9832 & 2363.9326 & 1182.4699 & 2362.9485 & 1181.9779 & 20 \\
\hline 5 & 672.2559 & 336.6316 & 655.2293 & 328.1183 & & & $\mathbf{P}$ & 2217.8958 & 1109.4515 & 2200.8692 & 1100.9383 & 2199.8852 & 1100.4462 & 19 \\
\hline 6 & 773.3035 & 387.1554 & 756.2770 & 378.6421 & 755.2930 & 378.1501 & $T$ & 2120.8430 & 1060.9251 & 2103.8165 & 1052.4119 & 2102.8324 & 1051.9199 & 18 \\
\hline 7 & 933.3342 & 467.1707 & 916.3076 & 458.6575 & 915.3236 & 458.1654 & $\mathrm{C}$ & 2019.7953 & 1010.4013 & 2002.7688 & 1001.8880 & 2001.7848 & 1001.3960 & 17 \\
\hline 8 & 1048.3611 & 524.6842 & 1031.3346 & 516.1709 & 1030.3506 & 515.6789 & D & 1859.7647 & 930.3860 & 1842.7381 & 921.8727 & 1841.7541 & 921.3807 & 16 \\
\hline 9 & 1176.4197 & 588.7135 & 1159.3932 & 580.2002 & 1158.4091 & 579.7082 & $\mathbf{Q}$ & 1744.7377 & 872.8725 & 1727.7112 & 864.3592 & 1726.7272 & 863.8672 & 15 \\
\hline 10 & 1289.5038 & 645.2555 & 1272.4772 & 636.7422 & 1271.4932 & 636.2502 & $\mathbf{L}$ & 1616.6792 & 808.8432 & 1599.6526 & 800.3299 & 1598.6686 & 799.8379 & 14 \\
\hline 11 & 1390.5514 & 695.7794 & 1373.5249 & 687.2661 & 1372.5409 & 686.7741 & $\mathbf{T}$ & 1503.5951 & 752.3012 & 1486.5685 & 743.7879 & 1485.5845 & 743.2959 & 13 \\
\hline 12 & 1550.5821 & 775.7947 & 1533.5555 & 767.2814 & 1532.5715 & 766.7894 & $\mathrm{C}$ & 1402.5474 & \begin{tabular}{l|l}
701.7773 \\
\end{tabular} & 1385.5209 & 693.2641 & 1384.5369 & 692.7721 & 12 \\
\hline 13 & 1621.6192 & 811.3132 & 1604.5927 & 802.8000 & 1603.6086 & 802.3080 & $\mathbf{A}$ & 1242.5168 & 621.7620 & 1225.4902 & 613.2487 & 1224.5062 & 612.7567 & 11 \\
\hline 14 & 1735.6621 & 868.3347 & 1718.6356 & 859.8214 & 1717.6516 & 859.3294 & $\mathbf{N}$ & $\begin{array}{l}1171.4797 \\
\end{array}$ & 586.2435 & 1154.4531 & 577.7302 & 1153.4691 & 577.2382 & 10 \\
\hline 15 & 1792.6836 & 896.8454 & 1775.6571 & 888.3322 & 1774.6730 & 887.8402 & G & 1057.4367 & 529.2220 & 1040.4102 & 520.7087 & 1039.4262 & 520.2167 & 9 \\
\hline 16 & 1863.7207 & 932.3640 & 1846.6942 & 923.8507 & 1845.7102 & 923.3587 & $\mathbf{A}$ & 1000.4153 & 500.7113 & 983.3887 & 492.1980 & 982.4047 & 491.7060 & 8 \\
\hline 17 & 2023.7514 & 1012.3793 & 2006.7248 & 1003.8660 & 2005.7408 & 1003.3740 & $\mathrm{C}$ & 929.3782 & 465.1927 & 912.3516 & 456.6794 & 911.3676 & 456.1874 & 7 \\
\hline 18 & 2186.8147 & 1093.9110 & 2169.7881 & 1085.3977 & 2168.8041 & 1084.9057 & $\mathbf{Y}$ & 769.3475 & 385.1774 & 752.3210 & 376.6641 & 751.3369 & 376.1721 & 6 \\
\hline
\end{tabular}




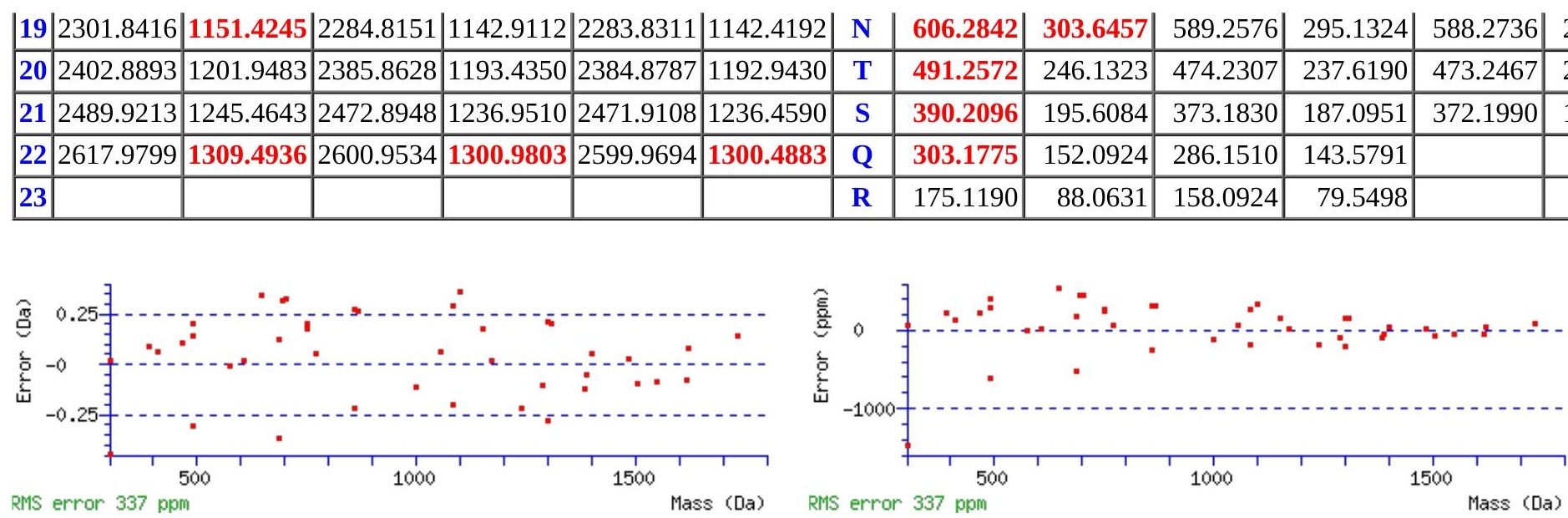

All matches to this query

\begin{tabular}{|l|l|l|l|}
\hline Score & Mr(calc): & Delta & \multicolumn{1}{|c|}{ Sequence } \\
\hline 62.3 & 2791.0843 & 0.0090 & NCHYPTCDQLTCANGACYNTSQR \\
\hline 33.0 & 2791.0843 & 0.0090 & NCHYPTCDQLTCANGACYNTSOR \\
\hline 8.3 & 2790.1003 & 0.9930 & NCHYPTCDQLTCANGACYNTSQR \\
\hline 6.5 & 2791.0843 & 0.0090 & NCHYPTCDOLTCANGACYNTSOR \\
\hline 6.2 & 2791.0817 & 0.0116 & DCIMHGYMSKMGNPFLTQWQR \\
\hline 2.8 & 2789.0750 & 2.0183 & SPATPVPPDSTLESPFEEMALVR \\
\hline 1.8 & 2791.0817 & 0.0116 & DCIMHGYMSKMGNPFLTQWQR \\
\hline 1.6 & 2789.0871 & 2.0062 & GDSTLQVSSGLNENITVNGGGWSEK \\
\hline 0.3 & 2789.0710 & 2.0224 & KNSVSSAMPETESYDAVEIIRK \\
\hline 0.3 & 2789.0710 & 2.0224 & KNSVSSAMPETESYDAVEIIRK \\
\hline
\end{tabular}

Spectrum No: 491; Query: 1061; Rank: 1

\section{Peptide View}

MS/MS Fragmentation of VGVDEVSPEKNSSKPK

Found in IPI00231423, Tax_Id=10116 Gene_Symbol=C9 C9 protein

Match to Query 1061: 1699.866492 from(567.629440,3+)

Title: 100101RatKid_NS_deglyco_19.473.473.3.dta

Data file K:INewmanPaper|Piliangl3SubProteomes\Piliang3SP\mgf5ppm\ERLIC_3SubProteomes5ppm.mgf

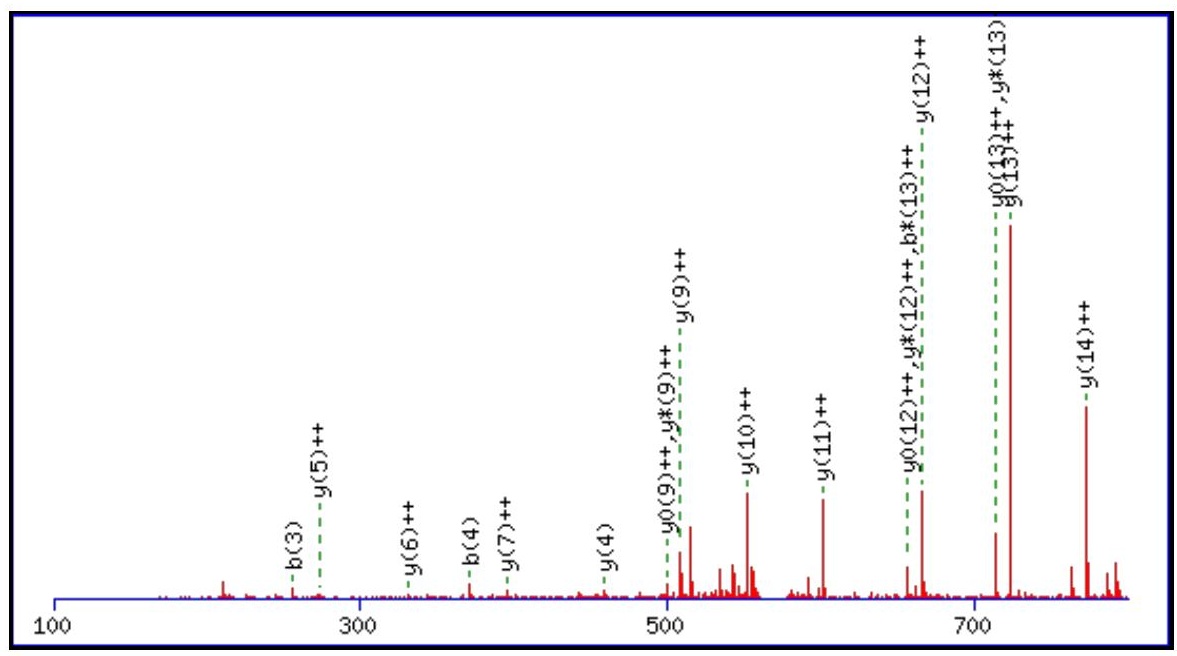



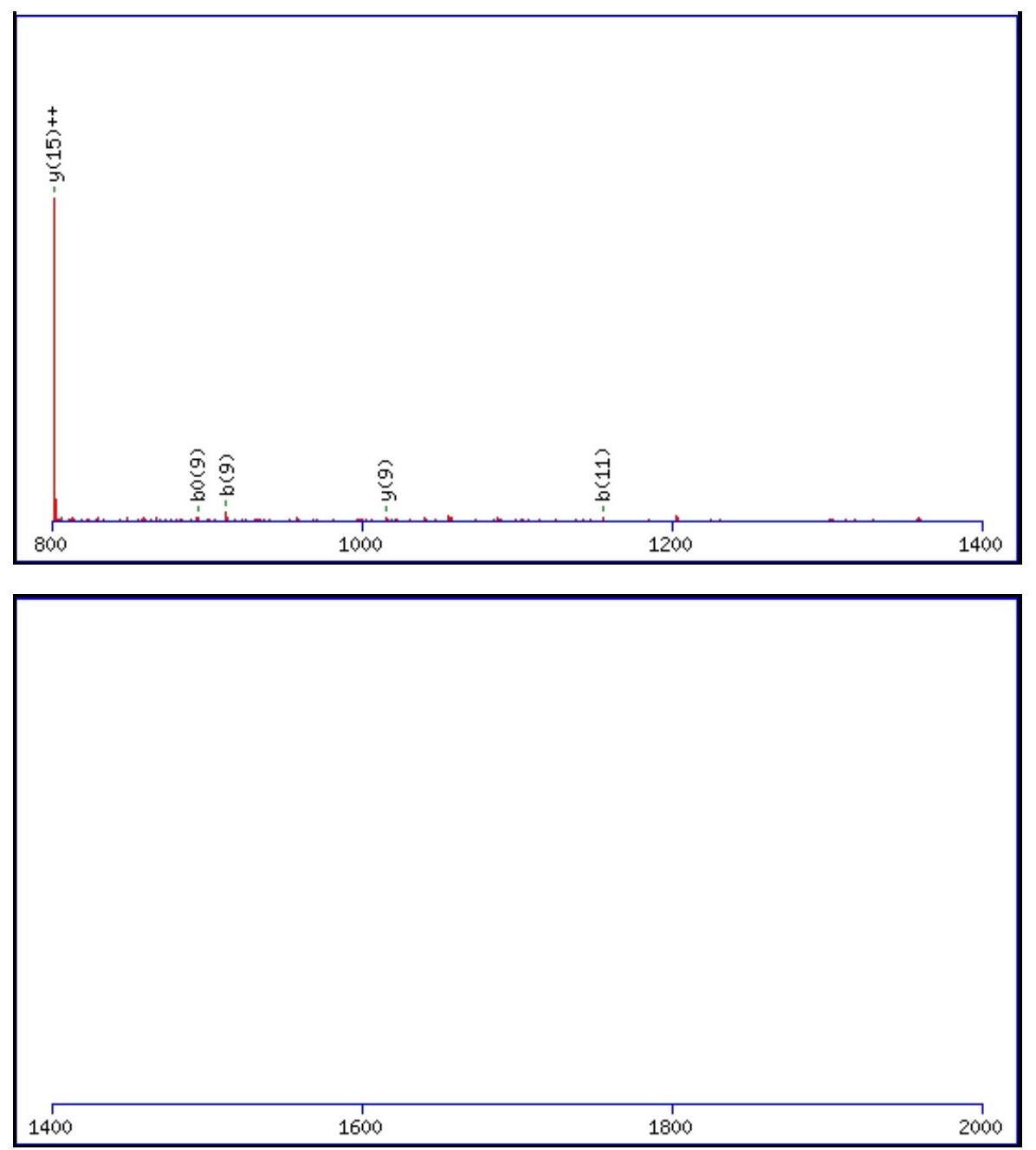

Monoisotopic mass of neutral peptide $\operatorname{Mr}($ calc): 1699.8628

Fixed modifications: Carbamidomethyl (C)

Variable modifications:

N11 : Deamidated_N (N)

Ions Score: 62 Expect: $8.4 \mathrm{e}-005$

Matches (Bold Red): 24/150 fragment ions using 41 most intense peaks

\begin{tabular}{|r|c|c|c|c|c|c|c|c|c|c|c|c|c|c|}
\hline$\#$ & $\mathbf{b}$ & $\mathbf{b}^{++}$ & $\mathbf{b}^{*}$ & $\mathbf{b}^{\mathbf{*}^{++}}$ & $\mathbf{b}^{\mathbf{0}}$ & $\mathbf{b}^{\mathbf{0 + +}}$ & $\mathbf{S e q}$ & $\mathbf{y}$ & $\mathbf{y}^{++}$ & $\mathbf{y}^{\mathbf{*}}$ & $\mathbf{y}^{\mathbf{*}^{++}}$ & $\mathbf{y}^{\mathbf{0}}$ & $\mathbf{y}^{\mathbf{0 + +}}$ & $\#$ \\
\hline $\mathbf{1}$ & 100.0757 & 50.5415 & & & & & $\mathbf{V}$ & & & & & & & $\mathbf{1 6}$ \\
\hline $\mathbf{2}$ & 157.0972 & 79.0522 & & & & & $\mathbf{G}$ & 1601.8017 & $\mathbf{8 0 1 . 4 0 4 5}$ & 1584.7752 & 792.8912 & 1583.7911 & 792.3992 & $\mathbf{1 5}$ \\
\hline $\mathbf{3}$ & $\mathbf{2 5 6 . 1 6 5 6}$ & 128.5864 & & & & & $\mathbf{V}$ & 1544.7802 & 772.8938 & 1527.7537 & 764.3805 & 1526.7697 & 763.8885 & $\mathbf{1 4}$ \\
\hline $\mathbf{4}$ & $\mathbf{3 7 1 . 1 9 2 5}$ & 186.0999 & & & 353.1819 & 177.0946 & $\mathbf{D}$ & 1445.7118 & 723.3595 & 1428.6853 & 714.8463 & 1427.7013 & 714.3543 & $\mathbf{1 3}$ \\
\hline $\mathbf{5}$ & 500.2351 & 250.6212 & & & 482.2245 & 241.6159 & $\mathbf{E}$ & 1330.6849 & $\mathbf{6 6 5 . 8 4 6 1}$ & 1313.6583 & $\mathbf{6 5 7 . 3 3 2 8}$ & 1312.6743 & $\mathbf{6 5 6 . 8 4 0 8}$ & $\mathbf{1 2}$ \\
\hline $\mathbf{6}$ & 599.3035 & 300.1554 & & & 581.2930 & 291.1501 & $\mathbf{V}$ & 1201.6423 & $\mathbf{6 0 1 . 3 2 4 8}$ & 1184.6157 & 592.8115 & 1183.6317 & 592.3195 & $\mathbf{1 1}$ \\
\hline $\mathbf{7}$ & 686.3355 & 343.6714 & & & 668.3250 & 334.6661 & $\mathbf{S}$ & 1102.5739 & 551.7906 & 1085.5473 & 543.2773 & 1084.5633 & 542.7853 & $\mathbf{1 0}$ \\
\hline $\mathbf{8}$ & 783.3883 & 392.1978 & & & 765.3777 & 383.1925 & $\mathbf{P}$ & $\mathbf{1 0 1 5 . 5 4 1 8}$ & $5 \mathbf{0 8 . 2 7 4 6}$ & 998.5153 & $\mathbf{4 9 9 . 7 6 1 3}$ & 997.5313 & $\mathbf{4 9 9 . 2 6 9 3}$ & $\mathbf{9}$ \\
\hline $\mathbf{9}$ & $\mathbf{9 1 2 . 4 3 0 9}$ & 456.7191 & & & $\mathbf{8 9 4 . 4 2 0 3}$ & 447.7138 & $\mathbf{E}$ & 918.4891 & 459.7482 & 901.4625 & 451.2349 & 900.4785 & 450.7429 & $\mathbf{8}$ \\
\hline $\mathbf{1 0}$ & 1040.5259 & 520.7666 & 1023.4993 & 512.2533 & 1022.5153 & 511.7613 & $\mathbf{K}$ & 789.4465 & 395.2269 & 772.4199 & 386.7136 & 771.4359 & 386.2216 & $\mathbf{7}$ \\
\hline $\mathbf{1 1}$ & $\mathbf{1 1 5 5 . 5 5 2 8}$ & 578.2800 & 1138.5263 & 569.7668 & 1137.5422 & 569.2748 & $\mathbf{N}$ & 661.3515 & 331.1794 & 644.3250 & 322.6661 & 643.3410 & 322.1741 & $\mathbf{6}$ \\
\hline $\mathbf{1 2}$ & 1242.5848 & 621.7961 & 1225.5583 & 613.2828 & 1224.5743 & 612.7908 & $\mathbf{S}$ & 546.3246 & 273.6659 & 529.2980 & 265.1527 & 528.3140 & 264.6606 & $\mathbf{5}$ \\
\hline $\mathbf{1 3}$ & 1329.6169 & 665.3121 & 1312.5903 & $\mathbf{6 5 6 . 7 9 8 8}$ & 1311.6063 & 656.3068 & $\mathbf{S}$ & $\mathbf{4 5 9 . 2 9 2 6}$ & 230.1499 & 442.2660 & 221.6366 & 441.2820 & 221.1446 & $\mathbf{4}$ \\
\hline $\mathbf{1 4}$ & 1457.7118 & 729.3595 & 1440.6853 & 720.8463 & 1439.7013 & 720.3543 & $\mathbf{K}$ & 372.2605 & 186.6339 & 355.2340 & 178.1206 & & & $\mathbf{3}$ \\
\hline $\mathbf{1 5}$ & 1554.7646 & 777.8859 & 1537.7380 & 769.3727 & 1536.7540 & 768.8806 & $\mathbf{P}$ & 244.1656 & 122.5864 & 227.1390 & 114.0731 & & & $\mathbf{2}$ \\
\hline $\mathbf{1 6}$ & & & & & & & $\mathbf{K}$ & 147.1128 & 74.0600 & 130.0863 & 65.5468 & & & $\mathbf{1}$ \\
\hline
\end{tabular}



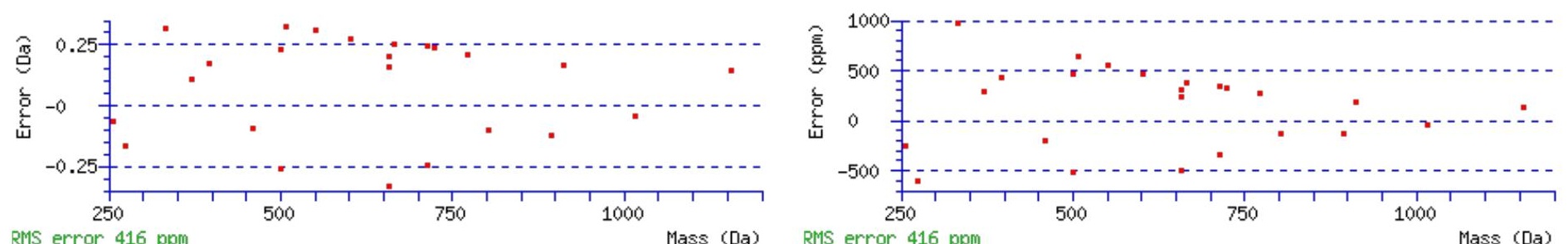

\section{All matches to this query}

\begin{tabular}{|l|l|c|l|}
\hline Score & Mr(calc): & Delta & \multicolumn{1}{c|}{ Sequence } \\
\hline 62.3 & 1699.8628 & 0.0037 & VGVDEVSPEKNSSKPK \\
\hline 7.3 & 1699.8580 & 0.0085 & NSAKVMVTNVTSLLK \\
\hline 5.1 & 1699.8586 & 0.0079 & $\underline{\text { SWLLLADIYIQSAK }}$ \\
\hline 4.7 & 1698.8463 & 1.0202 & RNRWMTIMLLLR \\
\hline 4.5 & 1699.8580 & 0.0085 & NSAKVMVTNVTSLLK \\
\hline 4.2 & 1699.8580 & 0.0085 & $\underline{\text { NSAKVMVTNVTSLLK }}$ \\
\hline 3.6 & 1699.8740 & -0.0075 & $\underline{\text { NTGVILANDANAERLK }}$ \\
\hline 2.3 & 1698.8788 & 0.9877 & VGVDEVSPEKNSSKPK \\
\hline 1.3 & 1699.8699 & -0.0034 & QGIFPVSYVEVVKR \\
\hline 1.1 & 1699.8740 & -0.0075 & $\underline{\text { NTGVILANDANAERLK }}$ \\
\hline
\end{tabular}

Spectrum No: 492; Query: 733; Rank: 1

\section{Peptide View}

\section{MS/MS Fragmentation of TNSSFVQGLVDHVK}

Found in IPI00195160, Tax_Id=10116 Gene_Symbol=Psap Sulfated glycoprotein 1 precursor

Match to Query 733: 1530.771008 from(766.392780,2+)

Title: 091008RatKidney_NH4Format01_18.3646.3646.2.dta

Data file K:INewmanPaper|Piliang|3SubProteomes\Piliang3SP\mgf5ppm\ERLIC_3SubProteomes5ppm.mgf

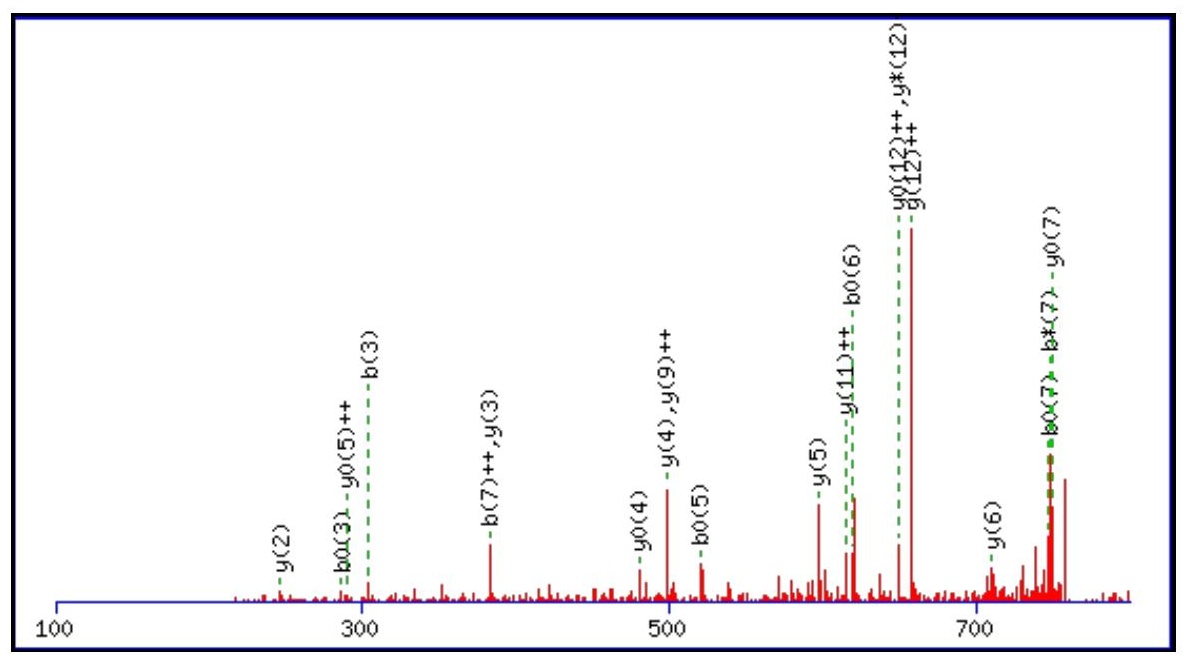



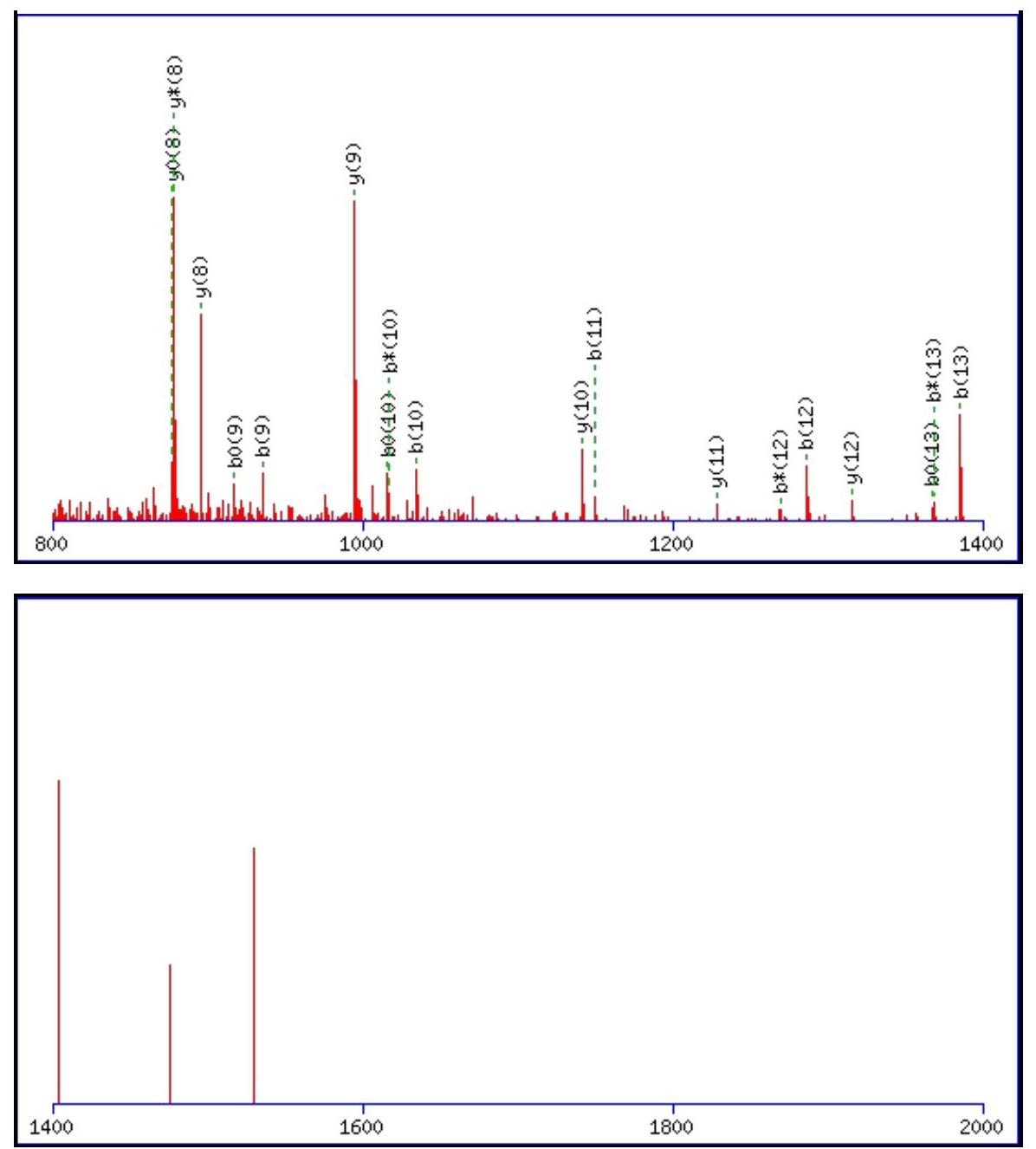

Monoisotopic mass of neutral peptide $\operatorname{Mr}($ calc): 1530.7678

Fixed modifications: Carbamidomethyl (C)

Variable modifications:

N2 : Deamidated_N (N)

Ions Score: 62 Expect: $8.4 \mathrm{e}-005$

Matches (Bold Red): 38/148 fragment ions using 68 most intense peaks

\begin{tabular}{|c|c|c|c|c|c|c|c|c|c|c|c|c|c|c|}
\hline \# & b & $\mathbf{b}^{++}$ & $\mathbf{b}^{*}$ & $\mathbf{b}^{*^{++}}$ & $\mathbf{b}^{0}$ & $\mathbf{b}^{0++}$ & Seq. & $\mathbf{y}$ & $\mathbf{y}^{++}$ & $\mathbf{y}^{*}$ & $\mathrm{y}^{*^{++}}$ & $\mathbf{y}^{\mathbf{0}}$ & $\mathbf{y}^{\mathbf{0 + +}}$ & \# \\
\hline 1 & 102.0550 & 51.5311 & & & 84.0444 & 42.5258 & $\mathbf{T}$ & & & & & & & 14 \\
\hline 2 & 217.0819 & 109.0446 & 200.0553 & 100.5313 & 199.0713 & 100.0393 & $\mathbf{N}$ & 1430.7274 & 715.8673 & 1413.7009 & 707.3541 & 1412.7168 & 706.8621 & 13 \\
\hline 3 & 304.1139 & 152.5606 & 287.0874 & 144.0473 & 286.1034 & 143.5553 & $\mathbf{S}$ & 1315.7005 & 658.3539 & 1298.6739 & 649.8406 & 1297.6899 & 649.3486 & 12 \\
\hline 4 & 391.1459 & 196.0766 & 374.1194 & 187.5633 & 373.1354 & 187.0713 & S & 1228.6684 & 614.8379 & 1211.6419 & 606.3246 & 1210.6579 & 605.8326 & 11 \\
\hline 5 & 538.2144 & 269.6108 & 521.1878 & 261.0975 & 520.2038 & 260.6055 & $\mathbf{F}$ & 1141.6364 & 571.3218 & 1124.6099 & 562.8086 & 1123.6259 & 562.3166 & 10 \\
\hline 6 & 637.2828 & 319.1450 & 620.2562 & 310.6318 & 619.2722 & 310.1397 & V & 994.5680 & 497.7876 & 977.5415 & 489.2744 & 976.5574 & 488.7824 & 9 \\
\hline 7 & 765.3414 & 383.1743 & 748.3148 & 374.6610 & 747.3308 & 374.1690 & $\mathbf{Q}$ & 895.4996 & 448.2534 & 878.4730 & 439.7402 & 877.4890 & 439.2482 & 8 \\
\hline 8 & 822.3628 & 411.6850 & 805.3363 & 403.1718 & 804.3523 & 402.6798 & G & 767.4410 & 384.2241 & 750.4145 & 375.7109 & 749.4305 & 375.2189 & 7 \\
\hline 9 & 935.4469 & 468.2271 & 918.4203 & 459.7138 & 917.4363 & 459.2218 & $\mathbf{L}$ & 710.4196 & 355.7134 & 693.3930 & 347.2001 & 692.4090 & 346.7081 & 6 \\
\hline 10 & 1034.5153 & 517.7613 & 1017.4887 & 509.2480 & 1016.5047 & 508.7560 & $\mathbf{V}$ & 597.3355 & 299.1714 & 580.3089 & 290.6581 & 579.3249 & 290.1661 & 5 \\
\hline 11 & 1149.5422 & 575.2748 & 1132.5157 & 566.7615 & 1131.5317 & 566.2695 & D & 498.2671 & 249.6372 & 481.2405 & 241.1239 & 480.2565 & 240.6319 & 4 \\
\hline 12 & 1286.6012 & 643.8042 & 1269.5746 & 635.2909 & 1268.5906 & 634.7989 & $\mathbf{H}$ & 383.2401 & 192.1237 & 366.2136 & 183.6104 & & & 3 \\
\hline 13 & 1385.6696 & 693.3384 & 1368.6430 & 684.8251 & 1367.6590 & 684.3331 & $\mathbf{V}$ & 246.1812 & 123.5942 & 229.1547 & 115.0810 & & & 2 \\
\hline 14 & & & & & & & $\mathbf{K}$ & 147.1128 & 74.0600 & 130.0863 & 65.5468 & & & 1 \\
\hline
\end{tabular}



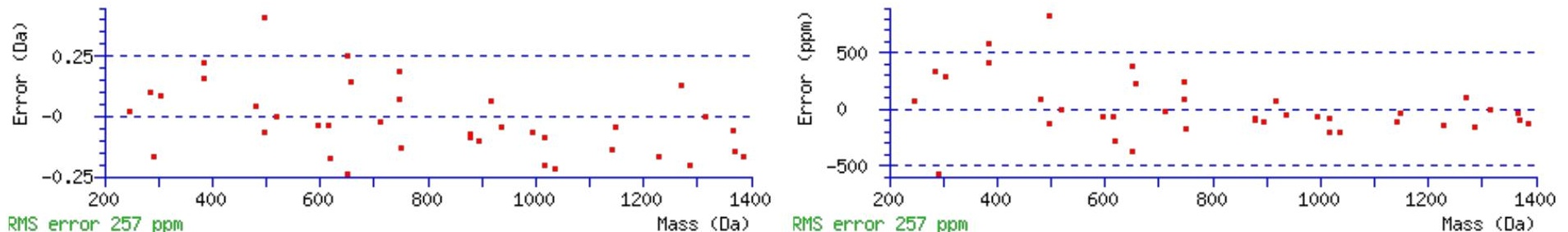

All matches to this query

\begin{tabular}{|l|c|c|l|}
\hline Score & Mr(calc): & Delta & \multicolumn{1}{c|}{ Sequence } \\
\hline 62.3 & 1530.7678 & 0.0032 & TNSSFVQGLVDHVK \\
\hline 5.4 & 1530.7687 & 0.0023 & GPFLSLAACVMPPR \\
\hline 3.8 & 1530.7777 & -0.0067 & $\underline{\text { ILGVDKNASEDEIK }}$ \\
\hline 1.5 & 1530.7684 & 0.0026 & $\underline{\text { ARGLSLTRVGLTK }}$ \\
\hline 1.5 & 1530.7684 & 0.0026 & ARGLSLTRVGLTK \\
\hline 0.7 & 1530.7646 & 0.0064 & $\underline{\text { RCLIDDREMPVK }}$ \\
\hline 0.1 & 1529.7677 & 1.0033 & $\underline{\text { AVDPKTRLLMYK }}$ \\
\hline
\end{tabular}

Spectrum No: 493; Query: 2049; Rank: 1

\section{Peptide View}

MS/MS Fragmentation of TGGTYGEDLGADYNLSQVCDGK

Found in IPI00200661, Tax_Id=10116 Gene_Symbol=Fasn Fatty acid synthase

Match to Query 2049: 2319.978168 from(1160.996360,2+)

Title: 091008RatKidney_NH4Format01_17.2996.2996.2.dta

Data file K:INewmanPaper|Piliang|3SubProteomes\Piliang3SP\mgf5ppm\ERLIC_3SubProteomes5ppm.mgf

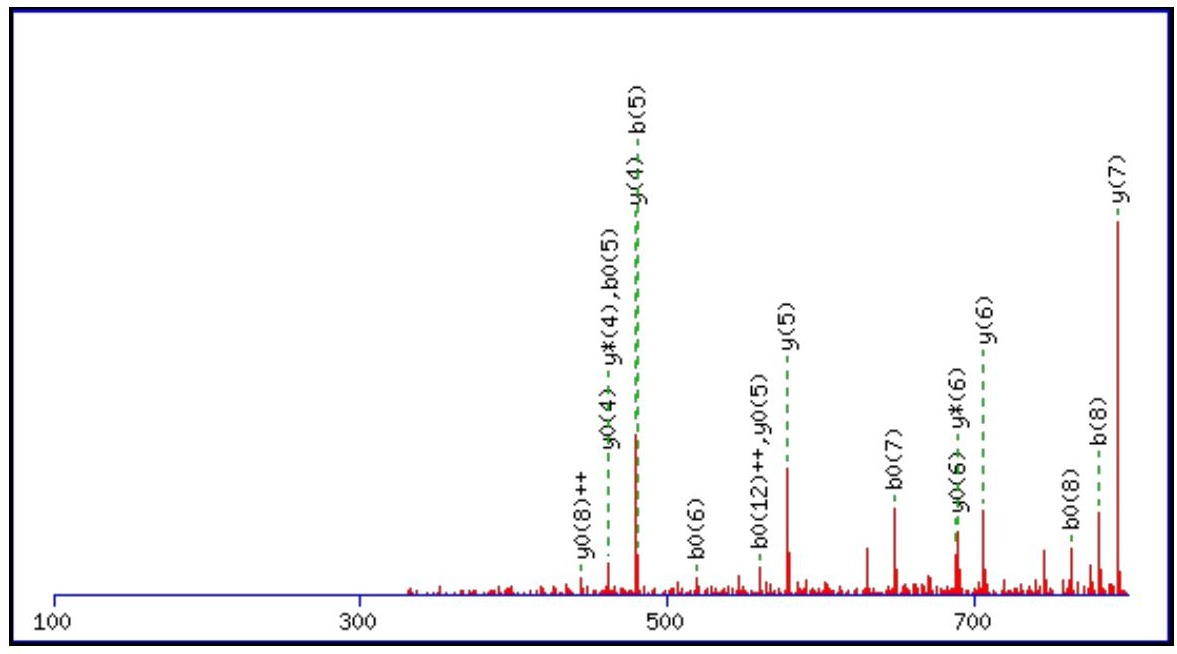



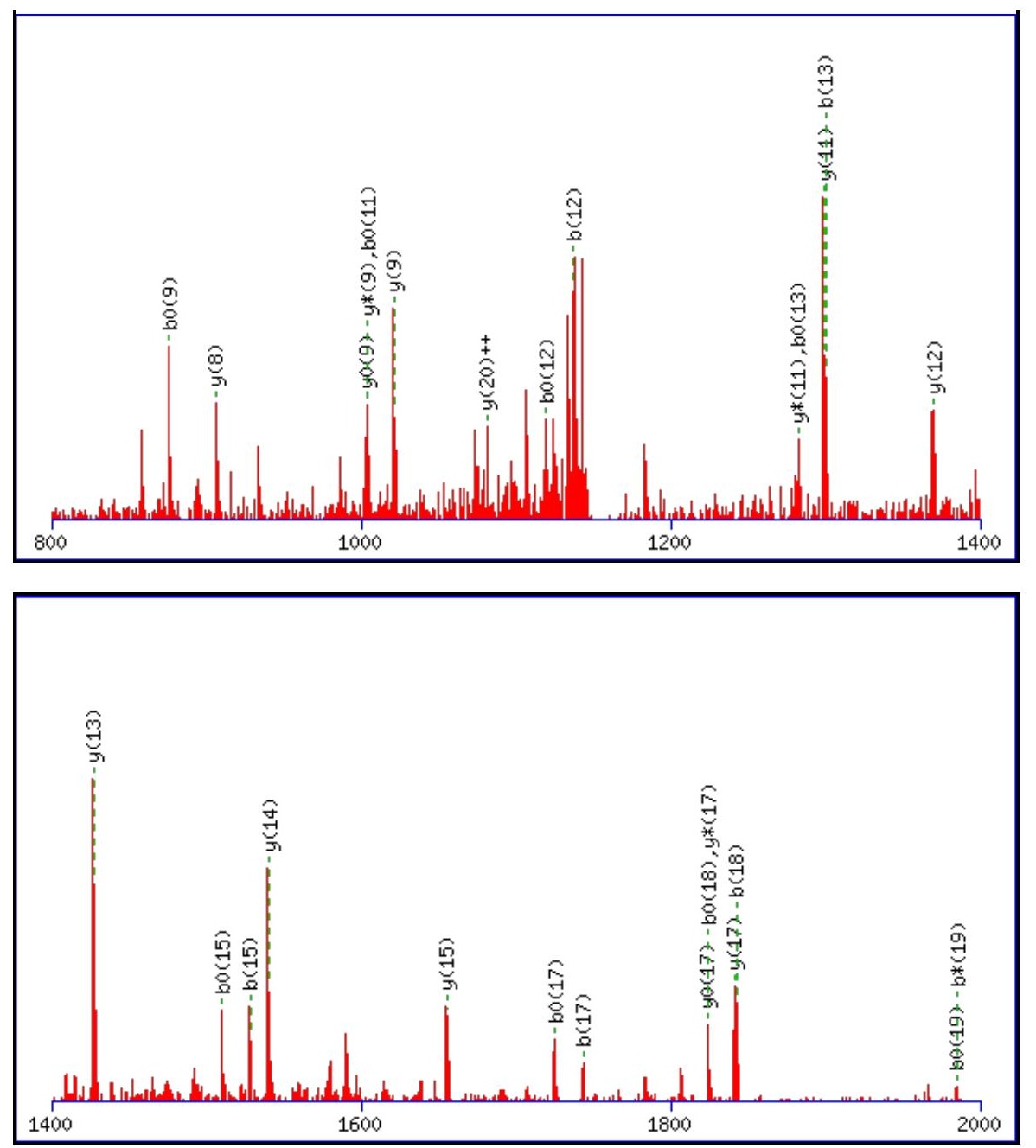

Monoisotopic mass of neutral peptide $\operatorname{Mr}($ calc): 2319.9801

Fixed modifications: Carbamidomethyl (C)

Variable modifications:

N14 : Deamidated_N (N)

Ions Score: 62 Expect: 0.00017

Matches (Bold Red): 45/222 fragment ions using 73 most intense peaks

\begin{tabular}{|c|c|c|c|c|c|c|c|c|c|c|c|c|c|c|}
\hline \# & b & $\mathbf{b}^{++}$ & b* & $\mathbf{b}^{*^{++}}$ & $\mathbf{b}^{\mathbf{0}}$ & $\mathbf{b}^{0++}$ & Seq. & $\mathbf{y}$ & $\mathrm{y}^{++}$ & $\mathbf{y}^{*}$ & $\mathrm{y}^{\mathrm{*}^{++}}$ & $\mathbf{y}^{0}$ & $\mathbf{y}^{\mathbf{0 + +}}$ & \# \\
\hline 1 & 102.0550 & 51.5311 & & & 84.0444 & 42.5258 & $\mathbf{T}$ & & & & & & & 22 \\
\hline 2 & 159.0764 & 80.0418 & & & 141.0659 & 71.0366 & $\mathbf{G}$ & 2219.9397 & 1110.4735 & 2202.9132 & 1101.9602 & 2201.9292 & 1101.4682 & 21 \\
\hline 4 & 317.1456 & 159.0764 & & & 299.1350 & 150.0711 & $\mathbf{T}$ & 2105.8968 & 1053.4520 & 2088.8703 & 1044.9388 & 2087.8862 & 1044.4468 & 19 \\
\hline 5 & 480.2089 & 240.6081 & & & 462.1983 & 231.6028 & $\mathbf{Y}$ & 2004.8491 & 1002.9282 & 1987.8226 & 994.4149 & 1986.8386 & 993.9229 & 18 \\
\hline 6 & 537.2304 & 269.1188 & & & 519.2198 & 260.1135 & G & 1841.7858 & 921.3965 & 1824.7592 & 912.8833 & 1823.7752 & 912.3913 & 17 \\
\hline 7 & 666.2729 & 333.6401 & & & 648.2624 & 324.6348 & $\mathbf{E}$ & 1784.7643 & 892.8858 & 1767.7378 & 884.3725 & 1766.7538 & 883.8805 & 16 \\
\hline 8 & 781.2999 & 391.1536 & & & 763.2893 & 382.1483 & D & 1655.7217 & 828.3645 & 1638.6952 & 819.8512 & 1637.7112 & 819.3592 & 15 \\
\hline 9 & 894.3840 & 447.6956 & & & 876.3734 & 438.6903 & $\mathbf{L}$ & 1540.6948 & 770.8510 & 1523.6682 & 762.3378 & 1522.6842 & 761.8458 & 14 \\
\hline 10 & 951.4054 & 476.2063 & & & 933.3949 & 467.2011 & G & 1427.6107 & 714.3090 & 1410.5842 & 705.7957 & 1409.6002 & 705.3037 & 13 \\
\hline 11 & 1022.4425 & 511.7249 & & & 1004.4320 & 502.7196 & A & 1370.5893 & 685.7983 & 1353.5627 & 677.2850 & 1352.5787 & 676.7930 & 12 \\
\hline 12 & 1137.4695 & 569.2384 & & & 1119.4589 & 560.2331 & D & 1299.5522 & 650.2797 & 1282.5256 & 641.7664 & 1281.5416 & 641.2744 & 11 \\
\hline 14 & 1415.5597 & 708.2835 & 1398.5332 & 699.7702 & 1397.5492 & 699.2782 & $\mathbf{N}$ & 1021.4619 & 511.2346 & 1004.4353 & 502.7213 & 1003.4513 & 502.2293 & 9 \\
\hline 15 & 1528.6438 & 764.8255 & 1511.6173 & 756.3123 & 1510.6332 & 755.8203 & $\mathbf{L}$ & 906.4349 & 453.7211 & 889.4084 & 445.2078 & 888.4244 & 444.7158 & 8 \\
\hline 16 & 1615.6758 & 808.3416 & 1598.6493 & 799.8283 & 1597.6653 & 799.3363 & $S$ & 793.3509 & 397.1791 & 776.3243 & 388.6658 & 775.3403 & 388.1738 & 7 \\
\hline 17 & 1743.7344 & 872.3708 & 1726.7079 & 863.8576 & 1725.7238 & 863.3656 & $\mathbf{Q}$ & 706.3189 & 353.6631 & 689.2923 & 345.1498 & 688.3083 & 344.6578 & 6 \\
\hline 18 & 1842.8028 & 921.9051 & 1825.7763 & 913.3918 & 1824.7923 & 912.8998 & $\mathbf{V}$ & 578.2603 & 289.6338 & 561.2337 & 281.1205 & 560.2497 & 280.6285 & 5 \\
\hline
\end{tabular}




\begin{tabular}{|r|r|r|r|r|r|r|r|r|r|r|r|r|r|r|}
$\mathbf{1 9}$ & 2002.8335 & 1001.9204 & $\mathbf{1 9 8 5 . 8 0 6 9}$ & 993.4071 & $\mathbf{1 9 8 4 . 8 2 2 9}$ & 992.9151 & $\mathbf{C}$ & $\mathbf{4 7 9 . 1 9 1 9}$ & 240.0996 & $\mathbf{4 6 2 . 1 6 5 3}$ & 231.5863 & $\mathbf{4 6 1 . 1 8 1 3}$ & 231.0943 & $\mathbf{4}$ \\
\hline $\mathbf{2 0}$ & 2117.8604 & 1059.4338 & 2100.8339 & 1050.9206 & 2099.8499 & 1050.4286 & D & 319.1612 & 160.0842 & 302.1347 & 151.5710 & 301.1506 & 151.0790 & $\mathbf{3}$ \\
\hline $\mathbf{2 1}$ & 2174.8819 & 1087.9446 & 2157.8553 & 1079.4313 & 2156.8713 & 1078.9393 & G & 204.1343 & 102.5708 & 187.1077 & 94.0575 & & & $\mathbf{2}$ \\
\hline $\mathbf{2 2}$ & & & & & & & K & 147.1128 & 74.0600 & 130.0863 & 65.5468 & & & $\mathbf{1}$ \\
\hline
\end{tabular}
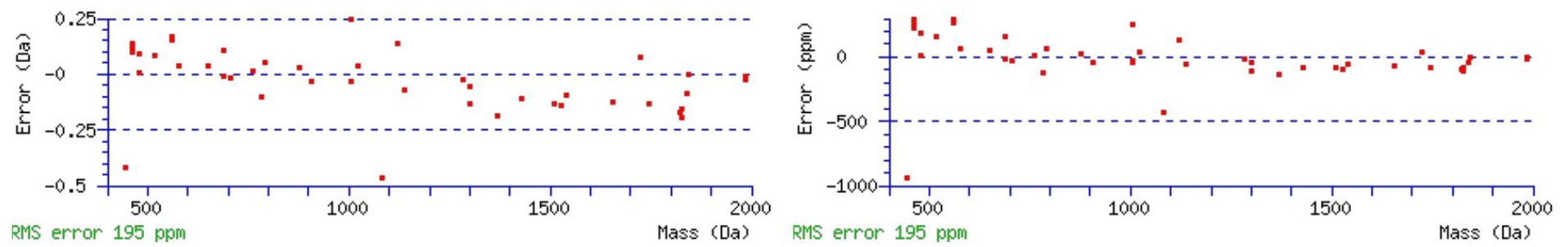

\section{All matches to this query}

\begin{tabular}{|l|l|l|l|}
\hline Score & Mr(calc): & Delta & \multicolumn{1}{c|}{ Sequence } \\
\hline 62.2 & 2319.9801 & -0.0020 & TGGTYGEDLGADYNLSQVCDGK \\
\hline 62.0 & 2318.9961 & 0.9820 & TGGTYGEDLGADYNLSQVCDGK \\
\hline 2.0 & 2318.9709 & 1.0073 & HVDLLEVAOETDGFSGSDLK \\
\hline 1.7 & 2318.9920 & 0.9862 & AESEEEREDLSALGSVLPTK \\
\hline 1.5 & 2317.9796 & 1.9985 & YLSLYNALTYYSETTVTR \\
\hline
\end{tabular}

Spectrum No: 494; Query: 383; Rank: 1

\section{Peptide View}

MS/MS Fragmentation of LNGTDPIVAVDSK

Found in IPI00369995, Tax_Id=10116 Gene_Symbol=Lrp1 similar to low density lipoprotein receptor-related protein 1

Match to Query 383: 1328.684728 from(665.349640,2+)

Title: 091008RatKidney_NH4Format01_24.1702.1702.2.dta

Data file K:INewmanPaper|Piliang|3SubProteomes\Piliang3SP\mgf5ppm\ERLIC_3SubProteomes5ppm.mgf

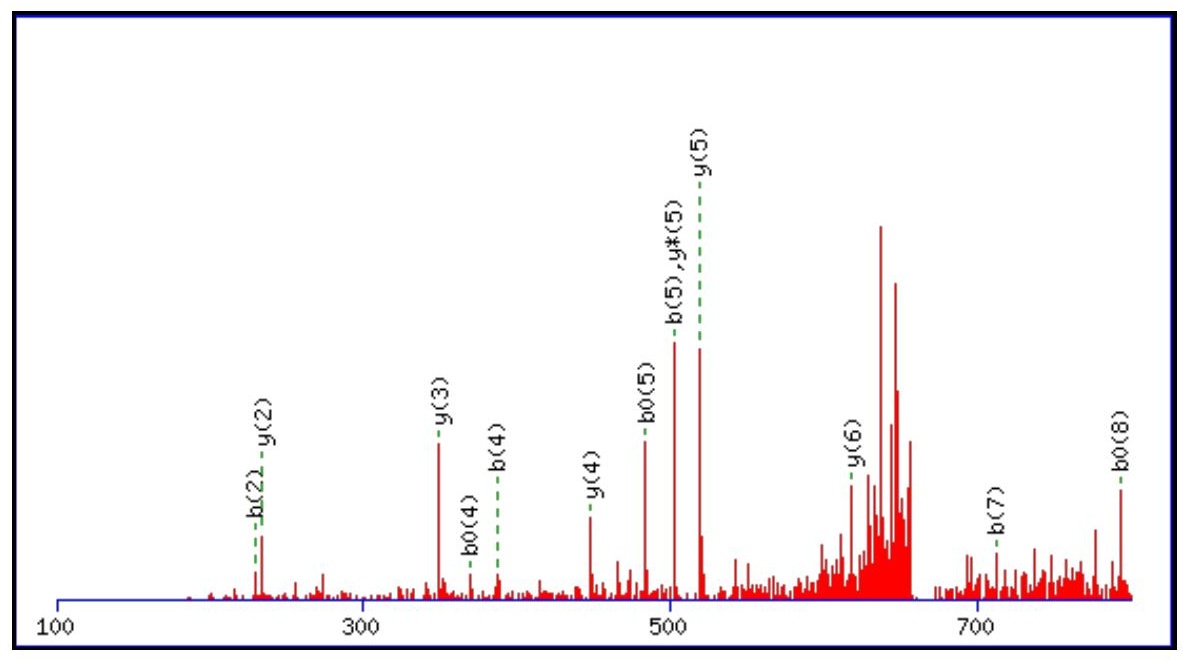



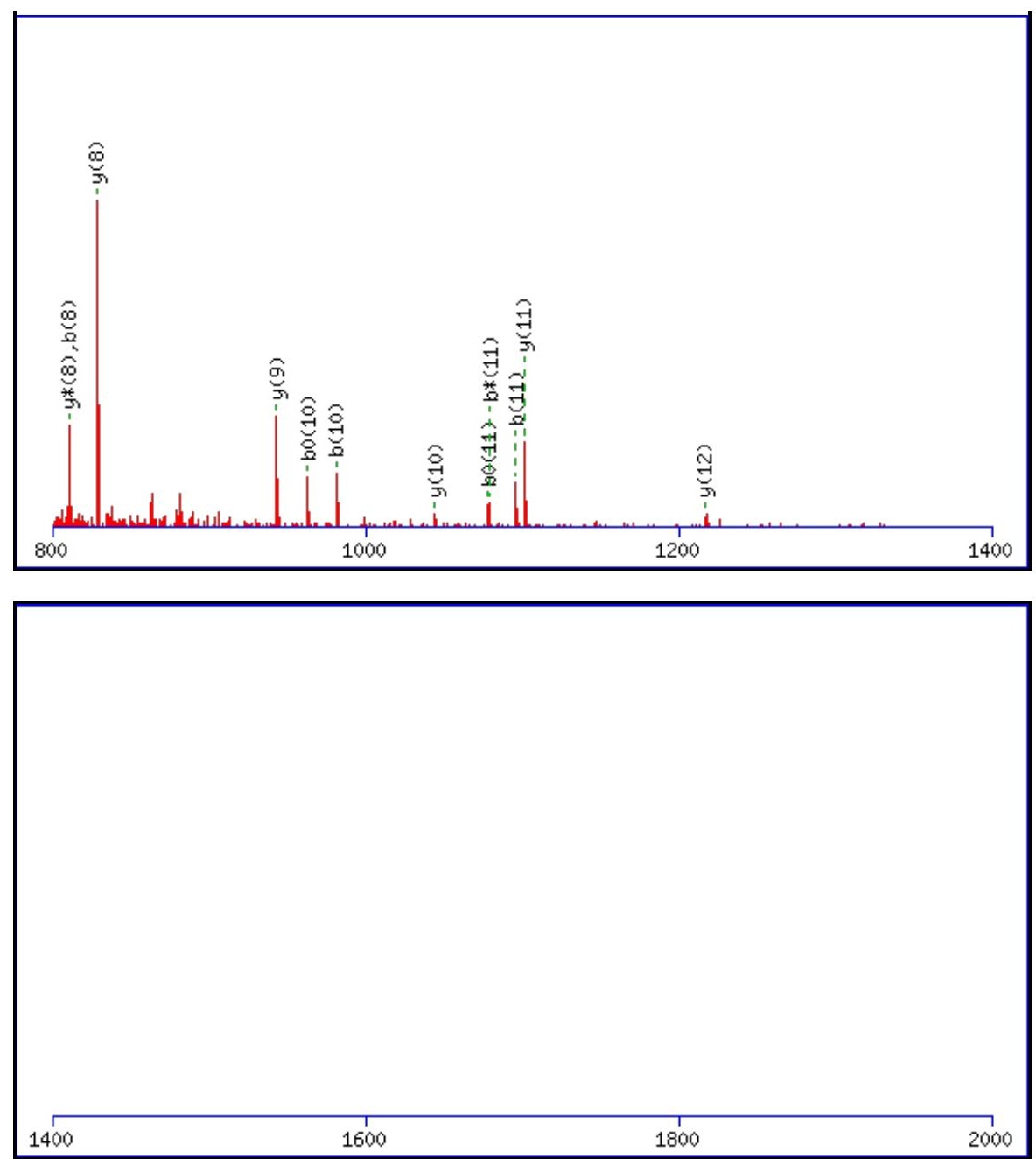

Monoisotopic mass of neutral peptide $\operatorname{Mr}($ calc): 1328.6824

Fixed modifications: Carbamidomethyl (C)

Variable modifications:

N2: Deamidated $\mathrm{N}(\mathrm{N})$

Ions Score: 62 Expect: $6.4 \mathrm{e}-005$

Matches (Bold Red): 25/134 fragment ions using 59 most intense peaks

\begin{tabular}{|c|c|c|c|c|c|c|c|c|c|c|c|c|c|c|}
\hline \# & b & $\mathbf{b}^{++}$ & b* & $\mathbf{b}^{*^{++}}$ & $\mathbf{b}^{0}$ & $\mathbf{b}^{0++}$ & Seq. & $\mathbf{y}$ & $\mathbf{y}^{++}$ & $\mathbf{y}^{*}$ & $\mathrm{y}^{*^{++}}$ & $\mathbf{y}^{\mathbf{0}}$ & $\mathbf{y}^{\mathbf{0 + +}}$ & \# \\
\hline 1 & 114.0913 & 57.5493 & & & & & $\mathbf{L}$ & & & & & & & 13 \\
\hline 2 & 229.1183 & 115.0628 & 212.0917 & 106.5495 & & & $\mathbf{N}$ & 1216.6056 & 608.8064 & 1199.5790 & 600.2931 & 1198.5950 & 599.8011 & 12 \\
\hline 3 & 286.1397 & 143.5735 & 269.1132 & 135.0602 & & & G & 1101.5786 & 551.2930 & 1084.5521 & 542.7797 & 1083.5681 & 542.2877 & 11 \\
\hline 4 & 387.1874 & 194.0973 & 370.1609 & 185.5841 & 369.1769 & 185.0921 & $\mathbf{T}$ & 1044.5572 & 522.7822 & 1027.5306 & 514.2689 & 1026.5466 & 513.7769 & 10 \\
\hline 5 & 502.2144 & 251.6108 & 485.1878 & 243.0975 & 484.2038 & 242.6055 & D & 943.5095 & 472.2584 & 926.4829 & 463.7451 & 925.4989 & 463.2531 & 9 \\
\hline 6 & 599.2671 & 300.1372 & 582.2406 & 291.6239 & 581.2566 & 291.1319 & $\mathbf{P}$ & 828.4825 & 414.7449 & 811.4560 & 406.2316 & 810.4720 & 405.7396 & 8 \\
\hline 7 & 712.3512 & 356.6792 & 695.3246 & 348.1660 & 694.3406 & 347.6740 & I & 731.4298 & 366.2185 & 714.4032 & 357.7053 & 713.4192 & 357.2132 & 7 \\
\hline 8 & 811.4196 & 406.2134 & 794.3931 & 397.7002 & 793.4090 & 397.2082 & $\mathbf{V}$ & 618.3457 & 309.6765 & 601.3192 & 301.1632 & 600.3352 & 300.6712 & 6 \\
\hline 9 & 882.4567 & 441.7320 & 865.4302 & 433.2187 & 864.4462 & 432.7267 & A & 519.2773 & 260.1423 & 502.2508 & 251.6290 & 501.2667 & 251.1370 & 5 \\
\hline 10 & 981.5251 & 491.2662 & 964.4986 & 482.7529 & 963.5146 & 482.2609 & $\mathbf{V}$ & 448.2402 & 224.6237 & 431.2136 & 216.1105 & 430.2296 & 215.6185 & 4 \\
\hline 11 & 1096.5521 & 548.7797 & 1079.5255 & 540.2664 & 1078.5415 & 539.7744 & D & 349.1718 & 175.0895 & 332.1452 & 166.5763 & 331.1612 & 166.0842 & 3 \\
\hline 12 & 1183.5841 & 592.2957 & 1166.5576 & 583.7824 & 1165.5735 & 583.2904 & $\mathrm{~S}$ & 234.1448 & 117.5761 & 217.1183 & 109.0628 & 216.1343 & 108.5708 & 2 \\
\hline 13 & & & & & & & $\mathbf{K}$ & 147.1128 & 74.0600 & 130.0863 & 65.5468 & & & 1 \\
\hline
\end{tabular}



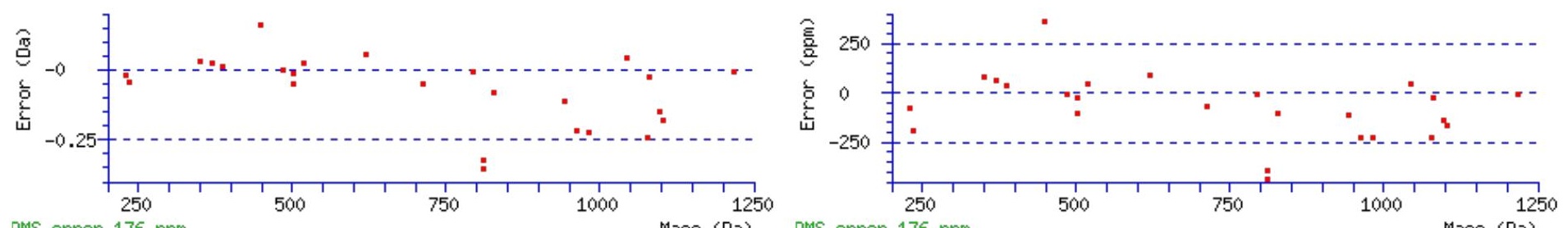

RIS error 176 ppm

Mass (Da) RMS error 176 ppm

\section{All matches to this query}

\begin{tabular}{|l|l|l|l|}
\hline Score & Mr(calc): & Delta & \multicolumn{1}{c|}{ Sequence } \\
\hline 62.2 & 1328.6824 & 0.0024 & LNGTDPIVAVDSK \\
\hline 4.6 & 1327.6772 & 1.0075 & VGQYSLYIGNSK \\
\hline 4.5 & 1328.6832 & 0.0015 & $\underline{\text { IIIMLENMHSK }}$ \\
\hline 3.6 & 1328.6949 & -0.0102 & $\underline{\text { RATHVPYRDSK }}$ \\
\hline 1.0 & 1328.6725 & 0.0123 & $\underline{\text { LAFSSTARFDSK }}$ \\
\hline 0.5 & 1328.6877 & -0.0030 & $\underline{\text { LDLYHWLNQK }}$ \\
\hline
\end{tabular}

Spectrum No: 495; Query: 138; Rank: 1

\section{Peptide View}

MS/MS Fragmentation of ITNYTVVYR

Found in IPI00231988, Tax_Id=10116 Gene_Symbol=Ptprf Leukocyte common antigen-related phosphatase precursor

Match to Query 138: 1128.582388 from(565.298470,2+)

Title: 100101RatKid_NS_deglyco_21.1888.1888.2.dta

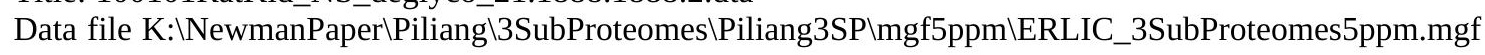

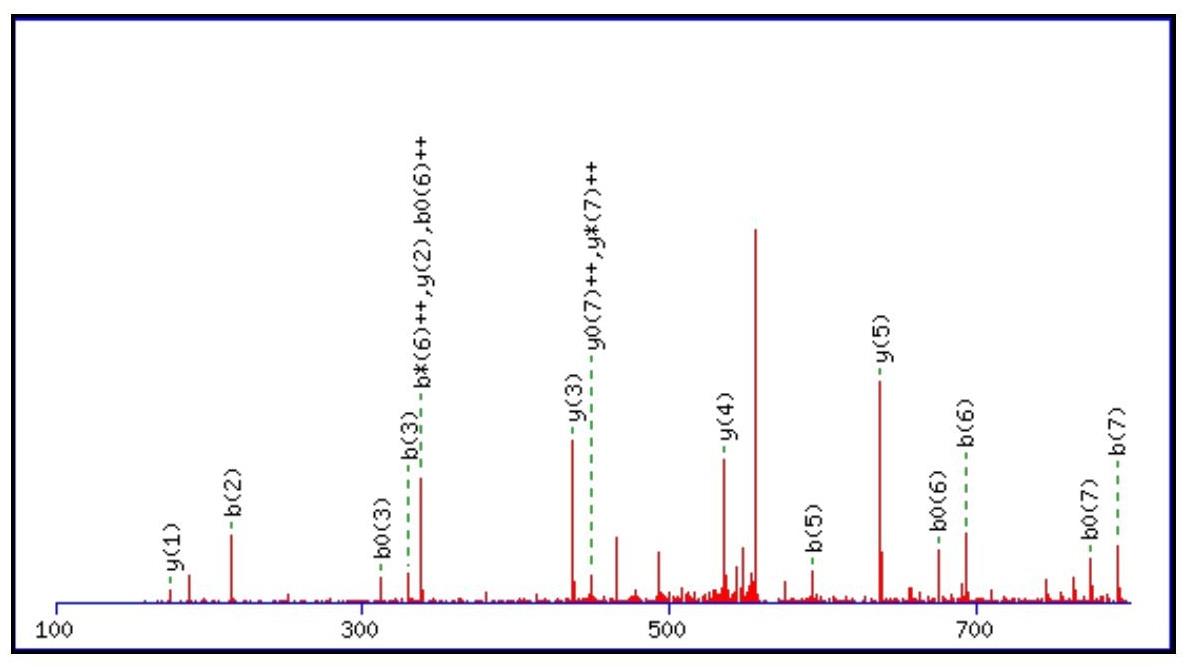



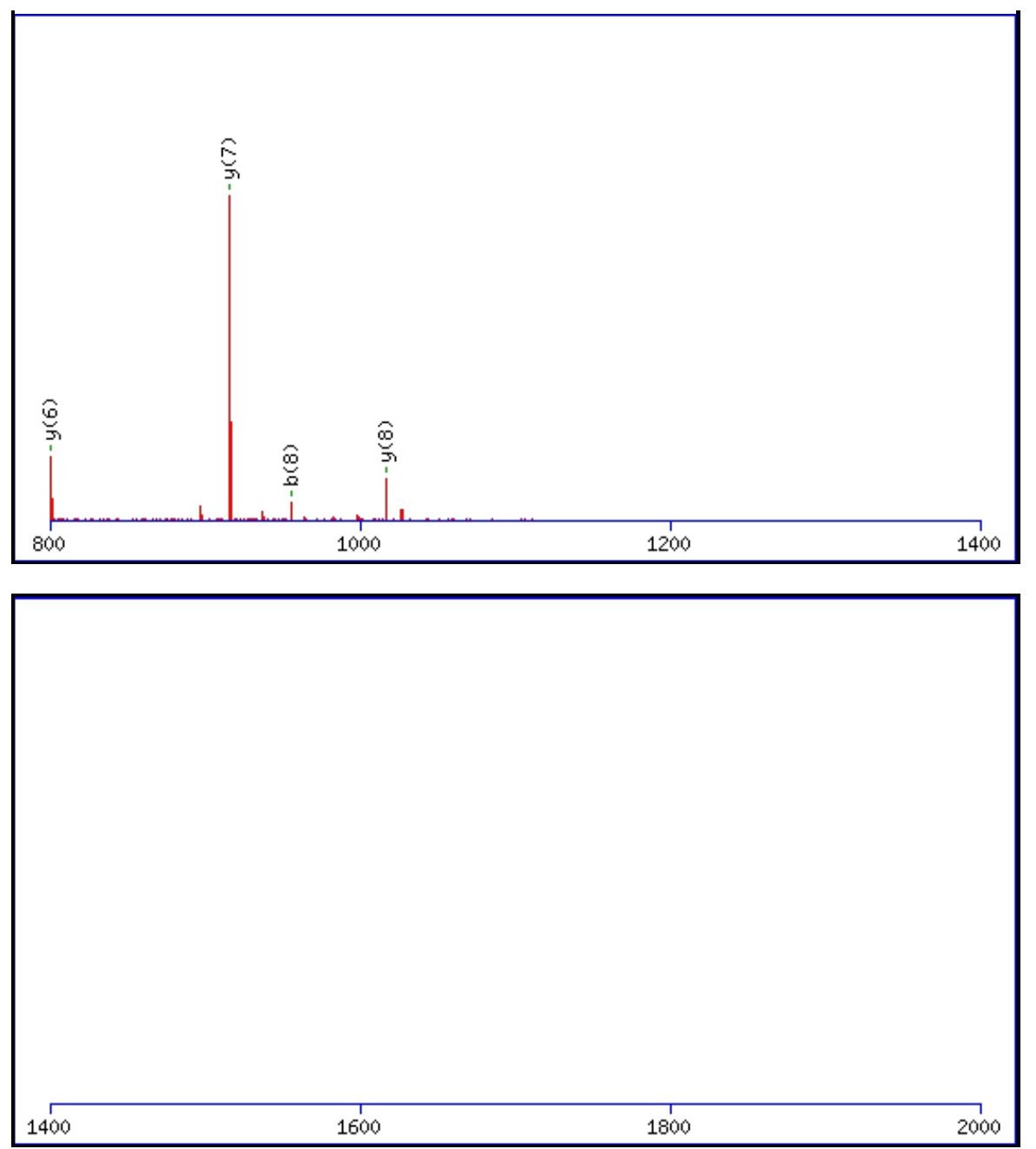

Monoisotopic mass of neutral peptide $\operatorname{Mr}($ calc): 1128.5815

Fixed modifications: Carbamidomethyl (C)

Variable modifications:

N3 : Deamidated $\mathrm{N}(\mathrm{N})$

Ions Score: 62 Expect: $5.6 \mathrm{e}-005$

Matches (Bold Red): 21/82 fragment ions using 31 most intense peaks

\begin{tabular}{|c|c|c|c|c|c|c|c|c|c|c|c|c|c|c|}
\hline$\#$ & $\mathbf{b}$ & $\mathbf{b}^{++}$ & $\mathbf{b}^{*}$ & $\mathbf{b}^{\boldsymbol{*}^{++}}$ & $\mathbf{b}^{\mathbf{0}}$ & $\mathbf{b}^{\mathbf{0 + +}}$ & $\mathbf{S e q}$ & $\mathbf{y}$ & $\mathbf{y}^{++}$ & $\mathbf{y}^{*}$ & $\mathbf{y}^{\boldsymbol{*}^{++}}$ & $\mathbf{y}^{\mathbf{0}}$ & $\mathbf{y}^{\mathbf{0 + +}}$ & $\#$ \\
\hline $\mathbf{1}$ & 114.0913 & 57.5493 & & & & & $\mathbf{I}$ & & & & & & & $\mathbf{9}$ \\
\hline $\mathbf{2}$ & $\mathbf{2 1 5 . 1 3 9 0}$ & 108.0731 & & & 197.1285 & 99.0679 & $\mathbf{T}$ & $\mathbf{1 0 1 6 . 5 0 4 7}$ & 508.7560 & 999.4782 & 500.2427 & 998.4942 & 499.7507 & $\mathbf{8}$ \\
\hline $\mathbf{3}$ & $\mathbf{3 3 0 . 1 6 6 0}$ & 165.5866 & 313.1394 & 157.0733 & 312.1554 & 156.5813 & $\mathbf{N}$ & $\mathbf{9 1 5 . 4 5 7 1}$ & 458.2322 & 898.4305 & $\mathbf{4 4 9 . 7 1 8 9}$ & 897.4465 & $\mathbf{4 4 9 . 2 2 6 9}$ & $\mathbf{7}$ \\
\hline $\mathbf{4}$ & 493.2293 & 247.1183 & 476.2027 & 238.6050 & 475.2187 & 238.1130 & $\mathbf{Y}$ & $\mathbf{8 0 0 . 4 3 0 1}$ & 400.7187 & 783.4036 & 392.2054 & 782.4196 & 391.7134 & $\mathbf{6}$ \\
\hline $\mathbf{5}$ & $\mathbf{5 9 4 . 2 7 7 0}$ & 297.6421 & 577.2504 & 289.1288 & 576.2664 & 288.6368 & $\mathbf{T}$ & $\mathbf{6 3 7 . 3 6 6 8}$ & 319.1870 & 620.3402 & 310.6738 & 619.3562 & 310.1817 & $\mathbf{5}$ \\
\hline $\mathbf{6}$ & $\mathbf{6 9 3 . 3 4 5 4}$ & 347.1763 & 676.3188 & 338.6631 & $\mathbf{6 7 5 . 3 3 4 8}$ & 338.1710 & $\mathbf{V}$ & $\mathbf{5 3 6 . 3 1 9 1}$ & 268.6632 & 519.2926 & 260.1499 & & & $\mathbf{4}$ \\
\hline $\mathbf{7}$ & $\mathbf{7 9 2 . 4 1 3 8}$ & 396.7105 & 775.3872 & 388.1973 & 774.4032 & 387.7053 & $\mathbf{V}$ & $\mathbf{4 3 7 . 2 5 0 7}$ & 219.1290 & 420.2241 & 210.6157 & & & $\mathbf{3}$ \\
\hline $\mathbf{8}$ & $\mathbf{9 5 5 . 4 7 7 1}$ & 478.2422 & 938.4506 & 469.7289 & 937.4666 & 469.2369 & $\mathbf{Y}$ & $\mathbf{3 3 8 . 1 8 2 3}$ & 169.5948 & 321.1557 & 161.0815 & & & $\mathbf{2}$ \\
\hline $\mathbf{9}$ & & & & & & & $\mathbf{R}$ & $\mathbf{1 7 5 . 1 1 9 0}$ & 88.0631 & 158.0924 & 79.5498 & & & $\mathbf{1}$ \\
\hline
\end{tabular}
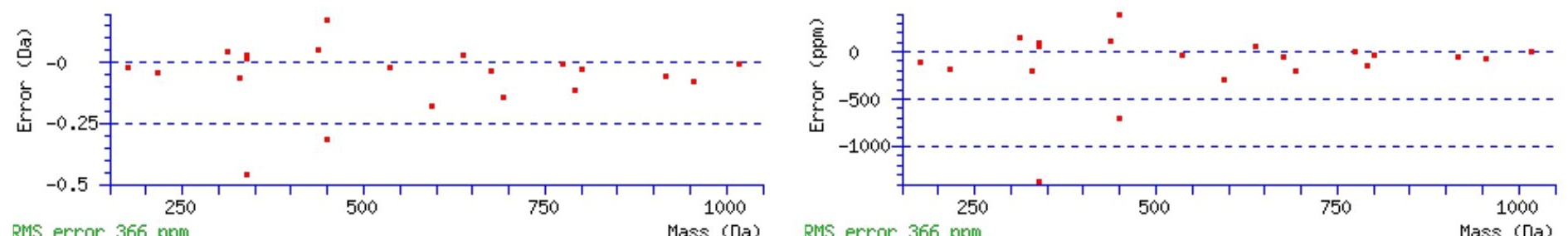

\section{All matches to this query}


Mascot Search Results: Peptide View

\begin{tabular}{|l|c|c|l|} 
Score & $\mathbf{M r}$ (calc): & Delta & \multicolumn{1}{|c|}{ Sequence } \\
\hline 62.1 & 1128.5815 & 0.0009 & ITNYTVVYR \\
\hline 9.0 & 1128.5805 & 0.0019 & LRSHPAQLK \\
\hline 4.6 & 1128.5775 & 0.0049 & VDPNESLSIR \\
\hline 3.0 & 1127.5740 & 1.0084 & LVIQSYLGR \\
\hline 2.3 & 1128.5927 & -0.0104 & LPQGLGEPYR \\
\hline 2.2 & 1127.5852 & 0.9972 & LTPLKHSPR \\
\hline 1.1 & 1128.5815 & 0.0009 & LTGPPPSFEGK \\
\hline
\end{tabular}

Spectrum No: 496; Query: 2403; Rank: 1

\section{Peptide View}

MS/MS Fragmentation of KVEEANKAEENGSEALMTLDDQANK

Found in IPI00212608, Tax_Id=10116 Gene_Symbol=Dab2 Isoform p82 of Disabled homolog 2

Match to Query 2403: 2734.268772 from(912.430200,3+)

Title: 100101RatKid_NS_deglyco_13.2652.2652.3.dta

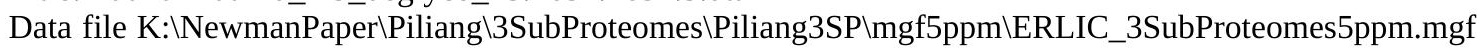
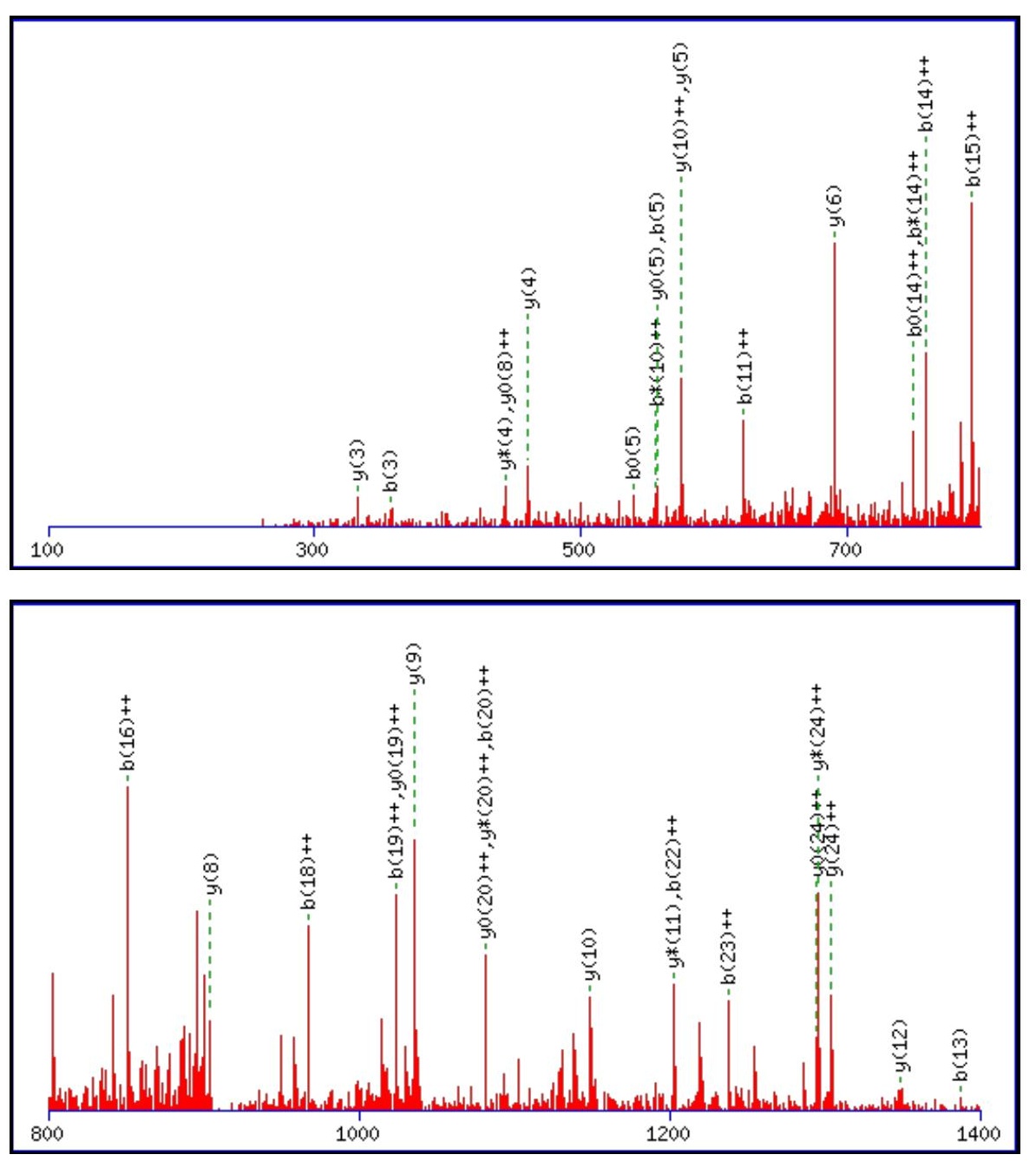


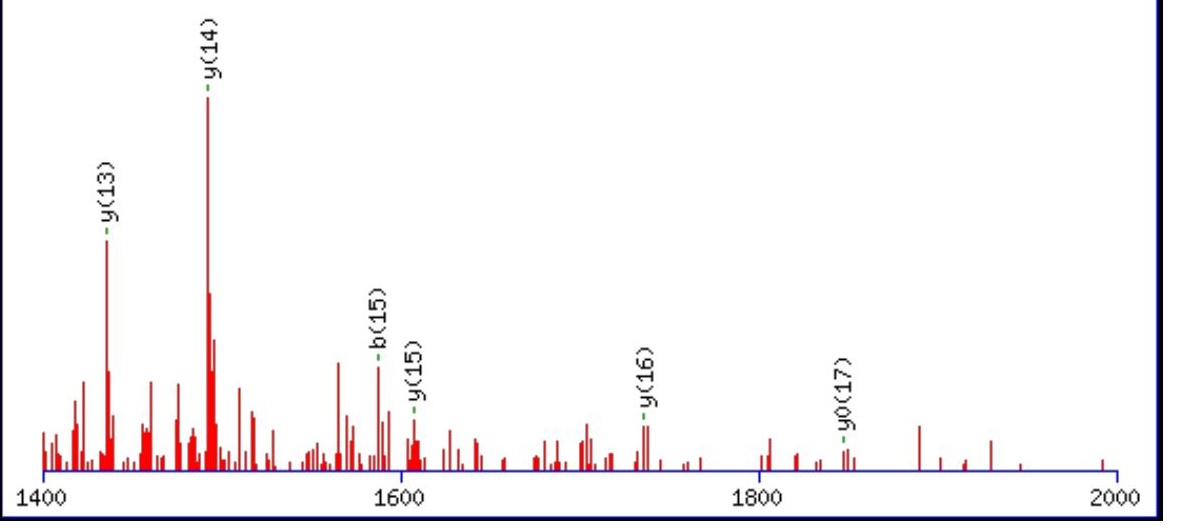

Monoisotopic mass of neutral peptide $\operatorname{Mr}($ calc): 2734.2603

Fixed modifications: Carbamidomethyl (C)

Variable modifications:

N11: Deamidated $\mathrm{N}(\mathrm{N})$

Ions Score: 62 Expect: 0.00021

Matches (Bold Red): 42/276 fragment ions using 59 most intense peaks

\begin{tabular}{|c|c|c|c|c|c|c|c|c|c|c|c|c|c|c|}
\hline \# & b & $\mathbf{b}^{++}$ & $\mathbf{b}^{*}$ & $\mathbf{b}^{*^{++}}$ & $\mathbf{b}^{\mathbf{0}}$ & & Seq. & $\mathbf{y}$ & & $\mathbf{y}^{*}$ & & $\mathbf{y}^{\mathbf{0}}$ & & \# \\
\hline 1 & 129.1022 & 65.0548 & 112.0757 & 56.5415 & & & $\mathbf{K}$ & & & & & & & 25 \\
\hline 2 & 228.1707 & 114.5890 & 211.1441 & 106.0757 & & & $\mathbf{V}$ & & & 461 & 5767 & 2589.1621 & 1295.0847 & 24 \\
\hline 3 & 357.2132 & 179.1103 & 340.1867 & 170.5970 & & & $\mathbf{E}$ & 2508.1042 & 1254.5558 & 2491.0777 & 1246.0425 & 2490.0937 & 5505 & 23 \\
\hline 4 & 486.2558 & 243.6316 & 469.2293 & 235.1183 & 468.2453 & 234.6263 & $\mathbf{E}$ & 2379.0616 & 1190.0345 & 2362.0351 & 1181.5212 & 2361.0511 & 1181.0292 & 22 \\
\hline 5 & 557.2930 & 279.1501 & 540.2664 & 270.6368 & 539.2824 & 270.1448 & A & 2250.0190 & 5132 & 2232.9925 & 1116.9999 & 2232.0085 & 1116.5079 & 21 \\
\hline 6 & 671.3359 & 336.1716 & 654.3093 & 327.6583 & 653.3253 & 327.1663 & $\mathbf{N}$ & 2178.9819 & 9946 & 9554 & 1081.4813 & 2160.9714 & 1080.9893 & 20 \\
\hline 7 & 799.4308 & 400.2191 & 782.4043 & 391.7058 & 781.4203 & 391.2138 & $\mathbf{K}$ & 2064.9390 & .9731 & 2047.9124 & 1024.4599 & 2046.9284 & 1023.9679 & 19 \\
\hline 8 & 870.4680 & 435.7376 & 853.4414 & 427.2243 & 852.4574 & 426.7323 & A & 1936.8440 & 968.9257 & 3175 & 960.4124 & 1918.8335 & 959.9204 & 18 \\
\hline 9 & 999.5106 & 500.2589 & 982.4840 & 491.7456 & 981.5000 & 491.2536 & $\mathbf{E}$ & 1865.8069 & 933.4071 & 1848.7804 & 8938 & 1847.7964 & 924.4018 & 17 \\
\hline 10 & 1128.5531 & 564.7802 & 1111.5266 & 556.2669 & 110.5426 & 555.7749 & $\mathbf{E}$ & 1736.7643 & 868.8858 & 1719.7378 & 860.3725 & 1718.7538 & 859.8805 & 16 \\
\hline 11 & 1243.5801 & 622.2937 & 226.5535 & 613.7804 & 1225.5695 & 613.2884 & $\mathbf{N}$ & 1607.7217 & 3645 & 1590 & 8512 & 1589.7112 & 795.3592 & 15 \\
\hline 12 & 1300.6015 & 650.8044 & 1283.5750 & 642.2911 & 1282.5910 & 641.7991 & G & 1492.6948 & 746.8510 & 1475.6683 & 738.3378 & 1474.6842 & 737.8458 & 14 \\
\hline 13 & 387.6336 & 694.3204 & 370.6070 & 685.8071 & 1369.6230 & 685.3151 & S & 1435.6733 & 718.3403 & 1418 & 709.8270 & 1417.6628 & 709.3350 & 13 \\
\hline 14 & 1516.6762 & 758.8417 & 1499.6496 & 750.3284 & 1498.6656 & 749.8364 & $\mathbf{E}$ & 1348.6413 & 674.8243 & 1331.6148 & 666.3110 & 1330.6307 & 665.8190 & 12 \\
\hline 15 & 1587.7133 & 794.3603 & 570.6867 & 785.8470 & 1569.7027 & 785.3550 & A & 1219.5987 & 610.3030 & 1202.5722 & 601.7897 & 1201.5881 & 601.2977 & 11 \\
\hline 16 & 1700.7973 & 850.9023 & 1683.7708 & 842.3890 & 1682.7868 & 841.8970 & $\mathbf{L}$ & 1148.5616 & 574.7844 & 1131.5351 & 566.2712 & 1130.5510 & 565.7792 & 10 \\
\hline 17 & 1831.8378 & 916.4226 & 1814.8113 & 907.9093 & 1813.8273 & 907.4173 & $\mathbf{M}$ & 1035.4775 & 518.2424 & 1018.4510 & 509.7291 & 1017.4670 & 509.2371 & 9 \\
\hline 18 & 1932.8855 & 966.9464 & 1915.8590 & 958.4331 & 1914.8749 & 957.9411 & $\mathbf{T}$ & 904.4371 & 452.7222 & 887.4105 & 444.2089 & 886.4265 & 443.7169 & 8 \\
\hline 19 & 2045.9696 & 1023.4884 & 2028.9430 & 1014.9751 & 2027.9590 & 1014.4831 & $\mathbf{L}$ & 803.3894 & 402.1983 & 786.3628 & 393.6850 & 785.3788 & 393.1930 & 7 \\
\hline 20 & 2160.9965 & 1081.0019 & 2143.9700 & 1072.4886 & 2142.9859 & 1071.9966 & D & 690.3053 & 345.6563 & 673.2788 & 337.1430 & 672.2947 & 336.6510 & 6 \\
\hline 21 & 2276.0235 & 1138.5154 & 2258.9969 & 1130.0021 & 2258.0129 & 1129.5101 & D & 575.2784 & 288.1428 & 558.2518 & 279.6295 & 557.2678 & 279.1375 & 5 \\
\hline 22 & 2404.0820 & 1202.5447 & 2387.0555 & 1194.0314 & 2386.0715 & 1193.5394 & $\mathbf{Q}$ & 460.2514 & 230.6293 & 443.2249 & 222.1161 & & & 4 \\
\hline 23 & 2475.1191 & 1238.0632 & 2458.0926 & 1229.5499 & 2457.1086 & 1229.0579 & A & 332.1928 & 166.6001 & 315.1663 & 158.0868 & & & 3 \\
\hline 24 & 2589.1621 & 1295.0847 & 2572.1355 & 1286.5714 & 2571.1515 & 1286.0794 & $\mathbf{N}$ & 261.1557 & 131.0815 & 244.1292 & 122.5682 & & & 2 \\
\hline 25 & & & & & & & $\mathbf{K}$ & 147.1128 & 74.0600 & 130.0863 & 65.5468 & & & 1 \\
\hline
\end{tabular}$$
\text { 妾 }
$$
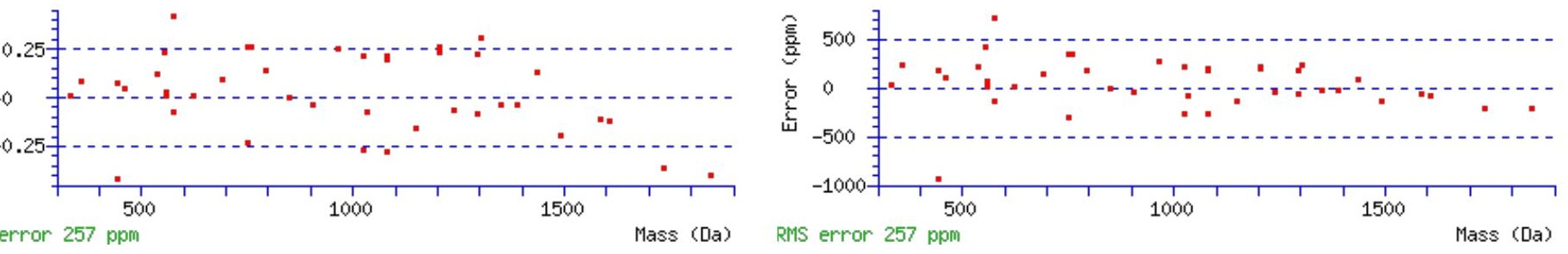


\section{All matches to this query}

\begin{tabular}{|l|c|c|l|}
\hline Score & Mr(calc): & Delta & \multicolumn{1}{|c|}{ Sequence } \\
\hline 62.1 & 2734.2603 & 0.0085 & $\underline{\text { KVEEANKAEENGSEALMTLDDQANK }}$ \\
\hline 54.4 & 2734.2603 & 0.0085 & KVEEANKAEENGSEALMTLDDQANK \\
\hline 33.4 & 2733.2762 & 0.9925 & $\underline{\text { KVEEANKAEENGSEALMTLDDQANK }}$ \\
\hline 27.7 & 2734.2603 & 0.0085 & $\underline{\text { KVEEANKAEENGSEALMTLDDQANK }}$ \\
\hline 5.5 & 2734.2544 & 0.0144 & FNEALNTPFEMNLSEEPGNPGLRR \\
\hline 3.3 & 2734.2585 & 0.0102 & $\underline{\text { TDDQNVNASLLLXKGNKNTLEYSK }}$ \\
\hline 1.5 & 2734.2901 & -0.0213 & ELQSSSLSLLLLCPSSAVNKNKGK \\
\hline 1.5 & 2734.2901 & -0.0213 & ELQSSSLSLLLLCPSSAVNKNKGK \\
\hline 1.5 & 2734.2901 & -0.0213 & ELQSSSLSLLLLCPSSAVNKNKGK \\
\hline 1.5 & 2734.2901 & -0.0213 & ELQSSSLSLLLLCPSSAVNKNKGK \\
\hline
\end{tabular}

Spectrum No: 497; Query: 996; Rank: 1

\section{Peptide View}

MS/MS Fragmentation of SHINCSVMEPTEEK

Found in IPI00193765, Tax_Id=10116 Gene_Symbol=Ctsc Dipeptidyl-peptidase 1 precursor

Match to Query 996: 1660.713868 from(831.364210,2+)

Title: 100101RatKid_NS_deglyco_20.1124.1124.2.dta

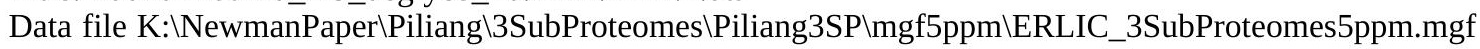

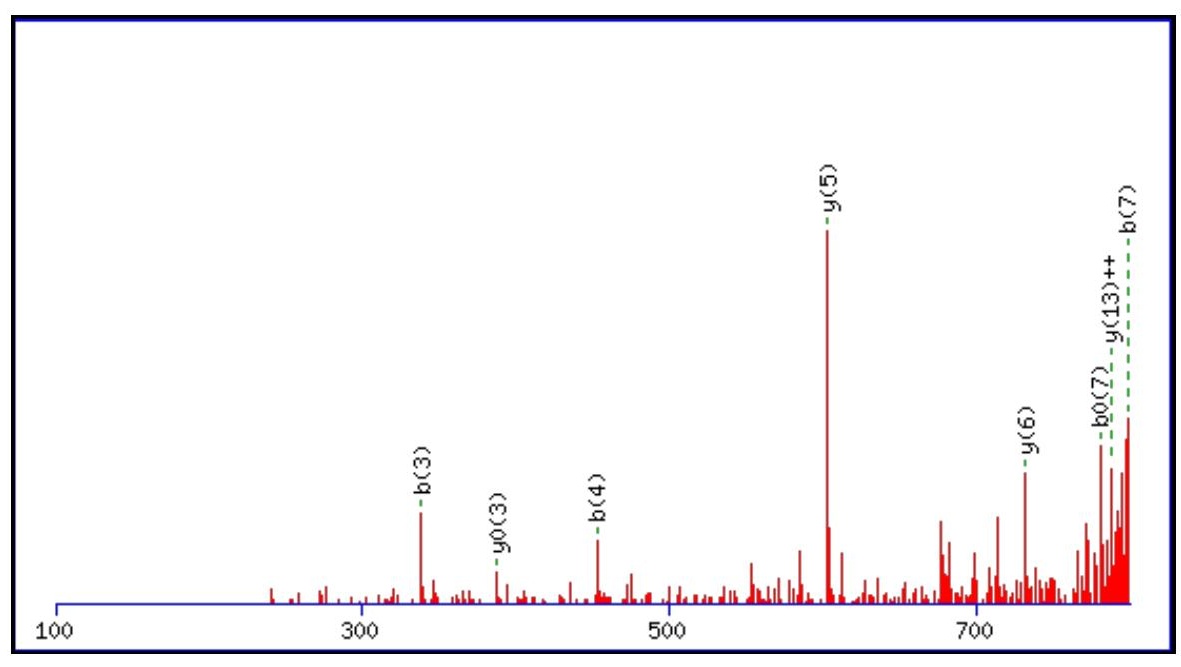



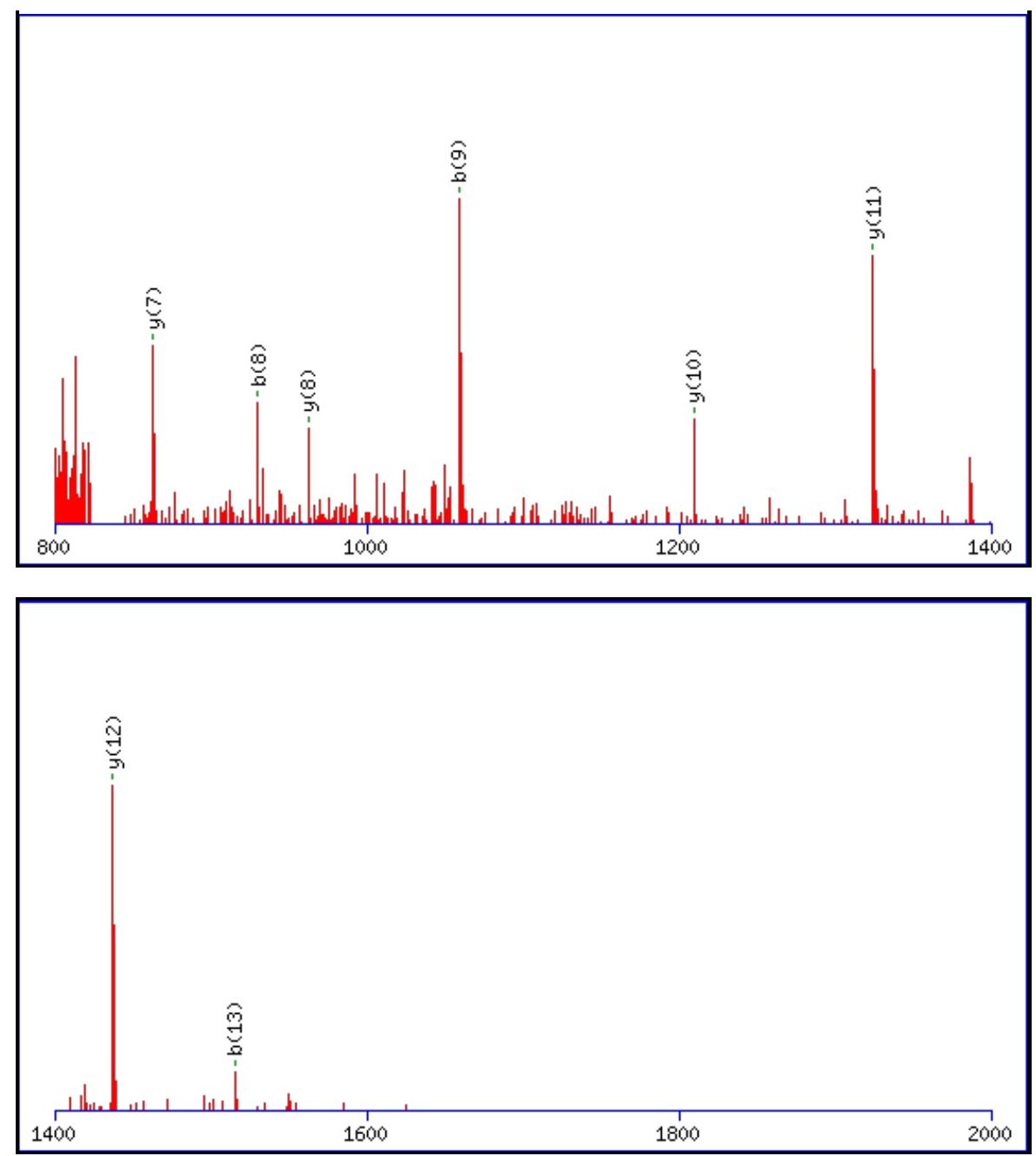

Monoisotopic mass of neutral peptide $\operatorname{Mr}($ calc): 1660.7072

Fixed modifications: Carbamidomethyl (C)

Variable modifications:

N4 : Deamidated_N (N)

Ions Score: 62 Expect: 0.00012

Matches (Bold Red): 16/148 fragment ions using 23 most intense peaks

\begin{tabular}{|c|c|c|c|c|c|c|c|c|c|c|c|c|c|c|}
\hline \# & b & $\mathbf{b}^{++}$ & $\mathbf{b}^{*}$ & $\mathbf{b}^{*^{++}}$ & $\mathbf{b}^{\mathbf{0}}$ & $\mathbf{b}^{0++}$ & Seq. & $\mathbf{y}$ & $\mathbf{y}^{++}$ & $\mathbf{y}^{*}$ & $\mathbf{y}^{*^{++}}$ & $\mathbf{y}^{\mathbf{0}}$ & $\mathbf{y}^{\mathbf{0}^{++}}$ & \# \\
\hline 1 & 88.0393 & 44.5233 & & & 70.0287 & 35.5180 & S & & & & & & & 14 \\
\hline 2 & 225.0982 & 113.0527 & & & 207.0877 & 104.0475 & H & 1574.6825 & 787.8449 & 1557.6560 & 779.3316 & 1556.6719 & 778.8396 & 13 \\
\hline 3 & 338.1823 & 169.5948 & & & 320.1717 & 160.5895 & I & 1437.6236 & 719.3154 & 1420.5971 & 710.8022 & 1419.6130 & 710.3102 & 12 \\
\hline 4 & 453.2092 & 227.1082 & 436.1827 & 218.5950 & 435.1987 & 218.1030 & $\mathbf{N}$ & 1324.5395 & 662.7734 & 1307.5130 & 654.2601 & 1306.5290 & 653.7681 & 11 \\
\hline 5 & 613.2399 & 307.1236 & 596.2133 & 298.6103 & 595.2293 & 298.1183 & $\mathrm{C}$ & 1209.5126 & 605.2599 & 1192.4861 & 596.7467 & 1191.5020 & 596.2547 & 10 \\
\hline 6 & 700.2719 & 350.6396 & 683.2453 & 342.1263 & 682.2613 & 341.6343 & S & 1049.4820 & 525.2446 & 1032.4554 & 516.7313 & 1031.4714 & 516.2393 & 9 \\
\hline 7 & 799.3403 & 400.1738 & 782.3138 & 391.6605 & 781.3297 & 391.1685 & $\mathbf{V}$ & 962.4499 & 481.7286 & 945.4234 & 473.2153 & 944.4394 & 472.7233 & 8 \\
\hline 8 & 930.3808 & 465.6940 & 913.3542 & 457.1808 & 912.3702 & 456.6888 & M & 863.3815 & 432.1944 & 846.3550 & 423.6811 & 845.3709 & 423.1891 & 7 \\
\hline 9 & 1059.4234 & 530.2153 & 1042.3968 & 521.7021 & 1041.4128 & 521.2100 & $E$ & 732.3410 & 366.6742 & 715.3145 & 358.1609 & 714.3305 & 357.6689 & 6 \\
\hline 10 & 1156.4761 & 578.7417 & 1139.4496 & 570.2284 & 1138.4656 & 569.7364 & $\mathbf{P}$ & 603.2984 & 302.1529 & 586.2719 & 293.6396 & 585.2879 & 293.1476 & 5 \\
\hline 11 & 1257.5238 & 629.2656 & 1240.4973 & 620.7523 & 1239.5133 & 620.2603 & $T$ & 506.2457 & 253.6265 & 489.2191 & 245.1132 & 488.2351 & 244.6212 & 4 \\
\hline 12 & 1386.5664 & 693.7868 & 1369.5399 & 685.2736 & 1368.5559 & 684.7816 & $E$ & 405.1980 & 203.1026 & 388.1714 & 194.5894 & 387.1874 & 194.0974 & 3 \\
\hline 13 & \begin{tabular}{|l|}
1515.6090 \\
\end{tabular} & 758.3081 & 1498.5825 & 749.7949 & 1497.5984 & 749.3029 & $\mathbf{E}$ & 276.1554 & 138.5813 & 259.1288 & 130.0681 & 258.1448 & 129.5761 & 2 \\
\hline 14 & & & & & & & $\mathbf{K}$ & 147.1128 & 74.0600 & 130.0863 & 65.5468 & & & 1 \\
\hline
\end{tabular}



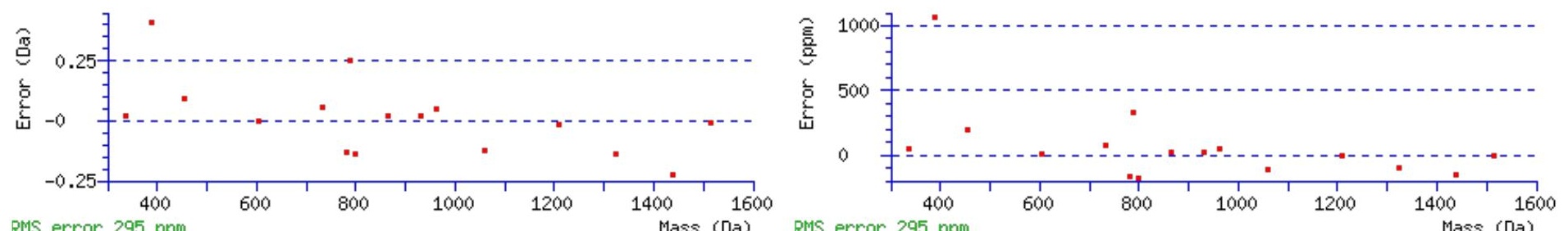

RMS error 295 ppm

Mass (Da) RNS error 295 ppm

\section{All matches to this query}

\begin{tabular}{|l|l|l|l|}
\hline Score & Mr(calc): & Delta & \multicolumn{1}{c|}{ Sequence } \\
\hline 62.1 & 1660.7072 & 0.0066 & SHINCSVMEPTEEK \\
\hline 17.9 & 1659.7232 & 0.9906 & SHINCSVMEPTEEK \\
\hline 2.2 & 1660.7110 & 0.0029 & SPAVSPSKASEDAKK \\
\hline 0.7 & 1660.7055 & 0.0083 & EKEPDLFLSSECK \\
\hline 0.2 & 1659.7110 & 1.0029 & MARKPTVCESETR \\
\hline 0.2 & 1659.7110 & 1.0029 & MARKPTVCESETR \\
\hline
\end{tabular}

Spectrum No: 498; Query: 2579; Rank: 1

\section{Peptide View}

MS/MS Fragmentation of EGTYSLPSSNFTVPDLELPSWLSTGNYR

Found in IPI00470260, Tax_Id=10116 Gene_Symbol=Gm2a GM2 ganglioside activator protein

Match to Query 2579: 3130.489752 from(1044.503860,3+)

Title: 100101RatKid_NS_deglyco_10.6892.6892.3.dta

Data file K:\NewmanPaper\Piliang \3SubProteomes\Piliang3SP \mgf5ppm\ERLIC_3SubProteomes5ppm.mgf

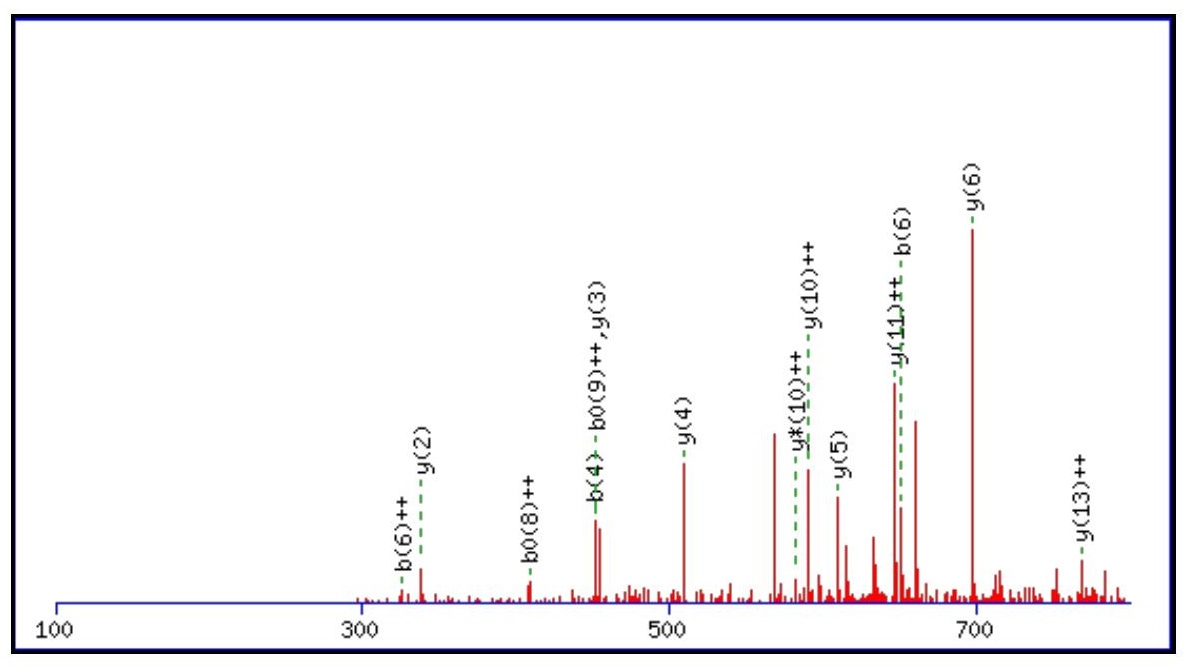



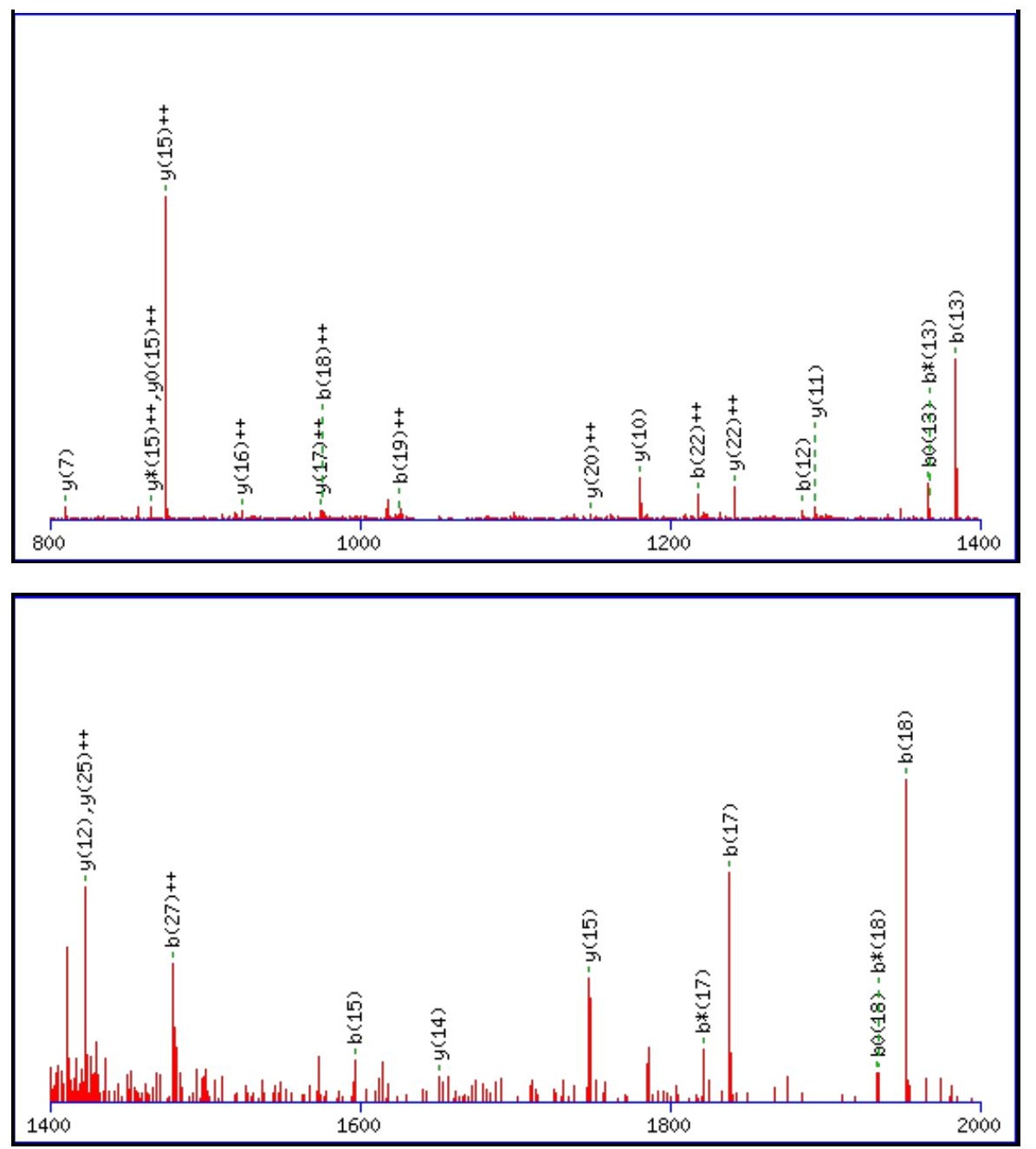

Monoisotopic mass of neutral peptide $\operatorname{Mr}($ calc): 3130.4771

Fixed modifications: Carbamidomethyl (C)

Variable modifications:

N10 : Deamidated_N (N)

Ions Score: 62 Expect: 0.00021

Matches (Bold Red): 42/298 fragment ions using 74 most intense peaks

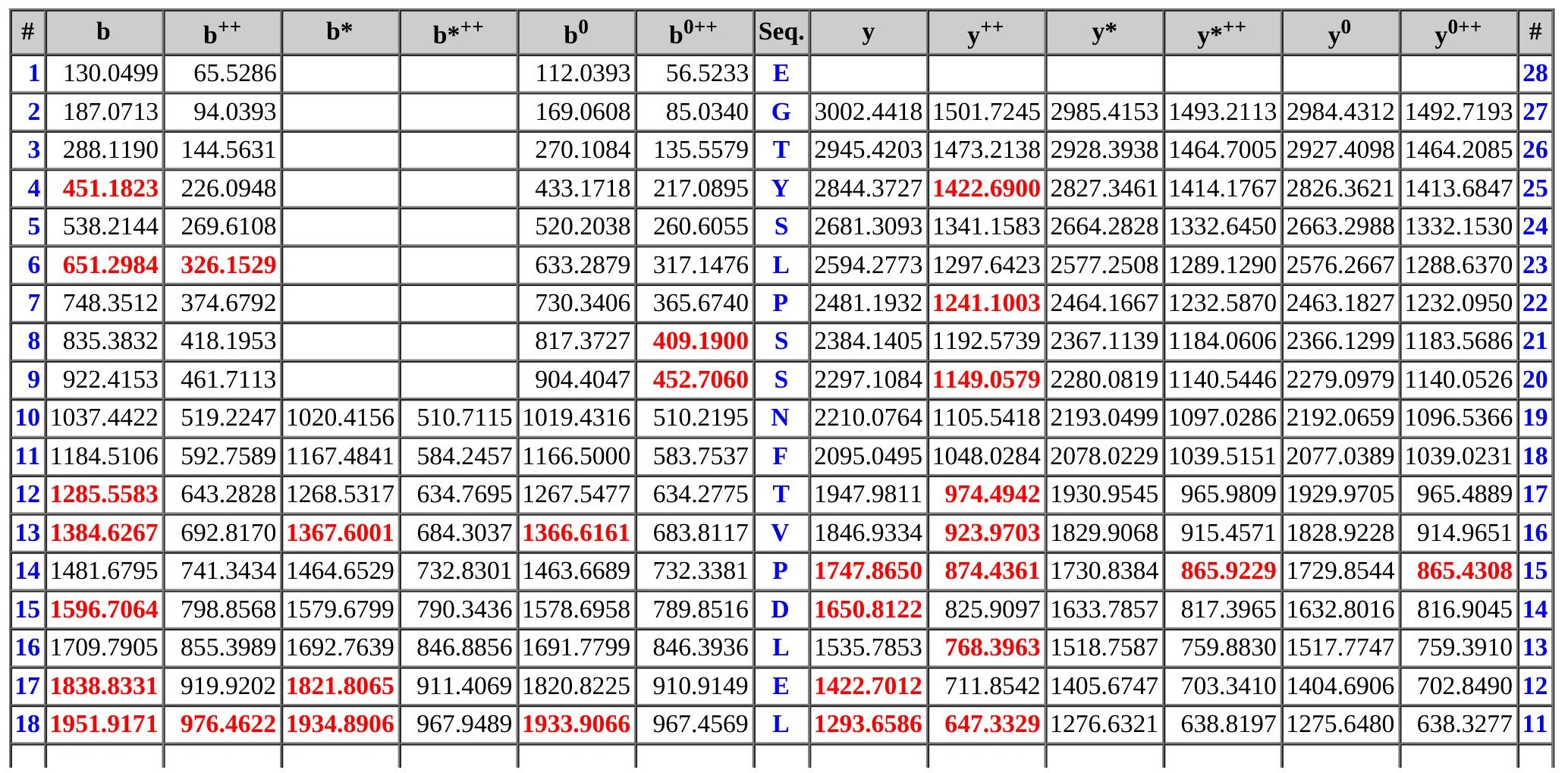




\begin{tabular}{|c|c|c|c|c|c|c|c|c|c|c|c|c|c|c|}
\hline 19 & 2048.9699 & |1024.9886 & |2031.9433 & 1016.4753 & |2030.9593| & 1015.9833 & $\mathbf{P}$ & 1180.5745 & 590.7909 & |1163.5480 & 582.2776 & $|1162.5640|$ & 581.7856 & \\
\hline 20 & 2136.0019 & 1068.5046 & 2118.9754 & 1059.9913 & 2117.9914 & 1059.4993 & S & 1083.5218 & 542.2645 & 1066.4952 & 533.7513 & 1065.5112 & 533.2592 & \\
\hline 21 & 2322.0812 & 1161.5443 & 2305.0547 & 1153.0310 & 2304.0707 & 1152.5390 & W & 996.4898 & 498.7485 & 979.4632 & 490.2352 & 978.4792 & 489.7432 & \\
\hline 22 & 2435.1653 & 1218.0863 & 2418.1387 & 1209.5730 & 2417.1547 & 1209.0810 & $\mathbf{L}$ & 810.4104 & 405.7089 & 793.3839 & 397.1956 & 792.3999 & 396.7036 & \\
\hline 23 & 2522.1973 & 1261.6023 & 2505.1708 & 1253.0890 & 2504.1868 & 1252.5970 & S & 697.3264 & 349.1668 & 680.2998 & 340.6536 & 679.3158 & 340.1615 & \\
\hline 24 & 2623.2450 & 1312.1261 & 2606.2185 & 1303.6129 & 2605.2344 & 1303.1209 & $T$ & 610.2944 & 305.6508 & 593.2678 & 297.1375 & 592.2838 & 296.6455 & \\
\hline 25 & 2680.2665 & 1340.6369 & 2663.2399 & 1332.1236 & |2662.2559| & 1331.6316 & G & 509.2467 & 255.1270 & 492.2201 & 246.6137 & & & \\
\hline 26 & 2794.3094 & 1397.6583 & 2777.2828 & 1389.1451 & 2776.2988 & 1388.6531 & $\mathbf{N}$ & 452.2252 & 226.6162 & 435.1987 & 218.1030 & & & \\
\hline 27 & 2957.3727 & 1479.1900 & 2940.3462 & 1470.6767 & 2939.3622 & 1470.1847 & $\mathbf{Y}$ & 338.1823 & 169.5948 & 321.1557 & 161.0815 & & & \\
\hline 28 & & & & & & & $\mathbf{R}$ & 175.1190 & 88.0631 & 158.0924 & 79.5498 & & & \\
\hline
\end{tabular}
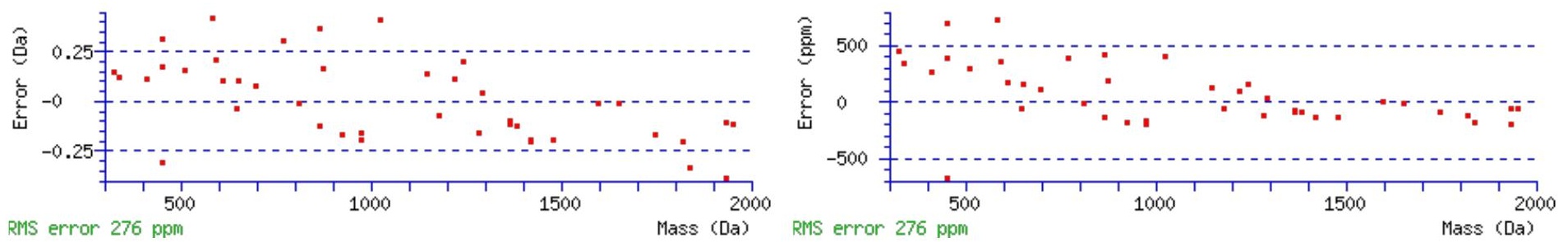

\section{All matches to this query}

\begin{tabular}{|l|l|l|l|}
\hline Score & Mr(calc): & Delta & \multicolumn{1}{|c|}{ Sequence } \\
\hline 62.1 & 3130.4771 & 0.0126 & EGTYSLPSSNFTVPDLELPSWLSTGNYR \\
\hline 35.9 & 3129.4931 & 0.9966 & EGTYSLPSSNFTVPDLELPSWLSTGNYR \\
\hline 7.8 & 3130.4771 & 0.0126 & EGTYSLPSSNFTVPDLELPSWLSTGNYR \\
\hline 5.9 & 3128.4872 & 2.0026 & TALLYNHSILSDKAKAFDGTSLFLSEK \\
\hline 5.9 & 3128.4872 & 2.0026 & TALLYNHSILSDKAKAFDGTSLFLSEK \\
\hline 5.7 & 3130.4695 & 0.0203 & TMSAESVHYNALLDLINREQNRWYK \\
\hline 5.0 & 3128.4914 & 1.9983 & HHSPHHSPHRKNQSVQTLSSHLAGVNR \\
\hline 4.2 & 3130.4695 & 0.0203 & TMSAESVHYNALLDLINREQNRWYK \\
\hline 4.2 & 3130.4695 & 0.0203 & TMSAESVHYNALLDLINREQNRWYK \\
\hline 4.0 & 3130.4678 & 0.0220 & HEYALPLTHSEPEYATPIVERHLLR \\
\hline
\end{tabular}

Spectrum No: 499; Query: 564; Rank: 1

\section{Peptide View}

MS/MS Fragmentation of TWSVFQNGTDKR

Found in IPI00205325, Tax_Id=10116 Gene_Symbol=Lrp2 Low-density lipoprotein receptor-related protein 2 precursor

Match to Query 564: 1438.680988 from(720.347770,2+)

Title: 091008RatKidney_NoSalt_24.1578.1578.2.dta

Data file K:INewmanPaper|Piliang|3SubProteomes\Piliang3SP\mgf5ppm\ERLIC_3SubProteomes5ppm.mgf 

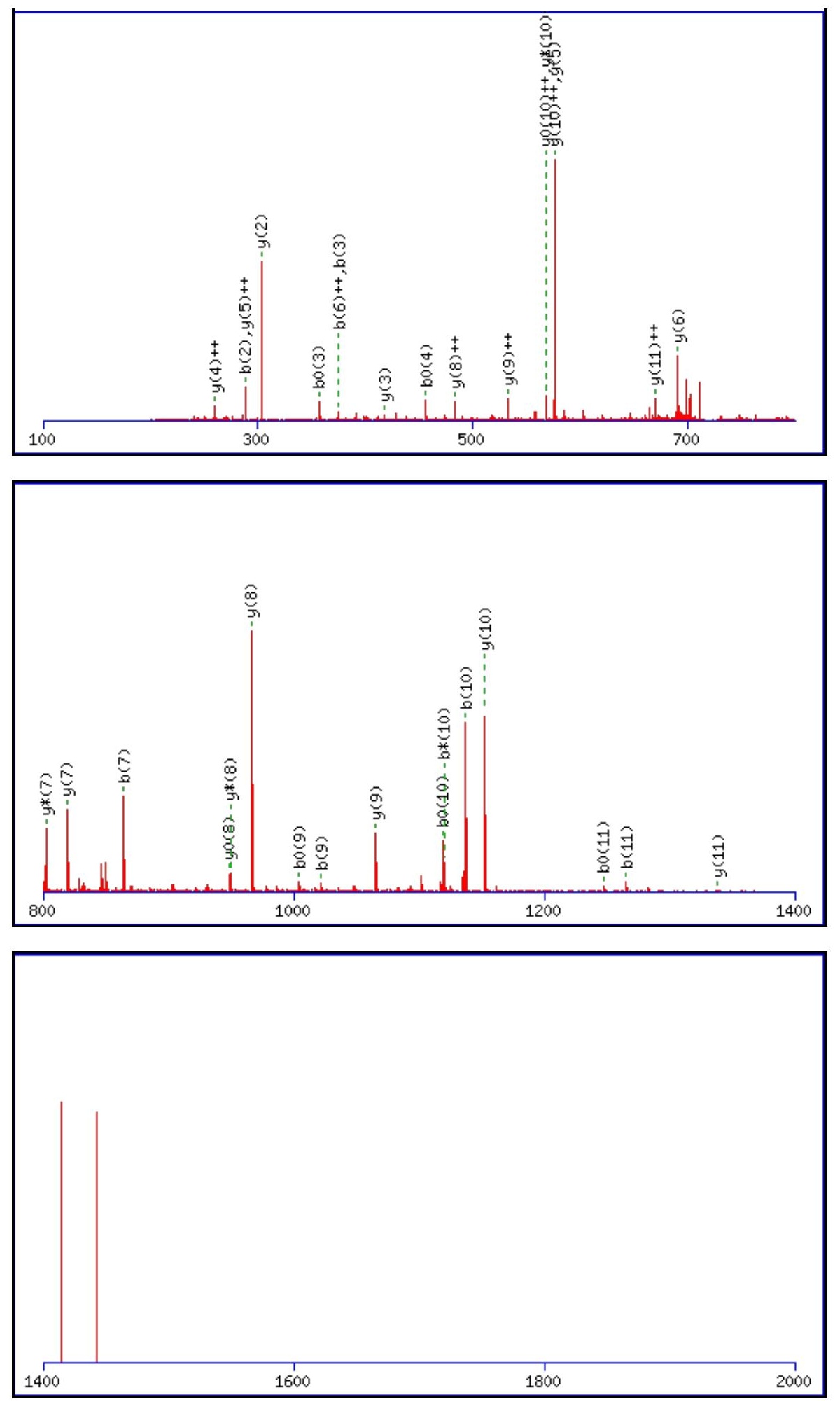

Monoisotopic mass of neutral peptide $\operatorname{Mr}($ calc): 1438.6841

Fixed modifications: Carbamidomethyl (C)

Variable modifications:

N7 : Deamidated_N (N)

Ions Score: 62 Expect: $9.3 \mathrm{e}-005$

Matches (Bold Red): 33/118 fragment ions using 56 most intense peaks

\begin{tabular}{|r|c|c|c|c|r|r|r|r|r|r|r|r|r|r|}
\hline$\#$ & $\mathbf{b}$ & $\mathbf{b}^{++}$ & $\mathbf{b}^{*}$ & $\mathbf{b}^{\mathbf{*}^{++}}$ & $\mathbf{b}^{\mathbf{0}}$ & $\mathbf{b}^{\mathbf{0 + +}}$ & Seq. & $\mathbf{y}$ & $\mathbf{y}^{++}$ & $\mathbf{y}^{\mathbf{*}}$ & $\mathbf{y}^{\boldsymbol{*}^{++}}$ & $\mathbf{y}^{\mathbf{0}}$ & $\mathbf{y}^{\mathbf{0 + +}}$ & $\#$ \\
\hline $\mathbf{1}$ & 102.0550 & 51.5311 & & & 84.0444 & 42.5258 & $\mathbf{T}$ & & & & & & & $\mathbf{1 2}$ \\
\hline $\mathbf{2}$ & $\mathbf{2 8 8 . 1 3 4 3}$ & 144.5708 & & & 270.1237 & 135.5655 & $\mathbf{W}$ & $\mathbf{1 3 3 8 . 6 4 3 7}$ & $\mathbf{6 6 9 . 8 2 5 5}$ & 1321.6171 & 661.3122 & 1320.6331 & 660.8202 & $\mathbf{1 1}$ \\
\hline $\mathbf{3}$ & $\mathbf{3 7 5 . 1 6 6 3}$ & 188.0868 & & & 357.1557 & 179.0815 & S & $\mathbf{1 1 5 2 . 5 6 4 4}$ & 576.7858 & 1135.5378 & $\mathbf{5 6 8 . 2 7 2 5}$ & 1134.5538 & $\mathbf{5 6 7 . 7 8 0 5}$ & $\mathbf{1 0}$ \\
\hline $\mathbf{4}$ & 474.2347 & 237.6210 & & & $\mathbf{4 5 6 . 2 2 4 1}$ & 228.6157 & $\mathbf{V}$ & $\mathbf{1 0 6 5 . 5 3 2 3}$ & 533.2698 & 1048.5058 & 524.7565 & 1047.5218 & 524.2645 & $\mathbf{9}$ \\
\hline $\mathbf{5}$ & 621.3031 & 311.1552 & & & 603.2926 & 302.1499 & $\mathbf{F}$ & $\mathbf{9 6 6 . 4 6 3 9}$ & $\mathbf{4 8 3 . 7 3 5 6}$ & $\mathbf{9 4 9 . 4 3 7 4}$ & $\mathbf{4 7 5 . 2 2 2 3}$ & $\mathbf{9 4 8 . 4 5 3 4}$ & 474.7303 & $\mathbf{8}$ \\
\hline
\end{tabular}




\begin{tabular}{|c|c|c|c|c|c|c|c|c|c|c|c|c|c|c|}
\hline 6 & 749.3617| & |375.1845 & 732.3352 & |366.6712 & 731.3511 & |366.1792 & $\mathbf{Q}$ & 819.3955 & 410.2014 & 802.3690 & 401.6881 & 801.3850 & |401.1961 & 7 \\
\hline 7 & 864.3886 & 432.6980 & 847.3621 & |424.1847 & 846.3781 & 423.6927 & $\mathbf{N}$ & 691.3369 & 346.1721 & 674.3104 & 337.6588 & 673.3264 & 337.1668 & 6 \\
\hline 8 & 921.4101 & 461.2087 & 904.3836 & 452.6954 & 903.3995 & 452.2034 & G & 576.3100 & 288.6586 & 559.2835 & 280.1454 & 558.2994 & 279.6534 & 5 \\
\hline 9 & 1022.4578 & 511.7325 & 1005.4312 & 503.2193 & 1004.4472 & 502.7272 & $\mathbf{T}$ & 519.2885 & 260.1479 & 502.2620 & 251.6346 & 501.2780 & 251.1426 & 4 \\
\hline 10 & 1137.4847 & 569.2460 & 1120.4582 & 560.7327 & 1119.4742 & 560.2407 & D & 418.2409 & 209.6241 & 401.2143 & 201.1108 & 400.2303 & 200.6188 & 3 \\
\hline 11 & 1265.5797 & 633.2935 & 1248.5531 & 624.7802 & 1247.5691 & 624.2882 & $\mathbf{K}$ & 303.2139 & 152.1106 & 286.1874 & 143.5973 & & & 2 \\
\hline 12 & & & & & & & $\mathbf{R}$ & 175.1190 & 88.0631 & 158.0924 & 79.5498 & & & 1 \\
\hline
\end{tabular}
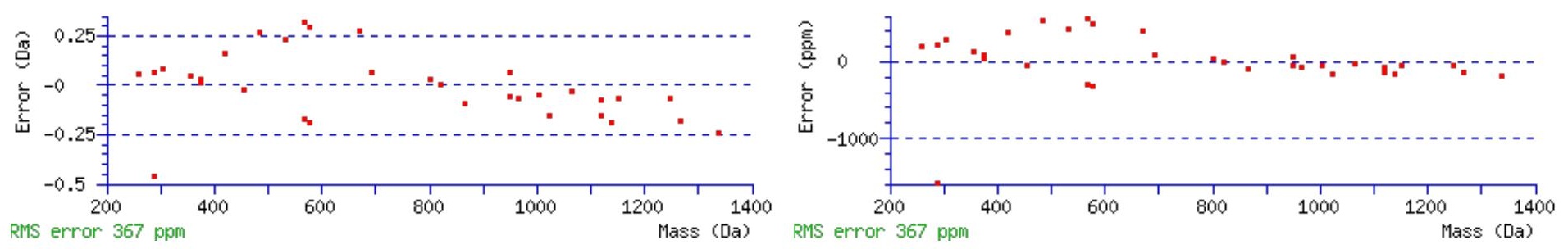

\section{All matches to this query}

\begin{tabular}{|l|l|l|l|}
\hline Score & Mr(calc): & Delta & \multicolumn{1}{c|}{ Sequence } \\
\hline 62.1 & 1438.6841 & -0.0031 & TWSVFQNGTDKR \\
\hline 16.1 & 1438.6817 & -0.0007 & QAVDTTVNGVSIR \\
\hline 16.1 & 1438.6817 & -0.0007 & QAVDTTVNGVSIR \\
\hline 8.2 & 1438.6718 & 0.0092 & SVDGYGRLHSLR \\
\hline 8.2 & 1438.6929 & -0.0119 & GLREGVTSGEAKR \\
\hline 7.7 & 1438.6817 & -0.0007 & EKLNGDTKEGLR \\
\hline 6.9 & 1438.6817 & -0.0007 & NKIAAEEQTAKR \\
\hline 6.7 & 1438.6752 & 0.0058 & ALSSPCARTQIR \\
\hline 6.5 & 1438.6857 & -0.0048 & NATVGVPTSPFLR \\
\hline 6.5 & 1438.6857 & -0.0048 & NATVGVPTSPFLR \\
\hline
\end{tabular}

Spectrum No: 500; Query: 2841; Rank: 1

\section{Peptide View}

MS/MS Fragmentation of EAGLPPNVIQFVPADGPTFGDTVTSSEHLCGINFTGSVPTFK

Found in IPI00475676, Tax_Id=10116 Gene_Symbol=Tas1r2 Delta-1-pyrroline-5-carboxylate dehydrogenase, mitochondrial precursor

Match to Query 2841: 4404.143472 from(1469.055100,3+)

Title: 100101RatKid_NS_deglyco_02.6670.6670.3.dta

Data file K:\NewmanPaper|Piliangl3SubProteomes\Piliang3SP\mgf5ppm\ERLIC_3SubProteomes5ppm.mgf 

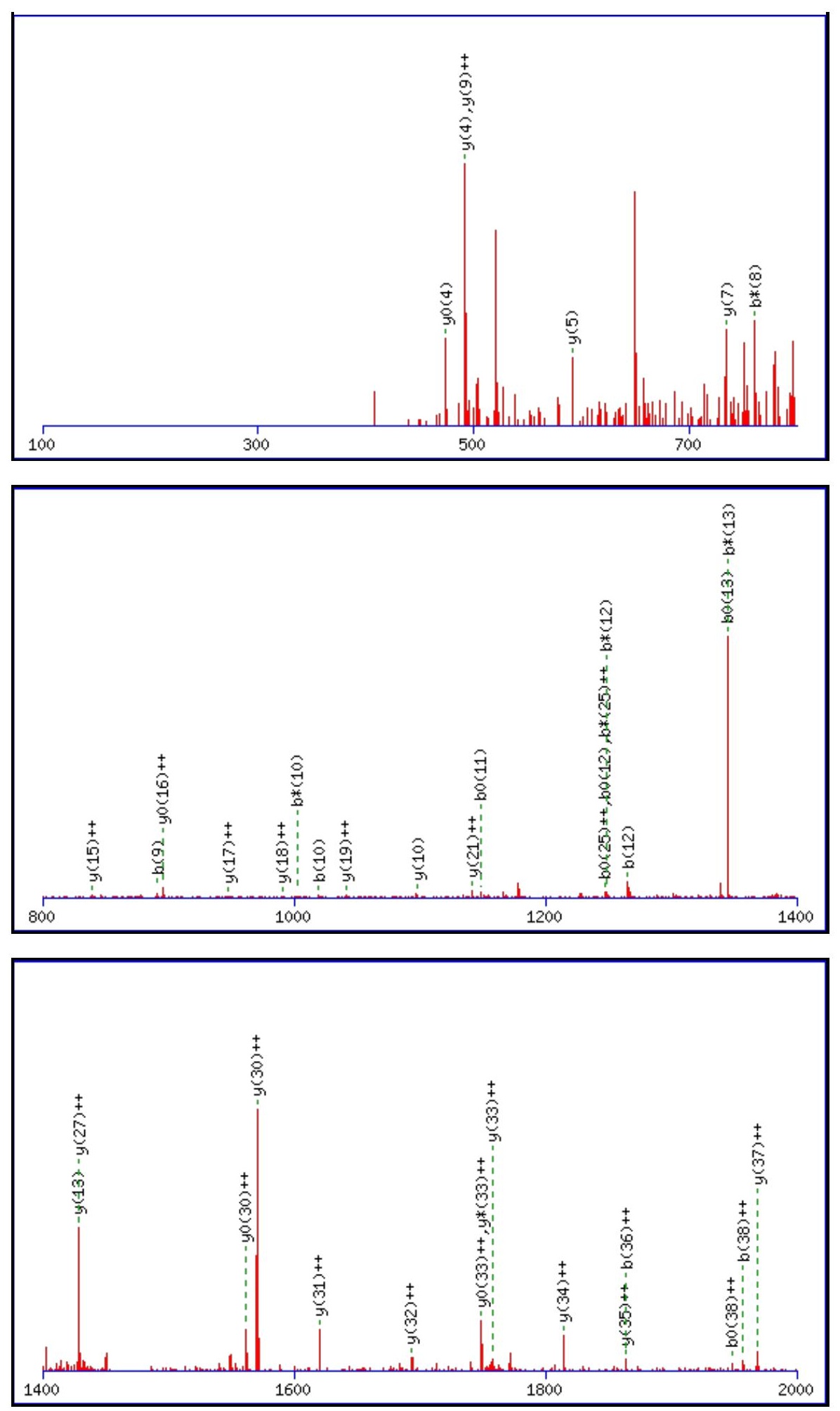

Monoisotopic mass of neutral peptide $\operatorname{Mr}($ calc): 4404.1261

Fixed modifications: Carbamidomethyl (C)

Variable modifications:

N33 : Deamidated N (N)

Ions Score: 62 Expect: 0.00017

Matches (Bold Red): 39/476 fragment ions using 67 most intense peaks

\begin{tabular}{|r|c|c|c|c|c|r|r|c|c|c|c|c|c|c|}
\hline$\#$ & $\mathbf{b}$ & $\mathbf{b}^{++}$ & $\mathbf{b}^{*}$ & $\mathbf{b}^{\boldsymbol{*}^{++}}$ & $\mathbf{b}^{\mathbf{0}}$ & $\mathbf{b}^{\mathbf{0 + +}}$ & Seq. & $\mathbf{y}$ & $\mathbf{y}^{++}$ & $\mathbf{y}^{\mathbf{*}}$ & $\mathbf{y}^{\mathbf{*}^{++}}$ & $\mathbf{y}^{\mathbf{0}}$ & $\mathbf{y}^{\mathbf{0 + +}}$ & $\#$ \\
\hline $\mathbf{1}$ & 130.0499 & 65.5286 & & & 112.0393 & 56.5233 & $\mathbf{E}$ & & & & & & & $\mathbf{4 2}$ \\
\hline $\mathbf{2}$ & 201.0870 & 101.0471 & & & 183.0764 & 92.0418 & $\mathbf{A}$ & 4276.0907 & 2138.5490 & 4259.0642 & 2130.0357 & 4258.0801 & 2129.5437 & $\mathbf{4 1}$ \\
\hline $\mathbf{3}$ & 258.1084 & 129.5579 & & & 240.0979 & 120.5526 & $\mathbf{G}$ & 4205.0536 & 2103.0304 & 4188.0270 & 2094.5172 & 4187.0430 & 2094.0251 & $\mathbf{4 0}$ \\
\hline $\mathbf{4}$ & 371.1925 & 186.0999 & & & 353.1819 & 177.0946 & $\mathbf{L}$ & 4148.0321 & 2074.5197 & 4131.0056 & 2066.0064 & 4130.0216 & 2065.5144 & $3 \mathbf{3 9}$ \\
\hline $\mathbf{5}$ & 468.2453 & 234.6263 & & & 450.2347 & 225.6210 & $\mathbf{P}$ & 4034.9481 & 2017.9777 & 4017.9215 & 2009.4644 & 4016.9375 & 2008.9724 & $\mathbf{3 8}$ \\
\hline
\end{tabular}




\begin{tabular}{|c|c|c|c|c|c|c|c|c|c|c|c|c|c|c|}
\hline & 565.2980 & 283.1527 & & & 547.2875 & & $\mathbf{P}$ & 3937.8953 & |1969.4513 & |3920.8687| & & 3919.8847| & $|1960.4460|$ & |37 \\
\hline 7 & 679.3410 & 340.1741 & 662.3144 & 331.6608 & 661.3304 & 331.1688 & $\mathbf{N}$ & 3840.8425 & 1920.9249 & 3823.8160 & 1912.4116 & 3822.8320 & 1911.9196 & 36 \\
\hline 8 & 778.4094 & 389.7083 & 761.3828 & 381.1951 & 760.3988 & 380.7030 & V & 3726.7996 & 1863.9034 & 3709.7731 & 1855.3902 & 3708.7890 & 1854.8982 & 35 \\
\hline 9 & 891.4934 & 446.2504 & 874.4669 & 437.7371 & 873.4829 & 437.2451 & I & 3627.7312 & 1814.3692 & 3610.7046 & 1805.8560 & 3609.7206 & 1805.3639 & 34 \\
\hline 10 & 1019.5520 & 510.2796 & 1002.5255 & 501.7664 & 1001.5415 & 501.2744 & $\mathbf{Q}$ & 3514.6471 & 1757.8272 & 3497.6206 & 1749.3139 & 3496.6366 & 1748.8219 & 33 \\
\hline 1 & 1166.6204 & 583.8139 & 1149.5939 & 575.3006 & 1148.6099 & 574.8086 & $\mathbf{F}$ & 3386.5885 & 1693.7979 & 3369.5620 & 1685.2846 & 3368.5780 & 1684.7926 & 32 \\
\hline 12 & 1265.6889 & 633.3481 & 1248.6623 & 624.8348 & 1247.6783 & 624.3428 & V & 3239.5201 & 1620.2637 & 3222.4936 & 1611.7504 & 3221.5096 & 1611.2584 & 31 \\
\hline 13 & 1362.7416 & 681.8744 & 1345.7151 & 673.3612 & 1344.7310 & 672.8692 & $\mathbf{P}$ & 3140.4517 & 1570.7295 & 3123.4252 & 1562.2162 & 3122.4412 & 1561.7242 & 30 \\
\hline 4 & 1433.7787 & 717.3930 & 1416.7522 & 708.8797 & 1415.7682 & 708.3877 & A & 3043.3990 & 1522.2031 & 3026.3724 & 1513.6898 & 3025.3884 & 1513.1978 & 29 \\
\hline 15 & 1548.8057 & 774.9065 & 1531.7791 & 766.3932 & 1530.7951 & 765.9012 & D & 2972.3618 & 1486.6846 & 2955.3353 & 1478.1713 & 2954.3513 & 1477.6793 & 28 \\
\hline 16 & 1605.8271 & 803.4172 & 1588.8006 & 794.9039 & 1587.8166 & 794.4119 & $\mathbf{G}$ & 3349 & 1429.1711 & 2840.3083 & 1420. & 2839.3243 & .1658 & 27 \\
\hline 17 & 1702.8799 & 851.9436 & 1685.8534 & 843.4303 & 1684.8693 & 842.9383 & $\mathbf{P}$ & 2800.3134 & 1400.6604 & 2783.2869 & 1392.1471 & 2782.3029 & 1391.6551 & 26 \\
\hline 18 & 1803.9276 & 902.4674 & 1786.9010 & 893.9542 & 1785.9170 & 893.4621 & $\mathbf{T}$ & 2703.2607 & 1352.1340 & 2686.2341 & 1343.6207 & 2685.2501 & 1343.1287 & 25 \\
\hline 9 & 1950.9960 & 976.0016 & 1933.9694 & 967. & 1932.9854 & 966.9964 & $\mathbf{F}$ & 2130 & & 2585.1864 & 969 & 024 & 6049 & 24 \\
\hline 20 & 2008.0175 & 1004.5124 & 1990.9909 & 995.9991 & 1990.0069 & 995.5071 & G & 2455.1446 & 1228.0759 & 2438.1180 & 1219.5627 & 2437.1340 & 1219.0706 & 23 \\
\hline 21 & 2123.0444 & 1062.0258 & 2106.0178 & 1053.5126 & 2105.0338 & 1053.0206 & D & 2398.1231 & 1199.5652 & 2381.0966 & 1191.0519 & 2380.1125 & 1190.5599 & 22 \\
\hline 22 & 2224 & 1112. & & 364 & 2206.0815 & 1103.5444 & $\mathbf{T}$ & 0962 & & 2266 & 384 & .0856 & 0464 & 21 \\
\hline 23 & 2323.1605 & 1162.0839 & 2306.1339 & 1153.5706 & 2305.1499 & 1153.0786 & $\mathbf{V}$ & 2182.0485 & 1091.5279 & 2165.0219 & 1083.0146 & 2164.0379 & 1082.5226 & 20 \\
\hline 24 & 2424.2082 & 1212.6077 & 2407.1816 & 1204.0944 & 2406.1976 & 1203.6024 & $\mathbf{T}$ & 2082.9801 & 1041.9937 & 2065.9535 & 1033.4804 & 2064.9695 & 1032.9884 & 19 \\
\hline 5 & 2511.2402 & 1256.1237 & 2494.2136 & 1247.6105 & 2493.2296 & 1247.1185 & $\mathrm{~S}$ & 1981.9324 & 991.4698 & 1964.9058 & 982.9566 & 1963.9218 & 4646 & 18 \\
\hline 26 & 2598.2722 & 1299.6398 & 2581.2457 & 1291.1265 & 2580.2617 & 1290.6345 & $\mathrm{~S}$ & 1894.9004 & 947.9538 & 1877.8738 & 939.4405 & 1876.8898 & 938.9485 & 17 \\
\hline 27 & 2727.3148 & 1364.1610 & 2710.2883 & 1355.6478 & 2709.3043 & 1355.1558 & $\mathbf{E}$ & 1807.8683 & 904.4378 & 1790.8418 & 895.9245 & 1789.8578 & 895.4325 & 16 \\
\hline 28 & 2864.3737 & 1432.6905 & 2847.3472 & 1424.1772 & 2846.3632 & 1423.6852 & $\mathbf{H}$ & 1678.8257 & 839.9165 & 1661.7992 & 831.4032 & 1660.8152 & 830.9112 & 15 \\
\hline 29 & 2977.4578 & 1489.2325 & 2960.4312 & 1480.7193 & 2959.4472 & 1480.2273 & $\mathbf{L}$ & 1541.7668 & 771.3871 & 1524.7403 & 762.8738 & 1523.7563 & 762.3818 & 14 \\
\hline 30 & 3137.4884 & 1569.2479 & 3120.4619 & 1560.7346 & 3119.4779 & 1560.2426 & $\mathrm{C}$ & 1428.6828 & 714.8450 & 1411.6562 & 706.3318 & 1410.6722 & 705.8397 & 13 \\
\hline 31 & 3194.5099 & 1597.7586 & 3177.4834 & 1589.2453 & 3176.4993 & 1588.7533 & G & 1268.6521 & 634.8297 & 1251.6256 & 626.3164 & 1250.6416 & 625.8244 & 12 \\
\hline 32 & 3307.5940 & 1654.3006 & 3290.5674 & 1645.7873 & 3289.5834 & 1645.2953 & I & 1211.6307 & 606.3190 & 1194.6041 & 597.8057 & 1193.6201 & 597.3137 & 11 \\
\hline 33 & 3422.6209 & 1711.8141 & 3405.5944 & 1703.3008 & 3404.6103 & 1702.8088 & $\mathbf{N}$ & 1098.5466 & 549.7769 & 1081.5200 & 541.2637 & 1080.5360 & 540.7717 & 10 \\
\hline 34 & 3569.6893 & 1785.3483 & 3552.6628 & 1776.8350 & 3551.6788 & 1776.3430 & $\mathbf{F}$ & 983.5197 & 492.2635 & 966.4931 & 483.7502 & 965.5091 & 483.2582 & 9 \\
\hline 35 & 3670.7370 & 1835.8721 & 3653.7105 & 1827.3589 & 3652.7264 & 1826.8669 & $T$ & 836.4512 & 418.7293 & 819.4247 & 410.2160 & 818.4407 & 409.7240 & 8 \\
\hline 36 & 3727.7585 & 1864.3829 & 3710.7319 & 1855.8696 & 3709.7479 & 1855.3776 & G & 735.4036 & 368.2054 & 718.3770 & 359.6921 & 717.3930 & 359.2001 & 7 \\
\hline 37 & 3814.7905 & 1907.8989 & 3797.7639 & 1899.3856 & 3796.7799 & 1898.8936 & $\mathrm{~S}$ & 678.3821 & 339.6947 & 661.3556 & 331.1814 & 660.3715 & 330.6894 & 6 \\
\hline 38 & 3913.8589 & 1957.4331 & 3896.8324 & 1948.9198 & 3895.8483 & 1948.4278 & V & 591.3501 & 296.1787 & 574.3235 & 287.6654 & 573.3395 & 287.1734 & 5 \\
\hline 39 & 4010.9117 & 2005.9595 & 3993.8851 & 1997.4462 & 3992.9011 & 1996.9542 & $\mathbf{P}$ & 492.2817 & 246.6445 & 475.2551 & 238.1312 & 474.2711 & 237.6392 & 4 \\
\hline 40 & 4111.9594 & 2056.4833 & 4094.9328 & 2047.9700 & 4093.9488 & 2047.4780 & $\mathbf{T}$ & 395.2289 & 198.1181 & 378.2023 & 189.6048 & 377.2183 & 189.1128 & 3 \\
\hline 41 & 4259.0278 & 2130.0175 & 4242.0012 & 2121.5042 & 4241.0172 & 2121.0122 & $\mathbf{F}$ & 294.1812 & 147.5942 & 277.1547 & 139.0810 & & & 2 \\
\hline 42 & & & & & & & $\mathbf{K}$ & 147.1128 & 74.0600 & 130.0863 & 65.5468 & & & 1 \\
\hline
\end{tabular}
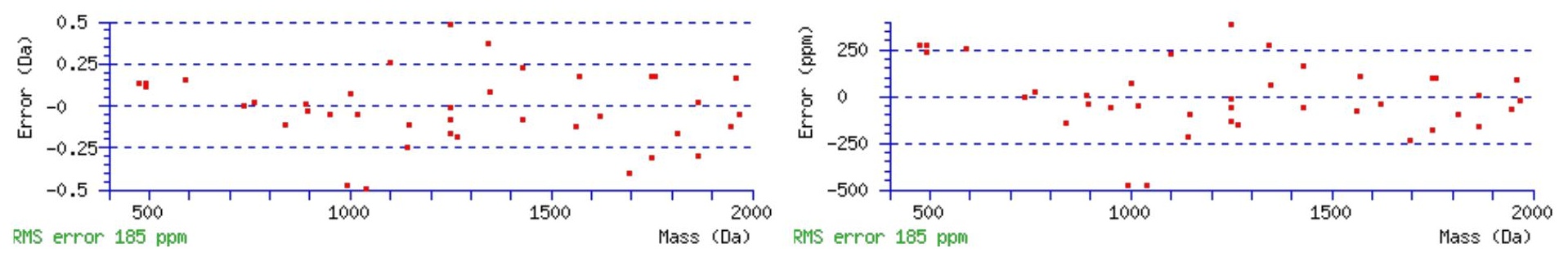

\section{All matches to this query}

\begin{tabular}{|l|l|c|l|}
\hline Score & Mr(calc): & Delta & \multicolumn{1}{c|}{ Sequence } \\
\hline 62.1 & 4404.1261 & 0.0174 & EAGLPPNVIQFVPADGPTFGDTVTSSEHLCGINFTGSVPTFK \\
\hline 53.1 & 4404.1261 & 0.0174 & EAGLPPNVIQFVPADGPTFGDTVTSSEHLCGINFTGSVPTFK \\
\hline 52.9 & 4403.1421 & 1.0014 & EAGLPPNVIQFVPADGPTFGDTVTSSEHLCGINFTGSVPTFK \\
\hline
\end{tabular}




\begin{tabular}{|l|l|l|l|}
\hline 10.1 & 4403.1219 & 1.0216 & SLVNMPADGVTVDPALLPPACLGALGDLSVDPPVQFRSFDR \\
\hline 7.1 & 4404.1820 & -0.0385 & LLQSLVTVELGNQRYXMSLVDCVFPPKVGSLSMITNSQK \\
\hline 5.7 & 4404.1051 & 0.0384 & ASHILASHSPQKPLFLYVAFQAVHTPLQSPREYLYR \\
\hline 5.6 & 4403.1219 & 1.0216 & SLVNMPADGVTVDPALLPPACLGALGDLSVDPPVQFRSFDR \\
\hline 4.5 & 4402.1526 & 1.9908 & RYIEYVCDMVAEEPITPHSKPMLVKSVVMTPVPLFSK \\
\hline 4.3 & 4402.1526 & 1.9908 & RYIEYVCDMVAEEPITPHSKPMLVKSVVMTPVPLFSK \\
\hline 3.7 & 4402.1138 & 2.0297 & RFHQPIYYLLGNLAAADLFAGMAYLFLMFHTGPRTAR \\
\hline
\end{tabular}

Spectrum No: 501; Query: 106; Rank: 1

\section{Peptide View}

MS/MS Fragmentation of IANISMEVGR

Found in IPI00363534, Tax_Id=10116 Gene_Symbol=Lama1 laminin, alpha 1

Match to Query 106: 1105.541408 from(553.777980,2+)

Title: 091008RatKidney_NH4Format01_27.1010.1010.2.dta

Data file K:INewmanPaper|Piliang|3SubProteomes\Piliang3SP\mgf5ppm\ERLIC_3SubProteomes5ppm.mgf
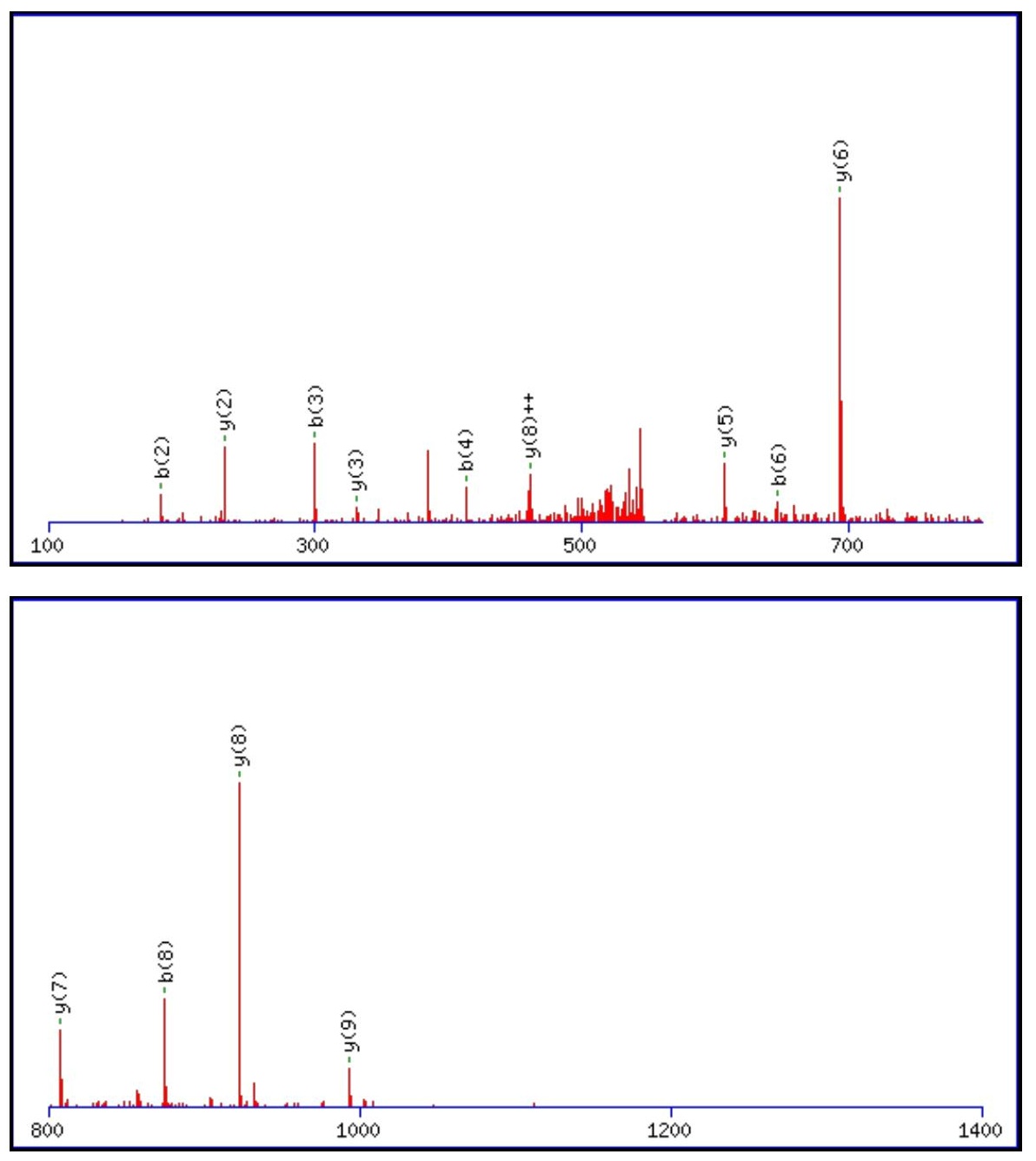


$1400 \quad 1600 \quad 1800 \quad 2000$

Monoisotopic mass of neutral peptide $\operatorname{Mr}($ calc): 1105.5437

Fixed modifications: Carbamidomethyl (C)

Variable modifications:

N3 : Deamidated_N (N)

M6 : Oxidation (M)

Ions Score: 62 Expect: $7.1 \mathrm{e}-005$

Matches (Bold Red): 13/90 fragment ions using 21 most intense peaks

\begin{tabular}{|r|c|c|c|c|c|c|c|c|c|c|c|c|c|c|}
\hline$\#$ & $\mathbf{b}$ & $\mathbf{b}^{++}$ & $\mathbf{b}^{*}$ & $\mathbf{b}^{\boldsymbol{*}^{++}}$ & $\mathbf{b}^{\mathbf{0}}$ & $\mathbf{b}^{\mathbf{0 + +}}$ & $\mathbf{S e q}$ & $\mathbf{y}$ & $\mathbf{y}^{++}$ & $\mathbf{y}^{\mathbf{*}}$ & $\mathbf{y}^{\mathbf{*}^{++}}$ & $\mathbf{y}^{\mathbf{0}}$ & $\mathbf{y}^{\mathbf{0 + +}}$ & $\#$ \\
\hline $\mathbf{1}$ & 114.0913 & 57.5493 & & & & & $\mathbf{I}$ & & & & & & & $\mathbf{1 0}$ \\
\hline $\mathbf{2}$ & $\mathbf{1 8 5 . 1 2 8 5}$ & 93.0679 & & & & & $\mathbf{A}$ & $\mathbf{9 9 3 . 4 6 7 0}$ & 497.2371 & 976.4404 & 488.7238 & 975.4564 & 488.2318 & $\mathbf{9}$ \\
\hline $\mathbf{3}$ & $\mathbf{3 0 0 . 1 5 5 4}$ & 150.5813 & 283.1288 & 142.0681 & & & $\mathbf{N}$ & $\mathbf{9 2 2 . 4 2 9 9}$ & $\mathbf{4 6 1 . 7 1 8 6}$ & 905.4033 & 453.2053 & 904.4193 & 452.7133 & $\mathbf{8}$ \\
\hline $\mathbf{4}$ & $\mathbf{4 1 3 . 2 3 9 5}$ & 207.1234 & 396.2129 & 198.6101 & & & $\mathbf{I}$ & $\mathbf{8 0 7 . 4 0 2 9}$ & 404.2051 & 790.3764 & 395.6918 & 789.3924 & 395.1998 & $\mathbf{7}$ \\
\hline $\mathbf{5}$ & 500.2715 & 250.6394 & 483.2449 & 242.1261 & 482.2609 & 241.6341 & $\mathbf{S}$ & $\mathbf{6 9 4 . 3 1 8 9}$ & 347.6631 & 677.2923 & 339.1498 & 676.3083 & 338.6578 & $\mathbf{6}$ \\
\hline $\mathbf{6}$ & $\mathbf{6 4 7 . 3 0 6 9}$ & 324.1571 & 630.2803 & 315.6438 & 629.2963 & 315.1518 & $\mathbf{M}$ & $\mathbf{6 0 7 . 2 8 6 8}$ & 304.1471 & 590.2603 & 295.6338 & 589.2763 & 295.1418 & $\mathbf{5}$ \\
\hline $\mathbf{7}$ & $\mathbf{7 7 6 . 3 4 9 5}$ & 388.6784 & 759.3229 & 380.1651 & 758.3389 & 379.6731 & $\mathbf{E}$ & 460.2514 & 230.6293 & 443.2249 & 222.1161 & 442.2409 & 221.6241 & $\mathbf{4}$ \\
\hline $\mathbf{8}$ & $\mathbf{8 7 5 . 4 1 7 9}$ & 438.2126 & 858.3913 & 429.6993 & 857.4073 & 429.2073 & $\mathbf{V}$ & 331.2088 & 166.1081 & 314.1823 & 157.5948 & & & 3 \\
\hline $\mathbf{9}$ & 932.4394 & 466.7233 & 915.4128 & 458.2100 & 914.4288 & 457.7180 & $\mathbf{G}$ & 232.1404 & 116.5738 & 215.1139 & 108.0606 & & & $\mathbf{2}$ \\
\hline $\mathbf{1 0}$ & & & & & & & $\mathbf{R}$ & $\mathbf{1 7 5 . 1 1 9 0}$ & 88.0631 & 158.0924 & 79.5498 & & & $\mathbf{1}$ \\
\hline
\end{tabular}
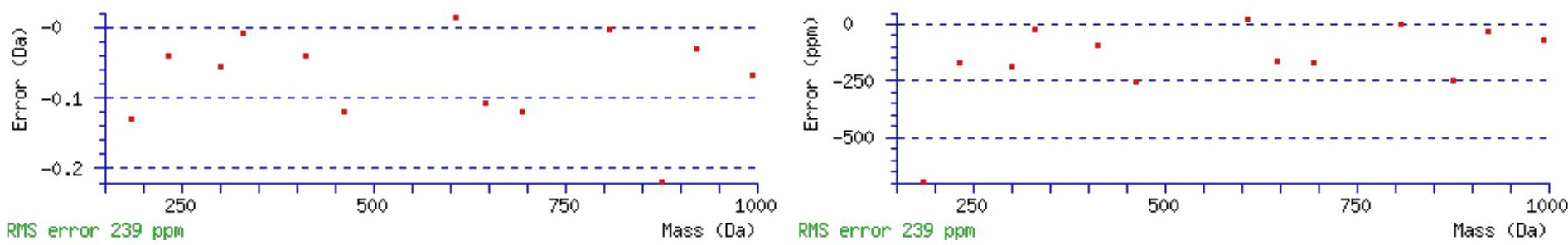

\section{All matches to this query}

\begin{tabular}{|l|l|l|l|}
\hline Score & Mr(calc): & Delta & \multicolumn{1}{|c|}{ Sequence } \\
\hline 62.0 & 1105.5437 & -0.0023 & IANISMEVGR \\
\hline 17.2 & 1105.5403 & 0.0011 & LANAAGFNAEK \\
\hline 15.2 & 1105.5404 & 0.0010 & LADAAENFQK \\
\hline 11.8 & 1105.5478 & -0.0064 & IALNMDWVK \\
\hline 8.5 & 1105.5338 & 0.0076 & LAQYMAHTR \\
\hline 7.8 & 1105.5394 & 0.0021 & IHVTSRTGR \\
\hline 4.1 & 1105.5393 & 0.0021 & SPAKRTGGPR \\
\hline 3.9 & 1105.5393 & 0.0021 & HSOKKSVGR \\
\hline 2.7 & 1105.5506 & -0.0092 & SAAPRRGVGR \\
\hline 1.7 & 1103.5393 & 2.0021 & VEAMLNDRR \\
\hline
\end{tabular}


Spectrum No: 502; Query: 1280; Rank: 1

\section{Peptide View}

MS/MS Fragmentation of IVSNASCTTNCLAPLAK

Found in IPI00361708, Tax_Id=10116 Gene_Symbol=- 36 kDa protein

Match to Query 1280: 1819.879148 from(910.946850,2+)

Title: 091008RatKidney_NH4Format01_06.2238.2238.2.dta

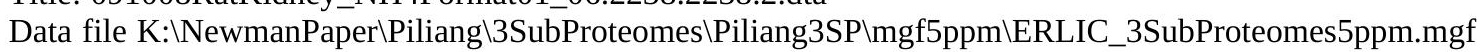
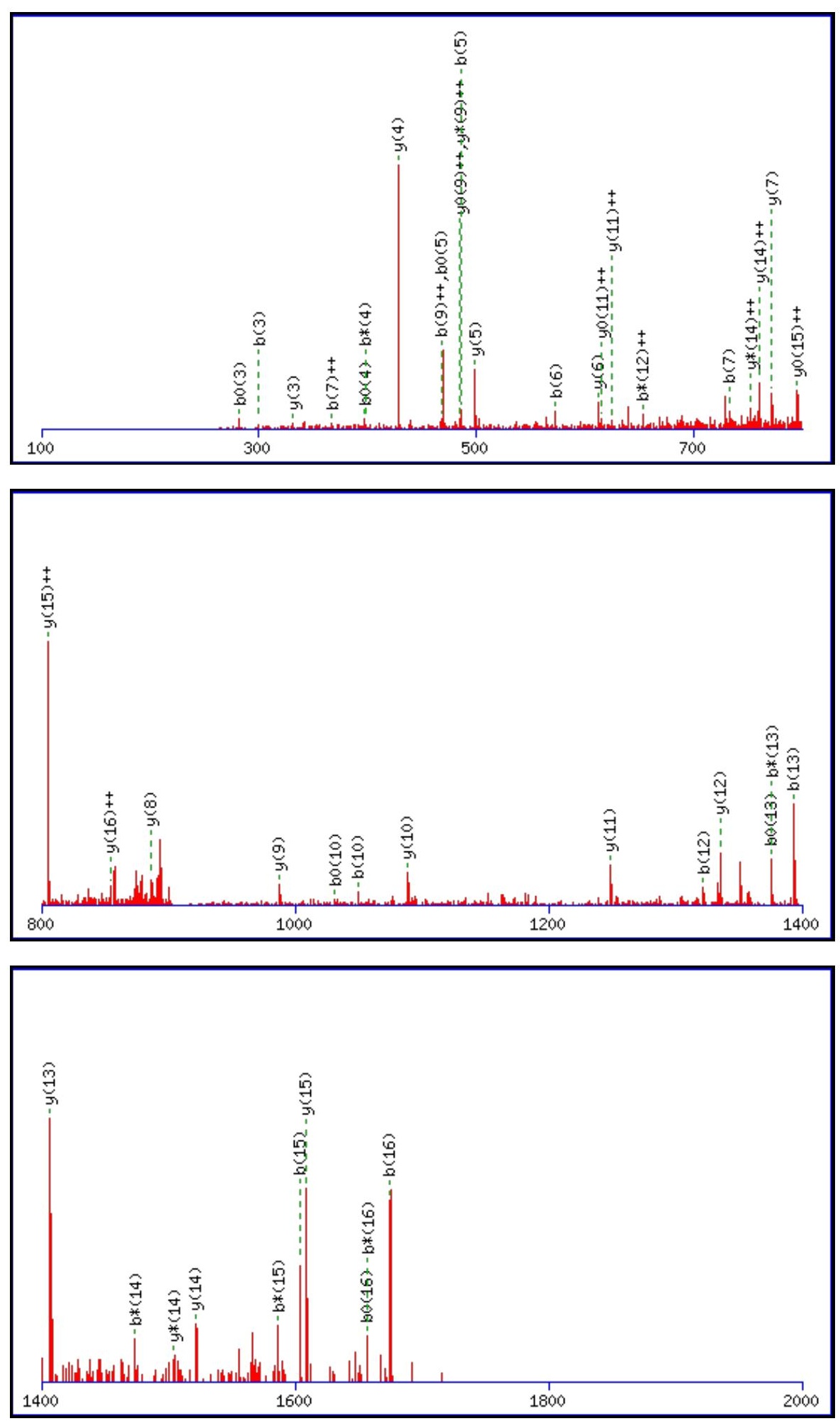
Monoisotopic mass of neutral peptide $\operatorname{Mr}($ calc): 1819.8808

Fixed modifications: Carbamidomethyl (C)

Variable modifications:

N4 : Deamidated_N (N)

Ions Score: 62 Expect: 0.00014

Matches (Bold Red): 46/166 fragment ions using 106 most intense peaks

\begin{tabular}{|c|c|c|c|c|c|c|c|c|c|c|c|c|c|c|}
\hline \# & b & $\mathbf{b}^{++}$ & $\mathbf{b}^{*}$ & $\mathbf{b}^{*^{++}}$ & $\mathbf{b}^{0}$ & $\mathbf{b}^{\mathbf{0 + +}}$ & Seq. & $\mathbf{y}$ & $\mathbf{y}^{++}$ & $\mathbf{y}^{*}$ & $\mathrm{y}^{\mathrm{*}^{++}}$ & $\mathbf{y}^{0}$ & $\mathbf{y}^{\mathbf{0 + +}}$ & $\#$ \\
\hline 1 & 114.0913 & 57.5493 & & & & & I & & & & & & & 17 \\
\hline 2 & 213.1598 & 107.0835 & & & & & $\mathbf{V}$ & 1707.8040 & 854.4057 & 1690.7775 & 845.8924 & 1689.7935 & 845.4004 & \\
\hline 3 & 300.1918 & 150.5995 & & & 282.1812 & 141.5942 & $\mathrm{~S}$ & 1608.7356 & 804.8714 & 1591.7091 & 796.3582 & 1590.7250 & 795.8662 & 1 \\
\hline 4 & 415.2187 & 208.1130 & 398.1922 & 199.5997 & 397.2082 & 199.1077 & $\mathbf{N}$ & 1521.7036 & 761.3554 & 1504.6770 & 752.8422 & 1503.6930 & 752.3501 & \\
\hline 5 & 486.2558 & 243.6316 & 469.2293 & 235.1183 & 468.2453 & 234.6263 & A & \begin{tabular}{|l}
1406.6766 \\
\end{tabular} & 703.8420 & 1389.6501 & 695.3287 & 1388.6661 & 694.8367 & \\
\hline 6 & 573.2879 & 287.1476 & 556.2613 & 278.6343 & 555.2773 & 278.1423 & $\mathrm{~S}$ & 1335.6395 & 668.3234 & 1318.6130 & 659.8101 & 1317.6290 & 659.3181 & 1 \\
\hline 7 & 733.3185 & 367.1629 & 716.2920 & 358.6496 & 715.3079 & 358.1576 & C & 1248.6075 & 624.8074 & 1231.5810 & 616.2941 & 1230.5969 & 615.8021 & \\
\hline 8 & 834.3662 & 417.6867 & 817.3396 & 409.1735 & 816.3556 & 408.6814 & $T$ & \begin{tabular}{|l|l|}
1088.5769 \\
\end{tabular} & 544.7921 & 1071.5503 & 536.2788 & 1070.5663 & 535.7868 & 1 \\
\hline 9 & 935.4139 & 468.2106 & 918.3873 & 459.6973 & 917.4033 & 459.2053 & $T$ & 987.5292 & 494.2682 & 970.5026 & 485.7550 & 969.5186 & 485.2629 & \\
\hline 10 & 1049.4568 & 525.2320 & 1032.4302 & 516.7188 & 1031.4462 & 516.2268 & $\mathbf{N}$ & 886.4815 & 443.7444 & 869.4550 & 435.2311 & & & \\
\hline 11 & 1209.4874 & 605.2474 & 1192.4609 & 596.7341 & 1191.4769 & 596.2421 & $\mathrm{C}$ & 772.4386 & 386.7229 & 755.4120 & 378.2096 & & & \\
\hline 12 & 1322.5715 & 661.7894 & 1305.5450 & 653.2761 & 1304.5609 & 652.7841 & $\mathbf{L}$ & 612.4079 & 306.7076 & 595.3814 & 298.1943 & & & \\
\hline 13 & 1393.6086 & 697.3079 & 1376.5821 & 688.7947 & 1375.5981 & 688.3027 & $\mathbf{A}$ & 499.3239 & 250.1656 & 482.2973 & 241.6523 & & & \\
\hline 14 & 1490.6614 & 745.8343 & 1473.6348 & 737.3211 & 1472.6508 & 736.8290 & $\mathbf{P}$ & 428.2867 & 214.6470 & 411.2602 & 206.1337 & & & \\
\hline 15 & 1603.7454 & 802.3764 & 1586.7189 & 793.8631 & 1585.7349 & 793.3711 & $\mathbf{L}$ & 331.2340 & 166.1206 & 314.2074 & 157.6074 & & & \\
\hline 16 & \begin{tabular}{|l|}
1674.7826 \\
\end{tabular} & 837.8949 & 1657.7560 & 829.3816 & 1656.7720 & 828.8896 & A & 218.1499 & 109.5786 & 201.1234 & 101.0653 & & & \\
\hline 17 & & & & & & & $\mathbf{K}$ & 147.1128 & 74.0600 & 130.0863 & 65.5468 & & & \\
\hline
\end{tabular}

.
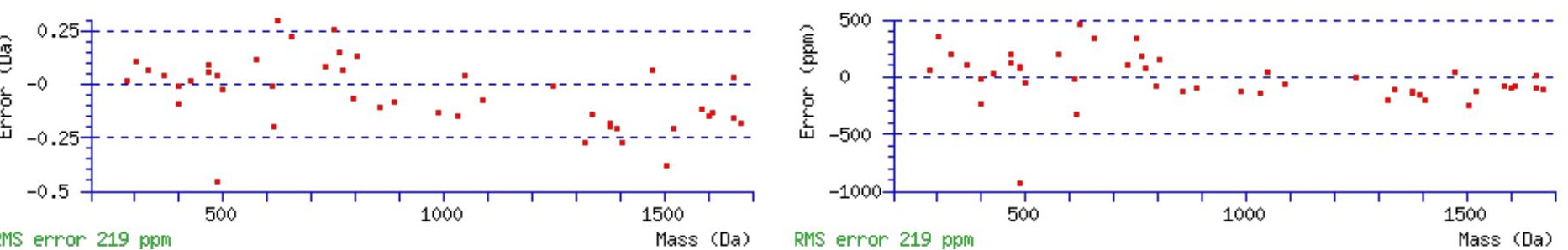

All matches to this query

\begin{tabular}{|l|l|l|l|}
\hline Score & Mr(calc): & Delta & \multicolumn{1}{c|}{ Sequence } \\
\hline 61.9 & 1819.8808 & -0.0017 & IVSNASCTTNCLAPLAK \\
\hline 48.3 & 1819.8808 & -0.0017 & IVSNASCTTNCLAPLAK \\
\hline 3.4 & 1818.8872 & 0.9920 & LSGKIKMTLEMLSEK \\
\hline 2.4 & 1818.8625 & 1.0166 & $\underline{\text { ISPSAATTNKNHLTQR }}$ \\
\hline 0.6 & 1817.8842 & 1.9949 & ELANEFTRMARDPPR \\
\hline 0.4 & 1818.8872 & 0.9920 & $\underline{\text { LSGKIKMTLEMLSEK }}$ \\
\hline
\end{tabular}

Spectrum No: 503; Query: 1053; Rank: 1

\section{Peptide View}

MS/MS Fragmentation of CDQCEENYFYNR

Found in IPI00363849, Tax_Id=10116 Gene_Symbol=Lamc1 similar to Laminin gamma-1 chain precursor

Match to Query 1053: 1697.614648 from(849.814600,2+)

Title: 100101RatKid_NS_deglyco_24.1916.1916.2.dta

Data file K:INewmanPaper|Piliang|3SubProteomes\Piliang3SP\mgf5ppm\ERLIC_3SubProteomes5ppm.mgf 

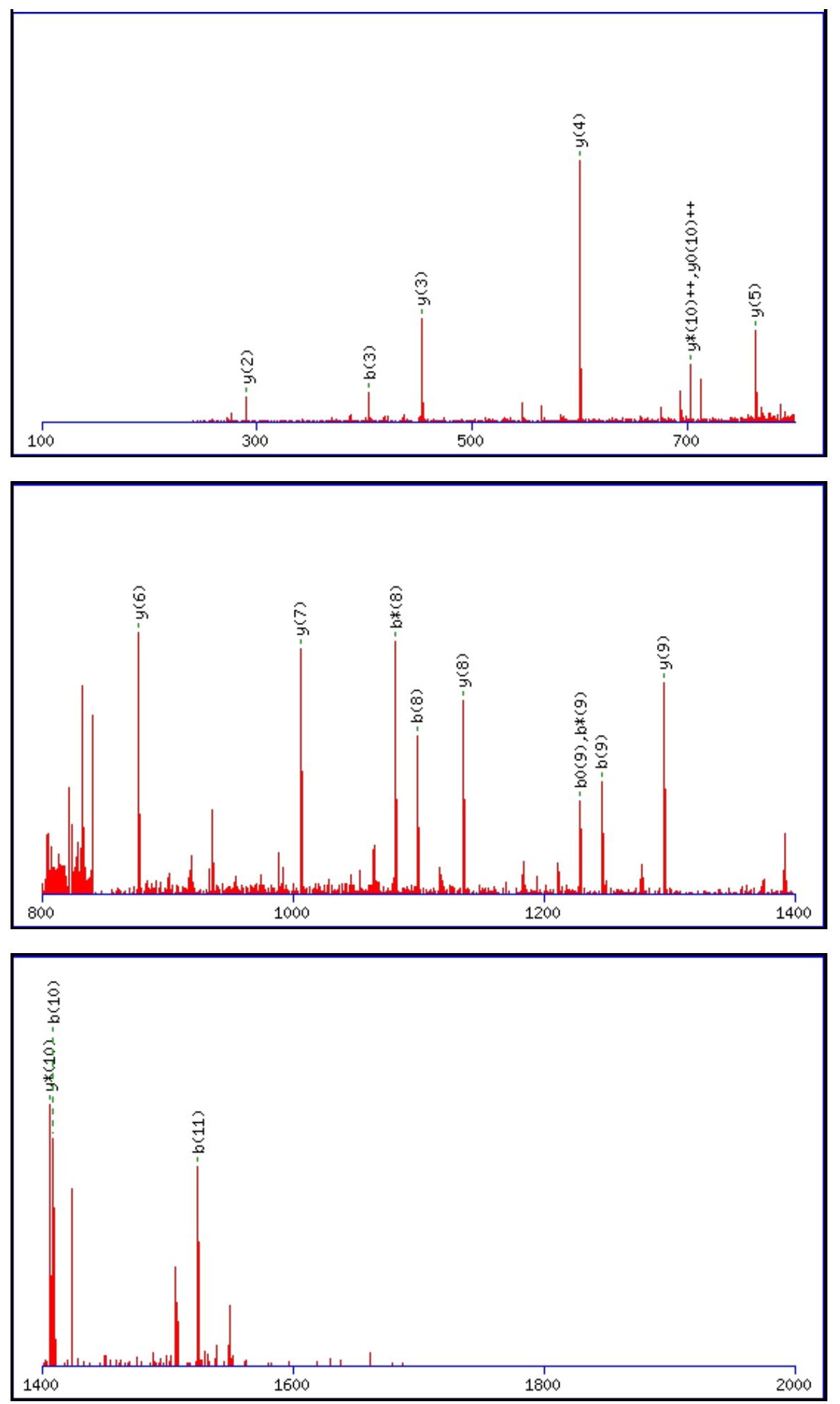

Monoisotopic mass of neutral peptide $\operatorname{Mr}($ calc): 1697.6086

Fixed modifications: Carbamidomethyl (C)

Variable modifications:

N11 : Deamidated N (N)

Ions Score: 62 Expect: 5 e- 005

Matches (Bold Red): 19/114 fragment ions using 24 most intense peaks

\begin{tabular}{|r|c|c|c|c|c|c|c|c|c|c|c|c|c|c|}
\hline$\#$ & $\mathbf{b}$ & $\mathbf{b}^{++}$ & $\mathbf{b}^{*}$ & $\mathbf{b}^{\boldsymbol{*}^{++}}$ & $\mathbf{b}^{\mathbf{0}}$ & $\mathbf{b}^{\mathbf{0 + +}}$ & Seq. & $\mathbf{y}$ & $\mathbf{y}^{++}$ & $\mathbf{y}^{\mathbf{*}}$ & $\mathbf{y}^{\boldsymbol{*}^{++}}$ & $\mathbf{y}^{\mathbf{0}}$ & $\mathbf{y}^{\mathbf{0 + +}}$ & $\#$ \\
\hline $\mathbf{1}$ & 161.0379 & 81.0226 & & & & & $\mathbf{C}$ & & & & & & & $\mathbf{1 2}$ \\
\hline $\mathbf{2}$ & 276.0649 & 138.5361 & & & 258.0543 & 129.5308 & $\mathbf{D}$ & 1538.5852 & 769.7963 & 1521.5587 & 761.2830 & 1520.5747 & 760.7910 & $\mathbf{1 1}$ \\
\hline $\mathbf{3}$ & $\mathbf{4 0 4 . 1 2 3 4}$ & 202.5654 & 387.0969 & 194.0521 & 386.1129 & 193.5601 & $\mathbf{Q}$ & 1423.5583 & 712.2828 & $\mathbf{1 4 0 6 . 5 3 1 8}$ & $\mathbf{7 0 3 . 7 6 9 5}$ & 1405.5477 & $\mathbf{7 0 3 . 2 7 7 5}$ & $\mathbf{1 0}$ \\
\hline $\mathbf{4}$ & 564.1541 & 282.5807 & 547.1275 & 274.0674 & 546.1435 & 273.5754 & $\mathbf{C}$ & $\mathbf{1 2 9 5 . 4 9 9 7}$ & 648.2535 & 1278.4732 & 639.7402 & 1277.4892 & 639.2482 & $\mathbf{9}$ \\
\hline $\mathbf{5}$ & 693.1967 & 347.1020 & 676.1701 & 338.5887 & 675.1861 & 338.0967 & $\mathbf{E}$ & $\mathbf{1 1 3 5 . 4 6 9 1}$ & 568.2382 & 1118.4425 & 559.7249 & 1117.4585 & 559.2329 & $\mathbf{8}$ \\
\hline
\end{tabular}




\begin{tabular}{|c|c|c|c|c|c|c|c|c|c|c|c|c|c|c|}
\hline 6 & 822.2393 & |411.6233 & 805.2127 & |403.1100 & 804.2287 & 402.6180| & E & |1006.4265 & |503.7169 & 989.3999| & 495.2036 & 988.4159 & |494.7116 & 7 \\
\hline 7 & 936.2822 & 468.6447 & 919.2557 & 460.1315 & 918.2716 & 459.6395 & $\mathbf{N}$ & 877.3839 & 439.1956 & 860.3573 & 430.6823 & & & 6 \\
\hline 8 & 1099.3455 & 550.1764 & 1082.3190 & 541.6631 & 1081.3350 & 541.1711 & $\mathbf{Y}$ & 763.3410 & 382.1741 & 746.3144 & 373.6608 & & & 5 \\
\hline 9 & 1246.4140 & 623.7106 & 1229.3874 & 615.1973 & 1228.4034 & 614.7053 & $\mathbf{F}$ & 600.2776 & 300.6425 & 583.2511 & 292.1292 & & & 4 \\
\hline 10 & 1409.4773 & 705.2423 & 1392.4507 & 696.7290 & 1391.4667 & 696.2370 & $\mathbf{Y}$ & 453.2092 & 227.1082 & 436.1827 & 218.5950 & & & 3 \\
\hline 11 & 1524.5042 & 762.7557 & 1507.4777 & 754.2425 & 1506.4937 & 753.7505 & $\mathbf{N}$ & 290.1459 & 145.5766 & 273.1193 & 137.0633 & & & 2 \\
\hline 12 & & & & & & & $\mathbf{R}$ & 175.1190 & 88.0631 & 158.0924 & 79.5498 & & & 1 \\
\hline
\end{tabular}
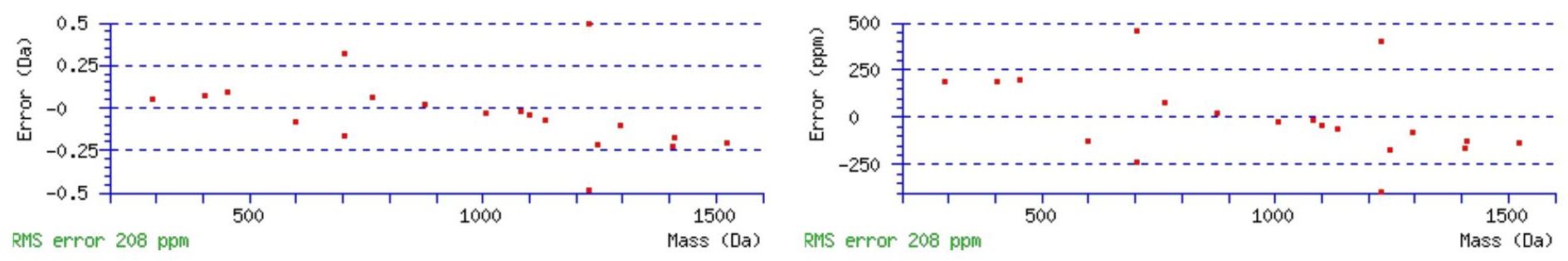

\section{All matches to this query}

\begin{tabular}{|l|l|l|l|}
\hline Score & Mr(calc): & Delta & Sequence \\
\hline 61.7 & 1697.6086 & 0.0061 & CDQCEENYFYNR \\
\hline 42.1 & 1697.6086 & 0.0061 & CDQCEENYFYNR \\
\hline 24.8 & 1696.6246 & 0.9901 & CDQCEENYFYNR \\
\hline 3.4 & 1697.6191 & -0.0044 & NQAMSSMIRNQEK \\
\hline 3.1 & 1697.6182 & -0.0036 & SQETSQNSKANSKGA \\
\hline 3.0 & 1697.6182 & -0.0036 & SQETSQNSKANSKGA \\
\hline 3.0 & 1697.6182 & -0.0036 & SQETSQNSKANSKGA \\
\hline
\end{tabular}

Spectrum No: 504; Query: 2388; Rank: 1

\section{Peptide View}

\section{MS/MS Fragmentation of HNNDTQHIWESDSNEFSVIADPR}

Found in IPI00365985, Tax_Id=10116 Gene_Symbol=Tra1_predicted 93 kDa protein

Match to Query 2388: 2711.192622 from(904.738150,3+)

Title: 100101RatKid_NS_deglyco_14.3521.3521.3.dta

Data file K:INewmanPaper|Piliangl3SubProteomes\Piliang3SP\mgf5ppm\ERLIC_3SubProteomes5ppm.mgf

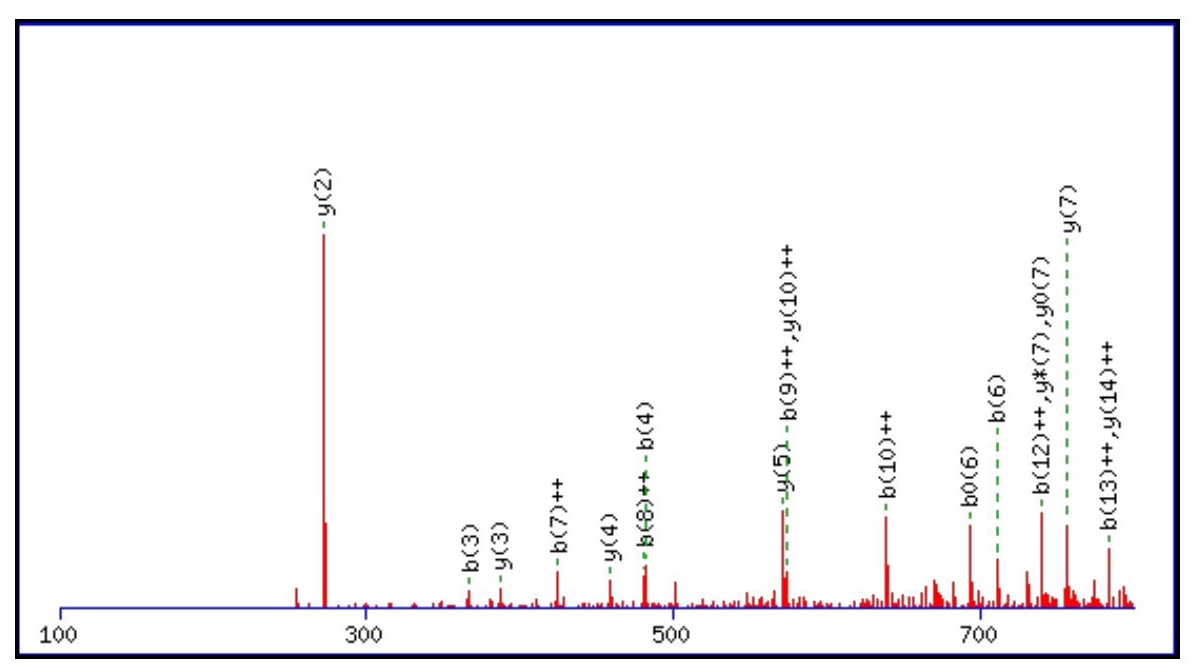



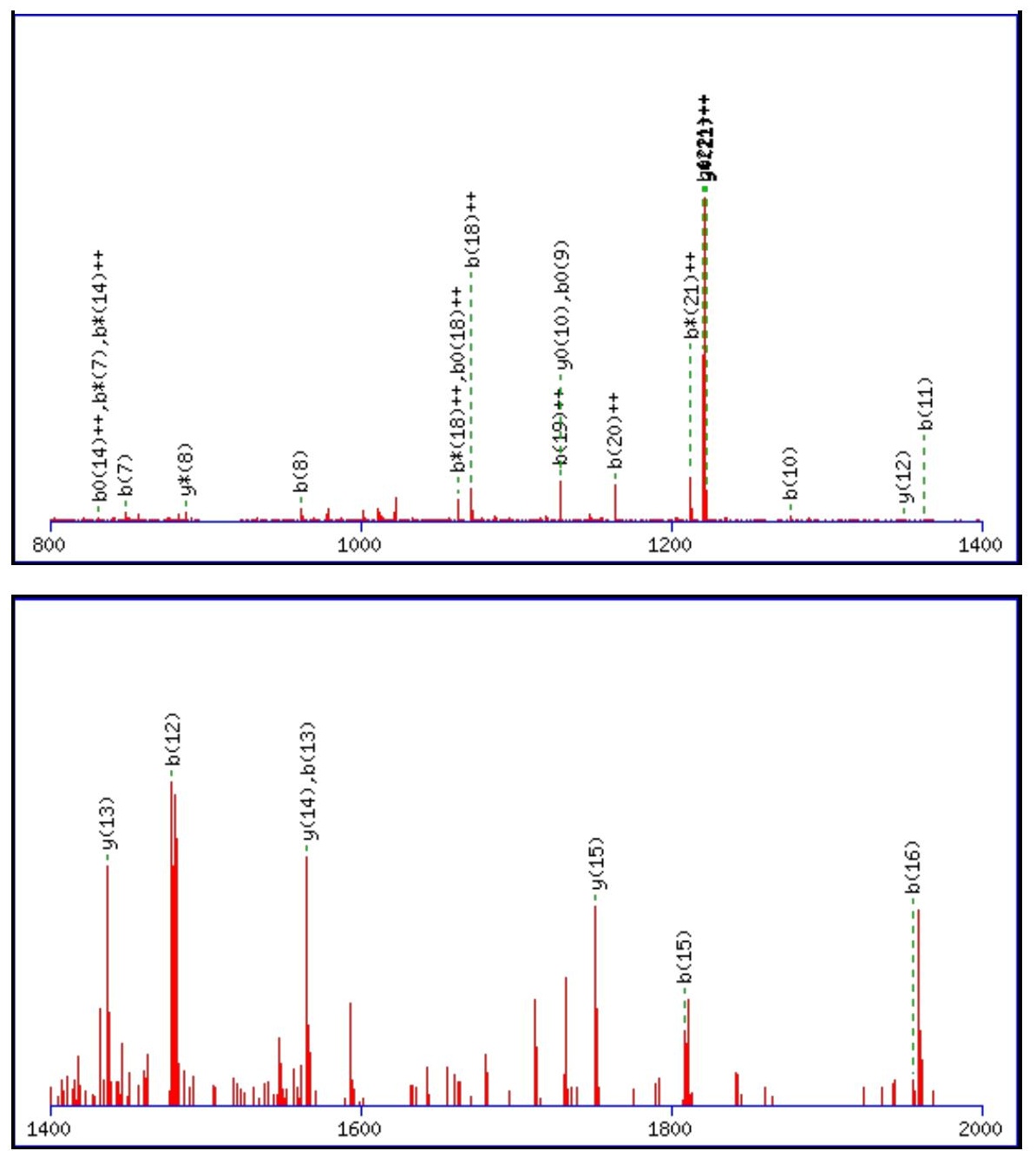

Monoisotopic mass of neutral peptide $\operatorname{Mr}($ calc): 2711.1848

Fixed modifications: Carbamidomethyl (C)

Variable modifications:

N3 : Deamidated_N (N)

Ions Score: 62 Expect: 0.00024

Matches (Bold Red): 46/252 fragment ions using 75 most intense peaks

\begin{tabular}{|c|c|c|c|c|c|c|c|c|c|c|c|c|c|c|}
\hline \# & b & $\mathbf{b}^{++}$ & $\mathbf{b}^{*}$ & $\mathbf{b}^{*^{++}}$ & $\mathbf{b}^{\mathbf{0}}$ & $\mathbf{b}^{0++}$ & Seq. & $\mathbf{y}$ & $\mathbf{y}^{++}$ & $\mathbf{y}^{*}$ & $\mathbf{y}^{*^{++}}$ & $\mathbf{y}^{\mathbf{0}}$ & $\mathbf{y}^{0++}$ & \# \\
\hline 1 & 138.0662 & 69.5367 & & & & & $\mathbf{H}$ & & & & & & & 23 \\
\hline 2 & 252.1091 & 126.5582 & 235.0826 & 118.0449 & & & $\mathbf{N}$ & 2575.1332 & 1288.0702 & 2558.1066 & & 2557.1226 & 1279.0649 & 22 \\
\hline 3 & 367.1361 & 184.0717 & 350.1095 & 175.5584 & & & $\mathbf{N}$ & 2461.0902 & 1231.0488 & 2444.0637 & 1222.5355 & 2443.0797 & 1222.0435 & 21 \\
\hline 4 & 482.1630 & 241.5851 & 465.1364 & 233.0719 & 464.1524 & 232.5799 & D & 2346.0633 & 1173.5353 & 2329.0367 & 1165.0220 & 2328.0527 & 1164.5300 & 20 \\
\hline 5 & 583.2107 & 292.1090 & 566.1841 & 283.5957 & 565.2001 & 283.1 & $\mathbf{T}$ & 2231.0364 & 1116.0218 & 2214.0098 & 1107.5085 & 2213.0258 & 1107.0165 & 19 \\
\hline 6 & 711.2693 & 356.1383 & 694.2427 & 347.6250 & 693.2587 & 347.1330 & $\mathbf{Q}$ & 2129.9887 & 1065.4980 & 2112.9621 & 1056.9847 & 2111.9781 & 1056.4927 & 18 \\
\hline 7 & 848.3282 & 424.6677 & 831.3016 & 416.1544 & 830.3176 & 415.6624 & $\mathbf{H}$ & 2001.9301 & 1001.4687 & 1984.9035 & 992.9554 & 1983.9195 & 992.4634 & 17 \\
\hline 8 & 961.4122 & 481.2098 & 944.3857 & 472.6965 & 943.4017 & 472.2045 & I & 1864.8712 & 932.9392 & 1847.8446 & 924.4260 & 1846.8606 & 923.9339 & 16 \\
\hline 9 & 1147.4915 & 574.2494 & 1130.4650 & 565.7361 & 1129.4810 & 565.2441 & W & 1751.7871 & 876.3972 & 1734.7606 & 867.8839 & 1733.7766 & 867.3919 & 15 \\
\hline 10 & 1276.5341 & 638.7707 & 1259.5076 & 630.2574 & 1258.5236 & 629.7654 & $\mathbf{E}$ & 1565.7078 & 783.3575 & 1548.6813 & 774.8443 & 1547.6972 & 774.3523 & 14 \\
\hline 11 & 1363.5662 & 682.2867 & 1346.5396 & 673.7734 & 1345.5556 & 673.2814 & S & 1436.6652 & 718.8362 & 1419.6387 & 710.3230 & 1418.6546 & 709.8310 & 13 \\
\hline 12 & 1478.5931 & 739.8002 & 1461.5666 & 731.2869 & 1460.5825 & 730.7949 & D & 1349.6332 & 675.3202 & 1332.6066 & 666.8070 & 1331.6226 & 666.3149 & 12 \\
\hline 13 & 1565.6251 & 783.3162 & 1548.5986 & 774.8029 & 1547.6146 & 774.3109 & S & 1234.6062 & 617.8068 & 1217.5797 & 609.2935 & 1216.5957 & 608.8015 & 11 \\
\hline 14 & 1679.6681 & 840.3377 & 1662.6415 & 831.8244 & 1661.6575 & 831.3324 & $\mathbf{N}$ & 1147.5742 & 574.2907 & 1130.5477 & 565.7775 & 1129.5636 & 565.2855 & 10 \\
\hline 15 & 1808.7107 & 904.8590 & 1791.6841 & 896.3457 & 1790.7001 & 895.8537 & $\mathbf{E}$ & 1033.5313 & 517.2693 & 1016.5047 & 508.7560 & 1015.5207 & 508.2640 & 9 \\
\hline 16 & 1955.7791 & 978.3932 & 1938.7525 & 969.8799 & 1937.7685 & 969.3879 & $\mathbf{F}$ & 904.4887 & 452.7480 & 887.4621 & 444.2347 & 886.4781 & 443.7427 & 8 \\
\hline 17 & 2042.8111 & 1021.9092 & 2025.7845 & 1013.3959 & 2024.8005 & 1012.9039 & S & 757.4203 & 379.2138 & 740.3937 & 370.7005 & 739.4097 & 370.2085 & 7 \\
\hline 18 & 2141.8795 & 1071.4434 & 2124.8530 & 1062.9301 & 2123.8689 & 1062.4381 & $\mathbf{V}$ & 670.3883 & 335.6978 & 653.3617 & 327.1845 & 652.3777 & 326.6925 & 6 \\
\hline
\end{tabular}




\begin{tabular}{|r|r|r|r|r|r|r|r|r|r|r|r|r|r|r|}
$\mathbf{1 9}$ & 2254.9636 & $\mathbf{1 1 2 7 . 9 8 5 4}$ & 2237.9370 & 1119.4721 & 2236.9530 & 1118.9801 & I & $\mathbf{5 7 1 . 3 1 9 8}$ & $\mathbf{2 8 6 . 1 6 3 6}$ & 554.2933 & 277.6503 & 553.3093 & $\mathbf{2 7 7 . 1 5 8 3}$ & $\mathbf{5}$ \\
\hline $\mathbf{2 0}$ & 2326.0007 & $\mathbf{1 1 6 3 . 5 0 4 0}$ & 2308.9741 & 1154.9907 & 2307.9901 & 1154.4987 & $\mathbf{A}$ & $\mathbf{4 5 8 . 2 3 5 8}$ & 229.6215 & 441.2092 & 221.1083 & 440.2252 & 220.6162 & $\mathbf{4}$ \\
\hline $\mathbf{2 1}$ & 2441.0276 & $\mathbf{1 2 2 1 . 0 1 7 5}$ & 2424.0011 & $\mathbf{1 2 1 2 . 5 0 4 2}$ & 2423.0171 & 1212.0122 & $\mathbf{D}$ & $\mathbf{3 8 7 . 1 9 8 7}$ & 194.1030 & 370.1721 & 185.5897 & 369.1881 & 185.0977 & $\mathbf{3}$ \\
\hline $\mathbf{2 2}$ & 2538.0804 & 1269.5438 & 2521.0538 & 1261.0306 & 2520.0698 & 1260.5386 & $\mathbf{P}$ & $\mathbf{2 7 2 . 1 7 1 7}$ & 136.5895 & 255.1452 & 128.0762 & & & $\mathbf{2}$ \\
\hline $\mathbf{2 3}$ & & & & & & & $\mathbf{R}$ & 175.1190 & 88.0631 & 158.0924 & $\mathbf{7 9 . 5 4 9 8}$ & & & $\mathbf{1}$ \\
\hline
\end{tabular}
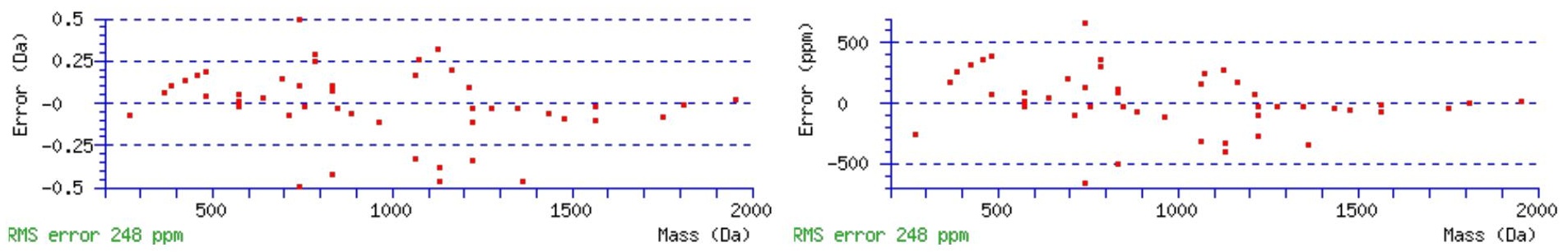

\section{All matches to this query}

\begin{tabular}{|l|c|c|l|}
\hline Score & Mr(calc): & Delta & \multicolumn{1}{c|}{ Sequence } \\
\hline 61.7 & 2711.1848 & 0.0078 & $\underline{\text { HNNDTQHIWESDSNEFSVIADPR }}$ \\
\hline 61.7 & 2711.1848 & 0.0078 & HNNDTQHIWESDSNEFSVIADPR \\
\hline 21.0 & 2711.1848 & 0.0078 & HNNDTQHIWESDSNEFSVIADPR \\
\hline 18.7 & 2710.2008 & 0.9918 & HNNDTOHIWESDSNEFSVIADPR \\
\hline 5.4 & 2711.2043 & -0.0117 & SELEGSMLSVLSAASTASRLLPPR \\
\hline 2.6 & 2710.1656 & 1.0271 & KELQSPASSFVMSSETMWESTPR \\
\hline 2.6 & 2710.1656 & 1.0271 & KELQSPASSFVMSSETMWESTPR \\
\hline 2.1 & 2711.1915 & 0.0012 & GSDVDTHAVSLEKGEGLILEHSMK \\
\hline 1.8 & 2711.2043 & -0.0117 & SELEGSMLSVLSAASTASRLLPPR \\
\hline 1.8 & 2711.2043 & -0.0117 & SELEGSMLSVLSAASTASRLLPPR \\
\hline
\end{tabular}

Spectrum No: 505; Query: 1403; Rank: 1

\section{Peptide View}

MS/MS Fragmentation of TSLQPMVSALNISLGGTGK

Found in IPI00198021, Tax_Id=10116 Gene_Symbol=Umod Uromodulin precursor

Match to Query 1403: 1889.976848 from(945.995700,2+)

Title: 091008RatKidney_NH4Format01_26.2986.2986.2.dta

Data file K:INewmanPaper|Piliang|3SubProteomes\Piliang3SP\mgf5ppm\ERLIC_3SubProteomes5ppm.mgf

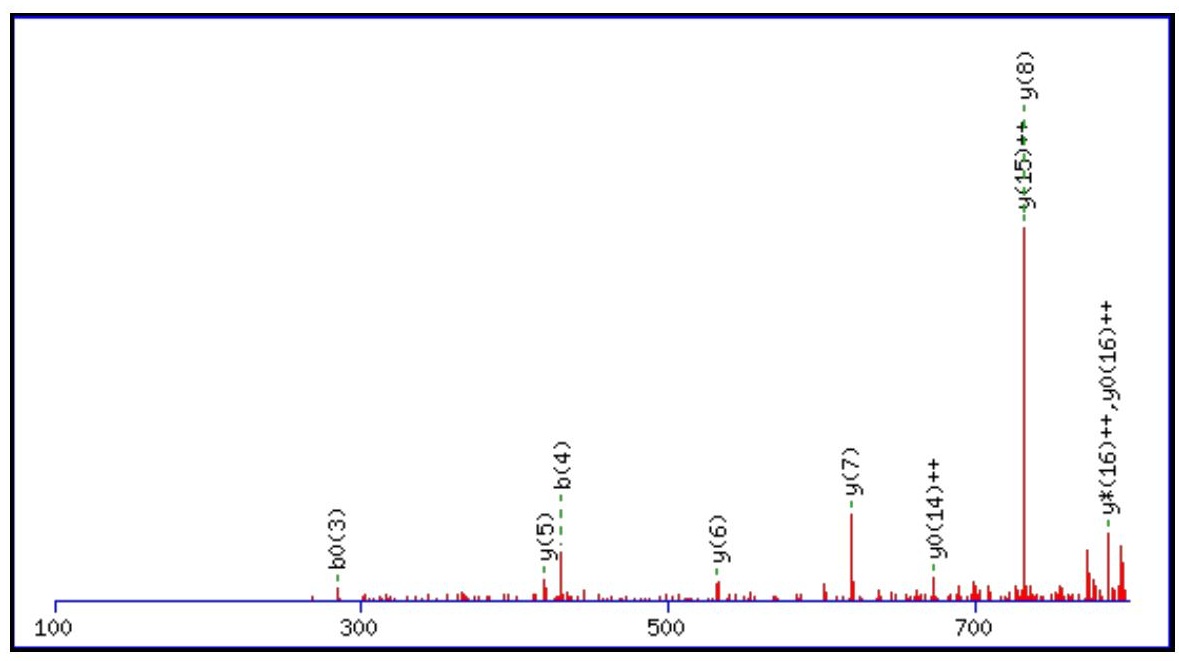




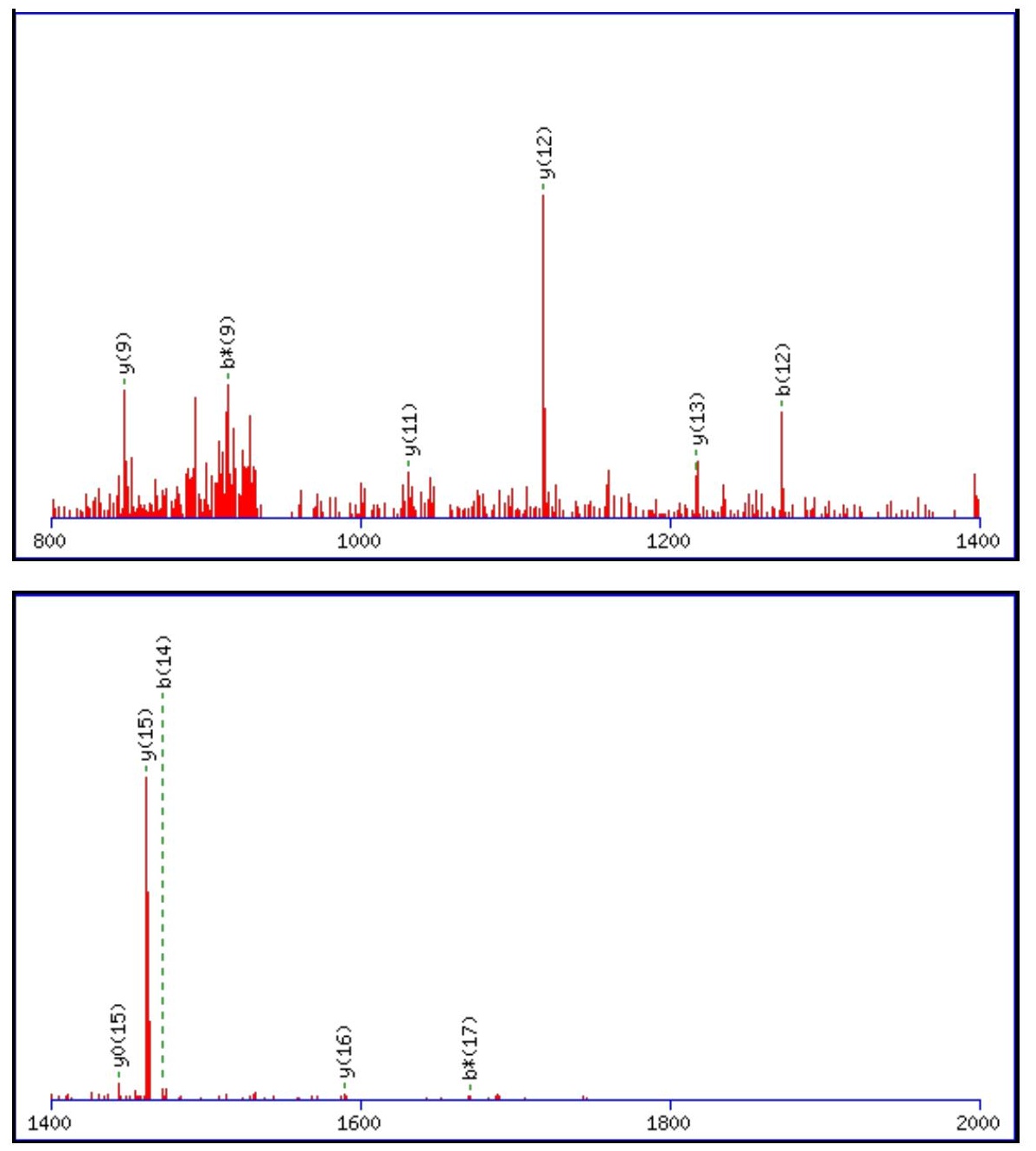

Monoisotopic mass of neutral peptide $\operatorname{Mr}($ calc): 1889.9768

Fixed modifications: Carbamidomethyl (C)

Variable modifications:

M6 : Oxidation (M)

N11: Deamidated_N (N)

Ions Score: 62 Expect: $9.7 \mathrm{e}-005$

Matches (Bold Red): 21/206 fragment ions using 48 most intense peaks

\begin{tabular}{|c|c|c|c|c|c|c|c|c|c|c|c|c|c|c|}
\hline \# & b & $\mathbf{b}^{++}$ & $\mathbf{b}^{*}$ & & $\mathbf{b}^{0}$ & & Seq. & $\mathbf{y}$ & & & & $\mathbf{y}^{\mathbf{0}}$ & & \# \\
\hline 1 & 102.0550 & & & & & 42.5258 & $\mathbf{T}$ & & & & & & & 19 \\
\hline 2 & 189.0870 & 95.0471 & & & 171.0764 & 86.0418 & S & & & & & & & 18 \\
\hline 3 & 302.1710 & 151.5892 & & & 284.1605 & 142.5839 & $\mathbf{L}$ & & & 1685.8778 & & 8938 & 842.9506 & 17 \\
\hline 4 & 430.2296 & 215.6185 & & & 412.2191 & 206.6132 & $\mathbf{Q}$ & 1589.8203 & 138 & 1572.7938 & & 8098 & 1085 & 16 \\
\hline 5 & 527.2 & 264.1448 & 58 & 16 & 509.2 & 1396 & $\mathbf{P}$ & & 45 & 352 & & 512 & 3792 & 15 \\
\hline 6 & 674.3178 & 337.6625 & 657.2912 & 329.1 & 656.3072 & 328.6573 & $\mathbf{M}$ & & & & & & 8529 & 14 \\
\hline 7 & 773.3862 & 387.1967 & 756.3 & 378. & 755.3 & 378.1915 & $\mathbf{V}$ & 736 & 404 & 1200. & 3272 & 6630 & 351 & 13 \\
\hline 8 & 860.4182 & 430.7128 & 843.3917 & 422.1 & 842.4077 & 421.7075 & S & & & 1101. & & 1100.5946 & 550.8009 & 12 \\
\hline 9 & 931.4553 & 466.2313 & 914.4288 & 457.7180 & 913.4448 & 457.2260 & A & 1031.5731 & 516.2902 & 1014.5466 & 507.7769 & 1013.5626 & 507.2849 & 11 \\
\hline 10. & 1044.5394 & 522.7733 & 027.5129 & 514.2601 & 026.5288 & 513.7681 & $\mathbf{L}$ & 960.5360 & 480. & 095 & 472 & 5255 & 471.7664 & 10 \\
\hline 11 & 1159.5663 & 580.2868 & 1142.5398 & 571.7735 & 1141.5558 & 571.2815 & $\mathbf{N}$ & 847.4520 & 424.2296 & 830.4254 & 415.7163 & 829.4414 & 415.2243 & 9 \\
\hline 12 & 1272.6504 & 636.8288 & 1255.6239 & 628.3156 & 1254.6398 & 627.8236 & I & & 366. & & & 145 & 357. & 8 \\
\hline 13 & 1359.6824 & 680.3449 & 1342.6559 & 671.8316 & 1341.6719 & 671.3396 & S & & 310.1741 & 602.3144 & 301.6608 & 601.3304 & 301.1688 & 7 \\
\hline 14 & 1472.7665 & 736.8869 & 1455.7400 & 728.3736 & 1454.7559 & 727.8816 & $\mathbf{L}$ & 532.3089 & 266.6581 & 515.2824 & 258.1448 & 514.2984 & 257.6528 & 6 \\
\hline 15 & 1529.7880 & 765.3976 & 1512.7614 & 756.8843 & 1511.7774 & 756.3923 & G & 419.2249 & 210.1161 & 402.1983 & 201.6028 & 401.2143 & 201.1108 & 5 \\
\hline 16 & 1586.8094 & 793.9084 & 1569.7829 & 785.3951 & 1568.7989 & 784.9031 & G & 362.2034 & 181.6053 & 345.1769 & 173.0921 & 344.1928 & 172.6001 & 4 \\
\hline 17 & 1687.8571 & 844.4322 & 1670.8306 & 835.9189 & 1669.8465 & 835.4269 & $\mathbf{T}$ & 305.1819 & 153.0946 & 288.1554 & 144.5813 & 287.1714 & 144.0893 & 3 \\
\hline 18 & 1744.8786 & 872.9429 & 1727.8520 & 864.4297 & 1726.8680 & 863.9376 & G & 204.1343 & 102.5708 & 187.1077 & 94.0575 & & & 2 \\
\hline
\end{tabular}




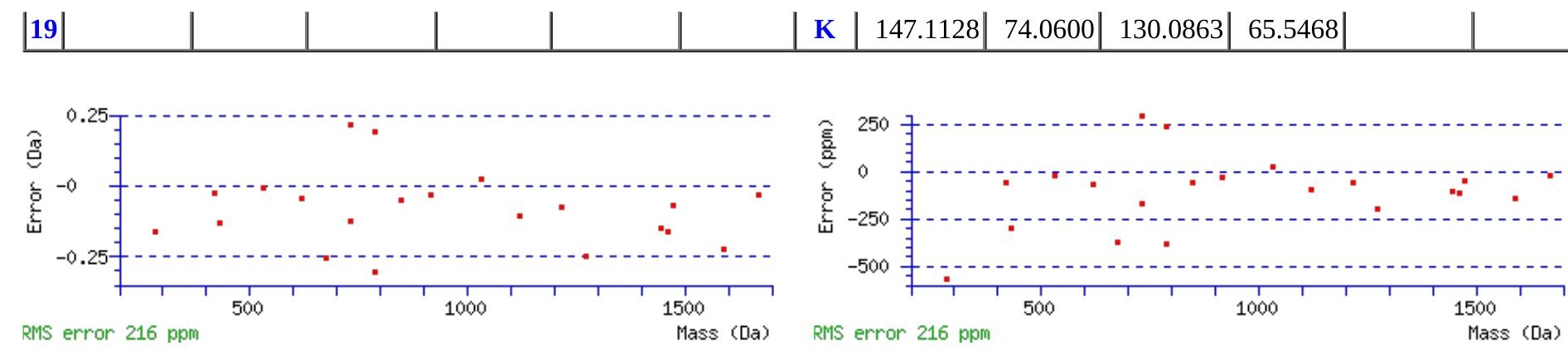

\section{All matches to this query}

\begin{tabular}{|l|l|l|l|}
\hline Score & Mr(calc): & Delta & \multicolumn{1}{|c|}{ Sequence } \\
\hline 61.5 & 1889.9768 & 0.0000 & TSLQPMVSALNISLGGTGK \\
\hline 1.9 & 1889.9669 & 0.0099 & TAELKETMGRASWSPVK \\
\hline 0.8 & 1887.9760 & 2.0008 & SIVKNWQQRYFVLK \\
\hline 0.8 & 1887.9625 & 2.0143 & VTVKELNLPTHPHCSR \\
\hline 0.7 & 1888.9772 & 0.9997 & VPSPRIDSRLADSLVGK \\
\hline 0.2 & 1888.9577 & 1.0191 & NGKGASTHCLKVNSFLR \\
\hline
\end{tabular}

Spectrum No: 506; Query: 345; Rank: 1

\section{Peptide View}

MS/MS Fragmentation of GSPGQTVNFTCK

Found in IPI00326536, Tax_Id=10116 Gene_Symbol=Ptpns1 Ptpns1 protein

Match to Query 345: 1295.584708 from(648.799630,2+)

Title: 100101RatKid_NS_deglyco_25.1185.1185.2.dta

Data file K:INewmanPaper|Piliang|3SubProteomes\Piliang3SP\mgf5ppm\ERLIC_3SubProteomes5ppm.mgf

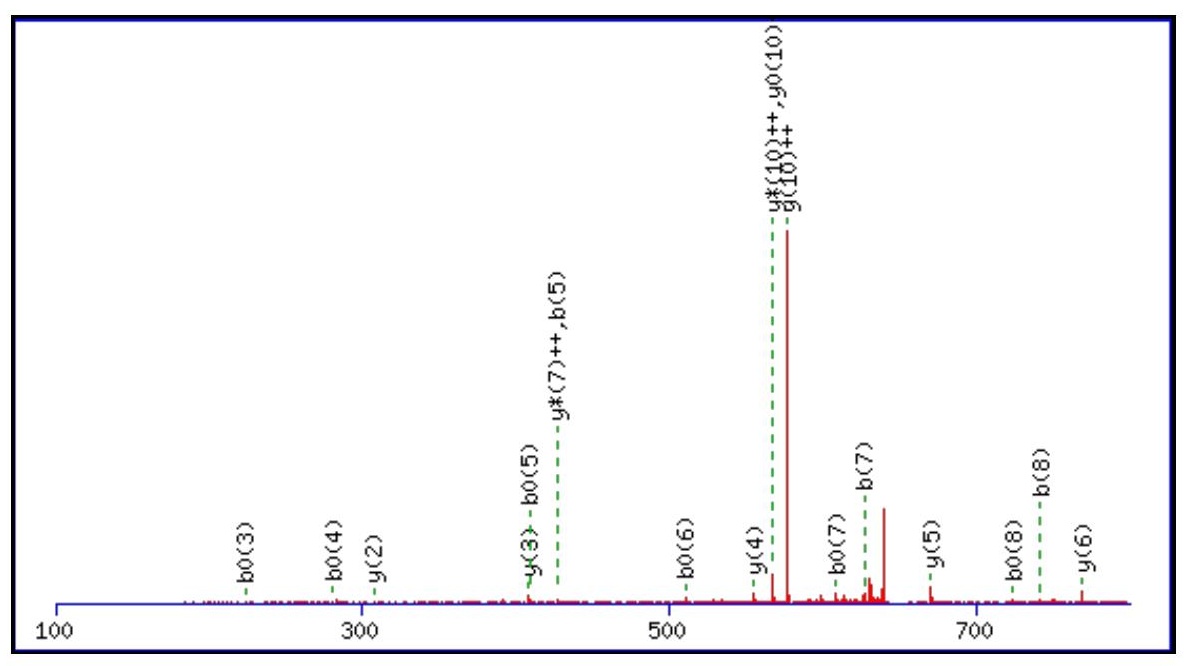



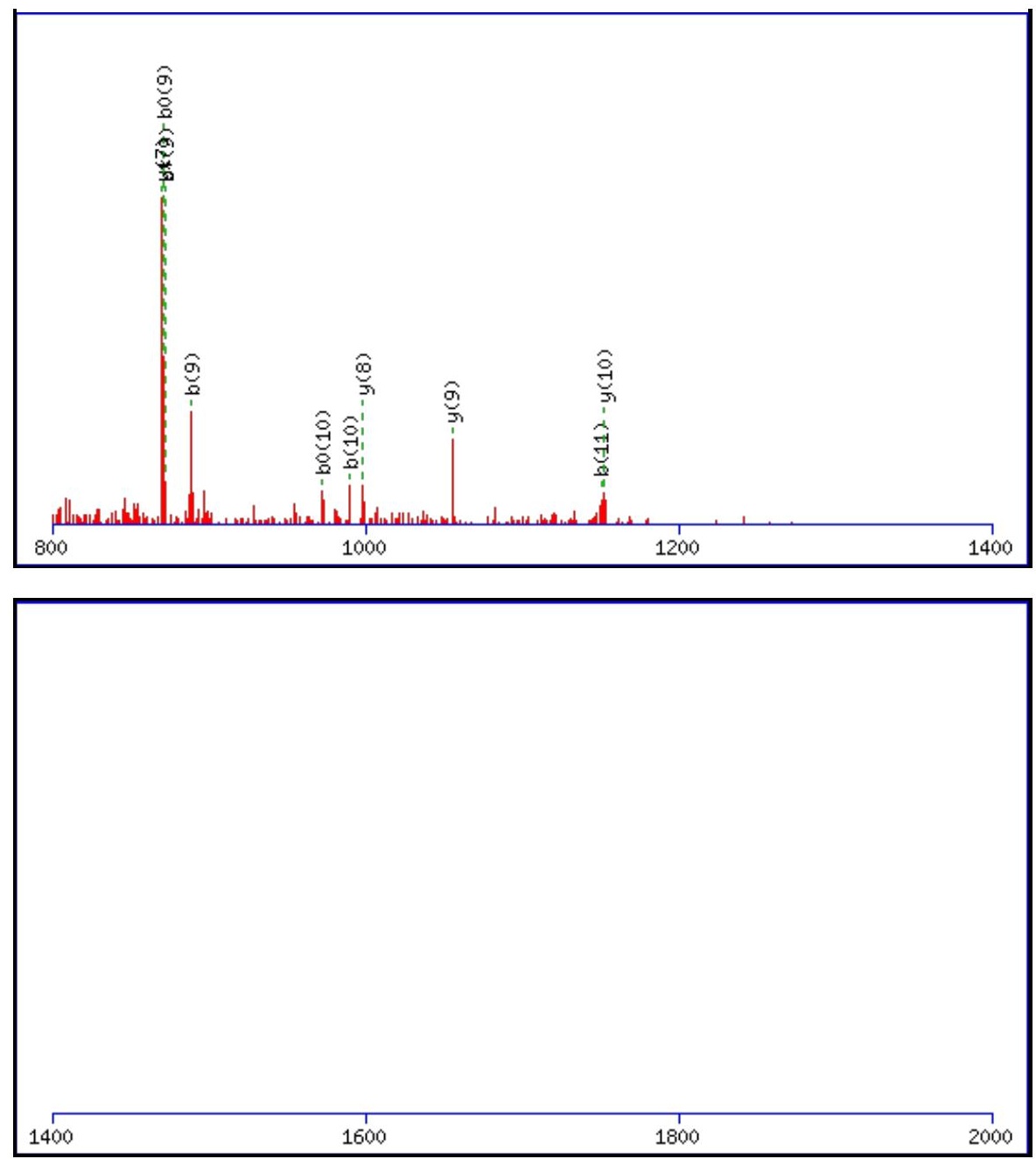

Monoisotopic mass of neutral peptide $\operatorname{Mr}($ calc): 1295.5816

Fixed modifications: Carbamidomethyl (C)

Variable modifications:

N8 : Deamidated_N (N)

Ions Score: 61 Expect: 8.8e- 005

Matches (Bold Red): 28/118 fragment ions using 58 most intense peaks

\begin{tabular}{|c|c|c|c|c|c|c|c|c|c|c|c|c|c|c|}
\hline$\#$ & b & $\mathbf{b}^{++}$ & $\mathbf{b}^{*}$ & $\mathbf{b}^{*^{++}}$ & $\mathbf{b}^{0}$ & $\mathbf{b}^{0++}$ & Seq. & $\mathbf{y}$ & $y^{++}$ & $\mathbf{y}^{*}$ & $\mathrm{y}^{*^{++}}$ & $\mathbf{y}^{\mathbf{0}}$ & $\mathbf{y}^{\mathbf{0 + +}}$ & \# \\
\hline 1 & 58.0287 & 29.5180 & & & & & G & & & & & & & 12 \\
\hline 2 & 145.0608 & 73.0340 & & & 127.0502 & 64.0287 & S & 1239.5674 & 620.2873 & 1222.5409 & 611.7741 & 1221.5568 & 611.2821 & 11 \\
\hline 3 & 242.1135 & 121.5604 & & & 224.1030 & 112.5551 & $\mathbf{P}$ & 1152.5354 & 576.7713 & 1135.5088 & 568.2581 & 1134.5248 & 567.7660 & 10 \\
\hline 4 & 299.1350 & 150.0711 & & & 281.1244 & 141.0659 & $\mathbf{G}$ & 1055.4826 & 528.2449 & 1038.4561 & 519.7317 & 1037.4721 & 519.2397 & 9 \\
\hline 5 & 427.1936 & 214.1004 & 410.1670 & 205.5872 & 409.1830 & 205.0951 & $\mathbf{Q}$ & 998.4612 & 499.7342 & 981.4346 & 491.2209 & 980.4506 & 490.7289 & 8 \\
\hline 6 & 528.2413 & 264.6243 & 511.2147 & 256.1110 & 510.2307 & 255.6190 & $\mathbf{T}$ & 870.4026 & 435.7049 & 853.3760 & 427.1917 & 852.3920 & 426.6996 & 7 \\
\hline 7 & 627.3097 & 314.1585 & 610.2831 & 305.6452 & 609.2991 & 305.1532 & $\mathbf{V}$ & 769.3549 & 385.1811 & 752.3283 & 376.6678 & 751.3443 & 376.1758 & 6 \\
\hline 8 & 742.3366 & 371.6719 & 725.3101 & 363.1587 & 724.3260 & 362.6667 & $\mathbf{N}$ & 670.2865 & 335.6469 & 653.2599 & 327.1336 & 652.2759 & 326.6416 & 5 \\
\hline 9 & 889.4050 & 445.2061 & 872.3785 & 436.6929 & 871.3945 & 436.2009 & $\mathbf{F}$ & 555.2595 & 278.1334 & 538.2330 & 269.6201 & 537.2490 & 269.1281 & 4 \\
\hline 10 & 990.4527 & 495.7300 & 973.4261 & 487.2167 & 972.4421 & 486.7247 & $\mathbf{T}$ & 408.1911 & 204.5992 & 391.1646 & 196.0859 & 390.1806 & 195.5939 & 3 \\
\hline 11 & 1150.4833 & 575.7453 & 1133.4568 & 567.2320 & 1132.4728 & 566.7400 & C & 307.1435 & 154.0754 & 290.1169 & 145.5621 & & & 2 \\
\hline 12 & & & & & & & $\mathbf{K}$ & 147.1128 & 74.0600 & 130.0863 & 65.5468 & & & 1 \\
\hline
\end{tabular}



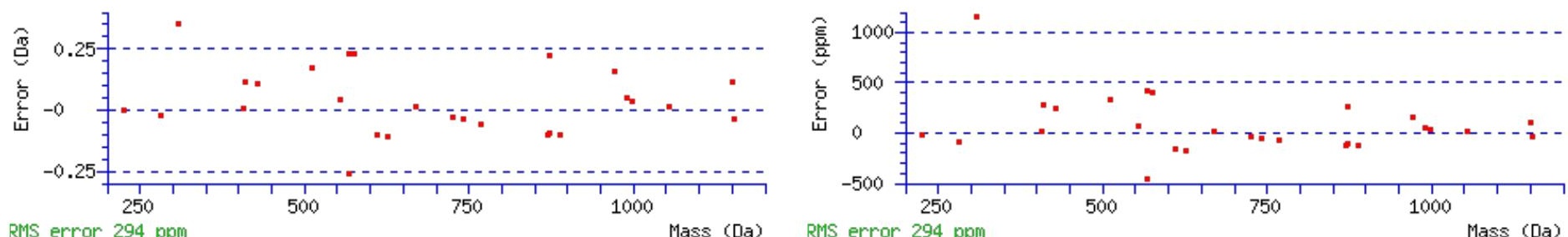

RNS error 294 ppm

Mass (Da) RMS error 294 ppm

\section{All matches to this query}

\begin{tabular}{|l|l|l|l|}
\hline Score & Mr(calc): & Delta & \multicolumn{1}{c|}{ Sequence } \\
\hline 61.4 & 1295.5816 & 0.0031 & GSPGQTVNFTCK \\
\hline 11.2 & 1295.5871 & -0.0024 & GSPSPASVSSGRK \\
\hline 9.4 & 1295.5871 & -0.0024 & $\underline{\text { GSPSPASVSSGRK }}$ \\
\hline 9.3 & 1295.5871 & -0.0024 & $\underline{\text { GSPSPASVSSGRK }}$ \\
\hline 9.3 & 1295.5751 & 0.0096 & $\underline{\text { NFMKTHNTCK }}$ \\
\hline 8.7 & 1294.5897 & 0.9950 & AADMGNLKAMEK \\
\hline 7.1 & 1295.5927 & -0.0080 & FNLTSVKSLK \\
\hline 4.4 & 1295.5871 & -0.0024 & GSPSPASVSSGRK \\
\hline 4.0 & 1295.5871 & -0.0024 & GSPSPASVSSGRK \\
\hline 4.0 & 1295.5807 & 0.0040 & SMMFFLXGKK \\
\hline
\end{tabular}

Spectrum No: 507; Query: 2112; Rank: 1

\section{Peptide View}

\section{MS/MS Fragmentation of KEDSCQLNYSEGPCLGMQQK}

Found in IPI00210900, Tax_Id=10116 Gene_Symbol=Ambp AMBP protein precursor

Match to Query 2112: 2372.015232 from(791.679020,3+)

Title: 100101RatKid_NS_deglyco_25.2006.2006.3.dta

Data file K:INewmanPaper|Piliangl3SubProteomes\Piliang3SP\mgf5ppm|ERLIC_3SubProteomes5ppm.mgf

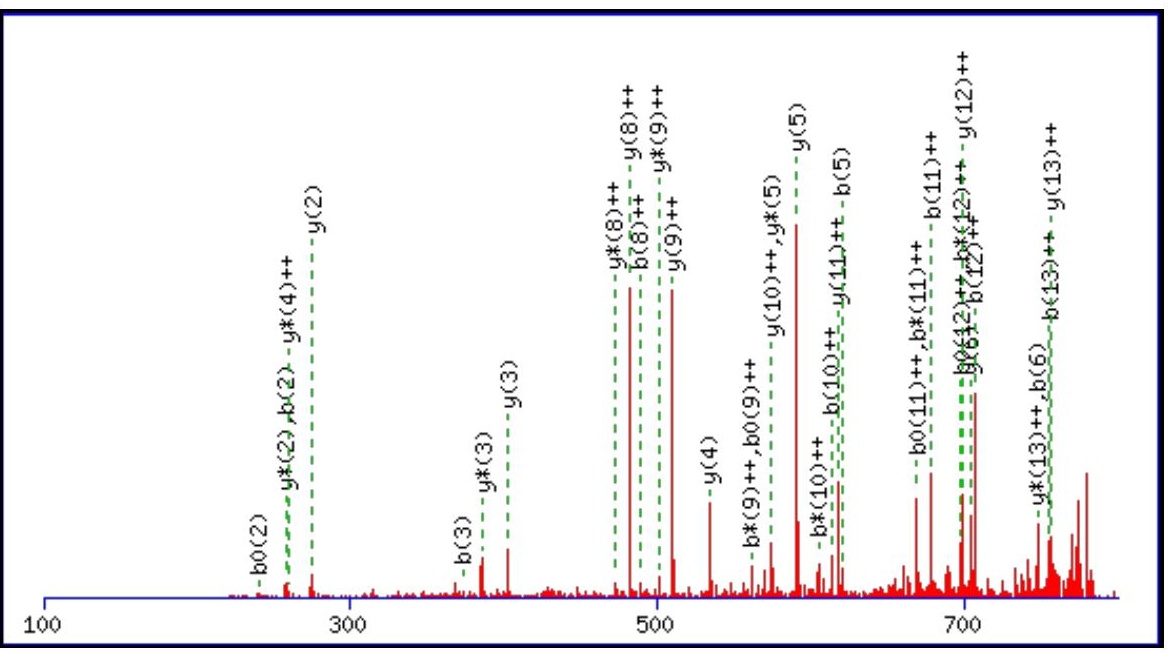



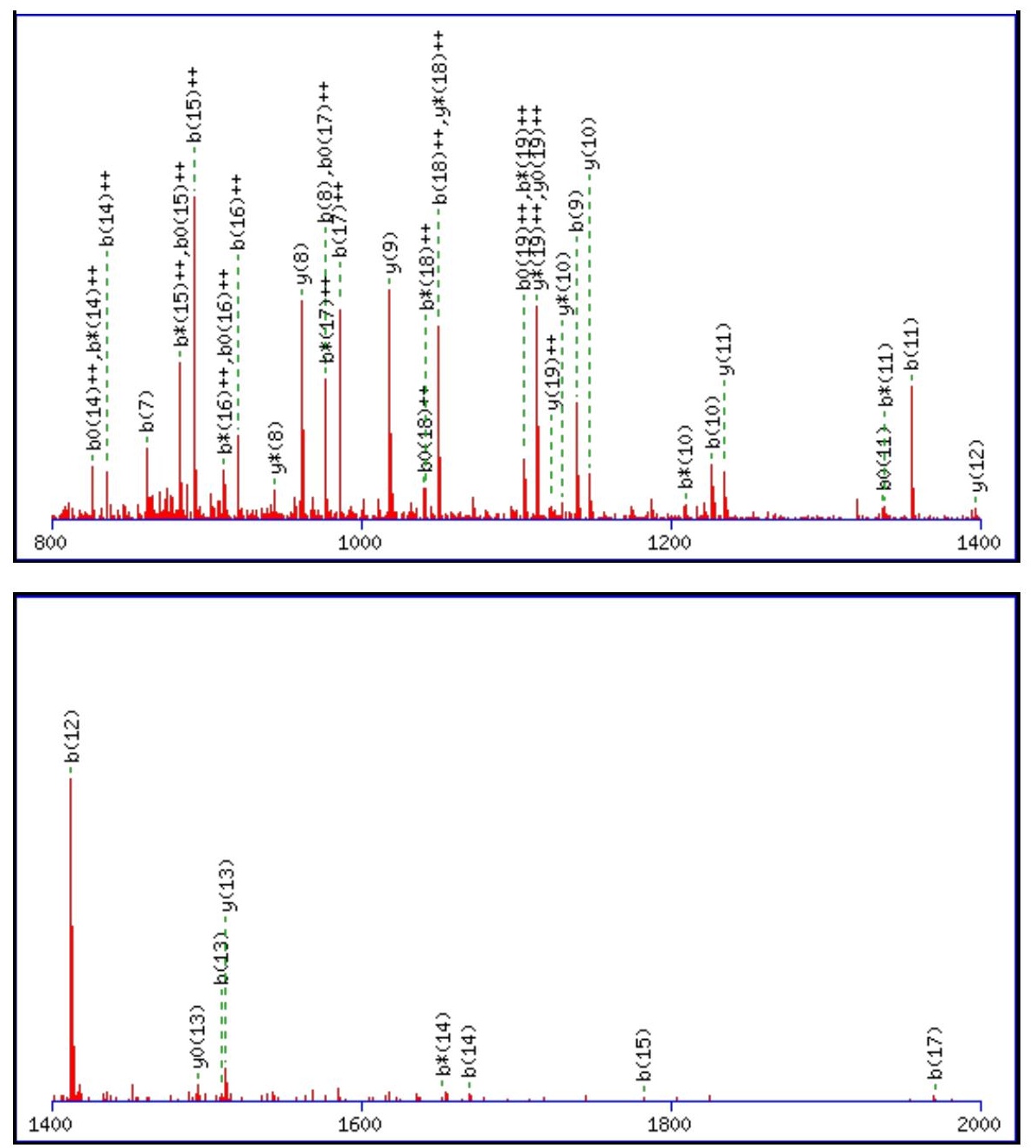

Monoisotopic mass of neutral peptide $\operatorname{Mr}($ calc): 2372.0083

Fixed modifications: Carbamidomethyl (C)

Variable modifications:

N8: Deamidated $\mathrm{N}(\mathrm{N})$

Ions Score: 61 Expect: 0.00023

Matches (Bold Red): 80/208 fragment ions using 148 most intense peaks

\begin{tabular}{|c|c|c|c|c|c|c|c|c|c|c|c|c|c|c|}
\hline \# & b & $\mathbf{b}^{++}$ & $\mathbf{b}^{*}$ & $\mathbf{b}^{*^{++}}$ & $\mathbf{b}^{\mathbf{0}}$ & $\mathbf{b}^{0++}$ & Seq. & $\mathbf{y}$ & $y^{++}$ & $\mathbf{y}^{*}$ & $\mathrm{y}^{\mathrm{*}^{++}}$ & $\mathbf{y}^{\mathbf{0}}$ & $\mathbf{y}^{0++}$ & \# \\
\hline 1 & 129.1022 & 65.0548 & 112.0757 & 56.5415 & & & $\mathbf{K}$ & & & & & & & 20 \\
\hline 2 & 258.1448 & 129.5761 & 241.1183 & 121.0628 & 240.1343 & 120.5708 & $\mathbf{E}$ & 2244.9206 & 1122.9639 & 2227.8940 & 1114.4507 & 2226.9100 & 1113.9587 & 19 \\
\hline 4 & 460.2038 & 230.6055 & 443.1773 & 222.0923 & 442.1932 & 221.6003 & $\mathrm{~S}$ & 2000.8511 & 1000.9292 & 1983.8245 & 992.4159 & 1982.8405 & 991.9239 & 17 \\
\hline 5 & 620.2345 & 310.6209 & 603.2079 & 302.1076 & 602.2239 & 301.6156 & $\mathrm{C}$ & 1913.8190 & 957.4132 & 1896.7925 & 948.8999 & 1895.8085 & 948.4079 & 16 \\
\hline 6 & 748.2930 & 374.6502 & 731.2665 & 366.1369 & 730.2825 & 365.6449 & $\mathbf{Q}$ & 1753.7884 & 877.3978 & 1736.7618 & 868.8846 & 1735.7778 & 868.3925 & 15 \\
\hline 7 & 861.3771 & 431.1922 & 844.3505 & 422.6789 & 843.3665 & 422.1869 & $\mathbf{L}$ & 1625.7298 & 813.3685 & 1608.7033 & 804.8553 & 1607.7192 & 804.3633 & 14 \\
\hline 8 & 976.4040 & 488.7057 & 959.3775 & 480.1924 & 958.3935 & 479.7004 & $\mathbf{N}$ & 1512.6457 & 756.8265 & 1495.6192 & 748.3132 & 1494.6352 & 747.8212 & 13 \\
\hline 9 & 1139.4674 & 570.2373 & 1122.4408 & 561.7240 & 1121.4568 & 561.2320 & $\mathbf{Y}$ & 1397.6188 & 699.3130 & 1380.5923 & 690.7998 & 1379.6082 & 690.3078 & 12 \\
\hline 10 & 1226.4994 & 613.7533 & 1209.4728 & 605.2401 & 1208.4888 & 604.7480 & $\mathrm{~S}$ & 1234.5555 & 617.7814 & 1217.5289 & 609.2681 & 1216.5449 & 608.7761 & 11 \\
\hline 11 & 1355.5420 & 678.2746 & 1338.5154 & 669.7614 & 1337.5314 & 669.2693 & $\mathbf{E}$ & 1147.5234 & 574.2654 & 1130.4969 & 565.7521 & 1129.5129 & 565.2601 & 10 \\
\hline 12 & 1412.5634 & 706.7854 & 1395.5369 & 698.2721 & 1394.5529 & 697.7801 & G & 1018.4809 & 509.7441 & 1001.4543 & 501.2308 & & & 9 \\
\hline 14 & 1669.6469 & 835.3271 & 1652.6203 & 826.8138 & 1651.6363 & 826.3218 & $\mathrm{C}$ & 864.4066 & 432.7069 & 847.3801 & 424.1937 & & & 7 \\
\hline 15 & 1782.7309 & 891.8691 & 1765.7044 & 883.3558 & 1764.7204 & 882.8638 & $\mathbf{L}$ & 704.3760 & 352.6916 & 687.3494 & 344.1784 & & & 6 \\
\hline 16 & 1839.7524 & 920.3798 & 1822.7258 & 911.8666 & 1821.7418 & 911.3745 & G & 591.2919 & 296.1496 & 574.2654 & 287.6363 & & & 5 \\
\hline 17 & 1970.7929 & 985.9001 & 1953.7663 & 977.3868 & 1952.7823 & 976.8948 & $\mathbf{M}$ & 534.2704 & 267.6389 & 517.2439 & 259.1256 & & & 4 \\
\hline 18 & 2098.8514 & 1049.9294 & 2081.8249 & 1041.4161 & 2080.8409 & 1040.9241 & $\mathbf{Q}$ & 403.2300 & 202.1186 & 386.2034 & 193.6053 & & & 3 \\
\hline
\end{tabular}




\begin{tabular}{|r|r|r|r|r|r|r|r|r|r|r|r|r|r|r|r|r|r|r|}
$\mathbf{1 9}$ & 2226.9100 & $\mathbf{1 1 1 3 . 9 5 8 7}$ & 2209.8835 & $\mathbf{1 1 0 5 . 4 4 5 4}$ & 2208.8995 & $\mathbf{1 1 0 4 . 9 5 3 4}$ & $\mathbf{Q}$ & $\mathbf{2 7 5 . 1 7 1 4}$ & 138.0893 & $\mathbf{2 5 8 . 1 4 4 8}$ & 129.5761 & & \\
\hline $\mathbf{2 0}$ & & & & & & & $\mathbf{K}$ & 147.1128 & 74.0600 & 130.0863 & 65.5468 & & & $\mathbf{1}$ \\
\hline
\end{tabular}
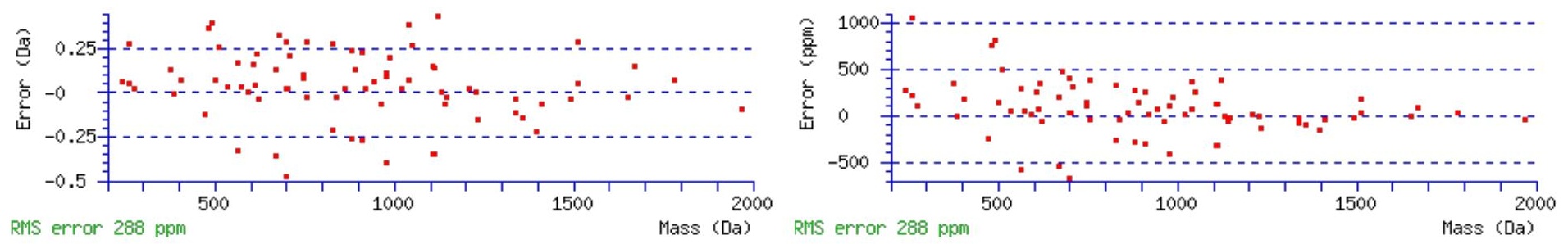

\section{All matches to this query}

\begin{tabular}{|l|l|l|l|}
\hline Score & Mr(calc): & Delta & \multicolumn{1}{|c|}{ Sequence } \\
\hline 61.4 & 2372.0083 & 0.0070 & KEDSCQLNYSEGPCLGMQQK \\
\hline 24.2 & 2371.0242 & 0.9910 & KEDSCQLNYSEGPCLGMQQK \\
\hline 14.7 & 2372.0169 & -0.0017 & QQGFLTDDLVSQEENGQQQK \\
\hline 5.0 & 2370.0085 & 2.0068 & VQRPEDASGGSSPSGTSKSDANR \\
\hline 5.0 & 2370.0085 & 2.0068 & VQRPEDASGGSSPSGTSKSDANR \\
\hline 4.8 & 2372.0169 & -0.0017 & QQGFLTDDLVSQEENGQQQK \\
\hline 4.7 & 2371.0080 & 1.0073 & MSSPNTWSTGSTVYSPVFSOK \\
\hline 3.3 & 2371.0080 & 1.0073 & MSSPNTWSTGSTVYSPVFSQK \\
\hline 2.6 & 2372.0235 & -0.0083 & DMVECMYGRNYQQGSYIVK \\
\hline 2.4 & 2370.9990 & 1.0162 & ENMTLQPKSFGSKCQLSEK \\
\hline
\end{tabular}

Spectrum No: 508; Query: 2516; Rank: 1

\section{Peptide View}

MS/MS Fragmentation of RHDSTEPSSTSSSSDPILDFNISLAVAK

Found in IPI00200898, Tax_Id=10116 Gene_Symbol=Slc9a3r1 Ezrin-radixin-moesin-binding phosphoprotein 50

Match to Query 2516: 2961.434016 from(741.365780,4+)

Title: 091008RatKidney_NH4Format01_15.5453.5453.4.dta

Data file K:INewmanPaper|Piliang \3SubProteomes\Piliang3SP\mgf5ppm\ERLIC_3SubProteomes5ppm.mgf

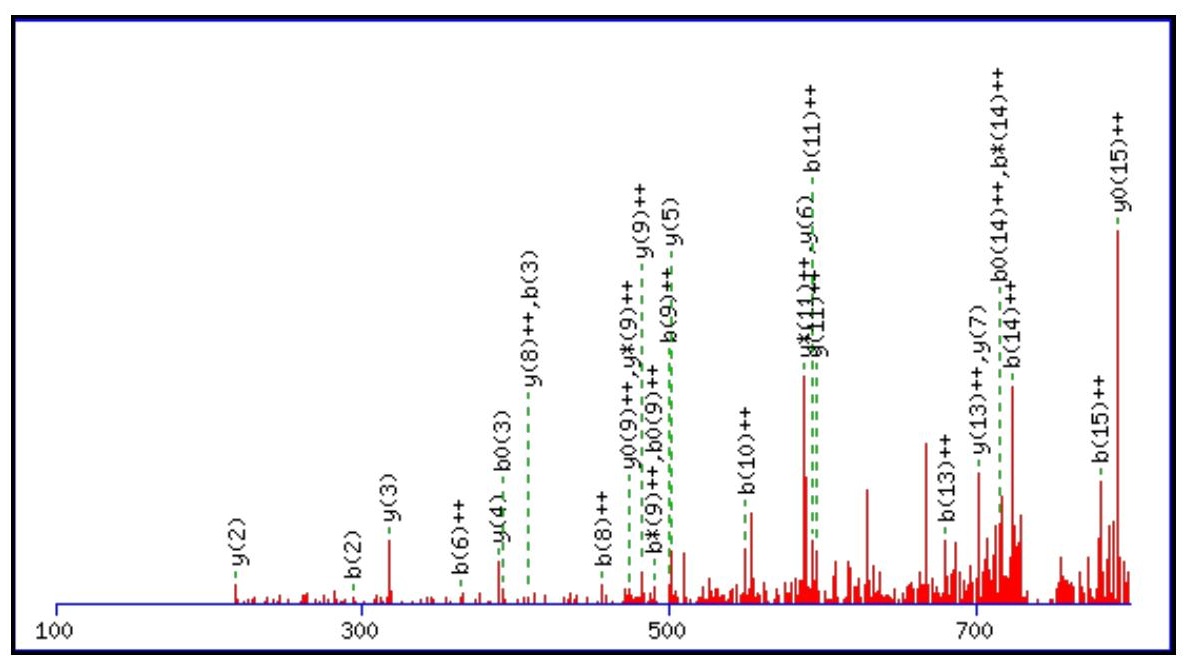



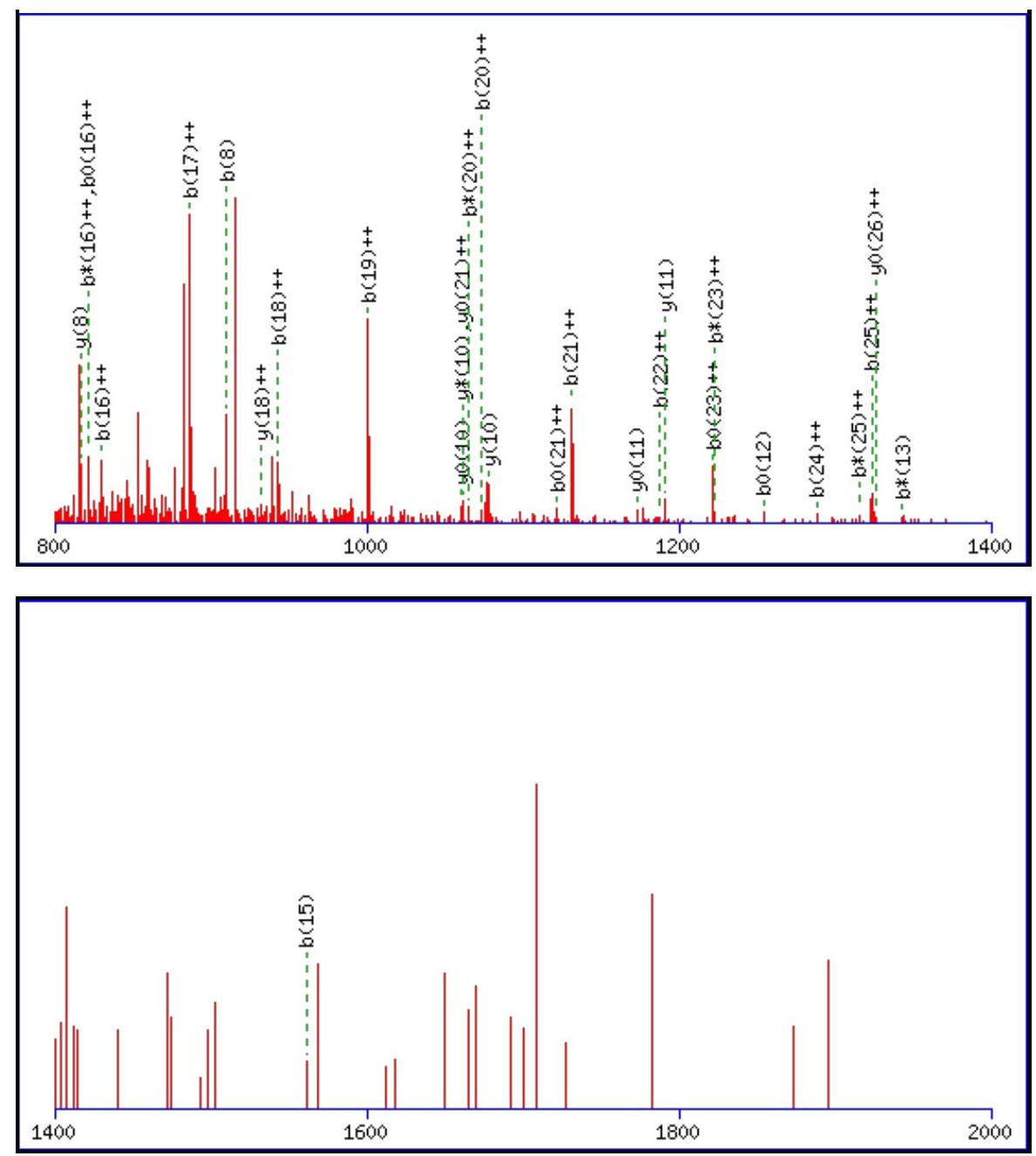

Monoisotopic mass of neutral peptide $\operatorname{Mr}($ calc): 2961.4203

Fixed modifications: Carbamidomethyl (C)

Variable modifications:

N21 : Deamidated $\mathrm{N}(\mathrm{N})$

Ions Score: 61 Expect: 0.00022

Matches (Bold Red): 58/310 fragment ions using 144 most intense peaks

\begin{tabular}{|c|c|c|c|c|c|c|c|c|c|c|c|c|c|c|}
\hline \# & b & $\mathbf{b}^{++}$ & $\mathbf{b}^{*}$ & $\mathbf{b}^{*^{++}}$ & $\mathbf{b}^{0}$ & & Seq. & $\mathbf{y}$ & $\mathbf{y}^{++}$ & $\mathbf{y}^{*}$ & $\mathbf{y}^{*^{++}}$ & $\mathbf{y}^{0}$ & $\mathbf{y}^{0++}$ & \# \\
\hline 1 & 157.1084 & 79.0578 & 140.0818 & 70.5446 & & & $\mathbf{R}$ & & & & & & & 28 \\
\hline 2 & 294.1673 & 147.5873 & 277.1408 & 139.0740 & & & H & 2806.3265 & 1403.6669 & 2789.2999 & .1536 & 2788.3159 & 1394.6616 & 27 \\
\hline 3 & 409.1942 & 205.1008 & 392.1677 & 196.5875 & 391.1837 & 196.0955 & D & 2669.2676 & 1335.1374 & 2652.2410 & 1326.6242 & 2651.2570 & 1326.1321 & 26 \\
\hline 4 & 496.2263 & 248.6168 & 479.1997 & 240.1035 & 478.2157 & 239. & S & 2554.2406 & .6240 & 2537.2141 & 1269.1107 & 2536.2301 & 1268.6187 & 25 \\
\hline 5 & 597.2739 & 299.1406 & 580.2474 & 290.6273 & 579.2634 & 290.1353 & $\mathbf{T}$ & 2467.2086 & 1234.1079 & 2450.1821 & 1225.5947 & 2449.1980 & 1225.1027 & 24 \\
\hline 6 & 726.3165 & 363.6619 & 709.2900 & 355.1486 & 708.3060 & 354.6566 & $\mathbf{E}$ & 2366.1609 & 1183.5841 & 2349.1344 & 1175.0708 & 2348.1504 & 1174.5788 & 23 \\
\hline 7 & 823.3693 & 412.1883 & 806.3428 & 403.6750 & 805.3587 & 403.1830 & $\mathbf{P}$ & 2237.1183 & 1119.0628 & 2220.0918 & 1110.5495 & 2219.1078 & 1110.0575 & 22 \\
\hline 8 & 910.4013 & 455.7043 & 893.3748 & 447.1910 & 892.3908 & 446.6990 & S & 2140.0656 & 1070.5364 & 2123.0390 & 1062.0232 & 2122.0550 & 1061.5311 & 21 \\
\hline 9 & 997.4334 & 499.2203 & 980.4068 & 490.7070 & 979.4228 & 490.2150 & $S$ & 2053.0335 & 1027.0204 & 2036.0070 & 1018.5071 & 2035.0230 & 1018.0151 & 20 \\
\hline 10 & 1098.4810 & 549.7442 & 1081.4545 & 541.2309 & 1080.4705 & 540.7389 & $\mathbf{T}$ & 1966.0015 & 983.5044 & 1948.9750 & 974.9911 & 1947.9910 & 974.4991 & 19 \\
\hline 11 & 1185.5131 & 593.2602 & 1168.4865 & 584.7469 & 1167.5025 & 584.2549 & $S$ & 1864.9538 & 932.9806 & 1847.9273 & 924.4673 & 1846.9433 & 923.9753 & 18 \\
\hline 12 & 1272.5451 & 636.7762 & 1255.5185 & 628.2629 & 1254.5345 & 627.7709 & $\mathrm{~S}$ & 1777.9218 & 889.4645 & 1760.8953 & 880.9513 & 1759.9112 & 880.4593 & 17 \\
\hline 13 & 1359.5771 & 680.2922 & 1342.5506 & 671.7789 & 1341.5666 & 671.2869 & $\mathrm{~S}$ & 1690.8898 & 845.9485 & 1673.8632 & 837.4353 & 1672.8792 & 836.9432 & 16 \\
\hline 14 & 1446.6092 & 723.8082 & 1429.5826 & 715.2949 & 1428.5986 & 714.8029 & $\mathrm{~S}$ & 1603.8578 & 802.4325 & 1586.8312 & 793.9192 & 1585.8472 & 793.4272 & 15 \\
\hline 15 & 1561.6361 & 781.3217 & 1544.6095 & 772.8084 & 1543.6255 & 772.3164 & D & 1516.8257 & 758.9165 & 1499.7992 & 750.4032 & 1498.8152 & 749.9112 & 14 \\
\hline 16 & 1658.6889 & 829.8481 & 1641.6623 & 821.3348 & 1640.6783 & 820.8428 & $\mathbf{P}$ & 1401.7988 & 701.4030 & 1384.7722 & 692.8898 & 1383.7882 & 692.3977 & 13 \\
\hline 17 & 1771.7729 & 886.3901 & 1754.7464 & 877.8768 & 1753.7624 & 877.3848 & I & 1304.7460 & 652.8766 & 1287.7195 & 644.3634 & 1286.7355 & 643.8714 & 12 \\
\hline 18 & 1884.8570 & 942.9321 & 1867.8304 & 934.4189 & 1866.8464 & 933.9268 & $\mathbf{L}$ & 1191.6620 & 596.3346 & 1174.6354 & 587.8213 & 1173.6514 & 587.3293 & 11 \\
\hline
\end{tabular}




\begin{tabular}{|c|c|c|c|c|c|c|c|c|c|c|c|c|c|c|}
\hline 19 & 1999.8839 & $\mid 1000.4456$ & |1982.8574 & 991.9323 & |1981.8734 & 991.4403 & D & 1078.5779 & 539.7926 & 1061.5513 & 531.2793 & 1060.5673 & 530.7873 & 10 \\
\hline 20 & 2146.9523 & 1073.9798 & 2129.9258 & 1065.4665 & 2128.9418 & 1064.9745 & $\mathbf{F}$ & 963.5510 & 482.2791 & 946.5244 & 473.7658 & 945.5404 & 473.2738 & 9 \\
\hline 21 & 2261.9793 & 1131.4933 & 2244.9527 & 1122.9800 & 2243.9687 & 1122.4880 & $\mathbf{N}$ & 816.4825 & 408.7449 & 799.4560 & 400.2316 & 798.4720 & 399.7396 & 8 \\
\hline 22 & 2375.0633 & 1188.0353 & 2358.0368 & 1179.5220 & 2357.0528 & 1179.0300 & I & 701.4556 & 351.2314 & 684.4291 & 342.7182 & 683.4450 & 342.2262 & 7 \\
\hline 23 & 2462.0954 & 1231.5513 & 2445.0688 & 1223.0380 & 2444.0848 & 1222.5460 & S & 588.3715 & 294.6894 & 571.3450 & 286.1761 & 570.3610 & 285.6841 & 6 \\
\hline 24 & 2575.1794 & 1288.0934 & 2558.1529 & 1279.5801 & 2557.1689 & |1279.0881 & L & 501.3395 & 251.1734 & 484.3130 & 242.6601 & & & 5 \\
\hline 25 & 2646.2166 & 1323.6119 & |2629.1900 & 1315.0986 & 2628.2060 & 1314.6066 & A & 388.2554 & 194.6314 & 371.2289 & 186.1181 & & & 4 \\
\hline 26 & 2745.2850 & 1373.1461 & 2728.2584 & 1364.6328 & 2727.2744 & 1364.1408 & V & 317.2183 & 159.1128 & 300.1918 & 150.5995 & & & 3 \\
\hline 27 & 2816.3221 & 1408.6647 & 2799.2955 & 1400.1514 & 2798.3115 & 1399.6594 & A & 218.1499 & 109.5786 & 201.1234 & 101.0653 & & & 2 \\
\hline 28 & & & & & & & $\mathbf{K}$ & 147.1128 & 74.0600 & 130.0863 & 65.5468 & & & 1 \\
\hline
\end{tabular}
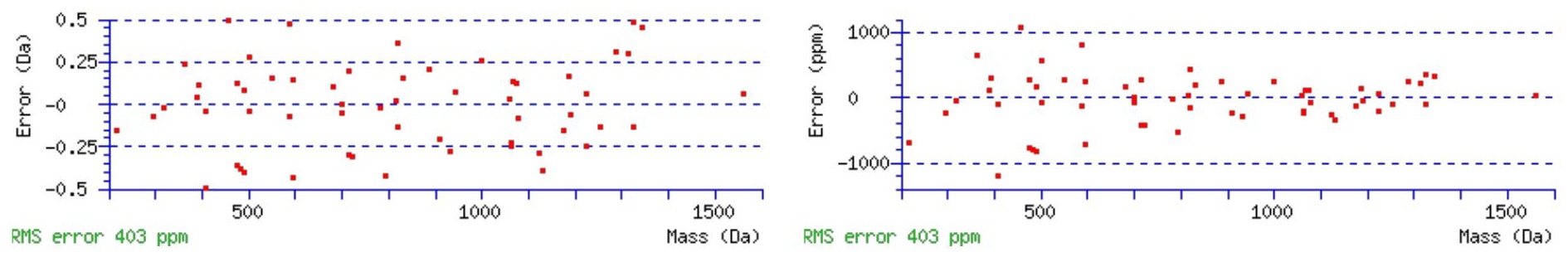

\section{All matches to this query}

\begin{tabular}{|l|l|l|l|}
\hline Score & Mr(calc): & Delta & \multicolumn{1}{|c|}{ Sequence } \\
\hline 61.4 & 2961.4203 & 0.0137 & RHDSTEPSSTSSSSDPILDFNISLAVAK \\
\hline 52.6 & 2960.4363 & 0.9977 & RHDSTEPSSTSSSSDPILDFNISLAVAK \\
\hline 6.9 & 2961.4632 & -0.029 & MSILGIIFIQGNSTSEEDMWKILKK \\
\hline 2.9 & 2960.4368 & 0.9972 & EGSPYGHLPNWRLLSVIVKCGDDLR \\
\hline 2.9 & 2960.4368 & 0.9972 & EGSPYGHLPNWRLLSVIVKCGDDLR \\
\hline 2.8 & 2961.4120 & 0.0221 & VAAALDQWPLWYDDWMDGQLVEKGR \\
\hline 1.6 & 2961.4228 & 0.0112 & NLQKMVDSVYSNILQMSDSLDLIQK \\
\hline 1.6 & 2961.4228 & 0.0112 & NLQKMVDSVYSNILQMSDSLDLIQK \\
\hline 1.4 & 2960.4538 & 0.9802 & FNIWGGSLSLGHPFGATGCRLVMAAANR \\
\hline 1.3 & 2961.4378 & -0.0038 & FNIWGGSLSLGHPFGATGCRLVMAAANR \\
\hline
\end{tabular}

Spectrum No: 509; Query: 205; Rank: 1

\section{Peptide View}

MS/MS Fragmentation of DALQNMSSSLK

Found in IPI00325312, Tax_Id=10116 Gene_Symbol=Prom1 prominin 1 isoform 1

Match to Query 205: 1193.559988 from(597.787270,2+)

Title: 100101RatKid_NS_deglyco_08.2715.2715.2.dta

Data file K:INewmanPaper|Piliangl3SubProteomes\Piliang3SP\mgf5ppm\ERLIC_3SubProteomes5ppm.mgf 

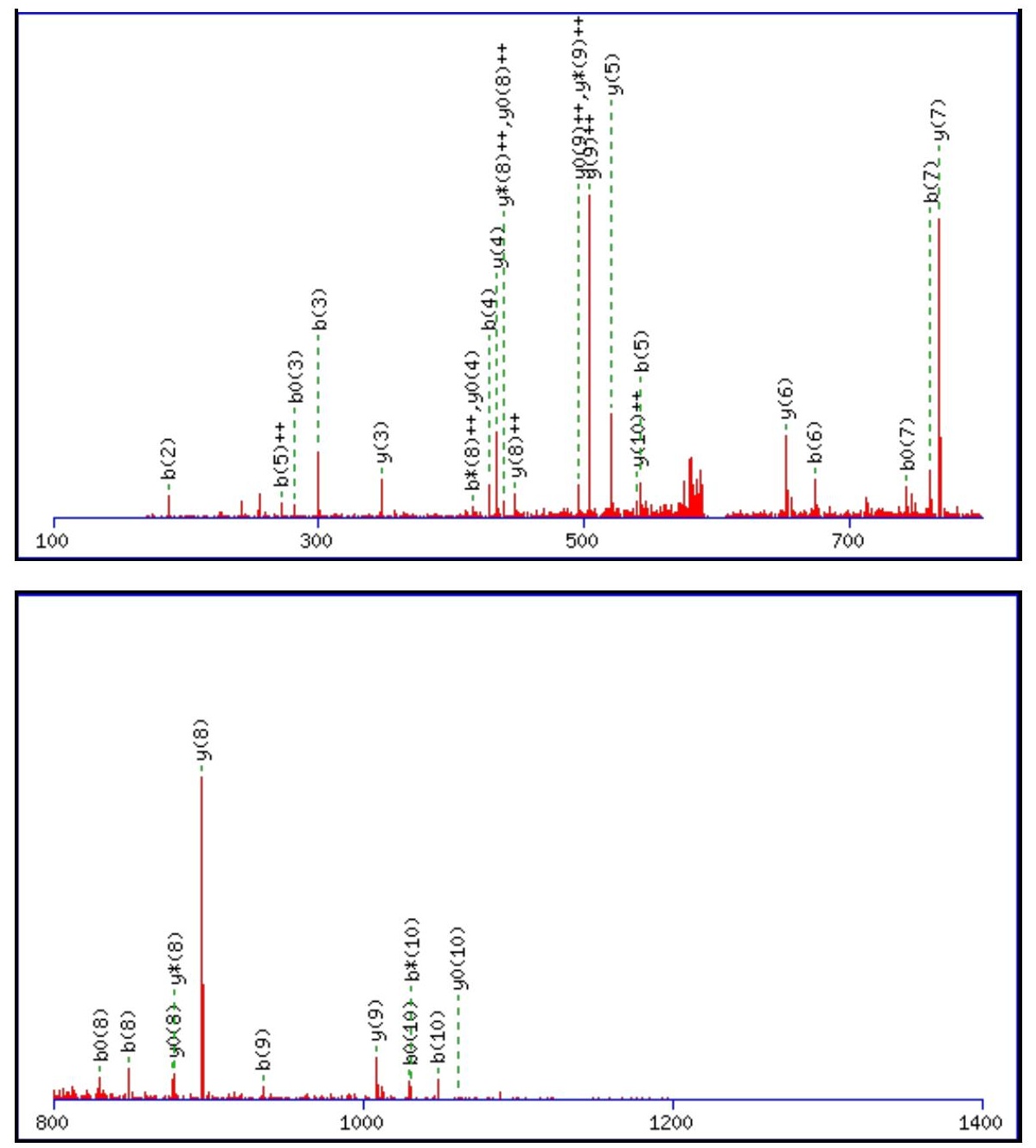

\begin{tabular}{rrrr}
\hline 00 & 1600 & 1800 & 2000 \\
\hline
\end{tabular}

Monoisotopic mass of neutral peptide $\operatorname{Mr}($ calc): 1193.5598

Fixed modifications: Carbamidomethyl (C)

Variable modifications:

N5 : Deamidated $\mathrm{N}(\mathrm{N})$

Ions Score: 61 Expect: $8.9 \mathrm{e}-005$

Matches (Bold Red): 34/110 fragment ions using 62 most intense peaks

\begin{tabular}{|r|c|c|c|c|r|r|r|r|r|r|r|r|r|r|}
\hline$\#$ & $\mathbf{b}$ & $\mathbf{b}^{++}$ & $\mathbf{b}^{*}$ & $\mathbf{b}^{\mathbf{*}^{++}}$ & \multicolumn{1}{|c|}{$\mathbf{b}^{\mathbf{0}}$} & \multicolumn{1}{|c|}{$\mathbf{b}^{\mathbf{0 + +}}$} & Seq. & $\mathbf{y}$ & $\mathbf{y}^{++}$ & $\mathbf{y}^{\mathbf{*}}$ & $\mathbf{y}^{\boldsymbol{*}^{++}}$ & $\mathbf{y}^{\mathbf{0}}$ & $\mathbf{y}^{\mathbf{0 + +}}$ & $\#$ \\
\hline $\mathbf{1}$ & 116.0342 & 58.5207 & & & 98.0237 & 49.5155 & $\mathbf{D}$ & & & & & & & $\mathbf{1 1}$ \\
\hline $\mathbf{2}$ & $\mathbf{1 8 7 . 0 7 1 3}$ & 94.0393 & & & 169.0608 & 85.0340 & $\mathbf{A}$ & 1079.5401 & 540.2737 & 1062.5136 & 531.7604 & $\mathbf{1 0 6 1 . 5 2 9 6}$ & 531.2684 & $\mathbf{1 0}$ \\
\hline $\mathbf{3}$ & $\mathbf{3 0 0 . 1 5 5 4}$ & 150.5813 & & & $\mathbf{2 8 2 . 1 4 4 8}$ & 141.5761 & $\mathbf{L}$ & $\mathbf{1 0 0 8 . 5 0 3 0}$ & 504.7551 & 991.4765 & $\mathbf{4 9 6 . 2 4 1 9}$ & 990.4924 & $\mathbf{4 9 5 . 7 4 9 9}$ & $\mathbf{9}$ \\
\hline $\mathbf{4}$ & $\mathbf{4 2 8 . 2 1 4 0}$ & 214.6106 & 411.1874 & 206.0974 & 410.2034 & 205.6053 & $\mathbf{Q}$ & $\mathbf{8 9 5 . 4 1 9 0}$ & $\mathbf{4 4 8 . 2 1 3 1}$ & $\mathbf{8 7 8 . 3 9 2 4}$ & $\mathbf{4 3 9 . 6 9 9 8}$ & $\mathbf{8 7 7 . 4 0 8 4}$ & $\mathbf{4 3 9 . 2 0 7 8}$ & $\mathbf{8}$ \\
\hline $\mathbf{5}$ & $\mathbf{5 4 3 . 2 4 0 9}$ & $\mathbf{2 7 2 . 1 2 4 1}$ & 526.2144 & 263.6108 & 525.2303 & 263.1188 & $\mathbf{N}$ & $\mathbf{7 6 7 . 3 6 0 4}$ & 384.1838 & 750.3338 & 375.6705 & $\mathbf{7 4 9 . 3 4 9 8}$ & 375.1785 & $\mathbf{7}$ \\
\hline
\end{tabular}




\begin{tabular}{|r|r|r|r|r|r|r|r|r|r|r|r|r|r|r|r|r|r|r|r|}
$\mathbf{6}$ & $\mathbf{6 7 4 . 2 8 1 4}$ & 337.6443 & 657.2548 & 329.1311 & 656.2708 & 328.6391 & $\mathbf{M}$ & $\mathbf{6 5 2 . 3 3 3 4}$ & 326.6704 & 635.3069 & 318.1571 & 634.3229 & 317.6651 & $\mathbf{6}$ \\
\hline $\mathbf{7}$ & $\mathbf{7 6 1 . 3 1 3 4}$ & 381.1604 & 744.2869 & 372.6471 & $\mathbf{7 4 3 . 3 0 2 9}$ & 372.1551 & S & $\mathbf{5 2 1 . 2 9 3 0}$ & 261.1501 & 504.2664 & 252.6368 & 503.2824 & 252.1448 & $\mathbf{5}$ \\
\hline $\mathbf{8}$ & $\mathbf{8 4 8 . 3 4 5 5}$ & 424.6764 & 831.3189 & $\mathbf{4 1 6 . 1 6 3 1}$ & $\mathbf{8 3 0 . 3 3 4 9}$ & 415.6711 & S & $\mathbf{4 3 4 . 2 6 0 9}$ & 217.6341 & 417.2344 & 209.1208 & $\mathbf{4 1 6 . 2 5 0 4}$ & 208.6288 & $\mathbf{4}$ \\
\hline $\mathbf{9}$ & $\mathbf{9 3 5 . 3 7 7 5}$ & 468.1924 & 918.3509 & 459.6791 & 917.3669 & 459.1871 & S & $\mathbf{3 4 7 . 2 2 8 9}$ & 174.1181 & 330.2023 & 165.6048 & 329.2183 & 165.1128 & $\mathbf{3}$ \\
\hline $\mathbf{1 0}$ & $\mathbf{1 0 4 8 . 4 6 1 5}$ & 524.7344 & $\mathbf{1 0 3 1 . 4 3 5 0}$ & 516.2211 & $\mathbf{1 0 3 0 . 4 5 1 0}$ & 515.7291 & $\mathbf{L}$ & 260.1969 & 130.6021 & 243.1703 & 122.0888 & & & \\
\hline $\mathbf{1 1}$ & & & & & & & K & 147.1128 & 74.0600 & 130.0863 & 65.5468 & & & & $\mathbf{1}$ \\
\hline
\end{tabular}
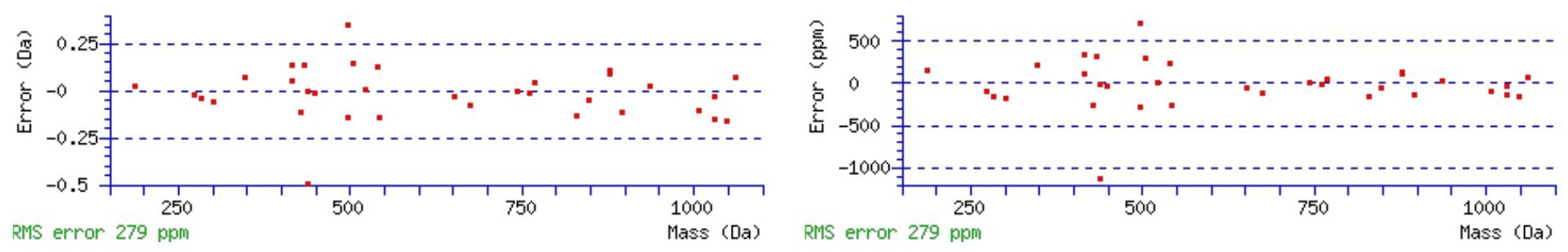

\section{All matches to this query}

\begin{tabular}{|l|l|l|l|}
\hline Score & Mr(calc): & Delta & \multicolumn{1}{|c|}{ Sequence } \\
\hline 61.3 & 1193.5598 & 0.0002 & DALQNMSSSLK \\
\hline 6.1 & 1193.5693 & -0.0093 & GEVGATTALAPK \\
\hline 6.1 & 1193.5693 & -0.0093 & GEVGATTALAPK \\
\hline 5.5 & 1192.5462 & 1.0138 & AEQRSGGRPR \\
\hline 5.1 & 1193.5645 & -0.0045 & EGLSKCGRCK \\
\hline 4.9 & 1193.5693 & -0.0093 & EAVKTLGGDPK \\
\hline 4.7 & 1192.5601 & 0.9999 & SIEEKSHKR \\
\hline 4.1 & 1193.5710 & -0.0110 & VSCNKKDDTK \\
\hline 3.4 & 1193.5532 & 0.0068 & NAEMKNAMRK \\
\hline 2.1 & 1193.5598 & 0.0002 & MTNSISDAVEK \\
\hline
\end{tabular}

Spectrum No: 510; Query: 343; Rank: 1

\section{Peptide View}

MS/MS Fragmentation of ELISNSSDALDK

Found in IPI00210566, Tax_Id=10116 Gene_Symbol=Hspca Heat shock protein HSP 90-alpha

Match to Query 343: 1291.613108 from(646.813830,2+)

Title: 091008RatKidney_NoSalt_21.1190.1190.2.dta

Data file K:INewmanPaper|Piliangl3SubProteomes\Piliang3SP\mgf5ppm\ERLIC_3SubProteomes5ppm.mgf

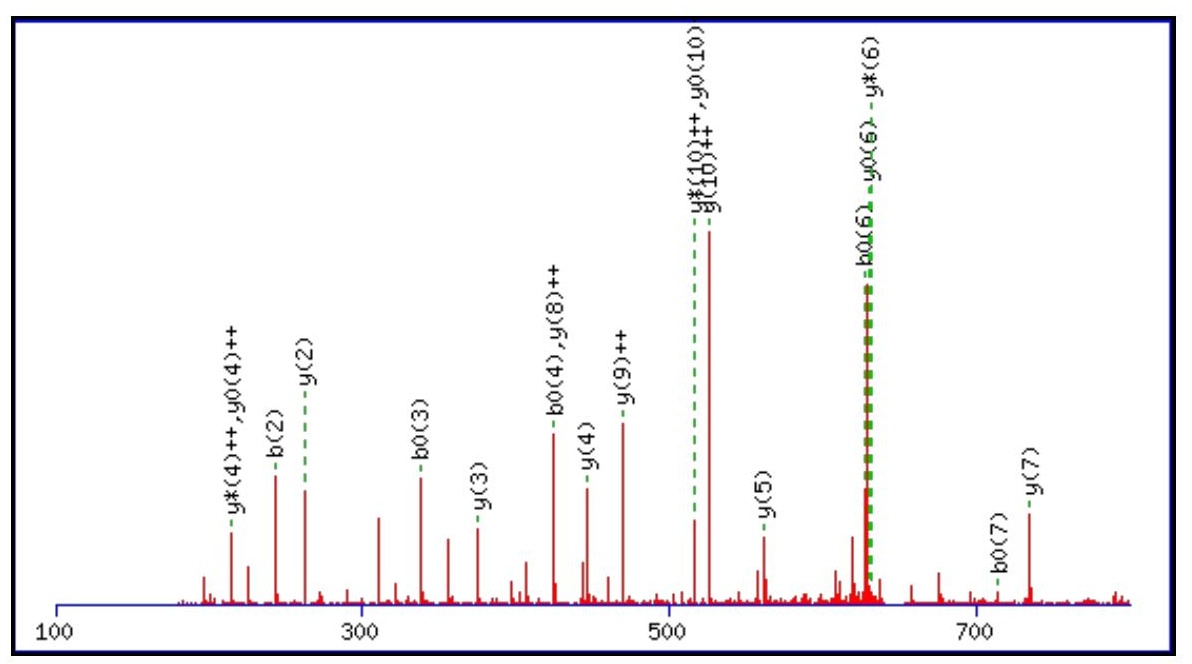




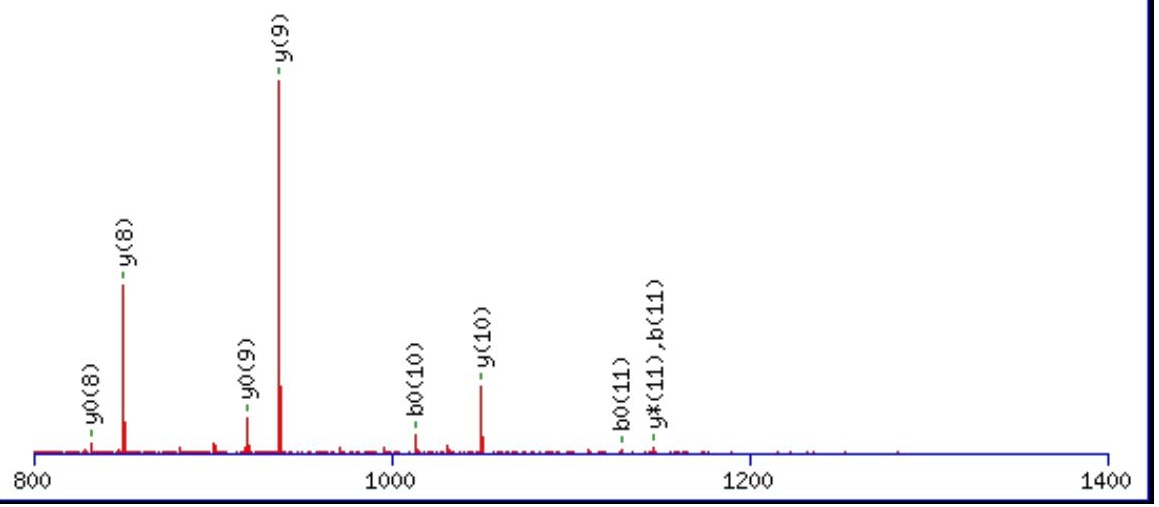

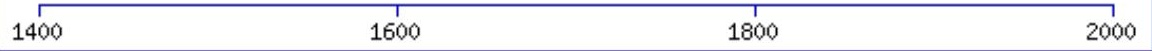

Monoisotopic mass of neutral peptide $\operatorname{Mr}$ (calc): 1291.6143

Fixed modifications: Carbamidomethyl (C)

Variable modifications:

N5 : Deamidated_N (N)

Ions Score: 61 Expect: $9.7 \mathrm{e}-005$

Matches (Bold Red): 28/122 fragment ions using 47 most intense peaks

\begin{tabular}{|c|c|c|c|c|c|c|c|c|c|c|c|c|c|c|}
\hline \# & b & $\mathbf{b}^{++}$ & $\mathbf{b}^{*}$ & $\mathbf{b}^{*^{++}}$ & $\mathbf{b}^{0}$ & $\mathbf{b}^{0++}$ & Seq. & $\mathbf{y}$ & $y^{++}$ & $\mathbf{y}^{*}$ & $\mathrm{y}^{*^{++}}$ & $\mathbf{y}^{0}$ & $\mathbf{y}^{0++}$ & \# \\
\hline 1 & 130.0499 & 65.5286 & & & 112.0393 & 56.5233 & $\mathbf{E}$ & & & & & & & 12 \\
\hline 2 & 243.1339 & 122.0706 & & & 225.1234 & 113.0653 & $\mathbf{L}$ & 1163.5790 & 582.2931 & 1146.5525 & 573.7799 & 1145.5685 & 573.2879 & 11 \\
\hline 3 & 356.2180 & 178.6126 & & & 338.2074 & 169.6074 & I & 1050.4950 & 525.7511 & 1033.4684 & 517.2378 & 1032.4844 & 516.7458 & 10 \\
\hline 4 & 443.2500 & 222.1287 & & & 425.2395 & 213.1234 & $\mathrm{~S}$ & 937.4109 & 469.2091 & 920.3843 & 460.6958 & 919.4003 & 460.2038 & 9 \\
\hline 5 & 558.2770 & 279.6421 & 541.2504 & 271.1288 & 540.2664 & 270.6368 & $\mathbf{N}$ & 850.3789 & 425.6931 & 833.3523 & 417.1798 & 832.3683 & 416.6878 & 8 \\
\hline 6 & 645.3090 & 323.1581 & 628.2824 & 314.6449 & 627.2984 & 314.1529 & S & 735.3519 & 368.1796 & 718.3254 & 359.6663 & 717.3414 & 359.1743 & 7 \\
\hline 7 & 732.3410 & 366.6741 & 715.3145 & 358.1609 & 714.3305 & 357.6689 & $S$ & 648.3199 & 324.6636 & 631.2933 & 316.1503 & 630.3093 & 315.6583 & 6 \\
\hline 8 & 847.3680 & 424.1876 & 830.3414 & 415.6743 & 829.3574 & 415.1823 & D & 561.2879 & 281.1476 & 544.2613 & 272.6343 & 543.2773 & 272.1423 & 5 \\
\hline 9 & 918.4051 & 459.7062 & 901.3785 & 451.1929 & 900.3945 & 450.7009 & A & 446.2609 & 223.6341 & 429.2344 & 215.1208 & 428.2504 & 214.6288 & 4 \\
\hline 10 & 1031.4891 & 516.2482 & 1014.4626 & 507.7349 & 1013.4786 & 507.2429 & $\mathbf{L}$ & 375.2238 & 188.1155 & 358.1973 & 179.6023 & 357.2132 & 179.1103 & 3 \\
\hline 11 & 1146.5161 & 573.7617 & 1129.4895 & 565.2484 & 1128.5055 & 564.7564 & D & 262.1397 & 131.5735 & 245.1132 & 123.0602 & 244.1292 & 122.5682 & 2 \\
\hline 12 & & & & & & & $\mathbf{K}$ & 147.1128 & 74.0600 & 130.0863 & 65.5468 & & & 1 \\
\hline
\end{tabular}



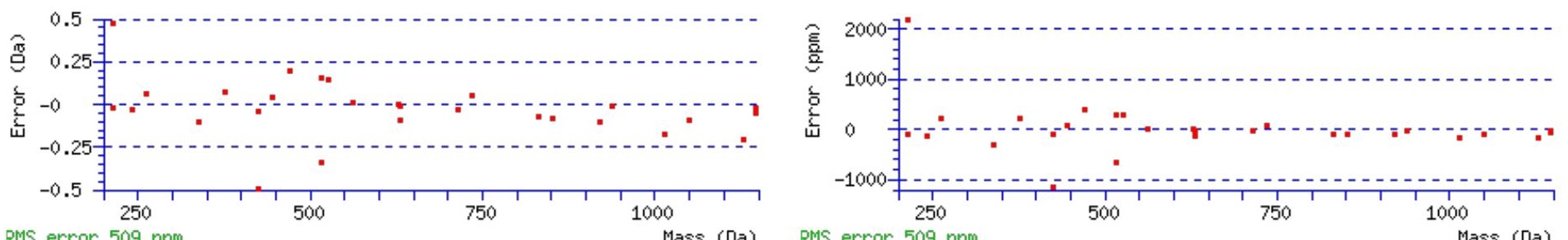

RIS error 509 ppm

Mass (Da) RMS error 509 ppm

Mass (Da)

\section{All matches to this query}

\begin{tabular}{|l|l|l|l|}
\hline Score & Mr(calc): & Delta & \multicolumn{1}{|c|}{ Sequence } \\
\hline 61.3 & 1291.6143 & -0.0012 & ELISNSSDALDK \\
\hline 12.7 & 1290.5986 & 1.0146 & LKLSTSEVVR \\
\hline 10.2 & 1289.6169 & 1.9962 & AALTIQQYFR \\
\hline 7.3 & 1291.6173 & -0.0042 & KTHNETELIK \\
\hline 4.1 & 1291.6060 & 0.0071 & EKSYTSIKEK \\
\hline 3.8 & 1290.5986 & 1.0146 & LKLSTSEVVR \\
\hline 3.8 & 1290.5986 & 1.0146 & LKLSTSEVVR \\
\hline 3.7 & 1291.6069 & 0.0062 & MLLYLMGLSR \\
\hline 3.6 & 1290.6138 & 0.9993 & QILPTIYKR \\
\hline 2.3 & 1290.6125 & 1.0006 & CTPETSNQLLK \\
\hline
\end{tabular}

Spectrum No: 511; Query: 1018; Rank: 1

\section{Peptide View}

\section{MS/MS Fragmentation of SHINCSVMEPTEEK}

Found in IPI00193765, Tax_Id=10116 Gene_Symbol=Ctsc Dipeptidyl-peptidase 1 precursor

Match to Query 1018: 1676.698528 from(839.356540,2+)

Title: 091008RatKidney_NH4Format01_24.622.622.2.dta

Data file K:INewmanPaper|Piliang|3SubProteomes\Piliang3SP\mgf5ppm\ERLIC_3SubProteomes5ppm.mgf

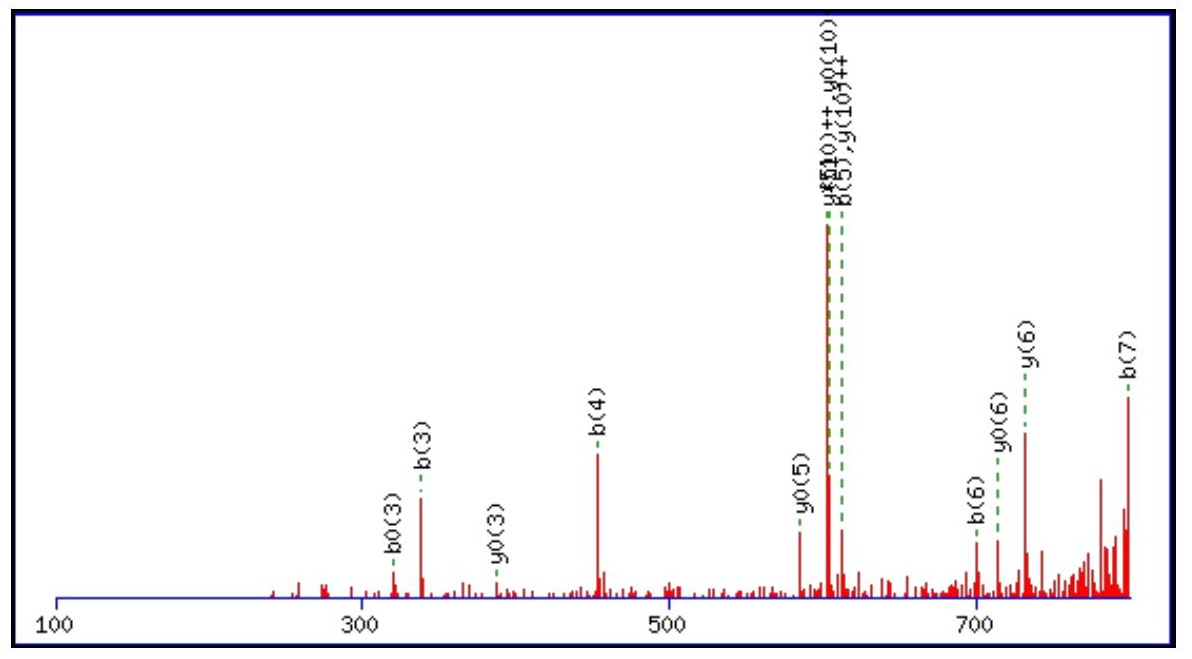



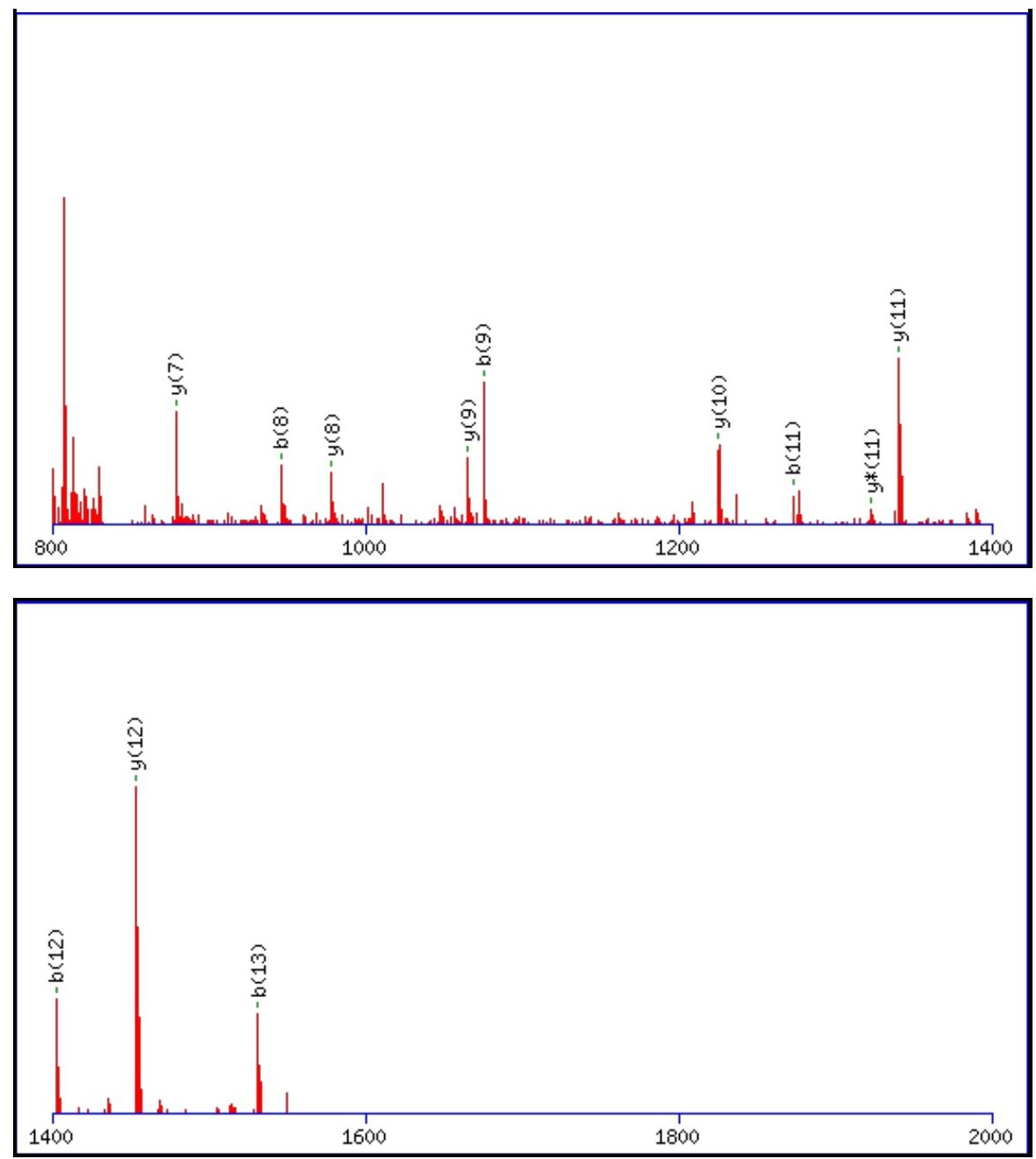

Monoisotopic mass of neutral peptide $\operatorname{Mr}($ calc): 1676.7022

Fixed modifications: Carbamidomethyl (C)

Variable modifications:

N4 : Deamidated_N (N)

M8 : Oxidation (M)

Ions Score: 61 Expect: 0.00013

Matches (Bold Red): 26/148 fragment ions using 44 most intense peaks

\begin{tabular}{|c|c|c|c|c|c|c|c|c|c|c|c|c|c|c|}
\hline \# & b & $\mathbf{b}^{++}$ & $\mathbf{b}^{*}$ & $\mathbf{b}^{*^{++}}$ & $\mathbf{b}^{0}$ & $\mathbf{b}^{\mathbf{0 + +}}$ & Seq. & $\mathbf{y}$ & $\mathbf{y}^{++}$ & $\mathbf{y}^{*}$ & $\mathrm{y}^{*^{++}}$ & $\mathbf{y}^{\mathbf{0}}$ & $\mathbf{y}^{\mathbf{0 + +}}$ & \# \\
\hline 1 & 88.0393 & 44.5233 & & & 70.0287 & 35.5180 & S & & & & & & & 14 \\
\hline 2 & 225.0982 & 113.0527 & & & 207.0877 & 104.0475 & $\mathbf{H}$ & 1590.6774 & 795.8424 & 1573.6509 & 787.3291 & 1572.6669 & 786.8371 & 13 \\
\hline 3 & 338.1823 & 169.5948 & & & 320.1717 & 160.5895 & I & 1453.6185 & 727.3129 & 1436.5920 & 718.7996 & 1435.6080 & 718.3076 & 12 \\
\hline 4 & 453.2092 & 227.1082 & 436.1827 & 218.5950 & 435.1987 & 218.1030 & $\mathbf{N}$ & 1340.5345 & 670.7709 & 1323.5079 & 662.2576 & 1322.5239 & 661.7656 & 11 \\
\hline 5 & 613.2399 & 307.1236 & 596.2133 & 298.6103 & 595.2293 & 298.1183 & $\mathbf{C}$ & 1225.5075 & 613.2574 & 1208.4810 & 604.7441 & 1207.4970 & 604.2521 & 10 \\
\hline 6 & 700.2719 & 350.6396 & 683.2453 & 342.1263 & 682.2613 & 341.6343 & $\mathrm{~S}$ & 1065.4769 & 533.2421 & 1048.4503 & 524.7288 & 1047.4663 & 524.2368 & 9 \\
\hline 7 & 799.3403 & 400.1738 & 782.3138 & 391.6605 & 781.3297 & 391.1685 & $\mathbf{V}$ & 978.4448 & 489.7261 & 961.4183 & 481.2128 & 960.4343 & 480.7208 & 8 \\
\hline 8 & 946.3757 & 473.6915 & 929.3492 & 465.1782 & 928.3651 & 464.6862 & $\mathbf{M}$ & 879.3764 & 440.1919 & 862.3499 & 431.6786 & 861.3659 & 431.1866 & 7 \\
\hline 9 & 1075.4183 & 538.2128 & 1058.3918 & 529.6995 & 1057.4077 & 529.2075 & $\mathbf{E}$ & 732.3410 & 366.6742 & 715.3145 & 358.1609 & 714.3305 & 357.6689 & 6 \\
\hline 10 & 1172.4711 & 586.7392 & 1155.4445 & 578.2259 & 1154.4605 & 577.7339 & $\mathbf{P}$ & 603.2984 & 302.1529 & 586.2719 & 293.6396 & 585.2879 & 293.1476 & 5 \\
\hline 11 & 1273.5187 & 637.2630 & 1256.4922 & 628.7497 & 1255.5082 & 628.2577 & $\mathbf{T}$ & 506.2457 & 253.6265 & 489.2191 & 245.1132 & 488.2351 & 244.6212 & 4 \\
\hline 12 & 1402.5613 & 701.7843 & 1385.5348 & 693.2710 & 1384.5508 & 692.7790 & $\mathbf{E}$ & 405.1980 & 203.1026 & 388.1714 & 194.5894 & 387.1874 & 194.0974 & 3 \\
\hline 13 & 1531.6039 & 766.3056 & 1514.5774 & 757.7923 & 1513.5934 & 757.3003 & $\mathbf{E}$ & 276.1554 & 138.5813 & 259.1288 & 130.0681 & 258.1448 & 129.5761 & 2 \\
\hline 14 & & & & & & & $\mathbf{K}$ & 147.1128 & 74.0600 & 130.0863 & 65.5468 & & & 1 \\
\hline
\end{tabular}



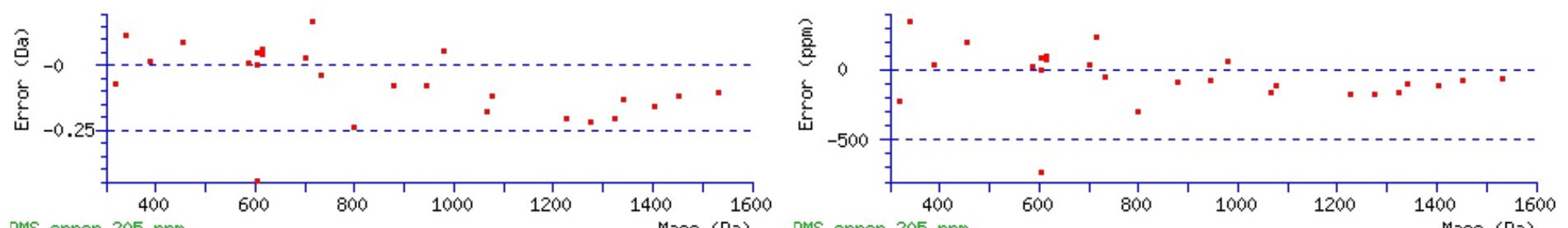

RIS error 205 ppm Mass (Da) RMS error $205 \mathrm{ppm}$

\section{All matches to this query}

\begin{tabular}{|l|l|l|l|}
\hline Score & Mr(calc): & Delta & \multicolumn{1}{c|}{ Sequence } \\
\hline 61.2 & 1676.7022 & -0.0036 & $\underline{\text { SHINCSVMEPTEEK }}$ \\
\hline 6.7 & 1674.6929 & 2.0057 & MGPAIERMGLSMDR \\
\hline 6.1 & 1674.6929 & 2.0057 & MGPAIERMGLSMDR \\
\hline 4.1 & 1676.7083 & -0.0098 & QFDAGTVNYEQPTK \\
\hline 1.3 & 1675.7048 & 0.9937 & TENFEXGLIYRK \\
\hline
\end{tabular}

Spectrum No: 512; Query: 2443; Rank: 1

\section{Peptide View}

MS/MS Fragmentation of CFANTVNLSFPSAQSLPASDTHLTVK

Found in IPI00326140, Tax_Id=10116 Gene_Symbol=Pzp Alpha-1-macroglobulin precursor

Match to Query 2443: 2805.360102 from(936.127310,3+)

Title: 091008RatKidney_NoSalt_25.4011.4011.3.dta

Data file K:\NewmanPaper\Piliang\3SubProteomes\Piliang3SP \mgf5ppm\ERLIC_3SubProteomes5ppm.mgf

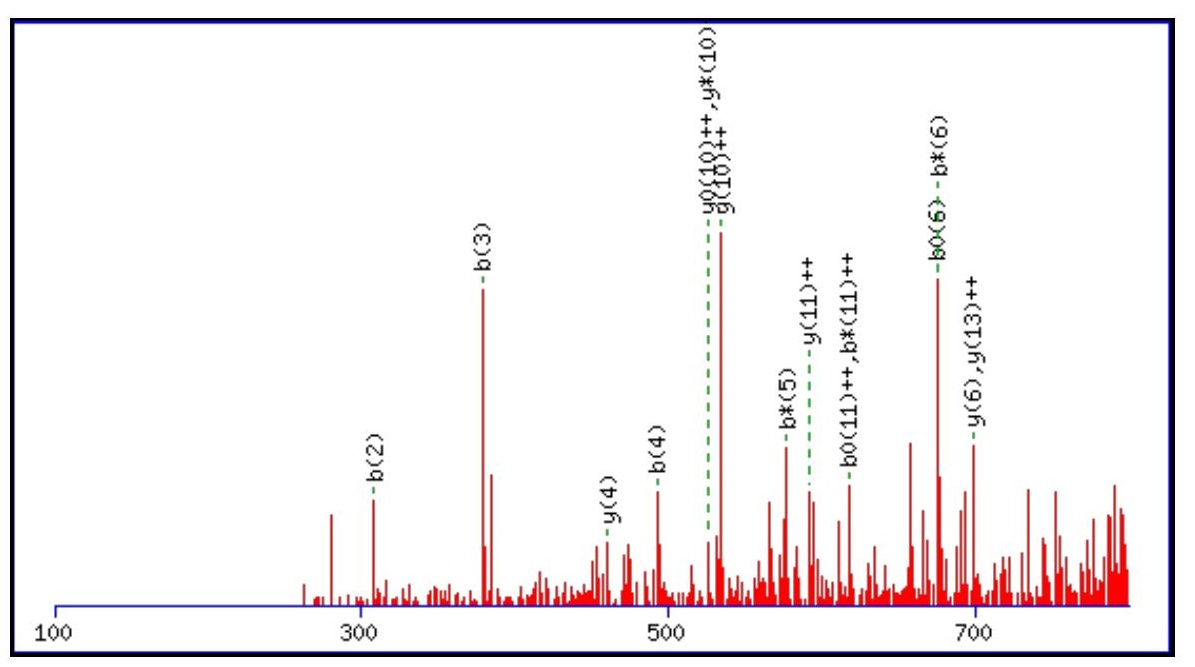



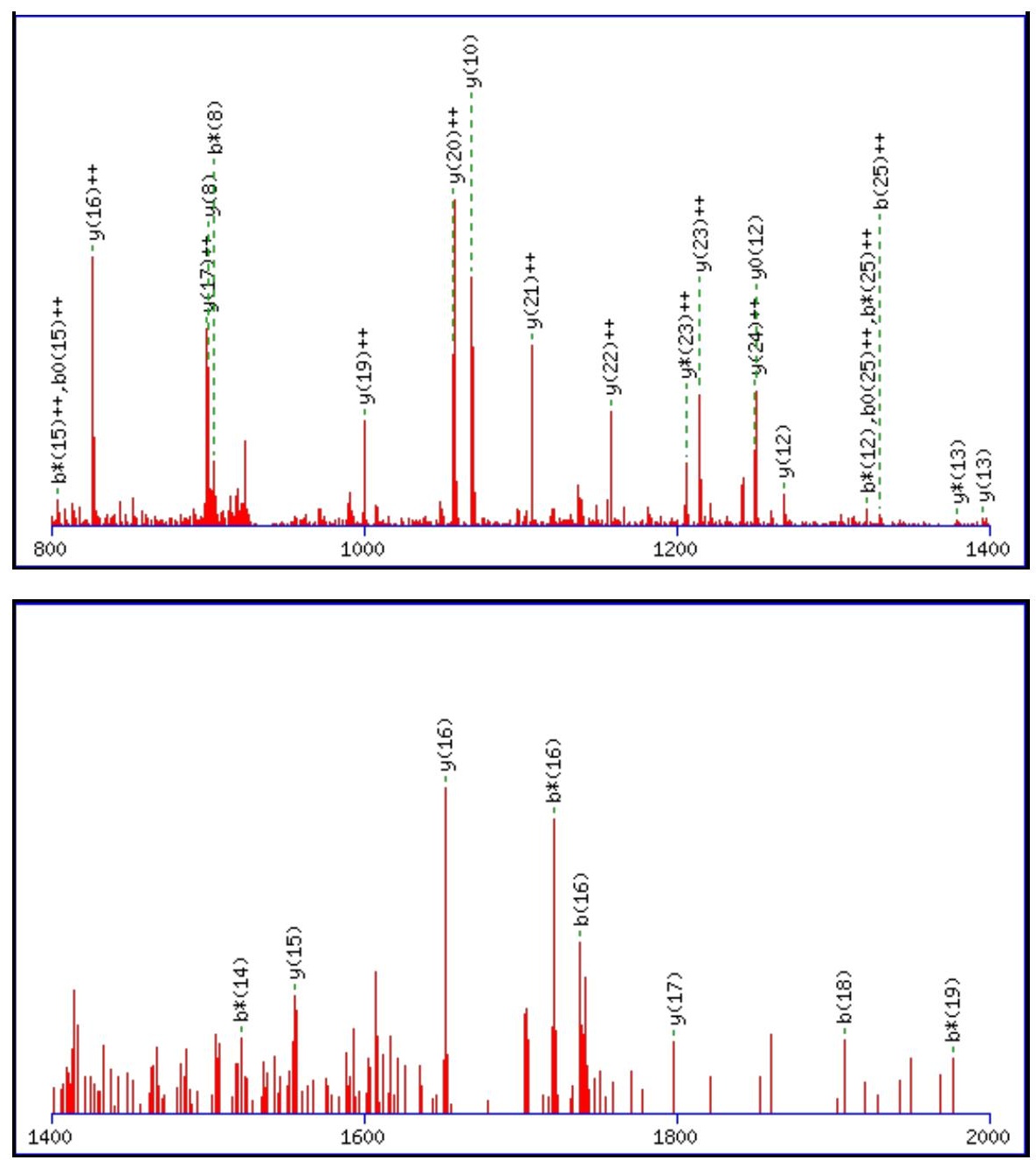

Monoisotopic mass of neutral peptide $\operatorname{Mr}($ calc): 2805.3643

Fixed modifications: Carbamidomethyl (C)

Variable modifications:

N7 : Deamidated_N (N)

Ions Score: 61 Expect: 0.00022

Matches (Bold Red): 45/282 fragment ions using 75 most intense peaks

\begin{tabular}{|c|c|c|c|c|c|c|c|c|c|c|c|c|c|c|}
\hline \# & b & $\mathbf{b}^{++}$ & $\mathbf{b}^{*}$ & $\mathbf{b}^{*^{++}}$ & $\mathbf{b}^{\mathbf{0}}$ & $\mathbf{b}^{0++}$ & Seq. & $\mathbf{y}$ & $y^{++}$ & $\mathbf{y}^{*}$ & $\mathbf{y}^{*^{++}}$ & $\mathbf{y}^{0}$ & $\mathbf{y}^{0++}$ & \# \\
\hline 1 & 161.0379 & 81.0226 & & & & & $\mathrm{C}$ & & & & & & & 26 \\
\hline 2 & 308.1063 & 154.5568 & & & & & $\mathbf{F}$ & 2646.3410 & 1323.6741 & 2629.3144 & 1315.1608 & 2628.3304 & 1314.6688 & 25 \\
\hline 3 & 379.1435 & 190.0754 & & & & & A & 2499.2726 & 1250.1399 & 2482.2460 & 1241.6266 & 2481.2620 & 1241.1346 & 24 \\
\hline 4 & 493.1864 & 247.0968 & 476.1598 & 238.5836 & & & $\mathbf{N}$ & 2428.2354 & 1214.6214 & 2411.2089 & 1206.1081 & 2410.2249 & 1205.6161 & 23 \\
\hline 5 & 594.2341 & 297.6207 & 577.2075 & 289.1074 & 576.2235 & 288.6154 & $\mathbf{T}$ & 2314.1925 & 1157.5999 & 2297.1660 & 1149.0866 & 2296.1819 & 1148.5946 & 22 \\
\hline 6 & 693.3025 & 347.1549 & 676.2759 & 338.6416 & 675.2919 & 338.1496 & V & 2213.1448 & 1107.0761 & 2196.1183 & 1098.5628 & 2195.1343 & 1098.0708 & 21 \\
\hline 7 & 808.3294 & 404.6683 & 791.3029 & 396.1551 & 790.3188 & 395.6631 & $\mathbf{N}$ & 2114.0764 & 1057.5418 & 2097.0499 & 1049.0286 & 2096.0659 & 1048.5366 & 20 \\
\hline 8 & 921.4135 & 461.2104 & 904.3869 & 452.6971 & 903.4029 & 452.2051 & $\mathbf{L}$ & 1999.0495 & 1000.0284 & 1982.0229 & 991.5151 & 1981.0389 & 991.0231 & 19 \\
\hline 9 & 1008.4455 & 504.7264 & 991.4190 & 496.2131 & 990.4349 & 495.7211 & $S$ & 1885.9654 & 943.4863 & 1868.9389 & 934.9731 & 1867.9549 & 934.4811 & 18 \\
\hline 10 & 1155.5139 & 578.2606 & 1138.4874 & 569.7473 & 1137.5034 & 569.2553 & $\mathbf{F}$ & 1798.9334 & 899.9703 & 1781.9068 & 891.4571 & 1780.9228 & 890.9651 & 17 \\
\hline 11 & 1252.5667 & 626.7870 & 1235.5401 & 618.2737 & 1234.5561 & 617.7817 & $\mathbf{P}$ & 1651.8650 & 826.4361 & 1634.8384 & 817.9229 & 1633.8544 & 817.4308 & 16 \\
\hline 12 & 1339.5987 & 670.3030 & 1322.5722 & 661.7897 & 1321.5881 & 661.2977 & $\mathrm{~S}$ & 1554.8122 & 777.9097 & 1537.7857 & 769.3965 & 1536.8016 & 768.9045 & 15 \\
\hline 13 & 1410.6358 & 705.8215 & 1393.6093 & 697.3083 & 1392.6253 & 696.8163 & A & 1467.7802 & 734.3937 & 1450.7536 & 725.8805 & 1449.7696 & 725.3884 & 14 \\
\hline 14 & 1538.6944 & 769.8508 & 1521.6679 & 761.3376 & 1520.6838 & 760.8456 & $\mathbf{Q}$ & 1396.7431 & 698.8752 & 1379.7165 & 690.3619 & 1378.7325 & 689.8699 & 13 \\
\hline 15 & 1625.7264 & 813.3669 & 1608.6999 & 804.8536 & 1607.7159 & 804.3616 & $S$ & 1268.6845 & 634.8459 & 1251.6579 & 626.3326 & 1250.6739 & 625.8406 & 12 \\
\hline 16 & 1738.8105 & 869.9089 & 1721.7839 & 861.3956 & 1720.7999 & 860.9036 & $\mathbf{L}$ & 1181.6525 & 591.3299 & 1164.6259 & 582.8166 & 1163.6419 & 582.3246 & 11 \\
\hline 17 & 1835.8633 & 918.4353 & 1818.8367 & 909.9220 & 1817.8527 & 909.4300 & $\mathbf{P}$ & 1068.5684 & 534.7878 & 1051.5419 & 526.2746 & 1050.5578 & 525.7826 & 10 \\
\hline 18 & 1906.9004 & 953.9538 & 1889.8738 & 945.4405 & 1888.8898 & 944.9485 & A & 971.5156 & 486.2615 & 954.4891 & 477.7482 & 953.5051 & 477.2562 & 9 \\
\hline
\end{tabular}




\begin{tabular}{|c|c|c|c|c|c|c|c|c|c|c|c|c|c|c|}
\hline 19| & |1993.9324 & 997.4698 & |1976.9058 & 988.9566 & |1975.9218 & 988.4646 & S & 900.4785 & 450.7429 & 883.4520 & 442.2296 & 882.4680 & 441.7376 & 8 \\
\hline 20 & 2108.9593 & 1054.9833 & 2091.9328 & 1046.4700 & 2090.9488 & 1045.9780 & D & 813.4465 & 407.2269 & 796.4199 & 398.7136 & 795.4359 & 398.2216 & 7 \\
\hline 21 & 2210.0070 & 1105.5071 & 2192.9805 & 1096.9939 & 2191.9965 & 1096.5019 & $\mathbf{T}$ & 698.4196 & 349.7134 & 681.3930 & 341.2001 & 680.4090 & 340.7081 & 6 \\
\hline 22 & 2347.0659 & 1174.0366 & 2330.0394 & 1165.5233 & 2329.0554 & 1165.0313 & $\mathbf{H}$ & 597.3719 & 299.1896 & 580.3453 & 290.6763 & 579.3613 & 290.1843 & 5 \\
\hline 23 & 2460.1500 & 1230.5786 & 2443.1234 & 1222.0654 & 2442.1394 & 1221.5734 & $\mathbf{L}$ & 460.3130 & 230.6601 & 443.2864 & 222.1468 & 442.3024 & 221.6548 & 4 \\
\hline 24 & 2561.1977 & 1281.1025 & 2544.1711 & 1272.5892 & 2543.1871 & 1272.0972 & $\mathbf{T}$ & 347.2289 & 174.1181 & 330.2023 & 165.6048 & 329.2183 & 165.1128 & 3 \\
\hline 25 & 2660.2661 & 1330.6367 & 2643.2395 & 1322.1234 & 2642.2555 & 1321.6314 & V & 246.1812 & 123.5942 & 229.1547 & 115.0810 & & & 2 \\
\hline 26 & & & & & & & $\mathbf{K}$ & 147.1128 & 74.0600 & 130.0863 & 65.5468 & & & 1 \\
\hline
\end{tabular}
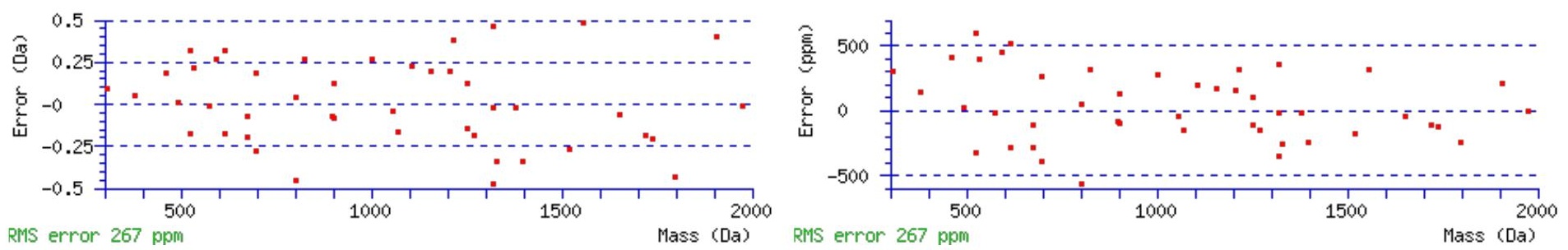

\section{All matches to this query}

\begin{tabular}{|l|l|l|l|}
\hline Score & Mr(calc): & Delta & \multicolumn{1}{c|}{ Sequence } \\
\hline 61.1 & 2805.3643 & -0.0042 & CFANTVNLSFPSAQSLPASDTHLTVK \\
\hline 46.2 & 2805.3643 & -0.0042 & CFANTVNLSFPSAQSLPASDTHLTVK \\
\hline 38.2 & 2804.3803 & 0.9798 & CFANTVNLSFPSAQSLPASDTHLTVK \\
\hline 5.4 & 2805.3578 & 0.0023 & MSSGNAKIGHPAPSFKATAVMPDGQFK \\
\hline 2.6 & 2805.3786 & -0.0185 & TSEMARLFGIQFLHVLTRGSQYR \\
\hline 2.6 & 2805.3786 & -0.0185 & TSEMARLFGIQFLHVLTRGSQYR \\
\hline 2.6 & 2805.3786 & -0.0185 & TSEMARLFGIQFLHVLTRGSQYR \\
\hline 0.8 & 2803.3755 & 1.9846 & EKYGIVDYMVEQSGPPSKEILTLK \\
\hline 0.1 & 2803.3463 & 2.0138 & MEQADLEVALSLHSLGLSLVNNENK \\
\hline
\end{tabular}

Spectrum No: 513; Query: 1796; Rank: 1

\section{Peptide View}

\section{MS/MS Fragmentation of RFSPNLNISESVIPAIIEK}

Found in IPI00195148, Tax_Id=10116 Gene_Symbol=Es2 Liver carboxylesterase 1 precursor

Match to Query 1796: 2127.160092 from(710.060640,3+)

Title: 091008RatKidney_NH4Format01_27.3356.3356.3.dta

Data file K:INewmanPaper|Piliangl3SubProteomes\Piliang3SP\mgf5ppm\ERLIC_3SubProteomes5ppm.mgf 

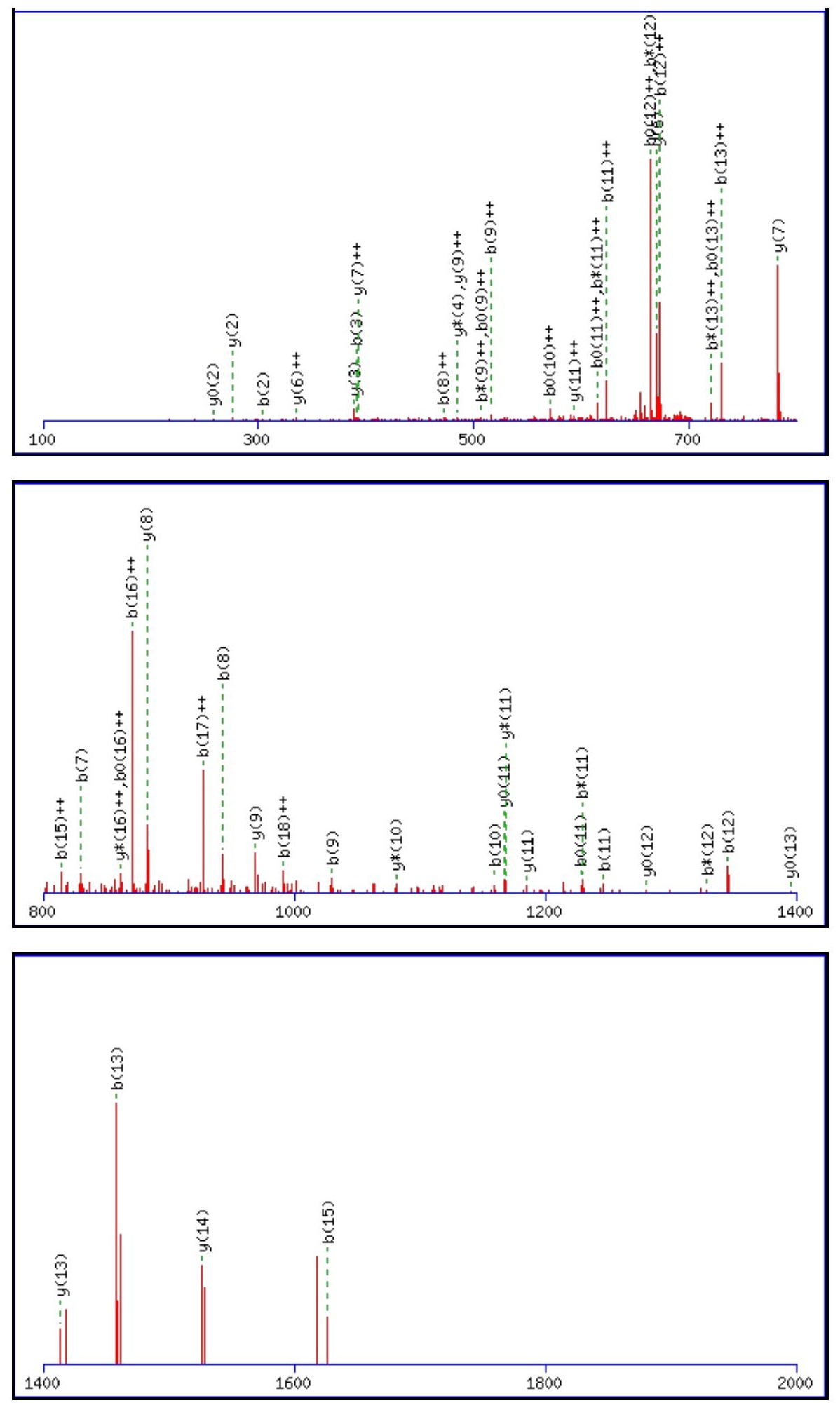

Monoisotopic mass of neutral peptide $\operatorname{Mr}($ calc): 2127.1575

Fixed modifications: Carbamidomethyl (C)

Variable modifications:

N7 : Deamidated_N (N)

Ions Score: 61 Expect: $4.4 \mathrm{e}-005$

Matches (Bold Red): 53/210 fragment ions using 84 most intense peaks

\begin{tabular}{|r|c|c|c|c|c|c|c|c|c|c|c|c|c|c|}
\hline$\#$ & $\mathbf{b}$ & $\mathbf{b}^{++}$ & $\mathbf{b}^{*}$ & $\mathbf{b}^{\boldsymbol{*}_{++}}$ & $\mathbf{b}^{\mathbf{0}}$ & $\mathbf{b}^{\mathbf{0 + +}}$ & $\mathbf{S e q}$ & $\mathbf{y}$ & $\mathbf{y}^{++}$ & $\mathbf{y}^{\mathbf{*}}$ & $\mathbf{y}^{\boldsymbol{*}^{++}}$ & $\mathbf{y}^{\mathbf{0}}$ & $\mathbf{y}^{\mathbf{0 + +}}$ & $\#$ \\
\hline $\mathbf{1}$ & 157.1084 & 79.0578 & 140.0818 & 70.5446 & & & $\mathbf{R}$ & & & & & & & $\mathbf{1 9}$ \\
\hline $\mathbf{2}$ & $\mathbf{3 0 4 . 1 7 6 8}$ & 152.5920 & 287.1503 & 144.0788 & & & $\mathbf{F}$ & 1972.0637 & 986.5355 & 1955.0372 & 978.0222 & 1954.0532 & 977.5302 & $\mathbf{1 8}$ \\
\hline $\mathbf{3}$ & $\mathbf{3 9 1 . 2 0 8 8}$ & 196.1081 & 374.1823 & 187.5948 & 373.1983 & 187.1028 & $\mathbf{S}$ & 1824.9953 & 913.0013 & 1807.9688 & 904.4880 & 1806.9847 & 903.9960 & $\mathbf{1 7}$ \\
\hline $\mathbf{4}$ & 488.2616 & 244.6344 & 471.2350 & 236.1212 & 470.2510 & 235.6292 & $\mathbf{P}$ & 1737.9633 & 869.4853 & 1720.9367 & $\mathbf{8 6 0 . 9 7 2 0}$ & 1719.9527 & 860.4800 & $\mathbf{1 6}$ \\
\hline $\mathbf{5}$ & 602.3045 & 301.6559 & 585.2780 & 293.1426 & 584.2940 & 292.6506 & $\mathbf{N}$ & 1640.9105 & 820.9589 & 1623.8840 & 812.4456 & 1622.9000 & 811.9536 & $\mathbf{1 5}$ \\
\hline
\end{tabular}




\begin{tabular}{|c|c|c|c|c|c|c|c|c|c|c|c|c|c|c|}
\hline 6 & 715.3886 & |358.1979 & 698.3620 & |349.6847 & 697.3780 & |349.1926 & L & 1526.8676 & |763.9374 & |1509.8410 & |755.4242 & |1508.8570 & |754.9322 & 14 \\
\hline 7 & 830.4155 & 415.7114 & 813.3890 & 407.1981 & 812.4050 & 406.7061 & $\mathbf{N}$ & 1413.7835 & 707.3954 & 1396.7570 & 698.8821 & 1395.7730 & 698.3901 & 13 \\
\hline 8 & 943.4996 & 472.2534 & 926.4730 & 463.7402 & 925.4890 & 463.2481 & I & 1298.7566 & 649.8819 & 1281.7300 & 641.3687 & 1280.7460 & 640.8767 & 12 \\
\hline 9 & 1030.5316 & 515.7694 & 1013.5051 & 507.2562 & 1012.5210 & 506.7642 & S & 1185.6725 & 593.3399 & 1168.6460 & 584.8266 & 1167.6620 & 584.3346 & 11 \\
\hline 10 & 1159.5742 & 580.2907 & 1142.5477 & 571.7775 & 1141.5636 & 571.2855 & $\mathbf{E}$ & 1098.6405 & 549.8239 & 1081.6140 & 541.3106 & 1080.6299 & 540.8186 & 10 \\
\hline 11 & 1246.6062 & 623.8068 & 1229.5797 & 615.2935 & 1228.5957 & 614.8015 & S & 969.5979 & 485.3026 & 952.5714 & 476.7893 & 951.5873 & 476.2973 & 9 \\
\hline 12 & 1345.6746 & 673.3410 & 1328.6481 & 664.8277 & 1327.6641 & 664.3357 & V & 882.5659 & 441.7866 & 865.5393 & 433.2733 & 864.5553 & 432.7813 & 8 \\
\hline 13 & 1458.7587 & 729.8830 & 1441.7322 & 721.3697 & 1440.7481 & 720.8777 & I & 783.4975 & 392.2524 & 766.4709 & 383.7391 & 765.4869 & 383.2471 & 7 \\
\hline 14 & 1555.8115 & 778.4094 & 1538.7849 & 769.8961 & 1537.8009 & 769.4041 & $\mathbf{F}$ & 670.4134 & 335.7103 & 653.3869 & |327.1971 & 652.4028 & 326.7051 & 6 \\
\hline 15 & 1626.8486 & 813.9279 & 1609.8220 & 805.4147 & 1608.8380 & 804.9227 & A & 573.3606 & 287.1840 & 556.3341 & 278.6707 & 555.3501 & 278.1787 & 5 \\
\hline 16 & 1739.9327 & 870.4700 & 1722.9061 & 861.9567 & 1721.9221 & 861.4647 & I & 502.3235 & 251.6654 & 485.2970 & 243.1521 & 484.3130 & 242.6601 & 4 \\
\hline 17 & 1853.0167 & 927.0120 & 1835.9902 & 918.4987 & 1835.0062 & 918.0067 & I & 389.2395 & 195.1234 & 372.2129 & 186.6101 & 371.2289 & 186.1181 & 3 \\
\hline 18 & 1982.0593 & 991.5333 & 1965.0328 & 983.0200 & 1964.0487 & 982.5280 & $\mathbf{E}$ & 276.1554 & 138.5813 & 259.1288 & 130.0681 & 258.1448 & 129.5761 & 2 \\
\hline 19 & & & & & & & $\mathbf{K}$ & 147.1128 & 74.0600 & 130.0863 & 65.5468 & & & 1 \\
\hline
\end{tabular}
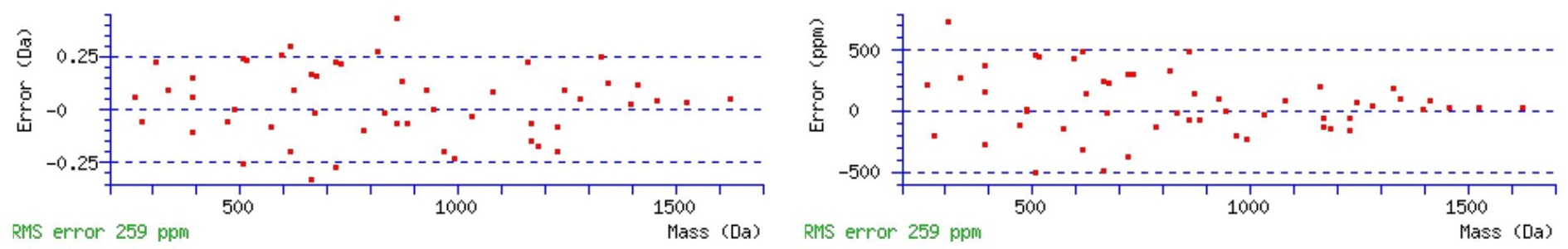

\section{All matches to this query}

\begin{tabular}{|l|l|l|l|}
\hline Score & Mr(calc): & Delta & \multicolumn{1}{|c|}{ Sequence } \\
\hline 61.1 & 2127.1575 & 0.0026 & RFSPNLNISESVIPAIIEK \\
\hline 55.4 & 2127.1575 & 0.0026 & RFSPNLNISESVIPAIIEK \\
\hline 15.6 & 2126.1575 & 1.0026 & LTTLLVCLVFSLATLVQR \\
\hline 15.6 & 2126.1575 & 1.0026 & LTTLLVCLVFSLATLVQR \\
\hline 8.1 & 2127.1414 & 0.0186 & HVVVELITCNVILPLISK \\
\hline 7.3 & 2126.1735 & 0.9866 & RFSPNLNISESVIPAIIEK \\
\hline 7.2 & 2127.1423 & 0.0178 & VDSDTSKPVTLSDLLVPVSR \\
\hline 5.5 & 2127.1453 & 0.0148 & EIQNAVQGVKHIKTLIEK \\
\hline 4.4 & 2127.1527 & 0.0074 & MKIISPVLSNLVFSRSIK \\
\hline 4.2 & 2127.1527 & 0.0074 & MKIISPVLSNLVFSRSIK \\
\hline
\end{tabular}

Spectrum No: 514; Query: 1384; Rank: 1

\section{Peptide View}

MS/MS Fragmentation of TLPLPLSTEFCVQQNK

Found in IPI00565969, Tax_Id=10116 Gene_Symbol=RGD1311652_predicted 56 kDa protein

Match to Query 1384: 1874.949068 from(938.481810,2+)

Title: 100101RatKid_NS_deglyco_25.3921.3921.2.dta

Data file K:INewmanPaper|Piliangl3SubProteomes\Piliang3SP\mgf5ppm\ERLIC_3SubProteomes5ppm.mgf 

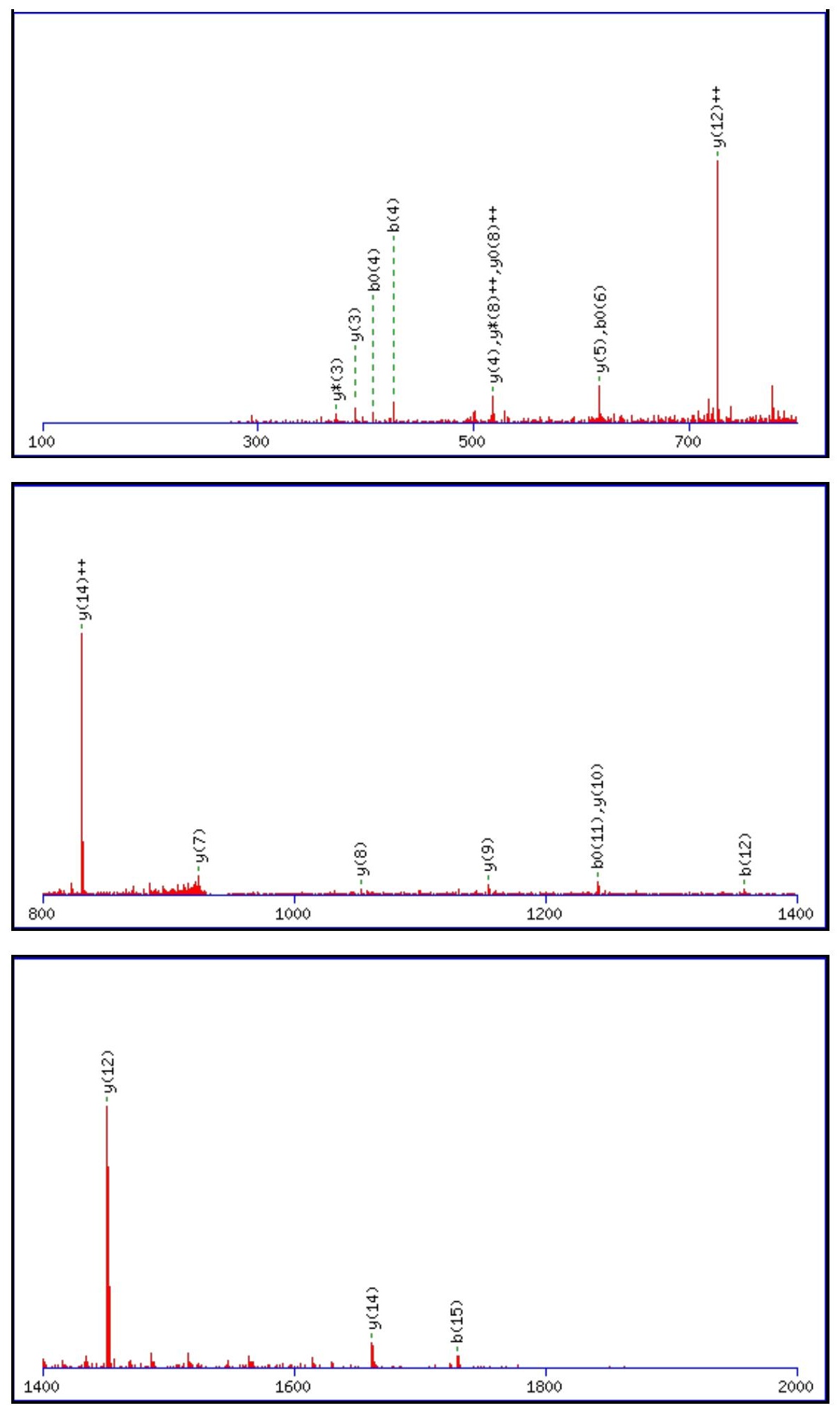

Monoisotopic mass of neutral peptide $\operatorname{Mr}($ calc): 1874.9448

Fixed modifications: Carbamidomethyl (C)

Variable modifications:

N15 : Deamidated_N (N)

Ions Score: 61 Expect: 0.00012

Matches (Bold Red): 20/142 fragment ions using 27 most intense peaks

\begin{tabular}{|r|c|c|c|c|r|r|r|r|c|c|c|c|c|c|}
\hline$\#$ & $\mathbf{b}$ & $\mathbf{b}^{++}$ & $\mathbf{b}^{*}$ & $\mathbf{b}^{\boldsymbol{*}^{++}}$ & $\mathbf{b}^{\mathbf{0}}$ & $\mathbf{b}^{\mathbf{0 + +}}$ & Seq. & $\mathbf{y}$ & $\mathbf{y}^{++}$ & $\mathbf{y}^{\mathbf{*}}$ & $\mathbf{y}^{\boldsymbol{*}^{++}}$ & $\mathbf{y}^{\mathbf{0}}$ & $\mathbf{y}^{\mathbf{0 + +}}$ & $\#$ \\
\hline $\mathbf{1}$ & 102.0550 & 51.5311 & & & 84.0444 & 42.5258 & $\mathbf{T}$ & & & & & & & $\mathbf{1 6}$ \\
\hline $\mathbf{2}$ & 215.1390 & 108.0731 & & & 197.1285 & 99.0679 & $\mathbf{L}$ & 1774.9044 & 887.9558 & 1757.8778 & 879.4426 & 1756.8938 & $\mathbf{8 7 8 . 9 5 0 6}$ & $\mathbf{1 5}$ \\
\hline $\mathbf{3}$ & 312.1918 & 156.5995 & & & 294.1812 & 147.5942 & $\mathbf{P}$ & $\mathbf{1 6 6 1 . 8 2 0 3}$ & $\mathbf{8 3 1 . 4 1 3 8}$ & 1644.7938 & 822.9005 & 1643.8098 & 822.4085 & $\mathbf{1 4}$ \\
\hline $\mathbf{4}$ & $\mathbf{4 2 5 . 2 7 5 8}$ & 213.1416 & & & $\mathbf{4 0 7 . 2 6 5 3}$ & 204.1363 & $\mathbf{L}$ & 1564.7676 & 782.8874 & 1547.7410 & 774.3741 & 1546.7570 & 773.8821 & $\mathbf{1 3}$ \\
\hline $\mathbf{5}$ & 522.3286 & 261.6679 & & & 504.3180 & 252.6627 & $\mathbf{P}$ & $\mathbf{1 4 5 1 . 6 8 3 5}$ & $\mathbf{7 2 6 . 3 4 5 4}$ & 1434.6570 & 717.8321 & 1433.6729 & 717.3401 & $\mathbf{1 2}$ \\
\hline
\end{tabular}




\begin{tabular}{|r|r|r|r|r|r|r|r|r|r|r|r|r|r|r|r|r|r|}
$\mathbf{6}$ & 635.4127 & 318.2100 & & & $\mathbf{6 1 7 . 4 0 2 1}$ & 309.2047 & L & 1354.6307 & 677.8190 & 1337.6042 & 669.3057 & 1336.6202 & 668.8137 & $\mathbf{1 1}$ \\
\hline $\mathbf{7}$ & 722.4447 & 361.7260 & & & 704.4341 & 352.7207 & S & $\mathbf{1 2 4 1 . 5 4 6 7}$ & 621.2770 & 1224.5201 & 612.7637 & 1223.5361 & 612.2717 & $\mathbf{1 0}$ \\
\hline $\mathbf{8}$ & 823.4924 & 412.2498 & & & 805.4818 & 403.2445 & T & $\mathbf{1 1 5 4 . 5 1 4 6}$ & 577.7610 & 1137.4881 & 569.2477 & 1136.5041 & 568.7557 & $\mathbf{9}$ \\
\hline $\mathbf{9}$ & 952.5350 & 476.7711 & & & 934.5244 & 467.7658 & E & $\mathbf{1 0 5 3 . 4 6 7 0}$ & 527.2371 & 1036.4404 & 518.7238 & 1035.4564 & $\mathbf{5 1 8 . 2 3 1 8}$ & $\mathbf{8}$ \\
\hline $\mathbf{1 0}$ & 1099.6034 & 550.3053 & & & 1081.5928 & 541.3001 & F & $\mathbf{9 2 4 . 4 2 4 4}$ & 462.7158 & 907.3978 & 454.2026 & & & & $\mathbf{7}$ \\
\hline $\mathbf{1 1}$ & 1259.6340 & 630.3207 & & & $\mathbf{1 2 4 1 . 6 2 3 5}$ & 621.3154 & C & 777.3560 & 389.1816 & 760.3294 & 380.6683 & & & & $\mathbf{6}$ \\
\hline $\mathbf{1 2}$ & $\mathbf{1 3 5 8 . 7 0 2 5}$ & 679.8549 & & & 1340.6919 & 670.8496 & V & $\mathbf{6 1 7 . 3 2 5 3}$ & 309.1663 & 600.2988 & 300.6530 & & & & $\mathbf{5}$ \\
\hline $\mathbf{1 3}$ & 1486.7610 & 743.8842 & 1469.7345 & 735.3709 & 1468.7505 & 734.8789 & Q & 518.2569 & 259.6321 & 501.2303 & 251.1188 & & & & $\mathbf{4}$ \\
\hline $\mathbf{1 4}$ & 1614.8196 & 807.9134 & 1597.7931 & 799.4002 & 1596.8090 & 798.9082 & Q & 390.1983 & 195.6028 & 373.1718 & 187.0895 & & & $\mathbf{3}$ \\
\hline $\mathbf{1 5}$ & $\mathbf{1 7 2 9 . 8 4 6 5}$ & 865.4269 & 1712.8200 & 856.9136 & 1711.8360 & 856.4216 & N & 262.1397 & 131.5735 & 245.1132 & 123.0602 & & & $\mathbf{2}$ \\
\hline $\mathbf{1 6}$ & & & & & & & K & 147.1128 & 74.0600 & 130.0863 & 65.5468 & & & $\mathbf{1}$ \\
\hline
\end{tabular}
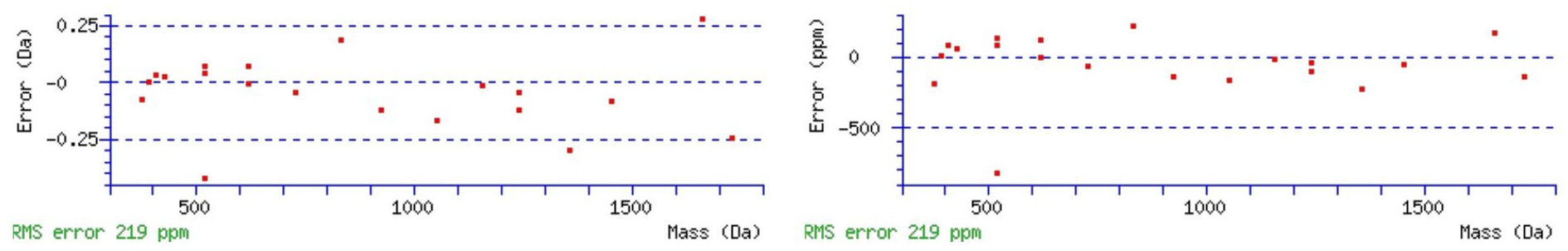

\section{All matches to this query}

\begin{tabular}{|l|l|l|l|}
\hline Score & Mr(calc): & Delta & \multicolumn{1}{|c|}{ Sequence } \\
\hline 61.1 & 1874.9448 & 0.0043 & TLPLPLSTEFCVQQNK \\
\hline 3.0 & 1873.9608 & 0.9883 & TLPLPLSTEFCVQQNK \\
\hline 2.2 & 1872.9308 & 2.0183 & MEKQNLTVVTEFILK \\
\hline 1.9 & 1872.9499 & 1.9992 & NVSFSYPSRPSAKILK \\
\hline 1.9 & 1872.9499 & 1.9992 & $\underline{\text { NVSFSYPSRPSAKILK }}$ \\
\hline 1.9 & 1873.9339 & 1.0152 & NVSFSYPSRPSAKILK \\
\hline 1.9 & 1873.9339 & 1.0152 & NVSFSYPSRPSAKILK \\
\hline 0.9 & 1873.9356 & 1.0135 & GRPIRITPDFSPENMK \\
\hline 0.3 & 1872.9532 & 1.9959 & LETKRLYCIILSER \\
\hline 0.2 & 1872.9243 & 2.0248 & $\underline{\text { IRTMVNLPVMSPFKK }}$ \\
\hline
\end{tabular}

Spectrum No: 515; Query: 85; Rank: 1

\section{Peptide View}

MS/MS Fragmentation of FNGSVSFFR

Found in IPI00369539, Tax_Id=10116 Gene_Symbol=Mfap4 Microfibrillar-associated protein 4

Match to Query 85: 1060.502108 from(531.258330,2+)

Title: 091008RatKidney_NH4Format01_23.2517.2517.2.dta

Data file K:INewmanPaper|Piliangl3SubProteomes\Piliang3SP\mgf5ppm\ERLIC_3SubProteomes5ppm.mgf 

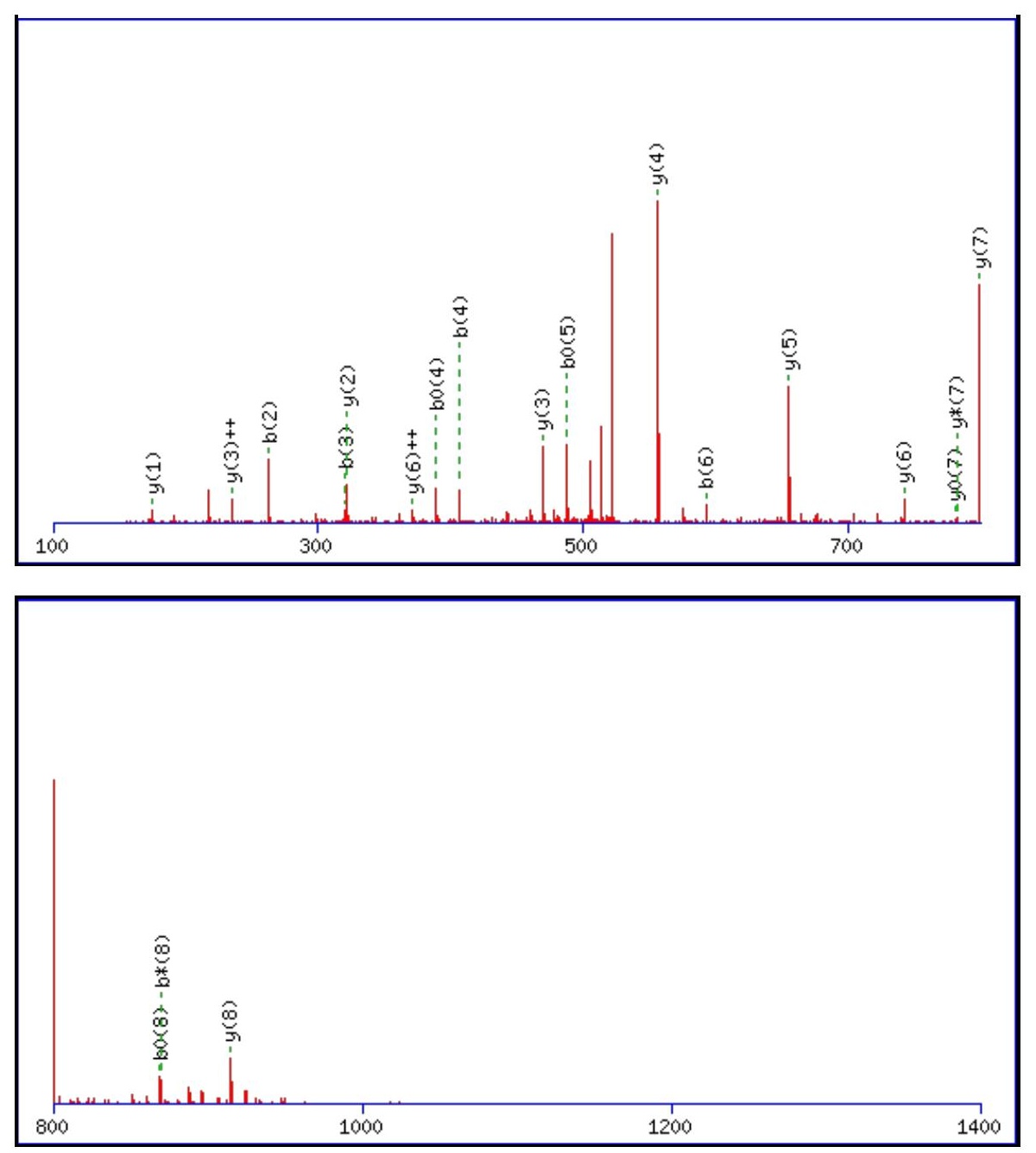

\begin{tabular}{rrrr}
\hline & 1 & 1 & 2000 \\
\hline
\end{tabular}

Monoisotopic mass of neutral peptide $\operatorname{Mr}($ calc): 1060.4978

Fixed modifications: Carbamidomethyl (C)

Variable modifications:

N2 : Deamidated_N (N)

Ions Score: 61 Expect: 0.0001

Matches (Bold Red): 20/82 fragment ions using 45 most intense peaks

\begin{tabular}{|c|c|c|c|c|c|c|c|c|c|c|c|c|c|c|}
\hline$\#$ & $\mathbf{b}$ & $\mathbf{b}^{++}$ & $\mathbf{b}^{*}$ & $\mathbf{b}^{*_{+}^{++}}$ & $\mathbf{b}^{\mathbf{0}}$ & $\mathbf{b}^{\mathbf{0 + +}}$ & $\mathbf{S e q}$ & $\mathbf{y}$ & $\mathbf{y}^{++}$ & $\mathbf{y}^{*}$ & $\mathbf{y}^{\mathbf{*}^{++}}$ & $\mathbf{y}^{\mathbf{0}}$ & $\mathbf{y}^{\mathbf{0}++}$ & $\#$ \\
\hline $\mathbf{1}$ & 148.0757 & 74.5415 & & & & & $\mathbf{F}$ & & & & & & & $\mathbf{9}$ \\
\hline $\mathbf{2}$ & $\mathbf{2 6 3 . 1 0 2 6}$ & 132.0550 & 246.0761 & 123.5417 & & & $\mathbf{N}$ & $\mathbf{9 1 4 . 4 3 6 6}$ & 457.7220 & 897.4101 & 449.2087 & 896.4261 & 448.7167 & $\mathbf{8}$ \\
\hline $\mathbf{3}$ & $\mathbf{3 2 0 . 1 2 4 1}$ & 160.5657 & 303.0975 & 152.0524 & & & $\mathbf{G}$ & $\mathbf{7 9 9 . 4 0 9 7}$ & 400.2085 & $\mathbf{7 8 2 . 3 8 3 2}$ & 391.6952 & $\mathbf{7 8 1 . 3 9 9 1}$ & 391.2032 & $\mathbf{7}$ \\
\hline $\mathbf{4}$ & $\mathbf{4 0 7 . 1 5 6 1}$ & 204.0817 & 390.1296 & 195.5684 & $\mathbf{3 8 9 . 1 4 5 6}$ & 195.0764 & $\mathbf{S}$ & $\mathbf{7 4 2 . 3 8 8 2}$ & $\mathbf{3 7 1 . 6 9 7 8}$ & 725.3617 & 363.1845 & 724.3777 & 362.6925 & $\mathbf{6}$ \\
\hline $\mathbf{5}$ & 506.2245 & 253.6159 & 489.1980 & 245.1026 & $\mathbf{4 8 8 . 2 1 4 0}$ & 244.6106 & $\mathbf{V}$ & $\mathbf{6 5 5 . 3 5 6 2}$ & 328.1817 & 638.3297 & 319.6685 & 637.3457 & 319.1765 & 5 \\
\hline
\end{tabular}




\begin{tabular}{|l|r|r|r|r|r|r|r|r|r|r|r|r|r|r|r|r|r|}
$\mathbf{6}$ & 593.2566 & 297.1319 & 576.2300 & 288.6186 & 575.2460 & 288.1266 & S & 556.2878 & 278.6475 & 539.2613 & 270.1343 & 538.2772 & 269.6423 & $\mathbf{4}$ \\
\hline $\mathbf{7}$ & 740.3250 & 370.6661 & 723.2984 & 362.1529 & 722.3144 & 361.6608 & F & $\mathbf{4 6 9 . 2 5 5 8}$ & 235.1315 & 452.2292 & 226.6183 & & & 3 \\
\hline $\mathbf{8}$ & 887.3934 & 444.2003 & $\mathbf{8 7 0 . 3 6 6 8}$ & 435.6871 & $\mathbf{8 6 9 . 3 8 2 8}$ & 435.1950 & $\mathbf{F}$ & $\mathbf{3 2 2 . 1 8 7 4}$ & 161.5973 & 305.1608 & 153.0840 & & & $\mathbf{2}$ \\
\hline $\mathbf{9}$ & & & & & & & R & $\mathbf{1 7 5 . 1 1 9 0}$ & 88.0631 & 158.0924 & 79.5498 & & & $\mathbf{1}$ \\
\hline
\end{tabular}
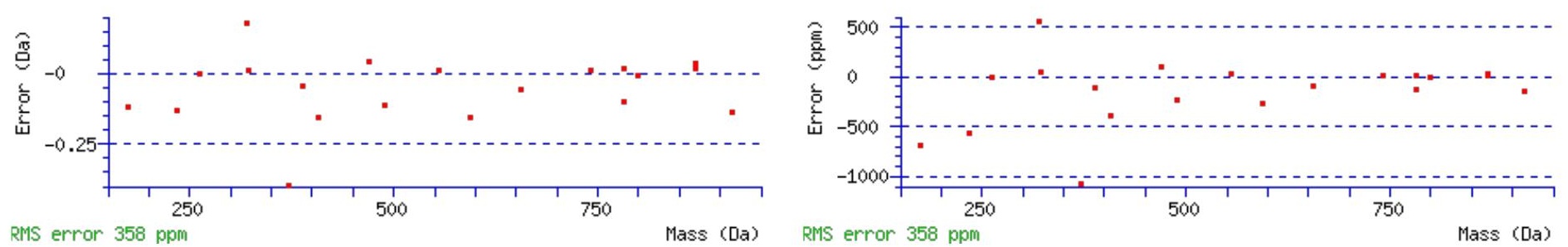

\section{All matches to this query}

\begin{tabular}{|l|l|l|l|}
\hline Score & Mr(calc): & Delta & \multicolumn{1}{|c|}{ Sequence } \\
\hline 61.0 & 1060.4978 & 0.0043 & FNGSVSFFR \\
\hline 9.0 & 1059.5075 & 0.9946 & VIVMLYNK \\
\hline 8.7 & 1059.5019 & 1.0002 & GLCPGASGVDK \\
\hline 8.1 & 1059.5026 & 0.9995 & FFFDVGSNK \\
\hline 7.6 & 1059.5002 & 1.0020 & VKFLSXSNK \\
\hline 6.4 & 1060.5107 & -0.0085 & FLKSPAYR \\
\hline 5.9 & 1060.4971 & 0.0050 & APGAGDSMSLR \\
\hline 5.6 & 1060.5037 & -0.0016 & LLSQDDQDK \\
\hline 3.6 & 1059.5002 & 1.0020 & VKFLSXSNK \\
\hline 2.9 & 1060.4938 & 0.0083 & VVYNEHGSR \\
\hline
\end{tabular}

Spectrum No: 516; Query: 1553; Rank: 1

\section{Peptide View}

MS/MS Fragmentation of EINISICPASQTSEHFR

Found in IPI00400747, Tax_Id=10116 Gene_Symbol=Man2b1 Mannosidase 2, alpha B1

Match to Query 1553: 1988.924508 from(995.469530,2+)

Title: 091008RatKidney_NH4Format01_24.2392.2392.2.dta

Data file K:INewmanPaper|Piliang|3SubProteomes\Piliang3SP\mgf5ppm\ERLIC_3SubProteomes5ppm.mgf

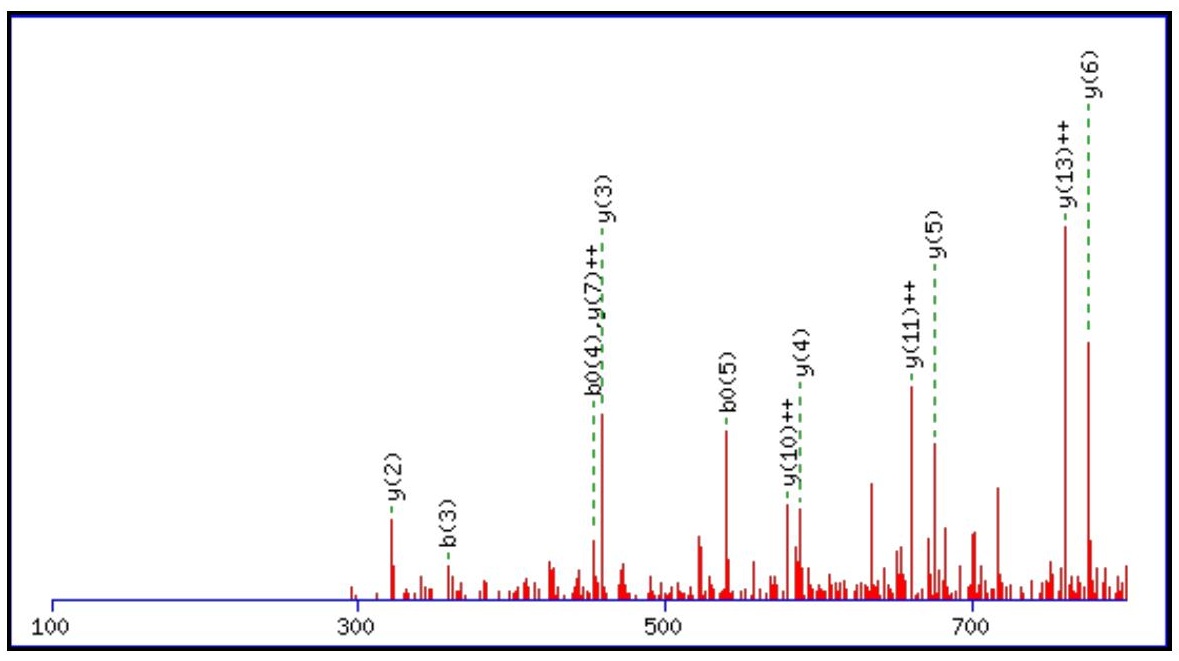



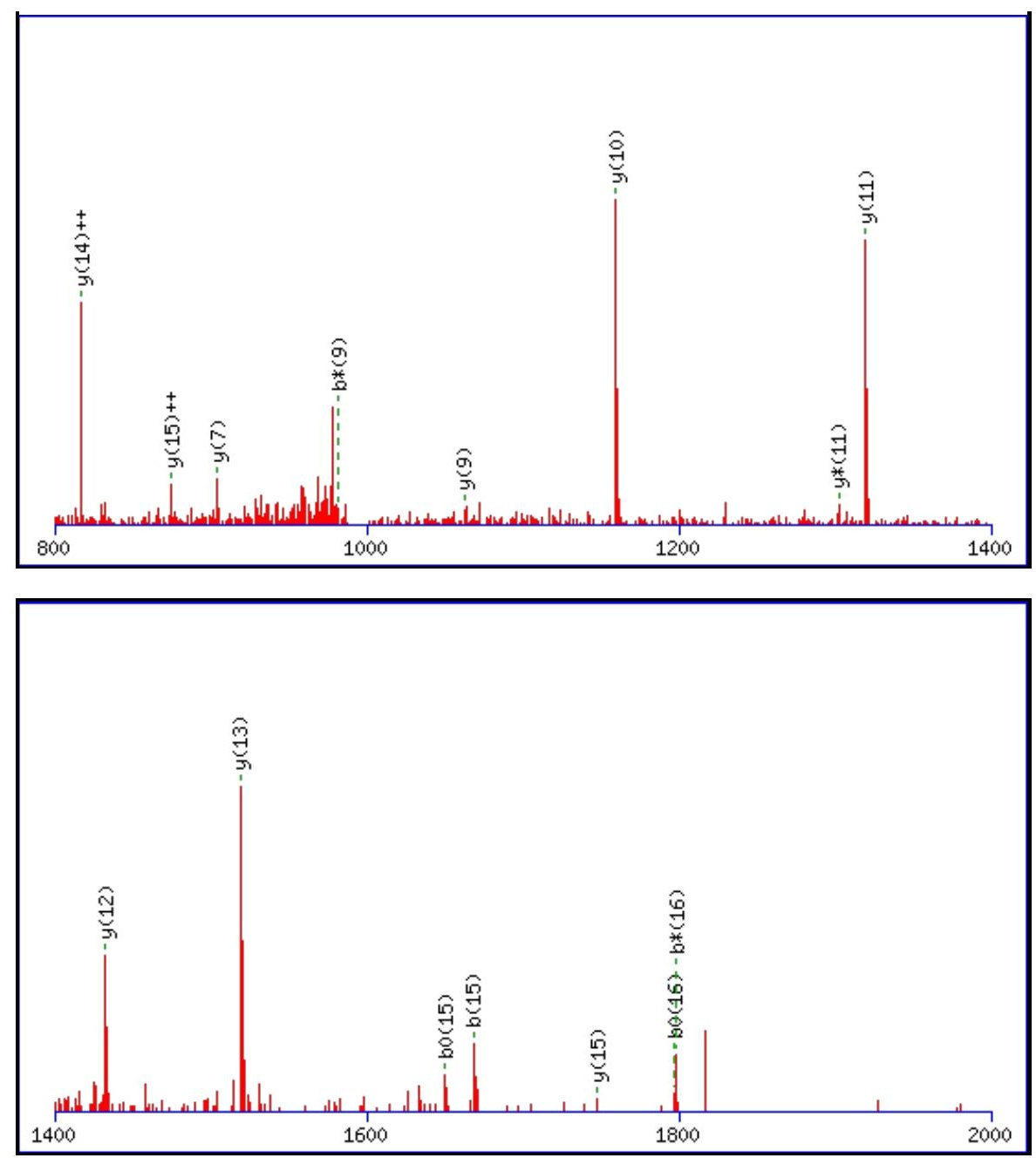

Monoisotopic mass of neutral peptide $\operatorname{Mr}($ calc): 1988.9262

Fixed modifications: Carbamidomethyl (C)

Variable modifications:

N3 : Deamidated $\mathrm{N}(\mathrm{N})$

Ions Score: 61 Expect: 0.00019

Matches (Bold Red): 27/182 fragment ions using 59 most intense peaks

\begin{tabular}{|c|c|c|c|c|c|c|c|c|c|c|c|c|c|c|}
\hline \# & b & $\mathbf{b}^{++}$ & b* & $\mathbf{b}^{*^{++}}$ & $\mathbf{b}^{0}$ & $\mathbf{b}^{0++}$ & Seq. & $\mathbf{y}$ & $\mathrm{y}^{++}$ & $\mathbf{y}^{*}$ & $\mathrm{y}^{*^{++}}$ & $\mathbf{y}^{0}$ & $y^{0++}$ & \# \\
\hline 1 & 130.0499 & 65.5286 & & & 112.0393 & 56.5233 & $\mathbf{E}$ & & & & & & & 17 \\
\hline 2 & 243.1339 & 122.0706 & & & 225.1234 & 113.0653 & I & & 930. & 1843.8 & 358 & .8803 & 921.9438 & 16 \\
\hline 3 & 358.1609 & 179.5841 & 341.1343 & 171.0708 & 340.1503 & 170.5788 & $\mathbf{N}$ & 1747.8068 & 874.4070 & 1730.7803 & 865.8938 & 1729.7962 & 865.4018 & 15 \\
\hline 4 & 471.2449 & 236.1261 & 454.2184 & 227.6128 & 453.2344 & 227.1208 & I & 1632.7799 & 816.8936 & 1615.7 & 808.3803 & 1614.7693 & 807.8883 & 14 \\
\hline 5 & 558.2770 & 279.6421 & 541.2504 & 271.1288 & 540.2664 & 270.6368 & S & 1519.6958 & 760.3515 & 1502.6693 & 751.8383 & 1501.6852 & 751.3463 & 13 \\
\hline 6 & 671.3610 & 336.1842 & 654.3345 & 327.6709 & 653.3505 & 327.1789 & I & 1432.6638 & 716.8355 & 1415.6372 & 708.3223 & 1414.6532 & 707.8302 & 12 \\
\hline 7 & 831.3917 & 416.1995 & 814.3651 & 407.6862 & 813.3811 & 407.1942 & C & 1319.5797 & 660.2935 & 1302.5532 & 651.7802 & 1301.5691 & 651.2882 & 11 \\
\hline 8 & 928.4444 & 464.7259 & 911.4179 & 456.2126 & 910.4339 & 455.7206 & $\mathbf{P}$ & 1159.5491 & 580.2782 & 1142.5225 & 571.7649 & 1141.5385 & 571.2729 & 10 \\
\hline 9 & 999.4816 & 500.2444 & 982.4550 & 491.7311 & 981.4710 & 491.2391 & A & 1062.4963 & 531.7518 & 1045.4697 & 523.2385 & 1044.4857 & 522.7465 & 9 \\
\hline 10 & 1086.5136 & 543.7604 & 1069.4870 & 535.2472 & 1068.5030 & 534.7551 & S & 991.4592 & 496.2332 & 974.4326 & 487.7200 & 973.4486 & 487.2279 & 8 \\
\hline 11 & 1214.5722 & 607.7897 & 1197.5456 & 599.2764 & 1196.5616 & 598.7844 & $\mathbf{Q}$ & 904.4272 & 452.7172 & 887.4006 & 444.2039 & 886.4166 & 443.7119 & 7 \\
\hline 12 & 1315.6198 & 658.3136 & 1298.5933 & 649.8003 & 1297.6093 & 649.3083 & $\mathbf{T}$ & 776.3686 & 388.6879 & 759.3420 & 380.1747 & 758.3580 & 379.6826 & 6 \\
\hline 13 & 1402.6519 & 701.8296 & 1385.6253 & 693.3163 & 1384.6413 & 692.8243 & S & 675.3209 & 338.1641 & 658.2944 & 329.6508 & 657.3103 & 329.1588 & 5 \\
\hline 14 & 1531.6945 & 766.3509 & 1514.6679 & 757.8376 & 1513.6839 & 757.3456 & $\mathbf{E}$ & 588.2889 & 294.6481 & 571.2623 & 286.1348 & 570.2783 & 285.6428 & 4 \\
\hline 15 & 1668.7534 & 834.8803 & 1651.7268 & 826.3670 & 1650.7428 & 825.8750 & H & 459.2463 & 230.1268 & 442.2197 & 221.6135 & & & 3 \\
\hline 16 & 1815.8218 & 908.4145 & 1798.7952 & 899.9013 & 1797.8112 & 899.4092 & $\mathbf{F}$ & 322.1874 & 161.5973 & 305.1608 & 153.0840 & & & 2 \\
\hline 17 & & & & & & & $\mathbf{R}$ & 175.1190 & 88.0631 & 158.0924 & 79.5498 & & & 1 \\
\hline
\end{tabular}



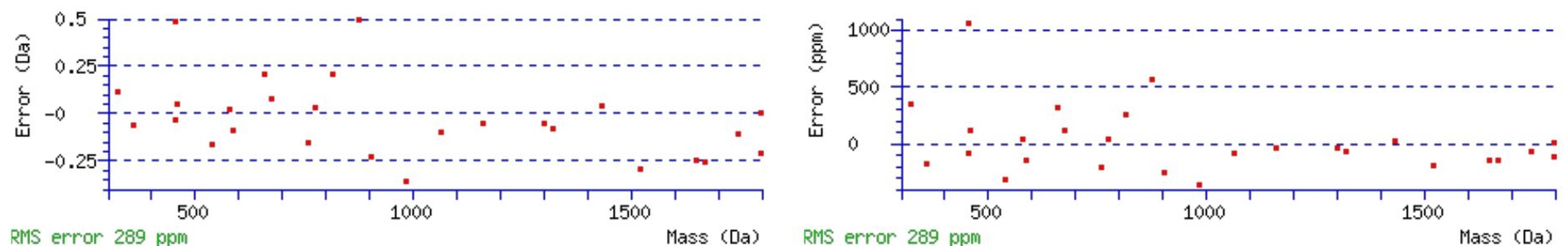

\section{All matches to this query}

\begin{tabular}{|l|l|l|l|}
\hline Score & Mr(calc): & Delta & \multicolumn{1}{c|}{ Sequence } \\
\hline 61.0 & 1988.9262 & -0.0017 & EINISICPASQTSEHFR \\
\hline 3.1 & 1986.9346 & 1.9899 & TPNGSLIPLHQGCKQTR \\
\hline 0.0 & 1988.9390 & -0.0145 & LGLDQRAHSLDMLSPEK \\
\hline
\end{tabular}

Spectrum No: 517; Query: 789; Rank: 1

\section{Peptide View}

\section{MS/MS Fragmentation of LHRLNASIADLQSK}

Found in IPI00190577, Tax_Id=10116 Gene_Symbol=Lama5 similar to Laminin alpha-5 chain precursor

Match to Query 789: 1565.856342 from(522.959390,3+)

Title: 100101RatKid_NS_deglyco_08.2020.2020.3.dta

Data file K:INewmanPaper|Piliangl3SubProteomes\Piliang3SP\mgf5ppm\ERLIC_3SubProteomes5ppm.mgf
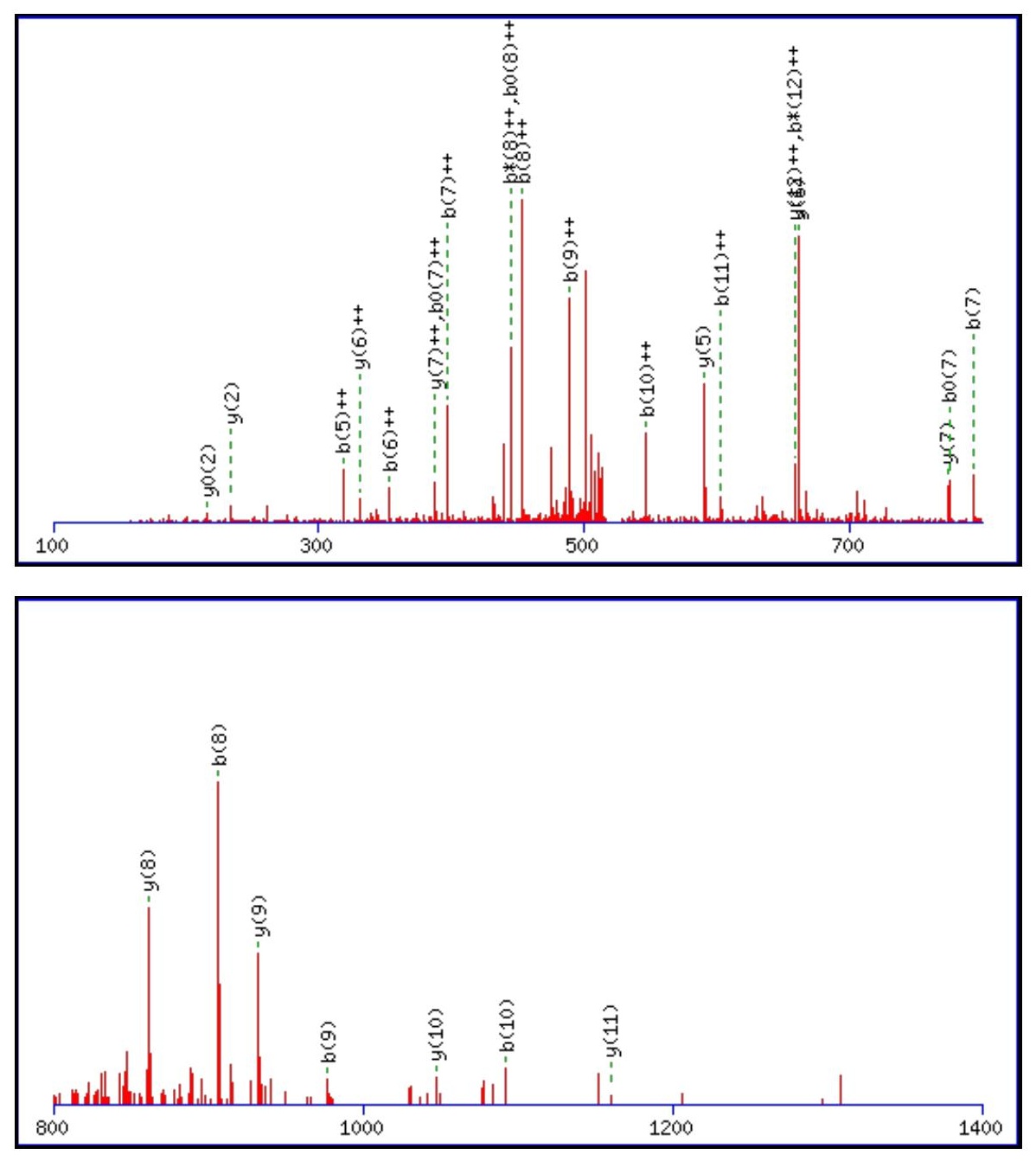


\begin{tabular}{rrrr}
\hline 1400 & 1600 & 1800 & 2000 \\
\hline
\end{tabular}

Monoisotopic mass of neutral peptide $\operatorname{Mr}($ calc): 1565.8525

Fixed modifications: Carbamidomethyl (C)

Variable modifications:

N5 : Deamidated_N (N)

Ions Score: 61 Expect: $5.4 \mathrm{e}-005$

Matches (Bold Red): 28/138 fragment ions using 38 most intense peaks

\begin{tabular}{|c|c|c|c|c|c|c|c|c|c|c|c|c|c|c|}
\hline \# & b & $\mathbf{b}^{++}$ & $\mathbf{b}^{*}$ & $\mathbf{b}^{*^{++}}$ & $\mathbf{b}^{\mathbf{0}}$ & $\mathbf{b}^{0++}$ & Seq. & $\mathbf{y}$ & $y^{++}$ & $\mathbf{y}^{*}$ & $\mathbf{y}^{*^{++}}$ & $\mathbf{y}^{0}$ & $\mathbf{y}^{\mathbf{0 + +}}$ & \# \\
\hline 1 & 114.0913 & 57.5493 & & & & & $\mathbf{L}$ & & & & & & & 14 \\
\hline 2 & 251.1503 & 126.0788 & & & & & $\mathbf{H}$ & 1453.7758 & 727.3915 & 1436.7492 & 718.8782 & 1435.7652 & 718.3862 & 13 \\
\hline 3 & 407.2514 & 204.1293 & 390.2248 & 195.6160 & & & $\mathbf{R}$ & 1316.7168 & 658.8621 & 1299.6903 & 650.3488 & 1298.7063 & 649.8568 & 12 \\
\hline 4 & 520.3354 & 260.6714 & 503.3089 & 252.1581 & & & $\mathbf{L}$ & 1160.6157 & 580.8115 & 1143.5892 & 572.2982 & 1142.6052 & 571.8062 & 11 \\
\hline 5 & 635.3624 & 318.1848 & 618.3358 & 309.6715 & & & $\mathbf{N}$ & 1047.5317 & 524.2695 & 1030.5051 & 515.7562 & 1029.5211 & 515.2642 & 10 \\
\hline 6 & 706.3995 & 353.7034 & 689.3729 & 345.1901 & & & A & 932.5047 & 466.7560 & 915.4782 & 458.2427 & 914.4942 & 457.7507 & 9 \\
\hline 7 & 793.4315 & 397.2194 & 776.4050 & 388.7061 & 775.4209 & 388.2141 & S & 861.4676 & 431.2374 & 844.4411 & 422.7242 & 843.4571 & 422.2322 & 8 \\
\hline 8 & 906.5156 & 453.7614 & 889.4890 & 445.2481 & 888.5050 & 444.7561 & I & 774.4356 & 387.7214 & 757.4090 & 379.2082 & 756.4250 & 378.7162 & 7 \\
\hline 9 & 977.5527 & 489.2800 & 960.5261 & 480.7667 & 959.5421 & 480.2747 & A & 661.3515 & 331.1794 & 644.3250 & 322.6661 & 643.3410 & 322.1741 & 6 \\
\hline 10 & 1092.5796 & 546.7935 & 1075.5531 & 538.2802 & 1074.5691 & 537.7882 & D & 590.3144 & 295.6608 & 573.2879 & 287.1476 & 572.3039 & 286.6556 & 5 \\
\hline 11 & 1205.6637 & 603.3355 & 1188.6371 & 594.8222 & 1187.6531 & 594.3302 & $\mathbf{L}$ & 475.2875 & 238.1474 & 458.2609 & 229.6341 & 457.2769 & 229.1421 & 4 \\
\hline 12 & 1333.7223 & 667.3648 & 1316.6957 & 658.8515 & 1315.7117 & 658.3595 & $\mathbf{Q}$ & 362.2034 & 181.6053 & 345.1769 & 173.0921 & 344.1928 & 172.6001 & 3 \\
\hline 13 & 1420.7543 & 710.8808 & 1403.7277 & 702.3675 & 1402.7437 & 701.8755 & $S$ & 234.1448 & 117.5761 & 217.1183 & 109.0628 & 216.1343 & 108.5708 & 2 \\
\hline 14 & & & & & & & $\mathbf{K}$ & 147.1128 & 74.0600 & 130.0863 & 65.5468 & & & 1 \\
\hline
\end{tabular}
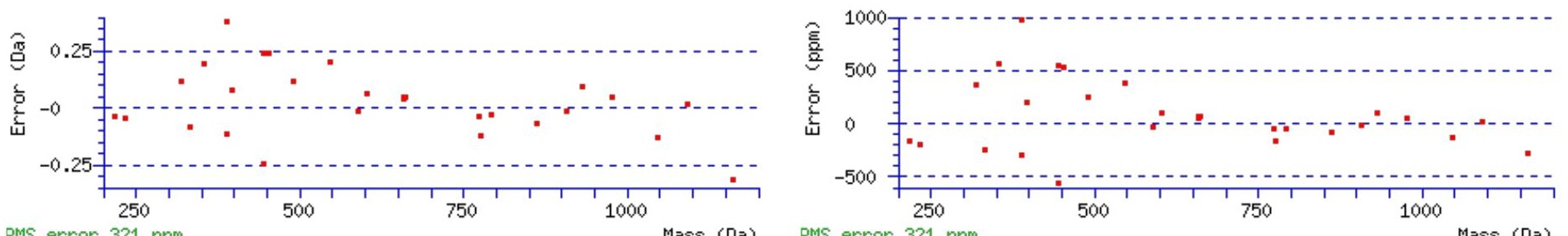

RMS error 321 ppm

Mass (Da) RMS error 321 ppm

All matches to this query

\begin{tabular}{|l|l|l|l|}
\hline Score & Mr(calc): & Delta & \multicolumn{1}{c|}{ Sequence } \\
\hline 60.9 & 1565.8525 & 0.0038 & LHRLNASIADLQSK \\
\hline 12.1 & 1564.8685 & 0.9878 & $\underline{\text { LHRLNASIADLQSK }}$ \\
\hline 7.4 & 1565.8413 & 0.0151 & STLKPNAPXPNSAAVK \\
\hline 5.6 & 1565.8413 & 0.0151 & NVXISLHLELANSK \\
\hline 5.6 & 1565.8413 & 0.0151 & NVXISLHLELANSK \\
\hline 2.6 & 1565.8413 & 0.0151 & STLKPNAPXPNSAAVK \\
\hline 1.2 & 1563.8620 & 1.9943 & $\underline{\text { ILHNGEELAKLEAK }}$ \\
\hline
\end{tabular}


Spectrum No: 518; Query: 2298; Rank: 1

\section{Peptide View}

MS/MS Fragmentation of DVLEDLNIKDLLTNQSDFSGNTK

Found in IPI00210824, Tax_Id=10116 Gene_Symbol=Serpina6 Corticosteroid-binding globulin precursor

Match to Query 2298: 2579.267022 from(860.762950,3+)

Title: 100101RatKid_NS_deglyco_25.5007.5007.3.dta

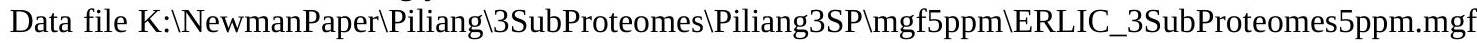
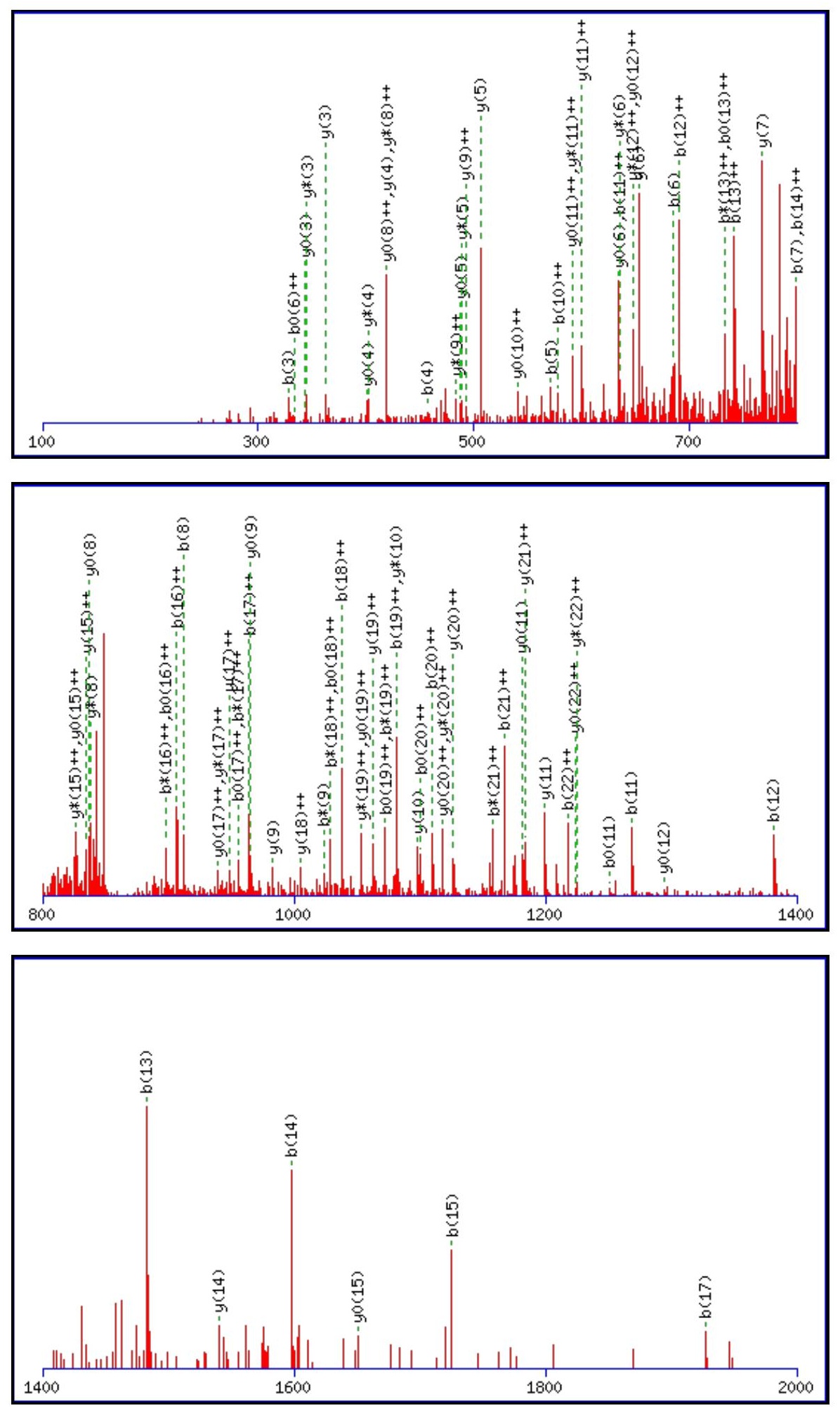
Monoisotopic mass of neutral peptide $\operatorname{Mr}($ calc): 2579.2602

Fixed modifications: Carbamidomethyl (C)

Variable modifications:

N14 : Deamidated_N (N)

Ions Score: 61 Expect: 0.00021

Matches (Bold Red): 89/250 fragment ions using 151 most intense peaks

\begin{tabular}{|c|c|c|c|c|c|c|c|c|c|c|c|c|c|c|}
\hline \# & b & $\mathbf{b}^{++}$ & $\mathbf{b}^{*}$ & $\mathbf{b}^{*^{++}}$ & $\mathbf{b}^{0}$ & $\mathbf{b}^{\mathbf{0 + +}}$ & Seq. & $\mathbf{y}$ & $\mathbf{y}^{++}$ & $\mathbf{y}^{*}$ & $\mathrm{y}^{*^{++}}$ & $\mathbf{y}^{\mathbf{0}}$ & $\mathbf{y}^{0++}$ & \# \\
\hline 1 & 116.0342 & 58.5207 & & & 98.0237 & 49.5155 & D & & & & & & & 23 \\
\hline 2 & 215.1026 & 108.0550 & & & 197.0921 & 99.0497 & V & 2465.2406 & 1233.1239 & 2448.2140 & 1224.6107 & 2447.2300 & 1224.1186 & 22 \\
\hline 3 & 328.1867 & 164.5970 & & & 310.1761 & 155.5917 & $\mathbf{L}$ & 2366.1722 & 1183.5897 & 2349.1456 & 1175.0764 & 2348.1616 & 1174.5844 & 21 \\
\hline 4 & 457.2293 & 229.1183 & & & 439.2187 & 220.1130 & $\mathbf{E}$ & 2253.0881 & 1127.0477 & 2236.0616 & 1118.5344 & 2235.0775 & 1118.0424 & 20 \\
\hline 5 & 572.2562 & 286.6318 & & & 554.2457 & 277.6265 & D & 2124.0455 & 1062.5264 & 2107.0190 & 1054.0131 & 2106.0349 & 1053.5211 & 19 \\
\hline 6 & 685.3403 & 343.1738 & & & 667.3297 & 334.1685 & $\mathbf{L}$ & 2009.0186 & 1005.0129 & 1991.9920 & 996.4996 & 1991.0080 & 996.0076 & 18 \\
\hline 7 & 799.3832 & 400.1953 & 782.3567 & 391.6820 & 781.3727 & 391.1900 & $\mathbf{N}$ & 1895.9345 & 948.4709 & 1878.9080 & 939.9576 & 1877.9239 & 939.4656 & 17 \\
\hline 8 & 912.4673 & 456.7373 & 895.4407 & 448.2240 & 894.4567 & 447.7320 & I & 1781.8916 & 891.4494 & 1764.8650 & 882.9362 & 1763.8810 & 882.4441 & 16 \\
\hline 9 & 1040.5623 & 520.7848 & 1023.5357 & 512.2715 & 1022.5517 & 511.7795 & $\mathbf{K}$ & 1668.8075 & 834.9074 & 1651.7810 & 826.3941 & 1650.7969 & 825.9021 & 15 \\
\hline 10 & 1155.5892 & 578.2982 & 1138.5626 & 569.7850 & 1137.5786 & 569.2930 & D & 1540.7126 & 770.8599 & 1523.6860 & 762.3466 & 1522.7020 & 761.8546 & 14 \\
\hline 11 & 1268.6733 & 634.8403 & 1251.6467 & 626.3270 & 1250.6627 & 625.8350 & $\mathbf{L}$ & 1425.6856 & 713.3464 & 1408.6591 & 704.8332 & 1407.6750 & 704.3412 & 13 \\
\hline 12 & 1381.7573 & 691.3823 & 1364.7308 & 682.8690 & 1363.7468 & 682.3770 & $\mathbf{L}$ & 1312.6015 & 656.8044 & 1295.5750 & 648.2911 & 1294.5910 & 647.7991 & 12 \\
\hline 13 & 1482.8050 & 741.9061 & 1465.7785 & 733.3929 & 1464.7944 & 732.9009 & $\mathbf{T}$ & 1199.5175 & 600.2624 & 1182.4909 & 591.7491 & 1181.5069 & 591.2571 & 11 \\
\hline 14 & 1597.8319 & 799.4196 & 1580.8054 & 790.9063 & 1579.8214 & 790.4143 & $\mathbf{N}$ & 1098.4698 & 549.7385 & 1081.4433 & 541.2253 & 1080.4592 & 540.7333 & 10 \\
\hline 15 & 1725.8905 & 863.4489 & 1708.8640 & 854.9356 & 1707.8799 & 854.4436 & $\mathbf{Q}$ & 983.4429 & 492.2251 & 966.4163 & 483.7118 & 965.4323 & 483.2198 & 9 \\
\hline 16 & 1812.9225 & 906.9649 & 1795.8960 & 898.4516 & 1794.9120 & 897.9596 & $\mathrm{~S}$ & 855.3843 & 428.1958 & 838.3577 & 419.6825 & 837.3737 & 419.1905 & 8 \\
\hline 17 & 1927.9495 & 964.4784 & 1910.9229 & 955.9651 & 1909.9389 & 955.4731 & D & 768.3523 & 384.6798 & 751.3257 & 376.1665 & 750.3417 & 375.6745 & 7 \\
\hline 18 & 2075.0179 & 1038.0126 & 2057.9914 & 1029.4993 & 2057.0073 & 1029.0073 & $\mathbf{F}$ & 653.3253 & 327.1663 & 636.2988 & 318.6530 & 635.3148 & 318.1610 & 6 \\
\hline 19 & 2162.0499 & 1081.5286 & 2145.0234 & 1073.0153 & 2144.0394 & 1072.5233 & $\mathrm{~S}$ & 506.2569 & 253.6321 & 489.2304 & 245.1188 & 488.2463 & 244.6268 & 5 \\
\hline 20 & 2219.0714 & 1110.0393 & 2202.0448 & 1101.5261 & 2201.0608 & 1101.0341 & G & 419.2249 & 210.1161 & 402.1983 & 201.6028 & 401.2143 & 201.1108 & 4 \\
\hline 21 & 2333.1143 & 1167.0608 & 2316.0878 & 1158.5475 & 2315.1038 & 1158.0555 & $\mathbf{N}$ & 362.2034 & 181.6053 & 345.1769 & 173.0921 & 344.1928 & 172.6001 & 3 \\
\hline 22 & 2434.1620 & 1217.5846 & 2417.1354 & 1209.0714 & 2416.1514 & 1208.5794 & $\mathbf{T}$ & 248.1605 & 124.5839 & 231.1339 & 116.0706 & 230.1499 & 115.5786 & 2 \\
\hline 23 & & & & & & & $\mathbf{K}$ & 147.1128 & 74.0600 & 130.0863 & 65.5468 & & & 1 \\
\hline
\end{tabular}
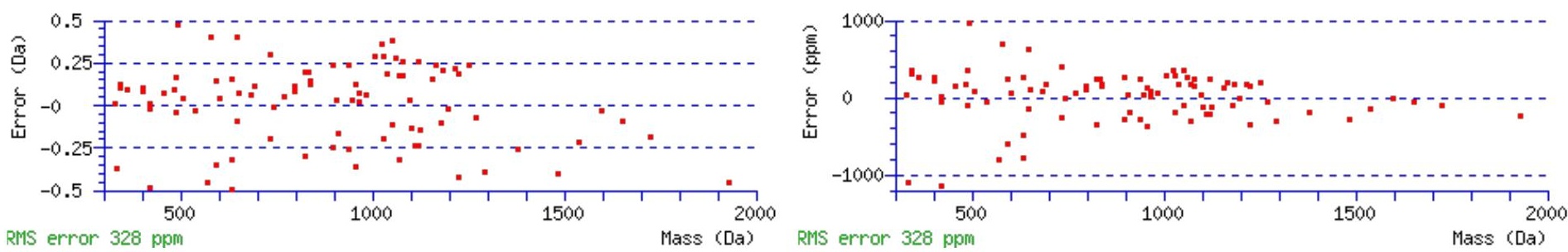

\section{All matches to this query}

\begin{tabular}{|l|l|l|l|}
\hline Score & Mr(calc): & Delta & \multicolumn{1}{|c|}{ Sequence } \\
\hline 60.8 & 2579.2602 & 0.0068 & DVLEDLNIKDLLTNQSDFSGNTK \\
\hline 48.3 & 2579.2602 & 0.0068 & DVLEDLNIKDLLTNQSDFSGNTK \\
\hline 34.5 & 2579.2602 & 0.0068 & DVLEDLNIKDLLTNQSDFSGNTK \\
\hline 20.3 & 2578.2762 & 0.9908 & DVLEDLNIKDLLTNQSDFSGNTK \\
\hline 2.6 & 2579.2755 & -0.0085 & GSEDSSYNPVFSALPETNILNVVK \\
\hline 2.6 & 2577.2741 & 1.9929 & $\underline{\text { TLLSVGGWSFGSERFSRIVSNAK }}$ \\
\hline 2.2 & 2577.2452 & 2.0218 & TSKRIEGTVDISVITNGIQATSK \\
\hline 1.9 & 2579.2744 & -0.0074 & $\underline{\text { NVNPNWSVNIKGGTTRTLAVASAK }}$ \\
\hline 1.8 & 2579.2744 & -0.0074 & $\underline{\text { NVNPNWSVNIKGGTTRTLAVASAK }}$ \\
\hline 1.7 & 2579.2744 & -0.0074 & NVNPNWSVNIKGGTTRTLAVASAK \\
\hline
\end{tabular}


Spectrum No: 519; Query: 2415; Rank: 1

\section{Peptide View}

MS/MS Fragmentation of KVEEANKAEENGSEALMTLDDQANK

Found in IPI00212608, Tax_Id=10116 Gene_Symbol=Dab2 Isoform p82 of Disabled homolog 2

Match to Query 2415: 2751.247842 from(918.089890,3+)

Title: 091008RatKidney_NH4Format02_24.2660.2660.3.dta

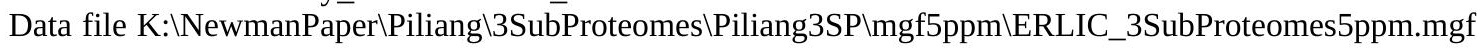
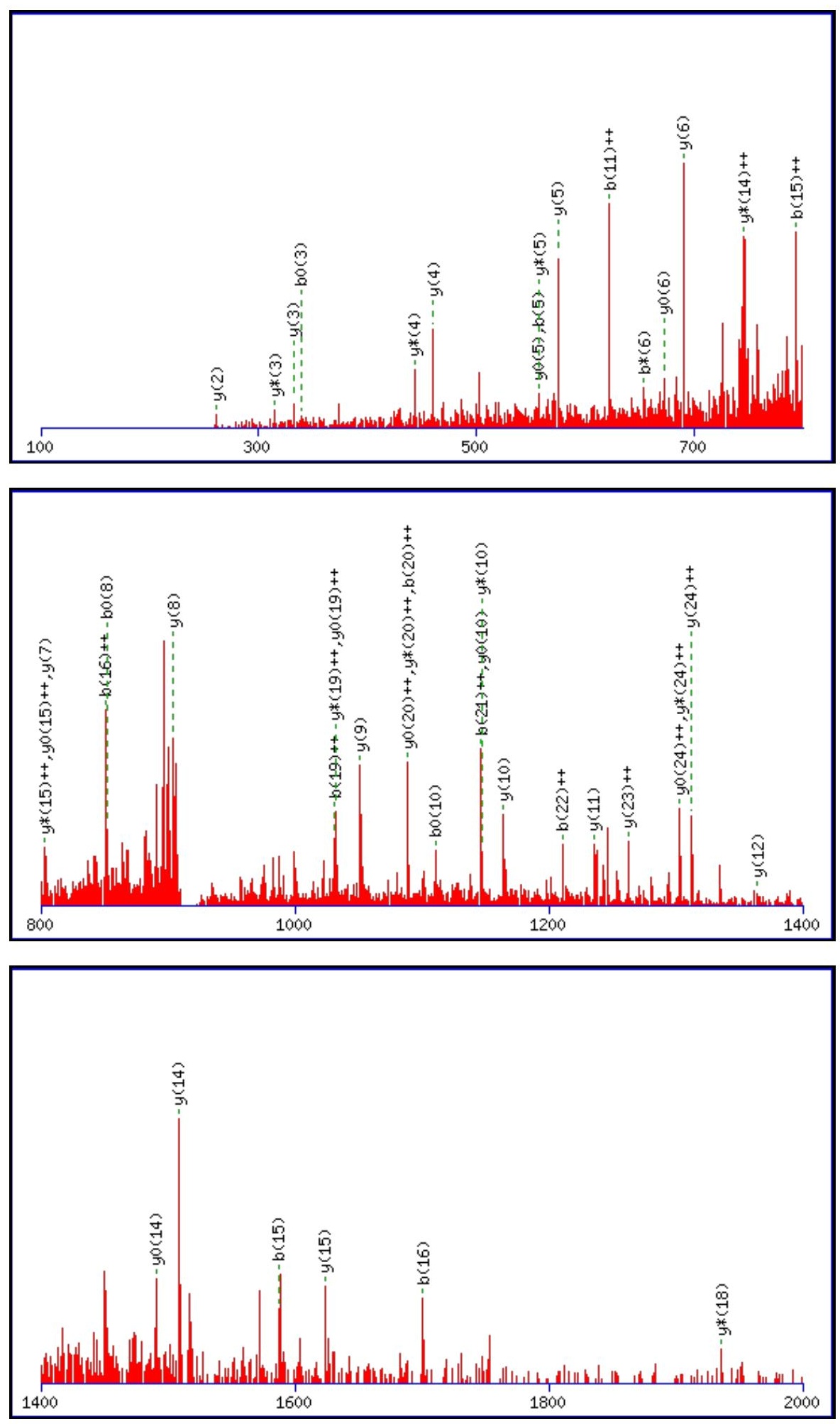

Monoisotopic mass of neutral peptide $\operatorname{Mr}($ calc): 2750.2552 


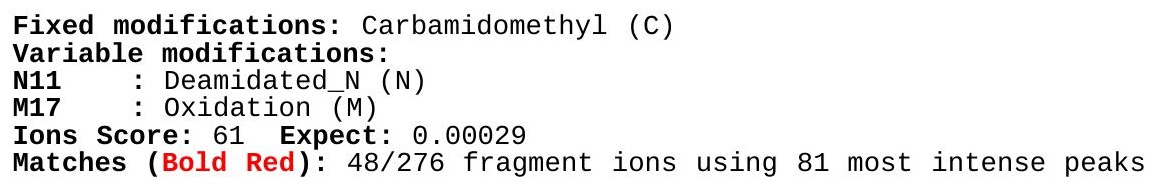

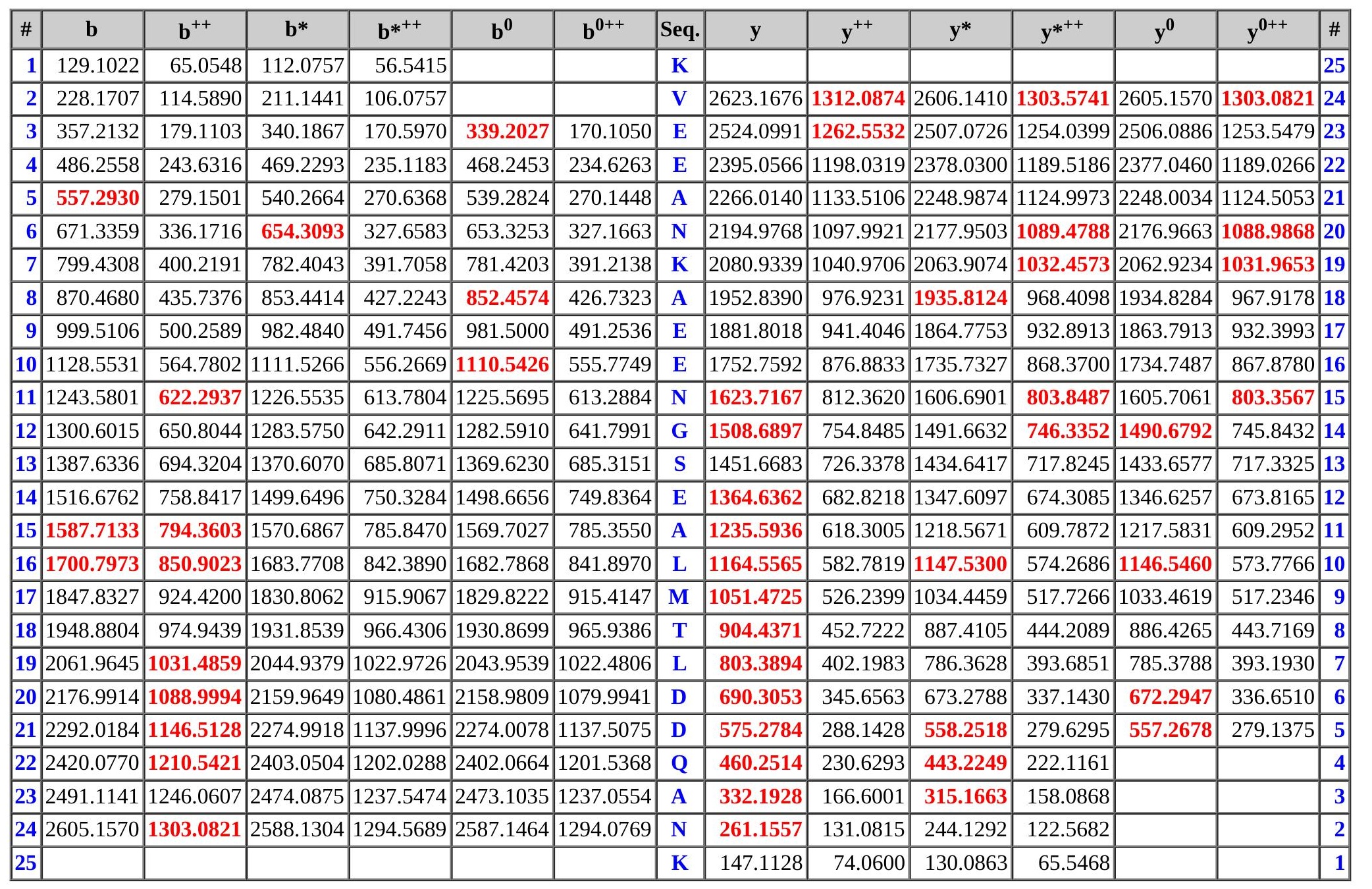
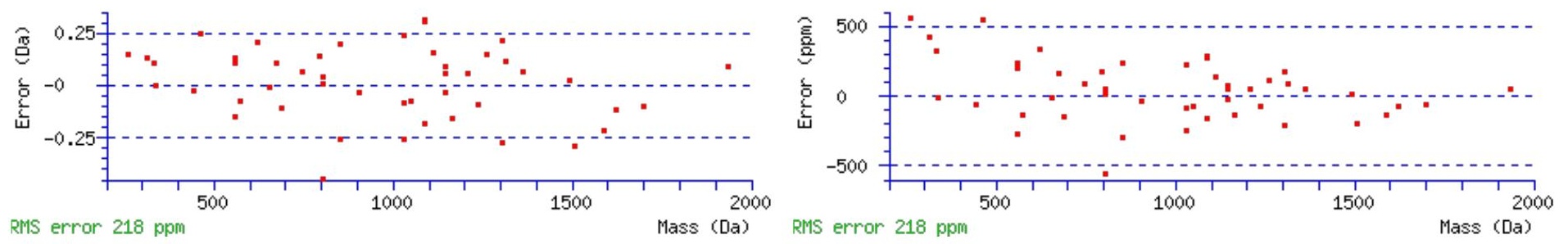

\section{All matches to this query}

\begin{tabular}{|l|l|l|l|}
\hline Score & Mr(calc): & Delta & \multicolumn{1}{|c|}{ Sequence } \\
\hline 60.8 & 2750.2552 & 0.9927 & KVEEANKAEENGSEALMTLDDQANK \\
\hline 58.1 & 2751.2392 & 0.0087 & $\underline{\text { KVEEANKAEENGSEALMTLDDQANK }}$ \\
\hline 56.8 & 2750.2552 & 0.9927 & $\underline{\text { KVEEANKAEENGSEALMTLDDQANK }}$ \\
\hline 28.6 & 2751.2392 & 0.0087 & $\underline{\text { KVEEANKAEENGSEALMTLDDQANK }}$ \\
\hline 26.4 & 2751.2392 & 0.0087 & $\underline{\text { KVEEANKAEENGSEALMTLDDQANK }}$ \\
\hline 3.5 & 2751.2629 & -0.0151 & $\underline{\text { AQVNSRKAQTLYNNIDTTIQNAK }}$ \\
\hline 3.5 & 2751.2629 & -0.0151 & $\underline{\text { AQVNSRKAQTLYNNIDTTIQNAK }}$ \\
\hline 3.4 & 2751.2652 & -0.0174 & QEDAMDMEPEIKQATMVSESEILK \\
\hline 2.3 & 2751.2282 & 0.0196 & AANQPEAVSTDELKSLLALTRER \\
\hline 1.8 & 2750.2510 & 0.9969 & $\underline{\text { ELTQVFEFQLASEDMKALDGLNR }}$
\end{tabular}


Spectrum No: 520; Query: 402; Rank: 1

\section{Peptide View}

MS/MS Fragmentation of NELMLNSSLMR

Found in IPI00554123, Tax_Id=10116 Gene_Symbol=Agrn Agrin precursor

Match to Query 402: 1339.610608 from(670.812580,2+)

Title: 091008RatKidney_NH4Format01_23.1717.1717.2.dta

Data file K:\NewmanPaper\Piliang \3SubProteomes $\backslash$ Piliang3SP $\backslash$ mgf5ppm\ERLIC_3SubProteomes5ppm.mgf
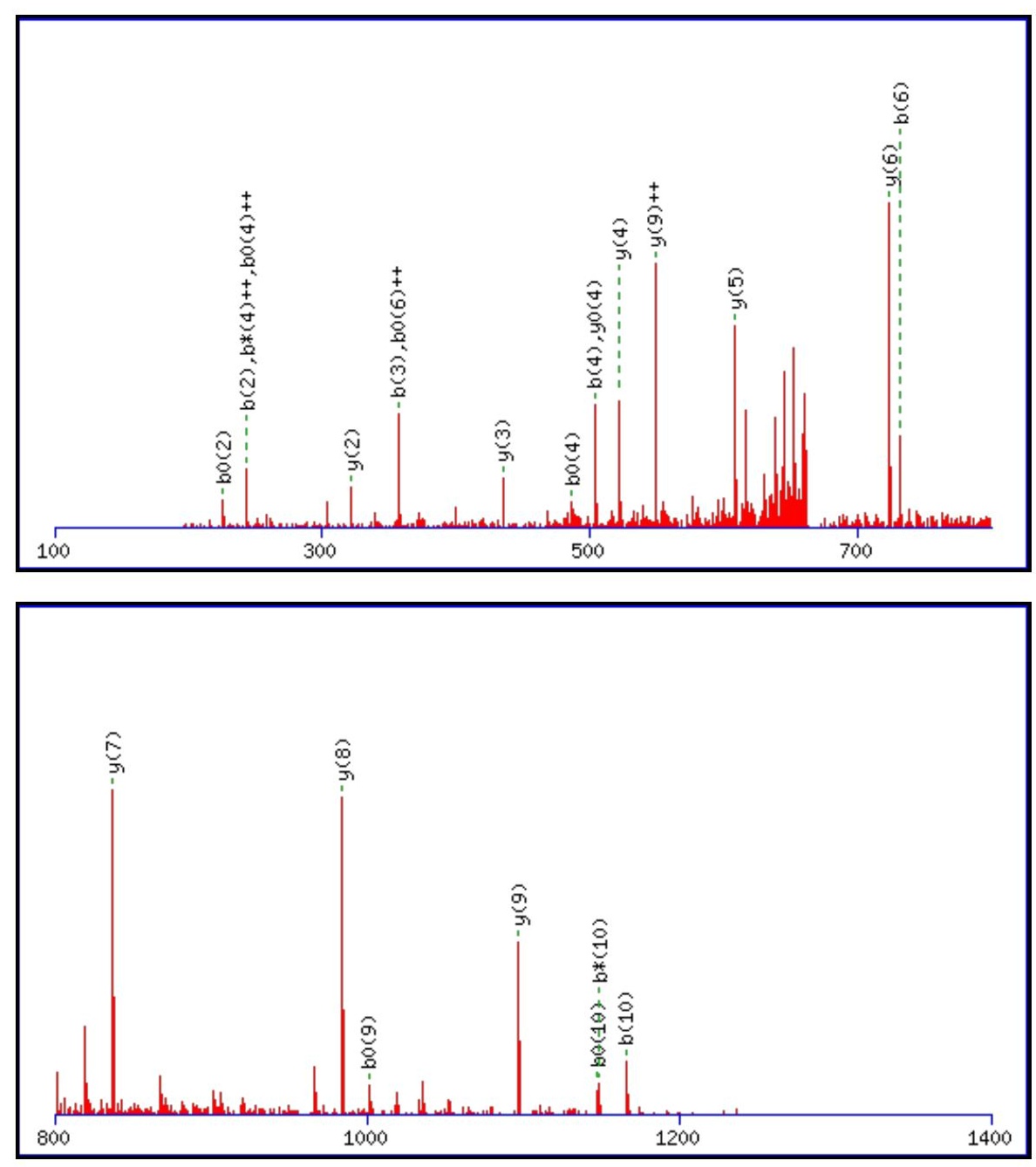
Monoisotopic mass of neutral peptide $\operatorname{Mr}($ calc): 1339.6111

Fixed modifications: Carbamidomethyl (C)

Variable modifications:

M4 : Oxidation (M)

N6 : Deamidated_N (N)

M10 : Oxidation (M)

Ions Score: 61 Expect : 0.00012

Matches (Bold Red): 23/112 fragment ions using 32 most intense peaks

\begin{tabular}{|r|c|c|c|c|c|c|c|c|c|c|c|c|c|c|}
\hline$\#$ & $\mathbf{b}$ & $\mathbf{b}^{++}$ & $\mathbf{b}^{*}$ & $\mathbf{b}^{\boldsymbol{*}_{++}}$ & $\mathbf{b}^{\mathbf{0}}$ & $\mathbf{b}^{\mathbf{0 + +}}$ & $\mathbf{S e q}$ & $\mathbf{y}$ & $\mathbf{y}^{++}$ & $\mathbf{y}^{\mathbf{*}}$ & $\mathbf{y}^{\boldsymbol{*}^{++}}$ & $\mathbf{y}^{\mathbf{0}}$ & $\mathbf{y}^{\mathbf{0 + +}}$ & $\#$ \\
\hline $\mathbf{1}$ & 115.0502 & 58.0287 & 98.0237 & 49.5155 & & & $\mathbf{N}$ & & & & & & & $\mathbf{1 1}$ \\
\hline $\mathbf{2}$ & $\mathbf{2 4 4 . 0 9 2 8}$ & 122.5500 & 227.0662 & 114.0368 & $\mathbf{2 2 6 . 0 8 2 2}$ & 113.5448 & $\mathbf{E}$ & 1226.5755 & 613.7914 & 1209.5490 & 605.2781 & 1208.5650 & 604.7861 & $\mathbf{1 0}$ \\
\hline $\mathbf{3}$ & $\mathbf{3 5 7 . 1 7 6 9}$ & 179.0921 & 340.1503 & 170.5788 & 339.1663 & 170.0868 & $\mathbf{L}$ & $\mathbf{1 0 9 7 . 5 3 2 9}$ & 549.2701 & 1080.5064 & 540.7568 & 1079.5224 & 540.2648 & $\mathbf{9}$ \\
\hline $\mathbf{4}$ & $\mathbf{5 0 4 . 2 1 2 3}$ & 252.6098 & 487.1857 & 244.0965 & $\mathbf{4 8 6 . 2 0 1 7}$ & $\mathbf{2 4 3 . 6 0 4 5}$ & $\mathbf{M}$ & $\mathbf{9 8 4 . 4 4 8 9}$ & 492.7281 & 967.4223 & 484.2148 & 966.4383 & 483.7228 & $\mathbf{8}$ \\
\hline $\mathbf{5}$ & 617.2963 & 309.1518 & 600.2698 & 300.6385 & 599.2858 & 300.1465 & $\mathbf{L}$ & $\mathbf{8 3 7 . 4 1 3 5}$ & 419.2104 & 820.3869 & 410.6971 & 819.4029 & 410.2051 & $\mathbf{7}$ \\
\hline $\mathbf{6}$ & $\mathbf{7 3 2 . 3 2 3 3}$ & 366.6653 & 715.2967 & 358.1520 & 714.3127 & 357.6600 & $\mathbf{N}$ & $\mathbf{7 2 4 . 3 2 9 4}$ & 362.6683 & 707.3029 & 354.1551 & 706.3188 & 353.6631 & $\mathbf{6}$ \\
\hline $\mathbf{7}$ & 819.3553 & 410.1813 & 802.3287 & 401.6680 & 801.3447 & 401.1760 & $\mathbf{S}$ & $\mathbf{6 0 9 . 3 0 2 5}$ & 305.1549 & 592.2759 & 296.6416 & 591.2919 & 296.1496 & $\mathbf{5}$ \\
\hline $\mathbf{8}$ & 906.3873 & 453.6973 & 889.3608 & 445.1840 & 888.3768 & 444.6920 & $\mathbf{S}$ & $\mathbf{5 2 2 . 2 7 0 4}$ & 261.6389 & 505.2439 & 253.1256 & $\mathbf{5 0 4 . 2 5 9 9}$ & 252.6336 & $\mathbf{4}$ \\
\hline $\mathbf{9}$ & 1019.4714 & 510.2393 & 1002.4448 & 501.7261 & $\mathbf{1 0 0 1 . 4 6 0 8}$ & 501.2340 & $\mathbf{L}$ & $\mathbf{4 3 5 . 2 3 8 4}$ & 218.1228 & 418.2119 & 209.6096 & & & $\mathbf{3}$ \\
\hline $\mathbf{1 0}$ & $\mathbf{1 1 6 6 . 5 0 6 8}$ & 583.7570 & $\mathbf{1 1 4 9 . 4 8 0 2}$ & 575.2438 & $\mathbf{1 1 4 8 . 4 9 6 2}$ & 574.7517 & $\mathbf{M}$ & 322.1544 & 161.5808 & 305.1278 & 153.0675 & & & $\mathbf{2}$ \\
\hline $\mathbf{1 1}$ & & & & & & & $\mathbf{R}$ & 175.1190 & 88.0631 & 158.0924 & 79.5498 & & & $\mathbf{1}$ \\
\hline
\end{tabular}
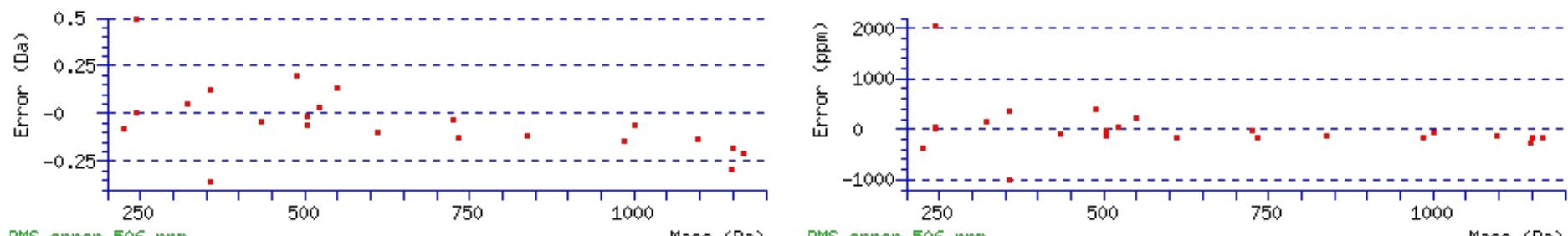

RMS error 506 ppm

All matches to this query

\begin{tabular}{|l|c|c|c|}
\hline Score & Mr(calc): & Delta & Sequence \\
\hline 60.7 & 1339.6111 & -0.0005 & $\underline{\text { NELMLNSSLMR }}$ \\
\hline 21.4 & 1339.6111 & -0.0005 & $\underline{\text { NELMLNSSLMR }}$ \\
\hline 9.8 & 1339.6020 & 0.0086 & $\underline{\text { NKVKQDSESPK }}$ \\
\hline 9.8 & 1339.6020 & 0.0086 & $\underline{\text { NKVKQDSESPK }}$ \\
\hline 8.6 & 1339.6061 & 0.0045 & $\underline{\text { GSPEYQELLPK }}$ \\
\hline 6.9 & 1337.6145 & 1.9961 & $\underline{\text { LKLFNSKGGSK }}$ \\
\hline 6.6 & 1338.6180 & 0.9926 & $\underline{\text { NKVKQDSESPK }}$ \\
\hline 6.6 & 1338.6180 & 0.9926 & $\underline{\text { NKVKQDSESPK }}$ \\
\hline 6.6 & 1339.6078 & 0.0028 & $\underline{\text { AERFEEGMEVK }}$ \\
\hline & & & \\
\hline
\end{tabular}




\section{\begin{tabular}{|l||l|l|l|}
6.1 & 1338.6180 & 0.9926 & EKDQIEAALSR \\
\hline
\end{tabular}}

Spectrum No: 521; Query: 1123; Rank: 1

\section{Peptide View}

MS/MS Fragmentation of TFNTSPGDIISPNFPK

Found in IPI00196620, Tax_Id=10116 Gene_Symbol=Cubn Cubilin precursor

Match to Query 1123: 1734.843468 from(868.429010,2+)

Title: 091008RatKidney_NH4Format01_24.3177.3177.2.dta

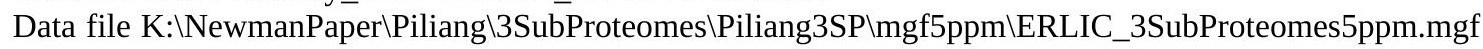
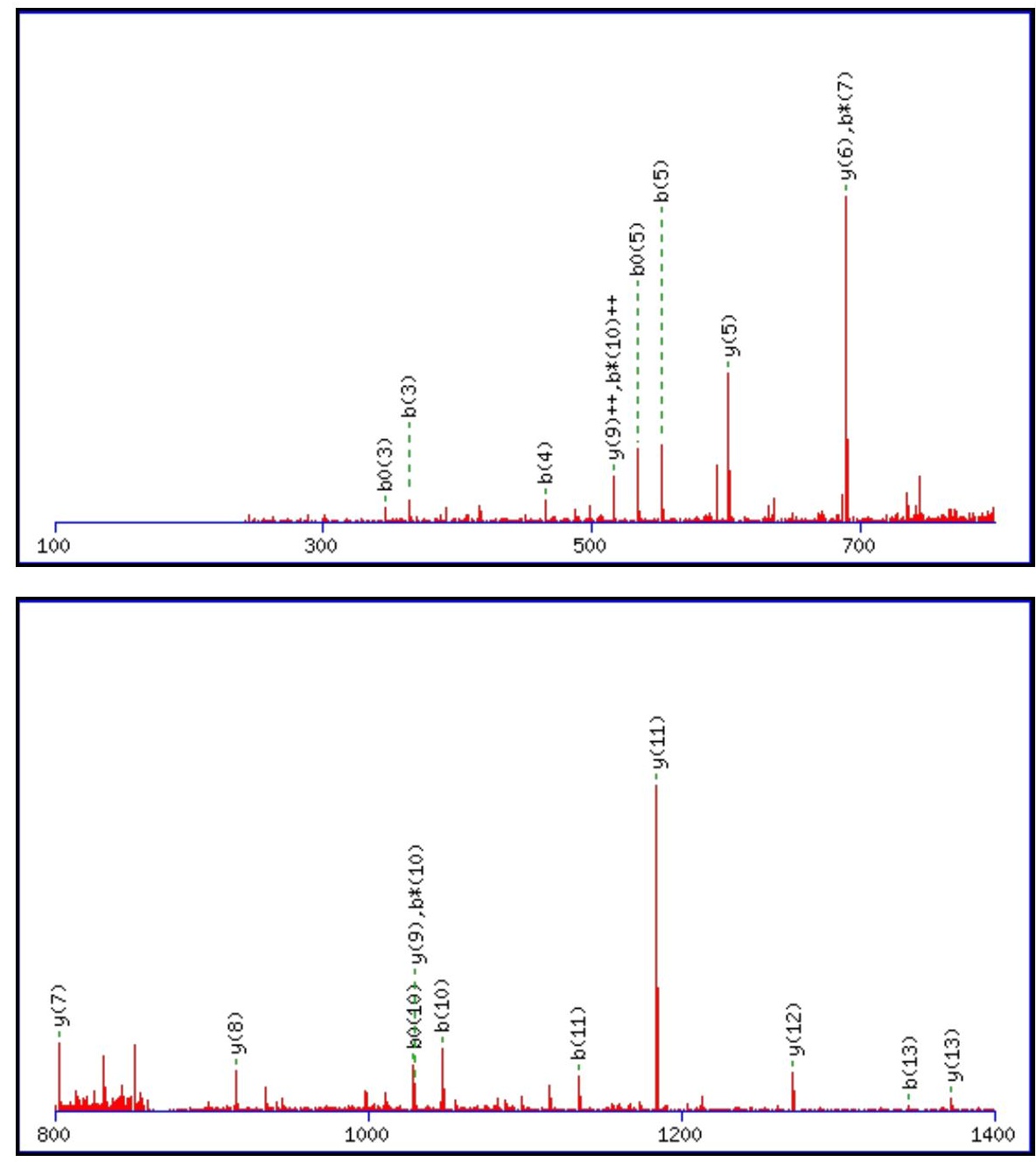


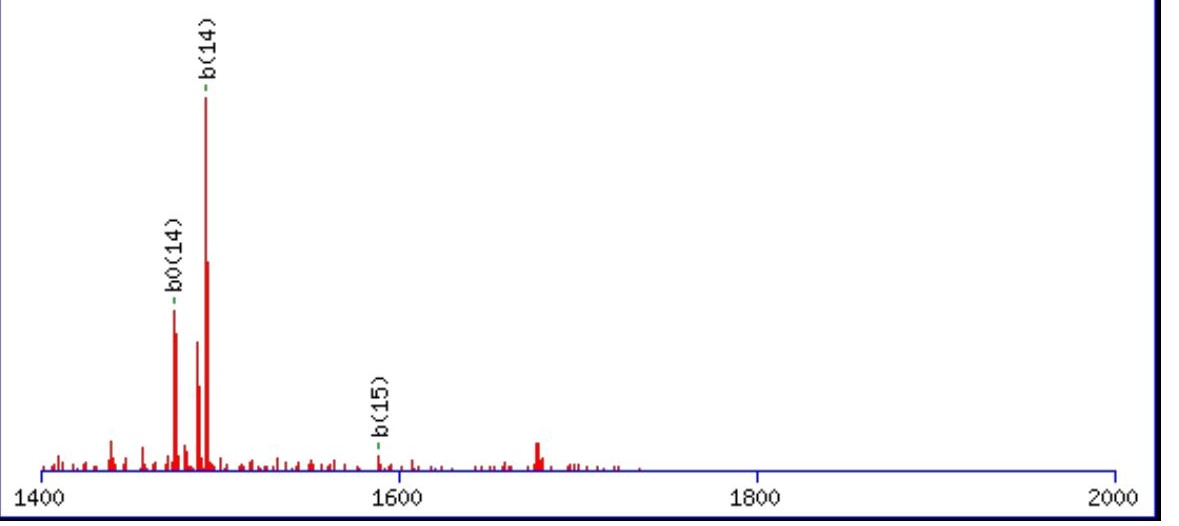

Monoisotopic mass of neutral peptide $\operatorname{Mr}($ calc): 1734.8465

Fixed modifications: Carbamidomethyl (C)

Variable modifications:

N3 : Deamidated_N (N)

Ions Score: 61 Expect: 0.00016

Matches (Bold Red): 24/166 fragment ions using 37 most intense peaks

\begin{tabular}{|c|c|c|c|c|c|c|c|c|c|c|c|c|c|c|}
\hline \# & b & $\mathbf{b}^{++}$ & $\mathbf{b}^{*}$ & $\mathbf{b}^{*^{++}}$ & $\mathbf{b}^{0}$ & $\mathbf{b}^{\mathbf{0 + +}}$ & Seq. & $\mathbf{y}$ & $\mathbf{y}^{++}$ & $\mathbf{y}^{*}$ & $\mathbf{y}^{*^{++}}$ & $\mathbf{y}^{0}$ & $\mathbf{y}^{0++}$ & \# \\
\hline 1 & 102.0550 & 51.5311 & & & 84.0444 & 42.5258 & $\mathbf{T}$ & & & & & & & 16 \\
\hline 2 & 249.1234 & 125.0653 & & & 231.1128 & 116.0600 & $\mathbf{F}$ & 1634.8061 & 817.9067 & 1617.7795 & 809.3934 & 1616.7955 & 808.9014 & 15 \\
\hline 3 & 364.1503 & 182.5788 & 347.1238 & 174.0655 & 346.1397 & 173.5735 & $\mathbf{N}$ & 1487.7376 & 744.3725 & 1470.7111 & 735.8592 & 1469.7271 & 735.3672 & 14 \\
\hline 4 & 465.1980 & 233.1026 & 448.1714 & 224.5894 & 447.1874 & 224.0973 & $\mathbf{T}$ & 1372.7107 & 686.8590 & 1355.6842 & 678.3457 & 1354.7001 & 677.8537 & 13 \\
\hline 5 & 552.2300 & 276.6186 & 535.2035 & 268.1054 & 534.2194 & 267.6134 & S & 1271.6630 & 636.3352 & 1254.6365 & 627.8219 & 1253.6525 & 627.3299 & 12 \\
\hline 6 & 649.2828 & 325.1450 & 632.2562 & 316.6318 & 631.2722 & 316.1397 & $\mathbf{P}$ & 1184.6310 & 592.8191 & 1167.6045 & 584.3059 & 1166.6204 & 583.8139 & 11 \\
\hline 7 & 706.3042 & 353.6558 & 689.2777 & 345.1425 & 688.2937 & 344.6505 & G & 1087.5782 & 544.2928 & 1070.5517 & 535.7795 & 1069.5677 & 535.2875 & 10 \\
\hline 8 & 821.3312 & 411.1692 & 804.3046 & 402.6560 & 803.3206 & 402.1639 & D & 1030.5568 & 515.7820 & 1013.5302 & 507.2687 & 1012.5462 & 506.7767 & 9 \\
\hline 9 & 934.4152 & 467.7113 & 917.3887 & 459.1980 & 916.4047 & 458.7060 & I & 915.5298 & 458.2686 & 898.5033 & 449.7553 & 897.5193 & 449.2633 & 8 \\
\hline 10 & 1047.4993 & 524.2533 & 1030.4728 & 515.7400 & 1029.4887 & 515.2480 & I & 802.4458 & 401.7265 & 785.4192 & 393.2132 & 784.4352 & 392.7212 & 7 \\
\hline 11 & 1134.5313 & 567.7693 & 1117.5048 & 559.2560 & 1116.5208 & 558.7640 & S & 689.3617 & 345.1845 & 672.3352 & 336.6712 & 671.3511 & 336.1792 & 6 \\
\hline 12 & 1231.5841 & 616.2957 & 1214.5576 & 607.7824 & 1213.5735 & 607.2904 & $\mathbf{P}$ & 602.3297 & 301.6685 & 585.3031 & 293.1552 & & & 5 \\
\hline 13 & 1345.6270 & 673.3172 & 1328.6005 & 664.8039 & 1327.6165 & 664.3119 & $\mathbf{N}$ & 505.2769 & 253.1421 & 488.2504 & 244.6288 & & & 4 \\
\hline 14 & 1492.6954 & 746.8514 & 1475.6689 & 738.3381 & 1474.6849 & 737.8461 & $\mathbf{F}$ & 391.2340 & 196.1206 & 374.2074 & 187.6074 & & & 3 \\
\hline 15 & 1589.7482 & 795.3777 & 1572.7217 & 786.8645 & 1571.7376 & 786.3725 & $\mathbf{P}$ & 244.1656 & 122.5864 & 227.1390 & 114.0731 & & & 2 \\
\hline 16 & & & & & & & $\mathbf{K}$ & 147.1128 & 74.0600 & 130.0863 & 65.5468 & & & 1 \\
\hline
\end{tabular}
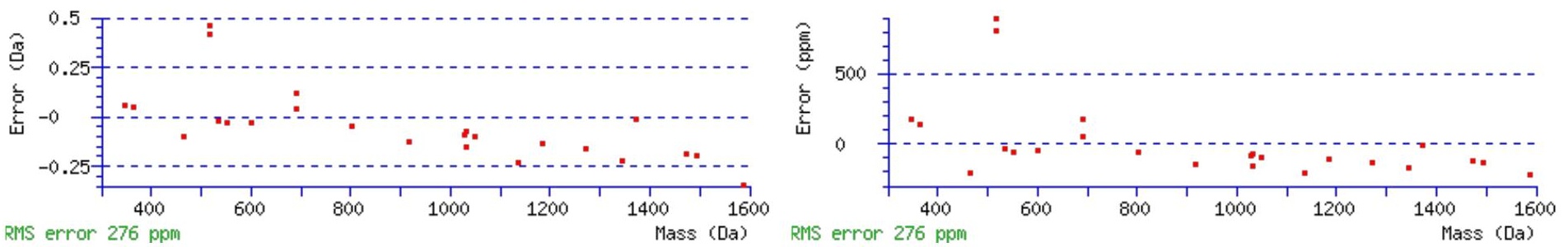

\section{All matches to this query}

\begin{tabular}{|l|l|l|l|}
\hline Score & Mr(calc): & Delta & \multicolumn{1}{c|}{ Sequence } \\
\hline 60.6 & 1734.8465 & -0.0030 & TFNTSPGDIISPNFPK \\
\hline 37.7 & 1734.8465 & -0.0030 & TFNTSPGDIISPNFPK \\
\hline 12.3 & 1734.8384 & 0.0051 & $\underline{\text { SSSNAATISIGGSGELQR }}$ \\
\hline 11.4 & 1733.8366 & 1.0068 & $\underline{\text { SGCEVRIPVVSSSSNR }}$ \\
\hline 8.5 & 1734.8271 & 0.0163 & $\underline{\text { GEKSLSSQEAKEAEDK }}$ \\
\hline & & &
\end{tabular}




\begin{tabular}{|l|l|l|l|}
6.1 & 1734.8570 & -0.0135 & $\underline{\text { SVIATSTKVDISKVK }}$ \\
\hline 6.1 & 1734.8570 & -0.0135 & $\underline{\text { SVIATSTKVDISKVK }}$ \\
\hline 6.1 & 1734.8570 & -0.0135 & SVIATSTKVDISKVK \\
\hline 6.1 & 1734.8570 & -0.0135 & $\underline{\text { SVIATSTKVDISKVK }}$ \\
\hline 5.6 & 1732.8526 & 1.9908 & $\underline{\text { SGCEVRIPVVSSSSNR }}$ \\
\hline
\end{tabular}

Spectrum No: 522; Query: 497; Rank: 1

\section{Peptide View}

MS/MS Fragmentation of QELNDSLQVAER

Found in IPI00198667, Tax_Id=10116 Gene_Symbol=Clu Clusterin precursor

Match to Query 497: 1401.671348 from(701.842950,2+)

Title: 091008RatKidney_NoSalt_41.1174.1174.2.dta

Data file K:INewmanPaper|Piliangl3SubProteomes\Piliang3SP\mgf5ppm\ERLIC_3SubProteomes5ppm.mgf
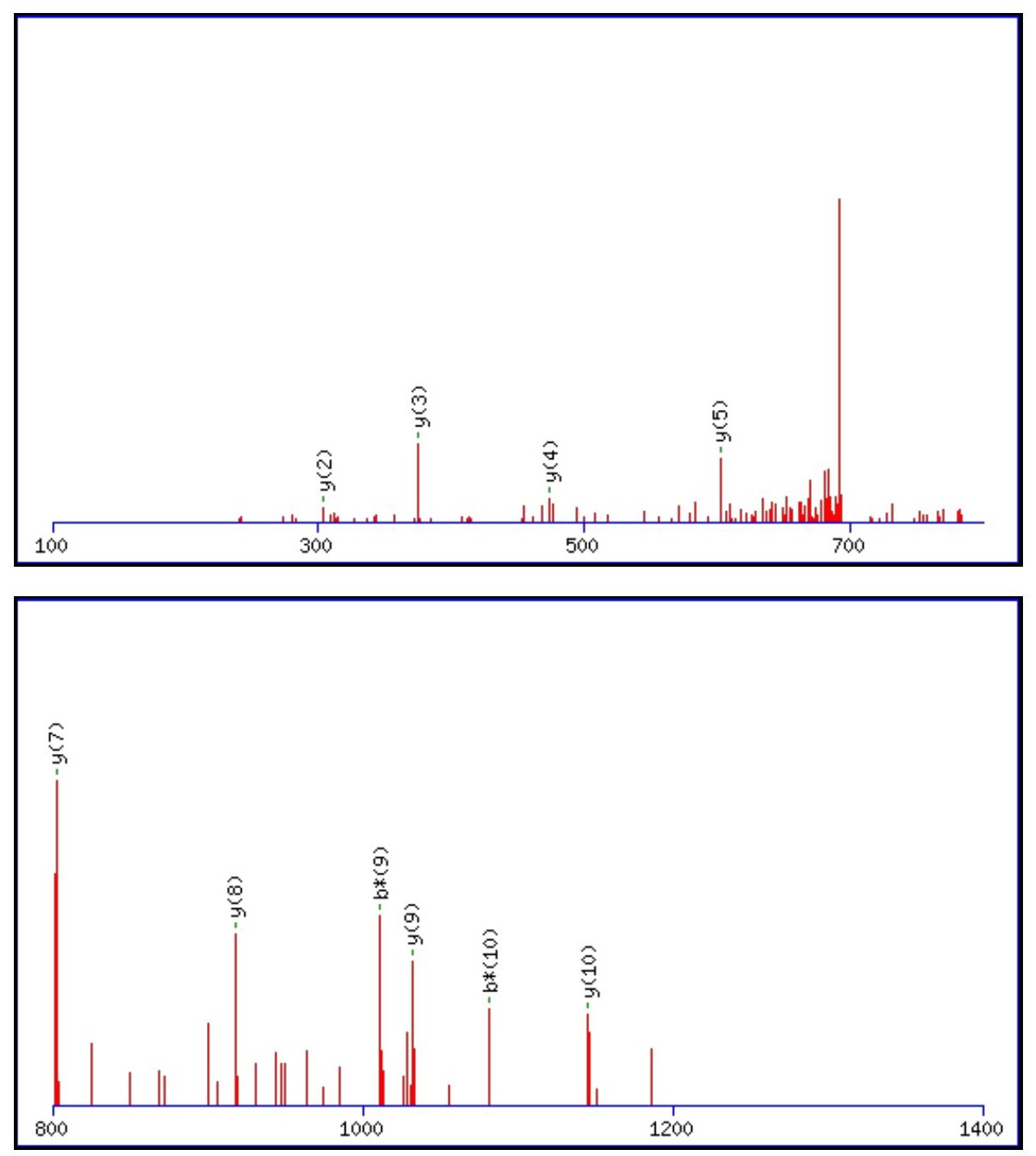


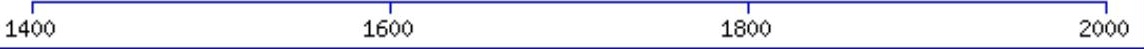

Monoisotopic mass of neutral peptide $\operatorname{Mr}($ calc): 1401.6736

Fixed modifications: Carbamidomethyl (C)

Variable modifications:

N4 : Deamidated_N (N)

Ions Score: 61 Expect: 0.00014

Matches (Bold Red): 10/128 fragment ions using 11 most intense peaks

\begin{tabular}{|c|c|c|c|c|c|c|c|c|c|c|c|c|c|c|}
\hline \# & b & $\mathbf{b}^{++}$ & $\mathbf{b}^{*}$ & $\mathbf{b}^{*^{++}}$ & $\mathbf{b}^{0}$ & $\mathbf{b}^{\mathbf{0 + +}}$ & Seq. & $\mathbf{y}$ & $\mathbf{y}^{++}$ & $\mathbf{y}^{*}$ & $\mathrm{y}^{*^{++}}$ & $\mathbf{y}^{\mathbf{0}}$ & $\mathbf{y}^{0++}$ & \# \\
\hline 1 & 129.0659 & 65.0366 & 112.0393 & 56.5233 & & & $\mathbf{Q}$ & & & & & & & 12 \\
\hline 2 & 258.1084 & 129.5579 & 241.0819 & 121.0446 & 240.0979 & 120.5526 & $\mathbf{E}$ & 1274.6223 & 637.8148 & 1257.5957 & 629.3015 & 1256.6117 & 628.8095 & 11 \\
\hline 3 & 371.1925 & 186.0999 & 354.1660 & 177.5866 & 353.1819 & 177.0946 & $\mathbf{L}$ & 1145.5797 & 573.2935 & 1128.5531 & 564.7802 & 1127.5691 & 564.2882 & 10 \\
\hline 4 & 486.2194 & 243.6134 & 469.1929 & 235.1001 & 468.2089 & 234.6081 & $\mathbf{N}$ & 1032.4956 & 516.7514 & 1015.4691 & 508.2382 & 1014.4851 & 507.7462 & 9 \\
\hline 5 & 601.2464 & 301.1268 & 584.2198 & 292.6136 & 583.2358 & 292.1216 & D & 917.4687 & 459.2380 & 900.4421 & 450.7247 & 899.4581 & 450.2327 & 8 \\
\hline 6 & 688.2784 & 344.6428 & 671.2519 & 336.1296 & 670.2679 & 335.6376 & S & 802.4417 & 401.7245 & 785.4152 & 393.2112 & 784.4312 & 392.7192 & 7 \\
\hline 7 & 801.3625 & 401.1849 & 784.3359 & 392.6716 & 783.3519 & 392.1796 & $\mathbf{L}$ & 715.4097 & 358.2085 & 698.3832 & 349.6952 & 697.3991 & 349.2032 & 6 \\
\hline 8 & 929.4211 & 465.2142 & 912.3945 & 456.7009 & 911.4105 & 456.2089 & $\mathbf{Q}$ & 602.3257 & 301.6665 & 585.2991 & 293.1532 & 584.3151 & 292.6612 & 5 \\
\hline 9 & 1028.4895 & 514.7484 & 1011.4629 & 506.2351 & 1010.4789 & 505.7431 & $\mathbf{V}$ & 474.2671 & 237.6372 & 457.2405 & 229.1239 & 456.2565 & 228.6319 & 4 \\
\hline 10 & 1099.5266 & 550.2669 & 1082.5000 & 541.7537 & 1081.5160 & 541.2616 & A & 375.1987 & 188.1030 & 358.1721 & 179.5897 & 357.1881 & 179.0977 & 3 \\
\hline 11 & 1228.5692 & 614.7882 & 1211.5426 & 606.2750 & 1210.5586 & 605.7829 & $\mathbf{E}$ & 304.1615 & 152.5844 & 287.1350 & 144.0711 & 286.1510 & 143.5791 & 2 \\
\hline 12 & & & & & & & $\mathbf{R}$ & 175.1190 & 88.0631 & 158.0924 & 79.5498 & & & 1 \\
\hline
\end{tabular}
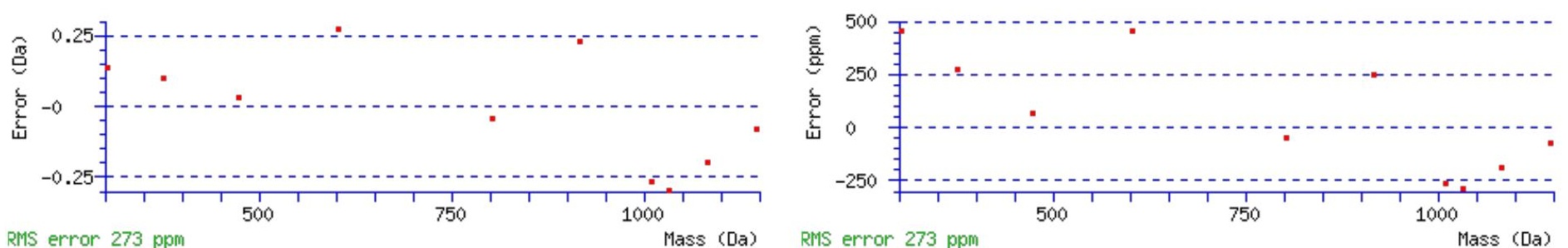

All matches to this query

\begin{tabular}{|l|l|l|l|}
\hline Score & Mr(calc): & Delta & \multicolumn{1}{c|}{ Sequence } \\
\hline 60.6 & 1401.6736 & -0.0022 & QELNDSLQVAER \\
\hline 9.9 & 1401.6727 & -0.0014 & VCSFVKQVVEK \\
\hline 9.7 & 1400.6700 & 1.0013 & NYILTNNLLSR \\
\hline 9.6 & 1400.6748 & 0.9966 & HLGHMGSKVSLR \\
\hline 4.5 & 1401.6727 & -0.0014 & VFQLISLMDTR \\
\hline 3.8 & 1401.6653 & 0.0060 & SFLGSISQQSIR \\
\hline 3.6 & 1401.6653 & 0.0060 & FGRLKSVNESGK \\
\hline 2.9 & 1399.6732 & 1.9982 & SAPEVNAPGAPAYR \\
\hline 2.2 & 1401.6653 & 0.0060 & FGRLKSVNESGK \\
\hline & & &
\end{tabular}




\begin{tabular}{|l|l|l|l|l|}
1.9 & 1401.6604 & 0.0109 & MSRLLPLLGSR \\
\hline
\end{tabular}

Spectrum No: 523; Query: 2133; Rank: 1

\section{Peptide View}

MS/MS Fragmentation of VLTLANFTTKDEGDYMCELR

Found in IPI00188956, Tax_Id=10116 Gene_Symbol=Thy1 Thy-1 membrane glycoprotein precursor

Match to Query 2133: 2392.094952 from(798.372260,3+)

Title: 100101RatKid_NS_deglyco_19.3893.3893.3.dta

Data file K:INewmanPaper|Piliang|3SubProteomes\Piliang3SP\mgf5ppm\ERLIC_3SubProteomes5ppm.mgf
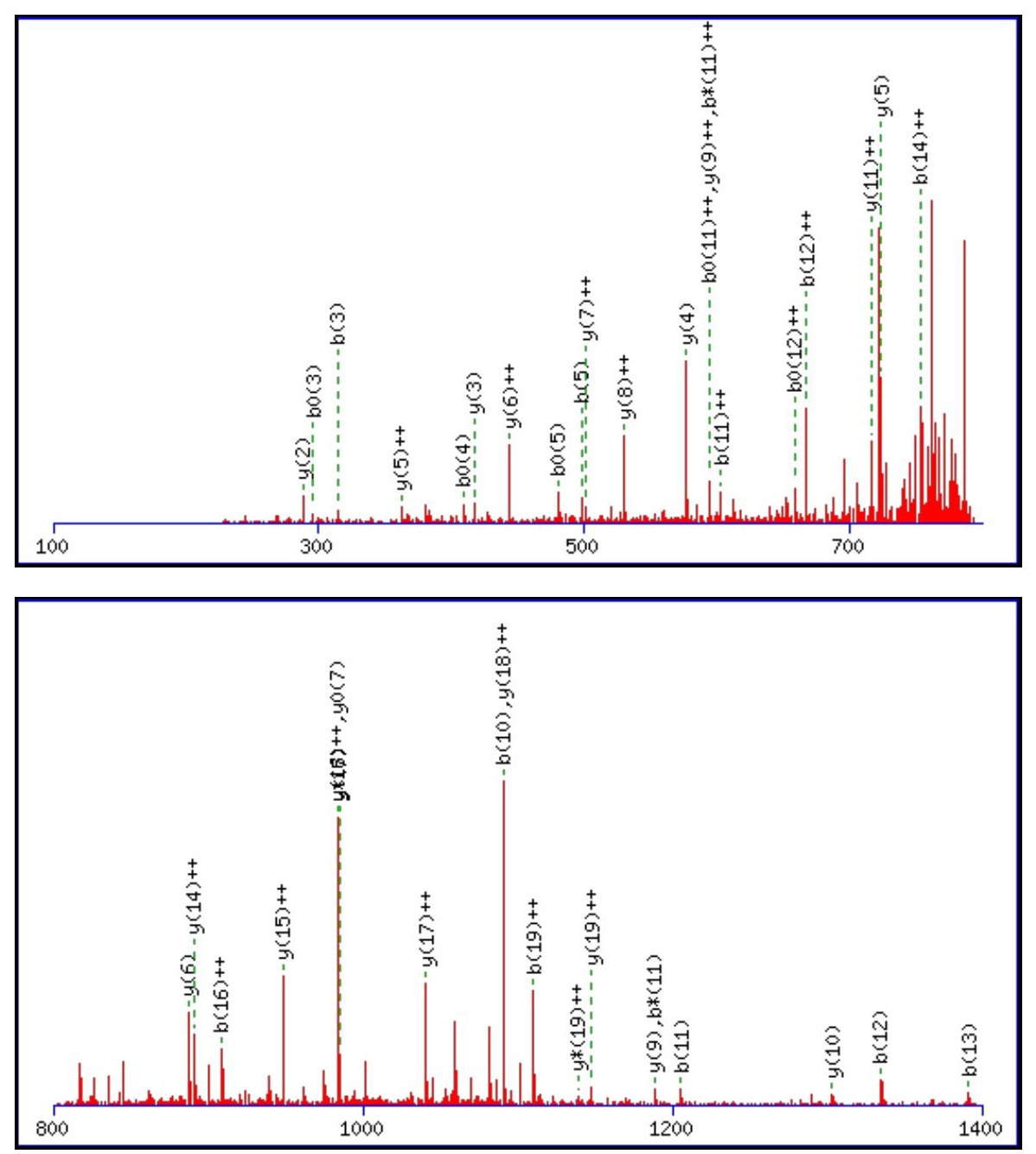


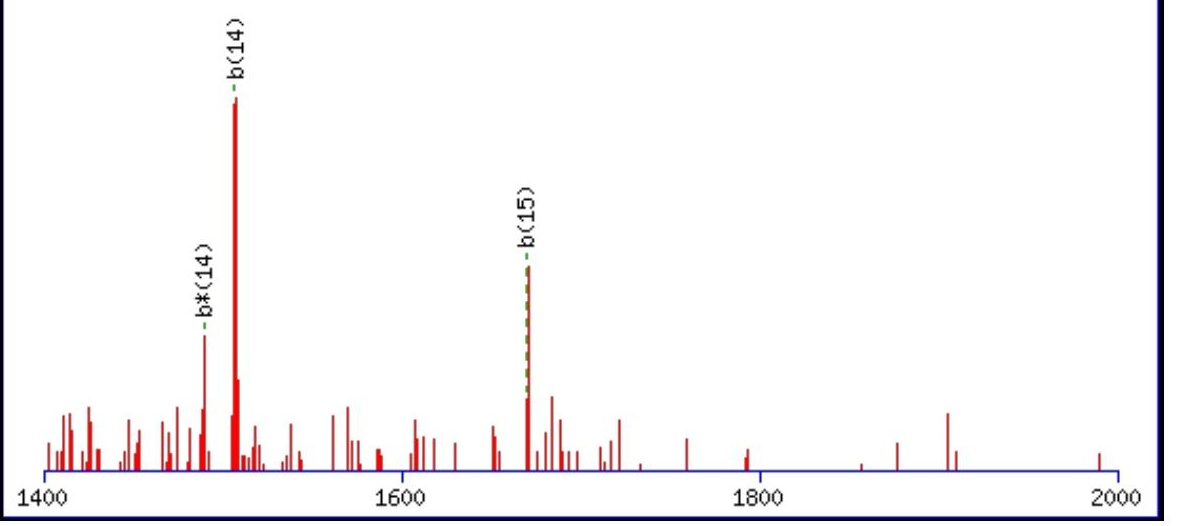

Monoisotopic mass of neutral peptide $\operatorname{Mr}($ calc): 2392.0927

Fixed modifications: Carbamidomethyl (C)

Variable modifications:

N6 : Deamidated_N (N)

M16 : Oxidation (M)

Ions Score: 60 Expect : 0.00027

Matches (Bold Red): 43/210 fragment ions using 84 most intense peaks

\begin{tabular}{|c|c|c|c|c|c|c|c|c|c|c|c|c|c|c|}
\hline \# & b & $\mathbf{b}^{++}$ & $\mathbf{b}^{*}$ & $\mathbf{b}^{*^{++}}$ & $\mathbf{b}^{\mathbf{0}}$ & $\mathbf{b}^{\mathbf{0 + +}}$ & Seq. & $\mathbf{y}$ & $\mathbf{y}^{++}$ & $\mathbf{y}^{*}$ & $\mathrm{y}^{*^{++}}$ & $\mathbf{y}^{\mathbf{0}}$ & $y^{0++}$ & \# \\
\hline 1 & 100.0757 & 50.5415 & & & & & $\mathbf{V}$ & & & & & & & 20 \\
\hline 2 & 213.1598 & 107.0835 & & & & & $\mathbf{L}$ & 2294.0315 & 1147.5194 & 2277.0050 & 1139.0061 & 2276.0210 & 1138.5141 & 19 \\
\hline 3 & 314.2074 & 157.6074 & & & 296.1969 & 148.6021 & $\mathbf{T}$ & 2180.9475 & 1090.9774 & 2163.9209 & 1082.4641 & 2162.9369 & 1081.9721 & 18 \\
\hline 4 & 427.2915 & 214.1494 & & & 409.2809 & 205.1441 & $\mathbf{L}$ & 2079.8998 & 1040.4535 & 2062.8732 & 1031.9403 & 2061.8892 & 1031.4482 & 17 \\
\hline 5 & 498.3286 & 249.6679 & & & 480.3180 & 240.6627 & A & 1966.8157 & 983.9115 & 1949.7892 & 975.3982 & 1948.8052 & 974.9062 & 16 \\
\hline 6 & 613.3555 & 307.1814 & 596.3290 & 298.6681 & 595.3450 & 298.1761 & $\mathbf{N}$ & 1895.7786 & 948.3929 & 1878.7521 & 939.8797 & 1877.7680 & 939.3877 & 15 \\
\hline 7 & 760.4240 & 380.7156 & 743.3974 & 372.2023 & 742.4134 & 371.7103 & $\mathbf{F}$ & 1780.7517 & 890.8795 & 1763.7251 & 882.3662 & 1762.7411 & 881.8742 & 14 \\
\hline 8 & 861.4716 & 431.2395 & 844.4451 & 422.7262 & 843.4611 & 422.2342 & $\mathbf{T}$ & 1633.6833 & 817.3453 & 1616.6567 & 808.8320 & 1615.6727 & 808.3400 & 13 \\
\hline 9 & 962.5193 & 481.7633 & 945.4928 & 473.2500 & 944.5088 & 472.7580 & $\mathbf{T}$ & 1532.6356 & 766.8214 & 1515.6090 & 758.3082 & 1514.6250 & 757.8161 & 12 \\
\hline 10 & 1090.6143 & 545.8108 & 1073.5877 & 537.2975 & 1072.6037 & 536.8055 & $\mathbf{K}$ & 1431.5879 & 716.2976 & 1414.5613 & 707.7843 & 1413.5773 & 707.2923 & 11 \\
\hline 11 & 1205.6412 & 603.3243 & 1188.6147 & 594.8110 & 1187.6307 & 594.3190 & D & 1303.4929 & 652.2501 & 1286.4664 & 643.7368 & 1285.4824 & 643.2448 & 10 \\
\hline 12 & 1334.6838 & 667.8455 & 1317.6573 & 659.3323 & 1316.6733 & 658.8403 & $\mathbf{E}$ & 1188.4660 & 594.7366 & 1171.4394 & 586.2234 & 1170.4554 & 585.7314 & 9 \\
\hline 13 & 1391.7053 & 696.3563 & 1374.6787 & 687.8430 & 1373.6947 & 687.3510 & $\mathbf{G}$ & 1059.4234 & 530.2153 & 1042.3968 & 521.7021 & 1041.4128 & 521.2101 & 8 \\
\hline 14 & 1506.7322 & 753.8698 & 1489.7057 & 745.3565 & 1488.7217 & 744.8645 & D & 1002.4019 & 501.7046 & 985.3754 & 493.1913 & 984.3914 & 492.6993 & 7 \\
\hline 15 & 1669.7956 & 835.4014 & 1652.7690 & 826.8881 & 1651.7850 & 826.3961 & $\mathbf{Y}$ & 887.3750 & 444.1911 & 870.3484 & 435.6779 & 869.3644 & 435.1859 & 6 \\
\hline 16 & 1816.8310 & 908.9191 & 1799.8044 & 900.4058 & 1798.8204 & 899.9138 & M & 724.3117 & 362.6595 & 707.2851 & 354.1462 & 706.3011 & 353.6542 & 5 \\
\hline 17 & 1976.8616 & 988.9344 & 1959.8351 & 980.4212 & 1958.8510 & 979.9292 & C & 577.2763 & 289.1418 & 560.2497 & 280.6285 & 559.2657 & 280.1365 & 4 \\
\hline 18 & 2105.9042 & 1053.4557 & 2088.8777 & 1044.9425 & 2087.8936 & 1044.4505 & $\mathbf{E}$ & 417.2456 & 209.1264 & 400.2191 & 200.6132 & 399.2350 & 200.1212 & 3 \\
\hline 19 & 2218.9883 & 1109.9978 & 2201.9617 & 1101.4845 & 2200.9777 & 1100.9925 & $\mathbf{L}$ & 288.2030 & 144.6051 & 271.1765 & 136.0919 & & & 2 \\
\hline 20 & & & & & & & $\mathbf{R}$ & 175.1190 & 88.0631 & 158.0924 & 79.5498 & & & 1 \\
\hline
\end{tabular}

$$
\text { 吾 }
$$

RMS error 238 ppm

\section{All matches to this query}

\begin{tabular}{|l|l|l|l|}
\hline Score & Mr(calc): & Delta & Sequence \\
\hline 60.5 & 2392.0927 & 0.0023 & VLTLANFTTKDEGDYMCELR \\
\hline
\end{tabular}

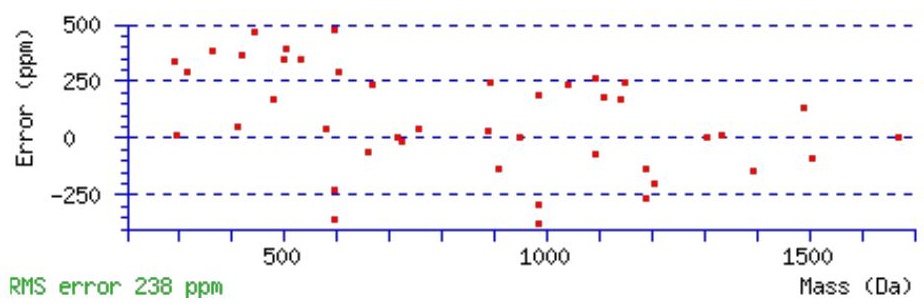

Mass (Da) RMS error 238 ppm
Mass (Da) 


\begin{tabular}{|l|l|l|l|}
28.8 & 2391.1086 & 0.9863 & VLTLANFTTKDEGDYMCELR \\
\hline 10.7 & 2392.0783 & 0.0166 & IRTSGECRHTLDYIWYSR \\
\hline 10.7 & 2392.0783 & 0.0166 & IRTSGECRHTLDYIWYSR \\
\hline 2.8 & 2392.0783 & 0.0166 & IRTSGECRHTLDYIWYSR \\
\hline 0.3 & 2392.0882 & 0.0068 & AIELDTGNRPREAYMEYLR \\
\hline
\end{tabular}

Spectrum No: 524; Query: 72; Rank: 1

\section{Peptide View}

MS/MS Fragmentation of TPGNASLSLR

Found in IPI00193010, Tax_Id=10116 Gene_Symbol=Slc23a3_predicted solute carrier family 23 (nucleobase transporters), member 3

Match to Query 72: 1015.530588 from(508.772570,2+)

Title: 100101RatKid_NS_deglyco_25.1367.1367.2.dta

Data file K:INewmanPaper|Piliangl3SubProteomes\Piliang3SP\mgf5ppm\ERLIC_3SubProteomes5ppm.mgf
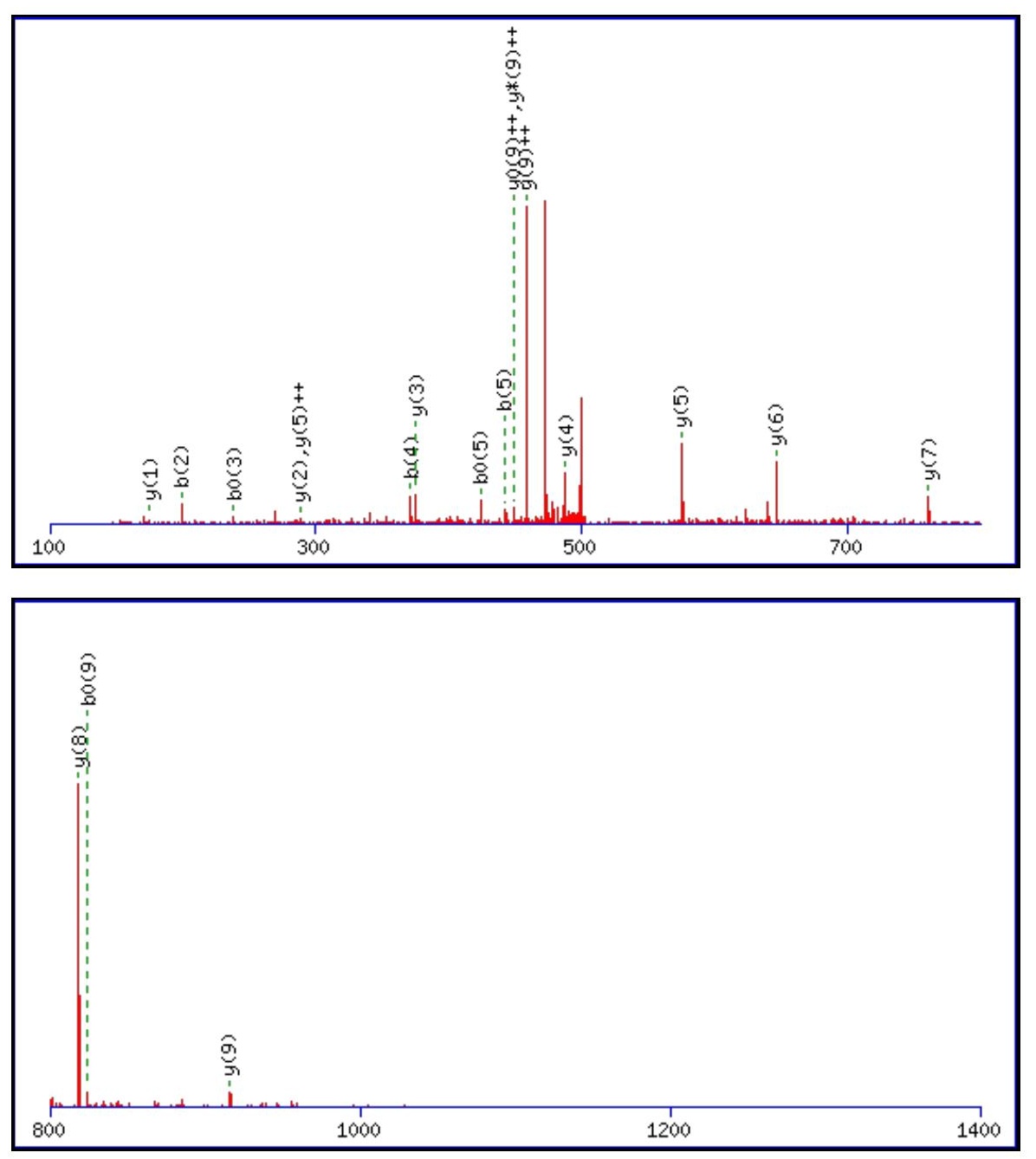


$1400 \quad 1600 \quad 1800 \quad 2000$

Monoisotopic mass of neutral peptide $\operatorname{Mr}($ calc): 1015.5298

Fixed modifications: Carbamidomethyl (C)

Variable modifications:

N4 : Deamidated_N (N)

Ions Score: 60 Expect: $7.3 \mathrm{e}-005$

Matches (Bold Red): 19/98 fragment ions using 43 most intense peaks

\begin{tabular}{|r|c|c|c|c|c|c|c|c|c|c|c|c|c|c|}
\hline$\#$ & $\mathbf{b}$ & $\mathbf{b}^{++}$ & $\mathbf{b}^{*}$ & $\mathbf{b}^{\mathbf{*}^{++}}$ & $\mathbf{b}^{\mathbf{0}}$ & $\mathbf{b}^{\mathbf{0 + +}}$ & $\mathbf{S e q}$ & $\mathbf{y}$ & $\mathbf{y}^{++}$ & $\mathbf{y}^{\mathbf{*}}$ & $\mathbf{y}^{\mathbf{*}^{++}}$ & $\mathbf{y}^{\mathbf{0}}$ & $\mathbf{y}^{\mathbf{0 + +}}$ & $\#$ \\
\hline $\mathbf{1}$ & 102.0550 & 51.5311 & & & 84.0444 & 42.5258 & $\mathbf{T}$ & & & & & & & $\mathbf{1 0}$ \\
\hline $\mathbf{2}$ & $\mathbf{1 9 9 . 1 0 7 7}$ & 100.0575 & & & 181.0972 & 91.0522 & $\mathbf{P}$ & $\mathbf{9 1 5 . 4 8 9 4}$ & $\mathbf{4 5 8 . 2 4 8 3}$ & 898.4629 & $\mathbf{4 4 9 . 7 3 5 1}$ & 897.4788 & $\mathbf{4 4 9 . 2 4 3 1}$ & $\mathbf{9}$ \\
\hline $\mathbf{3}$ & 256.1292 & 128.5682 & & & 238.1186 & 119.5629 & $\mathbf{G}$ & $\mathbf{8 1 8 . 4 3 6 7}$ & 409.7220 & 801.4101 & 401.2087 & 800.4261 & 400.7167 & $\mathbf{8}$ \\
\hline $\mathbf{4}$ & 371.1561 & 186.0817 & 354.1296 & 177.5684 & 353.1456 & 177.0764 & $\mathbf{N}$ & $\mathbf{7 6 1 . 4 1 5 2}$ & 381.2112 & 744.3886 & 372.6980 & 743.4046 & 372.2059 & $\mathbf{7}$ \\
\hline $\mathbf{5}$ & $\mathbf{4 4 2 . 1 9 3 2}$ & 221.6003 & 425.1667 & 213.0870 & 424.1827 & 212.5950 & $\mathbf{A}$ & $\mathbf{6 4 6 . 3 8 8 3}$ & 323.6978 & 629.3617 & 315.1845 & 628.3777 & 314.6925 & $\mathbf{6}$ \\
\hline $\mathbf{6}$ & 529.2253 & 265.1163 & 512.1987 & 256.6030 & 511.2147 & 256.1110 & $\mathbf{S}$ & 575.3511 & $\mathbf{2 8 8 . 1 7 9 2}$ & 558.3246 & 279.6659 & 557.3406 & 279.1739 & $\mathbf{5}$ \\
\hline $\mathbf{7}$ & 642.3093 & 321.6583 & 625.2828 & 313.1450 & 624.2988 & 312.6530 & $\mathbf{L}$ & $\mathbf{4 8 8 . 3 1 9 1}$ & 244.6632 & 471.2926 & 236.1499 & 470.3085 & 235.6579 & $\mathbf{4}$ \\
\hline $\mathbf{8}$ & 729.3414 & 365.1743 & 712.3148 & 356.6610 & 711.3308 & 356.1690 & $\mathbf{S}$ & 375.2350 & 188.1212 & 358.2085 & 179.6079 & 357.2245 & 179.1159 & 3 \\
\hline $\mathbf{9}$ & 842.4254 & 421.7163 & 825.3989 & 413.2031 & $\mathbf{8 2 4 . 4 1 4 9}$ & 412.7111 & $\mathbf{L}$ & $\mathbf{2 8 8 . 2 0 3 0}$ & 144.6051 & 271.1765 & 136.0919 & & & $\mathbf{2}$ \\
\hline $\mathbf{1 0}$ & & & & & & & $\mathbf{R}$ & $\mathbf{1 7 5 . 1 1 9 0}$ & 88.0631 & 158.0924 & 79.5498 & & & $\mathbf{1}$ \\
\hline
\end{tabular}
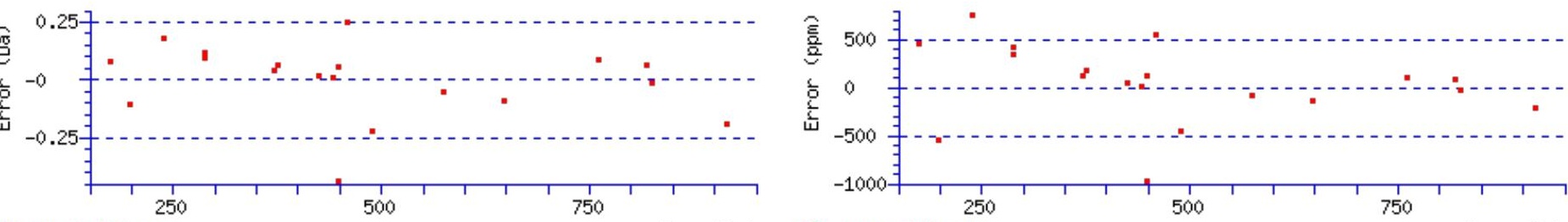

RMS error $395 \mathrm{ppm}$

Mass (Da) RMS error 395 ppm

Mass (Da)

\section{All matches to this query}

\begin{tabular}{|l|l|l|l|}
\hline Score & Mr(calc): & Delta & \multicolumn{1}{|c|}{ Sequence } \\
\hline 60.4 & 1015.5298 & 0.0008 & TPGNASLSLR \\
\hline 9.2 & 1014.5233 & 1.0073 & VDPESVEIK \\
\hline 4.4 & 1013.5142 & 2.0164 & VPTTGAPESR \\
\hline 4.3 & 1013.5328 & 1.9978 & VTAPCTPLR \\
\hline 2.6 & 1014.5233 & 1.0073 & NPDLSILSGV \\
\hline 2.0 & 1015.5298 & 0.0008 & LKEAGNINR \\
\hline 1.8 & 1015.5298 & 0.0008 & GLLSDQDLR \\
\hline 0.6 & 1014.5280 & 1.0026 & VPMDERLR \\
\hline 0.6 & 1013.5311 & 1.9995 & VLFLSTVR \\
\hline
\end{tabular}




\section{Peptide View}

MS/MS Fragmentation of VNNSSLIGLGYTQTLKPGIK

Found in IPI00365813, Tax_Id=10116 Gene_Symbol=RGD1565338_predicted similar to Voltage-dependent anion-selective channel protein 1

Match to Query 1750: 2103.152802 from(702.058210,3+)

Title: 091008RatKidney_NoSalt_05.2761.2761.3.dta

Data file K:\NewmanPaper \Piliang \3SubProteomes \Piliang3SP \mgf5ppm\ERLIC_3SubProteomes5ppm.mgf
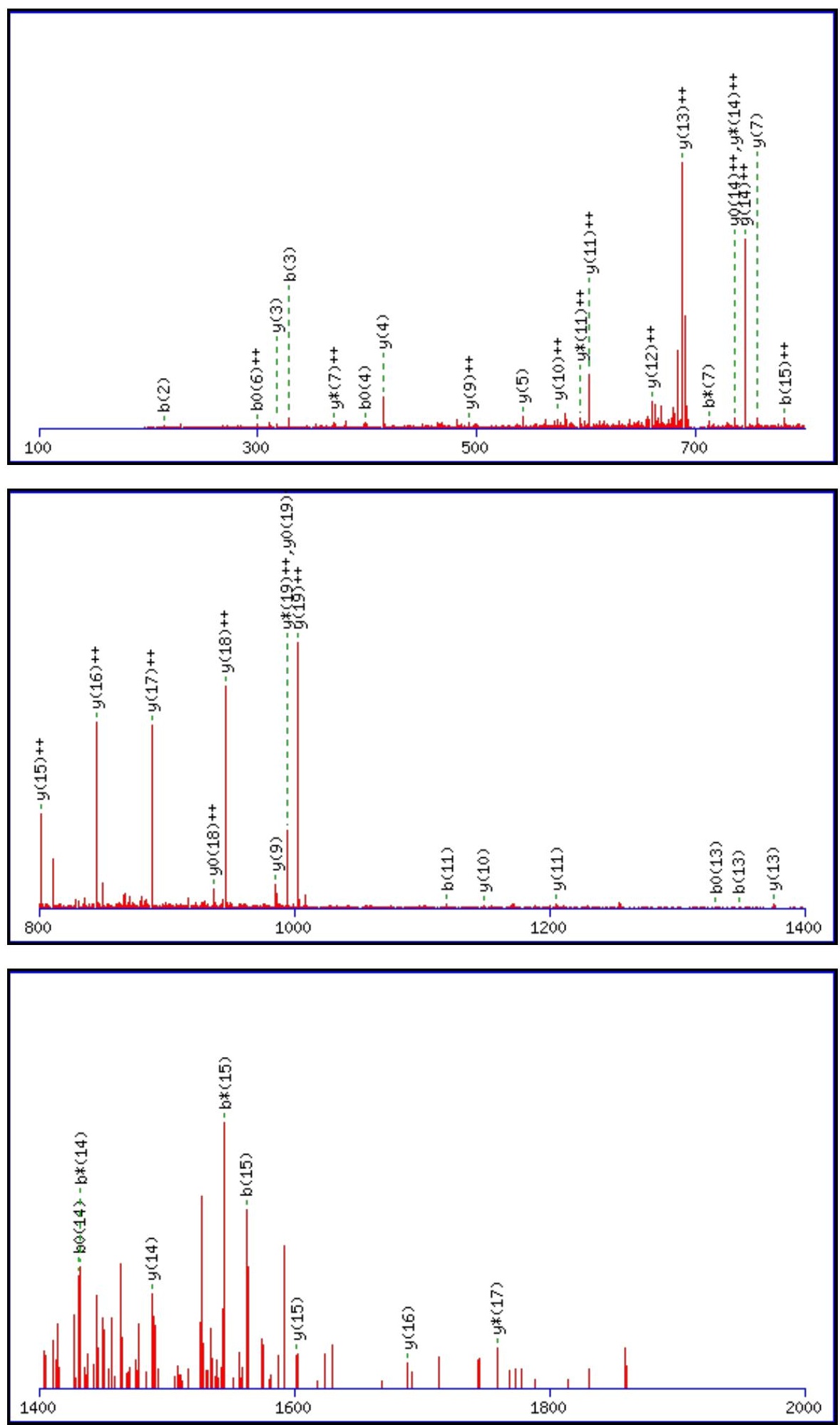

Monoisotopic mass of neutral peptide $\operatorname{Mr}($ calc): 2103.1575

Fixed modifications: Carbamidomethyl (C)

Variable modifications: 
N3 : Deamidated_N (N)

Ions Score: 60 Expect: $5.1 \mathrm{e}-005$

Matches (Bold Red): 43/208 fragment ions using 92 most intense peaks

\begin{tabular}{|c|c|c|c|c|c|c|c|c|c|c|c|c|c|c|}
\hline \# & b & $\mathbf{b}^{++}$ & b* & $\mathbf{b}^{*^{++}}$ & $\mathbf{b}^{\mathbf{0}}$ & $\mathbf{b}^{0++}$ & Seq. & $\mathbf{y}$ & $\mathbf{y}^{++}$ & $\mathbf{y}^{*}$ & $\mathrm{y}^{*^{++}}$ & $\mathbf{y}^{\mathbf{0}}$ & $\mathbf{y}^{\mathbf{0 + +}}$ & \# \\
\hline 1 & 100.0757 & 50.5415 & & & & & V & & & & & & & 20 \\
\hline 2 & 214.1186 & 107.5629 & 197.0921 & 99.0497 & & & $\mathbf{N}$ & 2005.0964 & 1003.0519 & 1988.0699 & 994.5386 & 1987.0859 & 994.0466 & 19 \\
\hline 3 & 329.1456 & 165.0764 & 312.1190 & 156.5631 & & & $\mathbf{N}$ & 1891.0535 & 946.0304 & 1874.0270 & 937.5171 & 1873.0429 & 937.0251 & 18 \\
\hline 4 & 416.1776 & 208.5924 & 399.1510 & 200.0792 & 398.1670 & 199.5871 & $\mathbf{S}$ & 1776.0266 & 888.5169 & 1759.0000 & 880.0036 & 1758.0160 & 879.5116 & 17 \\
\hline 5 & 503.2096 & 252.1084 & 486.1831 & 243.5952 & 485.1990 & 243.1032 & S & 1688.9945 & 845.0009 & 1671.9680 & 836.4876 & 1670.9840 & 835.9956 & 16 \\
\hline 6 & 616.2937 & 308.6505 & 599.2671 & 300.1372 & 598.2831 & 299.6452 & $\mathbf{L}$ & 1601.9625 & 801.4849 & 1584.9360 & 792.9716 & 1583.9519 & 792.4796 & 15 \\
\hline 7 & 729.3777 & 365.1925 & 712.3512 & 356.6792 & 711.3672 & 356.1872 & I & 1488.8784 & 744.9429 & 1471.8519 & 736.4296 & 1470.8679 & 735.9376 & 14 \\
\hline 8 & 786.3992 & 393.7032 & 769.3727 & 385.1900 & 768.3886 & 384.6980 & G & 1375.7944 & 688.4008 & 1358.7678 & 679.8876 & 1357.7838 & 679.3955 & 13 \\
\hline 9 & 899.4833 & 450.2453 & 882.4567 & 441.7320 & 881.4727 & 441.2400 & $\mathbf{L}$ & 1318.7729 & 659.8901 & 1301.7464 & 651.3768 & 1300.7624 & 650.8848 & 12 \\
\hline 10 & 956.5047 & 478.7560 & 939.4782 & 470.2427 & 938.4942 & 469.7507 & G & 1205.6889 & 603.3481 & 1188.6623 & 594.8348 & 1187.6783 & 594.3428 & 11 \\
\hline 11 & 1119.5681 & 560.2877 & 1102.5415 & 551.7744 & 1101.5575 & 551.2824 & $\mathbf{Y}$ & 1148.6674 & 574.8373 & 1131.6408 & 566.3241 & 1130.6568 & 565.8320 & 10 \\
\hline 12 & 1220.6157 & 610.8115 & 1203.5892 & 602.2982 & 1202.6052 & 601.8062 & $\mathbf{T}$ & 985.6041 & 493.3057 & 968.5775 & 484.7924 & 967.5935 & 484.3004 & 9 \\
\hline 13 & 1348.6743 & 674.8408 & 1331.6478 & 666.3275 & 1330.6637 & 665.8355 & $\mathbf{Q}$ & 884.5564 & 442.7818 & 867.5298 & 434.2686 & 866.5458 & 433.7765 & 8 \\
\hline 14 & 1449.7220 & 725.3646 & 1432.6954 & 716.8514 & 1431.7114 & 716.3594 & $\mathbf{T}$ & 756.4978 & 378.7525 & 739.4713 & 370.2393 & 738.4872 & 369.7473 & 7 \\
\hline 15 & 1562.8061 & 781.9067 & 1545.7795 & 773.3934 & 1544.7955 & 772.9014 & $\mathbf{L}$ & 655.4501 & 328.2287 & 638.4236 & 319.7154 & & & 6 \\
\hline 16 & 1690.9010 & 845.9541 & 1673.8745 & 837.4409 & 1672.8905 & 836.9489 & $\mathbf{K}$ & 542.3661 & 271.6867 & 525.3395 & 263.1734 & & & 5 \\
\hline 17 & 1787.9538 & 894.4805 & 1770.9272 & 885.9673 & 1769.9432 & 885.4752 & $\mathbf{P}$ & 414.2711 & 207.6392 & 397.2445 & 199.1259 & & & 4 \\
\hline 18 & 1844.9752 & 922.9913 & 1827.9487 & 914.4780 & 1826.9647 & 913.9860 & G & 317.2183 & 159.1128 & 300.1918 & 150.5995 & & & 3 \\
\hline 19 & 1958.0593 & 979.5333 & 1941.0328 & 971.0200 & 1940.0487 & 970.5280 & I & 260.1969 & 130.6021 & 243.1703 & 122.0888 & & & 2 \\
\hline 20 & & & & & & & $\mathbf{K}$ & 147.1128 & 74.0600 & 130.0863 & 65.5468 & & & 1 \\
\hline
\end{tabular}
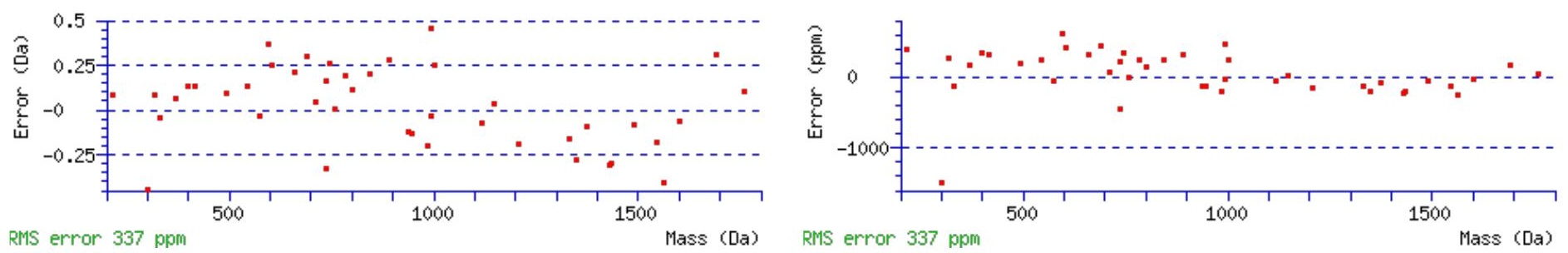

\section{All matches to this query}

\begin{tabular}{|l|l|l|l|}
\hline Score & Mr(calc): & Delta & \multicolumn{1}{|c|}{ Sequence } \\
\hline 60.4 & 2103.1575 & -0.0047 & VNNSSLIGLGYTQTLKPGIK \\
\hline 60.4 & 2103.1575 & -0.0047 & VNNSSLIGLGYTQTLKPGIK \\
\hline 27.4 & 2103.1324 & 0.0204 & VNNSSLIGVGYTQTLRPGVK \\
\hline 27.4 & 2103.1324 & 0.0204 & VNNSSLIGVGYTQTLRPGVK \\
\hline 14.5 & 2102.1484 & 1.0044 & VNNSSLIGVGYTQTLRPGVK \\
\hline 4.1 & 2101.1565 & 1.9963 & AQEVGKILTMVTVENLXRK \\
\hline 2.8 & 2101.1452 & 2.0076 & LENGKPLSMSEQTGLLKIK \\
\hline 2.4 & 2103.1549 & -0.0021 & VNLTRLHTLGDNLLDPRR \\
\hline 2.2 & 2103.1477 & 0.0051 & VKWTFLSGDREVEVLVAR \\
\hline 0.3 & 2102.1653 & 0.9875 & XLLLLGLGLGLGSQPPPPGPR \\
\hline
\end{tabular}

Spectrum No: 526; Query: 124; Rank: 1

\section{Peptide View}




\section{MS/MS Fragmentation of SAEGTFFINK}

Found in IPI00394353, Tax_Id=10116 Gene_Symbol=Hexa Beta-hexosaminidase alpha chain precursor

Match to Query 124: 1113.536968 from(557.775760,2+)

Title: 100101RatKid_NS_deglyco_15.2585.2585.2.dta

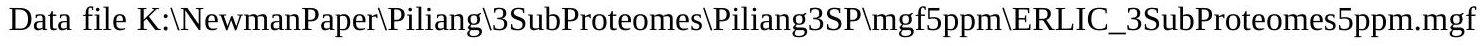
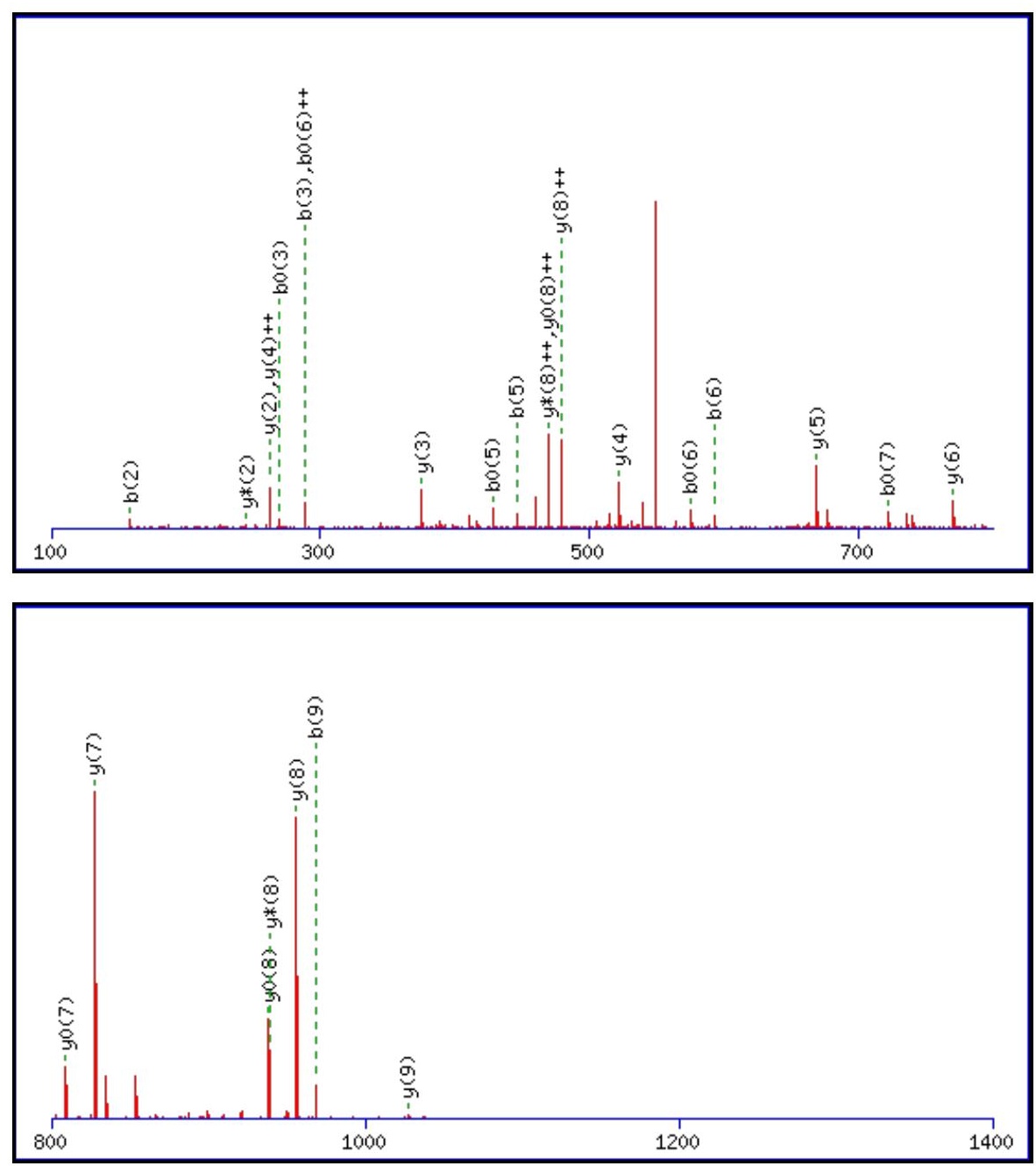

\begin{tabular}{rrrr}
\hline & 1 & 1 & 2000 \\
1400 & 1600 & 1800 & 2000 \\
\hline
\end{tabular}

Monoisotopic mass of neutral peptide $\operatorname{Mr}($ calc): 1113.5342

Fixed modifications: Carbamidomethyl (C)

Variable modifications:

N9 : Deamidated_N (N)

Ions Score: 60 Expect: 0.0001

Matches (Bold Red): 26/82 fragment ions using 38 most intense peaks

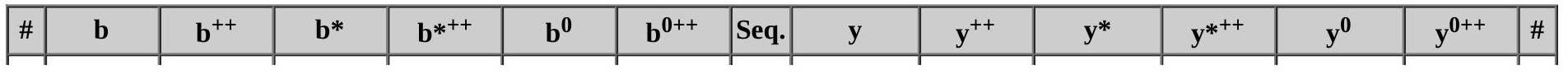




\begin{tabular}{|c|c|c|c|c|c|c|c|c|c|c|c|c|c|c|}
\hline 1] & 88.0393 & 44.5233 & & & 70.0287 & 35.5180| & $S$ & & & & & & & 10 \\
\hline 2 & 159.0764 & 80.0418 & & & 141.0659 & 71.0366 & A & 1027.5095 & 514.2584 & 1010.4829 & 505.7451 & 1009.4989 & 505.2531 & 9 \\
\hline 3 & 288.1190 & 144.5631 & & & 270.1084 & 135.5579 & $\mathbf{E}$ & 956.4724 & 478.7398 & 939.4458 & 470.2265 & 938.4618 & 469.7345 & 8 \\
\hline 4 & 345.1405 & 173.0739 & & & 327.1299 & 164.0686 & G & 827.4298 & 414.2185 & 810.4032 & 405.7053 & 809.4192 & 405.2132 & 7 \\
\hline 5 & 446.1882 & 223.5977 & & & 428.1776 & 214.5924 & $T$ & 770.4083 & 385.7078 & 753.3818 & 377.1945 & 752.3977 & 376.7025 & 6 \\
\hline 6 & 593.2566 & 297.1319 & & & 575.2460 & 288.1266 & $\mathbf{F}$ & 669.3606 & 335.1840 & 652.3341 & 326.6707 & & & 5 \\
\hline 7 & 740.3250 & 370.6661 & & & 722.3144 & 361.6608 & $\mathbf{F}$ & 522.2922 & 261.6497 & 505.2657 & 253.1365 & & & 4 \\
\hline 8 & 853.4090 & 427.2082 & & & 835.3985 & 418.2029 & I & 375.2238 & 188.1155 & 358.1973 & 179.6023 & & & 3 \\
\hline 9 & 968.4360 & 484.7216 & 951.4094 & 476.2084 & 950.4254 & 475.7163 & $\mathbf{N}$ & 262.1397 & 131.5735 & 245.1132 & 123.0602 & & & 2 \\
\hline 10 & & & & & & & $\mathbf{K}$ & 147.1128 & 74.0600 & 130.0863 & 65.5468 & & & 1 \\
\hline
\end{tabular}
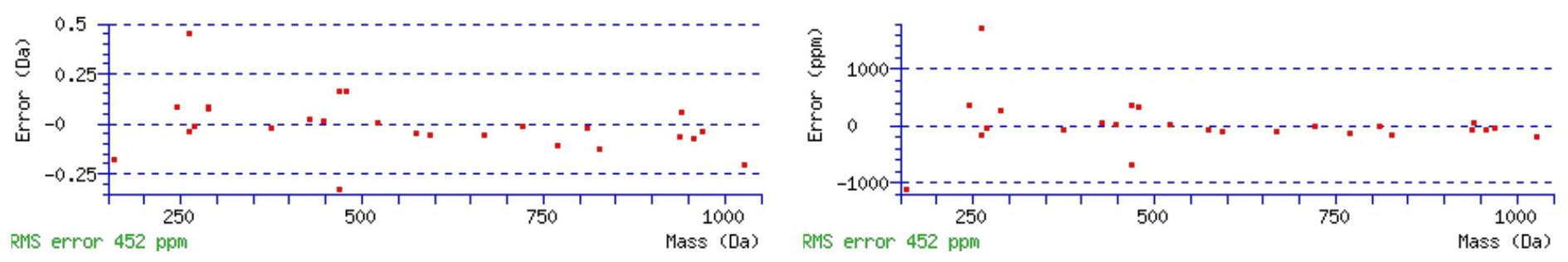

\section{All matches to this query}

\begin{tabular}{|l|l|c|l|}
\hline Score & Mr(calc): & Delta & \multicolumn{1}{|c|}{ Sequence } \\
\hline 60.3 & 1113.5342 & 0.0027 & SAEGTFFINK \\
\hline 19.8 & 1111.5379 & 1.9991 & REFMMRSR \\
\hline 9.1 & 1111.5274 & 2.0095 & REGETVTLK \\
\hline 8.1 & 1113.5315 & 0.0054 & NHNRFDPSK \\
\hline 5.5 & 1112.5240 & 1.0130 & RLNHIHSR \\
\hline 5.4 & 1113.5454 & -0.0085 & VYNYESAIR \\
\hline 5.0 & 1111.5274 & 2.0095 & RATEILSDK \\
\hline 4.8 & 1112.5240 & 1.0130 & RSQSPFRR \\
\hline 4.5 & 1112.5274 & 1.0096 & RMRLXSSR \\
\hline 3.8 & 1112.5324 & 1.0045 & EWYMIISR \\
\hline
\end{tabular}

Spectrum No: 527; Query: 640; Rank: 1

\section{Peptide View}

MS/MS Fragmentation of HAEDKFNETTER

Found in IPI00207668, Tax_Id=10116 Gene_Symbol=Afm Afamin precursor

Match to Query 640: 1476.648848 from(739.331700,2+)

Title: 100101RatKid_NS_deglyco_23.318.318.2.dta

Data file K:INewmanPaper|Piliangl3SubProteomes\Piliang3SP\mgf5ppm\ERLIC_3SubProteomes5ppm.mgf 

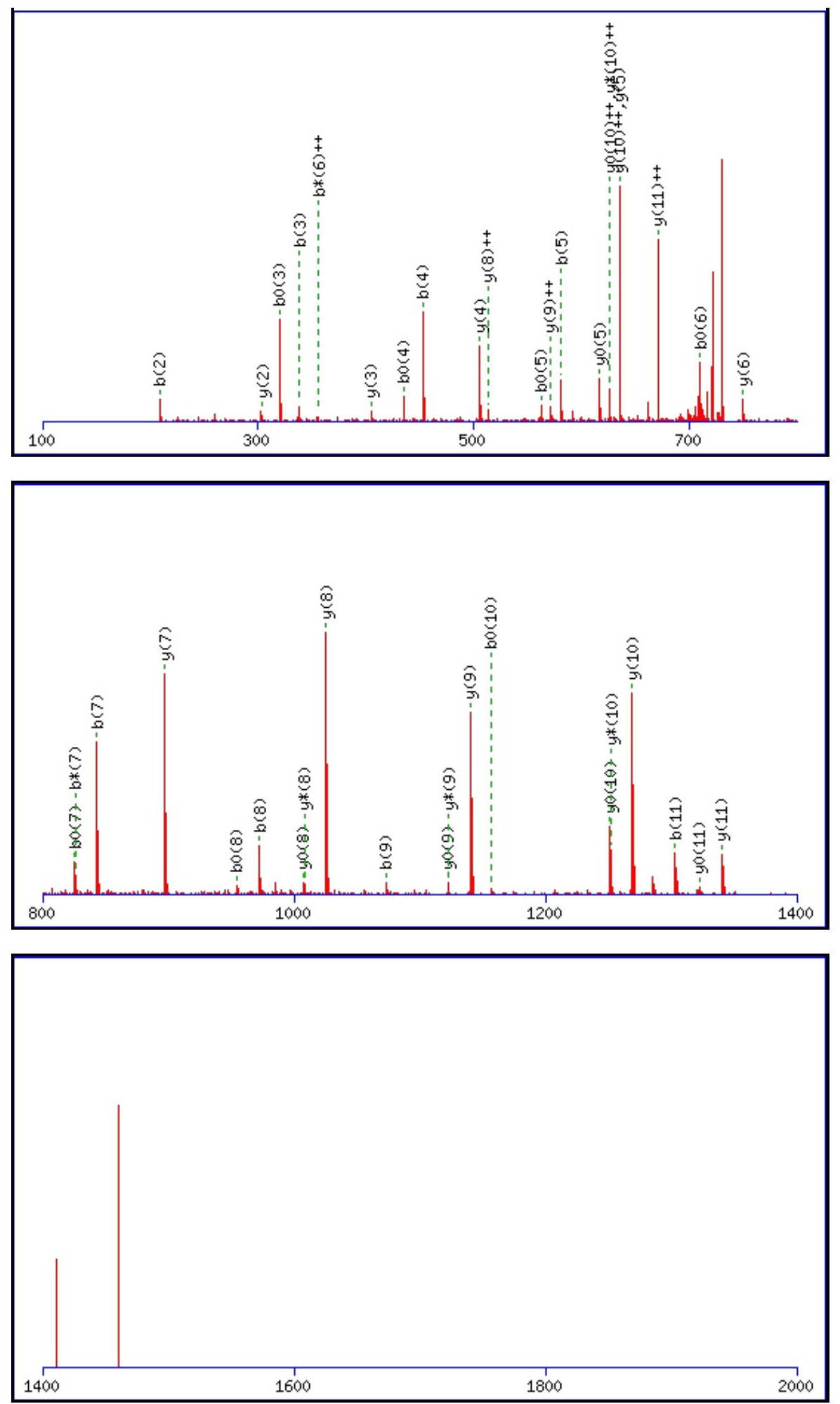

Monoisotopic mass of neutral peptide $\operatorname{Mr}($ calc): 1476.6481

Fixed modifications: Carbamidomethyl (C)

Variable modifications:

N7 : Deamidated_N (N)

Ions Score: 60 Expect: 0.00016

Matches (Bold Red): 41/118 fragment ions using 80 most intense peaks

\begin{tabular}{|r|c|c|c|c|c|c|c|c|c|c|c|c|c|c|}
\hline$\#$ & $\mathbf{b}$ & $\mathbf{b}^{++}$ & $\mathbf{b}^{*}$ & $\mathbf{b}^{\mathbf{*}^{++}}$ & $\mathbf{b}^{\mathbf{0}}$ & $\mathbf{b}^{\mathbf{0 + +}}$ & Seq. & $\mathbf{y}$ & $\mathbf{y}^{++}$ & $\mathbf{y}^{\mathbf{*}}$ & $\mathbf{y}^{\boldsymbol{*}^{++}}$ & $\mathbf{y}^{\mathbf{0}}$ & $\mathbf{y}^{\mathbf{0 + +}}$ & $\#$ \\
\hline $\mathbf{1}$ & 138.0662 & 69.5367 & & & & & $\mathbf{H}$ & & & & & & & $\mathbf{1 2}$ \\
\hline $\mathbf{2}$ & $\mathbf{2 0 9 . 1 0 3 3}$ & 105.0553 & & & & & $\mathbf{A}$ & $\mathbf{1 3 4 0 . 5 9 6 5}$ & $\mathbf{6 7 0 . 8 0 1 9}$ & 1323.5699 & 662.2886 & $\mathbf{1 3 2 2 . 5 8 5 9}$ & 661.7966 & $\mathbf{1 1}$ \\
\hline $\mathbf{3}$ & 338.1459 & 169.5766 & & & 320.1353 & 160.5713 & $\mathbf{E}$ & $\mathbf{1 2 6 9 . 5 5 9 3}$ & $\mathbf{6 3 5 . 2 8 3 3}$ & $\mathbf{1 2 5 2 . 5 3 2 8}$ & $\mathbf{6 2 6 . 7 7 0 0}$ & $\mathbf{1 2 5 1 . 5 4 8 8}$ & $\mathbf{6 2 6 . 2 7 8 0}$ & $\mathbf{1 0}$ \\
\hline $\mathbf{4}$ & $\mathbf{4 5 3 . 1 7 2 8}$ & 227.0901 & & & $\mathbf{4 3 5 . 1 6 2 3}$ & 218.0848 & $\mathbf{D}$ & $\mathbf{1 1 4 0 . 5 1 6 8}$ & 570.7620 & $\mathbf{1 1 2 3 . 4 9 0 2}$ & 562.2487 & $\mathbf{1 1 2 2 . 5 0 6 2}$ & 561.7567 & $\mathbf{9}$ \\
\hline $\mathbf{5}$ & $\mathbf{5 8 1 . 2 6 7 8}$ & 291.1375 & 564.2413 & 282.6243 & 563.2572 & 282.1323 & $\mathbf{K}$ & $\mathbf{1 0 2 5 . 4 8 9 8}$ & $\mathbf{5 1 3 . 2 4 8 5}$ & $\mathbf{1 0 0 8 . 4 6 3 3}$ & 504.7353 & $\mathbf{1 0 0 7 . 4 7 9 2}$ & 504.2433 & $\mathbf{8}$ \\
\hline
\end{tabular}




\begin{tabular}{|c|c|c|c|c|c|c|c|c|c|c|c|c|c|c|}
\hline 6 & 728.3362 & |364.6717 & 711.3097 & |356.1585 & 710.3257 & |355.6665 & $\mathbf{F}$ & 897.3948 & |449.2011 & 880.3683 & |440.6878 & 879.3843 & |440.1958 & 7 \\
\hline 7 & 843.3632 & 422.1852 & 826.3366 & 413.6719 & 825.3526 & 413.1799 & $\mathbf{N}$ & 750.3264 & 375.6669 & 733.2999 & 367.1536 & 732.3159 & |366.6616 & 6 \\
\hline 8 & 972.4057 & 486.7065 & 955.3792 & 478.1932 & 954.3952 & 477.7012 & $\mathbf{E}$ & 635.2995 & |318.1534 & 618.2729 & 309.6401 & 617.2889 & 309.1481 & 5 \\
\hline 9 & 1073.4534 & 537.2303 & 1056.4269 & 528.7171 & 1055.4429 & 528.2251 & $\mathbf{T}$ & 506.2569 & 253.6321 & 489.2304 & 245.1188 & 488.2463 & 244.6268 & 4 \\
\hline 10 & 1174.5011 & 587.7542 & 1157.4746 & 579.2409 & 1156.4905 & 578.7489 & $\mathrm{~T}$ & 405.2092 & 203.1083 & 388.1827 & $\mid 194.5950$ & 387.1987 & 194.1030 & 3 \\
\hline 11 & 1303.5437 & 652.2755 & 1286.5171 & 643.7622 & 1285.5331 & 643.2702 & $\mathbf{E}$ & 304.1615 & 152.5844 & 287.1350 & 144.0711 & 286.1510 & 143.5791 & 2 \\
\hline 12 & & & & & & & $\mathbf{R}$ & 175.1190 & 88.0631 & 158.0924 & 79.5498 & & & 1 \\
\hline
\end{tabular}
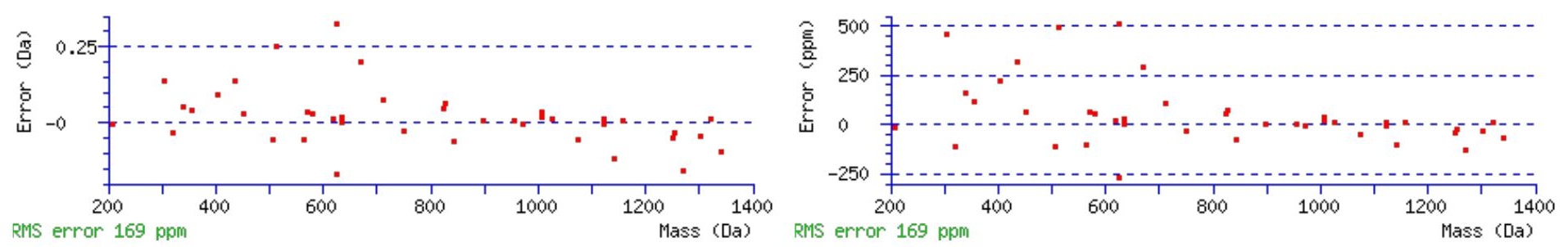

\section{All matches to this query}

\begin{tabular}{|l|c|c|l|}
\hline Score & Mr(calc): & Delta & \multicolumn{1}{c|}{ Sequence } \\
\hline 60.2 & 1476.6481 & 0.0008 & HAEDKFNETTER \\
\hline 21.2 & 1476.6358 & 0.0130 & HSLDASOGTATGPR \\
\hline 6.8 & 1476.6358 & 0.0130 & HSLDASQGTATGPR \\
\hline 6.8 & 1476.6358 & 0.0130 & HSLDASOGTATGPR \\
\hline 1.5 & 1476.6545 & -0.0056 & DHVTKPTAMGQGR \\
\hline 1.4 & 1476.6375 & 0.0114 & GDLSQRAKDLSK \\
\hline
\end{tabular}

Spectrum No: 528; Query: 2497; Rank: 1

\section{Peptide View}

MS/MS Fragmentation of VPFIFNINPATTNFTGSCQPQTAQLR

Found in IPI00212730, Tax_Id=10116 Gene_Symbol=Lamp2 Lysosome-associated membrane glycoprotein 2 precursor

Match to Query 2497: 2923.434552 from(975.485460,3+)

Title: 100101RatKid_NS_deglyco_21.5101.5101.3.dta

Data file K:INewmanPaper|Piliangl3SubProteomes\Piliang3SP\mgf5ppm\ERLIC_3SubProteomes5ppm.mgf

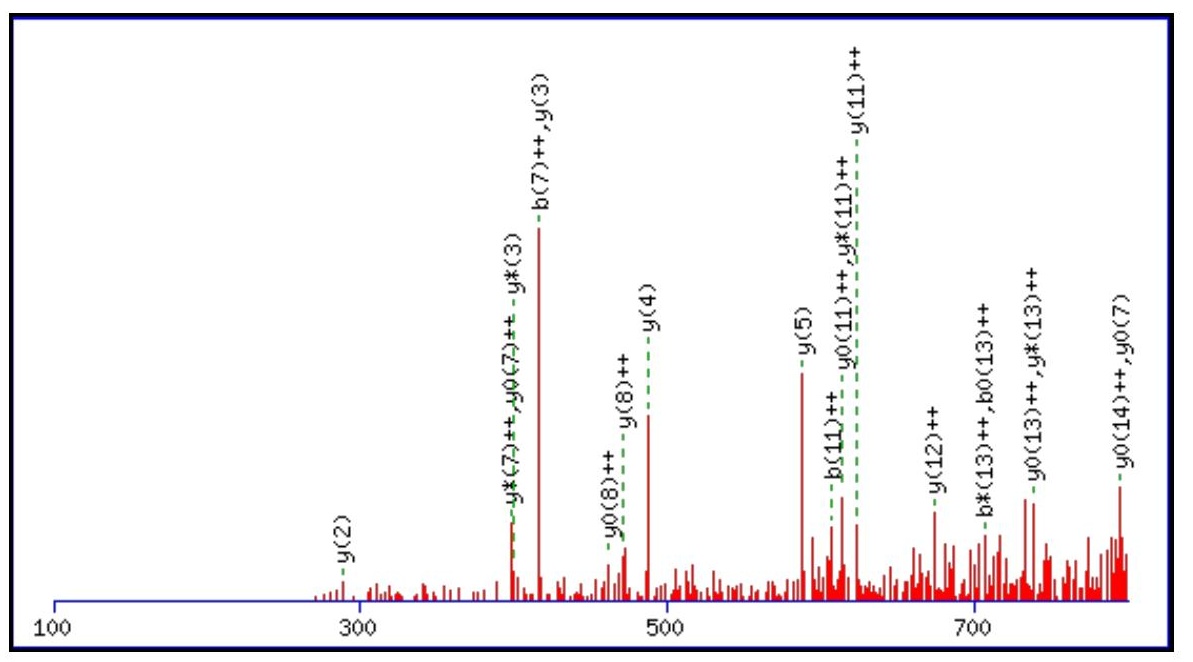



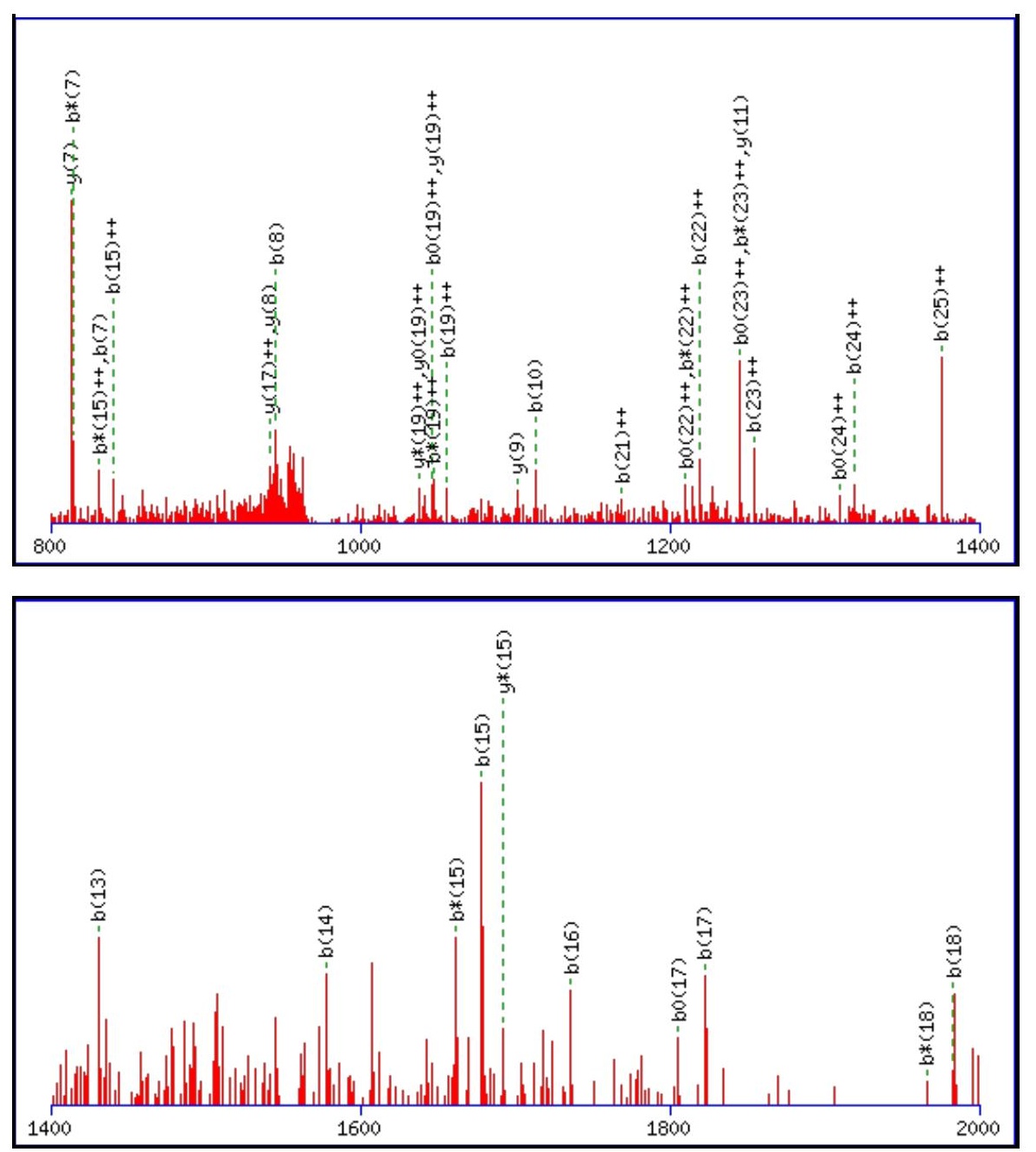

Monoisotopic mass of neutral peptide $\operatorname{Mr}($ calc): 2922.4334

Fixed modifications: Carbamidomethyl (C)

Variable modifications:

N13 : Deamidated_N (N)

Ions Score: 60 Expect: 0.00026

Matches (Bold Red): 58/262 fragment ions using 79 most intense peaks

\begin{tabular}{|c|c|c|c|c|c|c|c|c|c|c|c|c|c|c|}
\hline \# & b & $\mathbf{b}^{++}$ & b* & $\mathbf{b}^{*^{++}}$ & $\mathbf{b}^{0}$ & $\mathbf{b}^{0++}$ & Seq. & $\mathbf{y}$ & $\mathbf{y}^{++}$ & $\mathbf{y}^{*}$ & $\mathrm{y}^{*^{++}}$ & $\mathbf{y}^{\mathbf{0}}$ & $y^{0++}$ & \# \\
\hline 1 & 100.0757 & 50.5415 & & & & & $\mathbf{V}$ & & & & & & & 26 \\
\hline 2 & 197.1285 & 99.0679 & & & & & $\mathbf{P}$ & 2824.3723 & 1412.6898 & 2807.3457 & 1404.1765 & 2806.3617 & 1403.6845 & 25 \\
\hline 3 & 344.1969 & 172.6021 & & & & & $\mathbf{F}$ & 2727.3195 & 1364.1634 & 2710.2930 & 1355.6501 & 2709.3090 & 1355.1581 & 24 \\
\hline 4 & 457.2809 & 229.1441 & & & & & I & 2580.2511 & 1290.6292 & 2563.2246 & 1282.1159 & 2562.2405 & 1281.6239 & 23 \\
\hline 5 & 604.3493 & 302.6783 & & & & & $\mathbf{F}$ & 2467.1670 & 1234.0872 & 2450.1405 & 1225.5739 & 2449.1565 & 1225.0819 & 22 \\
\hline 6 & 718.3923 & 359.6998 & 701.3657 & 351.1865 & & & $\mathbf{N}$ & 2320.0986 & 1160.5530 & 2303.0721 & 1152.0397 & 2302.0881 & 1151.5477 & 21 \\
\hline 7 & 831.4763 & 416.2418 & 814.4498 & 407.7285 & & & I & 2206.0557 & 1103.5315 & 2189.0292 & 1095.0182 & 2188.0451 & 1094.5262 & 20 \\
\hline 8 & 945.5193 & 473.2633 & 928.4927 & 464.7500 & & & $\mathbf{N}$ & 2092.9716 & 1046.9895 & 2075.9451 & 1038.4762 & 2074.9611 & 1037.9842 & 19 \\
\hline 9 & 1042.5720 & 521.7897 & 1025.5455 & 513.2764 & & & $\mathbf{P}$ & 1978.9287 & 989.9680 & 1961.9022 & 981.4547 & 1960.9181 & 980.9627 & 18 \\
\hline 10 & 1113.6091 & 557.3082 & 1096.5826 & 548.7949 & & & A & 1881.8759 & 941.4416 & 1864.8494 & 932.9283 & 1863.8654 & 932.4363 & 17 \\
\hline 11 & 1214.6568 & 607.8320 & 1197.6303 & 599.3188 & 1196.6463 & 598.8268 & $\mathbf{T}$ & 1810.8388 & 905.9231 & 1793.8123 & 897.4098 & 1792.8283 & 896.9178 & 16 \\
\hline 12 & 1315.7045 & 658.3559 & 1298.6780 & 649.8426 & 1297.6939 & 649.3506 & $\mathbf{T}$ & 1709.7912 & 855.3992 & 1692.7646 & 846.8859 & 1691.7806 & 846.3939 & 15 \\
\hline 13 & 1430.7314 & 715.8694 & 1413.7049 & 707.3561 & 1412.7209 & 706.8641 & $\mathbf{N}$ & 1608.7435 & 804.8754 & 1591.7169 & 796.3621 & 1590.7329 & 795.8701 & 14 \\
\hline 14 & 1577.7998 & 789.4036 & 1560.7733 & 780.8903 & 1559.7893 & 780.3983 & $\mathbf{F}$ & 1493.7165 & 747.3619 & 1476.6900 & 738.8486 & 1475.7060 & 738.3566 & 13 \\
\hline 15 & 1678.8475 & 839.9274 & 1661.8210 & 831.4141 & 1660.8370 & 830.9221 & $\mathbf{T}$ & 1346.6481 & 673.8277 & 1329.6216 & 665.3144 & 1328.6376 & 664.8224 & 12 \\
\hline 16 & $\mathbf{1 7 3 5 . 8 6 9 0}$ & 868.4381 & 1718.8424 & 859.9249 & 1717.8584 & 859.4329 & G & 1245.6004 & 623.3039 & 1228.5739 & 614.7906 & 1227.5899 & 614.2986 & 11 \\
\hline 17 & 1822.9010 & 911.9541 & 1805.8745 & 903.4409 & 1804.8905 & 902.9489 & S & 1188.5790 & 594.7931 & 1171.5524 & 586.2799 & 1170.5684 & 585.7878 & 10 \\
\hline 18 & 1982.9317 & 991.9695 & 1965.9051 & 983.4562 & 1964.9211 & 982.9642 & C & 1101.5470 & 551.2771 & 1084.5204 & 542.7638 & 1083.5364 & 542.2718 & 9 \\
\hline
\end{tabular}




\begin{tabular}{|c|c|c|c|c|c|c|c|c|c|c|c|c|c|c|}
\hline 19 & 2110.9902 & |1055.9988 & |2093.9637 & |1047.4855| & |2092.9797 & |1046.9935 & $\mathbf{Q}$ & 941.5163 & 471.2618 & 924.4898| & 462.7485 & 923.5057| & 462.2565 & 8 \\
\hline 20 & 2208.0430 & 1104.5251 & 2191.0165 & 1096.0119 & 2190.0324 & 1095.5199 & $\mathbf{P}$ & 813.4577 & 407.2325 & 796.4312 & 398.7192 & 795.4472 & 398.2272 & 7 \\
\hline 21 & 2336.1016 & 1168.5544 & 2319.0750 & 1160.0412 & 2318.0910 & 1159.5492 & $\mathbf{Q}$ & 716.4050 & 358.7061 & 699.3784 & 350.1928 & 698.3944 & 349.7008 & 6 \\
\hline 22 & 2437.1493 & 1219.0783 & 2420.1227 & 1210.5650 & 2419.1387 & 1210.0730 & $\mathbf{T}$ & 588.3464 & 294.6768 & 571.3198 & 286.1636 & 570.3358 & 285.6715 & 5 \\
\hline 23 & 2508.1864 & 1254.5968 & 2491.1598 & 1246.0836 & 2490.1758 & 1245.5915 & A & 487.2987 & 244.1530 & 470.2722 & 235.6397 & & & 4 \\
\hline 24 & 2636.2450 & 1318.6261 & 2619.2184 & 1310.1128 & 2618.2344 & 1309.6208 & $\bar{Q}$ & 416.2616 & 208.6344 & 399.2350 & 200.1212 & & & 3 \\
\hline 25 & 2749.3290 & 1375.1682 & 2732.3025 & 1366.6549 & 2731.3185 & 1366.1629 & $\mathbf{L}$ & 288.2030 & 144.6051 & 271.1765 & 136.0919 & & & 2 \\
\hline 26 & & & & & & & $\mathbf{R}$ & 175.1190 & 88.0631 & 158.0924 & 79.5498 & & & 1 \\
\hline
\end{tabular}
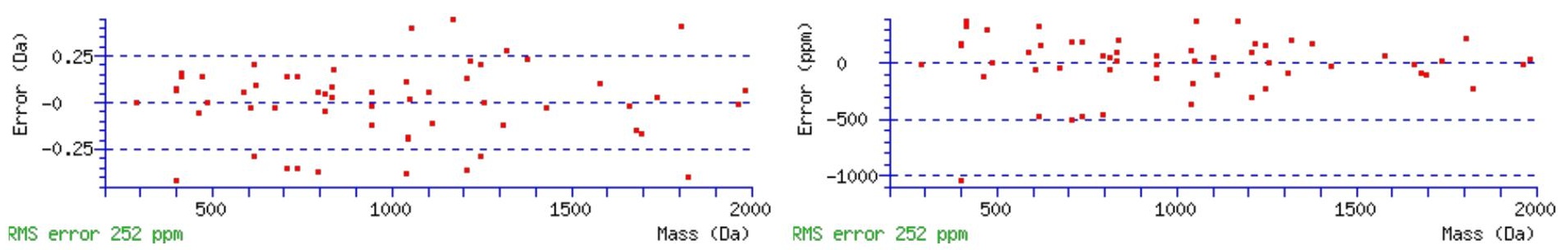

\section{All matches to this query}

\begin{tabular}{|l|l|l|l|}
\hline Score & Mr(calc): & Delta & \multicolumn{1}{|c|}{ Sequence } \\
\hline 60.1 & 2922.4334 & 1.0011 & VPFIFNINPATTNFTGSCQPQTAQLR \\
\hline 39.4 & 2922.4334 & 1.0011 & VPFIFNINPATTNFTGSCQPQTAQLR \\
\hline 34.7 & 2922.4334 & 1.0011 & VPFIFNINPATTNFTGSCQPQTAQLR \\
\hline 33.2 & 2923.4174 & 0.0171 & VPFIFNINPATTNFTGSCQPQTAQLR \\
\hline 28.9 & 2921.4494 & 1.9851 & VPFIFNINPATTNFTGSCQPQTAQLR \\
\hline 28.2 & 2923.4174 & 0.0171 & VPFIFNINPATTNFTGSCQPQTAQLR \\
\hline 25.5 & 2923.4174 & 0.0171 & VPFIFNINPATTNFTGSCQPQTAQLR \\
\hline 3.5 & 2923.4189 & 0.0157 & NTFAERLSAVEAIANAISVVSSNGPGNR \\
\hline 3.5 & 2922.4277 & 1.0069 & VNLTFRTVYPDPRGAPGDTSAELPLR \\
\hline 3.5 & 2922.4277 & 1.0069 & VNLTFRTVYPDPRGAPGDTSAELPLR \\
\hline
\end{tabular}

Spectrum No: 529; Query: 2471; Rank: 1

\section{Peptide View}

MS/MS Fragmentation of REEAIQQVADALQYLQNISGDIFSR

Found in IPI00388036, Tax_Id=10116 Gene_Symbol=ORF19 similar to CXYorf1-related protein

Match to Query 2471: 2864.439882 from(955.820570,3+)

Title: 091008RatKidney_NH4Format01_11.6624.6624.3.dta

Data file K:INewmanPaper|Piliangl3SubProteomes\Piliang3SP\mgf5ppm\ERLIC_3SubProteomes5ppm.mgf 

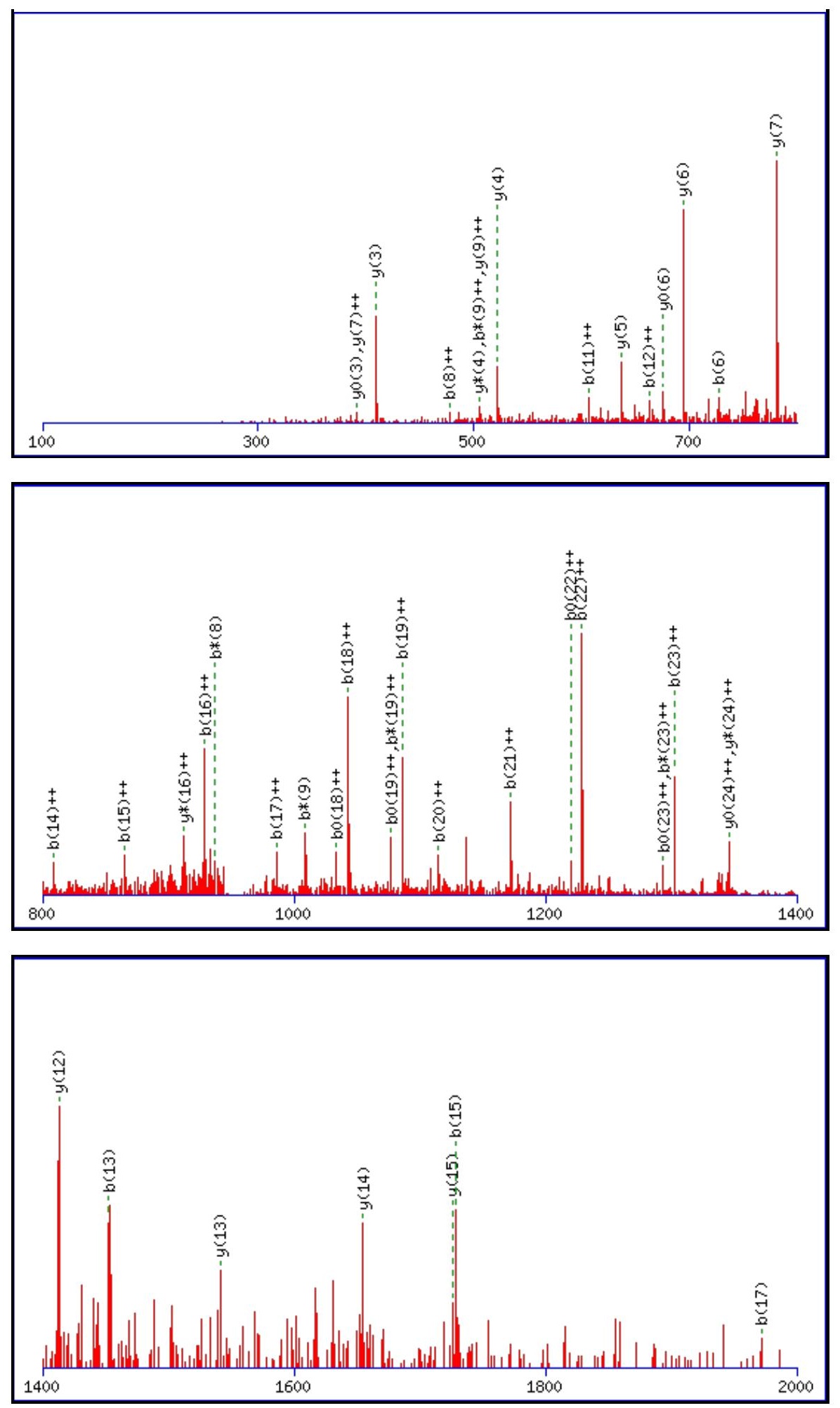

Monoisotopic mass of neutral peptide $\operatorname{Mr}($ calc): 2864.4304

Fixed modifications: Carbamidomethyl (C)

Variable modifications:

N17 : Deamidated_N (N)

Ions Score: 60 Expect: 0.00021

Matches (Bold Red): 44/284 fragment ions using 91 most intense peaks

\begin{tabular}{|r|c|c|c|c|c|c|c|c|c|c|c|c|c|c|}
\hline$\#$ & $\mathbf{b}$ & $\mathbf{b}^{++}$ & $\mathbf{b}^{*}$ & $\mathbf{b}^{\boldsymbol{*}^{++}}$ & $\mathbf{b}^{\mathbf{0}}$ & $\mathbf{b}^{\mathbf{0 + +}}$ & Seq. & $\mathbf{y}$ & $\mathbf{y}^{++}$ & $\mathbf{y}^{\mathbf{*}}$ & $\mathbf{y}^{\mathbf{*}^{++}}$ & $\mathbf{y}^{\mathbf{0}}$ & $\mathbf{y}^{\mathbf{0 + +}}$ & $\#$ \\
\hline $\mathbf{1}$ & 157.1084 & 79.0578 & 140.0818 & 70.5446 & & & $\mathbf{R}$ & & & & & & & $\mathbf{2 5}$ \\
\hline $\mathbf{2}$ & 286.1510 & 143.5791 & 269.1244 & 135.0659 & 268.1404 & 134.5738 & $\mathbf{E}$ & 2709.3366 & 1355.1719 & 2692.3101 & $\mathbf{1 3 4 6 . 6 5 8 7}$ & 2691.3260 & $\mathbf{1 3 4 6 . 1 6 6 7}$ & $\mathbf{2 4}$ \\
\hline $\mathbf{3}$ & 415.1936 & 208.1004 & 398.1670 & 199.5872 & 397.1830 & 199.0951 & $\mathbf{E}$ & 2580.2940 & 1290.6506 & 2563.2675 & 1282.1374 & 2562.2835 & 1281.6454 & $\mathbf{2 3}$ \\
\hline $\mathbf{4}$ & 486.2307 & 243.6190 & 469.2041 & 235.1057 & 468.2201 & 234.6137 & $\mathbf{A}$ & 2451.2514 & 1226.1294 & 2434.2249 & 1217.6161 & 2433.2409 & 1217.1241 & $\mathbf{2 2}$ \\
\hline $\mathbf{5}$ & 599.3148 & 300.1610 & 582.2882 & 291.6477 & 581.3042 & 291.1557 & $\mathbf{I}$ & 2380.2143 & 1190.6108 & 2363.1878 & 1182.0975 & 2362.2037 & 1181.6055 & $\mathbf{2 1}$ \\
\hline
\end{tabular}




\begin{tabular}{|c|c|c|c|c|c|c|c|c|c|c|c|c|c|c|}
\hline 6 & 727.3733 & 364.1903 & 710.3468 & 355.6770 & 709.3628 & 355.1850 & $\mathbf{Q}$ & |2267.1302 & 1134.0688 & |2250.1037 & 1125.5555 & |2249.1197 & 1125.0635 & |20| \\
\hline 7 & 855.4319 & 428.2196 & 838.4054 & 419.7063 & 837.4213 & 419.2143 & $\mathbf{Q}$ & 2139.0717 & 1070.0395 & 2122.0451 & 1061.5262 & 2121.0611 & 1061.0342 & 19 \\
\hline 8 & 954.5003 & 477.7538 & 937.4738 & 469.2405 & 936.4898 & 468.7485 & V & 2011.0131 & 1006.0102 & 1993.9865 & 997.4969 & 1993.0025 & 997.0049 & 18 \\
\hline 9 & 1025.5374 & 513.2724 & 1008.5109 & 504.7591 & 1007.5269 & 504.2671 & A & 1911.9447 & 956.4760 & 1894.9181 & 947.9627 & 1893.9341 & 947.4707 & 17 \\
\hline 10 & 1140.5644 & 570.7858 & 1123.5378 & 562.2726 & 1122.5538 & 561.7805 & D & 1840.9076 & 920.9574 & 1823.8810 & 912.4441 & 1822.8970 & 911.9521 & 16 \\
\hline 11 & 1211.6015 & 606.3044 & 1194.5749 & 597.7911 & 1193.5909 & 597.2991 & A & 1725.8806 & 863.4439 & 1708.8541 & 854.9307 & 1707.8701 & 854.4387 & 15 \\
\hline 12 & 1324.6856 & 662.8464 & 1307.6590 & 654.3331 & 1306.6750 & 653.8411 & $\mathbf{L}$ & 1654.8435 & 827.9254 & 1637.8170 & 819.4121 & 1636.8329 & 818.9201 & 14 \\
\hline 13 & 1452.7441 & 726.8757 & 1435.7176 & 718.3624 & 1434.7336 & 717.8704 & $\mathbf{Q}$ & 1541.7594 & 771.3834 & 1524.7329 & 762.8701 & 1523.7489 & 762.3781 & 13 \\
\hline 14 & 1615.8075 & 808.4074 & 1598.7809 & 799.8941 & 1597.7969 & 799.4021 & $\mathbf{Y}$ & 1413.7009 & 707.3541 & 1396.6743 & 698.8408 & 1395.6903 & 698.3488 & 12 \\
\hline 15 & 1728.8915 & 864.9494 & 1711.8650 & 856.4361 & 1710.8810 & 855.9441 & $\mathbf{L}$ & 1250.6375 & 625.8224 & 1233.6110 & 617.3091 & 1232.6270 & 616.8171 & 11 \\
\hline 16 & 1856.9501 & 928.9787 & 1839.9236 & 920.4654 & 1838.9395 & 919.9734 & $\mathbf{Q}$ & 1137.5535 & 569.2804 & 1120.5269 & 560.7671 & 1119.5429 & 560.2751 & 10 \\
\hline 17 & 1971.9770 & 986.4922 & 1954.9505 & 977.9789 & 1953.9665 & 977.4869 & $\mathbf{N}$ & 1009.4949 & 505.2511 & 992.4683 & 496.7378 & 991.4843 & 496.2458 & 9 \\
\hline 18 & 2085.0611 & 1043.0342 & 2068.0346 & 1034.5209 & 2067.0505 & 1034.0289 & I & 894.4680 & 447.7376 & 877.4414 & 439.2243 & 876.4574 & 438.7323 & 8 \\
\hline 19 & 2172.0931 & 1086.5502 & 2155.0666 & 1078.0369 & 2154.0826 & 1077.5449 & S & 781.3839 & 391.1956 & 764.3573 & 382.6823 & 763.3733 & 382.1903 & 7 \\
\hline 21 & 2344.1415 & 1172.5744 & 2327.1150 & 1164.0611 & 2326.1310 & 1163.5691 & D & 637.3304 & 319.1688 & 620.3039 & 310.6556 & 619.3198 & 310.1636 & 5 \\
\hline 22 & 2457.2256 & 1229.1164 & 2440.1991 & 1220.6032 & 2439.2150 & 1220.1112 & I & 522.3035 & 261.6554 & 505.2769 & 253.1421 & 504.2929 & 252.6501 & 4 \\
\hline 23 & 2604.2940 & 1302.6506 & 2587.2675 & 1294.1374 & 2586.2835 & 1293.6454 & $\mathbf{F}$ & 409.2194 & 205.1133 & 392.1928 & 196.6001 & 391.2088 & 196.1081 & 3 \\
\hline 24 & 2691.3260 & 1346.1667 & 2674.2995 & 1337.6534 & 2673.3155 & 1337.1614 & S & 262.1510 & 131.5791 & 245.1244 & 123.0659 & 244.1404 & 122.5738 & 2 \\
\hline 25 & & & & & & & $\mathbf{R}$ & 175.1190 & 88.0631 & 158.0924 & 79.5498 & & & 1 \\
\hline
\end{tabular}
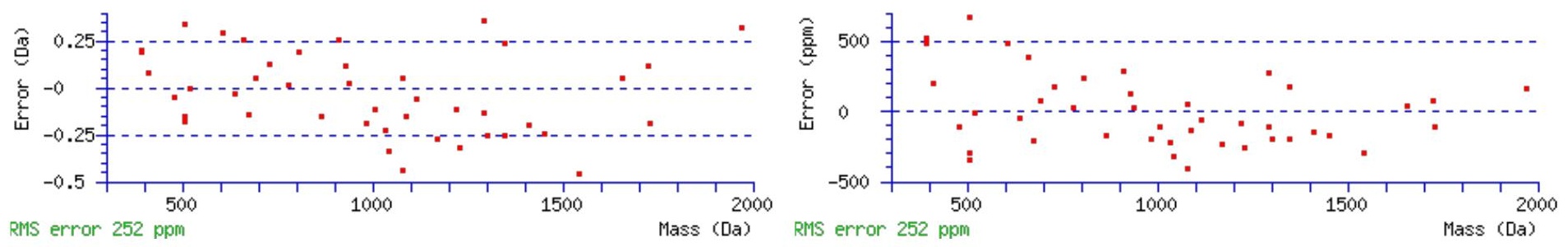

\section{All matches to this query}

\begin{tabular}{|l|l|l|l|}
\hline Score & Mr(calc): & Delta & \multicolumn{1}{|c|}{ Sequence } \\
\hline 60.0 & 2864.4304 & 0.0095 & REEAIQQVADALQYLQNISGDIFSR \\
\hline 48.2 & 2863.4464 & 0.9935 & REEAIQQVADALQYLQNISGDIFSR \\
\hline 3.3 & 2863.4481 & 0.9918 & QESVLGSIPLLSFRVAAVQPSDNISR \\
\hline 1.6 & 2863.4410 & 0.9989 & DLETLRTTLDNTTSNTKAELQALASR \\
\hline 1.6 & 2864.4130 & 0.0269 & TNIILLGDSIGDLTMADGVPGVQNILK \\
\hline 1.3 & 2863.4265 & 1.0134 & QVVSLSYTMVTPMLNPLIYSLRNK \\
\hline 0.8 & 2862.4528 & 1.9871 & EKSRGGLPGVGLSQDNTIILNPQFLK \\
\hline 0.8 & 2863.4317 & 1.0082 & TPPMSSVKWLLPNGTVLSHASRHPR \\
\hline 0.8 & 2863.4317 & 1.0082 & TPPMSSVKWLLPNGTVLSHASRHPR \\
\hline 0.4 & 2863.4265 & 1.0134 & QVVSLSYTMVTPMLNPLIYSLRNK \\
\hline
\end{tabular}

Spectrum No: 530; Query: 835; Rank: 1

\section{Peptide View}

MS/MS Fragmentation of EVTPVDLPNCTLVK

Found in IPI00231264, Tax_Id=10116 Gene_Symbol=Pon1 Serum paraoxonase/arylesterase 1

Match to Query 835: 1584.806448 from(793.410500,2+)

Title: 091008RatKidney_NoSalt_34.2149.2149.2.dta 


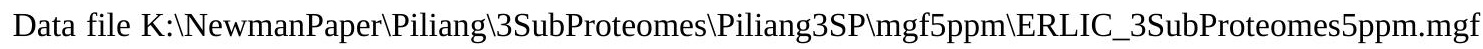
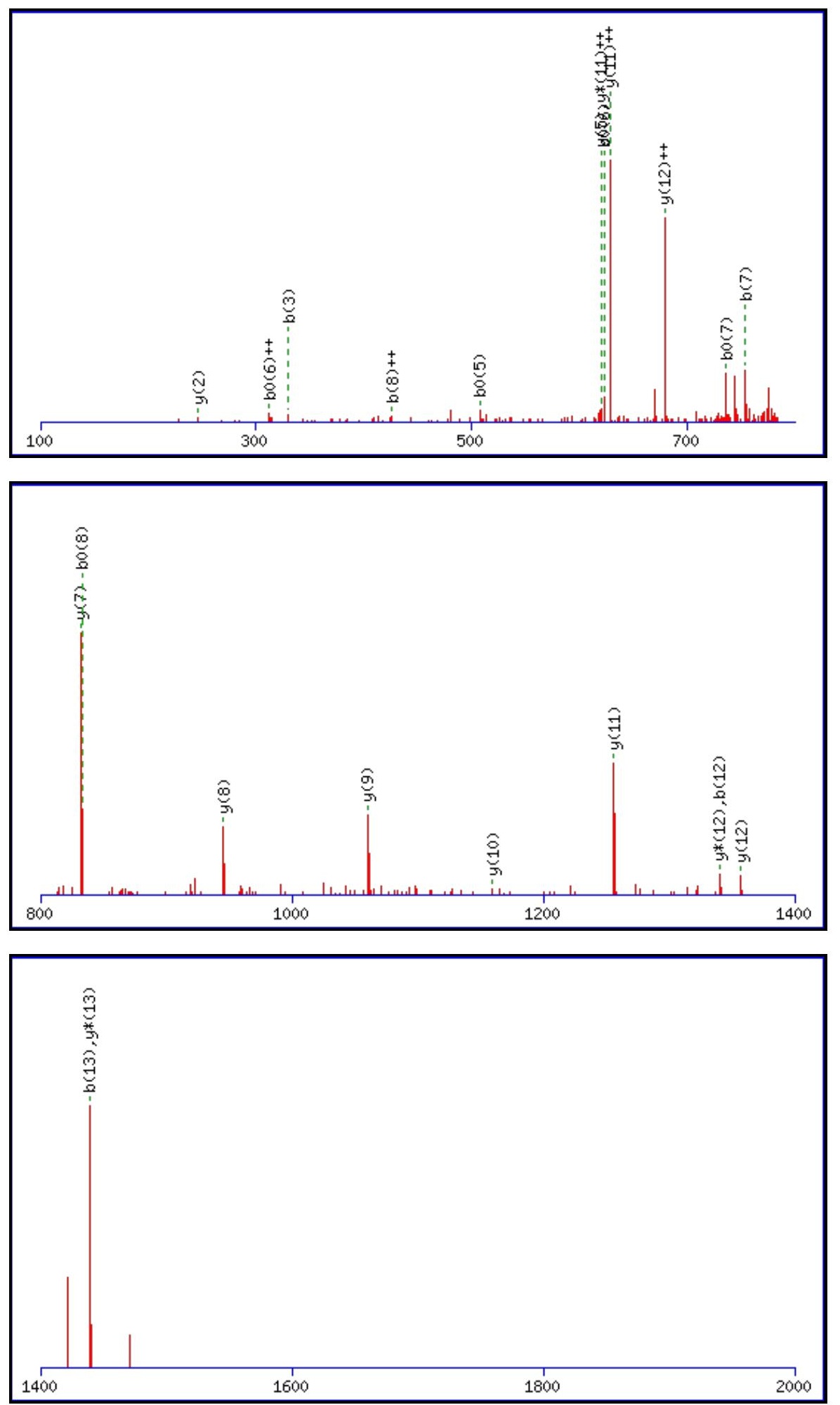

Monoisotopic mass of neutral peptide $\operatorname{Mr}($ calc): 1584.8069

Fixed modifications: Carbamidomethyl (C)

Variable modifications:

N9 : Deamidated_N (N)

Ions Score: 60 Expect: 0.00014

Matches (Bold Red): 24/134 fragment ions using 31 most intense peaks

\begin{tabular}{|c|c|c|c|c|c|c|c|c|c|c|c|c|c|c|}
\hline$\#$ & $\mathbf{b}$ & $\mathbf{b}^{++}$ & $\mathbf{b}^{*}$ & $\mathbf{b}^{\boldsymbol{*}^{++}}$ & $\mathbf{b}^{\mathbf{0}}$ & $\mathbf{b}^{\mathbf{0}+}$ & Seq. & $\mathbf{y}$ & $\mathbf{y}^{++}$ & $\mathbf{y}^{\mathbf{*}}$ & $\mathbf{y}^{\boldsymbol{*}^{++}}$ & $\mathbf{y}^{\mathbf{0}}$ & $\mathbf{y}^{\mathbf{0 + +}}$ & $\#$ \\
\hline $\mathbf{1}$ & 130.0499 & 65.5286 & & & 112.0393 & 56.5233 & $\mathbf{E}$ & & & & & & & $\mathbf{1 4}$ \\
\hline $\mathbf{2}$ & 229.1183 & 115.0628 & & & 211.1077 & 106.0575 & $\mathbf{V}$ & 1456.7716 & 728.8894 & $\mathbf{1 4 3 9 . 7 4 5 0}$ & 720.3762 & 1438.7610 & 719.8841 & $\mathbf{1 3}$ \\
\hline $\mathbf{3}$ & $\mathbf{3 3 0 . 1 6 6 0}$ & 165.5866 & & & 312.1554 & 156.5813 & $\mathbf{T}$ & $\mathbf{1 3 5 7 . 7 0 3 2}$ & $\mathbf{6 7 9 . 3 5 5 2}$ & $\mathbf{1 3 4 0 . 6 7 6 6}$ & 670.8420 & 1339.6926 & 670.3499 & $\mathbf{1 2}$ \\
\hline $\mathbf{4}$ & 427.2187 & 214.1130 & & & 409.2082 & 205.1077 & $\mathbf{P}$ & $\mathbf{1 2 5 6 . 6 5 5 5}$ & $\mathbf{6 2 8 . 8 3 1 4}$ & 1239.6289 & $\mathbf{6 2 0 . 3 1 8 1}$ & 1238.6449 & 619.8261 & $\mathbf{1 1}$
\end{tabular}




\begin{tabular}{|c|c|c|c|c|c|c|c|c|c|c|c|c|c|c|}
\hline 5 & 526.2871 & 263.6472 & & & 508.2766 & 254.6419 & $\mathbf{V}$ & 1159.6027 & 580.3050 & 1142.5762 & 571.7917 & 1141.5922 & 571.2997 & 10 \\
\hline 6 & 641.3141 & 321.1607 & & & 623.3035 & 312.1554 & D & 1060.5343 & 530.7708 & 1043.5078 & 522.2575 & 1042.5238 & 521.7655 & 9 \\
\hline 7 & 754.3981 & 377.7027 & & & 736.3876 & 368.6974 & $\mathbf{L}$ & 945.5074 & 473.2573 & 928.4808 & 464.7441 & 927.4968 & 464.2520 & 8 \\
\hline 8 & 851.4509 & 426.2291 & & & 833.4403 & 417.2238 & $\mathbf{P}$ & 832.4233 & 416.7153 & 815.3968 & 408.2020 & 814.4127 & 407.7100 & 7 \\
\hline 9 & 966.4778 & 483.7426 & 949.4513 & 475.2293 & 948.4673 & 474.7373 & $\mathbf{N}$ & 735.3705 & 368.1889 & 718.3440 & 359.6756 & 717.3600 & 359.1836 & 6 \\
\hline 10 & 1126.5085 & 563.7579 & 1109.4819 & 555.2446 & 1108.4979 & 554.7526 & C & 620.3436 & 310.6754 & 603.3171 & 302.1622 & 602.3330 & 301.6702 & 5 \\
\hline 11 & 1227.5562 & 614.2817 & 1210.5296 & 605.7685 & 1209.5456 & 605.2764 & $\mathbf{T}$ & 460.3130 & 230.6601 & 443.2864 & 222.1468 & 442.3024 & 221.6548 & 4 \\
\hline 12 & $\mid 1340.6402$ & 670.8238 & 1323.6137 & 662.3105 & 1322.6297 & 661.8185 & $\mathbf{L}$ & 359.2653 & 180.1363 & 342.2387 & 171.6230 & & & 3 \\
\hline 13 & 1439.7087 & 720.3580 & 1422.6821 & 711.8447 & 1421.6981 & 711.3527 & V & 246.1812 & 123.5942 & 229.1547 & 115.0810 & & & 2 \\
\hline 14 & & & & & & & $\mathbf{K}$ & 147.1128 & 74.0600 & 130.0863 & 65.5468 & & & 1 \\
\hline
\end{tabular}
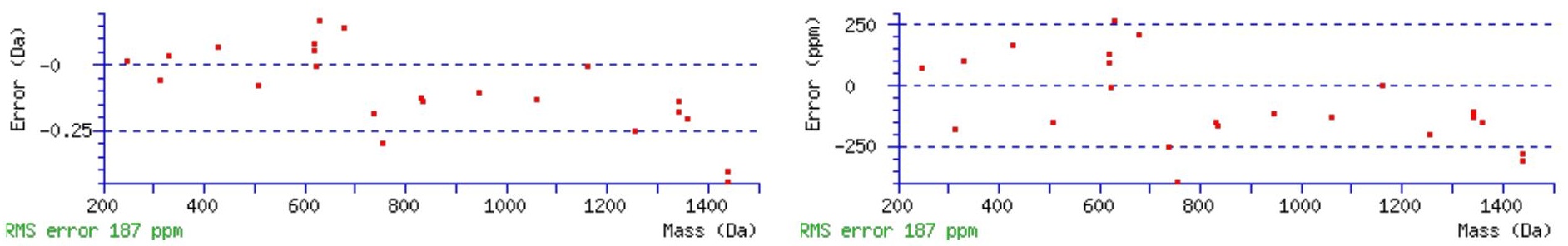

\section{All matches to this query}

\begin{tabular}{|l|l|l|l|}
\hline Score & Mr(calc): & Delta & \multicolumn{1}{c|}{ Sequence } \\
\hline 59.9 & 1584.8069 & -0.0005 & EVTPVDLPNCTLVK \\
\hline 11.2 & 1582.8154 & 1.9911 & $\underline{\text { ILTMVTVENLXRK }}$ \\
\hline 7.8 & 1582.7991 & 2.0073 & $\underline{\text { ILTNDWEDHLTVK }}$ \\
\hline 7.0 & 1584.8219 & -0.0155 & $\underline{\text { NSQSSKKANPTPQAK }}$ \\
\hline 6.3 & 1582.8154 & 1.9911 & $\underline{\text { ILTMVTVENLXRK }}$ \\
\hline 6.2 & 1584.8148 & -0.0083 & EVTADSTLAGFHLPK \\
\hline 6.0 & 1584.8137 & -0.0073 & FTGALALSRNTRAK \\
\hline 5.7 & 1583.8106 & 0.9959 & $\underline{\text { KKGINTLINIMSR }}$ \\
\hline 5.1 & 1584.8154 & -0.0090 & VKGQRAGGTPNMAAAR \\
\hline 4.9 & 1584.7912 & 0.0152 & NLLSVAYKNVVGAR \\
\hline
\end{tabular}

Spectrum No: 531; Query: 1173; Rank: 1

\section{Peptide View}

MS/MS Fragmentation of GAVSILCNSSGNCQCK

Found in IPI00357926, Tax_Id=10116 Gene_Symbol=Egfl5_predicted EGF-like-domain, multiple 5

Match to Query 1173: 1754.739608 from(878.377080,2+)

Title: 100101RatKid_NS_deglyco_23.1616.1616.2.dta

Data file K:\NewmanPaper $\backslash$ Piliang $\backslash 3$ SubProteomes $\backslash$ Piliang3SP $\backslash$ mgf5ppm\ERLIC_3SubProteomes5ppm.mgf 

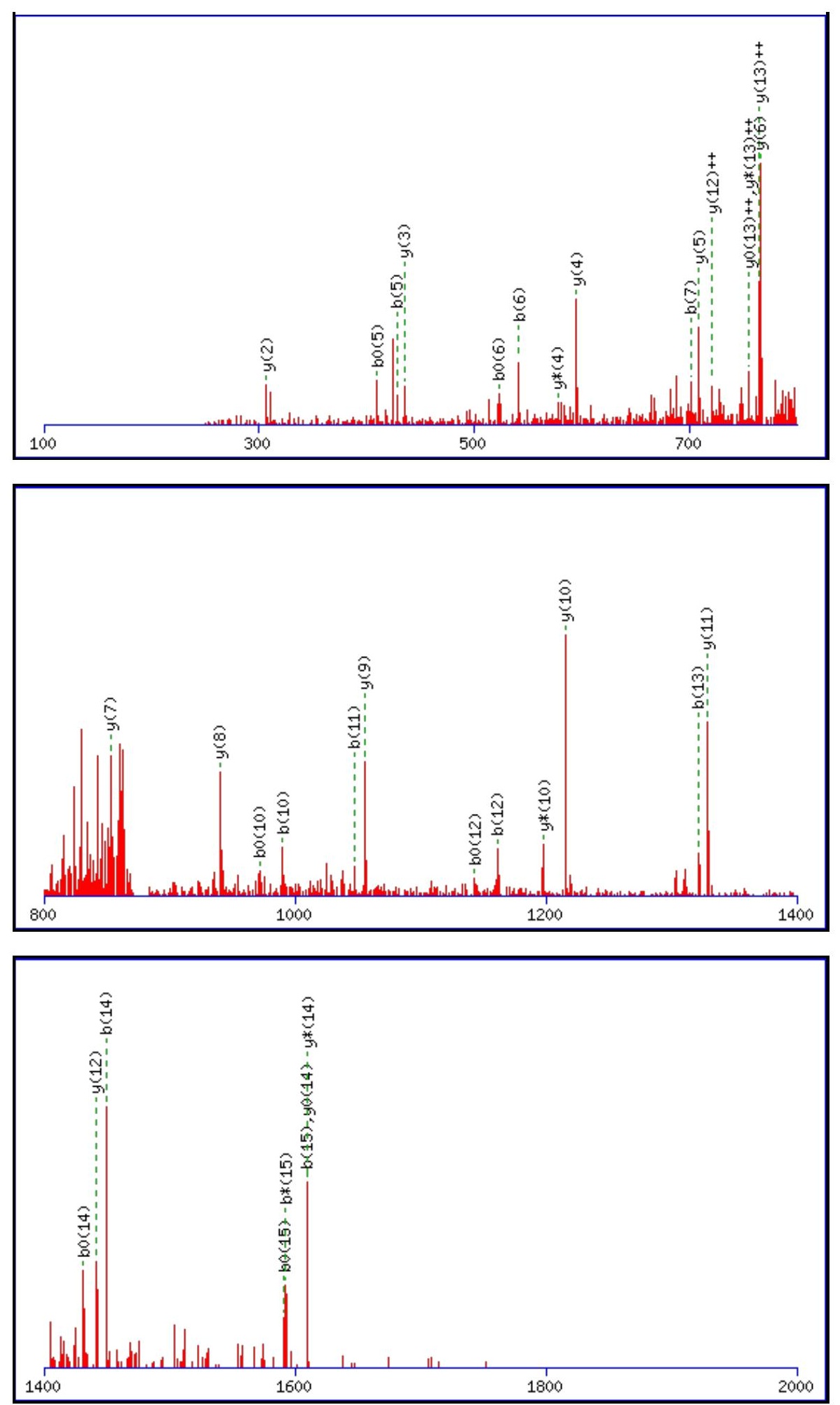

Monoisotopic mass of neutral peptide $\operatorname{Mr}($ calc): 1754.7386

Fixed modifications: Carbamidomethyl (C)

Variable modifications:

N8: : Deamidated_N (N)

Ions Score: 60 Expect: 0.00018

Matches (Bold Red): 35/148 fragment ions using 66 most intense peaks

\begin{tabular}{|r|r|r|c|c|c|c|c|c|c|c|c|c|c|c|}
\hline$\#$ & $\mathbf{b}$ & $\mathbf{b}^{++}$ & $\mathbf{b}^{*}$ & $\mathbf{b}^{\boldsymbol{*}^{++}}$ & $\mathbf{b}^{\mathbf{0}}$ & $\mathbf{b}^{\mathbf{0 + +}}$ & Seq. & $\mathbf{y}$ & $\mathbf{y}^{++}$ & $\mathbf{y}^{\mathbf{*}}$ & $\mathbf{y}^{\boldsymbol{*}^{++}}$ & $\mathbf{y}^{\mathbf{0}}$ & $\mathbf{y}^{\mathbf{0 + +}}$ & $\#$ \\
\hline $\mathbf{1}$ & 58.0287 & 29.5180 & & & & & $\mathbf{G}$ & & & & & & & $\mathbf{1 6}$ \\
\hline $\mathbf{2}$ & 129.0659 & 65.0366 & & & & & A & 1698.7244 & 849.8658 & 1681.6978 & 841.3526 & 1680.7138 & 840.8606 & $\mathbf{1 5}$ \\
\hline 3 & 228.1343 & 114.5708 & & & & & $\mathbf{V}$ & 1627.6873 & 814.3473 & $\mathbf{1 6 1 0 . 6 6 0 7}$ & 805.8340 & $\mathbf{1 6 0 9 . 6 7 6 7}$ & 805.3420 & $\mathbf{1 4}$ \\
\hline $\mathbf{4}$ & 315.1663 & 158.0868 & & & 297.1557 & 149.0815 & S & 1528.6189 & 764.8131 & 1511.5923 & 756.2998 & 1510.6083 & 755.8078 & $\mathbf{1 3}$ \\
\hline $\mathbf{5}$ & $\mathbf{4 2 8 . 2 5 0 4}$ & 214.6288 & & & $\mathbf{4 1 0 . 2 3 9 8}$ & 205.6235 & $\mathbf{I}$ & $\mathbf{1 4 4 1 . 5 8 6 8}$ & $\mathbf{7 2 1 . 2 9 7 1}$ & 1424.5603 & 712.7838 & 1423.5763 & 712.2918 & $\mathbf{1 2}$ \\
\hline
\end{tabular}




\begin{tabular}{|c|c|c|c|c|c|c|c|c|c|c|c|c|c|c|}
\hline 6 & 541.3344 & 271.1709 & & & 523.3239 & |262.1656 | & $\mathbf{L}$ & 1328.5028 & 664.7550 & 1311.4762 & 656.2418 & 1310.4922 & 655.7497 & 11 \\
\hline 7 & 701.3651 & 351.1862 & & & 683.3545 & 342.1809 & $\mathrm{C}$ & 1215.4187 & 608.2130 & 1198.3922 & 599.6997 & 1197.4081 & 599.2077 & 10 \\
\hline 8 & 816.3920 & 408.6996 & 799.3655 & 400.1864 & 798.3814 & 399.6944 & $\mathbf{N}$ & 1055.3881 & 528.1977 & 1038.3615 & 519.6844 & 1037.3775 & 519.1924 & 9 \\
\hline 9 & 903.4240 & 452.2157 & 886.3975 & 443.7024 & 885.4135 & 443.2104 & S & 940.3611 & 470.6842 & 923.3346 & 462.1709 & 922.3506 & 461.6789 & 8 \\
\hline 10 & 990.4561 & 495.7317 & 973.4295 & 487.2184 & 972.4455 & 486.7264 & $\mathbf{S}$ & 853.3291 & 427.1682 & 836.3025 & 418.6549 & 835.3185 & 418.1629 & 7 \\
\hline 11 & 1047.4775 & 524.2424 & 1030.4510 & 515.7291 & 1029.4670 & 515.2371 & G & 766.2971 & 383.6522 & 749.2705 & 375.1389 & & & 6 \\
\hline 12 & 1161.5205 & 581.2639 & 1144.4939 & 572.7506 & 1143.5099 & 572.2586 & $\mathbf{N}$ & 709.2756 & 355.1414 & 692.2491 & 346.6282 & & & 5 \\
\hline 13 & 1321.5511 & 661.2792 & 1304.5246 & 652.7659 & 1303.5405 & 652.2739 & C & 595.2327 & 298.1200 & 578.2061 & 289.6067 & & & 4 \\
\hline 14 & 1449.6097 & 725.3085 & 1432.5831 & 716.7952 & 1431.5991 & 716.3032 & $\mathbf{Q}$ & 435.2020 & 218.1047 & 418.1755 & 209.5914 & & & 3 \\
\hline 15 & 1609.6403 & 805.3238 & 1592.6138 & 796.8105 & 1591.6298 & 796.3185 & C & 307.1435 & 154.0754 & 290.1169 & 145.5621 & & & 2 \\
\hline 16 & & & & & & & K & 147.1128 & 74.0600 & 130.0863 & 65.5468 & & & 1 \\
\hline
\end{tabular}
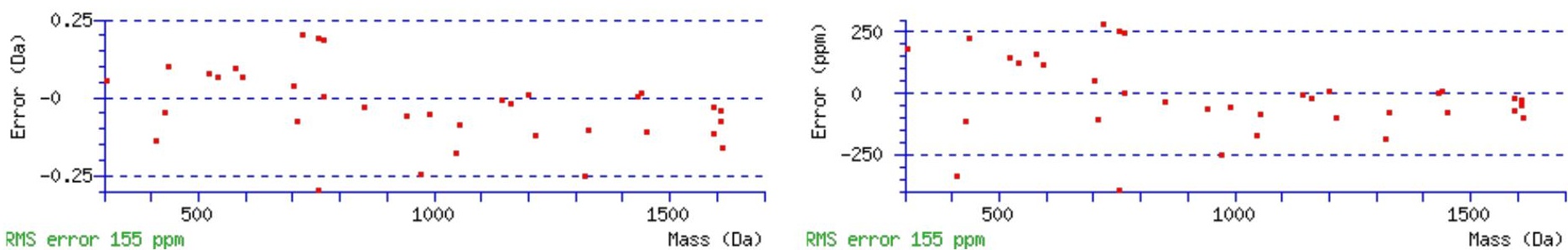

\section{All matches to this query}

\begin{tabular}{|l|l|l|l|}
\hline Score & Mr(calc): & Delta & \multicolumn{1}{c|}{ Sequence } \\
\hline 59.9 & 1754.7386 & 0.0010 & $\underline{\text { GAVSILCNSSGNCQCK }}$ \\
\hline 24.0 & 1754.7386 & 0.0010 & $\underline{\text { GAVSILCNSSGNCQCK }}$ \\
\hline 10.1 & 1754.7406 & -0.0010 & $\underline{\text { KTVXAKPQINSSNK }}$ \\
\hline 5.5 & 1754.7406 & -0.0010 & $\underline{\text { KTVXAKPQINSSNK }}$ \\
\hline 4.8 & 1754.7390 & 0.0006 & $\underline{\text { RGIAGSSGSSVSNFLR }}$ \\
\hline 4.2 & 1754.7390 & 0.0006 & $\underline{\text { RGIAGSSGSSVSNFLR }}$ \\
\hline 2.6 & 1754.7256 & 0.0140 & $\underline{\text { TNMEEKFMATNLSK }}$ \\
\hline 1.6 & 1754.7390 & 0.0006 & $\underline{\text { RGIAGSSGSSVSNFLR }}$ \\
\hline 1.2 & 1754.7390 & 0.0006 & $\underline{\text { RGIAGSSGSSVSNFLR }}$ \\
\hline
\end{tabular}

Spectrum No: 532; Query: 2345; Rank: 1

\section{Peptide View}

MS/MS Fragmentation of VEFDDKGNVVTSYGNPILLNSTIR

Found in IPI00204348, Tax_Id=10116 Gene_Symbol=Nt5e 5'-nucleotidase precursor

Match to Query 2345: 2651.352042 from(884.791290,3+)

Title: 100101RatKid_NS_deglyco_13.4562.4562.3.dta

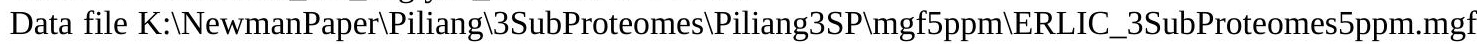



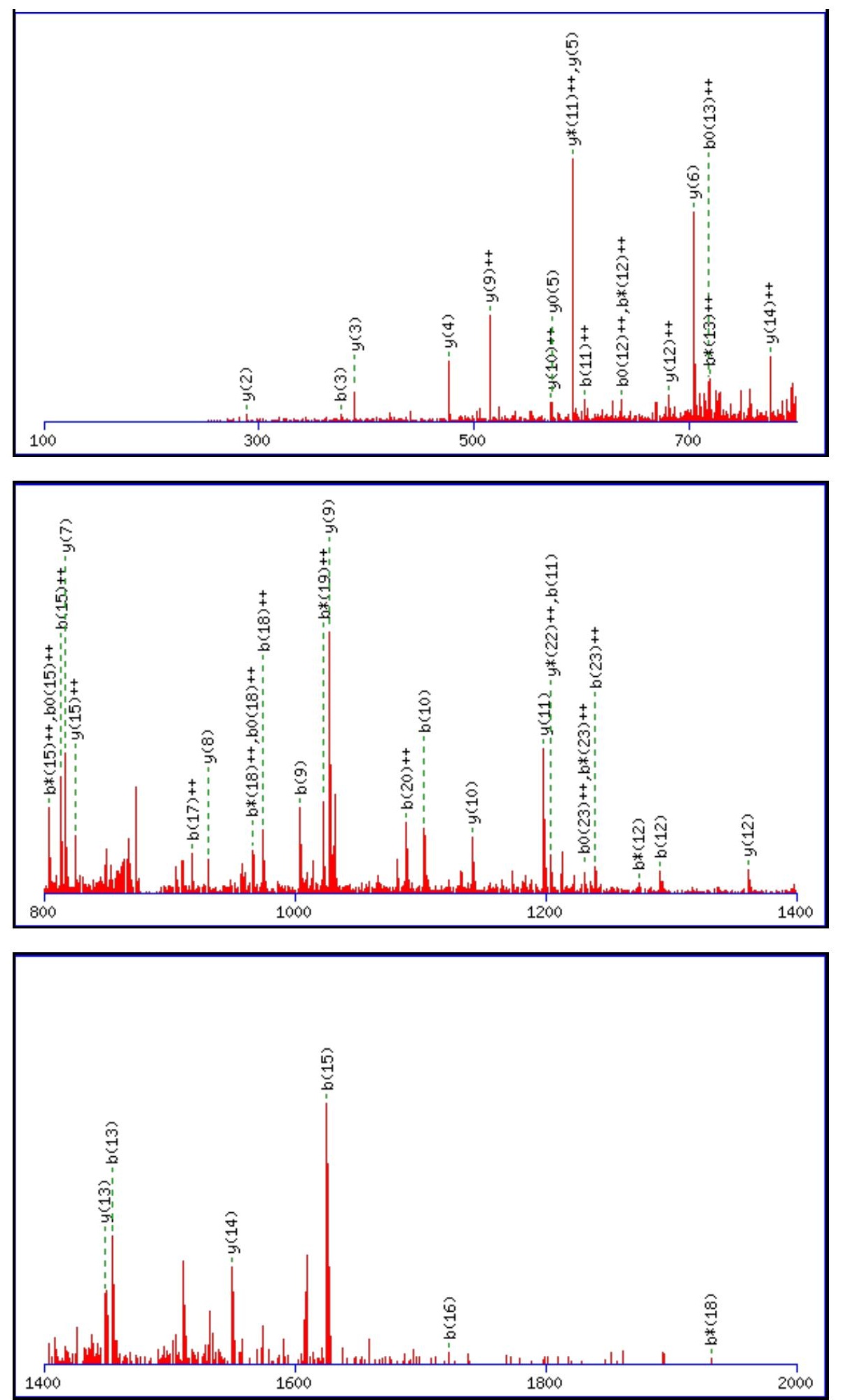

Monoisotopic mass of neutral peptide $\operatorname{Mr}($ calc): 2651.3443

Fixed modifications: Carbamidomethyl (C)

Variable modifications:

N20 : Deamidated N ( N)

Ions Score: 60 Expect: 0.00015

Matches (Bold Red): 48/260 fragment ions using 89 most intense peaks

\begin{tabular}{|c|c|c|c|c|c|c|c|c|c|c|c|c|c|c|}
\hline$\#$ & $\mathbf{b}$ & $\mathbf{b}^{++}$ & $\mathbf{b}^{*}$ & $\mathbf{b}^{\boldsymbol{*}^{++}}$ & $\mathbf{b}^{\mathbf{0}}$ & $\mathbf{b}^{\mathbf{0 + +}}$ & Seq. & $\mathbf{y}$ & $\mathbf{y}^{++}$ & $\mathbf{y}^{\mathbf{*}}$ & $\mathbf{y}^{\mathbf{*}^{++}}$ & $\mathbf{y}^{\mathbf{0}}$ & $\mathbf{y}^{\mathbf{0 + +}}$ & $\#$ \\
\hline $\mathbf{1}$ & 100.0757 & 50.5415 & & & & & $\mathbf{V}$ & & & & & & & $\mathbf{2 4}$ \\
\hline $\mathbf{2}$ & 229.1183 & 115.0628 & & & 211.1077 & 106.0575 & $\mathbf{E}$ & 2553.2831 & 1277.1452 & 2536.2566 & 1268.6319 & 2535.2726 & 1268.1399 & $\mathbf{2 3}$ \\
\hline $\mathbf{3}$ & $\mathbf{3 7 6 . 1 8 6 7}$ & 188.5970 & & & 358.1761 & 179.5917 & $\mathbf{F}$ & 2424.2405 & 1212.6239 & 2407.2140 & $\mathbf{1 2 0 4 . 1 1 0 6}$ & 2406.2300 & 1203.6186 & 22 \\
\hline $\mathbf{4}$ & 491.2136 & 246.1105 & & & 473.2031 & 237.1052 & $\mathbf{D}$ & 2277.1721 & 1139.0897 & 2260.1456 & 1130.5764 & 2259.1615 & 1130.0844 & $\mathbf{2 1}$ \\
\hline $\mathbf{5}$ & 606.2406 & 303.6239 & & & 588.2300 & 294.6186 & $\mathbf{D}$ & 2162.1452 & 1081.5762 & 2145.1186 & 1073.0629 & 2144.1346 & 1072.5709 & $\mathbf{2 0}$ \\
\hline
\end{tabular}




\begin{tabular}{|c|c|c|c|c|c|c|c|c|c|c|c|c|c|c|}
\hline 6 & 734.3355 & 367.6714 & 717.3090 & 359.1581 & 716.3250 & 358.6661 & K & 047.1182 & & & & & & \\
\hline 7 & 791.3570 & 396.1821 & 774.3305 & 387.6689 & 773.3464 & 387.1769 & G & 1919.0233 & 960.0153 & 1901.9967 & 951.5020 & 1901.0127 & 951.0100 & 18 \\
\hline 8 & 905. & 453. & 888. & & & & $\mathbf{N}$ & & & & & & & \\
\hline 9 & 684 & 502.7378 & 987.4418 & 494.2 & 986. & 493.7 & V & 9589 & 1831 & $1 / 30$. & 9698 & 9483 & 865.4778 & 6 \\
\hline 10 & 103.5368 & 552.2720 & 1086 & 543.7 & 108 & & V & & & & & & & 5 \\
\hline 11 & 844 & 602.7 & 1187. & 594. & 1186 & 593. & $\mathbf{T}$ & 3220 & 147 & 153 & 9014 & 115 & 4094 & 4 \\
\hline 12 & 291.6165 & 646.3119 & 1274. & 637. & 1273 & 637. & $\mathbf{S}$ & 744 & 724 & 143 & & & 855 & 3 \\
\hline 13 & 454.6798 & 727. & 437. & 719. & 436 & 718 & $\mathbf{Y}$ & 423 & 748 & 134 & 615 & & 695 & 12 \\
\hline 14 & 1511.7013 & 756.3543 & 494.6747 & 747.8410 & 400.0507 & 747.3 & U & & & & & & 590.8379 & 11 \\
\hline 15 & 625.7442 & 813.3757 & 1608.7176 & 804.8625 & 1607.7336 & 804. & $\mathbf{N}$ & 1141.6575 & 3324 & 310 & 3191 & 1123 & 562.3271 & 10 \\
\hline 16 & 722.7970 & 861.9021 & 1705.7 & 853.3888 & 1704.7864 & 852.8 & $\mathbf{P}$ & 1027. & 51 & 1010 & 7977 & 1009 & 505.3057 & 9 \\
\hline 17 & 1835.8810 & 918.4441 & 1818.8545 & 909.9309 & 1817.8705 & 909. & I & 930.5619 & 465.7846 & 913. & 457.2713 & 513 & 456.7793 & 8 \\
\hline 18 & 948.9651 & 974.9862 & 1931.9385 & 966.4 & 1930. & 965. & $\mathbf{L}$ & & $40 s$ & 80 & 400. & & 400.2372 & 7 \\
\hline 19 & 2062.0491 & 1031.5282 & 2045.0226 & 1023.0149 & 2044.0386 & 1022.5229 & $\mathbf{L}$ & 704.3937 & 352.7005 & 687.3672 & 344.1872 & 686.3832 & 343.6952 & 6 \\
\hline 20 & 2177.0761 & 1089.0417 & 2160.0495 & 1080.5284 & 2159.0655 & 1080.0364 & $\mathbf{N}$ & 591.3097 & 296.1585 & 2831 & 287.6452 & 991 & 287.1532 & 5 \\
\hline 21 & 2264.1081 & 1132.5577 & 2247.0816 & 1124.0444 & 2246.0975 & 1123.5524 & $\mathbf{S}$ & 476.2827 & 238.6450 & 459.2562 & 230. & 458.2722 & 229.6397 & 4 \\
\hline 22 & 2365.1558 & 1183.0815 & 2348.1292 & 1174.5683 & 2347.1452 & 1174.0763 & $\mathbf{T}$ & 389.2507 & 195.1290 & 372.2241 & 186.6157 & 371.2401 & 186.1237 & 3 \\
\hline & 2478.2399 & 1239.6236 & 2461.2133 & 1231.1103 & 2460.2293 & 1230.6183 & I & 288.2030 & 144.6051 & 271.1765 & 136.0919 & & & 2 \\
\hline 4 & & & & & & & $\mathbf{R}$ & 175.1190 & 88.0631 & 158.0924 & 79.5498 & & & 1 \\
\hline
\end{tabular}
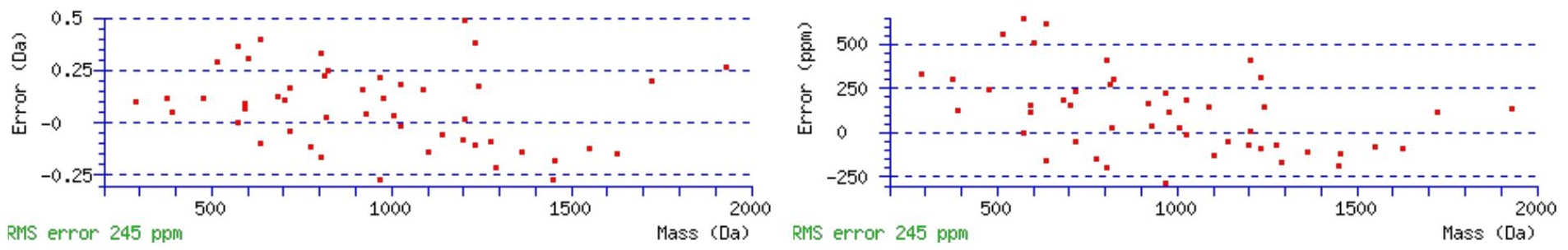

\section{All matches to this query}

\begin{tabular}{|l|l|l|l|}
\hline Score & Mr(calc): & Delta & \multicolumn{1}{|c|}{ Sequence } \\
\hline 59.9 & 2651.3443 & 0.0078 & $\underline{\text { VEFDDKGNVVTSYGNPILLNSTIR }}$ \\
\hline 37.5 & 2651.3443 & 0.0078 & VEFDDKGNVVTSYGNPILLNSTIR \\
\hline 23.9 & 2651.3443 & 0.0078 & $\underline{\text { VEFDDKGNVVTSYGNPILLNSTIR }}$ \\
\hline 17.0 & 2650.3602 & 0.9918 & $\underline{\text { VEFDDKGNVVTSYGNPILLNSTIR }}$ \\
\hline 7.5 & 2651.3571 & -0.0051 & $\underline{\text { AGKLLDYLQENPKGLDTLVESIR }}$ \\
\hline 7.5 & 2651.3571 & -0.0051 & $\underline{\text { AGKLLDYLQENPKGLDTLVESIR }}$ \\
\hline 3.2 & 2651.3571 & -0.0051 & $\underline{\text { AGKLLDYLQENPKGLDTLVESIR }}$ \\
\hline 2.4 & 2650.3570 & 0.9951 & $\underline{\text { RYLTLLSDVLVVSSKLMNIGLR }}$ \\
\hline 2.4 & 2650.3570 & 0.9951 & $\underline{\text { RYLTLLSDVLVVSSKLMNIGLR }}$ \\
\hline 2.4 & 2650.3570 & 0.9951 & $\underline{\text { RYLTLLSDVLVVSSKLMNIGLR }}$ \\
\hline
\end{tabular}

Spectrum No: 533; Query: 2174; Rank: 1

\section{Peptide View}

MS/MS Fragmentation of LTLVPGKNITCQDPIIGIGEPGK

Found in IPI00205333, Tax_Id=10116 Gene_Symbol=Gpr116 Probable G-protein coupled receptor 116 precursor

Match to Query 2174: 2420.308152 from(807.776660,3+)

Title: 100101RatKid_NS_deglyco_23.3947.3947.3.dta

Data file K:\NewmanPaper\Piliang \3SubProteomes \Piliang3SP \mgf5ppm\ERLIC_3SubProteomes5ppm.mgf 

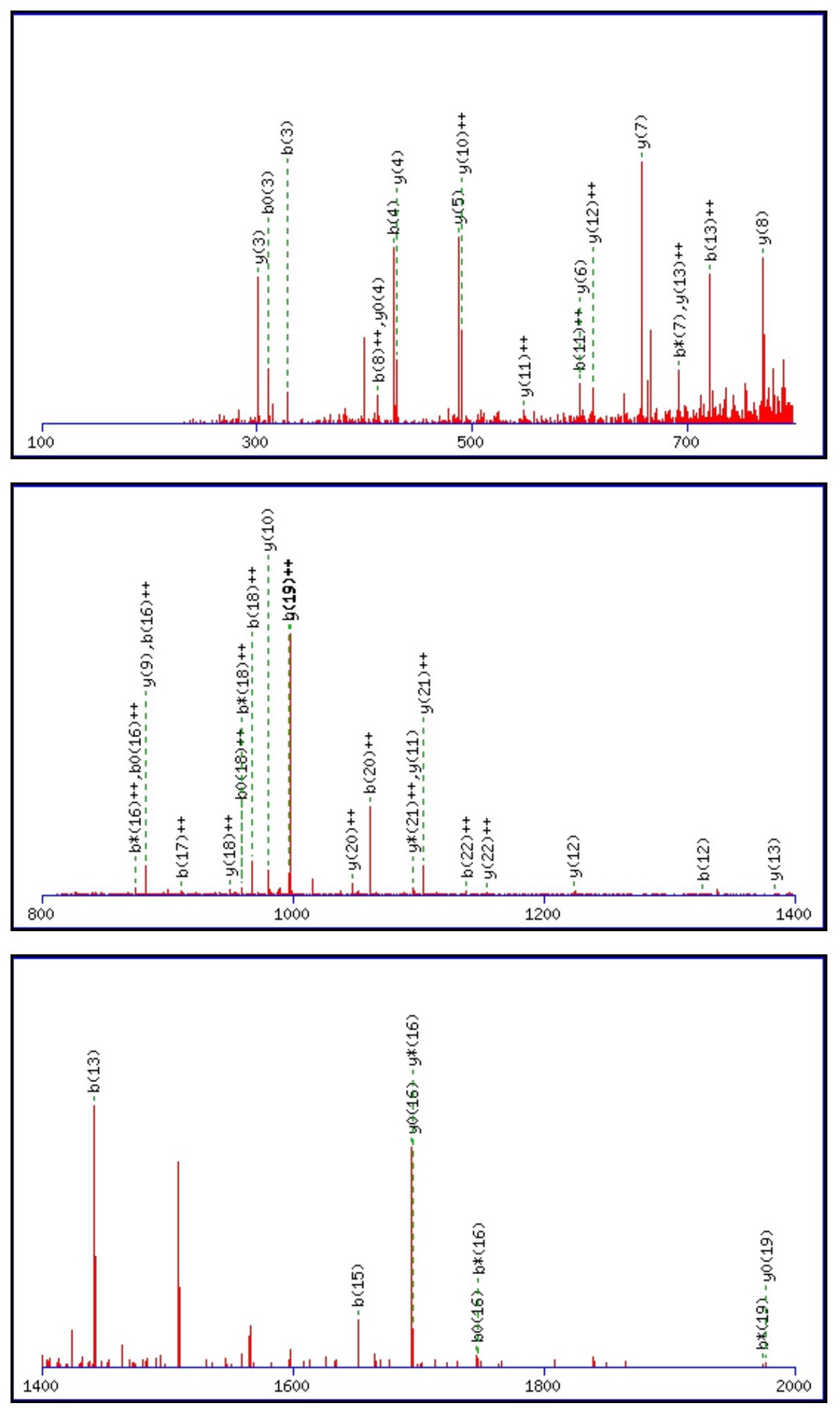

Monoisotopic mass of neutral peptide $\operatorname{Mr}$ (calc): 2420.2985

Fixed modifications: Carbamidomethyl (C)

Variable modifications:

N8 : Deamidated_N (N)

Ions Score: 60 Expect: $6.1 \mathrm{e}-005$

Matches (Bold Red): 48/244 fragment ions using 75 most intense peaks

\begin{tabular}{|c|c|c|c|c|c|c|c|c|c|c|c|c|c|c|}
\hline \# & b & $\mathbf{b}^{++}$ & b* & $\mathbf{b}^{*^{++}}$ & $\mathbf{b}^{0}$ & $\mathbf{b}^{\mathbf{0}^{++}}$ & Seq. & $\mathbf{y}$ & $\mathbf{y}^{++}$ & $\mathbf{y}^{*}$ & $\mathrm{y}^{*^{++}}$ & $\mathbf{y}^{0}$ & $\mathbf{y}^{0++}$ & \# \\
\hline 1 & 114.0913 & 57.5493 & & & & & $\mathbf{L}$ & & & & & & & 23 \\
\hline 2 & 215.1390 & 108.0731 & & & 197.1285 & 99.0679 & $\mathbf{T}$ & 2308.2217 & 1154.6145 & 2291.1952 & 1146.1012 & 2290.2111 & 1145.6092 & 22 \\
\hline 3 & 328.2231 & 164.6152 & & & 310.2125 & 155.6099 & $\mathbf{L}$ & 2207.1740 & \begin{tabular}{|l|}
1104.0907 \\
\end{tabular} & 2190.1475 & 1095.5774 & 2189.1635 & 1095.0854 & 21 \\
\hline 4 & 427.2915 & 214.1494 & & & 409.2809 & 205.1441 & $\mathbf{V}$ & 2094.0900 & 1047.5486 & 2077.0634 & 1039.0353 & 2076.0794 & 1038.5433 & 20 \\
\hline 5 & 524.3443 & 262.6758 & & & 506.3337 & 253.6705 & $\mathbf{P}$ & 1995.0215 & 998.0144 & 1977.9950 & 989.5011 & 1977.0110 & 989.0091 & 19 \\
\hline
\end{tabular}




\begin{tabular}{|c|c|c|c|c|c|c|c|c|c|c|c|c|c|c|}
\hline & 581.3657 & 291.1865 & & & 563.3552 & 282.1812 & G & & 949.4880 & & & & 940.4827| & 18 \\
\hline 7 & 709.4607 & 355.2340 & 692.4341 & 346.7207 & 691.4501 & 346.2287 & $\mathbf{K}$ & 1840.9473 & 920.9773 & 1823.9208 & 912.4640 & 1822.9368 & 911.9720 & 17 \\
\hline 8 & 824.4876 & 412.7475 & 807.4611 & 404.2342 & 806.4771 & 403.7422 & $\mathbf{N}$ & 1712.8524 & 856.9298 & 1695.8258 & 848.4165 & 1694.8418 & 847.9245 & 16 \\
\hline 9 & 937.5717 & 469.2895 & 920.5451 & 460.7762 & 919.5611 & 460.2842 & I & 1597.8254 & 799.4163 & 1580.7989 & 790.9031 & 1579.8149 & 790.4111 & 15 \\
\hline 10 & 1038.6194 & 519.8133 & 1021.5928 & 511.3000 & 1020.6088 & 510.8080 & $\mathbf{T}$ & 1484.7414 & 742.8743 & 1467.7148 & 734.3610 & 1466.7308 & 733.8690 & 14 \\
\hline 11 & 1198.6500 & 599.8286 & 1181.6235 & 591.3154 & 1180.6395 & 590.8234 & C & 1383.6937 & 692.3505 & 1366.6671 & 683.8372 & 1365.6831 & 683.3452 & 13 \\
\hline 12 & 1326.7086 & 663.8579 & 1309.6820 & 655.3447 & 1308.6980 & 654.8527 & $\mathbf{Q}$ & 1223.6630 & 612.3352 & 1206.6365 & 603.8219 & 1205.6525 & 603.3299 & 12 \\
\hline 13 & 1441.7355 & 721.3714 & 1424.7090 & 712.8581 & 1423.7250 & 712.3661 & D & 1095.6045 & 548.3059 & 1078.5779 & 539.7926 & 1077.5939 & 539.3006 & 11 \\
\hline 14 & 1538.7883 & 769.8978 & 1521.7618 & 761.3845 & 1520.7777 & 760.8925 & $\mathbf{P}$ & $\mathbf{9 8 0 . 5 7 7 5}$ & 490.7924 & 963.5510 & 482.2791 & 962.5669 & 481.7871 & 10 \\
\hline 15 & 1651.8724 & 826.4398 & 1634.8458 & 817.9265 & 1633.8618 & 817.4345 & I & 883.5247 & 442.2660 & 866.4982 & 433.7527 & 865.5142 & 433.2607 & 9 \\
\hline 16 & 1764.9564 & 882.9819 & 1747.9299 & 874.4686 & 1746.9459 & 873.9766 & I & 770.4407 & 385.7240 & 753.4141 & 377.2107 & 752.4301 & 376.7187 & 8 \\
\hline 17 & 1821.9779 & 911.4926 & 1804.9513 & 902.9793 & 1803.9673 & 902.4873 & G & 657.3566 & 329.1819 & 640.3301 & 320.6687 & 639.3461 & 320.1767 & 7 \\
\hline 18 & 1935.0620 & 968.0346 & 1918.0354 & 959.5213 & 1917.0514 & 959.0293 & I & 600.3352 & 300.6712 & 583.3086 & 292.1579 & 582.3246 & 291.6659 & 6 \\
\hline 19 & 1992.0834 & 996.5453 & 1975.0569 & 988.0321 & 1974.0729 & 987.5401 & G & 487.2511 & 244.1292 & 470.2245 & 235.6159 & 469.2405 & 235.1239 & 5 \\
\hline 20 & 2121.1260 & 1061.0666 & 2104.0995 & 1052.5534 & 2103.1154 & 1052.0614 & $E$ & 430.2296 & 215.6185 & 413.2031 & 207.1052 & 412.2191 & 206.6132 & 4 \\
\hline 21 & 2218.1788 & 1109.5930 & 2201.1522 & 1101.0798 & 2200.1682 & 1100.5877 & $\mathbf{P}$ & 301.1870 & 151.0972 & 284.1605 & 142.5839 & & & 3 \\
\hline 22 & 2275.2002 & 1138.1038 & 2258.1737 & 1129.5905 & 2257.1897 & 1129.0985 & G & 204.1343 & 102.5708 & 187.1077 & 94.0575 & & & 2 \\
\hline 23 & & & & & & & $\mathbf{K}$ & 147.1128 & 74.0600 & 130.0863 & 65.5468 & & & 1 \\
\hline
\end{tabular}
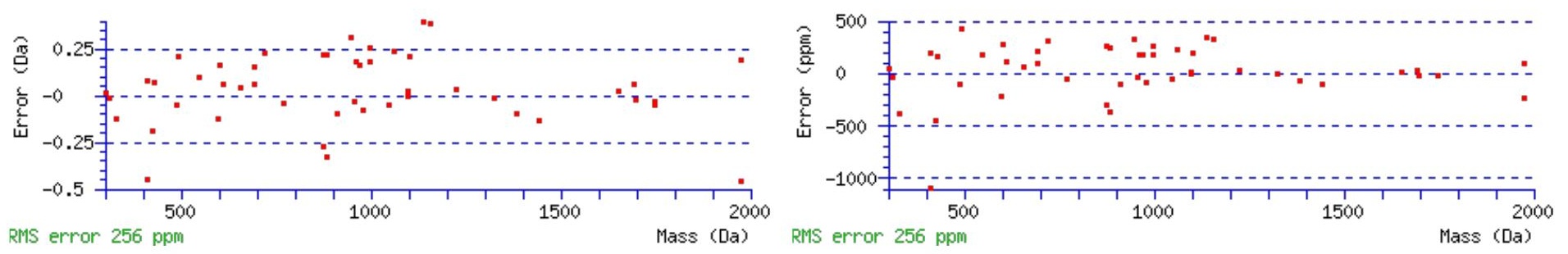

\section{All matches to this query}

\begin{tabular}{|l|r|r|l|}
\hline Score & Mr(calc): & Delta & \multicolumn{1}{|c|}{ Sequence } \\
\hline 59.9 & 2420.2985 & 0.0097 & LTLVPGKNITCQDPIIGIGEPGK \\
\hline 36.9 & 2419.3145 & 0.9937 & LTLVPGKNITCQDPIIGIGEPGK \\
\hline 3.3 & 2418.2954 & 2.0128 & HNIIYVNGSVPGHRNCLVKIK \\
\hline 1.9 & 2418.2883 & 2.0199 & KQISQLNALITLLIGNLSAGDR \\
\hline 1.6 & 2420.3117 & -0.0036 & VPLHLPLPVHWETLXDWKAR \\
\hline 1.5 & 2419.3158 & 0.9924 & EINHPELHMVLSNLAAILIHR \\
\hline 1.3 & 2420.2845 & 0.0237 & RKLNQLLLPLLQGPDITLSK \\
\hline 1.0 & 2420.2884 & 0.0197 & AILEGADRGLGGALGGLLGGGGQAGAGR \\
\hline 0.5 & 2420.2845 & 0.0236 & AMLNLHNGQNDTIALLLDVARK \\
\hline 0.5 & 2420.2845 & 0.0236 & AMLNLHNGQNDTIALLLDVARK \\
\hline
\end{tabular}

Spectrum No: 534; Query: 2490; Rank: 1

\section{Peptide View}

MS/MS Fragmentation of ENGTVTAANASTLNDGAAAVVLMTAEAAQR

Found in IPI00324302, Tax_Id=10116 Gene_Symbol=Acat1 Acetyl-CoA acetyltransferase, mitochondrial precursor

Match to Query 2490: 2917.411182 from(973.477670,3+)

Title: 091008RatKidney_NoSalt_17.6182.6182.3.dta

Data file K:INewmanPaper|Piliang|3SubProteomes\Piliang3SP\mgf5ppm\ERLIC_3SubProteomes5ppm.mgf 

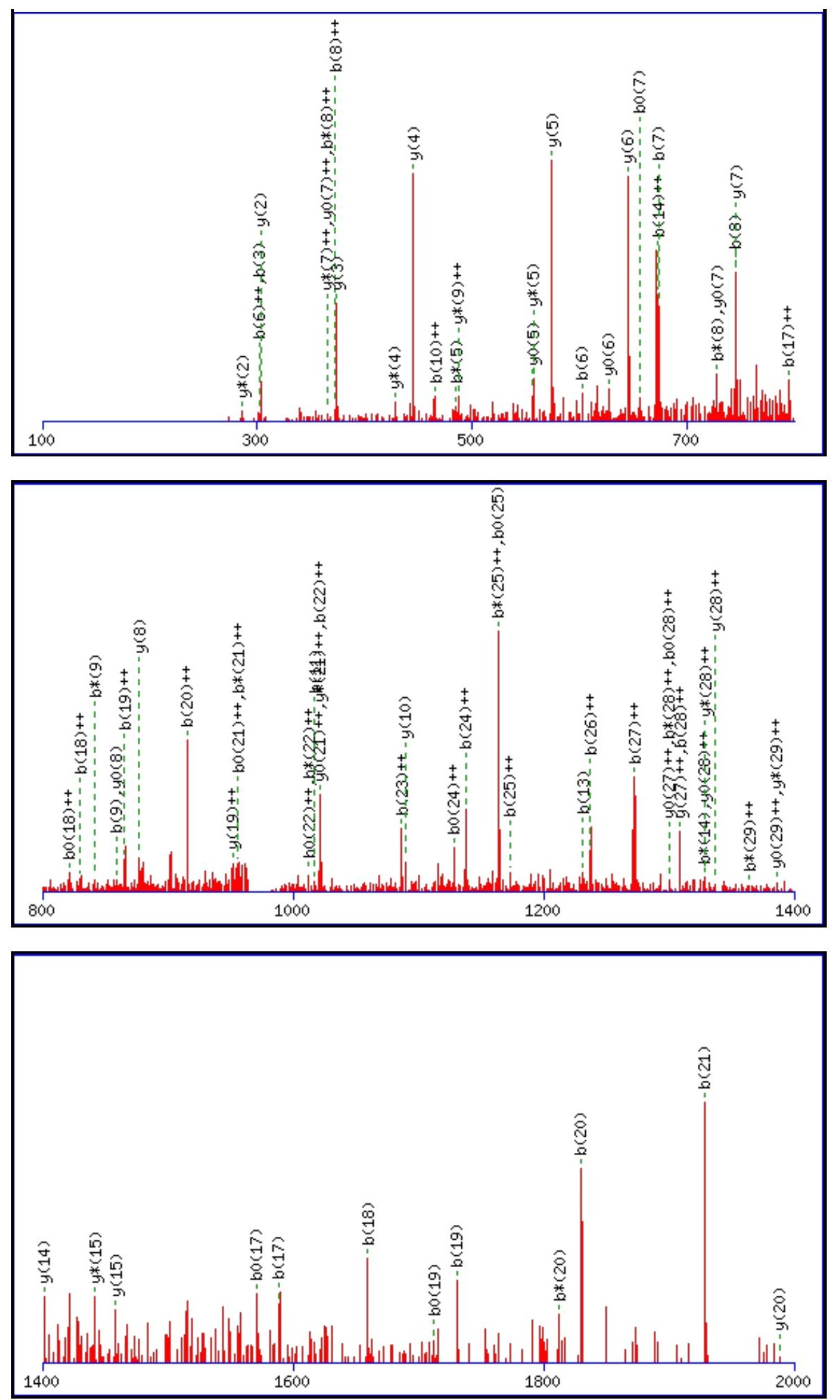

Monoisotopic mass of neutral peptide $\operatorname{Mr}($ calc): 2917.4087

Fixed modifications: Carbamidomethyl (C)

Variable modifications:

N2 : Deamidated_N (N)

Ions Score: 60 Expect: 0.0003

Matches (Bold Red): 79/338 fragment ions using 166 most intense peaks

\begin{tabular}{|c|c|c|c|c|c|c|c|c|c|c|c|c|c|c|}
\hline$\#$ & $\mathbf{b}$ & $\mathbf{b}^{++}$ & $\mathbf{b}^{*}$ & $\mathbf{b}^{\boldsymbol{*}^{++}}$ & $\mathbf{b}^{\mathbf{0}}$ & $\mathbf{b}^{\mathbf{0 + +}}$ & Seq. & $\mathbf{y}$ & $\mathbf{y}^{++}$ & $\mathbf{y}^{\mathbf{*}}$ & $\mathbf{y}^{\mathbf{*}^{++}}$ & $\mathbf{y}^{\mathbf{0}}$ & $\mathbf{y}^{\mathbf{0 + +}}$ & $\#$ \\
\hline $\mathbf{1}$ & 130.0499 & 65.5286 & & & 112.0393 & 56.5233 & $\mathbf{E}$ & & & & & & & $\mathbf{3 0}$ \\
\hline $\mathbf{2}$ & 245.0768 & 123.0420 & 228.0503 & 114.5288 & 227.0662 & 114.0368 & $\mathbf{N}$ & 2789.3734 & 1395.1903 & 2772.3469 & $\mathbf{1 3 8 6 . 6 7 7 1}$ & 2771.3628 & $\mathbf{1 3 8 6 . 1 8 5 1}$ & $\mathbf{2 9}$ \\
\hline $\mathbf{3}$ & $\mathbf{3 0 2 . 0 9 8 3}$ & 151.5528 & 285.0717 & 143.0395 & 284.0877 & 142.5475 & $\mathbf{G}$ & 2674.3465 & $\mathbf{1 3 3 7 . 6 7 6 9}$ & 2657.3199 & $\mathbf{1 3 2 9 . 1 6 3 6}$ & 2656.3359 & $\mathbf{1 3 2 8 . 6 7 1 6}$ & $\mathbf{2 8}$ \\
\hline $\mathbf{4}$ & 403.1459 & 202.0766 & 386.1194 & 193.5633 & 385.1354 & 193.0713 & $\mathbf{T}$ & 2617.3250 & $\mathbf{1 3 0 9 . 1 6 6 1}$ & 2600.2985 & 1300.6529 & 2599.3144 & $\mathbf{1 3 0 0 . 1 6 0 9}$ & $\mathbf{2 7}$ \\
\hline $\mathbf{5}$ & 502.2144 & 251.6108 & $\mathbf{4 8 5 . 1 8 7 8}$ & 243.0975 & 484.2038 & 242.6055 & $\mathbf{V}$ & 2516.2773 & 1258.6423 & 2499.2508 & 1250.1290 & 2498.2668 & 1249.6370 & $\mathbf{2 6}$ \\
\hline
\end{tabular}




\begin{tabular}{|c|c|c|c|c|c|c|c|c|c|c|c|c|c|c|}
\hline 6 & 603.2620 & 302.1347 & 586.2355 & 293.6214 & 585.2515 & 293.1294| & $\mathbf{T}$ & |2417.2089 & |1209.1081 & |2400.1824| & 1200.5948 & |2399.1984| & 1200.1028 & |25| \\
\hline 7 & 674.2992 & 337.6532 & 657.2726 & 329.1399 & 656.2886 & 328.6479 & A & 2316.1612 & 1158.5843 & 2299.1347 & 1150.0710 & 2298.1507 & 1149.5790 & 24 \\
\hline 8 & 745.3363 & 373.1718 & 728.3097 & 364.6585 & 727.3257 & 364.1665 & A & 2245.1241 & 1123.0657 & 2228.0976 & 1114.5524 & 2227.1136 & 1114.0604 & 3 \\
\hline 9 & 859.3792 & 430.1932 & 842.3526 & 421.6800 & 841.3686 & 421.1880 & $\mathbf{N}$ & 2174.0870 & 1087.5471 & 2157.0605 & 1079.0339 & 2156.0764 & 1078.5419 & 22 \\
\hline 10 & 930.4163 & 465.7118 & 913.3898 & 457.1985 & 912.4057 & 456.7065 & A & 2060.0441 & 1030.5257 & 2043.0175 & 1022.0124 & 2042.0335 & 1021.5204 & 21 \\
\hline 11 & 1017.4483 & 509.2278 & 1000.4218 & 500.7145 & 999.4378 & 500.2225 & S & 1989.0070 & 995.0071 & 1971.9804 & 986.4938 & 1970.9964 & 986.0018 & 20 \\
\hline 12 & 1118.4960 & 559.7516 & 1101.4695 & 551.2384 & 1100.4855 & 550.7464 & $\mathbf{T}$ & 1901.9749 & 951.4911 & 1884.9484 & 942.9778 & 1883.9644 & 942.4858 & 19 \\
\hline 13 & 1231.5801 & 616.2937 & 1214.5535 & 607.7804 & 1213.5695 & 607.2884 & $\mathbf{L}$ & 1800.9273 & 900.9673 & 1783.9007 & 892.4540 & 1782.9167 & 891.9620 & 18 \\
\hline 14 & 1345.6230 & 673.3151 & 1328.5965 & 664.8019 & 1327.6124 & 664.3099 & $\mathbf{N}$ & 1687.8432 & 844.4252 & 1670.8166 & 835.9120 & 1669.8326 & 835.4200 & 17 \\
\hline 15 & 1460.6500 & 730.8286 & 1443.6234 & 722.3153 & 1442.6394 & 721.8233 & D & 1573.8003 & 787.4038 & 1556.7737 & 778.8905 & 1555.7897 & 778.3985 & 16 \\
\hline 16 & 1517.6714 & 759.3393 & 1500.6449 & 750.8261 & 1499.6608 & 750.3341 & G & 1458.7733 & 729.8903 & 1441.7468 & 721.3770 & 1440.7628 & 720.8850 & 15 \\
\hline 17 & 1588.7085 & 794.8579 & 1571.6820 & 786.3446 & 1570.6980 & 785.8526 & A & 1401.7519 & 701.3796 & 1384.7253 & 692.8663 & 1383.7413 & 692.3743 & 14 \\
\hline 18 & 1659.7456 & 830.3765 & 1642.7191 & 821.8632 & 1641.7351 & 821.3712 & A & 1330.7147 & 665.8610 & 1313.6882 & 657.3477 & 1312.7042 & 656.8557 & 13 \\
\hline 19 & 1730.7828 & 865.8950 & 1713.7562 & 857.3817 & 1712.7722 & 856.8897 & A & 1259.6776 & 630.3425 & 1242.6511 & 621.8292 & 1241.6671 & 621.3372 & 12 \\
\hline 21 & 1928.9196 & 964.9634 & 1911.8930 & 956.4502 & 1910.9090 & 955.9581 & $\mathbf{V}$ & 1089.5721 & 545.2897 & 1072.5456 & 536.7764 & 1071.5615 & 536.2844 & 10 \\
\hline 22 & 2042.0036 & 1021.5055 & 2024.9771 & 1012.9922 & 2023.9931 & 1012.5002 & $\mathbf{L}$ & 990.5037 & 495.7555 & 973.4771 & 487.2422 & 972.4931 & 486.7502 & 9 \\
\hline 23 & 2173.0441 & 1087.0257 & 2156.0176 & 1078.5124 & 2155.0336 & 1078.0204 & $\mathbf{M}$ & 877.4196 & 439.2135 & 860.3931 & 430.7002 & 859.4091 & 430.2082 & 8 \\
\hline 24 & 2274.0918 & 1137.5495 & 2257.0653 & 1129.0363 & 2256.0812 & 1128.5443 & $\mathbf{T}$ & 746.3791 & 373.6932 & 729.3526 & 365.1799 & 728.3686 & 364.6879 & 7 \\
\hline 25 & 2345.1289 & 1173.0681 & 2328.1024 & 1164.5548 & 2327.1184 & 1164.0628 & A & 645.3315 & 323.1694 & 628.3049 & 314.6561 & 627.3209 & 314.1641 & 6 \\
\hline 26 & 2474.1715 & 1237.5894 & 2457.1450 & 1229.0761 & 2456.1610 & 1228.5841 & $\mathbf{E}$ & 574.2944 & 287.6508 & 557.2678 & 279.1375 & 556.2838 & 278.6455 & 5 \\
\hline 27 & 2545.2086 & 1273.1080 & 2528.1821 & 1264.5947 & 2527.1981 & 1264.1027 & A & 445.2518 & 223.1295 & 428.2252 & 214.6162 & & & 4 \\
\hline 28 & 2616.2457 & 1308.6265 & 2599.2192 & 1300.1132 & 2598.2352 & 1299.6212 & A & 374.2146 & 187.6110 & 357.1881 & 179.0977 & & & 3 \\
\hline 29 & 2744.3043 & 1372.6558 & 2727.2778 & 1364.1425 & 2726.2938 & 1363.6505 & $\mathbf{Q}$ & 303.1775 & 152.0924 & 286.1510 & 143.5791 & & & 2 \\
\hline 30 & & & & & & & $\mathbf{R}$ & 175.1190 & 88.0631 & 158.0924 & 79.5498 & & & 1 \\
\hline
\end{tabular}
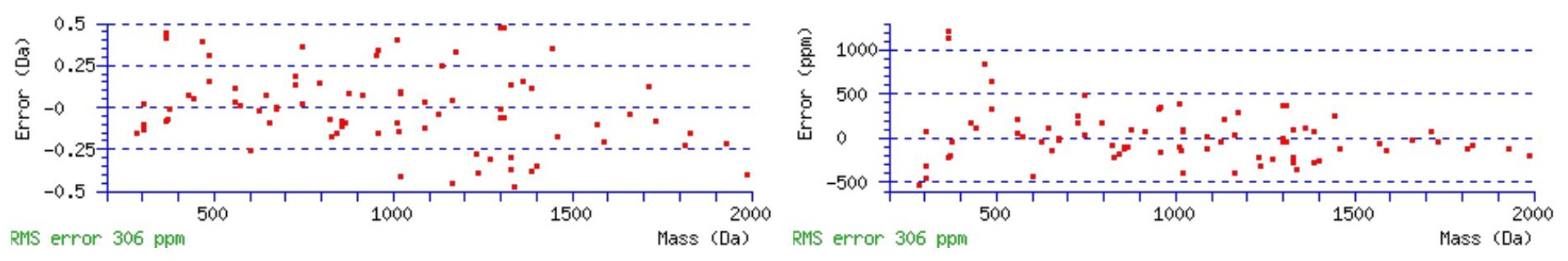

\section{All matches to this query}

\begin{tabular}{|l|l|l|l|}
\hline Score & Mr(calc): & Delta & \multicolumn{1}{|c|}{ Sequence } \\
\hline 59.8 & 2917.4087 & 0.0025 & ENGTVTAANASTLNDGAAAVVLMTAEAAQR \\
\hline 54.4 & 2917.4087 & 0.0025 & ENGTVTAANASTLNDGAAAVVLMTAEAAQR \\
\hline 49.3 & 2917.4087 & 0.0025 & ENGTVTAANASTLNDGAAAVVLMTAEAAQR \\
\hline 20.9 & 2916.4247 & 0.9865 & ENGTVTAANASTLNDGAAAVVLMTAEAAQR \\
\hline 9.4 & 2917.4157 & -0.0045 & FGLGLAVAGGMVNSALYNVDARNRAVGK \\
\hline 8.7 & 2917.4157 & -0.0045 & FGLGLAVAGGMVNSALYNVDARNRAVGK \\
\hline 7.6 & 2917.4157 & -0.0045 & FGLGLAVAGGMVNSALYNVDARNRAVGK \\
\hline 5.1 & 2917.4157 & -0.0045 & FGLGLAVAGGMVNSALYNVDARNRAVGK \\
\hline 5.0 & 2915.3922 & 2.0190 & VEERTPYIVVALQECERMNILTR \\
\hline 3.6 & 2917.4157 & -0.0045 & FGLGLAVAGGMVNSALYNVDARNRAVGK \\
\hline
\end{tabular}

Spectrum No: 535; Query: 2172; Rank: 1 


\section{Peptide View}

\section{MS/MS Fragmentation of QTQVGIVQYGENVTHEFNLNK}

Found in IPI00324585, Tax_Id=10116 Gene_Symbol=Itga1 Integrin alpha-1 precursor

Match to Query 2172: 2418.188952 from(807.070260,3+)

Title: 100101RatKid_NS_deglyco_18.4608.4608.3.dta

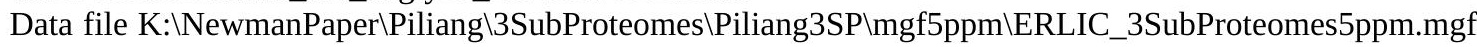
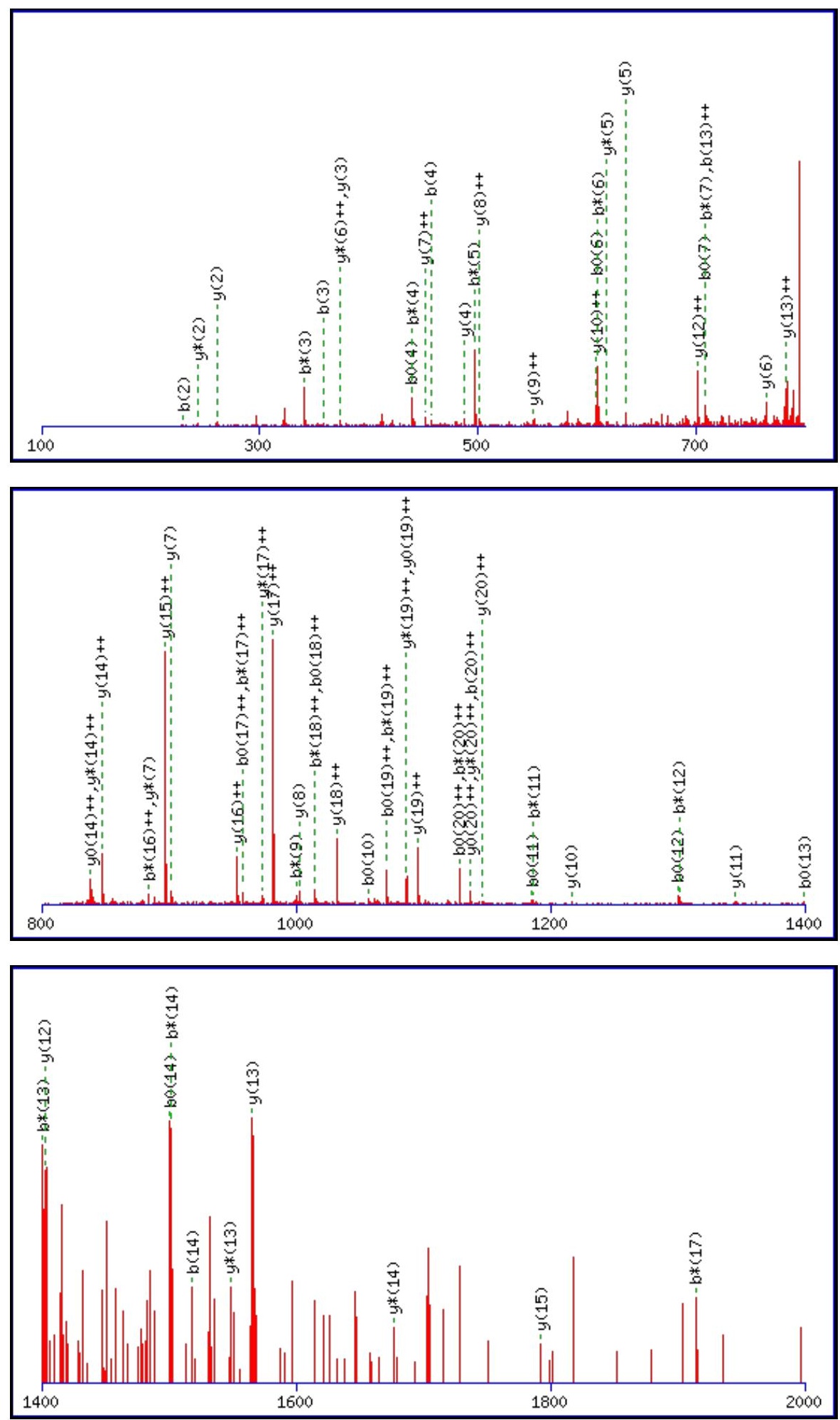

Monoisotopic mass of neutral peptide $\operatorname{Mr}($ calc): 2418.1815

Fixed modifications: Carbamidomethyl (C)

Variable modifications:

N12 : Deamidated_N (N)

Ions Score: 60 Expect: 0.00026

Matches (Bold Red): 72/228 fragment ions using 164 most intense peaks 


\begin{tabular}{|c|c|c|c|c|c|c|c|c|c|c|c|c|c|c|}
\hline \# & b & $\mathbf{b}^{++}$ & b* & $\mathbf{b}^{*++}$ & $\mathbf{b}^{0}$ & $\mathbf{b}^{0++}$ & Seq. & $\mathbf{y}$ & $\mathbf{y}^{++}$ & $\mathrm{y}^{*}$ & $\mathrm{y}^{*^{++}}$ & $\mathbf{y}^{0}$ & $y^{0++}$ & \# \\
\hline 1 & 129.0659 & 65.0366 & 112.0393 & 56.5233 & & & $\mathbf{Q}$ & & & & & & & 21 \\
\hline 2 & 230.1135 & 115.5604 & 213.0870 & 107.0471 & 212.1030 & 106.5551 & $T$ & 2291.1302 & 1146.0688 & 2274.1037 & 1137.5555 & 2273.1197 & 1137.0635 & 20 \\
\hline 3 & 358.1721 & 179.5897 & 341.1456 & 171.0764 & 340.1615 & 170.5844 & $\mathbf{Q}$ & 2190.0826 & 1095.5449 & 2173.0560 & 1087.0316 & 2172.0720 & 1086.5396 & 19 \\
\hline 4 & 457.2405 & 229.1239 & 440.2140 & 220.6106 & 439.2300 & 220.1186 & $\mathbf{V}$ & 2062.0240 & 1031.5156 & 2044.9974 & 1023.0024 & 2044.0134 & 1022.5104 & 18 \\
\hline 5 & 514.2620 & 257.6346 & 497.2354 & 249.1214 & 496.2514 & 248.6294 & G & 1962.9556 & 981.9814 & 1945.9290 & \begin{tabular}{|l|}
973.4682 \\
\end{tabular} & 1944.9450 & 972.9761 & 17 \\
\hline 6 & 627.3461 & 314.1767 & 610.3195 & 305.6634 & 609.3355 & 305.1714 & $\mathbf{I}$ & 1905.9341 & 953.4707 & 1888.9076 & 944.9574 & 1887.9235 & 944.4654 & 16 \\
\hline 7 & 726.4145 & 363.7109 & 709.3879 & 355.1976 & 708.4039 & 354.7056 & V & 1792.8500 & 896.9287 & 1775.8235 & 888.4154 & 1774.8395 & 887.9234 & 15 \\
\hline 8 & 854.4 & 427.7 & 837. & 419. & 625 & 418.7349 & $\mathbf{Q}$ & 1693.7816 & 3945 & 551 & 838.8812 & 1675.7711 & 838.3892 & 14 \\
\hline 9 & 1017.5364 & 509.2718 & 1000.5098 & 500.7586 & 999.5258 & 500.2665 & $\mathbf{Y}$ & 1565.7231 & 783.3652 & 1548.6965 & 774.8519 & 1547.7125 & 774.3599 & 13 \\
\hline 101 & 1074.5578 & 537.7826 & 1057.5313 & 529.2693 & 1056.5473 & 528.7773 & G & 1402.6597 & 701.8335 & 1385.6332 & 693.3202 & 1384.6492 & 692.8282 & 12 \\
\hline 111 & 1203.6004 & 602.3039 & 1186.5739 & 593.7906 & 1185.5899 & 593.2986 & $\mathbf{E}$ & 1345.6383 & 673.3228 & 1328.6117 & 664.8095 & 1327.6277 & 664.3175 & 11 \\
\hline \begin{tabular}{l|l}
12 & 1 \\
\end{tabular} & 1318.6274 & 659.8173 & 1301.6008 & 651.3040 & 1300.6168 & 650.8120 & $\mathbf{N}$ & 1216.5957 & 608.8015 & 1199.5691 & 600.2882 & 1198.5851 & 599.7962 & 10 \\
\hline 131 & 1417.6958 & 709.3515 & 1400.6692 & 700.8383 & 1399.6852 & 700.3462 & $\mathbf{V}$ & 1101.5687 & 551.2880 & 1084.5422 & 542.7747 & 1083.5582 & 542.2827 & 9 \\
\hline 141 & \begin{tabular}{|l|}
1518.7435 \\
\end{tabular} & 759.8754 & \begin{tabular}{|l|}
1501.7169 \\
\end{tabular} & 751.3621 & 1500.7329 & 750.8701 & $T$ & 1002.5003 & 501.7538 & 985.4738 & 493.2405 & 984.4898 & 492.7485 & 8 \\
\hline $15 \mid 1$ & 1655.8024 & 828.4048 & 1638.7758 & 819.8915 & 1637.7918 & 819.3995 & $\mathbf{H}$ & 901.4526 & 451.2300 & 884.4261 & 442.7167 & 883.4421 & 442.2247 & 7 \\
\hline \begin{tabular}{l|l}
6 & 1 \\
\end{tabular} & 1784.8450 & 892.9261 & 1767.8184 & 884.4128 & 1766.8344 & 883.9208 & $\mathbf{E}$ & 764.3937 & 382.7005 & 747.3672 & 374.1872 & 746.3832 & 373.6952 & 6 \\
\hline 171 & 1931.9134 & 966.4603 & \begin{tabular}{|l|}
1914.8868 \\
\end{tabular} & 957.9471 & 1913.9028 & 957.4550 & $\mathbf{F}$ & 635.3511 & 318.1792 & 618.3246 & 309.6659 & & & 5 \\
\hline 18 & 2045.9563 & 1023.4818 & 2028.9298 & 1014.9685 & 2027.9457 & 1014.4765 & $\mathbf{N}$ & 488.2827 & 244.6450 & 471.2562 & 236.1317 & & & 4 \\
\hline 192 & 2159.0404 & 1080.0238 & 2142.0138 & \begin{tabular}{|l|}
1071.5105 \\
\end{tabular} & 2141.0298 & \begin{tabular}{|l|}
1071.0185 \\
\end{tabular} & $\mathbf{L}$ & 374.2398 & 187.6235 & 357.2132 & 179.1103 & & & 3 \\
\hline 202 & 2273.0833 & 1137.0453 & 2256.0567 & 1128.5320 & 2255.0727 & 1128.0400 & $\mathbf{N}$ & 261.1557 & 131.0815 & 244.1292 & 122.5682 & & & 2 \\
\hline 21 & & & & & & & $\mathbf{K}$ & 147.1128 & 74.0600 & 130.0863 & 65.5468 & & & 1 \\
\hline
\end{tabular}
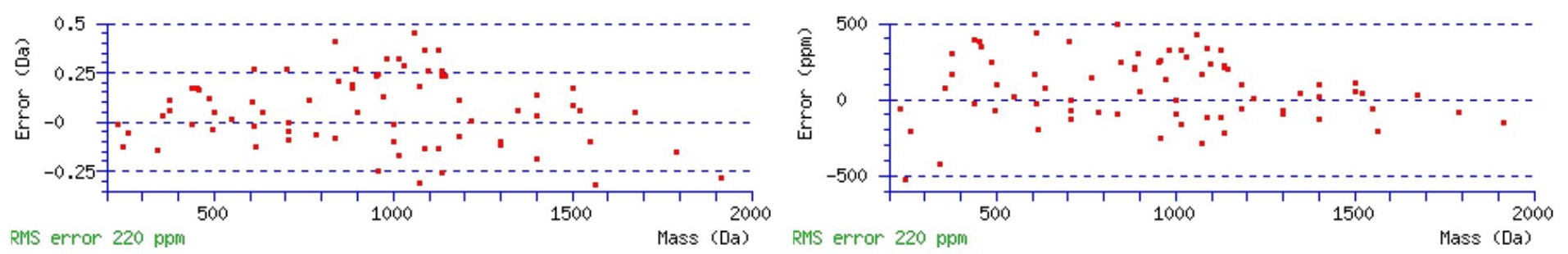

\section{All matches to this query}

\begin{tabular}{|l|c|c|l|}
\hline Score & Mr(calc): & Delta & \multicolumn{1}{|c|}{ Sequence } \\
\hline 59.5 & 2418.1815 & 0.0074 & QTQVGIVQYGENVTHEFNLNK \\
\hline 25.4 & 2418.1815 & 0.0074 & QTQVGIVQYGENVTHEFNLNK \\
\hline 23.6 & 2418.1815 & 0.0074 & QTQVGIVQYGENVTHEFNLNK \\
\hline 9.0 & 2417.2032 & 0.9858 & DGKGSTIYLWEFLLALLQDR \\
\hline 9.0 & 2417.2032 & 0.9858 & DGKGSTIYLWEFLLALLQDR \\
\hline 4.3 & 2418.2004 & -0.0115 & SLLSLNIESNFISSTGLMAVLK \\
\hline 4.3 & 2418.2004 & -0.0115 & SLLSLNIESNFISSTGLMAVLK \\
\hline 4.2 & 2418.2004 & -0.0115 & SLLSLNIESNFISSTGLMAVLK \\
\hline 4.2 & 2418.2004 & -0.0115 & SLLSLNIESNFISSTGLMAVLK \\
\hline 2.9 & 2418.1821 & 0.0068 & REALRPSSKQISYVIQAQGK \\
\hline
\end{tabular}

Spectrum No: 536; Query: 1655; Rank: 1

\section{Peptide View}

MS/MS Fragmentation of RVLYLPAYNCTLRPVSK 
Found in IPI00212708, Tax_Id=10116 Gene_Symbol=Fetub Fetub protein

Match to Query 1655: 2050.102122 from(684.374650,3+)

Title: 091008RatKidney_NoSalt_23.2434.2434.3.dta

Data file K:\NewmanPaper|Piliang|3SubProteomes\Piliang3SP\mgf5ppm\ERLIC_3SubProteomes5ppm.mgf
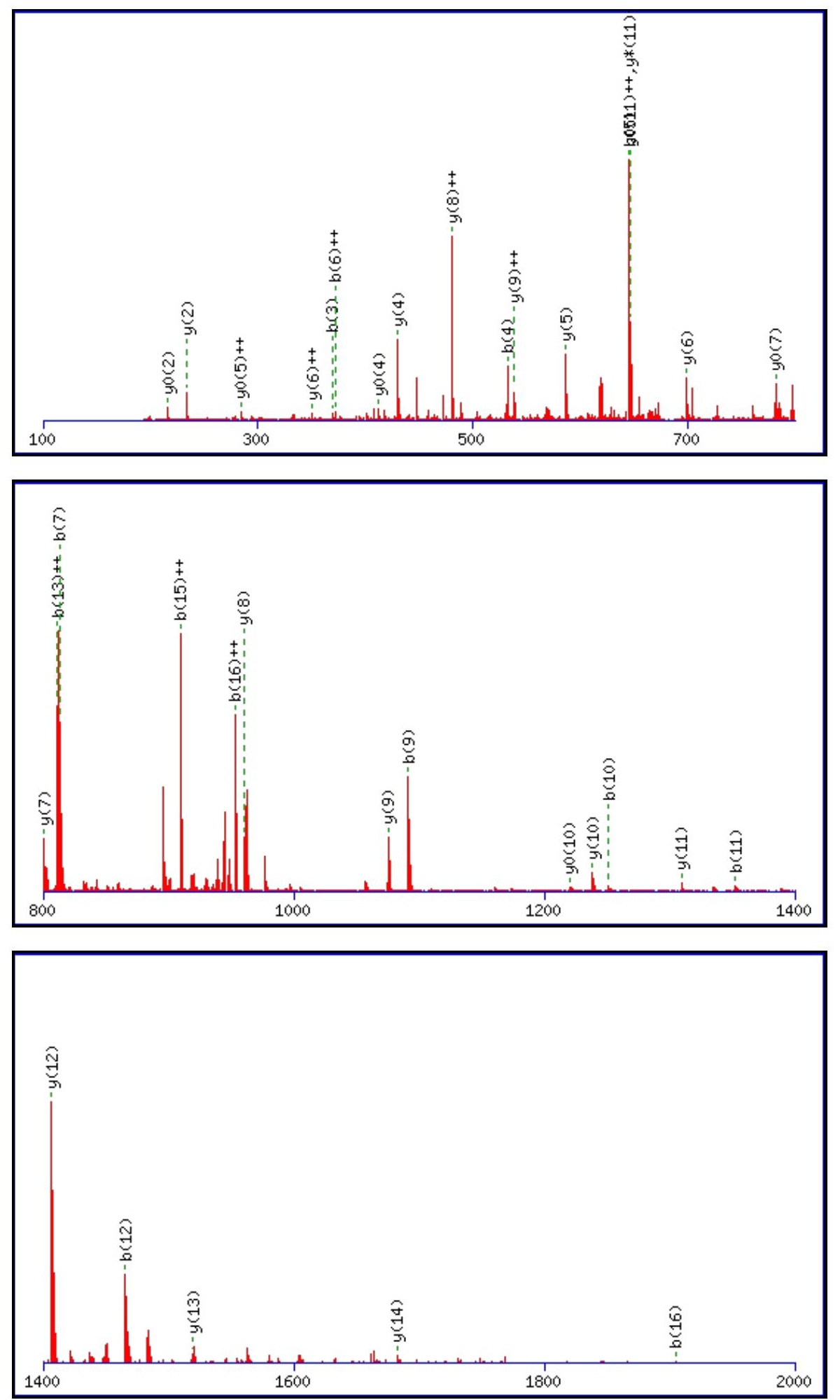

Monoisotopic mass of neutral peptide $\operatorname{Mr}$ (calc): 2050.1033

Fixed modifications: Carbamidomethyl (C)

Variable modifications:

N9 : Deamidated_N (N)

Ions Score: 59 Expect: $7.9 \mathrm{e}-005$

Matches (Bold Red): 35/170 fragment ions using 73 most intense peaks

\begin{tabular}{|c|c|c|c|c|c|c|c|c|c|c|c|c|c|c|}
\hline$\#$ & $\mathbf{b}$ & $\mathbf{b}^{++}$ & $\mathbf{b}^{*}$ & $\mathbf{b}^{\boldsymbol{*}^{++}}$ & $\mathbf{b}^{\mathbf{0}}$ & $\mathbf{b}^{\mathbf{0 + +}}$ & Seq. & $\mathbf{y}$ & $\mathbf{y}^{++}$ & $\mathbf{y}^{\mathbf{*}}$ & $\mathbf{y}^{\boldsymbol{*}^{++}}$ & $\mathbf{y}^{\mathbf{0}}$ & $\mathbf{y}^{\mathbf{0 + +}}$ & $\#$ \\
\hline $\mathbf{1}$ & 157.1084 & 79.0578 & 140.0818 & 70.5446 & & & $\mathbf{R}$ & & & & & & & $\mathbf{1 7}$ \\
\hline
\end{tabular}




\begin{tabular}{|c|c|c|c|c|c|c|c|c|c|c|c|c|c|c|}
\hline 2 & 256.1768 & 128.5920 & 239.1503 & 120.0788 & & & $\mathbf{V}$ & 1895.0095 & 948.0084 & 1877.9830 & 939.4951 & 1876.9990 & 939.0031 & 16 \\
\hline 3 & 369.2609 & 185.1341 & 352.2343 & 176.6208 & & & $\mathbf{L}$ & 1795.9411 & 898.4742 & 1778.9146 & 889.9609 & 1777.9305 & 889.4689 & 15 \\
\hline 4 & 532.3242 & 266.6657 & 515.2976 & 258.1525 & & & $\mathbf{Y}$ & 1682.8570 & 841.9322 & 1665.8305 & 833.4189 & 1664.8465 & 832.9269 & 14 \\
\hline 5 & 645.4083 & 323.2078 & 628.3817 & 314.6945 & & & $\mathbf{L}$ & 1519.7937 & 760.4005 & 1502.7672 & 751.8872 & 1501.7832 & 751.3952 & 13 \\
\hline 6 & 742.4610 & 371.7341 & 725.4345 & 363.2209 & & & $\mathbf{P}$ & 1406.7097 & 703.8585 & 1389.6831 & 695.3452 & 1388.6991 & 694.8532 & 12 \\
\hline 7 & 813.4981 & 407.2527 & 796.4716 & 398.7394 & & & A & 1309.6569 & 655.3321 & 1292.6303 & 646.8188 & 1291.6463 & 646.3268 & 11 \\
\hline 8 & 976.5615 & 488.7844 & 959.5349 & 480.2711 & & & $\mathbf{Y}$ & 1238.6198 & 619.8135 & 1221.5932 & 611.3003 & 1220.6092 & 610.8082 & 10 \\
\hline 9 & 1091.5884 & 546.2978 & 1074.5619 & 537.7846 & & & $\mathbf{N}$ & 1075.5564 & 538.2819 & 1058.5299 & 529.7686 & 1057.5459 & 529.2766 & 9 \\
\hline 10 & 1251.6191 & 626.3132 & 1234.5925 & 617.7999 & & & C & 960.5295 & 480.7684 & 943.5030 & 472.2551 & 942.5189 & 471.7631 & 8 \\
\hline 11 & 1352.6667 & 676.8370 & 1335.6402 & 668.3237 & 1334.6562 & 667.8317 & $\mathbf{T}$ & 800.4989 & 400.7531 & 783.4723 & 392.2398 & 782.4883 & 391.7478 & 7 \\
\hline 12 & 1465.7508 & 733.3790 & 1448.7242 & 724.8658 & 1447.7402 & 724.3738 & $\mathbf{L}$ & 699.4512 & 350.2292 & 682.4246 & 341.7160 & 681.4406 & 341.2239 & 6 \\
\hline 13 & 1621.8519 & 811.4296 & 1604.8254 & 802.9163 & 1603.8413 & 802.4243 & $\mathbf{R}$ & 586.3671 & 293.6872 & 569.3406 & 285.1739 & 568.3566 & 284.6819 & 5 \\
\hline 14 & 1718.9047 & 859.9560 & 1701.8781 & 851.4427 & 1700.8941 & 850.9507 & $\mathbf{P}$ & 430.2660 & 215.6366 & 413.2395 & 207.1234 & 412.2554 & 206.6314 & 4 \\
\hline 15 & 1817.9731 & 909.4902 & 1800.9465 & 900.9769 & 1799.9625 & 900.4849 & $\mathbf{V}$ & 333.2132 & 167.1103 & 316.1867 & 158.5970 & 315.2027 & 158.1050 & 3 \\
\hline 16 & 1905.0051 & 953.0062 & 1887.9786 & 944.4929 & 1886.9945 & 944.0009 & S & 234.1448 & 117.5761 & 217.1183 & 109.0628 & 216.1343 & 108.5708 & 2 \\
\hline 17 & & & & & & & K & 147.1128 & 74.0600 & 130.0863 & 65.5468 & & & 1 \\
\hline
\end{tabular}

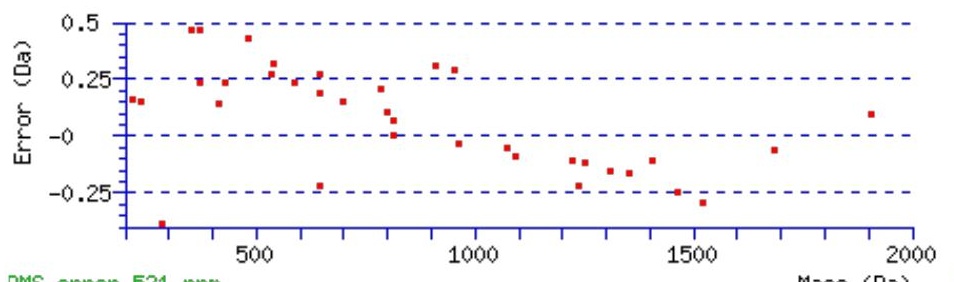

RMS error 521 ppm

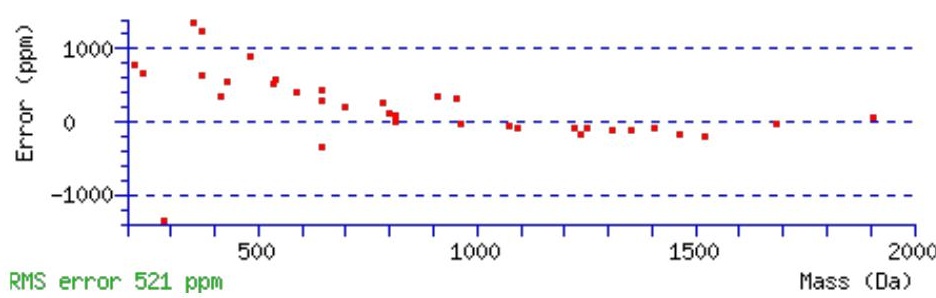

Mass (Da)

\section{All matches to this query}

\begin{tabular}{|l|l|l|l|}
\hline Score & Mr(calc): & Delta & \multicolumn{1}{c|}{ Sequence } \\
\hline 59.5 & 2050.1033 & -0.0012 & RVLYLPAYNCTLRPVSK \\
\hline 7.9 & 2049.1106 & 0.9916 & RSLKTILTYAEEDLELR \\
\hline 1.9 & 2050.1032 & -0.0010 & VVTDNAAAATRHAQTRLQK \\
\hline
\end{tabular}

Spectrum No: 537; Query: 1616; Rank: 1

\section{Peptide View}

MS/MS Fragmentation of DEYPSVLMYLNATQPER

Found in IPI00207180, Tax_Id=10116 Gene_Symbol=Slc6a18 Sodium- and chloride-dependent transporter XTRP2

Match to Query 1616: 2025.938708 from(1013.976630,2+)

Title: 100101RatKid_NS_deglyco_24.4874.4874.2.dta

Data file K:\NewmanPaper\Piliang \3SubProteomes \Piliang3SP $\backslash$ mgf5ppm\ERLIC_3SubProteomes5ppm.mgf 

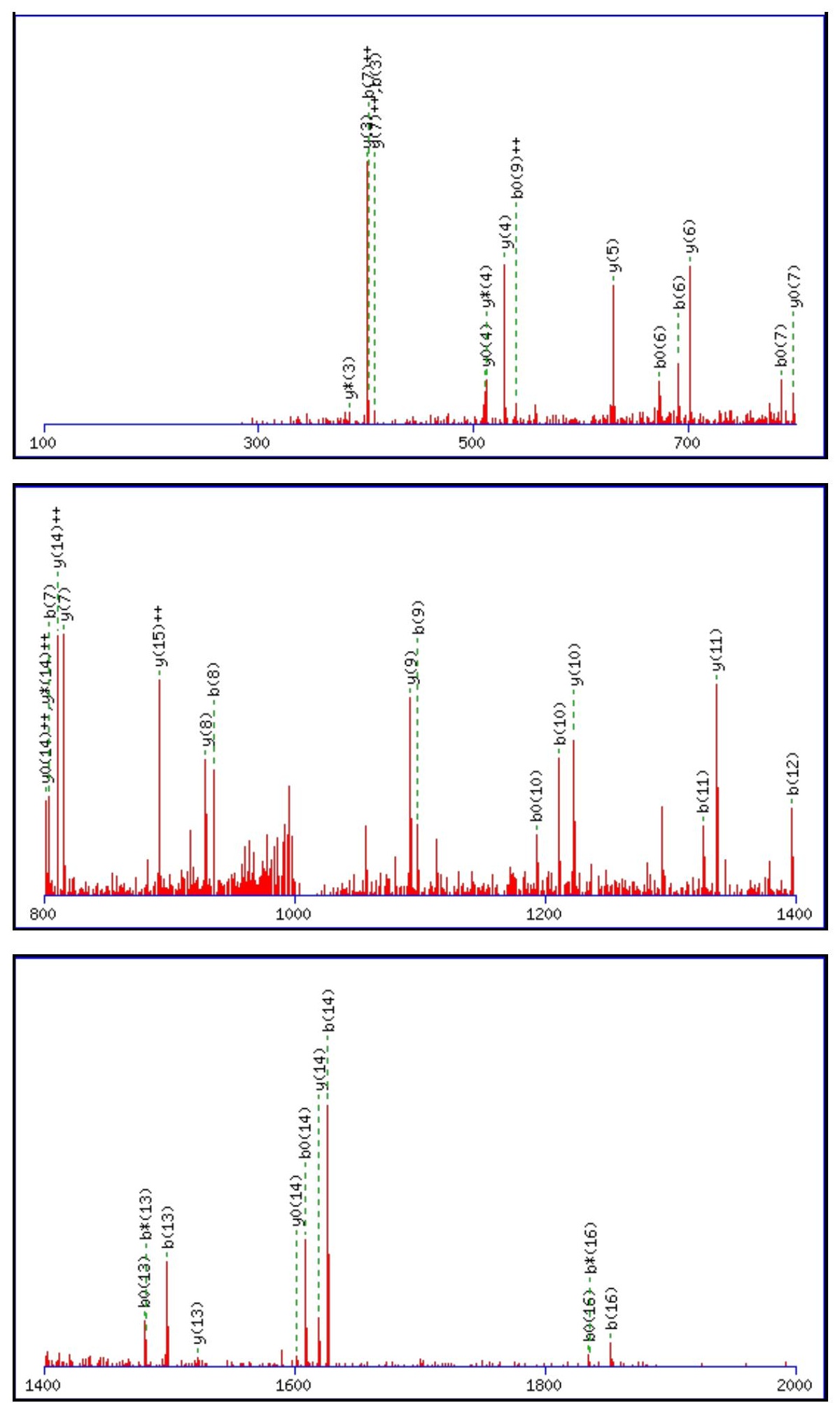

Monoisotopic mass of neutral peptide $\operatorname{Mr}($ calc): 2025.9353

Fixed modifications: Carbamidomethyl (C)

Variable modifications:

N11 : Deamidated_N (N)

Ions Score: 59 Expect: 0.00028

Matches (Bold Red): 42/170 fragment ions using 81 most intense peaks

\begin{tabular}{|r|c|c|c|c|r|r|r|r|c|c|c|c|c|c|}
\hline$\#$ & $\mathbf{b}$ & $\mathbf{b}^{++}$ & $\mathbf{b}^{*}$ & $\mathbf{b}^{\mathbf{*}^{++}}$ & $\mathbf{b}^{\mathbf{0}}$ & $\mathbf{b}^{\mathbf{0 + +}}$ & Seq. & $\mathbf{y}$ & $\mathbf{y}^{++}$ & $\mathbf{y}^{\mathbf{*}}$ & $\mathbf{y}^{\boldsymbol{*}^{++}}$ & $\mathbf{y}^{\mathbf{0}}$ & $\mathbf{y}^{\mathbf{0 + +}}$ & $\#$ \\
\hline $\mathbf{1}$ & 116.0342 & 58.5207 & & & 98.0237 & 49.5155 & $\mathbf{D}$ & & & & & & & $\mathbf{1 7}$ \\
\hline $\mathbf{2}$ & 245.0768 & 123.0420 & & & 227.0662 & 114.0368 & $\mathbf{E}$ & 1911.9157 & 956.4615 & 1894.8891 & 947.9482 & 1893.9051 & 947.4562 & $\mathbf{1 6}$ \\
\hline $\mathbf{3}$ & $\mathbf{4 0 8 . 1 4 0 1}$ & 204.5737 & & & 390.1296 & 195.5684 & $\mathbf{Y}$ & 1782.8731 & $\mathbf{8 9 1 . 9 4 0 2}$ & 1765.8465 & 883.4269 & 1764.8625 & 882.9349 & $\mathbf{1 5}$ \\
\hline $\mathbf{4}$ & 505.1929 & 253.1001 & & & 487.1823 & 244.0948 & $\mathbf{P}$ & $\mathbf{1 6 1 9 . 8 0 9 8}$ & $\mathbf{8 1 0 . 4 0 8 5}$ & 1602.7832 & $\mathbf{8 0 1 . 8 9 5 2}$ & $\mathbf{1 6 0 1 . 7 9 9 2}$ & $\mathbf{8 0 1 . 4 0 3 2}$ & $\mathbf{1 4}$ \\
\hline $\mathbf{5}$ & 592.2249 & 296.6161 & & & 574.2144 & 287.6108 & $\mathbf{S}$ & $\mathbf{1 5 2 2 . 7 5 7 0}$ & 761.8821 & 1505.7305 & 753.3689 & 1504.7464 & 752.8769 & $\mathbf{1 3}$ \\
\hline
\end{tabular}




\begin{tabular}{|c|c|c|c|c|c|c|c|c|c|c|c|c|c|c|}
\hline 6 & 691.2933 & |346.1503 & & & 673.2828 & |337.1450| & V & |1435.7250 & |718.3661 & |1418.6984 & 709.8528 & 1417.7144 & |709.3608| & 12 \\
\hline 7 & 804.3774 & 402.6923 & & & 786.3668 & |393.6871 & $\mathbf{L}$ & |1336.6566 & 668.8319 & 1319.6300 & 660.3186 & 1318.6460 & 659.8266 & 11 \\
\hline 8 & 935.4179 & 468.2126 & & & 917.4073 & 459.2073 & M & 1223.5725 & 612.2899 & 1206.5459 & 603.7766 & 1205.5619 & 603.2846 & 10 \\
\hline 9 & 1098.4812 & 549.7443 & & & 1080.4707 & 540.7390 & $\mathbf{Y}$ & 1092.5320 & 546.7696 & 1075.5055 & 538.2564 & 1074.5214 & 537.7644 & 9 \\
\hline 10 & 1211.5653 & 606.2863 & & & 1193.5547 & 597.2810 & L & 929.4687 & 465.2380 & 912.4421 & 456.7247 & 911.4581 & 456.2327 & 8 \\
\hline 11 & 1326.5922 & 663.7998 & 1309.5657 & 655.2865 & 1308.5817 & 654.7945 & $\mathbf{N}$ & 816.3846 & 408.6959 & 799.3581 & 400.1827 & 798.3741 & 399.6907 & 7 \\
\hline 12 & 1397.6293 & 699.3183 & 1380.6028 & 690.8050 & 1379.6188 & 690.3130 & A & 701.3577 & 351.1825 & 684.3311 & 342.6692 & 683.3471 & 342.1772 & 6 \\
\hline 13 & 1498.6770 & 749.8421 & 1481.6505 & 741.3289 & 1480.6665 & 740.8369 & $\mathbf{T}$ & 630.3206 & 315.6639 & 613.2940 & 307.1506 & 612.3100 & 306.6586 & 5 \\
\hline 14 & 1626.7356 & 813.8714 & 1609.7090 & 805.3582 & 1608.7250 & 804.8662 & $\mathbf{Q}$ & 529.2729 & 265.1401 & 512.2463 & 256.6268 & 511.2623 & 256.1348 & 4 \\
\hline 15 & 1723.7884 & 862.3978 & 1706.7618 & 853.8845 & 1705.7778 & 853.3925 & $\mathbf{P}$ & 401.2143 & 201.1108 & 384.1878 & 192.5975 & 383.2037 & 192.1055 & 3 \\
\hline 16 & 1852.8310 & 926.9191 & 1835.8044 & 918.4058 & 1834.8204 & 917.9138 & E & 304.1615 & 152.5844 & 287.1350 & 144.0711 & 286.1510 & 143.5791 & 2 \\
\hline 17 & & & & & & & $\mathbf{R}$ & 175.1190 & 88.0631 & 158.0924 & 79.5498 & & & 1 \\
\hline
\end{tabular}
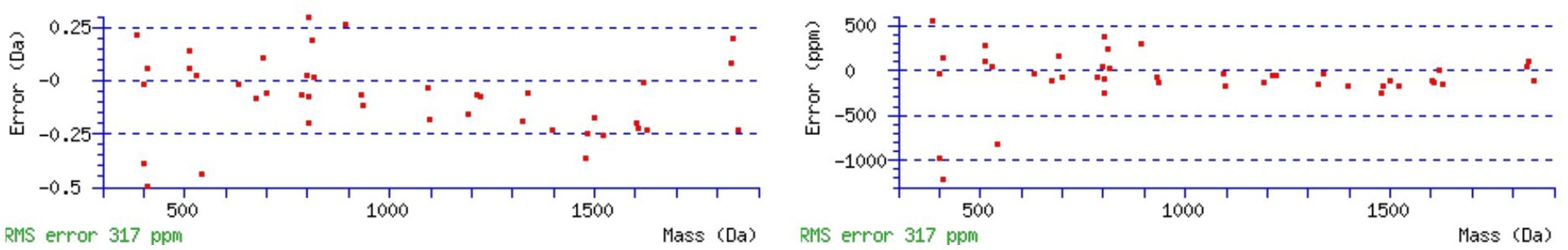

\section{All matches to this query}

\begin{tabular}{|l|l|l|l|}
\hline Score & Mr(calc): & Delta & \multicolumn{1}{|c|}{ Sequence } \\
\hline 59.5 & 2025.9353 & 0.0034 & DEYPSVLMYLNATQPER \\
\hline 19.8 & 2024.9513 & 0.9874 & DEYPSVLMYLNATQPER \\
\hline 9.4 & 2025.9303 & 0.0084 & TALDECLRDGVIGSRER \\
\hline 7.8 & 2025.9288 & 0.0100 & DVLLTDFLPTMAVYIR \\
\hline 5.5 & 2025.9269 & 0.0118 & QIDNTGSSTYRPPPRTR \\
\hline 4.6 & 2025.9269 & 0.0118 & QIDNTGSSTYRPPPRTR \\
\hline 3.7 & 2025.9359 & 0.0028 & YMRDTGLTALTNLKLR \\
\hline 1.5 & 2025.9359 & 0.0028 & YMRDTGLTALTNLKLR \\
\hline 0.1 & 2025.9238 & 0.0150 & MDQTSASILCRRPQQR \\
\hline 0.1 & 2025.9238 & 0.0150 & MDQTSASILCRRPQQR \\
\hline
\end{tabular}

Spectrum No: 538; Query: 1023; Rank: 1

\section{Peptide View}

MS/MS Fragmentation of GDSGGALVFLDNETQR

Found in IPI00359149, Tax_Id=10116 Gene_Symbol=Masp2 Isoform 1 of Mannan-binding lectin serine protease 2 precursor

Match to Query 1023: 1678.786708 from(840.400630,2+)

Title: 100101RatKid_NS_deglyco_25.3370.3370.2.dta

Data file K:INewmanPaper|Piliang|3SubProteomes\Piliang3SP\mgf5ppm\ERLIC_3SubProteomes5ppm.mgf 

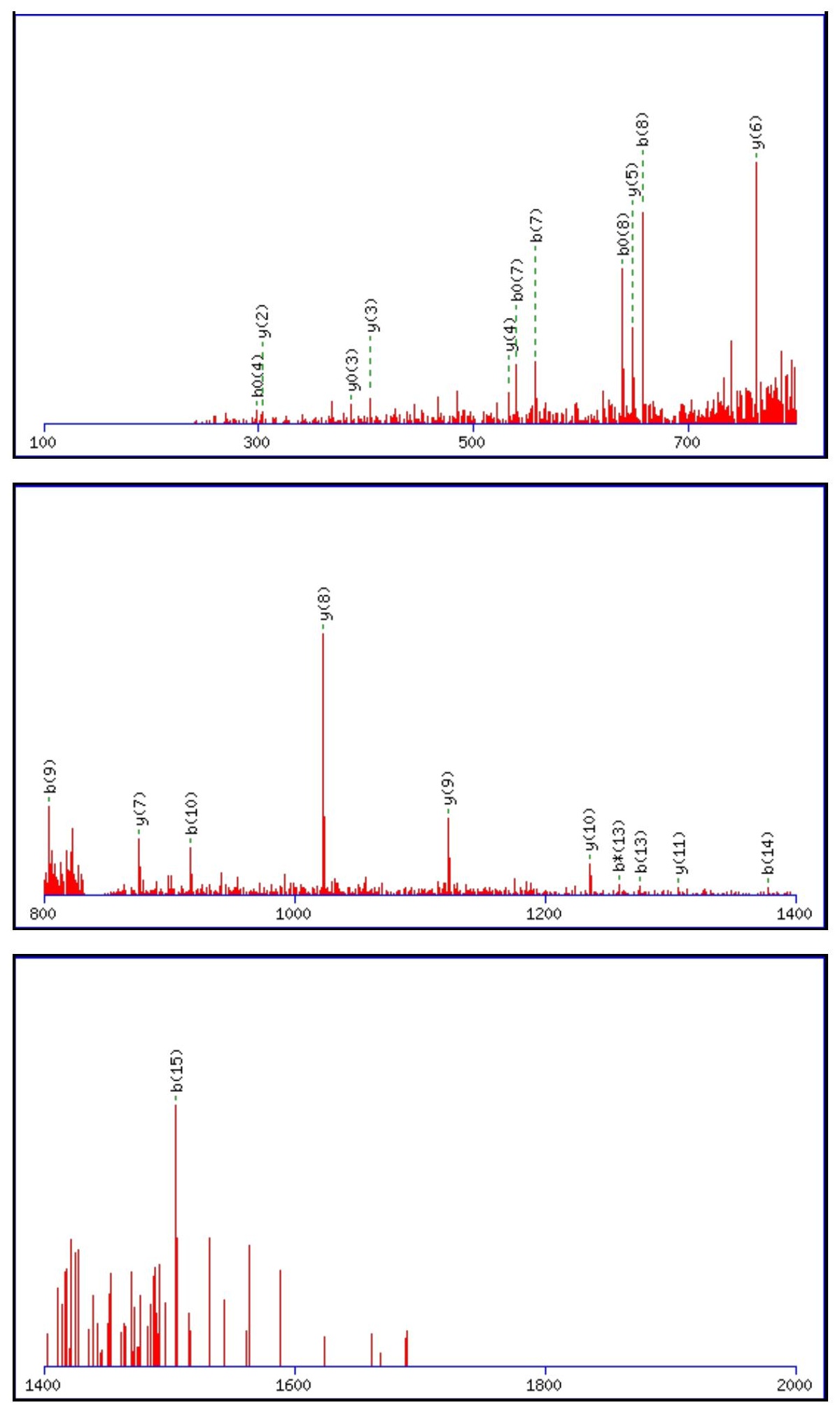

Monoisotopic mass of neutral peptide $\operatorname{Mr}($ calc): 1678.7798

Fixed modifications: Carbamidomethyl (C)

Variable modifications:

N12 : Deamidated_N (N)

Ions Score: 59 Expect: 0.00023

Matches (Bold Red): 22/152 fragment ions using 49 most intense peaks

\begin{tabular}{|r|c|c|c|c|c|c|c|c|c|c|c|c|c|c|}
\hline$\#$ & $\mathbf{b}$ & $\mathbf{b}^{++}$ & $\mathbf{b}^{*}$ & $\mathbf{b}^{\boldsymbol{*}^{++}}$ & $\mathbf{b}^{\mathbf{0}}$ & $\mathbf{b}^{\mathbf{0 + +}}$ & Seq. & $\mathbf{y}$ & $\mathbf{y}^{++}$ & $\mathbf{y}^{\mathbf{*}}$ & $\mathbf{y}^{\boldsymbol{*}^{++}}$ & $\mathbf{y}^{\mathbf{0}}$ & $\mathbf{y}^{\mathbf{0 + +}}$ & $\#$ \\
\hline $\mathbf{1}$ & 58.0287 & 29.5180 & & & & & $\mathbf{G}$ & & & & & & & $\mathbf{1 6}$ \\
\hline $\mathbf{2}$ & 173.0557 & 87.0315 & & & 155.0451 & 78.0262 & $\mathbf{D}$ & 1622.7657 & 811.8865 & 1605.7391 & 803.3732 & 1604.7551 & 802.8812 & $\mathbf{1 5}$ \\
\hline 3 & 260.0877 & 130.5475 & & & 242.0771 & 121.5422 & S & 1507.7387 & 754.3730 & 1490.7122 & 745.8597 & 1489.7281 & 745.3677 & $\mathbf{1 4}$ \\
\hline $\mathbf{4}$ & 317.1092 & 159.0582 & & & 299.0986 & 150.0529 & G & 1420.7067 & 710.8570 & 1403.6801 & 702.3437 & 1402.6961 & 701.8517 & $\mathbf{1 3}$ \\
\hline $\mathbf{5}$ & 374.1306 & 187.5690 & & & 356.1201 & 178.5637 & $\mathbf{G}$ & 1363.6852 & 682.3462 & 1346.6587 & 673.8330 & 1345.6746 & 673.3410 & $\mathbf{1 2}$ \\
\hline
\end{tabular}




\begin{tabular}{|r|r|r|r|r|r|r|r|r|r|r|r|r|r|r|r|r|}
$\mathbf{6}$ & 445.1678 & 223.0875 & & & 427.1572 & 214.0822 & A & $\mathbf{1 3 0 6 . 6 6 3 8}$ & 653.8355 & 1289.6372 & 645.3222 & 1288.6532 & 644.8302 & $\mathbf{1 1}$ \\
\hline $\mathbf{7}$ & $\mathbf{5 5 8 . 2 5 1 8}$ & 279.6295 & & & 540.2413 & 270.6243 & L & $\mathbf{1 2 3 5 . 6 2 6 6}$ & 618.3170 & 1218.6001 & 609.8037 & 1217.6161 & 609.3117 & $\mathbf{1 0}$ \\
\hline $\mathbf{8}$ & $\mathbf{6 5 7 . 3 2 0 2}$ & 329.1638 & & & $\mathbf{6 3 9 . 3 0 9 7}$ & 320.1585 & V & $\mathbf{1 1 2 2 . 5 4 2 6}$ & 561.7749 & 1105.5160 & 553.2617 & 1104.5320 & 552.7696 & $\mathbf{9}$ \\
\hline $\mathbf{9}$ & $\mathbf{8 0 4 . 3 8 8 6}$ & 402.6980 & & & 786.3781 & 393.6927 & F & $\mathbf{1 0 2 3 . 4 7 4 2}$ & 512.2407 & 1006.4476 & 503.7274 & 1005.4636 & 503.2354 & $\mathbf{8}$ \\
\hline $\mathbf{1 0}$ & $\mathbf{9 1 7 . 4 7 2 7}$ & 459.2400 & & & 899.4621 & 450.2347 & L & $\mathbf{8 7 6 . 4 0 5 7}$ & 438.7065 & 859.3792 & 430.1932 & 858.3952 & 429.7012 & $\mathbf{7}$ \\
\hline $\mathbf{1 1}$ & 1032.4997 & 516.7535 & & & 1014.4891 & 507.7482 & D & 763.3217 & 382.1645 & 746.2951 & 373.6512 & 745.3111 & 373.1592 & $\mathbf{6}$ \\
\hline $\mathbf{1 2}$ & 1147.5266 & 574.2669 & 1130.5000 & 565.7537 & 1129.5160 & 565.2616 & N & $\mathbf{6 4 8 . 2 9 4 7}$ & 324.6510 & 631.2682 & 316.1377 & 630.2842 & 315.6457 & $\mathbf{5}$ \\
\hline $\mathbf{1 3}$ & $\mathbf{1 2 7 6 . 5 6 9 2}$ & 638.7882 & $\mathbf{1 2 5 9 . 5 4 2 6}$ & 630.2750 & 1258.5586 & 629.7829 & E & 533.2678 & 267.1375 & 516.2413 & 258.6243 & 515.2572 & 258.1323 & $\mathbf{4}$ \\
\hline $\mathbf{1 4}$ & $\mathbf{1 3 7 7 . 6 1 6 9}$ & 689.3121 & 1360.5903 & 680.7988 & 1359.6063 & 680.3068 & T & $\mathbf{4 0 4 . 2 2 5 2}$ & 202.6162 & 387.1987 & 194.1030 & $\mathbf{3 8 6 . 2 1 4 6}$ & 193.6110 & $\mathbf{3}$ \\
\hline $\mathbf{1 5}$ & $\mathbf{1 5 0 5 . 6 7 5 4}$ & 753.3414 & 1488.6489 & 744.8281 & 1487.6649 & 744.3361 & $\mathbf{Q}$ & $\mathbf{3 0 3 . 1 7 7 5}$ & 152.0924 & 286.1510 & 143.5791 & & & & $\mathbf{2}$ \\
\hline $\mathbf{1 6}$ & & & & & & & $\mathbf{R}$ & 175.1190 & 88.0631 & 158.0924 & 79.5498 & & & $\mathbf{1}$ \\
\hline
\end{tabular}
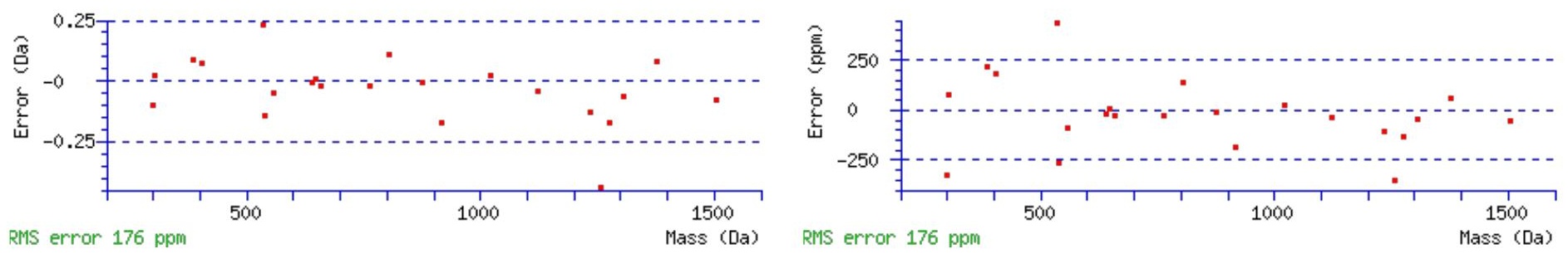

\section{All matches to this query}

\begin{tabular}{|l|l|l|l|}
\hline Score & Mr(calc): & Delta & \multicolumn{1}{c|}{ Sequence } \\
\hline 59.5 & 1678.7798 & 0.0069 & GDSGGALVFLDNETQR \\
\hline 10.2 & 1677.7958 & 0.9909 & GDSGGALVFLDNETQR \\
\hline 8.7 & 1676.7900 & 1.9967 & AXQTTPRNAGNCTRK \\
\hline 1.4 & 1678.7716 & 0.0151 & ETSLDHPYEKPRK \\
\hline 1.4 & 1678.7716 & 0.0151 & ETSLDHPYEKPRK \\
\hline 0.6 & 1678.7997 & -0.0130 & FWRSQQKVVITK \\
\hline 0.2 & 1678.7902 & -0.0035 & GNYLEMKNKFLAR \\
\hline
\end{tabular}

Spectrum No: 539; Query: 730; Rank: 1

\section{Peptide View}

MS/MS Fragmentation of AVAQGNLSSADVQAAK

Found in IPI00188924, Tax_Id=10116 Gene_Symbol=Uqcrc2 Cytochrome b-c1 complex subunit 2, mitochondrial precursor

Match to Query 730: 1529.768268 from(765.891410,2+)

Title: 091008RatKidney_NH4Format01_08.1146.1146.2.dta

Data file K:INewmanPaper|Piliang|3SubProteomes\Piliang3SP\mgf5ppm\ERLIC_3SubProteomes5ppm.mgf 

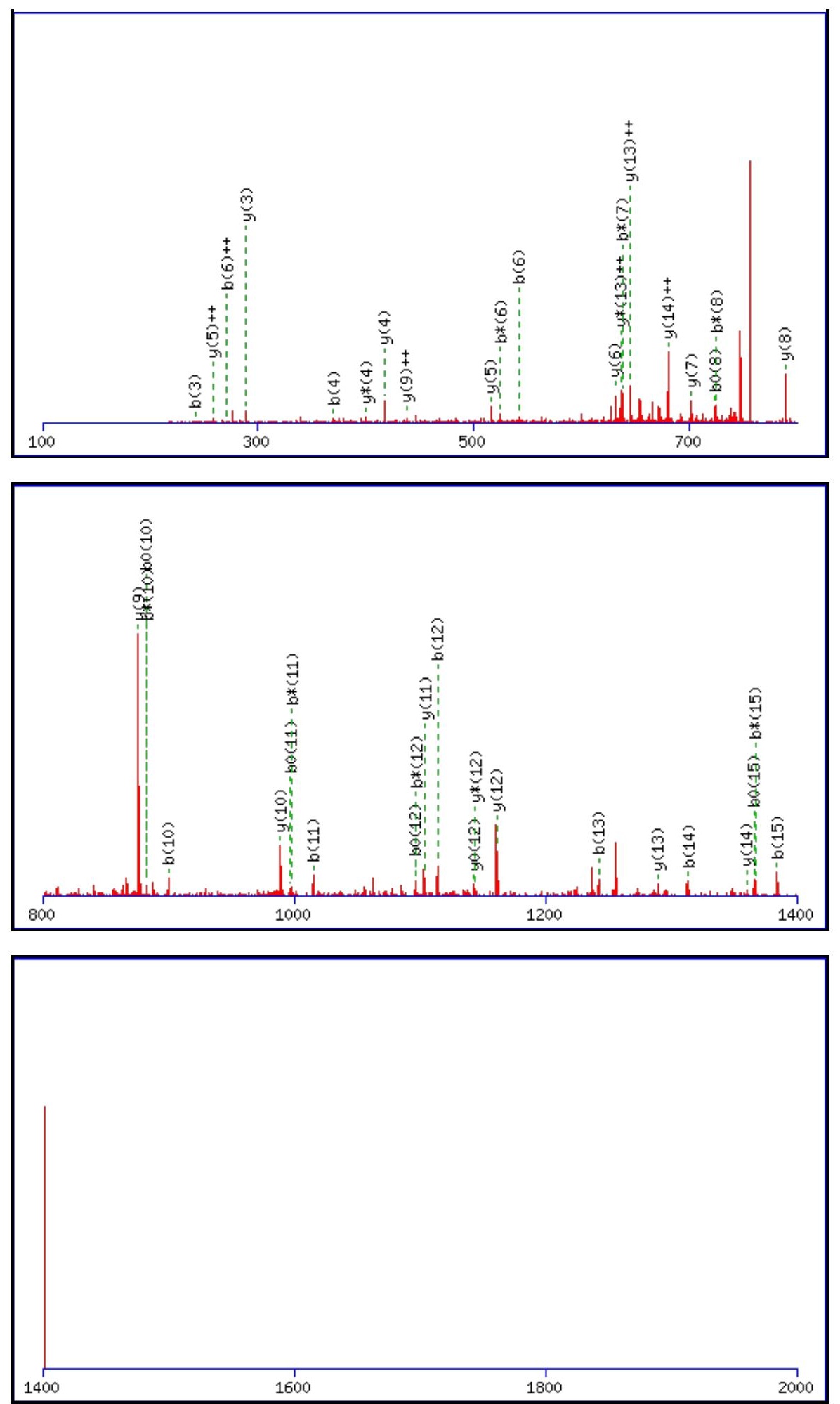

Monoisotopic mass of neutral peptide $\operatorname{Mr}($ calc): 1529.7685

Fixed modifications: Carbamidomethyl (C)

Variable modifications:

N6

Ions Score: 59 Expect: 0.00016

Matches (Bold Red): 42/150 fragment ions using 102 most intense peaks

\begin{tabular}{|r|c|c|c|c|c|c|c|c|c|c|c|c|c|c|}
\hline$\#$ & $\mathbf{b}$ & $\mathbf{b}^{++}$ & $\mathbf{b}^{*}$ & $\mathbf{b}^{\boldsymbol{*}^{++}}$ & $\mathbf{b}^{\mathbf{0}}$ & $\mathbf{b}^{\mathbf{0 + +}}$ & Seq. & $\mathbf{y}$ & $\mathbf{y}^{++}$ & $\mathbf{y}^{\mathbf{*}}$ & $\mathbf{y}^{\boldsymbol{*}^{++}}$ & $\mathbf{y}^{\mathbf{0}}$ & $\mathbf{y}^{\mathbf{0 + +}}$ & $\#$ \\
\hline $\mathbf{1}$ & 72.0444 & 36.5258 & & & & & $\mathbf{A}$ & & & & & & & $\mathbf{1 6}$ \\
\hline $\mathbf{2}$ & 171.1128 & 86.0600 & & & & & $\mathbf{V}$ & 1459.7387 & 730.3730 & 1442.7122 & 721.8597 & 1441.7281 & 721.3677 & $\mathbf{1 5}$ \\
\hline $\mathbf{3}$ & $\mathbf{2 4 2 . 1 4 9 9}$ & 121.5786 & & & & & A & $\mathbf{1 3 6 0 . 6 7 0 3}$ & $\mathbf{6 8 0 . 8 3 8 8}$ & 1343.6437 & 672.3255 & 1342.6597 & 671.8335 & $\mathbf{1 4}$ \\
\hline $\mathbf{4}$ & $\mathbf{3 7 0 . 2 0 8 5}$ & 185.6079 & 353.1819 & 177.0946 & & & $\mathbf{Q}$ & $\mathbf{1 2 8 9 . 6 3 3 2}$ & $\mathbf{6 4 5 . 3 2 0 2}$ & 1272.6066 & $\mathbf{6 3 6 . 8 0 7 0}$ & 1271.6226 & 636.3149 & $\mathbf{1 3}$ \\
\hline $\mathbf{5}$ & 427.2300 & 214.1186 & 410.2034 & 205.6053 & & & $\mathbf{G}$ & $\mathbf{1 1 6 1 . 5 7 4 6}$ & 581.2909 & $\mathbf{1 1 4 4 . 5 4 8 1}$ & 572.7777 & $\mathbf{1 1 4 3 . 5 6 4 0}$ & 572.2857 & $\mathbf{1 2}$ \\
\hline
\end{tabular}




\begin{tabular}{|c|c|c|c|c|c|c|c|c|c|c|c|c|c|c|}
\hline 6 & 542.2569 & |271.6321 & 525.2303 & |263.1188 & & & $\mathbf{N}$ & |1104.5531| & 552.7802 & 1087.5266 & 544.2669 & 1086.5426 & 543.7749| & 11 \\
\hline 7 & 655.3410 & 328.1741 & 638.3144 & 319.6608 & & & $\mathbf{L}$ & 989.5262 & 495.2667 & 972.4997 & 486.7535 & 971.5156 & 486.2615 & 10 \\
\hline 8 & 742.3730 & 371.6901 & 725.3464 & 363.1769 & 724.3624 & 362.6848 & S & 876.4421 & 438.7247 & 859.4156 & 430.2114 & 858.4316 & 429.7194 & 9 \\
\hline 9 & 829.4050 & 415.2061 & 812.3785 & 406.6929 & 811.3945 & 406.2009 & $\mathrm{~S}$ & 789.4101 & 395.2087 & 772.3836 & 386.6954 & 771.3995 & 386.2034 & 8 \\
\hline 10 & 900.4421 & 450.7247 & 883.4156 & 442.2114 & 882.4316 & 441.7194 & A & 702.3781 & 351.6927 & 685.3515 & 343.1794 & 684.3675 & 342.6874 & 7 \\
\hline 11 & 1015.4691 & 508.2382 & 998.4425 & 499.7249 & 997.4585 & 499.2329 & D & 631.3410 & 316.1741 & 614.3144 & 307.6608 & 613.3304 & 307.1688 & 6 \\
\hline 12 & 1114.5375 & 557.7724 & 1097.5109 & 549.2591 & 1096.5269 & 548.7671 & V & 516.3140 & 258.6607 & 499.2875 & 250.1474 & & & 5 \\
\hline 13 & 1242.5961 & 621.8017 & 1225.5695 & 613.2884 & 1224.5855 & 612.7964 & $\mathbf{Q}$ & 417.2456 & 209.1264 & 400.2191 & 200.6132 & & & 4 \\
\hline 14 & 1313.6332 & 657.3202 & 1296.6066 & 648.8070 & 1295.6226 & 648.3149 & A & 289.1870 & 145.0972 & 272.1605 & 136.5839 & & & 3 \\
\hline 15 & 1384.6703 & 692.8388 & 1367.6437 & 684.3255 & 1366.6597 & 683.8335 & A & 218.1499 & 109.5786 & 201.1234 & 101.0653 & & & 2 \\
\hline 16 & & & & & & & $\mathbf{K}$ & 147.1128 & 74.0600 & 130.0863 & 65.5468 & & & 1 \\
\hline
\end{tabular}
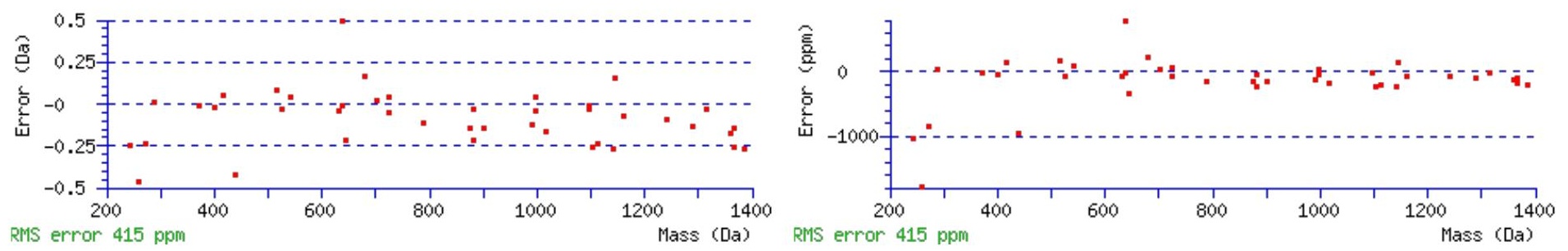

\section{All matches to this query}

\begin{tabular}{|l|l|l|l|}
\hline Score & Mr(calc): & Delta & \multicolumn{1}{|c|}{ Sequence } \\
\hline 59.5 & 1529.7685 & -0.0003 & AVAQGNLSSADVQAAK \\
\hline 7.1 & 1529.7789 & -0.0106 & MTVLRNLPHLQK \\
\hline 6.6 & 1529.7739 & -0.0056 & AVSQFSWGHRVEK \\
\hline 5.9 & 1528.7724 & 0.9958 & QFLGXVPIMVKSK \\
\hline 5.7 & 1529.7715 & -0.0033 & RTLFQTKNSVTR \\
\hline 2.9 & 1528.7650 & 1.0033 & TINPSKYQTIRK \\
\hline 2.4 & 1528.7650 & 1.0033 & LLGYSRDELQKK \\
\hline 2.4 & 1528.7650 & 1.0033 & LLGYSRDELQKK \\
\hline 1.5 & 1529.7715 & -0.0032 & VLSAPPKESRPNR \\
\hline 1.0 & 1528.7511 & 1.0172 & SLASHPTREPKAR \\
\hline
\end{tabular}

Spectrum No: 540; Query: 92; Rank: 1

\section{Peptide View}

MS/MS Fragmentation of LTFTKNTTR

Found in IPI00206336, Tax_Id=10116 Gene_Symbol=Lamp1 Lysosome-associated membrane glycoprotein 1 precursor

Match to Query 92: 1081.574188 from(541.794370,2+)

Title: 091008RatKidney_NH4Format01_23.492.492.2.dta

Data file K:INewmanPaper|Piliangl3SubProteomes\Piliang3SP\mgf5ppm\ERLIC_3SubProteomes5ppm.mgf 

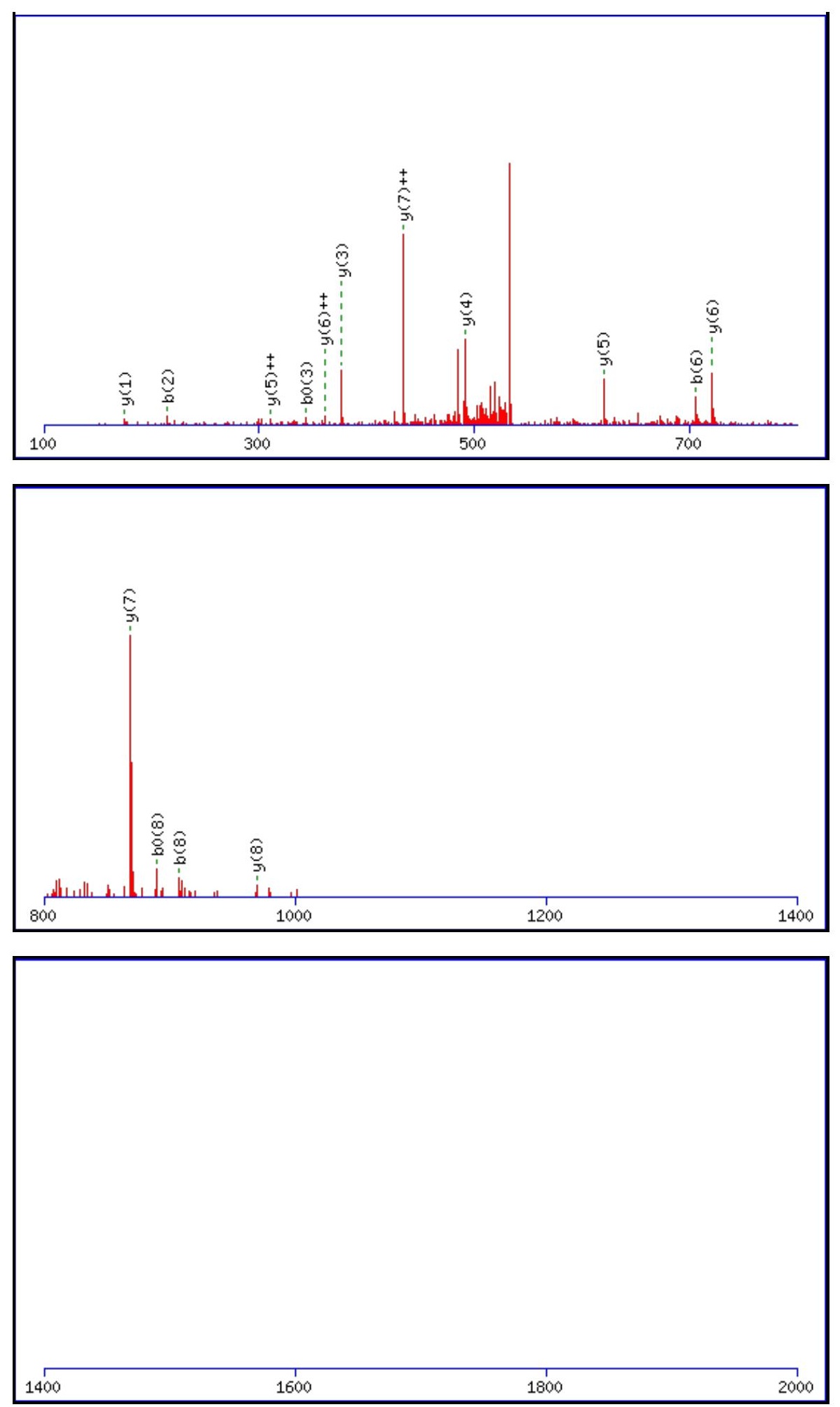

Monoisotopic mass of neutral peptide $\operatorname{Mr}($ calc): 1081.5768

Fixed modifications: Carbamidomethyl (C)

Variable modifications:

N6: Deamidated $\mathrm{N}(\mathrm{N})$

Ions Score: 59 Expect: $8.6 \mathrm{e}-005$

Matches (Bold Red): 15/84 fragment ions using 25 most intense peaks

\begin{tabular}{|c|c|c|c|c|c|c|c|c|c|c|c|c|c|c|}
\hline$\#$ & $\mathbf{b}$ & $\mathbf{b}^{++}$ & $\mathbf{b}^{*}$ & $\mathbf{b}^{*_{+}^{++}}$ & $\mathbf{b}^{\mathbf{0}}$ & $\mathbf{b}^{\mathbf{0 + +}}$ & $\mathbf{S e q}$ & $\mathbf{y}$ & $\mathbf{y}^{++}$ & $\mathbf{y}^{*}$ & $\mathbf{y}^{\mathbf{*}^{++}}$ & $\mathbf{y}^{\mathbf{0}}$ & $\mathbf{y}^{\mathbf{0}++}$ & $\#$ \\
\hline $\mathbf{1}$ & 114.0913 & 57.5493 & & & & & $\mathbf{L}$ & & & & & & & $\mathbf{9}$ \\
\hline $\mathbf{2}$ & $\mathbf{2 1 5 . 1 3 9 0}$ & 108.0731 & & & 197.1285 & 99.0679 & $\mathbf{T}$ & $\mathbf{9 6 9 . 5 0 0 0}$ & 485.2536 & 952.4734 & 476.7404 & 951.4894 & 476.2483 & $\mathbf{8}$ \\
\hline $\mathbf{3}$ & 362.2074 & 181.6074 & & & 344.1969 & 172.6021 & $\mathbf{F}$ & $\mathbf{8 6 8 . 4 5 2 3}$ & $\mathbf{4 3 4 . 7 2 9 8}$ & 851.4258 & 426.2165 & 850.4417 & 425.7245 & $\mathbf{7}$ \\
\hline $\mathbf{4}$ & 463.2551 & 232.1312 & & & 445.2445 & 223.1259 & $\mathbf{T}$ & $\mathbf{7 2 1 . 3 8 3 9}$ & $\mathbf{3 6 1 . 1 9 5 6}$ & 704.3573 & 352.6823 & 703.3733 & 352.1903 & $\mathbf{6}$ \\
\hline $\mathbf{5}$ & 591.3501 & 296.1787 & 574.3235 & 287.6654 & 573.3395 & 287.1734 & $\mathbf{K}$ & $\mathbf{6 2 0 . 3 3 6 2}$ & $\mathbf{3 1 0 . 6 7 1 7}$ & 603.3097 & 302.1585 & 602.3256 & 301.6665 & 5 \\
\hline
\end{tabular}




\begin{tabular}{|l|l|l|l|r|r|r|r|r|r|r|r|r|r|r|r|}
$\mathbf{6}$ & 706.3770 & 353.6921 & 689.3505 & 345.1789 & 688.3664 & 344.6869 & $\mathbf{N}$ & $\mathbf{4 9 2 . 2 4 1 2}$ & 246.6243 & 475.2147 & 238.1110 & 474.2307 & 237.6190 & $\mathbf{4}$ \\
\hline $\mathbf{7}$ & 807.4247 & 404.2160 & 790.3981 & 395.7027 & 789.4141 & 395.2107 & $\mathbf{T}$ & 377.2143 & 189.1108 & 360.1878 & 180.5975 & 359.2037 & 180.1055 & 3 \\
\hline $\mathbf{8}$ & $\mathbf{9 0 8 . 4 7 2 4}$ & 454.7398 & 891.4458 & 446.2265 & $\mathbf{8 9 0 . 4 6 1 8}$ & 445.7345 & $\mathbf{T}$ & 276.1666 & 138.5870 & 259.1401 & 130.0737 & 258.1561 & 129.5817 & 2 \\
\hline $\mathbf{9}$ & & & & & & & $\mathbf{R}$ & $\mathbf{1 7 5 . 1 1 9 0}$ & 88.0631 & 158.0924 & 79.5498 & & & $\mathbf{1}$ \\
\hline
\end{tabular}
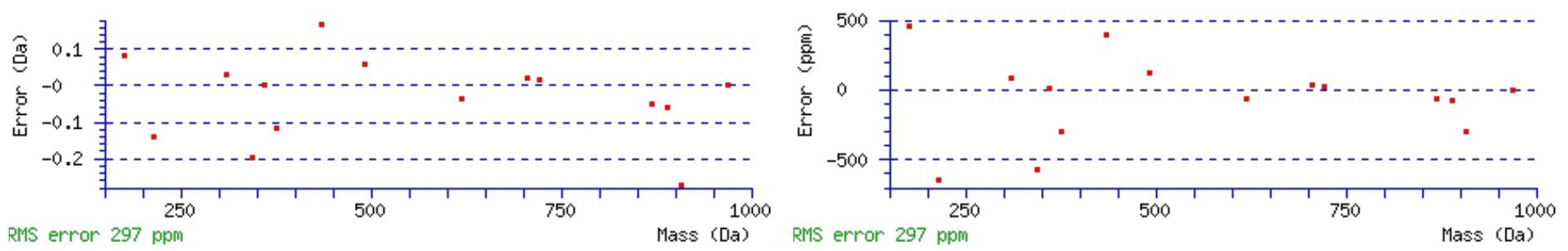

\section{All matches to this query}

\begin{tabular}{|l|l|l|l|}
\hline Score & $\mathbf{M r}$ (calc): & Delta & \multicolumn{1}{|c|}{ Sequence } \\
\hline 59.4 & 1081.5768 & -0.0026 & LTFTKNTTR \\
\hline 8.8 & 1081.5768 & -0.0026 & $\underline{\text { ITDYSISKR }}$ \\
\hline 7.2 & 1081.5645 & 0.0097 & LTLVSRGTR \\
\hline 6.7 & 1079.5645 & 2.0097 & $\underline{\text { TKLSTNLMR }}$ \\
\hline 6.6 & 1081.5645 & 0.0097 & LTLVSRGTR \\
\hline 6.2 & 1079.5611 & 2.0131 & VIQYLSSNR \\
\hline 5.8 & 1079.5740 & 2.0002 & $\underline{\text { LASLLGLASR }}$ \\
\hline 2.6 & 1081.5784 & -0.0042 & $\underline{\text { ITKTEVAIK }}$ \\
\hline 2.6 & 1081.5784 & -0.0042 & ITSTLQVLK \\
\hline 2.6 & 1081.5784 & -0.0042 & $\underline{\text { ITSTLQVLK }}$ \\
\hline
\end{tabular}

Spectrum No: 541; Query: 1035; Rank: 1

\section{Peptide View}

MS/MS Fragmentation of ERSWPAVGNCTAALR

Found in IPI00195516, Tax_Id=10116 Gene_Symbol=Hpx Hemopexin precursor

Match to Query 1035: 1687.806612 from(563.609480,3+)

Title: 091008RatKidney_NoSalt_27.1982.1982.3.dta

Data file K:INewmanPaper|Piliang|3SubProteomes\Piliang3SP\mgf5ppm\ERLIC_3SubProteomes5ppm.mgf

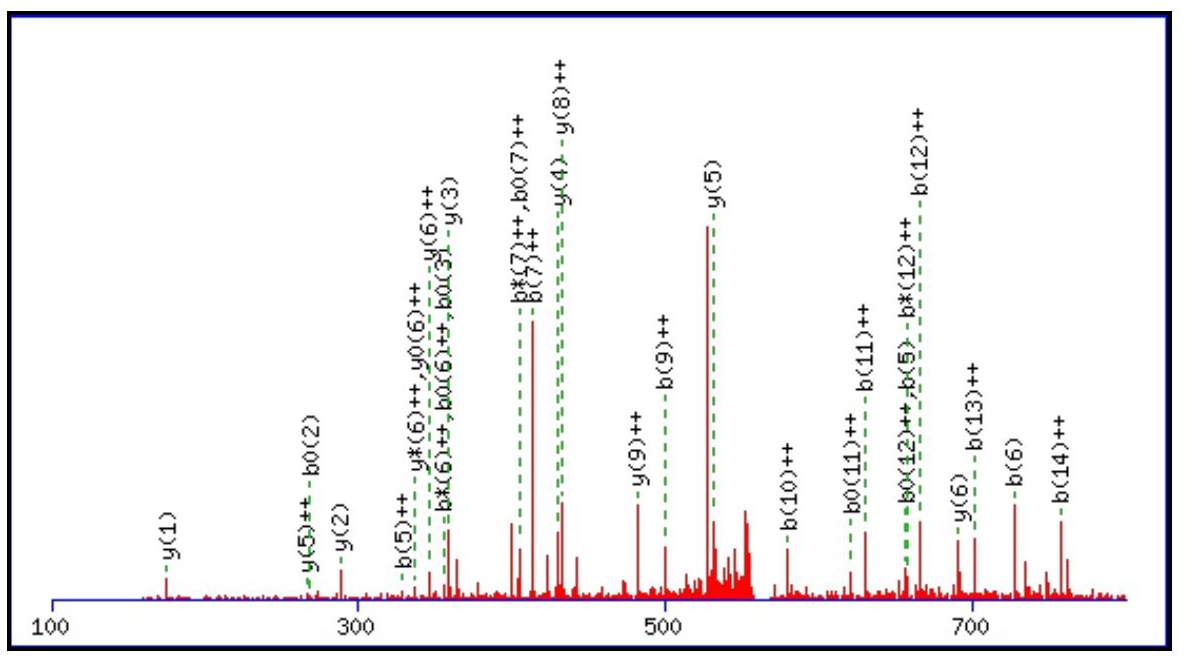



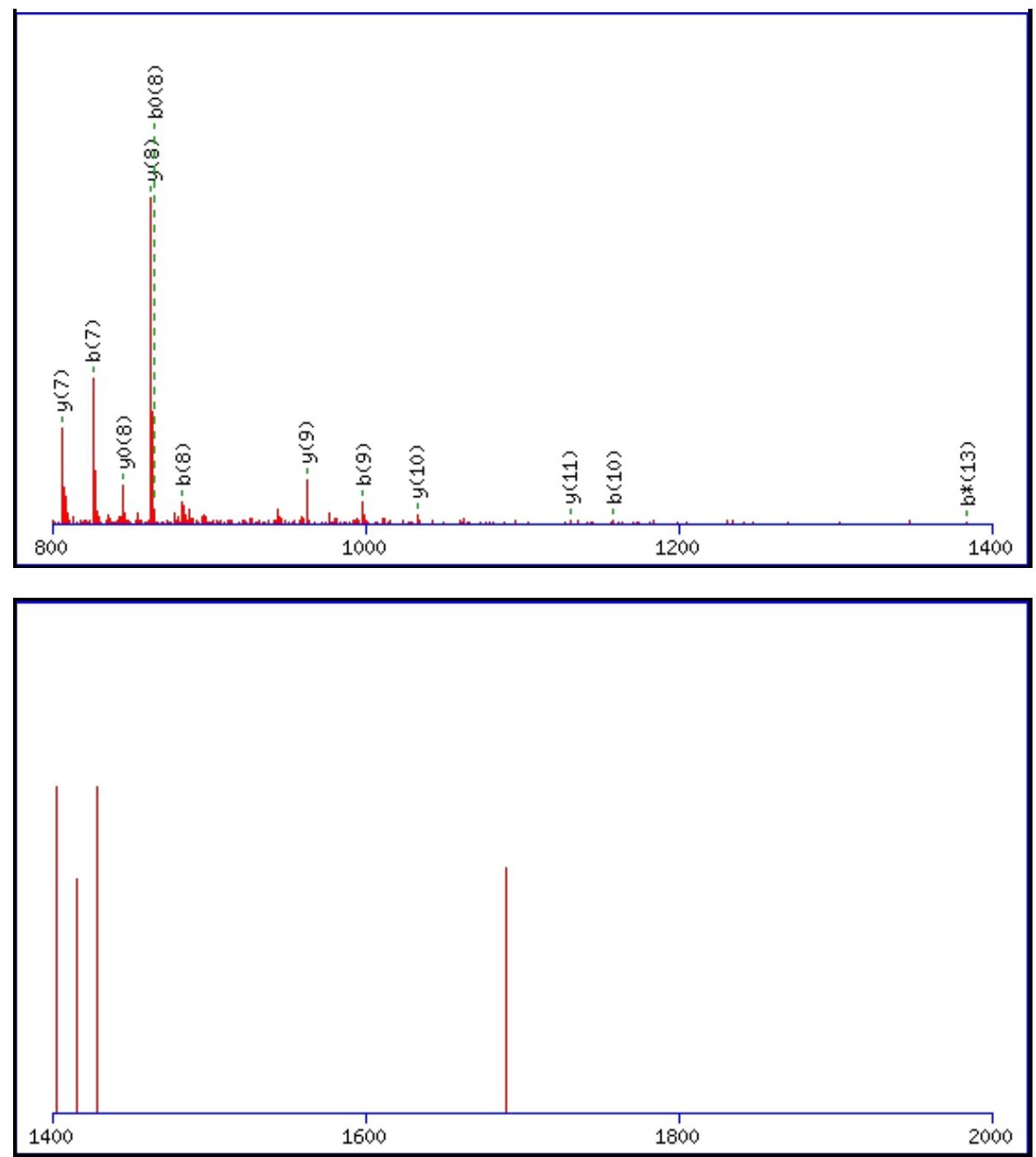

Monoisotopic mass of neutral peptide $\operatorname{Mr}($ calc): 1687.8100

Fixed modifications: Carbamidomethyl (C)

Variable modifications:

N9 : Deamidated_N (N)

Ions Score: 59 Expect: 0.00022

Matches (Bold Red): 43/158 fragment ions using 79 most intense peaks

\begin{tabular}{|c|c|c|c|c|c|c|c|c|c|c|c|c|c|c|}
\hline \# & $\mathbf{b}$ & $\mathbf{b}^{++}$ & $\mathbf{b}^{*}$ & $\mathbf{b}^{*^{++}}$ & $\mathbf{b}^{0}$ & $\mathbf{b}^{0++}$ & Seq. & $\mathbf{y}$ & $y^{++}$ & $\mathbf{y}^{*}$ & $\mathbf{y}^{*^{++}}$ & $\mathbf{y}^{0}$ & $\mathbf{y}^{0++}$ & \# \\
\hline 1 & 130.0499 & 65.5286 & & & 112.0393 & 56.5233 & $\mathbf{E}$ & & & & & & & 15 \\
\hline 2 & 286.1510 & 143.5791 & 269.1244 & 135.0659 & 268.1404 & 134.5738 & $\mathbf{R}$ & 1559.7747 & 780.3910 & 1542.7482 & 771.8777 & 1541.7642 & 771.3857 & 14 \\
\hline 3 & 373.1830 & 187.0951 & 356.1565 & 178.5819 & 355.1724 & 178.0899 & S & 1403.6736 & 702.3404 & 1386.6471 & 693.8272 & 1385.6630 & 693.3352 & 13 \\
\hline 4 & 559.2623 & 280.1348 & 542.2358 & 271.6215 & 541.2518 & 271.1295 & $\mathbf{W}$ & 1316.6416 & 658.8244 & 1299.6150 & 650.3112 & 1298.6310 & 649.8191 & 12 \\
\hline 5 & 656.3151 & 328.6612 & 639.2885 & 320.1479 & 638.3045 & 319.6559 & $\mathbf{P}$ & 1130.5623 & 565.7848 & 1113.5357 & 557.2715 & 1112.5517 & 556.7795 & 11 \\
\hline 6 & 727.3522 & 364.1797 & 710.3257 & 355.6665 & 709.3416 & 355.1745 & A & 1033.5095 & 517.2584 & 1016.4830 & 508.7451 & 1015.4989 & 508.2531 & 10 \\
\hline 7 & 826.4206 & 413.7139 & 809.3941 & 405.2007 & 808.4100 & 404.7087 & $\mathbf{V}$ & 962.4724 & 481.7398 & 945.4458 & 473.2266 & 944.4618 & 472.7345 & 9 \\
\hline 8 & 883.4421 & 442.2247 & 866.4155 & 433.7114 & 865.4315 & 433.2194 & $\mathbf{G}$ & 863.4040 & 432.2056 & 846.3774 & 423.6923 & 845.3934 & 423.2003 & 8 \\
\hline 9 & 998.4690 & 499.7381 & 981.4425 & 491.2249 & 980.4584 & 490.7329 & $\mathbf{N}$ & 806.3825 & 403.6949 & 789.3560 & 395.1816 & 788.3719 & 394.6896 & 7 \\
\hline 10 & 1158.4997 & 579.7535 & 1141.4731 & 571.2402 & 1140.4891 & 570.7482 & $\mathrm{C}$ & 691.3556 & 346.1814 & 674.3290 & 337.6681 & 673.3450 & 337.1761 & 6 \\
\hline 11 & 1259.5473 & 630.2773 & 1242.5208 & 621.7640 & 1241.5368 & 621.2720 & $\mathbf{T}$ & 531.3249 & 266.1661 & 514.2984 & 257.6528 & 513.3144 & 257.1608 & 5 \\
\hline 12 & 1330.5845 & 665.7959 & 1313.5579 & 657.2826 & 1312.5739 & 656.7906 & A & 430.2772 & 215.6423 & 413.2507 & 207.1290 & & & 4 \\
\hline 13 & 1401.6216 & 701.3144 & 1384.5950 & 692.8011 & 1383.6110 & 692.3091 & A & 359.2401 & 180.1237 & 342.2136 & 171.6104 & & & 3 \\
\hline 14 & 1514.7056 & 757.8565 & 1497.6791 & 749.3432 & 1496.6951 & 748.8512 & $\mathbf{L}$ & 288.2030 & 144.6051 & 271.1765 & 136.0919 & & & 2 \\
\hline 15 & & & & & & & $\mathbf{R}$ & 175.1190 & 88.0631 & 158.0924 & 79.5498 & & & 1 \\
\hline
\end{tabular}



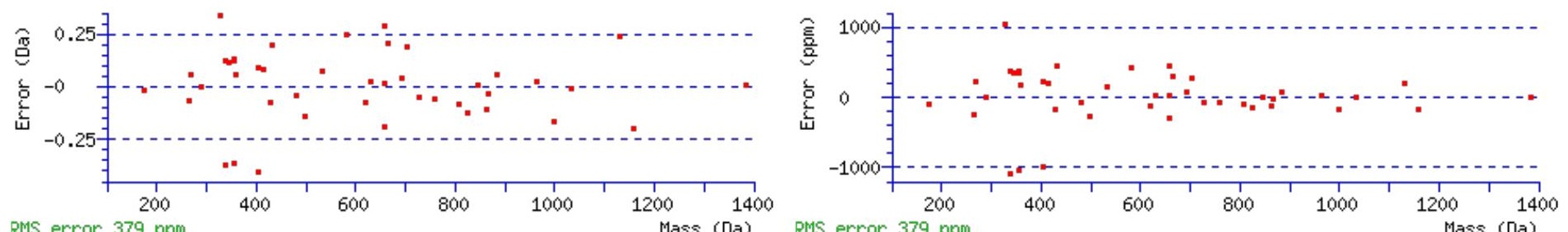

RMS error 379 ppm Mass (Da) RNS error $379 \mathrm{ppm}$

\section{All matches to this query}

\begin{tabular}{|l|l|l|l|}
\hline Score & Mr(calc): & Delta & \multicolumn{1}{c|}{ Sequence } \\
\hline 59.4 & 1687.8100 & -0.0034 & ERSWPAVGNCTAALR \\
\hline 14.0 & 1685.8025 & 2.0041 & QLTYEKVDLNNIR \\
\hline 14.0 & 1685.8025 & 2.0041 & QLTYEKVDLNNIR \\
\hline 8.0 & 1687.7917 & 0.0149 & $\underline{\text { HGHSRNGQAHVPRR }}$ \\
\hline 6.1 & 1686.8025 & 1.0041 & $\underline{\text { ICGVDRTQYRAIR }}$ \\
\hline 6.0 & 1685.8025 & 2.0041 & QLTYEKVDLNNIR \\
\hline 6.0 & 1685.8025 & 2.0041 & QLTYEKVDLNNIR \\
\hline 4.9 & 1686.7978 & 1.0088 & DGIFANSASSKLLER \\
\hline 4.3 & 1686.8198 & 0.9868 & VSMLKMDERTLLR \\
\hline 3.9 & 1687.7910 & 0.0157 & VLDAESMHDCVSVVK \\
\hline
\end{tabular}

Spectrum No: 542; Query: 2328; Rank: 1

\section{Peptide View}

MS/MS Fragmentation of VLASLDSAQHFLTSNLSSIVIGETK

Found in IPI00325312, Tax_Id=10116 Gene_Symbol=Prom 1 prominin 1 isoform 1

Match to Query 2328: 2630.379342 from(877.800390,3+)

Title: 091008RatKidney_NH4Format01_24.4550.4550.3.dta

Data file K:\NewmanPaper \Piliang \3SubProteomes\Piliang3SP \mgf5ppm\ERLIC_3SubProteomes5ppm.mgf

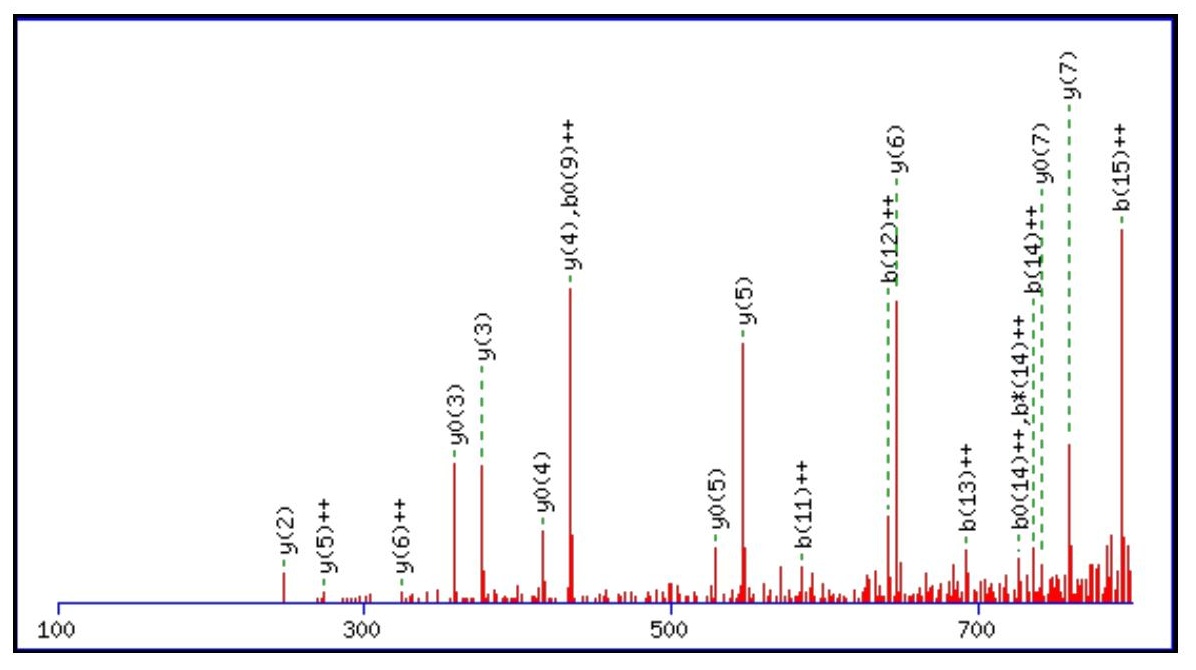



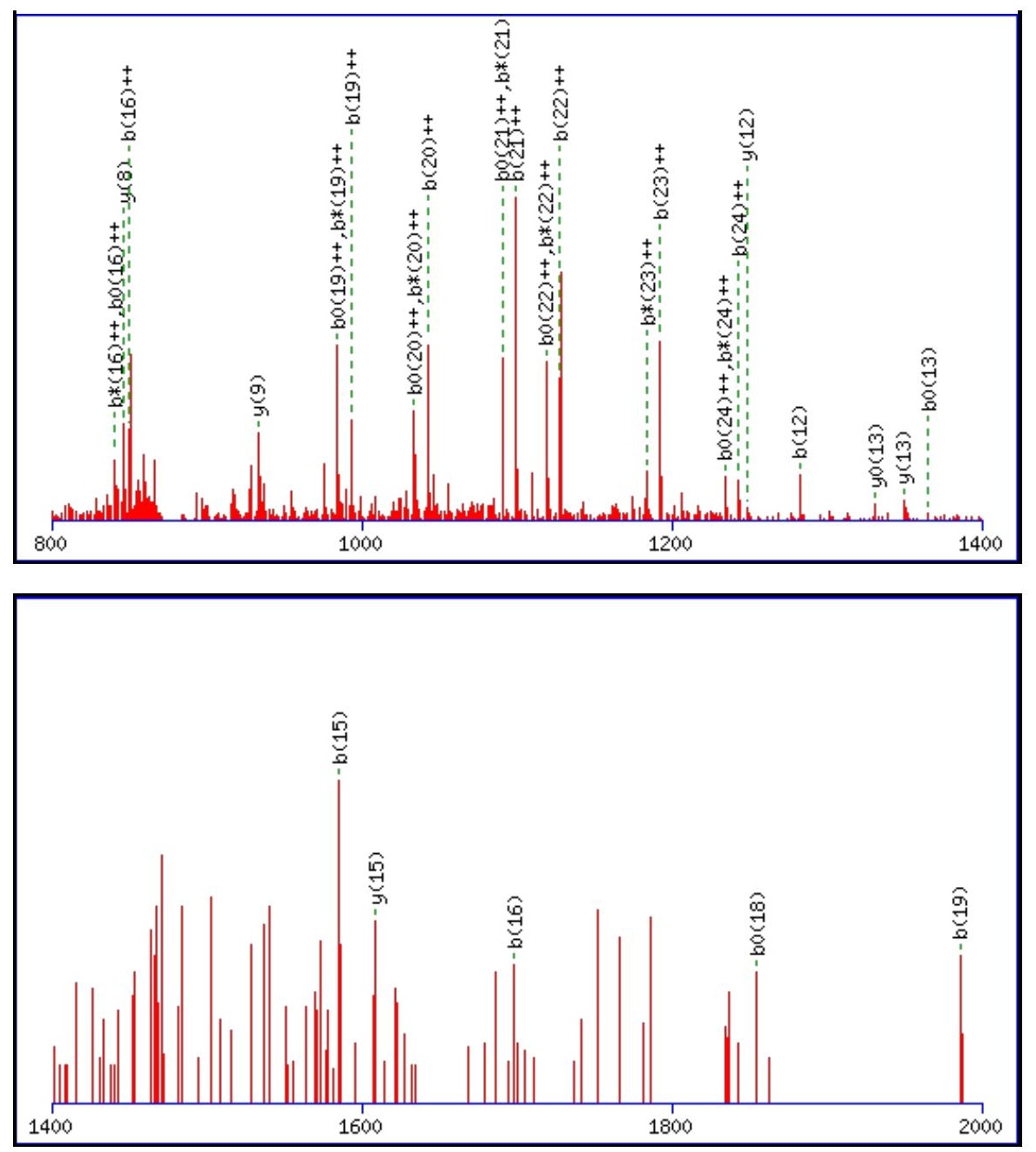

Monoisotopic mass of neutral peptide $\operatorname{Mr}($ calc): 2630.3803

Fixed modifications: Carbamidomethyl (C)

Variable modifications:

N15 : Deamidated_N (N)

Ions Score: 59 Expect: 0.00013

Matches (Bold Red): 52/264 fragment ions using 89 most intense peaks

\begin{tabular}{|c|c|c|c|c|c|c|c|c|c|c|c|c|c|c|}
\hline \# & b & $\mathbf{b}^{++}$ & b* & $\mathbf{b}^{*^{++}}$ & $\mathbf{b}^{\mathbf{0}}$ & $\mathbf{b}^{0++}$ & Seq. & $\mathbf{y}$ & $\mathbf{y}^{++}$ & $\mathbf{y}^{*}$ & $\mathrm{y}^{*^{++}}$ & $\mathbf{y}^{\mathbf{0}}$ & $\mathbf{y}^{0++}$ & \# \\
\hline 1 & 100.0757 & 50.5415 & & & & & $\mathbf{V}$ & & & & & & & 25 \\
\hline 2 & 213.1598 & 107.0835 & & & & & $\mathbf{L}$ & 2532.3192 & 1266.6632 & 2515.2926 & 1258.1499 & 2514.3086 & 1257.6579 & 24 \\
\hline 3 & 284.1969 & 142.6021 & & & & & A & 2419.2351 & 1210.1212 & 2402.2086 & 1201.6079 & 2401.2245 & 1201.1159 & 23 \\
\hline 4 & 371.2289 & 186.1181 & & & 353.2183 & 177.1128 & S & 2348.1980 & 1174.6026 & 2331.1714 & 1166.0894 & 2330.1874 & 1165.5974 & 22 \\
\hline 5 & 484.3130 & 242.6601 & & & 466.3024 & 233.6548 & $\mathbf{L}$ & 2261.1660 & 1131.0866 & 2244.1394 & 1122.5733 & 2243.1554 & 1122.0813 & 21 \\
\hline 6 & 599.3399 & 300.1736 & & & 581.3293 & 291.1683 & D & 2148.0819 & 1074.5446 & 2131.0553 & 1066.0313 & 2130.0713 & 1065.5393 & 20 \\
\hline 7 & 686.3719 & 343.6896 & & & 668.3614 & 334.6843 & S & 2033.0550 & 1017.0311 & 2016.0284 & 1008.5178 & 2015.0444 & 1008.0258 & 19 \\
\hline 8 & 757.4090 & 379.2082 & & & 739.3985 & 370.2029 & A & 1946.0229 & 973.5151 & 1928.9964 & 965.0018 & 1928.0124 & 964.5098 & 18 \\
\hline 9 & 885.4676 & 443.2374 & 868.4411 & 434.7242 & 867.4571 & 434.2322 & $\mathbf{Q}$ & 1874.9858 & 937.9965 & 1857.9593 & 929.4833 & 1856.9752 & 928.9913 & 17 \\
\hline 10 & 1022.5265 & 511.7669 & 1005.5000 & 503.2536 & 1004.5160 & 502.7616 & $\mathbf{H}$ & 1746.9272 & 873.9673 & 1729.9007 & 865.4540 & 1728.9167 & 864.9620 & 16 \\
\hline 11 & 1169.5949 & 585.3011 & 1152.5684 & 576.7878 & 1151.5844 & 576.2958 & $\mathbf{F}$ & 1609.8683 & 805.4378 & 1592.8418 & 796.9245 & 1591.8578 & 796.4325 & 15 \\
\hline 12 & 1282.6790 & 641.8431 & 1265.6525 & 633.3299 & 1264.6684 & 632.8379 & $\mathbf{L}$ & 1462.7999 & 731.9036 & 1445.7734 & 723.3903 & 1444.7893 & 722.8983 & 14 \\
\hline 13 & 1383.7267 & 692.3670 & 1366.7001 & 683.8537 & 1365.7161 & 683.3617 & $\mathbf{T}$ & 1349.7158 & 675.3616 & 1332.6893 & 666.8483 & 1331.7053 & 666.3563 & 13 \\
\hline 14 & 1470.7587 & 735.8830 & 1453.7322 & 727.3697 & 1452.7482 & 726.8777 & $\mathrm{~S}$ & 1248.6682 & 624.8377 & 1231.6416 & 616.3244 & 1230.6576 & 615.8324 & 12 \\
\hline 15 & 1585.7857 & 793.3965 & 1568.7591 & 784.8832 & 1567.7751 & 784.3912 & $\mathbf{N}$ & 1161.6361 & 581.3217 & 1144.6096 & 572.8084 & 1143.6256 & 572.3164 & 11 \\
\hline 16 & 1698.8697 & 849.9385 & 1681.8432 & 841.4252 & 1680.8592 & 840.9332 & $\mathbf{L}$ & 1046.6092 & 523.8082 & 1029.5827 & 515.2950 & 1028.5986 & 514.8030 & 10 \\
\hline 17 & 1785.9017 & 893.4545 & 1768.8752 & 884.9412 & 1767.8912 & 884.4492 & S & 933.5251 & 467.2662 & 916.4986 & 458.7529 & 915.5146 & 458.2609 & 9 \\
\hline 18 & 1872.9338 & 936.9705 & 1855.9072 & 928.4573 & 1854.9232 & 927.9652 & $S$ & 846.4931 & 423.7502 & 829.4666 & 415.2369 & 828.4825 & 414.7449 & 8 \\
\hline
\end{tabular}




\begin{tabular}{|c|c|c|c|c|c|c|c|c|c|c|c|c|c|c|}
\hline 19 & |1986.0178 & 993.5126 & 1968. & 984.9993 & |1968.0073| & 984.5073 & I & 759.4611| & 2342 & 4345 & & & & \\
\hline 20 & 2085.0863 & \begin{tabular}{|l|}
1043.0468 \\
\end{tabular} & 2068.0597 & 1034.5335 & 2067.0757 & 1034.0415 & V & 646.3770 & 323.6921 & 629.3505 & 315.1789 & 628.3665 & 314.6869 & \\
\hline 21 & 2198.1703 & 1099.5888 & 2181.1438 & 1091.0755 & 2180.1598 & 1090.5835 & I & 547.3086 & 274.1579 & 530.2821 & 265.6447 & 529.2980 & 265.1527 & \\
\hline 22 & 2255.1918 & 1128.0995 & 2238.1652 & 1119.5863 & 2237.1812 & 1119.0942 & G & 434.2245 & 217.6159 & 417.1980 & 209.1026 & 416.2140 & 208.6106 & \\
\hline 23 & 2384.2344 & 1192.6208 & 2367.2078 & 1184.1076 & 2366.2238 & 1183.6155 & $\mathbf{E}$ & 377.2031 & 189.1052 & 360.1765 & 180.5919 & 359.1925 & 180.0999 & \\
\hline 24 & 2485.2821 & 1243.1447 & 2468.2555 & 1234.6314 & 2467.2715 & 1234.1394 & $T$ & 248.1605 & 124.5839 & 231.1339 & 116.0706 & 230.1499 & 115.5786 & \\
\hline 25 & & & & & & & $\mathbf{K}$ & 147.1128 & 74.0600 & 130.0863 & 65.5468 & & & \\
\hline
\end{tabular}
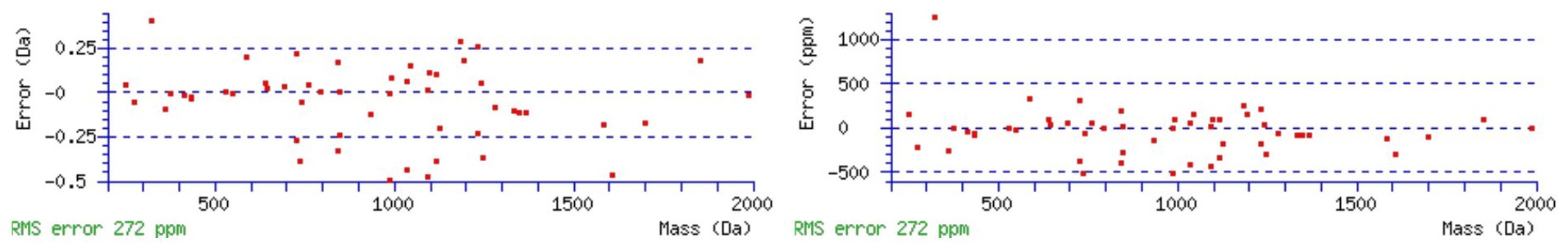

\section{All matches to this query}

\begin{tabular}{|l|l|l|l|}
\hline Score & Mr(calc): & Delta & \multicolumn{1}{c|}{ Sequence } \\
\hline 59.4 & 2630.3803 & -0.0010 & VLASLDSAQHFLTSNLSSIVIGETK \\
\hline 14.9 & 2629.3963 & 0.9831 & VLASLDSAOHFLTSNLSSIVIGETK \\
\hline 2.7 & 2628.3903 & 1.9891 & YVAICFPLRYTTILTHQIVIK \\
\hline 0.4 & 2629.3922 & 0.9871 & EAVSKEDLARATLVTITNNIGSVAR \\
\hline
\end{tabular}

Spectrum No: 543; Query: 2844; Rank: 1

\section{Peptide View}

MS/MS Fragmentation of IIGVDPEGSILAEPEELNQTEQTAYEVEGIGYDFIPTVLDR

Found in IPI00214299, Tax_Id=10116 Gene_Symbol=Cbs Isoform I of Cystathionine beta-synthase

Match to Query 2844: 4520.206936 from(1131.059010,4+)

Title: 091008RatKidney_NH4Format01_10.6231.6231.4.dta

Data file K:INewmanPaper|Piliang|3SubProteomes\Piliang3SP\mgf5ppm\ERLIC_3SubProteomes5ppm.mgf

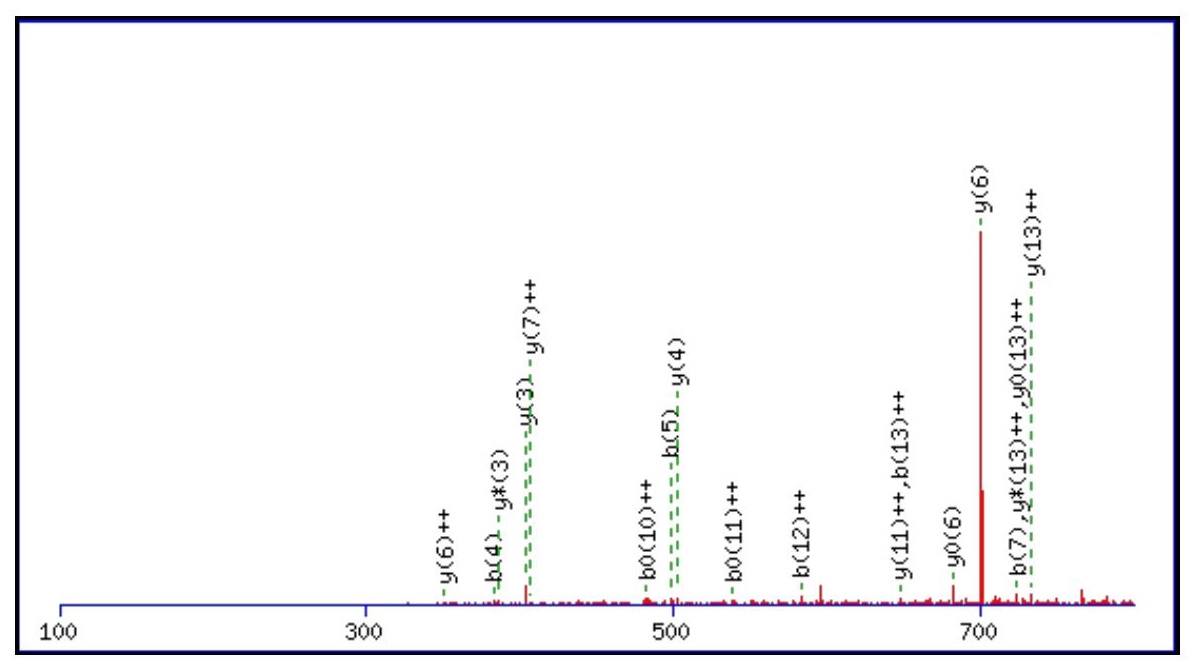



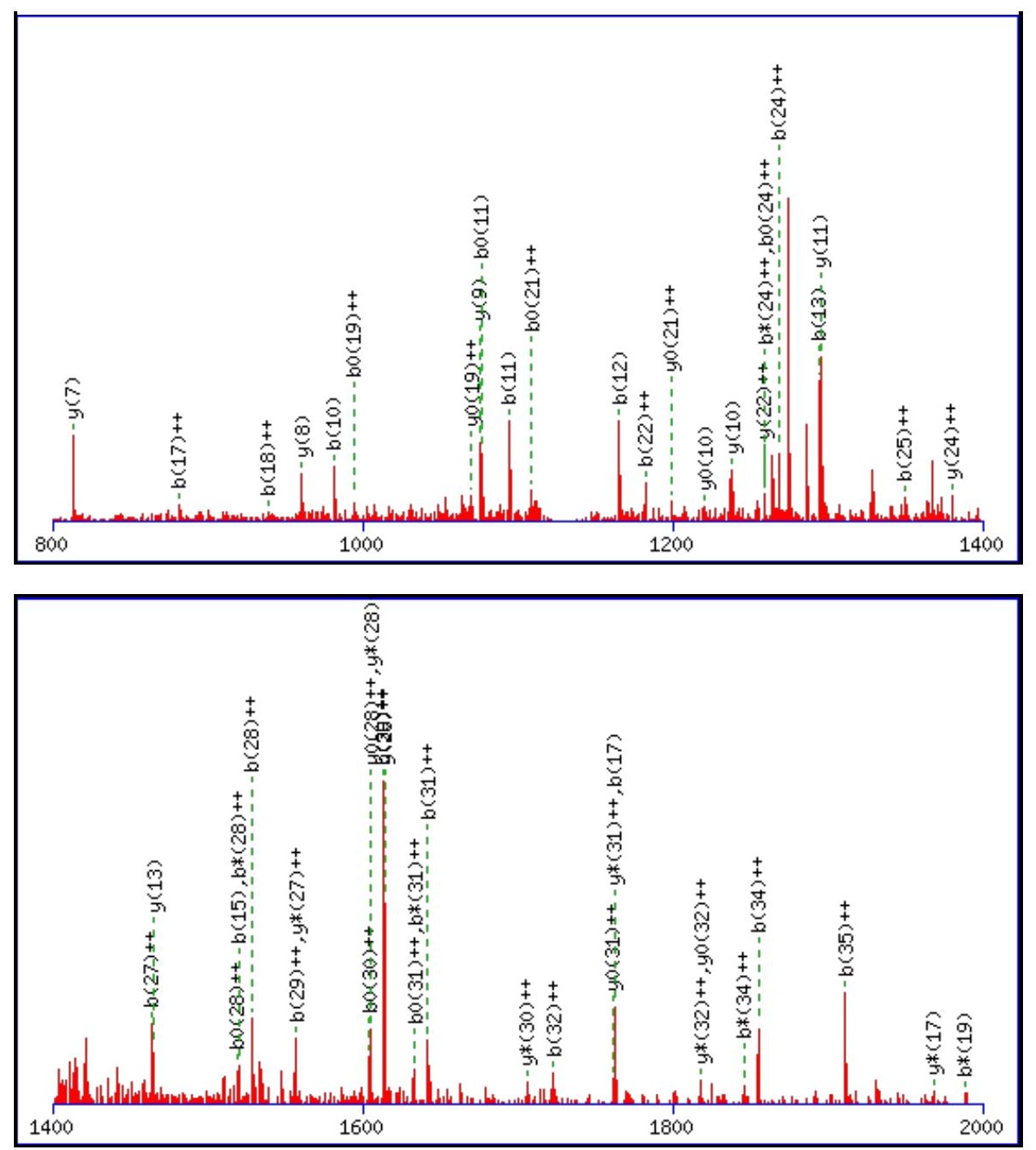

Monoisotopic mass of neutral peptide $\operatorname{Mr}($ calc): 4520.2010

Fixed modifications: Carbamidomethyl (C)

Variable modifications:

N18 : Deamidated_N (N)

Ions Score: 59 Expect: 0.00034

Matches (Bold Red): 71/436 fragment ions using 124 most intense peaks

\begin{tabular}{|c|c|c|c|c|c|c|c|c|c|c|c|c|c|c|}
\hline \# & b & $\mathbf{b}^{++}$ & b* & $\mathbf{b}^{*^{++}}$ & $\mathbf{b}^{0}$ & $\mathbf{b}^{0++}$ & Seq. & $\mathbf{y}$ & $\mathbf{y}^{++}$ & $\mathbf{y}^{*}$ & $\mathrm{y}^{\mathrm{*}^{++}}$ & $\mathbf{y}^{\mathbf{0}}$ & $\mathbf{y}^{0++}$ & \# \\
\hline 1 & 114.0913 & 57.5493 & & & & & I & & & & & & & 41 \\
\hline 2 & 227.1754 & 114.0913 & & & & & I & 4408.1242 & 2204.5658 & 4391.0977 & 2196.0525 & 4390.1137 & 2195.5605 & 40 \\
\hline 3 & 284.1969 & 142.6021 & & & & & G & 4295.0402 & 2148.0237 & 4278.0136 & 2139.5104 & 4277.0296 & 2139.0184 & 439 \\
\hline 4 & 383.2653 & 192.1363 & & & & & $\mathbf{V}$ & 4238.0187 & 2119.5130 & 4220.9922 & 2110.9997 & 4220.0081 & 2110.5077 & 738 \\
\hline 5 & 498.2922 & 249.6498 & & & 480.2817 & 240.6445 & D & 4138.9503 & 2069.9788 & 4121.9237 & 2061.4655 & 4120.9397 & 2060.9735 & 37 \\
\hline 6 & 595.3450 & 298.1761 & & & 577.3344 & 289.1709 & $\mathbf{P}$ & 4023.9233 & 2012.4653 & 4006.8968 & 2003.9520 & 4005.9128 & 2003.4600 & 36 \\
\hline 7 & 724.3876 & 362.6974 & & & 706.3770 & 353.6921 & $\mathbf{E}$ & 3926.8706 & 1963.9389 & 3909.8440 & 1955.4257 & 3908.8600 & 1954.9336 & 35 \\
\hline 8 & 781.4090 & 391.2082 & & & 763.3985 & 382.2029 & G & 3797.8280 & 1899.4176 & 3780.8014 & 1890.9044 & 3779.8174 & 1890.4123 & 34 \\
\hline 9 & 868.4411 & 434.7242 & & & 850.4305 & 425.7189 & S & 3740.8065 & 1870.9069 & 3723.7800 & 1862.3936 & 3722.7960 & 1861.9016 & 33 \\
\hline 10 & 981.5251 & 491.2662 & & & 963.5146 & 482.2609 & I & 3653.7745 & 1827.3909 & 3636.7479 & 1818.8776 & 3635.7639 & 1818.3856 & 32 \\
\hline 11 & 1094.6092 & 547.8082 & & & 1076.5986 & 538.8030 & $\mathbf{L}$ & 3540.6904 & 1770.8489 & 3523.6639 & 1762.3356 & 3522.6799 & 1761.8436 & 31 \\
\hline 12 & 1165.6463 & 583.3268 & & & 1147.6358 & 574.3215 & A & 3427.6064 & 1714.3068 & 3410.5798 & 1705.7935 & 3409.5958 & 1705.3015 & 30 \\
\hline 13 & 1294.6889 & 647.8481 & & & 1276.6783 & 638.8428 & $\mathbf{E}$ & 3356.5693 & 1678.7883 & 3339.5427 & 1670.2750 & 3338.5587 & 1669.7830 & 29 \\
\hline 14 & 1391.7417 & 696.3745 & & & 1373.7311 & 687.3692 & $\mathbf{P}$ & 3227.5267 & 1614.2670 & 3210.5001 & 1605.7537 & 3209.5161 & 1605.2617 & 28 \\
\hline 15 & 1520.7843 & 760.8958 & & & 1502.7737 & 751.8905 & $\mathbf{E}$ & 3130.4739 & 1565.7406 & 3113.4473 & 1557.2273 & 3112.4633 & 1556.7353 & 27 \\
\hline 16 & 1649.8269 & 825.4171 & & & 1631.8163 & 816.4118 & $\mathbf{E}$ & 3001.4313 & 1501.2193 & 2984.4048 & 1492.7060 & 2983.4207 & 1492.2140 & 26 \\
\hline 17 & 1762.9109 & 881.9591 & & & 1744.9004 & 872.9538 & $\mathbf{L}$ & 2872.3887 & 1436.6980 & 2855.3622 & 1428.1847 & 2854.3781 & 1427.6927 & 25 \\
\hline 18 & 1877.9379 & 939.4726 & 1860.9113 & 930.9593 & 1859.9273 & 930.4673 & $\mathbf{N}$ & 2759.3046 & 1380.1560 & 2742.2781 & 1371.6427 & 2741.2941 & 1371.1507 & 24 \\
\hline
\end{tabular}




\begin{tabular}{|c|c|c|c|c|c|c|c|c|c|c|c|c|c|c|}
\hline 19 & 2005.9964 & |1003.5019 & & 994.9886 & |1987.9859 & 994.4966 & $\mathbf{Q}$ & & & |2627.2512| & & |2626.2671 & |1313.6372 & 23 \\
\hline 20 & 2107.0441 & 1054.0257 & 2090.0176 & 1045.5124 & 2089.0336 & 1045.0204 & $T$ & 2516.2191 & 1258.6132 & 2499.1926 & 1250.0999 & 2498.2086 & 1249.6079 & 22 \\
\hline 21 & 2236.0867 & 1118.5470 & 2219.0602 & 1110.0337 & 2218.0761 & 1109.5417 & $\mathbf{E}$ & 2415.1715 & 1208.0894 & 2398.1449 & 1199.5761 & 2397.1609 & 1199.0841 & 21 \\
\hline 22 & 2364.1453 & 1182.5763 & 2347.1187 & 1174.0630 & 2346.1347 & 1173.5710 & $\mathbf{Q}$ & 2286.1289 & 1143.5681 & 2269.1023 & 1135.0548 & 2268.1183 & 1134.5628 & 20 \\
\hline 23 & 2465.1930 & 1233.1001 & 2448.1664 & 1224.5868 & 2447.1824 & 1224.0948 & $\mathbf{T}$ & 2158.0703 & 1079.5388 & |2141.0437 & 1071.0255 & 2140.0597 & 1070.5335 & 19 \\
\hline 24 & 2536.2301 & 1268.6187 & 2519.2035 & 1260.1054 & 2518.2195 & $\mid 1259.6134$ & A & 2057.0226 & 1029.0149 & 2039.9961 & 1020.5017 & 2039.0120 & 1020.0097 & 18 \\
\hline 25 & 2699.2934 & 1350.1503 & 2682.2669 & 1341.6371 & 2681.2828 & 1341.1451 & $\mathbf{Y}$ & 1985.9855 & 993.4964 & 1968.9589 & 984.9831 & 1967.9749 & 984.4911 & 17 \\
\hline 26 & 2828.3360 & 1414.6716 & 2811.3095 & 1406.1584 & 2810.3254 & 1405.6664 & E & 1822.9222 & 911.9647 & 1805.8956 & 903.4514 & 1804.9116 & 902.9594 & 16 \\
\hline 27 & 2927.4044 & 1464.2058 & 2910.3779 & 1455.6926 & 2909.3939 & 1455.2006 & V & 1693.8796 & 847.4434 & 1676.8530 & 838.9301 & 1675.8690 & 838.4381 & 15 \\
\hline 28 & 3056.4470 & 1528.7271 & 3039.4205 & 1520.2139 & 3038.4364 & 1519.7219 & E & 1594.8112 & 797.9092 & 1577.7846 & 789.3959 & 1576.8006 & 788.9039 & 14 \\
\hline 29 & 3113.4685 & 1557.2379 & 3096.4419 & 1548.7246 & 3095.4579 & 1548.2326 & G & 1465.7686 & 733.3879 & 1448.7420 & 724.8746 & 1447.7580 & 724.3826 & 13 \\
\hline 30 & 3226.5525 & 1613.7799 & 3209.5260 & 1605.2666 & 3208.5420 & 1604.7746 & I & 1408.7471 & 704.8772 & 1391.7205 & 696.3639 & 1390.7365 & 695.8719 & 12 \\
\hline 31 & 3283.5740 & 1642.2906 & 3266.5475 & 1633.7774 & 3265.5634 & 1633.2854 & G & 1295.6630 & 648.3352 & 1278.6365 & 639.8219 & 1277.6525 & 639.3299 & 11 \\
\hline 32 & 3446.6373 & 1723.8223 & 3429.6108 & 1715.3090 & 3428.6268 & 1714.8170 & $\mathbf{Y}$ & 1238.6416 & 619.8244 & 1221.6150 & 611.3111 & 1220.6310 & 610.8191 & 10 \\
\hline 33 & 3561.6643 & 1781.3358 & 3544.6377 & 1772.8225 & 3543.6537 & 1772.3305 & D & 1075.5782 & 538.2928 & 1058.5517 & 529.7795 & 1057.5677 & 529.2875 & 9 \\
\hline 34 & 3708.7327 & 1854.8700 & 3691.7061 & 1846.3567 & 3690.7221 & 1845.8647 & $\mathbf{F}$ & 960.5513 & 480.7793 & 943.5247 & 472.2660 & 942.5407 & 471.7740 & 8 \\
\hline 35 & 3821.8168 & 1911.4120 & 3804.7902 & 1902.8987 & 3803.8062 & 1902.4067 & I & 813.4829 & 407.2451 & 796.4563 & 398.7318 & 795.4723 & 398.2398 & 7 \\
\hline 36 & 3918.8695 & 1959.9384 & 3901.8430 & 1951.4251 & 3900.8590 & 1950.9331 & $\mathbf{P}$ & 700.3988 & 350.7030 & 683.3723 & 342.1898 & 682.3883 & 341.6978 & 6 \\
\hline 37 & 4019.9172 & 2010.4622 & 4002.8906 & 2001.9490 & 4001.9066 & 2001.4570 & $\mathrm{~T}$ & 603.3461 & 302.1767 & 586.3195 & 293.6634 & 585.3355 & 293.1714 & 5 \\
\hline 38 & 4118.9856 & 2059.9964 & 4101.9591 & 2051.4832 & 4100.9750 & 2050.9912 & V & 502.2984 & 251.6528 & 485.2718 & 243.1395 & 484.2878 & 242.6475 & 4 \\
\hline 39 & 4232.0697 & 2116.5385 & 4215.0431 & 2108.0252 & 4214.0591 & 2107.5332 & $\mathbf{L}$ & 403.2300 & 202.1186 & 386.2034 & 193.6053 & 385.2194 & 193.1133 & 3 \\
\hline 40 & 4347.0966 & 2174.0519 & 4330.0701 & 2165.5387 & 4329.0861 & 2165.0467 & D & 290.1459 & 145.5766 & 273.1193 & 137.0633 & 272.1353 & 136.5713 & 2 \\
\hline 41 & & & & & & & $\mathbf{R}$ & 175.1190 & 88.0631 & 158.0924 & 79.5498 & & & 1 \\
\hline
\end{tabular}
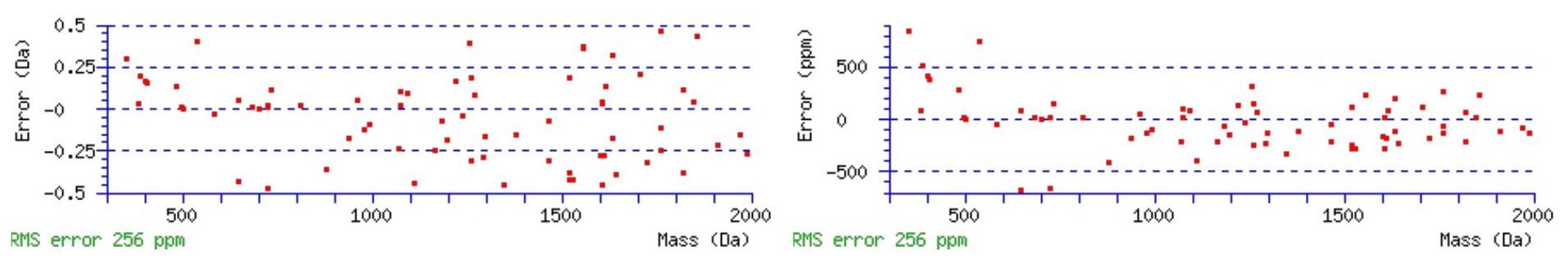

\section{All matches to this query}

\begin{tabular}{|l|l|c|l|}
\hline Score & Mr(calc): & Delta & \multicolumn{1}{c|}{ Sequence } \\
\hline 59.2 & 4520.2010 & 0.0059 & IIGVDPEGSILAEPEELNQTEQTAYEVEGIGYDFIPTVLDR \\
\hline 49.6 & 4519.2170 & 0.9900 & IIGVDPEGSILAEPEELNQTEQTAYEVEGIGYDFIPTVLDR \\
\hline 3.9 & 4518.1662 & 2.0408 & VEVTQOVLQTLPEENYRVLHFLTAFLVQVSAHSYQNR \\
\hline 3.4 & 4520.2077 & -0.0007 & TVLDVGAGTGILSIFCAQAGARRVYAVEASAIWQQAQEVVR \\
\hline 3.2 & 4518.2205 & 1.9864 & QWFNLNSLLTGPELISDTYLALFLAOLQQEGYSIFVVK \\
\hline 2.8 & 4519.1973 & 1.0097 & NAVPTLILADLHSAEHLKSMAMDFIILHASEVSKTLEWK \\
\hline 2.8 & 4519.1973 & 1.0097 & NAVPTLILADLHSAEHLKSMAMDFILHASEVSKTLEWK \\
\hline 2.8 & 4519.1973 & 1.0097 & NAVPTLILADLHSAEHLKSMAMDFIILHASEVSKTLEWK \\
\hline 2.8 & 4518.1662 & 2.0408 & VEVTQQVLQTLPEENYRVLHFLTAFLVQVSAHSYQNR \\
\hline 2.6 & 4520.1867 & 0.0202 & AGGGNTGWLWMSSCNLGLPVLSISFLIWLLLAAPGAQAAGYK \\
\hline
\end{tabular}

Spectrum No: 544; Query: 2688; Rank: 1

\section{Peptide View}

MS/MS Fragmentation of SCGECIQAGPNCGWCTNTTFLQEGMPTSAR 
Found in IPI00191681, Tax_Id=10116 Gene_Symbol=Itgb1 Integrin beta-1 precursor

Match to Query 2688: 3406.394142 from(1136.471990,3+)

Title: 100101RatKid_NS_deglyco_22.3597.3597.3.dta

Data file K:INewmanPaper|Piliang|3SubProteomes\Piliang3SP\mgf5ppm\ERLIC_3SubProteomes5ppm.mgf
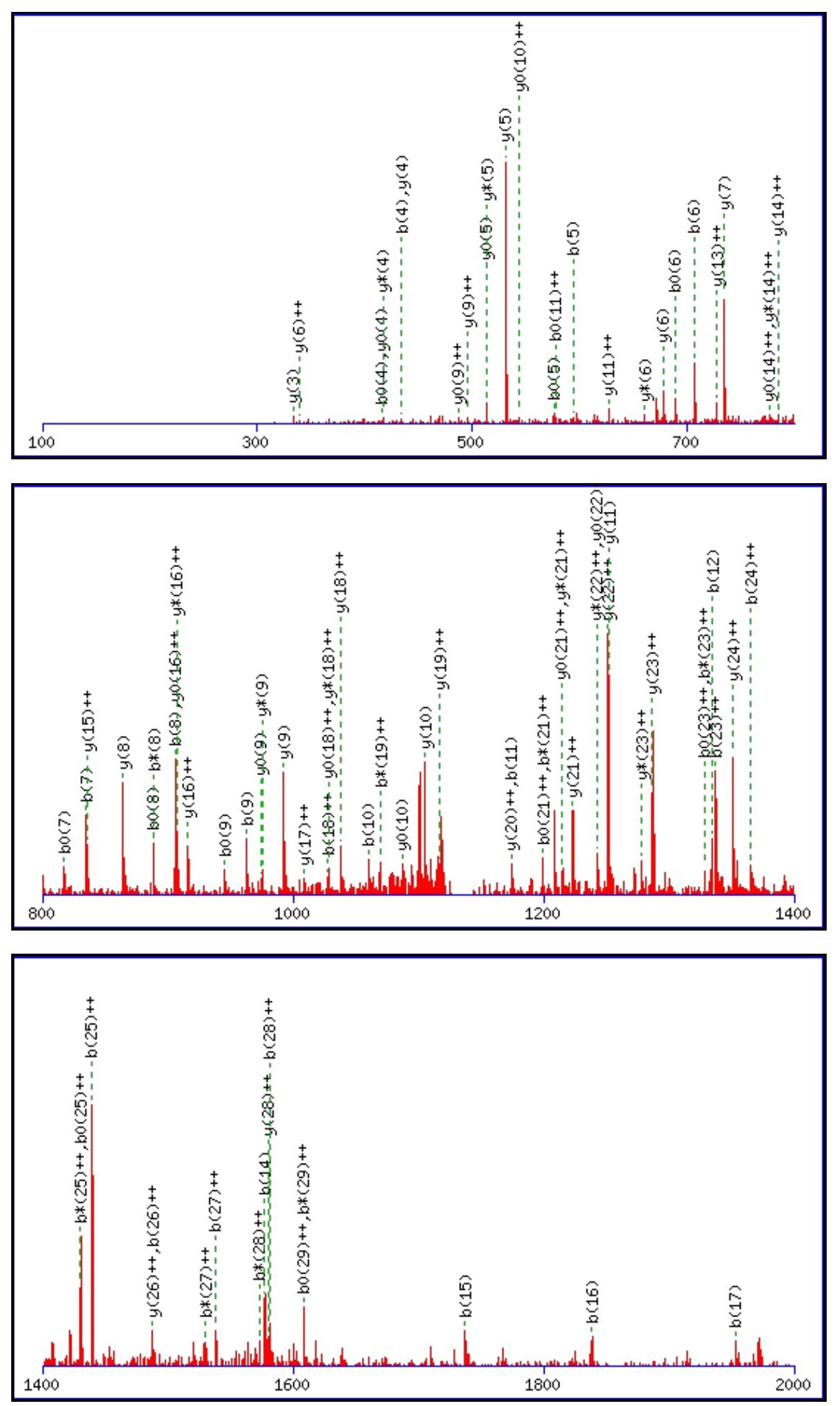

Monoisotopic mass of neutral peptide $\operatorname{Mr}($ calc): 3406.3781

Fixed modifications: Carbamidomethyl (C)

Variable modifications:

N17 : Deamidated_N (N)

M25 : Oxidation (M)

Ions Score: 59 Expect: 0.00047

Matches (Bold Red): 86/332 fragment ions using 172 most intense peaks

\begin{tabular}{|l|l|l|l|l|l|l|l|l|l|l|l|l|l|l|}
\hline$\#$ & $\mathbf{b}$ & $\mathbf{b}^{++}$ & $\mathbf{b}^{*}$ & $\mathbf{b}^{\boldsymbol{*}^{++}}$ & $\mathbf{b}^{\mathbf{0}}$ & $\mathbf{b}^{\mathbf{0}+}$ & Seq. & $\mathbf{y}$ & $\mathbf{y}^{++}$ & $\mathbf{y}^{*}$ & $\mathbf{y}^{*^{++}}$ & $\mathbf{y}^{\mathbf{0}}$ & $\mathbf{y}^{\mathbf{0}}$ & $\#$ \\
\hline
\end{tabular}




\begin{tabular}{|c|c|c|c|c|c|c|c|c|c|c|c|c|c|c|}
\hline & 88.0393 & 44.5233 & & & 70.0287 & 35.5180 & S & & & & & & & 30 \\
\hline 2 & 248.0700 & 124.5386 & & & 230.0594 & 115.5333 & $\mathrm{C}$ & 3320.3534 & 1660.6803 & 3303.3268 & 1652.1670 & 3302.3428 & 1651.6750 & 29 \\
\hline 3 & 305.0914 & 153.0493 & & & 287.0809 & 144.0441 & G & 3160.3227 & 1580.6650 & 3143.2962 & 1572.1517 & 3142.3122 & 1571.6597 & 28 \\
\hline 4 & 434.1340 & 217.5706 & & & 416.1234 & 208.5654 & $\mathbf{E}$ & 3103.3013 & 1552.1543 & 3086.2747 & 1543.6410 & 3085.2907 & $\mid 1543.1490$ & 27 \\
\hline 5 & 594.1647 & 297.5860 & & & 576.1541 & 288.5807 & $\mathrm{C}$ & 2974.2587 & 1487.6330 & 2957.2321 & 1479.1197 & 2956.2481 & 1478.6277| & 26 \\
\hline 6 & 707.2487 & 354.1280 & & & 689.2382 & 345.1227 & I & 2814.2280 & 1407.6176 & 2797.2015 & 1399.1044 & 2796.2174 & 1398.6124 & 25 \\
\hline 7 & 835.3073 & 418.1573 & 818.2808 & 409.6440 & 817.2967 & 409.1520 & $\mathbf{Q}$ & 2701.1439 & 1351.0756 & 2684.1174 & 1342.5623 & 2683.1334 & 1342.0703 & 24 \\
\hline 8 & 906.3444 & 453.6758 & 889.3179 & 445.1626 & 888.3338 & 444.6706 & A & 2573.0854 & \begin{tabular}{|l}
1287.0463 \\
\end{tabular} & 2556.0588 & 1278.5330 & 2555.0748 & 1278.0410 & 23 \\
\hline 9 & 963.3659 & 482.1866 & 946.3393 & 473.6733 & 945.3553 & 473.1813 & G & 2502.0483 & 1251.5278 & 2485.0217 & 1243.0145 & 2484.0377 & 1242.5225 & 22 \\
\hline 10 & 1060.4186 & 530.7130 & 1043.3921 & 522.1997 & 1042.4081 & 521.7077 & $\mathbf{P}$ & 2445.0268 & 1223.0170 & 2428.0002 & 1214.5038 & 2427.0162 & 1214.0118 & 21 \\
\hline 11 & 1174.4616 & 587.7344 & 1157.4350 & 579.2211 & 1156.4510 & 578.7291 & $\mathbf{N}$ & 2347.9740 & 1174.4907 & 2330.9475 & 1165.9774 & 2329.9635 & 1165.4854 & 20 \\
\hline 12 & \begin{tabular}{|l|}
1334.4922 \\
\end{tabular} & 667.7497 & 1317.4657 & 659.2365 & 1316.4817 & 658.7445 & $\mathrm{C}$ & 2233.9311 & 1117.4692 & 2216.9046 & 1108.9559 & 2215.9205 & 1108.4639 & 19 \\
\hline 13 & 1391.5137 & 696.2605 & 1374.4871 & 687.7472 & 1373.5031 & 687.2552 & G & 2073.9005 & 1037.4539 & 2056.8739 & 1028.9406 & 2055.8899 & 1028.4486 & 18 \\
\hline 14 & 1577.5930 & 789.3001 & 1560.5664 & 780.7869 & 1559.5824 & 780.2949 & $\mathbf{W}$ & 2016.8790 & 1008.9431 & 1999.8524 & 1000.4299 & 1998.8684 & 999.9379 & 17 \\
\hline 15 & 1737.6236 & 869.3155 & 1720.5971 & 860.8022 & 1719.6131 & 860.3102 & $\mathrm{C}$ & 1830.7997 & 915.9035 & 1813.7731 & 907.3902 & 1812.7891 & 906.8982 & 16 \\
\hline 16 & 1838.6713 & 919.8393 & 1821.6448 & 911.3260 & 1820.6608 & 910.8340 & $T$ & 1670.7690 & 835.8882 & 1653.7425 & 827.3749 & 1652.7585 & 826.8829 & 15 \\
\hline 17 & 1953.6983 & 977.3528 & 1936.6717 & 968.8395 & 1935.6877 & 968.3475 & $\mathbf{N}$ & 1569.7213 & 785.3643 & 1552.6948 & 776.8510 & 1551.7108 & 776.3590 & 14 \\
\hline 18 & 2054.7459 & 1027.8766 & 2037.7194 & 1019.3633 & 2036.7354 & 1018.8713 & $\mathbf{T}$ & 1454.6944 & 727.8508 & 1437.6679 & 719.3376 & 1436.6838 & 718.8456 & 13 \\
\hline 19 & 2155.7936 & 1078.4004 & 2138.7671 & \begin{tabular}{|l}
1069.8872 \\
\end{tabular} & 2137.7831 & 1069.3952 & $\mathbf{T}$ & 1353.6467 & 677.3270 & 1336.6202 & 668.8137 & 1335.6362 & 668.3217 & 12 \\
\hline 20 & 2302.8620 & 1151.9347 & 2285.8355 & 1143.4214 & 2284.8515 & 1142.9294 & $\mathbf{F}$ & 1252.5991 & 626.8032 & 1235.5725 & 618.2899 & 1234.5885 & 617.7979 & 11 \\
\hline 21 & 2415.9461 & 1208.4767 & 2398.9195 & 1199.9634 & 2397.9355 & 1199.4714 & $\mathbf{L}$ & 1105.5306 & 553.2690 & 1088.5041 & 544.7557 & 1087.5201 & 544.2637 & 10 \\
\hline 22 & 2544.0047 & 1272.5060 & 2526.9781 & 1263.9927 & 2525.9941 & 1263.5007 & $\mathbf{Q}$ & 992.4466 & 496.7269 & 975.4200 & 488.2137 & 974.4360 & 487.7216 & 9 \\
\hline 23 & 2673.0473 & \begin{tabular}{|l|l|}
1337.0273 \\
\end{tabular} & 2656.0207 & \begin{tabular}{|l}
1328.5140 \\
\end{tabular} & 2655.0367 & 1328.0220 & $\mathbf{E}$ & 864.3880 & 432.6976 & 847.3614 & 424.1844 & 846.3774 & 423.6924 & 8 \\
\hline 24 & 2730.0687 & \begin{tabular}{|l|l|}
1365.5380 \\
\end{tabular} & 2713.0422 & 1357.0247 & 2712.0582 & 1356.5327 & G & 735.3454 & 368.1763 & 718.3189 & 359.6631 & 717.3348 & 359.1711 & 7 \\
\hline 25 & 2877.1041 & \begin{tabular}{|l|l|}
1439.0557 \\
\end{tabular} & 2860.0776 & 1430.5424 & 2859.0936 & 1430.0504 & M & 678.3239 & 339.6656 & 661.2974 & 331.1523 & 660.3134 & 330.6603 & 6 \\
\hline 26 & 2974.1569 & \begin{tabular}{|l|l|}
487.5821 \\
\end{tabular} & 2957.1303 & 1479.0688 & 2956.1463 & 1478.5768 & $\mathbf{P}$ & 531.2885 & 266.1479 & 514.2620 & 257.6346 & 513.2780 & 257.1426 & 5 \\
\hline 27 & 3075.2046 & \begin{tabular}{|l|l|}
1538.1059 \\
\end{tabular} & 3058.1780 & \begin{tabular}{|l|}
1529.5927 \\
\end{tabular} & 3057.1940 & 1529.1006 & $\mathbf{T}$ & 434.2358 & 217.6215 & 417.2092 & 209.1083 & 416.2252 & 208.6162 & 4 \\
\hline 28 & 3162.2366 & 1581.6219 & 3145.2101 & 1573.1087 & 3144.2260 & 1572.6167 & S & 333.1881 & 167.0977 & 316.1615 & 158.5844 & 315.1775 & 158.0924 & 3 \\
\hline 29 & 3233.2737 & 1617.1405 & 3216.2472 & 1608.6272 & 3215.2632 & 1608.1352 & A & 246.1561 & 123.5817 & 229.1295 & 115.0684 & & & 2 \\
\hline 30 & & & & & & & $\mathbf{R}$ & 175.1190 & 88.0631 & 158.0924 & 79.5498 & & & 1 \\
\hline
\end{tabular}
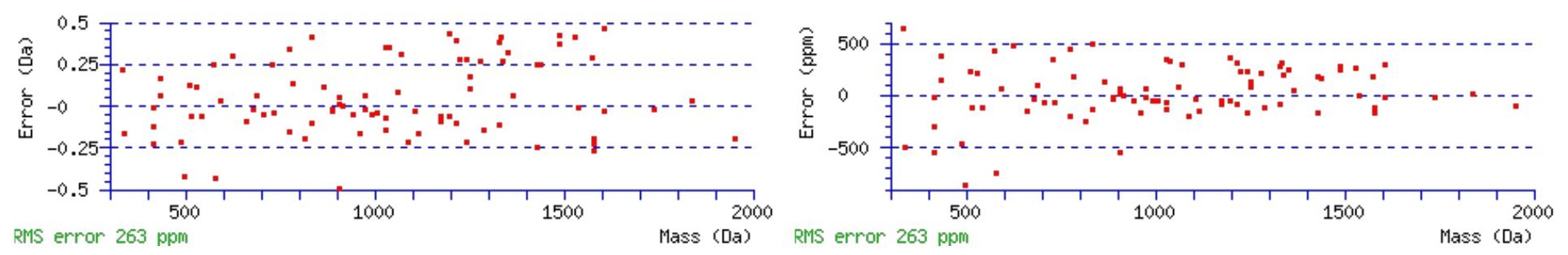

\section{All matches to this query}

\begin{tabular}{|l|r|r|l|}
\hline Score & Mr(calc): & Delta & \multicolumn{1}{c|}{ Sequence } \\
\hline 59.2 & 3406.3781 & 0.0160 & SCGECIQAGPNCGWCTNTTFLQEGMPTSAR \\
\hline 54.1 & 3406.3781 & 0.0160 & SCGECIQAGPNCGWCTNTTFLQEGMPTSAR \\
\hline 41.4 & 3405.3941 & 1.0000 & SCGECIQAGPNCGWCTNTTFLQEGMPTSAR \\
\hline 1.8 & 3405.4043 & 0.9898 & HYLVKCPQNSSGETVTSVTSLSPLQPQK \\
\hline 1.5 & 3404.3569 & 2.0373 & RIYGTWEHNMINHLQVFSYFDTKGK \\
\hline 1.5 & 3404.3569 & 2.0373 & RIYGTWEHNMINHLQVFSYFDTKGK \\
\hline 1.4 & 3406.4143 & -0.020 & ATAAASGTTSSSNTVVAGQDSFPDPEESKILK \\
\hline 1.2 & 3404.3569 & 2.0373 & $\underline{\text { RIYGTWEHNMINHLQVFSYFDTKGK }}$ \\
\hline 0.8 & 3406.4204 & -0.0263 & MDSSAPETTSMNTTDSGVNCLCAICGDRATGK \\
\hline 0.5 & 3405.4004 & 0.9937 & ATLFPTQVEELLCNLNSILYDTVKMR \\
\hline
\end{tabular}


Spectrum No: 545; Query: 30; Rank: 1

\section{Peptide View}

MS/MS Fragmentation of QLALNTTR

Found in IPI00471666, Tax_Id=10116 Gene_Symbol=MGC94145 Uncharacterized protein C1orf179 homolog

Match to Query 30: 916.496868 from(459.255710,2+)

Title: 091008RatKidney_NH4Format01_27.693.693.2.dta

Data file K:\NewmanPaper \Piliang \3SubProteomes \Piliang3SP \mgf5ppm\ERLIC_3SubProteomes5ppm.mgf
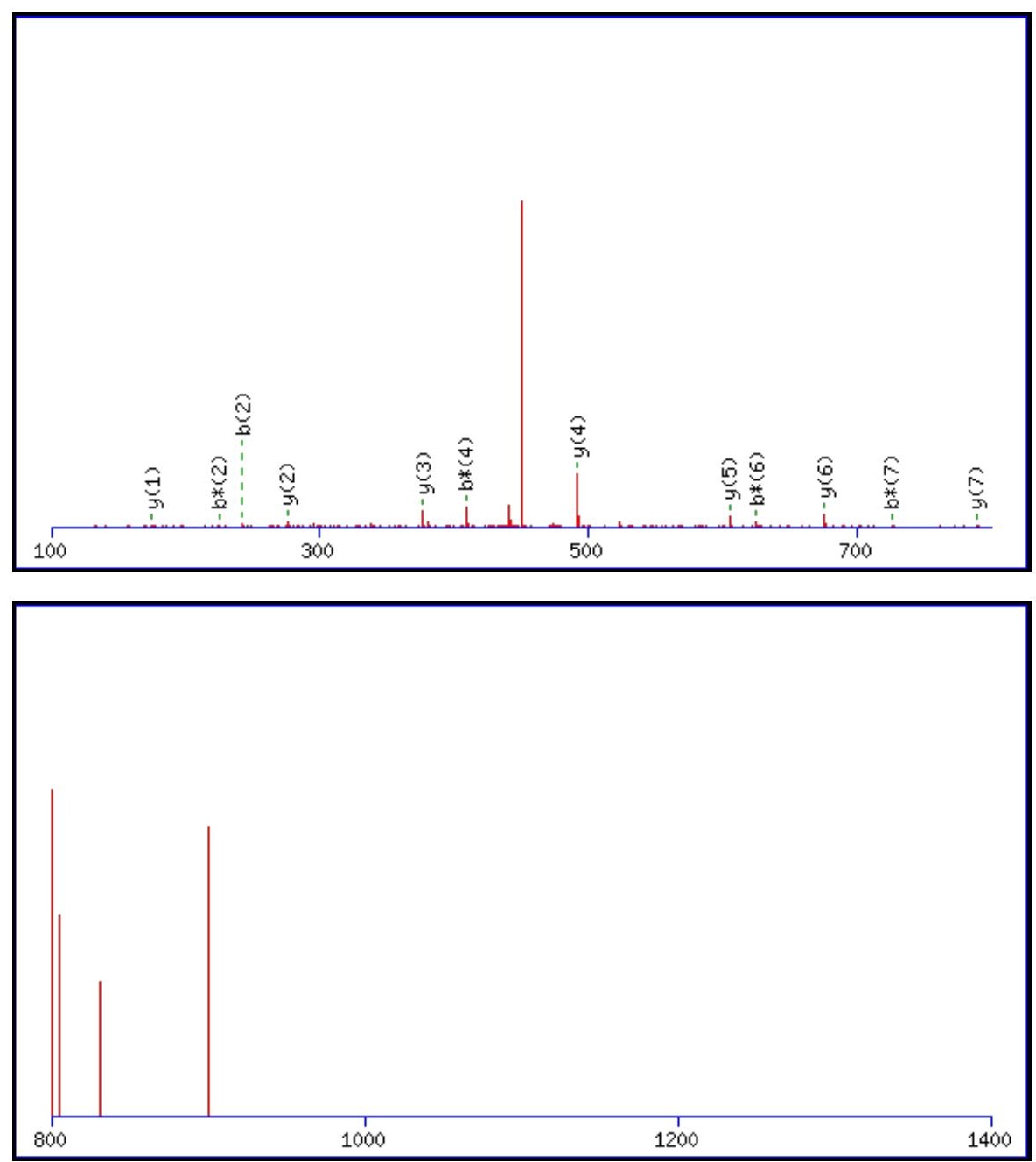


\begin{tabular}{rrrr}
\hline 1400 & 1600 & 1800 & 2000
\end{tabular}

Monoisotopic mass of neutral peptide $\operatorname{Mr}($ calc): 916.4978

Fixed modifications: Carbamidomethyl (C)

Variable modifications:

N5 : Deamidated_N (N)

Ions Score: 59 Expect: 0.00013

Matches (Bold Red): 12/72 fragment ions using 21 most intense peaks

\begin{tabular}{|c|c|c|c|c|c|c|c|c|c|c|c|c|c|c|}
\hline${ }^{+}$ & b & $\mathbf{b}^{++}$ & $\mathbf{b}^{*}$ & $\mathbf{b}^{*^{++}}$ & $\mathbf{b}^{\mathbf{0}}$ & $\mathbf{b}^{\mathbf{0 + +}}$ & Seq. & $\mathbf{y}$ & $\mathbf{y}^{++}$ & $\mathbf{y}^{*}$ & $\mathrm{y}^{\mathrm{*}^{++}}$ & $\mathbf{y}^{\mathbf{0}}$ & $y^{0++}$ & \# \\
\hline 1 & 129.0659 & 65.0366 & 112.0393 & 56.5233 & & & $\mathbf{Q}$ & & & & & & & 8 \\
\hline 2 & 242.1499 & 121.5786 & 225.1234 & 113.0653 & & & $\mathbf{L}$ & 789.4465 & 395.2269 & 772.4199 & 386.7136 & 771.4359 & 386.2216 & 7 \\
\hline 3 & 313.1870 & 157.0972 & 296.1605 & 148.5839 & & & A & 676.3624 & 338.6849 & 659.3359 & 330.1716 & 658.3519 & 329.6796 & \\
\hline 4 & 426.2711 & 213.6392 & 409.2445 & 205.1259 & & & $\mathbf{L}$ & 605.3253 & 303.1663 & 588.2988 & 294.6530 & 587.3147 & 294.1610 & \\
\hline 5 & 541.2980 & 271.1527 & 524.2715 & 262.6394 & & & $\mathbf{N}$ & 492.2412 & 246.6243 & 475.2147 & 238.1110 & 474.2307 & 237.6190 & 4 \\
\hline 6 & 642.3457 & 321.6765 & 625.3192 & 313.1632 & 624.3351 & 312.6712 & $T$ & 377.2143 & 189.1108 & 360.1878 & 180.5975 & 359.2037 & 180.1055 & \\
\hline 7 & 743.3934 & 372.2003 & 726.3668 & 363.6871 & 725.3828 & 363.1951 & $\mathbf{T}$ & 276.1666 & 138.5870 & 259.1401 & 130.0737 & 258.1561 & 129.5817 & 2 \\
\hline & & & & & & & $\mathbf{R}$ & 175.1190 & 88.0631 & 158.0924 & 79.5498 & & & 1 \\
\hline
\end{tabular}
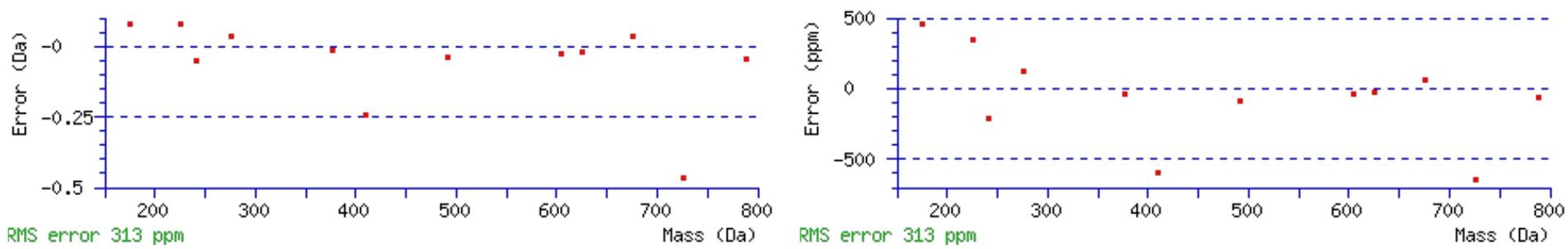

\section{All matches to this query}

\begin{tabular}{|l|l|l|l|}
\hline Score & Mr(calc): & Delta & \multicolumn{1}{|c|}{ Sequence } \\
\hline 59.2 & 916.4978 & -0.0009 & QLALNTTR \\
\hline 19.1 & 916.4978 & -0.0009 & KLDREEK \\
\hline 19.1 & 916.4977 & -0.0009 & KLNREEK \\
\hline 17.9 & 915.5025 & 0.9943 & IPTNITTR \\
\hline 16.8 & 916.5052 & -0.0083 & QLLLDMGK \\
\hline 11.2 & 916.4951 & 0.0018 & RRQSNTR \\
\hline 11.2 & 916.4978 & -0.0009 & VGSSPITTR \\
\hline 11.1 & 916.5025 & -0.0056 & KLCRNAR \\
\hline 11.1 & 916.4978 & -0.0009 & KLEEDKR \\
\hline 11.1 & 916.4977 & -0.0009 & KLKEENR \\
\hline
\end{tabular}

Spectrum No: 546; Query: 2035; Rank: 1 


\section{Peptide View}

MS/MS Fragmentation of RPYIVPILWLNETGTIGDEK

Found in IPI00231197, Tax_Id=10116 Gene_Symbol=Cd36 Platelet glycoprotein 4

Match to Query 2035: 2314.227372 from(772.416400,3+)

Title: 091008RatKidney_NoSalt_17.5830.5830.3.dta

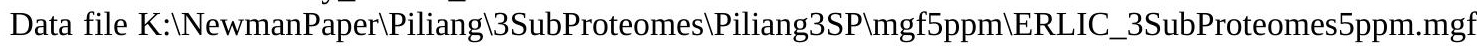
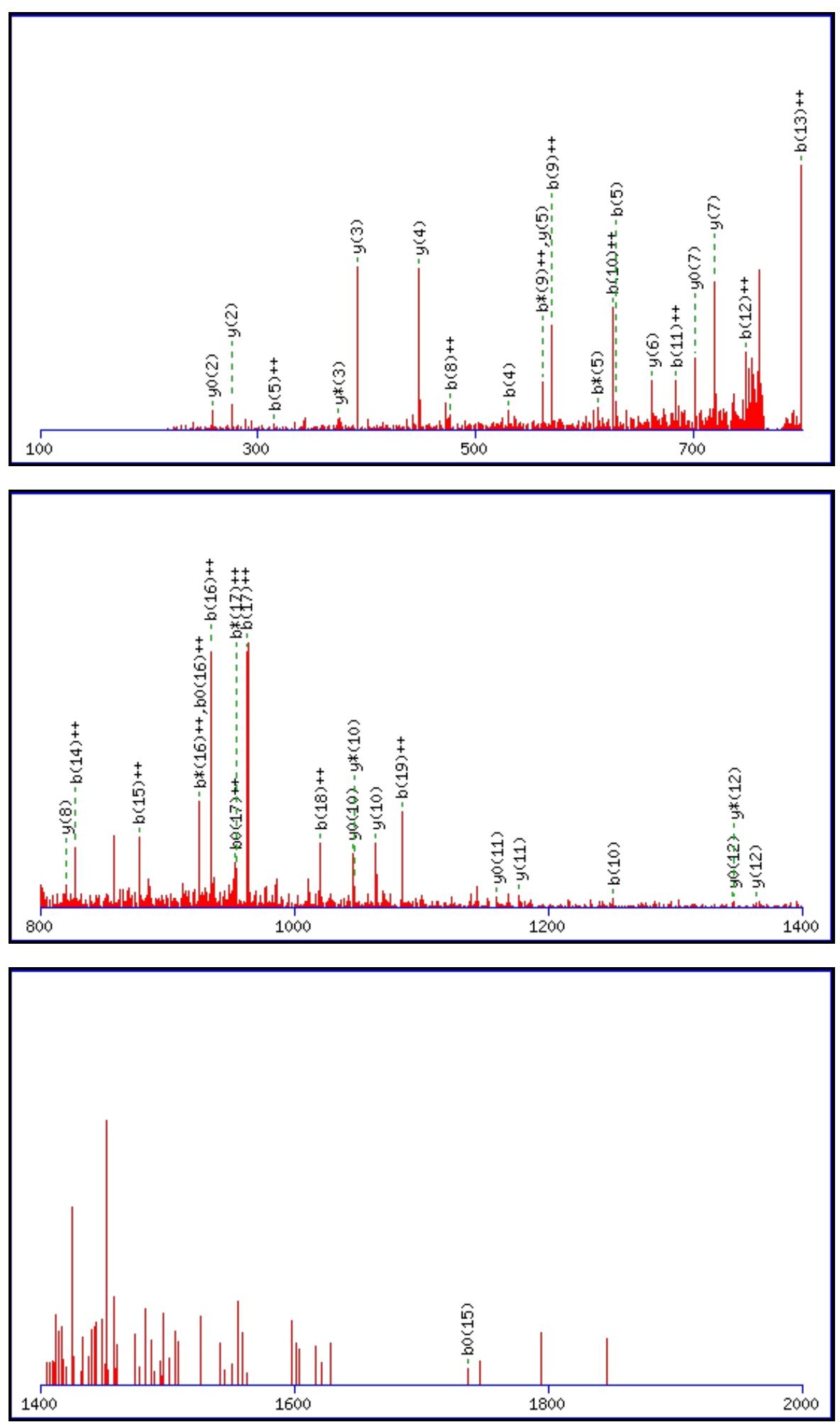

Monoisotopic mass of neutral peptide $\operatorname{Mr}($ calc): 2314.2209

Fixed modifications: Carbamidomethyl (C)

Variable modifications:

N11 : Deamidated_N (N)

Ions Score: 59 Expect : 0.00018 
Matches (Bold Red): 41/204 fragment ions using 94 most intense peaks

\begin{tabular}{|c|c|c|c|c|c|c|c|c|c|c|c|c|c|c|}
\hline \# & b & $\mathbf{b}^{++}$ & $\mathbf{b}^{*}$ & $\mathbf{b}^{*^{++}}$ & $\mathbf{b}^{\mathbf{0}}$ & $\mathbf{b}^{\mathbf{0 + +}}$ & Seq. & $\mathbf{y}$ & $\mathbf{y}^{++}$ & $\mathbf{y}^{*}$ & $\mathrm{y}^{\mathrm{*}^{++}}$ & $\mathbf{y}^{0}$ & $\mathbf{y}^{0^{++}}$ & \# \\
\hline 1 & 157.1084 & 79.0578 & 140.0818 & 70.5446 & & & $\mathbf{R}$ & & & & & & & 20 \\
\hline 2 & 254.1612 & 127.5842 & 237.1346 & 119.0709 & & & $\mathbf{P}$ & 2159.1271 & 1080.0672 & 2142.1005 & 1071.5539 & 2141.1165 & 1071.0619 & 19 \\
\hline 3 & 417.2245 & 209.1159 & 400.1979 & 200.6026 & & & $\mathbf{Y}$ & 2062.0743 & 1031.5408 & 2045.0477 & 1023.0275 & 2044.0637 & 1022.5355 & 18 \\
\hline 4 & 530.3085 & 265.6579 & 513.2820 & 257.1446 & & & I & 1899.0110 & 950.0091 & 1881.9844 & 941.4958 & 1881.0004 & 941.0038 & 17 \\
\hline 5 & 629.3770 & 315.1921 & 612.3504 & 306.6788 & & & $\mathrm{~V}$ & 1785.9269 & 893.4671 & 1768.9004 & 884.9538 & 1767.9163 & 884.4618 & 16 \\
\hline 6 & 726.4297 & 363.7185 & 709.4032 & 355.2052 & & & $\mathbf{P}$ & 1686.8585 & 843.9329 & 1669.8319 & 835.4196 & 1668.8479 & 834.9276 & 15 \\
\hline 7 & 839.5138 & 420.2605 & 822.4872 & 411.7473 & & & I & 1589.8057 & 795.4065 & 1572.7792 & 786.8932 & 1571.7952 & 786.4012 & 14 \\
\hline 8 & 952.5979 & 476.8026 & 935.5713 & 468.2893 & & & $\mathbf{L}$ & 1476.7217 & 738.8645 & 1459.6951 & 730.3512 & 1458.7111 & 729.8592 & 13 \\
\hline 9 & 1138.6772 & 569.8422 & 1121.6506 & 561.3289 & & & $\mathbf{W}$ & 1363.6376 & 682.3224 & 1346.6110 & 673.8092 & 1345.6270 & 673.3172 & 12 \\
\hline 10 & 1251.7612 & 626.3843 & 1234.7347 & 617.8710 & & & $\mathbf{L}$ & 1177.5583 & 589.2828 & 1160.5317 & 580.7695 & 1159.5477 & 580.2775 & 11 \\
\hline 11 & 1366.7882 & 683.8977 & 1349.7616 & 675.3844 & & & $\mathbf{N}$ & 1064.4742 & 532.7407 & 1047.4477 & 524.2275 & 1046.4637 & 523.7355 & 10 \\
\hline 12 & 1495.8308 & 748.4190 & 1478.8042 & 739.9057 & 1477.8202 & 739.4137 & $\mathbf{E}$ & 949.4473 & 475.2273 & 932.4207 & 466.7140 & 931.4367 & 466.2220 & 9 \\
\hline 13 & 1596.8784 & 798.9429 & 1579.8519 & 790.4296 & 1578.8679 & 789.9376 & $T$ & 820.4047 & 410.7060 & 803.3781 & 402.1927 & 802.3941 & 401.7007 & 8 \\
\hline 14 & 1653.8999 & 827.4536 & 1636.8734 & 818.9403 & 1635.8893 & 818.4483 & G & 719.3570 & 360.1821 & 702.3305 & 351.6689 & 701.3464 & 351.1769 & 7 \\
\hline 15 & 1754.9476 & 877.9774 & 1737.9210 & 869.4642 & 1736.9370 & 868.9721 & $\mathbf{T}$ & 662.3355 & 331.6714 & 645.3090 & 323.1581 & 644.3250 & 322.6661 & 6 \\
\hline 16 & 1868.0316 & 934.5195 & 1851.0051 & 926.0062 & 1850.0211 & 925.5142 & $\mathbf{I}$ & 561.2879 & 281.1476 & 544.2613 & 272.6343 & 543.2773 & 272.1423 & 5 \\
\hline 17 & 1925.0531 & 963.0302 & 1908.0266 & 954.5169 & 1907.0425 & 954.0249 & G & 448.2038 & 224.6055 & 431.1773 & 216.0923 & 430.1932 & 215.6003 & 4 \\
\hline 18 & 2040.0800 & 1020.5437 & 2023.0535 & 1012.0304 & 2022.0695 & 1011.5384 & D & 391.1823 & 196.0948 & 374.1558 & 187.5815 & 373.1718 & 187.0895 & 3 \\
\hline 19 & 2169.1226 & 1085.0650 & 2152.0961 & 1076.5517 & 2151.1121 & 1076.0597 & E & 276.1554 & 138.5813 & 259.1288 & 130.0681 & 258.1448 & 129.5761 & 2 \\
\hline 20 & & & & & & & K & 147.1128 & 74.0600 & 130.0863 & 65.5468 & & & 1 \\
\hline
\end{tabular}
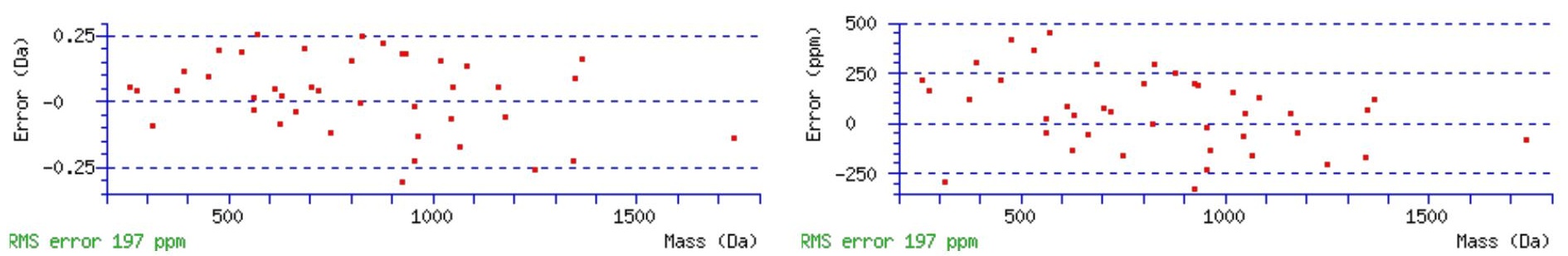

\section{All matches to this query}

\begin{tabular}{|l|l|l|l|}
\hline Score & Mr(calc): & Delta & \multicolumn{1}{c|}{ Sequence } \\
\hline 59.1 & 2314.2209 & 0.0065 & RPYIVPILWLNETGTIGDEK \\
\hline 18.1 & 2313.2369 & 0.9905 & RPYIVPILWLNETGTIGDEK \\
\hline 0.4 & 2314.2142 & 0.0132 & VDVRPATRQNLSQFEAQARK \\
\hline
\end{tabular}

Spectrum No: 547; Query: 55; Rank: 1

\section{Peptide View}

MS/MS Fragmentation of YMSLALNR

Found in IPI00367674, Tax_Id=10116 Gene_Symbol=Gla galactosidase, alpha

Match to Query 55: 983.477008 from(492.745780,2+)

Title: 091008RatKidney_NH4Format01_26.977.977.2.dta

Data file K:INewmanPaper|Piliang|3SubProteomes\Piliang3SP\mgf5ppm\ERLIC_3SubProteomes5ppm.mgf 

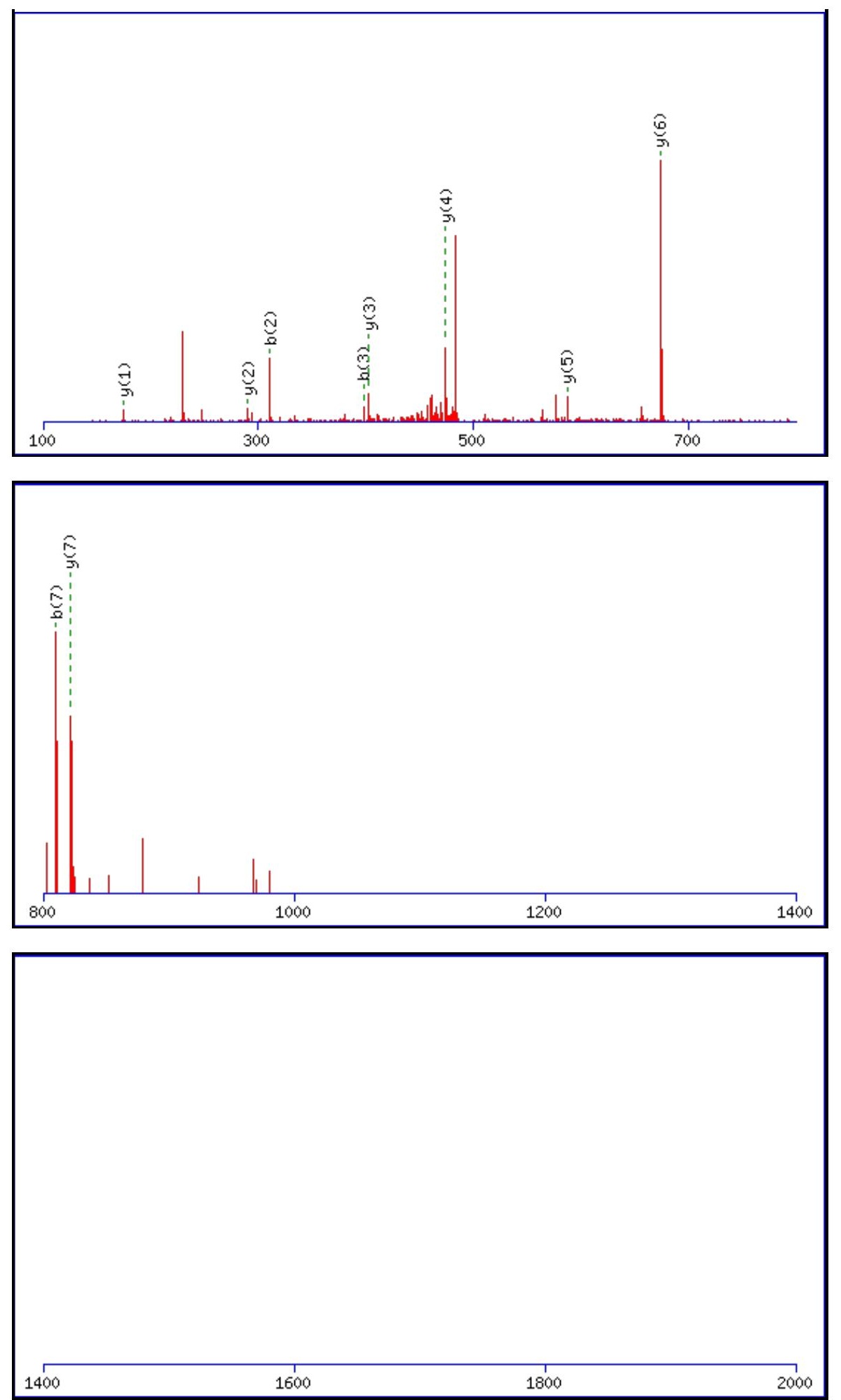

Monoisotopic mass of neutral peptide $\operatorname{Mr}($ calc): 983.4746

Fixed modifications: Carbamidomethyl (C)

Variable modifications:

M2 : Oxidation (M)

N7 : Deamidated_N (N)

Ions Score: 59 Expect: 0.00014

Matches (Bold Red): 10/58 fragment ions using 20 most intense peaks

\begin{tabular}{|c|c|c|c|c|c|c|c|c|c|c|c|c|c|c|}
\hline$\#$ & $\mathbf{b}$ & $\mathbf{b}^{++}$ & $\mathbf{b}^{*}$ & $\mathbf{b}^{*^{++}}$ & $\mathbf{b}^{\mathbf{0}}$ & $\mathbf{b}^{\mathbf{0 + +}}$ & Seq. & $\mathbf{y}$ & $\mathbf{y}^{++}$ & $\mathbf{y}^{\mathbf{*}}$ & $\mathbf{y}^{\boldsymbol{*}^{++}}$ & $\mathbf{y}^{\mathbf{0}}$ & $\mathbf{y}^{\mathbf{0}++}$ & $\#$ \\
\hline $\mathbf{1}$ & 164.0706 & 82.5389 & & & & & $\mathbf{Y}$ & & & & & & & $\mathbf{8}$ \\
\hline $\mathbf{2}$ & 311.1060 & 156.0566 & & & & & $\mathbf{M}$ & $\mathbf{8 2 1 . 4 1 8 6}$ & 411.2129 & 804.3920 & 402.6996 & 803.4080 & 402.2076 & 7 \\
\hline $\mathbf{3}$ & $3 \mathbf{3 9 8 . 1 3 8 0}$ & 199.5727 & & & 380.1275 & 190.5674 & $\mathbf{S}$ & $\mathbf{6 7 4 . 3 8 3 2}$ & 337.6952 & 657.3566 & 329.1819 & 656.3726 & 328.6899 & $\mathbf{6}$ \\
\hline $\mathbf{4}$ & 511.2221 & 256.1147 & & & 493.2115 & 247.1094 & $\mathbf{L}$ & $5 \mathbf{8 8 7 . 3 5 1 1}$ & 294.1792 & 570.3246 & 285.6659 & & & 5 \\
\hline & & & & & & & & & & & & & &
\end{tabular}


Mascot Search Results: Peptide View

\begin{tabular}{|l|l|l|l|l|l|l|l|l|l|l|l|l|l|l|l|}
$\mathbf{5}$ & 582.2592 & 291.6332 & & & 564.2487 & 282.6280 & $\mathbf{A}$ & $\mathbf{4 7 4 . 2 6 7 1}$ & 237.6372 & 457.2405 & 229.1239 & & & $\mathbf{4}$ \\
\hline $\mathbf{6}$ & 695.3433 & 348.1753 & & & 677.3327 & 339.1700 & $\mathbf{L}$ & $\mathbf{4 0 3 . 2 3 0 0}$ & 202.1186 & 386.2034 & 193.6053 & & & $\mathbf{3}$ \\
\hline $\mathbf{7}$ & $\mathbf{8 1 0 . 3 7 0 2}$ & 405.6887 & 793.3437 & 397.1755 & 792.3596 & 396.6835 & $\mathbf{N}$ & $\mathbf{2 9 0 . 1 4 5 9}$ & 145.5766 & 273.1193 & 137.0633 & & & $\mathbf{2}$ \\
\hline $\mathbf{8}$ & & & & & & & $\mathbf{R}$ & $\mathbf{1 7 5 . 1 1 9 0}$ & 88.0631 & 158.0924 & 79.5498 & & & $\mathbf{1}$ \\
\hline
\end{tabular}
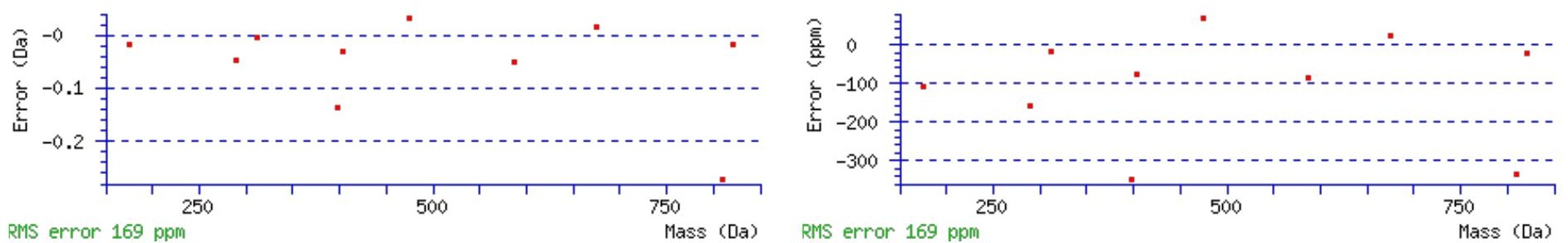

\section{All matches to this query}

\begin{tabular}{|l|l|l|l|}
\hline Score & Mr(calc): & Delta & \multicolumn{1}{|c|}{ Sequence } \\
\hline 59.0 & 983.4746 & 0.0024 & YMSLALNR \\
\hline 20.2 & 983.4746 & 0.0024 & YMILGTDR \\
\hline 13.0 & 983.4726 & 0.0044 & FYPHHQR \\
\hline 11.3 & 983.4712 & 0.0058 & YFEEIQR \\
\hline 10.3 & 982.4832 & 0.9938 & YSRSSLNR \\
\hline 10.0 & 983.4689 & 0.0082 & VLSISTER \\
\hline 10.0 & 983.4689 & 0.0082 & VLSISTER \\
\hline 7.7 & 981.4740 & 2.0030 & GNHSQGGVAR \\
\hline 7.3 & 983.4688 & 0.0082 & ETSKALQK \\
\hline 6.6 & 983.4712 & 0.0058 & NGYVFINR \\
\hline
\end{tabular}

Spectrum No: 548; Query: 1211; Rank: 1

\section{Peptide View}

MS/MS Fragmentation of DDDIAALVVDNGSGMCK

Found in IPI00189819, Tax_Id=10116 Gene_Symbol=Actb Actin, cytoplasmic 1

Match to Query 1211: 1780.768628 from(891.391590,2+)

Title: 091008RatKidney_NH4Format02_25.3525.3525.2.dta

Data file K:INewmanPaper|Piliang|3SubProteomes\Piliang3SP\mgf5ppm\ERLIC_3SubProteomes5ppm.mgf

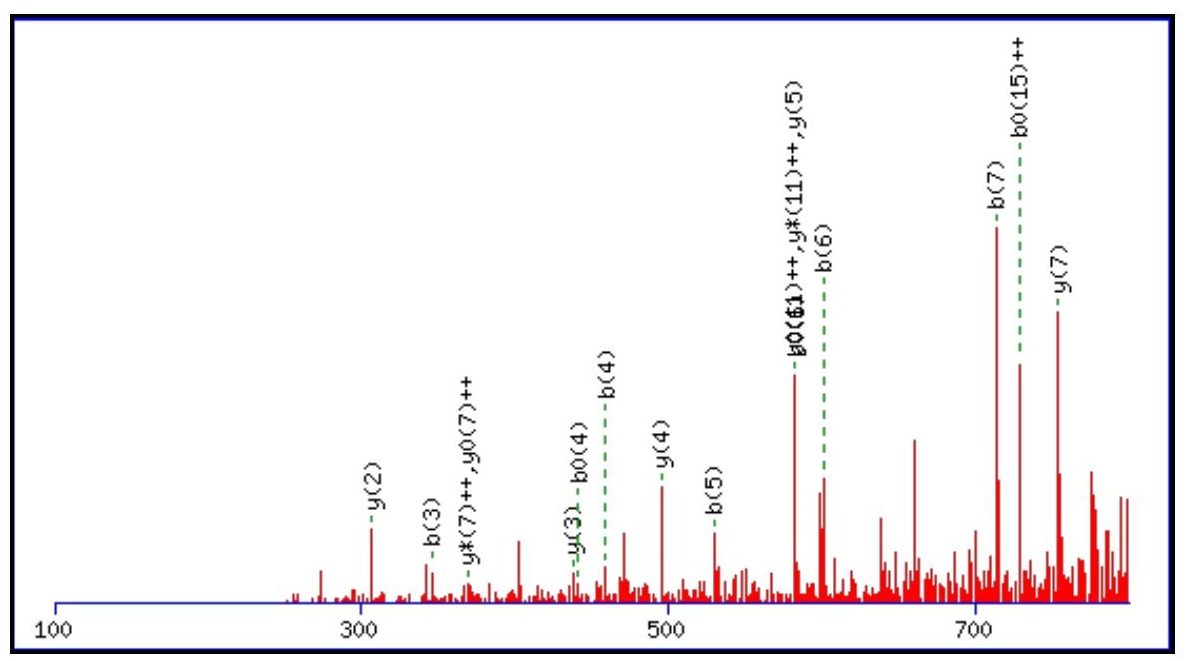



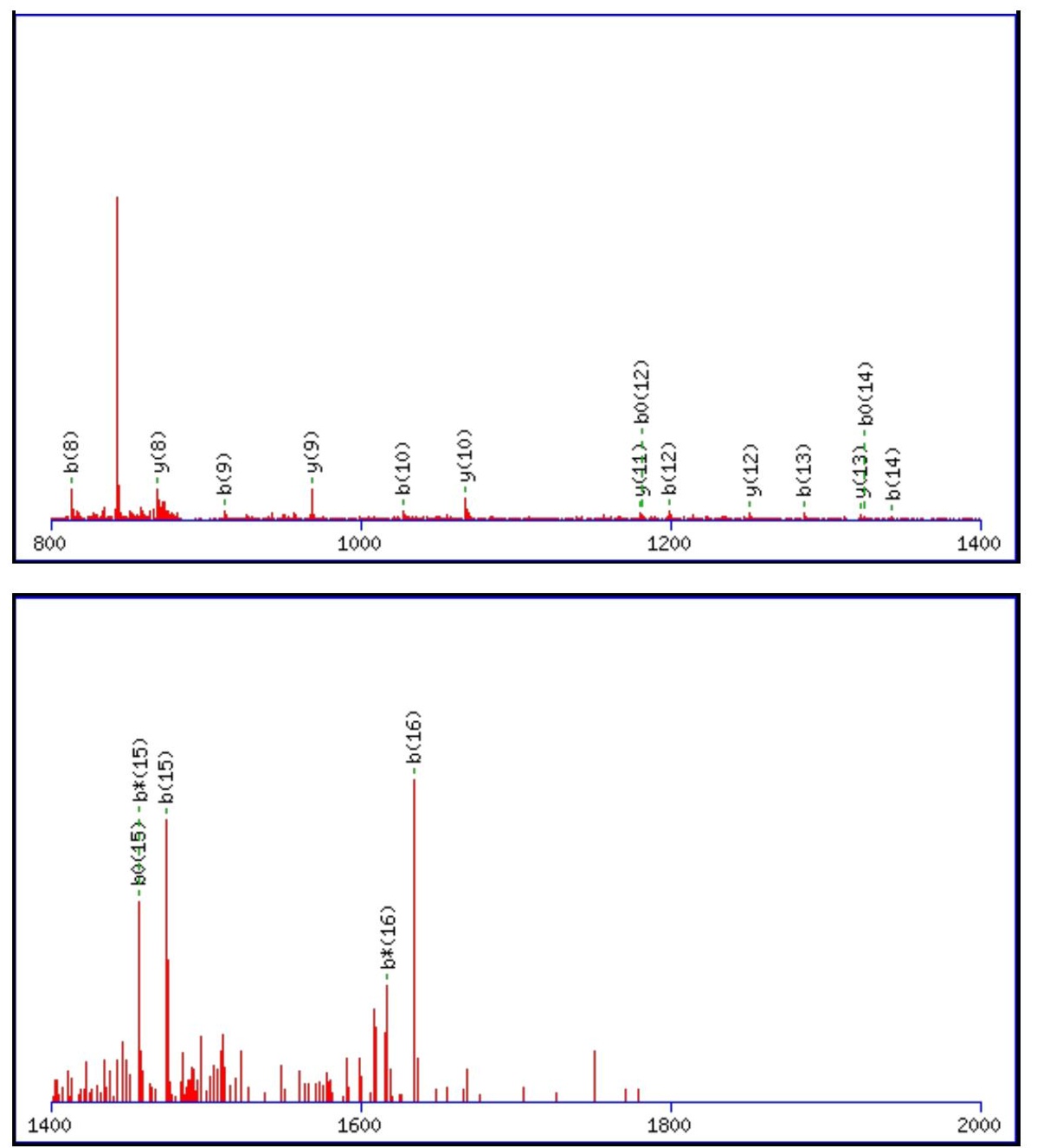

Monoisotopic mass of neutral peptide $\operatorname{Mr}($ calc): 1779.7655

Fixed modifications: Carbamidomethyl (C)

Variable modifications:

N11 : Deamidated_N (N)

Ions Score: 59 Expect: 0.00026

Matches (Bold Red): 36/164 fragment ions using 76 most intense peaks

\begin{tabular}{|c|c|c|c|c|c|c|c|c|c|c|c|c|c|c|}
\hline \# & b & $\mathbf{b}^{++}$ & $\mathbf{b}^{*}$ & $\mathbf{b}^{*^{++}}$ & $\mathbf{b}^{\mathbf{0}}$ & $\mathbf{b}^{\mathbf{0 + +}}$ & Seq. & $\mathbf{y}$ & $\mathbf{y}^{++}$ & $\mathbf{y}^{*}$ & $\mathrm{y}^{*^{++}}$ & $\mathbf{y}^{0}$ & $\mathbf{y}^{\mathbf{0}^{++}}$ & \# \\
\hline 1 & 116.0342 & 58.5207 & & & 98.0237 & 49.5155 & D & & & & & & & 17 \\
\hline 2 & 231.0612 & 116.0342 & & & 213.0506 & 107.0289 & D & 1665.7458 & 833.3766 & 1648.7193 & 824.8633 & 1647.7353 & 824.3713 & 16 \\
\hline 3 & 346.0881 & 173.5477 & & & 328.0775 & 164.5424 & D & 1550.7189 & 775.8631 & 1533.6924 & 767.3498 & 1532.7083 & 766.8578 & 15 \\
\hline 4 & 459.1722 & 230.0897 & & & 441.1616 & 221.0844 & I & 1435.6920 & 718.3496 & 1418.6654 & 709.8363 & 1417.6814 & 709.3443 & 14 \\
\hline 5 & 530.2093 & 265.6083 & & & 512.1987 & 256.6030 & $\mathbf{A}$ & 1322.6079 & 661.8076 & 1305.5813 & 653.2943 & 1304.5973 & 652.8023 & 13 \\
\hline 6 & 601.2464 & 301.1268 & & & 583.2358 & |292.1216 & A & 1251.5708 & 626.2890 & 1234.5442 & 617.7758 & 1233.5602 & 617.2837 & 12 \\
\hline 7 & 714.3305 & 357.6689 & & & 696.3199 & 348.6636 & $\mathbf{L}$ & 1180.5337 & 590.7705 & 1163.5071 & 582.2572 & 1162.5231 & 581.7652 & 11 \\
\hline 8 & 813.3989 & 407.2031 & & & 795.3883 & 398.1978 & $\mathbf{V}$ & 1067.4496 & 534.2284 & 1050.4231 & 525.7152 & 1049.4390 & 525.2232 & 10 \\
\hline 9 & \begin{tabular}{|l|}
912.4673 \\
\end{tabular} & 456.7373 & & & 894.4567 & 447.7320 & $\mathrm{~V}$ & 968.3812 & 484.6942 & 951.3546 & 476.1810 & 950.3706 & 475.6889 & 9 \\
\hline 10 & 1027.4942 & 514.2508 & & & 1009.4837 & 505.2455 & D & 869.3128 & 435.1600 & 852.2862 & 426.6468 & 851.3022 & 426.1547 & 8 \\
\hline 11 & 1142.5212 & 571.7642 & 1125.4946 & 563.2509 & 1124.5106 & 562.7589 & $\mathbf{N}$ & 754.2858 & 377.6466 & 737.2593 & 369.1333 & 736.2753 & 368.6413 & 7 \\
\hline 12 & 1199.5426 & 600.2750 & 1182.5161 & 591.7617 & 1181.5321 & 591.2697 & G & 639.2589 & 320.1331 & 622.2323 & 311.6198 & 621.2483 & 311.1278 & 6 \\
\hline 13 & 1286.5747 & 643.7910 & 1269.5481 & 635.2777 & 1268.5641 & 634.7857 & $S$ & 582.2374 & 291.6224 & 565.2109 & 283.1091 & 564.2269 & 282.6171 & 5 \\
\hline 14 & 1343.5961 & 672.3017 & 1326.5696 & 663.7884 & 1325.5856 & 663.2964 & G & 495.2054 & 248.1063 & 478.1789 & 239.5931 & & & 4 \\
\hline 15 & 1474.6366 & 737.8219 & 1457.6101 & 729.3087 & 1456.6260 & 728.8167 & $\mathbf{M}$ & 438.1839 & 219.5956 & 421.1574 & 211.0823 & & & 3 \\
\hline 16 & 1634.6673 & 817.8373 & 1617.6407 & 809.3240 & 1616.6567 & 808.8320 & C & 307.1435 & 154.0754 & 290.1169 & 145.5621 & & & 2 \\
\hline 17 & & & & & & & $\mathbf{K}$ & 147.1128 & 74.0600 & 130.0863 & 65.5468 & & & 1 \\
\hline
\end{tabular}



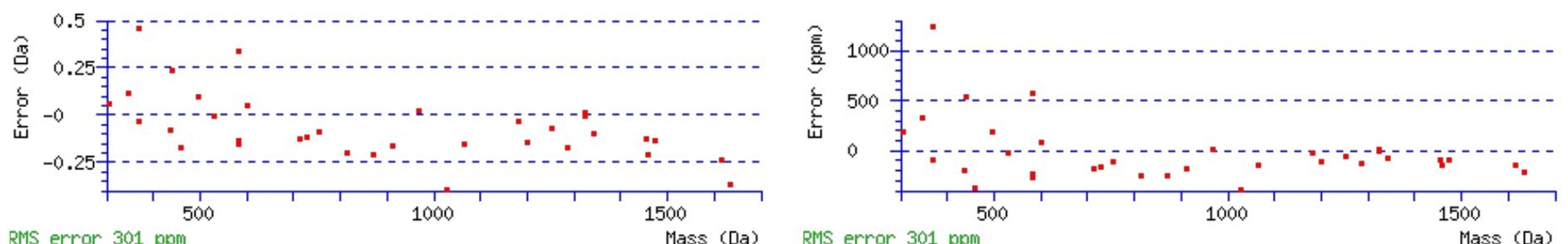

RMS error 301 ppm

\section{All matches to this query}

\begin{tabular}{|l|l|l|l|}
\hline Score & Mr(calc): & Delta & \multicolumn{1}{|c|}{ Sequence } \\
\hline 59.0 & 1779.7655 & 1.0031 & DDDIAALVVDNGSGMCK \\
\hline 11.2 & 1779.7706 & 0.9981 & LENHSTHSIRNIAK \\
\hline 4.9 & 1779.7706 & 0.9981 & LENHSTHSIRNIAK \\
\hline 3.0 & 1780.7546 & 0.0141 & LENHSTHSIRNIAK \\
\hline 1.3 & 1779.7766 & 0.9920 & KMELLPETSSLTQK \\
\hline 1.3 & 1779.7766 & 0.9920 & KMELLPETSSLTQK \\
\hline 0.7 & 1778.7723 & 1.9963 & AKKQEEEPQDDPASK \\
\hline 0.7 & 1779.7766 & 0.9920 & KMELLPETSSLTQK \\
\hline 0.5 & 1779.7481 & 1.0205 & ESGTTAWQLINSSVK \\
\hline 0.5 & 1779.7481 & 1.0205 & ESGTTAWQLINSSVK \\
\hline
\end{tabular}

Spectrum No: 549; Query: 604; Rank: 1

\section{Peptide View}

MS/MS Fragmentation of LQVTLYNCSFGR

Found in IPI00370450, Tax_Id=10116 Gene_Symbol=Plxnb2 plexin B2

Match to Query 604: 1457.698828 from(729.856690,2+)

Title: 091008RatKidney_NoSalt_25.3019.3019.2.dta

Data file K:INewmanPaper|Piliang\3SubProteomes\Piliang3SP\mgf5ppm\ERLIC_3SubProteomes5ppm.mgf

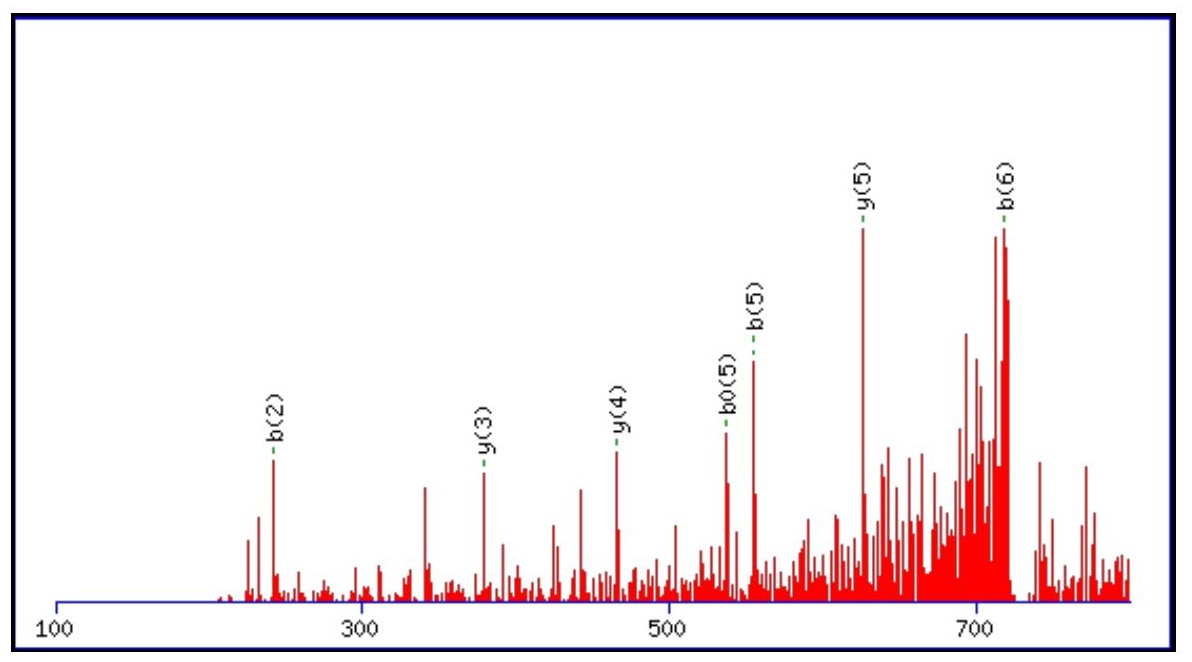



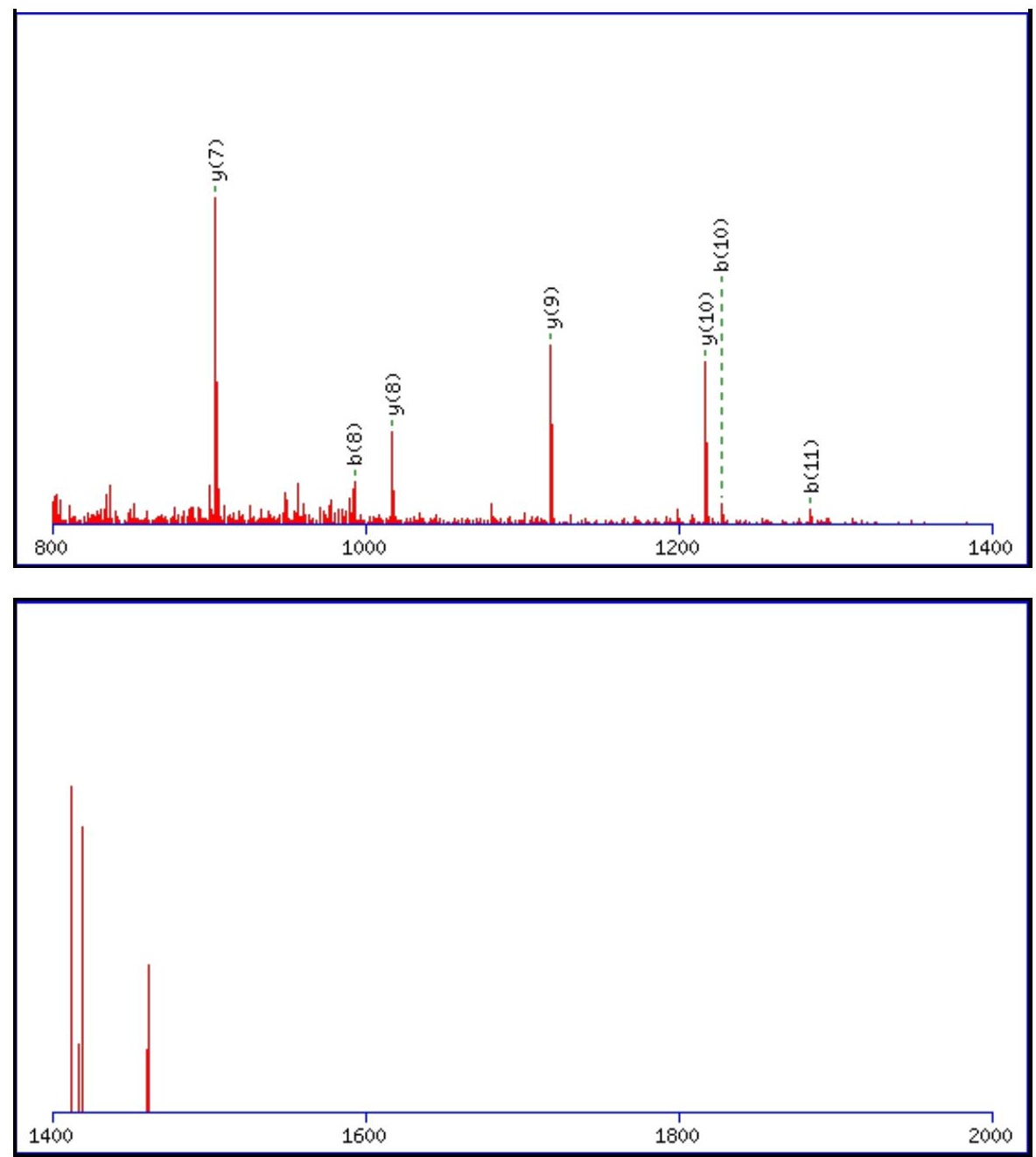

Monoisotopic mass of neutral peptide $\operatorname{Mr}($ calc): 1457.6973

Fixed modifications: Carbamidomethyl (C)

Variable modifications:

N7 : Deamidated $\mathrm{N}(\mathrm{N})$

Ions Score: 59 Expect: 0.00021

Matches (Bold Red): 14/118 fragment ions using 24 most intense peaks

\begin{tabular}{|c|c|c|c|c|c|c|c|c|c|c|c|c|c|c|}
\hline \# & b & $\mathbf{b}^{++}$ & b* & $\mathbf{b}^{*^{++}}$ & $\mathbf{b}^{0}$ & $\mathbf{b}^{0++}$ & Seq. & $\mathbf{y}$ & $\mathrm{y}^{++}$ & $\mathbf{y}^{*}$ & $\mathrm{y}^{*^{++}}$ & $\mathbf{y}^{0}$ & $y^{0++}$ & \# \\
\hline 1 & 114.0913 & 57.5493 & & & & & $\mathbf{L}$ & & & & & & & 12 \\
\hline 2 & 242.1499 & 121.5786 & 225.1234 & 113.0653 & & & $\mathbf{Q}$ & 1345.6205 & 673.3139 & 1328.5940 & 664.8006 & 1327.6099 & 664.3086 & 11 \\
\hline 3 & 341.2183 & 171.1128 & 324.1918 & 162.5995 & & & V & 1217.5619 & 609.2846 & 1200.5354 & 600.7713 & 1199.5514 & 600.2793 & 10 \\
\hline 4 & 442.2660 & 221.6366 & 425.2395 & 213.1234 & 424.2554 & 212.6314 & $\mathbf{T}$ & 1118.4935 & 559.7504 & 1101.4670 & 551.2371 & 1100.4830 & 550.7451 & 9 \\
\hline 5 & 555.3501 & 278.1787 & 538.3235 & 269.6654 & 537.3395 & 269.1734 & $\mathbf{L}$ & 1017.4458 & 509.2266 & 1000.4193 & 500.7133 & 999.4353 & 500.2213 & 8 \\
\hline 6 & 718.4134 & 359.7103 & 701.3869 & 351.1971 & 700.4028 & 350.7051 & $\mathbf{Y}$ & 904.3618 & 452.6845 & 887.3352 & 444.1712 & 886.3512 & 443.6792 & 7 \\
\hline 7 & 833.4403 & 417.2238 & 816.4138 & 408.7105 & 815.4298 & 408.2185 & $\mathbf{N}$ & 741.2984 & 371.1529 & 724.2719 & 362.6396 & 723.2879 & 362.1476 & 6 \\
\hline 8 & 993.4710 & 497.2391 & 976.4444 & 488.7259 & 975.4604 & 488.2339 & $\mathrm{C}$ & 626.2715 & 313.6394 & 609.2450 & 305.1261 & 608.2609 & 304.6341 & 5 \\
\hline 9 & 1080.5030 & 540.7551 & 1063.4765 & 532.2419 & 1062.4925 & 531.7499 & $\mathrm{~S}$ & 466.2409 & 233.6241 & 449.2143 & 225.1108 & 448.2303 & 224.6188 & 4 \\
\hline 10 & 1227.5714 & 614.2894 & 1210.5449 & 605.7761 & 1209.5609 & 605.2841 & $\mathbf{F}$ & 379.2088 & 190.1081 & 362.1823 & 181.5948 & & & 3 \\
\hline 11 & 1284.5929 & 642.8001 & 1267.5663 & 634.2868 & 1266.5823 & 633.7948 & G & 232.1404 & 116.5738 & 215.1139 & 108.0606 & & & 2 \\
\hline 12 & & & & & & & $\mathbf{R}$ & 175.1190 & 88.0631 & 158.0924 & 79.5498 & & & 1 \\
\hline
\end{tabular}



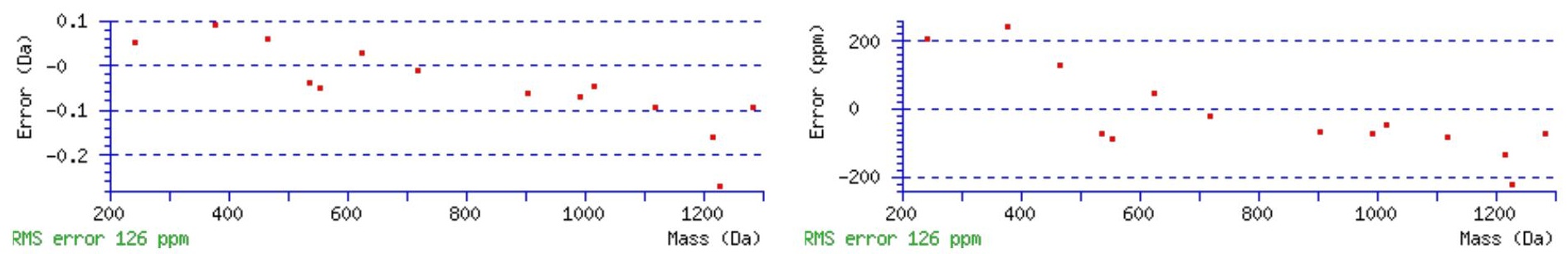

\section{All matches to this query}

\begin{tabular}{|l|l|c|c|}
\hline Score & Mr(calc): & Delta & Sequence \\
\hline 59.0 & 1457.6973 & 0.0015 & LQVTLYNCSFGR \\
\hline 7.9 & 1457.7085 & -0.0097 & IQVEHPQMTFGR \\
\hline
\end{tabular}

Spectrum No: 550; Query: 338; Rank: 1

\section{Peptide View}

MS/MS Fragmentation of LLNLTSPEATAR

Found in IPI00515854, Tax_Id=10116 Gene_Symbol=Entpd2 Ectonucleoside triphosphate diphosphohydrolase 2

Match to Query 338: 1285.686188 from(643.850370,2+)

Title: 091008RatKidney_NH4Format01_29.1566.1566.2.dta

Data file K:INewmanPaper|Piliangl3SubProteomes\Piliang3SP\mgf5ppm\ERLIC_3SubProteomes5ppm.mgf
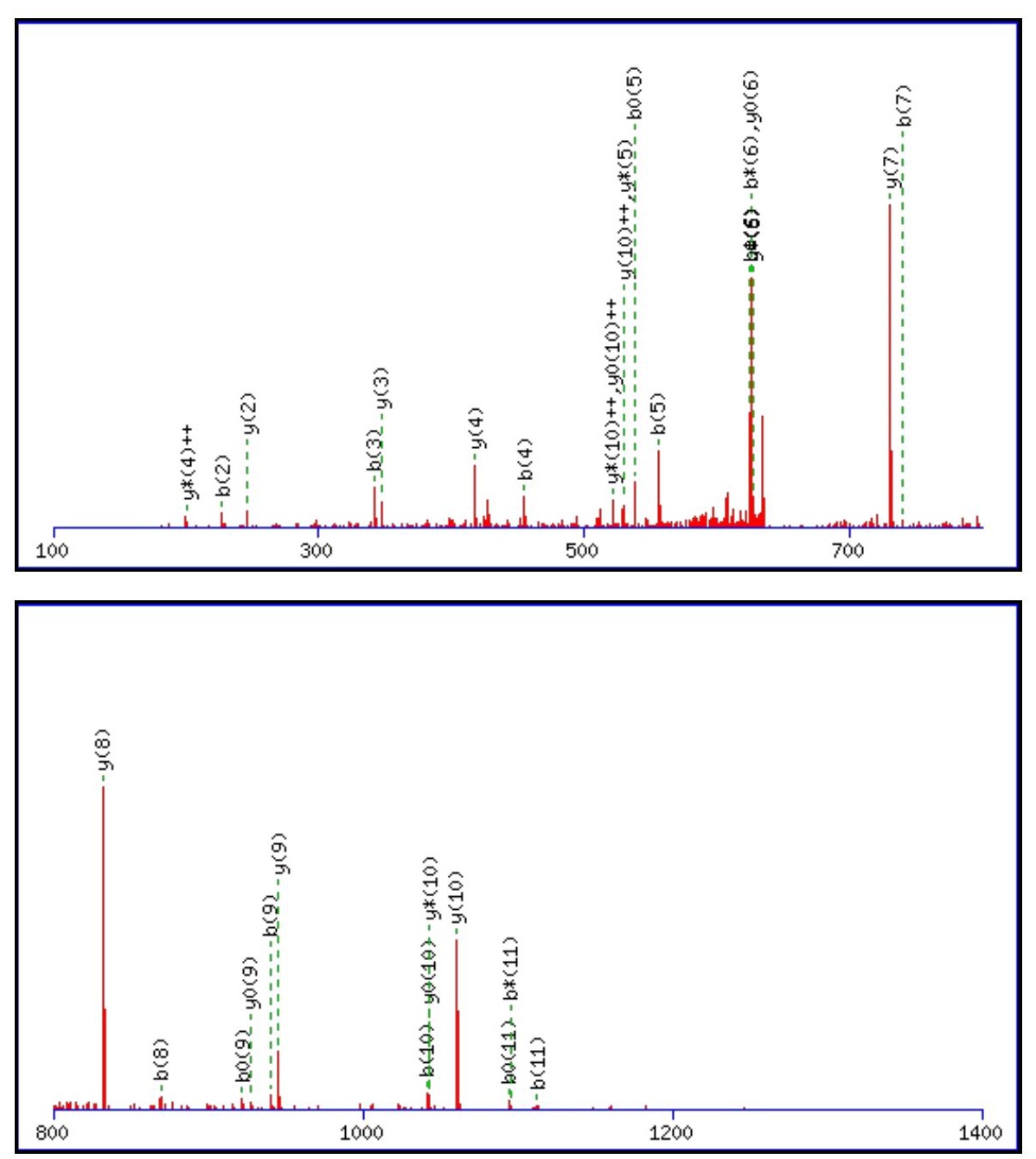


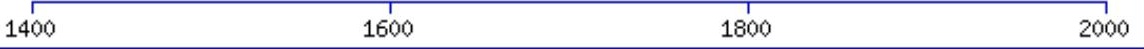

Monoisotopic mass of neutral peptide $\operatorname{Mr}($ calc): 1285.6877

Fixed modifications: Carbamidomethyl (C)

Variable modifications:

N3 : Deamidated_N (N)

Ions Score: 59 Expect: 0.00012

Matches (Bold Red): 32/116 fragment ions using 54 most intense peaks

\begin{tabular}{|r|c|c|c|c|c|c|c|c|c|c|c|c|c|c|}
\hline$\#$ & $\mathbf{b}$ & $\mathbf{b}^{++}$ & $\mathbf{b}^{*}$ & $\mathbf{b}^{\mathbf{*}^{++}}$ & $\mathbf{b}^{\mathbf{0}}$ & $\mathbf{b}^{\mathbf{0 + +}}$ & $\mathbf{S e q}$ & $\mathbf{y}$ & $\mathbf{y}^{++}$ & $\mathbf{y}^{\mathbf{*}}$ & $\mathbf{y}^{\mathbf{*}^{++}}$ & $\mathbf{y}^{\mathbf{0}}$ & $\mathbf{y}^{\mathbf{0 + +}}$ & $\#$ \\
\hline $\mathbf{1}$ & 114.0913 & 57.5493 & & & & & $\mathbf{L}$ & & & & & & & $\mathbf{1 2}$ \\
\hline $\mathbf{2}$ & $\mathbf{2 2 7 . 1 7 5 4}$ & 114.0913 & & & & & $\mathbf{L}$ & 1173.6110 & 587.3091 & 1156.5844 & 578.7959 & 1155.6004 & 578.3038 & $\mathbf{1 1}$ \\
\hline $\mathbf{3}$ & $\mathbf{3 4 2 . 2 0 2 3}$ & 171.6048 & 325.1758 & 163.0915 & & & $\mathbf{N}$ & $\mathbf{1 0 6 0 . 5 2 6 9}$ & 530.7671 & $\mathbf{1 0 4 3 . 5 0 0 4}$ & $\mathbf{5 2 2 . 2 5 3 8}$ & $\mathbf{1 0 4 2 . 5 1 6 4}$ & $\mathbf{5 2 1 . 7 6 1 8}$ & $\mathbf{1 0}$ \\
\hline $\mathbf{4}$ & $\mathbf{4 5 5 . 2 8 6 4}$ & 228.1468 & 438.2599 & 219.6336 & & & $\mathbf{L}$ & $\mathbf{9 4 5 . 5 0 0 0}$ & 473.2536 & 928.4734 & 464.7404 & $\mathbf{9 2 7 . 4 8 9 4}$ & 464.2483 & $\mathbf{9}$ \\
\hline $\mathbf{5}$ & $\mathbf{5 5 6 . 3 3 4 1}$ & 278.6707 & 539.3075 & 270.1574 & 538.3235 & 269.6654 & $\mathbf{T}$ & $\mathbf{8 3 2 . 4 1 5 9}$ & 416.7116 & 815.3894 & 408.1983 & 814.4054 & 407.7063 & $\mathbf{8}$ \\
\hline $\mathbf{6}$ & 643.3661 & 322.1867 & $\mathbf{6 2 6 . 3 3 9 6}$ & 313.6734 & $\mathbf{6 2 5 . 3 5 5 5}$ & 313.1814 & S & 731.3682 & 366.1878 & 714.3417 & 357.6745 & 713.3577 & 357.1825 & $\mathbf{7}$ \\
\hline $\mathbf{7}$ & $\mathbf{7 4 0 . 4 1 8 9}$ & 370.7131 & 723.3923 & 362.1998 & 722.4083 & 361.7078 & $\mathbf{P}$ & 644.3362 & 322.6717 & $\mathbf{6 2 7 . 3 0 9 7}$ & 314.1585 & $\mathbf{6 2 6 . 3 2 5 7}$ & 313.6665 & $\mathbf{6}$ \\
\hline $\mathbf{8}$ & $\mathbf{8 6 9 . 4 6 1 5}$ & 435.2344 & 852.4349 & 426.7211 & 851.4509 & 426.2291 & $\mathbf{E}$ & 547.2835 & 274.1454 & 530.2569 & 265.6321 & 529.2729 & 265.1401 & $\mathbf{5}$ \\
\hline $\mathbf{9}$ & $\mathbf{9 4 0 . 4 9 8 6}$ & 470.7529 & 923.4720 & 462.2397 & $\mathbf{9 2 2 . 4 8 8 0}$ & 461.7476 & $\mathbf{A}$ & $\mathbf{4 1 8 . 2 4 0 9}$ & 209.6241 & 401.2143 & $\mathbf{2 0 1 . 1 1 0 8}$ & 400.2303 & 200.6188 & $\mathbf{4}$ \\
\hline $\mathbf{1 0}$ & $\mathbf{1 0 4 1 . 5 4 6 3}$ & 521.2768 & 1024.5197 & 512.7635 & 1023.5357 & 512.2715 & $\mathbf{T}$ & 347.2037 & 174.1055 & 330.1772 & 165.5922 & 329.1932 & 165.1002 & $\mathbf{3}$ \\
\hline $\mathbf{1 1}$ & $\mathbf{1 1 1 2 . 5 8 3 4}$ & 556.7953 & $\mathbf{1 0 9 5 . 5 5 6 8}$ & 548.2821 & $\mathbf{1 0 9 4 . 5 7 2 8}$ & 547.7900 & $\mathbf{A}$ & $\mathbf{2 4 6 . 1 5 6 1}$ & 123.5817 & 229.1295 & 115.0684 & & & $\mathbf{2}$ \\
\hline $\mathbf{1 2}$ & & & & & & & $\mathbf{R}$ & 175.1190 & 88.0631 & 158.0924 & 79.5498 & & & $\mathbf{1}$ \\
\hline
\end{tabular}
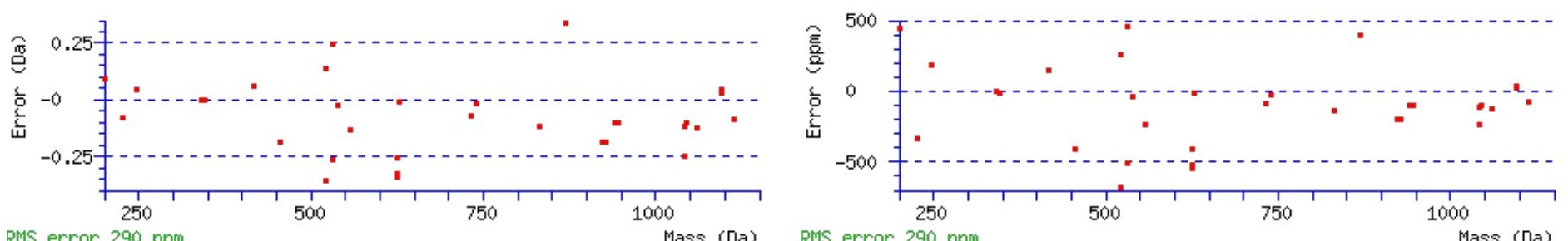

RMS error $290 \mathrm{ppm}$

Mass (Da) RMS error $290 \mathrm{ppm}$

\section{All matches to this query}

\begin{tabular}{|l|l|l|l|}
\hline Score & Mr(calc): & Delta & \multicolumn{1}{c|}{ Sequence } \\
\hline 59.0 & 1285.6877 & -0.0016 & LLNLTSPEATAR \\
\hline 7.6 & 1285.6891 & -0.0029 & ILIDNWNRSR \\
\hline 7.5 & 1284.6714 & 1.0148 & VLEDFVEIHGK \\
\hline 6.0 & 1285.6795 & 0.0067 & AAGAAVPKPTPVK \\
\hline 5.6 & 1283.6807 & 2.0055 & TAAPSGRGGRGAAR \\
\hline 4.7 & 1285.6795 & 0.0067 & LVESFRKTVK \\
\hline 4.5 & 1285.6738 & 0.0124 & ARGGLGAEEASLR \\
\hline 3.4 & 1285.6990 & -0.0128 & RNADVNLKNLK \\
\hline 3.2 & 1285.6738 & 0.0123 & LIQEQTRQDR \\
\hline & & & \\
\hline
\end{tabular}




\section{|2.9 ||1285.6990|-0.0128|GLNIALVNGTTGR}

Spectrum No: 551; Query: 160; Rank: 1

\section{Peptide View}

MS/MS Fragmentation of LNVTSPDLFR

Found in IPI00190577, Tax_Id=10116 Gene_Symbol=Lama5 similar to Laminin alpha-5 chain precursor

Match to Query 160: 1161.604488 from(581.809520,2+)

Title: 100101RatKid_NS_deglyco_21.3122.3122.2.dta

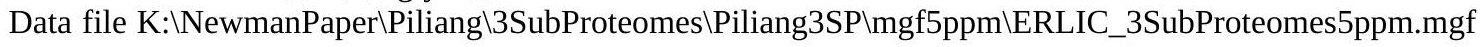
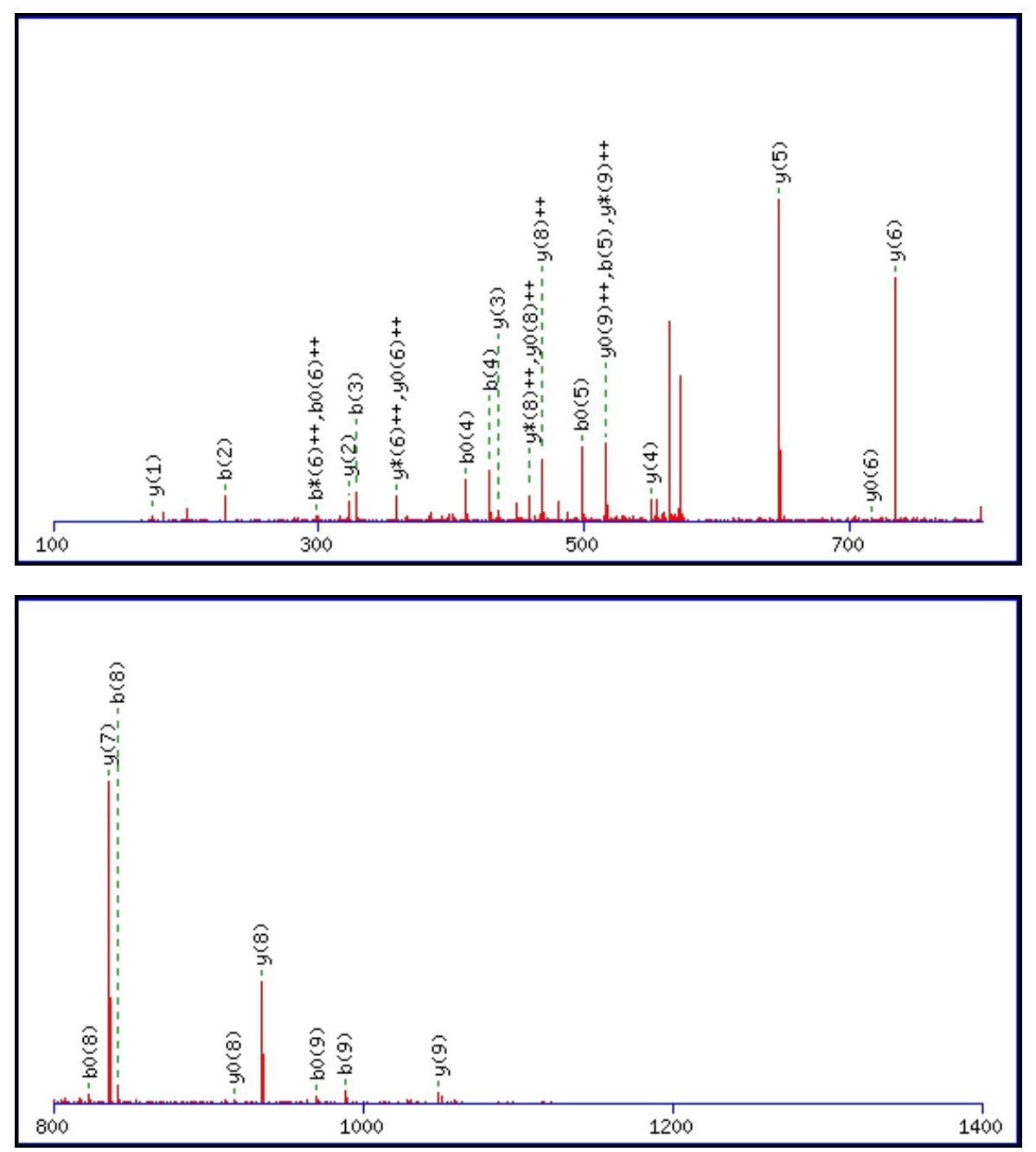
Spectrum No: 552; Query: 181; Rank: 1

\section{Peptide View}

MS/MS Fragmentation of DGVANVSIEDR

Found in IPI00231643, Tax_Id=10116 Gene_Symbol=Sod1 Superoxide dismutase

Match to Query 181: 1174.543168 from(588.278860,2+)

Title: 091008RatKidney_NoSalt_23.948.948.2.dta

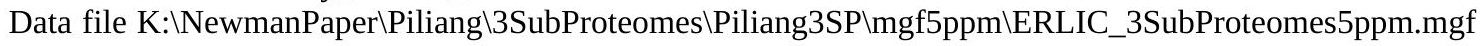
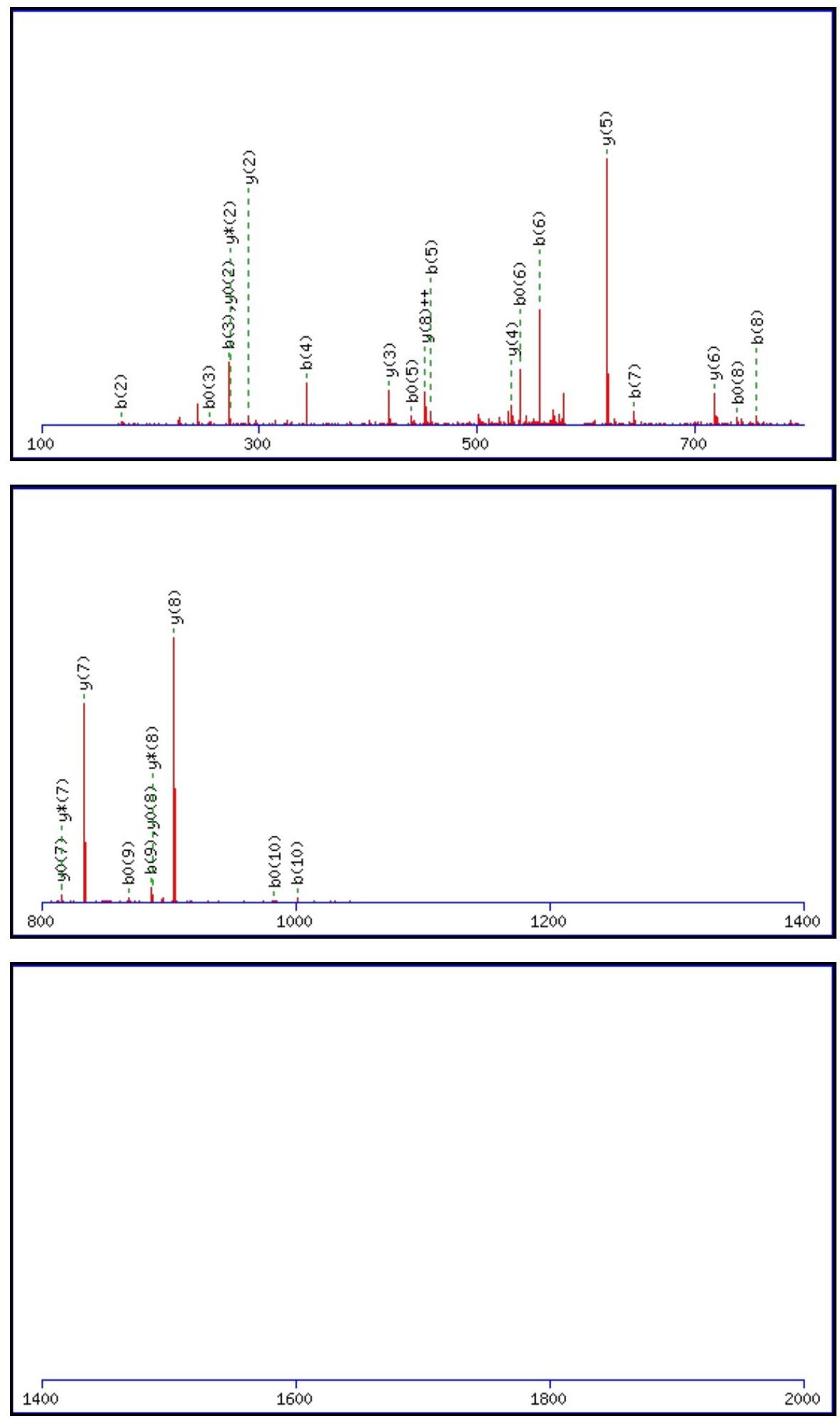

Monoisotopic mass of neutral peptide $\operatorname{Mr}($ calc): 1174.5466 
Fixed modifications: Carbamidomethyl (C)

Variable modifications:

N5: : Deamidated $\mathrm{N}(\mathrm{N})$

Ions Score: 59 Expect: 0.00015

Matches (Bold Red): 29/110 fragment ions using 54 most intense peaks

\begin{tabular}{|c|c|c|c|c|c|c|c|c|c|c|c|c|c|c|}
\hline$\#$ & b & $\mathbf{b}^{++}$ & $\mathbf{b}^{*}$ & $\mathbf{b}^{*^{++}}$ & $\mathbf{b}^{0}$ & $\mathbf{b}^{0++}$ & Seq. & $\mathbf{y}$ & $\mathbf{y}^{++}$ & $\mathbf{y}^{*}$ & $\mathrm{y}^{*^{++}}$ & $\mathbf{y}^{\mathbf{0}}$ & $y^{0++}$ & $\#$ \\
\hline 1 & 116.0342 & 58.5207 & & & 98.0237 & 49.5155 & D & & & & & & & 11 \\
\hline 2 & 173.0557 & 87.0315 & & & 155.0451 & 78.0262 & G & 1060.5269 & 530.7671 & 1043.5004 & 522.2538 & 1042.5164 & 521.7618 & 10 \\
\hline 3 & 272.1241 & 136.5657 & & & 254.1135 & 127.5604 & $\mathbf{V}$ & 1003.5055 & 502.2564 & 986.4789 & 493.7431 & 985.4949 & 493.2511 & 9 \\
\hline 4 & 343.1612 & 172.0842 & & & 325.1506 & 163.0790 & A & 904.4370 & 452.7222 & 887.4105 & 444.2089 & 886.4265 & 443.7169 & 8 \\
\hline 5 & 458.1881 & 229.5977 & 441.1616 & 221.0844 & 440.1776 & 220.5924 & $\mathbf{N}$ & 833.3999 & 417.2036 & 816.3734 & 408.6903 & 815.3894 & 408.1983 & 7 \\
\hline 6 & 557.2566 & 279.1319 & 540.2300 & 270.6186 & 539.2460 & 270.1266 & V & 718.3730 & 359.6901 & 701.3464 & 351.1769 & 700.3624 & 350.6849 & 6 \\
\hline 7 & 644.2886 & 322.6479 & 627.2620 & 314.1347 & 626.2780 & 313.6427 & $\mathrm{~S}$ & 619.3046 & 310.1559 & 602.2780 & 301.6427 & 601.2940 & 301.1506 & 5 \\
\hline 8 & 757.3727 & 379.1900 & 740.3461 & 370.6767 & \begin{tabular}{|l|}
739.3621 \\
\end{tabular} & 370.1847 & $\mathbf{I}$ & 532.2726 & 266.6399 & 515.2460 & 258.1266 & 514.2620 & 257.6346 & 4 \\
\hline 9 & \begin{tabular}{|l|}
886.4152 \\
\end{tabular} & 443.7113 & 869.3887 & 435.1980 & \begin{tabular}{|l|}
868.4047 \\
\end{tabular} & 434.7060 & $\mathbf{E}$ & 419.1885 & 210.0979 & 402.1619 & 201.5846 & 401.1779 & 201.0926 & 3 \\
\hline 10 & 1001.4422 & 501.2247 & 984.4156 & 492.7115 & 983.4316 & 492.2195 & D & 290.1459 & 145.5766 & 273.1193 & 137.0633 & 272.1353 & 136.5713 & 2 \\
\hline 11 & & & & & & & $\mathbf{R}$ & 175.1190 & 88.0631 & 158.0924 & 79.5498 & & & 1 \\
\hline
\end{tabular}
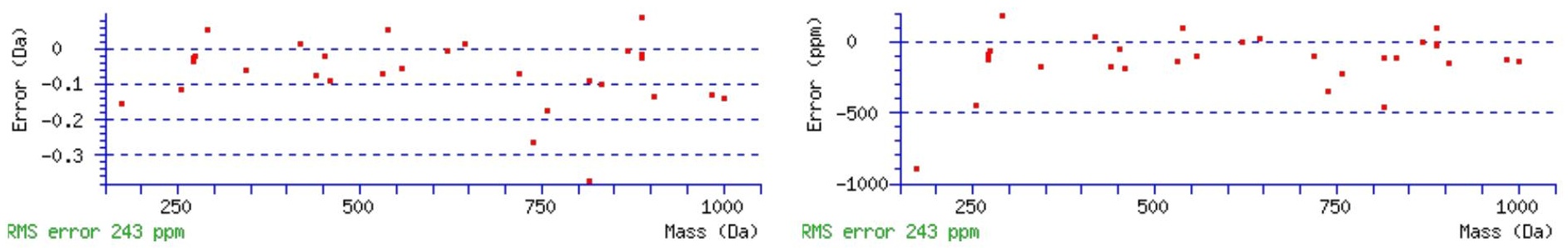

\section{All matches to this query}

\begin{tabular}{|l|l|l|l|}
\hline Score & Mr(calc): & Delta & \multicolumn{1}{|c|}{ Sequence } \\
\hline 58.9 & 1174.5466 & -0.0034 & DGVANVSIEDR \\
\hline 14.4 & 1174.5424 & 0.0008 & EVTFSFLPR \\
\hline 10.8 & 1174.5466 & -0.0034 & ELNTVNDVDR \\
\hline 9.4 & 1173.5292 & 1.0140 & ATAQLSHNPR \\
\hline 8.4 & 1174.5495 & -0.0064 & KNVPEKHSR \\
\hline 6.5 & 1174.5383 & 0.0048 & ERGTPPVDPK \\
\hline 4.8 & 1174.5327 & 0.0105 & EKDRDNQDR \\
\hline 3.5 & 1174.5401 & 0.0031 & ATVPAGSANCAR \\
\hline 2.1 & 1174.5326 & 0.0105 & EDENRNKNR \\
\hline 1.3 & 1174.5474 & -0.0043 & LMMDPLGQNR \\
\hline
\end{tabular}

Spectrum No: 553; Query: 857; Rank: 1

\section{Peptide View}

MS/MS Fragmentation of DFVEIWENHTSGR

Found in IPI00196620, Tax_Id=10116 Gene_Symbol=Cubn Cubilin precursor

Match to Query 857: 1589.716208 from(795.865380,2+)

Title: 100101RatKid_NS_deglyco_14.3807.3807.2.dta

Data file K:INewmanPaper|Piliangl3SubProteomes\Piliang3SP\mgf5ppm\ERLIC_3SubProteomes5ppm.mgf 

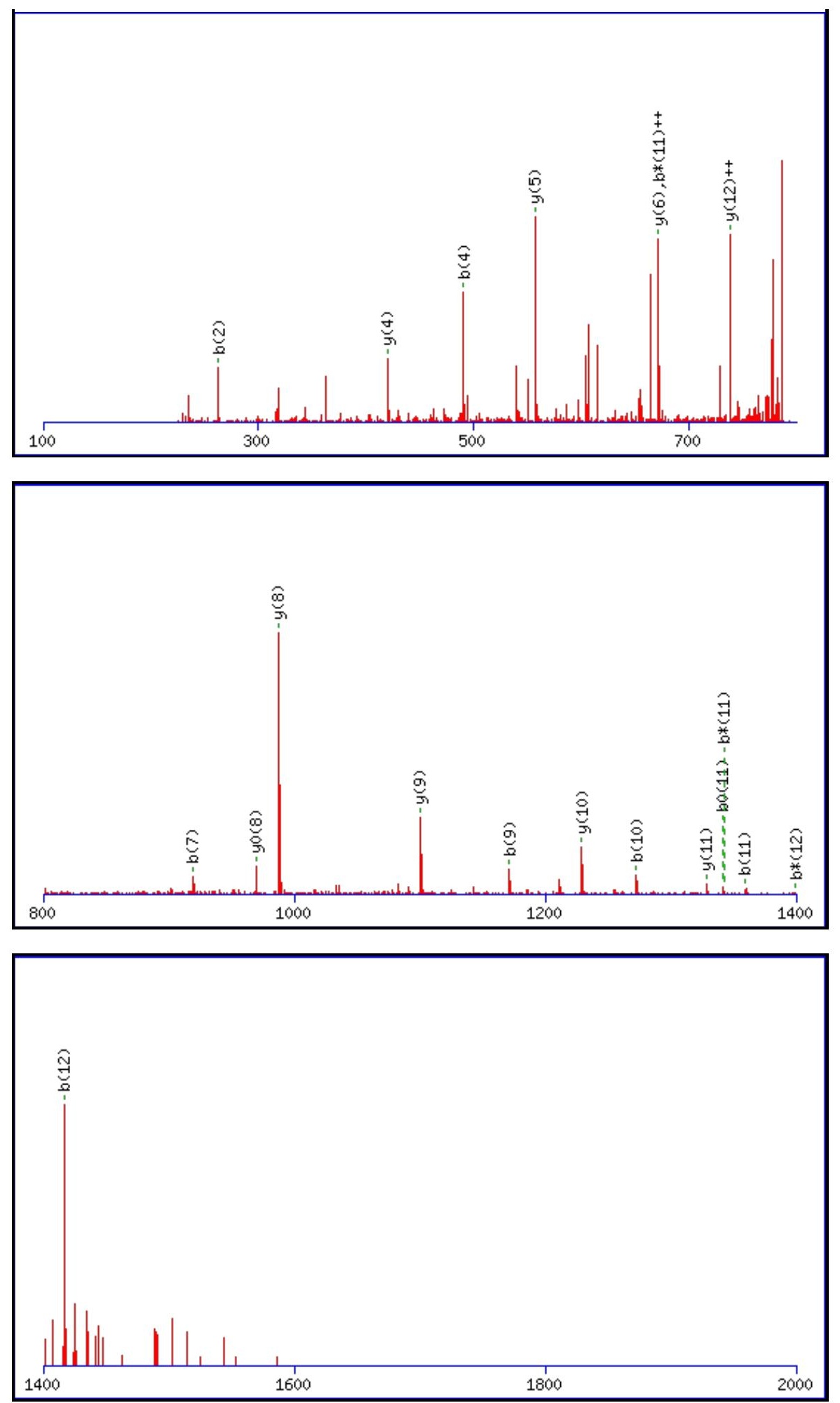

Monoisotopic mass of neutral peptide $\operatorname{Mr}($ calc): 1589.7110

Fixed modifications: Carbamidomethyl (C)

Variable modifications:

N8 : Deamidated $\mathrm{N}(\mathrm{N})$

Ions Score: 59 Expect: 0.00028

Matches (Bold Red): 20/126 fragment ions using 40 most intense peaks

\begin{tabular}{|r|c|c|c|c|r|r|r|r|c|c|c|c|c|c|}
\hline$\#$ & $\mathbf{b}$ & $\mathbf{b}^{++}$ & $\mathbf{b}^{*}$ & $\mathbf{b}^{\boldsymbol{*}^{++}}$ & $\mathbf{b}^{\mathbf{0}}$ & $\mathbf{b}^{\mathbf{0 + +}}$ & Seq. & $\mathbf{y}$ & $\mathbf{y}^{++}$ & $\mathbf{y}^{\mathbf{*}}$ & $\mathbf{y}^{\boldsymbol{*}^{++}}$ & $\mathbf{y}^{\mathbf{0}}$ & $\mathbf{y}^{\mathbf{0 + +}}$ & $\#$ \\
\hline $\mathbf{1}$ & 116.0342 & 58.5207 & & & 98.0237 & 49.5155 & $\mathbf{D}$ & & & & & & & $\mathbf{1 3}$ \\
\hline $\mathbf{2}$ & $\mathbf{2 6 3 . 1 0 2 6}$ & 132.0550 & & & 245.0921 & 123.0497 & $\mathbf{F}$ & 1475.6914 & 738.3493 & 1458.6648 & 729.8360 & 1457.6808 & 729.3440 & $\mathbf{1 2}$ \\
\hline $\mathbf{3}$ & 362.1710 & 181.5892 & & & 344.1605 & 172.5839 & $\mathbf{V}$ & $\mathbf{1 3 2 8 . 6 2 2 9}$ & 664.8151 & 1311.5964 & 656.3018 & 1310.6124 & 655.8098 & $\mathbf{1 1}$ \\
\hline $\mathbf{4}$ & $\mathbf{4 9 1 . 2 1 3 6}$ & 246.1105 & & & 473.2031 & 237.1052 & $\mathbf{E}$ & $\mathbf{1 2 2 9 . 5 5 4 5}$ & 615.2809 & 1212.5280 & 606.7676 & 1211.5440 & 606.2756 & $\mathbf{1 0}$ \\
\hline $\mathbf{5}$ & 604.2977 & 302.6525 & & & 586.2871 & 293.6472 & $\mathbf{I}$ & $\mathbf{1 1 0 0 . 5 1 1 9}$ & 550.7596 & 1083.4854 & 542.2463 & 1082.5014 & 541.7543 & $\mathbf{9}$ \\
\hline
\end{tabular}




\begin{tabular}{|r|r|r|r|r|r|r|r|r|r|r|r|r|r|r|r|r|r|}
$\mathbf{6}$ & 790.3770 & 395.6921 & & & 772.3665 & 386.6869 & $\mathbf{W}$ & $\mathbf{9 8 7 . 4 2 7 9}$ & $\mathbf{4 9 4 . 2 1 7 6}$ & $\mathbf{9 7 0 . 4 0 1 3}$ & 485.7043 & $\mathbf{9 6 9 . 4 1 7 3}$ & $\mathbf{4 8 5 . 2 1 2 3}$ & $\mathbf{8}$ \\
\hline $\mathbf{7}$ & $\mathbf{9 1 9 . 4 1 9 6}$ & 460.2134 & & & 901.4090 & 451.2082 & E & 801.3486 & 401.1779 & 784.3220 & 392.6646 & 783.3380 & 392.1726 & $\mathbf{7}$ \\
\hline $\mathbf{8}$ & 1034.4465 & 517.7269 & 1017.4200 & 509.2136 & 1016.4360 & 508.7216 & N & $\mathbf{6 7 2 . 3 0 6 0}$ & 336.6566 & 655.2794 & 328.1433 & 654.2954 & 327.6513 & $\mathbf{6}$ \\
\hline $\mathbf{9}$ & $\mathbf{1 1 7 1 . 5 0 5 5}$ & 586.2564 & 1154.4789 & 577.7431 & 1153.4949 & 577.2511 & H & 557.2790 & 279.1432 & 540.2525 & 270.6299 & 539.2685 & 270.1379 & $\mathbf{5}$ \\
\hline $\mathbf{1 0}$ & $\mathbf{1 2 7 2 . 5 5 3 1}$ & 636.7802 & 1255.5266 & 628.2669 & 1254.5426 & 627.7749 & T & $\mathbf{4 2 0 . 2 2 0 1}$ & 210.6137 & 403.1936 & 202.1004 & 402.2096 & 201.6084 & $\mathbf{4}$ \\
\hline $\mathbf{1 1}$ & $\mathbf{1 3 5 9 . 5 8 5 2}$ & 680.2962 & $\mathbf{1 3 4 2 . 5 5 8 6}$ & $\mathbf{6 7 1 . 7 8 2 9}$ & $\mathbf{1 3 4 1 . 5 7 4 6}$ & 671.2909 & S & 319.1724 & 160.0899 & 302.1459 & 151.5766 & 301.1619 & 151.0846 & $\mathbf{3}$ \\
\hline $\mathbf{1 2}$ & $\mathbf{1 4 1 6 . 6 0 6 6}$ & $\mathbf{7 0 8 . 8 0 7 0}$ & $\mathbf{1 3 9 9 . 5 8 0 1}$ & $\mathbf{7 0 0 . 2 9 3 7}$ & 1398.5961 & 699.8017 & G & 232.1404 & 116.5738 & 215.1139 & 108.0606 & & & $\mathbf{2}$ \\
\hline $\mathbf{1 3}$ & & & & & & & R & 175.1190 & 88.0631 & 158.0924 & $\mathbf{7 9 . 5 4 9 8}$ & & & $\mathbf{1}$ \\
\hline
\end{tabular}
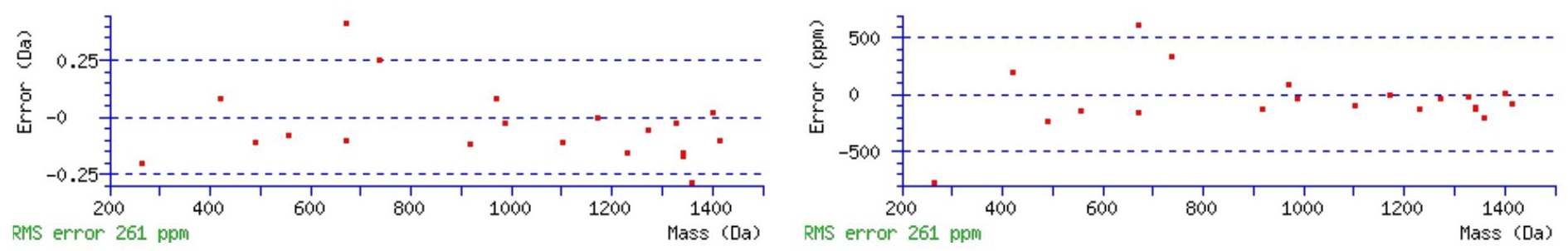

\section{All matches to this query}

\begin{tabular}{|l|l|c|l|}
\hline Score & Mr(calc): & Delta & \multicolumn{1}{c|}{ Sequence } \\
\hline 58.8 & 1589.7110 & 0.0052 & DFVEIWENHTSGR \\
\hline 13.9 & 1588.7270 & 0.9892 & DFVEIWENHTSGR \\
\hline 8.6 & 1589.7062 & 0.0100 & SCIQWLAVSFSGR \\
\hline 5.7 & 1587.6977 & 2.0185 & HTHEMRVLEGQR \\
\hline 3.4 & 1589.7281 & -0.0119 & RDSELQEQNETNK \\
\hline 3.3 & 1589.7120 & 0.0042 & SMKGAGTDEKTLTR \\
\hline 2.5 & 1587.7004 & 2.0158 & MARYSLDPETPTK \\
\hline 2.5 & 1587.6970 & 2.0192 & YPPNSESKVSWSK \\
\hline 2.2 & 1587.7103 & 2.0060 & LNETLEEMKSVAK \\
\hline 1.6 & 1587.7198 & 1.9964 & $\underline{\text { LGPEKSINTEVLK }}$ \\
\hline
\end{tabular}

Spectrum No: 554; Query: 2398; Rank: 1

\section{Peptide View}

MS/MS Fragmentation of TDDEVVQREEEAIQLDGLNASQIR

Found in IPI00365985, Tax_Id=10116 Gene_Symbol=Tra1_predicted 93 kDa protein

Match to Query 2398: 2728.320942 from(910.447590,3+)

Title: 100101RatKid_NS_deglyco_15.4468.4468.3.dta

Data file K:INewmanPaper|Piliang|3SubProteomes\Piliang3SP\mgf5ppm\ERLIC_3SubProteomes5ppm.mgf 

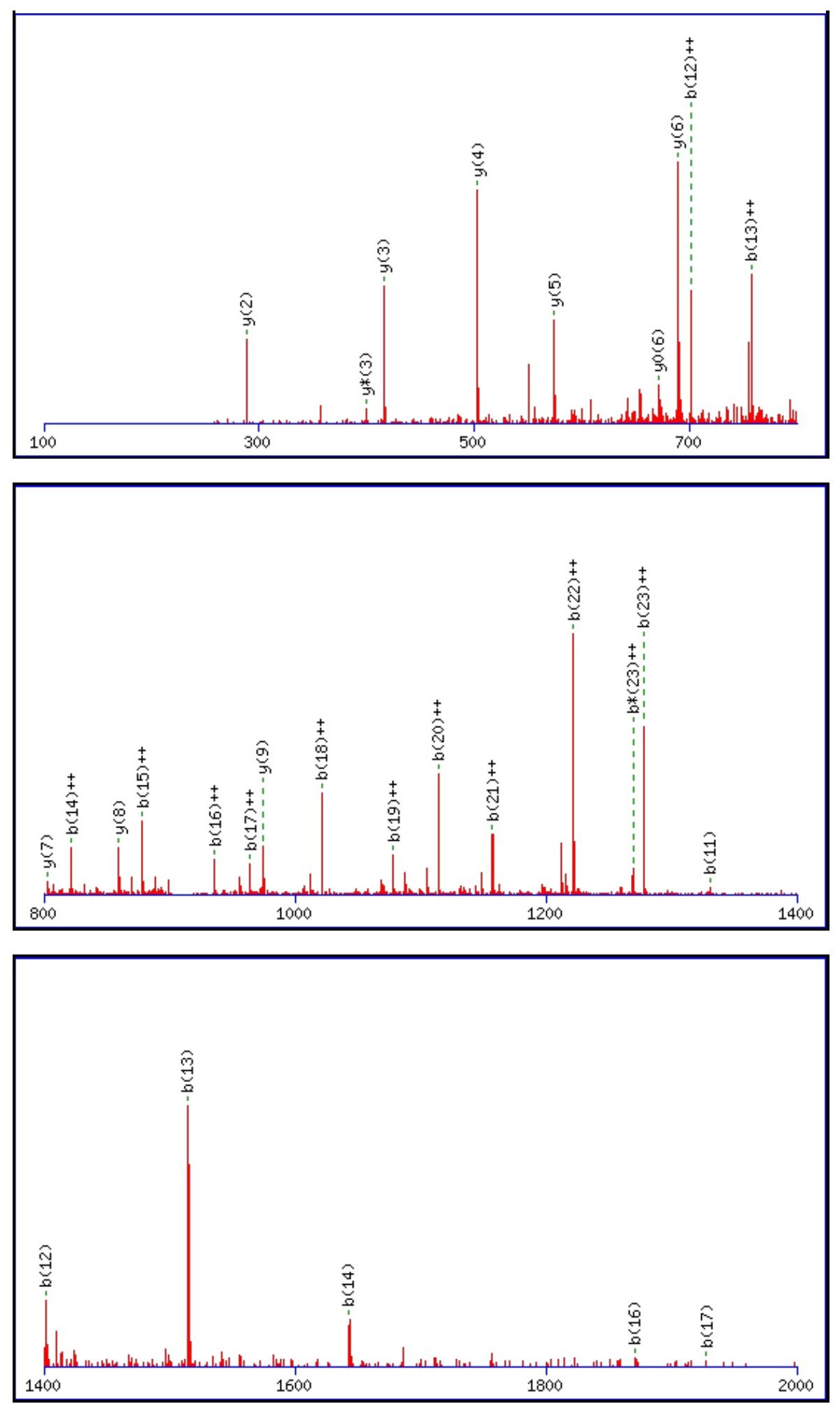

Monoisotopic mass of neutral peptide $\operatorname{Mr}($ calc): 2728.3151

Fixed modifications: Carbamidomethyl (C)

Variable modifications:

N19 : Deamidated_N (N)

Ions Score: 59 Expect: 0.00035

Matches (Bold Red): 29/258 fragment ions using 71 most intense peaks

\begin{tabular}{|c|c|c|c|c|c|c|c|c|c|c|c|c|c|c|}
\hline$\#$ & b & $\mathbf{b}^{++}$ & $\mathbf{b}^{*}$ & $\mathbf{b}^{*^{++}}$ & $\mathbf{b}^{\mathbf{0}}$ & $\mathbf{b}^{0++}$ & Seq. & $\mathbf{y}$ & $y^{++}$ & $\mathbf{y}^{*}$ & $y^{*^{++}}$ & $\mathbf{y}^{0}$ & $y^{0++}$ & $\#$ \\
\hline 1 & 102.0550 & 51.5311 & & & 84.0444 & 42.5258 & $\mathbf{T}$ & & & & & & & 24 \\
\hline 2 & 217.0819 & 109.0446 & & & 199.0713 & 100.0393 & D & 2628.2747 & 1314.6410 & 2611.2482 & 1306.1277 & 2610.2642 & 1305.6357 & 23 \\
\hline 3 & 332.1088 & 166.5581 & & & 314.0983 & 157.5528 & D & 2513.2478 & 1257.1275 & 2496.2212 & 1248.6143 & 2495.2372 & 1248.1223 & 22 \\
\hline 4 & 461.1514 & 231.0794 & & & 443.1409 & 222.0741 & $\mathbf{E}$ & 2398.2209 & 1199.6141 & 2381.1943 & 1191.1008 & 2380.2103 & 1190.6088 & 21 \\
\hline 5 & 560.2198 & 280.6136 & & & 542.2093 & 271.6083 & $\mathbf{V}$ & 2269.1783 & 1135.0928 & 2252.1517 & 1126.5795 & 2251.1677 & 1126.0875 & 20 \\
\hline
\end{tabular}




\begin{tabular}{|c|c|c|c|c|c|c|c|c|c|c|c|c|c|c|}
\hline 6 & 659.2883 & 330.1478 & & & 641.2777 & 321.1425 & V & 2170.1098 & $|1085.5586|$ & |2153.0833| & 1077.0453 & |2152.0993| & |1076.5533|1 & |19| \\
\hline 7 & 787.3468 & 394.1771 & 770.3203 & 385.6638 & 769.3363 & 385.1718 & $\mathbf{Q}$ & 2071.0414 & 1036.0244 & 2054.0149 & 1027.5111 & 2053.0309 & 1027.0191 & 18 \\
\hline 8 & 943.4480 & 472.2276 & 926.4214 & 463.7143 & 925.4374 & 463.2223 & $\mathbf{R}$ & 1942.9829 & 971.9951 & 1925.9563 & 963.4818 & 1924.9723 & 962.9898 & 17 \\
\hline 9 & 1072.4905 & 536.7489 & 1055.4640 & 528.2356 & 1054.4800 & 527.7436 & $\mathbf{E}$ & 1786.8817 & 893.9445 & 1769.8552 & 885.4312 & 1768.8712 & 884.9392 & 16 \\
\hline 10 & 1201.5331 & 601.2702 & 1184.5066 & 592.7569 & |1183.5226 & 592.2649 & $\mathbf{E}$ & 1657.8391 & 829.4232 & 1640.8126 & 820.9099 & 1639.8286 & 820.4179 & 15 \\
\hline 11 & 1330.5757 & 665.7915 & 1313.5492 & 657.2782 & 1312.5652 & 656.7862 & $\bar{E}$ & 1528.7966 & 764.9019 & 1511.7700 & 756.3886 & 1510.7860 & 755.8966 & 14 \\
\hline 12 & 1401.6128 & 701.3101 & 1384.5863 & 692.7968 & 1383.6023 & 692.3048 & A & 1399.7540 & 700.3806 & 1382.7274 & 691.8673 & 1381.7434 & 691.3753 & 13 \\
\hline 13 & 1514.6969 & 757.8521 & 1497.6704 & 749.3388 & 1496.6863 & 748.8468 & I & 1328.7168 & 664.8621 & 1311.6903 & 656.3488 & 1310.7063 & 655.8568 & 12 \\
\hline 14 & 1642.7555 & 821.8814 & 1625.7289 & 813.3681 & 1624.7449 & 812.8761 & $\mathbf{Q}$ & 1215.6328 & 608.3200 & 1198.6062 & 599.8068 & 1197.6222 & 599.3147 & 11 \\
\hline 15 & 1755.8396 & 878.4234 & 1738.8130 & 869.9101 & 1737.8290 & 869.4181 & $\mathbf{L}$ & 1087.5742 & 544.2907 & 1070.5477 & 535.7775 & 1069.5636 & 535.2855 & 10 \\
\hline 16 & 1870.8665 & 935.9369 & 1853.8399 & 927.4236 & 1852.8559 & 926.9316 & D & 974.4901 & 487.7487 & 957.4636 & 479.2354 & 956.4796 & 478.7434 & 9 \\
\hline 17 & 1927.8880 & 964.4476 & 1910.8614 & 955.9343 & 1909.8774 & 955.4423 & G & 859.4632 & 430.2352 & 842.4367 & 421.7220 & 841.4526 & 421.2300 & 8 \\
\hline 182 & 2040.9720 & 1020.9896 & 2023.9455 & 1012.4764 & 2022.9615 & 1011.9844 & $\mathbf{L}$ & 802.4417 & 401.7245 & 785.4152 & 393.2112 & 784.4312 & 392.7192 & 7 \\
\hline 192 & 2155.9990 & 1078.5031 & 2138.9724 & 1069.9898 & 2137.9884 & 1069.4978 & $\mathbf{N}$ & 689.3577 & 345.1825 & 672.3311 & 336.6692 & 671.3471 & 336.1772 & 6 \\
\hline 202 & 2227.0361 & 1114.0217 & 2210.0095 & 1105.5084 & 2209.0255 & 1105.0164 & A & 574.3307 & 287.6690 & 557.3042 & 279.1557 & 556.3202 & 278.6637 & 5 \\
\hline 212 & 2314.0681 & 1157.5377 & 2297.0415 & 1149.0244 & 2296.0575 & 1148.5324 & $\mathbf{S}$ & 503.2936 & 252.1504 & 486.2671 & 243.6372 & 485.2831 & 243.1452 & 4 \\
\hline 222 & 2442.1267 & 1221.5670 & 2425.1001 & 1213.0537 & 2424.1161 & 1212.5617 & $\mathbf{Q}$ & 416.2616 & 208.6344 & 399.2350 & 200.1212 & & & 3 \\
\hline 232 & 2555.2107 & 1278.1090 & 2538.1842 & 1269.5957 & 2537.2002 & 1269.1037 & I & 288.2030 & 144.6051 & 271.1765 & 136.0919 & & & 2 \\
\hline 24 & & & & & & & $\mathbf{R}$ & 175.1190 & 88.0631 & 158.0924 & 79.5498 & & & 1 \\
\hline
\end{tabular}
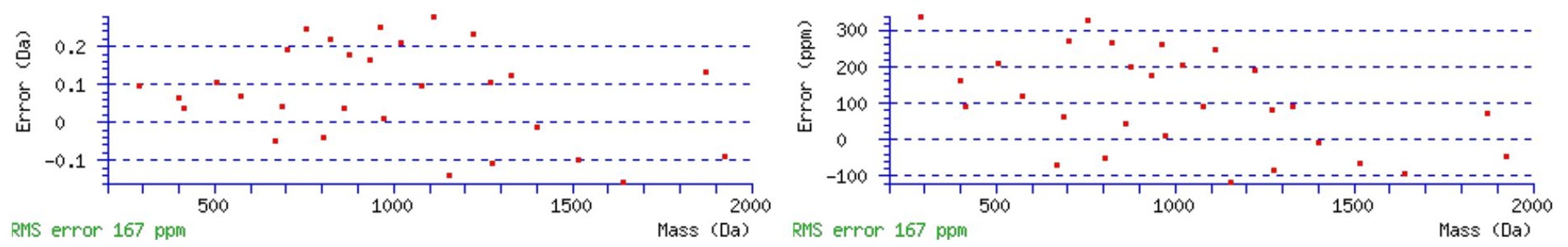

\section{All matches to this query}

\begin{tabular}{|l|c|c|c|}
\hline Score & Mr(calc): & Delta & \multicolumn{1}{c|}{ Sequence } \\
\hline 58.8 & 2728.3151 & 0.0058 & TDDEVVQREEEAIQLDGLNASQIR \\
\hline 25.0 & 2727.3311 & 0.9898 & TDDEVVQREEEAIQLDGLNASQIR \\
\hline 5.5 & 2728.2996 & 0.0213 & FEKSVYEADLAENSAPGTPILQLR \\
\hline 3.6 & 2728.3354 & -0.0145 & VKDLLCTSSSTPGESLETKNLTLR \\
\hline 3.6 & 2728.3354 & -0.0145 & VKDLLCTSSSTPGESLETKNLTLR \\
\hline 3.6 & 2728.3354 & -0.0145 & VKDLLCTSSSTPGESLETKNLTLR \\
\hline 3.6 & 2728.3354 & -0.0145 & VKDLLCTSSSTPGESLETKNLTLR \\
\hline 3.6 & 2728.3354 & -0.0145 & VKDLLCTSSSTPGESLETKNLTLR \\
\hline 3.5 & 2728.3354 & -0.0145 & VKDLLCTSSSTPGESLETKNLTLR \\
\hline 2.8 & 2728.2996 & 0.0213 & FEKSVYEADLAENSAPGTPILQLR \\
\hline
\end{tabular}

Spectrum No: 555; Query: 2390; Rank: 1

\section{Peptide View}

MS/MS Fragmentation of YIAENGTDPINNQPLSEEQLIDIK

Found in IPI00204772, Tax_Id=10116 Gene_Symbol=Prpf19 Pre-mRNA-processing factor 19

Match to Query 2390: 2715.340568 from(1358.677560,2+)

Title: 100101RatKid_NS_deglyco_14.4454.4454.2.dta

Data file K:INewmanPaper|Piliangl3SubProteomes\Piliang3SP\mgf5ppm\ERLIC_3SubProteomes5ppm.mgf 

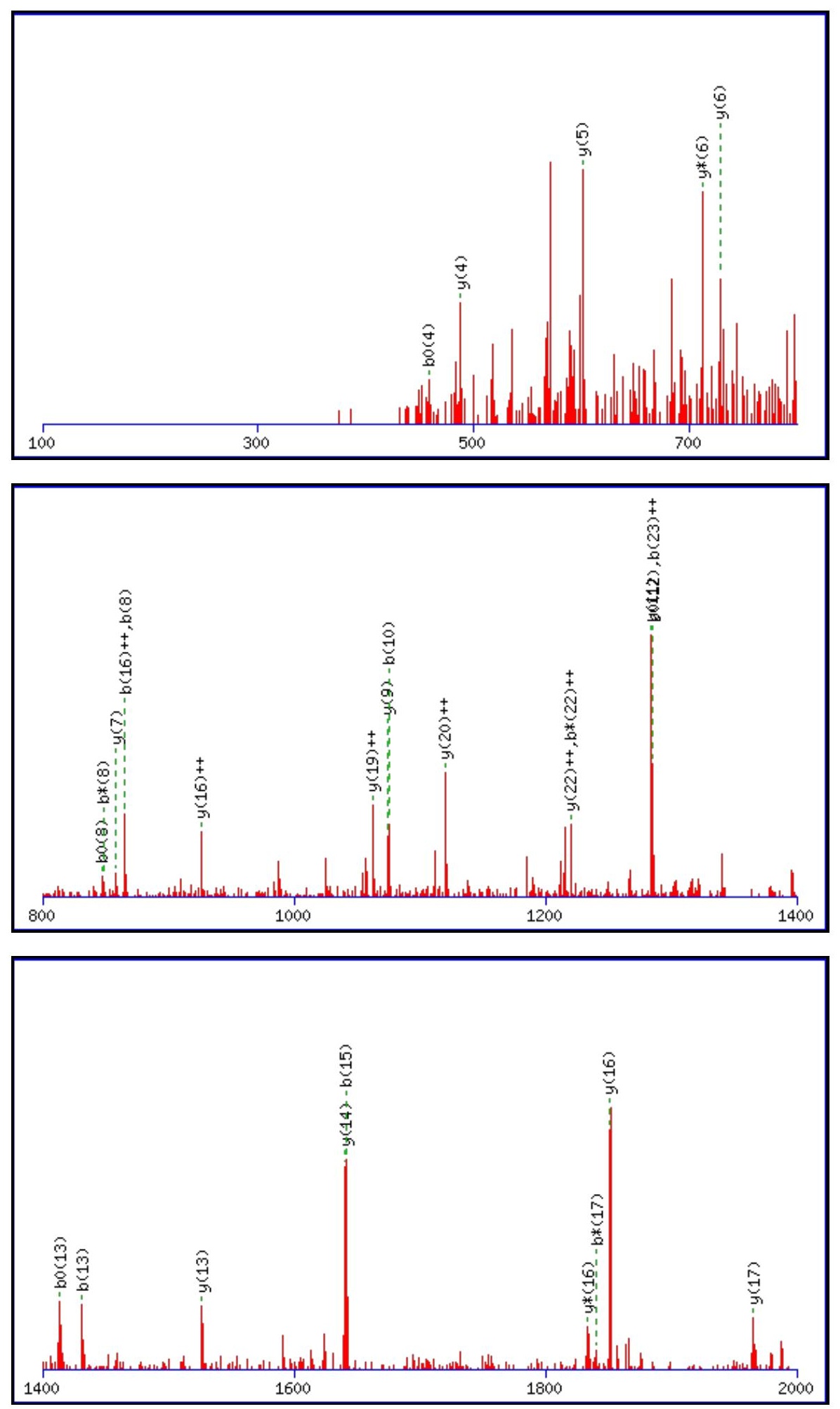

Monoisotopic mass of neutral peptide $\operatorname{Mr}$ (calc): 2714.3286

Fixed modifications: Carbamidomethyl (C)

Variable modifications:

N5 : Deamidated_N (N)

Ions Score: 59 Expect: $\odot .00031$

Matches (Bold Red): 29/258 fragment ions using 42 most intense peaks

\begin{tabular}{|c|c|c|c|c|c|c|c|c|c|c|c|c|c|c|}
\hline$\#$ & $\mathbf{b}$ & $\mathbf{b}^{++}$ & $\mathbf{b}^{*}$ & $\mathbf{b}^{\boldsymbol{*}^{++}}$ & $\mathbf{b}^{\mathbf{0}}$ & $\mathbf{b}^{\mathbf{0 + +}}$ & Seq. & $\mathbf{y}$ & $\mathbf{y}^{++}$ & $\mathbf{y}^{\mathbf{*}}$ & $\mathbf{y}^{\mathbf{*}^{++}}$ & $\mathbf{y}^{\mathbf{0}}$ & $\mathbf{y}^{\mathbf{0 + +}}$ & $\#$ \\
\hline $\mathbf{1}$ & 164.0706 & 82.5389 & & & & & $\mathbf{Y}$ & & & & & & & $\mathbf{2 4}$ \\
\hline $\mathbf{2}$ & 277.1547 & 139.0810 & & & & & $\mathbf{I}$ & 2552.2726 & 1276.6399 & 2535.2461 & 1268.1267 & 2534.2620 & 1267.6347 & $\mathbf{2 3}$ \\
\hline $\mathbf{3}$ & 348.1918 & 174.5995 & & & & & $\mathbf{A}$ & 2439.1885 & $\mathbf{1 2 2 0 . 0 9 7 9}$ & 2422.1620 & 1211.5846 & 2421.1780 & 1211.0926 & $\mathbf{2 2}$ \\
\hline $\mathbf{4}$ & 477.2344 & 239.1208 & & & $\mathbf{4 5 9 . 2 2 3 8}$ & 230.1155 & $\mathbf{E}$ & 2368.1514 & 1184.5794 & 2351.1249 & 1176.0661 & 2350.1409 & 1175.5741 & $\mathbf{2 1}$ \\
\hline $\mathbf{5}$ & 592.2613 & 296.6343 & 575.2348 & 288.1210 & 574.2507 & 287.6290 & $\mathbf{N}$ & 2239.1088 & $\mathbf{1 1 2 0 . 0 5 8 1}$ & 2222.0823 & 1111.5448 & 2221.0983 & 1111.0528 & $\mathbf{2 0}$ \\
\hline
\end{tabular}




\begin{tabular}{|c|c|c|c|c|c|c|c|c|c|c|c|c|c|c|}
\hline 6 & 649.2828 & 325.1450 & 632.2562 & 316.6318 & 631.2722 & 316.1397 & G & 0819| - - & 1062.5446 & |2107.0554| & 1054.0313 & 2106.0713 & 1053.5393 & $\mid 19$ \\
\hline 7 & 750.3305 & 375.6689 & 733.3039 & 367.1556 & 732.3199 & 366.6636 & $T$ & 2067.0604 & 1034.0339 & 2050.0339 & 1025.5206 & 2049.0499 & 1025.0286 & 18 \\
\hline 8 & 865.3574 & 433.1823 & 848.3308 & 424.6691 & 847.3468 & 424.1771 & D & 1966.0128 & 983.5100 & 1948.9862 & 974.9967 & 1948.0022 & 974.5047 & 17 \\
\hline 9 & 962.4102 & 481.7087 & 945.3836 & 473.1954 & 944.3996 & 472.7034 & $\mathbf{P}$ & 1850.9858 & 925.9965 & 1833.9593 & 917.4833 & 1832.9753 & 916.9913 & 16 \\
\hline 10 & 1075.4942 & 538.2508 & 1058.4677 & 529.7375 & 1057.4837 & 529.2455 & I & 1753.9331 & 877.4702 & 1736.9065 & 868.9569 & 1735.9225 & 868.4649 & 15 \\
\hline 11 & 1189.5372 & 595.2722 & 1172.5106 & 586.7589 & 1171.5266 & 586.2669 & $\mathbf{N}$ & 1640.8490 & 820.9281 & 1623.8224 & 812.4149 & 1622.8384 & 811.9229 & 14 \\
\hline 12 & 1303.5801 & 652.2937 & 1286.5535 & 643.7804 & 1285.5695 & 643.2884 & $\mathbf{N}$ & 1526.8061 & 763.9067 & 1509.7795 & 755.3934 & 1508.7955 & 754.9014 & 13 \\
\hline 13 & 1431.6387 & 716.3230 & 1414.6121 & 707.8097 & 1413.6281 & 707.3177 & $\mathbf{Q}$ & 1412.7631 & 706.8852 & 1395.7366 & 698.3719 & 1394.7526 & 697.8799 & 12 \\
\hline 14 & 1528.6914 & 764.8493 & 1511.6649 & 756.3361 & 1510.6809 & 755.8441 & $\mathbf{P}$ & 1284.7046 & 642.8559 & 1267.6780 & 634.3426 & 1266.6940 & 633.8506 & 11 \\
\hline 15 & 1641.7755 & 821.3914 & 1624.7489 & 812.8781 & 1623.7649 & 812.3861 & $\mathbf{L}$ & 1187.6518 & 594.3295 & 1170.6252 & 585.8163 & 1169.6412 & 585.3243 & 10 \\
\hline 16 & 1728.8075 & 864.9074 & 1711.7810 & 856.3941 & 1710.7969 & 855.9021 & $S$ & 1074.5677 & 537.7875 & 1057.5412 & 529.2742 & 1056.5572 & 528.7822 & 9 \\
\hline 17 & 1857.8501 & 929.4287 & 1840.8236 & 920.9154 & 1839.8395 & 920.4234 & $\mathbf{E}$ & 987.5357 & 494.2715 & 970.5092 & 485.7582 & 969.5251 & 485.2662 & 8 \\
\hline 18 & 1986.8927 & 993.9500 & 1969.8662 & 985.4367 & 1968.8821 & 984.9447 & $\mathbf{E}$ & 858.4931 & 429.7502 & 841.4666 & 421.2369 & 840.4825 & 420.7449 & 7 \\
\hline 19 & 2114.9513 & 1057.9793 & 2097.9247 & 1049.4660 & 2096.9407 & 1048.9740 & $\mathbf{Q}$ & 729.4505 & 365.2289 & 712.4240 & 356.7156 & 711.4400 & 356.2236 & 6 \\
\hline 20 & 2228.0353 & 1114.5213 & 2211.0088 & 1106.0080 & 2210.0248 & 1105.5160 & $\mathbf{L}$ & 601.3919 & 301.1996 & 584.3654 & 292.6863 & 583.3814 & 292.1943 & 5 \\
\hline 21 & 2341.1194 & 1171.0633 & 2324.0929 & 1162.5501 & 2323.1088 & 1162.0581 & I & 488.3079 & 244.6576 & 471.2813 & 236.1443 & 470.2973 & 235.6523 & 4 \\
\hline 22 & 2456.1463 & 1228.5768 & 2439.1198 & 1220.0635 & 2438.1358 & 1219.5715 & D & 375.2238 & 188.1155 & 358.1973 & 179.6023 & 357.2132 & 179.1103 & 3 \\
\hline 23 & 2569.2304 & 1285.1188 & 2552.2039 & 1276.6056 & 2551.2198 & 1276.1136 & I & 260.1969 & 130.6021 & 243.1703 & 122.0888 & & & 2 \\
\hline 24 & & & & & & & $\mathbf{K}$ & 147.1128 & 74.0600 & 130.0863 & 65.5468 & & & 1 \\
\hline
\end{tabular}
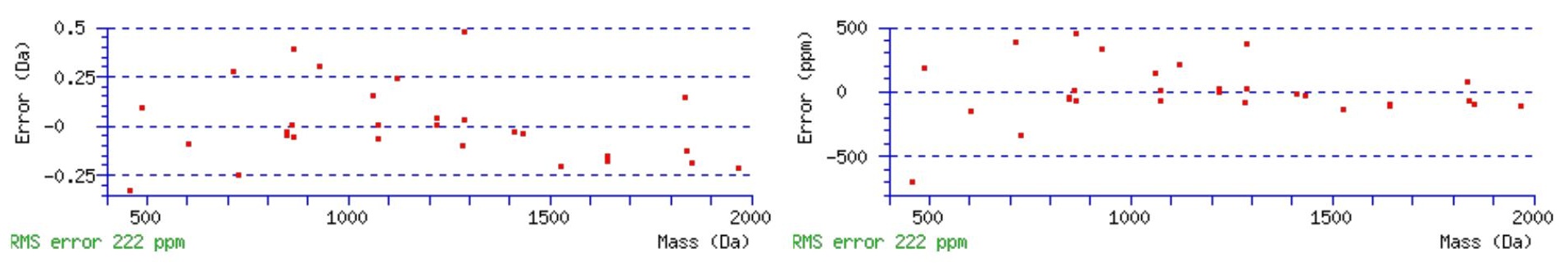

\section{All matches to this query}

\begin{tabular}{|l|l|c|l|}
\hline Score & Mr(calc): & Delta & \multicolumn{1}{|c|}{ Sequence } \\
\hline 58.7 & 2714.3286 & 1.0120 & YIAENGTDPINNQPLSEEQLIDIK \\
\hline 54.1 & 2714.3286 & 1.0120 & YIAENGTDPINNQPLSEEQLIDIK \\
\hline 54.1 & 2714.3286 & 1.0120 & YIAENGTDPINNQPLSEEQLIDIK \\
\hline 49.5 & 2713.3446 & 1.9960 & YIAENGTDPINNQPLSEEQLIDIK \\
\hline 2.8 & 2713.3455 & 1.9951 & ENQMTPTNLAVCLAPSLFHLNTLK \\
\hline 2.8 & 2713.3455 & 1.9951 & ENQMTPTNLAVCLAPSLFHLNTLK \\
\hline 2.1 & 2713.3605 & 1.9801 & NWDPAOLSRTTQGLTALLLSLKK \\
\hline 2.1 & 2713.3605 & 1.9801 & NWDPAQLSRTTQGLTALLLSLKK \\
\hline 2.1 & 2713.3605 & 1.9801 & NWDPAOLSRTTOGLTALLLSLKK \\
\hline 2.0 & 2713.3480 & 1.9926 & LAEETDGVIVTNEQIHILMNNSKK \\
\hline
\end{tabular}

Spectrum No: 556; Query: 577; Rank: 1

\section{Peptide View}

MS/MS Fragmentation of KICGNETLFPIR

Found in IPI00196620, Tax_Id=10116 Gene_Symbol=Cubn Cubilin precursor

Match to Query 577: 1447.752128 from(724.883340,2+)

Title: 100101RatKid_NS_deglyco_16.2845.2845.2.dta

Data file K:INewmanPaper|Piliangl3SubProteomes\Piliang3SP\mgf5ppm\ERLIC_3SubProteomes5ppm.mgf 

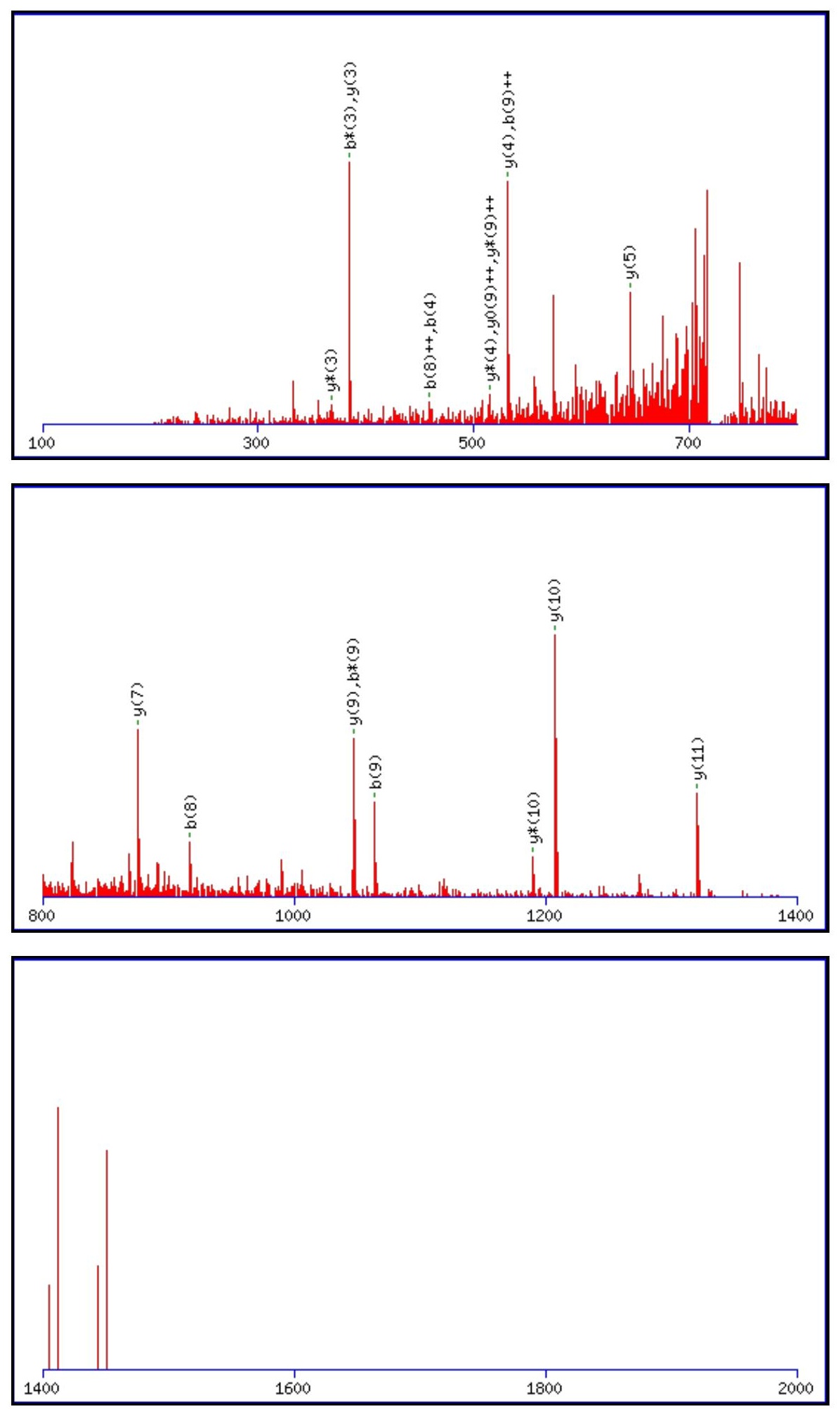

Monoisotopic mass of neutral peptide $\operatorname{Mr}($ calc): 1447.7493 Fixed modifications: Carbamidomethyl (C)

Variable modifications:

N5 : Deamidated_N (N)

Ions Score: 59 Expect: 0.00017

Matches (Bold Red): 19/112 fragment ions using 31 most intense peaks

\begin{tabular}{|r|c|c|c|c|c|c|c|c|c|c|c|c|c|c|}
\hline$\#$ & $\mathbf{b}$ & $\mathbf{b}^{++}$ & $\mathbf{b}^{*}$ & $\mathbf{b}^{\boldsymbol{*}^{++}}$ & $\mathbf{b}^{\mathbf{0}}$ & $\mathbf{b}^{\mathbf{0 + +}}$ & Seq. & $\mathbf{y}$ & $\mathbf{y}^{++}$ & $\mathbf{y}^{\mathbf{*}}$ & $\mathbf{y}^{\boldsymbol{*}^{++}}$ & $\mathbf{y}^{\mathbf{0}}$ & $\mathbf{y}^{\mathbf{0 + +}}$ & $\#$ \\
\hline $\mathbf{1}$ & 129.1022 & 65.0548 & 112.0757 & 56.5415 & & & $\mathbf{K}$ & & & & & & & $\mathbf{1 2}$ \\
\hline $\mathbf{2}$ & 242.1863 & 121.5968 & 225.1598 & 113.0835 & & & $\mathbf{I}$ & $\mathbf{1 3 2 0 . 6 6 1 6}$ & 660.8345 & 1303.6351 & 652.3212 & 1302.6511 & 651.8292 & $\mathbf{1 1}$ \\
\hline $\mathbf{3}$ & 402.2170 & 201.6121 & 385.1904 & 193.0988 & & & $\mathbf{C}$ & $\mathbf{1 2 0 7 . 5 7 7 6}$ & 604.2924 & $\mathbf{1 1 9 0 . 5 5 1 0}$ & 595.7792 & 1189.5670 & 595.2871 & $\mathbf{1 0}$ \\
\hline $\mathbf{4}$ & $\mathbf{4 5 9 . 2 3 8 4}$ & 230.1228 & 442.2119 & 221.6096 & & & $\mathbf{G}$ & $\mathbf{1 0 4 7 . 5 4 6 9}$ & 524.2771 & 1030.5204 & $\mathbf{5 1 5 . 7 6 3 8}$ & 1029.5364 & $\mathbf{5 1 5 . 2 7 1 8}$ & $\mathbf{9}$ \\
\hline $\mathbf{5}$ & 574.2654 & 287.6363 & 557.2388 & 279.1230 & & & $\mathbf{N}$ & 990.5255 & 495.7664 & 973.4989 & 487.2531 & 972.5149 & 486.7611 & $\mathbf{8}$ \\
\hline
\end{tabular}




\begin{tabular}{|c|c|c|c|c|c|c|c|c|c|c|c|c|c|c|}
\hline 6 & 703.3079| & |352.1576 & 686.2814 & |343.6443 & 685.2974 & 343.1523 & E & 875.4985 & |438.2529 & 858.4720 & 429.7396 & 857.4880 & $|429.2476|$ & 7 \\
\hline 7 & 804.3556 & 402.6815 & 787.3291 & |394.1682 & 786.3451 & 393.6762 & $\mathbf{T}$ & 746.4559 & |373.7316 & 729.4294 & 365.2183 & 728.4454 & 364.7263 & 6 \\
\hline 8 & 917.4397 & 459.2235 & 900.4131 & 450.7102 & 899.4291 & 450.2182 & L & 645.4083 & 323.2078 & 628.3817 & 314.6945 & & & 5 \\
\hline 9 & |1064.5081 & 532.7577 & 1047.4816 & 524.2444 & 1046.4975 & 523.7524 & $\mathbf{F}$ & 532.3242 & 266.6657 & 515.2976 & 258.1525 & & & 4 \\
\hline 10 & 1161.5609 & 581.2841 & 1144.5343 & 572.7708 & 1143.5503 & 572.2788 & $\mathbf{P}$ & 385.2558 & |193.1315 & 368.2292 & 184.6183 & & & 3 \\
\hline 11 & 1274.6449 & 637.8261 & 1257.6184 & 629.3128 & 1256.6344 & 628.8208 & I & 288.2030 & 144.6051 & 271.1765 & 136.0919 & & & 2 \\
\hline 12 & & & & & & & $\mathbf{R}$ & 175.1190 & 88.0631 & 158.0924 & 79.5498 & & & 1 \\
\hline
\end{tabular}
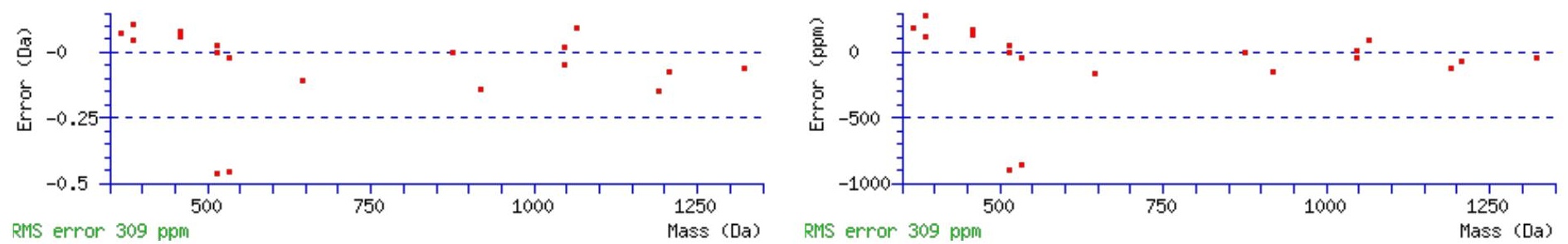

\section{All matches to this query}

\begin{tabular}{|l|l|c|l|}
\hline Score & Mr(calc): & Delta & \multicolumn{1}{|c|}{ Sequence } \\
\hline 58.6 & 1447.7493 & 0.0028 & KICGNETLFPIR \\
\hline 3.3 & 1447.7419 & 0.0102 & KRVGDNDFLEVR \\
\hline 0.9 & 1447.7419 & 0.0102 & KRNFSNSIDLPR \\
\hline 0.8 & 1447.7409 & 0.0112 & RTSRQLGAPAVGR \\
\hline
\end{tabular}

Spectrum No: 557; Query: 1098; Rank: 1

\section{Peptide View}

\section{MS/MS Fragmentation of LDELKNLTSQFQER}

Found in IPI00363534, Tax_Id=10116 Gene_Symbol=Lama1 laminin, alpha 1

Match to Query 1098: 1720.862022 from(574.627950,3+)

Title: 091008RatKidney_NH4Format01_24.2948.2948.3.dta

Data file K:INewmanPaper|Piliangl3SubProteomes\Piliang3SP\mgf5ppm\ERLIC_3SubProteomes5ppm.mgf

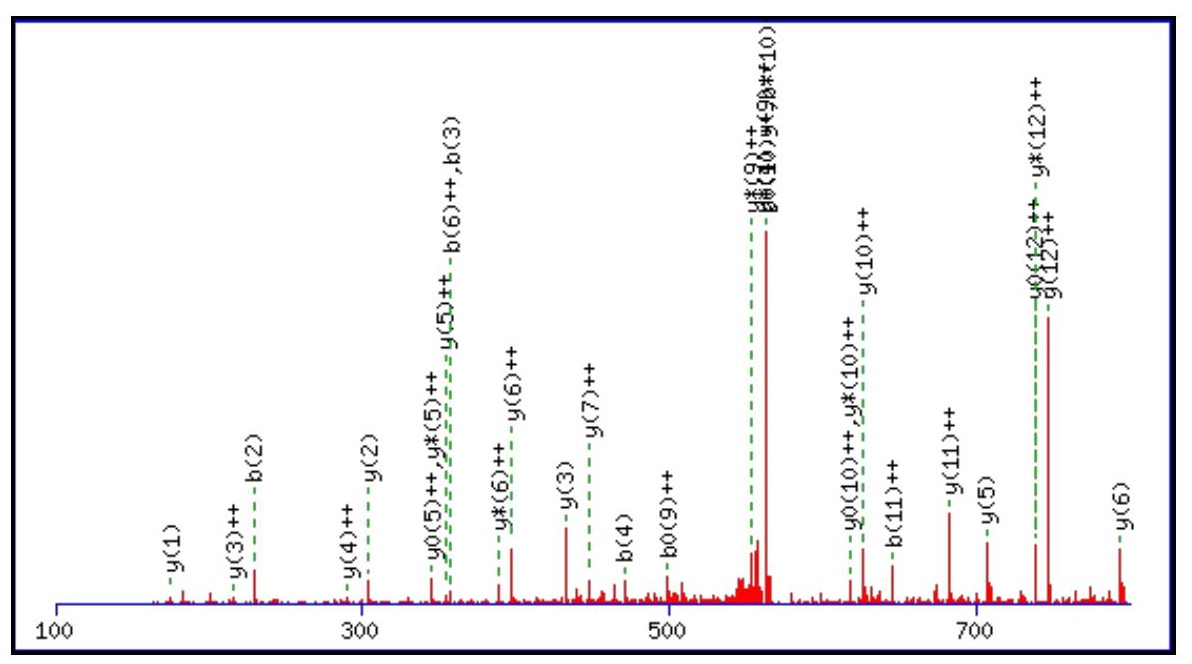



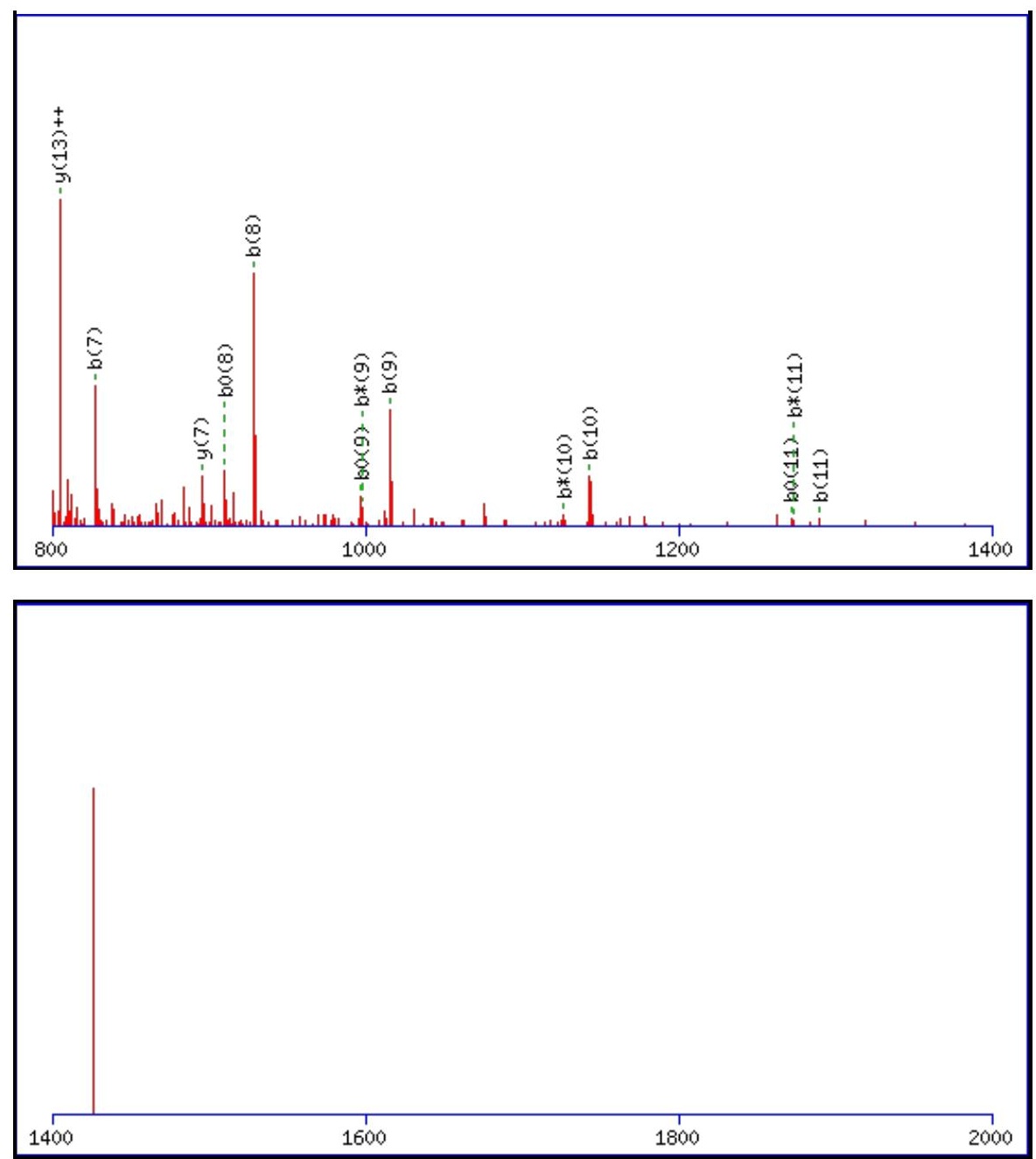

Monoisotopic mass of neutral peptide $\operatorname{Mr}($ calc): 1720.8631

Fixed modifications: Carbamidomethyl (C)

Variable modifications:

N6 : Deamidated_N (N)

Ions Score: 59 Expect: 0.00026

Matches (Bold Red): 44/144 fragment ions using 66 most intense peaks

\begin{tabular}{|c|c|c|c|c|c|c|c|c|c|c|c|c|c|c|}
\hline \# & b & $\mathbf{b}^{++}$ & $\mathbf{b}^{*}$ & $\mathbf{b}^{*^{++}}$ & $\mathbf{b}^{\mathbf{0}}$ & $\mathbf{b}^{0++}$ & Seq. & $\mathbf{y}$ & $\mathbf{y}^{++}$ & $\mathbf{y}^{*}$ & $\mathrm{y}^{*^{++}}$ & $\mathbf{y}^{\mathbf{0}}$ & $\mathbf{y}^{\mathbf{0}^{++}}$ & \# \\
\hline 1 & 114.0913 & 57.5493 & & & & & $\mathbf{L}$ & & & & & & & 14 \\
\hline 2 & 229.1183 & 115.0628 & & & 211.1077 & 106.0575 & D & 1608.7864 & 804.8968 & 1591.7598 & 796.3836 & 1590.7758 & 795.8915 & 13 \\
\hline 3 & 358.1609 & 179.5841 & & & 340.1503 & 170.5788 & E & 1493.7594 & 747.3834 & 1476.7329 & 738.8701 & 1475.7489 & 738.3781 & 12 \\
\hline 4 & 471.2449 & 236.1261 & & & 453.2344 & 227.1208 & $\mathbf{L}$ & 1364.7168 & 682.8621 & 1347.6903 & 674.3488 & 1346.7063 & 673.8568 & 11 \\
\hline 5 & 599.3399 & 300.1736 & 582.3134 & 291.6603 & 581.3293 & 291.1683 & $\mathbf{K}$ & 1251.6328 & 626.3200 & 1234.6062 & 617.8068 & 1233.6222 & 617.3147 & 10 \\
\hline 6 & 714.3668 & 357.6871 & 697.3403 & 349.1738 & 696.3563 & 348.6818 & $\mathbf{N}$ & 1123.5378 & 562.2725 & 1106.5113 & 553.7593 & 1105.5273 & 553.2673 & 9 \\
\hline 7 & 827.4509 & 414.2291 & 810.4244 & 405.7158 & 809.4403 & 405.2238 & $\mathbf{L}$ & 1008.5109 & 504.7591 & 991.4843 & 496.2458 & 990.5003 & 495.7538 & 8 \\
\hline 8 & 928.4986 & 464.7529 & 911.4720 & 456.2397 & 910.4880 & 455.7476 & $\mathbf{T}$ & 895.4268 & 448.2170 & 878.4003 & 439.7038 & 877.4163 & 439.2118 & 7 \\
\hline 9 & 1015.5306 & 508.2689 & 998.5041 & 499.7557 & 997.5200 & 499.2637 & $\mathrm{~S}$ & 794.3791 & 397.6932 & 777.3526 & 389.1799 & 776.3686 & 388.6879 & 6 \\
\hline 10 & 1143.5892 & 572.2982 & \begin{tabular}{|l}
1126.5626 \\
\end{tabular} & 563.7850 & 1125.5786 & 563.2929 & $\mathbf{Q}$ & 707.3471 & 354.1772 & 690.3206 & 345.6639 & 689.3366 & 345.1719 & 5 \\
\hline 11 & 1290.6576 & 645.8324 & 1273.6311 & 637.3192 & 1272.6470 & 636.8272 & $\mathbf{F}$ & 579.2885 & 290.1479 & 562.2620 & 281.6346 & 561.2780 & 281.1426 & 4 \\
\hline 12 & 1418.7162 & 709.8617 & 1401.6896 & 701.3485 & 1400.7056 & 700.8564 & $\mathbf{Q}$ & 432.2201 & 216.6137 & 415.1936 & 208.1004 & 414.2096 & 207.6084 & 3 \\
\hline 13 & 1547.7588 & 774.3830 & 1530.7322 & 765.8698 & 1529.7482 & 765.3777 & $\mathbf{E}$ & 304.1615 & 152.5844 & 287.1350 & 144.0711 & 286.1510 & 143.5791 & 2 \\
\hline 14 & & & & & & & $\mathbf{R}$ & 175.1190 & 88.0631 & 158.0924 & 79.5498 & & & 1 \\
\hline
\end{tabular}



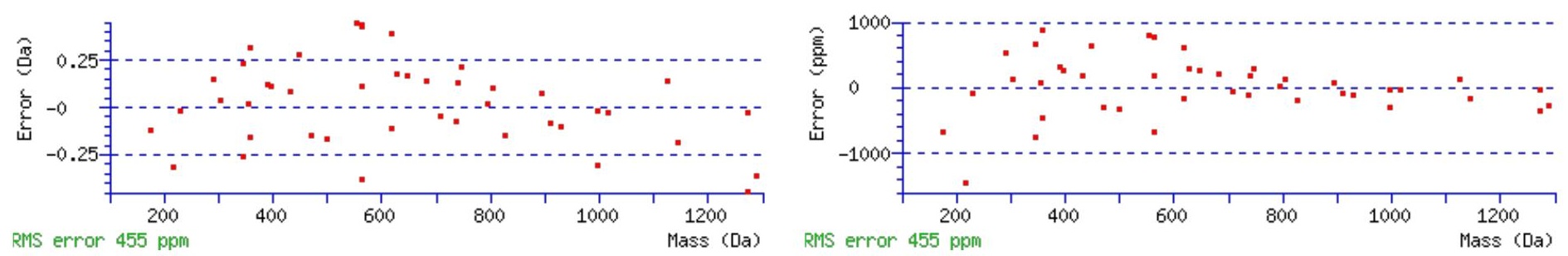

\section{All matches to this query}

\begin{tabular}{|l|l|l|l|}
\hline Score & Mr(calc): & Delta & \multicolumn{1}{c|}{ Sequence } \\
\hline 58.6 & 1720.8631 & -0.0011 & LDELKNLTSQFQER \\
\hline 8.7 & 1720.8477 & 0.0143 & KVYLEDGFAYVPLK \\
\hline 3.1 & 1720.8640 & -0.0020 & NLEIKNCMAKLWGK \\
\hline 2.9 & 1718.8426 & 2.0194 & LLNQQVHSLTVEMK \\
\hline 2.9 & 1718.8426 & 2.0194 & LLNQQVHSLTVEMK \\
\hline 2.9 & 1720.8640 & -0.0020 & NLEIKNCMAKLWGK \\
\hline 2.9 & 1718.8716 & 1.9904 & QQNKLKDLSPIAER \\
\hline 2.9 & 1719.8556 & 1.0064 & QQNKLKDLSPIAER \\
\hline 0.9 & 1720.8679 & -0.0058 & NQQVKQAFSDLMRR \\
\hline 0.4 & 1720.8784 & -0.0164 & LNGDWFSIVLASDKR \\
\hline
\end{tabular}

Spectrum No: 558; Query: 2046; Rank: 1

\section{Peptide View}

MS/MS Fragmentation of CVWLECPLPDASNITNVTVK

Found in IPI00372689, Tax_Id=10116 Gene_Symbol=Itga3_predicted 117 kDa protein

Match to Query 2046: 2318.103788 from(1160.059170,2+)

Title: 100101RatKid_NS_deglyco_16.5074.5074.2.dta

Data file K:INewmanPaper|Piliangl3SubProteomes\Piliang3SP\mgf5ppm\ERLIC_3SubProteomes5ppm.mgf

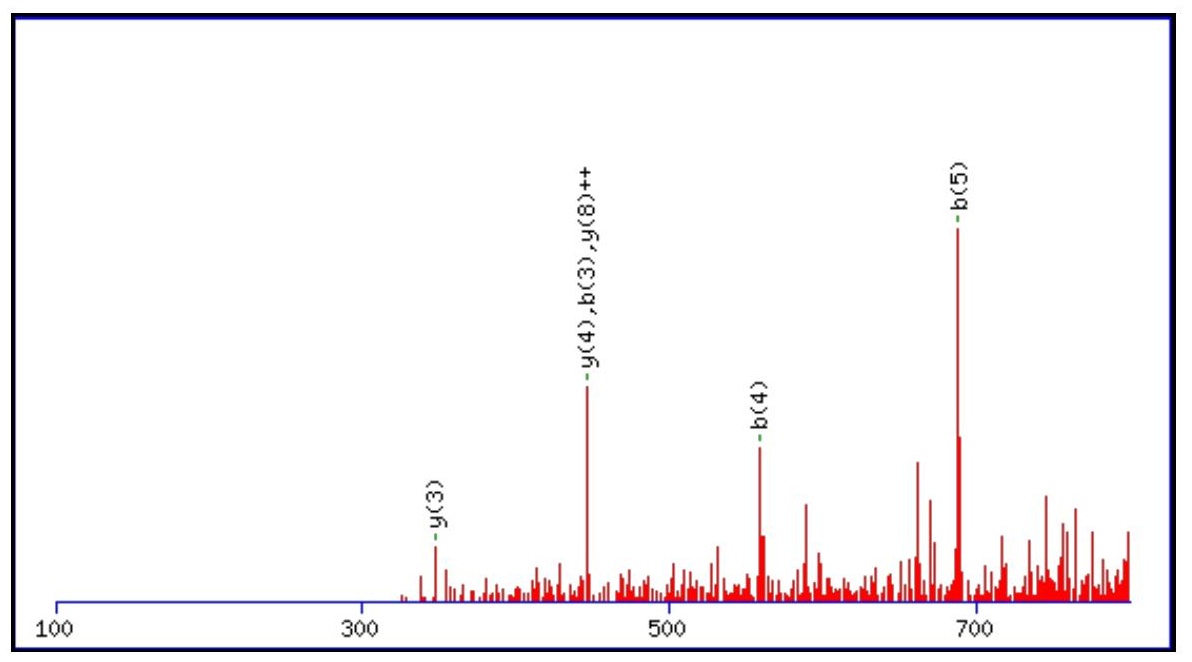



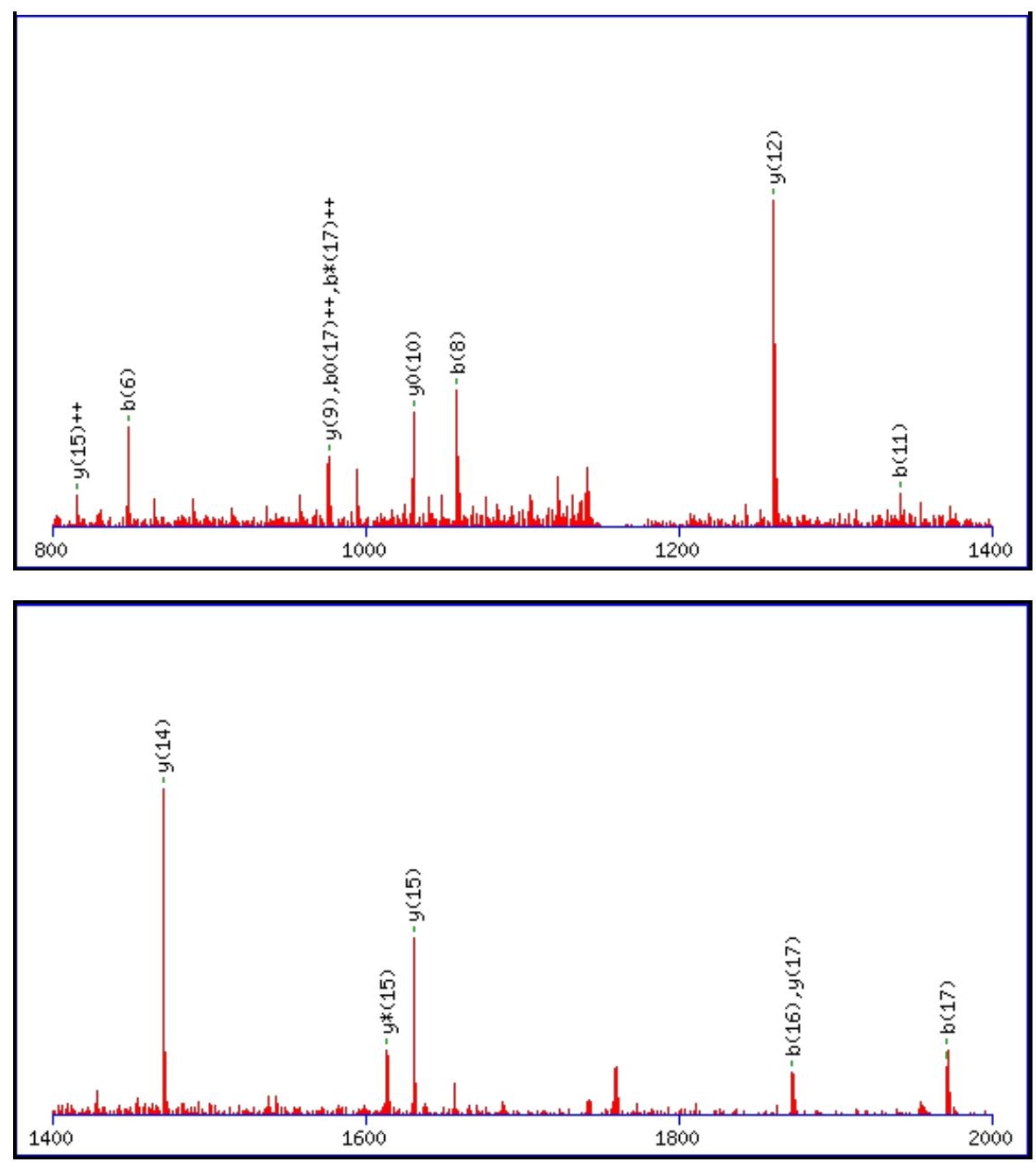

Monoisotopic mass of neutral peptide $\operatorname{Mr}($ calc): 2317.0970

Fixed modifications: Carbamidomethyl (C)

Variable modifications:

N13 : Deamidated_N (N)

N16 : Deamidated_N (N)

Ions Score: 59 Expect: 0.00039

Matches (Bold Red): 21/192 fragment ions using 24 most intense peaks

\begin{tabular}{|c|c|c|c|c|c|c|c|c|c|c|c|c|c|c|}
\hline \# & b & $\mathbf{b}^{++}$ & b* & $\mathbf{b}^{*^{++}}$ & $\mathbf{b}^{0}$ & $\mathbf{b}^{0++}$ & Seq. & $\mathbf{y}$ & $y^{++}$ & $\mathbf{y}^{*}$ & $\mathbf{y}^{*^{++}}$ & $\mathbf{y}^{0}$ & $y^{0++}$ & $\#$ \\
\hline 1 & 161.0379 & 81.0226 & & & & & $\mathrm{C}$ & & & & & & & 20 \\
\hline 2 & 260.1063 & 130.5568 & & & & & V & 2158.0736 & 1079.5405 & 2141.0471 & 1071.0272 & 2140.0631 & 1070.5352 & 19 \\
\hline 3 & 446.1857 & 223.5965 & & & & & $\mathbf{W}$ & 2059.0052 & 1030.0062 & 2041.9787 & 1021.4930 & 2040.9947 & 1021.0010 & 18 \\
\hline 4 & 559.2697 & 280.1385 & & & & & $\mathbf{L}$ & 1872.9259 & 936.9666 & 1855.8994 & 928.4533 & 1854.9153 & 927.9613 & 17 \\
\hline 5 & 688.3123 & 344.6598 & & & 670.3017 & 335.6545 & $\mathbf{E}$ & 1759.8418 & 880.4246 & 1742.8153 & 871.9113 & 1741.8313 & 871.4193 & 16 \\
\hline 6 & 848.3430 & 424.6751 & & & 830.3324 & 415.6698 & C & 1630.7993 & 815.9033 & 1613.7727 & 807.3900 & 1612.7887 & 806.8980 & 15 \\
\hline 7 & 945.3957 & 473.2015 & & & 927.3852 & 464.1962 & $\mathbf{P}$ & 1470.7686 & 735.8879 & 1453.7421 & 727.3747 & 1452.7580 & 726.8827 & 14 \\
\hline 8 & 1058.4798 & 529.7435 & & & 1040.4692 & 520.7382 & $\mathbf{L}$ & 1373.7158 & 687.3616 & 1356.6893 & 678.8483 & 1355.7053 & 678.3563 & 13 \\
\hline 9 & 1155.5326 & 578.2699 & & & 1137.5220 & 569.2646 & $\mathbf{P}$ & 1260.6318 & 630.8195 & 1243.6052 & 622.3063 & 1242.6212 & 621.8142 & 12 \\
\hline 10 & 1270.5595 & 635.7834 & & & 1252.5489 & 626.7781 & D & 1163.5790 & 582.2931 & 1146.5525 & 573.7799 & 1145.5684 & 573.2879 & 11 \\
\hline 11 & 1341.5966 & 671.3019 & & & 1323.5860 & 662.2967 & $\mathbf{A}$ & 1048.5521 & 524.7797 & 1031.5255 & 516.2664 & 1030.5415 & 515.7744 & 10 \\
\hline 12 & 1428.6286 & 714.8180 & & & 1410.6181 & 705.8127 & $S$ & 977.5150 & 489.2611 & 960.4884 & 480.7478 & 959.5044 & 480.2558 & 9 \\
\hline 13 & 1543.6556 & 772.3314 & 1526.6290 & 763.8181 & 1525.6450 & 763.3261 & $\mathbf{N}$ & 890.4829 & 445.7451 & 873.4564 & 437.2318 & 872.4724 & 436.7398 & 8 \\
\hline 14 & 1656.7396 & 828.8735 & 1639.7131 & 820.3602 & 1638.7291 & 819.8682 & I & 775.4560 & 388.2316 & 758.4294 & 379.7184 & 757.4454 & 379.2264 & 7 \\
\hline 15 & 1757.7873 & 879.3973 & 1740.7608 & 870.8840 & 1739.7767 & 870.3920 & $T$ & 662.3719 & 331.6896 & 645.3454 & 323.1763 & 644.3614 & 322.6843 & 6 \\
\hline 16 & 1872.8143 & 936.9108 & 1855.7877 & 928.3975 & 1854.8037 & 927.9055 & $\mathbf{N}$ & 561.3242 & 281.1658 & 544.2977 & 272.6525 & 543.3137 & 272.1605 & 5 \\
\hline 17 & 1971.8827 & 986.4450 & 1954.8561 & 977.9317 & 1953.8721 & 977.4397 & $\bar{V}$ & 446.2973 & 223.6523 & 429.2708 & 215.1390 & 428.2867 & 214.6470 & 4 \\
\hline 18 & 2072.9303 & 1036.9688 & 2055.9038 & 1028.4555 & 2054.9198 & 1027.9635 & $\mathbf{T}$ & 347.2289 & 174.1181 & 330.2023 & 165.6048 & 329.2183 & 165.1128 & 3 \\
\hline
\end{tabular}




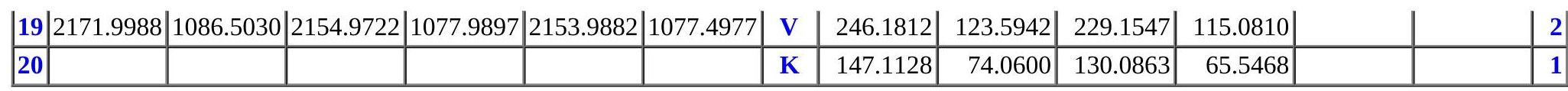
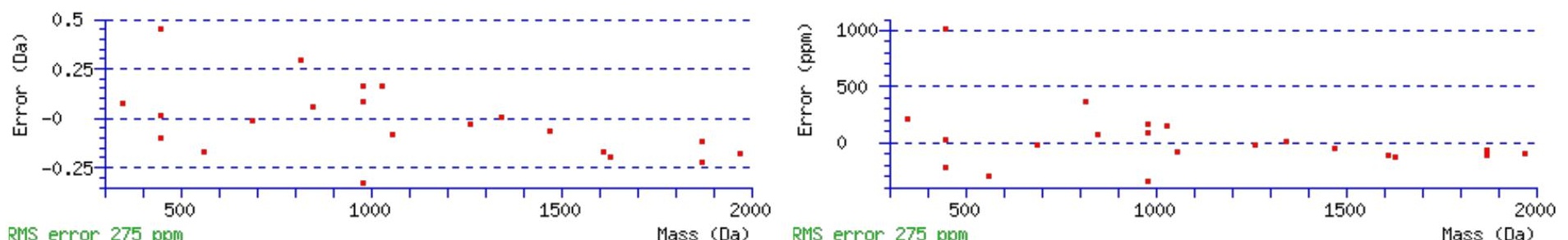

RMS error $275 \mathrm{ppm}$ Mass (Da) RMS error 275 ppm

\section{All matches to this query}

\begin{tabular}{|l|l|l|l|}
\hline Score & Mr(calc): & Delta & \multicolumn{1}{|c|}{ Sequence } \\
\hline 58.5 & 2317.0970 & 1.0068 & CVWLECPLPDASNITNVTVK \\
\hline 27.9 & 2316.1130 & 1.9908 & CVWLECPLPDASNITNVTVK \\
\hline 20.5 & 2316.1130 & 1.9908 & CVWLECPLPDASNITNVTVK \\
\hline 9.8 & 2318.0895 & 0.0143 & MAGSVLENIQSVLQKTWVR \\
\hline 6.5 & 2317.0975 & 1.0063 & ETRSDHLTVVNAFEGWEEAK \\
\hline 6.1 & 2316.1185 & 1.9853 & MAKDNQSRVTEFILAGLTDK \\
\hline 6.0 & 2316.0816 & 2.0222 & DMVVMLLSMLEGNVVNGTIGK \\
\hline 4.4 & 2316.1185 & 1.9853 & MAKDNQSRVTEFILAGLTDK \\
\hline 3.6 & 2317.0773 & 1.0265 & SLMLKQGSDERTQTFNLPR \\
\hline 3.5 & 2317.1025 & 1.0013 & MAKDNQSRVTEFILAGLTDK \\
\hline
\end{tabular}

Spectrum No: 559; Query: 407; Rank: 1

\section{Peptide View}

MS/MS Fragmentation of NNASTDYDLSDK

Found in IPI00395285, Tax_Id=10116 Gene_Symbol=Rpl3 60S ribosomal protein L3

Match to Query 407: 1342.554468 from(672.284510,2+)

Title: 091008RatKidney_NH4Format01_21.1019.1019.2.dta

Data file K:\NewmanPaper \Piliang \3SubProteomes\Piliang3SP \mgf5ppm\ERLIC_3SubProteomes5ppm.mgf

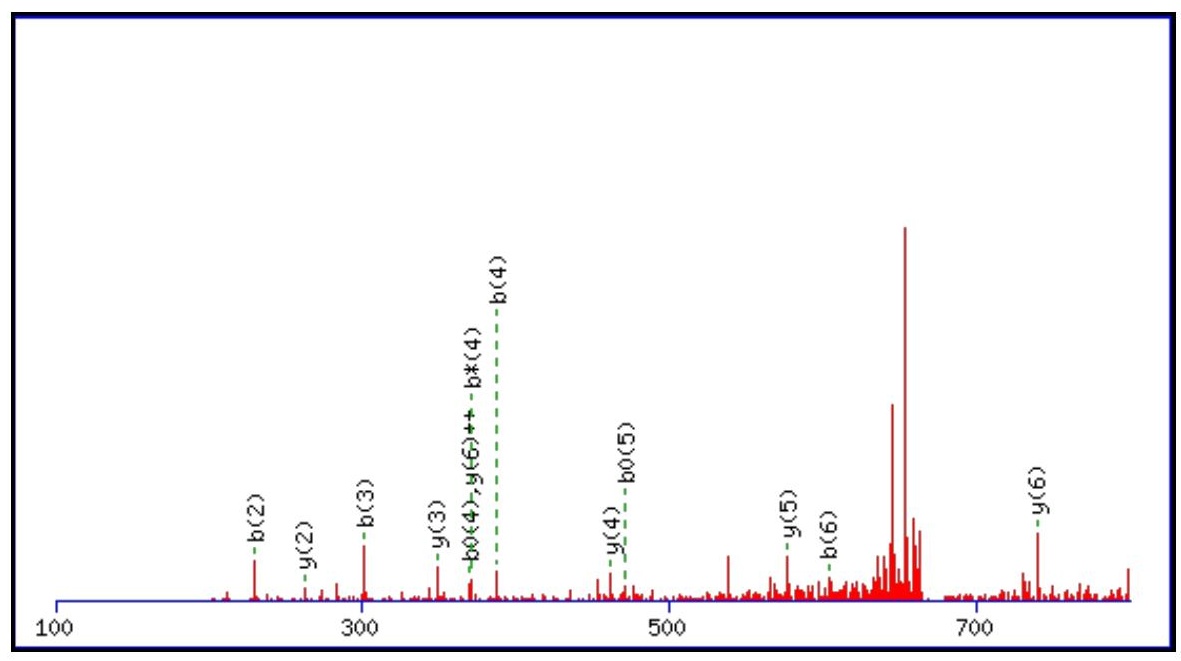



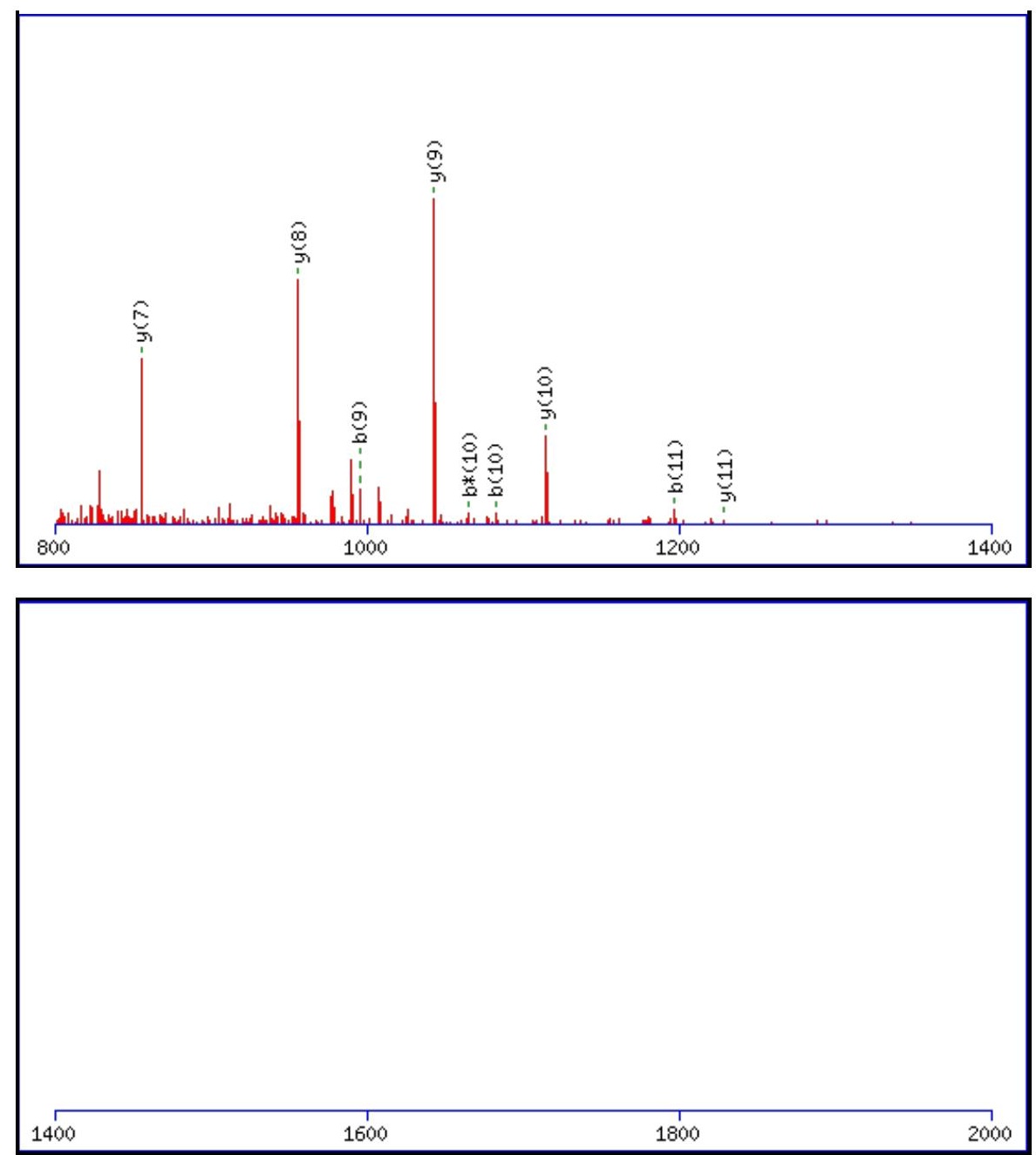

Monoisotopic mass of neutral peptide $\operatorname{Mr}($ calc): 1342.5524

Fixed modifications: Carbamidomethyl (C)

Variable modifications:

N2 : Deamidated $\mathrm{N}(\mathrm{N})$

Ions Score: 58 Expect: 0.00017

Matches (Bold Red): 22/124 fragment ions using 59 most intense peaks

\begin{tabular}{|c|c|c|c|c|c|c|c|c|c|c|c|c|c|c|}
\hline$\#$ & b & $\mathbf{b}^{++}$ & b* & $\mathbf{b}^{*^{++}}$ & $\mathbf{b}^{0}$ & $\mathbf{b}^{0++}$ & Seq. & $\mathbf{y}$ & $y^{++}$ & $\mathbf{y}^{*}$ & $\mathrm{y}^{*^{++}}$ & $\mathbf{y}^{0}$ & $\mathbf{y}^{0++}$ & \# \\
\hline 1 & 115.0502 & 58.0287 & 98.0237 & 49.5155 & & & $\mathbf{N}$ & & & & & & & 12 \\
\hline 2 & 230.0771 & 115.5422 & 213.0506 & 107.0289 & & & $\mathbf{N}$ & 1229.5168 & 615.2620 & 1212.4903 & 606.7488 & 1211.5062 & 606.2568 & 11 \\
\hline 3 & 301.1143 & 151.0608 & 284.0877 & 142.5475 & & & A & 1114.4899 & 557.7486 & 1097.4633 & 549.2353 & 1096.4793 & 548.7433 & 10 \\
\hline 4 & 388.1463 & 194.5768 & 371.1197 & 186.0635 & 370.1357 & 185.5715 & S & 1043.4528 & 522.2300 & 1026.4262 & 513.7167 & 1025.4422 & 513.2247 & 9 \\
\hline 5 & 489.1940 & 245.1006 & 472.1674 & 236.5873 & 471.1834 & 236.0953 & $\mathbf{T}$ & 956.4207 & 478.7140 & 939.3942 & 470.2007 & 938.4102 & 469.7087 & 8 \\
\hline 6 & 604.2209 & 302.6141 & 587.1944 & 294.1008 & 586.2103 & 293.6088 & D & 855.3731 & 428.1902 & 838.3465 & 419.6769 & 837.3625 & 419.1849 & 7 \\
\hline 7 & 767.2842 & 384.1458 & 750.2577 & 375.6325 & 749.2737 & 375.1405 & $\mathbf{Y}$ & 740.3461 & 370.6767 & 723.3196 & 362.1634 & 722.3355 & 361.6714 & 6 \\
\hline 8 & 882.3112 & 441.6592 & 865.2846 & 433.1460 & 864.3006 & 432.6539 & D & 577.2828 & 289.1450 & 560.2562 & 280.6318 & 559.2722 & 280.1397 & 5 \\
\hline 9 & 995.3952 & 498.2013 & 978.3687 & 489.6880 & 977.3847 & 489.1960 & $\mathbf{L}$ & 462.2558 & 231.6316 & 445.2293 & 223.1183 & 444.2453 & 222.6263 & 4 \\
\hline 10 & 1082.4273 & 541.7173 & 1065.4007 & 533.2040 & 1064.4167 & 532.7120 & $\mathrm{~S}$ & 349.1718 & 175.0895 & 332.1452 & 166.5763 & 331.1612 & 166.0842 & 3 \\
\hline 11 & 1197.4542 & 599.2307 & 1180.4277 & 590.7175 & 1179.4436 & 590.2255 & D & 262.1397 & 131.5735 & 245.1132 & 123.0602 & 244.1292 & 122.5682 & 2 \\
\hline 12 & & & & & & & $\mathbf{K}$ & 147.1128 & 74.0600 & 130.0863 & 65.5468 & & & 1 \\
\hline
\end{tabular}



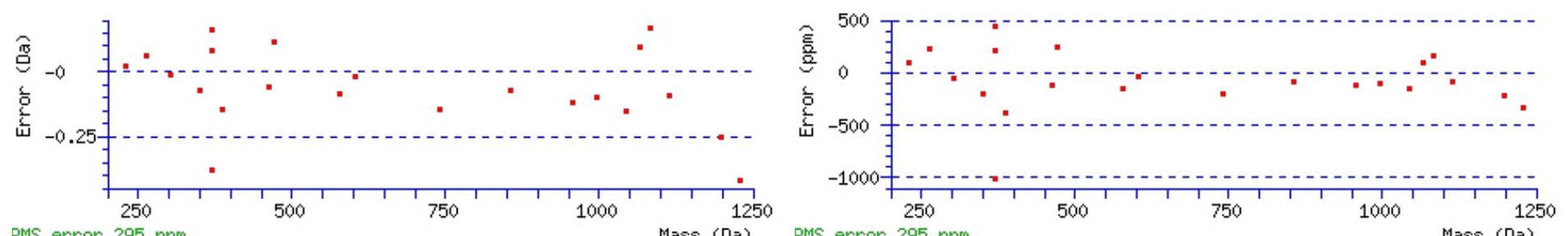

RMS error $295 \mathrm{ppm}$

Mass (Da) RMS error 295 ppm

Mass (Da)

\section{All matches to this query}

\begin{tabular}{|l|l|c|c|}
\hline Score & Mr(calc): & Delta & Sequence \\
\hline 58.4 & 1342.5524 & 0.0020 & NNASTDYDLSDK \\
\hline 50.6 & 1342.5524 & 0.0020 & NNASTDYDLSDK \\
\hline 11.9 & 1342.5654 & -0.0109 & QATAVVETDSDK \\
\hline 6.0 & 1342.5654 & -0.0109 & QATAVVETDSDK \\
\hline 0.1 & 1340.5609 & 1.9936 & QAEGAQSKADEK \\
\hline
\end{tabular}

Spectrum No: 560; Query: 470; Rank: 1

\section{Peptide View}

MS/MS Fragmentation of IFIFNQTGIEAK

Found in IPI00367013, Tax_Id=10116 Gene_Symbol=RGD1304696 Thioredoxin domain-containing protein 15 precursor

Match to Query 470: 1380.725948 from(691.370250,2+)

Title: 091008RatKidney_NH4Format01_24.3182.3182.2.dta

Data file K:INewmanPaper|Piliang|3SubProteomes\Piliang3SP\mgf5ppm\ERLIC_3SubProteomes5ppm.mgf

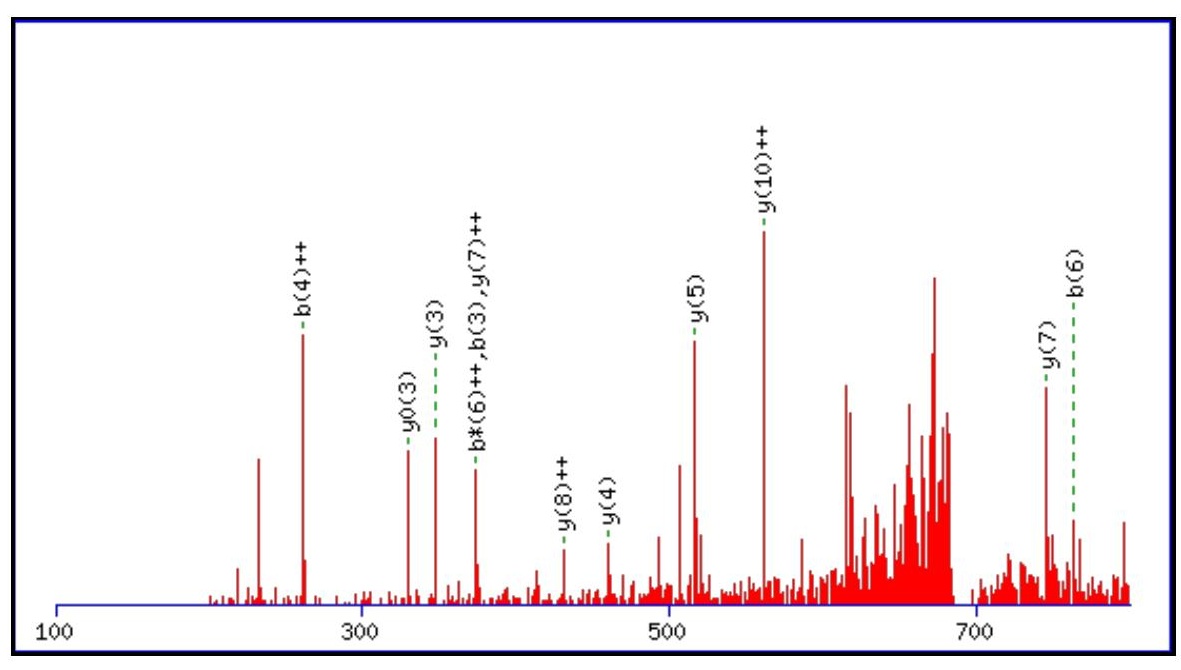



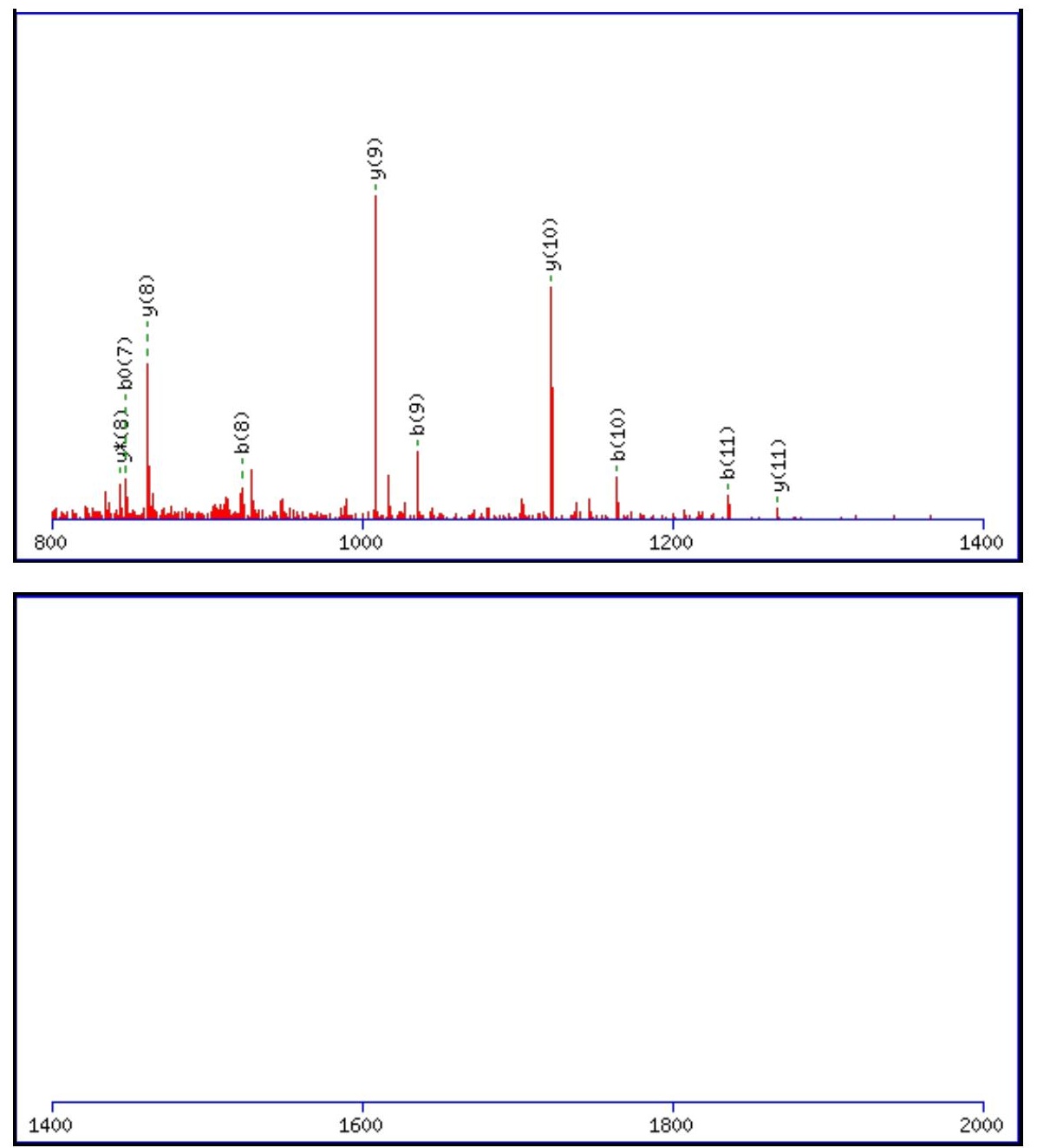

Monoisotopic mass of neutral peptide $\operatorname{Mr}($ calc): 1380.7289

Fixed modifications: Carbamidomethyl (C)

Variable modifications:

N5: Deamidated $\mathrm{N}(\mathrm{N})$

Ions Score: 58 Expect: 0.00016

Matches (Bold Red): 23/108 fragment ions using 37 most intense peaks

\begin{tabular}{|r|c|c|c|c|c|c|c|c|c|c|c|c|c|c|}
\hline$\#$ & $\mathbf{b}$ & $\mathbf{b}^{++}$ & $\mathbf{b}^{*}$ & $\mathbf{b}^{\mathbf{*}^{++}}$ & $\mathbf{b}^{\mathbf{0}}$ & $\mathbf{b}^{\mathbf{0 + +}}$ & $\mathbf{S e q}$ & $\mathbf{y}$ & $\mathbf{y}^{++}$ & $\mathbf{y}^{\mathbf{*}}$ & $\mathbf{y}^{\mathbf{*}^{++}}$ & $\mathbf{y}^{\mathbf{0}}$ & $\mathbf{y}^{\mathbf{0 + +}}$ & $\#$ \\
\hline $\mathbf{1}$ & 114.0913 & 57.5493 & & & & & $\mathbf{I}$ & & & & & & & $\mathbf{1 2}$ \\
\hline $\mathbf{2}$ & $\mathbf{2 6 1 . 1 5 9 8}$ & 131.0835 & & & & & $\mathbf{F}$ & $\mathbf{1 2 6 8 . 6 5 2 1}$ & 634.8297 & 1251.6256 & 626.3164 & 1250.6416 & 625.8244 & $\mathbf{1 1}$ \\
\hline $\mathbf{3}$ & $\mathbf{3 7 4 . 2 4 3 8}$ & 187.6255 & & & & & $\mathbf{I}$ & $\mathbf{1 1 2 1 . 5 8 3 7}$ & $\mathbf{5 6 1 . 2 9 5 5}$ & 1104.5572 & 552.7822 & 1103.5731 & 552.2902 & $\mathbf{1 0}$ \\
\hline $\mathbf{4}$ & 521.3122 & $\mathbf{2 6 1 . 1 5 9 8}$ & & & & & $\mathbf{F}$ & $\mathbf{1 0 0 8 . 4 9 9 6}$ & 504.7535 & 991.4731 & 496.2402 & 990.4891 & 495.7482 & $\mathbf{9}$ \\
\hline $\mathbf{5}$ & 636.3392 & 318.6732 & 619.3126 & 310.1599 & & & $\mathbf{N}$ & $\mathbf{8 6 1 . 4 3 1 2}$ & $\mathbf{4 3 1 . 2 1 9 3}$ & $\mathbf{8 4 4 . 4 0 4 7}$ & 422.7060 & 843.4207 & 422.2140 & $\mathbf{8}$ \\
\hline $\mathbf{6}$ & $\mathbf{7 6 4 . 3 9 7 7}$ & 382.7025 & 747.3712 & 374.1892 & & & $\mathbf{Q}$ & $\mathbf{7 4 6 . 4 0 4 3}$ & 373.7058 & 729.3777 & 365.1925 & 728.3937 & 364.7005 & $\mathbf{7}$ \\
\hline $\mathbf{7}$ & 865.4454 & 433.2264 & 848.4189 & 424.7131 & $\mathbf{8 4 7 . 4 3 4 9}$ & 424.2211 & $\mathbf{T}$ & 618.3457 & 309.6765 & 601.3192 & 301.1632 & 600.3352 & 300.6712 & $\mathbf{6}$ \\
\hline $\mathbf{8}$ & $\mathbf{9 2 2 . 4 6 6 9}$ & 461.7371 & 905.4403 & 453.2238 & 904.4563 & 452.7318 & $\mathbf{G}$ & $\mathbf{5 1 7 . 2 9 8 0}$ & 259.1527 & 500.2715 & 250.6394 & 499.2875 & 250.1474 & $\mathbf{5}$ \\
\hline $\mathbf{9}$ & $\mathbf{1 0 3 5 . 5 5 1 0}$ & 518.2791 & 1018.5244 & 509.7658 & 1017.5404 & 509.2738 & $\mathbf{I}$ & $\mathbf{4 6 0 . 2 7 6 6}$ & 230.6419 & 443.2500 & 222.1287 & 442.2660 & 221.6366 & $\mathbf{4}$ \\
\hline $\mathbf{1 0}$ & $\mathbf{1 1 6 4 . 5 9 3 5}$ & 582.8004 & 1147.5670 & 574.2871 & 1146.5830 & 573.7951 & $\mathbf{E}$ & 347.1925 & 174.0999 & 330.1660 & 165.5866 & 329.1819 & 165.0946 & $\mathbf{3}$ \\
\hline $\mathbf{1 1}$ & $\mathbf{1 2 3 5 . 6 3 0 7}$ & 618.3190 & 1218.6041 & 609.8057 & 1217.6201 & 609.3137 & $\mathbf{A}$ & 218.1499 & 109.5786 & 201.1234 & 101.0653 & & & $\mathbf{2}$ \\
\hline $\mathbf{1 2}$ & & & & & & & $\mathbf{K}$ & 147.1128 & 74.0600 & 130.0863 & 65.5468 & & & $\mathbf{1}$ \\
\hline
\end{tabular}



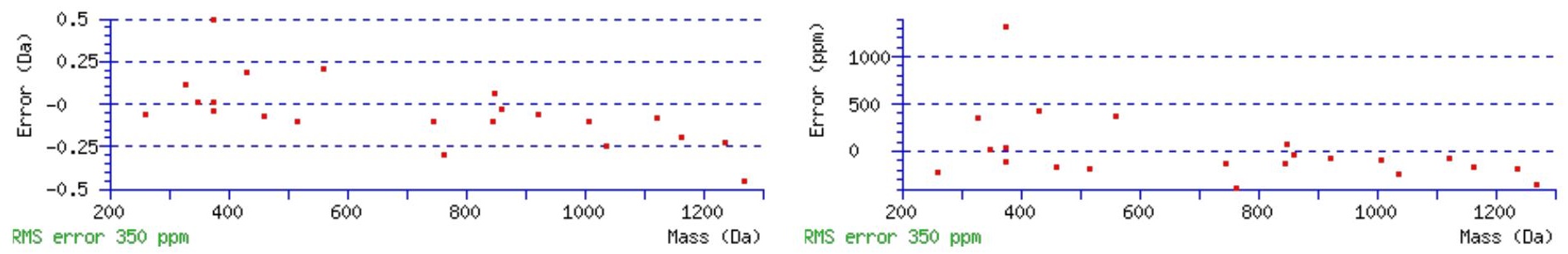

\section{All matches to this query}

\begin{tabular}{|l|c|c|l|}
\hline Score & Mr(calc): & Delta & \multicolumn{1}{|c|}{ Sequence } \\
\hline 58.0 & 1380.7289 & -0.0029 & IFIFNQTGIEAK \\
\hline 15.2 & 1380.7258 & 0.0002 & FMVPRTIMIGGK \\
\hline 11.0 & 1379.7330 & 0.9930 & ASLEKSASSMLLK \\
\hline 8.5 & 1380.7377 & -0.0118 & KILVSLSTGAANK \\
\hline 8.5 & 1380.7377 & -0.0118 & KILVSLSTGAANK \\
\hline 7.9 & 1380.7257 & 0.0002 & FIIMTKCNNIK \\
\hline 7.8 & 1379.7214 & 1.0046 & FLSPLQQNLLK \\
\hline 7.2 & 1379.7305 & 0.9954 & KCLCEXLFILK \\
\hline 7.0 & 1380.7370 & -0.0110 & VCRGYLIKMGGK \\
\hline 6.6 & 1380.7249 & 0.0011 & KDKSEFVSSLNK \\
\hline
\end{tabular}

Spectrum No: 561; Query: 1682; Rank: 1

\section{Peptide View}

MS/MS Fragmentation of VMELEKEIANATTKPEDR

Found in IPI00231789, Tax_Id=10116 Gene_Symbol=Mme Neprilysin

Match to Query 1682: 2074.023432 from(692.348420,3+)

Title: 091008RatKidney_NoSalt_23.2657.2657.3.dta

Data file K:INewmanPaper|Piliangl3SubProteomes\Piliang3SP\mgf5ppm|ERLIC_3SubProteomes5ppm.mgf

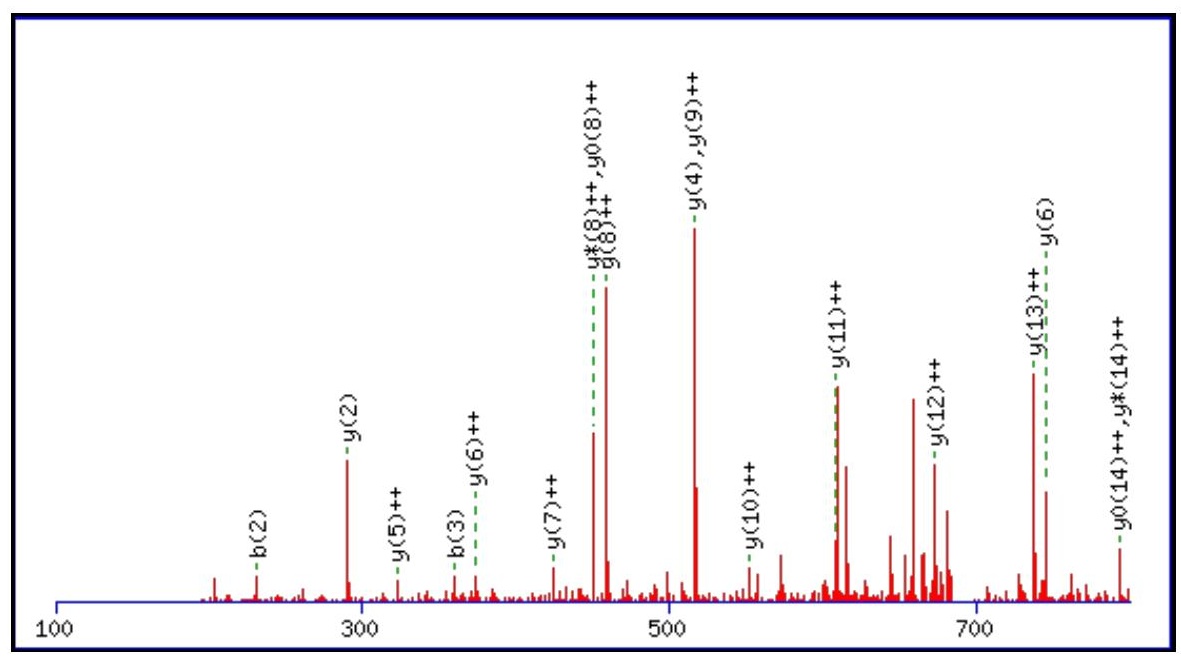



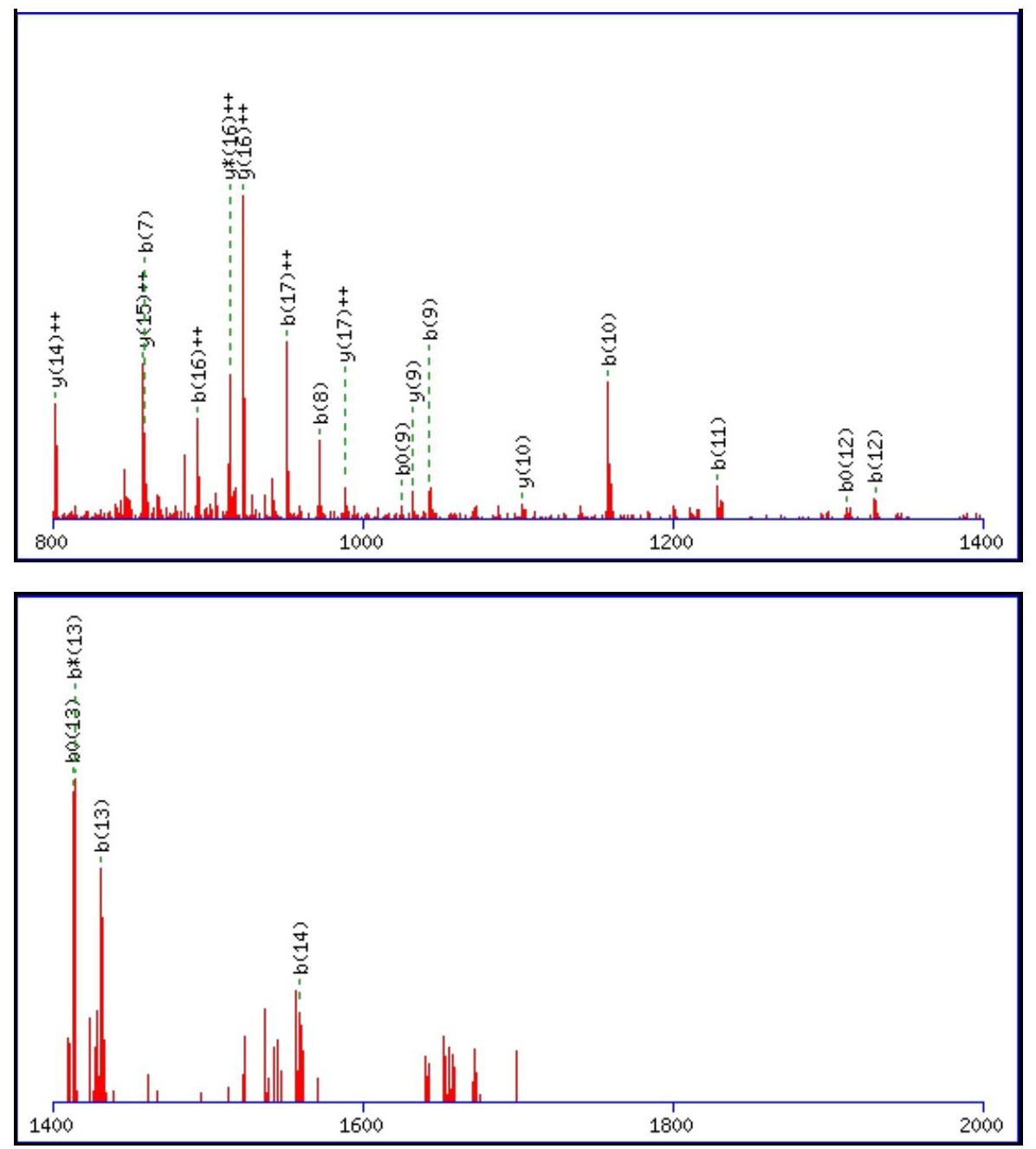

Monoisotopic mass of neutral peptide $\operatorname{Mr}($ calc): 2074.0252

Fixed modifications: Carbamidomethyl (C)

Variable modifications:

N10 : Deamidated_N (N)

Ions Score: 58 Expect: 0.00031

Matches (Bold Red): 39/188 fragment ions using 69 most intense peaks

\begin{tabular}{|c|c|c|c|c|c|c|c|c|c|c|c|c|c|c|}
\hline \# & b & $\mathbf{b}^{++}$ & $\mathbf{b}^{*}$ & $\mathbf{b}^{*^{++}}$ & $\mathbf{b}^{0}$ & & Seq. & $\mathbf{y}$ & $\mathbf{y}^{++}$ & $\mathbf{y}^{*}$ & $\mathbf{y}^{*^{++}}$ & $\mathbf{y}^{0}$ & $y^{0++}$ & \# \\
\hline 1 & 100.0757 & 50.5415 & & & & & $\mathbf{V}$ & & & & & & & 18 \\
\hline 2 & 231.1162 & 116.0617 & & & & & $\mathbf{M}$ & 1975.9641 & 988.4857 & 1958.9375 & 979.9724 & 1957 & 979.4804 & 17 \\
\hline 3 & 360.1588 & 180.5830 & & & 342.1482 & 171.5777 & $\mathbf{E}$ & 1844.9236 & 922.9654 & 1827.8971 & 914.4522 & 1826.9130 & 913.9602 & 16 \\
\hline 4 & 473.2428 & 237.1251 & & & 455.2323 & 228.1198 & $\mathbf{L}$ & 1715.8810 & 858.4441 & 1698.8545 & 849.9309 & 1697.8704 & 849.4389 & 15 \\
\hline 5 & 602.2854 & 301.6464 & & & 584.2749 & 292.6411 & $\mathbf{E}$ & 1602.7970 & 801.9021 & 1585.7704 & 793.3888 & 1584.7864 & 792.8968 & 14 \\
\hline 6 & 730.3804 & 365.6938 & 713.3538 & 357.1806 & 712.3698 & 356.6885 & $\mathbf{K}$ & 1473.7544 & 737.3808 & 1456.7278 & 728.8675 & 1455.7438 & 728.3755 & 13 \\
\hline 7 & 859.4230 & 430.2151 & 842.3964 & 421.7019 & 841.4124 & 421.2098 & $\mathbf{E}$ & 1345.6594 & 673.3333 & 1328.6328 & 664.8201 & 1327.6488 & 664.3281 & 12 \\
\hline 8 & 972.5070 & 486.7572 & 955.4805 & 478.2439 & 954.4965 & 477.7519 & I & 1216.6168 & 608.8120 & 1199.5903 & 600.2988 & 1198.6062 & 599.8068 & 11 \\
\hline 9 & 1043.5442 & 522.2757 & 1026.5176 & 513.7624 & 1025.5336 & 513.2704 & A & 1103.5327 & 552.2700 & 1086.5062 & 543.7567 & 1085.5222 & 543.2647 & 10 \\
\hline 10 & 1158.5711 & 579.7892 & 1141.5445 & 571.2759 & 1140.5605 & 570.7839 & $\mathbf{N}$ & 1032.4956 & 516.7514 & 1015.4691 & 508.2382 & 1014.4851 & 507.7462 & 9 \\
\hline 11 & 1229.6082 & 615.3077 & 1212.5817 & 606.7945 & 1211.5976 & 606.3025 & A & 917.4687 & 459.2380 & 900.4421 & 450.7247 & 899.4581 & 450.2327 & 8 \\
\hline 12 & 1330.6559 & 665.8316 & 1313.6293 & 657.3183 & 1312.6453 & 656.8263 & $\mathbf{T}$ & 846.4316 & 423.7194 & 829.4050 & 415.2062 & 828.4210 & 414.7141 & 7 \\
\hline 13 & 1431.7036 & 716.3554 & 1414.6770 & 707.8421 & 1413.6930 & 707.3501 & $\mathbf{T}$ & 745.3839 & 373.1956 & 728.3573 & 364.6823 & 727.3733 & 364.1903 & 6 \\
\hline 14 & 1559.7985 & 780.4029 & 1542.7720 & 771.8896 & 1541.7880 & 771.3976 & $\mathbf{K}$ & 644.3362 & 322.6717 & 627.3097 & 314.1585 & 626.3257 & 313.6665 & 5 \\
\hline 15 & 1656.8513 & 828.9293 & 1639.8247 & 820.4160 & 1638.8407 & 819.9240 & $\mathbf{P}$ & 516.2413 & 258.6243 & 499.2147 & 250.1110 & 498.2307 & 249.6190 & 4 \\
\hline 16 & 1785.8939 & 893.4506 & 1768.8673 & 884.9373 & 1767.8833 & 884.4453 & $\mathbf{E}$ & 419.1885 & 210.0979 & 402.1619 & 201.5846 & 401.1779 & 201.0926 & 3 \\
\hline 17 & 1900.9208 & 950.9641 & 1883.8943 & 942.4508 & 1882.9103 & 941.9588 & D & 290.1459 & 145.5766 & 273.1193 & 137.0633 & 272.1353 & 136.5713 & 2 \\
\hline 18 & & & & & & & $\mathbf{R}$ & 175.1190 & 88.0631 & 158.0924 & 79.5498 & & & 1 \\
\hline
\end{tabular}



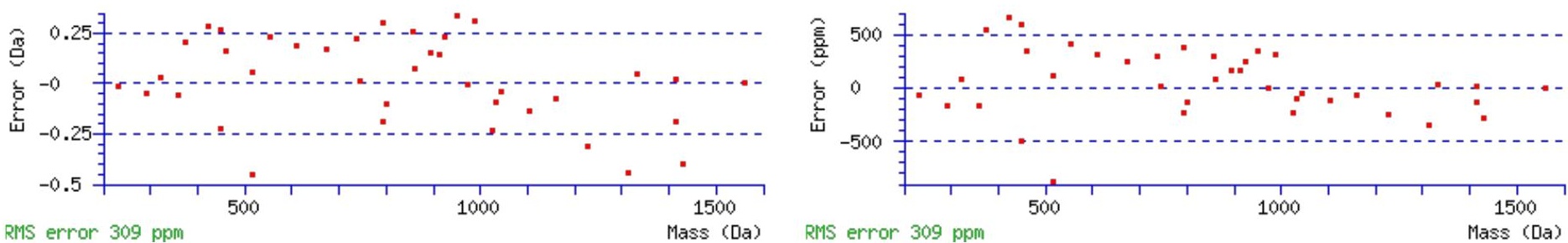

\section{All matches to this query}

\begin{tabular}{|l|l|l|l|}
\hline Score & Mr(calc): & Delta & \multicolumn{1}{|c|}{ Sequence } \\
\hline 58.0 & 2074.0252 & -0.0017 & VMELEKEIANATTKPEDR \\
\hline 10.8 & 2072.0238 & 1.9996 & YINQVGSTCLALRGVRGK \\
\hline 10.0 & 2074.0113 & 0.0122 & CQALERKNSATPSELNEK \\
\hline 7.8 & 2074.0136 & 0.0098 & LQPAYAGSYLVVTAASLDR \\
\hline 7.8 & 2074.0136 & 0.0098 & LQPAYAGSYLVVTAASLDR \\
\hline 4.8 & 2074.0166 & 0.0069 & YIISTQRPGHLNLFKK \\
\hline 4.7 & 2073.0207 & 1.0027 & GQRAMVTVQATLGLSILR \\
\hline 2.8 & 2072.0327 & 1.9907 & EVQLEKAGHFSWKDVNGK \\
\hline 1.8 & 2074.0143 & 0.0091 & VPGLANGVKCGSPRQLQGR \\
\hline 1.4 & 2073.0273 & 0.9962 & QCASAVQRQEEQTNALLK \\
\hline
\end{tabular}

Spectrum No: 562; Query: 2125; Rank: 1

\section{Peptide View}

MS/MS Fragmentation of KEDSCQLNYSEGPCLGMQQK

Found in IPI00210900, Tax_Id=10116 Gene_Symbol=Ambp AMBP protein precursor

Match to Query 2125: 2388.001902 from(797.007910,3+)

Title: 091008RatKidney_NoSalt_32.1260.1260.3.dta

Data file K:INewmanPaper|Piliang|3SubProteomes\Piliang3SP\mgf5ppm\ERLIC_3SubProteomes5ppm.mgf

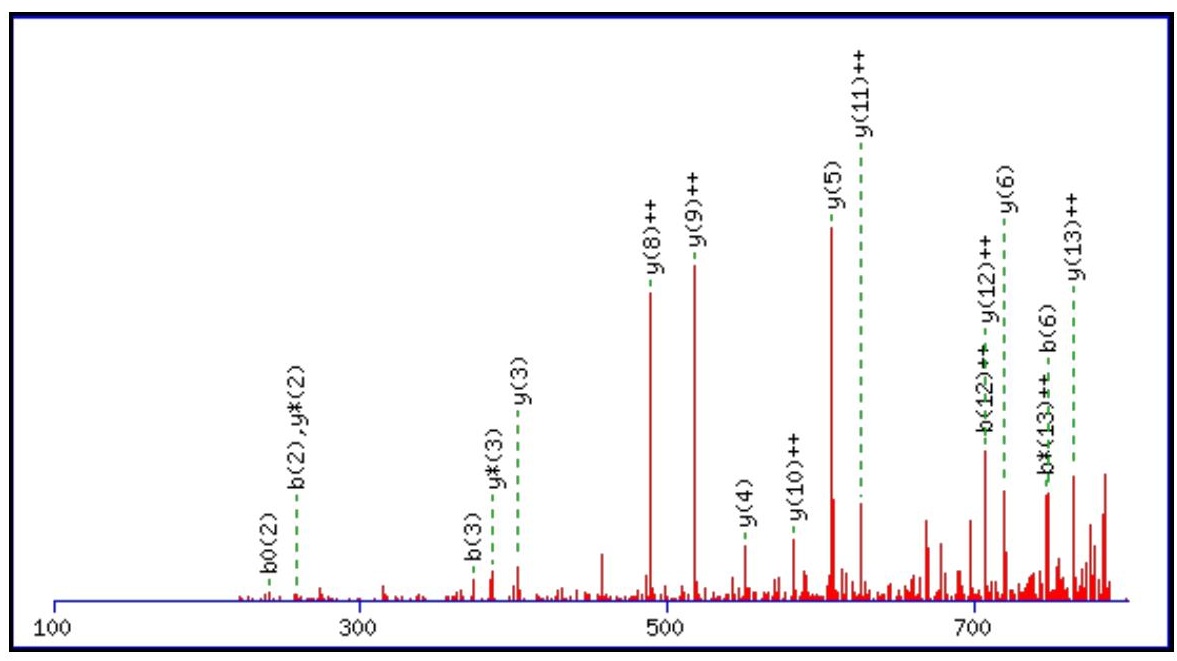



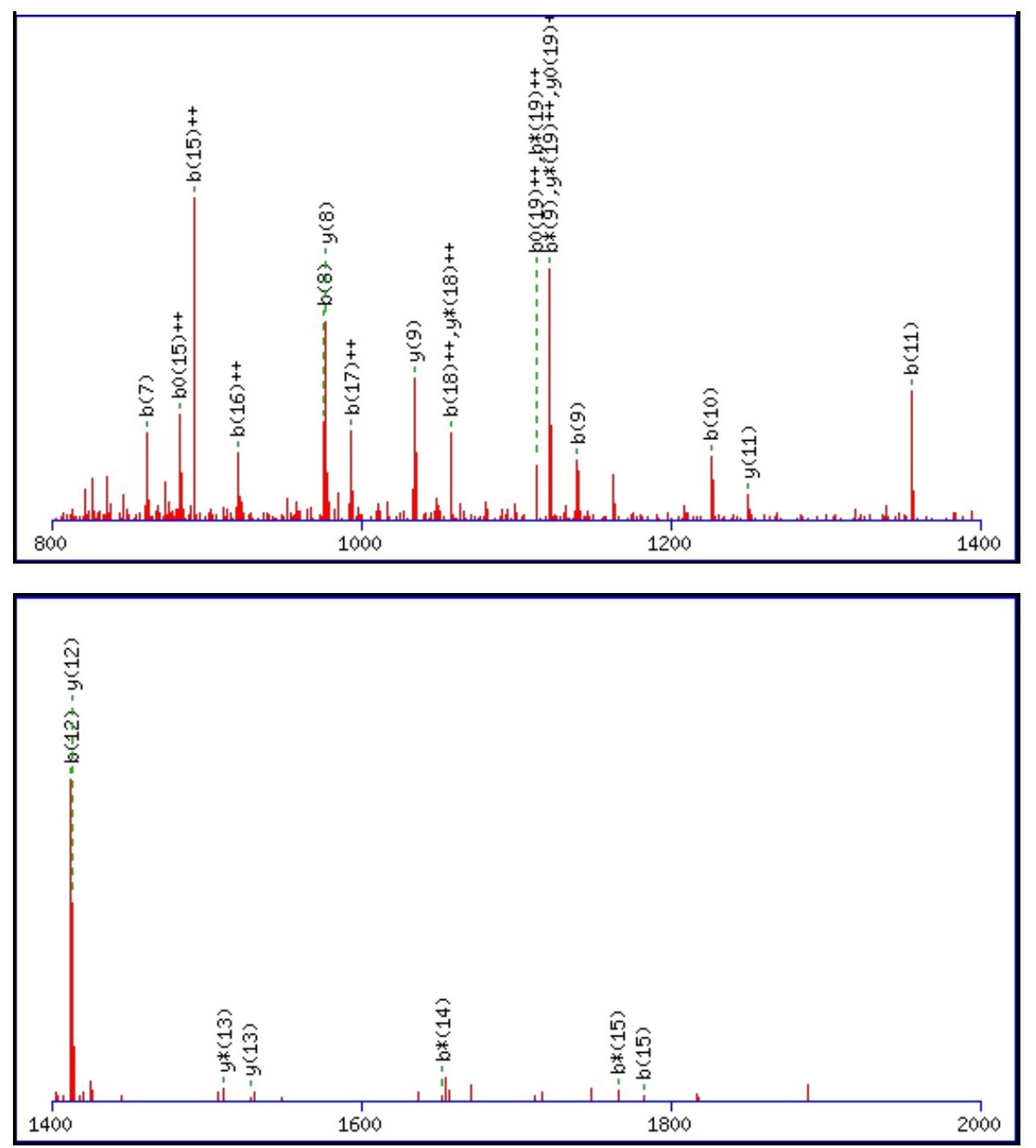

Monoisotopic mass of neutral peptide $\operatorname{Mr}($ calc): 2388.0032

Fixed modifications: Carbamidomethyl (C)

Variable modifications:

N8 : Deamidated_N (N)

M17 : Oxidation (M)

Ions Score: 58 Expect : $\odot .00047$

Matches (Bold Red): 45/208 fragment ions using 68 most intense peaks

\begin{tabular}{|c|c|c|c|c|c|c|c|c|c|c|c|c|c|c|}
\hline \# & b & $\mathbf{b}^{++}$ & $\mathbf{b}^{*}$ & $\mathbf{b}^{*++}$ & $\mathbf{b}^{0}$ & & Seq. & $\mathbf{y}$ & & $\mathbf{y}^{*}$ & $\mathbf{y}^{*^{++}}$ & $\mathbf{y}^{\mathbf{0}}$ & & \# \\
\hline 1 & 129.1022 & 65.0548 & 112.0757 & 56.5415 & & & $\mathbf{K}$ & & & & & & & 20 \\
\hline 2 & 258.1448 & 129.5761 & 241.1183 & 121.0628 & 240.1343 & 120.5708 & $\mathbf{E}$ & 2260.9155 & 1130.9614 & 2243.8890 & 4481 & 2242.9049 & 9561 & 19 \\
\hline 3 & 373.1718 & 187.0895 & 356.1452 & 178.5763 & 355.1612 & 178.0842 & D & 2131.8729 & 1066.4401 & 2114.8464 & 1057.9268 & 2113.8624 & 1057.4348 & 18 \\
\hline 4 & 460.2038 & 230.6055 & 443.1773 & 222.0923 & 442.1932 & 221.6003 & $\mathrm{~S}$ & 2016.8460 & 1008.9266 & 1999.8194 & 1000.4134 & 1998.8354 & 999.9213 & 17 \\
\hline 5 & 620.2345 & 310.6209 & 603.2079 & 302.1 & 602.2239 & 301.6156 & C & 1929.8139 & 965.4106 & 1912.7874 & 956.8973 & 1911.8034 & 956.4053 & 16 \\
\hline 6 & 748.2930 & 374.6502 & 731.2665 & 366.1369 & 730.2825 & 365.6449 & $\mathbf{Q}$ & 1769.7833 & 885.3953 & 1752.7567 & 876.8820 & 1751.7727 & 876.3900 & 15 \\
\hline 7 & 861.3771 & 431.1922 & 844.3505 & 422.6789 & 843.3665 & 422.1869 & $\mathbf{L}$ & 1641.7247 & 821.3660 & 1624.6982 & 812.8527 & 1623.7142 & 812.3607 & 14 \\
\hline 8 & 976.4040 & 488.7057 & 959.3775 & 480.1924 & 958.3935 & 479.7004 & $\mathbf{N}$ & 1528.6407 & 764.8240 & 1511.6141 & 756.3107 & 1510.6301 & 755.8187 & 13 \\
\hline 9 & 1139.4674 & 570.2373 & 1122.4408 & 561.7240 & 1121.4568 & 561.2320 & $\mathbf{Y}$ & 1413.6137 & 707.3105 & 1396.5872 & 698.7972 & 1395.6032 & 698.3052 & 12 \\
\hline 10 & 1226.4994 & 613.7533 & 1209.4728 & 605.2401 & 1208.4888 & 604.7480 & $\mathrm{~S}$ & 1250.5504 & 625.7788 & 1233.5238 & 617.2656 & 1232.5398 & 616.7736 & 11 \\
\hline 11 & 1355.5420 & 678.2746 & 1338.5154 & 669.7614 & 1337.5314 & 669.2693 & $\mathbf{E}$ & 1163.5184 & 582.2628 & 1146.4918 & 573.7495 & 1145.5078 & 573.2575 & 10 \\
\hline 12 & 1412.5634 & 706.7854 & 1395.5369 & 698.2721 & 1394.5529 & 697.7801 & G & 1034.4758 & 517.7415 & 1017.4492 & 509.2282 & & & 9 \\
\hline 13 & 1509.6162 & 755.3117 & 1492.5897 & 746.7985 & 1491.6056 & 746.3065 & $\mathbf{P}$ & 977.4543 & 489.2308 & 960.4278 & 480.7175 & & & 8 \\
\hline 14 & 1669.6469 & 835.3271 & 1652.6203 & 826.8138 & 1651.6363 & 826.3218 & C & 880.4015 & 440.7044 & 863.3750 & 432.1911 & & & 7 \\
\hline 15 & 1782.7309 & 891.8691 & 1765.7044 & 883.3558 & 1764.7204 & 882.8638 & $\mathbf{L}$ & 720.3709 & 360.6891 & 703.3443 & 352.1758 & & & 6 \\
\hline 16 & 1839.7524 & 920.3798 & 1822.7258 & 911.8666 & 1821.7418 & 911.3745 & $\mathbf{G}$ & 607.2868 & 304.1471 & 590.2603 & 295.6338 & & & 5 \\
\hline 17 & 1986.7878 & 993.8975 & 1969.7612 & 985.3843 & 1968.7772 & 984.8922 & $\mathbf{M}$ & 550.2654 & 275.6363 & 533.2388 & 267.1230 & & & 4 \\
\hline 18 & 2114.8464 & 1057.9268 & 2097.8198 & 1049.4135 & 2096.8358 & 1048.9215 & $\mathbf{Q}$ & 403.2300 & 202.1186 & 386.2034 & 193.6053 & & & 3 \\
\hline
\end{tabular}




\begin{tabular}{|l|l|l|l|l|l|r|r|r|r|r|r|r|r|r||}
$\mathbf{1 9}$ & 2242.9049 & $\mathbf{1 1 2 1 . 9 5 6 1}$ & 2225.8784 & $\mathbf{1 1 1 3 . 4 4 2 8}$ & 2224.8944 & $\mathbf{1 1 1 2 . 9 5 0 8}$ & $\mathbf{Q}$ & 275.1714 & 138.0893 & $\mathbf{2 5 8 . 1 4 4 8}$ & 129.5761 & & & $\mathbf{2}$ \\
\hline $\mathbf{2 0}$ & & & & & & & $\mathbf{K}$ & 147.1128 & 74.0600 & 130.0863 & 65.5468 & & & $\mathbf{1}$ \\
\hline
\end{tabular}
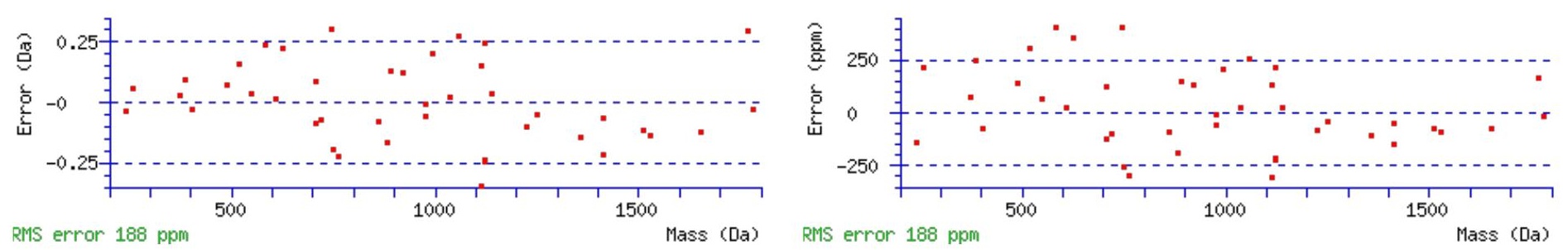

\section{All matches to this query}

\begin{tabular}{|l|l|l|l|}
\hline Score & Mr(calc): & Delta & \multicolumn{1}{|c|}{ Sequence } \\
\hline 58.0 & 2388.0032 & -0.0013 & KEDSCQLNYSEGPCLGMQQK \\
\hline 25.1 & 2387.0192 & 0.9827 & KEDSCQLNYSEGPCLGMQQK \\
\hline 4.5 & 2385.9790 & 2.0229 & VTNAGTRTVSVDAASGIQMPR \\
\hline 3.5 & 2387.0073 & 0.9946 & LGSSGSQVSNPGSRPYARXTK \\
\hline 3.4 & 2388.0247 & -0.0228 & KLLHNSDDQSLKSETEEVR \\
\hline 2.7 & 2385.9992 & 2.0027 & LDSLKSTGSSGAFSHAGSQFSR \\
\hline 2.3 & 2388.0063 & -0.0044 & DQAGEYECSAENDVSFPDVKK \\
\hline 2.1 & 2387.9980 & 0.0039 & QYVYIAVTDYLPISSTSTK \\
\hline 2.1 & 2387.9980 & 0.0039 & QYVYIAVTDYLPISSTSTK \\
\hline 2.1 & 2387.9980 & 0.0039 & QYVYIAVTDYLPISSTSTK \\
\hline
\end{tabular}

Spectrum No: 563; Query: 2852; Rank: 1

\section{Peptide View}

MS/MS Fragmentation of YCAAPTEPVIHNGSQGTGTNGSEISDSDYQAEYPDEYHGEYPDEYPR

Found in IPI00205566, Tax_Id=10116 Gene_Symbol=Cnn3 Calponin-3

Match to Query 2852: 5268.193136 from(1318.055560,4+)

Title: 100101RatKid_NS_deglyco_18.3466.3466.4.dta

Data file K:INewmanPaper|Piliangl3SubProteomes\Piliang3SP\mgf5ppm\ERLIC_3SubProteomes5ppm.mgf

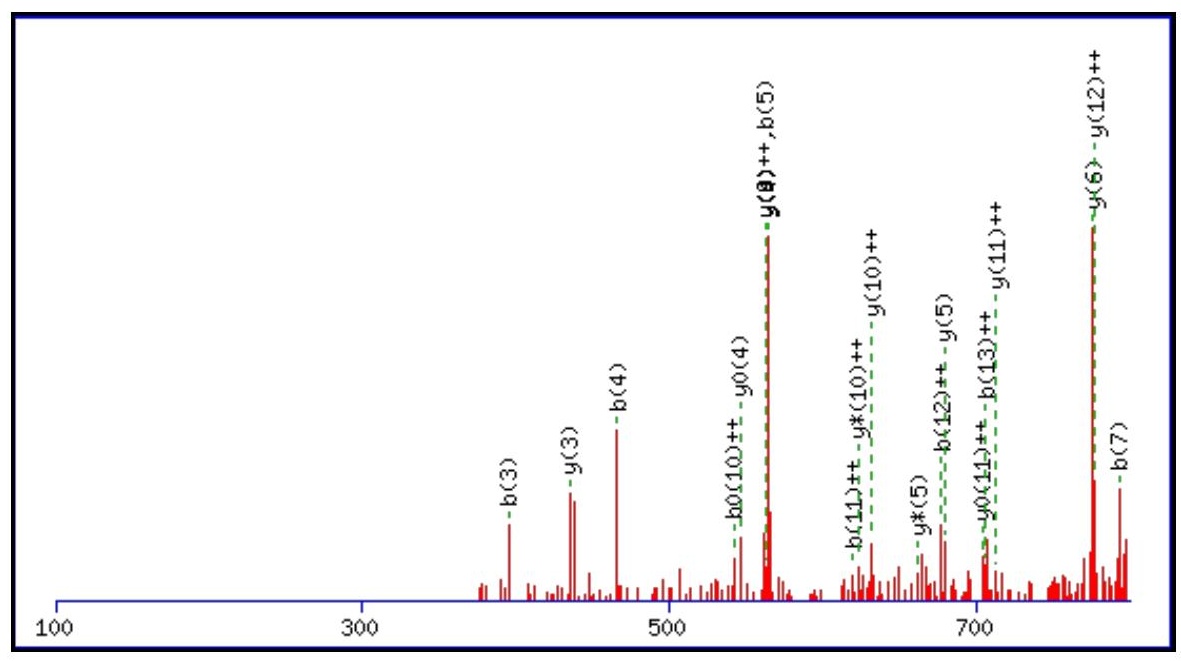



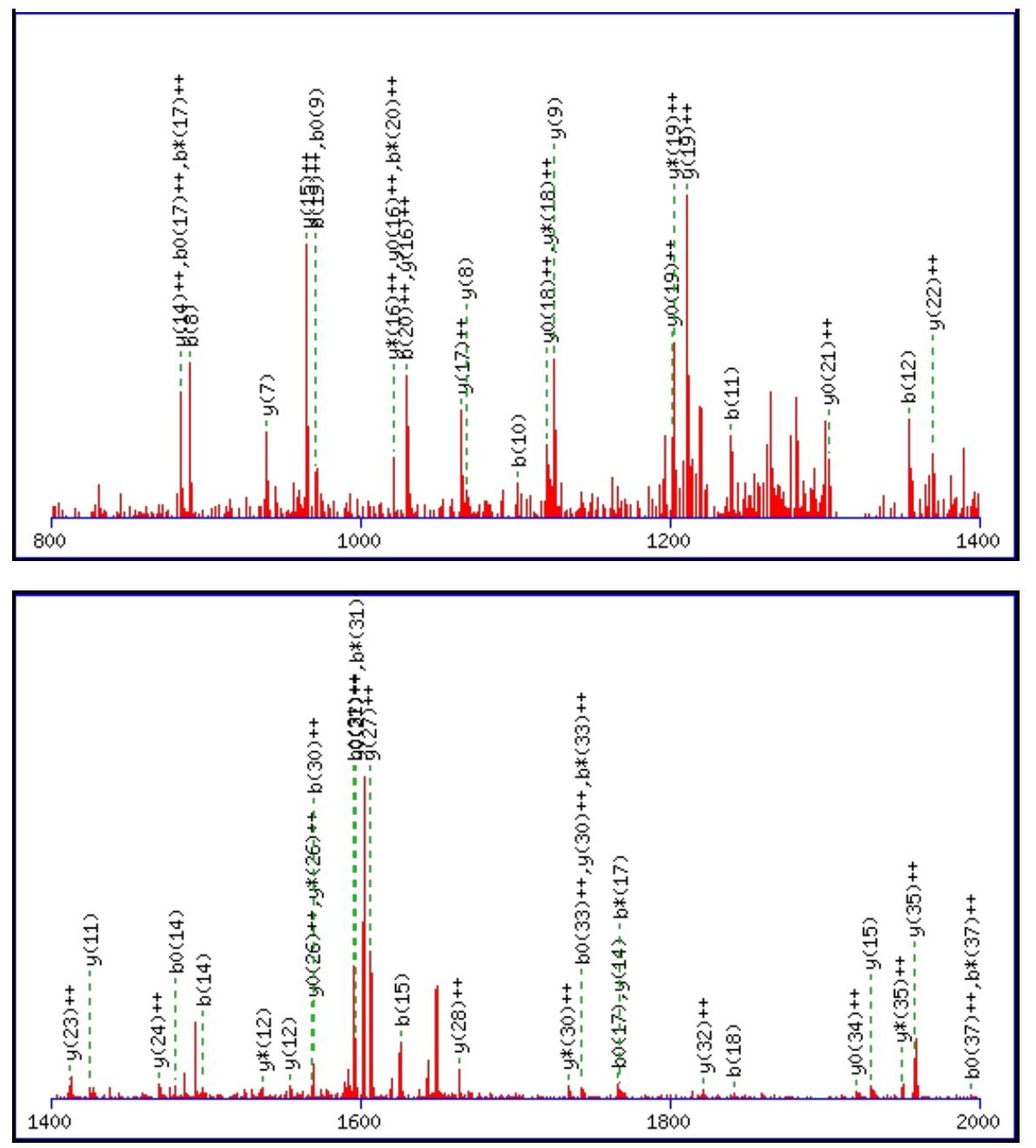

Monoisotopic mass of neutral peptide $\operatorname{Mr}($ calc): 5267.1646

Fixed modifications: Carbamidomethyl (C)

Variable modifications:

N12 : Deamidated_N (N)

N20 : Deamidated_N $(\mathrm{N})$

Ions Score: 58 Expect: 0.0009

Matches (Bold Red): 78/514 fragment ions using 159 most intense peaks

\begin{tabular}{|c|c|c|c|c|c|c|c|c|c|c|c|c|c|c|}
\hline \# & b & $\mathbf{b}^{++}$ & $\mathbf{b}^{*}$ & $\mathbf{b}^{*^{++}}$ & $\mathbf{b}^{0}$ & $\mathbf{b}^{0++}$ & Seq. & $\mathbf{y}$ & $\mathbf{y}^{++}$ & $\mathbf{y}^{*}$ & $\mathrm{y}^{*^{++}}$ & $\mathbf{y}^{0}$ & $\mathbf{y}^{\mathbf{0 + +}}$ & \# \\
\hline 1 & 164.0706 & 82.5389 & & & & & $\mathbf{Y}$ & & & & & & & 47 \\
\hline 2 & 324.1013 & 162.5543 & & & & & C & 5105.1086 & 2553.0579 & 5088.0821 & & 5087.0980 & 2544.0527 & 46 \\
\hline 3 & 395.1384 & 198.0728 & & & & & A & 4945.0 & 2473. & 4928.0514 & 2464 & 4927.0674 & 0373 & 45 \\
\hline 4 & 466.1755 & 233.5914 & & & & & A & 4874.0408 & 2437.5241 & 4857.0143 & 2429.0108 & 4856.0303 & 2428.5188 & 44 \\
\hline 5 & 563.2282 & 282.1178 & & & & & $\mathbf{P}$ & 4803.0037 & 2402.0055 & 4785.9772 & 2393.4922 & 4784.9932 & 2393.0002 & 43 \\
\hline 6 & 664.2759 & 332.6416 & & & 646.2654 & 63 & $\mathbf{T}$ & 4705.9510 & 2353.4791 & 4688.9244 & 2344.9658 & 4687.9404 & 2344.4738 & 42 \\
\hline 7 & 793.3185 & 397.1629 & & & 775.3080 & 388.1576 & $\mathbf{E}$ & 4604.9033 & 2302.9553 & 4587.8767 & 2294.4420 & 4586.8927 & 2293.9500 & 41 \\
\hline 8 & 890.3713 & 445.6893 & & & 872.3607 & 436.6840 & $\mathbf{P}$ & 4475.8607 & 2238.4340 & 4458.8341 & 2229.9207 & 4457.8501 & 2229.4287 & 40 \\
\hline 9 & 989.4397 & 495.2235 & & & 971.4291 & 486.2182 & $\mathbf{V}$ & 4378.8079 & 2189. & 4361.7814 & 2181.3943 & 4360.7974 & 2180.9023 & 39 \\
\hline 10 & 1102.5238 & 551.7655 & & & 1084.5132 & 542.7602 & I & 4279.7395 & 2140.3734 & 4262.7130 & 2131.8601 & 4261.7289 & 2131.3681 & 38 \\
\hline 11 & 1239.5827 & 620.2950 & & & 1221.5721 & 611.2897 & H & 4166.6554 & 2083.8314 & 4149.6289 & 2075.3181 & 4148.6449 & 2074.8261 & 37 \\
\hline 12 & 1354.6096 & 677.8084 & 31 & & 336.5990 & 668.8032 & $\mathbf{N}$ & 4029.5965 & 2015.3019 & 4012.5700 & 2006.7886 & 4011.5860 & 2006.2966 & 36 \\
\hline 13 & 1411.6311 & 706.3192 & 394.6045 & 697.8 & 1393.6205 & 697.3139 & G & 3914.5696 & 1957.7884 & 3897.5430 & 1949.2752 & 3896.5590 & 1948.7832 & 35 \\
\hline 14 & 1498.6631 & 749.8352 & 1481.6366 & 741.3219 & 1480.6525 & 740.8299 & S & 3857.5481 & 1929.2777 & 3840.5216 & 1920.7644 & 3839.5376 & 1920.2724 & 34 \\
\hline 15 & \begin{tabular}{|l|}
1626.7217 \\
\end{tabular} & 813.8645 & 1609.6951 & 805.3512 & 1608.7111 & 804.8592 & $\mathbf{Q}$ & 3770.5161 & 1885.7617 & 3753.4896 & 1877.2484 & 3752.5055 & 1876.7564 & 33 \\
\hline 16 & 1683.7431 & 842.3752 & 1666.7166 & 833.8619 & 1665.7326 & 833.3699 & G & 3642.4575 & 1821.7324 & 3625.4310 & 1813.2191 & 3624.4470 & 1812.7271 & 32 \\
\hline 17 & 1784.7908 & 892.8990 & 1767.7643 & 884.3858 & 1766.7803 & 883.8938 & $T$ & 3585.4361 & 1793.2217 & 3568.4095 & 1784.7084 & 3567.4255 & 1784.2164 & 31 \\
\hline 18 & 1841.8123 & 921.4098 & 1824.7857 & 912.8965 & 1823.8017 & 912.4045 & G & 3484.3884 & 1742.6978 & 3467.3618 & 1734.1846 & 3466.3778 & 1733.6925 & 30 \\
\hline
\end{tabular}




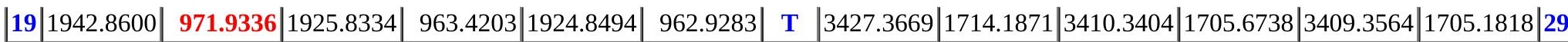
\begin{tabular}{|l|l|l|l|l|l|l|l|l|l|l|l|l|l|l|l|l|}
\hline 20 & 2057.8869 & $\mathbf{1 0 2 9 . 4 4 7 1}$ & 2040.8604 & $\mathbf{1 0 2 0 . 9 3 3 8}$ & 2039.8763 & 1020.4418 & $\mathbf{N}$ & 3326.3192 & $\mathbf{1 6 6 3 . 6 6 3 3}$ & 3309.2927 & 1655.1500 & 3308.3087 & 1654.6580 & $\mathbf{2 8}$ \\
\hline
\end{tabular} \begin{tabular}{|l|l|l|l|l|l|l|l|l|l|l|l|l|l|l|l|}
\hline 21 & 2114.9084 & 1057.9578 & 2097.8818 & 1049.4445 & 2096.8978 & 1048.9525 & G & 3211.2923 & $\mathbf{1 6 0 6 . 1 4 9 8}$ & 3194.2658 & $\mathbf{1 5 9 7 . 6 3 6 5}$ & 3193.2817 & $\mathbf{1 5 9 7 . 1 4 4 5}$ & $\mathbf{2 7}$ \\
\hline
\end{tabular} \begin{tabular}{|l|l|l|l|l|l|l|l|l|l|l|l|l|l|l|l|}
22 & 2201.9404 & 1101.4738 & 2184.9138 & 1092.9606 & 2183.9298 & 1092.4686 & S & 3154.2708 & 1577.6391 & 3137.2443 & $\mathbf{1 5 6 9 . 1 2 5 8}$ & 3136.2603 & $\mathbf{1 5 6 8 . 6 3 3 8}$ & $\mathbf{2 6}$ \\
\hline
\end{tabular} \begin{tabular}{|l|l|l|l|l|l|l|l|l|l|l|l|l|l|l|l|}
\hline 23 & 2330.9830 & 1165.9951 & 2313.9564 & 1157.4819 & 2312.9724 & 1156.9898 & E & 3067.2388 & 1534.1230 & 3050.2123 & 1525.6098 & 3049.2282 & 1525.1178 & 25 \\
\hline
\end{tabular}

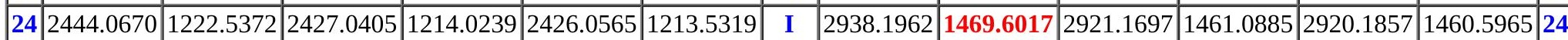
\begin{tabular}{|l|l|l|l|l|l|l|l|l|l|l|l|l|l|l|l|}
\hline 25 & 2531.0991 & 1266.0532 & 2514.0725 & 1257.5399 & 2513.0885 & 1257.0479 & S & 2825.1122 & $\mathbf{1 4 1 3 . 0 5 9 7}$ & 2808.0856 & 1404.5464 & 2807.1016 & 1404.0544 & 23 \\
\hline
\end{tabular} \begin{tabular}{|l|l|l|l|l|l|l|l|l|l|l|l|l|l|l|l|}
\hline $\mathbf{2 6}$ & 2646.1260 & 1323.5666 & 2629.0995 & 1315.0534 & 2628.1155 & 1314.5614 & D & 2738.0801 & $\mathbf{1 3 6 9 . 5 4 3 7}$ & 2721.0536 & 1361.0304 & 2720.0696 & 1360.5384 & 22 \\
\hline
\end{tabular} \begin{tabular}{|l|l|l|l|l|l|l|l|l|l|l|l|l|l|l|l|l|}
\hline 27 & 2733.1580 & 1367.0827 & 2716.1315 & 1358.5694 & 2715.1475 & 1358.0774 & S & 2623.0532 & 1312.0302 & 2606.0266 & 1303.5170 & 2605.0426 & $\mathbf{1 3 0 3 . 0 2 4 9}$ & $\mathbf{2 1}$ \\
\hline
\end{tabular} \begin{tabular}{|l|l|l|l|l|l|l|l|l|l|l|l|l|l|l|l|l|}
\hline $\mathbf{2 8}$ & 2848.1850 & 1424.5961 & 2831.1584 & 1416.0829 & 2830.1744 & 1415.5909 & D & 2536.0212 & 1268.5142 & 2518.9946 & 1260.0009 & 2518.0106 & 1259.5089 & $\mathbf{2 0}$ \\
\hline
\end{tabular} \begin{tabular}{|l|l|l|l|l|l|l|l|l|l|l|l|l|l|l|l|}
\hline 29 & 3011.2483 & 1506.1278 & 2994.2218 & 1497.6145 & 2993.2378 & 1497.1225 & Y & 2420.9942 & $\mathbf{1 2 1 1 . 0 0 0 7}$ & 2403.9677 & $\mathbf{1 2 0 2 . 4 8 7 5}$ & 2402.9837 & $\mathbf{1 2 0 1 . 9 9 5 5}$ & $\mathbf{1 9}$ \\
\hline
\end{tabular} \begin{tabular}{|l|l|l|l|l|l|l|l|l|l|l|l|l|l|l|l|}
\hline 30 & 3139.3069 & $\mathbf{1 5 7 0 . 1 5 7 1}$ & 3122.2803 & 1561.6438 & 3121.2963 & 1561.1518 & $\mathbf{Q}$ & 2257.9309 & 1129.4691 & 2240.9043 & $\mathbf{1 1 2 0 . 9 5 5 8}$ & 2239.9203 & $\mathbf{1 1 2 0 . 4 6 3 8}$ & $\mathbf{1 8}$ \\
\hline
\end{tabular} \begin{tabular}{|l|l|l|l|l|l|l|l|l|l|l|l|l|l|l|}
\hline 31 & 3210.3440 & 1605.6756 & 3193.3175 & $\mathbf{1 5 9 7 . 1 6 2 4}$ & 3192.3334 & $\mathbf{1 5 9 6 . 6 7 0 4}$ & A & 2129.8723 & $\mathbf{1 0 6 5 . 4 3 9 8}$ & 2112.8458 & 1056.9265 & 2111.8617 & 1056.4345 & $\mathbf{1 7}$ \\
\hline
\end{tabular} \begin{tabular}{|l|l|l|l|l|l|l|l|l|l|l|l|l|l|l|l|}
\hline 32 & 3339.3866 & 1670.1969 & 3322.3601 & 1661.6837 & 3321.3760 & 1661.1917 & E & 2058.8352 & $\mathbf{1 0 2 9 . 9 2 1 2}$ & 2041.8086 & $\mathbf{1 0 2 1 . 4 0 8 0}$ & 2040.8246 & $\mathbf{1 0 2 0 . 9 1 6 0}$ & $\mathbf{1 6}$ \\
\hline
\end{tabular} \begin{tabular}{|l|l|l|l|l|l|l|l|l|l|l|l|l|l|l|}
\hline 33 & 3502.4499 & 1751.7286 & 3485.4234 & $\mathbf{1 7 4 3 . 2 1 5 3}$ & 3484.4394 & $\mathbf{1 7 4 2 . 7 2 3 3}$ & $\mathbf{Y}$ & $\mathbf{1 9 2 9 . 7 9 2 6}$ & $\mathbf{9 6 5 . 3 9 9 9}$ & $\mathbf{1 9 1 2 . 7 6 6 1}$ & $\mathbf{9 5 6 . 8 8 6 7}$ & 1911.7820 & 956.3947 & $\mathbf{1 5}$ \\
\hline
\end{tabular}

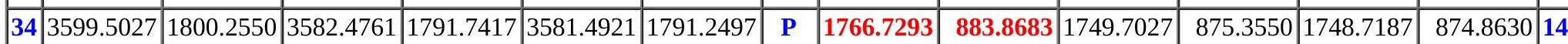
\begin{tabular}{|l|l|l|l|l|l|l|l|l|l|l|l|l|l|l|l|l|}
\hline 35 & 3714.5296 & 1857.7685 & 3697.5031 & 1849.2552 & 3696.5191 & 1848.7632 & D & 1669.6765 & 835.3419 & 1652.6500 & 826.8286 & 1651.6659 & 826.3366 & 13 \\
\hline
\end{tabular} \begin{tabular}{|l|l|l|l|l|l|l|l|l|l|l|l|l|l|l|l|}
\hline 36 & 3843.5722 & 1922.2898 & 3826.5457 & 1913.7765 & 3825.5617 & 1913.2845 & E & $\mathbf{1 5 5 4 . 6 4 9 6}$ & $\mathbf{7 7 7 . 8 2 8 4}$ & $\mathbf{1 5 3 7 . 6 2 3 0}$ & $\mathbf{7 6 9 . 3 1 5 1}$ & 1536.6390 & 768.8231 & $\mathbf{1 2}$ \\
\hline
\end{tabular}

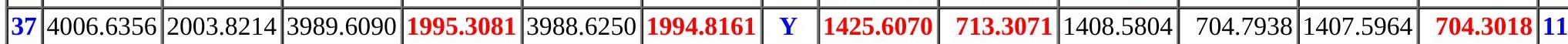
\begin{tabular}{|l|l|l|l|l|l|l|l|l|l|l|l|l|l|l|l|}
\hline 38 & 4143.6945 & 2072.3509 & 4126.6679 & 2063.8376 & 4125.6839 & 2063.3456 & H & 1262.5436 & $\mathbf{6 3 1 . 7 7 5 5}$ & 1245.5171 & $\mathbf{6 2 3 . 2 6 2 2}$ & 1244.5331 & 622.7702 & $\mathbf{1 0}$ \\
\hline
\end{tabular} \begin{tabular}{|l|l|l|l|l|l|l|l|l|l|l|l|l|l|l|l|}
\hline 39 & 4200.7159 & 2100.8616 & 4183.6894 & 2092.3483 & 4182.7054 & 2091.8563 & G & $\mathbf{1 1 2 5 . 4 8 4 7}$ & $\mathbf{5 6 3 . 2 4 6 0}$ & 1108.4582 & 554.7327 & 1107.4742 & 554.2407 & $\mathbf{9}$ \\
\hline
\end{tabular} \begin{tabular}{|l|l|l|l|l|l|l|l|l|l|l|l|l|l|l|l|}
\hline $\mathbf{4 0}$ & 4329.7585 & 2165.3829 & 4312.7320 & 2156.8696 & 4311.7480 & 2156.3776 & E & $\mathbf{1 0 6 8 . 4 6 3 3}$ & 534.7353 & 1051.4367 & 526.2220 & 1050.4527 & 525.7300 & $\mathbf{8}$ \\
\hline
\end{tabular} \begin{tabular}{|l|l|l|l|l|l|l|l|l|l|l|l|l|l|l|l|l|}
\hline 41 & 4492.8219 & 2246.9146 & 4475.7953 & 2238.4013 & 4474.8113 & 2237.9093 & Y & $\mathbf{9 3 9 . 4 2 0 7}$ & 470.2140 & 922.3941 & 461.7007 & 921.4101 & 461.2087 & 7 \\
\hline
\end{tabular} \begin{tabular}{|l|l|l|l|l|l|l|l|l|l|l|l|l|l|l|l|l|l|}
\hline 42 & 4589.8746 & 2295.4410 & 4572.8481 & 2286.9277 & 4571.8641 & 2286.4357 & P & 776.3573 & 388.6823 & 759.3308 & 380.1690 & 758.3468 & 379.6770 & $\mathbf{6}$ \\
\hline
\end{tabular} \begin{tabular}{|l|l|l|l|l|l|l|l|l|l|l|l|l|l|l|l|}
\hline 43 & 4704.9016 & 2352.9544 & 4687.8750 & 2344.4411 & 4686.8910 & 2343.9491 & D & $\mathbf{6 7 9 . 3 0 4 6}$ & 340.1559 & $\mathbf{6 6 2 . 2 7 8 0}$ & 331.6427 & 661.2940 & 331.1506 & $\mathbf{5}$ \\
\hline
\end{tabular} \begin{tabular}{|l|l|l|l|l|l|l|l|l|l|l|l|l|l|l|l|l|}
\hline 44 & 4833.9442 & 2417.4757 & 4816.9176 & 2408.9624 & 4815.9336 & 2408.4704 & E & 564.2776 & 282.6425 & 547.2511 & 274.1292 & 546.2671 & 273.6372 & $\mathbf{4}$ \\
\hline
\end{tabular} \begin{tabular}{|l|l|l|l|l|l|l|l|l|l|}
\hline 45 & 4997.0075 & 2499.0074 & 4979.9809 & 2490.4941 & 4978.9969 & 2490.0021 & Y \\
\hline
\end{tabular} \begin{tabular}{|l|l|l|l|l|l|l|l|l|}
\hline 46 & 5094.0603 & 2547.5338 & 5077.0337 & 2539.0205 & 5076.0497 & 2538.5285 & P \\
\hline
\end{tabular} 47

\begin{tabular}{l|l|l|l|l|l|}
$\mathbf{P}$ & 272.1717 & 136.5895 & 255.1452 & 128.0762 \\
\hline
\end{tabular}

\begin{tabular}{l|r|r|r|r|r|}
$\mathbf{R}$ & 175.1190 & 88.0631 & 158.0924 & 79.5498 \\
\hline
\end{tabular}
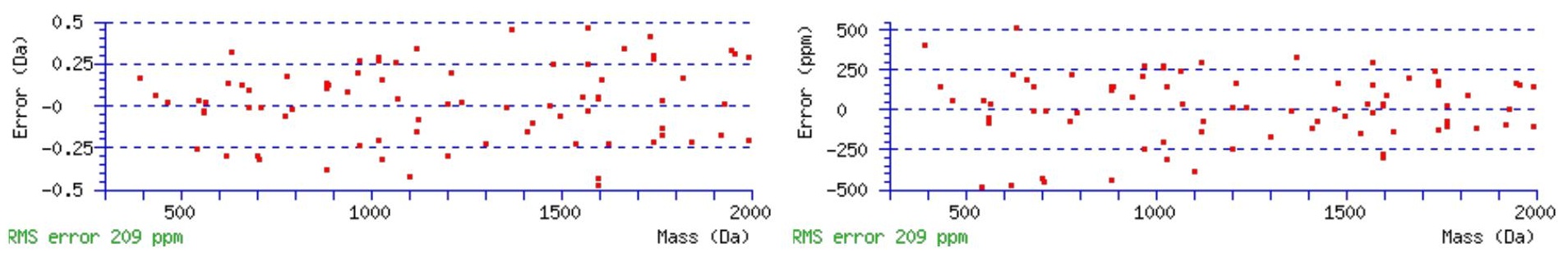

\section{All matches to this query}

\begin{tabular}{|l|c|c|l|}
\hline Score & Mr(calc): & Delta & \multicolumn{1}{c|}{ Sequence } \\
\hline 58.0 & 5267.1646 & 1.0285 & YCAAPTEPVIHNGSOGTGTNGSEISDSDYQAEYPDEYHGEYPDEYPR \\
\hline 49.1 & 5266.1806 & 2.0125 & YCAAPTEPVIHNGSQGTGTNGSEISDSDYQAEYPDEYHGEYPDEYPR \\
\hline 48.6 & 5266.1806 & 2.0125 & YCAAPTEPVIHNGSQGTGTNGSEISDSDYQAEYPDEYHGEYPDEYPR \\
\hline 3.4 & 5268.2444 & -0.0513 & MGLAASLQREKGQLTTVTVANVGSSADNVFTTSVANAASISGHVLSGR \\
\hline 3.3 & 5268.1473 & 0.0458 & ESVENPNWTEFIQTGRISIXGAGFLNCILETFASTFLRQGAQK \\
\hline 3.2 & 5268.1533 & 0.0398 & MSESEVGKDTTPEPSPANGTGPGPEWGLCPGPPTVGTDTSGASGLGTPRR \\
\hline 3.2 & 5268.1692 & 0.0239 & LGTFTVLFNPWQQGDDVFMSNHAERQEYVEEDSGIIYVGSTNR \\
\hline 3.2 & 5266.1880 & 2.0051 & RSHTAPPTFKDYVMDENPSMEVTSEPSQPTHIMTTDSTHAEETSR \\
\hline 3.2 & 5267.1720 & 1.0211 & RSHTAPPTFKDYVMDENPSMEVTSEPSQPTHIMTTDSTHAEETSR \\
\hline 3.1 & 5268.1473 & 0.0458 & ESVENPNWTEFIQTGRISIXGAGFLNCILETFASTFLRQGAOK \\
\hline
\end{tabular}


Spectrum No: 564; Query: 2408; Rank: 1

\section{Peptide View}

MS/MS Fragmentation of ISDTNITAIPQGLPTSISELHLDGNK

Found in IPI00199861, Tax_Id=10116 Gene_Symbol=Dcn Decorin precursor

Match to Query 2408: 2734.409532 from(912.477120,3+)

Title: 100101RatKid_NS_deglyco_18.4795.4795.3.dta

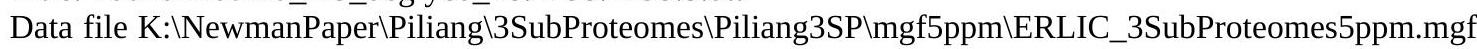
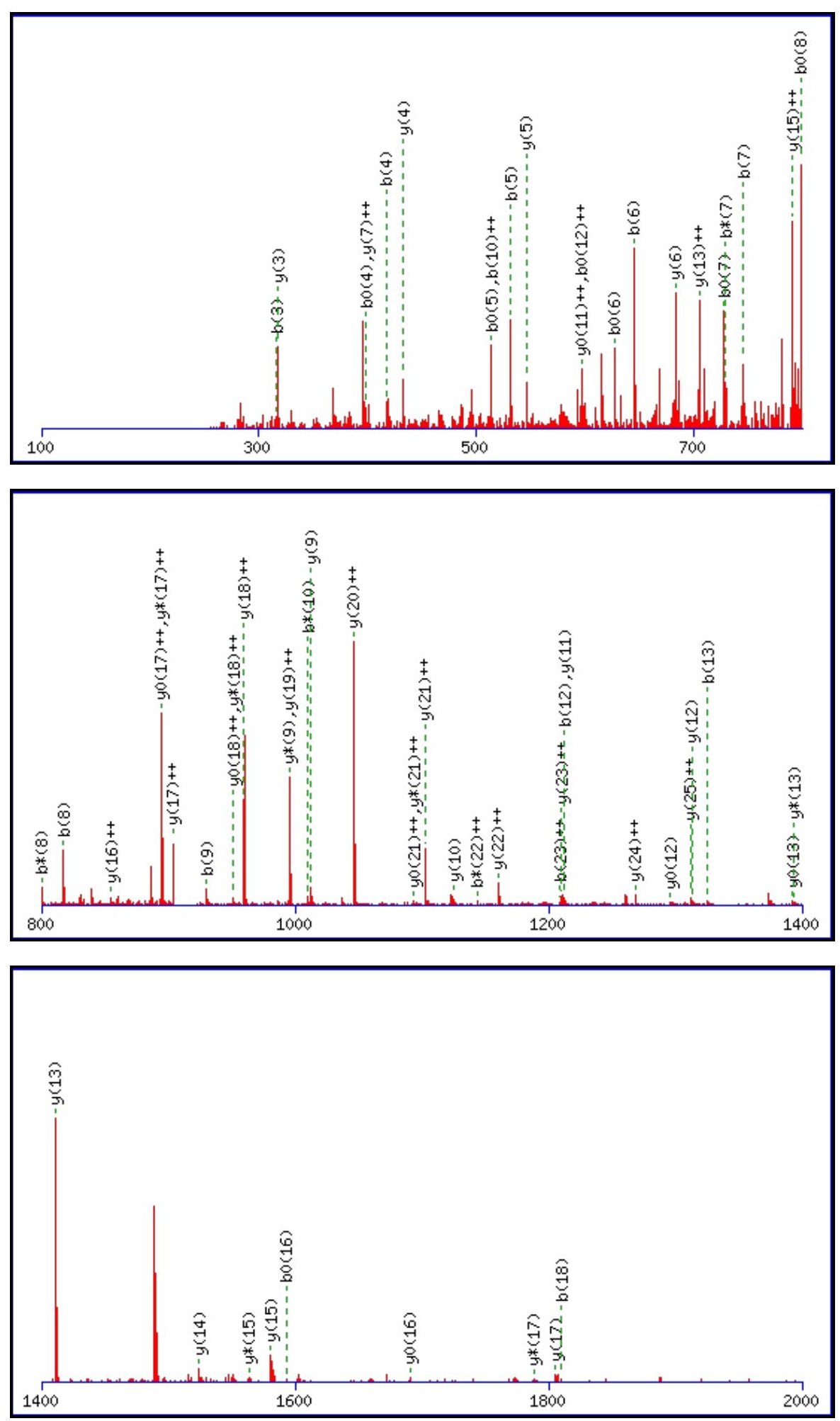
Monoisotopic mass of neutral peptide $\operatorname{Mr}($ calc): 2734.4025

Fixed modifications: Carbamidomethyl (C)

Variable modifications:

N5 : Deamidated_N (N)

Ions Score: 58 Expect: $\odot .00023$

Matches (Bold Red): 62/284 fragment ions using 153 most intense peaks

\begin{tabular}{|c|c|c|c|c|c|c|c|c|c|c|c|c|c|c|}
\hline \# & b & & $\mathbf{b}^{*}$ & & $\mathbf{b}^{\mathbf{0}}$ & & & $\mathbf{y}$ & & $\mathbf{y}^{*}$ & & $\mathbf{y}^{0}$ & $\mathbf{y}^{0++}$ & \# \\
\hline 1 & 114.0913 & 57.5493 & & & & & & & & & & & & 26 \\
\hline 2 & 201. & & & & & & & & & & & & & 25 \\
\hline 3 & 316.1503 & 158.5788 & & & & 149.5735 & D & & 505 & 2518.2671 & & 2517.2831 & & 24 \\
\hline 4 & 417.1980 & & & & & & & & & & & & & 23 \\
\hline 5 & 532. & 266. & & & & & I & & & & & 085 & & 22 \\
\hline 6 & 645.3090 & 323.1 & 628.2 & 19 & 627.2984 & 29 & 1 & 2204.1921 & 1102. & 2187.1656 & 0864 & 1816 & 944 & 21 \\
\hline 7 & 746.3567 & 373.6820 & & & & & 1 & & & 815 & & & & 420 \\
\hline 8 & 817. & 409. & & & & & A & & & 197 & & 197 & & 19 \\
\hline 9 & 930.4778 & 465.7426 & 913. & 457. & 912.4673 & 456.7373 & 1 & 1919.0233 & 0153 & 1901 & 5020 & 1901 & 0100 & 18 \\
\hline 10 & 1027.5306 & 589 & & & 200 & & & & & & & & & 17 \\
\hline 11 & 92 & 578. & & & 36 & & Q & 1708.8864 & & & & & & 16 \\
\hline 12 & \begin{tabular}{|l|}
1212.6107 \\
\end{tabular} & 606.8090 & 95.5841 & 598. & 1194.6001 & 597.8 & G & 1580.8279 & 176 & 1563 & 4043 & 1562 & 123 & 15 \\
\hline 13 & 25.6947 & 663.3 & 82 & 77 & 342 & 457 & $\mathbf{L}$ & 1523. & & 1506 & & 150 & 016 & 14 \\
\hline 14 & 422.7475 & 711.8774 & 05.7209 & 703.3641 & 1404.7369 & & $\mathbf{P}$ & & & & & 1392.7118 & 8595 & 13 \\
\hline 15 & 523.7952 & 762.4012 & 06.7686 & 753.8879 & 1505.7846 & 753.3959 & $T$ & 1313.6696 & 3384 & 1296.6430 & 8251 & 1295 & 3331 & 12 \\
\hline 16 & 10.8272 & 805.9 & 06 & 797. & 66 & 796. & $\mathrm{~S}$ & 1212. & 146 & 1195 & 013 & 1194 & 3093 & 11 \\
\hline 17 & 1723.9113 & 862.4593 & 47 & 853 & & & & & & & & & & 10 \\
\hline 18 & 1810.9433 & 905.9753 & 793.9167 & 897.4620 & 1792.9327 & 896.9700 & $\mathrm{~S}$ & \begin{tabular}{|l|l|}
1012.5058 \\
\end{tabular} & 506.7565 & \begin{tabular}{|l|}
995.4792 \\
\end{tabular} & 498.2433 & 4952 & 497.7513 & 9 \\
\hline 19 & 339.9859 & 970.4966 & 722.9593 & 961. & 1921.9753 & 961.4913 & 1 & 925.4 & 463.2405 & 4472 & 7272 & 907.4632 & 454.2352 & 8 \\
\hline 20 & 2053.0699 & 1027.0386 & 2036.0434 & 18.5253 & 2035.0594 & 1018.0333 & & & & & & & 7139 & 7 \\
\hline 21 & 2190.1288 & 1095.5681 & 2173.1023 & 1087.0548 & 2172.1183 & 1086.5628 & $\mathbf{H}$ & 683.3471 & 342.1772 & 666.3206 & 333.6639 & 665.3365 & 333.1719 & 6 \\
\hline 22 & 2303.2129 & 1152.1101 & 2286.1864 & 143.5968 & 2285.2023 & 1143.1048 & $\mathbf{L}$ & 546.2882 & 273.6477 & 529.2617 & 265.1345 & 528.2776 & 6425 & 5 \\
\hline $23:$ & 2418.2399 & 1209.6236 & 2401.2133 & 1201.1103 & 2400.2293 & 1200.6183 & D & 433.2041 & 217.1057 & 416.1776 & 208.5924 & 415.1936 & 208.1004 & 4 \\
\hline 24 & 2475.2613 & 1238.1343 & 2458.2348 & 1229.6210 & 2457.2508 & 1229.1290 & G & 318.1772 & 159.5922 & 301.1506 & 151.0790 & & & 3 \\
\hline $25:$ & 2589.3042 & 1295.1558 & 2572.2777 & 1286.6425 & 2571.2937 & 1286.1505 & $\mathbf{N}$ & 261.1557 & 131.0815 & 244.1292 & 122.5682 & & & 2 \\
\hline 26 & & & & & & & $\mathbf{K}$ & 147.1128 & 74.0600 & 130.0863 & 65.5468 & & & \\
\hline
\end{tabular}
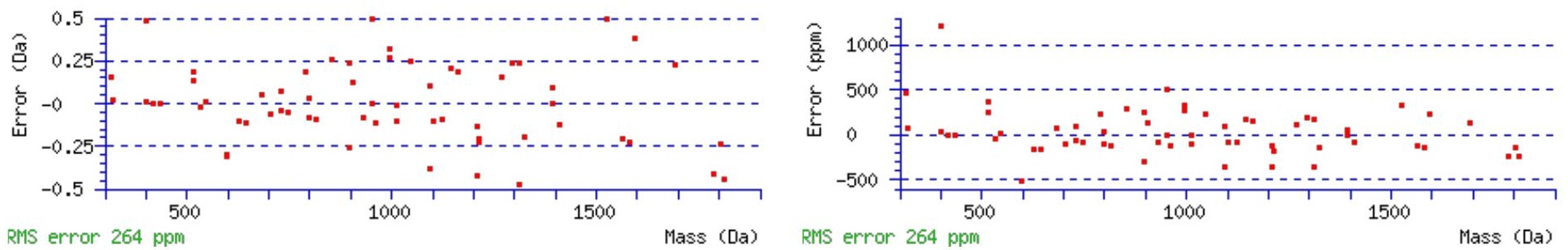

\section{All matches to this query}

\begin{tabular}{|l|l|l|l|}
\hline Score & Mr(calc): & Delta & \multicolumn{1}{c|}{ Sequence } \\
\hline 58.0 & 2734.4025 & 0.0071 & $\underline{\text { ISDTNITAIPQGLPTSISELHLDGNK }}$ \\
\hline 29.1 & 2733.4185 & 0.9911 & $\underline{\text { ISDTNITAIPQGLPTSISELHLDGNK }}$ \\
\hline 27.9 & 2734.4025 & 0.0071 & $\underline{\text { ISDTNITAIPQGLPTSISELHLDGNK }}$ \\
\hline 6.2 & 2733.4119 & 0.9977 & TLTLLITLLLLALHTQAESPQER \\
\hline 2.2 & 2733.4119 & 0.9977 & TLTLLITLLLLALHTQAESPQER \\
\hline 2.0 & 2732.4169 & 1.9927 & MVSVVYTFITPILNPLIYSMRNK \\
\hline 1.6 & 2734.4337 & -0.0242 & THQLFCQEVLKLRPGEPGIVSNGR \\
\hline 0.4 & 2734.4032 & 0.0063 & TLGIIMGVFTLCWLPFFLANVVK \\
\hline & & & \\
\hline
\end{tabular}




\section{\begin{tabular}{|l||l|l|l|l|}
0.1 & 2733.4119 & 0.9977 & TLTLLITLLLLALHTQAESPQER
\end{tabular}}

Spectrum No: 565; Query: 2339; Rank: 1

\section{Peptide View}

MS/MS Fragmentation of DPAVGNISSTCYQPRPDSYHLLR

Found in IPI00201120, Tax_Id=10116 Gene_Symbol=Slc5a2 Low affinity Na-dependent glucose transporter SGLT2 delta e trans

Match to Query 2339: 2646.257952 from(883.093260,3+)

Title: 100101RatKid_NS_deglyco_25.2734.2734.3.dta

Data file K:\NewmanPaper\Piliang $\backslash 3$ SubProteomes $\backslash$ Piliang3SP $\backslash$ mgf5ppm\ERLIC_3SubProteomes5ppm.mgf
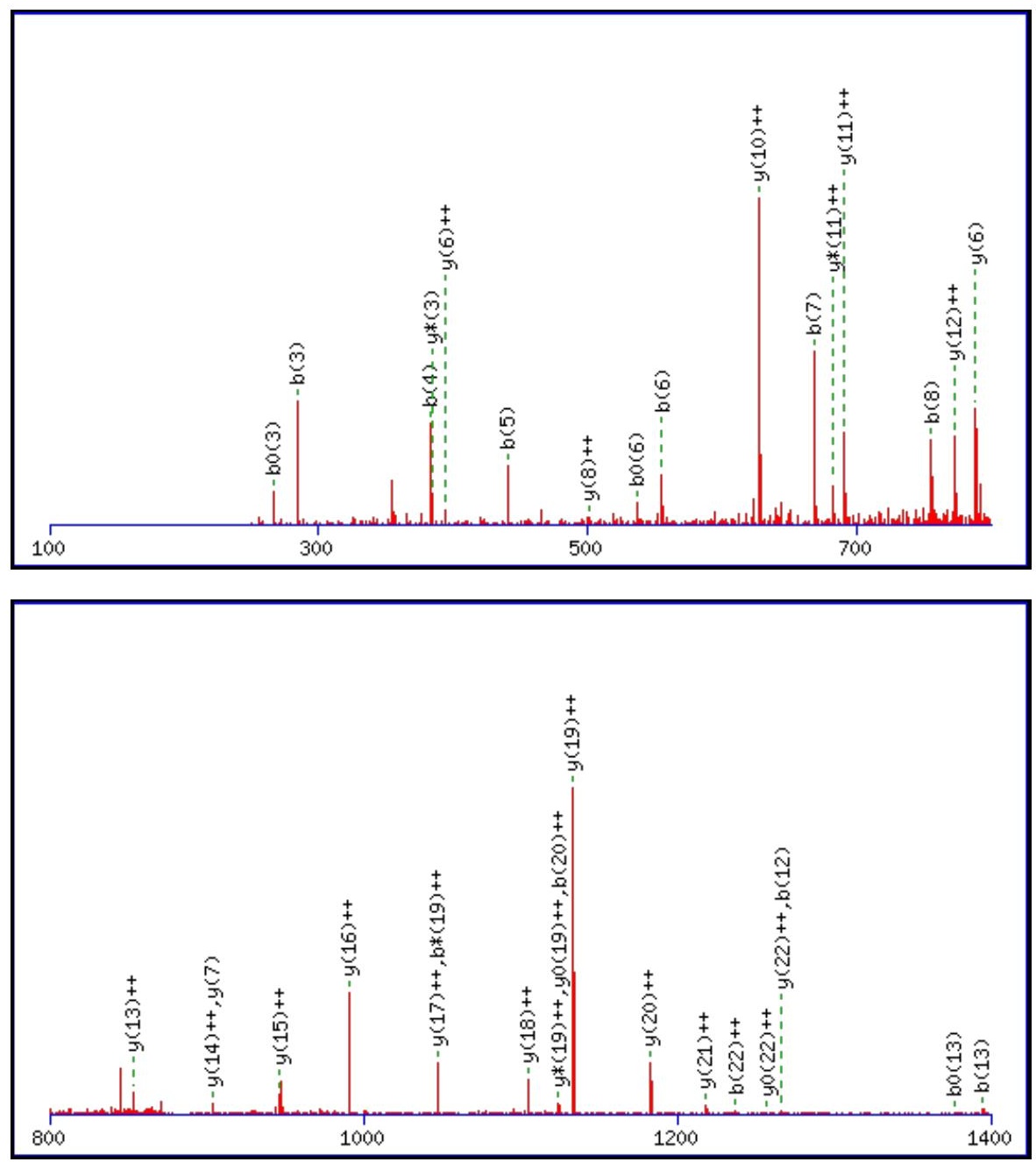


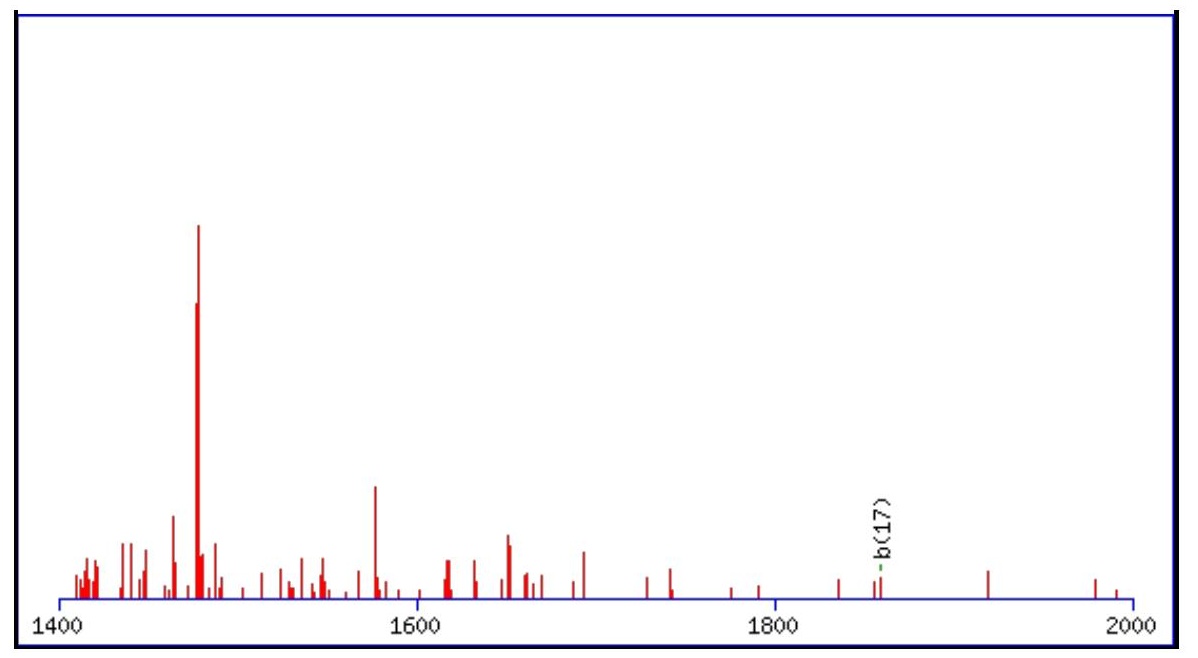

Monoisotopic mass of neutral peptide $\operatorname{Mr}($ calc): 2646.2496

Fixed modifications: Carbamidomethyl (C)

Variable modifications:

N6: Deamidated $\mathrm{N}(\mathrm{N})$

Ions Score: 58 Expect: 0.00049

Matches (Bold Red): 37/244 fragment ions using 91 most intense peaks

\begin{tabular}{|c|c|c|c|c|c|c|c|c|c|c|c|c|c|c|}
\hline \# & b & $\mathbf{b}^{++}$ & $\mathbf{b}^{*}$ & $\mathbf{b}^{*^{++}}$ & $\mathbf{b}^{0}$ & $\mathbf{b}^{0++}$ & Seq. & $\mathbf{y}$ & $y^{++}$ & $\mathbf{y}^{*}$ & $\mathrm{y}^{\mathrm{*}^{++}}$ & $\mathbf{y}^{0}$ & $y^{0++}$ & \# \\
\hline 1 & 116.0342 & 58.5207 & & & 98.0237 & 49.5155 & D & & & & & & & 23 \\
\hline 2 & 213.0870 & 107.0471 & & & 195.0764 & 98.0418 & $\mathbf{P}$ & 2532.2300 & 1266.6186 & 2515.2034 & 1258.1054 & 2514.2194 & 1257.6133 & 22 \\
\hline 3 & 284.1241 & 142.5657 & & & 266.1135 & 133.5604 & A & 2435.1772 & 1218.0922 & 2418.1507 & 1209.5790 & 2417.1666 & 1209.0870 & 21 \\
\hline 4 & 383.1925 & 192.0999 & & & 365.1819 & 183.0946 & V & 2364.1401 & 1182.5737 & 2347.1136 & 1174.0604 & 2346.1295 & 1173.5684 & 20 \\
\hline 5 & 440.2140 & 220.6106 & & & 422.2034 & 211.6053 & G & 2265.0717 & 1133.0395 & 2248.0451 & 1124.5262 & 2247.0611 & 1124.0342 & 19 \\
\hline 6 & 555.2409 & 278.1241 & 538.2144 & 269.6108 & 537.2303 & 269.1188 & $\mathbf{N}$ & 2208.0502 & 1104.5287 & 2191.0237 & 1096.0155 & 2190.0397 & 1095.5235 & 18 \\
\hline 7 & 668.3250 & 334.6661 & 651.2984 & 326.1529 & 650.3144 & 325.6608 & I & 2093.0233 & 1047.0153 & 2075.9967 & 1038.5020 & 2075.0127 & 1038.0100 & 17 \\
\hline 8 & 755.3570 & 378.1821 & 738.3305 & 369.6689 & 737.3464 & 369.1769 & S & 1979.9392 & 990.4732 & 1962.9127 & 981.9600 & 1961.9287 & 981.4680 & 16 \\
\hline 9 & 842.3890 & 421.6982 & 825.3625 & 413.1849 & 824.3785 & 412.6929 & S & 1892.9072 & 946.9572 & 1875.8806 & 938.4440 & 1874.8966 & 937.9520 & 15 \\
\hline 10 & 943.4367 & 472.2220 & 926.4102 & 463.7087 & 925.4261 & 463.2167 & $\mathbf{T}$ & 1805.8752 & 903.4412 & 1788.8486 & 894.9279 & 1787.8646 & 894.4359 & 14 \\
\hline 11 & 1103.4674 & 552.2373 & 1086.4408 & 543.7240 & 1085.4568 & 543.2320 & C & 1704.8275 & 852.9174 & 1687.8009 & 844.4041 & 1686.8169 & 843.9121 & 13 \\
\hline 12 & 1266.5307 & 633.7690 & 1249.5041 & 625.2557 & 1248.5201 & 624.7637 & $\mathbf{Y}$ & 1544.7968 & 772.9021 & 1527.7703 & 764.3888 & 1526.7863 & 763.8968 & 12 \\
\hline 13 & 1394.5893 & 697.7983 & 1377.5627 & 689.2850 & 1376.5787 & 688.7930 & $\mathbf{Q}$ & 1381.7335 & 691.3704 & 1364.7070 & 682.8571 & 1363.7229 & 682.3651 & 11 \\
\hline 14 & 1491.6420 & 746.3247 & 1474.6155 & 737.8114 & 1473.6315 & 737.3194 & $\mathbf{P}$ & 1253.6749 & 627.3411 & 1236.6484 & 618.8278 & 1235.6644 & 618.3358 & 10 \\
\hline 15 & 1647.7431 & 824.3752 & 1630.7166 & 815.8619 & 1629.7326 & 815.3699 & $\mathbf{R}$ & 1156.6222 & 578.8147 & 1139.5956 & 570.3014 & 1138.6116 & 569.8094 & 9 \\
\hline 16 & 1744.7959 & 872.9016 & 1727.7694 & 864.3883 & 1726.7853 & 863.8963 & $\mathbf{P}$ & 1000.5211 & 500.7642 & 983.4945 & 492.2509 & 982.5105 & 491.7589 & 8 \\
\hline 17 & 1859.8228 & 930.4151 & 1842.7963 & 921.9018 & 1841.8123 & 921.4098 & D & 903.4683 & 452.2378 & 886.4417 & 443.7245 & 885.4577 & 443.2325 & 7 \\
\hline 18 & 1946.8549 & 973.9311 & 1929.8283 & 965.4178 & 1928.8443 & 964.9258 & S & 788.4413 & 394.7243 & 771.4148 & 386.2110 & 770.4308 & 385.7190 & 6 \\
\hline 19 & 2109.9182 & 1055.4627 & 2092.8917 & 1046.9495 & 2091.9076 & 1046.4575 & $\mathbf{Y}$ & 701.4093 & 351.2083 & 684.3828 & 342.6950 & & & 5 \\
\hline 20 & 2246.9771 & 1123.9922 & 2229.9506 & 1115.4789 & 2228.9666 & 1114.9869 & $\mathbf{H}$ & 538.3460 & 269.6766 & 521.3194 & 261.1634 & & & 4 \\
\hline 21 & 2360.0612 & 1180.5342 & 2343.0346 & 1172.0210 & 2342.0506 & 1171.5289 & $\mathbf{L}$ & 401.2871 & 201.1472 & 384.2605 & 192.6339 & & & 3 \\
\hline 22 & 2473.1452 & 1237.0763 & 2456.1187 & 1228.5630 & 2455.1347 & 1228.0710 & $\mathbf{L}$ & 288.2030 & 144.6051 & 271.1765 & 136.0919 & & & 2 \\
\hline 23 & & & & & & & $\mathbf{R}$ & 175.1190 & 88.0631 & 158.0924 & 79.5498 & & & 1 \\
\hline
\end{tabular}

$$
\text { 跣 }
$$

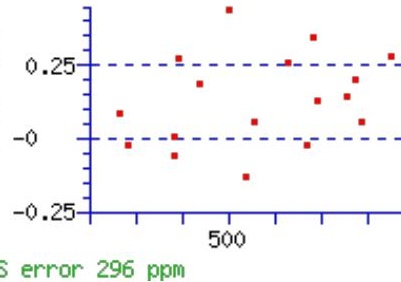

$:$

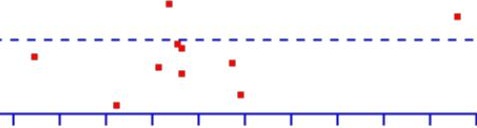

1000

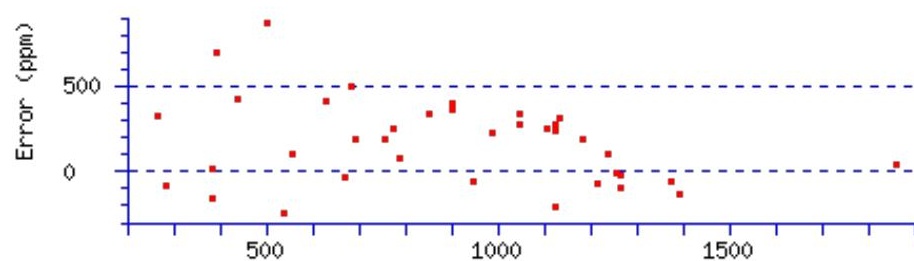

Mass (Da)

All matches to this query 


\begin{tabular}{|l|c|c|l|}
\hline Score & Mr(calc): & Delta & \multicolumn{1}{|c|}{ Sequence } \\
\hline 58.0 & 2646.2496 & 0.0083 & DPAVGNISSTCYQPRPDSYHLLR \\
\hline 23.9 & 2645.2656 & 0.9923 & DPAVGNISSTCYQPRPDSYHLLR \\
\hline 9.0 & 2646.2357 & 0.0223 & DNVITPLLRFQSVHSGNPQVHK \\
\hline 5.7 & 2646.2357 & 0.0223 & DNVITPLLRFQSVHSGNPQVHK \\
\hline 5.0 & 2646.2479 & 0.0100 & SITRSYYRNSVGGFLVFDITNR \\
\hline 3.7 & 2646.2357 & 0.0223 & DNVITPLLRFQSVHSGNPQVHK \\
\hline 3.5 & 2646.2439 & 0.0141 & DSVRQIIFSSLFSPQGNKADDSR \\
\hline 3.1 & 2646.2586 & -0.0007 & SLTVVYTVITPMLNPLIYTLR \\
\hline 3.0 & 2646.2319 & 0.0260 & VFGNLSQDHDVCNFLMQKNVHK \\
\hline 3.0 & 2646.2319 & 0.0260 & VFGNLSQDHDVCNFLMQKNVHK \\
\hline
\end{tabular}

Spectrum No: 566; Query: 1192; Rank: 1

\section{Peptide View}

MS/MS Fragmentation of GGSPLGALSWLNETQLK

Found in IPI00764756, Tax_Id=10116 Gene_Symbol=LOC684529 similar to cathepsin O

Match to Query 1192: 1770.922328 from(886.468440,2+)

Title: 091008RatKidney_NH4Format01_17.5000.5000.2.dta

Data file K:\NewmanPaper\Piliang\3SubProteomes\Piliang3SP\mgf5ppm\ERLIC_3SubProteomes5ppm.mgf
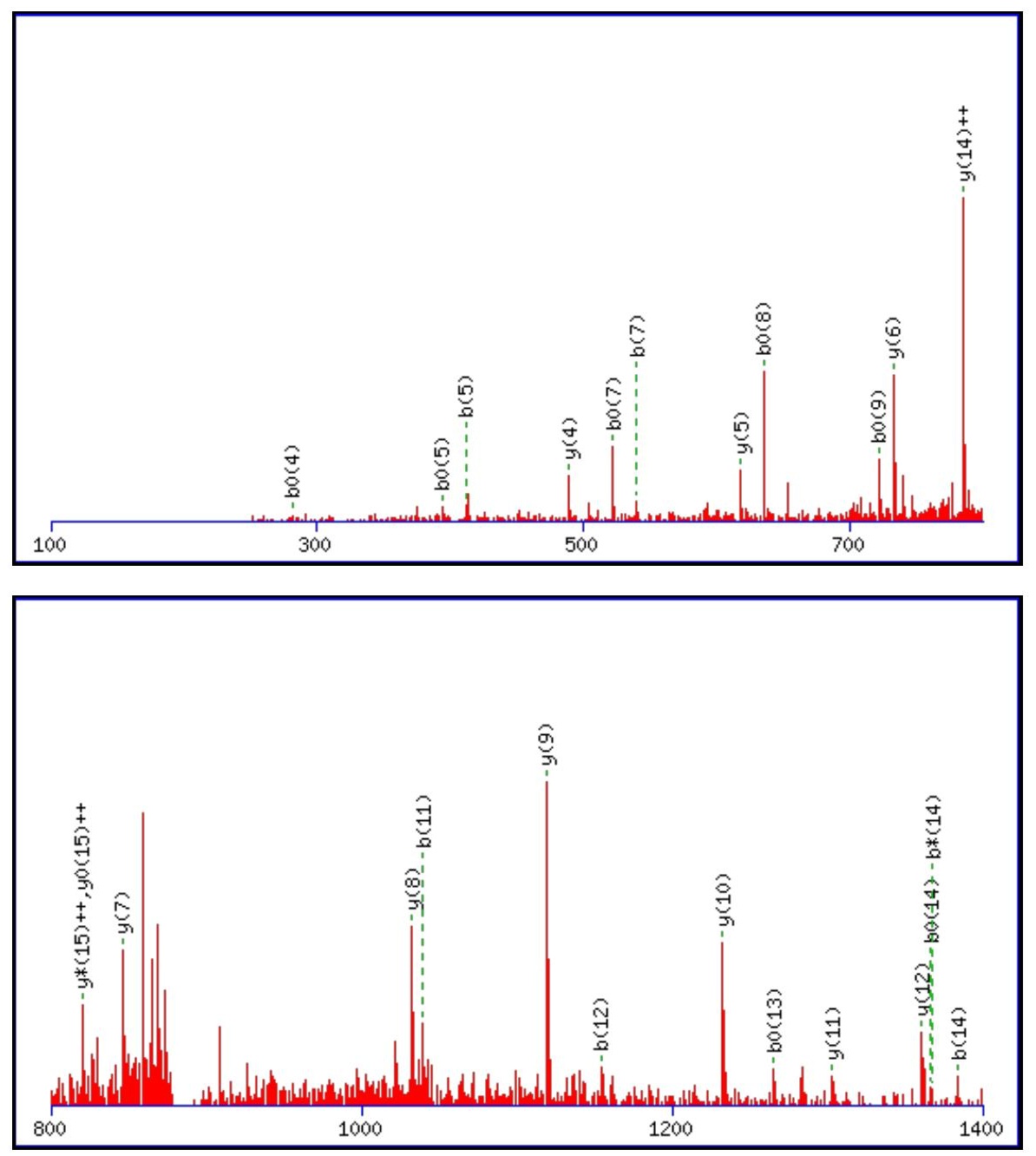


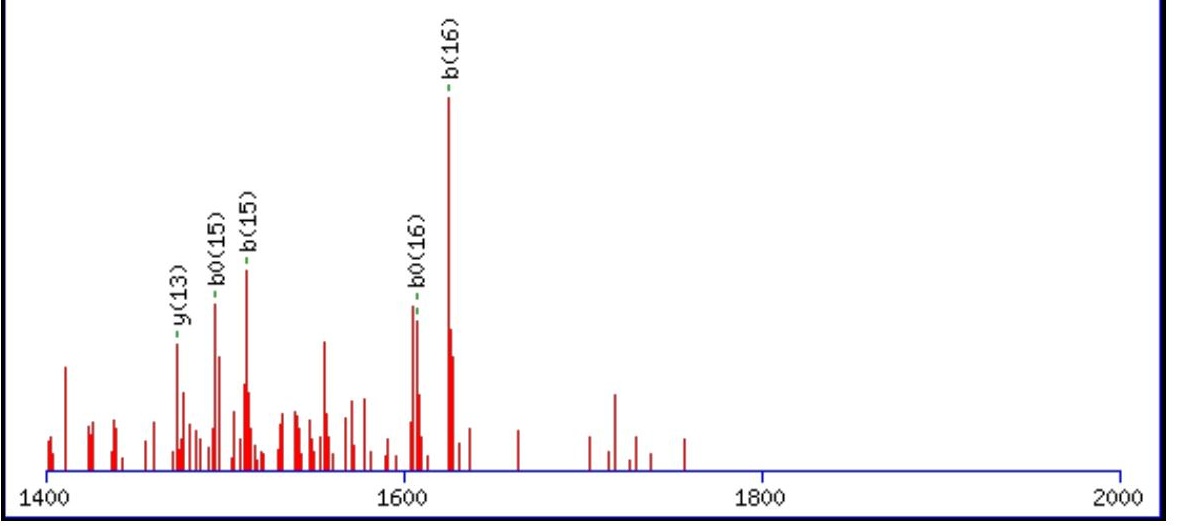

Monoisotopic mass of neutral peptide $\operatorname{Mr}($ calc): 1770.9152

Fixed modifications: Carbamidomethyl (C)

Variable modifications:

N12 : Deamidated_N (N)

Ions Score: 58 Expect: 0.00021

Matches (Bold Red): 30/160 fragment ions using 55 most intense peaks

\begin{tabular}{|c|c|c|c|c|c|c|c|c|c|c|c|c|c|c|}
\hline \# & b & $\mathbf{b}^{++}$ & $\mathbf{b}^{*}$ & $\mathbf{b}^{*^{++}}$ & $\mathbf{b}^{0}$ & $\mathbf{b}^{0++}$ & Seq. & $\mathbf{y}$ & $\mathbf{y}^{++}$ & $\mathrm{y}^{*}$ & $\mathrm{y}^{*^{++}}$ & $\mathbf{y}^{0}$ & $y^{0++}$ & $\#$ \\
\hline 1 & 58.0287 & 29.5180 & & & & & $\mathbf{G}$ & & & & & & & 17 \\
\hline 2 & 115.0502 & 58.0287 & & & & & G & 1714.9010 & 857.9541 & 1697.8745 & 849.4409 & 1696.8905 & 848.9489 & 16 \\
\hline 3 & 202.0822 & 101.5448 & & & 184.0717 & 92.5395 & $\mathrm{~S}$ & 1657.8796 & 829.4434 & 1640.8530 & 820.9301 & 1639.8690 & 820.4381 & 15 \\
\hline 4 & 299.1350 & 150.0711 & & & 281.1244 & 141.0659 & $\mathbf{P}$ & 1570.8475 & 785.9274 & 1553.8210 & 777.4141 & 1552.8370 & 776.9221 & 14 \\
\hline 5 & 412.2191 & 206.6132 & & & 394.2085 & 197.6079 & $\mathbf{L}$ & 1473.7948 & 737.4010 & 1456.7682 & 728.8877 & 1455.7842 & 728.3957 & 13 \\
\hline 6 & 469.2405 & 235.1239 & & & 451.2300 & 226.1186 & G & 1360.7107 & 680.8590 & 1343.6842 & 672.3457 & 1342.7001 & 671.8537 & 12 \\
\hline 7 & 540.2776 & 270.6425 & & & 522.2671 & 261.6372 & $\mathbf{A}$ & 1303.6892 & 652.3483 & 1286.6627 & 643.8350 & 1285.6787 & 643.3430 & 11 \\
\hline 8 & 653.3617 & 327.1845 & & & 635.3511 & 318.1792 & $\mathbf{L}$ & 1232.6521 & 616.8297 & 1215.6256 & 608.3164 & 1214.6416 & 607.8244 & 10 \\
\hline 9 & 740.3937 & 370.7005 & & & 722.3832 & 361.6952 & $S$ & 1119.5681 & 560.2877 & 1102.5415 & 551.7744 & 1101.5575 & 551.2824 & 9 \\
\hline 10 & 926.4730 & 463.7402 & & & 908.4625 & 454.7349 & W & 1032.5360 & 516.7717 & 1015.5095 & 508.2584 & 1014.5255 & 507.7664 & 8 \\
\hline 11 & 1039.5571 & 520.2822 & & & 1021.5465 & 511.2769 & $\mathbf{L}$ & 846.4567 & 423.7320 & 829.4302 & 415.2187 & 828.4462 & 414.7267 & 7 \\
\hline 12 & 1154.5840 & 577.7957 & 1137.5575 & 569.2824 & 1136.5735 & 568.7904 & $\mathbf{N}$ & 733.3727 & 367.1900 & 716.3461 & 358.6767 & 715.3621 & 358.1847 & 6 \\
\hline 13 & 1283.6266 & 642.3170 & 1266.6001 & 633.8037 & 1265.6161 & 633.3117 & $\mathbf{E}$ & 618.3457 & 309.6765 & 601.3192 & 301.1632 & 600.3352 & 300.6712 & 5 \\
\hline 14 & 1384.6743 & 692.8408 & 1367.6478 & 684.3275 & \begin{tabular}{|l|}
1366.6637 \\
\end{tabular} & 683.8355 & $T$ & 489.3031 & 245.1552 & 472.2766 & 236.6419 & 471.2926 & 236.1499 & 4 \\
\hline 15 & 1512.7329 & 756.8701 & 1495.7063 & 748.3568 & 1494.7223 & 747.8648 & $\mathbf{Q}$ & 388.2554 & 194.6314 & 371.2289 & 186.1181 & & & 3 \\
\hline 16 & 1625.8170 & 813.4121 & 1608.7904 & 804.8988 & 1607.8064 & 804.4068 & $\mathbf{L}$ & 260.1969 & 130.6021 & 243.1703 & 122.0888 & & & 2 \\
\hline 17 & & & & & & & $\mathbf{K}$ & 147.1128 & 74.0600 & 130.0863 & 65.5468 & & & 1 \\
\hline
\end{tabular}
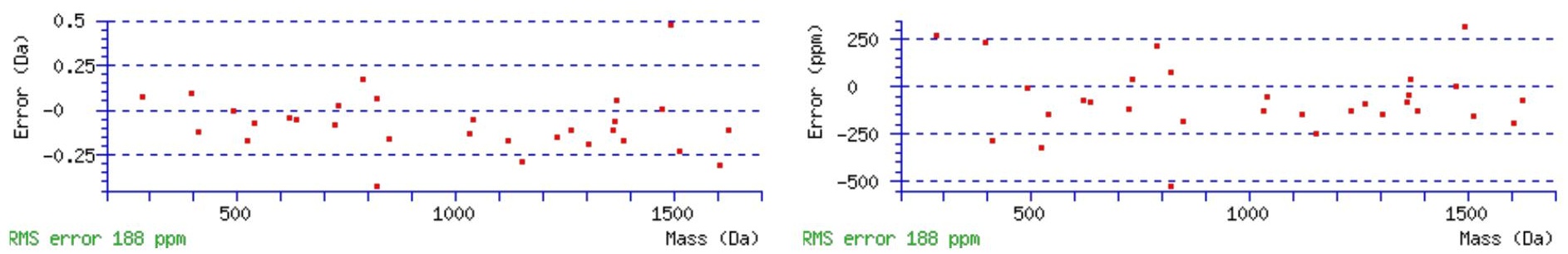

\section{All matches to this query}

\begin{tabular}{|l|l|c|l|}
\hline Score & Mr(calc): & Delta & \multicolumn{1}{c|}{ Sequence } \\
\hline 57.9 & 1770.9152 & 0.0071 & GGSPLGALSWLNETQLK \\
\hline 3.7 & 1770.9264 & -0.0041 & NGIGIHEHASIDLPGIK \\
\hline 3.7 & 1769.9312 & 0.9911 & GGSPLGALSWLNETQLK \\
\hline 0.1 & 1770.9103 & 0.0120 & LSDIGFPMARSKLLK \\
\hline
\end{tabular}


Spectrum No: 567; Query: 1241; Rank: 1

\section{Peptide View}

MS/MS Fragmentation of DNFTLIPEGTNGTEER

Found in IPI00870112, Tax_Id=10116 Gene_Symbol=Dpysl2 Dihydropyrimidinase-related protein 2

Match to Query 1241: 1792.810588 from(897.412570,2+)

Title: 091008RatKidney_NoSalt_22.2603.2603.2.dta

Data file K:\NewmanPaper\Piliang \3SubProteomes $\backslash$ Piliang3SP $\backslash$ mgf5ppm\ERLIC_3SubProteomes5ppm.mgf
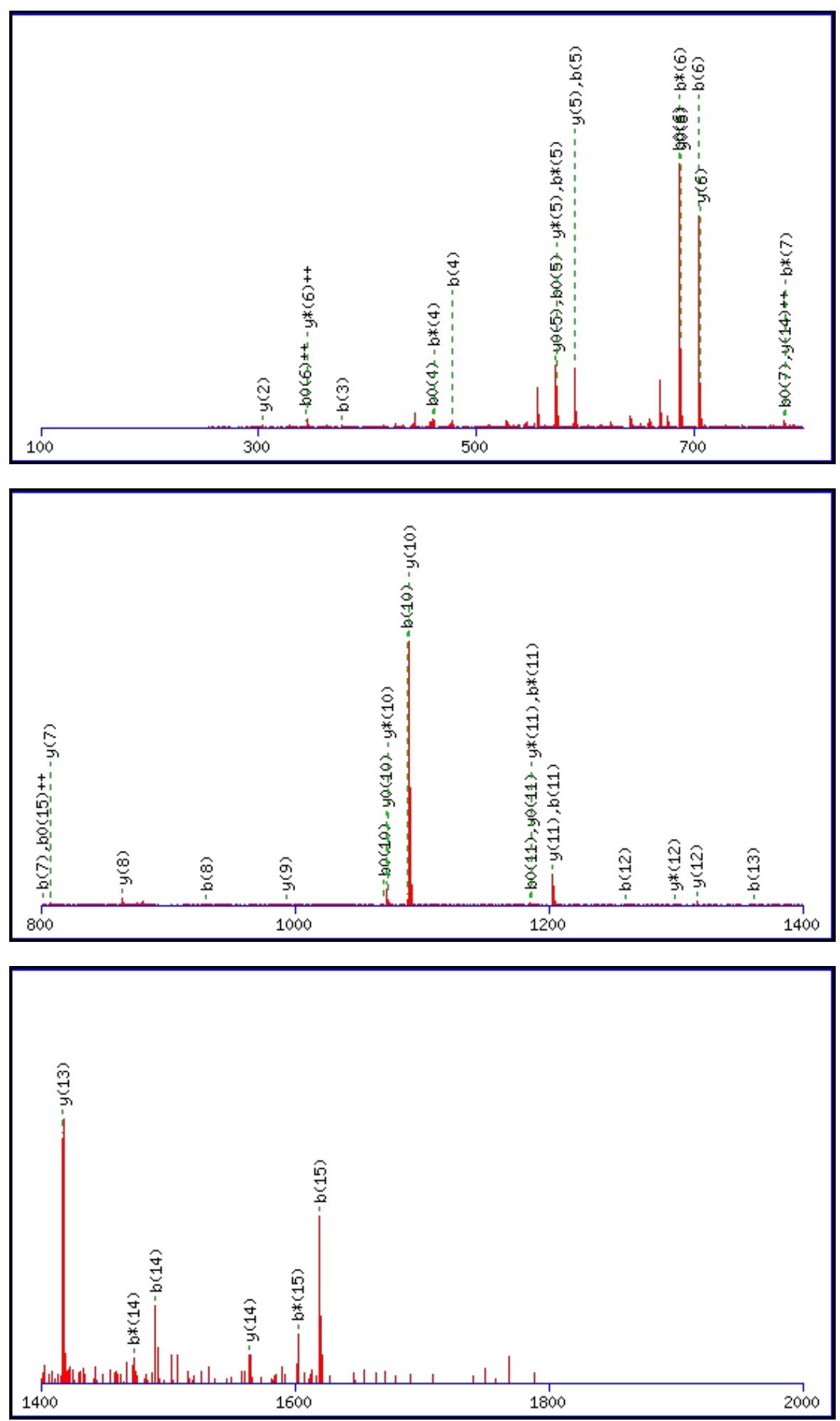
Monoisotopic mass of neutral peptide $\operatorname{Mr}($ calc): 1792.8115

Fixed modifications: Carbamidomethyl (C)

Variable modifications:

N11 : Deamidated_N (N)

Ions Score: 58 Expect: 0.00037

Matches (Bold Red): 48/176 fragment ions using 108 most intense peaks

\begin{tabular}{|c|c|c|c|c|c|c|c|c|c|c|c|c|c|c|}
\hline \# & b & $\mathbf{b}^{++}$ & b* & & $\mathbf{b}^{\mathbf{0}}$ & & Seq. & $\mathbf{y}$ & & $\mathbf{y}^{*}$ & & $\mathbf{y}^{\mathbf{0}}$ & & \# \\
\hline 1 & 116.0342 & 58.5207 & & & 98.0237 & 49.5155 & D & & & & & & & 16 \\
\hline 2 & 230.0771 & 115.5422 & 213.0506 & 107.0289 & 212.0666 & 106.5369 & $\mathbf{N}$ & & & 1661.7653 & & 1660.7813 & 830.8943 & 15 \\
\hline 3 & 377.1456 & 189.0764 & 360.1190 & 180.5631 & 359.1350 & 180.0711 & $\mathbf{F}$ & 1564.7489 & 782.8781 & 1547.7224 & & 1546.7384 & 773.8728 & 14 \\
\hline 4 & 478.1932 & 239.6003 & 461.1667 & 231.0870 & 460.1827 & 230.5950 & $\mathbf{T}$ & 1417.6805 & 709.3439 & 1400.6540 & 700.8306 & 1399.6700 & 700.3386 & 13 \\
\hline 5 & 591.2773 & 296.1423 & 574.2508 & 287.6290 & 573.2667 & 287.1370 & $\mathbf{L}$ & 1316.6328 & 658.8201 & 1299.6063 & 650.3068 & 1298.6223 & 649.8148 & 12 \\
\hline 6 & 704.3614 & 352.6843 & 687.3348 & 344.1710 & 686.3508 & 343.6790 & I & 488 & 602.2780 & 1186.5222 & 593.7648 & 1185 & 593.2727 & 11 \\
\hline 7 & 801.4141 & 401.2107 & 784.3876 & 392.6974 & 783.4036 & 392.2054 & $\mathbf{P}$ & 1090.4647 & 545.7360 & 1073.4382 & 537.2227 & 1072.4542 & 536.7307 & 10 \\
\hline 8 & 930.4567 & 465.7320 & 913.4302 & 457.2187 & 912.4462 & 456.7267 & $\mathbf{E}$ & 993.4120 & 497.2096 & 976.3854 & 488.6963 & 975.4014 & 488.2043 & 9 \\
\hline 9 & 987.4782 & 494.2427 & 970.4516 & 485.7295 & 969.4676 & 485.2374 & G & 864.3694 & 432.6883 & 847.3428 & 424.1750 & 846.3588 & 423.6830 & 8 \\
\hline 10 & 1088.5259 & 544.7666 & 1071.4993 & 536.2533 & 1070.5153 & 535.7613 & $\mathbf{T}$ & 807.3479 & 404.1776 & 790.3213 & 395.6643 & 789.3373 & 395.1723 & 7 \\
\hline 11 & 1203.5528 & 602.2800 & 1186.5263 & 593.7668 & 1185.5422 & 593.2748 & $\mathbf{N}$ & 706.3002 & 353.6537 & 689.2737 & 345.1405 & 688.2897 & 344.6485 & 6 \\
\hline 12 & 1260.5743 & 630.7908 & 1243.5477 & 622.2775 & 1242.5637 & 621.7855 & G & 591.2733 & 296.1403 & 574.2467 & 287.6270 & 573.2627 & 287.1350 & 5 \\
\hline 13 & 1361.6219 & 681.3146 & 1344.5954 & 672.8013 & 1343.6114 & 672.3093 & $\mathbf{T}$ & 534.2518 & 267.6295 & 517.2253 & 259.1163 & 516.2413 & 258.6243 & 4 \\
\hline 14 & 1490.6645 & 745.8359 & 1473.6380 & 737.3226 & 1472.6540 & 736.8306 & $\mathbf{E}$ & 433.2041 & 217.1057 & 416.1776 & 208.5924 & 415.1936 & 208.1004 & 3 \\
\hline 15 & 1619.7071 & 810.3572 & 1602.6806 & 801.8439 & 1601.6966 & 801.3519 & $\mathbf{E}$ & 304.1615 & 152.5844 & 287.1350 & 144.0711 & 286.1510 & 143.5791 & 2 \\
\hline 16 & & & & & & & $\mathbf{R}$ & 175.1190 & 88.0631 & 158.0924 & 79.5498 & & & 1 \\
\hline
\end{tabular}
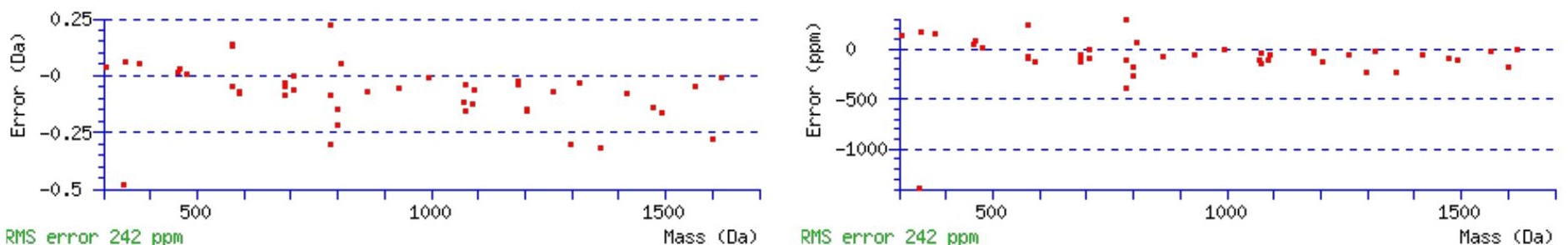

All matches to this query

\begin{tabular}{|l|l|l|l|}
\hline Score & Mr(calc): & Delta & \multicolumn{1}{c|}{ Sequence } \\
\hline 57.8 & 1792.8115 & -0.0009 & $\underline{\text { DNFTLIPEGTNGTEER }}$ \\
\hline 13.9 & 1792.8115 & -0.0009 & DNFTLIPEGTNGTEER \\
\hline 9.9 & 1792.8083 & 0.0023 & $\underline{\text { QTCLKTVVLLTDNK }}$ \\
\hline 5.0 & 1792.8259 & -0.0153 & $\underline{\text { MSLKPFTYPFPETR }}$ \\
\hline 3.6 & 1792.7978 & 0.0128 & $\underline{\text { WLYMDSNYLDTLSR }}$ \\
\hline 3.3 & 1790.8096 & 2.0010 & $\underline{\text { VEYVIKCDMSALQR }}$ \\
\hline 3.0 & 1791.8192 & 0.9913 & $\underline{\text { RANNTXYGLAAGVFTK }}$ \\
\hline 2.4 & 1791.8243 & 0.9863 & $\underline{\text { QTCLKTVVLLTDNK }}$ \\
\hline 2.4 & 1791.8015 & 1.0090 & VDDLVWHSVGLQCGK \\
\hline 2.4 & 1790.8087 & 2.0019 & $\underline{\text { NSPATPPNTLNLXSEK }}$ \\
\hline
\end{tabular}

Spectrum No: 568; Query: 2677; Rank: 1

\section{Peptide View}

MS/MS Fragmentation of TQYACPPFNVTECSIQDEAGNSIDLSSLSR

Found in IPI00332022, Tax_Id=10116 Gene_Symbol=Igf2r Mannose 6-phosphate/insulin-like growth factor II receptor 
Match to Query 2677: 3360.504672 from(1121.175500,3+)

Title: 100101RatKid_NS_deglyco_23.4794.4794.3.dta

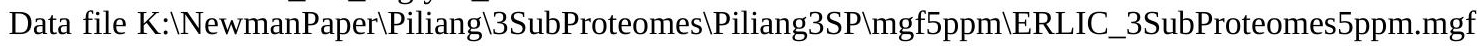
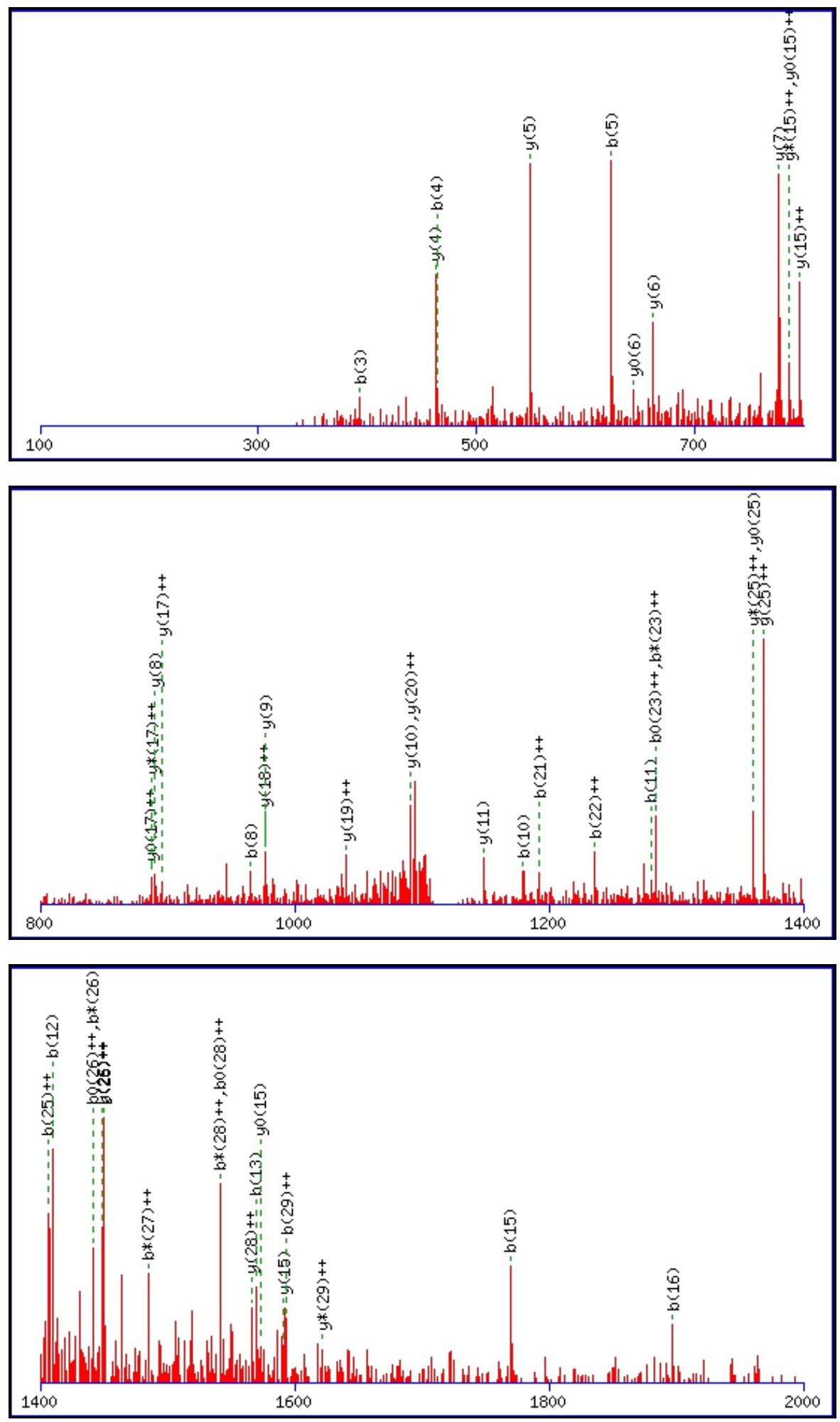

Monoisotopic mass of neutral peptide $\operatorname{Mr}($ calc): 3359.4922

Fixed modifications: Carbamidomethyl (C)

Variable modifications:

N9: Deamidated_N (N)

Ions Score: 58 Expect: 0.00076

Matches (Bold Red): 48/344 fragment ions using 79 most intense peaks

\begin{tabular}{|c|c|c|c|c|c|c|c|c|c|c|c|c|c|c|}
\hline \# & b & $\mathbf{b}^{++}$ & b* & $\mathbf{b}^{*^{++}}$ & $\mathbf{b}^{\mathbf{0}}$ & $\mathbf{b}^{\mathbf{0 + +}}$ & Seq. & $\mathbf{y}$ & $\mathbf{y}^{++}$ & $\mathbf{y}^{*}$ & $\mathbf{y}^{*^{++}}$ & $\mathbf{y}^{\mathbf{0}}$ & $\mathbf{y}^{0++}$ & \# \\
\hline 1 & 102.0550 & 51.5311 & & & 84.0444 & 42.5258 & $\mathbf{T}$ & & & & & & & 30 \\
\hline 2 & 230.1135 & 115.5604 & 213.0870 & 107.0471 & 212.1030 & 106.5551 & $\mathbf{Q}$ & 3259.4518 & 1630.2295 & 3242.4252 & 1621.7163 & 3241.4412 & 1621.2243 & 29 \\
\hline
\end{tabular}




\begin{tabular}{|c|c|c|c|c|c|c|c|c|c|c|c|c|c|c|}
\hline & & & & & & & Y & & & & & & & \\
\hline 4 & 140 & 106 & 447.1874 & 974 & 34 & 53 & & 299 & 686 & & 553 & & & \\
\hline 5 & & & & & & & & & & & & & & \\
\hline 6 & & & & & & & & & & & & & & \\
\hline 7 & & & & & & & & & & & & & & \\
\hline 8 & 965. & 483.2129 & 948. & 474. & 80 & & & & & 1300 & & & & \\
\hline 9 & 1080. & & 190 & & 1062 & & & & & & & & & \\
\hline 10 & 39 & & 74 & & & & & & & & & & & \\
\hline 1 & 1280.5616 & 640 & 350 & 632 & 1262. & 631. & $\mathbf{1}$ & 28 & & & & & & \\
\hline 2 & 042 & & 76 & & & & & & & & & & & \\
\hline 3 & 48 & & 33 & 776 & 155 & & & & & & & & & \\
\hline 4 & 1656.6669 & 828.8 & 403 & 820. & 1638. & 819 & & 719 & & 454 & 263 & 613 & 343 & \\
\hline 5 & 09 & & 44 & & 104 & & & & & & & & & \\
\hline 16 & 95 & & 30 & 51 & 89 & & & & & & & & & \\
\hline 7 & 2012.8365 & 06.9219 & 1995.8099 & 086 & 1994.8 & 166 & & 6972 & 523 & 707 & 390 & 144 & 3470 & \\
\hline 18 & 90 & & 25 & 99 & 885 & & & 134 & & & & & & \\
\hline 19 & 2212.9162 & & 2195. & & 2194. & & & & & & & & & \\
\hline 20 & 2269.9376 & 35.4724 & 2252. & 592 & 2251. & 672 & G & 906 & 989 & 640 & 857 & 800 & 9937 & \\
\hline 21 & 2383 & 39 & 2366 & 06 & 2365 & 386 & $\mathbf{N}$ & 109 & & 5426 & & 586 & 2829 & \\
\hline 22 & 2471. & 36.0099 & 2453.9860 & 27.4967 & 2453.0020 & 0046 & & & & & & & 615 & \\
\hline 23 & 2584.0966 & 1292.5520 & 2567.0701 & 1284.0387 & 2566.0861 & 1283.5467 & I & 4942 & 7507 & 4676 & 2374 & 4836 & .7454 & \\
\hline 24 & 2699. & 654 & 2682. & 522 & 130 & 601 & & 101 & 087 & 3836 & 6954 & 3995 & 2034 & \\
\hline 252 & 2812.2076 & 1406.6075 & 2795.1811 & 398.0942 & 2794.1971 & 1397.6022 & $\mathbf{H}$ & & & & & & & \\
\hline 26 & 2899.2397 & 450.1235 & 2882.2131 & 102 & 2881.2291 & 182 & $\mathrm{~S}$ & 2991 & 1532 & 2726 & & 2885 & 479 & \\
\hline 272 & 2986.2717 & 1493.6395 & 2969.2452 & 1485.1262 & 2968.2611 & 1484.6342 & 3 & 2671 & 6372 & 2405 & 239 & 2565 & 3319 & \\
\hline 283 & 3099.3558 & 1550.1815 & 3082.3292 & 1541.6682 & 3081.3452 & 1541.1762 & $\mathbf{L}$ & & & 2085 & & & 159 & \\
\hline 293 & 3186.3878 & 1593.6975 & 3169.3612 & 1585.1843 & 3168.3772 & 1584.6923 & $\mathrm{~S}$ & & 131.5791 & 245.1244 & & & & \\
\hline 30 & & & & & & & $\mathbf{R}$ & 175.1190 & 0631 & 0924 & .5498 & & & \\
\hline
\end{tabular}
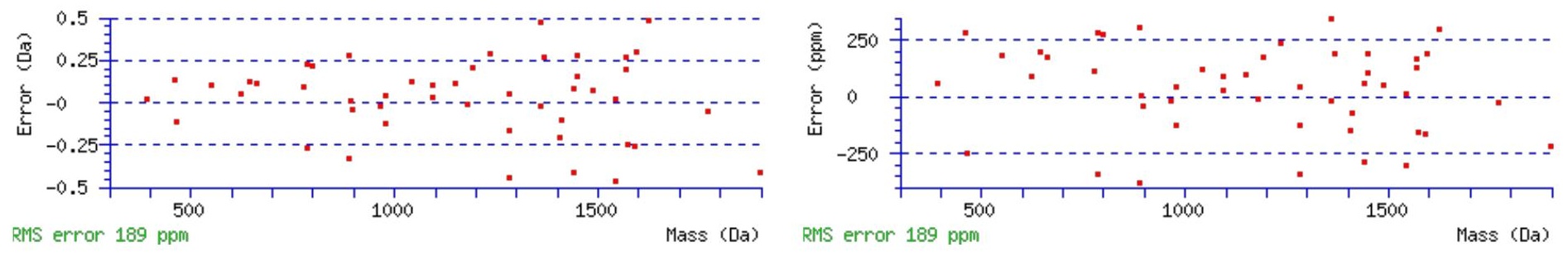

\section{All matches to this query}

\begin{tabular}{|l|l|l|l|}
\hline Score & Mr(calc): & Delta & \multicolumn{1}{|c|}{ Sequence } \\
\hline 57.8 & 3359.4922 & 1.0125 & TQYACPPFNVTECSIQDEAGNSIDLSSLSR \\
\hline 45.6 & 3360.4762 & 0.0285 & TQYACPPFNVTECSIQDEAGNSIDLSSLSR \\
\hline 29.8 & 3359.4922 & 1.0125 & TQYACPPFNVTECSIQDEAGNSIDLSSLSR \\
\hline 26.6 & 3358.5082 & 1.9965 & TQYACPPFNVTECSIQDEAGNSIDLSSLSR \\
\hline 8.8 & 3359.5018 & 1.0028 & LSIILALMGGSKVVILDEPSSGMDPMSRR \\
\hline 5.7 & 3359.4710 & 1.0337 & VPVDVAYQRGYFDQMLNSILLDPSDDTK \\
\hline 4.1 & 3359.5018 & 1.0028 & $\underline{\text { LSIILALMGGSKVVILDEPSSGMDPMSRR }}$ \\
\hline 3.0 & 3358.4870 & 2.0177 & VPVDVAYQRGYFDQMLNSILLDPSDDTK \\
\hline 3.0 & 3358.4870 & 2.0177 & VPVDVAYQRGYFDQMLNSILLDPSDDTK \\
\hline 2.6 & 3359.4710 & 1.0337 & $\underline{\text { VPVDVAYQRGYFDQMLNSILLDPSDDTK }}$ \\
\hline
\end{tabular}


Spectrum No: 569; Query: 393; Rank: 1

\section{Peptide View}

MS/MS Fragmentation of YYNYTLSINGK

Found in IPI00366226, Tax_Id=10116 Gene_Symbol=Gns Glucosamine (N-acetyl)-6-sulfatase

Match to Query 393: 1335.635348 from(668.824950,2+)

Title: 091008RatKidney_NH4Format01_23.2133.2133.2.dta

Data file K:\NewmanPaper\Piliang \3SubProteomes $\backslash$ Piliang3SP $\backslash$ mgf5ppm\ERLIC_3SubProteomes5ppm.mgf
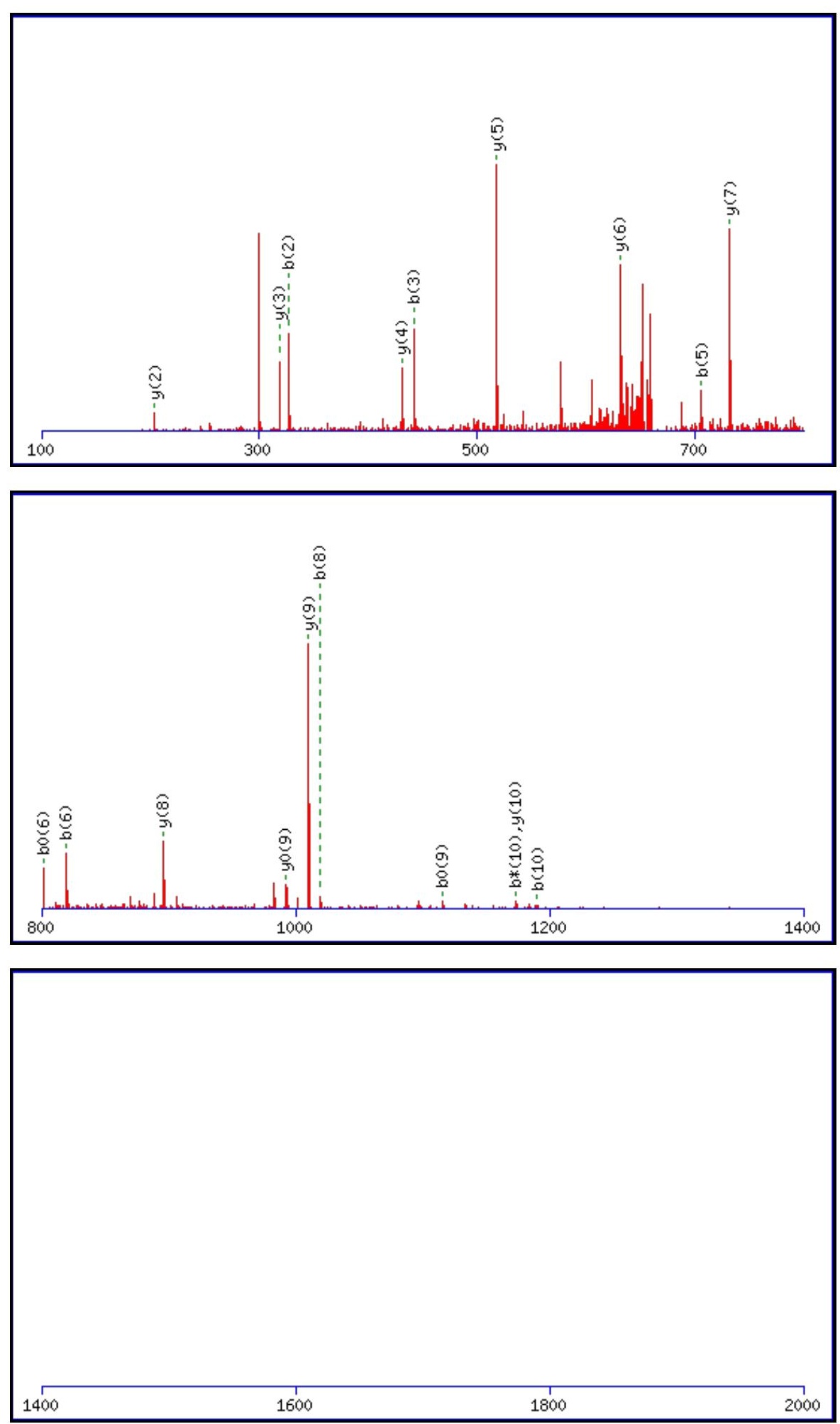

Monoisotopic mass of neutral peptide $\operatorname{Mr}($ calc): 1335.6346

Fixed modifications: Carbamidomethyl (C) 
Variable modifications:

N3 : Deamidated_N (N)

Ions Score: 57 Expect: $\odot .00024$

Matches (Bold Red): 19/10๑ fragment ions using 40 most intense peaks

\begin{tabular}{|r|c|c|c|c|c|c|c|c|c|c|c|c|c|c|}
\hline$\#$ & $\mathbf{b}$ & $\mathbf{b}^{++}$ & $\mathbf{b}^{*}$ & $\mathbf{b}^{\mathbf{*}^{++}}$ & $\mathbf{b}^{\mathbf{0}}$ & $\mathbf{b}^{\mathbf{0 + +}}$ & $\mathbf{S e q}$ & $\mathbf{y}$ & $\mathbf{y}^{++}$ & $\mathbf{y}^{\mathbf{*}}$ & $\mathbf{y}^{\mathbf{*}^{++}}$ & $\mathbf{y}^{\mathbf{0}}$ & $\mathbf{y}^{\mathbf{0 + +}}$ & $\#$ \\
\hline $\mathbf{1}$ & 164.0706 & 82.5389 & & & & & $\mathbf{Y}$ & & & & & & & $\mathbf{1 1}$ \\
\hline $\mathbf{2}$ & $\mathbf{3 2 7 . 1 3 3 9}$ & 164.0706 & & & & & $\mathbf{Y}$ & $\mathbf{1 1 7 3 . 5 7 8 6}$ & 587.2930 & 1156.5521 & 578.7797 & 1155.5681 & 578.2877 & $\mathbf{1 0}$ \\
\hline $\mathbf{3}$ & $\mathbf{4 4 2 . 1 6 0 9}$ & 221.5841 & 425.1343 & 213.0708 & & & $\mathbf{N}$ & $\mathbf{1 0 1 0 . 5 1 5 3}$ & 505.7613 & 993.4887 & 497.2480 & $\mathbf{9 9 2 . 5 0 4 7}$ & 496.7560 & $\mathbf{9}$ \\
\hline $\mathbf{4}$ & 605.2242 & 303.1157 & 588.1977 & 294.6025 & & & $\mathbf{Y}$ & $\mathbf{8 9 5 . 4 8 8 4}$ & 448.2478 & 878.4618 & 439.7345 & 877.4778 & 439.2425 & $\mathbf{8}$ \\
\hline $\mathbf{5}$ & $\mathbf{7 0 6 . 2 7 1 9}$ & 353.6396 & 689.2453 & 345.1263 & 688.2613 & 344.6343 & $\mathbf{T}$ & $\mathbf{7 3 2 . 4 2 5 0}$ & 366.7162 & 715.3985 & 358.2029 & 714.4145 & 357.7109 & $\mathbf{7}$ \\
\hline $\mathbf{6}$ & $\mathbf{8 1 9 . 3 5 5 9}$ & 410.1816 & 802.3294 & 401.6683 & $\mathbf{8 0 1 . 3 4 5 4}$ & 401.1763 & $\mathbf{L}$ & $\mathbf{6 3 1 . 3 7 7 4}$ & 316.1923 & 614.3508 & 307.6790 & 613.3668 & 307.1870 & $\mathbf{6}$ \\
\hline $\mathbf{7}$ & 906.3880 & 453.6976 & 889.3614 & 445.1843 & 888.3774 & 444.6923 & $\mathbf{S}$ & $\mathbf{5 1 8 . 2 9 3 3}$ & 259.6503 & 501.2667 & 251.1370 & 500.2827 & 250.6450 & $\mathbf{5}$ \\
\hline $\mathbf{8}$ & $\mathbf{1 0 1 9 . 4 7 2 0}$ & 510.2397 & 1002.4455 & 501.7264 & 1001.4615 & 501.2344 & $\mathbf{I}$ & $\mathbf{4 3 1 . 2 6 1 3}$ & 216.1343 & 414.2347 & 207.6210 & & & $\mathbf{4}$ \\
\hline $\mathbf{9}$ & 1133.5150 & 567.2611 & 1116.4884 & 558.7478 & $\mathbf{1 1 1 5 . 5 0 4 4}$ & 558.2558 & $\mathbf{N}$ & $\mathbf{3 1 8 . 1 7 7 2}$ & 159.5922 & 301.1506 & 151.0790 & & & $\mathbf{3}$ \\
\hline $\mathbf{1 0}$ & $\mathbf{1 1 9 0 . 5 3 6 4}$ & 595.7719 & $\mathbf{1 1 7 3 . 5 0 9 9}$ & 587.2586 & 1172.5259 & 586.7666 & $\mathbf{G}$ & $\mathbf{2 0 4 . 1 3 4 3}$ & 102.5708 & 187.1077 & 94.0575 & & & $\mathbf{2}$ \\
\hline $\mathbf{1 1}$ & & & & & & & $\mathbf{K}$ & 147.1128 & 74.0600 & 130.0863 & 65.5468 & & & $\mathbf{1}$ \\
\hline
\end{tabular}
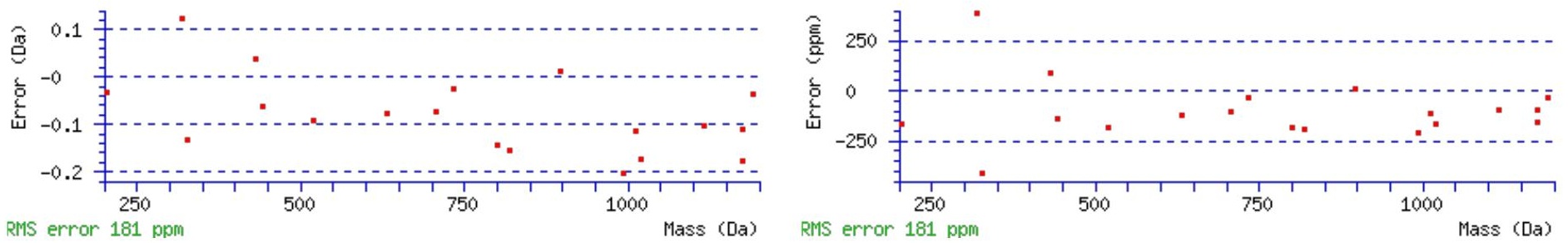

RMS error 181 ppm

Mass (Da) RMS error 181 ppn

\section{All matches to this query}

\begin{tabular}{|l|l|c|l|}
\hline Score & Mr(calc): & Delta & \multicolumn{1}{c|}{ Sequence } \\
\hline 57.5 & 1335.6346 & 0.0007 & YYNYTLSINGK \\
\hline 17.9 & 1335.6346 & 0.0007 & YYNYTLSINGK \\
\hline 6.0 & 1335.6279 & 0.0074 & NAASSSTSNWRR \\
\hline 1.9 & 1335.6444 & -0.0090 & MKYKTSLVMR \\
\hline
\end{tabular}

Spectrum No: 570; Query: 257; Rank: 1

\section{Peptide View}

MS/MS Fragmentation of LPVTAVNTTMR

Found in IPI00197684, Tax_Id=10116 Gene_Symbol=Xpnpep2 Membrane-bound aminopeptidase P

Match to Query 257: 1218.629228 from(610.321890,2+)

Title: 091008RatKidney_NH4Format01_26.902.902.2.dta

Data file K:\NewmanPaper\Piliang \3SubProteomes \Piliang3SP $\backslash$ mgf5ppm\ERLIC_3SubProteomes5ppm.mgf 

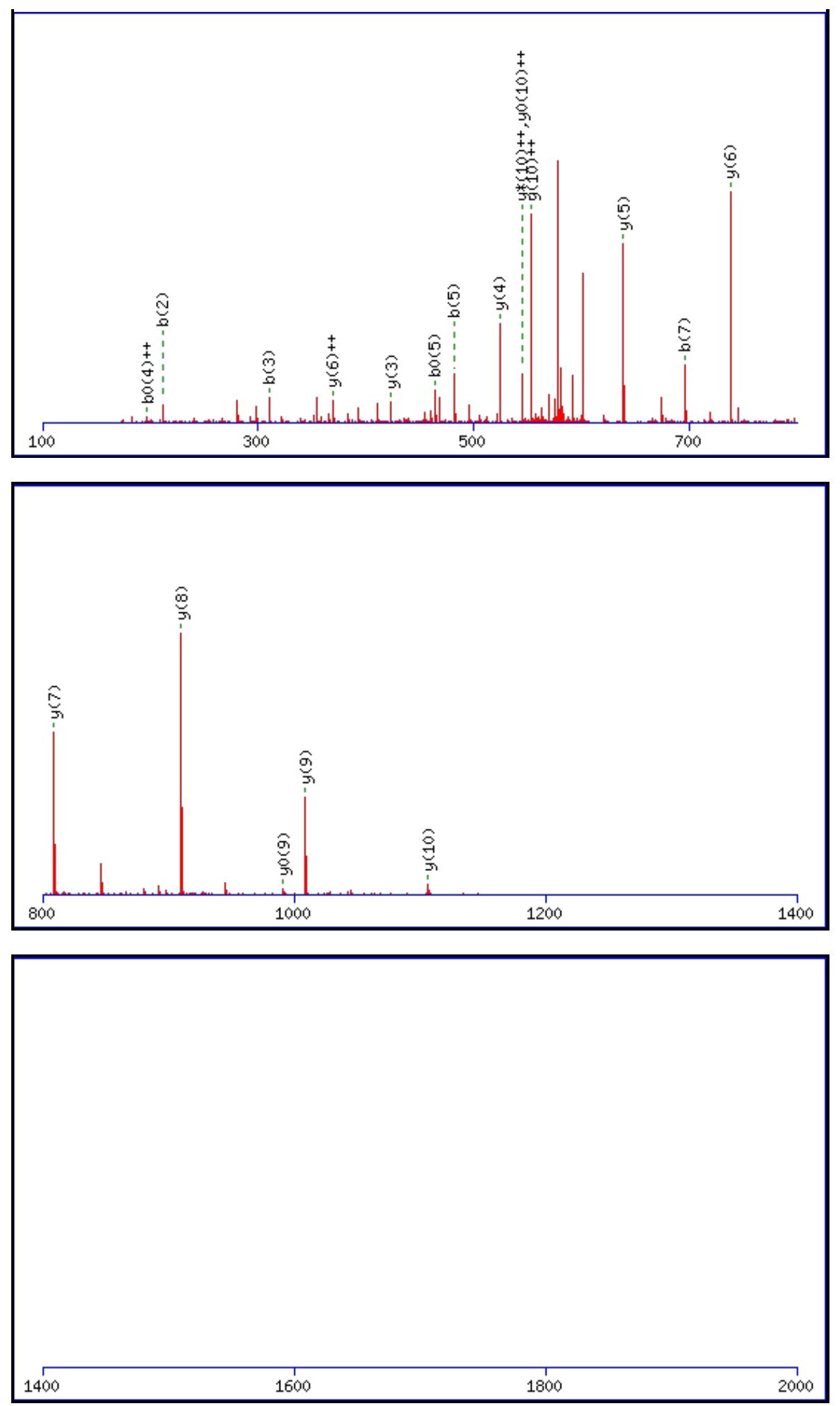

Monoisotopic mass of neutral peptide $\operatorname{Mr}($ calc): 1218.6278

Fixed modifications: Carbamidomethyl (C)

Variable modifications:

N7 : Deamidated_N (N)

M10 : Oxidation (M)

Ions Score: 58 Expect : 0.00026

Matches (Bold Red): 19/98 fragment ions using 32 most intense peaks

\begin{tabular}{|r|c|c|c|c|c|c|c|c|c|c|c|c|c|c|}
\hline$\#$ & $\mathbf{b}$ & $\mathbf{b}^{++}$ & $\mathbf{b}^{*}$ & $\mathbf{b}^{\boldsymbol{*}_{+}^{++}}$ & $\mathbf{b}^{\mathbf{0}}$ & $\mathbf{b}^{\mathbf{0 + +}}$ & Seq. & $\mathbf{y}$ & $\mathbf{y}^{++}$ & $\mathbf{y}^{\mathbf{*}}$ & $\mathbf{y}^{\mathbf{*}^{++}}$ & $\mathbf{y}^{\mathbf{0}}$ & $\mathbf{y}^{\mathbf{0}++}$ & $\#$ \\
\hline $\mathbf{1}$ & 114.0913 & 57.5493 & & & & & $\mathbf{L}$ & & & & & & & $\mathbf{1 1}$ \\
\hline $\mathbf{2}$ & $\mathbf{2 1 1 . 1 4 4 1}$ & 106.0757 & & & & & $\mathbf{P}$ & $\mathbf{1 1 0 6 . 5 5 1 0}$ & 553.7792 & 1089.5245 & $\mathbf{5 4 5 . 2 6 5 9}$ & 1088.5405 & $\mathbf{5 4 4 . 7 7 3 9}$ & $\mathbf{1 0}$ \\
\hline $\mathbf{3}$ & $\mathbf{3 1 0 . 2 1 2 5}$ & 155.6099 & & & & & $\mathbf{V}$ & $\mathbf{1 0 0 9 . 4 9 8 3}$ & 505.2528 & 992.4717 & 496.7395 & $\mathbf{9 9 1 . 4 8 7 7}$ & 496.2475 & $\mathbf{9}$ \\
\hline $\mathbf{4}$ & 411.2602 & 206.1337 & & & 393.2496 & $\mathbf{1 9 7 . 1 2 8 5}$ & $\mathbf{T}$ & $\mathbf{9 1 0 . 4 2 9 9}$ & 455.7186 & 893.4033 & 447.2053 & 892.4193 & 446.7133 & $\mathbf{8}$ \\
\hline & & & & & & & & & & & & & &
\end{tabular}




\begin{tabular}{|r|r|r|r|r|r|r|r|r|r|r|r|r|r|r|r|r|}
$\mathbf{5}$ & $\mathbf{4 8 2 . 2 9 7 3}$ & 241.6523 & & & $\mathbf{4 6 4 . 2 8 6 7}$ & 232.6470 & $\mathbf{A}$ & $\mathbf{8 0 9 . 3 8 2 2}$ & 405.1947 & 792.3556 & 396.6815 & 791.3716 & 396.1894 & $\mathbf{7}$ \\
\hline $\mathbf{6}$ & 581.3657 & 291.1865 & & & 563.3552 & 282.1812 & $\mathbf{V}$ & $\mathbf{7 3 8 . 3 4 5 1}$ & $\mathbf{3 6 9 . 6 7 6 2}$ & 721.3185 & 361.1629 & $\mathbf{7 2 0 . 3 3 4 5}$ & 360.6709 & $\mathbf{6}$ \\
\hline $\mathbf{7}$ & $\mathbf{6 9 6 . 3 9 2 7}$ & 348.7000 & 679.3661 & 340.1867 & 678.3821 & 339.6947 & $\mathbf{N}$ & $\mathbf{6 3 9 . 2 7 6 7}$ & 320.1420 & 622.2501 & 311.6287 & 621.2661 & 311.1367 & $\mathbf{5}$ \\
\hline $\mathbf{8}$ & 797.4403 & 399.2238 & 780.4138 & 390.7105 & 779.4298 & 390.2185 & $\mathbf{T}$ & 524.2497 & 262.6285 & 507.2232 & 254.1152 & 506.2391 & 253.6232 & $\mathbf{4}$ \\
\hline $\mathbf{9}$ & 898.4880 & 449.7476 & 881.4615 & 441.2344 & 880.4775 & 440.7424 & $\mathbf{T}$ & $\mathbf{4 2 3 . 2 0 2 0}$ & 212.1047 & 406.1755 & 203.5914 & 405.1915 & 203.0994 & $\mathbf{3}$ \\
\hline $\mathbf{1 0}$ & 1045.5234 & 523.2653 & 1028.4969 & 514.7521 & 1027.5129 & 514.2601 & $\mathbf{M}$ & 322.1544 & 161.5808 & 305.1278 & 153.0675 & & & & $\mathbf{2}$ \\
\hline $\mathbf{1 1}$ & & & & & & & $\mathbf{R}$ & 175.1190 & 88.0631 & 158.0924 & 79.5498 & & & $\mathbf{1}$ \\
\hline
\end{tabular}
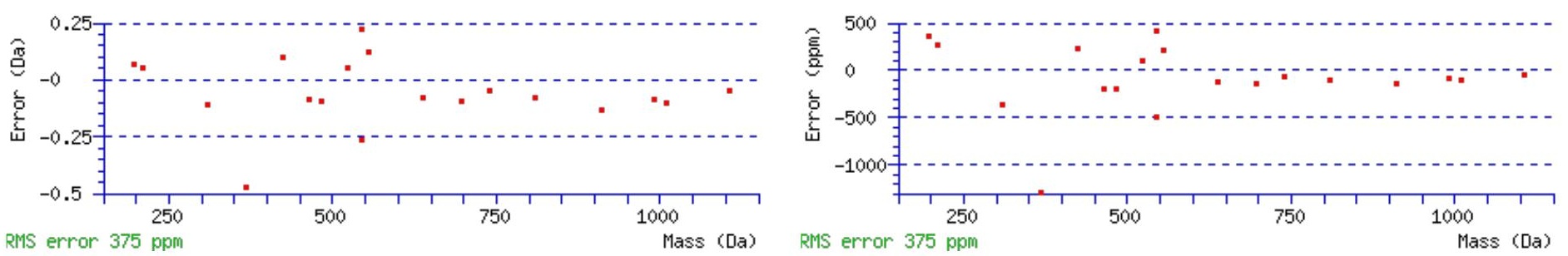

All matches to this query

\begin{tabular}{|l|l|l|l|}
\hline Score & Mr(calc): & Delta & \multicolumn{1}{|c|}{ Sequence } \\
\hline 57.5 & 1218.6278 & 0.0014 & LPVTAVNTTMR \\
\hline 3.7 & 1218.6278 & 0.0014 & INTVAMNSLVR \\
\hline 2.3 & 1218.6397 & -0.0105 & PVTLGYWDIR \\
\hline
\end{tabular}

Spectrum No: 571; Query: 2438; Rank: 1

\section{Peptide View}

MS/MS Fragmentation of LVIYLEHNLEKNSTKEEILAALEK

Found in IPI00195160, Tax_Id=10116 Gene_Symbol=Psap Sulfated glycoprotein 1 precursor

Match to Query 2438: 2797.518642 from(933.513490,3+)

Title: 091008RatKidney_NoSalt_13.4731.4731.3.dta

Data file K:INewmanPaper|Piliangl3SubProteomes\Piliang3SP\mgf5ppm\ERLIC_3SubProteomes5ppm.mgf

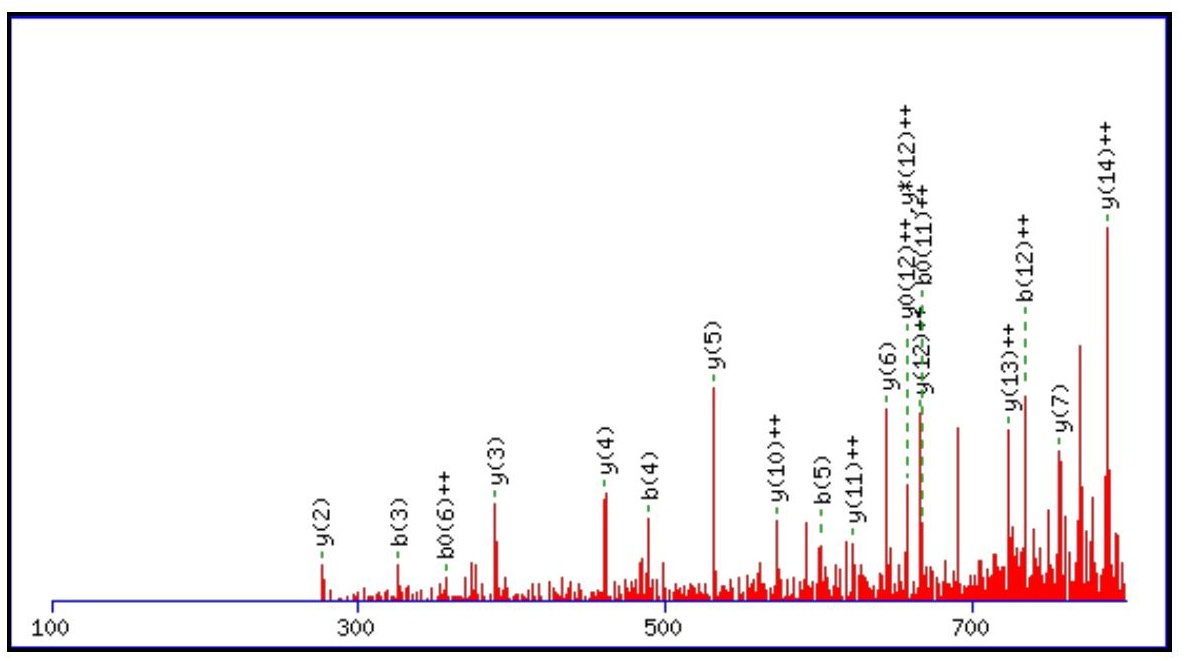



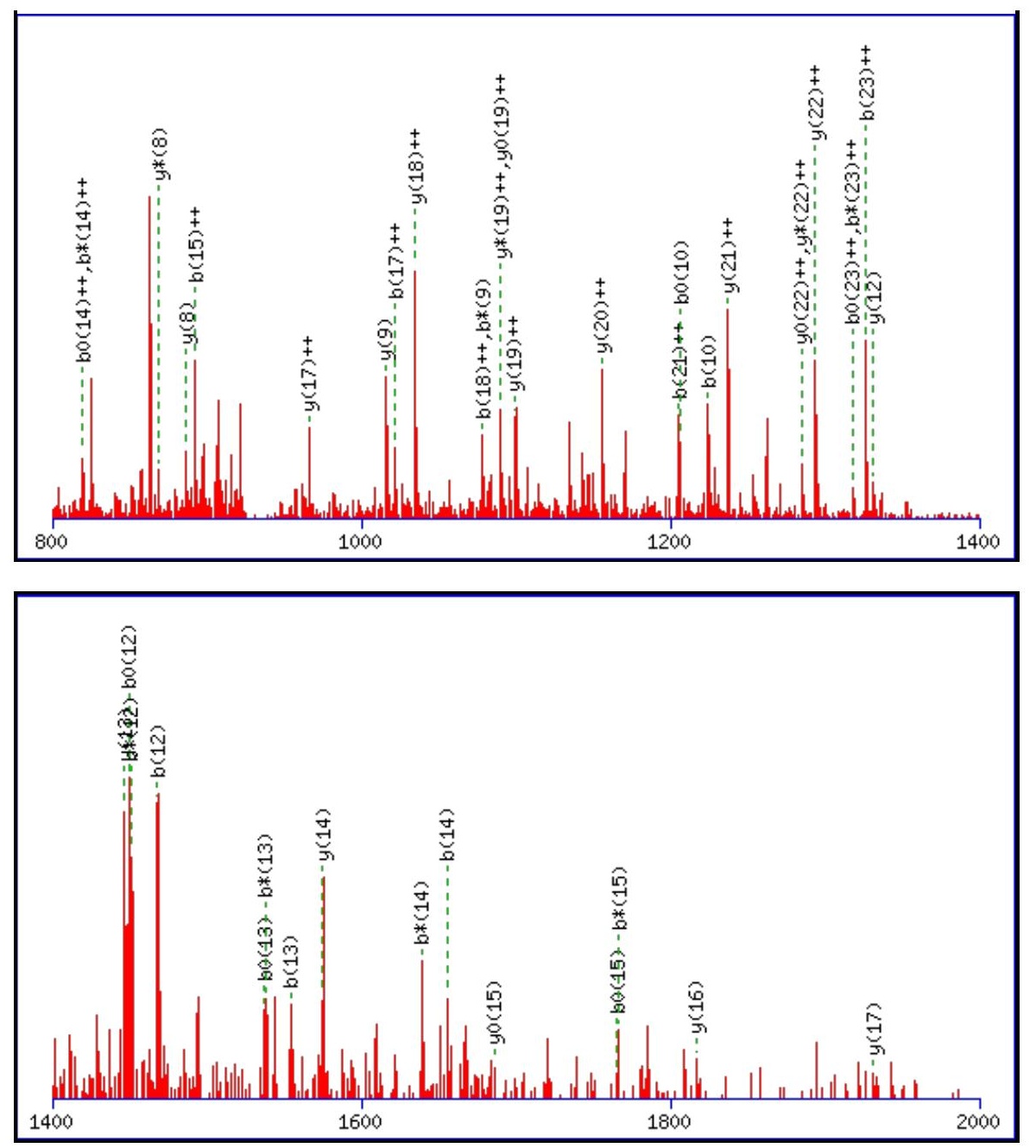

Monoisotopic mass of neutral peptide $\operatorname{Mr}($ calc): 2797.5112

Fixed modifications: Carbamidomethyl (C)

Variable modifications:

N12 : Deamidated_N (N)

Ions Score: 57 Expect: $7.9 \mathrm{e}-005$

Matches (Bold Red): 60/250 fragment ions using 142 most intense peaks

\begin{tabular}{|c|c|c|c|c|c|c|c|c|c|c|c|c|c|c|}
\hline \# & b & $\mathbf{b}^{++}$ & $\mathbf{b}^{*}$ & $\mathbf{b}^{*++}$ & $\mathbf{b}^{\mathbf{0}}$ & $\mathbf{b}^{\mathbf{0 + +}}$ & Seq. & $\mathbf{y}$ & $\mathbf{y}^{++}$ & $\mathbf{y}^{*}$ & $\mathrm{y}^{\mathrm{*}^{++}}$ & $\mathbf{y}^{0}$ & $y^{0++}$ & \# \\
\hline 1 & \begin{tabular}{|l|}
114.0913 \\
\end{tabular} & 57.5493 & & & & & $\mathbf{L}$ & & & & & & & 24 \\
\hline 2 & 213.1598 & 107.0835 & & & & & V & 2685.4345 & 1343.2209 & 2668.4080 & 1334.7076 & 2667.4240 & 1334.2156 & 23 \\
\hline 3 & 326.2438 & 163.6255 & & & & & I & 2586.3661 & 1293.6867 & 2569.3396 & 1285.1734 & 2568.3556 & 1284.6814 & 22 \\
\hline 4 & \begin{tabular}{|l|}
489.3071 \\
\end{tabular} & 245.1572 & & & & & $\mathbf{Y}$ & 2473.2821 & 1237.1447 & 2456.2555 & 1228.6314 & 2455.2715 & 1228.1394 & 21 \\
\hline 5 & 602.3912 & 301.6992 & & & & & $\mathbf{L}$ & 2310.2187 & 1155.6130 & 2293.1922 & 1147.0997 & 2292.2082 & 1146.6077 & 20 \\
\hline 6 & 731.4338 & 366.2205 & & & 713.4232 & 357.2153 & $\mathbf{E}$ & 2197.1347 & 1099.0710 & 2180.1081 & 1090.5577 & 2179.1241 & 1090.0657 & 19 \\
\hline 7 & 868.4927 & 434.7500 & & & 850.4822 & 425.7447 & H & 2068.0921 & 1034.5497 & 2051.0655 & 1026.0364 & 2050.0815 & 1025.5444 & 18 \\
\hline 8 & 982.5356 & 491.7715 & 965.5091 & 483.2582 & 964.5251 & 482.7662 & $\mathbf{N}$ & 1931.0332 & 966.0202 & 1914.0066 & 957.5069 & 1913.0226 & 957.0149 & 17 \\
\hline 9 & 1095.6197 & 548.3135 & 1078.5932 & 539.8002 & 1077.6091 & 539.3082 & $\mathbf{L}$ & 1816.9902 & 908.9988 & 1799.9637 & 900.4855 & 1798.9797 & 899.9935 & 16 \\
\hline 10 & 1224.6623 & 612.8348 & 1207.6358 & 604.3215 & 1206.6517 & 603.8295 & $\mathbf{E}$ & 1703.9062 & 852.4567 & 1686.8796 & 843.9434 & 1685.8956 & 843.4514 & 15 \\
\hline 11 & 1352.7573 & 676.8823 & 1335.7307 & 668.3690 & 1334.7467 & 667.8770 & $\mathbf{K}$ & 1574.8636 & 787.9354 & 1557.8370 & 779.4221 & 1556.8530 & 778.9301 & 14 \\
\hline 12 & 1467.7842 & 734.3957 & 1450.7576 & 725.8825 & 1449.7736 & 725.3905 & $\mathbf{N}$ & 1446.7686 & 723.8879 & 1429.7421 & 715.3747 & 1428.7580 & 714.8827 & 13 \\
\hline 13 & 1554.8162 & 777.9118 & \begin{tabular}{|l|}
1537.7897 \\
\end{tabular} & 769.3985 & \begin{tabular}{|l}
1536.8057 \\
\end{tabular} & 768.9065 & $S$ & 1331.7417 & 666.3745 & 1314.7151 & 657.8612 & 1313.7311 & 657.3692 & 12 \\
\hline 14 & \begin{tabular}{|l|}
1655.8639 \\
\end{tabular} & 828.4356 & \begin{tabular}{|l|}
1638.8374 \\
\end{tabular} & 819.9223 & 1637.8533 & 819.4303 & $T$ & 1244.7096 & 622.8585 & 1227.6831 & 614.3452 & 1226.6991 & 613.8532 & 11 \\
\hline 15 & 1783.9589 & 892.4831 & 1766.9323 & 883.9698 & 1765.9483 & 883.4778 & K & 1143.6620 & 572.3346 & 1126.6354 & 563.8213 & 1125.6514 & 563.3293 & 10 \\
\hline 16 & 1913.0015 & 957.0044 & 1895.9749 & 948.4911 & 1894.9909 & 947.9991 & $\mathbf{E}$ & 1015.5670 & 508.2871 & 998.5405 & 499.7739 & 997.5564 & 499.2819 & 9 \\
\hline 17 & 2042.0441 & 1021.5257 & 2025.0175 & 1013.0124 & 2024.0335 & 1012.5204 & $\mathbf{E}$ & 886.5244 & 443.7658 & 869.4979 & 435.2526 & 868.5138 & 434.7606 & 8 \\
\hline 18 & 2155.1281 & 1078.0677 & 2138.1016 & 1069.5544 & 2137.1176 & 1069.0624 & I & 757.4818 & 379.2445 & 740.4553 & 370.7313 & 739.4713 & 370.2393 & 7 \\
\hline
\end{tabular}




\begin{tabular}{|c|c|c|c|c|c|c|c|c|c|c|c|c|c|c|}
\hline 19 & |2268.2122 & |1134.6097 & |2251.1856 & |1126.0965 & |2250.2016 & |1125.6044 & $\mathbf{L}$ & 644.3978 & 322.7025 & 627.3712 & 314.1892 & 626.3872 & 313.6972 & 6 \\
\hline 20 & 2339.2493 & 1170.1283 & 2322.2227 & 1161.6150 & 2321.2387 & 1161.1230 & A & 531.3137 & 266.1605 & 514.2871 & 257.6472 & 513.3031 & 257.1552 & 5 \\
\hline 21 & 2410.2864 & 1205.6468 & 2393.2599 & 1197.1336 & 2392.2758 & 1196.6416 & A & 460.2766 & 230.6419 & 443.2500 & 222.1287 & 442.2660 & 221.6366 & 4 \\
\hline 22 & 2523.3705 & 1262.1889 & 2506.3439 & 1253.6756 & 2505.3599 & 1253.1836 & $\mathbf{L}$ & 389.2395 & 195.1234 & 372.2129 & 186.6101 & 371.2289 & 186.1181 & 3 \\
\hline 23 & 2652.4131 & 1326.7102 & 2635.3865 & 1318.1969 & 2634.4025 & 1317.7049 & $\mathbf{E}$ & 276.1554 & 138.5813 & 259.1288 & 130.0681 & 258.1448 & 129.5761 & 2 \\
\hline 24 & & & & & & & $\mathbf{K}$ & 147.1128 & 74.0600 & 130.0863 & 65.5468 & & & 1 \\
\hline
\end{tabular}
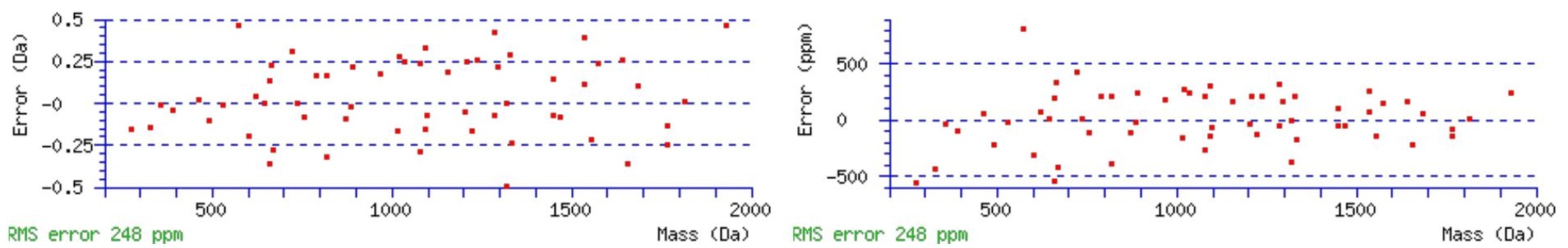

\section{All matches to this query}

\begin{tabular}{|l|l|l|l|}
\hline Score & Mr(calc): & Delta & \multicolumn{1}{|c|}{ Sequence } \\
\hline 57.4 & 2797.5112 & 0.0074 & LVIYLEHNLEKNSTKEEILAALEK \\
\hline 34.4 & 2797.5112 & 0.0074 & LVIYLEHNLEKNSTKEEILAALEK \\
\hline 8.3 & 2796.5272 & 0.9914 & $\underline{\text { LVIYLEHNLEKNSTKEEILAALEK }}$ \\
\hline
\end{tabular}

Spectrum No: 572; Query: 826; Rank: 1

\section{Peptide View}

MS/MS Fragmentation of VYGQNINNETWSR

Found in IPI00361557, Tax_Id=10116 Gene_Symbol=Cnnm2 94 kDa protein

Match to Query 826: 1580.726408 from(791.370480,2+)

Title: 100101RatKid_NS_deglyco_23.1982.1982.2.dta

Data file K:INewmanPaper|Piliangl3SubProteomes\Piliang3SP\mgf5ppm\ERLIC_3SubProteomes5ppm.mgf

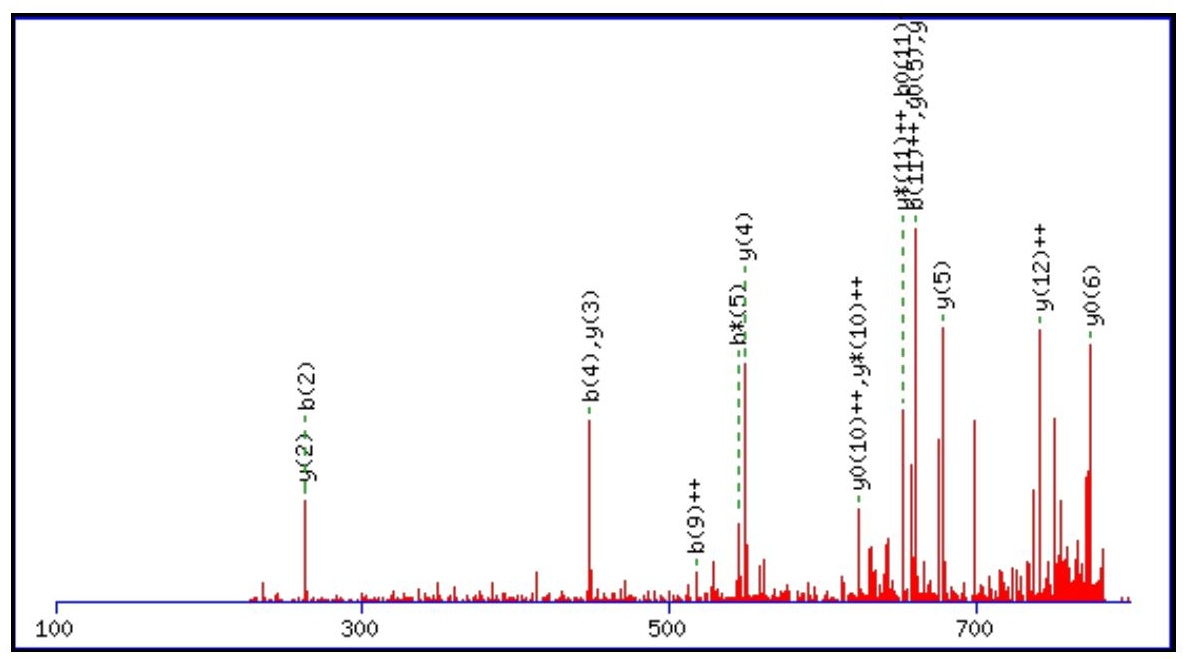



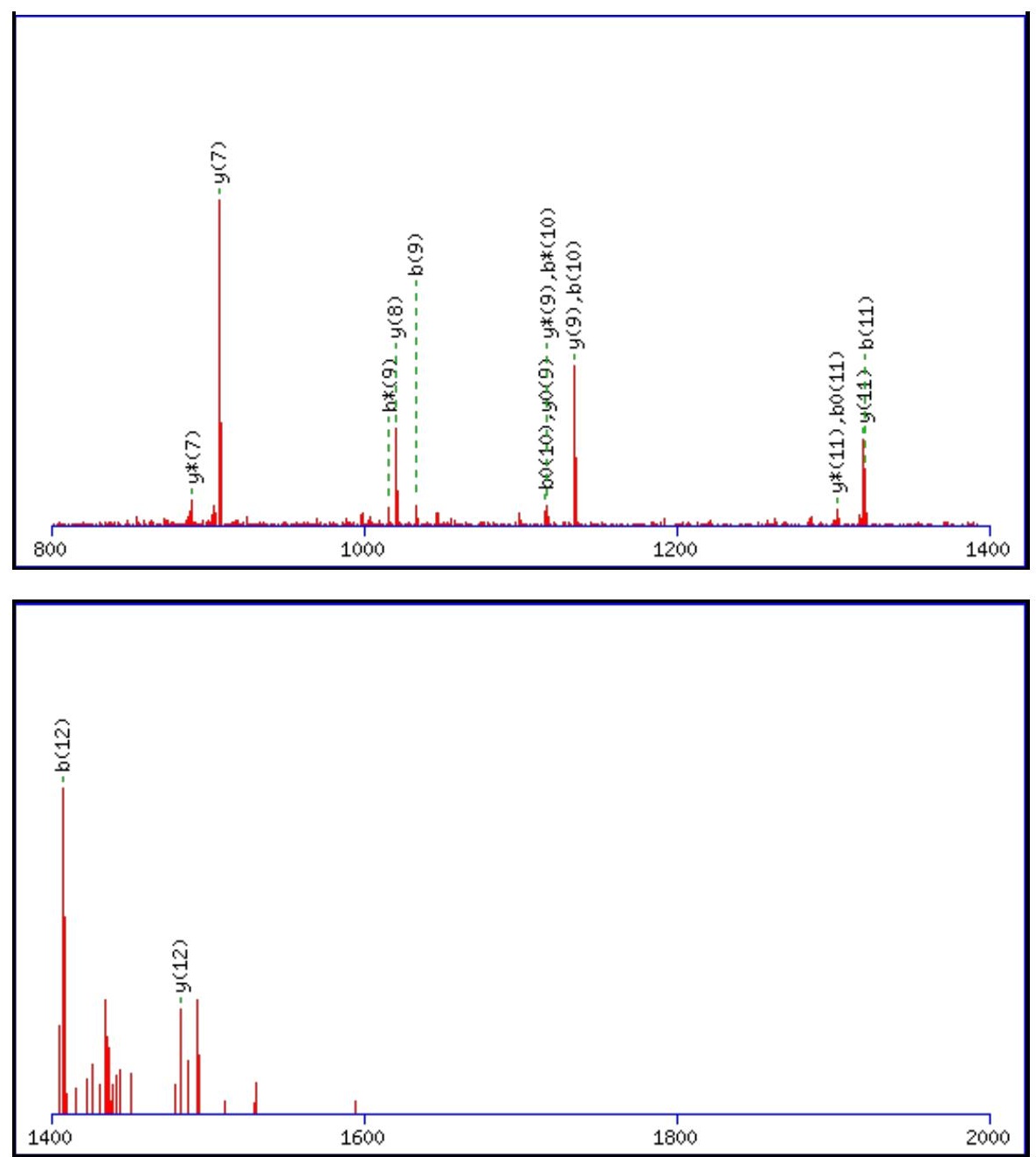

Monoisotopic mass of neutral peptide $\operatorname{Mr}($ calc): 1580.7219

Fixed modifications: Carbamidomethyl (C)

Variable modifications:

N8 : Deamidated $\mathrm{N}(\mathrm{N})$

Ions Score: 57 Expect: 0.00036

Matches (Bold Red): 35/120 fragment ions using 47 most intense peaks

\begin{tabular}{|c|c|c|c|c|c|c|c|c|c|c|c|c|c|c|}
\hline \# & b & $\mathbf{b}^{++}$ & b* & $\mathbf{b}^{*^{++}}$ & $\mathbf{b}^{0}$ & $\mathbf{b}^{0++}$ & Seq. & $\mathbf{y}$ & $y^{++}$ & $\mathbf{y}^{*}$ & $\mathbf{y}^{*^{++}}$ & $\mathbf{y}^{\mathbf{0}}$ & $y^{0++}$ & \# \\
\hline 1 & 100.0757 & 50.5415 & & & & & $\mathbf{V}$ & & & & & & & 13 \\
\hline 2 & 263.1390 & 132.0731 & & & & & $\mathbf{Y}$ & 1482.6608 & 741.8340 & 1465.6342 & 733.3208 & 1464.6502 & 732.8288 & 12 \\
\hline 3 & 320.1605 & 160.5839 & & & & & G & 1319.5975 & 660.3024 & 1302.5709 & 651.7891 & 1301.5869 & 651.2971 & 11 \\
\hline 4 & 448.2191 & 224.6132 & 431.1925 & 216.0999 & & & $\mathbf{Q}$ & 1262.5760 & 631.7916 & 1245.5494 & 623.2784 & 1244.5654 & 622.7864 & 10 \\
\hline 5 & 562.2620 & 281.6346 & 545.2354 & 273.1214 & & & $\mathbf{N}$ & 1134.5174 & 567.7623 & 1117.4909 & 559.2491 & 1116.5069 & 558.7571 & 9 \\
\hline 6 & 675.3461 & 338.1767 & 658.3195 & 329.6634 & & & I & 1020.4745 & 510.7409 & 1003.4479 & 502.2276 & 1002.4639 & 501.7356 & 8 \\
\hline 7 & 789.3890 & 395.1981 & 772.3624 & 386.6849 & & & $\mathbf{N}$ & 907.3904 & 454.1989 & 890.3639 & 445.6856 & 889.3799 & 445.1936 & 7 \\
\hline 8 & 904.4159 & 452.7116 & 887.3894 & 444.1983 & & & $\mathbf{N}$ & 793.3475 & 397.1774 & 776.3210 & 388.6641 & 775.3369 & 388.1721 & 6 \\
\hline 9 & 1033.4585 & 517.2329 & 1016.4320 & 508.7196 & 1015.4479 & 508.2276 & $\mathbf{E}$ & 678.3206 & 339.6639 & 661.2940 & 331.1506 & 660.3100 & 330.6586 & 5 \\
\hline 10 & 1134.5062 & 567.7567 & 1117.4796 & 559.2435 & 1116.4956 & 558.7514 & $\mathbf{T}$ & 549.2780 & 275.1426 & 532.2514 & 266.6293 & 531.2674 & 266.1373 & 4 \\
\hline 11 & 1320.5855 & 660.7964 & 1303.5590 & 652.2831 & 1302.5749 & 651.7911 & W & 448.2303 & 224.6188 & 431.2037 & 216.1055 & 430.2197 & 215.6135 & 3 \\
\hline 12 & 1407.6175 & 704.3124 & 1390.5910 & 695.7991 & 1389.6070 & 695.3071 & S & 262.1510 & 131.5791 & 245.1244 & 123.0659 & 244.1404 & 122.5738 & 2 \\
\hline 13 & & & & & & & $\mathbf{R}$ & 175.1190 & 88.0631 & 158.0924 & 79.5498 & & & 1 \\
\hline
\end{tabular}



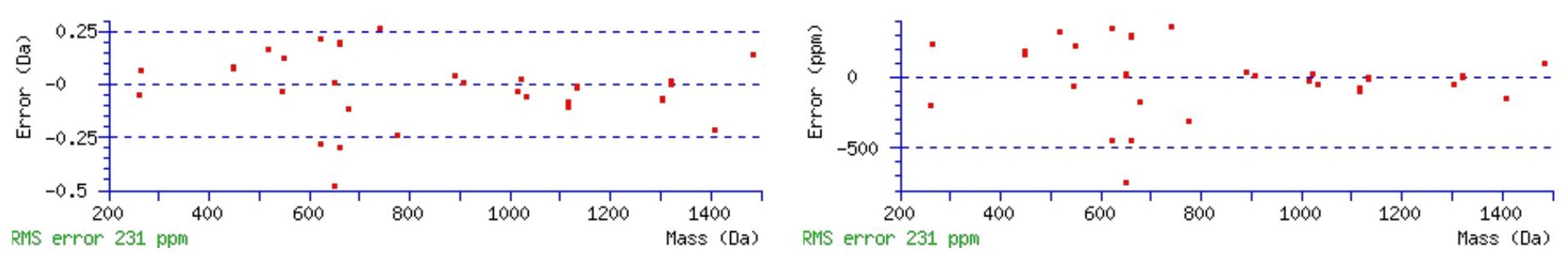

\section{All matches to this query}

\begin{tabular}{|l|l|l|l|}
\hline Score & Mr(calc): & Delta & \multicolumn{1}{|c|}{ Sequence } \\
\hline 57.1 & 1580.7219 & 0.0045 & VYGQNINNETWSR \\
\hline 57.1 & 1580.7219 & 0.0045 & VYGQNINNETWSR \\
\hline 34.5 & 1580.7219 & 0.0045 & VYGQNINNETWSR \\
\hline 12.5 & 1579.7379 & 0.9885 & VYGQNINNETWSR \\
\hline 12.1 & 1580.7154 & 0.0110 & MNNWESRSLSWR \\
\hline 10.3 & 1580.7174 & 0.0090 & SLDQVTDMMIANSR \\
\hline 5.3 & 1580.7276 & -0.0012 & NFSNFVLDKFLR \\
\hline 5.1 & 1579.7334 & 0.9930 & SLDQVTDMMIANSR \\
\hline 4.7 & 1580.7399 & -0.0135 & RMTERDVETICR \\
\hline 3.5 & 1579.7083 & 1.0181 & MNQSQRMAPVGSDK \\
\hline
\end{tabular}

Spectrum No: 573; Query: 543; Rank: 1

\section{Peptide View}

\section{MS/MS Fragmentation of KNLSYEEAPDHK}

Found in IPI00471800, Tax_Id=10116 Gene_Symbol=Cdh16 Cadherin 16

Match to Query 543: 1430.670282 from(477.897370,3+)

Title: 100101RatKid_NS_deglyco_21.487.487.3.dta

Data file K:INewmanPaperlPiliangl3SubProteomes\Piliang3SP\mgf5ppm\ERLIC_3SubProteomes5ppm.mgf

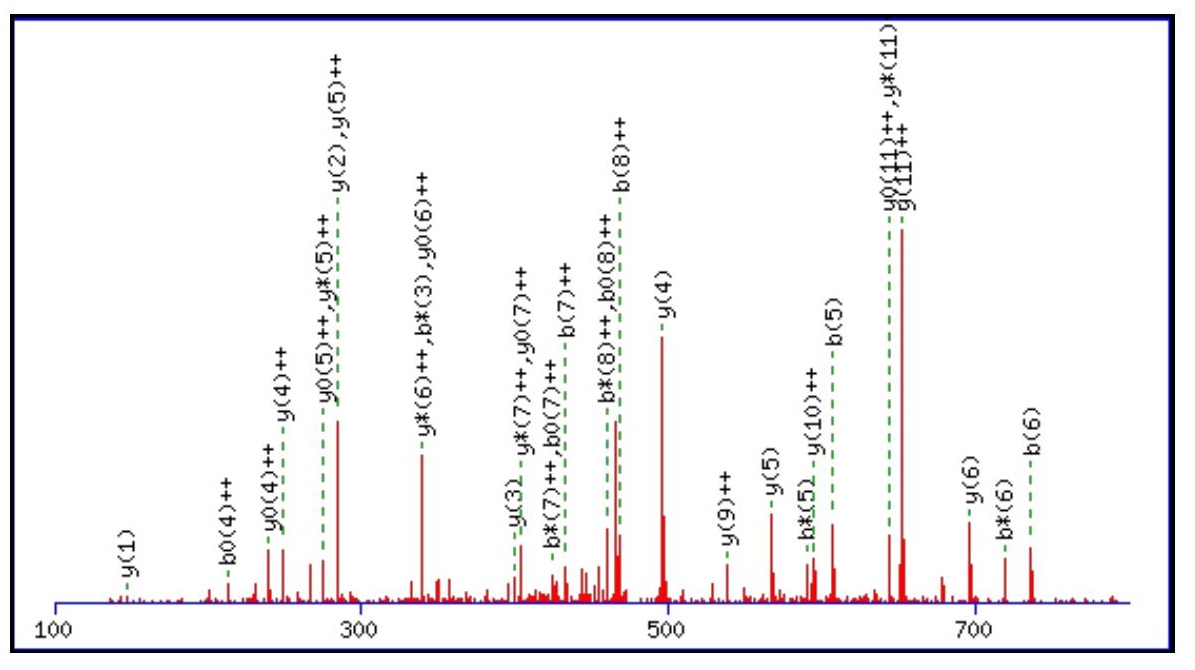



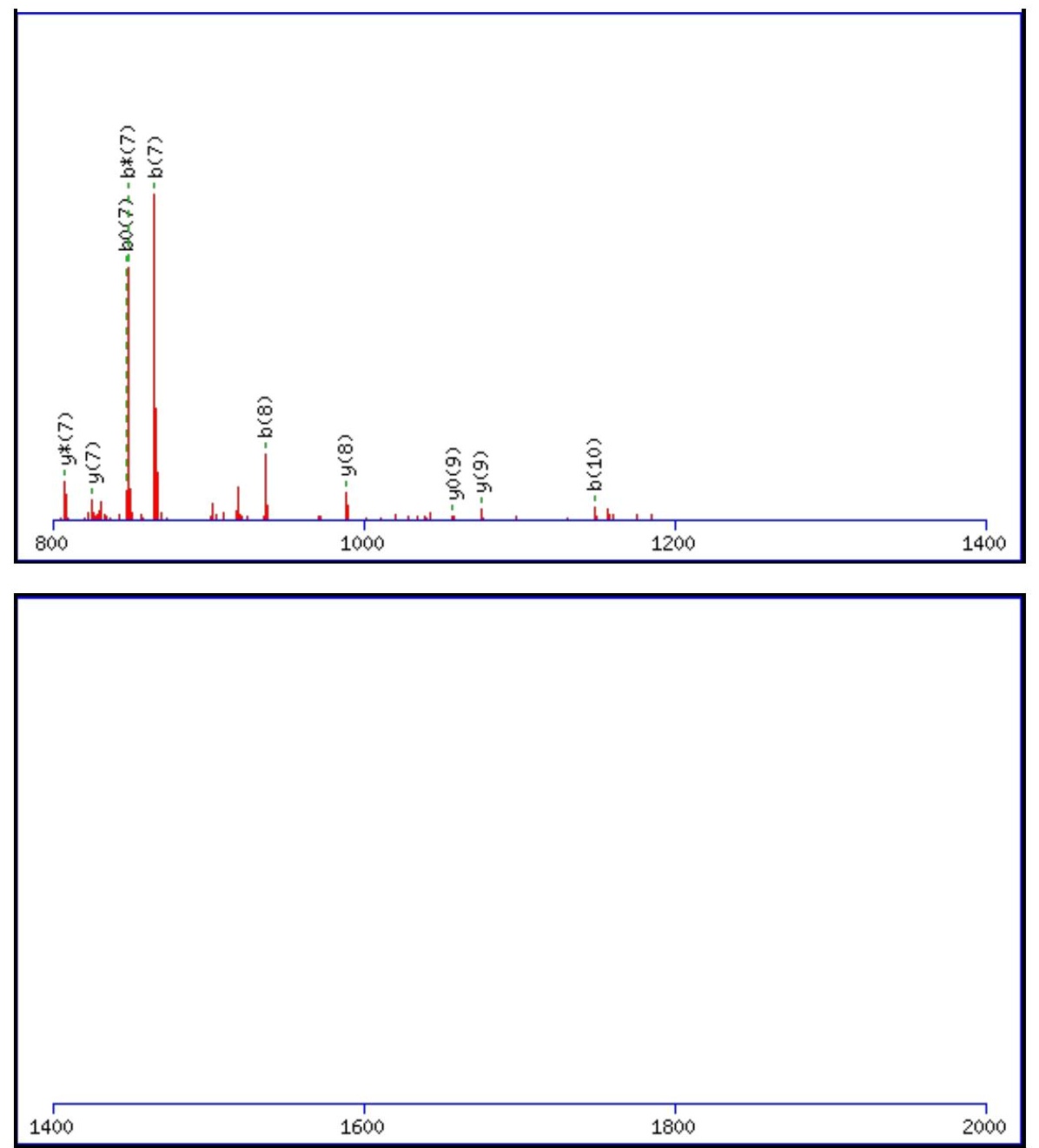

Monoisotopic mass of neutral peptide $\operatorname{Mr}($ calc): 1430.6677

Fixed modifications: Carbamidomethyl (C)

Variable modifications:

N2 : Deamidated $\mathrm{N}(\mathrm{N})$

Ions Score: 57 Expect: 0.00031

Matches (Bold Red): 43/122 fragment ions using 58 most intense peaks

\begin{tabular}{|c|c|c|c|c|c|c|c|c|c|c|c|c|c|c|}
\hline$\#$ & b & $\mathbf{b}^{++}$ & b* & $\mathbf{b}^{*^{++}}$ & $\mathbf{b}^{0}$ & $\mathbf{b}^{0++}$ & Seq. & $\mathbf{y}$ & $y^{++}$ & $\mathbf{y}^{*}$ & $\mathrm{y}^{*^{++}}$ & $\mathbf{y}^{0}$ & $\mathbf{y}^{\mathbf{0 + +}}$ & \# \\
\hline 1 & 129.1022 & 65.0548 & 112.0757 & 56.5415 & & & $\mathbf{K}$ & & & & & & & 12 \\
\hline 2 & 244.1292 & 122.5682 & 227.1026 & 114.0550 & & & $\mathbf{N}$ & 1303.5801 & 652.2937 & 1286.5535 & 643.7804 & 1285.5695 & 643.2884 & 11 \\
\hline 3 & 357.2132 & 179.1103 & 340.1867 & 170.5970 & & & $\mathbf{L}$ & 1188.5531 & 594.7802 & 1171.5266 & 586.2669 & 1170.5426 & 585.7749 & 10 \\
\hline 4 & 444.2453 & 222.6263 & 427.2187 & 214.1130 & 426.2347 & 213.6210 & S & 1075.4691 & 538.2382 & 1058.4425 & 529.7249 & 1057.4585 & 529.2329 & 9 \\
\hline 5 & 607.3086 & 304.1579 & 590.2820 & 295.6447 & 589.2980 & 295.1527 & $\mathbf{Y}$ & 988.4371 & 494.7222 & 971.4105 & 486.2089 & 970.4265 & 485.7169 & 8 \\
\hline 6 & 736.3512 & 368.6792 & 719.3246 & 360.1660 & 718.3406 & 359.6740 & $\mathbf{E}$ & 825.3737 & 413.1905 & 808.3472 & 404.6772 & 807.3632 & 404.1852 & 7 \\
\hline 7 & 865.3938 & 433.2005 & 848.3672 & 424.6873 & 847.3832 & 424.1952 & $\mathbf{E}$ & 696.3311 & 348.6692 & 679.3046 & 340.1559 & 678.3206 & 339.6639 & 6 \\
\hline 8 & 936.4309 & 468.7191 & 919.4043 & 460.2058 & 918.4203 & 459.7138 & A & 567.2885 & 284.1479 & 550.2620 & 275.6346 & 549.2780 & 275.1426 & 5 \\
\hline 9 & 1033.4837 & 517.2455 & 1016.4571 & 508.7322 & 1015.4731 & 508.2402 & $\mathbf{P}$ & 496.2514 & 248.6293 & 479.2249 & 240.1161 & 478.2409 & 239.6241 & 4 \\
\hline 10 & 1148.5106 & 574.7589 & 1131.4841 & 566.2457 & 1130.5000 & 565.7537 & D & 399.1987 & 200.1030 & 382.1721 & 191.5897 & 381.1881 & 191.0977 & 3 \\
\hline 11 & 1285.5695 & 643.2884 & 1268.5430 & 634.7751 & 1267.5590 & 634.2831 & $\mathbf{H}$ & 284.1717 & 142.5895 & 267.1452 & 134.0762 & & & 2 \\
\hline 12 & & & & & & & $\mathbf{K}$ & 147.1128 & 74.0600 & 130.0863 & 65.5468 & & & 1 \\
\hline
\end{tabular}



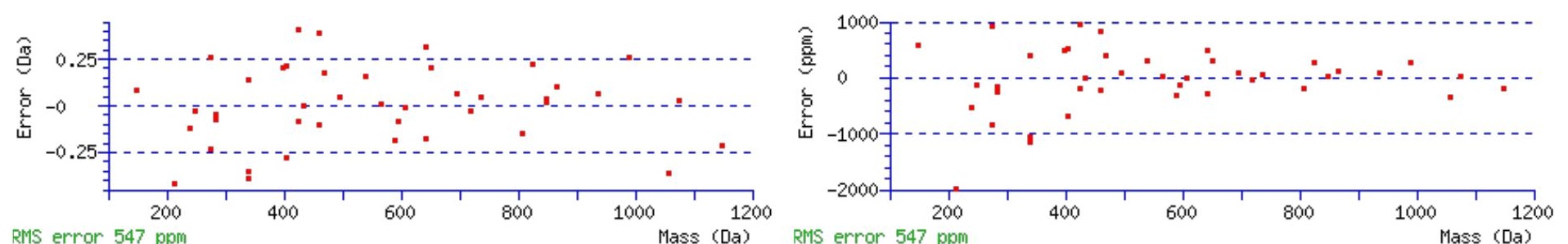

RNS error 547 ppm

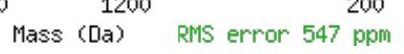

\section{All matches to this query}

\begin{tabular}{|l|l|l|l|}
\hline Score & Mr(calc): & Delta & \multicolumn{1}{c|}{ Sequence } \\
\hline 57.0 & 1430.6677 & 0.0026 & $\underline{\text { KNLSYEEAPDHK }}$ \\
\hline 8.3 & 1428.6762 & 1.9941 & $\underline{\text { KTTGIPREEYR }}$ \\
\hline 7.5 & 1428.6551 & 2.0152 & $\underline{\text { QHEGLATFYRK }}$ \\
\hline 6.5 & 1430.6579 & 0.0124 & $\underline{\text { RSWHEESPFEK }}$ \\
\hline 4.9 & 1430.6667 & 0.0035 & $\underline{\text { SLNTSLHVHSTR }}$ \\
\hline 4.5 & 1430.6840 & -0.0137 & $\underline{\text { KVLGSQMTSEKK }}$ \\
\hline 3.8 & 1430.6707 & -0.0005 & $\underline{\text { AFHNPSILSTHK }}$ \\
\hline 3.6 & 1429.6715 & 0.9988 & $\underline{\text { SSNGRLLGYEVR }}$ \\
\hline 3.6 & 1429.6715 & 0.9988 & $\underline{\text { SSNGRLLGYEVR }}$ \\
\hline 2.7 & 1428.6762 & 1.9940 & $\underline{\text { SVPGTTLESFRR }}$ \\
\hline
\end{tabular}

Spectrum No: 574; Query: 1614; Rank: 1

\section{Peptide View}

MS/MS Fragmentation of GWKENPVEFDSLFNESK

Found in IPI00360085, Tax_Id=10116 Gene_Symbol=Pign_predicted Phosphatidylinositol glycan class N

Match to Query 1614: 2025.933522 from(676.318450,3+)

Title: 091008RatKidney_NoSalt_19.3749.3749.3.dta

Data file K:\NewmanPaper \Piliang \3SubProteomes \Piliang3SP \mgf5ppm\ERLIC_3SubProteomes5ppm.mgf

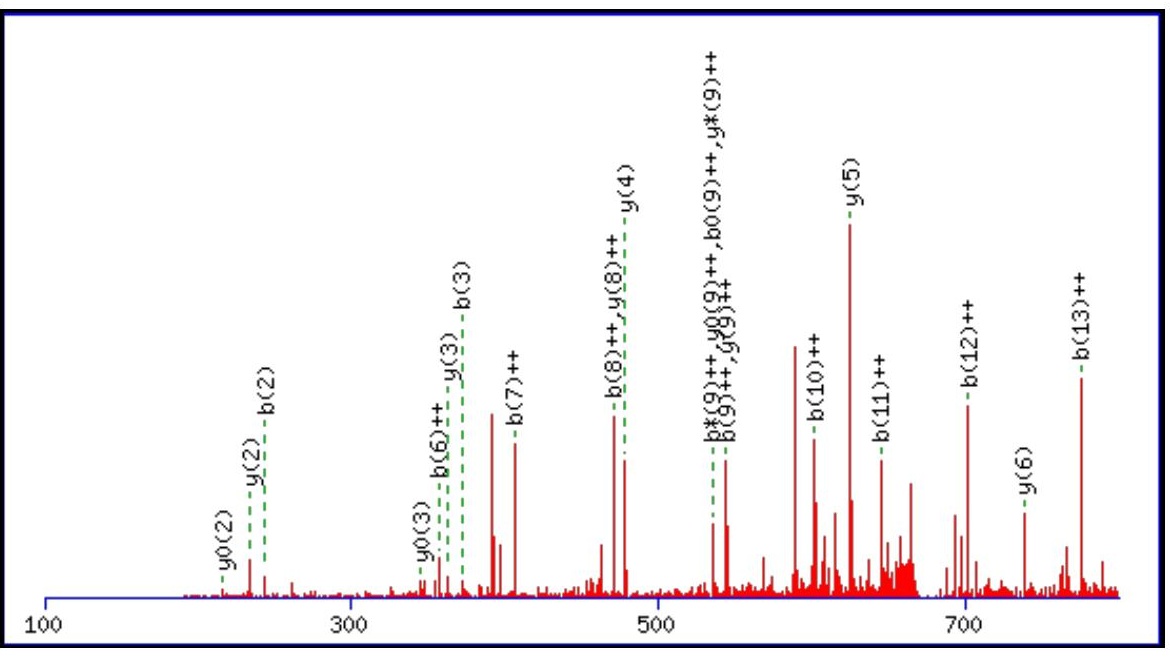



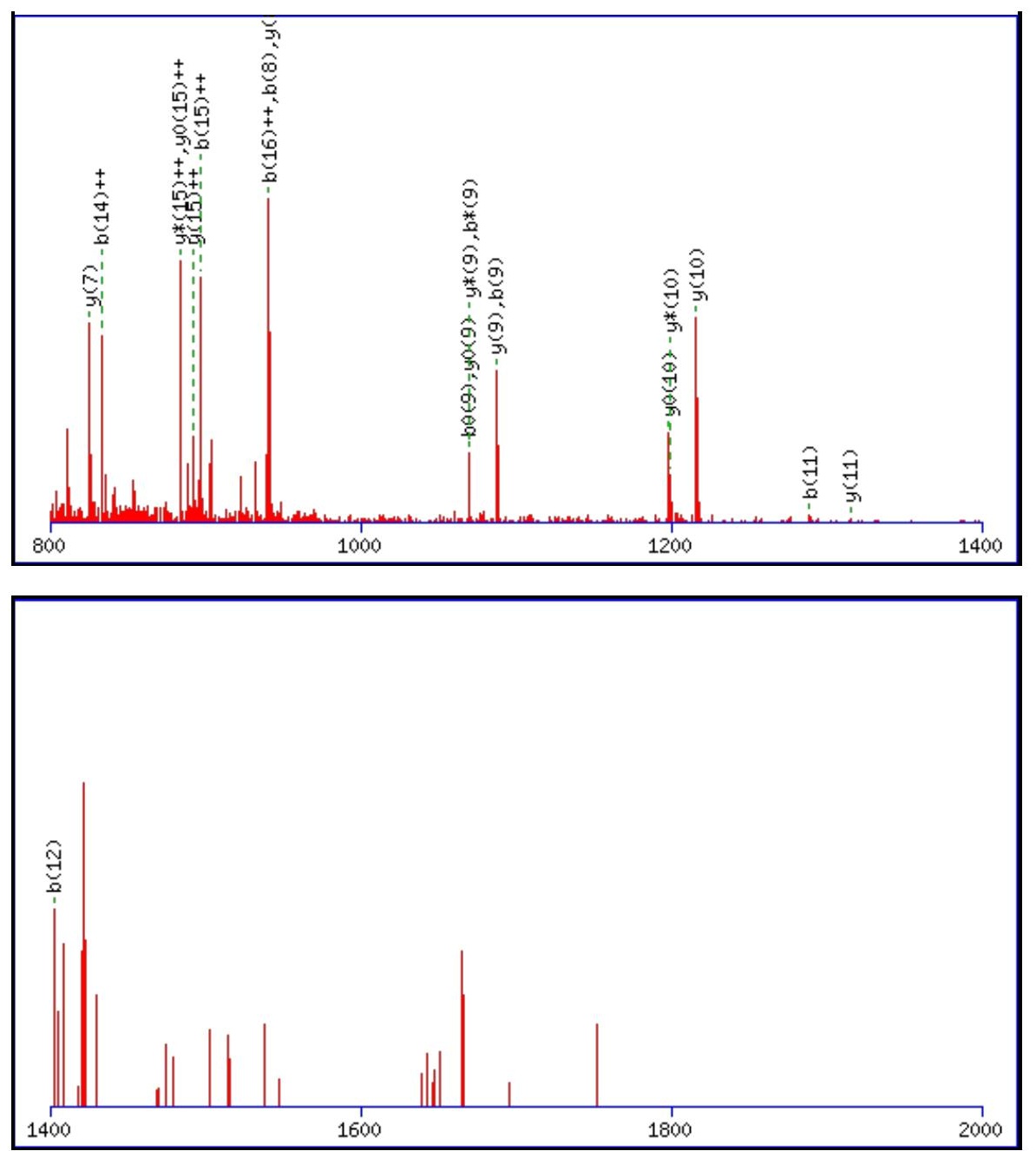

Monoisotopic mass of neutral peptide $\operatorname{Mr}($ calc): 2025.9320

Fixed modifications: Carbamidomethyl (C)

Variable modifications:

N14 : Deamidated_N (N)

Ions Score: 57 Expect: 0.00051

Matches (Bold Red): 44/180 fragment ions using 75 most intense peaks

\begin{tabular}{|c|c|c|c|c|c|c|c|c|c|c|c|c|c|c|}
\hline \# & b & $\mathbf{b}^{++}$ & $\mathbf{b}^{*}$ & $\mathbf{b}^{*^{++}}$ & $\mathbf{b}^{\mathbf{0}}$ & $\mathbf{b}^{\mathbf{0 + +}}$ & Seq. & $\mathbf{y}$ & $\mathbf{y}^{++}$ & $\mathbf{y}^{*}$ & $\mathrm{y}^{*^{++}}$ & $\mathbf{y}^{0}$ & $\mathbf{y}^{\mathbf{0}^{++}}$ & \# \\
\hline 1 & 58.0287 & 29.5180 & & & & & G & & & & & & & 17 \\
\hline 2 & 244.1081 & 122.5577 & & & & & $\mathbf{W}$ & |1969.9178 & 985.4625 & |1952.8912 & 976.9493 & 1951.9072 & 976.4573 & 16 \\
\hline 3 & 372.2030 & 186.6051 & 355.1765 & 178.0919 & & & $\mathbf{K}$ & 1783.8385 & 892.4229 & 1766.8119 & 883.9096 & 1765.8279 & 883.4176 & 15 \\
\hline 4 & 501.2456 & 251.1264 & 484.2191 & 242.6132 & 483.2350 & 242.1212 & $\mathbf{E}$ & 1655.7435 & 828.3754 & 1638.7170 & 819.8621 & 1637.7330 & 819.3701 & 14 \\
\hline 5 & 615.2885 & 308.1479 & 598.2620 & 299.6346 & 597.2780 & 299.1426 & $\mathbf{N}$ & 1526.7009 & 763.8541 & 1509.6744 & 755.3408 & 1508.6904 & 754.8488 & 13 \\
\hline 6 & 712.3413 & 356.6743 & 695.3148 & 348.1610 & 694.3307 & 347.6690 & $\mathbf{P}$ & 1412.6580 & 706.8326 & 1395.6314 & 698.3194 & 1394.6474 & 697.8274 & 12 \\
\hline 7 & 811.4097 & 406.2085 & 794.3832 & 397.6952 & 793.3991 & 397.2032 & $\mathbf{V}$ & 1315.6052 & 658.3063 & 1298.5787 & 649.7930 & 1297.5947 & 649.3010 & 11 \\
\hline 8 & 940.4523 & 470.7298 & 923.4258 & 462.2165 & 922.4417 & 461.7245 & $\mathbf{E}$ & 1216.5368 & 608.7720 & 1199.5103 & 600.2588 & 1198.5263 & 599.7668 & 10 \\
\hline 9 & 1087.5207 & 544.2640 & 1070.4942 & 535.7507 & 1069.5102 & 535.2587 & $\mathbf{F}$ & 1087.4942 & 544.2508 & 1070.4677 & 535.7375 & 1069.4837 & 535.2455 & 9 \\
\hline 10 & 1202.5477 & 601.7775 & 1185.5211 & 593.2642 & 1184.5371 & 592.7722 & D & \begin{tabular}{|l|}
940.4258 \\
\end{tabular} & 470.7165 & 923.3993 & 462.2033 & 922.4152 & 461.7113 & 8 \\
\hline 11 & \begin{tabular}{|l|}
1289.5797 \\
\end{tabular} & 645.2935 & 1272.5531 & 636.7802 & 1271.5691 & 636.2882 & $\mathrm{~S}$ & 825.3989 & 413.2031 & 808.3723 & 404.6898 & 807.3883 & 404.1978 & 7 \\
\hline 12 & 1402.6638 & 701.8355 & 1385.6372 & 693.3222 & 1384.6532 & 692.8302 & $\mathbf{L}$ & 738.3668 & 369.6871 & 721.3403 & 361.1738 & 720.3563 & 360.6818 & 6 \\
\hline 13 & 1549.7322 & 775.3697 & 1532.7056 & 766.8564 & 1531.7216 & 766.3644 & $\mathbf{F}$ & 625.2828 & 313.1450 & 608.2562 & 304.6318 & 607.2722 & 304.1397 & 5 \\
\hline 14 & 1664.7591 & 832.8832 & 1647.7326 & 824.3699 & 1646.7485 & 823.8779 & $\mathbf{N}$ & 478.2144 & 239.6108 & 461.1878 & 231.0975 & 460.2038 & 230.6055 & 4 \\
\hline 15 & 1793.8017 & 897.4045 & 1776.7752 & 888.8912 & 1775.7911 & 888.3992 & $E$ & 363.1874 & 182.0974 & 346.1609 & 173.5841 & 345.1769 & 173.0921 & 3 \\
\hline 16 & 1880.8337 & 940.9205 & 1863.8072 & 932.4072 & 1862.8232 & 931.9152 & $\mathrm{~S}$ & 234.1448 & 117.5761 & 217.1183 & 109.0628 & 216.1343 & 108.5708 & 2 \\
\hline 17 & & & & & & & $\mathbf{K}$ & 147.1128 & 74.0600 & 130.0863 & 65.5468 & & & 1 \\
\hline
\end{tabular}



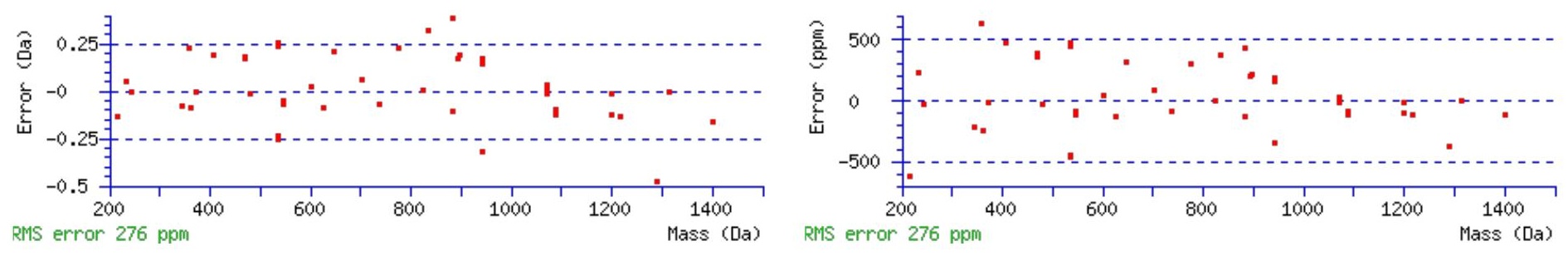

\section{All matches to this query}

\begin{tabular}{|l|l|l|l|}
\hline Score & Mr(calc): & Delta & \multicolumn{1}{|c|}{ Sequence } \\
\hline 57.0 & 2025.9320 & 0.0016 & GWKENPVEFDSLFNESK \\
\hline 39.2 & 2025.9320 & 0.0016 & GWKENPVEFDSLFNESK \\
\hline 28.8 & 2024.9480 & 0.9856 & GWKENPVEFDSLFNESK \\
\hline 9.2 & 2025.9200 & 0.0135 & SKEENGLAEENSAKELFM \\
\hline 5.4 & 2025.9287 & 0.0048 & FVAISNPLLYTVAMSPK \\
\hline 1.7 & 2025.9377 & -0.0042 & ESMRKVSLHGVXQDMSK \\
\hline 1.1 & 2024.9343 & 0.9992 & YISQEEQSTAALTLYTK \\
\hline 0.6 & 2025.9427 & -0.0092 & VMGFGLYLMDGSVSNIYK \\
\hline 0.3 & 2024.9191 & 1.0145 & LQNELDNVSTLLEEAEK \\
\hline 0.3 & 2024.9191 & 1.0145 & LQNELDNVSTLLEEAEK \\
\hline
\end{tabular}

Spectrum No: 575; Query: 2141; Rank: 1

\section{Peptide View}

MS/MS Fragmentation of EQNYTCLVEHEGLPEPLSQR

Found in IPI00393441, Tax_Id=10116 Gene_Symbol=RT1-CE2 MHC class I protein

Match to Query 2141: 2399.108928 from(1200.561740,2+)

Title: 100101RatKid_NS_deglyco_24.3335.3335.2.dta

Data file K:INewmanPaper|Piliangl3SubProteomes\Piliang3SP\mgf5ppm|ERLIC_3SubProteomes5ppm.mgf

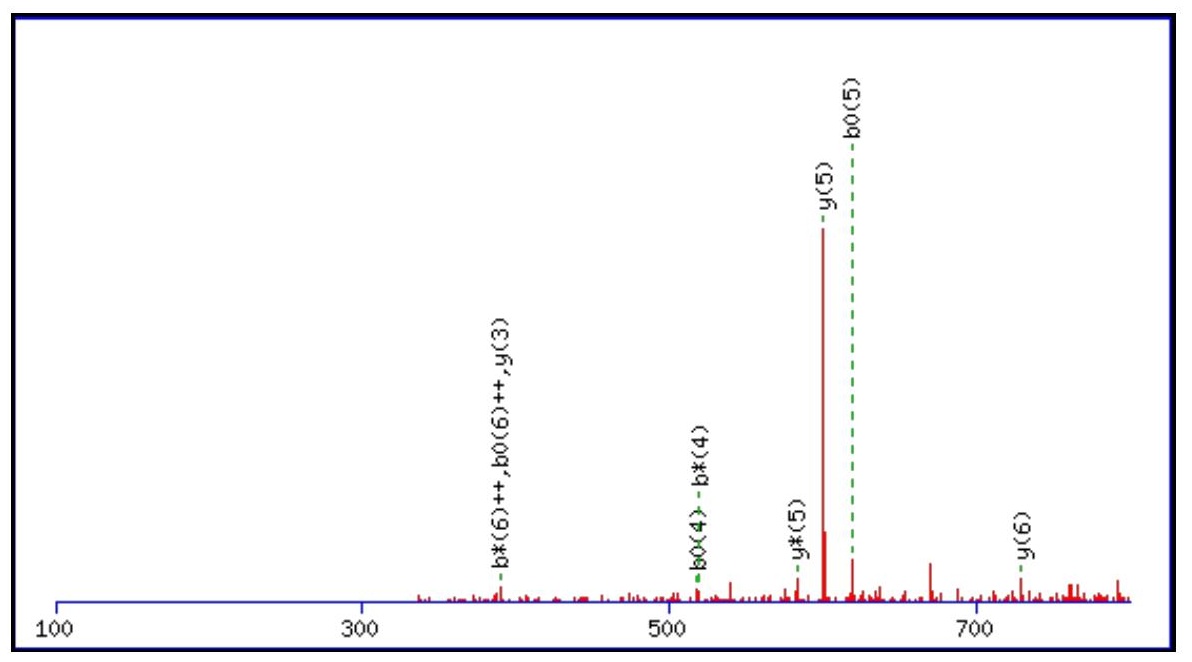



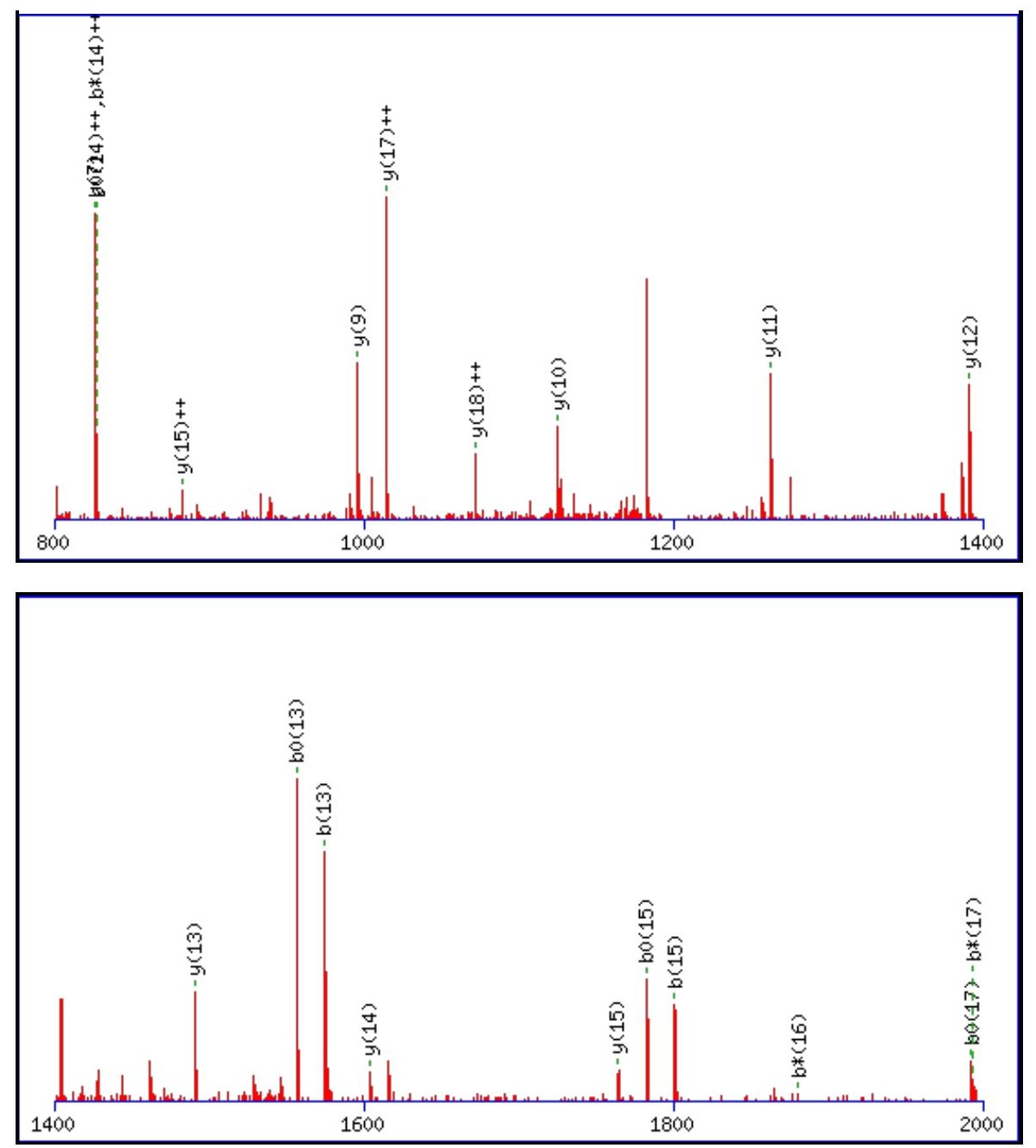

Monoisotopic mass of neutral peptide $\operatorname{Mr}($ calc): 2399.1063

Fixed modifications: Carbamidomethyl (C)

Variable modifications:

N3 : Deamidated_N (N)

Ions Score: 57 Expect: 0.00062

Matches (Bold Red): 29/222 fragment ions using 54 most intense peaks

\begin{tabular}{|c|c|c|c|c|c|c|c|c|c|c|c|c|c|c|}
\hline \# & b & $\mathbf{b}^{++}$ & $\mathbf{b}^{*}$ & $\mathbf{b}^{*^{++}}$ & $\mathbf{b}^{\mathbf{0}}$ & $\mathbf{b}^{0++}$ & Seq. & $\mathbf{y}$ & $\mathrm{y}^{++}$ & $\mathbf{y}^{*}$ & $\mathbf{y}^{*^{++}}$ & $\mathbf{y}^{0}$ & $\mathbf{y}^{0++}$ & \# \\
\hline 1 & 130.0499 & 65.5286 & & & 112.0393 & 56.5233 & $\mathbf{E}$ & & & & & & & 20 \\
\hline 2 & 258.1084 & 129.5579 & 241.0819 & 121.0446 & 240.0979 & 120.5526 & $\mathbf{Q}$ & 2271.0710 & 1136.0391 & 2254.0445 & 1127.5259 & 2253.0605 & 1127.0339 & 19 \\
\hline 4 & 536.1987 & 268.6030 & 519.1722 & 260.0897 & 518.1881 & 259.5977 & $\mathbf{Y}$ & 2027.9855 & 1014.4964 & 2010.9590 & 1005.9831 & 2009.9749 & 1005.4911 & 17 \\
\hline 5 & 637.2464 & 319.1268 & 620.2198 & 310.6136 & 619.2358 & 310.1216 & $\mathbf{T}$ & 1864.9222 & 932.9647 & 1847.8956 & 924.4515 & 1846.9116 & 923.9594 & 16 \\
\hline 6 & 797.2770 & 399.1422 & 780.2505 & 390.6289 & 779.2665 & 390.1369 & C & 1763.8745 & 882.4409 & 1746.8479 & 873.9276 & 1745.8639 & 873.4356 & 15 \\
\hline 7 & 910.3611 & 455.6842 & 893.3346 & 447.1709 & 892.3505 & 446.6789 & $\mathbf{L}$ & 1603.8438 & 802.4256 & 1586.8173 & 793.9123 & 1585.8333 & 793.4203 & 14 \\
\hline 8 & 1009.4295 & 505.2184 & 992.4030 & 496.7051 & 991.4190 & 496.2131 & V & 1490.7598 & 745.8835 & 1473.7332 & 737.3703 & 1472.7492 & 736.8782 & 13 \\
\hline 9 & 1138.4721 & 569.7397 & 1121.4456 & 561.2264 & 1120.4615 & 560.7344 & $\mathbf{E}$ & 1391.6914 & 696.3493 & 1374.6648 & 687.8360 & 1373.6808 & 687.3440 & 12 \\
\hline 10 & 1275.5310 & 638.2691 & 1258.5045 & 629.7559 & 1257.5205 & 629.2639 & $\mathbf{H}$ & 1262.6488 & 631.8280 & 1245.6222 & 623.3148 & 1244.6382 & 622.8227 & 11 \\
\hline 11 & 1404.5736 & 702.7904 & 1387.5471 & 694.2772 & 1386.5631 & 693.7852 & $\mathbf{E}$ & 1125.5899 & 563.2986 & 1108.5633 & 554.7853 & 1107.5793 & 554.2933 & 10 \\
\hline 12 & 1461.5951 & 731.3012 & 1444.5685 & 722.7879 & 1443.5845 & 722.2959 & G & 996.5473 & 498.7773 & 979.5207 & 490.2640 & 978.5367 & 489.7720 & 9 \\
\hline 14 & 1671.7319 & 836.3696 & 1654.7054 & 827.8563 & 1653.7213 & 827.3643 & $\mathbf{P}$ & 826.4417 & 413.7245 & 809.4152 & 405.2112 & 808.4312 & 404.7192 & 7 \\
\hline 15 & 1800.7745 & 900.8909 & 1783.7480 & 892.3776 & 1782.7639 & 891.8856 & $\mathbf{E}$ & 729.3890 & 365.1981 & 712.3624 & 356.6849 & 711.3784 & 356.1928 & 6 \\
\hline 16 & 1897.8273 & 949.4173 & 1880.8007 & 940.9040 & 1879.8167 & 940.4120 & $\mathbf{P}$ & 600.3464 & 300.6768 & 583.3198 & 292.1636 & 582.3358 & 291.6715 & 5 \\
\hline 17 & 2010.9113 & 1005.9593 & 1993.8848 & 997.4460 & 1992.9008 & 996.9540 & $\mathbf{L}$ & 503.2936 & 252.1504 & 486.2671 & 243.6372 & 485.2831 & 243.1452 & 4 \\
\hline 18 & 2097.9434 & 1049.4753 & 2080.9168 & 1040.9620 & 2079.9328 & 1040.4700 & S & 390.2096 & 195.6084 & 373.1830 & 187.0951 & 372.1990 & 186.6031 & 3 \\
\hline
\end{tabular}




\begin{tabular}{|r|r|r|r|r|r|r|r|r|r|r|r|r|r|r|r|r|r|}
$\mathbf{1 9}$ & 2226.0019 & 1113.5046 & 2208.9754 & 1104.9913 & 2207.9914 & 1104.4993 & $\mathbf{Q}$ & 303.1775 & 152.0924 & 286.1510 & 143.5791 & & \\
\hline $\mathbf{2 0}$ & & & & & & & $\mathbf{R}$ & 175.1190 & 88.0631 & 158.0924 & 79.5498 & & & $\mathbf{1}$ \\
\hline
\end{tabular}
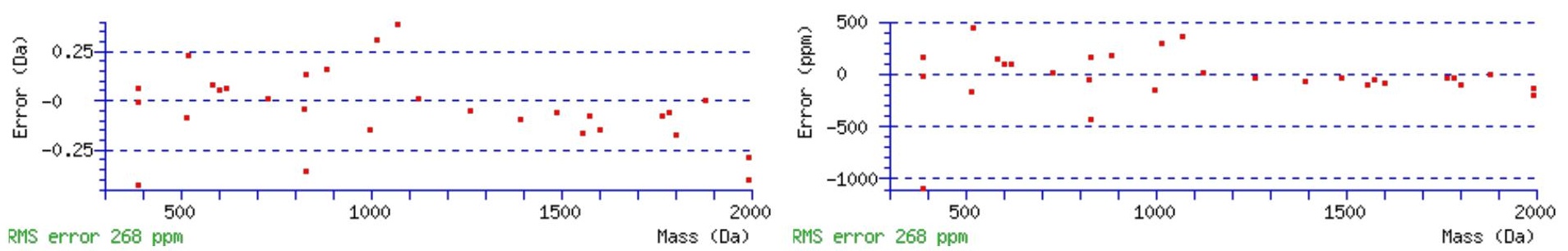

\section{All matches to this query}

\begin{tabular}{|l|l|c|l|}
\hline Score & Mr(calc): & Delta & \multicolumn{1}{|c|}{ Sequence } \\
\hline 57.0 & 2399.1063 & 0.0026 & EQNYTCLVEHEGLPEPLSQR \\
\hline 34.6 & 2398.1223 & 0.9866 & EQNYTCLVEHEGLPEPLSQR \\
\hline 5.6 & 2398.0862 & 1.0228 & EEEIPENANTEKISMETLLK \\
\hline 5.4 & 2398.0862 & 1.0228 & EEEIPENANTEKISMETLLK \\
\hline 5.3 & 2399.0924 & 0.0166 & FSGVAGEVPSNLSVPVAVGSGPGR \\
\hline 4.5 & 2399.0924 & 0.0166 & FSGVAGEVPSNLSVPVAVGSGPGR \\
\hline 3.5 & 2397.1022 & 2.0068 & EEEIPENANTEKISMETLLK \\
\hline 1.9 & 2397.0946 & 2.0143 & VPTTGIIEYPFDLENIIFR \\
\hline 1.6 & 2399.1262 & -0.0173 & VLVFPMEGSHWLSIRDVVR \\
\hline 1.3 & 2399.1110 & -0.0021 & MPTPSSQPTSMSHRSYQPPQR \\
\hline
\end{tabular}

Spectrum No: 576; Query: 334; Rank: 1

\section{Peptide View}

MS/MS Fragmentation of GLFTNGSCADIK

Found in IPI00190846, Tax_Id=10116 Gene_Symbol=Casr Extracellular calcium-sensing receptor precursor

Match to Query 334: 1282.589748 from(642.302150,2+)

Title: 100101RatKid_NS_deglyco_23.2256.2256.2.dta

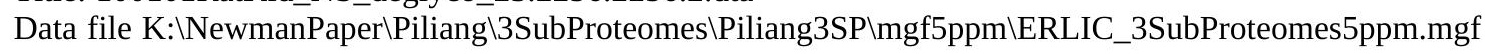

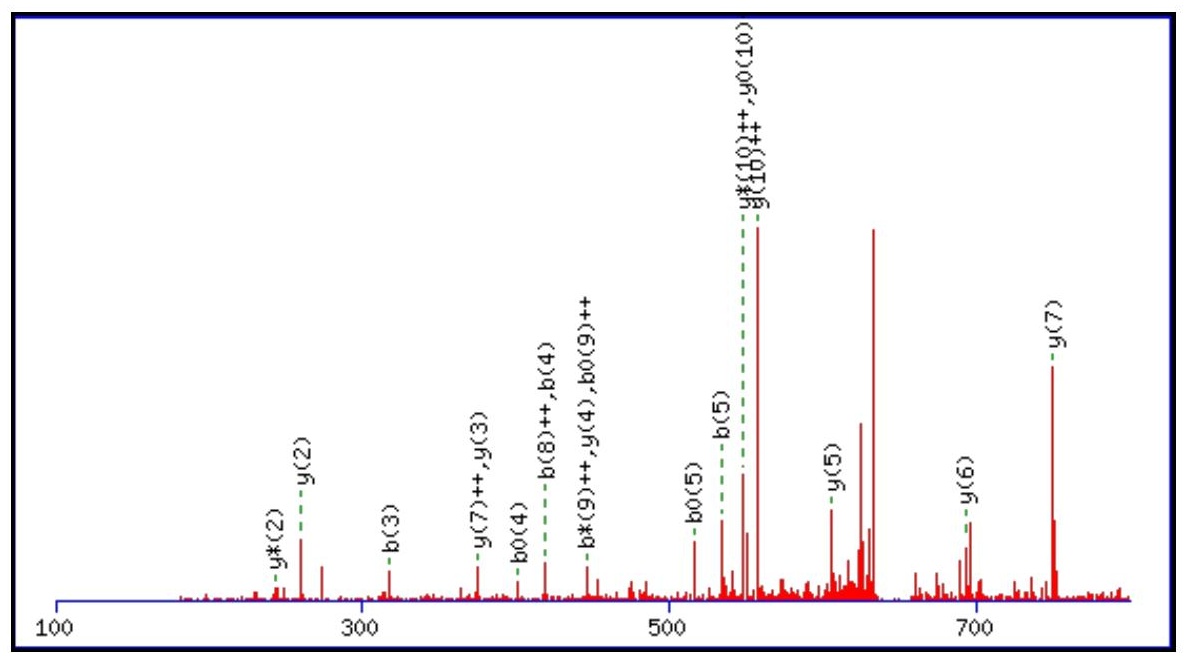



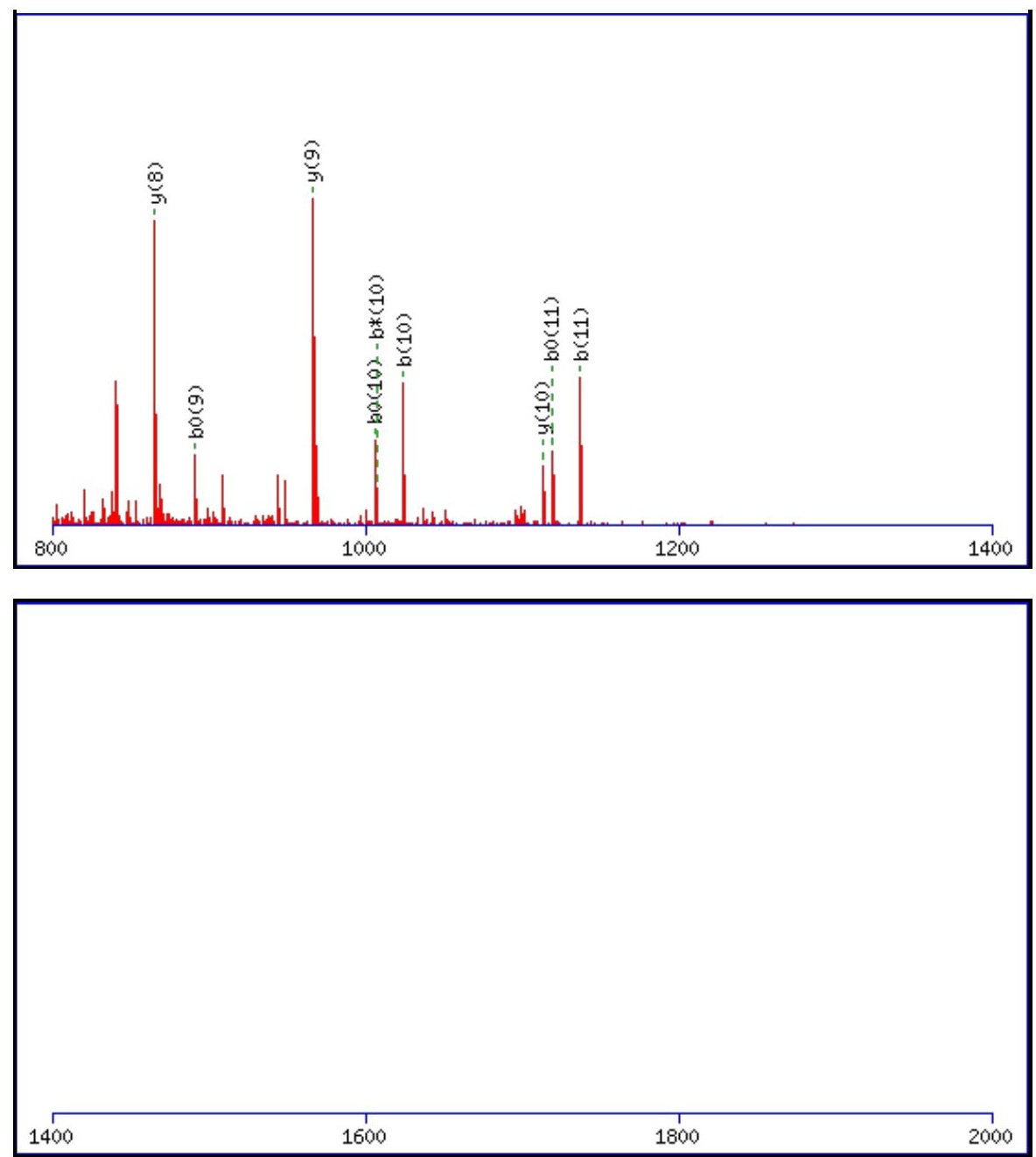

Monoisotopic mass of neutral peptide $\operatorname{Mr}($ calc): 1282.5863

Fixed modifications: Carbamidomethyl (C)

Variable modifications:

N5 : Deamidated_N (N)

Ions Score: 57 Expect: 0.00033

Matches (Bold Red): 28/114 fragment ions using 50 most intense peaks

\begin{tabular}{|c|c|c|c|c|c|c|c|c|c|c|c|c|c|c|}
\hline \# & b & $\mathbf{b}^{++}$ & b* & $\mathbf{b}^{*^{++}}$ & $\mathbf{b}^{0}$ & $\mathbf{b}^{0++}$ & Seq. & $\mathbf{y}$ & $\mathrm{y}^{++}$ & $\mathbf{y}^{*}$ & $\mathrm{y}^{*^{++}}$ & $\mathbf{y}^{0}$ & $\mathbf{y}^{0++}$ & \# \\
\hline 1 & 58.0287 & 29.5180 & & & & & G & & & & & & & 12 \\
\hline 2 & 171.1128 & 86.0600 & & & & & $\mathbf{L}$ & 1226.5722 & 613.7897 & 1209.5456 & 605.2764 & 1208.5616 & 604.7844 & 11 \\
\hline 3 & 318.1812 & 159.5942 & & & & & $\mathbf{F}$ & 1113.4881 & 557.2477 & 1096.4615 & 548.7344 & 1095.4775 & 548.2424 & 10 \\
\hline 4 & 419.2289 & 210.1181 & & & 401.2183 & 201.1128 & $\mathbf{T}$ & 966.4197 & 483.7135 & 949.3931 & 475.2002 & 948.4091 & 474.7082 & 9 \\
\hline 5 & 534.2558 & 267.6316 & 517.2293 & 259.1183 & 516.2453 & 258.6263 & $\mathbf{N}$ & 865.3720 & 433.1896 & 848.3455 & 424.6764 & 847.3614 & 424.1844 & 8 \\
\hline 6 & 591.2773 & 296.1423 & 574.2507 & 287.6290 & 573.2667 & 287.1370 & $\mathbf{G}$ & 750.3451 & 375.6762 & 733.3185 & 367.1629 & 732.3345 & 366.6709 & 7 \\
\hline 7 & 678.3093 & 339.6583 & 661.2828 & 331.1450 & 660.2988 & 330.6530 & $\mathrm{~S}$ & 693.3236 & 347.1654 & 676.2971 & 338.6522 & 675.3130 & 338.1602 & 6 \\
\hline 8 & 838.3400 & 419.6736 & 821.3134 & 411.1604 & 820.3294 & 410.6683 & C & 606.2916 & 303.6494 & 589.2650 & 295.1362 & 588.2810 & 294.6441 & 5 \\
\hline 9 & 909.3771 & 455.1922 & 892.3505 & 446.6789 & 891.3665 & 446.1869 & A & 446.2609 & 223.6341 & 429.2344 & 215.1208 & 428.2504 & 214.6288 & 4 \\
\hline 10 & 1024.4040 & 512.7057 & 1007.3775 & 504.1924 & 1006.3935 & 503.7004 & D & 375.2238 & 188.1155 & 358.1973 & 179.6023 & 357.2132 & 179.1103 & 3 \\
\hline 11 & 1137.4881 & 569.2477 & 1120.4615 & 560.7344 & 1119.4775 & 560.2424 & I & 260.1969 & 130.6021 & 243.1703 & 122.0888 & & & 2 \\
\hline 12 & & & & & & & $\mathbf{K}$ & 147.1128 & 74.0600 & 130.0863 & 65.5468 & & & 1 \\
\hline
\end{tabular}



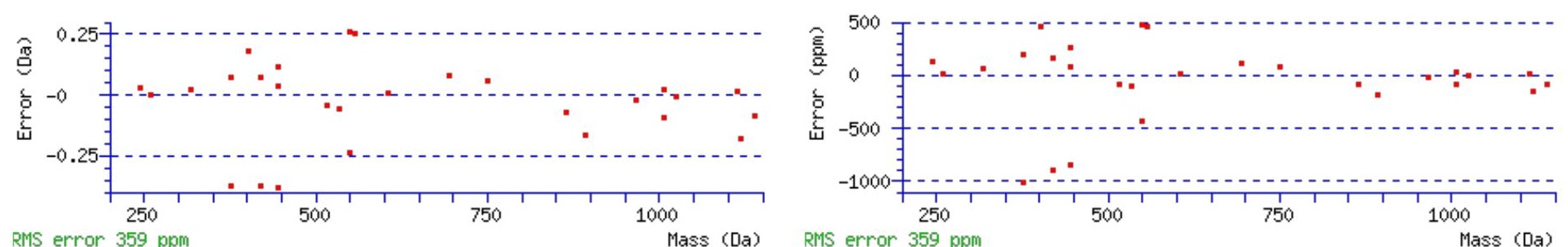

RMS error 359 ppm

\section{All matches to this query}

\begin{tabular}{|l|l|c|l|}
\hline Score & Mr(calc): & Delta & \multicolumn{1}{c|}{ Sequence } \\
\hline 56.9 & 1282.5863 & 0.0034 & GLFTNGSCADIK \\
\hline 17.1 & 1282.5976 & -0.0078 & ASTFQNTCNLK \\
\hline 14.2 & 1282.5798 & 0.0099 & VCDGCFRELK \\
\hline 9.0 & 1282.5992 & -0.0095 & MVKQASEGPLK \\
\hline 9.0 & 1282.5992 & -0.0095 & VMKQASEGPLK \\
\hline 7.0 & 1282.5815 & 0.0083 & CVMGAGSIPAIK \\
\hline 6.6 & 1282.5806 & 0.0092 & QGSNSIEINIK \\
\hline 5.7 & 1281.5941 & 0.9957 & CLAWPLSSLR \\
\hline 5.7 & 1282.5958 & -0.0061 & NTALGVISNWK \\
\hline 5.7 & 1282.6016 & -0.0119 & GSWACSIFDLK \\
\hline
\end{tabular}

Spectrum No: 577; Query: 130; Rank: 1

\section{Peptide View}

MS/MS Fragmentation of QANDSLTVLR

Found in IPI00363534, Tax_Id=10116 Gene_Symbol=Lama1 laminin, alpha 1

Match to Query 130: 1116.579348 from(559.296950,2+)

Title: 100101RatKid_NS_deglyco_23.1764.1764.2.dta

Data file K:INewmanPaper|Piliangl3SubProteomes\Piliang3SP\mgf5ppm\ERLIC_3SubProteomes5ppm.mgf

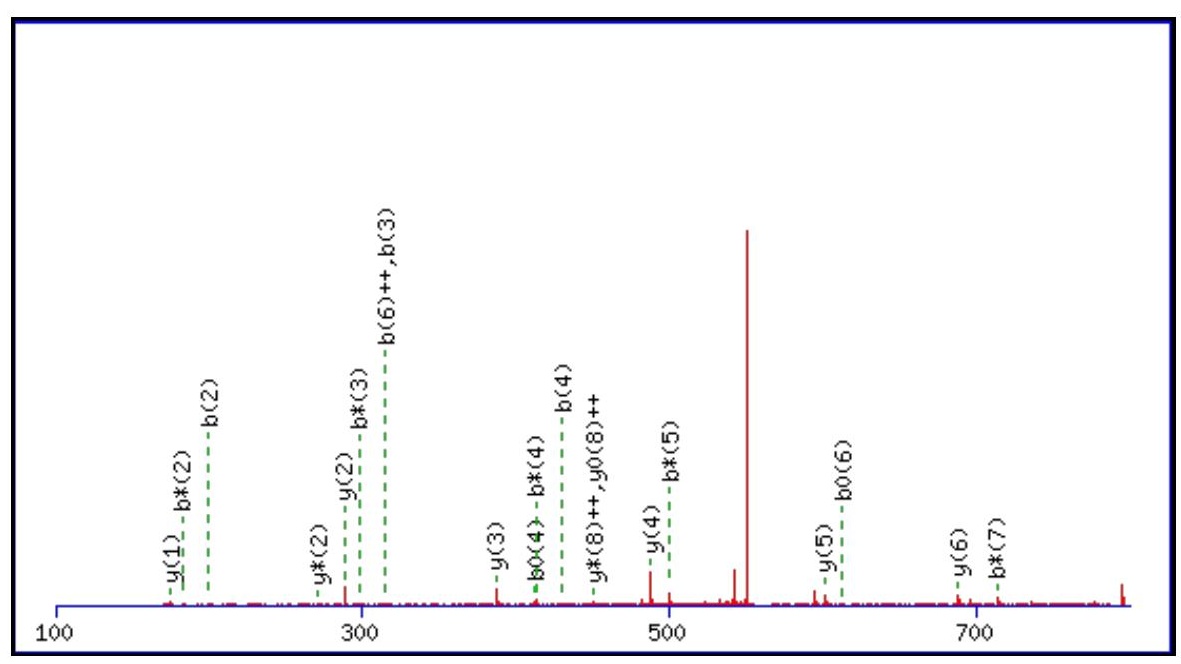



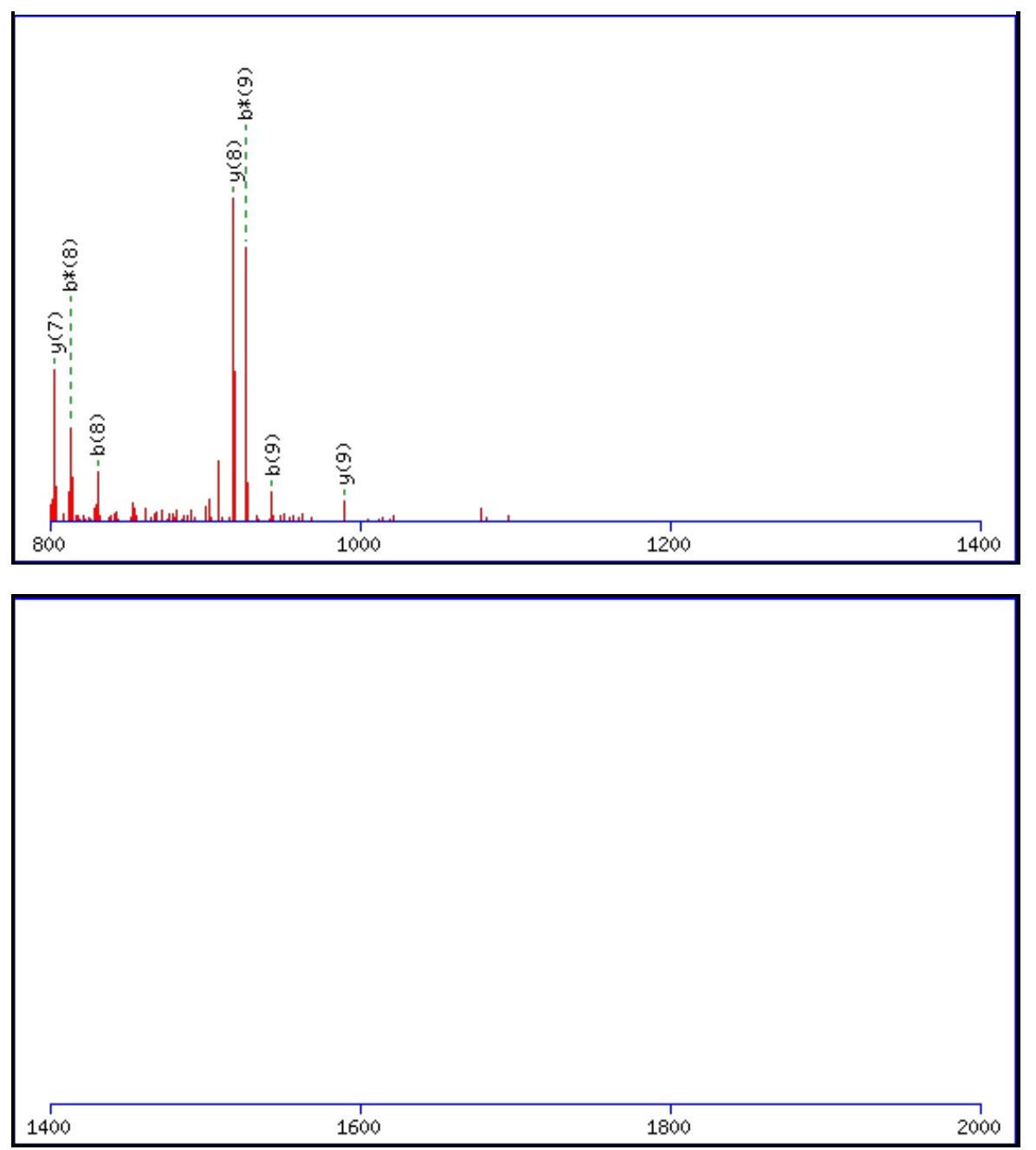

Monoisotopic mass of neutral peptide $\operatorname{Mr}($ calc): 1116.5775

Fixed modifications: Carbamidomethyl (C)

Variable modifications:

N3 : Deamidated_N (N)

Ions Score: 57 Expect: 0.00022

Matches (Bold Red): 27/96 fragment ions using 58 most intense peaks

\begin{tabular}{|r|c|c|c|c|c|c|c|c|c|c|c|c|c|c|}
\hline$\#$ & $\mathbf{b}$ & $\mathbf{b}^{++}$ & $\mathbf{b}^{*}$ & $\mathbf{b}^{\mathbf{*}^{++}}$ & $\mathbf{b}^{\mathbf{0}}$ & $\mathbf{b}^{\mathbf{0 + +}}$ & $\mathbf{S e q}$ & $\mathbf{y}$ & $\mathbf{y}^{++}$ & $\mathbf{y}^{*}$ & $\mathbf{y}^{\mathbf{*}^{++}}$ & $\mathbf{y}^{\mathbf{0}}$ & $\mathbf{y}^{\mathbf{0 + +}}$ & $\#$ \\
\hline $\mathbf{1}$ & 129.0659 & 65.0366 & 112.0393 & 56.5233 & & & $\mathbf{Q}$ & & & & & & & $\mathbf{1 0}$ \\
\hline $\mathbf{2}$ & $\mathbf{2 0 0 . 1 0 3 0}$ & 100.5551 & $\mathbf{1 8 3 . 0 7 6 4}$ & 92.0418 & & & $\mathbf{A}$ & $\mathbf{9 8 9 . 5 2 6 2}$ & 495.2667 & $\mathbf{9 7 2 . 4 9 9 6}$ & $\mathbf{4 8 6 . 7 5 3 5}$ & $\mathbf{9 7 1 . 5 1 5 6}$ & $\mathbf{4 8 6 . 2 6 1 5}$ & $\mathbf{9}$ \\
\hline $\mathbf{3}$ & 315.1299 & 158.0686 & $\mathbf{2 9 8 . 1 0 3 4}$ & 149.5553 & & & $\mathbf{N}$ & $\mathbf{9 1 8 . 4 8 9 1}$ & 459.7482 & 901.4625 & $\mathbf{4 5 1 . 2 3 4 9}$ & 900.4785 & $\mathbf{4 5 0 . 7 4 2 9}$ & $\mathbf{8}$ \\
\hline $\mathbf{4}$ & $\mathbf{4 3 0 . 1 5 6 8}$ & 215.5821 & $\mathbf{4 1 3 . 1 3 0 3}$ & 207.0688 & $\mathbf{4 1 2 . 1 4 6 3}$ & 206.5768 & $\mathbf{D}$ & $\mathbf{8 0 3 . 4 6 2 1}$ & 402.2347 & 786.4356 & 393.7214 & 785.4516 & 393.2294 & $\mathbf{7}$ \\
\hline $\mathbf{5}$ & 517.1889 & 259.0981 & $\mathbf{5 0 0 . 1 6 2 3}$ & 250.5848 & 499.1783 & 250.0928 & $\mathbf{S}$ & $\mathbf{6 8 8 . 4 3 5 2}$ & 344.7212 & 671.4087 & 336.2080 & 670.4246 & 335.7160 & $\mathbf{6}$ \\
\hline $\mathbf{6}$ & 630.2729 & 315.6401 & 613.2464 & 307.1268 & $\mathbf{6 1 2 . 2 6 2 4}$ & 306.6348 & $\mathbf{L}$ & $\mathbf{6 0 1 . 4 0 3 2}$ & 301.2052 & 584.3766 & 292.6920 & 583.3926 & 292.1999 & $\mathbf{5}$ \\
\hline $\mathbf{7}$ & $\mathbf{7 3 1 . 3 2 0 6}$ & 366.1639 & $\mathbf{7 1 4 . 2 9 4 1}$ & 357.6507 & 713.3101 & 357.1587 & $\mathbf{T}$ & $\mathbf{4 8 8 . 3 1 9 1}$ & 244.6632 & 471.2926 & 236.1499 & 470.3085 & 235.6579 & $\mathbf{4}$ \\
\hline $\mathbf{8}$ & $\mathbf{8 3 0 . 3 8 9 0}$ & 415.6982 & $\mathbf{8 1 3 . 3 6 2 5}$ & 407.1849 & 812.3785 & 406.6929 & $\mathbf{V}$ & $\mathbf{3 8 7 . 2 7 1 4}$ & 194.1394 & 370.2449 & 185.6261 & & & $\mathbf{3}$ \\
\hline $\mathbf{9}$ & $\mathbf{9 4 3 . 4 7 3 1}$ & 472.2402 & $\mathbf{9 2 6 . 4 4 6 5}$ & 463.7269 & 925.4625 & 463.2349 & $\mathbf{L}$ & $\mathbf{2 8 8 . 2 0 3 0}$ & 144.6051 & $\mathbf{2 7 1 . 1 7 6 5}$ & 136.0919 & & & $\mathbf{2}$ \\
\hline $\mathbf{1 0}$ & & & & & & & $\mathbf{R}$ & $\mathbf{1 7 5 . 1 1 9 0}$ & 88.0631 & 158.0924 & 79.5498 & & & $\mathbf{1}$ \\
\hline
\end{tabular}

$$
\text { 产 }
$$

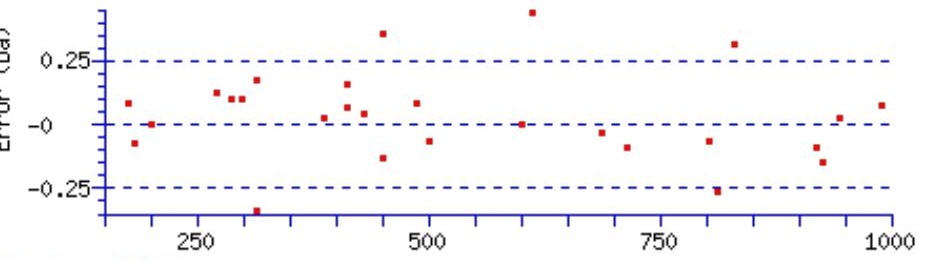

RMS error 386 ppm

\section{All matches to this query}

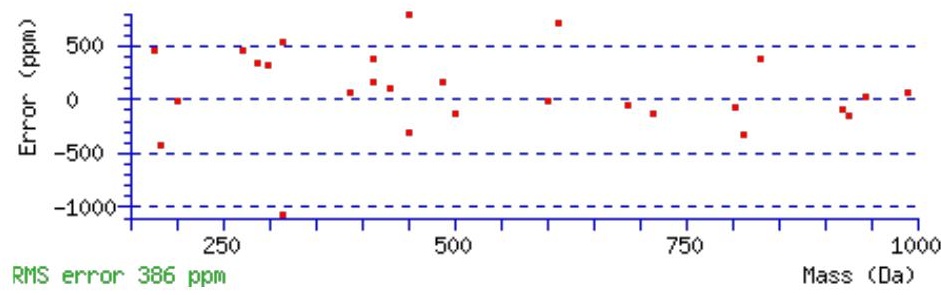

file:///R//wwwroot/SupportingData/3SubProteomes/ERLIC_3SubProteo_Glyco/ERLIC_3SubProteo_Glyco_003.html[4/27/2010 12:23:22 PM] 


\begin{tabular}{|l|c|c|l|}
\hline Score & Mr(calc): & Delta & \multicolumn{1}{c|}{ Sequence } \\
\hline 56.9 & 1116.5775 & 0.0019 & QANDSLTVLR \\
\hline 22.7 & 1115.5852 & 0.9941 & QLISAHVLR \\
\hline 14.7 & 1116.5692 & 0.0101 & KKSYVGSLR \\
\hline 13.0 & 1116.5692 & 0.0101 & KKSYVGSLR \\
\hline 13.0 & 1116.5887 & -0.0094 & QNTLRESLR \\
\hline 12.9 & 1115.5723 & 1.0070 & KNEGVNWLR \\
\hline 12.9 & 1115.5740 & 1.0053 & KYGVTTIVR \\
\hline 12.9 & 1115.5740 & 1.0053 & KYGVTTLVR \\
\hline 12.8 & 1116.5692 & 0.0101 & KKSYVGSLR \\
\hline 12.8 & 1116.5790 & 0.0003 & $\underline{\text { KPMYFFLR }}$ \\
\hline
\end{tabular}

Spectrum No: 578; Query: 2341; Rank: 1

\section{Peptide View}

MS/MS Fragmentation of STGIDAIHPNKNEFNHNFSVSYR

Found in IPI00208659, Tax_Id=10116 Gene_Symbol=Cfh Complement inhibitory factor H

Match to Query 2341: 2648.251392 from(883.757740,3+)

Title: 100101RatKid_NS_deglyco_08.2740.2740.3.dta

Data file K:INewmanPaper|Piliangl3SubProteomes\Piliang3SP\mgf5ppm\ERLIC_3SubProteomes5ppm.mgf
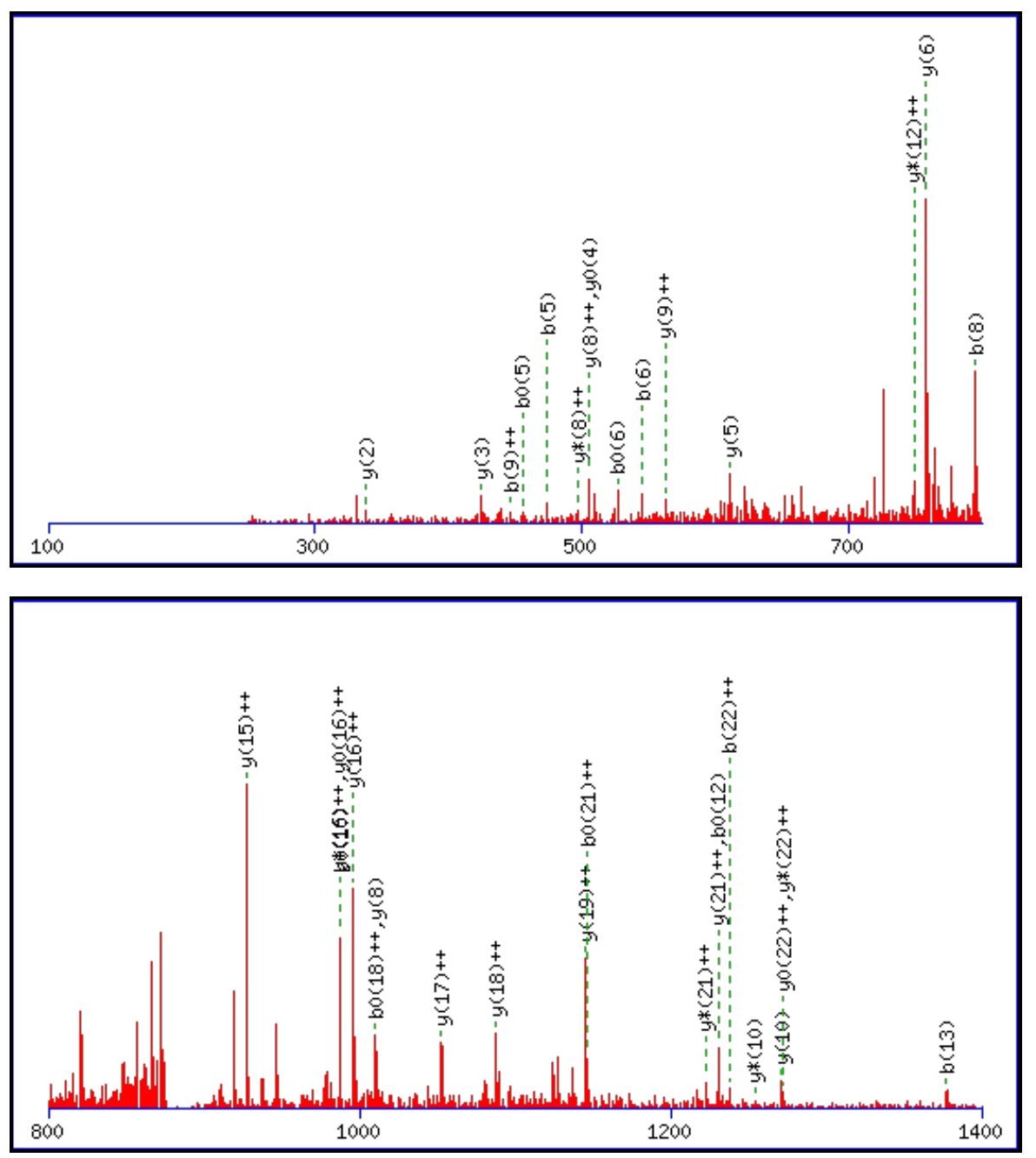


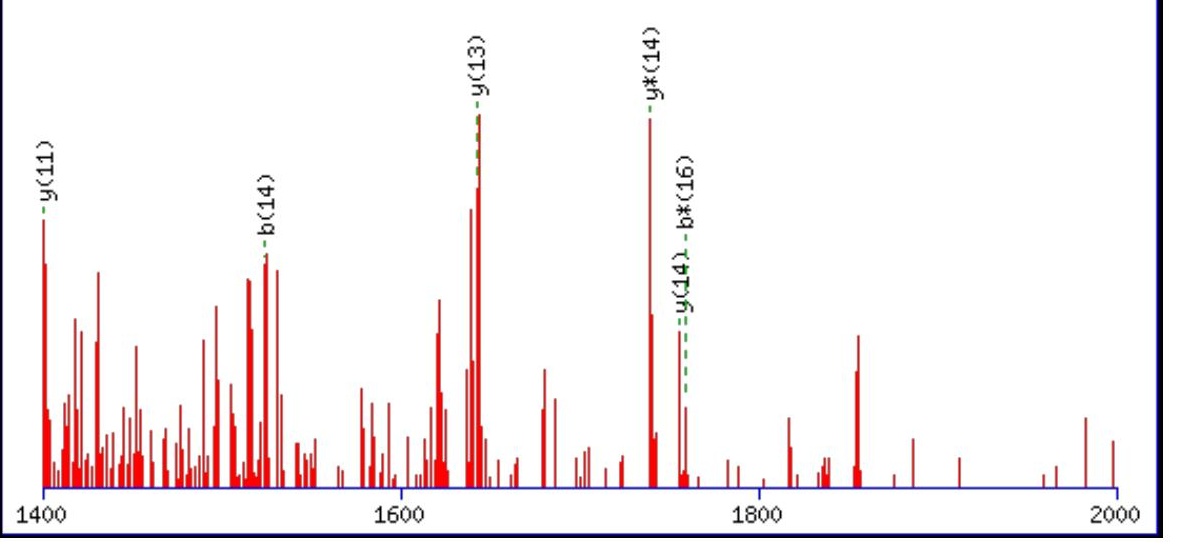

Monoisotopic mass of neutral peptide $\operatorname{Mr}($ calc): 2647.2415

Fixed modifications: Carbamidomethyl (C)

Variable modifications:

N17 : Deamidated $\mathrm{N}(\mathrm{N})$

Ions Score: 57 Expect: 0.00068

Matches (Bold Red): 41/242 fragment ions using 67 most intense peaks

\begin{tabular}{|c|c|c|c|c|c|c|c|c|c|c|c|c|c|c|}
\hline \# & b & $\mathbf{b}^{++}$ & b* & $\mathbf{b}^{*^{++}}$ & $\mathbf{b}^{\mathbf{0}}$ & $\mathbf{b}^{\mathbf{0 + +}}$ & Seq. & $\mathbf{y}$ & $\mathbf{y}^{++}$ & $\mathbf{y}^{*}$ & $\mathrm{y}^{*^{++}}$ & $\mathbf{y}^{0}$ & $\mathbf{y}^{\mathbf{0 + +}}$ & \# \\
\hline 1 & 88.0393 & 44.5233 & & & 70.0287 & 35.5180 & S & & & & & & & 23 \\
\hline 2 & 189.0870 & 95.0471 & & & 171.0764 & 86.0418 & $\mathbf{T}$ & 2561.2168 & 1281.1120 & 2544.1902 & 1272.5987 & 2543.2062 & 1272.1067 & 22 \\
\hline 3 & 246.1084 & 123.5579 & & & 228.0979 & 114.5526 & G & 2460.1691 & 1230.5882 & 2443.1425 & 1222.0749 & 2442.1585 & 1221.5829 & 21 \\
\hline 4 & 359.1925 & 180.0999 & & & 341.1819 & 171.0946 & I & 2403.1476 & 1202.0775 & 2386.1211 & 1193.5642 & 2385.1371 & 1193.0722 & 20 \\
\hline 5 & 474.2195 & 237.6134 & & & 456.2089 & 228.6081 & D & 2290.0636 & 1145.5354 & 2273.0370 & 1137.0221 & 2272.0530 & 1136.5301 & 19 \\
\hline 6 & 545.2566 & 273.1319 & & & 527.2460 & 264.1266 & A & 2175.0366 & 1088.0219 & 2158.0101 & 1079.5087 & 2157.0261 & 1079.0167 & 18 \\
\hline 7 & 658.3406 & 329.6740 & & & 640.3301 & 320.6687 & I & 2103.9995 & 1052.5034 & 2086.9730 & 1043.9901 & 2085.9889 & 1043.4981 & 17 \\
\hline 8 & 795.3995 & 398.2034 & & & 777.3890 & 389.1981 & $\mathbf{H}$ & 1990.9154 & 995.9614 & 1973.8889 & 987.4481 & 1972.9049 & 986.9561 & 16 \\
\hline 9 & 892.4523 & 446.7298 & & & 874.4417 & 437.7245 & $\mathbf{P}$ & 1853.8565 & 927.4319 & 1836.8300 & 918.9186 & 1835.8460 & 918.4266 & 15 \\
\hline 10 & 1006.4952 & 503.7513 & 989.4687 & 495.2380 & 988.4847 & 494.7460 & $\mathbf{N}$ & 1756.8038 & 878.9055 & 1739.7772 & 870.3922 & 1738.7932 & 869.9002 & 14 \\
\hline 11 & 1134.5902 & 567.7987 & 1117.5636 & 559.2855 & 1116.5796 & 558.7935 & $\mathbf{K}$ & 1642.7608 & 821.8841 & 1625.7343 & 813.3708 & 1624.7503 & 812.8788 & 13 \\
\hline 12 & 1248.6331 & 624.8202 & 1231.6066 & 616.3069 & 1230.6226 & 615.8149 & $\mathbf{N}$ & 1514.6659 & 757.8366 & 1497.6393 & 749.3233 & 1496.6553 & 748.8313 & 12 \\
\hline 13 & 1377.6757 & 689.3415 & 1360.6492 & 680.8282 & 1359.6652 & 680.3362 & $\mathbf{E}$ & 1400.6229 & 700.8151 & 1383.5964 & 692.3018 & 1382.6124 & 691.8098 & 11 \\
\hline 14 & 1524.7441 & 762.8757 & 1507.7176 & 754.3624 & 1506.7336 & 753.8704 & $\mathbf{F}$ & 1271.5804 & 636.2938 & 1254.5538 & 627.7805 & 1253.5698 & 627.2885 & 10 \\
\hline 15 & 1638.7871 & 819.8972 & 1621.7605 & 811.3839 & 1620.7765 & 810.8919 & $\mathbf{N}$ & 1124.5119 & 562.7596 & 1107.4854 & 554.2463 & 1106.5014 & 553.7543 & 9 \\
\hline 16 & 1775.8460 & 888.4266 & 1758.8194 & 879.9133 & 1757.8354 & 879.4213 & H & 1010.4690 & 505.7381 & 993.4425 & 497.2249 & 992.4584 & 496.7329 & 8 \\
\hline 17 & 1890.8729 & 945.9401 & 1873.8464 & 937.4268 & 1872.8623 & 936.9348 & $\mathbf{N}$ & 873.4101 & 437.2087 & 856.3836 & 428.6954 & 855.3995 & 428.2034 & 7 \\
\hline 18 & 2037.9413 & 1019.4743 & 2020.9148 & 1010.9610 & 2019.9308 & 1010.4690 & $\mathbf{F}$ & 758.3832 & 379.6952 & 741.3566 & 371.1819 & 740.3726 & 370.6899 & 6 \\
\hline 19 & 2124.9733 & 1062.9903 & 2107.9468 & 1054.4770 & 2106.9628 & 1053.9850 & S & 611.3148 & 306.1610 & 594.2882 & 297.6477 & 593.3042 & 297.1557 & 5 \\
\hline 20 & 2224.0418 & 1112.5245 & 2207.0152 & 1104.0112 & 2206.0312 & 1103.5192 & V & 524.2827 & 262.6450 & 507.2562 & 254.1317 & 506.2722 & 253.6397 & 4 \\
\hline 21 & 2311.0738 & 1156.0405 & 2294.0472 & 1147.5273 & 2293.0632 & 1147.0353 & $\mathbf{S}$ & 425.2143 & 213.1108 & 408.1878 & 204.5975 & 407.2037 & 204.1055 & 3 \\
\hline 22 & 2474.1371 & 1237.5722 & 2457.1106 & 1229.0589 & 2456.1266 & 1228.5669 & $\mathbf{Y}$ & 338.1823 & 169.5948 & 321.1557 & 161.0815 & & & 2 \\
\hline 23 & & & & & & & $\mathbf{R}$ & 175.1190 & 88.0631 & 158.0924 & 79.5498 & & & 1 \\
\hline
\end{tabular}
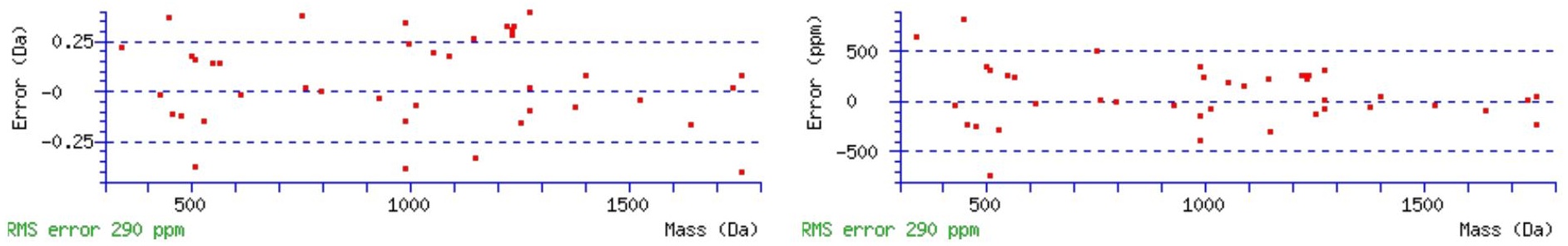

\section{All matches to this query}




\begin{tabular}{|l|l|l|l|}
\hline Score & Mr(calc): & Delta & Sequence \\
\hline 56.8 & 2647.2415 & 1.0099 & STGIDAIHPNKNEFNHNFSVSYR \\
\hline 56.8 & 2648.2255 & 0.0259 & STGIDAIHPNKNEFNHNFSVSYR \\
\hline 54.3 & 2648.2255 & 0.0259 & STGIDAIHPNKNEFNHNFSVSYR \\
\hline 53.3 & 2648.2255 & 0.0259 & $\underline{\text { STGIDAIHPNKNEFNHNFSVSYR }}$ \\
\hline 50.9 & 2648.2255 & 0.0259 & $\underline{\text { STGIDAIHPNKNEFNHNFSVSYR }}$ \\
\hline 50.4 & 2647.2415 & 1.0099 & $\underline{\text { STGIDAIHPNKNEFNHNFSVSYR }}$ \\
\hline 47.9 & 2648.2255 & 0.0259 & $\underline{\text { STGIDAIHPNKNEFNHNFSVSYR }}$ \\
\hline 33.8 & 2647.2415 & 1.0099 & $\underline{\text { STGIDAIHPNKNEFNHNFSVSYR }}$ \\
\hline 27.0 & 2647.2415 & 1.0099 & $\underline{\text { STGIDAIHPNKNEFNHNFSVSYR }}$ \\
\hline 26.2 & 2648.2255 & 0.0259 & $\underline{\text { STGIDAIHPNKNEFNHNFSVSYR }}$ \\
\hline
\end{tabular}

Spectrum No: 579; Query: 618; Rank: 1

\section{Peptide View}

MS/MS Fragmentation of NMSSEFYATQLR

Found in IPI00206254, Tax_Id=10116 Gene_Symbol=Ggt1 Gamma-glutamyltranspeptidase 1 precursor

Match to Query 618: 1462.637528 from(732.326040,2+)

Title: 091008RatKidney_NH4Format01_26.1825.1825.2.dta

Data file K:INewmanPaper\Piliangl3SubProteomes\Piliang3SP\mgf5ppm\ERLIC_3SubProteomes5ppm.mgf
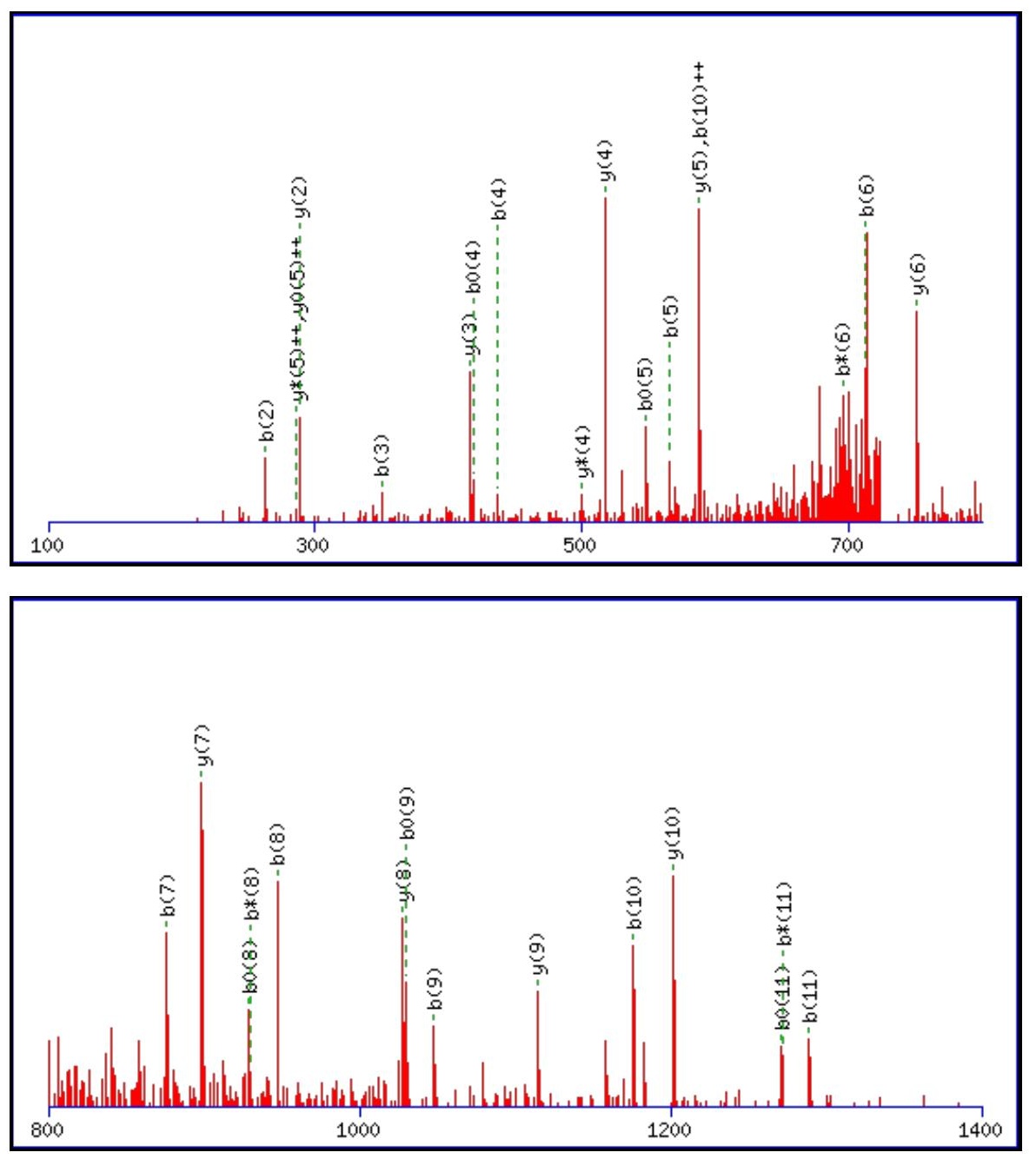


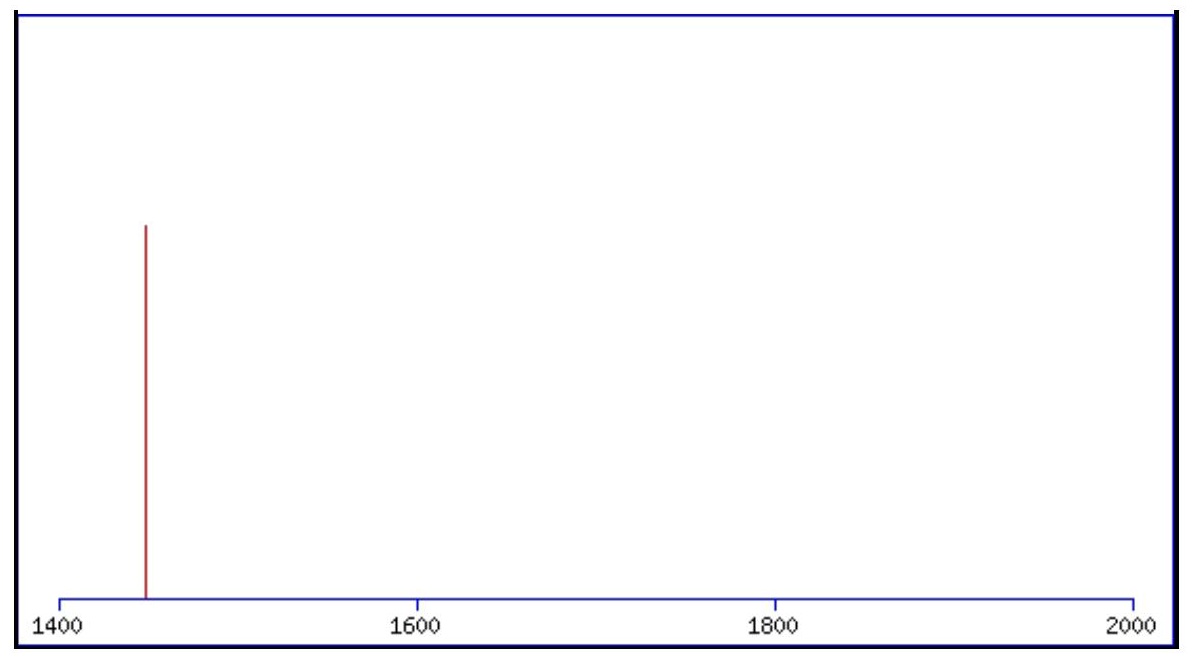

Monoisotopic mass of neutral peptide $\operatorname{Mr}($ calc): 1462.6398

Fixed modifications: Carbamidomethyl (C)

Variable modifications:

N1 : Deamidated_N (N)

M2 : Oxidation (M)

Ions Score: 57 Expect : 0.00032

Matches (Bold Red): 31/122 fragment ions using 66 most intense peaks

\begin{tabular}{|c|c|c|c|c|c|c|c|c|c|c|c|c|c|c|}
\hline \# & b & $\mathbf{b}^{++}$ & $\mathbf{b}^{*}$ & $\mathbf{b}^{*^{++}}$ & $\mathbf{b}^{0}$ & $\mathbf{b}^{0++}$ & Seq. & $\mathbf{y}$ & $y^{++}$ & $\mathbf{y}^{*}$ & $\mathrm{y}^{*^{++}}$ & $\mathbf{y}^{0}$ & $\mathbf{y}^{0++}$ & \# \\
\hline 1 & 116.0342 & 58.5207 & 99.0077 & 50.0075 & & & $\mathbf{N}$ & & & & & & & 12 \\
\hline 2 & 263.0696 & 132.0384 & 246.0431 & 123.5252 & & & $\mathbf{M}$ & 1348.6202 & 674.8137 & 1331.5936 & 666.3005 & 1330.6096 & 665.8084 & 11 \\
\hline 3 & 350.1016 & 175.5545 & 333.0751 & 167.0412 & 332.0911 & 166.5492 & S & 1201.5848 & 601.2960 & 1184.5582 & 592.7828 & 1183.5742 & 592.2907 & 10 \\
\hline 4 & 437.1337 & 219.0705 & 420.1071 & 210.5572 & 419.1231 & 210.0652 & $\mathrm{~S}$ & 1114.5528 & 557.7800 & 1097.5262 & 549.2667 & 1096.5422 & 548.7747 & 9 \\
\hline 5 & 566.1763 & 283.5918 & 549.1497 & 275.0785 & 548.1657 & 274.5865 & $\mathbf{E}$ & 1027.5207 & 514.2640 & 1010.4942 & 505.7507 & 1009.5102 & 505.2587 & 8 \\
\hline 6 & 713.2447 & 357.1260 & 696.2181 & 348.6127 & 695.2341 & 348.1207 & $\mathbf{F}$ & 898.4781 & 449.7427 & 881.4516 & 441.2294 & 880.4676 & 440.7374 & 7 \\
\hline 7 & 876.3080 & 438.6576 & 859.2815 & 430.1444 & 858.2974 & 429.6524 & $\bar{Y}$ & 751.4097 & 376.2085 & 734.3832 & 367.6952 & 733.3992 & 367.2032 & 6 \\
\hline 8 & 947.3451 & 474.1762 & 930.3186 & 465.6629 & 929.3346 & 465.1709 & A & 588.3464 & 294.6768 & 571.3198 & 286.1636 & 570.3358 & 285.6715 & 5 \\
\hline 9 & 1048.3928 & 524.7000 & 1031.3663 & 516.1868 & 1030.3822 & 515.6948 & $\mathbf{T}$ & 517.3093 & 259.1583 & 500.2827 & 250.6450 & 499.2987 & 250.1530 & 4 \\
\hline 10 & 1176.4514 & 588.7293 & 1159.4248 & 580.2161 & 1158.4408 & 579.7240 & $\mathbf{Q}$ & 416.2616 & 208.6344 & 399.2350 & 200.1212 & & & 3 \\
\hline 11 & 1289.5354 & 645.2714 & 1272.5089 & 636.7581 & 1271.5249 & 636.2661 & $\mathbf{L}$ & 288.2030 & 144.6051 & 271.1765 & 136.0919 & & & 2 \\
\hline 12 & & & & & & & $\mathbf{R}$ & 175.1190 & 88.0631 & 158.0924 & 79.5498 & & & 1 \\
\hline
\end{tabular}

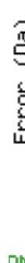
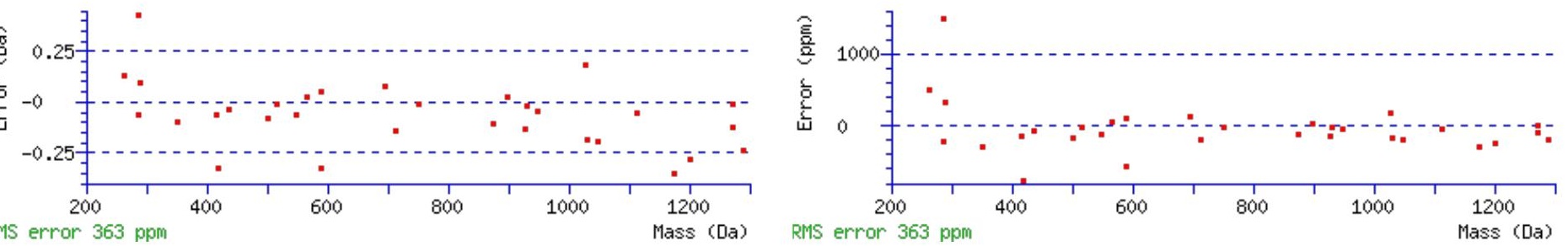

\section{All matches to this query}

\begin{tabular}{|l|l|l|l|}
\hline Score & Mr(calc): & Delta & \multicolumn{1}{|c|}{ Sequence } \\
\hline 56.7 & 1462.6398 & -0.0023 & NMSSEFYATQLR \\
\hline 9.7 & 1462.6469 & -0.0094 & ESAIASTEVKLR \\
\hline 6.1 & 1462.6483 & -0.0108 & FRGRDLXPSLR \\
\hline 5.9 & 1462.6299 & 0.0077 & YTAVSSFIFLR \\
\hline 5.9 & 1462.6299 & 0.0077 & YTAVSSFIFLR \\
\hline 4.9 & 1462.6314 & 0.0062 & SNRPNNNPGGSLR \\
\hline 4.9 & 1462.6314 & 0.0062 & SNRPNNNPGGSLR \\
\hline 4.9 & 1462.6469 & -0.0094 & ESAIASTEVKLR \\
\hline 4.9 & 1462.6469 & -0.0094 & ESAIASTEVKLR \\
\hline
\end{tabular}




\section{5 |1462.6388|-0.0013|NSPHMQNKGTLR}

Spectrum No: 580; Query: 388; Rank: 1

\section{Peptide View}

MS/MS Fragmentation of EEGDSWVLNGTK

Found in IPI00231359, Tax_Id=10116 Gene_Symbol=Acads Acetyl-Coenzyme A dehydrogenase, short chain

Match to Query 388: 1334.597868 from(668.306210,2+)

Title: 091008RatKidney_NoSalt_21.1707.1707.2.dta

Data file K:\NewmanPaper\Piliang \3SubProteomes \Piliang3SP $\backslash$ mgf5ppm\ERLIC_3SubProteomes5ppm.mgf
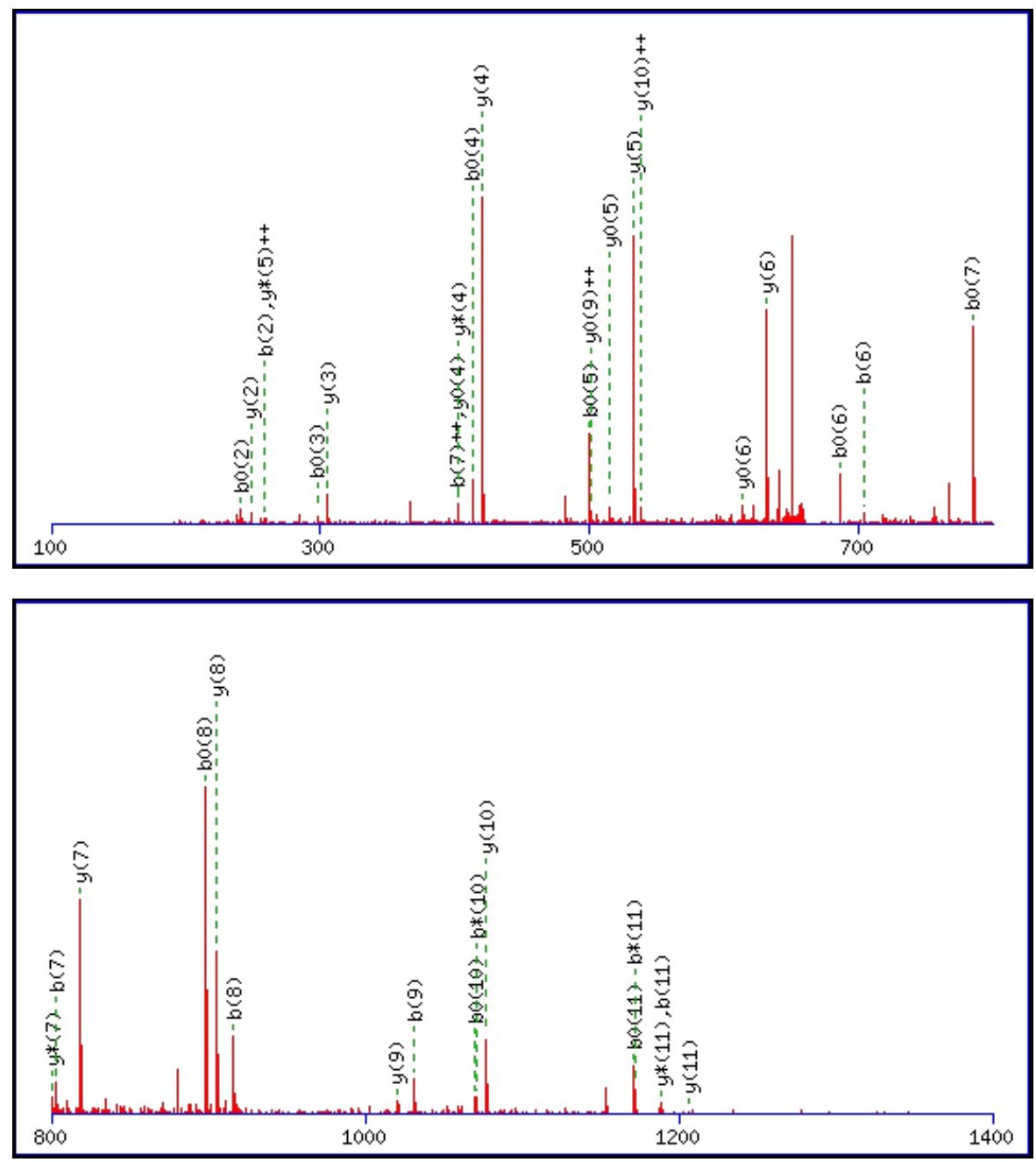
Monoisotopic mass of neutral peptide $\operatorname{Mr}($ calc): 1334.5990

Fixed modifications: Carbamidomethyl (C)

Variable modifications:

N9 : Deamidated_N (N)

Ions Score: 57 Expect: 0.00032

Matches (Bold Red): 37/114 fragment ions using 74 most intense peaks

\begin{tabular}{|r|c|c|c|c|c|c|c|c|c|c|c|c|c|c|}
\hline$\#$ & $\mathbf{b}$ & $\mathbf{b}^{++}$ & $\mathbf{b}^{*}$ & $\mathbf{b}^{\mathbf{*}^{++}}$ & $\mathbf{b}^{\mathbf{0}}$ & $\mathbf{b}^{\mathbf{0 + +}}$ & $\mathbf{S e q}$ & $\mathbf{y}$ & $\mathbf{y}^{++}$ & $\mathbf{y}^{\mathbf{*}}$ & $\mathbf{y}^{\mathbf{*}^{++}}$ & $\mathbf{y}^{\mathbf{0}}$ & $\mathbf{y}^{\mathbf{0 + +}}$ & $\#$ \\
\hline $\mathbf{1}$ & 130.0499 & 65.5286 & & & 112.0393 & 56.5233 & $\mathbf{E}$ & & & & & & & $\mathbf{1 2}$ \\
\hline $\mathbf{2}$ & $\mathbf{2 5 9 . 0 9 2 5}$ & 130.0499 & & & $\mathbf{2 4 1 . 0 8 1 9}$ & 121.0446 & $\mathbf{E}$ & $\mathbf{1 2 0 6 . 5 6 3 7}$ & 603.7855 & $\mathbf{1 1 8 9 . 5 3 7 2}$ & 595.2722 & 1188.5531 & 594.7802 & $\mathbf{1 1}$ \\
\hline $\mathbf{3}$ & 316.1139 & 158.5606 & & & $\mathbf{2 9 8 . 1 0 3 4}$ & 149.5553 & $\mathbf{G}$ & $\mathbf{1 0 7 7 . 5 2 1 1}$ & 539.2642 & 1060.4946 & 530.7509 & 1059.5105 & 530.2589 & $\mathbf{1 0}$ \\
\hline $\mathbf{4}$ & 431.1409 & 216.0741 & & & $\mathbf{4 1 3 . 1 3 0 3}$ & 207.0688 & $\mathbf{D}$ & $\mathbf{1 0 2 0 . 4 9 9 6}$ & 510.7535 & 1003.4731 & 502.2402 & 1002.4891 & $\mathbf{5 0 1 . 7 4 8 2}$ & $\mathbf{9}$ \\
\hline $\mathbf{5}$ & 518.1729 & 259.5901 & & & $\mathbf{5 0 0 . 1 6 2 3}$ & 250.5848 & $\mathbf{S}$ & $\mathbf{9 0 5 . 4 7 2 7}$ & 453.2400 & 888.4462 & 444.7267 & 887.4621 & 444.2347 & $\mathbf{8}$ \\
\hline $\mathbf{6}$ & $\mathbf{7 0 4 . 2 5 2 2}$ & 352.6297 & & & $\mathbf{6 8 6 . 2 4 1 6}$ & 343.6245 & $\mathbf{W}$ & $\mathbf{8 1 8 . 4 4 0 7}$ & 409.7240 & $\mathbf{8 0 1 . 4 1 4 1}$ & 401.2107 & 800.4301 & 400.7187 & $\mathbf{7}$ \\
\hline $\mathbf{7}$ & $\mathbf{8 0 3 . 3 2 0 6}$ & $\mathbf{4 0 2 . 1 6 4 0}$ & & & $\mathbf{7 8 5 . 3 1 0 1}$ & 393.1587 & $\mathbf{V}$ & $\mathbf{6 3 2 . 3 6 1 4}$ & 316.6843 & 615.3348 & 308.1710 & $\mathbf{6 1 4 . 3 5 0 8}$ & 307.6790 & $\mathbf{6}$ \\
\hline $\mathbf{8}$ & $\mathbf{9 1 6 . 4 0 4 7}$ & 458.7060 & & & $\mathbf{8 9 8 . 3 9 4 1}$ & 449.7007 & $\mathbf{L}$ & 533.2929 & 267.1501 & 516.2664 & $\mathbf{2 5 8 . 6 3 6 8}$ & $\mathbf{5 1 5 . 2 8 2 4}$ & 258.1448 & $\mathbf{5}$ \\
\hline $\mathbf{9}$ & $\mathbf{1 0 3 1 . 4 3 1 6}$ & 516.2195 & 1014.4051 & 507.7062 & 1013.4211 & 507.2142 & $\mathbf{N}$ & $\mathbf{4 2 0 . 2 0 8 9}$ & 210.6081 & $\mathbf{4 0 3 . 1 8 2 3}$ & 202.0948 & $\mathbf{4 0 2 . 1 9 8 3}$ & 201.6028 & $\mathbf{4}$ \\
\hline $\mathbf{1 0}$ & 1088.4531 & 544.7302 & $\mathbf{1 0 7 1 . 4 2 6 5}$ & 536.2169 & $\mathbf{1 0 7 0 . 4 4 2 5}$ & 535.7249 & $\mathbf{G}$ & $\mathbf{3 0 5 . 1 8 1 9}$ & 153.0946 & 288.1554 & 144.5813 & 287.1714 & 144.0893 & $\mathbf{3}$ \\
\hline $\mathbf{1 1}$ & $\mathbf{1 1 8 9 . 5 0 0 8}$ & 595.2540 & $\mathbf{1 1 7 2 . 4 7 4 2}$ & 586.7407 & $\mathbf{1 1 7 1 . 4 9 0 2}$ & 586.2487 & $\mathbf{T}$ & $\mathbf{2 4 8 . 1 6 0 5}$ & 124.5839 & 231.1339 & 116.0706 & 230.1499 & 115.5786 & $\mathbf{2}$ \\
\hline $\mathbf{1 2}$ & & & & & & & $\mathbf{K}$ & 147.1128 & 74.0600 & 130.0863 & 65.5468 & & & $\mathbf{1}$ \\
\hline
\end{tabular}

$$
\text { 璦 }
$$

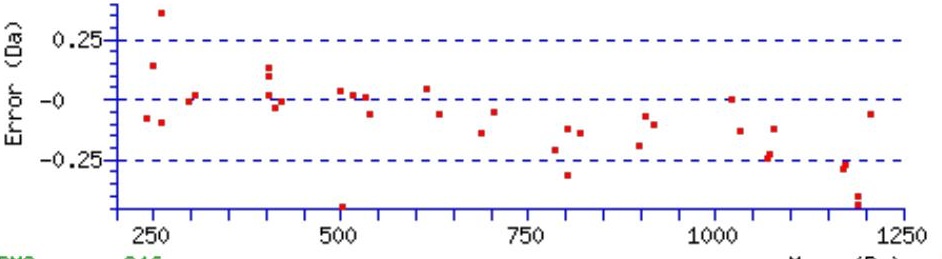

RIS error 346 ppm

\section{All matches to this query}

\begin{tabular}{|l|l|l|l|}
\hline Score & Mr(calc): & Delta & \multicolumn{1}{c|}{ Sequence } \\
\hline 56.5 & 1334.5990 & -0.0011 & EEGDSWVLNGTK \\
\hline 6.2 & 1334.6054 & -0.0075 & $\underline{\text { KLFSGATMASSR }}$ \\
\hline 2.7 & 1333.5915 & 1.0064 & $\underline{\text { FLASSDTLTSGR }}$ \\
\hline 2.6 & 1333.5915 & 1.0064 & $\underline{\text { FLASSDTLTSGR }}$ \\
\hline 0.4 & 1333.6028 & 0.9951 & $\underline{\text { HTVDDGLDVRK }}$ \\
\hline 0.4 & 1333.5915 & 1.0064 & $\underline{\text { FLASSDTLTSGR }}$ \\
\hline
\end{tabular}

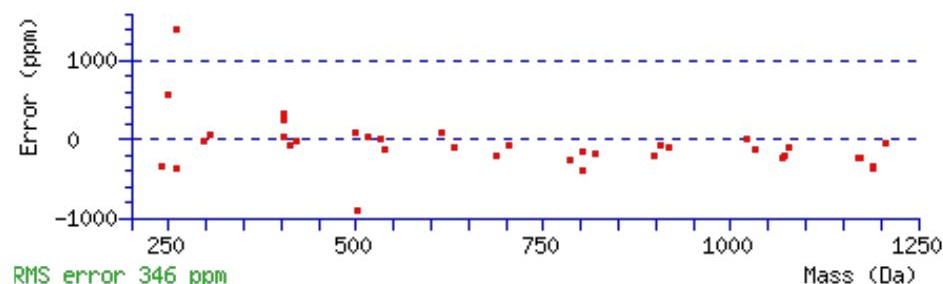

Mass (Da) 


\section{Peptide View}

MS/MS Fragmentation of GDEKENITAEALELSLK

Found in IPI00192302, Tax_Id=10116 Gene_Symbol=Itih3 99 kDa protein

Match to Query 1360: 1859.936768 from(930.975660,2+)

Title: 091008RatKidney_NH4Format01_27.2699.2699.2.dta

Data file K: \NewmanPaper\Piliang \3SubProteomes \Piliang3SP $\backslash$ mgf5ppm\ERLIC_3SubProteomes5ppm.mgf
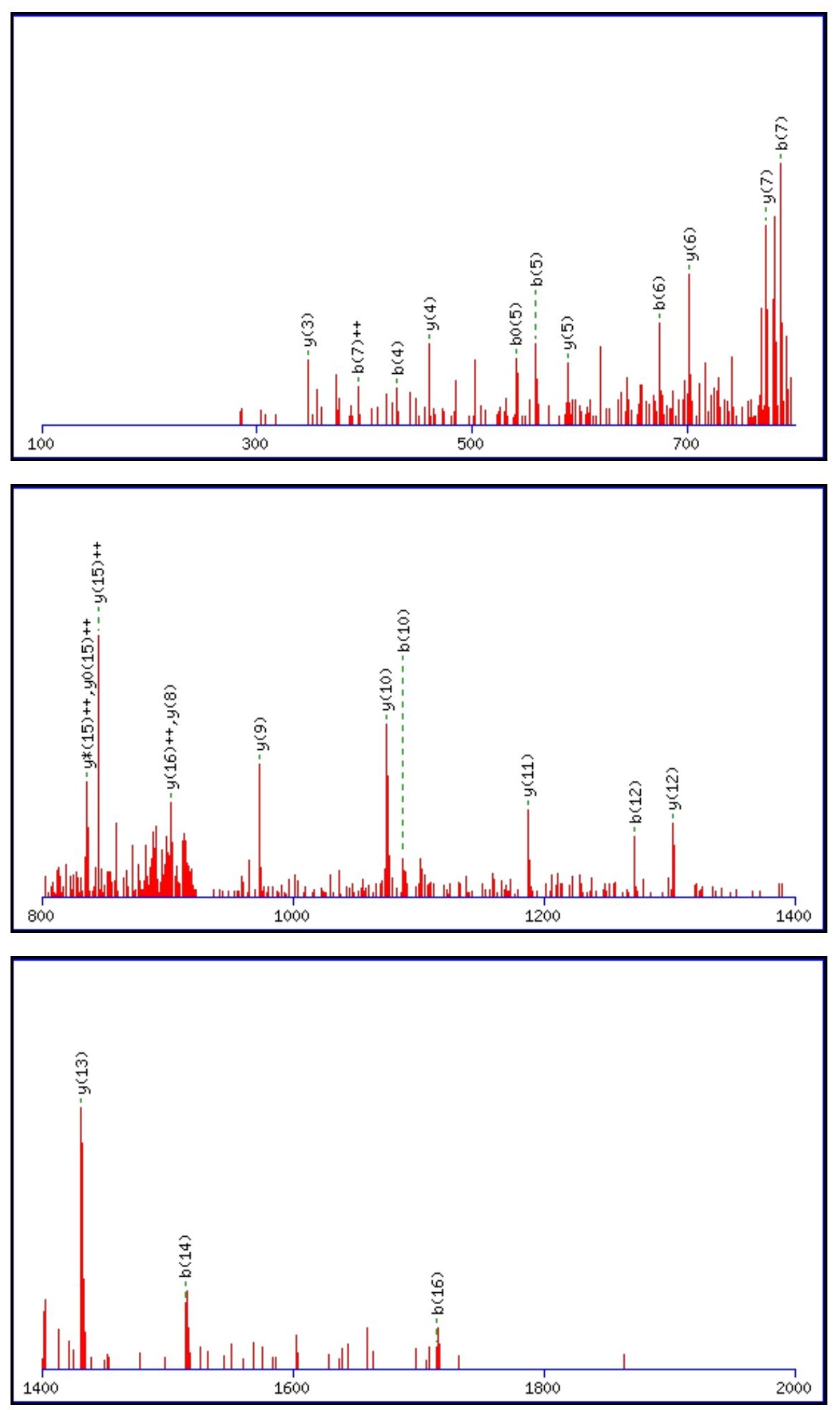

Monoisotopic mass of neutral peptide $\operatorname{Mr}($ calc): 1859.9363

Fixed modifications: Carbamidomethyl (C)

Variable modifications:

N6 : Deamidated_N (N)

Ions Score: 56 Expect: 0.0004 
Matches (Bold Red): 25/180 fragment ions using 49 most intense peaks

\begin{tabular}{|c|c|c|c|c|c|c|c|c|c|c|c|c|c|c|}
\hline \# & b & $\mathbf{b}^{++}$ & $\mathbf{b}^{*}$ & $\mathbf{b}^{*++}$ & $\mathbf{b}^{0}$ & $\mathbf{b}^{0++}$ & Seq. & $\mathbf{y}$ & $y^{++}$ & $\mathbf{y}^{*}$ & $\mathrm{y}^{*++}$ & $\mathbf{y}^{\mathbf{0}}$ & $y^{0++}$ & $\#$ \\
\hline 1 & 58.0287 & 29.5180 & & & & & G & & & & & & & 17 \\
\hline 2 & 173.0557 & 87.0315 & & & 155.0451 & 78.0262 & D & 1803.9222 & 902.4647 & 1786.8957 & 893.9515 & 1785.9116 & 893.4595 & 16 \\
\hline 3 & 302.0983 & 151.5528 & & & 284.0877 & 142.5475 & $\mathbf{E}$ & 1688.8953 & 844.9513 & 1671.8687 & 836.4380 & 1670.8847 & 835.9460 & 15 \\
\hline 4 & 430.1932 & 215.6003 & 413.1667 & 207.0870 & 412.1827 & 206.5950 & $\mathbf{K}$ & 1559.8527 & 780.4300 & 1542.8261 & 771.9167 & 1541.8421 & 771.4247 & 14 \\
\hline 5 & 559.2358 & 280.1216 & 542.2093 & 271.6083 & 541.2253 & 271.1163 & $\mathbf{E}$ & 1431.7577 & 716.3825 & 1414.7312 & 707.8692 & 1413.7471 & 707.3772 & 13 \\
\hline 6 & 674.2628 & 337.6350 & 657.2362 & 329.1217 & 656.2522 & 328.6297 & $\mathbf{N}$ & 1302.7151 & 651.8612 & 1285.6886 & 643.3479 & 1284.7046 & 642.8559 & 12 \\
\hline 7 & 787.3468 & 394.1771 & 770.3203 & 385.6638 & 769.3363 & 385.1718 & I & 1187.6882 & 594.3477 & 1170.6616 & 585.8345 & 1169.6776 & 585.3424 & 11 \\
\hline 8 & 888.3945 & 444.7009 & 871.3680 & 436.1876 & 870.3839 & 435.6956 & $T$ & \begin{tabular}{|l|}
1074.6041 \\
\end{tabular} & 537.8057 & 1057.5776 & 529.2924 & 1056.5936 & 528.8004 & 10 \\
\hline 9 & 959.4316 & 480.2195 & 942.4051 & 471.7062 & 941.4211 & 471.2142 & A & 973.5564 & 487.2819 & 956.5299 & 478.7686 & 955.5459 & 478.2766 & 9 \\
\hline 10 & 1088.4742 & 544.7407 & 1071.4477 & 536.2275 & 1070.4637 & 535.7355 & $\mathbf{E}$ & 902.5193 & 451.7633 & 885.4928 & 443.2500 & 884.5088 & 442.7580 & 8 \\
\hline 11 & 1159.5113 & 580.2593 & 1142.4848 & 571.7460 & 1141.5008 & 571.2540 & $\mathbf{A}$ & 773.4767 & 387.2420 & 756.4502 & 378.7287 & 755.4662 & 378.2367 & 7 \\
\hline 12 & 1272.5954 & 636.8013 & 1255.5688 & 628.2881 & 1254.5848 & 627.7961 & $\mathbf{L}$ & 702.4396 & 351.7234 & 685.4131 & 343.2102 & 684.4291 & 342.7182 & 6 \\
\hline 13 & 1401.6380 & 701.3226 & 1384.6114 & 692.8094 & 1383.6274 & 692.3174 & $\mathbf{E}$ & 589.3556 & 295.1814 & 572.3290 & 286.6681 & 571.3450 & 286.1761 & 5 \\
\hline 14 & 1514.7221 & 757.8647 & 1497.6955 & 749.3514 & 1496.7115 & 748.8594 & $\mathbf{L}$ & 460.3130 & 230.6601 & 443.2864 & 222.1468 & 442.3024 & 221.6548 & 4 \\
\hline 15 & 1601.7541 & 801.3807 & 1584.7275 & 792.8674 & 1583.7435 & 792.3754 & $S$ & 347.2289 & 174.1181 & 330.2023 & 165.6048 & 329.2183 & 165.1128 & 3 \\
\hline 16 & 1714.8381 & 857.9227 & 1697.8116 & 849.4094 & 1696.8276 & 848.9174 & $\mathbf{L}$ & 260.1969 & 130.6021 & 243.1703 & 122.0888 & & & 2 \\
\hline \begin{tabular}{|l|l}
17 & \\
\end{tabular} & & & & & & & $\mathbf{K}$ & 147.1128 & 74.0600 & 130.0863 & 65.5468 & & & 1 \\
\hline
\end{tabular}
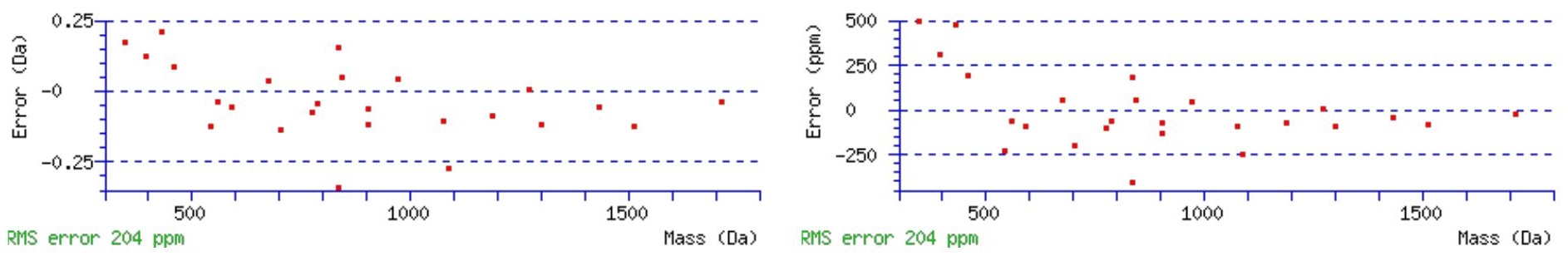

\section{All matches to this query}

\begin{tabular}{|l|l|l|l|}
\hline Score & Mr(calc): & Delta & \multicolumn{1}{|c|}{ Sequence } \\
\hline 56.3 & 1859.9363 & 0.0004 & GDEKENITAEALELSLK \\
\hline 5.5 & 1859.9364 & 0.0004 & EEGIIDSSDKDIVAEIK \\
\hline 2.9 & 1859.9216 & 0.0152 & MVKVTFNSALAQKEAK \\
\hline 2.6 & 1859.9311 & 0.0056 & KQHSLTLAPVLTPPAK \\
\hline
\end{tabular}

Spectrum No: 582; Query: 1562; Rank: 1

\section{Peptide View}

MS/MS Fragmentation of VNASTTDPNSTVEQSALTR

Found in IPI00365542, Tax_Id=10116 Gene_Symbol=Lamb1_predicted laminin, beta 1

Match to Query 1562: 1990.947188 from(996.480870,2+)

Title: 100101RatKid_NS_deglyco_27.1470.1470.2.dta

Data file K:INewmanPaper|Piliangl3SubProteomes\Piliang3SP\mgf5ppm\ERLIC_3SubProteomes5ppm.mgf 

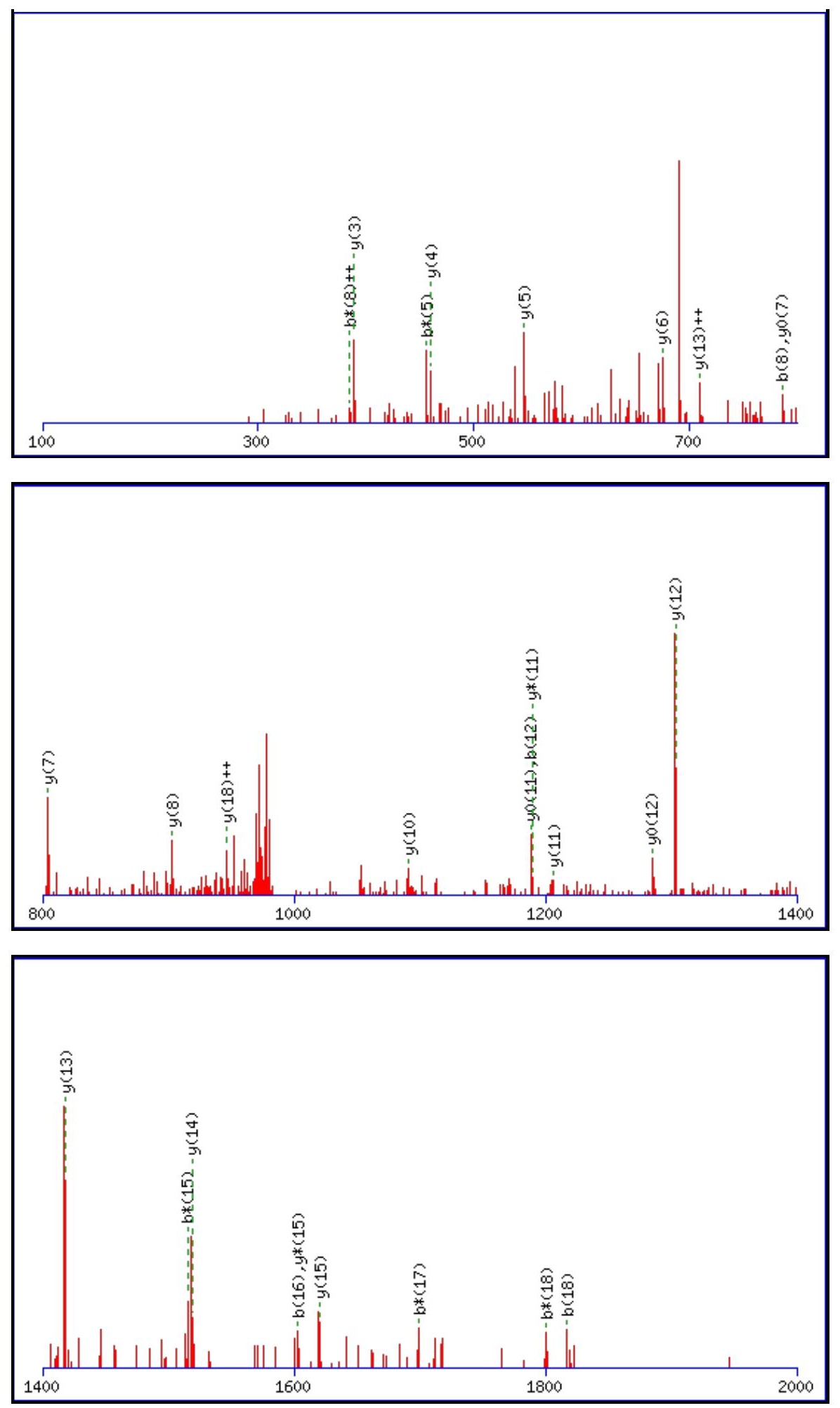

Monoisotopic mass of neutral peptide $\operatorname{Mr}($ calc): 1990.9443

Fixed modifications: Carbamidomethyl (C)

Variable modifications:

N9 : Deamidated $\mathrm{N}(\mathrm{N})$

Ions Score: 56 Expect: 0.00058

Matches (Bold Red): 28/206 fragment ions using 57 most intense peaks

\begin{tabular}{|r|c|c|c|c|c|c|c|c|c|c|c|c|c|c|}
\hline$\#$ & $\mathbf{b}$ & $\mathbf{b}^{++}$ & $\mathbf{b}^{*}$ & $\mathbf{b}^{\boldsymbol{*}^{++}}$ & $\mathbf{b}^{\mathbf{0}}$ & $\mathbf{b}^{\mathbf{0 + +}}$ & $\mathbf{S e q}$ & $\mathbf{y}$ & $\mathbf{y}^{++}$ & $\mathbf{y}^{\mathbf{*}}$ & $\mathbf{y}^{\boldsymbol{*}^{++}}$ & $\mathbf{y}^{\mathbf{0}}$ & $\mathbf{y}^{\mathbf{0 + +}}$ & $\#$ \\
\hline $\mathbf{1}$ & 100.0757 & 50.5415 & & & & & $\mathbf{V}$ & & & & & & & $\mathbf{1 9}$ \\
\hline $\mathbf{2}$ & 214.1186 & 107.5629 & 197.0921 & 99.0497 & & & $\mathbf{N}$ & 1892.8832 & $\mathbf{9 4 6 . 9 4 5 2}$ & 1875.8567 & 938.4320 & 1874.8726 & 937.9400 & $\mathbf{1 8}$ \\
\hline 3 & 285.1557 & 143.0815 & 268.1292 & 134.5682 & & & A & 1778.8403 & 889.9238 & 1761.8137 & 881.4105 & 1760.8297 & 880.9185 & $\mathbf{1 7}$ \\
\hline $\mathbf{4}$ & 372.1878 & 186.5975 & 355.1612 & 178.0842 & 354.1772 & 177.5922 & S & 1707.8032 & 854.4052 & 1690.7766 & 845.8919 & 1689.7926 & 845.3999 & $\mathbf{1 6}$ \\
\hline $\mathbf{5}$ & 473.2354 & 237.1214 & $\mathbf{4 5 6 . 2 0 8 9}$ & 228.6081 & 455.2249 & 228.1161 & $\mathbf{T}$ & $\mathbf{1 6 2 0 . 7 7 1 1}$ & 810.8892 & $\mathbf{1 6 0 3 . 7 4 4 6}$ & 802.3759 & 1602.7606 & 801.8839 & $\mathbf{1 5}$ \\
\hline
\end{tabular}




\begin{tabular}{|c|c|c|c|c|c|c|c|c|c|c|c|c|c|c|}
\hline 6 & 574.2831 & |287.6452 | & 557.2566 & |279.1319 & 556.2726 & |278.6399| & $\mathbf{T}$ & |1519.7235 & 760.3654 & |1502.6969 & |751.8521 & |1501.7129| & |751.3601| & 14 \\
\hline 7 & 689.3101 & 345.1587 & 672.2835 & 336.6454 & 671.2995 & 336.1534 & D & 1418.6758 & 709.8415 & 1401.6492 & 701.3282 & 1400.6652 & 700.8362 & 13 \\
\hline 8 & 786.3628 & 393.6851 & 769.3363 & 385.1718 & 768.3523 & 384.6798 & $\mathbf{P}$ & 1303.6488 & 652.3281 & 1286.6223 & 643.8148 & 1285.6383 & 643.3228 & 2 \\
\hline 9 & 901.3898 & 451.1985 & 884.3632 & 442.6852 & 883.3792 & 442.1932 & $\mathbf{N}$ & 1206.5961 & 603.8017 & 1189.5695 & 595.2884 & 1188.5855 & 594.7964 & 1 \\
\hline 10 & 988.4218 & 494.7145 & 971.3952 & 486.2013 & 970.4112 & 485.7092 & S & 1091.5691 & 546.2882 & 1074.5426 & 537.7749 & 1073.5586 & 537.2829 & 0 \\
\hline 11 & 1089.4695 & 545.2384 & 1072.4429 & 536.7251 & 1071.4589 & 536.2331 & $\mathbf{T}$ & 1004.5371 & 502.7722 & 987.5106 & 494.2589 & 986.5265 & 493.7669 & 9 \\
\hline 12 & 1188.5379 & 594.7726 & 1171.5113 & 586.2593 & 1170.5273 & 585.7673 & V & 903.4894 & 452.2483 & 886.4629 & 443.7351 & 885.4789 & 443.2431 & 8 \\
\hline 13 & 1317.5805 & 659.2939 & 1300.5539 & 650.7806 & 1299.5699 & 650.2886 & $\mathbf{E}$ & 804.4210 & 402.7141 & 787.3945 & 394.2009 & 786.4104 & 393.7089 & 7 \\
\hline 14 & 1445.6391 & 723.3232 & 1428.6125 & 714.8099 & 1427.6285 & 714.3179 & $\mathbf{Q}$ & 675.3784 & 338.1928 & 658.3519 & 329.6796 & 657.3679 & 329.1876 & 6 \\
\hline 15 & 1532.6711 & 766.8392 & 1515.6445 & 758.3259 & 1514.6605 & 757.8339 & S & 547.3198 & 274.1636 & 530.2933 & 265.6503 & 529.3093 & 265.1583 & 5 \\
\hline 16 & 1603.7082 & 802.3577 & 1586.6816 & 793.8445 & 1585.6976 & 793.3525 & A & 460.2878 & 230.6475 & 443.2613 & 222.1343 & 442.2772 & 221.6423 & 4 \\
\hline 17 & 1716.7923 & 858.8998 & 1699.7657 & 850.3865 & 1698.7817 & 849.8945 & $\mathbf{L}$ & 389.2507 & 195.1290 & 372.2241 & 186.6157 & 371.2401 & 186.1237 & 3 \\
\hline 18 & 1817.8399 & 909.4236 & 1800.8134 & 900.9103 & 1799.8294 & 900.4183 & $\mathbf{T}$ & 276.1666 & 138.5870 & 259.1401 & 130.0737 & 258.1561 & 129.5817 & 2 \\
\hline 19 & & & & & & & $\mathbf{R}$ & 175.1190 & 88.0631 & 158.0924 & 79.5498 & & & 1 \\
\hline
\end{tabular}
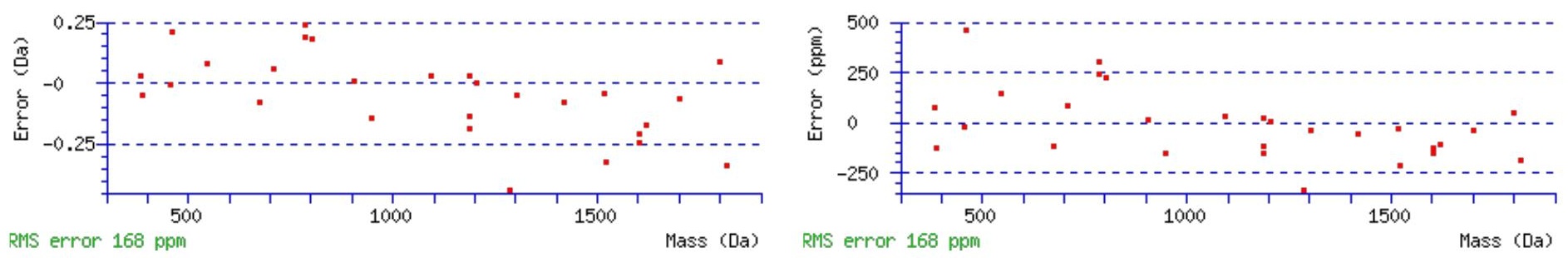

\section{All matches to this query}

\begin{tabular}{|l|c|c|l|}
\hline Score & Mr(calc): & Delta & \multicolumn{1}{|c|}{ Sequence } \\
\hline 56.2 & 1990.9443 & 0.0029 & VNASTTDPNSTVEQSALTR \\
\hline 52.1 & 1990.9443 & 0.0029 & VNASTTDPNSTVEQSALTR \\
\hline 37.5 & 1989.9603 & 0.9869 & VNASTTDPNSTVEQSALTR \\
\hline 2.6 & 1990.9408 & 0.0064 & $\underline{\text { LLCESSNRALSLQHAGR }}$ \\
\hline 0.1 & 1990.9625 & -0.0154 & DQFANLHILRRSSEPK \\
\hline
\end{tabular}

Spectrum No: 583; Query: 1717; Rank: 1

\section{Peptide View}

MS/MS Fragmentation of VMELEKEIANATTKPEDR

Found in IPI00231789, Tax_Id=10116 Gene_Symbol=Mme Neprilysin

Match to Query 1717: 2090.019372 from(697.680400,3+)

Title: 091008RatKidney_NH4Format01_24.1993.1993.3.dta

Data file K:INewmanPaper\Piliang $\mid 3 S$ ubProteomes\Piliang3SP\mgf5ppm\ERLIC_3SubProteomes5ppm.mgf 

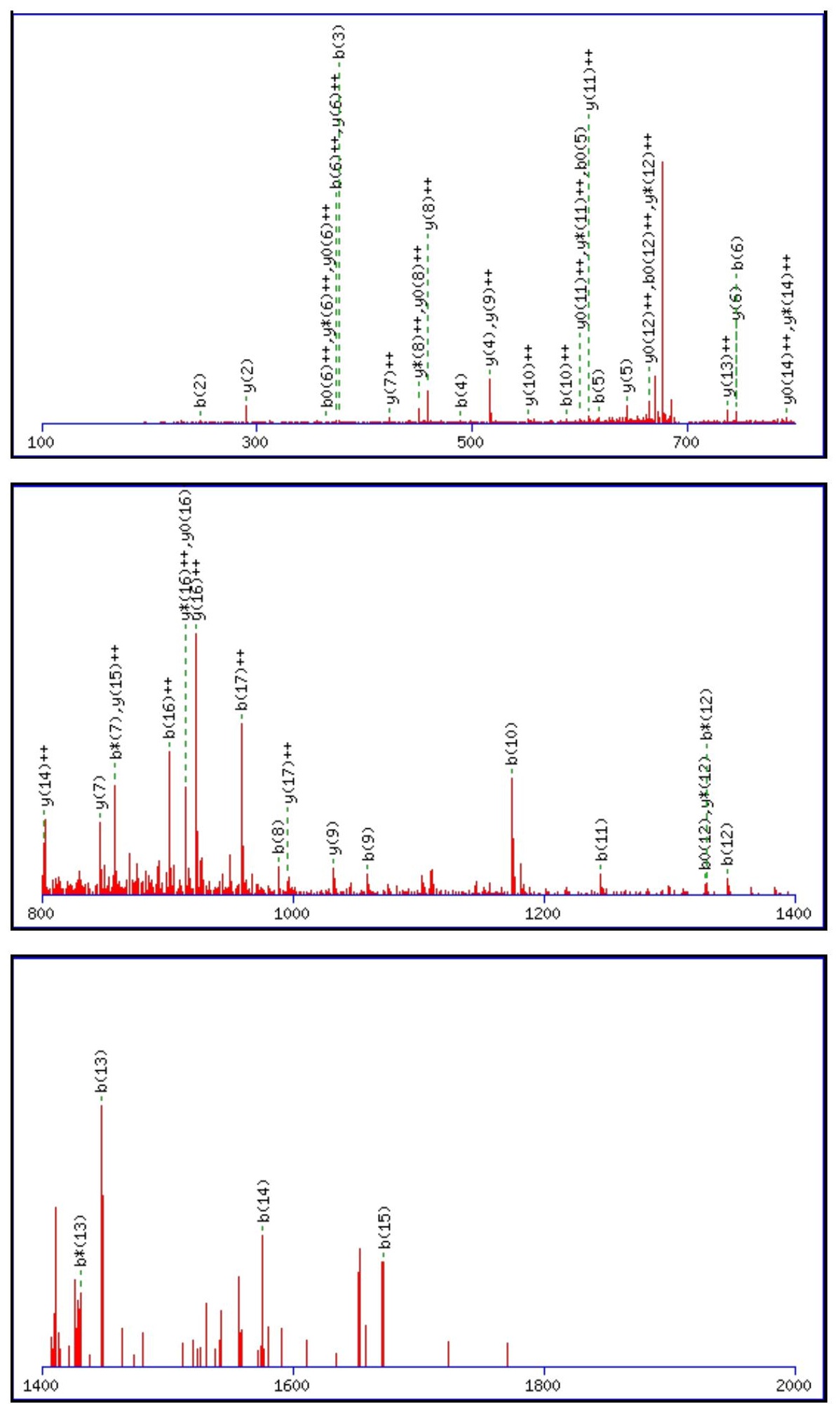

Monoisotopic mass of neutral peptide $\operatorname{Mr}($ calc): 2090.0201

Fixed modifications: Carbamidomethyl (C)

Variable modifications:

M2 : Oxidation $(\mathrm{M})$

N10 : Deamidated_N (N)

Ions Score: 56 Expect: 0.0005

Matches (Bold Red): 54/188 fragment ions using 94 most intense peaks

\begin{tabular}{|c|c|c|c|c|c|c|c|c|c|c|c|c|c|c|}
\hline$\#$ & $\mathbf{b}$ & $\mathbf{b}^{++}$ & $\mathbf{b}^{*}$ & $\mathbf{b}^{\boldsymbol{*}_{+}^{++}}$ & $\mathbf{b}^{\mathbf{0}}$ & $\mathbf{b}^{\mathbf{0}+}$ & $\mathbf{S e q}$ & $\mathbf{y}$ & $\mathbf{y}^{++}$ & $\mathbf{y}^{\mathbf{*}}$ & $\mathbf{y}^{\mathbf{*}^{++}}$ & $\mathbf{y}^{\mathbf{0}}$ & $\mathbf{y}^{\mathbf{0}+}$ & $\#$ \\
\hline $\mathbf{1}$ & 100.0757 & 50.5415 & & & & & $\mathbf{V}$ & & & & & & & $\mathbf{1 8}$ \\
\hline $\mathbf{2}$ & $\mathbf{2 4 7 . 1 1 1 1}$ & 124.0592 & & & & & $\mathbf{M}$ & 1991.9590 & $\mathbf{9 9 6 . 4 8 3 1}$ & 1974.9325 & 987.9699 & 1973.9484 & 987.4779 & $\mathbf{1 7}$ \\
\hline $\mathbf{3}$ & 376.1537 & 188.5805 & & & 358.1431 & 179.5752 & $\mathbf{E}$ & 1844.9236 & $\mathbf{9 2 2 . 9 6 5 4}$ & 1827.8971 & $\mathbf{9 1 4 . 4 5 2 2}$ & 1826.9130 & $\mathbf{9 1 3 . 9 6 0 2}$ & $\mathbf{1 6}$ \\
\hline $\mathbf{4}$ & $\mathbf{4 8 9 . 2 3 7 8}$ & 245.1225 & & & 471.2272 & 236.1172 & $\mathbf{L}$ & 1715.8810 & $\mathbf{8 5 8 . 4 4 4 1}$ & 1698.8545 & 849.9309 & 1697.8704 & 849.4389 & $\mathbf{1 5}$ \\
\hline & & & & & & & & & & & & & &
\end{tabular}




\begin{tabular}{|c|c|c|c|c|c|c|c|c|c|c|c|c|c|c|}
\hline & 618.2803 & & & & & & E & & & 1585 & & & & 14 \\
\hline 6 & 746.3753 & 373.6913 & 729.3488 & 365.1780 & 728.3647 & 364.6860 & $\mathbf{K}$ & 1473.7544 & 737.3808 & 1456.7278 & 728.8675 & 1455.7438 & 728.3755 & 13 \\
\hline 7 & 875.4179 & 438.2126 & 858.3914 & 429.6993 & 857.4073 & 429.2073 & $\mathbf{E}$ & 1345.6594 & 673.3333 & 1328.6328 & 664.8201 & 1327.6488 & 664.3281 & 12 \\
\hline 8 & 988.5020 & 494.7546 & 971.4754 & 486.2413 & 970.4914 & 485.7493 & I & 1216.6168 & 608.8120 & 1199.5903 & 600.2988 & 1198.6062 & 599.8068 & 11 \\
\hline 9 & 59.5391 & 530.2732 & 1042.5125 & 521.7599 & 1041.5285 & 521.2679 & A & 1103.5327 & 552.2700 & 1086.5062 & 543.7567 & 1085.5222 & 543.2647 & 10 \\
\hline 10 & |74.5660 & 587.7866 & 157.5395 & 579.2734 & 1156.5554 & 578.7814 & $\mathbf{N}$ & 1032.4956 & 516.7514 & 1015.4691 & 508.2382 & 1014.4851 & 507.7462 & 9 \\
\hline 11 & 1245.6031 & 623.3052 & 1228.5766 & 614.7919 & 1227.5926 & 614.2999 & A & 917.4687 & 459.2380 & 900.4421 & 450.7247 & 899.4581 & 450.2327 & 8 \\
\hline 12 & 1346.6508 & 673.8290 & 1329.6243 & 665.3158 & 1328.6402 & 664.8238 & $\mathbf{T}$ & 846.4316 & 423.7194 & 829.4050 & 415.2062 & 828.4210 & 414.7141 & 7 \\
\hline 13 & 447.6985 & 724.3529 & 1430.6719 & 715.8396 & 1429.6879 & 715.3476 & $\mathbf{T}$ & 745.3839 & 373.1956 & 728.3573 & 364.6823 & 727.3733 & 364.1903 & 6 \\
\hline 14 & 1575.7934 & 788.4004 & 1558.7669 & 779.8871 & 1557.7829 & 779.3951 & $\mathbf{K}$ & 644.3362 & 322.6717 & 627.3097 & 314.1585 & 626.3257 & 313.6665 & 5 \\
\hline 15 & 1672.8462 & 836.9267 & 1655.8197 & 828.4135 & 1654.8356 & 827.9215 & $\mathbf{P}$ & 516.2413 & 258.6243 & 499.2147 & 250.1110 & 498.2307 & 249.6190 & 4 \\
\hline 16 & 1801.8888 & 901.4480 & 1784.8623 & 892.9348 & 1783.8782 & 892.4428 & E & 419.1885 & 210.0979 & 402.1619 & 201.5846 & 401.1779 & 201.0926 & 3 \\
\hline 17 & 1916.9157 & 958.9615 & 1899.8892 & 950.4482 & 1898.9052 & 949.9562 & D & 290.1459 & 145.5766 & 273.1193 & 137.0633 & 272.1353 & 136.5713 & 2 \\
\hline 18 & & & & & & & $\mathbf{R}$ & 175.1190 & 88.0631 & 158.0924 & 79.5498 & & & 1 \\
\hline
\end{tabular}
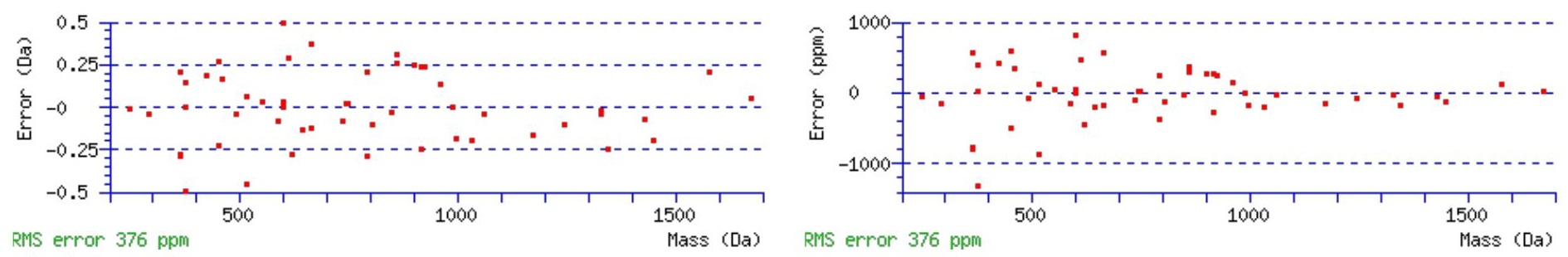

\section{All matches to this query}

\begin{tabular}{|l|c|c|l|}
\hline Score & Mr(calc): & Delta & \multicolumn{1}{c|}{ Sequence } \\
\hline 56.2 & 2090.0201 & -0.0007 & VMELEKEIANATTKPEDR \\
\hline 21.3 & 2089.0361 & 0.9833 & VMELEKEIANATTKPEDR \\
\hline 10.5 & 2089.0092 & 1.0101 & LHQDEKAVLVNNITTGEK \\
\hline 10.5 & 2089.0092 & 1.0101 & LHQDEKAVLVNNITTGEK \\
\hline 7.7 & 2088.0182 & 2.0012 & MIEDTMTLLSLLGRIMR \\
\hline 6.3 & 2088.0252 & 1.9941 & LHQDEKAVLVNNITTGEK \\
\hline 6.3 & 2088.0252 & 1.9941 & LHQDEKAVLVNNITTGEK \\
\hline 3.0 & 2087.9929 & 2.0265 & SPYYQELVGQSQLQLQK \\
\hline 3.0 & 2089.0352 & 0.9842 & ISLYKRMIDLMEQLEK \\
\hline 2.8 & 2088.0088 & 2.0106 & ECRKTFIQIGHLNQHK \\
\hline
\end{tabular}

Spectrum No: 584; Query: 1187; Rank: 1

\section{Peptide View}

MS/MS Fragmentation of KDDALLETTDGFNTTK

Found in IPI00212546, Tax_Id=10116 Gene_Symbol=Emb Embigin precursor

Match to Query 1187: 1768.838532 from(590.620120,3+)

Title: 100101RatKid_NS_deglyco_26.2263.2263.3.dta

Data file K:INewmanPaper|Piliang 3 SUbProteomes\Piliang3SP\mgf5ppm\ERLIC_3SubProteomes5ppm.mgf 

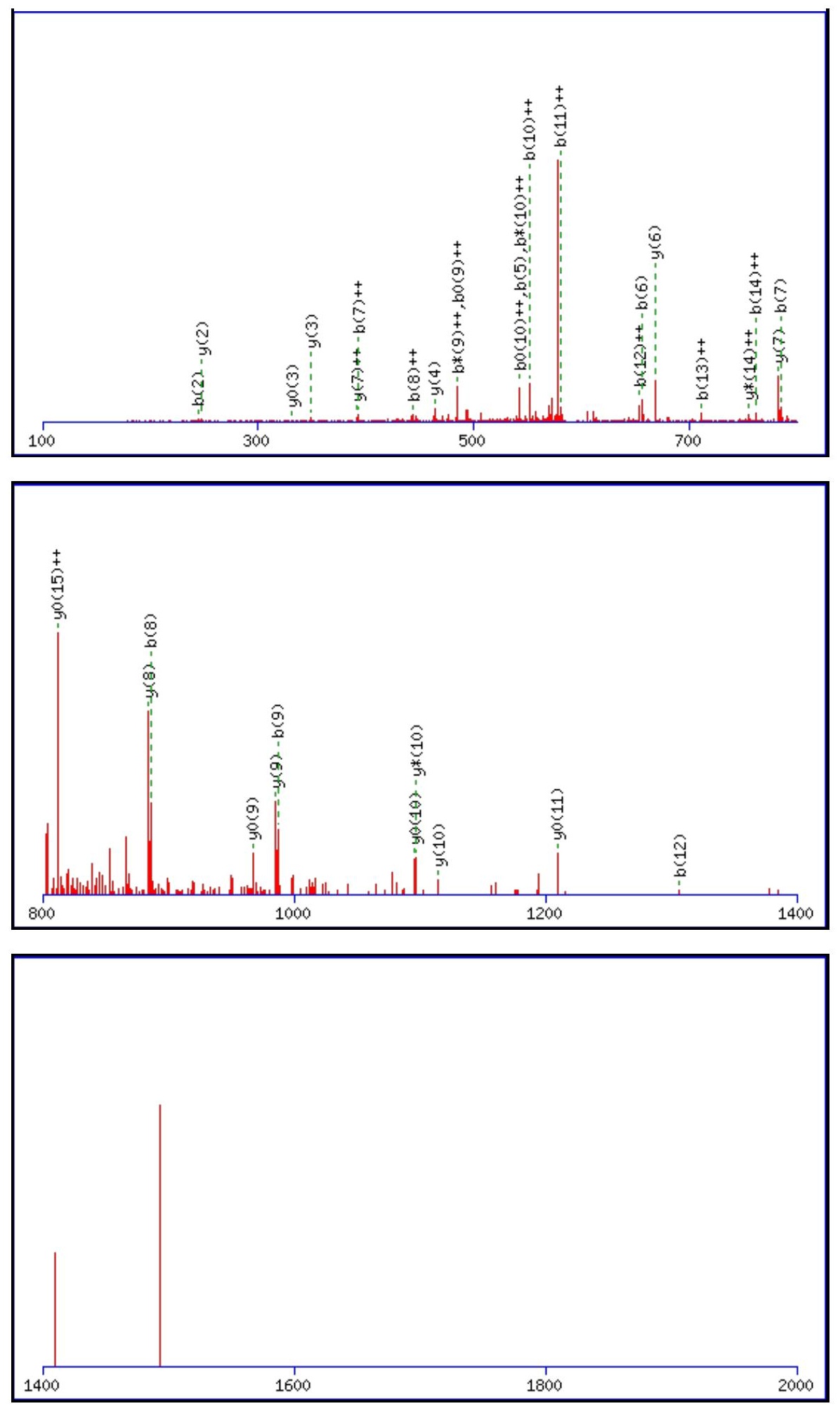

Monoisotopic mass of neutral peptide $\operatorname{Mr}($ calc): 1768.8367

Fixed modifications: Carbamidomethyl (C)

Variable modifications:

N13 : Deamidated_N (N)

Ions Score: 56 Expect: 0.00054

Matches (Bold Red): 35/176 fragment ions using 55 most intense peaks

\begin{tabular}{|r|c|c|c|c|c|c|c|c|c|c|c|c|c|c|}
\hline$\#$ & $\mathbf{b}$ & $\mathbf{b}^{++}$ & $\mathbf{b}^{*}$ & $\mathbf{b}^{\boldsymbol{*}_{++}}$ & $\mathbf{b}^{\mathbf{0}}$ & $\mathbf{b}^{\mathbf{0 + +}}$ & Seq. & $\mathbf{y}$ & $\mathbf{y}^{++}$ & $\mathbf{y}^{\mathbf{*}}$ & $\mathbf{y}^{\boldsymbol{*}^{++}}$ & $\mathbf{y}^{\mathbf{0}}$ & $\mathbf{y}^{\mathbf{0 + +}}$ & $\#$ \\
\hline $\mathbf{1}$ & 129.1022 & 65.0548 & 112.0757 & 56.5415 & & & $\mathbf{K}$ & & & & & & & $\mathbf{1 6}$ \\
\hline $\mathbf{2}$ & $\mathbf{2 4 4 . 1 2 9 2}$ & 122.5682 & 227.1026 & 114.0550 & 226.1186 & 113.5629 & $\mathbf{D}$ & 1641.7490 & 821.3781 & 1624.7224 & 812.8649 & 1623.7384 & $\mathbf{8 1 2 . 3 7 2 9}$ & $\mathbf{1 5}$ \\
\hline $\mathbf{3}$ & 359.1561 & 180.0817 & 342.1296 & 171.5684 & 341.1456 & 171.0764 & $\mathbf{D}$ & 1526.7221 & 763.8647 & 1509.6955 & 755.3514 & 1508.7115 & 754.8594 & $\mathbf{1 4}$ \\
\hline $\mathbf{4}$ & 430.1932 & 215.6003 & 413.1667 & 207.0870 & 412.1827 & 206.5950 & $\mathbf{A}$ & 1411.6951 & 706.3512 & 1394.6686 & 697.8379 & 1393.6845 & 697.3459 & $\mathbf{1 3}$ \\
\hline $\mathbf{5}$ & $\mathbf{5 4 3 . 2 7 7 3}$ & 272.1423 & 526.2508 & 263.6290 & 525.2667 & 263.1370 & $\mathbf{L}$ & 1340.6580 & 670.8326 & 1323.6314 & 662.3194 & 1322.6474 & 661.8274 & $\mathbf{1 2}$ \\
\hline
\end{tabular}




\begin{tabular}{|c|c|c|c|c|c|c|c|c|c|c|c|c|c|c|}
\hline 6 & 656.3614 & 328.6843 & 639.3348 & |320.1710 & 638.3508 & 19.6790 & & & & & & & & 11 \\
\hline 7 & 785.4040 & 393.2056 & 768.3774 & 384.6923 & 767.3934 & 384.2003 & $\mathbf{E}$ & 1114.4899 & 557.7486 & 1097.4633 & 549.2353 & 1096.4793 & 548.7433 & 10 \\
\hline 8 & 886.4516 & 443.7295 & 869.4251 & 435.2162 & 868.4411 & 434.7242 & $\mathbf{T}$ & 985.4473 & 493.2273 & 968.4207 & 484.7140 & 967.4367 & 484.2220 & 9 \\
\hline 9 & 987.4993 & 494.2533 & 970.4728 & 485.7400 & 969.4888 & 485.2480 & $\mathbf{T}$ & 884.3996 & 442.7034 & 867.3730 & 902 & 890 & 433. & 8 \\
\hline 10 & 102.5263 & 551.7668 & 1085.4997 & 543.2535 & 1084.5157 & 542.7615 & D & 783.3519 & 392.1796 & 3254 & 383.6663 & 414 & 383. & 7 \\
\hline 11 & 1159.5477 & 580.2775 & 1142.5212 & 571.7642 & 1141.5372 & 571.2722 & G & 668.3250 & 334.6661 & 651.2984 & 326.1529 & 650.3144 & 325.6608 & 6 \\
\hline 12 & 306.6161 & 653.8117 & 1289.5896 & 645.2984 & 1288.6056 & 644.8064 & $\mathbf{F}$ & 611.3035 & 306.1554 & 594.2770 & 297.6421 & 3.2929 & 297.1501 & 5 \\
\hline 13 & 1421.6431 & 711.3252 & 1404.6165 & 702.8119 & 1403.6325 & 702.3199 & $\mathbf{N}$ & 464.2351 & 232.6212 & 447.2085 & 224.1079 & 446.2245 & 223.6159 & 4 \\
\hline 14 & 1522.6908 & 761.8490 & 1505.6642 & 753.3357 & 1504.6802 & 752.8437 & $\mathbf{T}$ & 349.2082 & 175.1077 & 332.1816 & 166.5944 & 331.1976 & 166.1024 & 3 \\
\hline 15 & 1623.7384 & 812.3729 & 1606.7119 & 803.8596 & 1605.7279 & 803.3676 & $\mathbf{T}$ & 248.1605 & 124.5839 & 231.1339 & 116.0706 & 230.1499 & 115.5786 & 2 \\
\hline 16 & & & & & & & I & 147.1128 & 74.0600 & 130.0863 & 65.5468 & & & 1 \\
\hline
\end{tabular}
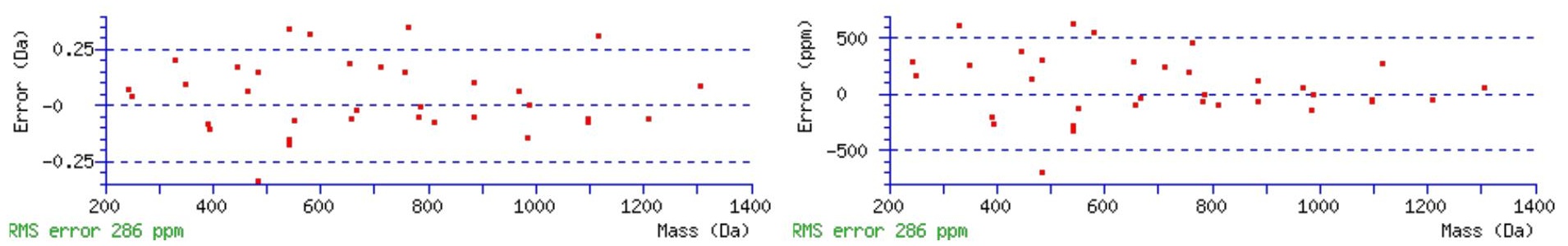

\section{All matches to this query}

\begin{tabular}{|l|l|l|l|}
\hline Score & Mr(calc): & Delta & \multicolumn{1}{|c|}{ Sequence } \\
\hline 56.1 & 1768.8367 & 0.0018 & KDDALLETTDGFNTTK \\
\hline 32.9 & 1767.8527 & 0.9859 & KDDALLETTDGFNTTK \\
\hline 8.7 & 1766.8274 & 2.0112 & LTPATKMNNKADGTPK \\
\hline 8.7 & 1766.8274 & 2.0112 & LTPATKMNNKADGTPK \\
\hline 6.5 & 1767.8478 & 0.9907 & CQTLPVSTPTAATLTK \\
\hline 4.3 & 1767.8477 & 0.9908 & NKCLQLLGNLGSLEK \\
\hline 3.9 & 1767.8403 & 0.9982 & RLETLLRNIDNSDK \\
\hline 3.5 & 1767.8378 & 1.0007 & MKNFLHNLRESLGK \\
\hline 3.4 & 1767.8516 & 0.9869 & IRRIGSGLEQNNTTK \\
\hline 3.0 & 1766.8492 & 1.9894 & IWAPTAEASTELTGLK \\
\hline
\end{tabular}

Spectrum No: 585; Query: 1297; Rank: 1

\section{Peptide View}

MS/MS Fragmentation of KVGVDEVSPEKNSSKPK

Found in IPI00231423, Tax_Id=10116 Gene_Symbol=C9 C9 protein

Match to Query 1297: 1827.962142 from(610.327990,3+)

Title: 100101RatKid_NS_deglyco_23.311.311.3.dta

Data file K:INewmanPaper|Piliangl3SubProteomes\Piliang3SP\mgf5ppm\ERLIC_3SubProteomes5ppm.mgf 

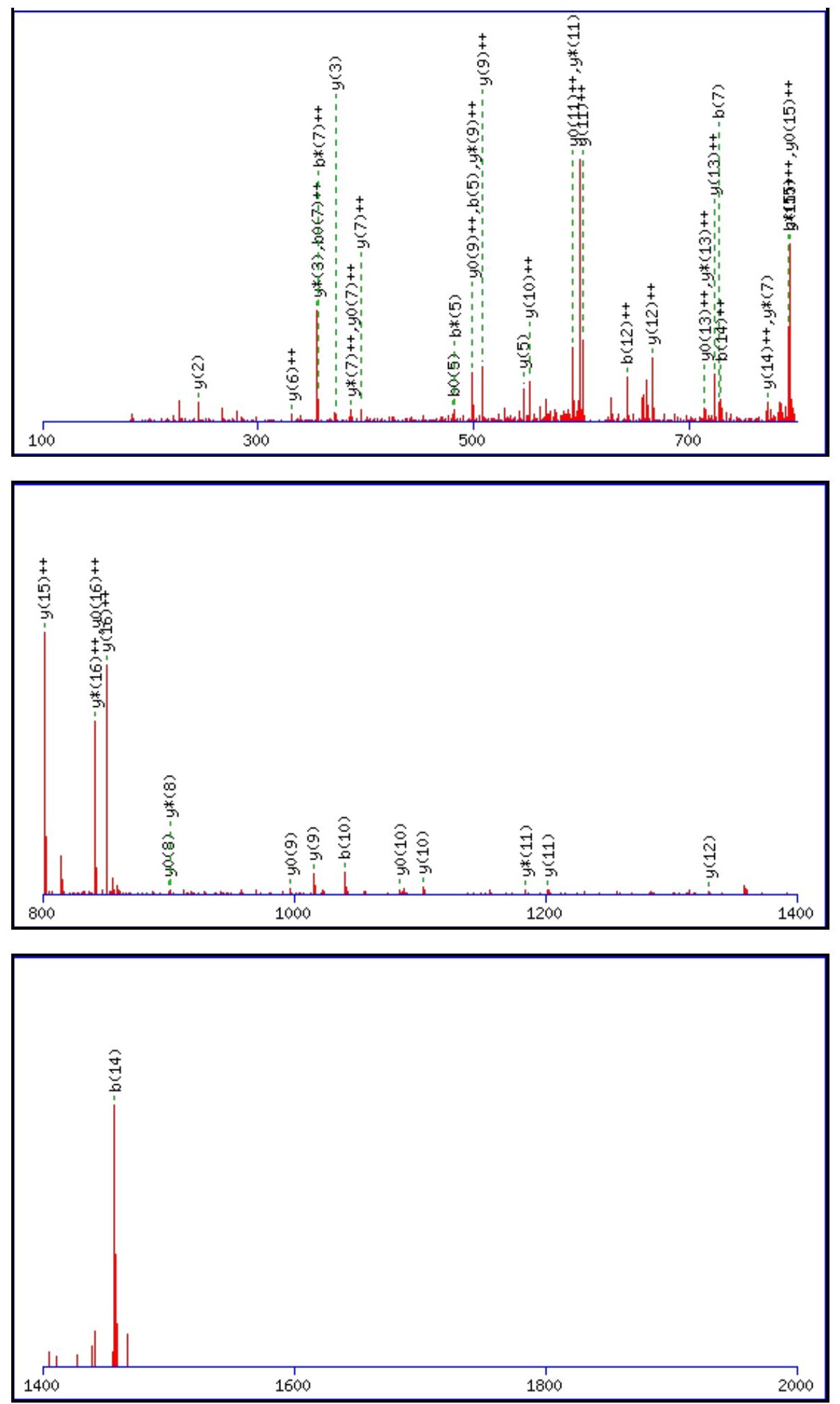

Monoisotopic mass of neutral peptide $\operatorname{Mr}($ calc): 1827.9578

Fixed modifications: Carbamidomethyl (C)

Variable modifications:

N12 : Deamidated_N (N)

Ions Score: 56 Expect: 0.00028

Matches (Bold Red): 48/178 fragment ions using 74 most intense peaks

\begin{tabular}{|r|c|c|c|c|c|c|c|c|c|c|c|c|c|c|}
\hline$\#$ & $\mathbf{b}$ & $\mathbf{b}^{++}$ & $\mathbf{b}^{*}$ & $\mathbf{b}^{\boldsymbol{*}_{++}}$ & $\mathbf{b}^{\mathbf{0}}$ & $\mathbf{b}^{\mathbf{0 + +}}$ & Seq. & $\mathbf{y}$ & $\mathbf{y}^{++}$ & $\mathbf{y}^{\mathbf{*}}$ & $\mathbf{y}^{\boldsymbol{*}^{++}}$ & $\mathbf{y}^{\mathbf{0}}$ & $\mathbf{y}^{\mathbf{0 + +}}$ & $\#$ \\
\hline $\mathbf{1}$ & 129.1022 & 65.0548 & 112.0757 & 56.5415 & & & $\mathbf{K}$ & & & & & & & $\mathbf{1 7}$ \\
\hline $\mathbf{2}$ & 228.1707 & 114.5890 & 211.1441 & 106.0757 & & & $\mathbf{V}$ & 1700.8701 & $\mathbf{8 5 0 . 9 3 8 7}$ & 1683.8436 & $\mathbf{8 4 2 . 4 2 5 4}$ & 1682.8595 & $\mathbf{8 4 1 . 9 3 3 4}$ & $\mathbf{1 6}$ \\
\hline $\mathbf{3}$ & 285.1921 & 143.0997 & 268.1656 & 134.5864 & & & $\mathbf{G}$ & 1601.8017 & $\mathbf{8 0 1 . 4 0 4 5}$ & 1584.7752 & $\mathbf{7 9 2 . 8 9 1 2}$ & 1583.7911 & $\mathbf{7 9 2 . 3 9 9 2}$ & $\mathbf{1 5}$ \\
\hline $\mathbf{4}$ & 384.2605 & 192.6339 & 367.2340 & 184.1206 & & & $\mathbf{V}$ & 1544.7802 & 772.8938 & 1527.7537 & 764.3805 & 1526.7697 & 763.8885 & $\mathbf{1 4}$ \\
\hline $\mathbf{5}$ & $\mathbf{4 9 9 . 2 8 7 5}$ & 250.1474 & $\mathbf{4 8 2 . 2 6 0 9}$ & 241.6341 & $\mathbf{4 8 1 . 2 7 6 9}$ & 241.1421 & $\mathbf{D}$ & 1445.7118 & 723.3595 & 1428.6853 & 714.8463 & 1427.7013 & $\mathbf{7 1 4 . 3 5 4 3}$ & $\mathbf{1 3}$ \\
\hline
\end{tabular}




\begin{tabular}{|c|c|c|c|c|c|c|c|c|c|c|c|c|c|c|}
\hline 6 & 628.3301 & |314.6687 & 611.3035 & 306.1554 & 610.3195 & |305.6634 & $\mathbf{E}$ & $|1330.6849|$ & 665.8461 & |1313.6583 & & 1312.6743 & & 12 \\
\hline 7 & 727.3985 & |364.2029 & 710.3719 & 355.6896 & 709.3879 & |355.1976 & V & 1201.6423 & 601.3248 & 1184.6157 & 592.8115 & 1183.6317 & 592.3195 & 11 \\
\hline 8 & 814.4305 & 407.7189 & 797.4040 & 399.2056 & 796.4199 & |398.7136 & S & 1102.5739 & 551.7906 & 1085.5473 & 543.2773 & 1084.5633 & 542.7853 & 10 \\
\hline 9 & 911.4833 & 456.2453 & 894.4567 & 447.7320 & 893.4727 & 447.2400 & $\mathbf{P}$ & 1015.5418 & 508.2746 & 998.5153 & 499.7613 & 997.5313 & 499.2693 & 9 \\
\hline 10 & 1040.5259 & 520.7666 & 1023.4993 & 512.2533 & 1022.5153 & 511.7613 & E & 918.4891 & 459.7482 & 901.4625 & 451.2349 & 900.4785 & 450.7429 & 8 \\
\hline 11 & 1168.6208 & 584.8141 & 1151.5943 & 576.3008 & 1150.6103 & 575.8088 & $\mathbf{K}$ & 789.4465 & 395.2269 & 772.4199 & 386.7136 & 771.4359 & 386.2216 & 7 \\
\hline 12 & 1283.6478 & 642.3275 & 1266.6212 & 633.8142 & 1265.6372 & 633.3222 & $\mathbf{N}$ & 661.3515 & 331.1794 & 644.3250 & 322.6661 & 643.3410 & 322.1741 & 6 \\
\hline 13 & 1370.6798 & 685.8435 & 1353.6532 & 677.3303 & 1352.6692 & 676.8383 & S & 546.3246 & 273.6659 & 529.2980 & 265.1527 & 528.3140 & 264.6606 & 5 \\
\hline 14 & 1457.7118 & 729.3595 & 1440.6853 & 720.8463 & 1439.7013 & 720.3543 & $S$ & 459.2926 & 230.1499 & 442.2660 & 221.6366 & 441.2820 & 221.1446 & 4 \\
\hline 15 & 1585.8068 & 793.4070 & 1568.7802 & 784.8938 & 1567.7962 & 784.4017 & $\mathbf{K}$ & 372.2605 & 186.6339 & 355.2340 & 178.1206 & & & 3 \\
\hline 16 & 1682.8595 & 841.9334 & 1665.8330 & 833.4201 & 1664.8490 & 832.9281 & $\mathbf{P}$ & 244.1656 & 122.5864 & 227.1390 & 114.0731 & & & 2 \\
\hline 17 & & & & & & & $\mathbf{K}$ & 147.1128 & 74.0600 & 130.0863 & 65.5468 & & & 1 \\
\hline
\end{tabular}
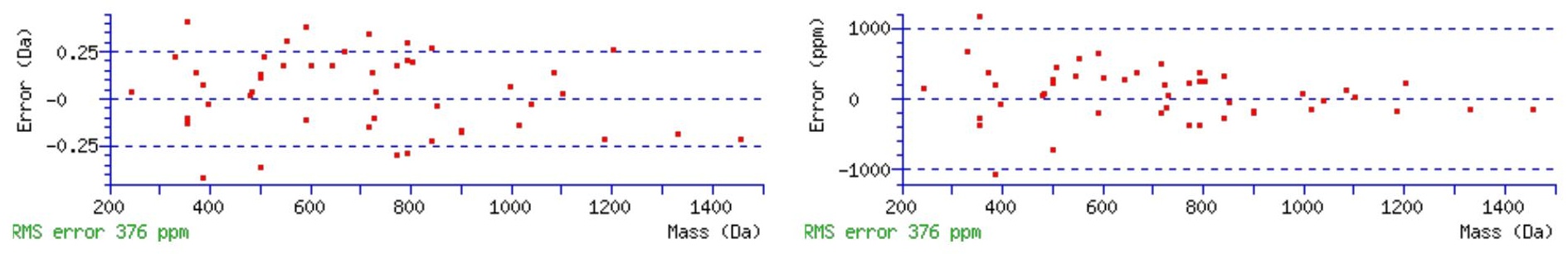

\section{All matches to this query}

\begin{tabular}{|l|l|l|l|}
\hline Score & Mr(calc): & Delta & \multicolumn{1}{c|}{ Sequence } \\
\hline 56.1 & 1827.9578 & 0.0044 & KVGVDEVSPEKNSSKPK \\
\hline 13.3 & 1827.9730 & -0.0109 & QELQEVVEFLKNPQK \\
\hline 13.1 & 1826.9738 & 0.9884 & KVGVDEVSPEKNSSKPK \\
\hline 6.8 & 1827.9744 & -0.0123 & GGLRWGVSPTLPQSFAR \\
\hline 3.5 & 1825.9448 & 2.0173 & RPNSGLWQRKGGWAGR \\
\hline 3.4 & 1826.9543 & 1.0078 & VATKGAVTPKPVPEPEK \\
\hline 1.0 & 1827.9802 & -0.0181 & KPKTGSSNQTQRSLAPK \\
\hline 0.6 & 1825.9608 & 2.0014 & QPMDLSSVISKIDLHK \\
\hline
\end{tabular}

Spectrum No: 586; Query: 1813; Rank: 1

\section{Peptide View}

MS/MS Fragmentation of VFNTSEDLSRVNATVQETK

Found in IPI00363534, Tax_Id=10116 Gene_Symbol=Lama1 laminin, alpha 1

Match to Query 1813: 2139.037962 from(714.019930,3+)

Title: 100101RatKid_NS_deglyco_23.3055.3055.3.dta

Data file K:INewmanPaper|Piliangl3SubProteomes\Piliang3SP\mgf5ppm\ERLIC_3SubProteomes5ppm.mgf 

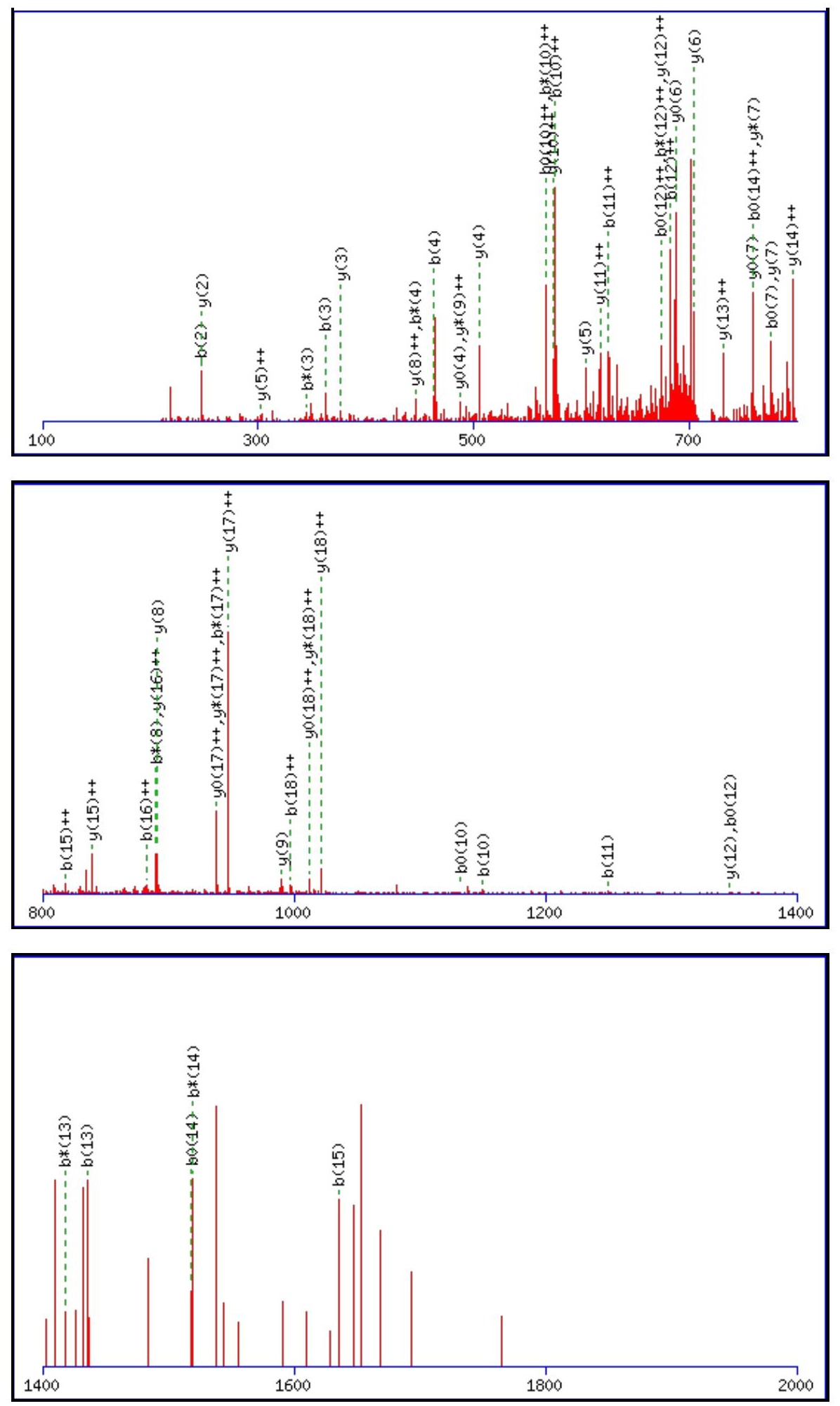

Monoisotopic mass of neutral peptide $\operatorname{Mr}($ calc): 2139.0331

Fixed modifications: Carbamidomethyl (C)

Variable modifications:

N3 : Deamidated_N (N)

N12 : Deamidated_N (N)

Ions Score: 56 Expect: 0.00061

Matches (Bold Red): 57/204 fragment ions using 97 most intense peaks

\begin{tabular}{|c|c|c|c|c|c|c|c|c|c|c|c|c|c|c|}
\hline$\#$ & $\mathbf{b}$ & $\mathbf{b}^{++}$ & $\mathbf{b}^{*}$ & $\mathbf{b}^{*_{++}}$ & $\mathbf{b}^{\mathbf{0}}$ & $\mathbf{b}^{\mathbf{0}+}$ & Seq. & $\mathbf{y}$ & $\mathbf{y}^{++}$ & $\mathbf{y}^{*}$ & $\mathbf{y}^{\mathbf{*}^{++}}$ & $\mathbf{y}^{\mathbf{0}}$ & $\mathbf{y}^{\mathbf{0}+}$ & $\#$ \\
\hline $\mathbf{1}$ & 100.0757 & 50.5415 & & & & & $\mathbf{V}$ & & & & & & & $\mathbf{1 9}$ \\
\hline $\mathbf{2}$ & $\mathbf{2 4 7 . 1 4 4 1}$ & 124.0757 & & & & & $\mathbf{F}$ & 2040.9720 & $\mathbf{1 0 2 0 . 9 8 9 6}$ & 2023.9455 & $\mathbf{1 0 1 2 . 4 7 6 4}$ & 2022.9614 & $\mathbf{1 0 1 1 . 9 8 4 4}$ & $\mathbf{1 8}$ \\
\hline $\mathbf{3}$ & $\mathbf{3 6 2 . 1 7 1 0}$ & 181.5892 & 345.1445 & 173.0759 & & & $\mathbf{N}$ & 1893.9036 & $\mathbf{9 4 7 . 4 5 5 4}$ & 1876.8770 & $\mathbf{9 3 8 . 9 4 2 2}$ & 1875.8930 & $\mathbf{9 3 8 . 4 5 0 2}$ & $\mathbf{1 7}$ \\
\hline $\mathbf{4}$ & $\mathbf{4 6 3 . 2 1 8 7}$ & 232.1130 & 446.1922 & 223.5997 & 445.2082 & 223.1077 & $\mathbf{T}$ & 1778.8767 & $\mathbf{8 8 9 . 9 4 2 0}$ & 1761.8501 & 881.4287 & 1760.8661 & 880.9367 & $\mathbf{1 6}$ \\
\hline & & & & & & & & & & & & & &
\end{tabular}




\begin{tabular}{|c|c|c|c|c|c|c|c|c|c|c|c|c|c|c|}
\hline & 550.2507 & |275.6290 & 242 & 267. & 532.2402 & |266.6237 & S & 1677. & 839.4181 & 166 & & & 830.4128 & 15 \\
\hline 6 & 679.2933 & 340.1503 & 662.2668 & 331.6370 & 661.2828 & 331.1450 & $\mathbf{E}$ & 1590.7969 & 795.9021 & 1573.7704 & 787.3888 & 1572.7864 & 786.8968 & 14 \\
\hline 7 & 794.3203 & 397.6638 & 777.2937 & 389.1505 & 776.3097 & 388.6585 & D & 1461.7544 & 731.3808 & 1444.7278 & 722.8675 & 1443.7438 & 722.3755 & 13 \\
\hline 8 & 907.4043 & 454.2058 & 890.3778 & 445.6925 & 889.3938 & 445.2005 & $\mathbf{L}$ & 1346.7274 & 673.8673 & 1329.7009 & 665.3541 & 1328.7168 & 664.8621 & 12 \\
\hline 9 & 994.4364 & 497.7218 & 977.4098 & 489.2086 & 976.4258 & |488.7165 & $S$ & 1233.6434 & 617.3253 & 1216.6168 & 608.8120 & 1215.6328 & 608.3200 & 11 \\
\hline 10 & 1150.5375 & 575.7724 & 1133.5109 & 567.2591 & 1132.5269 & 566.7671 & $\mathbf{R}$ & 1146.6113 & 573.8093 & 1129.5848 & 565.2960 & 1128.6008 & 564.8040 & 10 \\
\hline 11 & 1249.6059 & 625.3066 & 1232.5794 & 616.7933 & 1231.5953 & 616.3013 & $\mathbf{V}$ & 990.5102 & 495.7587 & 973.4837 & 487.2455 & 972.4996 & 486.7535 & 9 \\
\hline 12 & 328 & 682.8 & 1347 & 674. & 1346 & 673.8148 & $\mathbf{N}$ & & & & 437.7113 & 312 & 2193 & 8 \\
\hline 13 & 1435.6700 & 718.3386 & 1418.6434 & 709.8253 & 1417.6594 & 709.3333 & A & 776.4149 & 388.7111 & 759.3883 & 380.1978 & 758.4043 & 379.7058 & 7 \\
\hline 14 & 1536.7176 & 768.8625 & 1519.6911 & 760.3492 & 1518.7071 & 759.8572 & $\mathbf{T}$ & 705.3777 & 353.1925 & 688.3512 & 344.6792 & 687.3672 & 344.1872 & 6 \\
\hline 15 & 1635.7860 & 818.3967 & 1618.7595 & 809.8834 & 1617.7755 & 809.3914 & $\mathrm{~V}$ & 604.3301 & 302.6687 & 587.3035 & 294.1554 & 586.3195 & 293.6634 & 5 \\
\hline 16 & 1763.8446 & 882.4259 & 1746.8181 & 873.9127 & 1745.8341 & 873.4207 & $\mathbf{Q}$ & 505.2617 & 253.1345 & 488.2351 & 244.6212 & 487.2511 & 244.1292 & 4 \\
\hline 17 & 1892.8872 & 946.9472 & 1875.8607 & 938.4340 & 1874.8766 & 937.9420 & $\mathbf{E}$ & 377.2031 & 189.1052 & 360.1765 & 180.5919 & 359.1925 & 180.0999 & 3 \\
\hline 18 & 1993.9349 & 997.4711 & 1976.9083 & 988.9578 & 1975.9243 & 988.4658 & $\mathbf{T}$ & 248.1605 & 124.5839 & 231.1339 & 116.0706 & 230.1499 & 115.5786 & 2 \\
\hline 19 & & & & & & & $\mathbf{K}$ & 147.1128 & 74.0600 & 130.0863 & 65.5468 & & & 1 \\
\hline
\end{tabular}
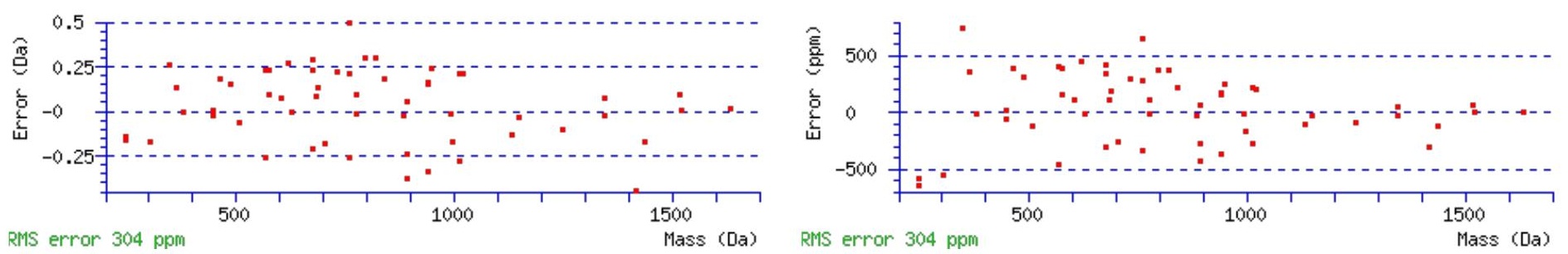

\section{All matches to this query}

\begin{tabular}{|l|c|c|l|}
\hline Score & Mr(calc): & Delta & \multicolumn{1}{|c|}{ Sequence } \\
\hline 56.0 & 2139.0331 & 0.0048 & VFNTSEDLSRVNATVQETK \\
\hline 26.0 & 2138.0491 & 0.9888 & VFNTSEDLSRVNATVQETK \\
\hline 16.3 & 2138.0491 & 0.9888 & VFNTSEDLSRVNATVQETK \\
\hline 5.8 & 2137.0296 & 2.0084 & NMSVQCIPSNTLLNLFNR \\
\hline 3.6 & 2139.0469 & -0.0089 & MVNEPRSGMLVLVIQAASK \\
\hline 2.6 & 2138.0136 & 1.0244 & NMSVQCIPSNTLLNLFNR \\
\hline 2.2 & 2138.0136 & 1.0244 & NMSVQCIPSNTLLNLFNR \\
\hline 2.0 & 2138.0136 & 1.0244 & NMSVQCIPSNTLLNLFNR \\
\hline 1.6 & 2138.0442 & 0.9937 & GQSRSLSVKMLLSTSTGYK \\
\hline 1.6 & 2139.0469 & -0.0089 & $\underline{\text { MVNEPRSGMLVLVIQAASK }}$ \\
\hline
\end{tabular}

Spectrum No: 587; Query: 410; Rank: 1

\section{Peptide View}

MS/MS Fragmentation of EIANATTKPEDR

Found in IPI00231789, Tax_Id=10116 Gene_Symbol=Mme Neprilysin

Match to Query 410: 1344.651628 from(673.333090,2+)

Title: 091008RatKidney_NH4Format01_26.226.226.2.dta

Data file K:INewmanPaper\Piliang $\mid 3 S$ SubProteomes\Piliang3SP\mgf5ppm\ERLIC_3SubProteomes5ppm.mgf 

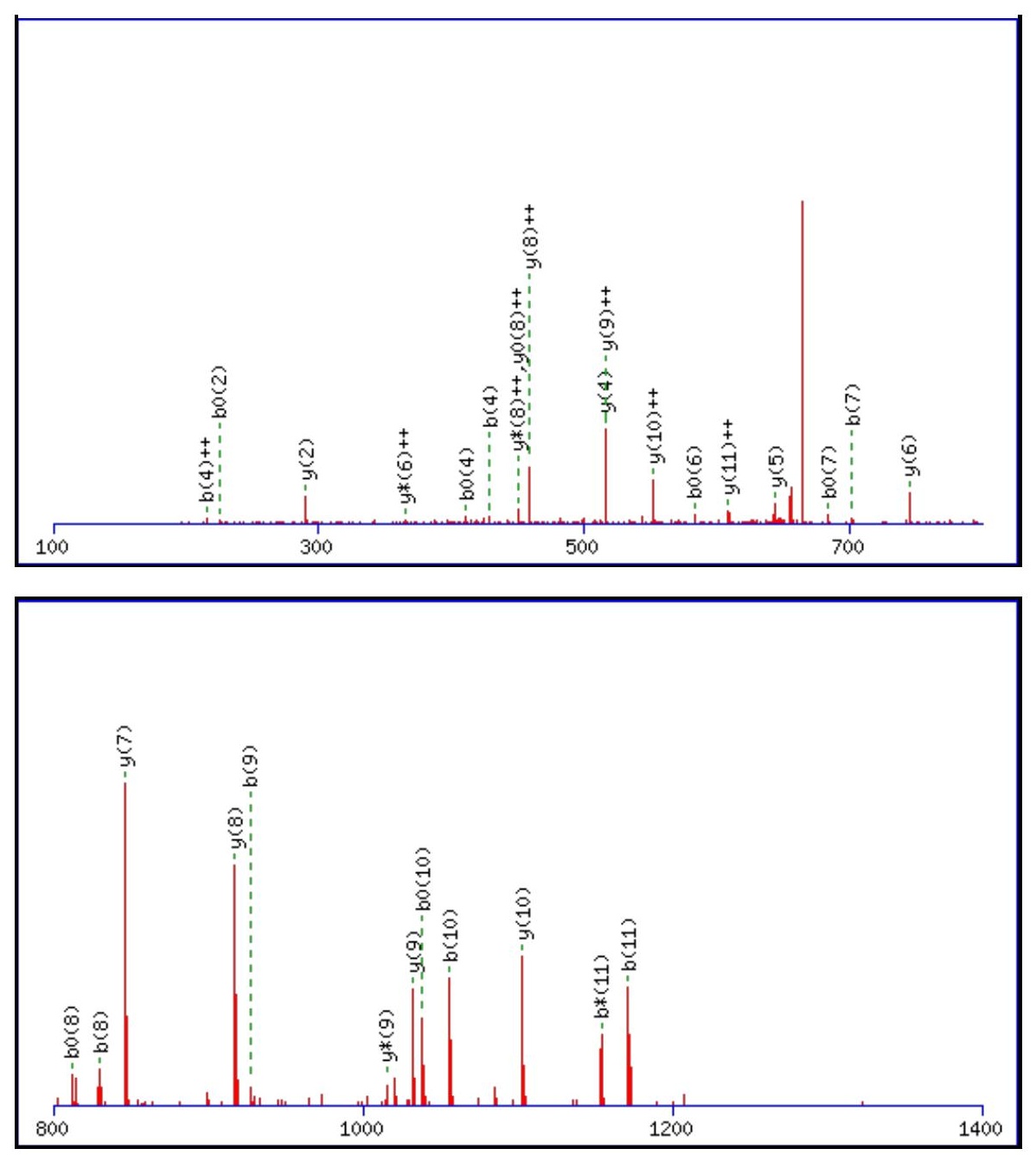

\begin{tabular}{rrrr}
\hline 00 & 1600 & 1800 & 2000 \\
\hline
\end{tabular}

Monoisotopic mass of neutral peptide $\operatorname{Mr}($ calc): 1344.6521

Fixed modifications: Carbamidomethyl (C)

Variable modifications:

N4 : Deamidated_N (N)

Ions Score: 56 Expect: 0.00038

Matches (Bold Red): 30/124 fragment ions using 52 most intense peaks

\begin{tabular}{|r|c|c|c|c|c|c|c|c|c|c|c|c|c|c|}
\hline$\#$ & $\mathbf{b}$ & $\mathbf{b}^{++}$ & $\mathbf{b}^{*}$ & $\mathbf{b}^{\boldsymbol{*}^{++}}$ & $\mathbf{b}^{\mathbf{0}}$ & $\mathbf{b}^{\mathbf{0 + +}}$ & Seq. & $\mathbf{y}$ & $\mathbf{y}^{++}$ & $\mathbf{y}^{\mathbf{*}}$ & $\mathbf{y}^{\boldsymbol{*}^{++}}$ & $\mathbf{y}^{\mathbf{0}}$ & $\mathbf{y}^{\mathbf{0 + +}}$ & $\#$ \\
\hline $\mathbf{1}$ & 130.0499 & 65.5286 & & & 112.0393 & 56.5233 & $\mathbf{E}$ & & & & & & & $\mathbf{1 2}$ \\
\hline $\mathbf{2}$ & 243.1339 & 122.0706 & & & 225.1234 & 113.0653 & $\mathbf{I}$ & 1216.6168 & $\mathbf{6 0 8 . 8 1 2 0}$ & 1199.5903 & 600.2988 & 1198.6062 & 599.8068 & $\mathbf{1 1}$ \\
\hline 3 & 314.1710 & 157.5892 & & & 296.1605 & 148.5839 & $\mathbf{A}$ & $\mathbf{1 1 0 3 . 5 3 2 7}$ & 552.2700 & 1086.5062 & 543.7567 & 1085.5222 & 543.2647 & $\mathbf{1 0}$ \\
\hline $\mathbf{4}$ & $\mathbf{4 2 9 . 1 9 8 0}$ & $\mathbf{2 1 5 . 1 0 2 6}$ & 412.1714 & 206.5894 & $\mathbf{4 1 1 . 1 8 7 4}$ & 206.0973 & $\mathbf{N}$ & $\mathbf{1 0 3 2 . 4 9 5 6}$ & $\mathbf{5 1 6 . 7 5 1 4}$ & $\mathbf{1 0 1 5 . 4 6 9 1}$ & 508.2382 & 1014.4851 & 507.7462 & $\mathbf{9}$ \\
\hline $\mathbf{5}$ & 500.2351 & 250.6212 & 483.2085 & 242.1079 & 482.2245 & 241.6159 & $\mathbf{A}$ & $\mathbf{9 1 7 . 4 6 8 7}$ & $\mathbf{4 5 9 . 2 3 8 0}$ & 900.4421 & $\mathbf{4 5 0 . 7 2 4 7}$ & 899.4581 & $\mathbf{4 5 0 . 2 3 2 7}$ & $\mathbf{8}$ \\
\hline
\end{tabular}




\begin{tabular}{|r|r|r|r|r|r|r|r|r|r|r|r|r|r|r|r|r|}
$\mathbf{6}$ & 601.2828 & 301.1450 & 584.2562 & 292.6318 & $\mathbf{5 8 3 . 2 7 2 2}$ & 292.1397 & $\mathbf{T}$ & $\mathbf{8 4 6 . 4 3 1 6}$ & 423.7194 & 829.4050 & 415.2062 & 828.4210 & 414.7141 & $\mathbf{7}$ \\
\hline $\mathbf{7}$ & $\mathbf{7 0 2 . 3 3 0 5}$ & 351.6689 & 685.3039 & 343.1556 & $\mathbf{6 8 4 . 3 1 9 9}$ & 342.6636 & $\mathbf{T}$ & $\mathbf{7 4 5 . 3 8 3 9}$ & 373.1956 & $\mathbf{7 2 8 . 3 5 7 3}$ & $\mathbf{3 6 4 . 6 8 2 3}$ & $\mathbf{7 2 7 . 3 7 3 3}$ & 364.1903 & $\mathbf{6}$ \\
\hline $\mathbf{8}$ & $\mathbf{8 3 0 . 4 2 5 4}$ & 415.7163 & 813.3989 & 407.2031 & $\mathbf{8 1 2 . 4 1 4 9}$ & 406.7111 & $\mathbf{K}$ & $\mathbf{6 4 4 . 3 3 6 2}$ & 322.6717 & 627.3097 & 314.1585 & 626.3257 & 313.6665 & $\mathbf{5}$ \\
\hline $\mathbf{9}$ & $\mathbf{9 2 7 . 4 7 8 2}$ & 464.2427 & 910.4516 & 455.7295 & 909.4676 & 455.2374 & $\mathbf{P}$ & $\mathbf{5 1 6 . 2 4 1 3}$ & 258.6243 & 499.2147 & 250.1110 & 498.2307 & 249.6190 & $\mathbf{4}$ \\
\hline $\mathbf{1 0}$ & $\mathbf{1 0 5 6 . 5 2 0 8}$ & 528.7640 & 1039.4942 & 520.2508 & $\mathbf{1 0 3 8 . 5 1 0 2}$ & 519.7587 & $\mathbf{E}$ & 419.1885 & 210.0979 & 402.1619 & 201.5846 & 401.1779 & 201.0926 & $\mathbf{3}$ \\
\hline $\mathbf{1 1}$ & $\mathbf{1 1 7 1 . 5 4 7 7}$ & 586.2775 & $\mathbf{1 1 5 4 . 5 2 1 2}$ & 577.7642 & 1153.5372 & 577.2722 & $\mathbf{D}$ & $\mathbf{2 9 0 . 1 4 5 9}$ & 145.5766 & 273.1193 & 137.0633 & 272.1353 & 136.5713 & $\mathbf{2}$ \\
\hline $\mathbf{1 2}$ & & & & & & & $\mathbf{R}$ & 175.1190 & 88.0631 & 158.0924 & 79.5498 & & & $\mathbf{1}$ \\
\hline
\end{tabular}
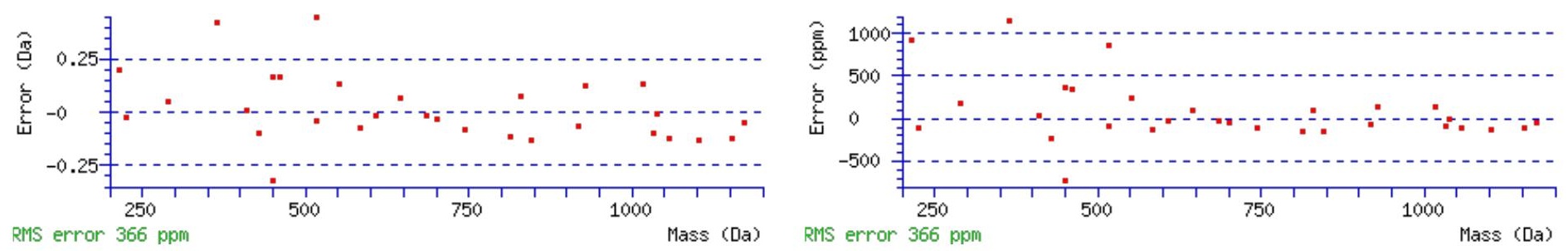

\section{All matches to this query}

\begin{tabular}{|l|l|l|l|}
\hline Score & Mr(calc): & Delta & \multicolumn{1}{|c|}{ Sequence } \\
\hline 56.0 & 1344.6521 & -0.0005 & EIANATTKPEDR \\
\hline 10.4 & 1344.6384 & 0.0132 & TLVMAVYDFDR \\
\hline 8.8 & 1343.6469 & 1.0047 & LQWEEEAAVNR \\
\hline 8.2 & 1344.6609 & -0.0092 & EFIRSTPPPDR \\
\hline 7.9 & 1344.6534 & -0.0018 & APGTQGXAGRPNR \\
\hline 7.1 & 1344.6633 & -0.0117 & ELAELRESQDR \\
\hline 7.0 & 1344.6417 & 0.0099 & LTSLMISMYDR \\
\hline 6.0 & 1344.6551 & -0.0035 & GLHLLQSGAVDR \\
\hline 5.9 & 1344.6422 & 0.0094 & YQHTATPSSPTR \\
\hline 5.1 & 1344.6521 & -0.0005 & LVINSGNGAVEDR \\
\hline
\end{tabular}

Spectrum No: 588; Query: 2782; Rank: 1

\section{Peptide View}

MS/MS Fragmentation of NSFGLAPAAPLQVHAPLSPNQTVEISLPLNTVGSVMK

Found in IPI00201713, Tax_Id=10116 Gene_Symbol=Ap1b1 Isoform B of AP-1 complex subunit beta-1

Match to Query 2782: 3798.009192 from(1267.010340,3+)

Title: 091008RatKidney_NoSalt_03.5384.5384.3.dta

Data file K:INewmanPaper\Piliang\3SubProteomes\Piliang3SP\mgf5ppm\ERLIC_3SubProteomes5ppm.mgf 

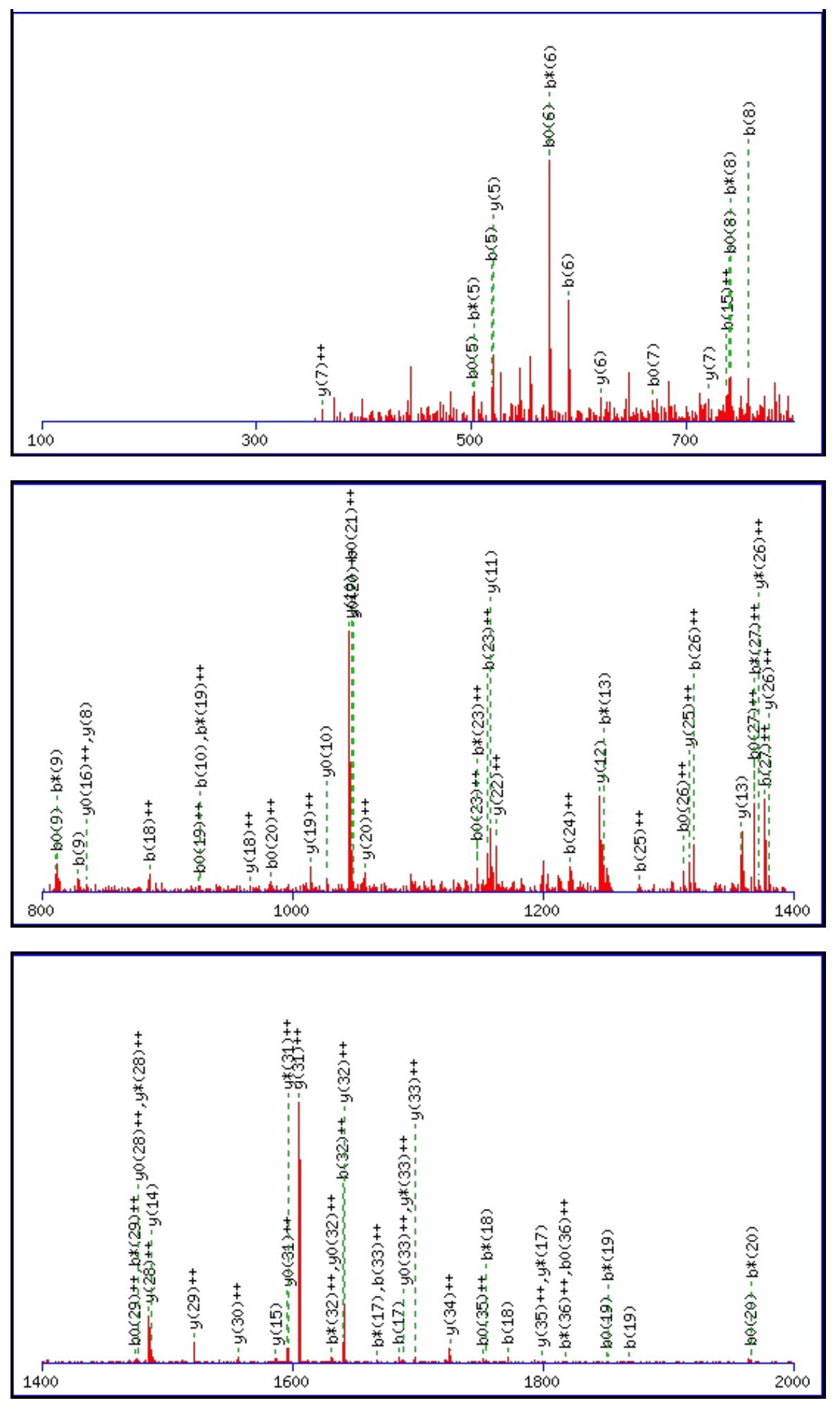

Monoisotopic mass of neutral peptide $\operatorname{Mr}($ calc): 3798.0026

Fixed modifications: Carbamidomethyl (C)

Variable modifications:

N20 : Deamidated_N (N)

Ions Score: 56 Expect: 0.00019

Matches (Bold Red): 85/424 fragment ions using 179 most intense peaks

\begin{tabular}{|c|c|c|c|c|c|c|c|c|c|c|c|c|c|c|}
\hline \# & b & $\mathbf{b}^{++}$ & $\mathbf{b}^{*}$ & $\mathbf{b}^{*^{++}}$ & $\mathbf{b}^{0}$ & $\mathbf{b}^{0++}$ & Seq. & $\mathbf{y}$ & $y^{++}$ & $\mathrm{y}^{*}$ & $\mathrm{y}^{*^{++}}$ & $\mathbf{y}^{0}$ & $y^{0++}$ & $\#$ \\
\hline 1 & 115.0502 & 58.0287 & 98.0237 & 49.5155 & & & $\mathbf{N}$ & & & & & & & 37 \\
\hline 2 & 202.0822 & 101.5448 & 185.0557 & 93.0315 & 184.0717 & 92.5395 & $S$ & 3684.9669 & 1842.9871 & 3667.9404 & 1834.4738 & 3666.9564 & 1833.9818 & 36 \\
\hline 3 & 349.1506 & 175.0790 & 332.1241 & 166.5657 & 331.1401 & 166.0737 & $\mathbf{F}$ & 3597.9349 & 1799.4711 & 3580.9084 & 1790.9578 & 3579.9243 & 1790.4658 & 35 \\
\hline 4 & 406.1721 & 203.5897 & 389.1456 & 195.0764 & 388.1615 & 194.5844 & G & 3450.8665 & 1725.9369 & 3433.8399 & 1717.4236 & 3432.8559 & 1716.9316 & 34 \\
\hline 5 & 519.2562 & 260.1317 & 502.2296 & 251.6185 & 501.2456 & 251.1264 & $\mathbf{L}$ & 3393.8450 & 1697.4262 & 3376.8185 & 1688.9129 & 3375.8345 & 1688.4209 & 33 \\
\hline
\end{tabular}




\begin{tabular}{|c|c|c|c|c|c|c|c|c|c|c|c|c|c|c|}
\hline & 590.2933 & 295.6503 & 573.2667 & 287.1370 & 572.2827 & 286.6450 & A & & |1640.8841 & & & |3262.7504| & 1631.8788 & 32 \\
\hline 7 & 687.3461 & 344.1767 & 670.3195 & 335.6634 & 669.3355 & 335.1714 & $\mathbf{P}$ & 3209.7239 & 1605.3656 & 3192.6973 & 1596.8523 & 3191.7133 & 1596.3603 & 31 \\
\hline 8 & 758.3832 & 379.6952 & 741.3566 & 371.1819 & 740.3726 & 370.6899 & A & 3112.6711 & 1556.8392 & 3095.6445 & 1548.3259 & 3094.6605 & 1547.8339 & 30 \\
\hline 9 & 829.4203 & 415.2138 & 812.3937 & 406.7005 & 811.4097 & 406.2085 & A & 3041.6340 & 1521.3206 & 3024.6074 & 1512.8074 & 3023.6234 & 1512.3153 & 29 \\
\hline 10 & 926.4730 & 463.7402 & 909.4465 & 455.2269 & 908.4625 & 454.7349 & $\mathbf{P}$ & 2970.5969 & 1485.8021 & 2953.5703 & 1477.2888 & 2952.5863 & 1476.7968 & 28 \\
\hline 111 & 1039.5571 & 520.2822 & 1022.5306 & 511.7689 & 1021.5465 & 511.2769 & $\mathbf{L}$ & 2873.5441 & 1437.2757 & 2856.5176 & 1428.7624 & 2855.5335 & 1428.2704 & 27 \\
\hline 121 & 1167.6157 & 584.3115 & 1150.5891 & 575.7982 & 1149.6051 & 575.3062 & $\mathbf{Q}$ & 2760.4600 & 1380.7337 & 2743.4335 & 1372.2204 & 2742.4495 & 1371.7284 & 26 \\
\hline 13 & 1266.6841 & 633.8457 & 1249.6576 & 625.3324 & 1248.6735 & 624.8404 & $\mathbf{V}$ & 2632.4015 & 1316.7044 & 2615.3749 & 1308.1911 & 2614.3909 & 1307.6991 & 25 \\
\hline 14 & 1403.7430 & 702.3751 & 1386.7165 & 693.8619 & 1385.7324 & 693.3699 & $\mathbf{H}$ & 2533.3330 & 1267.1702 & 2516.3065 & 1258.6569 & 2515.3225 & 1258.1649 & 24 \\
\hline 151 & 1474.7801 & 737.8937 & 1457.7536 & 729.3804 & 1456.7696 & 728.8884 & A & 2396.2741 & 1198.6407 & 2379.2476 & 1190.1274 & 2378.2636 & 1189.6354 & 23 \\
\hline 16 & 1571.8329 & 786.4201 & 1554.8063 & 777.9068 & 1553.8223 & 777.4148 & $\mathbf{P}$ & 2325.2370 & 1163.1221 & 2308.2105 & 1154.6089 & 2307.2265 & 1154.1169 & 22 \\
\hline 171 & 1684.9170 & 842.9621 & \begin{tabular}{|l|}
1667.8904 \\
\end{tabular} & 834.4488 & 1666.9064 & 833.9568 & $\mathbf{L}$ & 2228.1843 & 1114.5958 & 2211.1577 & 1106.0825 & 2210.1737 & 1105.5905 & 1 \\
\hline 18 & 1771.9490 & 886.4781 & 1754.9224 & 877.9649 & 1753.9384 & 877.4728 & $\mathrm{~S}$ & 2115.1002 & 1058.0537 & 2098.0736 & 1049.5405 & 2097.0896 & 1049.0485 & 20 \\
\hline 19 & 1869.0017 & 935.0 & 1851.9752 & 926.4912 & 1850.9912 & 925.9992 & $\mathbf{P}$ & 2028.0682 & 1014.5377 & 2011.0416 & 1006.0244 & 2010.0576 & 1005.5324 & 19 \\
\hline 201 & 1984.0287 & 992.5180 & 1967.0021 & 984.0047 & 1966.0181 & 983.5127 & $\mathbf{N}$ & 1931.0154 & 966.0113 & 1913.9889 & 957.4981 & 1913.0048 & 957.0061 & 18 \\
\hline 212 & 2112.0873 & 1056.5473 & 2095.0607 & 1048.0340 & 2094.0767 & 1047.5420 & $\mathbf{Q}$ & 1815.9885 & 908.4979 & 1798.9619 & 899.9846 & 1797.9779 & 899.4926 & 17 \\
\hline 222 & 2213.1349 & 1107.0711 & 2196.1084 & 1098.5578 & 2195.1244 & 1098.0658 & $T$ & 1687.9299 & 844.4686 & 1670.9033 & 835.9553 & 1669.9193 & 835.4633 & 16 \\
\hline 23 & 2312.2034 & 1156.6053 & 2295.1768 & 1148.0920 & 2294.1928 & 1147.6000 & V & 1586.8822 & 793.9447 & 1569.8557 & 785.4315 & 1568.8716 & 784.9395 & 5 \\
\hline 24 & 2441.2459 & 1221.1266 & 2424.2194 & 1212.6133 & 2423.2354 & 1212.1213 & $\mathbf{E}$ & 1487.8138 & 744.4105 & 1470.7872 & 735.8973 & 1469.8032 & 735.4053 & 4 \\
\hline 252 & 2554.3300 & 1277.6686 & 2537.3035 & 1269.1554 & 2536.3194 & 1268.6634 & I & 1358.7712 & 679.8892 & 1341.7447 & 671.3760 & 1340.7606 & 670.8840 & 13 \\
\hline \begin{tabular}{l|l}
26 & 2 \\
\end{tabular} & 2641.3620 & 1321.1847 & 2624.3355 & 1312.6714 & 2623.3515 & 1312.1794 & $S$ & 1245.6871 & 623.3472 & 1228.6606 & 614.8339 & 1227.6766 & 614.3419 & 12 \\
\hline 27 & 2754.4461 & \begin{tabular}{|l|}
1377.7267 \\
\end{tabular} & 2737.4196 & 1369.2134 & 2736.4355 & \begin{tabular}{|l|}
1368.7214 \\
\end{tabular} & $\mathbf{L}$ & 1158.6551 & 579.8312 & 1141.6286 & 571.3179 & 1140.6445 & 570.8259 & 11 \\
\hline 282 & 2851.4989 & 1426.2531 & 2834.4723 & 1417.7398 & 2833.4883 & 1417.2478 & $\mathbf{P}$ & 1045.5710 & 523.2892 & 1028.5445 & 514.7759 & 1027.5605 & 514.2839 & 10 \\
\hline$\left.29\right|^{2}$ & 2964.5829 & 1482.7951 & 2947.5564 & 1474.2818 & 2946.5724 & 1473.7898 & $\mathbf{L}$ & 948.5183 & 474.7628 & 931.4917 & 466.2495 & 930.5077 & 465.7575 & 9 \\
\hline 30 & 3078.6259 & 1539.8166 & 3061.5993 & 1531.3033 & 3060.6153 & 1530.8113 & $\mathbf{N}$ & 835.4342 & 418.2207 & 818.4077 & 409.7075 & 817.4236 & 409.2155 & 8 \\
\hline 313 & 3179.6735 & 1590.3404 & 3162.6470 & 1581.8271 & 3161.6630 & 1581.3351 & $T$ & 721.3913 & 361.1993 & 704.3647 & 352.6860 & 703.3807 & 352.1940 & 7 \\
\hline 323 & 3278.7419 & 1639.8746 & 3261.7154 & 1631.3613 & 3260.7314 & 1630.8693 & $\mathbf{V}$ & 620.3436 & 310.6754 & 603.3171 & 302.1622 & 602.3330 & 301.6702 & 6 \\
\hline 333 & 3335.7634 & \begin{tabular}{|l|}
1668.3853 \\
\end{tabular} & 3318.7369 & 1659.8721 & 3317.7528 & 1659.3801 & G & 521.2752 & 261.1412 & 504.2486 & 252.6280 & 503.2646 & 252.1360 & 5 \\
\hline 343 & 3422.7954 & 1711.9014 & 3405.7689 & 1703.3881 & 3404.7849 & 1702.8961 & $\mathrm{~S}$ & 464.2537 & 232.6305 & 447.2272 & 224.1172 & 446.2432 & 223.6252 & 4 \\
\hline 353 & 3521.8639 & 1761.4356 & 3504.8373 & 1752.9223 & 3503.8533 & 1752.4303 & $\mathbf{V}$ & 377.2217 & 189.1145 & 360.1952 & 180.6012 & & & 3 \\
\hline 363 & 3652.9043 & 1826.9558 & 3635.8778 & 1818.4425 & 3634.8938 & $\mid 1817.9505$ & $\mathbf{M}$ & 278.1533 & 139.5803 & 261.1267 & 131.0670 & & & 2 \\
\hline 37 & & & & & & & $\mathbf{K}$ & 147.1128 & 74.0600 & 130.0863 & 65.5468 & & & 1 \\
\hline
\end{tabular}
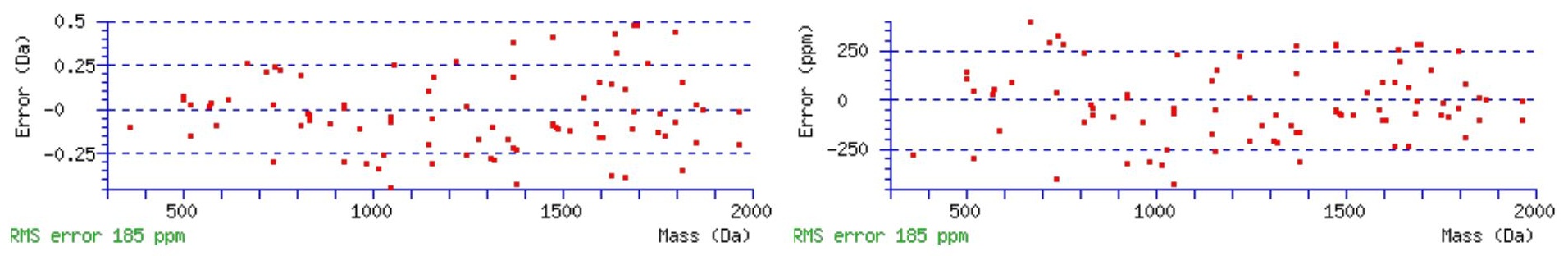

\section{All matches to this query}

\begin{tabular}{|l|l|l|l|}
\hline Score & Mr(calc): & Delta & \multicolumn{1}{|c|}{ Sequence } \\
\hline 56.0 & 3798.0026 & 0.0066 & NSFGLAPAAPLQVHAPLSPNQTVEISLPLNTVGSVMK \\
\hline 55.8 & 3798.0026 & 0.0066 & NSFGLAPAAPLQVHAPLSPNQTVEISLPLNTVGSVMK \\
\hline 38.3 & 3797.0186 & 0.9906 & NSFGLAPAAPLQVHAPLSPNQTVEISLPLNTVGSVMK \\
\hline 33.6 & 3798.0026 & 0.0066 & NSFGLAPAAPLQVHAPLSPNQTVEISLPLNTVGSVMK \\
\hline 1.0 & 3797.9977 & 0.0115 & NQSELVHIICMITSLLNPSVHSSILILGNFKLK \\
\hline 1.0 & 3797.9977 & 0.0115 & NQSELVHIICMITSLLNPSVHSSILILGNFKLK \\
\hline 1.0 & 3797.9977 & 0.0115 & NQSELVHIICMITSLLNPSVHSSILILGNFKLK \\
\hline 1.0 & 3797.9977 & 0.0115 & NQSELVHIICMITSLLNPSVHSSILILGNFKLK \\
\hline
\end{tabular}


Spectrum No: 589; Query: 2031; Rank: 1

\section{Peptide View}

MS/MS Fragmentation of NATYSYVLDDPDPDDGFNYK

Found in IPI00776882, Tax_Id=10116 Gene_Symbol=Calu calumenin isoform a

Match to Query 2031: 2309.972248 from(1155.993400,2+)

Title: 091008RatKidney_NoSalt_24.3641.3641.2.dta

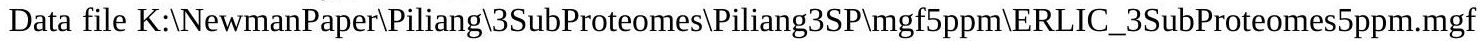
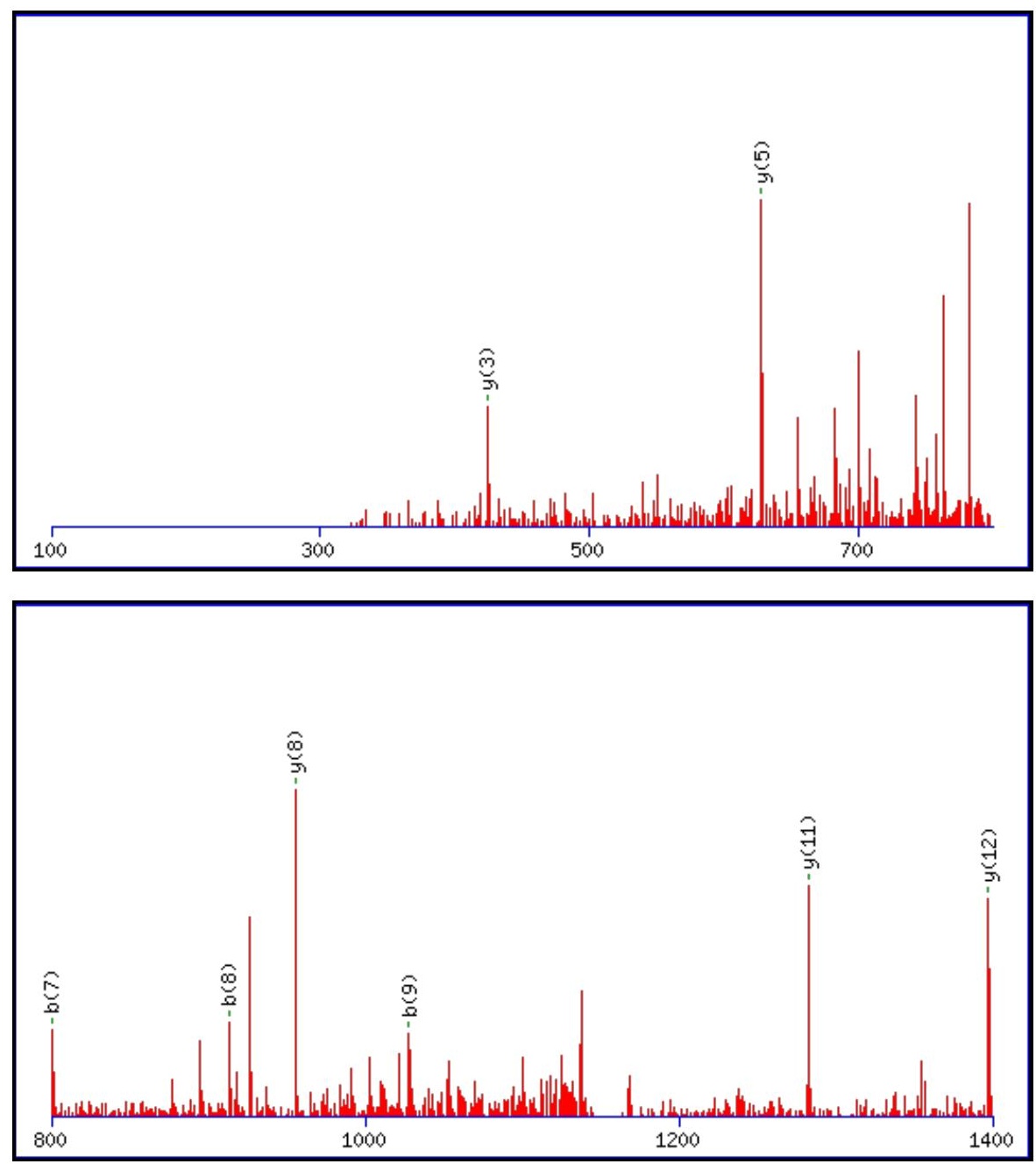


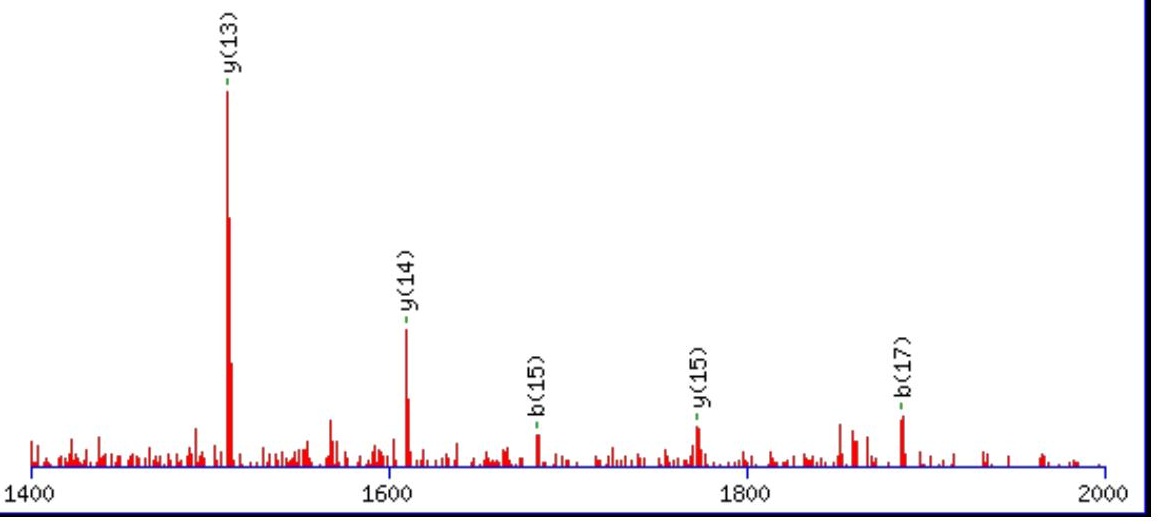

Monoisotopic mass of neutral peptide $\operatorname{Mr}($ calc): 2308.9648

Fixed modifications: Carbamidomethyl (C)

Variable modifications:

N1 : Deamidated $\mathrm{N}(\mathrm{N})$

Ions Score: 56 Expect: 0.00072

Matches (Bold Red): 13/214 fragment ions using 24 most intense peaks

\begin{tabular}{|c|c|c|c|c|c|c|c|c|c|c|c|c|c|c|}
\hline \# & b & $\mathbf{b}^{++}$ & b* & $\mathbf{b}^{*^{++}}$ & $\mathbf{b}^{0}$ & $\mathbf{b}^{0++}$ & Seq. & $\mathbf{y}$ & $y^{++}$ & $\mathbf{y}^{*}$ & $\mathbf{y}^{*^{++}}$ & $\mathbf{y}^{0}$ & $\mathbf{y}^{0++}$ & \# \\
\hline 1 & 116.0342 & 58.5207 & 99.0077 & 50.0075 & & & $\mathbf{N}$ & & & & & & & 20 \\
\hline 2 & 187.0713 & 94.0393 & 170.0448 & 85.5260 & & & $\mathbf{A}$ & 2194.9451 & 1097.9762 & 2177.9186 & 1089.4629 & 2176.9346 & 1088.9709 & 19 \\
\hline 3 & 288.1190 & 144.5631 & 271.0925 & 136.0499 & 270.1084 & 135.5579 & $\mathbf{T}$ & 2123.9080 & 1062.4576 & 2106.8815 & 1053.9444 & 2105.8975 & 1053.4524 & 18 \\
\hline 4 & 451.1823 & 226.0948 & 434.1558 & 217.5815 & 433.1718 & 217.0895 & $\mathbf{Y}$ & 2022.8603 & 1011.9338 & 2005.8338 & 1003.4205 & 2004.8498 & 1002.9285 & 17 \\
\hline 5 & 538.2144 & 269.6108 & 521.1878 & 261.0975 & 520.2038 & 260.6055 & S & 1859.7970 & 930.4021 & 1842.7705 & 921.8889 & 1841.7865 & 921.3969 & 16 \\
\hline 6 & 701.2777 & 351.1425 & 684.2511 & 342.6292 & 683.2671 & 342.1372 & $\mathbf{Y}$ & 1772.7650 & 886.8861 & 1755.7384 & 878.3729 & 1754.7544 & 877.8808 & 15 \\
\hline 7 & 800.3461 & 400.6767 & 783.3196 & 392.1634 & 782.3355 & 391.6714 & $\mathbf{V}$ & 1609.7017 & 805.3545 & 1592.6751 & 796.8412 & 1591.6911 & 796.3492 & 14 \\
\hline 8 & 913.4302 & 457.2187 & 896.4036 & 448.7054 & 895.4196 & 448.2134 & $\mathbf{L}$ & 1510.6332 & 755.8203 & 1493.6067 & 747.3070 & 1492.6227 & 746.8150 & 13 \\
\hline 9 & 1028.4571 & 514.7322 & 1011.4306 & 506.2189 & 1010.4465 & 505.7269 & D & 1397.5492 & 699.2782 & 1380.5226 & 690.7650 & 1379.5386 & 690.2729 & 12 \\
\hline 10 & 1143.4841 & 572.2457 & 1126.4575 & 563.7324 & 1125.4735 & 563.2404 & D & 1282.5222 & 641.7648 & 1265.4957 & 633.2515 & 1264.5117 & 632.7595 & 11 \\
\hline 11 & 1240.5368 & 620.7720 & 1223.5103 & 612.2588 & 1222.5263 & 611.7668 & $\mathbf{P}$ & 1167.4953 & 584.2513 & 1150.4687 & 575.7380 & 1149.4847 & 575.2460 & 10 \\
\hline 12 & 1355.5638 & 678.2855 & 1338.5372 & 669.7722 & 1337.5532 & 669.2802 & D & 1070.4425 & 535.7249 & 1053.4160 & 527.2116 & 1052.4320 & 526.7196 & 9 \\
\hline 13 & 1452.6165 & 726.8119 & 1435.5900 & 718.2986 & 1434.6060 & 717.8066 & $\mathbf{P}$ & 955.4156 & 478.2114 & 938.3890 & 469.6982 & 937.4050 & 469.2061 & 8 \\
\hline 14 & 1567.6435 & 784.3254 & 1550.6169 & 775.8121 & 1549.6329 & 775.3201 & D & 858.3628 & 429.6851 & 841.3363 & 421.1718 & 840.3523 & 420.6798 & 7 \\
\hline 15 & 1682.6704 & 841.8388 & 1665.6439 & 833.3256 & 1664.6598 & 832.8336 & D & 743.3359 & 372.1716 & 726.3093 & 363.6583 & 725.3253 & 363.1663 & 6 \\
\hline 16 & 1739.6919 & 870.3496 & 1722.6653 & 861.8363 & 1721.6813 & 861.3443 & G & 628.3089 & 314.6581 & 611.2824 & 306.1448 & & & 5 \\
\hline 17 & 1886.7603 & 943.8838 & 1869.7337 & 935.3705 & 1868.7497 & 934.8785 & $\mathbf{F}$ & 571.2875 & 286.1474 & 554.2609 & 277.6341 & & & 4 \\
\hline 18 & 2000.8032 & 1000.9052 & 1983.7767 & 992.3920 & 1982.7927 & 991.9000 & $\mathbf{N}$ & 424.2191 & 212.6132 & 407.1925 & 204.0999 & & & 3 \\
\hline 19 & 2163.8665 & 1082.4369 & 2146.8400 & 1073.9236 & 2145.8560 & 1073.4316 & $\mathbf{Y}$ & 310.1761 & 155.5917 & 293.1496 & 147.0784 & & & 2 \\
\hline 20 & & & & & & & $\mathbf{K}$ & 147.1128 & 74.0600 & 130.0863 & 65.5468 & & & 1 \\
\hline
\end{tabular}
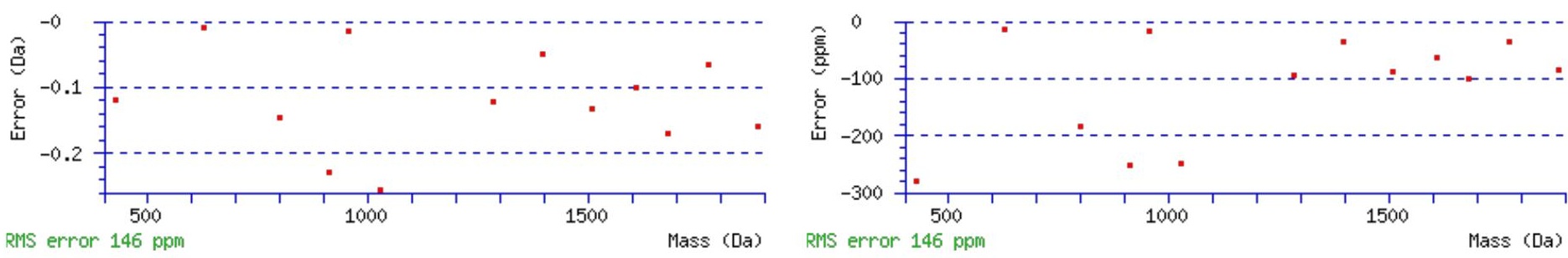

\section{All matches to this query}

\begin{tabular}{|l|l|l|l|}
\hline Score & Mr(calc): & Delta & \multicolumn{1}{c|}{ Sequence } \\
\hline 55.9 & 2308.9648 & 1.0075 & NATYSYVLDDPDPDDGFNYK \\
\hline & & &
\end{tabular}




\begin{tabular}{|l|l|l|l|}
39.6 & 2307.9808 & 1.9915 & NATYSYVLDDPDPDDGFNYK \\
\hline 31.1 & 2308.9648 & 1.0074 & NVTYGTYLDDPDPDDGFNYK \\
\hline 19.2 & 2307.9808 & 1.9914 & NVTYGTYLDDPDPDDGFNYK \\
\hline 7.1 & 2308.9648 & 1.0075 & NATYSYVLDDPDPDDGFNYK \\
\hline 6.2 & 2307.9653 & 2.0070 & MGGSSGALYGLFLTAAAQPLK \\
\hline 5.6 & 2308.9825 & 0.9898 & SGELRKEINSNTEQLQSSK \\
\hline 5.6 & 2308.9825 & 0.9898 & SGELRKEINSNTEQLQSSK \\
\hline 5.6 & 2308.9825 & 0.9898 & SGELRKEINSNTEQLQSSK \\
\hline 5.6 & 2308.9825 & 0.9898 & SGELRKEINSNTEQLQSSK \\
\hline
\end{tabular}

Spectrum No: 590; Query: 375; Rank: 1

\section{Peptide View}

MS/MS Fragmentation of NITAFNETLFR

Found in IPI00363550, Tax_Id=10116 Gene_Symbol=Tfrc similar to transferrin receptor

Match to Query 375: 1325.658548 from(663.836550,2+)

Title: 091008RatKidney_NH4Format01_24.3404.3404.2.dta

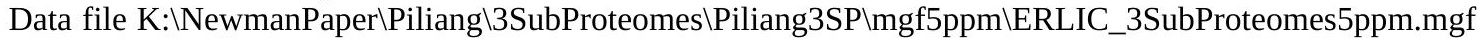
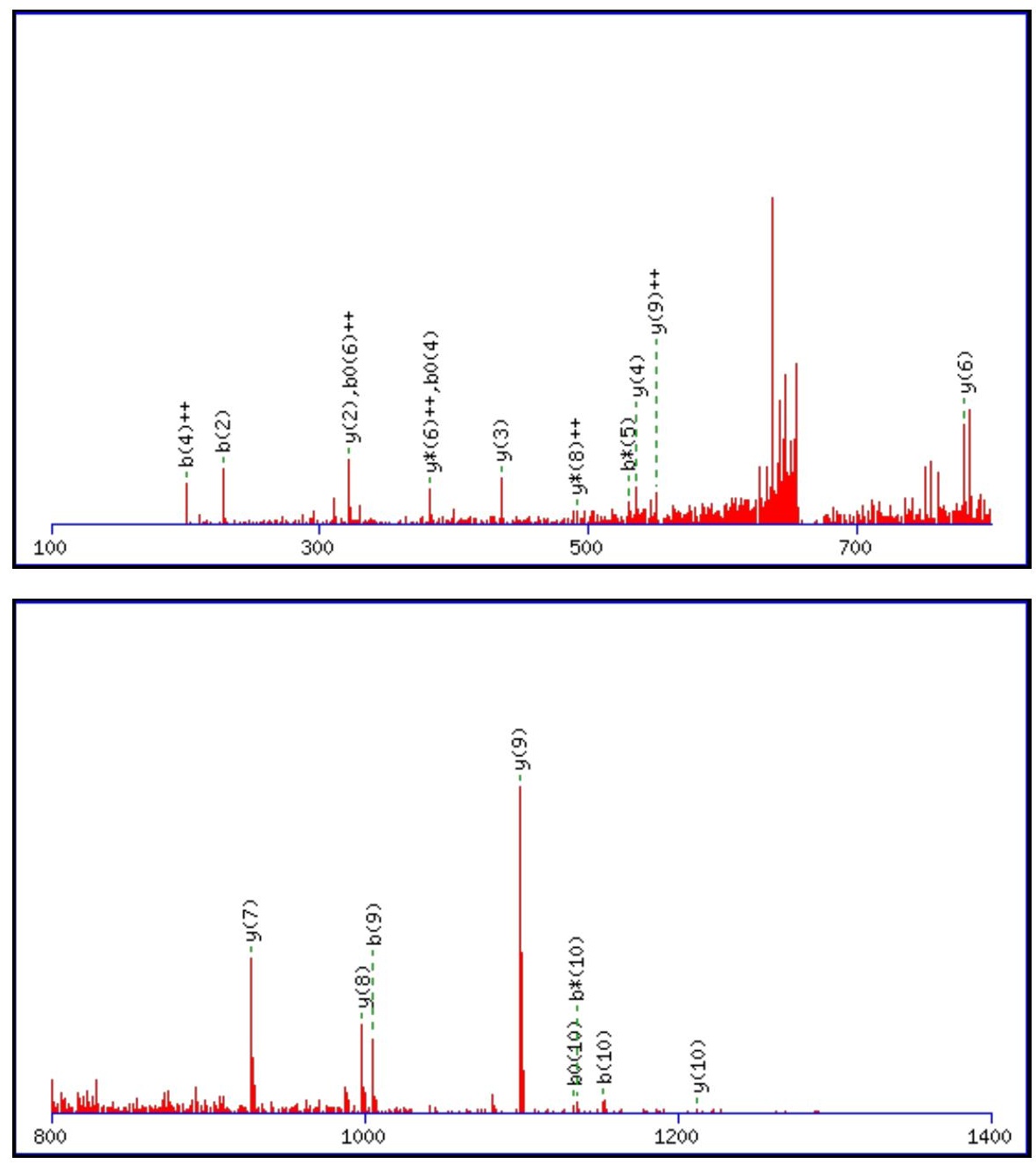


$1400 \quad 1600 \quad 1800 \quad 2000$

Monoisotopic mass of neutral peptide $\operatorname{Mr}($ calc): 1325.6615

Fixed modifications: Carbamidomethyl (C)

Variable modifications:

N6: Deamidated $\mathrm{N}(\mathrm{N})$

Ions Score: 56 Expect: 0.00036

Matches (Bold Red): 20/110 fragment ions using 42 most intense peaks

\begin{tabular}{|c|c|c|c|c|c|c|c|c|c|c|c|c|c|c|}
\hline \# & b & $\mathbf{b}^{++}$ & $\mathbf{b}^{*}$ & $\mathbf{b}^{*^{++}}$ & $\mathbf{b}^{0}$ & $\mathbf{b}^{0++}$ & Seq. & $\mathbf{y}$ & $\mathbf{y}^{++}$ & $\mathrm{y}^{*}$ & $\mathrm{y}^{*^{++}}$ & $\mathbf{y}^{0}$ & $y^{0++}$ & \# \\
\hline 1 & 115.0502 & 58.0287 & 98.0237 & 49.5155 & & & $\mathbf{N}$ & & & & & & & 11 \\
\hline 2 & 228.1343 & 114.5708 & 211.1077 & 106.0575 & & & I & 1212.6259 & 606.8166 & 1195.5994 & 598.3033 & 1194.6153 & 597.8113 & 10 \\
\hline 3 & 329.1819 & 165.0946 & 312.1554 & 156.5813 & 311.1714 & 156.0893 & $\mathbf{T}$ & 1099.5418 & 550.2746 & 1082.5153 & 541.7613 & 1081.5313 & 541.2693 & 9 \\
\hline 4 & 400.2191 & 200.6132 & 383.1925 & 192.0999 & 382.2085 & 191.6079 & A & 998.4942 & 499.7507 & 981.4676 & 491.2374 & 980.4836 & 490.7454 & 8 \\
\hline 5 & 547.2875 & 274.1474 & 530.2609 & 265.6341 & 529.2769 & 265.1421 & F & 927.4571 & 464.2322 & 910.4305 & 455.7189 & 909.4465 & 455.2269 & 7 \\
\hline 6 & 662.3144 & 331.6608 & 645.2879 & 323.1476 & 644.3038 & 322.6556 & $\mathbf{N}$ & 780.3886 & 390.6980 & 763.3621 & 382.1847 & 762.3781 & 381.6927 & 6 \\
\hline 7 & 791.3570 & 396.1821 & 774.3305 & 387.6689 & 773.3464 & 387.1769 & $\mathbf{E}$ & 665.3617 & 333.1845 & 648.3352 & 324.6712 & 647.3511 & 324.1792 & 5 \\
\hline 8 & 892.4047 & 446.7060 & 875.3781 & 438.1927 & 874.3941 & 437.7007 & $\mathbf{T}$ & 536.3191 & 268.6632 & 519.2926 & 260.1499 & 518.3085 & 259.6579 & 4 \\
\hline 9 & 1005.4887 & 503.2480 & 988.4622 & 494.7347 & 987.4782 & 494.2427 & $\mathbf{L}$ & 435.2714 & 218.1394 & 418.2449 & 209.6261 & & & 3 \\
\hline 10 & 1152.5572 & 576.7822 & 1135.5306 & 568.2689 & 1134.5466 & 567.7769 & $\mathbf{F}$ & 322.1874 & 161.5973 & 305.1608 & 153.0840 & & & 2 \\
\hline 11 & & & & & & & $\mathbf{R}$ & 175.1190 & 88.0631 & 158.0924 & 79.5498 & & & 1 \\
\hline
\end{tabular}
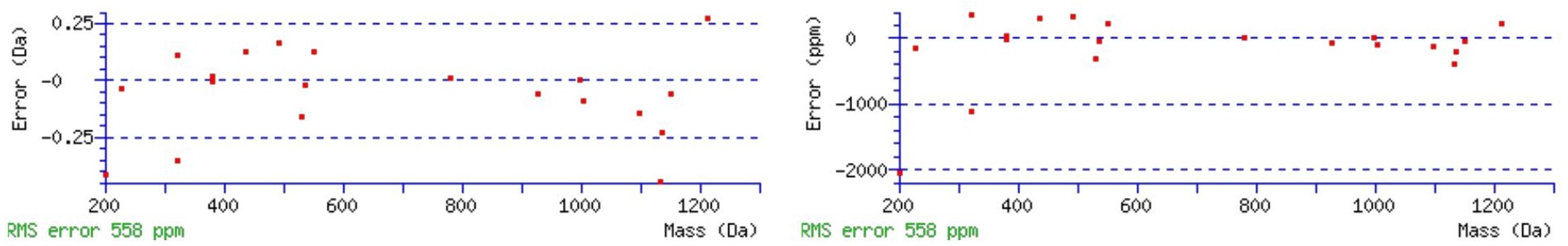

\section{All matches to this query}

\begin{tabular}{|l|c|c|l|}
\hline Score & Mr(calc): & Delta & \multicolumn{1}{c|}{ Sequence } \\
\hline 55.8 & 1325.6615 & -0.0030 & NITAFNETLFR \\
\hline 3.0 & 1325.6589 & -0.0003 & ARGSGGWAGTPGPR \\
\hline 2.8 & 1324.6540 & 1.0045 & LLGNGLKYPNR \\
\hline 2.8 & 1324.6639 & 0.9946 & KVAIISVEGNSK \\
\hline 1.4 & 1325.6615 & -0.0030 & NITAFNETLFR \\
\hline 1.2 & 1324.6639 & 0.9946 & LSAELLKDGATK \\
\hline 1.0 & 1325.6493 & 0.0093 & VKYTKDHTVR \\
\hline 1.0 & 1324.6468 & 1.0117 & LLYFYKPSSK \\
\hline 1.0 & 1324.6468 & 1.0117 & LLYFYKPSSK \\
\hline
\end{tabular}


Spectrum No: 591; Query: 18; Rank: 1

\section{Peptide View}

MS/MS Fragmentation of ANATIEVK

Found in IPI00326260, Tax_Id=10116 Gene_Symbol=Sdfr1 Isoform 2 of Neuroplastin precursor

Match to Query 18: 845.451648 from(423.733100,2+)

Title: 100101RatKid_NS_deglyco_15.647.647.2.dta

Data file K:\NewmanPaper\Piliang \3SubProteomes \Piliang3SP $\backslash$ mgf5ppm\ERLIC_3SubProteomes5ppm.mgf
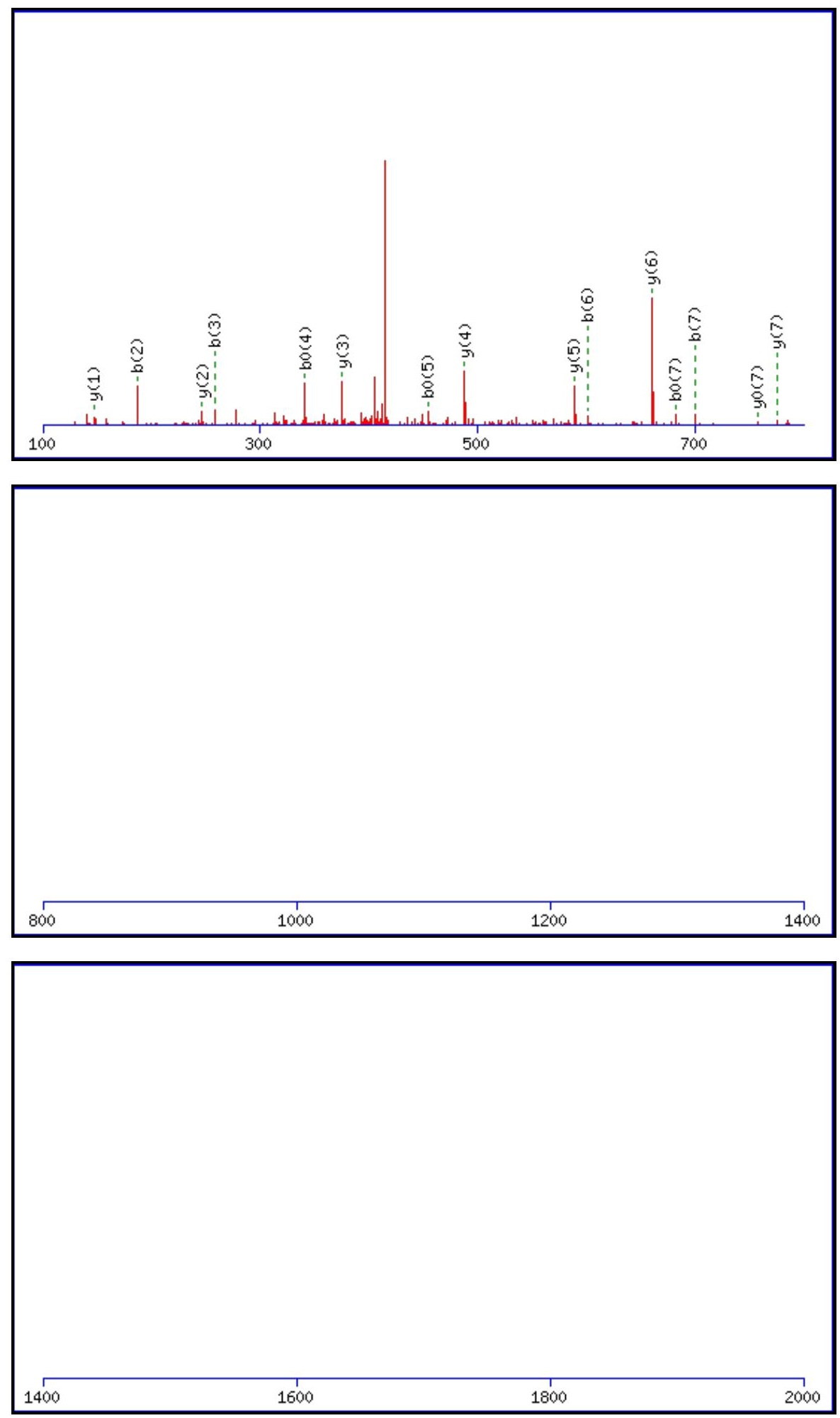

Monoisotopic mass of neutral peptide $\operatorname{Mr}($ calc): 845.4494 
Fixed modifications: Carbamidomethyl (C)

Variable modifications:

N2 : Deamidated_N (N)

Ions Score: 56 Expect: 0.00025

Matches (Bold Red): 15/72 fragment ions using 29 most intense peaks

\begin{tabular}{|c|c|c|c|c|c|c|c|c|c|c|c|c|c|c|}
\hline$\#$ & $\mathbf{b}$ & $\mathbf{b}^{++}$ & $\mathbf{b}^{*}$ & $\mathbf{b}^{*^{++}}$ & $\mathbf{b}^{\mathbf{0}}$ & $\mathbf{b}^{\mathbf{0 + +}}$ & Seq. & $\mathbf{y}$ & $\mathbf{y}^{++}$ & $\mathbf{y}^{*}$ & $\mathbf{y}^{\boldsymbol{*}^{++}}$ & $\mathbf{y}^{\mathbf{0}}$ & $\mathbf{y}^{\mathbf{0}++}$ & $\#$ \\
\hline $\mathbf{1}$ & 72.0444 & 36.5258 & & & & & $\mathbf{A}$ & & & & & & & $\mathbf{8}$ \\
\hline $\mathbf{2}$ & $\mathbf{1 8 7 . 0 7 1 3}$ & 94.0393 & 170.0448 & 85.5260 & & & $\mathbf{N}$ & $\mathbf{7 7 5 . 4 1 9 6}$ & 388.2134 & 758.3931 & 379.7002 & 757.4090 & 379.2082 & $\mathbf{7}$ \\
\hline $\mathbf{3}$ & $\mathbf{2 5 8 . 1 0 8 4}$ & 129.5579 & 241.0819 & 121.0446 & & & $\mathbf{A}$ & $\mathbf{6 6 0 . 3 9 2 7}$ & 330.7000 & 643.3661 & 322.1867 & 642.3821 & 321.6947 & $\mathbf{6}$ \\
\hline $\mathbf{4}$ & 359.1561 & 180.0817 & 342.1296 & 171.5684 & $\mathbf{3 4 1 . 1 4 5 6}$ & 171.0764 & $\mathbf{T}$ & $\mathbf{5 8 9 . 3 5 5 6}$ & 295.1814 & 572.3290 & 286.6681 & 571.3450 & 286.1761 & $\mathbf{5}$ \\
\hline $\mathbf{5}$ & 472.2402 & 236.6237 & 455.2136 & 228.1105 & $\mathbf{4 5 4 . 2 2 9 6}$ & 227.6184 & $\mathbf{I}$ & $\mathbf{4 8 8 . 3 0 7 9}$ & 244.6576 & 471.2813 & 236.1443 & 470.2973 & 235.6523 & $\mathbf{4}$ \\
\hline $\mathbf{6}$ & $\mathbf{6 0 1 . 2 8 2 8}$ & 301.1450 & 584.2562 & 292.6318 & 583.2722 & 292.1397 & $\mathbf{E}$ & $\mathbf{3 7 5 . 2 2 3 8}$ & 188.1155 & 358.1973 & 179.6023 & 357.2132 & 179.1103 & $\mathbf{3}$ \\
\hline $\mathbf{7}$ & $\mathbf{7 0 0 . 3 5 1 2}$ & 350.6792 & 683.3246 & 342.1660 & $\mathbf{6 8 2 . 3 4 0 6}$ & 341.6740 & $\mathbf{V}$ & $\mathbf{2 4 6 . 1 8 1 2}$ & 123.5942 & 229.1547 & 115.0810 & & & $\mathbf{2}$ \\
\hline $\mathbf{8}$ & & & & & & & $\mathbf{K}$ & $\mathbf{1 4 7 . 1 1 2 8}$ & 74.0600 & 130.0863 & 65.5468 & & & $\mathbf{1}$ \\
\hline
\end{tabular}
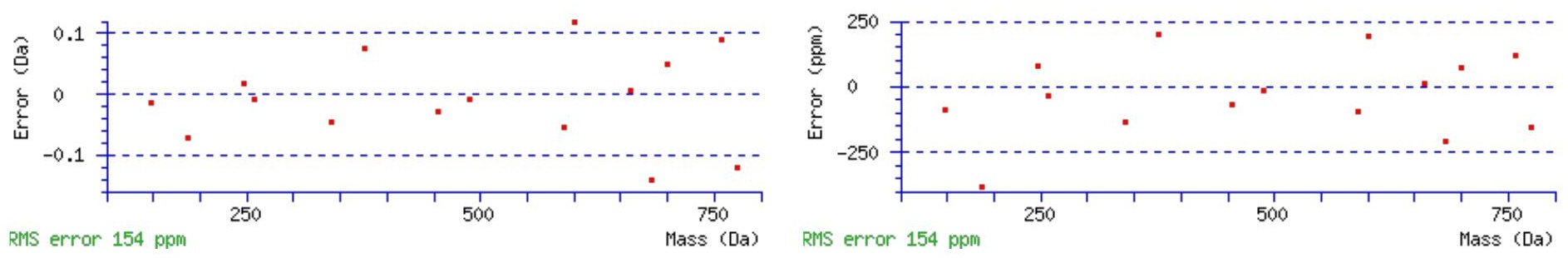

\section{All matches to this query}

\begin{tabular}{|l|l|l|l|}
\hline Score & Mr(calc): & Delta & Sequence \\
\hline 55.8 & 845.4494 & 0.0022 & ANATIEVK \\
\hline 16.6 & 845.4494 & 0.0022 & EKVNEVK \\
\hline 11.6 & 843.4491 & 2.0026 & GEGLPFPK \\
\hline 10.4 & 845.4494 & 0.0022 & EKEDVVK \\
\hline 10.3 & 845.4494 & 0.0022 & EATLGEVK \\
\hline 9.9 & 845.4494 & 0.0022 & TGAELDIK \\
\hline 8.8 & 845.4494 & 0.0022 & VVNASEVK \\
\hline 8.4 & 845.4494 & 0.0022 & AGELISEK \\
\hline 8.2 & 845.4508 & 0.0009 & RTTHGFK \\
\hline 8.2 & 845.4494 & 0.0022 & EKVDINK \\
\hline
\end{tabular}

Spectrum No: 592; Query: 61; Rank: 1

\section{Peptide View}

MS/MS Fragmentation of IDQNITYK

Found in IPI00372786, Tax_Id=10116 Gene_Symbol=Nid2 similar to nidogen 2 isoform 2

Match to Query 61: 994.495588 from(498.255070,2+)

Title: 091008RatKidney_NoSalt_27.654.654.2.dta

Data file K:INewmanPaper|Piliang|3SubProteomes\Piliang3SP\mgf5ppm\ERLIC_3SubProteomes5ppm.mgf 

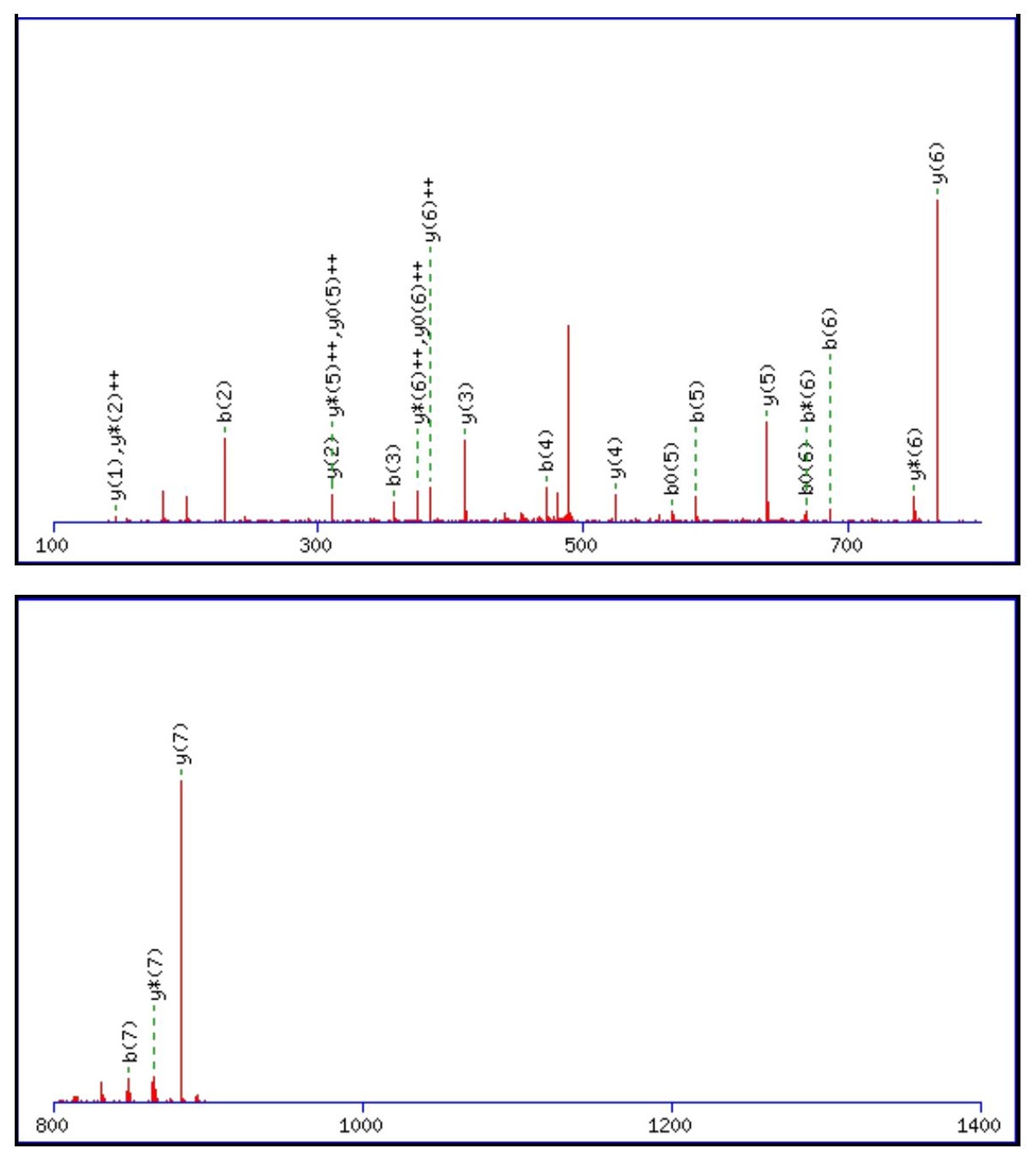

\begin{tabular}{rrrr}
\hline 00 & 1600 & 1800 & 2000 \\
\hline
\end{tabular}

Monoisotopic mass of neutral peptide $\operatorname{Mr}($ calc): 994.4971

Fixed modifications: Carbamidomethyl (C)

Variable modifications:

N4 : Deamidated_N (N)

Ions Score: 56 Expect: 0.00026

Matches (Bold Red): 24/74 fragment ions using 32 most intense peaks

\begin{tabular}{|c|c|c|c|c|c|c|c|c|c|c|c|c|c|c|}
\hline$\#$ & $\mathbf{b}$ & $\mathbf{b}^{++}$ & $\mathbf{b}^{*}$ & $\mathbf{b}^{\boldsymbol{*}^{++}}$ & $\mathbf{b}^{\mathbf{0}}$ & $\mathbf{b}^{\mathbf{0}+}$ & $\mathbf{S e q}$ & $\mathbf{y}$ & $\mathbf{y}^{++}$ & $\mathbf{y}^{*}$ & $\mathbf{y}^{\boldsymbol{*}^{++}}$ & $\mathbf{y}^{\mathbf{0}}$ & $\mathbf{y}^{\mathbf{0}}$ & $\#$ \\
\hline $\mathbf{1}$ & 114.0913 & 57.5493 & & & & & $\mathbf{I}$ & & & & & & & $\mathbf{8}$ \\
\hline $\mathbf{2}$ & 229.1183 & 115.0628 & & & 211.1077 & 106.0575 & $\mathbf{D}$ & $\mathbf{8 8 2 . 4 2 0 3}$ & 441.7138 & $\mathbf{8 6 5 . 3 9 3 8}$ & 433.2005 & 864.4098 & 432.7085 & $\mathbf{7}$ \\
\hline $\mathbf{3}$ & 357.1769 & 179.0921 & 340.1503 & 170.5788 & 339.1663 & 170.0868 & $\mathbf{Q}$ & $\mathbf{7 6 7 . 3 9 3 4}$ & $\mathbf{3 8 4 . 2 0 0 3}$ & $\mathbf{7 5 0 . 3 6 6 8}$ & 375.6871 & 749.3828 & $\mathbf{3 7 5 . 1 9 5 1}$ & $\mathbf{6}$ \\
\hline $\mathbf{4}$ & $\mathbf{4 7 2 . 2 0 3 8}$ & 236.6055 & 455.1772 & 228.0923 & 454.1932 & 227.6003 & $\mathbf{N}$ & $\mathbf{6 3 9 . 3 3 4 8}$ & 320.1710 & 622.3083 & 311.6578 & 621.3242 & $\mathbf{3 1 1 . 1 6 5 8}$ & $\mathbf{5}$ \\
\hline $\mathbf{5}$ & $\mathbf{5 8 5 . 2 8 7 9}$ & 293.1476 & 568.2613 & 284.6343 & $\mathbf{5 6 7 . 2 7 7 3}$ & 284.1423 & $\mathbf{I}$ & $\mathbf{5 2 4 . 3 0 7 9}$ & 262.6576 & 507.2813 & 254.1443 & 506.2973 & 253.6523 & $\mathbf{4}$ \\
\hline
\end{tabular}




\begin{tabular}{|l|l|l|l|l|l|l|l|l|l|l|l|l|l|l|l|l|l|l|}
$\mathbf{6}$ & $\mathbf{6 8 6 . 3 3 5 5}$ & 343.6714 & $\mathbf{6 6 9 . 3 0 9 0}$ & 335.1581 & $\mathbf{6 6 8 . 3 2 5 0}$ & 334.6661 & $\mathbf{T}$ & $\mathbf{4 1 1 . 2 2 3 8}$ & 206.1155 & 394.1973 & 197.6023 & 393.2132 & 197.1103 & $\mathbf{3}$ \\
\hline $\mathbf{7}$ & $\mathbf{8 4 9 . 3 9 8 9}$ & 425.2031 & 832.3723 & 416.6898 & $\mathbf{8 3 1 . 3 8 8 3}$ & $\mathbf{4 1 6 . 1 9 7 8}$ & $\mathbf{Y}$ & $\mathbf{3 1 0 . 1 7 6 1}$ & $\mathbf{1 5 5 . 5 9 1 7}$ & 293.1496 & $\mathbf{1 4 7 . 0 7 8 4}$ & & & $\mathbf{2}$ \\
\hline $\mathbf{8}$ & & & & & & & $\mathbf{K}$ & $\mathbf{1 4 7 . 1 1 2 8}$ & $\mathbf{7 4 . 0 6 0 0}$ & 130.0863 & 65.5468 & & & $\mathbf{1}$ \\
\hline
\end{tabular}
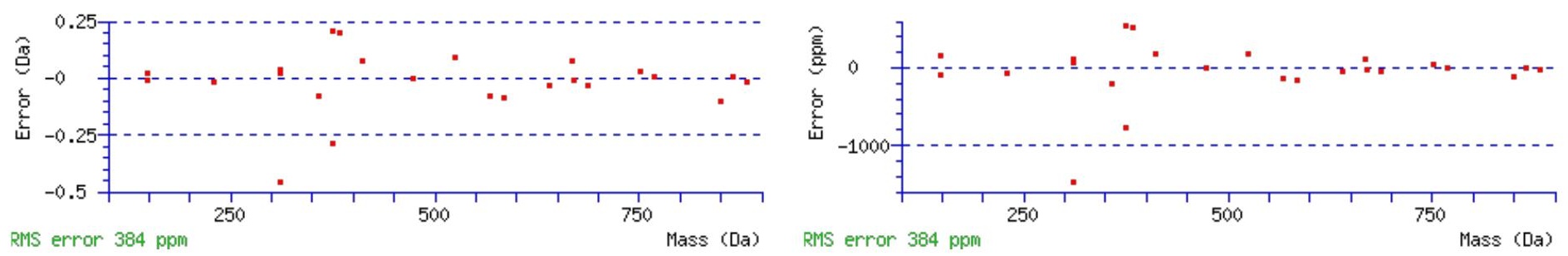

\section{All matches to this query}

\begin{tabular}{|l|l|l|l|}
\hline Score & Mr(calc): & Delta & \multicolumn{1}{|c|}{ Sequence } \\
\hline 55.8 & 994.4971 & -0.0015 & IDQNITYK \\
\hline 14.4 & 994.4971 & -0.0015 & ILDENTYK \\
\hline 13.1 & 994.5011 & -0.0055 & IDNYIPIF \\
\hline 12.4 & 994.4961 & -0.0005 & $\underline{\text { RAOVSOVK }}$ \\
\hline 11.6 & 993.4896 & 1.0060 & QTVTQPLK \\
\hline 10.7 & 994.4971 & -0.0015 & LQSENLYK \\
\hline 10.6 & 994.4961 & -0.0005 & EVTAALRR \\
\hline 10.2 & 993.4896 & 1.0060 & EPAGKIATK \\
\hline 9.9 & 994.4922 & 0.0034 & IPVTLNMK \\
\hline 9.7 & 994.4987 & -0.0032 & IDSLIELL \\
\hline
\end{tabular}

Spectrum No: 593; Query: 1767; Rank: 1

\section{Peptide View}

MS/MS Fragmentation of DDAGNYTCIASNEPQGQIR

Found in IPI00373753, Tax_Id=10116 Gene_Symbol=Ptk7_predicted 97 kDa protein

Match to Query 1767: 2108.917268 from(1055.465910,2+)

Title: 100101RatKid_NS_deglyco_24.2090.2090.2.dta

Data file K:INewmanPaper|Piliangl3SubProteomes\Piliang3SP\mgf5ppm\ERLIC_3SubProteomes5ppm.mgf

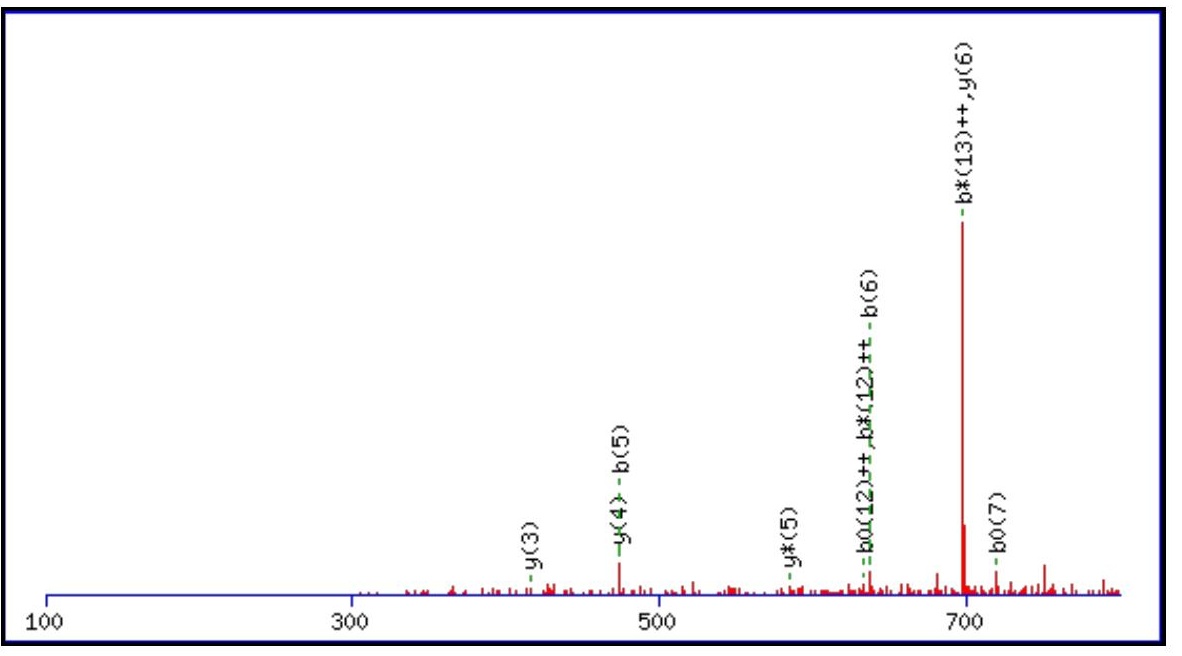



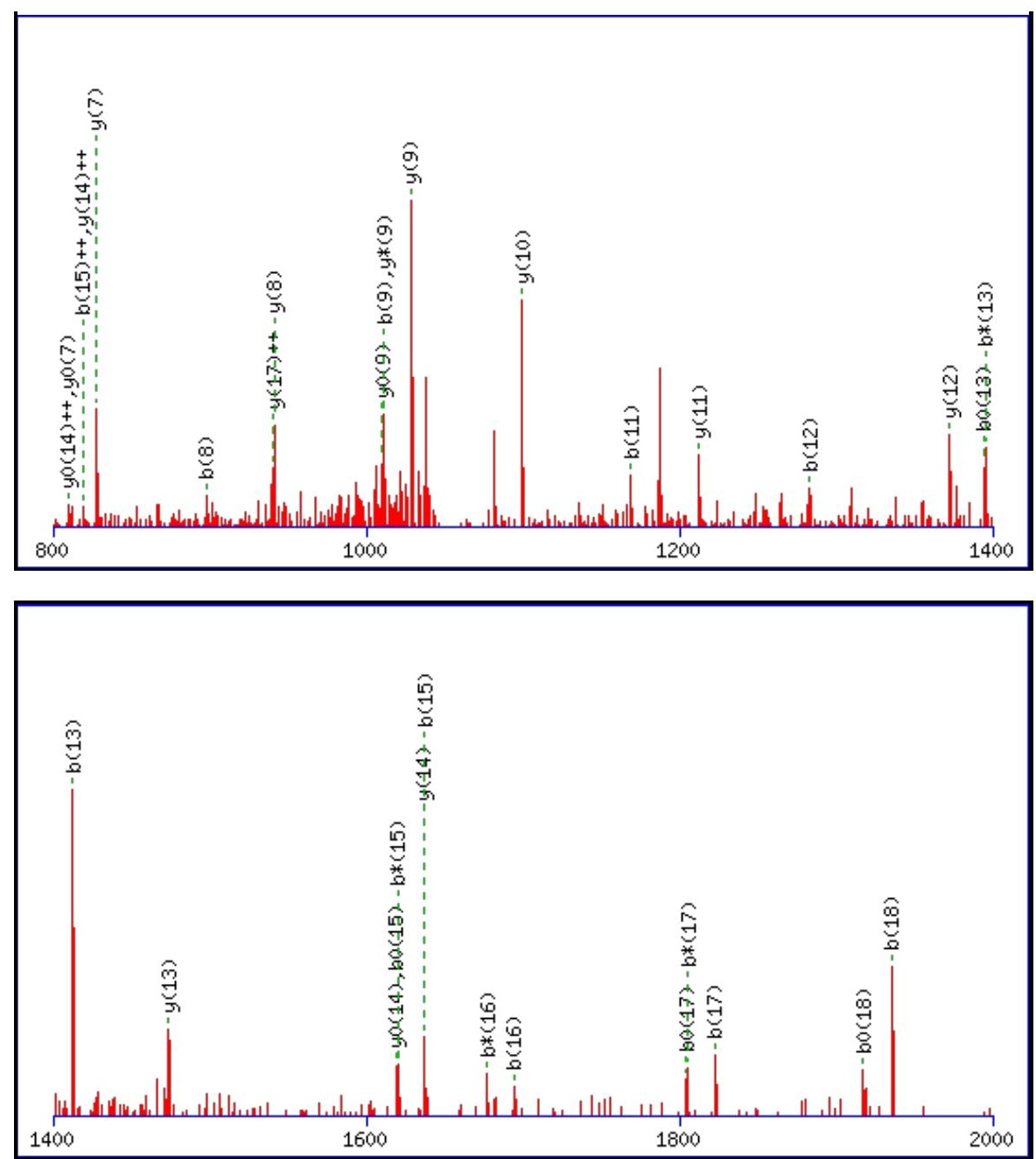

Monoisotopic mass of neutral peptide $\operatorname{Mr}($ calc): 2108.9069

Fixed modifications: Carbamidomethyl (C)

Variable modifications:

N5 : Deamidated_N (N)

Ions Score: 56 Expect: 0.00072

Matches (Bold Red): 43/196 fragment ions using 72 most intense peaks

\begin{tabular}{|c|c|c|c|c|c|c|c|c|c|c|c|c|c|c|}
\hline \# & b & $\mathbf{b}^{++}$ & $\mathbf{b}^{*}$ & $\mathbf{b}^{*^{++}}$ & $\mathbf{b}^{0}$ & $\mathbf{b}^{\mathbf{0 + +}}$ & Seq. & $\mathbf{y}$ & $y^{++}$ & $\mathbf{y}^{*}$ & $\mathrm{y}^{*^{++}}$ & $\mathbf{y}^{\mathbf{0}}$ & $\mathbf{y}^{0++}$ & \# \\
\hline 1 & 116.0342 & 58.5207 & & & 98.0237 & 49.5155 & D & & & & & & & 19 \\
\hline 2 & 231.0612 & 116.0342 & & & 213.0506 & 107.0289 & D & 1994.8872 & 997.9473 & 1977.8607 & 989.4340 & 1976.8767 & 988.9420 & 18 \\
\hline 3 & 302.0983 & 151.5528 & & & 284.0877 & 142.5475 & A & 1879.8603 & 940.4338 & 1862.8337 & 931.9205 & 1861.8497 & 931.4285 & 17 \\
\hline 4 & 359.1197 & 180.0635 & & & 341.1092 & 171.0582 & G & 1808.8232 & 904.9152 & 1791.7966 & 896.4020 & 1790.8126 & 895.9099 & 16 \\
\hline 5 & 474.1467 & 237.5770 & 457.1201 & 229.0637 & 456.1361 & 228.5717 & $\mathbf{N}$ & 1751.8017 & 876.4045 & 1734.7752 & 867.8912 & 1733.7912 & 867.3992 & 15 \\
\hline 6 & 637.2100 & 319.1086 & 620.1835 & 310.5954 & 619.1994 & 310.1034 & $\mathbf{Y}$ & 1636.7748 & 818.8910 & 1619.7482 & 810.3778 & 1618.7642 & 809.8857 & 14 \\
\hline 7 & 738.2577 & 369.6325 & 721.2311 & 361.1192 & 720.2471 & 360.6272 & $\mathbf{T}$ & 1473.7115 & 737.3594 & 1456.6849 & 728.8461 & 1455.7009 & 728.3541 & 13 \\
\hline 8 & 898.2883 & 449.6478 & 881.2618 & 441.1345 & 880.2778 & 440.6425 & C & 1372.6638 & 686.8355 & 1355.6372 & 678.3223 & 1354.6532 & 677.8302 & 12 \\
\hline 9 & 1011.3724 & 506.1898 & 994.3458 & 497.6766 & 993.3618 & 497.1846 & I & 1212.6331 & 606.8202 & 1195.6066 & 598.3069 & 1194.6226 & 597.8149 & 11 \\
\hline 10 & 1082.4095 & 541.7084 & 1065.3830 & 533.1951 & 1064.3989 & 532.7031 & A & 1099.5491 & 550.2782 & 1082.5225 & 541.7649 & 1081.5385 & 541.2729 & 10 \\
\hline 11 & 1169.4415 & 585.2244 & 1152.4150 & 576.7111 & 1151.4310 & 576.2191 & S & 1028.5119 & 514.7596 & 1011.4854 & 506.2463 & 1010.5014 & 505.7543 & 9 \\
\hline 12 & 1283.4845 & 642.2459 & 1266.4579 & 633.7326 & 1265.4739 & 633.2406 & $\mathbf{N}$ & 941.4799 & 471.2436 & 924.4534 & 462.7303 & 923.4694 & 462.2383 & 8 \\
\hline 13 & 1412.5271 & 706.7672 & 1395.5005 & 698.2539 & 1394.5165 & 697.7619 & $\mathbf{E}$ & 827.4370 & 414.2221 & 810.4104 & 405.7089 & 809.4264 & 405.2169 & 7 \\
\hline 14 & 1509.5798 & 755.2935 & 1492.5533 & 746.7803 & 1491.5693 & 746.2883 & $\mathbf{P}$ & 698.3944 & 349.7008 & 681.3679 & 341.1876 & & & 6 \\
\hline 15 & 1637.6384 & 819.3228 & 1620.6119 & 810.8096 & 1619.6278 & 810.3176 & $\mathbf{Q}$ & 601.3416 & 301.1745 & 584.3151 & 292.6612 & & & 5 \\
\hline 16 & 1694.6599 & 847.8336 & 1677.6333 & 839.3203 & 1676.6493 & 838.8283 & $\mathbf{G}$ & 473.2831 & 237.1452 & 456.2565 & 228.6319 & & & 4 \\
\hline 17 & 1822.7184 & 911.8629 & 1805.6919 & 903.3496 & 1804.7079 & 902.8576 & $\mathbf{Q}$ & 416.2616 & 208.6344 & 399.2350 & 200.1212 & & & 3 \\
\hline 18 & 1935.8025 & 968.4049 & 1918.7760 & 959.8916 & 1917.7919 & 959.3996 & I & 288.2030 & 144.6051 & 271.1765 & 136.0919 & & & 2 \\
\hline
\end{tabular}




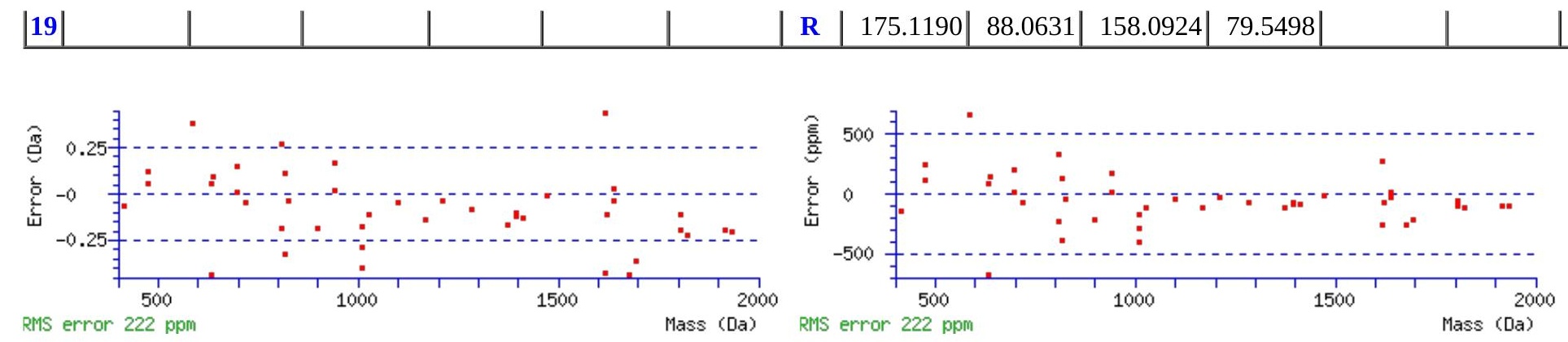

\section{All matches to this query}

\begin{tabular}{|l|c|c|l|}
\hline Score & Mr(calc): & Delta & \multicolumn{1}{|c|}{ Sequence } \\
\hline 55.5 & 2108.9069 & 0.0104 & DDAGNYTCIASNEPQGQIR \\
\hline 35.7 & 2108.9069 & 0.0104 & DDAGNYTCIASNEPQGQIR \\
\hline 33.1 & 2107.9229 & 0.9944 & DDAGNYTCIASNEPQGQIR \\
\hline 7.0 & 2108.9140 & 0.0033 & SVVRKDSSGSISEQLSNR \\
\hline 5.5 & 2106.9098 & 2.0074 & TLPVFCNVDGIIETNQK \\
\hline 2.6 & 2106.9180 & 1.9992 & GDLALSGNLSNLYAMTQDK \\
\hline 2.0 & 2108.9247 & -0.0074 & WMKEQTGSICQYLVMR \\
\hline 1.3 & 2107.8985 & 1.0187 & FOQHCSDLMGTLKVIR \\
\hline 0.5 & 2106.8919 & 2.0254 & AIGCAGVVRSGGSDQERTK \\
\hline
\end{tabular}

Spectrum No: 594; Query: 420; Rank: 1

\section{Peptide View}

MS/MS Fragmentation of HYVNLTNGLYR

Found in IPI00765231, Tax_Id=10116 Gene_Symbol=RGD1564063_predicted hypothetical protein LOC498226

Match to Query 420: 1349.670748 from(675.842650,2+)

Title: 091008RatKidney_NH4Format01_23.1847.1847.2.dta

Data file K:INewmanPaper|Piliang|3SubProteomes\Piliang3SP\mgf5ppm|ERLIC_3SubProteomes5ppm.mgf

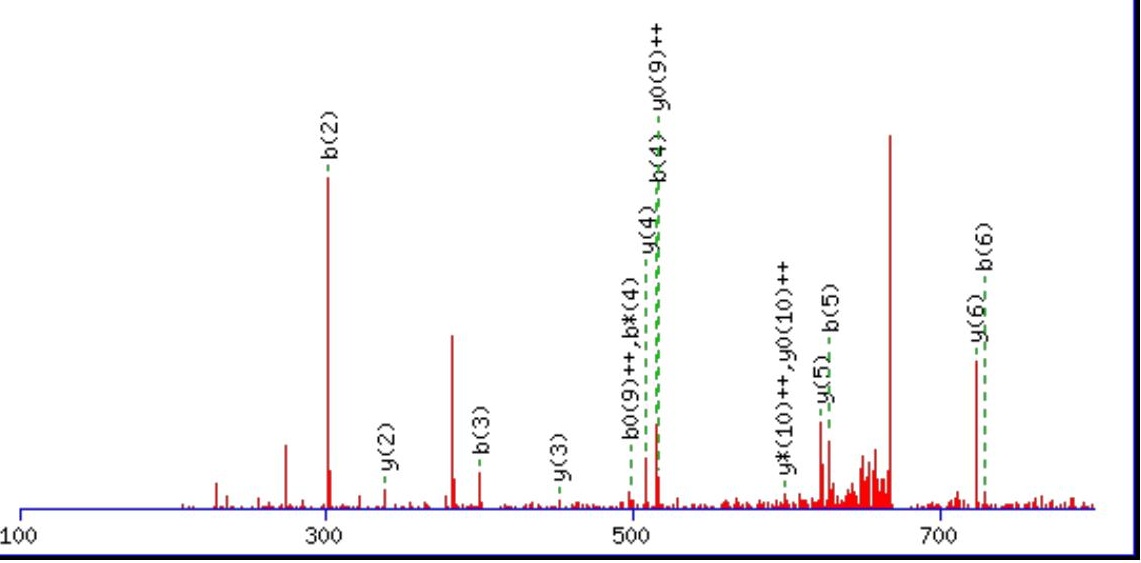




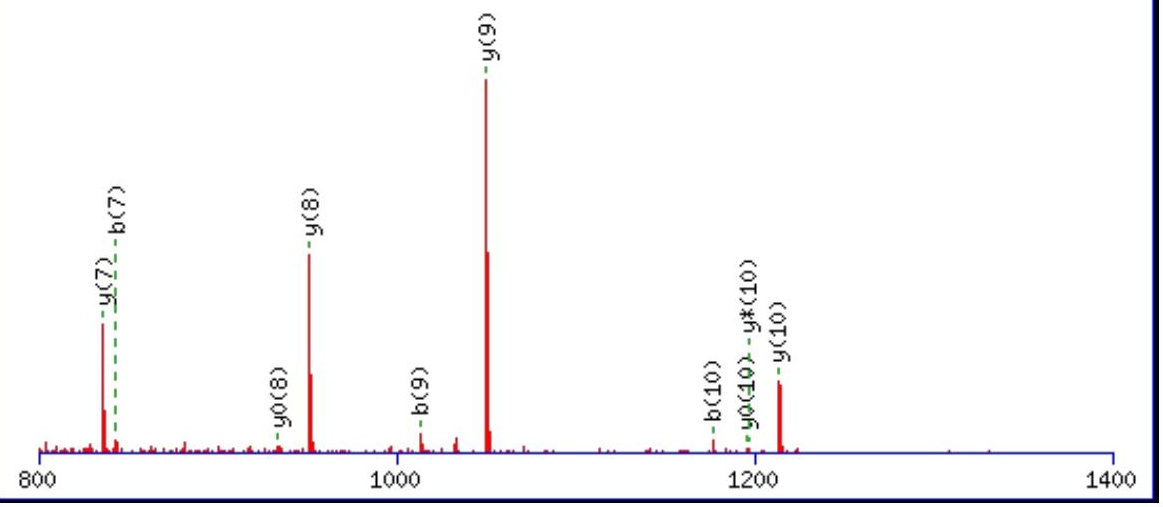

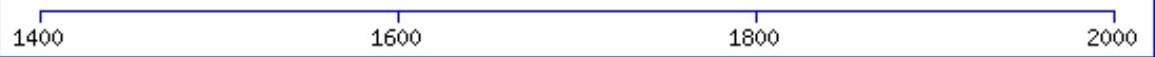

Monoisotopic mass of neutral peptide $\operatorname{Mr}($ calc): 1349.6728

Fixed modifications: Carbamidomethyl (C)

Variable modifications:

N4 : Deamidated_N (N)

Ions Score: 55 Expect: 0.00041

Matches (Bold Red): 25/94 fragment ions using 47 most intense peaks

\begin{tabular}{|c|c|c|c|c|c|c|c|c|c|c|c|c|c|c|}
\hline \# & b & $\mathbf{b}^{++}$ & $\mathbf{b}^{*}$ & $\mathbf{b}^{*++}$ & $\mathbf{b}^{0}$ & $\mathbf{b}^{\mathbf{0 + +}}$ & Seq. & $\mathbf{y}$ & $\mathbf{y}^{++}$ & $\mathbf{y}^{*}$ & $\mathbf{y}^{*^{++}}$ & $\mathbf{y}^{0}$ & $\mathbf{y}^{0++}$ & \# \\
\hline 1 & 138.0662 & 69.5367 & & & & & $\mathbf{H}$ & & & & & & & 11 \\
\hline 2 & 301.1295 & 151.0684 & & & & & $\mathbf{Y}$ & 1213.6212 & 607.3142 & 1196.5946 & 598.8009 & 1195.6106 & 598.3089 & 10 \\
\hline 3 & 400.1979 & 200.6026 & & & & & $\mathbf{V}$ & 1050.5578 & 525.7826 & 1033.5313 & 517.2693 & 1032.5473 & 516.7773 & 9 \\
\hline 4 & 515.2249 & 258.1161 & 498.1983 & 249.6028 & & & $\mathbf{N}$ & 951.4894 & 476.2483 & 934.4629 & 467.7351 & 933.4788 & 467.2431 & 8 \\
\hline 5 & 628.3089 & 314.6581 & 611.2824 & 306.1448 & & & $\mathbf{L}$ & 836.4625 & 418.7349 & 819.4359 & 410.2216 & 818.4519 & 409.7296 & 7 \\
\hline 6 & 729.3566 & 365.1819 & 712.3301 & 356.6687 & 711.3460 & 356.1767 & $\mathbf{T}$ & 723.3784 & 362.1928 & 706.3519 & 353.6796 & 705.3678 & 353.1876 & 6 \\
\hline 7 & 843.3995 & 422.2034 & 826.3730 & 413.6901 & 825.3890 & 413.1981 & $\mathbf{N}$ & 622.3307 & 311.6690 & 605.3042 & 303.1557 & & & 5 \\
\hline 8 & 900.4210 & 450.7141 & 883.3945 & 442.2009 & 882.4104 & 441.7089 & G & 508.2878 & 254.6475 & 491.2613 & 246.1343 & & & 4 \\
\hline 9 & 1013.5051 & 507.2562 & 996.4785 & 498.7429 & 995.4945 & 498.2509 & $\mathbf{L}$ & 451.2663 & 226.1368 & 434.2398 & 217.6235 & & & 3 \\
\hline 10 & 1176.5684 & 588.7878 & 1159.5418 & 580.2746 & 1158.5578 & 579.7826 & $\mathbf{Y}$ & 338.1823 & 169.5948 & 321.1557 & 161.0815 & & & 2 \\
\hline 11 & & & & & & & $\mathbf{R}$ & 175.1190 & 88.0631 & 158.0924 & 79.5498 & & & 1 \\
\hline
\end{tabular}

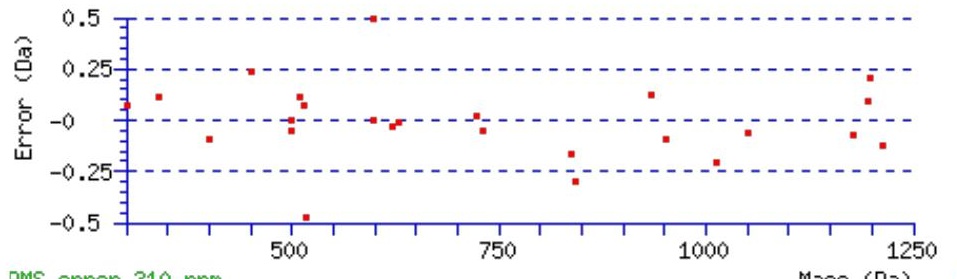

RMS error 310 ppm

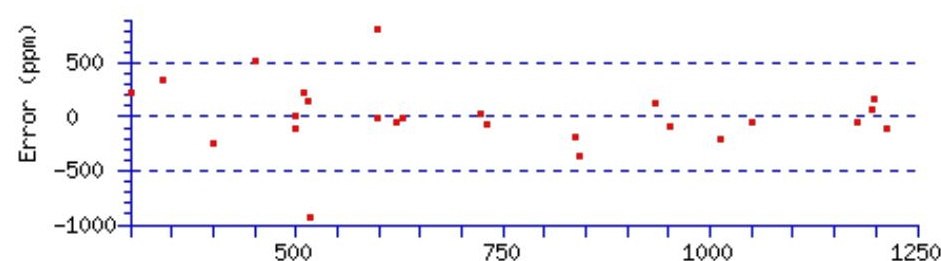

RMS error 310 ppm
Mass (Da) 


\section{All matches to this query}

\begin{tabular}{|l|l|l|l|}
\hline Score & Mr(calc): & Delta & \multicolumn{1}{|c|}{ Sequence } \\
\hline 55.5 & 1349.6728 & -0.0020 & HYVNLTNGLYR \\
\hline 40.9 & 1349.6728 & -0.0020 & $\underline{\text { HYVNLTNGLYR }}$ \\
\hline 9.0 & 1349.6647 & 0.0060 & RSRNTLGSTNSR \\
\hline 6.9 & 1349.6795 & -0.0088 & AVLACINISSMR \\
\hline 6.4 & 1349.6622 & 0.0085 & $\underline{\text { HYCKSISSRGR }}$ \\
\hline 4.6 & 1349.6609 & 0.0099 & $\underline{\text { NSLAELKDSMSR }}$ \\
\hline 4.4 & 1349.6817 & -0.0109 & TVVVASAQRSPR \\
\hline 2.9 & 1349.6704 & 0.0004 & INATLETKQPR \\
\hline 1.3 & 1349.6575 & 0.0132 & GRGETPTSYTGPK \\
\hline 0.3 & 1349.6745 & -0.0037 & $\underline{\text { GSLGIAGFPGIPGK }}$ \\
\hline
\end{tabular}

Spectrum No: 595; Query: 290; Rank: 1

\section{Peptide View}

MS/MS Fragmentation of FINYNQTVSR

Found in IPI00359123, Tax_Id=10116 Gene_Symbol=RGD1310427_predicted hypothetical protein LOC362643

Match to Query 290: 1241.607108 from(621.810830,2+)

Title: 091008RatKidney_NH4Format01_25.1348.1348.2.dta

Data file K:\NewmanPaper\Piliang \3SubProteomes \Piliang3SP \mgf5ppm\ERLIC_3SubProteomes5ppm.mgf
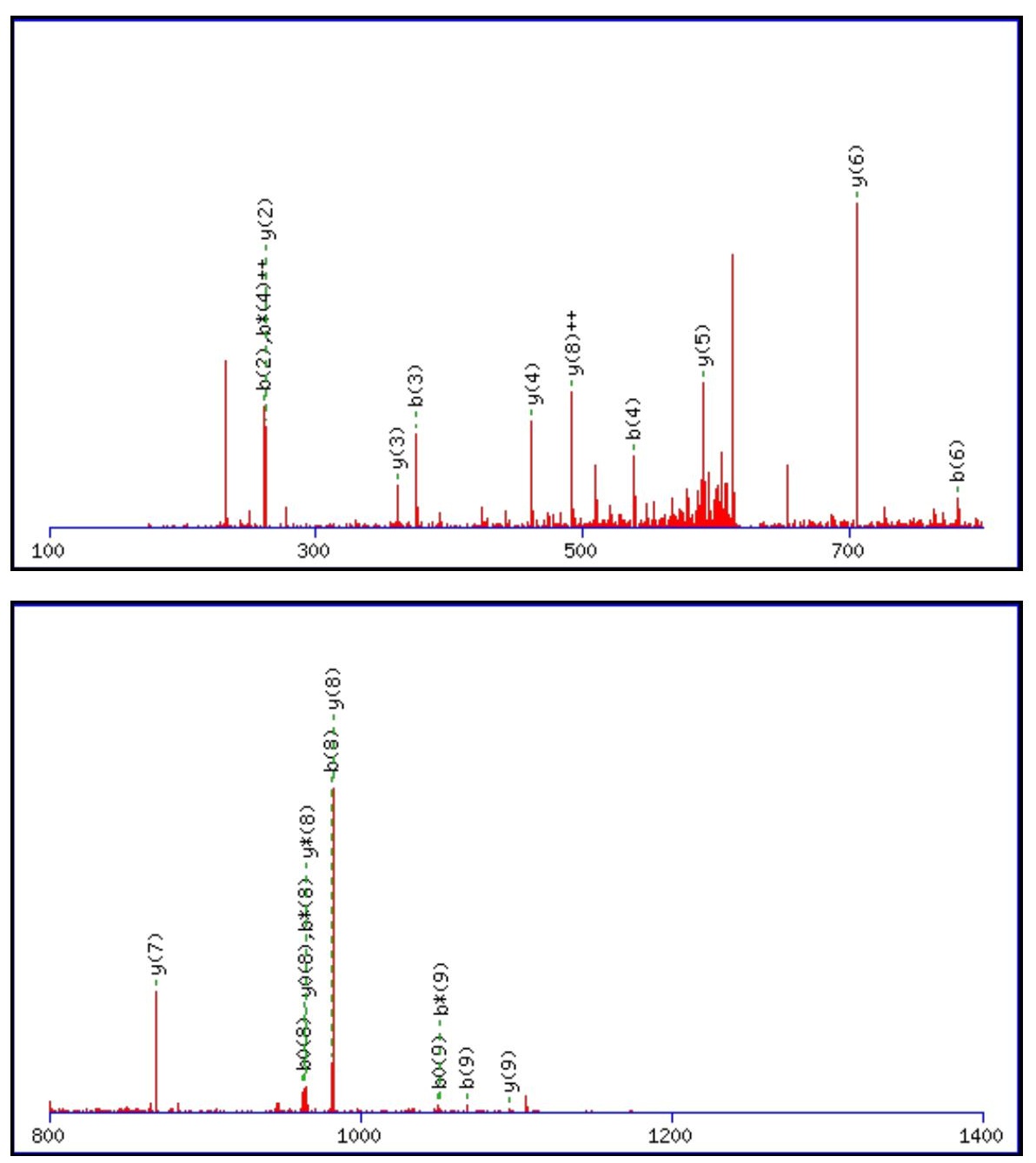


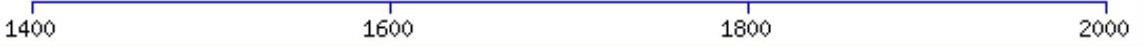

Monoisotopic mass of neutral peptide $\operatorname{Mr}($ calc): 1241.6040

Fixed modifications: Carbamidomethyl (C)

Variable modifications:

N5 : Deamidated_N (N)

Ions Score: 55 Expect: 0.00036

Matches (Bold Red): 22/90 fragment ions using 35 most intense peaks

\begin{tabular}{|r|c|c|c|c|c|c|c|c|c|c|c|c|c|c|}
\hline$\#$ & $\mathbf{b}$ & $\mathbf{b}^{++}$ & $\mathbf{b}^{*}$ & $\mathbf{b}^{\mathbf{*}^{++}}$ & $\mathbf{b}^{\mathbf{0}}$ & $\mathbf{b}^{\mathbf{0 + +}}$ & $\mathbf{S e q}$ & $\mathbf{y}$ & $\mathbf{y}^{++}$ & $\mathbf{y}^{\mathbf{*}}$ & $\mathbf{y}^{\mathbf{*}^{++}}$ & $\mathbf{y}^{\mathbf{0}}$ & $\mathbf{y}^{\mathbf{0 + +}}$ & $\#$ \\
\hline $\mathbf{1}$ & 148.0757 & 74.5415 & & & & & $\mathbf{F}$ & & & & & & & $\mathbf{1 0}$ \\
\hline $\mathbf{2}$ & $\mathbf{2 6 1 . 1 5 9 8}$ & 131.0835 & & & & & $\mathbf{I}$ & $\mathbf{1 0 9 5 . 5 4 2 9}$ & 548.2751 & 1078.5164 & 539.7618 & 1077.5323 & 539.2698 & $\mathbf{9}$ \\
\hline $\mathbf{3}$ & $\mathbf{3 7 5 . 2 0 2 7}$ & 188.1050 & 358.1761 & 179.5917 & & & $\mathbf{N}$ & $\mathbf{9 8 2 . 4 5 8 8}$ & $\mathbf{4 9 1 . 7 3 3 1}$ & $\mathbf{9 6 5 . 4 3 2 3}$ & 483.2198 & $\mathbf{9 6 4 . 4 4 8 3}$ & 482.7278 & $\mathbf{8}$ \\
\hline $\mathbf{4}$ & $\mathbf{5 3 8 . 2 6 6 0}$ & 269.6366 & 521.2395 & $\mathbf{2 6 1 . 1 2 3 4}$ & & & $\mathbf{Y}$ & $\mathbf{8 6 8 . 4 1 5 9}$ & 434.7116 & 851.3894 & 426.1983 & 850.4054 & 425.7063 & $\mathbf{7}$ \\
\hline $\mathbf{5}$ & 653.2929 & 327.1501 & 636.2664 & 318.6368 & & & $\mathbf{N}$ & $\mathbf{7 0 5 . 3 5 2 6}$ & 353.1799 & 688.3260 & 344.6667 & 687.3420 & 344.1746 & $\mathbf{6}$ \\
\hline $\mathbf{6}$ & $\mathbf{7 8 1 . 3 5 1 5}$ & 391.1794 & 764.3250 & 382.6661 & & & $\mathbf{Q}$ & $\mathbf{5 9 0 . 3 2 5 7}$ & 295.6665 & 573.2991 & 287.1532 & 572.3151 & 286.6612 & $\mathbf{5}$ \\
\hline $\mathbf{7}$ & $\mathbf{8 8 2 . 3 9 9 2}$ & 441.7032 & 865.3727 & 433.1900 & 864.3886 & 432.6980 & $\mathbf{T}$ & $\mathbf{4 6 2 . 2 6 7 1}$ & 231.6372 & 445.2405 & 223.1239 & 444.2565 & 222.6319 & $\mathbf{4}$ \\
\hline $\mathbf{8}$ & $\mathbf{9 8 1 . 4 6 7 6}$ & 491.2374 & $\mathbf{9 6 4 . 4 4 1 1}$ & 482.7242 & $\mathbf{9 6 3 . 4 5 7 1}$ & 482.2322 & $\mathbf{V}$ & $3 \mathbf{3 6 1 . 2 1 9 4}$ & 181.1133 & 344.1928 & 172.6001 & 343.2088 & 172.1081 & $\mathbf{3}$ \\
\hline $\mathbf{9}$ & $\mathbf{1 0 6 8 . 4 9 9 6}$ & 534.7535 & $\mathbf{1 0 5 1 . 4 7 3 1}$ & 526.2402 & $\mathbf{1 0 5 0 . 4 8 9 1}$ & 525.7482 & $\mathbf{S}$ & $\mathbf{2 6 2 . 1 5 1 0}$ & 131.5791 & 245.1244 & 123.0659 & 244.1404 & 122.5738 & $\mathbf{2}$ \\
\hline $\mathbf{1 0}$ & & & & & & & $\mathbf{R}$ & 175.1190 & 88.0631 & 158.0924 & 79.5498 & & & $\mathbf{1}$ \\
\hline
\end{tabular}

$$
\begin{aligned}
& \text {. } \\
& \begin{array}{ll}
2 & 0.1 \\
2 & -0 \\
\frac{5}{2} & -0.1 \\
-0.2 &
\end{array}
\end{aligned}
$$$$
\text { 青- - }
$$

error 226 ppm

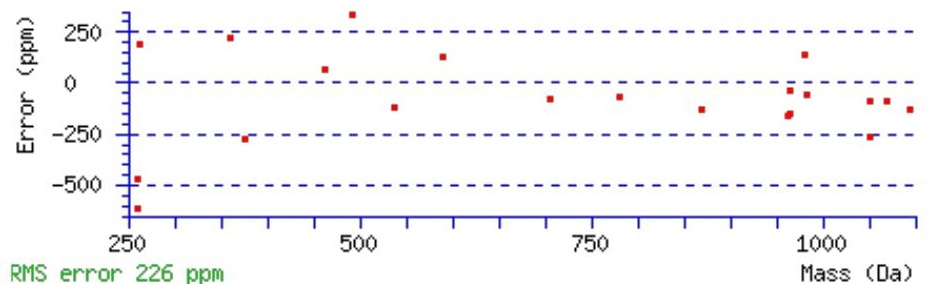

RMS error 226 ppm

\section{All matches to this query}

\begin{tabular}{|l|l|l|l|}
\hline Score & Mr(calc): & Delta & \multicolumn{1}{|c|}{ Sequence } \\
\hline 55.5 & 1241.6040 & 0.0031 & FINYNQTVSR \\
\hline 40.5 & 1241.6040 & 0.0031 & FINYNQTVSR \\
\hline 10.2 & 1240.6138 & 0.9933 & MTSATSPIILK \\
\hline 9.5 & 1240.6009 & 1.0062 & MLISIFSNGNK \\
\hline 9.5 & 1240.6009 & 1.0062 & MLISLFSNGNK \\
\hline 8.7 & 1239.5956 & 2.0115 & GNSAQHSPSSLR \\
\hline 8.2 & 1241.6035 & 0.0036 & MENLSMFLIK \\
\hline 7.8 & 1241.6169 & -0.0098 & FNQILTALSR \\
\hline 7.3 & 1240.6064 & 1.0007 & DSNKSQKLLK \\
\hline 7.1 & 1241.6187 & -0.0115 & CVEKHVEVSR \\
\hline
\end{tabular}


Spectrum No: 596; Query: 2414; Rank: 1

\section{Peptide View}

\section{MS/MS Fragmentation of LCESDHFCVLDVISTGDPSVGNATR}

Found in IPI00191919, Tax_Id=10116 Gene_Symbol=Susd2_predicted sushi domain containing 2

Match to Query 2414: 2749.240332 from(917.420720,3+)

Title: 100101RatKid_NS_deglyco_21.4458.4458.3.dta

Data file K:INewmanPaper|Piliang|3SubProteomes\Piliang3SP\mgf5ppm\ERLIC_3SubProteomes5ppm.mgf
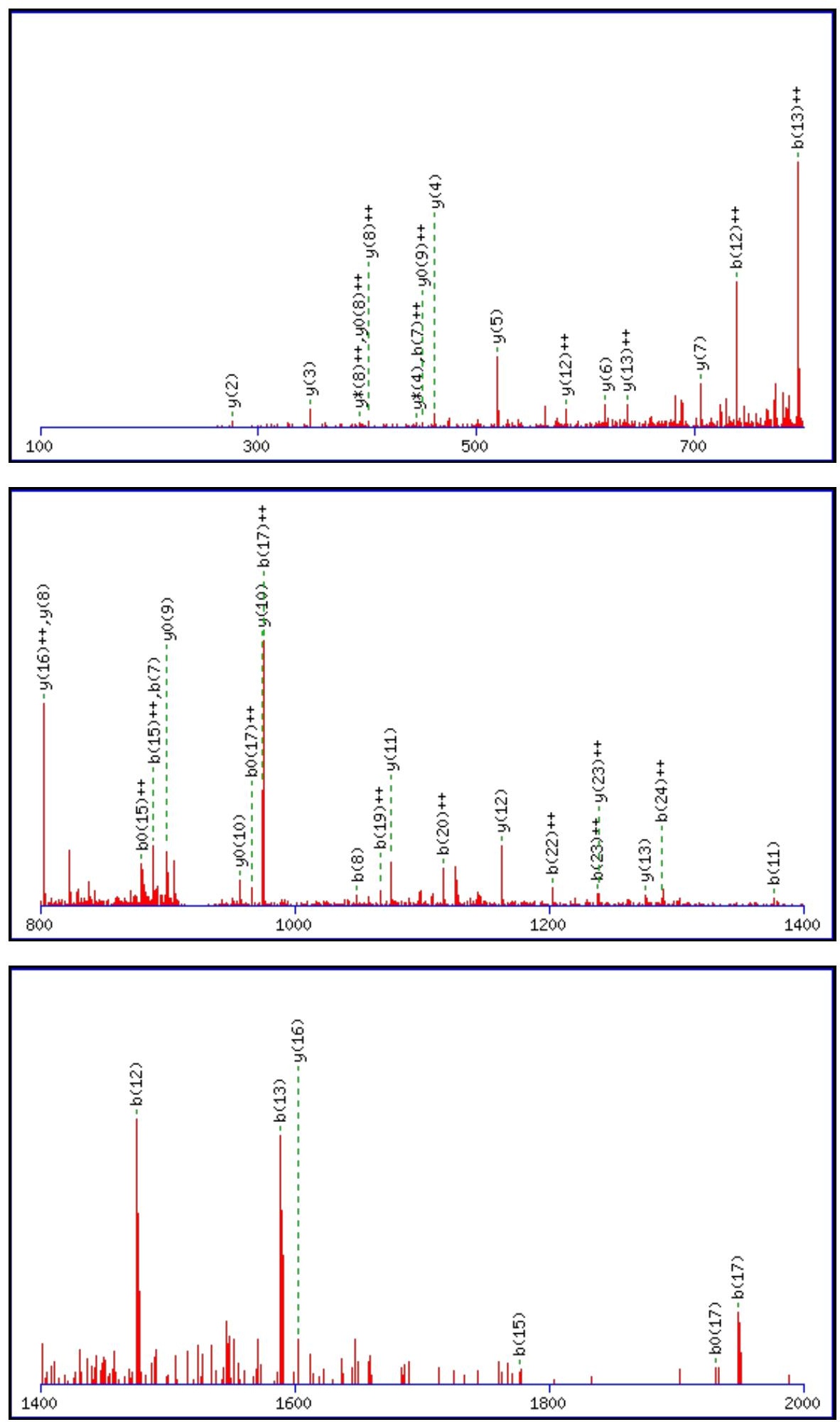

2749.2324 
Monoisotopic mass of neutral peptide $\operatorname{Mr}$ (calc):

Fixed modifications: Carbamidomethyl (C)

Variable modifications:

N22 : Deamidated_N (N)

Ions Score: 55 Expect: $\odot .001$

Matches (Bold Red): 43/240 fragment ions using 86 most intense peaks

\begin{tabular}{|c|c|c|c|c|c|c|c|c|c|c|c|c|c|c|}
\hline \# & b & $\mathbf{b}^{++}$ & $\mathbf{b}^{*}$ & $\mathbf{b}^{*^{++}}$ & $\mathbf{b}^{0}$ & $\mathbf{b}^{0++}$ & Seq. & $\mathbf{y}$ & $\mathrm{y}^{++}$ & $\mathbf{y}^{*}$ & $\mathbf{y}^{*^{++}}$ & $\mathbf{y}^{\mathbf{0}}$ & $\mathbf{y}^{\mathbf{0 + +}}$ & \# \\
\hline 1 & 114.0913 & 57.5493 & & & & & $\mathbf{L}$ & & & & & & & 25 \\
\hline 2 & 274.1220 & 137.5646 & & & & & C & 2637.1556 & 1319.0814 & 2620.1290 & 1310.5681 & 2619.1450 & 1310.0761 & 24 \\
\hline 3 & 403.1646 & 202.0859 & & & 385.1540 & 193.0806 & $\mathbf{E}$ & 2477.1249 & 1239.0661 & 2460.0984 & 1230.5528 & 2459.1143 & 1230.0608 & 23 \\
\hline 4 & 490.1966 & 245.6019 & & & 472.1860 & 236.5967 & $S$ & 2348.0823 & 1174.5448 & 2331.0558 & 1166.0315 & 2330.0717 & 1165.5395 & 22 \\
\hline 5 & 605.2236 & 303.1154 & & & 587.2130 & 294.1101 & D & 2261.0503 & 1131.0288 & 2244.0237 & 1122.5155 & 2243.0397 & 1122.0235 & 21 \\
\hline 6 & 742.2825 & 371.6449 & & & 724.2719 & 362.6396 & $\mathbf{H}$ & 2146.0233 & 1073.5153 & 2128.9968 & 1065.0020 & 2128.0128 & 1064.5100 & 20 \\
\hline 7 & 889.3509 & 445.1791 & & & 871.3403 & 436.1738 & $\mathbf{F}$ & 2008.9644 & 1004.9859 & 1991.9379 & 996.4726 & 1990.9539 & 995.9806 & 19 \\
\hline 8 & 1049.3815 & 525.1944 & & & 1031.3710 & 516.1891 & $\mathrm{C}$ & 1861.8960 & 931.4516 & 1844.8695 & 922.9384 & 1843.8854 & 922.4464 & 18 \\
\hline 9 & 1148.4499 & 574.7286 & & & 1130.4394 & 565.7233 & V & 1701.8654 & 851.4363 & 1684.8388 & 842.9230 & 1683.8548 & 842.4310 & 17 \\
\hline 10 & 1261.5340 & 631.2706 & & & 1243.5234 & 622.2654 & $\mathbf{L}$ & 1602.7969 & 801.9021 & 1585.7704 & 793.3888 & 1584.7864 & 792.8968 & 16 \\
\hline 11 & 1376.5609 & 688.7841 & & & 1358.5504 & 679.7788 & D & 1489.7129 & 745.3601 & 1472.6863 & 736.8468 & 1471.7023 & 736.3548 & 15 \\
\hline 12 & 1475.6294 & 738.3183 & & & 1457.6188 & 729.3130 & $\mathbf{V}$ & 1374.6859 & 687.8466 & 1357.6594 & 679.3333 & 1356.6754 & 678.8413 & 14 \\
\hline 13 & 1588.7134 & 794.8604 & & & 1570.7029 & 785.8551 & I & 1275.6175 & 638.3124 & 1258.5910 & 629.7991 & 1257.6070 & 629.3071 & 13 \\
\hline 14 & 1675.7455 & 838.3764 & & & 1657.7349 & 829.3711 & $S$ & 1162.5335 & 581.7704 & 1145.5069 & 573.2571 & 1144.5229 & 572.7651 & 12 \\
\hline 15 & 1776.7931 & 888.9002 & & & 1758.7826 & 879.8949 & $\mathbf{T}$ & 1075.5014 & 538.2544 & 1058.4749 & 529.7411 & 1057.4909 & 529.2491 & 11 \\
\hline 16 & 1833.8146 & 917.4109 & & & 1815.8040 & 908.4057 & $\mathbf{G}$ & 974.4538 & 487.7305 & 957.4272 & 479.2172 & 956.4432 & 478.7252 & 10 \\
\hline 17 & 1948.8415 & 974.9244 & & & 1930.8310 & 965.9191 & D & 917.4323 & 459.2198 & 900.4057 & 450.7065 & 899.4217 & 450.2145 & 9 \\
\hline 18 & 2045.8943 & 1023.4508 & & & 2027.8837 & 1014.4455 & $\mathbf{P}$ & 802.4054 & 401.7063 & 785.3788 & 393.1930 & 784.3948 & 392.7010 & 8 \\
\hline 19 & 2132.9263 & 1066.9668 & & & 2114.9158 & 1057.9615 & $\mathrm{~S}$ & 705.3526 & 353.1799 & 688.3260 & 344.6667 & 687.3420 & 344.1746 & 7 \\
\hline 20 & 2231.9947 & 1116.5010 & & & 2213.9842 & 1107.4957 & $\mathbf{V}$ & 618.3206 & 309.6639 & 601.2940 & 301.1506 & 600.3100 & 300.6586 & 6 \\
\hline 21 & 2289.0162 & 1145.0117 & & & 2271.0056 & 1136.0065 & G & 519.2521 & 260.1297 & 502.2256 & 251.6164 & 501.2416 & 251.1244 & 5 \\
\hline 22 & 2404.0431 & 1202.5252 & 2387.0166 & 1194.0119 & 2386.0326 & 1193.5199 & $\mathbf{N}$ & 462.2307 & 231.6190 & 445.2041 & 223.1057 & 444.2201 & 222.6137 & 4 \\
\hline 23 & 2475.0803 & 1238.0438 & 2458.0537 & 1229.5305 & 2457.0697 & 1229.0385 & A & 347.2037 & 174.1055 & 330.1772 & 165.5922 & 329.1932 & 165.1002 & 3 \\
\hline 24 & 2576.1279 & 1288.5676 & 2559.1014 & 1280.0543 & 2558.1174 & 1279.5623 & $\mathbf{T}$ & 276.1666 & 138.5870 & 259.1401 & 130.0737 & 258.1561 & 129.5817 & 2 \\
\hline 25 & & & & & & & $\mathbf{R}$ & 175.1190 & 88.0631 & 158.0924 & 79.5498 & & & 1 \\
\hline
\end{tabular}
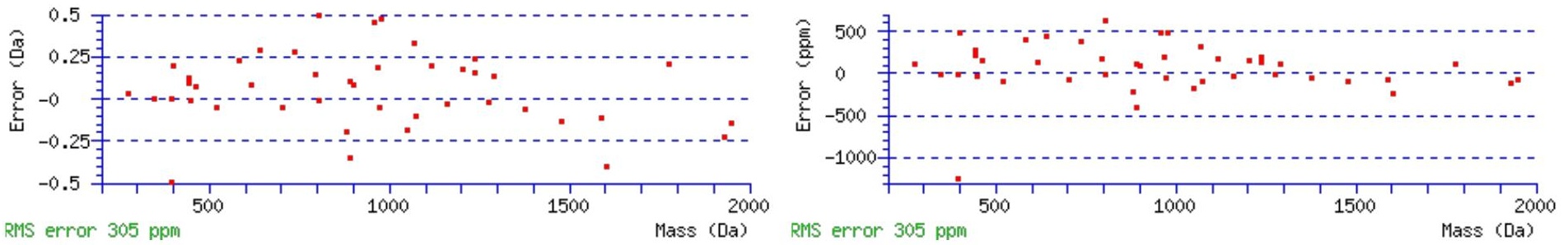

\section{All matches to this query}

\begin{tabular}{|l|c|c|l|}
\hline Score & Mr(calc): & Delta & \multicolumn{1}{c|}{ Sequence } \\
\hline 55.4 & 2749.2324 & 0.0080 & $\underline{\text { LCESDHFCVLDVISTGDPSVGNATR }}$ \\
\hline 10.8 & 2748.2484 & 0.9920 & $\underline{\text { LCESDHFCVLDVISTGDPSVGNATR }}$ \\
\hline 8.4 & 2748.2308 & 1.0095 & $\underline{\text { TTIEEDNIMMKNPDLSVGTTMAIK }}$ \\
\hline 6.1 & 2749.2517 & -0.0114 & SESGGLGVSMVEYVLSSSPADKLDSR \\
\hline 5.9 & 2749.2136 & 0.0268 & SNSLPSTPAVKLESLEESNPEYAK \\
\hline 5.9 & 2749.2136 & 0.0268 & SNSLPSTPAVKLESLEESNPEYAK \\
\hline 5.9 & 2748.2308 & 1.0095 & TTIEEDNIMMKNPDLSVGTTMAIK \\
\hline 5.5 & 2749.2419 & -0.0015 & DENFTLKHVGPGVLSMANAGPNTDGK \\
\hline 5.0 & 2748.2578 & 0.9825 & DENFTLKHVGPGVLSMANAGPNTDGK \\
\hline 4.3 & 2749.2239 & 0.0164 & $\underline{\text { MTHTPAAGSGAARSVSNGNTEAAGGVAAR }}$ \\
\hline
\end{tabular}


Spectrum No: 597; Query: 2176; Rank: 1

\section{Peptide View}

MS/MS Fragmentation of VVMDIPYELWNETSAEVADLK

Found in IPI00371736, Tax_Id=10116 Gene_Symbol=LOC685174 similar to trinucleotide repeat containing 5

Match to Query 2176: 2423.167632 from(808.729820,3+)

Title: 091008RatKidney_NoSalt_19.6582.6582.3.dta

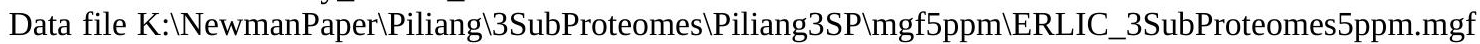
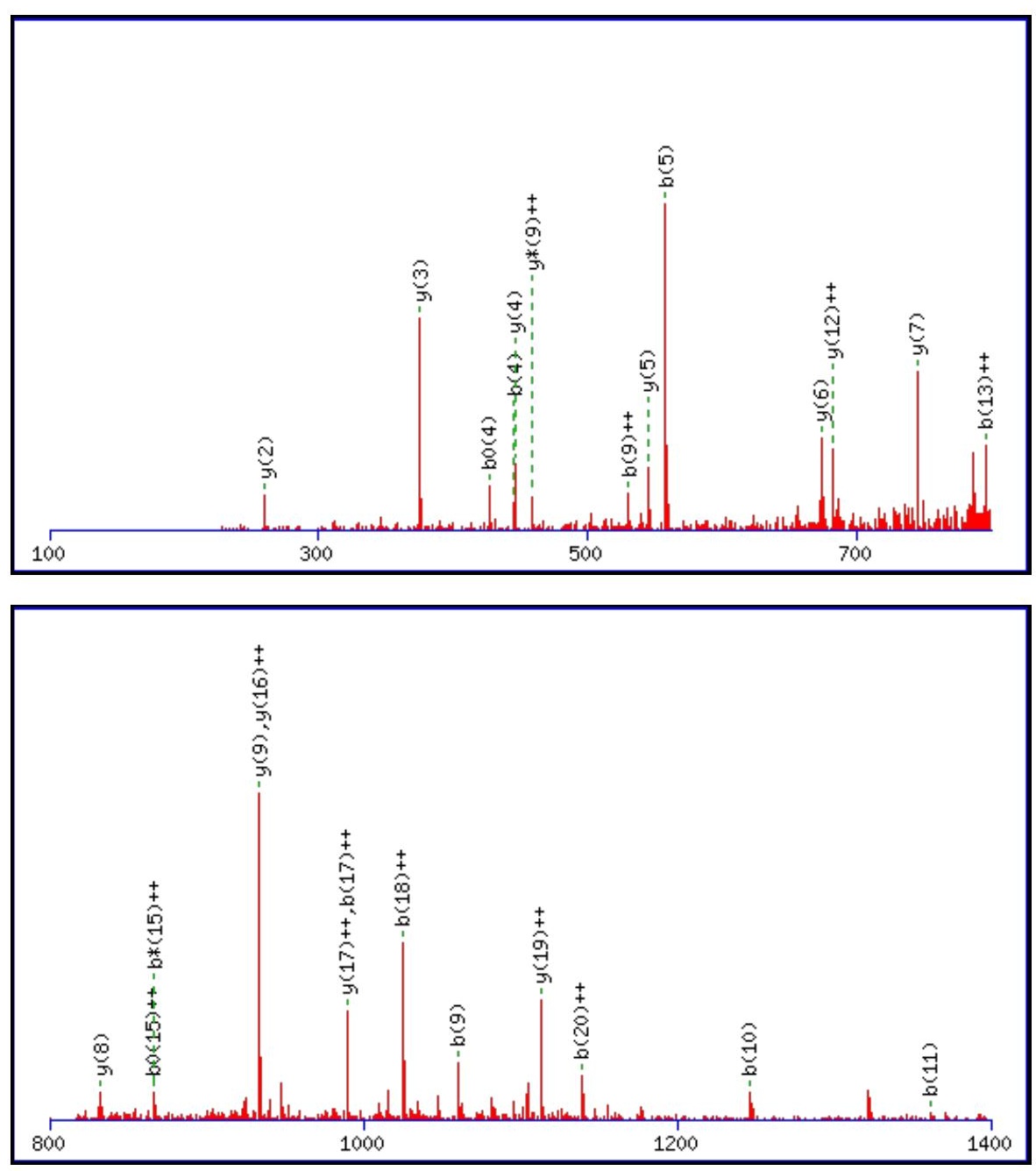


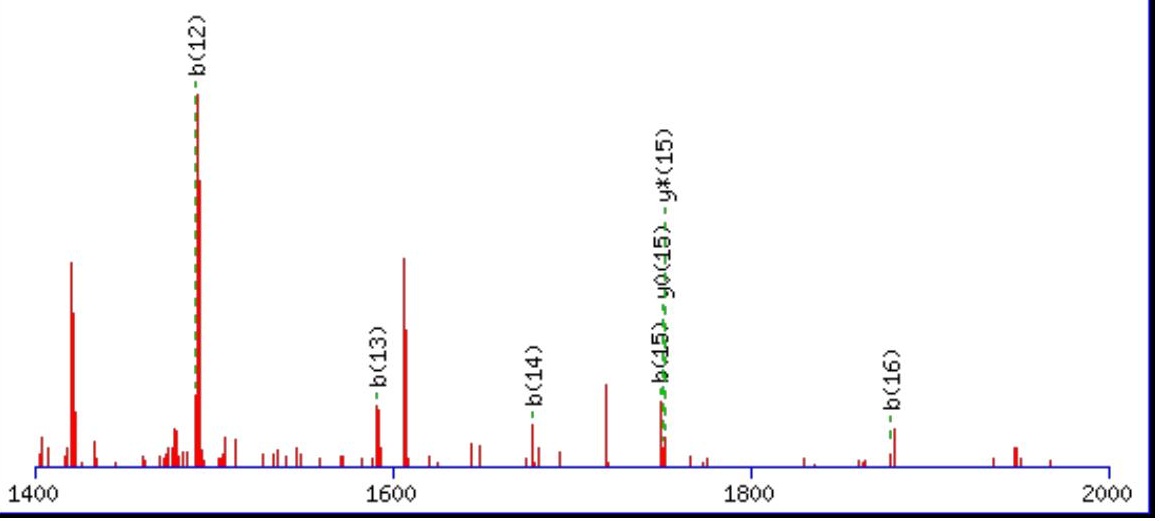

Monoisotopic mass of neutral peptide $\operatorname{Mr}($ calc): 2422.1614

Fixed modifications: Carbamidomethyl (C)

Variable modifications:

N11 : Deamidated_N (N)

Ions Score: 55 Expect: 0.00081

Matches (Bold Red): 33/210 fragment ions using 59 most intense peaks

\begin{tabular}{|c|c|c|c|c|c|c|c|c|c|c|c|c|c|c|}
\hline \# & b & $\mathbf{b}^{++}$ & $\mathbf{b}^{*}$ & $\mathbf{b}^{*^{++}}$ & $\mathbf{b}^{0}$ & $\mathbf{b}^{\mathbf{0 + +}}$ & Seq. & $\mathbf{y}$ & $\mathbf{y}^{++}$ & $\mathbf{y}^{*}$ & $\mathbf{y}^{*^{++}}$ & $\mathbf{y}^{0}$ & $\mathbf{y}^{\mathbf{0 + +}}$ & \# \\
\hline 1 & 100.0757 & 50.5415 & & & & & $\mathbf{V}$ & & & & & & & 21 \\
\hline 2 & 199.1441 & 100.0757 & & & & & V & 2324.1003 & 1162.5538 & 2307.0737 & 1154.0405 & 2306.0897 & 1153.5485 & 20 \\
\hline 3 & 330.1846 & 165.5959 & & & & & $\mathbf{M}$ & 2225.0318 & 1113.0196 & 2208.0053 & 1104.5063 & 2207.0213 & 1104.0143 & 19 \\
\hline 4 & 445.2115 & 223.1094 & & & 427.2010 & 214.1041 & D & 2093.9914 & 1047.4993 & 2076.9648 & 1038.9860 & 2075.9808 & 1038.4940 & 18 \\
\hline 5 & 558.2956 & 279.6514 & & & 540.2850 & 270.6462 & I & 1978.9644 & 989.9858 & 1961.9379 & 981.4726 & 1960.9538 & 980.9806 & 17 \\
\hline 6 & 655.3484 & 328.1778 & & & 637.3378 & 319.1725 & $\mathbf{P}$ & 1865.8803 & 933.4438 & 1848.8538 & 924.9305 & 1847.8698 & 924.4385 & 16 \\
\hline 7 & 818.4117 & 409.7095 & & & 800.4011 & 400.7042 & $\mathbf{Y}$ & 1768.8276 & 884.9174 & 1751.8010 & 876.4042 & 1750.8170 & 875.9121 & 15 \\
\hline 8 & 947.4543 & 474.2308 & & & 929.4437 & 465.2255 & $\mathbf{E}$ & 1605.7643 & 803.3858 & 1588.7377 & 794.8725 & 1587.7537 & 794.3805 & 14 \\
\hline 9 & 1060.5383 & 530.7728 & & & 1042.5278 & 521.7675 & $\mathbf{L}$ & 1476.7217 & 738.8645 & 1459.6951 & 730.3512 & 1458.7111 & 729.8592 & 13 \\
\hline 10 & 1246.6177 & 623.8125 & & & 1228.6071 & 614.8072 & W & 1363.6376 & 682.3224 & 1346.6110 & 673.8092 & 1345.6270 & 673.3172 & 12 \\
\hline 11 & 1361.6446 & 681.3259 & 1344.6180 & 672.8127 & 1343.6340 & 672.3207 & $\mathbf{N}$ & 1177.5583 & 589.2828 & 1160.5317 & 580.7695 & 1159.5477 & 580.2775 & 11 \\
\hline 12 & 1490.6872 & 745.8472 & 1473.6606 & 737.3340 & 1472.6766 & 736.8419 & $\mathbf{E}$ & 1062.5313 & 531.7693 & 1045.5048 & 523.2560 & 1044.5208 & 522.7640 & 10 \\
\hline 13 & 1591.7349 & 796.3711 & 1574.7083 & 787.8578 & 1573.7243 & 787.3658 & $\mathbf{T}$ & 933.4888 & 467.2480 & 916.4622 & 458.7347 & 915.4782 & 458.2427 & 9 \\
\hline 14 & 1678.7669 & 839.8871 & 1661.7403 & 831.3738 & 1660.7563 & 830.8818 & $S$ & 832.4411 & 416.7242 & 815.4145 & 408.2109 & 814.4305 & 407.7189 & 8 \\
\hline 15 & 1749.8040 & 875.4056 & 1732.7775 & 866.8924 & 1731.7934 & 866.4004 & A & 745.4090 & 373.2082 & 728.3825 & 364.6949 & 727.3985 & 364.2029 & 7 \\
\hline 16 & 1878.8466 & 939.9269 & 1861.8201 & 931.4137 & 1860.8360 & 930.9217 & $\mathbf{E}$ & 674.3719 & 337.6896 & 657.3454 & 329.1763 & 656.3614 & 328.6843 & 6 \\
\hline 17 & 1977.9150 & 989.4611 & 1960.8885 & 980.9479 & 1959.9045 & 980.4559 & $\mathbf{V}$ & 545.3293 & 273.1683 & 528.3028 & 264.6550 & 527.3188 & 264.1630 & 5 \\
\hline 18 & 2048.9521 & 1024.9797 & 2031.9256 & 1016.4664 & 2030.9416 & 1015.9744 & A & 446.2609 & 223.6341 & 429.2344 & 215.1208 & 428.2504 & 214.6288 & 4 \\
\hline 19 & 2163.9791 & 1082.4932 & 2146.9525 & 1073.9799 & 2145.9685 & 1073.4879 & D & 375.2238 & 188.1155 & 358.1973 & 179.6023 & 357.2132 & 179.1103 & 3 \\
\hline 20 & 2277.0631 & 1139.0352 & 2260.0366 & 1130.5219 & 2259.0526 & 1130.0299 & $\mathbf{L}$ & 260.1969 & 130.6021 & 243.1703 & 122.0888 & & & 2 \\
\hline 21 & & & & & & & $\mathbf{K}$ & 147.1128 & 74.0600 & 130.0863 & 65.5468 & & & 1 \\
\hline
\end{tabular}

$$
\text { 営 }
$$

$$
\text { 蒠 }
$$
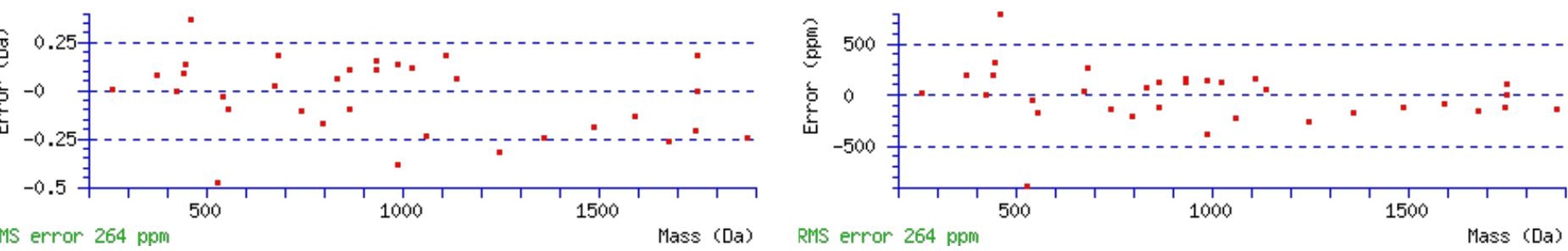

\section{All matches to this query}

\section{Score $\operatorname{Mr}$ (calc): $\quad$ Delta}




\begin{tabular}{|l|l|l|l|}
55.3 & 2422.1614 & 1.0063 & VVMDIPYELWNETSAEVADLK \\
\hline 21.0 & 2421.1774 & 1.9903 & VVMDIPYELWNETSAEVADLK \\
\hline 3.9 & 2423.1441 & 0.0235 & FQVDKVNFHMFDVGGQRDER \\
\hline 3.4 & 2423.1791 & -0.0114 & FWPLMLTNKNTDLESGGNTRK \\
\hline 3.4 & 2423.1767 & -0.0090 & KAKSLEAELMQLQEDLAAAER \\
\hline 2.6 & 2422.1682 & 0.9994 & $\underline{\text { LEAGRFGQLTYVRNYQGELK }}$ \\
\hline 2.4 & 2422.1457 & 1.0219 & KAKNNPDPTIYPVLDWNDIK \\
\hline 1.5 & 2423.1457 & 0.0219 & MAATSARWNHVWVGTETGILK \\
\hline 1.5 & 2423.1457 & 0.0219 & MAATSARWNHVWVGTETGILK \\
\hline 1.1 & 2423.1791 & -0.0114 & FWPLMLTNKNTDLESGGNTRK \\
\hline
\end{tabular}

Spectrum No: 598; Query: 177; Rank: 1

\section{Peptide View}

MS/MS Fragmentation of EEQYNSTFR

Found in IPI00202440, Tax_Id=10116 Gene_Symbol=Igh-1a Igh-1a protein

Match to Query 177: 1173.498548 from(587.756550,2+)

Title: 091008RatKidney_NH4Format01_25.934.934.2.dta

Data file K:\NewmanPaper\Piliang \3SubProteomes \Piliang3SP $\backslash$ mgf5ppm\ERLIC_3SubProteomes5ppm.mgf
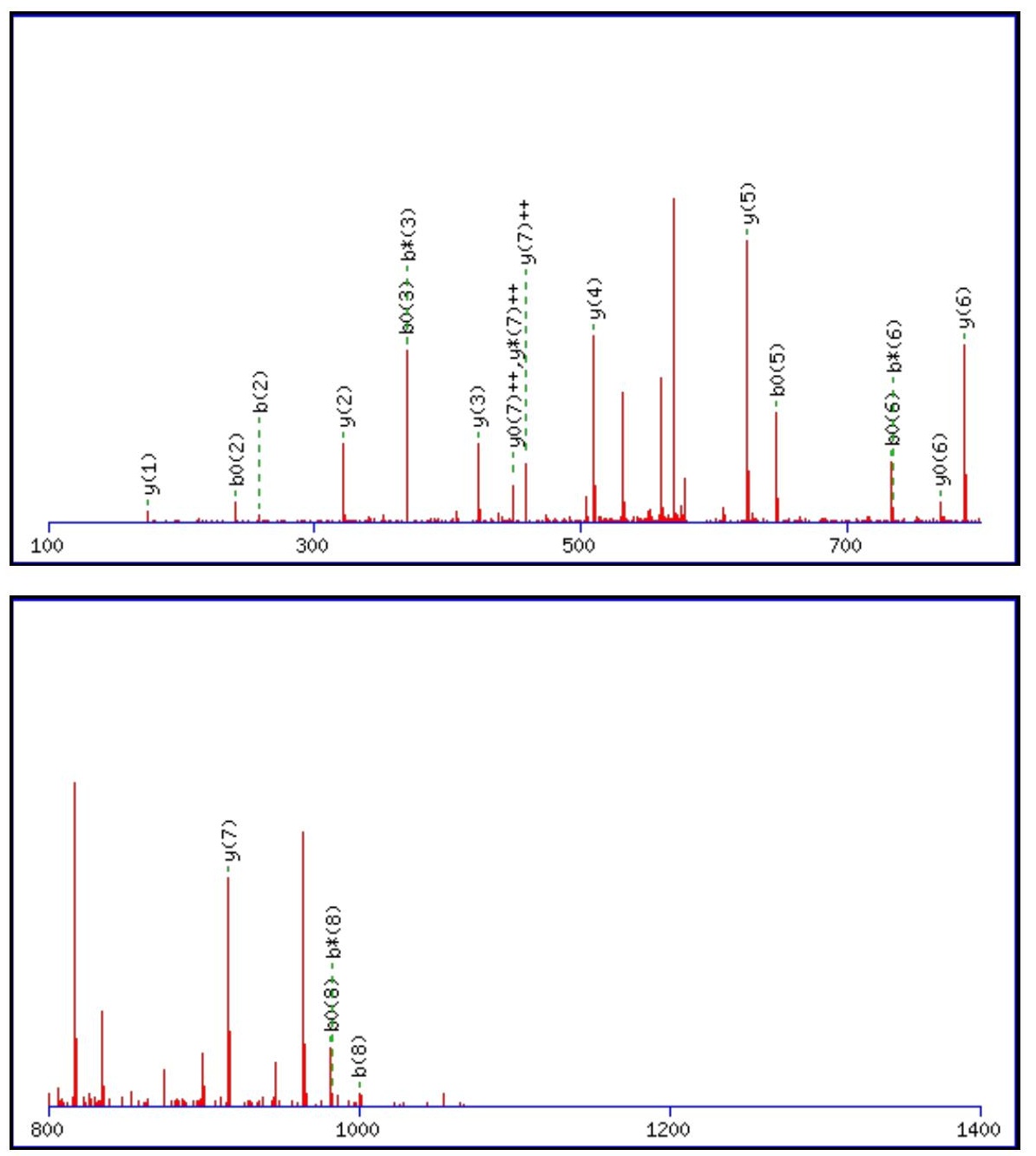


\begin{tabular}{rrrr}
\hline 1400 & 1600 & 1800 & 2000 \\
\hline
\end{tabular}

Monoisotopic mass of neutral peptide $\operatorname{Mr}($ calc): 1173.4938

Fixed modifications: Carbamidomethyl (C)

Variable modifications:

N5 : Deamidated_N (N)

Ions Score: 55 Expect: 0.00028

Matches (Bold Red): 21/88 fragment ions using 29 most intense peaks

\begin{tabular}{|c|c|c|c|c|c|c|c|c|c|c|c|c|c|}
\hline \# & b & $\mathbf{b}^{++}$ & $\mathbf{b}^{*}$ & $\mathbf{b}^{*^{++}}$ & $\mathbf{b}^{0}$ & $\mathbf{b}^{0++}$ & Seq. & $\mathbf{y}$ & $\mathbf{y}^{++}$ & $y^{*}$ & $\mathrm{y}^{*^{++}}$ & $\mathbf{y}^{0}$ & $y^{0++}$ \\
\hline 1 & 130.0499 & 65.5286 & & & 112.0393 & 56.5233 & E & & & & & & \\
\hline 2 & 259.0925 & 130.0499 & & & 241.0819 & 121.0446 & E & 1045.4585 & 523.2329 & 1028.4320 & 514.7196 & 1027.4479 & 514.2276 \\
\hline 3 & 387.1510 & 194.0792 & 370.1245 & 185.5659 & 369.1405 & 185.0739 & $\mathbf{Q}$ & 916.4159 & 458.7116 & 899.3894 & 450.1983 & 898.4054 & 449.7063 \\
\hline 4 & 550.2144 & 275.6108 & 533.1878 & 267.0975 & 532.2038 & 266.6055 & $\mathbf{Y}$ & 788.3573 & 394.6823 & 771.3308 & 386.1690 & 770.3468 & 385.6770 \\
\hline 5 & 665.2413 & 333.1243 & 648.2148 & 324.6110 & 647.2307 & 324.1190 & $\mathbf{N}$ & 625.2940 & 313.1506 & 608.2675 & 304.6374 & 607.2834 & 304.1454 \\
\hline 6 & 752.2733 & 376.6403 & 735.2468 & 368.1270 & 734.2628 & 367.6350 & S & 510.2671 & 255.6372 & 493.2405 & 247.1239 & 492.2565 & 246.6319 \\
\hline 7 & 853.3210 & 427.1641 & 836.2945 & 418.6509 & 835.3104 & 418.1589 & $\mathbf{T}$ & 423.2350 & 212.1212 & 406.2085 & 203.6079 & 405.2245 & 203.1159 \\
\hline 8 & 1000.3894 & 500.6984 & 983.3629 & 492.1851 & 982.3789 & 491.6931 & $\mathbf{F}$ & 322.1874 & 161.5973 & 305.1608 & 153.0840 & & \\
\hline 9 & & & & & & & $\mathbf{R}$ & 175.1190 & 88.0631 & 158.0924 & 79.5498 & & \\
\hline
\end{tabular}
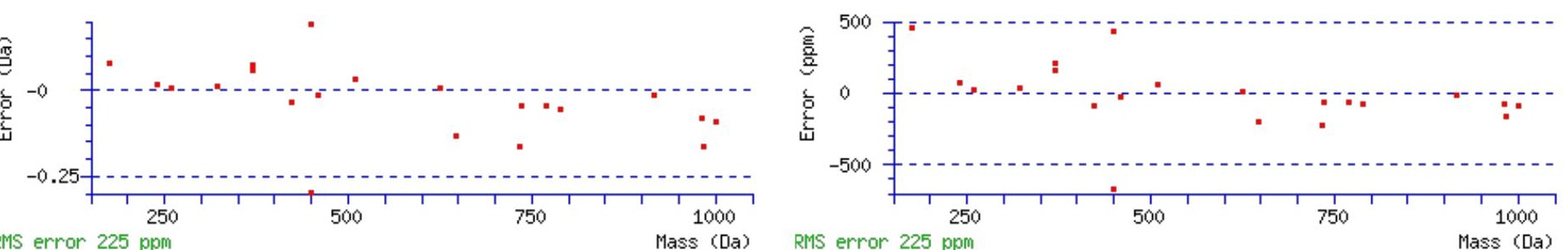

All matches to this query

\begin{tabular}{|l|l|l|l|}
\hline Score & Mr(calc): & Delta & \multicolumn{1}{|c|}{ Sequence } \\
\hline 55.3 & 1173.4938 & 0.0047 & EEQYNSTFR \\
\hline 7.0 & 1173.4873 & 0.0112 & WDPEPNCTR \\
\hline 5.2 & 1173.4988 & -0.0003 & EEKMETLSK \\
\hline 5.2 & 1173.4988 & -0.0003 & EEKMETLSK \\
\hline 4.3 & 1173.5035 & -0.0050 & EKKCCTLR \\
\hline 3.8 & 1172.4897 & 1.0089 & EKAGEGSMLR \\
\hline 3.0 & 1171.5001 & 1.9984 & KYMNSEPMR \\
\hline 2.9 & 1173.5036 & -0.0050 & MTPRLGTMR \\
\hline 2.8 & 1173.4890 & 0.0096 & VPQTDMTFR \\
\hline 2.7 & 1173.4898 & 0.0088 & QDNESNVDPR \\
\hline
\end{tabular}


Spectrum No: 599; Query: 1529; Rank: 1

\section{Peptide View}

MS/MS Fragmentation of RQDPVSWNKTFEDISR

Found in IPI00191437, Tax_Id=10116 Gene_Symbol=- 64 kDa protein

Match to Query 1529: 1977.953262 from(660.325030,3+)

Title: 091008RatKidney_NH4Format01_23.2429.2429.3.dta

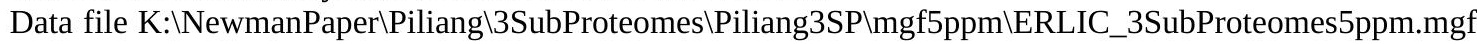
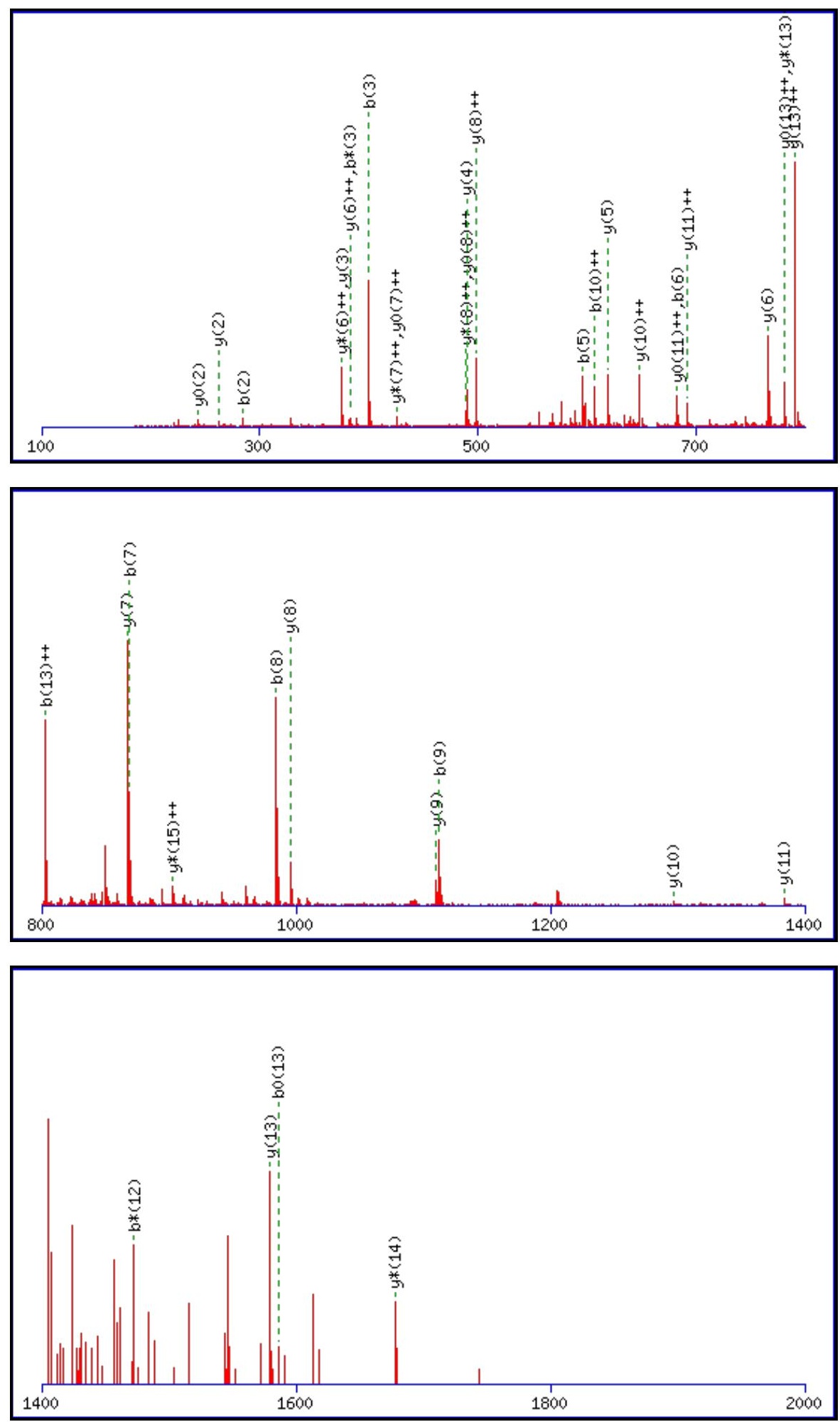

Monoisotopic mass of neutral peptide $\operatorname{Mr}($ calc): 1977.9544

Fixed modifications: Carbamidomethyl (C)

Variable modifications: 
N8 : Deamidated_N (N)

Ions Score: 55 Expect: 0.00066

Matches (Bold Red): 39/174 fragment ions using 67 most intense peaks

\begin{tabular}{|c|c|c|c|c|c|c|c|c|c|c|c|c|c|c|}
\hline \# & b & $\mathbf{b}^{++}$ & $\mathbf{b}^{*}$ & $\mathbf{b}^{*^{++}}$ & $\mathbf{b}^{\mathbf{0}}$ & & Seq. & $\mathbf{y}$ & $\mathbf{y}^{++}$ & $\mathbf{y}^{*}$ & $\mathbf{y}^{*^{++}}$ & $\mathbf{y}^{0}$ & & \# \\
\hline 1 & 157.1084 & 79.0578 & 140.0818 & 70.5446 & & & $\mathbf{R}$ & & & & & & & 16 \\
\hline 2 & 285.1670 & 143.0871 & 268.1404 & 134.5738 & & & $\mathbf{Q}$ & & & 1805.8341 & & 1804.8500 & 902.9287 & 15 \\
\hline 3 & 400.1939 & 200.6006 & 383.1674 & 192.0873 & 382.1833 & & D & & & 1677.7755 & & 1676.7915 & 838.8994 & 14 \\
\hline 4 & 497.2467 & 249.1270 & 480.2201 & 240.6137 & 479.2361 & 240.1217 & $\mathbf{P}$ & 1579.7751 & 790.3912 & 1562.7485 & 781.8779 & 1561.7645 & 781.3859 & 13 \\
\hline 5 & 596.3151 & 298.6612 & 579.2885 & 290.1479 & 578.3045 & 289.6559 & V & 1482.7223 & 741.8648 & 1465.6958 & 733.3515 & 1464.7118 & 732.8595 & 12 \\
\hline 6 & 683.3471 & 342.1772 & 666.3206 & 333.6639 & 665.3365 & 333.1719 & S & 1383.6539 & 692.3306 & 1366.6274 & 683.8173 & 1365.6433 & 683.3253 & 11 \\
\hline 7 & 869.4264 & 435.2169 & 852.3999 & 426.7036 & 851.4159 & 426.2116 & W & 1296.6219 & 648.8146 & 1279.5953 & 640.3013 & 1278.6113 & 639.8093 & 10 \\
\hline 8 & 984.4534 & 492.7303 & 967.4268 & 484.2170 & 966.4428 & 483.7250 & $\mathbf{N}$ & 426 & 555.7749 & 1093.5160 & 547.2616 & 1092.5320 & 546.7696 & 9 \\
\hline 9 & 1112.5483 & 556.7778 & 1095.5218 & 548.2645 & 1094.5378 & 547.7725 & $\mathbf{K}$ & 995.5156 & 498.2615 & 978.4891 & 489.7482 & 977.5051 & 489.2562 & 8 \\
\hline 10 & 1213.5960 & 607.3016 & 1196.5695 & 598.7884 & 1195.5854 & 598.2964 & $\mathbf{T}$ & 867.4207 & 434.2140 & 850.3941 & 425.7007 & 849.4101 & 425.2087 & 7 \\
\hline 11 & 1360.6644 & 680.8358 & 1343.6379 & 672.3226 & 1342.6539 & 671.8306 & $\mathbf{F}$ & 766.3730 & 383.6901 & 749.3464 & 375.1769 & 748.3624 & 374.6849 & 6 \\
\hline 12 & 1489.7070 & 745.3571 & 1472.6805 & 736.8439 & 1471.6964 & 736.3519 & $\mathbf{E}$ & 619.3046 & 310.1559 & 602.2780 & 301.6427 & 601.2940 & 301.1506 & 5 \\
\hline 13 & 1604.7340 & 802.8706 & 1587.7074 & 794.3573 & 1586.7234 & 793.8653 & D & 490.2620 & 245.6346 & 473.2354 & 237.1214 & 472.2514 & 236.6293 & 4 \\
\hline 14 & 1717.8180 & 859.4126 & 1700.7915 & 850.8994 & 1699.8075 & 850.4074 & I & 375.2350 & 188.1212 & 358.2085 & 179.6079 & 357.2245 & 179.1159 & 3 \\
\hline 15 & 1804.8500 & 902.9287 & 1787.8235 & 894.4154 & 1786.8395 & 893.9234 & S & 262.1510 & 131.5791 & 245.1244 & 123.0659 & 244.1404 & 122.5738 & 2 \\
\hline 16 & & & & & & & $\mathbf{R}$ & 175.1190 & 88.0631 & 158.0924 & 79.5498 & & & 1 \\
\hline
\end{tabular}
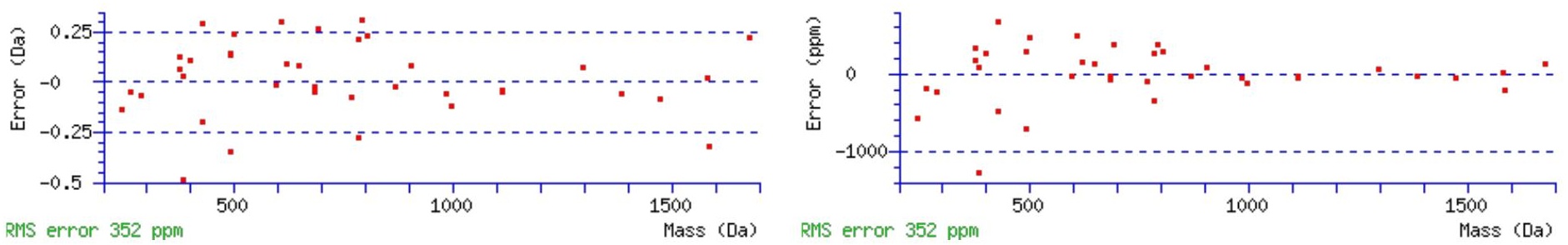

\section{All matches to this query}

\begin{tabular}{|l|l|l|l|}
\hline Score & Mr(calc): & Delta & \multicolumn{1}{c|}{ Sequence } \\
\hline 55.2 & 1977.9544 & -0.0012 & RQDPVSWNKTFEDISR \\
\hline 2.6 & 1975.9517 & 2.0016 & $\underline{\text { VLSLSQALATEASQWHR }}$ \\
\hline 2.3 & 1977.9601 & -0.0068 & $\underline{\text { IGAASLWALTYNYQKAK }}$ \\
\hline 2.3 & 1977.9601 & -0.0068 & IGAASLWALTYNYQKAK \\
\hline 0.9 & 1977.9537 & -0.0004 & $\underline{\text { SISTSTKLVNGQKNITK }}$ \\
\hline
\end{tabular}

Spectrum No: 600; Query: 1529; Rank: 1

\section{Peptide View}

\section{MS/MS Fragmentation of RQDPVSWNKTFEDISR}

Found in IPI00191437, Tax_Id=10116 Gene_Symbol=- 64 kDa protein

Match to Query 1529: 1977.953262 from(660.325030,3+)

Title: 091008RatKidney_NH4Format01_23.2429.2429.3.dta

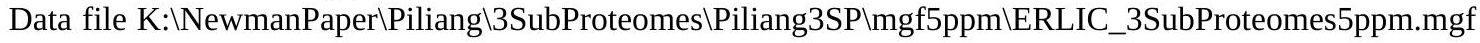



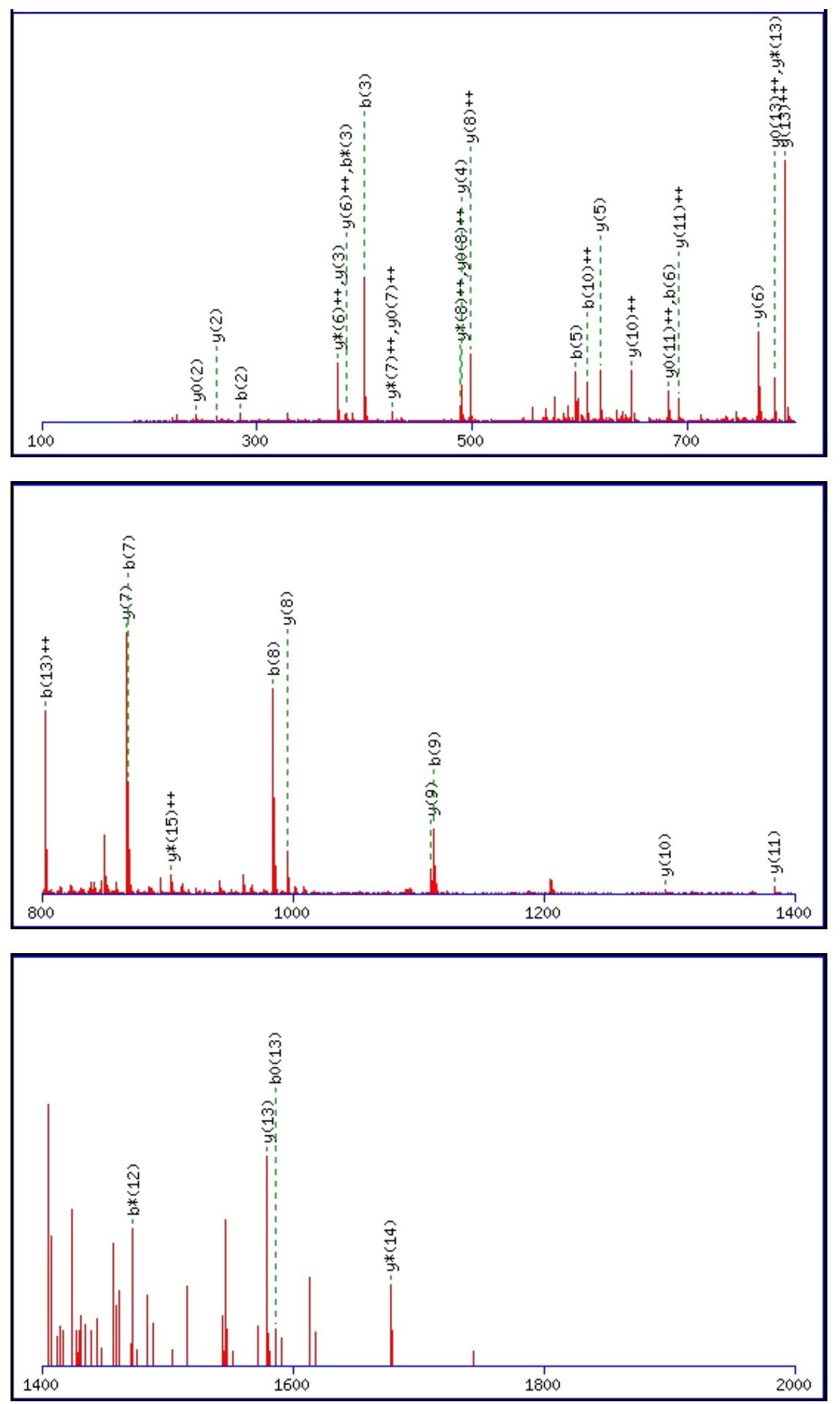

Monoisotopic mass of neutral peptide $\operatorname{Mr}($ calc): 1977.9544

Fixed modifications: Carbamidomethyl (C)

Variable modifications:

N8: Deamidated $\mathrm{N}(\mathrm{N})$

Ions Score: 55 Expect: 0.00066

Matches (Bold Red): 39/174 fragment ions using 67 most intense peaks

\begin{tabular}{|r|c|c|c|c|c|c|c|c|c|c|c|c|c|c|}
\hline$\#$ & $\mathbf{b}$ & $\mathbf{b}^{++}$ & $\mathbf{b}^{*}$ & $\mathbf{b}^{\boldsymbol{*}_{++}}$ & $\mathbf{b}^{\mathbf{0}}$ & $\mathbf{b}^{\mathbf{0 + +}}$ & Seq. & $\mathbf{y}$ & $\mathbf{y}^{++}$ & $\mathbf{y}^{\mathbf{*}}$ & $\mathbf{y}^{\boldsymbol{*}^{++}}$ & $\mathbf{y}^{\mathbf{0}}$ & $\mathbf{y}^{\mathbf{0 + +}}$ & $\#$ \\
\hline $\mathbf{1}$ & 157.1084 & 79.0578 & 140.0818 & 70.5446 & & & $\mathbf{R}$ & & & & & & & $\mathbf{1 6}$ \\
\hline $\mathbf{2}$ & $\mathbf{2 8 5 . 1 6 7 0}$ & 143.0871 & 268.1404 & 134.5738 & & & $\mathbf{Q}$ & 1822.8606 & 911.9339 & 1805.8341 & $\mathbf{9 0 3 . 4 2 0 7}$ & 1804.8500 & 902.9287 & $\mathbf{1 5}$ \\
\hline $\mathbf{3}$ & $\mathbf{4 0 0 . 1 9 3 9}$ & 200.6006 & 383.1674 & 192.0873 & 382.1833 & 191.5953 & $\mathbf{D}$ & 1694.8020 & 847.9047 & $\mathbf{1 6 7 7 . 7 7 5 5}$ & 839.3914 & 1676.7915 & 838.8994 & $\mathbf{1 4}$ \\
\hline $\mathbf{4}$ & 497.2467 & 249.1270 & 480.2201 & 240.6137 & 479.2361 & 240.1217 & $\mathbf{P}$ & $\mathbf{1 5 7 9 . 7 7 5 1}$ & $\mathbf{7 9 0 . 3 9 1 2}$ & 1562.7485 & $\mathbf{7 8 1 . 8 7 7 9}$ & 1561.7645 & $\mathbf{7 8 1 . 3 8 5 9}$ & $\mathbf{1 3}$ \\
\hline $\mathbf{5}$ & $\mathbf{5 9 6 . 3 1 5 1}$ & 298.6612 & 579.2885 & 290.1479 & 578.3045 & 289.6559 & $\mathbf{V}$ & 1482.7223 & 741.8648 & 1465.6958 & 733.3515 & 1464.7118 & 732.8595 & $\mathbf{1 2}$ \\
\hline
\end{tabular}




\begin{tabular}{|c|c|c|c|c|c|c|c|c|c|c|c|c|c|c|}
\hline 6 & 683.3471 & |342.1772 & 666.3206 & 333.6639 & 665.3365 & |333.1719 & $S$ & 1383.6539 & |692.3306 & |1366.6274 & 683.8173 & 1365.6433 & 683.3253 & 11 \\
\hline 7 & 869.4264 & 435.2169 & 852.3999 & 426.7036 & 851.4159 & 426.2116 & W & 1296.6219 & 648.8146 & 1279.5953 & 640.3013 & 1278.6113 & 639.8093 & 10 \\
\hline 8 & 984.4534 & 492.7303 & 967.4268 & 484.2170 & 966.4428 & 483.7250 & $\mathbf{N}$ & 1110.5426 & 555.7749 & 1093.5160 & 547.2616 & 1092.5320 & 546.7696 & 9 \\
\hline 9 & 1112.5483 & 556.7778 & 1095.5218 & 548.2645 & 1094.5378 & 547.7725 & $\mathbf{K}$ & 995.5156 & 498.2615 & 978.4891 & 489.7482 & 977.5051 & 489.2562 & 8 \\
\hline 10 & 1213.5960 & 607.3016 & 1196.5695 & 598.7884 & 1195.5854 & 598.2964 & $\mathbf{T}$ & 867.4207 & 434.2140 & 850.3941 & 425.7007 & 849.4101 & 425.2087 & 7 \\
\hline 11 & 1360.6644 & 680.8358 & 1343.6379 & 672.3226 & 1342.6539 & 671.8306 & $\mathbf{F}$ & 766.3730 & 383.6901 & 749.3464 & 375.1769 & 748.3624 & 374.6849 & 6 \\
\hline 12 & 1489.7070 & 745.3571 & 1472.6805 & 736.8439 & 1471.6964 & 736.3519 & $\mathbf{E}$ & 619.3046 & 310.1559 & 602.2780 & 301.6427 & 601.2940 & 301.1506 & 5 \\
\hline 13 & 1604.7340 & 802.8706 & 1587.7074 & 794.3573 & 1586.7234 & 793.8653 & D & 490.2620 & 245.6346 & 473.2354 & 237.1214 & 472.2514 & 236.6293 & 4 \\
\hline 14 & 1717.8180 & 859.4126 & 1700.7915 & 850.8994 & 1699.8075 & 850.4074 & I & 375.2350 & |188.1212 & 358.2085 & 179.6079 & 357.2245 & 179.1159 & 3 \\
\hline 15 & 1804.8500 & 902.9287 & 1787.8235 & 894.4154 & 1786.8395 & 893.9234 & $\mathrm{~S}$ & 262.1510 & 131.5791 & 245.1244 & 123.0659 & 244.1404 & 122.5738 & 2 \\
\hline 16 & & & & & & & $\mathbf{R}$ & 175.1190 & 88.0631 & 158.0924 & 79.5498 & & & 1 \\
\hline
\end{tabular}
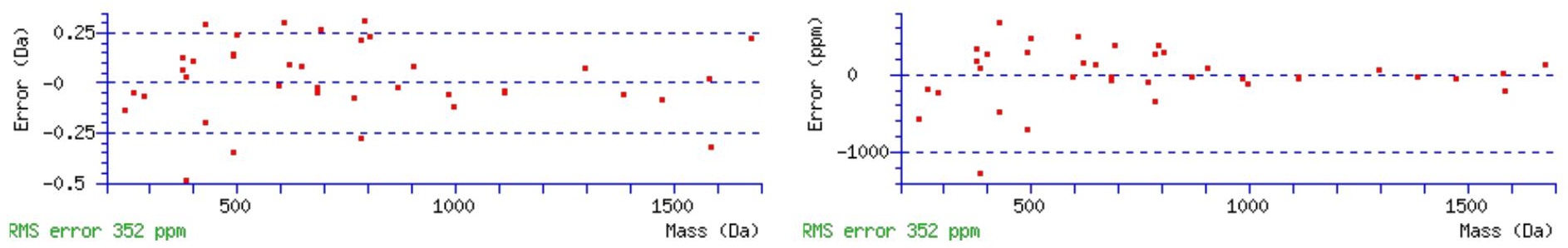

\section{All matches to this query}

\begin{tabular}{|l|l|l|l|}
\hline Score & Mr(calc): & Delta & \multicolumn{1}{c|}{ Sequence } \\
\hline 55.2 & 1977.9544 & -0.0012 & RQDPVSWNKTFEDISR \\
\hline 2.6 & 1975.9517 & 2.0016 & VLSLSQALATEASQWHR \\
\hline 2.3 & 1977.9601 & -0.0068 & IGAASLWALTYNYQKAK \\
\hline 2.3 & 1977.9601 & -0.0068 & IGAASLWALTYNYOKAK \\
\hline 0.9 & 1977.9537 & -0.0004 & SISTSTKLVNGQKNITK \\
\hline
\end{tabular}

Mascot: http://www.matrixscience.com/ 


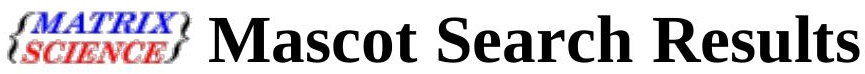

Results Generated by a Script Modified from Mascot Pepitde View

by Newman Sze, School of Biological Sciences, Nanyang Technological University

Spectrum No: 601; Query: 554; Rank: 1

\section{Peptide View}

MS/MS Fragmentation of LSNITNIGPLDMK

Found in IPI00421319, Tax_Id=10116 Gene_Symbol=Tmem106b LRRGT00101

Match to Query 554: 1431.726608 from(716.870580,2+)

Title: 091008RatKidney_NH4Format01_23.2195.2195.2.dta

Data file K:INewmanPaper|Piliang|3SubProteomes\Piliang3SP\mgf5ppm\ERLIC_3SubProteomes5ppm.mgf
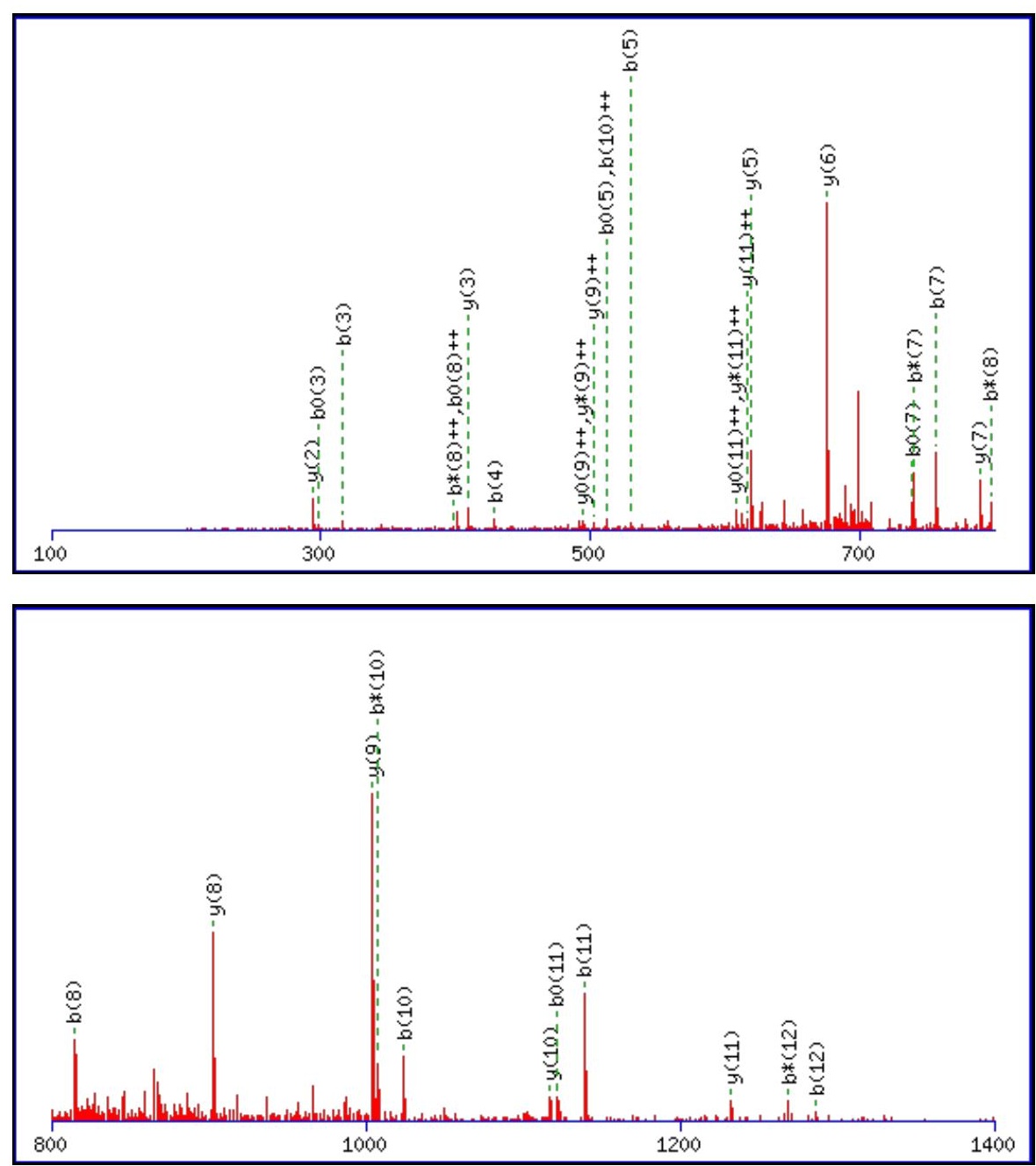


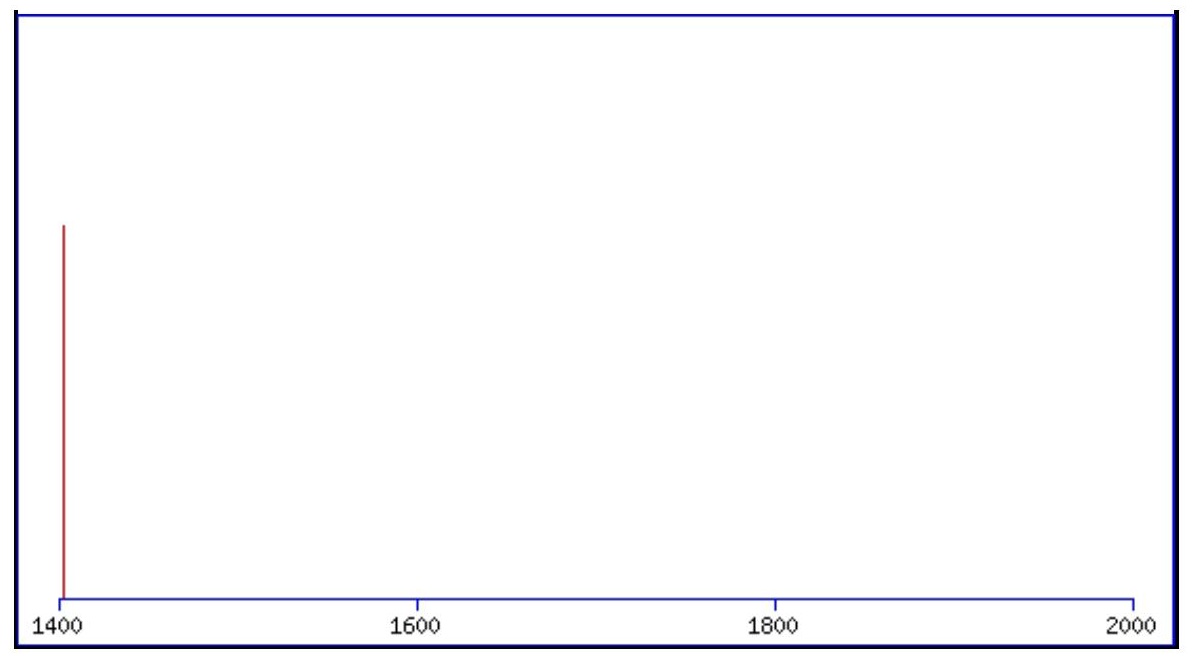

Monoisotopic mass of neutral peptide $\operatorname{Mr}($ calc): 1431.7279

Fixed modifications: Carbamidomethyl (C)

Variable modifications:

N3 : Deamidated_N (N)

M12 : Oxidation (M)

Ions Score: 55 Expect : 0.00041

Matches (Bold Red): 34/134 fragment ions using 55 most intense peaks

\begin{tabular}{|c|c|c|c|c|c|c|c|c|c|c|c|c|c|c|}
\hline \# & b & $\mathbf{b}^{++}$ & $\mathbf{b}^{*}$ & $\mathbf{b}^{*^{++}}$ & $\mathbf{b}^{0}$ & $\mathbf{b}^{0++}$ & Seq. & $\mathbf{y}$ & $y^{++}$ & $\mathbf{y}^{*}$ & $\mathrm{y}^{*^{++}}$ & $\mathbf{y}^{0}$ & $\mathbf{y}^{0++}$ & \# \\
\hline 1 & 114.0913 & 57.5493 & & & & & $\mathbf{L}$ & & & & & & & 13 \\
\hline 2 & 201.1234 & 101.0653 & & & 183.1128 & 92.0600 & $S$ & 1319.6511 & 660.3292 & 1302.6246 & 651.8159 & 1301.6406 & 651.3239 & 12 \\
\hline 3 & 316.1503 & 158.5788 & 299.1238 & 150.0655 & 298.1397 & 149.5735 & $\mathbf{N}$ & 1232.6191 & 616.8132 & 1215.5926 & 608.2999 & 1214.6085 & 607.8079 & 11 \\
\hline 4 & 429.2344 & 215.1208 & 412.2078 & 206.6075 & 411.2238 & 206.1155 & I & 1117.5922 & 559.2997 & 1100.5656 & 550.7865 & 1099.5816 & 550.2944 & 10 \\
\hline 5 & 530.2820 & 265.6447 & 513.2555 & 257.1314 & 512.2715 & 256.6394 & $\mathbf{T}$ & 1004.5081 & 502.7577 & 987.4816 & 494.2444 & 986.4975 & 493.7524 & 9 \\
\hline 6 & 644.3250 & 322.6661 & 627.2984 & 314.1529 & 626.3144 & 313.6608 & $\mathbf{N}$ & 903.4604 & 452.2339 & 886.4339 & 443.7206 & 885.4499 & 443.2286 & 8 \\
\hline 7 & 757.4090 & 379.2082 & 740.3825 & 370.6949 & 739.3985 & 370.2029 & I & 789.4175 & 395.2124 & 772.3910 & 386.6991 & 771.4069 & 386.2071 & 7 \\
\hline 8 & 814.4305 & 407.7189 & 797.4040 & 399.2056 & 796.4199 & 398.7136 & G & 676.3334 & 338.6704 & 659.3069 & 330.1571 & 658.3229 & 329.6651 & 6 \\
\hline 9 & 911.4833 & 456.2453 & 894.4567 & 447.7320 & 893.4727 & 447.2400 & $\mathbf{P}$ & 619.3120 & 310.1596 & 602.2854 & 301.6464 & 601.3014 & 301.1543 & 5 \\
\hline 10 & 1024.5673 & 512.7873 & 1007.5408 & 504.2740 & 1006.5568 & 503.7820 & $\mathbf{L}$ & 522.2592 & 261.6332 & 505.2327 & 253.1200 & 504.2487 & 252.6280 & 4 \\
\hline 11 & 1139.5943 & 570.3008 & 1122.5677 & 561.7875 & 1121.5837 & 561.2955 & D & 409.1752 & 205.0912 & 392.1486 & 196.5779 & 391.1646 & 196.0859 & 3 \\
\hline 12 & 1286.6297 & 643.8185 & 1269.6031 & 635.3052 & 1268.6191 & 634.8132 & $\mathbf{M}$ & 294.1482 & 147.5777 & 277.1217 & 139.0645 & & & 2 \\
\hline 13 & & & & & & & $\mathbf{K}$ & 147.1128 & 74.0600 & 130.0863 & 65.5468 & & & 1 \\
\hline
\end{tabular}
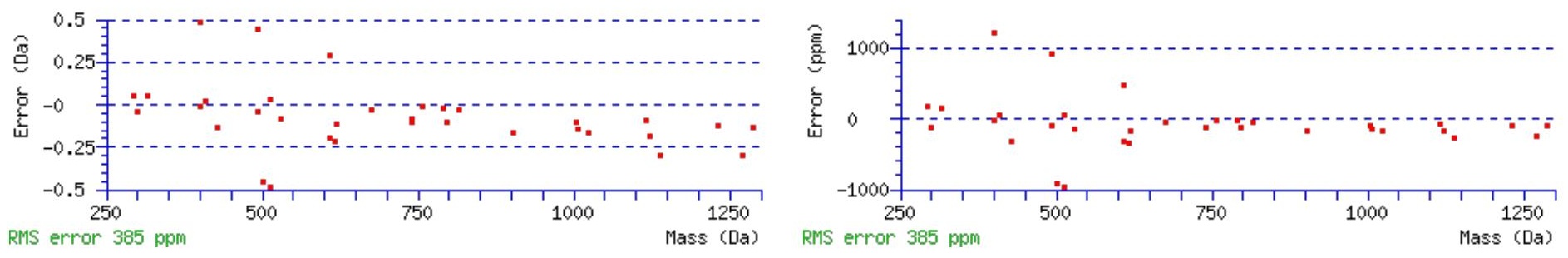

\section{All matches to this query}

\begin{tabular}{|l|l|l|l|}
\hline Score & Mr(calc): & Delta & \multicolumn{1}{c|}{ Sequence } \\
\hline 55.2 & 1431.7279 & -0.0013 & LSNITNIGPLDMK \\
\hline 45.7 & 1431.7279 & -0.0013 & LSNITNIGPLDMK \\
\hline 5.5 & 1431.7252 & 0.0014 & NLISSXLQRGQR \\
\hline 0.8 & 1431.7205 & 0.0061 & ISIDSIQEVSEGR \\
\hline 0.8 & 1431.7163 & 0.0103 & DVLPTPTVVHFK \\
\hline 0.6 & 1429.7218 & 2.0048 & VNTLFTLAKQSK \\
\hline 0.4 & 1431.7309 & -0.0043 & $\underline{\text { AMLSLKSRGYVK }}$ \\
\hline
\end{tabular}


Spectrum No: 602; Query: 2196; Rank: 1

\section{Peptide View}

MS/MS Fragmentation of VATNPQGLVGTENTTSEMDPSHR

Found in IPI00358014, Tax_Id=10116 Gene_Symbol=Btd Biotinidase precursor

Match to Query 2196: 2441.119902 from(814.713910,3+)

Title: 100101RatKid_NS_deglyco_21.2026.2026.3.dta

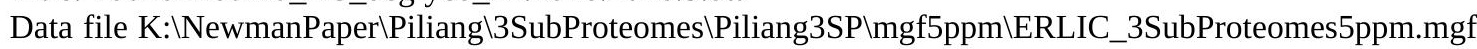
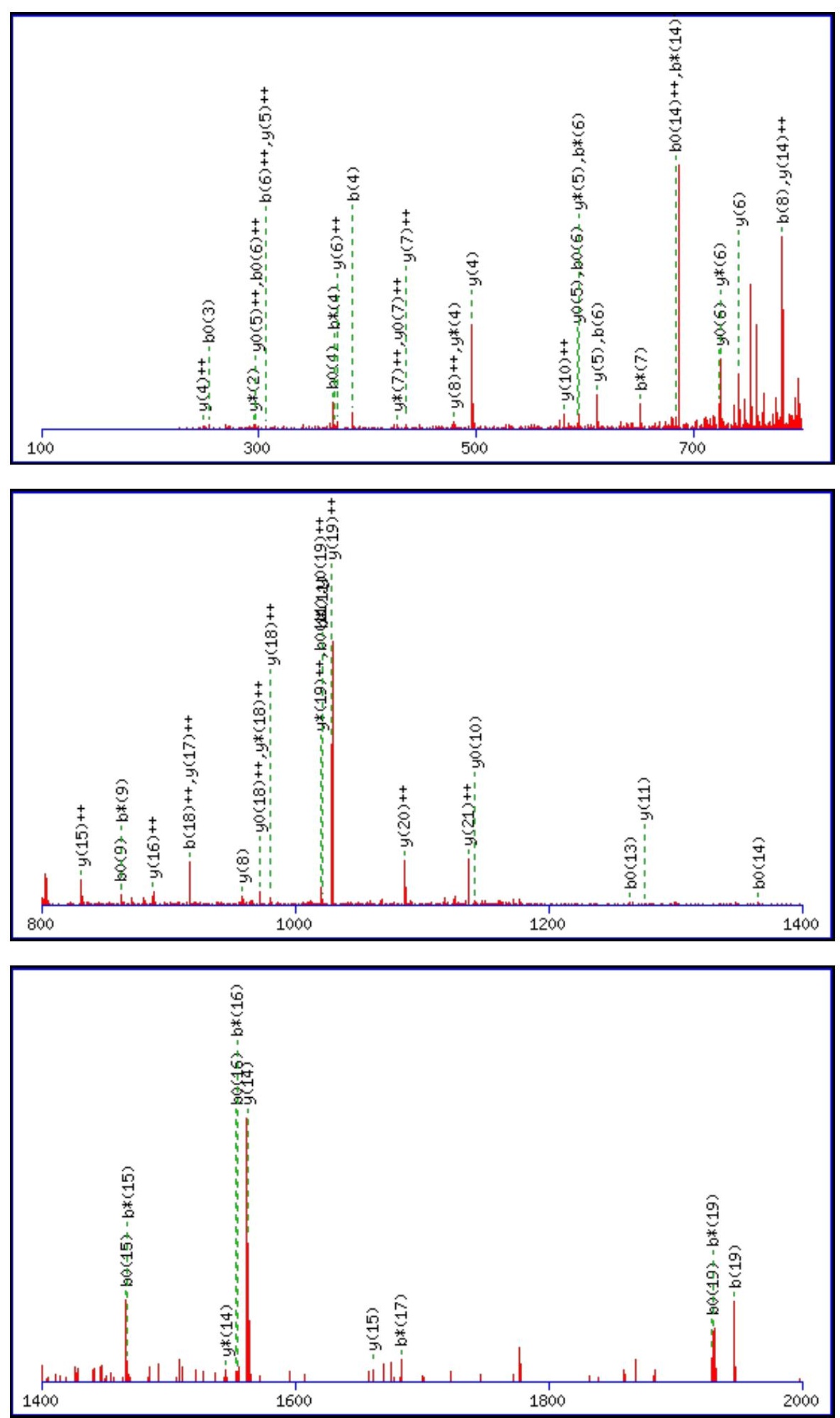
Monoisotopic mass of neutral peptide $\operatorname{Mr}($ calc): 2441.1129

Fixed modifications: Carbamidomethyl (C)

Variable modifications:

N13 : Deamidated_N (N)

Ions Score: 55 Expect: 0.00095

Matches (Bold Red): 64/250 fragment ions using 128 most intense peaks

\begin{tabular}{|c|c|c|c|c|c|c|c|c|c|c|c|c|c|c|}
\hline \# & b & & $\mathbf{b}^{*}$ & & $\mathbf{b}^{\mathbf{0}}$ & & Seq. & $\mathbf{y}$ & & & & & & \# \\
\hline 1 & 100.0757 & 50.5415 & & & & & V & & & & & & & 23 \\
\hline 2 & 171.1128 & 86.0600 & & & & & A & & & 2326.0252 & & & & 22 \\
\hline 3 & 272.1605 & 136.5839 & & & 254.1499 & 127.5786 & $\mathbf{T}$ & 2272.0146 & 1136.5110 & 2254.9881 & 1127.9977 & 2254.0041 & 5057 & 21 \\
\hline 4 & 386.2034 & 193.6053 & 369.1769 & 185.0921 & 368.1928 & 184.6001 & $\mathbf{N}$ & 2170.9669 & 1085.9871 & 9404 & 1077.4738 & 2152.9564 & 1076.9818 & 20 \\
\hline 5 & 483.2562 & 242.1317 & 466.2296 & 233.6185 & 465.2456 & 233.1264 & $\mathbf{P}$ & 2056.9240 & 1028.9656 & 2039.8975 & 1020.4524 & 2038.9135 & 1019.9604 & 19 \\
\hline 6 & 611.3148 & 306.1610 & 594.2 & 297.6477 & 593.3042 & 297.1557 & $\mathbf{Q}$ & 1959.8713 & 980.4393 & 1942.8447 & 971.9260 & 1941.8607 & 971.4340 & 18 \\
\hline 7 & 668.3362 & 334.6717 & 651.3097 & 326.1585 & 650.3257 & 325.6665 & $\mathbf{G}$ & 1831.8127 & 916.4100 & 1814.7861 & 907.8967 & 1813.8021 & 907.4047 & 17 \\
\hline 8 & 781.4203 & 391.2138 & 764.3937 & 382.7005 & 763.4097 & 382.2085 & $\mathbf{L}$ & 1774.7912 & 887.8992 & 1757.7647 & 879.3860 & 1756.7806 & 878.8940 & 16 \\
\hline 9 & 880.4887 & 440.7480 & 863.4621 & 432.2347 & 862.4781 & 431.7427 & $\mathbf{V}$ & 1661.7071 & 831.3572 & 1644.6806 & 822.8439 & 1643.6966 & 822.3519 & 15 \\
\hline 10 & 937.5102 & 469.2587 & 920.4836 & 460.7454 & 919.4996 & 460.2534 & G & 1562.6387 & 781.8230 & 1545.6122 & 773.3097 & 1544.6282 & 772.8177 & 14 \\
\hline 11 & 1038.5578 & 519.7826 & 1021.5313 & 511.2693 & 1020.5473 & 510.7773 & $\mathbf{T}$ & 1505.6173 & 753.3123 & 1488.5907 & 744.7990 & 1487.6067 & 744.3070 & 13 \\
\hline 12 & 1167.6004 & 584.3039 & 1150.5739 & 575.7906 & 1149.5899 & 575.2986 & $\mathbf{E}$ & 1404.5696 & 702.7884 & 1387.5430 & 694.2752 & 1386.5590 & 693.7832 & 12 \\
\hline 13 & 1282.6274 & 641.8173 & 1265.6008 & 633.3040 & 1264.6168 & 632.8120 & $\mathbf{N}$ & 1275.5270 & 638.2671 & 1258. & .7539 & 1257.5164 & 2619 & 11 \\
\hline 14 & 1383.6750 & 692.3412 & 1366.6485 & 683.8279 & 1365.6645 & 683.3359 & $\mathbf{T}$ & 1160.5001 & 580.7537 & 1143.4735 & 572.2404 & 1142.4895 & 571.7484 & 10 \\
\hline 15 & 1484.7227 & 742.8650 & \begin{tabular}{|l|}
1467.6962 \\
\end{tabular} & 734.3517 & 1466.7122 & 733.8597 & $\mathbf{T}$ & 1059.4524 & 530.2298 & 1042.4258 & 521.7166 & 1041.4418 & 245 & 9 \\
\hline 16 & 1571.7548 & 786.3810 & 1554.7282 & 777.8677 & 1553.7442 & 777.3757 & S & 958.4047 & 479.7060 & 941.3782 & 471.1927 & 940.3941 & 470.7007 & 8 \\
\hline 17 & 1700.7973 & 850.9023 & 1683.7708 & 842.3890 & 1682.7868 & 841.8970 & $\mathbf{E}$ & 871.3727 & 436.1900 & 854.3461 & 427.6767 & 853.3621 & 427.1847 & 7 \\
\hline 18 & 1831.8378 & 916.4226 & 1814.8113 & 907.9093 & 1813.8273 & 907.4173 & $\mathbf{M}$ & 742.3301 & 371.6687 & 725.3035 & 363.1554 & 724.3195 & 362.6634 & 6 \\
\hline 19 & 1946.8648 & 973.9360 & \begin{tabular}{|l|}
1929.8382 \\
\end{tabular} & 965.4228 & 1928.8542 & 964.9307 & D & 611.2896 & 306.1484 & 594.2630 & 297.6352 & 593.2790 & 297.1432 & 5 \\
\hline 20 & 2043.9175 & 1022.4624 & 2026.8910 & 1013.9491 & 2025.9070 & 1013.4571 & $\mathbf{P}$ & 496.2627 & 248.6350 & 479.2361 & 240.1217 & 478.2521 & 239.6297 & 4 \\
\hline 21 & 2130.9496 & 1065.9784 & 2113.9230 & 1057.4651 & 2112.9390 & 1056.9731 & S & 399.2099 & 200.1086 & 382.1833 & 191.5953 & 381.1993 & 191.1033 & 3 \\
\hline 22 & 2268.0085 & 1134.5079 & 2250.9819 & 1125.9946 & 2249.9979 & 1125.5026 & $\mathbf{H}$ & 312.1779 & 156.5926 & 295.1513 & 148.0793 & & & 2 \\
\hline 23 & & & & & & & $\mathbf{R}$ & 175.1190 & 88.0631 & 158.0924 & 79.5498 & & & 1 \\
\hline
\end{tabular}
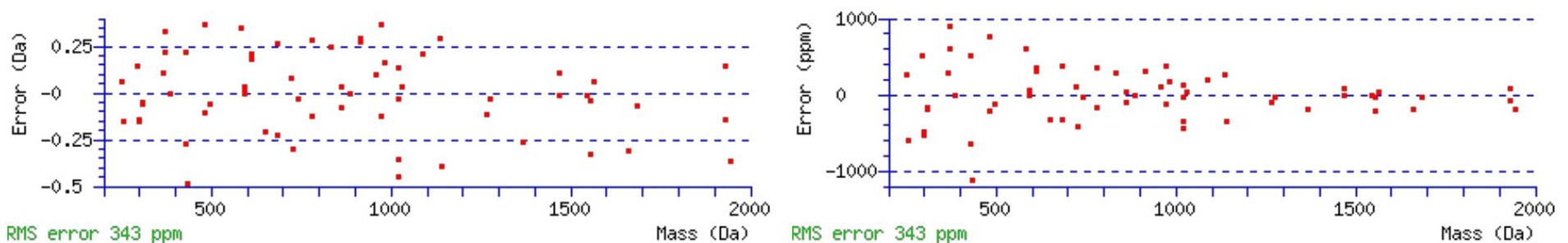

\section{All matches to this query}

\begin{tabular}{|l|l|l|l|}
\hline Score & Mr(calc): & Delta & \multicolumn{1}{|c|}{ Sequence } \\
\hline 55.1 & 2441.1129 & 0.0070 & VATNPQGLVGTENTTSEMDPSHR \\
\hline 29.4 & 2441.1129 & 0.0070 & VATNPQGLVGTENTTSEMDPSHR \\
\hline 14.2 & 2440.1289 & 0.9910 & VATNPQGLVGTENTTSEMDPSHR \\
\hline 5.3 & 2441.1240 & -0.0041 & SKSDLAVSNISPPSPDSKSLPR \\
\hline 3.0 & 2441.1087 & 0.0112 & NCDFSFTGLQHVTDKLITHK \\
\hline 3.0 & 2441.1087 & 0.0112 & NCDFSFTGLQHVTDKLITHK \\
\hline 2.8 & 2441.1033 & 0.0166 & NVDSNLANLIMNEIVDNGTAVK \\
\hline 1.8 & 2440.0993 & 1.0206 & SGSAEPTNNHANGTQNKPRQPR \\
\hline 1.4 & 2441.1240 & -0.0041 & SKSDLAVSNISPPSPDSKSLPR \\
\hline 0.9 & 2439.0930 & 2.0269 & GFEYRTQFTIGMGNLGSHLSK \\
\hline
\end{tabular}


Spectrum No: 603; Query: 87; Rank: 1

\section{Peptide View}

MS/MS Fragmentation of MDGSLNFNR

Found in IPI00202651, Tax_Id=10116 Gene_Symbol=Fga Isoform 1 of Fibrinogen alpha chain precursor

Match to Query 87: 1069.446728 from(535.730640,2+)

Title: 091008RatKidney_NH4Format01_30.790.790.2.dta

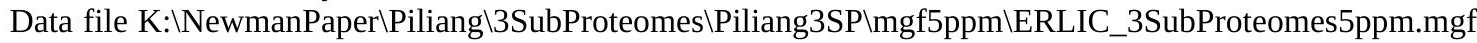
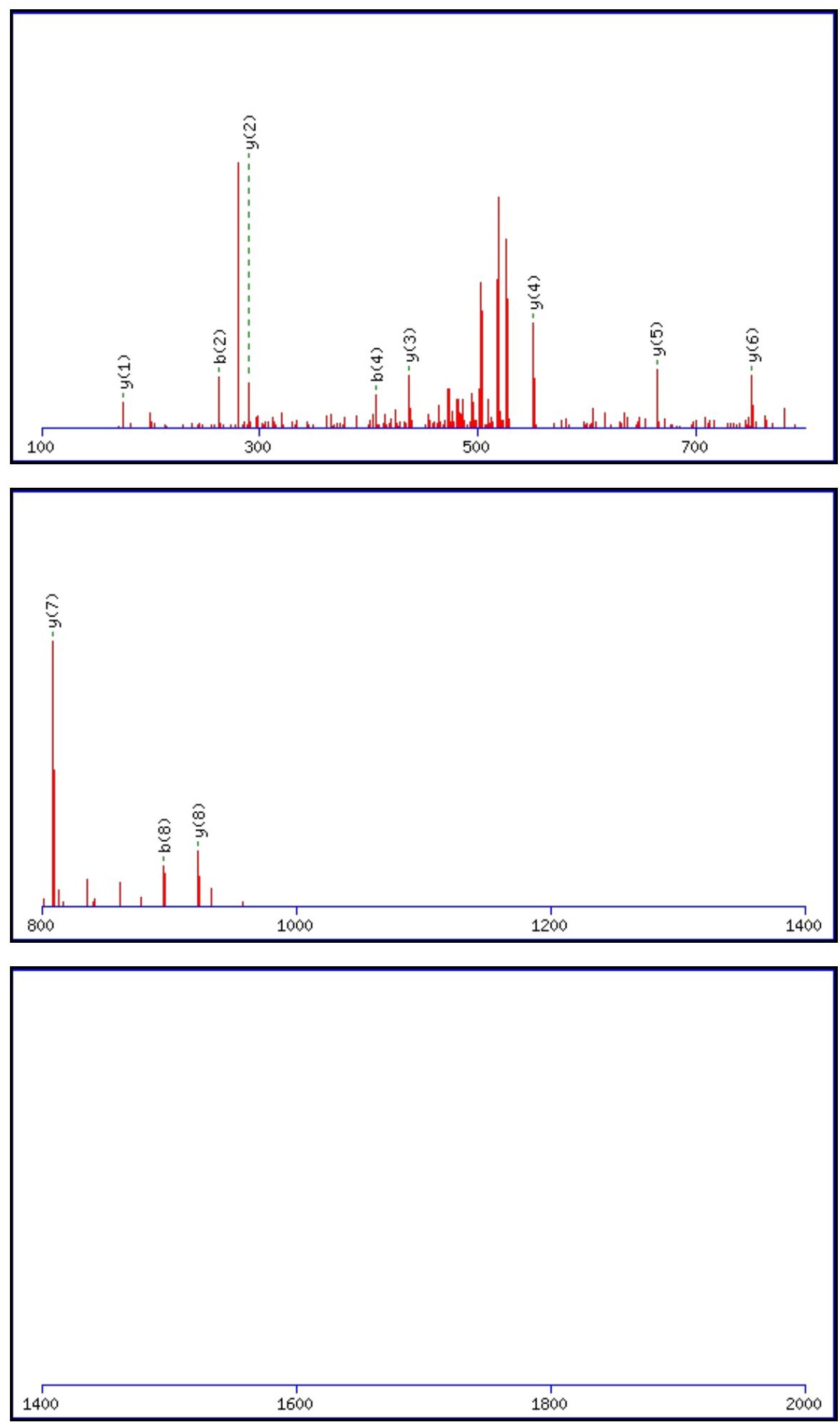

Monoisotopic mass of neutral peptide $\operatorname{Mr}($ calc): 1069.4498 


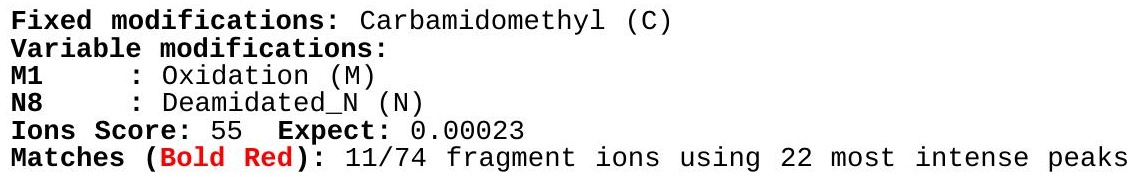

\begin{tabular}{|c|c|c|c|c|c|c|c|c|c|c|c|c|c|c|}
\hline$\#$ & $\mathbf{b}$ & $\mathbf{b}^{++}$ & $\mathbf{b}^{*}$ & $\mathbf{b}^{\boldsymbol{*}^{++}}$ & $\mathbf{b}^{\mathbf{0}}$ & $\mathbf{b}^{\mathbf{0 + +}}$ & $\mathbf{S e q}$ & $\mathbf{y}$ & $\mathbf{y}^{++}$ & $\mathbf{y}^{*}$ & $\mathbf{y}^{\boldsymbol{*}^{++}}$ & $\mathbf{y}^{\mathbf{0}}$ & $\mathbf{y}^{\mathbf{0 + +}}$ & $\#$ \\
\hline $\mathbf{1}$ & $\mathbf{1 4 8 . 0 4 2 7}$ & $\mathbf{7 4 . 5 2 5 0}$ & & & & & $\mathbf{M}$ & & & & & & & $\mathbf{9}$ \\
\hline $\mathbf{2}$ & $\mathbf{2 6 3 . 0 6 9 6}$ & 132.0384 & & & 245.0591 & 123.0332 & $\mathbf{D}$ & $\mathbf{9 2 3 . 4 2 1 7}$ & 462.2145 & 906.3952 & 453.7012 & 905.4112 & 453.2092 & $\mathbf{8}$ \\
\hline $\mathbf{3}$ & 320.0911 & 160.5492 & & & 302.0805 & 151.5439 & $\mathbf{G}$ & $\mathbf{8 0 8 . 3 9 4 8}$ & 404.7010 & 791.3682 & 396.1878 & 790.3842 & 395.6957 & $\mathbf{7}$ \\
\hline $\mathbf{4}$ & $\mathbf{4 0 7 . 1 2 3 1}$ & 204.0652 & & & 389.1125 & 195.0599 & $\mathbf{S}$ & 751.3733 & 376.1903 & 734.3468 & 367.6770 & 733.3628 & 367.1850 & $\mathbf{6}$ \\
\hline $\mathbf{5}$ & 520.2072 & 260.6072 & & & 502.1966 & 251.6019 & $\mathbf{L}$ & $\mathbf{6 6 4 . 3 4 1 3}$ & 332.6743 & 647.3147 & 324.1610 & & & $\mathbf{5}$ \\
\hline $\mathbf{6}$ & 634.2501 & 317.6287 & 617.2236 & 309.1154 & 616.2395 & 308.6234 & $\mathbf{N}$ & $\mathbf{5 5 1 . 2 5 7 2}$ & 276.1323 & 534.2307 & 267.6190 & & & $\mathbf{4}$ \\
\hline $\mathbf{7}$ & 781.3185 & 391.1629 & 764.2920 & 382.6496 & 763.3080 & 382.1576 & $\mathbf{F}$ & $\mathbf{4 3 7 . 2 1 4 3}$ & 219.1108 & 420.1878 & 210.5975 & & & $\mathbf{3}$ \\
\hline $\mathbf{8}$ & $\mathbf{8 9 6 . 3 4 5 5}$ & 448.6764 & 879.3189 & 440.1631 & 878.3349 & 439.6711 & $\mathbf{N}$ & $\mathbf{2 9 0 . 1 4 5 9}$ & 145.5766 & 273.1193 & 137.0633 & & & $\mathbf{2}$ \\
\hline $\mathbf{9}$ & & & & & & & $\mathbf{R}$ & $\mathbf{1 7 5 . 1 1 9 0}$ & 88.0631 & 158.0924 & 79.5498 & & & $\mathbf{1}$ \\
\hline
\end{tabular}
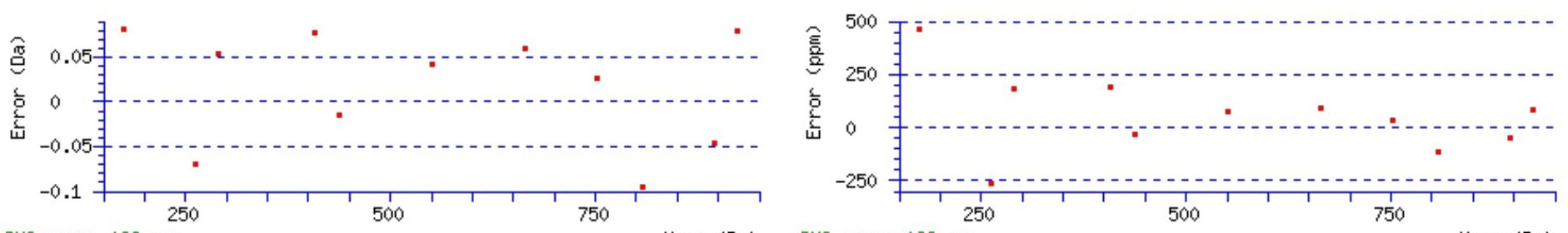

RMS error 189 ppm

\section{All matches to this query}

\begin{tabular}{|l|c|c|l|}
\hline Score & Mr(calc): & Delta & \multicolumn{1}{c|}{ Sequence } \\
\hline 55.1 & 1069.4498 & -0.0031 & MDGSLNFNR \\
\hline 27.1 & 1069.4498 & -0.0031 & MDGSLNFNR \\
\hline 10.2 & 1068.4489 & 0.9979 & VTPQSANSGK \\
\hline 10.1 & 1069.4441 & 0.0027 & SLEAAAESGR \\
\hline 9.2 & 1069.4498 & -0.0031 & AFAADTGMNR \\
\hline 9.1 & 1069.4467 & 0.0000 & MMIGRCNR \\
\hline 8.9 & 1067.4376 & 2.0092 & VCGSTMEER \\
\hline 8.3 & 1069.4441 & 0.0026 & SLDKNNDGK \\
\hline 7.5 & 1068.4488 & 0.9979 & KQATPSNNK \\
\hline 6.2 & 1069.4376 & 0.0092 & LSSCPRNR \\
\hline
\end{tabular}

Spectrum No: 604; Query: 2800; Rank: 1

\section{Peptide View}

MS/MS Fragmentation of YIEQFKDTLNTSVATSIAGFFAEPIQGVNGVVQYPK

Found in IPI00213584, Tax_Id=10116 Gene_Symbol=Agxt2 Alanine--glyoxylate aminotransferase 2, mitochondrial precursor

Match to Query 2800: 3932.004222 from(1311.675350,3+)

Title: 091008RatKidney_NH4Format01_06.5433.5433.3.dta

Data file K:INewmanPaper|Piliangl3SubProteomes\Piliang3SP\mgf5ppm\ERLIC_3SubProteomes5ppm.mgf 

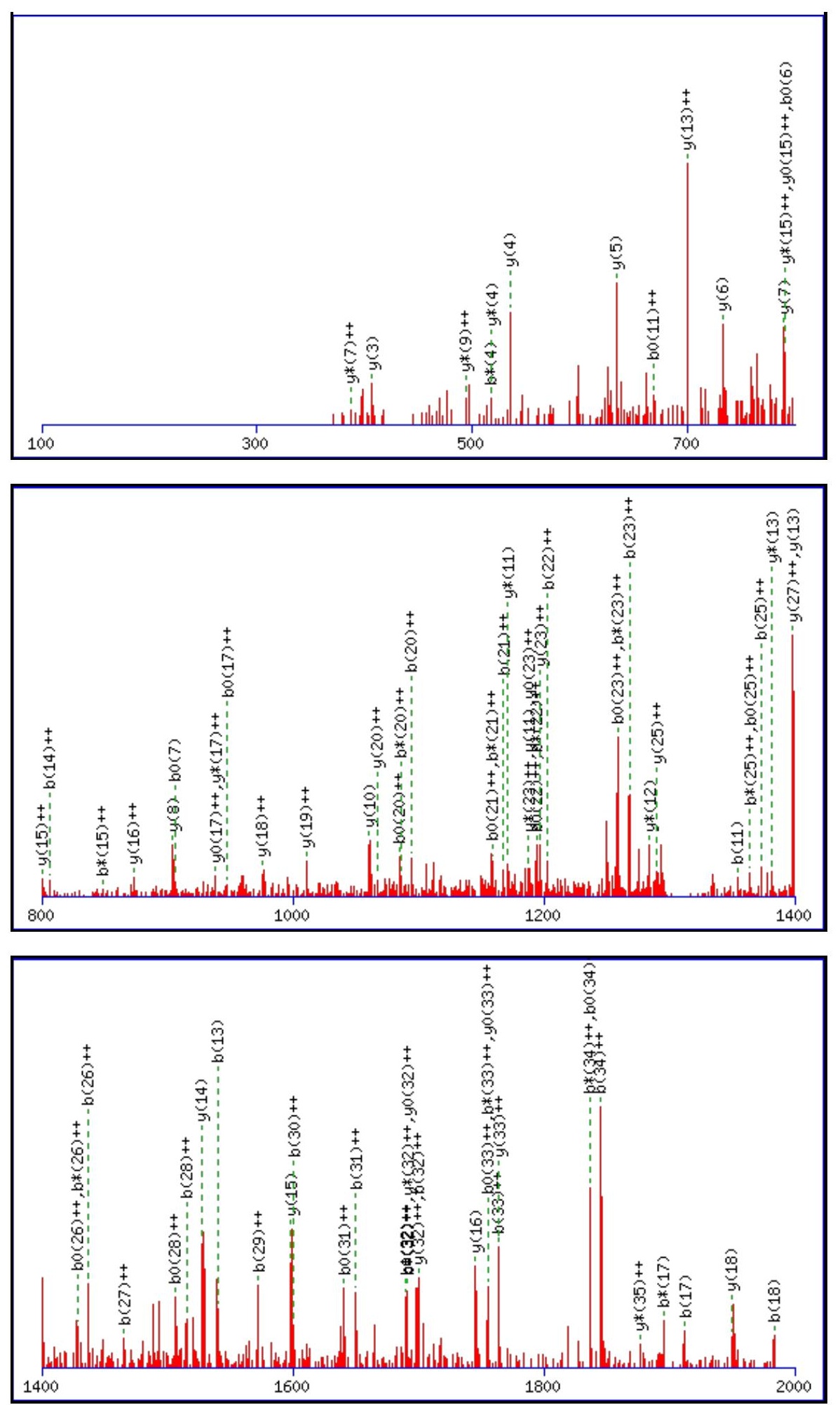

Monoisotopic mass of neutral peptide $\operatorname{Mr}($ calc): 3931.9884

Fixed modifications: Carbamidomethyl (C)

Variable modifications:

N10 : Deamidated_N (N)

Ions Score: 55 Expect: 0.00044

Matches (Bold Red): 86/384 fragment ions using 180 most intense peaks

\begin{tabular}{|c|c|c|c|c|c|c|c|c|c|c|c|c|c|c|}
\hline$\#$ & $\mathbf{b}$ & $\mathbf{b}^{++}$ & $\mathbf{b}^{*}$ & $\mathbf{b}^{\boldsymbol{*}^{++}}$ & $\mathbf{b}^{\mathbf{0}}$ & $\mathbf{b}^{\mathbf{0 + +}}$ & Seq. & $\mathbf{y}$ & $\mathbf{y}^{++}$ & $\mathbf{y}^{\mathbf{*}}$ & $\mathbf{y}^{\mathbf{*}^{++}}$ & $\mathbf{y}^{\mathbf{0}}$ & $\mathbf{y}^{\mathbf{0 + +}}$ & $\#$ \\
\hline $\mathbf{1}$ & 164.0706 & 82.5389 & & & & & $\mathbf{Y}$ & & & & & & & 36 \\
\hline $\mathbf{2}$ & 277.1547 & 139.0810 & & & & & $\mathbf{I}$ & 3769.9323 & 1885.4698 & 3752.9058 & $\mathbf{1 8 7 6 . 9 5 6 5}$ & 3751.9218 & 1876.4645 & $\mathbf{3 5}$ \\
\hline $\mathbf{3}$ & 406.1973 & 203.6023 & & & 388.1867 & 194.5970 & $\mathbf{E}$ & 3656.8483 & 1828.9278 & 3639.8217 & 1820.4145 & 3638.8377 & 1819.9225 & 34 \\
\hline $\mathbf{4}$ & 534.2558 & 267.6316 & 517.2293 & 259.1183 & 516.2453 & 258.6263 & $\mathbf{Q}$ & 3527.8057 & $\mathbf{1 7 6 4 . 4 0 6 5}$ & 3510.7791 & 1755.8932 & 3509.7951 & $\mathbf{1 7 5 5 . 4 0 1 2}$ & $\mathbf{3 3}$ \\
\hline $\mathbf{5}$ & 681.3243 & 341.1658 & 664.2977 & 332.6525 & 663.3137 & 332.1605 & $\mathbf{F}$ & 3399.7471 & $\mathbf{1 7 0 0 . 3 7 7 2}$ & 3382.7205 & $\mathbf{1 6 9 1 . 8 6 3 9}$ & 3381.7365 & $\mathbf{1 6 9 1 . 3 7 1 9}$ & $\mathbf{3 2}$ \\
\hline
\end{tabular}




\begin{tabular}{|c|c|c|c|c|c|c|c|c|c|c|c|c|c|c|}
\hline 6 & 809.4192 & 405.2132 & 792.3927 & 396.7000 & 791.4087 & 396.2080 & $\mathbf{K}$ & 3252.6787 & $|1626.8430|$ & 3235.6521 & & 3234.6681 & 1617.8377| & |31 \\
\hline 7 & 924.4462 & 462.7267 & 907.4196 & 454.2134 & 906.4356 & 453.7214 & D & 3124.5837 & 1562.7955 & 3107.5572 & 1554.2822 & 3106.5732 & 1553.7902 & 30 \\
\hline 8 & 1025.4938 & 513.2506 & 1008.4673 & 504.7373 & 1007.4833 & 504.2453 & $\mathbf{T}$ & 3009.5568 & 1505.2820 & 2992.5302 & 1496.7688 & 2991.5462 & 1496.2767 & 29 \\
\hline 9 & 1138.5779 & 569.7926 & 1121.5514 & 561.2793 & 1120.5673 & 560.7873 & L & 2908.5091 & 1454.7582 & 2891.4825 & 1446.2449 & 2890.4985 & 1445.7529 & 28 \\
\hline 10 & 1253.6048 & 627.3061 & 1236.5783 & 618.7928 & 1235.5943 & 618.3008 & $\mathbf{N}$ & 2795.4250 & 1398.2162 & 2778.3985 & $\mid 1389.7029$ & 2777.4145 & 1389.2109 & 27 \\
\hline 11 & 1354.6525 & 677.8299 & 1337.6260 & 669.3166 & 1336.6420 & 668.8246 & $T$ & 2680.3981 & 1340.7027 & 2663.3715 & 1332.1894 & 2662.3875 & 1331.6974 & 26 \\
\hline 12 & 1441.6845 & 721.3459 & 1424.6580 & 712.8326 & 1423.6740 & 712.3406 & S & 2579.3504 & 1290.1788 & 2562.3239 & 1281.6656 & 2561.3399 & 1281.1736 & 5 \\
\hline 13 & 1540.7530 & 770.8801 & 1523.7264 & 762.3668 & 1522.7424 & 761.8748 & V & 2492.3184 & 1246.6628 & 2475.2918 & 1238.1496 & 2474.3078 & 1237.6576 & 24 \\
\hline 14 & 1611.7901 & 806.3987 & 1594.7635 & 797.8854 & 1593.7795 & 797.3934 & A & 2393.2500 & 1197.1286 & 2376.2234 & 1188.6154 & 2375.2394 & 1188.1233 & 23 \\
\hline 15 & 1712.8378 & 856.9225 & 1695.8112 & 848.4092 & 1694.8272 & 847.9172 & $T$ & 2322.2129 & 1161.6101 & 2305.1863 & $\mid 1153.0968$ & 2304.2023 & 1152.6048 & 22 \\
\hline 16 & 1799.8698 & 900.4385 & 1782.8432 & 891.9253 & 1781.8592 & 891.4332 & S & 2221.1652 & 1111.0862 & 2204.1386 & 1102.5730 & 2203.1546 & 1102.0809 & 21 \\
\hline 17 & 1912.9538 & 956.9806 & 1895.9273 & 948.4673 & 1894.9433 & 947.9753 & $\mathbf{I}$ & 2134.1332 & 1067.5702 & 2117.1066 & $\mid 1059.0569$ & 2116.1226 & 1058.5649 & 20 \\
\hline 18 & 1983.9910 & 992.4991 & 1966.9644 & 983.9858 & 1965.9804 & 983.4938 & A & 2021.0491 & 1011.0282 & 2004.0225 & 1002.5149 & 2003.0385 & 1002.0229 & 19 \\
\hline 19 & 2041.0124 & 1021.0098 & 2023.9859 & 1012.4966 & 2023.0019 & 1012.0046 & G & 1950.0120 & 975.5096 & 1932.9854 & 966.9964 & 1932.0014 & 966.5043 & 18 \\
\hline 20 & 2188.0808 & 1094.5441 & 2171.0543 & 1086.0308 & 2170.0703 & 1085.5388 & $\mathbf{F}$ & 1892.9905 & 946.9989 & 1875.9640 & 938.4856 & 1874.9799 & 937.9936 & 17 \\
\hline 21 & 2335.1493 & 1168.0783 & 2318.1227 & 1159.5650 & 2317.1387 & 1159.0730 & $\mathbf{F}$ & 1745.9221 & 873.4647 & 1728.8956 & 864.9514 & 1727.9115 & 864.4594 & 16 \\
\hline 22 & 2406.1864 & 1203.5968 & 2389.1598 & 1195.0835 & 2388.1758 & 1194.5915 & A & 1598.8537 & 799.9305 & 1581.8271 & 791.4172 & 1580.8431 & 790.9252 & 15 \\
\hline 23 & 2535.2290 & 1268.1181 & 2518.2024 & 1259.6048 & 2517.2184 & 1259.1128 & $\mathbf{E}$ & 1527.8166 & 764.4119 & 1510.7900 & 755.8986 & 1509.8060 & 755.4066 & 14 \\
\hline 24 & 2632.2817 & 1316.6445 & 2615.2552 & 1308.1312 & 2614.2712 & 1307.6392 & $\mathbf{P}$ & 1398.7740 & 699.8906 & 1381.7474 & 691.3774 & & & 13 \\
\hline 25 & 2745.3658 & 1373.1865 & 2728.3392 & 1364.6733 & 2727.3552 & 1364.1812 & I & 1301.7212 & 651.3642 & 1284.6947 & 642.8510 & & & 12 \\
\hline 26 & 2873.4244 & 1437.2158 & 2856.3978 & 1428.7025 & 2855.4138 & 1428.2105 & $\mathbf{Q}$ & 1188.6371 & 594.8222 & 1171.6106 & 586.3089 & & & 11 \\
\hline 27 & 2930.4458 & \begin{tabular}{|l|}
1465.7266 \\
\end{tabular} & 2913.4193 & 1457.2133 & 2912.4353 & 1456.7213 & G & 1060.5786 & 530.7929 & 1043.5520 & 522.2796 & & & 10 \\
\hline 28 & 3029.5142 & 1515.2608 & 3012.4877 & 1506.7475 & 3011.5037 & 1506.2555 & $\mathbf{V}$ & 1003.5571 & 502.2822 & 986.5306 & 493.7689 & & & 9 \\
\hline 29 & 3143.5572 & 1572.2822 & 3126.5306 & 1563.7689 & 3125.5466 & 1563.2769 & $\mathbf{N}$ & 904.4887 & 452.7480 & 887.4621 & 444.2347 & & & 8 \\
\hline 30 & 3200.5786 & 1600.7930 & 3183.5521 & 1592.2797 & 3182.5681 & 1591.7877 & G & 790.4458 & 395.7265 & 773.4192 & 387.2132 & & & 7 \\
\hline 31 & 3299.6470 & 1650.3272 & 3282.6205 & 1641.8139 & 3281.6365 & 1641.3219 & V & 733.4243 & 367.2158 & 716.3978 & 358.7025 & & & 6 \\
\hline 32 & 3398.7155 & 1699.8614 & 3381.6889 & 1691.3481 & 3380.7049 & 1690.8561 & $\mathbf{V}$ & 634.3559 & 317.6816 & 617.3293 & 309.1683 & & & 5 \\
\hline 33 & 3526.7740 & 1763.8907 & 3509.7475 & 1755.3774 & 3508.7635 & 1754.8854 & $\mathbf{Q}$ & 535.2875 & 268.1474 & 518.2609 & 259.6341 & & & 4 \\
\hline 34 & 3689.8374 & 1845.4223 & 3672.8108 & \begin{tabular}{|l}
1836.9090 \\
\end{tabular} & 3671.8268 & 1836.4170 & $\mathbf{Y}$ & 407.2289 & 204.1181 & 390.2023 & 195.6048 & & & 3 \\
\hline 35 & 3786.8901 & 1893.9487 & 3769.8636 & 1885.4354 & 3768.8796 & 1884.9434 & $\mathbf{P}$ & 244.1656 & 122.5864 & 227.1390 & 114.0731 & & & 2 \\
\hline 36 & & & & & & & $\mathbf{K}$ & 147.1128 & 74.0600 & 130.0863 & 65.5468 & & & 1 \\
\hline
\end{tabular}
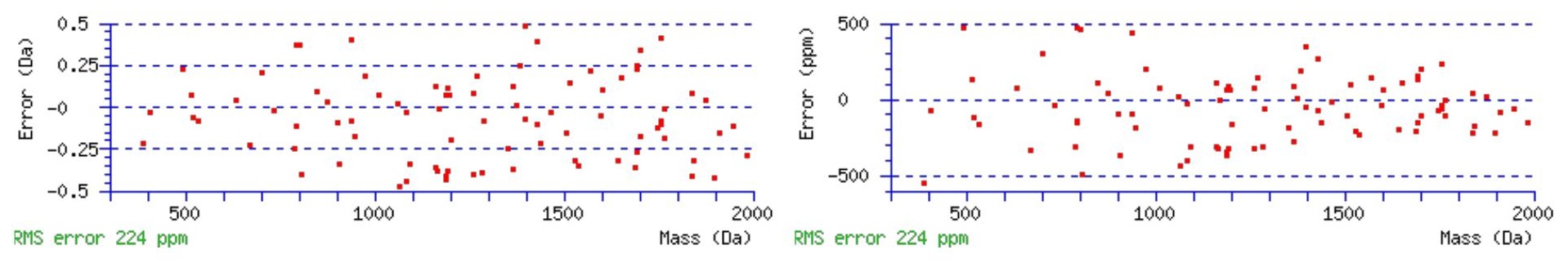

\section{All matches to this query}

\begin{tabular}{|l|l|l|l|}
\hline Score & Mr(calc): & Delta & \multicolumn{1}{|c|}{ Sequence } \\
\hline 55.1 & 3931.9884 & 0.0158 & YIEQFKDTLNTSVATSIAGFFAEPIQGVNGVVQYPK \\
\hline 46.7 & 3931.9884 & 0.0158 & YIEQFKDTLNTSVATSIAGFFAEPIQGVNGVVQYPK \\
\hline 38.9 & 3931.0044 & 0.9998 & YIEQFKDTLNTSVATSIAGFFAEPIQGVNGVVQYPK \\
\hline 4.4 & 3931.9942 & 0.0100 & VDGIAAWIHASHAKAARPEETADHNIRPQTWKAQR \\
\hline 1.6 & 3931.9920 & 0.0122 & WLPGRRALALXLSELALTGSEPAFPSGSWRPLSCR \\
\hline 1.6 & 3930.0128 & 1.9915 & WLPGRRALALXLSELALTGSEPAFPSGSWRPLSCR \\
\hline
\end{tabular}


Spectrum No: 605; Query: 341; Rank: 1

\section{Peptide View}

MS/MS Fragmentation of VFQNCSELYK

Found in IPI00368780, Tax_Id=10116 Gene_Symbol=Tmem87a_predicted similar to CG17660-PA

Match to Query 341: 1287.583408 from(644.798980,2+)

Title: 100101RatKid_NS_deglyco_25.1713.1713.2.dta

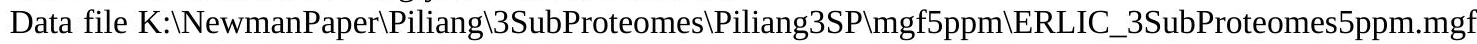
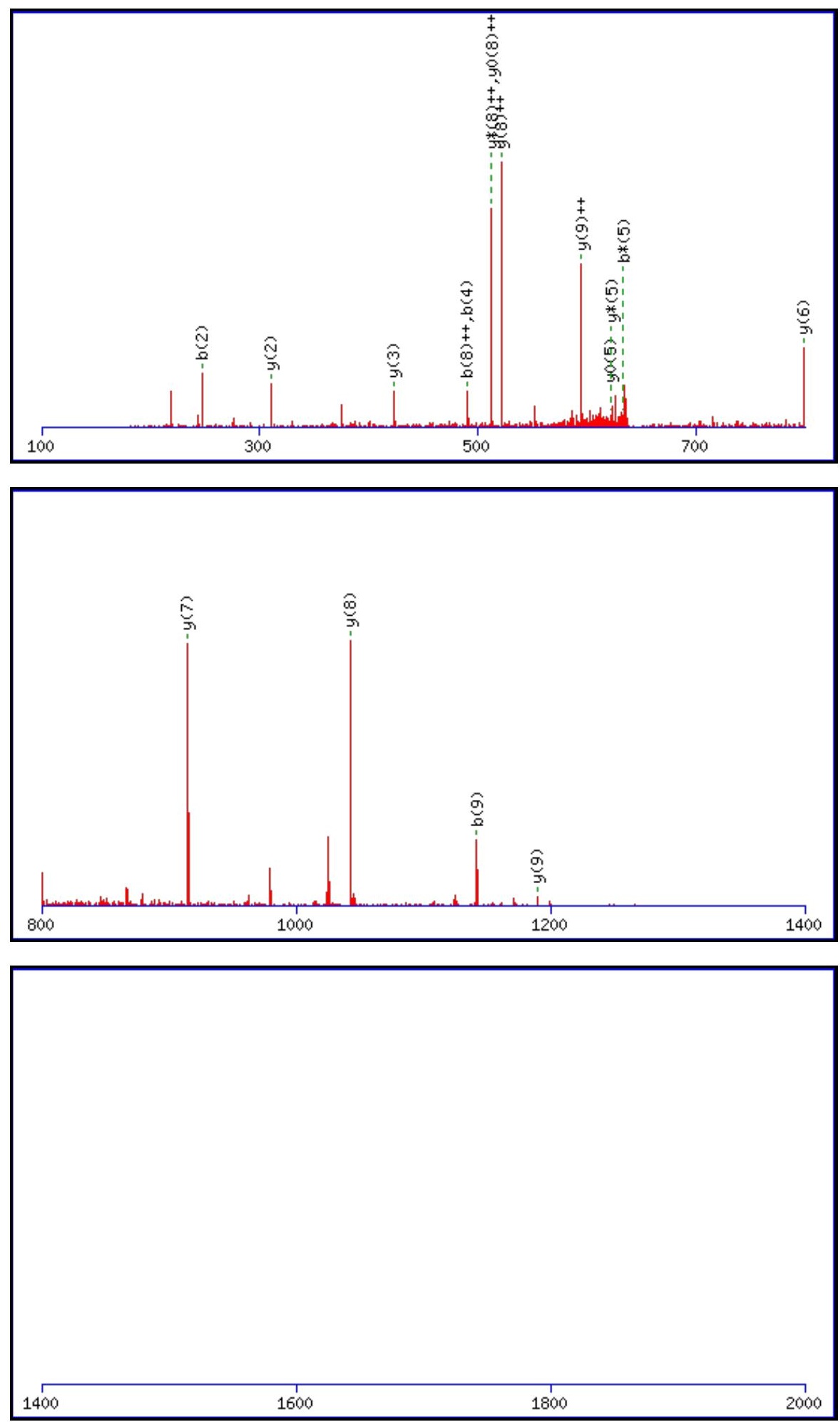

Monoisotopic mass of neutral peptide $\operatorname{Mr}($ calc): 1287.5805

Fixed modifications: Carbamidomethyl (C)

Variable modifications: 
N4 : Deamidated_N (N)

Ions Score: 55 Expect: 0.00045

Matches (Bold Red): 17/88 fragment ions using 36 most intense peaks

\begin{tabular}{|c|c|c|c|c|c|c|c|c|c|c|c|c|c|c|}
\hline \# & b & $\mathbf{b}^{++}$ & b* & $\mathbf{b}^{*^{++}}$ & $\mathbf{b}^{\mathbf{0}}$ & $\mathbf{b}^{0++}$ & Seq. & $\mathbf{y}$ & $y^{++}$ & $\mathbf{y}^{*}$ & $\mathbf{y}^{*^{++}}$ & $\mathbf{y}^{0}$ & $\mathbf{y}^{\mathbf{0 + +}}$ & \# \\
\hline 1 & 100.0757 & 50.5415 & & & & & V & & & & & & & 10 \\
\hline 2 & 247.1441 & 124.0757 & & & & & $\mathbf{F}$ & 1189.5194 & 595.2633 & 1172.4928 & 586.7501 & 1171.5088 & 586.2581 & 9 \\
\hline 3 & 375.2027 & 188.1050 & 358.1761 & 179.5917 & & & $\mathbf{Q}$ & 1042.4510 & 521.7291 & 1025.4244 & 513.2159 & 1024.4404 & 512.7238 & 8 \\
\hline 4 & 490.2296 & 245.6184 & 473.2031 & 237.1052 & & & $\mathbf{N}$ & 914.3924 & 457.6998 & 897.3659 & 449.1866 & 896.3818 & 448.6946 & 7 \\
\hline 5 & 650.2603 & 325.6338 & 633.2337 & 317.1205 & & & C & 799.3655 & 400.1864 & 782.3389 & 391.6731 & 781.3549 & 391.1811 & 6 \\
\hline 6 & 737.2923 & 369.1498 & 720.2657 & 360.6365 & 719.2817 & 360.1445 & S & 639.3348 & 320.1710 & 622.3083 & 311.6578 & 621.3243 & 311.1658 & 5 \\
\hline 7 & 866.3349 & 433.6711 & 849.3083 & 425.1578 & 848.3243 & 424.6658 & $\mathbf{E}$ & 552.3028 & 276.6550 & 535.2762 & 268.1418 & 534.2922 & 267.6498 & 4 \\
\hline 8 & 979.4190 & 490.2131 & 962.3924 & 481.6998 & 961.4084 & 481.2078 & $\mathbf{L}$ & 423.2602 & 212.1337 & 406.2336 & 203.6205 & & & 3 \\
\hline 9 & 1142.4823 & 571.7448 & 1125.4557 & 563.2315 & 1124.4717 & 562.7395 & $\mathbf{Y}$ & 310.1761 & 155.5917 & 293.1496 & 147.0784 & & & 2 \\
\hline 10 & & & & & & & $\mathbf{K}$ & 147.1128 & 74.0600 & 130.0863 & 65.5468 & & & 1 \\
\hline
\end{tabular}
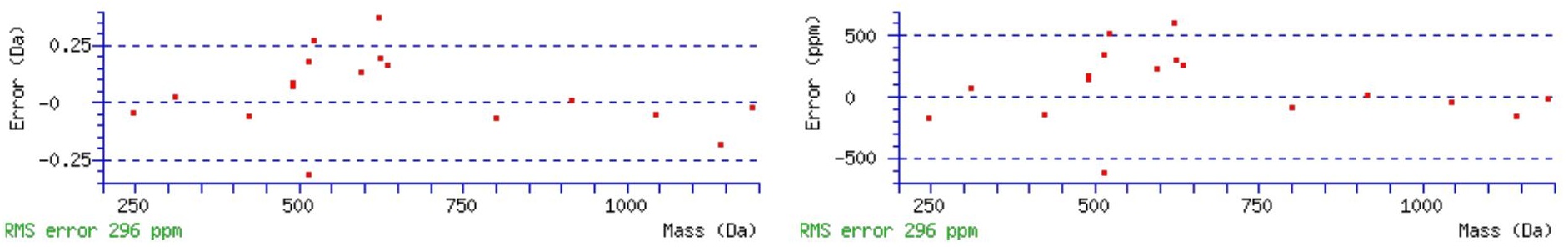

\section{All matches to this query}

\begin{tabular}{|l|c|c|l|}
\hline Score & Mr(calc): & Delta & \multicolumn{1}{c|}{ Sequence } \\
\hline 55.0 & 1287.5805 & 0.0029 & VFQNCSELYK \\
\hline 9.3 & 1285.5778 & 2.0056 & FVMTIENPAGK \\
\hline 9.3 & 1285.5778 & 2.0056 & $\underline{\text { QITMVPFDQK }}$ \\
\hline 8.8 & 1287.5894 & -0.0060 & $\underline{\text { EMRSSLDKVK }}$ \\
\hline 8.8 & 1287.5894 & -0.0060 & EMRSSLDKVK \\
\hline 8.4 & 1286.5795 & 1.0039 & $\underline{\text { FQTLINDLDK }}$ \\
\hline 8.2 & 1287.5723 & 0.0111 & VFVFDSVHMK \\
\hline 7.6 & 1286.5908 & 0.9926 & $\underline{\text { FQTEIQTVNK }}$ \\
\hline 6.9 & 1285.5849 & 1.9985 & $\underline{\text { AKRASEMASQK }}$ \\
\hline 6.5 & 1287.5748 & 0.0086 & $\underline{\text { LQSLSQEFEK }}$ \\
\hline
\end{tabular}

Spectrum No: 606; Query: 1417; Rank: 1

\section{Peptide View}

MS/MS Fragmentation of AADPPAENSSAPEAEQGGAE

Found in IPI00551815, Tax_Id=10116 Gene_Symbol=Ybx1-ps3 Ybx1 protein

Match to Query 1417: 1897.782528 from(949.898540,2+)

Title: 091008RatKidney_NH4Format02_27.855.855.2.dta

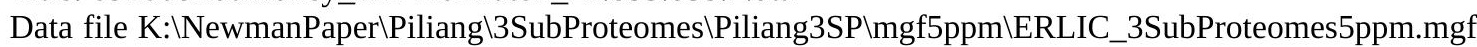



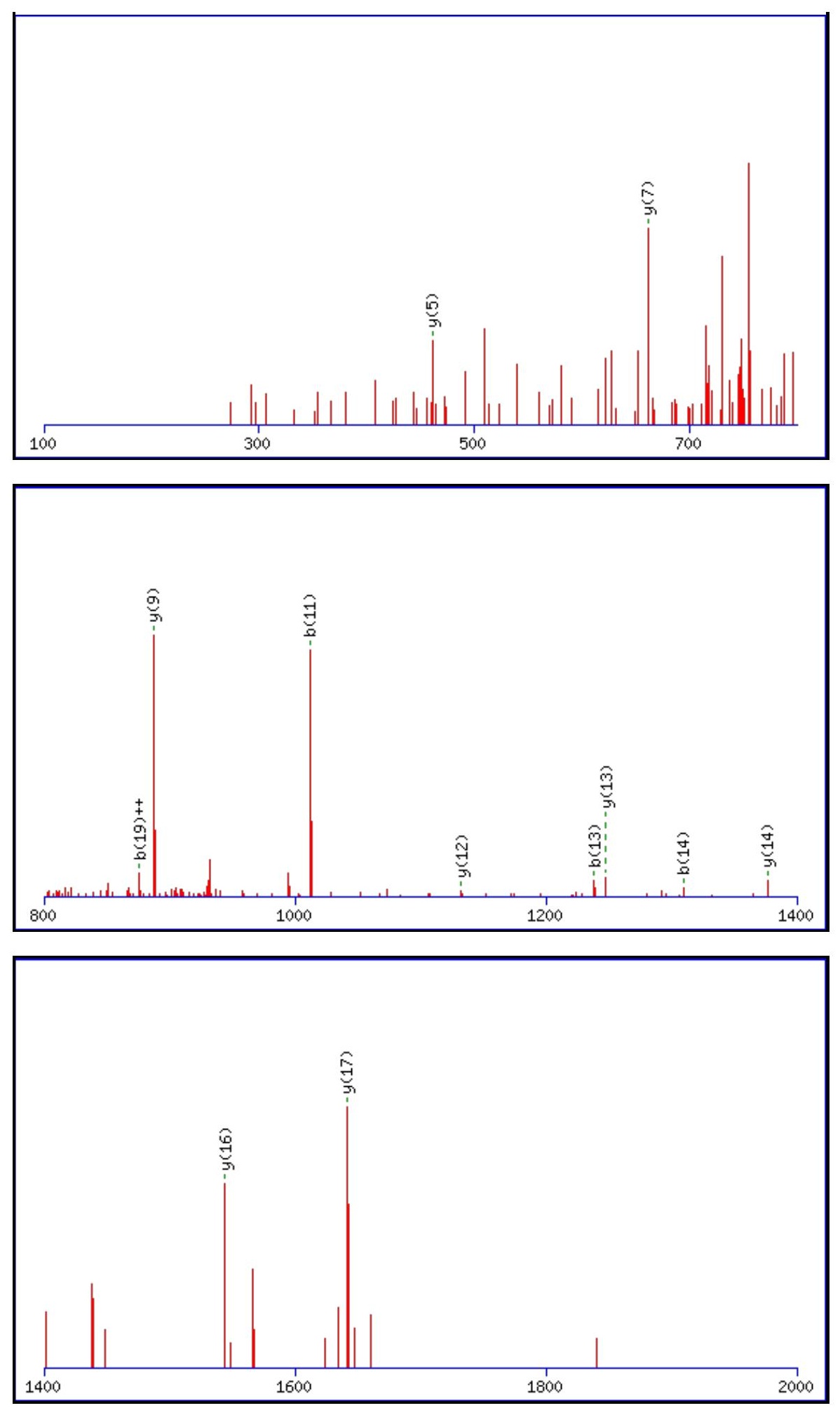

Monoisotopic mass of neutral peptide $\operatorname{Mr}($ calc): 1897.7813

Fixed modifications: Carbamidomethyl (C)

Variable modifications:

N8: Deamidated_N (N)

Ions Score: 55 Expect: 0.00058

Matches (Bold Red): 12/202 fragment ions using 24 most intense peaks

\begin{tabular}{|r|c|c|c|c|c|c|c|c|c|c|c|c|c|c|}
\hline$\#$ & $\mathbf{b}$ & $\mathbf{b}^{++}$ & $\mathbf{b}^{*}$ & $\mathbf{b}^{\boldsymbol{*}^{++}}$ & $\mathbf{b}^{\mathbf{0}}$ & $\mathbf{b}^{\mathbf{0 + +}}$ & Seq. & $\mathbf{y}$ & $\mathbf{y}^{++}$ & $\mathbf{y}^{\mathbf{*}}$ & $\mathbf{y}^{\boldsymbol{*}^{++}}$ & $\mathbf{y}^{\mathbf{0}}$ & $\mathbf{y}^{\mathbf{0 + +}}$ & $\#$ \\
\hline $\mathbf{1}$ & 72.0444 & 36.5258 & & & & & $\mathbf{A}$ & & & & & & & $\mathbf{2 0}$ \\
\hline $\mathbf{2}$ & 143.0815 & 72.0444 & & & & & $\mathbf{A}$ & 1827.7515 & 914.3794 & 1810.7250 & 905.8661 & 1809.7410 & 905.3741 & $\mathbf{1 9}$ \\
\hline 3 & 258.1084 & 129.5579 & & & 240.0979 & 120.5526 & $\mathbf{D}$ & 1756.7144 & 878.8608 & 1739.6879 & 870.3476 & 1738.7038 & 869.8556 & $\mathbf{1 8}$ \\
\hline $\mathbf{4}$ & 355.1612 & 178.0842 & & & 337.1506 & 169.0790 & $\mathbf{P}$ & $\mathbf{1 6 4 1 . 6 8 7 5}$ & 821.3474 & 1624.6609 & 812.8341 & 1623.6769 & 812.3421 & $\mathbf{1 7}$ \\
\hline $\mathbf{5}$ & 452.2140 & 226.6106 & & & 434.2034 & 217.6053 & $\mathbf{P}$ & $\mathbf{1 5 4 4 . 6 3 4 7}$ & 772.8210 & 1527.6081 & 764.3077 & 1526.6241 & 763.8157 & $\mathbf{1 6}$ \\
\hline
\end{tabular}




\begin{tabular}{|c|c|c|c|c|c|c|c|c|c|c|c|c|c|c|}
\hline 6 & 523.2511 & 262.1292 & & & 505.2405 & |253.1239 & A & |1447.5819| & |724.2946 & |1430.5554 & 715.7813 & 1429.5714 & |715.2893 & 15 \\
\hline 7 & 652.2937 & 326.6505 & & & 634.2831 & 317.6452 & $\mathbf{E}$ & 1376.5448 & 688.7760 & 1359.5183 & 680.2628 & 1358.5343 & 679.7708 & 14 \\
\hline 8 & 767.3206 & 384.1639 & 750.2941 & 375.6507 & 749.3101 & 375.1587 & $\mathbf{N}$ & 1247.5022 & 624.2547 & 1230.4757 & 615.7415 & 1229.4917 & 615.2495 & 13 \\
\hline 9 & 854.3526 & 427.6800 & 837.3261 & 419.1667 & 836.3421 & 418.6747 & S & 1132.4753 & 566.7413 & 1115.4487 & 558.2280 & 1114.4647 & 557.7360 & 12 \\
\hline 10 & 941.3847 & 471.1960 & 924.3581 & 462.6827 & 923.3741 & 462.1907 & S & 1045.4433 & 523.2253 & 1028.4167 & 514.7120 & 1027.4327 & 514.2200 & 11 \\
\hline 11 & 1012.4218 & 506.7145 & 995.3952 & 498.2013 & 994.4112 & 497.7092 & A & 958.4112 & 479.7093 & 941.3847 & 471.1960 & 940.4007 & 470.7040 & 0 \\
\hline 12 & 1109.4746 & 555.2409 & 1092.4480 & 546.7276 & 1091.4640 & 546.2356 & $\mathbf{P}$ & 887.3741 & 444.1907 & 870.3476 & 435.6774 & 869.3636 & 435.1854 & 9 \\
\hline 13 & 1238.5171 & 619.7622 & 1221.4906 & 611.2489 & 1220.5066 & 610.7569 & $\mathbf{E}$ & 790.3214 & 395.6643 & 773.2948 & 387.1510 & 772.3108 & 386.6590 & 8 \\
\hline 14 & 1309.5543 & 655.2808 & 1292.5277 & 646.7675 & 1291.5437 & 646.2755 & A & 661.2788 & 331.1430 & 644.2522 & 322.6297 & 643.2682 & 322.1377 & 7 \\
\hline 15 & 1438.5969 & 719.8021 & 1421.5703 & 711.2888 & 1420.5863 & 710.7968 & $\mathbf{E}$ & 590.2416 & 295.6245 & 573.2151 & 287.1112 & 572.2311 & 286.6192 & 6 \\
\hline 16 & 1566.6554 & 783.8314 & 1549.6289 & 775.3181 & 1548.6449 & 774.8261 & $\mathbf{Q}$ & 461.1991 & 231.1032 & 444.1725 & 222.5899 & 443.1885 & 222.0979 & 5 \\
\hline 17 & 1623.6769 & 812.3421 & 1606.6503 & 803.8288 & 1605.6663 & 803.3368 & G & 333.1405 & 167.0739 & & & 315.1299 & 158.0686 & 4 \\
\hline 18 & 1680.6984 & 840.8528 & 1663.6718 & 832.3395 & 1662.6878 & 831.8475 & G & 276.1190 & 138.5631 & & & 258.1084 & 129.5579 & 3 \\
\hline 19 & 1751.7355 & 876.3714 & 1734.7089 & 867.8581 & 1733.7249 & 867.3661 & A & 219.0975 & 110.0524 & & & 201.0870 & 101.0471 & 2 \\
\hline 20 & & & & & & & E & 148.0604 & 74.5339 & & & 130.0499 & 65.5286 & 1 \\
\hline
\end{tabular}
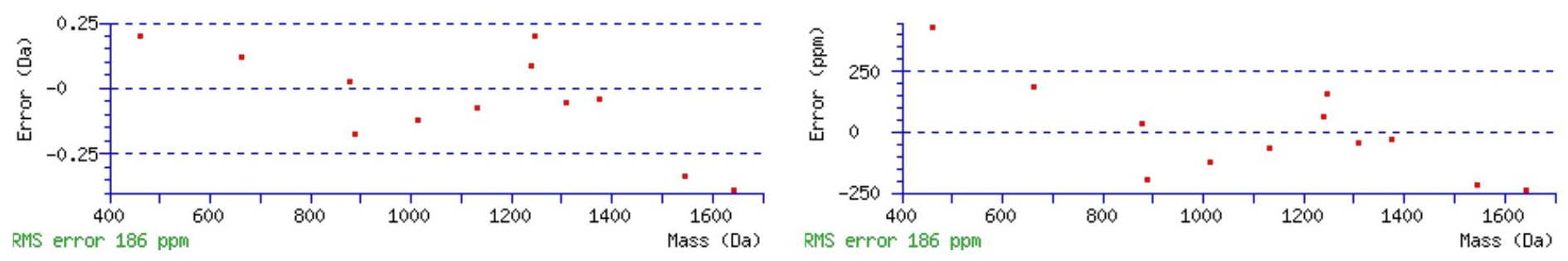

\section{All matches to this query}

\begin{tabular}{|l|l|l|l|}
\hline Score & Mr(calc): & Delta & \multicolumn{1}{|c|}{ Sequence } \\
\hline 54.9 & 1897.7813 & 0.0012 & AADPPAENSSAPEAEQGGAE \\
\hline 5.1 & 1896.7973 & 0.9852 & AADPPAENSSAPEAEQGGAE \\
\hline 3.6 & 1897.7884 & -0.0058 & EKVFTSDSDASGWAYR \\
\hline 3.6 & 1897.7884 & -0.0058 & EKVFTSDSDASGWAYR \\
\hline 1.1 & 1897.7794 & 0.0031 & LMTAQNVASYRTPDR \\
\hline 0.8 & 1897.7951 & -0.0126 & AELPVTEMPGDALCSGR \\
\hline 0.8 & 1897.7811 & 0.0014 & KSPALSTSAPMSPTKR \\
\hline 0.7 & 1895.7915 & 1.9911 & SGNSSKPSSTDLKVSDK \\
\hline 0.6 & 1897.7794 & 0.0031 & LMTAQNVASYRTPDR \\
\hline
\end{tabular}

Spectrum No: 607; Query: 2194; Rank: 1

\section{Peptide View}

MS/MS Fragmentation of LGLSFNSITVVENGSLANVPHLR

Found in IPI00199861, Tax_Id=10116 Gene_Symbol=Dcn Decorin precursor

Match to Query 2194: 2437.302402 from(813.441410,3+)

Title: 100101RatKid_NS_deglyco_14.5352.5352.3.dta

Data file K:INewmanPaper|Piliang 13 SubProteomes\Piliang3SP\mgf5ppm\ERLIC_3SubProteomes5ppm.mgf 

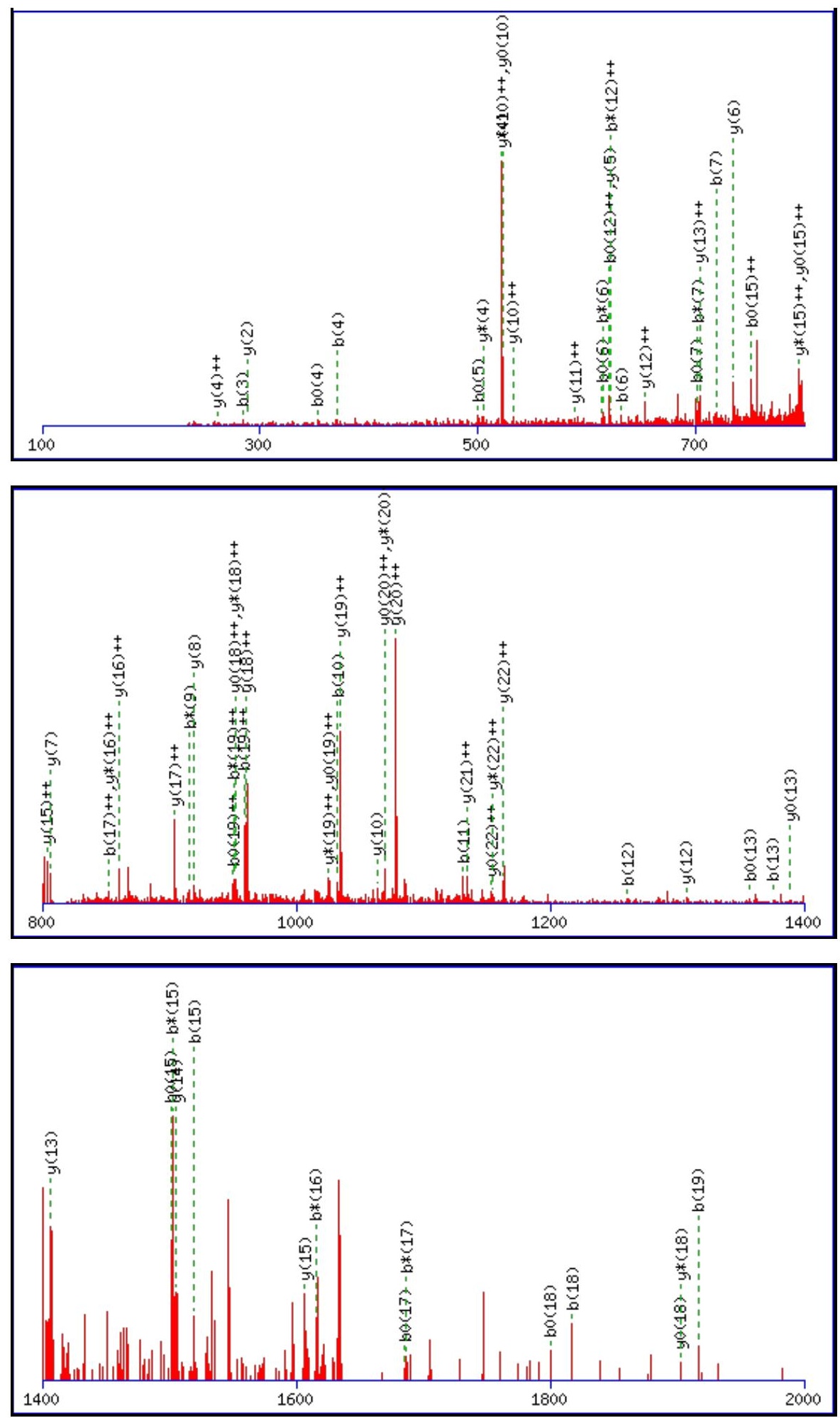

Monoisotopic mass of neutral peptide $\operatorname{Mr}($ calc): 2437.2965

Fixed modifications: Carbamidomethyl (C)

Variable modifications:

N13 : Deamidated_N (N)

Ions Score: 55 Expect: 0.00023

Matches (Bold Red): 73/232 fragment ions using 172 most intense peaks

\begin{tabular}{|r|c|c|c|c|c|c|c|c|c|c|c|c|c|c|}
\hline$\#$ & $\mathbf{b}$ & $\mathbf{b}^{++}$ & $\mathbf{b}^{*}$ & $\mathbf{b}^{\mathbf{*}^{++}}$ & $\mathbf{b}^{\mathbf{0}}$ & $\mathbf{b}^{\mathbf{0 + +}}$ & Seq. & $\mathbf{y}$ & $\mathbf{y}^{++}$ & $\mathbf{y}^{\mathbf{*}}$ & $\mathbf{y}^{\mathbf{*}^{++}}$ & $\mathbf{y}^{\mathbf{0}}$ & $\mathbf{y}^{\mathbf{0}+}$ & $\#$ \\
\hline $\mathbf{1}$ & 114.0913 & 57.5493 & & & & & $\mathbf{L}$ & & & & & & & $\mathbf{2 3}$ \\
\hline $\mathbf{2}$ & 171.1128 & 86.0600 & & & & & $\mathbf{G}$ & 2325.2197 & $\mathbf{1 1 6 3 . 1 1 3 5}$ & 2308.1932 & $\mathbf{1 1 5 4 . 6 0 0 2}$ & 2307.2092 & $\mathbf{1 1 5 4 . 1 0 8 2}$ & $\mathbf{2 2}$ \\
\hline $\mathbf{3}$ & $\mathbf{2 8 4 . 1 9 6 9}$ & 142.6021 & & & & & L & 2268.1983 & $\mathbf{1 1 3 4 . 6 0 2 8}$ & 2251.1717 & 1126.0895 & 2250.1877 & 1125.5975 & $\mathbf{2 1}$ \\
\hline $\mathbf{4}$ & $\mathbf{3 7 1 . 2 2 8 9}$ & 186.1181 & & & 353.2183 & 177.1128 & S & 2155.1142 & $\mathbf{1 0 7 8 . 0 6 0 7}$ & 2138.0877 & $\mathbf{1 0 6 9 . 5 4 7 5}$ & 2137.1036 & $\mathbf{1 0 6 9 . 0 5 5 5}$ & $\mathbf{2 0}$ \\
\hline $\mathbf{5}$ & 518.2973 & 259.6523 & & & $\mathbf{5 0 0 . 2 8 6 7}$ & 250.6470 & $\mathbf{F}$ & 2068.0822 & $\mathbf{1 0 3 4 . 5 4 4 7}$ & 2051.0556 & $\mathbf{1 0 2 6 . 0 3 1 4}$ & 2050.0716 & $\mathbf{1 0 2 5 . 5 3 9 4}$ & $\mathbf{1 9}$ \\
\hline
\end{tabular}




\begin{tabular}{|c|c|c|c|c|c|c|c|c|c|c|c|c|c|c|}
\hline 6 & 632.3402 & 316.6738 & 615.3137 & 308.1605 & 614.3297 & 307.6685 & $\mathbf{N}$ & |1921.0138| & 961.0105 & |1903.9872 & 952.4972 & |1903.0032| & 952.0052 & 18 \\
\hline 7 & 719.3723 & 360.1898 & 702.3457 & 351.6765 & 701.3617 & 351.1845 & S & 1806.9708 & 903.9891 & 1789.9443 & 895.4758 & 1788.9603 & 894.9838 & 17 \\
\hline 8 & 832.4563 & 416.7318 & 815.4298 & 408.2185 & 814.4458 & 407.7265 & I & 1719.9388 & 860.4730 & 1702.9123 & 851.9598 & 1701.9282 & 851.4678 & 16 \\
\hline 9 & 933.5040 & 467.2556 & 916.4775 & 458.7424 & 915.4934 & 458.2504 & $\mathbf{T}$ & 1606.8547 & 803.9310 & 1589.8282 & 795.4177 & 1588.8442 & 794.9257 & 15 \\
\hline 10 & 1032.5724 & 516.7898 & 1015.5459 & 508.2766 & 1014.5619 & 507.7846 & $\mathbf{V}$ & 1505.8071 & 753.4072 & 1488.7805 & 744.8939 & 1487.7965 & 744.4019 & 14 \\
\hline 11 & 1131.6408 & 566.3241 & 1114.6143 & 557.8108 & 1113.6303 & 557.3188 & $\mathbf{V}$ & 1406.7386 & 703.8730 & 1389.7121 & 695.3597 & 1388.7281 & 694.8677 & 13 \\
\hline 12 & 1260.6834 & 630.8454 & 1243.6569 & 622.3321 & 1242.6729 & 621.8401 & $\mathbf{E}$ & 1307.6702 & 654.3388 & 1290.6437 & 645.8255 & 1289.6597 & 645.3335 & 12 \\
\hline 13 & 1375.7104 & 688.3588 & 1358.6838 & 679.8455 & 1357.6998 & 679.3535 & $\mathbf{N}$ & 1178.6276 & 589.8175 & 1161.6011 & 581.3042 & 1160.6171 & 580.8122 & 11 \\
\hline 14 & 1432.7318 & 716.8696 & 1415.7053 & 708.3563 & 1414.7213 & 707.8643 & G & 1063.6007 & 532.3040 & 1046.5742 & 523.7907 & 1045.5901 & 523.2987 & 10 \\
\hline 15 & 1519.7639 & 760.3856 & 1502.7373 & 751.8723 & 1501.7533 & 751.3803 & $\mathbf{S}$ & 1006.5792 & 503.7933 & 989.5527 & 495.2800 & 988.5687 & 494.7880 & 9 \\
\hline 16 & 1632.8479 & 816.9276 & 1615.8214 & 808.4143 & 1614.8374 & 807.9223 & $\mathbf{L}$ & 919.5472 & 460.2772 & 902.5207 & 451.7640 & & & 8 \\
\hline 17 & 1703.8850 & 852.4462 & 1686.8585 & 843.9329 & 1685.8745 & 843.4409 & A & 806.4631 & 403.7352 & 789.4366 & 395.2219 & & & 7 \\
\hline 18 & 1817.9280 & 909.4676 & 1800.9014 & 900.9543 & 1799.9174 & 900.4623 & $\mathbf{N}$ & 735.4260 & 368.2167 & 718.3995 & 359.7034 & & & 6 \\
\hline 19 & 1916.9964 & 959.0018 & 1899.9698 & 950.4886 & 1898.9858 & 949.9965 & V & 621.3831 & 311.1952 & 604.3566 & 302.6819 & & & 5 \\
\hline 21 & 2151.1081 & 1076.0577 & 2134.0815 & 1067.5444 & 2133.0975 & 1067.0524 & $\mathbf{H}$ & 425.2619 & 213.1346 & 408.2354 & 204.6213 & & & 3 \\
\hline 22 & 2264.1921 & 1132.5997 & 2247.1656 & 1124.0864 & 2246.1816 & 1123.5944 & $\mathbf{L}$ & 288.2030 & 144.6051 & 271.1765 & 136.0919 & & & 2 \\
\hline 23 & & & & & & & $\mathbf{R}$ & 175.1190 & 88.0631 & 158.0924 & 79.5498 & & & 1 \\
\hline
\end{tabular}
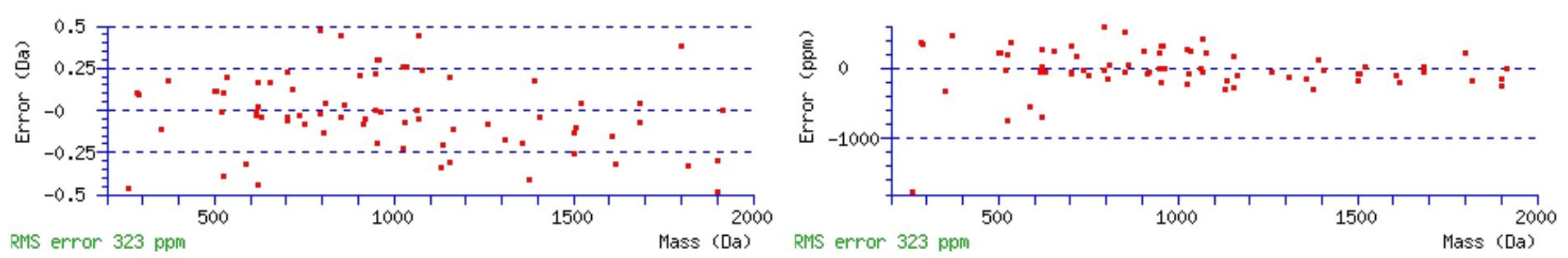

\section{All matches to this query}

\begin{tabular}{|l|l|c|c|}
\hline Score & Mr(calc): & Delta & Sequence \\
\hline 54.8 & 2437.2965 & 0.0059 & LGLSFNSITVVENGSLANVPHLR \\
\hline 43.3 & 2437.2965 & 0.0059 & LGLSFNSITVVENGSLANVPHLR \\
\hline 18.2 & 2437.2965 & 0.0059 & LGLSFNSITVVENGSLANVPHLR \\
\hline 16.1 & 2436.3125 & 0.9899 & LGLSFNSITVVENGSLANVPHLR \\
\hline
\end{tabular}

Spectrum No: 608; Query: 2428; Rank: 1

\section{Peptide View}

MS/MS Fragmentation of SENGLEFTSSGSANTETTKVNGSLETK

Found in IPI00421874, Tax_Id=10116 Gene_Symbol=Vdac1 Voltage-dependent anion-selective channel protein 1

Match to Query 2428: 2788.294182 from(930.438670,3+)

Title: 100101RatKid_NS_deglyco_13.2271.2271.3.dta

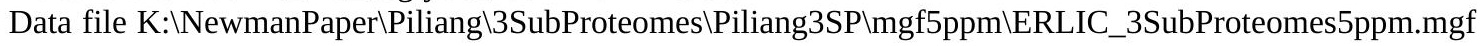



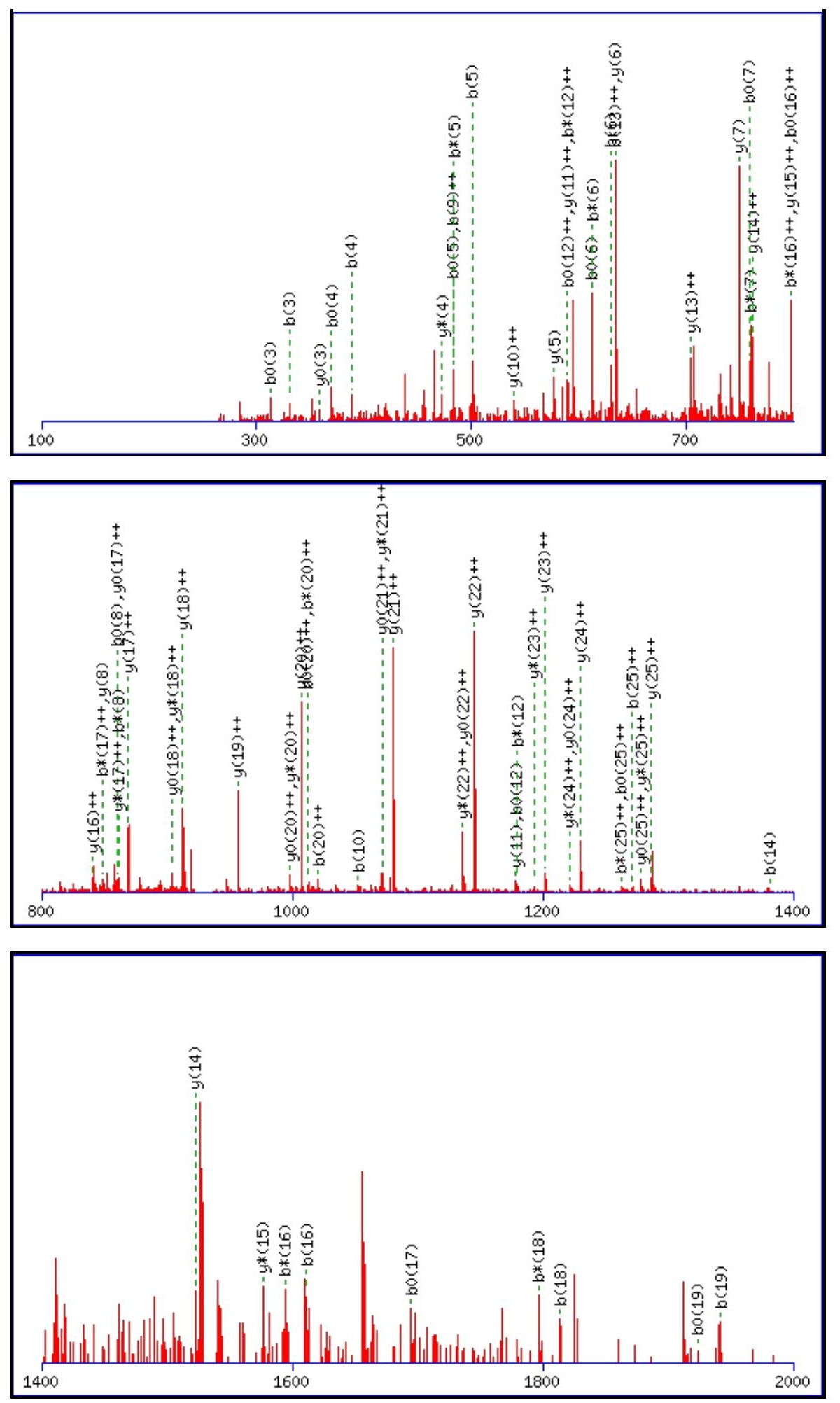

Monoisotopic mass of neutral peptide $\operatorname{Mr}($ calc): 2788.2886

Fixed modifications: Carbamidomethyl (C)

Variable modifications:

N21 : Deamidated_N (N)

Ions Score: 55 Expect: $\odot .0013$

Matches (Bold Red): 77/306 fragment ions using 144 most intense peaks

\begin{tabular}{|c|c|c|c|c|c|c|c|c|c|c|c|c|c|c|}
\hline \# & b & $\mathbf{b}^{++}$ & b* & $\mathbf{b}^{*^{++}}$ & $\mathbf{b}^{0}$ & $\mathbf{b}^{0++}$ & Seq. & $\mathbf{y}$ & $y^{++}$ & $\mathrm{y}^{*}$ & $y^{*^{++}}$ & $\mathbf{y}^{0}$ & $y^{0++}$ & \# \\
\hline 1 & 88.0393 & 44.5233 & & & 70.0287 & 35.5180 & $\mathbf{S}$ & & & & & & & 27 \\
\hline 2 & 217.0819 & 109.0446 & & & 199.0713 & 100.0393 & E & 2702.2639 & 1351.6356 & 2685.2373 & 1343.1223 & 2684.2533 & 1342.6303 & 26 \\
\hline 3 & 331.1248 & 166.0660 & 314.0983 & 157.5528 & 313.1143 & 157.0608 & $\mathbf{N}$ & 2573.2213 & 1287.1143 & 2556.1948 & 1278.6010 & 2555.2107 & 1278.1090 & 25 \\
\hline 4 & 388.1463 & 194.5768 & 371.1197 & 186.0635 & 370.1357 & 185.5715 & G & 2459.1784 & 1230.0928 & 2442.1518 & 1221.5796 & 2441.1678 & 1221.0875 & 24 \\
\hline 5 & 501.2304 & 251.1188 & 484.2038 & 242.6055 & 483.2198 & 242.1135 & $\mathbf{L}$ & 2402.1569 & 1201.5821 & 2385.1304 & 1193.0688 & 2384.1463 & 1192.5768 & 23 \\
\hline
\end{tabular}




\begin{tabular}{|c|c|c|c|c|c|c|c|c|c|c|c|c|c|c|}
\hline 6 & 630.2729 & 315.6401 & 613.2464 & 307.1268 & 612.2624 & 306.6348 & $\mathbf{E}$ & |2289.0728 | & |1145.0401 & 2272.0463 & |1136.5268 & |2271.0623| & $\mid 1136.0348$ & 22 \\
\hline 7 & 777.3414 & 389.1743 & 760.3148 & 380.6610 & 759.3308 & 380.1690 & $\mathbf{F}$ & 2160.0303 & 1080.5188 & 2143.0037 & 1072.0055 & 2142.0197 & 1071.5135 & 21 \\
\hline 8 & 878.3890 & 439.6982 & 861.3625 & 431.1849 & 860.3785 & 430.6929 & $\mathbf{T}$ & 9618 & 1006.9846 & 1995. & 998.4713 & 1994.9513 & 997.9793 & 0 \\
\hline 9 & 965.4211 & 483.2142 & 948.3945 & 474.7009 & 947.4105 & 474.2089 & S & 1911.9142 & 956.4607 & 1894.8876 & 947.9474 & 1893.9036 & 947.4554 & 19 \\
\hline 10 & 1052.4531 & 526.7302 & 1035.4265 & 518.2169 & 1034.4425 & 517.7249 & $\mathbf{S}$ & 1824.8821 & 912.9447 & 1807.8556 & 904.4314 & 1806.8716 & 903.9394 & 18 \\
\hline 11 & 1109.4746 & 555.2409 & 1092.4480 & 546.7276 & 1091.4640 & 546.2356 & G & 1737.8501 & 869.4287 & 1720.8236 & 860.9154 & 1719.8395 & 860.4234 & 17 \\
\hline 12 & 1196.5066 & 598.7569 & 1179.4800 & 590.2437 & 1178.4960 & 589.7516 & $\mathbf{S}$ & 1680.8286 & 840.9180 & 1663.8021 & 832.4047 & 8181 & 831.9127 & 16 \\
\hline 13 & 1267.5437 & 634.2755 & 1250.5172 & 625.7622 & 1249.5331 & 625.2702 & A & 1593.7966 & 797.4019 & 1576.7701 & 788.8887 & 1575.7861 & 788.3967 & 15 \\
\hline 14 & 1381.5866 & 691.2970 & 1364.5601 & 682.7837 & 1363.5761 & 682.2917 & $\mathbf{N}$ & 1522.7595 & 761.8834 & 1505.7330 & 753.3701 & 1504.7489 & 752.8781 & 14 \\
\hline 15 & 1482.6343 & 741.8208 & 1465.6078 & 733.3075 & 1464.6237 & 732.8155 & $\mathbf{T}$ & 1408.7166 & 704.8619 & 1391.6900 & 696.3487 & 1390.7060 & 566 & 13 \\
\hline 16 & 1611.6769 & 806.3421 & 1594.6503 & 797.8288 & 1593.6663 & 797.3368 & $\mathbf{E}$ & 1307.6689 & 654.3381 & 1290.6423 & 645.8248 & 1289.6583 & 645.3328 & 12 \\
\hline 17 & 1712.7246 & 856.8659 & 1695.6980 & 848.3527 & 1694.7140 & 847.8606 & $\mathbf{T}$ & 1178.6263 & 589.8168 & 1161.5998 & 581.3035 & 1160.6157 & 580.8115 & 11 \\
\hline 18 & 1813.7723 & 907.3898 & 1796.7457 & 898.8765 & 1795.7617 & 898.3845 & $\mathbf{T}$ & 1077.5786 & 539.2930 & 1060.5521 & 530.7797 & 1059.5681 & 530.2877 & 10 \\
\hline 19 & 1941.8672 & 971.4372 & 1924.8407 & 962.9240 & 1923.8567 & 962.4320 & $\mathbf{K}$ & 976.5309 & 488.7691 & 959.5044 & 480.2558 & 958.5204 & 479.7638 & \\
\hline 20 & 2040.9356 & 1020.9715 & 2023.9091 & 1012.4582 & 2022.9251 & 1011.9662 & $\mathbf{V}$ & 848.4360 & 424.7216 & 831.4094 & 416.2084 & 830.4254 & 415.7163 & 8 \\
\hline 21 & 2155.9626 & 1078.4849 & 2138.9360 & 1069.9716 & 2137.9520 & 1069.4796 & $\mathbf{N}$ & 749.3676 & 375.1874 & 732.3410 & 366.6741 & 731.3570 & 366.1821 & 7 \\
\hline 22 & 2212.9840 & 1106.9957 & 2195.9575 & 1098.4824 & 2194.9735 & 1097.9904 & G & 634.3406 & 317.6740 & 617.3141 & 309.1607 & 616.3301 & 308.6687 & 6 \\
\hline 23 & 2300.0161 & 1150.5117 & 2282.9895 & 1141.9984 & 2282.0055 & 1141.5064 & S & 577.3192 & 289.1632 & 560.2926 & 280.6499 & 559.3086 & 280.1579 & 5 \\
\hline 24 & 2413.1001 & 1207.0537 & 2396.0736 & 1198.5404 & 2395.0896 & 1198.0484 & $\mathbf{L}$ & 490.2871 & 245.6472 & 473.2606 & 237.1339 & 472.2766 & 236.6419 & 4 \\
\hline 25 & 2542.1427 & 1271.5750 & 2525.1162 & 1263.0617 & 2524.1322 & 1262.5697 & $\mathbf{E}$ & 377.2031 & 189.1052 & 360.1765 & 180.5919 & 359.1925 & 180.0999 & 3 \\
\hline 26 & 2643.1904 & 1322.0988 & 2626.1638 & 1313.5856 & 2625.1798 & 1313.0936 & $\mathbf{T}$ & 248.1605 & 124.5839 & 231.1339 & 116.0706 & 230.1499 & 115.5786 & 2 \\
\hline 27 & & & & & & & $\mathbf{K}$ & 147.1128 & 74.0600 & 130.0863 & 65.5468 & & & 1 \\
\hline
\end{tabular}
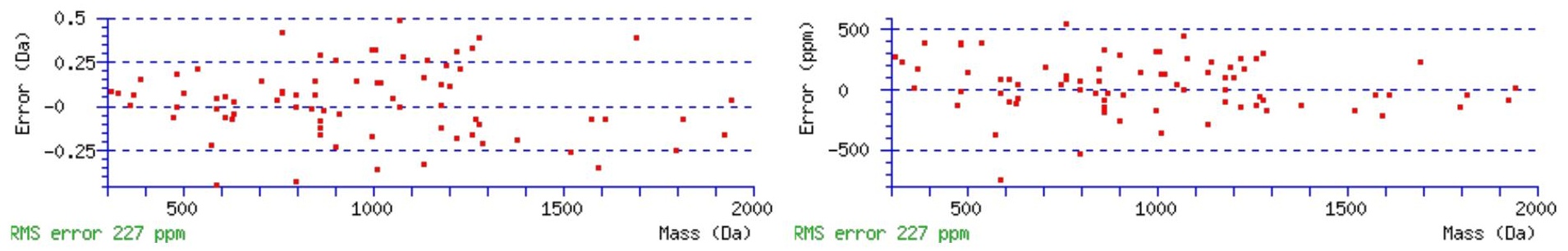

\section{All matches to this query}

\begin{tabular}{|l|l|l|l|}
\hline Score & Mr(calc): & Delta & \multicolumn{1}{|c|}{ Sequence } \\
\hline 54.8 & 2788.2886 & 0.0055 & $\underline{\text { SENGLEFTSSGSANTETTKVNGSLETK }}$ \\
\hline 33.5 & 2788.2886 & 0.0055 & $\underline{\text { SENGLEFTSSGSANTETTKVNGSLETK }}$ \\
\hline 13.0 & 2787.3046 & 0.9896 & $\underline{\text { SENGLEFTSSGSANTETTKVNGSLETK }}$ \\
\hline 11.2 & 2786.3071 & 1.9871 & $\underline{\text { GRILHDGAYSLTLQGLGIHSLGLR }}$ \\
\hline 8.8 & 2786.3071 & 1.9871 & GRILHDGAYSLTLQGLGIHSLGLR \\
\hline 8.8 & 2786.3071 & 1.9871 & $\underline{\text { GRILHDGAYSLTLQGLGIHSLGLR }}$ \\
\hline 6.2 & 2788.2924 & 0.0018 & TTPGKLTKEELYTVLAMVAVTQR \\
\hline 6.2 & 2788.2924 & 0.0018 & TTPGKLTKEELYTVLAMVAVTQR \\
\hline 5.5 & 2786.2850 & 2.0092 & TQVELLEPPTPELKRTLDSMNSK \\
\hline 4.4 & 2788.2713 & 0.0229 & SIAVLNTSVAPMLNPFIYSLRNK \\
\hline
\end{tabular}

Spectrum No: 609; Query: 2312; Rank: 1

\section{Peptide View}

MS/MS Fragmentation of LTLEELDEIIKNASGIYAEIDGAK

Found in IPI00361106, Tax_Id=10116 Gene_Symbol=RGD1560062_predicted hypothetical protein LOC309816 
Match to Query 2312: 2605.347402 from(869.456410,3+)

Title: 100101RatKid_NS_deglyco_13.7449.7449.3.dta

Data file K:INewmanPaper|Piliang|3SubProteomes\Piliang3SP\mgf5ppm\ERLIC_3SubProteomes5ppm.mgf
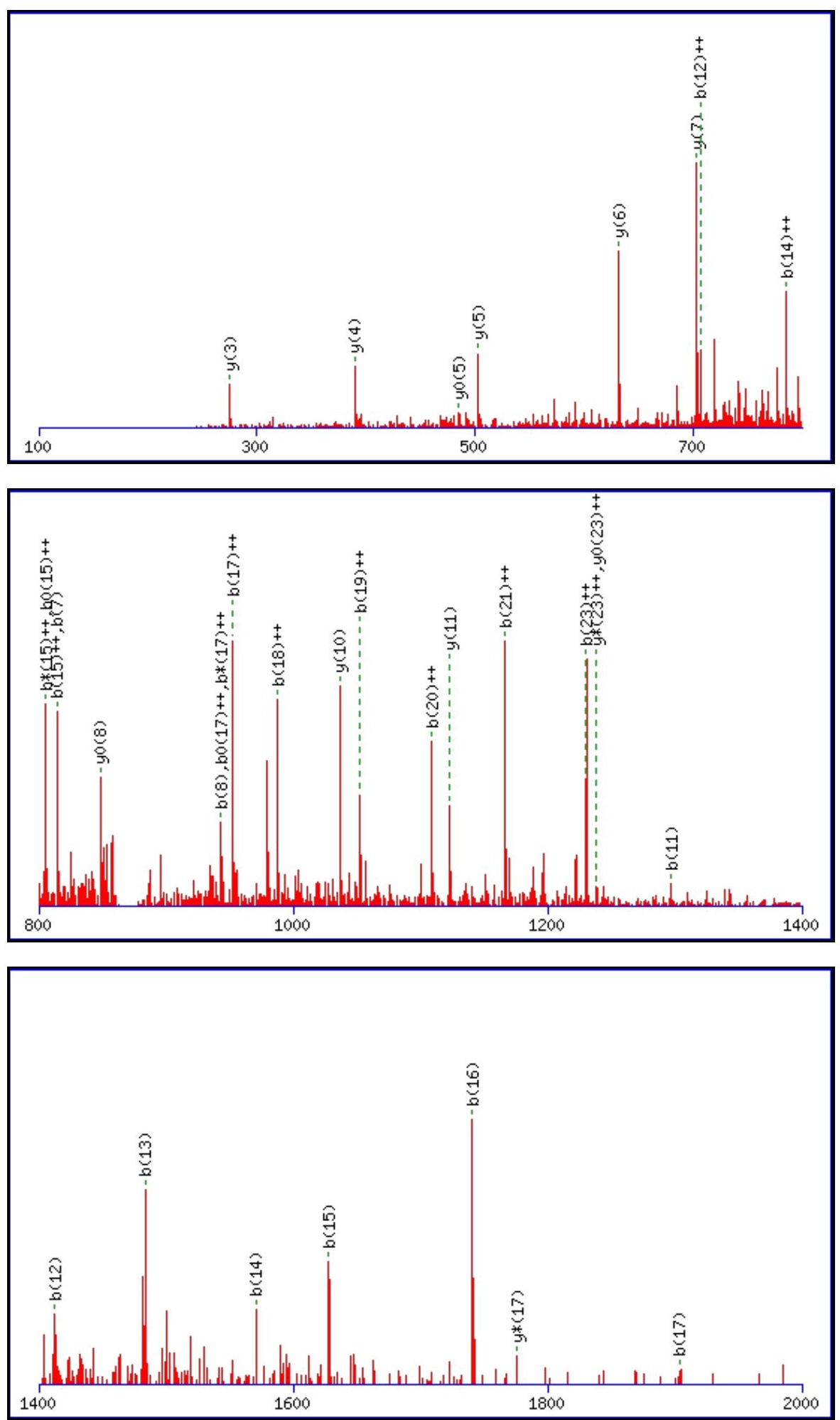

Monoisotopic mass of neutral peptide $\operatorname{Mr}$ (calc): 2605.3374

Fixed modifications: Carbamidomethyl (C)

Variable modifications:

N12 : Deamidated_N (N)

Ions Score: 55 Expect: 0.00043

Matches (Bold Red): 34/248 fragment ions using 55 most intense peaks

\begin{tabular}{|c|c|c|c|c|c|c|c|c|c|c|c|c|c|c|}
\hline$\#$ & $\mathbf{b}$ & $\mathbf{b}^{++}$ & $\mathbf{b}^{*}$ & $\mathbf{b}^{*^{++}}$ & $\mathbf{b}^{\mathbf{0}}$ & $\mathbf{b}^{\mathbf{0}+}$ & Seq. & $\mathbf{y}$ & $\mathbf{y}^{++}$ & $\mathbf{y}^{*}$ & $\mathbf{y}^{\boldsymbol{*}^{++}}$ & $\mathbf{y}^{\mathbf{0}}$ & $\mathbf{y}^{\mathbf{0 + +}}$ & $\#$ \\
\hline $\mathbf{1}$ & 114.0913 & 57.5493 & & & & & $\mathbf{L}$ & & & & & & & 24 \\
\hline $\mathbf{2}$ & 215.1390 & 108.0731 & & & 197.1285 & 99.0679 & $\mathbf{T}$ & 2493.2607 & 1247.1340 & 2476.2341 & $\mathbf{1 2 3 8 . 6 2 0 7}$ & 2475.2501 & 1238.1287 & 23 \\
\hline
\end{tabular}




\begin{tabular}{|c|c|c|c|c|c|c|c|c|c|c|c|c|c|c|}
\hline & 328.2231 & 164.6152 & & & 310.2125 & 155.6099| & $\mathbf{L}$ & |2392.2130 & 1196.6101 & |2375.1864| & |1188.0968 & 2374.2024 & $|1187.6048|$ & $22 \mid$ \\
\hline 4 & 457.2657 & 229.1365 & & & 439.2551 & 220.1312 & $\mathbf{E}$ & 2279.1289 & 1140.0681 & 2262.1024 & 1131.5548 & 2261.1183 & 1131.0628 & 21 \\
\hline 5 & 586.3083 & 293.6578 & & & 568.2977 & 284.6525 & E & 2150.0863 & 1075.5468 & 2133.0598 & 1067.0335 & 2132.0757 & 1066.5415 & 20 \\
\hline 6 & 699.3923 & 350.1998 & & & 681.3818 & 341.1945 & $\mathbf{L}$ & 2021.0437 & 1011.0255 & 2004.0172 & 1002.5122 & 2003.0332 & 1002.0202 & 9 \\
\hline 7 & 814.4193 & 407.7133 & & & 796.4087 & 398.7080 & D & 1907.9597 & 954.4835 & 1890.9331 & 945.9702 & 1889.9491 & 945.4782 & 18 \\
\hline 8 & 943.4619 & 472.2346 & & & 925.4513 & 463.2293 & $\mathbf{E}$ & 1792.9327 & 896.9700 & \begin{tabular}{|l|}
1775.9062 \\
\end{tabular} & 888.4567 & 1774.9221 & 887.9647 & 17 \\
\hline 9 & 1056.5459 & 528.7766 & & & 1038.5354 & 519.7713 & I & 1663.8901 & 832.4487 & 1646.8636 & 823.9354 & 1645.8796 & 823.4434 & 16 \\
\hline 10 & 1169.6300 & 585.3186 & & & 1151.6194 & 576.3134 & I & 1550.8061 & 775.9067 & 1533.7795 & 767.3934 & 1532.7955 & 766.9014 & 15 \\
\hline 11 & 1297.7250 & 649.3661 & 1280.6984 & 640.8528 & 1279.7144 & 640.3608 & $\mathbf{K}$ & 1437.7220 & 719.3646 & 1420.6954 & 710.8514 & 1419.7114 & 710.3594 & 14 \\
\hline 12 & 1412.7519 & 706.8796 & 1395.7253 & 698.3663 & 1394.7413 & 697.8743 & $\mathbf{N}$ & 1309.6270 & 655.3172 & 1292.6005 & 646.8039 & 1291.6165 & 646.3119 & 13 \\
\hline 13 & 1483.7890 & 742.3981 & 1466. & 733.8849 & 1465.7784 & 733.3929 & A & 1194.6001 & 597.8037 & 1177.5735 & 589.2904 & 1176.5895 & 588.7984 & 12 \\
\hline 14 & 1570.8210 & 785.9142 & 1553.7945 & 777.4009 & 1552.8105 & 776.9089 & $\mathrm{~S}$ & 1123.5630 & 562.2851 & 1106.5364 & 553.7719 & 1105.5524 & 553.2798 & 11 \\
\hline 15 & 1627.8425 & 814.4249 & 1610.8160 & 805.9116 & 1609.8319 & 805.4196 & G & 1036.5310 & 518.7691 & 1019.5044 & 510.2558 & 1018.5204 & 509.7638 & 10 \\
\hline 16 & 1740.9266 & 870.9669 & 1723.9000 & 862.4536 & 1722.9160 & 861.9616 & I & 979.5095 & 490.2584 & 962.4829 & 481.7451 & 961.4989 & 481.2531 & 9 \\
\hline 17 & 1903.9899 & 952.4986 & 1886.9633 & 943.9853 & 1885.9793 & 943.4933 & $\mathbf{Y}$ & 866.4254 & 433.7164 & 849.3989 & 425.2031 & 848.4149 & 424.7111 & 8 \\
\hline 18 & 1975.0270 & 988.0171 & 1958.0005 & 979.5039 & 1957.0164 & 979.0119 & A & 703.3621 & 352.1847 & 686.3355 & 343.6714 & 685.3515 & 343.1794 & 7 \\
\hline 19 & 2104.0696 & 1052.5384 & 2087.0431 & 1044.0252 & 2086.0590 & 1043.5332 & $\mathbf{E}$ & 632.3250 & 316.6661 & 615.2984 & 308.1529 & 614.3144 & 307.6608 & 6 \\
\hline 20 & 2217.1537 & 1109.0805 & 2200.1271 & 1100.5672 & 2199.1431 & 1100.0752 & I & 503.2824 & 252.1448 & 486.2558 & 243.6316 & 485.2718 & 243.1396 & 5 \\
\hline 21 & 2332.1806 & 1166.5939 & 2315.1541 & 1158.0807 & 2314.1700 & 1157.5887 & D & 390.1983 & 195.6028 & 373.1718 & 187.0895 & 372.1878 & 186.5975 & 4 \\
\hline 22 & 2389.2021 & 1195.1047 & 2372.1755 & 1186.5914 & 2371.1915 & 1186.0994 & G & 275.1714 & 138.0893 & 258.1448 & 129.5761 & & & 3 \\
\hline 23 & 2460.2392 & 1230.6232 & 2443.2126 & 1222.1100 & 2442.2286 & 1221.6179 & A & 218.1499 & 109.5786 & 201.1234 & 101.0653 & & & 2 \\
\hline 24 & & & & & & & $\mathbf{K}$ & 147.1128 & 74.0600 & 130.0863 & 65.5468 & & & 1 \\
\hline
\end{tabular}
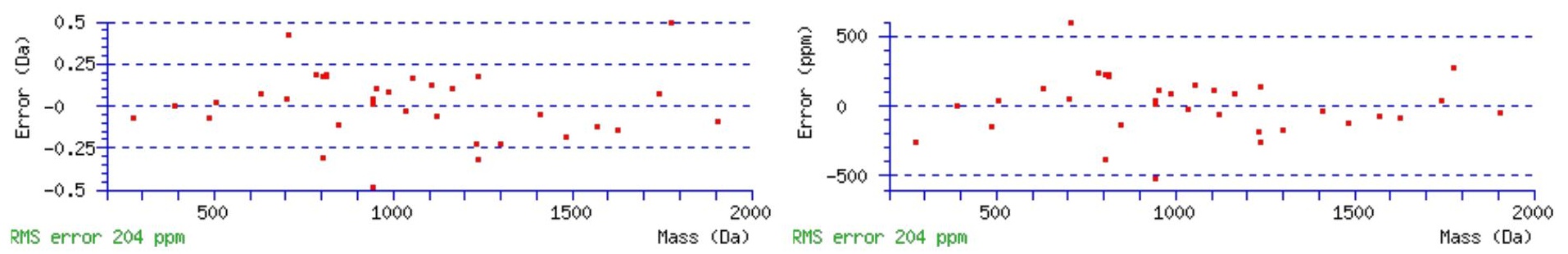

\section{All matches to this query}

\begin{tabular}{|l|l|l|l|}
\hline Score & Mr(calc): & Delta & \multicolumn{1}{|c|}{ Sequence } \\
\hline 54.8 & 2605.3374 & 0.0100 & LTLEELDEIIKNASGIYAEIDGAK \\
\hline 15.5 & 2604.3534 & 0.9940 & LTLEELDEIIKNASGIYAEIDGAK \\
\hline 5.5 & 2603.3555 & 1.9919 & ENGGGVPPGVAGAGAAAAALADEKALALGK \\
\hline 2.5 & 2605.3364 & 0.0110 & ITLSQVGDVLRALGTNPTNAEVKK \\
\hline 2.4 & 2603.3207 & 2.0267 & KEGSSLVTIGESPVPLQEIGTLNR \\
\hline 1.5 & 2604.3420 & 1.0054 & VMNATAYGISKTGVSIQVAVKMLK \\
\hline 1.5 & 2604.3420 & 1.0054 & VMNATAYGISKTGVSIQVAVKMLK \\
\hline 1.5 & 2605.3260 & 0.0214 & VMNATAYGISKTGVSIQVAVKMLK \\
\hline 1.5 & 2605.3260 & 0.0214 & VMNATAYGISKTGVSIQVAVKMLK \\
\hline 1.1 & 2604.3420 & 1.0054 & VMNATAYGISKTGVSIQVAVKMLK \\
\hline
\end{tabular}

Spectrum No: 610; Query: 2409; Rank: 1

\section{Peptide View}

MS/MS Fragmentation of IYNFSAPILTLSQPEVSEGDQVTVK

Found in IPI00197164, Tax_Id=10116 Gene_Symbol=Icam1 Intercellular adhesion molecule 1 precursor 
Match to Query 2409: 2735.386528 from(1368.700540,2+)

Title: 100101RatKid_NS_deglyco_21.4959.4959.2.dta

Data file K:INewmanPaper|Piliang|3SubProteomes\Piliang3SP\mgf5ppm\ERLIC_3SubProteomes5ppm.mgf
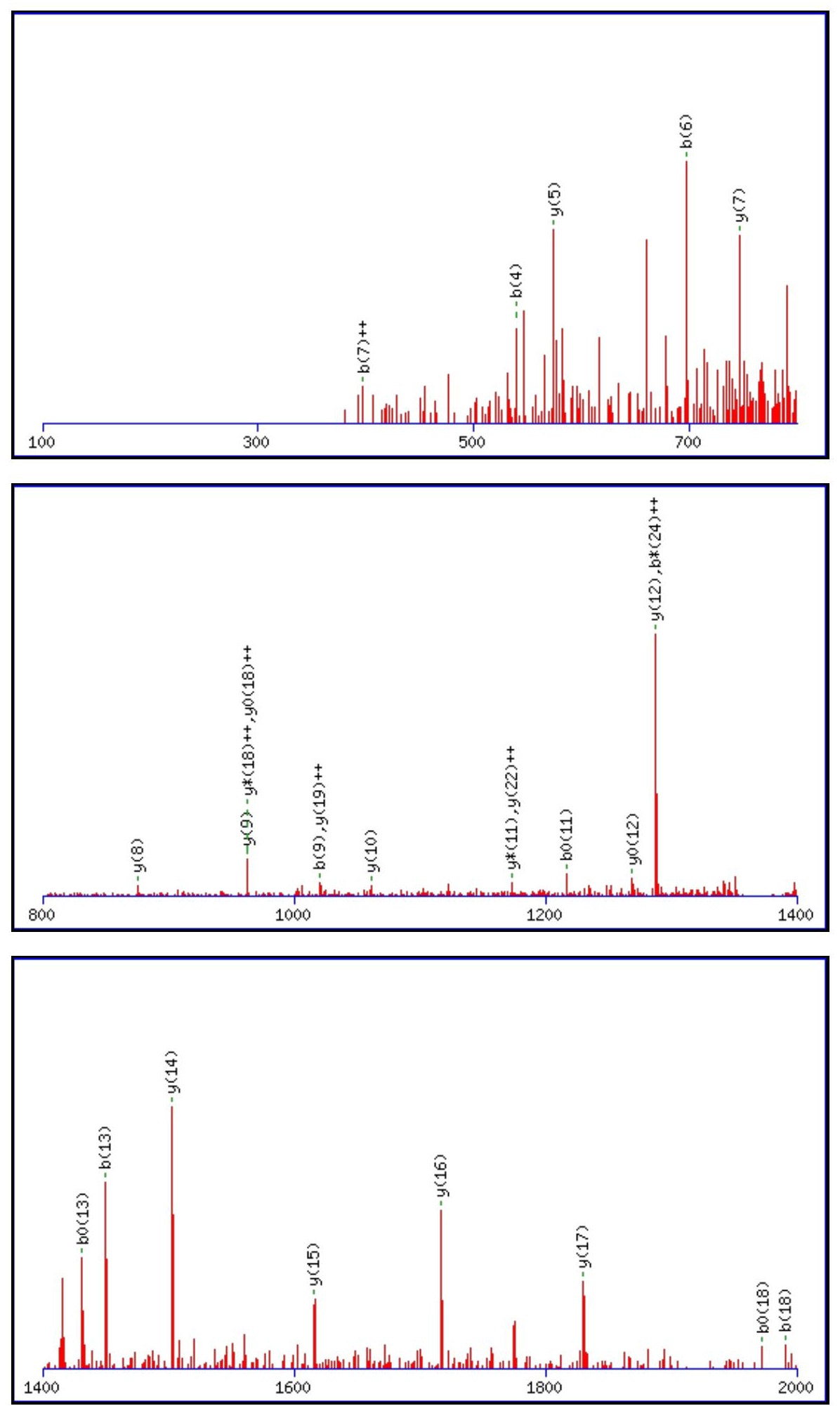

Monoisotopic mass of neutral peptide $\operatorname{Mr}($ calc): 2735.3905

Fixed modifications: Carbamidomethyl (C)

Variable modifications:

N3 : Deamidated_N (N)

Ions Score: 55 Expect: 0.00063

Matches (Bold Red): 26/272 fragment ions using 36 most intense peaks

\begin{tabular}{|c|c|c|c|c|c|c|c|c|c|c|c|c|c|c|}
\hline$\#$ & $\mathbf{b}$ & $\mathbf{b}^{++}$ & $\mathbf{b}^{*}$ & $\mathbf{b}^{*^{++}}$ & $\mathbf{b}^{\mathbf{0}}$ & $\mathbf{b}^{\mathbf{0 + +}}$ & Seq. & $\mathbf{y}$ & $\mathbf{y}^{++}$ & $\mathbf{y}^{*}$ & $\mathbf{y}^{\boldsymbol{*}^{++}}$ & $\mathbf{y}^{\mathbf{0}}$ & $\mathbf{y}^{\mathbf{0 + +}}$ & $\#$ \\
\hline $\mathbf{1}$ & 114.0913 & 57.5493 & & & & & $\mathbf{I}$ & & & & & & & 25 \\
\hline $\mathbf{2}$ & 277.1547 & 139.0810 & & & & & $\mathbf{Y}$ & 2623.3137 & 1312.1605 & 2606.2872 & 1303.6472 & 2605.3032 & 1303.1552 & 24 \\
\hline
\end{tabular}




\begin{tabular}{|c|c|c|c|c|c|c|c|c|c|c|c|c|c|c|}
\hline 3 & 392.1816 & 196.5944 & 375.1551 & 188.0812 & & & $\mathbf{N}$ & 2460.2504 & |1230.6288 & 2443.2239 & |1222.1156 & |2442.2399 & 1221.6236 & 23 \\
\hline 4 & 539.2500 & 270.1286 & 522.2235 & 261.6154 & & & $\mathbf{F}$ & 2345.2235 & 1173.1154 & 2328.1969 & 1164.6021 & 2327.2129 & 1164.1101 & 22 \\
\hline 5 & 626.2820 & 313.6447 & 609.2555 & 305.1314 & 608.2715 & 304.6394 & $\mathrm{~S}$ & 2198.1551 & 1099.5812 & 2181.1285 & 1091.0679 & 2180.1445 & 1090.5759 & 21 \\
\hline 6 & 697.3192 & 349.1632 & 680.2926 & 340.6499 & 679.3086 & 340.1579 & A & 2111.1230 & 1056.0652 & 2094.0965 & 1047.5519 & 2093.1125 & 1047.0599 & 20 \\
\hline 7 & 794.3719 & 397.6896 & 777.3454 & 389.1763 & 776.3614 & 388.6843 & $\mathbf{P}$ & 2040.0859 & 1020.5466 & 2023.0594 & 1012.0333 & |2022.0754 & 1011.5413 & 19 \\
\hline 8 & 907.4560 & 454.2316 & 890.4294 & 445.7184 & 889.4454 & 445.2264 & I & 1943.0332 & 972.0202 & 1926.0066 & 963.5069 & 1925.0226 & 963.0149 & 18 \\
\hline 9 & 1020.5401 & 510.7737 & 1003.5135 & 502.2604 & 1002.5295 & 501.7684 & $\mathbf{L}$ & 1829.9491 & 915.4782 & 1812.9226 & 906.9649 & 1811.9385 & 906.4729 & 17 \\
\hline 10 & 1121.5877 & 561.2975 & 1104.5612 & 552.7842 & 1103.5772 & 552.2922 & $\mathbf{T}$ & 1716.8650 & 858.9362 & 1699.8385 & 850.4229 & 1698.8545 & 849.9309 & 16 \\
\hline 11 & 1234.6718 & 617.8395 & 1217.6452 & 609.3263 & \begin{tabular}{|l}
1216.6612 \\
\end{tabular} & 608.8343 & $\mathbf{L}$ & 1615.8174 & 808.4123 & 1598.7908 & 799.8990 & 1597.8068 & 799.4070 & 15 \\
\hline 12 & 1321.7038 & 661.3555 & 1304.6773 & 652.8423 & 1303.6933 & 652.3503 & $S$ & 1502.7333 & 751.8703 & 1485.7067 & 743.3570 & 1484.7227 & 742.8650 & 4 \\
\hline 13 & 1449.7624 & 725.3848 & 1432.7359 & 716.8716 & 1431.7518 & 716.3796 & $\mathbf{Q}$ & 1415.7013 & 708.3543 & 1398.6747 & 699.8410 & 1397.6907 & 699.3490 & 13 \\
\hline 14 & 1546.8152 & 773.9112 & 1529.7886 & 765.3979 & 1528.8046 & 764.9059 & $\mathbf{P}$ & 1287.6427 & 644.3250 & 1270.6161 & 635.8117 & 1269.6321 & 635.3197 & 12 \\
\hline 15 & 1675.8578 & 838.4325 & 1658.8312 & 829.9192 & 1657.8472 & 829.4272 & $\mathbf{E}$ & 1190.5899 & 595.7986 & 1173.5634 & 587.2853 & 1172.5794 & 586.7933 & 11 \\
\hline 16 & 1774.9262 & 887.9667 & 1757.8996 & 879.4535 & 1756.9156 & 878.9614 & $\mathbf{V}$ & 1061.5473 & 531.2773 & 1044.5208 & 522.7640 & 1043.5368 & 522.2720 & 10 \\
\hline 17 & 1861.9582 & 931.4827 & 1844.9317 & 922.9695 & 1843.9476 & 922.4775 & $\mathrm{~S}$ & 962.4789 & 481.7431 & 945.4524 & 473.2298 & 944.4684 & 472.7378 & 9 \\
\hline 18 & 1991.0008 & 996.0040 & 1973.9742 & 987.4908 & \begin{tabular}{|l|l|}
1972.9902 \\
\end{tabular} & 986.9988 & $\mathbf{E}$ & 875.4469 & 438.2271 & 858.4203 & 429.7138 & 857.4363 & 429.2218 & 8 \\
\hline 19 & 2048.0223 & 1024.5148 & 2030.9957 & 1016.0015 & 2030.0117 & 1015.5095 & G & 746.4043 & 373.7058 & 729.3777 & 365.1925 & 728.3937 & 364.7005 & 7 \\
\hline $20:$ & 2163.0492 & 1082.0282 & 2146.0227 & 1073.5150 & 2145.0386 & 1073.0230 & D & 689.3828 & 345.1951 & 672.3563 & 336.6818 & 671.3723 & 336.1898 & 6 \\
\hline 21 & 2291.1078 & 1146.0575 & 2274.0812 & 1137.5443 & 2273.0972 & 1137.0522 & $\mathbf{Q}$ & 574.3559 & 287.6816 & 557.3293 & 279.1683 & 556.3453 & 278.6763 & 5 \\
\hline 22 & 2390.1762 & 1195.5917 & 2373.1496 & 1187.0785 & 2372.1656 & 1186.5865 & V & 446.2973 & 223.6523 & 429.2708 & 215.1390 & 428.2867 & 214.6470 & 4 \\
\hline 23 & 2491.2239 & 1246.1156 & 2474.1973 & 1237.6023 & 2473.2133 & 1237.1103 & $\mathbf{T}$ & 347.2289 & 174.1181 & 330.2023 & 165.6048 & 329.2183 & 165.1128 & 3 \\
\hline 24 & 2590.2923 & 1295.6498 & 2573.2657 & 1287.1365 & 2572.2817 & 1286.6445 & V & 246.1812 & 123.5942 & 229.1547 & 115.0810 & & & 2 \\
\hline 25 & & & & & & & $\mathbf{K}$ & 147.1128 & 74.0600 & 130.0863 & 65.5468 & & & 1 \\
\hline
\end{tabular}
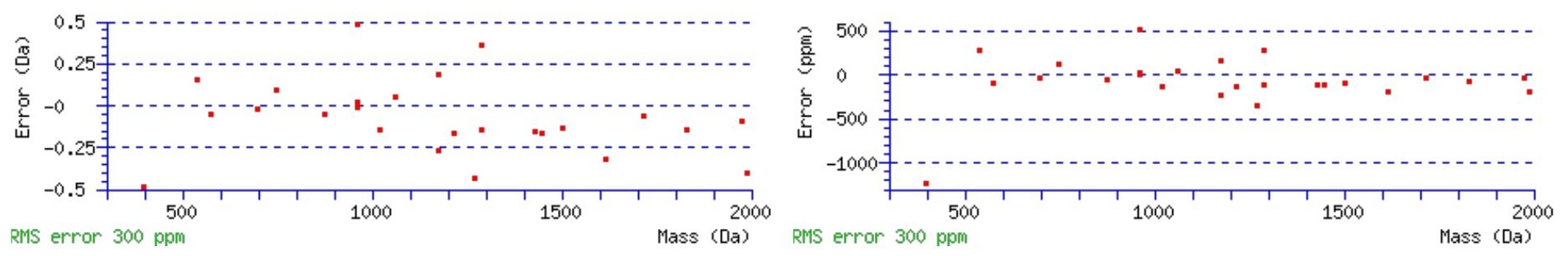

\section{All matches to this query}

\begin{tabular}{|l|c|c|l|}
\hline Score & Mr(calc): & Delta & \multicolumn{1}{c|}{ Sequence } \\
\hline 54.7 & 2735.3905 & -0.0040 & IYNFSAPILTLSQPEVSEGDQVTVK \\
\hline 42.2 & 2734.4065 & 0.9800 & IYNFSAPILTLSQPEVSEGDQVTVK \\
\hline 2.5 & 2735.3605 & 0.0260 & IIAVNNISLGIPAGECFGLLGVNGAGK \\
\hline 1.5 & 2733.3908 & 1.9957 & LLQVFQEECAQTYQVSLVTHGKR \\
\hline 1.1 & 2733.3537 & 2.0328 & LVPASFEDPEFKSSFNQSLSLYTK \\
\hline 0.7 & 2734.3870 & 0.9995 & AEYFLVLAVLNSGTNPIIYTLTNK \\
\hline 0.3 & 2735.3621 & 0.0244 & THIDAIILALPEDLQGILLKTGMT \\
\hline
\end{tabular}

Spectrum No: 611; Query: 191; Rank: 1

\section{Peptide View}

MS/MS Fragmentation of YEQLQNETR

Found in IPI00201276, Tax_Id=10116 Gene_Symbol=Acp2 Lysosomal acid phosphatase precursor

Match to Query 191: 1180.534228 from(591.274390,2+)

Title: 091008RatKidney_NH4Format01_24.610.610.2.dta 

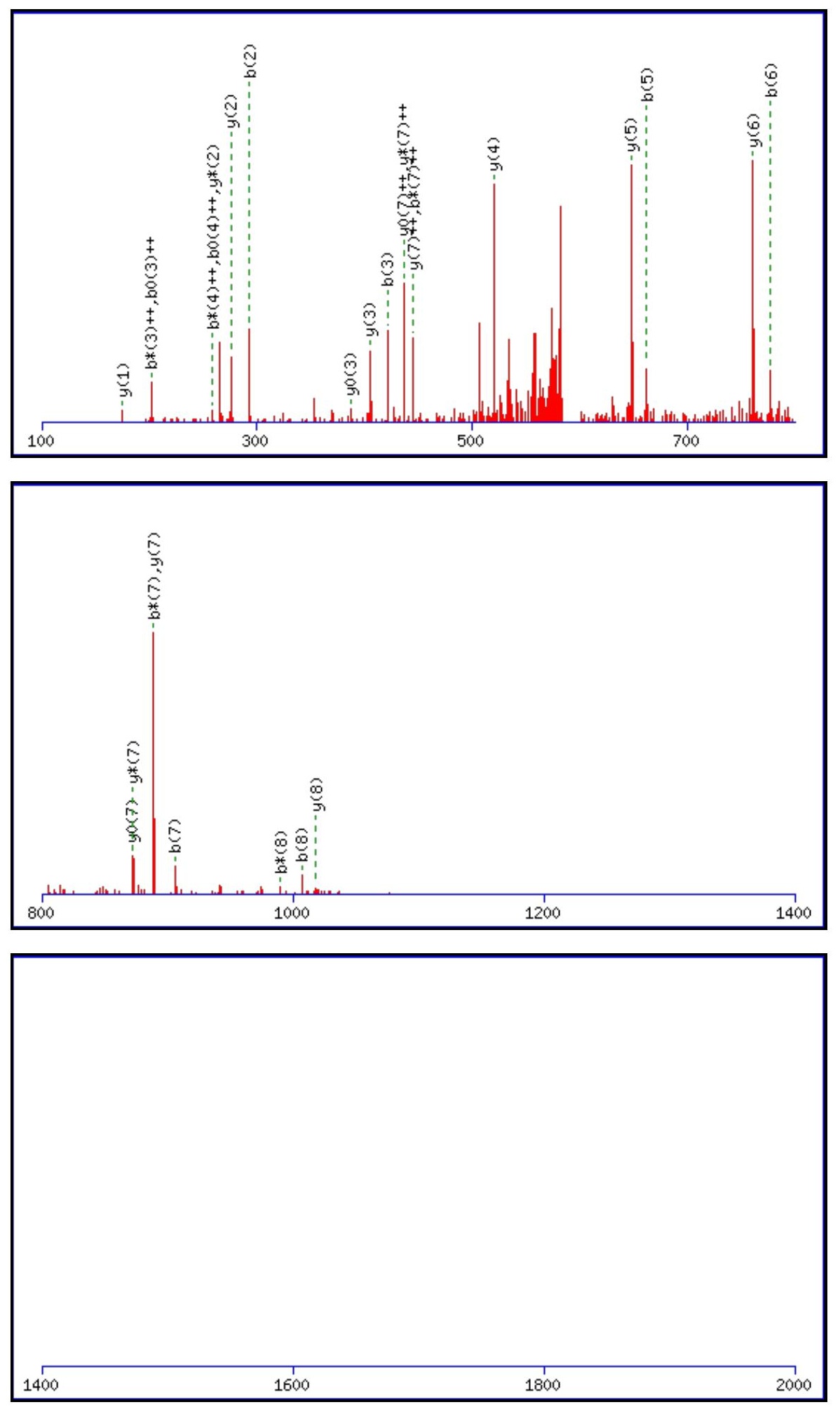

Monoisotopic mass of neutral peptide $\operatorname{Mr}($ calc): 1180.5360

Fixed modifications: Carbamidomethyl (C)

Variable modifications:

N6 : Deamidated_N (N)

Ions Score: 55 Expect: 0.00035

Matches (Bold Red): 28/88 fragment ions using 49 most intense peaks

\begin{tabular}{|c|c|c|c|c|c|c|c|c|c|c|c|c|c|c|}
\hline$\#$ & $\mathbf{b}$ & $\mathbf{b}^{++}$ & $\mathbf{b}^{*}$ & $\mathbf{b}^{\boldsymbol{*}^{++}}$ & $\mathbf{b}^{\mathbf{0}}$ & $\mathbf{b}^{\mathbf{0}+}$ & $\mathbf{S e q}$ & $\mathbf{y}$ & $\mathbf{y}^{++}$ & $\mathbf{y}^{*}$ & $\mathbf{y}^{\boldsymbol{*}^{++}}$ & $\mathbf{y}^{\mathbf{0}}$ & $\mathbf{y}^{\mathbf{0 + +}}$ & $\#$ \\
\hline $\mathbf{1}$ & 164.0706 & 82.5389 & & & & & $\mathbf{Y}$ & & & & & & & $\mathbf{9}$ \\
\hline $\mathbf{2}$ & $\mathbf{2 9 3 . 1 1 3 2}$ & 147.0602 & & & 275.1026 & 138.0550 & $\mathbf{E}$ & $\mathbf{1 0 1 8 . 4 8 0 0}$ & 509.7436 & 1001.4534 & 501.2303 & 1000.4694 & 500.7383 & $\mathbf{8}$ \\
\hline $\mathbf{3}$ & $\mathbf{4 2 1 . 1 7 1 8}$ & 211.0895 & 404.1452 & $\mathbf{2 0 2 . 5 7 6 3}$ & 403.1612 & $\mathbf{2 0 2 . 0 8 4 2}$ & $\mathbf{Q}$ & $\mathbf{8 8 9 . 4 3 7 4}$ & $\mathbf{4 4 5 . 2 2 2 3}$ & $\mathbf{8 7 2 . 4 1 0 8}$ & $\mathbf{4 3 6 . 7 0 9 1}$ & $\mathbf{8 7 1 . 4 2 6 8}$ & $\mathbf{4 3 6 . 2 1 7 0}$ & $\mathbf{7}$ \\
\hline $\mathbf{4}$ & 534.2558 & 267.6316 & 517.2293 & 259.1183 & 516.2453 & 258.6263 & $\mathbf{L}$ & $\mathbf{7 6 1 . 3 7 8 8}$ & 381.1930 & 744.3523 & 372.6798 & 743.3682 & 372.1878 & $\mathbf{6}$ \\
\hline
\end{tabular}




\begin{tabular}{|c|c|c|c|c|c|c|c|c|c|c|c|c|c|c|}
\hline 5 & 662.3144 & 331.6608 & 645.2879 & 323.1476 & 644.3039 & 322.6556 & $\mathbf{Q}$ & 648.2947 & 324.6510 & 631.2682 & 316.1377 & 630.2842 & 315.6457 & 5 \\
\hline 6 & 777.3414 & 389.1743 & 760.3148 & 380.6610 & 759.3308 & 380.1690 & $\mathbf{N}$ & 520.2362 & 260.6217 & 503.2096 & 252.1084 & 502.2256 & 251.6164 & 4 \\
\hline 7 & 906.3839 & 453.6956 & 889.3574 & 445.1823 & 888.3734 & 444.6903 & $\mathbf{E}$ & 405.2092 & 203.1082 & 388.1827 & 194.5950 & 387.1987 & 194.1030 & 3 \\
\hline 8 & 1007.4316 & 504.2195 & 990.4051 & 495.7062 & 989.4211 & 495.2142 & $\mathbf{T}$ & 276.1666 & 138.5870 & 259.1401 & 130.0737 & 258.1561 & 129.5817 & 2 \\
\hline 9 & & & & & & & $\mathbf{R}$ & 175.1190 & 88.0631 & 158.0924 & 79.5498 & & & \\
\hline
\end{tabular}
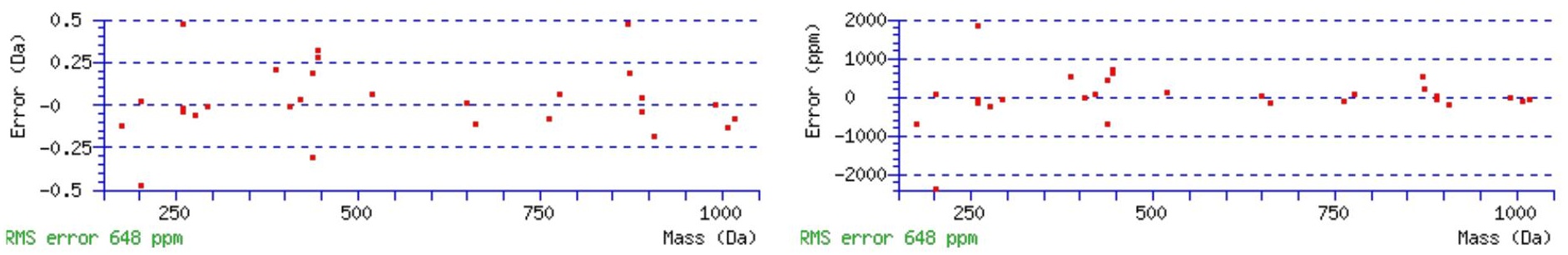

\section{All matches to this query}

\begin{tabular}{|l|l|l|l|}
\hline Score & Mr(calc): & Delta & \multicolumn{1}{|c|}{ Sequence } \\
\hline 54.6 & 1180.5360 & -0.0018 & YEQLQNETR \\
\hline 8.8 & 1178.5350 & 1.9993 & LCEEKSGNSR \\
\hline 6.1 & 1180.5335 & 0.0007 & MWSLNNWSK \\
\hline 4.7 & 1180.5406 & -0.0064 & SKSGIKVFR \\
\hline 4.2 & 1178.5276 & 2.0067 & GKSSGRSDDNR \\
\hline 4.2 & 1178.5276 & 2.0067 & SRGTNGSETNR \\
\hline 3.0 & 1179.5367 & 0.9975 & TGNNSTKAETR \\
\hline 2.9 & 1180.5311 & 0.0031 & CNVIITQPR \\
\hline 2.8 & 1180.5237 & 0.0105 & LPSQNERTR \\
\hline 2.5 & 1180.5377 & -0.0034 & LPQAVSTEEK \\
\hline
\end{tabular}

Spectrum No: 612; Query: 2847; Rank: 1

\section{Peptide View}

MS/MS Fragmentation of TCGMGVCSVLNCEYQCHQTPFGGECFCPPGHIINSNDSR

Found in IPI00205325, Tax_Id=10116 Gene_Symbol=Lrp2 Low-density lipoprotein receptor-related protein 2 precursor

Match to Query 2847: 4545.847576 from(1137.469170,4+)

Title: 100101RatKid_NS_deglyco_16.4337.4337.4.dta

Data file K:\NewmanPaper\Piliang $\backslash 3$ SubProteomes $\backslash$ Piliang3SP $\backslash$ mgf5ppm\ERLIC_3SubProteomes5ppm.mgf

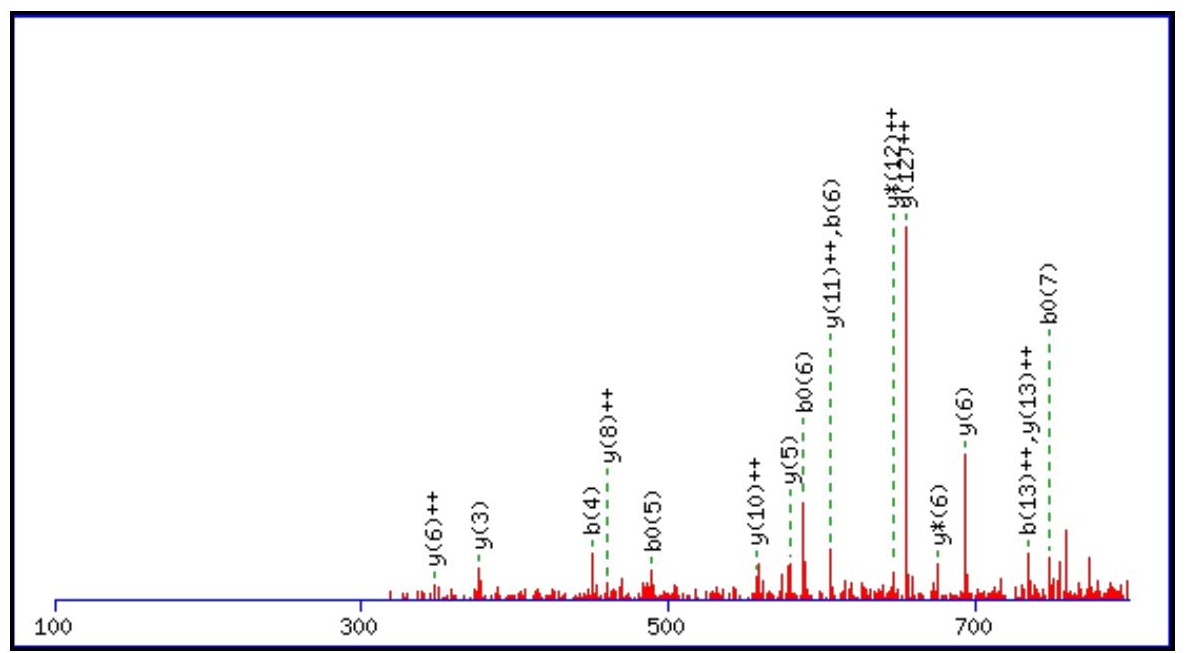



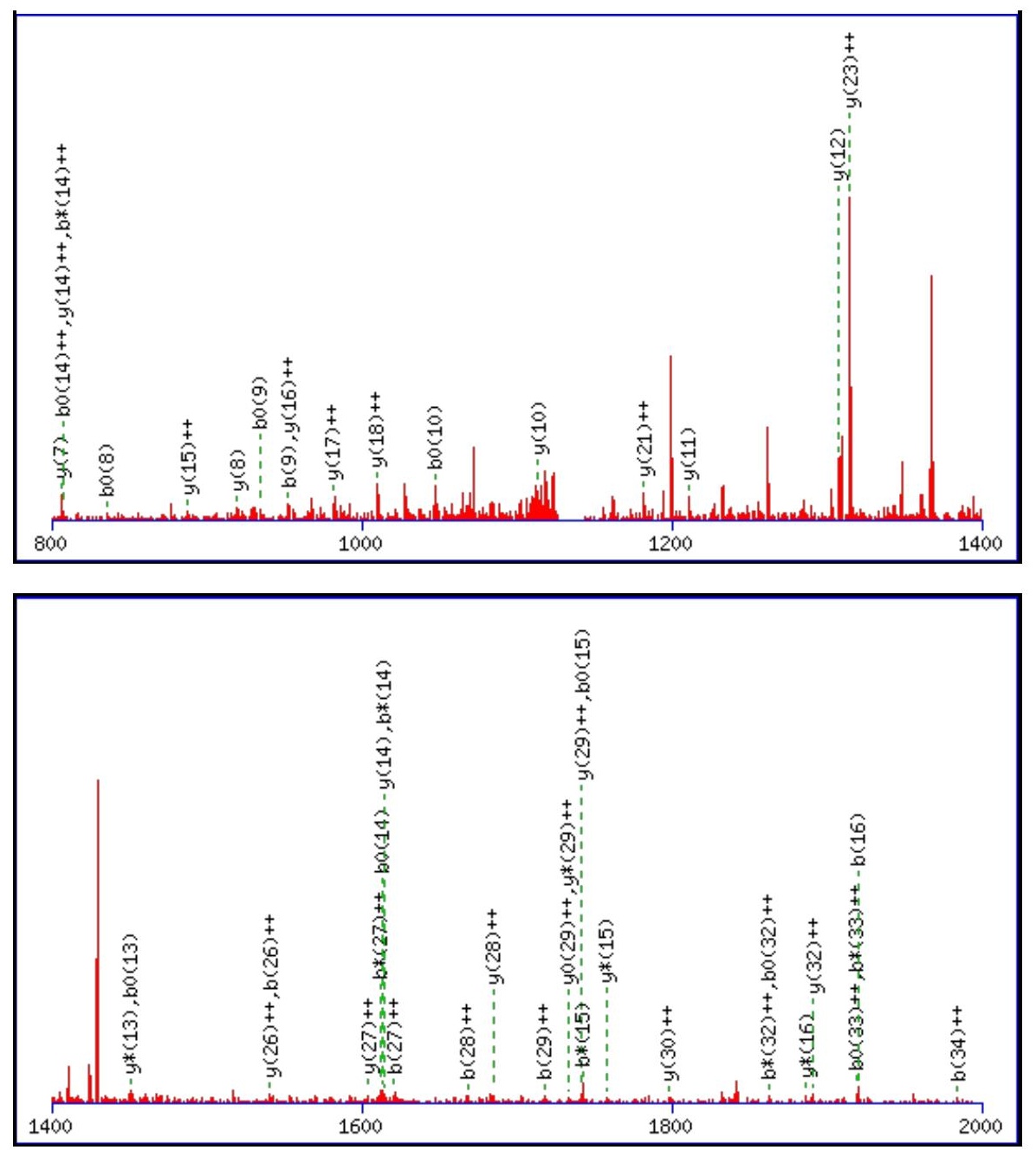

Monoisotopic mass of neutral peptide $\operatorname{Mr}($ calc): 4545.8422

Fixed modifications: Carbamidomethyl (C)

Variable modifications:

N36 : Deamidated_N (N)

Ions Score: 55 Expect: 0.0017

Matches (Bold Red): 63/434 fragment ions using 147 most intense peaks

\begin{tabular}{|c|c|c|c|c|c|c|c|c|c|c|c|c|c|c|}
\hline \# & b & $\mathbf{b}^{++}$ & b* & $\mathbf{b}^{*^{++}}$ & $\mathbf{b}^{0}$ & $\mathbf{b}^{\mathbf{0 + +}}$ & Seq. & $\mathbf{y}$ & $y^{++}$ & $\mathbf{y}^{*}$ & $\mathrm{y}^{*^{++}}$ & $\mathbf{y}^{\mathbf{0}}$ & $\mathbf{y}^{0++}$ & \# \\
\hline 1 & 102.0550 & 51.5311 & & & 84.0444 & 42.5258 & $\mathbf{T}$ & & & & & & & 39 \\
\hline 2 & 262.0856 & 131.5464 & & & 244.0750 & 122.5412 & $\mathrm{C}$ & 4445.8018 & 2223.4045 & 4428.7752 & 2214.8913 & 4427.7912 & 2214.3992 & 38 \\
\hline 3 & 319.1071 & 160.0572 & & & 301.0965 & 151.0519 & G & 4285.7711 & 2143.3892 & 4268.7446 & 2134.8759 & 4267.7606 & 2134.3839 & 37 \\
\hline 4 & 450.1476 & 225.5774 & & & 432.1370 & 216.5721 & $\mathbf{M}$ & 4228.7497 & 2114.8785 & 4211.7231 & 2106.3652 & 4210.7391 & 2105.8732 & 36 \\
\hline 5 & 507.1690 & 254.0881 & & & 489.1585 & 245.0829 & G & 4097.7092 & 2049.3582 & 4080.6826 & 2040.8450 & 4079.6986 & 2040.3529 & 35 \\
\hline 6 & 606.2374 & 303.6224 & & & 588.2269 & 294.6171 & $\mathbf{V}$ & 4040.6877 & 2020.8475 & 4023.6612 & 2012.3342 & 4022.6772 & 2011.8422 & 34 \\
\hline 7 & 766.2681 & 383.6377 & & & 748.2575 & 374.6324 & $\mathrm{C}$ & 3941.6193 & 1971.3133 & 3924.5928 & 1962.8000 & 3923.6087 & 1962.3080 & 33 \\
\hline 8 & 853.3001 & 427.1537 & & & 835.2895 & 418.1484 & $\mathrm{~S}$ & 3781.5887 & 1891.2980 & 3764.5621 & 1882.7847 & 3763.5781 & 1882.2927 & 32 \\
\hline 9 & 952.3685 & 476.6879 & & & 934.3580 & 467.6826 & $\mathbf{V}$ & 3694.5566 & 1847.7820 & 3677.5301 & 1839.2687 & 3676.5461 & 1838.7767 & 31 \\
\hline 10 & 1065.4526 & 533.2299 & & & 1047.4420 & 524.2246 & $\mathbf{L}$ & 3595.4882 & 1798.2477 & 3578.4617 & 1789.7345 & 3577.4776 & 1789.2425 & 30 \\
\hline 11 & 1179.4955 & 590.2514 & 1162.4690 & 581.7381 & 1161.4849 & 581.2461 & $\mathbf{N}$ & 3482.4042 & 1741.7057 & 3465.3776 & 1733.1924 & 3464.3936 & 1732.7004 & 29 \\
\hline 12 & 1339.5262 & 670.2667 & 1322.4996 & 661.7534 & 1321.5156 & 661.2614 & C & 3368.3612 & 1684.6842 & 3351.3347 & 1676.1710 & 3350.3507 & 1675.6790 & 28 \\
\hline 13 & 1468.5688 & 734.7880 & 1451.5422 & 726.2747 & 1450.5582 & 725.7827 & $\mathbf{E}$ & 3208.3306 & 1604.6689 & 3191.3040 & 1596.1557 & 3190.3200 & 1595.6636 & 27 \\
\hline 14 & 1631.6321 & 816.3197 & 1614.6055 & 807.8064 & 1613.6215 & 807.3144 & $\mathbf{Y}$ & 3079.2880 & 1540.1476 & 3062.2614 & 1531.6344 & 3061.2774 & 1531.1423 & 26 \\
\hline 15 & 1759.6907 & 880.3490 & 1742.6641 & 871.8357 & 1741.6801 & 871.3437 & $\mathbf{Q}$ & 2916.2247 & 1458.6160 & 2899.1981 & 1450.1027 & 2898.2141 & 1449.6107 & 725 \\
\hline 16 & 1919.7213 & 960.3643 & 1902.6948 & 951.8510 & 1901.7107 & 951.3590 & $\mathrm{C}$ & 2788.1661 & 1394.5867 & 2771.1395 & 1386.0734 & 2770.1555 & 1385.5814 & 24 \\
\hline 17 & 2056.7802 & 1028.8937 & 2039.7537 & 1020.3805 & 2038.7697 & 1019.8885 & H & 2628.1354 & 1314.5714 & 2611.1089 & 1306.0581 & 2610.1249 & 1305.5661 & 23 \\
\hline 18 & 2184.8388 & 1092.9230 & 2167.8123 & 1084.4098 & 2166.8282 & 1083.9178 & $\mathbf{Q}$ & 2491.0765 & 1246.0419 & 2474.0500 & 1237.5286 & 2473.0659 & 1237.0366 & 22 \\
\hline
\end{tabular}




\begin{tabular}{|c|c|c|c|c|c|c|c|c|c|c|c|c|c|c|}
\hline 19 & |2285.8865| & |1143.4469 & |2268.8599 & 1134.9336 & |2267.8759 & |1134.4416 & $T$ & & 1182.0126 & & & |2345.0074 & 0073 & $3 \mid 21$ \\
\hline 20 & 2382.9392 & 1191.9733 & 2365.9127 & 1183.4600 & 2364.9287 & 1182.9680 & $\mathbf{P}$ & 2261.9703 & 1131.4888 & 2244.9437 & 1122.9755 & 2243.9597 & 1122.4835 & 20 \\
\hline 21 & 2530.0077 & 1265.5075 & 2512.9811 & 1256.9942 & 2511.9971 & 1256.5022 & $\mathbf{F}$ & 2164.9175 & 1082.9624 & 2147.8909 & 1074.4491 & 2146.9069 & 1073.9571 & 19 \\
\hline 22 & 2587.0291 & 1294.0182 & 2570.0026 & 1285.5049 & 2569.0186 & 1285.0129 & G & 2017.8491 & 1009.4282 & 2000.8225 & 1000.9149 & 1999.8385 & 1000.4229 & 18 \\
\hline 23 & 2644.0506 & 1322.5289 & 2627.0240 & 1314.0157 & 2626.0400 & 1313.5236 & G & 1960.8276 & 980.9174 & 1943.8011 & 972.4042 & 1942.8170 & 971.9122 & 17 \\
\hline 24 & 2773.0932 & 1387.0502 & 2756.0666 & 1378.5370 & 2755.0826 & 1378.0449 & E & 1903.8062 & 952.4067 & 1886.7796 & 943.8934 & 1885.7956 & 943.4014 & 16 \\
\hline 25 & 2933.1238 & 1467.0656 & 2916.0973 & 1458.5523 & 2915.1133 & 1458.0603 & C & 1774.7636 & 887.8854 & 1757.7370 & 879.3721 & 1756.7530 & 878.8801 & 15 \\
\hline 26 & 3080.1922 & 1540.5998 & 3063.1657 & 1532.0865 & 3062.1817 & 1531.5945 & $\mathbf{F}$ & 1614.7329 & 807.8701 & 1597.7064 & 799.3568 & 1596.7223 & 798.8648 & 14 \\
\hline 27 & 3240.2229 & 1620.6151 & 3223.1963 & 1612.1018 & 3222.2123 & 1611.6098 & C & 1467.6645 & 734.3359 & \begin{tabular}{|l|l|}
1450.6379 \\
\end{tabular} & 725.8226 & 1449.6539 & 725.3306 & 13 \\
\hline 28 & 3337.2757 & 1669.1415 & 3320.2491 & 1660.6282 & 3319.2651 & 1660.1362 & $\mathbf{P}$ & 1307.6338 & 654.3206 & 1290.6073 & 645.8073 & 1289.6233 & 645.3153 & 12 \\
\hline 29 & 3434.3284 & 1717.6678 & 3417.3019 & 1709.1546 & 3416.3 & 1708.6626 & $\mathbf{P}$ & 1210.5811 & 605.7942 & 1193.5545 & 597.2809 & 1192.5705 & 596.7889 & 11 \\
\hline 30 & 3491.3499 & 1746.1786 & 3474.3233 & 1737.6653 & 3473.3393 & 1737.1733 & G & 1113.5283 & 557.2678 & 1096.5018 & 548.7545 & 1095.5178 & 548.2625 & 10 \\
\hline 31 & 3628.4088 & 1814.7080 & 3611.3822 & 1806.1948 & 3610.3982 & 1805.7028 & H & 1056.5069 & 528.7571 & 1039.4803 & 520.2438 & 1038.4963 & 519.7518 & 9 \\
\hline 32 & 3741.4929 & 1871.2501 & 3724.4663 & 1862.7368 & 3723.4823 & 1862.2448 & I & 919.4479 & 460.2276 & 902.4214 & 451.7143 & 901.4374 & 451.2223 & 8 \\
\hline 33 & 3854.5769 & 1927.7921 & 3837.5504 & 1919.2788 & 3836.5664 & 1918.7868 & I & 806.3639 & 403.6856 & 789.3373 & 395.1723 & 788.3533 & 394.6803 & 7 \\
\hline 34 & 3968.6198 & 1984.8136 & 3951.5933 & 1976.3003 & 3950.6093 & 1975.8083 & $\mathbf{N}$ & 693.2798 & 347.1435 & 676.2533 & 338.6303 & 675.2692 & 338.1383 & 6 \\
\hline 35 & 4055.6519 & 2028.3296 & 4038.6253 & 2019.8163 & 4037.6413 & 2019.3243 & $\mathrm{~S}$ & 579.2369 & 290.1221 & 562.2103 & 281.6088 & 561.2263 & 281.1168 & 5 \\
\hline 36 & 4170.6788 & 2085.8430 & 4153.6523 & 2077.3298 & 4152.6682 & 2076.8378 & $\mathbf{N}$ & 492.2049 & 246.6061 & 475.1783 & 238.0928 & 474.1943 & 237.6008 & 4 \\
\hline 37 & 4285.7058 & 2143.3565 & 4268.6792 & 2134.8432 & 4267.6952 & 2134.3512 & D & 377.1779 & 189.0926 & 360.1514 & 180.5793 & 359.1674 & 180.0873 & 3 \\
\hline 38 & 4372.7378 & 2186.8725 & 4355.7112 & 2178.3593 & 4354.7272 & 2177.8672 & $\mathrm{~S}$ & 262.1510 & 131.5791 & 245.1244 & 123.0659 & 244.1404 & 122.5738 & 2 \\
\hline 39 & & & & & & & $\mathbf{R}$ & 175.1190 & 88.0631 & 158.0924 & 79.5498 & & & 1 \\
\hline
\end{tabular}
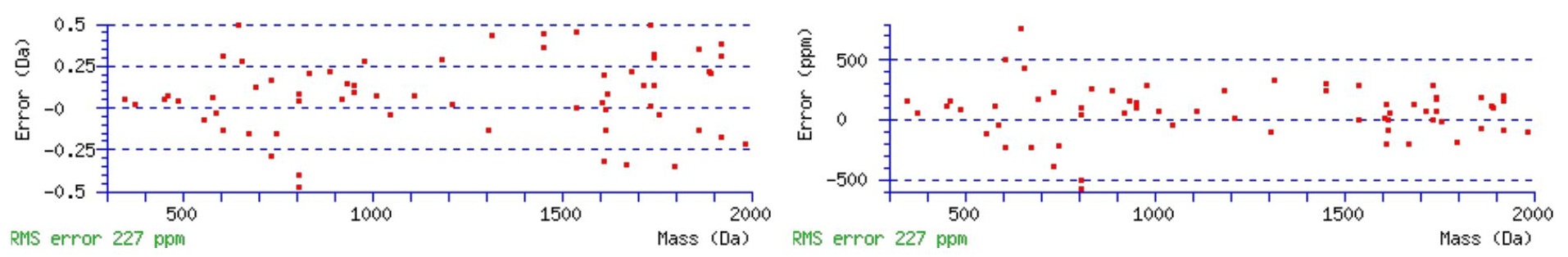

\section{All matches to this query}

\begin{tabular}{|l|l|c|l|}
\hline Score & Mr(calc): & Delta & \multicolumn{1}{c|}{ Sequence } \\
\hline 54.7 & 4545.8422 & 0.0053 & TCGMGVCSVLNCEYQCHQTPFGGECFCPPGHIINSNDSR \\
\hline 53.7 & 4545.8422 & 0.0053 & TCGMGVCSVLNCEYQCHQTPFGGECFCPPGHIINSNDSR \\
\hline 5.6 & 4544.8735 & 0.9741 & IDRTMIGEPMNFVHLTHIGSGEMGAGDGLAMTGAVQEQMR \\
\hline 5.6 & 4544.8735 & 0.9741 & IDRTMIGEPMNFVHLTHIGSGEMGAGDGLAMTGAVQEQMR \\
\hline 5.4 & 4543.8300 & 2.0175 & DALVMHDTSMVMGMDMGMDMGNSEQQNQGPGLQRSTSRTR \\
\hline 5.4 & 4543.8300 & 2.0175 & DALVMHDTSMVMGMDMGMDMGNSEQQNQGPGLQRSTSRTR \\
\hline 4.8 & 4545.8575 & -0.0099 & IDRTMIGEPMNFVHLTHIGSGEMGAGDGLAMTGAVQEQMR \\
\hline 4.2 & 4544.8735 & 0.9741 & IDRTMIGEPMNFVHLTHIGSGEMGAGDGLAMTGAVQEQMR \\
\hline 3.3 & 4545.8575 & -0.0099 & IDRTMIGEPMNFVHLTHIGSGEMGAGDGLAMTGAVQEQMR \\
\hline 3.3 & 4545.8575 & -0.0099 & IDRTMIGEPMNFVHLTHIGSGEMGAGDGLAMTGAVQEQMR \\
\hline
\end{tabular}

Spectrum No: 613; Query: 354; Rank: 1

\section{Peptide View}

MS/MS Fragmentation of TLSPTGNISSAPR

Found in IPI00192818, Tax_Id=10116 Gene_Symbol=Unc84a 102 kDa protein

Match to Query 354: 1300.660948 from(651.337750,2+) 
Title: 091008RatKidney_NH4Format01_24.1171.1171.2.dta

Data file K:\NewmanPaper\Piliang \3SubProteomes\Piliang3SP \mgf5ppm\ERLIC_3SubProteomes5ppm.mgf
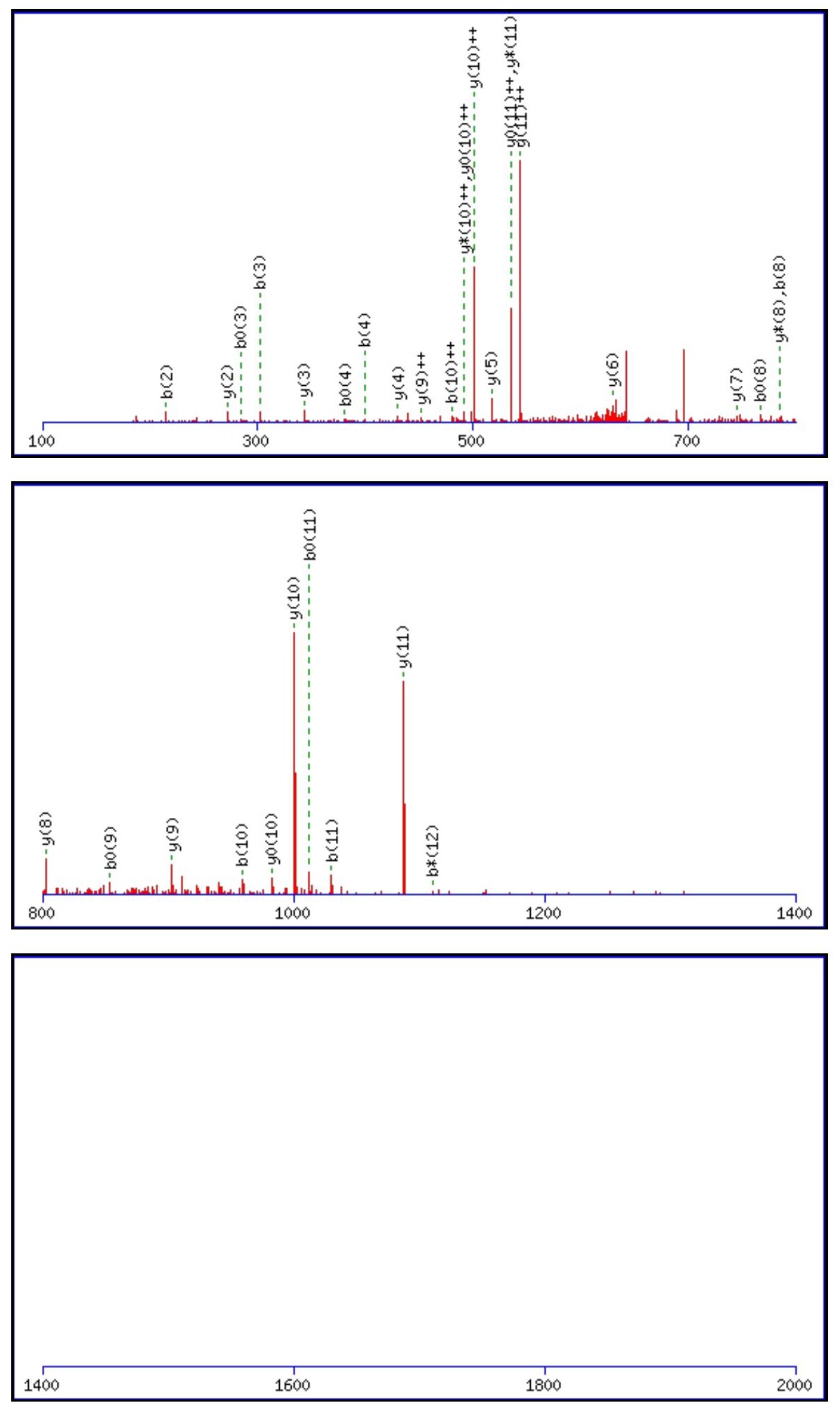

Monoisotopic mass of neutral peptide $\operatorname{Mr}($ calc): 1300.6623

Fixed modifications: Carbamidomethyl (C)

Variable modifications:

N7 : Deamidated N (N)

Ions Score: 55 Expect: 0.00045

Matches (Bold Red): 32/126 fragment ions using 71 most intense peaks

\begin{tabular}{|r|c|c|c|c|r|r|r|r|c|c|c|c|c|c|}
\hline$\#$ & $\mathbf{b}$ & $\mathbf{b}^{++}$ & $\mathbf{b}^{*}$ & $\mathbf{b}^{\boldsymbol{*}^{++}}$ & $\mathbf{b}^{\mathbf{0}}$ & $\mathbf{b}^{\mathbf{0 + +}}$ & Seq. & $\mathbf{y}$ & $\mathbf{y}^{++}$ & $\mathbf{y}^{\mathbf{*}}$ & $\mathbf{y}^{\boldsymbol{*}^{++}}$ & $\mathbf{y}^{\mathbf{0}}$ & $\mathbf{y}^{\mathbf{0 + +}}$ & $\#$ \\
\hline $\mathbf{1}$ & 102.0550 & 51.5311 & & & 84.0444 & 42.5258 & $\mathbf{T}$ & & & & & & & $\mathbf{1 3}$ \\
\hline $\mathbf{2}$ & $\mathbf{2 1 5 . 1 3 9 0}$ & 108.0731 & & & 197.1285 & 99.0679 & L & 1200.6219 & 600.8146 & 1183.5953 & 592.3013 & 1182.6113 & 591.8093 & $\mathbf{1 2}$ \\
\hline 3 & $\mathbf{3 0 2 . 1 7 1 0}$ & 151.5892 & & & $\mathbf{2 8 4 . 1 6 0 5}$ & 142.5839 & S & $\mathbf{1 0 8 7 . 5 3 7 8}$ & 544.2725 & 1070.5113 & 535.7593 & 1069.5273 & 535.2673 & $\mathbf{1 1}$ \\
\hline
\end{tabular}




\begin{tabular}{|c|c|c|c|c|c|c|c|c|c|c|c|c|c|c|}
\hline 4 & 399.2238 & |200.1155 & & & 381.2132 & |191.1103 & $\mathbf{P}$ & $\mid 1000.5058$ & |500.7565 & 983.4792 & 492.2433 & 982.4952 & 491.7513 & 10 \\
\hline 5 & 500.2715 & 250.6394 & & & 482.2609 & 241.6341 & $\mathbf{T}$ & 903.4530 & 452.2302 & 886.4265 & 443.7169 & 885.4425 & 443.2249 & 9 \\
\hline 6 & 557.2930 & 279.1501 & & & 539.2824 & 270.1448 & G & 802.4054 & 401.7063 & 785.3788 & 393.1930 & 784.3948 & 392.7010 & 8 \\
\hline 7 & 672.3199 & 336.6636 & 655.2933 & 328.1503 & 654.3093 & 327.6583 & $\mathbf{N}$ & 745.3839 & 373.1956 & 728.3573 & 364.6823 & 727.3733 & 364.1903 & 7 \\
\hline 8 & 785.4040 & 393.2056 & 768.3774 & 384.6923 & 767.3934 & 384.2003 & I & 630.3569 & 315.6821 & 613.3304 & 307.1688 & 612.3464 & 306.6768 & 6 \\
\hline 9 & 872.4360 & 436.7216 & 855.4094 & 428.2084 & 854.4254 & 427.7163 & S & 517.2729 & 259.1401 & 500.2463 & 250.6268 & 499.2623 & 250.1348 & 5 \\
\hline 10 & 959.4680 & 480.2376 & 942.4415 & 471.7244 & 941.4574 & 471.2324 & S & 430.2409 & 215.6241 & 413.2143 & 207.1108 & 412.2303 & 206.6188 & 4 \\
\hline 11 & 1030.5051 & 515.7562 & 1013.4786 & 507.2429 & 1012.4946 & 506.7509 & A & 343.2088 & 172.1081 & 326.1823 & 163.5948 & & & 3 \\
\hline 12 & 1127.5579 & 564.2826 & 1110.5313 & 555.7693 & 1109.5473 & 555.2773 & $\mathbf{P}$ & 272.1717 & 136.5895 & 255.1452 & 128.0762 & & & 2 \\
\hline 13 & & & & & & & $\mathbf{R}$ & 175.1190 & 88.0631 & 158.0924 & 79.5498 & & & 1 \\
\hline
\end{tabular}
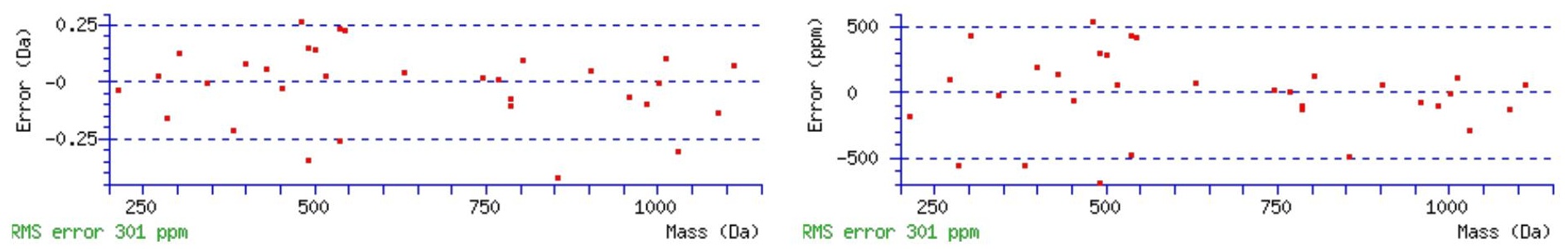

\section{All matches to this query}

\begin{tabular}{|l|l|l|l|}
\hline Score & Mr(calc): & Delta & \multicolumn{1}{|c|}{ Sequence } \\
\hline 54.6 & 1300.6623 & -0.0013 & \\
\hline 8.5 & 1300.6540 & 0.0069 & VNSTGNISSAPR \\
\hline 7.8 & 1300.6540 & 0.0069 & VNSTYLVRLR \\
\hline 7.8 & 1300.6735 & -0.0126 & DVSGSRPDLSLR \\
\hline 4.1 & 1299.6475 & 1.0134 & NAIEKFAVTVK \\
\hline 3.4 & 1299.6700 & 0.9909 & VNSTYLVRLR \\
\hline 2.6 & 1299.6700 & 0.9909 & VNSTYLVRLR \\
\hline 2.3 & 1298.6578 & 2.0031 & NNAQAEKNGKPK \\
\hline 1.6 & 1298.6635 & 1.9974 & NAIEKFAVTVK \\
\hline 1.5 & 1300.6696 & -0.0087 & VNPAASMEIIQK \\
\hline
\end{tabular}

Spectrum No: 614; Query: 2361; Rank: 1

\section{Peptide View}

MS/MS Fragmentation of WKVPFNPNDTFESEFYLDEKR

Found in IPI00200591, Tax_Id=10116 Gene_Symbol=LOC299282 Serine protease inhibitor A3L precursor

Match to Query 2361: 2661.248412 from(888.090080,3+)

Title: 100101RatKid_NS_deglyco_20.4434.4434.3.dta

Data file K:INewmanPaper|Piliangl3SubProteomes\Piliang3SP\mgf5ppm\ERLIC_3SubProteomes5ppm.mgf 

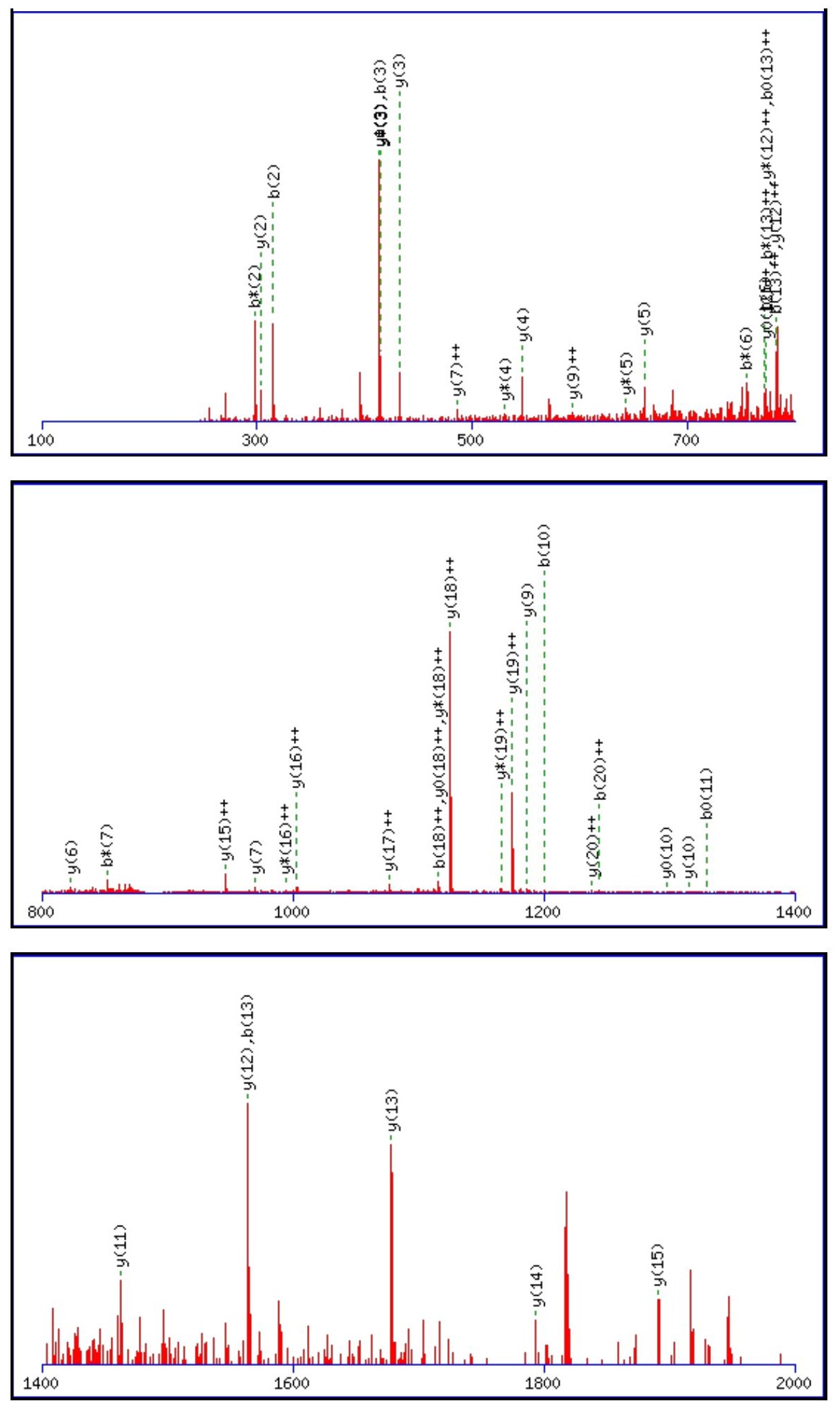

Monoisotopic mass of neutral peptide $\operatorname{Mr}($ calc): 2661.2387

Fixed modifications: Carbamidomethyl (C)

Variable modifications:

N8 : Deamidated $\mathrm{N}(\mathrm{N})$

Ions Score: 55 Expect: 0.0011

Matches (Bold Red): 47/218 fragment ions using 90 most intense peaks

\begin{tabular}{|c|c|c|c|c|c|c|c|c|c|c|c|c|c|c|}
\hline$\#$ & $\mathbf{b}$ & $\mathbf{b}^{++}$ & $\mathbf{b}^{*}$ & $\mathbf{b}^{\boldsymbol{*}^{++}}$ & $\mathbf{b}^{\mathbf{0}}$ & $\mathbf{b}^{\mathbf{0 + +}}$ & $\mathbf{S e q}$ & $\mathbf{y}$ & $\mathbf{y}^{++}$ & $\mathbf{y}^{*}$ & $\mathbf{y}^{\boldsymbol{*}^{++}}$ & $\mathbf{y}^{\mathbf{0}}$ & $\mathbf{y}^{\mathbf{0 + +}}$ & $\#$ \\
\hline $\mathbf{1}$ & $\mathbf{1} 87.0866$ & 94.0469 & & & & & $\mathbf{W}$ & & & & & & & $\mathbf{2 1}$ \\
\hline $\mathbf{2}$ & $\mathbf{3 1 5 . 1 8 1 6}$ & 158.0944 & $\mathbf{2 9 8 . 1 5 5 0}$ & 149.5811 & & & $\mathbf{K}$ & 2476.1667 & $\mathbf{1 2 3 8 . 5 8 7 0}$ & 2459.1401 & 1230.0737 & 2458.1561 & 1229.5817 & $\mathbf{2 0}$ \\
\hline $\mathbf{3}$ & $\mathbf{4 1 4 . 2 5 0 0}$ & 207.6286 & 397.2234 & 199.1153 & & & $\mathbf{V}$ & 2348.0717 & $\mathbf{1 1 7 4 . 5 3 9 5}$ & 2331.0452 & $\mathbf{1 1 6 6 . 0 2 6 2}$ & 2330.0612 & 1165.5342 & $\mathbf{1 9}$ \\
\hline $\mathbf{4}$ & 511.3027 & 256.1550 & 494.2762 & 247.6417 & & & $\mathbf{P}$ & 2249.0033 & $\mathbf{1 1 2 5 . 0 0 5 3}$ & 2231.9768 & $\mathbf{1 1 1 6 . 4 9 2 0}$ & 2230.9927 & $\mathbf{1 1 1 6 . 0 0 0 0}$ & $\mathbf{1 8}$ \\
\hline $\mathbf{5}$ & 658.3711 & 329.6892 & 641.3446 & 321.1759 & & & $\mathbf{F}$ & 2151.9505 & $\mathbf{1 0 7 6 . 4 7 8 9}$ & 2134.9240 & 1067.9656 & 2133.9400 & 1067.4736 & $\mathbf{1 7}$ \\
\hline
\end{tabular}




\begin{tabular}{|c|c|c|c|c|c|c|c|c|c|c|c|c|c|c|}
\hline & 772.4141 & 386.7107 & 755.3875 & 378.1974 & & & $\mathbf{N}$ & 2004.8821 & $|1002.9447|$ & |1987.8556 | & 994.4314 & & 993.9394 & $\mid 16$ \\
\hline 7 & 869.4668 & 435.2371 & 852.4403 & 426.7238 & & & $\mathbf{P}$ & 1890.8392 & 945.9232 & 1873.8127 & 937.4100 & 1872.8286 & 936.9180 & 15 \\
\hline 8 & 984.4938 & 492.7505 & 967.4672 & 484.2372 & & & $\mathbf{N}$ & 1793.7864 & 897.3969 & 1776.7599 & 888.8836 & 1775.7759 & 888.3916 & 14 \\
\hline 9 & 1099.5207 & 550.2640 & 1082.4942 & 541.7507 & 1081.5101 & 541.2587 & D & 1678.7595 & 839.8834 & 1661.7330 & 831.3701 & 1660.7489 & 830.8781 & 13 \\
\hline 10 & 1200.5684 & 600.7878 & 1183.5418 & 592.2746 & 1182.5578 & 591.7826 & $\mathrm{~T}$ & 1563.7326 & 782.3699 & 1546.7060 & 773.8566 & 1545.7220 & 773.3646 & 12 \\
\hline 11 & 1347.6368 & 674.3220 & 1330.6103 & 665.8088 & 1329.6262 & 665.3168 & $\mathbf{F}$ & 1462.6849 & 731.8461 & 1445.6583 & 723.3328 & 1444.6743 & 722.8408 & 11 \\
\hline 12 & 1476.6794 & 738.8433 & 1459.6529 & 730.3301 & 1458.6688 & 729.8381 & $\mathbf{E}$ & 1315.6165 & 658.3119 & 1298.5899 & 649.7986 & 1297.6059 & 649.3066 & 10 \\
\hline 13 & 1563.7114 & 782.3594 & 1546.6849 & 773.8461 & 1545.7009 & 773.3541 & S & 1186.5739 & 593.7906 & 1169.5473 & 585.2773 & 1168.5633 & 584.7853 & 9 \\
\hline 14 & 1692.7540 & 846.8806 & 1675.7275 & 838.3674 & 1674.7435 & 837.8754 & $\mathbf{E}$ & 1099.5419 & 550.2746 & 1082.5153 & 541.7613 & 1081.5313 & 541.2693 & 8 \\
\hline 15 & 1839.8224 & 920.4149 & 1822.7959 & 911.9016 & 1821.8119 & 911.4096 & $\mathbf{F}$ & 970.4993 & 485.7533 & 953.4727 & 477.2400 & 952.4887 & 476.7480 & 7 \\
\hline 16 & 2002.8858 & 1001.9465 & 1985.8592 & 993.4332 & 1984.8752 & 992.9412 & $\mathbf{Y}$ & 823.4308 & 412.2191 & 806.4043 & 403.7058 & 805.4203 & 403.2138 & 6 \\
\hline 17 & 2115.9698 & 1058.4886 & 2098.9433 & 1049.9753 & 2097.9593 & 1049.4833 & L & 660.3675 & 330.6874 & 643.3410 & 322.1741 & 642.3569 & 321.6821 & 5 \\
\hline 18 & 2230.9968 & 1116.0020 & 2213.9702 & 1107.4887 & 2212.9862 & 1106.9967 & D & 547.2835 & 274.1454 & 530.2569 & 265.6321 & 529.2729 & 265.1401 & 4 \\
\hline 19 & 2360.0394 & 1180.5233 & 2343.0128 & 1172.0100 & 2342.0288 & 1171.5180 & $\mathbf{E}$ & 432.2565 & 216.6319 & 415.2300 & 208.1186 & 414.2459 & 207.6266 & 3 \\
\hline 20 & 2488.1343 & 1244.5708 & 2471.1078 & 1236.0575 & 2470.1238 & 1235.5655 & $\mathbf{K}$ & 303.2139 & 152.1106 & 286.1874 & 143.5973 & & & 2 \\
\hline 21 & & & & & & & $\mathbf{R}$ & 175.1190 & 88.0631 & 158.0924 & 79.5498 & & & 1 \\
\hline
\end{tabular}
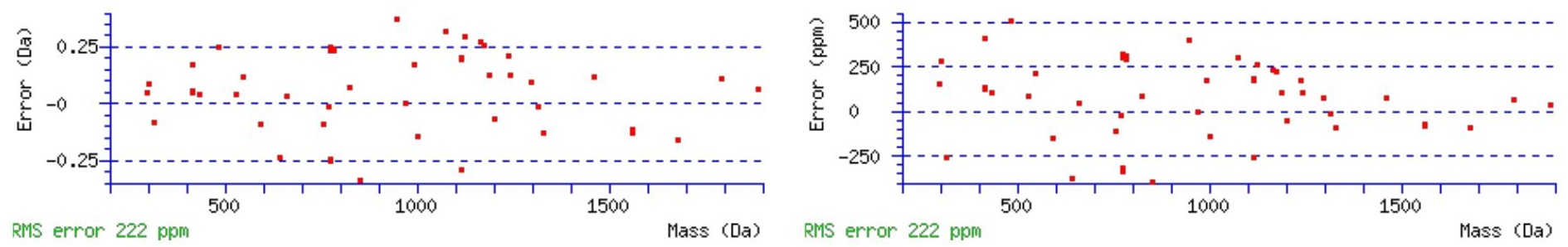

\section{All matches to this query}

\begin{tabular}{|l|l|l|l|}
\hline Score & Mr(calc): & Delta & \multicolumn{1}{|c|}{ Sequence } \\
\hline 54.6 & 2661.2387 & 0.0097 & WKVPFNPNDTFESEFYLDEKR \\
\hline 40.1 & 2661.2387 & 0.0097 & WKVPFNPNDTFESEFYLDEKR \\
\hline 27.6 & 2660.2547 & 0.9937 & WKVPFNPNDTFESEFYLDEKR \\
\hline 7.8 & 2661.2305 & 0.0179 & RWNCSTVDNLSVFGRVMQIGSR \\
\hline 4.9 & 2661.2305 & 0.0179 & RWNCSTVDNLSVFGRVMQIGSR \\
\hline 3.7 & 2659.2568 & 1.9916 & RHTDFSSGYFSSRWGPLLEDFR \\
\hline 2.9 & 2660.2590 & 0.9894 & ALLSSVTLGLSTSLGLLSVVAFCL \\
\hline 2.9 & 2659.2271 & 2.0214 & DVVKRDGQMFNLFLSLSFNLR \\
\hline 2.9 & 2659.2271 & 2.0214 & DVVKRDGQMFNLFLSLSFNLR \\
\hline 2.9 & 2660.2546 & 0.9938 & LTLSYNRRGDLAIHLISPMGTR \\
\hline
\end{tabular}

Spectrum No: 615; Query: 2300; Rank: 1

\section{Peptide View}

MS/MS Fragmentation of RIYYSDFSNQTINSMAEDGSNR

Found in IPI00205325, Tax_Id=10116 Gene_Symbol=Lrp2 Low-density lipoprotein receptor-related protein 2 precursor

Match to Query 2300: 2584.122252 from(862.381360,3+)

Title: 100101RatKid_NS_deglyco_22.2398.2398.3.dta

Data file K:INewmanPaper|Piliangl3SubProteomes\Piliang3SP\mgf5ppm|ERLIC_3SubProteomes5ppm.mgf 

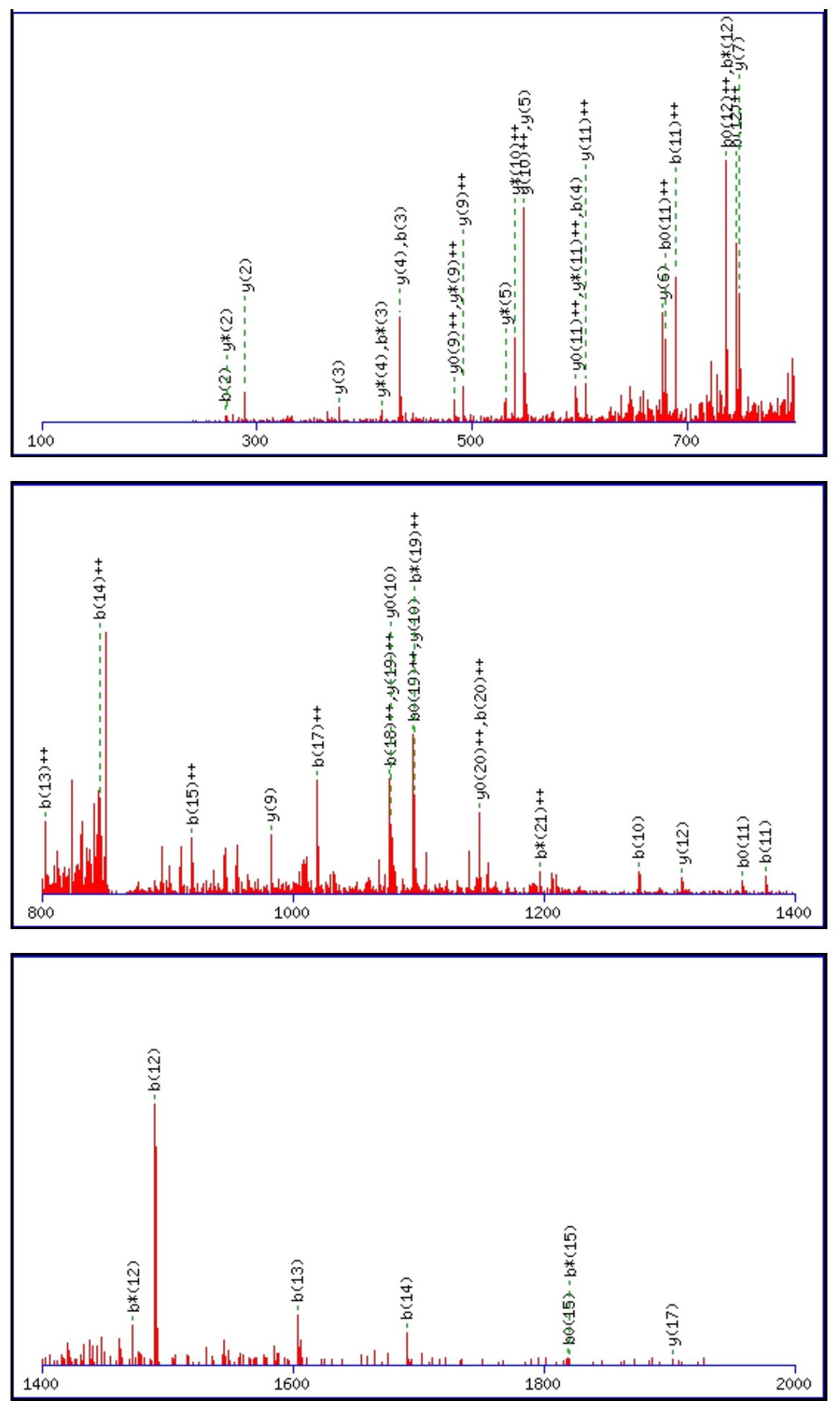

Monoisotopic mass of neutral peptide $\operatorname{Mr}($ calc): 2584.1136

Fixed modifications: Carbamidomethyl (C)

Variable modifications:

N9 : Deamidated_N (N)

M15 : Oxidation (M)

Ions Score: 54 Expect : 0.0012

Matches (Bold Red): 51/240 fragment ions using 71 most intense peaks

\begin{tabular}{|c|c|c|c|c|c|c|c|c|c|c|c|c|c|c|}
\hline$\#$ & $\mathbf{b}$ & \multicolumn{1}{|c|}{$\mathbf{b}^{++}$} & $\mathbf{b}^{*}$ & $\mathbf{b}^{*^{++}}$ & $\mathbf{b}^{\mathbf{0}}$ & $\mathbf{b}^{\mathbf{0}+}$ & Seq. & $\mathbf{y}$ & $\mathbf{y}^{++}$ & $\mathbf{y}^{*}$ & $\mathbf{y}^{*^{++}}$ & $\mathbf{y}^{\mathbf{0}}$ & $\mathbf{y}^{\mathbf{0}+}$ & $\#$ \\
\hline $\mathbf{1}$ & 157.1084 & 79.0578 & 140.0818 & 70.5446 & & & $\mathbf{R}$ & & & & & & & $\mathbf{2 2}$ \\
\hline $\mathbf{2}$ & $\mathbf{2 7 0 . 1 9 2 5}$ & 135.5999 & 253.1659 & 127.0866 & & & $\mathbf{I}$ & 2429.0198 & 1215.0135 & 2411.9932 & 1206.5002 & 2411.0092 & 1206.0082 & $\mathbf{2 1}$ \\
\hline $\mathbf{3}$ & $\mathbf{4 3 3 . 2 5 5 8}$ & 217.1315 & $4 \mathbf{4 1 6 . 2 2 9 2}$ & 208.6183 & & & $\mathbf{Y}$ & 2315.9357 & 1158.4715 & 2298.9092 & 1149.9582 & 2297.9251 & $\mathbf{1 1 4 9 . 4 6 6 2}$ & $\mathbf{2 0}$ \\
\hline $\mathbf{4}$ & $\mathbf{5 9 6 . 3 1 9 1}$ & 298.6632 & 579.2926 & 290.1499 & & & $\mathbf{Y}$ & 2152.8724 & $\mathbf{1 0 7 6 . 9 3 9 8}$ & 2135.8458 & 1068.4266 & 2134.8618 & 1067.9345 & $\mathbf{1 9}$ \\
\hline & & & & & & & & & & & & & &
\end{tabular}




\begin{tabular}{|c|c|c|c|c|c|c|c|c|c|c|c|c|c|c|}
\hline & 683.3511 & 342.1792 & 666.3246 & 659 & 665 & 1739 & $\mathbf{S}$ & 3090 & 995.4082 & & & & & \\
\hline 6 & 798.3781 & 399.6927 & 781.3515 & 391.1794 & 780.3675 & 390.6874 & D & 1902.7770 & 951.8921 & 1885.7505 & 943.3789 & 1884.7665 & 942.8869 & 17 \\
\hline 7 & 945.4465 & 473.2269 & 928.4199 & 464.7136 & 927.4359 & 464.2216 & & 1787.7501 & 894.3787 & 1770.7235 & 885.8654 & 1769.7395 & & \\
\hline 8 & 1032.4785 & 16.7429 & 1015.4 & & 1014.4680 & & & 1640.6817 & & & 812.3312 & & 8392 & \\
\hline 9 & 1147.5055 & 574.2564 & 1130.4789 & 565.7431 & 4949 & 565.2511 & & 496 & 777.3285 & 6231 & 768.8152 & 391 & 3232 & \\
\hline 10 & 1275.5640 & 638.2857 & 1258.5375 & 629.7724 & 1257.5535 & 629.2804 & $\mathbf{Q}$ & 1438.6227 & 719.8150 & 1421.5962 & 711.3017 & 1420.6121 & 710.8097 & 3 \\
\hline 1 & 1376.6117 & 688.8 & 852 & 680.2962 & 1358. & & $\mathbf{T}$ & 641 & 655.7857 & 5376 & & & & \\
\hline 2 & 1489.6958 & 745.3 & 1472.6692 & 736. & 1471. & 736.3462 & & 164 & 605. & 4899 & .7486 & & 566 & \\
\hline 13 & 1603.7387 & 802.3730 & 1586.7122 & 793.8597 & 1585.7281 & 793.3677 & $\mathbf{N}$ & $\mid 1096.4324$ & 548.7198 & 1079.4058 & 540.2066 & 1078.4218 & 539.7145 & 10 \\
\hline 4 & 1690.7707 & 845.8890 & 1673.7442 & 837.3757 & 1672.7602 & 836. & & 3895 & 491.6984 & 3629 & 1851 & 789 & 931 & \\
\hline 15 & 1837.8061 & 919.4067 & 1820.7796 & 910.8934 & 1819.7956 & 910.4014 & & 895.3574 & 448.1824 & & & & 1771 & 8 \\
\hline 16 & 1908.8433 & 954.9253 & 1891.8167 & 946.4120 & 1890.8327 & 945.9200 & & 748.3220 & 374.6646 & 731.2955 & 366.1514 & 730.3115 & 365.6594 & 7 \\
\hline 17 & 2037.8858 & 1019.4466 & 2020.8593 & 010.9333 & 2019.8753 & 1010.4413 & & 677.2849 & 339.1461 & 660.2584 & 330.6328 & 659.2743 & 330.1408 & 6 \\
\hline 18 & 2152.9128 & 1076.9600 & 2135.8862 & 068.4468 & 2134.9022 & 1067.9547 & & 8.2423 & 274.6248 & .2158 & 1115 & 2317 & 195 & 5 \\
\hline 19 & 2209.9343 & 1105.4708 & 2192.9077 & 1096.9575 & 2191.9237 & 1096.4655 & & 433.2154 & 217.1113 & 416.1888 & 208.5980 & 415.2048 & 208.1060 & 4 \\
\hline 20 & 2296.9663 & \begin{tabular}{|l|l|}
1148.9868 \\
\end{tabular} & 2279.9397 & 1140.4735 & 2278.9557 & 1139.9815 & S & 376.1939 & 188.6006 & 359.1674 & 180.0873 & 3.1833 & 179.5953 & 3 \\
\hline 21 & 2411.0092 & 1206.0082 & 2393.9827 & 1197.4950 & 2392.9986 & 1197.0030 & $\mathbf{N}$ & 289.1619 & 145.0846 & .1353 & 136.5713 & & & 2 \\
\hline 4 & & & & & & & $\mathbf{R}$ & 175.1190 & 88.0631 & 158.0924 & 79.5498 & & & \\
\hline
\end{tabular}
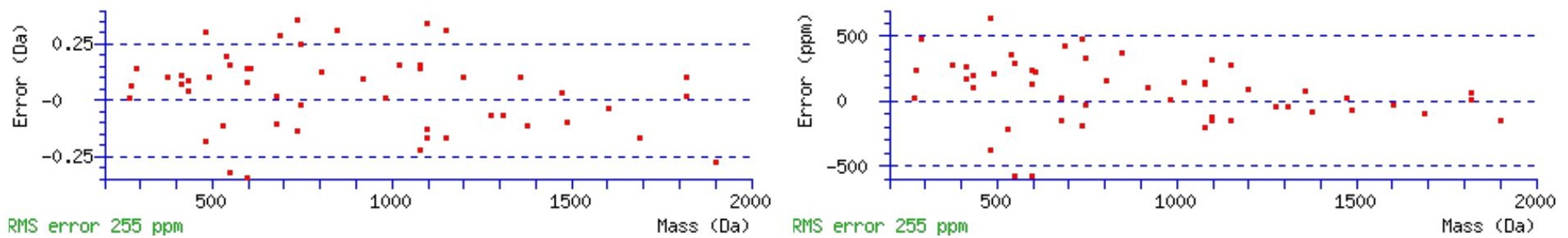

\section{All matches to this query}

\begin{tabular}{|l|l|l|l|}
\hline Score & Mr(calc): & Delta & \multicolumn{1}{|c|}{ Sequence } \\
\hline 54.4 & 2584.1136 & 0.0087 & RIYYSDFSNQTINSMAEDGSNR \\
\hline 39.6 & 2584.1136 & 0.0087 & RIYYSDFSNQTINSMAEDGSNR \\
\hline 24.5 & 2583.1296 & 0.9927 & RIYYSDFSNQTINSMAEDGSNR \\
\hline 9.6 & 2583.1329 & 0.9894 & TLMSLDTDGPVSKPDAPVQAEPR \\
\hline 9.6 & 2583.1329 & 0.9894 & TLMSLDTDGPVSKPDAPVQAEPR \\
\hline 5.4 & 2584.1136 & 0.0087 & RIYYSDFSNQTINSMAEDGSNR \\
\hline 4.9 & 2584.1435 & -0.0212 & NCTDKNTFLHTSFADVVGACGNR \\
\hline 4.4 & 2584.1435 & -0.0212 & NCTDKNTFLHTSFADVVGACGNR \\
\hline 3.3 & 2582.0978 & 2.0244 & NAWGNLSYADLITRAIESSPDK \\
\hline 3.3 & 2582.0978 & 2.0244 & NAWGNLSYADLITRAIESSPDK \\
\hline
\end{tabular}

Spectrum No: 616; Query: 1157; Rank: 1

\section{Peptide View}

MS/MS Fragmentation of LDLPVNTSIPNVTEIK

Found in IPI00324585, Tax_Id=10116 Gene_Symbol=Itga1 Integrin alpha-1 precursor

Match to Query 1157: 1752.951928 from(877.483240,2+)

Title: 091008RatKidney_NoSalt_30.3403.3403.2.dta

Data file K:INewmanPaper|Piliang|3SubProteomes\Piliang3SP\mgf5ppm\ERLIC_3SubProteomes5ppm.mgf 

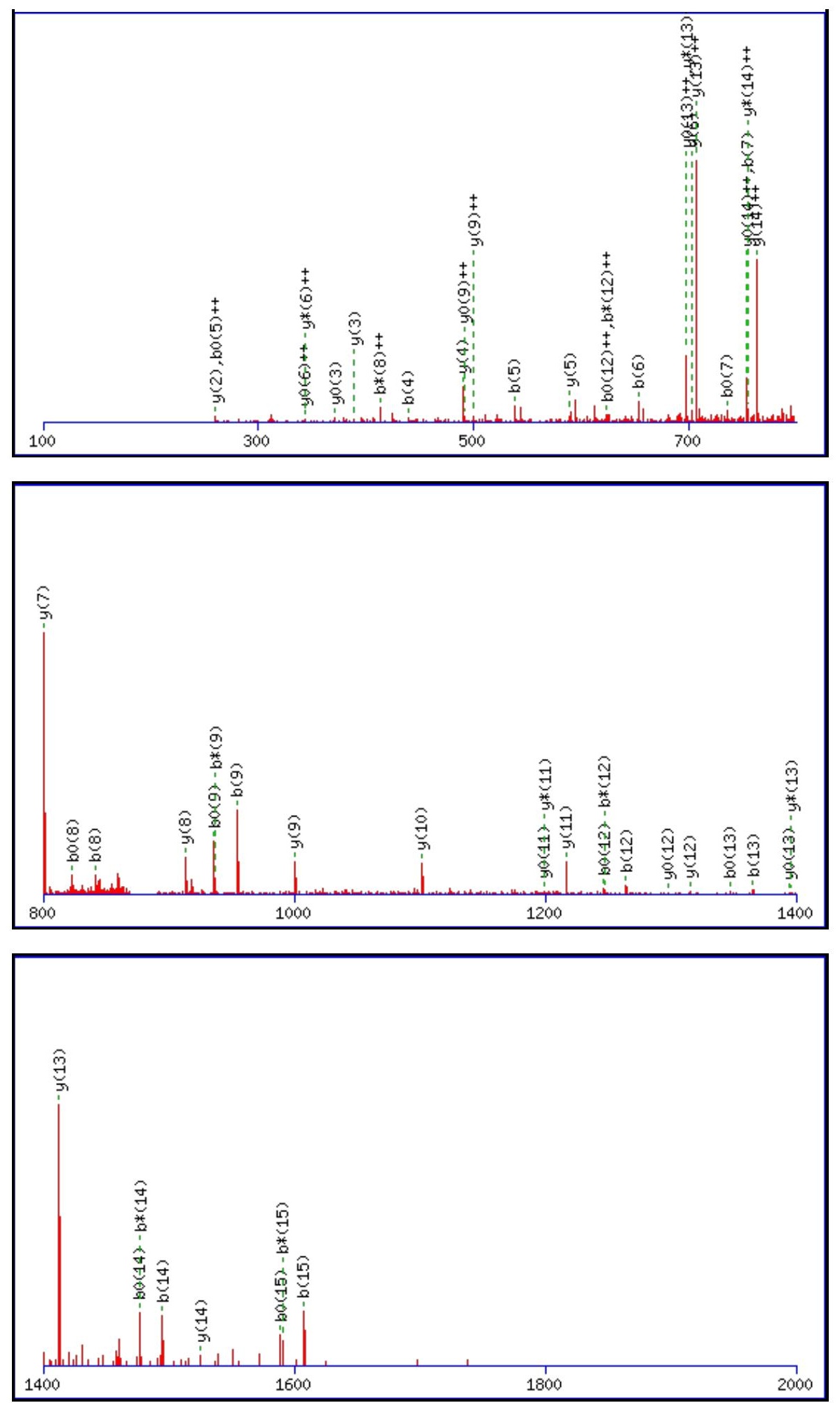

Monoisotopic mass of neutral peptide $\operatorname{Mr}($ calc): 1752.9509

Fixed modifications: Carbamidomethyl (C)

Variable modifications:

N6

Ions Score: 54 Expect: 0.00026

Matches (Bold Red): 54/164 fragment ions using 142 most intense peaks

\begin{tabular}{|r|c|c|c|c|c|c|c|c|c|c|c|c|c|c|}
\hline$\#$ & $\mathbf{b}$ & $\mathbf{b}^{++}$ & $\mathbf{b}^{*}$ & $\mathbf{b}^{\mathbf{*}^{++}}$ & $\mathbf{b}^{\mathbf{0}}$ & $\mathbf{b}^{\mathbf{0 + +}}$ & Seq. & $\mathbf{y}$ & $\mathbf{y}^{++}$ & $\mathbf{y}^{\mathbf{*}}$ & $\mathbf{y}^{\boldsymbol{*}^{++}}$ & $\mathbf{y}^{\mathbf{0}}$ & $\mathbf{y}^{\mathbf{0 + +}}$ & $\#$ \\
\hline $\mathbf{1}$ & 114.0913 & 57.5493 & & & & & $\mathbf{L}$ & & & & & & & $\mathbf{1 6}$ \\
\hline $\mathbf{2}$ & 229.1183 & 115.0628 & & & 211.1077 & 106.0575 & $\mathbf{D}$ & 1640.8741 & 820.9407 & 1623.8476 & 812.4274 & 1622.8636 & 811.9354 & $\mathbf{1 5}$ \\
\hline 3 & 342.2023 & 171.6048 & & & 324.1918 & 162.5995 & $\mathbf{L}$ & $\mathbf{1 5 2 5 . 8 4 7 2}$ & $\mathbf{7 6 3 . 4 2 7 2}$ & 1508.8206 & 754.9140 & 1507.8366 & 754.4220 & $\mathbf{1 4}$ \\
\hline $\mathbf{4}$ & $\mathbf{4 3 9 . 2 5 5 1}$ & 220.1312 & & & 421.2445 & 211.1259 & $\mathbf{P}$ & $\mathbf{1 4 1 2 . 7 6 3 1}$ & $\mathbf{7 0 6 . 8 8 5 2}$ & $\mathbf{1 3 9 5 . 7 3 6 6}$ & $\mathbf{6 9 8 . 3 7 1 9}$ & $\mathbf{1 3 9 4 . 7 5 2 6}$ & $\mathbf{6 9 7 . 8 7 9 9}$ & $\mathbf{1 3}$ \\
\hline $\mathbf{5}$ & 538.3235 & 269.6654 & & & 520.3130 & $\mathbf{2 6 0 . 6 6 0 1}$ & $\mathbf{V}$ & $\mathbf{1 3 1 5 . 7 1 0 4}$ & 658.3588 & 1298.6838 & 649.8455 & $\mathbf{1 2 9 7 . 6 9 9 8}$ & 649.3535 & $\mathbf{1 2}$ \\
\hline
\end{tabular}




\begin{tabular}{|c|c|c|c|c|c|c|c|c|c|c|c|c|c|c|}
\hline 6 & 653.3505 & |327.1789 & 636.3239 & |318.6656 & 635.3399| & |318.1736 & $\mathbf{N}$ & $\mid 1216.6420$ & |608.8246 & |1199.6154 & 600.3113 & 1198.6314 & |599.8193| & \\
\hline 7 & 754.3981 & 377.7027 & 737.3716 & 369.1894 & 736.3876 & 368.6974 & $\mathbf{T}$ & 1101.6150 & 551.3111 & 1084.5885 & 542.7979 & 1083.6045 & 542.3059 & 10 \\
\hline 8 & 841.4302 & 421.2187 & 824.4036 & 412.7054 & 823.4196 & 412.2134 & S & 1000.5673 & 500.7873 & 983.5408 & 492.2740 & 982.5568 & 491.7820 & 9 \\
\hline 9 & 954.5142 & 477.7608 & 937.4877 & 469.2475 & 936.5037 & 468.7555 & I & 913.5353 & 457.2713 & 896.5088 & 448.7580 & 895.5247 & 448.2660 & 8 \\
\hline 10 & 1051.5670 & 526.2871 & 1034.5404 & 517.7739 & 1033.5564 & 517.2819 & $\mathbf{P}$ & 800.4512 & 400.7293 & 783.4247 & 392.2160 & 782.4407 & 391.7240 & 7 \\
\hline 11 & 1165.6099 & 583.3086 & 1148.5834 & 574.7953 & 1147.5994 & 574.3033 & $\mathbf{N}$ & 703.3985 & 352.2029 & 686.3719 & 343.6896 & 685.3879 & 343.1976 & 6 \\
\hline 12 & 1264.6783 & 632.8428 & 1247.6518 & 624.3295 & 1246.6678 & 623.8375 & V & 589.3556 & 295.1814 & 572.3290 & 286.6681 & 571.3450 & 286.1761 & 5 \\
\hline 13 & 1365.7260 & 683.3666 & 1348.6995 & 674.8534 & 1347.7155 & 674.3614 & $T$ & 490.2871 & 245.6472 & 473.2606 & 237.1339 & 472.2766 & 236.6419 & 4 \\
\hline 14 & 1494.7686 & 747.8879 & 1477.7421 & 739.3747 & 1476.7580 & 738.8827 & $\mathbf{E}$ & 389.2395 & 195.1234 & 372.2129 & 186.6101 & 371.2289 & 186.1181 & 3 \\
\hline 15 & 1607.8527 & 804.4300 & 1590.8261 & 795.9167 & 1589.8421 & 795.4247 & I & 260.1969 & 130.6021 & 243.1703 & 122.0888 & & & 2 \\
\hline 16 & & & & & & & $\mathbf{K}$ & 147.1128 & 74.0600 & 130.0863 & 65.5468 & & & 1 \\
\hline
\end{tabular}
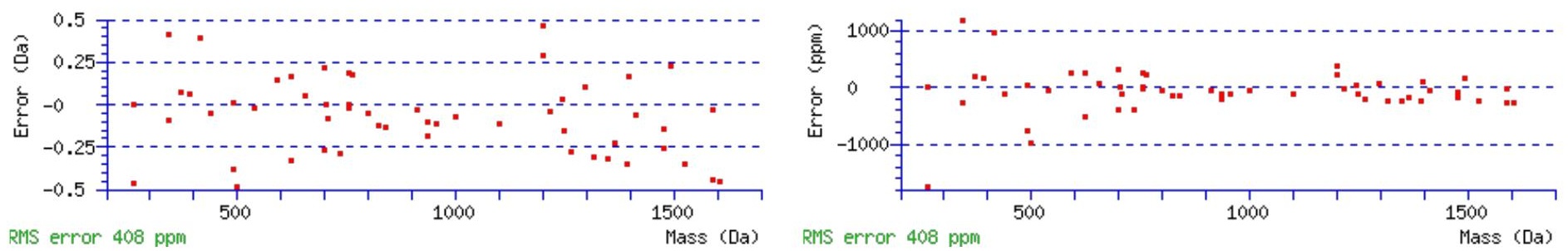

\section{All matches to this query}

\begin{tabular}{|l|l|c|c|}
\hline Score & Mr(calc): & Delta & Sequence \\
\hline 54.3 & 1752.9509 & 0.0010 & $\underline{\text { LDLPVNTSIPNVTEIK }}$ \\
\hline 38.4 & 1752.9509 & 0.0010 & $\underline{\text { LDLPVNTSIPNVTEIK }}$ \\
\hline 7.9 & 1752.9596 & -0.0077 & $\underline{\text { ALFQLRALSSYPIMK }}$ \\
\hline
\end{tabular}

Spectrum No: 617; Query: 1207; Rank: 1

\section{Peptide View}

\section{MS/MS Fragmentation of SYIFIYDGNKNSTTR}

Found in IPI00470238, Tax_Id=10116 Gene_Symbol=Cd47 Isoform 2 of Leukocyte surface antigen CD47 precursor

Match to Query 1207: 1778.854328 from(890.434440,2+)

Title: 100101RatKid_NS_deglyco_24.2387.2387.2.dta

Data file K:INewmanPaper|Piliangl3SubProteomes\Piliang3SP\mgf5ppm\ERLIC_3SubProteomes5ppm.mgf

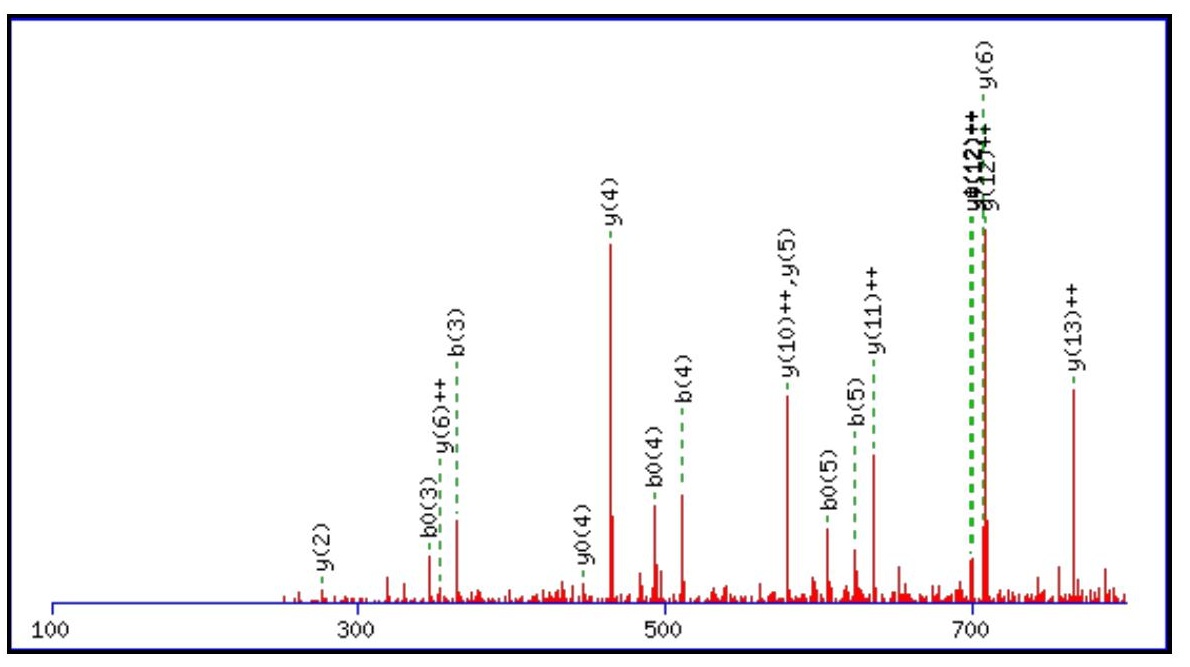



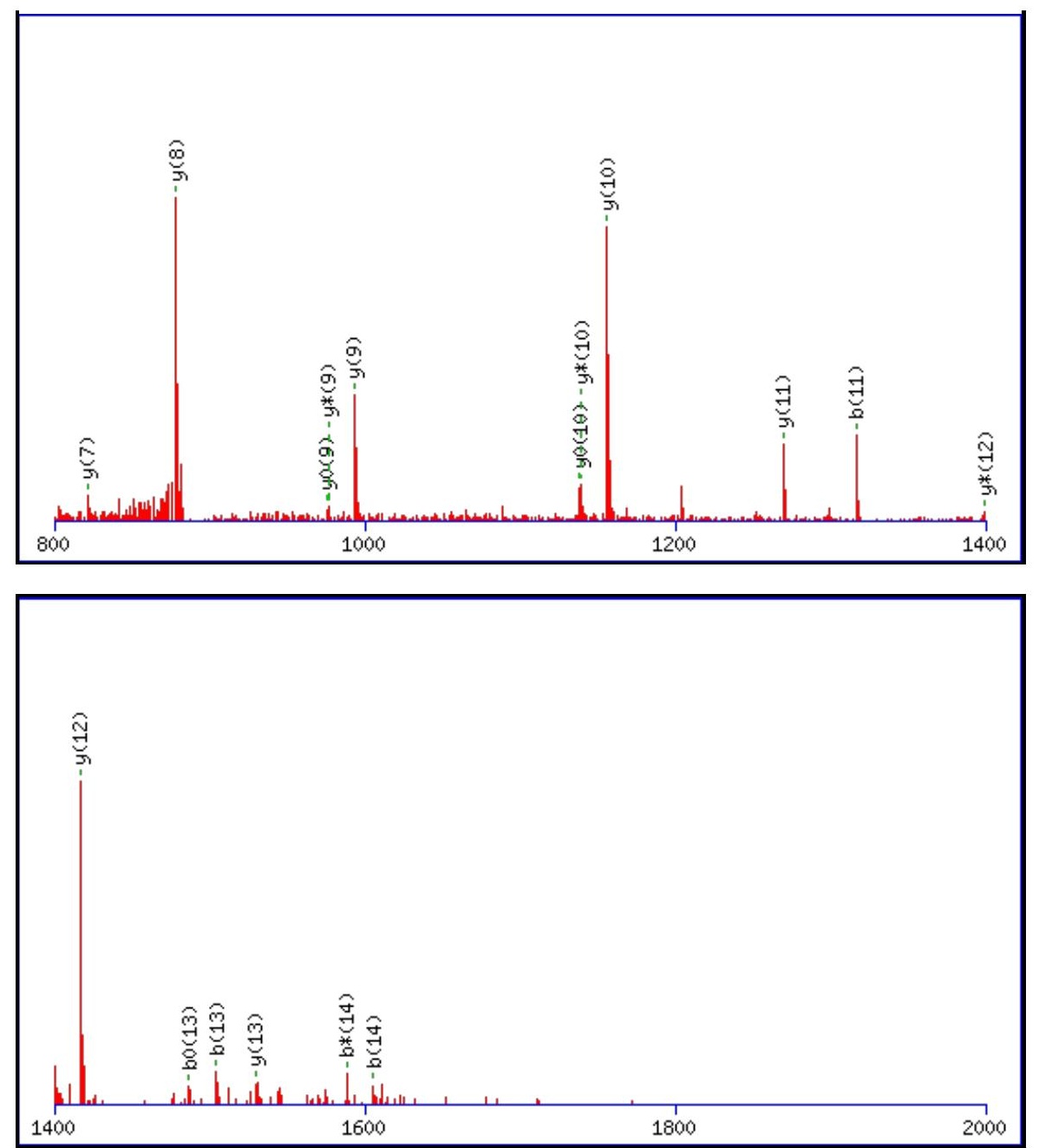

Monoisotopic mass of neutral peptide $\operatorname{Mr}($ calc): 1778.8475

Fixed modifications: Carbamidomethyl (C)

Variable modifications:

N11 : Deamidated_N (N)

Ions Score: 54 Expect: 0.00074

Matches (Bold Red): 35/150 fragment ions using 83 most intense peaks

\begin{tabular}{|c|c|c|c|c|c|c|c|c|c|c|c|c|c|c|}
\hline \# & b & $\mathbf{b}^{++}$ & $\mathbf{b}^{*}$ & $\mathbf{b}^{*^{++}}$ & $\mathbf{b}^{0}$ & $\mathbf{b}^{0++}$ & Seq. & $\mathbf{y}$ & $\mathbf{y}^{++}$ & $\mathbf{y}^{*}$ & $\mathbf{y}^{*^{++}}$ & $\mathbf{y}^{0}$ & $\mathbf{y}^{0++}$ & \# \\
\hline 1 & 88.0393 & 44.5233 & & & 70.0287 & 35.5180 & $\mathrm{~S}$ & & & & & & & 15 \\
\hline 2 & 251.1026 & 126.0550 & & & 233.0921 & 117.0497 & $\mathbf{Y}$ & 1692.8228 & 846.9150 & 1675.7962 & 838.4017 & 1674.8122 & 837.9097 & 14 \\
\hline 3 & 364.1867 & 182.5970 & & & 346.1761 & 173.5917 & I & 1529.7594 & 765.3834 & 1512.7329 & 756.8701 & 1511.7489 & 756.3781 & 13 \\
\hline 4 & 511.2551 & 256.1312 & & & 493.2445 & 247.1259 & $\mathbf{F}$ & 1416.6754 & 708.8413 & 1399.6488 & 700.3281 & 1398.6648 & 699.8360 & 12 \\
\hline 5 & 624.3392 & 312.6732 & & & 606.3286 & 303.6679 & I & 1269.6070 & 635.3071 & 1252.5804 & 626.7938 & 1251.5964 & 626.3018 & 11 \\
\hline 6 & 787.4025 & 394.2049 & & & 769.3919 & 385.1996 & $\mathbf{Y}$ & 1156.5229 & 578.7651 & 1139.4964 & 570.2518 & 1138.5123 & 569.7598 & 10 \\
\hline 7 & 902.4294 & 451.7184 & & & 884.4189 & 442.7131 & D & 993.4596 & 497.2334 & 976.4330 & 488.7201 & 975.4490 & 488.2281 & 9 \\
\hline 8 & 959.4509 & 480.2291 & & & 941.4403 & 471.2238 & G & 878.4326 & 439.7200 & 861.4061 & 431.2067 & 860.4221 & 430.7147 & 8 \\
\hline 9 & 1073.4938 & 537.2506 & 1056.4673 & 528.7373 & 1055.4833 & 528.2453 & $\mathbf{N}$ & 821.4112 & 411.2092 & 804.3846 & 402.6959 & 803.4006 & 402.2039 & 7 \\
\hline 10 & 1201.5888 & 601.2980 & 1184.5623 & 592.7848 & 1183.5782 & 592.2928 & $\mathbf{K}$ & 707.3682 & 354.1878 & 690.3417 & 345.6745 & 689.3577 & 345.1825 & 6 \\
\hline 11 & 1316.6157 & 658.8115 & 1299.5892 & 650.2982 & 1298.6052 & 649.8062 & $\mathbf{N}$ & 579.2733 & 290.1403 & 562.2467 & 281.6270 & 561.2627 & 281.1350 & 5 \\
\hline 12 & 1403.6478 & 702.3275 & 1386.6212 & 693.8142 & 1385.6372 & 693.3222 & $S$ & 464.2463 & 232.6268 & 447.2198 & 224.1135 & 446.2358 & 223.6215 & 4 \\
\hline 13 & 1504.6954 & 752.8514 & 1487.6689 & 744.3381 & 1486.6849 & 743.8461 & $\mathbf{T}$ & 377.2143 & 189.1108 & 360.1878 & 180.5975 & 359.2037 & 180.1055 & 3 \\
\hline 14 & 1605.7431 & 803.3752 & 1588.7166 & 794.8619 & 1587.7326 & 794.3699 & $T$ & 276.1666 & 138.5870 & 259.1401 & 130.0737 & 258.1561 & 129.5817 & 2 \\
\hline 15 & & & & & & & $\mathbf{R}$ & 175.1190 & 88.0631 & 158.0924 & 79.5498 & & & 1 \\
\hline
\end{tabular}



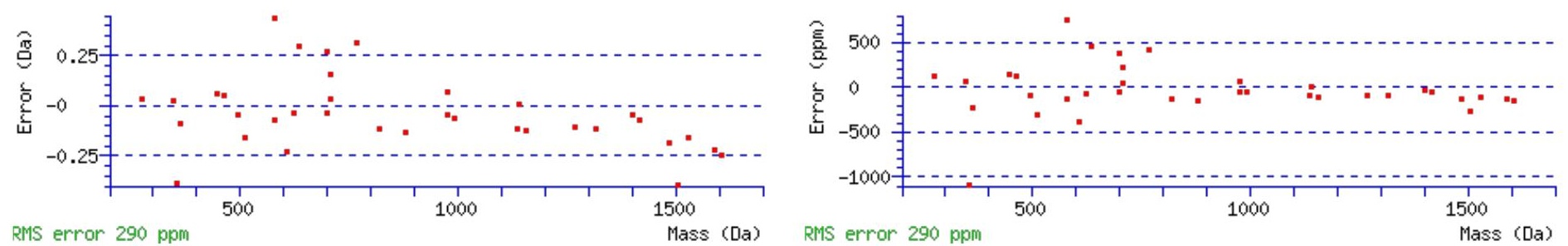

\section{All matches to this query}

\begin{tabular}{|l|l|c|l|}
\hline Score & Mr(calc): & Delta & \multicolumn{1}{c|}{ Sequence } \\
\hline 54.1 & 1778.8475 & 0.0068 & SYIFIYDGNKNSTTR \\
\hline 36.4 & 1778.8475 & 0.0068 & SYIFIYDGNKNSTTR \\
\hline 1.0 & 1778.8410 & 0.0133 & DFTMYLGYLRNQSR \\
\hline 0.8 & 1778.8703 & -0.0160 & DTAPVLEGLKGEEITK \\
\hline 0.4 & 1778.8427 & 0.0117 & CQGYIIFVVSLGSTR \\
\hline
\end{tabular}

Spectrum No: 618; Query: 745; Rank: 1

\section{Peptide View}

MS/MS Fragmentation of TIPHHQYNATINK

Found in IPI00211201, Tax_Id=10116 Gene_Symbol=F9 similar to coagulation factor IX

Match to Query 745: 1536.770168 from(769.392360,2+)

Title: 100101RatKid_NS_deglyco_19.508.508.2.dta

Data file K:\NewmanPaper|Piliangl3SubProteomes\Piliang3SP\mgf5ppm\ERLIC_3SubProteomes5ppm.mgf

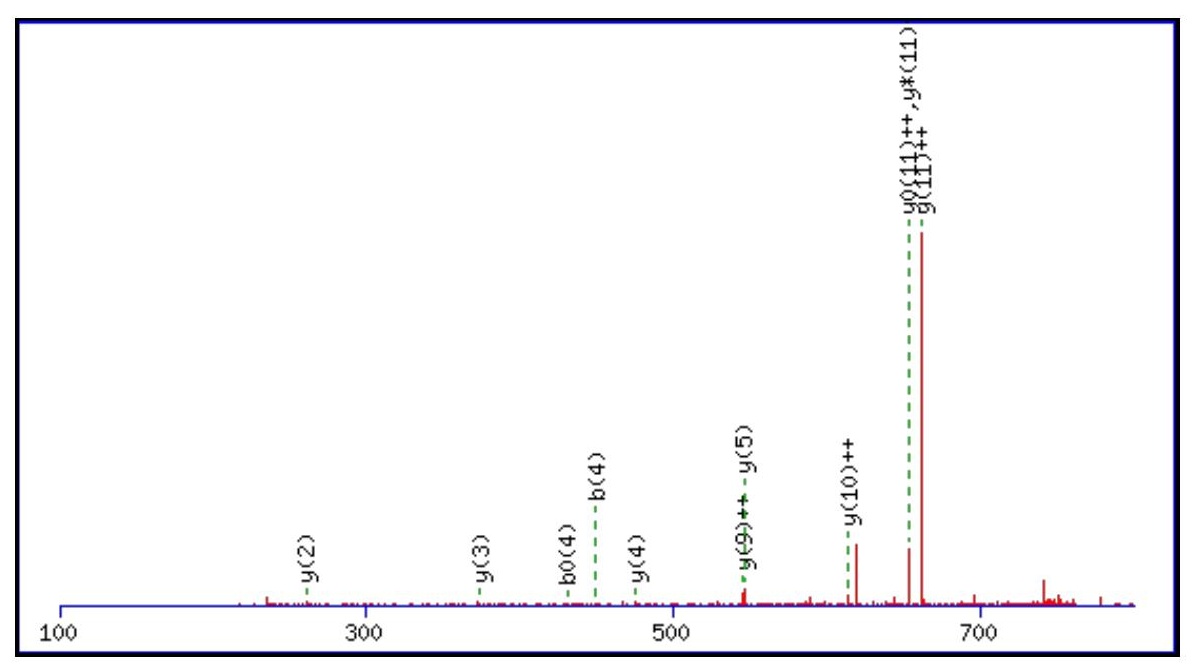



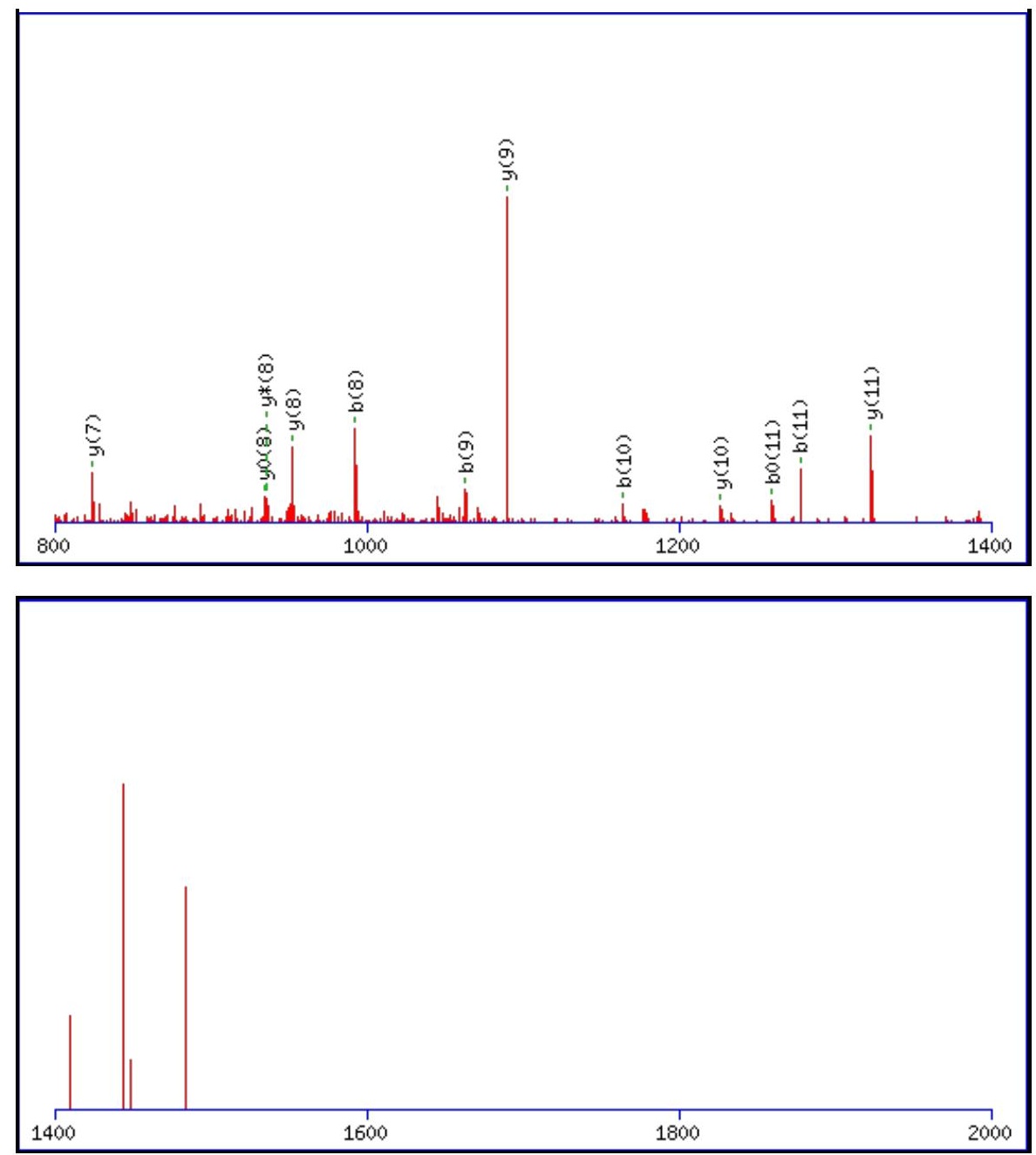

Monoisotopic mass of neutral peptide $\operatorname{Mr}($ calc): 1536.7685

Fixed modifications: Carbamidomethyl (C)

Variable modifications:

N8: : Deamidated $\mathrm{N}(\mathrm{N})$

Ions Score: 54 Expect: 0.00058

Matches (Bold Red): 23/128 fragment ions using 43 most intense peaks

\begin{tabular}{|c|c|c|c|c|c|c|c|c|c|c|c|c|c|c|}
\hline \# & b & $\mathbf{b}^{++}$ & $\mathbf{b}^{*}$ & $\mathbf{b}^{*^{++}}$ & $\mathbf{b}^{0}$ & $\mathbf{b}^{0++}$ & Seq. & $\mathbf{y}$ & $\mathbf{y}^{++}$ & $\mathbf{y}^{*}$ & $\mathrm{y}^{*^{++}}$ & $\mathbf{y}^{\mathbf{0}}$ & $\mathbf{y}^{0++}$ & \# \\
\hline 1 & 102.0550 & 51.5311 & & & 84.0444 & 42.5258 & $\mathbf{T}$ & & & & & & & 13 \\
\hline 2 & 215.1390 & 108.0731 & & & 197.1285 & 99.0679 & I & 1436.7281 & 718.8677 & 1419.7015 & 710.3544 & 1418.7175 & 709.8624 & 12 \\
\hline 3 & 312.1918 & 156.5995 & & & 294.1812 & 147.5942 & $\mathbf{P}$ & 1323.6440 & 662.3256 & 1306.6175 & 653.8124 & 1305.6335 & 653.3204 & 11 \\
\hline 4 & 449.2507 & 225.1290 & & & 431.2401 & 216.1237 & $\mathbf{H}$ & 1226.5913 & 613.7993 & 1209.5647 & 605.2860 & 1208.5807 & 604.7940 & 10 \\
\hline 5 & 586.3096 & 293.6584 & & & 568.2990 & 284.6532 & $\mathbf{H}$ & 1089.5323 & 545.2698 & 1072.5058 & 536.7565 & 1071.5218 & 536.2645 & 9 \\
\hline 6 & 714.3682 & 357.6877 & 697.3416 & 349.1745 & 696.3576 & 348.6824 & $\mathbf{Q}$ & 952.4734 & 476.7404 & 935.4469 & 468.2271 & 934.4629 & 467.7351 & 8 \\
\hline 7 & 877.4315 & 439.2194 & 860.4050 & 430.7061 & 859.4209 & 430.2141 & $\mathbf{Y}$ & 824.4149 & 412.7111 & 807.3883 & 404.1978 & 806.4043 & 403.7058 & 7 \\
\hline 8 & 992.4585 & 496.7329 & 975.4319 & 488.2196 & 974.4479 & 487.7276 & $\mathbf{N}$ & 661.3515 & 331.1794 & 644.3250 & 322.6661 & 643.3410 & 322.1741 & 6 \\
\hline 9 & 1063.4956 & 532.2514 & 1046.4690 & 523.7381 & 1045.4850 & 523.2461 & A & 546.3246 & 273.6659 & 529.2980 & 265.1527 & 528.3140 & 264.6606 & 5 \\
\hline 10 & 1164.5432 & 582.7753 & 1147.5167 & 574.2620 & 1146.5327 & 573.7700 & $\mathbf{T}$ & 475.2875 & 238.1474 & 458.2609 & 229.6341 & 457.2769 & 229.1421 & 4 \\
\hline 11 & 1277.6273 & 639.3173 & 1260.6008 & 630.8040 & 1259.6167 & 630.3120 & I & 374.2398 & 187.6235 & 357.2132 & 179.1103 & & & 3 \\
\hline 12 & 1391.6702 & 696.3388 & 1374.6437 & 687.8255 & 1373.6597 & 687.3335 & $\mathbf{N}$ & 261.1557 & 131.0815 & 244.1292 & 122.5682 & & & 2 \\
\hline 13 & & & & & & & $\mathbf{K}$ & 147.1128 & 74.0600 & 130.0863 & 65.5468 & & & 1 \\
\hline
\end{tabular}



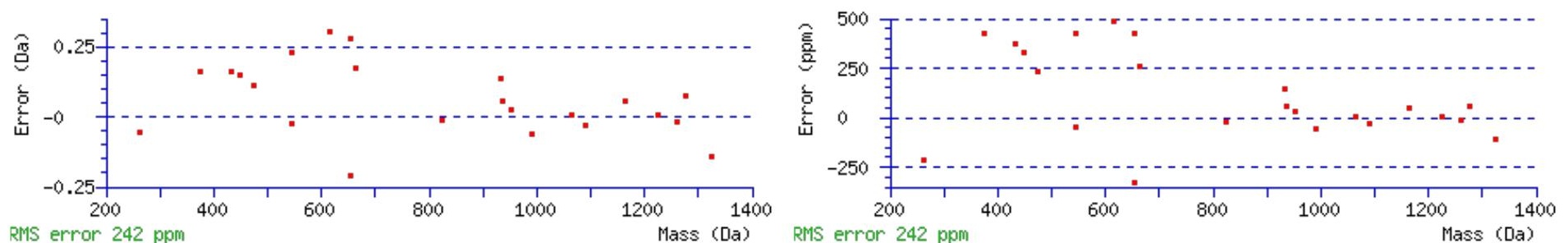

All matches to this query

\begin{tabular}{|l|l|l|l|}
\hline Score & Mr(calc): & Delta & \multicolumn{1}{c|}{ Sequence } \\
\hline 54.1 & 1536.7685 & 0.0017 & TIPHHQYNATINK \\
\hline 20.1 & 1536.7685 & 0.0017 & TIPHHQYNATINK \\
\hline 17.8 & 1536.7831 & -0.0129 & EMTSHRNHIATLK \\
\hline 11.4 & 1534.7540 & 2.0162 & LSMGTSLVFIMLK \\
\hline 10.4 & 1534.7540 & 2.0162 & LSMGTSLVFIMLK \\
\hline 3.9 & 1536.7711 & -0.0010 & VNDFEIADILYPK \\
\hline 1.9 & 1534.7740 & 1.9962 & $\underline{\text { HGELELDIPGAQTR }}$ \\
\hline 1.9 & 1534.7540 & 2.0162 & $\underline{\text { LSMGTSLVFIMLK }}$ \\
\hline 1.9 & 1534.7540 & 2.0162 & $\underline{\text { LSMGTSLVFIMLK }}$ \\
\hline 1.6 & 1535.7596 & 1.0106 & $\underline{\text { NKVNILPSDTNIK }}$ \\
\hline
\end{tabular}

Spectrum No: 619; Query: 2272; Rank: 1

\section{Peptide View}

MS/MS Fragmentation of AACAVRPQEVNMVNGTLTNPVTGK

Found in IPI00332022, Tax_Id=10116 Gene_Symbol=Igf2r Mannose 6-phosphate/insulin-like growth factor II receptor

Match to Query 2272: 2527.260912 from(843.427580,3+)

Title: 100101RatKid_NS_deglyco_23.2958.2958.3.dta

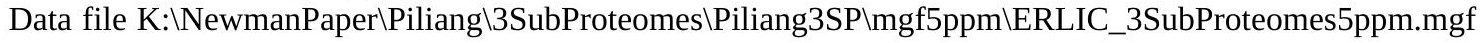

\begin{tabular}{|c|c|c|c|c|c|c|c|c|c|}
\hline & 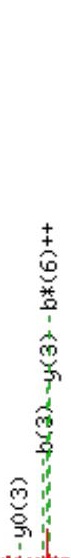 & $\begin{array}{l} \\
\\
\end{array}$ & 总产 & 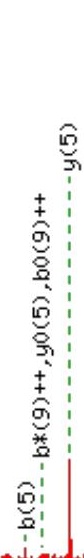 & 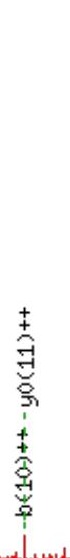 & 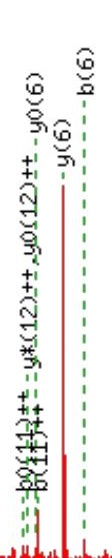 & 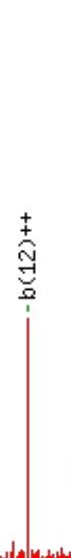 & 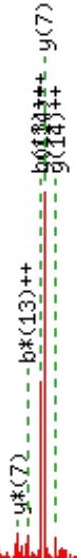 & 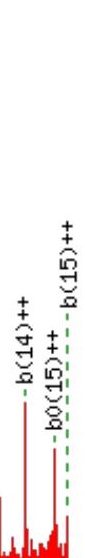 \\
\hline 100 & 300 & & & 500 & & & & 700 & \\
\hline
\end{tabular}



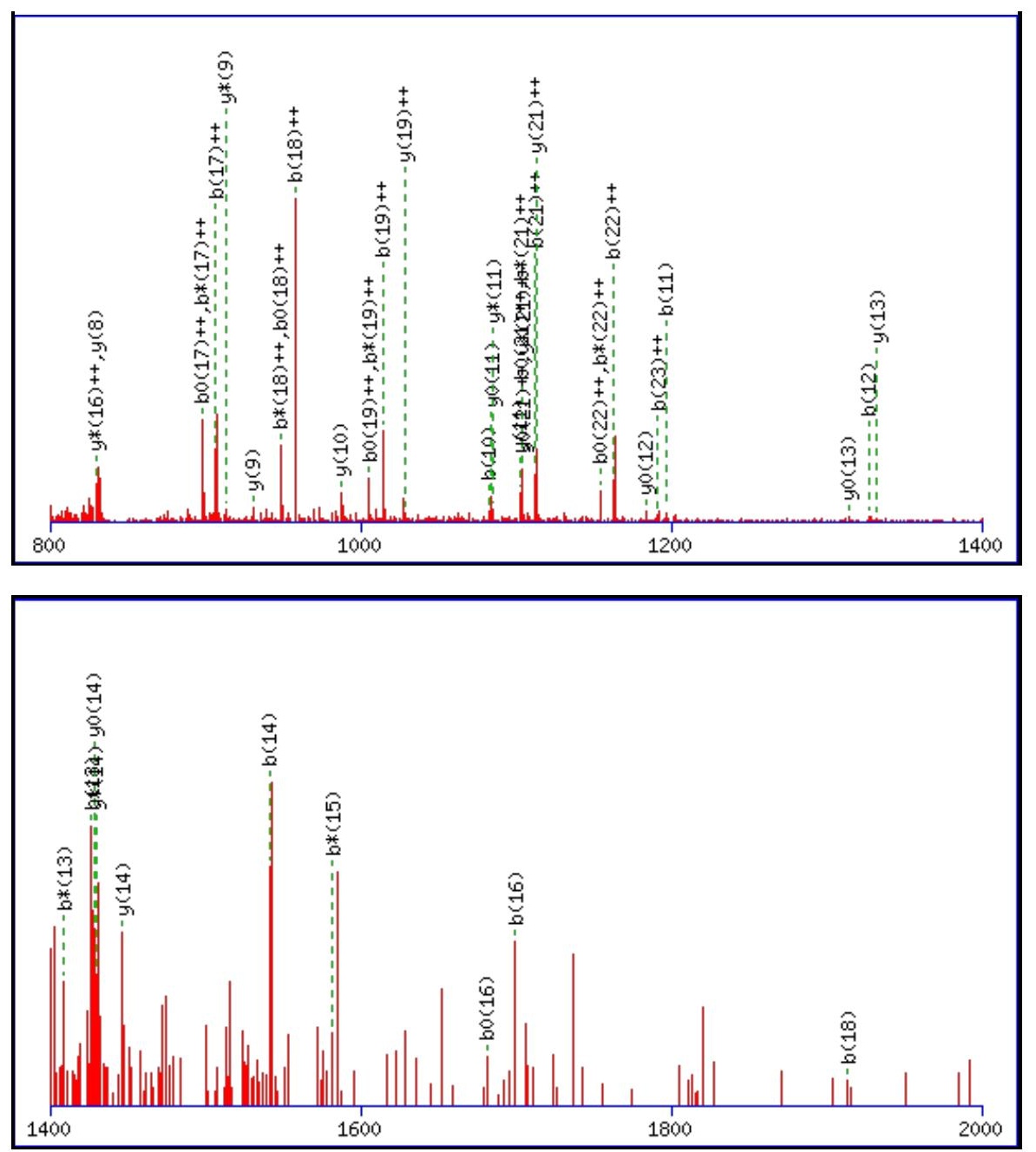

Monoisotopic mass of neutral peptide $\operatorname{Mr}($ calc): 2527.2523

Fixed modifications: Carbamidomethyl (C)

Variable modifications:

N14 : Deamidated_N (N)

Ions Score: 54 Expect: 0.00082

Matches (Bold Red): 77/246 fragment ions using 154 most intense peaks

\begin{tabular}{|c|c|c|c|c|c|c|c|c|c|c|c|c|c|c|}
\hline \# & b & $\mathbf{b}^{++}$ & $\mathbf{b}^{*}$ & $\mathbf{b}^{*^{++}}$ & $\mathbf{b}^{0}$ & $\mathbf{b}^{0++}$ & Seq. & $\mathbf{y}$ & $y^{++}$ & $\mathbf{y}^{*}$ & $\mathbf{y}^{*^{++}}$ & $\mathbf{y}^{\mathbf{0}}$ & $\mathbf{y}^{0++}$ & \# \\
\hline 1 & 72.0444 & 36.5258 & & & & & A & & & & & & & 24 \\
\hline 2 & 143.0815 & 72.0444 & & & & & A & 2457.2225 & 1229.1149 & 2440.1959 & 1220.6016 & 2439.2119 & 1220.1096 & 23 \\
\hline 3 & 303.1122 & 152.0597 & & & & & $\mathrm{C}$ & 2386.1853 & 1193.5963 & 2369.1588 & 1185.0830 & 2368.1748 & 1184.5910 & 22 \\
\hline 4 & 374.1493 & 187.5783 & & & & & A & 2226.1547 & 1113.5810 & 2209.1281 & 1105.0677 & 2208.1441 & 1104.5757 & 21 \\
\hline 5 & 473.2177 & 237.1125 & & & & & $\mathbf{V}$ & 2155.1176 & 1078.0624 & 2138.0910 & 1069.5492 & 2137.1070 & 1069.0571 & 20 \\
\hline 6 & 629.3188 & 315.1630 & 612.2922 & 306.6498 & & & $\mathbf{R}$ & 2056.0492 & 1028.5282 & 2039.0226 & 1020.0149 & 2038.0386 & 1019.5229 & 19 \\
\hline 7 & 726.3716 & 363.6894 & 709.3450 & 355.1761 & & & $\mathbf{P}$ & 1899.9480 & 950.4777 & 1882.9215 & 941.9644 & 1881.9375 & 941.4724 & 18 \\
\hline 8 & 854.4301 & 427.7187 & 837.4036 & 419.2054 & & & $\mathbf{Q}$ & 1802.8953 & 901.9513 & 1785.8687 & 893.4380 & 1784.8847 & 892.9460 & 17 \\
\hline 9 & 983.4727 & 492.2400 & 966.4462 & 483.7267 & 965.4622 & 483.2347 & $\mathbf{E}$ & 1674.8367 & 837.9220 & 1657.8102 & 829.4087 & 1656.8261 & 828.9167 & 16 \\
\hline 10 & 1082.5411 & 541.7742 & 1065.5146 & 533.2609 & 1064.5306 & 532.7689 & $\mathbf{V}$ & 1545.7941 & 773.4007 & 1528.7676 & 764.8874 & 1527.7835 & 764.3954 & 15 \\
\hline 11 & 1196.5841 & 598.7957 & 1179.5575 & 590.2824 & 1178.5735 & 589.7904 & $\overline{\mathbf{N}}$ & 1446.7257 & 723.8665 & 1429.6992 & 715.3532 & 1428.7151 & 714.8612 & 14 \\
\hline 12 & 1327.6246 & 664.3159 & 1310.5980 & 655.8026 & 1309.6140 & 655.3106 & $\mathbf{M}$ & 1332.6828 & 666.8450 & 1315.6562 & 658.3317 & 1314.6722 & 657.8397 & 13 \\
\hline 13 & 1426.6930 & 713.8501 & 1409.6664 & 705.3368 & 1408.6824 & 704.8448 & $\mathrm{~V}$ & 1201.6423 & 601.3248 & 1184.6157 & 592.8115 & 1183.6317 & 592.3195 & 12 \\
\hline 14 & 1541.7199 & 771.3636 & 1524.6934 & 762.8503 & 1523.7093 & 762.3583 & $\mathbf{N}$ & 1102.5739 & 551.7906 & 1085.5473 & 543.2773 & 1084.5633 & 542.7853 & 11 \\
\hline 15 & 1598.7414 & 799.8743 & 1581.7148 & 791.3610 & 1580.7308 & 790.8690 & G & 987.5469 & 494.2771 & 970.5204 & 485.7638 & 969.5364 & 485.2718 & 10 \\
\hline 16 & 1699.7890 & 850.3982 & 1682.7625 & 841.8849 & 1681.7785 & 841.3929 & $\mathbf{T}$ & 930.5255 & 465.7664 & 913.4989 & 457.2531 & 912.5149 & 456.7611 & 9 \\
\hline 17 & 1812.8731 & 906.9402 & 1795.8466 & 898.4269 & 1794.8625 & 897.9349 & $\mathbf{L}$ & 829.4778 & 415.2425 & 812.4512 & 406.7293 & 811.4672 & 406.2373 & 8 \\
\hline 18 & 1913.9208 & 957.4640 & 1896.8942 & 948.9508 & 1895.9102 & 948.4588 & $\mathbf{T}$ & 716.3937 & 358.7005 & 699.3672 & 350.1872 & 698.3832 & 349.6952 & 7 \\
\hline
\end{tabular}




\begin{tabular}{|r|r|r|r|r|r|r|r|r|r|r|r|r|r|r|r|r|}
$\mathbf{1 9}$ & 2027.9637 & $\mathbf{1 0 1 4 . 4 8 5 5}$ & 2010.9372 & $\mathbf{1 0 0 5 . 9 7 2 2}$ & $\mathbf{2 0 0 9 . 9 5 3 2}$ & $\mathbf{1 0 0 5 . 4 8 0 2}$ & $\mathbf{N}$ & $\mathbf{6 1 5 . 3 4 6 1}$ & 308.1767 & 598.3195 & $\mathbf{2 9 9 . 6 6 3 4}$ & $\mathbf{5 9 7 . 3 3 5 5}$ & $\mathbf{2 9 9 . 1 7 1 4}$ & $\mathbf{6}$ \\
\hline $\mathbf{2 0}$ & 2125.0165 & 1063.0119 & 2107.9899 & 1054.4986 & 2107.0059 & 1054.0066 & $\mathbf{P}$ & $\mathbf{5 0 1 . 3 0 3 1}$ & 251.1552 & 484.2766 & 242.6419 & $\mathbf{4 8 3 . 2 9 2 6}$ & 242.1499 & $\mathbf{5}$ \\
\hline $\mathbf{2 1}$ & 2224.0849 & $\mathbf{1 1 1 2 . 5 4 6 1}$ & 2207.0583 & $\mathbf{1 1 0 4 . 0 3 2 8}$ & 2206.0743 & $\mathbf{1 1 0 3 . 5 4 0 8}$ & $\mathbf{V}$ & $\mathbf{4 0 4 . 2 5 0 4}$ & 202.6288 & 387.2238 & 194.1155 & 386.2398 & 193.6235 & $\mathbf{4}$ \\
\hline $\mathbf{2 2}$ & 2325.1326 & $\mathbf{1 1 6 3 . 0 6 9 9}$ & 2308.1060 & $\mathbf{1 1 5 4 . 5 5 6 6}$ & 2307.1220 & $\mathbf{1 1 5 4 . 0 6 4 6}$ & $\mathbf{T}$ & $\mathbf{3 0 5 . 1 8 1 9}$ & 153.0946 & 288.1554 & 144.5813 & $\mathbf{2 8 7 . 1 7 1 4}$ & 144.0893 & $\mathbf{3}$ \\
\hline $\mathbf{2 3}$ & 2382.1540 & $\mathbf{1 1 9 1 . 5 8 0 7}$ & 2365.1275 & 1183.0674 & 2364.1435 & $\mathbf{1 1 8 2 . 5 7 5 4}$ & $\mathbf{G}$ & 204.1343 & 102.5708 & 187.1077 & 94.0575 & & & $\mathbf{2}$ \\
\hline $\mathbf{2 4}$ & & & & & & & $\mathbf{K}$ & 147.1128 & 74.0600 & 130.0863 & 65.5468 & & & $\mathbf{1}$ \\
\hline
\end{tabular}
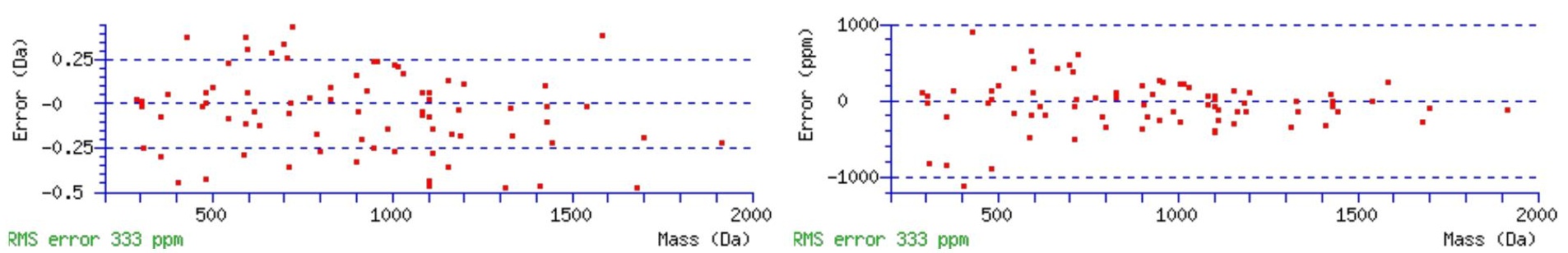

\section{All matches to this query}

\begin{tabular}{|l|l|l|l|}
\hline Score & Mr(calc): & Delta & \multicolumn{1}{|c|}{ Sequence } \\
\hline 54.1 & 2527.2523 & 0.0086 & AACAVRPQEVNMVNGTLTNPVTGK \\
\hline 49.0 & 2527.2523 & 0.0086 & AACAVRPQEVNMVNGTLTNPVTGK \\
\hline 38.9 & 2527.2523 & 0.0086 & AACAVRPOEVNMVNGTLTNPVTGK \\
\hline 37.5 & 2526.2683 & 0.9926 & AACAVRPQEVNMVNGTLTNPVTGK \\
\hline 6.5 & 2527.2596 & 0.0013 & MLKERLEMAEDPQQQMGAPVVK \\
\hline 4.1 & 2526.2666 & 0.9944 & STGILGGQGKMPTVVSPELYRTR \\
\hline 3.3 & 2525.2696 & 1.9913 & AQTVVLTITYMLNPTPNAGNHNR \\
\hline 3.3 & 2525.2696 & 1.9913 & AQTVVLTITYMLNPTPNAGNHNR \\
\hline 3.3 & 2525.2696 & 1.9913 & AQTVVLTITYMLNPTPNAGNHNR \\
\hline 3.3 & 2525.2696 & 1.9913 & AQTVVLTITYMLNPTPNAGNHNR \\
\hline
\end{tabular}

Spectrum No: 620; Query: 2781; Rank: 1

\section{Peptide View}

MS/MS Fragmentation of MLVDFEQLQPGDSVIQNASNSGVGQAVIQIASALGLK

Found in IPI00210183, Tax_Id=10116 Gene_Symbol=Mecr Trans-2-enoyl-CoA reductase, mitochondrial precursor

Match to Query 2781: 3797.964372 from(1266.995400,3+)

Title: 091008RatKidney_NoSalt_12.7779.7779.3.dta

Data file K:INewmanPaper|Piliang|3SubProteomes\Piliang3SP\mgf5ppm\ERLIC_3SubProteomes5ppm.mgf

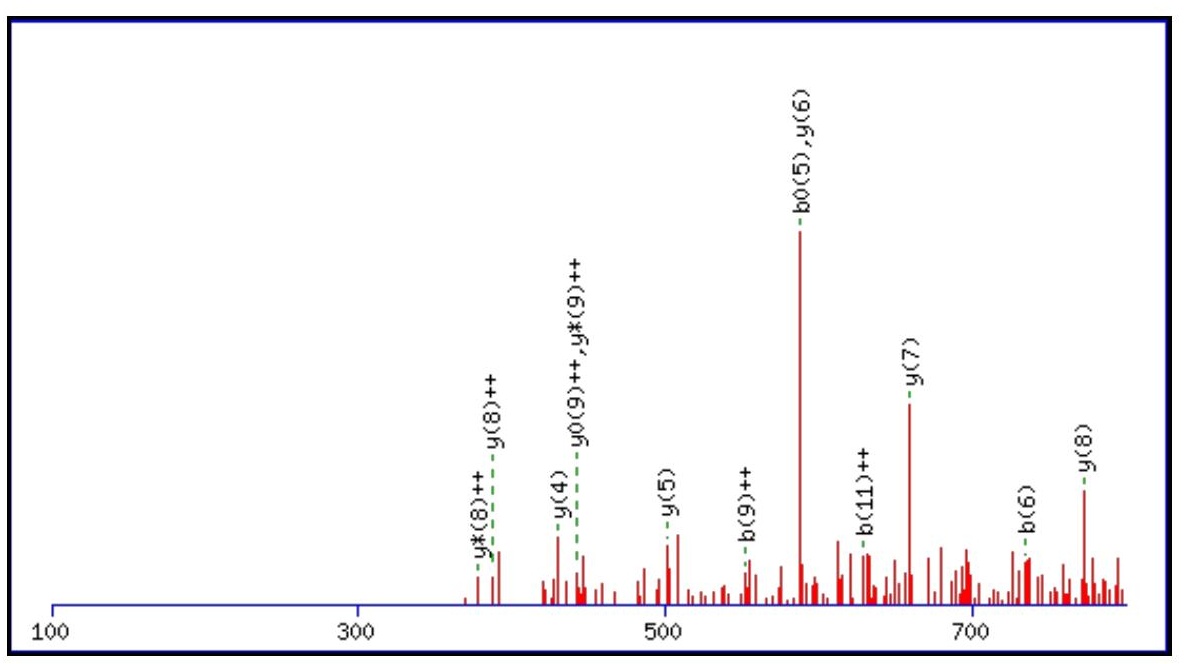



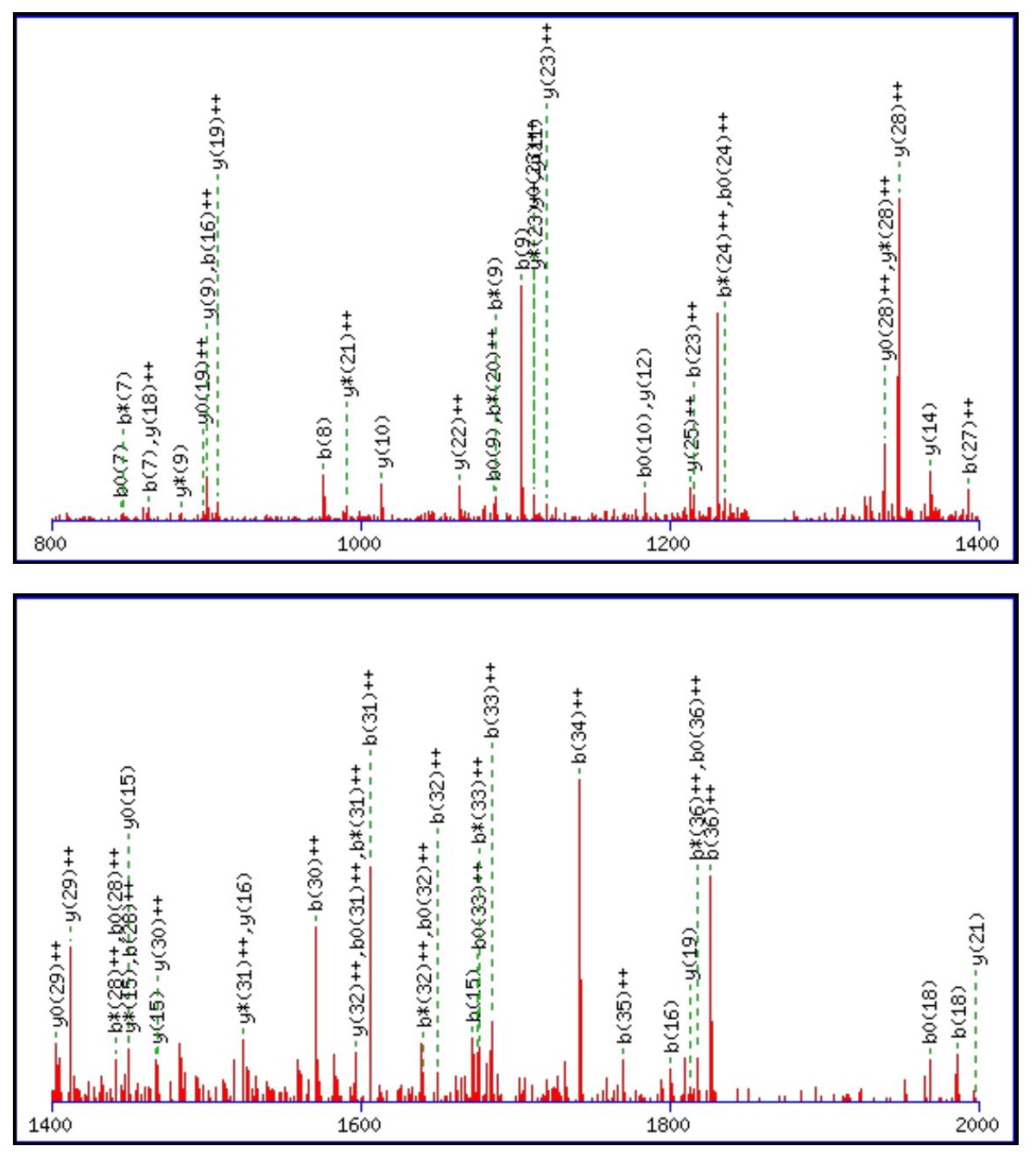

Monoisotopic mass of neutral peptide $\operatorname{Mr}($ calc): 3797.9509

Fixed modifications: Carbamidomethyl (C)

Variable modifications:

N17 : Deamidated_N (N)

Ions Score: 54 Expect: 0.00044

Matches (Bold Red): 78/404 fragment ions using 158 most intense peaks

\begin{tabular}{|c|c|c|c|c|c|c|c|c|c|c|c|c|c|c|}
\hline \# & b & $\mathbf{b}^{++}$ & b* $^{*}$ & $\mathbf{b}^{*^{++}}$ & $\mathbf{b}^{\mathbf{0}}$ & $\mathbf{b}^{0++}$ & Seq. & $\mathbf{y}$ & $y^{++}$ & $\mathrm{y}^{*}$ & $\mathrm{y}^{\mathrm{*}^{++}}$ & $\mathbf{y}^{\mathbf{0}}$ & $y^{0++}$ & \# \\
\hline 1 & 132.0478 & 66.5275 & & & & & M & & & & & & & 37 \\
\hline 2 & 245.1318 & 123.0696 & & & & & L & 3667.9177 & 1834.4625 & 3650.8912 & 1825.9492 & 3649.9072 & 1825.4572 & 36 \\
\hline 3 & 344.2002 & 172.6038 & & & & & $\mathbf{V}$ & 3554.8337 & 1777.9205 & 3537.8071 & 1769.4072 & 3536.8231 & 1768.9152 & 35 \\
\hline 4 & 459.2272 & 230.1172 & & & 441.2166 & 221.1119 & D & 3455.7653 & 1728.3863 & 3438.7387 & 1719.8730 & 3437.7547 & 1719.3810 & 34 \\
\hline 5 & 606.2956 & 303.6514 & & & 588.2850 & 294.6462 & $\mathbf{F}$ & 3340.7383 & 1670.8728 & 3323.7118 & 1662.3595 & 3322.7278 & 1661.8675 & 33 \\
\hline 6 & 735.3382 & 368.1727 & & & 717.3276 & 359.1675 & $\mathbf{E}$ & 3193.6699 & 1597.3386 & 3176.6434 & 1588.8253 & 3175.6593 & 1588.3333 & 32 \\
\hline 7 & 863.3968 & 432.2020 & 846.3702 & 423.6887 & 845.3862 & 423.1967 & $\mathbf{Q}$ & 3064.6273 & 1532.8173 & 3047.6008 & 1524.3040 & 3046.6167 & 1523.8120 & 31 \\
\hline 8 & 976.4808 & 488.7441 & 959.4543 & 480.2308 & 958.4703 & 479.7388 & $\mathbf{L}$ & 2936.5687 & 1468.7880 & 2919.5422 & 1460.2747 & 2918.5582 & 1459.7827 & 30 \\
\hline 9 & 1104.5394 & 552.7733 & 1087.5129 & 544.2601 & 1086.5288 & 543.7681 & $\mathbf{Q}$ & 2823.4847 & 1412.2460 & 2806.4581 & 1403.7327 & 2805.4741 & 1403.2407 & 29 \\
\hline 10 & 1201.5922 & 601.2997 & 1184.5656 & 592.7865 & 1183.5816 & 592.2944 & $\mathbf{P}$ & 2695.4261 & 1348.2167 & 2678.3995 & 1339.7034 & 2677.4155 & 1339.2114 & 28 \\
\hline 11 & 1258.6136 & 629.8105 & 1241.5871 & 621. & 1240.6031 & 620.8052 & G & 2598.3733 & 1299.6903 & 2581.3468 & 1291.1770 & 2580.3628 & 1290.6850 & 27 \\
\hline 12 & 1373.6406 & 687.3239 & 1356.6140 & 678.8107 & 1355.6300 & 678.3186 & D & 2541.3519 & 1271.1796 & 2524.3253 & 1262.6663 & 2523.3413 & 1262.1743 & 26 \\
\hline 13 & 1460.6726 & 730.8399 & 1443.6461 & 722.3267 & 1442.6620 & 721.8347 & $S$ & 2426.3249 & 1213.6661 & 2409.2984 & 1205.1528 & 2408.3144 & 1204.6608 & 25 \\
\hline 141 & 1559.7410 & 780.3741 & 1542.7145 & 771.8609 & 1541.7305 & 771.3689 & $\mathrm{~V}$ & 2339.2929 & 1170.1501 & 2322.2663 & 1161.6368 & 2321.2823 & 1161.1448 & 24 \\
\hline 15 & 1672.8251 & 836.9162 & 1655.7985 & 828.4029 & 1654.8145 & 827.9109 & I & 2240.2245 & 1120.6159 & 2223.1979 & 1112.1026 & 2222.2139 & 1111.6106 & 23 \\
\hline 16 & 1800.8837 & 900.9455 & 1783.8571 & 892.4322 & 1782.8731 & 891.9402 & $\mathbf{Q}$ & 2127.1404 & 1064.0738 & 2110.1139 & 1055.5606 & 2109.1299 & 1055.0686 & 22 \\
\hline 17 & 1915.9106 & 958.4589 & 1898.8841 & 949.9457 & 1897.9000 & 949.4537 & $\mathbf{N}$ & 1999.0818 & 1000.0446 & 1982.0553 & 991.5313 & 1981.0713 & 991.0393 & 21 \\
\hline 18 & 1986.9477 & 993.9775 & 1969.9212 & 985.4642 & 1968.9371 & 984.9722 & A & 1884.0549 & 942.5311 & 1867.0284 & 934.0178 & 1866.0443 & 933.5258 & \\
\hline
\end{tabular}




\begin{tabular}{|c|c|c|c|c|c|c|c|c|c|c|c|c|c|c|}
\hline 19 & |2073.9797 & |1037.4935 & & & & & $\mathrm{S}$ & 1813.0178 & 907.0125 & |1795.9912 & 898.4993 & |1795.0072| & 898.0073 & $\mid 19$ \\
\hline 20 & 2188.0227 & 1094.5150 & 2170.9961 & 1086.0017 & 2170.0121 & 1085.5097 & $\mathbf{N}$ & 1725.9858 & 863.4965 & 1708.9592 & 854.9832 & 1707.9752 & 854.4912 & 8 \\
\hline 21 & 2275.0547 & 1138.0310 & 2258.0281 & 1129.5177 & 2257.0441 & 1129.0257 & S & 1611.9428 & 806.4751 & 1594.9163 & 797.9618 & 1593.9323 & 797.4698 & 17 \\
\hline 22 & 2332.0762 & 1166.5417 & 2315.0496 & 1158.0284 & 2314.0656 & 1157.5364 & G & 1524.9108 & 762.9590 & 1507.8843 & 754.4458 & 1506.9002 & 753.9538 & 16 \\
\hline 23 & 2431.1446 & 1216.0759 & 2414.1180 & 1207.5627 & 2413.1340 & 1207.0706 & V & 1467.8893 & 734.4483 & 1450.8628 & 725.9350 & 1449.8788 & 725.4430 & 5 \\
\hline 24 & 2488.1660 & 1244.5867 & 2471.1395 & 1236.0734 & 2470.1555 & 1235.5814 & G & 1368.8209 & 684.9141 & 1351.7944 & 676.4008 & 1350.8104 & 675.9088 & 14 \\
\hline 25 & 2616.2246 & 1308.6159 & 2599.1981 & 1300.1027 & 2598.2141 & 1299.6107 & $\mathbf{Q}$ & 1311.7995 & 656.4034 & 1294.7729 & 647.8901 & 1293.7889 & 647.3981 & 13 \\
\hline 26 & 2687.2617 & 1344.1345 & 2670.2352 & 1335.6212 & 2669.2512 & 1335.1292 & A & 1183.7409 & 592.3741 & 1166.7143 & 583.8608 & 1165.7303 & 583.3688 & 12 \\
\hline 27 & 2786.3301 & 1393.6687 & 2769.3036 & 1385.1554 & 2768.3196 & 1384.6634 & V & 1112.7038 & 556.8555 & 1095.6772 & 548.3422 & 1094.6932 & 547.8502 & 11 \\
\hline 28 & 2899.4142 & 1450.2107 & 2882.3877 & 1441.6975 & 2881.4036 & 1441.2055 & I & 1013.6354 & 507.3213 & 996.6088 & 498.8080 & 995.6248 & 498.3160 & 10 \\
\hline 29 & 3027.4728 & 1514.2400 & 3010.4462 & 1505.7268 & 3009.4622 & 1505.2347 & Q & 900.5513 & 450.7793 & 883.5247 & 442.2660 & 882.5407 & 441.7740 & 9 \\
\hline 30 & 3140.5569 & 1570.7821 & 3123.5303 & 1562.2688 & 3122.5463 & 1561.7768 & I & 772.4927 & 386.7500 & 755.4662 & 378.2367 & 754.4822 & 377.7447 & 8 \\
\hline 31 & 3211.5940 & 1606.3006 & 3194.5674 & 1597.7873 & 3193.5834 & 1597.2953 & A & 659.4087 & 330.2080 & 642.3821 & 321.6947 & 641.3981 & 321.2027 & 7 \\
\hline 32 & 3298.6260 & 1649.8166 & 3281.5994 & 1641.3034 & 3280.6154 & 1640.8114 & S & 588.3715 & 294.6894 & 571.3450 & 286.1761 & 570.3610 & 285.6841 & 6 \\
\hline 33 & 3369.6631 & 1685.3352 & 3352.6366 & \begin{tabular}{|l|}
1676.8219 \\
\end{tabular} & 3351.6525 & 1676.3299 & $\mathbf{A}$ & 501.3395 & 251.1734 & 484.3130 & 242.6601 & & & 5 \\
\hline 34 & 3482.7472 & 1741.8772 & 3465.7206 & 1733.3639 & 3464.7366 & 1732.8719 & L & 430.3024 & 215.6548 & 413.2758 & 207.1416 & & & 4 \\
\hline 35 & 3539.7686 & 1770.3880 & 3522.7421 & 1761.8747 & 3521.7581 & 1761.3827 & G & 317.2183 & 159.1128 & 300.1918 & 150.5995 & & & 3 \\
\hline 36 & 3652.8527 & 1826.9300 & 3635.8262 & 1818.4167 & 3634.8421 & 1817.9247 & L & 260.1969 & 130.6021 & 243.1703 & 122.0888 & & & 2 \\
\hline 37 & & & & & & & $\mathbf{K}$ & 147.1128 & 74.0600 & 130.0863 & 65.5468 & & & 1 \\
\hline
\end{tabular}
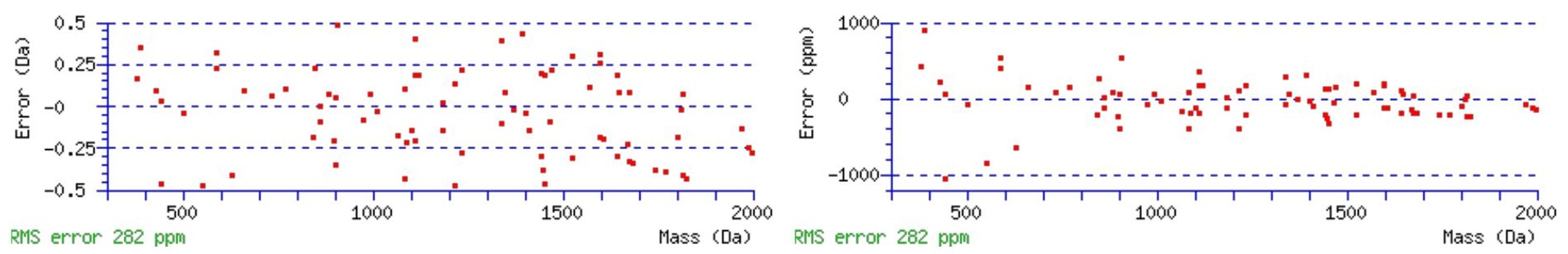

\section{All matches to this query}

\begin{tabular}{|l|l|l|l|}
\hline Score & Mr(calc): & Delta & \multicolumn{1}{c|}{ Sequence } \\
\hline 54.0 & 3797.9509 & 0.0134 & MLVDFEQLQPGDSVIQNASNSGVGQAVIQIASALGLK \\
\hline 50.9 & 3797.9509 & 0.0134 & MLVDFEQLQPGDSVIQNASNSGVGQAVIQIASALGLK \\
\hline 42.7 & 3796.9669 & 0.9974 & MLVDFEQLQPGDSVIQNASNSGVGQAVIQIASALGLK \\
\hline 4.4 & 3796.9692 & 0.9951 & ITNITDYTALVSWTIVDGYSISSIIIRYKVQGK \\
\hline 3.1 & 3796.9692 & 0.9951 & ITNITDYTALVSWTIVDGYSISSIIIRYKVQGK \\
\hline 2.3 & 3796.9692 & 0.9951 & ITNITDYTALVSWTIVDGYSISSIIIRYKVQGK \\
\hline 1.7 & 3796.9692 & 0.9951 & ITNITDYTALVSWTIVDGYSISSIIIRYKVQGK \\
\hline 1.5 & 3797.9532 & 0.0111 & ITNITDYTALVSWTIVDGYSISSIIIRYKVQGK \\
\hline 1.4 & 3797.9532 & 0.0111 & ITNITDYTALVSWTIVDGYSISSIIIRYKVQGK \\
\hline 1.4 & 3797.9532 & 0.0111 & ITNITDYTALVSWTIVDGYSISSIIIRYKVQGK \\
\hline
\end{tabular}

Spectrum No: 621; Query: 331; Rank: 1

\section{Peptide View}

MS/MS Fragmentation of MTLSQDNSTLR

Found in IPI00199607, Tax_Id=10116 Gene_Symbol=Ceacam1_v4;Ceacam1_v1;Ceacam1;Ceacam1_v3 carcinoembryonic antigen-related cell adhesion molecule isoform 2

Match to Query 331: 1281.588728 from(641.801640,2+)

Title: 091008RatKidney_NoSalt_31.646.646.2.dta 

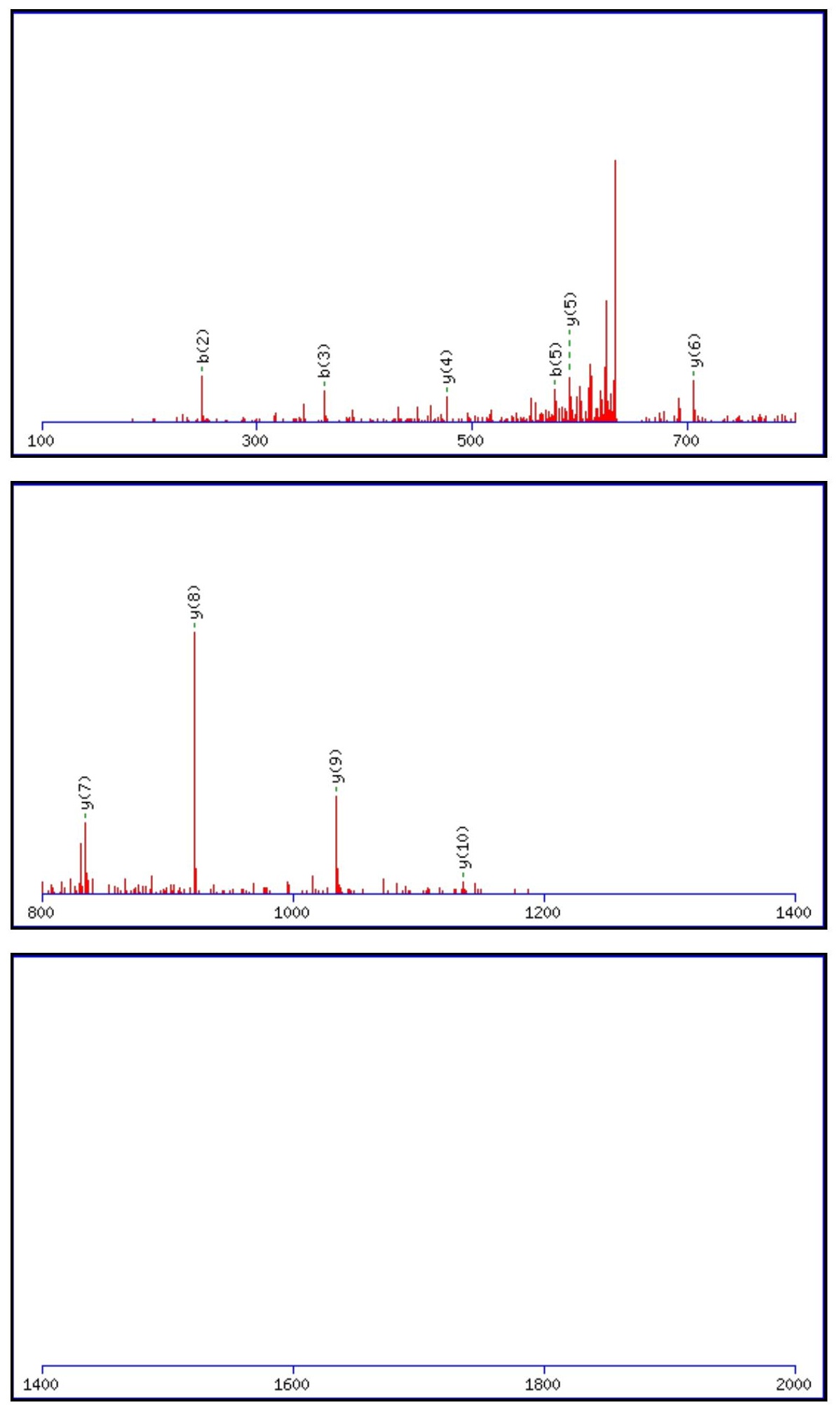

Monoisotopic mass of neutral peptide $\operatorname{Mr}($ calc): 1281.5871

Fixed modifications: Carbamidomethyl (C)

Variable modifications:

M1 : Oxidation (M)

N7 : Deamidated_N $(\mathrm{N})$

Ions Score: 54 Expect: 0.0006

Matches (Bold Red): 10/106 fragment ions using 27 most intense peaks

\begin{tabular}{|r|c|c|c|c|c|c|c|c|c|c|c|c|c|c|c|}
\hline$\#$ & $\mathbf{b}$ & $\mathbf{b}^{++}$ & $\mathbf{b}^{*}$ & $\mathbf{b}^{\boldsymbol{*}^{++}}$ & $\mathbf{b}^{\mathbf{0}}$ & $\mathbf{b}^{\mathbf{0}+}$ & Seq. & $\mathbf{y}$ & $\mathbf{y}^{++}$ & $\mathbf{y}^{\mathbf{*}}$ & $\mathbf{y}^{\boldsymbol{*}^{++}}$ & $\mathbf{y}^{\mathbf{0}}$ & $\mathbf{y}^{\mathbf{0 + +}}$ & $\#$ \\
\hline $\mathbf{1}$ & 148.0427 & 74.5250 & & & & & $\mathbf{M}$ & & & & & & & $\mathbf{1 1}$ \\
\hline $\mathbf{2}$ & $\mathbf{2 4 9 . 0 9 0 4}$ & 125.0488 & & & 231.0798 & 116.0435 & $\mathbf{T}$ & $\mathbf{1 1 3 5 . 5 5 9 0}$ & 568.2831 & 1118.5324 & 559.7698 & 1117.5484 & 559.2778 & $\mathbf{1 0}$ \\
\hline 3 & 362.1744 & 181.5908 & & & 344.1639 & 172.5856 & $\mathbf{L}$ & $\mathbf{1 0 3 4 . 5 1 1 3}$ & 517.7593 & 1017.4847 & 509.2460 & 1016.5007 & 508.7540 & $\mathbf{9}$ \\
\hline
\end{tabular}




\begin{tabular}{|r|r|r|r|r|r|r|r|r|r|r|r|r|r|r|r|r|}
$\mathbf{4}$ & 449.2065 & 225.1069 & & & 431.1959 & 216.1016 & S & $\mathbf{9 2 1 . 4 2 7 2}$ & 461.2172 & 904.4007 & 452.7040 & 903.4166 & 452.2120 & $\mathbf{8}$ \\
\hline $\mathbf{5}$ & $\mathbf{5 7 7 . 2 6 5 0}$ & 289.1362 & 560.2385 & 280.6229 & 559.2545 & 280.1309 & $\mathbf{Q}$ & $\mathbf{8 3 4 . 3 9 5 2}$ & 417.7012 & 817.3686 & 409.1880 & 816.3846 & 408.6959 & $\mathbf{7}$ \\
\hline $\mathbf{6}$ & 692.2920 & 346.6496 & 675.2654 & 338.1363 & 674.2814 & 337.6443 & D & $\mathbf{7 0 6 . 3 3 6 6}$ & 353.6719 & 689.3101 & 345.1587 & 688.3260 & 344.6667 & $\mathbf{6}$ \\
\hline $\mathbf{7}$ & 807.3189 & 404.1631 & 790.2924 & 395.6498 & 789.3083 & 395.1578 & $\mathbf{N}$ & $\mathbf{5 9 1 . 3 0 9 7}$ & 296.1585 & 574.2831 & 287.6452 & 573.2991 & 287.1532 & $\mathbf{5}$ \\
\hline $\mathbf{8}$ & 894.3509 & 447.6791 & 877.3244 & 439.1658 & 876.3404 & 438.6738 & S & $\mathbf{4 7 6 . 2 8 2 7}$ & 238.6450 & 459.2562 & 230.1317 & 458.2722 & 229.6397 & $\mathbf{4}$ \\
\hline $\mathbf{9}$ & 995.3986 & 498.2029 & 978.3721 & 489.6897 & 977.3880 & 489.1977 & T & 389.2507 & 195.1290 & 372.2241 & 186.6157 & 371.2401 & 186.1237 & $\mathbf{3}$ \\
\hline $\mathbf{1 0}$ & 1108.4827 & 554.7450 & 1091.4561 & 546.2317 & 1090.4721 & 545.7397 & L & 288.2030 & 144.6051 & 271.1765 & 136.0919 & & & \\
\hline $\mathbf{1 1}$ & & & & & & & R & 175.1190 & 88.0631 & 158.0924 & 79.5498 & & & & $\mathbf{1}$ \\
\hline
\end{tabular}
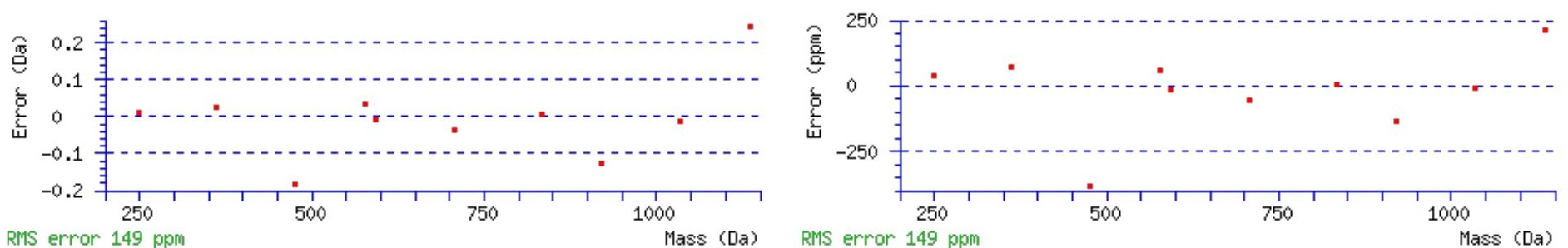

\section{All matches to this query}

\begin{tabular}{|l|l|l|l|}
\hline Score & Mr(calc): & Delta & \multicolumn{1}{|c|}{ Sequence } \\
\hline 54.0 & 1281.5871 & 0.0017 & MTLSODNSTLR \\
\hline 6.5 & 1281.5966 & -0.0079 & VENSQVTVAVR \\
\hline 4.8 & 1280.5809 & 1.0078 & HSRSCGRLTK \\
\hline 3.7 & 1280.5918 & 0.9969 & VSDATGQMNLTK \\
\hline 3.5 & 1281.6006 & -0.0119 & FTPPSTALSPGK \\
\hline 1.8 & 1280.5904 & 0.9983 & LSSLPRXRR \\
\hline 0.7 & 1280.5819 & 1.0068 & KPCQSFSENGK \\
\hline 0.3 & 1280.5809 & 1.0078 & HSRSCGRLTK \\
\hline
\end{tabular}

Spectrum No: 622; Query: 200; Rank: 1

\section{Peptide View}

MS/MS Fragmentation of EKIEQNGSMR

Found in IPI00554102, Tax_Id=10116 Gene_Symbol=LOC685482;LOC259246;LOC298116;LOC259245 similar to alpha2u globulin

Match to Query 200: 1191.551688 from(596.783120,2+)

Title: 091008RatKidney_NH4Format02_20.335.335.2.dta

Data file K:INewmanPaper|Piliangl3SubProteomes\Piliang3SP\mgf5ppm\ERLIC_3SubProteomes5ppm.mgf

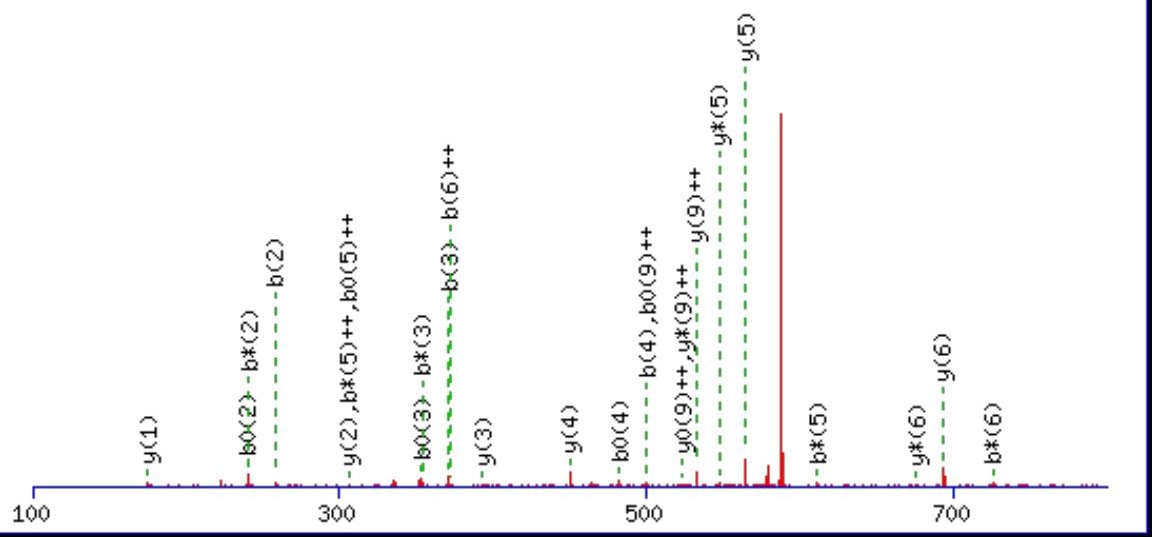




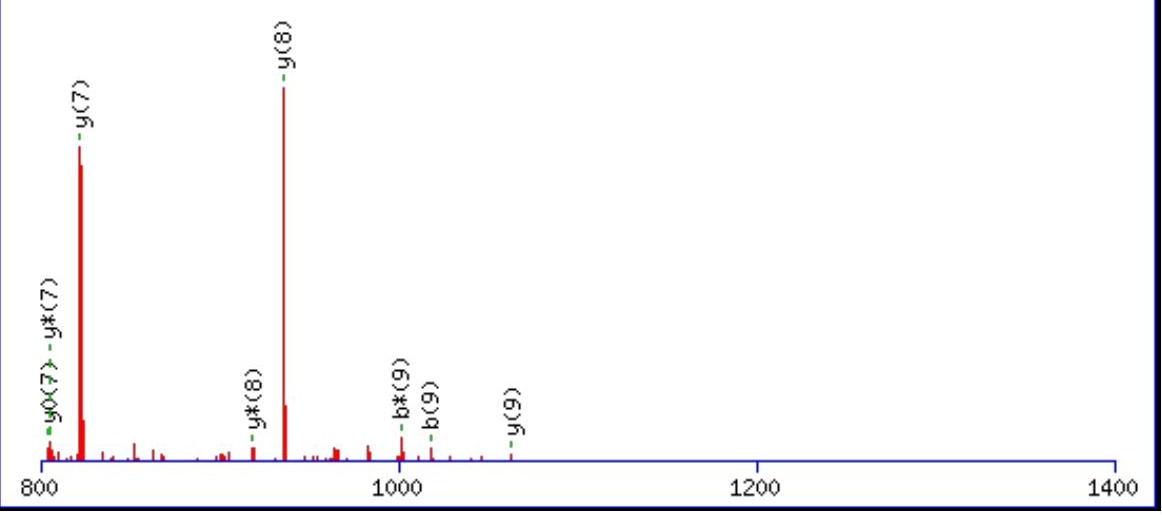

\begin{tabular}{|c|c|c|c|}
\hline 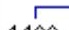 & 1 & 1 & 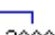 \\
\hline 1400 & 1600 & 1800 & 2000 \\
\hline
\end{tabular}

Monoisotopic mass of neutral peptide $\operatorname{Mr}($ calc): 1191.5553

Fixed modifications: Carbamidomethyl (C)

Variable modifications:

N6 : Deamidated_N (N)

Ions Score: 54 Expect: 0.00052

Matches (Bold Red): 33/102 fragment ions using 65 most intense peaks

\begin{tabular}{|c|c|c|c|c|c|c|c|c|c|c|c|c|c|c|}
\hline \# & b & $\mathbf{b}^{++}$ & $\mathbf{b}^{*}$ & $\mathbf{b}^{*^{++}}$ & $\mathbf{b}^{\mathbf{0}}$ & $\mathbf{b}^{\mathbf{0 + +}}$ & Seq. & $\mathbf{y}$ & $\mathbf{y}^{++}$ & $\mathbf{y}^{*}$ & $\mathrm{y}^{*^{++}}$ & $\mathbf{y}^{\mathbf{0}}$ & $\mathbf{y}^{\mathbf{0}^{++}}$ & \# \\
\hline 1 & 130.0499 & 65.5286 & & & 112.0393 & 56.5233 & $\mathbf{E}$ & & & & & & & 10 \\
\hline 2 & 258.1448 & 129.5761 & 241.1183 & 121.0628 & 240.1343 & 120.5708 & $\mathbf{K}$ & 1063.5201 & 532.2637 & 1046.4935 & 523.7504 & 1045.5095 & 523.2584 & 9 \\
\hline 3 & 371.2289 & |186.1181 & 354.2023 & |177.6048 & 353.2183 & 177.1128 & I & 935.4251 & 468.2162 & 918.3986 & 459.7029 & 917.4145 & 459.2109 & 8 \\
\hline 4 & 500.2715 & 250.6394 & 483.2449 & 242.1261 & 482.2609 & 241.6341 & E & 822.3410 & 411.6742 & 805.3145 & 403.1609 & $\mathbf{8 0 4 . 3 3 0 5}$ & 402.6689 & 7 \\
\hline 5 & 628.3301 & 314.6687 & 611.3035 & 306.1554 & 610.3195 & 305.6634 & $\mathbf{Q}$ & 693.2984 & 347.1529 & 676.2719 & 338.6396 & 675.2879 & 338.1476 & 6 \\
\hline 6 & 743.3570 & 372.1821 & 726.3305 & |363.6689 & 725.3464 & 363.1769 & $\mathbf{N}$ & 565.2399 & 283.1236 & 548.2133 & 274.6103 & 547.2293 & 274.1183 & 5 \\
\hline 7 & 800.3785 & 400.6929 & 783.3519 & 392.1796 & 782.3679 & 391.6876 & G & 450.2129 & 225.6101 & 433.1864 & 217.0968 & 432.2024 & 216.6048 & 4 \\
\hline 8 & 887.4105 & 444.2089 & 870.3839 & 435.6956 & 869.3999 & 435.2036 & $\mathrm{~S}$ & 393.1915 & |197.0994 & 376.1649 & 188.5861 & 375.1809 & 188.0941 & 3 \\
\hline 9 & 1018.4510 & 509.7291 & 1001.4244 & 501.2159 & 1000.4404 & 500.7238 & M & 306.1594 & 153.5834 & 289.1329 & 145.0701 & & & 2 \\
\hline 10 & & & & & & & $\mathbf{R}$ & 175.1190 & 88.0631 & 158.0924 & 79.5498 & & & 1 \\
\hline
\end{tabular}
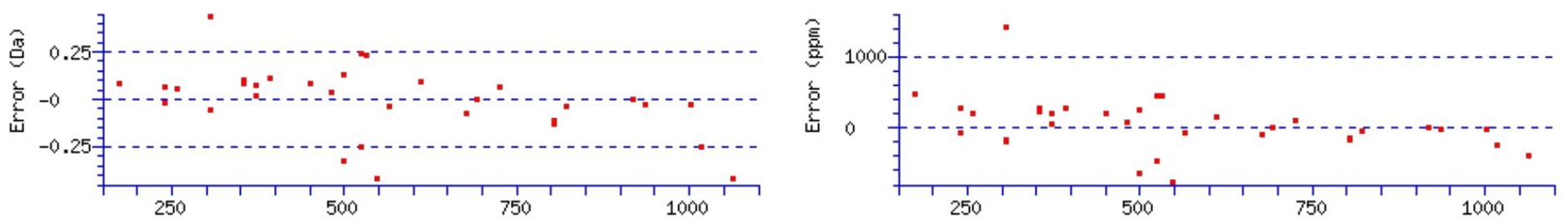

RMS error $379 \mathrm{ppm}$

Mass (Da) Rils error 379 ppm 


\section{All matches to this query}

\begin{tabular}{|l|l|l|l|}
\hline Score & Mr(calc): & Delta & \multicolumn{1}{|c|}{ Sequence } \\
\hline 54.0 & 1191.5553 & -0.0037 & EKIEQNGSMR \\
\hline 53.1 & 1191.5553 & -0.0037 & EKIEENGSMR \\
\hline 19.5 & 1191.5554 & -0.0037 & QELDLNSSMR \\
\hline 10.8 & 1190.5536 & 0.9981 & QMKPKEDCR \\
\hline 7.7 & 1191.5554 & -0.0037 & SSPGLSLDTCR \\
\hline 7.2 & 1190.5536 & 0.9981 & QKMQHMSSAK \\
\hline 7.2 & 1191.5590 & -0.0073 & KWSWPGVPR \\
\hline 7.1 & 1189.5427 & 2.0090 & RLERSYMR \\
\hline 6.4 & 1191.5454 & 0.0063 & GSPKLLYVR \\
\hline 6.3 & 1191.5554 & -0.0037 & QLEATLNSCR \\
\hline
\end{tabular}

Spectrum No: 623; Query: 423; Rank: 1

\section{Peptide View}

MS/MS Fragmentation of YQTLNCSVNVR

Found in IPI00205166, Tax_Id=10116 Gene_Symbol=Itga6 similar to integrin alpha 6 isoform 2

Match to Query 423: 1353.633368 from(677.823960,2+)

Title: 091008RatKidney_NH4Format01_23.1311.1311.2.dta

Data file K:INewmanPaper|Piliang|3SubProteomes\Piliang3SP\mgf5ppm\ERLIC_3SubProteomes5ppm.mgf
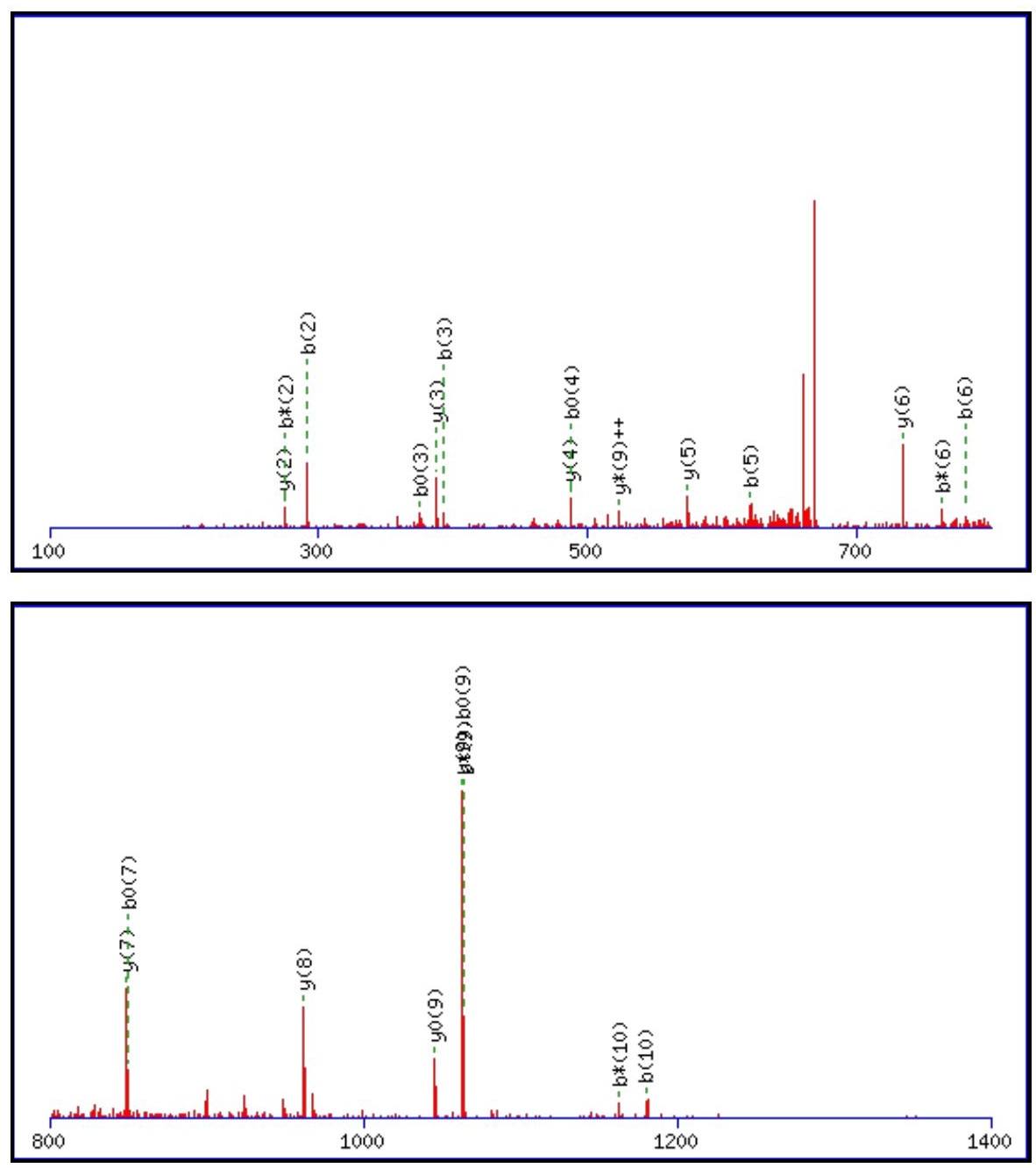


\begin{tabular}{|c|c|c|c|}
\hline 1400 & 1600 & 1800 & 2000 \\
\hline
\end{tabular}

Monoisotopic mass of neutral peptide $\operatorname{Mr}($ calc): 1353.6347

Fixed modifications: Carbamidomethyl (C)

Variable modifications:

N5 : Deamidated_N (N)

Ions Score: 54 Expect: 0.00063

Matches (Bold Red): 23/106 fragment ions using 46 most intense peaks

\begin{tabular}{|c|c|c|c|c|c|c|c|c|c|c|c|c|c|c|}
\hline \# & b & $\mathbf{b}^{++}$ & b* & $\mathbf{b}^{*^{++}}$ & $\mathbf{b}^{0}$ & $\mathbf{b}^{0++}$ & Seq. & $\mathbf{y}$ & $\mathbf{y}^{++}$ & $\mathbf{y}^{*}$ & $\mathbf{y}^{*^{++}}$ & $\mathbf{y}^{\mathbf{0}}$ & $\mathbf{y}^{0++}$ & \# \\
\hline 1 & 164.0706 & 82.5389 & & & & & $\mathbf{Y}$ & & & & & & & 11 \\
\hline 2 & 292.1292 & 146.5682 & 275.1026 & 138.0550 & & & $\mathbf{Q}$ & 1191.5786 & 596.2930 & 1174.5521 & 587.7797 & 1173.5681 & 587.2877 & 10 \\
\hline 3 & 393.1769 & 197.0921 & 376.1503 & 188.5788 & 375.1663 & 188.0868 & $\mathbf{T}$ & 1063.5201 & 532.2637 & 1046.4935 & 523.7504 & 1045.5095 & 523.2584 & 9 \\
\hline 4 & 506.2609 & 253.6341 & 489.2344 & 245.1208 & 488.2504 & 244.6288 & $\mathbf{L}$ & 962.4724 & 481.7398 & 945.4458 & 473.2266 & 944.4618 & 472.7345 & 8 \\
\hline 5 & 621.2879 & 311.1476 & 604.2613 & 302.6343 & 603.2773 & 302.1423 & $\mathbf{N}$ & 849.3883 & 425.1978 & 832.3618 & 416.6845 & 831.3778 & 416.1925 & 7 \\
\hline 6 & 781.3185 & 391.1629 & 764.2920 & 382.6496 & 763.3079 & 382.1576 & C & 734.3614 & 367.6843 & 717.3348 & 359.1711 & 716.3508 & 358.6790 & 6 \\
\hline 7 & 868.3505 & 434.6789 & 851.3240 & 426.1656 & 850.3400 & 425.6736 & S & 574.3307 & 287.6690 & 557.3042 & 279.1557 & 556.3202 & 278.6637 & 5 \\
\hline 8 & 967.4190 & 484.2131 & 950.3924 & 475.6998 & 949.4084 & 475.2078 & $\mathbf{V}$ & 487.2987 & 244.1530 & 470.2722 & 235.6397 & & & 4 \\
\hline 9 & 1081.4619 & 541.2346 & 1064.4353 & 532.7213 & 1063.4513 & 532.2293 & $\mathbf{N}$ & 388.2303 & 194.6188 & 371.2037 & 186.1055 & & & 3 \\
\hline 10 & 1180.5303 & 590.7688 & 1163.5037 & 582.2555 & 1162.5197 & 581.7635 & V & 274.1874 & 137.5973 & 257.1608 & 129.0840 & & & 2 \\
\hline 11 & & & & & & & $\mathbf{R}$ & 175.1190 & 88.0631 & 158.0924 & 79.5498 & & & 1 \\
\hline
\end{tabular}
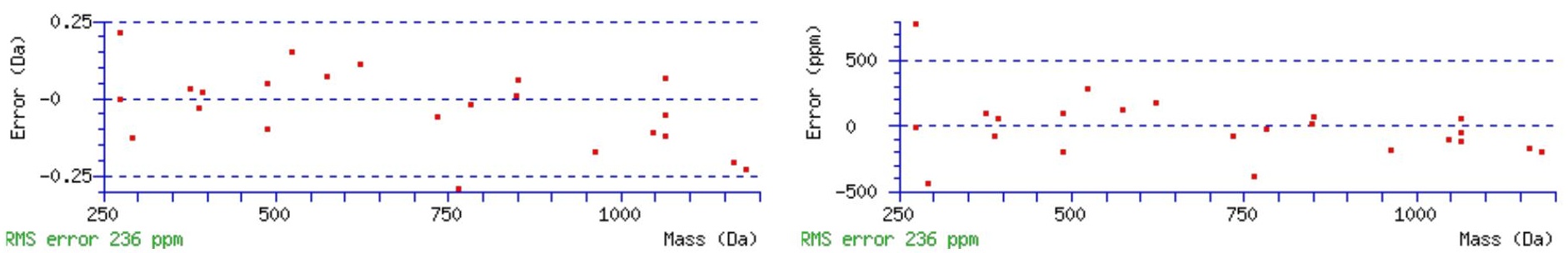

\section{All matches to this query}

\begin{tabular}{|l|l|l|l|}
\hline Score & Mr(calc): & Delta & \multicolumn{1}{c|}{ Sequence } \\
\hline 54.0 & 1353.6347 & -0.0013 & YQTLNCSVNVR \\
\hline 29.0 & 1353.6347 & -0.0013 & YQTLNCSVNVR \\
\hline 15.2 & 1352.6282 & 1.0052 & $\underline{\text { EVYLDCALNQK }}$ \\
\hline 7.0 & 1351.6376 & 1.9957 & $\underline{\text { QYTMNPKAMPR }}$ \\
\hline 4.0 & 1353.6402 & -0.0068 & $\underline{\text { SGQIERSKNVR }}$ \\
\hline 3.5 & 1353.6347 & -0.0013 & $\underline{\text { VKDDNYNLAMR }}$ \\
\hline 1.5 & 1352.6316 & 1.0018 & $\underline{\text { MATSDLMSELQK }}$ \\
\hline 0.9 & 1353.6289 & 0.0044 & VNRESVNSIQK \\
\hline 0.3 & 1353.6412 & -0.0078 & VKLETYNDESR \\
\hline
\end{tabular}


Spectrum No: 624; Query: 273; Rank: 1

\section{Peptide View}

MS/MS Fragmentation of MLEAYNLTEK

Found in IPI00189714, Tax_Id=10116 Gene_Symbol=Col12a1 similar to Collagen alpha-1(XII) chain precursor

Match to Query 273: 1227.571508 from(614.793030,2+)

Title: 100101RatKid_NS_deglyco_21.1875.1875.2.dta

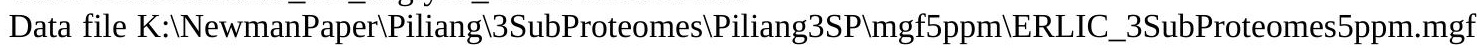
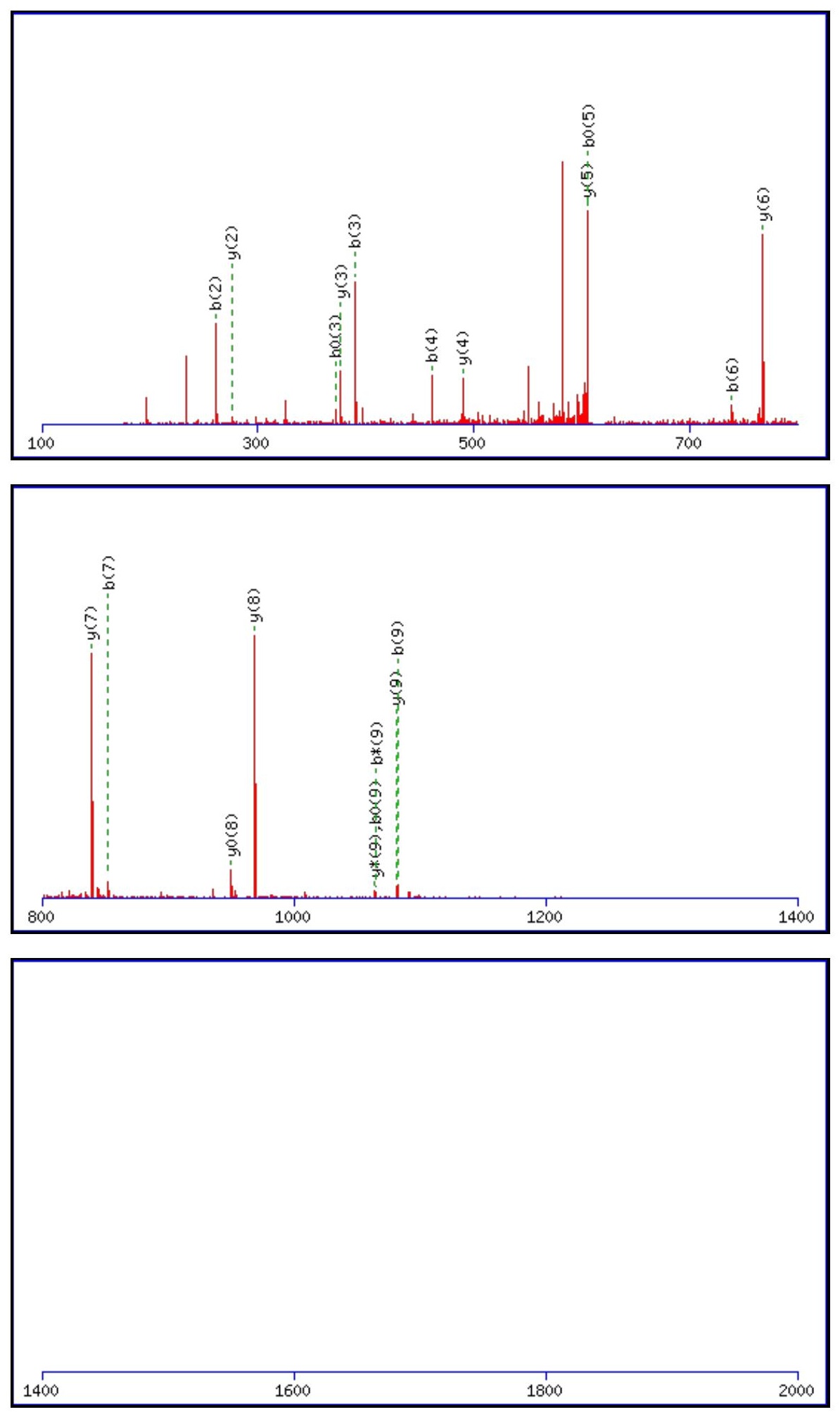

Monoisotopic mass of neutral peptide $\operatorname{Mr}($ calc): 1227.5693 


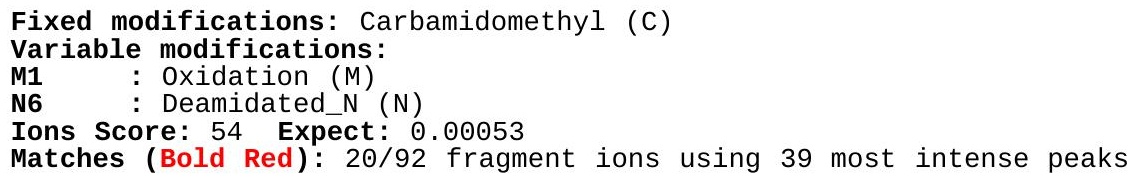

\begin{tabular}{|r|c|c|c|c|c|c|c|c|c|c|c|c|c|c|}
\hline$\#$ & $\mathbf{b}$ & $\mathbf{b}^{++}$ & $\mathbf{b}^{*}$ & $\mathbf{b}^{\mathbf{*}^{++}}$ & $\mathbf{b}^{\mathbf{0}}$ & $\mathbf{b}^{\mathbf{0 + +}}$ & $\mathbf{S e q}$ & $\mathbf{y}$ & $\mathbf{y}^{++}$ & $\mathbf{y}^{\mathbf{*}}$ & $\mathbf{y}^{\mathbf{*}^{++}}$ & $\mathbf{y}^{\mathbf{0}}$ & $\mathbf{y}^{\mathbf{0 + +}}$ & $\#$ \\
\hline $\mathbf{1}$ & 148.0427 & 74.5250 & & & & & $\mathbf{M}$ & & & & & & & $\mathbf{1 0}$ \\
\hline $\mathbf{2}$ & $\mathbf{2 6 1 . 1 2 6 7}$ & 131.0670 & & & & & $\mathbf{L}$ & $\mathbf{1 0 8 1 . 5 4 1 2}$ & 541.2742 & $\mathbf{1 0 6 4 . 5 1 4 6}$ & 532.7610 & 1063.5306 & 532.2689 & $\mathbf{9}$ \\
\hline $\mathbf{3}$ & $\mathbf{3 9 0 . 1 6 9 3}$ & 195.5883 & & & 372.1588 & 186.5830 & $\mathbf{E}$ & $\mathbf{9 6 8 . 4 5 7 1}$ & 484.7322 & 951.4306 & 476.2189 & $\mathbf{9 5 0 . 4 4 6 5}$ & 475.7269 & $\mathbf{8}$ \\
\hline $\mathbf{4}$ & $\mathbf{4 6 1 . 2 0 6 5}$ & 231.1069 & & & 443.1959 & 222.1016 & $\mathbf{A}$ & $\mathbf{8 3 9 . 4 1 4 5}$ & 420.2109 & 822.3880 & 411.6976 & 821.4040 & 411.2056 & $\mathbf{7}$ \\
\hline $\mathbf{5}$ & 624.2698 & 312.6385 & & & $\mathbf{6 0 6 . 2 5 9 2}$ & 303.6332 & $\mathbf{Y}$ & $\mathbf{7 6 8 . 3 7 7 4}$ & 384.6923 & 751.3509 & 376.1791 & 750.3668 & 375.6871 & $\mathbf{6}$ \\
\hline $\mathbf{6}$ & $\mathbf{7 3 9 . 2 9 6 7}$ & 370.1520 & 722.2702 & 361.6387 & 721.2862 & 361.1467 & $\mathbf{N}$ & $\mathbf{6 0 5 . 3 1 4 1}$ & 303.1607 & 588.2875 & 294.6474 & 587.3035 & 294.1554 & $\mathbf{5}$ \\
\hline $\mathbf{7}$ & $\mathbf{8 5 2 . 3 8 0 8}$ & 426.6940 & 835.3542 & 418.1808 & 834.3702 & 417.6887 & $\mathbf{L}$ & $\mathbf{4 9 0 . 2 8 7 1}$ & 245.6472 & 473.2606 & 237.1339 & 472.2766 & 236.6419 & $\mathbf{4}$ \\
\hline $\mathbf{8}$ & 953.4285 & 477.2179 & 936.4019 & 468.7046 & 935.4179 & 468.2126 & $\mathbf{T}$ & $\mathbf{3 7 7 . 2 0 3 1}$ & 189.1052 & 360.1765 & 180.5919 & 359.1925 & 180.0999 & $\mathbf{3}$ \\
\hline $\mathbf{9}$ & $\mathbf{1 0 8 2 . 4 7 1 1}$ & 541.7392 & $\mathbf{1 0 6 5 . 4 4 4 5}$ & 533.2259 & $\mathbf{1 0 6 4 . 4 6 0 5}$ & 532.7339 & $\mathbf{E}$ & $\mathbf{2 7 6 . 1 5 5 4}$ & 138.5813 & 259.1288 & 130.0681 & 258.1448 & 129.5761 & $\mathbf{2}$ \\
\hline $\mathbf{1 0}$ & & & & & & & $\mathbf{K}$ & 147.1128 & 74.0600 & 130.0863 & 65.5468 & & & $\mathbf{1}$ \\
\hline
\end{tabular}
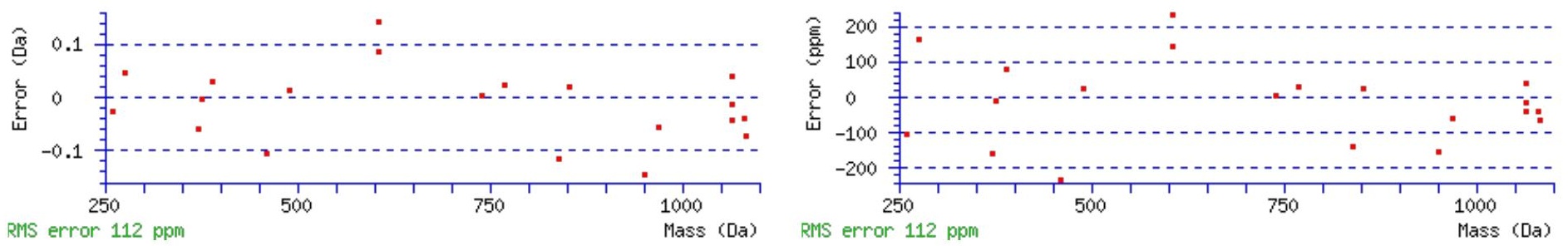

All matches to this query

\begin{tabular}{|l|l|l|l|}
\hline Score & Mr(calc): & Delta & \multicolumn{1}{|c|}{ Sequence } \\
\hline 54.0 & 1227.5693 & 0.0022 & MLEAYNLTEK \\
\hline 24.2 & 1227.5693 & 0.0022 & EMESLYSELK \\
\hline 21.1 & 1227.5693 & 0.0022 & EIMFSDITEK \\
\hline 18.9 & 1227.5788 & -0.0073 & NIPVLETAYK \\
\hline 9.2 & 1226.5584 & 1.0131 & EYPSQSPVLK \\
\hline 5.9 & 1227.5788 & -0.0073 & YPVNSVNILK \\
\hline 5.6 & 1227.5682 & 0.0033 & METEIKRNK \\
\hline 4.6 & 1227.5740 & -0.0025 & LYMSCIAQSR \\
\hline 4.5 & 1227.5748 & -0.0032 & DEKSAKTLEK \\
\hline 4.3 & 1226.5584 & 1.0131 & EYPSOSPVLK \\
\hline
\end{tabular}

Spectrum No: 625; Query: 270; Rank: 1

\section{Peptide View}

MS/MS Fragmentation of QAANLTQDLPR

Found in IPI00370977, Tax_Id=10116 Gene_Symbol=LOC689765 similar to beta 3-glycosyltransferase-like

Match to Query 270: 1226.627648 from(614.321100,2+)

Title: 100101RatKid_NS_deglyco_27.1795.1795.2.dta

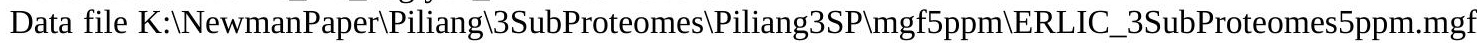



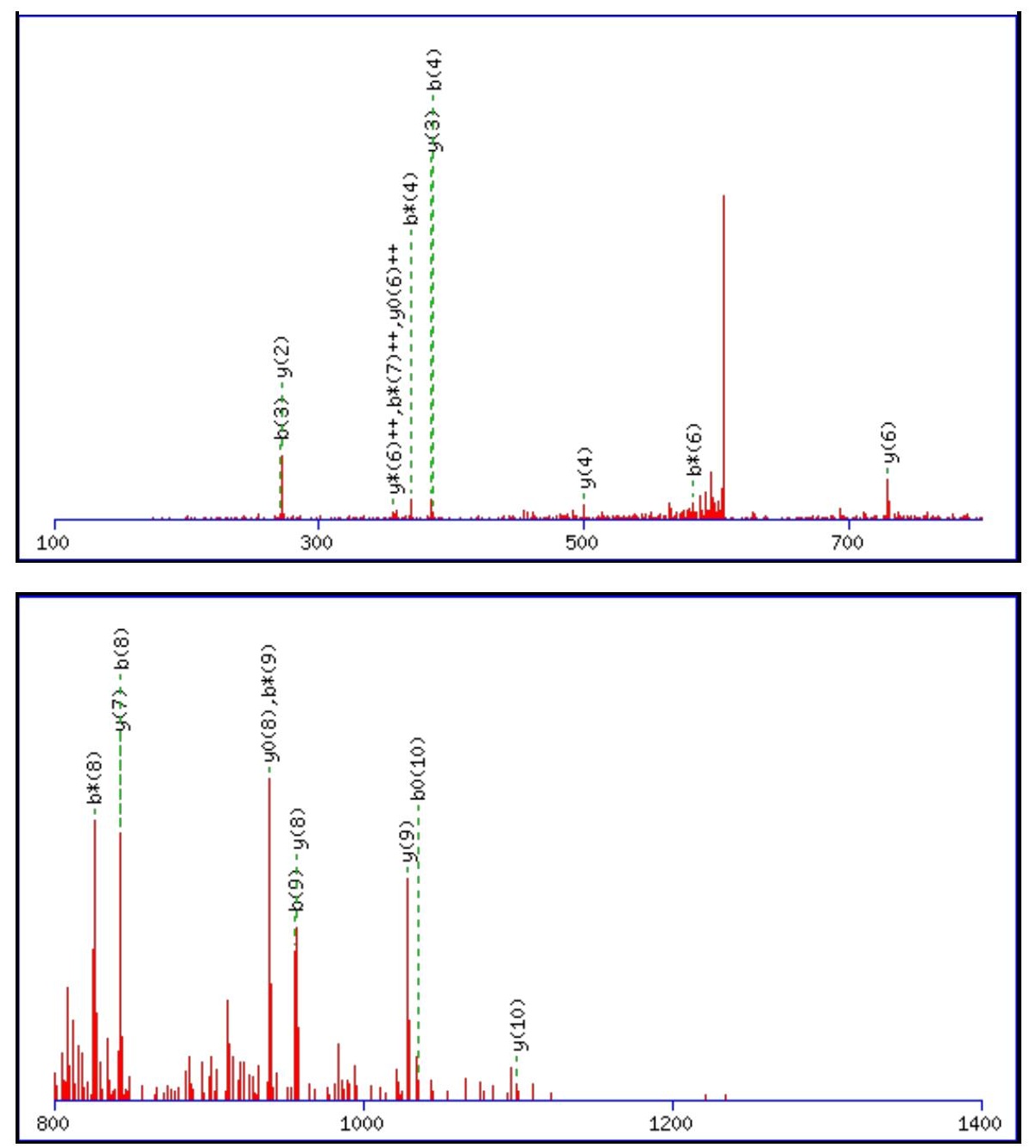

\begin{tabular}{rrrr}
\hline 00 & 1600 & 1800 & 2000 \\
\hline
\end{tabular}

Monoisotopic mass of neutral peptide $\operatorname{Mr}($ calc): 1226.6255

Fixed modifications: Carbamidomethyl (C)

Variable modifications:

N4 : Deamidated_N (N)

Ions Score: 54 Expect: 0.00043

Matches (Bold Red): 21/104 fragment ions using 46 most intense peaks

\begin{tabular}{|r|c|c|c|c|c|c|c|c|c|c|c|c|c|c|}
\hline$\#$ & $\mathbf{b}$ & $\mathbf{b}^{++}$ & $\mathbf{b}^{*}$ & $\mathbf{b}^{\boldsymbol{*}_{++}}$ & $\mathbf{b}^{\mathbf{0}}$ & $\mathbf{b}^{\mathbf{0 + +}}$ & Seq. & $\mathbf{y}$ & $\mathbf{y}^{++}$ & $\mathbf{y}^{\mathbf{*}}$ & $\mathbf{y}^{\boldsymbol{*}^{++}}$ & $\mathbf{y}^{\mathbf{0}}$ & $\mathbf{y}^{\mathbf{0 + +}}$ & $\#$ \\
\hline $\mathbf{1}$ & 129.0659 & 65.0366 & 112.0393 & 56.5233 & & & $\mathbf{Q}$ & & & & & & & $\mathbf{1 1}$ \\
\hline $\mathbf{2}$ & 200.1030 & 100.5551 & 183.0764 & 92.0418 & & & $\mathbf{A}$ & $\mathbf{1 0 9 9 . 5 7 4 2}$ & 550.2907 & 1082.5477 & 541.7775 & 1081.5636 & 541.2855 & $\mathbf{1 0}$ \\
\hline $\mathbf{3}$ & $\mathbf{2 7 1 . 1 4 0 1}$ & 136.0737 & 254.1135 & 127.5604 & & & $\mathbf{A}$ & $\mathbf{1 0 2 8 . 5 3 7 1}$ & 514.7722 & 1011.5105 & 506.2589 & 1010.5265 & 505.7669 & $\mathbf{9}$ \\
\hline $\mathbf{4}$ & $\mathbf{3 8 6 . 1 6 7 0}$ & 193.5871 & 369.1405 & 185.0739 & & & $\mathbf{N}$ & $\mathbf{9 5 7 . 5 0 0 0}$ & 479.2536 & 940.4734 & 470.7404 & $\mathbf{9 3 9 . 4 8 9 4}$ & 470.2483 & $\mathbf{8}$ \\
\hline $\mathbf{5}$ & 499.2511 & 250.1292 & 482.2245 & 241.6159 & & & $\mathbf{L}$ & $\mathbf{8 4 2 . 4 7 3 0}$ & 421.7402 & 825.4465 & 413.2269 & 824.4625 & 412.7349 & $\mathbf{7}$ \\
\hline
\end{tabular}




\begin{tabular}{|r|r|r|r|r|r|r|r|r|r|r|r|r|r|r|r|r|r|r|r|}
$\mathbf{6}$ & 600.2988 & 300.6530 & 583.2722 & 292.1397 & 582.2882 & 291.6477 & $\mathbf{T}$ & $\mathbf{7 2 9 . 3 8 9 0}$ & 365.1981 & 712.3624 & 356.6849 & 711.3784 & 356.1928 & $\mathbf{6}$ \\
\hline $\mathbf{7}$ & $\mathbf{7 2 8 . 3 5 7 3}$ & 364.6823 & 711.3308 & 356.1690 & 710.3468 & 355.6770 & $\mathbf{Q}$ & 628.3413 & 314.6743 & 611.3148 & 306.1610 & 610.3307 & 305.6690 & $\mathbf{5}$ \\
\hline $\mathbf{8}$ & $\mathbf{8 4 3 . 3 8 4 3}$ & 422.1958 & $\mathbf{8 2 6 . 3 5 7 7}$ & 413.6825 & 825.3737 & 413.1905 & $\mathbf{D}$ & $\mathbf{5 0 0 . 2 8 2 7}$ & 250.6450 & 483.2562 & 242.1317 & 482.2722 & 241.6397 & $\mathbf{4}$ \\
\hline $\mathbf{9}$ & $\mathbf{9 5 6 . 4 6 8 3}$ & 478.7378 & $\mathbf{9 3 9 . 4 4 1 8}$ & 470.2245 & 938.4578 & 469.7325 & $\mathbf{L}$ & $\mathbf{3 8 5 . 2 5 5 8}$ & 193.1315 & 368.2292 & 184.6183 & & & & $\mathbf{3}$ \\
\hline $\mathbf{1 0}$ & 1053.5211 & 527.2642 & 1036.4946 & 518.7509 & $\mathbf{1 0 3 5 . 5 1 0 5}$ & 518.2589 & $\mathbf{P}$ & $\mathbf{2 7 2 . 1 7 1 7}$ & 136.5895 & 255.1452 & 128.0762 & & & & $\mathbf{2}$ \\
\hline $\mathbf{1 1}$ & & & & & & & $\mathbf{R}$ & 175.1190 & 88.0631 & 158.0924 & 79.5498 & & & \\
\hline
\end{tabular}
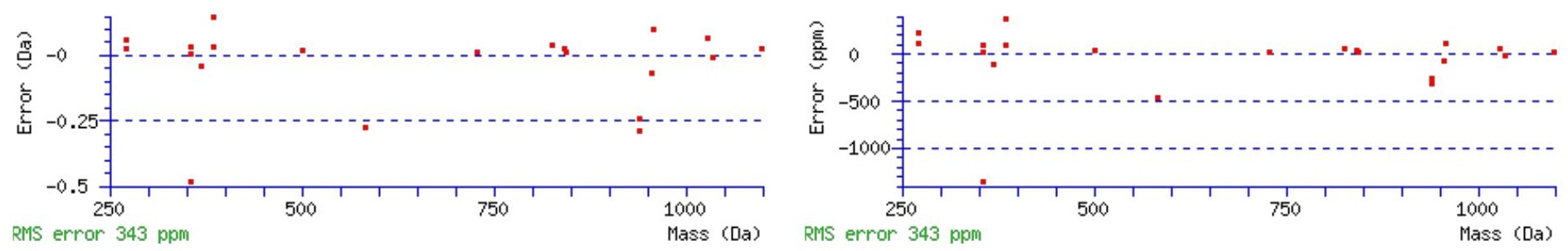

\section{All matches to this query}

\begin{tabular}{|l|l|r|l|}
\hline Score & Mr(calc): & Delta & \multicolumn{1}{|c|}{ Sequence } \\
\hline 53.9 & 1226.6255 & 0.0022 & QAANLTQDLPR \\
\hline 6.2 & 1226.6172 & 0.0104 & SFQGKKSIPR \\
\hline 5.4 & 1225.6332 & 0.9944 & RKTQFTPLR \\
\hline 4.3 & 1225.6141 & 1.0135 & MSSLTPLLLR \\
\hline 2.6 & 1224.6227 & 2.0049 & SLRPATGTKSK \\
\hline 1.7 & 1224.6268 & 2.0009 & QTLFITGLPR \\
\hline 1.7 & 1225.6203 & 1.0073 & FRPNPLNNPR \\
\hline 1.6 & 1226.6172 & 0.0104 & SFQGKKSIPR \\
\hline 1.4 & 1226.6302 & -0.0025 & RMKEGAHINR \\
\hline 1.3 & 1226.6329 & -0.0052 & IASIMALSSXR \\
\hline
\end{tabular}

Spectrum No: 626; Query: 1048; Rank: 1

\section{Peptide View}

MS/MS Fragmentation of VNGSCVDVDECATGGR

Found in IPI00204867, Tax_Id=10116 Gene_Symbol=Ltbp4 178 kDa protein

Match to Query 1048: 1695.686468 from(848.850510,2+)

Title: 100101RatKid_NS_deglyco_24.1110.1110.2.dta

Data file K:INewmanPaper|Piliangl3SubProteomes\Piliang3SP\mgf5ppm\ERLIC_3SubProteomes5ppm.mgf

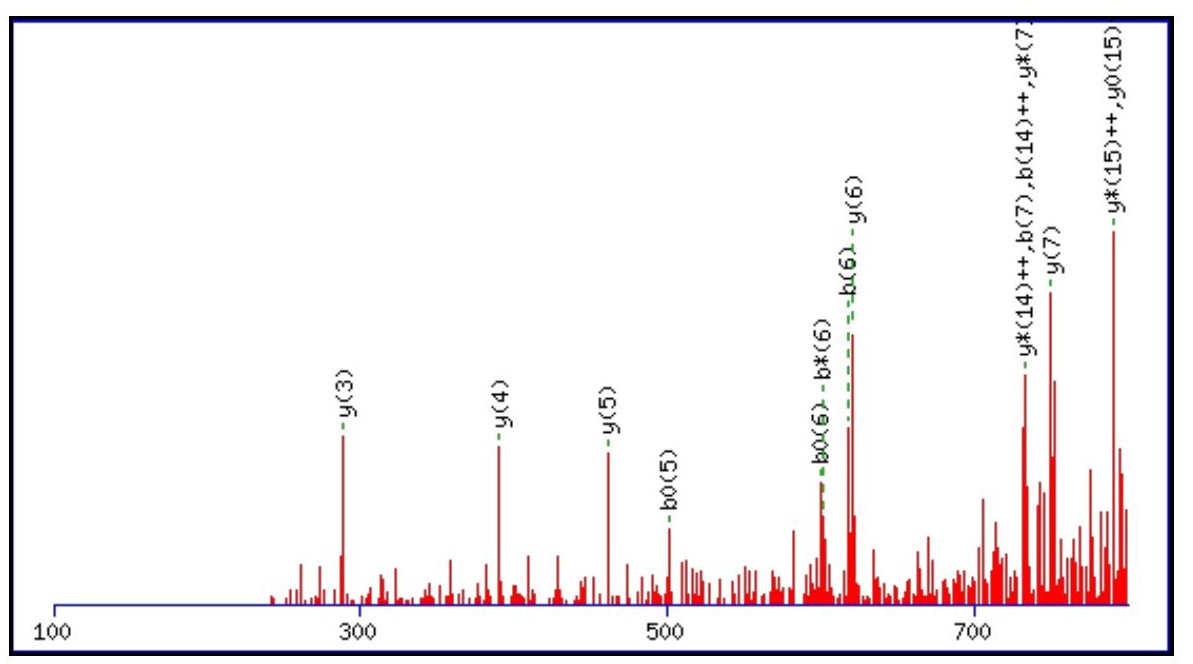



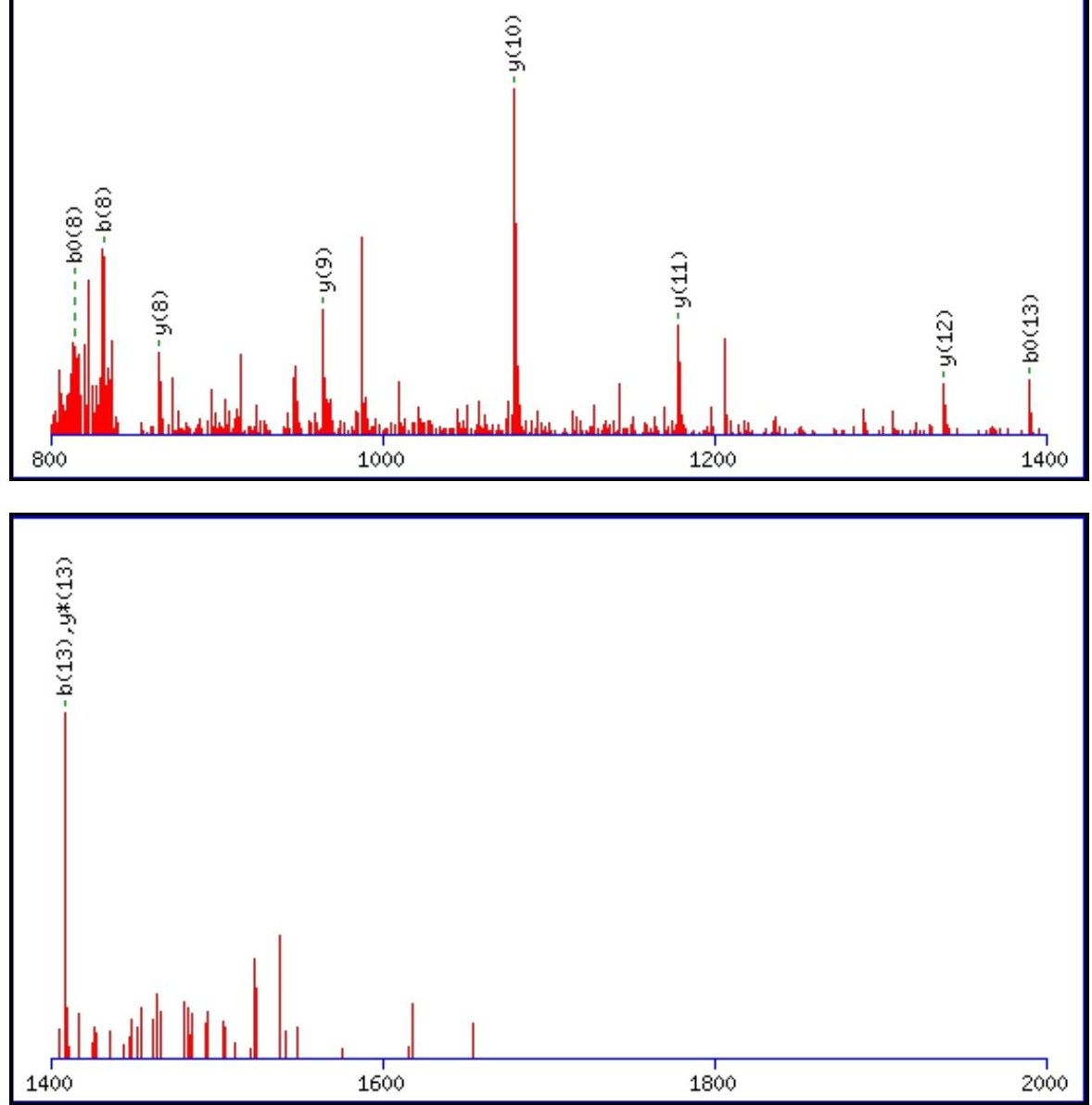

Monoisotopic mass of neutral peptide $\operatorname{Mr}($ calc): 1695.6829

Fixed modifications: Carbamidomethyl (C)

Variable modifications:

N2 : Deamidated_N (N)

Ions Score: 54 Expect: 0.00067

Matches (Bold Red): 25/166 fragment ions using 41 most intense peaks

\begin{tabular}{|c|c|c|c|c|c|c|c|c|c|c|c|c|c|c|}
\hline \# & b & $\mathbf{b}^{++}$ & $\mathbf{b}^{*}$ & $\mathbf{b}^{*^{++}}$ & $\mathbf{b}^{0}$ & $\mathbf{b}^{0++}$ & Seq. & $\mathbf{y}$ & $y^{++}$ & $\mathbf{y}^{*}$ & $\mathrm{y}^{*^{++}}$ & $\mathbf{y}^{0}$ & $\mathbf{y}^{0++}$ & \# \\
\hline 1 & 100.0757 & 50.5415 & & & & & V & & & & & & & 16 \\
\hline 2 & 215.1026 & 108.0550 & 198.0761 & 99.5417 & & & $\mathbf{N}$ & 1597.6217 & 799.3145 & 1580.5952 & 790.8012 & 1579.6111 & 790.3092 & 15 \\
\hline 3 & 272.1241 & 136.5657 & 255.0975 & 128.0524 & & & G & 1482.5948 & 741.8010 & 1465.5682 & 733.2877 & 1464.5842 & 732.7957 & 14 \\
\hline 4 & 359.1561 & 180.0817 & 342.1296 & 171.5684 & 341.1456 & & $S$ & 1425.5733 & 713.2903 & 1408.5468 & 704.7770 & 1407.5627 & 704.2850 & 13 \\
\hline 5 & 519.1868 & 260.0970 & 502.1602 & 251.5837 & 501.1762 & 251.0917 & C & 1338.5413 & 669.7743 & 1321.5147 & 661.2610 & 1320.5307 & 660.7690 & 12 \\
\hline 6 & 618.2552 & 309.6312 & 601.2286 & 301.1180 & 600.2446 & 300.6259 & $\mathbf{V}$ & 1178.5106 & 589.7590 & 1161.4841 & 581.2457 & 1160.5001 & 580.7537 & 11 \\
\hline 7 & 733.2821 & 367.1447 & 716.2556 & 358.6314 & 715.2716 & 358.1394 & D & 1079.4422 & 540.2247 & 1062.4157 & 531.7115 & 1061.4316 & 531.2195 & 10 \\
\hline 8 & 832.3505 & 416.6789 & 815.3240 & 408.1656 & 814.3400 & 407.6736 & $\mathbf{V}$ & 964.4153 & 482.7113 & 947.3887 & 474.1980 & 946.4047 & 473.7060 & 9 \\
\hline 9 & 947.3775 & 474.1924 & 930.3509 & 465.6791 & 929.3669 & 465.1871 & D & 865.3469 & 433.1771 & 848.3203 & 424.6638 & 847.3363 & 424.1718 & 8 \\
\hline 10 & 1076.4201 & 538.7137 & 1059.3935 & 530.2004 & 1058.4095 & 529.7084 & $\mathbf{E}$ & 750.3199 & 375.6636 & 733.2934 & 367.1503 & 732.3093 & 366.6583 & 7 \\
\hline 11 & 1236.4507 & 618.7290 & 1219.4242 & 610.2157 & 1218.4402 & 609.7237 & $\mathrm{C}$ & 621.2773 & 311.1423 & 604.2508 & 302.6290 & 603.2668 & 302.1370 & 6 \\
\hline 12 & 1307.4878 & 654.2476 & 1290.4613 & 645.7343 & 1289.4773 & 645.2423 & A & 461.2467 & 231.1270 & 444.2201 & 222.6137 & 443.2361 & 222.1217 & 5 \\
\hline 13 & 1408.5355 & 704.7714 & 1391.5090 & 696.2581 & 1390.5250 & 695.7661 & $\mathbf{T}$ & 390.2096 & 195.6084 & 373.1830 & 187.0951 & 372.1990 & 186.6031 & 4 \\
\hline 14 & 1465.5570 & 733.2821 & 1448.5304 & 724.7689 & 1447.5464 & 724.2768 & G & 289.1619 & 145.0846 & 272.1353 & 136.5713 & & & 3 \\
\hline 15 & 1522.5784 & 761.7929 & 1505.5519 & 753.2796 & 1504.5679 & 752.7876 & G & 232.1404 & 116.5738 & 215.1139 & 108.0606 & & & 2 \\
\hline 16 & & & & & & & $\mathbf{R}$ & 175.1190 & 88.0631 & 158.0924 & 79.5498 & & & 1 \\
\hline
\end{tabular}



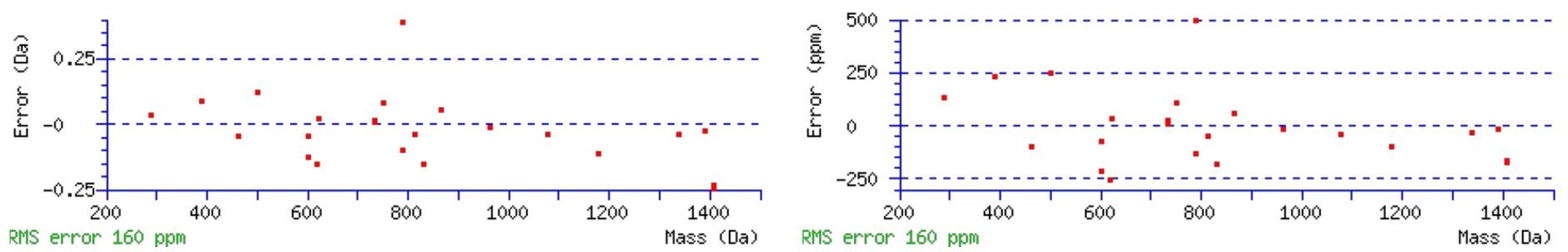

\section{All matches to this query}

\begin{tabular}{|l|l|l|l|}
\hline Score & Mr(calc): & Delta & \multicolumn{1}{c|}{ Sequence } \\
\hline 53.7 & 1695.6829 & 0.0036 & VNGSCVDVDECATGGR \\
\hline 52.3 & 1694.6989 & 0.9876 & VNGSCVDVDECATGGR \\
\hline 7.2 & 1694.6711 & 1.0154 & CKATFYTSRVMR \\
\hline 1.6 & 1695.6841 & 0.0024 & SRTVQYSPVCPSR \\
\hline
\end{tabular}

Spectrum No: 627; Query: 985; Rank: 1

\section{Peptide View}

MS/MS Fragmentation of ENPVEFDSLFNESK

Found in IPI00360085, Tax_Id=10116 Gene_Symbol=Pign_predicted Phosphatidylinositol glycan class N

Match to Query 985: 1654.738708 from(828.376630,2+)

Title: 100101RatKid_NS_deglyco_08.4144.4144.2.dta

Data file K:\NewmanPaper|Piliang|3SubProteomes\Piliang3SP\mgf5ppm\ERLIC_3SubProteomes5ppm.mgf

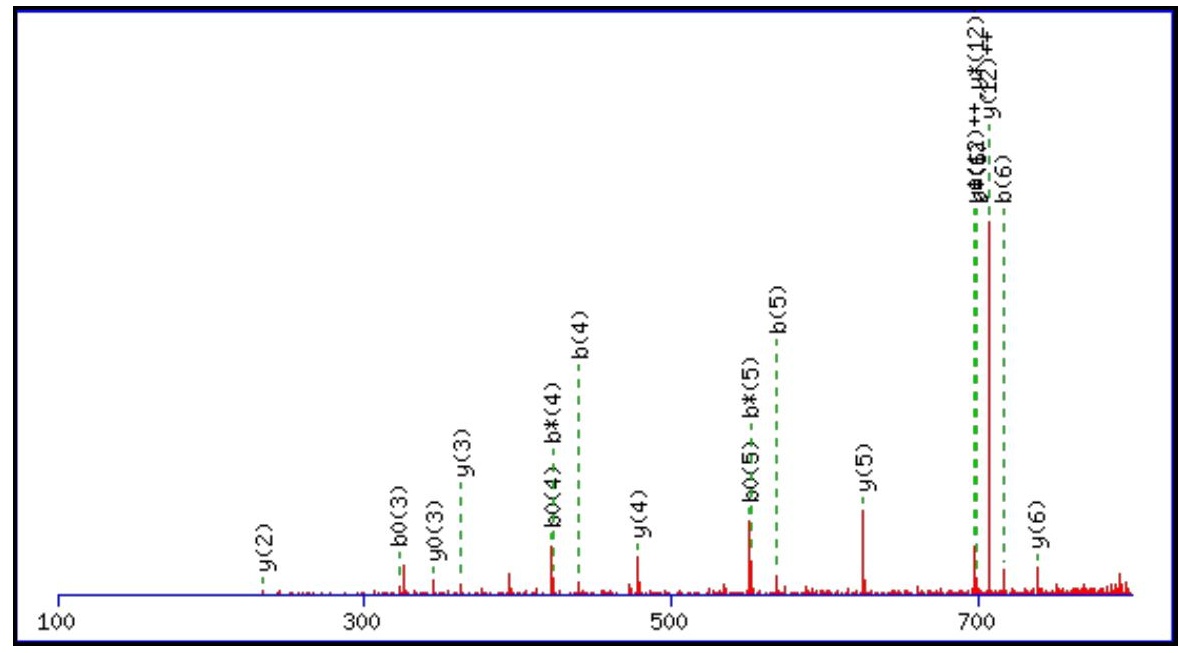



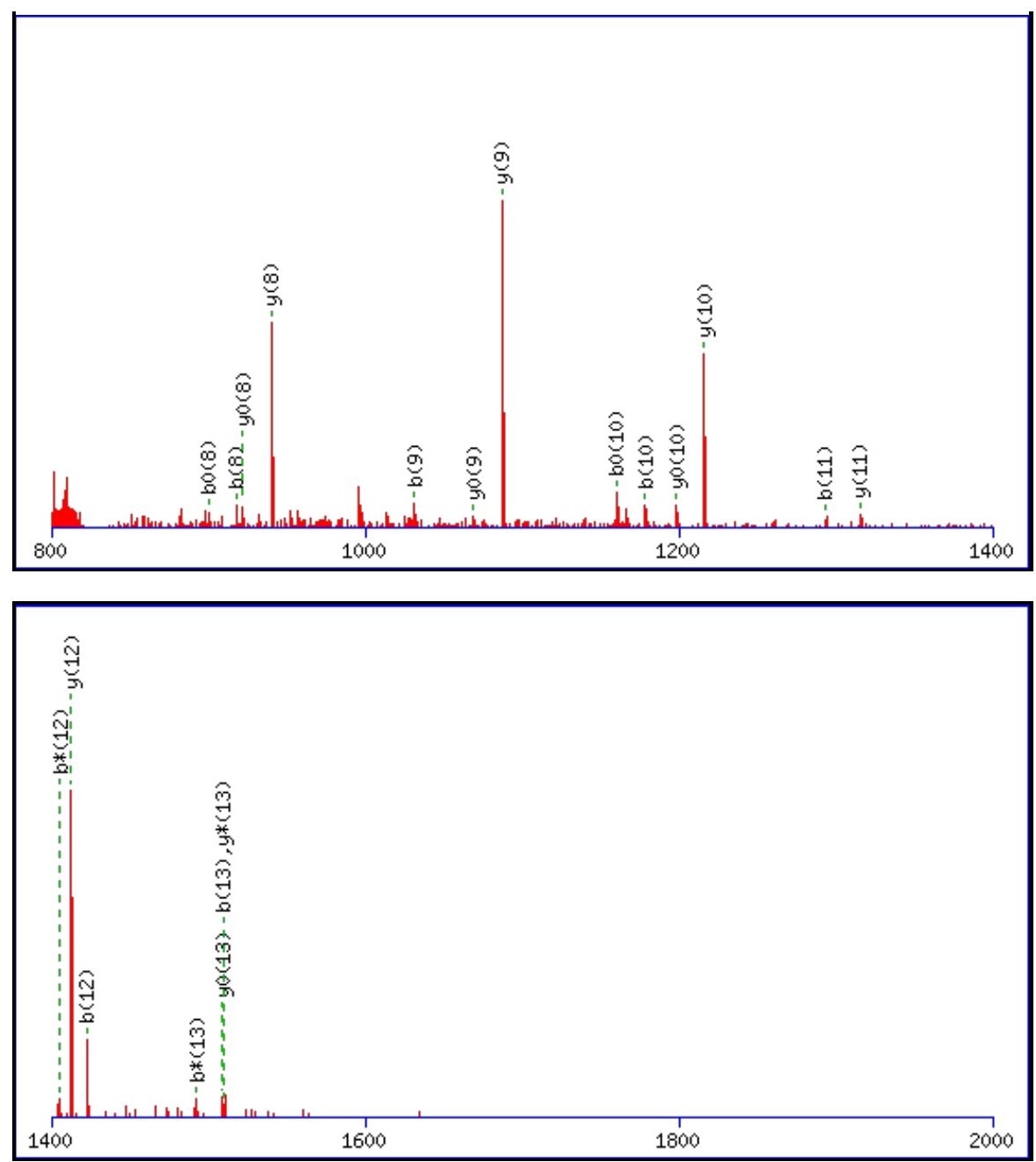

Monoisotopic mass of neutral peptide $\operatorname{Mr}($ calc): 1654.7362

Fixed modifications: Carbamidomethyl (C)

Variable modifications:

N11 : Deamidated_N (N)

Ions Score: 54 Expect: 0.00084

Matches (Bold Red): 39/152 fragment ions using 75 most intense peaks

\begin{tabular}{|c|c|c|c|c|c|c|c|c|c|c|c|c|c|c|}
\hline \# & b & $\mathbf{b}^{++}$ & $\mathbf{b}^{*}$ & $\mathbf{b}^{*^{++}}$ & $\mathbf{b}^{0}$ & $\mathbf{b}^{\mathbf{0 + +}}$ & Seq. & $\mathbf{y}$ & $\mathbf{y}^{++}$ & $\mathbf{y}^{*}$ & $\mathrm{y}^{*^{++}}$ & $\mathbf{y}^{\mathbf{0}}$ & $\mathbf{y}^{\mathbf{0 + +}}$ & \# \\
\hline 1 & 130.0499 & 65.5286 & & & 112.0393 & 56.5233 & $\mathbf{E}$ & & & & & & & 14 \\
\hline 2 & 244.0928 & 122.5500 & 227.0662 & 114.0368 & 226.0822 & 113.5448 & $\mathbf{N}$ & 1526.7009 & 763.8541 & 1509.6744 & 755.3408 & 1508.6904 & 754.8488 & 13 \\
\hline 3 & 341.1456 & 171.0764 & 324.1190 & 162.5631 & 323.1350 & 162.0711 & $\mathbf{P}$ & 1412.6580 & 706.8326 & 1395.6314 & 698.3194 & 1394.6474 & 697.8274 & 12 \\
\hline 4 & 440.2140 & 220.6106 & 423.1874 & 212.0974 & 422.2034 & 211.6053 & $\mathbf{V}$ & 1315.6052 & 658.3063 & 1298.5787 & 649.7930 & 1297.5947 & 649.3010 & 11 \\
\hline 5 & 569.2566 & 285.1319 & 552.2300 & 276.6186 & 551.2460 & 276.1266 & $\mathbf{E}$ & 1216.5368 & 608.7720 & 1199.5103 & 600.2588 & 1198.5263 & 599.7668 & 10 \\
\hline 6 & 716.3250 & 358.6661 & 699.2984 & 350.1529 & 698.3144 & 349.6608 & $\mathbf{F}$ & 1087.4942 & 544.2508 & 1070.4677 & 535.7375 & 1069.4837 & 535.2455 & 9 \\
\hline 7 & 831.3519 & 416.1796 & 814.3254 & 407.6663 & 813.3414 & 407.1743 & D & 940.4258 & 470.7165 & 923.3993 & 462.2033 & 922.4152 & 461.7113 & 8 \\
\hline 8 & 918.3840 & 459.6956 & 901.3574 & 451.1823 & 900.3734 & 450.6903 & S & 825.3989 & 413.2031 & 808.3723 & 404.6898 & 807.3883 & 404.1978 & 7 \\
\hline 9 & 1031.4680 & 516.2376 & 1014.4415 & 507.7244 & 1013.4575 & 507.2324 & $\mathbf{L}$ & 738.3668 & 369.6871 & 721.3403 & 361.1738 & 720.3563 & 360.6818 & 6 \\
\hline 10 & 1178.5364 & 589.7719 & 1161.5099 & 581.2586 & 1160.5259 & 580.7666 & $\mathbf{F}$ & 625.2828 & 313.1450 & 608.2562 & 304.6318 & 607.2722 & 304.1397 & 5 \\
\hline 11 & 1293.5634 & 647.2853 & 1276.5368 & 638.7720 & 1275.5528 & 638.2800 & $\mathbf{N}$ & 478.2144 & 239.6108 & 461.1878 & 231.0975 & 460.2038 & 230.6055 & 4 \\
\hline 12 & 1422.6060 & 711.8066 & 1405.5794 & 703.2933 & 1404.5954 & 702.8013 & $\mathbf{E}$ & 363.1874 & 182.0974 & 346.1609 & 173.5841 & 345.1769 & 173.0921 & 3 \\
\hline 13 & 1509.6380 & 755.3226 & 1492.6114 & 746.8094 & 1491.6274 & 746.3173 & S & 234.1448 & 117.5761 & 217.1183 & 109.0628 & 216.1343 & 108.5708 & 2 \\
\hline 14 & & & & & & & $\mathbf{K}$ & 147.1128 & 74.0600 & 130.0863 & 65.5468 & & & 1 \\
\hline
\end{tabular}



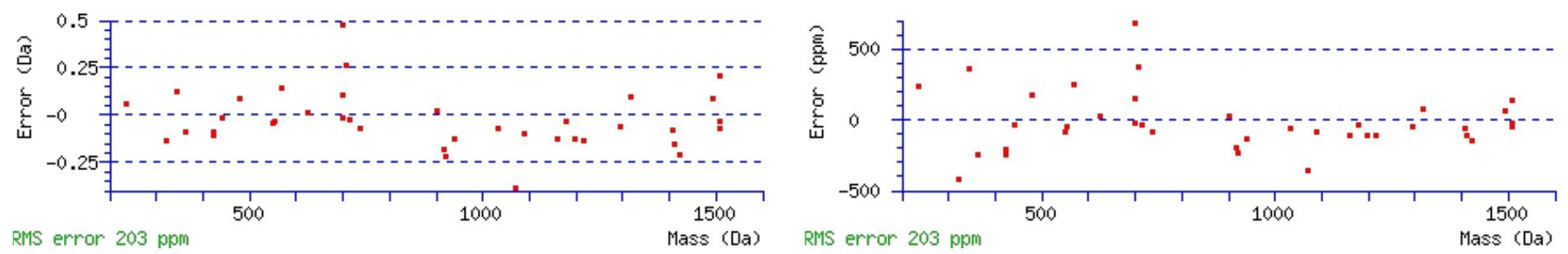

\section{All matches to this query}

\begin{tabular}{|l|l|l|l|}
\hline Score & Mr(calc): & Delta & \multicolumn{1}{|c|}{ Sequence } \\
\hline 53.6 & 1654.7362 & 0.0025 & ENPVEFDSLFNESK \\
\hline 5.6 & 1654.7538 & -0.0151 & GRTVMIEQSWGSPK \\
\hline 5.1 & 1653.7303 & 1.0084 & IKAASELYIETEK \\
\hline 3.9 & 1654.7538 & -0.0151 & MRPSVESGFPPVTR \\
\hline 0.8 & 1652.7407 & 1.9980 & LAGSSAASSPNGQGLQK \\
\hline 0.8 & 1652.7407 & 1.9980 & LAGSSAASSPNGQGLQK \\
\hline 0.7 & 1653.7246 & 1.0141 & LEEAEGALERNESK \\
\hline
\end{tabular}

Spectrum No: 628; Query: 2363; Rank: 1

\section{Peptide View}

MS/MS Fragmentation of SLDLDPSKTNVSGGAIALGHPLGGSGSR

Found in IPI00201413, Tax_Id=10116 Gene_Symbol=Acaa2 3-ketoacyl-CoA thiolase, mitochondrial

Match to Query 2363: 2663.363322 from(888.795050,3+)

Title: 091008RatKidney_NoSalt_10.2653.2653.3.dta

Data file K:INewmanPaper|Piliangl3SubProteomes\Piliang3SP\mgf5ppm\ERLIC_3SubProteomes5ppm.mgf

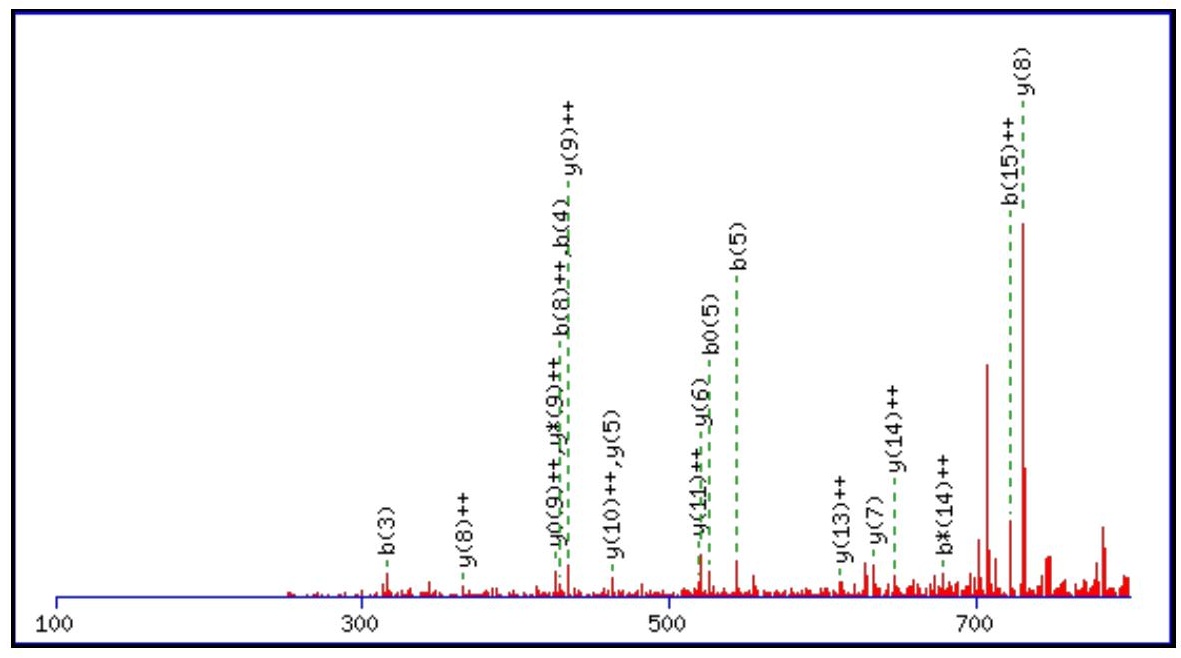



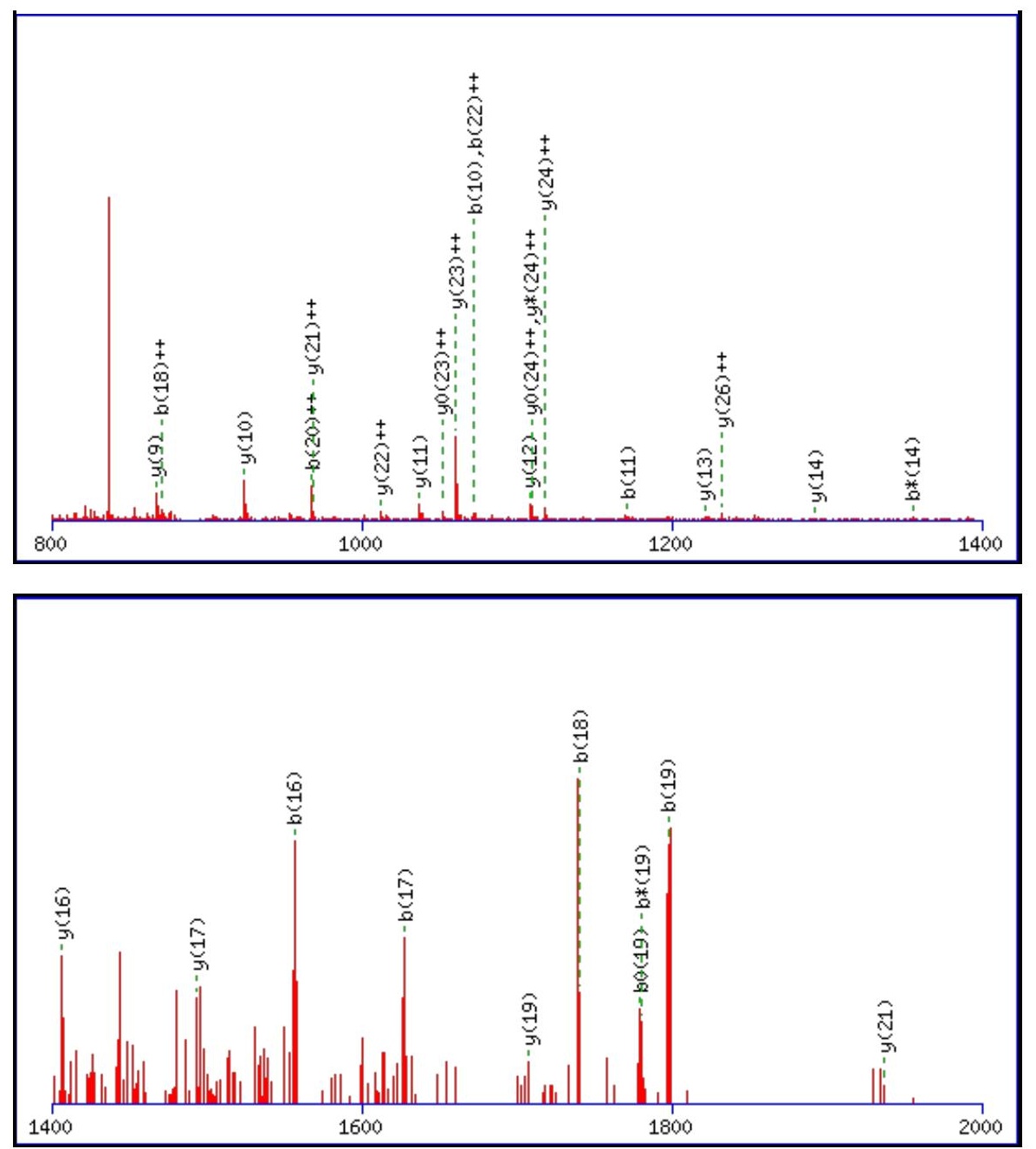

Monoisotopic mass of neutral peptide $\operatorname{Mr}($ calc): 2663.3515

Fixed modifications: Carbamidomethyl (C)

Variable modifications:

N10 : Deamidated_N (N)

Ions Score: 54 Expect: 0.00068

Matches (Bold Red): 49/308 fragment ions using 105 most intense peaks

\begin{tabular}{|c|c|c|c|c|c|c|c|c|c|c|c|c|c|c|}
\hline \# & b & $\mathbf{b}^{++}$ & $\mathbf{b}^{*}$ & $\mathbf{b}^{*++}$ & $\mathbf{b}^{0}$ & $\mathbf{b}^{\mathbf{0 + +}}$ & Seq. & $\mathbf{y}$ & $\mathbf{y}^{++}$ & $\mathbf{y}^{*}$ & $\mathbf{y}^{*^{++}}$ & $\mathbf{y}^{0}$ & $\mathbf{y}^{0++}$ & \# \\
\hline 1 & 88.0393 & 44.5233 & & & 70.0287 & 35.5180 & $S$ & & & & & & & 28 \\
\hline 2 & 201.1234 & 101.0653 & & & 183.1128 & 92.0600 & $\mathbf{L}$ & 2577.3267 & 1289.1670 & 2560.3002 & 1280.6537 & 2559.3161 & 1280.1617 & 27 \\
\hline 3 & 316.1503 & 158.5788 & & & 298.1397 & 149.5735 & D & 2464.2426 & 1232.6250 & 2447.2161 & 1224.1117 & 2446.2321 & 1223.6197 & 26 \\
\hline 4 & 429.2344 & 215.1208 & & & 411.2238 & 206.1155 & $\mathbf{L}$ & 2349.2157 & 1175.1115 & 2332.1892 & 1166.5982 & 2331.2051 & 1166.1062 & 25 \\
\hline 5 & 544.2613 & 272.6343 & & & 526.2508 & 263.6290 & D & 2236.1316 & 1118.5695 & 2219.1051 & 1110.0562 & 2218.1211 & 1109.5642 & 24 \\
\hline 6 & 641.3141 & 321.1607 & & & 623.3035 & 312.1554 & $\mathbf{P}$ & 2121.1047 & 1061.0560 & 2104.0782 & 1052.5427 & 2103.0941 & 1052.0507 & 23 \\
\hline 7 & 728.3461 & 364.6767 & & & 710.3355 & 355.6714 & $\mathrm{~S}$ & 2024.0519 & 1012.5296 & 2007.0254 & 1004.0163 & 2006.0414 & 1003.5243 & 22 \\
\hline 8 & 856.4411 & 428.7242 & 839.4145 & 420.2109 & 838.4305 & 419.7189 & $\mathbf{K}$ & 1937.0199 & 969.0136 & 1919.9934 & 960.5003 & 1919.0093 & 960.0083 & 21 \\
\hline 9 & 957.4888 & 479.2480 & 940.4622 & 470.7347 & 939.4782 & 470.2427 & $\mathbf{T}$ & 1808.9249 & 904.9661 & 1791.8984 & 896.4528 & 1790.9144 & 895.9608 & 20 \\
\hline 10 & 1072.5157 & 536.7615 & 1055.4891 & 528.2482 & 1054.5051 & 527.7562 & $\mathbf{N}$ & 1707.8773 & 854.4423 & 1690.8507 & 845.9290 & 1689.8667 & 845.4370 & 19 \\
\hline 11 & 1171.5841 & 586.2957 & 1154.5576 & 577.7824 & 1153.5735 & 577.2904 & $\mathbf{V}$ & 1592.8503 & 796.9288 & 1575.8238 & 788.4155 & 1574.8398 & 787.9235 & 18 \\
\hline 12 & 1258.6161 & 629.8117 & 1241.5896 & 621.2984 & 1240.6056 & 620.8064 & $\mathrm{~S}$ & 1493.7819 & 747.3946 & 1476.7554 & 738.8813 & 1475.7714 & 738.3893 & 17 \\
\hline 13 & 1315.6376 & 658.3224 & 1298.6110 & 649.8092 & 1297.6270 & 649.3172 & G & 1406.7499 & 703.8786 & 1389.7233 & 695.3653 & 1388.7393 & 694.8733 & 16 \\
\hline 14 & 1372.6591 & 686.8332 & 1355.6325 & 678.3199 & 1354.6485 & 677.8279 & G & 1349.7284 & 675.3678 & 1332.7019 & 666.8546 & 1331.7179 & 666.3626 & 15 \\
\hline 15 & 1443.6962 & 722.3517 & 1426.6696 & 713.8384 & 1425.6856 & 713.3464 & $\mathbf{A}$ & 1292.7070 & 646.8571 & 1275.6804 & 638.3438 & 1274.6964 & 637.8518 & 14 \\
\hline 16 & \begin{tabular}{|l|}
1556.7802 \\
\end{tabular} & 778.8938 & 1539.7537 & 770.3805 & 1538.7697 & 769.8885 & I & \begin{tabular}{|l}
1221.6698 \\
\end{tabular} & 611.3386 & 1204.6433 & 602.8253 & 1203.6593 & 602.3333 & 13 \\
\hline 17 & 1627.8174 & 814.4123 & 1610.7908 & 805.8990 & 1609.8068 & 805.4070 & A & 1108.5858 & 554.7965 & 1091.5592 & 546.2833 & 1090.5752 & 545.7912 & 12 \\
\hline 18 & 1740.9014 & 870.9543 & 1723.8749 & 862.4411 & 1722.8908 & 861.9491 & $\mathbf{L}$ & 1037.5487 & 519.2780 & 1020.5221 & 510.7647 & 1019.5381 & 510.2727 & 11 \\
\hline
\end{tabular}




\begin{tabular}{|l|r|r|r|r|r|r|r|r|r|r|r|r|r|r|r|}
$\mathbf{1 9}$ & $\mathbf{1 7 9 7 . 9 2 2 9}$ & $\mathbf{8 9 9 . 4 6 5 1}$ & $\mathbf{1 7 8 0 . 8 9 6 3}$ & 890.9518 & $\mathbf{1 7 7 9 . 9 1 2 3}$ & $\mathbf{8 9 0 . 4 5 9 8}$ & $\mathbf{G}$ & $\mathbf{9 2 4 . 4 6 4 6}$ & $\mathbf{4 6 2 . 7 3 5 9}$ & $\mathbf{9 0 7 . 4 3 8 1}$ & $\mathbf{4 5 4 . 2 2 2 7}$ & $\mathbf{9 0 6 . 4 5 4 0}$ & $\mathbf{4 5 3 . 7 3 0 7}$ & $\mathbf{1 0}$ \\
\hline $\mathbf{2 0}$ & 1934.9818 & $\mathbf{9 6 7 . 9 9 4 5}$ & 1917.9552 & 959.4813 & 1916.9712 & 958.9893 & H & $\mathbf{8 6 7 . 4 4 3 1}$ & $\mathbf{4 3 4 . 2 2 5 2}$ & 850.4166 & $\mathbf{4 2 5 . 7 1 1 9}$ & $\mathbf{8 4 9 . 4 3 2 6}$ & $\mathbf{4 2 5 . 2 1 9 9}$ & $\mathbf{9}$ \\
\hline $\mathbf{2 1}$ & 2032.0346 & 1016.5209 & 2015.0080 & 1008.0076 & 2014.0240 & 1007.5156 & $\mathbf{P}$ & $\mathbf{7 3 0 . 3 8 4 2}$ & $\mathbf{3 6 5 . 6 9 5 8}$ & 713.3577 & 357.1825 & 712.3737 & 356.6905 & $\mathbf{8}$ \\
\hline $\mathbf{2 2}$ & 2145.1186 & $\mathbf{1 0 7 3 . 0 6 2 9}$ & 2128.0921 & 1064.5497 & 2127.1081 & 1064.0577 & L & $\mathbf{6 3 3 . 3 3 1 5}$ & 317.1694 & 616.3049 & 308.6561 & 615.3209 & 308.1641 & $\mathbf{7}$ \\
\hline $\mathbf{2 3}$ & 2202.1401 & 1101.5737 & 2185.1135 & 1093.0604 & 2184.1295 & 1092.5684 & G & $\mathbf{5 2 0 . 2 4 7 4}$ & 260.6273 & 503.2209 & 252.1141 & 502.2368 & 251.6221 & $\mathbf{6}$ \\
\hline $\mathbf{2 4}$ & 2259.1615 & 1130.0844 & 2242.1350 & 1121.5711 & 2241.1510 & 1121.0791 & G & $\mathbf{4 6 3 . 2 2 5 9}$ & 232.1166 & 446.1994 & 223.6033 & 445.2154 & 223.1113 & $\mathbf{5}$ \\
\hline $\mathbf{2 5}$ & 2346.1936 & 1173.6004 & 2329.1670 & 1165.0872 & 2328.1830 & 1164.5951 & S & 406.2045 & 203.6059 & 389.1779 & 195.0926 & 388.1939 & 194.6006 & $\mathbf{4}$ \\
\hline $\mathbf{2 6}$ & 2403.2150 & 1202.1112 & 2386.1885 & 1193.5979 & 2385.2045 & 1193.1059 & G & 319.1724 & 160.0899 & 302.1459 & 151.5766 & 301.1619 & 151.0846 & $\mathbf{3}$ \\
\hline $\mathbf{2 7}$ & 2490.2471 & 1245.6272 & 2473.2205 & 1237.1139 & 2472.2365 & 1236.6219 & S & 262.1510 & 131.5791 & 245.1244 & 123.0659 & 244.1404 & 122.5738 & $\mathbf{2}$ \\
\hline $\mathbf{2 8}$ & & & & & & & R & 175.1190 & 88.0631 & 158.0924 & 79.5498 & & & & $\mathbf{1}$ \\
\hline
\end{tabular}
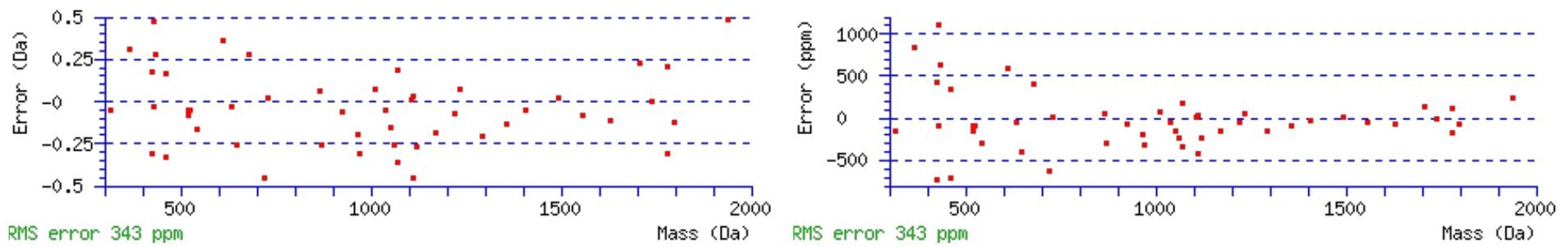

\section{All matches to this query}

\begin{tabular}{|l|c|c|l|}
\hline Score & Mr(calc): & Delta & \multicolumn{1}{|c|}{ Sequence } \\
\hline 53.6 & 2663.3515 & 0.0119 & SLDLDPSKTNVSGGAIALGHPLGGSGSR \\
\hline 38.2 & 2662.3675 & 0.9959 & SLDLDPSKTNVSGGAIALGHPLGGSGSR \\
\hline 1.6 & 2663.3766 & -0.0133 & LTVSKTTSSSITLSWLPGDNGGSSIR \\
\hline 1.0 & 2661.3316 & 2.0317 & FKASLTQNFSILPNQDKSIFQR \\
\hline 0.9 & 2661.3316 & 2.0317 & FKASLTQNFSILPNQDKSIFQR \\
\hline 0.8 & 2663.3377 & 0.0257 & TGIPVKAEPVPKPPPETAVPPGTPK \\
\hline 0.8 & 2662.3344 & 1.0289 & KALECYFLLCFTFIFLKIEK \\
\hline 0.7 & 2661.3796 & 1.9837 & TMSRPVQGIPTYVLLNTSGISSPAR \\
\hline
\end{tabular}

Spectrum No: 629; Query: 2553; Rank: 1

\section{Peptide View}

MS/MS Fragmentation of TVTLGGVFQLGNWSELNSVHDHNTIWK

Found in IPI00326225, Tax_Id=10116 Gene_Symbol=Dao1 D-amino-acid oxidase

Match to Query 2553: 3052.514532 from(1018.512120,3+)

Title: 091008RatKidney_NoSalt_04.5068.5068.3.dta

Data file K:INewmanPaper|Piliang|3SubProteomes\Piliang3SP\mgf5ppm\ERLIC_3SubProteomes5ppm.mgf 

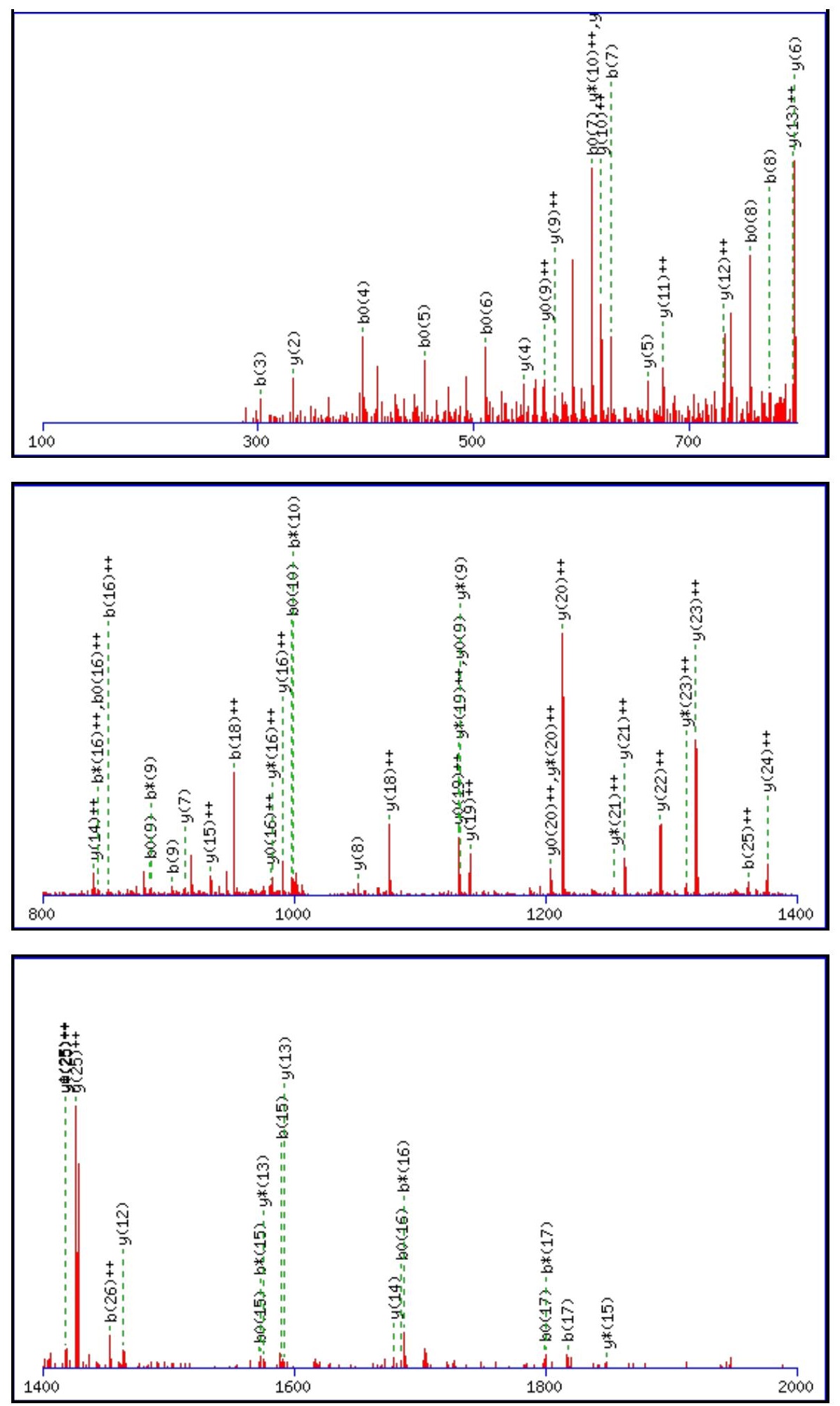

Monoisotopic mass of neutral peptide $\operatorname{Mr}($ calc): 3052.5043

Fixed modifications: Carbamidomethyl (C)

Variable modifications:

N12 : Deamidated_N (N)

Ions Score: 53 Expect: 0.00099

Matches (Bold Red): 69/290 fragment ions using 175 most intense peaks

\begin{tabular}{|c|c|c|c|c|c|c|c|c|c|c|c|c|c|c|}
\hline \# & b & $\mathbf{b}^{++}$ & $\mathbf{b}^{*}$ & $\mathbf{b}^{*^{++}}$ & $\mathbf{b}^{\mathbf{0}}$ & $\mathbf{b}^{\mathbf{0 + +}}$ & Seq. & $\mathbf{y}$ & $y^{++}$ & $\mathbf{y}^{*}$ & $\mathrm{y}^{*^{++}}$ & $\mathbf{y}^{\mathbf{0}}$ & $y^{0++}$ & \# \\
\hline 1 & 102.0550 & 51.5311 & & & 84.0444 & 42.5258 & $\mathbf{T}$ & & & & & & & 27 \\
\hline 2 & 201.1234 & 101.0653 & & & 183.1128 & 92.0600 & V & 2952.4639 & 1476.7356 & 2935.4373 & 1468.2223 & 2934.4533 & 1467.7303 & 26 \\
\hline 3 & 302.1710 & 151.5892 & & & 284.1605 & 142.5839 & $\mathbf{T}$ & 2853.3955 & 1427.2014 & 2836.3689 & 1418.6881 & 2835.3849 & 1418.1961 & 25 \\
\hline 4 & 415.2551 & 208.1312 & & & 397.2445 & 199.1259 & $\mathbf{L}$ & 2752.3478 & 1376.6775 & 2735.3212 & 1368.1643 & 2734.3372 & 1367.6722 & 24 \\
\hline 5 & 472.2766 & 236.6419 & & & 454.2660 & 227.6366 & G & 2639.2637 & 1320.1355 & 2622.2372 & 1311.6222 & 2621.2532 & 1311.1302 & 23 \\
\hline
\end{tabular}




\begin{tabular}{|c|c|c|c|c|c|c|c|c|c|c|c|c|c|c|}
\hline 6 & 529.2980 & 265.1527 & & & 511.2875 & 256.1474 & G & 2582.2423 & 1291.6248 & |2565.2157| & 1283.1115 & |2564.2317| & 1282.6195 & 22 \\
\hline 7 & 628.3665 & 314.6869 & & & 610.3559 & 305.6816 & V & 2525.2208 & 1263.1140 & 2508.1942 & 1254.6008 & 2507.2102 & 1254.1088 & 21 \\
\hline 8 & 775.4349 & 388.2211 & & & 757.4243 & 379.2158 & F & 2426.1524 & 1213.5798 & 2409.1258 & 1205.0666 & 2408.1418 & 1204.5745 & 20 \\
\hline 9 & 903.4934 & 452.2504 & 886.4669 & 443.7371 & 885.4829 & 443.2451 & Q & 2279.0840 & 1140.0456 & 2262.0574 & 1131.5323 & 2261.0734 & 1131.0403 & 9 \\
\hline 10 & 1016.5775 & 508.7924 & 999.5510 & 500.2791 & 998.5669 & 499.7871 & L & 2151.0254 & 1076.0163 & |2133.9988 & 1067.5031 & 2133.0148 & 1067.0110 & 18 \\
\hline 11 & 1073.5990 & 537.3031 & 1056.5724 & 528.7899 & 1055.5884 & 528.2978 & G & 2037.9413 & 1019.4743 & 2020.9148 & 1010.9610 & 2019.9308 & 1010.4690 & 17 \\
\hline 12 & 1188.6259 & 594.8166 & 1171.5994 & 586.3033 & 1170.6153 & 585.8113 & $\mathbf{N}$ & 1980.9199 & 990.9636 & 1963.8933 & 982.4503 & 1962.9093 & 981.9583 & 16 \\
\hline 13 & 1374.7052 & 687.8562 & 1357.6787 & 679.3430 & 1356.6947 & 678.8510 & W & 1865.8929 & 933.4501 & 1848.8664 & 924.9368 & 1847.8824 & 924.4448 & 15 \\
\hline 14 & 1461.7373 & 731.3723 & 1444.7107 & 722.8590 & 1443.7267 & 722.3670 & $S$ & \begin{tabular}{|l|l|}
1679.8136 \\
\end{tabular} & 840.4104 & 1662.7871 & 831.8972 & 1661.8030 & 831.4052 & 14 \\
\hline 15 & 1590.7798 & 795.8936 & 1573.7533 & 787.3803 & 1572.7693 & 786.8883 & $\mathbf{E}$ & 1592.7816 & 796.8944 & 1575.7550 & 788.3812 & 1574.7710 & 787.8891 & 13 \\
\hline 16 & 1703.8639 & 852.4356 & 1686.8374 & 843.9223 & 1685.8533 & 843.4303 & $\mathbf{L}$ & 1463.7390 & 732.3731 & 1446.7124 & 723.8599 & 1445.7284 & 723.3678 & 12 \\
\hline 17 & \begin{tabular}{|l|}
1817.9068 \\
\end{tabular} & 909.4571 & \begin{tabular}{|l|l|}
1800.8803 \\
\end{tabular} & 900.9438 & 1799.8963 & 900.4518 & $\mathbf{N}$ & 1350.6549 & 675.8311 & 1333.6284 & 667.3178 & 1332.6444 & 666.8258 & 11 \\
\hline 18 & 1904.9389 & 952.9731 & 1887.9123 & 944.4598 & 1886.9283 & 943.9678 & S & 1236.6120 & 618.8096 & 1219.5854 & 610.2964 & 1218.6014 & 609.8044 & 10 \\
\hline 19 & 2004.0073 & 1002.5073 & 1986.9807 & 993.9940 & 1985.9967 & 993.5020 & $\mathbf{V}$ & 1149.5800 & 575.2936 & 1132.5534 & 566.7803 & 1131.5694 & 566.2883 & 9 \\
\hline 20 & 2141.0662 & 1071.0367 & 2124.0396 & 1062.5235 & 2123.0556 & 1062.0314 & $\mathbf{H}$ & 1050.5116 & 525.7594 & 1033.4850 & 517.2461 & 1032.5010 & 516.7541 & 8 \\
\hline 21 & 2256.0931 & 1128.5502 & 2239.0666 & 1120.0369 & 2238.0826 & 1119.5449 & D & 913.4526 & 457.2300 & 896.4261 & 448.7167 & 895.4421 & 448.2247 & 7 \\
\hline 22 & 2393.1520 & 1197.0797 & 2376.1255 & 1188.5664 & 2375.1415 & 1188.0744 & H & 798.4257 & 399.7165 & 781.3992 & 391.2032 & 780.4151 & 390.7112 & 6 \\
\hline 23 & 2507.1950 & 1254.1011 & 2490.1684 & 1245.5878 & 2489.1844 & 1245.0958 & $\mathbf{N}$ & 661.3668 & 331.1870 & 644.3402 & 322.6738 & 643.3562 & 322.1817 & 5 \\
\hline 24 & 2608.2426 & 1304.6250 & 2591.2161 & 1296.1117 & 2590.2321 & 1295.6197 & $\mathbf{T}$ & 547.3239 & 274.1656 & 530.2973 & 265.6523 & 529.3133 & 265.1603 & 4 \\
\hline 25 & 2721.3267 & 1361.1670 & 2704.3002 & 1352.6537 & 2703.3161 & 1352.1617 & I & 446.2762 & 223.6417 & 429.2496 & 215.1285 & & & 3 \\
\hline 26 & 2907.4060 & 1454.2067 & 2890.3795 & 1445.6934 & 2889.3955 & 1445.2014 & W & 333.1921 & 167.0997 & 316.1656 & 158.5864 & & & 2 \\
\hline 27 & & & & & & & $\mathbf{K}$ & 147.1128 & 74.0600 & 130.0863 & 65.5468 & & & 1 \\
\hline
\end{tabular}
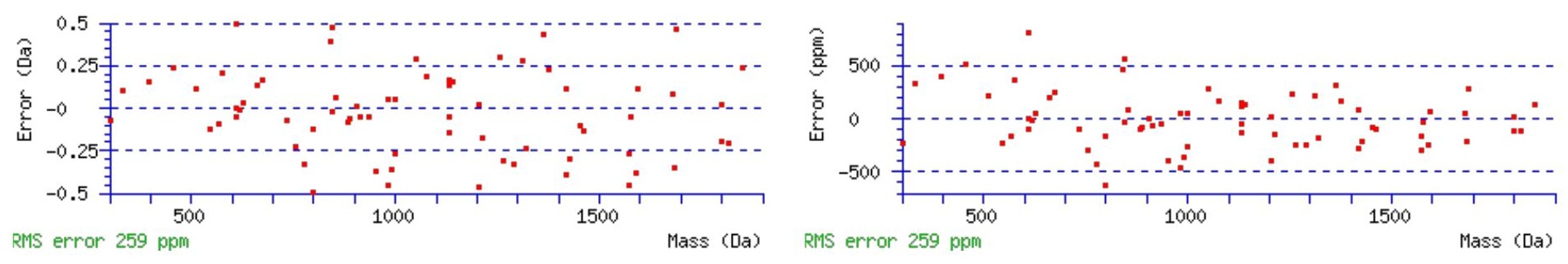

\section{All matches to this query}

\begin{tabular}{|l|c|c|l|}
\hline Score & Mr(calc): & Delta & \multicolumn{1}{|c|}{ Sequence } \\
\hline 53.4 & 3052.5043 & 0.0102 & TVTLGGVFQLGNWSELNSVHDHNTIWK \\
\hline 49.7 & 3052.5043 & 0.0102 & TVTLGGVFQLGNWSELNSVHDHNTIWK \\
\hline 48.5 & 3051.5203 & 0.9942 & TVTLGGVFQLGNWSELNSVHDHNTIWK \\
\hline 40.7 & 3052.5043 & 0.0102 & TVTLGGVFQLGNWSELNSVHDHNTIWK \\
\hline 1.9 & 3052.4910 & 0.0236 & QLEEKINEYSSIAAKNAELEQELMEK \\
\hline 1.1 & 3050.5059 & 2.0086 & ESFFPLTEGNLHTIOSLCPFLSKEEK \\
\hline 0.8 & 3052.5189 & -0.0043 & NSPPGRLAFPAAPPPHSACNLPTPSTELR \\
\hline 0.8 & 3050.5374 & 1.9771 & KTSAASLMYTVLPPMLNPFIYSLRNK \\
\hline 0.8 & 3050.5374 & 1.9771 & KTSAASLMYTVLPPMLNPFIYSLRNK \\
\hline 0.4 & 3050.5300 & 1.9845 & SVWGVLCHTPSFNETSILNKLLRAVK \\
\hline
\end{tabular}

Spectrum No: 630; Query: 39; Rank: 1

\section{Peptide View}

MS/MS Fragmentation of IEENGSMR

Found in IPI00191711, Tax_Id=10116 Gene_Symbol=LOC259246;LOC298116;LOC259245 Major urinary protein precursor 
Match to Query 39: 935.400108 from(468.707330,2+)

Title: 091008RatKidney_NoSalt_16.312.312.2.dta

Data file K:INewmanPaper|Piliang|3SubProteomes\Piliang3SP\mgf5ppm\ERLIC_3SubProteomes5ppm.mgf
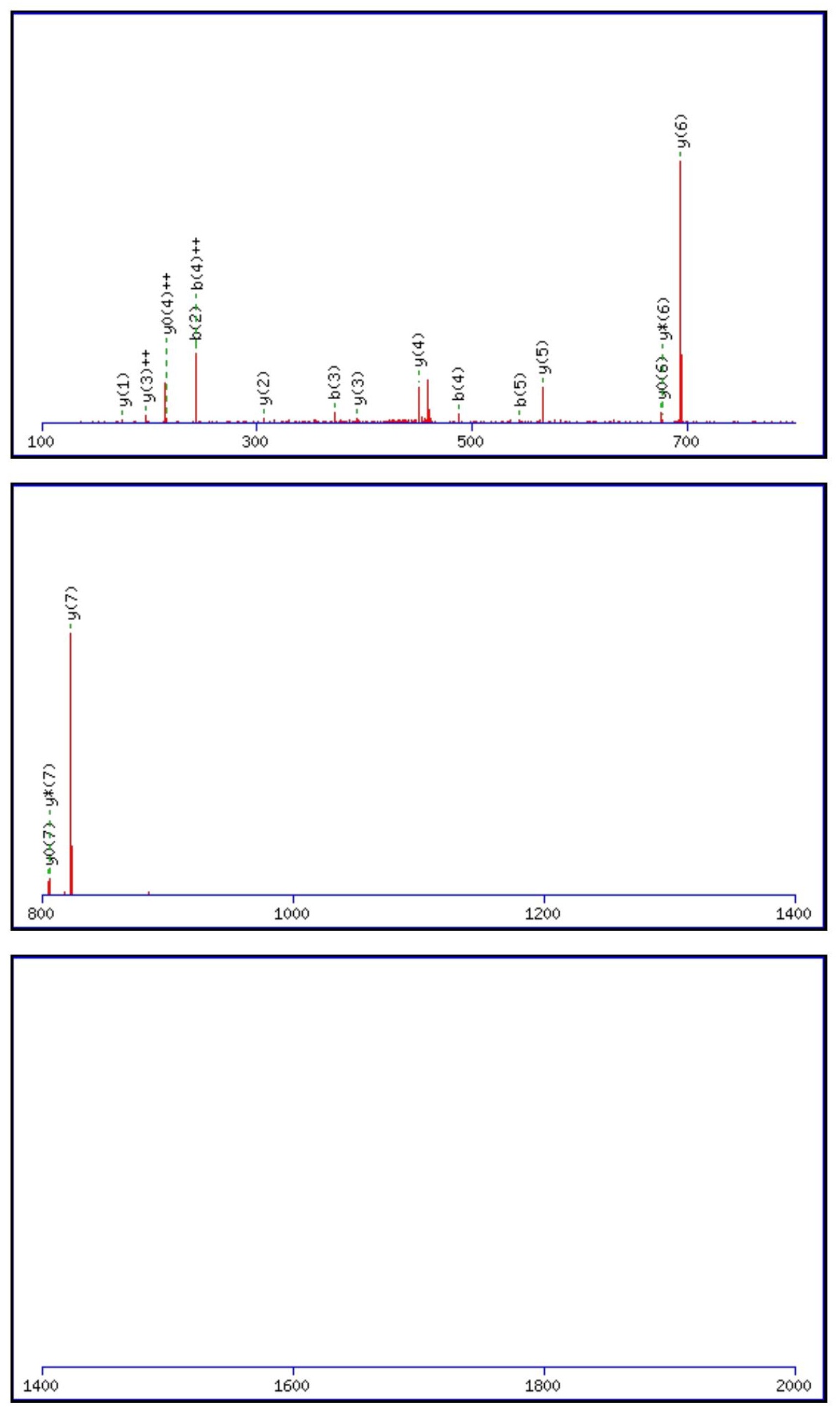

Monoisotopic mass of neutral peptide $\operatorname{Mr}($ calc) : 935.4018

Fixed modifications: Carbamidomethyl (C)

Variable modifications:

N4 : Deamidated_N ( N )

Ions Score: 53 Expect: 0.00028

Matches (Bold Red): 18/72 fragment ions using 33 most intense peaks

\begin{tabular}{|c|c|c|c|c|c|c|c|c|c|c|c|c|c|c|}
\hline$\#$ & $\mathbf{b}$ & $\mathbf{b}^{++}$ & $\mathbf{b}^{*}$ & $\mathbf{b}^{*_{+}^{++}}$ & $\mathbf{b}^{\mathbf{0}}$ & $\mathbf{b}^{\mathbf{0 + +}}$ & Seq. & $\mathbf{y}$ & $\mathbf{y}^{++}$ & $\mathbf{y}^{*}$ & $\mathbf{y}^{\mathbf{*}^{++}}$ & $\mathbf{y}^{\mathbf{0}}$ & $\mathbf{y}^{\mathbf{0}+}$ & $\#$ \\
\hline $\mathbf{1}$ & 114.0913 & 57.5493 & & & & & $\mathbf{I}$ & & & & & & & $\mathbf{8}$ \\
\hline $\mathbf{2}$ & 243.1339 & 122.0706 & & & 225.1234 & 113.0653 & $\mathbf{E}$ & $\mathbf{8 2 3 . 3 2 5 1}$ & 412.1662 & $\mathbf{8 0 6 . 2 9 8 5}$ & 403.6529 & $\mathbf{8 0 5 . 3 1 4 5}$ & 403.1609 & 7 \\
\hline
\end{tabular}




\begin{tabular}{|l|l|l|l|l|l|l|l|l|l|l|l|l|l|l|l|}
$\mathbf{3}$ & 372.1765 & 186.5919 & & & 354.1660 & 177.5866 & $\mathbf{E}$ & $\mathbf{6 9 4 . 2 8 2 5}$ & 347.6449 & $\mathbf{6 7 7 . 2 5 5 9}$ & 339.1316 & $\mathbf{6 7 6 . 2 7 1 9}$ & 338.6396 & $\mathbf{6}$ \\
\hline $\mathbf{4}$ & $\mathbf{4 8 7 . 2 0 3 5}$ & $\mathbf{2 4 4 . 1 0 5 4}$ & 470.1769 & 235.5921 & 469.1929 & 235.1001 & $\mathbf{N}$ & $\mathbf{5 6 5 . 2 3 9 9}$ & 283.1236 & 548.2133 & 274.6103 & 547.2293 & 274.1183 & $\mathbf{5}$ \\
\hline $\mathbf{5}$ & 544.2249 & 272.6161 & 527.1984 & 264.1028 & 526.2144 & 263.6108 & $\mathbf{G}$ & $\mathbf{4 5 0 . 2 1 2 9}$ & 225.6101 & 433.1864 & 217.0968 & 432.2024 & $\mathbf{2 1 6 . 6 0 4 8}$ & $\mathbf{4}$ \\
\hline $\mathbf{6}$ & 631.2570 & 316.1321 & 614.2304 & 307.6188 & 613.2464 & 307.1268 & $\mathbf{S}$ & $\mathbf{3 9 3 . 1 9 1 5}$ & $\mathbf{1 9 7 . 0 9 9 4}$ & 376.1649 & 188.5861 & 375.1809 & 188.0941 & $\mathbf{3}$ \\
\hline $\mathbf{7}$ & 762.2974 & 381.6524 & 745.2709 & 373.1391 & 744.2869 & 372.6471 & $\mathbf{M}$ & $\mathbf{3 0 6 . 1 5 9 4}$ & 153.5834 & 289.1329 & 145.0701 & & & $\mathbf{2}$ \\
\hline $\mathbf{8}$ & & & & & & & $\mathbf{R}$ & $\mathbf{1 7 5 . 1 1 9 0}$ & 88.0631 & 158.0924 & 79.5498 & & & $\mathbf{1}$ \\
\hline
\end{tabular}
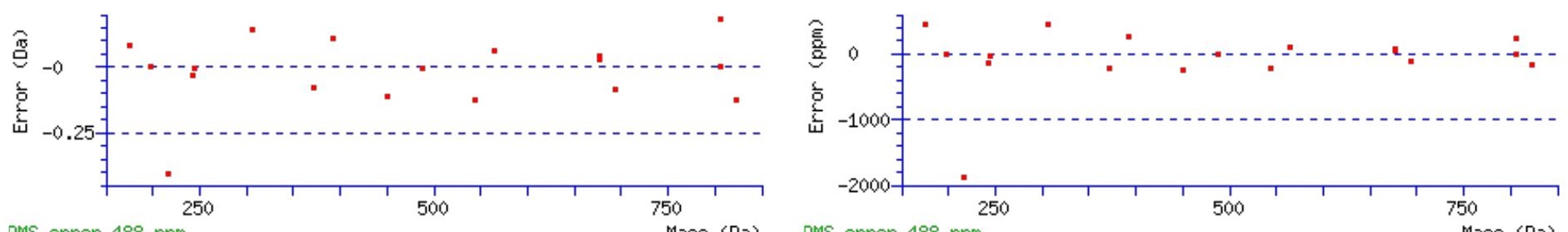

RMS error 488 ppm

\section{All matches to this query}

\begin{tabular}{|l|l|l|l|}
\hline Score & Mr(calc): & Delta & \multicolumn{1}{|c|}{ Sequence } \\
\hline 53.3 & 935.4018 & -0.0017 & $\underline{\text { IEENGSMR }}$ \\
\hline 22.2 & 935.4018 & -0.0017 & $\underline{\text { LEQDMER }}$ \\
\hline 6.7 & 935.4048 & -0.0047 & MLTHQAR \\
\hline 5.5 & 934.3950 & 1.0052 & QIEYFR \\
\hline 5.5 & 934.3983 & 1.0018 & LKEYMR \\
\hline 3.7 & 935.4018 & -0.0017 & LNMDEAAR \\
\hline 3.5 & 935.3920 & 0.0082 & QDWGMGAR \\
\hline 2.7 & 933.3974 & 2.0027 & ELDNQCR \\
\hline 2.1 & 933.3974 & 2.0027 & QIDQCNR \\
\hline 2.0 & 935.4084 & -0.0082 & ELDESDTK \\
\hline
\end{tabular}

Spectrum No: 631; Query: 101; Rank: 1

\section{Peptide View}

MS/MS Fragmentation of GNLTLEGVHR

Found in IPI00192310, Tax_Id=10116 Gene_Symbol=Bcam Lutheran antigen

Match to Query 101: 1095.568488 from(548.791520,2+)

Title: 100101RatKid_NS_deglyco_19.1278.1278.2.dta

Data file K:INewmanPaper|Piliangl3SubProteomes\Piliang3SP\mgf5ppm\ERLIC_3SubProteomes5ppm.mgf

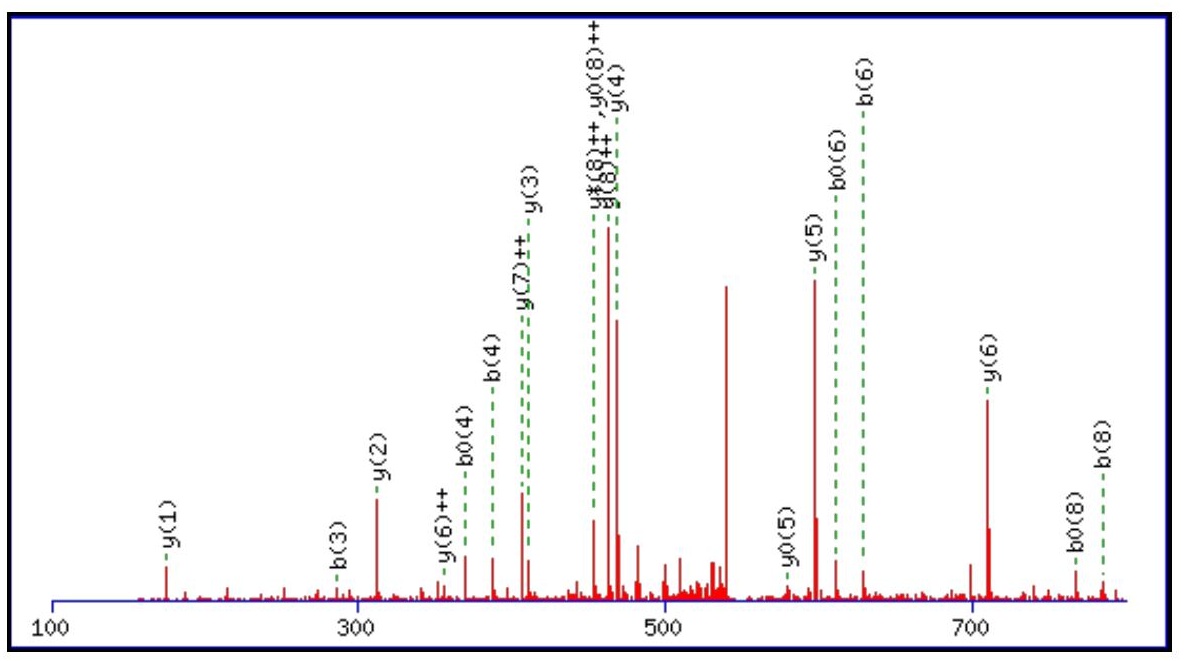




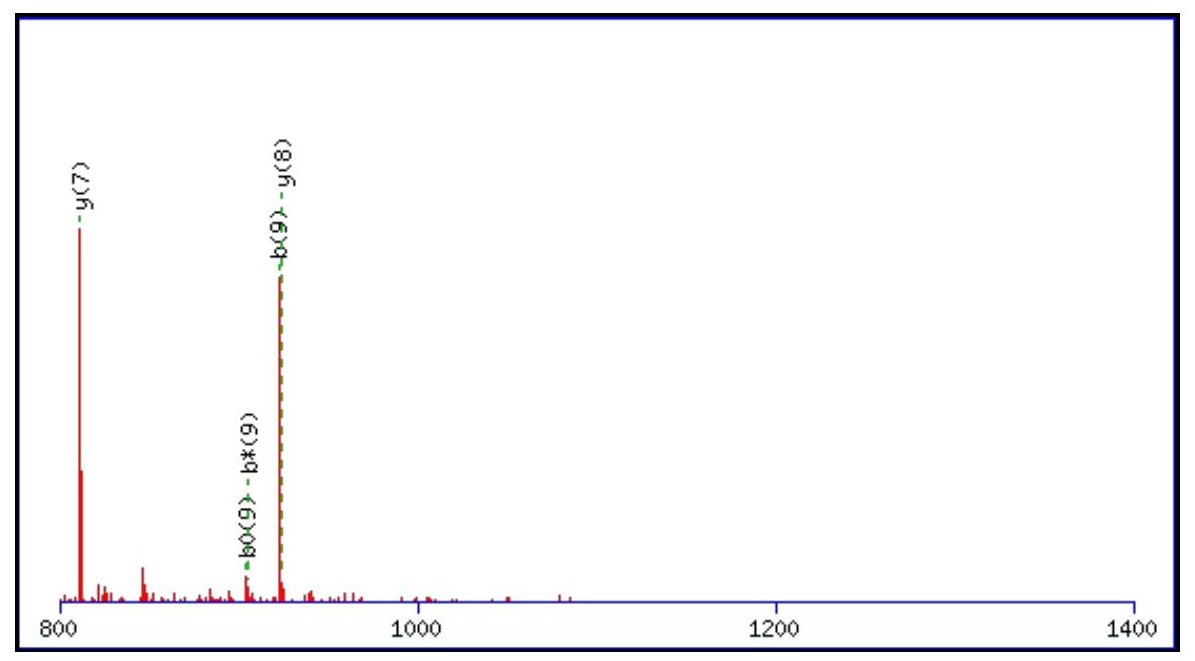

\begin{tabular}{|c|c|c|c|}
\hline 1400 & 1600 & 1800 & 2000 \\
\hline
\end{tabular}

Monoisotopic mass of neutral peptide $\operatorname{Mr}($ calc): 1095.5673

Fixed modifications: Carbamidomethyl (C)

Variable modifications:

N2 : Deamidated_N (N)

Ions Score: 53 Expect: 0.00053

Matches (Bold Red): 24/92 fragment ions using 50 most intense peaks

\begin{tabular}{|c|c|c|c|c|c|c|c|c|c|c|c|c|c|c|}
\hline$\#$ & b & $\mathbf{b}^{++}$ & b* & $\mathbf{b}^{*^{++}}$ & $\mathbf{b}^{\mathbf{0}}$ & $\mathbf{b}^{0++}$ & Seq. & $\mathbf{y}$ & $y^{++}$ & $\mathbf{y}^{*}$ & $\mathbf{y}^{*^{++}}$ & $\mathbf{y}^{0}$ & $\mathbf{y}^{0++}$ & \# \\
\hline 1 & 58.0287 & 29.5180 & & & & & G & & & & & & & 10 \\
\hline 2 & 173.0557 & 87.0315 & 156.0291 & 78.5182 & & & $\mathbf{N}$ & 1039.5531 & 520.2802 & 1022.5265 & 511.7669 & 1021.5425 & 511.2749 & 9 \\
\hline 3 & 286.1397 & 143.5735 & 269.1132 & 135.0602 & & & $\mathbf{L}$ & 924.5261 & 462.7667 & 907.4996 & 454.2534 & 906.5156 & 453.7614 & 8 \\
\hline 4 & 387.1874 & 194.0973 & 370.1609 & 185.5841 & 369.1769 & 185.0921 & $\mathbf{T}$ & 811.4421 & 406.2247 & 794.4155 & 397.7114 & 793.4315 & 397.2194 & 7 \\
\hline 5 & 500.2715 & 250.6394 & 483.2449 & 242.1261 & 482.2609 & 241.6341 & $\mathbf{L}$ & 710.3944 & 355.7008 & 693.3678 & 347.1876 & 692.3838 & 346.6956 & 6 \\
\hline 6 & 629.3141 & 315.1607 & 612.2875 & 306.6474 & 611.3035 & 306.1554 & $\mathbf{E}$ & 597.3103 & 299.1588 & 580.2838 & 290.6455 & 579.2998 & 290.1535 & 5 \\
\hline 7 & 686.3355 & 343.6714 & 669.3090 & 335.1581 & 668.3250 & 334.6661 & $\mathbf{G}$ & 468.2677 & 234.6375 & 451.2412 & 226.1242 & & & 4 \\
\hline 8 & 785.4040 & 393.2056 & \begin{tabular}{|l|l|}
768.3774 \\
\end{tabular} & 384.6923 & 767.3934 & 384.2003 & $\mathbf{V}$ & 411.2463 & 206.1268 & 394.2197 & 197.6135 & & & 3 \\
\hline 9 & 922.4629 & 461.7351 & 905.4363 & 453.2218 & 904.4523 & 452.7298 & $\mathbf{H}$ & 312.1779 & 156.5926 & 295.1513 & 148.0793 & & & 2 \\
\hline 10 & & & & & & & $\mathbf{R}$ & 175.1190 & 88.0631 & 158.0924 & 79.5498 & & & 1 \\
\hline
\end{tabular}

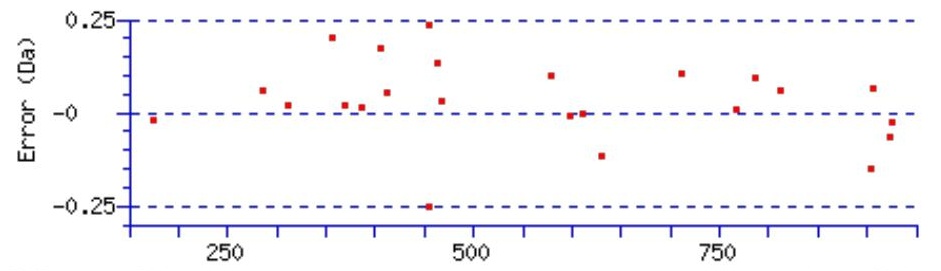

RNS error 241 ppm

Mass (Da) RMS error 241 ppm

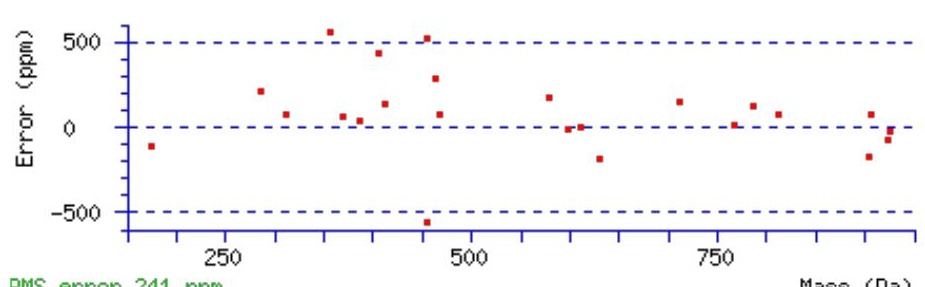




\section{All matches to this query}

\begin{tabular}{|l|l|l|l|}
\hline Score & Mr(calc): & Delta & \multicolumn{1}{c|}{ Sequence } \\
\hline 53.3 & 1095.5673 & 0.0012 & GNLTLEGVHR \\
\hline 15.2 & 1093.5628 & 2.0057 & ATVEPERHR \\
\hline 12.0 & 1095.5686 & -0.0001 & WSNLQRHR \\
\hline 11.1 & 1095.5673 & 0.0012 & AEEGSHVVLR \\
\hline 11.0 & 1094.5720 & 0.9965 & $\underline{\text { NGTLIFTSSR }}$ \\
\hline 9.8 & 1095.5689 & -0.0004 & $\underline{\text { IVSSIQTLR }}$ \\
\hline 9.1 & 1095.5736 & -0.0051 & $\underline{\text { RRMSVIVR }}$ \\
\hline 9.1 & 1095.5785 & -0.0100 & $\underline{\text { SVEEALRHR }}$ \\
\hline 8.4 & 1095.5590 & 0.0095 & SWILRSVR \\
\hline 7.3 & 1093.5533 & 2.0152 & $\underline{\text { GLITENVIR }}$ \\
\hline
\end{tabular}

Spectrum No: 632; Query: 1163; Rank: 1

\section{Peptide View}

MS/MS Fragmentation of LDLPVNTSIPNVTEIK

Found in IPI00324585, Tax_Id=10116 Gene_Symbol=Itga1 Integrin alpha-1 precursor

Match to Query 1163: 1753.940788 from(877.977670,2+)

Title: 100101RatKid_NS_deglyco_25.4120.4120.2.dta

Data file K:INewmanPaper|Piliang|3SubProteomes\Piliang3SP\mgf5ppm\ERLIC_3SubProteomes5ppm.mgf
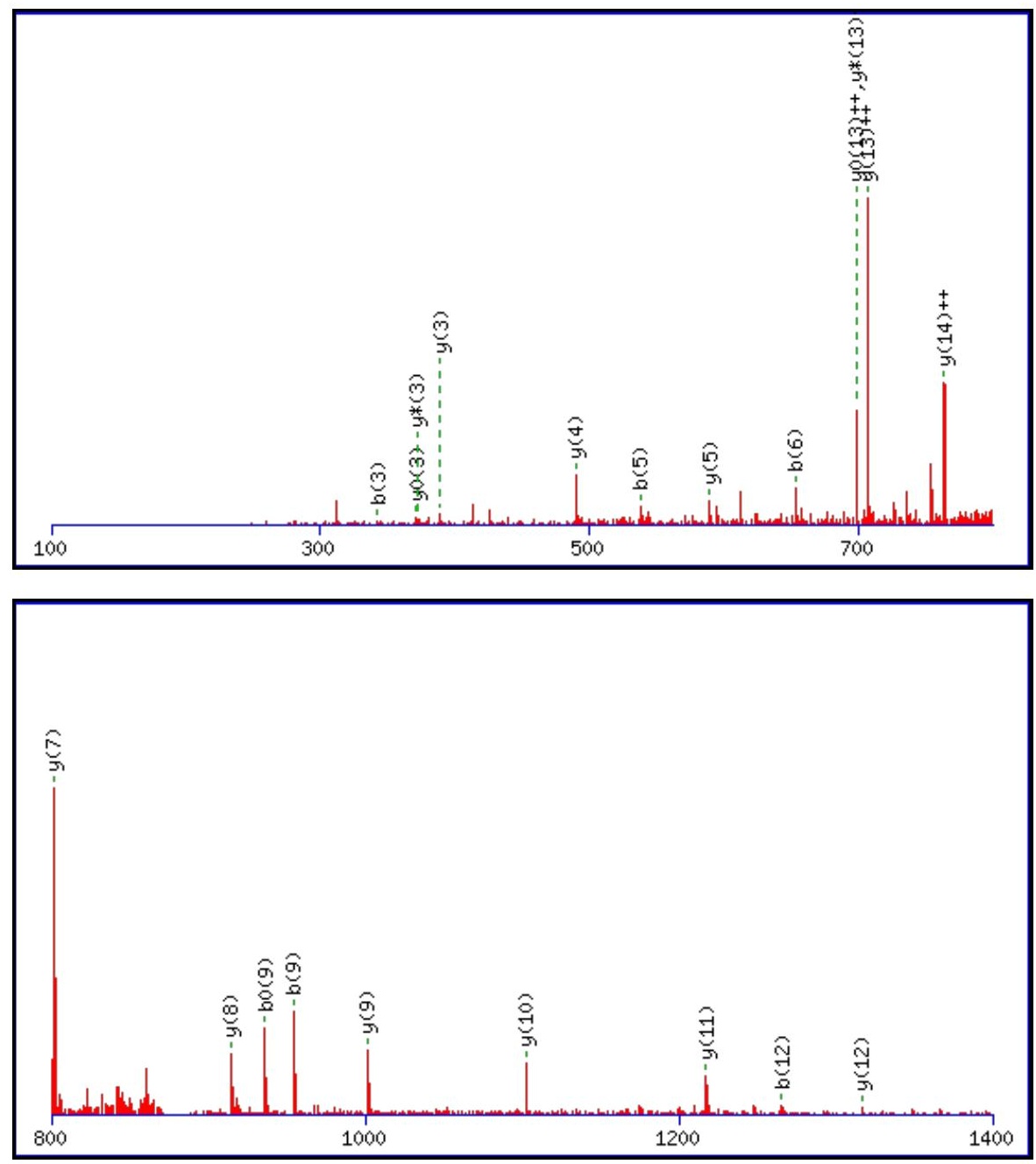


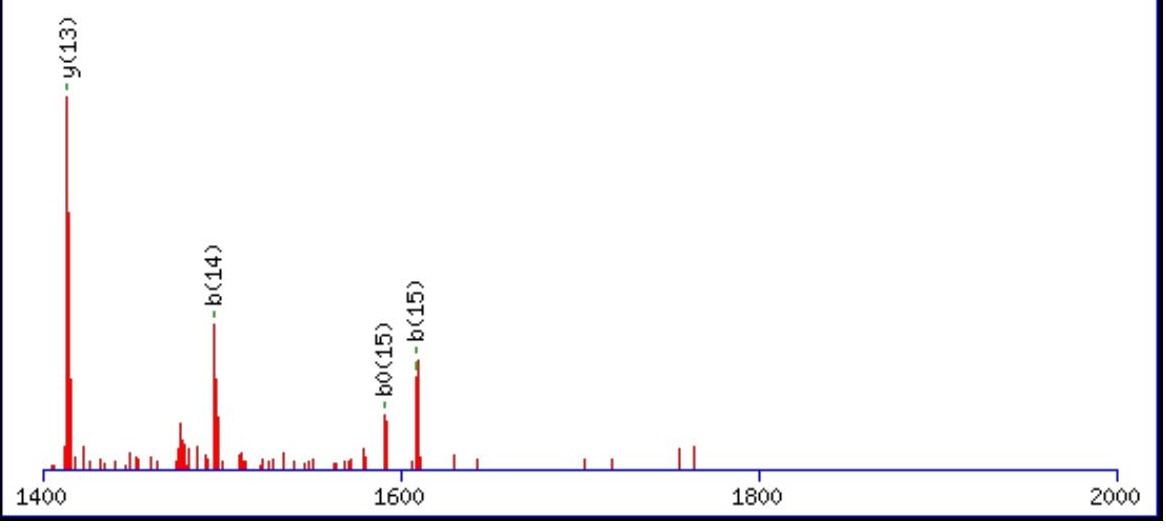

Monoisotopic mass of neutral peptide $\operatorname{Mr}($ calc): 1753.9349

Fixed modifications: Carbamidomethyl (C)

Variable modifications:

N6 : Deamidated_N (N)

N11 : Deamidated_N (N)

Ions Score: 53 Expect: 0.00042

Matches (Bold Red): 25/164 fragment ions using 55 most intense peaks

\begin{tabular}{|c|c|c|c|c|c|c|c|c|c|c|c|c|c|c|}
\hline \# & b & $\mathbf{b}^{++}$ & b* & $\mathbf{b}^{*^{++}}$ & $\mathbf{b}^{0}$ & $\mathbf{b}^{0++}$ & Seq. & $\mathbf{y}$ & $\mathbf{y}^{++}$ & $\mathbf{y}^{*}$ & $\mathbf{y}^{*^{++}}$ & $\mathbf{y}^{\mathbf{0}}$ & $\mathbf{y}^{0++}$ & \# \\
\hline 1 & 114.0913 & 57.5493 & & & & & $\mathbf{L}$ & & & & & & & 16 \\
\hline 2 & 229.1183 & 115.0628 & & & 211.1077 & 106.0575 & D & 1641.8581 & 821.4327 & 1624.8316 & 812.9194 & 1623.8476 & 812.4274 & 15 \\
\hline 3 & 342.2023 & 171.6048 & & & 324.1918 & 162.5995 & $\mathbf{L}$ & 1526.8312 & 763.9192 & 1509.8047 & 755.4060 & 1508.8206 & 754.9140 & 14 \\
\hline 4 & 439.2551 & 220.1312 & & & 421.2445 & 211.1259 & $\mathbf{P}$ & 1413.7471 & 707.3772 & 1396.7206 & 698.8639 & 1395.7366 & 698.3719 & 13 \\
\hline 5 & 538.3235 & 269.6654 & & & 520.3130 & 260.6601 & V & 1316.6944 & 658.8508 & 1299.6678 & 650.3376 & 1298.6838 & 649.8455 & 12 \\
\hline 6 & 653.3505 & 327.1789 & 636.3239 & 318.6656 & 635.3399 & 318.1736 & $\mathbf{N}$ & 1217.6260 & 609.3166 & 1200.5994 & 600.8033 & 1199.6154 & 600.3113 & 11 \\
\hline 7 & 754.3981 & 377.7027 & 737.3716 & 369.1894 & 736.3876 & 368.6974 & $\mathbf{T}$ & 1102.5990 & 551.8032 & 1085.5725 & 543.2899 & 1084.5885 & 542.7979 & 10 \\
\hline 8 & 841.4302 & 421.2187 & 824.4036 & 412.7054 & 823.4196 & 412.2134 & $S$ & 1001.5513 & 501.2793 & 984.5248 & 492.7660 & 983.5408 & 492.2740 & 9 \\
\hline 9 & 954.5142 & 477.7608 & 937.4877 & 469.2475 & 936.5037 & 468.7555 & I & 914.5193 & 457.7633 & 897.4928 & 449.2500 & 896.5088 & 448.7580 & 8 \\
\hline 10 & 1051.5670 & 526.2871 & 1034.5404 & 517.7739 & 1033.5564 & 517.2819 & $\mathbf{P}$ & 801.4353 & 401.2213 & 784.4087 & 392.7080 & 783.4247 & 392.2160 & 7 \\
\hline 11 & 1166.5939 & 583.8006 & 1149.5674 & 575.2873 & 1148.5834 & 574.7953 & $\mathbf{N}$ & 704.3825 & 352.6949 & 687.3559 & 344.1816 & 686.3719 & 343.6896 & 6 \\
\hline 12 & 1265.6623 & 633.3348 & 1248.6358 & 624.8215 & 1247.6518 & 624.3295 & $\mathbf{V}$ & 589.3556 & 295.1814 & 572.3290 & 286.6681 & 571.3450 & 286.1761 & 5 \\
\hline 13 & 1366.7100 & 683.8587 & 1349.6835 & 675.3454 & 1348.6995 & 674.8534 & $\mathbf{T}$ & 490.2871 & 245.6472 & 473.2606 & 237.1339 & 472.2766 & 236.6419 & 4 \\
\hline 14 & 1495.7526 & 748.3799 & 1478.7261 & 739.8667 & 1477.7421 & 739.3747 & $\mathbf{E}$ & 389.2395 & 195.1234 & 372.2129 & 186.6101 & 371.2289 & 186.1181 & 3 \\
\hline 15 & 1608.8367 & 804.9220 & 1591.8101 & 796.4087 & 1590.8261 & 795.9167 & I & 260.1969 & 130.6021 & 243.1703 & 122.0888 & & & 2 \\
\hline 16 & & & & & & & $\mathbf{K}$ & 147.1128 & 74.0600 & 130.0863 & 65.5468 & & & 1 \\
\hline
\end{tabular}

$$
\text { RN }
$$
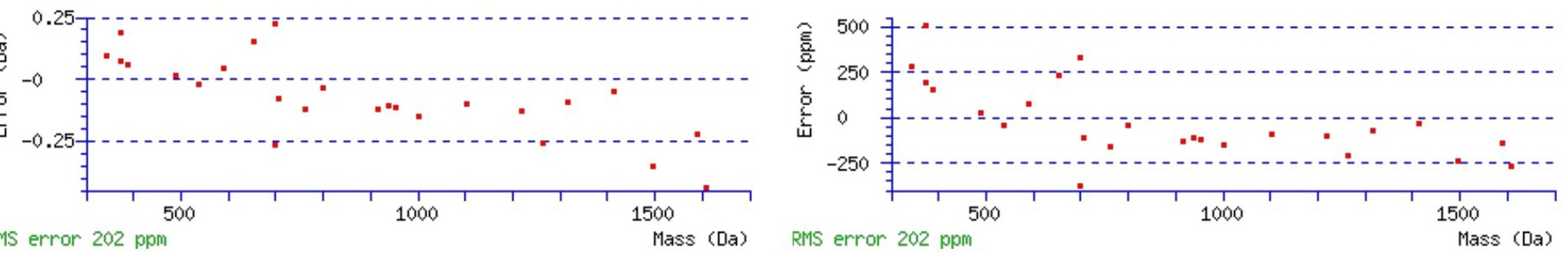

All matches to this query

\begin{tabular}{|l|l|l|l|}
\hline Score & Mr(calc): & Delta & \multicolumn{1}{c|}{ Sequence } \\
\hline 53.2 & 1753.9349 & 0.0059 & LDLPVNTSIPNVTEIK \\
\hline 13.3 & 1752.9509 & 0.9899 & LDLPVNTSIPNVTEIK \\
\hline 12.7 & 1752.9509 & 0.9899 & LDLPVNTSIPNVTEIK \\
\hline 2.3 & 1753.9574 & -0.0166 & DLLRAQGLGDIIDNLK \\
\hline 1.9 & 1753.9535 & -0.0127 & $\underline{\text { IDLETMSYIKTISLK }}$ \\
\hline
\end{tabular}




\section{\begin{tabular}{|l|l|l|l|}
\hline 0.2 & 1753.9322 & 0.0086 & QPVLSQTEARELVER \\
\hline
\end{tabular}}

Spectrum No: 633; Query: 303; Rank: 1

\section{Peptide View}

MS/MS Fragmentation of VNNSLHSQISR

Found in IPI00363849, Tax_Id=10116 Gene_Symbol=Lamc1 similar to Laminin gamma-1 chain precursor

Match to Query 303: 1254.630388 from(628.322470,2+)

Title: 091008RatKidney_NH4Format01_23.426.426.2.dta

Data file K:\NewmanPaper\Piliang \3SubProteomes $\backslash$ Piliang3SP $\backslash$ mgf5ppm\ERLIC_3SubProteomes5ppm.mgf
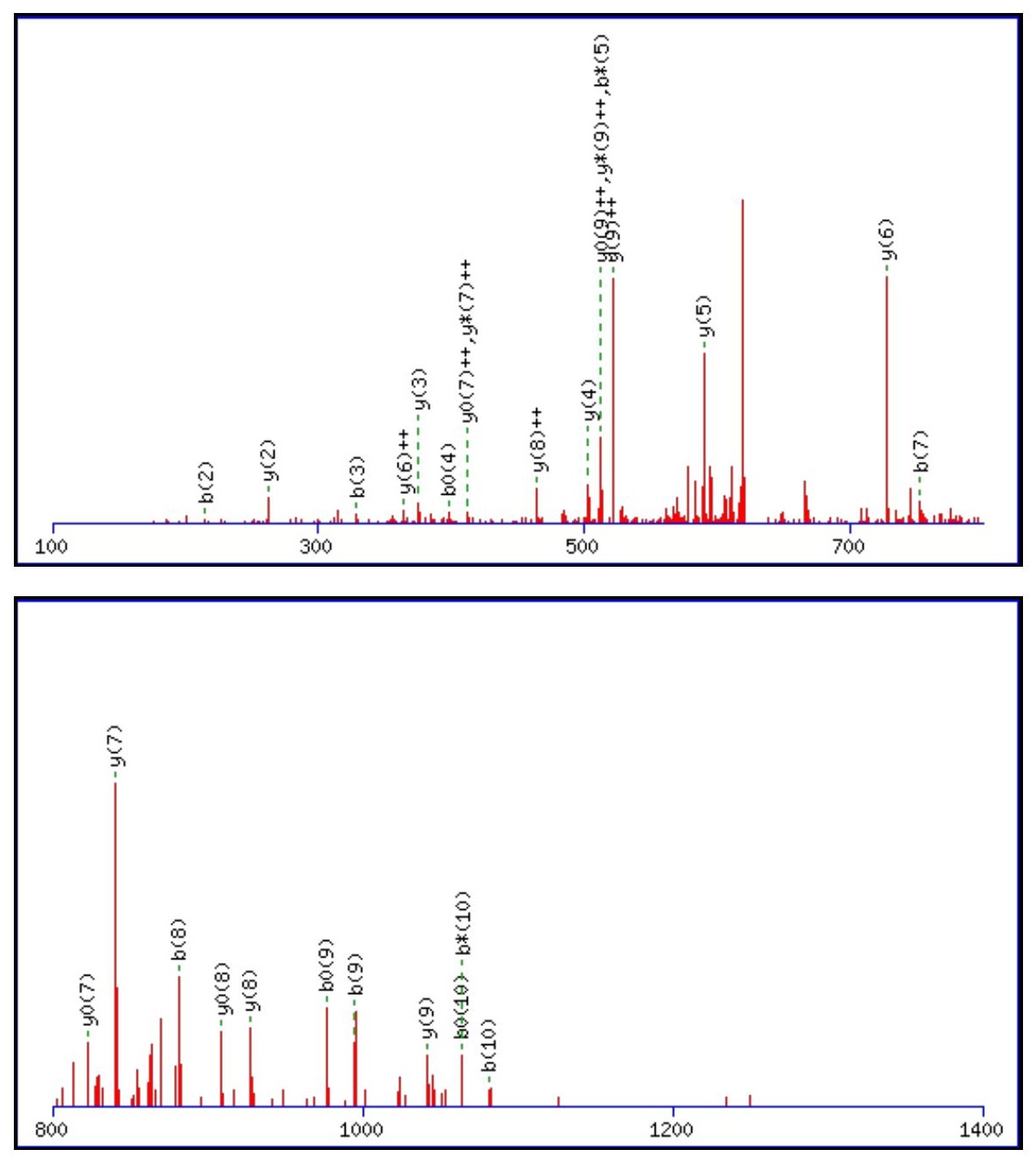


$1400 \quad 1600 \quad 1800 \quad 2000$

Monoisotopic mass of neutral peptide $\operatorname{Mr}($ calc): 1254.6316

Fixed modifications: Carbamidomethyl (C)

Variable modifications:

N2 : Deamidated $\mathrm{N}(\mathrm{N})$

Ions Score: 53 Expect: 0.00062

Matches (Bold Red): 28/110 fragment ions using 48 most intense peaks

\begin{tabular}{|r|c|c|c|c|c|c|c|c|c|c|c|c|c|c|}
\hline$\#$ & $\mathbf{b}$ & $\mathbf{b}^{++}$ & $\mathbf{b}^{*}$ & $\mathbf{b}^{\boldsymbol{*}^{++}}$ & $\mathbf{b}^{\mathbf{0}}$ & $\mathbf{b}^{\mathbf{0}+}$ & $\mathbf{S e q}$ & $\mathbf{y}$ & $\mathbf{y}^{++}$ & $\mathbf{y}^{*}$ & $\mathbf{y}^{\mathbf{*}^{++}}$ & $\mathbf{y}^{\mathbf{0}}$ & $\mathbf{y}^{\mathbf{0 + +}}$ & $\#$ \\
\hline $\mathbf{1}$ & 100.0757 & 50.5415 & & & & & $\mathbf{V}$ & & & & & & & $\mathbf{1 1}$ \\
\hline $\mathbf{2}$ & $\mathbf{2 1 5 . 1 0 2 6}$ & 108.0550 & 198.0761 & 99.5417 & & & $\mathbf{N}$ & 1156.5705 & 578.7889 & 1139.5440 & 570.2756 & 1138.5600 & 569.7836 & $\mathbf{1 0}$ \\
\hline $\mathbf{3}$ & $\mathbf{3 2 9 . 1 4 5 6}$ & 165.0764 & 312.1190 & 156.5631 & & & $\mathbf{N}$ & $\mathbf{1 0 4 1 . 5 4 3 6}$ & $\mathbf{5 2 1 . 2 7 5 4}$ & 1024.5170 & 512.7622 & 1023.5330 & $\mathbf{5 1 2 . 2 7 0 1}$ & $\mathbf{9}$ \\
\hline $\mathbf{4}$ & 416.1776 & 208.5924 & 399.1510 & 200.0792 & 398.1670 & 199.5871 & $\mathbf{S}$ & $\mathbf{9 2 7 . 5 0 0 7}$ & $\mathbf{4 6 4 . 2 5 4 0}$ & 910.4741 & 455.7407 & $\mathbf{9 0 9 . 4 9 0 1}$ & 455.2487 & $\mathbf{8}$ \\
\hline $\mathbf{5}$ & 529.2616 & 265.1345 & 512.2351 & 256.6212 & 511.2511 & 256.1292 & $\mathbf{L}$ & $\mathbf{8 4 0 . 4 6 8 6}$ & 420.7380 & 823.4421 & $\mathbf{4 1 2 . 2 2 4 7}$ & $\mathbf{8 2 2 . 4 5 8 1}$ & $\mathbf{4 1 1 . 7 3 2 7}$ & $\mathbf{7}$ \\
\hline $\mathbf{6}$ & 666.3206 & 333.6639 & 649.2940 & 325.1506 & 648.3100 & 324.6586 & $\mathbf{H}$ & $\mathbf{7 2 7 . 3 8 4 6}$ & 364.1959 & 710.3580 & 355.6826 & 709.3740 & 355.1906 & $\mathbf{6}$ \\
\hline $\mathbf{7}$ & $\mathbf{7 5 3 . 3 5 2 6}$ & 377.1799 & 736.3260 & 368.6667 & 735.3420 & 368.1746 & $\mathbf{S}$ & $\mathbf{5 9 0 . 3 2 5 7}$ & 295.6665 & 573.2991 & 287.1532 & 572.3151 & 286.6612 & $\mathbf{5}$ \\
\hline $\mathbf{8}$ & $\mathbf{8 8 1 . 4 1 1 2}$ & 441.2092 & 864.3846 & 432.6959 & 863.4006 & 432.2039 & $\mathbf{Q}$ & $\mathbf{5 0 3 . 2 9 3 6}$ & 252.1504 & 486.2671 & 243.6372 & 485.2831 & 243.1452 & $\mathbf{4}$ \\
\hline $\mathbf{9}$ & $\mathbf{9 9 4 . 4 9 5 2}$ & 497.7513 & 977.4687 & 489.2380 & $\mathbf{9 7 6 . 4 8 4 7}$ & 488.7460 & $\mathbf{I}$ & 375.2350 & 188.1212 & 358.2085 & 179.6079 & 357.2245 & 179.1159 & $\mathbf{3}$ \\
\hline $\mathbf{1 0}$ & $\mathbf{1 0 8 1 . 5 2 7 3}$ & 541.2673 & $\mathbf{1 0 6 4 . 5 0 0 7}$ & 532.7540 & $\mathbf{1 0 6 3 . 5 1 6 7}$ & 532.2620 & $\mathbf{S}$ & $\mathbf{2 6 2 . 1 5 1 0}$ & 131.5791 & 245.1244 & 123.0659 & 244.1404 & 122.5738 & $\mathbf{2}$ \\
\hline $\mathbf{1 1}$ & & & & & & & $\mathbf{R}$ & 175.1190 & 88.0631 & 158.0924 & 79.5498 & & & $\mathbf{1}$ \\
\hline
\end{tabular}
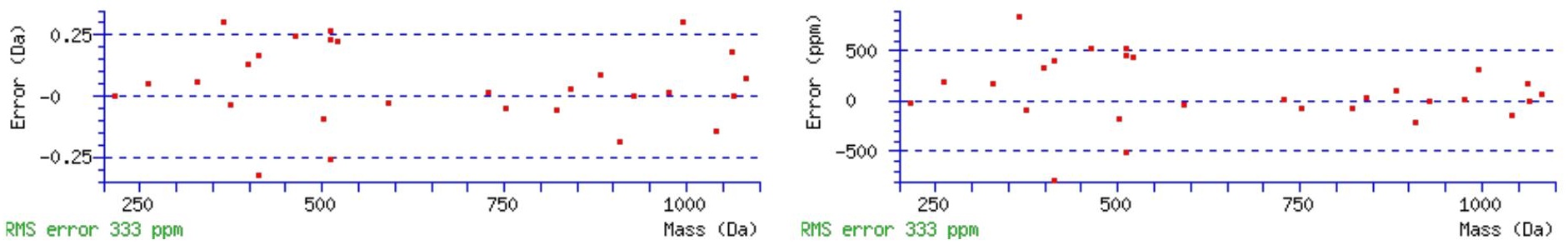

\section{All matches to this query}

\begin{tabular}{|l|l|l|l|}
\hline Score & Mr(calc): & Delta & \multicolumn{1}{c|}{ Sequence } \\
\hline 52.9 & 1254.6316 & -0.0013 & VNNSLHSQISR \\
\hline 43.7 & 1254.6316 & -0.0013 & VNNSLHSQISR \\
\hline 6.9 & 1254.6390 & -0.0086 & KEKMGFGSISR \\
\hline 6.0 & 1254.6218 & 0.0086 & RQPQPHHNEL \\
\hline 4.1 & 1254.6316 & -0.0012 & QASKAEHELSR \\
\hline 3.7 & 1254.6278 & 0.0026 & EKNMYKTVDK \\
\hline 2.5 & 1254.6268 & 0.0036 & ALARSGMKIGR \\
\hline 2.1 & 1254.6357 & -0.0053 & LHGXNFPVISR \\
\hline 2.1 & 1254.6220 & 0.0083 & SEIISIASLSR \\
\hline 1.9 & 1252.6316 & 1.9988 & LDSPILSSTLK \\
\hline
\end{tabular}


Spectrum No: 634; Query: 2340; Rank: 1

\section{Peptide View}

MS/MS Fragmentation of KENSSEICSNNGECVCGQCVCR

Found in IPI00191681, Tax_Id=10116 Gene_Symbol=Itgb1 Integrin beta-1 precursor

Match to Query 2340: 2647.025682 from(883.349170,3+)

Title: 100101RatKid_NS_deglyco_16.1209.1209.3.dta

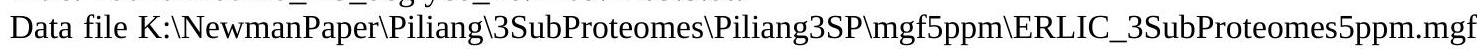
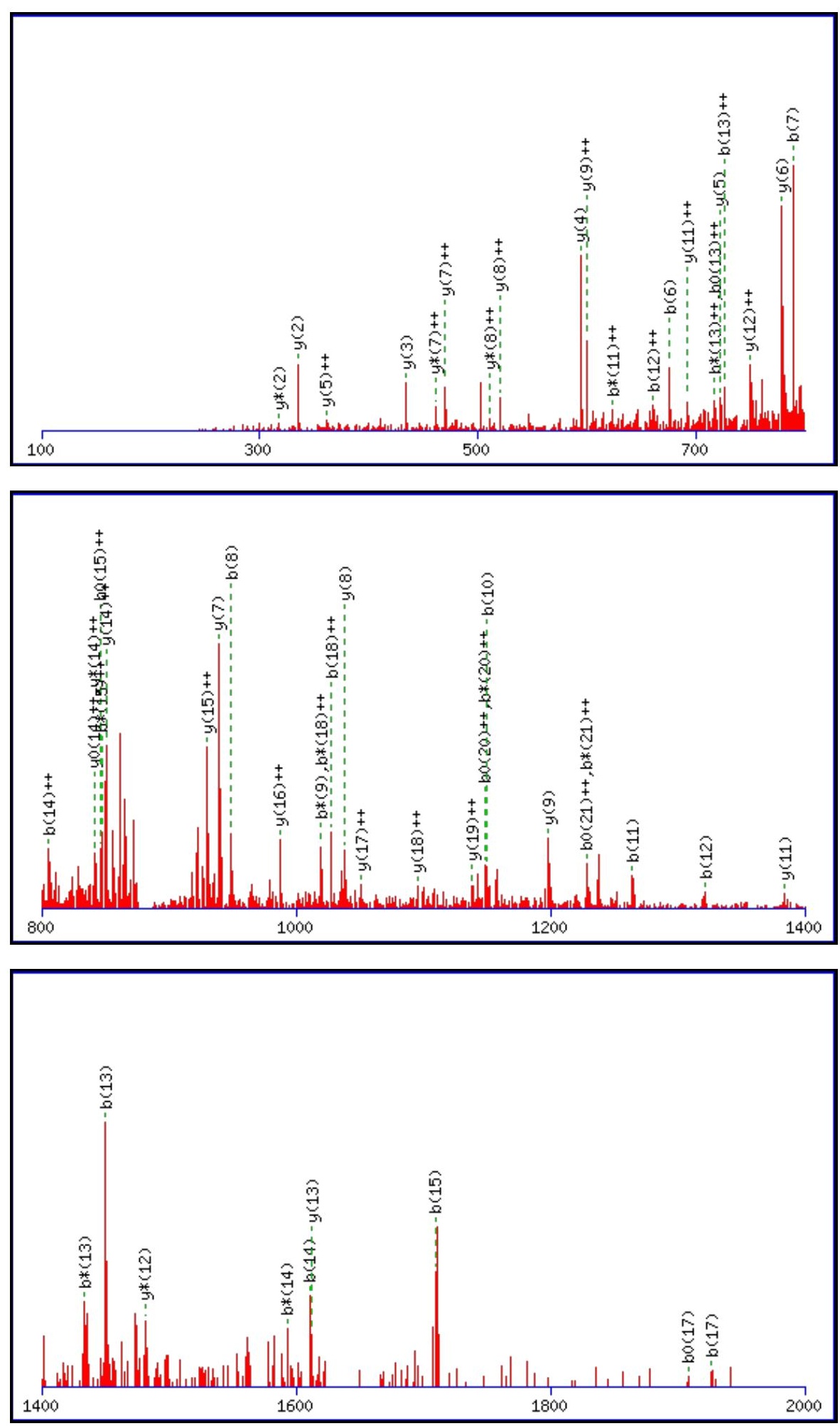
Monoisotopic mass of neutral peptide $\operatorname{Mr}($ calc): 2647.0189

Fixed modifications: Carbamidomethyl (C)

Variable modifications:

N3 : Deamidated_N (N)

Ions Score: 53 Expect: 0.0011

Matches (Bold Red): 56/232 fragment ions using 110 most intense peaks

\begin{tabular}{|c|c|c|c|c|c|c|c|c|c|c|c|c|c|c|}
\hline \# & b & $\mathbf{b}^{++}$ & $\mathbf{b}^{*}$ & $\mathbf{b}^{*^{++}}$ & $\mathbf{b}^{0}$ & $\mathbf{b}^{0++}$ & Seq. & $\mathbf{y}$ & $\mathbf{y}^{++}$ & $\mathbf{y}^{*}$ & $\mathrm{y}^{\mathrm{*}^{++}}$ & $\mathbf{y}^{0}$ & $\mathbf{y}^{\mathbf{0 + +}}$ & $\#$ \\
\hline 1 & 129.1022 & 65.0548 & 112.0757 & 56.5415 & & & $\mathbf{K}$ & & & & & & & 22 \\
\hline 2 & 258.1448 & 129.5761 & 241.1183 & 121.0628 & 240.1343 & 120.5708 & $\mathbf{E}$ & 2519.9312 & 1260.4693 & 2502.9047 & 1251.9560 & 2501.9207 & 1251.4640 & 21 \\
\hline 3 & 373.1718 & 187.0895 & 356.1452 & 178.5762 & 355.1612 & 178.0842 & $\mathbf{N}$ & 2390.8887 & 1195.9480 & 2373.8621 & 1187.4347 & 2372.8781 & 1186.9427 & 20 \\
\hline 4 & 460.2038 & 230.6055 & 443.1772 & 222.0923 & 442.1932 & 221.6003 & $\mathrm{~S}$ & 2275.8617 & 1138.4345 & 2258.8352 & 1129.9212 & 2257.8512 & 1129.4292 & 19 \\
\hline 5 & 547.2358 & 274.1216 & 530.2093 & 265.6083 & 529.2253 & 265.1163 & $\mathrm{~S}$ & 2188.8297 & 1094.9185 & 2171.8031 & 1086.4052 & 2170.8191 & 1085.9132 & 18 \\
\hline 6 & 676.2784 & 338.6428 & 659.2519 & 330.1296 & 658.2679 & 329.6376 & $E$ & 2101.7977 & 1051.4025 & 2084.7711 & 1042.8892 & 2083.7871 & 1042.3972 & 17 \\
\hline 7 & 789.3625 & 395.1849 & 772.3359 & 386.6716 & 771.3519 & 386.1796 & I & 1972.7551 & 986.8812 & 1955.7285 & 978.3679 & 1954.7445 & 977.8759 & 16 \\
\hline 8 & 949.3931 & 475.2002 & 932.3666 & 466.6869 & 931.3826 & 466.1949 & $\mathrm{C}$ & 1859.6710 & 930.3391 & 1842.6445 & 921.8259 & 1841.6604 & 921.3339 & 15 \\
\hline 9 & 1036.4252 & 518.7162 & \begin{tabular}{|l|}
1019.3986 \\
\end{tabular} & 510.2029 & 1018.4146 & 509.7109 & $\mathrm{~S}$ & 1699.6404 & 850.3238 & 1682.6138 & 841.8105 & 1681.6298 & 841.3185 & 14 \\
\hline 10 & 1150.4681 & 575.7377 & 1133.4415 & 567.2244 & 1132.4575 & 566.7324 & $\mathbf{N}$ & 1612.6083 & 806.8078 & 1595.5818 & 798.2945 & 1594.5978 & 797.8025 & 13 \\
\hline 11 & 1264.5110 & 632.7591 & 1247.4845 & 624.2459 & 1246.5004 & 623.7539 & $\mathbf{N}$ & 1498.5654 & 749.7863 & 1481.5389 & 741.2731 & 1480.5548 & 740.7811 & 12 \\
\hline 12 & 1321.5325 & 661.2699 & 1304.5059 & 652.7566 & 1303.5219 & 652.2646 & G & 1384.5225 & 692.7649 & 1367.4959 & 684.2516 & 1366.5119 & 683.7596 & 11 \\
\hline 131 & 50.5751 & 725.7912 & 33.5485 & 717. & 332.5645 & 716.7859 & $\mathbf{E}$ & 1327.5010 & 664.2541 & 1310.4745 & 655.7409 & 1309.4904 & 655.2489 & 10 \\
\hline 141 & 1610.6057 & 805.8065 & 1593.5792 & 797.2932 & 1592.5952 & 796.8012 & $\mathrm{C}$ & 1198.4584 & 599.7328 & 1181.4319 & 591.2196 & & & 9 \\
\hline 15 & 1709.6741 & 855.3407 & 1692.6476 & 846.8274 & 1691.6636 & 846.3354 & V & 1038.4278 & 519.7175 & 1021.4012 & 511.2042 & & & 8 \\
\hline 161 & 1869.7048 & 935.3560 & 1852.6782 & 926.8428 & 1851.6942 & 926.3507 & $\mathrm{C}$ & 939.3594 & 470.1833 & 922.3328 & 461.6700 & & & 7 \\
\hline 17 & 1926.7262 & 963.8668 & 1909.6997 & 955.3535 & 1908.7157 & 954.8615 & $\mathbf{G}$ & 779.3287 & 390.1680 & 762.3022 & 381.6547 & & & 6 \\
\hline 182 & 2054.7848 & 1027.8960 & 2037.7583 & 1019.3828 & 2036.7743 & 1018.8908 & $\mathbf{Q}$ & 722.3072 & 361.6573 & 705.2807 & 353.1440 & & & 5 \\
\hline 192 & 2214.8155 & 1107.9114 & 2197.7889 & 1099.3981 & 2196.8049 & 1098.9061 & $\mathrm{C}$ & 594.2487 & 297.6280 & 577.2221 & 289.1147 & & & 4 \\
\hline 202 & 2313.8839 & 1157.4456 & 2296.8573 & 1148.9323 & 2295.8733 & 1148.4403 & $\mathbf{V}$ & 434.2180 & 217.6126 & 417.1915 & 209.0994 & & & 3 \\
\hline 212 & 2473.9145 & 1237.4609 & 2456.8880 & 1228.9476 & 2455.9040 & 1228.4556 & $\mathrm{C}$ & 335.1496 & 168.0784 & 318.1231 & 159.5652 & & & 2 \\
\hline 22 & & & & & & & $\mathbf{R}$ & 175.1190 & 88.0631 & 158.0924 & 79.5498 & & & 1 \\
\hline
\end{tabular}
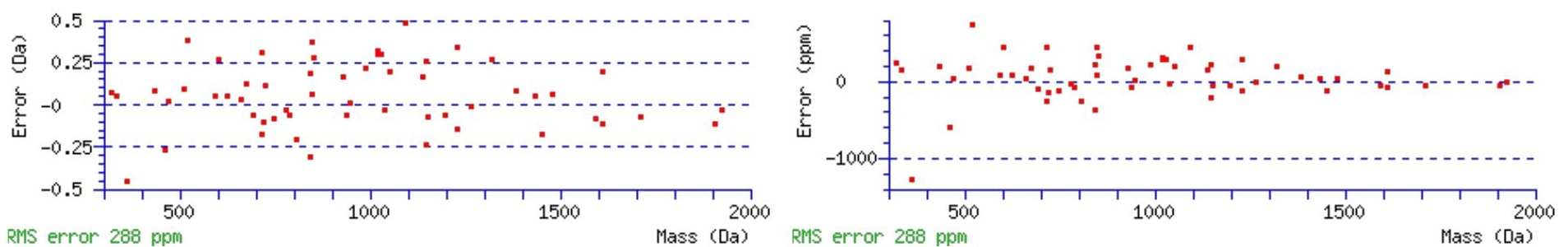

\section{All matches to this query}

\begin{tabular}{|l|l|l|l|}
\hline Score & Mr(calc): & Delta & \multicolumn{1}{c|}{ Sequence } \\
\hline 52.9 & 2647.0189 & 0.0067 & KENSSEICSNNGECVCGQCVCR \\
\hline 43.6 & 2647.0189 & 0.0067 & KENSSEICSNNGECVCGQCVCR \\
\hline 42.7 & 2647.0189 & 0.0067 & KENSSEICSNNGECVCGQCVCR \\
\hline 32.2 & 2646.0349 & 0.9908 & KENSSEICSNNGECVCGQCVCR \\
\hline 4.1 & 2647.0289 & -0.0033 & YSSIVTESFVIKATVFMAVR \\
\hline 1.1 & 2647.0372 & -0.0115 & TXDLTISINVLYNYLEANPK \\
\hline
\end{tabular}

Spectrum No: 635; Query: 36; Rank: 1

\section{Peptide View}




\section{MS/MS Fragmentation of IEQNGSMR}

Found in IPI00554102, Tax_Id=10116 Gene_Symbol=LOC685482;LOC259246;LOC298116;LOC259245 similar to alpha2u globulin

Match to Query 36: 934.415928 from(468.215240,2+)

Title: 091008RatKidney_NoSalt_14.238.238.2.dta

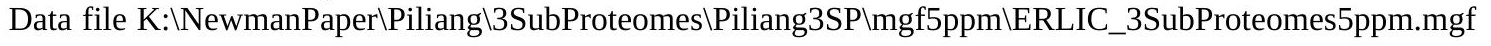
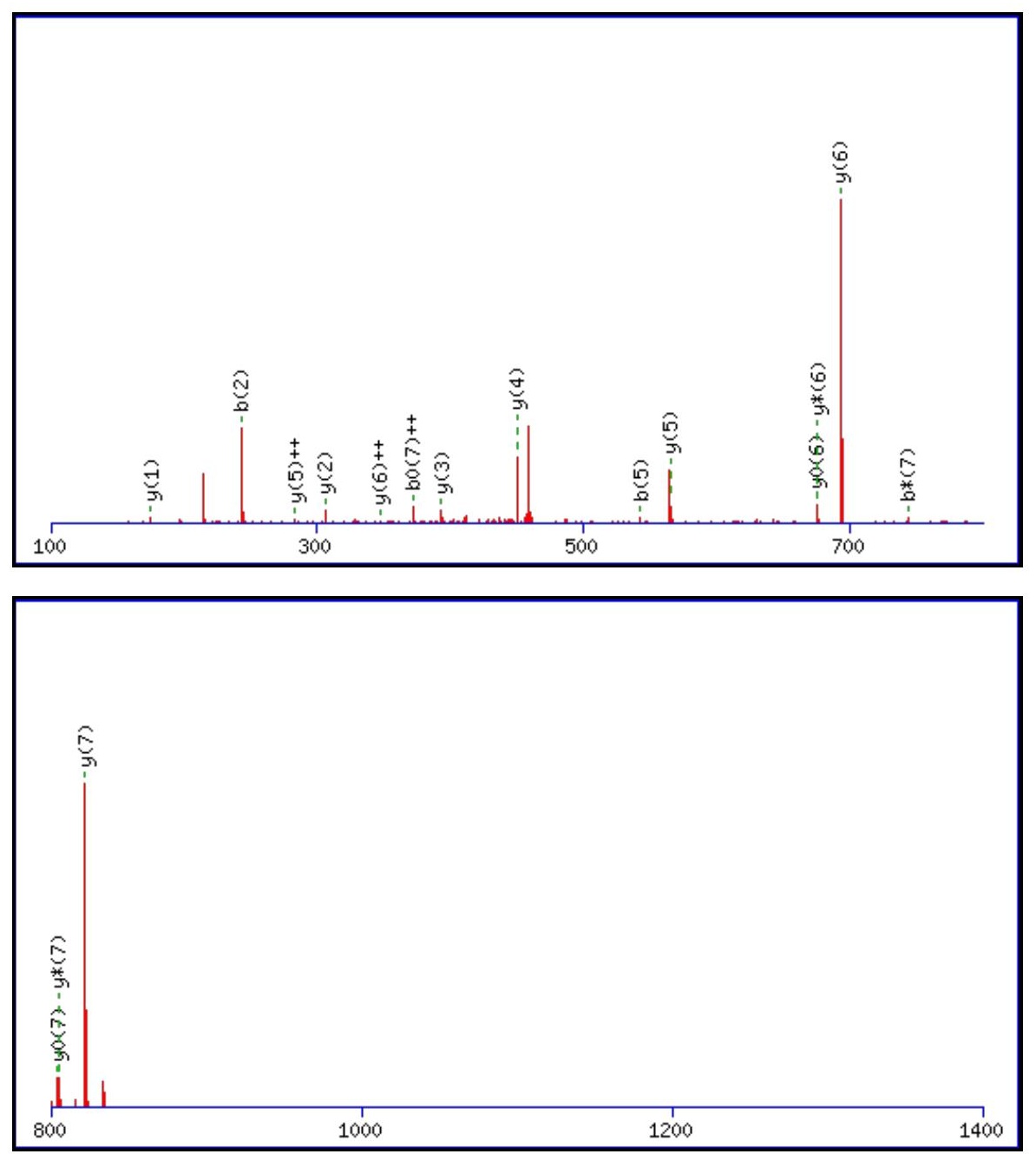

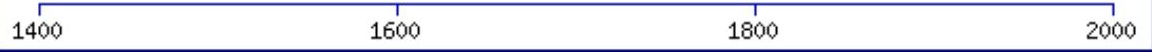

Monoisotopic mass of neutral peptide $\operatorname{Mr}($ calc): 934.4178

Fixed modifications: Carbamidomethyl (C)

Variable modifications:

N4 : Deamidated_N (N)

Ions Score: 53 Expect: 0.0004

Matches (Bold Red): 17/74 fragment ions using 29 most intense peaks

\begin{tabular}{|c|c|c|c|c|c|c|c|c|c|c|c|c|c|c|}
\hline$\#$ & $\mathbf{b}$ & $\mathbf{b}^{++}$ & $\mathbf{b}^{*}$ & $\mathbf{b}^{*++}$ & $\mathbf{b}^{0}$ & $\mathbf{b}^{\mathbf{0 + +}}$ & Seq. & $\mathbf{y}$ & $\mathrm{y}^{++}$ & $\mathbf{y}^{*}$ & $\mathbf{y}^{*^{++}}$ & $\mathbf{y}^{\mathbf{0}}$ & $\mathbf{y}^{\mathbf{0 + +}}$ & $\#$ \\
\hline
\end{tabular}


Mascot Search Results: Peptide View

\begin{tabular}{|c|c|c|c|c|c|c|c|c|c|c|c|c|c|c|}
\hline & 114.0913 & 57.5493 & & & & & I & & & & & & & \\
\hline F & 243.1339 & 122.0706 & & & 225.1234 & 113.0653 & E & 822.3410 & 411.6742 & 805.3145 & 403.1609 & 804.3305 & 402.6689 & \\
\hline p & 371.1925 & 186.0999 & 354.1660 & 177.5866 & 353.1819 & 177.0946 & $\mathbf{Q}$ & 693.2984 & 347.1529 & 676.2719 & 338.6396 & 675.2879 & 338.1476 & \\
\hline 4 & 486.2194 & 243.6134 & 469.1929 & 235.1001 & 468.2089 & 234.6081 & $\mathbf{N}$ & 565.2399 & 283.1236 & 548.2133 & 274.6103 & 547.2293 & 274.1183 & \\
\hline U & 543.2409 & 272.1241 & 526.2144 & 263.6108 & 525.2303 & 263.1188 & G & 450.2129 & 225.6101 & 433.1864 & 217.0968 & 432.2024 & 216.6048 & \\
\hline 6 & 630.2729 & 315.6401 & 613.2464 & 307.1268 & 612.2624 & 306.6348 & $\mathrm{~S}$ & 393.1915 & 197.0994 & 376.1649 & 188.5861 & 375.1809 & 188.0941 & \\
\hline 7 & 761.3134 & 381.1604 & 744.2869 & 372.6471 & 743.3029 & 372.1551 & M & 306.1594 & 153.5834 & 289.1329 & 145.0701 & & & \\
\hline & & & & & & & $\mathbf{R}$ & 175.1190 & 88.0631 & 158.0924 & 79.5498 & & & \\
\hline
\end{tabular}
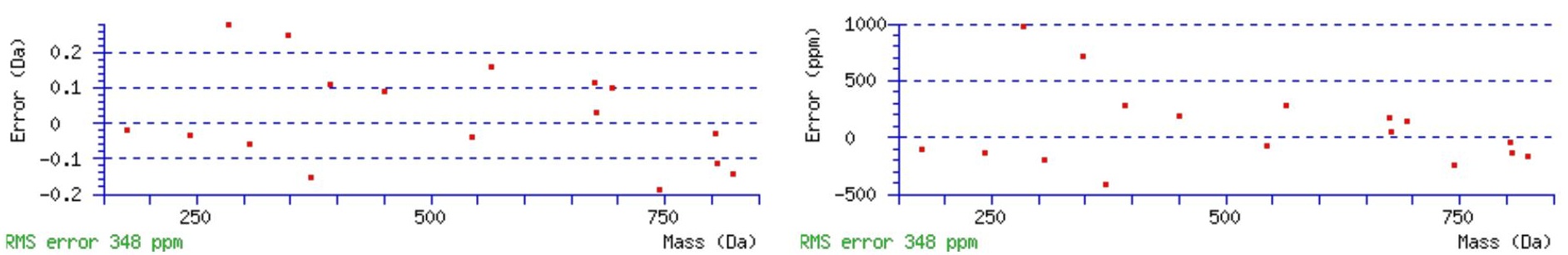

\section{All matches to this query}

\begin{tabular}{|l|l|l|l|}
\hline Score & Mr(calc): & Delta & Sequence \\
\hline 52.7 & 934.4178 & -0.0019 & IEQNGSMR \\
\hline 49.3 & 934.4178 & -0.0019 & IEENGSMR \\
\hline 20.0 & 934.4161 & -0.0001 & LEELHSK \\
\hline 9.0 & 934.4178 & -0.0019 & KLEDCDR \\
\hline 7.4 & 934.4178 & -0.0019 & IQQDSGCK \\
\hline 5.4 & 933.4109 & 1.0050 & QLQFYR \\
\hline 5.3 & 934.4178 & -0.0019 & NKEQCEK \\
\hline 5.0 & 933.4208 & 0.9951 & KLYESSK \\
\hline 5.0 & 933.4109 & 1.0050 & QLYQFR \\
\hline 4.7 & 934.4178 & -0.0019 & LEDCDRK \\
\hline
\end{tabular}

Spectrum No: 636; Query: 1645; Rank: 1

\section{Peptide View}

MS/MS Fragmentation of HAETIYNISMNHPQEMK

Found in IPI00190555, Tax_Id=10116 Gene_Symbol=Folh1 Glutamate carboxypeptidase 2

Match to Query 1645: 2042.927832 from(681.983220,3+)

Title: 100101RatKid_NS_deglyco_15.2300.2300.3.dta

Data file K:INewmanPaper|Piliang|3SubProteomes\Piliang3SP\mgf5ppm\ERLIC_3SubProteomes5ppm.mgf 

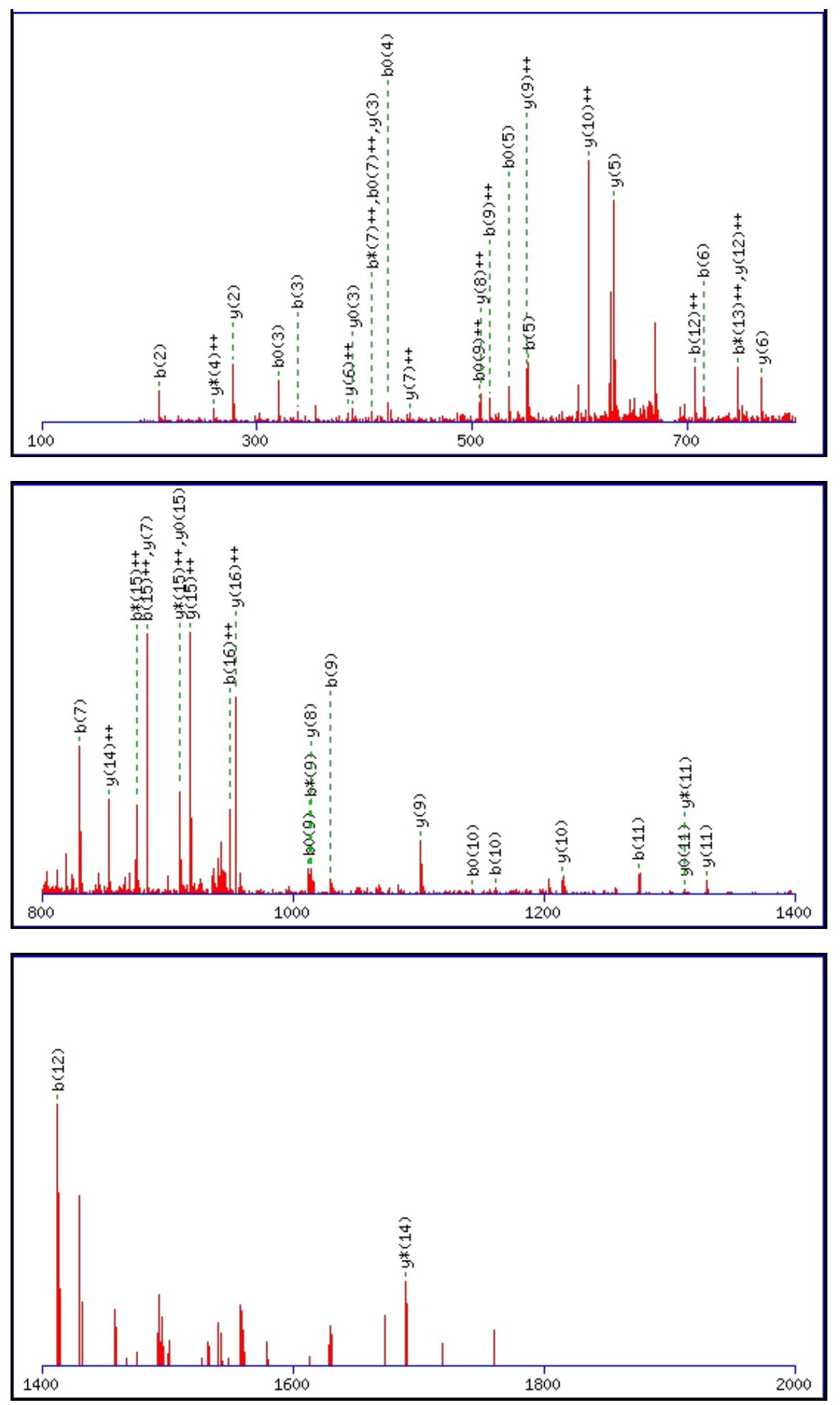

Monoisotopic mass of neutral peptide $\operatorname{Mr}($ calc): 2042.9189

Fixed modifications: Carbamidomethyl (C)

Variable modifications:

N7 : Deamidated $\mathrm{N}(\mathrm{N})$

Ions Score: 53 Expect: 0.0013

Matches (Bold Red): 49/172 fragment ions using 81 most intense peaks

\begin{tabular}{|r|c|c|c|c|c|c|c|c|c|c|c|c|c|c|}
\hline$\#$ & $\mathbf{b}$ & $\mathbf{b}^{++}$ & $\mathbf{b}^{*}$ & $\mathbf{b}^{\mathbf{*}^{++}}$ & $\mathbf{b}^{\mathbf{0}}$ & $\mathbf{b}^{\mathbf{0 + +}}$ & Seq. & $\mathbf{y}$ & $\mathbf{y}^{++}$ & $\mathbf{y}^{\mathbf{*}}$ & $\mathbf{y}^{\boldsymbol{*}^{++}}$ & $\mathbf{y}^{\mathbf{0}}$ & $\mathbf{y}^{\mathbf{0 + +}}$ & $\#$ \\
\hline $\mathbf{1}$ & 138.0662 & 69.5367 & & & & & $\mathbf{H}$ & & & & & & & $\mathbf{1 7}$ \\
\hline $\mathbf{2}$ & $\mathbf{2 0 9 . 1 0 3 3}$ & 105.0553 & & & & & A & 1906.8674 & $\mathbf{9 5 3 . 9 3 7 3}$ & 1889.8408 & 945.4240 & 1888.8568 & 944.9320 & $\mathbf{1 6}$ \\
\hline $\mathbf{3}$ & 338.1459 & 169.5766 & & & 320.1353 & 160.5713 & $\mathbf{E}$ & 1835.8302 & $\mathbf{9 1 8 . 4 1 8 8}$ & 1818.8037 & $\mathbf{9 0 9 . 9 0 5 5}$ & 1817.8197 & $\mathbf{9 0 9 . 4 1 3 5}$ & $\mathbf{1 5}$ \\
\hline $\mathbf{4}$ & 439.1936 & 220.1004 & & & $\mathbf{4 2 1 . 1 8 3 0}$ & 211.0951 & $\mathbf{T}$ & 1706.7876 & $\mathbf{8 5 3 . 8 9 7 5}$ & $\mathbf{1 6 8 9 . 7 6 1 1}$ & 845.3842 & 1688.7771 & 844.8922 & $\mathbf{1 4}$ \\
\hline $\mathbf{5}$ & $\mathbf{5 5 2 . 2 7 7 6}$ & 276.6425 & & & 534.2671 & 267.6372 & $\mathbf{I}$ & 1605.7400 & 803.3736 & 1588.7134 & 794.8603 & 1587.7294 & 794.3683 & $\mathbf{1 3}$ \\
\hline
\end{tabular}




\begin{tabular}{|c|c|c|c|c|c|c|c|c|c|c|c|c|c|c|}
\hline 6 & 715.3410 & |358.1741| & & & 697.3304 & 349.1688 & $\mathbf{Y}$ & 6559 & 746.8316 & & 738.3183 & 1474.6453 & 737.8263 & 12 \\
\hline 7 & 830.3679 & 415.6876 & 813.3414 & 407.1743 & 812.3573 & 406.6823 & $\mathbf{N}$ & 1329.5926 & 665.2999 & 1312.5660 & 656.7867 & 1311.5820 & 656.2946 & 11 \\
\hline 8 & 943.4520 & 472.2296 & 926.4254 & 463.7163 & 925.4414 & 463.2243 & I & 1214.5656 & 607.7865 & 1197.5391 & 599.2732 & 1196.5551 & 598.7812 & 10 \\
\hline 9 & 1030.4840 & 515.7456 & 1013.4574 & 507.2324 & 1012.4734 & 506.7404 & S & 1101.4816 & 551.2444 & 1084.4550 & 542.7312 & 1083.4710 & 542.2391 & 9 \\
\hline 10 & 1161.5245 & 581.2659 & 1144.4979 & 572.7526 & 1143.5139 & 572.2606 & M & 1014.4495 & 507.7284 & 997.4230 & 499.2151 & 996.4390 & 498.7231 & 8 \\
\hline 11 & 1275.5674 & 638.2873 & 1258.5409 & 629.7741 & 1257.5568 & 629.2821 & $\mathbf{N}$ & 883.4091 & 442.2082 & 866.3825 & 433.6949 & 865.3985 & 433.2029 & 7 \\
\hline 12 & 1412.6263 & 706.8168 & 1395.5998 & 698.3035 & 1394.6158 & 697.8115 & H & 769.3661 & 385.1867 & 752.3396 & 376.6734 & 751.3556 & 376.1814 & 6 \\
\hline 13 & 1509.6791 & 755.3432 & 1492.6525 & 746.8299 & 1491.6685 & 746.3379 & $\mathbf{P}$ & 632.3072 & 316.6573 & 615.2807 & 308.1440 & 614.2967 & 307.6520 & 5 \\
\hline 14 & 1637.7377 & 819.3725 & 1620.7111 & 810.8592 & 1619.7271 & 810.3672 & $\mathbf{Q}$ & 535.2545 & 268.1309 & 518.2279 & 259.6176 & 517.2439 & 259.1256 & 4 \\
\hline 15 & 1766.7803 & 883.8938 & 1749.7537 & 875.3805 & 1748.7697 & 874.8885 & $\mathbf{E}$ & 407.1959 & 204.1016 & 390.1693 & 195.5883 & 389.1853 & 195.0963 & 3 \\
\hline 16 & 1897.8207 & 949.4140 & 1880.7942 & 940.9007 & 1879.8102 & 940.4087 & M & 278.1533 & 139.5803 & 261.1267 & 131.0670 & & & 2 \\
\hline 17 & & & & & & & $\mathbf{K}$ & 147.1128 & 74.0600 & 130.0863 & 65.5468 & & & $\overline{1}$ \\
\hline
\end{tabular}
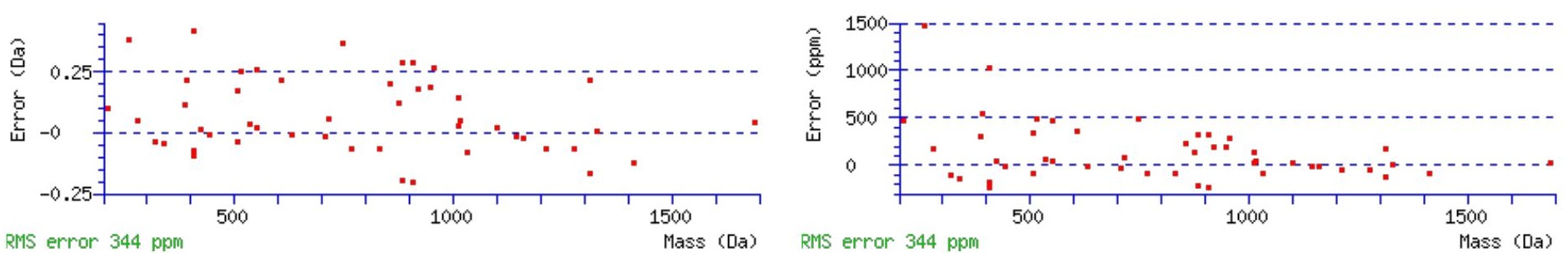

All matches to this query

\begin{tabular}{|l|l|l|l|}
\hline Score & Mr(calc): & Delta & \multicolumn{1}{|c|}{ Sequence } \\
\hline 52.6 & 2042.9189 & 0.0089 & HAETIYNISMNHPQEMK \\
\hline 48.8 & 2042.9189 & 0.0089 & HAETIYNISMNHPQEMK \\
\hline 23.5 & 2041.9349 & 0.9929 & HAETIYNISMNHPQEMK \\
\hline 7.1 & 2042.9231 & 0.0048 & $\underline{\text { LRDTENDSMAKIAELEK }}$ \\
\hline 6.3 & 2041.9057 & 1.0221 & LETVGSVFSRTRDLMR \\
\hline 3.5 & 2040.9265 & 2.0013 & TWNVGSSNRNKAENLLR \\
\hline 1.7 & 2040.9050 & 2.0228 & AYDLAGSCKGVKVMFNGK \\
\hline
\end{tabular}

Spectrum No: 637; Query: 89; Rank: 1

\section{Peptide View}

MS/MS Fragmentation of INVTEVYNK

Found in IPI00371761, Tax_Id=10116 Gene_Symbol=Enpp4_predicted ectonucleotide pyrophosphatase/phosphodiesterase 4

Match to Query 89: 1079.550768 from(540.782660,2+)

Title: 100101RatKid_NS_deglyco_27.1514.1514.2.dta

Data file K:INewmanPaper|Piliang|3SubProteomes\Piliang3SP\mgf5ppm\ERLIC_3SubProteomes5ppm.mgf 

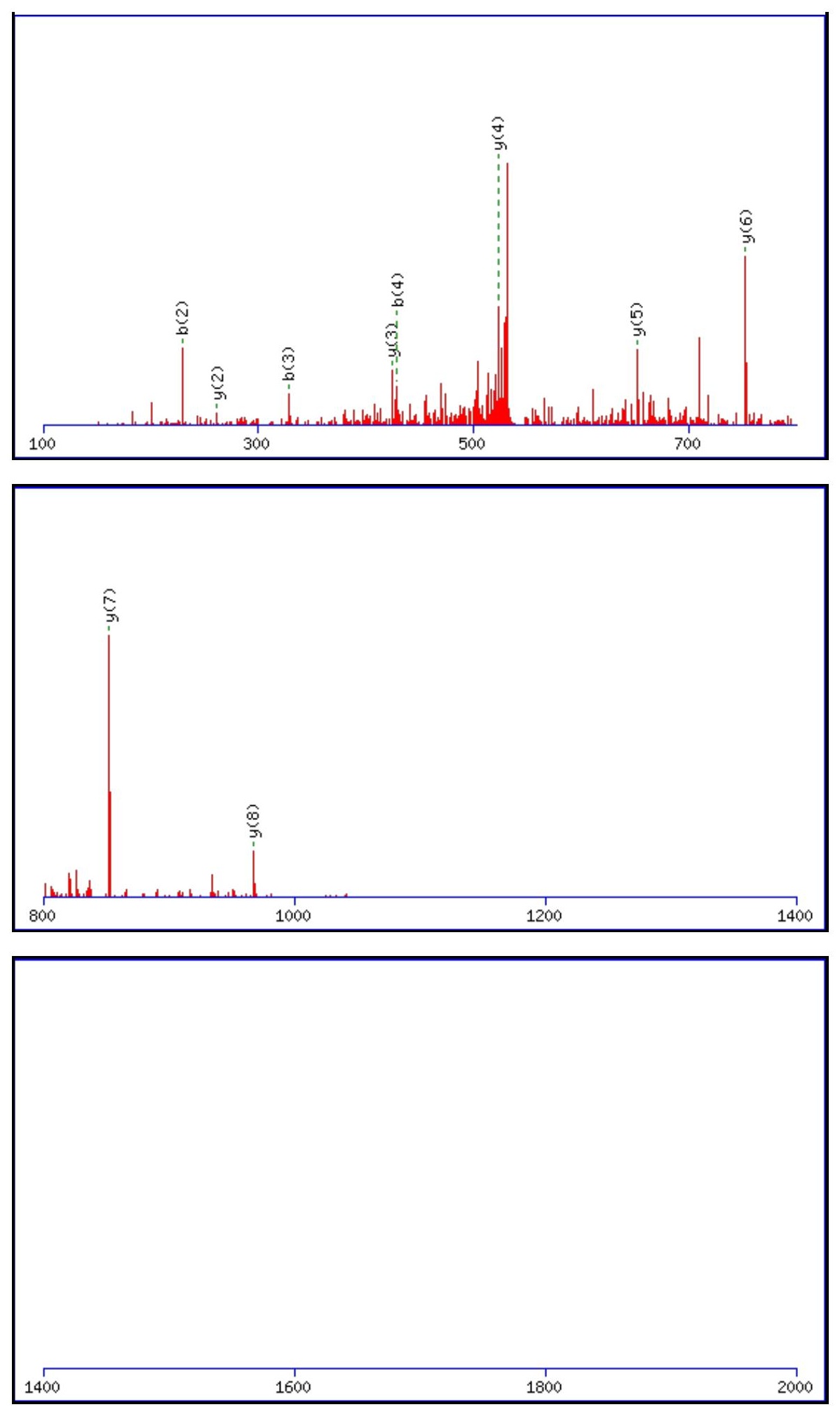

Monoisotopic mass of neutral peptide $\operatorname{Mr}($ calc): 1079.5499

Fixed modifications: Carbamidomethyl (C)

Variable modifications:

N2 : Deamidated N $(\mathrm{N})$

Ions Score: 52 Expect: 0.00059

Matches (Bold Red): 10/80 fragment ions using 23 most intense peaks

\begin{tabular}{|c|c|c|c|c|c|c|c|c|c|c|c|c|c|c|}
\hline$\#$ & $\mathbf{b}$ & $\mathbf{b}^{++}$ & $\mathbf{b}^{*}$ & $\mathbf{b}^{*_{+}^{++}}$ & $\mathbf{b}^{\mathbf{0}}$ & $\mathbf{b}^{\mathbf{0 + +}}$ & Seq. & $\mathbf{y}$ & $\mathbf{y}^{++}$ & $\mathbf{y}^{*}$ & $\mathbf{y}^{\mathbf{*}^{++}}$ & $\mathbf{y}^{\mathbf{0}}$ & $\mathbf{y}^{\mathbf{0}++}$ & $\#$ \\
\hline $\mathbf{1}$ & 114.0913 & 57.5493 & & & & & $\mathbf{I}$ & & & & & & & $\mathbf{9}$ \\
\hline $\mathbf{2}$ & $\mathbf{2 2 9 . 1 1 8 3}$ & 115.0628 & 212.0917 & 106.5495 & & & $\mathbf{N}$ & $\mathbf{9 6 7 . 4 7 3 1}$ & 484.2402 & 950.4465 & 475.7269 & 949.4625 & 475.2349 & $\mathbf{8}$ \\
\hline $\mathbf{3}$ & 328.1867 & 164.5970 & 311.1601 & 156.0837 & & & $\mathbf{V}$ & $\mathbf{8 5 2 . 4 4 6 2}$ & 426.7267 & 835.4196 & 418.2134 & 834.4356 & 417.7214 & $\mathbf{7}$ \\
\hline $\mathbf{4}$ & $\mathbf{4 2 9 . 2 3 4 4}$ & 215.1208 & 412.2078 & 206.6075 & 411.2238 & 206.1155 & $\mathbf{T}$ & 753.3777 & 377.1925 & 736.3512 & 368.6792 & 735.3672 & 368.1872 & $\mathbf{6}$ \\
\hline $\mathbf{5}$ & 558.2770 & 279.6421 & 541.2504 & 271.1288 & 540.2664 & 270.6368 & $\mathbf{E}$ & $\mathbf{6 5 2 . 3 3 0 1}$ & 326.6687 & 635.3035 & 318.1554 & 634.3195 & 317.6634 & 5 \\
\hline
\end{tabular}




\begin{tabular}{|l|l|l|l|l|l|l|l|l|l|l|l|l|l|l|l|}
$\mathbf{6}$ & 657.3454 & 329.1763 & 640.3188 & 320.6631 & 639.3348 & 320.1710 & $\mathbf{V}$ & $\mathbf{5 2 3 . 2 8 7 5}$ & 262.1474 & 506.2609 & 253.6341 & & & $\mathbf{4}$ \\
\hline $\mathbf{7}$ & 820.4087 & 410.7080 & 803.3822 & 402.1947 & 802.3981 & 401.7027 & $\mathbf{Y}$ & $\mathbf{4 2 4 . 2 1 9 1}$ & 212.6132 & 407.1925 & 204.0999 & & & $\mathbf{3}$ \\
\hline $\mathbf{8}$ & 934.4516 & 467.7295 & 917.4251 & 459.2162 & 916.4411 & 458.7242 & $\mathbf{N}$ & $\mathbf{2 6 1 . 1 5 5 7}$ & 131.0815 & 244.1292 & 122.5682 & & & $\mathbf{2}$ \\
\hline $\mathbf{9}$ & & & & & & & $\mathbf{K}$ & 147.1128 & 74.0600 & 130.0863 & 65.5468 & & & $\mathbf{1}$ \\
\hline
\end{tabular}
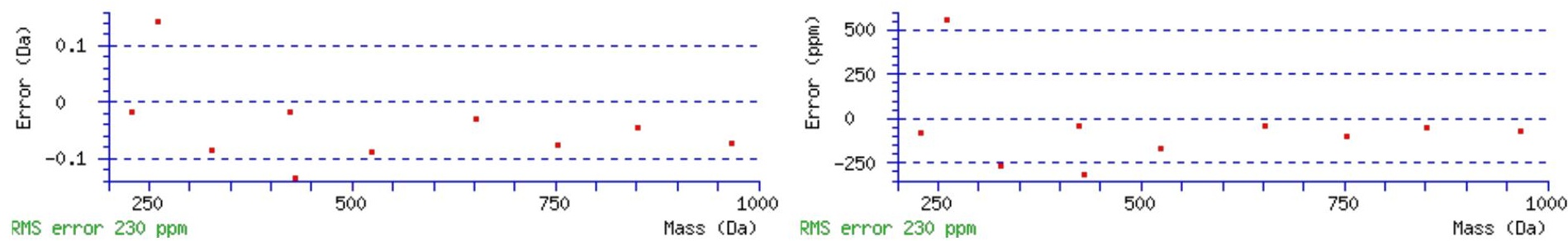

\section{All matches to this query}

\begin{tabular}{|l|l|l|l|}
\hline Score & Mr(calc): & Delta & \multicolumn{1}{|c|}{ Sequence } \\
\hline 52.5 & 1079.5499 & 0.0009 & INVTEVYNK \\
\hline 15.6 & 1079.5488 & 0.0019 & INVAVTRAR \\
\hline 10.6 & 1079.5499 & 0.0009 & LNQLESTFK \\
\hline 10.4 & 1079.5488 & 0.0019 & EVVQLTRR \\
\hline 8.2 & 1079.5532 & -0.0025 & LNXSSSIVMK \\
\hline 7.8 & 1079.5611 & -0.0103 & INSIYNAKR \\
\hline 7.1 & 1079.5499 & 0.0009 & DLVSXYQEK \\
\hline 6.3 & 1079.5533 & -0.0025 & VDLVTMATSK \\
\hline 5.1 & 1079.5433 & 0.0074 & IPMGKNYNK \\
\hline 3.9 & 1079.5499 & 0.0009 & NLISQETFK \\
\hline
\end{tabular}

Spectrum No: 638; Query: 2587; Rank: 1

\section{Peptide View}

\section{MS/MS Fragmentation of FNDFDVAPSNLCAHDFLEVFDGPSIGNR}

Found in IPI00196620, Tax_Id=10116 Gene_Symbol=Cubn Cubilin precursor

Match to Query 2587: 3153.426912 from(1052.149580,3+)

Title: 100101RatKid_NS_deglyco_19.5673.5673.3.dta

Data file K:INewmanPaper|Piliangl3SubProteomes\Piliang3SP\mgf5ppm\ERLIC_3SubProteomes5ppm.mgf

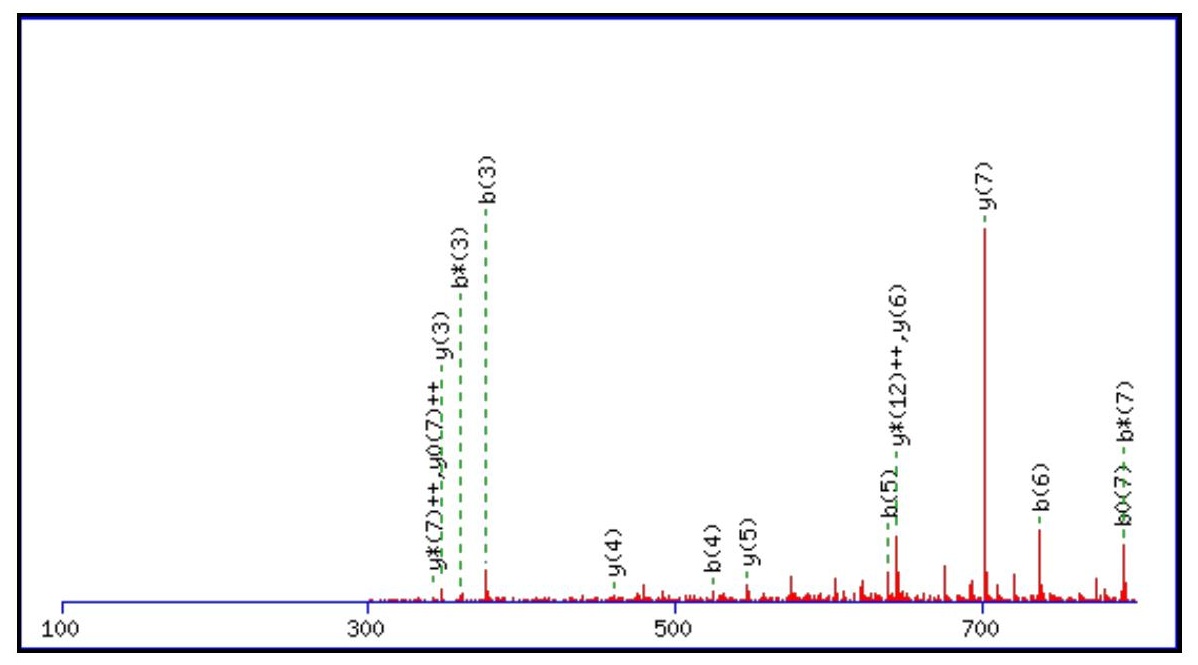



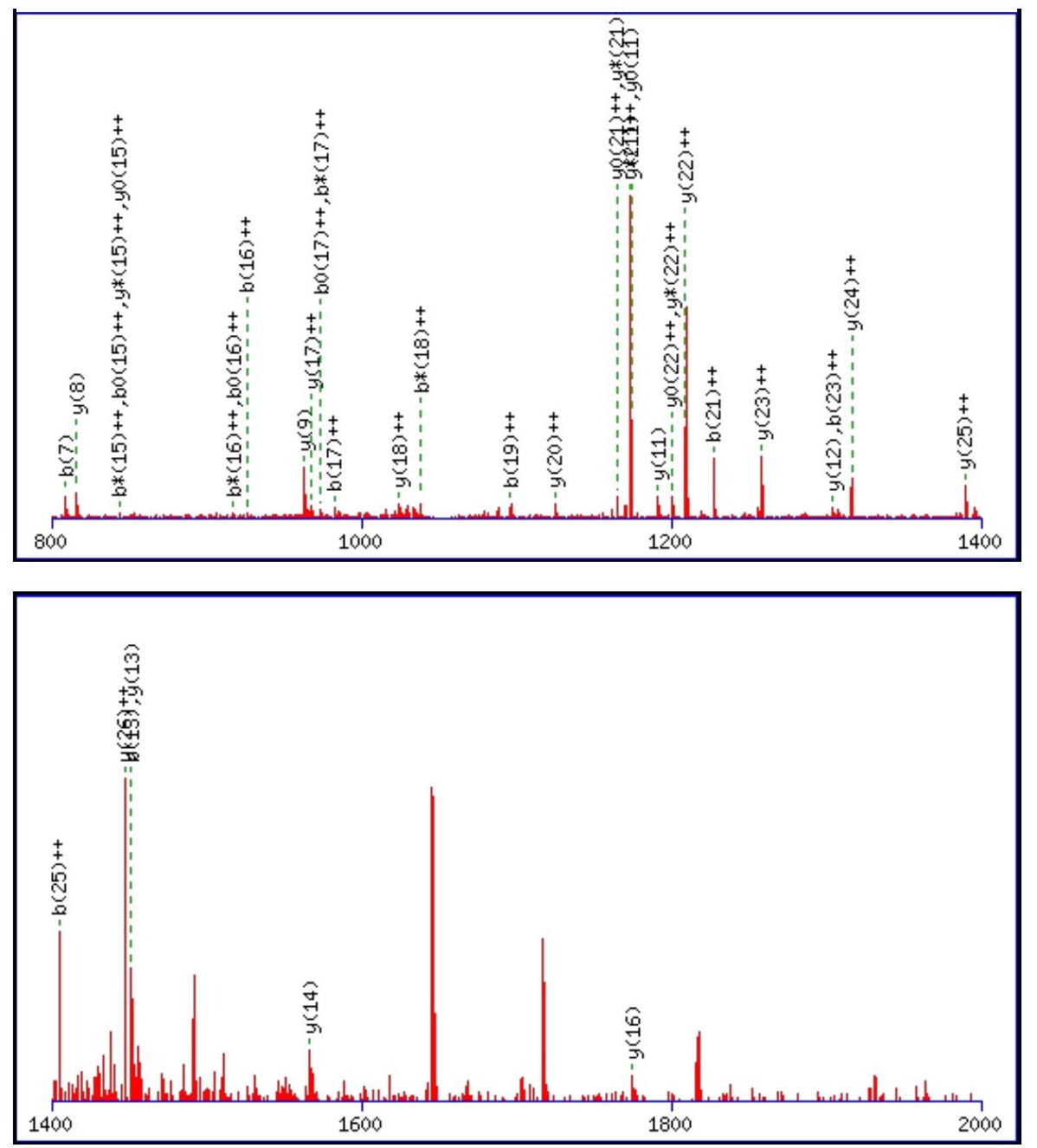

Monoisotopic mass of neutral peptide $\operatorname{Mr}($ calc): 3153.4138

Fixed modifications: Carbamidomethyl (C)

Variable modifications:

N27 : Deamidated_N (N)

Ions Score: 52 Expect: $\odot .0025$

Matches (Bold Red): 54/310 fragment ions using 104 most intense peaks

\begin{tabular}{|c|c|c|c|c|c|c|c|c|c|c|c|c|c|c|}
\hline \# & b & $\mathbf{b}^{++}$ & $\mathbf{b}^{*}$ & $\mathbf{b}^{*^{++}}$ & $\mathbf{b}^{0}$ & $\mathbf{b}^{0++}$ & Seq. & $\mathbf{y}$ & $\mathbf{y}^{++}$ & $\mathbf{y}^{*}$ & $\mathrm{y}^{*^{++}}$ & $\mathbf{y}^{\mathbf{0}}$ & $\mathbf{y}^{0++}$ & \# \\
\hline 1 & 148.0757 & 74.5415 & & & & & $\mathbf{F}$ & & & & & & & 28 \\
\hline 2 & 262.1186 & 131.5629 & 245.0921 & 123.0497 & & & $\mathbf{N}$ & 3007.3527 & 1504.1800 & 2990.3261 & 1495.6667 & 2989.3421 & 1495.1747 & 27 \\
\hline 4 & 524.2140 & 262.6106 & 507.1874 & 254.0974 & 506.2034 & 253.6053 & $\mathbf{F}$ & 2778.2828 & 1389.6450 & 2761.2563 & 1381.1318 & 2760.2722 & 1380.6398 & 25 \\
\hline 5 & 639.2409 & 320.1241 & 622.2144 & 311.6108 & 621.2304 & 311.1188 & D & 2631.2144 & 1316.1108 & 2614.1878 & 1307.5976 & 2613.2038 & 1307.1055 & 24 \\
\hline 6 & 738.3093 & 369.6583 & 721.2828 & 361.1450 & 720.2988 & 360.6530 & V & 2516.1874 & 1258.5974 & 2499.1609 & 1250.0841 & 2498.1769 & 1249.5921 & 23 \\
\hline 7 & 809.3464 & 405.1769 & 792.3199 & 396.6636 & 791.3359 & 396.1716 & A & 2417.1190 & 1209.0632 & 2400.0925 & 1200.5499 & 2399.1085 & 1200.0579 & 22 \\
\hline 8 & 906.3992 & 453.7032 & 889.3727 & 445.1900 & 888.3886 & 444.6980 & $\mathbf{P}$ & 2346.0819 & 1173.5446 & 2329.0554 & 1165.0313 & 2328.0713 & 1164.5393 & 21 \\
\hline 9 & 993.4312 & 497.2193 & 976.4047 & 488.7060 & 975.4207 & 488.2140 & $\mathrm{~S}$ & 2249.0292 & 1125.0182 & 2232.0026 & 1116.5049 & 2231.0186 & 1116.0129 & 20 \\
\hline 10 & 1107.4742 & 554.2407 & 1090.4476 & 545.7274 & 1089.4636 & 545.2354 & $\mathbf{N}$ & 2161.9971 & 1081.5022 & 2144.9706 & 1072.9889 & 2143.9866 & 1072.4969 & 19 \\
\hline 11 & 1220.5582 & 610.7828 & 1203.5317 & 602.2695 & 1202.5477 & 601.7775 & $\mathbf{L}$ & 2047.9542 & 1024.4807 & 2030.9276 & 1015.9675 & 2029.9436 & 1015.4755 & 18 \\
\hline 12 & 1380.5889 & 690.7981 & 1363.5623 & 682.2848 & 1362.5783 & 681.7928 & $\mathrm{C}$ & 1934.8701 & 967.9387 & 1917.8436 & 959.4254 & 1916.8596 & 958.9334 & 17 \\
\hline 14 & 1588.6849 & 794.8461 & 1571.6584 & 786.3328 & 1570.6743 & 785.8408 & $\mathbf{H}$ & 1703.8024 & 852.4048 & 1686.7758 & 843.8915 & 1685.7918 & 843.3995 & 15 \\
\hline 15 & 1703.7118 & 852.3596 & 1686.6853 & 843.8463 & 1685.7013 & 843.3543 & D & 1566.7435 & 783.8754 & 1549.7169 & 775.3621 & 1548.7329 & 774.8701 & 14 \\
\hline 16 & 1850.7803 & 925.8938 & 1833.7537 & 917.3805 & 1832.7697 & 916.8885 & $\mathbf{F}$ & 1451.7165 & 726.3619 & 1434.6900 & 717.8486 & 1433.7059 & 717.3566 & 13 \\
\hline 17 & 1963.8643 & 982.4358 & 1946.8378 & 973.9225 & 1945.8538 & 973.4305 & $\mathbf{L}$ & 1304.6481 & 652.8277 & 1287.6216 & 644.3144 & 1286.6375 & 643.8224 & 12 \\
\hline 18 & 2092.9069 & 1046.9571 & 2075.8804 & 1038.4438 & 2074.8964 & 1037.9518 & $\mathbf{E}$ & 1191.5640 & 596.2857 & 1174.5375 & 587.7724 & 1173.5535 & 587.2804 & 11 \\
\hline
\end{tabular}




\begin{tabular}{|c|c|c|c|c|c|c|c|c|c|c|c|c|c|c|}
\hline 19 & 2191.9753 & |1096.4913 & |2174.9488 & |1087.9780| & |2173.9648 & $\mid 1087.4860$ & V & 1062.5214 & 531.7644 & 1045.4949| & 523.2511 & 1044.5109 & 522.7591 & 10 \\
\hline 20 & 2339.0437 & 1170.0255 & 2322.0172 & 1161.5122 & 2321.0332 & 1161.0202 & $\mathbf{F}$ & 963.4530 & 482.2302 & 946.4265 & 473.7169 & 945.4425 & 473.2249 & 9 \\
\hline 21 & 2454.0707 & 1227.5390 & 2437.0441 & 1219.0257 & 2436.0601 & 1218.5337 & D & 816.3846 & 408.6959 & 799.3581 & 400.1827 & 798.3740 & 399.6907 & 8 \\
\hline 22 & 2511.0922 & 1256.0497 & 2494.0656 & 1247.5364 & 2493.0816 & 1247.0444 & G & 701.3577 & 351.1825 & 684.3311 & 342.6692 & 683.3471 & 342.1772 & 7 \\
\hline 23 & 2608.1449 & 1304.5761 & 2591.1184 & \begin{tabular}{|l|}
1296.0628 \\
\end{tabular} & 2590.1344 & 1295.5708 & $\mathbf{P}$ & 644.3362 & 322.6717 & 627.3097 & 314.1585 & 626.3256 & 313.6665 & 6 \\
\hline 24 & 2695.1769 & 1348.0921 & 2678.1504 & 1339.5788 & 2677.1664 & 1339.0868 & $\mathrm{~S}$ & 547.2834 & 274.1454 & 530.2569 & 265.6321 & 529.2729 & 265.1401 & 5 \\
\hline 25 & 2808.2610 & 1404.6341 & 2791.2345 & 1396.1209 & 2790.2504 & 1395.6289 & I & 460.2514 & 230.6293 & 443.2249 & 222.1161 & & & 4 \\
\hline 26 & 2865.2825 & 1433.1449 & 2848.2559 & 1424.6316 & 2847.2719 & 1424.1396 & G & 347.1674 & 174.0873 & 330.1408 & 165.5740 & & & 3 \\
\hline 27 & 2980.3094 & 1490.6583 & 2963.2829 & 1482.1451 & 2962.2988 & 1481.6531 & $\mathbf{N}$ & 290.1459 & 145.5766 & 273.1193 & 137.0633 & & & 2 \\
\hline 28 & & & & & & & $\mathbf{R}$ & 175.1190 & 88.0631 & 158.0924 & 79.5498 & & & 1 \\
\hline
\end{tabular}
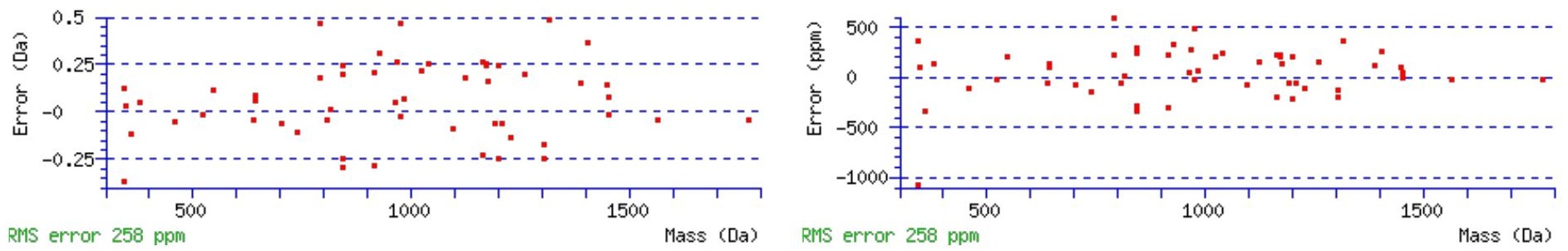

\section{All matches to this query}

\begin{tabular}{|l|l|l|l|}
\hline Score & Mr(calc): & Delta & \multicolumn{1}{c|}{ Sequence } \\
\hline 52.4 & 3153.4138 & 0.0131 & FNDFDVAPSNLCAHDFLEVFDGPSIGNR \\
\hline 15.6 & 3153.4138 & 0.0131 & FNDFDVAPSNLCAHDFLEVFDGPSIGNR \\
\hline 6.0 & 3152.4236 & 1.0034 & GFAFVEYEVPEAAQLALEQMNSVMLGGR \\
\hline 6.0 & 3152.4296 & 0.9973 & AMAPSSLLLLLAAALAPTQTRAGSHSLR \\
\hline 6.0 & 3152.4296 & 0.9973 & AMAPSSLLLLLAAALAPTQTRAGSHSLR \\
\hline 5.5 & 3153.4160 & 0.0109 & GEGSLLFEAASMLLWGHTGLSLELRAR \\
\hline 4.5 & 3151.4484 & 1.9785 & ITMQNQLQQNMQEGLQITSASFQLIK \\
\hline 4.2 & 3151.4030 & 2.0239 & GSDCGIVNVNIPTSGAEIGGAFGGEKHTGGGR \\
\hline 4.2 & 3151.4030 & 2.0239 & GSDCGIVNVNIPTSGAEIGGAFGGEKHTGGGR \\
\hline 4.2 & 3151.4030 & 2.0239 & GSDCGIVNVNIPTSGAEIGGAFGGEKHTGGGR \\
\hline
\end{tabular}

Spectrum No: 639; Query: 2334; Rank: 1

\section{Peptide View}

MS/MS Fragmentation of LEDLTTPYVRDEELNCTVVELK

Found in IPI00211075, Tax_Id=10116 Gene_Symbol=Serpina3n Serine protease inhibitor A3N precursor

Match to Query 2334: 2636.296362 from(879.772730,3+)

Title: 100101RatKid_NS_deglyco_21.3891.3891.3.dta

Data file K:INewmanPaper|Piliangl3SubProteomes\Piliang3SP\mgf5ppm\ERLIC_3SubProteomes5ppm.mgf 

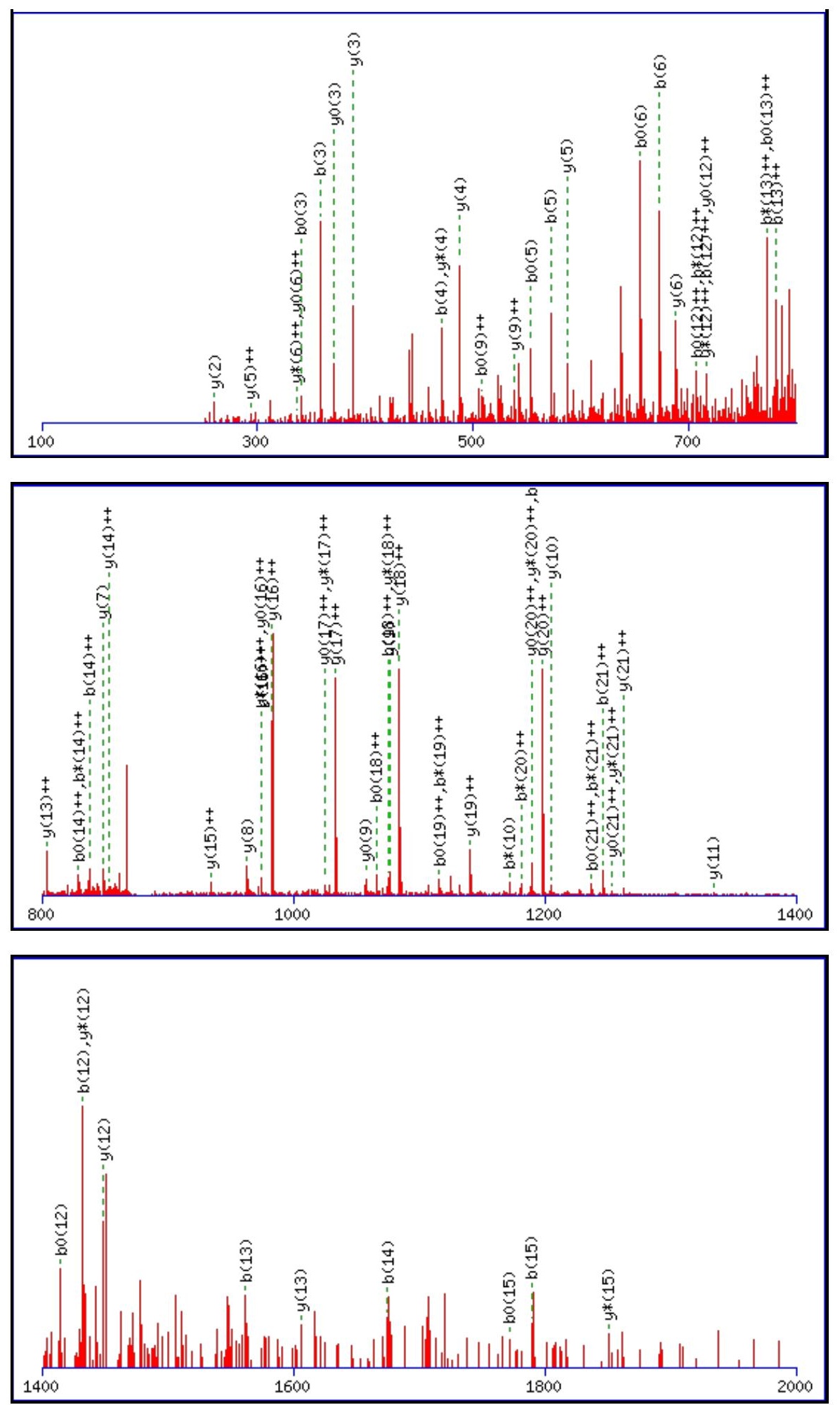

Monoisotopic mass of neutral peptide $\operatorname{Mr}($ calc): 2636.2891

Fixed modifications: Carbamidomethyl (C)

Variable modifications:

N15 : Deamidated N (N)

Ions Score: 52 Expect: 0.0014

Matches (Bold Red): 75/228 fragment ions using 178 most intense peaks

\begin{tabular}{|c|c|c|c|c|c|c|c|c|c|c|c|c|c|c|}
\hline$\#$ & $\mathbf{b}$ & $\mathbf{b}^{++}$ & $\mathbf{b}^{*}$ & $\mathbf{b}^{\boldsymbol{*}^{++}}$ & $\mathbf{b}^{\mathbf{0}}$ & $\mathbf{b}^{\mathbf{0 + +}}$ & Seq. & $\mathbf{y}$ & $\mathbf{y}^{++}$ & $\mathbf{y}^{\mathbf{*}}$ & $\mathbf{y}^{\mathbf{*}^{++}}$ & $\mathbf{y}^{\mathbf{0}}$ & $\mathbf{y}^{\mathbf{0 + +}}$ & $\#$ \\
\hline $\mathbf{1}$ & 114.0913 & 57.5493 & & & & & $\mathbf{L}$ & & & & & & & $\mathbf{2 2}$ \\
\hline $\mathbf{2}$ & 243.1339 & 122.0706 & & & 225.1234 & 113.0653 & $\mathbf{E}$ & 2524.2123 & $\mathbf{1 2 6 2 . 6 0 9 8}$ & 2507.1858 & $\mathbf{1 2 5 4 . 0 9 6 5}$ & 2506.2018 & $\mathbf{1 2 5 3 . 6 0 4 5}$ & $\mathbf{2 1}$ \\
\hline $\mathbf{3}$ & $\mathbf{3 5 8 . 1 6 0 9}$ & 179.5841 & & & 340.1503 & 170.5788 & $\mathbf{D}$ & 2395.1697 & $\mathbf{1 1 9 8 . 0 8 8 5}$ & 2378.1432 & $\mathbf{1 1 8 9 . 5 7 5 2}$ & 2377.1592 & $\mathbf{1 1 8 9 . 0 8 3 2}$ & $\mathbf{2 0}$ \\
\hline $\mathbf{4}$ & $\mathbf{4 7 1 . 2 4 4 9}$ & 236.1261 & & & 453.2344 & 227.1208 & L & 2280.1428 & $\mathbf{1 1 4 0 . 5 7 5 0}$ & 2263.1162 & 1132.0618 & 2262.1322 & 1131.5697 & $\mathbf{1 9}$ \\
\hline $\mathbf{5}$ & $\mathbf{5 7 2 . 2 9 2 6}$ & 286.6499 & & & 554.2821 & 277.6447 & $\mathbf{T}$ & 2167.0587 & $\mathbf{1 0 8 4 . 0 3 3 0}$ & 2150.0322 & $\mathbf{1 0 7 5 . 5 1 9 7}$ & 2149.0482 & 1075.0277 & $\mathbf{1 8}$ \\
\hline
\end{tabular}




\begin{tabular}{|c|c|c|c|c|c|c|c|c|c|c|c|c|c|c|}
\hline 6 & 673.3403 & 337.1738 & & & 655.3297 & 328.1685 & $\mathbf{T}$ & 2066.0110 & 1033.5092 & |2048.9845 & 1024.9959 & |2048.0005| & & |17| \\
\hline 7 & 770.3931 & 385.7002 & & & 752.3825 & 376.6949 & $\mathbf{P}$ & 1964.9634 & 982.9853 & 1947.9368 & 974.4720 & 1946.9528 & 973.9800 & 16 \\
\hline 8 & 933.4564 & 467.2318 & & & 915.4458 & 458.2266 & $\mathbf{Y}$ & 1867.9106 & 934.4589 & 1850.8841 & 925.9457 & 1849.9000 & 925.4537 & 15 \\
\hline 9 & 1032.5248 & 516.7660 & & & 1014.5142 & 507.7608 & $\mathbf{V}$ & 1704.8473 & 852.9273 & 1687.8207 & 844.4140 & 1686.8367 & 843.9220 & 14 \\
\hline 10 & 1188.6259 & 594.8166 & 1171.5994 & 586.3033 & 1170.6154 & 585.8113 & $\mathbf{R}$ & 1605.7789 & 803.3931 & 1588.7523 & 794.8798 & 1587.7683 & 794.3878 & 13 \\
\hline 11 & 1303.6529 & 652.3301 & 1286.6263 & 643.8168 & 1285.6423 & 643.3248 & D & 1449.6777 & 725.3425 & 1432.6512 & 716.8292 & 1431.6672 & 716.3372 & 12 \\
\hline 12 & 1432.6955 & 716.8514 & 1415.6689 & 708.3381 & 1414.6849 & 707.8461 & $\mathbf{E}$ & 1334.6508 & 667.8290 & 1317.6243 & 659.3158 & 1316.6402 & 658.8238 & 11 \\
\hline 13 & 1561.7380 & 781.3727 & 1544.7115 & 772.8594 & 1543.7275 & 772.3674 & $\mathbf{E}$ & 1205.6082 & 603.3077 & 1188.5817 & 594.7945 & 1187.5976 & 594.3025 & 10 \\
\hline 14 & 1674.8221 & 837.9147 & 1657.7956 & 829.4014 & 1656.8115 & 828.9094 & $\mathbf{L}$ & 1076.5656 & 538.7864 & 1059.5391 & 530.2732 & 1058.5551 & 529.7812 & 9 \\
\hline 15 & \begin{tabular}{|l|}
1789.8490 \\
\end{tabular} & 895.4282 & 1772.8225 & 886.9149 & 1771.8385 & 886.4229 & $\mathbf{N}$ & 963.4816 & 482.2444 & 946.4550 & 473.7311 & 945.4710 & 473.2391 & 8 \\
\hline 16 & 1949.8797 & 975.4435 & 1932.8531 & 966.9302 & 1931.8691 & 966.4382 & $\mathrm{C}$ & 848.4546 & 424.7309 & 831.4281 & 416.2177 & 830.4441 & 415.7257 & 7 \\
\hline 17 & 2050.9274 & 1025.9673 & 2033.9008 & 1017.4541 & 2032.9168 & 1016.9620 & $T$ & 688.4240 & 344.7156 & 671.3974 & 336.2023 & 670.4134 & 335.7103 & 6 \\
\hline 18 & 2149.9958 & 1075.5015 & 2132.9692 & 1066.9883 & 2131.9852 & 1066.4962 & V & 587.3763 & 294.1918 & 570.3497 & 285.6785 & 569.3657 & 285.1865 & 5 \\
\hline 19 & 2249.0642 & 1125.0357 & 2232.0377 & 1116.5225 & 2231.0536 & \begin{tabular}{|l|}
1116.0305 \\
\end{tabular} & $\mathbf{V}$ & 488.3079 & 244.6576 & 471.2813 & 236.1443 & 470.2973 & 235.6523 & 4 \\
\hline 20 & 2378.1068 & 1189.5570 & 2361.0802 & \begin{tabular}{|l}
1181.0438 \\
\end{tabular} & 2360.0962 & 1180.5518 & $\mathbf{E}$ & 389.2395 & 195.1234 & 372.2129 & 186.6101 & 371.2289 & 186.1181 & 3 \\
\hline 21 & 2491.1909 & 1246.0991 & 2474.1643 & 1237.5858 & 2473.1803 & 1237.0938 & $\mathbf{L}$ & 260.1969 & 130.6021 & 243.1703 & 122.0888 & & & 2 \\
\hline 22 & & & & & & & $\mathbf{K}$ & 147.1128 & 74.0600 & 130.0863 & 65.5468 & & & 1 \\
\hline
\end{tabular}
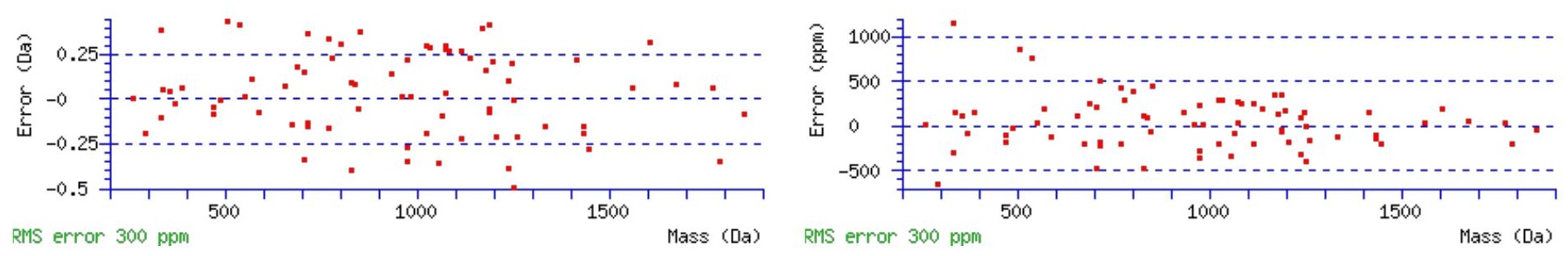

\section{All matches to this query}

\begin{tabular}{|l|l|c|l|}
\hline Score & Mr(calc): & Delta & \multicolumn{1}{|c|}{ Sequence } \\
\hline 52.2 & 2636.2891 & 0.0073 & LEDLTTPYVRDEELNCTVVELK \\
\hline 11.1 & 2635.3051 & 0.9913 & LEDLTTPYVRDEELNCTVVELK \\
\hline 4.5 & 2635.2693 & 1.0270 & TVTGSGMAVTRTIPSLTLTSTPLR \\
\hline 2.2 & 2636.3210 & -0.0247 & LEDLASLVYLNESSVLHTLRQR \\
\hline 2.2 & 2636.3210 & -0.0247 & LEDLASLVYLNESSVLHTLRQR \\
\hline 1.1 & 2636.2735 & 0.0229 & EIAVGDSEGQIVIYDVGEQIAVPR \\
\hline 1.1 & 2635.2676 & 1.0288 & IKNLSLQLKNQGPEESEAICQR \\
\hline 0.9 & 2636.3210 & -0.0247 & LEDLASLVYLNESSVLHTLRQR \\
\hline 0.9 & 2636.3210 & -0.0247 & LEDLASLVYLNESSVLHTLROR \\
\hline 0.9 & 2635.2771 & 1.0192 & LKGEAATSAASEGAKHVPAGQLAALK \\
\hline
\end{tabular}

Spectrum No: 640; Query: 1883; Rank: 1

\section{Peptide View}

MS/MS Fragmentation of AYIFERNQSVGDPTVDLIR

Found in IPI00231478, Tax_Id=10116 Gene_Symbol=Scarb2 Lysosome membrane protein 2

Match to Query 1883: 2193.113232 from(732.045020,3+)

Title: 100101RatKid_NS_deglyco_08.3797.3797.3.dta

Data file K:INewmanPaper|Piliangl3SubProteomes\Piliang3SP\mgf5ppm\ERLIC_3SubProteomes5ppm.mgf 

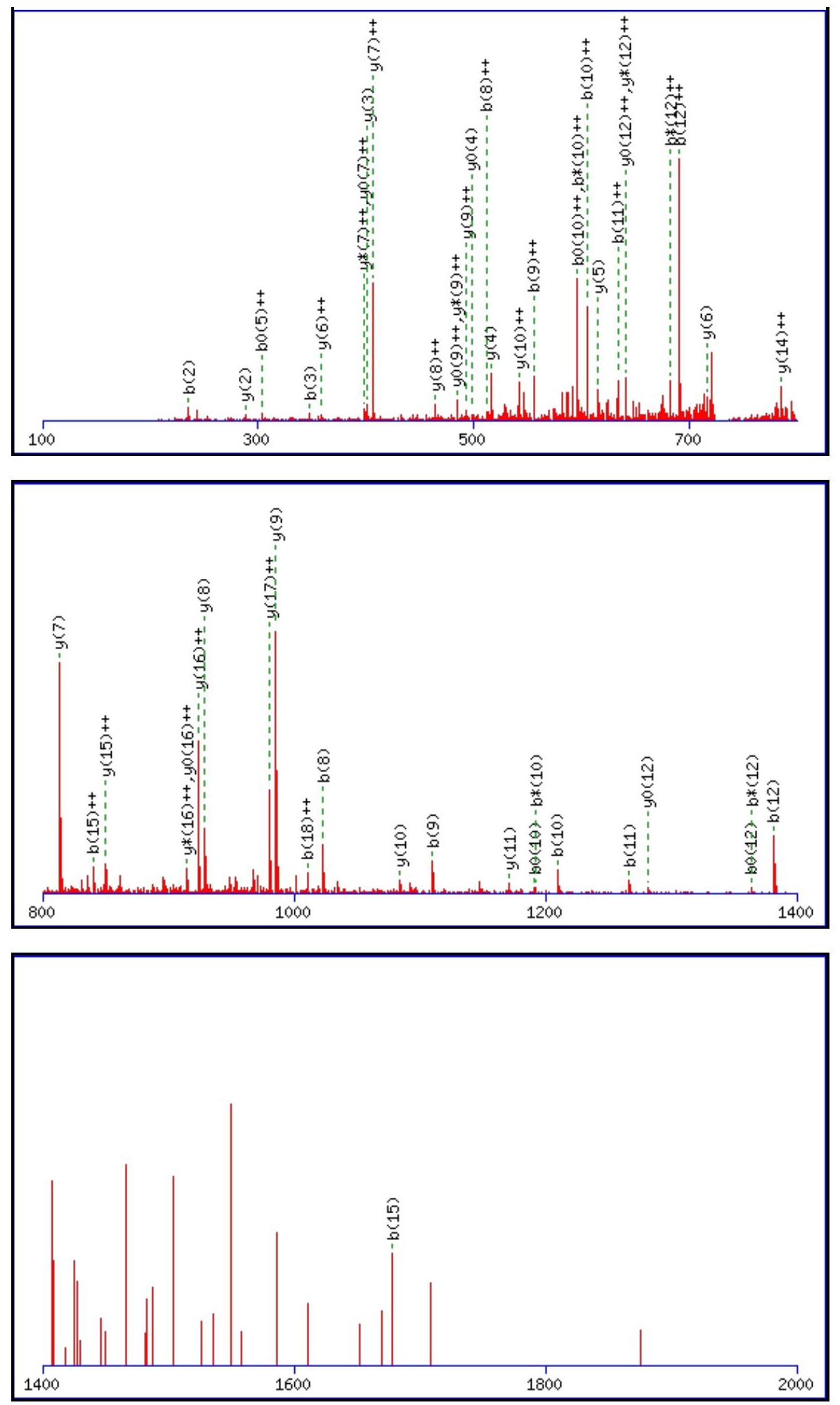

Monoisotopic mass of neutral peptide $\operatorname{Mr}($ calc): 2193.1066

Fixed modifications: Carbamidomethyl (C)

Variable modifications:

N7 : Deamidated $\mathrm{N}(\mathrm{N})$

Ions Score: 52 Expect: 0.001

Matches (Bold Red): 52/192 fragment ions using 94 most intense peaks

\begin{tabular}{|r|c|c|c|c|c|r|r|r|r|r|r|r|r|r|}
\hline$\#$ & $\mathbf{b}$ & $\mathbf{b}^{++}$ & $\mathbf{b}^{*}$ & $\mathbf{b}^{\boldsymbol{*}^{++}}$ & $\mathbf{b}^{\mathbf{0}}$ & $\mathbf{b}^{\mathbf{0 + +}}$ & Seq. & $\mathbf{y}$ & $\mathbf{y}^{++}$ & $\mathbf{y}^{\mathbf{*}}$ & $\mathbf{y}^{\mathbf{*}^{++}}$ & $\mathbf{y}^{\mathbf{0}}$ & $\mathbf{y}^{\mathbf{0 + +}}$ & $\#$ \\
\hline $\mathbf{1}$ & $\mathbf{7 2 . 0 4 4 4}$ & 36.5258 & & & & & $\mathbf{A}$ & & & & & & & $\mathbf{1 9}$ \\
\hline $\mathbf{2}$ & $\mathbf{2 3 5 . 1 0 7 7}$ & 118.0575 & & & & & $\mathbf{Y}$ & 2123.0768 & 1062.0420 & 2106.0502 & 1053.5287 & 2105.0662 & 1053.0367 & $\mathbf{1 8}$ \\
\hline 3 & 348.1918 & 174.5995 & & & & & $\mathbf{I}$ & 1960.0134 & $\mathbf{9 8 0 . 5 1 0 4}$ & 1942.9869 & 971.9971 & 1942.0029 & 971.5051 & $\mathbf{1 7}$ \\
\hline $\mathbf{4}$ & 495.2602 & 248.1337 & & & & & $\mathbf{F}$ & 1846.9294 & $\mathbf{9 2 3 . 9 6 8 3}$ & 1829.9028 & $\mathbf{9 1 5 . 4 5 5 0}$ & $\mathbf{1 8 2 8 . 9 1 8 8}$ & $\mathbf{9 1 4 . 9 6 3 0}$ & $\mathbf{1 6}$ \\
\hline $\mathbf{5}$ & 624.3028 & 312.6550 & & & 606.2922 & $\mathbf{3 0 3 . 6 4 9 8}$ & $\mathbf{E}$ & 1699.8609 & $\mathbf{8 5 0 . 4 3 4 1}$ & 1682.8344 & 841.9208 & 1681.8504 & 841.4288 & $\mathbf{1 5}$ \\
\hline
\end{tabular}




\begin{tabular}{|c|c|c|c|c|c|c|c|c|c|c|c|c|c|c|}
\hline 6 & 780.4039 & 390.7056 & 763.3774 & 382.1923 & 762.3933 & 381.7003 & $\mathbf{R}$ & |1570.8184| & 785.9128 & |1553.7918 & 777.3995 & |1552.8078| & 776.9075 & 14 \\
\hline 7 & 895.4308 & 448.2191 & 878.4043 & 439.7058 & 877.4203 & 439.2138 & $\mathbf{N}$ & 1414.7172 & 707.8623 & 1397.6907 & 699.3490 & 1396.7067 & 698.8570 & 13 \\
\hline 8 & 1023.4894 & 512.2483 & 1006.4629 & 503.7351 & 1005.4789 & 503.2431 & $\mathbf{Q}$ & 1299.6903 & 650.3488 & 1282.6638 & 641.8355 & 1281.6797 & 641.3435 & 12 \\
\hline 9 & 1110.5214 & 555.7644 & 1093.4949 & 547.2511 & 1092.5109 & 546.7591 & S & 1171.6317 & 586.3195 & 1154.6052 & 577.8062 & 1153.6212 & 577.3142 & 11 \\
\hline 10 & 1209.5899 & 605.2986 & 1192.5633 & 596.7853 & 1191.5793 & 596.2933 & V & 1084.5997 & 542.8035 & 1067.5732 & 534.2902 & 1066.5891 & 533.7982 & 10 \\
\hline 11 & 1266.6113 & 633.8093 & 1249.5848 & 625.2960 & 1248.6008 & 624.8040 & G & 985.5313 & 493.2693 & 968.5047 & 484.7560 & 967.5207 & 484.2640 & 9 \\
\hline 12 & 1381.6383 & 691.3228 & 1364.6117 & 682.8095 & 1363.6277 & 682.3175 & D & 928.5098 & 464.7585 & 911.4833 & 456.2453 & 910.4993 & 455.7533 & 8 \\
\hline 13 & 1478.6910 & 739.8492 & 1461.6645 & 731.3359 & 1460.6805 & 730.8439 & $\mathbf{P}$ & 813.4829 & 407.2451 & 796.4563 & 398.7318 & 795.4723 & 398.2398 & 7 \\
\hline 14 & 1579.7387 & 790.3730 & 1562.7122 & 781.8597 & 1561.7281 & 781.3677 & $\mathbf{T}$ & 716.4301 & 358.7187 & 699.4036 & 350.2054 & 698.4196 & 349.7134 & 6 \\
\hline 15 & 1678.8071 & 839.9072 & 1661.7806 & 831.3939 & 1660.7966 & 830.9019 & $\mathbf{V}$ & 615.3824 & 308.1949 & 598.3559 & 299.6816 & 597.3719 & 299.1896 & 5 \\
\hline 16 & 1793.8341 & 897.4207 & 1776.8075 & 888.9074 & 1775.8235 & 888.4154 & D & 516.3140 & 258.6606 & 499.2875 & 250.1474 & 498.3035 & 249.6554 & 4 \\
\hline 17 & 1906.9181 & 953.9627 & 1889.8916 & 945.4494 & 1888.9076 & 944.9574 & $\mathbf{L}$ & 401.2871 & 201.1472 & 384.2605 & 192.6339 & & & 3 \\
\hline 18 & 2020.0022 & 1010.5047 & 2002.9756 & 1001.9915 & 2001.9916 & 1001.4995 & I & 288.2030 & 144.6051 & 271.1765 & 136.0919 & & & 2 \\
\hline 19 & & & & & & & $\mathbf{R}$ & 175.1190 & 88.0631 & 158.0924 & 79.5498 & & & 1 \\
\hline
\end{tabular}
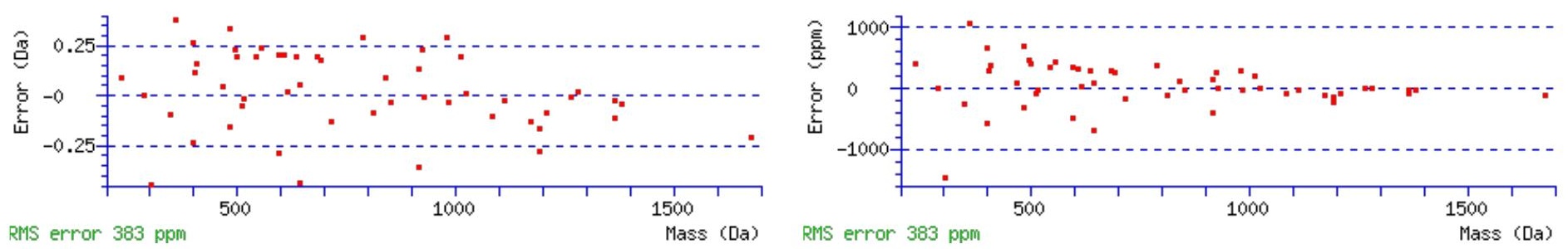

\section{All matches to this query}

\begin{tabular}{|l|l|l|l|}
\hline Score & Mr(calc): & Delta & \multicolumn{1}{|c|}{ Sequence } \\
\hline 52.1 & 2193.1066 & 0.0066 & AYIFERNQSVGDPTVDLIR \\
\hline 19.0 & 2192.1226 & 0.9907 & AYIFERNQSVGDPTVDLIR \\
\hline 3.4 & 2191.0894 & 2.0238 & GTLGGMFGMLKGLVGSKSLSR \\
\hline 2.5 & 2191.1137 & 1.9995 & LPVTSLGLAASTNGSSITPTPK \\
\hline 1.8 & 2191.1137 & 1.9995 & LPVTSLGLAASTNGSSITPTPK \\
\hline 0.3 & 2193.0943 & 0.0189 & TLPGAPAGFSGIGRVTALDASR \\
\hline
\end{tabular}

Spectrum No: 641; Query: 41; Rank: 1

\section{Peptide View}

MS/MS Fragmentation of VNVTNEFK

Found in IPI00196620, Tax_Id=10116 Gene_Symbol=Cubn Cubilin precursor

Match to Query 41: 950.469188 from(476.241870,2+)

Title: 091008RatKidney_NH4Format02_26.1248.1248.2.dta

Data file K:INewmanPaper|Piliang|3SubProteomes\Piliang3SP\mgf5ppm\ERLIC_3SubProteomes5ppm.mgf 

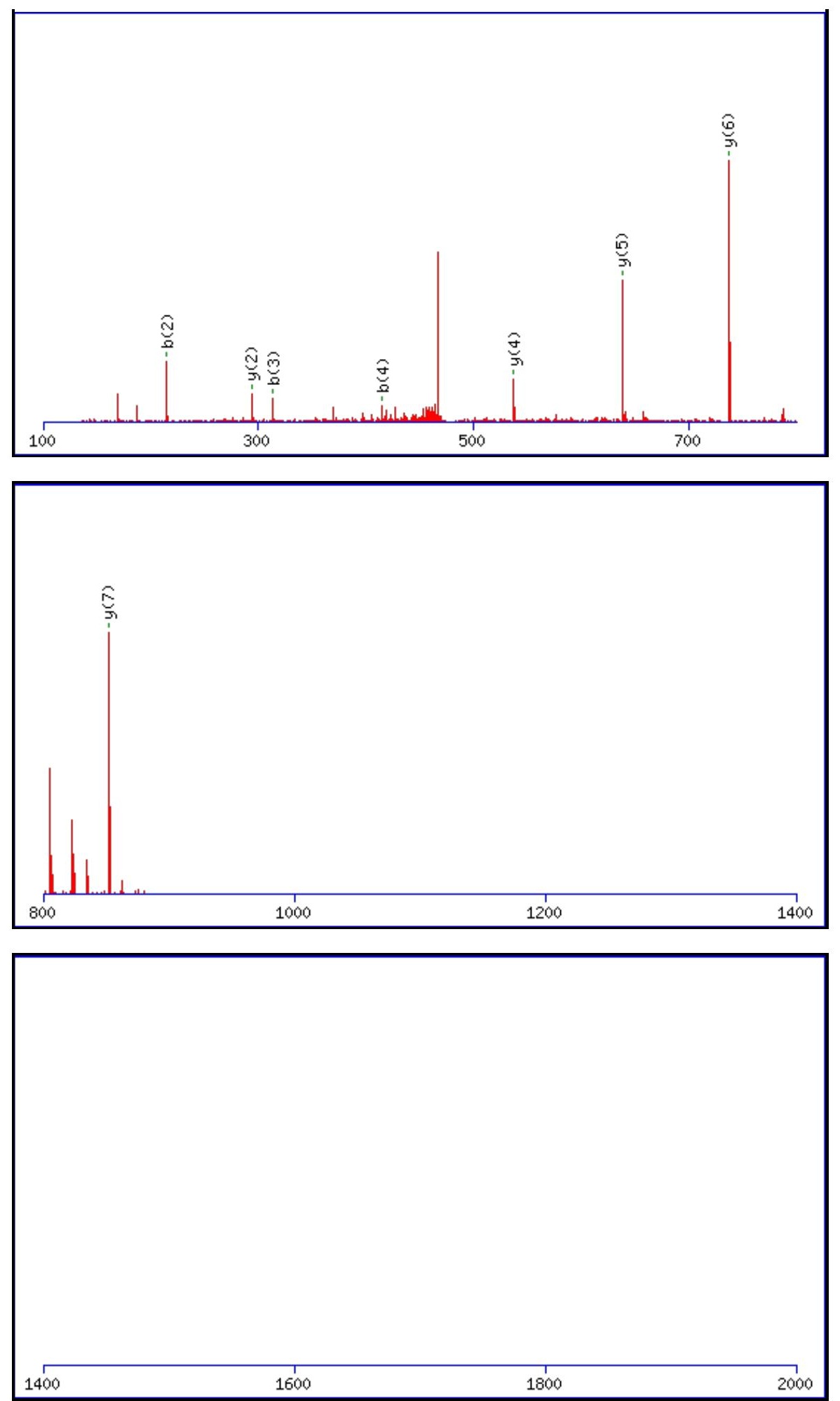

Monoisotopic mass of neutral peptide $\operatorname{Mr}($ calc): 950.4709

Fixed modifications: Carbamidomethyl (C)

Variable modifications:

N2 : Deamidated N $(\mathrm{N})$

Ions Score: 52 Expect: 0.00056

Matches (Bold Red): 8/72 fragment ions using 12 most intense peaks

\begin{tabular}{|c|c|c|c|c|c|c|c|c|c|c|c|c|c|c|}
\hline$\#$ & $\mathbf{b}$ & $\mathbf{b}^{++}$ & $\mathbf{b}^{*}$ & $\mathbf{b}^{\boldsymbol{*}^{++}}$ & $\mathbf{b}^{\mathbf{0}}$ & $\mathbf{b}^{\mathbf{0}+}$ & $\mathbf{S e q}$ & $\mathbf{y}$ & $\mathbf{y}^{++}$ & $\mathbf{y}^{*}$ & $\mathbf{y}^{\boldsymbol{*}^{++}}$ & $\mathbf{y}^{\mathbf{0}}$ & $\mathbf{y}^{\mathbf{0}++}$ & $\#$ \\
\hline $\mathbf{1}$ & 100.0757 & 50.5415 & & & & & $\mathbf{V}$ & & & & & & & $\mathbf{8}$ \\
\hline $\mathbf{2}$ & $\mathbf{2 1 5 . 1 0 2 6}$ & 108.0550 & 198.0761 & 99.5417 & & & $\mathbf{N}$ & $\mathbf{8 5 2 . 4 0 9 8}$ & 426.7085 & 835.3832 & 418.1952 & 834.3992 & 417.7032 & $\mathbf{7}$ \\
\hline $\mathbf{3}$ & 314.1710 & 157.5892 & 297.1445 & 149.0759 & & & $\mathbf{V}$ & 737.3828 & 369.1951 & 720.3563 & 360.6818 & 719.3723 & 360.1898 & $\mathbf{6}$ \\
\hline $\mathbf{4}$ & $\mathbf{4 1 5 . 2 1 8 7}$ & 208.1130 & 398.1922 & 199.5997 & 397.2082 & 199.1077 & $\mathbf{T}$ & $\mathbf{6 3 8 . 3 1 4 4}$ & 319.6608 & 621.2879 & 311.1476 & 620.3039 & 310.6556 & $\mathbf{5}$ \\
\hline $\mathbf{5}$ & 529.2616 & 265.1345 & 512.2351 & 256.6212 & 511.2511 & 256.1292 & $\mathbf{N}$ & 537.2667 & 269.1370 & 520.2402 & 260.6237 & 519.2562 & 260.1317 & $\mathbf{4}$ \\
\hline
\end{tabular}




\begin{tabular}{|l|l|l|l|l|l|l|l|l|l|l|l|l|l|l|l|l|l|}
$\mathbf{6}$ & 658.3042 & 329.6558 & 641.2777 & 321.1425 & 640.2937 & 320.6505 & E & 423.2238 & 212.1155 & 406.1973 & 203.6023 & 405.2132 & 203.1103 & $\mathbf{3}$ \\
\hline $\mathbf{7}$ & 805.3727 & 403.1900 & 788.3461 & 394.6767 & 787.3621 & 394.1847 & F & 294.1812 & 147.5942 & 277.1547 & 139.0810 & & & $\mathbf{2}$ \\
\hline $\mathbf{8}$ & & & & & & & K & 147.1128 & 74.0600 & 130.0863 & 65.5468 & & & $\mathbf{1}$ \\
\hline
\end{tabular}
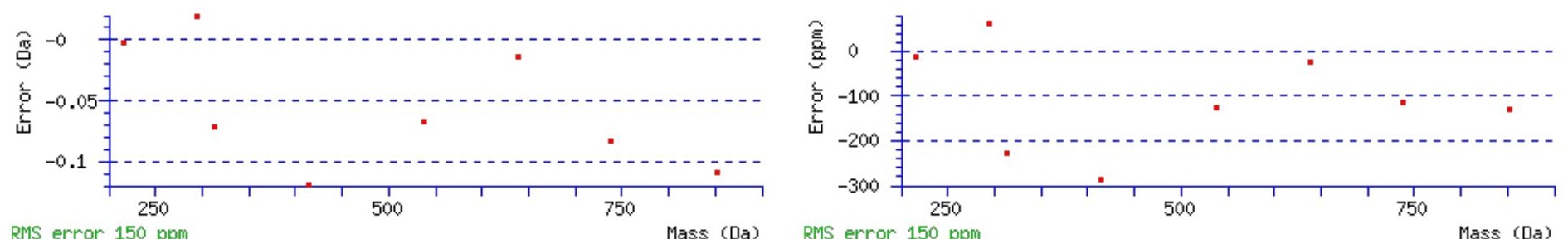

RMS error 150 ppm

\section{All matches to this query}

\begin{tabular}{|l|l|l|l|}
\hline Score & Mr(calc): & Delta & \multicolumn{1}{|c|}{ Sequence } \\
\hline 52.1 & 950.4709 & -0.0017 & VNVTNEFK \\
\hline 26.2 & 950.4709 & -0.0017 & VNVTNEFK \\
\hline 9.1 & 950.4644 & 0.0048 & WIDRSMK \\
\hline 6.1 & 950.4709 & -0.0017 & NVQFTVDK \\
\hline 5.7 & 950.4743 & -0.0051 & LTAVEGSMK \\
\hline 2.6 & 950.4709 & -0.0017 & QTLEDAFK \\
\hline 2.5 & 950.4644 & 0.0048 & NLRSWMK \\
\hline 2.5 & 950.4626 & 0.0065 & EKSFKSK \\
\hline 2.3 & 950.4709 & -0.0017 & DSIINDFK \\
\hline 2.2 & 950.4743 & -0.0051 & AATVLDSMK \\
\hline
\end{tabular}

Spectrum No: 642; Query: 1285; Rank: 1

\section{Peptide View}

MS/MS Fragmentation of QDPVSWNETFEDISR

Found in IPI00765413, Tax_Id=10116 Gene_Symbol=- 58 kDa protein

Match to Query 1285: 1822.807988 from(912.411270,2+)

Title: 100101RatKid_NS_deglyco_18.4367.4367.2.dta

Data file K:\NewmanPaper|Piliangl3SubProteomes\Piliang3SP\mgf5ppm\ERLIC_3SubProteomes5ppm.mgf

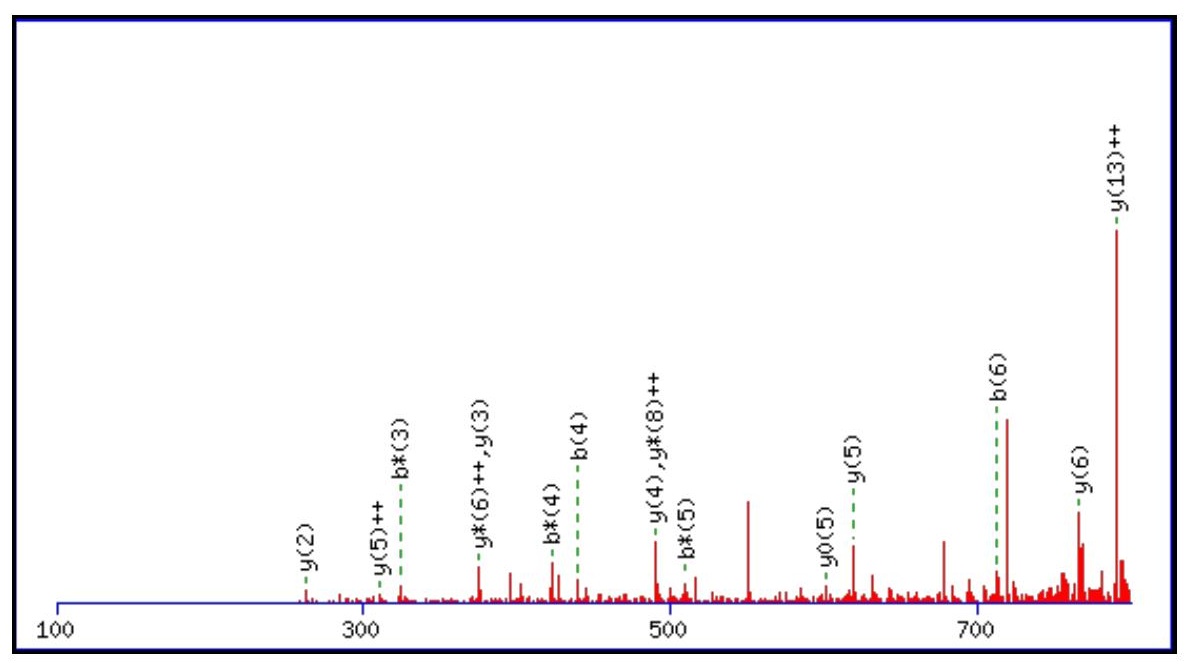



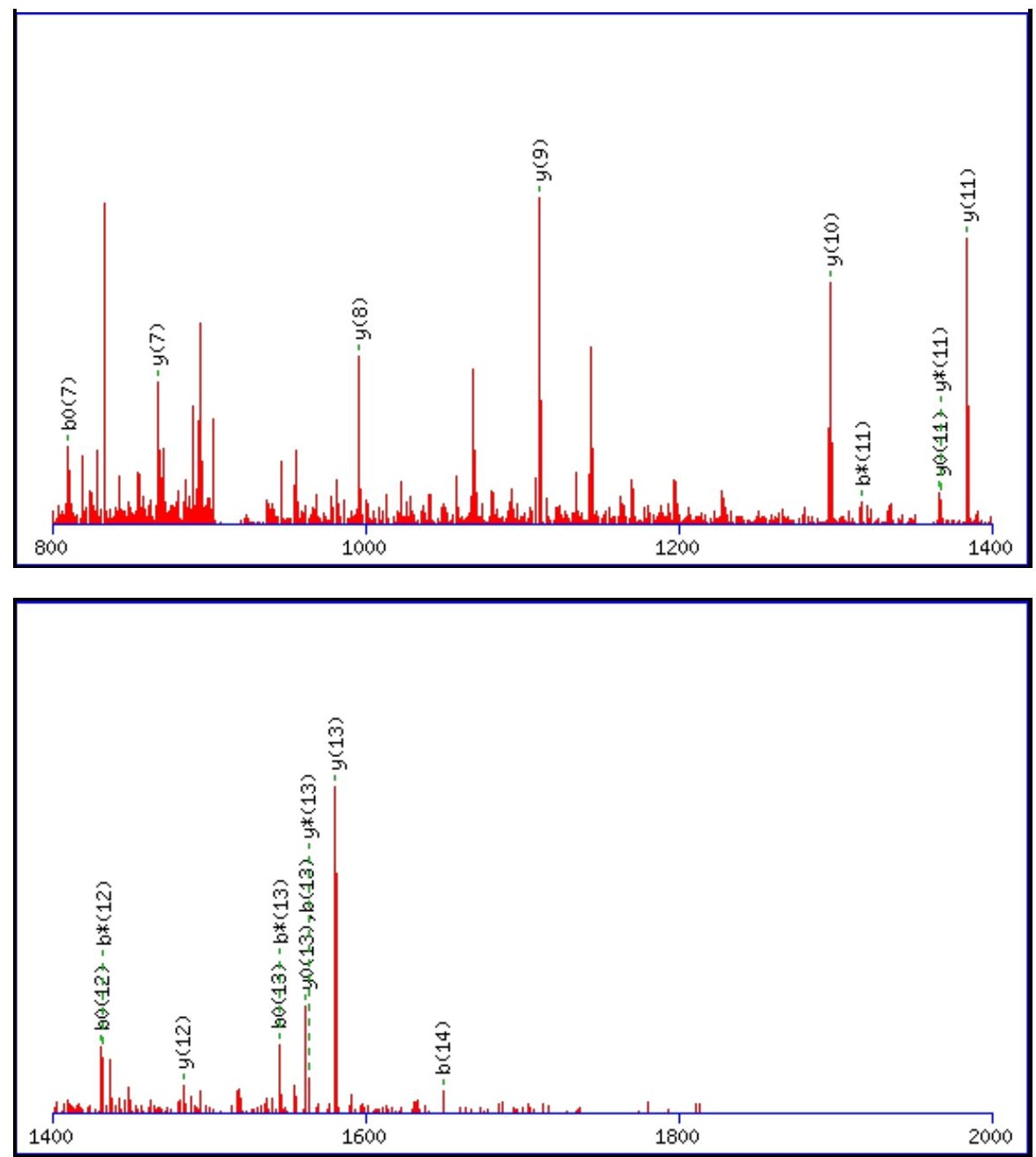

Monoisotopic mass of neutral peptide $\operatorname{Mr}($ calc): 1822.8010

Fixed modifications: Carbamidomethyl (C)

Variable modifications:

N7 : Deamidated $\mathrm{N}(\mathrm{N})$

Ions Score: 52 Expect: 0.0013

Matches (Bold Red): 34/164 fragment ions using 85 most intense peaks

\begin{tabular}{|c|c|c|c|c|c|c|c|c|c|c|c|c|c|c|}
\hline \# & b & $\mathbf{b}^{++}$ & $\mathbf{b}^{*}$ & $\mathbf{b}^{*^{++}}$ & $\mathbf{b}^{0}$ & $\mathbf{b}^{0++}$ & Seq. & $\mathbf{y}$ & $\mathbf{y}^{++}$ & $\mathbf{y}^{*}$ & $\mathbf{y}^{*^{++}}$ & $\mathbf{y}^{0}$ & & \# \\
\hline 1 & 129.0659 & 65.0366 & 112.0393 & 56.5233 & & & $\mathbf{Q}$ & & & & & & & 15 \\
\hline 2 & 244.0928 & 122.5500 & 227.0662 & 114.0368 & 226.0822 & 113.5448 & D & 1695.7497 & 848.3785 & 1678.7231 & & 1677.7391 & 839.3732 & 14 \\
\hline 3 & 341.1456 & 171.0764 & 324.1190 & 162.5631 & 323.1350 & 162.0711 & $\mathbf{P}$ & 1580.7227 & 790.8650 & 1563.6962 & 782.3517 & 1562.7122 & 781.8597 & 13 \\
\hline 4 & 440.2140 & 220.6106 & 423.1874 & 212.0974 & 422.2034 & 211.6053 & V & 1483.6700 & 742.3386 & 1466.6434 & 733.8253 & 1465.6594 & 733.3333 & 12 \\
\hline 5 & 527.2460 & 264.1266 & 510.2195 & 255.6134 & 509.2354 & 255.1214 & S & 1384.6015 & 692.8044 & 1367.5750 & 684.2911 & 1366.5910 & 683.7991 & 11 \\
\hline 6 & 713.3253 & 357.1663 & 696.2988 & 348.6530 & 695.3148 & 348.1610 & $\mathbf{W}$ & 1297.5695 & 649.2884 & 1280.5430 & 640.7751 & 1279.5590 & 640.2831 & 10 \\
\hline 7 & 828.3523 & 414.6798 & 811.3257 & 406.1665 & 810.3417 & 405.6745 & $\mathbf{N}$ & 1111.4902 & 556.2487 & 1094.4637 & 547.7355 & 1093.4796 & 547.2435 & 9 \\
\hline 8 & 957.3948 & 479.2011 & 940.3683 & 470.6878 & 939.3843 & 470.1958 & $\mathbf{E}$ & 996.4633 & 498.7353 & 979.4367 & 490.2220 & 978.4527 & 489.7300 & 8 \\
\hline 9 & 1058.4425 & 529.7249 & 1041.4160 & 521.2116 & 1040.4320 & 520.7196 & $\mathbf{T}$ & 867.4207 & 434.2140 & 850.3941 & 425.7007 & 849.4101 & 425.2087 & 7 \\
\hline 10 & 1205.5109 & 603.2591 & 1188.4844 & 594.7458 & 1187.5004 & 594.2538 & $\mathbf{F}$ & 766.3730 & 383.6901 & 749.3464 & 375.1769 & 748.3624 & 374.6849 & 6 \\
\hline 11 & 1334.5535 & 667.7804 & 1317.5270 & 659.2671 & 1316.5430 & 658.7751 & $\mathbf{E}$ & 619.3046 & 310.1559 & 602.2780 & 301.6427 & 601.2940 & 301.1506 & 5 \\
\hline 12 & 1449.5805 & 725.2939 & 1432.5539 & 716.7806 & 1431.5699 & 716.2886 & D & 490.2620 & 245.6346 & 473.2354 & 237.1214 & 472.2514 & 236.6293 & 4 \\
\hline 13 & 1562.6645 & 781.8359 & 1545.6380 & 773.3226 & 1544.6540 & 772.8306 & I & 375.2350 & 188.1212 & 358.2085 & 179.6079 & 357.2245 & 179.1159 & 3 \\
\hline 14 & 1649.6966 & 825.3519 & 1632.6700 & 816.8386 & 1631.6860 & 816.3466 & S & 262.1510 & 131.5791 & 245.1244 & 123.0659 & 244.1404 & 122.5738 & 2 \\
\hline 15 & & & & & & & $\mathbf{R}$ & 175.1190 & 88.0631 & 158.0924 & 79.5498 & & & 1 \\
\hline
\end{tabular}



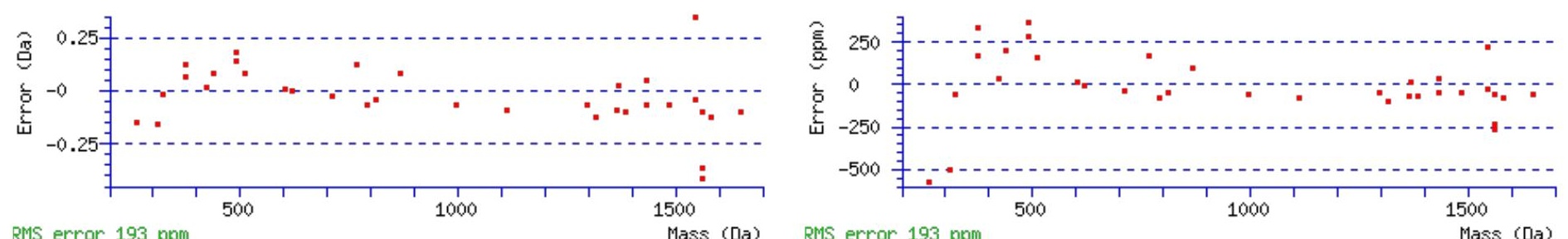

RMS error 193 ppm Mass (Da) RMS error 193 ppm

\section{All matches to this query}

\begin{tabular}{|l|l|c|l|}
\hline Score & Mr(calc): & Delta & Sequence \\
\hline 52.1 & 1822.8010 & 0.0070 & QDPVSWNETFEDISR \\
\hline 14.9 & 1821.8170 & 0.9910 & QDPVSWNETFEDISR \\
\hline 3.3 & 1821.8063 & 1.0017 & IQQQTNTYIITPSR \\
\hline 1.6 & 1821.8063 & 1.0017 & IQQQTNTYIITPSR \\
\hline
\end{tabular}

Spectrum No: 643; Query: 1238; Rank: 1

\section{Peptide View}

\section{MS/MS Fragmentation of LWSSNLTTNMDFAFK}

Found in IPI00394353, Tax_Id=10116 Gene_Symbol=Hexa Beta-hexosaminidase alpha chain precursor

Match to Query 1238: 1790.819048 from(896.416800,2+)

Title: 091008RatKidney_NH4Format01_23.3271.3271.2.dta

Data file K:INewmanPaper|Piliangl3SubProteomes\Piliang3SP\mgf5ppm\ERLIC_3SubProteomes5ppm.mgf

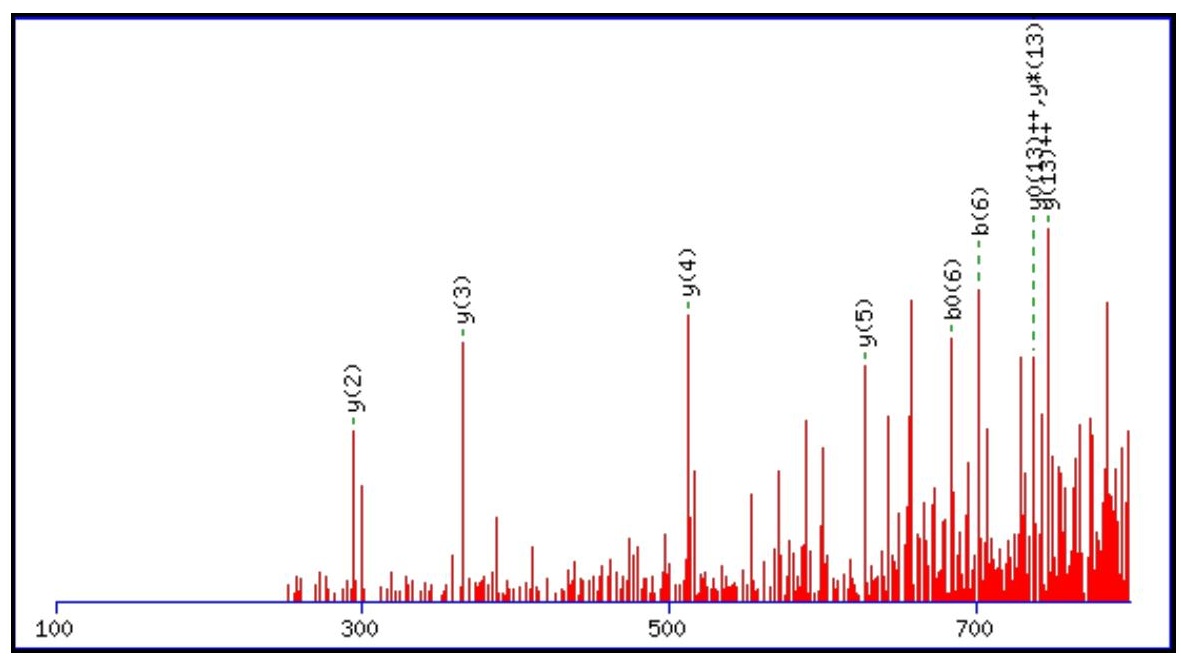



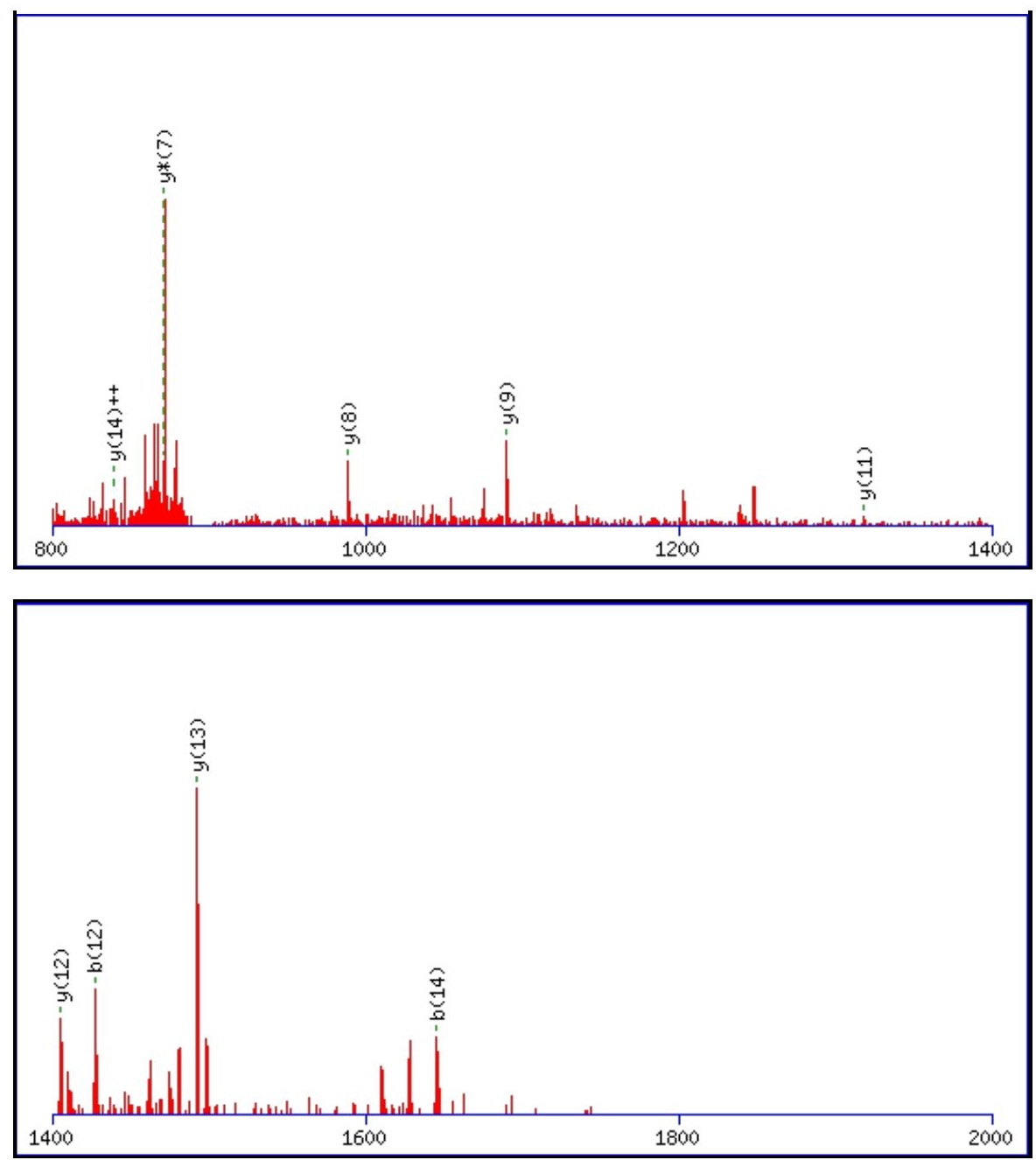

Monoisotopic mass of neutral peptide $\operatorname{Mr}($ calc): 1790.8185

Fixed modifications: Carbamidomethyl (C)

Variable modifications:

N5 : Deamidated_N (N)

M10 : Oxidation $(\mathrm{M})$

Ions Score: 52 Expect : 0.0014

Matches (Bold Red): 18/148 fragment ions using 36 most intense peaks

\begin{tabular}{|c|c|c|c|c|c|c|c|c|c|c|c|c|c|c|}
\hline \# & b & $\mathbf{b}^{++}$ & $\mathbf{b}^{*}$ & $\mathbf{b}^{*^{++}}$ & $\mathbf{b}^{\mathbf{0}}$ & $\mathbf{b}^{\mathbf{0 + +}}$ & Seq. & $\mathbf{y}$ & $\mathbf{y}^{++}$ & $\mathbf{y}^{*}$ & $\mathrm{y}^{*^{++}}$ & $\mathbf{y}^{\mathbf{0}}$ & $\mathbf{y}^{\mathbf{0 + +}}$ & \# \\
\hline 1 & 114.0913 & 57.5493 & & & & & $\mathbf{L}$ & & & & & & & 15 \\
\hline 2 & 300.1707 & 150.5890 & & & & & $\mathbf{W}$ & 1678.7417 & 839.8745 & 1661.7152 & 831.3612 & 1660.7312 & 830.8692 & 14 \\
\hline 3 & 387.2027 & 194.1050 & & & 369.1921 & 185.0997 & S & 1492.6624 & 746.8349 & 1475.6359 & 738.3216 & 1474.6519 & 737.8296 & 13 \\
\hline 4 & 474.2347 & 237.6210 & & & 456.2241 & 228.6157 & S & 1405.6304 & 703.3188 & 1388.6039 & 694.8056 & 1387.6198 & 694.3136 & 12 \\
\hline 5 & 589.2616 & 295.1345 & 572.2351 & 286.6212 & 571.2511 & 286.1292 & $\mathbf{N}$ & 1318.5984 & 659.8028 & 1301.5718 & 651.2896 & 1300.5878 & 650.7975 & 11 \\
\hline 6 & 702.3457 & 351.6765 & 685.3192 & 343.1632 & 684.3351 & 342.6712 & $\mathbf{L}$ & 1203.5714 & 602.2894 & 1186.5449 & 593.7761 & 1185.5609 & 593.2841 & 10 \\
\hline 7 & 803.3934 & 402.2003 & 786.3668 & 393.6871 & 785.3828 & 393.1950 & $\mathbf{T}$ & 1090.4874 & 545.7473 & 1073.4608 & 537.2341 & 1072.4768 & 536.7420 & 9 \\
\hline 8 & 904.4411 & 452.7242 & 887.4145 & 444.2109 & 886.4305 & 443.7189 & $\mathbf{T}$ & 989.4397 & 495.2235 & 972.4132 & 486.7102 & 971.4291 & 486.2182 & 8 \\
\hline 9 & 1018.4840 & 509.7456 & 1001.4574 & 501.2324 & 1000.4734 & 500.7404 & $\mathbf{N}$ & 888.3920 & 444.6996 & 871.3655 & 436.1864 & 870.3815 & 435.6944 & 7 \\
\hline 10 & 1165.5194 & 583.2633 & 1148.4928 & 574.7501 & 1147.5088 & 574.2581 & $\mathbf{M}$ & 774.3491 & 387.6782 & 757.3225 & 379.1649 & 756.3385 & 378.6729 & 6 \\
\hline 11 & 1280.5463 & 640.7768 & 1263.5198 & 632.2635 & 1262.5358 & 631.7715 & D & 627.3137 & 314.1605 & 610.2871 & 305.6472 & 609.3031 & 305.1552 & 5 \\
\hline 12 & 1427.6148 & 714.3110 & 1410.5882 & 705.7977 & 1409.6042 & 705.3057 & $\mathbf{F}$ & 512.2867 & 256.6470 & 495.2602 & 248.1337 & & & 4 \\
\hline 13 & 1498.6519 & 749.8296 & 1481.6253 & 741.3163 & 1480.6413 & 740.8243 & A & 365.2183 & 183.1128 & 348.1918 & 174.5995 & & & 3 \\
\hline 14 & 1645.7203 & 823.3638 & 1628.6937 & 814.8505 & 1627.7097 & 814.3585 & $\mathbf{F}$ & 294.1812 & 147.5942 & 277.1547 & 139.0810 & & & 2 \\
\hline 15 & & & & & & & $\mathbf{K}$ & 147.1128 & 74.0600 & 130.0863 & 65.5468 & & & 1 \\
\hline
\end{tabular}



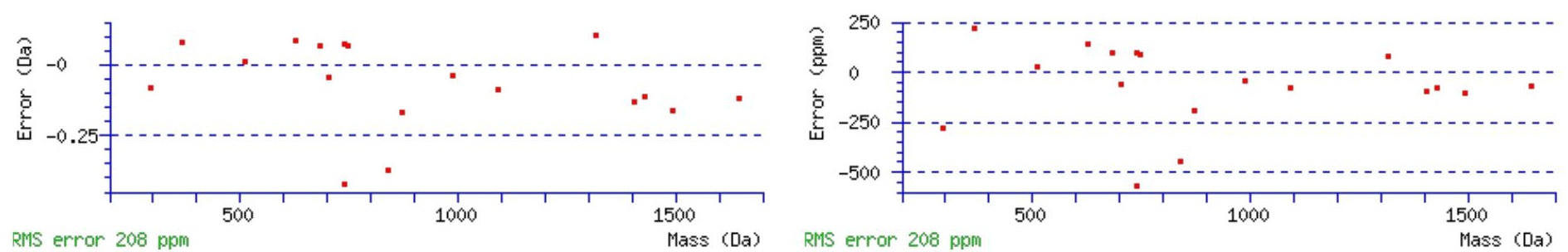

RNS error $208 \mathrm{ppm}$

\section{All matches to this query}

\begin{tabular}{|l|l|l|l|}
\hline Score & Mr(calc): & Delta & Sequence \\
\hline 52.0 & 1790.8185 & 0.0005 & LWSSNLTTNMDFAFK \\
\hline 49.7 & 1790.8185 & 0.0005 & $\underline{\text { LWSSNLTTNMDFAFK }}$ \\
\hline 11.5 & 1790.8195 & -0.0004 & EEKITTVAEMASKMK \\
\hline 4.9 & 1790.8195 & -0.0004 & $\underline{\text { EEKITTVAEMASKMK }}$ \\
\hline 3.0 & 1790.8039 & 0.0152 & $\underline{\text { KSSEQLLGSMPVSLR }}$ \\
\hline 3.0 & 1790.8039 & 0.0152 & $\underline{\text { KSSEQLLGSMPVSLR }}$ \\
\hline
\end{tabular}

Spectrum No: 644; Query: 2018; Rank: 1

\section{Peptide View}

MS/MS Fragmentation of VTNLTQEQCQDPSKVPNESK

Found in IPI00192495, Tax_Id=10116 Gene_Symbol=Ncstn Isoform 1 of Nicastrin precursor

Match to Query 2018: 2302.082742 from(768.368190,3+)

Title: 100101RatKid_NS_deglyco_21.1117.1117.3.dta

Data file K:\NewmanPaper\Piliang \3SubProteomes\Piliang3SP \mgf5ppm\ERLIC_3SubProteomes5ppm.mgf

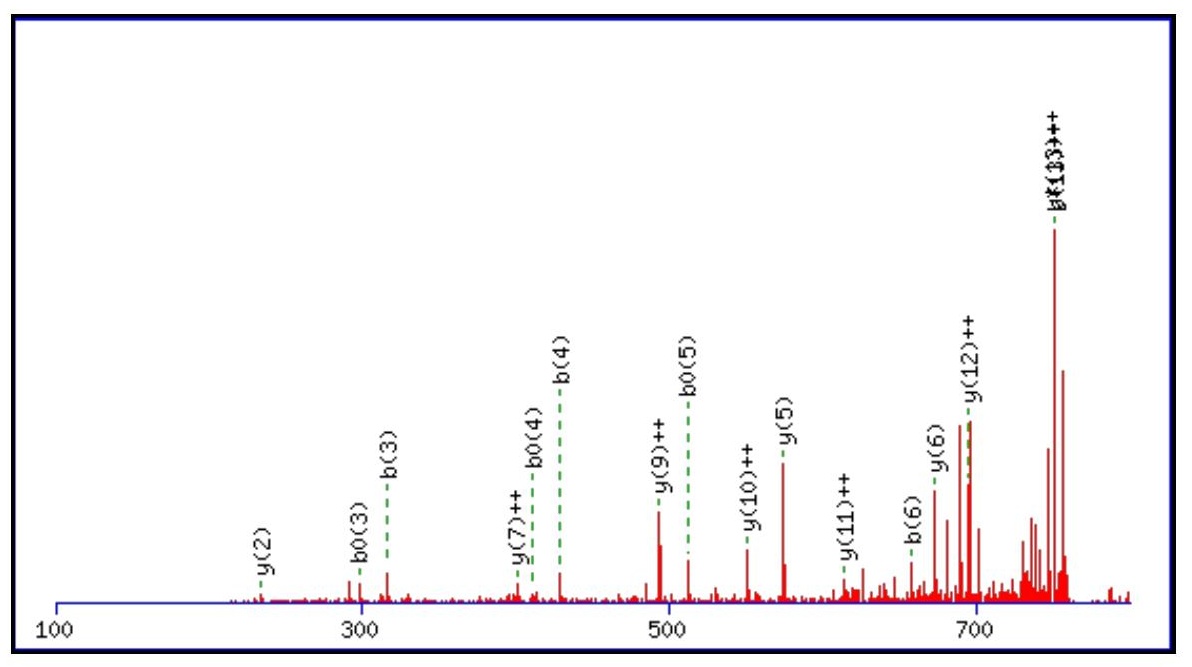



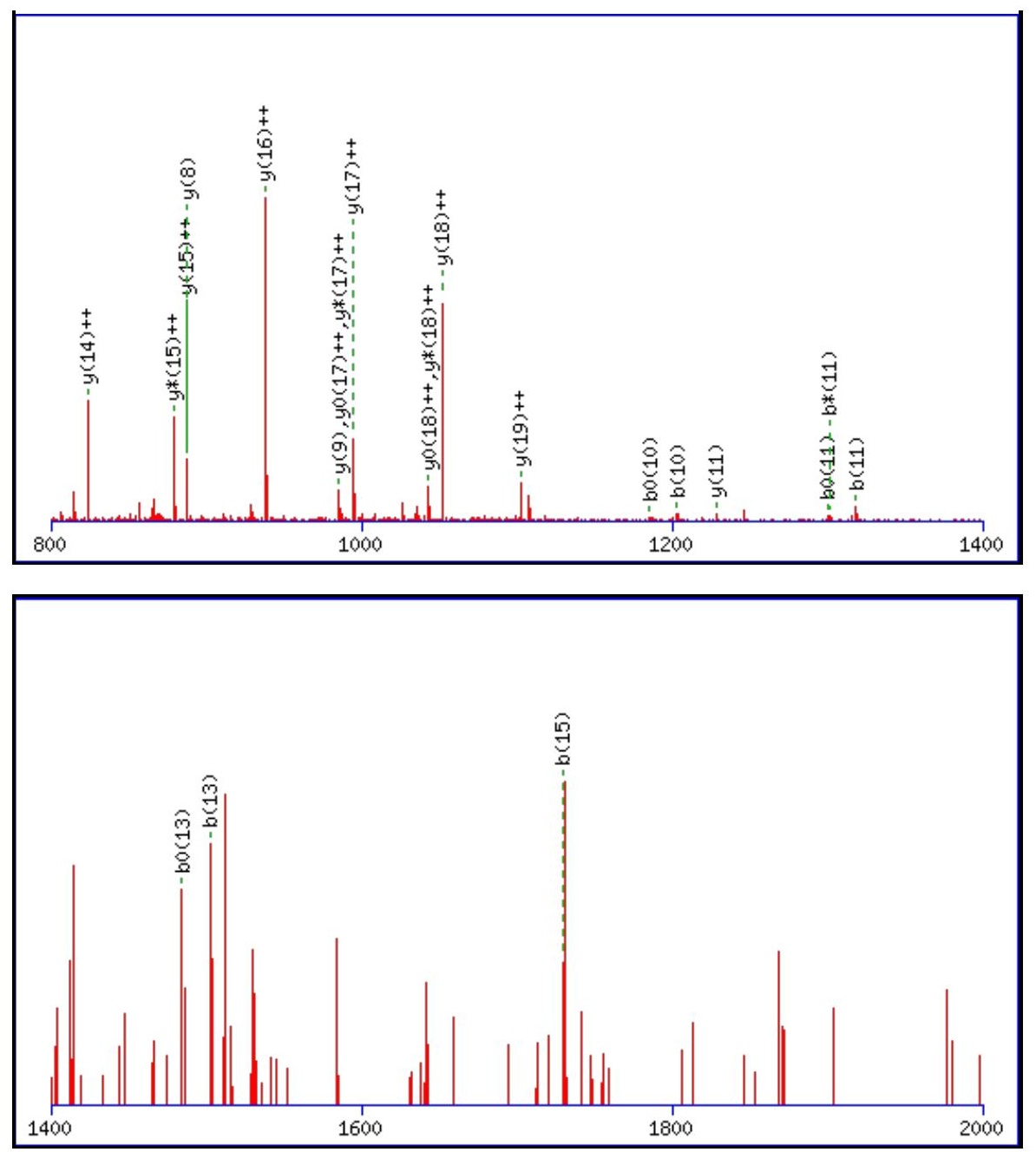

Monoisotopic mass of neutral peptide $\operatorname{Mr}($ calc): 2302.0747

Fixed modifications: Carbamidomethyl (C)

Variable modifications:

N3 : Deamidated $\mathrm{N}(\mathrm{N})$

Ions Score: 52 Expect: 0.0018

Matches (Bold Red): 38/220 fragment ions using 79 most intense peaks

\begin{tabular}{|c|c|c|c|c|c|c|c|c|c|c|c|c|c|c|}
\hline \# & b & $\mathbf{b}^{++}$ & $\mathbf{b}^{*}$ & $\mathbf{b}^{*^{++}}$ & $\mathbf{b}^{\mathbf{0}}$ & $\mathbf{b}^{\mathbf{0 + +}}$ & Seq. & $\mathbf{y}$ & $\mathbf{y}^{++}$ & $\mathbf{y}^{*}$ & $\mathbf{y}^{*^{++}}$ & $\mathbf{y}^{\mathbf{0}}$ & $\mathbf{y}^{\mathbf{0 + +}}$ & \# \\
\hline 1 & 100.0757 & 50.5415 & & & & & V & & & & & & & 20 \\
\hline 2 & 201.1234 & 101.0653 & & & 183.1128 & 92.0600 & $\mathbf{T}$ & 2204.0136 & 1102.5104 & 2186.9870 & 1093.9971 & 2186.0030 & 1093.5051 & 19 \\
\hline 3 & 316.1503 & 158.5788 & 299.1238 & 150.0655 & 298.1397 & 149.5735 & $\mathbf{N}$ & 2102.9659 & 1051.9866 & 2085.9393 & 1043.4733 & 2084.9553 & 1042.9813 & 18 \\
\hline 4 & 429.2344 & 215.1208 & 412.2078 & 206.6075 & 411.2238 & 206.1155 & $\mathbf{L}$ & 1987.9389 & 994.4731 & 1970.9124 & 985.9598 & 1969.9284 & 985.4678 & 17 \\
\hline 5 & 530.2820 & 265.6447 & 513.2555 & 257.1314 & 512.2715 & 256.6394 & $\mathbf{T}$ & 1874.8549 & 937.9311 & 1857.8283 & 929.4178 & 1856.8443 & 928.9258 & 16 \\
\hline 6 & 658.3406 & 329.6740 & 641.3141 & 321.1607 & 640.3301 & 320.6687 & $\mathbf{Q}$ & 1773.8072 & 887.4072 & 1756.7807 & 878.8940 & 1755.7966 & 878.4020 & 15 \\
\hline 7 & 787.3832 & 394.1952 & 770.3567 & 385.6820 & 769.3727 & 385.1900 & $\mathbf{E}$ & 1645.7486 & 823.3780 & 1628.7221 & 814.8647 & 1627.7381 & 814.3727 & 14 \\
\hline 8 & 915.4418 & 458.2245 & 898.4152 & 449.7113 & 897.4312 & 449.2193 & $\mathbf{Q}$ & 1516.7060 & 758.8567 & 1499.6795 & 750.3434 & 1498.6955 & 749.8514 & 13 \\
\hline 9 & 1075.4724 & 538.2399 & 1058.4459 & 529.7266 & 1057.4619 & 529.2346 & C & 1388.6475 & 694.8274 & 1371.6209 & 686.3141 & 1370.6369 & 685.8221 & 12 \\
\hline 10 & 1203.5310 & 602.2692 & 1186.5045 & 593.7559 & 1185.5205 & 593.2639 & $\mathbf{Q}$ & 1228.6168 & 614.8120 & 1211.5903 & 606.2988 & 1210.6062 & 605.8068 & 11 \\
\hline 11 & 1318.5580 & 659.7826 & 1301.5314 & 651.2693 & 1300.5474 & 650.7773 & D & 1100.5582 & 550.7828 & 1083.5317 & 542.2695 & 1082.5477 & 541.7775 & 10 \\
\hline 12 & 1415.6107 & 708.3090 & 1398.5842 & 699.7957 & 1397.6002 & 699.3037 & $\mathbf{P}$ & 985.5313 & 493.2693 & 968.5047 & 484.7560 & 967.5207 & 484.2640 & 9 \\
\hline 13 & 1502.6428 & 751.8250 & 1485.6162 & 743.3117 & 1484.6322 & 742.8197 & $\mathrm{~S}$ & 888.4785 & 444.7429 & 871.4520 & 436.2296 & 870.4680 & 435.7376 & 8 \\
\hline 14 & 1630.7377 & 815.8725 & 1613.7112 & 807.3592 & 1612.7272 & 806.8672 & $\mathbf{K}$ & 801.4465 & 401.2269 & 784.4199 & 392.7136 & 783.4359 & 392.2216 & 7 \\
\hline 15 & 1729.8061 & 865.4067 & 1712.7796 & 856.8934 & 1711.7956 & 856.4014 & V & 673.3515 & 337.1794 & 656.3250 & 328.6661 & 655.3410 & 328.1741 & 6 \\
\hline 16 & 1826.8589 & 913.9331 & 1809.8324 & 905.4198 & 1808.8483 & 904.9278 & $\mathbf{P}$ & 574.2831 & 287.6452 & 557.2566 & 279.1319 & 556.2726 & 278.6399 & 5 \\
\hline 17 & 1940.9018 & 970.9546 & 1923.8753 & 962.4413 & 1922.8913 & 961.9493 & $\mathbf{N}$ & 477.2304 & 239.1188 & 460.2038 & 230.6055 & 459.2198 & 230.1135 & 4 \\
\hline 18 & 2069.9444 & 1035.4758 & 2052.9179 & 1026.9626 & 2051.9339 & 1026.4706 & $\mathbf{E}$ & 363.1874 & 182.0974 & 346.1609 & 173.5841 & 345.1769 & 173.0921 & 3 \\
\hline
\end{tabular}




\begin{tabular}{|c|c|c|c|c|c|c|c|c|c|c|c|c|c|}
\hline 19 & |2156.9764 & |1078.9919 & 2139.9499 & 1070.4786 & 2138.9659 & |1069.9866 & S & 234.1448 & 117.5761 & 217.1183 & 109.0628 & 216.1343 & 108.5708 \\
\hline 20 & & & & & & & $\mathbf{K}$ & 147.1128 & 74.0600 & 130.0863 & 65.5468 & & \\
\hline
\end{tabular}
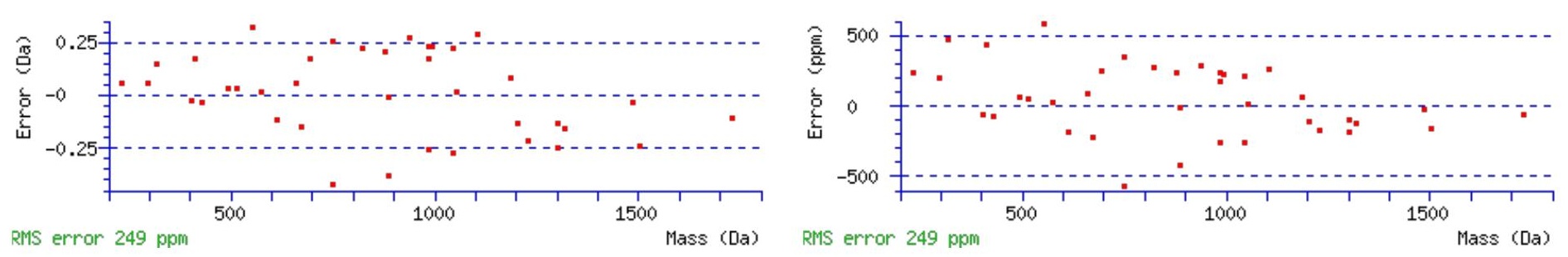

\section{All matches to this query}

\begin{tabular}{|l|l|c|l|}
\hline Score & Mr(calc): & Delta & \multicolumn{1}{|c|}{ Sequence } \\
\hline 52.0 & 2302.0747 & 0.0080 & VTNLTQEQCQDPSKVPNESK \\
\hline 24.4 & 2301.0907 & 0.9921 & VTNLTQEQCQDPSKVPNESK \\
\hline 17.6 & 2302.0747 & 0.0080 & VTNLTQEQCQDPSKVPNESK \\
\hline 4.3 & 2300.0636 & 2.0191 & QIPAVQGNMINLETKNTRL \\
\hline 1.1 & 2302.1028 & -0.0201 & ESLTKLMATLRNTNPNFVR \\
\hline 0.1 & 2300.0636 & 2.0191 & QIPAVQGNMINLETKNTRL \\
\hline
\end{tabular}

Spectrum No: 645; Query: 456; Rank: 1

\section{Peptide View}

\section{MS/MS Fragmentation of DAVRNLTEVVPR}

Found in IPI00361106, Tax_Id=10116 Gene_Symbol=RGD1560062_predicted hypothetical protein LOC309816

Match to Query 456: 1368.734652 from(457.252160,3+)

Title: 091008RatKidney_NH4Format01_23.2289.2289.3.dta

Data file K:\NewmanPaper\Piliang \3SubProteomes $\backslash P i l i a n g 3 S P \backslash m g f 5 p p m \backslash E R L I C \_3 S u b P r o t e o m e s 5 p p m . m g f$

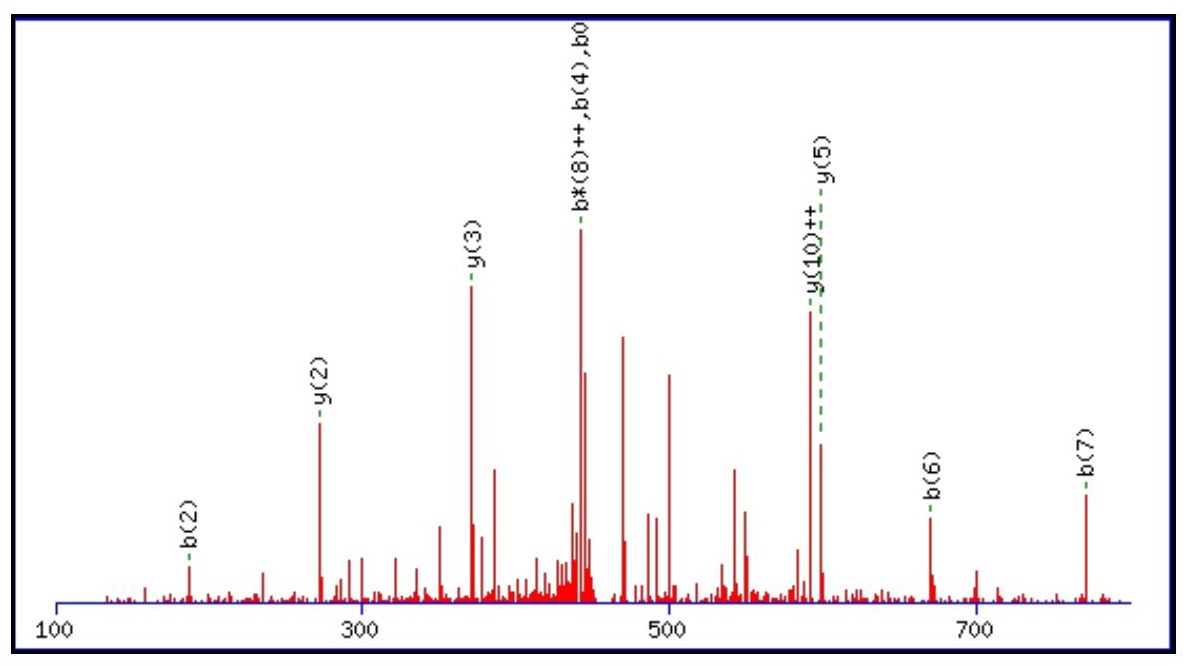



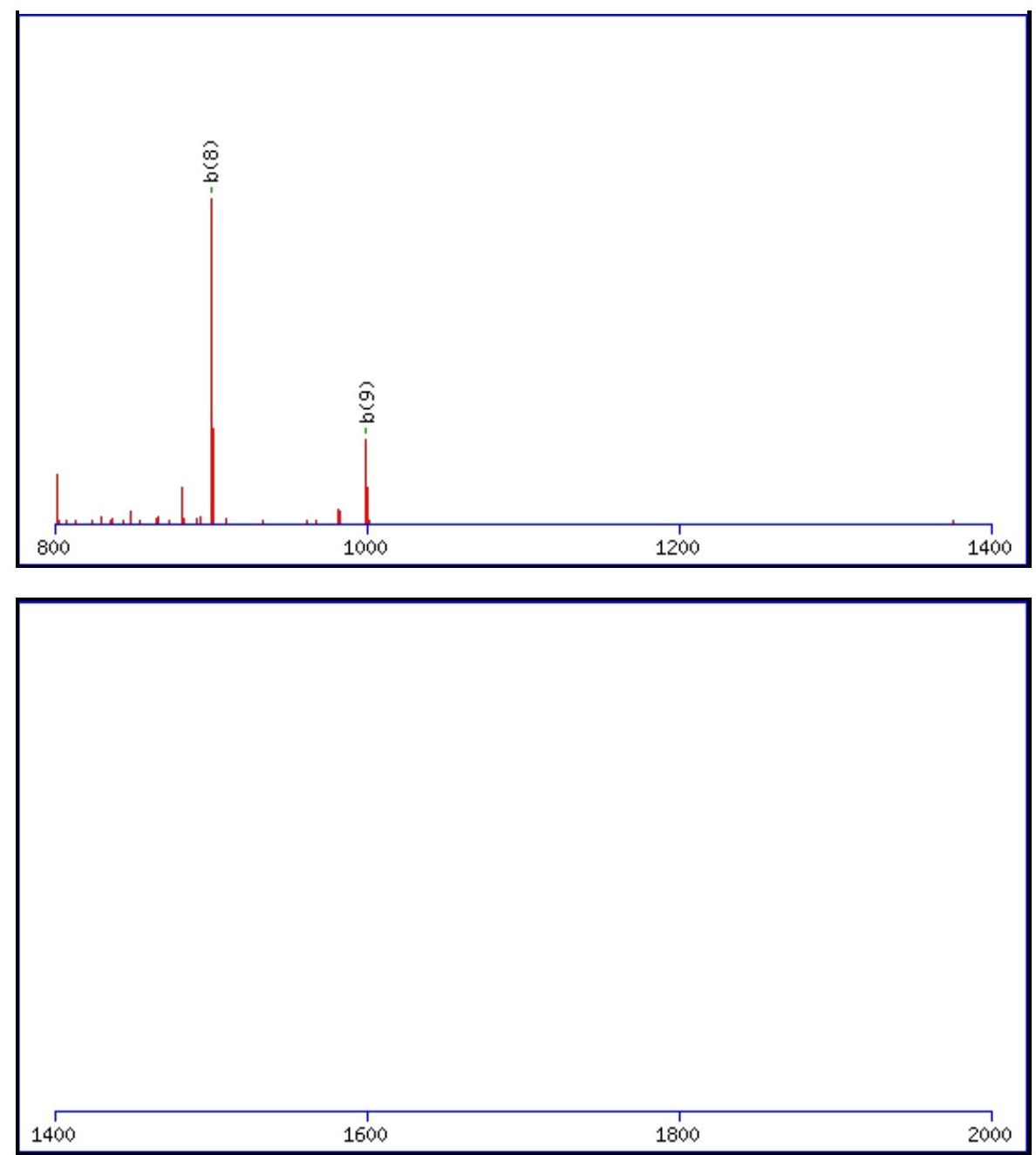

Monoisotopic mass of neutral peptide $\operatorname{Mr}($ calc): 1368.7361

Fixed modifications: Carbamidomethyl (C)

Variable modifications:

N5: Deamidated $\mathrm{N}(\mathrm{N})$

Ions Score: 52 Expect: 0.00052

Matches (Bold Red): 12/118 fragment ions using 11 most intense peaks

\begin{tabular}{|c|c|c|c|c|c|c|c|c|c|c|c|c|c|c|}
\hline \# & b & $\mathbf{b}^{++}$ & $\mathbf{b}^{*}$ & $\mathbf{b}^{*^{++}}$ & $\mathbf{b}^{0}$ & $\mathbf{b}^{0++}$ & Seq. & $\mathbf{y}$ & $y^{++}$ & $\mathbf{y}^{*}$ & $\mathrm{y}^{*^{++}}$ & $\mathbf{y}^{0}$ & $\mathbf{y}^{0++}$ & \# \\
\hline 1 & 116.0342 & 58.5207 & & & 98.0237 & 49.5155 & D & & & & & & & 12 \\
\hline 2 & 187.0713 & 94.0393 & & & 169.0608 & 85.0340 & A & 1254.7165 & 627.8619 & 1237.6899 & 619.3486 & 1236.7059 & 618.8566 & 11 \\
\hline 3 & 286.1397 & 143.5735 & & & 268.1292 & 134.5682 & $\mathbf{V}$ & 1183.6793 & 592.3433 & 1166.6528 & 583.8300 & 1165.6688 & 583.3380 & 10 \\
\hline 4 & 442.2409 & 221.6241 & 425.2143 & 213.1108 & 424.2303 & 212.6188 & $\mathbf{R}$ & 1084.6109 & 542.8091 & 1067.5844 & 534.2958 & 1066.6004 & 533.8038 & 9 \\
\hline 5 & 557.2678 & 279.1375 & 540.2412 & 270.6243 & 539.2572 & 270.1323 & $\mathbf{N}$ & 928.5098 & 464.7585 & 911.4833 & 456.2453 & 910.4993 & 455.7533 & 8 \\
\hline 6 & 670.3519 & 335.6796 & 653.3253 & 327.1663 & 652.3413 & 326.6743 & $\mathbf{L}$ & 813.4829 & 407.2451 & 796.4563 & 398.7318 & 795.4723 & 398.2398 & 7 \\
\hline 7 & 771.3995 & 386.2034 & 754.3730 & 377.6901 & 753.3890 & 377.1981 & $\mathbf{T}$ & 700.3988 & 350.7030 & 683.3723 & 342.1898 & 682.3883 & 341.6978 & 6 \\
\hline 8 & 900.4421 & 450.7247 & 883.4156 & 442.2114 & 882.4316 & 441.7194 & $\mathbf{E}$ & 599.3511 & 300.1792 & 582.3246 & 291.6659 & 581.3406 & 291.1739 & 5 \\
\hline 9 & 999.5105 & 500.2589 & 982.4840 & 491.7456 & 981.5000 & 491.2536 & $\mathbf{V}$ & 470.3085 & 235.6579 & 453.2820 & 227.1446 & & & 4 \\
\hline 10 & 1098.5790 & 549.7931 & 1081.5524 & 541.2798 & 1080.5684 & 540.7878 & $\mathbf{V}$ & 371.2401 & 186.1237 & 354.2136 & 177.6104 & & & 3 \\
\hline 11 & 1195.6317 & 598.3195 & 1178.6052 & 589.8062 & 1177.6212 & 589.3142 & $\mathbf{P}$ & 272.1717 & 136.5895 & 255.1452 & 128.0762 & & & 2 \\
\hline 12 & & & & & & & $\mathbf{R}$ & 175.1190 & 88.0631 & 158.0924 & 79.5498 & & & 1 \\
\hline
\end{tabular}



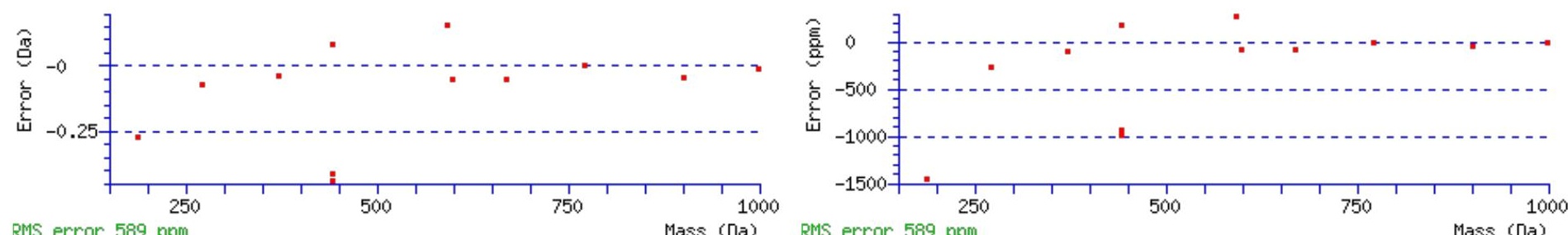

RMS error 589 ppm Mass (Da) RMS error 589 ppm

\section{All matches to this query}

\begin{tabular}{|l|l|l|l|}
\hline Score & Mr(calc): & Delta & \multicolumn{1}{c|}{ Sequence } \\
\hline 52.0 & 1368.7361 & -0.0015 & DAVRNLTEVVPR \\
\hline 13.5 & 1368.7361 & -0.0015 & NTGLITVQGPVDR \\
\hline 8.8 & 1368.7361 & -0.0015 & EQLNQTTLVAPR \\
\hline 7.3 & 1366.7221 & 2.0125 & KKLQTQVLDSK \\
\hline 5.0 & 1366.7235 & 2.0112 & WVRTTVTIRR \\
\hline 4.2 & 1367.7408 & 0.9938 & AASSISSAPKPPAGK \\
\hline 3.9 & 1368.7222 & 0.0125 & GNSLAGRPEGKQR \\
\hline 3.2 & 1367.7269 & 1.0077 & NGEQLQATPRVR \\
\hline 2.5 & 1368.7361 & -0.0014 & DAANLLNDALAIR \\
\hline 1.2 & 1366.7374 & 1.9973 & GIKKPFTEVIR \\
\hline
\end{tabular}

Spectrum No: 646; Query: 1587; Rank: 1

\section{Peptide View}

\section{MS/MS Fragmentation of TLGETSLFSNLTNLQTLR}

Found in IPI00400619, Tax_Id=10116 Gene_Symbol=Tlr2 Toll-like receptor 2 variant 2

Match to Query 1587: 2008.042328 from(1005.028440,2+)

Title: 091008RatKidney_NoSalt_29.4462.4462.2.dta

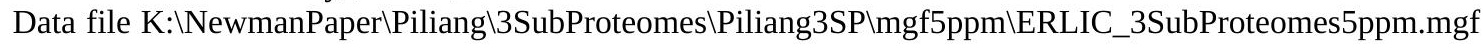

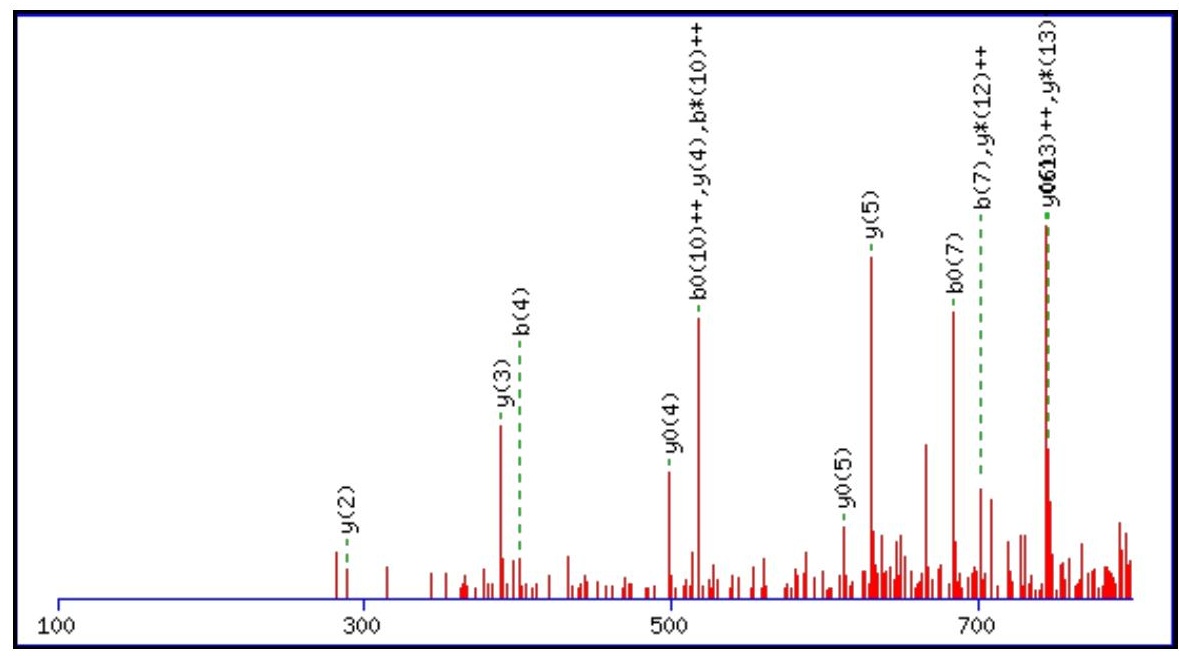



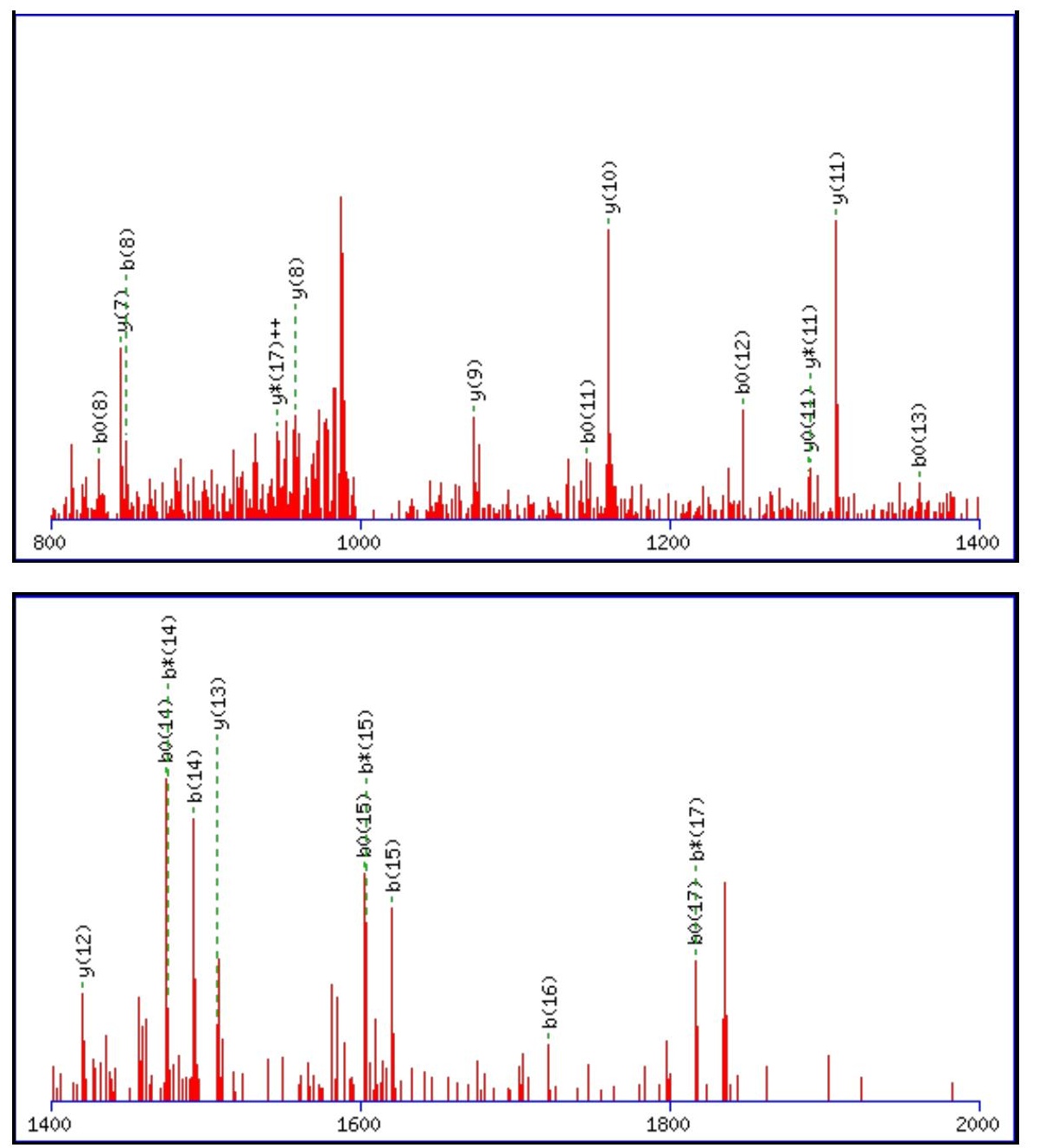

Monoisotopic mass of neutral peptide $\operatorname{Mr}($ calc): 2008.0477

Fixed modifications: Carbamidomethyl (C)

Variable modifications:

N10 : Deamidated_N (N)

Ions Score: 52 Expect: 0.00088

Matches (Bold Red): 39/182 fragment ions using 83 most intense peaks

\begin{tabular}{|c|c|c|c|c|c|c|c|c|c|c|c|c|c|c|}
\hline \# & b & $\mathbf{b}^{++}$ & $\mathbf{b}^{*}$ & $\mathbf{b}^{*^{++}}$ & $\mathbf{b}^{0}$ & $\mathbf{b}^{0++}$ & Seq. & $\mathbf{y}$ & $\mathbf{y}^{++}$ & $\mathbf{y}^{*}$ & $\mathbf{y}^{*^{++}}$ & $\mathbf{y}^{0}$ & $\mathbf{y}^{0++}$ & \# \\
\hline 1 & 102.0550 & 51.5311 & & & 84.0444 & 42.5258 & $\mathbf{T}$ & & & & & & & 18 \\
\hline 2 & 215.1390 & 108.0731 & & & 197.1285 & 99.0679 & $\mathbf{L}$ & 1908.0073 & 954.5073 & 1890.9807 & & 1889.9967 & 945.5020 & 17 \\
\hline 3 & 272.1605 & 136.5839 & & & 254.1499 & 127.5786 & G & 1794.9232 & 897.9652 & 1777.8967 & 889.4520 & 1776.9126 & 888.9600 & 16 \\
\hline 4 & 401.2031 & 201.1052 & & & 383.1925 & 192.0999 & $\mathbf{E}$ & 1737.9017 & 869.4545 & 1720.8752 & 860.9412 & 1719.8912 & 860.4492 & 15 \\
\hline 5 & 502.2508 & 251.6290 & & & 484.2402 & 242.6237 & $\mathbf{T}$ & 1608.8592 & 804.9332 & 1591.8326 & 796.4199 & 1590.8486 & 795.9279 & 14 \\
\hline 6 & 589.2828 & 295.1450 & & & 571.2722 & 286.1397 & S & 1507.8115 & 754.4094 & 1490.7849 & 745.8961 & 1489.8009 & 745.4041 & 13 \\
\hline 7 & 702.3668 & 351.6871 & & & 684.3563 & 342.6818 & $\mathbf{L}$ & 1420.7794 & 710.8934 & 1403.7529 & 702.3801 & 1402.7689 & 701.8881 & 12 \\
\hline 8 & 849.4353 & 425.2213 & & & 831.4247 & 416.2160 & $\mathbf{F}$ & 1307.6954 & 654.3513 & 1290.6688 & 645.8381 & 1289.6848 & 645.3460 & 11 \\
\hline 9 & 936.4673 & 468.7373 & & & 918.4567 & 459.7320 & $\mathrm{~S}$ & 1160.6270 & 580.8171 & 1143.6004 & 572.3038 & 1142.6164 & 571.8118 & 10 \\
\hline 10 & 1051.4942 & 526.2508 & 1034.4677 & 517.7375 & 1033.4837 & 517.2455 & $\mathbf{N}$ & 1073.5949 & 537.3011 & 1056.5684 & 528.7878 & 1055.5844 & 528.2958 & 9 \\
\hline 11 & 1164.5783 & 582.7928 & 1147.5517 & 574.2795 & 1146.5677 & 573.7875 & $\mathbf{L}$ & 958.5680 & 479.7876 & 941.5415 & 471.2744 & 940.5574 & 470.7824 & 8 \\
\hline 12 & 1265.6260 & 633.3166 & 1248.5994 & 624.8033 & 1247.6154 & 624.3113 & $\mathbf{T}$ & 845.4839 & 423.2456 & 828.4574 & 414.7323 & 827.4734 & 414.2403 & 7 \\
\hline 13 & 1379.6689 & 690.3381 & 1362.6423 & 681.8248 & 1361.6583 & 681.3328 & $\mathbf{N}$ & 744.4363 & 372.7218 & 727.4097 & 364.2085 & 726.4257 & 363.7165 & 6 \\
\hline 14 & 1492.7530 & 746.8801 & 1475.7264 & 738.3668 & 1474.7424 & 737.8748 & $\mathbf{L}$ & 630.3933 & 315.7003 & 613.3668 & 307.1870 & 612.3828 & 306.6950 & 5 \\
\hline 15 & 1620.8115 & 810.9094 & 1603.7850 & 802.3961 & 1602.8010 & 801.9041 & $\mathbf{Q}$ & 517.3093 & 259.1583 & 500.2827 & 250.6450 & 499.2987 & 250.1530 & 4 \\
\hline 16 & 1721.8592 & 861.4332 & 1704.8327 & 852.9200 & 1703.8487 & 852.4280 & $\mathbf{T}$ & 389.2507 & 195.1290 & 372.2241 & 186.6157 & 371.2401 & 186.1237 & 3 \\
\hline 17 & 1834.9433 & 917.9753 & 1817.9167 & 909.4620 & 1816.9327 & 908.9700 & L & 288.2030 & 144.6051 & 271.1765 & 136.0919 & & & 2 \\
\hline 18 & & & & & & & $\mathbf{R}$ & 175.1190 & 88.0631 & 158.0924 & 79.5498 & & & 1 \\
\hline
\end{tabular}



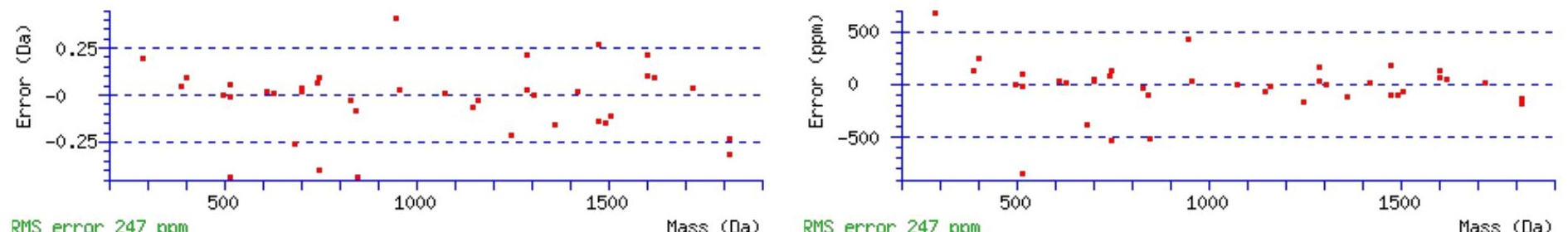

\section{All matches to this query}

\begin{tabular}{|l|l|l|l|}
\hline Score & Mr(calc): & Delta & \multicolumn{1}{|c|}{ Sequence } \\
\hline 51.8 & 2008.0477 & -0.0053 & TLGETSLFSNLTNLQTLR \\
\hline 34.8 & 2008.0477 & -0.0053 & TLGETSLFSNLTNLQTLR \\
\hline 9.6 & 2007.0434 & 0.9989 & LDCKIAGSLPMRVSWFK \\
\hline 2.3 & 2008.0411 & 0.0012 & MTLEKPPLTHSQLDNIR \\
\hline 1.5 & 2007.0376 & 1.0047 & AMNAAGNLKPKLPFLSVR \\
\hline
\end{tabular}

Spectrum No: 647; Query: 1852; Rank: 1

\section{Peptide View}

MS/MS Fragmentation of DWSQNMYFNCSEDNPSR

Found in IPI00200092, Tax_Id=10116 Gene_Symbol=RGD1560915_predicted hypothetical protein LOC500065

Match to Query 1852: 2166.815128 from(1084.414840,2+)

Title: 100101RatKid_NS_deglyco_27.2305.2305.2.dta

Data file K:INewmanPaper|Piliangl3SubProteomes\Piliang3SP\mgf5ppm\ERLIC_3SubProteomes5ppm.mgf

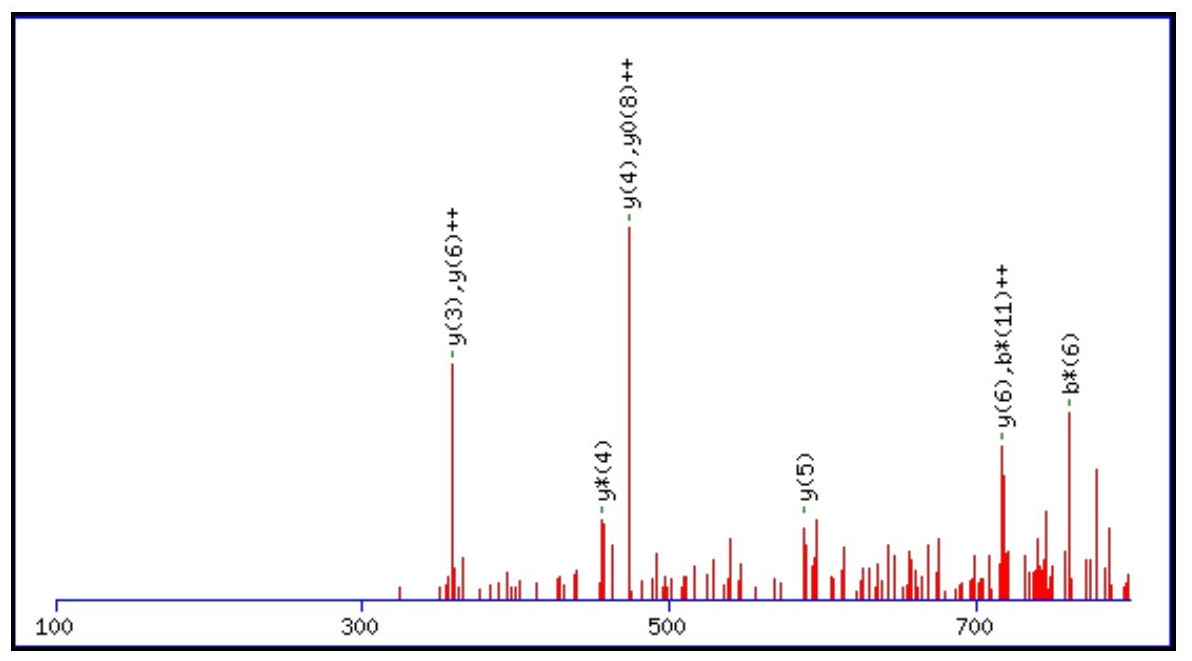



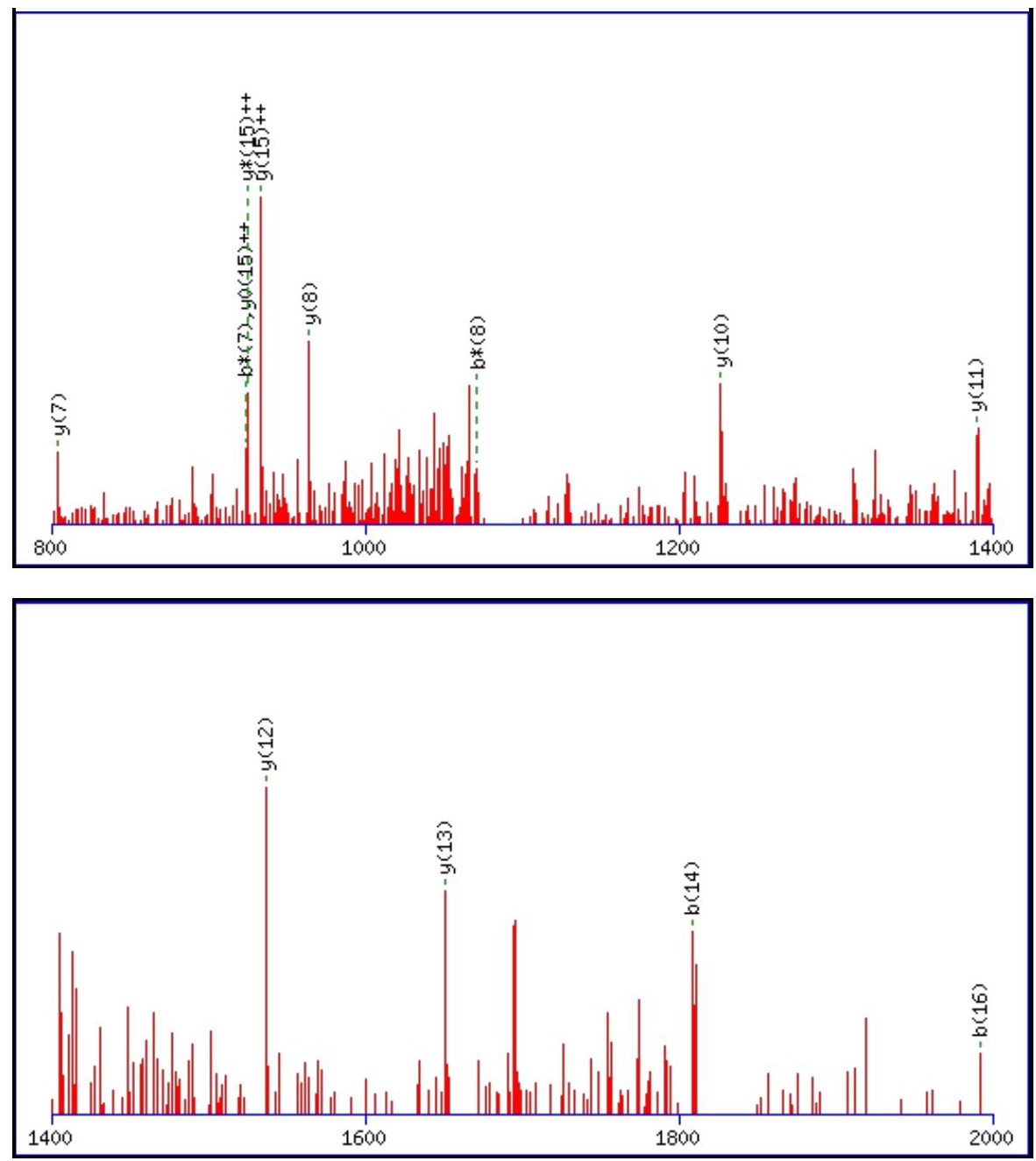

Monoisotopic mass of neutral peptide $\operatorname{Mr}($ calc): 2165.8055

Fixed modifications: Carbamidomethyl (C)

Variable modifications:

M6 : Oxidation (M)

N9 : Deamidated $\mathrm{N}(\mathrm{N})$

Ions Score: 52 Expect: 0.00092

Matches (Bold Red): 22/184 fragment ions using 41 most intense peaks

\begin{tabular}{|c|c|c|c|c|c|c|c|c|c|c|c|c|c|c|}
\hline \# & b & $\mathbf{b}^{++}$ & $\mathbf{b}^{*}$ & $\mathbf{b}^{*^{++}}$ & $\mathbf{b}^{0}$ & $\mathbf{b}^{\mathbf{0 + +}}$ & Seq. & $\mathbf{y}$ & & $\mathbf{y}^{*}$ & $\mathbf{y}^{*^{++}}$ & $\mathbf{y}^{\mathbf{0}}$ & & \# \\
\hline 1 & 116.0342 & 58.5207 & & & & 49.5155 & D & & & & & & & 17 \\
\hline 2 & 302.1135 & 151.5604 & & & 284.1030 & 142.5551 & $\mathbf{W}$ & & & & & & & 16 \\
\hline 3 & 389.1456 & 195.0764 & & & 371.1350 & 186.0711 & S & 1865.7065 & & 1848.6800 & 3436 & 1847.6959 & 924.3516 & 15 \\
\hline 4 & 517.2041 & 259.1057 & 500.1776 & 250. & 499.1936 & 250.1004 & $\mathbf{Q}$ & 1778.6745 & 889.8409 & 1761.6479 & 881.3276 & 1760.6639 & 880.8356 & 14 \\
\hline 5 & 631.2471 & 316.1272 & 614.2205 & 307.6 & 613.2 & 307.1219 & $\mathbf{N}$ & 1650.6159 & 825.8116 & 1633.5893 & 2983 & 1632.6053 & 816.8063 & 13 \\
\hline 6 & 778.2825 & 389.6449 & 761.2559 & 381.1316 & 760.2719 & 380.6396 & $\mathbf{M}$ & 1536.5730 & 768.7901 & 1519.5464 & 760.2768 & 1518.5624 & 759.7848 & 12 \\
\hline 7 & 941.3458 & 471.1765 & 924.3192 & 462.6633 & 923.3352 & 462.1713 & $\mathbf{Y}$ & 1389.5376 & 695.2724 & 1372.5110 & 686.7591 & 1371.5270 & 686.2671 & 11 \\
\hline 8 & 1088.4142 & 544.7107 & 1071.3877 & 536.1975 & 1070.4036 & 535.7055 & $\mathbf{F}$ & 1226.4742 & 613.7408 & 1209.4477 & 605.2275 & 1208.4637 & 604.7355 & 10 \\
\hline 9 & 1203.4411 & 602.2242 & 1186.4146 & 593.7109 & 1185.4306 & 593.2189 & $\mathbf{N}$ & 1079.4058 & 540.2065 & 1062.3793 & 531.6933 & 1061.3953 & 531.2013 & 9 \\
\hline 10 & 1363.4718 & 682.2395 & 1346.4452 & 673.7263 & 1345.4612 & 673.2343 & C & 964.3789 & 482.6931 & 947.3523 & 798 & 946.3683 & 473.6878 & 8 \\
\hline 11 & 1450.5038 & 725.7556 & 1433.4773 & 717.2423 & 1432.4933 & 716.7503 & S & 804.3482 & 402.6778 & 787.3217 & 394.1645 & 786.3377 & 393.6725 & 7 \\
\hline 12 & 1579.5464 & 790.2768 & 1562.5199 & 781.7636 & 1561.5359 & 781.2716 & $\mathbf{E}$ & 717.3162 & 359.1617 & 700.2897 & 350.6485 & 699.3056 & 350.1565 & 6 \\
\hline 13 & 1694.5734 & 847.7903 & 1677.5468 & 839.2770 & 1676.5628 & 838.7850 & D & 588.2736 & 294.6404 & 571.2471 & 286.1272 & 570.2630 & 285.6352 & 5 \\
\hline 14 & 1808.6163 & 904.8118 & 1791.5897 & 896.2985 & 1790.6057 & 895.8065 & $\mathbf{N}$ & 473.2467 & 237.1270 & 456.2201 & 228.6137 & 455.2361 & 228.1217 & 4 \\
\hline 15 & 1905.6691 & 953.3382 & 1888.6425 & 944.8249 & 1887.6585 & 944.3329 & $\mathbf{P}$ & 359.2037 & 180.1055 & 342.1772 & 171.5922 & 341.1932 & 171.1002 & 3 \\
\hline 16 & 1992.7011 & 996.8542 & 1975.6745 & 988.3409 & 1974.6905 & 987.8489 & S & 262.1510 & 131.5791 & 245.1244 & 123.0659 & 244.1404 & 122.5738 & 2 \\
\hline 17 & & & & & & & $\mathbf{R}$ & 175.1190 & 88.0631 & 158.0924 & 79.5498 & & & 1 \\
\hline
\end{tabular}



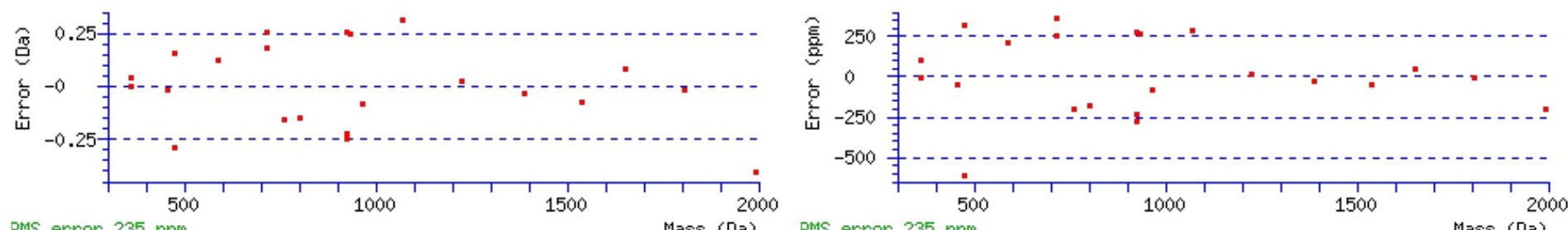

RMS error 235 ppm Mass (Da) RMS error 235 ppm

\section{All matches to this query}

\begin{tabular}{|l|l|l|l|}
\hline Score & Mr(calc): & Delta & Sequence \\
\hline 51.8 & 2165.8055 & 1.0097 & DWSQNMYFNCSEDNPSR \\
\hline 22.0 & 2165.8055 & 1.0097 & DWSQNMYFNCSEDNPSR \\
\hline 17.1 & 2165.8055 & 1.0097 & DWSQNMYFNCSEDNPSR \\
\hline 6.2 & 2164.8215 & 1.9937 & DWSQNMYFNCSEDNPSR \\
\hline
\end{tabular}

Spectrum No: 648; Query: 301; Rank: 1

\section{Peptide View}

\section{MS/MS Fragmentation of IAYSNNGSDWK}

Found in IPI00212307, Tax_Id=10116 Gene_Symbol=Nrp1 Neuropilin-1 precursor

Match to Query 301: 1254.554768 from(628.284660,2+)

Title: 100101RatKid_NS_deglyco_25.1410.1410.2.dta

Data file K:\NewmanPaper\Piliang \3SubProteomes\Piliang3SP \mgf5ppm\ERLIC_3SubProteomes5ppm.mgf

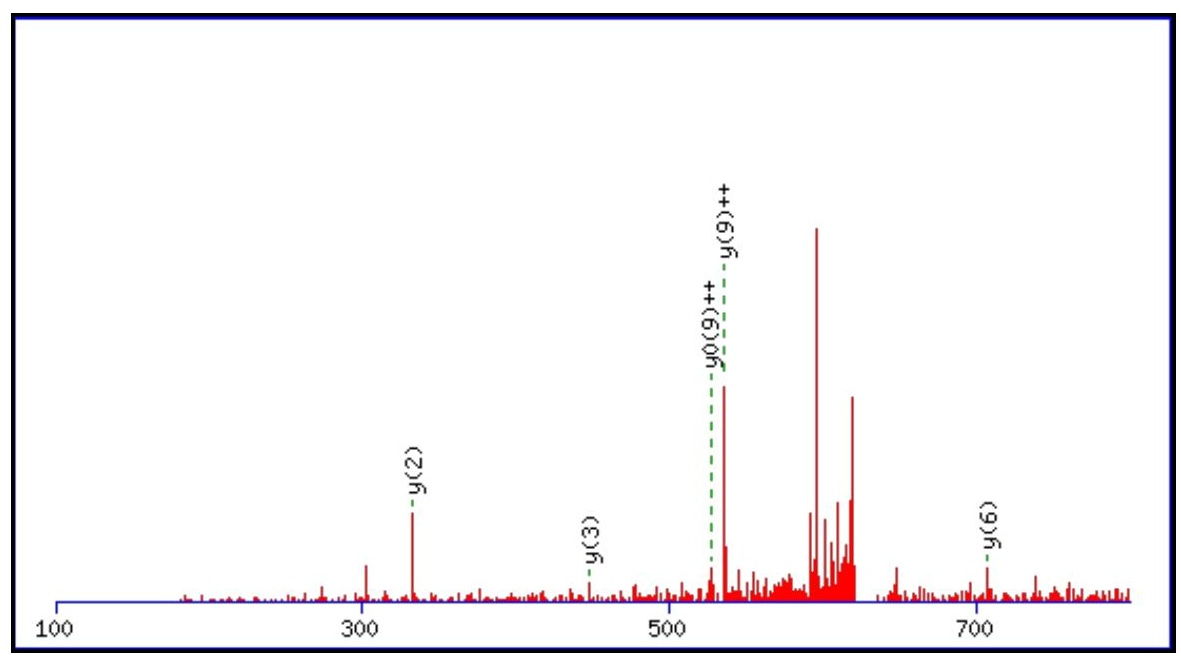




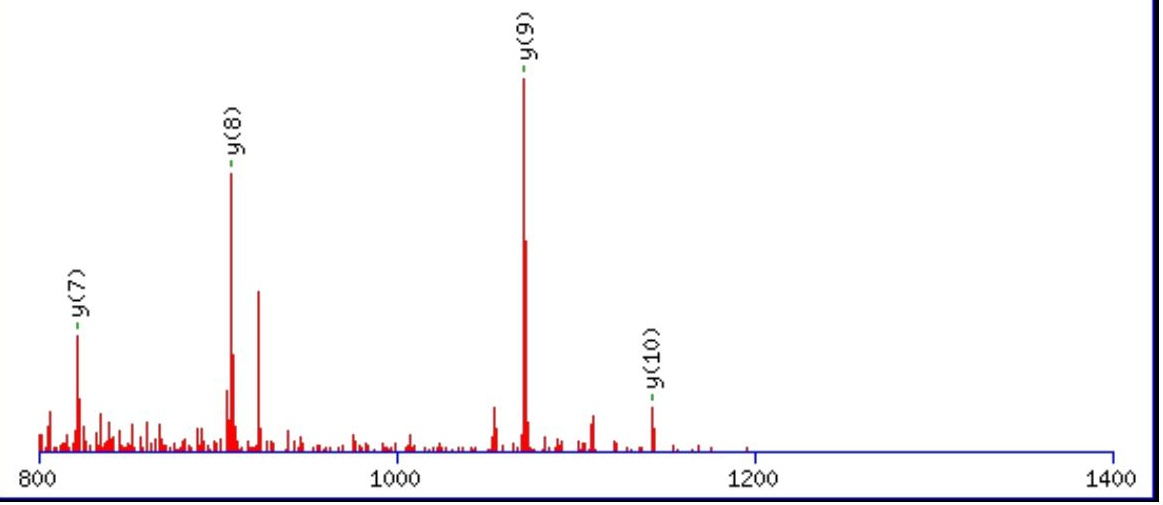

\begin{tabular}{rrr}
\hline 1600 & 1800 & 2000
\end{tabular}

Monoisotopic mass of neutral peptide $\operatorname{Mr}($ calc): 1254.5517

Fixed modifications: Carbamidomethyl (C)

Variable modifications:

N6 : Deamidated_N (N)

Ions Score: 52 Expect: $\odot .00077$

Matches (Bold Red): 9/102 fragment ions using 34 most intense peaks

\begin{tabular}{|c|c|c|c|c|c|c|c|c|c|c|c|c|c|c|}
\hline \# & b & $\mathbf{b}^{++}$ & $\mathbf{b}^{*}$ & $\mathbf{b}^{*^{++}}$ & $\mathbf{b}^{0}$ & $\mathbf{b}^{\mathbf{0 + +}}$ & Seq. & $\mathbf{y}$ & $y^{++}$ & $\mathbf{y}^{*}$ & $\mathrm{y}^{*^{++}}$ & $\mathbf{y}^{0}$ & $\mathbf{y}^{0++}$ & \# \\
\hline 1 & 114.0913 & 57.5493 & & & & & I & & & & & & & 11 \\
\hline 2 & 185.1285 & 93.0679 & & & & & A & 1142.4749 & 571.7411 & 1125.4483 & 563.2278 & 1124.4643 & 562.7358 & 10 \\
\hline 3 & 348.1918 & 174.5995 & & & & & $\mathbf{Y}$ & 1071.4378 & 536.2225 & 1054.4112 & 527.7092 & 1053.4272 & 527.2172 & 9 \\
\hline 4 & 435.2238 & 218.1155 & & & 417.2132 & 209.1103 & S & 908.3744 & 454.6909 & 891.3479 & 446.1776 & 890.3639 & 445.6856 & 8 \\
\hline 5 & 549.2667 & 275.1370 & 532.2402 & 266.6237 & 531.2562 & 266.1317 & $\mathbf{N}$ & 821.3424 & 411.1748 & 804.3159 & 402.6616 & 803.3319 & 402.1696 & 7 \\
\hline 6 & 664.2937 & 332.6505 & 647.2671 & 324.1372 & 646.2831 & 323.6452 & $\mathbf{N}$ & 707.2995 & 354.1534 & 690.2729 & 345.6401 & 689.2889 & 345.1481 & 6 \\
\hline 7 & 721.3151 & 361.1612 & 704.2886 & 352.6479 & 703.3046 & 352.1559 & G & 592.2726 & 296.6399 & 575.2460 & 288.1266 & 574.2620 & 287.6346 & 5 \\
\hline 8 & 808.3472 & 404.6772 & 791.3206 & 396.1639 & 790.3366 & 395.6719 & $\mathrm{~S}$ & 535.2511 & 268.1292 & 518.2245 & 259.6159 & 517.2405 & 259.1239 & 4 \\
\hline 9 & 923.3741 & 462.1907 & 906.3476 & 453.6774 & 905.3635 & 453.1854 & D & 448.2191 & 224.6132 & 431.1925 & 216.0999 & 430.2085 & 215.6079 & 3 \\
\hline 10 & 1109.4534 & 555.2303 & 1092.4269 & 546.7171 & 1091.4429 & 546.2251 & $\mathbf{W}$ & 333.1921 & 167.0997 & 316.1656 & 158.5864 & & & 2 \\
\hline 11 & & & & & & & $\mathbf{K}$ & 147.1128 & 74.0600 & 130.0863 & 65.5468 & & & 1 \\
\hline
\end{tabular}
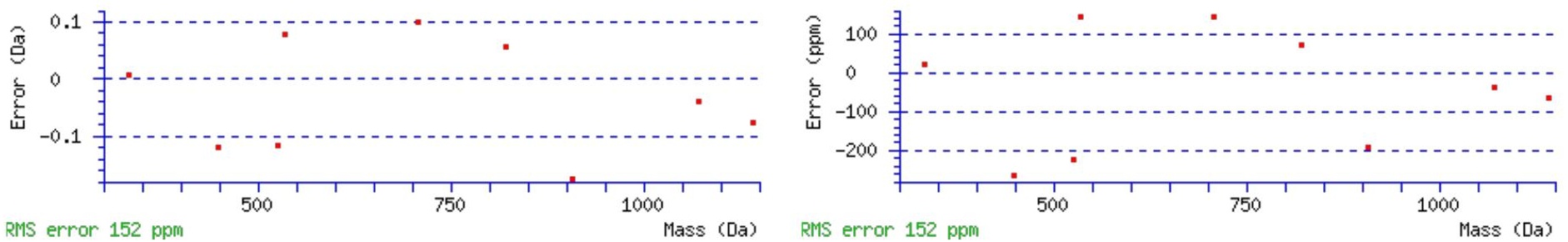


\section{All matches to this query}

\begin{tabular}{|l|l|l|l|}
\hline Score & Mr(calc): & Delta & Sequence \\
\hline 51.8 & 1254.5517 & 0.0031 & IAYSNNGSDWK \\
\hline 37.0 & 1254.5517 & 0.0031 & IAYSNNGSDWK \\
\hline
\end{tabular}

Spectrum No: 649; Query: 436; Rank: 1

\section{Peptide View}

MS/MS Fragmentation of TVLISKNVTKPR

Found in IPI00205325, Tax_Id=10116 Gene_Symbol=Lrp2 Low-density lipoprotein receptor-related protein 2 precursor

Match to Query 436: 1355.814612 from(452.945480,3+)

Title: 100101RatKid_NS_deglyco_19.806.806.3.dta

Data file K:INewmanPaper|Piliang|3SubProteomes\Piliang3SP\mgf5ppm\ERLIC_3SubProteomes5ppm.mgf
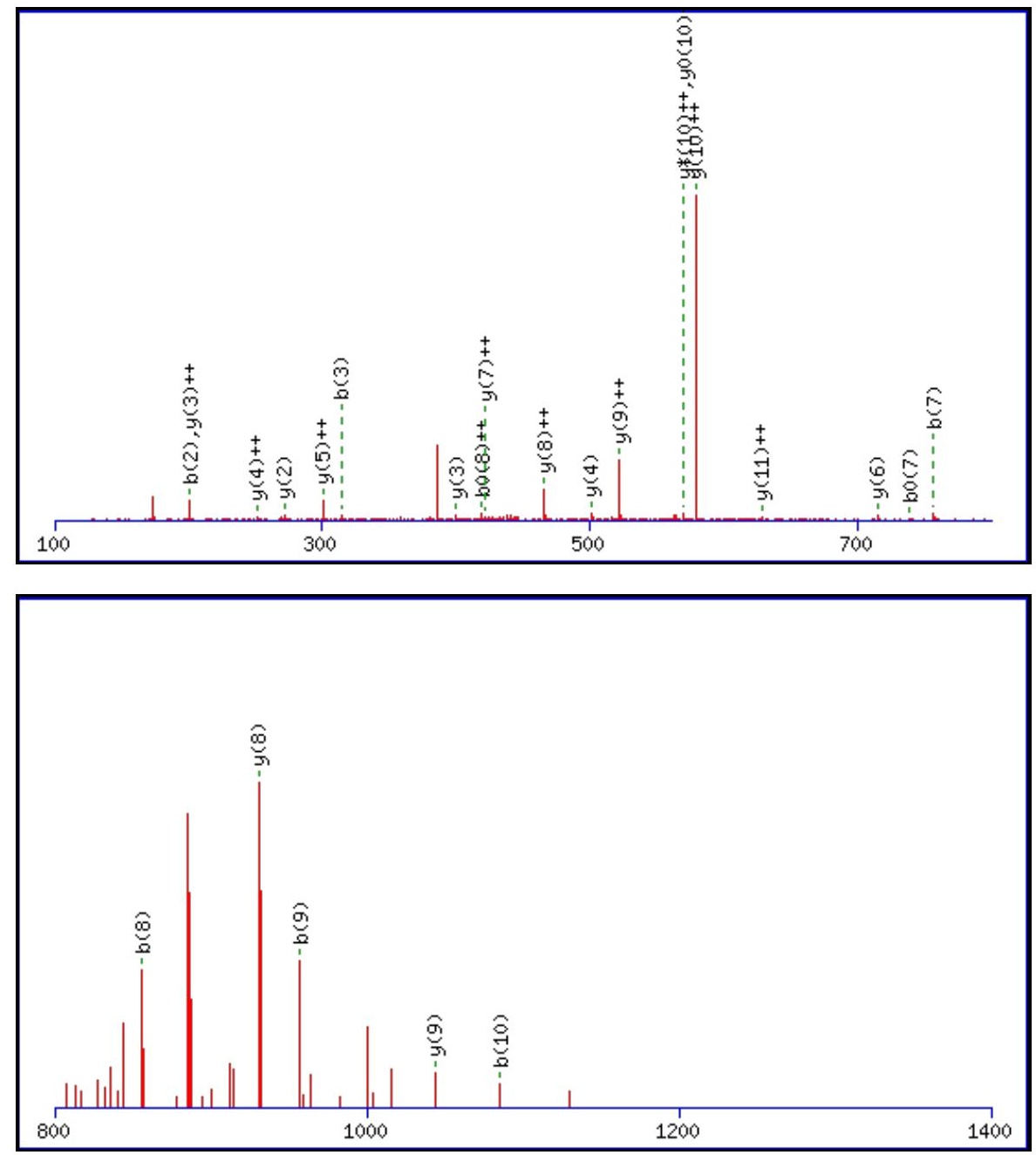


$1400 \quad 1600 \quad 1800 \quad 2000$

Monoisotopic mass of neutral peptide $\operatorname{Mr}($ calc): 1355.8136

Fixed modifications: Carbamidomethyl (C)

Variable modifications:

N7 : Deamidated_N (N)

Ions Score: 52 Expect: 0.00015

Matches (Bold Red): 24/116 fragment ions using $4 \odot$ most intense peaks

\begin{tabular}{|c|c|c|c|c|c|c|c|c|c|c|c|c|c|c|}
\hline \# & b & $\mathbf{b}^{++}$ & $\mathbf{b}^{*}$ & $\mathbf{b}^{*^{++}}$ & $\mathbf{b}^{0}$ & $\mathbf{b}^{0++}$ & Seq. & $\mathbf{y}$ & $\mathbf{y}^{++}$ & $\mathrm{y}^{*}$ & $\mathrm{y}^{*^{++}}$ & $\mathbf{y}^{\mathbf{0}}$ & $y^{0++}$ & \# \\
\hline 1 & 102.0550 & 51.5311 & & & 84.0444 & 42.5258 & $T$ & & & & & & & 12 \\
\hline 2 & 201.1234 & 101.0653 & & & 183.1128 & 92.0600 & V & 1255.7732 & 628.3903 & 1238.7467 & 619.8770 & 1237.7627 & 619.3850 & 11 \\
\hline 3 & 314.2074 & 157.6074 & & & 296.1969 & 148.6021 & L & 1156.7048 & 578.8561 & 1139.6783 & 570.3428 & 1138.6943 & 569.8508 & 10 \\
\hline 4 & 427.2915 & 214.1494 & & & 409.2809 & 205.1441 & I & 1043.6208 & 522.3140 & 1026.5942 & 513.8007 & 1025.6102 & 513.3087 & 9 \\
\hline 5 & 514.3235 & 257.6654 & & & 496.3130 & 248.6601 & $\mathrm{~S}$ & 930.5367 & 465.7720 & 913.5101 & 457.2587 & 912.5261 & 456.7667 & 8 \\
\hline 6 & 642.4185 & 321.7129 & 625.3919 & 313.1996 & 624.4079 & 312.7076 & K & 843.5047 & 422.2560 & 826.4781 & 413.7427 & 825.4941 & 413.2507 & 7 \\
\hline 7 & 757.4454 & 379.2264 & 740.4189 & 370.7131 & 739.4349 & 370.2211 & $\mathbf{N}$ & 715.4097 & 358.2085 & 698.3832 & 349.6952 & 697.3991 & 349.2032 & 6 \\
\hline 8 & 856.5138 & 428.7606 & 839.4873 & 420.2473 & 838.5033 & 419.7553 & V & 600.3828 & 300.6950 & 583.3562 & 292.1817 & 582.3722 & 291.6897 & 5 \\
\hline 9 & 957.5615 & 479.2844 & 940.5350 & 470.7711 & 939.5510 & 470.2791 & $\mathbf{T}$ & 501.3144 & 251.1608 & 484.2878 & 242.6475 & 483.3038 & 242.1555 & 4 \\
\hline 10 & 1085.6565 & 543.3319 & 1068.6299 & 534.8186 & 1067.6459 & 534.3266 & $\mathbf{K}$ & 400.2667 & 200.6370 & 383.2401 & 192.1237 & & & 3 \\
\hline 11 & 1182.7092 & 591.8583 & 1165.6827 & 583.3450 & 1164.6987 & 582.8530 & $\mathbf{P}$ & 272.1717 & 136.5895 & 255.1452 & 128.0762 & & & 2 \\
\hline 12 & & & & & & & $\mathbf{R}$ & 175.1190 & 88.0631 & 158.0924 & 79.5498 & & & 1 \\
\hline
\end{tabular}
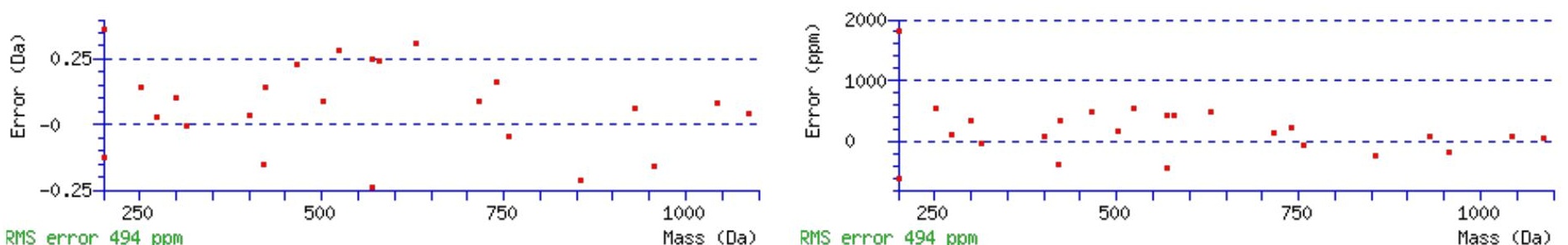

All matches to this query

\begin{tabular}{|l|l|l|l|}
\hline Score & Mr(calc): & Delta & \multicolumn{1}{|c|}{ Sequence } \\
\hline 51.7 & 1355.8136 & 0.0010 & TVLISKNVTKPR \\
\hline 9.6 & 1354.8044 & 1.0102 & AKIIARVSIDNR \\
\hline 2.5 & 1355.8136 & 0.0010 & AKLDISAIKEIR \\
\hline 2.5 & 1354.8045 & 1.0102 & AQLRRVNVTVAK \\
\hline
\end{tabular}

Spectrum No: 650; Query: 1607; Rank: 1

\section{Peptide View}




\section{MS/MS Fragmentation of DFISHDLGPALANSSHDVK}

Found in IPI00764168, Tax_Id=10116 Gene_Symbol=LOC684536 similar to Glucosylceramidase precursor (Beta-glucocerebrosidase) (Acid betaglucosidase) (D-glucosyl-N-acylsphingosine glucohydrolase) isoform 1

Match to Query 1607: 2022.965592 from(675.329140,3+)

Title: 100101RatKid_NS_deglyco_23.3043.3043.3.dta

Data file K:INewmanPaper|Piliang|3SubProteomes\Piliang3SP\mgf5ppm\ERLIC_3SubProteomes5ppm.mgf
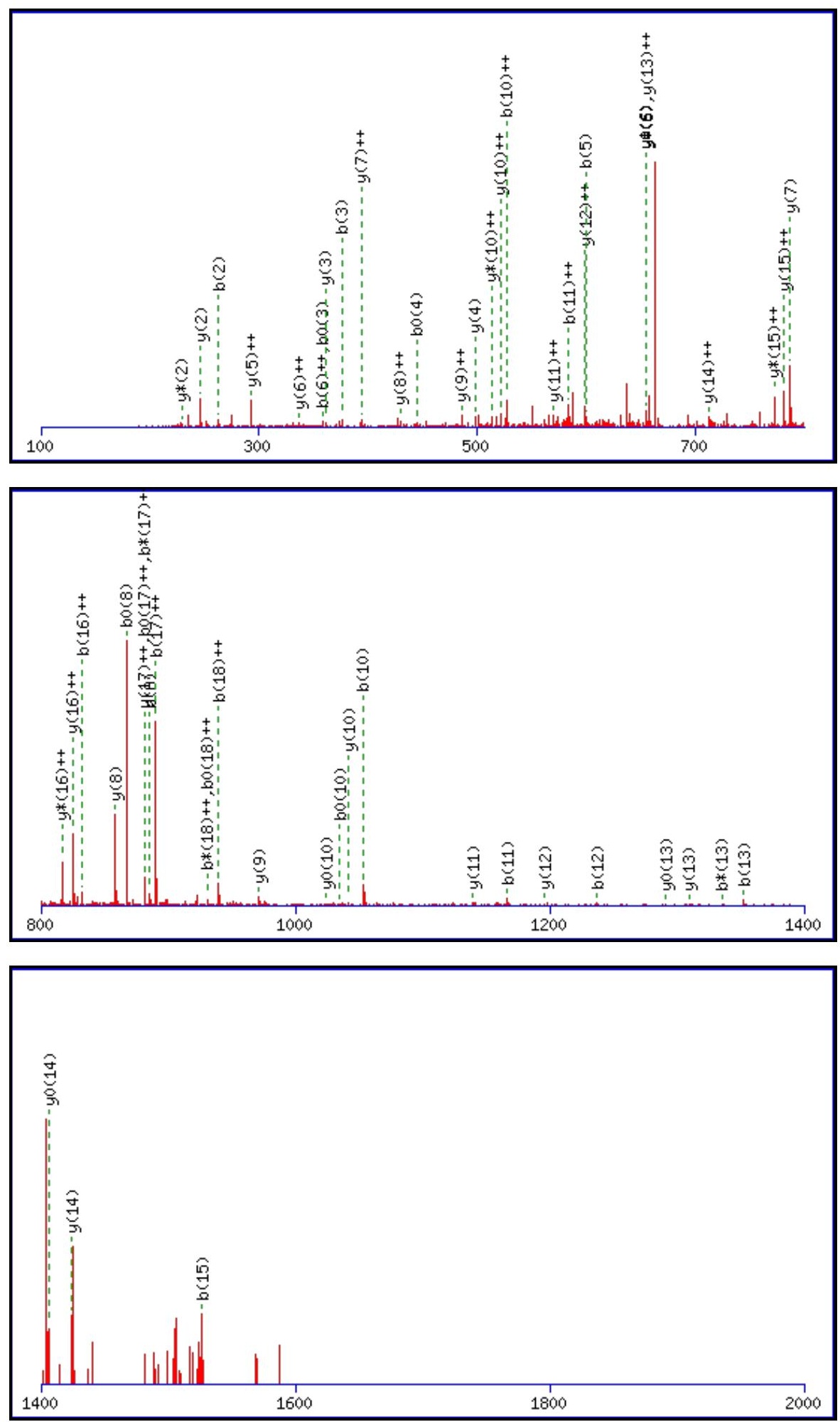

Monoisotopic mass of neutral peptide $\operatorname{Mr}($ calc): 2022.9647

Fixed modifications: Carbamidomethyl (C)

Variable modifications:

N13

: Deamidated_N ( N)

Ions Score: 52 Expect: 0.0016

Matches (Bold Red): 57/188 fragment ions using 144 most intense peaks 


\begin{tabular}{|c|c|c|c|c|c|c|c|c|c|c|c|c|c|c|}
\hline \# & b & $\mathbf{b}^{++}$ & $\mathbf{b}^{*}$ & $\mathbf{b}^{*^{++}}$ & $\mathbf{b}^{\mathbf{0}}$ & $\mathbf{b}^{\mathbf{0 + +}}$ & Seq. & $\mathbf{y}$ & $\mathbf{y}^{++}$ & $\mathbf{y}^{*}$ & $\mathrm{y}^{*^{++}}$ & $\mathbf{y}^{0}$ & $\mathbf{y}^{0^{++}}$ & \# \\
\hline 1 & 116.0342 & 58.5207 & & & 98.0237 & 49.5155 & D & & & & & & & 19 \\
\hline 2 & 263.1026 & 132.0550 & & & 245.0921 & 123.0497 & $\mathbf{F}$ & 1908.9450 & 954.9761 & 1891.9185 & 946.4629 & 1890.9344 & 945.9709 & 18 \\
\hline 3 & 376.1867 & 188.5970 & & & 358.1761 & 179.5917 & I & 1761.8766 & 881.4419 & 1744.8500 & 872.9287 & 1743.8660 & 872.4367 & 17 \\
\hline 4 & 463.2187 & 232.1130 & & & 445.2082 & 223.1077 & $\mathrm{~S}$ & 1648.7925 & 824.8999 & 1631.7660 & 816.3866 & 1630.7820 & 815.8946 & 16 \\
\hline 5 & 600.2776 & 300.6425 & & & 582.2671 & 291.6372 & $\mathbf{H}$ & 1561.7605 & 781.3839 & 1544.7340 & 772.8706 & 1543.7499 & 772.3786 & 15 \\
\hline 6 & 715.3046 & 358.1559 & & & 697.2940 & 349.1506 & D & 1424.7016 & 712.8544 & 1407.6750 & 704.3412 & 1406.6910 & 703.8492 & 14 \\
\hline 7 & 828.3886 & 414.6980 & & & 810.3781 & 405.6927 & $\mathbf{L}$ & \begin{tabular}{|l}
1309.6746 \\
\end{tabular} & 655.3410 & 1292.6481 & 646.8277 & 1291.6641 & 646.3357 & 13 \\
\hline 8 & 885.4101 & 443.2087 & & & 867.3995 & 434.2034 & G & 1196.5906 & 598.7989 & 1179.5640 & 590.2857 & 1178.5800 & 589.7936 & 12 \\
\hline 9 & 982.4629 & 491.7351 & & & 964.4523 & 482.7298 & $\mathbf{P}$ & 1139.5691 & 570.2882 & 1122.5426 & 561.7749 & 1121.5586 & 561.2829 & 11 \\
\hline 10 & 1053.5000 & 527.2536 & & & $\mathbf{1 0 3 5 . 4 8 9 4}$ & 518.2483 & $\mathbf{A}$ & 1042.5164 & 521.7618 & 1025.4898 & 513.2485 & 1024.5058 & 512.7565 & 10 \\
\hline 11 & 1166.5840 & 583.7957 & & & 1148.5735 & 574.7904 & $\mathbf{L}$ & 971.4792 & 486.2433 & 954.4527 & 477.7300 & 953.4687 & 477.2380 & 9 \\
\hline 12 & 1237.6212 & 619.3142 & & & 1219.6106 & 610.3089 & $\mathbf{A}$ & 858.3952 & 429.7012 & 841.3686 & 421.1880 & 840.3846 & 420.6959 & 8 \\
\hline 13 & 1352.6481 & 676.8277 & \begin{tabular}{|l}
1335.6216 \\
\end{tabular} & 668.3144 & 1334.6375 & 667.8224 & $\mathbf{N}$ & 787.3581 & 394.1827 & 770.3315 & 385.6694 & 769.3475 & 385.1774 & 7 \\
\hline 14 & 1439.6801 & 720.3437 & 1422.6536 & 711.8304 & 1421.6696 & 711.3384 & $\mathrm{~S}$ & 672.3311 & 336.6692 & 655.3046 & 328.1559 & 654.3206 & 327.6639 & 6 \\
\hline 15 & 1526.7122 & 763.8597 & 1509.6856 & 755.3464 & 1508.7016 & 754.8544 & $\mathrm{~S}$ & 585.2991 & 293.1532 & 568.2726 & 284.6399 & 567.2885 & 284.1479 & 5 \\
\hline 16 & 1663.7711 & 832.3892 & 1646.7445 & 823.8759 & 1645.7605 & 823.3839 & $\mathbf{H}$ & 498.2671 & 249.6372 & 481.2405 & 241.1239 & 480.2565 & 240.6319 & 4 \\
\hline 17 & 1778.7980 & \begin{tabular}{|l|}
889.9026 \\
\end{tabular} & 1761.7715 & 881.3894 & 1760.7874 & \begin{tabular}{|l|}
880.8974 \\
\end{tabular} & D & 361.2082 & 181.1077 & 344.1816 & 172.5944 & 343.1976 & 172.1024 & 3 \\
\hline 18 & 1877.8664 & 939.4369 & 1860.8399 & 930.9236 & 1859.8559 & 930.4316 & V & 246.1812 & 123.5942 & 229.1547 & 115.0810 & & & 2 \\
\hline 19 & & & & & & & $\mathbf{K}$ & 147.1128 & 74.0600 & 130.0863 & 65.5468 & & & 1 \\
\hline
\end{tabular}
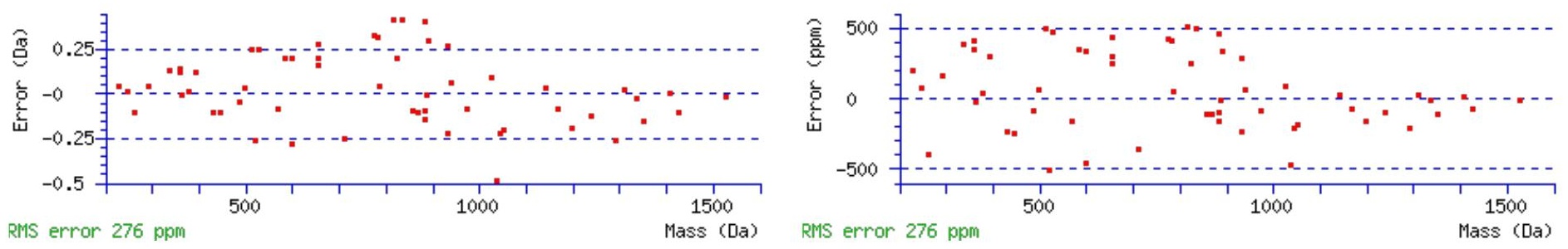

\section{All matches to this query}

\begin{tabular}{|l|l|l|l|}
\hline Score & Mr(calc): & Delta & \multicolumn{1}{|c|}{ Sequence } \\
\hline 51.6 & 2022.9647 & 0.0009 & DFISHDLGPALANSSHDVK \\
\hline 8.2 & 2021.9807 & 0.9849 & DFISHDLGPALANSSHDVK \\
\hline 3.7 & 2022.9581 & 0.0075 & ITKPFFAAVSDGKVQOK \\
\hline 3.0 & 2020.9483 & 2.0173 & EIRSTVTVNTIDLGSKK \\
\hline 1.4 & 2021.9588 & 1.0068 & STQLLANYQNLLRVTK \\
\hline 1.0 & 2020.9636 & 2.0020 & VNVGKQFTDNAIGNLCDR \\
\hline 1.0 & 2020.9636 & 2.0020 & VNVGKQFTDNAIGNLCDR \\
\hline 1.0 & 2021.9476 & 1.0179 & VNVGKQFTDNAIGNLCDR \\
\hline 1.0 & 2020.9636 & 2.0020 & VNVGKQFTDNAIGNLCDR \\
\hline 1.0 & 2021.9476 & 1.0179 & VNVGKQFTDNAIGNLCDR \\
\hline
\end{tabular}

Spectrum No: 651; Query: 2389; Rank: 1

\section{Peptide View}

MS/MS Fragmentation of IMSVVDPNHSGLVTFQAFIDFMSR

Found in IPI00213463, Tax_Id=10116 Gene_Symbol=Actn4 Alpha-actinin-4

Match to Query 2389: 2711.317152 from(904.779660,3+)

Title: 091008RatKidney_NoSalt_06.6795.6795.3.dta 

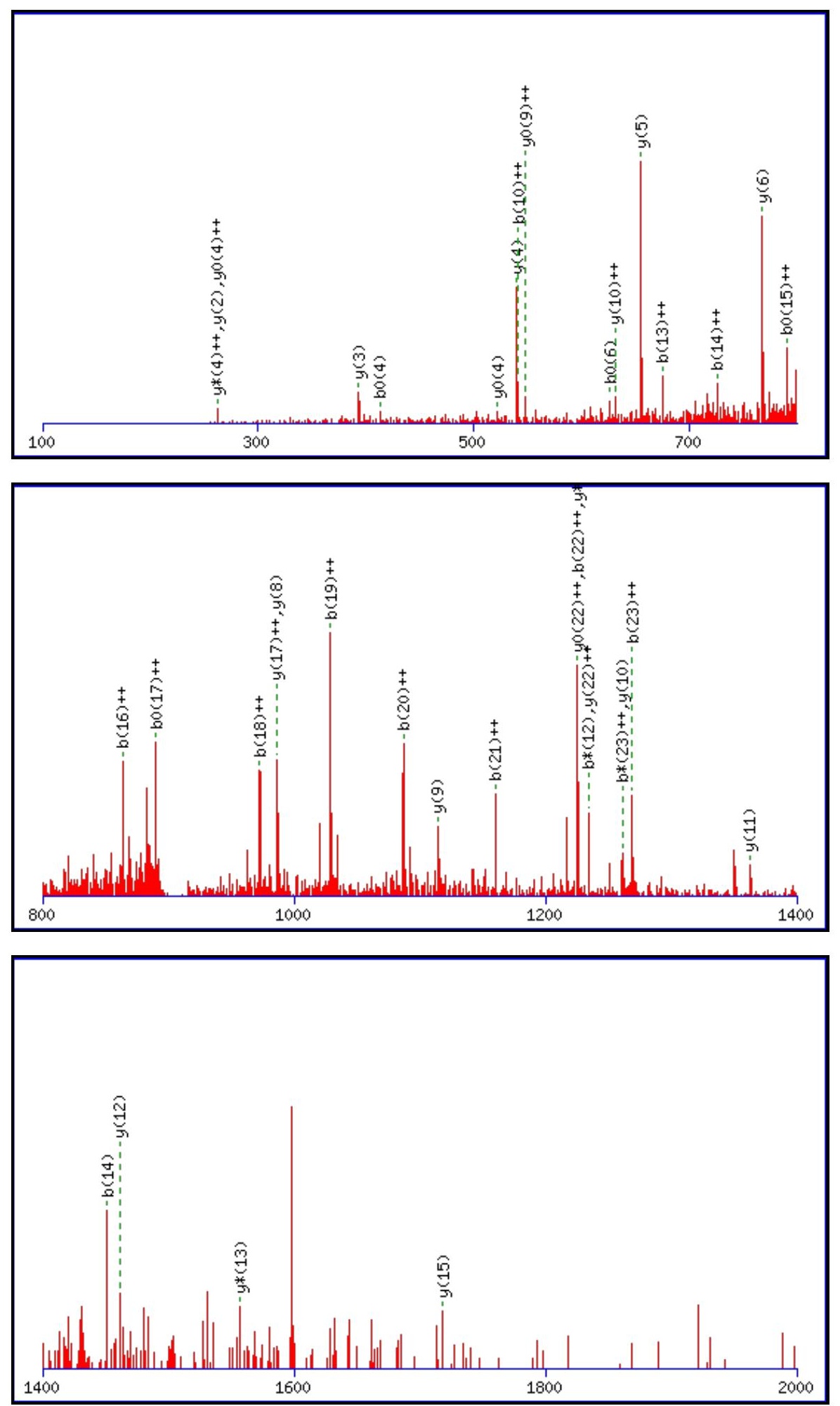

Monoisotopic mass of neutral peptide $\operatorname{Mr}($ calc): 2711.3088

Fixed modifications: Carbamidomethyl (C)

Variable modifications:

N8 : Deamidated_N (N)

Ions Score: 52 Expect: 0.0019

Matches (Bold Red): 38/256 fragment ions using 58 most intense peaks

\begin{tabular}{|c|c|c|c|c|c|c|c|c|c|c|c|c|c|c|}
\hline$\#$ & $\mathbf{b}$ & $\mathbf{b}^{++}$ & $\mathbf{b}^{*}$ & $\mathbf{b}^{\boldsymbol{*}^{++}}$ & $\mathbf{b}^{\mathbf{0}}$ & $\mathbf{b}^{\mathbf{0}+}$ & Seq. & $\mathbf{y}$ & $\mathbf{y}^{++}$ & $\mathbf{y}^{*}$ & $\mathbf{y}^{\mathbf{*}^{++}}$ & $\mathbf{y}^{\mathbf{0}}$ & $\mathbf{y}^{\mathbf{0}+}$ & $\#$ \\
\hline $\mathbf{1}$ & 114.0913 & 57.5493 & & & & & $\mathbf{I}$ & & & & & & & $\mathbf{2 4}$ \\
\hline $\mathbf{2}$ & 245.1318 & 123.0696 & & & & & $\mathbf{M}$ & 2599.2320 & 1300.1196 & 2582.2054 & 1291.6063 & 2581.2214 & 1291.1143 & $\mathbf{2 3}$ \\
\hline 3 & 332.1639 & 166.5856 & & & 314.1533 & 157.5803 & S & 2468.1915 & $\mathbf{1 2 3 4 . 5 9 9 4}$ & 2451.1649 & $\mathbf{1 2 2 6 . 0 8 6 1}$ & 2450.1809 & $\mathbf{1 2 2 5 . 5 9 4 1}$ & $\mathbf{2 2}$ \\
\hline $\mathbf{4}$ & 431.2323 & 216.1198 & & & $\mathbf{4 1 3 . 2 2 1 7}$ & 207.1145 & $\mathbf{V}$ & 2381.1594 & 1191.0834 & 2364.1329 & 1182.5701 & 2363.1489 & 1182.0781 & $\mathbf{2 1}$
\end{tabular}




\begin{tabular}{|c|c|c|c|c|c|c|c|c|c|c|c|c|c|c|}
\hline 5 & 530.3007 & 265.6540 & & & 512.2901 & 256.6487 & V & 2282.0910 & 1141.5491 & 2265.0645 & 1133.0359 & 2264.0805 & 1132.5439 & \\
\hline 6 & 645.3276 & 323.1675 & & & 627.3171 & 314.1622 & D & 2183.0226 & 1092.0149 & 2165.9961 & 1083.5017 & 2165.0120 & 1083.0097 & 9 \\
\hline 7 & 742.3804 & 371.6938 & & & 724.3698 & 362.6885 & $\mathbf{P}$ & 2067.9957 & 1034.5015 & 2050.9691 & 1025.9882 & 2049.9851 & 1025.4962 & \\
\hline 8 & 857.4073 & 429.2073 & 840.3808 & 420.6940 & 839.3968 & 420.2020 & $\mathbf{N}$ & 1970.9429 & 985.9751 & |1953.9164 & 977.4618 & 1952.9323 & 976.9698 & 17 \\
\hline 9 & 994.4662 & 497.7368 & 977.4397 & 489.2235 & 976.4557 & 488.7315 & $\mathbf{H}$ & 1855.9160 & 928.4616 & 1838.8894 & 919.9483 & 1837.9054 & 919.4563 & 16 \\
\hline 10 & 1081.4983 & 541.2528 & 1064.4717 & 532.7395 & 1063.4877 & 532.2475 & $S$ & 1718.8571 & 859.9322 & 1701.8305 & 851.4189 & 1700.8465 & 850.9269 & 15 \\
\hline 11 & 1138.5197 & 569.7635 & 1121.4932 & 561.2502 & 1120.5092 & 560.7582 & G & 1631.8250 & 816.4162 & 1614.7985 & 807.9029 & 1613.8145 & 807.4109 & 4 \\
\hline 12 & 1251.6038 & 626.3055 & 1234.5772 & 617.7923 & 1233.5932 & 617.3003 & $\mathbf{L}$ & 1574.8036 & 787.9054 & 1557.7770 & 779.3921 & 1556.7930 & 778.9001 & 13 \\
\hline 13 & 1350.6722 & 675.8397 & 1333.6457 & 667.3265 & 1332.6616 & 666.8345 & $\mathbf{V}$ & 1461.7195 & 731.3634 & 1444.6930 & 722.8501 & 1443.7089 & 722.3581 & 12 \\
\hline 14 & 1451.7199 & 726.3636 & 1434.6933 & 717.8503 & 1433.7093 & 717.3583 & $\mathbf{T}$ & 1362.6511 & & 1345.6245 & 673.3159 & 1344.6405 & 672.8239 & \\
\hline 15 & 1598.7883 & 799.8978 & 1581.7618 & 791.3845 & 1580.7777 & 790.8925 & $\mathbf{F}$ & 1261.6034 & 631.3053 & 1244.5769 & 622.7921 & 1243.5928 & 622.3001 & 10 \\
\hline 16 & 1726.8469 & 863.9271 & 1709.8203 & 855.4138 & 1708.8363 & 854.9218 & Q & 1114.5350 & 557.7711 & 1097.5084 & 549.2579 & 1096.5244 & 548.7659 & 9 \\
\hline 17 & 1797.8840 & 899.4456 & 1780.8574 & 890.9324 & 1779.8734 & 890.4404 & A & 986.4764 & 493.7418 & 969.4499 & 485.2286 & 968.4658 & 484.7366 & 8 \\
\hline 18 & 1944.9524 & 972.9798 & 1927.9259 & 964.4666 & 1926.9418 & 963.9746 & $\mathbf{F}$ & 915.4393 & 458.2233 & 898.4128 & 449.7100 & 897.4287 & 449.2180 & 7 \\
\hline 19 & 2058.0365 & 1029.5219 & 2041.0099 & 1021.0086 & 2040.0259 & 1020.5166 & I & 768.3709 & 384.6891 & 751.3443 & 376.1758 & 750.3603 & 375.6838 & 6 \\
\hline 20 & 2173.0634 & \begin{tabular}{|l|l|}
1087.0353 \\
\end{tabular} & 2156.0369 & 1078.5221 & 2155.0528 & 1078.0301 & D & 655.2868 & 328.1470 & 638.2603 & 319.6338 & 637.2763 & 319.1418 & 5 \\
\hline 21 & 2320.1318 & \begin{tabular}{|l|l|}
1160.5696 \\
\end{tabular} & 2303.1053 & 1152.0563 & 2302.1213 & 1151.5643 & $\mathbf{F}$ & 540.2599 & 270.6336 & 523.2333 & 262.1203 & 522.2493 & 261.6283 & 4 \\
\hline 22 & 2451.1723 & \begin{tabular}{|l|l|}
1226.0898 \\
\end{tabular} & 2434.1458 & 1217.5765 & 2433.1617 & 1217.0845 & M & 393.1915 & 197.0994 & 376.1649 & 188.5861 & 375.1809 & 188.0941 & 3 \\
\hline 23 & 2538.2043 & 1269.6058 & 2521.1778 & 1261.0925 & 2520.1938 & 1260.6005 & $\mathrm{~S}$ & 262.1510 & 131.5791 & 245.1244 & 123.0659 & 244.1404 & 122.5738 & 2 \\
\hline 24 & & & & & & & $\mathbf{R}$ & 175.1190 & 88.0631 & 158.0924 & 79.5498 & & & 1 \\
\hline
\end{tabular}
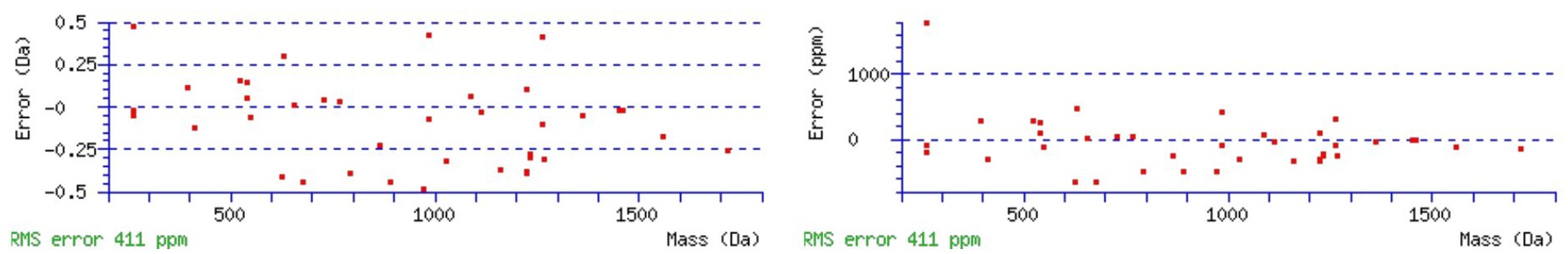

\section{All matches to this query}

\begin{tabular}{|l|l|l|l|}
\hline Score & Mr(calc): & Delta & \multicolumn{1}{|c|}{ Sequence } \\
\hline 51.6 & 2711.3088 & 0.0084 & IMSVVDPNHSGLVTFQAFIDFMSR \\
\hline 45.7 & 2710.3248 & 0.9924 & IMSVVDPNHSGLVTFQAFIDFMSR \\
\hline 6.5 & 2711.3159 & 0.0012 & SKVDLQHFCGYVTMLNASSQLASR \\
\hline 3.2 & 2711.3128 & 0.0043 & LNSLLKNLKFMNPGGDISLNPSEK \\
\hline 2.6 & 2711.2989 & 0.0183 & SNMNALQASLPKLSIKEHLYNSR \\
\hline 2.6 & 2711.2989 & 0.0183 & SNMNALQASLPKLSIKEHLYNSR \\
\hline 2.6 & 2711.3241 & -0.0069 & SILQNSWTEVMDLILKPRSGAEK \\
\hline 2.5 & 2710.3401 & 0.9771 & SILQNSWTEVMDLILKPRSGAEK \\
\hline 1.6 & 2711.3320 & -0.0148 & GHLGLVANPLKENLEPFOVSPESR \\
\hline 1.6 & 2711.3320 & -0.0148 & GHLGLVANPLKENLEPFQVSPESR \\
\hline
\end{tabular}

Spectrum No: 652; Query: 459; Rank: 1

\section{Peptide View}

MS/MS Fragmentation of NILPNITTSYLK

Found in IPI00192845, Tax_Id=10116 Gene_Symbol=P2rx4 40 kDa protein

Match to Query 459: 1376.753288 from(689.383920,2+)

Title: 091008RatKidney_NH4Format01_24.3364.3364.2.dta 

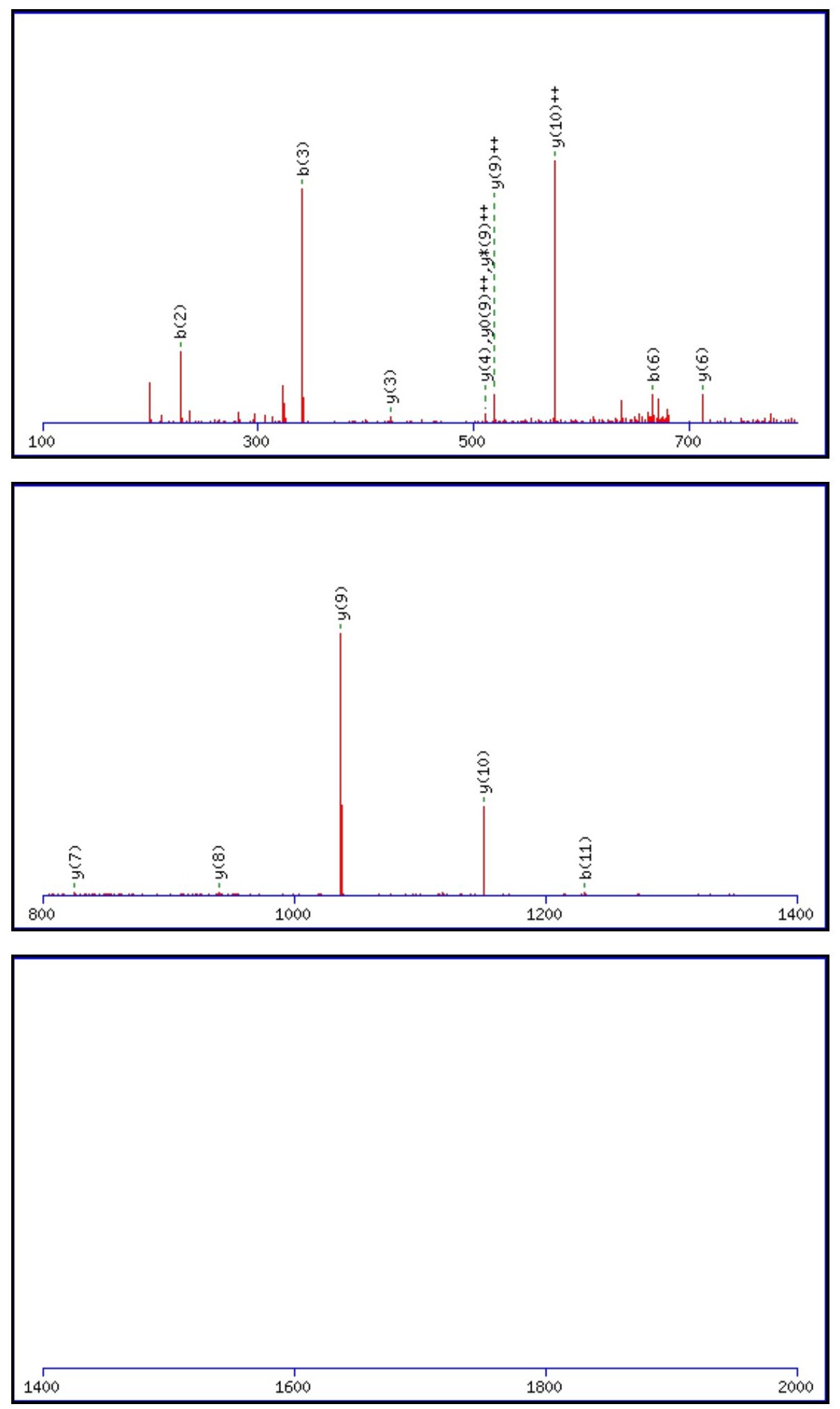

Monoisotopic mass of neutral peptide $\operatorname{Mr}($ calc): 1376.7551

Fixed modifications: Carbamidomethyl (C)

Variable modifications:

N5 : Deamidated_N (N)

Ions Score: 51 Expect: 0.00056

Matches (Bold Red): 15/114 fragment ions using 18 most intense peaks

\begin{tabular}{|r|c|c|c|c|c|c|c|c|c|c|c|c|c|c|}
\hline$\#$ & $\mathbf{b}$ & $\mathbf{b}^{++}$ & $\mathbf{b}^{*}$ & $\mathbf{b}^{*^{++}}$ & $\mathbf{b}^{\mathbf{0}}$ & $\mathbf{b}^{\mathbf{0}+}$ & Seq. & $\mathbf{y}$ & $\mathbf{y}^{++}$ & $\mathbf{y}^{*}$ & $\mathbf{y}^{\boldsymbol{*}^{++}}$ & $\mathbf{y}^{\mathbf{0}}$ & $\mathbf{y}^{\mathbf{0 + +}}$ & $\#$ \\
\hline $\mathbf{1}$ & 115.0502 & 58.0287 & 98.0237 & 49.5155 & & & $\mathbf{N}$ & & & & & & & $\mathbf{1 2}$ \\
\hline $\mathbf{2}$ & $\mathbf{2 2 8 . 1 3 4 3}$ & 114.5708 & 211.1077 & 106.0575 & & & $\mathbf{I}$ & 1263.7195 & 632.3634 & 1246.6929 & 623.8501 & 1245.7089 & 623.3581 & $\mathbf{1 1}$ \\
\hline $\mathbf{3}$ & 341.2183 & 171.1128 & 324.1918 & 162.5995 & & & $\mathbf{L}$ & $\mathbf{1 1 5 0 . 6 3 5 4}$ & 575.8213 & 1133.6089 & 567.3081 & 1132.6248 & 566.8161 & $\mathbf{1 0}$ \\
\hline $\mathbf{4}$ & 438.2711 & 219.6392 & 421.2445 & 211.1259 & & & $\mathbf{P}$ & $\mathbf{1 0 3 7 . 5 5 1 3}$ & $\mathbf{5 1 9 . 2 7 9 3}$ & 1020.5248 & $\mathbf{5 1 0 . 7 6 6 0}$ & 1019.5408 & $\mathbf{5 1 0 . 2 7 4 0}$ & $\mathbf{9}$
\end{tabular}




\begin{tabular}{|c|c|c|c|c|c|c|c|c|c|c|c|c|c|c|}
\hline 5 & 553.2980 & 277.1527 & 536.2715 & 268.6394 & & & $\mathbf{N}$ & 940.4986 & 470.7529 & 923.4720 & 462.2397 & 922.4880 & 461.7476 & 8 \\
\hline 6 & 666.3821 & 333.6947 & 649.3555 & 325.1814 & & & I & 825.4716 & 413.2395 & 808.4451 & 404.7262 & 807.4611 & 404.2342 & 7 \\
\hline 7 & 767.4298 & 384.2185 & 750.4032 & 375.7053 & 749.4192 & 375.2132 & $\mathbf{T}$ & 712.3876 & 356.6974 & 695.3610 & 348.1842 & 694.3770 & 347.6921 & 6 \\
\hline 8 & 868.4775 & 434.7424 & 851.4509 & 426.2291 & 850.4669 & 425.7371 & $\mathbf{T}$ & 611.3399 & 306.1736 & 594.3134 & 297.6603 & 593.3293 & 297.1683 & 5 \\
\hline 9 & 955.5095 & 478.2584 & 938.4829 & 469.7451 & 937.4989 & 469.2531 & S & 510.2922 & 255.6498 & 493.2657 & 247.1365 & 492.2817 & 246.6445 & 4 \\
\hline 10 & 1118.5728 & 559.7900 & 1101.5463 & 551.2768 & 1100.5622 & 550.7848 & $\mathbf{Y}$ & 423.2602 & 212.1337 & 406.2336 & 203.6205 & & & 3 \\
\hline 11 & 1231.6569 & 616.3321 & 1214.6303 & 607.8188 & 1213.6463 & 607.3268 & $\mathbf{L}$ & 260.1969 & 130.6021 & 243.1703 & 122.0888 & & & 2 \\
\hline 12 & & & & & & & $\mathbf{K}$ & 147.1128 & 74.0600 & 130.0863 & 65.5468 & & & 1 \\
\hline
\end{tabular}
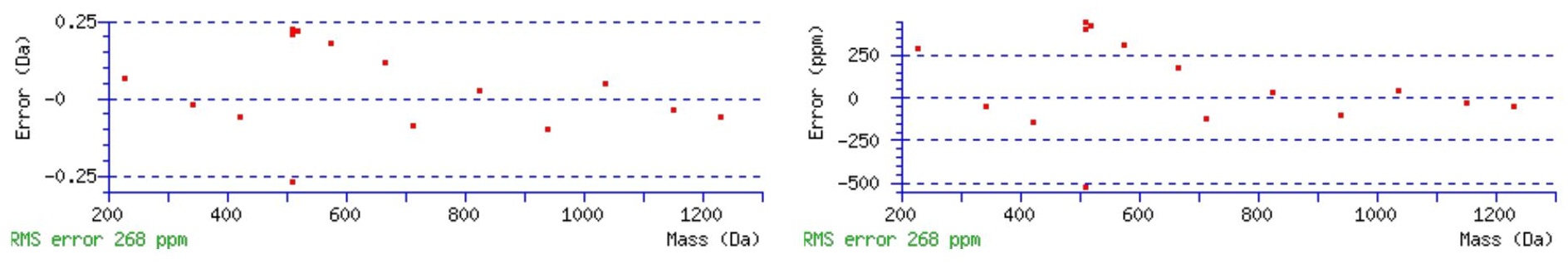

\section{All matches to this query}

\begin{tabular}{|l|l|l|l|}
\hline Score & Mr(calc): & Delta & \multicolumn{1}{c|}{ Sequence } \\
\hline 51.3 & 1376.7551 & -0.0018 & NILPNITTSYLK \\
\hline 13.5 & 1376.7428 & 0.0105 & KVLKQASEGPLK \\
\hline 13.5 & 1376.7581 & -0.0048 & VKINWLKPATK \\
\hline 12.2 & 1376.7565 & -0.0032 & QVLQHPWITQK \\
\hline 10.2 & 1375.7411 & 1.0122 & KLPPRVCVSLK \\
\hline 10.0 & 1375.7476 & 1.0057 & QLDLQLPLKTK \\
\hline 9.7 & 1375.7588 & 0.9945 & LLLSREILSPR \\
\hline 5.5 & 1376.7663 & -0.0131 & VKLEVEIATYGR \\
\hline 4.7 & 1376.7412 & 0.0121 & ARELARIEEYK \\
\hline 4.0 & 1375.7588 & 0.9945 & LLLSREILSPR \\
\hline
\end{tabular}

Spectrum No: 653; Query: 2318; Rank: 1

\section{Peptide View}

MS/MS Fragmentation of AFENVTDLQWLILDHNLLENSK

Found in IPI00206403, Tax_Id=10116 Gene_Symbol=Lum Lumican precursor

Match to Query 2318: 2612.320332 from(871.780720,3+)

Title: 100101RatKid_NS_deglyco_13.6502.6502.3.dta

Data file K:INewmanPaper|Piliang|3SubProteomes\Piliang3SP\mgf5ppm\ERLIC_3SubProteomes5ppm.mgf 

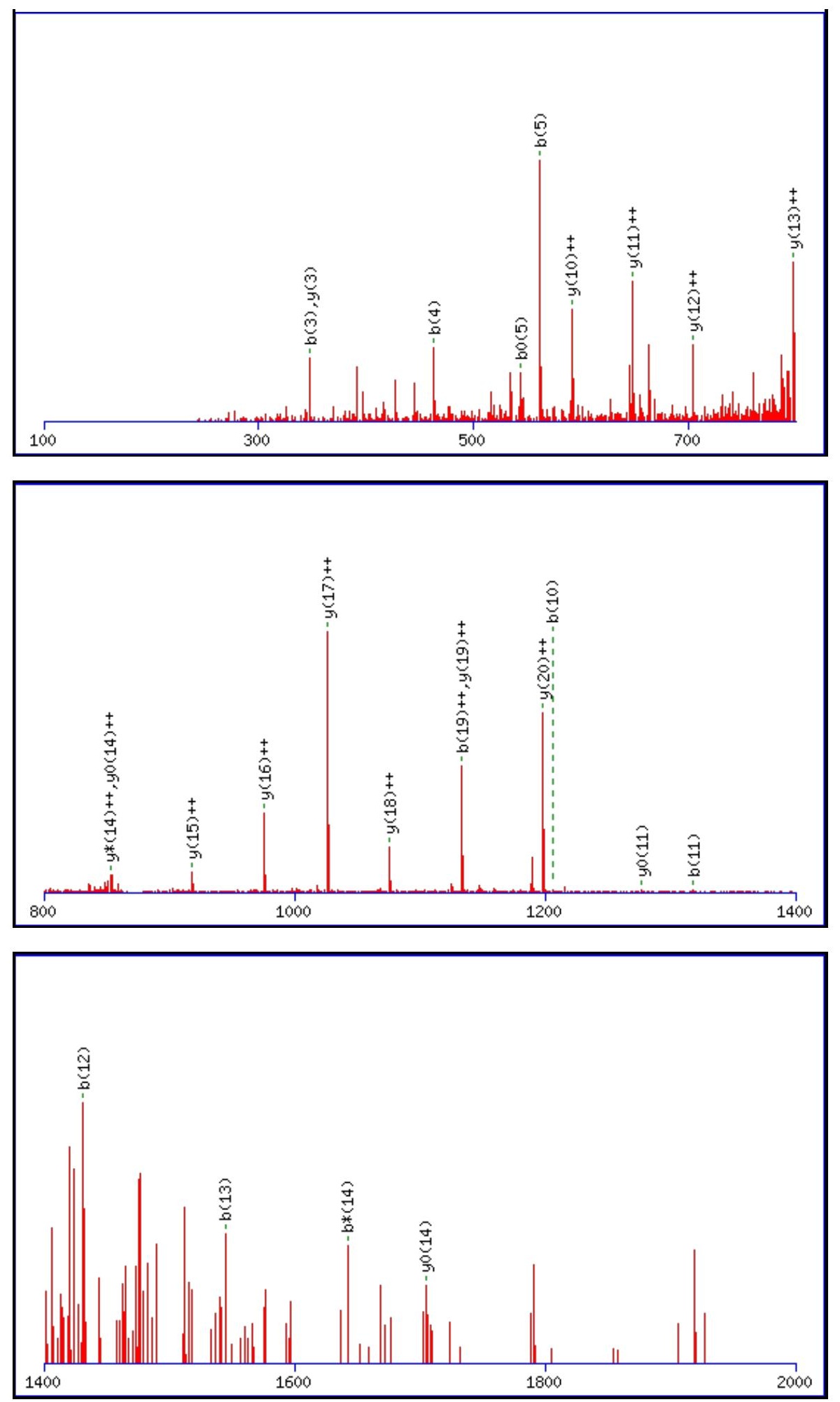

Monoisotopic mass of neutral peptide $\operatorname{Mr}($ calc): 2612.3122

Fixed modifications: Carbamidomethyl (C)

Variable modifications:

N4 : Deamidated $\mathrm{N}(\mathrm{N})$

Ions Score: 51 Expect: 0.0016

Matches (Bold Red): 25/240 fragment ions using 45 most intense peaks

\begin{tabular}{|r|c|c|c|c|c|c|c|c|c|c|c|c|c|c|}
\hline$\#$ & $\mathbf{b}$ & $\mathbf{b}^{++}$ & $\mathbf{b}^{*}$ & $\mathbf{b}^{\boldsymbol{*}^{++}}$ & $\mathbf{b}^{\mathbf{0}}$ & $\mathbf{b}^{\mathbf{0 + +}}$ & $\mathbf{S e q}$ & $\mathbf{y}$ & $\mathbf{y}^{++}$ & $\mathbf{y}^{\mathbf{*}}$ & $\mathbf{y}^{\mathbf{*}^{++}}$ & $\mathbf{y}^{\mathbf{0}}$ & $\mathbf{y}^{\mathbf{0 + +}}$ & $\#$ \\
\hline $\mathbf{1}$ & $\mathbf{7 2 . 0 4 4 4}$ & 36.5258 & & & & & $\mathbf{A}$ & & & & & & & $\mathbf{2 2}$ \\
\hline $\mathbf{2}$ & 219.1128 & 110.0600 & & & & & $\mathbf{F}$ & 2542.2824 & 1271.6448 & 2525.2558 & 1263.1316 & 2524.2718 & 1262.6395 & $\mathbf{2 1}$ \\
\hline $\mathbf{3}$ & $\mathbf{3 4 8 . 1 5 5 4}$ & 174.5813 & & & 330.1448 & 165.5761 & $\mathbf{E}$ & 2395.2140 & $\mathbf{1 1 9 8 . 1 1 0 6}$ & 2378.1874 & 1189.5974 & 2377.2034 & 1189.1053 & $\mathbf{2 0}$ \\
\hline $\mathbf{4}$ & $\mathbf{4 6 3 . 1 8 2 3}$ & 232.0948 & 446.1558 & 223.5815 & 445.1718 & 223.0895 & $\mathbf{N}$ & 2266.1714 & $\mathbf{1 1 3 3 . 5 8 9 3}$ & 2249.1448 & 1125.0761 & 2248.1608 & 1124.5840 & $\mathbf{1 9}$ \\
\hline $\mathbf{5}$ & $\mathbf{5 6 2 . 2 5 0 7}$ & 281.6290 & 545.2242 & 273.1157 & 544.2402 & 272.6237 & $\mathbf{V}$ & 2151.1444 & $\mathbf{1 0 7 6 . 0 7 5 9}$ & 2134.1179 & 1067.5626 & 2133.1339 & 1067.0706 & $\mathbf{1 8}$ \\
\hline
\end{tabular}




\begin{tabular}{|c|c|c|c|c|c|c|c|c|c|c|c|c|c|c|}
\hline 6 & 663.2984 & 332.1529 & 646.2719 & 323.6396 & 645.2879| & 323.1476 & $\mathbf{T}$ & 2052.0760 & |1026.5417 & |2035.0495 & 1018.0284 & |2034.0655 & 1017.5364 & 17 \\
\hline 7 & 778.3254 & 389.6663 & 761.2988 & 381.1530 & 760.3148 & 380.6610 & D & 1951.0284 & 976.0178 & 1934.0018 & 967.5045 & 1933.0178 & 967.0125 & 16 \\
\hline 8 & 891.4094 & 446.2084 & 874.3829 & 437.6951 & 873.3989 & 437.2031 & $\mathbf{L}$ & 1836.0014 & 918.5043 & 1818.9749 & 909.9911 & 1817.9908 & 909.4991 & 15 \\
\hline 9 & 1019.4680 & 510.2376 & 1002.4415 & 501.7244 & 1001.4574 & 501.2324 & $\mathbf{Q}$ & 1722.9173 & 861.9623 & 1705.8908 & 853.4490 & 1704.9068 & 852.9570 & 14 \\
\hline 10 & 1205.5473 & 603.2773 & 1188.5208 & 594.7640 & 1187.5368 & 594.2720 & W & 1594.8588 & 797.9330 & 1577.8322 & 789.4197 & 1576.8482 & 788.9277 & 13 \\
\hline 11 & 1318.6314 & 659.8193 & 1301.6048 & 651.3061 & 1300.6208 & 650.8140 & $\mathbf{L}$ & 1408.7795 & 704.8934 & 1391.7529 & 696.3801 & 1390.7689 & 695.8881 & 12 \\
\hline 12 & 1431.7155 & 716.3614 & 1414.6889 & 707.8481 & 1413.7049 & 707.3561 & I & 1295.6954 & 648.3513 & 1278.6688 & 639.8381 & 1277.6848 & 639.3461 & 11 \\
\hline 13 & 1544.7995 & 772.9034 & 1527.7730 & 764.3901 & 1526.7890 & 763.8981 & $\mathbf{L}$ & 1182.6113 & 591.8093 & 1165.5848 & 583.2960 & 1164.6008 & 582.8040 & 10 \\
\hline 14 & 1659.8265 & 830.4169 & 1642.7999 & 821.9036 & 1641.8159 & 821.4116 & D & 1069.5273 & 535.2673 & 1052.5007 & 526.7540 & 1051.5167 & 526.2620 & 9 \\
\hline 15 & 1796.8854 & 898.9463 & 1779.8588 & 890.4330 & 1778.8748 & 889.9410 & $\mathbf{H}$ & 954.5003 & 477.7538 & 937.4738 & 469.2405 & 936.4898 & 468.7485 & 8 \\
\hline 16 & 1910.9283 & 955.9678 & 1893.9017 & 947.4545 & 1892.9177 & 946.9625 & $\mathbf{N}$ & 817.4414 & 409.2243 & 800.4149 & 400.7111 & 799.4308 & 400.2191 & 7 \\
\hline 17 & 2024.0124 & 1012.5098 & 2006.9858 & 1003.9965 & 2006.0018 & 1003.5045 & $\mathbf{L}$ & 703.3985 & 352.2029 & 686.3719 & 343.6896 & 685.3879 & 343.1976 & 6 \\
\hline 18 & 2137.0964 & 1069.0519 & 2120.0699 & 1060.5386 & 2119.0859 & 1060.0466 & $\mathbf{L}$ & 590.3144 & 295.6608 & 573.2879 & 287.1476 & 572.3039 & 286.6556 & 5 \\
\hline 19 & 2266.1390 & 1133.5731 & 2249.1125 & 1125.0599 & 2248.1285 & 1124.5679 & $\mathbf{E}$ & 477.2304 & 239.1188 & 460.2038 & 230.6055 & 459.2198 & 230.1135 & 4 \\
\hline 21 & 2467.2140 & 1234.1106 & 2450.1874 & 1225.5974 & 2449.2034 & 1225.1053 & S & 234.1448 & 117.5761 & 217.1183 & 109.0628 & 216.1343 & 108.5708 & 2 \\
\hline 22 & & & & & & & K & 147.1128 & 74.0600 & 130.0863 & 65.5468 & & & 1 \\
\hline
\end{tabular}
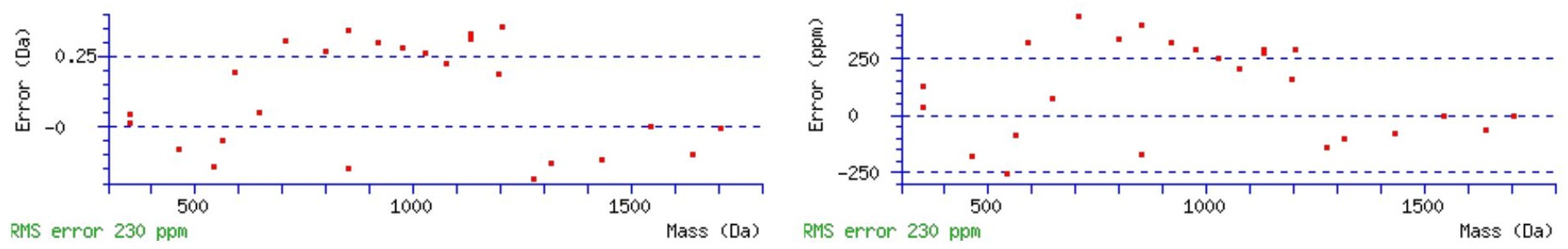

\section{All matches to this query}

\begin{tabular}{|l|l|c|l|}
\hline Score & Mr(calc): & Delta & \multicolumn{1}{c|}{ Sequence } \\
\hline 51.1 & 2612.3122 & 0.0081 & $\underline{\text { AFENVTDLQWLILDHNLLENSK }}$ \\
\hline 36.8 & 2612.3122 & 0.0081 & AFENVTDLQWLILDHNLLENSK \\
\hline 36.8 & 2612.3122 & 0.0081 & $\underline{\text { AFENVTDLQWLILDHNLLENSK }}$ \\
\hline 19.4 & 2611.3282 & 0.9921 & $\underline{\text { AFENVTDLQWLILDHNLLENSK }}$ \\
\hline 15.5 & 2612.3285 & -0.0082 & VLGPAVIFKVSANIQNMTTADVTK \\
\hline 14.2 & 2612.3285 & -0.0082 & VLGPAVIFKVSANIQNMTTADVTK \\
\hline 5.6 & 2611.3380 & 0.9823 & $\underline{\text { ANTRVDHLLNTLEELLPYELEK }}$ \\
\hline 3.5 & 2612.3152 & 0.0051 & LYSISPWAXTLTLQTSRWLPGR \\
\hline 3.4 & 2610.3033 & 2.0170 & GAAEMVLQTFSASKGETGPMVAATLK \\
\hline 2.9 & 2612.3098 & 0.0105 & LKNLPDINTLEVAGDIQLTHVQT \\
\hline
\end{tabular}

Spectrum No: 654; Query: 1015; Rank: 1

\section{Peptide View}

MS/MS Fragmentation of QDFNVTDVSLLEHR

Found in IPI00198021, Tax_Id=10116 Gene_Symbol=Umod Uromodulin precursor

Match to Query 1015: 1672.806702 from(558.609510,3+)

Title: 100101RatKid_NS_deglyco_28.2970.2970.3.dta

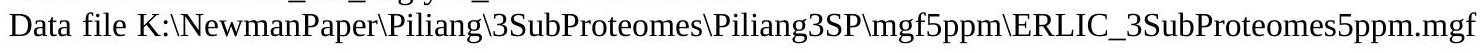



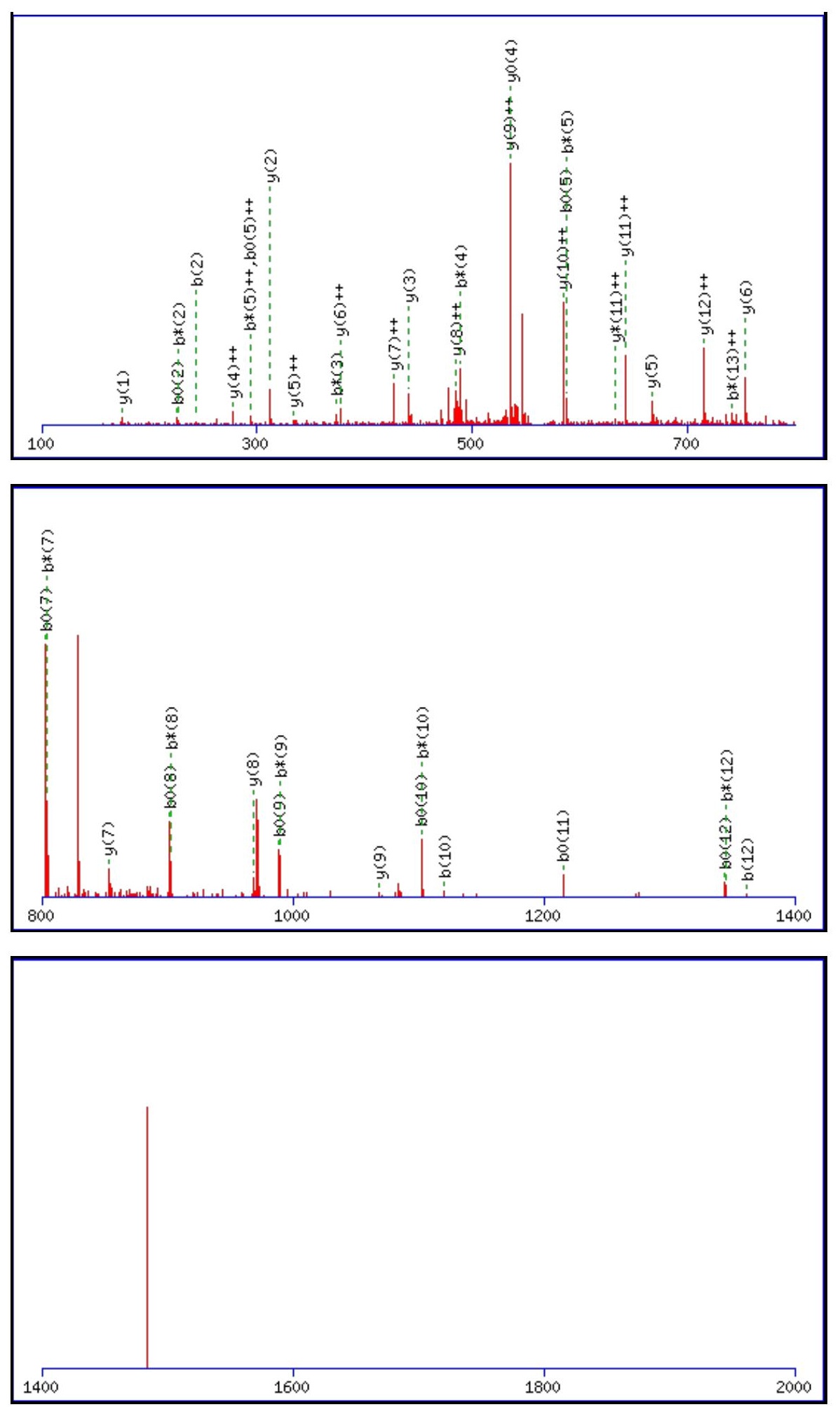

Monoisotopic mass of neutral peptide $\operatorname{Mr}($ calc): 1672.8057

Fixed modifications: Carbamidomethyl (C)

Variable modifications:

N4 : Deamidated $\mathrm{N}(\mathrm{N})$

Ions Score: 51 Expect: $\odot .0013$

Matches (Bold Red): 42/150 fragment ions using 81 most intense peaks

\begin{tabular}{|r|c|c|c|c|c|c|c|c|c|c|c|c|c|c|}
\hline$\#$ & $\mathbf{b}$ & $\mathbf{b}^{++}$ & $\mathbf{b}^{*}$ & $\mathbf{b}^{\boldsymbol{*}_{++}}$ & $\mathbf{b}^{\mathbf{0}}$ & $\mathbf{b}^{\mathbf{0 + +}}$ & $\mathbf{S e q}$ & $\mathbf{y}$ & $\mathbf{y}^{++}$ & $\mathbf{y}^{\mathbf{*}}$ & $\mathbf{y}^{\boldsymbol{*}^{++}}$ & $\mathbf{y}^{\mathbf{0}}$ & $\mathbf{y}^{\mathbf{0 + +}}$ & $\#$ \\
\hline $\mathbf{1}$ & 129.0659 & 65.0366 & 112.0393 & 56.5233 & & & $\mathbf{Q}$ & & & & & & & $\mathbf{1 4}$ \\
\hline $\mathbf{2}$ & $\mathbf{2 4 4 . 0 9 2 8}$ & 122.5500 & 227.0662 & 114.0368 & $\mathbf{2 2 6 . 0 8 2 2}$ & 113.5448 & $\mathbf{D}$ & 1545.7544 & 773.3808 & 1528.7278 & 764.8675 & 1527.7438 & 764.3755 & $\mathbf{1 3}$ \\
\hline 3 & 391.1612 & 196.0842 & 374.1347 & 187.5710 & 373.1506 & 187.0790 & $\mathbf{F}$ & 1430.7274 & 715.8673 & 1413.7009 & 707.3541 & 1412.7168 & 706.8621 & $\mathbf{1 2}$ \\
\hline $\mathbf{4}$ & 506.1881 & 253.5977 & $\mathbf{4 8 9 . 1 6 1 6}$ & 245.0844 & 488.1776 & 244.5924 & $\mathbf{N}$ & 1283.6590 & $\mathbf{6 4 2 . 3 3 3 1}$ & 1266.6324 & $\mathbf{6 3 3 . 8 1 9 9}$ & 1265.6484 & 633.3279 & $\mathbf{1 1}$ \\
\hline $\mathbf{5}$ & 605.2566 & 303.1319 & 588.2300 & $\mathbf{2 9 4 . 6 1 8 6}$ & $\mathbf{5 8 7 . 2 4 6 0}$ & $\mathbf{2 9 4 . 1 2 6 6}$ & $\mathbf{V}$ & 1168.6321 & $\mathbf{5 8 4 . 8 1 9 7}$ & 1151.6055 & 576.3064 & 1150.6215 & 575.8144 & $\mathbf{1 0}$ \\
\hline
\end{tabular}




\begin{tabular}{|c|c|c|c|c|c|c|c|c|c|c|c|c|c|c|}
\hline 6 & 706.3042 & |353.6558| & 689.2777 & |345.1425 & 688.2937 & |344.6505 & 1 & |1069.5636 & & & |526.7722 & & 2802 & 9 \\
\hline 7 & 821.3312 & 411.1692 & 804.3046 & 402.6560 & 803.3206 & 402.1639 & D & 968.5160 & 484.7616 & 951.4894 & 476.2483 & 950.5054 & 475.7563 & 8 \\
\hline 8 & 920.3996 & 460.7034 & 903.3730 & 452.1902 & 902.3890 & 451.6982 & V & 853.4890 & 427.2482 & 836.4625 & 418.7349 & 835.4785 & 418.2429 & 7 \\
\hline 9 & 1007.4316 & 504.2195 & 990.4051 & 495.7062 & 989.4211 & 495.2142 & S & 754.4206 & 377.7139 & 737.3941 & 369.2007 & 736.4100 & 368.7087 & 6 \\
\hline 10 & 1120.5157 & 560.7615 & 1103.4891 & 552.2482 & 1102.5051 & 551.7562 & $\mathbf{L}$ & 667.3886 & 334.1979 & 650.3620 & 325.6847 & 649.3780 & 325.1926 & 5 \\
\hline 11 & 1233.5998 & 617.3035 & 1216.5732 & 608.7902 & 1215.5892 & 608.2982 & $\mathbf{L}$ & 554.3045 & 277.6559 & 537.2780 & 269.1426 & 536.2940 & 268.6506 & 4 \\
\hline 12 & 1362.6423 & 681.8248 & 1345.6158 & 673.3115 & 1344.6318 & 672.8195 & $\mathbf{E}$ & 441.2205 & 221.1139 & 424.1939 & 212.6006 & 423.2099 & 212.1086 & 3 \\
\hline 13 & 1499.7013 & 750.3543 & 1482.6747 & 741.8410 & 1481.6907 & 741.3490 & H & 312.1779 & 156.5926 & 295.1513 & 148.0793 & & & 2 \\
\hline 14 & & & & & & & $\mathbf{R}$ & 175.1190 & 88.0631 & 158.0924 & 79.5498 & & & 1 \\
\hline
\end{tabular}
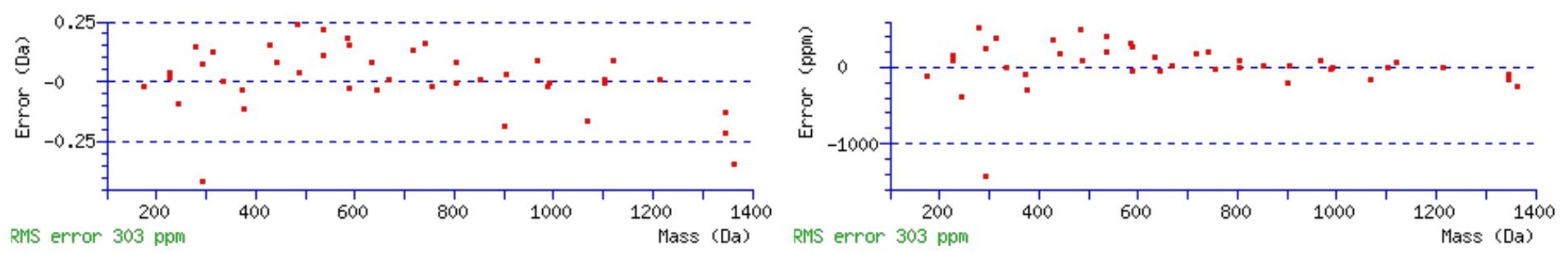

\section{All matches to this query}

\begin{tabular}{|l|l|c|l|}
\hline Score & Mr(calc): & Delta & \multicolumn{1}{|c|}{ Sequence } \\
\hline 51.1 & 1672.8057 & 0.0010 & QDFNVTDVSLLEHR \\
\hline 13.5 & 1671.8055 & 1.0012 & MLETLSWKDKVAR \\
\hline 10.4 & 1672.7944 & 0.0123 & KPTEDPANDTVDFPK \\
\hline 10.4 & 1671.8055 & 1.0012 & MLETLSWKDKVAR \\
\hline 6.4 & 1672.8203 & -0.0135 & VNDCILRVNEADVR \\
\hline 6.1 & 1670.8103 & 1.9964 & YLGLLAESCLLSPR \\
\hline 6.0 & 1671.8168 & 0.9899 & VMEQTFDRLIGKR \\
\hline 5.5 & 1672.7964 & 0.0103 & QEGCLGHSHRSLHR \\
\hline 5.3 & 1670.7986 & 2.0081 & IFKVTKYWASLR \\
\hline 3.5 & 1670.7950 & 2.0117 & RDTMLGVSIVLENK \\
\hline
\end{tabular}

Spectrum No: 655; Query: 2635; Rank: 1

\section{Peptide View}

MS/MS Fragmentation of AWSDGSHLMPIVNTSLGWPNGLAIDWSASR

Found in IPI00205325, Tax_Id=10116 Gene_Symbol=Lrp2 Low-density lipoprotein receptor-related protein 2 precursor

Match to Query 2635: 3238.549032 from(1080.523620,3+)

Title: 100101RatKid_NS_deglyco_13.6688.6688.3.dta

Data file K:INewmanPaper|Piliang|3SubProteomes\Piliang3SP\mgf5ppm\ERLIC_3SubProteomes5ppm.mgf 

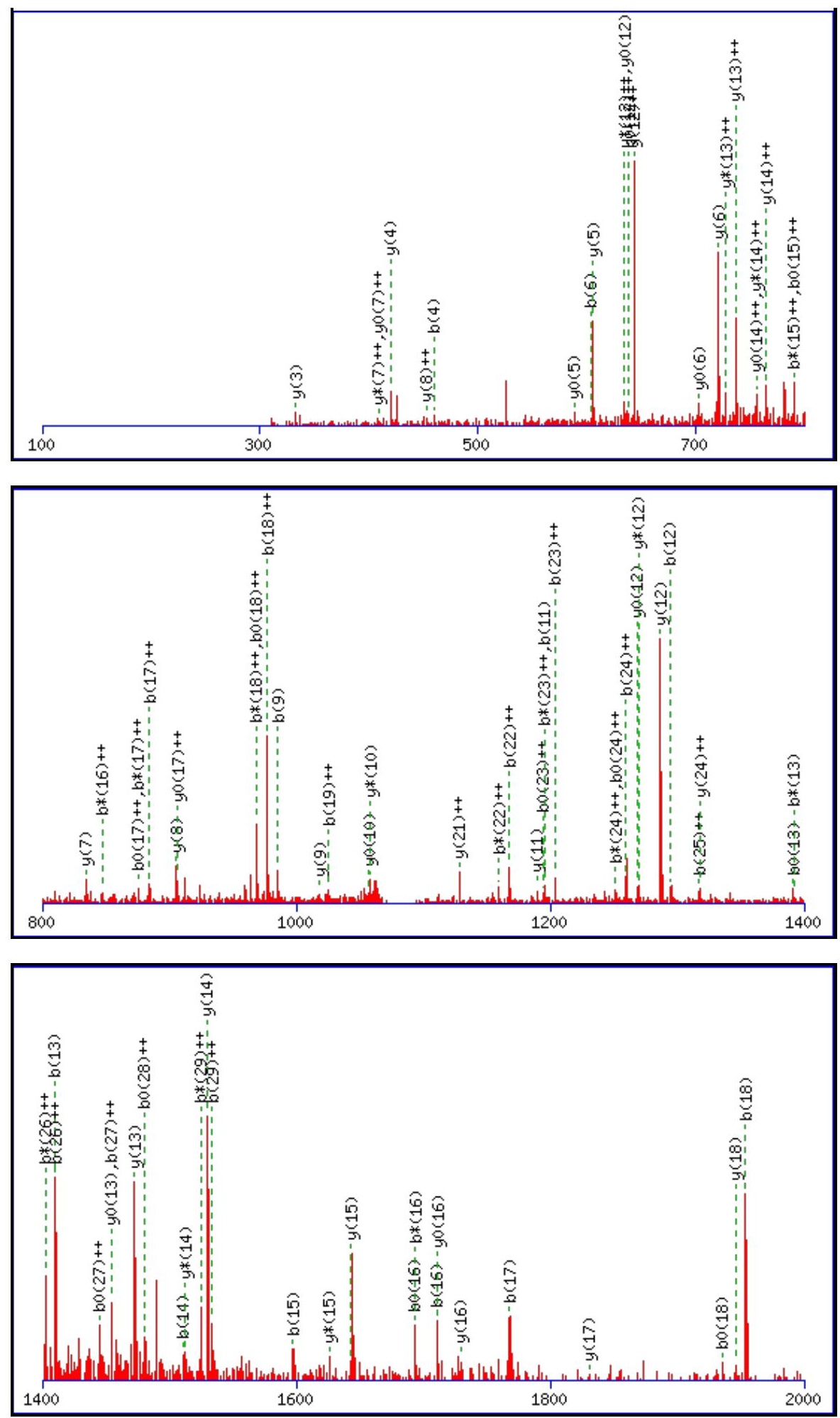

Monoisotopic mass of neutral peptide $\operatorname{Mr}($ calc): 3238.5506

Fixed modifications: Carbamidomethyl (C)

Variable modifications:

N13 : Deamidated_N (N)

Ions Score: 51 Expect: 0.0027

Matches (Bold Red): 82/318 fragment ions using 175 most intense peaks

\begin{tabular}{|r|c|c|c|c|c|c|c|c|c|c|c|c|c|c|}
\hline$\#$ & $\mathbf{b}$ & $\mathbf{b}^{++}$ & $\mathbf{b}^{*}$ & $\mathbf{b}^{\boldsymbol{*}^{++}}$ & $\mathbf{b}^{\mathbf{0}}$ & $\mathbf{b}^{\mathbf{0 + +}}$ & Seq. & $\mathbf{y}$ & $\mathbf{y}^{++}$ & $\mathbf{y}^{\mathbf{*}}$ & $\mathbf{y}^{\mathbf{*}^{++}}$ & $\mathbf{y}^{\mathbf{0}}$ & $\mathbf{y}^{\mathbf{0 + +}}$ & $\#$ \\
\hline $\mathbf{1}$ & $\mathbf{7 2 . 0 4 4 4}$ & 36.5258 & & & & & $\mathbf{A}$ & & & & & & & $\mathbf{3 0}$ \\
\hline $\mathbf{2}$ & 258.1237 & 129.5655 & & & & & $\mathbf{W}$ & 3168.5207 & 1584.7640 & 3151.4942 & 1576.2507 & 3150.5102 & 1575.7587 & $\mathbf{2 9}$ \\
\hline 3 & 345.1557 & 173.0815 & & & 327.1452 & 164.0762 & S & 2982.4414 & 1491.7244 & 2965.4149 & 1483.2111 & 2964.4309 & 1482.7191 & $\mathbf{2 8}$ \\
\hline $\mathbf{4}$ & $\mathbf{4 6 0 . 1 8 2 7}$ & 230.5950 & & & 442.1721 & 221.5897 & $\mathbf{D}$ & 2895.4094 & 1448.2083 & 2878.3828 & 1439.6951 & 2877.3988 & 1439.2031 & 27 \\
\hline $\mathbf{5}$ & 517.2041 & 259.1057 & & & 499.1936 & 250.1004 & $\mathbf{G}$ & 2780.3825 & 1390.6949 & 2763.3559 & 1382.1816 & 2762.3719 & 1381.6896 & $\mathbf{2 6}$ \\
\hline
\end{tabular}




\begin{tabular}{|c|c|c|c|c|c|c|c|c|c|c|c|c|c|c|}
\hline & 604.2362 & 302.6217 & & & 586.2256 & 293.6164| & $\mathbf{S}$ & 2723.3610 & |1362.1841|2 & |2706.3344| & |1353.6709 & |2705.3504| & | & \\
\hline 7 & 741.2951 & 371.1512 & & & 723.2845 & 362.1459 & $\mathbf{H}$ & 2636.3290 & 1318.6681 & 2619.3024 & 1310.1548 & 2618.3184 & 1309.6628 & 4 \\
\hline 8 & 854.3791 & 427.6932 & & & 836.3686 & 418.6879 & $\mathbf{L}$ & 2499.2701 & 1250.1387 & 2482.2435 & 1241.6254 & 2481.2595 & 1241.1334 & 23 \\
\hline 9 & 985.4196 & 493.2135 & & & 967.4091 & 484.2082 & $\mathbf{M}$ & 2386.1860 & 1193.5966 & 2369.1594 & 1185.0834 & 2368.1754 & 1184.5913 & \\
\hline 10 & 1082.4724 & 541.7398 & & & 1064.4618 & 532.7346 & $\mathbf{P}$ & 2255.1455 & 1128.0764 & 2238.1190 & 1119.5631 & 2237.1349 & 1119.0711 & 1 \\
\hline 11 & 1195.5565 & 598.2819 & & & 1177.5459 & 589.2766 & I & 2158.0927 & 1079.5500 & 2141.0662 & 1071.0367 & 2140.0822 & 1070.5447 & 7 \\
\hline 12 & 1294.6249 & 647.8161 & & & 1276.6143 & 638.8108 & $\mathbf{V}$ & 2045.0087 & 1023.0080 & 2027.9821 & 1014.4947 & 2026.9981 & 1014.0027 & \\
\hline 13 & 1409.6518 & 705.3295 & 1392.6253 & 696.8163 & 1391.6412 & 696.3243 & $\mathbf{N}$ & 1945.9403 & 973.4738 & 1928.9137 & 964.9605 & 1927.9297 & 964.4685 & \\
\hline 14 & 1510.6995 & 755.8534 & 1493.6729 & 747.3401 & 1492.6889 & 746.8481 & $\mathbf{T}$ & 1830.9133 & 915.9603 & 1813.8868 & 907.4470 & 1812.9028 & 906.9550 & 17 \\
\hline 15 & 1597.7315 & 799.3694 & 1580.7050 & 790.8561 & 1579.7209 & 790.3641 & $\mathrm{~S}$ & 1729.8656 & 865.4365 & 1712.8391 & 856.9232 & 1711.8551 & 856.4312 & ? \\
\hline 16 & 1710.8156 & 855.9114 & & & 1692.8050 & 846.9061 & $\mathbf{L}$ & 1642.8336 & 821.9204 & 1625.8071 & 813.4072 & 1624.8231 & 9152 & \\
\hline 17 & 1767.8370 & 884.4222 & 1750.8105 & 875.9089 & 1749.8265 & 875.4169 & G & 1529.7496 & 765.3784 & 1512.7230 & 756.8651 & 1511.7390 & 756.3731 & it \\
\hline 18 & 1953.9164 & 977.4618 & 1936.8898 & 968.9485 & 1935.9058 & 968.4565 & $\mathbf{W}$ & 1472.7281 & 736.8677 & 1455.7015 & 728.3544 & 1454.7175 & 727.8624 & 13 \\
\hline 19 & 2050.9691 & \begin{tabular}{|l|}
1025.9882 \\
\end{tabular} & 2033.9426 & 1017.4749 & 2032.9586 & 1016.9829 & $\mathbf{P}$ & 1286.6488 & 643.8280 & 1269.6222 & 635.3148 & 1268.6382 & 634.8227 & \\
\hline 20 & 2165.0120 & 1083.0097 & 2147.9855 & 1074.4964 & 2147.0015 & 1074.0044 & $\mathbf{N}$ & 1189.5960 & 595.3016 & 1172.5695 & 586.7884 & 1171.5854 & 586.2964 & 1 \\
\hline 21 & 2222.0335 & 1111.5204 & 2205.0070 & 1103.0071 & 2204.0229 & 1102.5151 & G & 1075.5531 & 538.2802 & 1058.5265 & 529.7669 & 1057.5425 & 529.2749 & 10 \\
\hline 22 & 2335.1176 & 1168.0624 & 2318.0910 & 1159.5491 & 2317.1070 & 1159.0571 & $\mathbf{L}$ & 1018.5316 & 509.7694 & 1001.5051 & 501.2562 & 1000.5211 & 500.7642 & 9 \\
\hline 23 & 2406.1547 & 1203.5810 & 2389.1281 & 1195.0677 & 2388.1441 & 1194.5757 & A & 905.4476 & 453.2274 & 888.4210 & 444.7141 & 887.4370 & 444.2221 & 8 \\
\hline 24 & 2519.2388 & 1260.1230 & 2502.2122 & 1251.6097 & 2501.2282 & 1251.1177 & $\mathbf{I}$ & 834.4104 & 417.7089 & 817.3839 & 409.1956 & 816.3999 & 408.7036 & 7 \\
\hline 25 & 2634.2657 & \begin{tabular}{|l|}
1317.6365 \\
\end{tabular} & 2617.2391 & 1309.1232 & 2616.2551 & 1308.6312 & D & 721.3264 & 361.1668 & 704.2998 & 352.6536 & 703.3158 & 352.1615 & 6 \\
\hline 26 & 2820.3450 & 1410.6761 & 2803.3185 & 1402.1629 & 2802.3344 & 1401.6709 & $\mathbf{W}$ & 606.2994 & 303.6534 & 589.2729 & 295.1401 & 588.2889 & 294.6481 & 5 \\
\hline 27 & 2907.3770 & \begin{tabular}{|l|}
1454.1922 \\
\end{tabular} & 2890.3505 & 1445.6789 & 2889.3665 & 1445.1869 & $\mathrm{~S}$ & 420.2201 & 210.6137 & 403.1936 & 202.1004 & 402.2096 & 201.6084 & 4 \\
\hline 28 & 2978.4141 & 1489.7107 & 2961.3876 & 1481.1974 & 2960.4036 & \begin{tabular}{|l|}
1480.7054 \\
\end{tabular} & A & 333.1881 & 167.0977 & 316.1615 & 158.5844 & 315.1775 & 158.0924 & 3 \\
\hline 29 & 3065.4462 & 1533.2267 & 3048.4196 & 1524.7135 & 3047.4356 & 1524.2214 & $S$ & 262.1510 & 131.5791 & 245.1244 & 123.0659 & 244.1404 & 122.5738 & 2 \\
\hline 30 & & & & & & & $\mathbf{R}$ & 175.1190 & 88.0631 & 158.0924 & 79.5498 & & & 1 \\
\hline
\end{tabular}
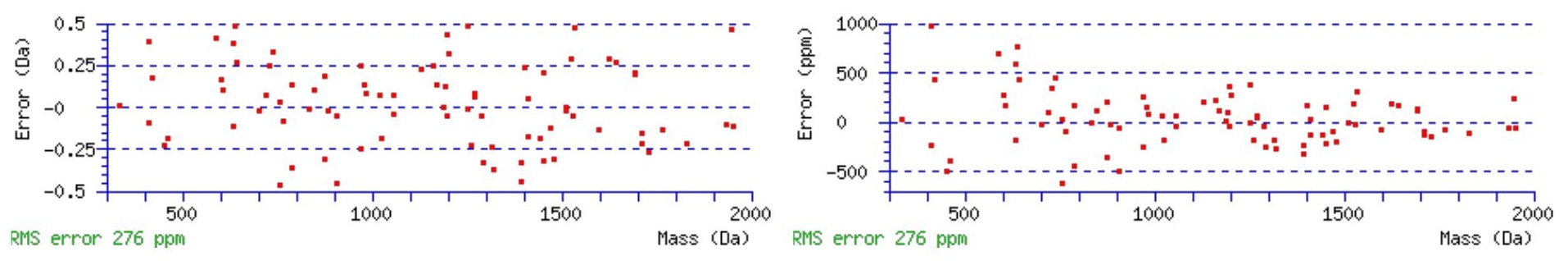

\section{All matches to this query}

\begin{tabular}{|l|l|l|l|}
\hline Score & Mr(calc): & Delta & \multicolumn{1}{c|}{ Sequence } \\
\hline 51.1 & 3238.5506 & -0.0015 & AWSDGSHLMPIVNTSLGWPNGLAIDWSASR \\
\hline 32.6 & 3237.5666 & 0.9825 & AWSDGSHLMPIVNTSLGWPNGLAIDWSASR \\
\hline 27.5 & 3238.5506 & -0.0015 & AWSDGSHLMPIVNTSLGWPNGLAIDWSASR \\
\hline 2.5 & 3238.5532 & -0.0042 & DLYEDVMLENYHNLVSIYRTFYPKAK \\
\hline 2.0 & 3236.5166 & 2.0324 & LPANSLPQEGDVVGITYDHVELNVYLNGK \\
\hline 1.3 & 3237.5216 & 1.0274 & KPQGPVNPSPERKGIEQLEMCQMPDNNK \\
\hline 1.3 & 3237.5216 & 1.0274 & KPQGPVNPSPERKGIEQLEMCQMPDNNK \\
\hline 1.2 & 3236.5376 & 2.0114 & KPQGPVNPSPERKGIEQLEMCQMPDNNK \\
\hline 0.6 & 3238.5321 & 0.0169 & TMKPGPGAHSPEKVTLNKPCAPTVTFGIK \\
\hline 0.6 & 3238.5321 & 0.0169 & TMKPGPGAHSPEKVTLNKPCAPTVTFGIK \\
\hline
\end{tabular}

Spectrum No: 656; Query: 2513; Rank: 1 


\section{Peptide View}

\section{MS/MS Fragmentation of DDFRPTWTLNQTEPVAGNYYPVNTR}

Found in IPI00400747, Tax_Id=10116 Gene_Symbol=Man2b1 Mannosidase 2, alpha B1

Match to Query 2513: 2954.389032 from(985.803620,3+)

Title: 100101RatKid_NS_deglyco_21.3936.3936.3.dta

Data file K:\NewmanPaper\Piliang \3SubProteomes \Piliang3SP $\backslash$ mgf5ppm\ERLIC_3SubProteomes5ppm.mgf
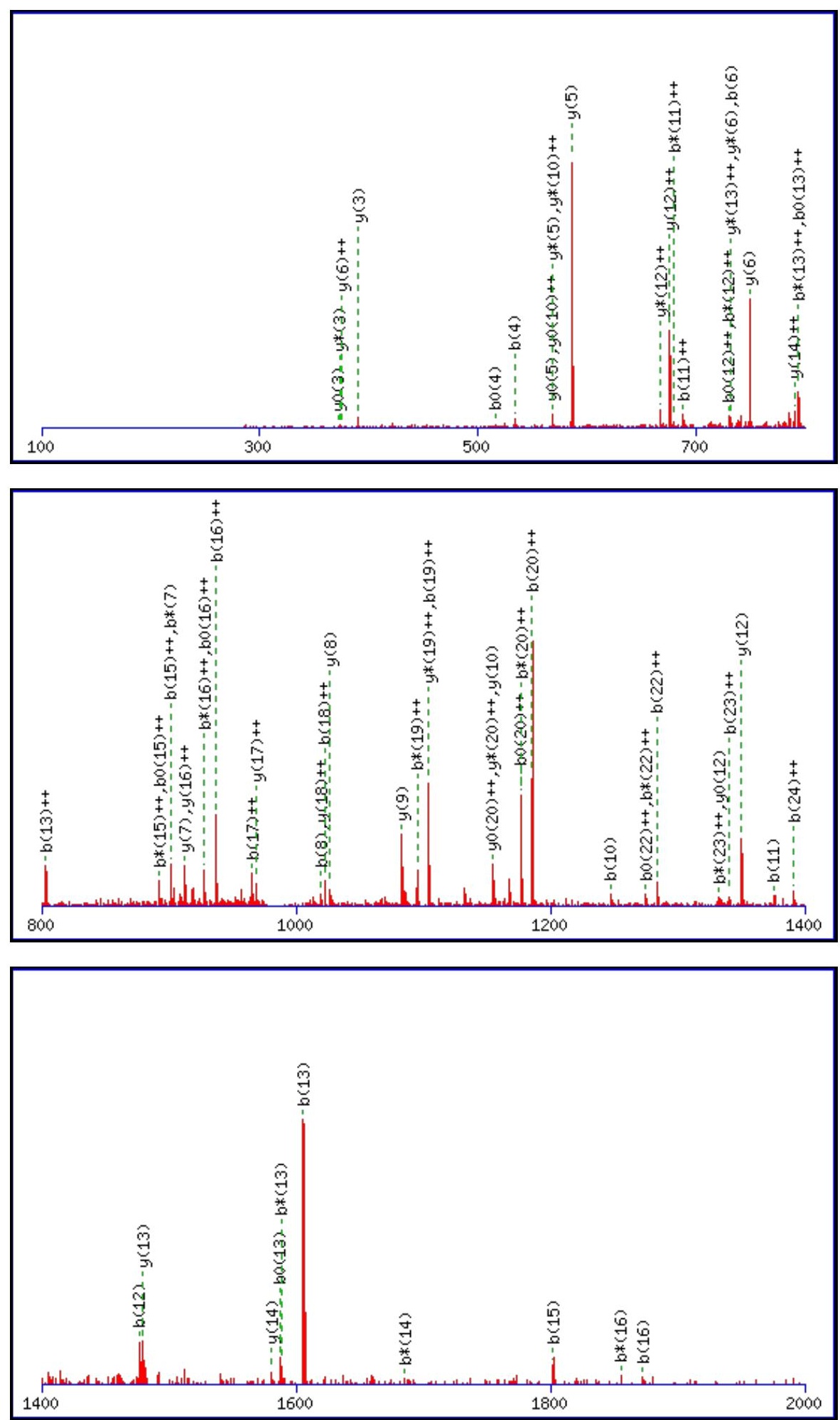

Monoisotopic mass of neutral peptide $\operatorname{Mr}($ calc): 2954.3835

Fixed modifications: Carbamidomethyl (C)

Variable modifications:

N10 : Deamidated_N (N)

Ions Score: 51 Expect: 0.0027

Matches (Bold Red): 70/280 fragment ions using 112 most intense peaks 


\begin{tabular}{|c|c|c|c|c|c|c|c|c|c|c|c|c|c|c|}
\hline \# & b & $\mathbf{b}^{++}$ & $\mathbf{b}^{*}$ & $\mathbf{b}^{*^{++}}$ & $\mathbf{b}^{\mathbf{0}}$ & $\mathbf{b}^{\mathbf{0 + +}}$ & Seq. & $\mathbf{y}$ & $\mathbf{y}^{++}$ & $\mathbf{y}^{*}$ & $\mathrm{y}^{*^{++}}$ & $\mathbf{y}^{0}$ & $\mathbf{y}^{\mathbf{0 + +}^{++}}$ & \# \\
\hline 1 & 116.0342 & 58.5207 & & & 98.0237 & 49.5155 & D & & & & & & & 25 \\
\hline 2 & 231.0612 & 116.0342 & & & 213.0506 & 107.0289 & D & 2840.3638 & 1420.6856 & 2823.3373 & 1412.1723 & 2822.3533 & 1411.6803 & 24 \\
\hline 3 & 378.1296 & 189.5684 & & & 360.1190 & 180.5631 & $\mathbf{F}$ & 2725.3369 & 1363.1721 & 2708.3103 & 1354.6588 & 2707.3263 & 1354.1668 & 23 \\
\hline 4 & 534.2307 & 267.6190 & 517.2041 & 259.1057 & 516.2201 & 258.6137 & $\mathbf{R}$ & 2578.2685 & 1289.6379 & 2561.2419 & 1281.1246 & 2560.2579 & 1280.6326 & 22 \\
\hline 5 & 631.2835 & 316.1454 & 614.2569 & 307.6321 & 613.2729 & 307.1401 & $\mathbf{P}$ & 2422.1674 & 1211.5873 & 2405.1408 & 1203.0740 & 2404.1568 & 1202.5820 & 21 \\
\hline 6 & 732.3311 & 366.6692 & 715.3046 & 358.1559 & 714.3206 & 357.6639 & $T$ & 2325.1146 & 1163.0609 & 2308.0880 & 1154.5477 & 2307.1040 & 1154.0557 & 20 \\
\hline 7 & 918.4104 & 459.7089 & 901.3839 & 451.1956 & 900.3999 & 450.7036 & W & 2224.0669 & 1112.5371 & 2207.0404 & 1104.0238 & 2206.0564 & 1103.5318 & 19 \\
\hline 8 & \begin{tabular}{|l|}
1019.4581 \\
\end{tabular} & 510.2327 & 1002.4316 & 501.7194 & 1001.4476 & 501.2274 & $T$ & 2037.9876 & \begin{tabular}{|l|l|}
1019.4974 \\
\end{tabular} & 2020.9611 & 1010.9842 & 2019.9770 & 1010.4922 & 18 \\
\hline 9 & 1132.5422 & 566.7747 & 1115.5156 & 558.2615 & 1114.5316 & 557.7694 & L & 1936.9399 & \begin{tabular}{|l|}
968.9736 \\
\end{tabular} & 1919.9134 & 960.4603 & 1918.9294 & 959.9683 & 17 \\
\hline 10 & 1247.5691 & 624.2882 & 1230.5426 & 615.7749 & 1229.5586 & 615.2829 & $\mathbf{N}$ & 1823.8559 & 912.4316 & 1806.8293 & 903.9183 & 1805.8453 & 903.4263 & 16 \\
\hline 11 & 1375.6277 & 688.3175 & 1358.6012 & 679.8042 & 1357.6171 & 679.3122 & $\mathbf{Q}$ & 1708.8289 & 854.9181 & 1691.8024 & 846.4048 & 1690.8184 & 845.9128 & 15 \\
\hline 12 & 1476.6754 & 738.8413 & 1459.6488 & 730.3281 & 1458.6648 & 729.8360 & $T$ & 1580.7703 & 790.8888 & 1563.7438 & 782.3755 & 1562.7598 & 781.8835 & 14 \\
\hline 13 & 1605.7180 & 803.3626 & 1588.6914 & 794.8493 & 1587.7074 & 794.3573 & $E$ & 1479.7227 & 740.3650 & 1462.6961 & 731.8517 & 1461.7121 & 731.3597 & 13 \\
\hline 14 & 1702.7707 & 851.8890 & 1685.7442 & 843.3757 & 1684.7602 & 842.8837 & $\mathbf{P}$ & 1350.6801 & 675.8437 & 1333.6535 & 667.3304 & 1332.6695 & 666.8384 & 12 \\
\hline 15 & 1801.8391 & 901.4232 & 1784.8126 & 892.9099 & 1783.8286 & 892.4179 & $\mathbf{V}$ & 1253.6273 & 627.3173 & 1236.6008 & 618.8040 & 1235.6167 & 618.3120 & 11 \\
\hline 16 & \begin{tabular}{|l|}
1872.8763 \\
\end{tabular} & 936.9418 & \begin{tabular}{|l}
1855.8497 \\
\end{tabular} & 928.4285 & 1854.8657 & 927.9365 & $\mathbf{A}$ & 1154.5589 & 577.7831 & 1137.5323 & 569.2698 & 1136.5483 & 568.7778 & 10 \\
\hline 17 & 1929.8977 & 965.4525 & 1912.8712 & 956.9392 & 1911.8872 & 956.4472 & $\mathbf{G}$ & 1083.5218 & 542.2645 & 1066.4952 & 533.7513 & 1065.5112 & 533.2592 & 9 \\
\hline 18 & 2043.9407 & 1022.4740 & 2026.9141 & 1013.9607 & 2025.9301 & 1013.4687 & $\mathbf{N}$ & 1026.5003 & 513.7538 & 1009.4738 & 505.2405 & 1008.4898 & 504.7485 & 8 \\
\hline $19:$ & 2207.0040 & 1104.0056 & 2189.9774 & 1095.4924 & 2188.9934 & 1095.0003 & $\mathbf{Y}$ & 912.4574 & 456.7323 & \begin{tabular}{|l|}
895.4308 \\
\end{tabular} & 448.2191 & 894.4468 & 447.7271 & 7 \\
\hline 20 & 2370.0673 & 1185.5373 & 2353.0408 & 1177.0240 & 2352.0567 & 1176.5320 & $\mathbf{Y}$ & 749.3941 & 375.2007 & 732.3675 & 366.6874 & 731.3835 & 366.1954 & 6 \\
\hline 21 & 2467.1201 & 1234.0637 & 2450.0935 & 1225.5504 & 2449.1095 & 1225.0584 & $\mathbf{P}$ & 586.3307 & 293.6690 & 569.3042 & 285.1557 & 568.3202 & 284.6637 & 5 \\
\hline $22:$ & 2566.1885 & 1283.5979 & 2549.1619 & 1275.0846 & 2548.1779 & 1274.5926 & $\mathbf{V}$ & 489.2780 & 245.1426 & 472.2514 & 236.6293 & 471.2674 & 236.1373 & 4 \\
\hline 23 & 2680.2314 & 1340.6193 & 2663.2049 & 1332.1061 & 2662.2209 & 1331.6141 & $\mathbf{N}$ & 390.2096 & 195.6084 & 373.1830 & 187.0951 & 372.1990 & 186.6031 & 3 \\
\hline $24:$ & 2781.2791 & 1391.1432 & 2764.2525 & 1382.6299 & 2763.2685 & 1382.1379 & $T$ & 276.1666 & 138.5870 & 259.1401 & 130.0737 & 258.1561 & 129.5817 & 2 \\
\hline 25 & & & & & & & $\mathbf{R}$ & 175.1190 & 88.0631 & 158.0924 & 79.5498 & & & 1 \\
\hline
\end{tabular}
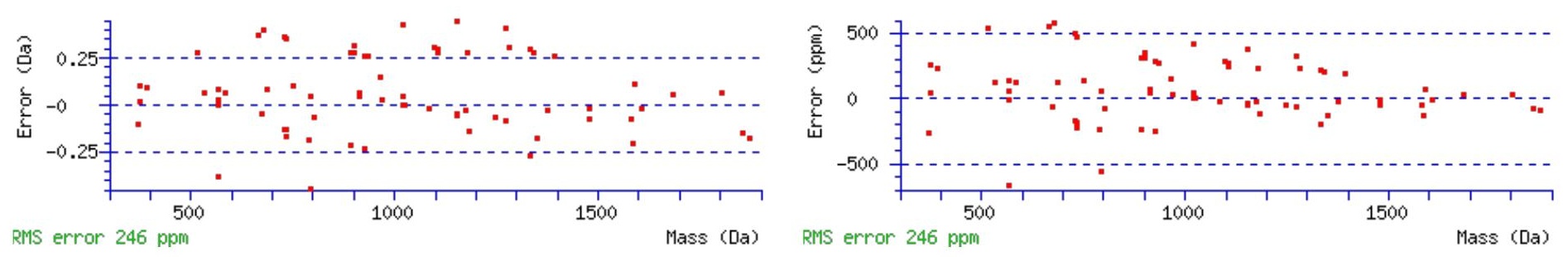

\section{All matches to this query}

\begin{tabular}{|l|l|c|l|}
\hline Score & Mr(calc): & Delta & \multicolumn{1}{|c|}{ Sequence } \\
\hline 51.0 & 2954.3835 & 0.0055 & DDFRPTWTLNQTEPVAGNYYPVNTR \\
\hline 20.6 & 2953.3995 & 0.9895 & DDFRPTWTLNQTEPVAGNYYPVNTR \\
\hline 18.1 & 2954.3835 & 0.0055 & DDFRPTWTLNQTEPVAGNYYPVNTR \\
\hline 10.8 & 2954.3835 & 0.0055 & DDFRPTWTLNQTEPVAGNYYPVNTR \\
\hline 6.2 & 2954.4062 & -0.0172 & TIQNAGYGAKVTSAHENLNSWIELKK \\
\hline 6.2 & 2954.4062 & -0.0172 & TIQNAGYGAKVTSAHENLNSWIELKK \\
\hline 4.0 & 2954.3940 & -0.0050 & KPRPLPQYGSKPTGNLGVTDLESSPR \\
\hline 3.3 & 2954.4018 & -0.0127 & SLMSISNAGSGLLVHSSTLTGVPIMEEK \\
\hline 1.5 & 2954.3689 & 0.0201 & DKVVALLYSIITPVLNPFIYSLR \\
\hline 0.8 & 2953.3695 & 1.0195 & $\underline{\text { IQDDIINLRSQQQSSHEGTRNILK }}$ \\
\hline
\end{tabular}

Spectrum No: 657; Query: 1182; Rank: 1 


\section{Peptide View}

MS/MS Fragmentation of NTENGSAPSTAPTDQNR

Found in IPI00369174, Tax_Id=10116 Gene_Symbol=Rnf126 Ring finger protein 126

Match to Query 1182: 1759.759908 from(880.887230,2+)

Title: 091008RatKidney_NH4Format01_21.348.348.2.dta

Data file K:INewmanPaper|Piliang|3SubProteomes\Piliang3SP\mgf5ppm\ERLIC_3SubProteomes5ppm.mgf
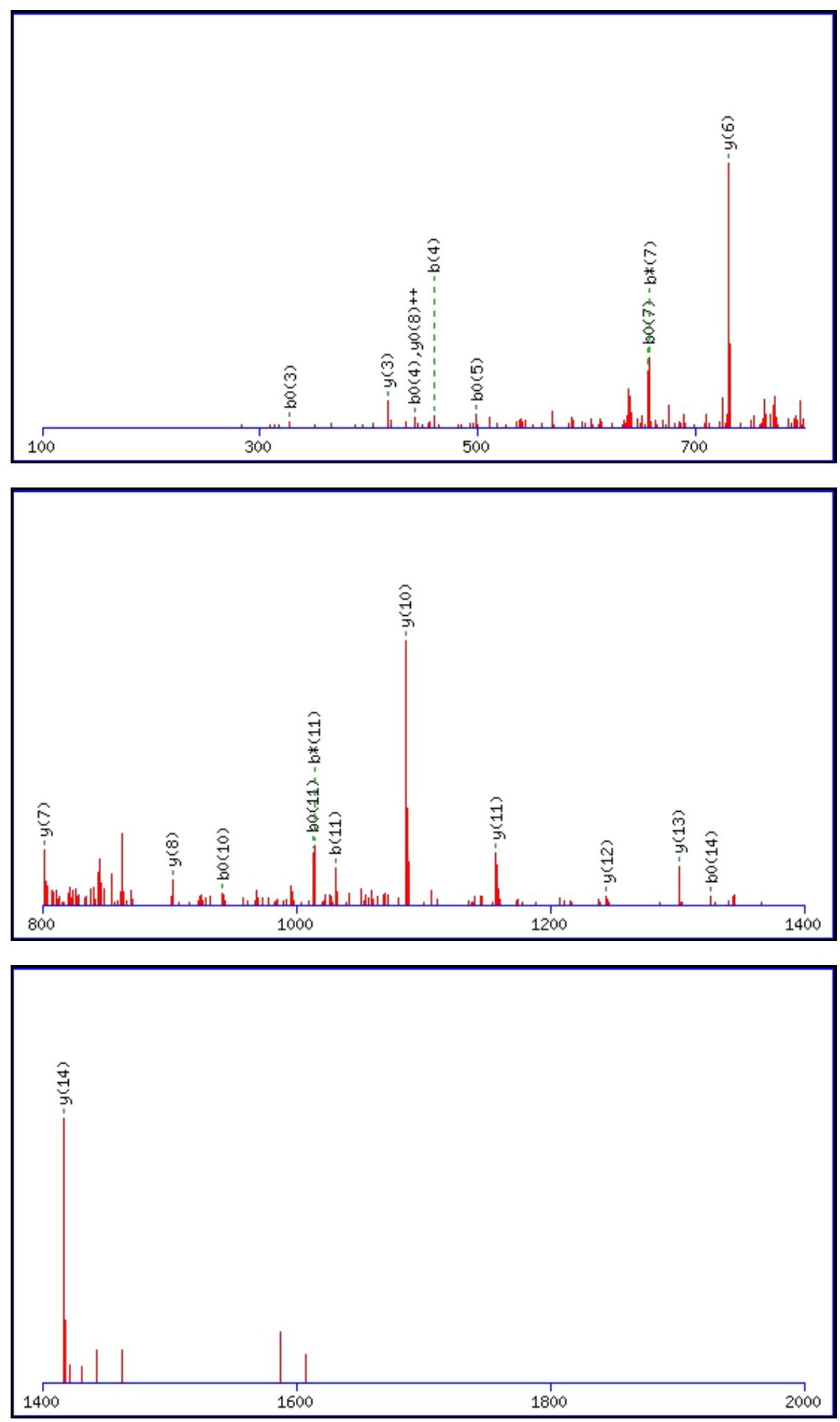

Monoisotopic mass of neutral peptide $\operatorname{Mr}($ calc): 1759.7609

Fixed modifications: Carbamidomethyl (C)

Variable modifications:

N4 : Deamidated_N (N) 
Ions Score: 51 Expect: $\odot .0016$

Matches (Bold Red): 21/184 fragment ions using 42 most intense peaks

\begin{tabular}{|c|c|c|c|c|c|c|c|c|c|c|c|c|c|c|}
\hline \# & b & $\mathbf{b}^{++}$ & $\mathbf{b}^{*}$ & $\mathbf{b}^{*^{++}}$ & $\mathbf{b}^{\mathbf{0}}$ & $\mathbf{b}^{0++}$ & Seq. & $\mathbf{y}$ & $\mathbf{y}^{++}$ & $\mathbf{y}^{*}$ & $\mathrm{y}^{\mathrm{*}^{++}}$ & $\mathbf{y}^{0}$ & $y^{0++}$ & \# \\
\hline 1 & 115.0502 & 58.0287 & 98.0237 & 49.5155 & & & $\mathbf{N}$ & & & & & & & 17 \\
\hline 2 & 216.0979 & 108.5526 & 199.0713 & 100.0393 & 198.0873 & 99.5473 & $\mathbf{T}$ & 1646.7252 & 823.8663 & 1629.6987 & 815.3530 & 1628.7147 & 814.8610 & 16 \\
\hline 3 & 345.1405 & 173.0739 & 328.1139 & 164.5606 & 327.1299 & 164.0686 & $\mathbf{E}$ & 1545.6776 & 773.3424 & $\mid 1528.6510$ & 764.8291 & 1527.6670 & 764.3371 & 15 \\
\hline 4 & 460.1674 & 230.5873 & 443.1409 & 222.0741 & 442.1568 & 221.5821 & $\mathbf{N}$ & 1416.6350 & 708.8211 & 1399.6084 & 700.3078 & 1398.6244 & 699.8158 & 14 \\
\hline 5 & 517.1889 & 259.0981 & 500.1623 & 250.5848 & 499.1783 & 250.0928 & G & 1301.6080 & 651.3077 & 1284.5815 & 642.7944 & 1283.5975 & 642.3024 & 13 \\
\hline 6 & 604.2209 & 302.6141 & 587.1944 & 294.1008 & 586.2103 & 293.6088 & S & 1244.5866 & 622.7969 & 1227.5600 & 614.2836 & 1226.5760 & 613.7916 & 12 \\
\hline 7 & 675.2580 & 338.1326 & 658.2315 & 329.6194 & 657.2475 & 329.1274 & A & 1157.5545 & 579.2809 & 1140.5280 & 570.7676 & 1139.5440 & 570.2756 & 11 \\
\hline 8 & 772.3108 & 386.6590 & 755.2842 & 378.1458 & 754.3002 & 377.6537 & $\mathbf{P}$ & \begin{tabular}{|l|}
1086.5174 \\
\end{tabular} & 543.7624 & 1069.4909 & 535.2491 & 1068.5069 & 534.7571 & 10 \\
\hline 9 & 859.3428 & 430.1750 & 842.3163 & 421.6618 & 841.3322 & 421.1698 & $\mathrm{~S}$ & 989.4647 & 495.2360 & \begin{tabular}{|c|}
972.4381 \\
\end{tabular} & 486.7227 & 971.4541 & 486.2307 & 9 \\
\hline 10 & 960.3905 & 480.6989 & 943.3639 & 472.1856 & 942.3799 & 471.6936 & $\mathbf{T}$ & 902.4326 & 451.7200 & 885.4061 & 443.2067 & 884.4221 & 442.7147 & 8 \\
\hline 11 & 1031.4276 & 516.2174 & 1014.4011 & 507.7042 & 1013.4170 & 507.2122 & A & 801.3850 & 401.1961 & 784.3584 & 392.6828 & 783.3744 & 392.1908 & 7 \\
\hline 12 & 1128.4804 & 564.7438 & 1111.4538 & 556.2305 & 1110.4698 & 555.7385 & $\mathbf{P}$ & 730.3478 & 365.6776 & 713.3213 & 357.1643 & 712.3373 & 356.6723 & 6 \\
\hline 13 & 1229.5280 & 615.2677 & 1212.5015 & 606.7544 & 1211.5175 & 606.2624 & $\mathbf{T}$ & 633.2951 & 317.1512 & 616.2685 & 308.6379 & 615.2845 & 308.1459 & 5 \\
\hline 14 & 1344.5550 & 672.7811 & 1327.5284 & 664.2679 & 1326.5444 & 663.7758 & D & 532.2474 & 266.6273 & 515.2209 & 258.1141 & 514.2368 & 257.6221 & 4 \\
\hline 15 & 1472.6136 & 736.8104 & 1455.5870 & 728.2971 & 1454.6030 & 727.8051 & $\mathbf{Q}$ & 417.2205 & 209.1139 & 400.1939 & 200.6006 & & & 3 \\
\hline 16 & 1586.6565 & 793.8319 & 1569.6299 & 785.3186 & 1568.6459 & 784.8266 & $\mathbf{N}$ & 289.1619 & 145.0846 & 272.1353 & 136.5713 & & & 2 \\
\hline 17 & & & & & & & $\mathbf{R}$ & 175.1190 & 88.0631 & 158.0924 & 79.5498 & & & 1 \\
\hline
\end{tabular}
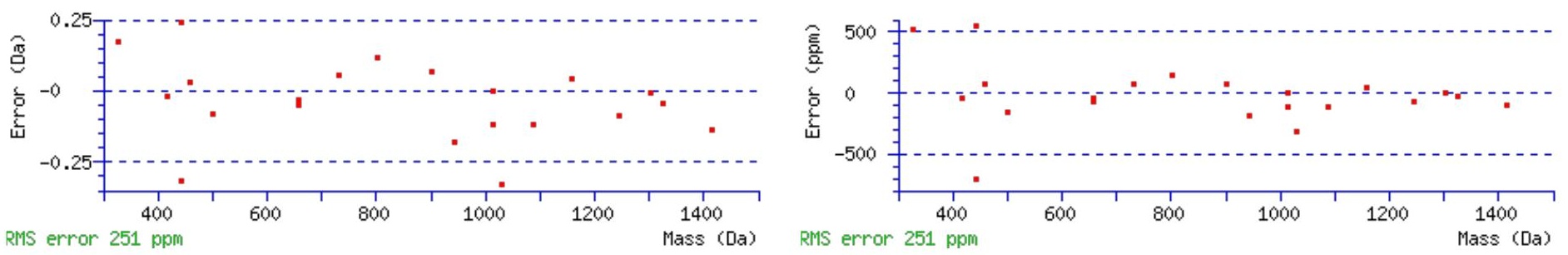

\section{All matches to this query}

\begin{tabular}{|l|l|l|l|}
\hline Score & Mr(calc): & Delta & \multicolumn{1}{|c|}{ Sequence } \\
\hline 51.0 & 1759.7609 & -0.0010 & NTENGSAPSTAPTDQNR \\
\hline 38.8 & 1759.7609 & -0.0010 & NTENGSAPSTAPTDQNR \\
\hline 10.4 & 1757.7498 & 2.0101 & EVHLKNTSRGSAGNK \\
\hline 6.0 & 1757.7404 & 2.0195 & MSVVTGGGEAAGGTSGGGAR \\
\hline 4.3 & 1759.7431 & 0.0168 & GPESVTQGDTLEVIR \\
\hline 3.2 & 1757.7404 & 2.0195 & MSVVTGGGEAAGGTSGGGAR \\
\hline 3.2 & 1757.7404 & 2.0195 & MSVVTGGGEAAGGTSGGGAR \\
\hline 1.8 & 1759.7609 & -0.0010 & NTENGSAPSTAPTDQNR \\
\hline 1.8 & 1757.7461 & 2.0138 & CAPGCSPGDPSQLQER \\
\hline 1.4 & 1757.7498 & 2.0101 & EVHLKNTSRGSAGNK \\
\hline
\end{tabular}

Spectrum No: 658; Query: 2809; Rank: 1

\section{Peptide View}

MS/MS Fragmentation of DKNDNTPYFLPNNQTFVIIPELVLPNQQVASVQAR

Found in IPI00362922, Tax_Id=10116 Gene_Symbol=RGD1560576_predicted similar to protocadherin LKC precursor

Match to Query 2809: 3983.055492 from(1328.692440,3+)

Title: 100101RatKid_NS_deglyco_21.5983.5983.3.dta 

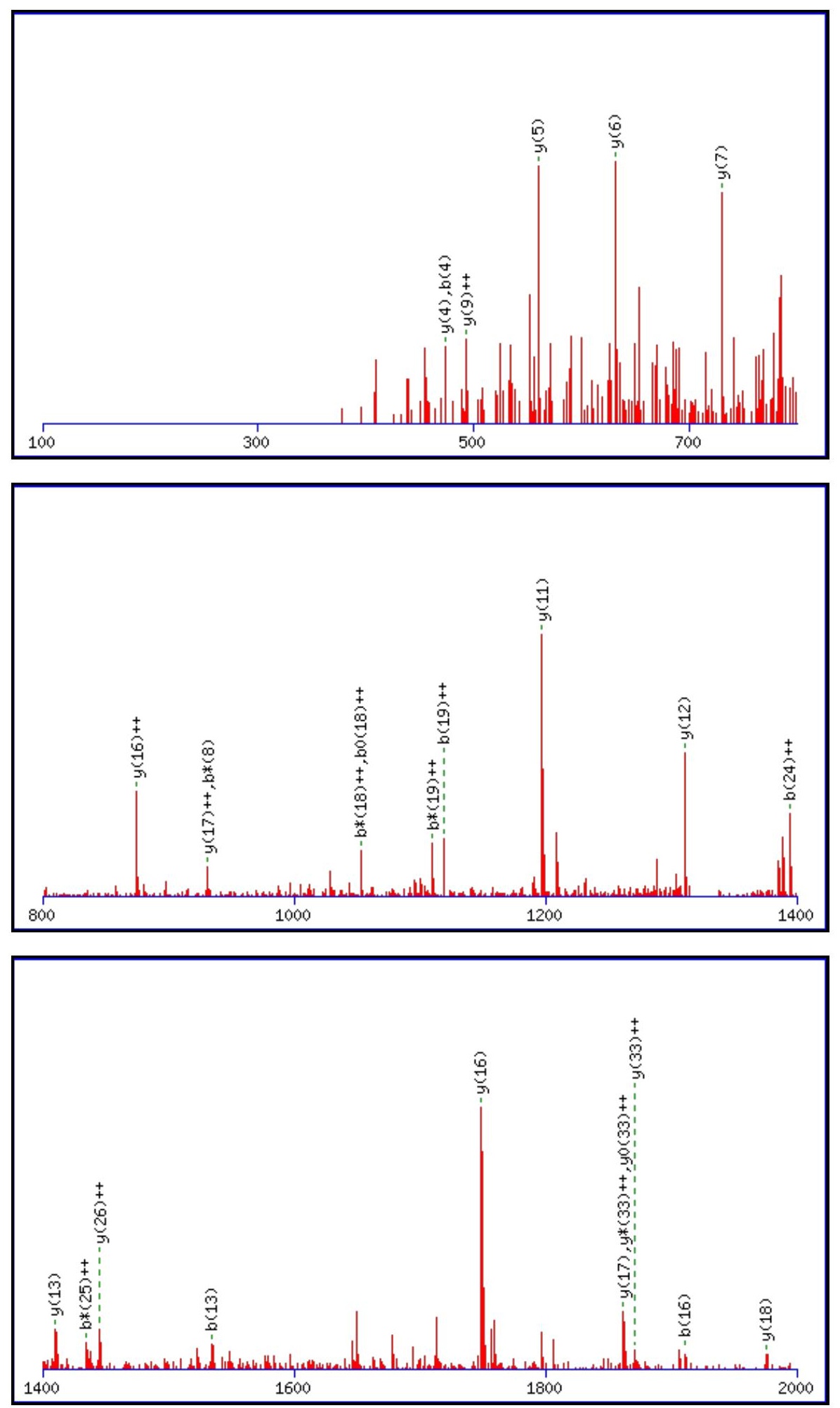

Monoisotopic mass of neutral peptide $\operatorname{Mr}($ calc): 3983.0429

Fixed modifications: Carbamidomethyl (C)

Variable modifications:

N13 : Deamidated_N (N)

Ions Score: 51 Expect: 0.00095

Matches (Bold Red): 27/398 fragment ions using 40 most intense peaks

\begin{tabular}{|c|c|c|c|c|c|c|c|c|c|c|c|c|c|c|}
\hline \# & b & $\mathbf{b}^{++}$ & $\mathbf{b}^{*}$ & $\mathbf{b}^{*++}$ & $\mathbf{b}^{0}$ & $\mathbf{b}^{\mathbf{0 + +}}$ & Seq. & $\mathbf{y}$ & $\mathbf{y}^{++}$ & $\mathbf{y}^{*}$ & $\mathbf{y}^{*^{++}}$ & $\mathbf{y}^{0}$ & $y^{0++}$ & \# \\
\hline 1 & 116.0342 & 58.5207 & & & 98.0237 & 49.5155 & D & & & & & & & 35 \\
\hline 2 & 244.1292 & 122.5682 & 227.1026 & 114.0550 & 226.1186 & 113.5629 & $\mathbf{K}$ & 3869.0232 & 1935.0152 & 3851.9967 & 1926.5020 & 3851.0126 & 1926.0100 & 34 \\
\hline 3 & 358.1721 & 179.5897 & 341.1456 & 171.0764 & 340.1615 & 170.5844 & $\mathbf{N}$ & 3740.9282 & 1870.9678 & 3723.9017 & 1862.4545 & 3722.9177 & 1861.9625 & 33 \\
\hline 4 & 473.1991 & 237.1032 & 456.1725 & 228.5899 & 455.1885 & 228.0979 & D & 3626.8853 & 1813.9463 & 3609.8588 & $\mid 1805.4330$ & 3608.8748 & 1804.9410 & 32 \\
\hline
\end{tabular}




\begin{tabular}{|c|c|c|c|c|c|c|c|c|c|c|c|c|c|c|}
\hline 5 & 587.2420 & 294.1246 & 570.2154 & 285.6114 & 569.2314 & 285.1193 & $\mathbf{N}$ & 3511.8584 & 1756.4328: & 3494.8318 & 1747.9196 & 3493.8478 & 1747.4275 & 31 \\
\hline 6 & 688.2897 & 344.6485 & 671.2631 & 336.1352 & 670.2791 & 335.6432 & $\mathbf{T}$ & 3397.8155 & 1699.4114 & 3380.7889 & 1690.8981 & 3379.8049 & 1690.4061 & 30 \\
\hline 7 & 785.3424 & 393.1748 & 768.3159 & 384.6616 & 767.3319 & 384.1696 & $\mathbf{P}$ & 3296.7678 & 1648.8875 & 3279.7412 & 1640.3742 & 3278.7572 & 1639.8822 & 29 \\
\hline 8 & 948.4058 & 474.7065 & 931.3792 & 466.1932 & 930.3952 & 465.7012 & $\mathbf{Y}$ & 3199.7150 & 1600.3611 & 3182.6885 & 1591.8479 & 3181.7044 & |1591.3559| & 28 \\
\hline 9 & 1095.4742 & 548.2407 & 1078.4476 & 539.7274 & 1077.4636 & 539.2354 & $\mathbf{F}$ & 3036.6517 & 1518.8295 & 3019.6251 & 1510.3162 & 3018.6411 & 1509.8242 & 27 \\
\hline 10 & 1208.5582 & 604.7828 & 1191.5317 & 596.2695 & 1190.5477 & 595.7775 & $\mathbf{L}$ & 2889.5833 & 1445.2953 & 2872.5567 & 1436.7820 & 2871.5727 & 1436.2900 & 26 \\
\hline 11 & 1305.6110 & 653.3091 & 1288.5844 & 644.7959 & 1287.6004 & 644.3039 & $\mathbf{P}$ & 2776.4992 & 1388.7532 & 2759.4727 & 1380.2400 & 2758.4886 & 1379.7480 & 25 \\
\hline 12 & 1419.6539 & 710.3306 & 1402.6274 & 701.8173 & 1401.6434 & 701.3253 & $\mathbf{N}$ & 2679.4464 & 1340.2269 & 2662.4199 & 1331.7136 & 2661.4359 & 1331.2216 & 24 \\
\hline 13 & 1534.6809 & 767.8441 & 1517.6543 & 759.3308 & 1516.6703 & 758.8388 & $\mathbf{N}$ & 2565.4035 & 1283.2054 & 2548.3770 & 1274.6921 & 2547.3929 & 1274.2001 & 23 \\
\hline 14 & 1662.7394 & 831.8734 & 1645.7129 & 823.3601 & 1644.7289 & 822.8681 & Q & 2450.3766 & 1225.6919 & 2433.3500 & 1217.1787 & 2432.3660 & 1216.6866 & 22 \\
\hline 15 & 1763.7871 & 882.3972 & 1746.7606 & 873.8839 & 1745.7765 & 873.3919 & $\mathbf{T}$ & 2322.3180 & 1161.6626 & 2305.2914 & 1153.1494 & 2304.3074 & 1152.6574 & 21 \\
\hline 16 & 1910.8555 & 955.9314 & 1893.8290 & 947.4181 & 1892.8450 & 946.9261 & $\mathbf{F}$ & 2221.2703 & 1111.1388 & 2204.2438 & 1102.6255 & 2203.2598 & 1335 & 20 \\
\hline 17. & 2009. & 1005.4656 & 1992.8974 & 996.9523 & 1991.9134 & 996.4603 & V & 2074.2019 & 1037.6046 & 2057.1754 & 1029.0913 & 2056.1913 & 1028.5993 & 19 \\
\hline 18 & 2123.0080 & 1062.0076 & 2105.9815 & 1053.4944 & 2104.9974 & 1053.0024 & $\mathbf{I}$ & 1975.1335 & 988.0704 & 1958.1069 & 979.5571 & 1957.1229 & 979.0651 & 18 \\
\hline 19 & 2236.0921 & 1118.5497 & 2219.0655 & 1110.0364 & 2218.0815 & 1109.5444 & $\mathbf{I}$ & 1862.0494 & 931.5284 & 1845.0229 & 923.0151 & 1844.0389 & 922.5231 & 17 \\
\hline 20 & 2333.1448 & 1167.0761 & 2316.1183 & 1158.5628 & 2315.1343 & 1158.0708 & $\mathbf{P}$ & 1748.9654 & 874.9863 & 1731.9388 & 866.4730 & 1730.9548 & 865.9810 & 16 \\
\hline 21 & 2462.1874 & 1231.5974 & 2445.1609 & 1223.0841 & 2444.1769 & 1222.5921 & $\mathbf{E}$ & 1651.9126 & 826.4599 & 1634.8860 & 817.9467 & 1633.9020 & 817.4547 & 15 \\
\hline 22 & 2575.2715 & 1288.1394 & 2558.2449 & 1279.6 & 2557.2609 & 1279.1341 & $\mathbf{L}$ & 1522.8700 & 761.9386 & 1505.8435 & 753.4254 & 1504.8594 & 752.9334 & 14 \\
\hline 23. & 2674.3399 & 1337.6736 & 2657.3134 & 1329.1603 & 2656.3293 & 1328.6683 & $\mathbf{V}$ & 1409.7859 & 705.3966 & 1392.7594 & 696.8833 & 1391.7754 & 696.3913 & 13 \\
\hline 24 & 2787.4240 & 1394.2156 & 2770.3974 & 1385.7023 & 2769.4134 & 1385.2103 & $\mathbf{L}$ & 1310.7175 & 655.8624 & 1293.6910 & 647.3491 & 1292.7070 & 646.8571 & 12 \\
\hline 25 & 2884.4767 & 1442.7420 & 2867.4502 & 1434.2287 & 2866.4662 & 1433.7367 & $\mathbf{P}$ & 1197.6335 & 599.3204 & 1180.6069 & 590.8071 & 1179.6229 & 590.3151 & 11 \\
\hline 26 & 2998.5197 & 1499.7635 & 2981.4931 & 1491.2502 & 2980.5091 & 1490.7582 & $\mathbf{N}$ & 1100.5807 & 550.7940 & 1083.5541 & 542.2807 & 1082.5701 & 541.7887 & 10 \\
\hline 27 & 3126.5782 & 1563.7928 & 3109.5517 & 1555.2795 & 3108.5677 & 1554.7875 & Q & 986.5378 & 493.7725 & 969.5112 & 485.2592 & 968.5272 & 484.7672 & 9 \\
\hline 28 & 3254.6368 & 1627.8220 & 3237.6103 & 1619.3088 & 3236.6263 & 1618.8168 & $\mathbf{Q}$ & 858.4792 & 429.7432 & 841.4526 & 421.2300 & 840.4686 & 420.7380 & 8 \\
\hline 29. & 3353.7052 & \begin{tabular}{|l|}
1677.3563 \\
\end{tabular} & 3336.6787 & 1668.8430 & 3335.6947 & 1668.3510 & V & 730.4206 & 365.7139 & 713.3941 & 357.2007 & 712.4100 & 356.7087 & 7 \\
\hline 30 & 3424.7423 & 1712.8748 & 3407.7158 & 1704.3615 & 3406.7318 & 1703.8695 & A & 631.3522 & 316.1797 & 614.3257 & 307.6665 & 613.3416 & 307.1745 & 6 \\
\hline 31. & 3511.7744 & 1756.3908 & 3494.7478 & 1747.8775 & 3493.7638 & 1747.3855 & S & 560.3151 & 280.6612 & 543.2885 & 272.1479 & 542.3045 & 271.6559 & 5 \\
\hline 32 & 3610.8428 & 1805.9250 & 3593.8162 & 1797.4118 & 3592.8322 & 1796.9197 & V & 473.2831 & 237.1452 & 456.2565 & 228.6319 & & & 4 \\
\hline 33 & 3738.9014 & 1869.9543 & 3721.8748 & 1861.4410 & 3720.8908 & 1860.9490 & Q & 374.2146 & 187.6110 & 357.1881 & 179.0977 & & & 3 \\
\hline 34 & 3809.9385 & 1905.4729 & 3792.9119 & 1896.9596 & 3791.9279 & 1896.4676 & A & 246.1561 & 123.5817 & 229.1295 & 115.0684 & & & 2 \\
\hline 35 & & & & & & & $\mathbf{R}$ & 175.1190 & 88.0631 & 158.0924 & 79.5498 & & & 1 \\
\hline
\end{tabular}
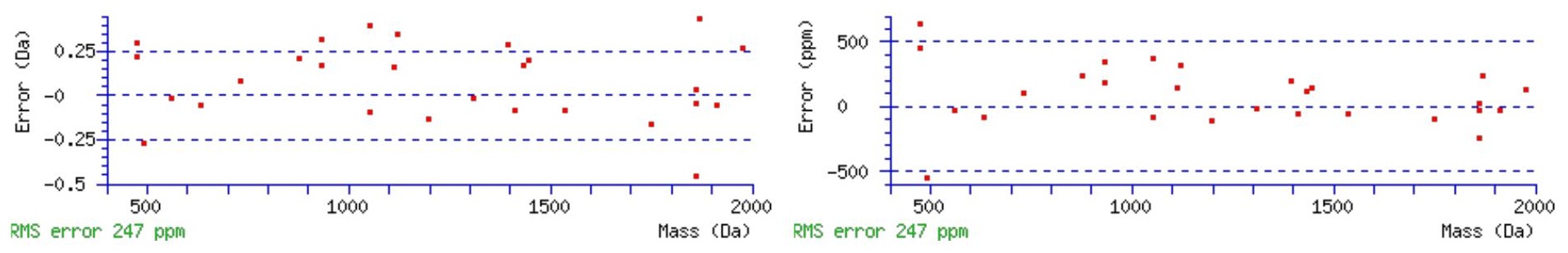

\section{All matches to this query}

\begin{tabular}{|l|c|c|l|}
\hline Score & Mr(calc): & Delta & \multicolumn{1}{c|}{ Sequence } \\
\hline 51.0 & 3983.0429 & 0.0126 & DKNDNTPYFLPNNQTFVIIPELVLPNQQVASVQAR \\
\hline 51.0 & 3983.0429 & 0.0126 & DKNDNTPYFLPNNQTFVIIPELVLPNQQVASVQAR \\
\hline 51.0 & 3983.0429 & 0.0126 & DKNDNTPYFLPNNQTFVIIPELVLPNQQVASVQAR \\
\hline 44.8 & 3983.0429 & 0.0126 & DKNDNTPYFLPNNQTFVIIPELVLPNQQVASVQAR \\
\hline 38.5 & 3982.0589 & 0.9966 & DKNDNTPYFLPNNQTFVIIPELVLPNQQVASVQAR \\
\hline 26.5 & 3983.0429 & 0.0126 & DKNDNTPYFLPNNQTFVIIPELVLPNQQVASVQAR \\
\hline 3.5 & 3983.0730 & -0.0175 & GGGGYGGSGDGYNGFGNDGGYGGSSSSSSYGSGRRF \\
\hline 3.2 & 3983.0384 & 0.0171 & TTTCISLQDAFDKLKPCPAGIIDQLSVIPNSNEPLK \\
\hline 3.0 & 3981.0851 & 1.9704 & DPNPVGLAPGTVASIILTLVLLVVLMVMCGPLIYKK \\
\hline
\end{tabular}


Spectrum No: 659; Query: 2564; Rank: 1

\section{Peptide View}

MS/MS Fragmentation of GSEEPPVFLEIHYTGSPNATEAPLVFVGK

Found in IPI00471530, Tax_Id=10116 Gene_Symbol=Lap3 Isoform 1 of Cytosol aminopeptidase

Match to Query 2564: 3085.538622 from(1029.520150,3+)

Title: 091008RatKidney_NH4Format02_17.4299.4299.3.dta

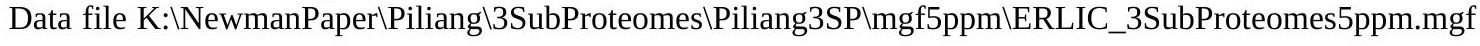
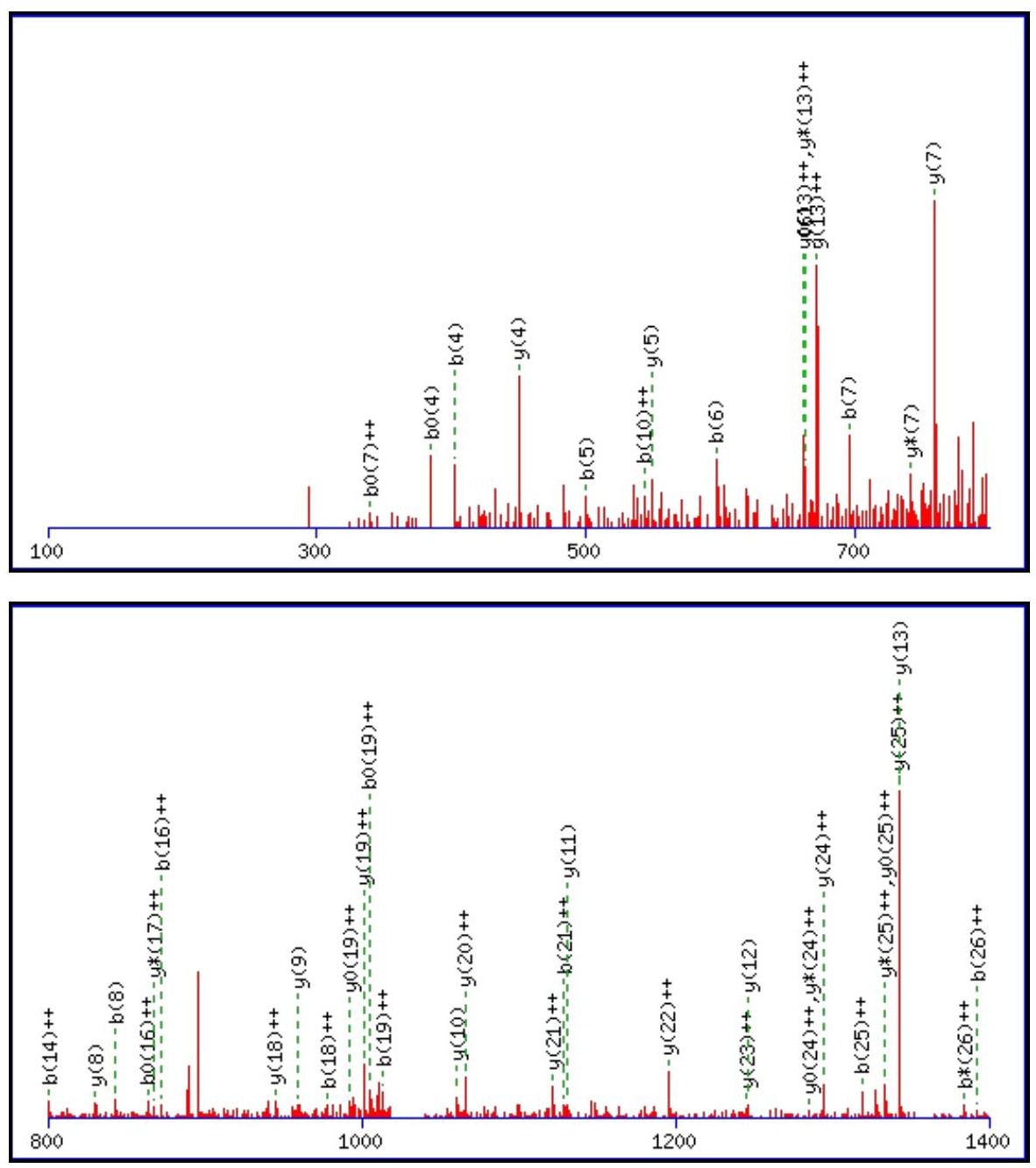


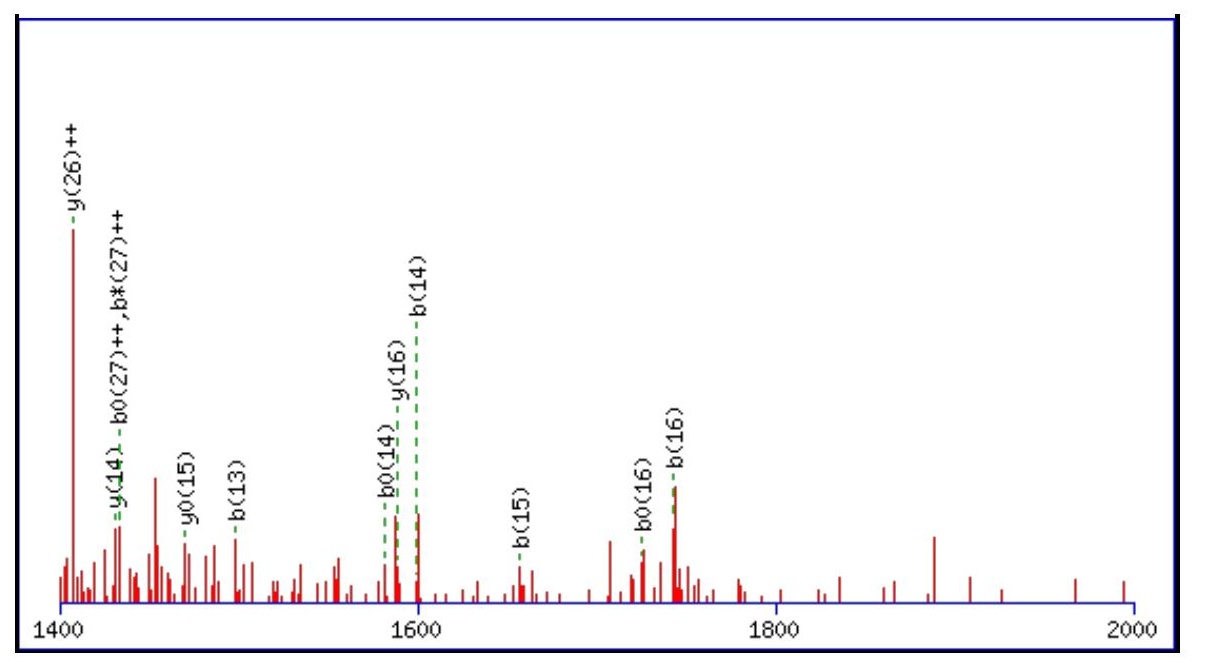

Monoisotopic mass of neutral peptide $\operatorname{Mr}($ calc): 3085.5284

Fixed modifications: Carbamidomethyl (C)

Variable modifications:

N18 : Deamidated_N (N)

Ions Score: 51 Expect: $\odot .0018$

Matches (Bold Red): 58/284 fragment ions using 160 most intense peaks

\begin{tabular}{|c|c|c|c|c|c|c|c|c|c|c|c|c|c|c|}
\hline \# & b & $\mathbf{b}^{++}$ & $\mathbf{b}^{*}$ & $\mathbf{b}^{*^{++}}$ & $\mathbf{b}^{\mathbf{0}}$ & $\mathbf{b}^{\mathbf{0}^{++}}$ & Seq. & $\mathbf{y}$ & $\mathbf{y}^{++}$ & $\mathbf{y}^{*}$ & $\mathrm{y}^{\mathrm{*}^{++}}$ & $\mathbf{y}^{0}$ & $\mathbf{y}^{\mathbf{0 + +}}$ & \# \\
\hline 1 & 58.0287 & 29.5180 & & & & & G & & & & & & & 29 \\
\hline 2 & 145.0608 & 73.0340 & & & 127.0502 & 64.0287 & $S$ & 3029.5142 & 1515.2608 & 3012.4877 & 1506.7475 & 3011.5037 & 1506.2555 & 28 \\
\hline 3 & 274.1034 & 137.5553 & & & 256.0928 & 128.5500 & $\mathbf{E}$ & 2942.4822 & 1471.7447 & |2925.4557| & 1463.2315 & 2924.4716 & 1462.7395 & 27 \\
\hline 4 & 403.1460 & 202.0766 & & & 385.1354 & 193.0713 & $\mathbf{E}$ & 2813.4396 & 1407.2234 & 2796.4131 & 1398.7102 & 2795.4291 & 1398.2182 & 26 \\
\hline 5 & 500.1987 & 250.6030 & & & 482.1882 & 241.5977 & $\mathbf{P}$ & 2684.3970 & 1342.7022 & 2667.3705 & 1334.1889 & 2666.3865 & 1333.6969 & 25 \\
\hline 6 & 597.2515 & 299.1294 & & & 579.2409 & 290.1241 & $\mathbf{P}$ & 2587.3443 & 1294.1758 & 2570.3177 & 1285.6625 & 2569.3337 & 1285.1705 & 24 \\
\hline 7 & 696.3199 & 348.6636 & & & 678.3093 & 339.6583 & $\mathbf{V}$ & 2490.2915 & 1245.6494 & 2473.2649 & 1237.1361 & 2472.2809 & 1236.6441 & 23 \\
\hline 8 & 843.3883 & 422.1978 & & & 825.3777 & 413.1925 & $\mathbf{F}$ & 2391.2231 & 1196.1152 & 2374.1965 & 1187.6019 & 2373.2125 & 1187.1099 & 22 \\
\hline 9 & 956.4724 & 478.7398 & & & 938.4618 & 469.7345 & L & 2244.1547 & 1122.5810 & 2227.1281 & 1114.0677 & 2226.1441 & 1113.5757 & 21 \\
\hline 10 & 1085.5150 & 543.2611 & & & 1067.5044 & 534.2558 & $\mathbf{E}$ & 2131.0706 & \begin{tabular}{|l}
1066.0389 \\
\end{tabular} & 2114.0441 & 1057.5257 & 2113.0600 & 1057.0337 & 20 \\
\hline 11 & 1198.5990 & 599.8032 & & & 1180.5885 & 590.7979 & I & 2002.0280 & 1001.5176 & 1985.0015 & 993.0044 & 1984.0174 & 992.5124 & 19 \\
\hline 12 & 1335.6579 & 668.3326 & & & 1317.6474 & 659.3273 & $\mathbf{H}$ & 1888.9440 & 944.9756 & 1871.9174 & 936.4623 & 1870.9334 & 935.9703 & 18 \\
\hline 13 & 1498.7213 & 749.8643 & & & 1480.7107 & 740.8590 & $\mathbf{Y}$ & 1751.8850 & 876.4462 & 1734.8585 & 867.9329 & 1733.8745 & 867.4409 & 17 \\
\hline 14 & 1599.7690 & 800.3881 & & & \begin{tabular}{|l|}
1581.7584 \\
\end{tabular} & 791.3828 & $T$ & \begin{tabular}{|l|}
1588.8217 \\
\end{tabular} & 794.9145 & \begin{tabular}{|l|}
1571.7952 \\
\end{tabular} & 786.4012 & 1570.8111 & 785.9092 & 16 \\
\hline 15 & 1656.7904 & 828.8988 & & & 1638.7798 & 819.8936 & G & 1487.7740 & 744.3907 & 1470.7475 & 735.8774 & 1469.7635 & 735.3854 & 15 \\
\hline 16 & 1743.8224 & 872.4149 & & & \begin{tabular}{|l|}
1725.8119 \\
\end{tabular} & 863.4096 & $S$ & \begin{tabular}{|l}
1430.7526 \\
\end{tabular} & 715.8799 & 1413.7260 & 707.3666 & 1412.7420 & 706.8746 & 14 \\
\hline 17 & 1840.8752 & 920.9412 & & & 1822.8646 & 911.9360 & $\mathbf{P}$ & 1343.7205 & 672.3639 & 1326.6940 & 663.8506 & 1325.7100 & 663.3586 & 13 \\
\hline 18 & 1955.9021 & 978.4547 & 1938.8756 & 969.9414 & 1937.8916 & 969.4494 & $\mathbf{N}$ & \begin{tabular}{|l|l|}
1246.6678 \\
\end{tabular} & 623.8375 & 1229.6412 & 615.3243 & 1228.6572 & 614.8322 & 12 \\
\hline 19 & 2026.9393 & \begin{tabular}{|l}
1013.9733 \\
\end{tabular} & 2009.9127 & 1005.4600 & 2008.9287 & 1004.9680 & A & 1131.6408 & 566.3241 & 1114.6143 & 557.8108 & 1113.6303 & 557.3188 & 11 \\
\hline 20 & 2127.9869 & 1064.4971 & 2110.9604 & 1055.9838 & 2109.9764 & 1055.4918 & $T$ & 1060.6037 & 530.8055 & 1043.5772 & 522.2922 & 1042.5932 & 521.8002 & 10 \\
\hline 21 & 2257.0295 & \begin{tabular}{|l}
1129.0184 \\
\end{tabular} & 2240.0030 & 1120.5051 & 2239.0190 & 1120.0131 & $\mathbf{E}$ & 959.5560 & 480.2817 & 942.5295 & 471.7684 & 941.5455 & 471.2764 & 9 \\
\hline 22 & 2328.0666 & 1164.5370 & 2311.0401 & 1156.0237 & 2310.0561 & 1155.5317 & A & 830.5135 & 415.7604 & 813.4869 & 407.2471 & & & 8 \\
\hline 23 & 2425.1194 & 1213.0633 & 2408.0929 & 1204.5501 & 2407.1088 & 1204.0581 & $\mathbf{P}$ & 759.4763 & 380.2418 & 742.4498 & 371.7285 & & & 7 \\
\hline 24 & 2538.2035 & 1269.6054 & 2521.1769 & 1261.0921 & 2520.1929 & 1260.6001 & $\mathbf{L}$ & 662.4236 & 331.7154 & 645.3970 & 323.2022 & & & 6 \\
\hline 25 & 2637.2719 & \begin{tabular}{|l|l|}
1319.1396 \\
\end{tabular} & 2620.2453 & 1310.6263 & 2619.2613 & 1310.1343 & V & 549.3395 & 275.1734 & 532.3130 & 266.6601 & & & 5 \\
\hline 26 & 2784.3403 & \begin{tabular}{|l}
1392.6738 \\
\end{tabular} & 2767.3137 & 1384.1605 & 2766.3297 & 1383.6685 & $\mathbf{F}$ & 450.2711 & 225.6392 & 433.2445 & 217.1259 & & & 4 \\
\hline 27 & 2883.4087 & 1442.2080 & 2866.3822 & \begin{tabular}{|l|}
1433.6947 \\
\end{tabular} & 2865.3981 & 1433.2027 & V & 303.2027 & 152.1050 & 286.1761 & 143.5917 & & & 3 \\
\hline 28 & 2940.4302 & 1470.7187 & 2923.4036 & 1462.2055 & 2922.4196 & 1461.7134 & G & 204.1343 & 102.5708 & 187.1077 & 94.0575 & & & 2 \\
\hline 29 & & & & & & & $\mathbf{K}$ & 147.1128 & 74.0600 & 130.0863 & 65.5468 & & & 1 \\
\hline
\end{tabular}



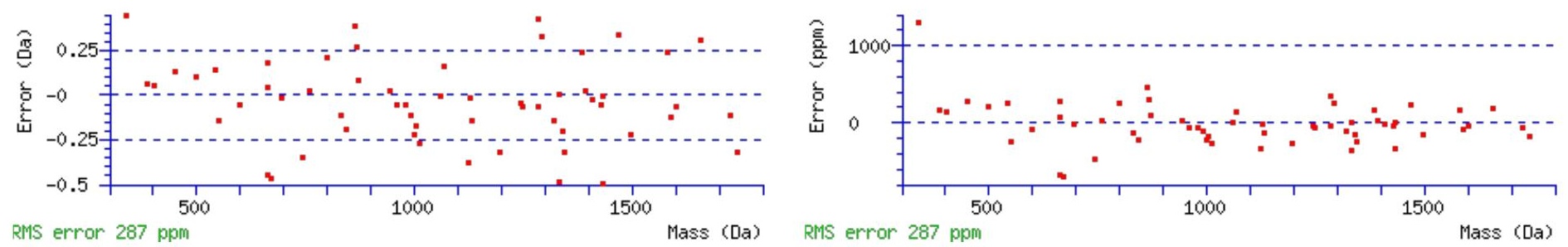

\section{All matches to this query}

\begin{tabular}{|l|l|l|l|}
\hline Score & Mr(calc): & Delta & \multicolumn{1}{|c|}{ Sequence } \\
\hline 50.9 & 3085.5284 & 0.0102 & GSEEPPVFLEIHYTGSPNATEAPLVFVGK \\
\hline 40.6 & 3084.5444 & 0.9942 & GSEEPPVFLEIHYTGSPNATEAPLVFVGK \\
\hline 2.8 & 3083.5416 & 1.9970 & EGTMSAHLGYPVVGWHIANKKPTLPKR \\
\hline 1.8 & 3083.5484 & 1.9903 & VVSVKCLLLGAGTLGCNVARTLMGWGVR \\
\hline 0.8 & 3085.5351 & 0.0035 & ATLFPTQVEELLCNLNSILYDTVKMR \\
\hline 0.5 & 3084.5620 & 0.9766 & LHLQSNHPDDTFLFPKLLQKMVDLR \\
\hline 0.5 & 3085.5140 & 0.0246 & VDIWSLGIMAIEMVEGEPPYLNENPLR \\
\hline 0.5 & 3085.5140 & 0.0246 & VDIWSLGIMAIEMVEGEPPYLNENPLR \\
\hline 0.2 & 3084.5620 & 0.9766 & LHLQSNHPDDTFLFPKLLQKMVDLR \\
\hline 0.2 & 3085.5460 & -0.0074 & LHLQSNHPDDTFLFPKLLQKMVDLR \\
\hline
\end{tabular}

Spectrum No: 660; Query: 1991; Rank: 1

\section{Peptide View}

MS/MS Fragmentation of LIFDGVDTVAEILFNNVTIGK

Found in IPI00388355, Tax_Id=10116 Gene_Symbol=Manba Isoform 1 of Beta-mannosidase precursor

Match to Query 1991: 2278.218102 from(760.413310,3+)

Title: 100101RatKid_NS_deglyco_17.8542.8542.3.dta

Data file K:INewmanPaper|Piliangl3SubProteomes\Piliang3SP\mgf5ppm\ERLIC_3SubProteomes5ppm.mgf

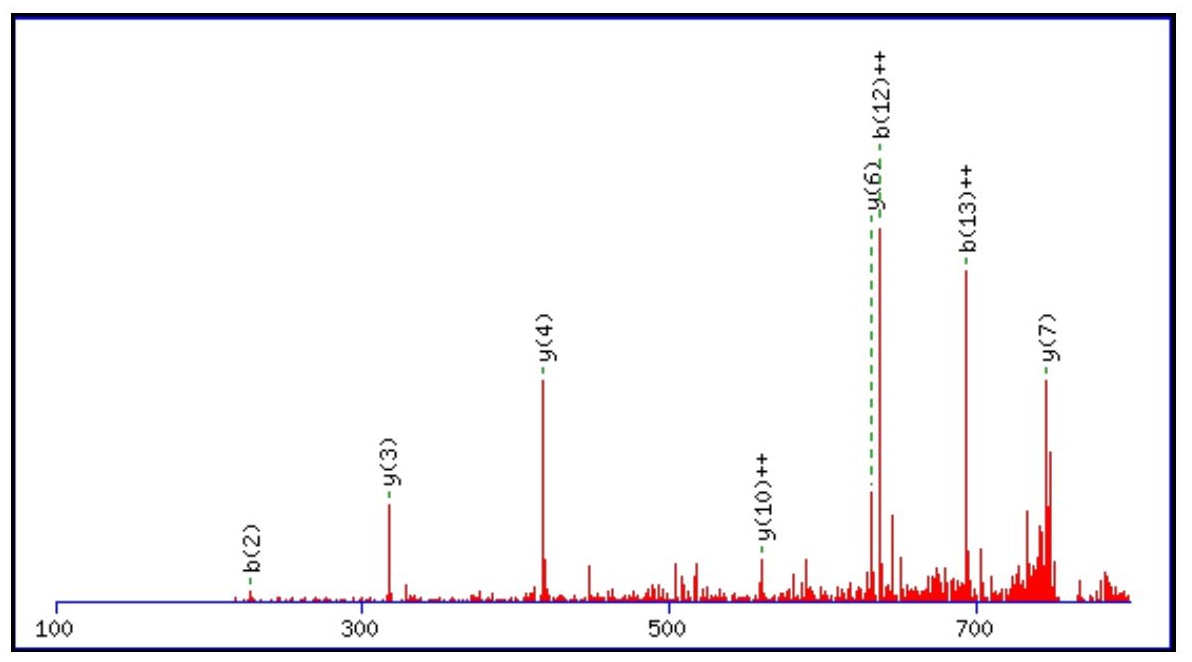



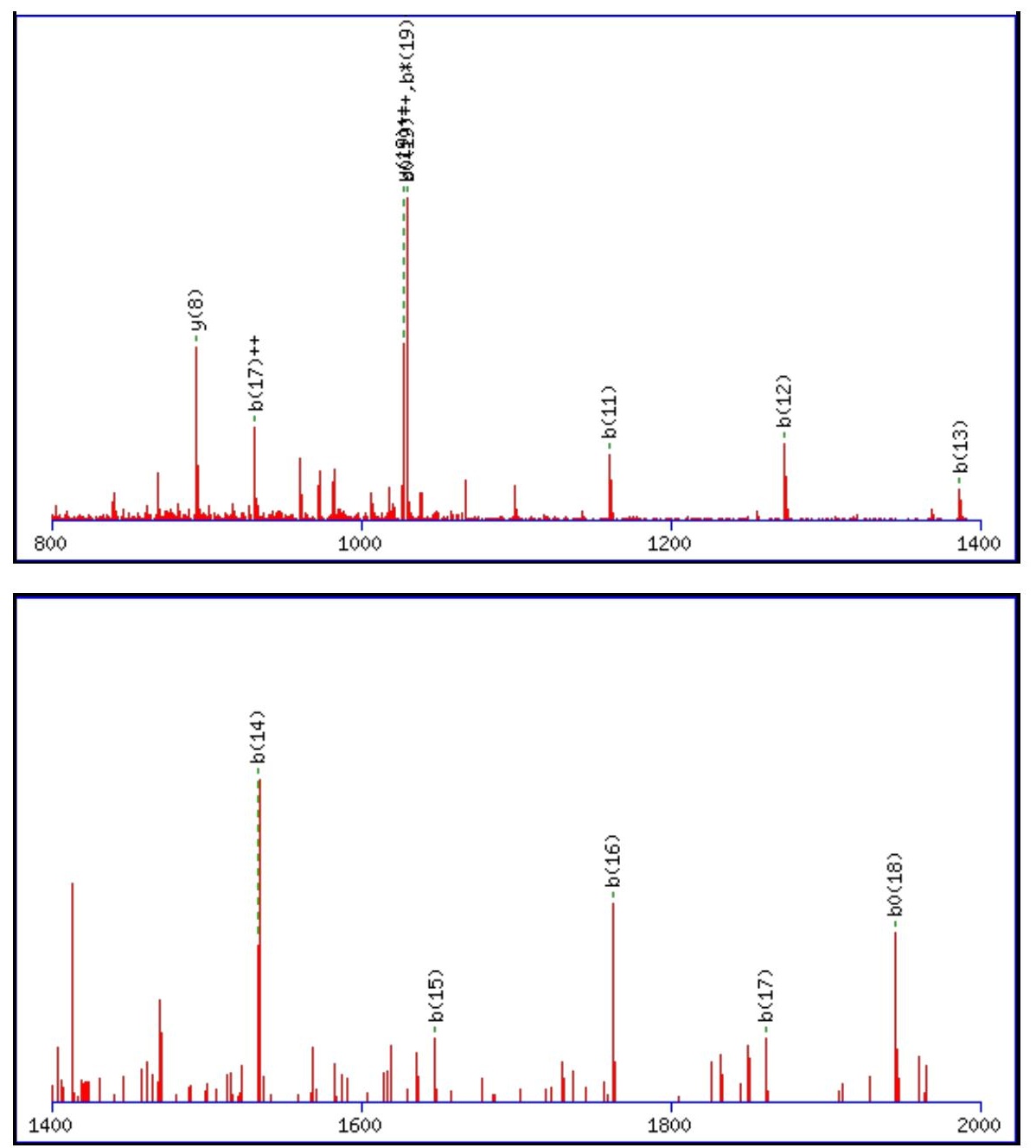

Monoisotopic mass of neutral peptide $\operatorname{Mr}($ calc): 2278.2097

Fixed modifications: Carbamidomethyl (C)

Variable modifications:

N16 : Deamidated_N (N)

Ions Score: 51 Expect: 0.00061

Matches (Bold Red): 21/200 fragment ions using 28 most intense peaks

\begin{tabular}{|c|c|c|c|c|c|c|c|c|c|c|c|c|c|c|}
\hline \# & b & $\mathbf{b}^{++}$ & b* & $\mathbf{b}^{*^{++}}$ & $\mathbf{b}^{\mathbf{0}}$ & $\mathbf{b}^{0++}$ & Seq. & $\mathbf{y}$ & $\mathbf{y}^{++}$ & $\mathbf{y}^{*}$ & $\mathrm{y}^{*^{++}}$ & $\mathbf{y}^{\mathbf{0}}$ & $\mathbf{y}^{0++}$ & \# \\
\hline 1 & 114.0913 & 57.5493 & & & & & $\mathbf{L}$ & & & & & & & 21 \\
\hline 2 & 227.1754 & 114.0913 & & & & & I & 2166.1329 & |1083.5701 & 2149.1063 & 1075.0568 & 2148.1223 & 1074.5648 & 20 \\
\hline 3 & 374.2438 & 187.6255 & & & & & $\mathbf{F}$ & 2053.0488 & 1027.0280 & 2036.0223 & 1018.5148 & 2035.0382 & 1018.0228 & 19 \\
\hline 4 & 489.2708 & 245.1390 & & & 471.2602 & 236.1337 & D & 1905.9804 & 953.4938 & 1888.9538 & 944.9806 & 1887.9698 & 944.4886 & 18 \\
\hline 5 & 546.2922 & 273.6498 & & & 528.2817 & 264.6445 & G & 1790.9535 & 895.9804 & 1773.9269 & 887.4671 & 1772.9429 & 886.9751 & 17 \\
\hline 6 & 645.3606 & 323.1840 & & & 627.3501 & 314.1787 & $\mathbf{V}$ & 1733.9320 & 867.4696 & 1716.9054 & 858.9564 & 1715.9214 & 858.4643 & 16 \\
\hline 7 & 760.3876 & 380.6974 & & & 742.3770 & 371.6921 & D & 1634.8636 & 817.9354 & 1617.8370 & 809.4222 & 1616.8530 & 808.9301 & 15 \\
\hline 8 & 861.4353 & 431.2213 & & & 843.4247 & 422.2160 & $\mathbf{T}$ & 1519.8366 & 760.4220 & 1502.8101 & 751.9087 & 1501.8261 & 751.4167 & 14 \\
\hline 9 & 960.5037 & 480.7555 & & & 942.4931 & 471.7502 & $\mathbf{V}$ & 1418.7890 & 709.8981 & 1401.7624 & 701.3848 & 1400.7784 & 700.8928 & 13 \\
\hline 10 & 1031.5408 & 516.2740 & & & 1013.5302 & 507.2687 & A & 1319.7205 & 660.3639 & 1302.6940 & 651.8506 & 1301.7100 & 651.3586 & 12 \\
\hline 11 & 1160.5834 & 580.7953 & & & 1142.5728 & 571.7900 & $\mathbf{E}$ & 1248.6834 & 624.8453 & 1231.6569 & 616.3321 & 1230.6729 & 615.8401 & 11 \\
\hline 12 & 1273.6674 & 637.3374 & & & 1255.6569 & 628.3321 & I & 1119.6408 & 560.3241 & 1102.6143 & 551.8108 & 1101.6303 & 551.3188 & 10 \\
\hline 13 & 1386.7515 & 693.8794 & & & 1368.7409 & 684.8741 & $\mathbf{L}$ & 1006.5568 & 503.7820 & 989.5302 & 495.2687 & 988.5462 & 494.7767 & 9 \\
\hline 14 & 1533.8199 & 767.4136 & & & 1515.8094 & 758.4083 & $\mathbf{F}$ & 893.4727 & 447.2400 & 876.4462 & 438.7267 & 875.4621 & 438.2347 & 8 \\
\hline 15 & 1647.8629 & 824.4351 & 1630.8363 & 815.9218 & 1629.8523 & 815.4298 & $\mathbf{N}$ & 746.4043 & 373.7058 & 729.3777 & 365.1925 & 728.3937 & 364.7005 & 7 \\
\hline 16 & 1762.8898 & 881.9485 & 1745.8632 & 873.4353 & 1744.8792 & 872.9432 & $\mathbf{N}$ & 632.3614 & 316.6843 & 615.3348 & 308.1710 & 614.3508 & 307.6790 & 6 \\
\hline 17 & 1861.9582 & 931.4827 & 1844.9317 & 922.9695 & 1843.9476 & 922.4775 & V & 517.3344 & 259.1709 & 500.3079 & 250.6576 & 499.3239 & 250.1656 & 5 \\
\hline 18 & 1963.0059 & 982.0066 & 1945.9793 & 973.4933 & 1944.9953 & 973.0013 & $\mathbf{T}$ & 418.2660 & 209.6366 & 401.2395 & 201.1234 & 400.2554 & 200.6314 & 4 \\
\hline
\end{tabular}




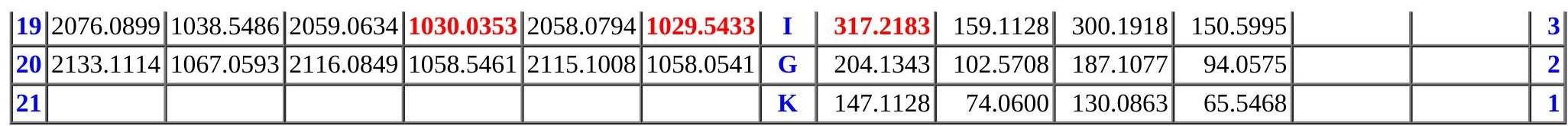
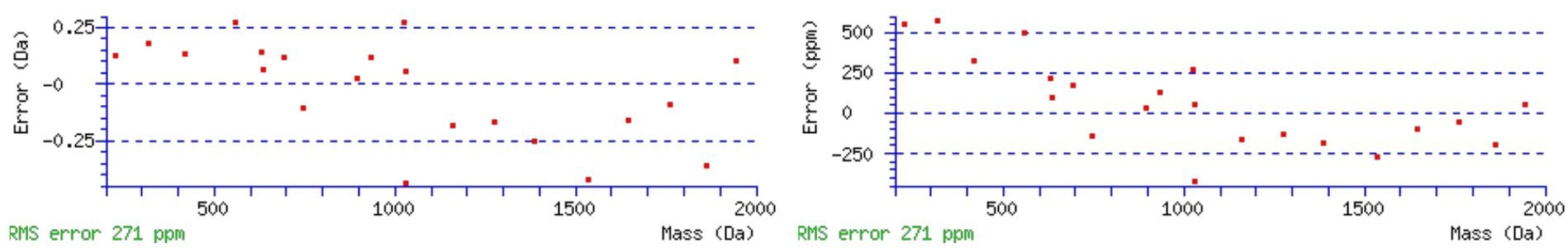

RMS error $271 \mathrm{ppm}$

Mass (Da) RMS error 271 ppm

\section{All matches to this query}

\begin{tabular}{|l|l|l|l|}
\hline Score & Mr(calc): & Delta & \multicolumn{1}{c|}{ Sequence } \\
\hline 50.8 & 2278.2097 & 0.0084 & LIFDGVDTVAEILFNNVTIGK \\
\hline 40.7 & 2278.2097 & 0.0084 & LIFDGVDTVAEILFNNVTIGK \\
\hline 20.3 & 2277.2257 & 0.9924 & LIFDGVDTVAEILFNNVTIGK \\
\hline 8.2 & 2277.2191 & 0.9990 & EAKIMRYDHLLFSLNAIVK \\
\hline 0.8 & 2276.1953 & 2.0228 & TFGAPRPYNSSAPSPFALAVVK \\
\hline
\end{tabular}

Spectrum No: 661; Query: 103; Rank: 1

\section{Peptide View}

\section{MS/MS Fragmentation of GNESIPVNLR}

Found in IPI00327398, Tax_Id=10116 Gene_Symbol=Enpep Isoform 1 of Glutamyl aminopeptidase

Match to Query 103: 1098.566328 from(550.290440,2+)

Title: 091008RatKidney_NH4Format01_27.1390.1390.2.dta

Data file K:\NewmanPaper\Piliang \3SubProteomes $\backslash P i l i a n g 3 S P \backslash m g f 5 p p m \backslash E R L I C \_3 S u b P r o t e o m e s 5 p p m . m g f$

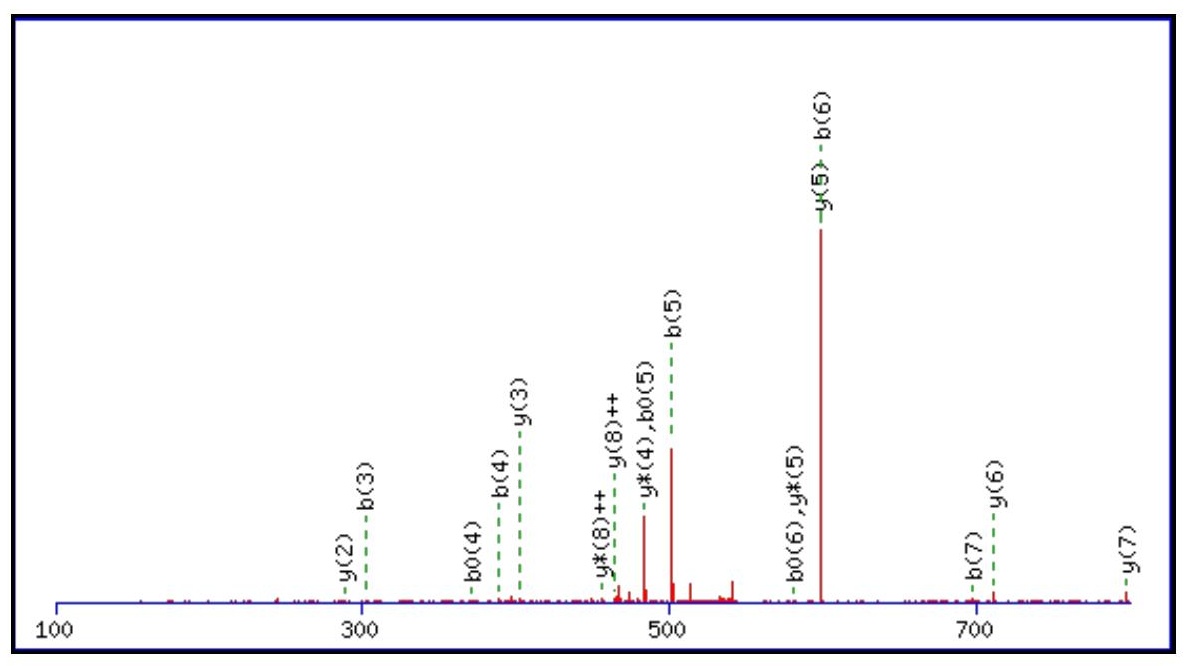




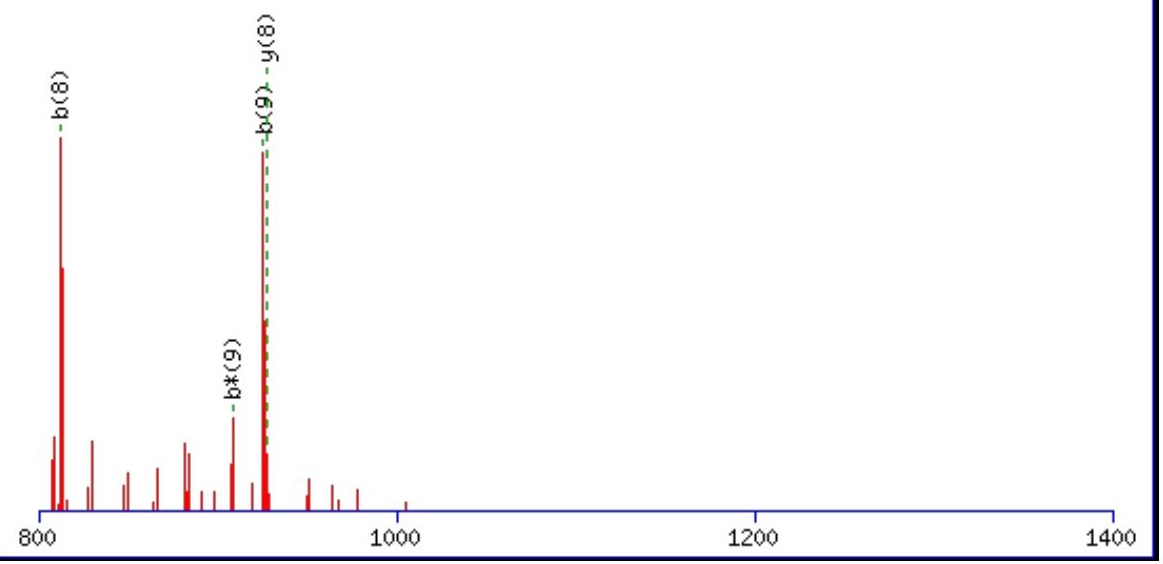

\begin{tabular}{rrr}
\hline 1600 & 1800 & 2000
\end{tabular}

Monoisotopic mass of neutral peptide $\operatorname{Mr}($ calc): 1098.5669 Fixed modifications: Carbamidomethyl (C)

Variable modifications:

N2 : Deamidated_N (N)

Ions Score: 51 Expect: 0.00068

Matches (Bold Red): 21/90 fragment ions using 40 most intense peaks

\begin{tabular}{|c|c|c|c|c|c|c|c|c|c|c|c|c|c|c|}
\hline \# & b & $\mathbf{b}^{++}$ & $\mathbf{b}^{*}$ & $\mathbf{b}^{*^{++}}$ & $\mathbf{b}^{\mathbf{0}}$ & $\mathbf{b}^{0++}$ & Seq. & $\mathbf{y}$ & $y^{++}$ & $\mathbf{y}^{*}$ & $\mathrm{y}^{\mathrm{*}^{++}}$ & $\mathbf{y}^{0}$ & $\mathbf{y}^{0++}$ & $\#$ \\
\hline 1 & 58.0287 & 29.5180 & & & & & G & & & & & & & 10 \\
\hline 2 & 173.0557 & 87.0315 & 156.0291 & 78.5182 & & & $\mathbf{N}$ & 1042.5527 & 521.7800 & 1025.5262 & 513.2667 & 1024.5422 & 512.7747 & 9 \\
\hline 3 & 302.0983 & 151.5528 & 285.0717 & 143.0395 & 284.0877 & 142.5475 & $\mathbf{E}$ & 927.5258 & 464.2665 & 910.4993 & 455.7533 & 909.5152 & 455.2613 & 8 \\
\hline 4 & 389.1303 & 195.0688 & 372.1037 & 186.5555 & 371.1197 & 186.0635 & S & 798.4832 & 399.7452 & 781.4567 & 391.2320 & 780.4726 & 390.7400 & 7 \\
\hline 5 & 502.2144 & 251.6108 & 485.1878 & 243.0975 & 484.2038 & 242.6055 & I & 711.4512 & 356.2292 & 694.4246 & 347.7160 & & & 6 \\
\hline 6 & 599.2671 & 300.1372 & 582.2406 & 291.6239 & 581.2566 & 291.1319 & $\mathbf{P}$ & 598.3671 & 299.6872 & 581.3406 & 291.1739 & & & 5 \\
\hline 7 & 698.3355 & 349.6714 & 681.3090 & 341.1581 & 680.3250 & 340.6661 & $\mathbf{V}$ & 501.3144 & 251.1608 & 484.2878 & 242.6475 & & & 4 \\
\hline 8 & 812.3785 & 406.6929 & 795.3519 & 398.1796 & 794.3679 & 397.6876 & $\mathbf{N}$ & 402.2459 & 201.6266 & 385.2194 & 193.1133 & & & 3 \\
\hline 9 & 925.4625 & 463.2349 & 908.4360 & 454.7216 & 907.4520 & 454.2296 & $\mathbf{L}$ & 288.2030 & 144.6051 & 271.1765 & 136.0919 & & & 2 \\
\hline 10 & & & & & & & $\mathbf{R}$ & 175.1190 & 88.0631 & 158.0924 & 79.5498 & & & 1 \\
\hline
\end{tabular}

$$
\text { 产 }
$$$$
\text { 产 }
$$

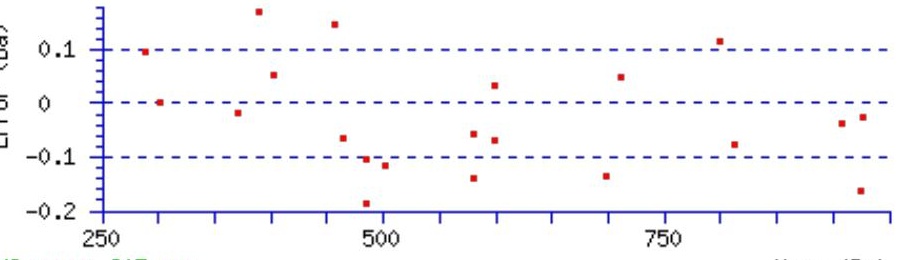

RMS error $205 \mathrm{ppm}$

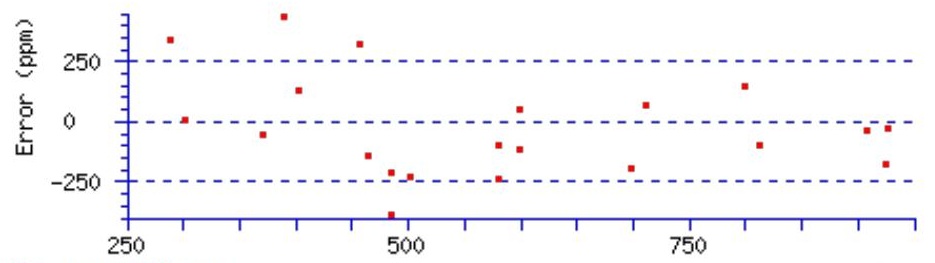

Mass (Da)

\section{All matches to this query}




\begin{tabular}{|l|l|l|l|}
\hline Score & Mr(calc): & Delta & \multicolumn{1}{|c|}{ Sequence } \\
\hline 50.9 & 1098.5669 & -0.0006 & GNESIPVNLR \\
\hline 27.5 & 1098.5669 & -0.0006 & GNESIPVNLR \\
\hline 11.4 & 1096.5641 & 2.0022 & SNSLISIKR \\
\hline 10.9 & 1098.5620 & 0.0043 & KLMLQSKR \\
\hline 8.1 & 1098.5669 & -0.0006 & DEGVSLPVQR \\
\hline 6.1 & 1096.5641 & 2.0022 & KVSSQSLLR \\
\hline 4.3 & 1098.5709 & -0.0046 & AYIYSXLNR \\
\hline 4.3 & 1098.5669 & -0.0006 & LDTNPGVIDR \\
\hline 2.8 & 1096.5488 & 2.0176 & $\underline{\text { MRYFDPLR }}$ \\
\hline 2.5 & 1096.5576 & 2.0087 & $\underline{\text { KCLLTRAR }}$ \\
\hline
\end{tabular}

Spectrum No: 662; Query: 2097; Rank: 1

\section{Peptide View}

MS/MS Fragmentation of YCGNSLPGNYSSAEGHSLWVR

Found in IPI00196620, Tax_Id=10116 Gene_Symbol=Cubn Cubilin precursor

Match to Query 2097: 2354.040162 from(785.687330,3+)

Title: 100101RatKid_NS_deglyco_16.3230.3230.3.dta

Data file K:INewmanPaper|Piliangl3SubProteomes\Piliang3SP\mgf5ppm\ERLIC_3SubProteomes5ppm.mgf
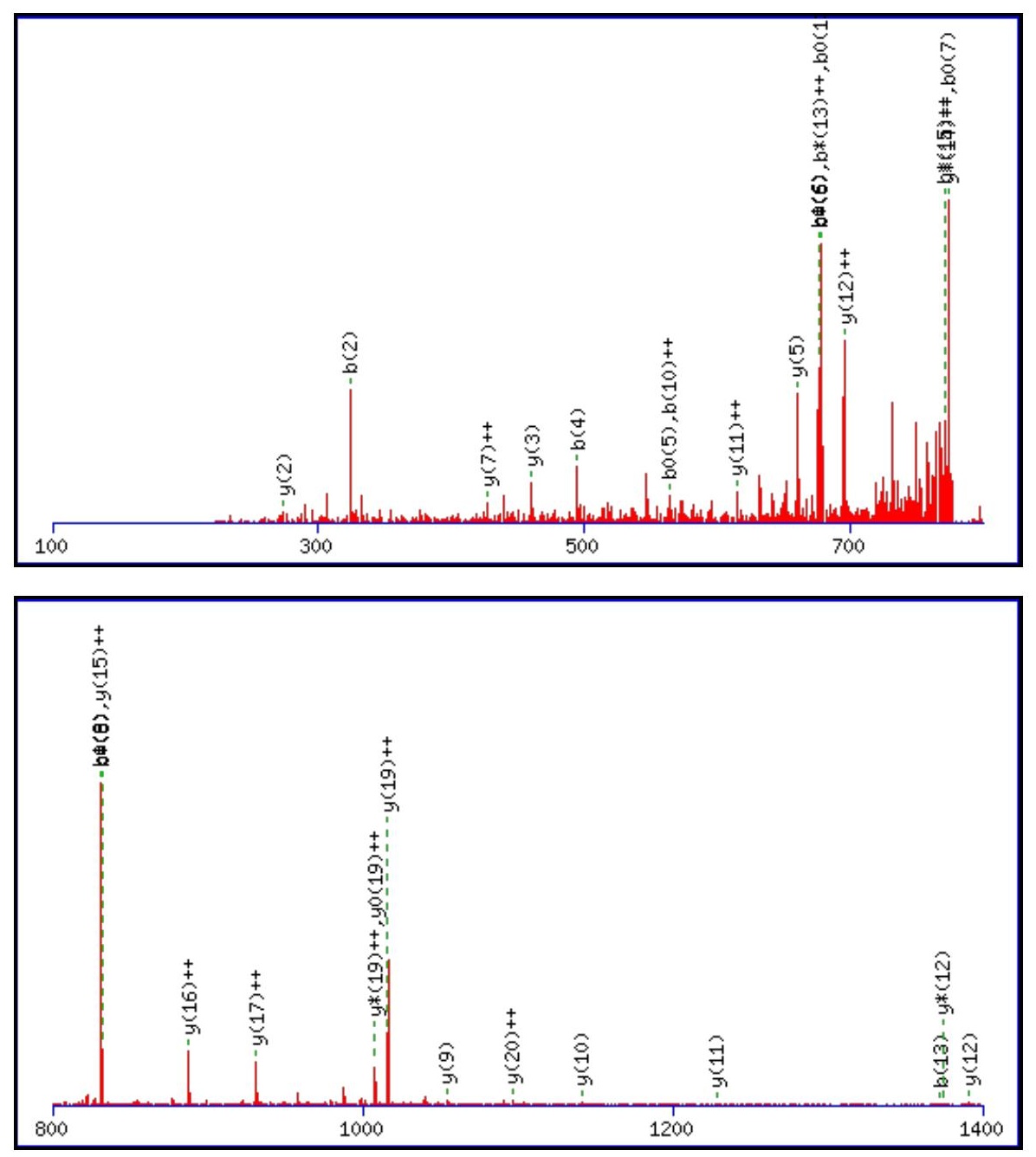


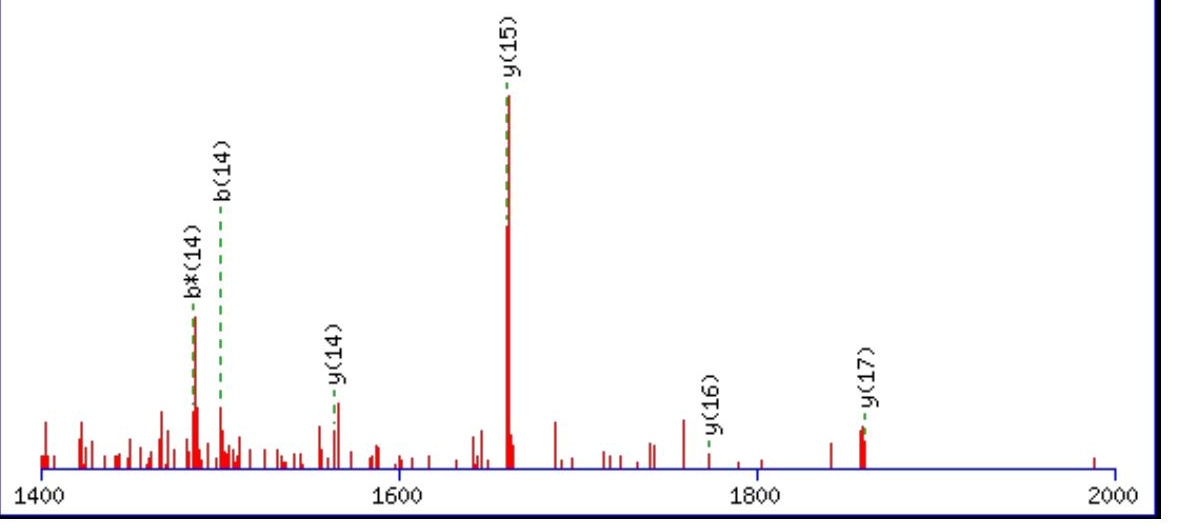

Monoisotopic mass of neutral peptide $\operatorname{Mr}($ calc): 2354.0386

Fixed modifications: Carbamidomethyl (C)

Variable modifications:

N9: Deamidated $\mathrm{N}(\mathrm{N})$

Ions Score: 51 Expect: 0.0025

Matches (Bold Red): 38/218 fragment ions using 78 most intense peaks

\begin{tabular}{|c|c|c|c|c|c|c|c|c|c|c|c|c|c|c|}
\hline \# & b & $\mathbf{b}^{++}$ & b* & $\mathbf{b}^{*^{++}}$ & $\mathbf{b}^{\mathbf{0}}$ & $\mathbf{b}^{0++}$ & Seq. & $\mathbf{y}$ & $y^{++}$ & $\mathbf{y}^{*}$ & $\mathbf{y}^{*^{++}}$ & $\mathbf{y}^{0}$ & $\mathbf{y}^{\mathbf{0 + +}}$ & \# \\
\hline 1 & 164.0706 & 82.5389 & & & & & $\mathbf{Y}$ & & & & & & & 21 \\
\hline 2 & 324.1013 & 162.5543 & & & & & C & 2191.9825 & 1096.4949 & 2174.9560 & 1087.9816 & 2173.9720 & 1087.4896 & 20 \\
\hline 3 & 381.1227 & 191.0650 & & & & & G & 2031.9519 & 1016.4796 & 2014.9253 & 1007.9663 & 2013.9413 & 1007.4743 & 19 \\
\hline 4 & 495.1656 & 248.0865 & 478.1391 & 239.5732 & & & $\mathbf{N}$ & 1974.9304 & 987.9688 & 1957.9039 & 979.4556 & 1956.9199 & 978.9636 & 18 \\
\hline 5 & 582.1977 & 291.6025 & 565.1711 & 283.0892 & 564.1871 & 282.5972 & S & 1860.8875 & 930.9474 & 1843.8609 & 922.4341 & 1842.8769 & 921.9421 & 17 \\
\hline 6 & 695.2817 & 348.1445 & 678.2552 & 339.6312 & 677.2712 & 339.1392 & $\mathbf{L}$ & 1773.8555 & 887.4314 & 1756.8289 & 878.9181 & 1755.8449 & 878.4261 & 16 \\
\hline 7 & 792.3345 & 396.6709 & 775.3080 & 388.1576 & 774.3239 & 387.6656 & $\mathbf{P}$ & 1660.7714 & 830.8893 & 1643.7449 & 822.3761 & 1642.7608 & 821.8841 & 15 \\
\hline 8 & 849.3560 & 425.1816 & 832.3294 & 416.6683 & 831.3454 & 416.1763 & G & 1563.7186 & 782.3630 & 1546.6921 & 773.8497 & 1545.7081 & 773.3577 & 14 \\
\hline 9 & 964.3829 & 482.6951 & 947.3564 & 474.1818 & 946.3723 & 473.6898 & $\mathbf{N}$ & 1506.6972 & 753.8522 & 1489.6706 & 745.3390 & 1488.6866 & 744.8469 & 13 \\
\hline 10 & 1127.4462 & 564.2268 & 1110.4197 & 555.7135 & 1109.4357 & 555.2215 & $\mathbf{Y}$ & 1391.6702 & 696.3388 & 1374.6437 & 687.8255 & 1373.6597 & 687.3335 & 12 \\
\hline 11 & 1214.4783 & 607.7428 & 1197.4517 & 599.2295 & 1196.4677 & 598.7375 & $\mathrm{~S}$ & 1228.6069 & 614.8071 & 1211.5804 & 606.2938 & 1210.5963 & 605.8018 & 11 \\
\hline 12 & 1301.5103 & 651.2588 & 1284.4837 & 642.7455 & 1283.4997 & 642.2535 & $S$ & 1141.5749 & 571.2911 & 1124.5483 & 562.7778 & 1123.5643 & 562.2858 & 10 \\
\hline 13 & 1372.5474 & 686.7773 & 1355.5209 & 678.2641 & 1354.5368 & 677.7721 & A & 1054.5429 & 527.7751 & 1037.5163 & 519.2618 & 1036.5323 & 518.7698 & 9 \\
\hline 14 & 1501.5900 & 751.2986 & 1484.5634 & 742.7854 & 1483.5794 & 742.2934 & $\mathbf{E}$ & 983.5057 & 492.2565 & 966.4792 & 483.7432 & 965.4952 & 483.2512 & 8 \\
\hline 15 & 1558.6115 & 779.8094 & 1541.5849 & 771.2961 & 1540.6009 & 770.8041 & G & 854.4631 & 427.7352 & 837.4366 & 419.2219 & 836.4526 & 418.7299 & 7 \\
\hline 16 & 1695.6704 & 848.3388 & 1678.6438 & 839.8255 & 1677.6598 & 839.3335 & $\mathbf{H}$ & 797.4417 & 399.2245 & 780.4151 & 390.7112 & 779.4311 & 390.2192 & 6 \\
\hline 17 & 1782.7024 & 891.8548 & 1765.6758 & 883.3416 & 1764.6918 & 882.8496 & S & 660.3828 & 330.6950 & 643.3562 & 322.1817 & 642.3722 & 321.6897 & 5 \\
\hline 18 & 1895.7865 & 948.3969 & 1878.7599 & 939.8836 & 1877.7759 & 939.3916 & $\mathbf{L}$ & 573.3507 & 287.1790 & 556.3242 & 278.6657 & & & 4 \\
\hline 19 & 2081.8658 & 1041.4365 & 2064.8392 & 1032.9233 & 2063.8552 & 1032.4312 & $\mathbf{W}$ & 460.2667 & 230.6370 & 443.2401 & 222.1237 & & & 3 \\
\hline 20 & 2180.9342 & 1090.9707 & 2163.9076 & 1082.4575 & 2162.9236 & 1081.9654 & $\mathbf{V}$ & 274.1874 & 137.5973 & 257.1608 & 129.0840 & & & 2 \\
\hline 21 & & & & & & & $\mathbf{R}$ & 175.1190 & 88.0631 & 158.0924 & 79.5498 & & & 1 \\
\hline
\end{tabular}
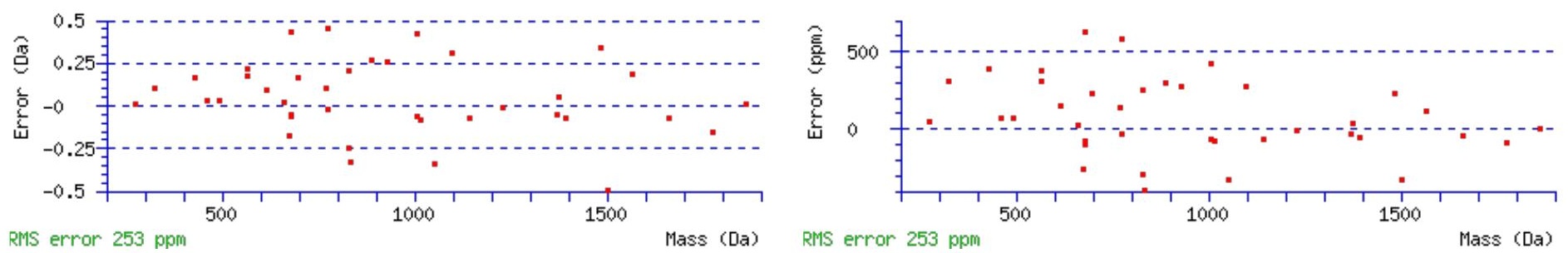

\section{All matches to this query}

Score $\operatorname{Mr}$ (calc): Delta

Sequence 


\begin{tabular}{|l|l|l|l|}
50.8 & 2354.0386 & 0.0016 & YCGNSLPGNYSSAEGHSLWVR \\
\hline 20.8 & 2354.0386 & 0.0016 & YCGNSLPGNYSSAEGHSLWVR \\
\hline 20.8 & 2353.0546 & 0.9856 & YCGNSLPGNYSSAEGHSLWVR \\
\hline 11.0 & 2352.0110 & 2.0292 & SGAVQADPGLLGYSVKADEMGK \\
\hline 4.7 & 2352.0432 & 1.9969 & ARFSVMDTGIPWTMPQTYR \\
\hline 4.7 & 2352.0432 & 1.9969 & ARFSVMDTGIPWTMPQTYR \\
\hline 2.8 & 2352.0324 & 2.0077 & RHGETLGWPEYTWTPNTAR \\
\hline 2.5 & 2354.0320 & 0.0082 & HNASSLLSPEGFFLGKMGFR \\
\hline 2.5 & 2354.0320 & 0.0082 & HNASSLLSPEGFFLGKMGFR \\
\hline 2.5 & 2354.0320 & 0.0082 & HNASSLLSPEGFFLGKMGFR \\
\hline
\end{tabular}

Spectrum No: 663; Query: 129; Rank: 1

\section{Peptide View}

MS/MS Fragmentation of NQTLENLQR

Found in IPI00199695, Tax_Id=10116 Gene_Symbol=Serpinf2 Serine (Or cysteine) peptidase inhibitor, clade F, member 2

Match to Query 129: 1115.553828 from(558.784190,2+)

Title: 091008RatKidney_NH4Format01_30.745.745.2.dta

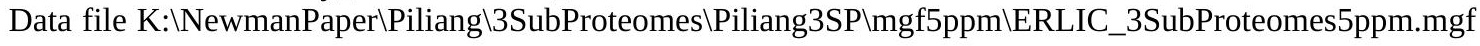
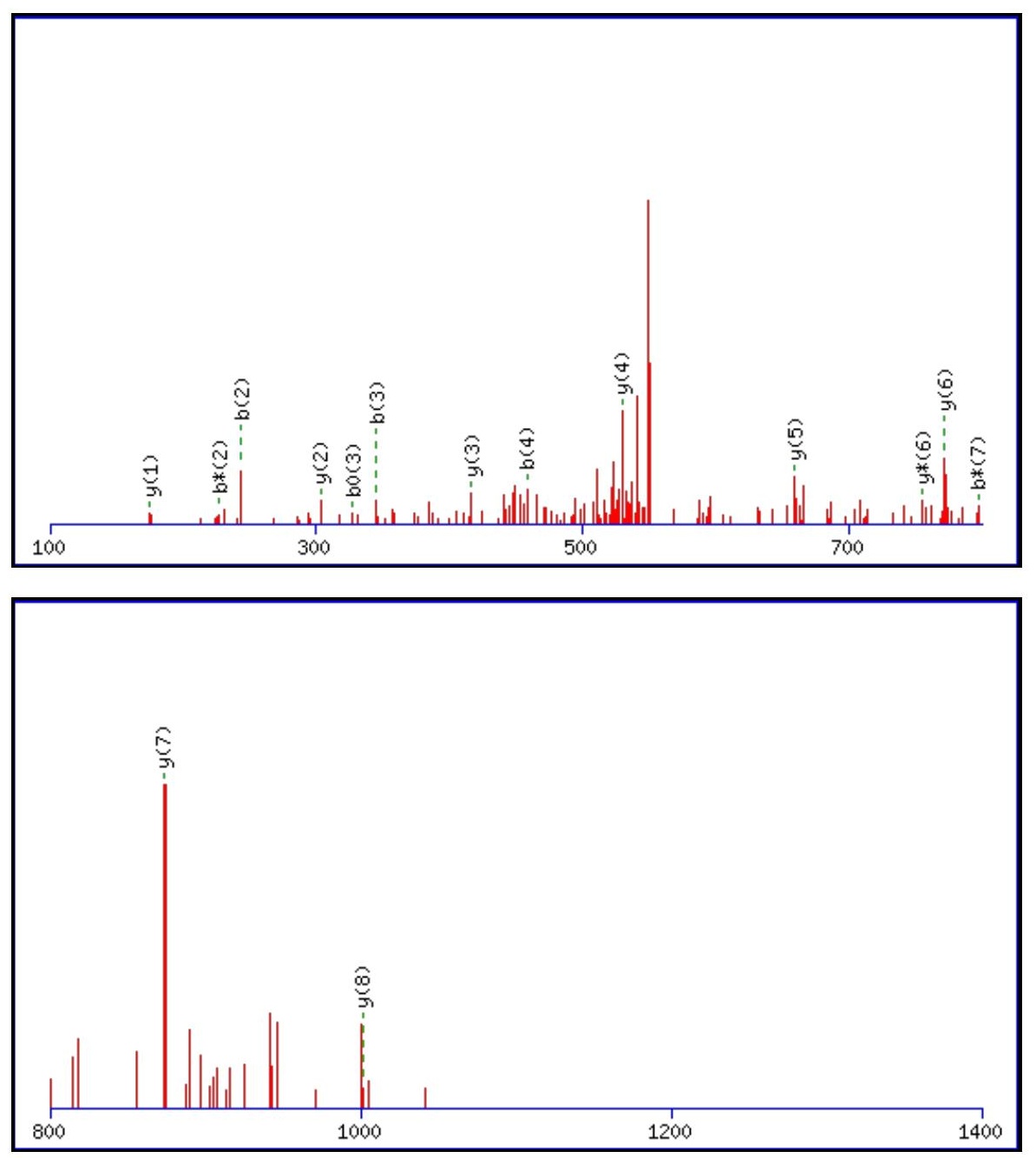


$1400 \quad 1600 \quad 1800 \quad 2000$

Monoisotopic mass of neutral peptide $\operatorname{Mr}($ calc): 1115.5571

Fixed modifications: Carbamidomethyl (C)

Variable modifications:

N1 : Deamidated_N (N)

Ions Score: 51 Expect: 0.00085

Matches (Bold Red): 15/84 fragment ions using 41 most intense peaks

\begin{tabular}{|c|c|c|c|c|c|c|c|c|c|c|c|c|c|c|}
\hline$\#$ & $\mathbf{b}$ & $\mathbf{b}^{++}$ & $\mathbf{b}^{*}$ & $\mathbf{b}^{\boldsymbol{*}^{++}}$ & $\mathbf{b}^{\mathbf{0}}$ & $\mathbf{b}^{\mathbf{0 + +}}$ & $\mathbf{S e q} \cdot$ & $\mathbf{y}$ & $\mathbf{y}^{++}$ & $\mathbf{y}^{*}$ & $\mathbf{y}^{\mathbf{*}^{++}}$ & $\mathbf{y}^{\mathbf{0}}$ & $\mathbf{y}^{\mathbf{0 + +}}$ & $\#$ \\
\hline $\mathbf{1}$ & $\mathbf{1 1 6 . 0 3 4 2}$ & 58.5207 & 99.0077 & 50.0075 & & & $\mathbf{N}$ & & & & & & & $\mathbf{9}$ \\
\hline $\mathbf{2}$ & $\mathbf{2 4 4 . 0 9 2 8}$ & 122.5500 & 227.0662 & 114.0368 & & & $\mathbf{Q}$ & $\mathbf{1 0 0 1 . 5 3 7 4}$ & 501.2724 & 984.5109 & 492.7591 & 983.5269 & 492.2671 & $\mathbf{8}$ \\
\hline $\mathbf{3}$ & $\mathbf{3 4 5 . 1 4 0 5}$ & 173.0739 & 328.1139 & 164.5606 & 327.1299 & 164.0686 & $\mathbf{T}$ & $\mathbf{8 7 3 . 4 7 8 9}$ & 437.2431 & 856.4523 & 428.7298 & 855.4683 & 428.2378 & $\mathbf{7}$ \\
\hline $\mathbf{4}$ & $\mathbf{4 5 8 . 2 2 4 5}$ & 229.6159 & 441.1980 & 221.1026 & 440.2140 & 220.6106 & $\mathbf{L}$ & 772.4312 & 386.7192 & 755.4046 & 378.2060 & 754.4206 & 377.7139 & $\mathbf{6}$ \\
\hline $\mathbf{5}$ & 587.2671 & 294.1372 & 570.2406 & 285.6239 & 569.2566 & 285.1319 & $\mathbf{E}$ & $\mathbf{6 5 9 . 3 4 7 1}$ & 330.1772 & 642.3206 & 321.6639 & 641.3365 & 321.1719 & $\mathbf{5}$ \\
\hline $\mathbf{6}$ & 701.3101 & 351.1587 & 684.2835 & 342.6454 & 683.2995 & 342.1534 & $\mathbf{N}$ & $\mathbf{5 3 0 . 3 0 4 5}$ & 265.6559 & 513.2780 & 257.1426 & & & $\mathbf{4}$ \\
\hline $\mathbf{7}$ & 814.3941 & 407.7007 & $7 \mathbf{9 7 . 3 6 7 6}$ & 399.1874 & 796.3836 & 398.6954 & $\mathbf{L}$ & $\mathbf{4 1 6 . 2 6 1 6}$ & 208.6344 & 399.2350 & 200.1212 & & & $\mathbf{3}$ \\
\hline $\mathbf{8}$ & 942.4527 & 471.7300 & 925.4261 & 463.2167 & 924.4421 & 462.7247 & $\mathbf{Q}$ & $\mathbf{3 0 3 . 1 7 7 5}$ & 152.0924 & 286.1510 & 143.5791 & & & $\mathbf{2}$ \\
\hline $\mathbf{9}$ & & & & & & & $\mathbf{R}$ & $\mathbf{1 7 5 . 1 1 9 0}$ & 88.0631 & 158.0924 & 79.5498 & & & $\mathbf{1}$ \\
\hline
\end{tabular}
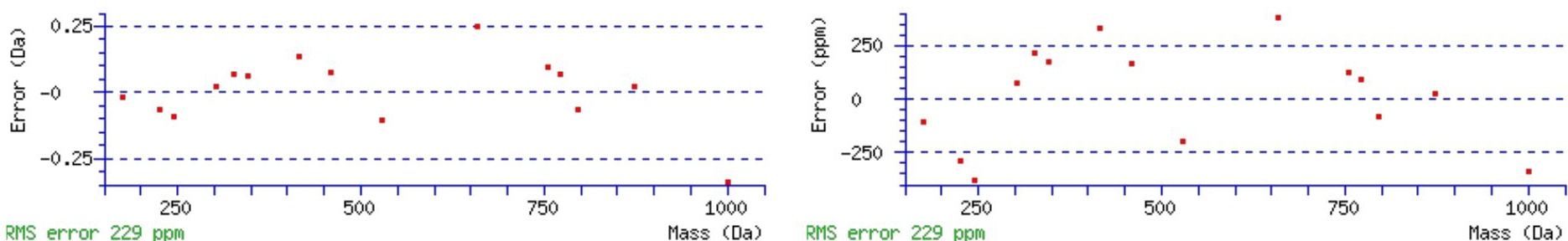

All matches to this query

\begin{tabular}{|l|l|l|l|}
\hline Score & Mr(calc): & Delta & \multicolumn{1}{|c|}{ Sequence } \\
\hline 50.7 & 1115.5571 & -0.0032 & NQTLENLQR \\
\hline 12.6 & 1115.5571 & -0.0032 & NQTLENLQR \\
\hline 9.4 & 1114.5407 & 1.0131 & DDIIEFAHR \\
\hline 9.2 & 1114.5506 & 1.0032 & NLDEQLSAPK \\
\hline 9.1 & 1114.5536 & 1.0003 & NKINVYKR \\
\hline 8.9 & 1114.5519 & 1.0019 & NFVSHLNQR \\
\hline 7.9 & 1115.5529 & 0.0010 & KYWAITVR \\
\hline 7.3 & 1115.5571 & -0.0033 & ANSANTNTVPK \\
\hline 7.2 & 1115.5472 & 0.0066 & GWRGNELQR \\
\hline 7.1 & 1115.5458 & 0.0080 & $\underline{\text { IIDENNLQR }}$ \\
\hline
\end{tabular}




\section{Peptide View}

MS/MS Fragmentation of LSREEGTSLSLENLSLNPYEGLLLQFPFVA

Found in IPI00211616, Tax_Id=10116 Gene_Symbol=Slc3a2 4F2 cell-surface antigen heavy chain

Match to Query 2666: 3336.713202 from(1113.245010,3+)

Title: 091008RatKidney_NH4Format01_06.5773.5773.3.dta

Data file K:\NewmanPaper \Piliang \3SubProteomes \Piliang3SP \mgf5ppm\ERLIC_3SubProteomes5ppm.mgf
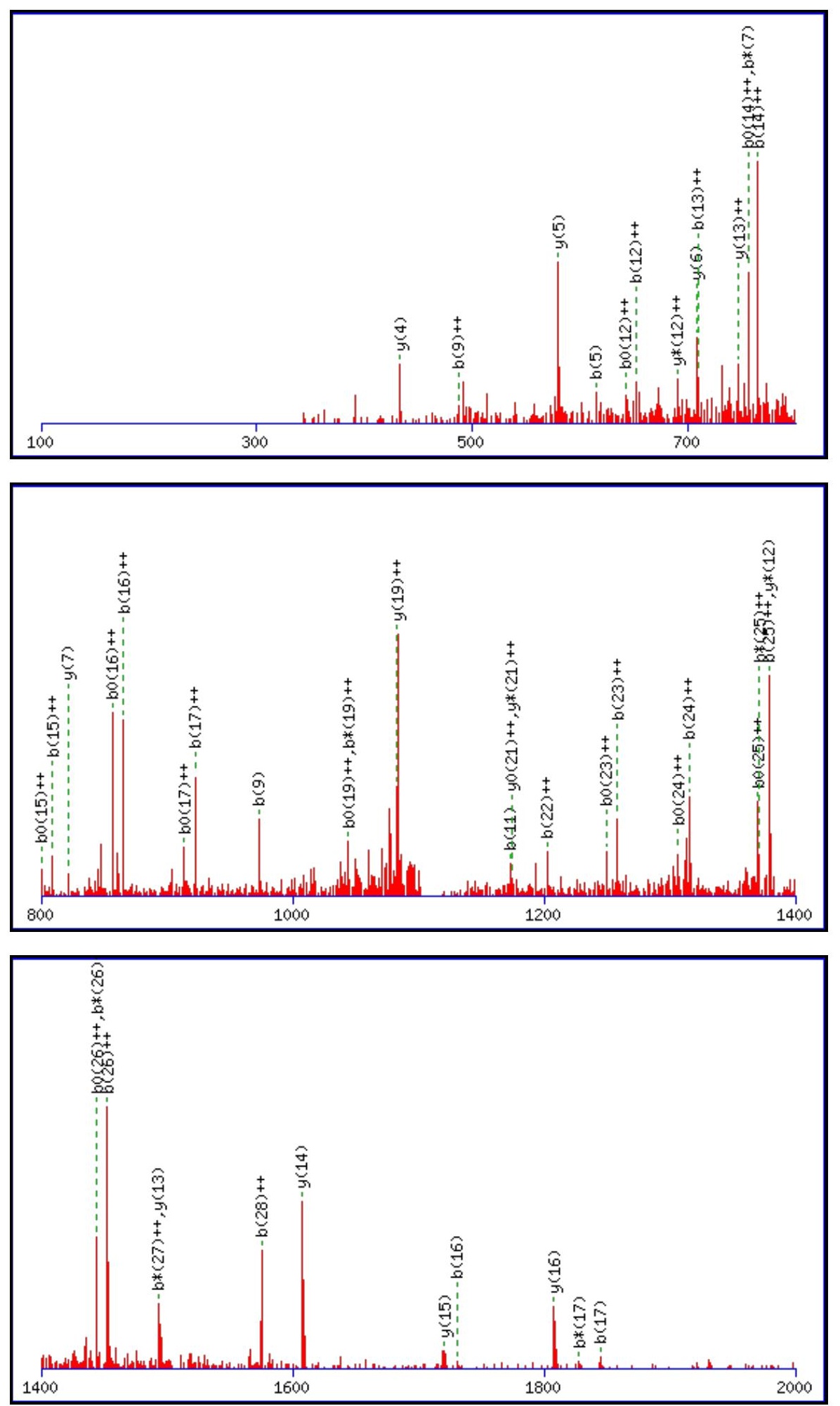

Monoisotopic mass of neutral peptide $\operatorname{Mr}($ calc): 3336.7129

Fixed modifications: Carbamidomethyl (C)

Variable modifications: 


\begin{tabular}{|c|c|c|c|c|c|c|c|c|c|c|c|c|c|c|}
\hline \# & b & $\mathbf{b}^{++}$ & $\mathbf{b}^{*}$ & $\mathbf{b}^{*^{++}}$ & $\mathbf{b}^{0}$ & $\mathbf{b}^{0++}$ & Seq. & $\mathbf{y}$ & $y^{++}$ & $\mathbf{y}^{*}$ & $\mathrm{y}^{\mathrm{*}^{++}}$ & $\mathbf{y}^{0}$ & $y^{0++}$ & $\#$ \\
\hline 1 & 114.0913 & 57.5493 & & & & & $\mathbf{L}$ & & & & & & & 30 \\
\hline 2 & 201.1234 & 101.0653 & & & 183.1128 & 92.0600 & $S$ & 3224.6361 & 1612.8217 & 3207.6096 & 1604.3084 & 3206.6256 & 1603.8164 & 29 \\
\hline 3 & 357.2245 & 179.1159 & 340.1979 & 170.6026 & 339.2139 & 170.1106 & $\mathbf{R}$ & 3137.6041 & 1569.3057 & 3120.5776 & 1560.7924 & 3119.5936 & 1560.3004 & 28 \\
\hline 4 & 486.2671 & 243.6372 & 469.2405 & 235.1239 & 468.2565 & 234.6319 & $\mathbf{E}$ & 2981.5030 & 1491.2551 & 2964.4765 & 1482.7419 & 2963.4924 & 1482.2499 & 27 \\
\hline 5 & 615.3097 & 308.1585 & 598.2831 & 299.6452 & 597.2991 & 299.1532 & $\mathbf{E}$ & 2852.4604 & 1426.7338 & 2835.4339 & 1418.2206 & 2834.4498 & 1417.7286 & 26 \\
\hline 6 & 672.3311 & 336.6692 & 655.3046 & 328.1559 & 654.3206 & 327.6639 & G & 2723.4178 & 1362.2125 & 2706.3913 & 1353.6993 & 2705.4073 & 1353.2073 & 25 \\
\hline 7 & 773.3788 & 387.1930 & 756.3523 & 378.6798 & 755.3682 & 378.1878 & $T$ & 2666.3964 & 1333.7018 & 2649.3698 & 1325.1885 & 2648.3858 & 1324.6965 & 24 \\
\hline 8 & 860.4108 & 430.7091 & 843.3843 & 422.1958 & 842.4003 & 421.7038 & $S$ & 2565.3487 & 1283.1780 & 2548.3221 & 1274.6647 & 2547.3381 & \begin{tabular}{|l|l}
1274.1727 \\
\end{tabular} & 23 \\
\hline 9 & 973.4949 & 487.2511 & 956.4684 & 478.7378 & 955.4843 & 478.2458 & $\mathbf{L}$ & 2478.3166 & 1239.6620 & 2461.2901 & 1231.1487 & 2460.3061 & 1230.6567 & 22 \\
\hline 10 & 1060.5269 & 530.7671 & 1043.5004 & 522.2538 & 1042.5164 & 521.7618 & $\mathrm{~S}$ & 2365.2326 & 1183.1199 & 2348.2060 & 1174.6067 & 2347.2220 & 1174.1146 & 21 \\
\hline 11 & 1173.6110 & 587.3091 & 1156.5844 & 578.7959 & 1155.6004 & 578.3039 & $\mathbf{L}$ & 2278.2006 & 1139.6039 & 2261.1740 & 1131.0906 & 2260.1900 & 1130.5986 & 20 \\
\hline 12 & 1302.6536 & 651.8304 & 1285.6270 & 643.3172 & 1284.6430 & 642.8251 & $\mathbf{E}$ & 2165.1165 & 1083.0619 & 2148.0899 & 1074.5486 & 2147.1059 & 1074.0566 & 19 \\
\hline 13 & 1417 & 709. & 1400. & & 1399. & & $\mathbf{N}$ & 2036.0 & 1018.5406 & 2019.0474 & 1010.0273 & 5633 & 1009.5353 & 18 \\
\hline 14 & 1530.7646 & 765.8859 & 1513.7380 & 757.3727 & 1512.7540 & 756.8806 & $\mathbf{L}$ & 1921.0470 & 961.0271 & 1904.0204 & 952.5138 & 1903.0364 & 952.0218 & 17 \\
\hline 15 & 1617.7966 & 809.4019 & 1600.7701 & 800.8887 & 1599.7860 & 800.3967 & $S$ & 1807.9629 & 904.4851 & 1790.9364 & 895.9718 & 1789.9523 & 895.4798 & 16 \\
\hline 16 & 1730.8 & 865. & 1713.8541 & 857.4307 & 1712.8701 & 856.9387 & $\mathbf{L}$ & 1720.9309 & 860.9691 & 1703.9043 & 852.4558 & 1702.9203 & 851.9638 & 15 \\
\hline 17 & \begin{tabular}{|l|}
1844.9236 \\
\end{tabular} & 922.9654 & 1827.8971 & 914.4522 & 1826.9130 & 913.9602 & $\mathbf{N}$ & \begin{tabular}{|l|}
1607.8468 \\
\end{tabular} & 804.4270 & 1590.8203 & 795.9138 & 1589.8362 & 795.4218 & 14 \\
\hline 18 & 1941.9764 & 971.4918 & 1924.9498 & 962.9785 & 1923.9658 & 962.4865 & $\mathbf{P}$ & 1493.8039 & 747.4056 & 1476.7773 & 738.8923 & 1475.7933 & 738.4003 & 13 \\
\hline 192 & 2105.0397 & 1053.0235 & 2088.0131 & 1044.5102 & 2087.0291 & \begin{tabular}{|l|}
1044.0182 \\
\end{tabular} & $\mathbf{Y}$ & 1396.7511 & 698.8792 & 1379.7246 & 690.3659 & 1378.7406 & 689.8739 & 12 \\
\hline 20 & 2234.0823 & 1117.5448 & 2217.0557 & 1109.0315 & 2216.0717 & 1108.5395 & $\mathbf{E}$ & 1233.6878 & 617.3475 & 1216.6612 & 608.8343 & 1215.6772 & 608.3422 & 11 \\
\hline 212 & 2291.1038 & 1146.0555 & 2274.0772 & 1137.5422 & 2273.0932 & 1137.0502 & G & 1104.6452 & 552.8262 & 1087.6186 & 544.3130 & & & 10 \\
\hline 22 & 2404.1878 & \begin{tabular}{|l|}
1202.5975 \\
\end{tabular} & 2387.1613 & 1194.0843 & 2386.1773 & 1193.5923 & $\mathbf{L}$ & 1047.6237 & 524.3155 & 1030.5972 & 515.8022 & & & 9 \\
\hline 23 & 2517.2719 & 1259.1396 & 2500.2453 & 1250.6263 & 2499.2613 & 1250.1343 & $\mathbf{L}$ & 934.5397 & 467.7735 & 917.5131 & 459.2602 & & & 8 \\
\hline $24:$ & 2630.3559 & \begin{tabular}{|l|}
1315.6816 \\
\end{tabular} & 2613.3294 & 1307.1683 & 2612.3454 & \begin{tabular}{|l|}
1306.6763 \\
\end{tabular} & $\mathbf{L}$ & 821.4556 & 411.2314 & 804.4291 & 402.7182 & & & 7 \\
\hline 25 & 2758.4145 & 1379.7109 & 2741.3880 & 1371.1976 & 2740.4040 & 1370.7056 & $\mathbf{Q}$ & 708.3715 & 354.6894 & 691.3450 & 346.1761 & & & 6 \\
\hline 26 & 2905.4829 & 1453.2451 & 2888.4564 & 1444.7318 & 2887.4724 & 1444.2398 & $\mathbf{F}$ & 580.3130 & 290.6601 & & & & & 5 \\
\hline 27 & 3002.5357 & 1501.7715 & 2985.5092 & \begin{tabular}{|l|}
1493.2582 \\
\end{tabular} & 2984.5251 & 1492.7662 & $\mathbf{P}$ & 433.2445 & 217.1259 & & & & & 4 \\
\hline 28 & 3149.6041 & \begin{tabular}{|l|}
1575.3057 \\
\end{tabular} & 3132.5776 & 1566.7924 & 3131.5936 & 1566.3004 & $\mathbf{F}$ & 336.1918 & 168.5995 & & & & & 3 \\
\hline 29 & 3248.6725 & 1624.8399 & 3231.6460 & 1616.3266 & 3230.6620 & 1615.8346 & $\mathrm{~V}$ & 189.1234 & 95.0653 & & & & & 2 \\
\hline 30 & & & & & & & A & 90.0550 & 45.5311 & & & & & 1 \\
\hline
\end{tabular}
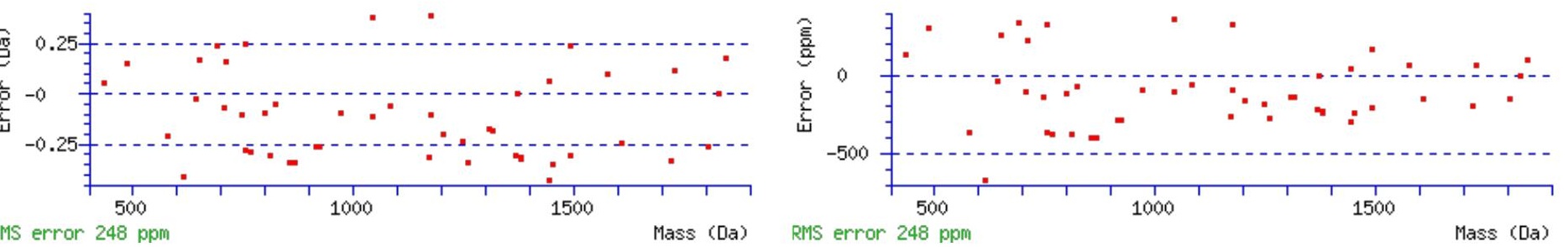

\section{All matches to this query}

\begin{tabular}{|l|c|c|l|}
\hline Score & Mr(calc): & Delta & \multicolumn{1}{c|}{ Sequence } \\
\hline 50.7 & 3336.7129 & 0.0003 & LSREEGTSLSLENLSLNPYEGLLLQFPFVA \\
\hline 43.4 & 3336.7129 & 0.0003 & LSREEGTSLSLENLSLNPYEGLLLQFPFVA \\
\hline 42.6 & 3335.7289 & 0.9843 & LSREEGTSLSLENLSLNPYEGLLLQFPFVA \\
\hline 13.0 & 3335.6919 & 1.0213 & DIYDQKLTLQPVDNSTISLQMGTNKVASQK \\
\hline 2.2 & 3335.6919 & 1.0213 & DIYDQKLTLQPVDNSTISLQMGTNKVASOK \\
\hline 1.1 & 3334.7079 & 2.0053 & DIYDQKLTLQPVDNSTISLQMGTNKVASQK \\
\hline
\end{tabular}


Spectrum No: 665; Query: 1031; Rank: 1

\section{Peptide View}

\section{MS/MS Fragmentation of VNYTEVAGTCNLWR}

Found in IPI00471757, Tax_Id=10116 Gene_Symbol=Naga Alpha-N-acetylgalactosaminidase precursor

Match to Query 1031: 1682.768708 from(842.391630,2+)

Title: 091008RatKidney_NH4Format01_23.2788.2788.2.dta

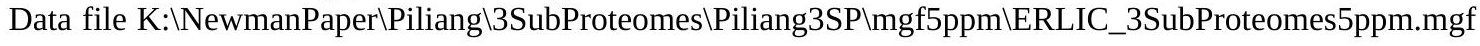
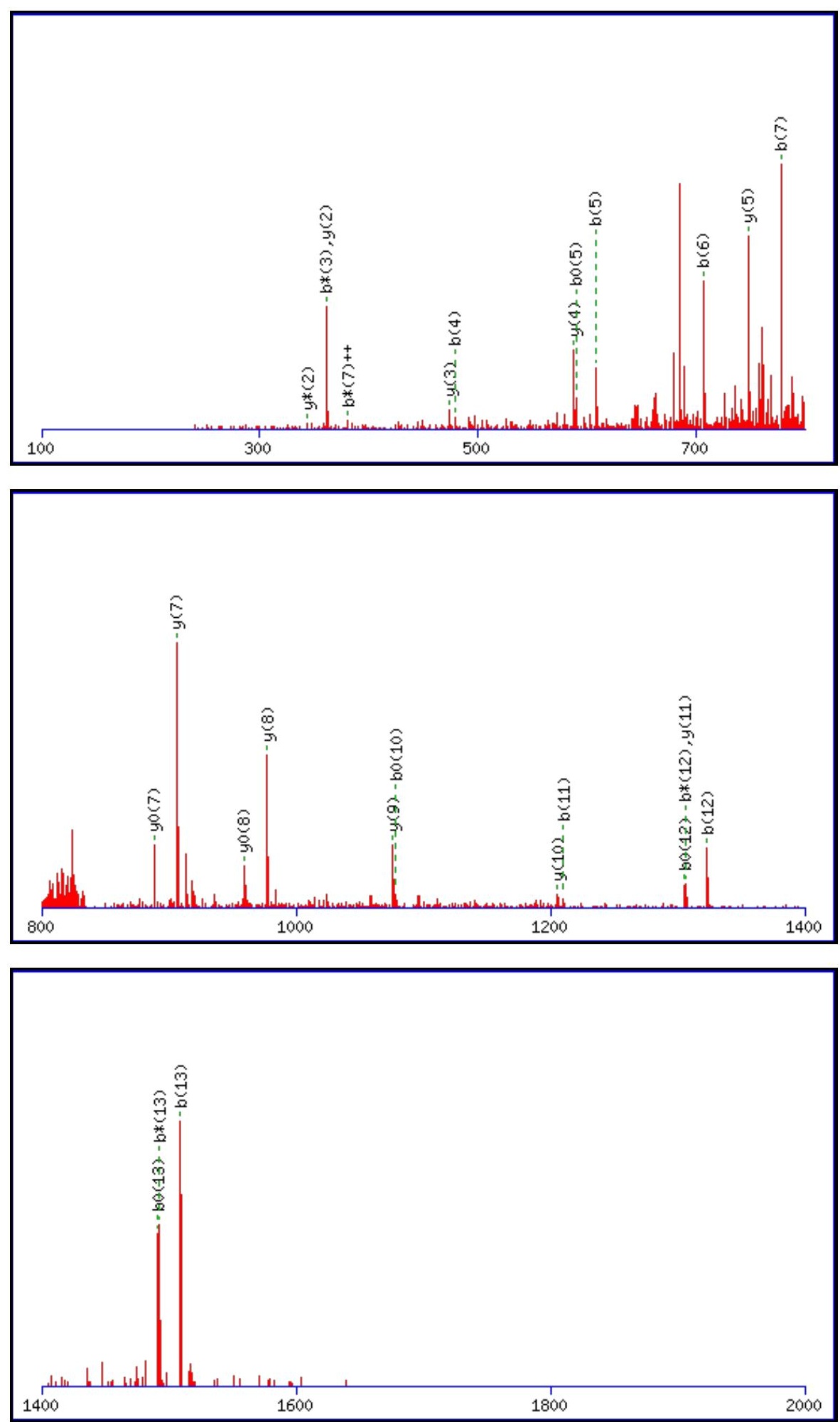
Monoisotopic mass of neutral peptide $\operatorname{Mr}($ calc): 1682.7722

Fixed modifications: Carbamidomethyl (C)

Variable modifications:

N2 : Deamidated $\mathrm{N}(\mathrm{N})$

Ions Score: 51 Expect: 0.0016

Matches (Bold Red): 27/138 fragment ions using 59 most intense peaks

\begin{tabular}{|c|c|c|c|c|c|c|c|c|c|c|c|c|c|c|}
\hline \# & b & $\mathbf{b}^{++}$ & $\mathbf{b}^{*}$ & $\mathbf{b}^{*^{++}}$ & $\mathbf{b}^{\mathbf{0}}$ & $\mathbf{b}^{\mathbf{0 + +}}$ & Seq. & $\mathbf{y}$ & $\mathbf{y}^{++}$ & $\mathbf{y}^{*}$ & $\mathrm{y}^{*^{++}}$ & $\mathbf{y}^{\mathbf{0}}$ & $\mathbf{y}^{0++}$ & $\#$ \\
\hline 1 & 100.0757 & 50.5415 & & & & & V & & & & & & & 14 \\
\hline 2 & 215.1026 & 108.0550 & 198.0761 & 99.5417 & & & $\mathbf{N}$ & 1584.7111 & 792.8592 & 1567.6846 & 784.3459 & 1566.7005 & 783.8539 & 13 \\
\hline 3 & 378.1660 & 189.5866 & 361.1394 & 181.0733 & & & $\mathbf{Y}$ & 1469.6842 & 735.3457 & 1452.6576 & 726.8325 & 1451.6736 & 726.3404 & 12 \\
\hline 4 & 479.2136 & 240.1105 & 462.1871 & 231.5972 & 461.2031 & 231.1052 & $\mathbf{T}$ & 1306.6208 & 653.8141 & 1289.5943 & 645.3008 & 1288.6103 & 644.8088 & 11 \\
\hline 5 & 608.2562 & 304.6318 & 591.2297 & 296.1185 & 590.2457 & 295.6265 & $\mathbf{E}$ & 1205.5732 & 603.2902 & 1188.5466 & 594.7769 & 1187.5626 & 594.2849 & 10 \\
\hline 6 & 707.3246 & 354.1660 & 690.2981 & 345.6527 & 689.3141 & 345.1607 & $\mathrm{~V}$ & 1076.5306 & 538.7689 & 1059.5040 & 530.2557 & 1058.5200 & 529.7636 & 9 \\
\hline 7 & 778.3618 & 389.6845 & 761.3352 & 381.1712 & 760.3512 & 380.6792 & A & 977.4622 & 489.2347 & 960.4356 & 480.7214 & 959.4516 & 480.2294 & 8 \\
\hline 8 & 835.3832 & 418.1952 & 818.3567 & 409.6820 & 817.3727 & 409.1900 & G & 906.4250 & 453.7162 & 889.3985 & 445.2029 & 888.4145 & 444.7109 & 7 \\
\hline 9 & 936.4309 & 468.7191 & 919.4043 & 460.2058 & 918.4203 & 459.7138 & $\mathbf{T}$ & 849.4036 & 425.2054 & 832.3770 & 416.6922 & 831.3930 & 416.2001 & 6 \\
\hline 10 & 1096.4615 & 548.7344 & 1079.4350 & 540.2211 & \begin{tabular}{|l|}
1078.4510 \\
\end{tabular} & 539.7291 & $\mathrm{C}$ & 748.3559 & 374.6816 & 731.3294 & 366.1683 & & & 5 \\
\hline 11 & 1210.5045 & 605.7559 & 1193.4779 & 597.2426 & 1192.4939 & 596.7506 & $\mathbf{N}$ & 588.3253 & 294.6663 & 571.2987 & 286.1530 & & & 2 \\
\hline 12 & 1323.5885 & 662.2979 & \begin{tabular}{|l}
1306.5620 \\
\end{tabular} & 653.7846 & \begin{tabular}{|l|}
1305.5780 \\
\end{tabular} & 653.2926 & $\mathbf{L}$ & 474.2823 & 237.6448 & 457.2558 & 229.1315 & & & 3 \\
\hline 13 & 1509.6679 & 755.3376 & 1492.6413 & 746.8243 & 1491.6573 & 746.3323 & W & 361.1983 & 181.1028 & 344.1717 & 172.5895 & & & 2 \\
\hline 14 & & & & & & & $\mathbf{R}$ & 175.1190 & 88.0631 & 158.0924 & 79.5498 & & & 1 \\
\hline
\end{tabular}
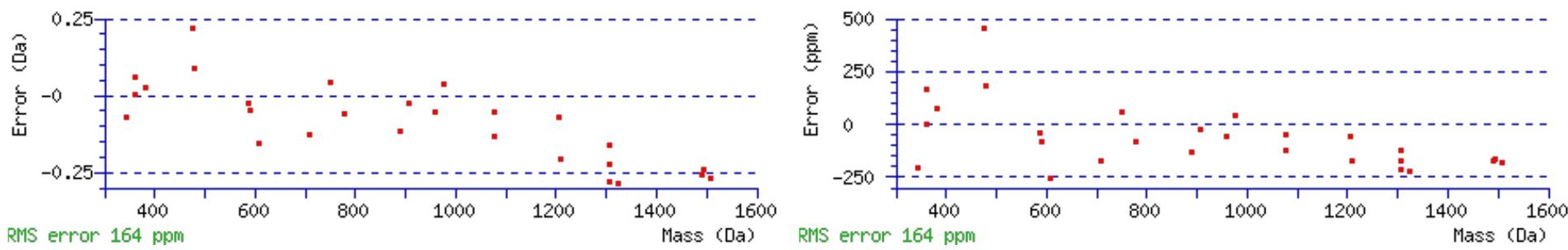

All matches to this query

\begin{tabular}{|l|l|l|l|}
\hline Score & Mr(calc): & Delta & \multicolumn{1}{c|}{ Sequence } \\
\hline 50.7 & 1682.7722 & -0.0035 & VNYTEVAGTCNLWR \\
\hline 21.6 & 1682.7722 & -0.0035 & VNYTEVAGTCNLWR \\
\hline 5.4 & 1682.7756 & -0.0069 & IVMNDPSYKENAMR \\
\hline 5.0 & 1680.7654 & 2.0033 & RLSGKNMEPNINAR \\
\hline 1.6 & 1682.7810 & -0.0123 & LALTLKVTGLGTTR \\
\hline 1.6 & 1682.7722 & -0.0035 & LTTIPNGAFVYLSK \\
\hline
\end{tabular}

Spectrum No: 666; Query: 2421; Rank: 1

\section{Peptide View}

MS/MS Fragmentation of QLITEEKIPNNTQWITWSQEGHK

Found in IPI00208422, Tax_Id=10116 Gene_Symbol=Dpp4 Dipeptidyl peptidase 4

Match to Query 2421: 2780.384232 from(927.802020,3+)

Title: 100101RatKid_NS_deglyco_19.3665.3665.3.dta

Data file K:INewmanPaper|Piliang|3SubProteomes\Piliang3SP\mgf5ppm\ERLIC_3SubProteomes5ppm.mgf 

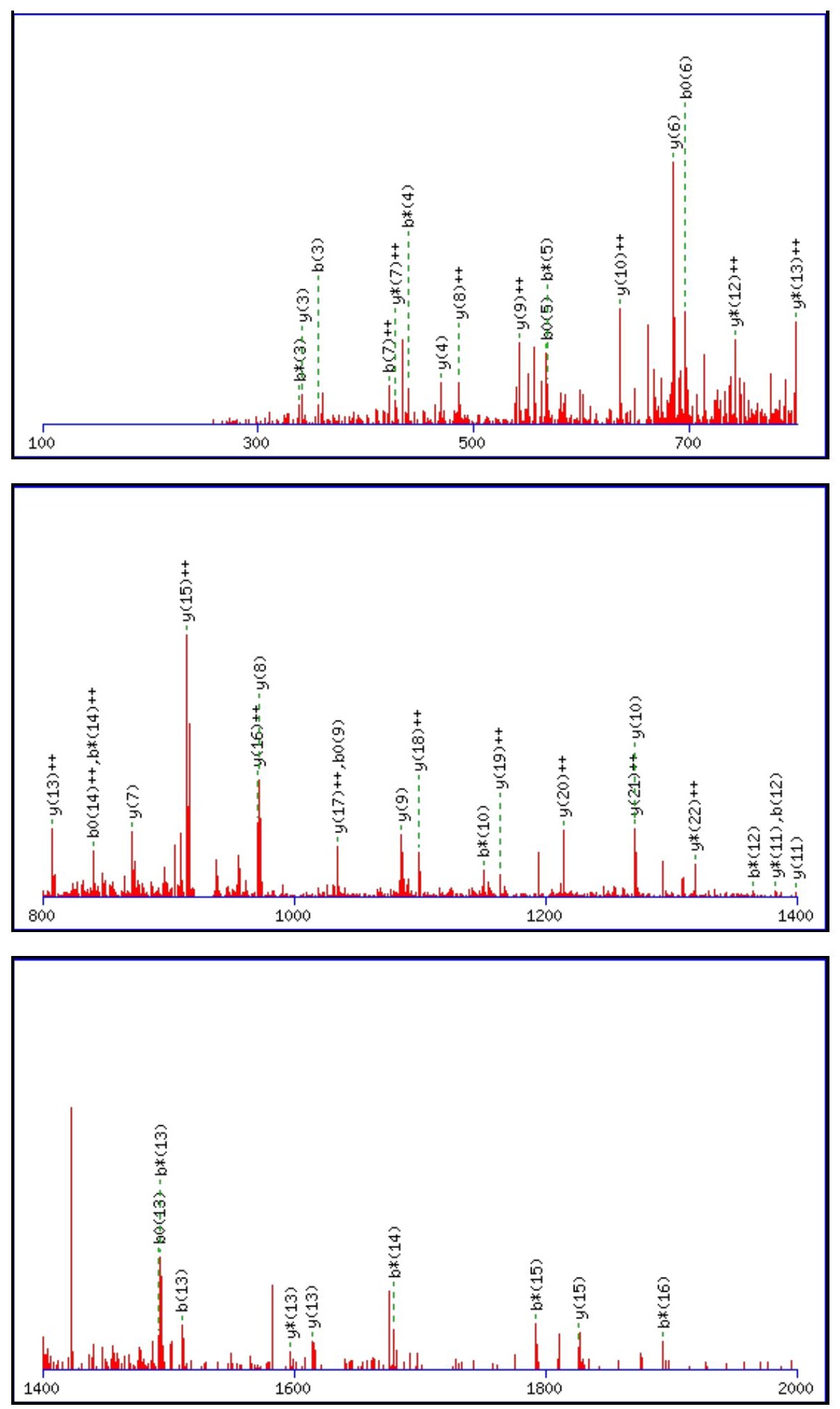

Monoisotopic mass of neutral peptide $\operatorname{Mr}($ calc): 2780.3769

Fixed modifications: Carbamidomethyl (C)

Variable modifications:

N10 : Deamidated_N (N)

Ions Score: 51 Expect: 0.0019

Matches (Bold Red): 46/252 fragment ions using 92 most intense peaks

\begin{tabular}{|c|c|c|c|c|c|c|c|c|c|c|c|c|c|c|}
\hline$\#$ & $\mathbf{b}$ & $\mathbf{b}^{++}$ & $\mathbf{b}^{*}$ & $\mathbf{b}^{\boldsymbol{*}^{++}}$ & $\mathbf{b}^{\mathbf{0}}$ & $\mathbf{b}^{\mathbf{0 + +}}$ & $\mathbf{S e q}$ & $\mathbf{y}$ & $\mathbf{y}^{++}$ & $\mathbf{y}^{\mathbf{*}}$ & $\mathbf{y}^{\mathbf{*}^{++}}$ & $\mathbf{y}^{\mathbf{0}}$ & $\mathbf{y}^{\mathbf{0 + +}}$ & $\#$ \\
\hline $\mathbf{1}$ & 129.0659 & 65.0366 & 112.0393 & 56.5233 & & & $\mathbf{Q}$ & & & & & & & $\mathbf{2 3}$ \\
\hline $\mathbf{2}$ & 242.1499 & 121.5786 & 225.1234 & 113.0653 & & & $\mathbf{L}$ & 2653.3257 & 1327.1665 & 2636.2991 & $\mathbf{1 3 1 8 . 6 5 3 2}$ & 2635.3151 & 1318.1612 & $\mathbf{2 2}$ \\
\hline $\mathbf{3}$ & $\mathbf{3 5 5 . 2 3 4 0}$ & 178.1206 & 338.2074 & 169.6074 & & & $\mathbf{I}$ & 2540.2416 & $\mathbf{1 2 7 0 . 6 2 4 4}$ & 2523.2150 & 1262.1112 & 2522.2310 & 1261.6191 & $\mathbf{2 1}$ \\
\hline $\mathbf{4}$ & 456.2817 & 228.6445 & $\mathbf{4 3 9 . 2 5 5 1}$ & 220.1312 & 438.2711 & 219.6392 & $\mathbf{T}$ & 2427.1575 & $\mathbf{1 2 1 4 . 0 8 2 4}$ & 2410.1310 & 1205.5691 & 2409.1470 & 1205.0771 & $\mathbf{2 0}$ \\
\hline $\mathbf{5}$ & 585.3243 & 293.1658 & $\mathbf{5 6 8 . 2 9 7 7}$ & 284.6525 & $\mathbf{5 6 7 . 3 1 3 7}$ & 284.1605 & $\mathbf{E}$ & 2326.1098 & $\mathbf{1 1 6 3 . 5 5 8 6}$ & 2309.0833 & 1155.0453 & 2308.0993 & 1154.5533 & $\mathbf{1 9}$ \\
\hline
\end{tabular}




\begin{tabular}{|c|c|c|c|c|c|c|c|c|c|c|c|c|c|c|}
\hline 6 & 714.3668 & 357.6871 & 697.3403 & 349.1738 & 696.3563 & & $\mathbf{E}$ & & 1099.0373 & |2180.0407| & 1090.5240 & |2179.0567 & $\mid$ & 18 \\
\hline 7 & 842.4618 & 421.7345 & 825.4353 & 413.2213 & 824.4512 & 412.7293 & $\mathbf{K}$ & 2068.0247 & 1034.5160 & 2050.9981 & 1026.0027 & 2050.0141 & 1025.5107 & 17 \\
\hline 8 & 955.5459 & 478.2766 & 938.5193 & 469.7633 & 937.5353 & 469.2713 & I & 1939.9297 & 970.4685 & |1922.9031 & 961.9552 & 1921.9191 & 961.4632 & 16 \\
\hline 9 & 1052.5986 & 526.8030 & 1035.5721 & 518.2897 & 1034.5881 & 517.7977 & $\mathbf{P}$ & 1826.8456 & 913.9265 & 1809.8191 & 905.4132 & 1808.8351 & 904.9212 & 15 \\
\hline 10 & 1167.6256 & 584.3164 & 1150.5990 & 575.8032 & 1149.6150 & 575.3111 & $\mathbf{N}$ & 1729.7929 & 865.4001 & 1712.7663 & 856.8868 & 1711.7823 & 856.3948 & 14 \\
\hline 11 & 1281.6685 & 641.3379 & 1264.6420 & 632.8246 & 1263.6579 & 632.3326 & $\mathbf{N}$ & 1614.7659 & 807.8866 & 1597.7394 & 799.3733 & 1596.7554 & 798.8813 & 13 \\
\hline 12 & 1382.7162 & 691.8617 & 1365.6896 & 683.3485 & 1364.7056 & 682.8564 & $T$ & 1500.7230 & 750.8651 & 1483.6965 & 742.3519 & 1482.7124 & 741.8599 & 12 \\
\hline 13 & 1510.7748 & 755.8910 & 1493.7482 & 747.3777 & 1492.7642 & 746.8857 & $\mathbf{Q}$ & 1399.6753 & 700.3413 & 1382.6488 & 691.8280 & 1381.6648 & 691.3360 & 11 \\
\hline 14 & 1696.8541 & 848.9307 & 1679.8275 & 840.4174 & 1678.8435 & 839.9254 & W & 1271.6167 & 636.3120 & 1254.5902 & 627.7987 & 1253.6062 & 627.3067 & 10 \\
\hline 15 & 1809.9381 & 905.4727 & 1792.9116 & 896.9594 & 1791.9276 & 896.4674 & I & 1085.5374 & 543.2724 & 1068.5109 & 534.7591 & 1067.5269 & 534.2671 & 9 \\
\hline 16 & 1910.9858 & 955.9965 & 1893.9593 & 947.4833 & 1892.9752 & 946.9913 & $T$ & 972.4534 & 486.7303 & 955.4268 & 478.2170 & 954.4428 & 477.7250 & 8 \\
\hline 17 & 2097.0651 & 1049.0362 & 2080.0386 & 1040.5229 & 2079.0546 & 1040.0309 & W & 871.4057 & 436.2065 & 854.3791 & 427.6932 & 853.3951 & 427.2012 & 7 \\
\hline 18 & 2184.0972 & 1092.5522 & 2167.0706 & 1084.0389 & 2166.0866 & 1083.5469 & S & 685.3264 & 343.1668 & 668.2998 & 334.6536 & 667.3158 & 334.1615 & 6 \\
\hline 19 & 2312.1557 & 1156.5815 & 2295.1292 & 1148.0682 & 2294.1452 & 1147.5762 & $\mathbf{Q}$ & 598.2944 & 299.6508 & 581.2678 & 291.1375 & 580.2838 & 290.6455 & 5 \\
\hline 20 & 2441.1983 & 1221.1028 & 2424.1718 & 1212.5895 & 2423.1878 & 1212.0975 & $\mathbf{E}$ & 470.2358 & 235.6215 & 453.2092 & 227.1083 & 452.2252 & 226.6162 & 4 \\
\hline 21 & 2498.2198 & 1249.6135 & 2481.1932 & 1241.1003 & 2480.2092 & 1240.6083 & G & 341.1932 & 171.1002 & 324.1666 & 162.5870 & & & 3 \\
\hline 22 & 2635.2787 & 1318.1430 & 2618.2522 & 1309.6297 & 2617.2681 & 1309.1377 & $\mathbf{H}$ & 284.1717 & 142.5895 & 267.1452 & 134.0762 & & & 2 \\
\hline 23 & & & & & & & K & 147.1128 & 74.0600 & 130.0863 & 65.5468 & & & 1 \\
\hline
\end{tabular}
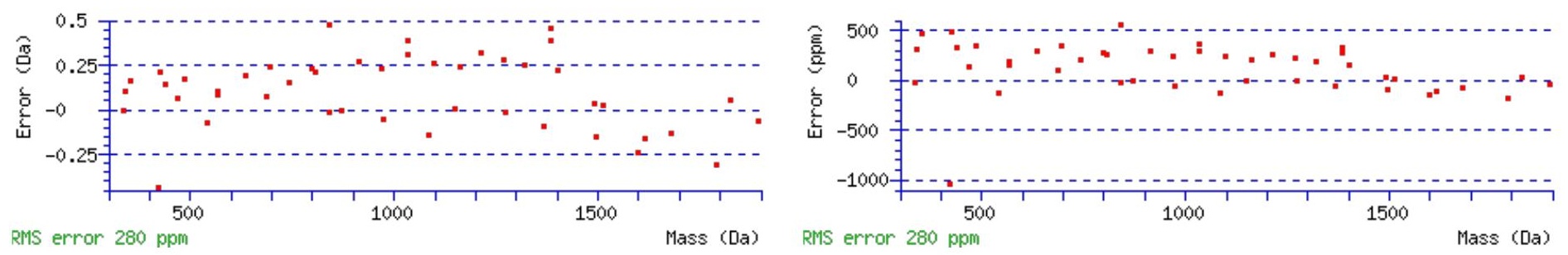

\section{All matches to this query}

\begin{tabular}{|l|l|c|l|}
\hline Score & Mr(calc): & Delta & \multicolumn{1}{|c|}{ Sequence } \\
\hline 50.6 & 2780.3769 & 0.0073 & QLITEEKIPNNTQWITWSQEGHK \\
\hline 49.6 & 2780.3769 & 0.0073 & QLITEEKIPNNTQWITWSQEGHK \\
\hline 18.0 & 2779.3929 & 0.9913 & QLITEEKIPNNTQWITWSQEGHK \\
\hline 2.4 & 2780.3987 & -0.0144 & ESIQINSGLLALGNVISALGDPRRK \\
\hline 0.1 & 2780.3873 & -0.0031 & EGWLPLHEAAYYGKLGCLKVLQR \\
\hline 0.1 & 2780.3873 & -0.0031 & EGWLPLHEAAYYGKLGCLKVLQR \\
\hline
\end{tabular}

Spectrum No: 667; Query: 465; Rank: 1

\section{Peptide View}

MS/MS Fragmentation of EALPFWMNSTGK

Found in IPI00358524, Tax_Id=10116 Gene_Symbol=Enpp6_predicted ectonucleotide pyrophosphatase/phosphodiesterase 6

Match to Query 465: 1380.641688 from(691.328120,2+)

Title: 100101RatKid_NS_deglyco_25.3812.3812.2.dta

Data file K:INewmanPaper|Piliangl3SubProteomes\Piliang3SP\mgf5ppm\ERLIC_3SubProteomes5ppm.mgf 

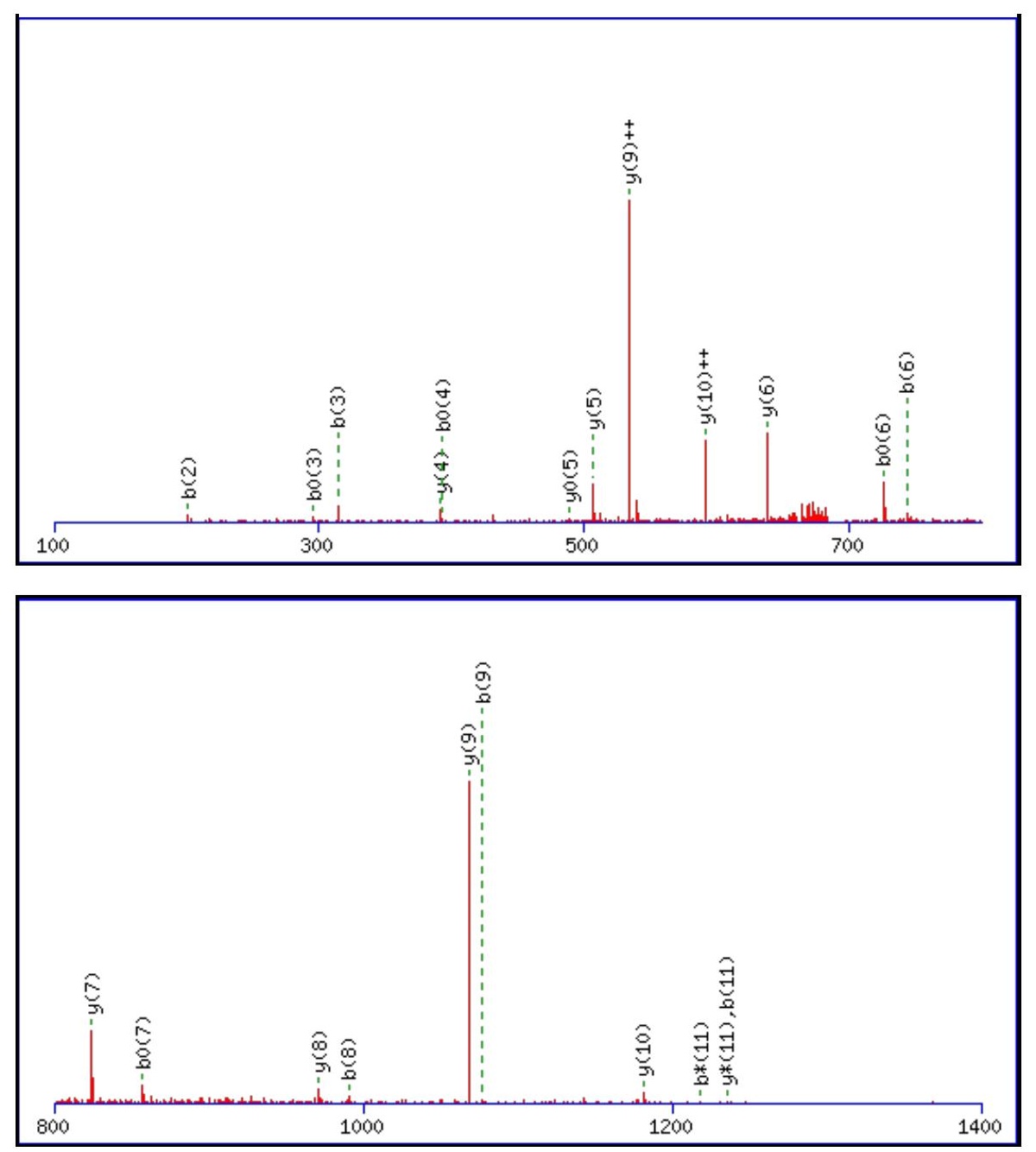

\begin{tabular}{rrrr}
\hline 00 & 1600 & 1800 & 2000 \\
\hline
\end{tabular}

Monoisotopic mass of neutral peptide $\operatorname{Mr}($ calc): 1380.6384

Fixed modifications: Carbamidomethyl (C)

Variable modifications:

N8: Deamidated $\mathrm{N}(\mathrm{N})$

Ions Score: 50 Expect: 0.0013

Matches (Bold Red): 22/114 fragment ions using 39 most intense peaks

\begin{tabular}{|r|c|c|c|c|c|c|c|c|c|c|c|c|c|c|}
\hline$\#$ & $\mathbf{b}$ & $\mathbf{b}^{++}$ & $\mathbf{b}^{*}$ & $\mathbf{b}^{\boldsymbol{*}^{++}}$ & $\mathbf{b}^{\mathbf{0}}$ & $\mathbf{b}^{\mathbf{0}++}$ & Seq. & $\mathbf{y}$ & $\mathbf{y}^{++}$ & $\mathbf{y}^{*}$ & $\mathbf{y}^{\mathbf{*}^{++}}$ & $\mathbf{y}^{\mathbf{0}}$ & $\mathbf{y}^{\mathbf{0 + +}}$ & $\#$ \\
\hline $\mathbf{1}$ & 130.0499 & 65.5286 & & & 112.0393 & 56.5233 & $\mathbf{E}$ & & & & & & & $\mathbf{1 2}$ \\
\hline $\mathbf{2}$ & $\mathbf{2 0 1 . 0 8 7 0}$ & 101.0471 & & & 183.0764 & 92.0418 & $\mathbf{A}$ & 1252.6031 & 626.8052 & $\mathbf{1 2 3 5 . 5 7 6 5}$ & 618.2919 & 1234.5925 & 617.7999 & $\mathbf{1 1}$ \\
\hline $\mathbf{3}$ & $\mathbf{3 1 4 . 1 7 1 0}$ & 157.5892 & & & $\mathbf{2 9 6 . 1 6 0 5}$ & 148.5839 & $\mathbf{L}$ & $\mathbf{1 1 8 1 . 5 6 6 0}$ & $\mathbf{5 9 1 . 2 8 6 6}$ & 1164.5394 & 582.7733 & 1163.5554 & 582.2813 & $\mathbf{1 0}$ \\
\hline $\mathbf{4}$ & 411.2238 & 206.1155 & & & 393.2132 & 197.1103 & $\mathbf{P}$ & $\mathbf{1 0 6 8 . 4 8 1 9}$ & 534.7446 & 1051.4553 & 526.2313 & 1050.4713 & 525.7393 & $\mathbf{9}$ \\
\hline $\mathbf{5}$ & 558.2922 & 279.6498 & & & 540.2817 & 270.6445 & $\mathbf{F}$ & $\mathbf{9 7 1 . 4 2 9 1}$ & 486.2182 & 954.4026 & 477.7049 & 953.4186 & 477.2129 & $\mathbf{8}$ \\
\hline
\end{tabular}




\begin{tabular}{|r|r|r|r|r|r|r|r|r|r|r|r|r|r|r|r|r|}
$\mathbf{6}$ & $\mathbf{7 4 4 . 3 7 1 5}$ & 372.6894 & & & $\mathbf{7 2 6 . 3 6 1 0}$ & 363.6841 & $\mathbf{W}$ & $\mathbf{8 2 4 . 3 6 0 7}$ & 412.6840 & 807.3342 & 404.1707 & 806.3501 & 403.6787 & $\mathbf{7}$ \\
\hline $\mathbf{7}$ & 875.4120 & 438.2096 & & & $\mathbf{8 5 7 . 4 0 1 5}$ & 429.2044 & $\mathbf{M}$ & $\mathbf{6 3 8 . 2 8 1 4}$ & 319.6443 & 621.2548 & 311.1311 & 620.2708 & 310.6391 & $\mathbf{6}$ \\
\hline $\mathbf{8}$ & $\mathbf{9 9 0 . 4 3 9 0}$ & 495.7231 & 973.4124 & 487.2098 & 972.4284 & 486.7178 & $\mathbf{N}$ & $\mathbf{5 0 7 . 2 4 0 9}$ & 254.1241 & 490.2144 & 245.6108 & $\mathbf{4 8 9 . 2 3 0 3}$ & 245.1188 & $\mathbf{5}$ \\
\hline $\mathbf{9}$ & $\mathbf{1 0 7 7 . 4 7 1 0}$ & 539.2391 & 1060.4444 & 530.7259 & 1059.4604 & 530.2338 & $\mathbf{S}$ & $\mathbf{3 9 2 . 2 1 4 0}$ & 196.6106 & 375.1874 & 188.0974 & 374.2034 & 187.6053 & $\mathbf{4}$ \\
\hline $\mathbf{1 0}$ & 1178.5187 & 589.7630 & 1161.4921 & 581.2497 & 1160.5081 & 580.7577 & $\mathbf{T}$ & 305.1819 & 153.0946 & 288.1554 & 144.5813 & 287.1714 & 144.0893 & $\mathbf{3}$ \\
\hline $\mathbf{1 1}$ & $\mathbf{1 2 3 5 . 5 4 0 1}$ & 618.2737 & $\mathbf{1 2 1 8 . 5 1 3 6}$ & 609.7604 & 1217.5296 & 609.2684 & $\mathbf{G}$ & 204.1343 & 102.5708 & 187.1077 & 94.0575 & & & & $\mathbf{2}$ \\
\hline $\mathbf{1 2}$ & & & & & & & $\mathbf{K}$ & 147.1128 & 74.0600 & 130.0863 & 65.5468 & & & $\mathbf{1}$ \\
\hline
\end{tabular}
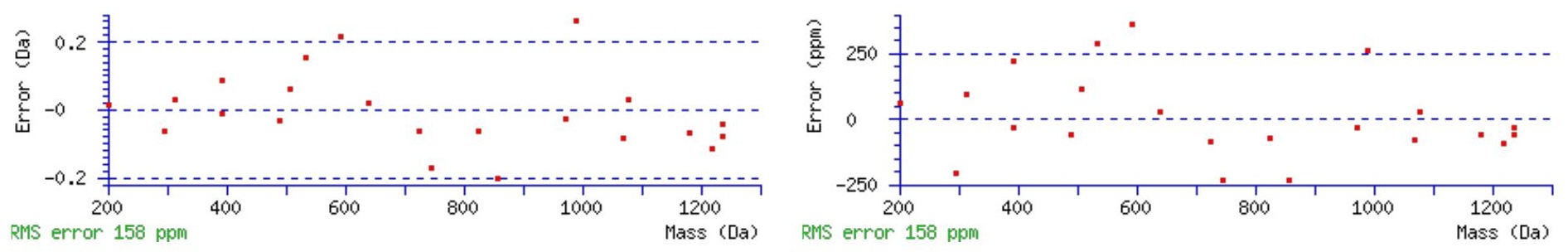

\section{All matches to this query}

\begin{tabular}{|l|l|l|l|}
\hline Score & Mr(calc): & Delta & \multicolumn{1}{|c|}{ Sequence } \\
\hline 50.5 & 1380.6384 & 0.0033 & EALPFWMNSTGK \\
\hline 20.9 & 1380.6400 & 0.0017 & SIIYYMVTSPK \\
\hline 17.9 & 1380.6360 & 0.0057 & EAMPIPKTPSSK \\
\hline 17.2 & 1380.6286 & 0.0131 & KSSQEGKPEPSK \\
\hline 13.1 & 1380.6343 & 0.0074 & VTLTSSPLYLK \\
\hline 13.1 & 1380.6343 & 0.0074 & VTLTSSPLYLK \\
\hline 13.1 & 1380.6343 & 0.0074 & VTLTSSPLYLK \\
\hline 12.5 & 1380.6439 & -0.0022 & VTIQPVXYNPR \\
\hline 11.6 & 1380.6400 & 0.0017 & SIIYYMVTSPK \\
\hline 11.6 & 1380.6286 & 0.0131 & SLLQSQGSPEQK \\
\hline
\end{tabular}

Spectrum No: 668; Query: 243; Rank: 1

\section{Peptide View}

MS/MS Fragmentation of EYNQTFGNLK

Found in IPI00196620, Tax_Id=10116 Gene_Symbol=Cubn Cubilin precursor

Match to Query 243: 1213.558408 from(607.786480,2+)

Title: 091008RatKidney_NH4Format01_24.1546.1546.2.dta

Data file K:INewmanPaper|Piliang|3SubProteomes\Piliang3SP\mgf5ppm\ERLIC_3SubProteomes5ppm.mgf 

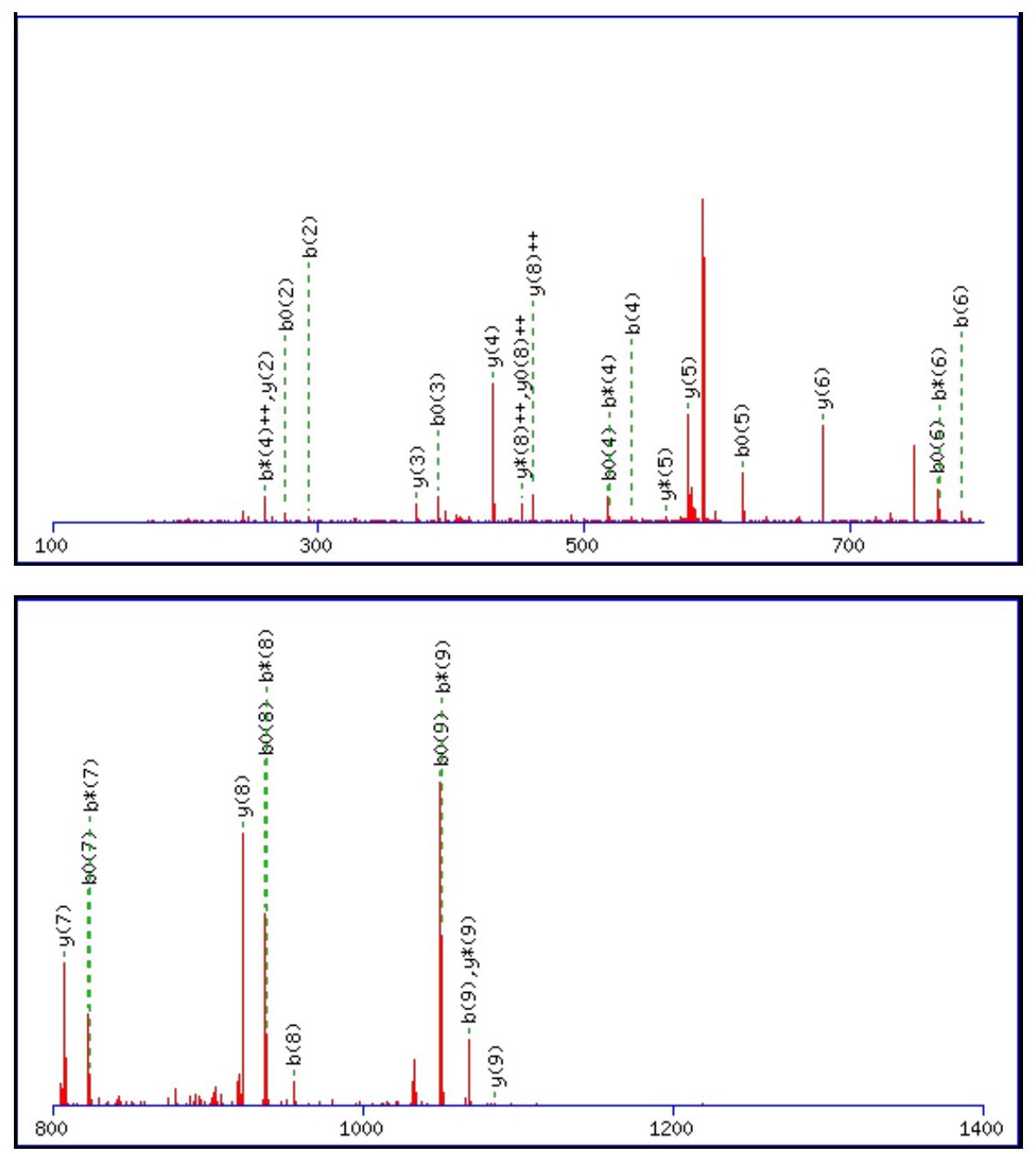

\begin{tabular}{rrrr}
\hline 00 & 1600 & 1800 & 2000 \\
\hline
\end{tabular}

Monoisotopic mass of neutral peptide $\operatorname{Mr}($ calc): 1213.5615

Fixed modifications: Carbamidomethyl (C)

Variable modifications:

N3 : Deamidated_N (N)

Ions Score: 50 Expect: 0.0011

Matches (Bold Red): 32/94 fragment ions using 55 most intense peaks

\begin{tabular}{|r|c|c|c|c|c|c|c|c|c|c|c|c|c|c|}
\hline$\#$ & $\mathbf{b}$ & $\mathbf{b}^{++}$ & $\mathbf{b}^{*}$ & $\mathbf{b}^{\boldsymbol{*}^{++}}$ & $\mathbf{b}^{\mathbf{0}}$ & $\mathbf{b}^{\mathbf{0 + +}}$ & Seq. & $\mathbf{y}$ & $\mathbf{y}^{++}$ & $\mathbf{y}^{\mathbf{*}}$ & $\mathbf{y}^{\boldsymbol{*}^{++}}$ & $\mathbf{y}^{\mathbf{0}}$ & $\mathbf{y}^{\mathbf{0 + +}}$ & $\#$ \\
\hline $\mathbf{1}$ & 130.0499 & 65.5286 & & & 112.0393 & 56.5233 & $\mathbf{E}$ & & & & & & & $\mathbf{1 0}$ \\
\hline $\mathbf{2}$ & $\mathbf{2 9 3 . 1 1 3 2}$ & 147.0602 & & & $\mathbf{2 7 5 . 1 0 2 6}$ & 138.0550 & $\mathbf{Y}$ & $\mathbf{1 0 8 5 . 5 2 6 2}$ & 543.2667 & $\mathbf{1 0 6 8 . 4 9 9 6}$ & 534.7535 & 1067.5156 & 534.2615 & $\mathbf{9}$ \\
\hline $\mathbf{3}$ & 408.1401 & 204.5737 & 391.1136 & 196.0604 & $\mathbf{3 9 0 . 1 2 9 6}$ & 195.5684 & $\mathbf{N}$ & $\mathbf{9 2 2 . 4 6 2 9}$ & $\mathbf{4 6 1 . 7 3 5 1}$ & 905.4363 & $\mathbf{4 5 3 . 2 2 1 8}$ & 904.4523 & $\mathbf{4 5 2 . 7 2 9 8}$ & $\mathbf{8}$ \\
\hline $\mathbf{4}$ & 536.1987 & 268.6030 & 519.1722 & $\mathbf{2 6 0 . 0 8 9 7}$ & $\mathbf{5 1 8 . 1 8 8 1}$ & 259.5977 & $\mathbf{Q}$ & $\mathbf{8 0 7 . 4 3 5 9}$ & 404.2216 & 790.4094 & 395.7083 & 789.4254 & 395.2163 & $\mathbf{7}$ \\
\hline $\mathbf{5}$ & 637.2464 & 319.1268 & 620.2198 & 310.6136 & $\mathbf{6 1 9 . 2 3 5 8}$ & 310.1216 & $\mathbf{T}$ & $\mathbf{6 7 9 . 3 7 7 4}$ & 340.1923 & 662.3508 & 331.6790 & 661.3668 & 331.1870 & $\mathbf{6}$ \\
\hline
\end{tabular}




\begin{tabular}{|c|c|c|c|c|c|c|c|c|c|c|c|c|}
\hline 6 & 784.3148 & $|392.6610|$ & 767.2883 & |384.1478| & 766.3042 & |383.6558 & $\mathbf{F}$ & 578.3297 & 289.6685 & 561.3031 & |281.1552 & 5 \\
\hline 7 & 841.3363 & 421.1718 & 824.3097 & 412.6585 & 823.3257 & 412.1665 & G & 431.2613 & 216.1343 & 414.2347 & 207.6210 & 4 \\
\hline 8 & 955.3792 & 478.1932 & 938.3526 & 469.6800 & 937.3686 & 469.1880 & $\mathbf{N}$ & 374.2398 & 187.6235 & 357.2132 & 179.1103 & 3 \\
\hline 9 & 1068.4633 & 534.7353 & 1051.4367 & 526.2220 & 1050.4527 & 525.7300 & $\mathbf{L}$ & 260.1969 & 130.6021 & 243.1703 & 122.0888 & 2 \\
\hline 10 & & & & & & & K & 147.1128 & 74.0600 & 130.0863 & 65.5468 & 1 \\
\hline
\end{tabular}
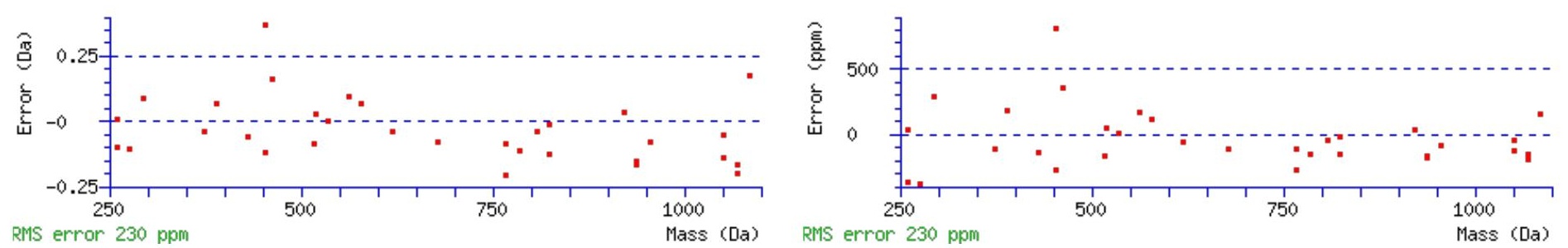

\section{All matches to this query}

\begin{tabular}{|l|l|l|l|}
\hline Score & Mr(calc): & Delta & \multicolumn{1}{|c|}{ Sequence } \\
\hline 50.5 & 1213.5615 & -0.0031 & EYNQTFGNLK \\
\hline 30.8 & 1213.5615 & -0.0031 & EYNQTFGNLK \\
\hline 17.3 & 1213.5615 & -0.0031 & EEDHAPEFIK \\
\hline 11.2 & 1213.5605 & -0.0020 & NSTPRFRQK \\
\hline 10.7 & 1213.5621 & -0.0037 & TKPSRQPLK \\
\hline 10.6 & 1213.5605 & -0.0020 & NSTPRFRQK \\
\hline 10.1 & 1213.5591 & -0.0007 & VLNSASDTSLK \\
\hline 8.8 & 1213.5591 & -0.0007 & VLNSASDTSLK \\
\hline 6.7 & 1212.5445 & 1.0139 & YTCEATNVAGK \\
\hline 5.9 & 1213.5526 & 0.0058 & MADIRTVGQK \\
\hline
\end{tabular}

Spectrum No: 669; Query: 1049; Rank: 1

\section{Peptide View}

MS/MS Fragmentation of GYYNQSEGGSHTIQR

Found in IPI00480739, Tax_Id=10116 Gene_Symbol=RT1-Aw2 Class I MHC

Match to Query 1049: 1696.747768 from(849.381160,2+)

Title: 100101RatKid_NS_deglyco_19.826.826.2.dta

Data file K:INewmanPaper|Piliangl3SubProteomes\Piliang3SP\mgf5ppm\ERLIC_3SubProteomes5ppm.mgf

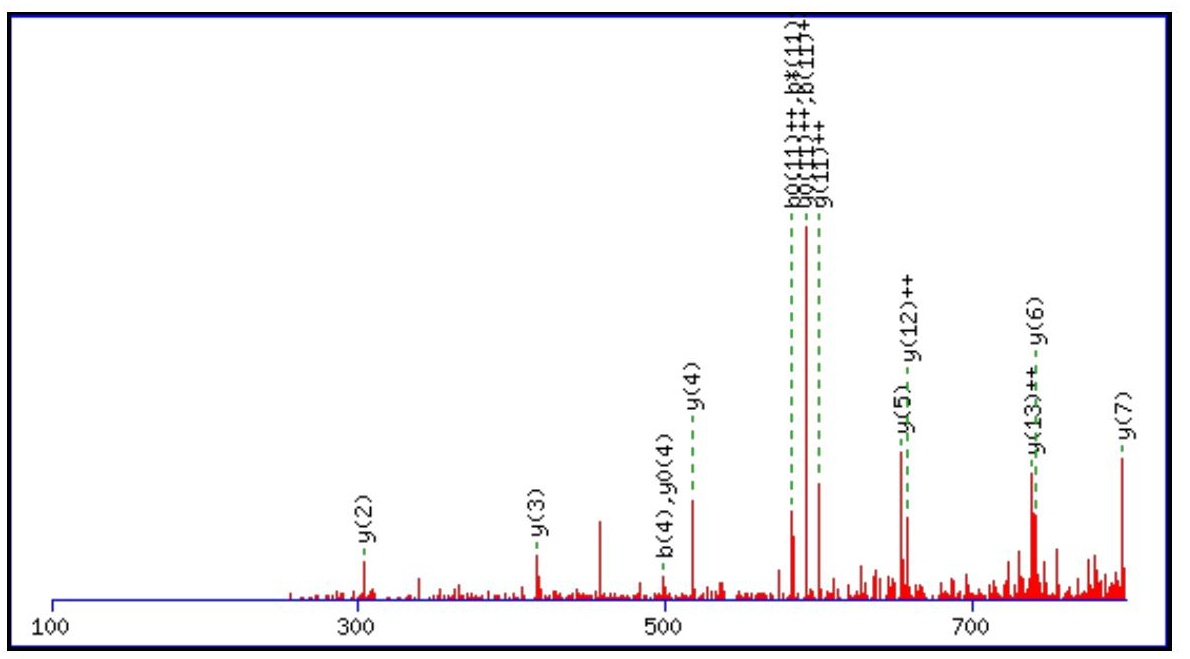



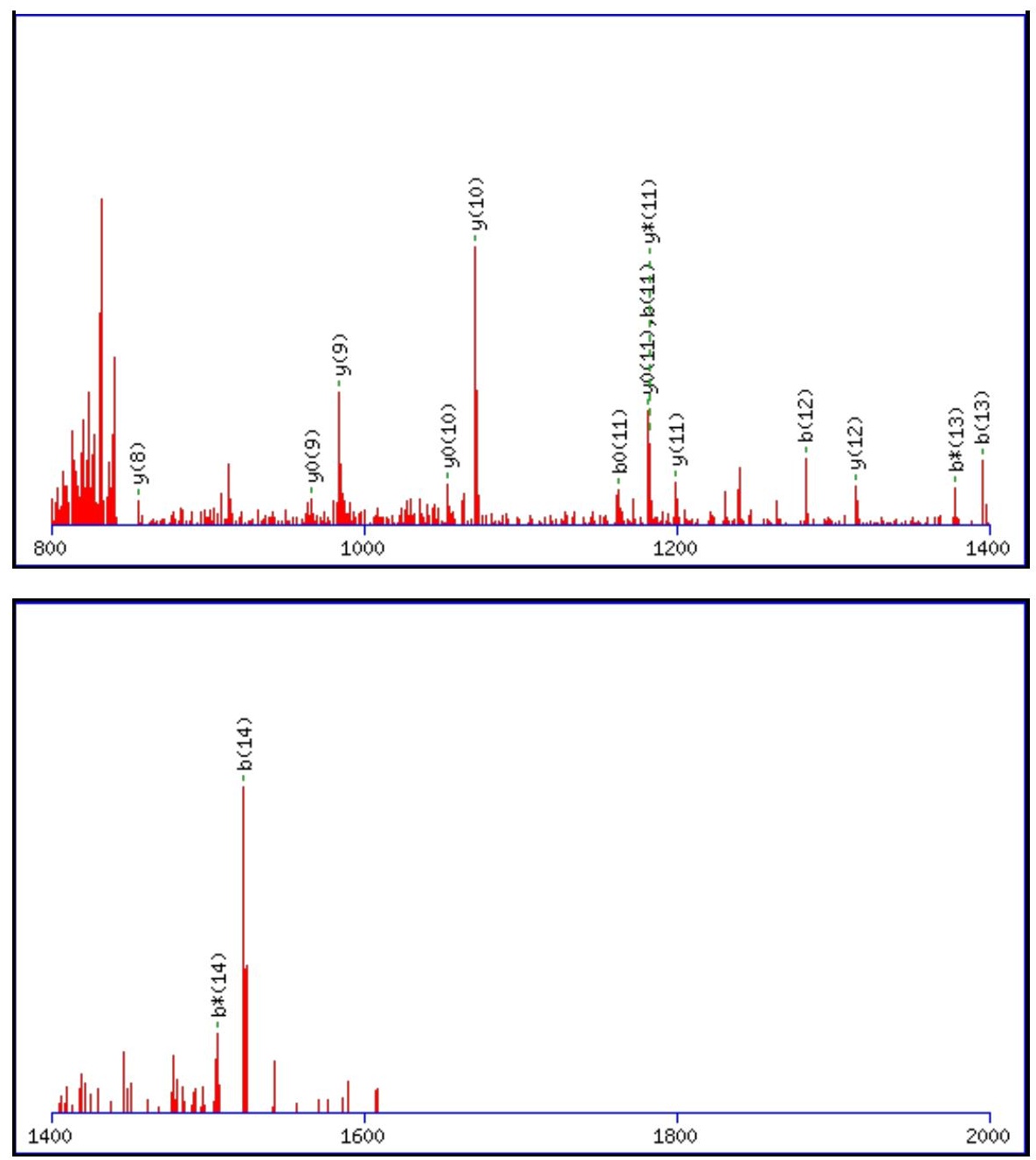

Monoisotopic mass of neutral peptide $\operatorname{Mr}($ calc): 1696.7441

Fixed modifications: Carbamidomethyl (C)

Variable modifications:

N4 : Deamidated $\mathrm{N}(\mathrm{N})$

Ions Score: 51 Expect: 0.0016

Matches (Bold Red): 32/146 fragment ions using 64 most intense peaks

\begin{tabular}{|c|c|c|c|c|c|c|c|c|c|c|c|c|c|c|}
\hline \# & b & $\mathbf{b}^{++}$ & $\mathbf{b}^{*}$ & $\mathbf{b}^{*^{++}}$ & $\mathbf{b}^{0}$ & $\mathbf{b}^{0++}$ & Seq. & $\mathbf{y}$ & $y^{++}$ & $\mathbf{y}^{*}$ & $\mathrm{y}^{*^{++}}$ & $\mathbf{y}^{\mathbf{0}}$ & $\mathbf{y}^{0++}$ & \# \\
\hline 1 & 58.0287 & 29.5180 & & & & & $\mathbf{G}$ & & & & & & & 15 \\
\hline 2 & 221.0921 & 111.0497 & & & & & $\mathbf{Y}$ & 1640.7299 & 820.8686 & 1623.7034 & 812.3553 & 1622.7194 & 811.8633 & 14 \\
\hline 3 & 384.1554 & 192.5813 & & & & & $\mathbf{Y}$ & 1477.6666 & 739.3369 & 1460.6401 & 730.8237 & 1459.6560 & 730.3317 & 13 \\
\hline 4 & 499.1823 & 250.0948 & 482.1558 & 241.5815 & & & $\mathbf{N}$ & 1314.6033 & 657.8053 & 1297.5767 & 649.2920 & 1296.5927 & 648.8000 & 12 \\
\hline 5 & 627.2409 & 314.1241 & 610.2144 & 305.6108 & & & $\mathbf{Q}$ & 1199.5763 & 600.2918 & 1182.5498 & 591.7785 & 1181.5658 & 591.2865 & 11 \\
\hline 6 & 714.2729 & 357.6401 & 697.2464 & 349.1268 & 696.2624 & 348.6348 & S & 1071.5178 & 536.2625 & 1054.4912 & 527.7492 & 1053.5072 & 527.2572 & 10 \\
\hline 7 & 843.3155 & 422.1614 & 826.2890 & 413.6481 & 825.3050 & 413.1561 & $\mathbf{E}$ & 984.4857 & 492.7465 & 967.4592 & 484.2332 & 966.4752 & 483.7412 & 9 \\
\hline 8 & 900.3370 & 450.6721 & 883.3104 & 442.1589 & 882.3264 & 441.6669 & G & 855.4431 & 428.2252 & 838.4166 & 419.7119 & 837.4326 & 419.2199 & 8 \\
\hline 9 & 957.3585 & 479.1829 & 940.3319 & 470.6696 & 939.3479 & 470.1776 & G & 798.4217 & 399.7145 & 781.3951 & 391.2012 & 780.4111 & 390.7092 & 7 \\
\hline 10 & 1044.3905 & 522.6989 & 1027.3639 & 514.1856 & 1026.3799 & 513.6936 & S & 741.4002 & 371.2037 & 724.3737 & 362.6905 & 723.3896 & 362.1985 & 6 \\
\hline 11 & 1181.4494 & 591.2283 & 1164.4229 & 582.7151 & 1163.4388 & 582.2231 & $\mathbf{H}$ & 654.3682 & 327.6877 & 637.3416 & 319.1745 & 636.3576 & 318.6824 & 5 \\
\hline 12 & 1282.4971 & 641.7522 & 1265.4705 & 633.2389 & 1264.4865 & 632.7469 & $\mathbf{T}$ & 517.3093 & 259.1583 & 500.2827 & 250.6450 & 499.2987 & 250.1530 & 4 \\
\hline 13 & 1395.5811 & 698.2942 & 1378.5546 & 689.7809 & 1377.5706 & 689.2889 & I & 416.2616 & 208.6344 & 399.2350 & 200.1212 & & & 3 \\
\hline 14 & 1523.6397 & 762.3235 & 1506.6132 & 753.8102 & 1505.6292 & 753.3182 & $\mathbf{Q}$ & 303.1775 & 152.0924 & 286.1510 & 143.5791 & & & 2 \\
\hline 15 & & & & & & & $\mathbf{R}$ & 175.1190 & 88.0631 & 158.0924 & 79.5498 & & & 1 \\
\hline
\end{tabular}




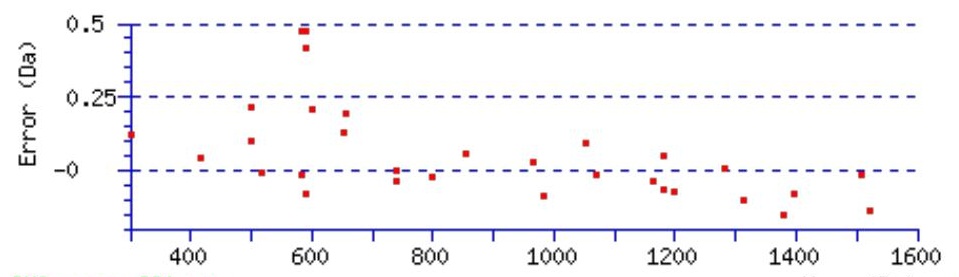

RMS error $281 \mathrm{ppm}$

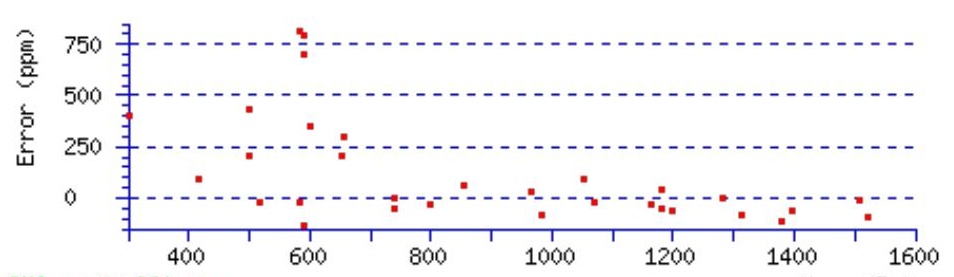

Mass (Da)

\section{All matches to this query}

\begin{tabular}{|l|c|c|l|}
\hline Score & Mr(calc): & Delta & \multicolumn{1}{c|}{ Sequence } \\
\hline 50.5 & 1696.7441 & 0.0037 & GYYNQSEGGSHTIQR \\
\hline 26.8 & 1695.7601 & 0.9877 & GYYNQSEGGSHTIQR \\
\hline 14.0 & 1696.7457 & 0.0020 & GNYIEVRTGEPPER \\
\hline 10.2 & 1694.7318 & 2.0160 & TIPGELTSYGTLQR \\
\hline 8.2 & 1696.7375 & 0.0103 & QYIVYPNRSLQR \\
\hline 8.2 & 1696.7375 & 0.0103 & QYIVYPNRSLQR \\
\hline 5.4 & 1696.7529 & -0.0052 & ADSNKTRIDEANQR \\
\hline 4.7 & 1696.7330 & 0.0148 & DASLGTMISVMLKR \\
\hline 4.7 & 1696.7330 & 0.0148 & DASLGTMISVMLKR \\
\hline 4.7 & 1696.7330 & 0.0148 & DASLGTMISVMLKR \\
\hline
\end{tabular}

Spectrum No: 670; Query: 1050; Rank: 1

\section{Peptide View}

MS/MS Fragmentation of NSSYFVEWIPNNVK

Found in IPI00195673, Tax_Id=10116 Gene_Symbol=Tubb6 Tubulin, beta 6

Match to Query 1050: 1696.805288 from(849.409920,2+)

Title: 091008RatKidney_NoSalt_09.5203.5203.2.dta

Data file K:INewmanPaper|Piliang|3SubProteomes\Piliang3SP\mgf5ppm\ERLIC_3SubProteomes5ppm.mgf

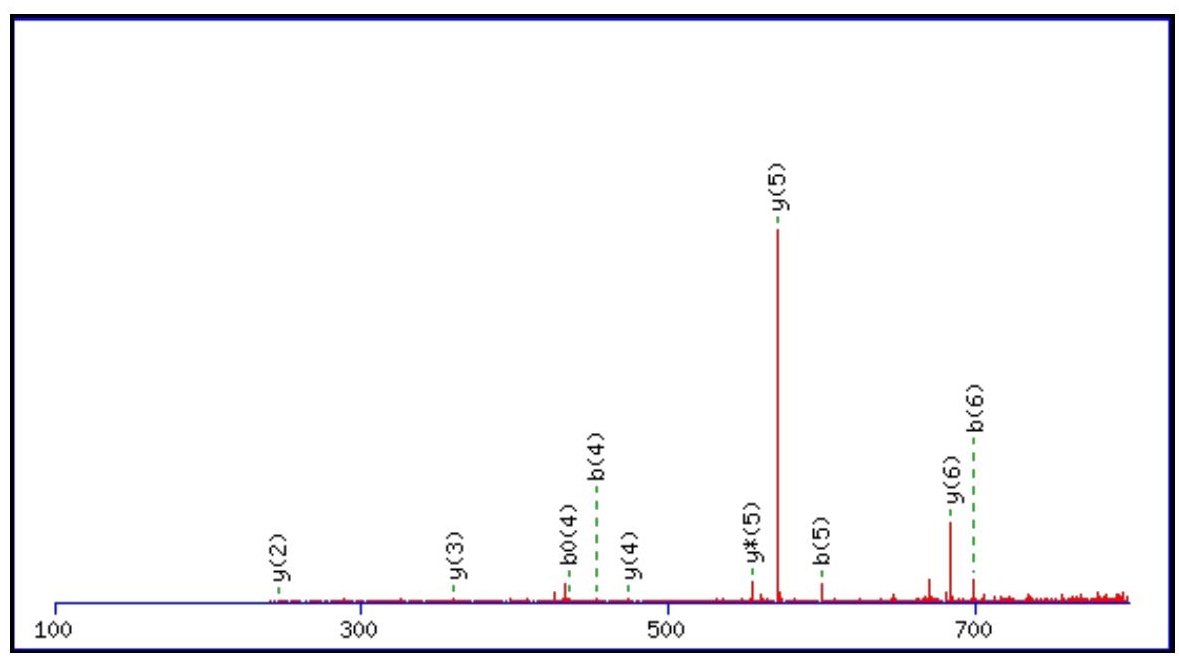



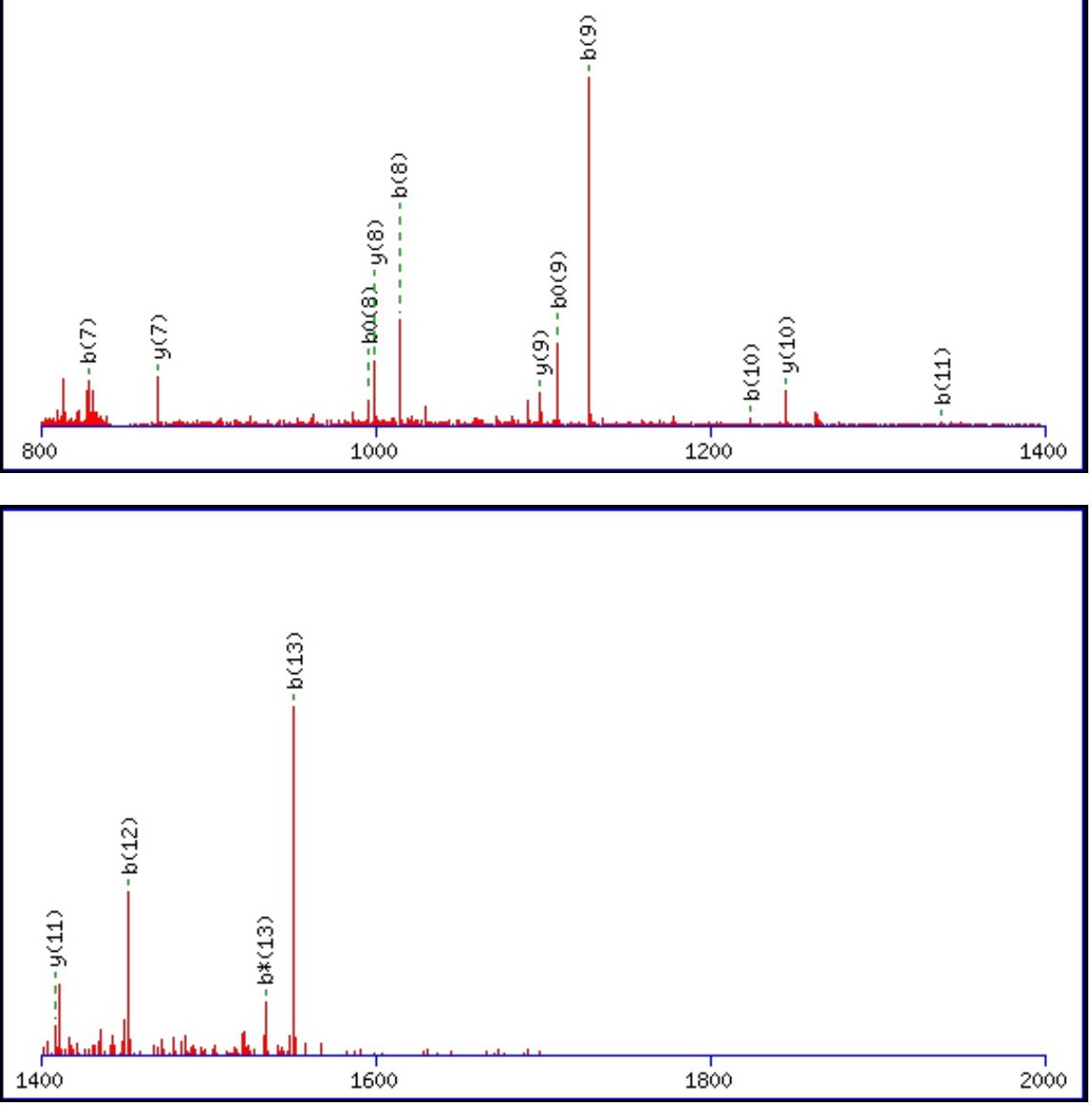

Monoisotopic mass of neutral peptide $\operatorname{Mr}($ calc): 1696.8097

Fixed modifications: Carbamidomethyl (C)

Variable modifications:

N1 : Deamidated $\mathrm{N}(\mathrm{N})$

Ions Score: 50 Expect : $\odot .0017$

Matches (Bold Red): 25/140 fragment ions using 62 most intense peaks

\begin{tabular}{|c|c|c|c|c|c|c|c|c|c|c|c|c|c|c|}
\hline \# & b & $\mathbf{b}^{++}$ & $\mathbf{b}^{*}$ & $\mathbf{b}^{*^{++}}$ & $\mathbf{b}^{0}$ & $\mathbf{b}^{\mathbf{0 + +}}$ & Seq. & $\mathbf{y}$ & $\mathbf{y}^{++}$ & $\mathbf{y}^{*}$ & $\mathrm{y}^{*^{++}}$ & $\mathbf{y}^{\mathbf{0}}$ & $\mathbf{y}^{\mathbf{0 + +}}$ & \# \\
\hline 1 & 116.0342 & 58.5207 & 99.0077 & 50.0075 & & & $\mathbf{N}$ & & & & & & & 14 \\
\hline 2 & 203.0662 & 102.0368 & 186.0397 & 93.5235 & 185.0557 & 93.0315 & S & 1582.7900 & 791.8986 & 1565.7635 & 783.3854 & 1564.7795 & 782.8934 & 13 \\
\hline 3 & 290.0983 & 145.5528 & 273.0717 & 137.0395 & 272.0877 & 136.5475 & S & 1495.7580 & 748.3826 & 1478.7314 & 739.8694 & 1477.7474 & 739.3774 & 12 \\
\hline 4 & 453.1616 & 227.0844 & 436.1350 & 218.5712 & 435.1510 & 218.0792 & $\mathbf{Y}$ & 1408.7260 & 704.8666 & 1391.6994 & 696.3533 & 1390.7154 & 695.8613 & 11 \\
\hline 5 & 600.2300 & 300.6186 & 583.2035 & 292.1054 & 582.2194 & 291.6134 & $\mathbf{F}$ & 1245.6626 & 623.3350 & 1228.6361 & 614.8217 & 1227.6521 & 614.3297 & 10 \\
\hline 6 & 699.2984 & 350.1529 & 682.2719 & 341.6396 & 681.2879 & 341.1476 & $\mathbf{V}$ & 1098.5942 & 549.8007 & 1081.5677 & 541.2875 & 1080.5837 & 540.7955 & 9 \\
\hline 7 & 828.3410 & 414.6741 & 811.3145 & 406.1609 & 810.3305 & 405.6689 & $\mathbf{E}$ & 999.5258 & 500.2665 & 982.4993 & 491.7533 & 981.5152 & 491.2613 & 8 \\
\hline 8 & 1014.4203 & 507.7138 & 997.3938 & 499.2005 & 996.4098 & 498.7085 & $\mathbf{W}$ & 870.4832 & 435.7452 & 853.4567 & 427.2320 & & & 7 \\
\hline 9 & 1127.5044 & 564.2558 & 1110.4778 & 555.7426 & 1109.4938 & 555.2506 & I & 684.4039 & 342.7056 & 667.3774 & 334.1923 & & & 6 \\
\hline 10 & 1224.5572 & 612.7822 & 1207.5306 & 604.2689 & 1206.5466 & 603.7769 & $\mathbf{P}$ & 571.3198 & 286.1636 & 554.2933 & 277.6503 & & & 5 \\
\hline 11 & 1338.6001 & 669.8037 & 1321.5735 & 661.2904 & 1320.5895 & 660.7984 & $\mathbf{N}$ & 474.2671 & 237.6372 & 457.2405 & 229.1239 & & & 4 \\
\hline 12 & 1452.6430 & 726.8251 & 1435.6165 & 718.3119 & 1434.6324 & 717.8199 & $\mathbf{N}$ & 360.2241 & 180.6157 & 343.1976 & 172.1024 & & & 3 \\
\hline 13 & 1551.7114 & 776.3594 & 1534.6849 & 767.8461 & 1533.7009 & 767.3541 & V & 246.1812 & 123.5942 & 229.1547 & 115.0810 & & & 2 \\
\hline 14 & & & & & & & $\mathbf{K}$ & 147.1128 & 74.0600 & 130.0863 & 65.5468 & & & 1 \\
\hline
\end{tabular}



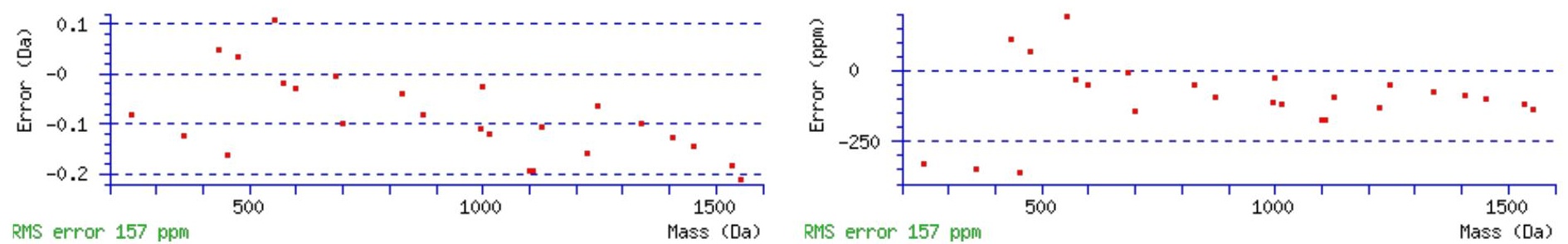

\section{All matches to this query}

\begin{tabular}{|l|l|l|l|}
\hline Score & Mr(calc): & Delta & \multicolumn{1}{|c|}{ Sequence } \\
\hline 50.4 & 1696.8097 & -0.0044 & NSSYFVEWIPNNVK \\
\hline 12.7 & 1696.7934 & 0.0119 & VHYNGRTQETSVVK \\
\hline 11.9 & 1696.7961 & 0.0092 & PEFLEDPSVLTKDK \\
\hline 8.4 & 1696.7934 & 0.0119 & VHYNGRTQETSVVK \\
\hline 7.6 & 1696.7934 & 0.0119 & VHYNGRTQETSVVK \\
\hline 6.1 & 1695.7885 & 1.0168 & SKLQNLSYTEILK \\
\hline 5.4 & 1696.8033 & 0.0020 & AANTGVGLSLQEGSVSK \\
\hline 5.4 & 1696.8033 & 0.0020 & AANTGVGLSLQEGSVSK \\
\hline 5.4 & 1696.8033 & 0.0020 & AANTGVGLSLQEGSVSK \\
\hline 5.4 & 1696.7920 & 0.0133 & DKAQKIVDEIDSEK \\
\hline
\end{tabular}

Spectrum No: 671; Query: 1050; Rank: 1

\section{Peptide View}

MS/MS Fragmentation of NSSYFVEWIPNNVK

Found in IPI00195673, Tax_Id=10116 Gene_Symbol=Tubb6 Tubulin, beta 6

Match to Query 1050: 1696.805288 from(849.409920,2+)

Title: 091008RatKidney_NoSalt_09.5203.5203.2.dta

Data file K:INewmanPaper|Piliang\3SubProteomes\Piliang3SP\mgf5ppm\ERLIC_3SubProteomes5ppm.mgf

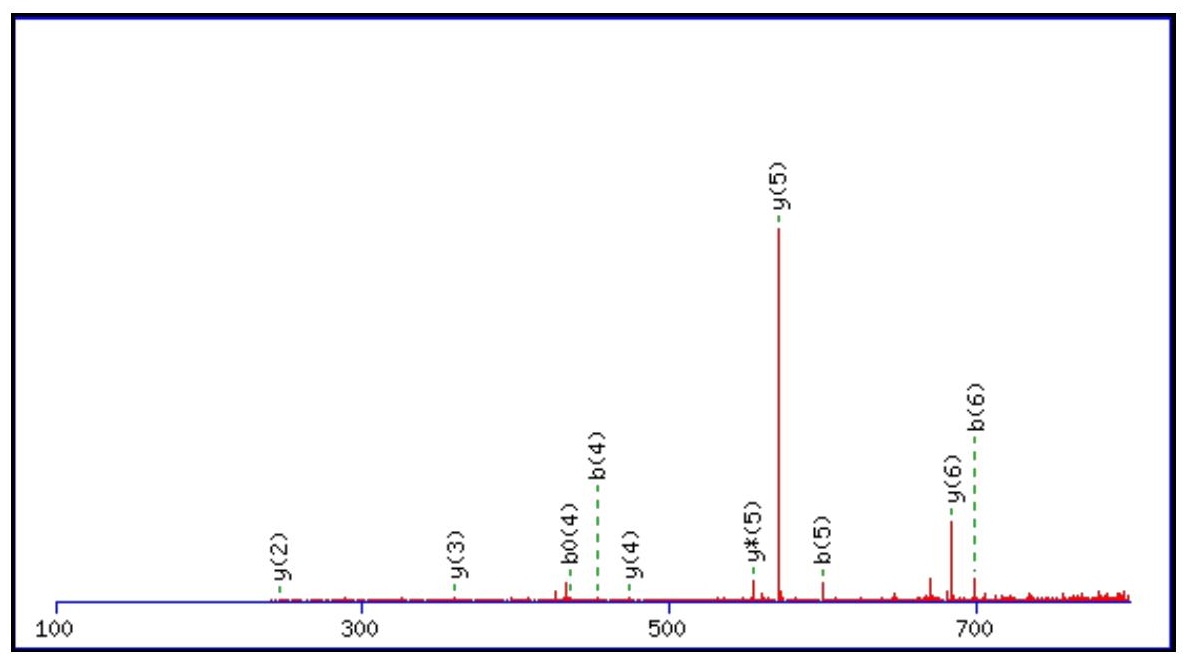



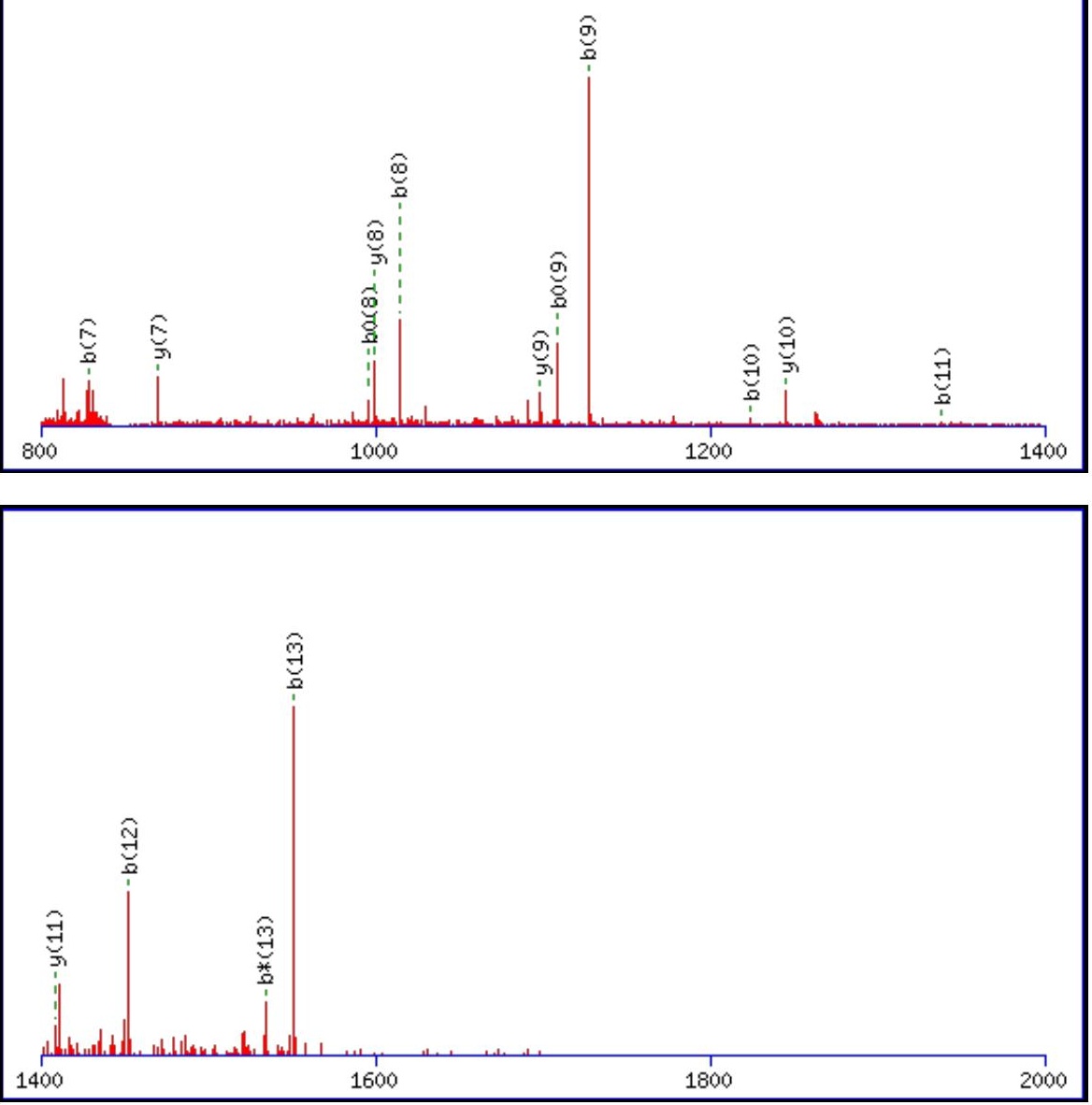

Monoisotopic mass of neutral peptide $\operatorname{Mr}($ calc): 1696.8097

Fixed modifications: Carbamidomethyl (C)

Variable modifications:

N1 : Deamidated $\mathrm{N}(\mathrm{N})$

Ions Score: 50 Expect : $\odot .0017$

Matches (Bold Red): 25/140 fragment ions using 62 most intense peaks

\begin{tabular}{|c|c|c|c|c|c|c|c|c|c|c|c|c|c|c|}
\hline \# & b & $\mathbf{b}^{++}$ & b* & $\mathbf{b}^{*^{++}}$ & $\mathbf{b}^{0}$ & $\mathbf{b}^{0++}$ & Seq. & $\mathbf{y}$ & $\mathbf{y}^{++}$ & $\mathbf{y}^{*}$ & $\mathrm{y}^{*^{++}}$ & $\mathbf{y}^{\mathbf{0}}$ & $\mathbf{y}^{0++}$ & \# \\
\hline 1 & 116.0342 & 58.5207 & 99.0077 & 50.0075 & & & $\mathbf{N}$ & & & & & & & 14 \\
\hline 2 & 203.0662 & 102.0368 & 186.0397 & 93.5235 & 185.0557 & 93.0315 & S & 1582.7900 & 791.8986 & 1565.7635 & 783.3854 & 1564.7795 & 782.8934 & 13 \\
\hline 3 & 290.0983 & 145.5528 & 273.0717 & 137.0395 & 272.0877 & 136.5475 & S & 1495.7580 & 748.3826 & 1478.7314 & 739.8694 & 1477.7474 & 739.3774 & 12 \\
\hline 4 & 453.1616 & 227.0844 & 436.1350 & 218.5712 & 435.1510 & 218.0792 & $\mathbf{Y}$ & 1408.7260 & 704.8666 & 1391.6994 & 696.3533 & 1390.7154 & 695.8613 & 11 \\
\hline 5 & 600.2300 & 300.6186 & 583.2035 & 292.1054 & 582.2194 & 291.6134 & $\mathbf{F}$ & 1245.6626 & 623.3350 & 1228.6361 & 614.8217 & 1227.6521 & 614.3297 & 10 \\
\hline 6 & 699.2984 & 350.1529 & 682.2719 & 341.6396 & 681.2879 & 341.1476 & $\mathbf{V}$ & 1098.5942 & 549.8007 & 1081.5677 & 541.2875 & 1080.5837 & 540.7955 & 9 \\
\hline 7 & 828.3410 & 414.6741 & 811.3145 & 406.1609 & 810.3305 & 405.6689 & $\mathbf{E}$ & 999.5258 & 500.2665 & 982.4993 & 491.7533 & 981.5152 & 491.2613 & 8 \\
\hline 8 & 1014.4203 & 507.7138 & 997.3938 & 499.2005 & 996.4098 & 498.7085 & W & 870.4832 & 435.7452 & 853.4567 & 427.2320 & & & 7 \\
\hline 9 & 1127.5044 & 564.2558 & 1110.4778 & 555.7426 & 1109.4938 & 555.2506 & I & 684.4039 & 342.7056 & 667.3774 & 334.1923 & & & 6 \\
\hline 10 & 1224.5572 & 612.7822 & 1207.5306 & 604.2689 & 1206.5466 & 603.7769 & $\mathbf{P}$ & 571.3198 & 286.1636 & 554.2933 & 277.6503 & & & 5 \\
\hline 11 & 1338.6001 & 669.8037 & 1321.5735 & 661.2904 & 1320.5895 & 660.7984 & $\mathbf{N}$ & 474.2671 & 237.6372 & 457.2405 & 229.1239 & & & 4 \\
\hline 12 & 1452.6430 & 726.8251 & 1435.6165 & 718.3119 & 1434.6324 & 717.8199 & $\mathbf{N}$ & 360.2241 & 180.6157 & 343.1976 & 172.1024 & & & 3 \\
\hline 13 & 1551.7114 & 776.3594 & 1534.6849 & 767.8461 & 1533.7009 & 767.3541 & V & 246.1812 & 123.5942 & 229.1547 & 115.0810 & & & 2 \\
\hline 14 & & & & & & & $\mathbf{K}$ & 147.1128 & 74.0600 & 130.0863 & 65.5468 & & & 1 \\
\hline
\end{tabular}



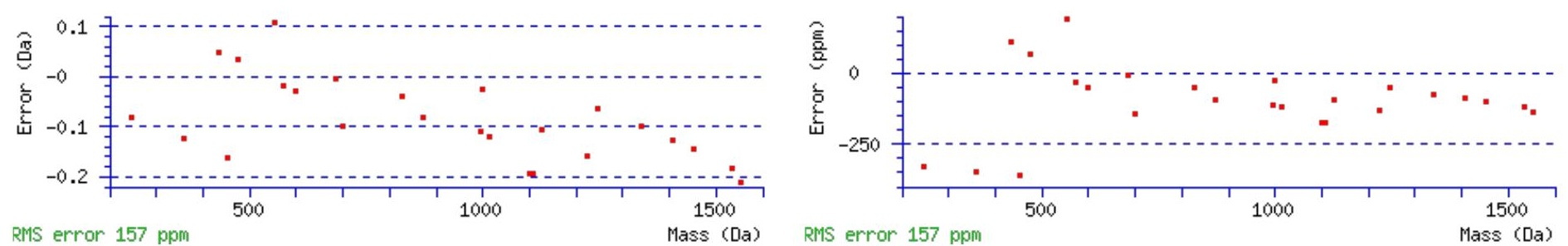

\section{All matches to this query}

\begin{tabular}{|l|l|l|l|}
\hline Score & Mr(calc): & Delta & \multicolumn{1}{|c|}{ Sequence } \\
\hline 50.4 & 1696.8097 & -0.0044 & NSSYFVEWIPNNVK \\
\hline 12.7 & 1696.7934 & 0.0119 & VHYNGRTQETSVVK \\
\hline 11.9 & 1696.7961 & 0.0092 & PEFLEDPSVLTKDK \\
\hline 8.4 & 1696.7934 & 0.0119 & VHYNGRTQETSVVK \\
\hline 7.6 & 1696.7934 & 0.0119 & VHYNGRTQETSVVK \\
\hline 6.1 & 1695.7885 & 1.0168 & SKLQNLSYTEILK \\
\hline 5.4 & 1696.8033 & 0.0020 & AANTGVGLSLQEGSVSK \\
\hline 5.4 & 1696.8033 & 0.0020 & AANTGVGLSLQEGSVSK \\
\hline 5.4 & 1696.8033 & 0.0020 & AANTGVGLSLQEGSVSK \\
\hline 5.4 & 1696.7920 & 0.0133 & DKAQKIVDEIDSEK \\
\hline
\end{tabular}

Spectrum No: 672; Query: 202; Rank: 1

\section{Peptide View}

\section{MS/MS Fragmentation of EKIEENGSMR}

Found in IPI00191711, Tax_Id=10116 Gene_Symbol=LOC259246;LOC298116;LOC259245 Major urinary protein precursor

Match to Query 202: 1192.537528 from(597.276040,2+)

Title: 091008RatKidney_NoSalt_16.475.475.2.dta

Data file K:INewmanPaper|Piliang\3SubProteomes\Piliang3SP\mgf5ppm\ERLIC_3SubProteomes5ppm.mgf

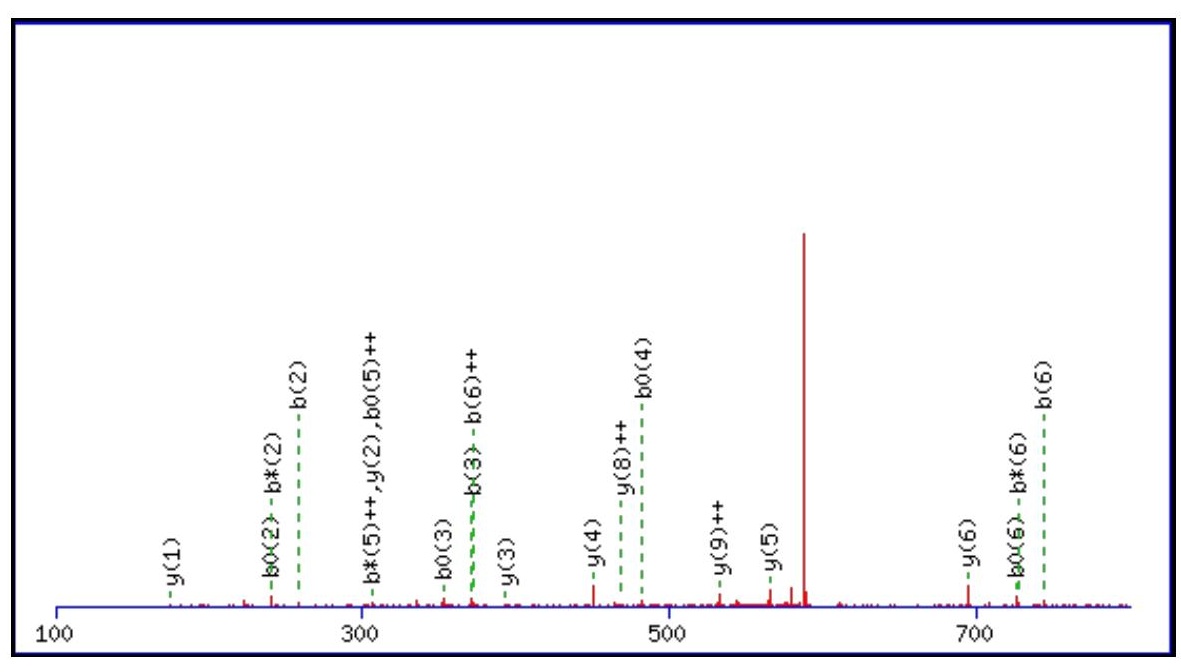



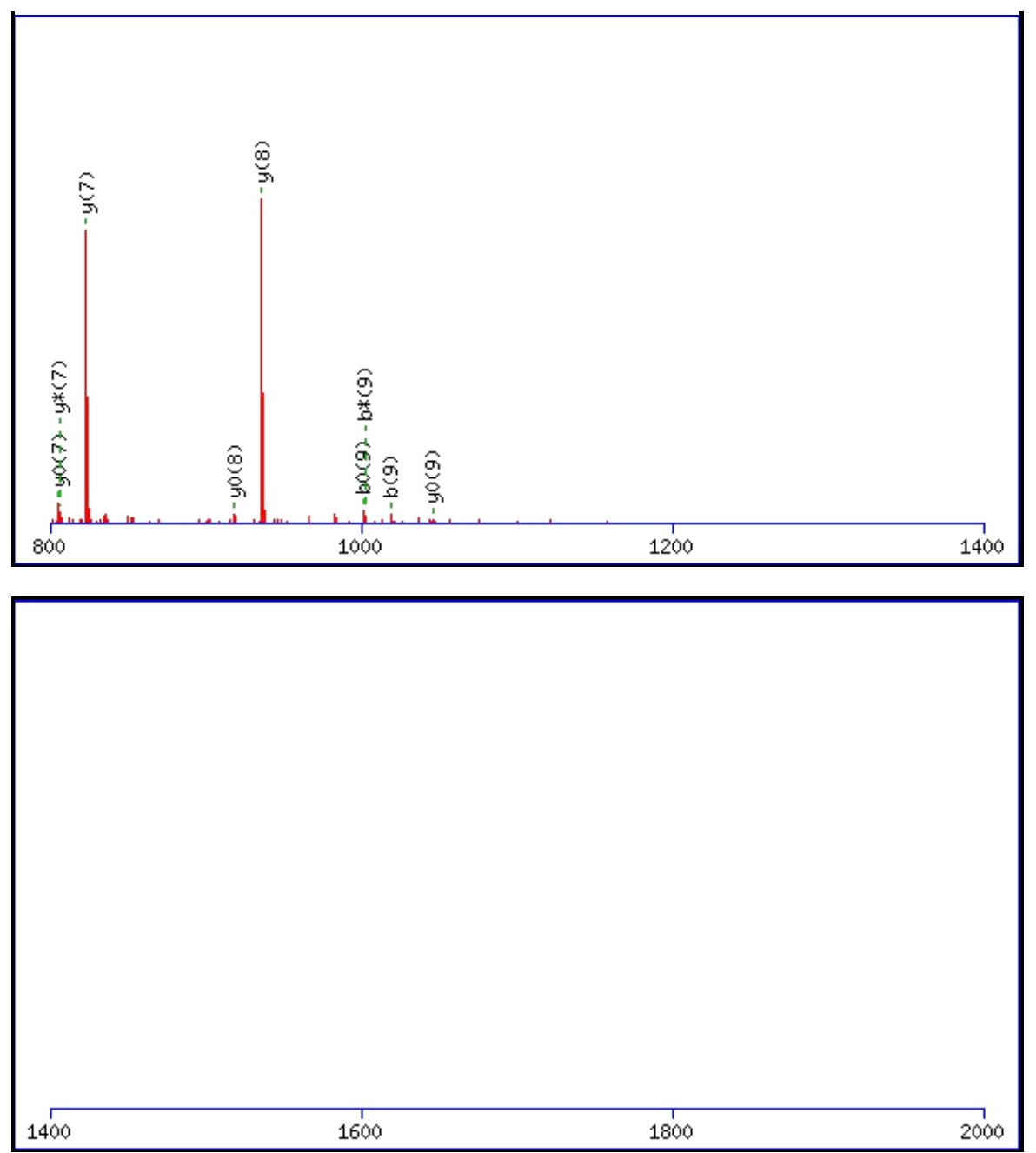

Monoisotopic mass of neutral peptide $\operatorname{Mr}($ calc): 1192.5394

Fixed modifications: Carbamidomethyl (C)

Variable modifications:

N6 : Deamidated_N (N)

Ions Score: 50 Expect : $\odot .001$

Matches (Bold Red): 29/102 fragment ions using 53 most intense peaks

\begin{tabular}{|c|c|c|c|c|c|c|c|c|c|c|c|c|c|c|}
\hline \# & b & $\mathbf{b}^{++}$ & $\mathbf{b}^{*}$ & $\mathbf{b}^{*^{++}}$ & $\mathbf{b}^{0}$ & $\mathbf{b}^{0++}$ & Seq. & $\mathbf{y}$ & $\mathbf{y}^{++}$ & $\mathbf{y}^{*}$ & $\mathrm{y}^{*^{++}}$ & $\mathbf{y}^{0}$ & $\mathbf{y}^{0++}$ & \# \\
\hline 1 & 130.0499 & 65.5286 & & & 112.0393 & 56.5233 & $\mathbf{E}$ & & & & & & & 10 \\
\hline 2 & 258.1448 & 129.5761 & 241.1183 & 121.0628 & 240.1343 & 120.5708 & $\mathbf{K}$ & 1064.5041 & 532.7557 & 1047.4775 & 524.2424 & 1046.4935 & 523.7504 & 9 \\
\hline 3 & 371.2289 & 186.1181 & 354.2023 & 177.6048 & 353.2183 & 177.1128 & I & 936.4091 & 468.7082 & 919.3826 & 460.1949 & 918.3986 & 459.7029 & 8 \\
\hline 4 & 500.2715 & 250.6394 & 483.2449 & 242.1261 & 482.2609 & 241.6341 & $\mathbf{E}$ & 823.3251 & 412.1662 & 806.2985 & 403.6529 & 805.3145 & 403.1609 & 7 \\
\hline 5 & 629.3141 & 315.1607 & 612.2875 & 306.6474 & 611.3035 & 306.1554 & $\mathbf{E}$ & 694.2825 & 347.6449 & 677.2559 & 339.1316 & 676.2719 & 338.6396 & 6 \\
\hline 6 & 744.3410 & 372.6741 & 727.3145 & 364.1609 & 726.3305 & 363.6689 & $\mathbf{N}$ & 565.2399 & 283.1236 & 548.2133 & 274.6103 & 547.2293 & 274.1183 & 5 \\
\hline 7 & 801.3625 & 401.1849 & 784.3359 & 392.6716 & 783.3519 & 392.1796 & $\mathbf{G}$ & 450.2129 & 225.6101 & 433.1864 & 217.0968 & 432.2024 & 216.6048 & 4 \\
\hline 8 & 888.3945 & 444.7009 & 871.3680 & 436.1876 & 870.3839 & 435.6956 & $\mathrm{~S}$ & 393.1915 & 197.0994 & 376.1649 & 188.5861 & 375.1809 & 188.0941 & 3 \\
\hline 9 & 1019.4350 & 510.2211 & 1002.4084 & 501.7079 & 1001.4244 & 501.2159 & $\mathbf{M}$ & 306.1594 & 153.5834 & 289.1329 & 145.0701 & & & 2 \\
\hline 10 & & & & & & & $\mathbf{R}$ & 175.1190 & 88.0631 & 158.0924 & 79.5498 & & & 1 \\
\hline
\end{tabular}
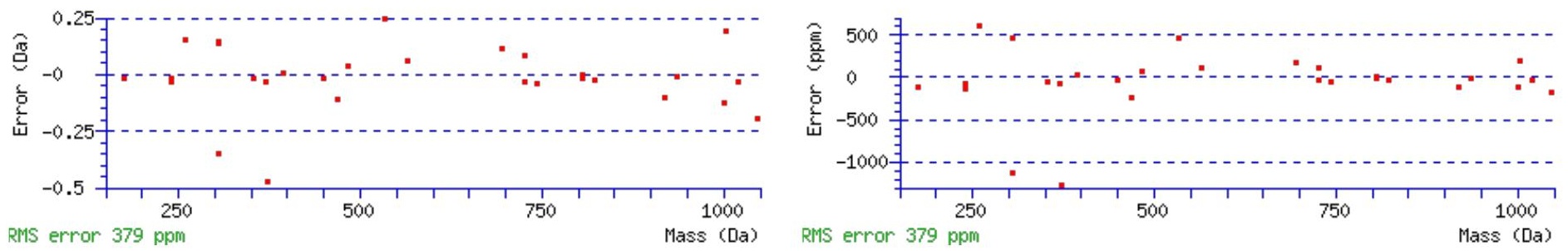

\section{All matches to this query}




\begin{tabular}{|l|l|l|l|}
\hline Score & Mr(calc): & Delta & \multicolumn{1}{|c|}{ Sequence } \\
\hline 50.4 & 1192.5394 & -0.0018 & EKIEENGSMR \\
\hline 25.0 & 1192.5394 & -0.0018 & QELDLNSSMR \\
\hline 13.0 & 1192.5489 & -0.0114 & SFSKSSTLTR \\
\hline 13.0 & 1192.5489 & -0.0114 & SFSKSSTLTR \\
\hline 13.0 & 1192.5489 & -0.0114 & SFSKSSTLTR \\
\hline 11.6 & 1192.5394 & -0.0019 & IEVANTDGSMR \\
\hline 10.4 & 1192.5295 & 0.0080 & HIFTASSDCR \\
\hline 9.4 & 1192.5424 & -0.0048 & GEAMPPLRSR \\
\hline 8.2 & 1192.5311 & 0.0064 & AFSEKTKMR \\
\hline 7.5 & 1192.5329 & 0.0047 & EKNLCQDMR \\
\hline
\end{tabular}

Spectrum No: 673; Query: 729; Rank: 1

\section{Peptide View}

MS/MS Fragmentation of LCDYENHFNTSK

Found in IPI00326242, Tax_Id=10116 Gene_Symbol=Clcn5 Chloride channel protein 5

Match to Query 729: 1527.632608 from(764.823580,2+)

Title: 100101RatKid_NS_deglyco_26.1300.1300.2.dta

Data file K:\NewmanPaper|Piliangl3SubProteomes\Piliang3SP\mgf5ppm\ERLIC_3SubProteomes5ppm.mgf
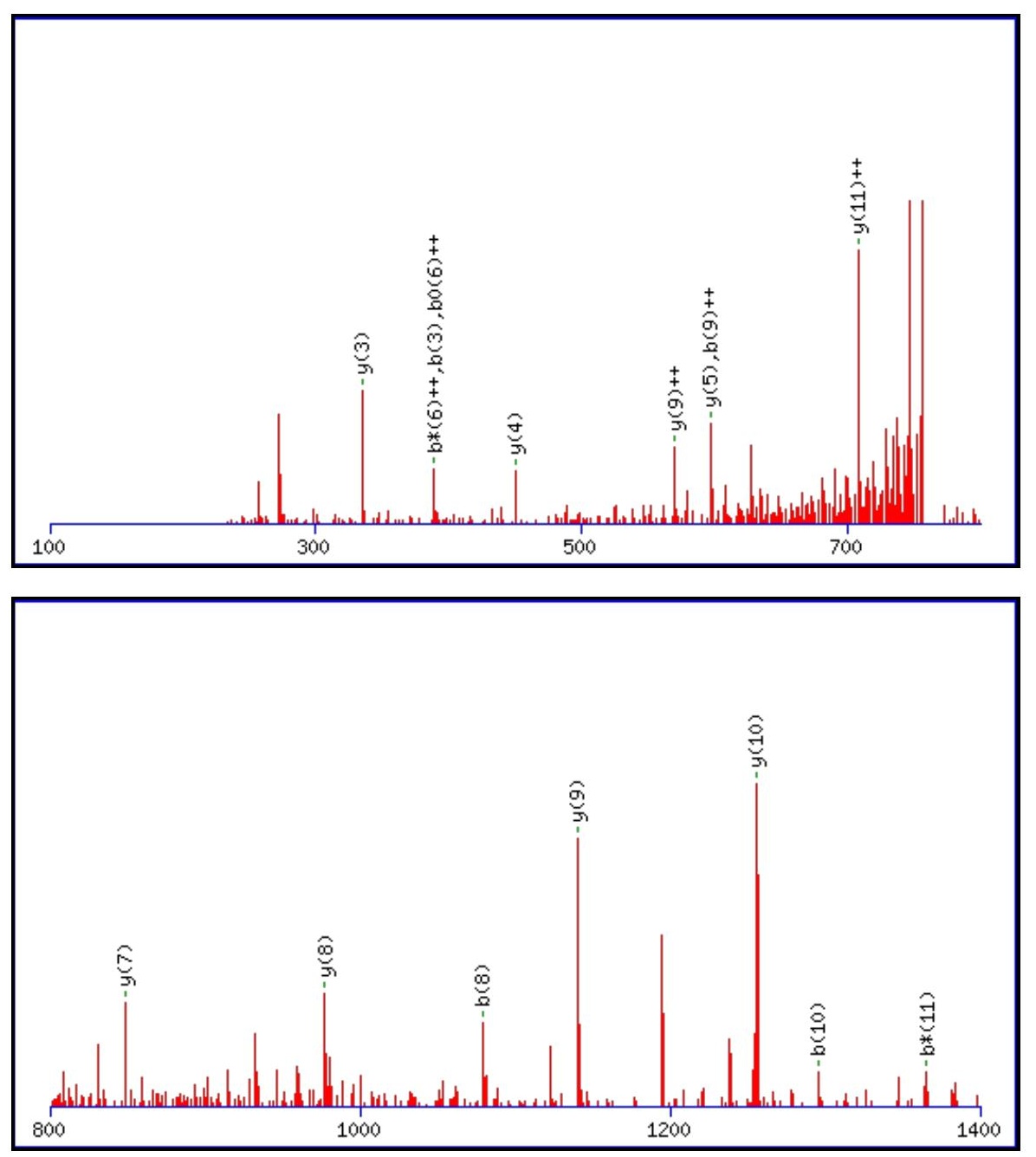


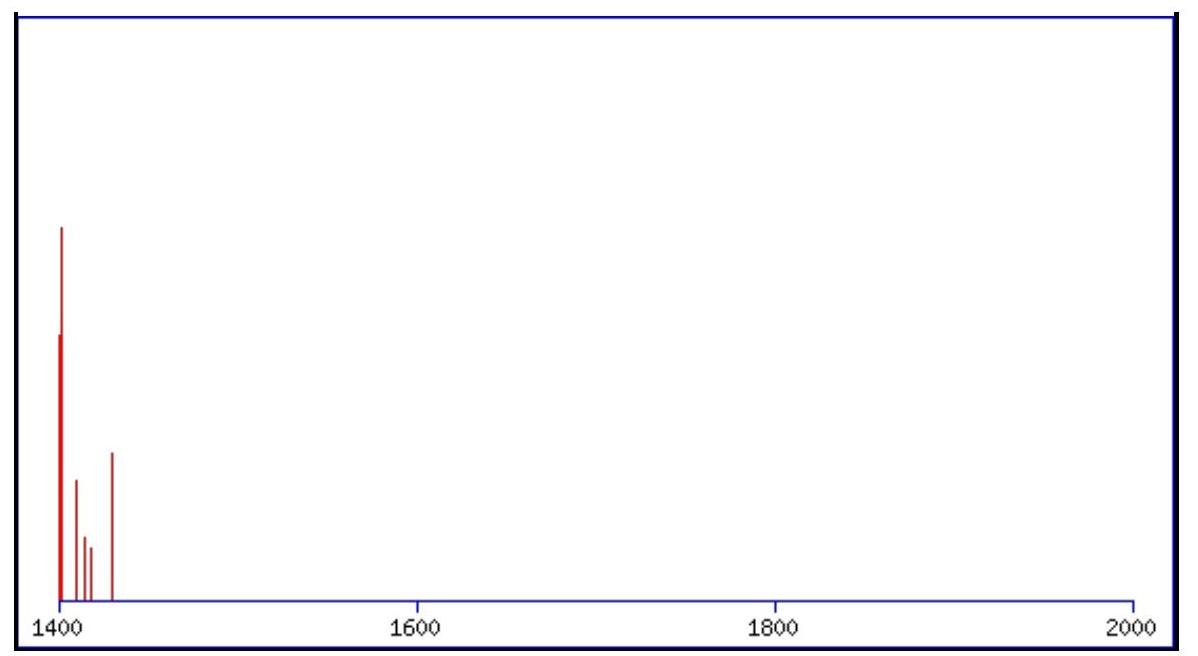

Monoisotopic mass of neutral peptide $\operatorname{Mr}($ calc): 1527.6300

Fixed modifications: Carbamidomethyl (C)

Variable modifications:

N9: Deamidated $\mathrm{N}(\mathrm{N})$

Ions Score: 50 Expect: 0.0011

Matches (Bold Red): 16/116 fragment ions using 18 most intense peaks

\begin{tabular}{|r|c|c|c|c|c|c|c|c|c|c|c|c|c|c|}
\hline$\#$ & $\mathbf{b}$ & $\mathbf{b}^{++}$ & $\mathbf{b}^{*}$ & $\mathbf{b}^{\mathbf{*}^{++}}$ & $\mathbf{b}^{\mathbf{0}}$ & $\mathbf{b}^{\mathbf{0 + +}}$ & $\mathbf{S e q}$ & $\mathbf{y}$ & $\mathbf{y}^{++}$ & $\mathbf{y}^{\mathbf{*}}$ & $\mathbf{y}^{\mathbf{*}^{++}}$ & $\mathbf{y}^{\mathbf{0}}$ & $\mathbf{y}^{\mathbf{0 + +}}$ & $\#$ \\
\hline $\mathbf{1}$ & 114.0913 & 57.5493 & & & & & $\mathbf{L}$ & & & & & & & $\mathbf{1 2}$ \\
\hline $\mathbf{2}$ & 274.1220 & 137.5646 & & & & & $\mathbf{C}$ & 1415.5532 & $\mathbf{7 0 8 . 2 8 0 2}$ & 1398.5267 & 699.7670 & 1397.5426 & 699.2750 & $\mathbf{1 1}$ \\
\hline $\mathbf{3}$ & $\mathbf{3 8 9 . 1 4 8 9}$ & 195.0781 & & & 371.1384 & 186.0728 & $\mathbf{D}$ & $\mathbf{1 2 5 5 . 5 2 2 6}$ & 628.2649 & 1238.4960 & 619.7516 & 1237.5120 & 619.2596 & $\mathbf{1 0}$ \\
\hline $\mathbf{4}$ & 552.2123 & 276.6098 & & & 534.2017 & 267.6045 & $\mathbf{Y}$ & $\mathbf{1 1 4 0 . 4 9 5 6}$ & $\mathbf{5 7 0 . 7 5 1 4}$ & 1123.4691 & 562.2382 & 1122.4851 & 561.7462 & $\mathbf{9}$ \\
\hline $\mathbf{5}$ & 681.2549 & 341.1311 & & & 663.2443 & 332.1258 & $\mathbf{E}$ & $\mathbf{9 7 7 . 4 3 2 3}$ & 489.2198 & 960.4057 & 480.7065 & 959.4217 & 480.2145 & $\mathbf{8}$ \\
\hline $\mathbf{6}$ & 795.2978 & 398.1525 & 778.2712 & 389.6393 & 777.2872 & 389.1472 & $\mathbf{N}$ & $\mathbf{8 4 8 . 3 8 9 7}$ & 424.6985 & 831.3632 & 416.1852 & 830.3791 & 415.6932 & $\mathbf{7}$ \\
\hline $\mathbf{7}$ & 932.3567 & 466.6820 & 915.3301 & 458.1687 & 914.3461 & 457.6767 & $\mathbf{H}$ & 734.3468 & 367.6770 & 717.3202 & 359.1638 & 716.3362 & 358.6717 & $\mathbf{6}$ \\
\hline $\mathbf{8}$ & $\mathbf{1 0 7 9 . 4 2 5 1}$ & 540.2162 & 1062.3986 & 531.7029 & 1061.4145 & 531.2109 & $\mathbf{F}$ & $\mathbf{5 9 7 . 2 8 7 9}$ & 299.1476 & 580.2613 & 290.6343 & 579.2773 & 290.1423 & $\mathbf{5}$ \\
\hline $\mathbf{9}$ & 1194.4520 & $\mathbf{5 9 7 . 7 2 9 7}$ & 1177.4255 & 589.2164 & 1176.4415 & 588.7244 & $\mathbf{N}$ & $\mathbf{4 5 0 . 2 1 9 4}$ & 225.6134 & 433.1929 & 217.1001 & 432.2089 & 216.6081 & $\mathbf{4}$ \\
\hline $\mathbf{1 0}$ & $\mathbf{1 2 9 5 . 4 9 9 7}$ & 648.2535 & 1278.4732 & 639.7402 & 1277.4892 & 639.2482 & $\mathbf{T}$ & $\mathbf{3 3 5 . 1 9 2 5}$ & 168.0999 & 318.1660 & 159.5866 & 317.1819 & 159.0946 & $\mathbf{3}$ \\
\hline $\mathbf{1 1}$ & 1382.5318 & 691.7695 & $\mathbf{1 3 6 5 . 5 0 5 2}$ & 683.2562 & 1364.5212 & 682.7642 & $\mathbf{S}$ & 234.1448 & 117.5761 & 217.1183 & 109.0628 & 216.1343 & 108.5708 & $\mathbf{2}$ \\
\hline $\mathbf{1 2}$ & & & & & & & $\mathbf{K}$ & 147.1128 & 74.0600 & 130.0863 & 65.5468 & & & $\mathbf{1}$ \\
\hline
\end{tabular}
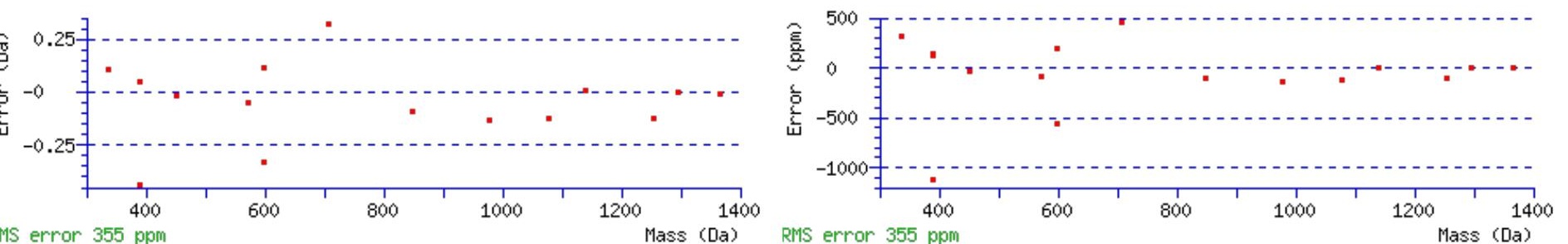

RMS error 355 ppm Mass (Da) RMS error $355 \mathrm{ppm}$

\section{All matches to this query}

\begin{tabular}{|l|l|l|l|}
\hline Score & Mr(calc): & Delta & \multicolumn{1}{c|}{ Sequence } \\
\hline 50.4 & 1527.6300 & 0.0026 & LCDYENHFNTSK \\
\hline 24.4 & 1527.6300 & 0.0026 & LCDYENHFNTSK \\
\hline 5.6 & 1525.6160 & 2.0166 & MGYTNLSYLNPR \\
\hline 4.0 & 1525.6160 & 2.0166 & MGYTNLSYLNPR \\
\hline 3.0 & 1526.6184 & 1.0142 & MXLSNSTETRDR \\
\hline 2.6 & 1527.6293 & 0.0033 & DSTAMSVKDQNCR \\
\hline 2.6 & 1525.6300 & 2.0026 & RDDRTHASRPR \\
\hline 1.6 & 1526.6151 & 1.0175 & NSSYPETGHGKNR \\
\hline 1.6 & 1526.6151 & 1.0175 & NSSYPETGHGKNR \\
\hline & & & \\
\hline
\end{tabular}




\section{\begin{tabular}{|l|l|l|l|}
0.7 & 1527.6178 & 0.0148 & FDGSPGQIGGGGPMR \\
\hline
\end{tabular}}

Spectrum No: 674; Query: 2480; Rank: 1

\section{Peptide View}

MS/MS Fragmentation of NDQDTWDYTNPNLSGQGDPGSNPNKR

Found in IPI00364061, Tax_Id=10116 Gene_Symbol=Hnrpl Hnrpl protein

Match to Query 2480: 2890.247502 from(964.423110,3+)

Title: 100101RatKid_NS_deglyco_15.2423.2423.3.dta

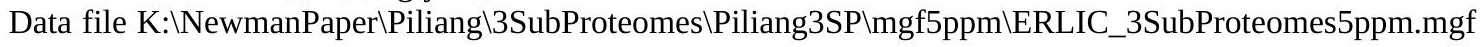
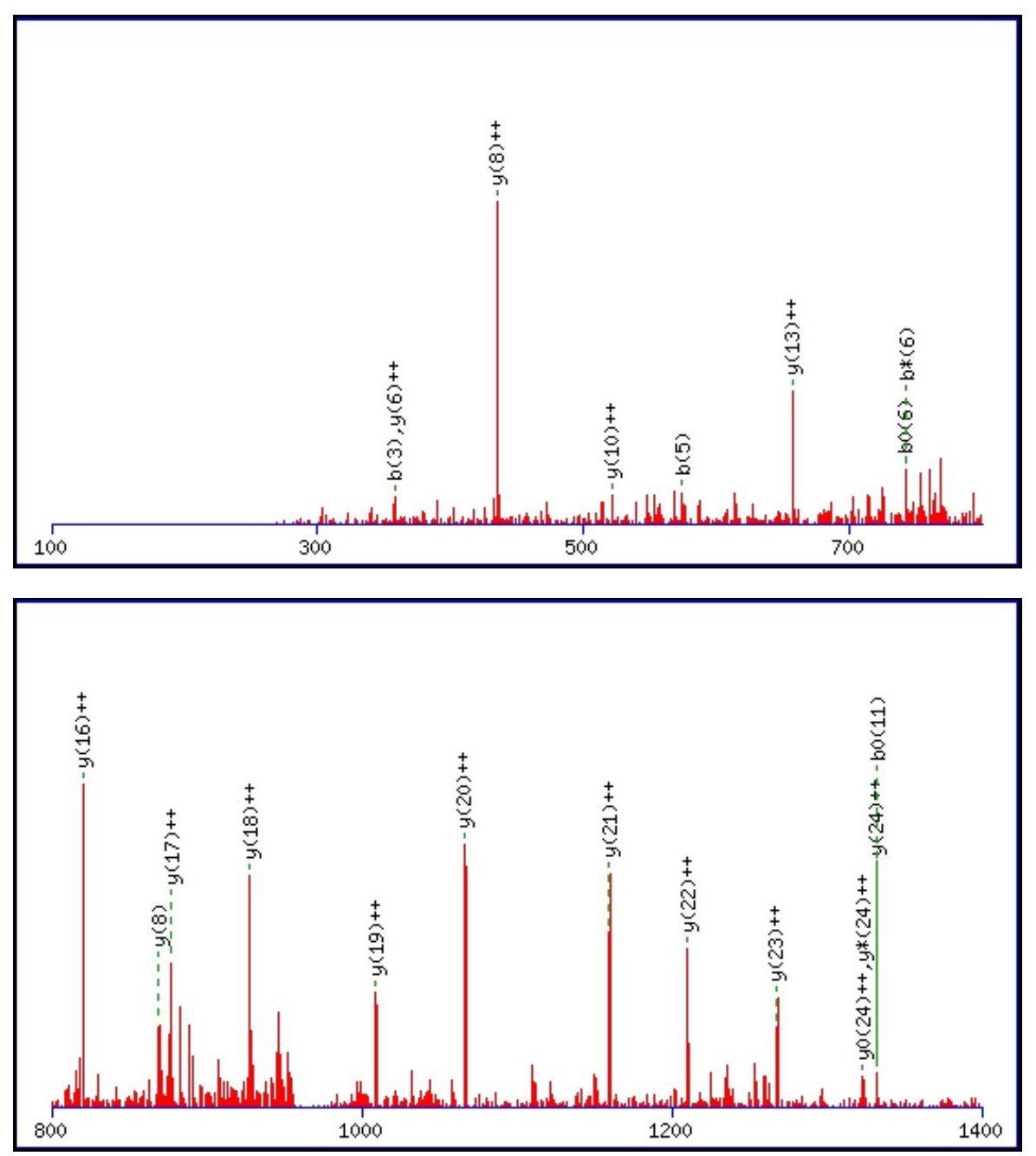


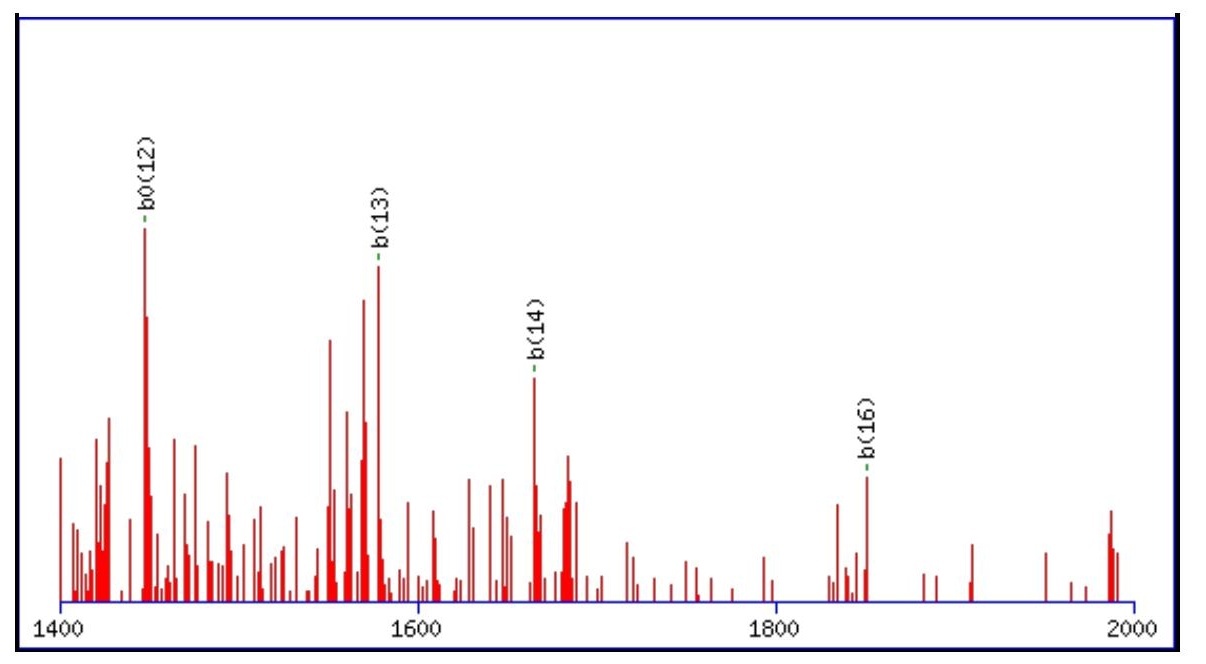

Monoisotopic mass of neutral peptide $\operatorname{Mr}($ calc): 2890.2390

Fixed modifications: Carbamidomethyl (C)

Variable modifications:

N12 : Deamidated $\mathrm{N}(\mathrm{N})$

Ions Score: 50 Expect: 0.0035

Matches (Bold Red): 25/288 fragment ions using 59 most intense peaks

\begin{tabular}{|c|c|c|c|c|c|c|c|c|c|c|c|c|c|c|}
\hline \# & b & $\mathbf{b}^{++}$ & $\mathbf{b}^{*}$ & $\mathbf{b}^{*^{++}}$ & $\mathbf{b}^{0}$ & & $q$ & $\mathbf{y}$ & & $\mathbf{y}^{*}$ & & $\mathbf{y}^{0}$ & & \# \\
\hline 1 & 115. & & & & & & $\mathbf{N}$ & & & & & & & 26 \\
\hline 2 & 230.0771 & 15.5422 & 213.0506 & & & & D & & & & & & & 25 \\
\hline 3 & 358.1357 & & & & & & Q & & & & & & & 24 \\
\hline 4 & 473.1627 & 50 & 456. & 17 & & & D & 78 & & 913 & 493 & 073 & 573 & 23 \\
\hline 5 & 574.2103 & 287.6088 & 557.1838 & 279.0955 & 556.1 & 278.6035 & $\mathbf{T}$ & 909 & 491 & 2402.0644 & 358 & 803 & & 22 \\
\hline 6 & 760.2897 & 80.6485 & & & & & W & & & & & & & 21 \\
\hline 7 & 875.3166 & 438.1 & 858. & 429. & 857 & 429 & D & & 856 & & & 533 & 105 & 20 \\
\hline 8 & 1038.3799 & 519.6936 & 21.3 & 511. & 694 & 510. & $\mathbf{Y}$ & 370 & 721 & 104 & 588 & 1998 & 9668 & 19 \\
\hline 9 & 776 & 74 & & & & & $\mathbf{T}$ & & & & & & & 18 \\
\hline 10 & 1253.4705 & 627.2389 & 236.4440 & 618.7256 & 1600 & 618.2 & $\mathbf{N}$ & 260 & 166 & 1735 & 4033 & 154 & 9113 & 17 \\
\hline 11 & 1350.5233 & 675.7653 & 333.4968 & 667.2520 & 127 & 666.7600 & $\mathbf{P}$ & 1638.7830 & 8952 & 1621 & 3819 & 1620 & 810.8899 & 16 \\
\hline 2 & 1465.5502 & 733.2 & 37 & & & & $\mathbf{N}$ & & & 152 & & & & 15 \\
\hline 3 & \begin{tabular}{|l|}
1578.6343 \\
\end{tabular} & 789.8208 & 1561.6078 & 781.3075 & 1560.6 & 780.8155 & $\mathbf{L}$ & 1426. & 3553 & 1409 & 420 & 1408 & 3500 & 14 \\
\hline 14 & 1665.6663 & 833.3368 & 548.6398 & 824.8235 & 647.6558 & 824.3315 & $\mathrm{~S}$ & 1313. & 3133 & 1296 . & 648.8000 & 1295 & 3080 & 13 \\
\hline 5 & 17 & 86 & 312 & & 772 & & G & & & 1209 & & 1208 & & 12 \\
\hline 6 & 850.7464 & 925.8768 & 33.7198 & 917.3635 & 332.7358 & 916. & $\mathbf{Q}$ & 1169. & 2865 & 1152 & & 115 & 812 & 11 \\
\hline 17 & 1907.7678 & 954.3876 & 1890.7413 & 945.8743 & 1889.7573 & 945.3823 & G & 1041.5072 & 521.2572 & 1024.4806 & .7440 & 1023.4966 & 2520 & 10 \\
\hline 18 & 22.7948 & 11.9010 & 05.7682 & 878 & 04.7842 & 8957 & D & 4857 & 492.7465 & 1592 & 2332 & 4752 & 483.7412 & ב \\
\hline 19 & 2119.8475 & 1060.4274 & 2102.8210 & 51.9141 & 2101.8370 & 1051.4221 & $\mathbf{P}$ & 869.4 & & & & & 2277 & \\
\hline 20 & 2176.8690 & 1088.9381 & 2159.8425 & 080.4249 & 2158.8584 & 1079.9329 & G & 772.4060 & 386.7067 & 755.3795 & .1934 & 754.3955 & 377.7014 & 7 \\
\hline 21 & 2263.9010 & 1132.4542 & 2246.8745 & 23.9409 & 2245.8905 & 1123.4489 & $\mathrm{~S}$ & 715.3846 & 358.1959 & 698.3580 & 6826 & 697. & 1906 & 6 \\
\hline 22 & 2377.9440 & 1189.4756 & 2360.9174 & 80.9623 & 2359.9334 & 1180.4703 & $\mathbf{N}$ & 628.3525 & 314.6799 & & & & & \\
\hline 23 & 2474.9967 & 1238.0020 & 2457.9702 & 229.4887 & 2456.9862 & 1228.9967 & $\mathbf{P}$ & 514.3096 & 257.6584 & 497.2831 & & & & \\
\hline $24:$ & 2589.0397 & 1295.0235 & 2572.0131 & 1286.5102 & 2571.0291 & 1286.0182 & $\mathbf{N}$ & 417.2568 & 209.1321 & 400.2303 & .6188 & & & \\
\hline 25 & 2717.1346 & 1359.0709 & 2700.1081 & 1350.5577 & 2699.1240 & 1350.0657 & $\mathbf{K}$ & 303.2139 & 152.1106 & 286.1874 & 143.5973 & & & 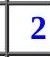 \\
\hline 26 & & & & & & & $\mathbf{R}$ & 175.1190 & 88.0631 & 158.0924 & 79.5498 & & & \\
\hline
\end{tabular}



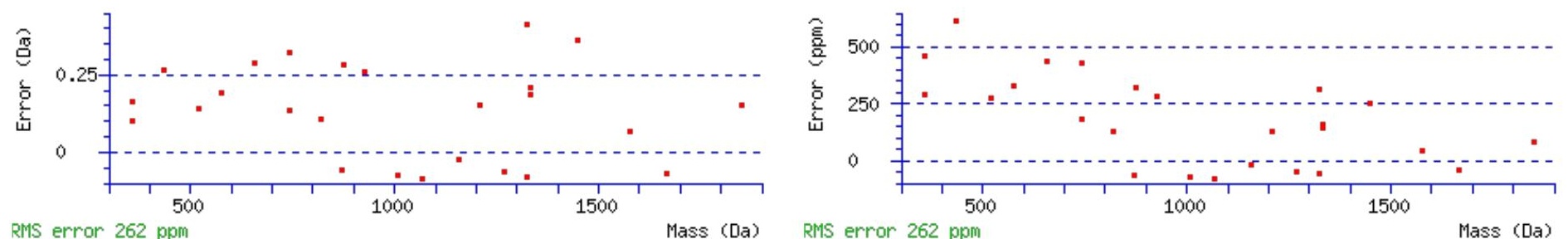

RIS error 262 ppm

\section{All matches to this query}

\begin{tabular}{|l|l|l|l|}
\hline Score & Mr(calc): & Delta & \multicolumn{1}{|c|}{ Sequence } \\
\hline 50.4 & 2890.2390 & 0.0085 & NDQDTWDYTNPNLSGQGDPGSNPNKR \\
\hline 49.3 & 2890.2390 & 0.0085 & NDQDTWDYTNPNLSGQGDPGSNPNKR \\
\hline 49.3 & 2890.2390 & 0.0085 & NDQDTWDYTNPNLSGQGDPGSNPNKR \\
\hline 40.8 & 2890.2390 & 0.0085 & NDQDTWDYTNPNLSGQGDPGSNPNKR \\
\hline 13.4 & 2889.2550 & 0.9925 & NDQDTWDYTNPNLSGQGDPGSNPNKR \\
\hline 13.2 & 2890.2390 & 0.0085 & NDQDTWDYTNPNLSGQGDPGSNPNKR \\
\hline 0.9 & 2889.2479 & 0.9996 & QFVLTAAHCNGXEITVTLGAHNMSK \\
\hline 0.9 & 2889.2479 & 0.9996 & QFVLTAAHCNGXEITVTLGAHNMSK \\
\hline 0.9 & 2889.2479 & 0.9996 & QFVLTAAHCNGXEITVTLGAHNMSK \\
\hline 0.9 & 2889.2479 & 0.9996 & QFVLTAAHCNGXEITVTLGAHNMSK \\
\hline
\end{tabular}

Spectrum No: 675; Query: 2281; Rank: 1

\section{Peptide View}

MS/MS Fragmentation of CDSGFALDSEERNCTDIDECR

Found in IPI00204006, Tax_Id=10116 Gene_Symbol=Fbn1 Fibrillin-1

Match to Query 2281: 2548.980552 from(850.667460,3+)

Title: 100101RatKid_NS_deglyco_18.2581.2581.3.dta

Data file K:INewmanPaper|Piliangl3SubProteomes\Piliang3SP\mgf5ppm\ERLIC_3SubProteomes5ppm.mgf

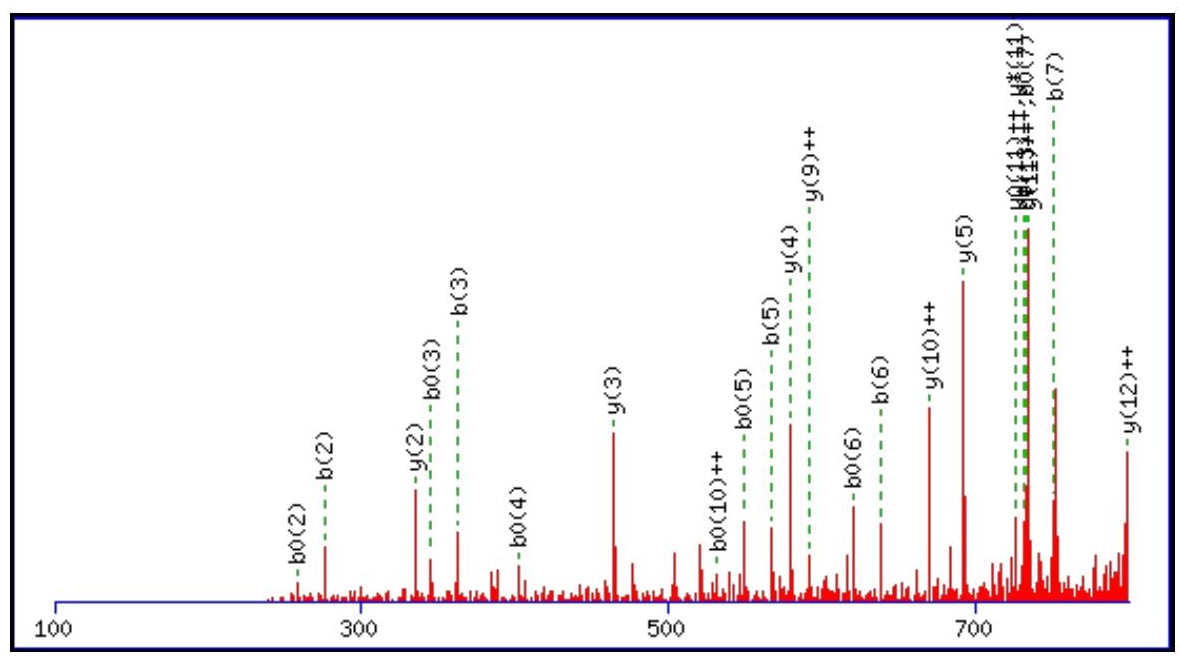



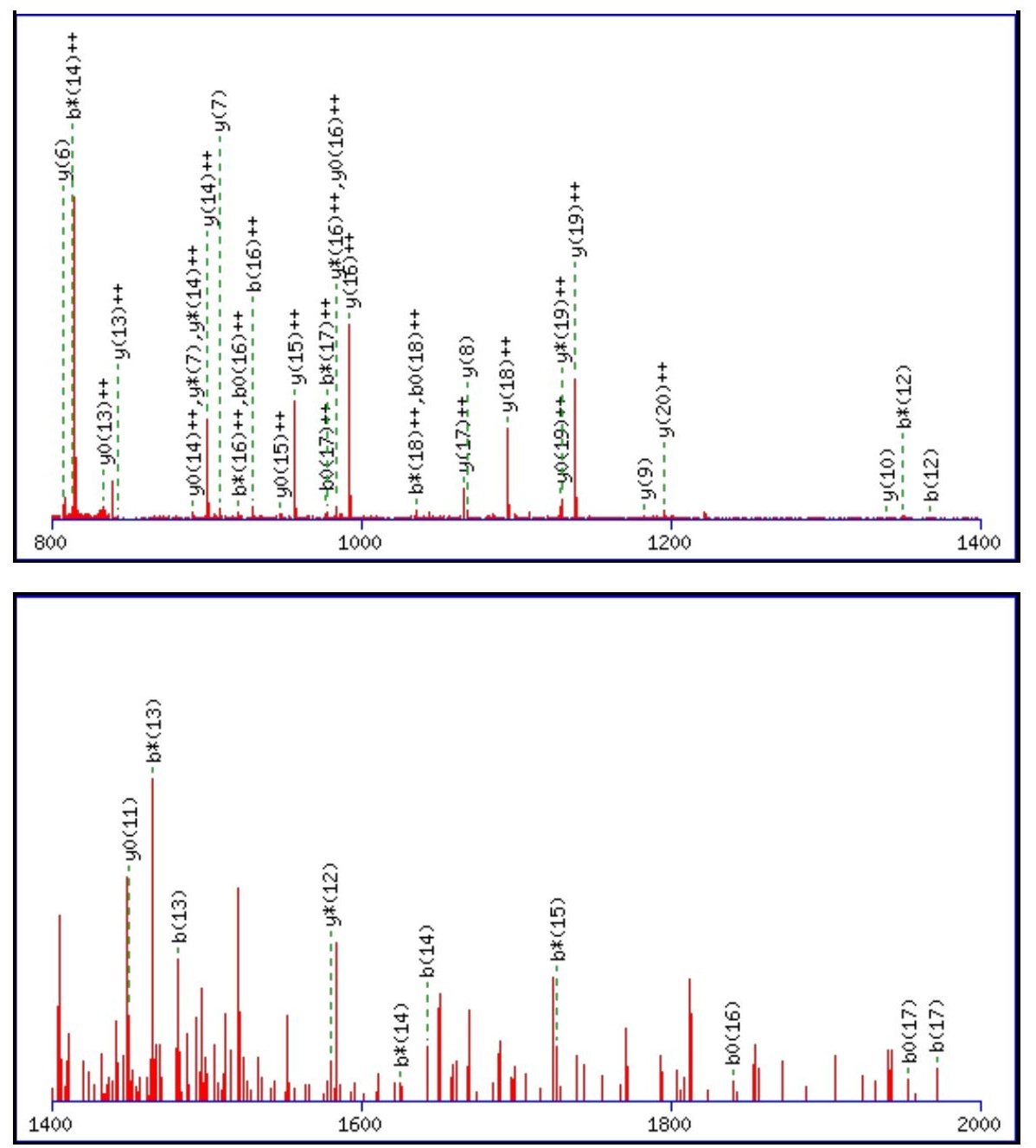

Monoisotopic mass of neutral peptide $\operatorname{Mr}($ calc): 2548.9741

Fixed modifications: Carbamidomethyl (C)

Variable modifications:

N13 : Deamidated_N (N)

Ions Score: 50 Expect: 0.0019

Matches (Bold Red): 66/212 fragment ions using 167 most intense peaks

\begin{tabular}{|c|c|c|c|c|c|c|c|c|c|c|c|c|c|c|}
\hline \# & b & $\mathbf{b}^{++}$ & $\mathbf{b}^{*}$ & $\mathbf{b}^{*^{++}}$ & $\mathbf{b}^{\mathbf{0}}$ & $\mathbf{b}^{\mathbf{0 + +}}$ & Seq. & $\mathbf{y}$ & $\mathbf{y}^{++}$ & $\mathbf{y}^{*}$ & $\mathbf{y}^{*^{++}}$ & $\mathbf{y}^{0}$ & $\mathbf{y}^{\mathbf{0 + +}}$ & \# \\
\hline 1 & 161.0379 & 81.0226 & & & & & C & & & & & & & 21 \\
\hline 2 & 276.0649 & 138.5361 & & & 258.0543 & 129.5308 & D & 2389.9507 & 1195.4790 & 2372.9242 & 1186.9657 & 2371.9401 & 1186.4737 & 20 \\
\hline 3 & 363.0969 & 182.0521 & & & 345.0863 & 173.0468 & $\mathrm{~S}$ & 2274.9238 & 1137.9655 & 2257.8972 & 1129.4522 & 2256.9132 & 1128.9602 & 19 \\
\hline 4 & 420.1184 & 210.5628 & & & 402.1078 & 201.5575 & G & 2187.8917 & 1094.4495 & 2170.8652 & 1085.9362 & |2169.8812 & 1085.4442 & 18 \\
\hline 5 & 567.1868 & 284.0970 & & & 549.1762 & 275.0917 & $\mathbf{F}$ & 2130.8703 & 1065.9388 & 2113.8437 & 1057.4255 & 2112.8597 & 1056.9335 & 17 \\
\hline 6 & 638.2239 & 319.6156 & & & 620.2133 & 310.6103 & A & 1983.8019 & 992.4046 & 1966.7753 & 983.8913 & 1965.7913 & 983.3993 & 16 \\
\hline 7 & 751.3080 & 376.1576 & & & 733.2974 & 367.1523 & $\mathbf{L}$ & 1912.7647 & 956.8860 & 1895.7382 & 948.3727 & 1894.7542 & 947.8807 & 15 \\
\hline 8 & 866.3349 & 433.6711 & & & 848.3243 & 424.6658 & D & 1799.6807 & 900.3440 & 1782.6541 & 891.8307 & 1781.6701 & 891.3387 & 14 \\
\hline 9 & 953.3669 & 477.1871 & & & 935.3564 & 468.1818 & $\mathrm{~S}$ & 1684.6537 & 842.8305 & 1667.6272 & 834.3172 & 1666.6432 & 833.8252 & 13 \\
\hline 10 & 1082.4095 & 541.7084 & & & 1064.3990 & 532.7031 & $\mathbf{E}$ & 1597.6217 & 799.3145 & 1580.5952 & 790.8012 & 1579.6111 & 790.3092 & 12 \\
\hline 11 & 1211.4521 & 606.2297 & & & 1193.4415 & 597.2244 & $\mathbf{E}$ & 1468.5791 & 734.7932 & 1451.5526 & 726.2799 & 1450.5685 & 725.7879 & 11 \\
\hline 12 & 1367.5532 & 684.2802 & 1350.5267 & 675.7670 & 1349.5427 & 675.2750 & $\mathbf{R}$ & 1339.5365 & 670.2719 & 1322.5100 & 661.7586 & 1321.5260 & 661.2666 & 10 \\
\hline 13 & \begin{tabular}{|l|}
1482.5802 \\
\end{tabular} & 741.7937 & \begin{tabular}{|l}
1465.5536 \\
\end{tabular} & 733.2804 & 1464.5696 & 732.7884 & $\mathbf{N}$ & 1183.4354 & 592.2213 & 1166.4089 & 583.7081 & 1165.4248 & 583.2161 & 9 \\
\hline 14 & 1642.6108 & 821.8090 & 1625.5843 & 813.2958 & 1624.6002 & 812.8038 & $\mathrm{C}$ & 1068.4085 & 534.7079 & 1051.3819 & 526.1946 & 1050.3979 & 525.7026 & 8 \\
\hline 15 & 1743.6585 & 872.3329 & 1726.6319 & 863.8196 & 1725.6479 & 863.3276 & $T$ & 908.3778 & 454.6925 & 891.3513 & 446.1793 & 890.3673 & 445.6873 & 7 \\
\hline 16 & 1858.6854 & 929.8464 & 1841.6589 & 921.3331 & 1840.6749 & 920.8411 & D & 807.3301 & 404.1687 & 790.3036 & 395.6554 & 789.3196 & 395.1634 & 6 \\
\hline 17 & 1971.7695 & 986.3884 & 1954.7429 & 977.8751 & 1953.7589 & 977.3831 & I & 692.3032 & 346.6552 & 675.2767 & 338.1420 & 674.2926 & 337.6500 & 5 \\
\hline 18 & 2086.7964 & 1043.9019 & 2069.7699 & 1035.3886 & 2068.7859 & 1034.8966 & D & 579.2191 & 290.1132 & 562.1926 & 281.5999 & 561.2086 & 281.1079 & 4 \\
\hline
\end{tabular}




\begin{tabular}{|r|r|r|r|r|r|r|r|r|r|r|r|r|r|r|r|}
$\mathbf{1 9}$ & 2215.8390 & 1108.4232 & 2198.8125 & 1099.9099 & 2197.8285 & 1099.4179 & $\mathbf{E}$ & $\mathbf{4 6 4 . 1 9 2 2}$ & 232.5997 & 447.1656 & 224.0865 & 446.1816 & 223.5945 & $\mathbf{3}$ \\
\hline $\mathbf{2 0}$ & 2375.8697 & 1188.4385 & 2358.8431 & 1179.9252 & 2357.8591 & 1179.4332 & $\mathbf{C}$ & $\mathbf{3 3 5 . 1 4 9 6}$ & 168.0784 & 318.1231 & 159.5652 & & & $\mathbf{2}$ \\
\hline $\mathbf{2 1}$ & & & & & & & $\mathbf{R}$ & 175.1190 & 88.0631 & 158.0924 & 79.5498 & & & $\mathbf{1}$ \\
\hline
\end{tabular}
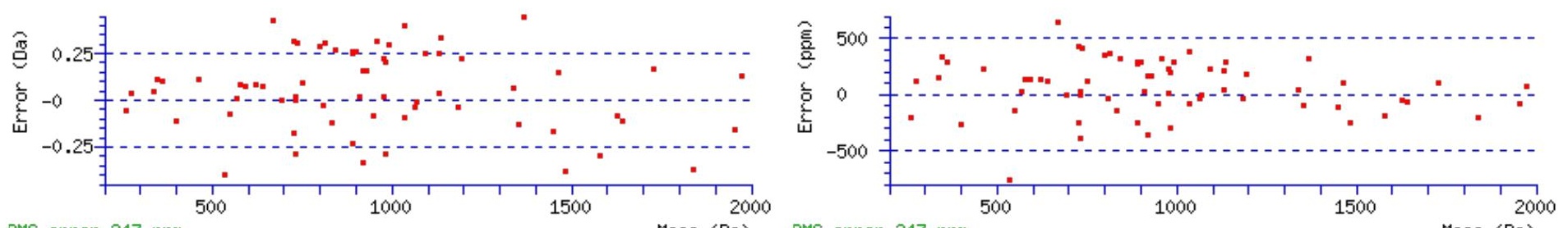

RMS error 247 ppm

Mass (Da) RMS error 247 ppm

Mass (Da)

\section{All matches to this query}

\begin{tabular}{|l|l|l|l|}
\hline Score & Mr(calc): & Delta & \multicolumn{1}{|c|}{ Sequence } \\
\hline 50.3 & 2548.9741 & 0.0065 & CDSGFALDSEERNCTDIDECR \\
\hline 6.2 & 2547.9901 & 0.9905 & CDSGFALDSEERNCTDIDECR \\
\hline 2.0 & 2546.9556 & 2.0249 & RNPSNRNQDCMPSSEPNSPAK \\
\hline 2.0 & 2546.9920 & 1.9886 & RNPSNRNQDCMPSSKPNSPAK \\
\hline 2.0 & 2546.9920 & 1.9886 & RNPSNRNQDCMPSSKPNSPAK \\
\hline 2.0 & 2546.9920 & 1.9886 & RNPSNRNQDCMPSSKPNSPAK \\
\hline 1.8 & 2546.9974 & 1.9831 & THTGEKPFSCPHCNRAFADR \\
\hline 1.8 & 2546.9974 & 1.9831 & THTGEKPFSCPHCNRAFADR \\
\hline 1.7 & 2546.9556 & 2.0249 & RNPSNRNQDCMPSSEPNSPAK \\
\hline 1.5 & 2548.9563 & 0.0243 & FLSGRESHSPDSAWRSYNGR \\
\hline
\end{tabular}

Spectrum No: 676; Query: 2253; Rank: 1

\section{Peptide View}

MS/MS Fragmentation of FNLTEITEEEIHQGFGHLLQR

Found in IPI00200591, Tax_Id=10116 Gene_Symbol=LOC299282 Serine protease inhibitor A3L precursor

Match to Query 2253: 2511.241842 from(838.087890,3+)

Title: 100101RatKid_NS_deglyco_08.5748.5748.3.dta

Data file K:\NewmanPaper|Piliangl3SubProteomes\Piliang3SP\mgf5ppm\ERLIC_3SubProteomes5ppm.mgf

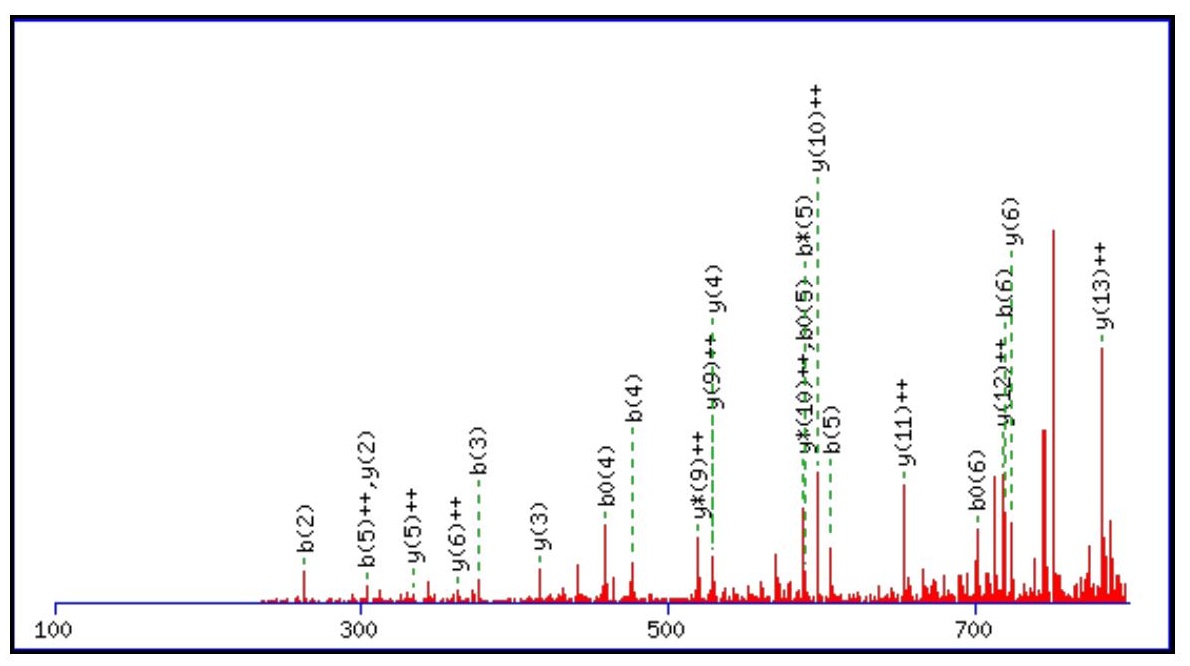



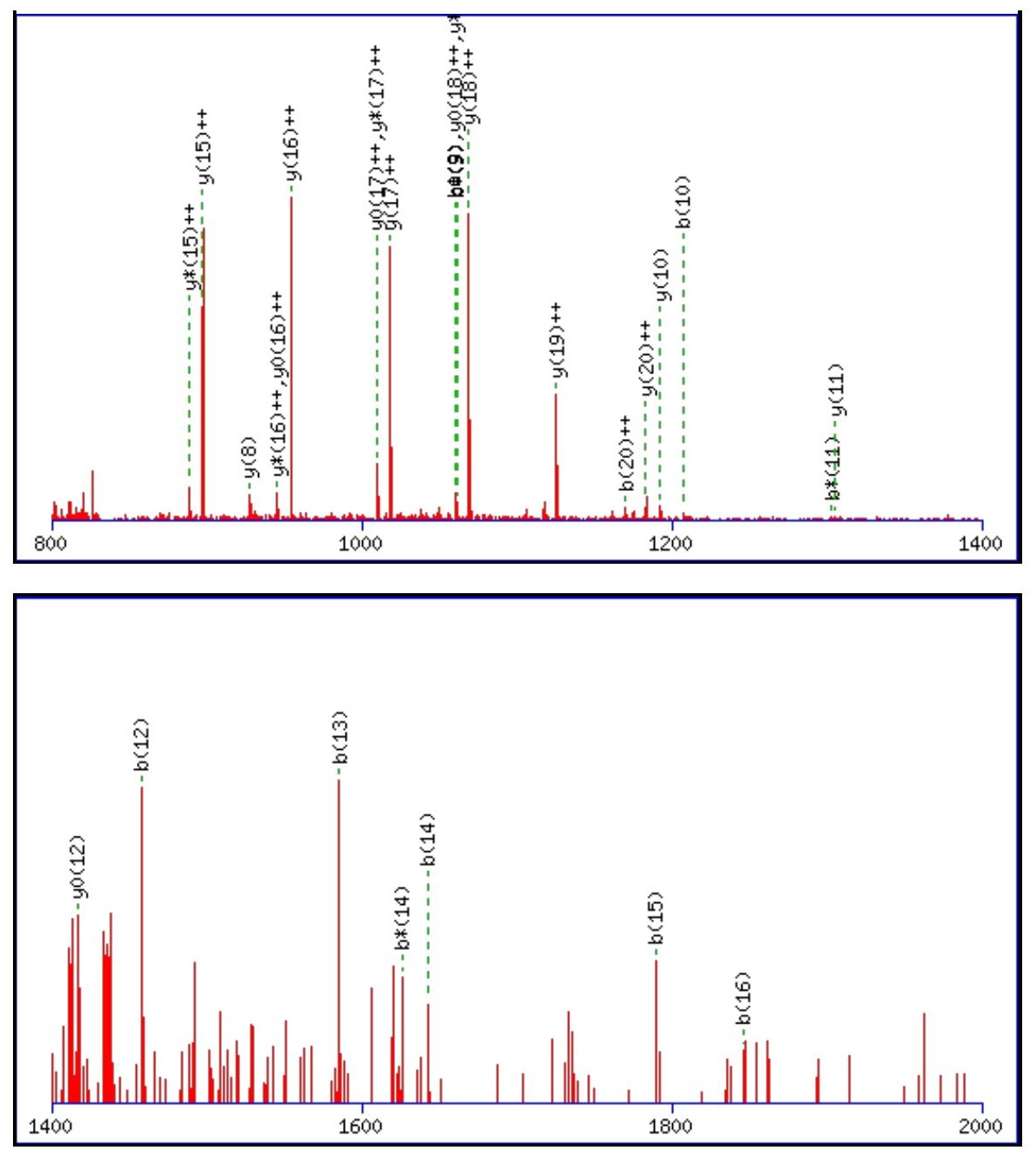

Monoisotopic mass of neutral peptide $\operatorname{Mr}($ calc): 2511.2394

Fixed modifications: Carbamidomethyl (C)

Variable modifications:

N2 : Deamidated $\mathrm{N}(\mathrm{N})$

Ions Score: 50 Expect: 0.0022

Matches (Bold Red): 51/210 fragment ions using 119 most intense peaks

\begin{tabular}{|c|c|c|c|c|c|c|c|c|c|c|c|c|c|c|}
\hline \# & b & $\mathbf{b}^{++}$ & $\mathbf{b}^{*}$ & $\mathbf{b}^{*^{++}}$ & $\mathbf{b}^{\mathbf{0}}$ & $\mathbf{b}^{\mathbf{0 + +}}$ & Seq. & $\mathbf{y}$ & $\mathbf{y}^{++}$ & $\mathbf{y}^{*}$ & $\mathbf{y}^{*^{++}}$ & $\mathbf{y}^{0}$ & $\mathbf{y}^{0++}$ & $\#$ \\
\hline 1 & 148.0757 & 74.5415 & & & & & $\mathbf{F}$ & & & & & & & 21 \\
\hline 2 & 263.1026 & 132.0550 & 246.0761 & 123.5417 & & & $\mathbf{N}$ & 2365.1783 & 1183.0928 & 2348.1517 & 1174.5795 & 2347.1677 & 1174.0875 & 20 \\
\hline 3 & 376.1867 & 188.5970 & 359.1601 & 180.0837 & & & $\mathbf{L}$ & 2250.1513 & 1125.5793 & 2233.1248 & 1117.0660 & 2232.1408 & 1116.5740 & 19 \\
\hline 4 & 477.2344 & 239.1208 & 460.2078 & 230.6075 & 459.2238 & 230.1155 & $T$ & 2137.0673 & 1069.0373 & 2120.0407 & 1060.5240 & 2119.0567 & 1060.0320 & 18 \\
\hline 5 & 606.2770 & 303.6421 & 589.2504 & 295.1288 & 588.2664 & 294.6368 & $E$ & 2036.0196 & 1018.5134 & 2018.9930 & 1010.0002 & 2018.0090 & 1009.5081 & 17 \\
\hline 6 & 719.3610 & 360.1842 & 702.3345 & 351.6709 & 701.3505 & 351.1789 & $\mathbf{I}$ & 1906.9770 & 953.9921 & 1889.9504 & 945.4789 & 1888.9664 & 944.9868 & 16 \\
\hline 7 & 820.4087 & 410.7080 & 803.3822 & 402.1947 & 802.3981 & 401.7027 & $\mathbf{T}$ & 1793.8929 & 897.4501 & 1776.8664 & 888.9368 & 1775.8824 & 888.4448 & 15 \\
\hline 8 & 949.4513 & 475.2293 & 932.4247 & 466.7160 & 931.4407 & 466.2240 & $\mathbf{E}$ & 1692.8452 & 846.9263 & 1675.8187 & 838.4130 & 1674.8347 & 837.9210 & 14 \\
\hline 9 & 1078.4939 & 539.7506 & 1061.4673 & 531.2373 & 1060.4833 & 530.7453 & $E$ & 1563.8027 & 782.4050 & 1546.7761 & 773.8917 & 1545.7921 & 773.3997 & 13 \\
\hline 10 & 1207.5365 & 604.2719 & 1190.5099 & 595.7586 & 1189.5259 & 595.2666 & $\mathbf{E}$ & 1434.7601 & 717.8837 & 1417.7335 & 709.3704 & 1416.7495 & 708.8784 & 12 \\
\hline 11 & 1320.6205 & 660.8139 & \begin{tabular}{|l|l|}
1303.5940 \\
\end{tabular} & 652.3006 & 1302.6100 & 651.8086 & I & 1305.7175 & 653.3624 & 1288.6909 & 644.8491 & & & 11 \\
\hline 12 & 1457.6795 & 729.3434 & 1440.6529 & 720.8301 & 1439.6689 & 720.3381 & $\mathbf{H}$ & 1192.6334 & 596.8203 & 1175.6069 & 588.3071 & & & 10 \\
\hline 13 & \begin{tabular}{|l}
1585.7380 \\
\end{tabular} & 793.3727 & 1568.7115 & 784.8594 & 1567.7275 & 784.3674 & $\mathbf{Q}$ & 1055.5745 & 528.2909 & 1038.5479 & 519.7776 & & & 9 \\
\hline 14 & 1642.7595 & 821.8834 & 1625.7330 & 813.3701 & 1624.7489 & 812.8781 & G & 927.5159 & 464.2616 & 910.4894 & 455.7483 & & & 8 \\
\hline 15 & 1789.8279 & 895.4176 & 1772.8014 & 886.9043 & 1771.8174 & 886.4123 & $\mathbf{F}$ & 870.4944 & 435.7509 & 853.4679 & 427.2376 & & & 7 \\
\hline 16 & 1846.8494 & 923.9283 & 1829.8228 & 915.4151 & 1828.8388 & 914.9230 & $\mathbf{G}$ & 723.4260 & 362.2167 & 706.3995 & 353.7034 & & & 6 \\
\hline 17 & 1983.9083 & 992.4578 & \begin{tabular}{|l}
1966.8817 \\
\end{tabular} & 983.9445 & 1965.8977 & 983.4525 & $\mathbf{H}$ & 666.4046 & 333.7059 & 649.3780 & 325.1926 & & & 5 \\
\hline 18 & 2096.9924 & 1048.9998 & 2079.9658 & 1040.4865 & 2078.9818 & 1039.9945 & $\mathbf{L}$ & 529.3457 & 265.1765 & 512.3191 & 256.6632 & & & 4 \\
\hline
\end{tabular}




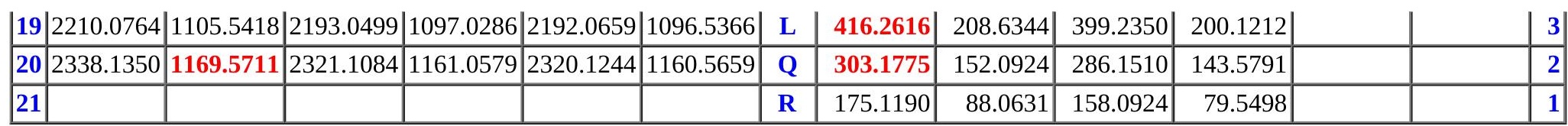
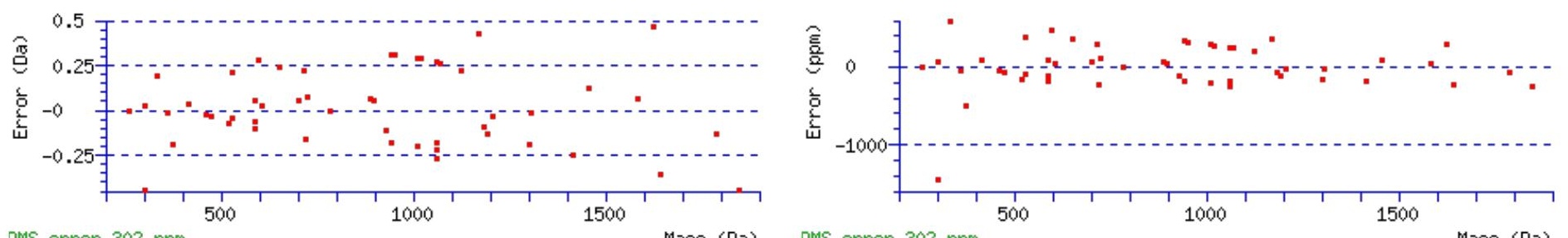

RMS error $302 \mathrm{ppm}$

Mass (Da) RMS error 302 ppm

Mass (Da)

\section{All matches to this query}

\begin{tabular}{|l|c|c|l|}
\hline Score & Mr(calc): & Delta & \multicolumn{1}{c|}{ Sequence } \\
\hline 50.3 & 2511.2394 & 0.0025 & FNLTEITEEEIHQGFGHLLQR \\
\hline 34.3 & 2510.2554 & 0.9865 & FNLTEITEEEIHQGFGHLLQR \\
\hline 12.1 & 2511.2354 & 0.0065 & YPEHSQKEGHLKTGTTTQLNDK \\
\hline 6.5 & 2511.2345 & 0.0073 & YVNVKQESLGHWSOGLKISMK \\
\hline 5.3 & 2511.2217 & 0.0201 & ETALEVGKGSDLNISSLSKSGSPR \\
\hline 4.7 & 2511.2386 & 0.0032 & SKLHVLLQNGKSLSELETDIK \\
\hline 4.7 & 2511.2386 & 0.0032 & SKLHVLLQNGKSLSELETDIK \\
\hline 4.7 & 2511.2386 & 0.0032 & SKLHVLLQNGKSLSELETDIK \\
\hline 3.9 & 2511.2467 & -0.0049 & VVYENAYGKFIGPHKIMATNNK \\
\hline 3.6 & 2511.2523 & -0.0104 & RPHLTVILVGDNPASHSYVLNK \\
\hline
\end{tabular}

Spectrum No: 677; Query: 2230; Rank: 1

\section{Peptide View}

MS/MS Fragmentation of SVANLSSDCLDTCDTGAIWPGLK

Found in IPI00369140, Tax_Id=10116 Gene_Symbol=RGD1309019_predicted 38 kDa protein

Match to Query 2230: 2481.129708 from(1241.572130,2+)

Title: 100101RatKid_NS_deglyco_25.4209.4209.2.dta

Data file K:INewmanPaper|Piliangl3SubProteomes\Piliang3SP\mgf5ppm\ERLIC_3SubProteomes5ppm.mgf

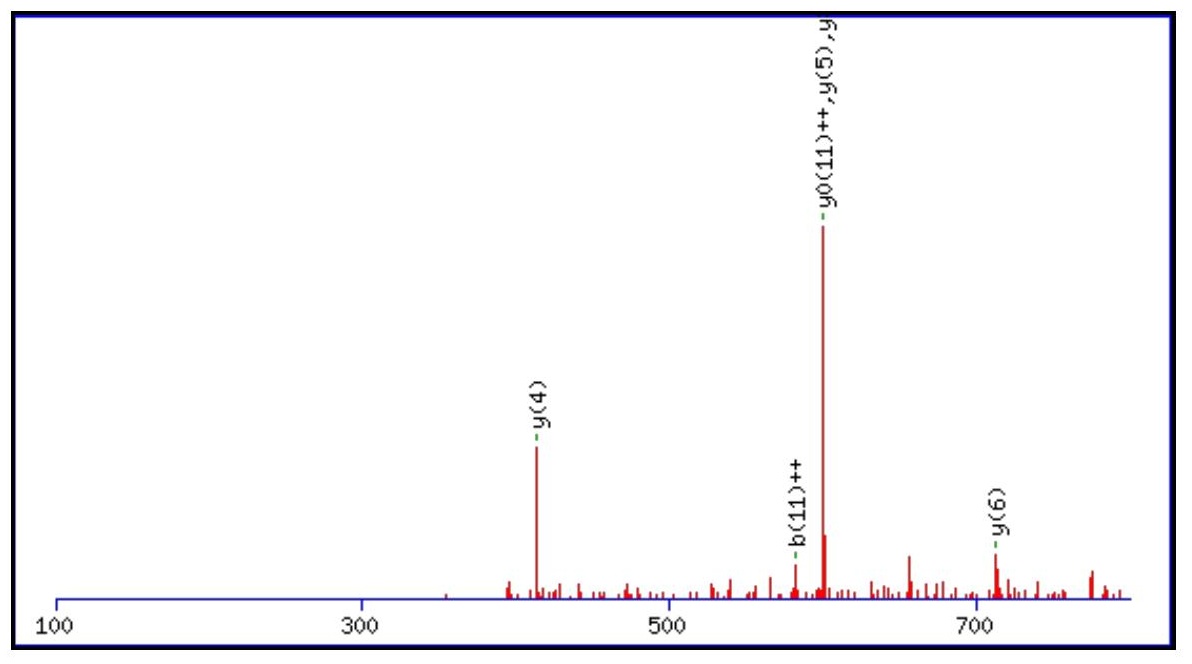



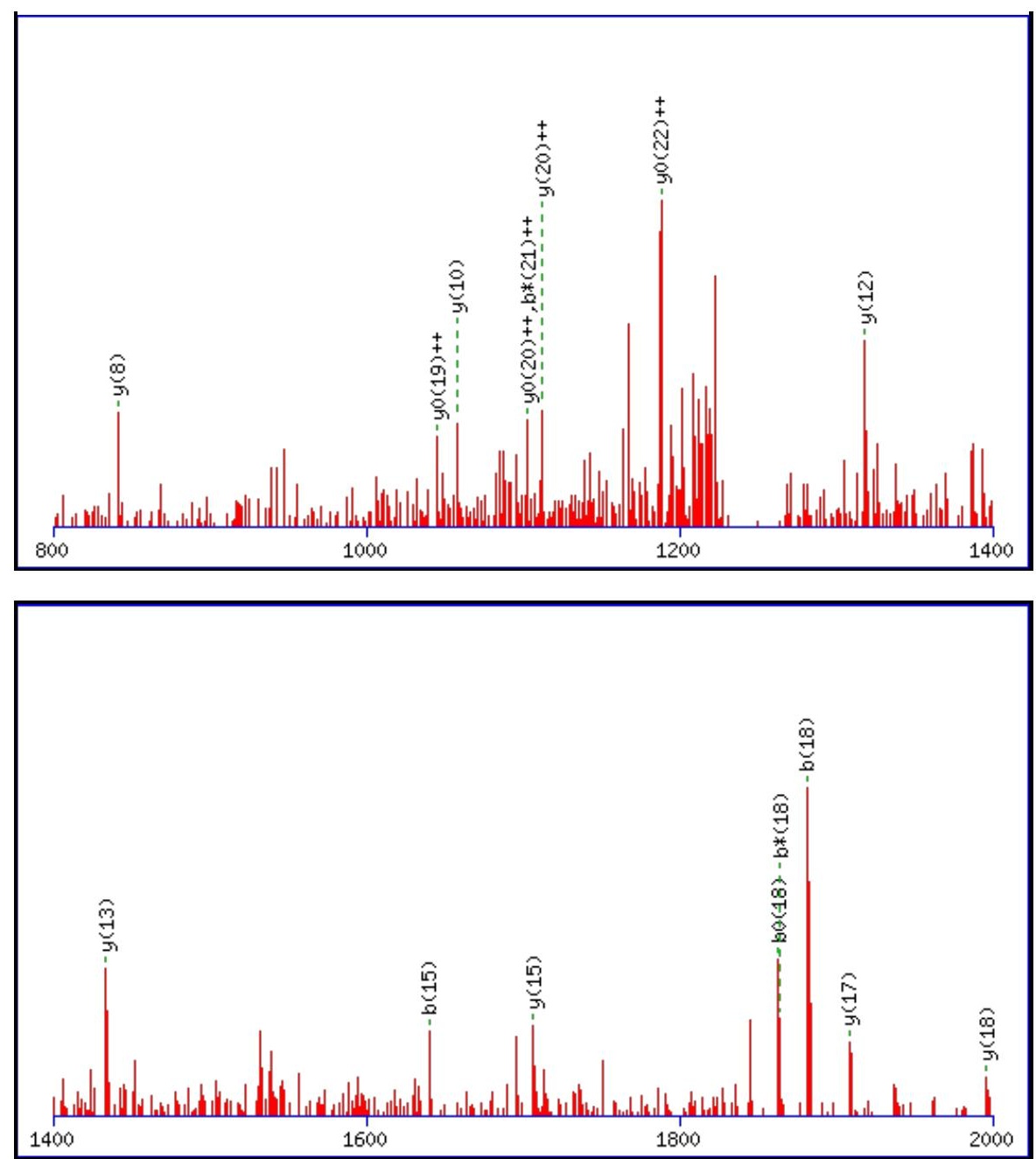

Monoisotopic mass of neutral peptide $\operatorname{Mr}($ calc): 2480.1199

Fixed modifications: Carbamidomethyl (C)

Variable modifications:

N4 : Deamidated $\mathrm{N}(\mathrm{N})$

Ions Score: 50 Expect : $\odot .003$

Matches (Bold Red): 22/242 fragment ions using 33 most intense peaks

\begin{tabular}{|c|c|c|c|c|c|c|c|c|c|c|c|c|c|c|}
\hline \# & b & $\mathbf{b}^{++}$ & $\mathbf{b}^{*}$ & $\mathbf{b}^{*^{++}}$ & $\mathbf{b}^{0}$ & $\mathbf{b}^{0++}$ & Seq. & $\mathbf{y}$ & $y^{++}$ & $\mathbf{y}^{*}$ & $\mathbf{y}^{*^{++}}$ & $\mathbf{y}^{0}$ & $\mathbf{y}^{0++}$ & \# \\
\hline 1 & 88.0393 & 44.5233 & & & 70.0287 & 35.5180 & S & & & & & & & 23 \\
\hline 2 & 187.1077 & 94.0575 & & & 169.0972 & 85.0522 & V & 2394.0952 & 1197.5512 & 2377.0686 & 1189.0380 & 2376.0846 & 1188.5459 & 22 \\
\hline 3 & 258.1448 & 129.5761 & & & 240.1343 & 120.5708 & A & 2295.0268 & 1148.0170 & 2278.0002 & 1139.5037 & 2277.0162 & 1139.0117 & 21 \\
\hline 4 & 373.1718 & 187.0895 & 356.1452 & 178.5762 & 355.1612 & 178.0842 & $\mathbf{N}$ & 2223.9897 & 1112.4985 & 2206.9631 & 1103.9852 & 2205.9791 & 1103.4932 & 20 \\
\hline 5 & 486.2558 & 243.6316 & 469.2293 & 235.1183 & 468.2453 & 234.6263 & $\mathbf{L}$ & 2108.9627 & 1054.9850 & 2091.9362 & 1046.4717 & 2090.9522 & 1045.9797 & 19 \\
\hline 6 & 573.2879 & 287.1476 & 556.2613 & 278.6343 & 555.2773 & 278.1423 & S & 1995.8787 & 998.4430 & 1978.8521 & 989.9297 & 1977.8681 & 989.4377 & 18 \\
\hline 7 & 660.3199 & 330.6636 & 643.2933 & 322.1503 & 642.3093 & 321.6583 & S & 1908.8466 & 954.9270 & 1891.8201 & 946.4137 & 1890.8361 & 945.9217 & 17 \\
\hline 8 & 775.3468 & 388.1771 & 758.3203 & 379.6638 & 757.3363 & 379.1718 & D & 1821.8146 & 911.4109 & 1804.7881 & 902.8977 & 1803.8040 & 902.4057 & 16 \\
\hline 9 & 935.3775 & 468.1924 & 918.3509 & 459.6791 & 917.3669 & 459.1871 & C & 1706.7877 & 853.8975 & 1689.7611 & 845.3842 & 1688.7771 & 844.8922 & 15 \\
\hline 10 & 1048.4615 & 524.7344 & 1031.4350 & 516.2211 & 1030.4510 & 515.7291 & $\mathbf{L}$ & 1546.7570 & 773.8821 & 1529.7305 & 765.3689 & 1528.7464 & 764.8769 & 14 \\
\hline 11 & 1163.4885 & 582.2479 & 1146.4619 & 573.7346 & 1145.4779 & 573.2426 & D & 1433.6729 & 717.3401 & 1416.6464 & 708.8268 & 1415.6624 & 708.3348 & 13 \\
\hline 12 & 1264.5362 & 632.7717 & 1247.5096 & 624.2584 & 1246.5256 & 623.7664 & $\mathbf{T}$ & 1318.6460 & 659.8266 & 1301.6195 & 651.3134 & 1300.6354 & 650.8214 & 12 \\
\hline 13 & 1424.5668 & 712.7870 & 1407.5403 & 704.2738 & 1406.5563 & 703.7818 & $\mathrm{C}$ & 1217.5983 & 609.3028 & 1200.5718 & 600.7895 & 1199.5878 & 600.2975 & 11 \\
\hline 14 & 1539.5938 & 770.3005 & 1522.5672 & 761.7872 & 1521.5832 & 761.2952 & D & 1057.5677 & 529.2875 & 1040.5411 & 520.7742 & 1039.5571 & 520.2822 & 10 \\
\hline 15 & 1640.6414 & 820.8244 & 1623.6149 & 812.3111 & 1622.6309 & 811.8191 & $\mathbf{T}$ & 942.5407 & 471.7740 & 925.5142 & 463.2607 & 924.5302 & 462.7687 & 9 \\
\hline 16 & 1697.6629 & 849.3351 & 1680.6364 & 840.8218 & 1679.6523 & 840.3298 & G & 841.4931 & 421.2502 & 824.4665 & 412.7369 & & & 8 \\
\hline 17 & 1768.7000 & 884.8536 & 1751.6735 & 876.3404 & 1750.6895 & 875.8484 & A & 784.4716 & 392.7394 & 767.4450 & 384.2262 & & & 7 \\
\hline 18 & 1881.7841 & 941.3957 & 1864.7575 & 932.8824 & 1863.7735 & 932.3904 & I & 713.4345 & 357.2209 & 696.4079 & 348.7076 & & & 6 \\
\hline
\end{tabular}




\begin{tabular}{|l|r|r|r|r|r|r|r|r|r|r|r|r|r|r|r|}
$\mathbf{1 9}$ & 2067.8634 & 1034.4353 & 2050.8368 & 1025.9221 & 2049.8528 & 1025.4301 & W & $\mathbf{6 0 0 . 3 5 0 4}$ & 300.6788 & 583.3239 & 292.1656 & & & $\mathbf{5}$ \\
\hline $\mathbf{2 0}$ & 2164.9162 & 1082.9617 & 2147.8896 & 1074.4484 & 2146.9056 & 1073.9564 & P & $\mathbf{4 1 4 . 2 7 1 1}$ & 207.6392 & 397.2445 & 199.1259 & & & $\mathbf{4}$ \\
\hline $\mathbf{2 1}$ & 2221.9376 & 1111.4724 & 2204.9111 & $\mathbf{1 1 0 2 . 9 5 9 2}$ & 2203.9271 & 1102.4672 & G & 317.2183 & 159.1128 & 300.1918 & 150.5995 & & & $\mathbf{3}$ \\
\hline $\mathbf{2 2}$ & 2335.0217 & 1168.0145 & 2317.9951 & 1159.5012 & 2317.0111 & 1159.0092 & L & 260.1969 & 130.6021 & 243.1703 & 122.0888 & & & $\mathbf{2}$ \\
\hline $\mathbf{2 3}$ & & & & & & & K & 147.1128 & 74.0600 & 130.0863 & 65.5468 & & & $\mathbf{1}$ \\
\hline
\end{tabular}
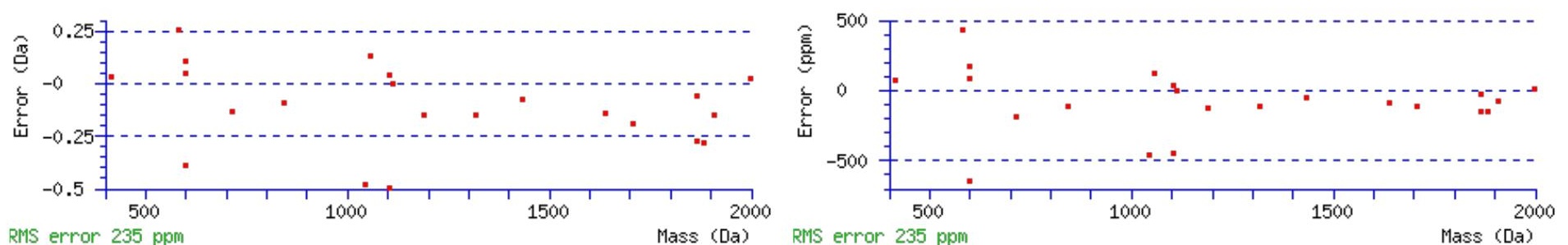

\section{All matches to this query}

\begin{tabular}{|l|l|l|l|}
\hline Score & Mr(calc): & Delta & \multicolumn{1}{|c|}{ Sequence } \\
\hline 50.2 & 2480.1199 & 1.0098 & SVANLSSDCLDTCDTGAIWPGLK \\
\hline 43.2 & 2479.1359 & 1.9938 & SVANLSSDCLDTCDTGAIWPGLK \\
\hline 0.8 & 2480.1185 & 1.0112 & WPRMTVVPTAHSLPSRSXQR \\
\hline 0.8 & 2480.1185 & 1.0112 & WPRMTVVPTAHSLPSRSXOR \\
\hline
\end{tabular}

Spectrum No: 678; Query: 1062; Rank: 1

\section{Peptide View}

MS/MS Fragmentation of NTTTYPPMCSQDAAK

Found in IPI00558154, Tax_Id=10116 Gene_Symbol=LOC100125372 60 kDa protein

Match to Query 1062: 1700.701248 from(851.357900,2+)

Title: 091008RatKidney_NH4Format01_25.720.720.2.dta

Data file K:INewmanPaper|Piliang|3SubProteomes\Piliang3SP\mgf5ppm\ERLIC_3SubProteomes5ppm.mgf

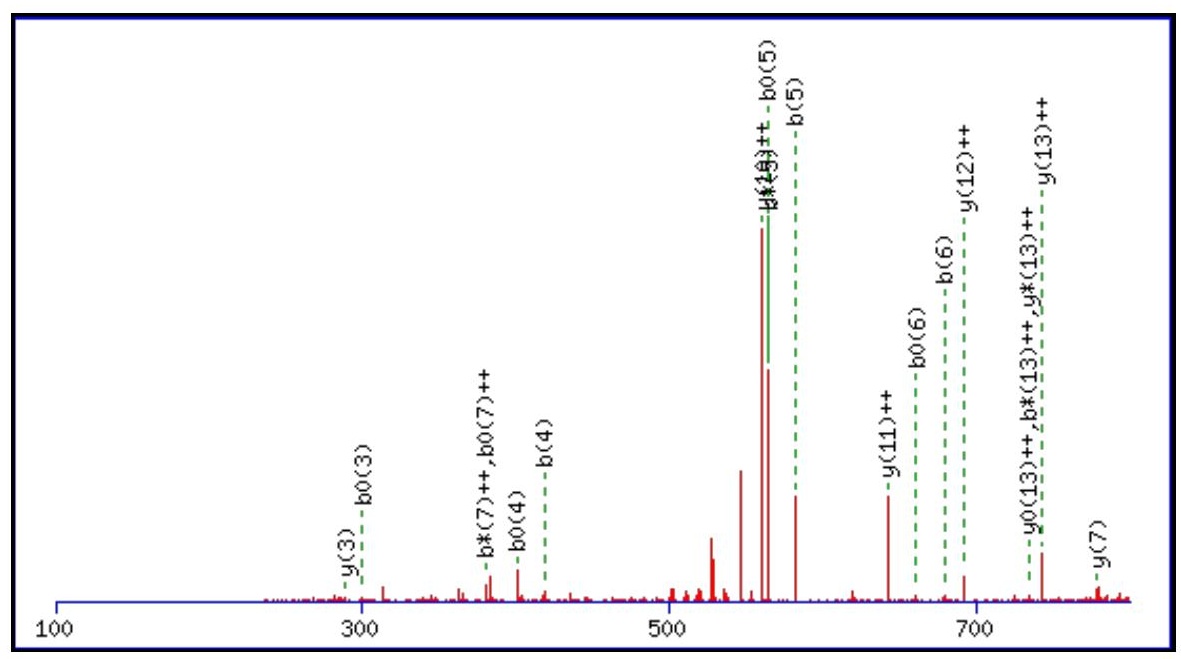



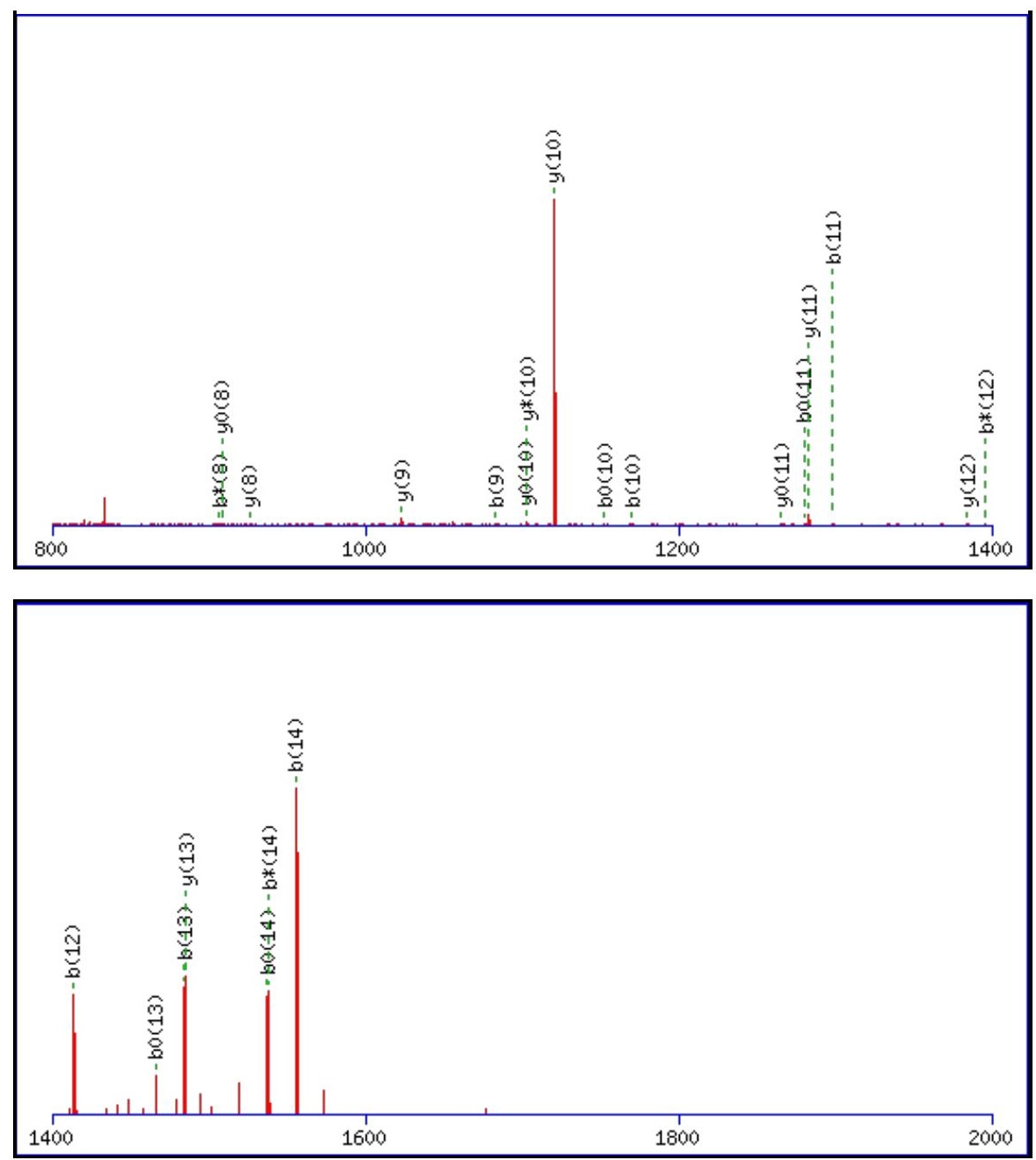

Monoisotopic mass of neutral peptide $\operatorname{Mr}($ calc): 1700.7022

Fixed modifications: Carbamidomethyl (C)

Variable modifications:

N1 : Deamidated_N (N)

M8 : Oxidation (M)

Ions Score: 50 Expect: 0.0017

Matches (Bold Red): 42/160 fragment ions using 90 most intense peaks

\begin{tabular}{|c|c|c|c|c|c|c|c|c|c|c|c|c|c|c|}
\hline \# & b & $\mathbf{b}^{++}$ & b* & $\mathbf{b}^{*^{++}}$ & $\mathbf{b}^{0}$ & $\mathbf{b}^{0++}$ & Seq. & $\mathbf{y}$ & $\mathbf{y}^{++}$ & $\mathbf{y}^{*}$ & $\mathbf{y}^{*^{++}}$ & $\mathbf{y}^{\mathbf{0}}$ & $y^{0++}$ & \# \\
\hline 1 & 116.0342 & 58.5207 & 99.0077 & 50.0075 & & & $\mathbf{N}$ & & & & & & & 15 \\
\hline 2 & 217.0819 & 109.0446 & 200.0553 & 100.5313 & 199.0713 & 100.0393 & $\mathbf{T}$ & 1586.6825 & 793.8449| & 1569.6560 & & 1568.6720 & 784.8396 & 14 \\
\hline 3 & 318.1296 & 159.5684 & 301.1030 & 151.0551 & 300.1190 & 150.5631 & $\mathbf{T}$ & 1485.6348 & 743.3211 & 1468.6083 & 734.8078 & 1467.6243 & 734.3158 & 13 \\
\hline 4 & 419.1772 & 210.0923 & 402.1507 & 201.5790 & 401.1667 & 201.0870 & $\mathbf{T}$ & 1384.5872 & 692.7972 & 1367.5606 & 684.2839 & 1366.5766 & 683.7919 & 12 \\
\hline 5 & 582.2406 & 291.6239 & 565.2140 & 283.1107 & 564.2300 & 282.6186 & $\mathbf{Y}$ & 1283.5395 & 642.2734 & 1266.5129 & 633.7601 & 1265.5289 & 633.2681 & 11 \\
\hline 6 & 679.2933 & 340.1503 & 662.2668 & 331.6370 & 661.2828 & 331.1450 & $\mathbf{P}$ & 1120.4762 & 560.7417 & 1103.4496 & 552.2284 & 1102.4656 & 551.7364 & 10 \\
\hline 7 & 776.3461 & 388.6767 & 759.3196 & 380.1634 & 758.3355 & 379.6714 & $\mathbf{P}$ & 1023.4234 & 512.2153 & 1006.3968 & 503.7021 & 1005.4128 & 503.2101 & 9 \\
\hline 8 & 923.3815 & 462.1944 & 906.3550 & 453.6811 & 905.3709 & 453.1891 & $\mathbf{M}$ & 926.3706 & 463.6890 & 909.3441 & 455.1757 & 908.3601 & 454.6837 & 8 \\
\hline 9 & 1083.4122 & 542.2097 & 1066.3856 & 533.6964 & 1065.4016 & 533.2044 & $\mathrm{C}$ & 779.3352 & 390.1713 & 762.3087 & 381.6580 & 761.3247 & 381.1660 & 7 \\
\hline 10 & 1170.4442 & 585.7257 & 1153.4176 & 577.2125 & 1152.4336 & 576.7204 & S & 619.3046 & 310.1559 & 602.2780 & 301.6427 & 601.2940 & 301.1506 & 6 \\
\hline 11 & 1298.5028 & 649.7550 & 1281.4762 & 641.2417 & 1280.4922 & 640.7497 & $\mathbf{Q}$ & 532.2726 & 266.6399 & 515.2460 & 258.1266 & 514.2620 & 257.6346 & 5 \\
\hline 12 & 1413.5297 & 707.2685 & 1396.5032 & 698.7552 & 1395.5191 & 698.2632 & D & 404.2140 & 202.6106 & 387.1874 & 194.0974 & 386.2034 & 193.6053 & 4 \\
\hline 13 & 1484.5668 & 742.7870 & 1467.5403 & 734.2738 & 1466.5563 & 733.7818 & A & 289.1870 & 145.0972 & 272.1605 & 136.5839 & & & 3 \\
\hline 14 & 1555.6039 & 778.3056 & 1538.5774 & 769.7923 & 1537.5934 & 769.3003 & A & 218.1499 & 109.5786 & 201.1234 & 101.0653 & & & 2 \\
\hline 15 & & & & & & & $\mathbf{K}$ & 147.1128 & 74.0600 & 130.0863 & 65.5468 & & & 1 \\
\hline
\end{tabular}



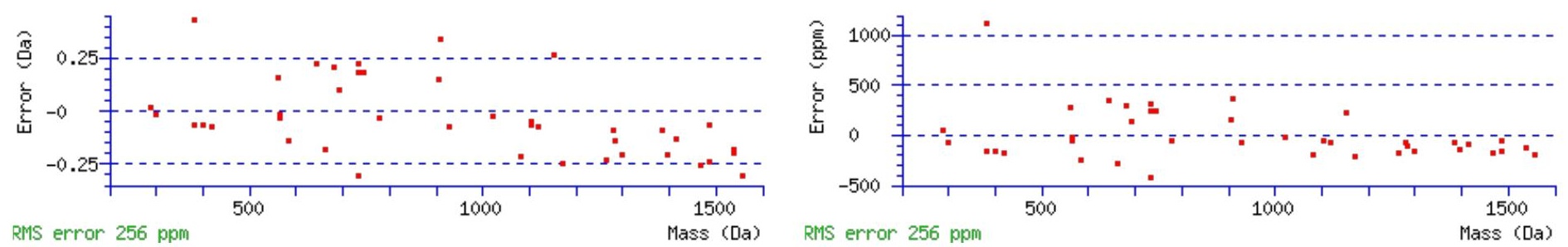

\section{All matches to this query}

\begin{tabular}{|l|l|l|l|}
\hline Score & Mr(calc): & Delta & \multicolumn{1}{c|}{ Sequence } \\
\hline 50.2 & 1700.7022 & -0.0009 & NTTTYPPMCSQDAAK \\
\hline 11.4 & 1698.6943 & 2.0069 & GFEFVAASAPISESK \\
\hline 11.4 & 1698.6943 & 2.0069 & GFEFVAASAPISESK \\
\hline 6.5 & 1698.7043 & 1.9970 & SQEGCPDFSSEITNK \\
\hline 5.6 & 1698.6985 & 2.0027 & SDQEAKPSTEDLGDK \\
\hline 4.0 & 1700.6964 & 0.0048 & EEQEKVVTNCQEK \\
\hline 2.5 & 1699.7120 & 0.9893 & RYTGKANAEFNLR \\
\hline 2.4 & 1700.7076 & -0.0063 & NLITKVQELTSSK \\
\hline 2.4 & 1700.7076 & -0.0063 & NLITKVQELTSSK \\
\hline 2.2 & 1698.6943 & 2.0069 & GFEFVAASAPISESK \\
\hline
\end{tabular}

Spectrum No: 679; Query: 1174; Rank: 1

\section{Peptide View}

\section{MS/MS Fragmentation of GNITEYQCHQYITK}

Found in IPI00207068, Tax_Id=10116 Gene_Symbol=Glg1 Golgi apparatus protein 1 precursor

Match to Query 1174: 1754.799508 from(878.407030,2+)

Title: 100101RatKid_NS_deglyco_19.1520.1520.2.dta

Data file K:INewmanPaper|Piliangl3SubProteomes\Piliang3SP\mgf5ppm\ERLIC_3SubProteomes5ppm.mgf

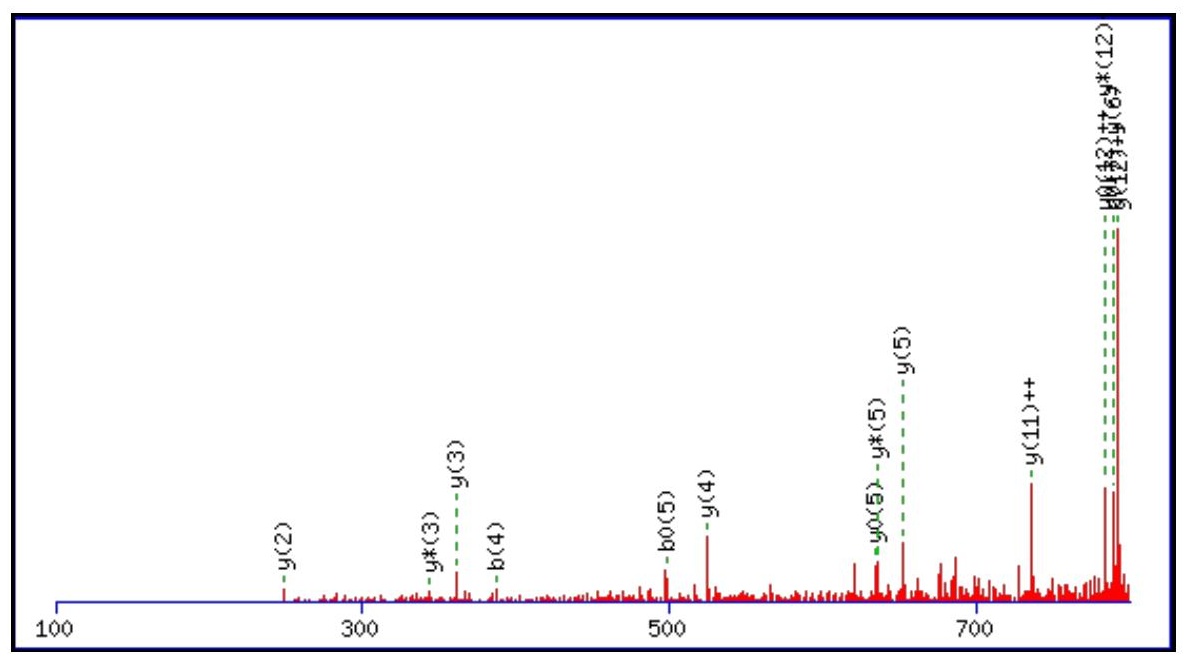



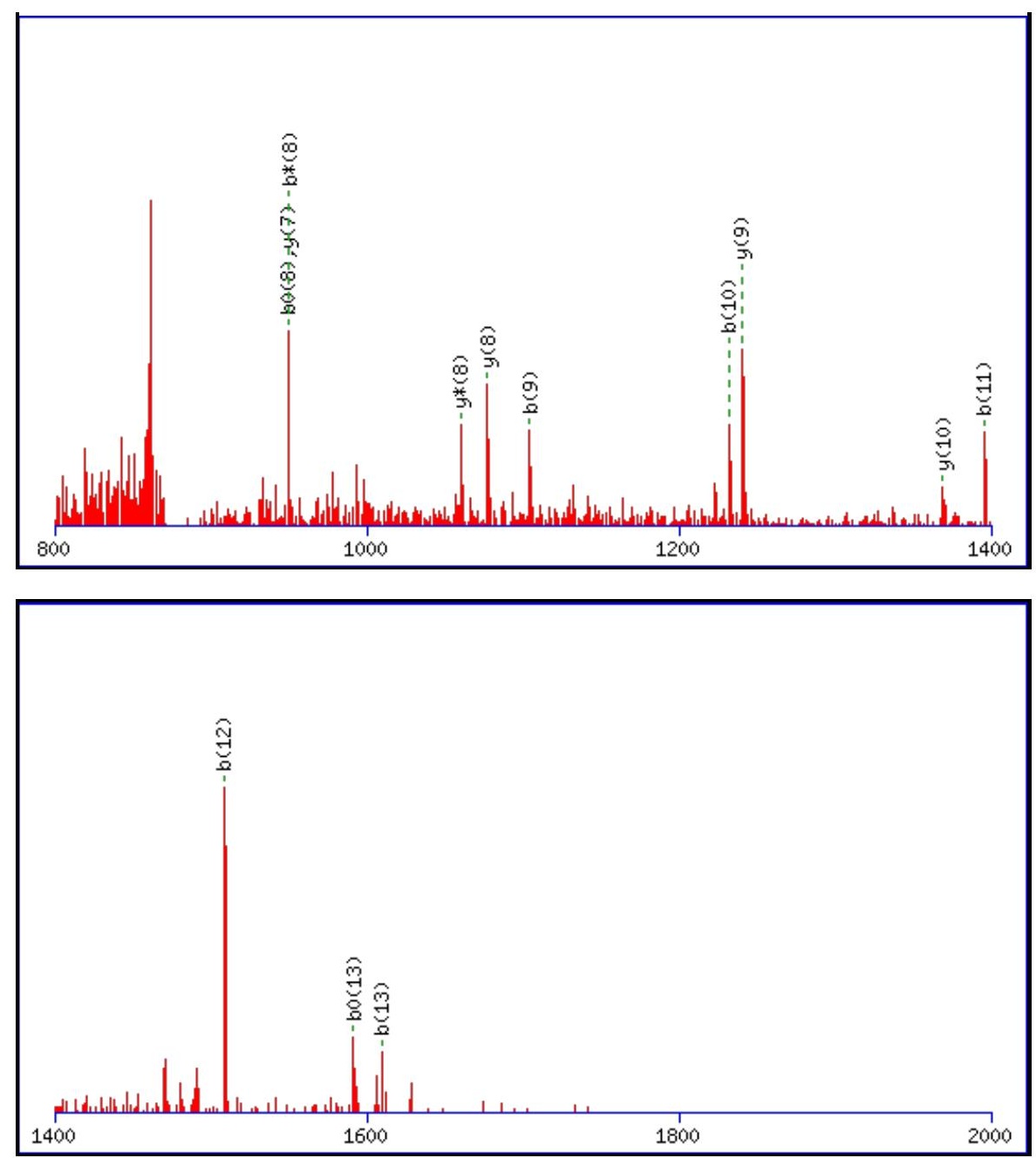

Monoisotopic mass of neutral peptide $\operatorname{Mr}($ calc): 1754.7934

Fixed modifications: Carbamidomethyl (C)

Variable modifications:

N2 : Deamidated $\mathrm{N}(\mathrm{N})$

Ions Score: 50 Expect : $\odot .002$

Matches (Bold Red): 28/146 fragment ions using 52 most intense peaks

\begin{tabular}{|c|c|c|c|c|c|c|c|c|c|c|c|c|c|c|}
\hline \# & b & $\mathbf{b}^{++}$ & b* & $\mathbf{b}^{*^{++}}$ & $\mathbf{b}^{\mathbf{0}}$ & $\mathbf{b}^{\mathbf{0 + +}}$ & Seq. & $\mathbf{y}$ & $\mathbf{y}^{++}$ & $\mathbf{y}^{*}$ & $\mathrm{y}^{*^{++}}$ & $\mathbf{y}^{0}$ & $\mathbf{y}^{0++}$ & \# \\
\hline 1 & 58.0287 & 29.5180 & & & & & $\mathbf{G}$ & & & & & & & 14 \\
\hline 2 & 173.0557 & 87.0315 & 156.0291 & 78.5182 & & & $\mathbf{N}$ & 1698.7792 & 849.8932 & 1681.7526 & 841.3800 & 1680.7686 & 840.8880 & 13 \\
\hline 3 & 286.1397 & 143.5735 & 269.1132 & 135.0602 & & & I & 1583.7523 & 792.3798 & 1566.7257 & 783.8665 & 1565.7417 & 783.3745 & 12 \\
\hline 4 & 387.1874 & 194.0973 & 370.1609 & 185.5841 & 369.1769 & 185.0921 & $\mathbf{T}$ & 1470.6682 & 735.8377 & 1453.6416 & 727.3245 & 1452.6576 & 726.8325 & 11 \\
\hline 5 & 516.2300 & 258.6186 & 499.2035 & 250.1054 & 498.2194 & 249.6134 & $\mathbf{E}$ & 1369.6205 & 685.3139 & 1352.5940 & 676.8006 & 1351.6100 & 676.3086 & 10 \\
\hline 6 & 679.2933 & 340.1503 & 662.2668 & 331.6370 & 661.2828 & 331.1450 & $\mathbf{Y}$ & 1240.5779 & 620.7926 & 1223.5514 & 612.2793 & 1222.5674 & 611.7873 & 9 \\
\hline 7 & 807.3519 & 404.1796 & 790.3254 & 395.6663 & 789.3414 & 395.1743 & $\mathbf{Q}$ & 1077.5146 & 539.2609 & 1060.4880 & 530.7477 & 1059.5040 & 530.2557 & 8 \\
\hline 8 & 967.3826 & 484.1949 & 950.3560 & 475.6816 & 949.3720 & 475.1896 & C & 949.4560 & 475.2316 & 932.4295 & 466.7184 & 931.4455 & 466.2264 & 7 \\
\hline 9 & 1104.4415 & 552.7244 & 1087.4149 & 544.2111 & 1086.4309 & 543.7191 & $\mathbf{H}$ & 789.4254 & 395.2163 & 772.3988 & 386.7030 & 771.4148 & 386.2110 & 6 \\
\hline 10 & 1232.5001 & 616.7537 & 1215.4735 & 608.2404 & 1214.4895 & 607.7484 & $\mathbf{Q}$ & 652.3665 & 326.6869 & 635.3399 & 318.1736 & 634.3559 & 317.6816 & 5 \\
\hline 11 & 1395.5634 & 698.2853 & 1378.5368 & 689.7721 & 1377.5528 & 689.2800 & $\mathbf{Y}$ & 524.3079 & 262.6576 & 507.2813 & 254.1443 & 506.2973 & 253.6523 & 4 \\
\hline 12 & 1508.6475 & 754.8274 & 1491.6209 & 746.3141 & 1490.6369 & 745.8221 & I & 361.2445 & 181.1259 & 344.2180 & 172.6126 & 343.2340 & 172.1206 & 3 \\
\hline 13 & 1609.6951 & 805.3512 & 1592.6686 & 796.8379 & 1591.6846 & 796.3459 & $\mathbf{T}$ & 248.1605 & 124.5839 & 231.1339 & 116.0706 & 230.1499 & 115.5786 & 2 \\
\hline 14 & & & & & & & $\mathbf{K}$ & 147.1128 & 74.0600 & 130.0863 & 65.5468 & & & 1 \\
\hline
\end{tabular}



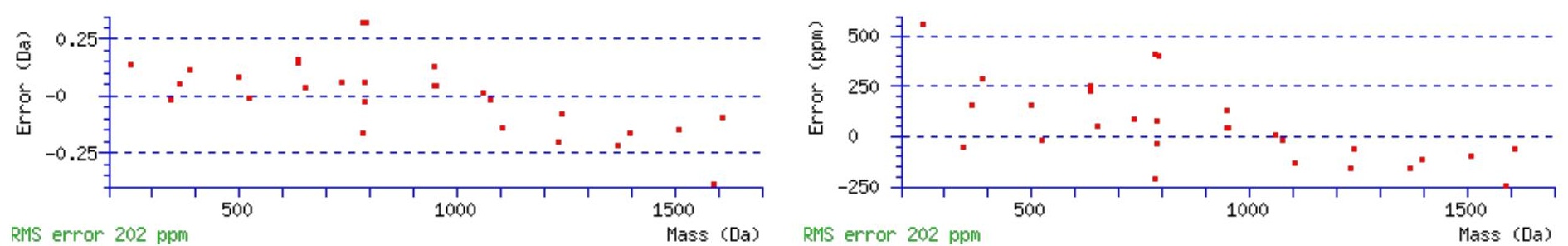

\section{All matches to this query}

\begin{tabular}{|l|l|l|l|}
\hline Score & Mr(calc): & Delta & Sequence \\
\hline 50.0 & 1754.7934 & 0.0061 & GNITEYQCHQYITK \\
\hline 32.3 & 1753.8094 & 0.9902 & GNITEYQCHQYITK \\
\hline 5.6 & 1752.7753 & 2.0242 & SGDVIYTGRKESMSK \\
\hline 5.6 & 1752.7753 & 2.0242 & SGDVIYTGRKESMSK \\
\hline
\end{tabular}

Spectrum No: 680; Query: 78; Rank: 1

\section{Peptide View}

MS/MS Fragmentation of QLNISTLVK

Found in IPI00204504, Tax_Id=10116 Gene_Symbol=Plvap Plasmalemma vesicle-associated protein

Match to Query 78: 1015.592348 from(508.803450,2+)

Title: 100101RatKid_NS_deglyco_21.2900.2900.2.dta

Data file K:INewmanPaper|Piliangl3SubProteomes\Piliang3SP\mgf5ppm\ERLIC_3SubProteomes5ppm.mgf

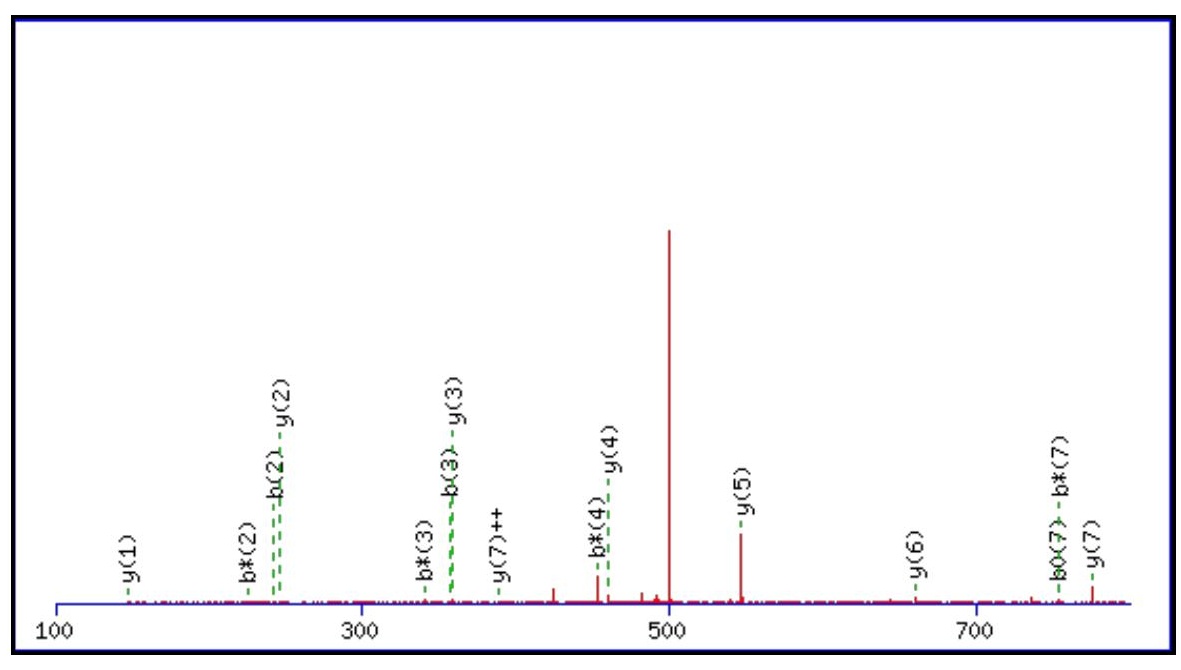



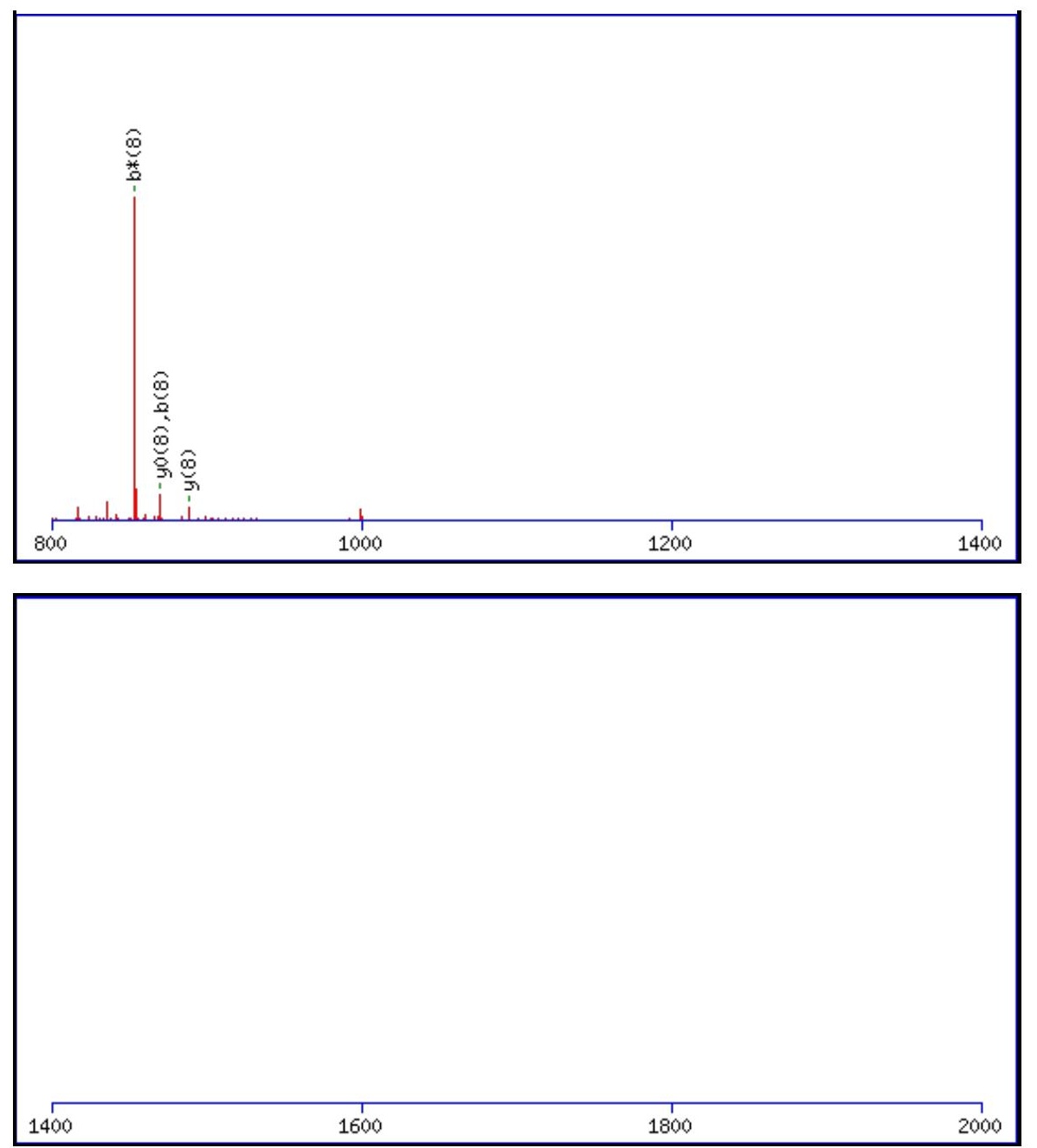

Monoisotopic mass of neutral peptide $\operatorname{Mr}($ calc): 1015.5913

Fixed modifications: Carbamidomethyl (C)

Variable modifications:

N3 : Deamidated_N (N)

Ions Score: 50 Expect: 0.00065

Matches (Bold Red): 19/82 fragment ions using 46 most intense peaks

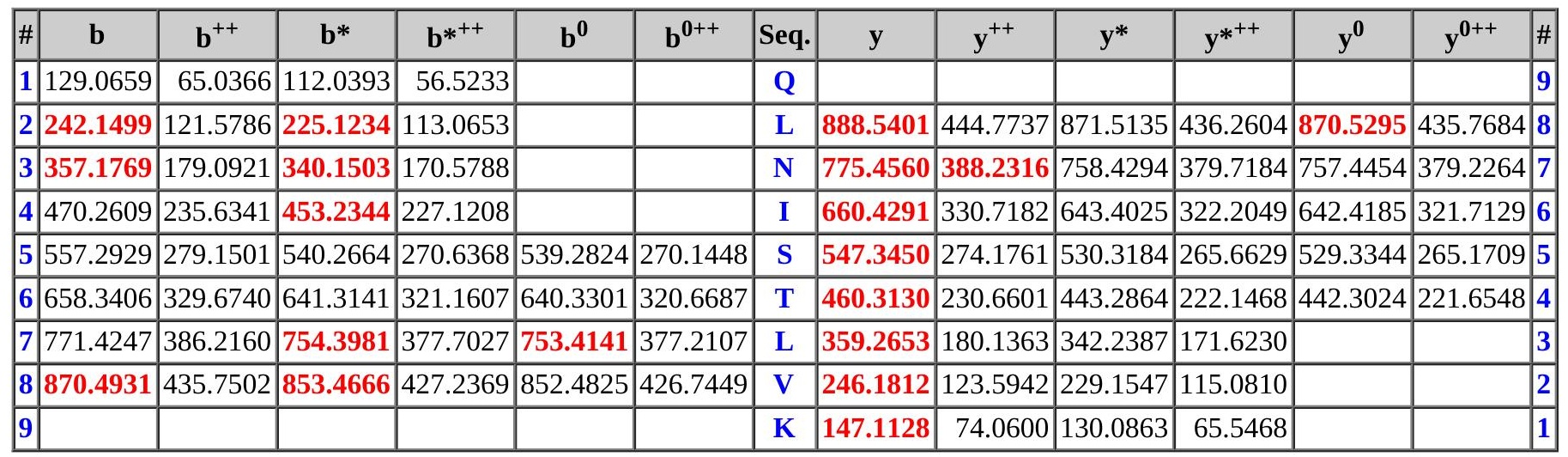
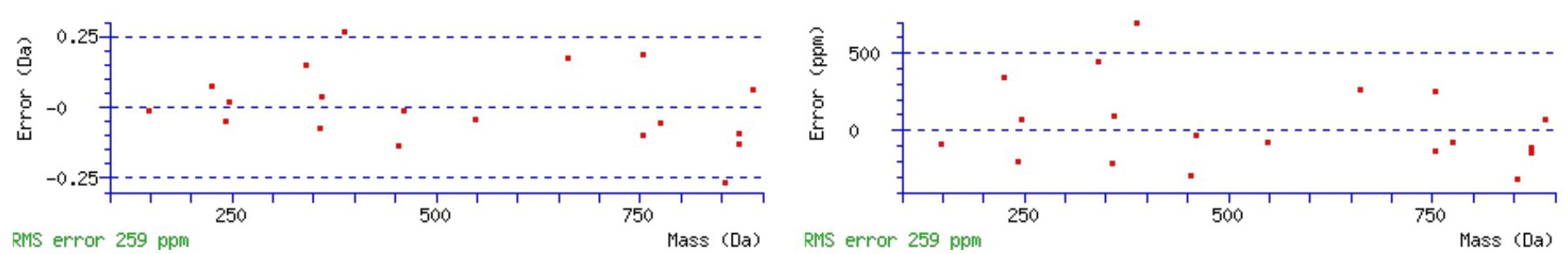

\section{All matches to this query}




\begin{tabular}{|l|l|l|l|} 
Score & Mr(calc): & Delta & \multicolumn{1}{|c|}{ Sequence } \\
\hline 50.0 & 1015.5913 & 0.0010 & QLNISTLVK \\
\hline 12.7 & 1015.5913 & 0.0010 & KINELTGIK \\
\hline 9.0 & 1015.5913 & 0.0010 & ELKELTGVK \\
\hline 9.0 & 1015.5914 & 0.0010 & IENVTTVLK \\
\hline 8.3 & 1015.5887 & 0.0037 & SGTGAARLRK \\
\hline 7.9 & 1015.5913 & 0.0010 & KLEELEKK \\
\hline 7.3 & 1015.5913 & 0.0010 & KLITDALNK \\
\hline 7.2 & 1015.5848 & 0.0075 & KIDQLMIR \\
\hline 6.4 & 1015.5961 & -0.0037 & KIRCVVNK \\
\hline 6.4 & 1015.5848 & 0.0076 & KLAALMQNK \\
\hline
\end{tabular}

Spectrum No: 681; Query: 2478; Rank: 1

\section{Peptide View}

MS/MS Fragmentation of KREDDEEEEGSIVNGSTTEDEEQTR

Found in IPI00209938, Tax_Id=10116 Gene_Symbol=Nexn Isoform 1 of Nexilin

Match to Query 2478: 2882.229856 from(721.564740,4+)

Title: 100101RatKid_NS_deglyco_20.969.969.4.dta

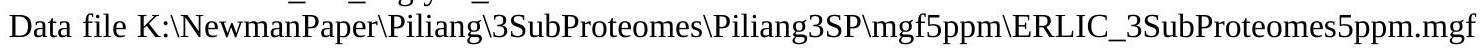
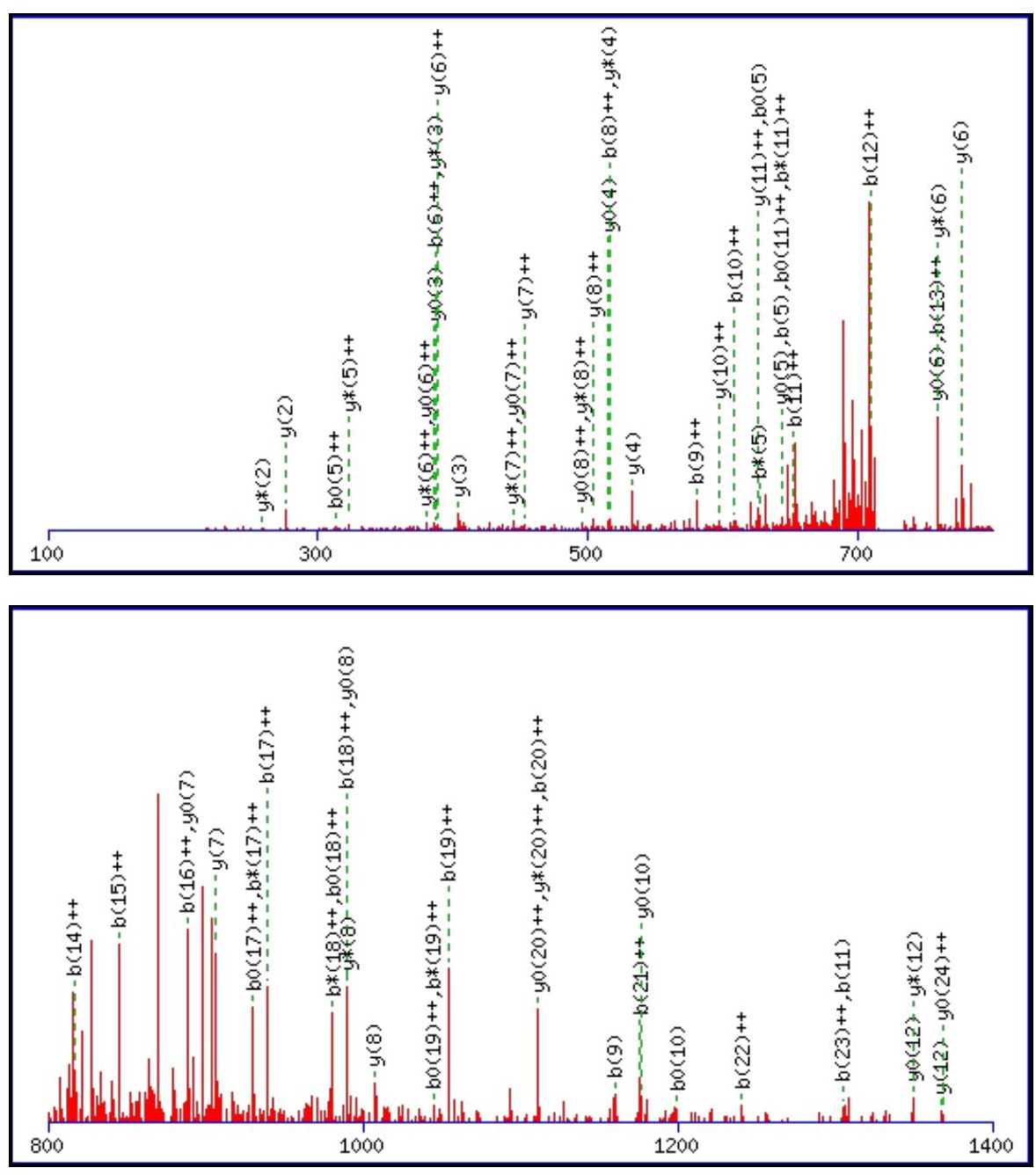


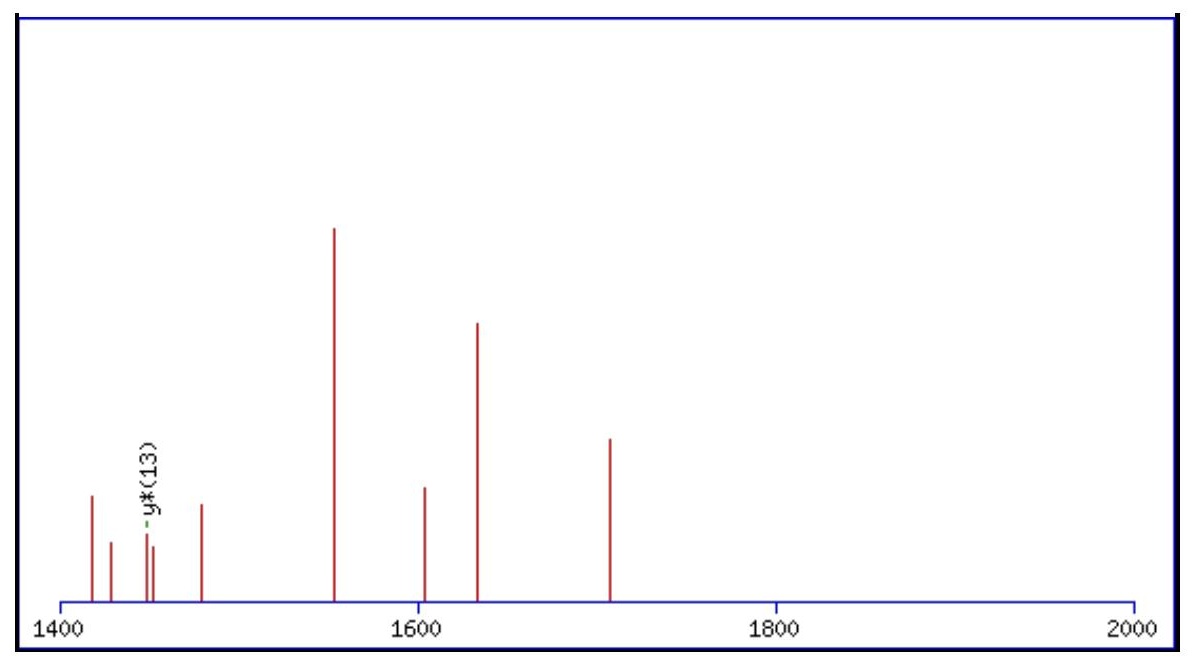

Monoisotopic mass of neutral peptide $\operatorname{Mr}($ calc): 2882.2173

Fixed modifications: Carbamidomethyl (C)

Variable modifications:

N14: Deamidated $\mathrm{N}(\mathrm{N})$

Ions Score: 50 Expect: 0.0035

Matches (Bold Red): 69/282 fragment ions using 140 most intense peaks

\begin{tabular}{|c|c|c|c|c|c|c|c|c|c|c|c|c|c|c|}
\hline \# & b & $\mathbf{b}^{++}$ & b* & $\mathbf{b}^{*^{++}}$ & $\mathbf{b}^{\mathbf{0}}$ & & Seq. & $\mathbf{y}$ & $\mathbf{y}^{++}$ & $\mathbf{y}^{*}$ & & $\mathbf{y}^{0}$ & & \# \\
\hline 1 & 129.1022 & 65.0548 & 112.0757 & 56.5415 & & & $\mathbf{K}$ & & & & & & & 25 \\
\hline 2 & 285.2034 & 43.1053 & 68.1768 & & & & $\mathbf{R}$ & & & & & & & 24 \\
\hline 3 & 414.2459 & 207.6266 & 397.2194 & 199.1133 & & & $\mathbf{E}$ & 0285 & 0179 & 2582.0020 & 1291.5046 & 2581.0180 & 0126 & 23 \\
\hline 4 & 529.2729 & 265.1401 & 512.2463 & 256.6268 & 511.2623 & 256.1348 & D & 2469.9859 & & 2452.9594 & 1226.9833 & 2451.9754 & & 22 \\
\hline 5 & 644.2998 & 322.6536 & 627.2733 & 314.1 & 893 & 313. & D & 590 & 9831 & 9325 & 4699 & 2336.9484 & 9779 & 21 \\
\hline 6 & 773.3424 & 387.1748 & 756.3159 & 378.6616 & 319 & 378.1696 & $\mathbf{E}$ & 321 & 4697 & 055 & 564 & 215 & & 20 \\
\hline 7 & 902.3850 & 451.6961 & 585 & 443.1829 & 745 & 442.6909 & $\mathbf{E}$ & 895 & 484 & 3629 & 4351 & 8789 & 1046.9431 & 19 \\
\hline 8 & 1031.4276 & 516.2174 & 014.4011 & 507.7042 & 013.4170 & 507.2122 & $\mathbf{E}$ & 1981.8469 & 4271 & 1964 & 138 & 3363 & 4218 & 18 \\
\hline 9 & 160.4702 & 580.7387 & 143.4437 & 572.2255 & 142.4596 & 571.7 & $\mathbf{E}$ & 3043 & 9058 & 777 & 925 & 1834 & 9005 & 17 \\
\hline 10 & 217.4917 & 609.2495 & 200.4651 & 600.7362 & 1199.4811 & 600.2442 & $\mathbf{G}$ & 1723. & 3845 & 1706 & 712 & 1705 & 3792 & 16 \\
\hline 11 & 304.5237 & 652.7655 & 287.4971 & 644.2522 & 131 & 643. 7 & $\mathrm{~S}$ & 7402 & 8737 & 1649 & 3605 & 1648 & 3685 & 15 \\
\hline 12 & 417.6078 & 709.3075 & 1400.5812 & 700.7942 & 972 & 700. & I & 082 & 3577 & 1562 & 3445 & 1561 & 3525 & 14 \\
\hline 13 & 516.6762 & 758.8417 & 1499.6496 & 750.3284 & 1498.6656 & 749.8364 & V & 1466.6241 & 733.8157 & 1449. & 3024 & 1448.6136 & 724.8104 & 13 \\
\hline 14 & 631.7031 & 816.3552 & 614.6766 & 807.8 & 925 & 807. & $\mathbf{N}$ & 1367 & 815 & 1350 & 682 & 1349 & .762 & 12 \\
\hline 15 & 1688.7246 & 844.8659 & 1671.6980 & 836.3526 & 1670.7140 & 835.8606 & $\mathbf{G}$ & 1252.5288 & 626.7680 & 1235.5022 & 2548 & 1234.5182 & 617.7627 & 11 \\
\hline 16 & 1775.7566 & 888.3819 & 758.7301 & 879.8687 & 1757.7460 & 879. & S & 1195 & 2573 & 4808 & 7440 & 1177 & 2520 & 10 \\
\hline 17 & 1876.8043 & 938.9058 & 1859.7777 & 930.3925 & 1858.7937 & 929.9005 & $\mathbf{T}$ & 1108.4753 & 554.7413 & 1091.4487 & 2280 & 1090.4647 & 545.7360 & 9 \\
\hline 18 & 1977.8520 & 989.4296 & 1960.8254 & 980.9163 & 1959.8414 & 980.4243 & $\mathbf{T}$ & 1007.4276 & 504.2174 & 990.4011 & 495.7042 & 4170 & 495.2122 & 8 \\
\hline 19 & 2106.8946 & 1053.9509 & 2089.8680 & 1045.4376 & 2088.8840 & 1044.9456 & $\mathbf{E}$ & 906.3799 & 453.6936 & 889 & 803 & 888.3694 & 444.6883 & 7 \\
\hline 20 & 2221.9215 & 1111.4644 & 2204.8949 & 1102.9511 & 2203.9109 & 1102.4591 & D & 777.3373 & 389.1723 & 760.3108 & 380.6590 & 759.3268 & 380.1670 & 6 \\
\hline 21 & 2350.9641 & 1175.9857 & 2333.9375 & 1167.4724 & 2332.9535 & 1166.9804 & $\mathbf{E}$ & 662.3104 & 331.6588 & 645.2838 & 1456 & 644.2998 & 322.6536 & 5 \\
\hline 22 & 2480.0067 & 1240.5070 & 2462.9801 & 1231.9937 & 2461.9961 & 1231.5017 & $\mathbf{E}$ & 533.2678 & 267.1375 & 516.2413 & 258.6243 & 515.2572 & 258.1323 & 4 \\
\hline 23 & 2608.0653 & 1304.5363 & 2591.0387 & 1296.0230 & 2590.0547 & 1295.5310 & $\mathbf{Q}$ & 404.2252 & 202.6162 & 387.1987 & 1030 & 386.2146 & 6110 & 3 \\
\hline 24 & 2709.1129 & 1355.0601 & 2692.0864 & 1346.5468 & 2691.1024 & 1346.0548 & $\mathbf{T}$ & 276.1666 & 138.5870 & 259.1401 & 130.0737 & 258.1561 & 129.5817 & 2 \\
\hline 25 & & & & & & & $\mathbf{R}$ & 175.1190 & 88.0631 & 158.0924 & 79.5498 & & & 1 \\
\hline
\end{tabular}
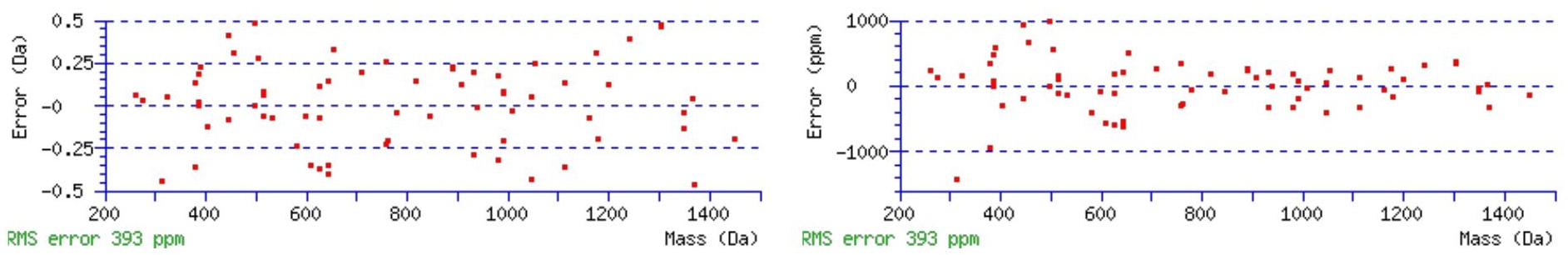


\section{All matches to this query}

\begin{tabular}{|l|l|l|l|}
\hline Score & Mr(calc): & Delta & \multicolumn{1}{|c|}{ Sequence } \\
\hline 50.0 & 2882.2173 & 0.0126 & KREDDEEEEGSIVNGSTTEDEEQTR \\
\hline 23.3 & 2881.2333 & 0.9966 & KREDDEEEEGSIVNGSTTEDEEQTR \\
\hline 8.2 & 2881.2526 & 0.9773 & EEMKKIMTHNQWSFVMSTVEWK \\
\hline 6.9 & 2881.2371 & 0.9928 & LMAVNVTYSSTGQIASIQRGTTSEK \\
\hline 4.3 & 2881.2371 & 0.9928 & LMAVNVTYSSTGQIASIQRGTTSEK \\
\hline 3.8 & 2882.2211 & 0.0088 & LMAVNVTYSSTGQIASIQRGTTSEK \\
\hline 3.2 & 2882.2211 & 0.0088 & LMAVNVTYSSTGQIASIQRGTTSEK \\
\hline 2.6 & 2882.2129 & 0.0170 & GYWFVLTAESLSWYKDDEEKEK \\
\hline 2.6 & 2882.2129 & 0.0170 & GYWFVLTAESLSWYKDDEEKEK \\
\hline 2.2 & 2882.2129 & 0.0170 & GYWFVLTAESLSWYKDDEEKEK \\
\hline
\end{tabular}

Spectrum No: 682; Query: 1948; Rank: 1

\section{Peptide View}

MS/MS Fragmentation of YHYNGTFLDGTLFDSSHNR

Found in IPI00215190, Tax_Id=10116 Gene_Symbol=Fkbp9 FK506-binding protein 9 precursor

Match to Query 1948: 2243.983332 from(749.001720,3+)

Title: 091008RatKidney_NH4Format01_23.3006.3006.3.dta

Data file K:\NewmanPaper\Piliang\3SubProteomes\Piliang3SP\mgf5ppm\ERLIC_3SubProteomes5ppm.mgf

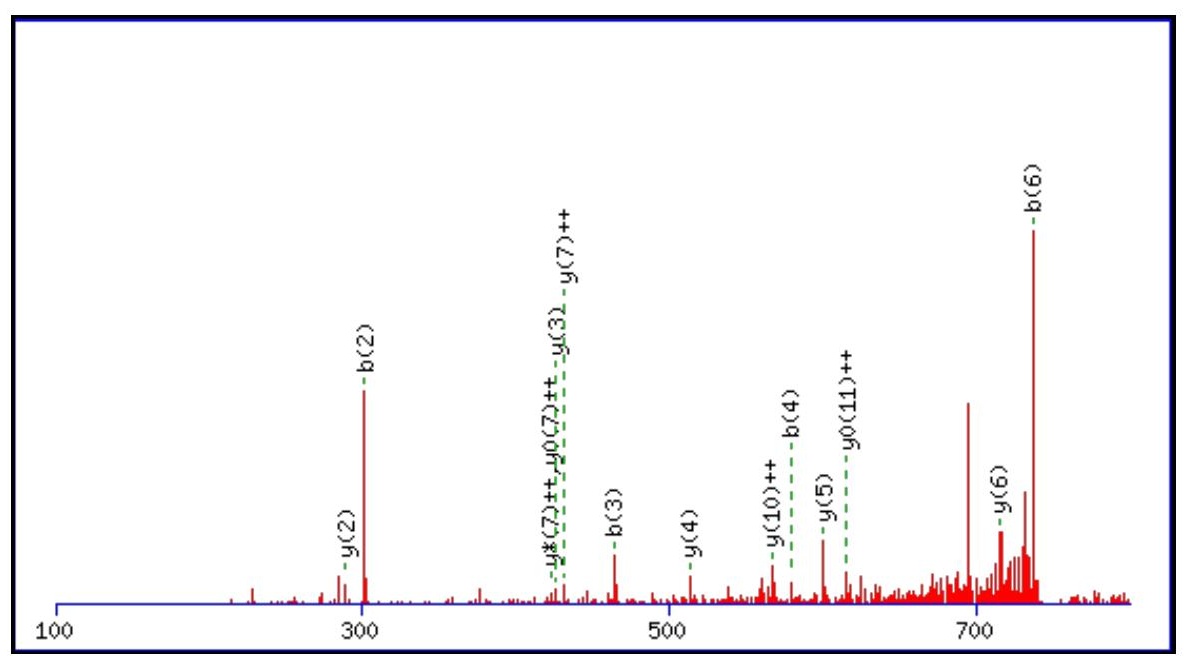



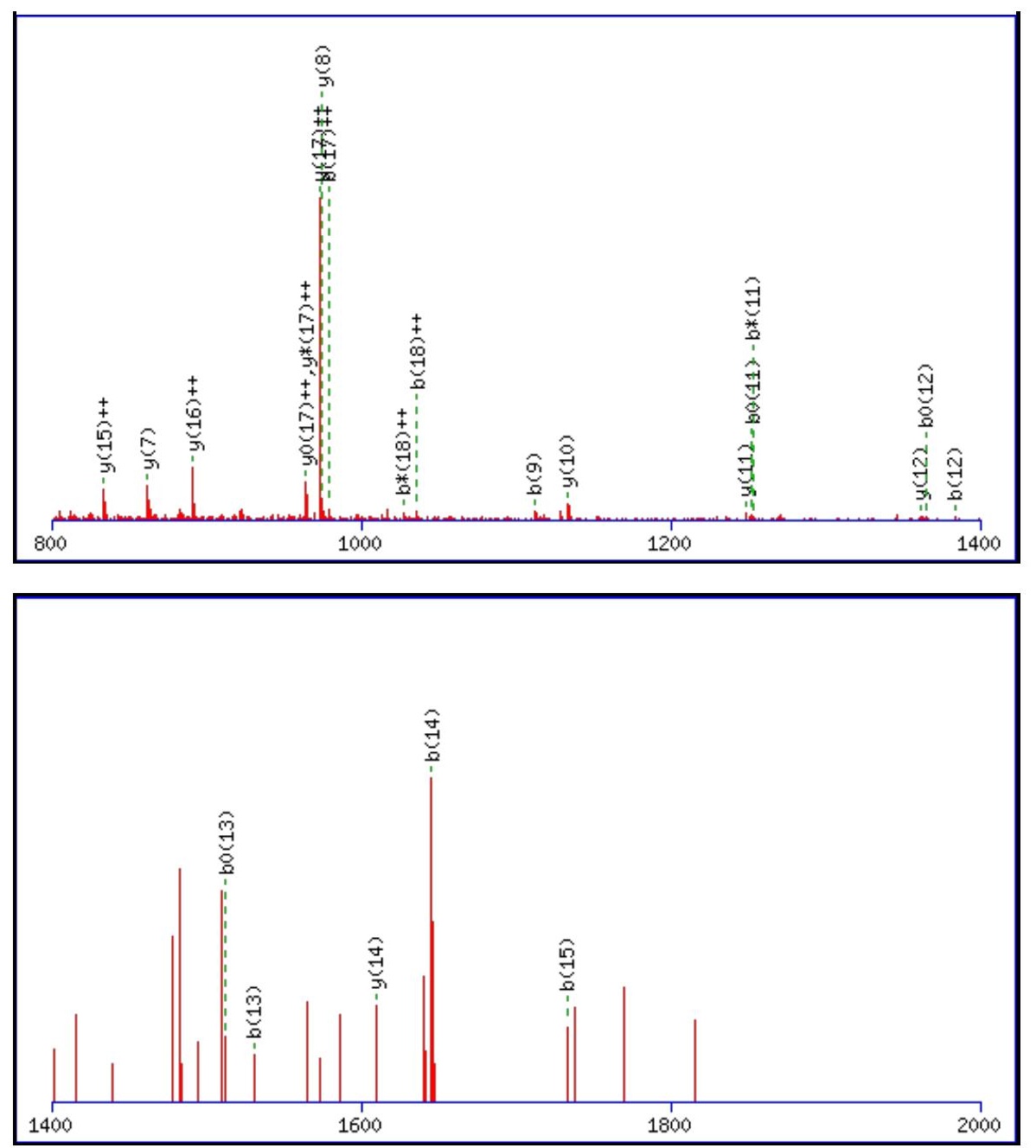

Monoisotopic mass of neutral peptide $\operatorname{Mr}($ calc): 2243.9872

Fixed modifications: Carbamidomethyl (C)

Variable modifications:

N4 : Deamidated_N (N)

Ions Score: 50 Expect: 0.0031

Matches (Bold Red): 37/194 fragment ions using 83 most intense peaks

\begin{tabular}{|c|c|c|c|c|c|c|c|c|c|c|c|c|c|c|}
\hline \# & b & $\mathbf{b}^{++}$ & b* & $\mathbf{b}^{*^{++}}$ & $\mathbf{b}^{0}$ & $\mathbf{b}^{\mathbf{0 + +}}$ & Seq. & $\mathbf{y}$ & $\mathbf{y}^{++}$ & $\mathbf{y}^{*}$ & $\mathbf{y}^{*^{++}}$ & $\mathbf{y}^{0}$ & $\mathbf{y}^{\mathbf{0 + +}}$ & \# \\
\hline 1 & 164.0706 & 82.5389 & & & & & $\mathbf{Y}$ & & & & & & & 19 \\
\hline 2 & 301.1295 & 151.0684 & & & & & $\mathbf{H}$ & 2081.9312 & 1041.4692 & 2064.9046 & 1032.9559 & 2063.9206 & 1032.4639 & 18 \\
\hline 3 & 464.1928 & 232.6001 & & & & & $\mathbf{Y}$ & 1944.8722 & 972.9398 & 1927.8457 & 964.4265 & 1926.8617 & 963.9345 & 17 \\
\hline 4 & 579.2198 & 290.1135 & 562.1932 & 281.6003 & & & $\mathbf{N}$ & 1781.8089 & 891.4081 & 1764.7824 & 882.8948 & 1763.7983 & 882.4028 & 16 \\
\hline 5 & 636.2412 & 318.6243 & 619.2147 & 310.1110 & & & $\mathbf{G}$ & 1666.7820 & 833.8946 & 1649.7554 & 825.3814 & 1648.7714 & 824.8893 & 15 \\
\hline 6 & 737.2889 & 369.1481 & 720.2624 & 360.6348 & 719.2784 & & $\mathbf{T}$ & 1609.7605 & 805.3839 & 1592.7340 & 796.8706 & 1591.7499 & 796.3786 & 14 \\
\hline 7 & 884.3573 & 442.6823 & 867.3308 & 434.1690 & 866.3468 & 433.6770 & $\mathbf{F}$ & 1508.7128 & 754.8601 & 1491.6863 & 746.3468 & 1490.7023 & 745.8548 & 13 \\
\hline 8 & 997.4414 & 499.2243 & 980.4149 & 490.7111 & 979.4308 & 490.2191 & $\mathbf{L}$ & 1361.6444 & 681.3258 & 1344.6179 & 672.8126 & 1343.6339 & 672.3206 & 12 \\
\hline 9 & 1112.4683 & 556.7378 & 1095.4418 & 548.2245 & 1094.4578 & 547.7325 & D & 1248.5604 & 624.7838 & 1231.5338 & 616.2705 & 1230.5498 & 615.7785 & 11 \\
\hline 10 & 1169.4898 & 585.2485 & 1152.4633 & 576.7353 & 1151.4792 & 576.2433 & G & 1133.5334 & 567.2703 & 1116.5069 & 558.7571 & 1115.5228 & 558.2651 & 10 \\
\hline 11 & 1270.5375 & 635.7724 & 1253.5109 & 627.2591 & 1252.5269 & 626.7671 & $\mathbf{T}$ & 1076.5119 & 538.7596 & 1059.4854 & 530.2463 & 1058.5014 & 529.7543 & 9 \\
\hline 12 & 1383.6216 & 692.3144 & 1366.5950 & 683.8011 & 1365.6110 & 683.3091 & $\mathbf{L}$ & 975.4643 & 488.2358 & 958.4377 & 479.7225 & 957.4537 & 479.2305 & 8 \\
\hline 13 & 1530.6900 & 765.8486 & 1513.6634 & 757.3353 & 1512.6794 & 756.8433 & $\mathbf{F}$ & 862.3802 & 431.6937 & 845.3537 & 423.1805 & 844.3696 & 422.6885 & 7 \\
\hline 14 & 1645.7169 & 823.3621 & 1628.6904 & 814.8488 & 1627.7063 & 814.3568 & D & 715.3118 & 358.1595 & 698.2852 & 349.6463 & 697.3012 & 349.1543 & 6 \\
\hline 15 & 1732.7489 & 866.8781 & 1715.7224 & 858.3648 & 1714.7384 & 857.8728 & $\mathrm{~S}$ & 600.2848 & 300.6461 & 583.2583 & 292.1328 & 582.2743 & 291.6408 & 5 \\
\hline 16 & 1819.7810 & 910.3941 & 1802.7544 & 901.8808 & 1801.7704 & 901.3888 & $\mathrm{~S}$ & 513.2528 & 257.1300 & 496.2263 & 248.6168 & 495.2423 & 248.1248 & 4 \\
\hline 17 & 1956.8399 & 978.9236 & 1939.8133 & 970.4103 & 1938.8293 & 969.9183 & $\mathbf{H}$ & 426.2208 & 213.6140 & 409.1942 & 205.1008 & & & 3 \\
\hline 18 & 2070.8828 & 1035.9450 & 2053.8563 & 1027.4318 & 2052.8722 & 1026.9398 & $\mathbf{N}$ & 289.1619 & 145.0846 & 272.1353 & 136.5713 & & & 2 \\
\hline
\end{tabular}




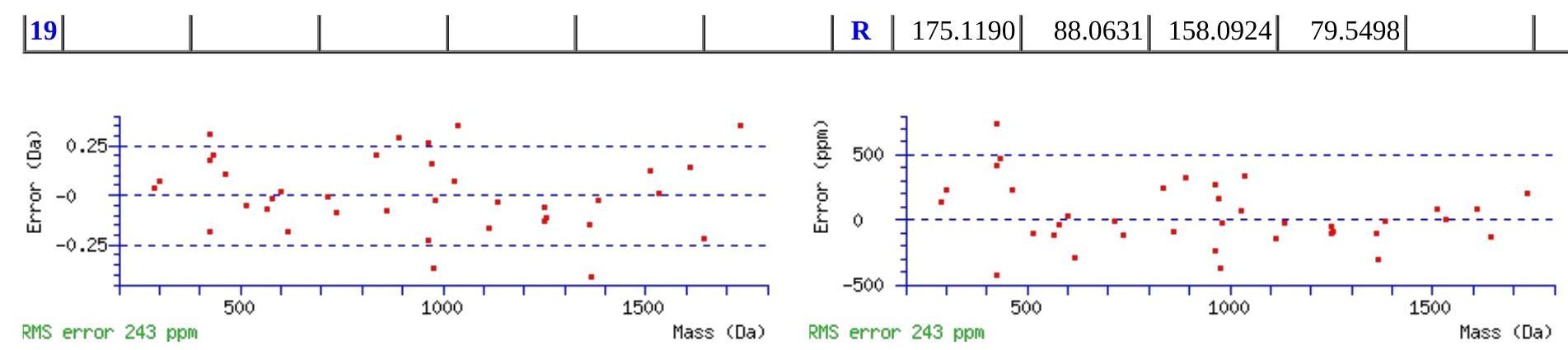

\section{All matches to this query}

\begin{tabular}{|l|l|l|l|}
\hline Score & Mr(calc): & Delta & \multicolumn{1}{|c|}{ Sequence } \\
\hline 49.8 & 2243.9872 & -0.0039 & YHYNGTFLDGTLFDSSHNR \\
\hline 16.4 & 2243.9872 & -0.0039 & YHYNGTFLDGTLFDSSHNR \\
\hline 1.8 & 2241.9717 & 2.0117 & VSQLYMNQKAWNTMVLR \\
\hline 1.2 & 2243.9758 & 0.0075 & EFVCHWGGCSRELRPFK \\
\hline 1.1 & 2241.9824 & 2.0009 & EPAVSVNATRSANILDNMNK \\
\hline 1.1 & 2241.9824 & 2.0009 & EPAVSVNATRSANILDNMNK \\
\hline 1.1 & 2241.9824 & 2.0009 & EPAVSVNATRSANILDNMNK \\
\hline 1.1 & 2241.9824 & 2.0009 & EPAVSVNATRSANILDNMNK \\
\hline 1.1 & 2243.9987 & -0.0154 & LADVLEETHYENGEYIIR \\
\hline 1.1 & 2243.9987 & -0.0154 & LADVLEETHYENGEYIIR \\
\hline
\end{tabular}

Spectrum No: 683; Query: 2489; Rank: 1

\section{Peptide View}

MS/MS Fragmentation of GTAEDANECLPCNCSGHSEECTFDR

Found in IPI00767923, Tax_Id=10116 Gene_Symbol=Lamc3_predicted laminin gamma 3

Match to Query 2489: 2916.078822 from(973.033550,3+)

Title: 100101RatKid_NS_deglyco_08.1889.1889.3.dta

Data file K:INewmanPaper|Piliangl3SubProteomes\Piliang3SP\mgf5ppm\ERLIC_3SubProteomes5ppm.mgf

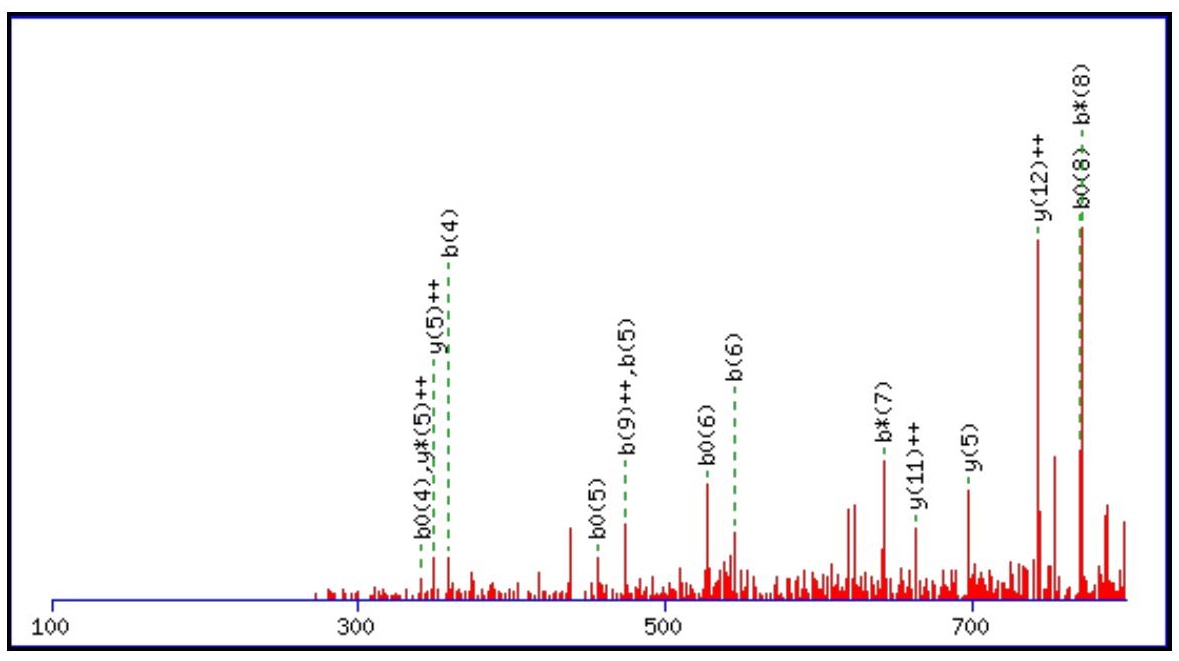



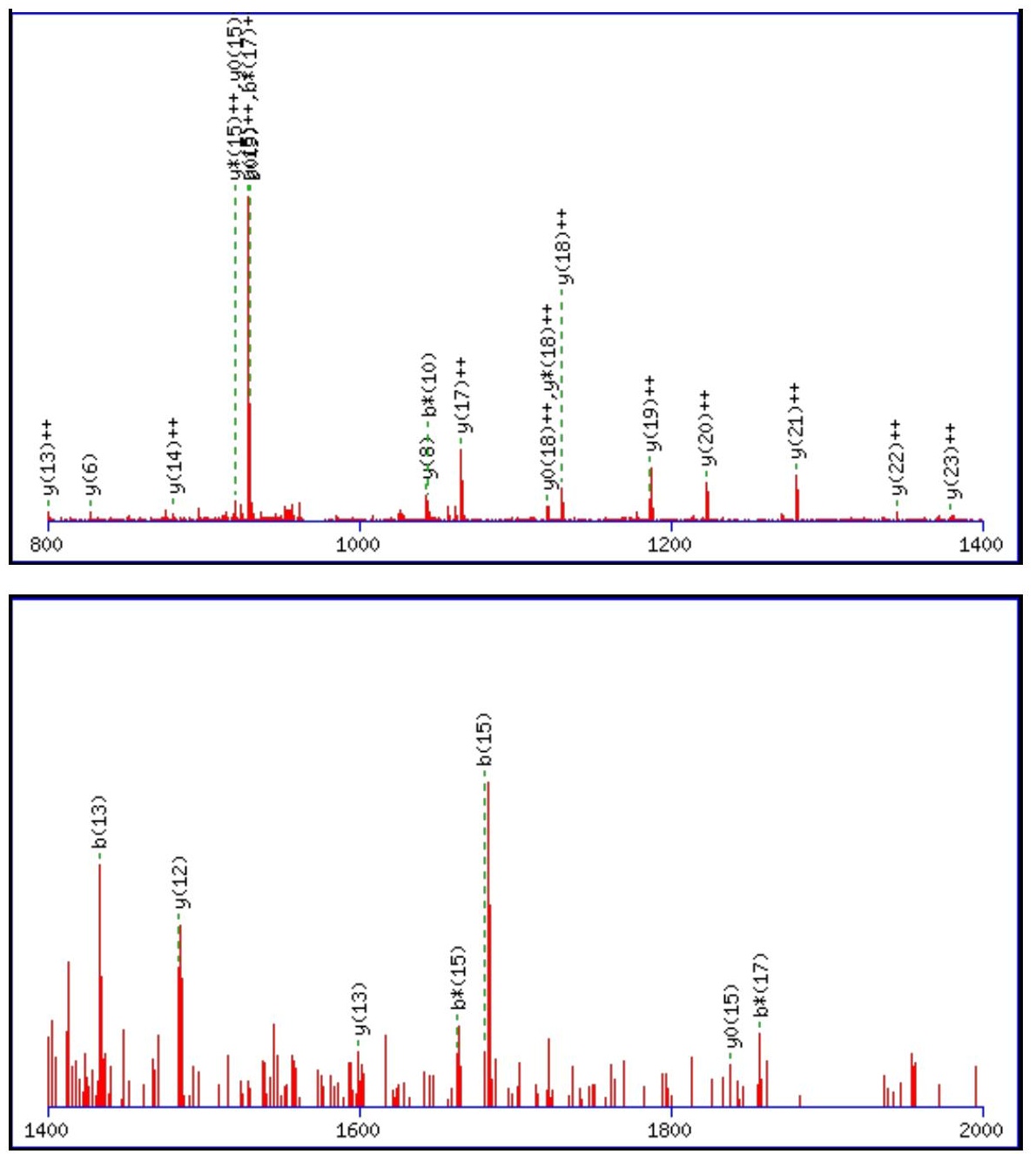

Monoisotopic mass of neutral peptide $\operatorname{Mr}($ calc): 2916.0691

Fixed modifications: Carbamidomethyl (C)

Variable modifications:

N13 : Deamidated_N (N)

Ions Score: 50 Expect: $\odot .0021$

Matches (Bold Red): 43/272 fragment ions using 78 most intense peaks

\begin{tabular}{|c|c|c|c|c|c|c|c|c|c|c|c|c|c|c|}
\hline \# & b & $\mathbf{b}^{++}$ & $\mathbf{b}^{*}$ & $\mathbf{b}^{*++}$ & $\mathbf{b}^{0}$ & $\mathbf{b}^{\mathbf{0 + +}}$ & Seq. & $\mathbf{y}$ & $y^{++}$ & $\mathbf{y}^{*}$ & $\mathbf{y}^{*^{++}}$ & $\mathbf{y}^{0}$ & $\mathbf{y}^{0++}$ & \# \\
\hline 1 & 58.0287 & 29.5180 & & & & & G & & & & & & & 25 \\
\hline 2 & 159.0764 & 80.0418 & & & 141.0659 & 71.0366 & $\mathbf{T}$ & 2860.0549 & 1430.5311 & 2843.0284 & 1422.0178 & 2842.0444 & 1421.5258 & 24 \\
\hline 3 & 230.1135 & 115.5604 & & & 212.1030 & 106.5551 & A & 2759.0072 & 1380.0073 & 2741.9807 & 1371.4940 & 2740.9967 & 1371.0020 & 23 \\
\hline 4 & 359.1561 & 180.0817 & & & 341.1456 & 171.0764 & $\mathbf{E}$ & 2687.9701 & 1344.4887 & 2670.9436 & 1335.9754 & 2669.9596 & 1335.4834 & 22 \\
\hline 5 & 474.1831 & 237.5952 & & & 456.1725 & 228.5899 & D & 2558.9275 & 1279.9674 & 2541.9010 & 1271.4541 & 2540.9170 & 1270.9621 & 21 \\
\hline 6 & 545.2202 & 273.1137 & & & 527.2096 & 264.1084 & A & 2443.9006 & 1222.4539 & 2426.8741 & 1213.9407 & 2425.8900 & 1213.4487 & 20 \\
\hline 7 & 659.2631 & 330.1352 & 642.2366 & 321.6219 & 641.2525 & 321.1299 & $\mathbf{N}$ & 2372.8635 & 1186.9354 & 2355.8369 & 1178.4221 & 2354.8529 & 1177.9301 & 19 \\
\hline 8 & 788.3057 & 394.6565 & 771.2792 & 386.1432 & 770.2951 & 385.6512 & $\mathbf{E}$ & 2258.8206 & 1129.9139 & 2241.7940 & 1121.4006 & 2240.8100 & 1120.9086 & 18 \\
\hline 9 & 948.3364 & 474.6718 & 931.3098 & 466.1585 & 930.3258 & 465.6665 & C & 2129.7780 & 1065.3926 & 2112.7514 & 1056.8793 & 2111.7674 & 1056.3873 & 17 \\
\hline 10 & 1061.4204 & 531.2138 & 1044.3939 & 522.7006 & 1043.4099 & 522.2086 & $\mathbf{L}$ & 1969.7473 & 985.3773 & 1952.7208 & 976.8640 & 1951.7368 & 976.3720 & 16 \\
\hline 11 & 1158.4732 & 579.7402 & 1141.4466 & 571.2270 & 1140.4626 & 570.7349 & $\mathbf{P}$ & 1856.6633 & 928.8353 & 1839.6367 & 920.3220 & 1838.6527 & 919.8300 & 15 \\
\hline 12 & 1318.5038 & 659.7556 & 1301.4773 & 651.2423 & 1300.4933 & 650.7503 & C & 1759.6105 & 880.3089 & 1742.5839 & 871.7956 & 1741.5999 & 871.3036 & 14 \\
\hline 13 & 1433.5308 & 717.2690 & 1416.5042 & 708.7557 & 1415.5202 & 708.2637 & $\mathbf{N}$ & 1599.5798 & 800.2936 & 1582.5533 & 791.7803 & 1581.5693 & 791.2883 & 13 \\
\hline 14 & 1593.5614 & 797.2843 & 1576.5349 & 788.7711 & 1575.5508 & 788.2791 & C & 1484.5529 & 742.7801 & 1467.5264 & 734.2668 & 1466.5423 & 733.7748 & 12 \\
\hline 15 & 1680.5934 & 840.8004 & 1663.5669 & 832.2871 & 1662.5829 & 831.7951 & $S$ & 1324.5223 & 662.7648 & 1307.4957 & 654.2515 & 1306.5117 & 653.7595 & 11 \\
\hline 16 & 1737.6149 & 869.3111 & 1720.5884 & 860.7978 & 1719.6043 & 860.3058 & $\mathbf{G}$ & 1237.4902 & 619.2488 & 1220.4637 & 610.7355 & 1219.4797 & 610.2435 & 10 \\
\hline 17 & 1874.6738 & 937.8405 & 1857.6473 & 929.3273 & 1856.6633 & 928.8353 & H & 1180.4688 & 590.7380 & 1163.4422 & 582.2247 & 1162.4582 & 581.7327 & 9 \\
\hline 18 & 1961.7058 & 981.3566 & 1944.6793 & 972.8433 & 1943.6953 & 972.3513 & $S$ & 1043.4099 & 522.2086 & 1026.3833 & 513.6953 & 1025.3993 & 513.2033 & 8 \\
\hline
\end{tabular}




\begin{tabular}{|c|c|c|c|c|c|c|c|c|c|c|c|c|c|}
\hline 19| & |2090.7484| & |1045.8779 & |2073.7219 & |1037.3646 & |2072.7379 & $|1036.8726|$ & $\mathbf{E}$ & 956.3778| & 478.6925 & 939.3513 & 470.1793 & 938.3673| & 469.6873 \\
\hline 20 & 2219.7910 & 1110.3992 & 2202.7645 & 1101.8859 & 2201.7805 & 1101.3939 & $\mathbf{E}$ & 827.3352 & 414.1713 & 810.3087 & 405.6580 & 809.3247 & 405.1660 \\
\hline 21 & 2379.8217 & 1190.4145 & 2362.7951 & 1181.9012 & 2361.8111 & 1181.4092 & C & 698.2926 & 349.6500 & 681.2661 & 341.1367 & 680.2821 & 340.6447 \\
\hline 22 & 2480.8694 & 1240.9383 & 2463.8428 & 1232.4250 & 2462.8588 & 1231.9330 & $\mathbf{T}$ & 538.2620 & 269.6346 & 521.2354 & 261.1214 & 520.2514 & 260.6293 \\
\hline 23 & 2627.9378 & 1314.4725 & 2610.9112 & 1305.9593 & 2609.9272 & 1305.4672 & $\mathbf{F}$ & 437.2143 & 219.1108 & 420.1878 & 210.5975 & 419.2037 & 210.1055 \\
\hline 24 & 2742.9647 & 1371.9860 & 2725.9382 & 1363.4727 & 2724.9542 & 1362.9807 & D & 290.1459 & 145.5766 & 273.1193 & 137.0633 & 272.1353 & 136.5713 \\
\hline 25 & & & & & & & $\mathbf{R}$ & 175.1190 & 88.0631 & 158.0924 & 79.5498 & & \\
\hline
\end{tabular}
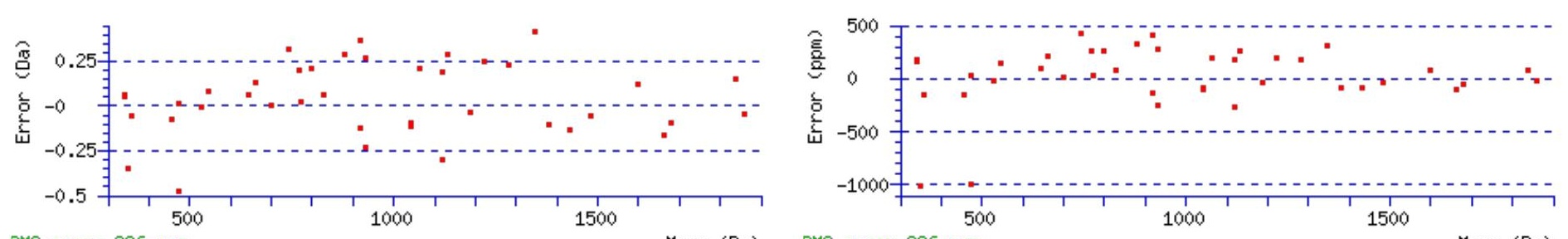

RNS error 286 ppm Mass (Da) RMS error 286 ppm

\section{All matches to this query}

\begin{tabular}{|l|l|l|l|}
\hline Score & Mr(calc): & Delta & \multicolumn{1}{|c|}{ Sequence } \\
\hline 49.8 & 2916.0691 & 0.0097 & GTAEDANECLPCNCSGHSEECTFDR \\
\hline 26.4 & 2916.0691 & 0.0097 & GTAEDANECLPCNCSGHSEECTFDR \\
\hline 12.5 & 2915.0851 & 0.9937 & GTAEDANECLPCNCSGHSEECTFDR \\
\hline 4.4 & 2915.0970 & 0.9818 & EPNPSVTGAQVSNGSNSGQMEEINTK \\
\hline 3.8 & 2916.0976 & -0.0187 & DQPPSDGHISSPLYISHPVSQKK \\
\hline 3.8 & 2916.0976 & -0.0187 & DQPPSDGHISSPLYISHPVSQKK \\
\hline 3.8 & 2916.0976 & -0.0187 & DQPPSDGHISSPLYISHPVSQKK \\
\hline 2.0 & 2916.1020 & -0.0232 & NGFGFRDSDLDICMTLEGHENAEK \\
\hline 1.8 & 2916.0772 & 0.0017 & YMTPSLNISEEDVTQANSEGKMAK \\
\hline 1.8 & 2916.1053 & -0.0265 & EESNSTGSTSSSEEDNHPKNIEADNR \\
\hline
\end{tabular}

Spectrum No: 684; Query: 562; Rank: 1

\section{Peptide View}

MS/MS Fragmentation of SLNLTEDVYKPR

Found in IPI00199448, Tax_Id=10116 Gene_Symbol=Lgals3bp Mama

Match to Query 562: 1434.736842 from(479.252890,3+)

Title: 100101RatKid_NS_deglyco_19.2371.2371.3.dta

Data file K:\NewmanPaper|Piliangl3SubProteomes\Piliang3SP\mgf5ppm\ERLIC_3SubProteomes5ppm.mgf 

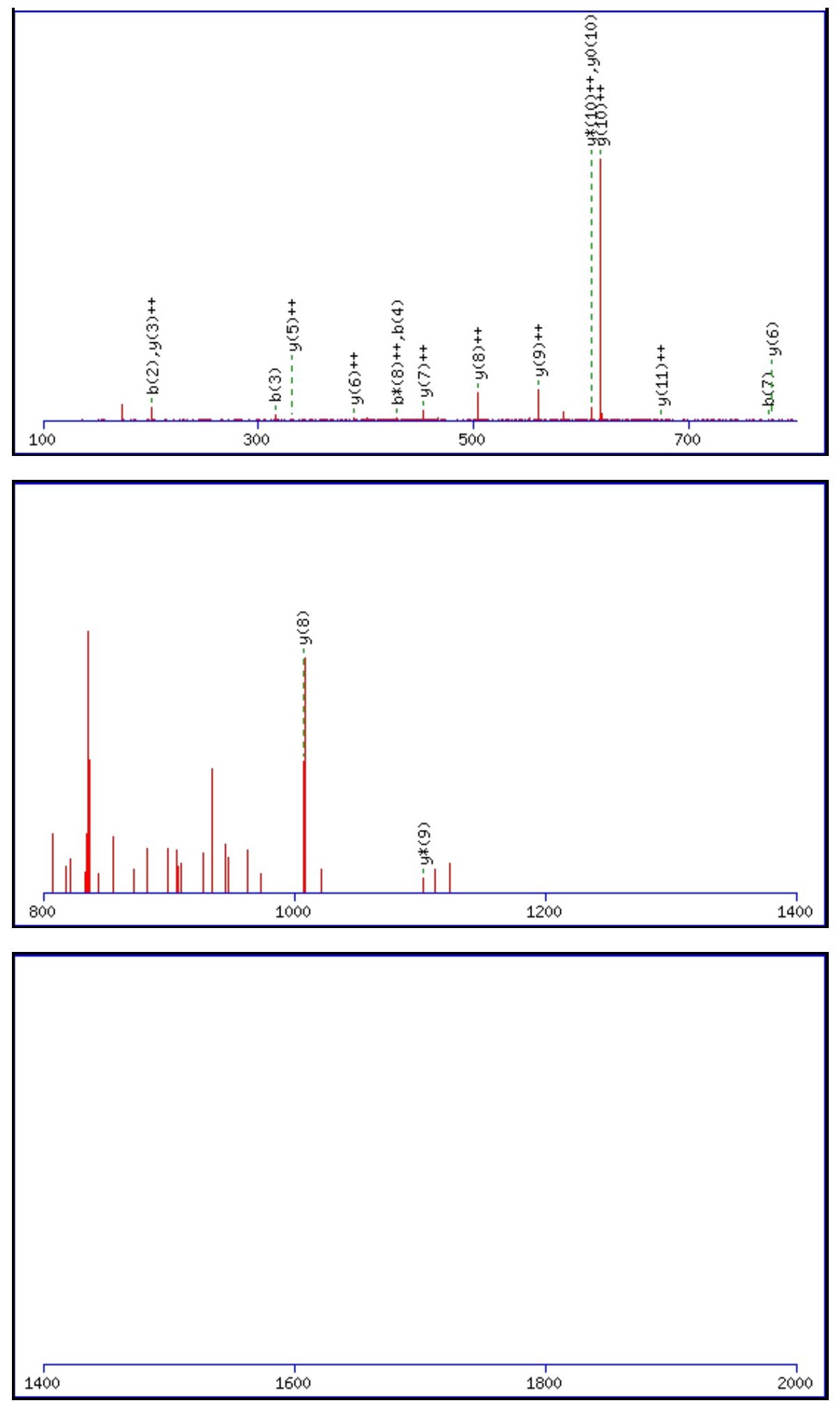

Monoisotopic mass of neutral peptide $\operatorname{Mr}($ calc): 1434.7354

Fixed modifications: Carbamidomethyl (C)

Variable modifications:

N3

Ions Score: 50 Expect: 0.0013

Matches (Bold Red): 18/118 fragment ions using 37 most intense peaks

\begin{tabular}{|c|c|c|c|c|c|c|c|c|c|c|c|c|c|c|}
\hline \# & b & $\mathbf{b}^{++}$ & $\mathbf{b}^{*}$ & $\mathbf{b}^{*^{++}}$ & $\mathbf{b}^{0}$ & $\mathbf{b}^{\mathbf{0 + +}}$ & Seq. & $\mathbf{y}$ & $\mathbf{y}^{++}$ & $\mathrm{y}^{*}$ & $\mathrm{y}^{*^{++}}$ & $\mathbf{y}^{0}$ & $y^{0++}$ & $\#$ \\
\hline 1 & 88.0393 & 44.5233 & & & 70.0287 & 35.5180 & $\mathbf{S}$ & & & & & & & 12 \\
\hline 2 & 201.1234 & 101.0653 & & & 183.1128 & 92.0600 & $\mathbf{L}$ & 1348.7107 & 674.8590 & 1331.6842 & 666.3457 & 1330.7001 & 665.8537 & 11 \\
\hline 3 & 316.1503 & 158.5788 & 299.1238 & 150.0655 & 298.1397 & 149.5735 & $\mathbf{N}$ & 1235.6266 & 618.3170 & 1218.6001 & 609.8037 & 1217.6161 & 609.3117 & 10 \\
\hline 4 & 429.2344 & 215.1208 & 412.2078 & 206.6075 & 411.2238 & 206.1155 & L & 1120.5997 & 560.8035 & 1103.5732 & 552.2902 & 1102.5891 & 551.7982 & 9 \\
\hline 5 & 530.2820 & 265.6447 & 513.2555 & 257.1314 & 512.2715 & 256.6394 & $\mathbf{T}$ & 1007.5156 & 504.2615 & 990.4891 & 495.7482 & 989.5051 & 495.2562 & 8 \\
\hline
\end{tabular}




\begin{tabular}{|c|c|c|c|c|c|c|c|c|c|c|c|c|c|c|}
\hline 6 & 659.3246 & |330.1660| & 642.2981 & |321.6527 & 641.3141 & |321.1607 & $\mathbf{E}$ & 906.4680 & |453.7376 & 889.4414 & |445.2243 & 888.4574 & |444.7323| & 7 \\
\hline 7 & 774.3516 & 387.6794 & 757.3250 & 379.1662 & 756.3410 & 378.6741 & D & 777.4254 & 389.2163 & 760.3988 & 380.7030 & 759.4148 & 380.2110 & 6 \\
\hline 8 & 873.4200 & 437.2136 & 856.3934 & 428.7004 & 855.4094 & 428.2084 & V & 662.3984 & 331.7028 & 645.3719 & 323.1896 & & & 5 \\
\hline 9 & 1036.4833 & 518.7453 & 1019.4568 & 510.2320 & 1018.4728 & 509.7400 & $\mathbf{Y}$ & 563.3300 & 282.1686 & 546.3035 & 273.6554 & & & 4 \\
\hline 10 & 1164.5783 & 582.7928 & 1147.5517 & 574.2795 & 1146.5677 & 573.7875 & $\mathbf{K}$ & 400.2667 & 200.6370 & 383.2401 & 192.1237 & & & 3 \\
\hline 11 & 1261.6311 & 631.3192 & 1244.6045 & 622.8059 & 1243.6205 & 622.3139 & $\mathbf{P}$ & 272.1717 & 136.5895 & 255.1452 & 128.0762 & & & 2 \\
\hline 12 & & & & & & & $\mathbf{R}$ & 175.1190 & 88.0631 & 158.0924 & 79.5498 & & & 1 \\
\hline
\end{tabular}
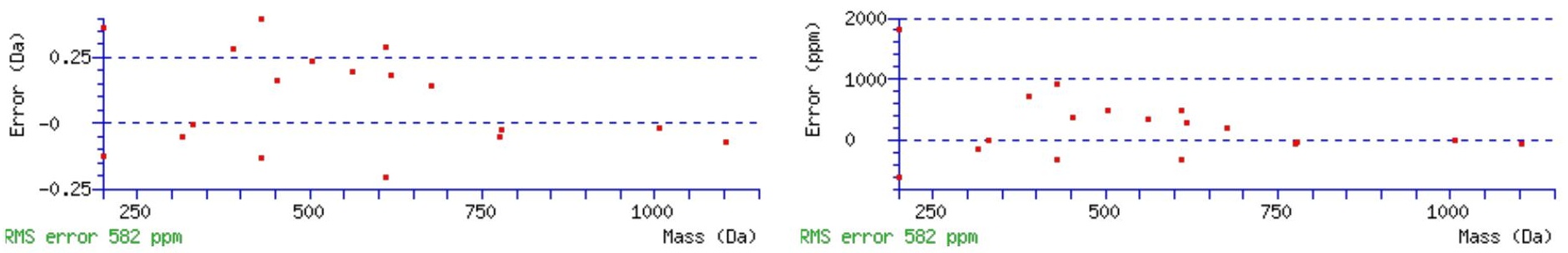

\section{All matches to this query}

\begin{tabular}{|l|l|l|l|}
\hline Score & Mr(calc): & Delta & \multicolumn{1}{c|}{ Sequence } \\
\hline 49.7 & 1434.7354 & 0.0014 & SLNLTEDVYKPR \\
\hline 11.2 & 1434.7500 & -0.0132 & SLGRMALGNVSSVK \\
\hline 9.7 & 1434.7467 & -0.0098 & $\underline{\text { LSDINQSVKFQR }}$ \\
\hline 5.9 & 1434.7500 & -0.0132 & $\underline{\text { SINEMLLSRLSR }}$ \\
\hline 4.5 & 1432.7439 & 1.9929 & VEVTPTVPRISR \\
\hline 4.5 & 1432.7439 & 1.9929 & $\underline{\text { VEVTPTVPRISR }}$ \\
\hline 4.3 & 1434.7394 & -0.0026 & $\underline{\text { WELTKYPELEK }}$ \\
\hline 4.0 & 1434.7388 & -0.0020 & $\underline{\text { MKGLNGKVSDLEK }}$ \\
\hline 3.9 & 1434.7500 & -0.0132 & $\underline{\text { LSNKNMKTQLTR }}$ \\
\hline 3.9 & 1434.7232 & 0.0137 & $\underline{\text { AEVEGPGRATKLK }}$ \\
\hline
\end{tabular}

Spectrum No: 685; Query: 1958; Rank: 1

\section{Peptide View}

MS/MS Fragmentation of GNNESQYDALITSNLVPMYK

Found in IPI00326462, Tax_Id=10116 Gene_Symbol=Enpp3 Ectonucleotide pyrophosphatase/phosphodiesterase family member 3

Match to Query 1958: 2257.061288 from(1129.537920,2+)

Title: 100101RatKid_NS_deglyco_21.4563.4563.2.dta

Data file K:\NewmanPaper\Piliang \3SubProteomes \Piliang3SP \mgf5ppm\ERLIC_3SubProteomes5ppm.mgf 

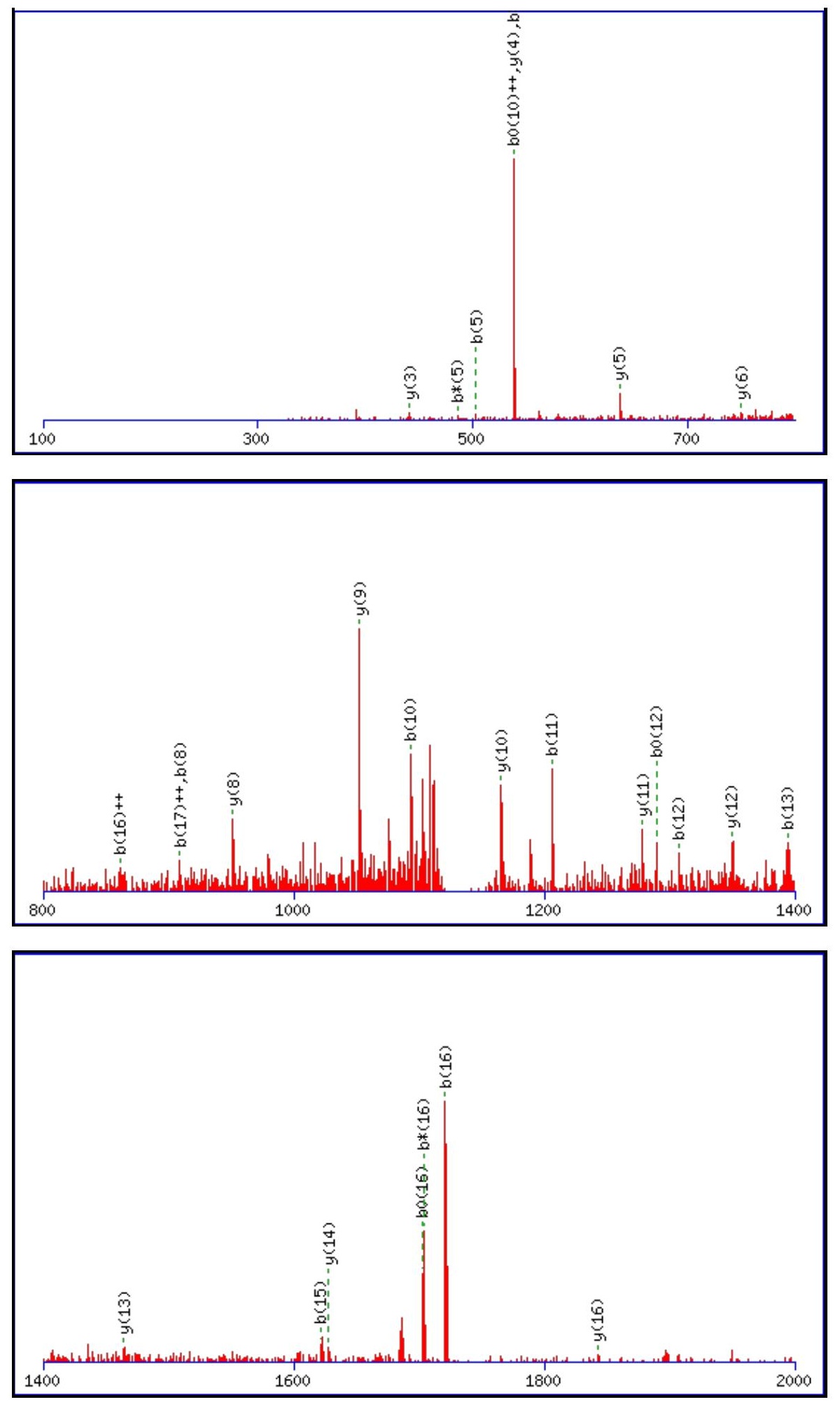

Monoisotopic mass of neutral peptide $\operatorname{Mr}($ calc): 2257.0572

Fixed modifications: Carbamidomethyl (C)

Variable modifications:

N3 : Deamidated $\mathrm{N}(\mathrm{N})$

Ions Score: 50 Expect : 0.003

Matches (Bold Red): 28/206 fragment ions using 62 most intense peaks

\begin{tabular}{|r|c|c|c|c|c|r|r|r|r|r|r|r|r|r|}
\hline$\#$ & $\mathbf{b}$ & \multicolumn{1}{|c|}{$\mathbf{b}^{++}$} & $\mathbf{b}^{*}$ & $\mathbf{b}^{\boldsymbol{*}^{++}}$ & $\mathbf{b}^{\mathbf{0}}$ & $\mathbf{b}^{\mathbf{0 + +}}$ & $\mathbf{S e q}$ & $\mathbf{y}$ & $\mathbf{y}^{++}$ & $\mathbf{y}^{*}$ & $\mathbf{y}^{\boldsymbol{*}^{++}}$ & $\mathbf{y}^{\mathbf{0}}$ & $\mathbf{y}^{\mathbf{0 + +}}$ & $\#$ \\
\hline $\mathbf{1}$ & 58.0287 & 29.5180 & & & & & $\mathbf{G}$ & & & & & & & $\mathbf{2 0}$ \\
\hline $\mathbf{2}$ & 172.0717 & 86.5395 & 155.0451 & 78.0262 & & & $\mathbf{N}$ & 2201.0431 & 1101.0252 & 2184.0165 & 1092.5119 & 2183.0325 & 1092.0199 & $\mathbf{1 9}$ \\
\hline $\mathbf{3}$ & 287.0986 & 144.0529 & 270.0721 & 135.5397 & & & $\mathbf{N}$ & 2087.0001 & 1044.0037 & 2069.9736 & 1035.4904 & 2068.9896 & 1034.9984 & $\mathbf{1 8}$ \\
\hline $\mathbf{4}$ & 416.1412 & 208.5742 & 399.1146 & 200.0610 & 398.1306 & 199.5690 & $\mathbf{E}$ & 1971.9732 & 986.4902 & 1954.9467 & 977.9770 & 1953.9626 & 977.4850 & $\mathbf{1 7}$ \\
\hline $\mathbf{5}$ & $\mathbf{5 0 3 . 1 7 3 2}$ & 252.0903 & $\mathbf{4 8 6 . 1 4 6 7}$ & 243.5770 & 485.1627 & 243.0850 & $\mathbf{S}$ & $\mathbf{1 8 4 2 . 9 3 0 6}$ & 921.9689 & 1825.9041 & 913.4557 & 1824.9200 & 912.9637 & $\mathbf{1 6}$ \\
\hline
\end{tabular}




\begin{tabular}{|c|c|c|c|c|c|c|c|c|c|c|c|c|c|c|}
\hline & 631.2318 & 316.1195 & 614.2053 & 307.6063 & 613.2212 & 307.1143 & $\mathbf{Q}$ & 1755.8986 & 878.4529 & |1738.8720 & 869.9397 & 1737.8880 & 869.4476 & 15 \\
\hline 7 & 794.2951 & 397.6512 & 777.2686 & 389.1379 & 776.2846 & 388.6459 & $\mathbf{Y}$ & 1627.8400 & 814.4236 & 1610.8135 & 805.9104 & 1609.8294 & 805.4184 & 14 \\
\hline 8 & 909.3221 & 455.1647 & 892.2955 & 446.6514 & 891.3115 & 446.1594 & D & 1464.7767 & 732.8920 & 1447.7501 & 724.3787 & 1446.7661 & 723.8867 & 13 \\
\hline 9 & 980.3592 & 490.6832 & 963.3326 & 482.1700 & 962.3486 & 481.6779 & A & 1349.7497 & 675.3785 & 1332.7232 & 666.8652 & 1331.7392 & 666.3732 & 12 \\
\hline 10 & 1093.4433 & 547.2253 & 1076.4167 & 538.7120 & 1075.4327 & 538.2200 & L & 1278.7126 & 639.8599 & 1261.6861 & 631.3467 & 1260.7021 & 630.8547 & 11 \\
\hline 11 & 1206.5273 & 603.7673 & 1189.5008 & 595.2540 & 1188.5168 & 594.7620 & I & 1165.6286 & 583.3179 & 1148.6020 & 574.8046 & 1147.6180 & 574.3126 & 10 \\
\hline 12 & 1307.5750 & 654.2911 & 1290.5484 & 645.7779 & 1289.5644 & 645.2859 & $\mathbf{T}$ & 1052.5445 & 526.7759 & 1035.5179 & 518.2626 & 1034.5339 & 517.7706 & 9 \\
\hline 13 & 1394.6070 & 697.8071 & 1377.5805 & 689.2939 & 1376.5965 & 688.8019 & $S$ & 951.4968 & 476.2520 & 934.4703 & 467.7388 & 933.4862 & 467.2468 & 8 \\
\hline 14 & 1508.6500 & 754.8286 & 1491.6234 & 746.3153 & 1490.6394 & 745.8233 & $\mathbf{N}$ & 864.4648 & 432.7360 & 847.4382 & 424.2228 & & & 7 \\
\hline 15 & 1621.7340 & 811.3706 & 1604.7075 & 802.8574 & 1603.7234 & 802.3654 & $\mathbf{L}$ & 750.4219 & 375.7146 & 733.3953 & 367.2013 & & & 6 \\
\hline 16 & 1720.8024 & 860.9049 & 1703.7759 & 852.3916 & 1702.7919 & 851.8996 & V & 637.3378 & 319.1725 & 620.3112 & 310.6593 & & & 5 \\
\hline 17 & 1817.8552 & 909.4312 & 1800.8286 & 900.9180 & 1799.8446 & 900.4260 & $\mathbf{P}$ & 538.2694 & 269.6383 & 521.2428 & 261.1251 & & & 4 \\
\hline 18 & 1948.8957 & 974.9515 & 1931.8691 & 966.4382 & 1930.8851 & 965.9462 & M & 441.2166 & 221.1119 & 424.1901 & 212.5987 & & & 3 \\
\hline 19 & 2111.9590 & 1056.4831 & 2094.9325 & 1047.9699 & 2093.9484 & 1047.4779 & $\mathbf{Y}$ & 310.1761 & 155.5917 & 293.1496 & 147.0784 & & & 2 \\
\hline 20 & & & & & & & $\mathbf{K}$ & 147.1128 & 74.0600 & 130.0863 & 65.5468 & & & 1 \\
\hline
\end{tabular}
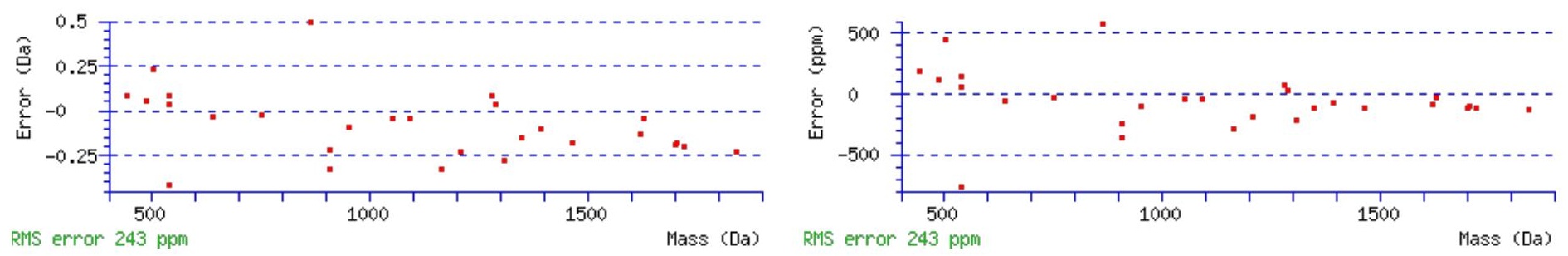

\section{All matches to this query}

\begin{tabular}{|l|l|c|l|}
\hline Score & Mr(calc): & Delta & \multicolumn{1}{c|}{ Sequence } \\
\hline 49.5 & 2257.0572 & 0.0041 & GNNESQYDALITSNLVPMYK \\
\hline 49.5 & 2257.0572 & 0.0041 & GNNESQYDALITSNLVPMYK \\
\hline 27.7 & 2256.0732 & 0.9881 & GNNESQYDALITSNLVPMYK \\
\hline 23.8 & 2257.0572 & 0.0041 & GNNESQYDALITSNLVPMYK \\
\hline 4.8 & 2257.0756 & -0.0143 & SKLEDIANAALAANAVTQVAK \\
\hline 2.7 & 2256.0585 & 1.0028 & SAHLQWMVVRNCSSFLIK \\
\hline 2.7 & 2256.0585 & 1.0028 & SAHLQWMVVRNCSSFLIK \\
\hline 1.0 & 2256.0580 & 1.0033 & HVQNQVDEVIDVMQENITK \\
\hline
\end{tabular}

Spectrum No: 686; Query: 1256; Rank: 1

\section{Peptide View}

\section{MS/MS Fragmentation of NVNRNISSLSEIFVGR}

Found in IPI00214328, Tax_Id=10116 Gene_Symbol=Naglt1 Sodium-dependent glucose transporter 1

Match to Query 1256: 1804.946112 from(602.655980,3+)

Title: 100101RatKid_NS_deglyco_26.3933.3933.3.dta

Data file K:INewmanPaper|Piliangl3SubProteomes\Piliang3SP\mgf5ppm\ERLIC_3SubProteomes5ppm.mgf 

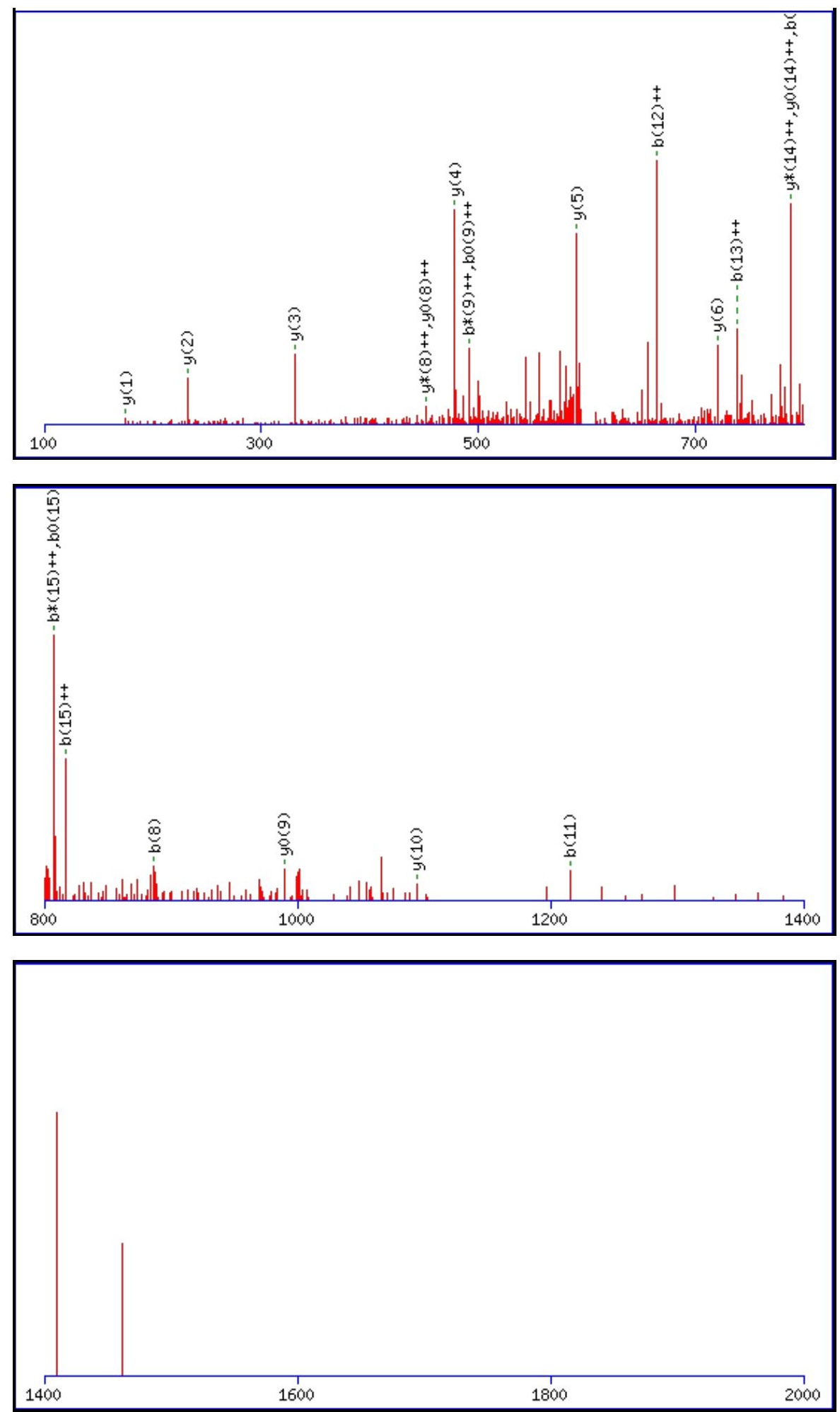

Monoisotopic mass of neutral peptide $\operatorname{Mr}($ calc): 1804.9431

Fixed modifications: Carbamidomethyl (C)

Variable modifications:

N5 : Deamidated $\mathrm{N}(\mathrm{N})$

Ions Score: 49 Expect : 0.0013

Matches (Bold Red): 23/158 fragment ions using 31 most intense peaks

\begin{tabular}{|r|c|c|c|c|c|c|c|c|c|c|c|c|c|c|}
\hline$\#$ & $\mathbf{b}$ & $\mathbf{b}^{++}$ & $\mathbf{b}^{*}$ & $\mathbf{b}^{\boldsymbol{*}_{++}}$ & $\mathbf{b}^{\mathbf{0}}$ & $\mathbf{b}^{\mathbf{0 + +}}$ & Seq. & $\mathbf{y}$ & $\mathbf{y}^{++}$ & $\mathbf{y}^{\mathbf{*}}$ & $\mathbf{y}^{\boldsymbol{*}^{++}}$ & $\mathbf{y}^{\mathbf{0}}$ & $\mathbf{y}^{\mathbf{0 + +}}$ & $\#$ \\
\hline $\mathbf{1}$ & 115.0502 & 58.0287 & 98.0237 & 49.5155 & & & $\mathbf{N}$ & & & & & & & $\mathbf{1 6}$ \\
\hline $\mathbf{2}$ & 214.1186 & 107.5629 & 197.0921 & 99.0497 & & & $\mathbf{V}$ & 1691.9075 & 846.4574 & 1674.8810 & 837.9441 & 1673.8969 & 837.4521 & $\mathbf{1 5}$ \\
\hline 3 & 328.1615 & 164.5844 & 311.1350 & 156.0711 & & & $\mathbf{N}$ & 1592.8391 & 796.9232 & 1575.8125 & 788.4099 & 1574.8285 & $\mathbf{7 8 7 . 9 1 7 9}$ & $\mathbf{1 4}$ \\
\hline $\mathbf{4}$ & 484.2627 & 242.6350 & 467.2361 & 234.1217 & & & $\mathbf{R}$ & 1478.7962 & 739.9017 & 1461.7696 & 731.3884 & 1460.7856 & 730.8964 & $\mathbf{1 3}$ \\
\hline $\mathbf{5}$ & 599.2896 & 300.1484 & 582.2630 & 291.6352 & & & $\mathbf{N}$ & 1322.6950 & 661.8512 & 1305.6685 & 653.3379 & 1304.6845 & 652.8459 & $\mathbf{1 2}$ \\
\hline
\end{tabular}




\begin{tabular}{|c|c|c|c|c|c|c|c|c|c|c|c|c|c|c|}
\hline 6 & 712.3737| & |356.6905 & 695.3471 & 348.1772 & & & I & |1207.6681| & 604.3377 & |1190.6416 & 595.8244 & 1189.6575 & |595.3324 & 11 \\
\hline 7 & 799.4057 & 400.2065 & 782.3791 & 391.6932 & 781.3951 & 391.2012 & S & 1094.5840 & 547.7957 & 1077.5575 & 539.2824 & 1076.5735 & 538.7904 & 10 \\
\hline 8 & 886.4377 & 443.7225 & 869.4112 & 435.2092 & 868.4271 & 434.7172 & S & 1007.5520 & 504.2796 & 990.5255 & 495.7664 & 989.5415 & 495.2744 & 9 \\
\hline 9 & 999.5218 & 500.2645 & 982.4952 & 491.7513 & 981.5112 & 491.2592 & $\mathbf{L}$ & 920.5200 & 460.7636 & 903.4934 & 452.2504 & 902.5094 & 451.7584 & 8 \\
\hline 10 & 1086.5538 & 543.7805 & 1069.5273 & 535.2673 & 1068.5432 & 534.7753 & S & 807.4359 & 404.2216 & 790.4094 & 395.7083 & 789.4254 & 395.2163 & 7 \\
\hline 11 & 1215.5964 & 608.3018 & 1198.5698 & 599.7886 & 1197.5858 & 599.2966 & $\mathbf{E}$ & 720.4039 & 360.7056 & 703.3774 & 352.1923 & 702.3933 & 351.7003 & 6 \\
\hline 12 & 1328.6805 & 664.8439 & 1311.6539 & 656.3306 & 1310.6699 & 655.8386 & I & 591.3613 & 296.1843 & 574.3348 & 287.6710 & & & 5 \\
\hline 13 & 1475.7489 & 738.3781 & 1458.7223 & 729.8648 & 1457.7383 & 729.3728 & $\mathbf{F}$ & 478.2772 & 239.6423 & 461.2507 & 231.1290 & & & 4 \\
\hline 14 & 1574.8173 & 787.9123 & 1557.7907 & 779.3990 & 1556.8067 & 778.9070 & V & 331.2088 & 166.1081 & 314.1823 & 157.5948 & & & 3 \\
\hline 15 & 1631.8388 & 816.4230 & 1614.8122 & 807.9097 & 1613.8282 & $\mathbf{8 0 7 . 4 1 7 7}$ & $\mathbf{G}$ & 232.1404 & 116.5738 & 215.1139 & 108.0606 & & & 2 \\
\hline 16 & & & & & & & $\mathbf{R}$ & 175.1190 & 88.0631 & 158.0924 & 79.5498 & & & 1 \\
\hline
\end{tabular}
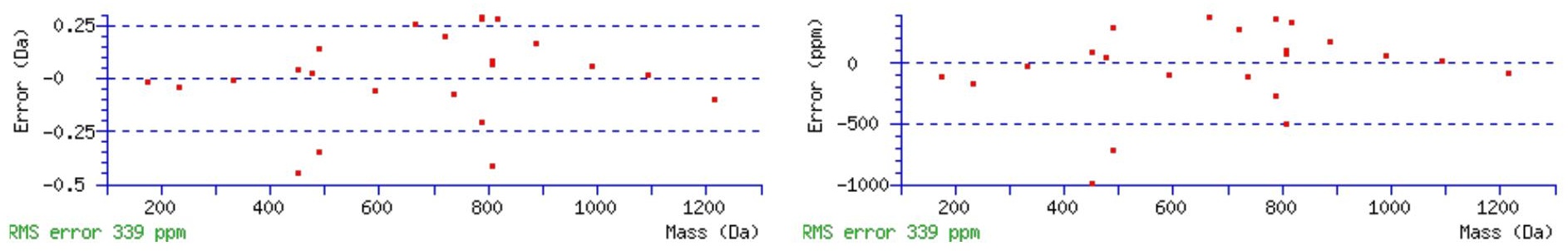

\section{All matches to this query}

\begin{tabular}{|l|l|l|l|}
\hline Score & Mr(calc): & Delta & \multicolumn{1}{|c|}{ Sequence } \\
\hline 49.5 & 1804.9431 & 0.0030 & NVNRNISSLSEIFVGR \\
\hline 49.5 & 1804.9431 & 0.0030 & NVNRNISSLSEIFVGR \\
\hline 49.5 & 1804.9431 & 0.0030 & NVNRNISSLSEIFVGR \\
\hline 37.8 & 1803.9591 & 0.9870 & NVNRNISSLSEIFVGR \\
\hline 10.5 & 1803.9481 & 0.9980 & NDFVIFLFTLMTSKK \\
\hline 8.5 & 1804.9349 & 0.0112 & VIPRRSPLSYPAVNR \\
\hline 8.1 & 1802.9216 & 2.0245 & DPVSRFLSNYFFRR \\
\hline 6.5 & 1804.9319 & 0.0142 & NQSFTDLLAELRAAQK \\
\hline 4.4 & 1804.9359 & 0.0102 & LAEEFLKSFPSDLPGR \\
\hline 3.5 & 1804.9292 & 0.0169 & LPKNEPQNPGANSARGR \\
\hline
\end{tabular}

Spectrum No: 687; Query: 627; Rank: 1

\section{Peptide View}

MS/MS Fragmentation of LTIVNSSVLDRPR

Found in IPI00763393, Tax_Id=10116 Gene_Symbol=Sorl1 similar to Sortilin-related receptor precursor (Sorting protein-related receptor containing LDLR class A repeats) (mSorLA) (SorLA-1) (Low-density lipoprotein receptor relative with 11 ligand-binding repeats) (LDLR relative with 11 ligand-binding re

Match to Query 627: 1469.823162 from(490.948330,3+)

Title: 100101RatKid_NS_deglyco_23.2478.2478.3.dta

Data file K:INewmanPaper|Piliang|3SubProteomes\Piliang3SP\mgf5ppm\ERLIC_3SubProteomes5ppm.mgf 

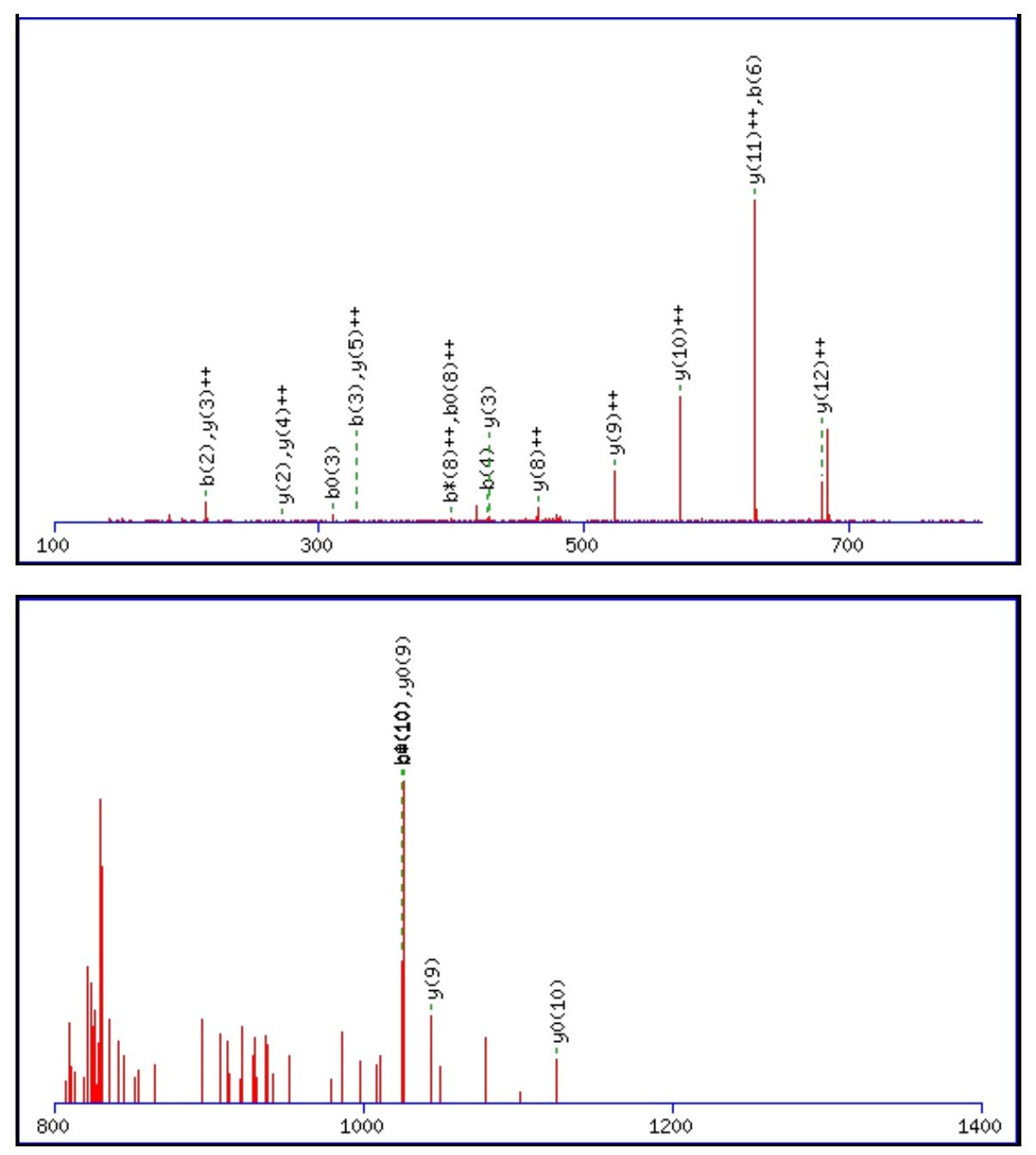

\begin{tabular}{rrrr}
\hline 00 & 1600 & 1800 & 2000 \\
\hline
\end{tabular}

Monoisotopic mass of neutral peptide $\operatorname{Mr}($ calc): 1469.8202

Fixed modifications: Carbamidomethyl (C)

Variable modifications:

N5 : Deamidated_N (N)

Ions Score: 49 Expect: 0.00065

Matches (Bold Red): 22/128 fragment ions using 33 most intense peaks

\begin{tabular}{|r|c|c|c|c|c|c|c|c|c|c|c|c|c|c|}
\hline$\#$ & $\mathbf{b}$ & $\mathbf{b}^{++}$ & $\mathbf{b}^{*}$ & $\mathbf{b}^{\boldsymbol{*}^{++}}$ & $\mathbf{b}^{\mathbf{0}}$ & $\mathbf{b}^{\mathbf{0 + +}}$ & Seq. & $\mathbf{y}$ & $\mathbf{y}^{++}$ & $\mathbf{y}^{\mathbf{*}}$ & $\mathbf{y}^{\boldsymbol{*}^{++}}$ & $\mathbf{y}^{\mathbf{0}}$ & $\mathbf{y}^{\mathbf{0 + +}}$ & $\#$ \\
\hline $\mathbf{1}$ & 114.0913 & 57.5493 & & & & & $\mathbf{L}$ & & & & & & & $\mathbf{1 3}$ \\
\hline $\mathbf{2}$ & $\mathbf{2 1 5 . 1 3 9 0}$ & 108.0731 & & & 197.1285 & 99.0679 & $\mathbf{T}$ & 1357.7434 & $\mathbf{6 7 9 . 3 7 5 3}$ & 1340.7168 & 670.8621 & 1339.7328 & 670.3701 & $\mathbf{1 2}$ \\
\hline $\mathbf{3}$ & $\mathbf{3 2 8 . 2 2 3 1}$ & 164.6152 & & & 310.2125 & 155.6099 & $\mathbf{I}$ & 1256.6957 & $\mathbf{6 2 8 . 8 5 1 5}$ & 1239.6692 & 620.3382 & 1238.6852 & 619.8462 & $\mathbf{1 1}$ \\
\hline $\mathbf{4}$ & $\mathbf{4 2 7 . 2 9 1 5}$ & 214.1494 & & & 409.2809 & 205.1441 & $\mathbf{V}$ & 1143.6117 & 572.3095 & 1126.5851 & 563.7962 & $\mathbf{1 1 2 5 . 6 0 1 1}$ & 563.3042 & $\mathbf{1 0}$ \\
\hline $\mathbf{5}$ & 542.3184 & 271.6629 & 525.2919 & 263.1496 & 524.3079 & 262.6576 & $\mathbf{N}$ & $\mathbf{1 0 4 4 . 5 4 3 2}$ & 522.7753 & 1027.5167 & 514.2620 & $\mathbf{1 0 2 6 . 5 3 2 7}$ & 513.7700 & $\mathbf{9}$ \\
\hline
\end{tabular}




\begin{tabular}{|c|c|c|c|c|c|c|c|c|c|c|c|c|c|c|}
\hline 6 & 629.3505 & |315.1789 & 612.3239 & |306.6656 & 611.3399 & |306.1736 & S & 929.5163 & |465.2618 & 912.4898 & |456.7485 & 911.5057 & |456.2565| & 8 \\
\hline 7 & 716.3825 & 358.6949 & 699.3559 & 350.1816 & 698.3719 & 349.6896 & S & 842.4843 & 421.7458 & 825.4577 & 413.2325 & 824.4737 & 412.7405 & 7 \\
\hline 8 & 815.4509 & 408.2291 & 798.4244 & 399.7158 & 797.4403 & 399.2238 & V & 755.4522 & 378.2298 & 738.4257 & 369.7165 & 737.4417 & 369.2245 & 6 \\
\hline 9 & 928.5350 & 464.7711 & 911.5084 & 456.2578 & 910.5244 & 455.7658 & $\mathbf{L}$ & 656.3838 & 328.6956 & 639.3573 & 320.1823 & 638.3733 & 319.6903 & 5 \\
\hline 10 & 1043.5619 & 522.2846 & 1026.5354 & 513.7713 & 1025.5513 & 513.2793 & D & 543.2998 & 272.1535 & 526.2732 & 263.6402 & 525.2892 & 263.1482 & 4 \\
\hline 11 & 1199.6630 & 600.3351 & 1182.6365 & 591.8219 & 1181.6525 & 591.3299 & $\mathbf{R}$ & 428.2728 & 214.6401 & 411.2463 & 206.1268 & & & 3 \\
\hline 12 & 1296.7158 & 648.8615 & 1279.6892 & 640.3483 & 1278.7052 & 639.8562 & $\mathbf{P}$ & 272.1717 & 136.5895 & 255.1452 & 128.0762 & & & 2 \\
\hline 13 & & & & & & & $\mathbf{R}$ & 175.1190 & 88.0631 & 158.0924 & 79.5498 & & & 1 \\
\hline
\end{tabular}
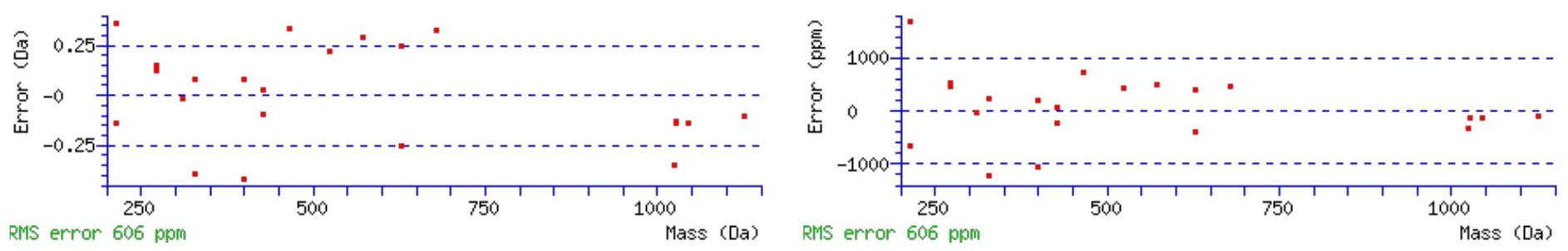

\section{All matches to this query}

\begin{tabular}{|l|c|c|l|}
\hline Score & Mr(calc): & Delta & \multicolumn{1}{c|}{ Sequence } \\
\hline 49.3 & 1469.8202 & 0.0030 & LTIVNSSVLDRPR \\
\hline 5.4 & 1469.8119 & 0.0112 & ITISIHNKLPVR \\
\hline 3.7 & 1467.8102 & 2.0130 & LLGASLVGALLFSK \\
\hline 3.6 & 1467.8102 & 2.0130 & LLILFNQSKLAK \\
\hline 2.7 & 1469.8314 & -0.0083 & AINQGGLTSVAVRGK \\
\hline 2.3 & 1468.8110 & 1.0122 & RGVLANALAGRDEK \\
\hline 2.2 & 1468.8323 & 0.9908 & DIASGLIGPLILCK \\
\hline 2.1 & 1468.8119 & 1.0113 & GRTLHCLMKVVR \\
\hline 2.0 & 1468.8054 & 1.0177 & VILEKATKVNFK \\
\hline 1.9 & 1468.8110 & 1.0121 & RGRGEVDAVTGKPK \\
\hline
\end{tabular}

Spectrum No: 688; Query: 1010; Rank: 1

\section{Peptide View}

MS/MS Fragmentation of GHVDPANDTFDIDPR

Found in IPI00370827, Tax_Id=10116 Gene_Symbol=Mcoln1_predicted mucolipin 1

Match to Query 1010: 1668.740022 from(557.253950,3+)

Title: 100101RatKid_NS_deglyco_27.1950.1950.3.dta

Data file K:INewmanPaper|Piliangl3SubProteomes\Piliang3SP\mgf5ppm\ERLIC_3SubProteomes5ppm.mgf 

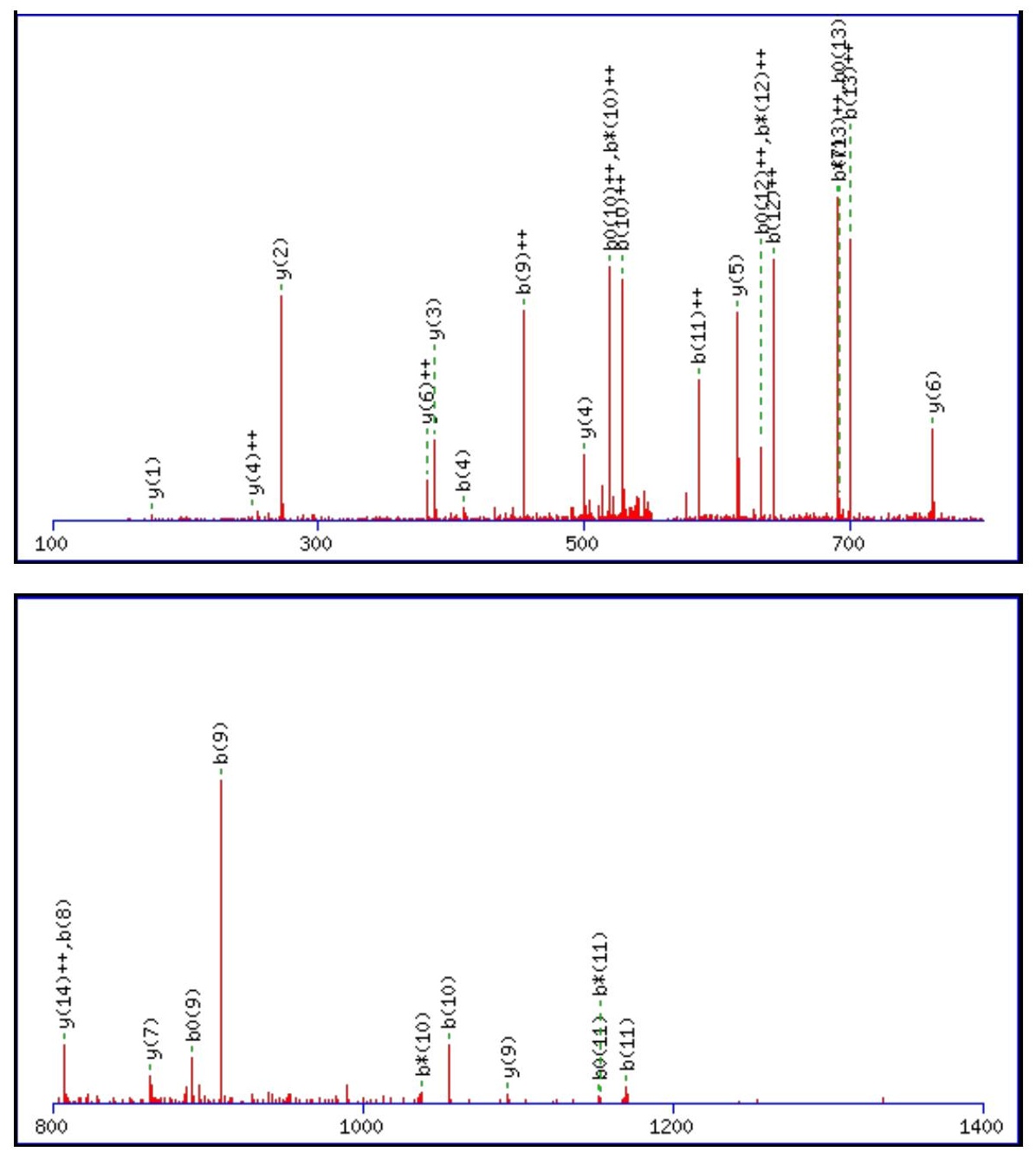

\begin{tabular}{rrrr}
\hline 1600 & 1600 & 1800 & 2000 \\
\hline
\end{tabular}

Monoisotopic mass of neutral peptide $\operatorname{Mr}($ calc): 1668.7380

Fixed modifications: Carbamidomethyl (C)

Variable modifications:

N7 : Deamidated_N (N)

Ions Score: 49 Expect: $\odot . \odot \odot 23$

Matches (Bold Red): 32/146 fragment ions using 45 most intense peaks

\begin{tabular}{|r|c|c|c|c|c|c|c|c|c|c|c|c|c|c|}
\hline$\#$ & $\mathbf{b}$ & $\mathbf{b}^{++}$ & $\mathbf{b}^{*}$ & $\mathbf{b}^{\boldsymbol{*}^{++}}$ & $\mathbf{b}^{\mathbf{0}}$ & $\mathbf{b}^{\mathbf{0 + +}}$ & Seq. & $\mathbf{y}$ & $\mathbf{y}^{++}$ & $\mathbf{y}^{\mathbf{*}}$ & $\mathbf{y}^{\boldsymbol{*}^{++}}$ & $\mathbf{y}^{\mathbf{0}}$ & $\mathbf{y}^{\mathbf{0 + +}}$ & $\#$ \\
\hline $\mathbf{1}$ & 58.0287 & 29.5180 & & & & & $\mathbf{G}$ & & & & & & & $\mathbf{1 5}$ \\
\hline $\mathbf{2}$ & 195.0877 & 98.0475 & & & & & $\mathbf{H}$ & 1612.7238 & $\mathbf{8 0 6 . 8 6 5 5}$ & 1595.6972 & 798.3523 & 1594.7132 & 797.8602 & $\mathbf{1 4}$ \\
\hline $\mathbf{3}$ & 294.1561 & 147.5817 & & & & & $\mathbf{V}$ & 1475.6649 & 738.3361 & 1458.6383 & 729.8228 & 1457.6543 & 729.3308 & $\mathbf{1 3}$ \\
\hline $\mathbf{4}$ & $\mathbf{4 0 9 . 1 8 3 0}$ & 205.0951 & & & 391.1724 & 196.0899 & $\mathbf{D}$ & 1376.5965 & 688.8019 & 1359.5699 & 680.2886 & 1358.5859 & 679.7966 & $\mathbf{1 2}$ \\
\hline $\mathbf{5}$ & 506.2358 & 253.6215 & & & 488.2252 & 244.6162 & $\mathbf{P}$ & 1261.5695 & 631.2884 & 1244.5430 & 622.7751 & 1243.5590 & 622.2831 & $\mathbf{1 1}$ \\
\hline
\end{tabular}




\begin{tabular}{|c|c|c|c|c|c|c|c|c|c|c|c|c|c|c|}
\hline 6 & 577.2729 & |289.1401 & & & 559.2623 & 280.1348 & A & 1164.5168 & 582.7620 & 1147.4902 & 574.2487 & 1146.5062 & |573.7567| & 10 \\
\hline 7 & 692.2998 & 346.6535 & 675.2733 & 338.1403 & 674.2893 & 337.6483 & $\mathbf{N}$ & 1093.4796 & 547.2435 & 1076.4531 & 538.7302 & 1075.4691 & 538.2382 & 9 \\
\hline 8 & 807.3268 & 404.1670 & 790.3002 & 395.6537 & 789.3162 & 395.1617 & D & 978.4527 & 489.7300 & 961.4262 & 481.2167 & 960.4421 & 480.7247 & 8 \\
\hline 9 & 908.3744 & |454.6909 & 891.3479 & 446.1776 & 890.3639 & 445.6856 & $\mathrm{~T}$ & 863.4258 & 432.2165 & 846.3992 & 423.7032 & 845.4152 & 423.2112 & 7 \\
\hline 10 & 1055.4429 & 528.2251 & |1038.4163 & 519.7118 & 1037.4323 & 519.2198 & $\mathbf{F}$ & 762.3781 & 381.6927 & 745.3515 & 373.1794 & 744.3675 & 372.6874 & 6 \\
\hline 11 & 1170.4698 & 585.7385 & 1153.4433 & 577.2253 & 1152.4592 & 576.7333 & D & 615.3097 & 308.1585 & 598.2831 & 299.6452 & 597.2991 & |299.1532 & 5 \\
\hline 12 & 1283.5539 & 642.2806 & |1266.5273 & 633.7673 & 1265.5433 & 633.2753 & I & 500.2827 & 250.6450 & 483.2562 & 242.1317 & 482.2722 & 241.6397 & 4 \\
\hline 13 & 1398.5808 & 699.7940 & 1381.5543 & 691.2808 & 1380.5702 & 690.7888 & D & 387.1987 & 194.1030 & 370.1721 & 185.5897 & 369.1881 & |185.0977 & 3 \\
\hline 14 & 1495.6336 & 748.3204 & 1478.6070 & 739.8072 & 1477.6230 & 739.3151 & $\mathbf{P}$ & 272.1717 & 136.5895 & 255.1452 & 128.0762 & & & 2 \\
\hline 15 & & & & & & & $\mathbf{R}$ & 175.1190 & 88.0631 & 158.0924 & 79.5498 & & & 1 \\
\hline
\end{tabular}
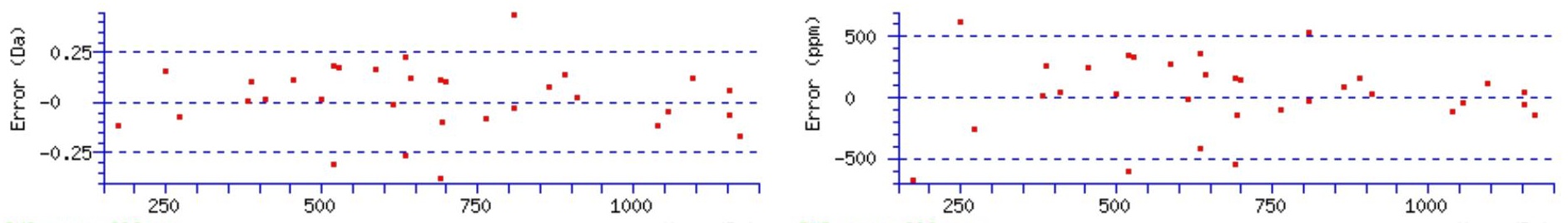

RMS error 296 ppm

Mass (Da) RIN error 296 ppm

\section{All matches to this query}

\begin{tabular}{|l|l|l|l|}
\hline Score & Mr(calc): & Delta & \multicolumn{1}{|c|}{ Sequence } \\
\hline 49.3 & 1668.7380 & 0.0020 & GHVDPANDTFDIDPR \\
\hline 11.8 & 1668.7542 & -0.0142 & GEVQQSAKELQNMK \\
\hline 9.1 & 1666.7498 & 1.9903 & QEAGKQKAMTQNPR \\
\hline 7.6 & 1667.7300 & 1.0101 & MAMPINVSDPDLLR \\
\hline 7.6 & 1667.7300 & 1.0101 & MAMPINVSDPDLLR \\
\hline 6.6 & 1668.7257 & 0.0143 & YVSHGATGKGNDQVR \\
\hline 6.3 & 1668.7290 & 0.0110 & ARSSAACANVLTPDR \\
\hline 6.3 & 1668.7290 & 0.0110 & ARSSAACANVLTPDR \\
\hline 5.7 & 1667.7387 & 1.0013 & RLDVEDSSFDQDSR \\
\hline 5.3 & 1666.7365 & 2.0035 & NSRLAQAWFNSHR \\
\hline
\end{tabular}

Spectrum No: 689; Query: 858; Rank: 1

\section{Peptide View}

MS/MS Fragmentation of SSSNQTLPDSVDWR

Found in IPI00210228, Tax_Id=10116 Gene_Symbol=Ctss Cathepsin S precursor

Match to Query 858: 1591.715488 from(796.865020,2+)

Title: 100101RatKid_NS_deglyco_24.2491.2491.2.dta

Data file K:INewmanPaper|Piliang|3SubProteomes\Piliang3SP\mgf5ppm\ERLIC_3SubProteomes5ppm.mgf 

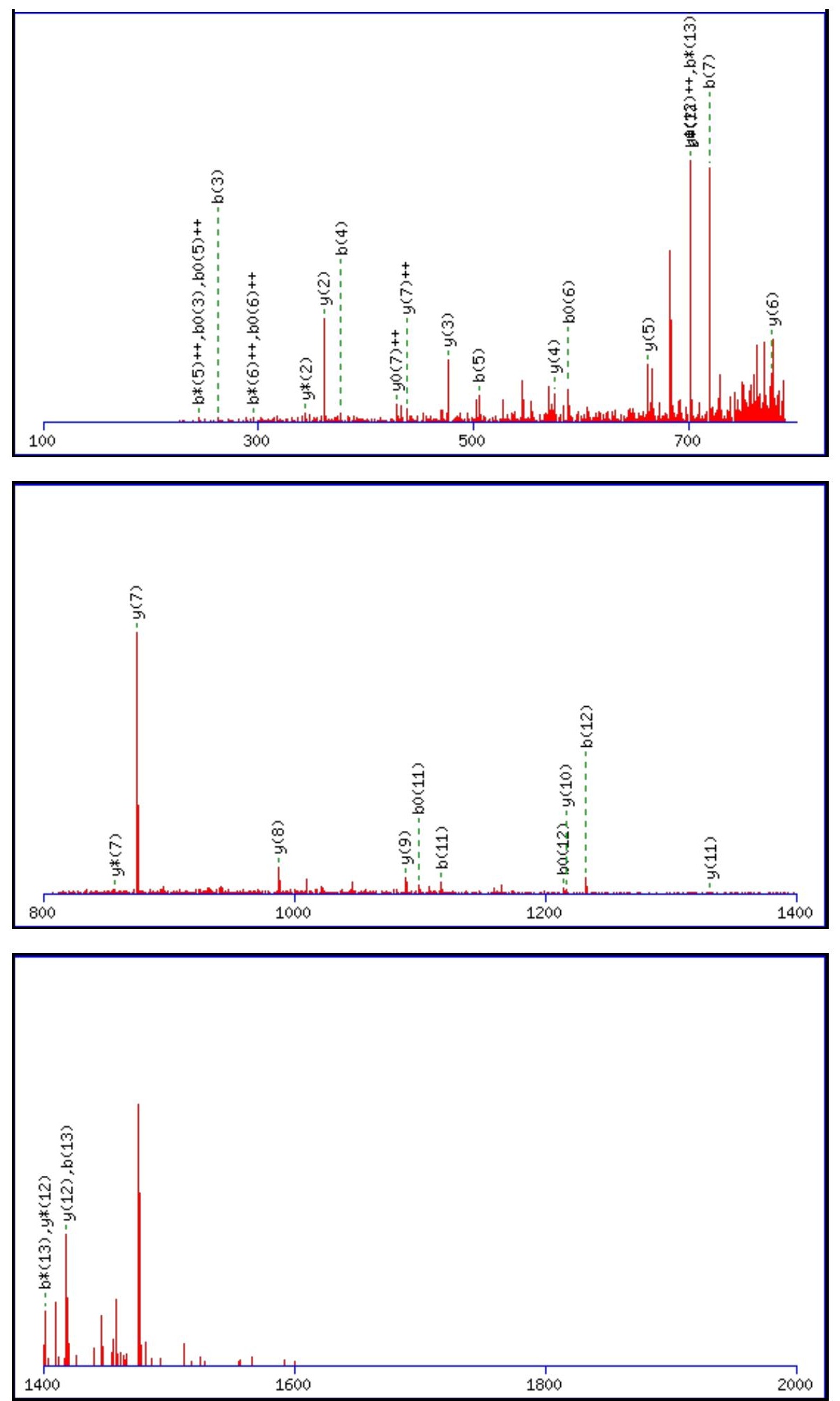

Monoisotopic mass of neutral peptide $\operatorname{Mr}($ calc): 1591.7114

Fixed modifications: Carbamidomethyl (C)

Variable modifications:

N4 : Deamidated_N (N)

Ions Score: 49 Expect: $\odot .0021$

Matches (Bold Red): 38/146 fragment ions using 98 most intense peaks

\begin{tabular}{|c|c|c|c|c|c|c|c|c|c|c|c|c|c|c|}
\hline \# & b & $\mathbf{b}^{++}$ & $\mathbf{b}^{*}$ & $\mathbf{b}^{*^{++}}$ & $\mathbf{b}^{0}$ & $\mathbf{b}^{\mathbf{0}^{++}}$ & Seq. & $\mathbf{y}$ & $\mathbf{y}^{++}$ & $\mathbf{y}^{*}$ & $\mathbf{y}^{*^{++}}$ & $\mathbf{y}^{0}$ & $\mathbf{y}^{\mathbf{0}^{++}}$ & \# \\
\hline 1 & 88.0393 & 44.5233 & & & 70.0287 & 35.5180 & S & & & & & & & 14 \\
\hline 2 & 175.0713 & 88.0393 & & & 157.0608 & 79.0340 & S & 1505.6867 & 753.3470 & 1488.6601 & 744.8337 & 1487.6761 & 744.3417 & 13 \\
\hline 3 & 262.1034 & |131.5553 & & & 244.0928 & 122.5500 & S & 1418.6546 & 709.8310 & | 1401.6281 & 701.3177 & 1400.6441 & 700.8257 & 12 \\
\hline 4 & 377.1303 & 189.0688 & 360.1037 & 180.5555 & 359.1197 & 180.0635 & $\mathbf{N}$ & 1331.6226 & 666.3149 & |1314.5961 & 657.8017 & 1313.6120 & 657.3097 & 11 \\
\hline 5 & 505.1889 & 253.0981 & 488.1623 & 244.5848 & 487.1783 & 244.0928 & $\mathbf{Q}$ & 1216.5957 & 608.8015 & 1199.5691 & 600.2882 & 1198.5851 & 599.7962 & 10 \\
\hline
\end{tabular}




\begin{tabular}{|c|c|c|c|c|c|c|c|c|c|c|c|c|c|c|}
\hline 6 & 606.2366 & |303.6219| & 589.2100 & |295.1086 & 588.2260 & |294.6166 & $\mathbf{T}$ & |1088.5371| & |544.7722| & |1071.5106 & |536.2589 & 1070.5265 & | 535.7669 & 9 \\
\hline 7 & 719.3206 & 360.1639 & 702.2941 & 351.6507 & 701.3101 & 351.1587 & $\mathbf{L}$ & 987.4894 & 494.2483 & 970.4629 & 485.7351 & 969.4789 & 485.2431 & 8 \\
\hline 8 & 816.3734 & 408.6903 & 799.3468 & 400.1771 & 798.3628 & 399.6850 & $\mathbf{P}$ & 874.4054 & 437.7063 & 857.3788 & 429.1930 & 856.3948 & 428.7010 & 7 \\
\hline 9 & 931.4003 & 466.2038 & 914.3738 & 457.6905 & 913.3898 & 457.1985 & D & 777.3526 & 389.1799 & 760.3260 & 380.6667 & 759.3420 & 380.1747 & 6 \\
\hline 10 & 1018.4324 & 509.7198 & 1001.4058 & 501.2065 & 1000.4218 & 500.7145 & S & 662.3257 & 331.6665 & 645.2991 & 323.1532 & 644.3151 & 322.6612 & 5 \\
\hline 11 & 1117.5008 & 559.2540 & 1100.4742 & 550.7407 & 1099.4902 & 550.2487 & V & 575.2936 & 288.1504 & 558.2671 & 279.6372 & 557.2831 & 279.1452 & 4 \\
\hline 12 & 1232.5277 & 616.7675 & 1215.5012 & 608.2542 & 1214.5171 & 607.7622 & D & 476.2252 & 238.6162 & 459.1987 & 230.1030 & 458.2146 & 229.6110 & 3 \\
\hline 13 & 1418.6070 & 709.8071 & 1401.5805 & 701.2939 & 1400.5965 & 700.8019 & W & 361.1983 & 181.1028 & 344.1717 & 172.5895 & & & 2 \\
\hline 14 & & & & & & & $\mathbf{R}$ & 175.1190 & 88.0631 & 158.0924 & 79.5498 & & & 1 \\
\hline
\end{tabular}
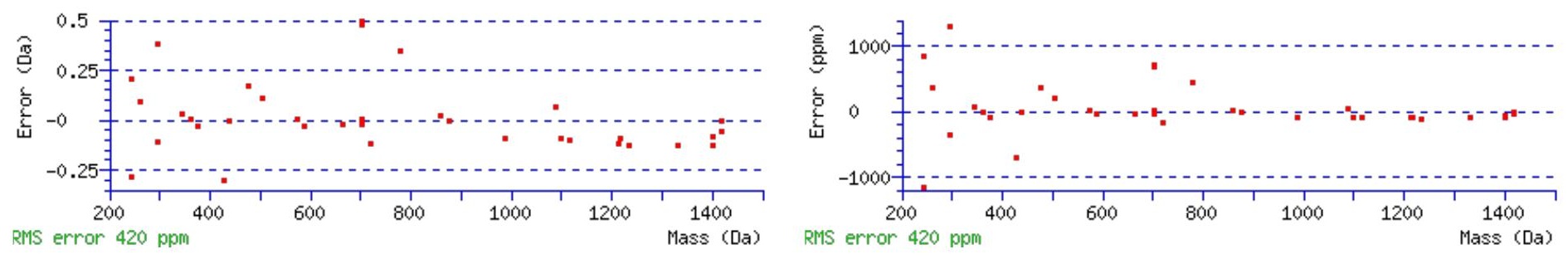

\section{All matches to this query}

\begin{tabular}{|l|l|c|l|}
\hline Score & Mr(calc): & Delta & \multicolumn{1}{|c|}{ Sequence } \\
\hline 49.2 & 1591.7114 & 0.0041 & SSSNQTLPDSVDWR \\
\hline 21.4 & 1590.7274 & 0.9881 & SSSNQTLPDSVDWR \\
\hline 9.9 & 1591.7048 & 0.0107 & $\underline{\text { IPTVNVGTFQSR }}$ \\
\hline 9.1 & 1591.7048 & 0.0107 & $\underline{\text { IPTVNVGTFQSR }}$ \\
\hline 8.8 & 1591.7227 & -0.0072 & DVFPQNETRSEGGR \\
\hline 7.2 & 1591.7300 & -0.0145 & QNTTLTIIWLTK \\
\hline 6.8 & 1591.7123 & 0.0032 & EACSLNPGMSALWR \\
\hline 6.0 & 1591.7243 & -0.0088 & NQHLAKKSEVNDK \\
\hline 4.8 & 1591.7300 & -0.0146 & GEGNETNNMVITWK \\
\hline 4.3 & 1591.7243 & -0.0088 & DKSPSEGDVTPPKR \\
\hline
\end{tabular}

Spectrum No: 690; Query: 64; Rank: 1

\section{Peptide View}

MS/MS Fragmentation of FLSYNVTR

Found in IPI00362369, Tax_Id=10116 Gene_Symbol=RGD1306248 similar to RIKEN cDNA 9630046K23

Match to Query 64: 999.500548 from(500.757550,2+)

Title: 091008RatKidney_NH4Format01_23.1576.1576.2.dta

Data file K:\NewmanPaper|Piliang|3SubProteomes\Piliang3SP\mgf5ppm\ERLIC_3SubProteomes5ppm.mgf 

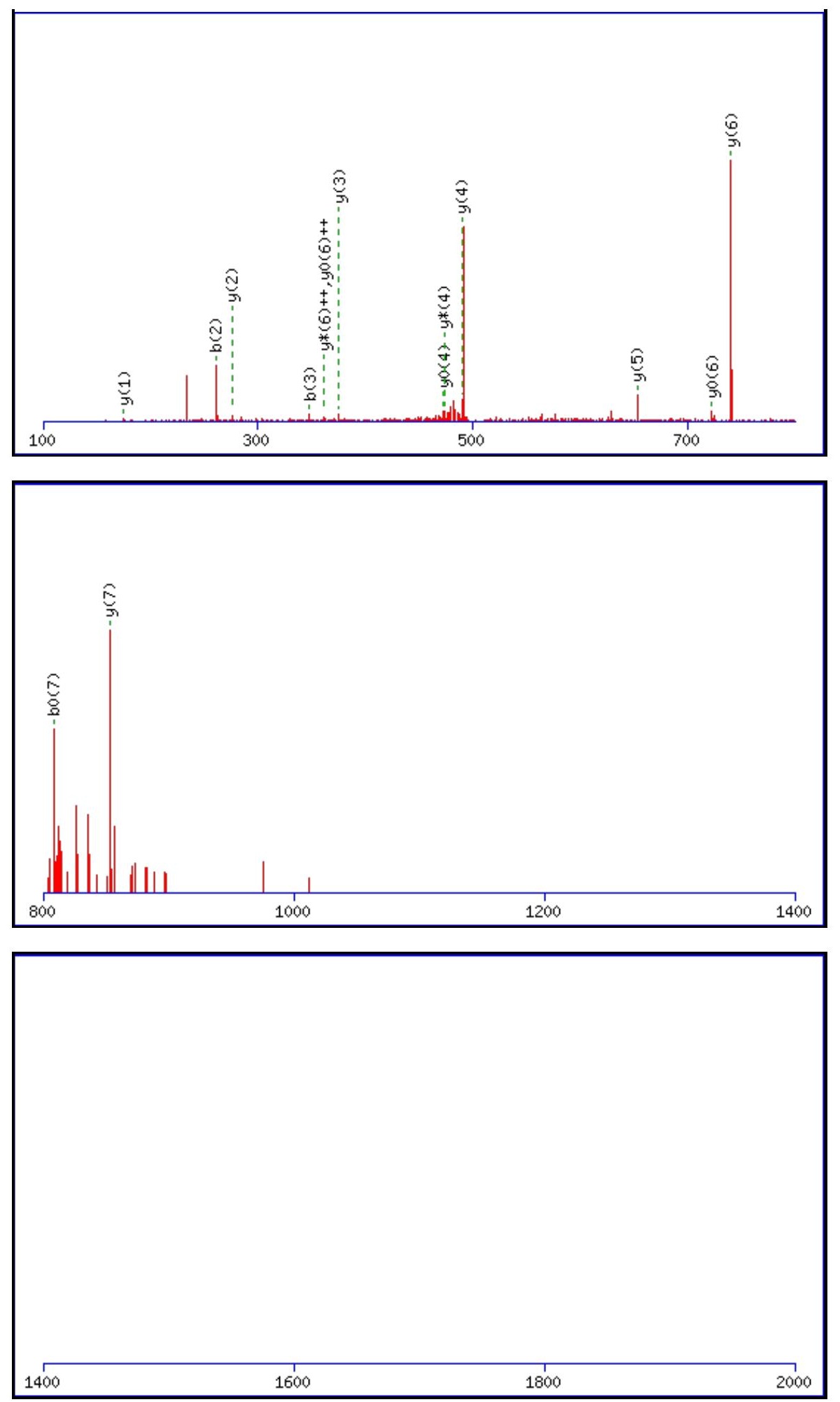

Monoisotopic mass of neutral peptide $\operatorname{Mr}($ calc): 999.5025

Fixed modifications: Carbamidomethyl (C)

Variable modifications:

N5 : Deamidated N (N)

Ions Score: 49 Expect: 0.00097

Matches (Bold Red): 15/70 fragment ions using 40 most intense peaks

\begin{tabular}{|c|c|c|c|c|c|c|c|c|c|c|c|c|c|c|}
\hline$\#$ & $\mathbf{b}$ & $\mathbf{b}^{++}$ & $\mathbf{b}^{*}$ & $\mathbf{b}^{\boldsymbol{*}^{++}}$ & $\mathbf{b}^{\mathbf{0}}$ & $\mathbf{b}^{\mathbf{0 + +}}$ & Seq. & $\mathbf{y}$ & $\mathbf{y}^{++}$ & $\mathbf{y}^{*}$ & $\mathbf{y}^{\boldsymbol{*}^{++}}$ & $\mathbf{y}^{\mathbf{0}}$ & $\mathbf{y}^{\mathbf{0 + +}}$ & $\#$ \\
\hline $\mathbf{1}$ & 148.0757 & 74.5415 & & & & & $\mathbf{F}$ & & & & & & & $\mathbf{8}$ \\
\hline $\mathbf{2}$ & $\mathbf{2 6 1 . 1 5 9 8}$ & 131.0835 & & & & & $\mathbf{L}$ & $\mathbf{8 5 3 . 4 4 1 4}$ & 427.2243 & 836.4149 & 418.7111 & 835.4308 & 418.2191 & $\mathbf{7}$ \\
\hline $\mathbf{3}$ & 348.1918 & 174.5995 & & & 330.1812 & 165.5942 & $\mathbf{S}$ & 740.3573 & 370.6823 & 723.3308 & 362.1690 & 722.3468 & $\mathbf{3 6 1 . 6 7 7 0}$ & $\mathbf{6}$ \\
\hline $\mathbf{4}$ & 511.2551 & 256.1312 & & & 493.2445 & 247.1259 & $\mathbf{Y}$ & $\mathbf{6 5 3 . 3 2 5 3}$ & 327.1663 & 636.2988 & 318.6530 & 635.3147 & 318.1610 & $\mathbf{5}$ \\
\hline $\mathbf{5}$ & 626.2820 & 313.6447 & 609.2555 & 305.1314 & 608.2715 & 304.6394 & $\mathbf{N}$ & $\mathbf{4 9 0 . 2 6 2 0}$ & 245.6346 & $\mathbf{4 7 3 . 2 3 5 4}$ & 237.1214 & $\mathbf{4 7 2 . 2 5 1 4}$ & 236.6293 & $\mathbf{4}$ \\
\hline
\end{tabular}




\begin{tabular}{|r|r|r|r|r|r|r|r|r|r|r|r|r|r|r|r|r|}
$\mathbf{6}$ & 725.3505 & 363.1789 & 708.3239 & 354.6656 & 707.3399 & 354.1736 & $\mathbf{V}$ & 375.2350 & 188.1212 & 358.2085 & 179.6079 & 357.2245 & 179.1159 & 3 \\
\hline $\mathbf{7}$ & 826.3981 & 413.7027 & 809.3716 & 405.1894 & $\mathbf{8 0 8 . 3 8 7 6}$ & $\mathbf{4 0 4 . 6 9 7 4}$ & $\mathbf{T}$ & $\mathbf{2 7 6 . 1 6 6 6}$ & 138.5870 & 259.1401 & 130.0737 & 258.1561 & 129.5817 & $\mathbf{2}$ \\
\hline $\mathbf{8}$ & & & & & & & $\mathbf{R}$ & $\mathbf{1 7 5 . 1 1 9 0}$ & 88.0631 & 158.0924 & 79.5498 & & & $\mathbf{1}$ \\
\hline
\end{tabular}
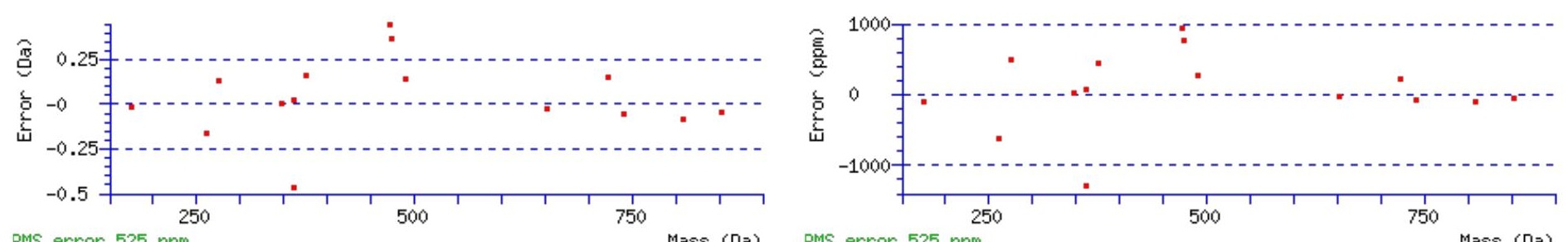

RMS error 525 ppm

\section{All matches to this query}

\begin{tabular}{|l|l|l|l|}
\hline Score & Mr(calc): & Delta & \multicolumn{1}{|c|}{ Sequence } \\
\hline 49.1 & 999.5025 & -0.0020 & FLSYNVTR \\
\hline 20.6 & 998.4984 & 1.0022 & KMATLVTR \\
\hline 11.9 & 998.4934 & 1.0072 & WQNSHSIK \\
\hline 10.8 & 998.5049 & 0.9957 & LKETTISK \\
\hline 10.8 & 998.5049 & 0.9957 & LKETTISK \\
\hline 10.3 & 999.4960 & 0.0045 & $\underline{\text { FLXGPPCPR }}$ \\
\hline 9.4 & 999.5097 & -0.0092 & ERAPGNSIR \\
\hline 8.0 & 998.4910 & 1.0096 & KNKVSTSR \\
\hline 8.0 & 998.4910 & 1.0096 & KNKVSTSR \\
\hline 7.5 & 999.5000 & 0.0005 & $\underline{\text { FLTMFWR }}$ \\
\hline
\end{tabular}

Spectrum No: 691; Query: 1478; Rank: 1

\section{Peptide View}

MS/MS Fragmentation of VWNSTFIEDYKDFDR

Found in IPI00372689, Tax_Id=10116 Gene_Symbol=Itga3_predicted 117 kDa protein

Match to Query 1478: 1934.871102 from(645.964310,3+)

Title: 100101RatKid_NS_deglyco_14.4453.4453.3.dta

Data file K:\NewmanPaper|Piliangl3SubProteomes\Piliang3SP\mgf5ppm\ERLIC_3SubProteomes5ppm.mgf

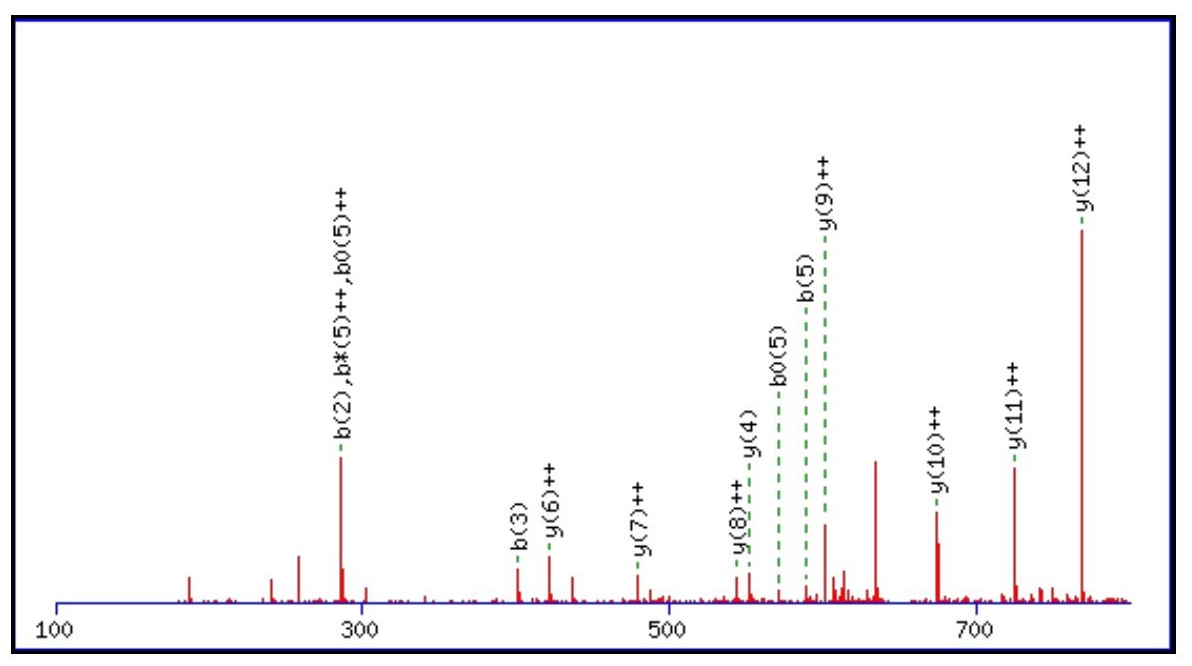



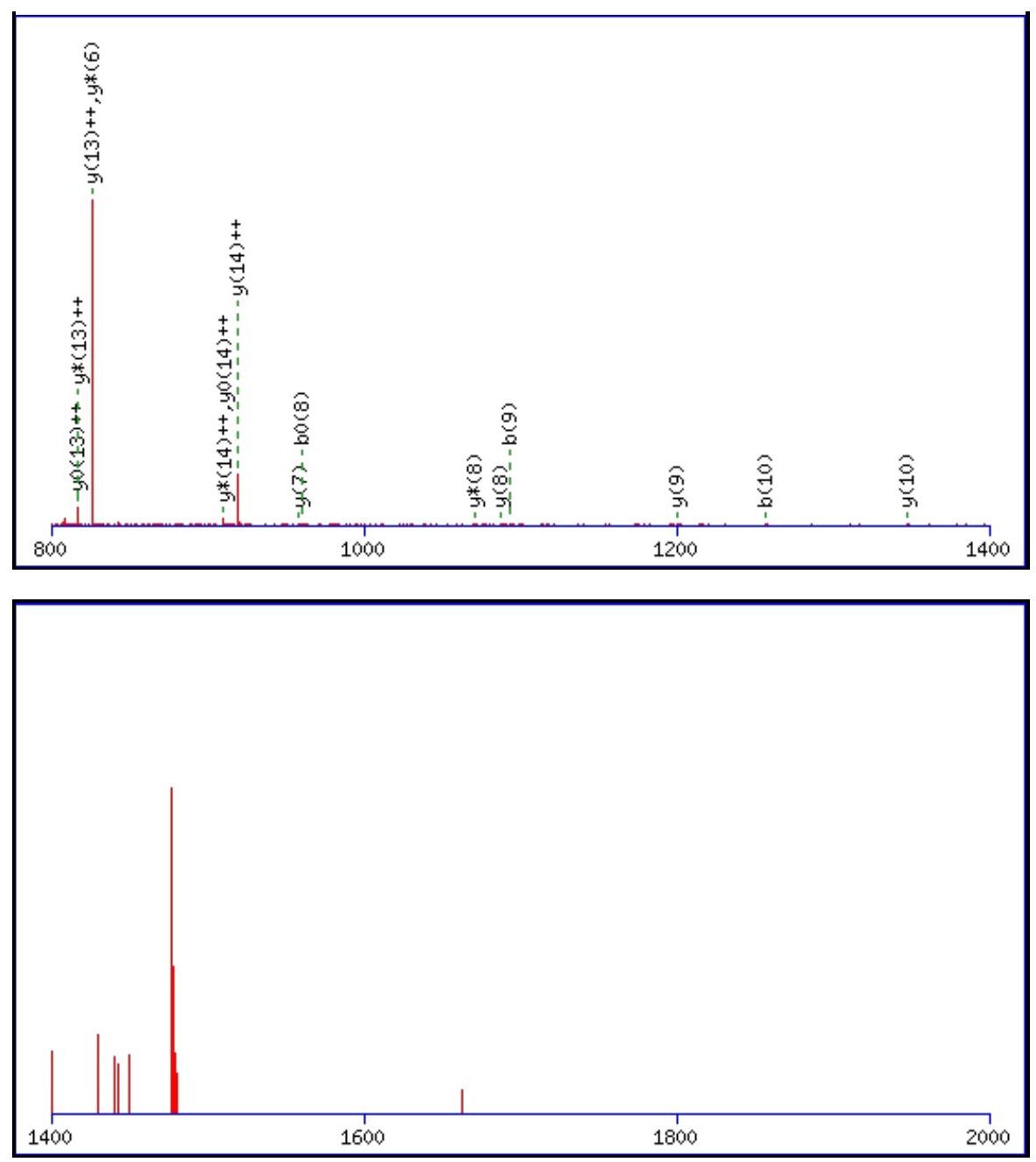

Monoisotopic mass of neutral peptide $\operatorname{Mr}($ calc): 1934.8687

Fixed modifications: Carbamidomethyl (C)

Variable modifications:

N3: Deamidated $\mathrm{N}(\mathrm{N})$

Ions Score: 49 Expect: 0.0029

Matches (Bold Red): 29/156 fragment ions using 54 most intense peaks

\begin{tabular}{|c|c|c|c|c|c|c|c|c|c|c|c|c|c|c|}
\hline \# & b & $\mathbf{b}^{++}$ & b* & $\mathbf{b}^{*^{++}}$ & $\mathbf{b}^{0}$ & $\mathbf{b}^{\mathbf{0 + +}}$ & Seq. & $\mathbf{y}$ & $y^{++}$ & $\mathbf{y}^{*}$ & $\mathbf{y}^{*^{++}}$ & $\mathbf{y}^{0}$ & $\mathbf{y}^{0++}$ & \# \\
\hline 1 & 100.0757 & 50.5415 & & & & & $\mathbf{V}$ & & & & & & & 15 \\
\hline 2 & 286.1550 & 143.5811 & & & & & $\mathbf{W}$ & 1836.8075 & 918.9074 & 1819.7810 & 910.3941 & 1818.7969 & 909.9021 & 14 \\
\hline 3 & 401.1819 & 201.0946 & 384.1554 & 192.5813 & & & $\mathbf{N}$ & 1650.7282 & 825.8677 & 1633.7017 & 817.3545 & 1632.7176 & 816.8625 & 13 \\
\hline 4 & 488.2140 & 244.6106 & 471.1874 & 236.0973 & 470.2034 & 235.6053 & $\mathbf{S}$ & 1535.7013 & 768.3543 & 1518.6747 & 759.8410 & 1517.6907 & 759.3490 & 12 \\
\hline 5 & 589.2616 & 295.1345 & 572.2351 & 286.6212 & 571.2511 & 286.1292 & $\mathbf{T}$ & 1448.6692 & 724.8383 & 1431.6427 & 716.3250 & 1430.6587 & 715.8330 & 11 \\
\hline 6 & 736.3301 & 368.6687 & 719.3035 & 360.1554 & 718.3195 & 359.6634 & $\mathbf{F}$ & 1347.6216 & 674.3144 & 1330.5950 & 665.8011 & 1329.6110 & 665.3091 & 10 \\
\hline 7 & 849.4141 & 425.2107 & 832.3876 & 416.6974 & 831.4036 & 416.2054 & I & 1200.5531 & 600.7802 & 1183.5266 & 592.2669 & 1182.5426 & 591.7749 & 9 \\
\hline 8 & 978.4567 & 489.7320 & 961.4302 & 481.2187 & 960.4462 & 480.7267 & $\mathbf{E}$ & 1087.4691 & 544.2382 & 1070.4425 & 535.7249 & 1069.4585 & 535.2329 & 8 \\
\hline 9 & 1093.4837 & 547.2455 & 1076.4571 & 538.7322 & 1075.4731 & 538.2402 & D & 958.4265 & 479.7169 & 941.3999 & 471.2036 & 940.4159 & 470.7116 & 7 \\
\hline 10 & 1256.5470 & 628.7771 & 1239.5204 & 620.2639 & 1238.5364 & 619.7719 & $\mathbf{Y}$ & 843.3995 & 422.2034 & 826.3730 & 413.6901 & 825.3890 & 413.1981 & 6 \\
\hline 11 & 1384.6420 & 692.8246 & 1367.6154 & 684.3113 & 1366.6314 & 683.8193 & $\mathbf{K}$ & 680.3362 & 340.6717 & 663.3097 & 332.1585 & 662.3257 & 331.6665 & 5 \\
\hline 12 & 1499.6689 & 750.3381 & 1482.6423 & 741.8248 & 1481.6583 & 741.3328 & D & 552.2413 & 276.6243 & 535.2147 & 268.1110 & 534.2307 & 267.6190 & 4 \\
\hline 13 & 1646.7373 & 823.8723 & 1629.7108 & 815.3590 & 1628.7267 & 814.8670 & $\mathbf{F}$ & 437.2143 & 219.1108 & 420.1878 & 210.5975 & 419.2037 & 210.1055 & 3 \\
\hline 14 & 1761.7643 & 881.3858 & 1744.7377 & 872.8725 & 1743.7537 & 872.3805 & D & 290.1459 & 145.5766 & 273.1193 & 137.0633 & 272.1353 & 136.5713 & 2 \\
\hline 15 & & & & & & & $\mathbf{R}$ & 175.1190 & 88.0631 & 158.0924 & 79.5498 & & & 1 \\
\hline
\end{tabular}



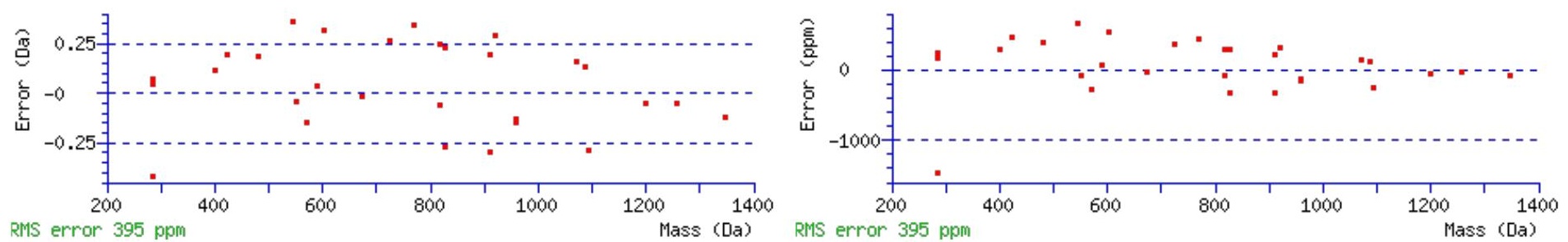

\section{All matches to this query}

\begin{tabular}{|l|l|l|l|}
\hline Score & Mr(calc): & Delta & \multicolumn{1}{|c|}{ Sequence } \\
\hline 48.9 & 1934.8687 & 0.0024 & VWNSTFIEDYKDFDR \\
\hline 23.4 & 1933.8846 & 0.9865 & VWNSTFIEDYKDFDR \\
\hline 7.0 & 1934.8750 & -0.0039 & VGKHFTLVDMPGYGYR \\
\hline 6.6 & 1934.8735 & -0.0024 & VSLEDHSSQGTLVNNVR \\
\hline 6.0 & 1934.8726 & -0.0015 & FMPRNGTGVLILSPTR \\
\hline 5.6 & 1934.8815 & -0.0104 & WEEAKTFYDNLSPKK \\
\hline 5.1 & 1933.8740 & 0.9971 & EGATPPIWYLTDKKR \\
\hline 5.0 & 1934.8639 & 0.0072 & SSLISSLEEEVSILNR \\
\hline 4.3 & 1933.8488 & 1.0223 & NRYSELVYLYRNGK \\
\hline 4.0 & 1932.8624 & 2.0087 & DSSRQPPTRLLAKPK \\
\hline
\end{tabular}

Spectrum No: 692; Query: 832; Rank: 1

\section{Peptide View}

MS/MS Fragmentation of EAGNHSSGAGLVQINK

Found in IPI00365669, Tax_Id=10116 Gene_Symbol=M6pr Cation-dependent mannose-6-phosphate receptor precursor

Match to Query 832: 1581.775812 from(528.265880,3+)

Title: 100101RatKid_NS_deglyco_24.1058.1058.3.dta

Data file K:INewmanPaper|Piliangl3SubProteomes\Piliang3SP\mgf5ppm|ERLIC_3SubProteomes5ppm.mgf

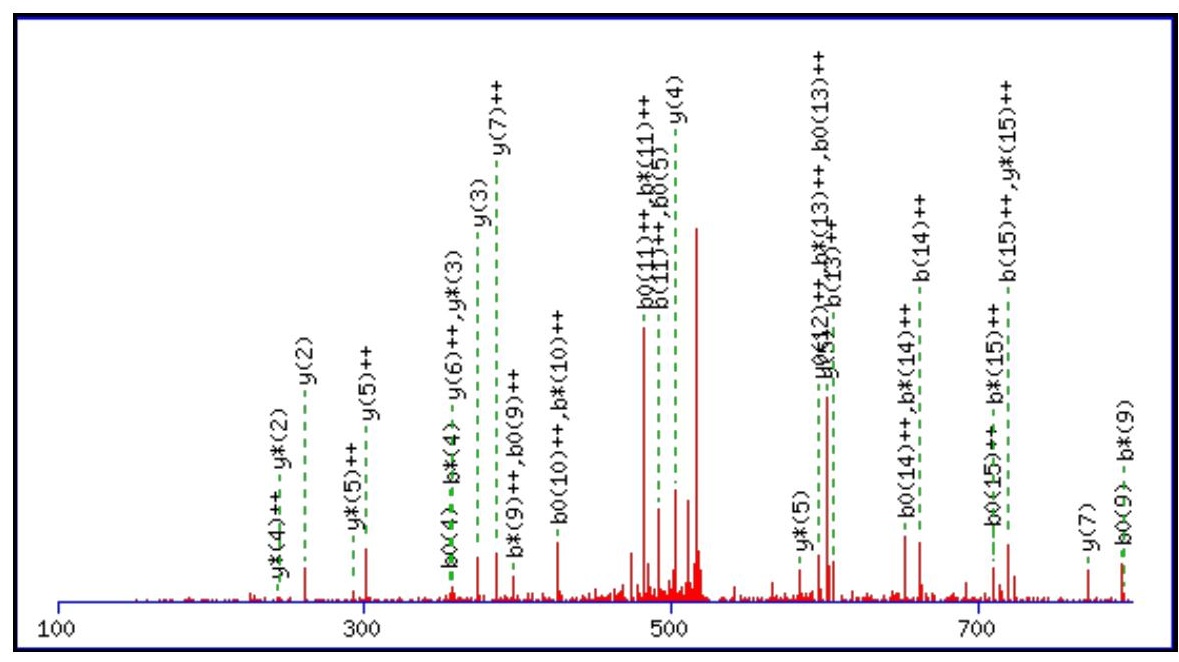




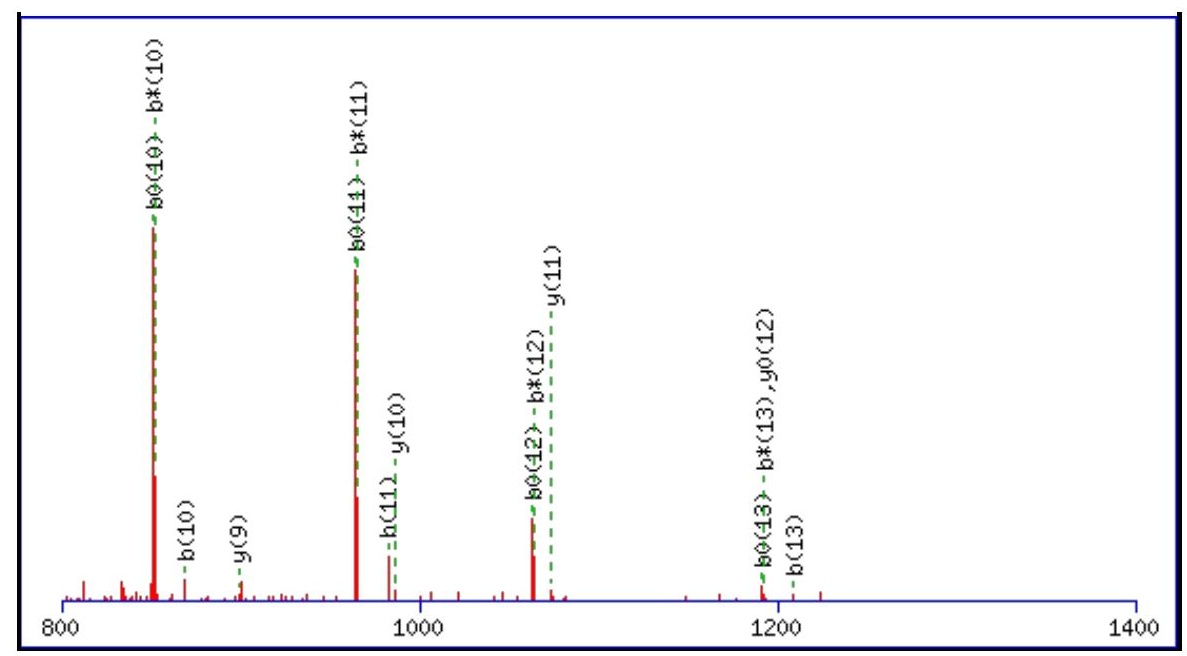

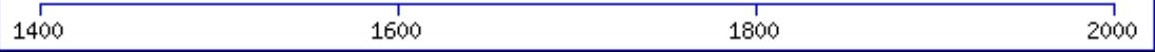

Monoisotopic mass of neutral peptide $\operatorname{Mr}($ calc): 1581.7747

Fixed modifications: Carbamidomethyl (C)

Variable modifications:

N4 : Deamidated_N (N)

Ions Score: 49 Expect: $\odot .0021$

Matches (Bold Red): 51/156 fragment ions using 60 most intense peaks

\begin{tabular}{|c|c|c|c|c|c|c|c|c|c|c|c|c|c|c|}
\hline \# & b & $\mathbf{b}^{++}$ & b* & $\mathbf{b}^{*^{++}}$ & $\mathbf{b}^{0}$ & $\mathbf{b}^{\mathbf{0 + +}}$ & Seq. & $\mathbf{y}$ & $y^{++}$ & $\mathbf{y}^{*}$ & $\mathbf{y}^{*^{++}}$ & $\mathbf{y}^{\mathbf{0}}$ & $\mathbf{y}^{0++}$ & \# \\
\hline 1 & 130.0499 & 65.5286 & & & 112.0393 & 56.5233 & $\mathbf{E}$ & & & & & & & 16 \\
\hline 2 & 201.0870 & 101.0471 & & & 183.0764 & 92.0418 & A & 1453.7394 & 727.3733 & 1436.7128 & 718.8601 & 1435.7288 & 718.3680 & 15 \\
\hline 3 & 258.1084 & 129.5579 & & & 240.0979 & 120.5526 & G & 1382.7023 & 691.8548 & 1365.6757 & 683.3415 & 1364.6917 & 682.8495 & 14 \\
\hline 4 & 373.1354 & 187.0713 & 356.1088 & 178.5581 & 355.1248 & 178.0660 & $\mathbf{N}$ & 1325.6808 & 663.3440 & 1308.6542 & 654.8308 & 1307.6702 & 654.3388 & 13 \\
\hline 5 & 510.1943 & 255.6008 & 493.1677 & 247.0875 & 492.1837 & 246.5955 & $\mathbf{H}$ & 1210.6539 & 605.8306 & 1193.6273 & 597.3173 & 1192.6433 & 596.8253 & 12 \\
\hline 6 & 597.2263 & 299.1168 & 580.1998 & 290.6035 & 579.2158 & 290.1115 & $\mathbf{S}$ & 1073.5949 & 537.3011 & 1056.5684 & 528.7878 & 1055.5844 & 528.2958 & 11 \\
\hline 7 & 684.2584 & 342.6328 & 667.2318 & 334.1195 & 666.2478 & 333.6275 & $S$ & 986.5629 & 493.7851 & 969.5364 & 485.2718 & 968.5524 & 484.7798 & 10 \\
\hline 8 & 741.2798 & 371.1435 & 724.2533 & 362.6303 & 723.2693 & 362.1383 & G & 899.5309 & 450.2691 & 882.5043 & 441.7558 & & & 9 \\
\hline 9 & 812.3169 & 406.6621 & 795.2904 & 398.1488 & 794.3064 & 397.6568 & A & 842.5094 & 421.7584 & 825.4829 & 413.2451 & & & 8 \\
\hline 10 & 869.3384 & 435.1728 & 852.3118 & 426.6596 & 851.3278 & 426.1676 & G & 771.4723 & 386.2398 & 754.4458 & 377.7265 & & & 7 \\
\hline 11 & 982.4225 & 491.7149 & 965.3959 & 483.2016 & 964.4119 & 482.7096 & $\mathbf{L}$ & 714.4509 & 357.7291 & 697.4243 & 349.2158 & & & 6 \\
\hline 12 & 1081.4909 & 541.2491 & 1064.4643 & 532.7358 & 1063.4803 & 532.2438 & $\mathbf{V}$ & 601.3668 & 301.1870 & 584.3402 & 292.6738 & & & 5 \\
\hline 13 & 1209.5494 & 605.2784 & 1192.5229 & 596.7651 & 1191.5389 & 596.2731 & $\mathbf{Q}$ & 502.2984 & 251.6528 & 485.2718 & 243.1395 & & & 4 \\
\hline 14 & 1322.6335 & 661.8204 & 1305.6070 & 653.3071 & 1304.6229 & 652.8151 & I & 374.2398 & 187.6235 & 357.2132 & 179.1103 & & & 3 \\
\hline 15 & 1436.6764 & 718.8419 & 1419.6499 & 710.3286 & 1418.6659 & 709.8366 & $\mathbf{N}$ & 261.1557 & 131.0815 & 244.1292 & 122.5682 & & & 2 \\
\hline 16 & & & & & & & $\mathbf{K}$ & 147.1128 & 74.0600 & 130.0863 & 65.5468 & & & 1 \\
\hline
\end{tabular}



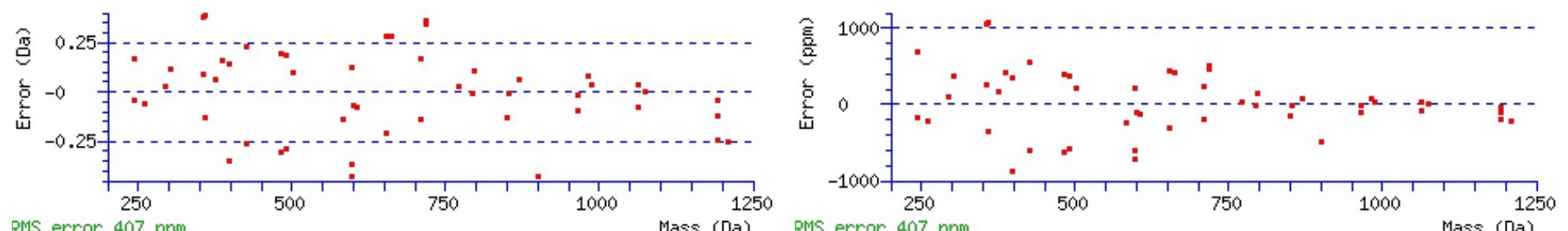

RMS error 407 ppm

Mass (Da) RMS error 407 ppm

Mass (Da)

\section{All matches to this query}

\begin{tabular}{|l|l|c|l|}
\hline Score & Mr(calc): & Delta & \multicolumn{1}{|c|}{ Sequence } \\
\hline 48.8 & 1581.7747 & 0.0011 & EAGNHSSGAGLVQINK \\
\hline 11.9 & 1581.7747 & 0.0011 & EAGNHSSGAGLVQINK \\
\hline 8.4 & 1581.7916 & -0.0158 & SDGRKFPELVLNK \\
\hline 8.1 & 1581.7664 & 0.0094 & ERLDQYPARLGGK \\
\hline 7.7 & 1580.7600 & 1.0158 & EVAWNLTSVDLVR \\
\hline 7.7 & 1580.7600 & 1.0158 & EVAWNLTSVDLVR \\
\hline 7.1 & 1580.7633 & 1.0125 & NKKDNPSAICLIK \\
\hline 6.8 & 1581.7643 & 0.0115 & LFLLNCVGQEMSR \\
\hline 6.0 & 1579.7586 & 2.0172 & SMGLEVVLMTGDNSK \\
\hline 5.8 & 1580.7572 & 1.0186 & LGIHEYSTNRRR \\
\hline
\end{tabular}

Spectrum No: 693; Query: 2305; Rank: 1

\section{Peptide View}

MS/MS Fragmentation of QLSCVANQNGSQADCELGNPFKR

Found in IPI00205166, Tax_Id=10116 Gene_Symbol=Itga6 similar to integrin alpha 6 isoform 2

Match to Query 2305: 2593.167852 from(865.396560,3+)

Title: 100101RatKid_NS_deglyco_18.2809.2809.3.dta

Data file K:INewmanPaper|Piliang|3SubProteomes\Piliang3SP\mgf5ppm\ERLIC_3SubProteomes5ppm.mgf

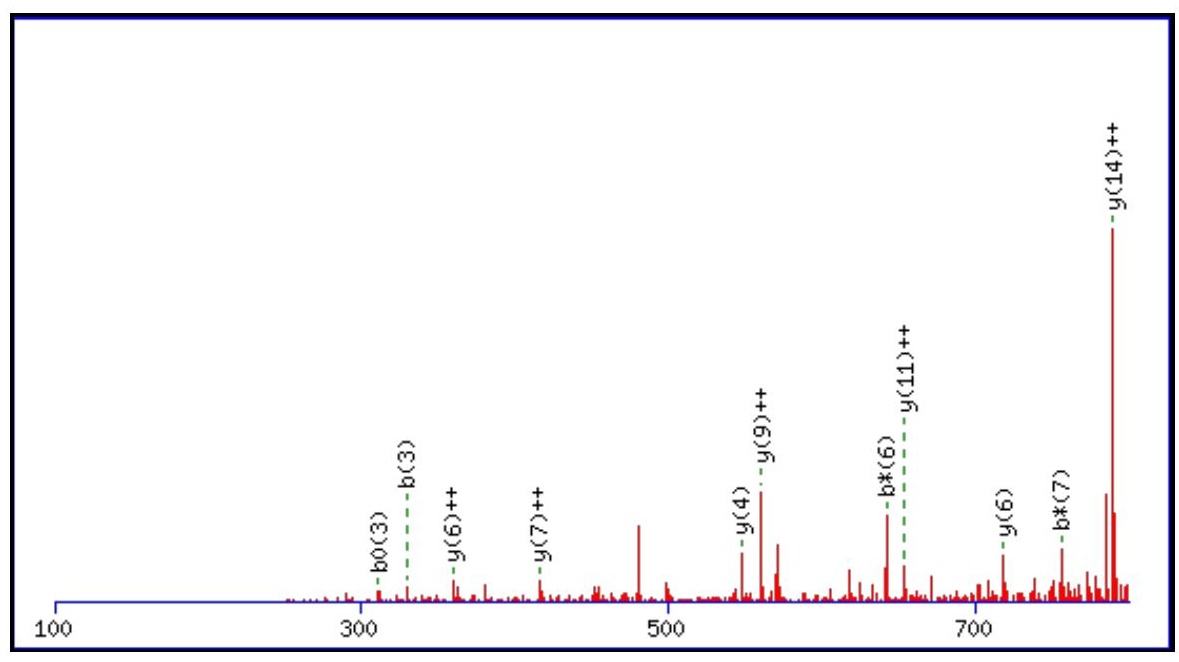



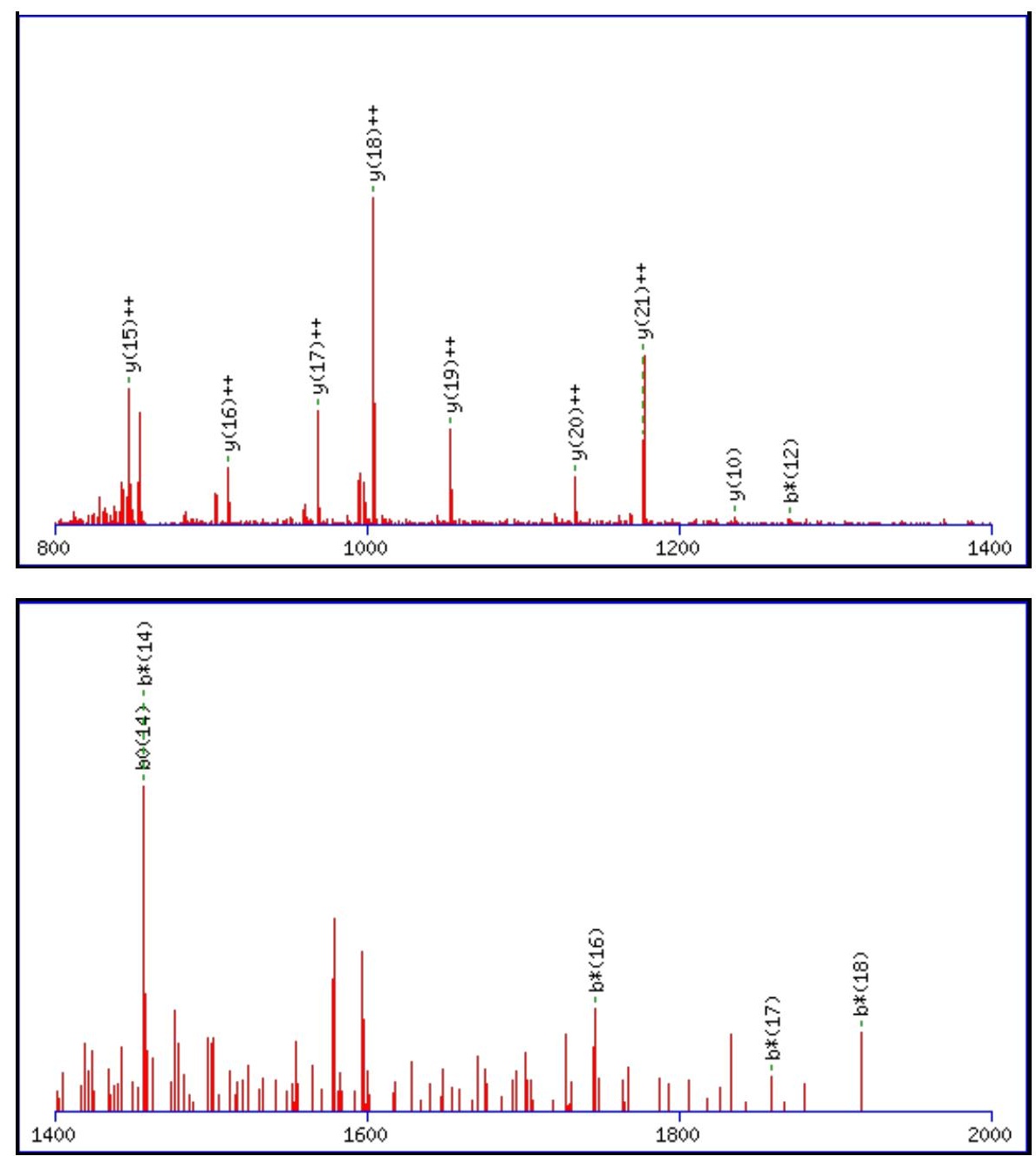

Monoisotopic mass of neutral peptide $\operatorname{Mr}($ calc): 2593.1649

Fixed modifications: Carbamidomethyl (C)

Variable modifications:

N9 : Deamidated_N (N)

Ions Score: 49 Expect: 0.0046

Matches (Bold Red): 25/246 fragment ions using 41 most intense peaks

\begin{tabular}{|c|c|c|c|c|c|c|c|c|c|c|c|c|c|c|}
\hline \# & b & $\mathbf{b}^{++}$ & $\mathbf{b}^{*}$ & $\mathbf{b}^{*^{++}}$ & $\mathbf{b}^{0}$ & $\mathbf{b}^{\mathbf{0 + +}}$ & Seq. & $\mathbf{y}$ & $\mathbf{y}^{++}$ & $\mathbf{y}^{*}$ & $\mathbf{y}^{*^{++}}$ & $\mathbf{y}^{0}$ & $\mathbf{y}^{0++}$ & \# \\
\hline 1 & 129.0659 & 65.0366 & 112.0393 & 56.5233 & & & $\mathbf{Q}$ & & & & & & & 23 \\
\hline 2 & 242.1499 & 121.5786 & 225.1234 & 113.0653 & & & $\mathbf{L}$ & 2466.1136 & 1233.5605 & 2449.0871 & 1225.0472 & 2448.1031 & 1224.5552 & 22 \\
\hline 3 & 329.1819 & 165.0946 & 312.1554 & 156.5813 & 311.1714 & 156.0893 & $\mathrm{~S}$ & 2353.0296 & 1177.0184 & 2336.0030 & 1168.5051 & 2335.0190 & 1168.0131 & 21 \\
\hline 4 & 489.2126 & 245.1099 & 472.1860 & 236.5967 & 471.2020 & 236.1047 & C & 2265.9975 & 1133.5024 & 2248.9710 & 1124.9891 & 2247.9870 & 1124.4971 & 20 \\
\hline 5 & 588.2810 & 294.6441 & 571.2545 & 286.1309 & 570.2704 & 285.6389 & V & 2105.9669 & 1053.4871 & 2088.9403 & 1044.9738 & 2087.9563 & 1044.4818 & 19 \\
\hline 6 & 659.3181 & 330.1627 & 642.2916 & 321.6494 & 641.3076 & 321.1574 & A & 2006.8985 & 1003.9529 & 1989.8719 & 995.4396 & 1988.8879 & 994.9476 & 18 \\
\hline 7 & 773.3610 & 387.1842 & 756.3345 & 378.6709 & 755.3505 & 378.1789 & $\mathbf{N}$ & 1935.8614 & 968.4343 & 1918.8348 & 959.9210 & 1917.8508 & 959.4290 & 17 \\
\hline 8 & 901.4196 & 451.2135 & 884.3931 & 442.7002 & 883.4091 & 442.2082 & $\mathbf{Q}$ & 1821.8184 & 911.4129 & 1804.7919 & 902.8996 & 1803.8079 & 902.4076 & 16 \\
\hline 9 & 1016.4466 & 508.7269 & 999.4200 & 500.2136 & 998.4360 & 499.7216 & $\mathbf{N}$ & 1693.7599 & 847.3836 & 1676.7333 & 838.8703 & 1675.7493 & 838.3783 & 15 \\
\hline 10 & 1073.4680 & 537.2377 & 1056.4415 & 528.7244 & 1055.4575 & 528.2324 & G & 1578.7329 & 789.8701 & 1561.7064 & 781.3568 & 1560.7224 & 780.8648 & 14 \\
\hline 11 & 1160.5001 & 580.7537 & 1143.4735 & 572.2404 & 1142.4895 & 571.7484 & $S$ & 1521.7115 & 761.3594 & 1504.6849 & 752.8461 & 1503.7009 & 752.3541 & 13 \\
\hline 12 & 1288.5586 & 644.7830 & 1271.5321 & 636.2697 & 1270.5481 & 635.7777 & $\mathbf{Q}$ & 1434.6794 & 717.8434 & 1417.6529 & 709.3301 & 1416.6689 & 708.8381 & 12 \\
\hline 13 & 1359.5957 & 680.3015 & 1342.5692 & 671.7882 & 1341.5852 & 671.2962 & A & 1306.6208 & 653.8141 & 1289.5943 & 645.3008 & 1288.6103 & 644.8088 & 11 \\
\hline 14 & 1474.6227 & 737.8150 & 1457.5961 & 729.3017 & 1456.6121 & 728.8097 & D & 1235.5837 & 618.2955 & 1218.5572 & 609.7822 & 1217.5732 & 609.2902 & 10 \\
\hline 15 & 1634.6533 & 817.8303 & 1617.6268 & 809.3170 & 1616.6428 & 808.8250 & C & 1120.5568 & 560.7820 & 1103.5302 & 552.2688 & 1102.5462 & 551.7768 & 9 \\
\hline 16 & 1763.6959 & 882.3516 & 1746.6694 & 873.8383 & 1745.6854 & 873.3463 & $\mathbf{E}$ & 960.5261 & 480.7667 & 943.4996 & 472.2534 & 942.5156 & 471.7614 & 8 \\
\hline 17 & 1876.7800 & 938.8936 & 1859.7534 & 930.3804 & 1858.7694 & 929.8884 & $\mathbf{L}$ & 831.4835 & 416.2454 & 814.4570 & 407.7321 & & & 7 \\
\hline 18 & 1933.8015 & 967.4044 & 1916.7749 & 958.8911 & 1915.7909 & 958.3991 & G & 718.3995 & 359.7034 & 701.3729 & 351.1901 & & & 6 \\
\hline
\end{tabular}




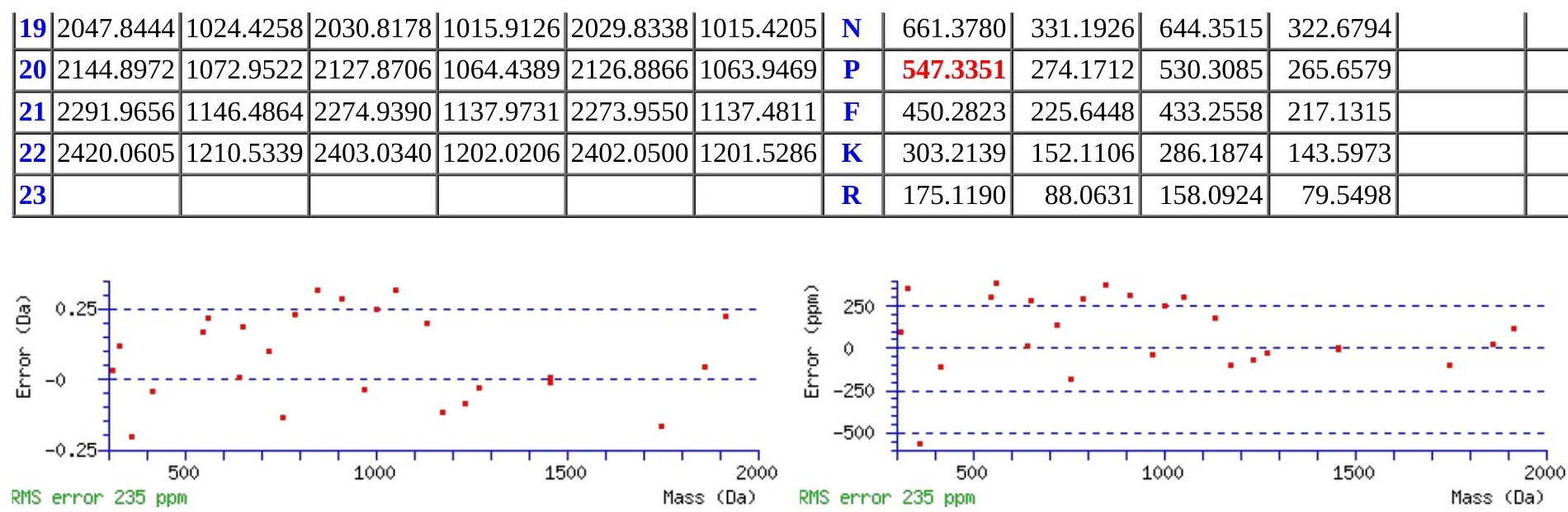

\section{All matches to this query}

\begin{tabular}{|l|l|l|l|}
\hline Score & Mr(calc): & Delta & \multicolumn{1}{c|}{ Sequence } \\
\hline 48.8 & 2593.1649 & 0.0029 & QLSCVANQNGSQADCELGNPFKR \\
\hline 39.0 & 2593.1649 & 0.0029 & QLSCVANQNGSQADCELGNPFKR \\
\hline 29.4 & 2593.1649 & 0.0029 & QLSCVANQNGSQADCELGNPFKR \\
\hline 7.5 & 2592.1809 & 0.9869 & QLSCVANQNGSQADCELGNPFKR \\
\hline 5.8 & 2592.1550 & 1.0128 & SHNNLKTGVWVTLGSEVFDVTK \\
\hline 5.8 & 2592.1550 & 1.0128 & SHNNLKTGVWVTLGSEVFDVTK \\
\hline 2.1 & 2592.1808 & 0.9870 & KLHGGNIPGSLSGGIVHNSMEVTK \\
\hline 1.2 & 2593.1704 & -0.0026 & NHLQSQKXERQTVMSGDGVSQR \\
\hline 0.6 & 2593.1437 & 0.0242 & AKNSRPPGTCPFYEELEALVR \\
\hline 0.1 & 2593.1883 & -0.0205 & MSQKFANTGSFIEREDLGKPNK \\
\hline
\end{tabular}

Spectrum No: 694; Query: 2626; Rank: 1

\section{Peptide View}

MS/MS Fragmentation of FDEFFSQGCAPGYKKNSTLCDLCIGPAK

Found in IPI00196656, Tax_Id=10116 Gene_Symbol=Srprb Ba1-667

Match to Query 2626: 3210.455622 from(1071.159150,3+)

Title: 100101RatKid_NS_deglyco_19.3730.3730.3.dta

Data file K:INewmanPaper|Piliangl3SubProteomes\Piliang3SP\mgf5ppm\ERLIC_3SubProteomes5ppm.mgf

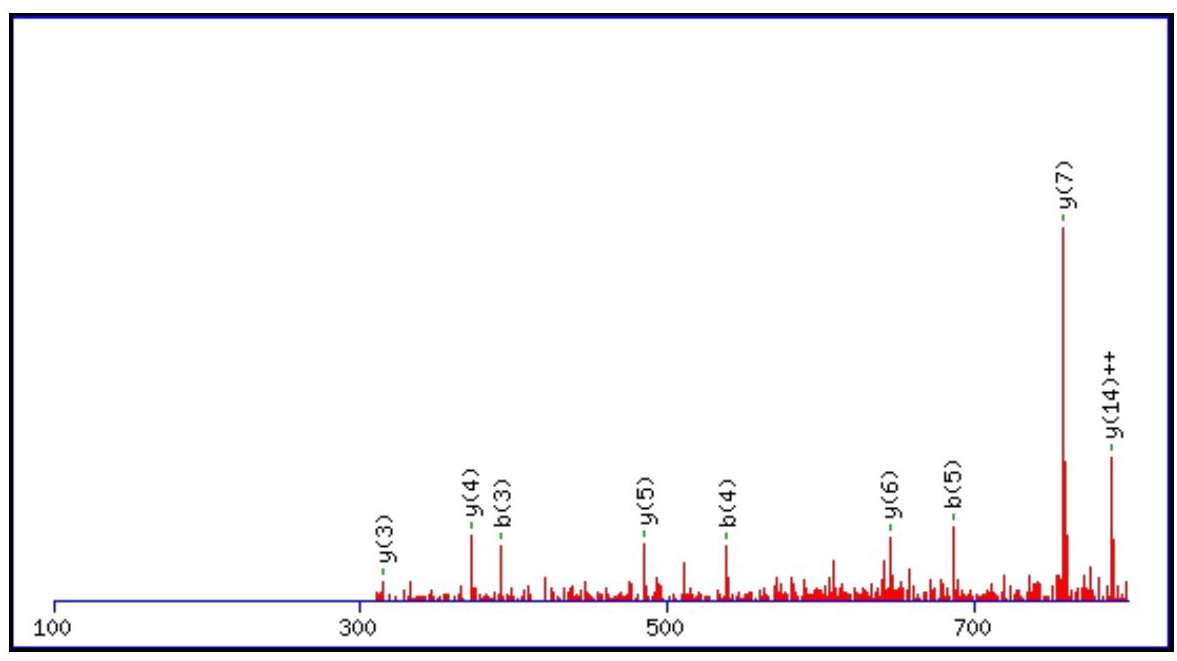



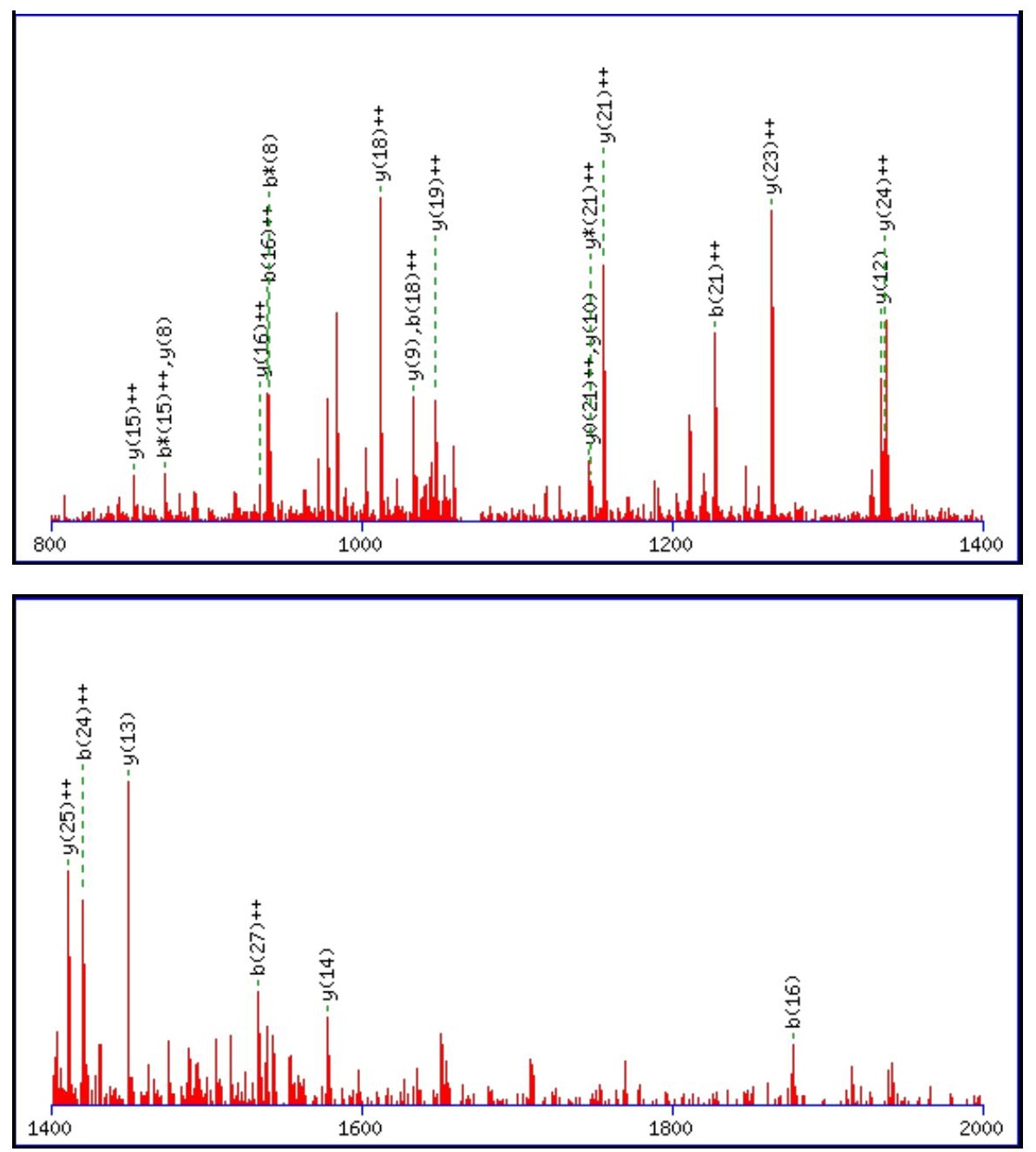

Monoisotopic mass of neutral peptide $\operatorname{Mr}($ calc): 3210.4460

Fixed modifications: Carbamidomethyl (C)

Variable modifications:

N16 : Deamidated_N (N)

Ions Score: 49 Expect: $\odot .0055$

Matches (Bold Red): 33/296 fragment ions using 73 most intense peaks

\begin{tabular}{|c|c|c|c|c|c|c|c|c|c|c|c|c|c|c|}
\hline \# & b & $\mathbf{b}^{++}$ & $\mathbf{b}^{*}$ & $\mathbf{b}^{*++}$ & $\mathbf{b}^{\mathbf{0}}$ & $\mathbf{b}^{\mathbf{0 + +}}$ & Seq. & $\mathbf{y}$ & $\mathbf{y}^{++}$ & $\mathbf{y}^{*}$ & $\mathrm{y}^{*^{++}}$ & $\mathbf{y}^{0}$ & $y^{0++}$ & \# \\
\hline 1 & 148.0757 & 74.5415 & & & & & $\mathbf{F}$ & & & & & & & 28 \\
\hline 2 & 263.1026 & 132.0550 & & & 245.0921 & 123.0497 & D & 3064.3849 & 1532.6961 & 3047.3584 & 1524.1828 & 3046.3743 & 1523.6908 & 27 \\
\hline 3 & 392.1452 & 196.5763 & & & 374.1347 & 187.5710 & $\mathbf{E}$ & 2949.3580 & 1475.1826 & 2932.3314 & 1466.6693 & 2931.3474 & 1466.1773 & 26 \\
\hline 4 & 539.2136 & 270.1105 & & & 521.2031 & 261.1052 & $\mathbf{F}$ & 2820.3154 & 1410.6613 & 2803.2888 & 1402.1480 & 2802.3048 & 1401.6560 & 25 \\
\hline 5 & 686.2821 & 343.6447 & & & 668.2715 & 334.6394 & $\mathbf{F}$ & 2673.2470 & 1337.1271 & 2656.2204 & 1328.6138 & 2655.2364 & 1328.1218 & 24 \\
\hline 6 & 773.3141 & 387.1607 & & & 755.3035 & 378.1554 & S & 2526.1785 & 1263.5929 & 2509.1520 & 1255.0796 & 2508.1680 & 1254.5876 & 23 \\
\hline 7 & 901.3727 & 451.1900 & 884.3461 & 442.6767 & 883.3621 & 442.1847 & $\mathbf{Q}$ & 2439.1465 & 1220.0769 & 2422.1200 & 1211.5636 & 2421.1359 & 1211.0716 & 22 \\
\hline 8 & 958.3941 & 479.7007 & 941.3676 & 471.1874 & 940.3836 & 470.6954 & $\mathbf{G}$ & 2311.0879 & 1156.0476 & 2294.0614 & 1147.5343 & 2293.0774 & 1147.0423 & 21 \\
\hline 9 & 1118.4248 & 559.7160 & 1101.3982 & 551.2028 & 1100.4142 & 550.7107 & $\mathrm{C}$ & 2254.0665 & 1127.5369 & 2237.0399 & 1119.0236 & 2236.0559 & 1118.5316 & 20 \\
\hline 10 & 1189.4619 & 595.2346 & 1172.4353 & 586.7213 & 1171.4513 & 586.2293 & A & 2094.0358 & 1047.5215 & 2077.0093 & 1039.0083 & 2076.0253 & 1038.5163 & 19 \\
\hline 11 & 1286.5147 & 643.7610 & 1269.4881 & 635.2477 & 1268.5041 & 634.7557 & $\mathbf{P}$ & 2022.9987 & 1012.0030 & 2005.9722 & 1003.4897 & 2004.9881 & 1002.9977 & 18 \\
\hline 12 & 1343.5361 & 672.2717 & 1326.5096 & 663.7584 & 1325.5255 & 663.2664 & G & 1925.9459 & 963.4766 & 1908.9194 & 954.9633 & 1907.9354 & 954.4713 & 17 \\
\hline 13 & 1506.5994 & 753.8034 & 1489.5729 & 745.2901 & 1488.5889 & 744.7981 & $\mathbf{Y}$ & 1868.9245 & 934.9659 & 1851.8979 & 926.4526 & 1850.9139 & 925.9606 & 16 \\
\hline 14 & 1634.6944 & 817.8508 & 1617.6679 & 809.3376 & 1616.6838 & 808.8456 & $\mathbf{K}$ & 1705.8611 & 853.4342 & 1688.8346 & 844.9209 & 1687.8506 & 844.4289 & 15 \\
\hline 15 & 1762.7894 & 881.8983 & 1745.7628 & 873.3850 & 1744.7788 & 872.8930 & K & 1577.7662 & 789.3867 & 1560.7396 & 780.8735 & 1559.7556 & 780.3814 & 14 \\
\hline 16 & 1877.8163 & 939.4118 & 1860.7898 & 930.8985 & 1859.8057 & 930.4065 & $\mathbf{N}$ & 1449.6712 & 725.3392 & 1432.6447 & 716.8260 & 1431.6607 & 716.3340 & 13 \\
\hline 17 & 1964.8483 & 982.9278 & 1947.8218 & \begin{tabular}{|c|}
974.4145 \\
\end{tabular} & 1946.8378 & 973.9225 & $\mathrm{~S}$ & 1334.6443 & 667.8258 & 1317.6177 & 659.3125 & 1316.6337 & 658.8205 & 12 \\
\hline 18 & 2065.8960 & 1033.4516 & 2048.8695 & 1024.9384 & 2047.8854 & 1024.4464 & $T$ & 1247.6123 & 624.3098 & 1230.5857 & 615.7965 & 1229.6017 & 615.3045 & 11 \\
\hline
\end{tabular}




\begin{tabular}{|r|r|r|r|r|r|r|r|r|r|r|r|r|r|r|r|r|r|}
$\mathbf{1 9}$ & 2178.9801 & 1089.9937 & 2161.9535 & 1081.4804 & 2160.9695 & 1080.9884 & L & $\mathbf{1 1 4 6 . 5 6 4 6}$ & 573.7859 & 1129.5380 & 565.2727 & 1128.5540 & 564.7806 & $\mathbf{1 0}$ \\
\hline $\mathbf{2 0}$ & 2339.0107 & 1170.0090 & 2321.9842 & 1161.4957 & 2321.0002 & 1161.0037 & C & $\mathbf{1 0 3 3 . 4 8 0 5}$ & 517.2439 & 1016.4540 & 508.7306 & 1015.4699 & 508.2386 & $\mathbf{9}$ \\
\hline $\mathbf{2 1}$ & 2454.0377 & $\mathbf{1 2 2 7 . 5 2 2 5}$ & 2437.0111 & 1219.0092 & 2436.0271 & 1218.5172 & $\mathbf{D}$ & $\mathbf{8 7 3 . 4 4 9 9}$ & 437.2286 & 856.4233 & 428.7153 & 855.4393 & 428.2233 & $\mathbf{8}$ \\
\hline $\mathbf{2 2}$ & 2567.1217 & 1284.0645 & 2550.0952 & 1275.5512 & 2549.1112 & 1275.0592 & L & $\mathbf{7 5 8 . 4 2 2 9}$ & 379.7151 & 741.3964 & 371.2018 & & & $\mathbf{7}$ \\
\hline $\mathbf{2 3}$ & 2727.1524 & 1364.0798 & 2710.1258 & 1355.5666 & 2709.1418 & 1355.0745 & C & $\mathbf{6 4 5 . 3 3 8 9}$ & 323.1731 & 628.3123 & 314.6598 & & & $\mathbf{6}$ \\
\hline $\mathbf{2 4}$ & 2840.2364 & $\mathbf{1 4 2 0 . 6 2 1 9}$ & 2823.2099 & 1412.1086 & 2822.2259 & 1411.6166 & I & $\mathbf{4 8 5 . 3 0 8 2}$ & 243.1577 & 468.2817 & 234.6445 & & & $\mathbf{5}$ \\
\hline $\mathbf{2 5}$ & 2897.2579 & 1449.1326 & 2880.2314 & 1440.6193 & 2879.2473 & 1440.1273 & G & 372.2241 & 186.6157 & 355.1976 & 178.1024 & & & $\mathbf{4}$ \\
\hline $\mathbf{2 6}$ & 2994.3107 & 1497.6590 & 2977.2841 & 1489.1457 & 2976.3001 & 1488.6537 & P & 315.2027 & 158.1050 & 298.1761 & 149.5917 & & & $\mathbf{3}$ \\
\hline $\mathbf{2 7}$ & 3065.3478 & $\mathbf{1 5 3 3 . 1 7 7 5}$ & 3048.3212 & 1524.6643 & 3047.3372 & 1524.1722 & A & 218.1499 & 109.5786 & 201.1234 & 101.0653 & & & $\mathbf{2}$ \\
\hline $\mathbf{2 8}$ & & & & & & & K & 147.1128 & 74.0600 & 130.0863 & 65.5468 & & & $\mathbf{1}$ \\
\hline
\end{tabular}
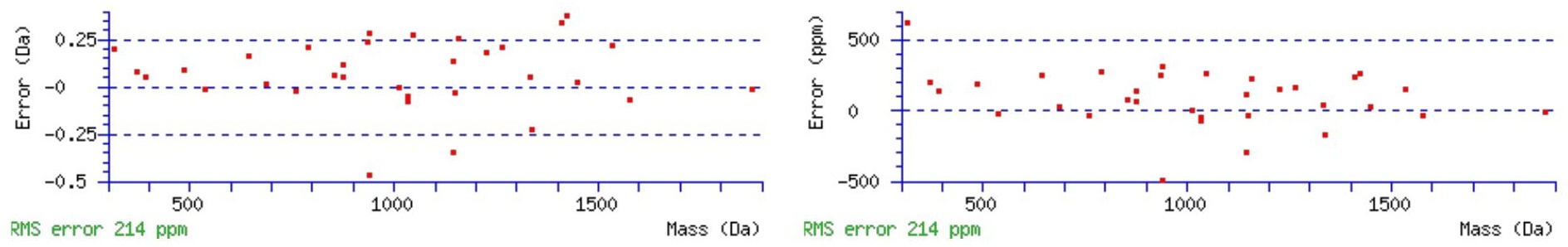

\section{All matches to this query}

\begin{tabular}{|l|c|c|l|}
\hline Score & Mr(calc): & Delta & \multicolumn{1}{|c|}{ Sequence } \\
\hline 48.8 & 3210.4460 & 0.0096 & FDEFFSQGCAPGYKKNSTLCDLCIGPAK \\
\hline 11.1 & 3209.4620 & 0.9936 & FDEFFSQGCAPGYKKNSTLCDLCIGPAK \\
\hline 9.3 & 3209.4342 & 1.0214 & EAGNINRSLSCLGQVITALVDVGNGKQR \\
\hline 6.7 & 3208.4502 & 2.0054 & EAGNINRSLSCLGQVITALVDVGNGKQR \\
\hline 5.8 & 3210.4651 & -0.0095 & TLDREAIPEYNVTIVATDRGKPPLSSK \\
\hline 5.2 & 3208.4244 & 2.0313 & VQAQLFAILTATAQEGGHNEGVETIKSR \\
\hline 2.2 & 3209.4345 & 1.0211 & LQDLFTIWTEESLMCVGCGAESSRRGK \\
\hline 1.0 & 3210.4509 & 0.0047 & GAEDDLNTVAAGTMTGMLYKCTGGLRGIAR \\
\hline 1.0 & 3210.4509 & 0.0047 & GAEDDLNTVAAGTMTGMLYKCTGGLRGIAR \\
\hline 0.8 & 3208.4186 & 2.0371 & CNYFSTEKNNYVQHVRTHTGERPYK \\
\hline
\end{tabular}

Spectrum No: 695; Query: 67; Rank: 1

\section{Peptide View}

MS/MS Fragmentation of LDGSTNFTR

Found in IPI00324102, Tax_Id=10116 Gene_Symbol=Fgl2 fibrinogen-like 2

Match to Query 67: 1010.465368 from(506.239960,2+)

Title: 091008RatKidney_NoSalt_27.701.701.2.dta

Data file K:INewmanPaper|Piliang|3SubProteomes\Piliang3SP\mgf5ppm\ERLIC_3SubProteomes5ppm.mgf 

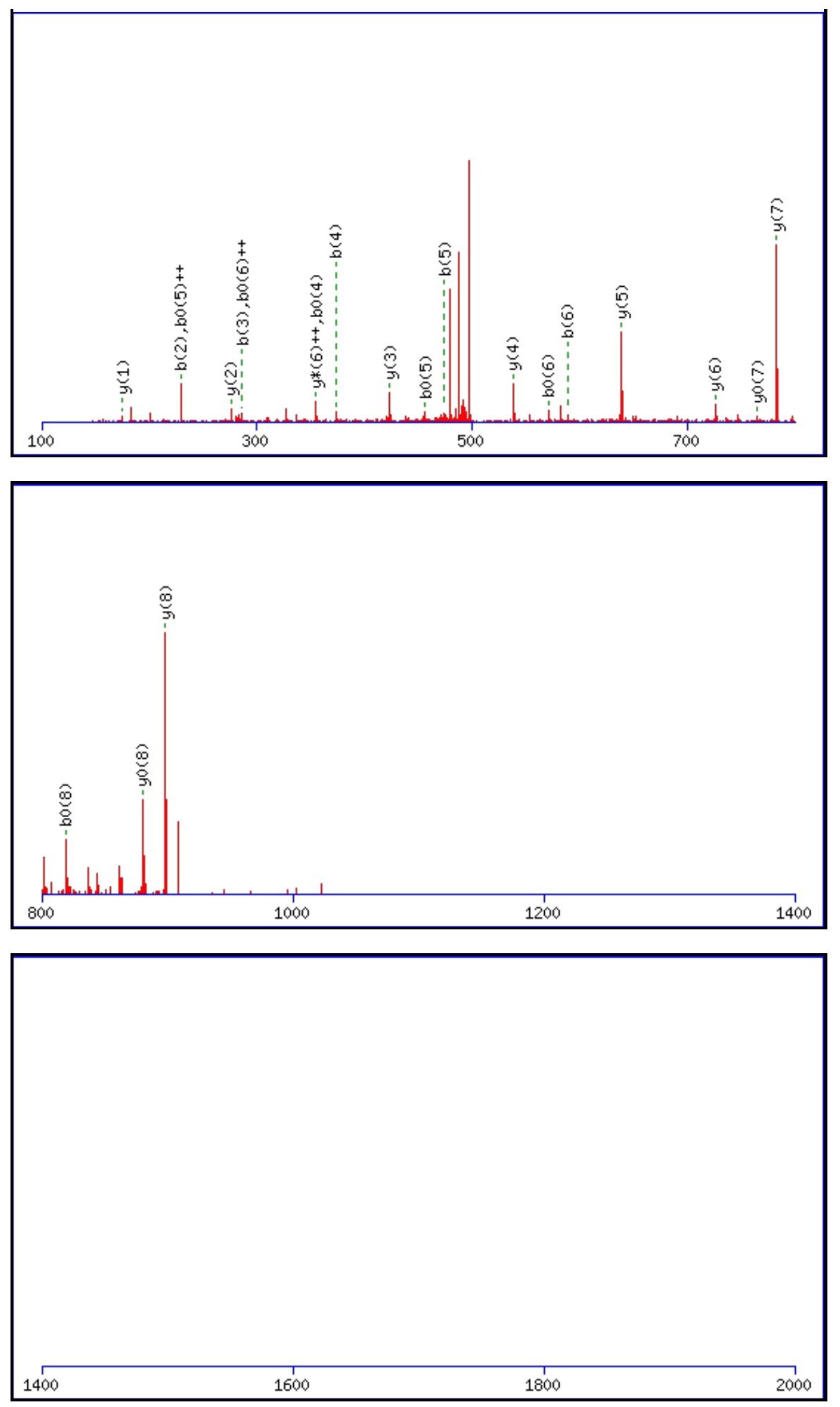

Monoisotopic mass of neutral peptide $\operatorname{Mr}($ calc): 1010.4669

Fixed modifications: Carbamidomethyl (C)

Variable modifications:

N6 : Deamidated_N (N)

Ions Score: 49 Expect: 0.0013

Matches (Bold Red): 22/82 fragment ions using 48 most intense peaks

\begin{tabular}{|c|c|c|c|c|c|c|c|c|c|c|c|c|c|}
\hline$\#$ & b & $\mathbf{b}^{++}$ & $\mathbf{b}^{*}$ & $\mathbf{b}^{*^{++}}$ & $\mathbf{b}^{\mathbf{0}}$ & $\mathbf{b}^{0++}$ & Seq. & $\mathbf{y}$ & $\mathbf{y}^{++}$ & $\mathbf{y}^{*}$ & $\mathbf{y}^{*^{++}}$ & $\mathbf{y}^{0}$ & $\mathbf{y}^{\mathbf{0 + +}}$ \\
\hline 1 & 114.0913 & 57.5493 & & & & & L & & & & & & \\
\hline 2 & 229.1183 & 115.0628 & & & 211.1077 & 106.0575 & D & 898.3901 & 449.6987 & 881.3635 & 441.1854 & 880.3795 & 440.6934 \\
\hline $3:$ & 286.1397 & 143.5735 & & & 268.1292 & 134.5682 & G & 783.3632 & 392.1852 & 766.3366 & 383.6719 & 765.3526 & 383.1799 \\
\hline 4 & 373.1718 & 187.0895 & & & 355.1612 & 178.0842 & S & 726.3417 & 363.6745 & |709.3151 & |355.1612 & |708.3311 & 354.6692 \\
\hline 5 & 474.2195 & 237.6134 & & & 456.2089 & 228.6081 & $\mathbf{T}$ & 639.3097 & 320.1585 & 622.2831 & 311.6452 & 621.2991 & 311.1532 \\
\hline
\end{tabular}




\begin{tabular}{|l|l|l|l|l|l|l|l|l|l|l|l|l|l|l|l|}
$\mathbf{6}$ & $\mathbf{5 8 9 . 2 4 6 4}$ & 295.1268 & 572.2198 & 286.6136 & $\mathbf{5 7 1 . 2 3 5 8}$ & $\mathbf{2 8 6 . 1 2 1 6}$ & $\mathbf{N}$ & $\mathbf{5 3 8 . 2 6 2 0}$ & 269.6346 & 521.2354 & 261.1214 & 520.2514 & 260.6293 & $\mathbf{4}$ \\
\hline $\mathbf{7}$ & 736.3148 & 368.6610 & 719.2883 & 360.1478 & 718.3042 & 359.6558 & $\mathbf{F}$ & $\mathbf{4 2 3 . 2 3 5 0}$ & 212.1212 & 406.2085 & 203.6079 & 405.2245 & 203.1159 & 3 \\
\hline $\mathbf{8}$ & 837.3625 & 419.1849 & 820.3359 & 410.6716 & $\mathbf{8 1 9 . 3 5 1 9}$ & 410.1796 & $\mathbf{T}$ & $\mathbf{2 7 6 . 1 6 6 6}$ & 138.5870 & 259.1401 & 130.0737 & 258.1561 & 129.5817 & $\mathbf{2}$ \\
\hline $\mathbf{9}$ & & & & & & & $\mathbf{R}$ & $\mathbf{1 7 5 . 1 1 9 0}$ & 88.0631 & 158.0924 & 79.5498 & & & $\mathbf{1}$ \\
\hline
\end{tabular}

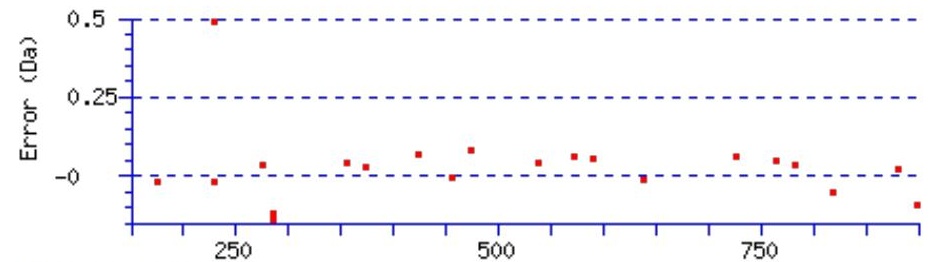

RMS error 486 ppm

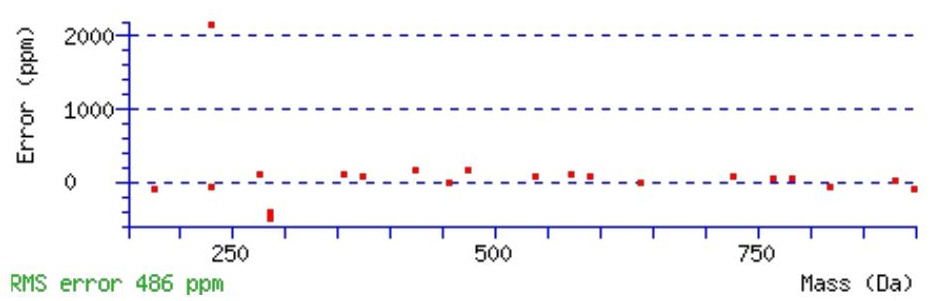

RMS error 486 ppm

\section{All matches to this query}

\begin{tabular}{|l|l|l|l|}
\hline Score & Mr(calc): & Delta & \multicolumn{1}{|c|}{ Sequence } \\
\hline 48.7 & 1010.4669 & -0.0015 & LDGSTNFTR \\
\hline 9.1 & 1010.4586 & 0.0067 & WSVADVVR \\
\hline 7.3 & 1010.4711 & -0.0057 & LICAMMTR \\
\hline 7.0 & 1010.4669 & -0.0015 & TSPHTPNEK \\
\hline 5.4 & 1010.4685 & -0.0032 & LSTSGIPEK \\
\hline 5.4 & 1010.4685 & -0.0032 & $\underline{\text { LSTSGIPEK }}$ \\
\hline 4.7 & 1010.4644 & 0.0010 & INVCYWR \\
\hline 3.6 & 1008.4641 & 2.0013 & ETNNPVKK \\
\hline 3.5 & 1008.4529 & 2.0125 & EPLVGSEAK \\
\hline 3.5 & 1010.4741 & -0.0087 & $\underline{\text { SSRSGSSSTR }}$ \\
\hline
\end{tabular}

Spectrum No: 696; Query: 481; Rank: 1

\section{Peptide View}

\section{MS/MS Fragmentation of YNYTEDPTILR}

Found in IPI00358371, Tax_Id=10116 Gene_Symbol=RGD1560871_predicted similar to plexin A1

Match to Query 481: 1384.649508 from(693.332030,2+)

Title: 091008RatKidney_NoSalt_31.1946.1946.2.dta

Data file K:INewmanPaper|Piliang|3SubProteomes\Piliang3SP\mgf5ppm\ERLIC_3SubProteomes5ppm.mgf

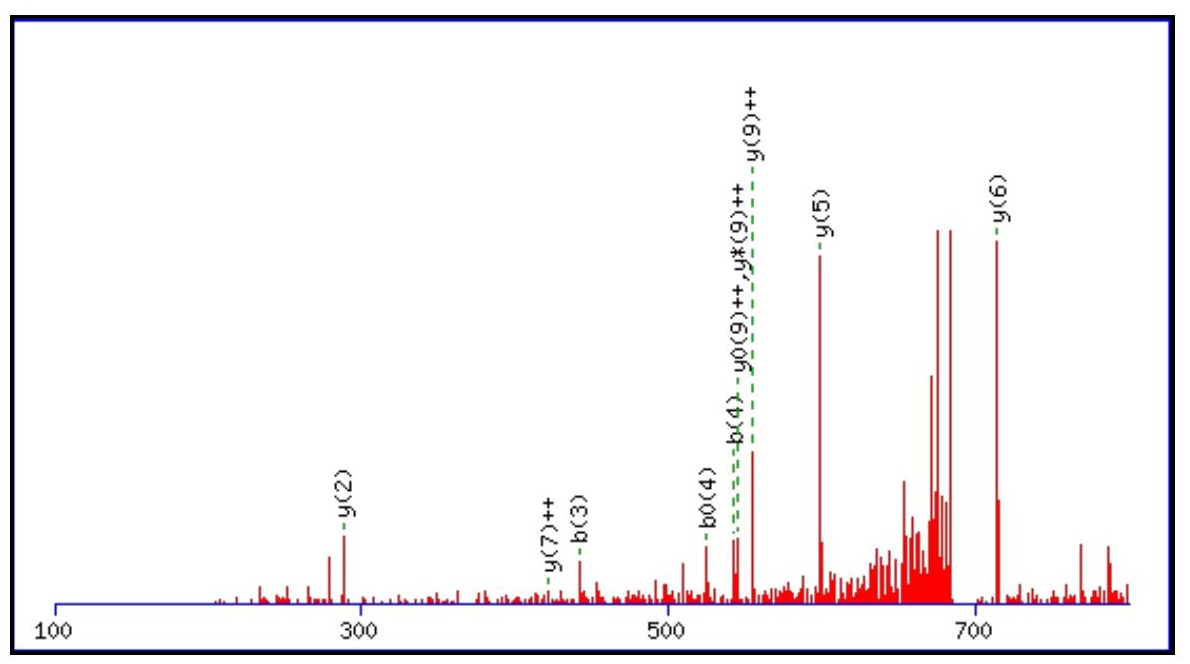



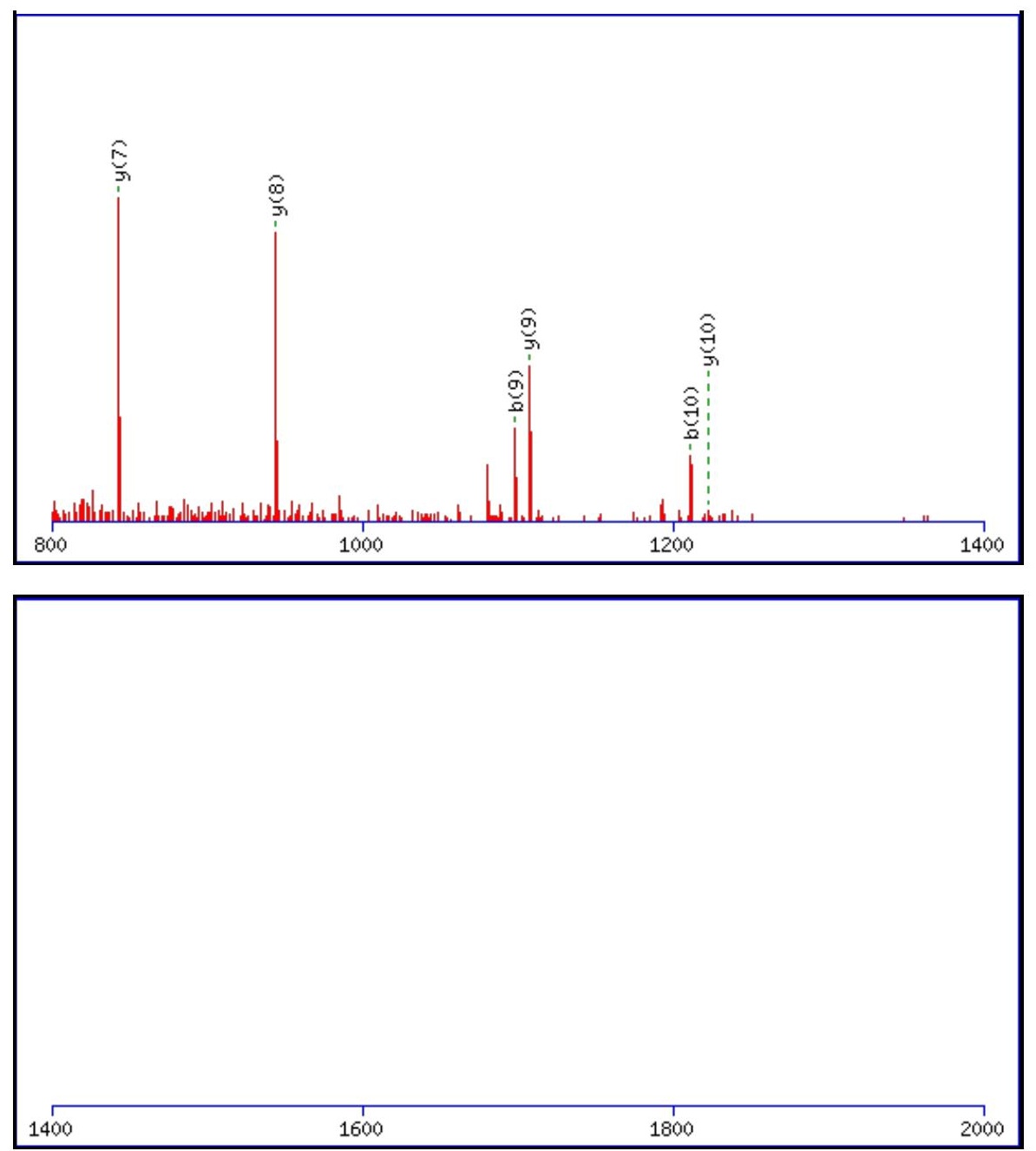

Monoisotopic mass of neutral peptide $\operatorname{Mr}($ calc): 1384.6510

Fixed modifications: Carbamidomethyl (C)

Variable modifications:

N2 : Deamidated_N (N)

Ions Score: 49 Expect: 0.0024

Matches (Bold Red): 16/106 fragment ions using 31 most intense peaks

\begin{tabular}{|r|c|c|c|c|c|c|c|c|c|c|c|c|c|c|}
\hline$\#$ & $\mathbf{b}$ & $\mathbf{b}^{++}$ & $\mathbf{b}^{*}$ & $\mathbf{b}^{\mathbf{*}^{++}}$ & $\mathbf{b}^{\mathbf{0}}$ & $\mathbf{b}^{\mathbf{0}+}$ & $\mathbf{S e q}$ & $\mathbf{y}$ & $\mathbf{y}^{++}$ & $\mathbf{y}^{*}$ & $\mathbf{y}^{\boldsymbol{*}^{++}}$ & $\mathbf{y}^{\mathbf{0}}$ & $\mathbf{y}^{\mathbf{0 + +}}$ & $\#$ \\
\hline $\mathbf{1}$ & 164.0706 & 82.5389 & & & & & $\mathbf{Y}$ & & & & & & & $\mathbf{1 1}$ \\
\hline $\mathbf{2}$ & 279.0975 & 140.0524 & 262.0710 & 131.5391 & & & $\mathbf{N}$ & $\mathbf{1 2 2 2 . 5 9 5 0}$ & 611.8011 & 1205.5685 & 603.2879 & 1204.5844 & 602.7959 & $\mathbf{1 0}$ \\
\hline $\mathbf{3}$ & $\mathbf{4 4 2 . 1 6 0 9}$ & 221.5841 & 425.1343 & 213.0708 & & & $\mathbf{Y}$ & $\mathbf{1 1 0 7 . 5 6 8 1}$ & 554.2877 & 1090.5415 & 545.7744 & 1089.5575 & $\mathbf{5 4 5 . 2 8 2 4}$ & $\mathbf{9}$ \\
\hline $\mathbf{4}$ & $\mathbf{5 4 3 . 2 0 8 5}$ & 272.1079 & 526.1820 & 263.5946 & 525.1980 & 263.1026 & $\mathbf{T}$ & $\mathbf{9 4 4 . 5 0 4 7}$ & 472.7560 & 927.4782 & 464.2427 & 926.4942 & 463.7507 & $\mathbf{8}$ \\
\hline $\mathbf{5}$ & 672.2511 & 336.6292 & 655.2246 & 328.1159 & 654.2406 & 327.6239 & $\mathbf{E}$ & $\mathbf{8 4 3 . 4 5 7 1}$ & $\mathbf{4 2 2 . 2 3 2 2}$ & 826.4305 & 413.7189 & 825.4465 & 413.2269 & $\mathbf{7}$ \\
\hline $\mathbf{6}$ & 787.2781 & 394.1427 & 770.2515 & 385.6294 & 769.2675 & 385.1374 & $\mathbf{D}$ & $\mathbf{7 1 4 . 4 1 4 5}$ & 357.7109 & 697.3879 & 349.1976 & 696.4039 & 348.7056 & $\mathbf{6}$ \\
\hline $\mathbf{7}$ & 884.3308 & 442.6691 & 867.3043 & 434.1558 & 866.3203 & 433.6638 & $\mathbf{P}$ & $\mathbf{5 9 9 . 3 8 7 5}$ & 300.1974 & 582.3610 & 291.6841 & 581.3770 & 291.1921 & $\mathbf{5}$ \\
\hline $\mathbf{8}$ & 985.3785 & 493.1929 & 968.3520 & 484.6796 & 967.3680 & 484.1876 & $\mathbf{T}$ & 502.3348 & 251.6710 & 485.3082 & 243.1577 & 484.3242 & 242.6657 & $\mathbf{4}$ \\
\hline $\mathbf{9}$ & $\mathbf{1 0 9 8 . 4 6 2 6}$ & 549.7349 & 1081.4360 & 541.2217 & 1080.4520 & 540.7297 & $\mathbf{I}$ & 401.2871 & 201.1472 & 384.2605 & 192.6339 & & & $\mathbf{3}$ \\
\hline $\mathbf{1 0}$ & $\mathbf{1 2 1 1 . 5 4 6 7}$ & 606.2770 & 1194.5201 & 597.7637 & 1193.5361 & 597.2717 & $\mathbf{L}$ & $\mathbf{2 8 8 . 2 0 3 0}$ & 144.6051 & 271.1765 & 136.0919 & & & $\mathbf{2}$ \\
\hline $\mathbf{1 1}$ & & & & & & & $\mathbf{R}$ & 175.1190 & 88.0631 & 158.0924 & 79.5498 & & & $\mathbf{1}$ \\
\hline
\end{tabular}
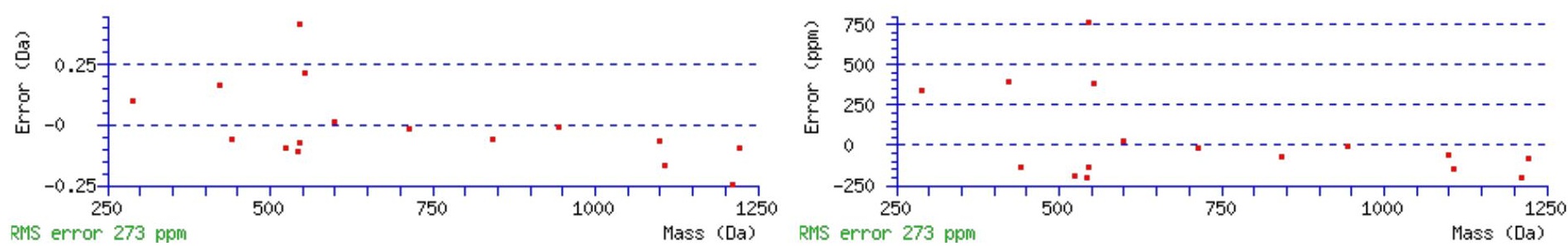


\section{All matches to this query}

\begin{tabular}{|l|l|l|l|}
\hline Score & Mr(calc): & Delta & \multicolumn{1}{|c|}{ Sequence } \\
\hline 48.6 & 1384.6510 & -0.0015 & YNYTEDPTILR \\
\hline 8.2 & 1384.6371 & 0.0124 & WAEETPEPDRR \\
\hline 7.4 & 1384.6558 & -0.0063 & MFFRQQDEIR \\
\hline 6.2 & 1384.6613 & -0.0117 & SVVHHLNGSSLR \\
\hline 6.2 & 1384.6613 & -0.0117 & SVVHHLNGSSLR \\
\hline 5.0 & 1383.6581 & 0.9914 & GMVATINSNRLK \\
\hline 3.0 & 1384.6421 & 0.0074 & NNGQLMKTIIR \\
\hline 2.7 & 1384.6388 & 0.0107 & VNNVDFTNIIR \\
\hline 2.7 & 1384.6388 & 0.0107 & VNNVDFTNIIR \\
\hline 2.5 & 1383.6435 & 1.0060 & VPEAGAFGTAEKK \\
\hline
\end{tabular}

Spectrum No: 697; Query: 1124; Rank: 1

\section{Peptide View}

MS/MS Fragmentation of IQNYSVMAICDYDK

Found in IPI00208422, Tax_Id=10116 Gene_Symbol=Dpp4 Dipeptidyl peptidase 4

Match to Query 1124: 1735.741708 from(868.878130,2+)

Title: 091008RatKidney_NoSalt_31.2137.2137.2.dta

Data file K:INewmanPaper|Piliangl3SubProteomes\Piliang3SP\mgf5ppm\ERLIC_3SubProteomes5ppm.mgf
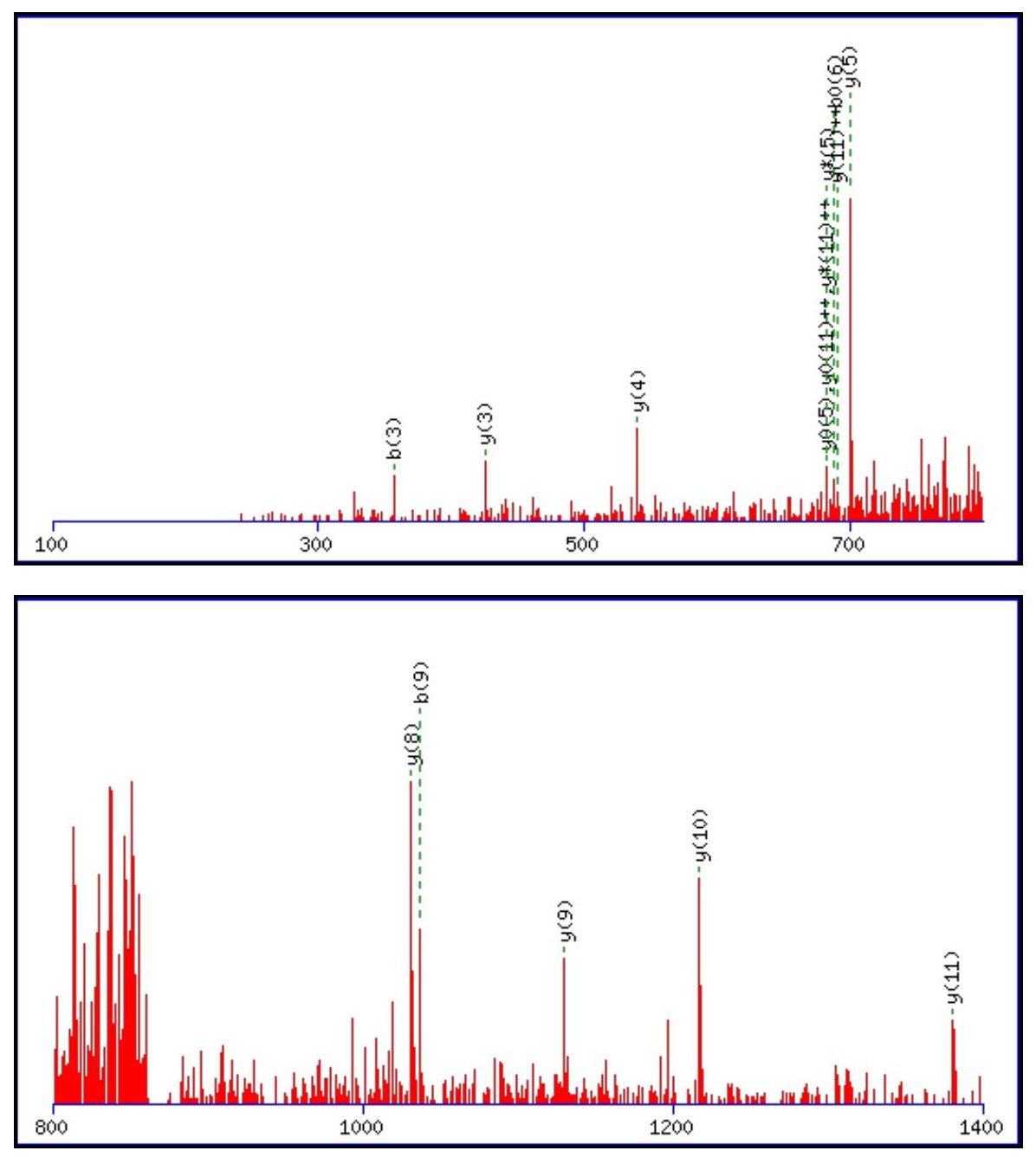


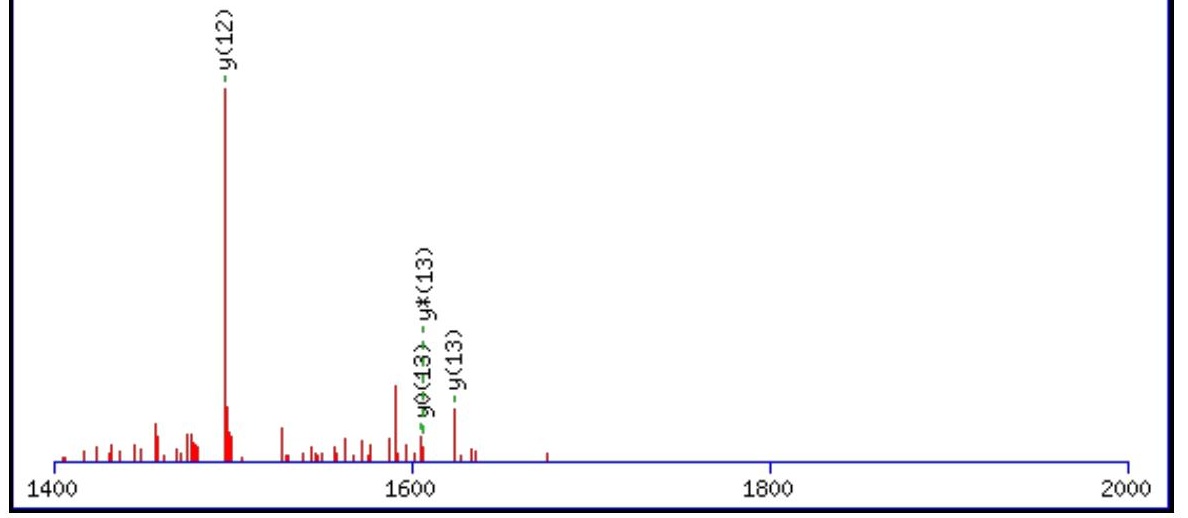

Monoisotopic mass of neutral peptide $\operatorname{Mr}($ calc): 1735.7433

Fixed modifications: Carbamidomethyl (C)

Variable modifications:

N3 : Deamidated_N (N)

M7 : Oxidation (M)

Ions Score: 49 Expect: 0.0025

Matches (Bold Red): 19/144 fragment ions using 29 most intense peaks

\begin{tabular}{|r|c|c|c|c|c|c|c|c|c|c|c|c|c|c|}
\hline$\#$ & $\mathbf{b}$ & $\mathbf{b}^{++}$ & $\mathbf{b}^{*}$ & $\mathbf{b}^{\boldsymbol{*}^{++}}$ & $\mathbf{b}^{\mathbf{0}}$ & $\mathbf{b}^{\mathbf{0 + +}}$ & $\mathbf{S e q}$ & $\mathbf{y}$ & $\mathbf{y}^{++}$ & $\mathbf{y}^{\mathbf{*}}$ & $\mathbf{y}^{\mathbf{*}^{++}}$ & $\mathbf{y}^{\mathbf{0}}$ & $\mathbf{y}^{\mathbf{0 + +}}$ & $\#$ \\
\hline $\mathbf{1}$ & 114.0913 & 57.5493 & & & & & $\mathbf{I}$ & & & & & & & $\mathbf{1 4}$ \\
\hline $\mathbf{2}$ & 242.1499 & 121.5786 & 225.1234 & 113.0653 & & & $\mathbf{Q}$ & $\mathbf{1 6 2 3 . 6 6 6 5}$ & 812.3369 & $\mathbf{1 6 0 6 . 6 4 0 0}$ & 803.8236 & $\mathbf{1 6 0 5 . 6 5 6 0}$ & $\mathbf{8 0 3 . 3 3 1 6}$ & $\mathbf{1 3}$ \\
\hline $\mathbf{3}$ & $\mathbf{3 5 7 . 1 7 6 9}$ & 179.0921 & 340.1503 & 170.5788 & & & $\mathbf{N}$ & $\mathbf{1 4 9 5 . 6 0 8 0}$ & 748.3076 & 1478.5814 & 739.7943 & 1477.5974 & 739.3023 & $\mathbf{1 2}$ \\
\hline $\mathbf{4}$ & 520.2402 & 260.6237 & 503.2136 & 252.1105 & & & $\mathbf{Y}$ & $\mathbf{1 3 8 0 . 5 8 1 0}$ & $\mathbf{6 9 0 . 7 9 4 1}$ & 1363.5545 & $\mathbf{6 8 2 . 2 8 0 9}$ & 1362.5705 & $\mathbf{6 8 1 . 7 8 8 9}$ & $\mathbf{1 1}$ \\
\hline $\mathbf{5}$ & 607.2722 & 304.1397 & 590.2457 & 295.6265 & 589.2616 & 295.1345 & $\mathbf{S}$ & $\mathbf{1 2 1 7 . 5 1 7 7}$ & 609.2625 & 1200.4911 & 600.7492 & 1199.5071 & 600.2572 & $\mathbf{1 0}$ \\
\hline $\mathbf{6}$ & 706.3406 & 353.6740 & 689.3141 & 345.1607 & $\mathbf{6 8 8 . 3 3 0 1}$ & 344.6687 & $\mathbf{V}$ & $\mathbf{1 1 3 0 . 4 8 5 7}$ & 565.7465 & 1113.4591 & 557.2332 & 1112.4751 & 556.7412 & $\mathbf{9}$ \\
\hline $\mathbf{7}$ & 853.3760 & 427.1917 & 836.3495 & 418.6784 & 835.3655 & 418.1864 & $\mathbf{M}$ & $\mathbf{1 0 3 1 . 4 1 7 3}$ & 516.2123 & 1014.3907 & 507.6990 & 1013.4067 & 507.2070 & $\mathbf{8}$ \\
\hline $\mathbf{8}$ & 924.4131 & 462.7102 & 907.3866 & 454.1969 & 906.4026 & 453.7049 & $\mathbf{A}$ & 884.3818 & 442.6946 & 867.3553 & 434.1813 & 866.3713 & 433.6893 & $\mathbf{7}$ \\
\hline $\mathbf{9}$ & $\mathbf{1 0 3 7 . 4 9 7 2}$ & 519.2522 & 1020.4707 & 510.7390 & 1019.4866 & 510.2470 & $\mathbf{I}$ & 813.3447 & 407.1760 & 796.3182 & 398.6627 & 795.3342 & 398.1707 & $\mathbf{6}$ \\
\hline $\mathbf{1 0}$ & 1197.5279 & 599.2676 & 1180.5013 & 590.7543 & 1179.5173 & 590.2623 & $\mathbf{C}$ & $\mathbf{7 0 0 . 2 6 0 7}$ & 350.6340 & $\mathbf{6 8 3 . 2 3 4 1}$ & 342.1207 & $\mathbf{6 8 2 . 2 5 0 1}$ & 341.6287 & $\mathbf{5}$ \\
\hline $\mathbf{1 1}$ & 1312.5548 & 656.7810 & 1295.5283 & 648.2678 & 1294.5442 & 647.7758 & $\mathbf{D}$ & $\mathbf{5 4 0 . 2 3 0 0}$ & 270.6186 & 523.2035 & 262.1054 & 522.2195 & 261.6134 & $\mathbf{4}$ \\
\hline $\mathbf{1 2}$ & 1475.6181 & 738.3127 & 1458.5916 & 729.7994 & 1457.6076 & 729.3074 & $\mathbf{Y}$ & $\mathbf{4 2 5 . 2 0 3 1}$ & 213.1052 & 408.1765 & 204.5919 & 407.1925 & 204.0999 & $\mathbf{3}$ \\
\hline $\mathbf{1 3}$ & 1590.6451 & 795.8262 & 1573.6185 & 787.3129 & 1572.6345 & 786.8209 & $\mathbf{D}$ & 262.1397 & 131.5735 & 245.1132 & 123.0602 & 244.1292 & 122.5682 & $\mathbf{2}$ \\
\hline $\mathbf{1 4}$ & & & & & & & $\mathbf{K}$ & 147.1128 & 74.0600 & 130.0863 & 65.5468 & & & $\mathbf{1}$ \\
\hline
\end{tabular}
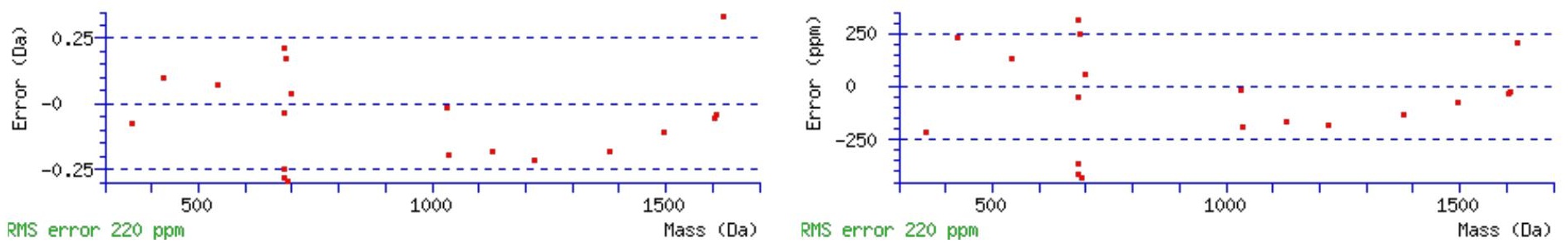

\section{All matches to this query}

\begin{tabular}{|l|l|l|l|}
\hline Score & Mr(calc): & Delta & \multicolumn{1}{c|}{ Sequence } \\
\hline 48.7 & 1735.7433 & -0.0016 & IQNYSVMAICDYDK \\
\hline 6.4 & 1735.7259 & 0.0158 & YTNYSIQVLAFTR \\
\hline 1.4 & 1735.7486 & -0.0069 & MLTNWFTFLLYK \\
\hline 1.3 & 1735.7511 & -0.0094 & LYLTGNYLQTVYK \\
\hline
\end{tabular}




\section{Peptide View}

MS/MS Fragmentation of WQMLDGGLLNITK

Found in IPI00188728, Tax_Id=10116 Gene_Symbol=Mfap3l Microfibrillar-associated protein 3-like precursor

Match to Query 662: 1488.766588 from(745.390570,2+)

Title: 100101RatKid_NS_deglyco_25.4582.4582.2.dta

Data file K:\NewmanPaper\Piliang \3SubProteomes \Piliang3SP \mgf5ppm\ERLIC_3SubProteomes5ppm.mgf
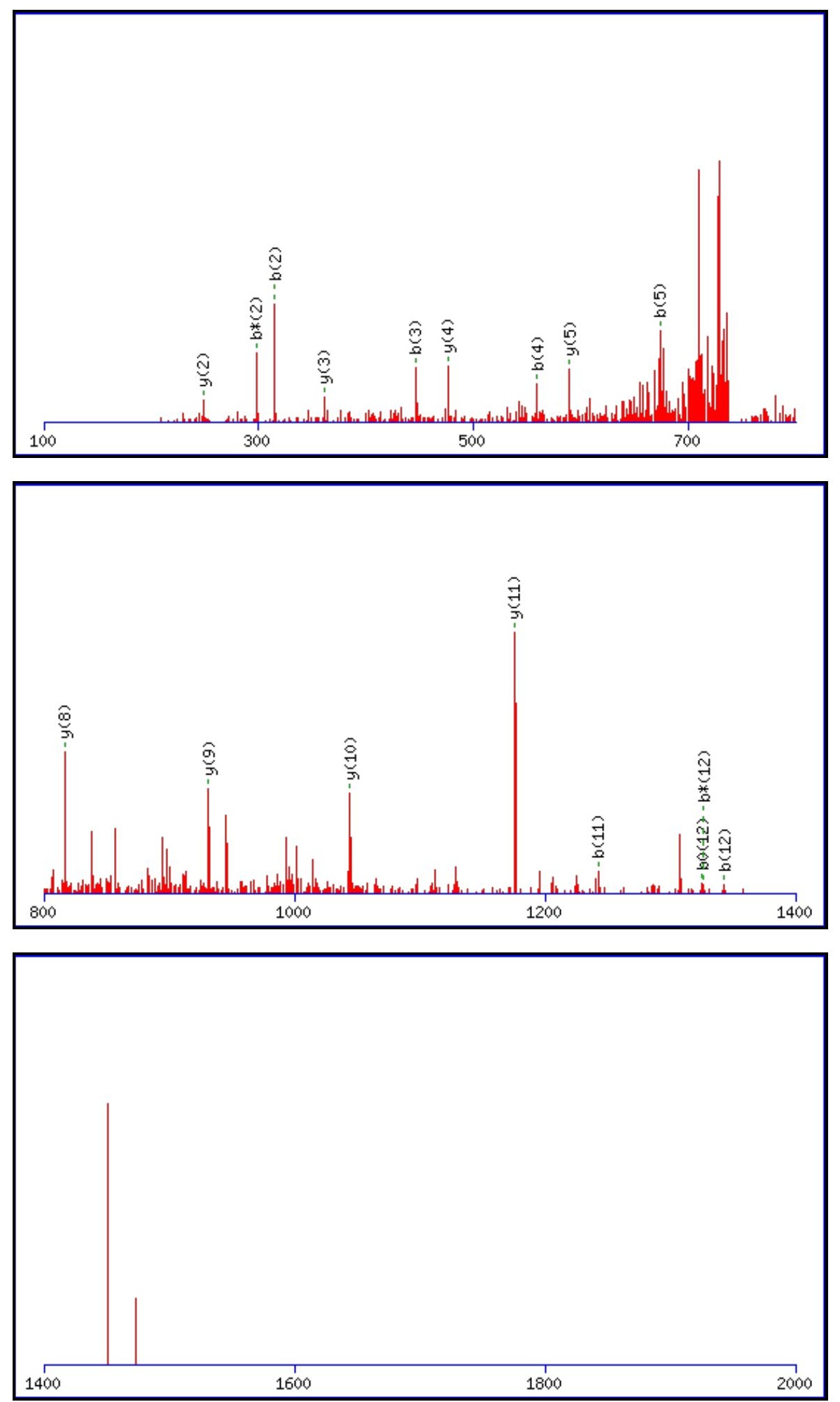

Monoisotopic mass of neutral peptide $\operatorname{Mr}($ calc): 1488.7646 Fixed modifications: Carbamidomethyl (C)

Variable modifications: 


\begin{tabular}{|c|c|c|c|c|c|c|c|c|c|c|c|c|c|c|}
\hline \# & b & $\mathbf{b}^{++}$ & $\mathbf{b}^{*}$ & $\mathbf{b}^{*^{++}}$ & $\mathbf{b}^{\mathbf{0}}$ & $\mathbf{b}^{\mathbf{0 + +}}$ & Seq. & $\mathbf{y}$ & $y^{++}$ & $\mathbf{y}^{*}$ & $\mathrm{y}^{*^{++}}$ & $\mathbf{y}^{\mathbf{0}}$ & $y^{0++}$ & \# \\
\hline 1 & 187.0866 & 94.0469 & & & & & W & & & & & & & 13 \\
\hline 2 & 315.1452 & 158.0762 & 298.1186 & 149.5629 & & & $\mathbf{Q}$ & 1303.6926 & 652.3499 & 1286.6661 & 643.8367 & 1285.6820 & 643.3447 & 12 \\
\hline 3 & 446.1857 & 223.5965 & 429.1591 & 215.0832 & & & M & 1175.6340 & 588.3207 & 1158.6075 & 579.8074 & 1157.6235 & 579.3154 & 11 \\
\hline 4 & 559.2697 & 280.1385 & 542.2432 & 271.6252 & & & $\mathbf{L}$ & 1044.5935 & 522.8004 & 1027.5670 & 514.2871 & 1026.5830 & 513.7951 & 10 \\
\hline 5 & 674.2967 & 337.6520 & 657.2701 & 329.1387 & 656.2861 & 328.6467 & D & 931.5095 & 466.2584 & 914.4829 & 457.7451 & 913.4989 & 457.2531 & 9 \\
\hline 6 & 731.3181 & 366.1627 & 714.2916 & 357.6494 & 713.3076 & 357.1574 & G & 816.4825 & 408.7449 & 799.4560 & 400.2316 & 798.4720 & 399.7396 & 8 \\
\hline 7 & 788.3396 & 394.6734 & 771.3130 & 386.1602 & 770.3290 & 385.6681 & G & 759.4611 & 380.2342 & 742.4345 & 371.7209 & 741.4505 & 371.2289 & 7 \\
\hline 8 & 901.4237 & 451.2155 & 884.3971 & 442.7022 & 883.4131 & 442.2102 & $\mathbf{L}$ & 702.4396 & 351.7234 & 685.4131 & 343.2102 & 684.4290 & 342.7182 & 6 \\
\hline 9 & 1014.5077 & 507.7575 & 997.4812 & 499.2442 & 996.4972 & 498.7522 & L & 589.3555 & 295.1814 & 572.3290 & 286.6681 & 571.3450 & 286.1761 & 5 \\
\hline 10 & 1129.5347 & 565.2710 & 1112.5081 & 556.7577 & 1111.5241 & 556.2657 & $\mathbf{N}$ & 476.2715 & 238.6394 & 459.2449 & 230.1261 & 458.2609 & 229.6341 & 4 \\
\hline 11 & 1242.6187 & 621.8130 & 1225.5922 & 613.2997 & 1224.6082 & 612.8077 & I & 361.2445 & 181.1259 & 344.2180 & 172.6126 & 343.2340 & 172.1206 & 3 \\
\hline 12 & 1343.6664 & 672.3368 & 1326.6398 & 663.8236 & 1325.6558 & 663.3316 & $\mathbf{T}$ & 248.1605 & 124.5839 & 231.1339 & 116.0706 & 230.1499 & 115.5786 & 2 \\
\hline 13 & & & & & & & $\mathbf{K}$ & 147.1128 & 74.0600 & 130.0863 & 65.5468 & & & 1 \\
\hline
\end{tabular}
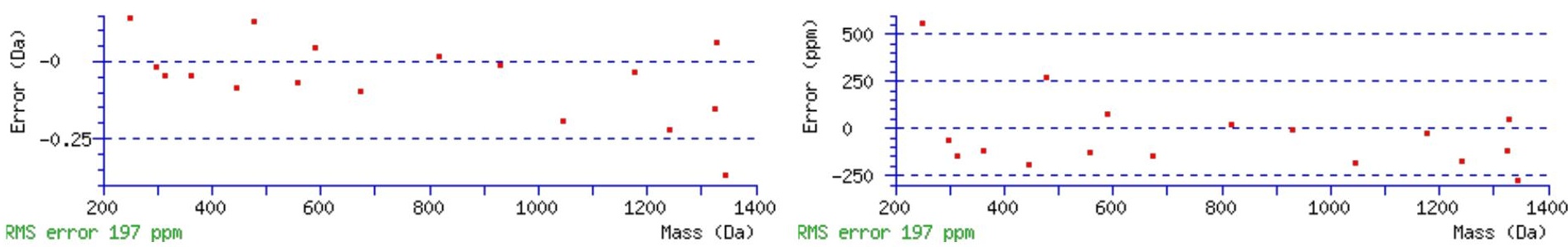

\section{All matches to this query}

\begin{tabular}{|l|l|l|l|}
\hline Score & Mr(calc): & Delta & \multicolumn{1}{|c|}{ Sequence } \\
\hline 48.5 & 1488.7646 & 0.0020 & WQMLDGGLLNITK \\
\hline 4.3 & 1488.7532 & 0.0134 & AENASQESKRELK \\
\hline 2.8 & 1486.7636 & 2.0030 & MPMALNASLSLPAR \\
\hline 2.5 & 1488.7741 & -0.0076 & YYNIKKVVGPTK \\
\hline 2.1 & 1486.7636 & 2.0030 & MPMALNASLSLPAR \\
\hline 1.3 & 1488.7684 & -0.0019 & FVRRNLNEPESK \\
\hline
\end{tabular}

Spectrum No: 699; Query: 1305; Rank: 1

\section{Peptide View}

MS/MS Fragmentation of DGQVINETSQHHDDLE

Found in IPI00230941, Tax_Id=10116 Gene_Symbol=Vim Vimentin

Match to Query 1305: 1836.771768 from(919.393160,2+)

Title: 091008RatKidney_NoSalt_21.884.884.2.dta

Data file K:INewmanPaper|Piliangl3SubProteomes\Piliang3SP\mgf5ppm\ERLIC_3SubProteomes5ppm.mgf 

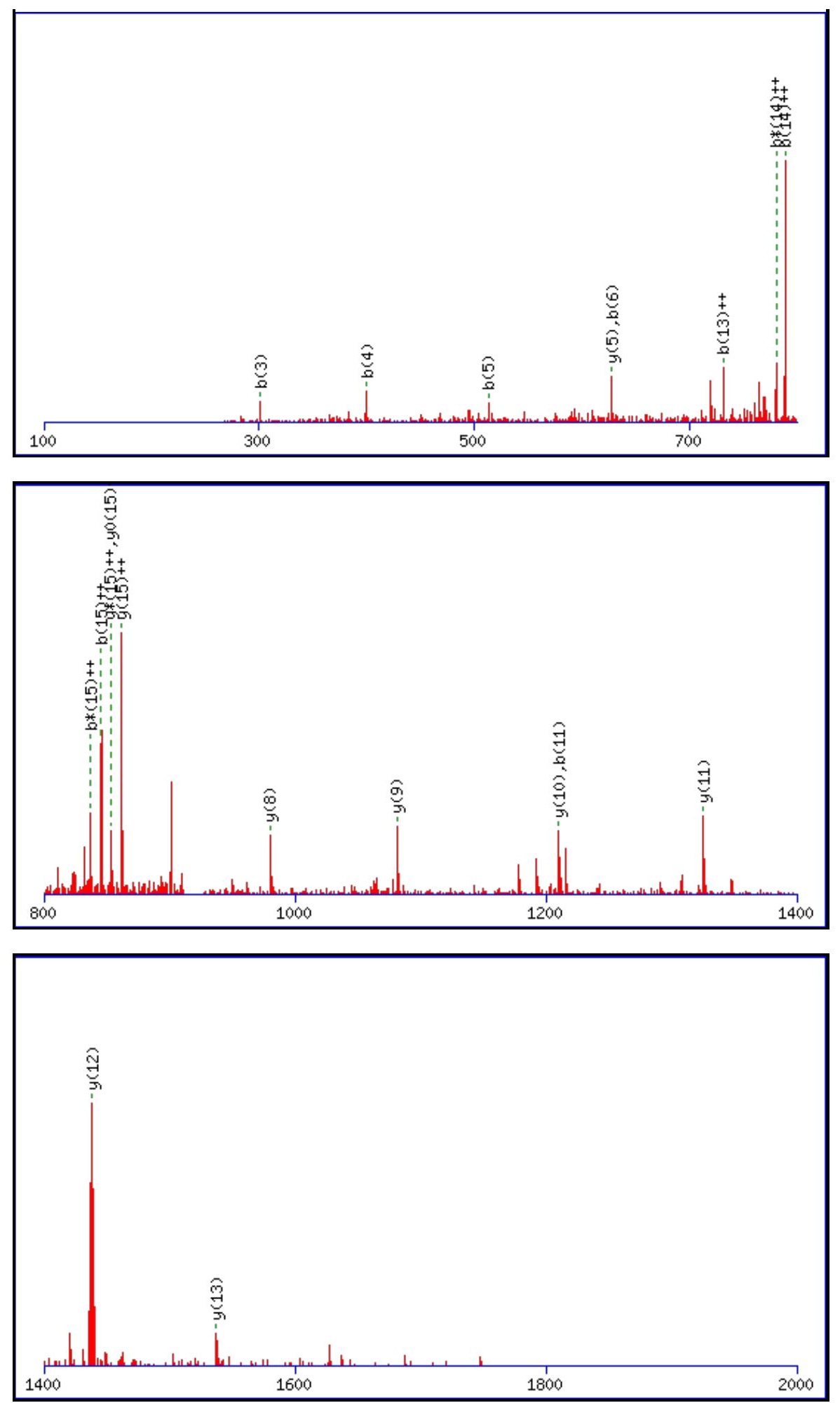

Monoisotopic mass of neutral peptide $\operatorname{Mr}($ calc): 1836.7762

Fixed modifications: Carbamidomethyl (C)

Variable modifications:

N6 : Deamidated $\mathrm{N}(\mathrm{N})$

Ions Score: 48 Expect: 0.0029

Matches (Bold Red): 20/164 fragment ions using 24 most intense peaks

\begin{tabular}{|r|c|c|c|c|c|c|c|c|c|c|c|c|c|c|}
\hline$\#$ & $\mathbf{b}$ & $\mathbf{b}^{++}$ & $\mathbf{b}^{*}$ & $\mathbf{b}^{\boldsymbol{*}^{++}}$ & $\mathbf{b}^{\mathbf{0}}$ & $\mathbf{b}^{\mathbf{0 + +}}$ & Seq. & $\mathbf{y}$ & $\mathbf{y}^{++}$ & $\mathbf{y}^{\mathbf{*}}$ & $\mathbf{y}^{\boldsymbol{*}^{++}}$ & $\mathbf{y}^{\mathbf{0}}$ & $\mathbf{y}^{\mathbf{0 + +}}$ & $\#$ \\
\hline $\mathbf{1}$ & 116.0342 & 58.5207 & & & 98.0237 & 49.5155 & $\mathbf{D}$ & & & & & & & $\mathbf{1 6}$ \\
\hline $\mathbf{2}$ & 173.0557 & 87.0315 & & & 155.0451 & 78.0262 & $\mathbf{G}$ & 1722.7565 & $\mathbf{8 6 1 . 8 8 1 9}$ & 1705.7300 & $\mathbf{8 5 3 . 3 6 8 6}$ & 1704.7460 & $\mathbf{8 5 2 . 8 7 6 6}$ & $\mathbf{1 5}$ \\
\hline $\mathbf{3}$ & $\mathbf{3 0 1 . 1 1 4 3}$ & 151.0608 & 284.0877 & 142.5475 & 283.1037 & 142.0555 & $\mathbf{Q}$ & 1665.7351 & 833.3712 & 1648.7085 & 824.8579 & 1647.7245 & 824.3659 & $\mathbf{1 4}$ \\
\hline $\mathbf{4}$ & $\mathbf{4 0 0 . 1 8 2 7}$ & 200.5950 & 383.1561 & 192.0817 & 382.1721 & 191.5897 & $\mathbf{V}$ & $\mathbf{1 5 3 7 . 6 7 6 5}$ & 769.3419 & 1520.6500 & 760.8286 & 1519.6659 & 760.3366 & $\mathbf{1 3}$ \\
\hline $\mathbf{5}$ & $\mathbf{5 1 3 . 2 6 6 7}$ & 257.1370 & 496.2402 & 248.6237 & 495.2562 & 248.1317 & $\mathbf{I}$ & $\mathbf{1 4 3 8 . 6 0 8 1}$ & 719.8077 & 1421.5815 & 711.2944 & 1420.5975 & 710.8024 & $\mathbf{1 2}$ \\
\hline
\end{tabular}




\begin{tabular}{|c|c|c|c|c|c|c|c|c|c|c|c|c|c|c|}
\hline 6 & 628.2937 & |314.6505 & 611.2671 & |306.1372 & 610.2831 & 305.6452 & $\mathbf{N}$ & |1325.5240 & |663.2656 & |1308.4975 & 654.7524 & 1307.5135 & 654.2604 & 11 \\
\hline 7 & 757.3363 & 379.1718 & 740.3097 & 370.6585 & 739.3257 & 370.1665 & $\mathbf{E}$ & 1210.4971 & 605.7522 & 1193.4705 & 597.2389 & 1192.4865 & 596.7469 & 10 \\
\hline 8 & 858.3839 & 429.6956 & 841.3574 & 421.1823 & 840.3734 & 420.6903 & $\mathbf{T}$ & 1081.4545 & 541.2309 & 1064.4279 & 532.7176 & 1063.4439 & 532.2256 & 9 \\
\hline 9 & 945.4160 & 473.2116 & 928.3894 & 464.6984 & 927.4054 & 464.2063 & $\mathrm{~S}$ & 980.4068 & 490.7070 & 963.3803 & 482.1938 & 962.3962 & 481.7018 & 8 \\
\hline 10 & 1073.4746 & 537.2409 & 1056.4480 & 528.7276 & 1055.4640 & 528.2356 & $\mathbf{Q}$ & 893.3748 & 447.1910 & 876.3482 & 438.6778 & 875.3642 & 438.1857 & 7 \\
\hline 11 & 1210.5335 & 605.7704 & 1193.5069 & 597.2571 & 1192.5229 & 596.7651 & $\mathbf{H}$ & 765.3162 & 383.1617 & & & 747.3056 & 374.1565 & 6 \\
\hline 12 & 1347.5924 & 674.2998 & 1330.5658 & 665.7866 & 1329.5818 & 665.2945 & $\mathbf{H}$ & 628.2573 & 314.6323 & & & 610.2467 & 305.6270 & 5 \\
\hline 13 & 1462.6193 & 731.8133 & 1445.5928 & 723.3000 & 1444.6088 & 722.8080 & D & 491.1984 & 246.1028 & & & 473.1878 & 237.0975 & 4 \\
\hline 14 & 1577.6463 & 789.3268 & 1560.6197 & 780.8135 & 1559.6357 & 780.3215 & D & 376.1714 & 188.5894 & & & 358.1609 & 179.5841 & 3 \\
\hline 15 & 1690.7303 & 845.8688 & 1673.7038 & 837.3555 & 1672.7198 & 836.8635 & L & 261.1445 & 131.0759 & & & 243.1339 & 122.0706 & 2 \\
\hline 16 & & & & & & & $\mathbf{E}$ & 148.0604 & 74.5339 & & & 130.0499 & 65.5286 & 1 \\
\hline
\end{tabular}
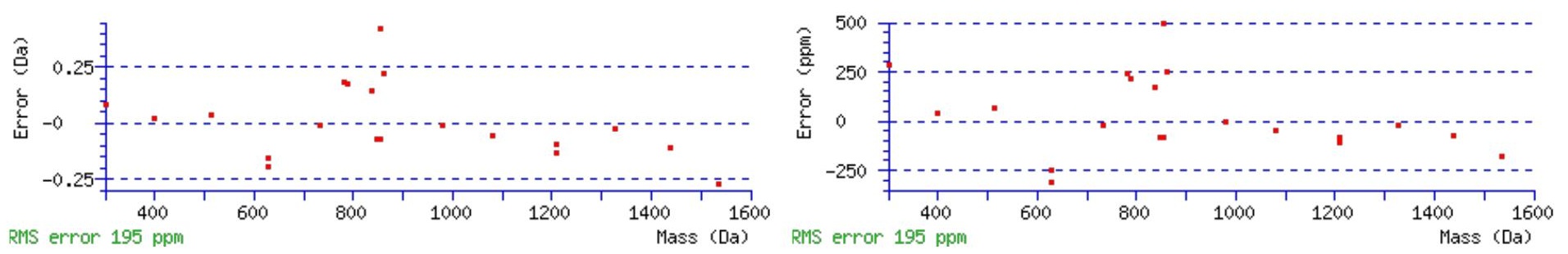

\section{All matches to this query}

\begin{tabular}{|l|c|c|l|}
\hline Score & Mr(calc): & Delta & Sequence \\
\hline 48.5 & 1836.7762 & -0.0044 & DGQVINETSQHHDDLE \\
\hline 0.9 & 1835.7574 & 1.0144 & ERKNETATFNNTDSK \\
\hline 0.9 & 1835.7574 & 1.0144 & ERKNETATFNNTDSK \\
\hline 0.9 & 1835.7574 & 1.0144 & ERKNETATFNNTDSK \\
\hline 0.9 & 1835.7574 & 1.0144 & ERKNETATFNNTDSK \\
\hline 0.9 & 1835.7574 & 1.0144 & ERKNETATFNNTDSK \\
\hline 0.9 & 1835.7574 & 1.0144 & ERKNETATFNNTDSK \\
\hline 0.3 & 1835.7615 & 1.0103 & LWNSSSGDNTLVNYGK \\
\hline 0.3 & 1835.7615 & 1.0103 & LWNSSSGDNTLVNYGK \\
\hline
\end{tabular}

Spectrum No: 700; Query: 1968; Rank: 1

\section{Peptide View}

MS/MS Fragmentation of QFWIFDVQNPEEVAKNSSK

Found in IPI00231197, Tax_Id=10116 Gene_Symbol=Cd36 Platelet glycoprotein 4

Match to Query 1968: 2266.096962 from(756.372930,3+)

Title: 100101RatKid_NS_deglyco_24.4554.4554.3.dta

Data file K:INewmanPaper|Piliangl3SubProteomes\Piliang3SP\mgf5ppm\ERLIC_3SubProteomes5ppm.mgf 

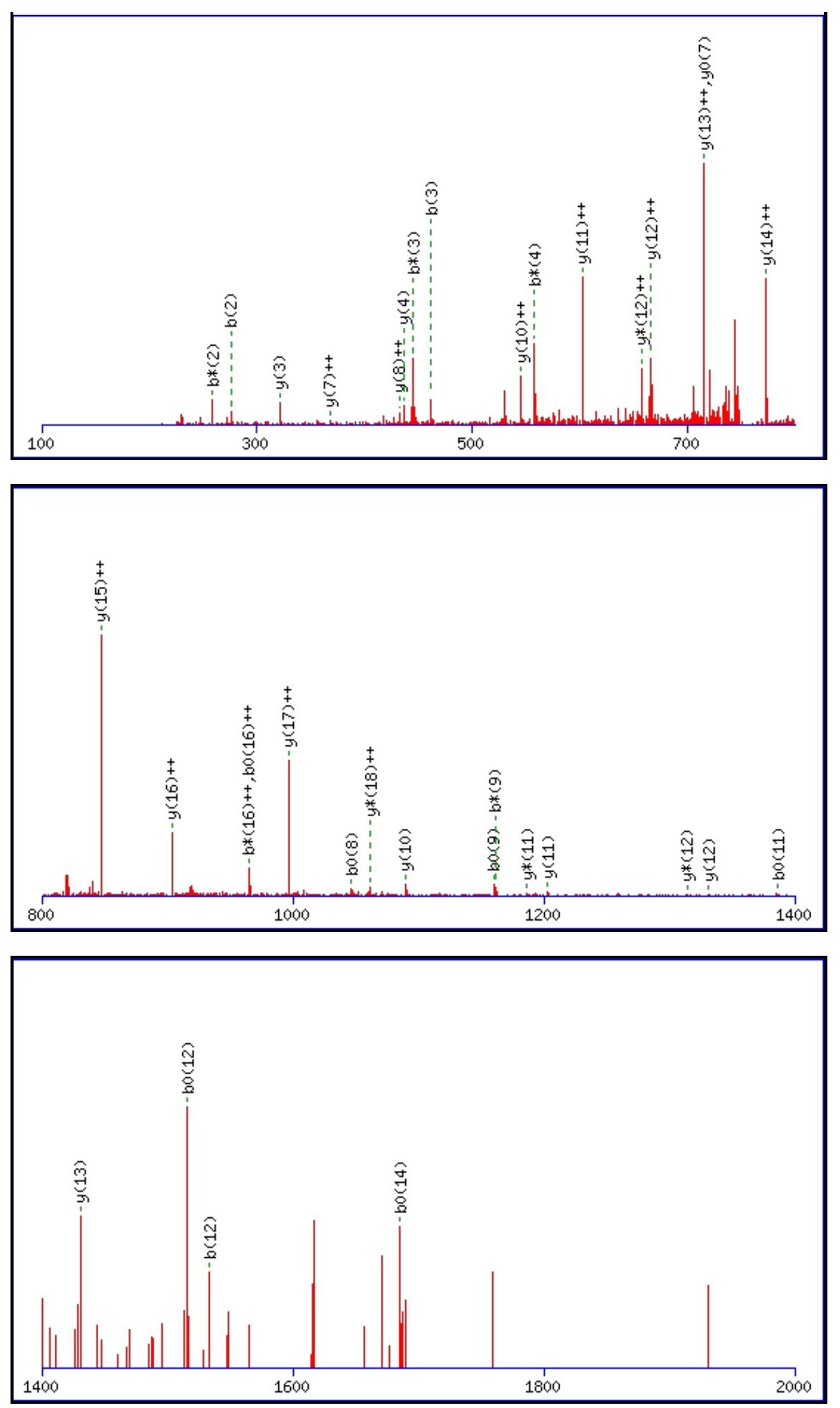

Monoisotopic mass of neutral peptide $\operatorname{Mr}($ calc): 2266.0906

Fixed modifications: Carbamidomethyl (C)

Variable modifications:

N16 : Deamidated N (N)

Ions Score: 48 Expect: 0.004

Matches (Bold Red): 35/204 fragment ions using 57 most intense peaks

\begin{tabular}{|r|c|c|c|c|c|r|r|r|r|r|r|r|r|r|}
\hline$\#$ & $\mathbf{b}$ & $\mathbf{b}^{++}$ & $\mathbf{b}^{*}$ & $\mathbf{b}^{\boldsymbol{*}^{++}}$ & $\mathbf{b}^{\mathbf{0}}$ & $\mathbf{b}^{\mathbf{0 + +}}$ & Seq. & $\mathbf{y}$ & $\mathbf{y}^{++}$ & $\mathbf{y}^{\mathbf{*}}$ & $\mathbf{y}^{\mathbf{*}^{++}}$ & $\mathbf{y}^{\mathbf{0}}$ & $\mathbf{y}^{\mathbf{0 + +}}$ & $\#$ \\
\hline $\mathbf{1}$ & 129.0659 & 65.0366 & 112.0393 & 56.5233 & & & $\mathbf{Q}$ & & & & & & & $\mathbf{1 9}$ \\
\hline $\mathbf{2}$ & $\mathbf{2 7 6 . 1 3 4 3}$ & 138.5708 & 259.1077 & 130.0575 & & & $\mathbf{F}$ & 2139.0393 & 1070.0233 & 2122.0128 & $\mathbf{1 0 6 1 . 5 1 0 0}$ & 2121.0287 & 1061.0180 & $\mathbf{1 8}$ \\
\hline $\mathbf{3}$ & $\mathbf{4 6 2 . 2 1 3 6}$ & 231.6104 & $\mathbf{4 4 5 . 1 8 7 0}$ & 223.0972 & & & $\mathbf{W}$ & 1991.9709 & $\mathbf{9 9 6 . 4 8 9 1}$ & 1974.9443 & 987.9758 & 1973.9603 & 987.4838 & $\mathbf{1 7}$ \\
\hline $\mathbf{4}$ & 575.2976 & 288.1525 & 558.2711 & 279.6392 & & & $\mathbf{I}$ & 1805.8916 & $\mathbf{9 0 3 . 4 4 9 4}$ & 1788.8650 & 894.9362 & $\mathbf{1 7 8 7 . 8 8 1 0}$ & 894.4441 & $\mathbf{1 6}$ \\
\hline $\mathbf{5}$ & 722.3661 & 361.6867 & 705.3395 & 353.1734 & & & $\mathbf{F}$ & 1692.8075 & $\mathbf{8 4 6 . 9 0 7 4}$ & 1675.7810 & 838.3941 & 1674.7969 & 837.9021 & $\mathbf{1 5}$ \\
\hline
\end{tabular}




\begin{tabular}{|c|c|c|c|c|c|c|c|c|c|c|c|c|c|c|}
\hline 6 & 837.3930 & 419.2001 & 820.3665 & 410.6869 & 819.3824 & 410.1949 & D & |1545.7391 & 773.3732 & |1528.7126 & 764.8599 & |1527.7285| & 764.3679| & 14 \\
\hline 7 & 936.4614 & 468.7343 & 919.4349 & 460.2211 & 918.4509 & 459.7291 & V & 1430.7122 & 715.8597 & 1413.6856 & 707.3464 & 1412.7016 & 706.8544 & 13 \\
\hline 8 & 1064.5200 & 532.7636 & 1047.4934 & 524.2504 & 1046.5094 & 523.7584 & $\mathbf{Q}$ & 1331.6437 & 666.3255 & 1314.6172 & 657.8122 & 1313.6332 & 657.3202 & 12 \\
\hline 9 & 1178.5629 & 589.7851 & 1161.5364 & 581.2718 & 1160.5524 & 580.7798 & $\mathbf{N}$ & 1203.5852 & 602.2962 & 1186.5586 & 593.7829 & 1185.5746 & 593.2909 & 11 \\
\hline 10 & 1275.6157 & 638.3115 & 1258.5891 & 629.7982 & 1257.6051 & 629.3062 & $\mathbf{P}$ & 1089.5422 & 545.2748 & 1072.5157 & 536.7615 & 1071.5317 & 536.2695 & 10 \\
\hline 11 & 1404.6583 & 702.8328 & 1387.6317 & 694.3195 & 1386.6477 & 693.8275 & $\mathbf{E}$ & 992.4895 & 496.7484 & 975.4629 & 488.2351 & 974.4789 & 487.7431 & 9 \\
\hline 12 & 1533.7009 & 767.3541 & 1516.6743 & 758.8408 & 1515.6903 & 758.3488 & $\mathbf{E}$ & 863.4469 & 432.2271 & 846.4203 & 423.7138 & 845.4363 & 423.2218 & 8 \\
\hline 13 & 1632.7693 & 816.8883 & 1615.7427 & 808.3750 & 1614.7587 & 807.8830 & $\mathbf{V}$ & 734.4043 & 367.7058 & 717.3777 & 359.1925 & 716.3937 & 358.7005 & 7 \\
\hline 14 & 1703.8064 & 852.4068 & 1686.7799 & 843.8936 & 1685.7958 & 843.4016 & A & 635.3359 & 318.1716 & 618.3093 & 309.6583 & 617.3253 & 309.1663 & 6 \\
\hline 15 & 1831.9014 & 916.4543 & 1814.8748 & 907.9410 & 1813.8908 & 907.4490 & $\mathbf{K}$ & 564.2988 & 282.6530 & 547.2722 & 274.1397 & 546.2882 & 273.6477 & 5 \\
\hline 16 & 1946.9283 & 973.9678 & 1929.9017 & 965.4545 & 1928.9177 & 964.9625 & $\mathbf{N}$ & 436.2038 & 218.6055 & 419.1772 & 210.0923 & 418.1932 & 209.6003 & 4 \\
\hline 17 & 2033.9603 & 1017.4838 & 2016.9338 & 1008.9705 & 2015.9498 & 1008.4785 & S & 321.1769 & 161.0921 & 304.1503 & 152.5788 & 303.1663 & 152.0868 & 3 \\
\hline 18 & 2120.9924 & 1060.9998 & 2103.9658 & 1052.4865 & 2102.9818 & 1051.9945 & S & 234.1448 & 117.5761 & 217.1183 & 109.0628 & 216.1343 & 108.5708 & 2 \\
\hline 19 & & & & & & & $\mathbf{K}$ & 147.1128 & 74.0600 & 130.0863 & 65.5468 & & & 1 \\
\hline
\end{tabular}
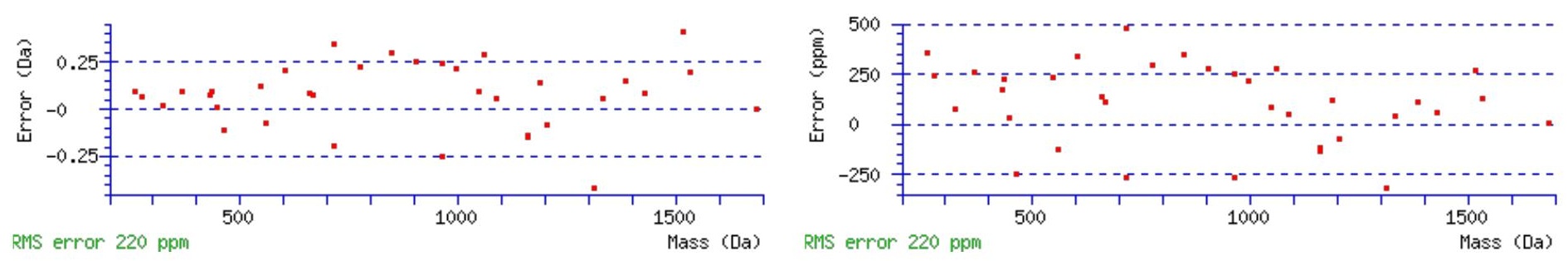

\section{All matches to this query}

\begin{tabular}{|l|l|l|l|}
\hline Score & Mr(calc): & Delta & \multicolumn{1}{|c|}{ Sequence } \\
\hline 48.4 & 2266.0906 & 0.0064 & QFWIFDVQNPEEVAKNSSK \\
\hline 34.4 & 2266.0906 & 0.0064 & QFWIFDVQNPEEVAKNSSK \\
\hline 5.3 & 2266.1038 & -0.0069 & MNKLYIGNLNESVTPADLEK \\
\hline 4.3 & 2264.0699 & 2.0271 & NGFTLHRNPIAQSTDGARTK \\
\hline 4.2 & 2264.0699 & 2.0271 & NGFTLHRNPIAQSTDGARTK \\
\hline 2.3 & 2266.1038 & -0.0069 & MNKLYIGNLNESVTPADLEK \\
\hline 2.2 & 2266.1038 & -0.0069 & MNKLYIGNLNESVTPADLEK \\
\hline 2.2 & 2265.1129 & 0.9840 & VNMIGSHVRYDIIHLFVR \\
\hline 2.1 & 2266.0753 & 0.0217 & ENYIDQEEVNKTNLIWTR \\
\hline 2.0 & 2264.0838 & 2.0131 & ATFDVTPRPAETVPTKSPDR \\
\hline
\end{tabular}

Spectrum No: 701; Query: 660; Rank: 1

\section{Peptide View}

MS/MS Fragmentation of DSYPDGNITWYR

Found in IPI00206188, Tax_Id=10116 Gene_Symbol=Alcam CD166 antigen precursor

Match to Query 660: 1486.637008 from(744.325780,2+)

Title: 091008RatKidney_NoSalt_28.2535.2535.2.dta

Data file K:INewmanPaper|Piliangl3SubProteomes\Piliang3SP\mgf5ppm\ERLIC_3SubProteomes5ppm.mgf 

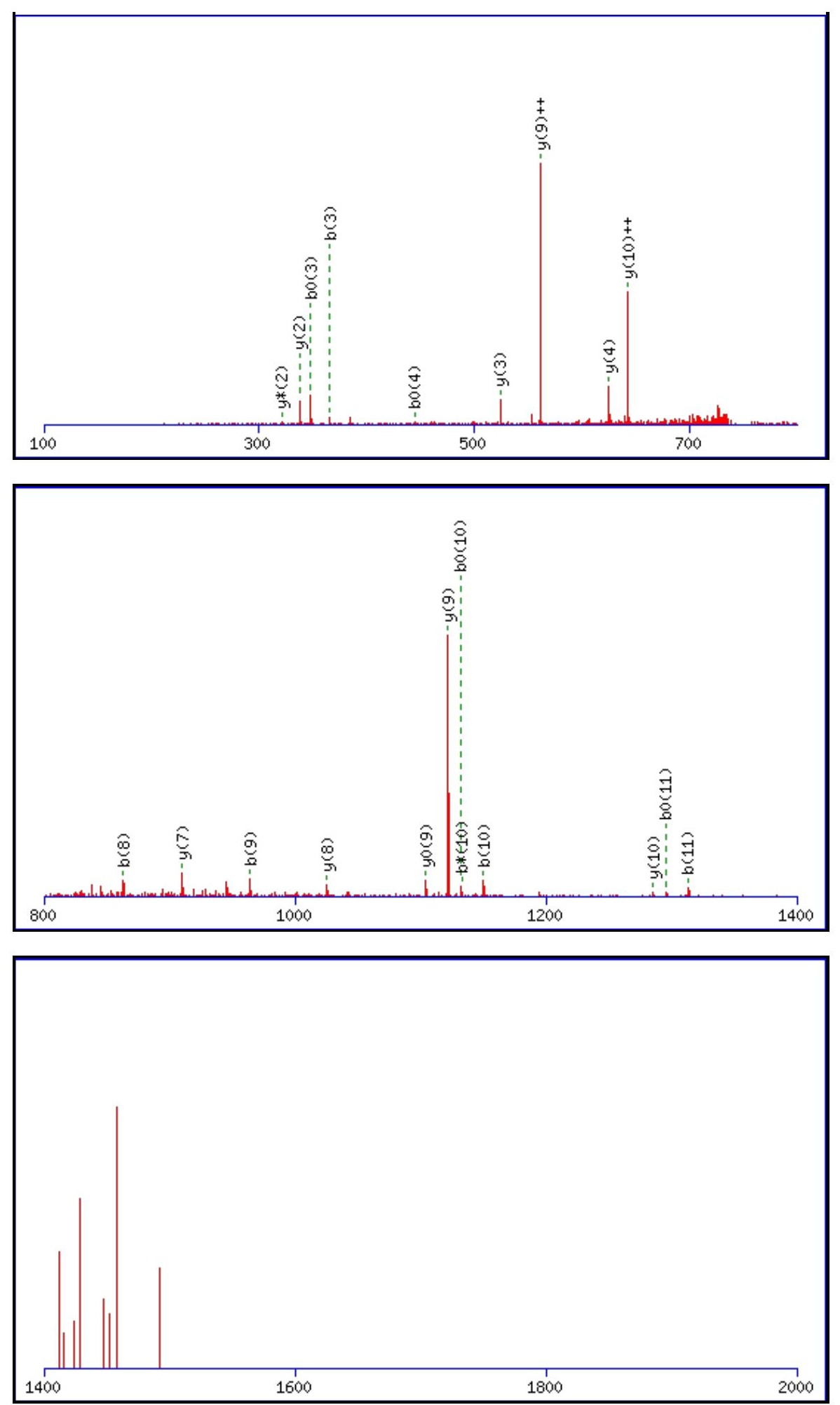

Monoisotopic mass of neutral peptide $\operatorname{Mr}($ calc): 1486.6365

Fixed modifications: Carbamidomethyl (C)

Variable modifications:

N7 : Deamidated $\mathrm{N}(\mathrm{N})$

Ions Score: 48 Expect: $\odot .0026$

Matches (Bold Red): 21/114 fragment ions using 36 most intense peaks

\begin{tabular}{|r|c|c|c|c|r|r|r|r|c|c|c|c|c|c|}
\hline$\#$ & $\mathbf{b}$ & $\mathbf{b}^{++}$ & $\mathbf{b}^{*}$ & $\mathbf{b}^{\boldsymbol{*}^{++}}$ & $\mathbf{b}^{\mathbf{0}}$ & $\mathbf{b}^{\mathbf{0 + +}}$ & Seq. & $\mathbf{y}$ & $\mathbf{y}^{++}$ & $\mathbf{y}^{\mathbf{*}}$ & $\mathbf{y}^{\boldsymbol{*}^{++}}$ & $\mathbf{y}^{\mathbf{0}}$ & $\mathbf{y}^{\mathbf{0 + +}}$ & $\#$ \\
\hline $\mathbf{1}$ & 116.0342 & 58.5207 & & & 98.0237 & 49.5155 & $\mathbf{D}$ & & & & & & & $\mathbf{1 2}$ \\
\hline $\mathbf{2}$ & 203.0662 & 102.0368 & & & 185.0557 & 93.0315 & $\mathbf{S}$ & 1372.6168 & 686.8120 & 1355.5903 & 678.2988 & 1354.6062 & 677.8068 & $\mathbf{1 1}$ \\
\hline $\mathbf{3}$ & $\mathbf{3 6 6 . 1 2 9 6}$ & 183.5684 & & & 348.1190 & 174.5631 & $\mathbf{Y}$ & $\mathbf{1 2 8 5 . 5 8 4 8}$ & $\mathbf{6 4 3 . 2 9 6 0}$ & 1268.5582 & 634.7828 & 1267.5742 & 634.2907 & $\mathbf{1 0}$ \\
\hline $\mathbf{4}$ & 463.1823 & 232.0948 & & & 445.1718 & 223.0895 & $\mathbf{P}$ & $\mathbf{1 1 2 2 . 5 2 1 4}$ & $\mathbf{5 6 1 . 7 6 4 4}$ & 1105.4949 & 553.2511 & $\mathbf{1 1 0 4 . 5 1 0 9}$ & 552.7591 & $\mathbf{9}$ \\
\hline $\mathbf{5}$ & 578.2093 & 289.6083 & & & 560.1987 & 280.6030 & $\mathbf{D}$ & $\mathbf{1 0 2 5 . 4 6 8 7}$ & 513.2380 & 1008.4421 & 504.7247 & 1007.4581 & 504.2327 & $\mathbf{8}$ \\
\hline
\end{tabular}




\begin{tabular}{|r|r|r|r|r|r|r|r|r|r|r|r|r|r|r|r|r|r|r|}
$\mathbf{6}$ & 635.2307 & 318.1190 & & & 617.2202 & 309.1137 & $\mathbf{G}$ & $\mathbf{9 1 0 . 4 4 1 7}$ & 455.7245 & 893.4152 & 447.2112 & 892.4312 & 446.7192 & $\mathbf{7}$ \\
\hline $\mathbf{7}$ & 750.2577 & 375.6325 & 733.2311 & 367.1192 & 732.2471 & 366.6272 & $\mathbf{N}$ & 853.4203 & 427.2138 & 836.3937 & 418.7005 & 835.4097 & 418.2085 & $\mathbf{6}$ \\
\hline $\mathbf{8}$ & $\mathbf{8 6 3 . 3 4 1 7}$ & 432.1745 & 846.3152 & 423.6612 & 845.3312 & 423.1692 & $\mathbf{I}$ & 738.3933 & 369.7003 & 721.3668 & 361.1870 & 720.3828 & 360.6950 & $\mathbf{5}$ \\
\hline $\mathbf{9}$ & $\mathbf{9 6 4 . 3 8 9 4}$ & 482.6984 & 947.3629 & 474.1851 & 946.3789 & 473.6931 & $\mathbf{T}$ & $\mathbf{6 2 5 . 3 0 9 3}$ & 313.1583 & 608.2827 & 304.6450 & 607.2987 & 304.1530 & $\mathbf{4}$ \\
\hline $\mathbf{1 0}$ & $\mathbf{1 1 5 0 . 4 6 8 7}$ & 575.7380 & $\mathbf{1 1 3 3 . 4 4 2 2}$ & 567.2247 & $\mathbf{1 1 3 2 . 4 5 8 2}$ & 566.7327 & $\mathbf{W}$ & 524.2616 & 262.6344 & 507.2350 & 254.1212 & & & & $\mathbf{3}$ \\
\hline $\mathbf{1 1}$ & $\mathbf{1 3 1 3 . 5 3 2 1}$ & 657.2697 & 1296.5055 & 648.7564 & $\mathbf{1 2 9 5 . 5 2 1 5}$ & 648.2644 & $\mathbf{Y}$ & $\mathbf{3 3 8 . 1 8 2 3}$ & 169.5948 & $\mathbf{3 2 1 . 1 5 5 7}$ & 161.0815 & & & & $\mathbf{2}$ \\
\hline $\mathbf{1 2}$ & & & & & & & $\mathbf{R}$ & 175.1190 & 88.0631 & 158.0924 & 79.5498 & & & $\mathbf{1}$ \\
\hline
\end{tabular}
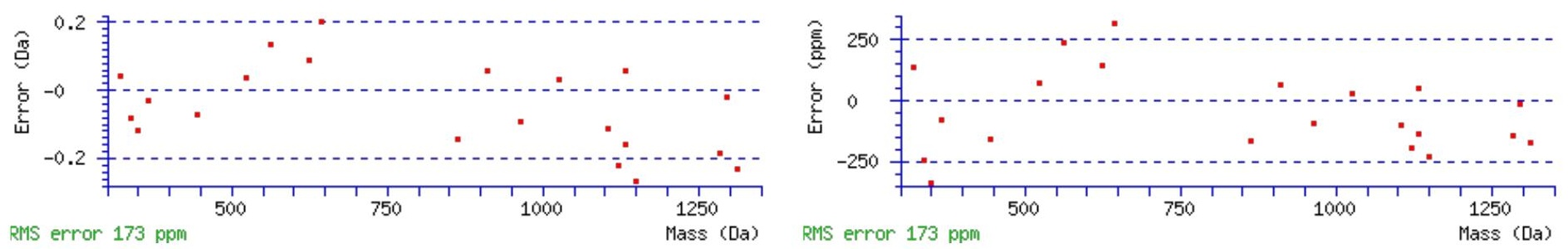

\section{All matches to this query}

\begin{tabular}{|l|l|l|l|}
\hline Score & Mr(calc): & Delta & \multicolumn{1}{|c|}{ Sequence } \\
\hline 48.4 & 1486.6365 & 0.0005 & DSYPDGNITWYR \\
\hline 1.8 & 1486.6234 & 0.0136 & STLTSVKNKLR \\
\hline 1.3 & 1484.6344 & 2.0026 & HKHPESQQQMR \\
\hline 1.3 & 1486.6259 & 0.0111 & YDSRSGTHNMYR \\
\hline 0.8 & 1484.6231 & 2.0139 & SLVGSWLKLSR \\
\hline 0.8 & 1484.6303 & 2.0067 & SSAVRTRXVLR \\
\hline 0.5 & 1486.6470 & -0.0100 & $\underline{\text { INHNENLESNMR }}$ \\
\hline
\end{tabular}

Spectrum No: 702; Query: 308; Rank: 1

\section{Peptide View}

\section{MS/MS Fragmentation of VGIPVTDENGTR}

Found in IPI00213735, Tax_Id=10116 Gene_Symbol=Sfxn1 Sideroflexin-1

Match to Query 308: 1257.623108 from(629.818830,2+)

Title: 100101RatKid_NS_deglyco_13.1456.1456.2.dta

Data file K:INewmanPaper|Piliangl3SubProteomes\Piliang3SP\mgf5ppm|ERLIC_3SubProteomes5ppm.mgf

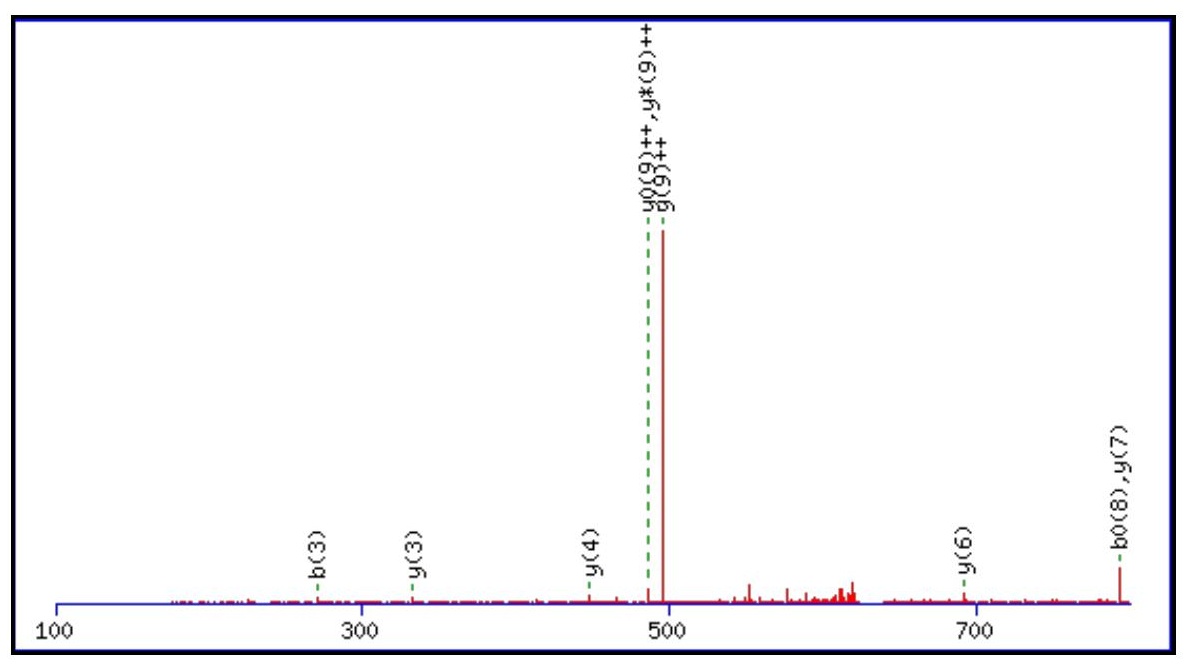



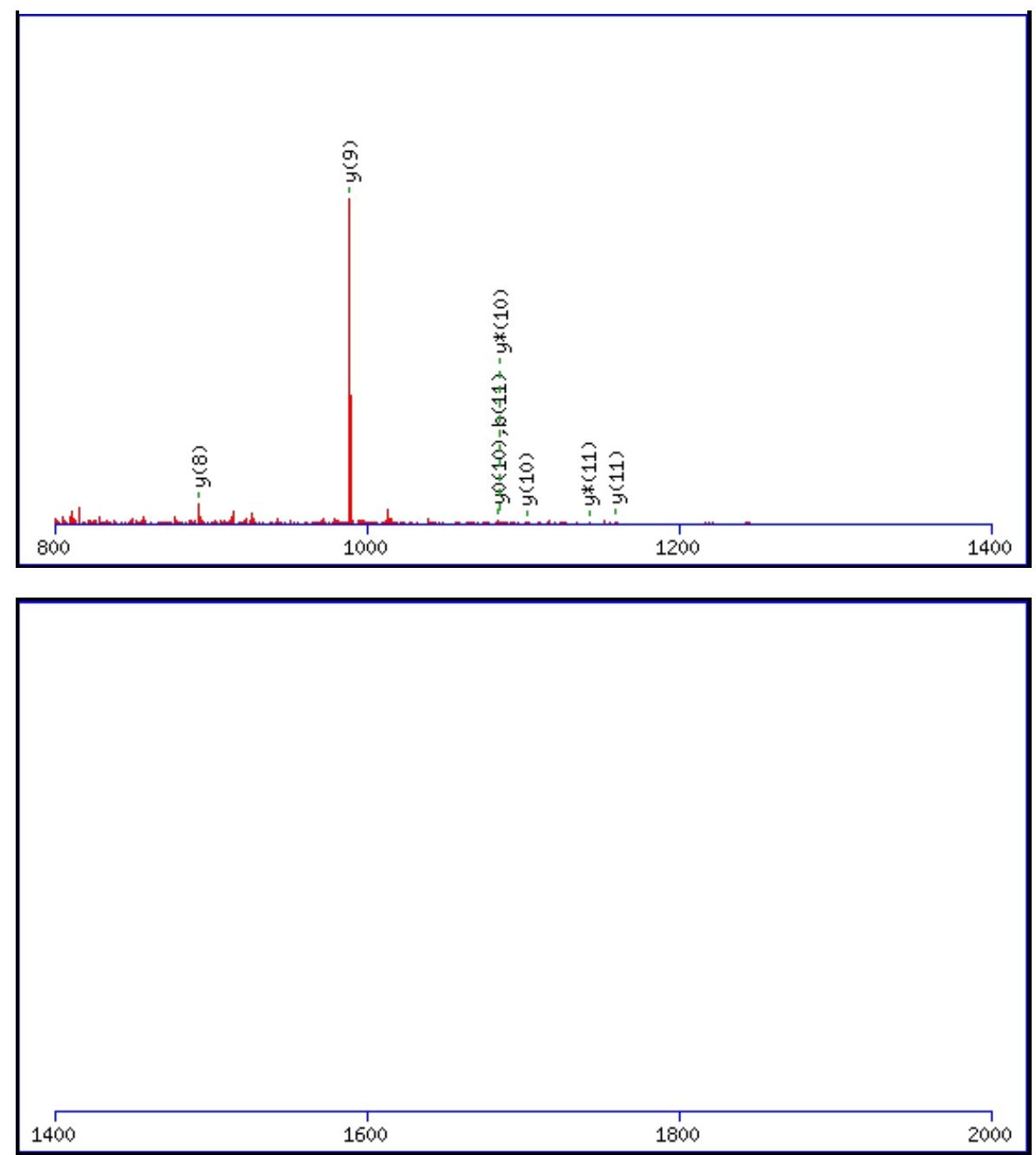

Monoisotopic mass of neutral peptide $\operatorname{Mr}($ calc): 1257.6201

Fixed modifications: Carbamidomethyl (C)

Variable modifications:

N9: Deamidated $\mathrm{N}(\mathrm{N})$

Ions Score: 48 Expect: 0.0018

Matches (Bold Red): 17/104 fragment ions using 33 most intense peaks

\begin{tabular}{|r|c|c|c|c|c|c|c|c|c|c|c|c|c|c|}
\hline$\#$ & $\mathbf{b}$ & $\mathbf{b}^{++}$ & $\mathbf{b}^{*}$ & $\mathbf{b}^{\mathbf{*}^{++}}$ & $\mathbf{b}^{\mathbf{0}}$ & $\mathbf{b}^{\mathbf{0 + +}}$ & $\mathbf{S e q}$ & $\mathbf{y}$ & $\mathbf{y}^{++}$ & $\mathbf{y}^{\mathbf{*}}$ & $\mathbf{y}^{\mathbf{*}^{++}}$ & $\mathbf{y}^{\mathbf{0}}$ & $\mathbf{y}^{\mathbf{0 + +}}$ & $\#$ \\
\hline $\mathbf{1}$ & 100.0757 & 50.5415 & & & & & $\mathbf{V}$ & & & & & & & $\mathbf{1 2}$ \\
\hline $\mathbf{2}$ & 157.0972 & 79.0522 & & & & & $\mathbf{G}$ & $\mathbf{1 1 5 9 . 5 5 9 0}$ & 580.2831 & $\mathbf{1 1 4 2 . 5 3 2 4}$ & 571.7698 & 1141.5484 & 571.2778 & $\mathbf{1 1}$ \\
\hline $\mathbf{3}$ & $\mathbf{2 7 0 . 1 8 1 2}$ & 135.5942 & & & & & $\mathbf{I}$ & $\mathbf{1 1 0 2 . 5 3 7 5}$ & 551.7724 & $\mathbf{1 0 8 5 . 5 1 0 9}$ & 543.2591 & $\mathbf{1 0 8 4 . 5 2 6 9}$ & 542.7671 & $\mathbf{1 0}$ \\
\hline $\mathbf{4}$ & 367.2340 & 184.1206 & & & & & $\mathbf{P}$ & $\mathbf{9 8 9 . 4 5 3 4}$ & $\mathbf{4 9 5 . 2 3 0 3}$ & 972.4269 & $\mathbf{4 8 6 . 7 1 7 1}$ & 971.4429 & $\mathbf{4 8 6 . 2 2 5 1}$ & $\mathbf{9}$ \\
\hline $\mathbf{5}$ & 466.3024 & 233.6548 & & & & & $\mathbf{V}$ & $\mathbf{8 9 2 . 4 0 0 7}$ & 446.7040 & 875.3741 & 438.1907 & 874.3901 & 437.6987 & $\mathbf{8}$ \\
\hline $\mathbf{6}$ & 567.3501 & 284.1787 & & & 549.3395 & 275.1734 & $\mathbf{T}$ & $\mathbf{7 9 3 . 3 3 2 2}$ & 397.1698 & 776.3057 & 388.6565 & 775.3217 & 388.1645 & $\mathbf{7}$ \\
\hline $\mathbf{7}$ & 682.3770 & 341.6921 & & & 664.3665 & 332.6869 & $\mathbf{D}$ & $\mathbf{6 9 2 . 2 8 4 6}$ & 346.6459 & 675.2580 & 338.1326 & 674.2740 & 337.6406 & $\mathbf{6}$ \\
\hline $\mathbf{8}$ & 811.4196 & 406.2134 & & & 793.4090 & 397.2082 & $\mathbf{E}$ & 577.2576 & 289.1325 & 560.2311 & 280.6192 & 559.2471 & 280.1272 & $\mathbf{5}$ \\
\hline $\mathbf{9}$ & 926.4465 & 463.7269 & 909.4200 & 455.2136 & 908.4360 & 454.7216 & $\mathbf{N}$ & $\mathbf{4 4 8 . 2 1 5 0}$ & 224.6112 & 431.1885 & 216.0979 & 430.2045 & 215.6059 & $\mathbf{4}$ \\
\hline $\mathbf{1 0}$ & 983.4680 & 492.2376 & 966.4415 & 483.7244 & 965.4574 & 483.2324 & $\mathbf{G}$ & 333.1881 & 167.0977 & 316.1615 & 158.5844 & 315.1775 & 158.0924 & $\mathbf{3}$ \\
\hline $\mathbf{1 1}$ & $\mathbf{1 0 8 4 . 5 1 5 7}$ & 542.7615 & 1067.4891 & 534.2482 & 1066.5051 & 533.7562 & $\mathbf{T}$ & 276.1666 & 138.5870 & 259.1401 & 130.0737 & 258.1561 & 129.5817 & $\mathbf{2}$ \\
\hline $\mathbf{1 2}$ & & & & & & & $\mathbf{R}$ & 175.1190 & 88.0631 & 158.0924 & 79.5498 & & & $\mathbf{1}$ \\
\hline
\end{tabular}




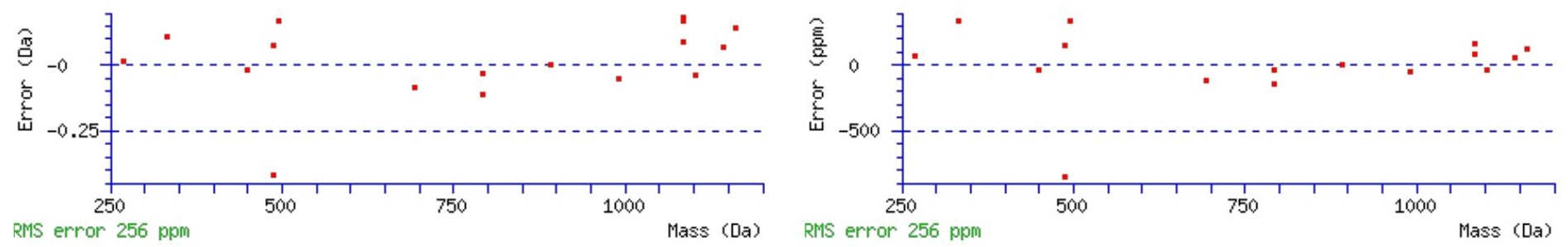

\section{All matches to this query}

\begin{tabular}{|l|l|l|l|}
\hline Score & Mr(calc): & Delta & \multicolumn{1}{|c|}{ Sequence } \\
\hline 48.2 & 1257.6201 & 0.0030 & VGIPVTDENGTR \\
\hline 8.0 & 1257.6231 & 0.0000 & VVASRQYTVR \\
\hline 6.9 & 1255.6173 & 2.0058 & KTGKTLQNISS \\
\hline 3.9 & 1255.6044 & 2.0187 & HLNLSSNNLDK \\
\hline 2.9 & 1257.6231 & 0.0000 & VVASRQYTVR \\
\hline 2.9 & 1257.6231 & 0.0000 & VVASRQYTVR \\
\hline 2.7 & 1256.6139 & 1.0092 & GGPGRSHVAALR \\
\hline 2.5 & 1257.6343 & -0.0112 & LRRTNYSLR \\
\hline 2.5 & 1257.6343 & -0.0112 & LRRTNYSLR \\
\hline 2.5 & 1257.6343 & -0.0112 & LRRTNYSLR \\
\hline
\end{tabular}

Spectrum No: 703; Query: 1980; Rank: 1

\section{Peptide View}

MS/MS Fragmentation of NASLAHVPDPYTQAFLTQAAR

Found in IPI00192504, Tax_Id=10116 Gene_Symbol=Mrc2 Macrophage mannose receptor 2 precursor

Match to Query 1980: 2271.126942 from(758.049590,3+)

Title: 091008RatKidney_NH4Format01_23.3207.3207.3.dta

Data file K:INewmanPaper|Piliang|3SubProteomes\Piliang3SP\mgf5ppm\ERLIC_3SubProteomes5ppm.mgf

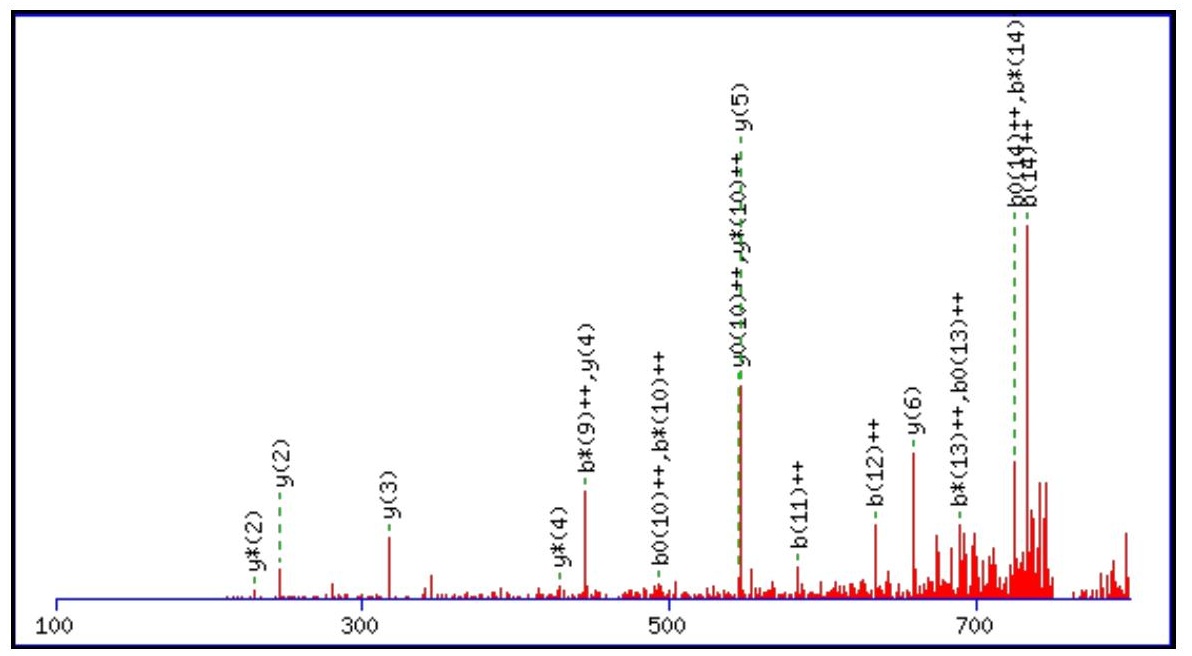



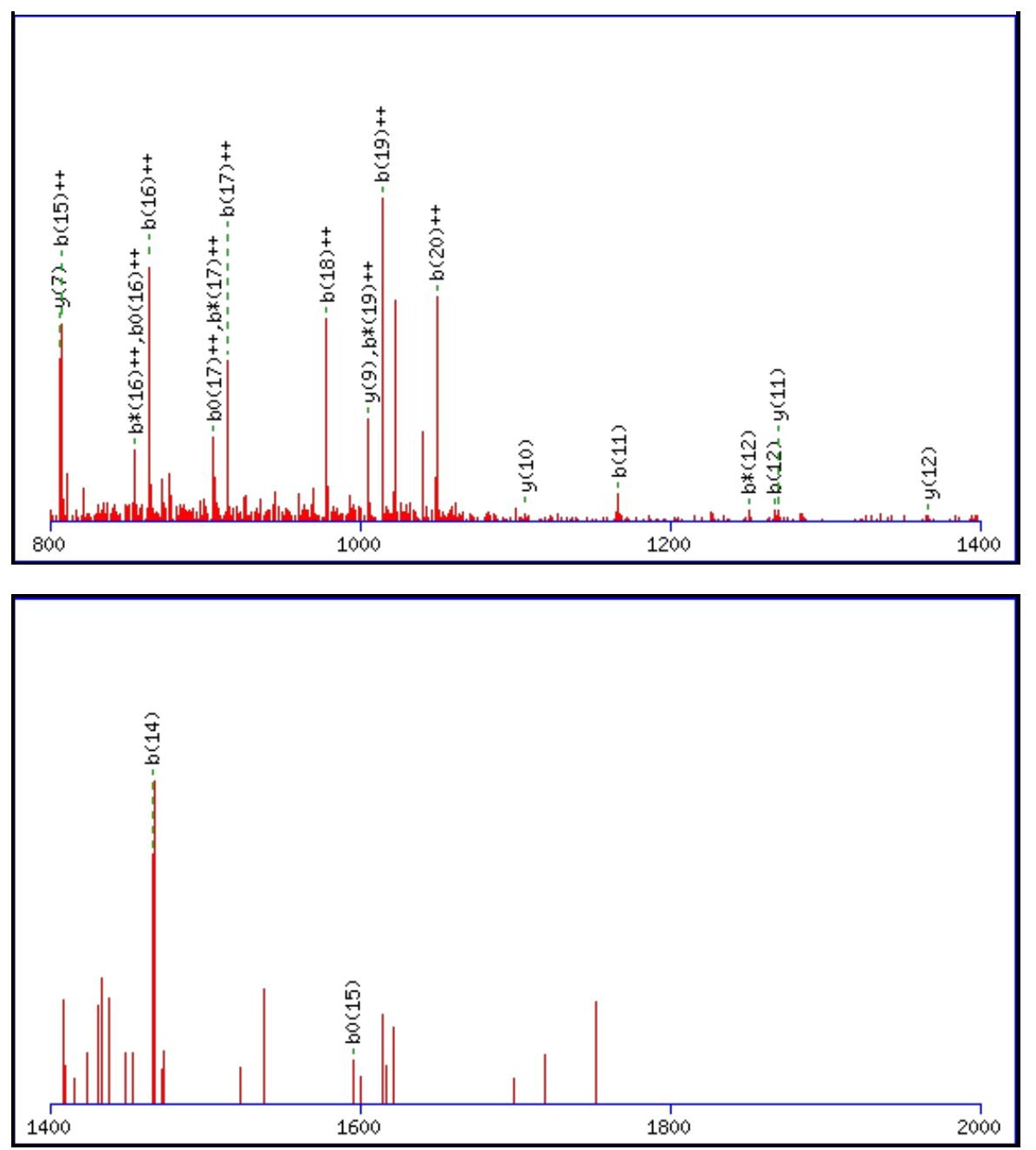

Monoisotopic mass of neutral peptide $\operatorname{Mr}($ calc): 2271.1284

Fixed modifications: Carbamidomethyl (C)

Variable modifications:

N1 : Deamidated $\mathrm{N}(\mathrm{N})$

Ions Score: 48 Expect: $\odot .0032$

Matches (Bold Red): 40/228 fragment ions using 68 most intense peaks

\begin{tabular}{|c|c|c|c|c|c|c|c|c|c|c|c|c|c|c|}
\hline \# & b & $\mathbf{b}^{++}$ & b* & $\mathbf{b}^{*^{++}}$ & $\mathbf{b}^{\mathbf{0}}$ & $\mathbf{b}^{\mathbf{0 + +}}$ & Seq. & $\mathbf{y}$ & $\mathbf{y}^{++}$ & $\mathbf{y}^{*}$ & $\mathbf{y}^{*^{++}}$ & $y^{0}$ & $\mathbf{y}^{0++}$ & \# \\
\hline 1 & 116.0342 & 58.5207 & 99.0077 & 50.0075 & & & $\mathbf{N}$ & & & & & & & 21 \\
\hline 2 & 187.0713 & 94.0393 & 170.0448 & 85.5260 & & & A & 2157.1087 & 1079.0580 & 2140.0822 & 1070.5447 & 2139.0982 & 1070.0527 & 20 \\
\hline 3 & 274.1034 & 137.5553 & 257.0768 & 129.0420 & 256.0928 & 128.5500 & $\mathrm{~S}$ & 2086.0716 & 1043.5394 & 2069.0451 & 1035.0262 & 2068.0611 & 1034.5342 & 19 \\
\hline 4 & 387.1874 & 194.0973 & 370.1609 & 185.5841 & 369.1769 & 185.0921 & $\mathbf{L}$ & 1999.0396 & 1000.0234 & 1982.0130 & 991.5102 & 1981.0290 & 991.0182 & 18 \\
\hline 5 & 458.2245 & 229.6159 & 441.1980 & 221.1026 & 440.2140 & 220.6106 & A & 1885.9555 & 943.4814 & 1868.9290 & 934.9681 & 1867.9450 & 934.4761 & 17 \\
\hline 6 & 595.2834 & 298.1454 & 578.2569 & 289.6321 & 577.2729 & 289.1401 & $\mathbf{H}$ & 1814.9184 & 907.9628 & 1797.8919 & 899.4496 & 1796.9078 & 898.9576 & 16 \\
\hline 7 & 694.3519 & 347.6796 & 677.3253 & 339.1663 & 676.3413 & 338.6743 & $\mathbf{V}$ & 1677.8595 & 839.4334 & 1660.8330 & 830.9201 & 1659.8489 & 830.4281 & 15 \\
\hline 8 & 791.4046 & 396.2059 & 774.3781 & 387.6927 & 773.3941 & 387.2007 & $\mathbf{P}$ & 1578.7911 & 789.8992 & 1561.7645 & 781.3859 & 1560.7805 & 780.8939 & 14 \\
\hline 9 & 906.4316 & 453.7194 & 889.4050 & 445.2061 & 888.4210 & 444.7141 & D & 1481.7383 & 741.3728 & 1464.7118 & 732.8595 & 1463.7278 & 732.3675 & 13 \\
\hline 10 & 1003.4843 & 502.2458 & 986.4578 & 493.7325 & 985.4738 & 493.2405 & $\mathbf{P}$ & 1366.7114 & 683.8593 & 1349.6848 & 675.3461 & 1348.7008 & 674.8540 & 12 \\
\hline 11 & 1166.5477 & 583.7775 & 1149.5211 & 575.2642 & 1148.5371 & 574.7722 & $\mathbf{Y}$ & 1269.6586 & 635.3329 & 1252.6321 & 626.8197 & 1251.6481 & 626.3277 & 11 \\
\hline 12 & 1267.5953 & 634.3013 & 1250.5688 & 625.7880 & 1249.5848 & 625.2960 & $\mathbf{T}$ & 1106.5953 & 553.8013 & 1089.5687 & 545.2880 & 1088.5847 & 544.7960 & 10 \\
\hline 13 & 1395.6539 & 698.3306 & 1378.6274 & 689.8173 & 1377.6434 & 689.3253 & $\mathbf{Q}$ & 1005.5476 & 503.2774 & 988.5211 & 494.7642 & 987.5370 & 494.2722 & 9 \\
\hline 14 & 1466.6910 & 733.8492 & 1449.6645 & 725.3359 & 1448.6805 & 724.8439 & A & 877.4890 & 439.2482 & 860.4625 & 430.7349 & 859.4785 & 430.2429 & 8 \\
\hline 15 & 1613.7594 & 807.3834 & 1596.7329 & 798.8701 & 1595.7489 & 798.3781 & $\mathbf{F}$ & 806.4519 & 403.7296 & 789.4254 & 395.2163 & 788.4414 & 394.7243 & 7 \\
\hline 16 & 1726.8435 & 863.9254 & 1709.8170 & 855.4121 & 1708.8329 & 854.9201 & $\mathbf{L}$ & 659.3835 & 330.1954 & 642.3570 & 321.6821 & 641.3729 & 321.1901 & 6 \\
\hline 17 & 1827.8912 & 914.4492 & 1810.8646 & 905.9360 & 1809.8806 & 905.4439 & $\mathbf{T}$ & 546.2994 & 273.6534 & 529.2729 & 265.1401 & 528.2889 & 264.6481 & 5 \\
\hline 18 & 1955.9498 & 978.4785 & 1938.9232 & 969.9652 & 1937.9392 & 969.4732 & $\mathbf{Q}$ & 445.2518 & 223.1295 & 428.2252 & 214.6162 & & & 4 \\
\hline
\end{tabular}




\begin{tabular}{|l|r|r|r|r|r|r|r|r|r|r|r|r|r|r|r|r|}
$\mathbf{1 9}$ & 2026.9869 & $\mathbf{1 0 1 3 . 9 9 7 1}$ & 2009.9603 & $\mathbf{1 0 0 5 . 4 8 3 8}$ & 2008.9763 & 1004.9918 & A & 317.1932 & 159.1002 & 300.1666 & 150.5870 & & & $\mathbf{3}$ \\
\hline $\mathbf{2 0}$ & 2098.0240 & $\mathbf{1 0 4 9 . 5 1 5 6}$ & 2080.9974 & 1041.0024 & 2080.0134 & 1040.5104 & A & $\mathbf{2 4 6 . 1 5 6 1}$ & 123.5817 & $\mathbf{2 2 9 . 1 2 9 5}$ & 115.0684 & & & $\mathbf{2}$ \\
\hline $\mathbf{2 1}$ & & & & & & & R & 175.1190 & 88.0631 & 158.0924 & 79.5498 & & & $\mathbf{1}$ \\
\hline
\end{tabular}
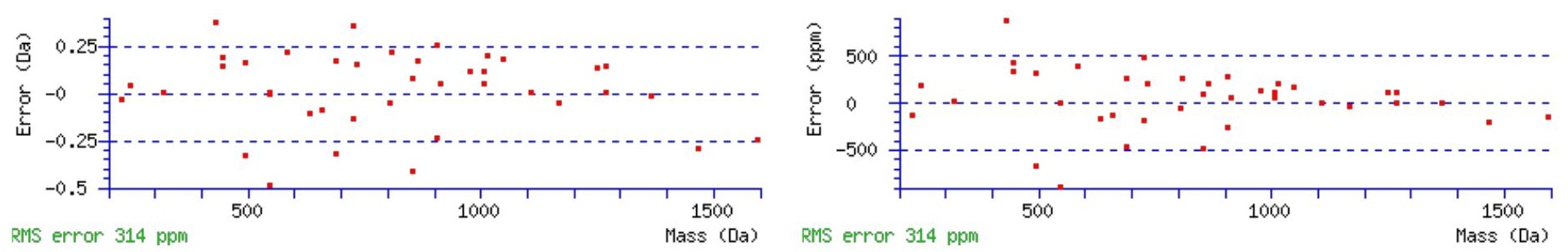

\section{All matches to this query}

\begin{tabular}{|l|l|l|l|}
\hline Score & Mr(calc): & Delta & \multicolumn{1}{|c|}{ Sequence } \\
\hline 48.1 & 2271.1284 & -0.0014 & NASLAHVPDPYTQAFLTQAAR \\
\hline 17.3 & 2270.1444 & 0.9826 & NASLAHVPDPYTQAFLTQAAR \\
\hline 8.1 & 2269.1177 & 2.0092 & DKKSYTPLHAAASSGMISVVK \\
\hline 8.1 & 2269.1177 & 2.0092 & DKKSYTPLHAAASSGMISVVK \\
\hline 8.1 & 2269.1177 & 2.0092 & DKKSYTPLHAAASSGMISVVK \\
\hline 6.8 & 2271.1177 & 0.0092 & NLYQLIRVATITIQAHTR \\
\hline 5.8 & 2269.1372 & 1.9897 & SIGAITNTNYGVCLDSTRSK \\
\hline 5.1 & 2271.1158 & 0.0111 & SFKEFLLSLDDSVDETEAVK \\
\hline 2.6 & 2269.1386 & 1.9883 & WGRVGKTGQHSLVACSGDLNK \\
\hline 2.5 & 2270.1235 & 1.0034 & QSSSSSLSYIFTVFAKTPIK \\
\hline
\end{tabular}

Spectrum No: 704; Query: 1952; Rank: 1

\section{Peptide View}

MS/MS Fragmentation of FNATSVNVGSGYFPEHGYFR

Found in IPI00358043, Tax_Id=10116 Gene_Symbol=Mmrn2_predicted multimerin 2

Match to Query 1952: 2249.023812 from(750.681880,3+)

Title: 100101RatKid_NS_deglyco_23.3851.3851.3.dta

Data file K:INewmanPaper|Piliangl3SubProteomes\Piliang3SP\mgf5ppm\ERLIC_3SubProteomes5ppm.mgf

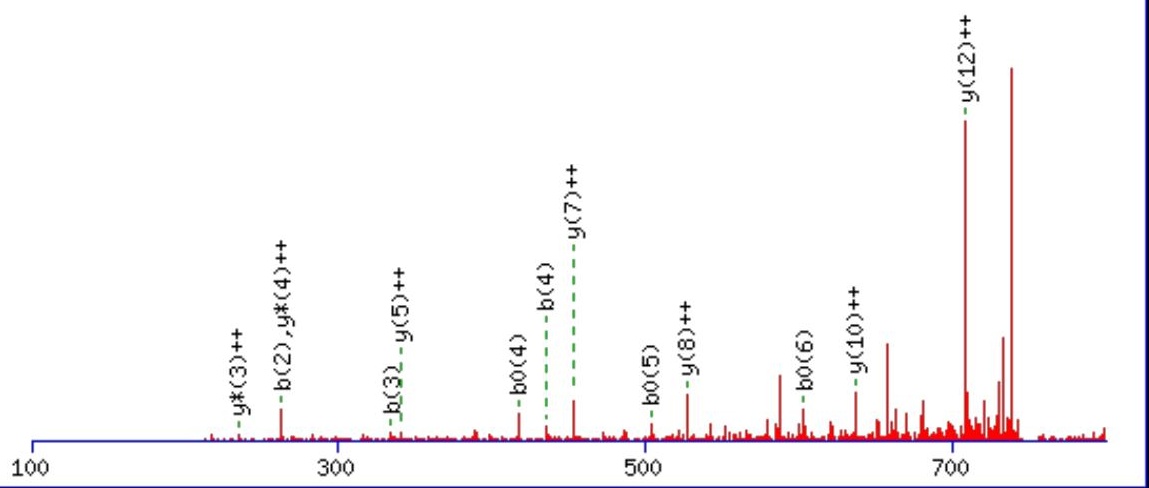



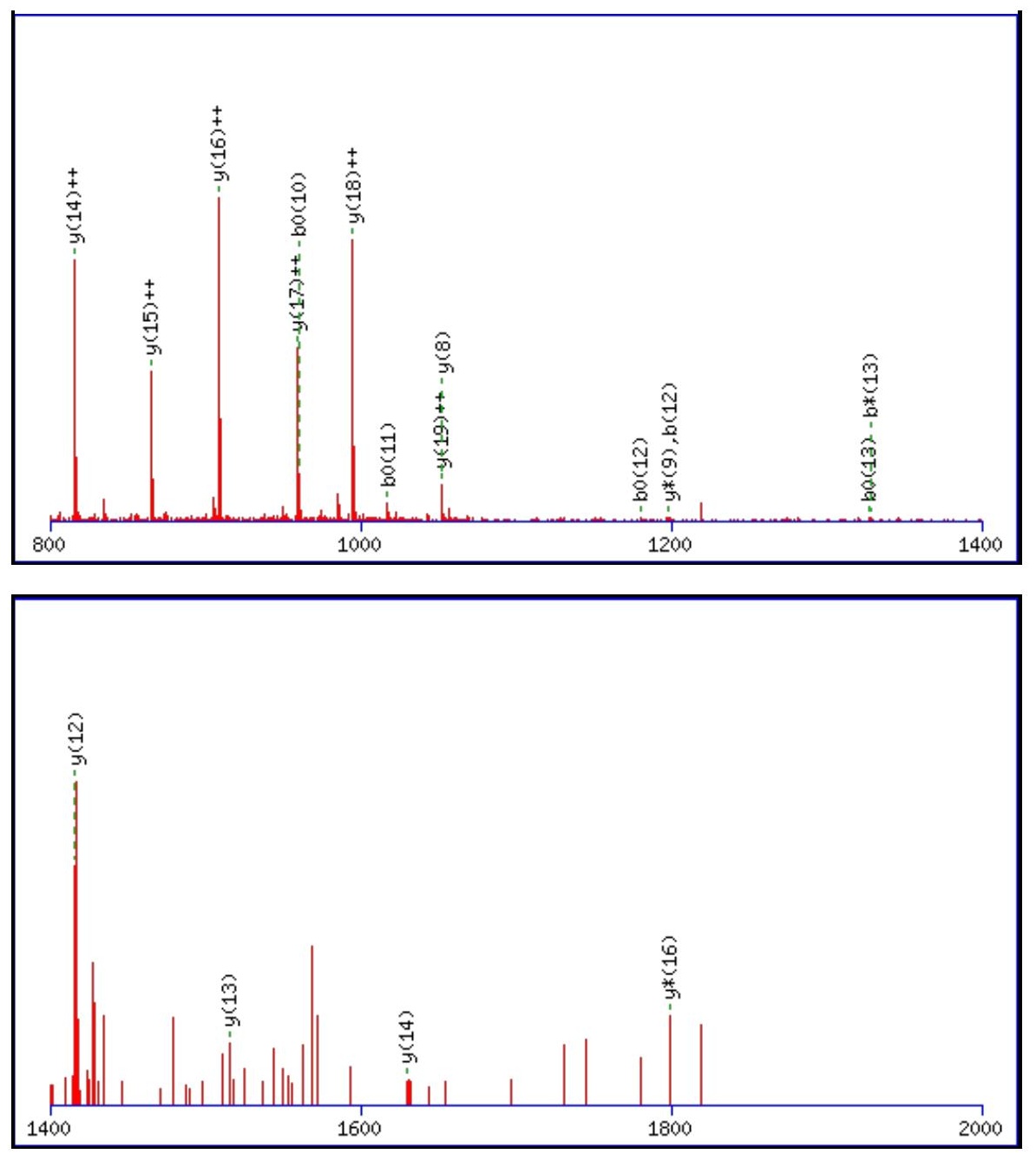

Monoisotopic mass of neutral peptide $\operatorname{Mr}($ calc): 2249.0178

Fixed modifications: Carbamidomethyl (C)

Variable modifications:

N2 : Deamidated $\mathrm{N}(\mathrm{N})$

Ions Score: 48 Expect: 0.005

Matches (Bold Red): 31/210 fragment ions using 69 most intense peaks

\begin{tabular}{|c|c|c|c|c|c|c|c|c|c|c|c|c|c|c|}
\hline \# & b & $\mathbf{b}^{++}$ & $\mathbf{b}^{*}$ & $\mathbf{b}^{*^{++}}$ & $\mathbf{b}^{\mathbf{0}}$ & $\mathbf{b}^{0++}$ & Seq. & $\mathbf{y}$ & $\mathbf{y}^{++}$ & $\mathbf{y}^{*}$ & $\mathrm{y}^{\mathrm{*}^{++}}$ & $\mathbf{y}^{\mathbf{0}}$ & $y^{0++}$ & \# \\
\hline 1 & 148.0757 & 74.5415 & & & & & $\mathbf{F}$ & & & & & & & 20 \\
\hline 2 & 263.1026 & 132.0550 & 246.0761 & 123.5417 & & & $\mathbf{N}$ & 2102.9566 & 1051.9820 & 2085.9301 & 1043.4687 & 2084.9461 & 1042.9767 & 19 \\
\hline 3 & 334.1397 & 167.5735 & 317.1132 & 159.0602 & & & A & 1987.9297 & 994.4685 & 1970.9032 & 985.9552 & 1969.9191 & 985.4632 & 18 \\
\hline 4 & 435.1874 & 218.0973 & 418.1609 & 209.5841 & 417.1769 & 209.0921 & $\mathbf{T}$ & 1916.8926 & 958.9499 & 1899.8660 & 950.4367 & 1898.8820 & 949.9447 & 17 \\
\hline 5 & 522.2194 & 261.6134 & 505.1929 & 253.1001 & 504.2089 & 252.6081 & $S$ & 1815.8449 & 908.4261 & 1798.8184 & 899.9128 & 1797.8343 & 899.4208 & 16 \\
\hline 6 & 621.2879 & 311.1476 & 604.2613 & 302.6343 & 603.2773 & 302.1423 & V & 1728.8129 & 864.9101 & 1711.7863 & 856.3968 & 1710.8023 & 855.9048 & 15 \\
\hline 7 & 735.3308 & 368.1690 & 718.3042 & 359.6558 & 717.3202 & 359.1637 & $\mathbf{N}$ & 1629.7445 & 815.3759 & 1612.7179 & 806.8626 & 1611.7339 & 806.3706 & 14 \\
\hline 8 & 834.3992 & 417.7032 & 817.3727 & 409.1900 & 816.3886 & 408.6980 & V & 1515.7015 & 758.3544 & 1498.6750 & 749.8411 & 1497.6910 & 749.3491 & 13 \\
\hline 9 & 891.4207 & 446.2140 & 874.3941 & 437.7007 & 873.4101 & 437.2087 & G & 1416.6331 & 708.8202 & 1399.6066 & 700.3069 & 1398.6226 & 699.8149 & 12 \\
\hline 10 & 978.4527 & 489.7300 & 961.4261 & 481.2167 & 960.4421 & 480.7247 & $S$ & 1359.6117 & 680.3095 & 1342.5851 & 671.7962 & 1341.6011 & 671.3042 & 11 \\
\hline 11 & 1035.4742 & 518.2407 & 1018.4476 & 509.7274 & 1017.4636 & 509.2354 & G & 1272.5796 & 636.7935 & 1255.5531 & 628.2802 & 1254.5691 & 627.7882 & 10 \\
\hline 12 & 1198.5375 & 599.7724 & 1181.5109 & 591.2591 & 1180.5269 & 590.7671 & $\mathbf{Y}$ & 1215.5582 & 608.2827 & 1198.5316 & 599.7694 & 1197.5476 & 599.2774 & 9 \\
\hline 13 & 1345.6059 & 673.3066 & 1328.5794 & 664.7933 & 1327.5953 & 664.3013 & $\mathbf{F}$ & 1052.4948 & 526.7511 & 1035.4683 & 518.2378 & 1034.4843 & 517.7458 & 8 \\
\hline 14 & 1442.6587 & 721.8330 & 1425.6321 & 713.3197 & 1424.6481 & 712.8277 & $\mathbf{P}$ & 905.4264 & 453.2169 & 888.3999 & 444.7036 & 887.4159 & 444.2116 & 7 \\
\hline 15 & 1571.7013 & 786.3543 & 1554.6747 & 777.8410 & 1553.6907 & 777.3490 & $\mathbf{E}$ & 808.3737 & 404.6905 & 791.3471 & 396.1772 & 790.3631 & 395.6852 & 6 \\
\hline 16 & 1708.7602 & 854.8837 & 1691.7336 & 846.3704 & 1690.7496 & 845.8784 & $\mathbf{H}$ & 679.3311 & 340.1692 & 662.3045 & 331.6559 & & & 5 \\
\hline 17 & 1765.7816 & 883.3945 & 1748.7551 & 874.8812 & 1747.7711 & 874.3892 & G & 542.2722 & 271.6397 & 525.2456 & 263.1264 & & & 4 \\
\hline 18 & 1928.8450 & 964.9261 & 1911.8184 & 956.4128 & 1910.8344 & 955.9208 & $\mathbf{Y}$ & 485.2507 & 243.1290 & 468.2241 & 234.6157 & & & 3 \\
\hline
\end{tabular}




\begin{tabular}{|r|r|r|r|r|r|r|r|r|r|r|r|r|r|r|r|}
$\mathbf{1 9}$ & 2075.9134 & 1038.4603 & 2058.8868 & 1029.9471 & 2057.9028 & 1029.4550 & $\mathbf{F}$ & 322.1874 & 161.5973 & 305.1608 & 153.0840 & & & $\mathbf{2}$ \\
\hline $\mathbf{2 0}$ & & & & & & & $\mathbf{R}$ & 175.1190 & 88.0631 & 158.0924 & 79.5498 & & & $\mathbf{1}$ \\
\hline
\end{tabular}
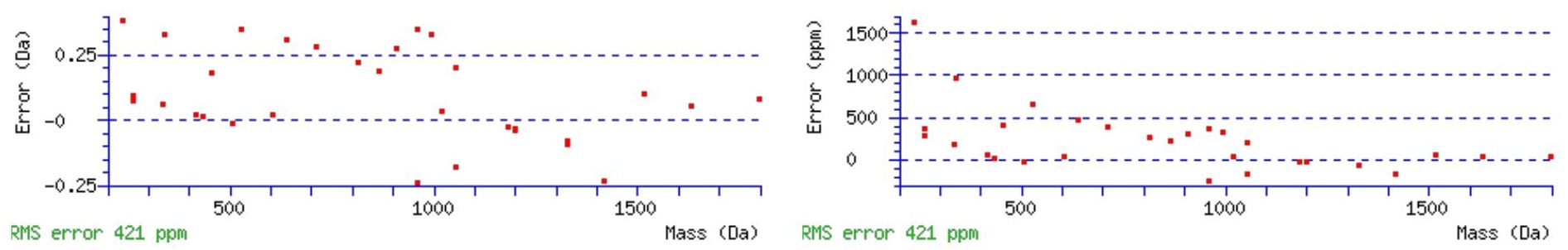

\section{All matches to this query}

\begin{tabular}{|l|l|l|l|}
\hline Score & Mr(calc): & Delta & \multicolumn{1}{|c|}{ Sequence } \\
\hline 48.1 & 2249.0178 & 0.0060 & FNATSVNVGSGYFPEHGYFR \\
\hline 36.3 & 2249.0178 & 0.0060 & FNATSVNVGSGYFPEHGYFR \\
\hline 35.3 & 2248.0338 & 0.9900 & FNATSVNVGSGYFPEHGYFR \\
\hline 5.6 & 2248.0057 & 1.0181 & YTAVAMPMLYNTRYSSKR \\
\hline 5.6 & 2248.0057 & 1.0181 & YTAVAMPMLYNTRYSSKR \\
\hline 4.5 & 2247.0274 & 1.9964 & YVAICLPLYYTRIMSPK \\
\hline 4.1 & 2248.0214 & 1.0024 & FDSMVMPVEMEAFEALKSR \\
\hline 4.1 & 2248.0214 & 1.0024 & FDSMVMPVEMEAFEALKSR \\
\hline 3.3 & 2247.0078 & 2.0160 & FREMTADQGLYMLARHGR \\
\hline 2.5 & 2248.0057 & 1.0181 & YTAVAMPMLYNTRYSSKR \\
\hline
\end{tabular}

Spectrum No: 705; Query: 1646; Rank: 1

\section{Peptide View}

MS/MS Fragmentation of ANQMVIPHNTTFQTEPAK

Found in IPI00324585, Tax_Id=10116 Gene_Symbol=Itga1 Integrin alpha-1 precursor

Match to Query 1646: 2042.967972 from(681.996600,3+)

Title: 091008RatKidney_NH4Format01_23.1296.1296.3.dta

Data file K:INewmanPaper|Piliang \3SubProteomes\Piliang3SP\mgf5ppm\ERLIC_3SubProteomes5ppm.mgf

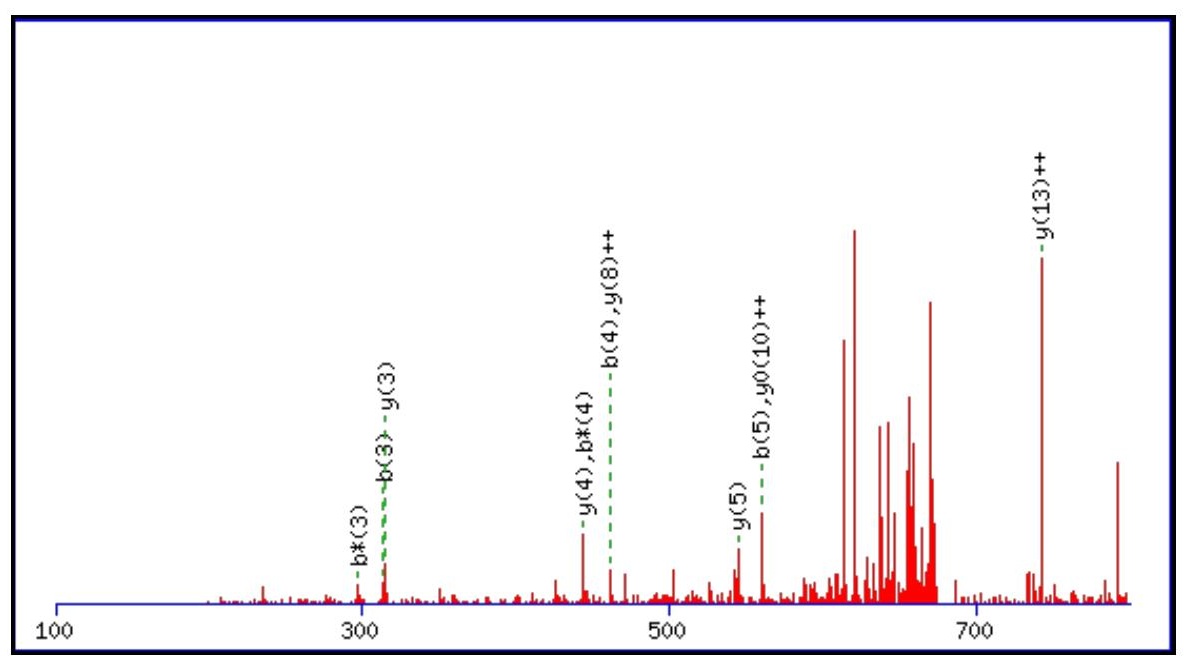



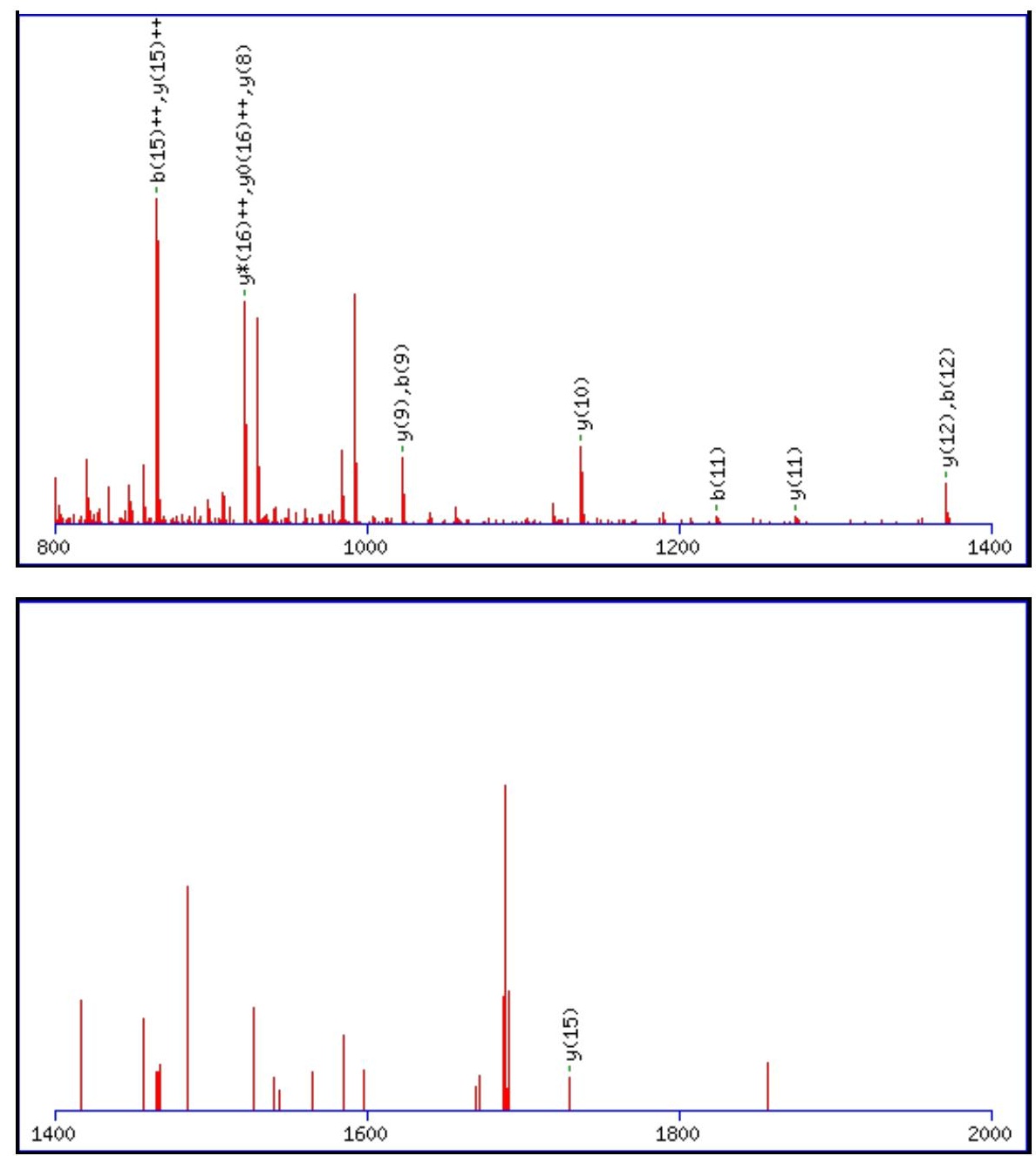

Monoisotopic mass of neutral peptide $\operatorname{Mr}($ calc): 2042.9731

Fixed modifications: Carbamidomethyl (C)

Variable modifications:

M4 : Oxidation (M)

N9 : Deamidated_N (N)

Ions Score: 48 Expect: $\odot .0038$

Matches (Bold Red): 24/178 fragment ions using 37 most intense peaks

\begin{tabular}{|c|c|c|c|c|c|c|c|c|c|c|c|c|c|c|}
\hline \# & b & $\mathbf{b}^{++}$ & $\mathbf{b}^{*}$ & $\mathbf{b}^{*^{++}}$ & $\mathbf{b}^{\mathbf{0}}$ & $\mathbf{b}^{\mathbf{0 + +}}$ & Seq. & $\mathbf{y}$ & $\mathbf{y}^{++}$ & $\mathbf{y}^{*}$ & $\mathbf{y}^{*^{++}}$ & $\mathbf{y}^{0}$ & $\mathbf{y}^{0++}$ & \# \\
\hline 1 & 72.0444 & 36.5258 & & & & & A & & & & & & & 18 \\
\hline 2 & 186.0873 & 93.5473 & 169.0608 & 85.0340 & & & $\mathbf{N}$ & 1972.9433 & 986.9753 & 1955.9168 & 978.4620 & 1954.9327 & 977.9700 & 17 \\
\hline 3 & 314.1459 & 157.5766 & 297.1193 & 149.0633 & & & $\mathbf{Q}$ & 1858.9004 & 929.9538 & 1841.8738 & 921.4406 & 1840.8898 & 920.9485 & 16 \\
\hline 4 & 461.1813 & 231.0943 & 444.1547 & 222.5810 & & & $\mathbf{M}$ & 1730.8418 & 865.9245 & 1713.8152 & 857.4113 & 1712.8312 & 856.9193 & 15 \\
\hline 5 & 560.2497 & 280.6285 & 543.2232 & 272.1152 & & & $\mathbf{V}$ & 1583.8064 & 792.4068 & 1566.7798 & 783.8936 & 1565.7958 & 783.4016 & 14 \\
\hline 6 & 673.3338 & 337.1705 & 656.3072 & 328.6573 & & & I & 1484.7380 & 742.8726 & 1467.7114 & 734.3594 & 1466.7274 & 733.8673 & 13 \\
\hline 7 & 770.3865 & 385.6969 & 753.3600 & 377.1836 & & & $\mathbf{P}$ & 1371.6539 & 686.3306 & 1354.6274 & 677.8173 & 1353.6434 & 677.3253 & 12 \\
\hline 8 & 907.4455 & 454.2264 & 890.4189 & 445.7131 & & & $\mathbf{H}$ & 1274.6012 & 637.8042 & 1257.5746 & 629.2909 & 1256.5906 & 628.7989 & 11 \\
\hline 9 & 1022.4724 & 511.7398 & 1005.4458 & 503.2266 & & & $\mathbf{N}$ & 1137.5422 & 569.2748 & 1120.5157 & 560.7615 & 1119.5317 & 560.2695 & 10 \\
\hline 10 & 1123.5201 & 562.2637 & 1106.4935 & 553.7504 & 1105.5095 & 553.2584 & $\mathbf{T}$ & 1022.5153 & 511.7613 & 1005.4888 & 503.2480 & 1004.5047 & 502.7560 & 9 \\
\hline 11 & 1224.5677 & 612.7875 & 1207.5412 & 604.2742 & 1206.5572 & 603.7822 & $\mathbf{T}$ & 921.4676 & 461.2375 & 904.4411 & 452.7242 & 903.4571 & 452.2322 & 8 \\
\hline 12 & 1371.6362 & 686.3217 & 1354.6096 & 677.8084 & 1353.6256 & 677.3164 & $\mathbf{F}$ & 820.4199 & 410.7136 & 803.3934 & 402.2003 & 802.4094 & 401.7083 & 7 \\
\hline 13 & 1499.6947 & 750.3510 & 1482.6682 & 741.8377 & 1481.6842 & 741.3457 & $\mathbf{Q}$ & 673.3515 & 337.1794 & 656.3250 & 328.6661 & 655.3410 & 328.1741 & 6 \\
\hline 14 & 1600.7424 & 800.8748 & 1583.7159 & 792.3616 & 1582.7319 & 791.8696 & $\mathbf{T}$ & 545.2930 & 273.1501 & 528.2664 & 264.6368 & 527.2824 & 264.1448 & 5 \\
\hline 15 & 1729.7850 & 865.3961 & 1712.7585 & 856.8829 & 1711.7744 & 856.3909 & $\mathbf{E}$ & 444.2453 & 222.6263 & 427.2187 & 214.1130 & 426.2347 & 213.6210 & 4 \\
\hline 16 & 1826.8378 & 913.9225 & 1809.8112 & 905.4093 & 1808.8272 & 904.9172 & $\mathbf{P}$ & 315.2027 & 158.1050 & 298.1761 & 149.5917 & & & 3 \\
\hline 17 & 1897.8749 & 949.4411 & 1880.8483 & 940.9278 & 1879.8643 & 940.4358 & A & 218.1499 & 109.5786 & 201.1234 & 101.0653 & & & 2 \\
\hline 18 & & & & & & & $\mathbf{K}$ & 147.1128 & 74.0600 & 130.0863 & 65.5468 & & & 1 \\
\hline
\end{tabular}



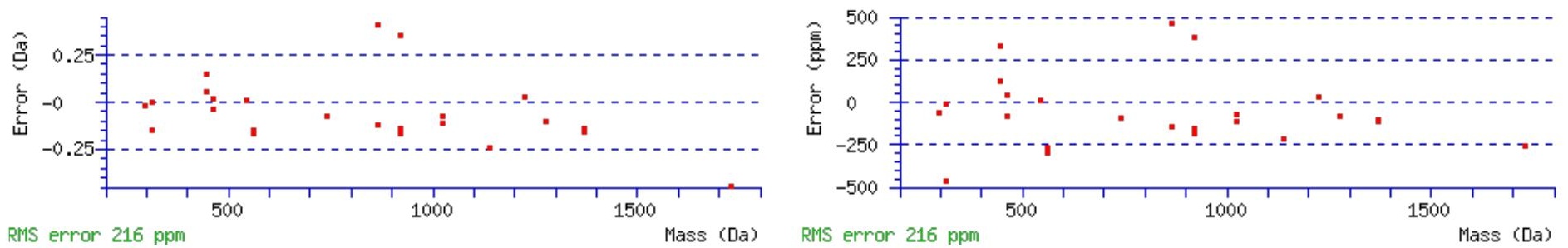

\section{All matches to this query}

\begin{tabular}{|l|l|l|l|}
\hline Score & Mr(calc): & Delta & \multicolumn{1}{c|}{ Sequence } \\
\hline 48.1 & 2042.9731 & -0.0052 & ANQMVIPHNTTFQTEPAK \\
\hline 12.6 & 2042.9731 & -0.0052 & ANQMVIPHNTTFQTEPAK \\
\hline 1.5 & 2040.9412 & 2.0268 & SGSLVTAGRIDREGLCDR \\
\hline 1.3 & 2040.9547 & 2.0132 & LSPFSTPVNVTSLARHR \\
\hline 1.3 & 2040.9547 & 2.0132 & LSPFSTPVNVTSLARHR \\
\hline 1.3 & 2040.9547 & 2.0132 & LSPFSTPVNVTSLARHR \\
\hline 1.3 & 2040.9547 & 2.0132 & LSPFSTPVNVTSLARHR \\
\hline 1.3 & 2041.9577 & 1.0102 & VLQCLDITVLSEERCK \\
\hline 0.8 & 2040.9412 & 2.0268 & SGSLVTAGRIDREGLCDR \\
\hline 0.3 & 2040.9799 & 1.9881 & LSYLLPSARPELGVGPGR \\
\hline
\end{tabular}

Spectrum No: 706; Query: 1402; Rank: 1

\section{Peptide View}

MS/MS Fragmentation of AQGAGFTNATTTDQHGLAK

Found in IPI00368704, Tax_Id=10116 Gene_Symbol=RGD1566313_predicted similar to Murinoglobulin 1 homolog

Match to Query 1402: 1888.893582 from(630.638470,3+)

Title: 100101RatKid_NS_deglyco_24.1101.1101.3.dta

Data file K:INewmanPaper|Piliang|3SubProteomes\Piliang3SP\mgf5ppm\ERLIC_3SubProteomes5ppm.mgf

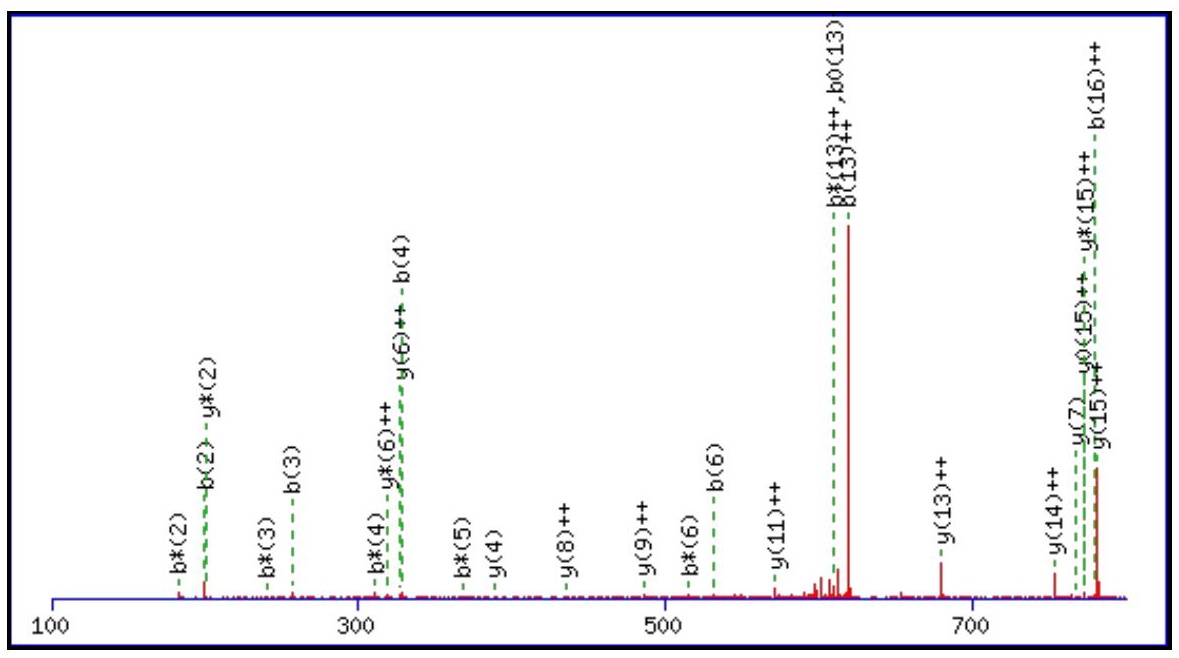



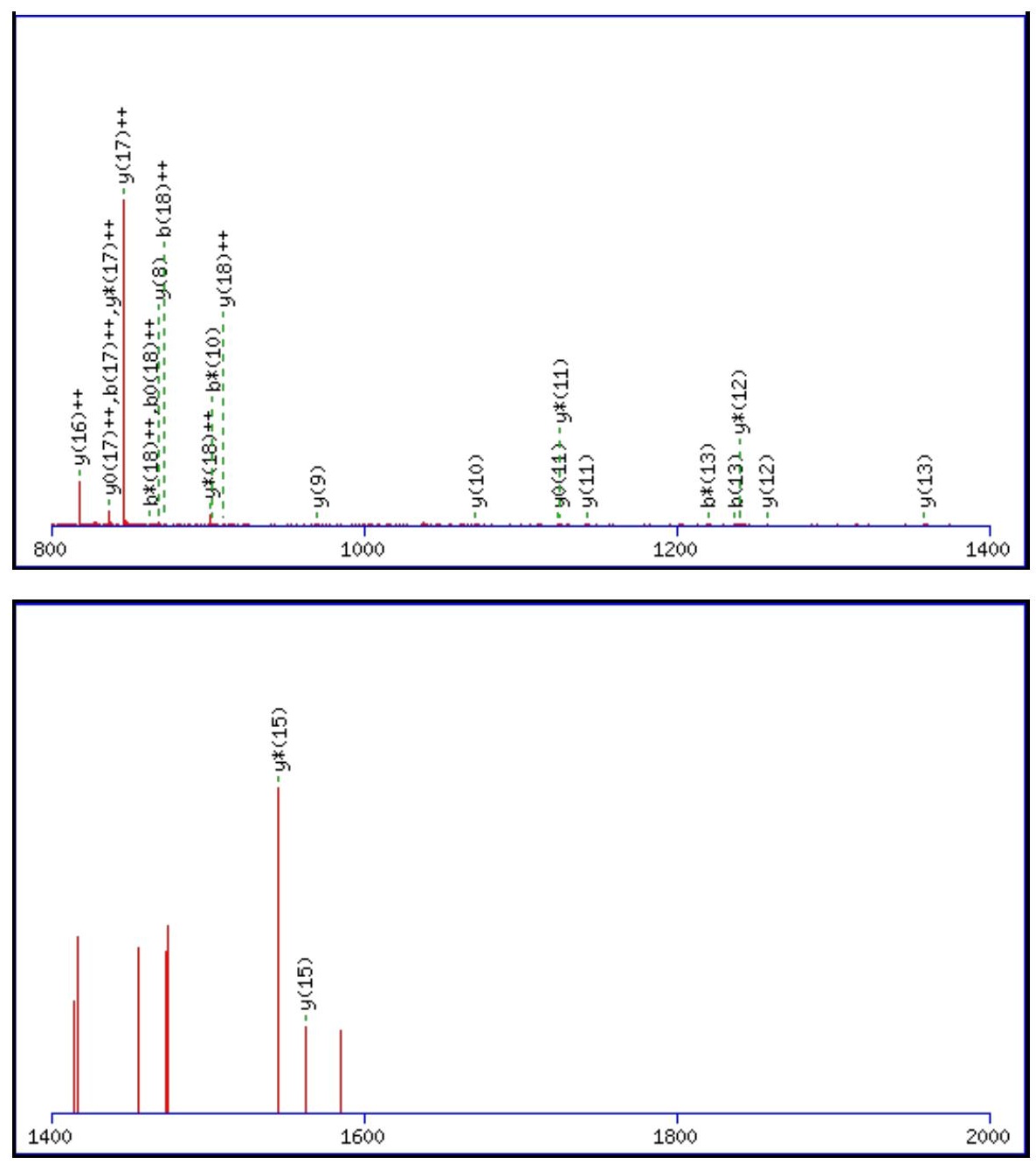

Monoisotopic mass of neutral peptide $\operatorname{Mr}($ calc): 1888.8915

Fixed modifications: Carbamidomethyl (C)

Variable modifications:

N8: Deamidated $\mathrm{N}(\mathrm{N})$

Ions Score: 48 Expect: 0.0039

Matches (Bold Red): 50/190 fragment ions using 105 most intense peaks

\begin{tabular}{|c|c|c|c|c|c|c|c|c|c|c|c|c|c|c|}
\hline \# & b & $\mathbf{b}^{++}$ & b* & $\mathbf{b}^{*^{++}}$ & $\mathbf{b}^{\mathbf{0}}$ & $\mathbf{b}^{0++}$ & Seq. & $\mathbf{y}$ & $\mathbf{y}^{++}$ & $\mathbf{y}^{*}$ & $\mathrm{y}^{*^{++}}$ & $\mathbf{y}^{0}$ & $\mathbf{y}^{0++}$ & \# \\
\hline 1 & 72.0444 & 36.5258 & & & & & A & & & & & & & 19 \\
\hline 2 & 200.1030 & 100.5551 & 183.0764 & 92.0418 & & & $\mathbf{Q}$ & 1818.8617 & 909.9345 & 1801.8351 & 901.4212 & 1800.8511 & 900.9292 & 18 \\
\hline 3 & 257.1244 & 129.0659 & 240.0979 & 120.5526 & & & $\mathbf{G}$ & 1690.8031 & 845.9052 & 1673.7766 & 837.3919 & 1672.7925 & 836.8999 & 17 \\
\hline 4 & 328.1615 & 164.5844 & 311.1350 & 156.0711 & & & A & 1633.7816 & 817.3945 & 1616.7551 & 808.8812 & 1615.7711 & 808.3892 & 16 \\
\hline 5 & 385.1830 & 193.0951 & 368.1565 & 184.5819 & & & G & 1562.7445 & 781.8759 & 1545.7180 & 773.3626 & 1544.7340 & 772.8706 & 15 \\
\hline 6 & 532.2514 & 266.6294 & 515.2249 & 258.1161 & & & $\mathbf{F}$ & 1505.7231 & 753.3652 & 1488.6965 & 744.8519 & 1487.7125 & 744.3599 & 14 \\
\hline 7 & 633.2991 & 317.1532 & 616.2726 & 308.6399 & 615.2885 & 308.1479 & $\mathbf{T}$ & 1358.6546 & 679.8310 & 1341.6281 & 671.3177 & 1340.6441 & 670.8257 & 13 \\
\hline 8 & 748.3260 & 374.6667 & 731.2995 & 366.1534 & 730.3155 & 365.6614 & $\mathbf{N}$ & 1257.6070 & 629.3071 & 1240.5804 & 620.7938 & 1239.5964 & 620.3018 & 12 \\
\hline 9 & 819.3632 & 410.1852 & 802.3366 & 401.6719 & 801.3526 & 401.1799 & A & 1142.5800 & 571.7937 & 1125.5535 & 563.2804 & 1124.5695 & 562.7884 & 11 \\
\hline 10 & 920.4108 & 460.7091 & 903.3843 & 452.1958 & 902.4003 & 451.7038 & $\mathbf{T}$ & 1071.5429 & 536.2751 & 1054.5164 & 527.7618 & 1053.5324 & 527.2698 & 10 \\
\hline 11 & 1021.4585 & 511.2329 & 1004.4320 & 502.7196 & 1003.4479 & 502.2276 & $\mathbf{T}$ & 970.4952 & 485.7513 & 953.4687 & 477.2380 & 952.4847 & 476.7460 & 9 \\
\hline 12 & 1122.5062 & 561.7567 & 1105.4796 & 553.2435 & 1104.4956 & 552.7515 & $\mathbf{T}$ & 869.4476 & 435.2274 & 852.4210 & 426.7141 & 851.4370 & 426.2221 & 8 \\
\hline 13 & 1237.5331 & 619.2702 & 1220.5066 & 610.7569 & 1219.5226 & 610.2649 & D & 768.3999 & 384.7036 & 751.3733 & 376.1903 & 750.3893 & 375.6983 & 7 \\
\hline 14 & 1365.5917 & 683.2995 & 1348.5652 & 674.7862 & 1347.5811 & 674.2942 & $\mathbf{Q}$ & 653.3729 & 327.1901 & 636.3464 & 318.6768 & & & 6 \\
\hline 15 & 1502.6506 & 751.8289 & 1485.6241 & 743.3157 & 1484.6401 & 742.8237 & H & 525.3144 & 263.1608 & 508.2878 & 254.6475 & & & 5 \\
\hline 16 & 1559.6721 & 780.3397 & 1542.6455 & 771.8264 & 1541.6615 & 771.3344 & G & 388.2554 & 194.6314 & 371.2289 & 186.1181 & & & 4 \\
\hline 17 & 1672.7562 & 836.8817 & 1655.7296 & 828.3684 & 1654.7456 & 827.8764 & $\mathbf{L}$ & 331.2340 & 166.1206 & 314.2074 & 157.6074 & & & 3 \\
\hline 18 & 1743.7933 & 872.4003 & 1726.7667 & 863.8870 & 1725.7827 & 863.3950 & A & 218.1499 & 109.5786 & 201.1234 & 101.0653 & & & 2 \\
\hline
\end{tabular}




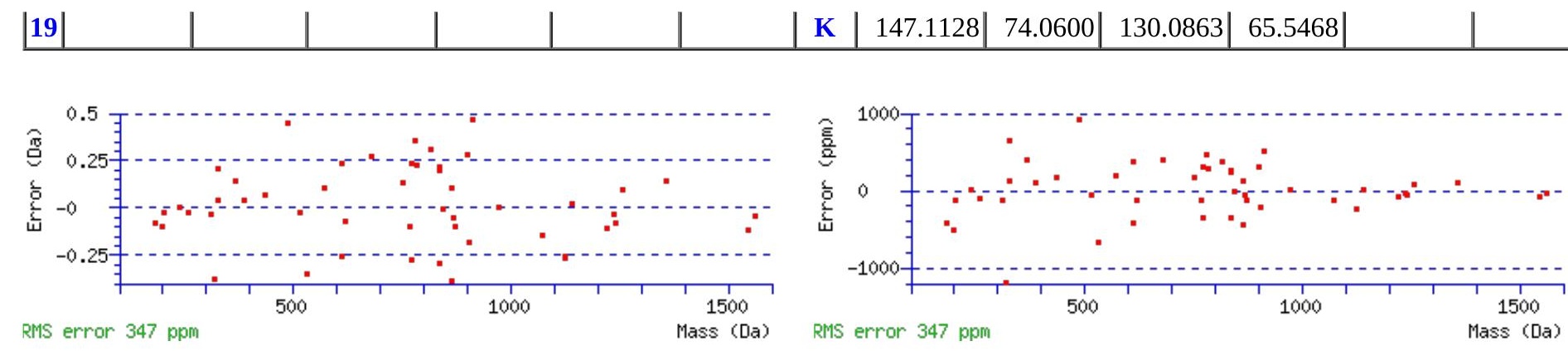

\section{All matches to this query}

\begin{tabular}{|l|l|l|l|}
\hline Score & Mr(calc): & Delta & \multicolumn{1}{|c|}{ Sequence } \\
\hline 48.0 & 1888.8915 & 0.0021 & AQGAGFTNATTTDQHGLAK \\
\hline 14.0 & 1887.9075 & 0.9861 & AQGAGFTNATTTDQHGLAK \\
\hline 10.8 & 1887.8728 & 1.0208 & GAQSGVNYSQGSLKDAVK \\
\hline 4.5 & 1887.8728 & 1.0208 & GAQSGVNYSQGSLKDAVK \\
\hline 4.4 & 1887.8880 & 1.0056 & FSFPGTTGSLAAAAAAAAAR \\
\hline 4.4 & 1887.8880 & 1.0056 & FSFPGTTGSLAAAAAAAAAR \\
\hline 4.0 & 1886.8887 & 2.0049 & ERFNAASSASKILQER \\
\hline 4.0 & 1886.8887 & 2.0049 & ERFNAASSASKILQER \\
\hline 2.5 & 1888.8906 & 0.0029 & TMRPSKYPDDIFLAR \\
\hline 2.5 & 1886.8726 & 2.0210 & QIATLHAQVTDMKKK \\
\hline
\end{tabular}

Spectrum No: 707; Query: 172; Rank: 1

\section{Peptide View}

MS/MS Fragmentation of DLDMFINASK

Found in IPI00194733, Tax_Id=10116 Gene_Symbol=Atrn Isoform 1 of Attractin precursor

Match to Query 172: 1169.525368 from(585.769960,2+)

Title: 091008RatKidney_NoSalt_32.2171.2171.2.dta

Data file K:INewmanPaper|Piliang|3SubProteomes\Piliang3SP\mgf5ppm\ERLIC_3SubProteomes5ppm.mgf

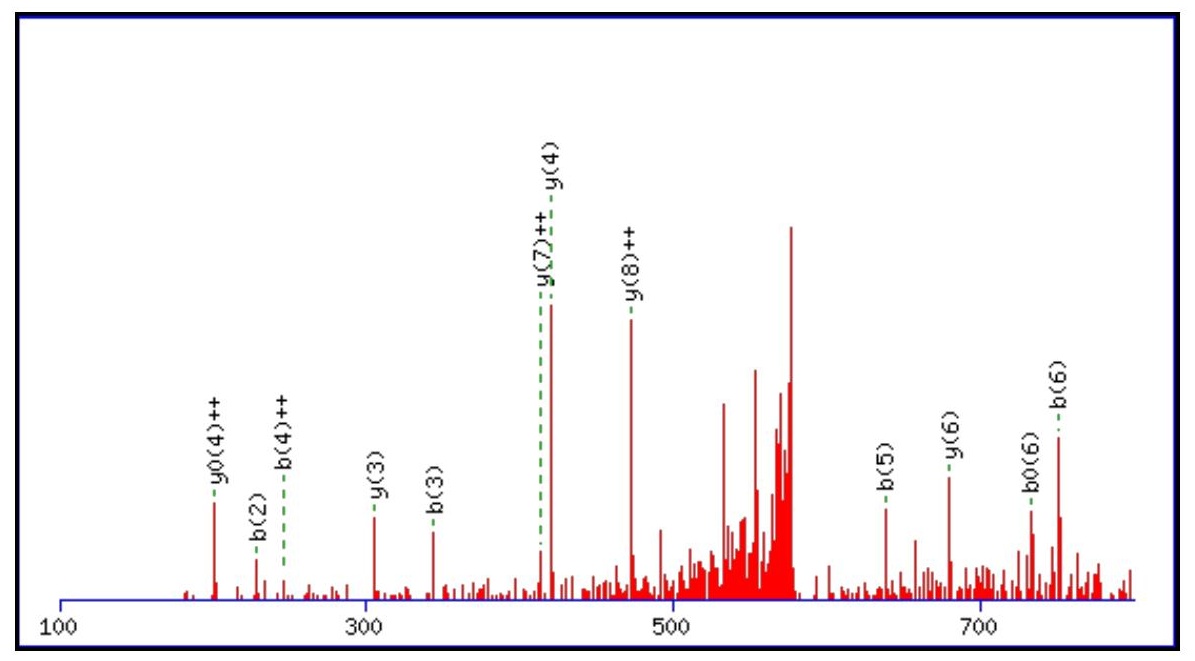




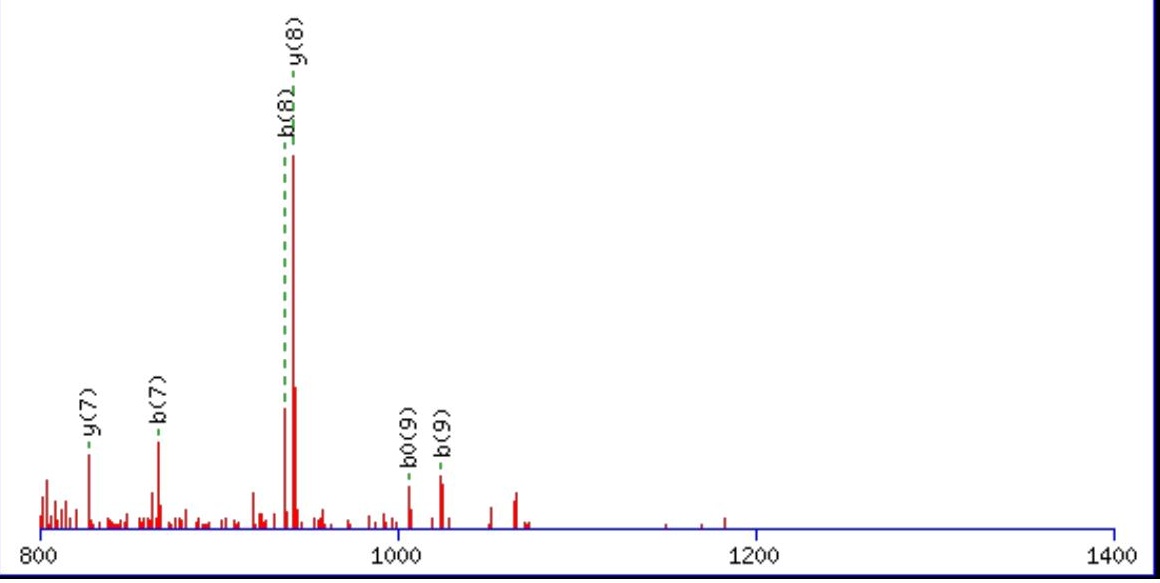

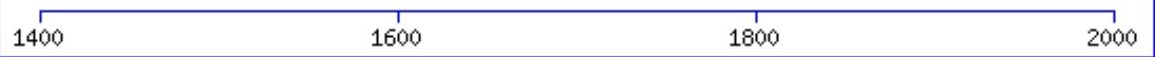

Monoisotopic mass of neutral peptide $\operatorname{Mr}($ calc): 1169.5274

Fixed modifications: Carbamidomethyl (C)

Variable modifications:

M4 : Oxidation (M)

N7 : Deamidated_N (N)

Ions Score: 48 Expect: 0.0021

Matches (Bold Red): 18/94 fragment ions using 38 most intense peaks

\begin{tabular}{|c|c|c|c|c|c|c|c|c|c|c|c|c|c|c|}
\hline \# & b & $\mathbf{b}^{++}$ & $\mathbf{b}^{*}$ & $\mathbf{b}^{*^{++}}$ & $\mathbf{b}^{\mathbf{0}}$ & $\mathbf{b}^{\mathbf{0 + +}}$ & Seq. & $\mathbf{y}$ & $\mathbf{y}^{++}$ & $\mathbf{y}^{*}$ & $\mathrm{y}^{*^{++}}$ & $\mathbf{y}^{0}$ & $\mathbf{y}^{\mathbf{0 + +}}$ & \# \\
\hline 1 & 116.0342 & 58.5207 & & & 98.0237 & 49.5155 & D & & & & & & & 10 \\
\hline 2 & 229.1183 & 115.0628 & & & 211.1077 & 106.0575 & $\mathbf{L}$ & 1055.5078 & 528.2575 & 1038.4812 & 519.7442 & 1037.4972 & 519.2522 & 9 \\
\hline 3 & 344.1452 & 172.5763 & & & 326.1347 & 163.5710 & D & 942.4237 & 471.7155 & 925.3972 & 463.2022 & 924.4131 & 462.7102 & 8 \\
\hline 4 & 491.1806 & 246.0940 & & & 473.1701 & 237.0887 & $\mathbf{M}$ & 827.3968 & 414.2020 & 810.3702 & 405.6887 & 809.3862 & 405.1967 & 7 \\
\hline 5 & 638.2490 & 319.6282 & & & 620.2385 & 310.6229 & $\mathbf{F}$ & 680.3614 & 340.6843 & 663.3348 & 332.1710 & 662.3508 & 331.6790 & 6 \\
\hline 6 & 751.3331 & 376.1702 & & & 733.3225 & 367.1649 & I & 533.2929 & 267.1501 & 516.2664 & 258.6368 & 515.2824 & 258.1448 & 5 \\
\hline 7 & 866.3600 & 433.6837 & 849.3335 & 425.1704 & 848.3495 & 424.6784 & $\mathbf{N}$ & 420.2089 & 210.6081 & 403.1823 & 202.0948 & 402.1983 & 201.6028 & 4 \\
\hline 8 & 937.3972 & 469.2022 & 920.3706 & 460.6889 & 919.3866 & 460.1969 & A & 305.1819 & 153.0946 & 288.1554 & 144.5813 & 287.1714 & 144.0893 & 3 \\
\hline 9 & 1024.4292 & 512.7182 & 1007.4026 & 504.2050 & 1006.4186 & 503.7129 & S & 234.1448 & 117.5761 & 217.1183 & 109.0628 & 216.1343 & 108.5708 & 2 \\
\hline 10 & & & & & & & $\mathbf{K}$ & 147.1128 & 74.0600 & 130.0863 & 65.5468 & & & 1 \\
\hline
\end{tabular}
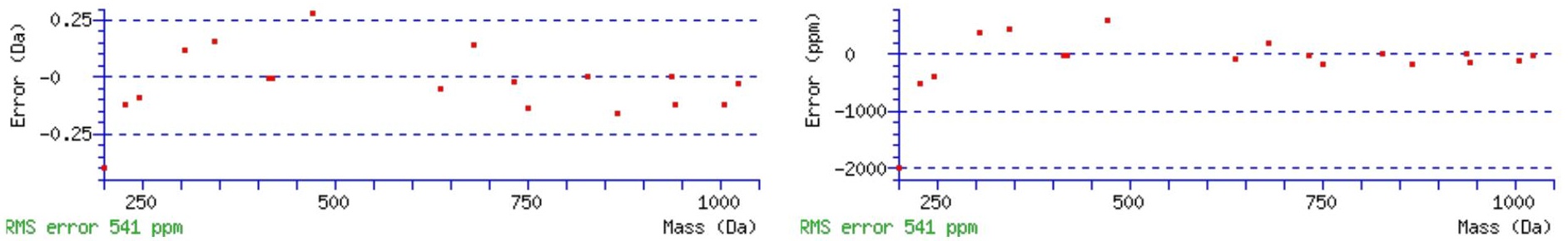


\section{All matches to this query}

\begin{tabular}{|l|l|l|l|}
\hline Score & Mr(calc): & Delta & \multicolumn{1}{c|}{ Sequence } \\
\hline 48.0 & 1169.5274 & -0.0021 & DLDMFINASK \\
\hline 5.3 & 1169.5329 & -0.0075 & VESNNLKGTK \\
\hline 5.3 & 1169.5329 & -0.0075 & VESNNLKGTK \\
\hline 2.8 & 1167.5172 & 2.0081 & KPSGLNGEASK \\
\hline 2.2 & 1167.5285 & 1.9969 & IIDAANQSTR \\
\hline 2.2 & 1167.5285 & 1.9969 & IIDAANQSTR \\
\hline 1.9 & 1169.5190 & 0.0064 & RSRSADSPSK \\
\hline 1.3 & 1169.5329 & -0.0075 & NLGSVSGELSK \\
\hline 1.3 & 1169.5329 & -0.0075 & NLGSVSGELSK \\
\hline 1.0 & 1169.5247 & 0.0007 & TSALLGAHLK \\
\hline
\end{tabular}

Spectrum No: 708; Query: 1749; Rank: 1

\section{Peptide View}

MS/MS Fragmentation of VFTAQTQPHEEQLNGTFR

Found in IPI00365922, Tax_Id=10116 Gene_Symbol=LOC362795 LOC362795 protein

Match to Query 1749: 2102.999112 from(702.006980,3+)

Title: 091008RatKidney_NH4Format01_23.1768.1768.3.dta

Data file K:INewmanPaper|Piliang|3SubProteomes\Piliang3SP\mgf5ppm\ERLIC_3SubProteomes5ppm.mgf
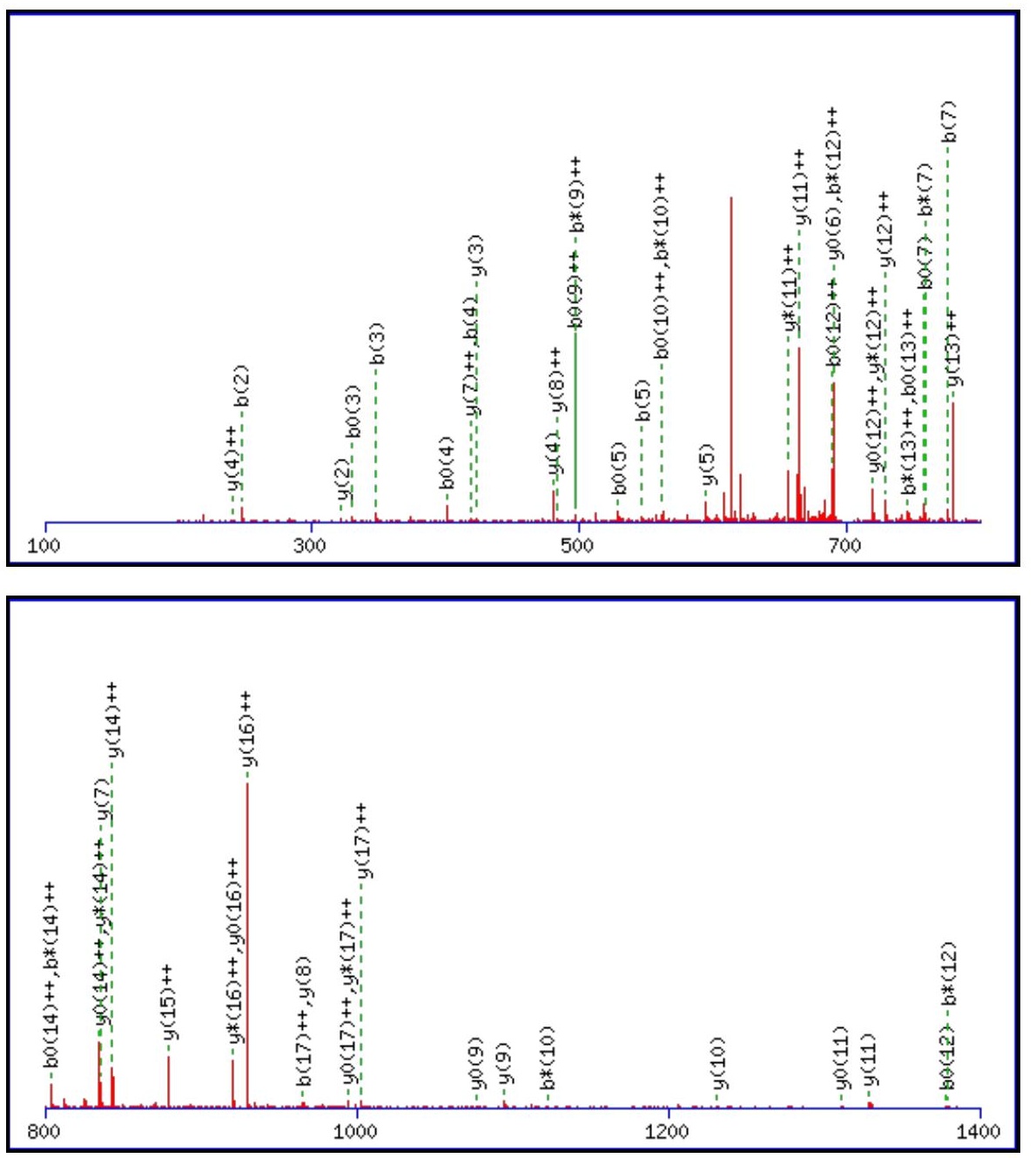


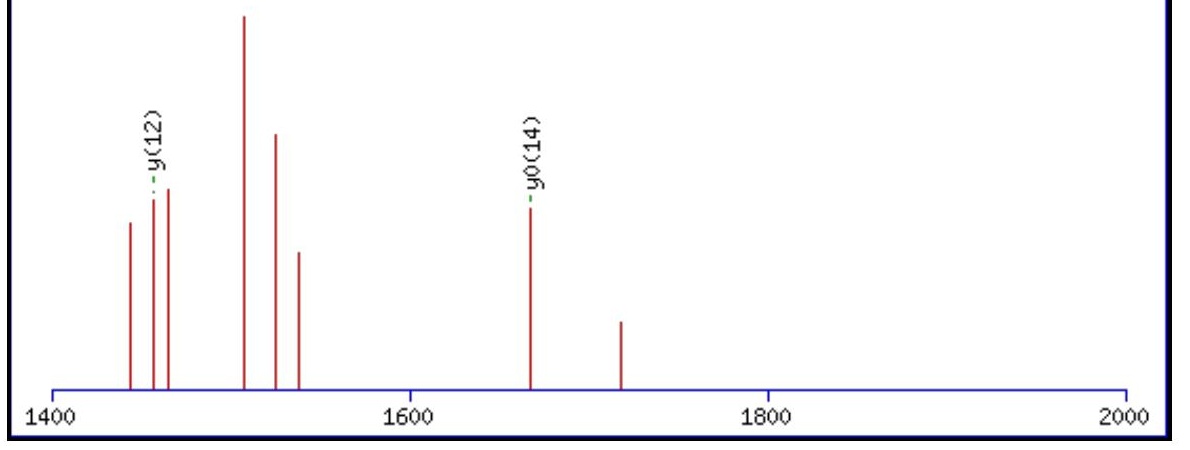

Monoisotopic mass of neutral peptide $\operatorname{Mr}($ calc): 2103.0021

Fixed modifications: Carbamidomethyl (C)

Variable modifications:

N14 : Deamidated_N (N)

Ions Score: 48 Expect : 0.004

Matches (Bold Red): 57/188 fragment ions using 106 most intense peaks

\begin{tabular}{|c|c|c|c|c|c|c|c|c|c|c|c|c|c|c|}
\hline \# & b & $\mathbf{b}^{++}$ & $\mathbf{b}^{*}$ & $\mathbf{b}^{*^{++}}$ & $\mathbf{b}^{\mathbf{0}}$ & & Seq. & $\mathbf{y}$ & $\mathbf{y}^{++}$ & $\mathbf{y}^{*}$ & $\mathbf{y}^{*^{++}}$ & $\mathbf{y}^{\mathbf{0}}$ & $\mathbf{y}^{\mathbf{0 + +}}$ & \# \\
\hline 1 & 100.0757 & 50.5415 & & & & & V & & & & & & & 18 \\
\hline 2 & 247.1441 & 124.0757 & & & & & $\mathbf{F}$ & 2004.9410 & 1002 & & & & & 17 \\
\hline 3 & 348.1918 & 174.5995 & & & 812 & 165.5942 & $\mathbf{T}$ & 1857.8726 & & 1840.8460 & 920.9267 & 1839.8620 & 920.4346 & 16 \\
\hline 4 & 419.2289 & 210.1181 & & & 401.2183 & 201.1128 & A & 1756.8249 & 878.9161 & 1739.7983 & 870.4028 & 1738.8143 & 869.9108 & 15 \\
\hline 5 & 547.2875 & 274.1474 & 530.2609 & 265.6 & 769 & 265.1421 & $\mathbf{Q}$ & 1685.7878 & 975 & 1668.7612 & 8843 & 1667.7772 & 834.3922 & 14 \\
\hline 6 & 648.3352 & 324.6712 & 631.3086 & 316.1579 & 630.3246 & 315.6659 & $\mathbf{T}$ & 1557.7292 & 779.3682 & 1540.7027 & 770.8550 & 1539.7186 & 770.3630 & 13 \\
\hline 7 & 776.3937 & 388.7005 & 759.3672 & 380.1872 & 758.3832 & 379.6952 & $\mathbf{Q}$ & 1456.6815 & 728.8444 & 1439.6550 & 720.3311 & 1438.6710 & 719.8391 & 12 \\
\hline 8 & 873.4465 & 437.2269 & 856.4199 & 428.7136 & 855.4359 & 428.2216 & $\mathbf{P}$ & 1328.6229 & 664.8151 & 1311.5964 & 656.3018 & 1310.6124 & 655.8098 & 11 \\
\hline 9 & 1010.5054 & 505.7563 & 993.4789 & 497.2431 & 992.4948 & 496.7511 & $\mathbf{H}$ & 1231.5702 & 616.2887 & 1214.5436 & 607.7755 & 1213.5596 & 607.2834 & 10 \\
\hline 10 & 1139.5480 & 570.2776 & 1122.5215 & 561.7644 & 121.5374 & 561.2724 & $\mathbf{E}$ & 1094.5113 & 547.7593 & 1077.4847 & 539.2460 & 1076.5007 & 538.7540 & 9 \\
\hline 11 & 1268.5906 & 634.7989 & 1251.5640 & 626.2857 & 1250.5800 & 625.7937 & $\mathbf{E}$ & 965.4687 & 483.2380 & 948.4421 & 474.7247 & 947.4581 & 474.2327 & 8 \\
\hline 12 & 1396.6492 & 698.8282 & 1379.6226 & 690.3149 & 1378.6386 & 689.8229 & $\mathbf{Q}$ & 836.4261 & 418.7167 & 819.3995 & 410.2034 & 818.4155 & 409.7114 & 7 \\
\hline 13 & 1509.7332 & 755.3703 & 1492.7067 & 746.8570 & 1491.7227 & 746.3650 & $\mathbf{L}$ & 708.3675 & 354.6874 & 691.3410 & 346.1741 & 690.3569 & 345.6821 & 6 \\
\hline 14 & 1624.7602 & 812.8837 & 1607.7336 & 804.3704 & 1606.7496 & 803.8784 & $\mathbf{N}$ & 595.2834 & 298.1454 & 578.2569 & 289.6321 & 577.2729 & 289.1401 & 5 \\
\hline 15 & 1681.7816 & 841.3945 & 1664.7551 & 832.8812 & 1663.7711 & 832.3892 & G & 480.2565 & 240.6319 & 463.2300 & 232.1186 & 462.2459 & 231.6266 & 4 \\
\hline 16 & 1782.8293 & 891.9183 & 1765.8028 & 883.4050 & 1764.8187 & 882.9130 & $\mathbf{T}$ & 423.2350 & 212.1212 & 406.2085 & 203.6079 & 405.2245 & 203.1159 & 3 \\
\hline 17 & 1929.8977 & 965.4525 & 1912.8712 & 956.9392 & 1911.8872 & 956.4472 & $\mathbf{F}$ & 322.1874 & 161.5973 & 305.1608 & 153.0840 & & & 2 \\
\hline 18 & & & & & & & $\mathbf{R}$ & 175.1190 & 88.0631 & 158.0924 & 79.5498 & & & 1 \\
\hline
\end{tabular}$$
\text { 政 }
$$
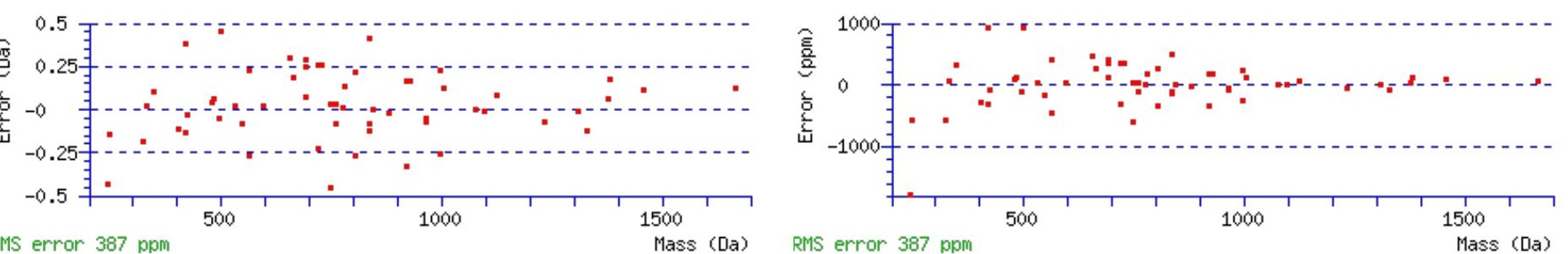

\section{All matches to this query}

\begin{tabular}{|l|c|c|l|}
\hline Score & Mr(calc): & Delta & \multicolumn{1}{c|}{ Sequence } \\
\hline 48.0 & 2103.0021 & -0.0030 & VFTAQTQPHEEQLNGTFR \\
\hline 3.5 & 2103.0167 & -0.0176 & VFLSOVIKSLDVGPNATR \\
\hline 2.8 & 2101.9804 & 1.0187 & WMSWLPVLVVSLMCSAK \\
\hline
\end{tabular}




\begin{tabular}{|l|l|l|l|}
2.5 & 2100.9737 & 2.0254 & KNTELVKVMGLSNYHCK \\
\hline 1.7 & 2102.0152 & 0.9839 & VLIRLTTAMCNEVASLSK \\
\hline 1.6 & 2103.0154 & -0.0163 & GGINLTATCPQSELDAETVK \\
\hline 1.5 & 2101.9861 & 1.0131 & LVPFTDLEELSEVFAEGK \\
\hline 1.3 & 2100.9915 & 2.0076 & AQPVNFSLMASLALDSRGK \\
\hline 1.3 & 2100.9915 & 2.0076 & AQPVNFSLMASLALDSRGK \\
\hline 1.2 & 2100.9993 & 1.9998 & KASSQREGNQTAFXGLWK \\
\hline
\end{tabular}

Spectrum No: 709; Query: 348; Rank: 1

\section{Peptide View}

MS/MS Fragmentation of DLAVDDDYTNR

Found in IPI00372689, Tax_Id=10116 Gene_Symbol=Itga3_predicted 117 kDa protein

Match to Query 348: 1296.544868 from(649.279710,2+)

Title: 091008RatKidney_NoSalt_35.1096.1096.2.dta

Data file K:\NewmanPaper\Piliang \3SubProteomes \Piliang3SP $\backslash$ mgf5ppm\ERLIC_3SubProteomes5ppm.mgf
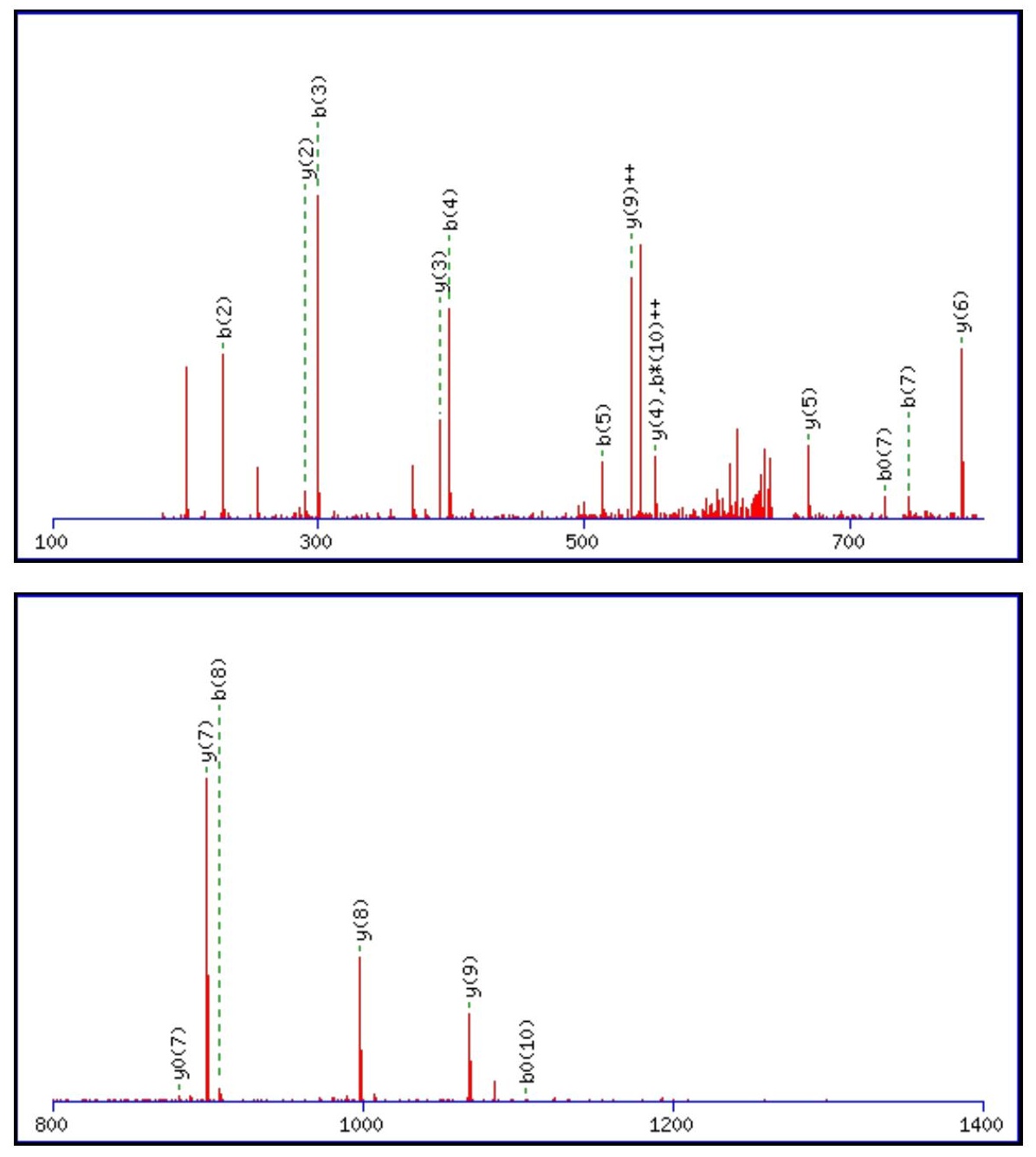


$1400 \quad 1600 \quad 1800 \quad 2000$

Monoisotopic mass of neutral peptide $\operatorname{Mr}($ calc): 1296.5470

Fixed modifications: Carbamidomethyl (C)

Variable modifications:

N10 : Deamidated_N (N)

Ions Score: 48 Expect: 0.0017

Matches (Bold Red): 19/98 fragment ions using 35 most intense peaks

\begin{tabular}{|c|c|c|c|c|c|c|c|c|c|c|c|c|c|c|}
\hline \# & b & $\mathbf{b}^{++}$ & $\mathbf{b}^{*}$ & $\mathbf{b}^{*^{++}}$ & $\mathbf{b}^{0}$ & $\mathbf{b}^{0++}$ & Seq. & $\mathbf{y}$ & $y^{++}$ & $\mathrm{y}^{*}$ & $y^{*^{++}}$ & $\mathbf{y}^{\mathbf{0}}$ & $y^{0++}$ & $\#$ \\
\hline 1 & 116.0342 & 58.5207 & & & 98.0237 & 49.5155 & D & & & & & & & 11 \\
\hline 2 & 229.1183 & 115.0628 & & & 211.1077 & 106.0575 & $\mathbf{L}$ & 1182.5273 & 591.7673 & 1165.5008 & 583.2540 & 1164.5168 & 582.7620 & 10 \\
\hline 3 & 300.1554 & 150.5813 & & & 282.1448 & 141.5761 & A & 1069.4433 & 535.2253 & 1052.4167 & 526.7120 & 1051.4327 & 526.2200 & 9 \\
\hline 4 & 399.2238 & 200.1155 & & & 381.2132 & 191.1103 & V & 998.4061 & 499.7067 & 981.3796 & 491.1934 & 980.3956 & 490.7014 & 8 \\
\hline 5 & 514.2508 & 257.6290 & & & 496.2402 & 248.6237 & D & 899.3377 & 450.1725 & 882.3112 & 441.6592 & 881.3272 & 441.1672 & 7 \\
\hline 6 & 629.2777 & 315.1425 & & & 611.2671 & 306.1372 & D & 784.3108 & 392.6590 & 767.2842 & 384.1458 & 766.3002 & 383.6537 & 6 \\
\hline 7 & 744.3046 & 372.6560 & & & 726.2941 & 363.6507 & D & 669.2838 & 335.1456 & 652.2573 & 326.6323 & 651.2733 & 326.1403 & 5 \\
\hline 8 & 907.3680 & 454.1876 & & & 889.3574 & 445.1823 & $\mathbf{Y}$ & 554.2569 & 277.6321 & 537.2303 & 269.1188 & 536.2463 & 268.6268 & 4 \\
\hline 9 & 1008.4156 & 504.7115 & & & 990.4051 & 495.7062 & $\mathbf{T}$ & 391.1936 & 196.1004 & 374.1670 & 187.5871 & 373.1830 & 187.0951 & 3 \\
\hline 10 & 1123.4426 & 562.2249 & 1106.4160 & 553.7117 & 1105.4320 & 553.2196 & $\mathbf{N}$ & 290.1459 & 145.5766 & 273.1193 & 137.0633 & & & 2 \\
\hline 11 & & & & & & & $\mathbf{R}$ & 175.1190 & 88.0631 & 158.0924 & 79.5498 & & & 1 \\
\hline
\end{tabular}
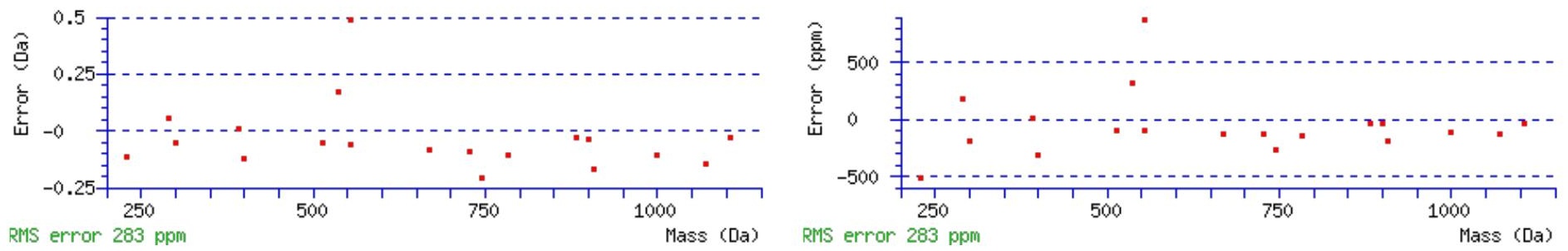

\section{All matches to this query}

\begin{tabular}{|l|l|l|l|}
\hline Score & Mr(calc): & Delta & \multicolumn{1}{c|}{ Sequence } \\
\hline 47.9 & 1296.5470 & -0.0021 & DLAVDDDYTNR \\
\hline 15.2 & 1296.5516 & -0.0068 & VLSTHSPPTAK \\
\hline 15.2 & 1296.5516 & -0.0068 & VLSTHSPPTAK \\
\hline 4.6 & 1295.5424 & 1.0024 & $\underline{\text { LSRGYSGLQR }}$ \\
\hline 2.4 & 1296.5459 & -0.0011 & SDPRDTAATQR \\
\hline 2.0 & 1296.5516 & -0.0068 & VLSTHSPPTAK \\
\hline 1.8 & 1295.5346 & 1.0103 & LSVGSSMRTAK \\
\hline 1.8 & 1295.5346 & 1.0103 & LSVGSSMRTAK \\
\hline 1.2 & 1296.5356 & 0.0093 & DIAHMTRCTL \\
\hline 1.1 & 1295.5346 & 1.0103 & $\underline{\text { LSVGSSMRTAK }}$ \\
\hline
\end{tabular}


Spectrum No: 710; Query: 1552; Rank: 1

\section{Peptide View}

MS/MS Fragmentation of KDGSQNFNQTWENYEK

Found in IPI00198903, Tax_Id=10116 Gene_Symbol=Angptl3 Angiopoietin-like 3

Match to Query 1552: 1987.860192 from(663.627340,3+)

Title: 100101RatKid_NS_deglyco_24.2286.2286.3.dta

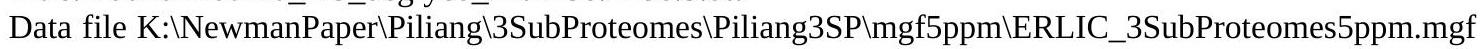
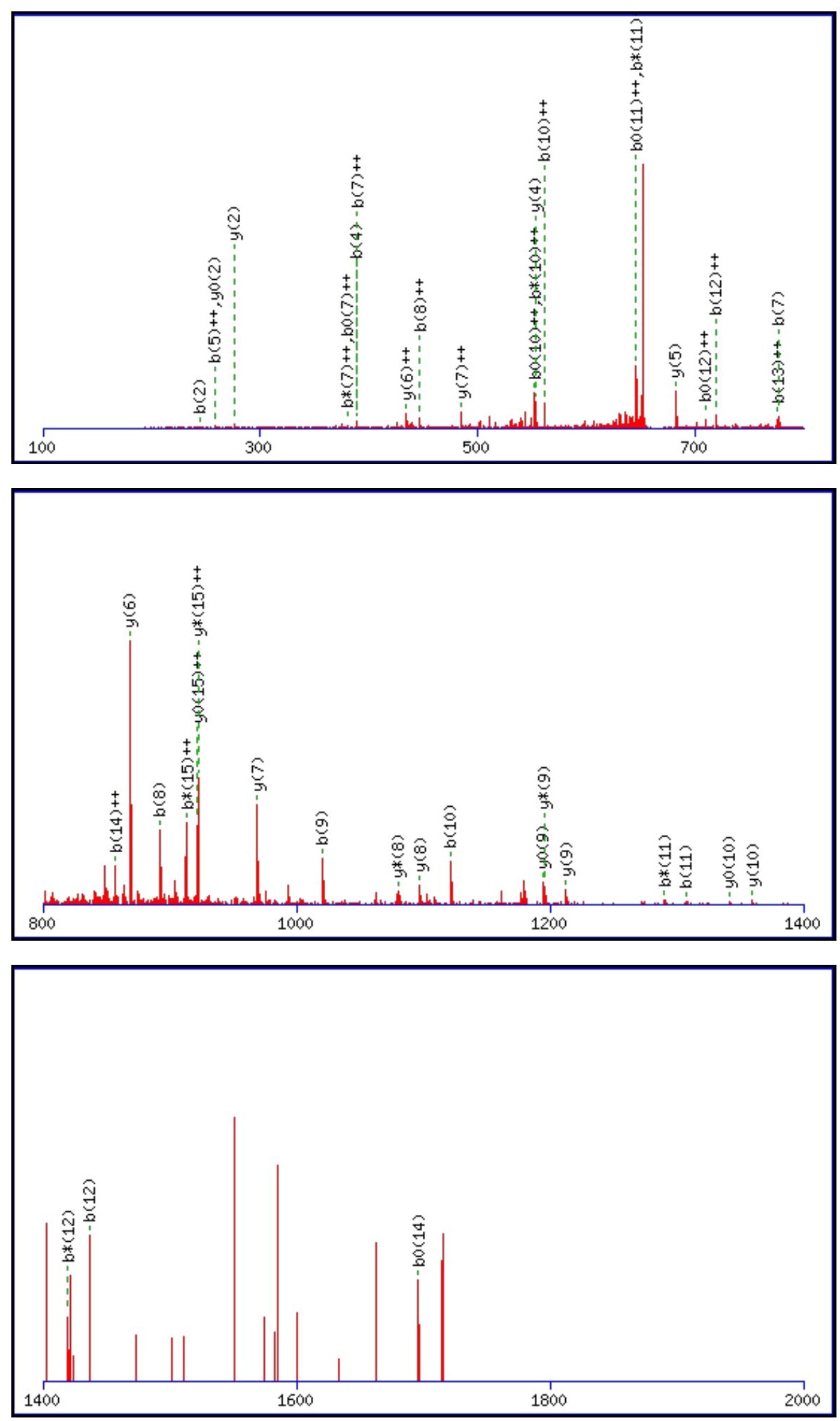
Monoisotopic mass of neutral peptide $\operatorname{Mr}($ calc): 1987.8548

Fixed modifications: Carbamidomethyl (C)

Variable modifications:

N8 : Deamidated_N (N)

Ions Score: 48 Expect: $\odot .0038$

Matches (Bold Red): 44/176 fragment ions using 72 most intense peaks

\begin{tabular}{|c|c|c|c|c|c|c|c|c|c|c|c|c|c|c|}
\hline \# & b & $\mathbf{b}^{++}$ & $\mathbf{b}^{*}$ & $\mathbf{b}^{*++}$ & $\mathbf{b}^{0}$ & $\mathbf{b}^{0++}$ & Seq. & $\mathbf{y}$ & $\mathbf{y}^{++}$ & $\mathbf{y}^{*}$ & $\mathbf{y}^{*^{++}}$ & $\mathbf{y}^{\mathbf{0}}$ & $y^{0++}$ & $\#$ \\
\hline 1 & 129.1022 & 65.0548 & 112.0757 & 56.5415 & & & $\mathbf{K}$ & & & & & & & 16 \\
\hline 2 & 244.1292 & 122.5682 & 227.1026 & 114.0550 & 226.1186 & 113.5629 & D & 1860.7671 & 930.8872 & 1843.7406 & 922.3739 & 1842.7565 & 921.8819 & 15 \\
\hline 3 & 301.1506 & 151.0790 & 284.1241 & 142.5657 & 283.1401 & 142.0737 & G & 1745.7402 & 873.3737 & 1728.7136 & 864.8604 & 1727.7296 & 864.3684 & 14 \\
\hline 4 & 388.1827 & 194.5950 & 371.1561 & 186.0817 & 370.1721 & 185.5897 & $\mathrm{~S}$ & 1688.7187 & 844.8630 & 1671.6922 & 836.3497 & 1670.7081 & 835.8577 & 13 \\
\hline 5 & 516.2413 & 258.6243 & 499.2147 & 250.1110 & 498.2307 & 249.6190 & $\mathbf{Q}$ & 1601.6867 & 801.3470 & 1584.6601 & 792.8337 & 1583.6761 & 792.3417 & 12 \\
\hline 6 & 630.2842 & 315.6457 & 613.2576 & 307.1325 & 612.2736 & 306.6404 & $\mathbf{N}$ & 1473.6281 & 737.3177 & 1456.6015 & 728.8044 & 1455.6175 & 728.3124 & 11 \\
\hline 7 & 777.3526 & 389.1799 & 760.3260 & 380.6667 & 759.3420 & 380.1747 & $\mathbf{F}$ & 1359.5852 & 680.2962 & 1342.5586 & 671.7829 & 1341.5746 & 671.2909 & 10 \\
\hline 8 & 892 & 446. & & & & & $\mathbf{N}$ & & & 1195.4902 & & 1194.5062 & 597.7567 & 9 \\
\hline 9 & 1020.4381 & 510.7227 & 1003.4116 & 502.2094 & 1002.4275 & 501.7174 & $\mathbf{Q}$ & 1097.4898 & 549.2485 & 1080.4633 & 540.7353 & 1079.4793 & 540.2433 & 8 \\
\hline 10 & 1121.4858 & 561.2465 & 1104.4592 & 552.7333 & 1103.4752 & 552.2412 & $\mathbf{T}$ & 969.4312 & 485.2193 & 952.4047 & 476.7060 & 951.4207 & 476.2140 & 7 \\
\hline 11 & \begin{tabular}{|l|}
1307.5651 \\
\end{tabular} & 654.2862 & 1290.5386 & 645.7729 & 1289.5545 & 645.2809 & $\mathbf{W}$ & 868.3836 & 434.6954 & 851.3570 & 426.1821 & 850.3730 & 425.6901 & 6 \\
\hline 12 & \begin{tabular}{|l|}
1436.6077 \\
\end{tabular} & \begin{tabular}{|l|l|}
718.8075 \\
\end{tabular} & 1419.5811 & 710.2942 & 1418.5971 & \begin{tabular}{|l|l|}
709.8022 \\
\end{tabular} & $\mathbf{E}$ & 682.3042 & 341.6558 & 665.2777 & 333.1425 & 664.2937 & 332.6505 & 5 \\
\hline 13 & 1550.6506 & \begin{tabular}{|l|}
775.8289 \\
\end{tabular} & 1533.6241 & 767.3157 & 1532.6401 & 766.8237 & $\mathbf{N}$ & 553.2617 & 277.1345 & 536.2351 & 268.6212 & 535.2511 & 268.1292 & 4 \\
\hline 14 & 1713.7139 & 857.3606 & 1696.6874 & 848.8473 & \begin{tabular}{|l|}
1695.7034 \\
\end{tabular} & 848.3553 & $\mathbf{Y}$ & 439.2187 & 220.1130 & 422.1922 & 211.5997 & 421.2082 & 211.1077 & 3 \\
\hline 15 & 1842.7565 & 921.8819 & 1825.7300 & 913.3686 & 1824.7460 & 912.8766 & $\mathbf{E}$ & 276.1554 & 138.5813 & 259.1288 & 130.0681 & 258.1448 & 129.5761 & 2 \\
\hline 16 & & & & & & & $\mathbf{K}$ & 147.1128 & 74.0600 & 130.0863 & 65.5468 & & & 1 \\
\hline
\end{tabular}
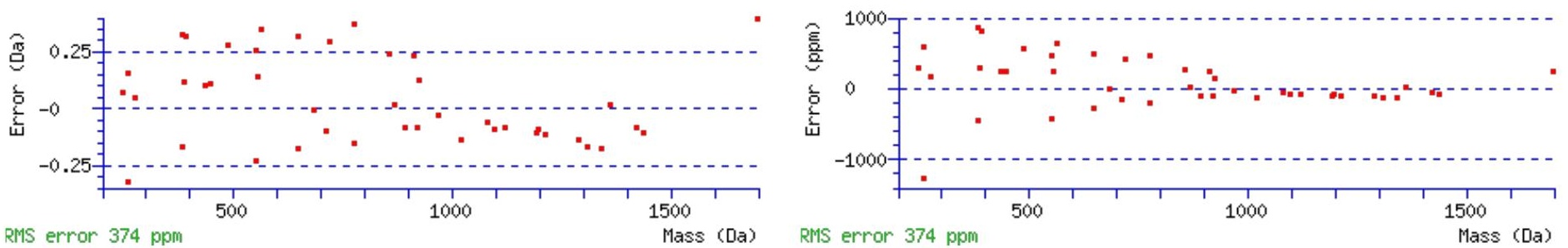

\section{All matches to this query}

\begin{tabular}{|l|l|l|l|}
\hline Score & Mr(calc): & Delta & \multicolumn{1}{|c|}{ Sequence } \\
\hline 47.7 & 1987.8548 & 0.0054 & KDGSQNFNQTWENYEK \\
\hline 41.8 & 1987.8548 & 0.0054 & KDGSQNFNQTWENYEK \\
\hline 19.6 & 1986.8708 & 0.9894 & KDGSQNFNQTWENYEK \\
\hline 16.0 & 1987.8548 & 0.0054 & KDGSQNFNQTWENYEK \\
\hline 13.3 & 1985.8675 & 1.9927 & KFLESTEFLLNNELK \\
\hline 6.0 & 1985.8520 & 2.0082 & DPKNPESTSGWAIANYR \\
\hline 4.4 & 1986.8561 & 1.0041 & RVTNDISPESSPGVGRR \\
\hline 4.4 & 1986.8561 & 1.0041 & $\underline{\text { RVTNDISPESSPGVGRR }}$ \\
\hline 4.4 & 1986.8601 & 1.0001 & $\underline{\text { YRPSHGDKTSNPEVLK }}$ \\
\hline 3.0 & 1987.8441 & 0.0160 & YRPSHGDKTSNPEVLK \\
\hline
\end{tabular}

Spectrum No: 711; Query: 2839; Rank: 1

\section{Peptide View}

MS/MS Fragmentation of LNQATVTSNRPGLFYGQCSEICGSNHSFMPIVLEMVPLK

Found in IPI00209908, Tax_Id=10116 Gene_Symbol=mt-Co2 Cytochrome c oxidase subunit 2 
Match to Query 2839: 4395.124692 from(1466.048840,3+)

Title: 091008RatKidney_NoSalt_04.5537.5537.3.dta

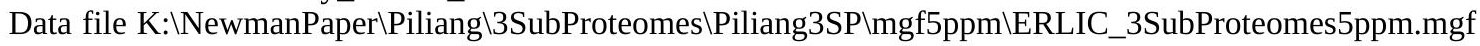
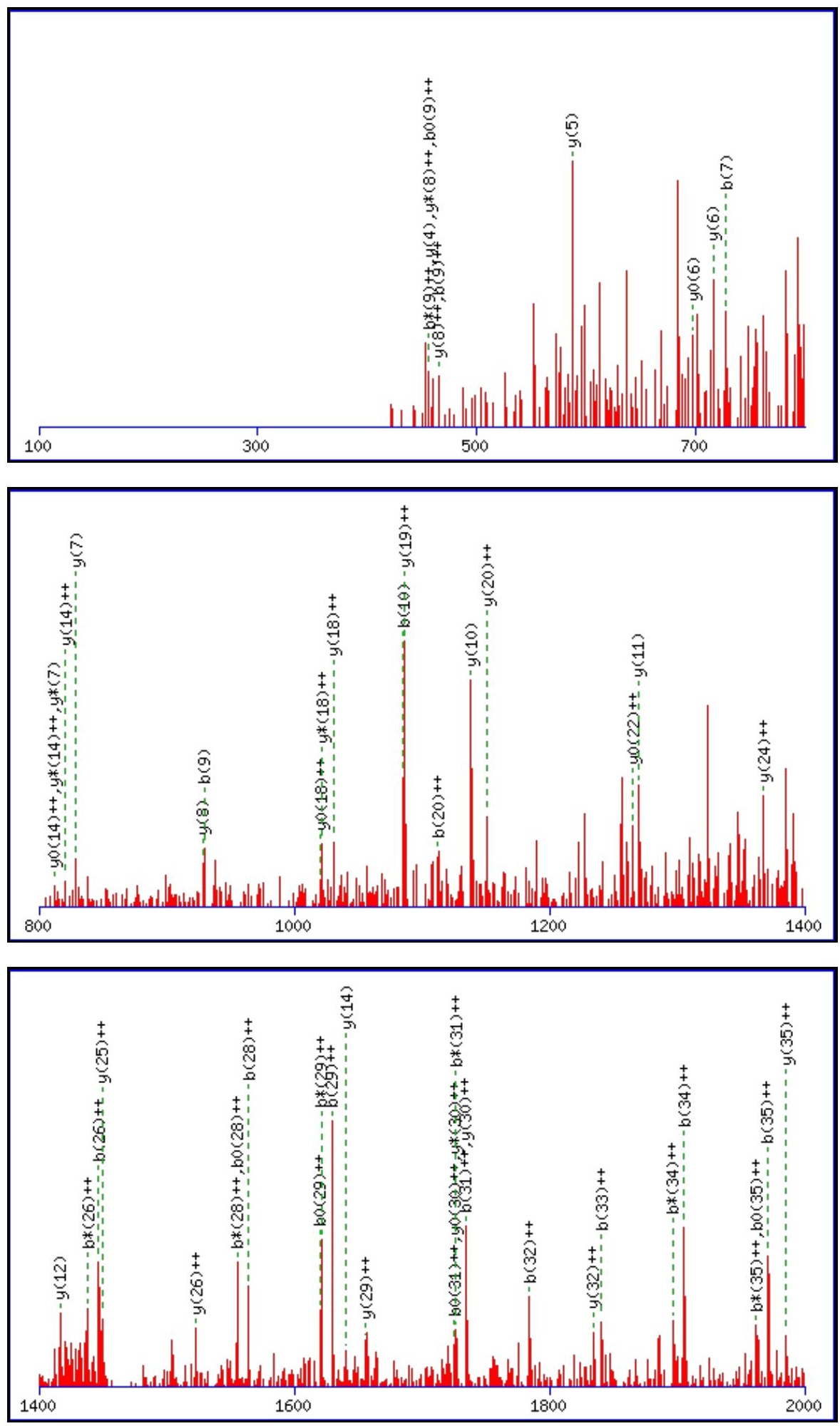

Monoisotopic mass of neutral peptide $\operatorname{Mr}($ calc): 4395.1160

Fixed modifications: Carbamidomethyl (C)

Variable modifications:

N25 : Deamidated_N (N)

Ions Score: 48 Expect: 0.0057

Matches (Bold Red): 56/436 fragment ions using 101 most intense peaks

\begin{tabular}{|c|c|c|c|c|c|c|c|c|c|c|c|c|c|c|}
\hline$\#$ & $\mathbf{b}$ & $\mathbf{b}^{++}$ & $\mathbf{b}^{*}$ & $\mathbf{b}^{\boldsymbol{*}^{++}}$ & $\mathbf{b}^{\mathbf{0}}$ & $\mathbf{b}^{\mathbf{0}+}$ & Seq. & $\mathbf{y}$ & $\mathbf{y}^{++}$ & $\mathbf{y}^{\mathbf{*}}$ & $\mathbf{y}^{\boldsymbol{*}^{++}}$ & $\mathbf{y}^{\mathbf{0}}$ & $\mathbf{y}^{\mathbf{0}++}$ & $\#$ \\
\hline $\mathbf{1}$ & 114.0913 & 57.5493 & & & & & $\mathbf{L}$ & & & & & & & 39 \\
\hline 2 & 228.1343 & 114.5708 & 211.1077 & 106.0575 & & & $\mathbf{N}$ & 4283.0392 & 2142.0232 & 4266.0127 & 2133.5100 & 4265.0286 & 2133.0180 & 38 \\
\hline
\end{tabular}




\begin{tabular}{|c|c|c|c|c|c|c|c|c|c|c|c|c|c|c|}
\hline & 356.1928 & 178.6001 & 339.1663 & 170.0868 & & & Q & 4168.9963 & 2085.0018 & |4151.9697 & 2076.4885 & 4150.9857 & 2075.9965 & 37 \\
\hline 4 & 427.2300 & 214.1186 & 410.2034 & 205.6053 & & & A & 4040.9377 & 2020.9725 & 4023.9112 & 2012.4592 & 4022.9271 & 2011.9672 & 36 \\
\hline 5 & 528.2776 & 264.6425 & 511.2511 & 256.1292 & 510.2671 & 255.6372 & $\mathbf{T}$ & 3969.9006 & 1985.4539 & 3952.8740 & 1976.9407 & 3951.8900 & 1976.4487 & 35 \\
\hline 6 & 627.3461 & 314.1767 & 610.3195 & 305.6634 & 609.3355 & 305.1714 & V & 3868.8529 & 1934.9301 & |3851.8264 & 1926.4168 & 3850.8424 & 1925.9248 & 34 \\
\hline 7 & 728.3937 & 364.7005 & 711.3672 & 356.1872 & 710.3832 & 355.6952 & $\mathbf{T}$ & 3769.7845 & 1885.3959 & 3752.7580 & 1876.8826 & 3751.7739 & 1876.3906 & 33 \\
\hline 8 & 815.4258 & 408.2165 & 798.3992 & 399.7032 & 797.4152 & 399.2112 & $\mathrm{~S}$ & 3668.7368 & 1834.8720 & 3651.7103 & 1826.3588 & 3650.7263 & 1825.8668 & 32 \\
\hline 9 & 929.4687 & 465.2380 & 912.4421 & 456.7247 & 911.4581 & 456.2327 & $\mathbf{N}$ & 3581.7048 & 1791.3560 & 3564.6782 & 1782.8428 & 3563.6942 & 1782.3508 & 31 \\
\hline 10 & 1085.5698 & 543.2885 & 1068.5432 & 534.7753 & 1067.5592 & 534.2833 & $\mathbf{R}$ & 3467.6619 & 1734.3346 & 3450.6353 & 1725.8213 & 3449.6513 & 1725.3293 & 30 \\
\hline 11 & 1182.6226 & 591.8149 & 1165.5960 & 583.3016 & 1164.6120 & 582.8096 & $\mathbf{P}$ & 3311.5608 & 1656.2840 & 3294.5342 & 1647.7707 & 3293.5502 & 1647.2787 & 29 \\
\hline 12 & 1239.6440 & 620.3257 & 1222.6175 & 611.8124 & 1221 & 204 & G & 5080 & 1607.7576 & |3197.4814 & 1599.2444 & 4974 & 7524 & 28 \\
\hline 13 & 1352.7281 & 676.8677 & 1335.7015 & 668.3544 & 1334.7175 & 667.8624 & $\mathbf{L}$ & 3157.4865 & 1579.2469 & 3140.4600 & 1570.7336 & 3139.4760 & 1570.2416 & 27 \\
\hline 14 & 1499.7965 & 750.4019 & 1482.7700 & 741.8886 & 1481.7859 & 741.3966 & F & 3044.4025 & 1522.7049 & 3027.3759 & 1514.1916 & 3026.3919 & 1513.6996 & 26 \\
\hline 15 & 1662.8598 & 831.9336 & 1645.8333 & 823.4203 & 1644.8493 & 822.9283 & $\mathbf{Y}$ & 2897.3341 & 1449.1707 & 2880.3075 & 5574 & 3235 & 1654 & 25 \\
\hline 16 & 1719.8813 & 860.4443 & 1702.8547 & 851.9310 & 1701.8707 & 851.4390 & G & 2734.2707 & 1367.6390 & 2717.2442 & 1359.1257 & 2716.2602 & 1358.6337 & 24 \\
\hline 17 & 1847.9399 & 924.4736 & 1830.9133 & 915.9603 & 1829.9293 & 915.4683 & $\mathbf{Q}$ & 2677.2493 & 1339.1283 & 2660.2227 & 1330.6150 & 2659.2387 & 1330.1230 & 23 \\
\hline 18 & 2007.9705 & 1004.4889 & 1990.9440 & 995.9756 & 1989.9600 & & C & 1907 & 1275.0990 & 2532.1641 & & 1801 & 0937 & 22 \\
\hline 19 & 2095.0026 & 1048.0049 & 2077.9760 & 1039.4916 & 2076.9920 & 1038.9996 & $\mathrm{~S}$ & 2389.1600 & 1195.0837 & 2372.1335 & 1186.5704 & 2371.1495 & 1186.0784 & 21 \\
\hline 20 & 2224.0451 & 1112.5262 & 2207.0186 & 1104.0129 & 2206.0346 & 1103.5209 & $\mathbf{E}$ & 2302.1280 & 1151.5676 & 2285.1015 & 1143.0544 & 2284.1174 & 1142.5624 & 20 \\
\hline 21 & 2337.1292 & 1169.0682 & 2320.1027 & 1160.5550 & 2319 & 1160.0630 & I & 2173.0854 & 1087 & 2156.0589 & 5331 & 0748 & 1078.0411 & 19 \\
\hline 22 & 2497.1599 & 1249.0836 & 2480.1333 & 1240.5703 & 2479.1493 & 1240.0783 & $\mathrm{C}$ & 2060.0013 & 1030.5043 & 2042.9748 & 1021.9910 & 2041.9908 & 1021.4990 & 18 \\
\hline 23 & 2554.1813 & 1277.5943 & 2537.1548 & 1269.0810 & 2536.1708 & 1268.5890 & $\mathbf{G}$ & 1899.9707 & 950.4890 & 1882.9441 & 941.9757 & 1881.9601 & 941.4837 & 17 \\
\hline 24 & 2641.2133 & 1321.1103 & 2624.1868 & 1312.5970 & 2623.2028 & 1312.1050 & $\mathrm{~S}$ & 1842.9492 & 921.9783 & 1825.9227 & 913.4650 & 1824.9387 & 912.9730 & 16 \\
\hline 25 & 2756.2403 & 1378.6238 & 2739.2137 & 1370.1105 & 2738.2297 & 1369.6185 & $\mathbf{N}$ & 1755.9172 & 878.4622 & 1738.8907 & 869.9490 & 1737.9066 & 869.4570 & 15 \\
\hline 26 & 2893.2992 & 1447.1532 & 2876.2726 & 1438.6400 & 2875.2886 & 1438.1480 & $\mathbf{H}$ & 1640.8903 & 820.9488 & 1623.8637 & 812.4355 & 1622.8797 & 811.9435 & 14 \\
\hline 27 & 2980.3312 & 1490.6693 & 2963.3047 & 1482.1560 & 2962.3207 & 1481.6640 & $\mathrm{~S}$ & 1503.8314 & 752.4193 & 1486.8048 & 743.9060 & 1485.8208 & 743.4140 & 13 \\
\hline 28 & 3127.3996 & 1564.2035 & 3110.3731 & 1555.6902 & 3109.3891 & 1555.1982 & $\mathbf{F}$ & 1416.7993 & 708.9033 & 1399.7728 & 700.3900 & 1398.7888 & 699.8980 & 12 \\
\hline 29 & 3258.4401 & \begin{tabular}{|l|}
1629.7237 \\
\end{tabular} & 3241.4136 & 1621.2104 & 3240.4296 & 1620.7184 & M & 1269.7309 & 635.3691 & 1252.7044 & 626.8558 & 1251.7203 & 626.3638 & 11 \\
\hline 30 & 3355.4929 & 1678.2501 & 3338.4663 & 1669.7368 & 3337.4823 & 1669.2448 & $\mathbf{P}$ & 1138.6904 & 569.8489 & 1121.6639 & 561.3356 & 1120.6799 & 560.8436 & 10 \\
\hline 31 & 3468.5770 & 1734.7921 & 3451.5504 & 1726.2788 & 3450.5664 & 1725.7868 & I & 1041.6377 & 521.3225 & 1024.6111 & 512.8092 & 1023.6271 & 512.3172 & 9 \\
\hline 32 & 3567.6454 & 1784.3263 & 3550.6188 & 1775.8130 & 3549.6348 & 1775.3210 & V & 928.5536 & 464.7804 & 911.5271 & 456.2672 & 910.5430 & 455.7752 & 8 \\
\hline 33 & 3680.7294 & 1840.8684 & 3663.7029 & 1832.3551 & 3662.7189 & 1831.8631 & $\mathbf{L}$ & 829.4852 & 415.2462 & 812.4586 & 406.7330 & 811.4746 & 406.2409 & 7 \\
\hline 34 & 3809.7720 & \begin{tabular}{|l|}
1905.3896 \\
\end{tabular} & 3792.7455 & \begin{tabular}{|l|}
1896.8764 \\
\end{tabular} & 3791.7615 & 1896.3844 & $\mathbf{E}$ & 716.4011 & 358.7042 & 699.3746 & 350.1909 & 698.3906 & 349.6989 & 6 \\
\hline 35 & 3940.8125 & 1970.9099 & 3923.7860 & 1962.3966 & 3922.8019 & 1961.9046 & M & 587.3585 & 294.1829 & 570.3320 & 285.6696 & & & 5 \\
\hline 36 & 4039.8809 & 2020.4441 & 4022.8544 & 2011.9308 & 4021.8704 & 2011.4388 & V & 456.3180 & 228.6627 & 439.2915 & 220.1494 & & & 4 \\
\hline 37 & 4136.9337 & 2068.9705 & 4119.9071 & 2060.4572 & 4118.9231 & 2059.9652 & $\mathbf{P}$ & 357.2496 & 179.1285 & 340.2231 & 170.6152 & & & 3 \\
\hline 38 & 4250.0177 & 2125.5125 & 4232.9912 & 2116.9992 & 4232.0072 & 2116.5072 & $\mathbf{L}$ & 260.1969 & 130.6021 & 243.1703 & 122.0888 & & & 2 \\
\hline 39 & & & & & & & $\mathbf{K}$ & 147.1128 & 74.0600 & 130.0863 & 65.5468 & & & 1 \\
\hline
\end{tabular}
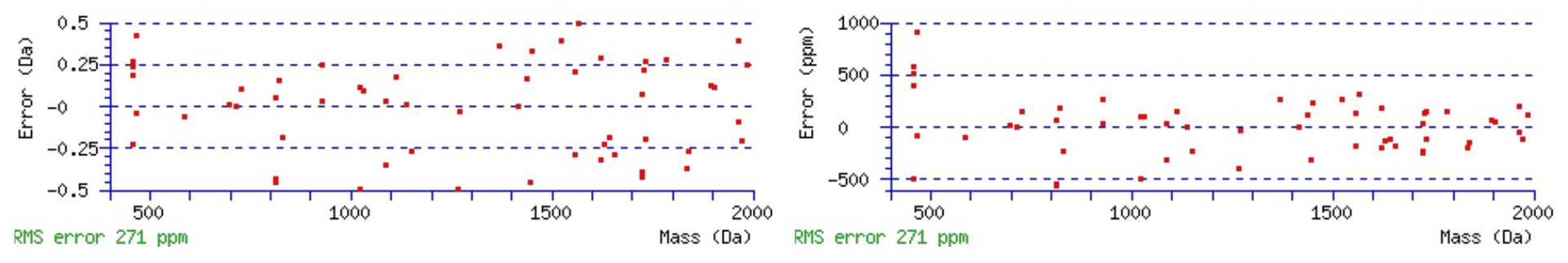

\section{All matches to this query}

\begin{tabular}{|l|l|l|l|}
\hline Score & Mr(calc): & Delta & \multicolumn{1}{c|}{ Sequence } \\
\hline 47.6 & 4395.1160 & 0.0087 & LNQATVTSNRPGLFYGQCSEICGSNHSFMPIVLEMVPLK \\
\hline 26.4 & 4395.1160 & 0.0087 & LNQATVTSNRPGLFYGQCSEICGSNHSFMPIVLEMVPLK \\
\hline 17.9 & 4394.1320 & 0.9927 & LNQATVTSNRPGLFYGQCSEICGSNHSFMPIVLEMVPLK \\
\hline
\end{tabular}




\begin{tabular}{|l|l|l|l|}
\hline 15.8 & 4395.1160 & 0.0087 & LNQATVTSNRPGLFYGQCSEICGSNHSFMPIVLEMVPLK \\
\hline 3.3 & 4394.0946 & 1.0301 & TNGIILVAYGVLGTQRYNGWVDQNSPVLLNEPVLSSMAK \\
\hline 2.6 & 4395.1184 & 0.0063 & SSMLPTMAAGLNSILFAINIDNKDLNGQSKFAPTVSDLLK \\
\hline 2.6 & 4395.1184 & 0.0063 & SSMLPTMAAGLNSILFAINIDNKDLNGQSKFAPTVSDLLK \\
\hline 2.6 & 4395.1184 & 0.0063 & SSMLPTMAAGLNSILFAINIDNKDLNGQSKFAPTVSDLLK \\
\hline 2.6 & 4395.1184 & 0.0063 & SSMLPTMAAGLNSILFAINIDNKDLNGQSKFAPTVSDLLK \\
\hline 2.6 & 4395.1184 & 0.0063 & SSMLPTMAAGLNSILFAINIDNKDLNGQSKFAPTVSDLLK \\
\hline
\end{tabular}

Spectrum No: 712; Query: 199; Rank: 1

\section{Peptide View}

MS/MS Fragmentation of RNWTETEVR

Found in IPI00327697, Tax_Id=10116 Gene_Symbol=Dpep1 Dipeptidase 1 precursor

Match to Query 199: 1190.570428 from(596.292490,2+)

Title: 100101RatKid_NS_deglyco_18.1282.1282.2.dta

Data file K:INewmanPaper|Piliangl3SubProteomes\Piliang3SP\mgf5ppm\ERLIC_3SubProteomes5ppm.mgf
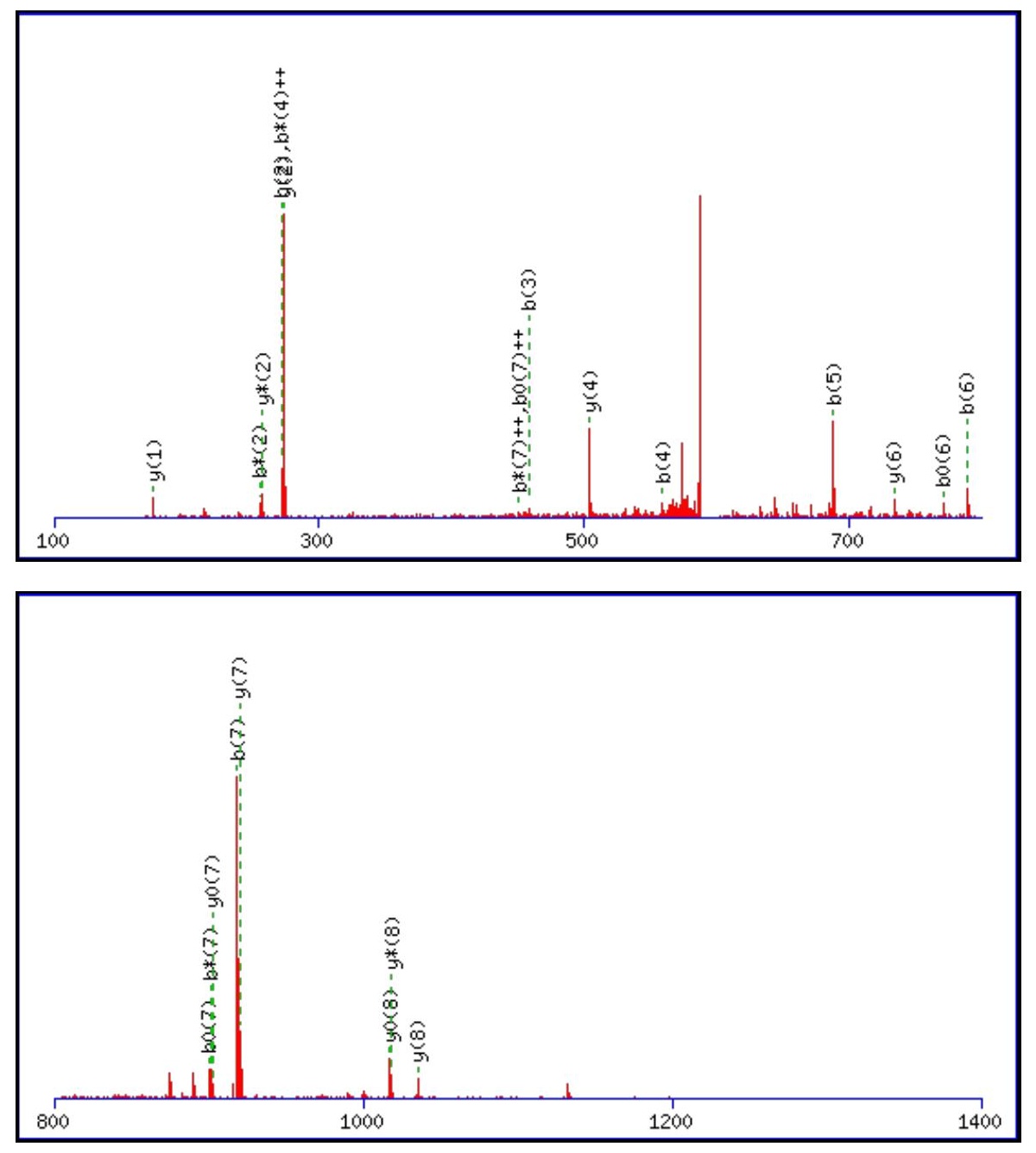


$1400 \quad 1600 \quad 1800 \quad 2000$

Monoisotopic mass of neutral peptide $\operatorname{Mr}($ calc): 1190.5680

Fixed modifications: Carbamidomethyl (C)

Variable modifications:

N2 : Deamidated $\mathrm{N}(\mathrm{N})$

Ions Score: 48 Expect: 0.0021

Matches (Bold Red): 24/86 fragment ions using 43 most intense peaks

\begin{tabular}{|c|c|c|c|c|c|c|c|c|c|c|c|c|c|c|}
\hline \# & b & $\mathbf{b}^{++}$ & $\mathbf{b}^{*}$ & $\mathbf{b}^{*^{++}}$ & $\mathbf{b}^{0}$ & $\mathbf{b}^{0++}$ & Seq. & $\mathbf{y}$ & $\mathbf{y}^{++}$ & $\mathrm{y}^{*}$ & $\mathrm{y}^{\mathrm{*}^{++}}$ & $\mathbf{y}^{\mathbf{0}}$ & $y^{0++}$ & $\#$ \\
\hline $\mathbf{1}$ & 157.1084 & 79.0578 & 140.0818 & 70.5446 & & & $\mathbf{R}$ & & & & & & & 9 \\
\hline 2 & 272.1353 & 136.5713 & 255.1088 & 128.0580 & & & $\mathbf{N}$ & 1035.4742 & 518.2407 & 1018.4476 & 509.7274 & 1017.4636 & 509.2354 & 8 \\
\hline 3 & 458.2146 & 229.6110 & 441.1881 & 221.0977 & & & W & 920.4472 & 460.7272 & 903.4207 & 452.2140 & 902.4367 & 451.7220 & 7 \\
\hline 4 & 559.2623 & 280.1348 & 542.2358 & 271.6215 & 541.2518 & 271.1295 & $\mathbf{T}$ & 734.3679 & 367.6876 & 717.3414 & 359.1743 & 716.3573 & 358.6823 & 6 \\
\hline 5 & 688.3049 & 344.6561 & 671.2784 & 336.1428 & 670.2943 & 335.6508 & E & 633.3202 & 317.1638 & 616.2937 & 308.6505 & 615.3097 & 308.1585 & 5 \\
\hline 6 & 789.3526 & 395.1799 & 772.3260 & 386.6667 & 771.3420 & 386.1746 & $\mathbf{T}$ & 504.2776 & 252.6425 & 487.2511 & 244.1292 & 486.2671 & 243.6372 & 4 \\
\hline 7 & 918.3952 & 459.7012 & 901.3686 & 451.1880 & 900.3846 & 450.6959 & $\mathbf{E}$ & 403.2300 & 202.1186 & 386.2034 & 193.6053 & 385.2194 & 193.1133 & 3 \\
\hline 0 & 1017.4636 & 509.2354 & 1000.4370 & 500.7222 & 999.4530 & 500.2302 & V & 274.1874 & 137.5973 & 257.1608 & 129.0840 & & & 2 \\
\hline 9 & & & & & & & $\mathbf{R}$ & 175.1190 & 88.0631 & 158.0924 & 79.5498 & & & 1 \\
\hline
\end{tabular}
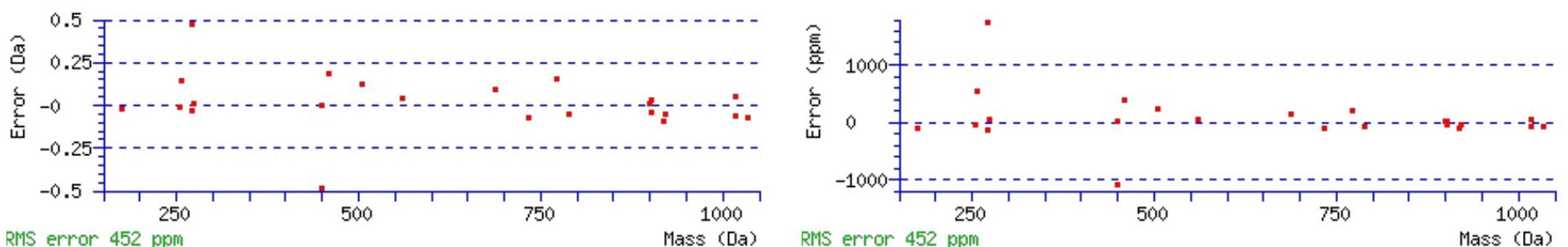

All matches to this query

\begin{tabular}{|l|l|l|l|}
\hline Score & Mr(calc): & Delta & \multicolumn{1}{|c|}{ Sequence } \\
\hline 47.6 & 1190.5680 & 0.0024 & RNWTETEVR \\
\hline 16.0 & 1188.5669 & 2.0035 & GNMQAQQSAVR \\
\hline 15.8 & 1190.5720 & -0.0016 & NQYPDIWVR \\
\hline 10.5 & 1190.5737 & -0.0032 & IFIATYEVR \\
\hline 10.4 & 1190.5710 & -0.0006 & FPNVHSRVR \\
\hline 9.5 & 1189.5717 & 0.9987 & GLGGTHASRVR \\
\hline 9.4 & 1189.5761 & 0.9943 & GDMPTLTDKGR \\
\hline 9.2 & 1190.5710 & -0.0006 & HKHTKGFTR \\
\hline 9.1 & 1188.5743 & 1.9961 & ISAMVTMQHR \\
\hline 8.9 & 1190.5737 & -0.0032 & IFIATYEVR \\
\hline
\end{tabular}


Spectrum No: 713; Query: 394; Rank: 1

\section{Peptide View}

MS/MS Fragmentation of DLCNATENFVR

Found in IPI00209975, Tax_Id=10116 Gene_Symbol=C4bpb C4b-binding protein beta chain precursor

Match to Query 394: 1338.585648 from(670.300100,2+)

Title: 091008RatKidney_NoSalt_32.1997.1997.2.dta

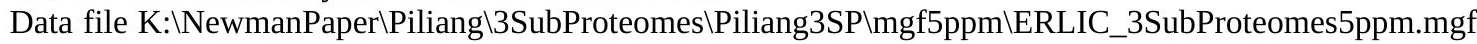
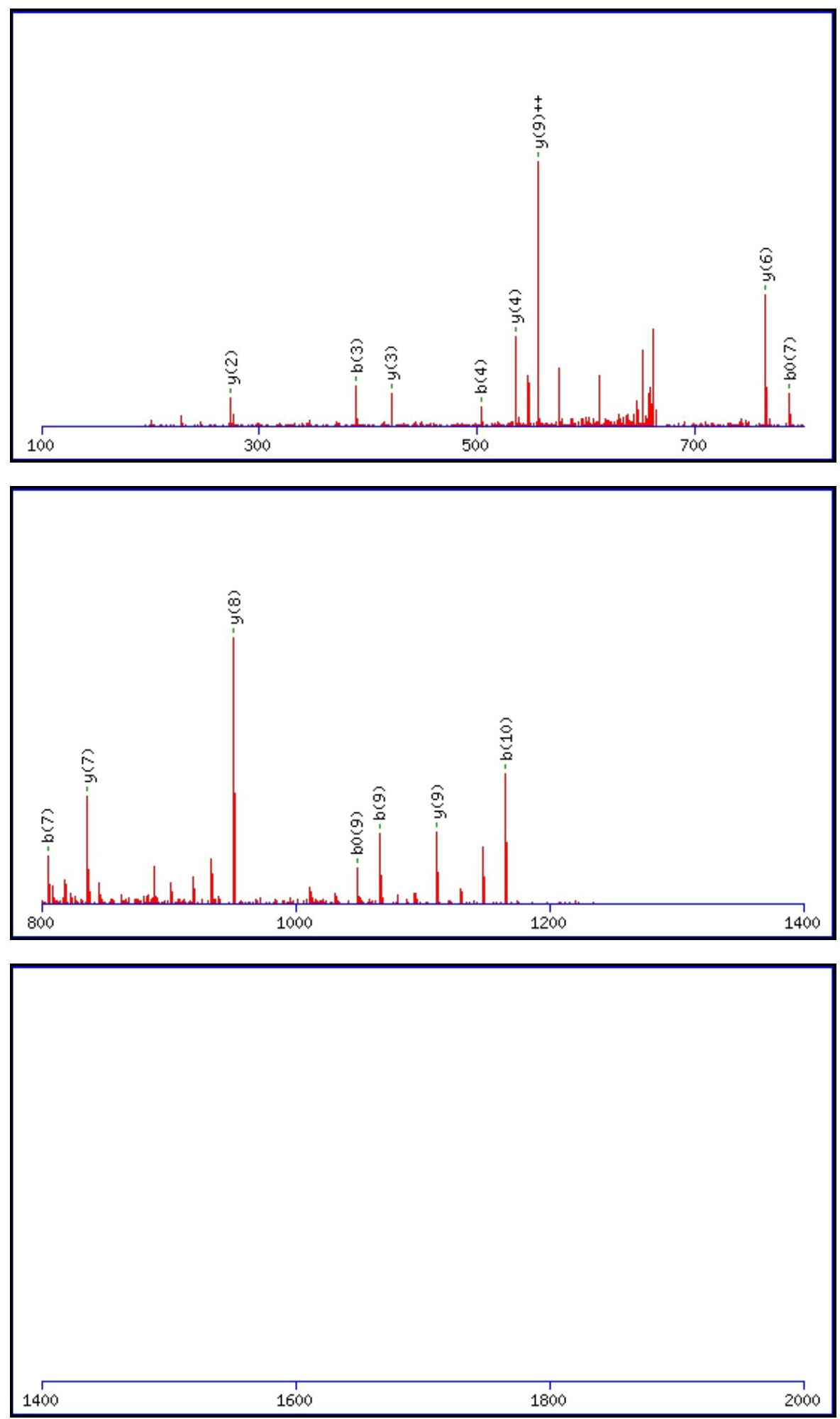

Monoisotopic mass of neutral peptide $\operatorname{Mr}($ calc): 1338.5874

Fixed modifications: Carbamidomethyl (C)

Variable modifications: 
N4 : Deamidated_N (N)

Ions Score: 48 Expect: 0.0024

Matches (Bold Red): 15/106 fragment ions using 27 most intense peaks

\begin{tabular}{|c|c|c|c|c|c|c|c|c|c|c|c|c|c|c|}
\hline \# & b & $\mathbf{b}^{++}$ & $\mathbf{b}^{*}$ & $\mathbf{b}^{*^{++}}$ & $\mathbf{b}^{0}$ & $\mathbf{b}^{0++}$ & Seq. & $\mathbf{y}$ & $y^{++}$ & $\mathbf{y}^{*}$ & $\mathrm{y}^{*^{++}}$ & $\mathbf{y}^{\mathbf{0}}$ & $\mathbf{y}^{0++}$ & \# \\
\hline 1 & 116.0342 & 58.5207 & & & 98.0237 & 49.5155 & D & & & & & & & 11 \\
\hline 2 & 229.1183 & 115.0628 & & & 211.1077 & 106.0575 & $\mathbf{L}$ & 1224.5677 & 612.7875 & 1207.5412 & 604.2742 & 1206.5572 & 603.7822 & 10 \\
\hline 3 & 389.1489 & 195.0781 & & & 371.1384 & 186.0728 & C & 1111.4837 & 556.2455 & 1094.4571 & 547.7322 & 1093.4731 & 547.2402 & 9 \\
\hline 4 & 504.1759 & 252.5916 & 487.1493 & 244.0783 & 486.1653 & 243.5863 & $\mathbf{N}$ & 951.4530 & 476.2302 & 934.4265 & 467.7169 & 933.4425 & 467.2249 & 8 \\
\hline 5 & 575.2130 & 288.1101 & 558.1864 & 279.5969 & 557.2024 & 279.1048 & A & 836.4261 & 418.7167 & 819.3995 & 410.2034 & 818.4155 & 409.7114 & 7 \\
\hline 6 & 676.2607 & 338.6340 & 659.2341 & 330.1207 & 658.2501 & 329.6287 & $\mathbf{T}$ & 765.3890 & 383.1981 & 748.3624 & 374.6849 & 747.3784 & 374.1928 & 6 \\
\hline 7 & 805.3033 & 403.1553 & 788.2767 & 394.6420 & 787.2927 & 394.1500 & $\mathbf{E}$ & 664.3413 & 332.6743 & 647.3148 & 324.1610 & 646.3307 & 323.6690 & 5 \\
\hline 8 & 919.3462 & 460.1767 & 902.3196 & 451.6635 & 901.3356 & 451.1714 & $\mathbf{N}$ & 535.2987 & 268.1530 & 518.2722 & 259.6397 & & & 4 \\
\hline 9 & 1066.4146 & 533.7109 & 1049.3880 & 525.1977 & 1048.4040 & 524.7057 & $\mathbf{F}$ & 421.2558 & 211.1315 & 404.2292 & 202.6183 & & & 3 \\
\hline 10 & 1165.4830 & 583.2451 & 1148.4565 & 574.7319 & 1147.4724 & 574.2399 & $\mathbf{V}$ & 274.1874 & 137.5973 & 257.1608 & 129.0840 & & & 2 \\
\hline 11 & & & & & & & $\mathbf{R}$ & 175.1190 & 88.0631 & 158.0924 & 79.5498 & & & 1 \\
\hline
\end{tabular}
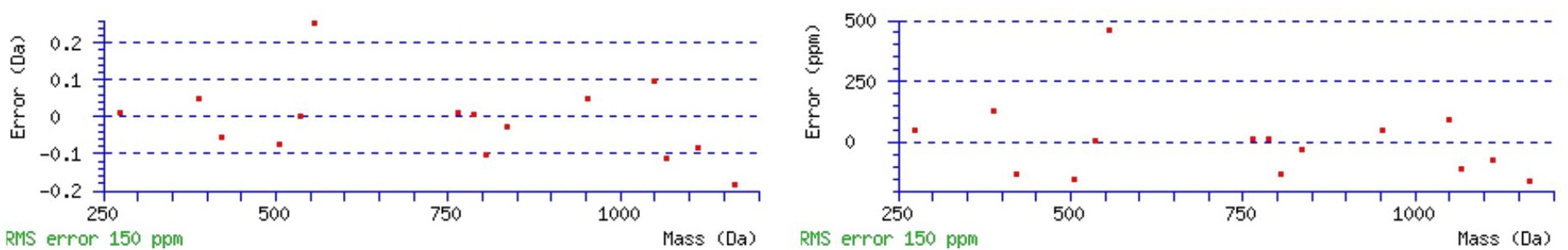

All matches to this query

\begin{tabular}{|l|l|l|l|}
\hline Score & Mr(calc): & Delta & \multicolumn{1}{c|}{ Sequence } \\
\hline 47.5 & 1338.5874 & -0.0018 & $\underline{\text { DLCNATENFVR }}$ \\
\hline 27.7 & 1338.5874 & -0.0018 & DLCNATENFVR \\
\hline 13.0 & 1337.5725 & 1.0132 & TGNRGSPVGAANR \\
\hline 10.1 & 1338.5904 & -0.0047 & $\underline{\text { RNLYHXPMVR }}$ \\
\hline 7.1 & 1338.5734 & 0.0122 & $\underline{\text { RTSFLEGTLR }}$ \\
\hline 6.3 & 1337.5725 & 1.0132 & TGNRGSPVGAANR \\
\hline 6.3 & 1336.5885 & 1.9972 & TGNRGSPVGAANR \\
\hline 6.0 & 1338.5908 & -0.0051 & GVMEGNCVSLTR \\
\hline 5.8 & 1338.5817 & 0.0040 & ELTTSSHDTIR \\
\hline 5.8 & 1338.5817 & 0.0040 & ELTTSSHDTIR \\
\hline
\end{tabular}

Spectrum No: 714; Query: 179; Rank: 1

\section{Peptide View}

\section{MS/MS Fragmentation of KNVTDLVEGAK}

Found in IPI00366496, Tax_Id=10116 Gene_Symbol=Slc44a2_predicted similar to CTL2 gene

Match to Query 179: 1173.625308 from(587.819930,2+)

Title: 100101RatKid_NS_deglyco_26.1499.1499.2.dta

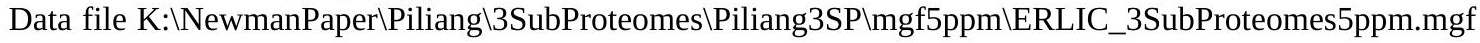



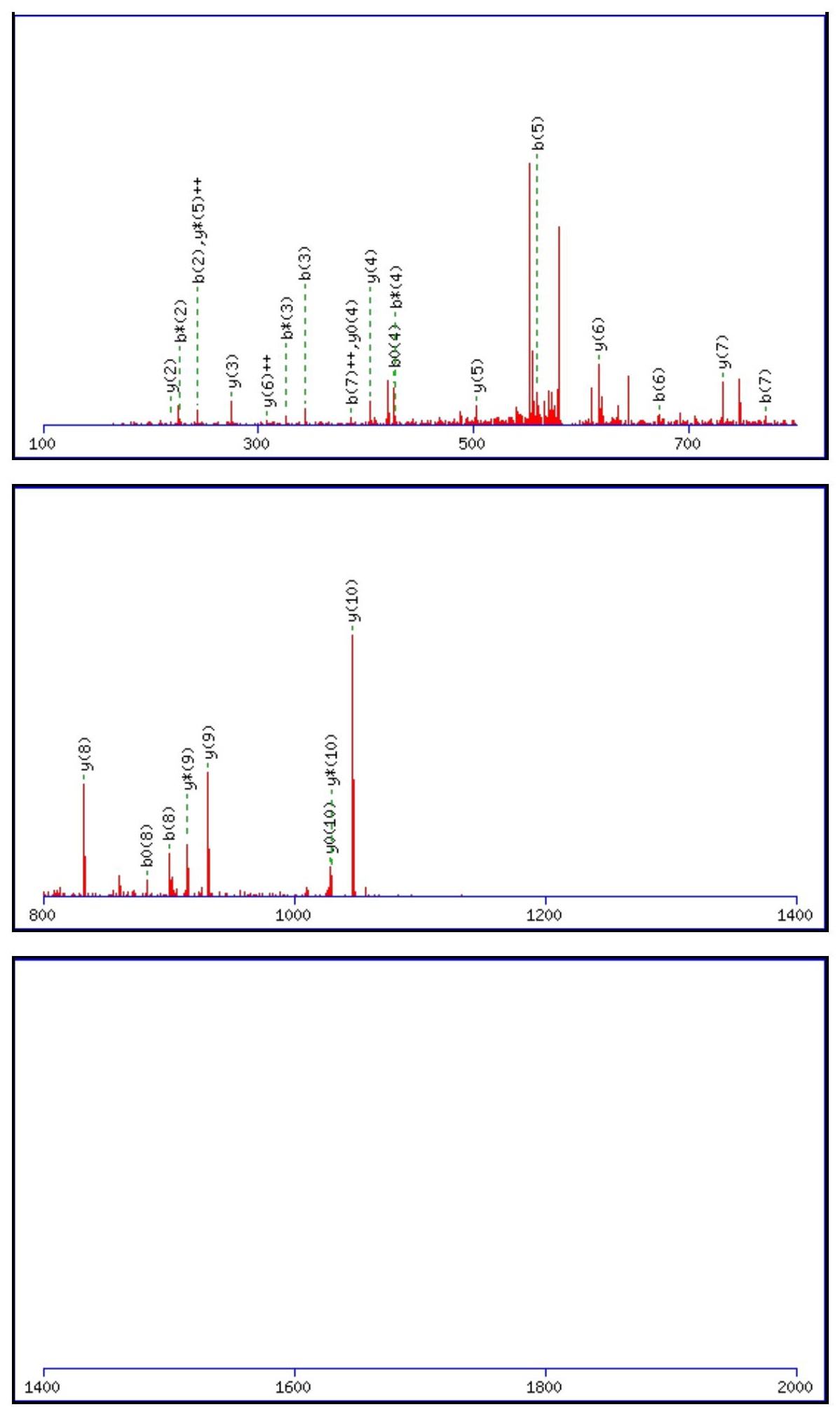

Monoisotopic mass of neutral peptide $\operatorname{Mr}($ calc): 1173.6241

Fixed modifications: Carbamidomethyl (C)

Variable modifications:

N2 : Deamidated $\mathrm{N}(\mathrm{N})$

Ions Score: 47 Expect: 0.002

Matches (Bold Red): 28/108 fragment ions using 65 most intense peaks

\begin{tabular}{|r|c|c|c|c|c|c|c|c|c|c|c|c|c|c|}
\hline$\#$ & $\mathbf{b}$ & $\mathbf{b}^{++}$ & $\mathbf{b}^{*}$ & $\mathbf{b}^{\boldsymbol{*}_{++}}$ & $\mathbf{b}^{\mathbf{0}}$ & $\mathbf{b}^{\mathbf{0 + +}}$ & $\mathbf{S e q}$ & $\mathbf{y}$ & $\mathbf{y}^{++}$ & $\mathbf{y}^{\mathbf{*}}$ & $\mathbf{y}^{\boldsymbol{*}^{++}}$ & $\mathbf{y}^{\mathbf{0}}$ & $\mathbf{y}^{\mathbf{0 + +}}$ & $\#$ \\
\hline $\mathbf{1}$ & 129.1022 & 65.0548 & 112.0757 & 56.5415 & & & $\mathbf{K}$ & & & & & & & $\mathbf{1 1}$ \\
\hline $\mathbf{2}$ & $\mathbf{2 4 4 . 1 2 9 2}$ & 122.5682 & 227.1026 & 114.0550 & & & $\mathbf{N}$ & $\mathbf{1 0 4 6 . 5 3 6 4}$ & 523.7719 & $\mathbf{1 0 2 9 . 5 0 9 9}$ & 515.2586 & $\mathbf{1 0 2 8 . 5 2 5 9}$ & 514.7666 & $\mathbf{1 0}$ \\
\hline $\mathbf{3}$ & $\mathbf{3 4 3 . 1 9 7 6}$ & 172.1024 & 326.1710 & 163.5892 & & & $\mathbf{V}$ & $\mathbf{9 3 1 . 5 0 9 5}$ & 466.2584 & $\mathbf{9 1 4 . 4 8 2 9}$ & 457.7451 & 913.4989 & 457.2531 & $\mathbf{9}$ \\
\hline $\mathbf{4}$ & 444.2453 & 222.6263 & 427.2187 & 214.1130 & $\mathbf{4 2 6 . 2 3 4 7}$ & 213.6210 & $\mathbf{T}$ & $\mathbf{8 3 2 . 4 4 1 1}$ & 416.7242 & 815.4145 & 408.2109 & 814.4305 & 407.7189 & $\mathbf{8}$ \\
\hline $\mathbf{5}$ & $\mathbf{5 5 9 . 2 7 2 2}$ & 280.1397 & 542.2457 & 271.6265 & 541.2616 & 271.1345 & $\mathbf{D}$ & $\mathbf{7 3 1 . 3 9 3 4}$ & 366.2003 & 714.3668 & 357.6871 & 713.3828 & 357.1951 & $\mathbf{7}$ \\
\hline
\end{tabular}




\begin{tabular}{|c|c|c|c|c|c|c|c|c|c|c|c|c|c|c|}
\hline 6 & 672.3563 & |336.6818| & 655.3297 & |328.1685 & 654.3457 & |327.6765 | & $\mathbf{L}$ & 616.3665 & |308.6869 & 599.3399 & |300.1736 & 598.3559| & |299.6816 | & 6 \\
\hline 7 & 771.4247 & 386.2160 & 754.3981 & 377.7027 & 753.4141 & 377.2107 & $\mathbf{V}$ & 503.2824 & 252.1448 & 486.2558 & 243.6316 & 485.2718 & 243.1396 & 5 \\
\hline 8 & 900.4673 & 450.7373 & 883.4407 & 442.2240 & 882.4567 & 441.7320 & $\mathbf{E}$ & 404.2140 & 202.6106 & 387.1874 & 194.0974 & 386.2034 & 193.6053 & 4 \\
\hline 9 & 957.4887 & 479.2480 & 940.4622 & 470.7347 & 939.4782 & 470.2427 & $\mathbf{G}$ & 275.1714 & 138.0893 & 258.1448 & 129.5761 & & & 3 \\
\hline 10 & 1028.5259 & 514.7666 & 1011.4993 & 506.2533 & 1010.5153 & 505.7613 & A & 218.1499 & 109.5786 & 201.1234 & 101.0653 & & & 2 \\
\hline 11 & & & & & & & $\mathbf{K}$ & 147.1128 & 74.0600 & 130.0863 & 65.5468 & & & 1 \\
\hline
\end{tabular}
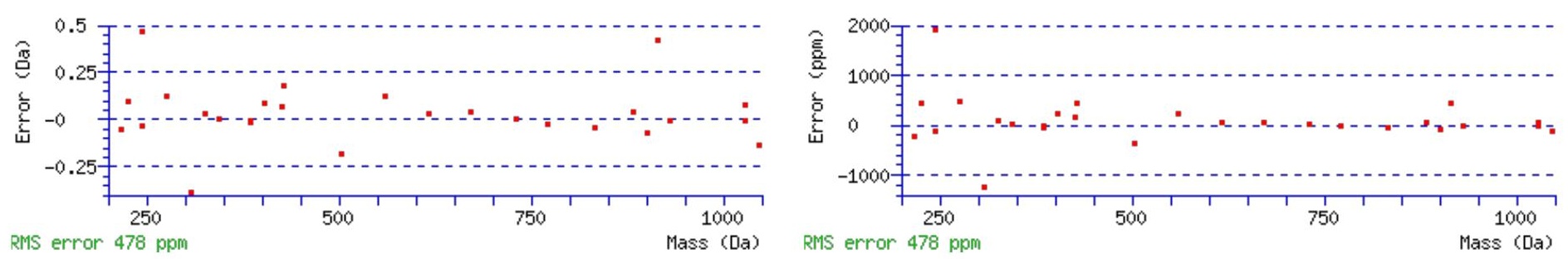

\section{All matches to this query}

\begin{tabular}{|l|l|l|l|}
\hline Score & Mr(calc): & Delta & \multicolumn{1}{c|}{ Sequence } \\
\hline 47.5 & 1173.6241 & 0.0012 & KNVTDLVEGAK \\
\hline 8.1 & 1173.6353 & -0.0100 & SSRTALNSLPK \\
\hline 5.7 & 1173.6353 & -0.0100 & VSKENVOKNK \\
\hline 4.6 & 1173.6250 & 0.0003 & VQMPSLKMPK \\
\hline 4.3 & 1173.6158 & 0.0095 & KLFSSSGLKK \\
\hline 3.9 & 1173.6176 & 0.0077 & MIRTNEAVPK \\
\hline 3.3 & 1173.6214 & 0.0039 & SRSLDRGLDR \\
\hline 3.2 & 1173.6353 & -0.0100 & SVQEKGKSSPK \\
\hline 3.1 & 1173.6295 & -0.0042 & QPHNLPPFPK \\
\hline 3.0 & 1171.6084 & 2.0169 & QIVNLSPANSK \\
\hline
\end{tabular}

Spectrum No: 715; Query: 556; Rank: 1

\section{Peptide View}

MS/MS Fragmentation of NQKDEVNETDLK

Found in IPI00211616, Tax_Id=10116 Gene_Symbol=Slc3a2 4F2 cell-surface antigen heavy chain

Match to Query 556: 1432.667228 from(717.340890,2+)

Title: 091008RatKidney_NoSalt_29.385.385.2.dta

Data file K:INewmanPaper|Piliang|3SubProteomes\Piliang3SP\mgf5ppm\ERLIC_3SubProteomes5ppm.mgf

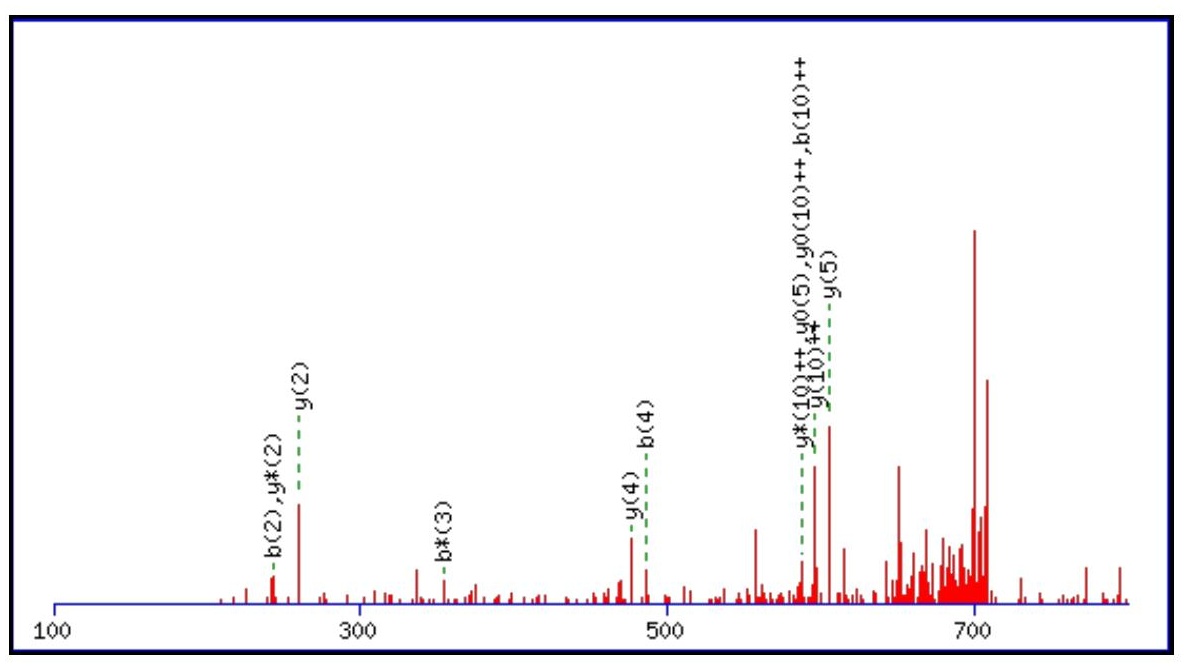



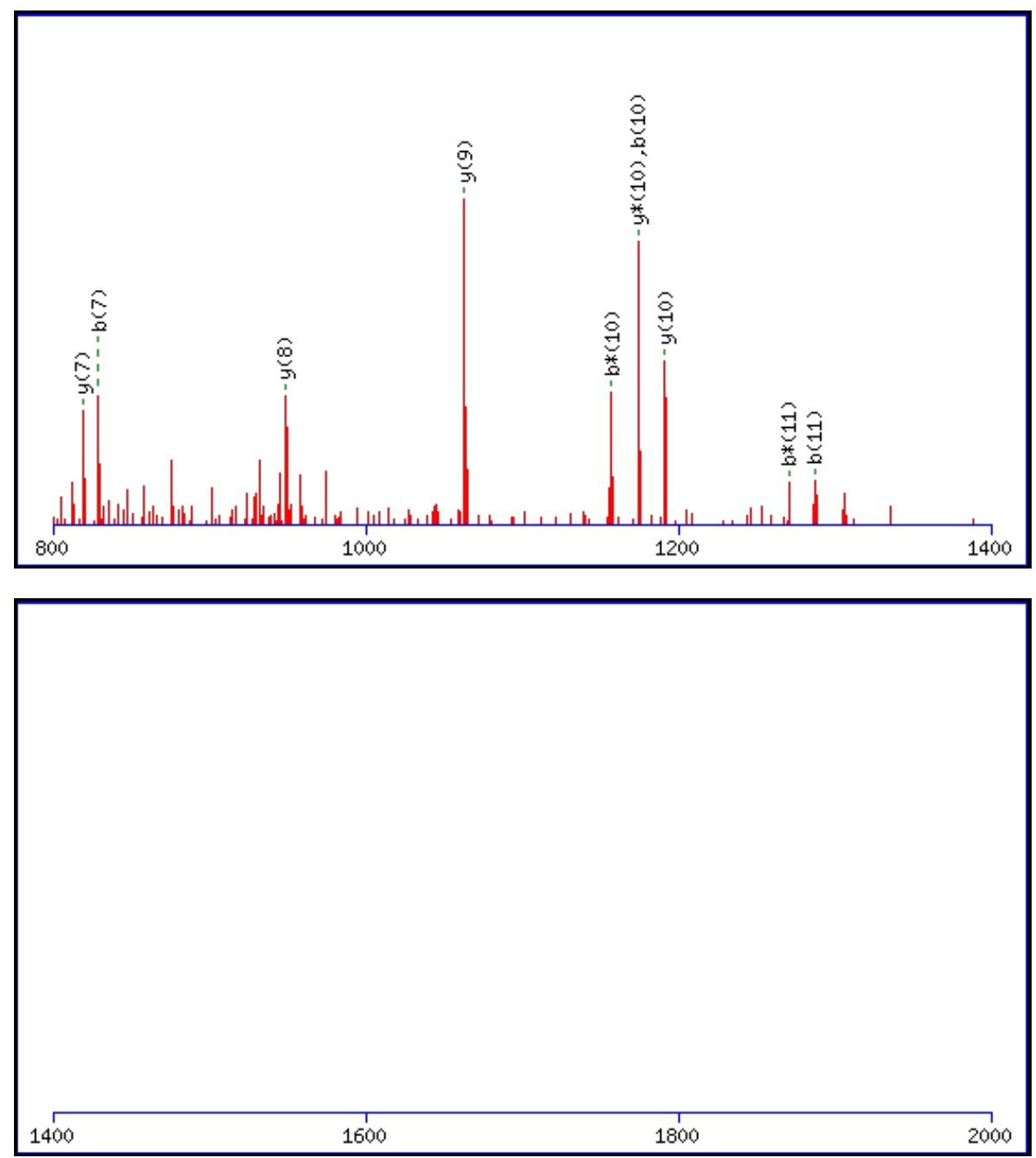

Monoisotopic mass of neutral peptide $\operatorname{Mr}($ calc): 1432.6681

Fixed modifications: Carbamidomethyl (C)

Variable modifications:

N7 : Deamidated_N (N)

Ions Score: 47 Expect: 0.0029

Matches (Bold Red): 22/122 fragment ions using 32 most intense peaks

\begin{tabular}{|r|c|c|c|c|c|c|c|c|c|c|c|c|c|c|}
\hline$\#$ & $\mathbf{b}$ & $\mathbf{b}^{++}$ & $\mathbf{b}^{*}$ & $\mathbf{b}^{\boldsymbol{*}^{++}}$ & $\mathbf{b}^{\mathbf{0}}$ & $\mathbf{b}^{\mathbf{0 + +}}$ & $\mathbf{S e q}$ & $\mathbf{y}$ & $\mathbf{y}^{++}$ & $\mathbf{y}^{*}$ & $\mathbf{y}^{\boldsymbol{*}^{++}}$ & $\mathbf{y}^{\mathbf{0}}$ & $\mathbf{y}^{\mathbf{0 + +}}$ & $\#$ \\
\hline $\mathbf{1}$ & 115.0502 & 58.0287 & 98.0237 & 49.5155 & & & $\mathbf{N}$ & & & & & & & $\mathbf{1 2}$ \\
\hline $\mathbf{2}$ & $\mathbf{2 4 3 . 1 0 8 8}$ & 122.0580 & 226.0822 & 113.5448 & & & $\mathbf{Q}$ & 1319.6325 & 660.3199 & 1302.6060 & 651.8066 & 1301.6219 & 651.3146 & $\mathbf{1 1}$ \\
\hline $\mathbf{3}$ & 371.2037 & 186.1055 & 354.1772 & 177.5922 & & & $\mathbf{K}$ & $\mathbf{1 1 9 1 . 5 7 3 9}$ & 596.2906 & $\mathbf{1 1 7 4 . 5 4 7 4}$ & $\mathbf{5 8 7 . 7 7 7 3}$ & 1173.5634 & $\mathbf{5 8 7 . 2 8 5 3}$ & $\mathbf{1 0}$ \\
\hline $\mathbf{4}$ & $\mathbf{4 8 6 . 2 3 0 7}$ & 243.6190 & 469.2041 & 235.1057 & 468.2201 & 234.6137 & $\mathbf{D}$ & $\mathbf{1 0 6 3 . 4 7 9 0}$ & 532.2431 & 1046.4524 & 523.7298 & 1045.4684 & 523.2378 & $\mathbf{9}$ \\
\hline $\mathbf{5}$ & 615.2733 & 308.1403 & 598.2467 & 299.6270 & 597.2627 & 299.1350 & $\mathbf{E}$ & $\mathbf{9 4 8 . 4 5 2 0}$ & 474.7297 & 931.4255 & 466.2164 & 930.4415 & 465.7244 & $\mathbf{8}$ \\
\hline $\mathbf{6}$ & 714.3417 & 357.6745 & 697.3151 & 349.1612 & 696.3311 & 348.6692 & $\mathbf{V}$ & $\mathbf{8 1 9 . 4 0 9 4}$ & 410.2084 & 802.3829 & 401.6951 & 801.3989 & 401.2031 & $\mathbf{7}$ \\
\hline $\mathbf{7}$ & $\mathbf{8 2 9 . 3 6 8 6}$ & 415.1880 & 812.3421 & 406.6747 & 811.3581 & 406.1827 & $\mathbf{N}$ & 720.3410 & 360.6741 & 703.3145 & 352.1609 & 702.3305 & 351.6689 & $\mathbf{6}$ \\
\hline $\mathbf{8}$ & 958.4112 & 479.7092 & 941.3847 & 471.1960 & 940.4007 & 470.7040 & $\mathbf{E}$ & $\mathbf{6 0 5 . 3 1 4 1}$ & 303.1607 & 588.2875 & 294.6474 & $\mathbf{5 8 7 . 3 0 3 5}$ & 294.1554 & $\mathbf{5}$ \\
\hline $\mathbf{9}$ & 1059.4589 & 530.2331 & 1042.4324 & 521.7198 & 1041.4483 & 521.2278 & $\mathbf{T}$ & $\mathbf{4 7 6 . 2 7 1 5}$ & 238.6394 & 459.2449 & 230.1261 & 458.2609 & 229.6341 & $\mathbf{4}$ \\
\hline $\mathbf{1 0}$ & $\mathbf{1 1 7 4 . 4 8 5 8}$ & $\mathbf{5 8 7 . 7 4 6 6}$ & $\mathbf{1 1 5 7 . 4 5 9 3}$ & 579.2333 & 1156.4753 & 578.7413 & $\mathbf{D}$ & 375.2238 & 188.1155 & 358.1973 & 179.6023 & 357.2132 & 179.1103 & $\mathbf{3}$ \\
\hline $\mathbf{1 1}$ & $\mathbf{1 2 8 7 . 5 6 9 9}$ & 644.2886 & $\mathbf{1 2 7 0 . 5 4 3 4}$ & 635.7753 & 1269.5593 & 635.2833 & $\mathbf{L}$ & $\mathbf{2 6 0 . 1 9 6 9}$ & 130.6021 & $\mathbf{2 4 3 . 1 7 0 3}$ & 122.0888 & & & $\mathbf{2}$ \\
\hline $\mathbf{1 2}$ & & & & & & & $\mathbf{K}$ & 147.1128 & 74.0600 & 130.0863 & 65.5468 & & & $\mathbf{1}$ \\
\hline
\end{tabular}



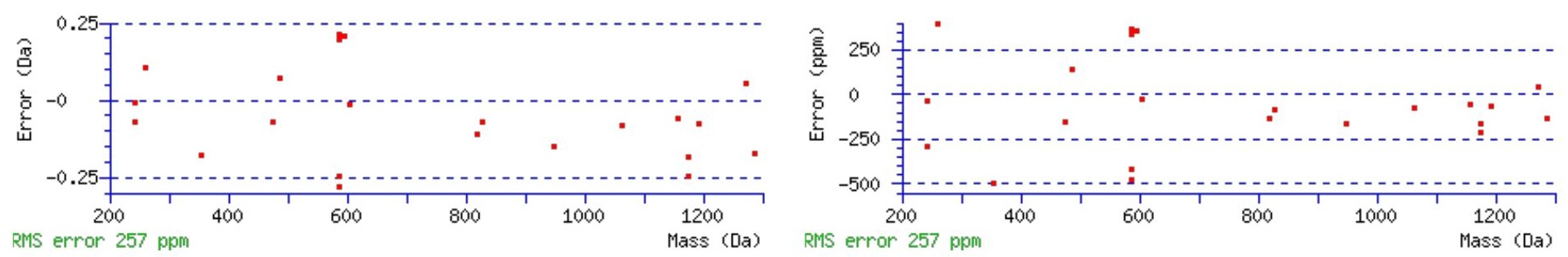

\section{All matches to this query}

\begin{tabular}{|l|l|l|l|}
\hline Score & Mr(calc): & Delta & \multicolumn{1}{c|}{ Sequence } \\
\hline 47.4 & 1432.6681 & -0.0009 & NQKDEVNETDLK \\
\hline 14.4 & 1432.6681 & -0.0009 & NQKDEVNETDLK \\
\hline 8.4 & 1432.6560 & 0.0112 & NNYEILTMLLK \\
\hline 6.6 & 1432.6673 & -0.0001 & LTFNEEIKTMK \\
\hline 6.4 & 1432.6615 & 0.0057 & ETVLSNGSLLIK \\
\hline 6.2 & 1432.6599 & 0.0073 & NPEKTSPNPELK \\
\hline 5.3 & 1432.6599 & 0.0073 & NPEKTSPNPELK \\
\hline 5.2 & 1432.6599 & 0.0073 & LEEVDLSGNHLK \\
\hline 5.0 & 1432.6615 & 0.0057 & ETVLSNGSLLIK \\
\hline 5.0 & 1432.6615 & 0.0057 & ETVLSNGSLLIK \\
\hline
\end{tabular}

Spectrum No: 716; Query: 377; Rank: 1

\section{Peptide View}

MS/MS Fragmentation of WSFSNGTSWQK

Found in IPI00558132, Tax_Id=10116 Gene_Symbol=Neu1 44 kDa protein

Match to Query 377: 1327.585268 from(664.799910,2+)

Title: 100101RatKid_NS_deglyco_27.2714.2714.2.dta

Data file K:INewmanPaper|Piliang|3SubProteomes\Piliang3SP\mgf5ppm\ERLIC_3SubProteomes5ppm.mgf

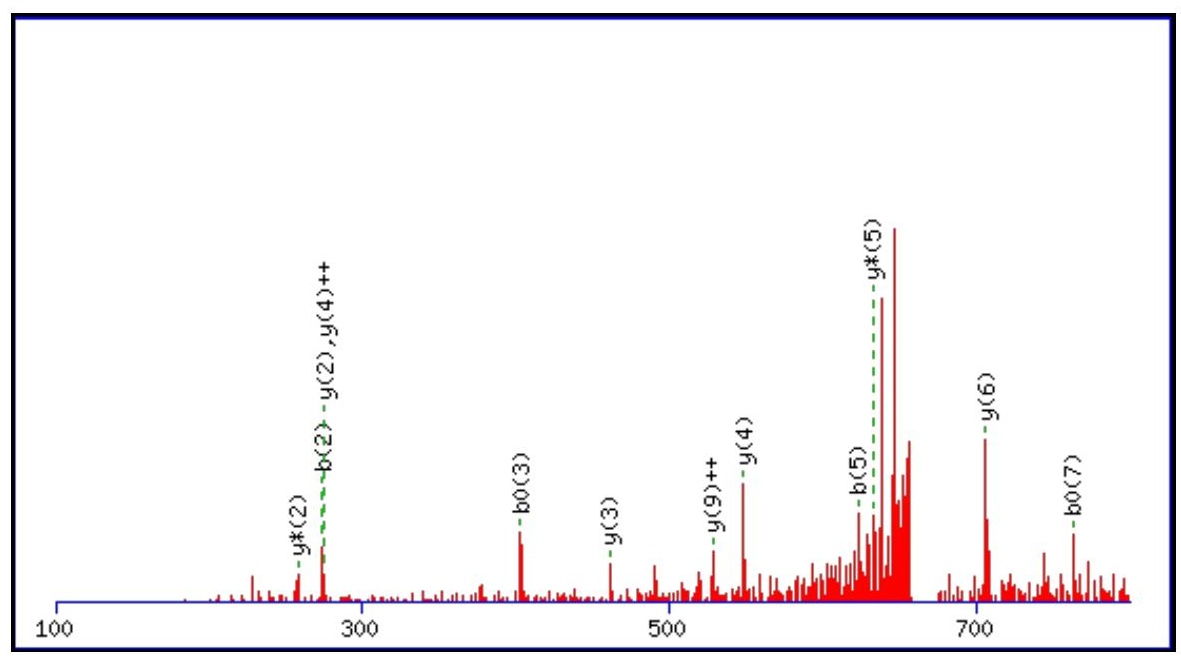




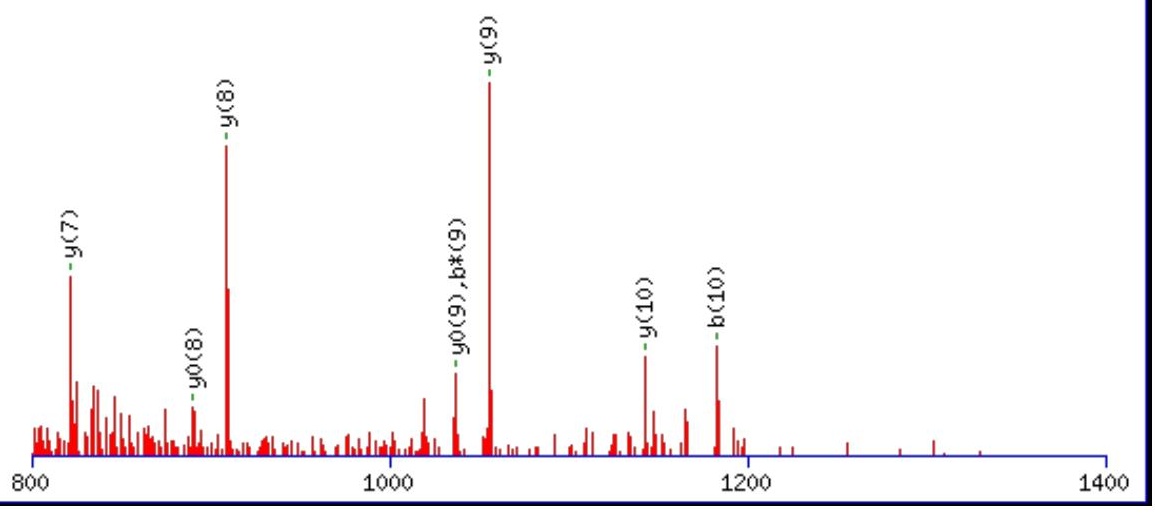

\begin{tabular}{rrr}
\hline 1600 & 1800 & 2000 \\
1400 & 1600
\end{tabular}

Monoisotopic mass of neutral peptide $\operatorname{Mr}($ calc): 1327.5833

Fixed modifications: Carbamidomethyl (C)

Variable modifications:

N5 : Deamidated_N (N)

Ions Score: 47 Expect: 0.0024

Matches (Bold Red): 20/104 fragment ions using 52 most intense peaks

\begin{tabular}{|c|c|c|c|c|c|c|c|c|c|c|c|c|c|c|}
\hline \# & b & $\mathbf{b}^{++}$ & b* & $\mathbf{b}^{*^{++}}$ & $\mathbf{b}^{0}$ & $\mathbf{b}^{0++}$ & Seq. & $\mathbf{y}$ & $\mathbf{y}^{++}$ & $\mathbf{y}^{*}$ & $\mathrm{y}^{*^{++}}$ & $\mathbf{y}^{0}$ & $\mathbf{y}^{\mathbf{0 + +}}$ & \# \\
\hline 1 & 187.0866 & 94.0469 & & & & & $\mathbf{W}$ & & & & & & & 11 \\
\hline 2 & 274.1186 & 137.5629 & & & 256.1081 & 128.5577 & S & 1142.5113 & 571.7593 & 1125.4847 & 563.2460 & 1124.5007 & 562.7540 & 10 \\
\hline 3 & 421.1870 & 211.0972 & & & 403.1765 & 202.0919 & $\mathbf{F}$ & 1055.4792 & 528.2433 & 1038.4527 & 519.7300 & 1037.4687 & 519.2380 & 9 \\
\hline 4 & 508.2191 & 254.6132 & & & 490.2085 & 245.6079 & S & 908.4108 & 454.7091 & 891.3843 & 446.1958 & 890.4003 & 445.7038 & 8 \\
\hline 5 & 623.2460 & 312.1266 & 606.2194 & 303.6134 & 605.2354 & 303.1214 & $\mathbf{N}$ & 821.3788 & 411.1930 & 804.3523 & 402.6798 & 803.3682 & 402.1878 & 7 \\
\hline 6 & 680.2675 & 340.6374 & 663.2409 & 332.1241 & 662.2569 & 331.6321 & G & 706.3519 & 353.6796 & 689.3253 & 345.1663 & 688.3413 & 344.6743 & 6 \\
\hline 7 & 781.3151 & 391.1612 & 764.2886 & 382.6479 & 763.3046 & 382.1559 & $\mathbf{T}$ & 649.3304 & 325.1688 & 632.3039 & 316.6556 & 631.3198 & 316.1636 & 5 \\
\hline 8 & 868.3472 & 434.6772 & 851.3206 & 426.1639 & 850.3366 & 425.6719 & $\mathrm{~S}$ & 548.2827 & 274.6450 & 531.2562 & 266.1317 & 530.2722 & 265.6397 & 4 \\
\hline 9 & 1054.4265 & 527.7169 & 1037.3999 & 519.2036 & 1036.4159 & 518.7116 & W & 461.2507 & 231.1290 & 444.2241 & 222.6157 & & & 3 \\
\hline 10 & 1182.4851 & 591.7462 & 1165.4585 & 583.2329 & 1164.4745 & 582.7409 & $\mathbf{Q}$ & 275.1714 & 138.0893 & 258.1448 & 129.5761 & & & 2 \\
\hline 11 & & & & & & & $\mathbf{K}$ & 147.1128 & 74.0600 & 130.0863 & 65.5468 & & & 1 \\
\hline
\end{tabular}
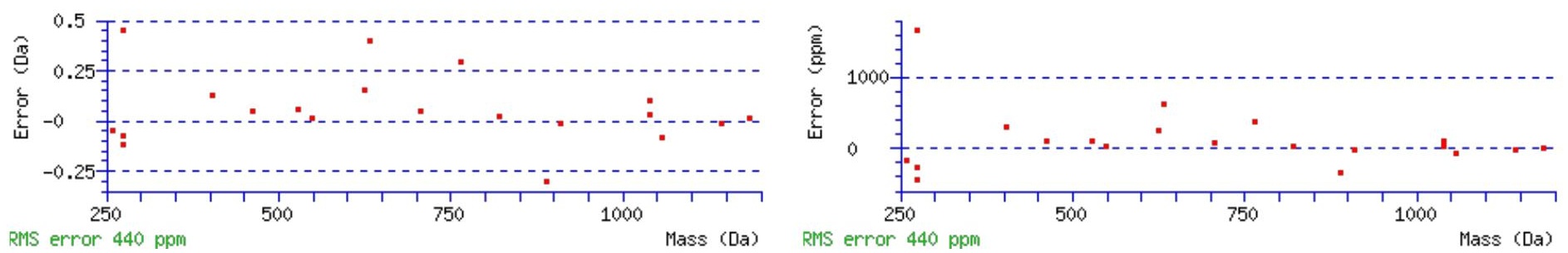


\section{All matches to this query}

\begin{tabular}{|l|l|l|l|}
\hline Score & Mr(calc): & Delta & \multicolumn{1}{c|}{ Sequence } \\
\hline 47.4 & 1327.5833 & 0.0020 & WSFSNGTSWQK \\
\hline 5.1 & 1327.5900 & -0.0048 & CLECEKAFSGK \\
\hline 5.0 & 1327.5723 & 0.0130 & LCNMFMEQQK \\
\hline 4.3 & 1327.5769 & 0.0084 & QAATSOSATEVR \\
\hline 4.3 & 1327.5769 & 0.0084 & QAATSQSATEVR \\
\hline 4.3 & 1327.5769 & 0.0084 & QAATSOSATEVR \\
\hline 1.4 & 1327.5955 & -0.0102 & $\underline{\text { SRLADMNATIR }}$ \\
\hline
\end{tabular}

Spectrum No: 717; Query: 1823; Rank: 1

\section{Peptide View}

MS/MS Fragmentation of GNSTLYPVASPSLQQLVLEK

Found in IPI00565110, Tax_Id=10116 Gene_Symbol=RGD1565147_predicted similar to N-acetylated alpha-linked acidic dipeptidase 2

Match to Query 1823: 2144.145708 from(1073.080130,2+)

Title: 100101RatKid_NS_deglyco_20.4416.4416.2.dta

Data file K:INewmanPaper|Piliangl3SubProteomes\Piliang3SP\mgf5ppm\ERLIC_3SubProteomes5ppm.mgf
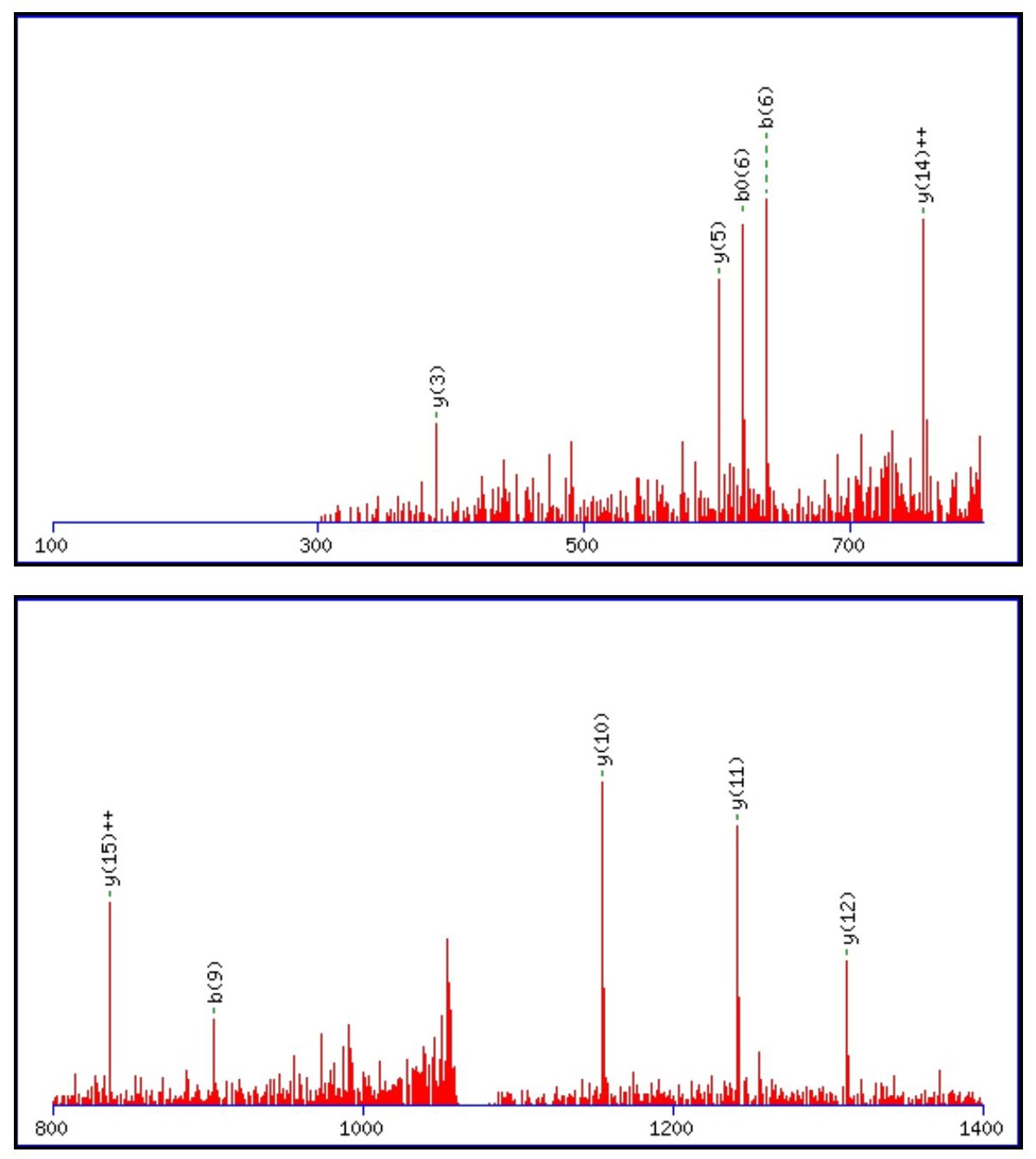


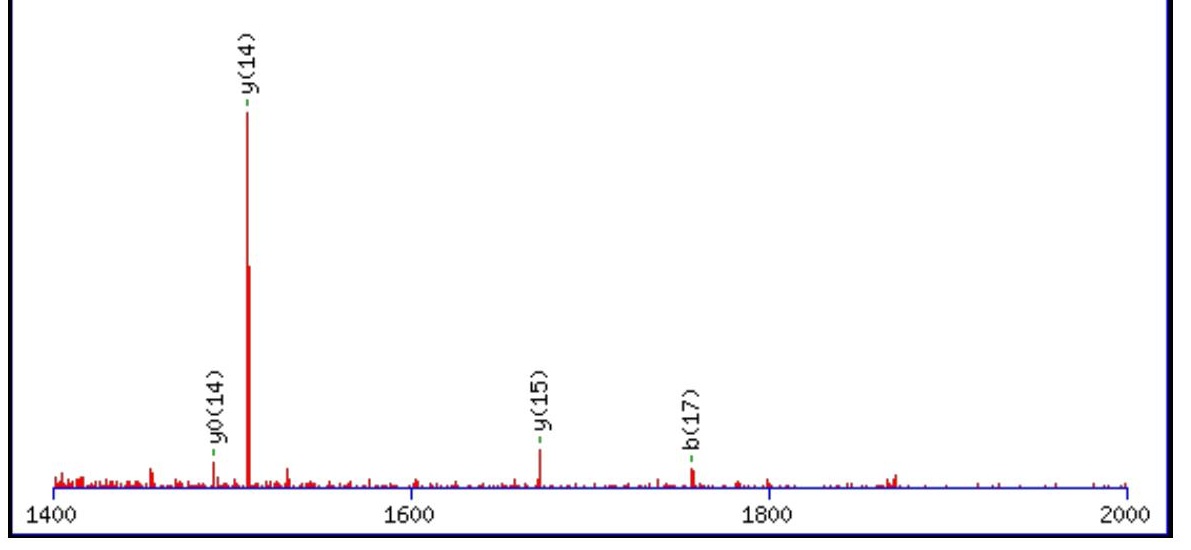

Monoisotopic mass of neutral peptide $\operatorname{Mr}($ calc): 2144.1365

Fixed modifications: Carbamidomethyl (C)

Variable modifications:

N2 : Deamidated $\mathrm{N}(\mathrm{N})$

Ions Score: 47 Expect: 0.0016

Matches (Bold Red): 14/220 fragment ions using 23 most intense peaks

\begin{tabular}{|c|c|c|c|c|c|c|c|c|c|c|c|c|c|c|}
\hline \# & b & $\mathbf{b}^{++}$ & $\mathbf{b}^{*}$ & $\mathbf{b}^{*^{++}}$ & $\mathbf{b}^{0}$ & $\mathbf{b}^{0++}$ & Seq. & $\mathbf{y}$ & $y^{++}$ & $\mathbf{y}^{*}$ & $\mathbf{y}^{*^{++}}$ & $\mathbf{y}^{0}$ & $\mathbf{y}^{\mathbf{0 + +}}$ & \# \\
\hline 1 & 58.0287 & 29.5180 & & & & & G & & & & & & & 20 \\
\hline 2 & 173.0557 & 87.0315 & 156.0291 & 78.5182 & & & $\mathbf{N}$ & 2088.1223 & 1044.5648 & 2071.0958 & 1036.0515 & 2070.1117 & 1035.5595 & 19 \\
\hline 3 & 260.0877 & 130.5475 & 243.0612 & 122.0342 & 242.0771 & 121.5422 & S & 1973.0954 & 987.0513 & 1956.0688 & 978.5380 & 1955.0848 & 978.0460 & 18 \\
\hline 4 & 361.1354 & 181.0713 & 344.1088 & 172.5581 & 343.1248 & 172.0660 & $\mathbf{T}$ & 1886.0633 & 943.5353 & 1869.0368 & 935.0220 & 1868.0528 & 934.5300 & 17 \\
\hline 5 & 474.2194 & 237.6134 & 457.1929 & 229.1001 & 456.2089 & 228.6081 & $\mathbf{L}$ & 1785.0157 & 893.0115 & 1767.9891 & 884.4982 & 1767.0051 & 884.0062 & 16 \\
\hline 6 & 637.2828 & 319.1450 & 620.2562 & 310.6318 & 619.2722 & 310.1397 & $\mathbf{Y}$ & 1671.9316 & 836.4694 & 1654.9051 & 827.9562 & 1653.9210 & 827.4642 & 15 \\
\hline 7 & 734.3355 & 367.6714 & 717.3090 & 359.1581 & 716.3250 & 358.6661 & $\mathbf{P}$ & 1508.8683 & 754.9378 & 1491.8417 & 746.4245 & 1490.8577 & 745.9325 & 14 \\
\hline 8 & 833.4040 & 417.2056 & 816.3774 & 408.6923 & 815.3934 & 408.2003 & $\mathbf{V}$ & 1411.8155 & 706.4114 & 1394.7890 & 697.8981 & 1393.8049 & 697.4061 & 13 \\
\hline 9 & 904.4411 & 452.7242 & 887.4145 & 444.2109 & 886.4305 & 443.7189 & A & 1312.7471 & 656.8772 & 1295.7205 & 648.3639 & 1294.7365 & 647.8719 & 12 \\
\hline 10 & 991.4731 & 496.2402 & 974.4465 & 487.7269 & 973.4625 & 487.2349 & $\mathbf{S}$ & 1241.7100 & 621.3586 & 1224.6834 & 612.8454 & 1223.6994 & 612.3533 & 11 \\
\hline 11 & 1088.5259 & 544.7666 & 1071.4993 & 536.2533 & 1070.5153 & 535.7613 & $\mathbf{P}$ & 1154.6780 & 577.8426 & 1137.6514 & 569.3293 & 1136.6674 & 568.8373 & 10 \\
\hline 12 & 1175.5579 & 588.2826 & 1158.5313 & 579.7693 & 1157.5473 & 579.2773 & $\mathrm{~S}$ & 1057.6252 & 529.3162 & 1040.5986 & 520.8030 & 1039.6146 & 520.3109 & 9 \\
\hline 13 & 1288.6420 & 644.8246 & 1271.6154 & 636.3113 & 1270.6314 & 635.8193 & $\mathbf{L}$ & 970.5932 & 485.8002 & 953.5666 & 477.2869 & 952.5826 & 476.7949 & 8 \\
\hline 14 & 1416.7005 & 708.8539 & 1399.6740 & 700.3406 & 1398.6900 & 699.8486 & $\mathbf{Q}$ & 857.5091 & 429.2582 & 840.4825 & 420.7449 & 839.4985 & 420.2529 & 7 \\
\hline 15 & 1544.7591 & 772.8832 & 1527.7326 & 764.3699 & 1526.7485 & 763.8779 & $\mathbf{Q}$ & 729.4505 & 365.2289 & 712.4240 & 356.7156 & 711.4400 & 356.2236 & 6 \\
\hline 16 & 1657.8432 & 829.4252 & 1640.8166 & 820.9119 & 1639.8326 & 820.4199 & $\mathbf{L}$ & 601.3919 & 301.1996 & 584.3654 & 292.6863 & 583.3814 & 292.1943 & 5 \\
\hline 17 & 1756.9116 & 878.9594 & 1739.8850 & 870.4462 & 1738.9010 & 869.9541 & $\mathbf{V}$ & 488.3079 & 244.6576 & 471.2813 & 236.1443 & 470.2973 & 235.6523 & 4 \\
\hline 18 & 1869.9956 & 935.5015 & 1852.9691 & 926.9882 & 1851.9851 & 926.4962 & $\mathbf{L}$ & 389.2395 & 195.1234 & 372.2129 & 186.6101 & 371.2289 & 186.1181 & 3 \\
\hline 19 & 1999.0382 & 1000.0228 & 1982.0117 & 991.5095 & 1981.0277 & 991.0175 & $\mathbf{E}$ & 276.1554 & 138.5813 & 259.1288 & 130.0681 & 258.1448 & 129.5761 & 2 \\
\hline 20 & & & & & & & $\mathbf{K}$ & 147.1128 & 74.0600 & 130.0863 & 65.5468 & & & 1 \\
\hline
\end{tabular}

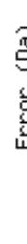
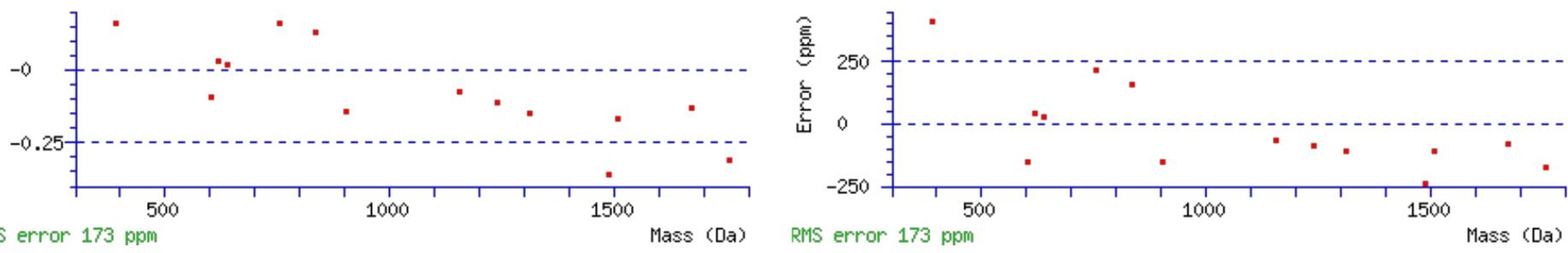

\section{All matches to this query}

\begin{tabular}{|l|c|c|c|}
\hline Score & Mr(calc): & Delta & Sequence \\
\hline 47.3 & 2144.1365 & 0.0092 & GNSTLYPVASPSLQQLVLEK \\
\hline & & & \\
\hline
\end{tabular}




\section{\begin{tabular}{|l|l|l|l|}
38.8 & 2143.1525 & 0.9932 & GNSTLYPVASPSLQQLVLEK \\
\hline
\end{tabular}}

Spectrum No: 718; Query: 12; Rank: 1

\section{Peptide View}

MS/MS Fragmentation of ALVNFTR

Found in IPI00210360, Tax_Id=10116 Gene_Symbol=Hspg2 RPD-I

Match to Query 12: 820.444888 from(411.229720,2+)

Title: 100101RatKid_NS_deglyco_25.1980.1980.2.dta

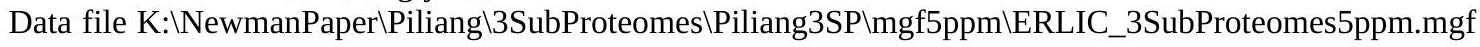
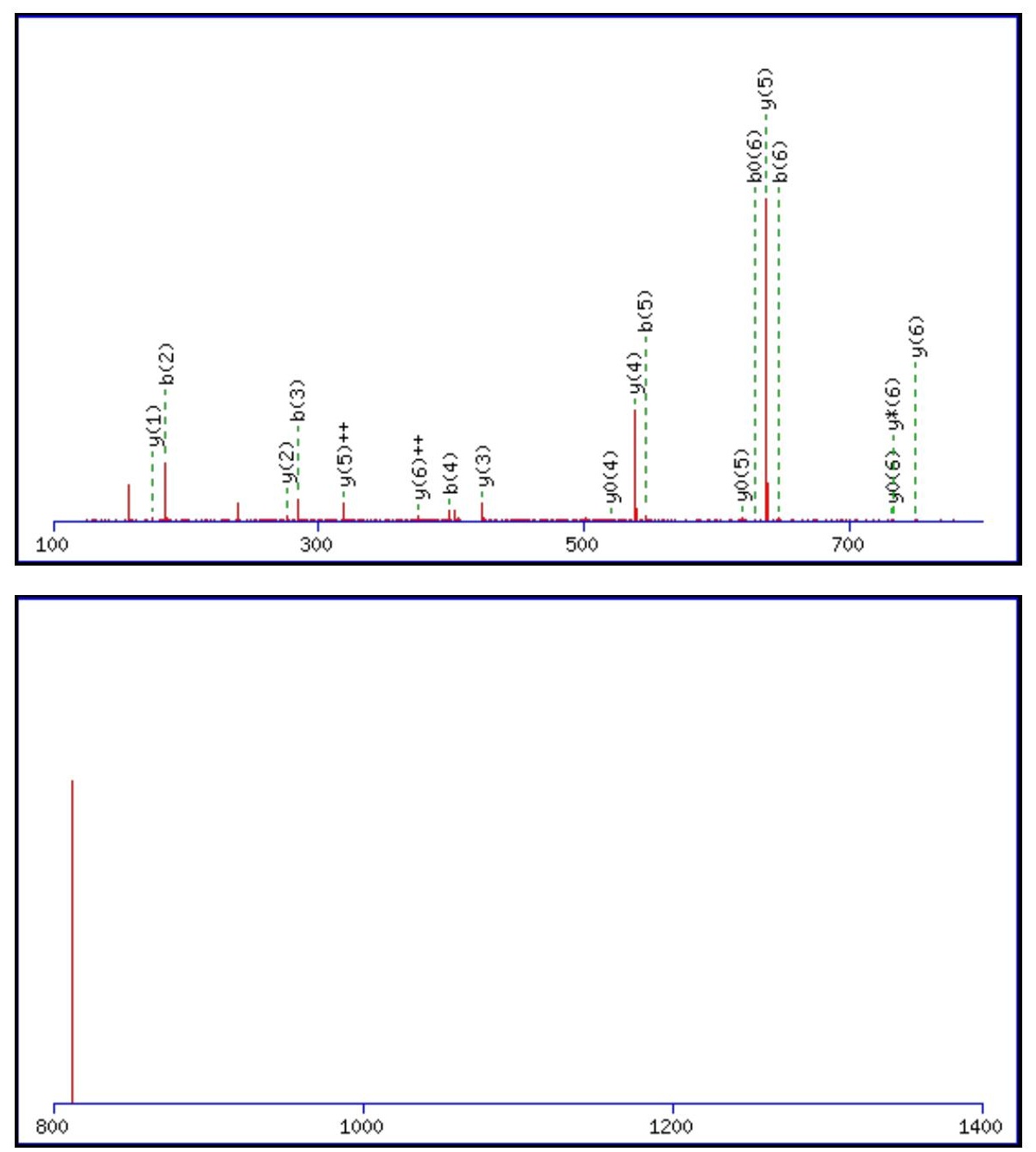
1400 1600

1800 2000

Monoisotopic mass of neutral peptide $\operatorname{Mr}($ calc): 820.4443

Fixed modifications: Carbamidomethyl (C)

Variable modifications:

N4 : Deamidated_N (N)

Ions Score: 47 Expect: 0.0014

Matches (Bold Red): 18/54 fragment ions using 37 most intense peaks

\begin{tabular}{|c|c|c|c|c|c|c|c|c|c|c|c|c|c|c|}
\hline \# & b & $\mathbf{b}^{++}$ & $\mathbf{b}^{*}$ & $\mathbf{b}^{*^{++}}$ & $\mathbf{b}^{0}$ & $\mathbf{b}^{0++}$ & Seq. & $\mathbf{y}$ & $\mathbf{y}^{++}$ & $\mathbf{y}^{*}$ & $\mathbf{y}^{*^{++}}$ & $\mathbf{y}^{0}$ & $\mathbf{y}^{0++}$ & \# \\
\hline 1 & 72.0444 & 36.5258 & & & & & A & & & & & & & 7 \\
\hline 2 & 185.1285 & 93.0679 & & & & & $\mathbf{L}$ & 750.4145 & 375.7109 & 733.3879 & 367.1976 & 732.4039 & 366.7056 & 66 \\
\hline 3 & 284.1969 & 142.6021 & & & & & V & 637.3304 & 319.1688 & 620.3038 & 310.6556 & 619.3198 & 310.1636 & 55 \\
\hline $4:$ & 399.2238 & 200.1155 & 382.1973 & 191.6023 & & & $\mathbf{N}$ & 538.2620 & 269.6346 & 521.2354 & 261.1214 & 520.2514 & 260.6293 & 34 \\
\hline 5 & 546.2922 & 273.6497 & 529.2657 & 265.1365 & & & $\mathbf{F}$ & 423.2350 & 212.1212 & 406.2085 & 203.6079 & 405.2245 & 203.1159 & $\begin{array}{ll}9 & 3 \\
\end{array}$ \\
\hline 6 & 647.3399 & 324.1736 & 630.3133 & 315.6603 & 629.3293 & 315.1683 & $T$ & 276.1666 & 138.5870 & 259.1401 & 130.0737 & 258.1561 & 129.5817 & 72 \\
\hline 7 & & & & & & & $\mathbf{R}$ & 175.1190 & 88.0631 & 158.0924 & 79.5498 & & & 1 \\
\hline
\end{tabular}

는
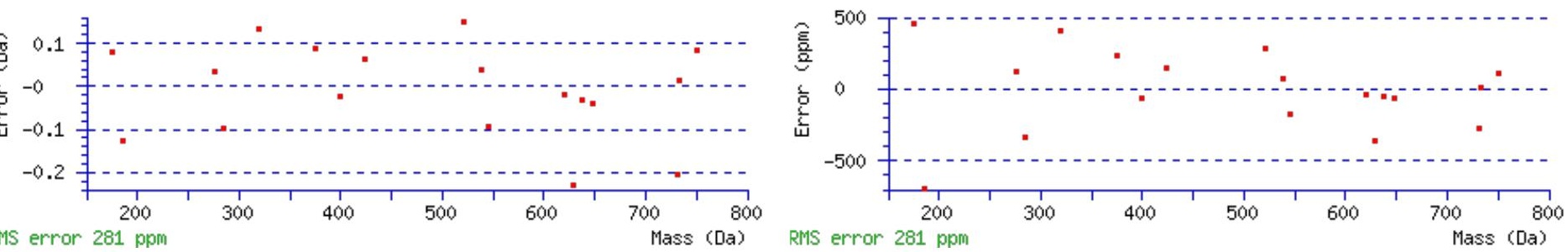

\section{All matches to this query}

\begin{tabular}{|l|l|l|l|}
\hline Score & Mr(calc): & Delta & Sequence \\
\hline 47.3 & 820.4443 & 0.0006 & $\underline{\text { ALVNFTR }}$ \\
\hline 13.9 & 820.4443 & 0.0006 & $\underline{\text { AIADYLR }}$ \\
\hline 13.9 & 820.4443 & 0.0006 & $\underline{\text { ALADYIR }}$ \\
\hline 10.8 & 820.4443 & 0.0006 & $\underline{\text { ALESVFR }}$ \\
\hline 10.4 & 820.4443 & 0.0006 & $\underline{\text { AIFVTDR }}$ \\
\hline 10.4 & 820.4443 & 0.0006 & $\underline{\text { ALLNXFR }}$ \\
\hline 9.2 & 820.4443 & 0.0006 & $\underline{\text { LAEYVAR }}$ \\
\hline 8.7 & 820.4459 & -0.0010 & $\underline{\text { AILLPSK }}$ \\
\hline 0.3 & 820.4443 & 0.0006 & $\underline{\text { RLEFEK }}$ \\
\hline
\end{tabular}

Spectrum No: 719; Query: 2057; Rank: 1

\section{Peptide View}




\section{MS/MS Fragmentation of DRVPDSCCINITVGCGNDFK}

Found in IPI00231686, Tax_Id=10116 Gene_Symbol=Cd63 CD63 antigen

Match to Query 2057: 2327.003172 from(776.675000,3+)

Title: 100101RatKid_NS_deglyco_23.3105.3105.3.dta

Data file K:\NewmanPaper\Piliang $\backslash 3$ SubProteomes $\backslash$ Piliang3SP $\backslash$ mgf5ppm\ERLIC_3SubProteomes5ppm.mgf
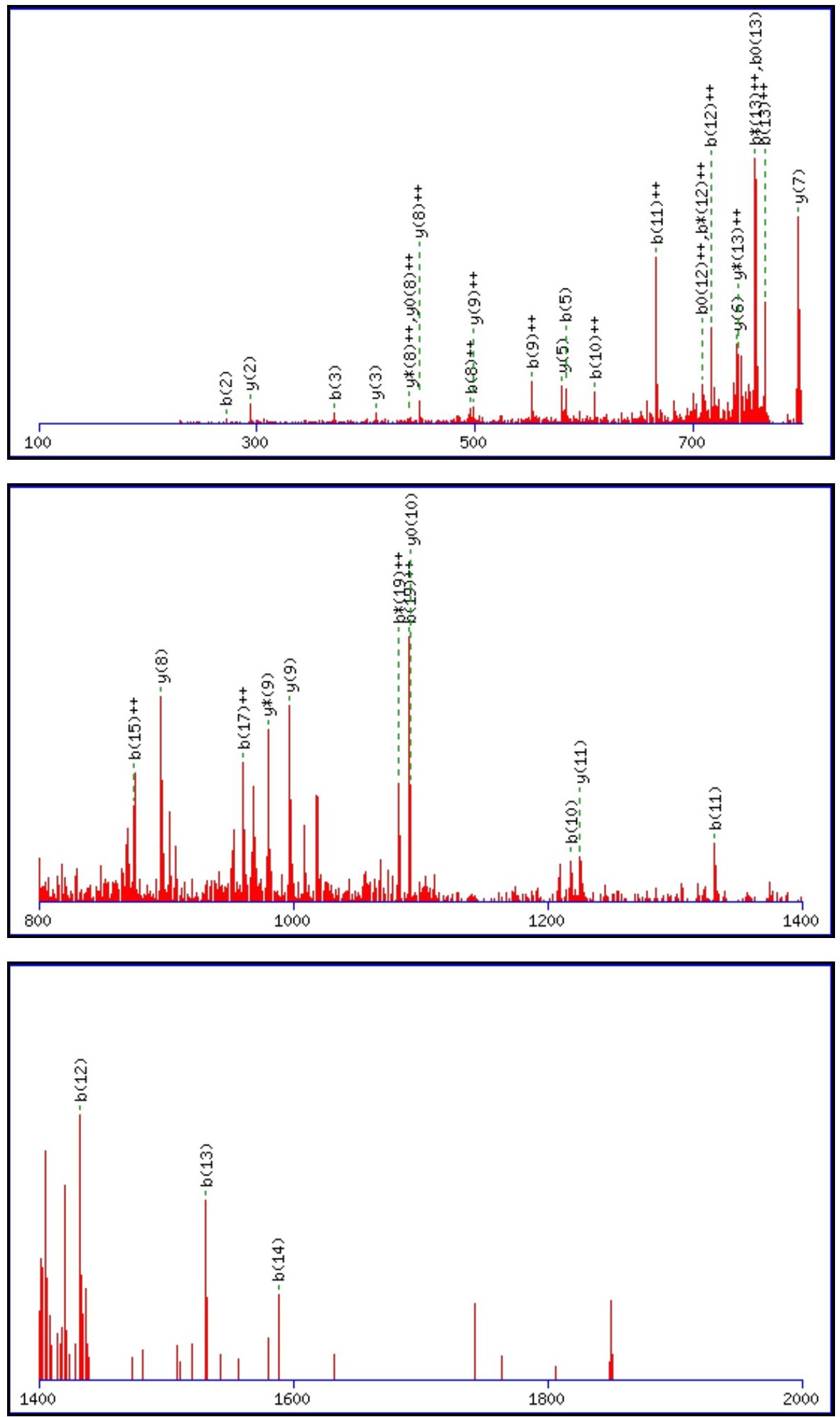

Monoisotopic mass of neutral peptide $\operatorname{Mr}($ calc): 2326.9981

Fixed modifications: Carbamidomethyl (C)

Variable modifications:

N10 : Deamidated_N (N)

Ions Score: 47 Expect : 0.0064

Matches (Bold Red): 37/222 fragment ions using 72 most intense peaks

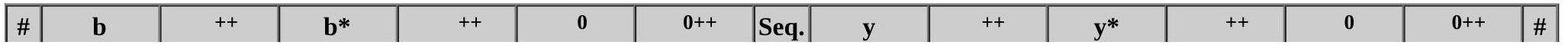




\begin{tabular}{|c|c|c|c|c|c|c|c|c|c|c|c|c|c|c|}
\hline & & b & & $\mathbf{b}^{*}$ & b & b & & & $\mathbf{y}$ & & & $\mathbf{y}$ & $\mathbf{y}$ & \\
\hline 1 & 116.0342 & 58.5207 & & & 98.0237 & 49.5155 & & & & & & & & 20 \\
\hline 2 & 272.1353 & 136.5713 & & & 254.1248 & & $\mathbf{R}$ & & & & & & 1097.9875 & 19 \\
\hline 3 & 371.2037 & 186.1055 & 354.1772 & 922 & 353.1932 & 177.1002 & $\mathbf{V}$ & 2056.8773 & 1028.9423 & 2039.8507 & 1020.4290 & 2038.8667 & $|1019.9370|$ & 18 \\
\hline 4 & 468.2565 & 234.6319 & 451.2300 & 226.1186 & 450.2459 & 225.6266 & $\mathbf{P}$ & & & & & & & 17 \\
\hline 5 & 583.2835 & 292.1454 & 566.2569 & 283.6321 & 565.2729 & 283.1401 & D & 1860.7561 & 930.8817 & 7295 & 922.3684 & 7455 & 921.8764 & 16 \\
\hline 6 & 670.3155 & 335.6614 & 653.2889 & 327.1481 & 652.3049 & 326.6561 & $\mathrm{~S}$ & .7291 & 873.3682 & 026 & & 186 & 629 & 15 \\
\hline 7 & 830.3461 & 415.6767 & 813.3196 & 407.1634 & 812.3356 & 406.6714 & C & 1658.6971 & 829.8522 & 1641.6706 & 821.3389 & 1640.6866 & 820.8469 & 14 \\
\hline 8 & 990.3768 & 495.6920 & 973.3502 & 487.1788 & 972.3662 & 486.6867 & C & 1498.6665 & 749.8369 & 6399 & 3236 & & 316 & 13 \\
\hline 9 & 1103.4608 & 552.2341 & 1086.4343 & 543.7208 & 1085.4503 & 543.2288 & I & 1338.6358 & 669.8215 & 1321.6093 & 083 & 132 & 63 & 12 \\
\hline 10 & 1218.4878 & 609.7475 & 1201.4612 & 601.2343 & 1200.4772 & 600.7422 & $\mathbf{N}$ & 1225.5518 & 613.2795 & 1208.5252 & 604.7662 & 1207.5412 & 604.2742 & 11 \\
\hline 11 & 1331.5718 & 666.2896 & 1314.5453 & 657.7 & 1313.5613 & 657.2843 & I & 1110.5248 & 555.7660 & 1093. & 547.2528 & 1092. & 608 & 10 \\
\hline 12 & 1432.6195 & 716.8134 & 1415.5930 & 708.3001 & 1414.6090 & 707.8081 & $\mathbf{T}$ & 997.4408 & 499.2240 & 980.4142 & 490.7107 & 979.4302 & 490.2187 & 9 \\
\hline 13 & 1531.6879 & 766.3476 & 1514.6614 & 757.8343 & 1513.6774 & 757.3423 & V & 896.3931 & 448.7002 & .3665 & 1869 & 878. & 439 & 8 \\
\hline 14 & 1588.7094 & 794.8583 & 1571.6828 & 786.3451 & 1570.6988 & 785.8531 & G & 797.3247 & 399.1660 & 780.2981 & 390.6527 & 779.3141 & 390.1607 & 7 \\
\hline 15 & 1748.7400 & 874.8737 & 1731.7135 & 866.3604 & 1730.7295 & 865.8684 & C & 740.3032 & 370.6552 & 723.2767 & 362.1420 & 722.2926 & 361.6500 & 6 \\
\hline 16 & 1805.7615 & 903.3844 & 1788.7350 & 894.8711 & 1787.7509 & 894.3791 & G & 580.2726 & 290.6399 & 563.2460 & 282.1266 & 562.2620 & 281.6346 & 5 \\
\hline 17 & 1919.8044 & 960.4059 & 1902.7779 & 951.8926 & 1901.7939 & 951.4006 & $\mathbf{N}$ & 523.2511 & 262.1292 & 506.2245 & 253.6159 & 505.2405 & 253.1239 & 4 \\
\hline 18 & 2034.8314 & 1017.9193 & 2017.8048 & 1009.4061 & 2016.8208 & 1008.9140 & D & 409.2082 & 205.1077 & 392.1816 & 196.5944 & 391.1976 & 196.1024 & 3 \\
\hline 19 : & 2181.8998 & 1091.4535 & 2164.8732 & 1082.9403 & 2163.8892 & 1082.4483 & $\mathbf{F}$ & 294.1812 & 147.5942 & 277.1547 & 139.0810 & & & 2 \\
\hline 20 & & & & & & & $\mathbf{K}$ & 147.1128 & 74.0600 & 130.0863 & 65.5468 & & & 1 \\
\hline
\end{tabular}
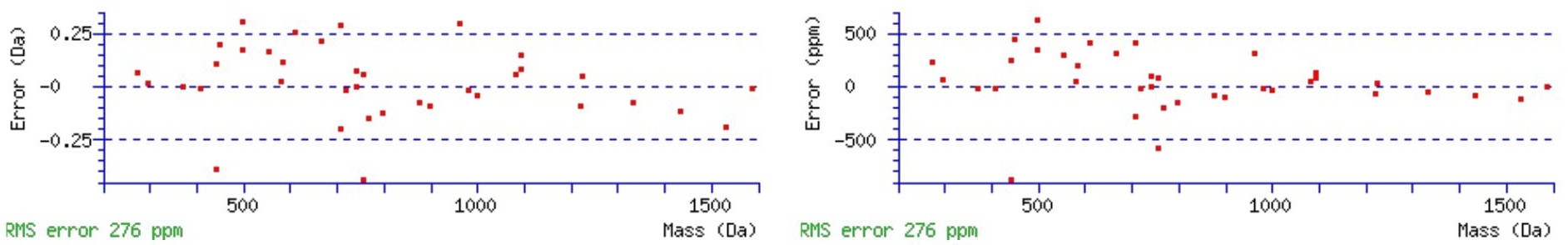

\section{All matches to this query}

\begin{tabular}{|l|l|l|l|}
\hline Score & Mr(calc): & Delta & \multicolumn{1}{c|}{ Sequence } \\
\hline 47.2 & 2326.9981 & 0.0051 & DRVPDSCCINITVGCGNDFK \\
\hline 19.6 & 2326.9981 & 0.0051 & DRVPDSCCINITVGCGNDFK \\
\hline 13.0 & 2326.0141 & 0.9891 & DRVPDSCCINITVGCGNDFK \\
\hline 5.6 & 2327.0264 & -0.0232 & FSSCVDTKGTQYETNSLDFK \\
\hline 4.8 & 2325.9970 & 1.0062 & VNGTMVTNSSHLEVVKLIK \\
\hline 4.2 & 2327.0018 & 0.0014 & SRKPNPGTTCDGVNPTQPIK \\
\hline 4.2 & 2327.0018 & 0.0014 & $\underline{\text { SRKPNPGTTCDGVNPTQPIK }}$ \\
\hline 3.4 & 2325.9970 & 1.0062 & VNGTMVTNSSHLEVVKLIK \\
\hline 3.3 & 2325.0130 & 1.9902 & VNGTMVTNSSHLEVVKLIK \\
\hline 3.2 & 2325.0130 & 1.9902 & VNGTMVTNSSHLEVVKLIK \\
\hline
\end{tabular}

Spectrum No: 720; Query: 2195; Rank: 1

\section{Peptide View}

MS/MS Fragmentation of FGAILAQGILDAGGHNVTISLQSR

Found in IPI00212512, Tax_Id=10116 Gene_Symbol=Psmd1 26S proteasome non-ATPase regulatory subunit 1

Match to Query 2195: 2438.303472 from(813.775100,3+) 
Title: 091008RatKidney_NoSalt_04.5897.5897.3.dta

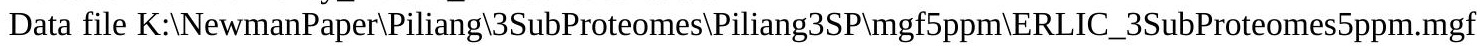
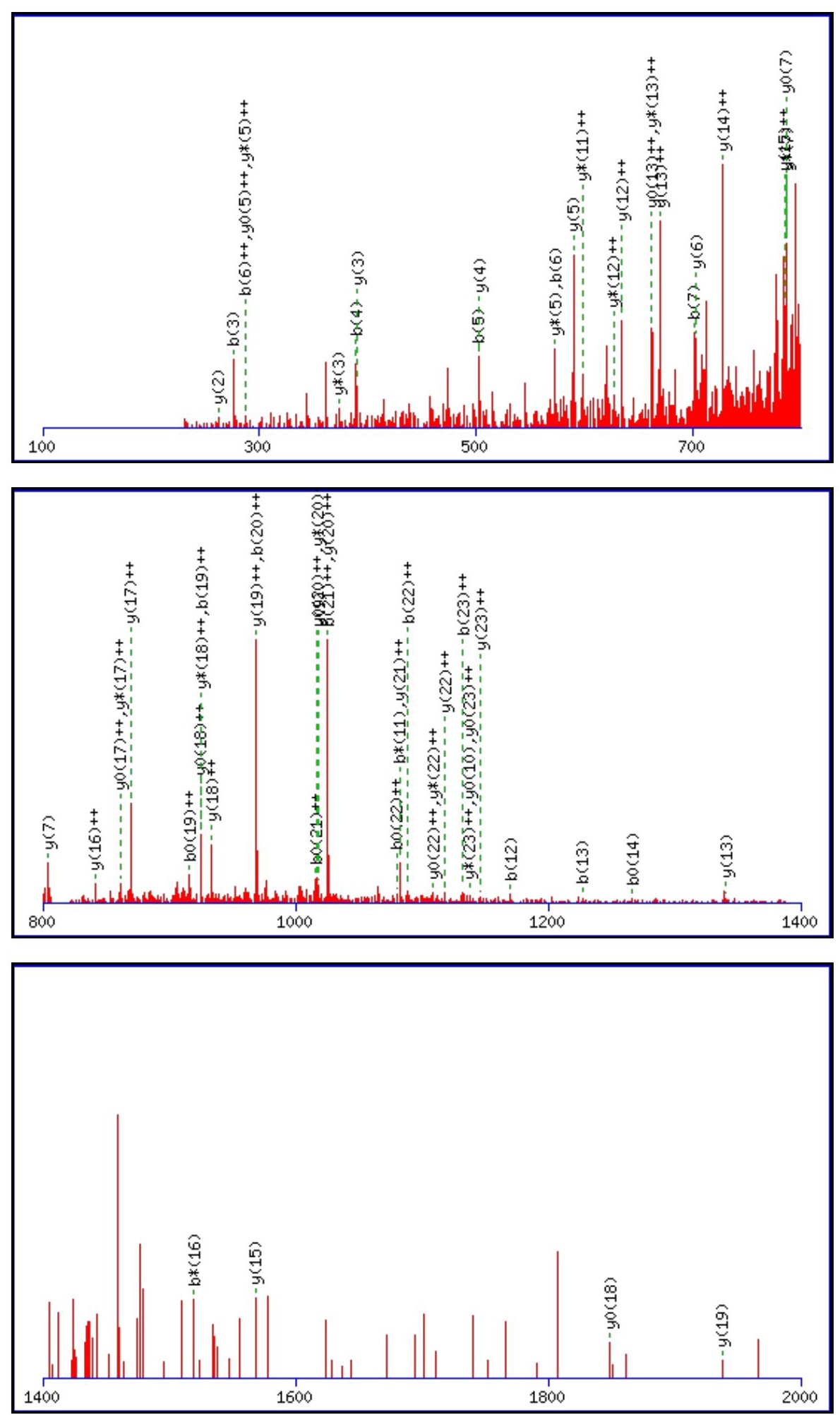

Monoisotopic mass of neutral peptide $\operatorname{Mr}($ calc): 2438.2918

Fixed modifications: Carbamidomethyl (C)

Variable modifications:

N16 : Deamidated N (N)

Ions Score: 47 Expect: 0.0014

Matches (Bold Red): 64/242 fragment ions using 151 most intense peaks

\begin{tabular}{|c|c|c|c|c|c|c|c|c|c|c|c|c|c|c|}
\hline \# & b & $\mathbf{b}^{++}$ & $\mathbf{b}^{*}$ & $\mathbf{b}^{*^{++}}$ & $\mathbf{b}^{\mathbf{0}}$ & $\mathbf{b}^{\mathbf{0 + +}^{++}}$ & Seq. & $\mathbf{y}$ & $\mathbf{y}^{++}$ & $\mathbf{y}^{*}$ & $\mathbf{y}^{*^{++}}$ & $\mathbf{y}^{0}$ & $\mathbf{y}^{\mathbf{0 + +}}$ & \# \\
\hline 1 & 148.0757 & 74.5415 & & & & & $\mathbf{F}$ & & & & & & & 24 \\
\hline 2 & 205.0972 & 103.0522 & & & & & G & 2292.2306 & 1146.6190 & 22275.2041 & 1138.1057 & 2274.2201 & 1137.6137 & 23 \\
\hline 3 & 276.1343 & 138.5708 & & & & & A & 2235.2092 & 1118.1082 & 2218.1826 & 1109.5949 & 2217.1986 & 1109.1029 & 22 \\
\hline
\end{tabular}




\begin{tabular}{|c|c|c|c|c|c|c|c|c|c|c|c|c|c|c|}
\hline & 389.2183 & 195.1128 & & & & & I & 2164.1721 & |1082.5897| & 2147.1455 & 1074.0764 & 2146.1615 & 1073.5844 & |21 \\
\hline 5 & 502.3024 & 251.6548 & & & & & $\mathbf{L}$ & 2051.0880 & 1026.0476 & 2034.0614 & 1017.5344 & 2033.0774 & 1017.0423 & 20 \\
\hline 6 & 573.3395 & 287.1734 & & & & & A & 1938.0039 & \begin{tabular}{|c|}
969.5056 \\
\end{tabular} & 1920.9774 & 960.9923 & 1919.9934 & 960.5003 & 19 \\
\hline 7 & 701.3981 & 351.2027 & 684.3715 & 342.6894 & & & $\mathbf{Q}$ & 1866.9668 & 933.9870 & 1849.9403 & 925.4738 & 1848.9562 & 924.9818 & 18 \\
\hline 8 & 758.4196 & 379.7134 & 741.3930 & 371.2001 & & & G & 1738.9082 & 869.9578 & 1721.8817 & 861.4445 & 1720.8977 & 860.9525 & 17 \\
\hline 9 & 871.5036 & 436.2554 & 854.4771 & 427.7422 & & & I & 1681.8868 & 841.4470 & 1664.8602 & 832.9337 & 1663.8762 & 832.4417 & 16 \\
\hline 10 & 984.5877 & 492.7975 & 967.5611 & 484.2842 & & & $\mathbf{L}$ & 1568.8027 & 784.9050 & 1551.7762 & 776.3917 & 1550.7921 & 775.8997 & 15 \\
\hline 11 & 1099.6146 & 550.3109 & 1082.5881 & 541.7977 & 1081.6041 & 541.3057 & D & 1455.7186 & 728.3630 & 1438.6921 & 719.8497 & 1437.7081 & 719.3577 & 14 \\
\hline 121 & 1170.6517 & 585.8295 & 1153.6252 & 577.3162 & 1152.6412 & 576.8242 & A & 1340.6917 & 670.8495 & 1323.6651 & 662.3362 & 1322.6811 & 661.8442 & 13 \\
\hline 13 & 1227.6732 & 614.3402 & 1210.6467 & 605.8270 & 1209.6626 & 605.3350 & G & 1269.6546 & 635.3309 & 1252.6280 & 626.8177 & 1251.6440 & 626.3256 & 12 \\
\hline 14 & 1284.6947 & 642.8510 & 1267.6681 & 634.3377 & 1266.6841 & 633.8457 & G & 1212.6331 & 606.8202 & 1195.6066 & 598.3069 & 1194.6226 & 597.8149 & 11 \\
\hline 15 & 1421.7536 & 711.3804 & 1404.7270 & 702.8672 & 1403.7430 & 702.3751 & $\mathbf{H}$ & 1155.6117 & 578.3095 & 1138.5851 & 569.7962 & 1137.6011 & 569.3042 & 10 \\
\hline 16 & 1536.7805 & 768.8939 & 1519.7540 & 760.3806 & 1518.7699 & 759.8886 & $\mathbf{N}$ & 1018.5527 & 509.7800 & 1001.5262 & 501.2667 & 1000.5422 & 500.7747 & 9 \\
\hline 171 & 1635.8489 & 818.4281 & 1618.8224 & 809.9148 & 1617.8384 & 809.4228 & $\mathrm{~V}$ & 903.5258 & 452.2665 & 886.4993 & 443.7533 & 885.5152 & 443.2613 & 8 \\
\hline 18 & 1736.8966 & 868.9519 & 1719.8701 & 860.4387 & 1718.8860 & 859.9467 & $T$ & 804.4574 & 402.7323 & 787.4308 & 394.2191 & 786.4468 & 393.7271 & 7 \\
\hline 19 & 1849.9807 & 925.4940 & 1832.9541 & 916.9807 & 1831.9701 & 916.4887 & $\mathbf{I}$ & 703.4097 & 352.2085 & 686.3832 & 343.6952 & 685.3991 & 343.2032 & 6 \\
\hline 201 & 1937.0127 & 969.0100 & 1919.9861 & 960.4967 & 1919.0021 & 960.0047 & $S$ & 590.3257 & 295.6665 & 573.2991 & 287.1532 & 572.3151 & 286.6612 & 5 \\
\hline 212 & 2050.0968 & 1025.5520 & 2033.0702 & \begin{tabular}{|l|}
1017.0387 \\
\end{tabular} & 2032.0862 & \begin{tabular}{|l|}
1016.5467 \\
\end{tabular} & $\mathbf{L}$ & 503.2936 & 252.1504 & 486.2671 & 243.6372 & 485.2831 & 243.1452 & 4 \\
\hline 22 & 2178.1553 & 1089.5813 & 2161.1288 & 1081.0680 & 2160.1448 & 1080.5760 & $\mathbf{Q}$ & 390.2096 & 195.6084 & 373.1830 & 187.0951 & 372.1990 & 186.6031 & 3 \\
\hline 232 & 2265.1874 & 1133.0973 & 2248.1608 & 1124.5840 & 2247.1768 & 1124.0920 & $\mathbf{S}$ & 262.1510 & 131.5791 & 245.1244 & 123.0659 & 244.1404 & 122.5738 & 2 \\
\hline 24 & & & & & & & $\mathbf{R}$ & 175.1190 & 88.0631 & 158.0924 & 79.5498 & & & 1 \\
\hline
\end{tabular}
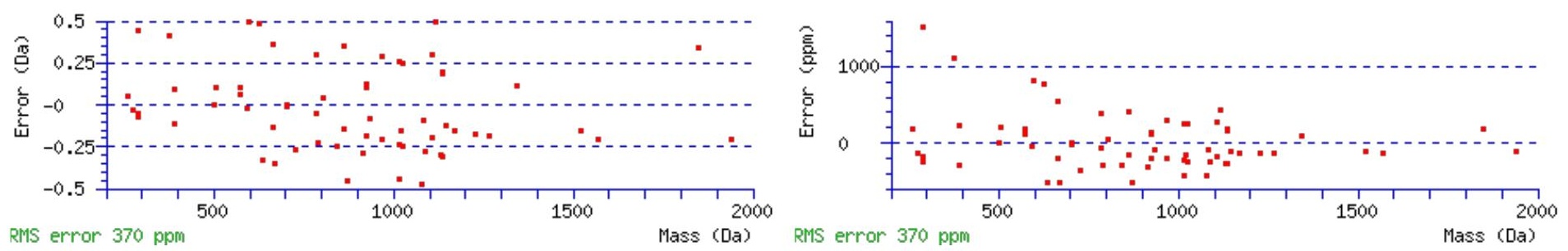

\section{All matches to this query}

\begin{tabular}{|l|l|l|l|}
\hline Score & Mr(calc): & Delta & \multicolumn{1}{|c|}{ Sequence } \\
\hline 47.2 & 2438.2918 & 0.0117 & FGAILAQGILDAGGHNVTISLQSR \\
\hline 40.0 & 2437.3078 & 0.9957 & FGAILAQGILDAGGHNVTISLQSR \\
\hline 10.2 & 2438.3243 & -0.0208 & GQTPLMLAVAYGHIDAVSLLLEK \\
\hline 8.8 & 2436.2972 & 2.0062 & TATINASASHSRLDDINPTVLIK \\
\hline 7.6 & 2437.2812 & 1.0222 & TATINASASHSRLDDINPTVLIK \\
\hline 6.5 & 2436.2934 & 2.0101 & MAKSFTSEDDLVKLQILNLGAK \\
\hline 3.9 & 2437.3050 & 0.9984 & NGAARPQGWGSRAOGLARTILTR \\
\hline 3.1 & 2437.3094 & 0.9941 & NQTVRGGTVEILFQNLSLIKK \\
\hline 3.1 & 2437.3094 & 0.9941 & NQTVRGGTVEILFQNLSLIKK \\
\hline 1.6 & 2438.2934 & 0.0101 & AASLKTFGIRLEEVLVNELTR \\
\hline
\end{tabular}

Spectrum No: 721; Query: 1266; Rank: 1

\section{Peptide View}

MS/MS Fragmentation of LWLPVNLTWADLEDK

Found in IPI00208183, Tax_Id=10116 Gene_Symbol=Lass2 Longevity assurance homolog 2

Match to Query 1266: 1812.936768 from(907.475660,2+) 
Title: 100101RatKid_NS_deglyco_13.6972.6972.2.dta

Data file K:\NewmanPaper\Piliang \3SubProteomes \Piliang3SP \mgf5ppm\ERLIC_3SubProteomes5ppm.mgf
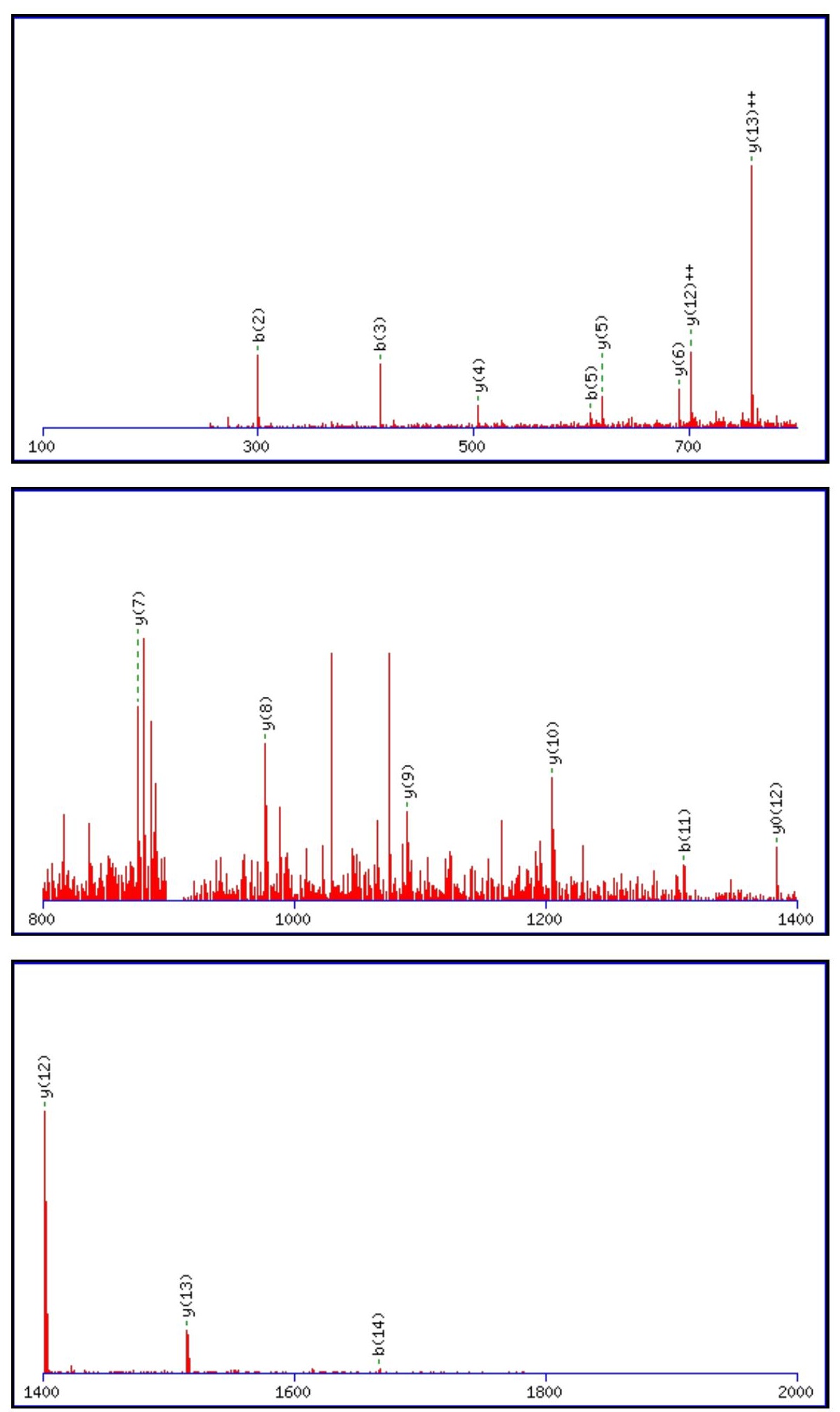

Monoisotopic mass of neutral peptide $\operatorname{Mr}($ calc): 1812.9298

Fixed modifications: Carbamidomethyl (C)

Variable modifications:

N6 : Deamidated_N (N)

Ions Score: 47 Expect : 0.0027

Matches (Bold Red): 17/142 fragment ions using 42 most intense peaks

\begin{tabular}{|r|c|c|c|c|c|c|c|c|c|c|c|c|c|c|}
\hline$\#$ & $\mathbf{b}$ & $\mathbf{b}^{++}$ & $\mathbf{b}^{*}$ & $\mathbf{b}^{\boldsymbol{*}^{++}}$ & $\mathbf{b}^{\mathbf{0}}$ & $\mathbf{b}^{\mathbf{0 + +}}$ & Seq. & $\mathbf{y}$ & $\mathbf{y}^{++}$ & $\mathbf{y}^{\mathbf{*}}$ & $\mathbf{y}^{\boldsymbol{*}^{++}}$ & $\mathbf{y}^{\mathbf{0}}$ & $\mathbf{y}^{\mathbf{0 + +}}$ & $\#$ \\
\hline $\mathbf{1}$ & 114.0913 & 57.5493 & & & & & $\mathbf{L}$ & & & & & & & $\mathbf{1 5}$ \\
\hline $\mathbf{2}$ & $\mathbf{3 0 0 . 1 7 0 7}$ & 150.5890 & & & & & $\mathbf{W}$ & 1700.8530 & 850.9301 & 1683.8265 & 842.4169 & 1682.8424 & 841.9249 & $\mathbf{1 4}$ \\
\hline 3 & $\mathbf{4 1 3 . 2 5 4 7}$ & 207.1310 & & & & & $\mathbf{L}$ & $\mathbf{1 5 1 4 . 7 7 3 7}$ & 757.8905 & 1497.7471 & 749.3772 & 1496.7631 & 748.8852 & $\mathbf{1 3}$ \\
\hline
\end{tabular}




\begin{tabular}{|r|r|r|r|r|r|r|r|r|r|r|r|r|r|r|r|}
$\mathbf{4}$ & 510.3075 & 255.6574 & & & & & $\mathbf{P}$ & $\mathbf{1 4 0 1 . 6 8 9 6}$ & $\mathbf{7 0 1 . 3 4 8 5}$ & 1384.6631 & 692.8352 & $\mathbf{1 3 8 3 . 6 7 9 1}$ & 692.3432 & $\mathbf{1 2}$ \\
\hline $\mathbf{5}$ & $\mathbf{6 0 9 . 3 7 5 9}$ & 305.1916 & & & & & V & 1304.6369 & 652.8221 & 1287.6103 & 644.3088 & 1286.6263 & 643.8168 & $\mathbf{1 1}$ \\
\hline $\mathbf{6}$ & 724.4028 & 362.7051 & 707.3763 & 354.1918 & & & N & $\mathbf{1 2 0 5 . 5 6 8 5}$ & 603.2879 & 1188.5419 & 594.7746 & 1187.5579 & 594.2826 & $\mathbf{1 0}$ \\
\hline $\mathbf{7}$ & 837.4869 & 419.2471 & 820.4603 & 410.7338 & & & L & $\mathbf{1 0 9 0 . 5 4 1 5}$ & 545.7744 & 1073.5150 & 537.2611 & 1072.5310 & 536.7691 & $\mathbf{9 9}$ \\
\hline $\mathbf{8}$ & 938.5346 & 469.7709 & 921.5080 & 461.2577 & 920.5240 & 460.7656 & T & $\mathbf{9 7 7 . 4 5 7 5}$ & 489.2324 & 960.4309 & 480.7191 & 959.4469 & 480.2271 & $\mathbf{8}$ \\
\hline $\mathbf{9}$ & 1124.6139 & 562.8106 & 1107.5873 & 554.2973 & 1106.6033 & 553.8053 & W & $\mathbf{8 7 6 . 4 0 9 8}$ & 438.7085 & 859.3832 & 430.1953 & 858.3992 & 429.7032 & $\mathbf{7}$ \\
\hline $\mathbf{1 0}$ & 1195.6510 & 598.3291 & 1178.6245 & 589.8159 & 1177.6404 & 589.3239 & A & $\mathbf{6 9 0 . 3 3 0 5}$ & 345.6689 & 673.3039 & 337.1556 & 672.3199 & 336.6636 & $\mathbf{6}$ \\
\hline $\mathbf{1 1}$ & $\mathbf{1 3 1 0 . 6 7 7 9}$ & 655.8426 & 1293.6514 & 647.3293 & 1292.6674 & 646.8373 & $\mathbf{D}$ & $\mathbf{6 1 9 . 2 9 3 3}$ & 310.1503 & 602.2668 & 301.6370 & 601.2828 & 301.1450 & $\mathbf{5}$ \\
\hline $\mathbf{1 2}$ & 1423.7620 & 712.3846 & 1406.7355 & 703.8714 & 1405.7514 & 703.3794 & L & $\mathbf{5 0 4 . 2 6 6 4}$ & 252.6368 & 487.2399 & 244.1236 & 486.2558 & 243.6316 & $\mathbf{4}$ \\
\hline $\mathbf{1 3}$ & 1552.8046 & 776.9059 & 1535.7781 & 768.3927 & 1534.7940 & 767.9007 & E & 391.1823 & 196.0948 & 374.1558 & 187.5815 & 373.1718 & 187.0895 & $\mathbf{3}$ \\
\hline $\mathbf{1 4}$ & $\mathbf{1 6 6 7 . 8 3 1 5}$ & 834.4194 & 1650.8050 & 825.9061 & 1649.8210 & 825.4141 & D & 262.1397 & 131.5735 & 245.1132 & 123.0602 & 244.1292 & 122.5682 & $\mathbf{2}$ \\
\hline $\mathbf{1 5}$ & & & & & & & K & 147.1128 & 74.0600 & 130.0863 & 65.5468 & & & & $\mathbf{1}$ \\
\hline
\end{tabular}
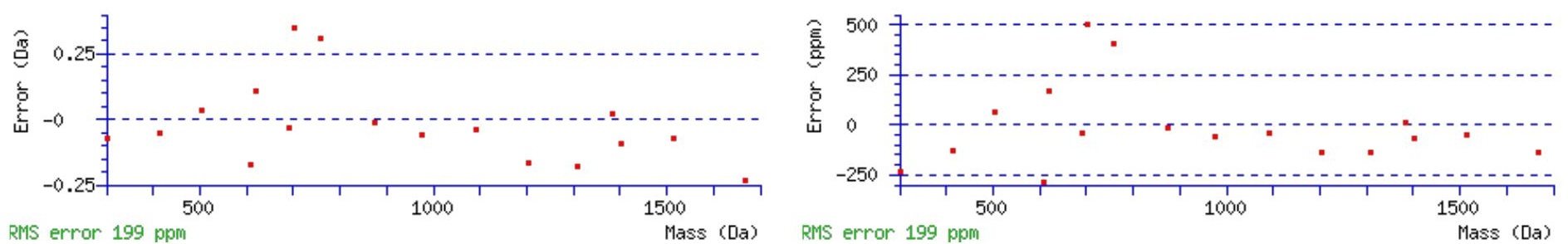

All matches to this query

\begin{tabular}{|l|l|l|l|}
\hline Score & Mr(calc): & Delta & \multicolumn{1}{|c|}{ Sequence } \\
\hline 47.1 & 1812.9298 & 0.0070 & LWLPVNLTWADLEDK \\
\hline 15.7 & 1811.9458 & 0.9910 & LWLPVNLTWADLEDK \\
\hline 3.0 & 1812.9274 & 0.0094 & TTPLIKKNLSNLNFK \\
\hline 2.3 & 1812.9499 & -0.0131 & TPTLKPNAPXPNSAAVK \\
\hline 0.9 & 1811.9393 & 0.9974 & SLIKLDLSRNGLSSTK \\
\hline 0.9 & 1811.9393 & 0.9974 & SLIKLDLSRNGLSSTK \\
\hline 0.3 & 1811.9393 & 0.9974 & SLIKLDLSRNGLSSTK \\
\hline 0.3 & 1811.9393 & 0.9974 & SLIKLDLSRNGLSSTK \\
\hline
\end{tabular}

Spectrum No: 722; Query: 309; Rank: 1

\section{Peptide View}

MS/MS Fragmentation of AHFSSLNLTLR

Found in IPI00324585, Tax_Id=10116 Gene_Symbol=Itga1 Integrin alpha-1 precursor

Match to Query 309: 1258.665188 from(630.339870,2+)

Title: 091008RatKidney_NH4Format01_23.2224.2224.2.dta

Data file K:INewmanPaper|Piliangl3SubProteomes\Piliang3SP\mgf5ppm\ERLIC_3SubProteomes5ppm.mgf 

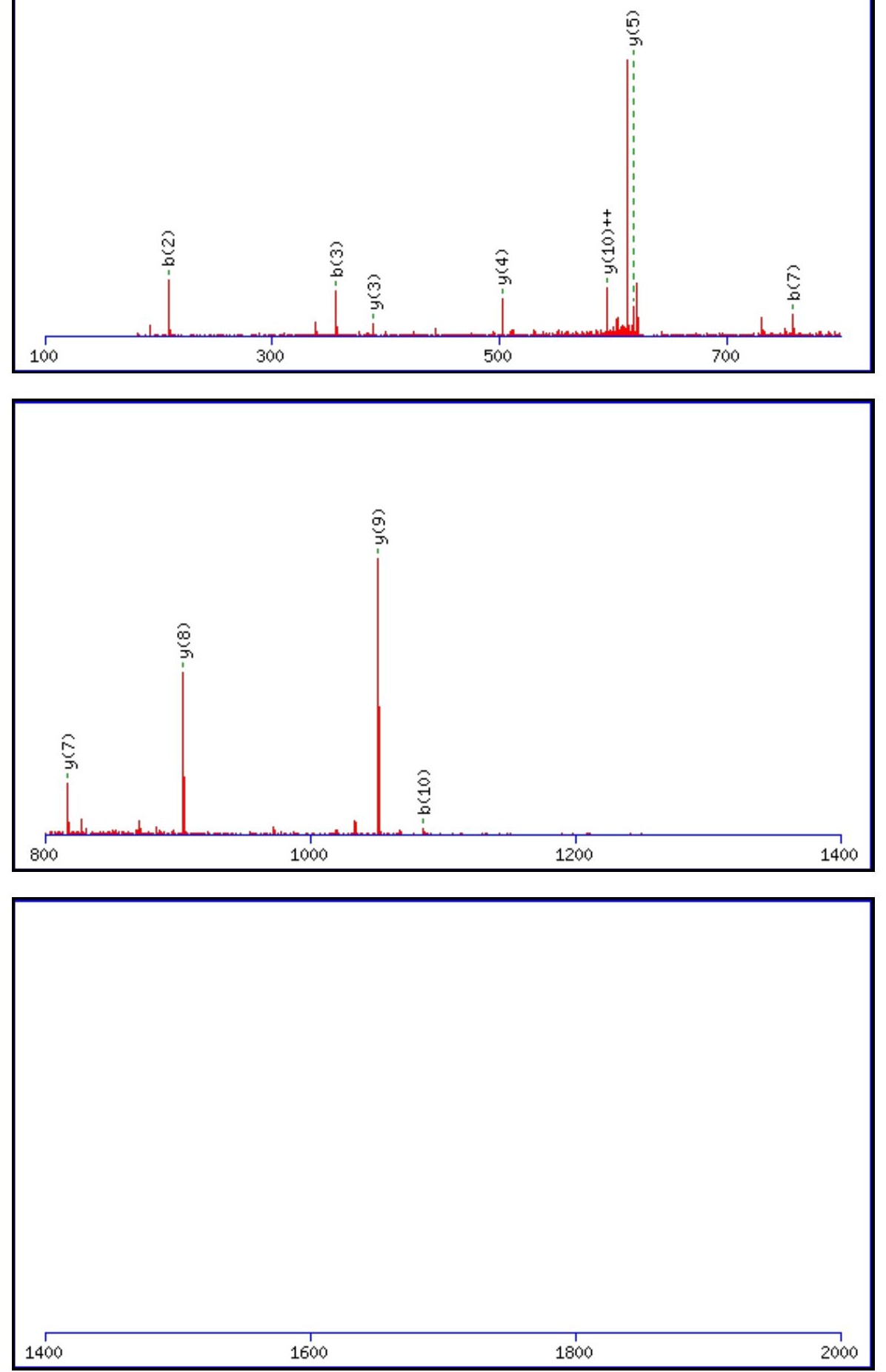

Monoisotopic mass of neutral peptide $\operatorname{Mr}($ calc): 1258.6670

Fixed modifications: Carbamidomethyl (C)

Variable modifications:

N7 : Deamidated N (N)

Ions Score: 47 Expect: 0.002

Matches (Bold Red): 11/98 fragment ions using 21 most intense peaks

\begin{tabular}{|r|c|c|c|c|c|c|c|c|c|c|c|c|c|c|}
\hline$\#$ & $\mathbf{b}$ & $\mathbf{b}^{++}$ & $\mathbf{b}^{*}$ & $\mathbf{b}^{\boldsymbol{*}^{++}}$ & $\mathbf{b}^{\mathbf{0}}$ & $\mathbf{b}^{\mathbf{0 + +}}$ & Seq. & $\mathbf{y}$ & $\mathbf{y}^{++}$ & $\mathbf{y}^{\mathbf{*}}$ & $\mathbf{y}^{\boldsymbol{*}^{++}}$ & $\mathbf{y}^{\mathbf{0}}$ & $\mathbf{y}^{\mathbf{0 + +}}$ & $\#$ \\
\hline $\mathbf{1}$ & $\mathbf{7 2 . 0 4 4 4}$ & 36.5258 & & & & & $\mathbf{A}$ & & & & & & & $\mathbf{1 1}$ \\
\hline $\mathbf{2}$ & $\mathbf{2 0 9 . 1 0 3 3}$ & 105.0553 & & & & & $\mathbf{H}$ & 1188.6371 & $5 \mathbf{9 4 . 8 2 2 2}$ & 1171.6106 & 586.3089 & 1170.6266 & 585.8169 & $\mathbf{1 0}$ \\
\hline $\mathbf{3}$ & $\mathbf{3 5 6 . 1 7 1 7}$ & 178.5895 & & & & & $\mathbf{F}$ & $\mathbf{1 0 5 1 . 5 7 8 2}$ & 526.2928 & 1034.5517 & 517.7795 & 1033.5677 & 517.2875 & $\mathbf{9}$ \\
\hline $\mathbf{4}$ & 443.2037 & 222.1055 & & & 425.1932 & 213.1002 & S & $\mathbf{9 0 4 . 5 0 9 8}$ & 452.7585 & 887.4833 & 444.2453 & 886.4992 & 443.7533 & $\mathbf{8}$ \\
\hline $\mathbf{5}$ & 530.2358 & 265.6215 & & & 512.2252 & 256.6162 & $\mathbf{S}$ & $\mathbf{8 1 7 . 4 7 7 8}$ & 409.2425 & 800.4512 & 400.7293 & 799.4672 & 400.2372 & $\mathbf{7}$ \\
\hline
\end{tabular}




\begin{tabular}{|r|r|r|r|r|r|r|r|r|r|r|r|r|r|r|r|r|r|}
$\mathbf{6}$ & 643.3198 & 322.1636 & & & 625.3093 & 313.1583 & L & 730.4458 & 365.7265 & 713.4192 & 357.2132 & 712.4352 & 356.7212 & $\mathbf{6}$ \\
\hline $\mathbf{7}$ & 758.3468 & 379.6770 & 741.3202 & 371.1637 & 740.3362 & 370.6717 & $\mathbf{N}$ & $\mathbf{6 1 7 . 3 6 1 7}$ & 309.1845 & 600.3351 & 300.6712 & 599.3511 & 300.1792 & $\mathbf{5}$ \\
\hline $\mathbf{8}$ & 871.4308 & 436.2191 & 854.4043 & 427.7058 & 853.4203 & 427.2138 & L & $\mathbf{5 0 2 . 3 3 4 8}$ & 251.6710 & 485.3082 & 243.1577 & 484.3242 & 242.6657 & $\mathbf{4}$ \\
\hline $\mathbf{9}$ & 972.4785 & 486.7429 & 955.4520 & 478.2296 & 954.4679 & 477.7376 & T & $\mathbf{3 8 9 . 2 5 0 7}$ & 195.1290 & 372.2241 & 186.6157 & 371.2401 & 186.1237 & $\mathbf{3}$ \\
\hline $\mathbf{1 0}$ & $\mathbf{1 0 8 5 . 5 6 2 6}$ & 543.2849 & 1068.5360 & 534.7717 & 1067.5520 & 534.2796 & L & 288.2030 & 144.6051 & 271.1765 & 136.0919 & & & & $\mathbf{2}$ \\
\hline $\mathbf{1 1}$ & & & & & & & R & 175.1190 & 88.0631 & 158.0924 & 79.5498 & & & \\
\hline
\end{tabular}
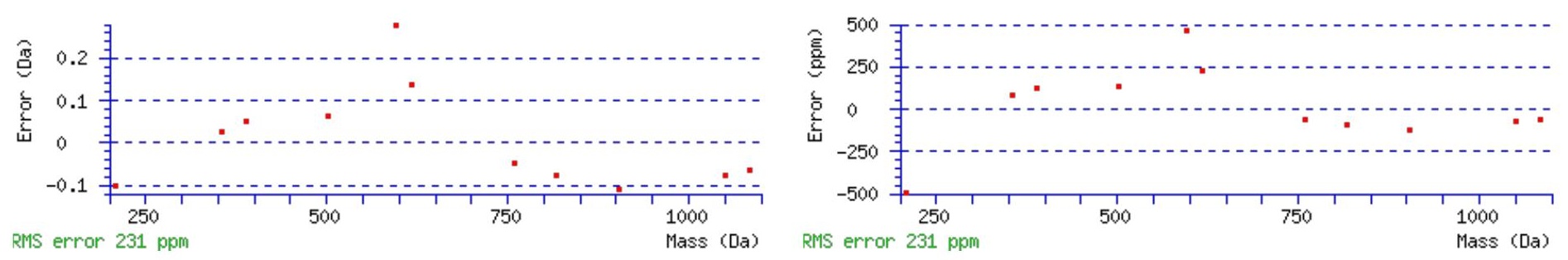

\section{All matches to this query}

\begin{tabular}{|l|l|l|l|}
\hline Score & Mr(calc): & Delta & \multicolumn{1}{|c|}{ Sequence } \\
\hline 47.1 & 1258.6670 & -0.0018 & AHFSSLNLTLR \\
\hline 7.8 & 1258.6621 & 0.0031 & KMLLLSFRR \\
\hline 5.7 & 1258.6670 & -0.0018 & TSXNWPALITR \\
\hline 4.7 & 1258.6557 & 0.0095 & EPNYLLSQPAK \\
\hline 2.7 & 1258.6670 & -0.0018 & KQWDSVVLER \\
\hline 2.2 & 1258.6591 & 0.0061 & MPKEQQELLK \\
\hline 1.2 & 1258.6659 & -0.0007 & HQRKAVALTR \\
\hline 0.8 & 1258.6629 & 0.0023 & QKVEDLRESR \\
\hline 0.6 & 1258.6768 & -0.0116 & EEASNILLNKK \\
\hline 0.4 & 1258.6638 & 0.0014 & $\underline{\text { ARKDMLLCPR }}$ \\
\hline
\end{tabular}

Spectrum No: 723; Query: 508; Rank: 1

\section{Peptide View}

MS/MS Fragmentation of EGNKTCEALLFK

Found in IPI00204504, Tax_Id=10116 Gene_Symbol=Plvap Plasmalemma vesicle-associated protein

Match to Query 508: 1409.688848 from(705.851700,2+)

Title: 100101RatKid_NS_deglyco_19.2008.2008.2.dta

Data file K:INewmanPaper|Piliangl3SubProteomes\Piliang3SP\mgf5ppm\ERLIC_3SubProteomes5ppm.mgf

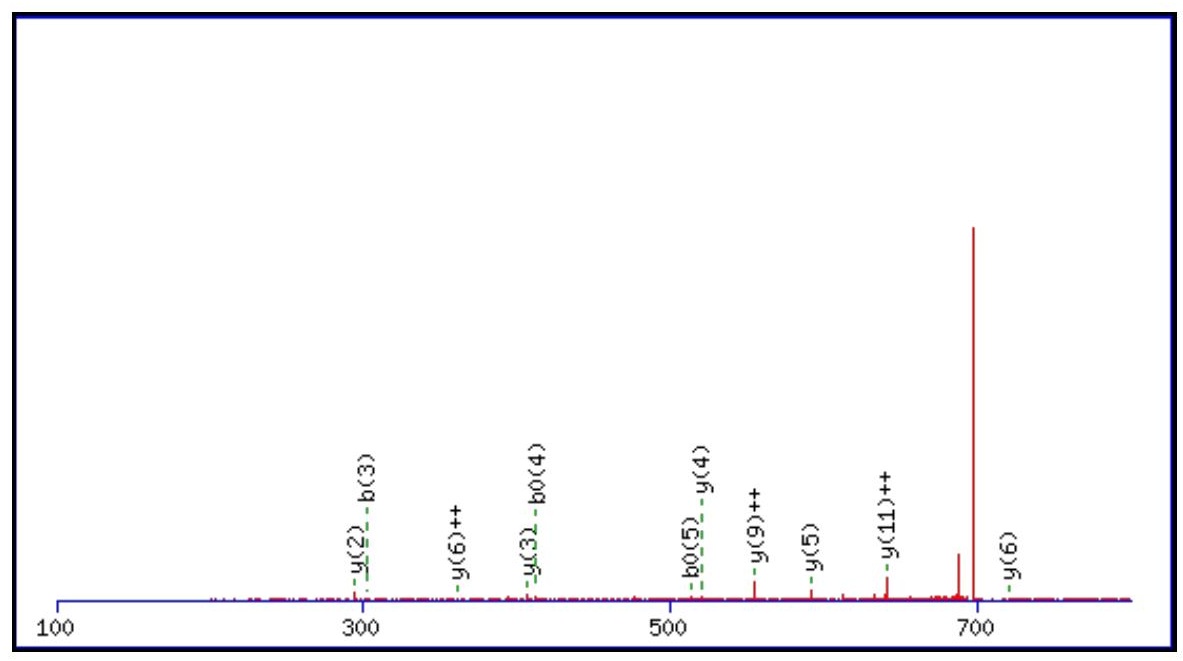



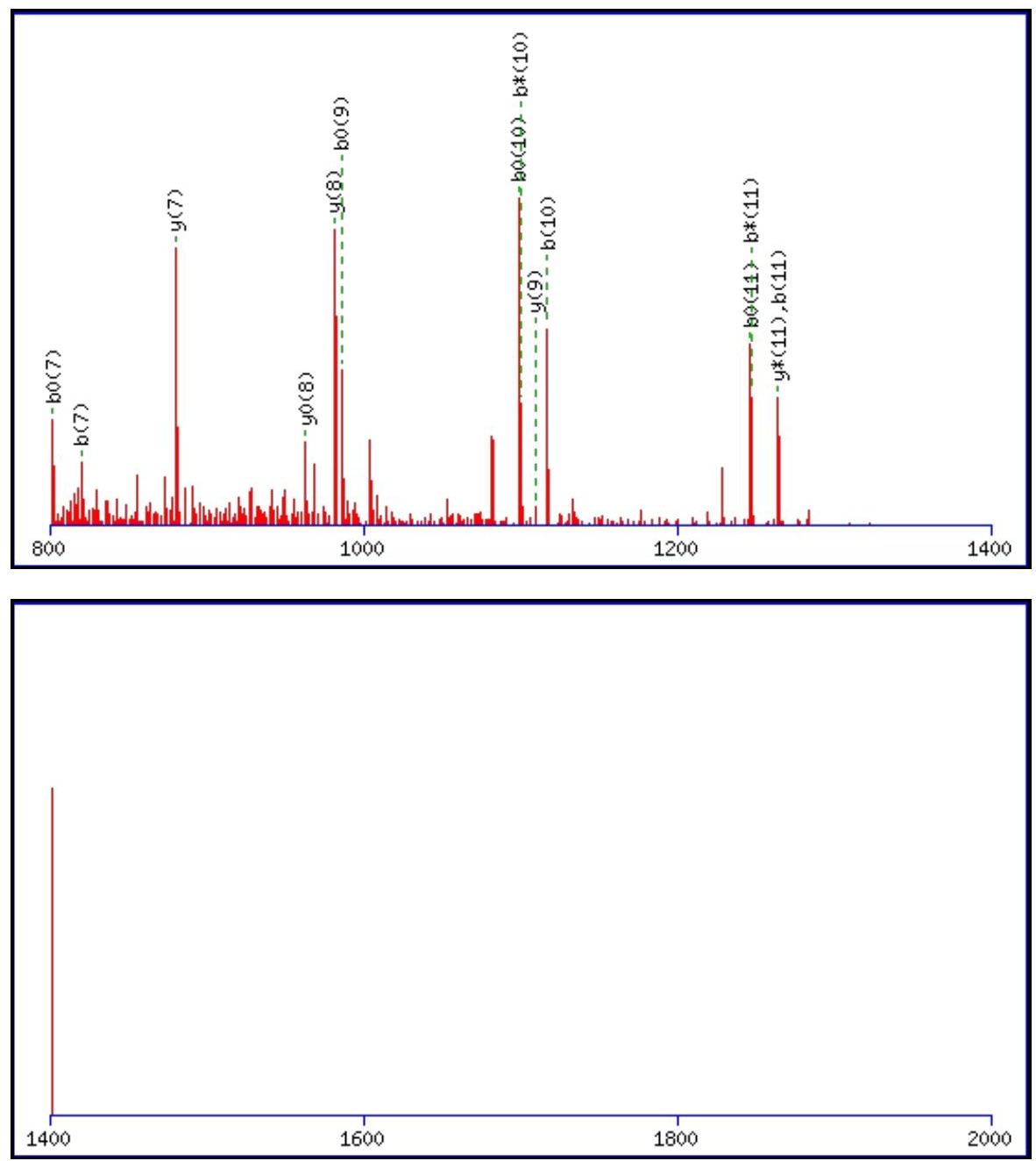

Monoisotopic mass of neutral peptide $\operatorname{Mr}($ calc): 1409.6860

Fixed modifications: Carbamidomethyl (C)

Variable modifications:

N3 : Deamidated_N (N)

Ions Score: 47 Expect : $\odot .003$

Matches (Bold Red): 25/118 fragment ions using 56 most intense peaks

\begin{tabular}{|r|c|c|c|c|c|c|c|c|c|c|c|c|c|c|}
\hline$\#$ & $\mathbf{b}$ & $\mathbf{b}^{++}$ & $\mathbf{b}^{*}$ & $\mathbf{b}^{\mathbf{*}^{++}}$ & $\mathbf{b}^{\mathbf{0}}$ & $\mathbf{b}^{\mathbf{0 + +}}$ & $\mathbf{S e q}$ & $\mathbf{y}$ & $\mathbf{y}^{++}$ & $\mathbf{y}^{*}$ & $\mathbf{y}^{\mathbf{*}^{++}}$ & $\mathbf{y}^{\mathbf{0}}$ & $\mathbf{y}^{\mathbf{0 + +}}$ & $\#$ \\
\hline $\mathbf{1}$ & 130.0499 & 65.5286 & & & 112.0393 & 56.5233 & $\mathbf{E}$ & & & & & & & $\mathbf{1 2}$ \\
\hline $\mathbf{2}$ & 187.0713 & 94.0393 & & & 169.0608 & 85.0340 & $\mathbf{G}$ & 1281.6507 & $\mathbf{6 4 1 . 3 2 9 0}$ & $\mathbf{1 2 6 4 . 6 2 4 2}$ & 632.8157 & 1263.6402 & 632.3237 & $\mathbf{1 1}$ \\
\hline $\mathbf{3}$ & $\mathbf{3 0 2 . 0 9 8 3}$ & 151.5528 & 285.0717 & 143.0395 & 284.0877 & 142.5475 & $\mathbf{N}$ & 1224.6293 & 612.8183 & 1207.6027 & 604.3050 & 1206.6187 & 603.8130 & $\mathbf{1 0}$ \\
\hline $\mathbf{4}$ & 430.1932 & 215.6003 & 413.1667 & 207.0870 & 412.1827 & 206.5950 & $\mathbf{K}$ & $\mathbf{1 1 0 9 . 6 0 2 3}$ & 555.3048 & 1092.5758 & 546.7915 & 1091.5918 & 546.2995 & $\mathbf{9}$ \\
\hline $\mathbf{5}$ & 531.2409 & 266.1241 & 514.2144 & 257.6108 & 513.2303 & 257.1188 & $\mathbf{T}$ & $\mathbf{9 8 1 . 5 0 7 4}$ & 491.2573 & 964.4808 & 482.7441 & $\mathbf{9 6 3 . 4 9 6 8}$ & 482.2520 & $\mathbf{8}$ \\
\hline $\mathbf{6}$ & 691.2716 & 346.1394 & 674.2450 & 337.6261 & 673.2610 & 337.1341 & $\mathbf{C}$ & $\mathbf{8 8 0 . 4 5 9 7}$ & 440.7335 & 863.4332 & 432.2202 & 862.4491 & 431.7282 & $\mathbf{7}$ \\
\hline $\mathbf{7}$ & $\mathbf{8 2 0 . 3 1 4 2}$ & 410.6607 & 803.2876 & 402.1474 & $\mathbf{8 0 2 . 3 0 3 6}$ & 401.6554 & $\mathbf{E}$ & $\mathbf{7 2 0 . 4 2 9 1}$ & $\mathbf{3 6 0 . 7 1 8 2}$ & 703.4025 & 352.2049 & 702.4185 & 351.7129 & $\mathbf{6}$ \\
\hline $\mathbf{8}$ & 891.3513 & 446.1793 & 874.3247 & 437.6660 & 873.3407 & 437.1740 & $\mathbf{A}$ & $5 \mathbf{5 9 1 . 3 8 6 5}$ & 296.1969 & 574.3599 & 287.6836 & & & $\mathbf{5}$ \\
\hline $\mathbf{9}$ & 1004.4353 & 502.7213 & 987.4088 & 494.2080 & $\mathbf{9 8 6 . 4 2 4 8}$ & 493.7160 & $\mathbf{L}$ & $5 \mathbf{5 2 0 . 3 4 9 3}$ & 260.6783 & 503.3228 & 252.1650 & & & $\mathbf{4}$ \\
\hline $\mathbf{1 0}$ & $\mathbf{1 1 1 7 . 5 1 9 4}$ & 559.2633 & $\mathbf{1 1 0 0 . 4 9 2 8}$ & 550.7501 & $\mathbf{1 0 9 9 . 5 0 8 8}$ & 550.2581 & $\mathbf{L}$ & $\mathbf{4 0 7 . 2 6 5 3}$ & 204.1363 & 390.2387 & 195.6230 & & & $\mathbf{3}$ \\
\hline $\mathbf{1 1}$ & $\mathbf{1 2 6 4 . 5 8 7 8}$ & 632.7975 & $\mathbf{1 2 4 7 . 5 6 1 3}$ & 624.2843 & $\mathbf{1 2 4 6 . 5 7 7 2}$ & 623.7923 & $\mathbf{F}$ & $\mathbf{2 9 4 . 1 8 1 2}$ & 147.5942 & 277.1547 & 139.0810 & & & $\mathbf{2}$ \\
\hline $\mathbf{1 2}$ & & & & & & & $\mathbf{K}$ & 147.1128 & 74.0600 & 130.0863 & 65.5468 & & & $\mathbf{1}$ \\
\hline
\end{tabular}



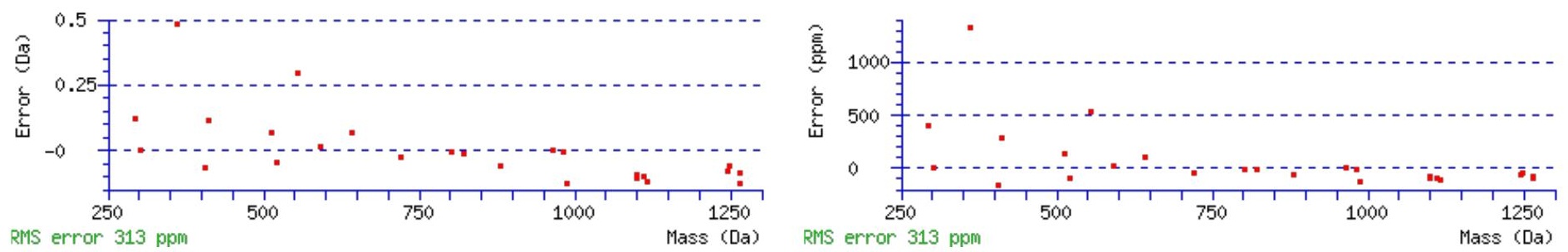

\section{All matches to this query}

\begin{tabular}{|l|l|l|l|}
\hline Score & Mr(calc): & Delta & \multicolumn{1}{c|}{ Sequence } \\
\hline 47.0 & 1409.6860 & 0.0028 & EGNKTCEALLFK \\
\hline 9.7 & 1408.6960 & 0.9929 & YQHTEPHSRVR \\
\hline 9.5 & 1409.6894 & -0.0005 & TMKSEDKAEIMK \\
\hline 7.1 & 1408.6722 & 1.0167 & VINLNDNTFTEK \\
\hline 6.1 & 1409.6939 & -0.0050 & YLEHLAAEAHEK \\
\hline 6.0 & 1408.6877 & 1.0012 & SXMTPMRSVPMK \\
\hline 6.0 & 1408.6877 & 1.0012 & SXMTPMRSVPMK \\
\hline 3.3 & 1409.6860 & 0.0028 & NGVEIEMSEKFK \\
\hline 3.1 & 1408.6834 & 1.0055 & YEITEQGKANQK \\
\hline 2.7 & 1408.6946 & 0.9942 & YEVRNKNDLTR \\
\hline
\end{tabular}

Spectrum No: 724; Query: 1628; Rank: 1

\section{Peptide View}

MS/MS Fragmentation of DDHFKEDPSWENMLNR

Found in IPI00372350, Tax_Id=10116 Gene_Symbol=Itfg3 Protein ITFG3

Match to Query 1628: 2032.862652 from(678.628160,3+)

Title: 100101RatKid_NS_deglyco_21.3100.3100.3.dta

Data file K:INewmanPaper|Piliangl3SubProteomes\Piliang3SP\mgf5ppm\ERLIC_3SubProteomes5ppm.mgf

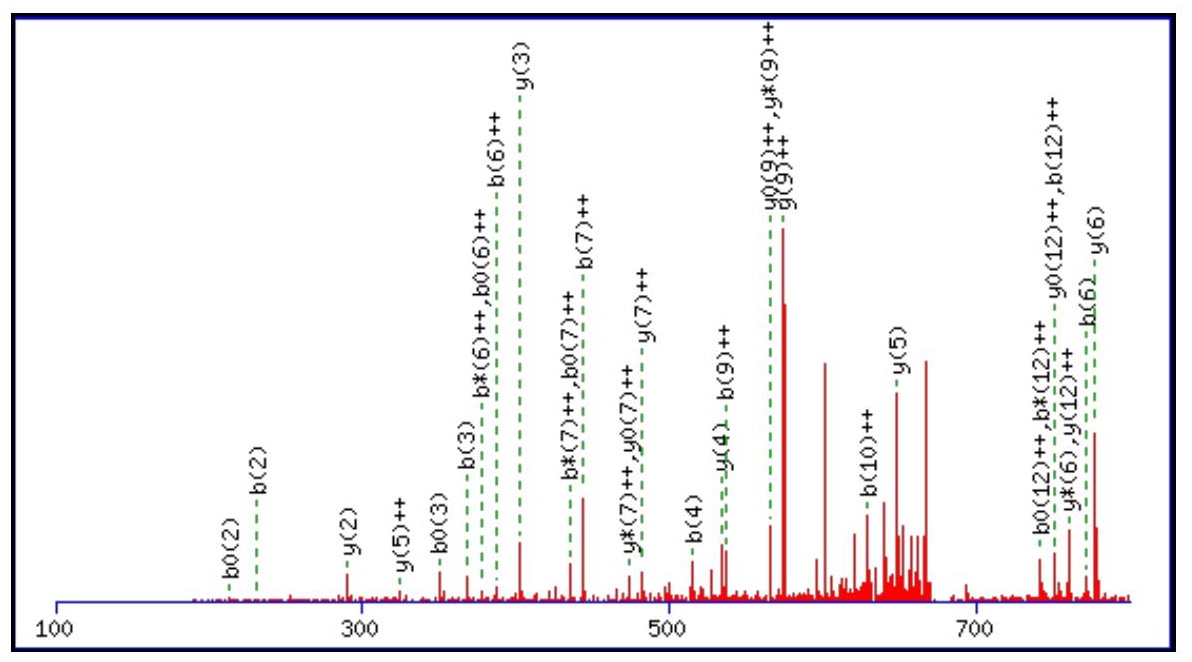



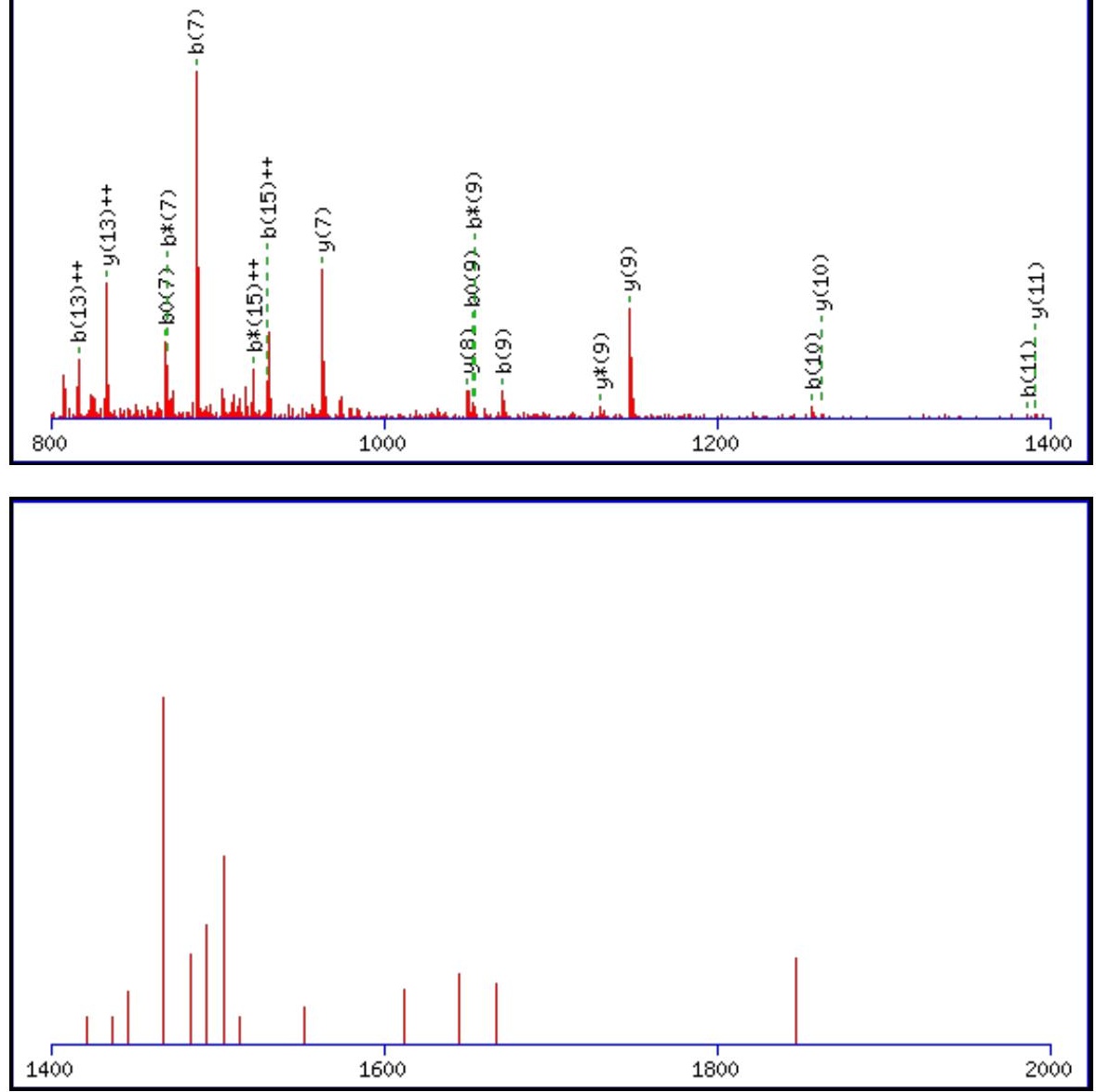

Monoisotopic mass of neutral peptide $\operatorname{Mr}($ calc): 2032.8585

Fixed modifications: Carbamidomethyl (C)

Variable modifications:

N15 : Deamidated_N (N)

Ions Score: 47 Expect: 0.0048

Matches (Bold Red): 50/162 fragment ions using 91 most intense peaks

\begin{tabular}{|c|c|c|c|c|c|c|c|c|c|c|c|c|c|c|}
\hline \# & b & $\mathbf{b}^{++}$ & $\mathbf{b}^{*}$ & $\mathbf{b}^{*^{++}}$ & $\mathbf{b}^{0}$ & $\mathbf{b}^{\mathbf{0 + +}}$ & Seq. & $\mathbf{y}$ & $\mathbf{y}^{++}$ & $\mathbf{y}^{*}$ & $\mathrm{y}^{*^{++}}$ & $\mathbf{y}^{\mathbf{0}}$ & $\mathbf{y}^{\mathbf{0 + +}}$ & \# \\
\hline 1 & 116.0342 & 58.5207 & & & 98.0237 & 49.5155 & D & & & & & & & 16 \\
\hline 2 & 231.0612 & 116.0342 & & & 213.0506 & 107.0289 & D & 1918.8388 & 959.9231 & 1901.8123 & 951.4098 & 1900.8283 & 950.9178 & 15 \\
\hline 3 & 368.1201 & 184.5637 & & & 350.1095 & 175.5584 & $\mathbf{H}$ & 1803.8119 & 902.4096 & 1786.7853 & 893.8963 & 1785.8013 & 893.4043 & 14 \\
\hline 4 & 515.1885 & 258.0979 & & & 497.1779 & 249.0926 & $\mathbf{F}$ & 1666.7530 & 833.8801 & 1649.7264 & 825.3669 & 1648.7424 & 824.8748 & 13 \\
\hline 5 & 643.2835 & 322.1454 & 626.2569 & 313.6321 & 625.2729 & 313.1401 & $\mathbf{K}$ & 1519.6846 & 760.3459 & 1502.6580 & 751.8326 & 1501.6740 & 751.3406 & 12 \\
\hline 6 & 772.3260 & 386.6667 & 755.2995 & 378.1534 & 754.3155 & 377.6614 & $\mathbf{E}$ & 1391.5896 & 696.2984 & 1374.5630 & 687.7852 & 1373.5790 & 687.2932 & 11 \\
\hline 7 & 887.3530 & 444.1801 & 870.3264 & 435.6669 & 869.3424 & 435.1748 & D & 1262.5470 & 631.7771 & 1245.5205 & 623.2639 & 1244.5364 & 622.7719 & 10 \\
\hline 8 & 984.4058 & 492.7065 & 967.3792 & 484.1932 & 966.3952 & 483.7012 & $\mathbf{P}$ & 1147.5201 & 574.2637 & 1130.4935 & 565.7504 & 1129.5095 & 565.2584 & 9 \\
\hline 9 & 1071.4378 & 536.2225 & 1054.4112 & 527.7093 & 1053.4272 & 527.2172 & S & 1050.4673 & 525.7373 & 1033.4407 & 517.2240 & 1032.4567 & 516.7320 & 8 \\
\hline 10 & 1257.5171 & 629.2622 & 1240.4905 & 620.7489 & 1239.5065 & 620.2569 & W & 963.4353 & 482.2213 & 946.4087 & 473.7080 & 945.4247 & 473.2160 & 7 \\
\hline 11 & 1386.5597 & 693.7835 & 1369.5331 & 685.2702 & 1368.5491 & 684.7782 & $\mathbf{E}$ & 777.3560 & 389.1816 & 760.3294 & 380.6683 & 759.3454 & 380.1763 & 6 \\
\hline 12 & 1500.6026 & 750.8049 & 1483.5761 & 742.2917 & 1482.5920 & 741.7997 & $\mathbf{N}$ & 648.3134 & 324.6603 & 631.2868 & 316.1470 & & & 5 \\
\hline 13 & 1631.6431 & 816.3252 & 1614.6165 & 807.8119 & 1613.6325 & 807.3199 & $\mathbf{M}$ & 534.2704 & 267.6389 & 517.2439 & 259.1256 & & & 4 \\
\hline 14 & 1744.7272 & 872.8672 & 1727.7006 & 864.3539 & 1726.7166 & 863.8619 & $\mathbf{L}$ & 403.2300 & 202.1186 & 386.2034 & 193.6053 & & & 3 \\
\hline 15 & 1859.7541 & 930.3807 & 1842.7275 & 921.8674 & 1841.7435 & 921.3754 & $\mathbf{N}$ & 290.1459 & 145.5766 & 273.1193 & 137.0633 & & & 2 \\
\hline 16 & & & & & & & $\mathbf{R}$ & 175.1190 & 88.0631 & 158.0924 & 79.5498 & & & 1 \\
\hline
\end{tabular}



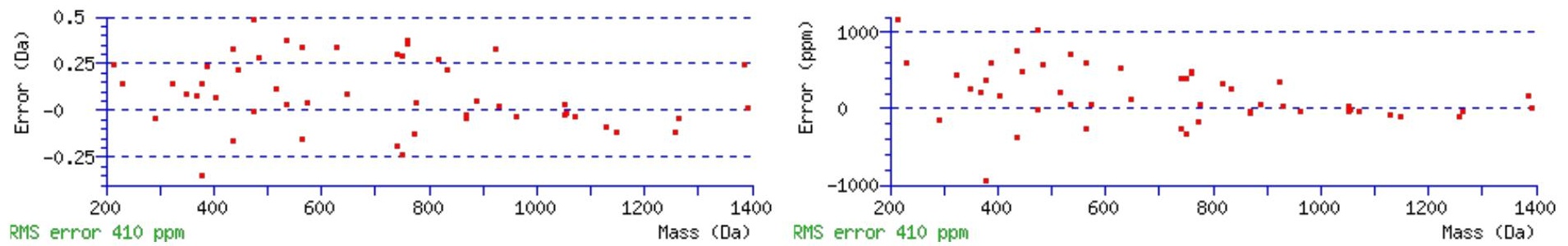

\section{All matches to this query}

\begin{tabular}{|l|c|c|c|}
\hline Score & Mr(calc): & Delta & \multicolumn{1}{c|}{ Sequence } \\
\hline 46.9 & 2032.8585 & 0.0042 & DDHFKEDPSWENMLNR \\
\hline 34.1 & 2032.8585 & 0.0042 & DDHFKEDPSWENMLNR \\
\hline 13.0 & 2031.8745 & 0.9882 & DDHFKEDPSWENMLNR \\
\hline 2.0 & 2032.8787 & -0.0160 & $\underline{\text { ILTPWLFKATMLTDK }}$ \\
\hline
\end{tabular}

Spectrum No: 725; Query: 32; Rank: 1

\section{Peptide View}

\section{MS/MS Fragmentation of GDGNLTWR}

Found in IPI00366226, Tax_Id=10116 Gene_Symbol=Gns Glucosamine (N-acetyl)-6-sulfatase

Match to Query 32: 918.419948 from(460.217250,2+)

Title: 091008RatKidney_NH4Format01_23.1169.1169.2.dta

Data file K:\NewmanPaper|Piliang|3SubProteomes\Piliang3SP\mgf5ppm\ERLIC_3SubProteomes5ppm.mgf

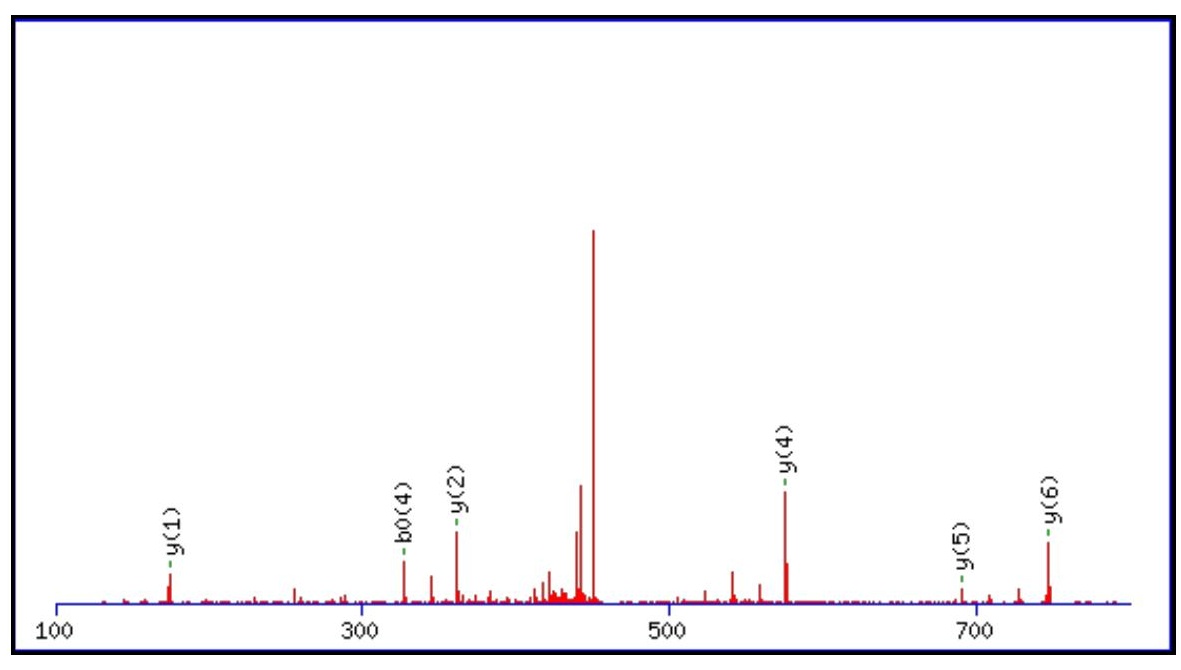



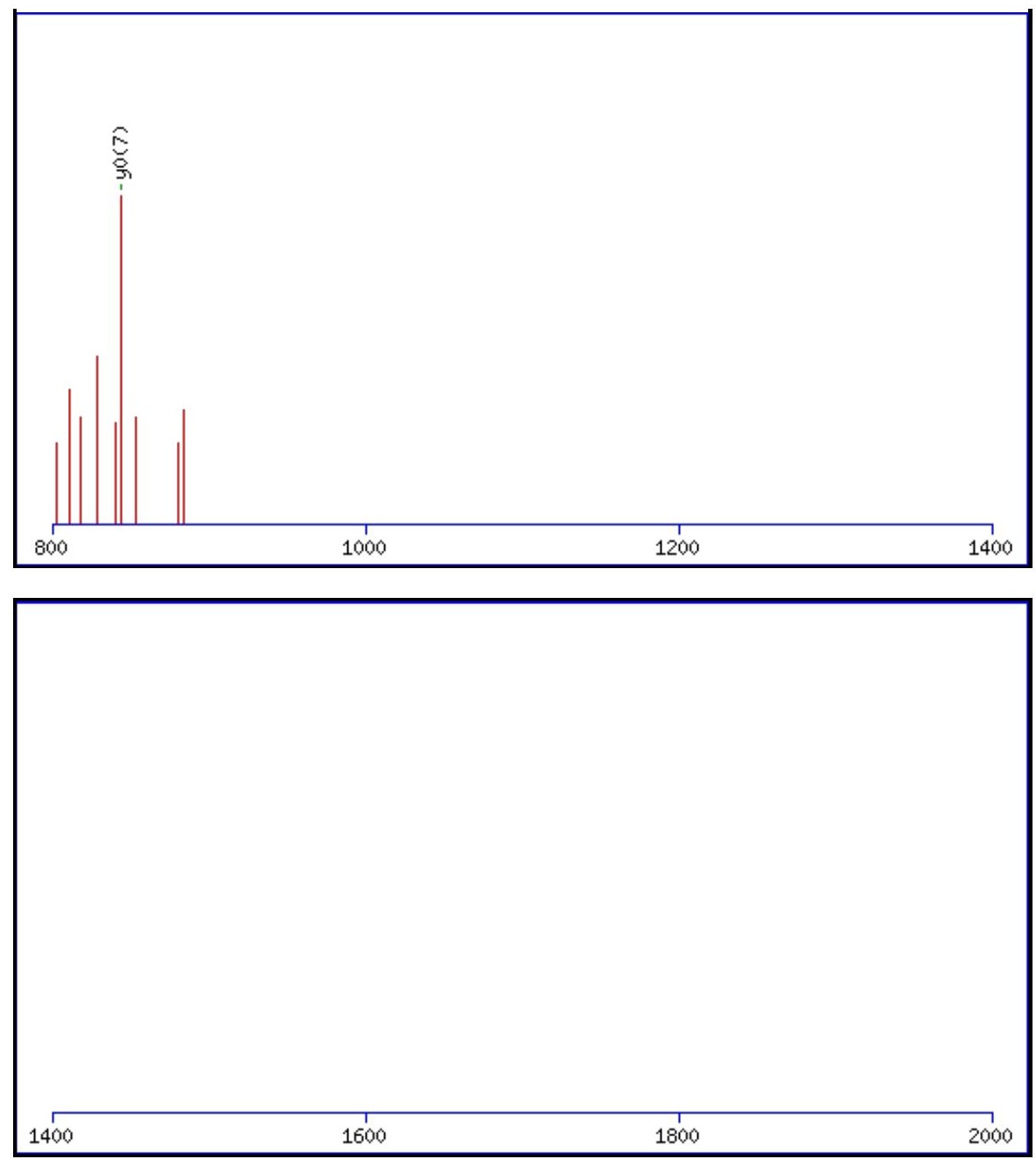

Monoisotopic mass of neutral peptide $\operatorname{Mr}($ calc): 918.4195

Fixed modifications: Carbamidomethyl (C)

Variable modifications:

N4 : Deamidated_N (N)

Ions Score: 47 Expect: 0.0015

Matches (Bold Red): 7/72 fragment ions using 11 most intense peaks

\begin{tabular}{|c|c|c|c|c|c|c|c|c|c|c|c|c|c|c|}
\hline$\#$ & $\mathbf{b}$ & $\mathbf{b}^{++}$ & $\mathbf{b}^{*}$ & $\mathbf{b}^{*^{++}}$ & $\mathbf{b}^{\mathbf{0}}$ & $\mathbf{b}^{\mathbf{0}+}$ & $\mathbf{S e q}$ & $\mathbf{y}$ & $\mathbf{y}^{++}$ & $\mathbf{y}^{*}$ & $\mathbf{y}^{\boldsymbol{*}^{++}}$ & $\mathbf{y}^{\mathbf{0}}$ & $\mathbf{y}^{\mathbf{0}++}$ & $\#$ \\
\hline $\mathbf{1}$ & 58.0287 & 29.5180 & & & & & $\mathbf{G}$ & & & & & & & $\mathbf{8}$ \\
\hline $\mathbf{2}$ & 173.0557 & 87.0315 & & & 155.0451 & 78.0262 & $\mathbf{D}$ & 862.4054 & 431.7063 & 845.3788 & 423.1930 & $\mathbf{8 4 4 . 3 9 4 8}$ & 422.7010 & $\mathbf{7}$ \\
\hline $\mathbf{3}$ & 230.0771 & 115.5422 & & & 212.0666 & 106.5369 & $\mathbf{G}$ & 747.3784 & 374.1928 & 730.3519 & 365.6796 & 729.3678 & 365.1876 & $\mathbf{6}$ \\
\hline $\mathbf{4}$ & 345.1041 & 173.0557 & 328.0775 & 164.5424 & 327.0935 & 164.0504 & $\mathbf{N}$ & $\mathbf{6 9 0 . 3 5 6 9}$ & 345.6821 & 673.3304 & 337.1688 & 672.3464 & 336.6768 & $\mathbf{5}$ \\
\hline $\mathbf{5}$ & 458.1881 & 229.5977 & 441.1616 & 221.0844 & 440.1776 & 220.5924 & $\mathbf{L}$ & 575.3300 & 288.1686 & 558.3035 & 279.6554 & 557.3194 & 279.1634 & $\mathbf{4}$ \\
\hline $\mathbf{6}$ & 559.2358 & 280.1216 & 542.2093 & 271.6083 & 541.2253 & 271.1163 & $\mathbf{T}$ & 462.2459 & 231.6266 & 445.2194 & 223.1133 & 444.2354 & 222.6213 & $\mathbf{3}$ \\
\hline $\mathbf{7}$ & 745.3151 & 373.1612 & 728.2886 & 364.6479 & 727.3046 & 364.1559 & $\mathbf{W}$ & $\mathbf{3 6 1 . 1 9 8 3}$ & 181.1028 & 344.1717 & 172.5895 & & & $\mathbf{2}$ \\
\hline $\mathbf{8}$ & & & & & & & $\mathbf{R}$ & $\mathbf{1 7 5 . 1 1 9 0}$ & 88.0631 & 158.0924 & 79.5498 & & & $\mathbf{1}$ \\
\hline
\end{tabular}
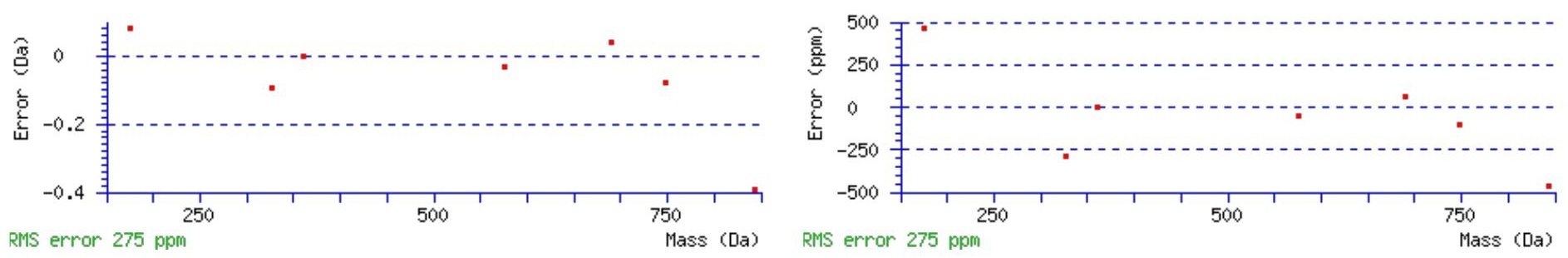

\section{All matches to this query}

\begin{tabular}{|l|l|l|l|}
\hline Score & $\operatorname{Mr}$ (calc): & Delta & Sequence \\
\hline
\end{tabular}




\begin{tabular}{|l|l|l|l|}
46.6 & 918.4195 & 0.0004 & GDGNLTWR \\
\hline 14.7 & 917.4202 & 0.9997 & VENDRER \\
\hline 14.3 & 917.4090 & 1.0109 & DLEEAEGR \\
\hline 13.8 & 917.4203 & 0.9997 & VEAGGGSGER \\
\hline 12.7 & 918.4212 & -0.0012 & TYVSTIR \\
\hline 11.8 & 916.4055 & 2.0144 & LGTDYIR \\
\hline 11.7 & 917.4203 & 0.9997 & DNGGTEGLR \\
\hline 10.8 & 918.4212 & -0.0012 & TYVSTIR \\
\hline 9.3 & 917.4090 & 1.0110 & GLEEENAR \\
\hline 9.1 & 917.4090 & 1.0109 & DDIDNVAR \\
\hline
\end{tabular}

Spectrum No: 726; Query: 632; Rank: 1

\section{Peptide View}

MS/MS Fragmentation of VFFNGSGQETVCK

Found in IPI00231848, Tax_Id=10116 Gene_Symbol=Egf Pro-epidermal growth factor precursor

Match to Query 632: 1472.658848 from(737.336700,2+)

Title: 091008RatKidney_NH4Format01_25.1655.1655.2.dta

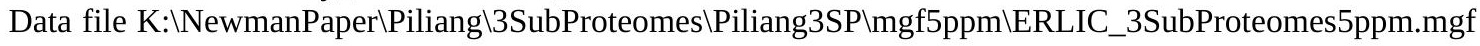
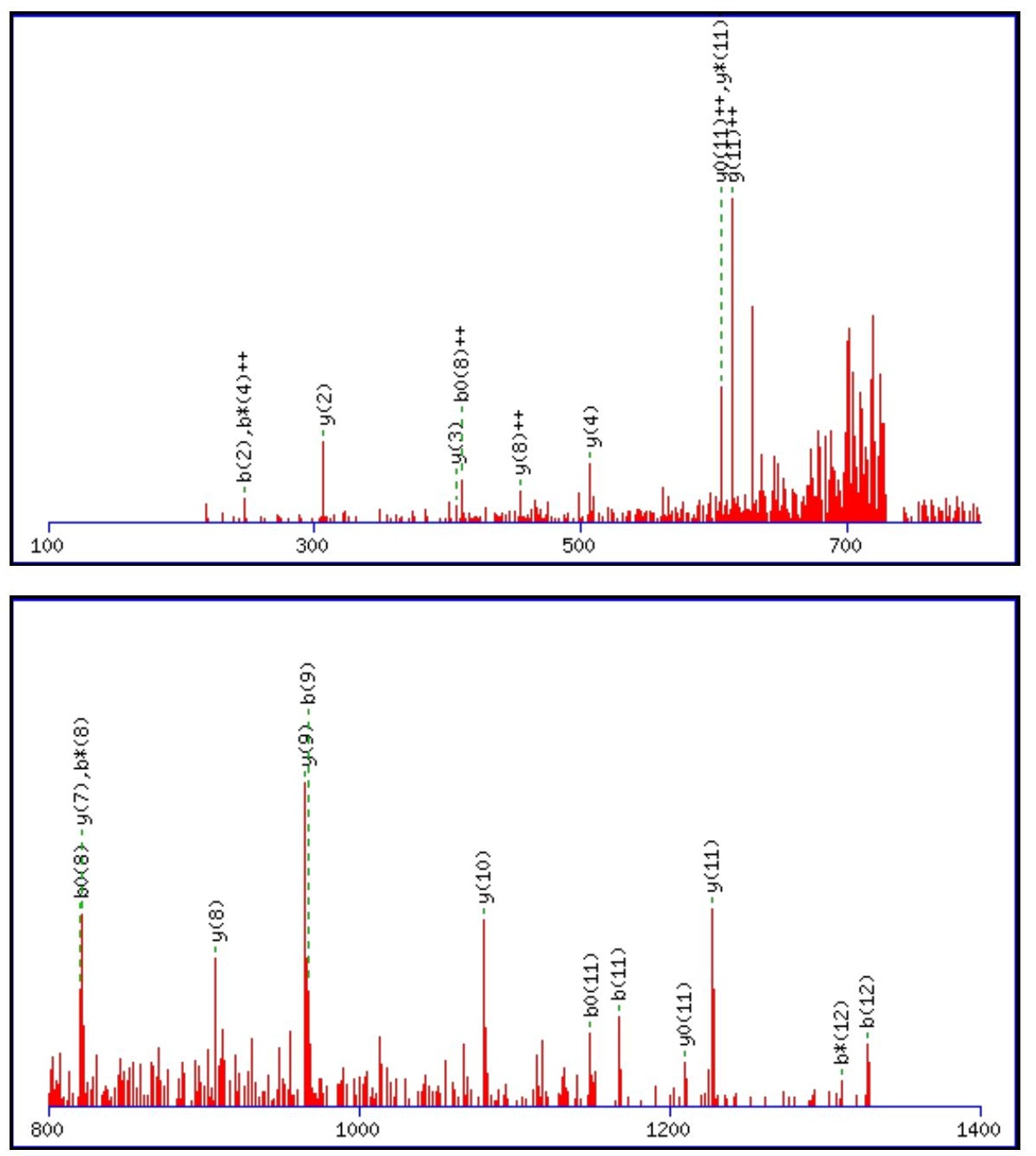


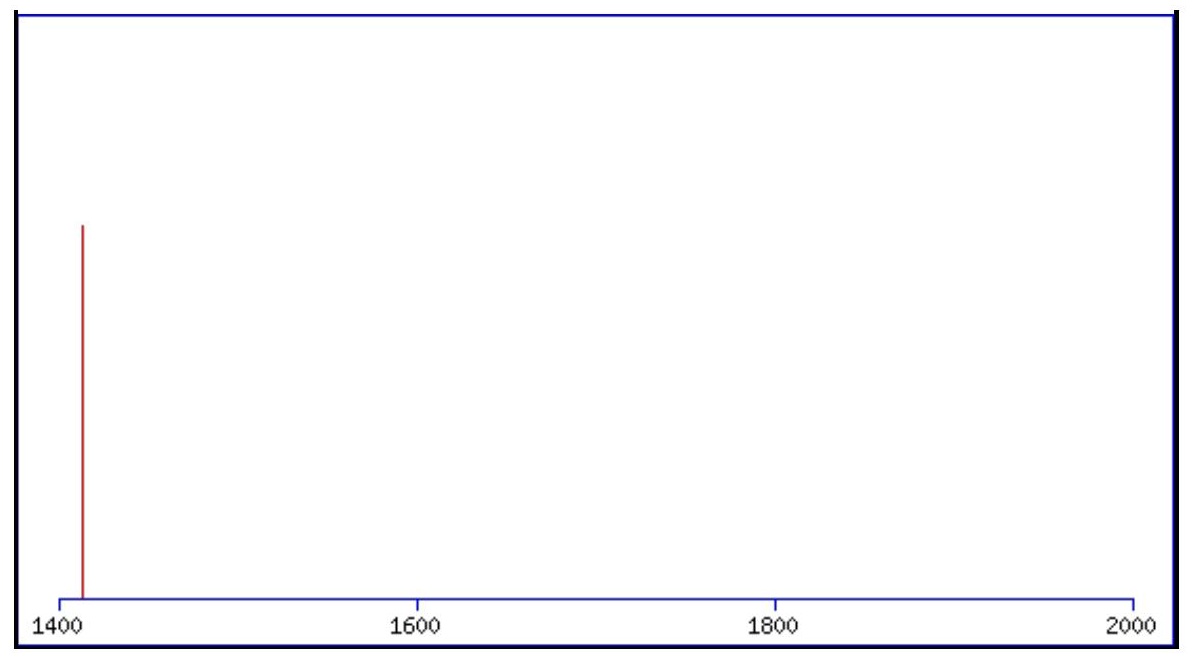

Monoisotopic mass of neutral peptide $\operatorname{Mr}($ calc): 1472.6606

Fixed modifications: Carbamidomethyl (C)

Variable modifications:

N4 : Deamidated_N (N)

Ions Score: 46 Expect: 0.0035

Matches (Bold Red): 23/122 fragment ions using 47 most intense peaks

\begin{tabular}{|r|c|c|c|c|c|c|c|c|c|c|c|c|c|c|}
\hline$\#$ & $\mathbf{b}$ & $\mathbf{b}^{++}$ & $\mathbf{b}^{*}$ & $\mathbf{b}^{\mathbf{*}^{++}}$ & $\mathbf{b}^{\mathbf{0}}$ & $\mathbf{b}^{\mathbf{0 + +}}$ & $\mathbf{S e q}$ & $\mathbf{y}$ & $\mathbf{y}^{++}$ & $\mathbf{y}^{\mathbf{*}}$ & $\mathbf{y}^{\mathbf{*}^{++}}$ & $\mathbf{y}^{\mathbf{0}}$ & $\mathbf{y}^{\mathbf{0 + +}}$ & $\#$ \\
\hline $\mathbf{1}$ & 100.0757 & 50.5415 & & & & & $\mathbf{V}$ & & & & & & & $\mathbf{1 3}$ \\
\hline $\mathbf{2}$ & $\mathbf{2 4 7 . 1 4 4 1}$ & 124.0757 & & & & & $\mathbf{F}$ & 1374.5994 & 687.8034 & 1357.5729 & 679.2901 & 1356.5889 & 678.7981 & $\mathbf{1 2}$ \\
\hline $\mathbf{3}$ & 394.2125 & 197.6099 & & & & & $\mathbf{F}$ & $\mathbf{1 2 2 7 . 5 3 1 0}$ & $\mathbf{6 1 4 . 2 6 9 1}$ & 1210.5045 & $\mathbf{6 0 5 . 7 5 5 9}$ & $\mathbf{1 2 0 9 . 5 2 0 5}$ & $\mathbf{6 0 5 . 2 6 3 9}$ & $\mathbf{1 1}$ \\
\hline $\mathbf{4}$ & 509.2395 & 255.1234 & 492.2129 & $2 \mathbf{2 4 6 . 6 1 0 1}$ & & & $\mathbf{N}$ & $\mathbf{1 0 8 0 . 4 6 2 6}$ & 540.7349 & 1063.4361 & 532.2217 & 1062.4520 & 531.7297 & $\mathbf{1 0}$ \\
\hline $\mathbf{5}$ & 566.2609 & 283.6341 & 549.2344 & 275.1208 & & & $\mathbf{G}$ & $\mathbf{9 6 5 . 4 3 5 7}$ & 483.2215 & 948.4091 & 474.7082 & 947.4251 & 474.2162 & $\mathbf{9}$ \\
\hline $\mathbf{6}$ & 653.2929 & 327.1501 & 636.2664 & 318.6368 & 635.2824 & 318.1448 & $\mathbf{S}$ & $\mathbf{9 0 8 . 4 1 4 2}$ & $\mathbf{4 5 4 . 7 1 0 7}$ & 891.3877 & 446.1975 & 890.4036 & 445.7055 & $\mathbf{8}$ \\
\hline $\mathbf{7}$ & 710.3144 & 355.6608 & 693.2879 & 347.1476 & 692.3038 & 346.6556 & $\mathbf{G}$ & $\mathbf{8 2 1 . 3 8 2 2}$ & 411.1947 & 804.3556 & 402.6815 & 803.3716 & 402.1894 & $\mathbf{7}$ \\
\hline $\mathbf{8}$ & 838.3730 & 419.6901 & $\mathbf{8 2 1 . 3 4 6 4}$ & 411.1769 & $\mathbf{8 2 0 . 3 6 2 4}$ & $\mathbf{4 1 0 . 6 8 4 8}$ & $\mathbf{Q}$ & 764.3607 & 382.6840 & 747.3342 & 374.1707 & 746.3502 & 373.6787 & $\mathbf{6}$ \\
\hline $\mathbf{9}$ & $\mathbf{9 6 7 . 4 1 5 6}$ & 484.2114 & 950.3890 & 475.6982 & 949.4050 & 475.2061 & $\mathbf{E}$ & 636.3021 & 318.6547 & 619.2756 & 310.1414 & 618.2916 & 309.6494 & $\mathbf{5}$ \\
\hline $\mathbf{1 0}$ & 1068.4633 & 534.7353 & 1051.4367 & 526.2220 & 1050.4527 & 525.7300 & $\mathbf{T}$ & $\mathbf{5 0 7 . 2 5 9 5}$ & 254.1334 & 490.2330 & 245.6201 & 489.2490 & 245.1281 & $\mathbf{4}$ \\
\hline $\mathbf{1 1}$ & $\mathbf{1 1 6 7 . 5 3 1 7}$ & 584.2695 & 1150.5051 & 575.7562 & $\mathbf{1 1 4 9 . 5 2 1 1}$ & 575.2642 & $\mathbf{V}$ & $\mathbf{4 0 6 . 2 1 1 9}$ & 203.6096 & 389.1853 & 195.0963 & & & $\mathbf{3}$ \\
\hline $\mathbf{1 2}$ & $\mathbf{1 3 2 7 . 5 6 2 3}$ & 664.2848 & $\mathbf{1 3 1 0 . 5 3 5 8}$ & 655.7715 & 1309.5518 & 655.2795 & $\mathbf{C}$ & $\mathbf{3 0 7 . 1 4 3 5}$ & 154.0754 & 290.1169 & 145.5621 & & & $\mathbf{2}$ \\
\hline $\mathbf{1 3}$ & & & & & & & $\mathbf{K}$ & 147.1128 & 74.0600 & 130.0863 & 65.5468 & & & $\mathbf{1}$ \\
\hline
\end{tabular}
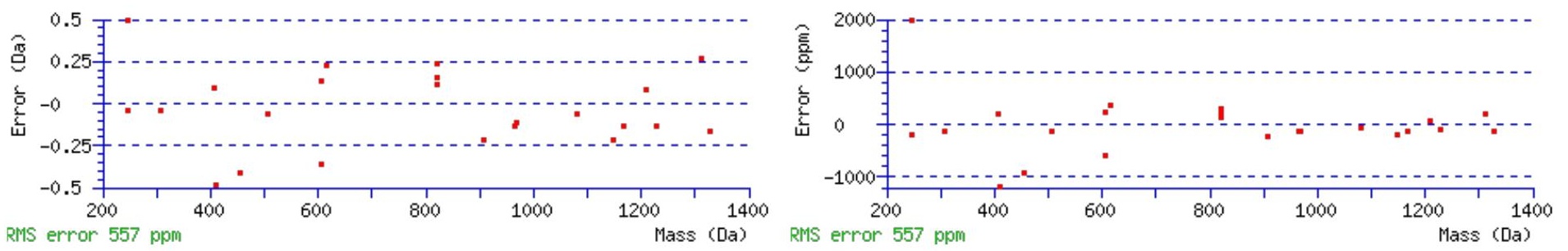

\section{All matches to this query}

\begin{tabular}{|l|l|l|l|}
\hline Score & Mr(calc): & Delta & \multicolumn{1}{c|}{ Sequence } \\
\hline 46.5 & 1472.6606 & -0.0018 & VFFNGSGQETVCK \\
\hline 14.7 & 1472.6500 & 0.0088 & CGTYNGTRGLSCK \\
\hline 4.5 & 1471.6629 & 0.9959 & EIVEAQSAATMVK \\
\hline 4.3 & 1471.6553 & 1.0035 & NYLSLAPLFFK \\
\hline 3.9 & 1472.6694 & -0.0106 & SVSDITRIQSCK \\
\hline 3.9 & 1472.6694 & -0.0106 & SVSDITRIQSCK \\
\hline 3.9 & 1472.6694 & -0.0106 & SVSDITRIQSCK \\
\hline 3.0 & 1472.6656 & -0.0067 & $\underline{\text { MDLLGESILVCK }}$ \\
\hline & & & \\
\hline
\end{tabular}




\begin{tabular}{|l|l|l|l|}
2.0 & 1472.6639 & -0.0051 & CALFNSVSSSLCK \\
\hline 1.6 & 1470.6578 & 2.0010 & FLTEVGNSHIMK \\
\hline
\end{tabular}

Spectrum No: 727; Query: 1301; Rank: 1

\section{Peptide View}

MS/MS Fragmentation of AVAYGEKNLTFQGPLPK

Found in IPI00767419, Tax_Id=10116 Gene_Symbol=Siae_predicted sialic acid acetylesterase

Match to Query 1301: 1832.966172 from(611.996000,3+)

Title: 091008RatKidney_NH4Format01_17.2632.2632.3.dta

Data file K:INewmanPaper|Piliangl3SubProteomes\Piliang3SP\mgf5ppm\ERLIC_3SubProteomes5ppm.mgf
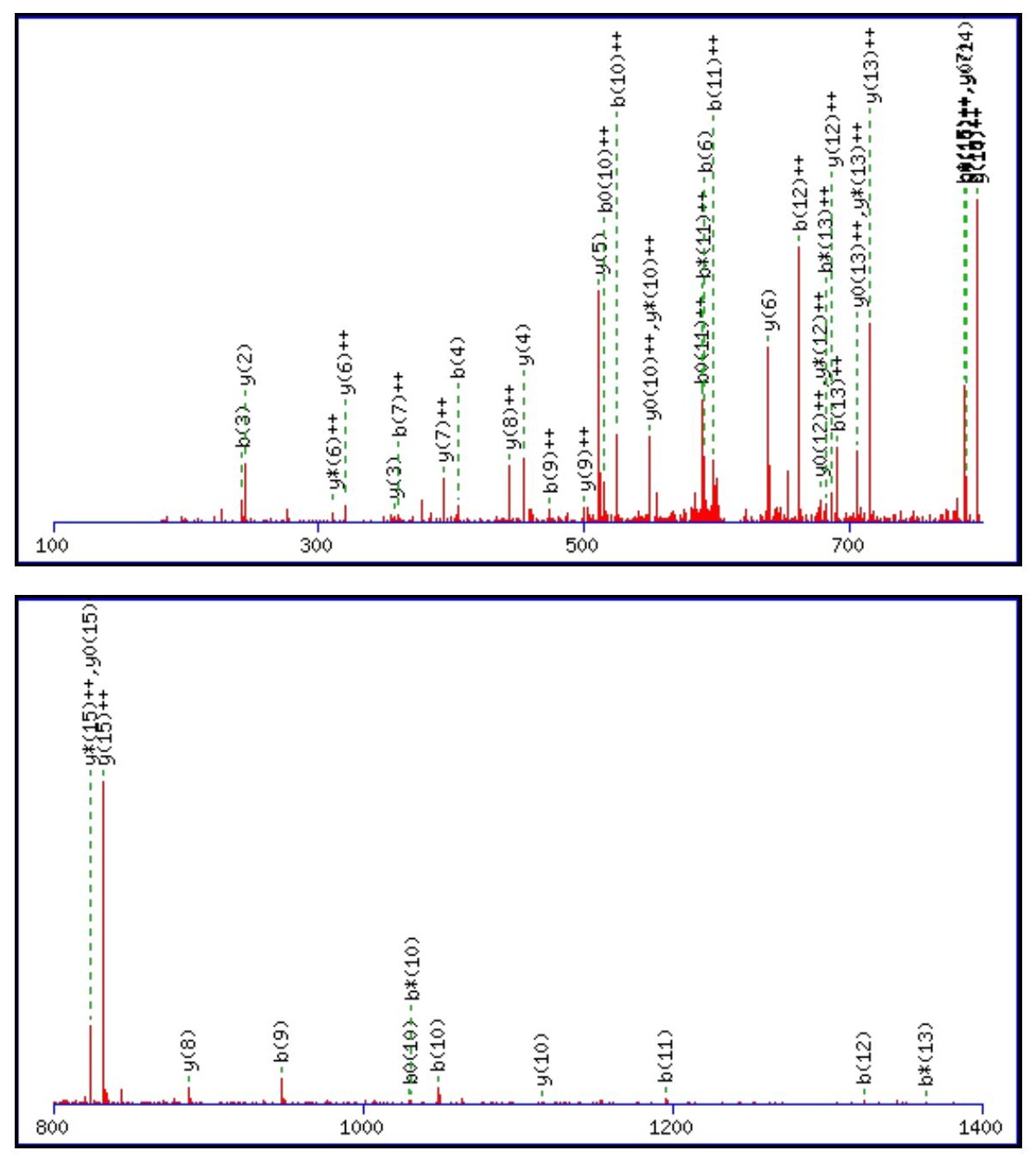


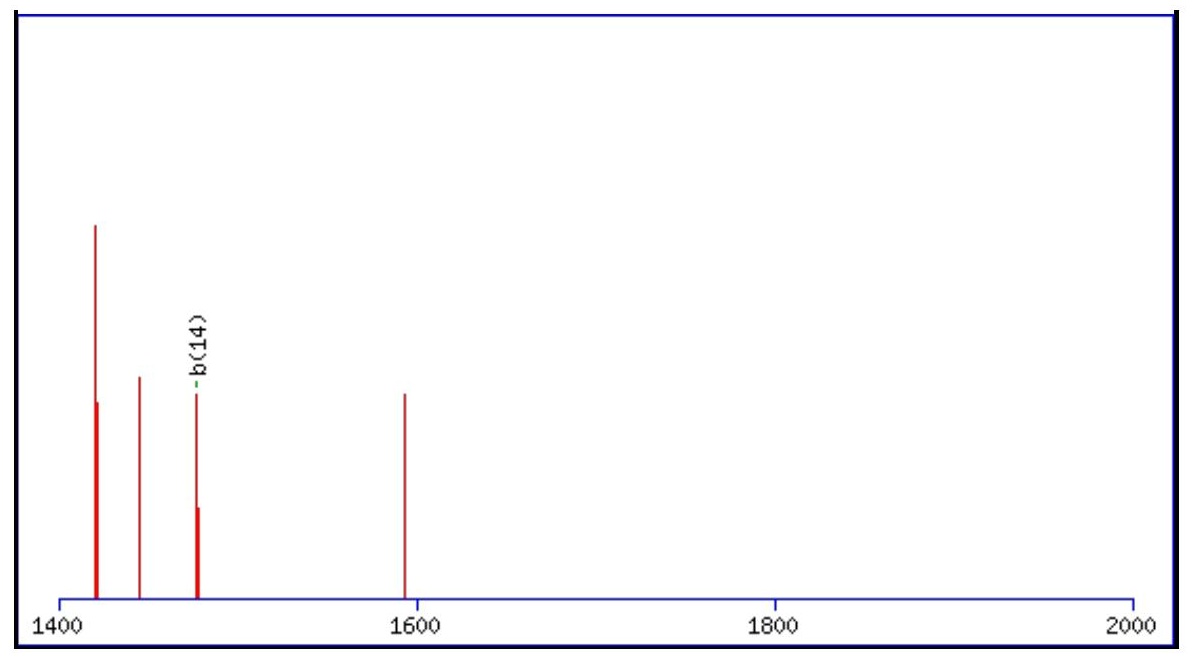

Monoisotopic mass of neutral peptide $\operatorname{Mr}($ calc): 1832.9672

Fixed modifications: Carbamidomethyl (C)

Variable modifications:

N8 : Deamidated_N (N)

Ions Score: 47 Expect: 0.0029

Matches (Bold Red): 51/156 fragment ions using 94 most intense peaks

\begin{tabular}{|c|c|c|c|c|c|c|c|c|c|c|c|c|c|c|}
\hline \# & b & $\mathbf{b}^{++}$ & $\mathbf{b}^{*}$ & $\mathbf{b}^{*^{++}}$ & $\mathbf{b}^{0}$ & $\mathbf{b}^{0++}$ & Seq. & $\mathbf{y}$ & $y^{++}$ & $\mathrm{y}^{*}$ & $\mathrm{y}^{*^{++}}$ & $\mathbf{y}^{0}$ & $y^{0++}$ & $\#$ \\
\hline 1 & 72.0444 & 36.5258 & & & & & A & & & & & & & 17 \\
\hline 2 & 171.1128 & 86.0600 & & & & & $\mathbf{V}$ & 1762.9374 & 881.9723 & 1745.9109 & 873.4591 & 1744.9268 & 872.9671 & 16 \\
\hline 3 & 242.1499 & 121.5786 & & & & & A & 1663.8690 & 832.4381 & 1646.8424 & 823.9249 & 1645.8584 & 823.4329 & 15 \\
\hline 4 & 405.2132 & 203.1103 & & & & & $\mathbf{Y}$ & 1592.8319 & 796.9196 & 1575.8053 & 788.4063 & 1574.8213 & 787.9143 & 14 \\
\hline 5 & 462.2347 & 231.6210 & & & & & G & 1429.7686 & \begin{tabular}{|l|l|}
715.3879 \\
\end{tabular} & 1412.7420 & 706.8746 & 1411.7580 & 706.3826 & 13 \\
\hline 6 & 591.2773 & 296.1423 & & & 573.2667 & 287.1370 & $\mathbf{E}$ & 1372.7471 & 686.8772 & 1355.7205 & 678.3639 & 1354.7365 & 677.8719 & 12 \\
\hline 7 & 719.3723 & 360.1898 & 702.3457 & 351.6765 & 701.3617 & 351.1845 & $\mathbf{K}$ & 1243.7045 & 622.3559 & 1226.6779 & 613.8426 & 1225.6939 & 613.3506 & 11 \\
\hline 8 & 834.3992 & 417.7032 & 817.3727 & 409.1900 & 816.3886 & 408.6980 & $\mathbf{N}$ & 1115.6095 & 558.3084 & 1098.5830 & 549.7951 & 1097.5990 & 549.3031 & 10 \\
\hline 9 & 947.4833 & 474.2453 & 930.4567 & 465.7320 & 929.4727 & 465.2400 & $\mathbf{L}$ & 1000.5826 & 500.7949 & 983.5560 & 492.2817 & 982.5720 & 491.7897 & 9 \\
\hline 10 & 1048.5309 & 524.7691 & 1031.5044 & 516.2558 & 1030.5204 & 515.7638 & $T$ & 887.4985 & 444.2529 & 870.4720 & 435.7396 & 869.4880 & 435.2476 & 8 \\
\hline 11 & 1195.5994 & 598.3033 & 1178.5728 & 589.7900 & 1177.5888 & 589.2980 & $\mathbf{F}$ & 786.4509 & 393.7291 & 769.4243 & 385.2158 & & & 7 \\
\hline 12 & 1323.6579 & 662.3326 & 1306.6314 & 653.8193 & 1305.6474 & 653.3273 & $\mathbf{Q}$ & 639.3824 & 320.1949 & 622.3559 & 311.6816 & & & 6 \\
\hline 13 & 1380.6794 & 690.8433 & 1363.6529 & 682.3301 & 1362.6688 & 681.8381 & G & 511.3239 & 256.1656 & 494.2973 & 247.6523 & & & 5 \\
\hline 14 & 1477.7322 & 739.3697 & 1460.7056 & 730.8564 & 1459.7216 & 730.3644 & $\mathbf{P}$ & 454.3024 & 227.6548 & 437.2758 & 219.1416 & & & 4 \\
\hline 15 & 1590.8162 & 795.9118 & 1573.7897 & 787.3985 & 1572.8057 & 786.9065 & $\mathbf{L}$ & 357.2496 & 179.1285 & 340.2231 & 170.6152 & & & 3 \\
\hline 16 & 1687.8690 & 844.4381 & 1670.8424 & 835.9249 & 1669.8584 & 835.4329 & $\mathbf{P}$ & 244.1656 & 122.5864 & 227.1390 & 114.0731 & & & 2 \\
\hline 17 & & & & & & & $\mathbf{K}$ & 147.1128 & 74.0600 & 130.0863 & 65.5468 & & & 1 \\
\hline
\end{tabular}
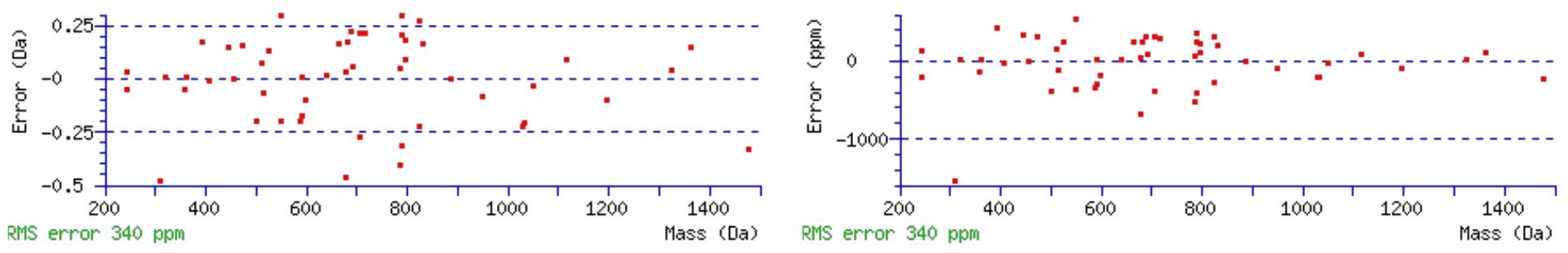

\section{All matches to this query}

\begin{tabular}{|l|l|l|l|}
\hline Score & Mr(calc): & Delta & \multicolumn{1}{c|}{ Sequence } \\
\hline 46.5 & 1832.9672 & -0.0011 & AVAYGEKNLTFQGPLPK \\
\hline 2.9 & 1830.9506 & 2.0156 & VGAPSPGPFLRGNTIIR \\
\hline 1.3 & 1832.9679 & -0.0017 & GLSRQMIGEFLGNRQK \\
\hline 0.2 & 1832.9495 & 0.0166 & QIVIGTPGTVLDWCFK \\
\hline
\end{tabular}


Spectrum No: 728; Query: 1467; Rank: 1

\section{Peptide View}

MS/MS Fragmentation of TCLLNETGDEPFQYKN

Found in IPI00324020, Tax_Id=10116 Gene_Symbol=Glul Glutamine synthetase

Match to Query 1467: 1928.842828 from(965.428690,2+)

Title: 091008RatKidney_NH4Format01_14.3282.3282.2.dta

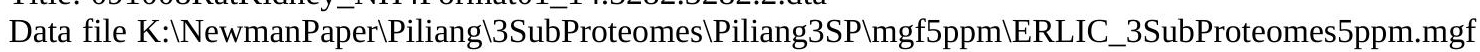
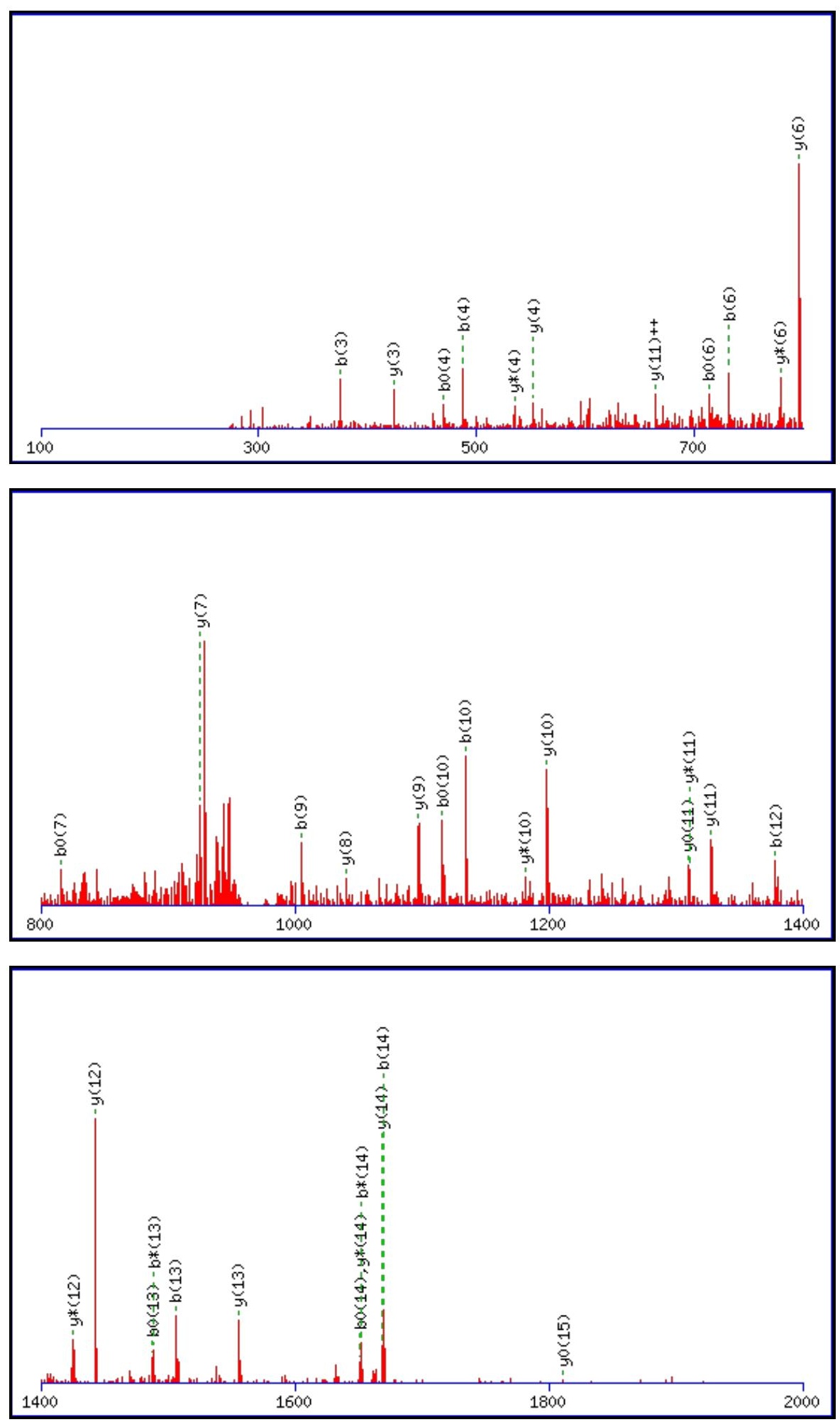
Monoisotopic mass of neutral peptide $\operatorname{Mr}($ calc): 1928.8462

Fixed modifications: Carbamidomethyl (C)

Variable modifications:

N5 : Deamidated_N (N)

Ions Score: 46 Expect: 0.0055

Matches (Bold Red): 36/160 fragment ions using 88 most intense peaks

\begin{tabular}{|c|c|c|c|c|c|c|c|c|c|c|c|c|c|c|}
\hline \# & b & $\mathbf{b}^{++}$ & $\mathbf{b}^{*}$ & $\mathbf{b}^{*^{++}}$ & $\mathbf{b}^{0}$ & $\mathbf{b}^{0++}$ & Seq. & $\mathbf{y}$ & $y^{++}$ & $\mathrm{y}^{*}$ & $\mathbf{y}^{*^{++}}$ & $\mathbf{y}^{0}$ & $y^{0++}$ & \# \\
\hline 1 & 102.0550 & 51.5311 & & & 84.0444 & 42.5258 & $\mathbf{T}$ & & & & & & & 16 \\
\hline 2 & 262.0856 & 131.5464 & & & 244.0750 & 122.5412 & C & 1828.8058 & 914.9065 & ؛ & 906.3933 & 1810.7952 & 905.9013 & 15 \\
\hline 3 & 375.1697 & 188.0885 & & & 357.1591 & 179.0832 & $\mathbf{L}$ & 1668.7752 & 834.8912 & 1651.7486 & 826.3779 & 1650.7646 & 825.8859 & 14 \\
\hline 4 & 488.2537 & 244.6305 & & & 470.2432 & 235.6252 & $\mathbf{L}$ & 1555.6911 & 778.3492 & 1538.6645 & 769.8359 & 1537.6805 & 769.3439 & 13 \\
\hline 5 & 603.2807 & 302.1440 & 586.2541 & 293.6307 & 585.2701 & 293.1387 & $\mathbf{N}$ & 1442.6070 & 721.8072 & 1425.5805 & 713.2939 & 1424.5965 & 712.8019 & 12 \\
\hline 6 & 732.3233 & 366.6653 & 715.2967 & 358.1520 & 714.3127 & 357.6600 & $\mathbf{E}$ & 1327.5801 & 664.2937 & 1310.5535 & 655.7804 & 1309.5695 & 655.2884 & 11 \\
\hline 7 & 833.3709 & 417.1891 & 816.3444 & 408.6758 & 815.3604 & 408.1838 & $\mathbf{T}$ & 1198.5375 & 599.7724 & 1181.5109 & 591.2591 & 1180.5269 & 590.7671 & 10 \\
\hline 8 & 890.3924 & 445.6998 & 873.3659 & 437.1866 & 872.3818 & 436.6946 & G & 1097.4898 & 549.2485 & 1080.4633 & 540.7353 & 1079.4793 & 540.2433 & 9 \\
\hline 9 & 1005.4193 & 503.2133 & 988.3928 & 494.7000 & 987.4088 & 494.2080 & D & 1040.4684 & 520.7378 & 1023.4418 & 512.2245 & 1022.4578 & 511.7325 & 8 \\
\hline 10 & 1134.4619 & 567.7346 & 1117.4354 & 559.2213 & 1116.4514 & 558.7293 & $\mathbf{E}$ & 925.4414 & 463.2243 & 908.4149 & 454.7111 & 907.4308 & 454.2191 & 7 \\
\hline 11 & 1231.5147 & 616.2610 & 1214.4882 & 607.7477 & 1213.5041 & 607.2557 & $\mathbf{P}$ & 796.3988 & 398.7030 & 779.3723 & 390.1898 & & & 6 \\
\hline 12 & 1378.5831 & 689.7952 & 1361.5566 & 681.2819 & 1360.5726 & 680.7899 & $\mathbf{F}$ & 699.3461 & 350.1767 & 682.3195 & 341.6634 & & & 5 \\
\hline 13 & 1506.6417 & 753.8245 & 1489.6151 & 745.3112 & \begin{tabular}{|l|}
1488.6311 \\
\end{tabular} & 744.8192 & $\mathbf{Q}$ & 552.2776 & 276.6425 & 535.2511 & 268.1292 & & & 4 \\
\hline 14 & \begin{tabular}{|l|}
1669.7050 \\
\end{tabular} & 835.3562 & 1652.6785 & 826.8429 & \begin{tabular}{|l|}
1651.6945 \\
\end{tabular} & 826.3509 & $\mathbf{Y}$ & 424.2191 & 212.6132 & 407.1925 & 204.0999 & & & 3 \\
\hline 15 & 1797.8000 & 899.4036 & 1780.7734 & 890.8904 & 1779.7894 & 890.3983 & $\mathbf{K}$ & 261.1557 & 131.0815 & 244.1292 & 122.5682 & & & 2 \\
\hline 16 & & & & & & & $\mathbf{N}$ & 133.0608 & 67.0340 & 116.0342 & 58.5207 & & & 1 \\
\hline
\end{tabular}
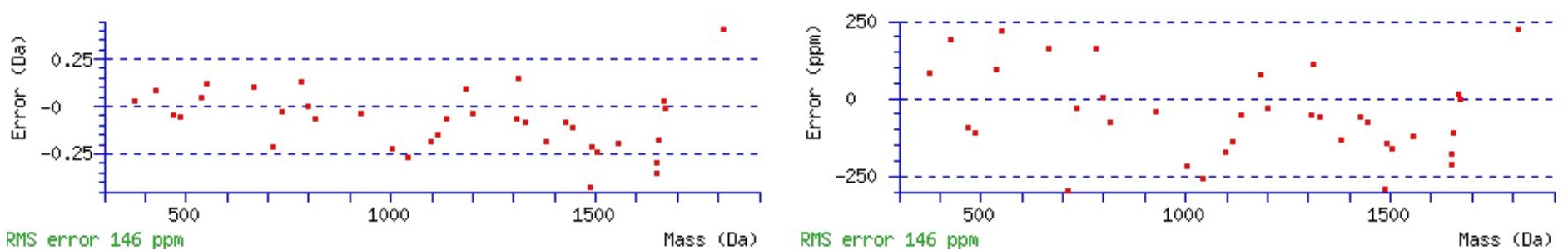

All matches to this query

\begin{tabular}{|l|l|l|l|}
\hline Score & Mr(calc): & Delta & \multicolumn{1}{c|}{ Sequence } \\
\hline 46.4 & 1928.8462 & -0.0034 & TCLLNETGDEPFQYKN \\
\hline 13.7 & 1928.8462 & -0.0034 & TCLLNETGDEPFQYKN \\
\hline 3.5 & 1928.8557 & -0.0128 & YANRYESDSYILNLK \\
\hline 1.4 & 1928.8557 & -0.0128 & YANRYESDSYILNLK \\
\hline 0.9 & 1926.8212 & 2.0216 & AHQKLHTGEKPYACK \\
\hline 0.7 & 1928.8557 & -0.0128 & YANRYESDSYILNLK \\
\hline 0.0 & 1927.8499 & 0.9930 & EMPINIQEAYRTPNR \\
\hline
\end{tabular}

Spectrum No: 729; Query: 1308; Rank: 1

\section{Peptide View}

MS/MS Fragmentation of KDTCAQECSHFNLTK

Found in IPI00191681, Tax_Id=10116 Gene_Symbol=Itgb1 Integrin beta-1 precursor

Match to Query 1308: 1838.798322 from(613.940050,3+)

Title: 100101RatKid_NS_deglyco_08.1193.1193.3.dta

Data file K:INewmanPaper|Piliang|3SubProteomes\Piliang3SP\mgf5ppm\ERLIC_3SubProteomes5ppm.mgf 

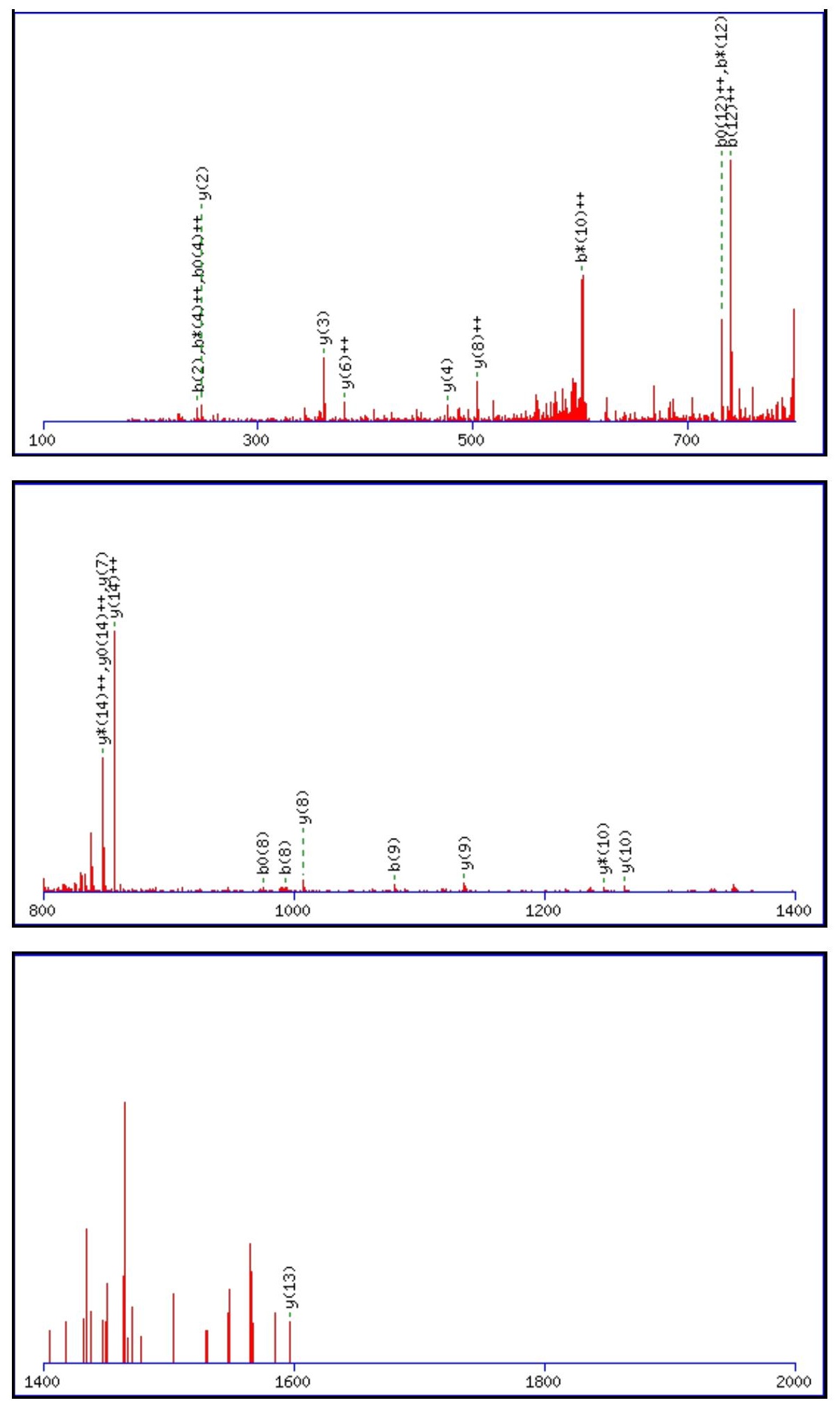

Monoisotopic mass of neutral peptide $\operatorname{Mr}($ calc): 1838.7927

Fixed modifications: Carbamidomethyl (C)

Variable modifications:

N12: Deamidated $\mathrm{N}(\mathrm{N})$

Ions Score: 46 Expect: 0.0051

Matches (Bold Red): 25/164 fragment ions using 42 most intense peaks

\begin{tabular}{|r|c|c|c|c|c|c|c|c|c|c|c|c|c|c|}
\hline$\#$ & $\mathbf{b}$ & $\mathbf{b}^{++}$ & $\mathbf{b}^{*}$ & $\mathbf{b}^{\boldsymbol{*}_{++}}$ & $\mathbf{b}^{\mathbf{0}}$ & $\mathbf{b}^{\mathbf{0 + +}}$ & Seq. & $\mathbf{y}$ & $\mathbf{y}^{++}$ & $\mathbf{y}^{\mathbf{*}}$ & $\mathbf{y}^{\boldsymbol{*}^{++}}$ & $\mathbf{y}^{\mathbf{0}}$ & $\mathbf{y}^{\mathbf{0 + +}}$ & $\#$ \\
\hline $\mathbf{1}$ & 129.1022 & 65.0548 & 112.0757 & 56.5415 & & & $\mathbf{K}$ & & & & & & & $\mathbf{1 5}$ \\
\hline $\mathbf{2}$ & $\mathbf{2 4 4 . 1 2 9 2}$ & 122.5682 & 227.1026 & 114.0550 & 226.1186 & 113.5629 & $\mathbf{D}$ & 1711.7050 & $\mathbf{8 5 6 . 3 5 6 2}$ & 1694.6785 & $\mathbf{8 4 7 . 8 4 2 9}$ & 1693.6945 & $\mathbf{8 4 7 . 3 5 0 9}$ & $\mathbf{1 4}$ \\
\hline $\mathbf{3}$ & 345.1769 & 173.0921 & 328.1503 & 164.5788 & 327.1663 & 164.0868 & $\mathbf{T}$ & $\mathbf{1 5 9 6 . 6 7 8 1}$ & 798.8427 & 1579.6516 & 790.3294 & 1578.6675 & 789.8374 & $\mathbf{1 3}$ \\
\hline $\mathbf{4}$ & 505.2075 & 253.1074 & 488.1810 & 244.5941 & 487.1969 & 244.1021 & $\mathbf{C}$ & 1495.6304 & 748.3188 & 1478.6039 & 739.8056 & 1477.6199 & 739.3136 & $\mathbf{1 2}$ \\
\hline $\mathbf{5}$ & 576.2446 & 288.6260 & 559.2181 & 280.1127 & 558.2341 & 279.6207 & $\mathbf{A}$ & 1335.5998 & 668.3035 & 1318.5732 & 659.7902 & 1317.5892 & 659.2982 & $\mathbf{1 1}$ \\
\hline
\end{tabular}




\begin{tabular}{|c|c|c|c|c|c|c|c|c|c|c|c|c|c|c|}
\hline 6 & 704.3032 & |352.6552 & 687.2767 & |344.1420 & 686.2926 & |343.6500| & $\mathbf{Q}$ & |1264.5627 & |632.7850| & |1247.5361 & |624.2717| & 1246.552 & |623.7797| & 10 \\
\hline 7 & 833.3458 & 417.1765 & 816.3192 & 408.6633 & 815.3352 & 408.1713 & $\mathbf{E}$ & 1136.5041 & 568.7557 & 1119.4775 & 560.2424 & 1118.4935 & 559.7504 & 9 \\
\hline 8 & 993.3764 & 497.1919 & 976.3499 & 488.6786 & 975.3659 & 488.1866 & C & 1007.4615 & 504.2344 & 990.4349 & 495.7211 & 989.4509 & 495.2291 & 8 \\
\hline 9 & 1080.4085 & 540.7079 & 1063.3819 & 532.1946 & 1062.3979 & 531.7026 & $\mathrm{~S}$ & 847.4308 & 424.2191 & 830.4043 & 415.7058 & 829.4203 & 415.2138 & 7 \\
\hline 10 & 1217.4674 & 609.2373 & 1200.4408 & 600.7241 & 1199.4568 & 600.2320 & $\mathbf{H}$ & 760.3988 & 380.7030 & 743.3723 & 372.1898 & 742.3882 & 371.6978 & 6 \\
\hline 11 & 1364.5358 & 682.7715 & 1347.5092 & 674.2583 & 1346.5252 & 673.7663 & $\mathbf{F}$ & 623.3399 & 312.1736 & 606.3133 & 303.6603 & 605.3293 & 303.1683 & 5 \\
\hline 12 & 1479.5627 & 740.2850 & 1462.5362 & 731.7717 & 1461.5522 & 731.2797 & $\mathbf{N}$ & 476.2715 & 238.6394 & 459.2449 & 230.1261 & 458.2609 & 229.6341 & 4 \\
\hline 13 & 1592.6468 & 796.8270 & 1575.6202 & 788.3138 & 1574.6362 & 787.8218 & $\mathbf{L}$ & 361.2445 & 181.1259 & 344.2180 & 172.6126 & 343.2340 & 172.1206 & 3 \\
\hline 14 & 1693.6945 & 847.3509 & 1676.6679 & 838.8376 & 1675.6839 & 838.3456 & $\mathbf{T}$ & 248.1605 & 124.5839 & 231.1339 & 116.0706 & 230.1499 & 115.5786 & 2 \\
\hline 15 & & & & & & & $\mathbf{K}$ & 147.1128 & 74.0600 & 130.0863 & 65.5468 & & & 1 \\
\hline
\end{tabular}
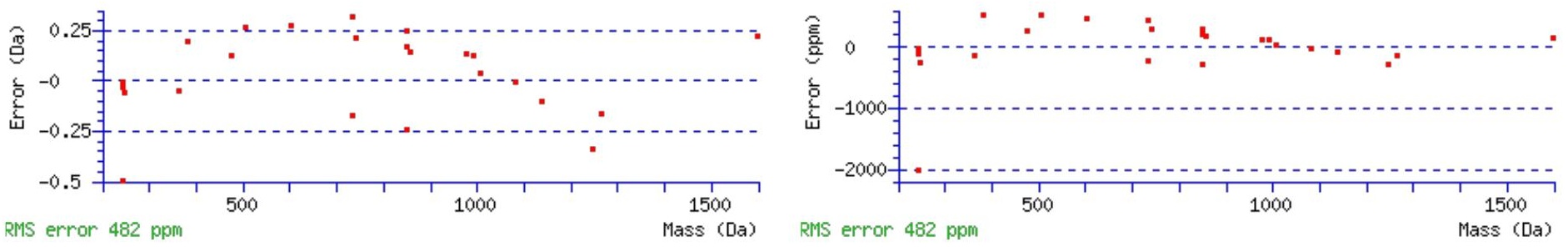

\section{All matches to this query}

\begin{tabular}{|l|l|l|l|}
\hline Score & Mr(calc): & Delta & \multicolumn{1}{c|}{ Sequence } \\
\hline 46.2 & 1838.7927 & 0.0056 & $\underline{\text { KDTCAQECSHFNLTK }}$ \\
\hline 3.8 & 1836.7865 & 2.0118 & YAASENVFNRQMVAR \\
\hline 3.3 & 1837.8087 & 0.9896 & $\underline{\text { KDTCAQECSHFNLTK }}$ \\
\hline 2.8 & 1836.7743 & 2.0240 & $\underline{\text { VSGTMYNTGRHVSLR }}$ \\
\hline 2.3 & 1838.8009 & -0.0026 & $\underline{\text { MEVEDGLGSPKPEDIK }}$ \\
\hline 1.9 & 1837.8047 & 0.9936 & $\underline{\text { EAGSNVDSSCVLRECR }}$ \\
\hline 0.2 & 1837.8004 & 0.9979 & $\underline{\text { LYSGTARYHMQMLR }}$ \\
\hline 0.2 & 1837.8004 & 0.9979 & $\underline{\text { LYSGTARYHMQMLR }}$ \\
\hline 0.1 & 1838.8005 & -0.0022 & $\underline{\text { AVTGFSGDGKAIWDKK }}$ \\
\hline
\end{tabular}

Spectrum No: 730; Query: 2453; Rank: 1

\section{Peptide View}

MS/MS Fragmentation of ENYAELLDDGFLKNITAQICIDKK

Found in IPI00371853, Tax_Id=10116 Gene_Symbol=Col6a1_predicted similar to Collagen alpha-1(VI) chain precursor

Match to Query 2453: 2811.410082 from(938.143970,3+)

Title: 091008RatKidney_NoSalt_16.6508.6508.3.dta

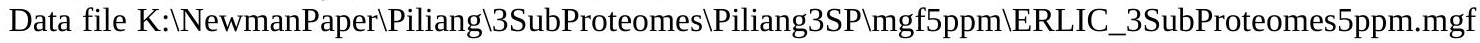



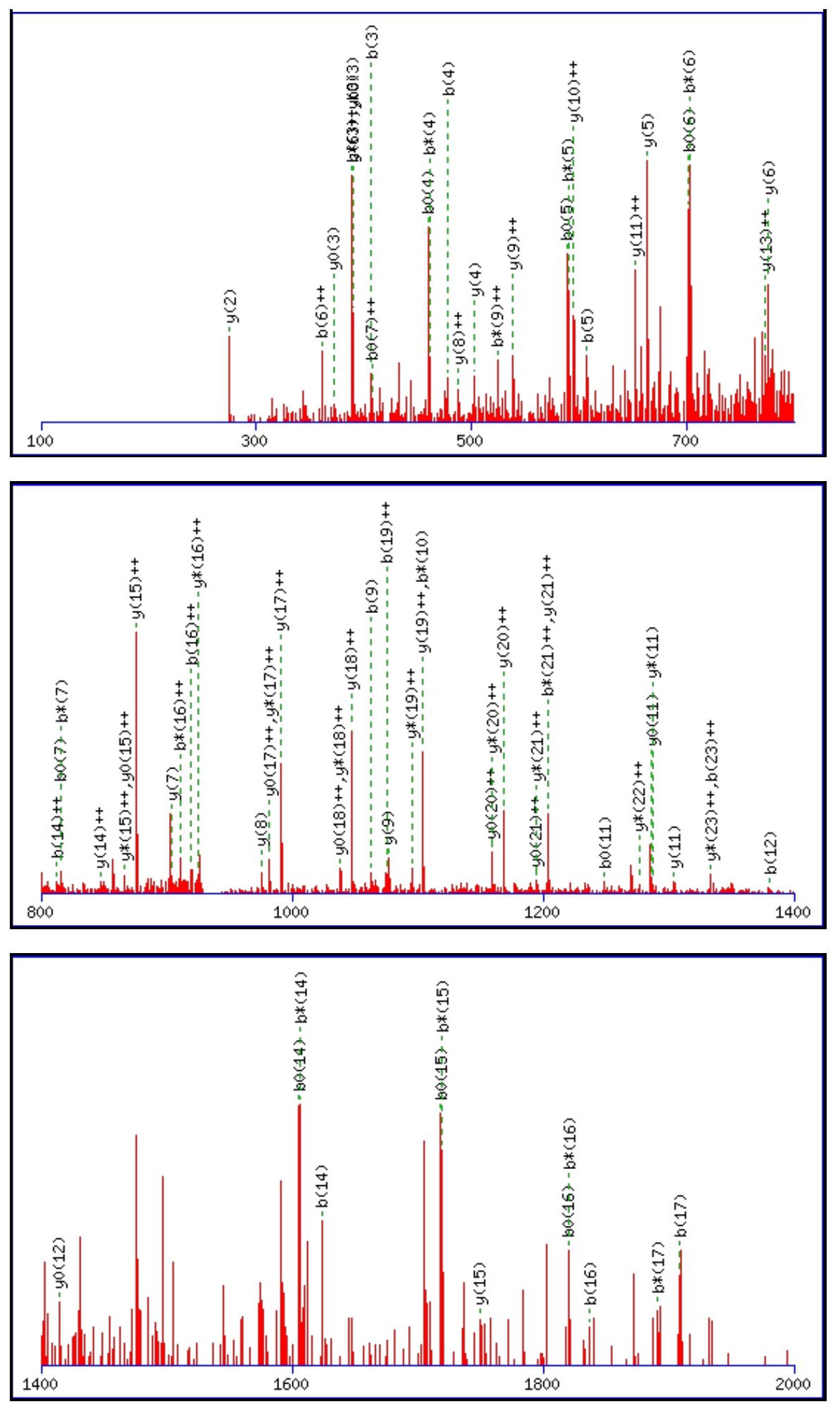

Monoisotopic mass of neutral peptide $\operatorname{Mr}($ calc): 2811.4000

Fixed modifications: Carbamidomethyl (C)

Variable modifications:

N14 : Deamidated N $(\mathrm{N})$

Ions Score: 46 Expect: 0.0052

Matches (Bold Red): 77/270 fragment ions using 173 most intense peaks

\begin{tabular}{|c|c|c|c|c|c|c|c|c|c|c|c|c|c|c|}
\hline \# & b & $\mathbf{b}^{++}$ & $\mathbf{b}^{*}$ & $\mathbf{b}^{*++}$ & $\mathbf{b}^{0}$ & $\mathbf{b}^{\mathbf{0}^{++}}$ & Seq. & $\mathbf{y}$ & $\mathbf{y}^{++}$ & $\mathbf{y}^{*}$ & $\mathbf{y}^{*^{++}}$ & $\mathbf{y}^{0}$ & $\mathbf{y}^{\mathbf{0}^{++}}$ & \# \\
\hline 1 & 130.0499 & 65.5286 & & & 112.0393 & 56.5233 & E & & & & & & & 24 \\
\hline 2 & 244.0928 & 122.5500 & 227.0662 & 114.0368 & 226.0822 & 113.5448 & $\mathbf{N}$ & 2683.3647 & 1342.1860 & 2666.3382 & 1333.6727 & 2665.3542 & 1333.1807 & 23 \\
\hline 3 & 407.1561 & 204.0817 & 390.1296 & 195.5684 & 389.1456 & 195.0764 & $\mathbf{Y}$ & 2569.3218 & 1285.1645 & 2552.2953 & 1276.6513 & 2551.3112 & 1276.1593 & 22 \\
\hline 4 & 478.1932 & 239.6003 & 461.1667 & 231.0870 & 460.1827 & 230.5950 & A & 2406.2585 & 1203.6329 & 2389.2319 & 1195.1196 & 2388.2479 & 1194.6276 & 21 \\
\hline 5 & 607.2358 & 304.1216 & 590.2093 & 295.6083 & 589.2253 & 295.1163 & E & 2335.2214 & 1168.1143 & 2318.1948 & 1159.6010 & 2317.2108 & 1159.1090 & 20 \\
\hline
\end{tabular}




\begin{tabular}{|c|c|c|c|c|c|c|c|c|c|c|c|c|c|c|}
\hline & 720.3199 & 636 & & & & & L & & & & & & 1094.5877 & 19 \\
\hline 7 & 833.4040 & 417.2056 & 816.3774 & 408.6923 & 815.3934 & 408.2003 & $\mathbf{L}$ & 2093.0947 & 1047.0510 & 2076.0682 & 1038.5377 & 2075.0841 & 1038.0457 & 18 \\
\hline 8 & 948.4309 & 474.7191 & 931.4044 & 466.2058 & 930.4203 & 465.7138 & D & 1980.0106 & 990.5090 & 1962.9841 & 981.9957 & 1962.0001 & .5037 & 7 \\
\hline 9 & 1063.4578 & 32.2326 & 1046.4313 & & 1045.4473 & & D & & & & 924.4822 & & 923.9902 & \\
\hline 10 & 1120.4793 & 560.7433 & 03.4528 & 300 & 1102.4687 & 551.7380 & G & 1749.9568 & 875.4820 & 1732.9302 & 866.9687 & 1731.9462 & 866.4767 & \\
\hline $11[1$ & 1267.5477 & 634.2775 & 1250.5212 & 625.7642 & 1249.5372 & 625.2722 & $\mathbf{F}$ & 1692.9353 & 846.9713 & 1675.9088 & 838.4580 & 1674.9247 & 837.9660 & 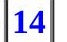 \\
\hline 121 & 1380.6318 & 690.8195 & 63. & 682.3063 & 1362.6212 & 681.8142 & $\mathbf{L}$ & 1545.8669 & 773.4371 & 1528.8403 & 764.9238 & 1527.8563 & 4318 & \\
\hline 3 & 1508.7268 & 754.8670 & 002 & 746. & 1490 & 617 & $\mathbf{K}$ & 1432 & 950 & & & & 3898 & \\
\hline 141 & 1623.7537 & 812.3805 & 1606.7271 & 803.8672 & 1605.7431 & 803.3752 & $\mathbf{N}$ & 1304.6879 & 652.8476 & 1287.6613 & 644.3343 & 1286.6773 & 643.8423 & 1 \\
\hline 15 & $1736 . \varepsilon$ & 868.9 & 1719.8112 & 860.4092 & 1718. & 172 & I & 1189. & 595.3341 & 1172. & 8208 & 1171 & 3288 & \\
\hline $16[1$ & 1837.8854 & 919.4464 & 1820.8589 & 910.9331 & 1819.8749 & & $T$ & 1076.5769 & & & & 1058.5663 & 529.7868 & \\
\hline 17 & 1908.9225 & 954.9649 & 1891.8960 & 946.4516 & 1890.9120 & 945.9596 & A & 975.5292 & 488.2682 & 958.5026 & 479.7550 & 957.5186 & 479.2629 & \\
\hline 18 & 2036.9811 & 18.9942 & 2019.9546 & 1010.4809 & 2018.9706 & 889 & $\mathbf{Q}$ & 904. & 452.7497 & 887.4655 & 444.2364 & 886 & 443.7444 & \\
\hline$\left.19\right|^{2}$ & 2150.0652 & 1075.5362 & 2133.0386 & 1067.0230 & 2132.0546 & 1066.5309 & I & 776.4335 & 388.7204 & 759.4069 & 380.2071 & 758.4229 & 379.7151 & \\
\hline 202 & 2310.0958 & 1155.5516 & 2293.0693 & 1147.0383 & 2292.0853 & 1146.5463 & C & 663.3494 & 332.1783 & 646.3229 & 323.6651 & 645.3389 & 323.1731 & \\
\hline 21 & 2423.1799 & 1212.0936 & 2406.1533 & 1203.5803 & 2405.1693 & 1203.0883 & I & 503.3188 & 252.1630 & 486.2922 & 243.6498 & 485.3082 & 243.1577 & \\
\hline $22 \mid 2$ & 2538.2068 & 1269.6071 & 2521.1803 & 1261.0938 & 2520.1963 & 1260.6018 & D & 390.2347 & 195.6210 & 373.2082 & 187.1077 & 372.2241 & 186.6157 & \\
\hline 232 & 2666.3018 & 1333.6545 & 2649.2753 & 1325.1413 & 2648.2912 & 1324.6493 & $\mathbf{K}$ & 275.2078 & 138.1075 & 258.1812 & 129.5942 & & & \\
\hline 24 & & & & & & & K & 147.1128 & 74.0600 & 130.0863 & 65.5468 & & & \\
\hline
\end{tabular}
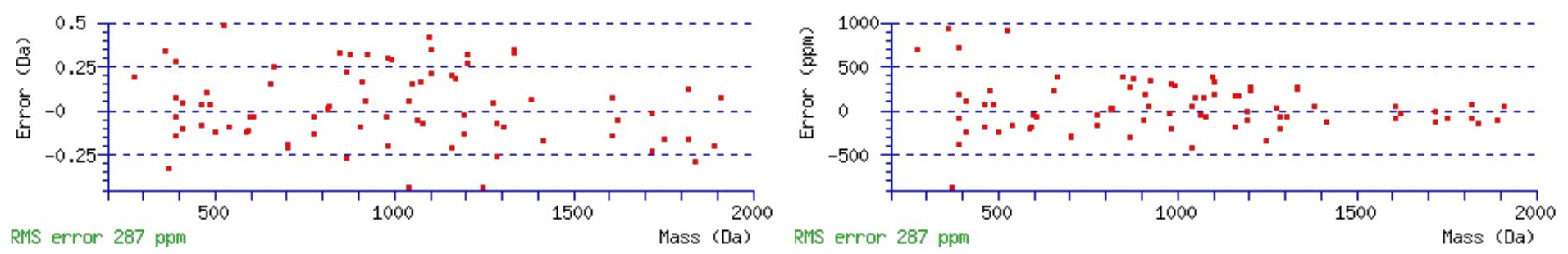

\section{All matches to this query}

\begin{tabular}{|l|l|l|l|}
\hline Score & Mr(calc): & Delta & \multicolumn{1}{|c|}{ Sequence } \\
\hline 46.1 & 2811.4000 & 0.0101 & ENYAELLDDGFLKNITAQICIDKK \\
\hline 14.6 & 2811.4000 & 0.0101 & ENYAELLDDGFLKNITAQICIDKK \\
\hline 10.7 & 2810.4160 & 0.9941 & ENYAELLDDGFLKNITAQICIDKK \\
\hline 6.5 & 2809.4138 & 1.9962 & SWNNSLIMKLLHGATKLLHFFSR \\
\hline 4.6 & 2809.4138 & 1.9962 & SWNNSLIMKLLHGATKLLHFFSR \\
\hline 4.0 & 2809.4142 & 1.9959 & NKITSVLYSVLMPTLNPLIYTLR \\
\hline 4.0 & 2809.4142 & 1.9959 & NKITSVLYSVLMPTLNPLIYTLR \\
\hline 4.0 & 2809.4142 & 1.9959 & NKITSVLYSVLMPTLNPLIYTLR \\
\hline 3.7 & 2810.3982 & 1.0119 & NKITSVLYSVLMPTLNPLIYTLR \\
\hline 3.7 & 2810.3982 & 1.0119 & NKITSVLYSVLMPTLNPLIYTLR \\
\hline
\end{tabular}

Spectrum No: 731; Query: 69; Rank: 1

\section{Peptide View}

MS/MS Fragmentation of SFLDCTNR

Found in IPI00205325, Tax_Id=10116 Gene_Symbol=Lrp2 Low-density lipoprotein receptor-related protein 2 precursor

Match to Query 69: 1012.426188 from(507.220370,2+)

Title: 091008RatKidney_NoSalt_32.1037.1037.2.dta

Data file K:INewmanPaper|Piliang|3SubProteomes\Piliang3SP\mgf5ppm\ERLIC_3SubProteomes5ppm.mgf 

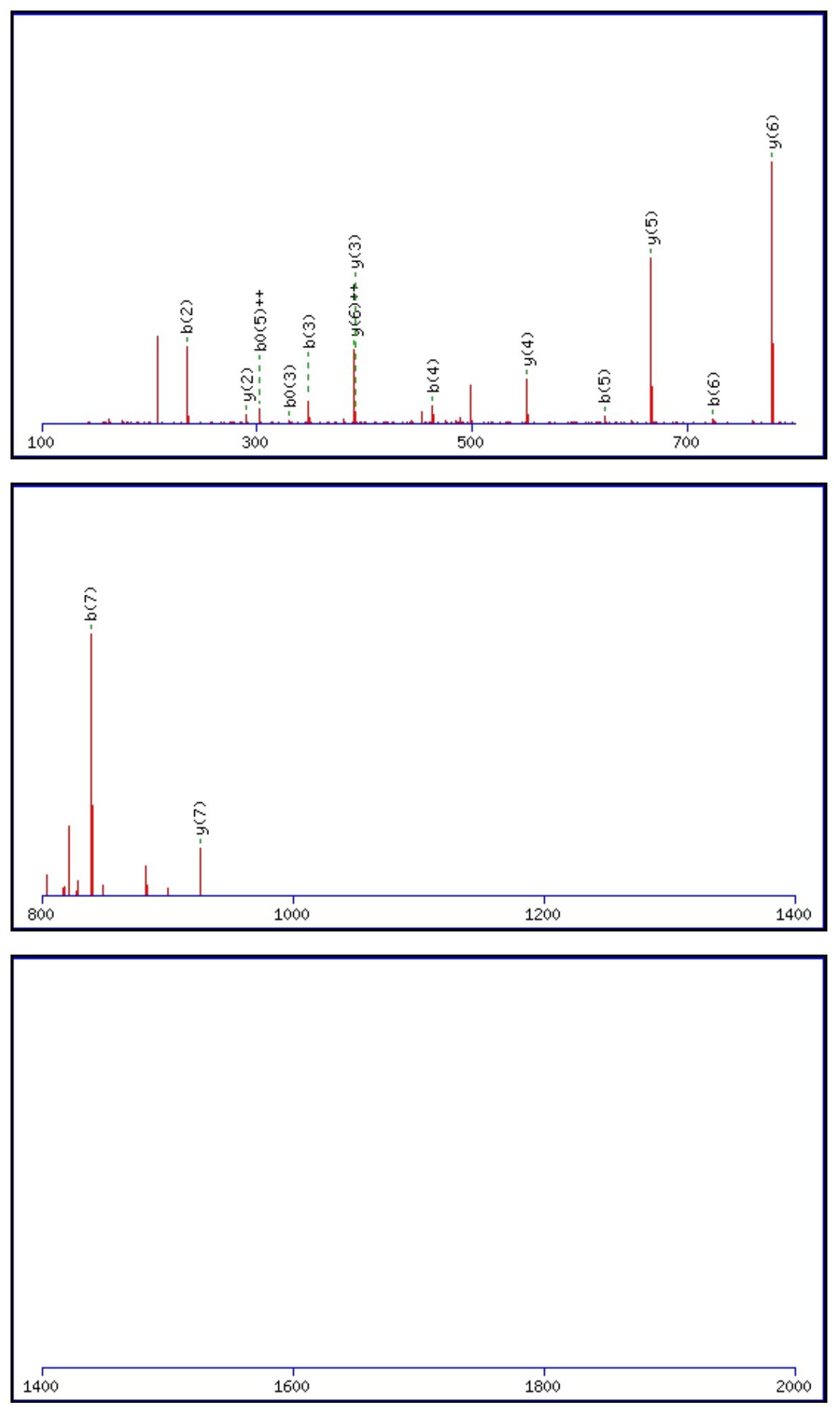

Monoisotopic mass of neutral peptide $\operatorname{Mr}$ (calc): 1012.4284

Fixed modifications: Carbamidomethyl (C)

Variable modifications:

N7 : Deamidated_N (N)

Ions Score: 46 Expect: 0.0017

Matches (Bold Red): 15/68 fragment ions using 25 most intense peaks

\begin{tabular}{|c|c|c|c|c|c|c|c|c|c|c|c|c|c|c|}
\hline$\#$ & $\mathbf{b}$ & $\mathbf{b}^{++}$ & $\mathbf{b}^{*}$ & $\mathbf{b}^{\boldsymbol{*}^{++}}$ & $\mathbf{b}^{\mathbf{0}}$ & $\mathbf{b}^{\mathbf{0}+}$ & Seq. & $\mathbf{y}$ & $\mathbf{y}^{++}$ & $\mathbf{y}^{*}$ & $\mathbf{y}^{\mathbf{*}^{++}}$ & $\mathbf{y}^{\mathbf{0}}$ & $\mathbf{y}^{\mathbf{0 + +}}$ & $\#$ \\
\hline $\mathbf{1}$ & $\mathbf{8 8 . 0 3 9 3}$ & 44.5233 & & & 70.0287 & 35.5180 & $\mathbf{S}$ & & & & & & & $\mathbf{8}$ \\
\hline $\mathbf{2}$ & 235.1077 & 118.0575 & & & 217.0972 & 109.0522 & $\mathbf{F}$ & $\mathbf{9 2 6 . 4 0 3 6}$ & 463.7055 & 909.3771 & 455.1922 & 908.3931 & 454.7002 & $\mathbf{7}$ \\
\hline $\mathbf{3}$ & 348.1918 & 174.5995 & & & 330.1812 & 165.5942 & $\mathbf{L}$ & 779.3352 & 390.1712 & 762.3087 & 381.6580 & 761.3247 & 381.1660 & $\mathbf{6}$ \\
\hline $\mathbf{4}$ & $\mathbf{4 6 3 . 2 1 8 7}$ & 232.1130 & & & 445.2082 & 223.1077 & $\mathbf{D}$ & $\mathbf{6 6 6 . 2 5 1 2}$ & 333.6292 & 649.2246 & 325.1159 & 648.2406 & 324.6239 & $\mathbf{5}$ \\
\hline $\mathbf{5}$ & $\mathbf{6 2 3 . 2 4 9 4}$ & 312.1283 & & & 605.2388 & 303.1230 & $\mathbf{C}$ & $\mathbf{5 5 1 . 2 2 4 2}$ & 276.1157 & 534.1977 & 267.6025 & 533.2137 & 267.1105 & $\mathbf{4}$ \\
\hline
\end{tabular}




\begin{tabular}{|r|r|r|r|r|r|r|r|r|r|r|r|r|r|r|r|r|r|r|}
\hline $\mathbf{6}$ & 724.2971 & 362.6522 & & & 706.2865 & 353.6469 & $\mathbf{T}$ & 391.1936 & 196.1004 & 374.1670 & 187.5871 & 373.1830 & 187.0951 & 3 \\
\hline $\mathbf{7}$ & $\mathbf{8 3 9 . 3 2 4 0}$ & 420.1656 & 822.2974 & 411.6524 & 821.3134 & 411.1604 & $\mathbf{N}$ & 290.1459 & 145.5766 & 273.1193 & 137.0633 & & & 2 \\
\hline $\mathbf{8}$ & & & & & & & R & 175.1190 & 88.0631 & 158.0924 & 79.5498 & & & $\mathbf{1}$ \\
\hline
\end{tabular}
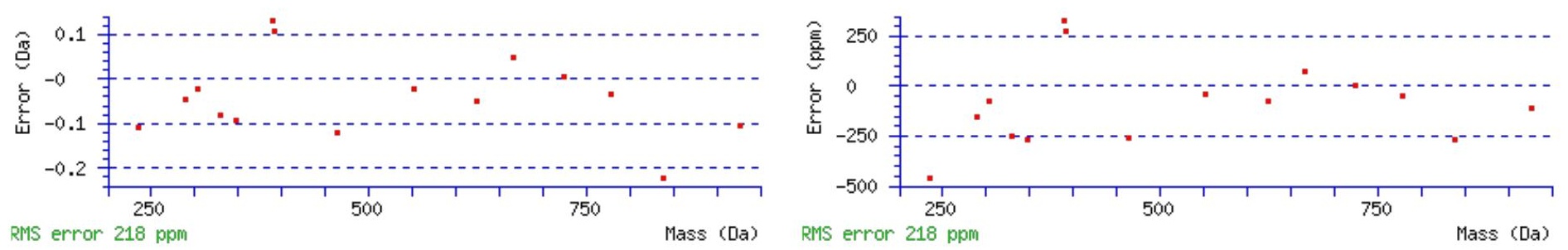

\section{All matches to this query}

\begin{tabular}{|l|l|l|l|}
\hline Score & Mr(calc): & Delta & \multicolumn{1}{|c|}{ Sequence } \\
\hline 45.9 & 1012.4284 & -0.0022 & SFLDCTNR \\
\hline 9.1 & 1012.4339 & -0.0077 & SISQQSGAR \\
\hline 6.8 & 1012.4358 & -0.0096 & AFEDCMLK \\
\hline 5.2 & 1010.4182 & 2.0080 & GSENGAALGR \\
\hline 3.7 & 1012.4284 & -0.0022 & HPMADNPSK \\
\hline 3.2 & 1011.4314 & 0.9948 & NPPYILNT \\
\hline 3.0 & 1012.4318 & -0.0056 & MAMSITGDR \\
\hline 2.1 & 1012.4318 & -0.0056 & MMTSVGTNR \\
\hline 1.9 & 1012.4235 & 0.0027 & ATIPMGMGR \\
\hline 1.1 & 1011.4136 & 1.0126 & MAQLYYK \\
\hline
\end{tabular}

Spectrum No: 732; Query: 1585; Rank: 1

\section{Peptide View}

MS/MS Fragmentation of DNSCVNPPHVPNATILTR

Found in IPI00208659, Tax_Id=10116 Gene_Symbol=Cfh Complement inhibitory factor H

Match to Query 1585: 2004.967428 from(1003.490990,2+)

Title: 091008RatKidney_NoSalt_28.2213.2213.2.dta

Data file K:INewmanPaper|Piliangl3SubProteomes\Piliang3SP\mgf5ppm\ERLIC_3SubProteomes5ppm.mgf

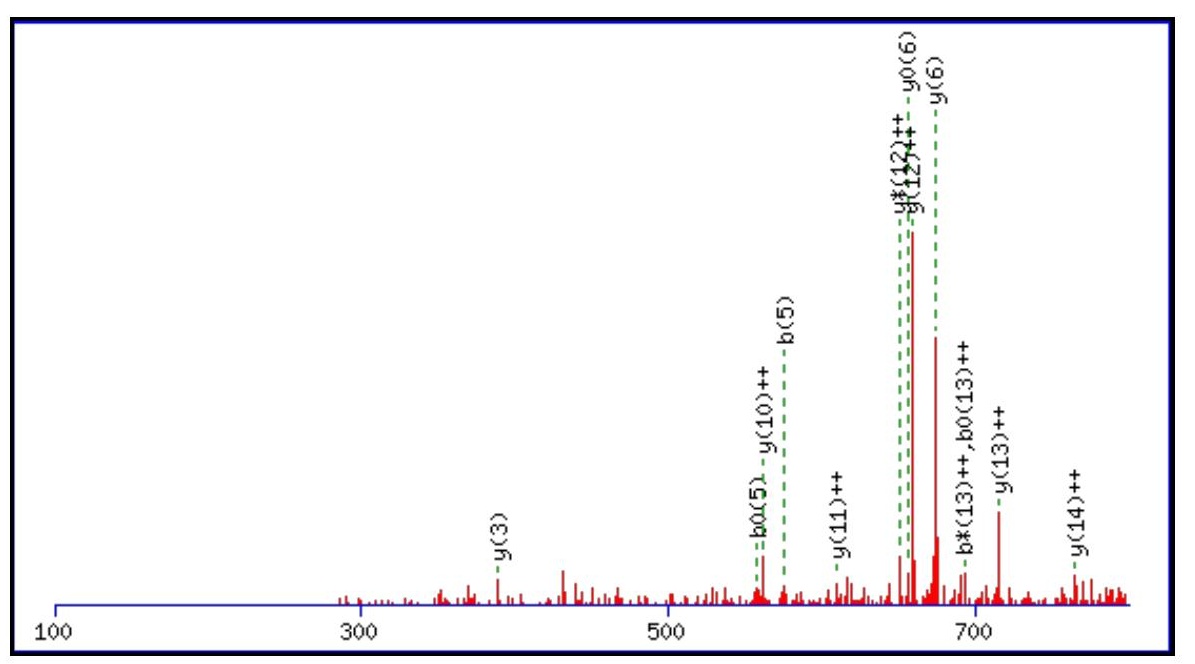



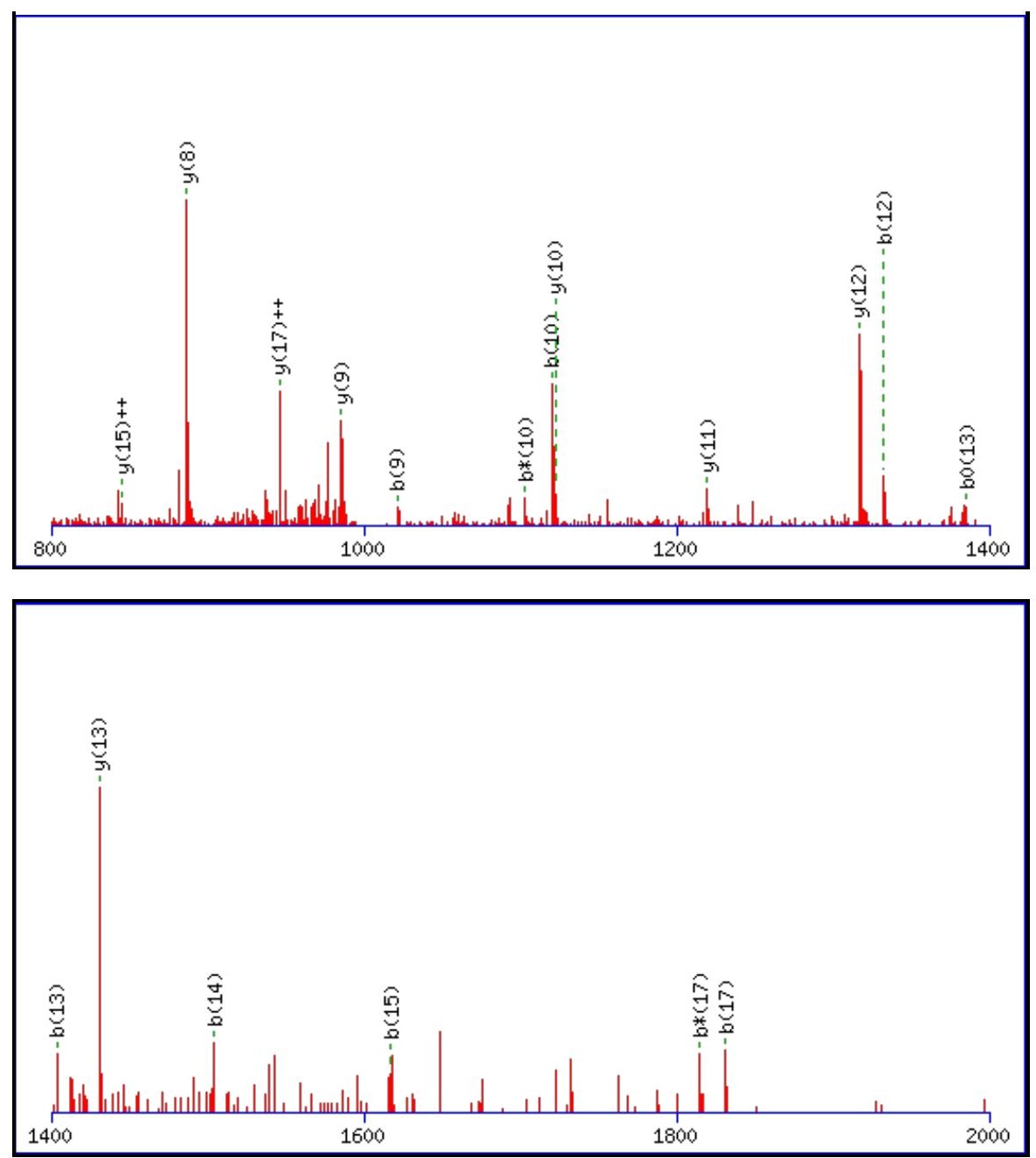

Monoisotopic mass of neutral peptide $\operatorname{Mr}($ calc): 2004.9687

Fixed modifications: Carbamidomethyl (C)

Variable modifications:

N12 : Deamidated_N (N)

Ions Score: 46 Expect: 0.0059

Matches (Bold Red): 31/200 fragment ions using 67 most intense peaks

\begin{tabular}{|c|c|c|c|c|c|c|c|c|c|c|c|c|c|c|}
\hline \# & b & $\mathbf{b}^{++}$ & $\mathbf{b}^{*}$ & $\mathbf{b}^{*++}$ & $\mathbf{b}^{0}$ & $\mathbf{b}^{\mathbf{0 + +}}$ & Seq. & $\mathbf{y}$ & $\mathbf{y}^{++}$ & $\mathbf{y}^{*}$ & $\mathbf{y}^{*^{++}}$ & $\mathbf{y}^{0}$ & $\mathbf{y}^{0++}$ & \# \\
\hline 1 & 116.0342 & 58.5207 & & & 98.0237 & 49.5155 & D & & & & & & & 18 \\
\hline 2 & 230.0771 & 115.5422 & 213.0506 & & 212.0666 & 106.5369 & $\mathbf{N}$ & & & & & 1872.9385 & & 17 \\
\hline 3 & 317.1092 & 59.0582 & 300.0826 & 150.5450 & 299.0986 & 150.0529 & S & 1776.9061 & 888.9567 & 1759.8796 & 880.4434 & 1758.8956 & 879.9514 & 16 \\
\hline 4 & 477.1398 & 239.0735 & 460.1133 & 230.5603 & 459.1293 & 230.0683 & C & 1689.8741 & 845.4407 & 3475 & 836.9274 & 1671.8635 & 836.4354 & 15 \\
\hline 5 & 576.2082 & 288.6078 & 559.1817 & 280.0945 & 558.1977 & 279.6025 & $\mathbf{V}$ & 1529.8434 & 254 & 8169 & 9121 & 1511.8329 & 756.4201 & 14 \\
\hline 6 & 690.2512 & 345.6292 & 673.2246 & 337.1159 & 672.2406 & 336.6239 & $\mathbf{N}$ & 1430.7750 & 715.8912 & 1413.7485 & 707.3779 & 1412.7645 & 706.8859 & 13 \\
\hline 7 & 787.3039 & 394.1556 & 770.2774 & 385.6423 & 769.2934 & 385.1503 & $\mathbf{P}$ & 1316. & 697 & 1299. & 564 & 1298.7215 & 649.8644 & 12 \\
\hline 8 & 884.3567 & 442.6820 & 867.3301 & 434.1687 & 866.3461 & 433.6767 & $\mathbf{P}$ & 1219.6793 & 610.3433 & 1202.6528 & 601.8300 & 1201.6688 & 601.3380 & 11 \\
\hline 9 & 1021.4156 & 511.2114 & 1004.3891 & 502.6982 & 1003.4050 & 502.2062 & $\mathbf{H}$ & 1122.6266 & 561.8169 & 1105.6000 & 553.3037 & 1104.6160 & 552.8116 & 10 \\
\hline 10 & 1120.4840 & 560.7456 & 1103.4575 & 552.2324 & 1102.4735 & 551.7404 & V & 985.5677 & 493.2875 & 968.5411 & 484.7742 & 967.5571 & 484.2822 & 9 \\
\hline 11 & 1217.5368 & 609.2720 & 1200.5102 & 600.7588 & 1199.5262 & 600.2667 & $\mathbf{P}$ & 886.4993 & 443.7533 & 869.4727 & 435.2400 & 868.4887 & 434.7480 & 8 \\
\hline 12 & 1332.5637 & 666.7855 & 1315.5372 & 658.2722 & 1314.5532 & 657.7802 & $\mathbf{N}$ & 789.4465 & 395.2269 & 772.4199 & 386.7136 & 771.4359 & 386.2216 & 7 \\
\hline 13 & 1403.6008 & 702.3041 & 1386.5743 & 693.7908 & 1385.5903 & 693.2988 & A & 674.4196 & 337.7134 & 657.3930 & 329.2001 & 656.4090 & 328.7081 & 6 \\
\hline 14 & 1504.6485 & 752.8279 & 1487.6220 & 744.3146 & 1486.6379 & 743.8226 & $\mathbf{T}$ & 603.3824 & 302.1949 & 586.3559 & 293.6816 & 585.3719 & 293.1896 & 5 \\
\hline 15 & 1617.7326 & 809.3699 & 1600.7060 & 800.8567 & 1599.7220 & 800.3646 & I & 502.3348 & 251.6710 & 485.3082 & 243.1577 & 484.3242 & 242.6657 & 4 \\
\hline 16 & 1730.8166 & 865.9120 & 1713.7901 & 857.3987 & 1712.8061 & 856.9067 & $\mathbf{L}$ & 389.2507 & 195.1290 & 372.2241 & 186.6157 & 371.2401 & 186.1237 & 3 \\
\hline 17 & 1831.8643 & 916.4358 & 1814.8378 & 907.9225 & 1813.8538 & 907.4305 & $\mathbf{T}$ & 276.1666 & 138.5870 & 259.1401 & 130.0737 & 258.1561 & 129.5817 & 2 \\
\hline 18 & & & & & & & $\mathbf{R}$ & 175.1190 & 88.0631 & 158.0924 & 79.5498 & & & 1 \\
\hline
\end{tabular}



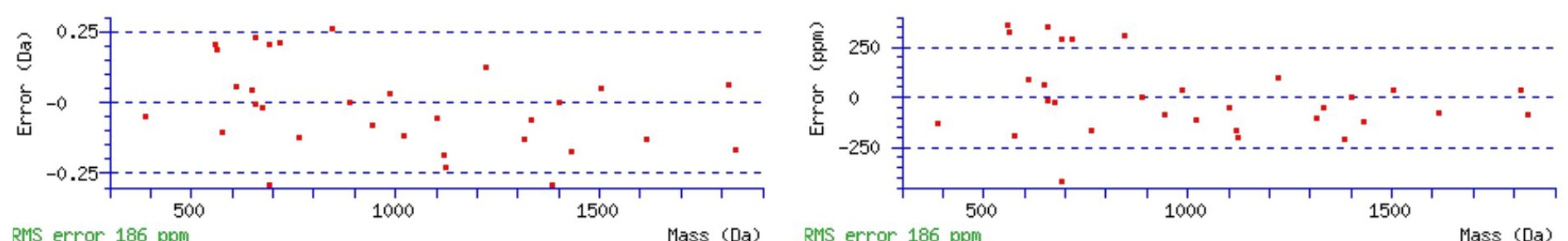

RMS error 186 ppm

Mass (Da) RMS error 186 ppm

Mass (Da)

\section{All matches to this query}

\begin{tabular}{|l|l|l|l|}
\hline Score & Mr(calc): & Delta & \multicolumn{1}{|c|}{ Sequence } \\
\hline 45.8 & 2004.9687 & -0.0013 & DNSCVNPPHVPNATILTR \\
\hline 18.8 & 2004.9687 & -0.0013 & DNSCVNPPHVPNATILTR \\
\hline 11.0 & 2004.9795 & -0.0120 & LCCQPLNTMNKKSPSVI \\
\hline 8.4 & 2003.9461 & 1.0213 & VGSLSMITNSQKXLTWK \\
\hline 6.5 & 2004.9490 & 0.0184 & SAEAGAAQAPGEXRGSVRVR \\
\hline 6.1 & 2004.9687 & -0.0013 & DNSCVNPPHVPNATILTR \\
\hline 3.6 & 2002.9758 & 1.9916 & TSTISSSALGVVMSQKATR \\
\hline 3.6 & 2002.9758 & 1.9916 & TSTISSSALGVVMSQKATR \\
\hline 1.1 & 2004.9826 & -0.0152 & LYYSMKGTEKDDSTLVR \\
\hline 0.5 & 2003.9501 & 1.0173 & TMYVIPFSMGPLGSPLAK \\
\hline
\end{tabular}

Spectrum No: 733; Query: 252; Rank: 1

\section{Peptide View}

MS/MS Fragmentation of AQAALDKANASR

Found in IPI00212868, Tax_Id=10116 Gene_Symbol=Lamb2 Laminin subunit beta-2 precursor

Match to Query 252: 1215.624162 from(406.215330,3+)

Title: 100101RatKid_NS_deglyco_20.224.224.3.dta

Data file K:INewmanPaper|Piliangl3SubProteomes\Piliang3SP\mgf5ppm\ERLIC_3SubProteomes5ppm.mgf

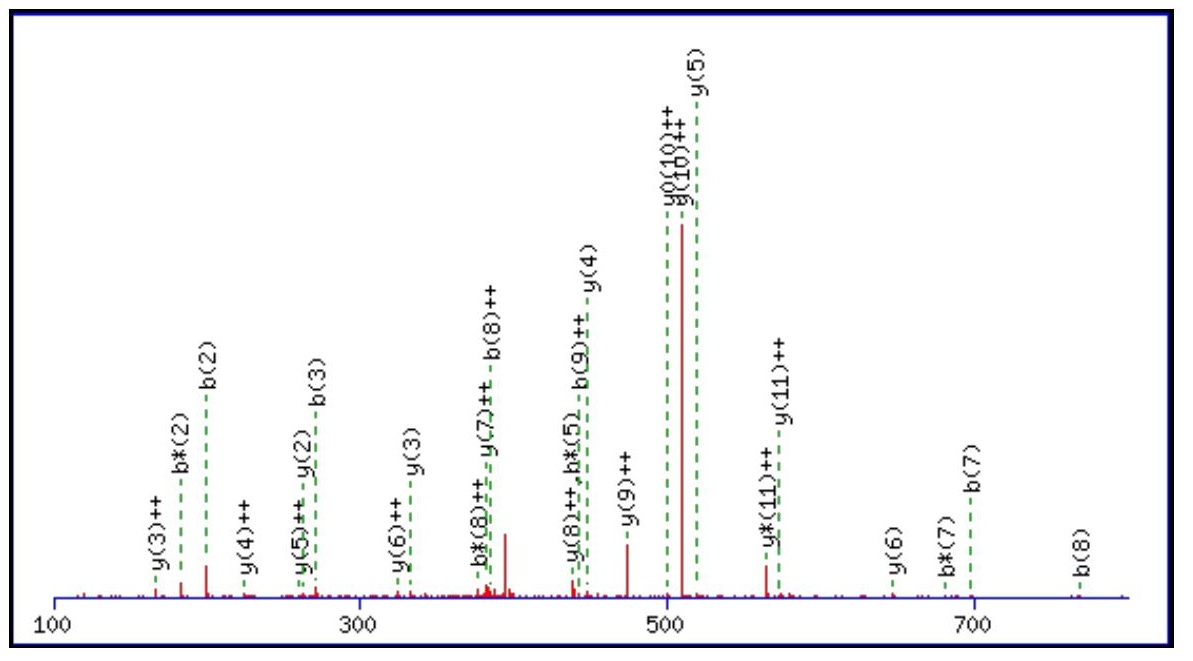



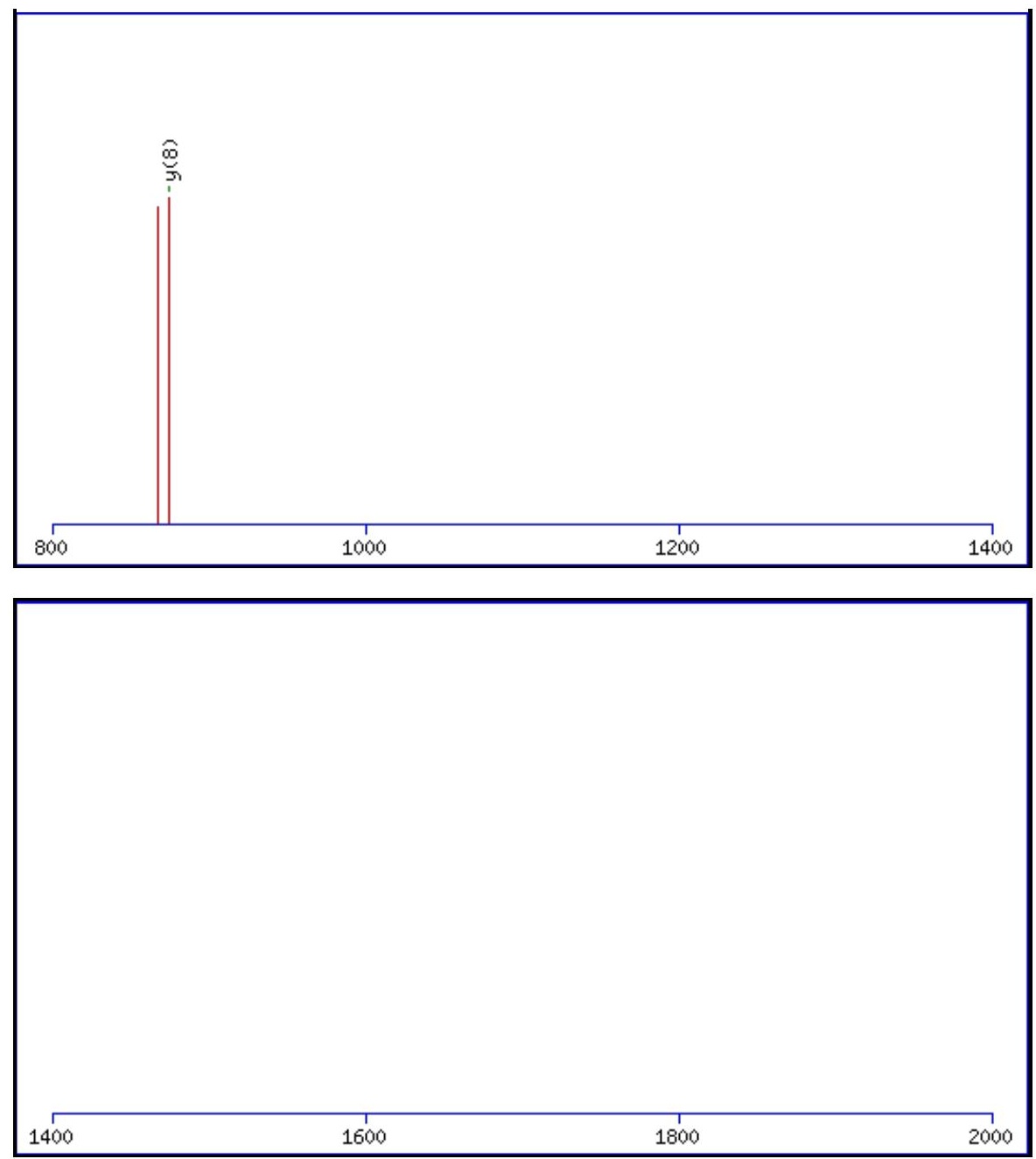

Monoisotopic mass of neutral peptide $\operatorname{Mr}($ calc): 1215.6207

Fixed modifications: Carbamidomethyl (C)

Variable modifications:

N9: Deamidated $\mathrm{N}(\mathrm{N})$

Ions Score: 46 Expect: 0.0028

Matches (Bold Red): 27/118 fragment ions using 61 most intense peaks

\begin{tabular}{|c|c|c|c|c|c|c|c|c|c|c|c|c|c|c|}
\hline \# & b & $\mathbf{b}^{++}$ & $\mathbf{b}^{*}$ & $\mathbf{b}^{*^{++}}$ & $\mathbf{b}^{0}$ & $\mathbf{b}^{0++}$ & Seq. & $\mathbf{y}$ & $\mathrm{y}^{++}$ & $\mathbf{y}^{*}$ & $\mathrm{y}^{*^{++}}$ & $\mathbf{y}^{0}$ & $y^{0++}$ & \# \\
\hline 1 & 72.0444 & 36.5258 & & & & & A & & & & & & & 12 \\
\hline 2 & 200.1030 & 100.5551 & 183.0764 & 92.0418 & & & $\mathbf{Q}$ & 1145.5909 & 573.2991 & 1128.5644 & 564.7858 & 1127.5804 & 564.2938 & 11 \\
\hline 3 & 271.1401 & 136.0737 & 254.1135 & 127.5604 & & & A & 1017.5323 & 509.2698 & 1000.5058 & 500.7565 & 999.5218 & 500.2645 & 10 \\
\hline 4 & 342.1772 & 171.5922 & 325.1506 & 163.0790 & & & A & 946.4952 & 473.7513 & 929.4687 & 465.2380 & 928.4847 & 464.7460 & 9 \\
\hline 5 & 455.2613 & 228.1343 & 438.2347 & 219.6210 & & & $\mathbf{L}$ & 875.4581 & 438.2327 & 858.4316 & 429.7194 & 857.4475 & 429.2274 & 8 \\
\hline 6 & 570.2882 & 285.6477 & 553.2617 & 277.1345 & 552.2776 & 276.6425 & D & 762.3741 & 381.6907 & 745.3475 & 373.1774 & 744.3635 & 372.6854 & 7 \\
\hline 7 & 698.3832 & 349.6952 & 681.3566 & 341.1819 & 680.3726 & 340.6899 & $\mathbf{K}$ & 647.3471 & 324.1772 & 630.3206 & 315.6639 & 629.3365 & 315.1719 & 6 \\
\hline 8 & 769.4203 & 385.2138 & 752.3937 & 376.7005 & 751.4097 & 376.2085 & A & 519.2521 & 260.1297 & 502.2256 & 251.6164 & 501.2416 & 251.1244 & 5 \\
\hline 9 & 884.4472 & 442.7272 & 867.4207 & 434.2140 & 866.4367 & 433.7220 & $\mathbf{N}$ & 448.2150 & 224.6112 & 431.1885 & 216.0979 & 430.2045 & 215.6059 & 4 \\
\hline 10 & 955.4843 & 478.2458 & 938.4578 & 469.7325 & 937.4738 & 469.2405 & A & 333.1881 & 167.0977 & 316.1615 & 158.5844 & 315.1775 & 158.0924 & 3 \\
\hline 11 & 1042.5164 & 521.7618 & 1025.4898 & 513.2485 & 1024.5058 & 512.7565 & S & 262.1510 & 131.5791 & 245.1244 & 123.0659 & 244.1404 & 122.5738 & 2 \\
\hline 12 & & & & & & & $\mathbf{R}$ & 175.1190 & 88.0631 & 158.0924 & 79.5498 & & & 1 \\
\hline
\end{tabular}



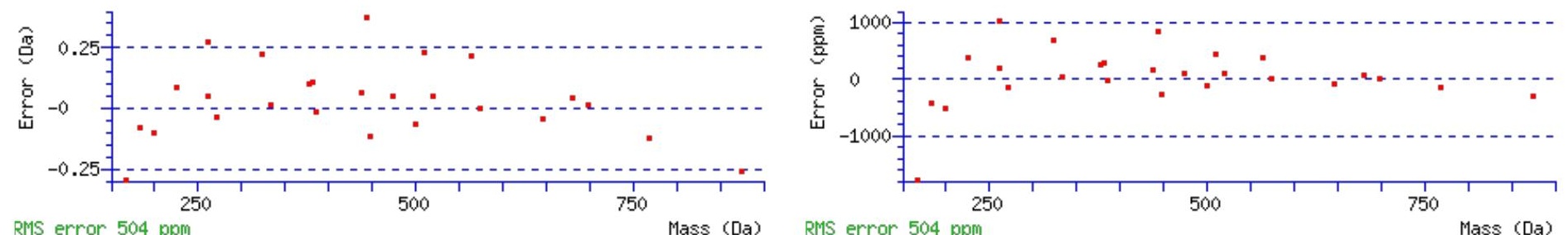

\section{All matches to this query}

\begin{tabular}{|l|l|l|l|}
\hline Score & Mr(calc): & Delta & \multicolumn{1}{|c|}{ Sequence } \\
\hline 45.8 & 1215.6207 & 0.0034 & AQAALDKANASR \\
\hline 6.5 & 1215.6264 & -0.0023 & TTFKVSNLVK \\
\hline 4.9 & 1215.6281 & -0.0040 & QATKMNLGPEK \\
\hline 4.7 & 1213.6220 & 2.0022 & AKKTFLGQNK \\
\hline 4.1 & 1215.6355 & -0.0114 & AKPMPSMTPLK \\
\hline 3.9 & 1214.6312 & 0.9930 & VTIINFTVTK \\
\hline 3.8 & 1215.6264 & -0.0023 & \\
\hline 2.7 & 1214.6100 & 1.0141 & YALLLVTPHK \\
\hline 2.1 & 1215.6237 & 0.0004 & TSPRPPGLRR \\
\hline 1.8 & 1215.6264 & -0.0022 & AKISVEKFSK \\
\hline
\end{tabular}

Spectrum No: 734; Query: 2335; Rank: 1

\section{Peptide View}

MS/MS Fragmentation of VVELLADIVQNISLEDSQIEKER

Found in IPI00471577, Tax_Id=10116 Gene_Symbol=Uqcrc1 Cytochrome b-c1 complex subunit 1, mitochondrial precursor

Match to Query 2335: 2640.393792 from(881.138540,3+)

Title: 100101RatKid_NS_deglyco_05.8541.8541.3.dta

Data file K:INewmanPaper|Piliangl3SubProteomes\Piliang3SP\mgf5ppm\ERLIC_3SubProteomes5ppm.mgf

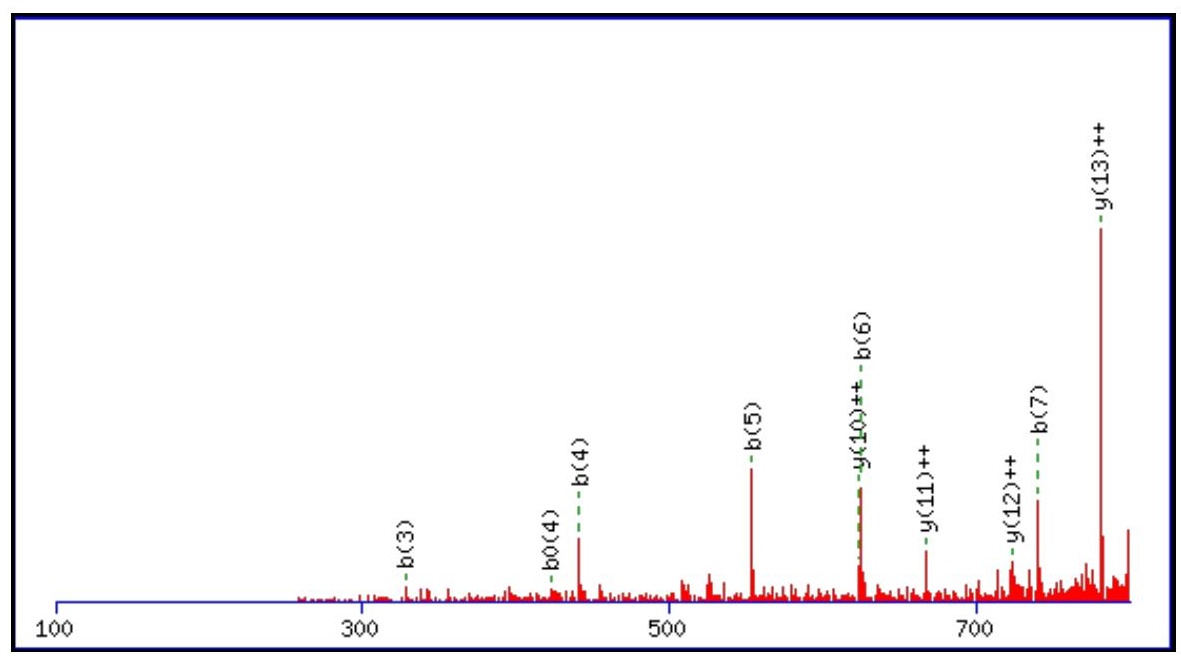



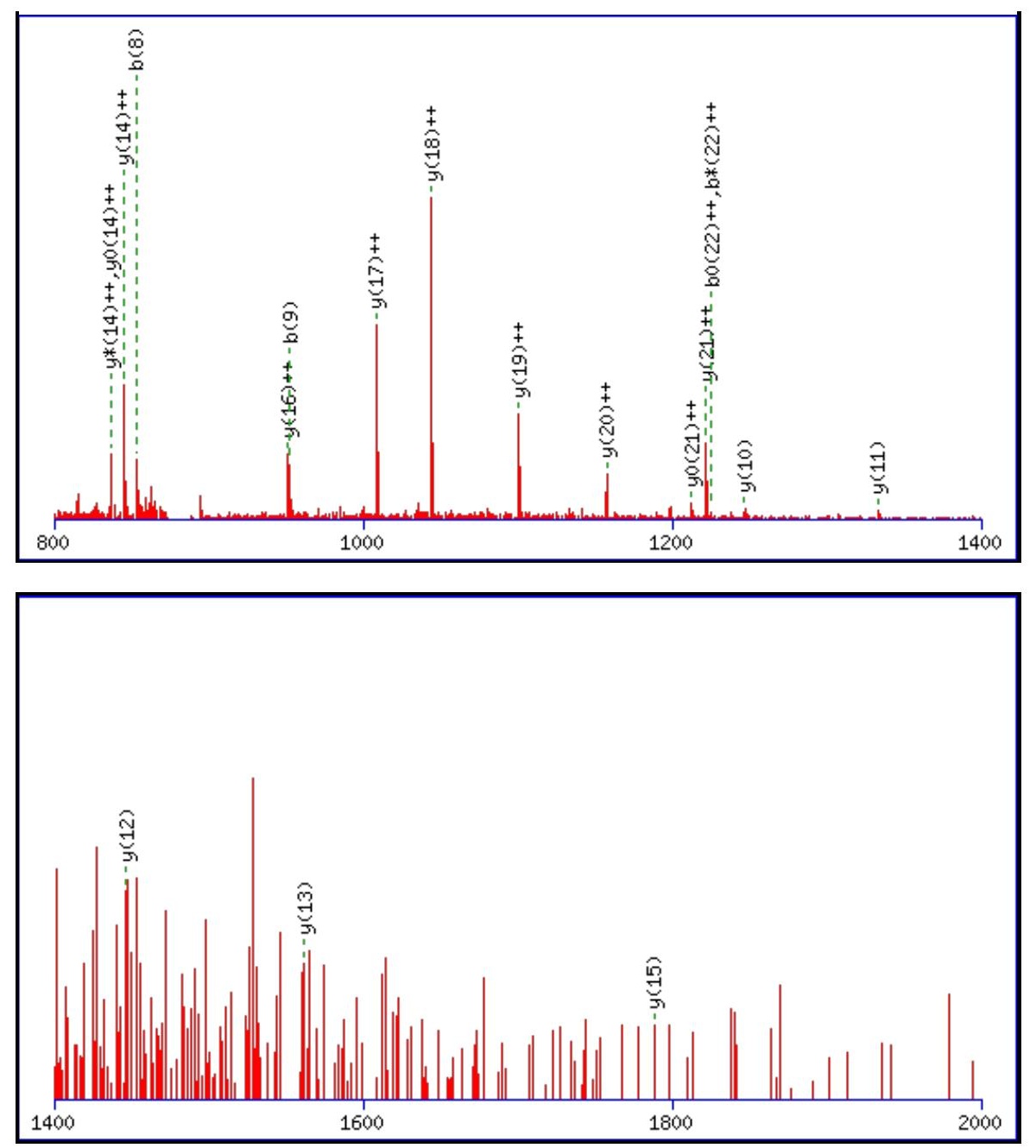

Monoisotopic mass of neutral peptide $\operatorname{Mr}($ calc): 2640.3857

Fixed modifications: Carbamidomethyl (C)

Variable modifications:

N11 : Deamidated_N (N)

Ions Score: 46 Expect: $\odot .0025$

Matches (Bold Red): 29/240 fragment ions using 70 most intense peaks

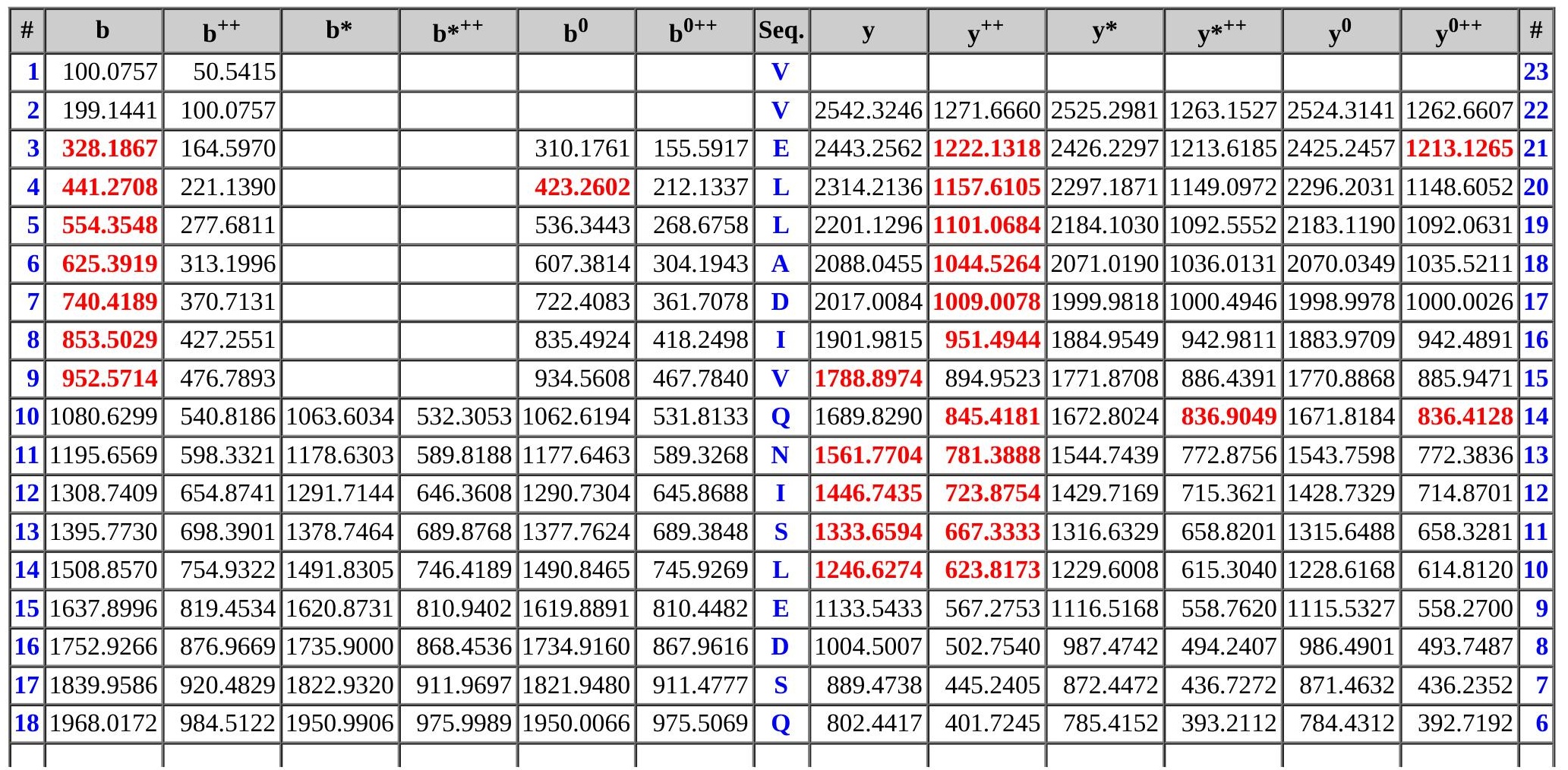




\begin{tabular}{|c|c|c|c|c|c|c|c|c|c|c|c|c|c|c|}
\hline 19| & |2081.1012 & |1041.0543 & |2064.0747 & |1032.5410| & |2063.0907 & |1032.0490 & I & 674.3832 & 337.6952 & 657.3566 & 329.1819 & 656.3726 & 328.6899| & 5 \\
\hline 20 & 2210.1438 & 1105.5756 & 2193.1173 & 1097.0623 & 2192.1333 & 1096.5703 & $\mathbf{E}$ & 561.2991 & 281.1532 & 544.2726 & 272.6399 & 543.2885 & 272.1479 & 4 \\
\hline 21 & 2338.2388 & 1169.6230 & 2321.2122 & 1161.1098 & 2320.2282 & 1160.6178 & $\mathbf{K}$ & 432.2565 & 216.6319 & 415.2300 & 208.1186 & 414.2459 & 207.6266 & 3 \\
\hline 22 & 2467.2814 & 1234.1443 & 2450.2548 & 1225.6311 & 2449.2708 & 1225.1390 & $\mathbf{E}$ & 304.1615 & 152.5844 & 287.1350 & 144.0711 & 286.1510 & 143.5791 & 2 \\
\hline 23 & & & & & & & $\mathbf{R}$ & 175.1190 & 88.0631 & 158.0924 & 79.5498 & & & 1 \\
\hline
\end{tabular}
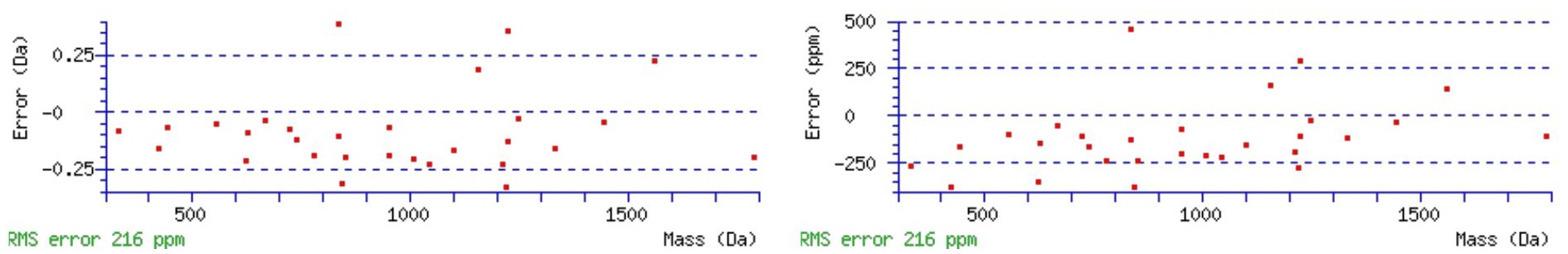

\section{All matches to this query}

\begin{tabular}{|l|l|l|l|}
\hline Score & Mr(calc): & Delta & \multicolumn{1}{c|}{ Sequence } \\
\hline 45.8 & 2640.3857 & 0.0080 & VVELLADIVQNISLEDSQIEKER \\
\hline 45.5 & 2639.4017 & 0.9921 & VVELLADIVQNISLEDSOIEKER \\
\hline 1.2 & 2639.3683 & 1.0255 & QALQLQGDDANSLHLLALLLSAQK \\
\hline 1.2 & 2639.3683 & 1.0255 & QALQLQGDDANSLHLLALLLSAQK \\
\hline 0.2 & 2640.4057 & -0.0119 & QQALGDHLDLASYLLKPIQRMSK \\
\hline
\end{tabular}

Spectrum No: 735; Query: 1592; Rank: 1

\section{Peptide View}

MS/MS Fragmentation of VNLTMFFNITADGEPLGR

Found in IPI00364439, Tax_Id=10116 Gene_Symbol=RGD1559682_predicted similar to Peptidyl-prolyl cis-trans isomerase A

Match to Query 1592: 2010.967688 from(1006.491120,2+)

Title: 100101RatKid_NS_deglyco_09.7557.7557.2.dta

Data file K:\NewmanPaper\Piliang \3SubProteomes\Piliang3SP \mgf5ppm\ERLIC_3SubProteomes5ppm.mgf

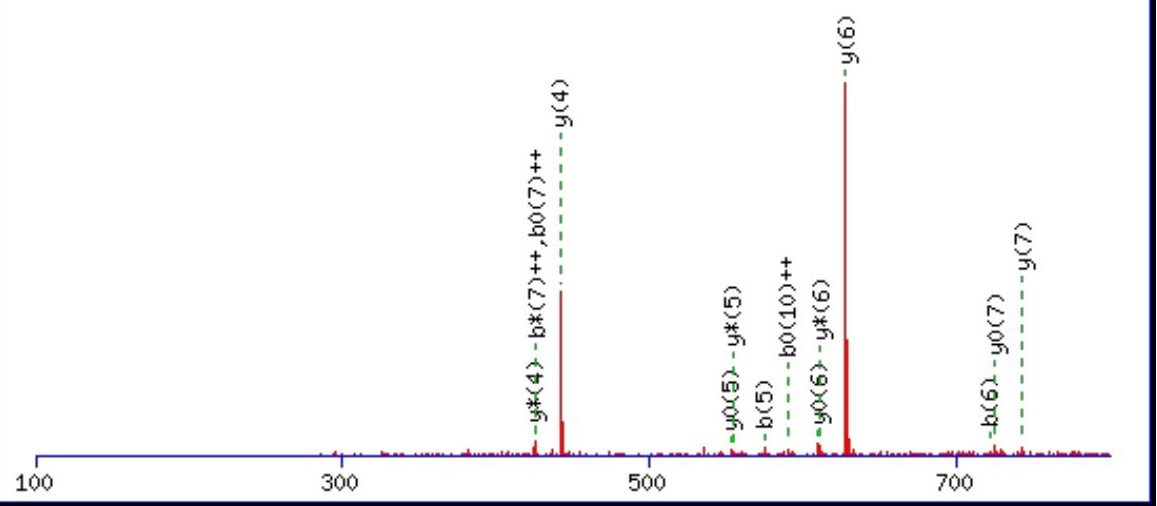



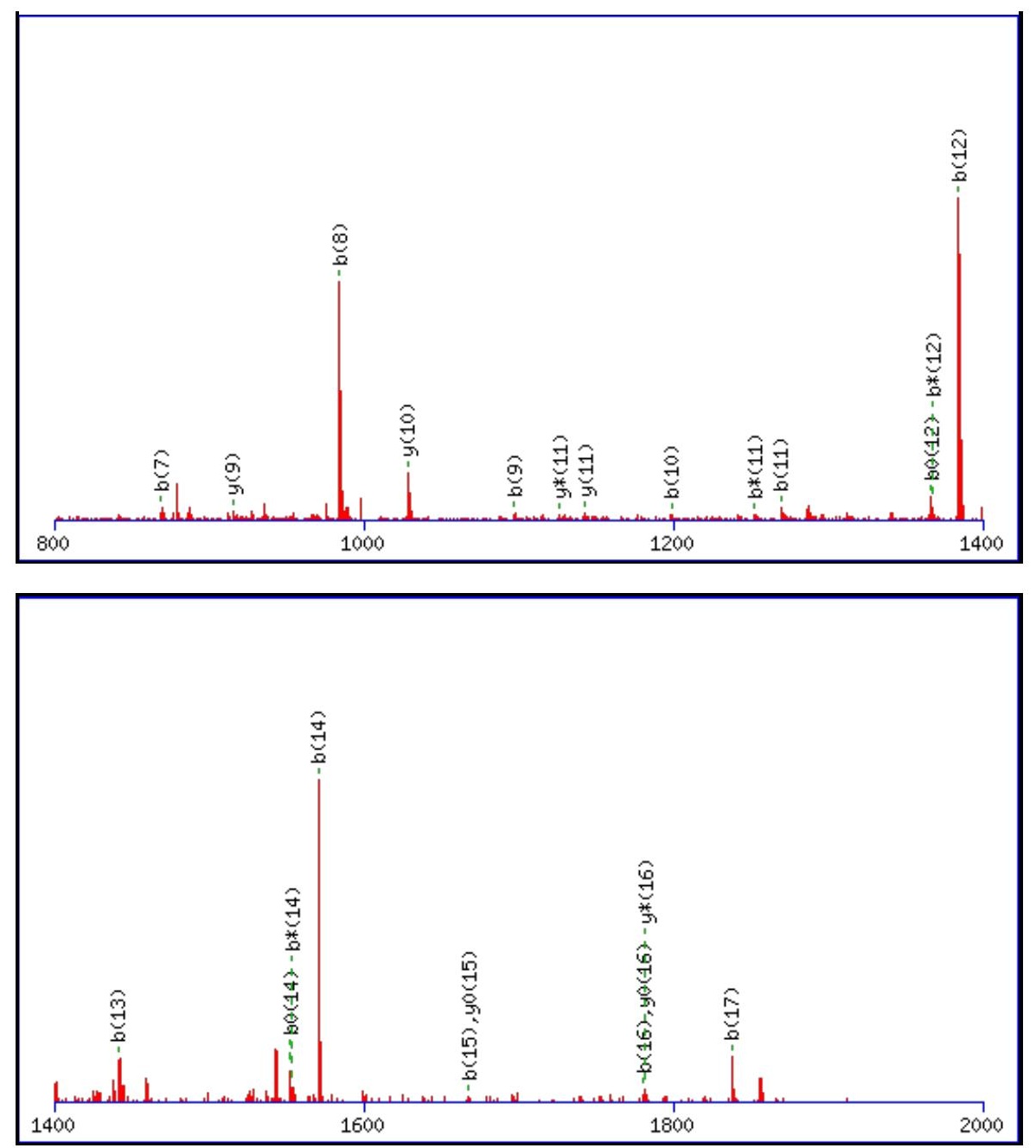

Monoisotopic mass of neutral peptide $\operatorname{Mr}($ calc): 2010.9721

Fixed modifications: Carbamidomethyl (C)

Variable modifications:

M5 : Oxidation (M)

N8 : Deamidated_N ( N

Ions Score: 46 Expect: $\odot .0071$

Matches (Bold Red): 37/188 fragment ions using 118 most intense peaks

\begin{tabular}{|c|c|c|c|c|c|c|c|c|c|c|c|c|c|c|}
\hline \# & b & $\mathbf{b}^{++}$ & $\mathbf{b}^{*}$ & $\mathbf{b}^{*^{++}}$ & $\mathbf{b}^{\mathbf{0}}$ & $\mathbf{b}^{\mathbf{0 + +}}$ & Seq. & $\mathbf{y}$ & $\mathbf{y}^{++}$ & $\mathbf{y}^{*}$ & $\mathbf{y}^{*^{++}}$ & $\mathbf{y}^{0}$ & $\mathbf{y}^{0++}$ & \# \\
\hline 1 & 100.0757 & 50.5415 & & & & & V & & & & & & & 18 \\
\hline 2 & 214.1186 & 107.5629 & 197.0921 & 99.0497 & & & $\mathbf{N}$ & 1912.9109 & 956.9591 & 1895.8844 & 948.4458 & 1894.9004 & 947.9538 & 17 \\
\hline 3 & 327.2027 & 164.1050 & 310.1761 & 155.5917 & & & $\mathbf{L}$ & 1798.8680 & 899.9376 & 1781.8415 & 891.4244 & 1780.8574 & 890.9324 & 16 \\
\hline 4 & 428.2504 & 214.6288 & 411.2238 & 206.1155 & & & $\mathbf{T}$ & 1685.7839 & 843.3956 & 1668.7574 & 834.8823 & 1667.7734 & 834.3903 & 15 \\
\hline 5 & 575.2858 & 288.1465 & 558.2592 & 279.6332 & 557.2752 & 279.1412 & $\mathbf{M}$ & 1584.7363 & 792.8718 & 1567.7097 & 784.3585 & 1566.7257 & 783.8665 & 14 \\
\hline 6 & 722.3542 & 361.6807 & 705.3276 & 353.1675 & 704.3436 & 352.6754 & $\mathbf{F}$ & 1437.7009 & 719.3541 & 1420.6743 & 710.8408 & 1419.6903 & 710.3488 & 13 \\
\hline 7 & 869.4226 & 435.2149 & 852.3960 & 426.7017 & 851.4120 & 426.2097 & $\mathbf{F}$ & 1290.6325 & 645.8199 & 1273.6059 & 637.3066 & 1272.6219 & 636.8146 & 12 \\
\hline 8 & 984.4495 & 492.7284 & 967.4230 & 484.2151 & 966.4390 & 483.7231 & $\mathbf{N}$ & 1143.5640 & 572.2857 & 1126.5375 & 563.7724 & 1125.5535 & 563.2804 & 11 \\
\hline 9 & 1097.5336 & 549.2704 & 1080.5070 & 540.7572 & 1079.5230 & 540.2652 & I & 1028.5371 & 514.7722 & 1011.5106 & 506.2589 & 1010.5265 & 505.7669 & 10 \\
\hline 10 & 1198.5813 & 599.7943 & 1181.5547 & 591.2810 & 1180.5707 & 590.7890 & $\mathbf{T}$ & 915.4530 & 458.2302 & 898.4265 & 449.7169 & 897.4425 & 449.2249 & 9 \\
\hline 11 & 1269.6184 & 635.3128 & 1252.5918 & 626.7996 & 1251.6078 & 626.3075 & A & 814.4054 & 407.7063 & 797.3788 & 399.1930 & 796.3948 & 398.7010 & 8 \\
\hline 12 & 1384.6453 & 692.8263 & 1367.6188 & 684.3130 & 1366.6348 & 683.8210 & D & 743.3682 & 372.1878 & 726.3417 & 363.6745 & 725.3577 & 363.1825 & 7 \\
\hline 13 & 1441.6668 & 721.3370 & 1424.6402 & 712.8238 & 1423.6562 & 712.3318 & $\mathbf{G}$ & 628.3413 & 314.6743 & 611.3148 & 306.1610 & 610.3307 & 305.6690 & 6 \\
\hline 14 & 1570.7094 & 785.8583 & 1553.6828 & 777.3451 & 1552.6988 & 776.8530 & $\mathbf{E}$ & 571.3198 & 286.1636 & 554.2933 & 277.6503 & 553.3093 & 277.1583 & 5 \\
\hline 15 & 1667.7621 & 834.3847 & 1650.7356 & 825.8714 & 1649.7516 & 825.3794 & $\mathbf{P}$ & 442.2772 & 221.6423 & 425.2507 & 213.1290 & & & 4 \\
\hline 16 & 1780.8462 & 890.9267 & 1763.8197 & 882.4135 & 1762.8356 & 881.9215 & $\mathbf{L}$ & 345.2245 & 173.1159 & 328.1979 & 164.6026 & & & 3 \\
\hline 17 & 1837.8677 & 919.4375 & 1820.8411 & 910.9242 & 1819.8571 & 910.4322 & G & 232.1404 & 116.5738 & 215.1139 & 108.0606 & & & 2 \\
\hline 18 & & & & & & & $\mathbf{R}$ & 175.1190 & 88.0631 & 158.0924 & 79.5498 & & & 1 \\
\hline
\end{tabular}



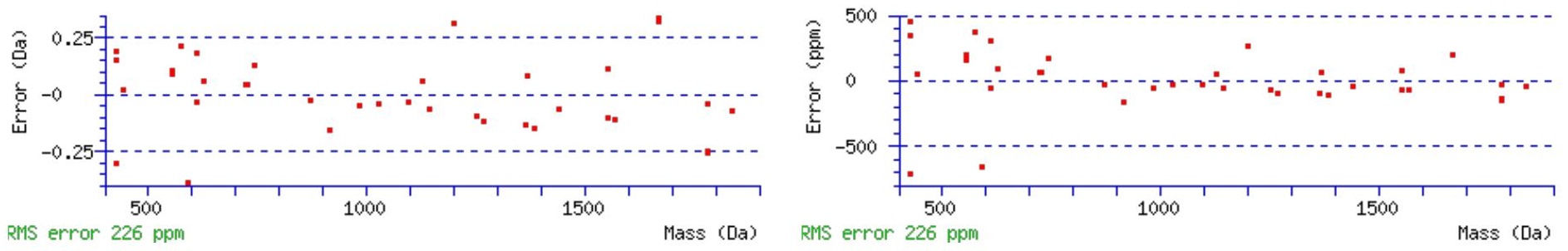

\section{All matches to this query}

\begin{tabular}{|l|l|l|l|}
\hline Score & Mr(calc): & Delta & \multicolumn{1}{|c|}{ Sequence } \\
\hline 45.7 & 2010.9721 & -0.0044 & VNLTMFFNITADGEPLGR \\
\hline 28.8 & 2010.9721 & -0.0044 & VNLTMFFNITADGEPLGR \\
\hline 11.5 & 2010.9832 & -0.0155 & YTPVSIYEEKLLLKR \\
\hline 11.5 & 2010.9832 & -0.0155 & YTPVSIYEEKLLLKR \\
\hline 5.8 & 2010.9557 & 0.0120 & NRVSAVDELAMATERLR \\
\hline 5.4 & 2010.9637 & 0.0040 & SSGLPDASLPGRPLGTGGHR \\
\hline 4.9 & 2010.9637 & 0.0040 & SSGLPDASLPGRPLGTGGHR \\
\hline 3.9 & 2008.9700 & 1.9977 & GRVVNISSMLGRMANPAR \\
\hline 3.9 & 2008.9700 & 1.9977 & GRVVNISSMLGRMANPAR \\
\hline 3.6 & 2009.9540 & 1.0137 & GRVVNISSMLGRMANPAR \\
\hline
\end{tabular}

Spectrum No: 736; Query: 2412; Rank: 1

\section{Peptide View}

MS/MS Fragmentation of STNVAVDSGGGLLKPTVASQNQSLPVAK

Found in IPI00194958, Tax_Id=10116 Gene_Symbol=Picalm Isoform 2 of Phosphatidylinositol-binding clathrin assembly protein

Match to Query 2412: 2738.450322 from(913.824050,3+)

Title: 091008RatKidney_NH4Format01_09.3022.3022.3.dta

Data file K:INewmanPaper|Piliangl3SubProteomes\Piliang3SP\mgf5ppm\ERLIC_3SubProteomes5ppm.mgf

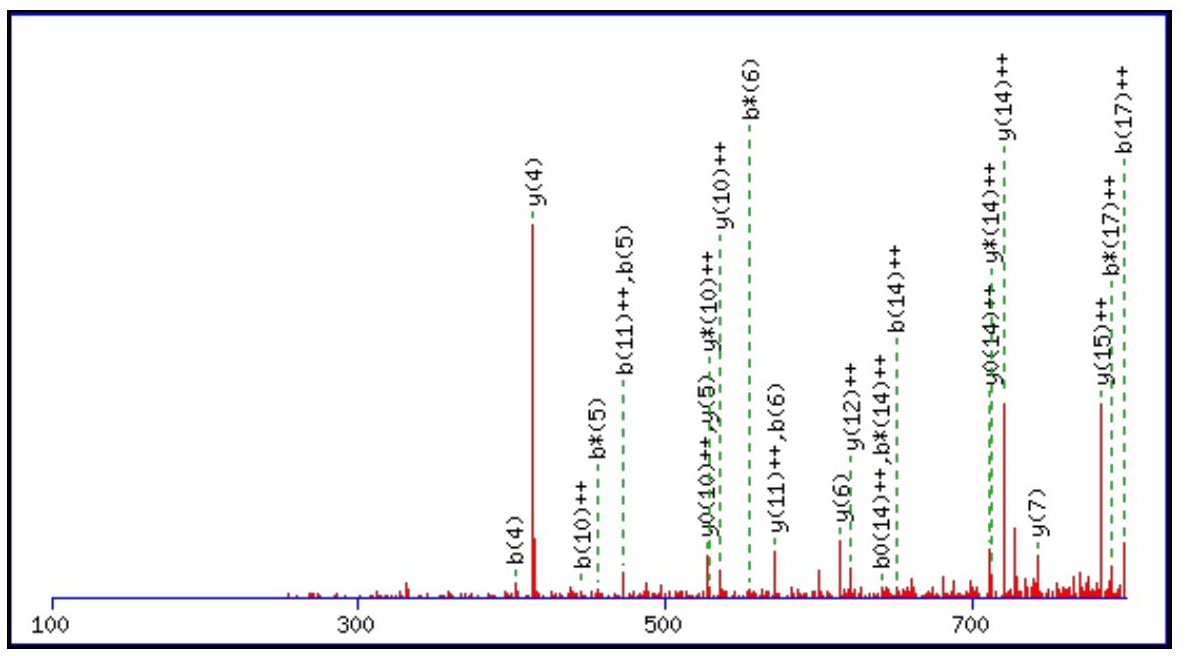



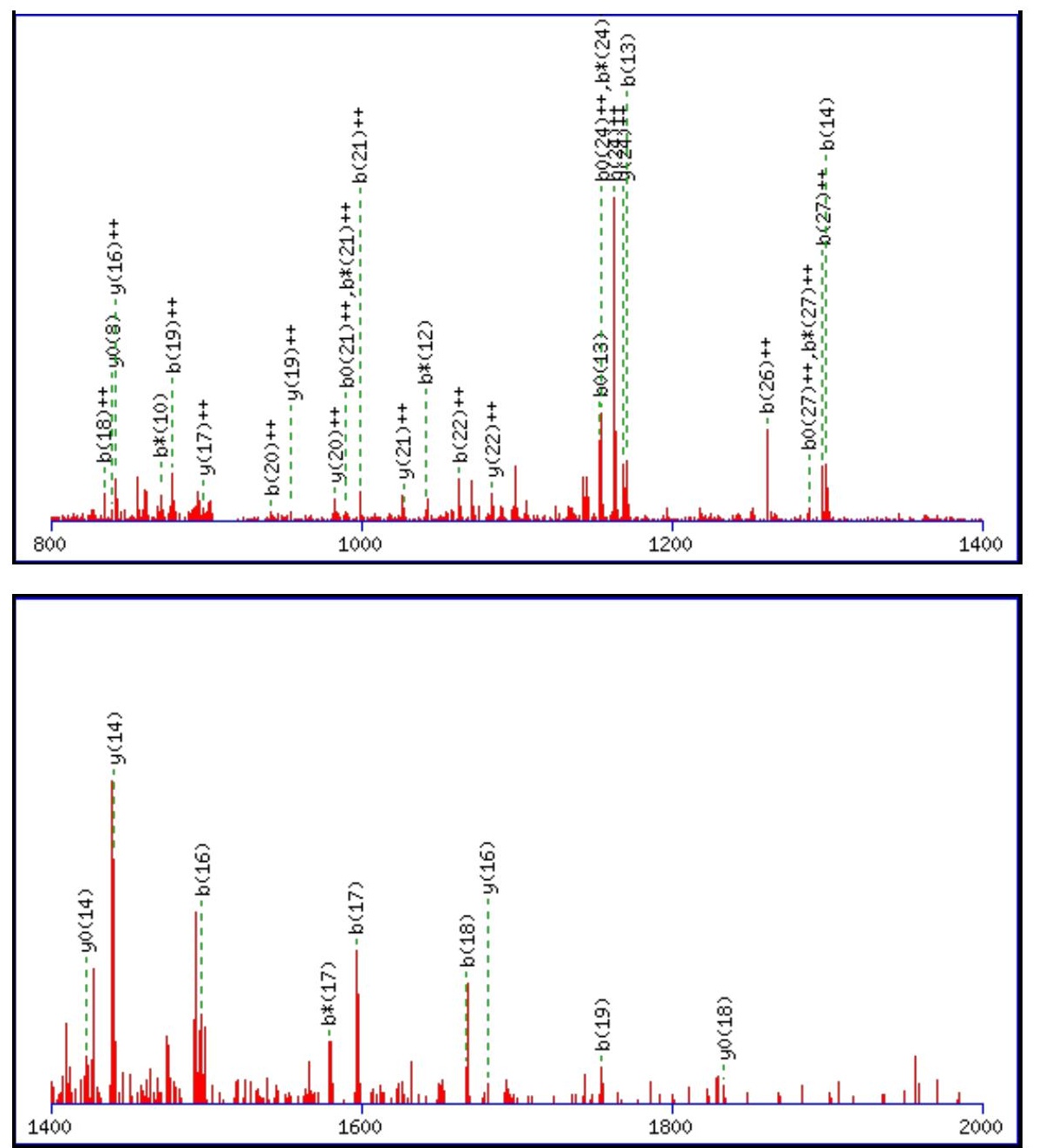

Monoisotopic mass of neutral peptide $\operatorname{Mr}($ calc): 2738.4450

Fixed modifications: Carbamidomethyl (C)

Variable modifications:

N21 : Deamidated_N (N)

Ions Score: 46 Expect: 0.0024

Matches (Bold Red): 62/310 fragment ions using 156 most intense peaks

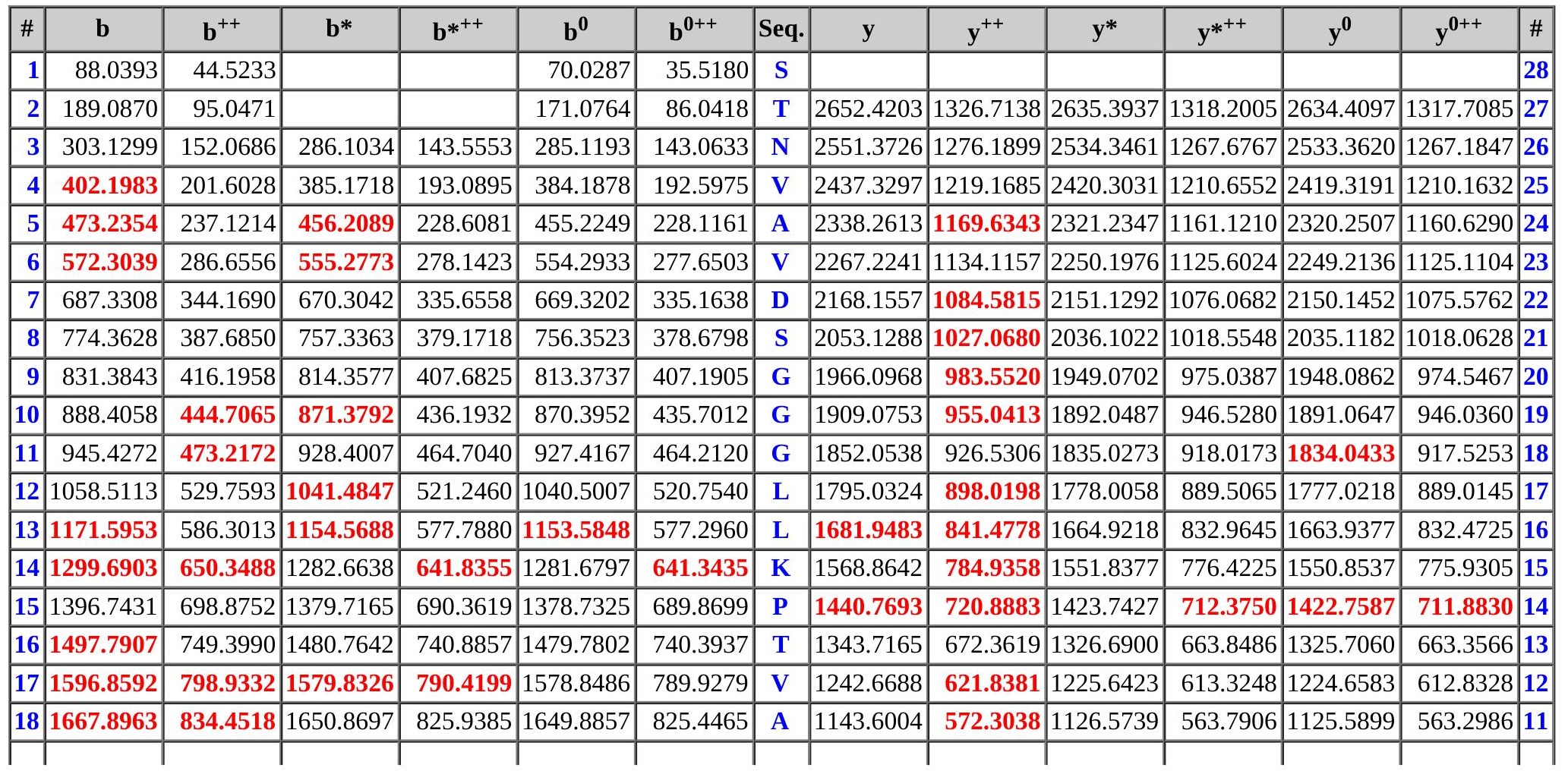




\begin{tabular}{|c|c|c|c|c|c|c|c|c|c|c|c|c|c|c|}
\hline 19 & 1754.9283 & 877.9678 & |1737.9018| & 869.4545 & |1736.9177| & 868.9625 & S & 1072.5633 & 536.7853 & 1055.5368 & 528.2720 & 1054.5527 & 527.7800 & \\
\hline 20 & 1882.9869 & 941.9971 & 1865.9603 & 933.4838 & 1864.9763 & 932.9918 & $\mathbf{Q}$ & 985.5313 & 493.2693 & 968.5047 & 484.7560 & 967.5207 & 484.2640 & \\
\hline 21 & 1998.0138 & 999.5105 & 1980.9873 & 990.9973 & 1980.0033 & 990.5053 & $\mathbf{N}$ & 857.4727 & 429.2400 & 840.4462 & 420.7267 & 839.4621 & 420.2347 & \\
\hline 22 & 2126.0724 & 1063.5398 & 2109.0458 & 1055.0266 & 2108.0618 & 1054.5346 & $\mathbf{Q}$ & 742.4458 & 371.7265 & 725.4192 & 363.2132 & 724.4352 & 362.7212 & \\
\hline 23 & 2213.1044 & 1107.0559 & 2196.0779 & 1098.5426 & 2195.0939 & 1098.0506 & S & 614.3872 & 307.6972 & 597.3606 & 299.1840 & 596.3766 & 298.6919 & \\
\hline 24 & 2326.1885 & 1163.5979 & 2309.1619 & 1155.0846 & 2308.1779 & 1154.5926 & L & 527.3552 & 264.1812 & 510.3286 & 255.6679 & & & \\
\hline 25 & 2423.2413 & 1212.1243 & 2406.2147 & 1203.6110 & 2405.2307| & 1203.1190 & $\mathbf{P}$ & 414.2711 & 207.6392 & 397.2445 & 199.1259 & & & \\
\hline 26 & 2522.3097 & 1261.6585 & 2505.2831 & 1253.1452 & 2504.2991 & 1252.6532 & V & 317.2183 & 159.1128 & 300.1918 & 150.5995 & & & \\
\hline 27 & 2593.3468 & 1297.1770 & 2576.3202 & 1288.6638 & 2575.3362 & 1288.1717 & A & 218.1499 & 109.5786 & 201.1234 & 101.0653 & & & \\
\hline 28 & & & & & & & $\mathbf{K}$ & 147.1128 & 74.0600 & 130.0863 & 65.5468 & & & \\
\hline
\end{tabular}
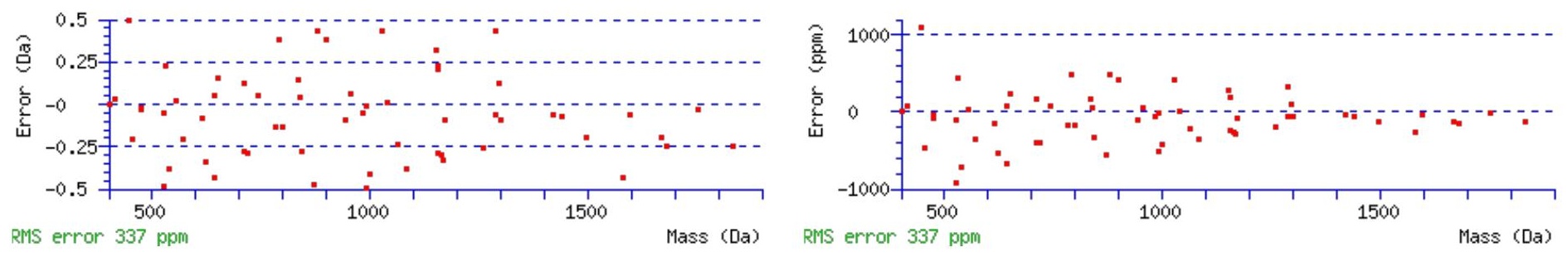

\section{All matches to this query}

\begin{tabular}{|l|l|c|l|}
\hline Score & Mr(calc): & Delta & \multicolumn{1}{c|}{ Sequence } \\
\hline 45.6 & 2738.4450 & 0.0053 & STNVAVDSGGGLLKPTVASONQSLPVAK \\
\hline 44.8 & 2737.4610 & 0.9893 & STNVAVDSGGGLLKPTVASQNQSLPVAK \\
\hline 37.7 & 2738.4450 & 0.0053 & STNVAVDSGGGLLKPTVASQNQSLPVAK \\
\hline 1.7 & 2737.4368 & 1.0136 & KLCCVQSPVPSQVINVRTIGQPTR \\
\hline 0.1 & 2738.4675 & -0.0171 & RIQSGLGALSRSHDTTSNTLAQLLAK \\
\hline 0.1 & 2737.4316 & 1.0187 & GHLEVVKLLVLTHGADPENYAVRK \\
\hline
\end{tabular}

Spectrum No: 737; Query: 26; Rank: 1

\section{Peptide View}

MS/MS Fragmentation of SFGNISER

Found in IPI00400738, Tax_Id=10116 Gene_Symbol=Galnt11 Polypeptide N-acetylgalactosaminyltransferase 11

Match to Query 26: 909.418188 from(455.716370,2+)

Title: 091008RatKidney_NoSalt_31.797.797.2.dta

Data file K:INewmanPaper|Piliangl3SubProteomes\Piliang3SP\mgf5ppm\ERLIC_3SubProteomes5ppm.mgf

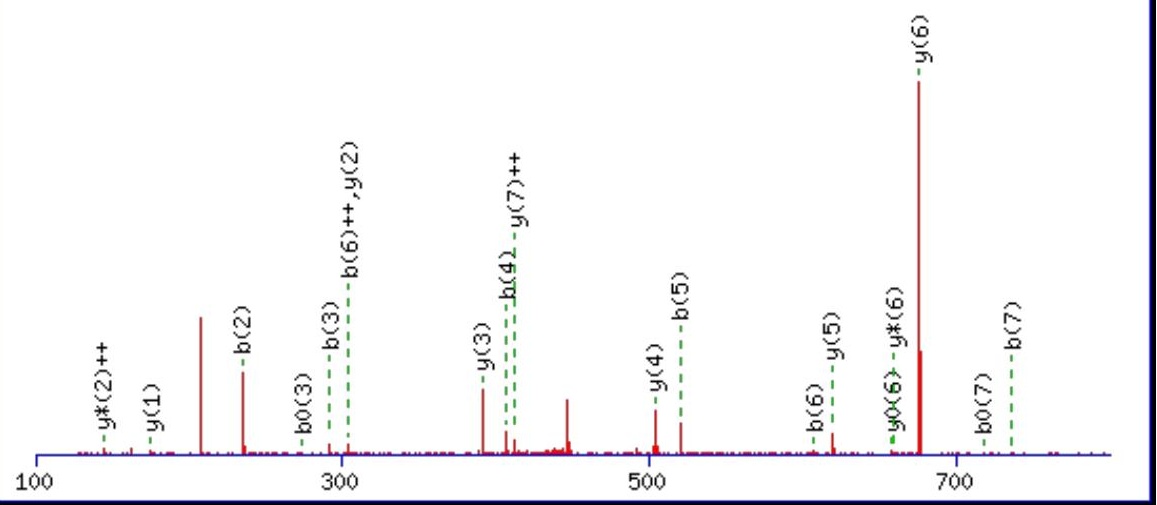



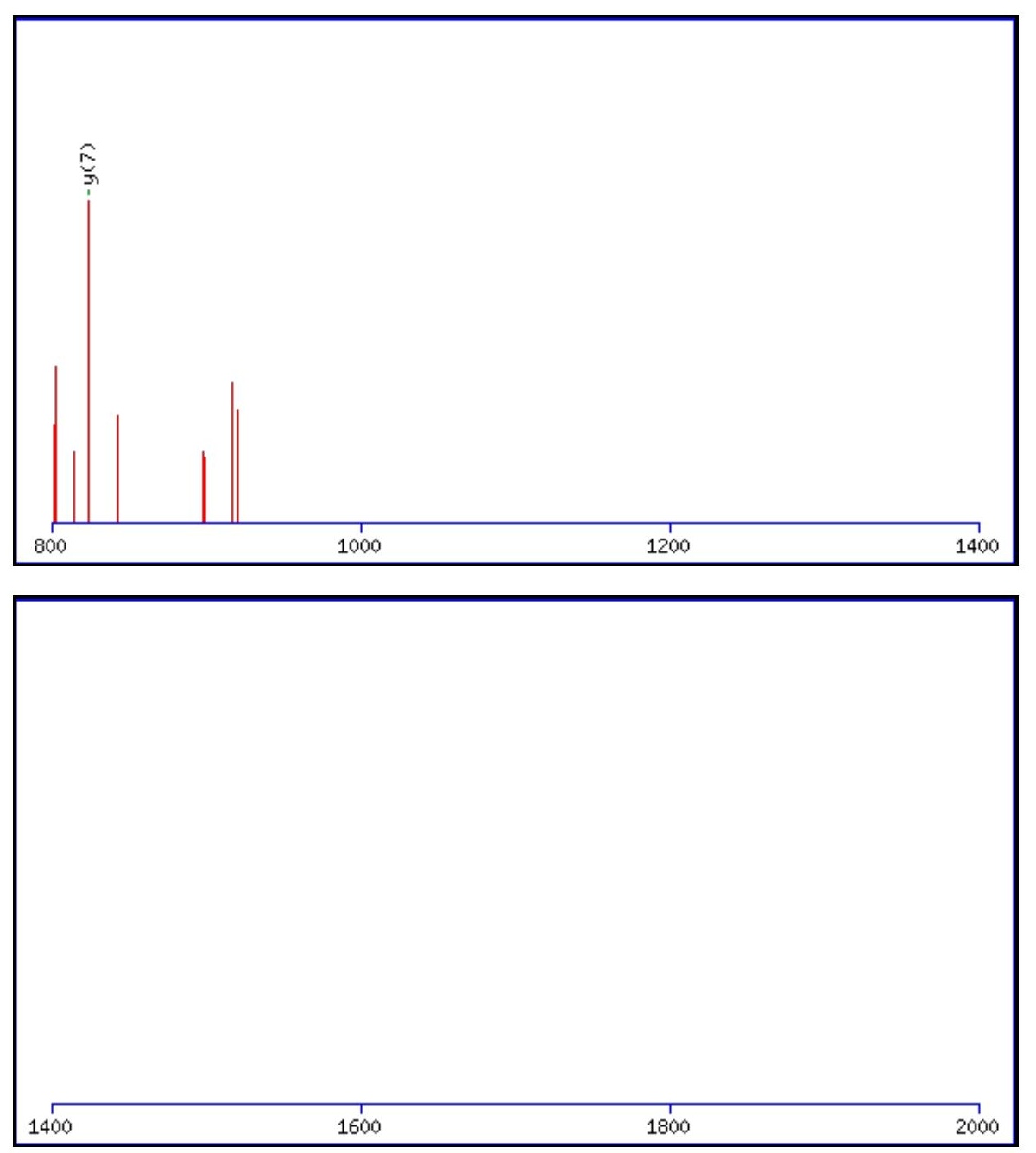

Monoisotopic mass of neutral peptide $\operatorname{Mr}($ calc): 909.4192

Fixed modifications: Carbamidomethyl (C)

Variable modifications:

N4 : Deamidated_N (N)

Ions Score: 45 Expect: 0.0021

Matches (Bold Red): 20/76 fragment ions using 42 most intense peaks

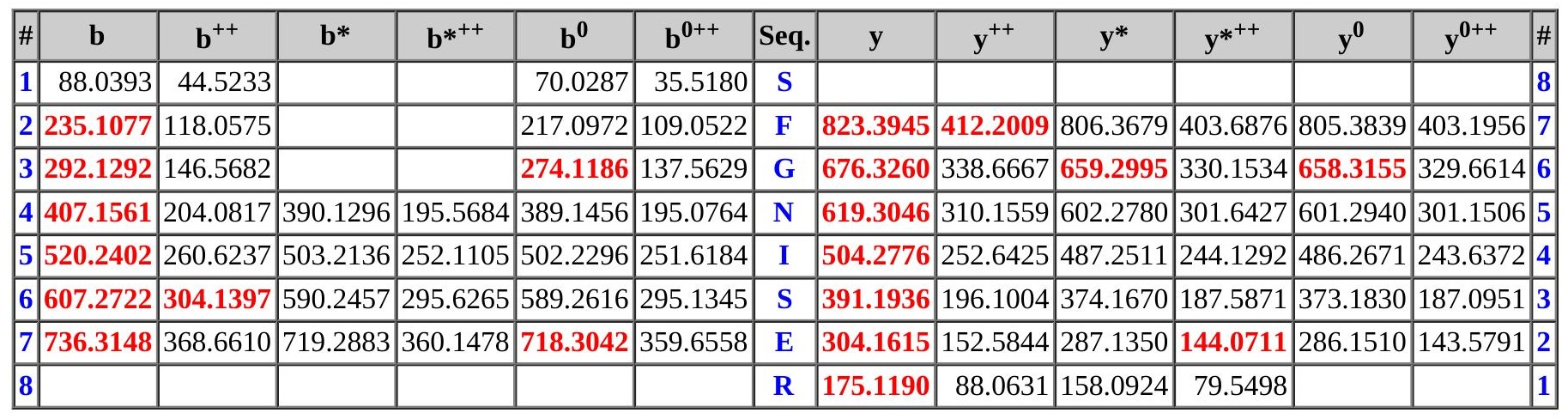
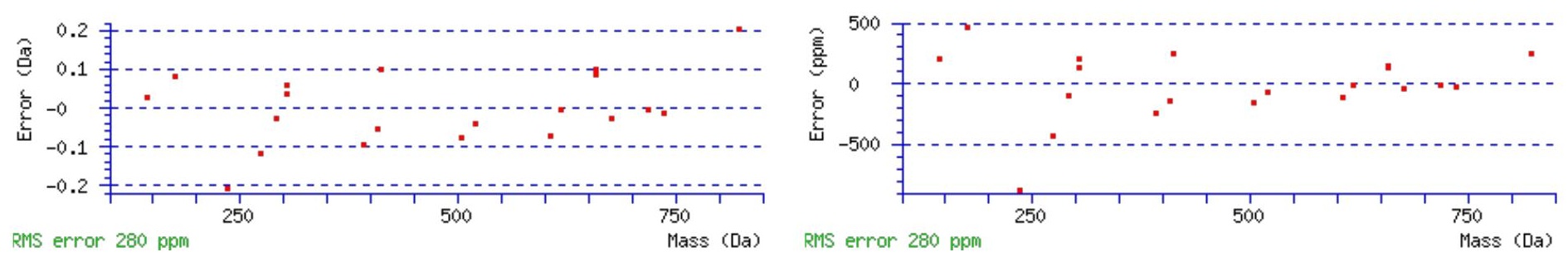

\section{All matches to this query}

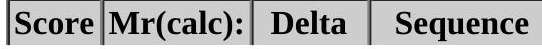




\begin{tabular}{|l|l|l|l|}
\hline 45.5 & 909.4192 & -0.0010 & SFGNISER \\
\hline 10.1 & 909.4192 & -0.0010 & ENYLSER \\
\hline 9.7 & 909.4192 & -0.0010 & SFRENEK \\
\hline 8.6 & 909.4192 & -0.0010 & SSFPTDTR \\
\hline 8.1 & 909.4192 & -0.0010 & FSKDEER \\
\hline 6.4 & 907.4069 & 2.0113 & $\underline{\text { MEQISER }}$ \\
\hline 5.2 & 907.4181 & 2.0001 & $\underline{\text { MTSERER }}$ \\
\hline 5.0 & 909.4192 & -0.0010 & YAADINSR \\
\hline 4.9 & 909.4192 & -0.0010 & KYNVNDR \\
\hline 4.7 & 909.4273 & -0.0091 & $\underline{\text { SMCGKRR }}$ \\
\hline
\end{tabular}

Spectrum No: 738; Query: 2116; Rank: 1

\section{Peptide View}

MS/MS Fragmentation of LKCDPAIKPHEELLILNLTR

Found in IPI00325860, Tax_Id=10116 Gene_Symbol=Podxl Podocalyxin precursor

Match to Query 2116: 2373.316902 from(792.112910,3+)

Title: 100101RatKid_NS_deglyco_08.3712.3712.3.dta

Data file K:INewmanPaper|Piliangl3SubProteomes\Piliang3SP\mgf5ppm\ERLIC_3SubProteomes5ppm.mgf
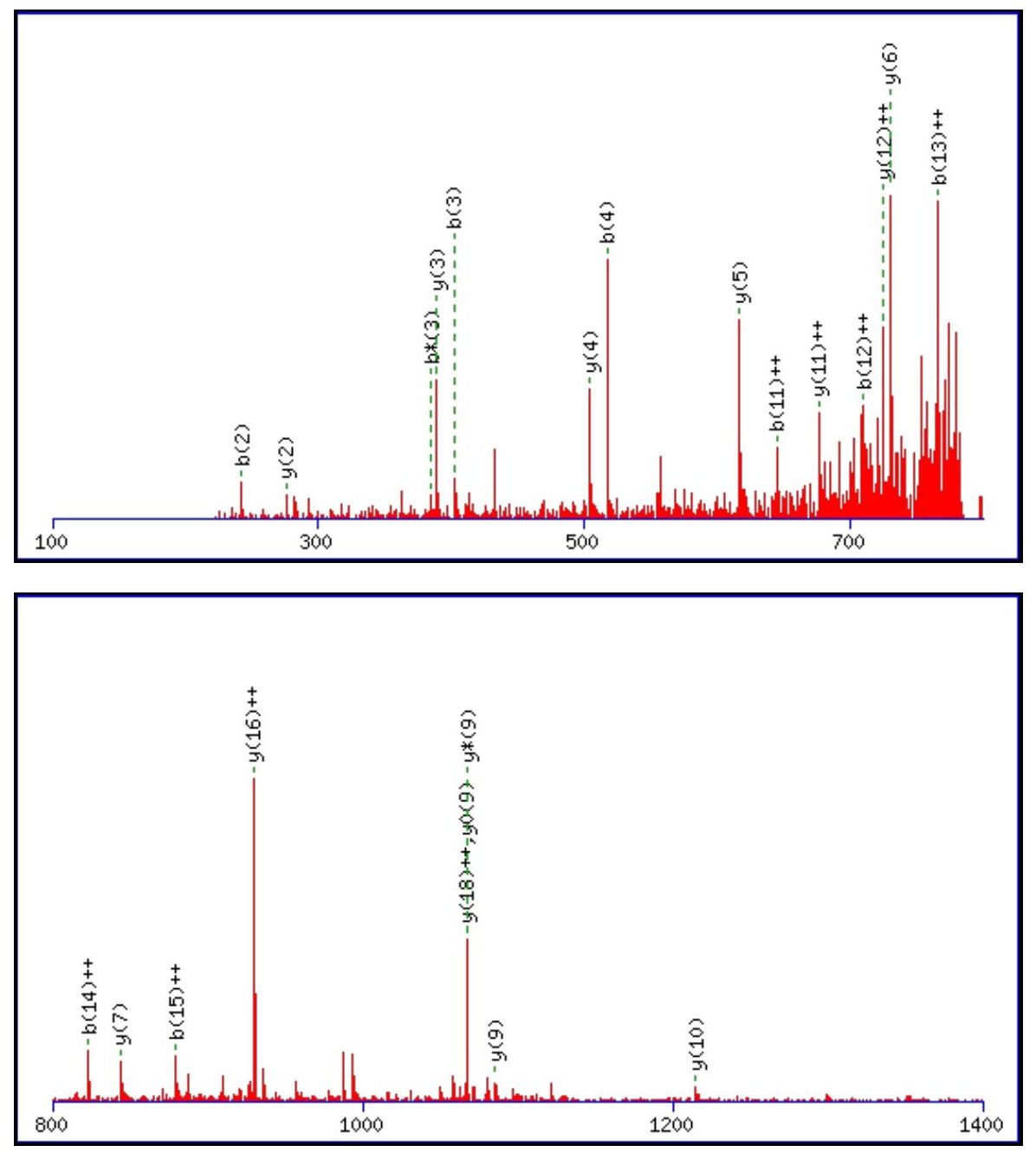


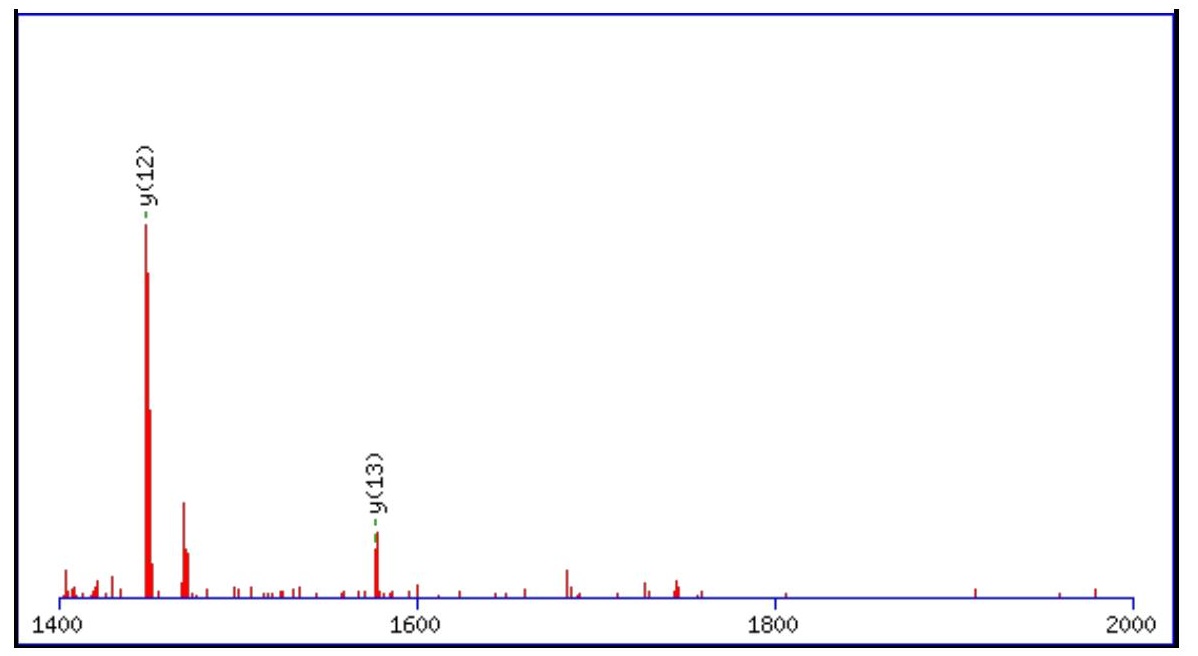

Monoisotopic mass of neutral peptide $\operatorname{Mr}($ calc): 2373.3089

Fixed modifications: Carbamidomethyl (C)

Variable modifications:

N17 : Deamidated_N ( N)

Ions Score: 45 Expect: 0.00083

Matches (Bold Red): 25/218 fragment ions using 51 most intense peaks

\begin{tabular}{|c|c|c|c|c|c|c|c|c|c|c|c|c|c|c|}
\hline \# & b & $\mathbf{b}^{++}$ & $\mathbf{b}^{*}$ & $\mathbf{b}^{*^{++}}$ & $\mathbf{b}^{\mathbf{0}}$ & $\mathbf{b}^{0++}$ & Seq. & $\mathbf{y}$ & $y^{++}$ & $\mathbf{y}^{*}$ & $\mathbf{y}^{*^{++}}$ & $\mathbf{y}^{0}$ & $\mathbf{y}^{0++}$ & \# \\
\hline 1 & 114.0913 & 57.5493 & & & & & $\mathbf{L}$ & & & & & & & 20 \\
\hline 2 & 242.1863 & 121.5968 & 225.1598 & 113.0835 & & & $\mathbf{K}$ & 2261.2322 & 1131.1197 & 2244.2057 & 1122.6065 & 2243.2216 & 1122.1145 & 19 \\
\hline 3 & 402.2170 & 201.6121 & 385.1904 & 193.0988 & & & C & 2133.1372 & 1067.0723 & 2116.1107 & 1058.5590 & 2115.1267 & 1058.0670 & 18 \\
\hline 4 & 517.2439 & 259.1256 & 500.2173 & 250.6123 & 499.2333 & 250.1203 & D & 1973.1066 & 987.0569 & 1956.0800 & 978.5437 & 1955.0960 & 978.0517 & 17 \\
\hline 5 & 614.2967 & 307.6520 & 597.2701 & 299.1387 & 596.2861 & 298.6467 & $\mathbf{P}$ & 1858.0797 & 929.5435 & 1841.0531 & 921.0302 & 1840.0691 & 920.5382 & 16 \\
\hline 6 & 685.3338 & 343.1705 & 668.3072 & 334.6573 & 667.3232 & 334.1652 & A & 1761.0269 & 881.0171 & 1744.0003 & 872.5038 & 1743.0163 & 872.0118 & 15 \\
\hline 7 & 798.4178 & 399.7126 & 781.3913 & 391.1993 & 780.4073 & 390.7073 & I & 1689.9898 & 845.4985 & 1672.9632 & 836.9853 & 1671.9792 & 836.4932 & 14 \\
\hline 8 & 926.5128 & 463.7600 & 909.4863 & 455.2468 & 908.5022 & 454.7548 & $\mathbf{K}$ & 1576.9057 & 788.9565 & 1559.8792 & 780.4432 & 1558.8951 & 779.9512 & 13 \\
\hline 9 & 1023.5656 & 512.2864 & 1006.5390 & 503.7731 & 1005.5550 & 503.2811 & $\mathbf{P}$ & 1448.8107 & 724.9090 & 1431.7842 & 716.3957 & 1430.8002 & 715.9037 & 12 \\
\hline 10 & 1160.6245 & 580.8159 & 1143.5979 & 572.3026 & 1142.6139 & 571.8106 & $\mathbf{H}$ & 1351.7580 & 676.3826 & 1334.7314 & 667.8694 & 1333.7474 & 667.3773 & 11 \\
\hline 11 & 1289.6671 & 645.3372 & 1272.6405 & 636.8239 & 1271.6565 & 636.3319 & $\mathbf{E}$ & 1214.6991 & 607.8532 & 1197.6725 & 599.3399 & 1196.6885 & 598.8479 & 10 \\
\hline 12 & 1418.7097 & 709.8585 & 1401.6831 & 701.3452 & 1400.6991 & 700.8532 & $\mathbf{E}$ & 1085.6565 & 543.3319 & 1068.6299 & 534.8186 & 1067.6459 & 534.3266 & 9 \\
\hline 13 & 1531.7937 & 766.4005 & 1514.7672 & 757.8872 & 1513.7832 & 757.3952 & $\mathbf{L}$ & 956.6139 & 478.8106 & 939.5873 & 470.2973 & 938.6033 & 469.8053 & 8 \\
\hline 14 & 1644.8778 & 822.9425 & 1627.8512 & 814.4293 & 1626.8672 & 813.9373 & $\mathbf{L}$ & 843.5298 & 422.2685 & 826.5033 & 413.7553 & 825.5193 & 413.2633 & 7 \\
\hline 15 & 1757.9619 & 879.4846 & 1740.9353 & 870.9713 & 1739.9513 & 870.4793 & I & 730.4458 & 365.7265 & 713.4192 & 357.2132 & 712.4352 & 356.7212 & 6 \\
\hline 16 & 1871.0459 & 936.0266 & 1854.0194 & 927.5133 & 1853.0354 & 927.0213 & $\mathbf{L}$ & 617.3617 & 309.1845 & 600.3351 & 300.6712 & 599.3511 & 300.1792 & 5 \\
\hline 17 & 1986.0729 & 993.5401 & 1969.0463 & 985.0268 & 1968.0623 & 984.5348 & $\mathbf{N}$ & 504.2776 & 252.6425 & 487.2511 & 244.1292 & 486.2671 & 243.6372 & 4 \\
\hline 18 & 2099.1569 & 1050.0821 & 2082.1304 & 1041.5688 & 2081.1464 & 1041.0768 & $\mathbf{L}$ & 389.2507 & 195.1290 & 372.2241 & 186.6157 & 371.2401 & 186.1237 & 3 \\
\hline 19 & 2200.2046 & 1100.6059 & 2183.1780 & 1092.0927 & 2182.1940 & 1091.6007 & $\mathbf{T}$ & 276.1666 & 138.5870 & 259.1401 & 130.0737 & 258.1561 & 129.5817 & 2 \\
\hline 20 & & & & & & & $\mathbf{R}$ & 175.1190 & 88.0631 & 158.0924 & 79.5498 & & & 1 \\
\hline
\end{tabular}

$$
\text { 的 }
$$

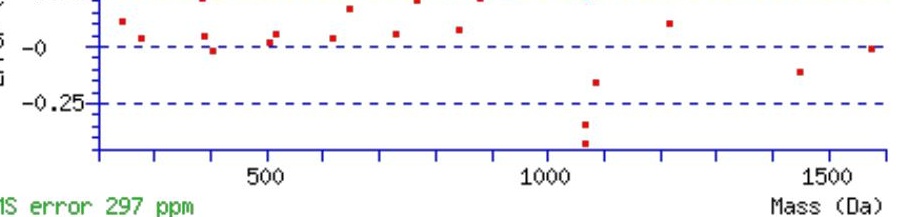

RMS error 297 ppm

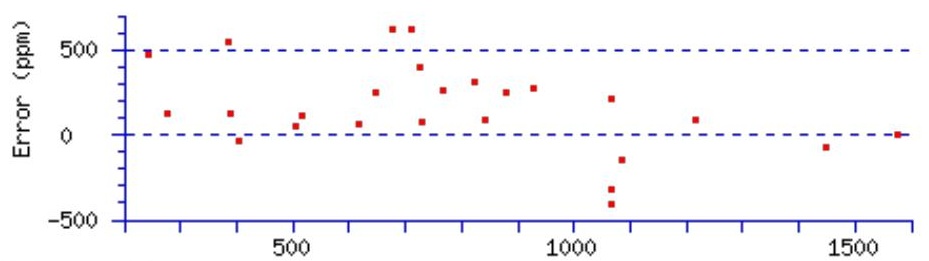

RMS error 297 ppm

\section{All matches to this query}

\begin{tabular}{|l|c|c|c|}
\hline Score & Mr(calc): & Delta & Sequence \\
\hline 45.4 & 2373.3089 & 0.0080 & LKCDPAIKPHEELLILNLTR \\
\hline & & & \\
\hline
\end{tabular}




\begin{tabular}{|l|l|l|l|}
1.8 & 2373.3308 & -0.0139 & $\underline{\text { IKQFPLTINKPSFSLTPIDSK }}$ \\
\hline 1.1 & 2372.3249 & 0.9920 & $\underline{\text { LKCDPAIKPHEELLILNLTR }}$ \\
\hline
\end{tabular}

Spectrum No: 739; Query: 2661; Rank: 1

\section{Peptide View}

MS/MS Fragmentation of LRPHFLSVCDPDFSQINCSEGYIQNYR

Found in IPI00205490, Tax_Id=10116 Gene_Symbol=Ppap2b Lipid phosphate phosphohydrolase 3

Match to Query 2661: 3316.522816 from(830.137980,4+)

Title: 100101RatKid_NS_deglyco_23.4161.4161.4.dta

Data file K:INewmanPaper|Piliangl3SubProteomes\Piliang3SP\mgf5ppm\ERLIC_3SubProteomes5ppm.mgf
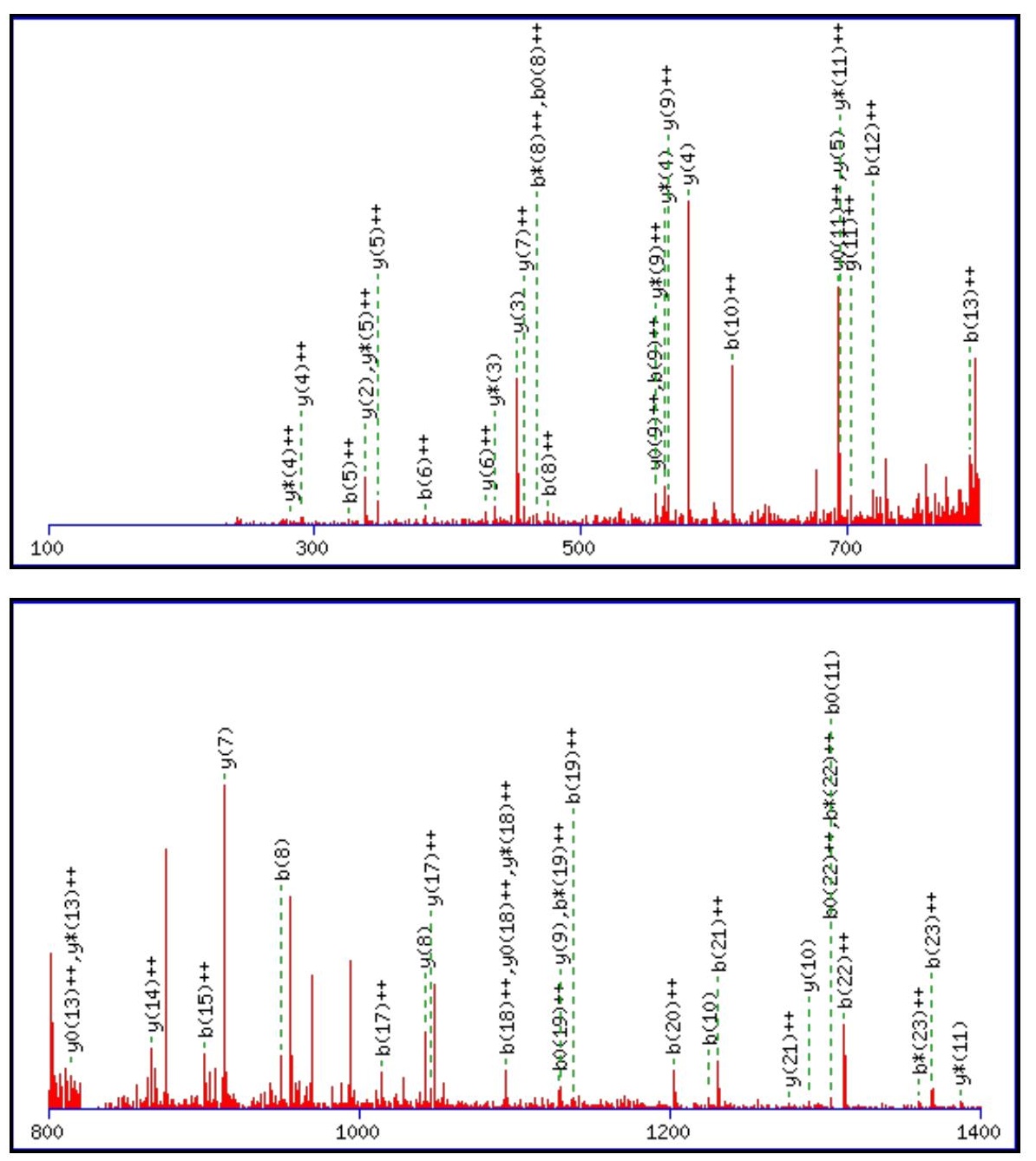


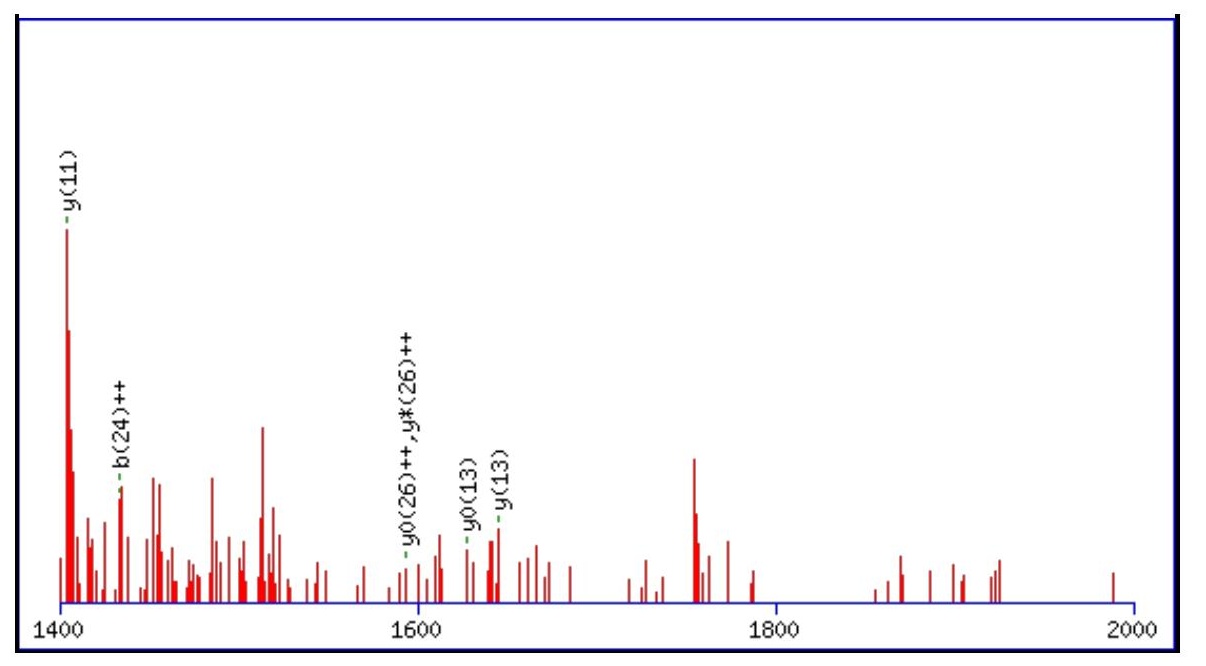

Monoisotopic mass of neutral peptide $\operatorname{Mr}($ calc): 3315.5077

Fixed modifications: Carbamidomethyl (C)

Variable modifications:

N17 : Deamidated_N (N)

Ions Score: 45 Expect: 0.013

Matches (Bold Red): 61/284 fragment ions using 140 most intense peaks

\begin{tabular}{|c|c|c|c|c|c|c|c|c|c|c|c|c|c|c|}
\hline \# & b & $\mathbf{b}^{++}$ & $\mathbf{b}^{*}$ & $\mathbf{b}^{*++}$ & $\mathbf{b}^{0}$ & $\mathbf{b}^{\mathbf{0 + +}}$ & Seq. & $\mathbf{y}$ & $\mathbf{y}^{++}$ & $\mathbf{y}^{*}$ & $\mathrm{y}^{\mathrm{*}^{++}}$ & $\mathbf{y}^{0}$ & $\mathbf{y}^{\mathbf{0 + +}}$ & \# \\
\hline 1 & 114.0913 & 57.5493 & & & & & $\mathbf{L}$ & & & & & & & 27 \\
\hline 2 & 270.1925 & 135.5999 & 253.1659 & 127.0866 & & & $\mathbf{R}$ & 3203.4309 & 1602.2191 & 3186.4044 & 1593.7058 & 3185.4204 & 1593.2138 & 26 \\
\hline 3 & 367.2452 & 184.1262 & 350.2187 & 175.6130 & & & $\mathbf{P}$ & 3047.3298 & 1524.1686 & 3030.3033 & 1515.6553 & 3029.3193 & 1515.1633 & 25 \\
\hline 4 & 504.3041 & 252.6557 & 487.2776 & 244.1424 & & & $\mathbf{H}$ & 2950.2771 & 1475.6422 & 2933.2505 & 1467.1289 & 2932.2665 & 1466.6369 & 24 \\
\hline 5 & 651.3725 & 326.1899 & 634.3460 & 317.6766 & & & $\mathbf{F}$ & 2813.2182 & 1407.1127 & 2796.1916 & 1398.5994 & 2795.2076 & 1398.1074 & 23 \\
\hline 6 & 764.4566 & 382.7319 & 747.4301 & 374.2187 & & & $\mathbf{L}$ & 2666.1497 & 1333.5785 & 2649.1232 & 1325.0652 & 2648.1392 & 1324.5732 & 22 \\
\hline 7 & 851.4886 & 426.2480 & 834.4621 & 417.7347 & 833.4781 & 417.2427 & $\mathrm{~S}$ & 2553.0657 & 1277.0365 & 2536.0391 & 1268.5232 & 2535.0551 & 1268.0312 & 21 \\
\hline 8 & 950.5570 & 475.7822 & 933.5305 & 467.2689 & 932.5465 & 466.7769 & V & 2466.0336 & 1233.5205 & 2449.0071 & 1225.0072 & 2448.0231 & 1224.5152 & 20 \\
\hline 9 & 1110.5877 & 555.7975 & 1093.5611 & 547.2842 & 1092.5771 & 546.7922 & C & 2366.9652 & 1183.9863 & 2349.9387 & 1175.4730 & 2348.9547 & 1174.9810 & 19 \\
\hline 10 & 1225.6146 & 613.3110 & 1208.5881 & 604.7977 & 1207.6041 & 604.3057 & D & 2206.9346 & 1103.9709 & 2189.9080 & 1095.4577 & 2188.9240 & 1094.9656 & 18 \\
\hline 11 & 1322.6674 & 661.8373 & 1305.6409 & 653.3241 & 1304.6568 & 652.8321 & $\mathbf{P}$ & 2091.9076 & 1046.4575 & 2074.8811 & 1037.9442 & 2073.8971 & 1037.4522 & 17 \\
\hline 12 & 1437.6943 & 719.3508 & 1420.6678 & 710.8375 & 1419.6838 & 710.3455 & D & 1994.8549 & 997.9311 & 1977.8283 & 989.4178 & 1976.8443 & 988.9258 & 16 \\
\hline 13 & 1584.7628 & 792.8850 & 1567.7362 & 784.3717 & 1566.7522 & 783.8797 & $\mathbf{F}$ & 1879.8279 & 940.4176 & 1862.8014 & 931.9043 & 1861.8174 & 931.4123 & 15 \\
\hline 14 & 1671.7948 & 836.4010 & 1654.7682 & 827.8878 & 1653.7842 & 827.3957 & $\mathrm{~S}$ & 1732.7595 & 866.8834 & 1715.7330 & 858.3701 & 1714.7490 & 857.8781 & 14 \\
\hline 15 & 1799.8534 & 900.4303 & 1782.8268 & 891.9170 & 1781.8428 & 891.4250 & $\mathbf{Q}$ & 1645.7275 & 823.3674 & 1628.7009 & 814.8541 & 1627.7169 & 814.3621 & 13 \\
\hline 16 & 1912.9374 & 956.9724 & 1895.9109 & 948.4591 & 1894.9269 & 947.9671 & I & \begin{tabular}{|l|}
1517.6689 \\
\end{tabular} & 759.3381 & 1500.6424 & 750.8248 & 1499.6583 & 750.3328 & 12 \\
\hline 17 & 2027.9644 & 1014.4858 & 2010.9378 & 1005.9725 & 2009.9538 & 1005.4805 & $\mathbf{N}$ & 1404.5848 & 702.7961 & 1387.5583 & 694.2828 & 1386.5743 & 693.7908 & 11 \\
\hline $18:$ & 2187.9950 & 1094.5011 & 2170.9685 & 1085.9879 & 2169.9844 & 1085.4959 & $\mathrm{C}$ & 1289.5579 & 645.2826 & 1272.5314 & 636.7693 & 1271.5473 & 636.2773 & 10 \\
\hline 19 & 2275.0270 & 1138.0172 & 2258.0005 & 1129.5039 & 2257.0165 & 1129.0119 & $\mathrm{~S}$ & 1129.5273 & 565.2673 & 1112.5007 & 556.7540 & 1111.5167 & 556.2620 & 9 \\
\hline 20 & 2404.0696 & 1202.5385 & 2387.0431 & 1194.0252 & 2386.0591 & 1193.5332 & $E$ & 1042.4952 & 521.7513 & 1025.4687 & 513.2380 & 1024.4847 & 512.7460 & 8 \\
\hline $21:$ & 2461.0911 & 1231.0492 & 2444.0646 & 1222.5359 & 2443.0805 & 1222.0439 & G & 913.4526 & 457.2300 & 896.4261 & 448.7167 & & & 7 \\
\hline $22:$ & 2624.1544 & 1312.5809 & 2607.1279 & 1304.0676 & 2606.1439 & 1303.5756 & $\mathbf{Y}$ & 856.4312 & 428.7192 & 839.4046 & 420.2060 & & & 6 \\
\hline 23 & 2737.2385 & 1369.1229 & 2720.2119 & 1360.6096 & 2719.2279 & 1360.1176 & I & 693.3679 & 347.1876 & 676.3413 & 338.6743 & & & 5 \\
\hline $24:$ & 2865.2971 & 1433.1522 & 2848.2705 & 1424.6389 & 2847.2865 & 1424.1469 & $\mathbf{Q}$ & 580.2838 & 290.6455 & 563.2572 & 282.1323 & & & 4 \\
\hline 25 & 2979.3400 & 1490.1736 & 2962.3134 & 1481.6604 & 2961.3294 & 1481.1684 & $\mathbf{N}$ & 452.2252 & 226.6162 & 435.1987 & 218.1030 & & & 3 \\
\hline 26 & 3142.4033 & 1571.7053 & 3125.3768 & 1563.1920 & 3124.3928 & 1562.7000 & $\mathbf{Y}$ & 338.1823 & 169.5948 & 321.1557 & 161.0815 & & & 2 \\
\hline 27 & & & & & & & $\mathbf{R}$ & 175.1190 & 88.0631 & 158.0924 & 79.5498 & & & 1 \\
\hline
\end{tabular}



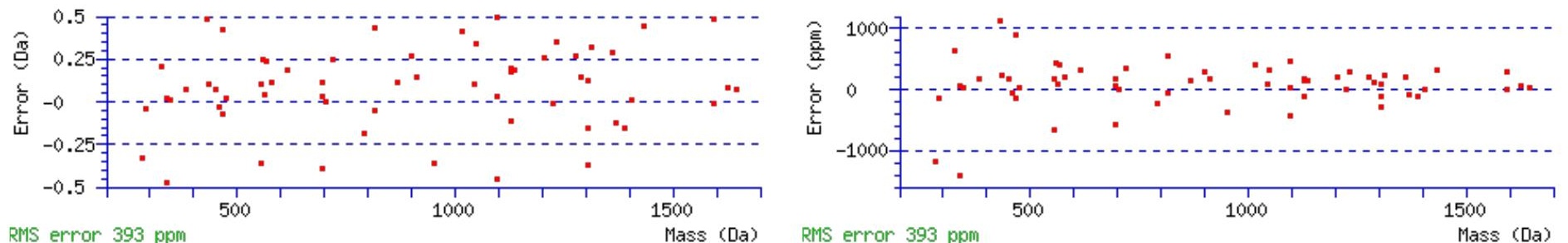

\section{All matches to this query}

\begin{tabular}{|l|l|r|l|}
\hline Score & Mr(calc): & Delta & \multicolumn{1}{|c|}{ Sequence } \\
\hline 45.4 & 3315.5077 & 1.0151 & LRPHFLSVCDPDFSQINCSEGYIQNYR \\
\hline 35.4 & 3316.4917 & 0.0311 & LRPHFLSVCDPDFSQINCSEGYIQNYR \\
\hline 18.0 & 3314.5237 & 1.9991 & LRPHFLSVCDPDFSQINCSEGYIQNYR \\
\hline 10.1 & 3315.5077 & 1.0151 & LRPHFLSVCDPDFSQINCSEGYIQNYR \\
\hline 3.6 & 3315.4879 & 1.0349 & KSSVIFIGSLVPCMENMITEERLNEVK \\
\hline 3.6 & 3315.4879 & 1.0349 & KSSVIFIGSLVPCMENMITEERLNEVK \\
\hline 3.4 & 3316.5046 & 0.0182 & KYSNSNVIMHETSQYHVQHLATFIMDK \\
\hline 3.4 & 3316.5046 & 0.0182 & KYSNSNVIMHETSQYHVQHLATFIMDK \\
\hline 2.6 & 3315.5311 & 0.9917 & YEMASNPLYRKPISTHTVDFAFNKFNK \\
\hline 2.5 & 3316.5232 & -0.0004 & LTIMQFMNHPWINQSMEVPQTPLHTAR \\
\hline
\end{tabular}

Spectrum No: 740; Query: 2556; Rank: 1

\section{Peptide View}

MS/MS Fragmentation of EFIANHPDYKQDSVITDEINYSLILK

Found in IPI00231862, Tax_Id=10116 Gene_Symbol=Gclc Glutamate--cysteine ligase catalytic subunit

Match to Query 2556: 3065.526222 from(1022.849350,3+)

Title: 091008RatKidney_NH4Format01_05.3857.3857.3.dta

Data file K:INewmanPaper|Piliang|3SubProteomes\Piliang3SP\mgf5ppm\ERLIC_3SubProteomes5ppm.mgf

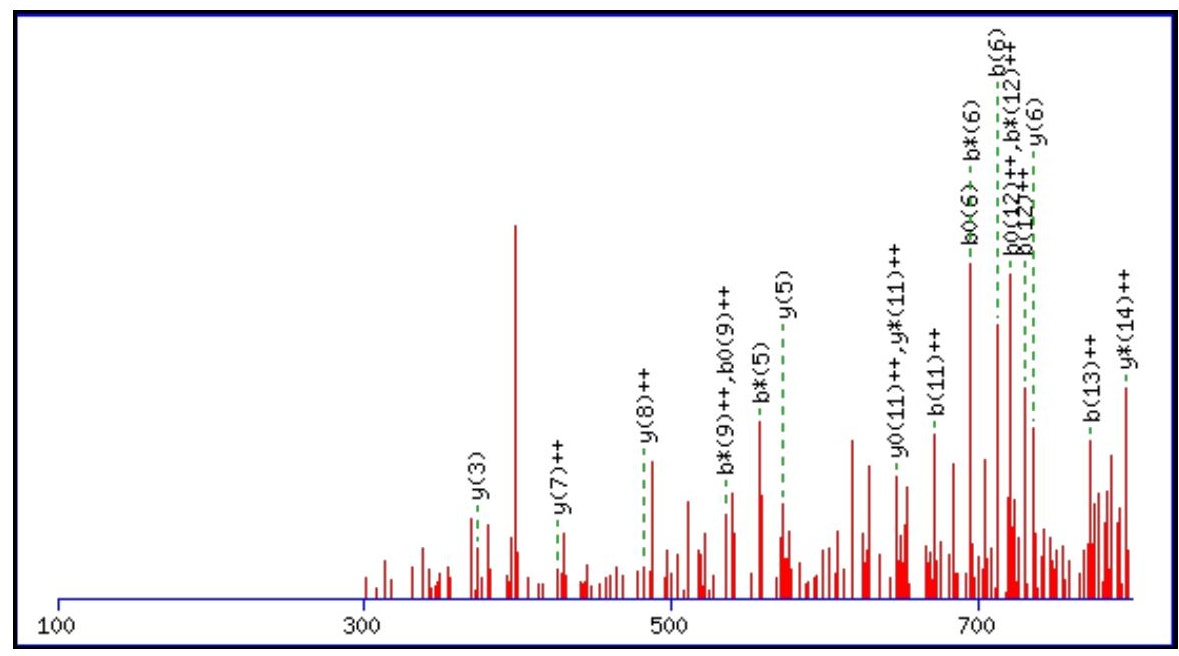



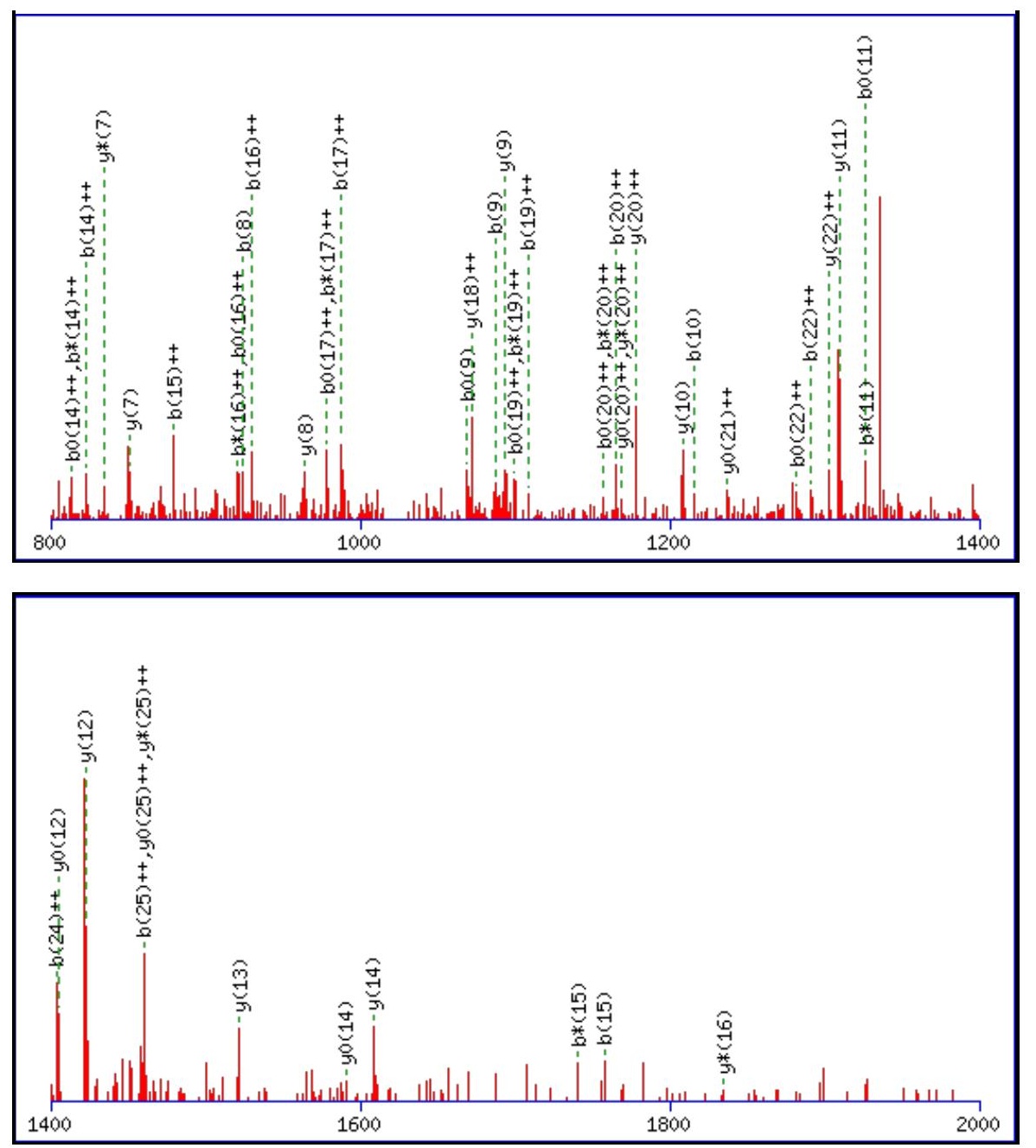

Monoisotopic mass of neutral peptide $\operatorname{Mr}($ calc): 3065.5233

Fixed modifications: Carbamidomethyl (C)

Variable modifications:

N20 : Deamidated_N (N)

Ions Score: 45 Expect: 0.0068

Matches (Bold Red): 67/284 fragment ions using 123 most intense peaks

\begin{tabular}{|c|c|c|c|c|c|c|c|c|c|c|c|c|c|c|}
\hline \# & b & $\mathbf{b}^{++}$ & b* & $\mathbf{b}^{*^{++}}$ & $\mathbf{b}^{\mathbf{0}}$ & $\mathbf{b}^{0++}$ & Seq. & $\mathbf{y}$ & $\mathbf{y}^{++}$ & $\mathbf{y}^{*}$ & $\mathrm{y}^{*^{++}}$ & $\mathbf{y}^{0}$ & $\mathbf{y}^{0++}$ & \# \\
\hline 1 & 130.0499 & 65.5286 & & & 112.0393 & 56.5233 & $\mathbf{E}$ & & & & & & & 26 \\
\hline 2 & 277.1183 & 139.0628 & & & 259.1077 & 130.0575 & $\mathbf{F}$ & 2937.4880 & 1469.2477 & 2920.4615 & 1460.7344 & 2919.4775 & 1460.2424 & 25 \\
\hline 3 & 390.2023 & 195.6048 & & & 372.1918 & 186.5995 & I & 2790.4196 & 1395.7134 & 2773.3931 & 1387.2002 & 2772.4090 & 1386.7082 & 24 \\
\hline 4 & 461.2395 & 231.1234 & & & 443.2289 & 222.1181 & A & 2677.3355 & 1339.1714 & 2660.3090 & 1330.6581 & 2659.3250 & 1330.1661 & 23 \\
\hline 5 & 575.2824 & 288.1448 & 558.2558 & 279.6316 & 557.2718 & 279.1395 & $\mathbf{N}$ & 2606.2984 & 1303.6529 & 2589.2719 & 1295.1396 & 2588.2879 & 1294.6476 & 22 \\
\hline 6 & 712.3413 & 356.6743 & 695.3148 & 348.1610 & 694.3307 & 347.6690 & $\mathbf{H}$ & 2492.2555 & 1246.6314 & 2475.2290 & 1238.1181 & 2474.2449 & 1237.6261 & 21 \\
\hline 7 & 809.3941 & 405.2007 & 792.3675 & 396.6874 & 791.3835 & 396.1954 & $\mathbf{P}$ & 2355.1966 & 1178.1019 & 2338.1700 & 1169.5887 & 2337.1860 & 1169.0967 & 20 \\
\hline 8 & 924.4210 & 462.7141 & 907.3945 & 454.2009 & 906.4104 & 453.7089 & D & 2258.1438 & 1129.5756 & 2241.1173 & 1121.0623 & 2240.1333 & 1120.5703 & 19 \\
\hline 9 & 1087.4843 & 544.2458 & 1070.4578 & 535.7325 & 1069.4738 & 535.2405 & $\mathbf{Y}$ & 2143.1169 & 1072.0621 & 2126.0903 & 1063.5488 & 2125.1063 & 1063.0568 & 18 \\
\hline 10 & 1215.5793 & 608.2933 & 1198.5528 & 599.7800 & 1197.5687 & 599.2880 & $\mathbf{K}$ & 1980.0536 & 990.5304 & 1963.0270 & 982.0171 & 1962.0430 & 981.5251 & 17 \\
\hline 11 & 1343.6379 & 672.3226 & 1326.6113 & 663.8093 & 1325.6273 & 663.3173 & $\mathbf{Q}$ & 1851.9586 & 926.4829 & 1834.9320 & 917.9697 & 1833.9480 & 917.4777 & 16 \\
\hline 12 & 1458.6648 & 729.8360 & 1441.6383 & 721.3228 & 1440.6543 & 720.8308 & D & 1723.9000 & 862.4536 & 1706.8735 & 853.9404 & 1705.8895 & 853.4484 & 15 \\
\hline 13 & 1545.6968 & 773.3521 & 1528.6703 & 764.8388 & 1527.6863 & 764.3468 & S & 1608.8731 & 804.9402 & 1591.8465 & 796.4269 & 1590.8625 & 795.9349 & 14 \\
\hline 14 & 1644.7653 & 822.8863 & 1627.7387 & 814.3730 & 1626.7547 & 813.8810 & $\mathbf{V}$ & 1521.8410 & 761.4242 & 1504.8145 & 752.9109 & 1503.8305 & 752.4189 & 13 \\
\hline 15 & 1757.8493 & 879.4283 & 1740.8228 & 870.9150 & 1739.8388 & 870.4230 & I & 1422.7726 & 711.8900 & 1405.7461 & 703.3767 & 1404.7621 & 702.8847 & 12 \\
\hline 16 & 1858.8970 & 929.9521 & 1841.8705 & 921.4389 & 1840.8864 & 920.9469 & $\mathbf{T}$ & 1309.6886 & 655.3479 & 1292.6620 & 646.8346 & 1291.6780 & 646.3426 & 11 \\
\hline 17 & 1973.9239 & 987.4656 & 1956.8974 & 978.9523 & 1955.9134 & 978.4603 & D & 1208.6409 & 604.8241 & 1191.6143 & 596.3108 & 1190.6303 & 595.8188 & 10 \\
\hline 18 & 2102.9665 & 1051.9869 & 2085.9400 & 1043.4736 & 2084.9560 & 1042.9816 & $\mathbf{E}$ & 1093.6139 & 547.3106 & 1076.5874 & 538.7973 & 1075.6034 & 538.3053 & 9 \\
\hline
\end{tabular}




\begin{tabular}{|c|c|c|c|c|c|c|c|c|c|c|c|c|c|c|}
\hline 19 & |2216.0506 & |1108.5289 & |2199.0241 & |1100.0157 & |2198.0400 & |1099.5237 & I & 964.5714 & 482.7893 & 947.5448 & 474.2760 & 946.5608 & 473.7840 & 8 \\
\hline 20 & 2331.0775 & 1166.0424 & 2314.0510 & 1157.5291 & 2313.0670 & 1157.0371 & $\mathbf{N}$ & 851.4873 & 426.2473 & 834.4607 & 417.7340 & 833.4767 & 417.2420 & 7 \\
\hline 21 & 2494.1409 & 1247.5741 & 2477.1143 & 1239.0608 & 2476.1303 & 1238.5688 & $\mathbf{Y}$ & 736.4604 & 368.7338 & 719.4338 & 360.2205 & 718.4498 & 359.7285 & 6 \\
\hline 22 & 2581.1729 & 1291.0901 & 2564.1463 & 1282.5768 & 2563.1623 & 1282.0848 & S & 573.3970 & 287.2022 & 556.3705 & 278.6889 & 555.3865 & 278.1969 & 5 \\
\hline 23 & 2694.2570 & 1347.6321 & 2677.2304 & 1339.1188 & 2676.2464 & 1338.6268 & L & 486.3650 & 243.6861 & 469.3384 & 235.1729 & & & 4 \\
\hline 24 & 2807.3410 & 1404.1742 & 2790.3145 & 1395.6609 & 2789.3305 & 1395.1689 & I & 373.2809 & 187.1441 & 356.2544 & 178.6308 & & & 3 \\
\hline 25 & 2920.4251 & 1460.7162 & 2903.3985 & 1452.2029 & 2902.4145 & 1451.7109 & $\mathbf{L}$ & 260.1969 & 130.6021 & 243.1703 & 122.0888 & & & 2 \\
\hline 26 & & & & & & & $\bar{K}$ & 147.1128 & 74.0600 & 130.0863 & 65.5468 & & & 1 \\
\hline
\end{tabular}
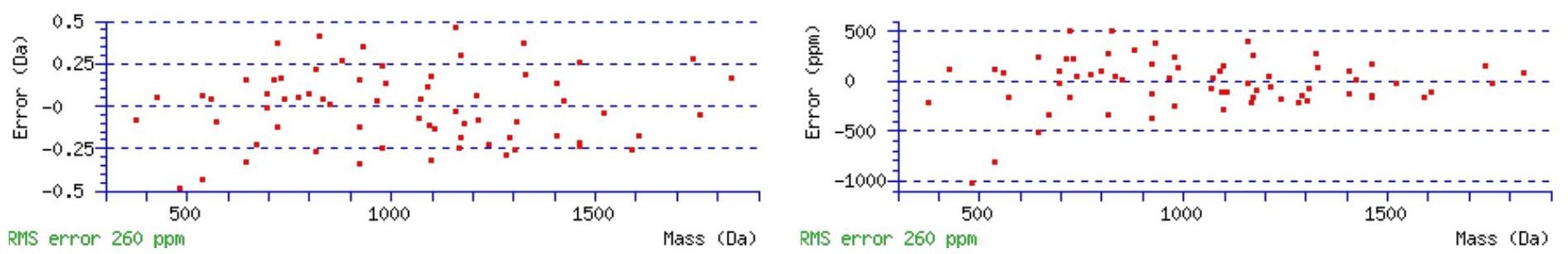

\section{All matches to this query}

\begin{tabular}{|l|l|l|l|}
\hline Score & Mr(calc): & Delta & \multicolumn{1}{|c|}{ Sequence } \\
\hline 45.4 & 3065.5233 & 0.0029 & EFIANHPDYKODSVITDEINYSLILK \\
\hline 33.7 & 3064.5393 & 0.9869 & EFIANHPDYKQDSVITDEINYSLILK \\
\hline 15.9 & 3065.5233 & 0.0029 & EFIANHPDYKODSVITDEINYSLILK \\
\hline 0.5 & 3064.4947 & 1.0316 & SQWLSEKFNLGLDIPNLPYLIDGSHK \\
\hline
\end{tabular}

Spectrum No: 741; Query: 2301; Rank: 1

\section{Peptide View}

MS/MS Fragmentation of VATNPQGLVGTENTTSEMDPSHRK

Found in IPI00358014, Tax_Id=10116 Gene_Symbol=Btd Biotinidase precursor

Match to Query 2301: 2585.195576 from(647.306170,4+)

Title: 091008RatKidney_NH4Format01_24.1114.1114.4.dta

Data file K:INewmanPaper|Piliang \3SubProteomes\Piliang3SP\mgf5ppm\ERLIC_3SubProteomes5ppm.mgf

\begin{tabular}{|c|c|c|c|c|c|c|c|}
\hline & 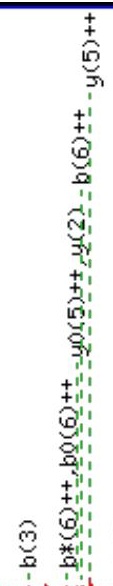 & 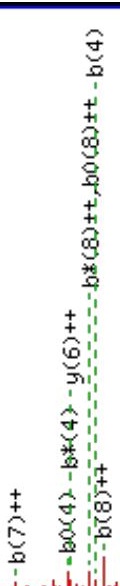 & 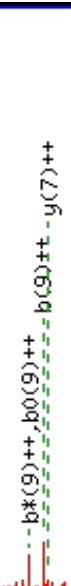 & 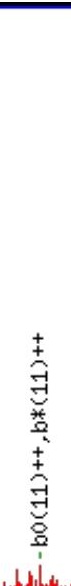 & 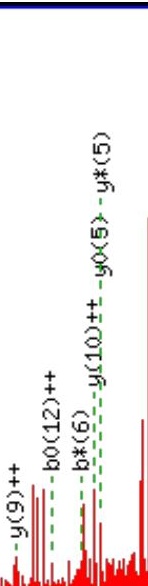 & 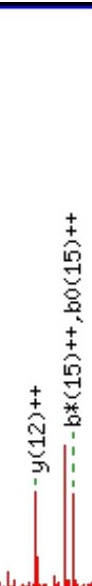 & 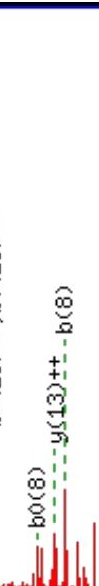 \\
\hline 100 & 300 & & & 500 & & 700 & \\
\hline
\end{tabular}



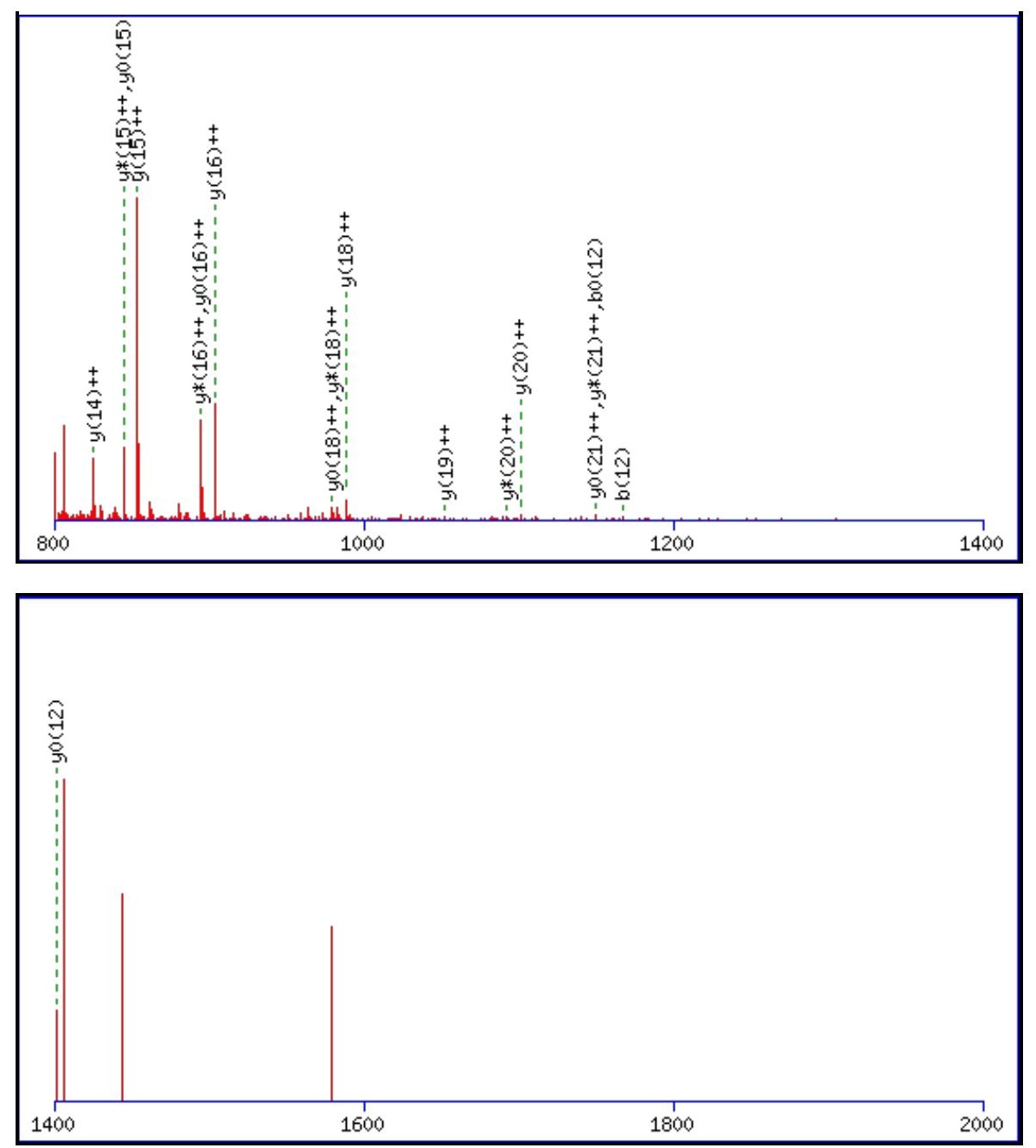

Monoisotopic mass of neutral peptide $\operatorname{Mr}($ calc): 2585.2028

Fixed modifications: Carbamidomethyl (C)

Variable modifications:

N13 : Deamidated_N (N)

M18 : Oxidation (M)

Ions Score: 45 Expect : 0.0094

Matches (Bold Red): 51/260 fragment ions using 94 most intense peaks

\begin{tabular}{|c|c|c|c|c|c|c|c|c|c|c|c|c|c|c|}
\hline \# & b & $\mathbf{b}^{++}$ & $\mathbf{b}^{*}$ & b* & $\mathbf{b}^{0}$ & & eq. & $\mathbf{y}$ & $y^{+}$ & $\mathbf{y}^{*}$ & & $\mathbf{y}^{0}$ & & \# \\
\hline 1 & 100.0757 & 50.5415 & & & & & $\mathrm{~V}$ & & & & & & & 24 \\
\hline 2 & 171.1128 & & & & & & A & & & & & & & 23 \\
\hline 3 & 272.1605 & 365030 & & & & & $\mathbf{T}$ & & 559 & 2399.0780 & & & 506 & 22 \\
\hline 4 & 386.2034 & 193.6053 & & & & & $\mathbf{N}$ & & & & & & & 21 \\
\hline 5 & 483.2562 & 242.1 & 466.2 & 233. & 465. & 204 & $\mathbf{P}$ & 139 & 106 & 874 & 973 & 0033 & 053 & 20 \\
\hline 6 & 611.3148 & 306.1610 & 594.2 & & 593.3 & & $\mathbf{Q}$ & & 842 & 2086 & 709 & 506 & 1043.4789 & 19 \\
\hline 7 & 668.3362 & 334.6717 & 651.3097 & 326.1585 & 650.3 & 325.6665 & G & 026 & 4549 & 1958 & 416 & 8920 & 496 & 18 \\
\hline 8 & 781.4203 & 391.2138 & 764.3937 & 382. & 763.4 & 382. & $\mathbf{L}$ & & 9442 & 1901 & & & 389 & 17 \\
\hline 9 & 880.4887 & 440.7480 & 863.4621 & 432.2347 & 862.4781 & 431.7427 & $\mathbf{V}$ & 1805.7970 & 903.4022 & 1788. & 8889 & 1787.7865 & 3969 & 16 \\
\hline 10 & 937.5102 & 469.2587 & 920.4836 & 460.7 & 919.4 & 460 & $\mathbf{G}$ & 1706.7286 & 8679 & 1689 & 547 & 1688.7181 & 8627 & 15 \\
\hline 11 & 1038.5578 & 519.7826 & 1021.5313 & 511.2693 & 1020.5473 & 510.7773 & $\mathbf{T}$ & 1649.7072 & 825.3572 & 1632.6806 & 816.8439 & 1631.6966 & 816.3519 & 14 \\
\hline 12 & 1167.6004 & 584.3039 & 150.5739 & 575.7 & 399 & & $\mathbf{E}$ & 1548. & 774.8334 & 1531. & 201 & 1530. & 281 & 13 \\
\hline 13 & 1282.6274 & 641.8173 & 1265.6008 & 633.3040 & 1264.6168 & 632.8120 & $\mathbf{N}$ & 1419.6169 & 710.3121 & 1402.5903 & 701.7988 & 1401.6063 & 701.3068 & 12 \\
\hline 14 & 1383.6750 & 692.3412 & 1366.6485 & 683.8279 & 1365.6645 & 683.3359 & $\mathbf{T}$ & 1304.5 & 652.7986 & 1287.5634 & 644.2853 & 1286. & 933 & 11 \\
\hline 15 & 1484.7227 & 742.8650 & 1467.6962 & 734.3517 & 1466.7122 & 733.8597 & $\mathbf{T}$ & 1203.5423 & 602.2748 & 1186.5157 & 593.7615 & 1185.5317 & 593.2695 & 10 \\
\hline 16 & 1571.7548 & 786.3810 & 1554.7282 & 777.8677 & 1553.7442 & 777.3757 & $\mathrm{~S}$ & 1102.4946 & 551.7509 & 1085.4680 & 543.2377 & 1084.4840 & 542.7456 & 9 \\
\hline 17 & 1700.7973 & 850.9023 & 1683.7708 & 842.3890 & 1682.7868 & 841.8970 & $\mathbf{E}$ & 1015.4626 & 508.2349 & 998.4360 & 499.7216 & 997.4520 & 499.2296 & 8 \\
\hline 18 & 1847.8327 & 924.4200 & 1830.8062 & 915.9067 & 1829.8222 & 915.4147 & $\mathbf{M}$ & 886.4200 & 443.7136 & 869.3934 & 435.2003 & 868.4094 & 434.7083 & 7 \\
\hline
\end{tabular}




\begin{tabular}{|l|r|r|r|r|r|r|r|r|r|r|r|r|r|r|r|r|}
$\mathbf{1 9}$ & 1962.8597 & 981.9335 & 1945.8331 & 973.4202 & 1944.8491 & 972.9282 & $\mathbf{D}$ & 739.3846 & 370.1959 & 722.3580 & 361.6826 & 721.3740 & 361.1906 & $\mathbf{6}$ \\
\hline $\mathbf{2 0}$ & 2059.9125 & 1030.4599 & 2042.8859 & 1021.9466 & 2041.9019 & 1021.4546 & $\mathbf{P}$ & 624.3576 & $\mathbf{3 1 2 . 6 8 2 4}$ & $\mathbf{6 0 7 . 3 3 1 1}$ & 304.1692 & $\mathbf{6 0 6 . 3 4 7 1}$ & $\mathbf{3 0 3 . 6 7 7 2}$ & $\mathbf{5}$ \\
\hline $\mathbf{2 1}$ & 2146.9445 & 1073.9759 & 2129.9179 & 1065.4626 & 2128.9339 & 1064.9706 & S & 527.3049 & 264.1561 & 510.2783 & 255.6428 & 509.2943 & 255.1508 & $\mathbf{4}$ \\
\hline $\mathbf{2 2}$ & 2284.0034 & 1142.5053 & 2266.9768 & 1133.9921 & 2265.9928 & 1133.5001 & $\mathbf{H}$ & 440.2728 & 220.6401 & 423.2463 & 212.1268 & & & $\mathbf{3}$ \\
\hline $\mathbf{2 3}$ & 2440.1045 & 1220.5559 & 2423.0780 & 1212.0426 & 2422.0939 & 1211.5506 & $\mathbf{R}$ & 303.2139 & 152.1106 & 286.1874 & 143.5973 & & & & $\mathbf{2}$ \\
\hline $\mathbf{2 4}$ & & & & & & & K & 147.1128 & 74.0600 & 130.0863 & 65.5468 & & & $\mathbf{1}$ \\
\hline
\end{tabular}
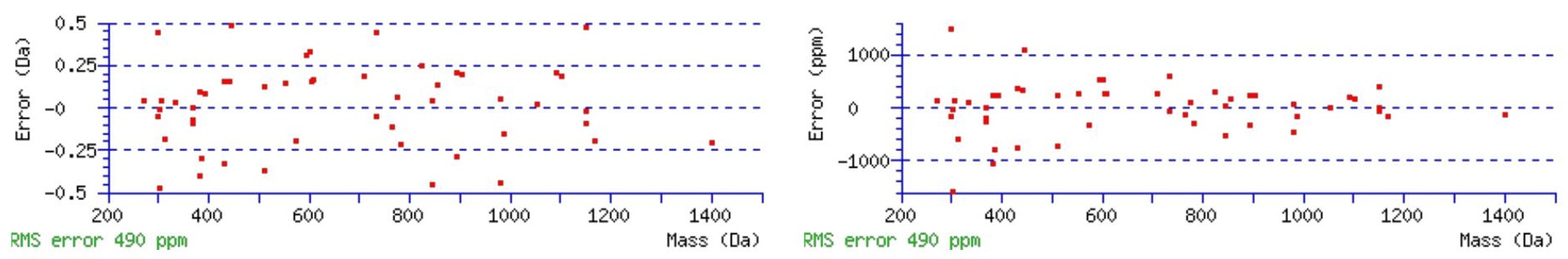

\section{All matches to this query}

\begin{tabular}{|l|l|l|l|}
\hline Score & Mr(calc): & Delta & \multicolumn{1}{|c|}{ Sequence } \\
\hline 45.4 & 2585.2028 & -0.0072 & VATNPQGLVGTENTTSEMDPSHRK \\
\hline 3.3 & 2585.1872 & 0.0084 & EAALVNTVFTPLLNPVIYTLR \\
\hline 3.1 & 2584.2145 & 0.9811 & MTSKGPEEEHPSVTLFRQYLR \\
\hline 2.7 & 2585.1872 & 0.0084 & EAALVNTVFTPLLNPVIYTLR \\
\hline 2.7 & 2585.1872 & 0.0084 & EAALVNTVFTPLLNPVIYTLR \\
\hline 2.4 & 2584.1999 & 0.9957 & KLALQYHPDKNPGNSQAAEFFK \\
\hline 2.3 & 2584.2022 & 0.9934 & MTLSPPHSPOGAAPRAPAEIPRK \\
\hline 2.3 & 2584.2105 & 0.9851 & MSRRFTVTSLPPAASAASADPESR \\
\hline 2.1 & 2585.2067 & -0.0111 & TYLHGKVPPGETLXVEETLVEK \\
\hline 2.1 & 2585.2067 & -0.0111 & TYLHGKVPPGETLXVEETLVEK \\
\hline
\end{tabular}

Spectrum No: 742; Query: 2676; Rank: 1

\section{Peptide View}

MS/MS Fragmentation of LLTHTLEGPSNNVTILQLQDWQDPDSWAR

Found in IPI00357957, Tax_Id=10116 Gene_Symbol=Procr Endothelial protein C receptor precursor

Match to Query 2676: 3347.648982 from(1116.890270,3+)

Title: 100101RatKid_NS_deglyco_24.5083.5083.3.dta

Data file K:\NewmanPaper|Piliang|3SubProteomes\Piliang3SP\mgf5ppm\ERLIC_3SubProteomes5ppm.mgf

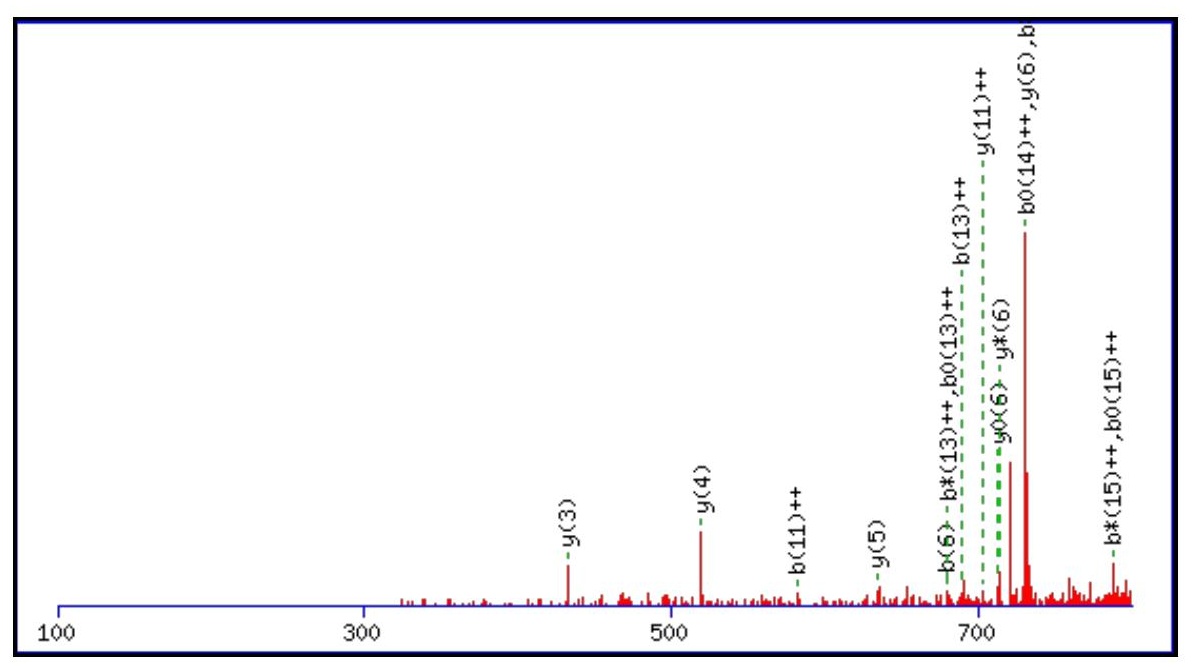



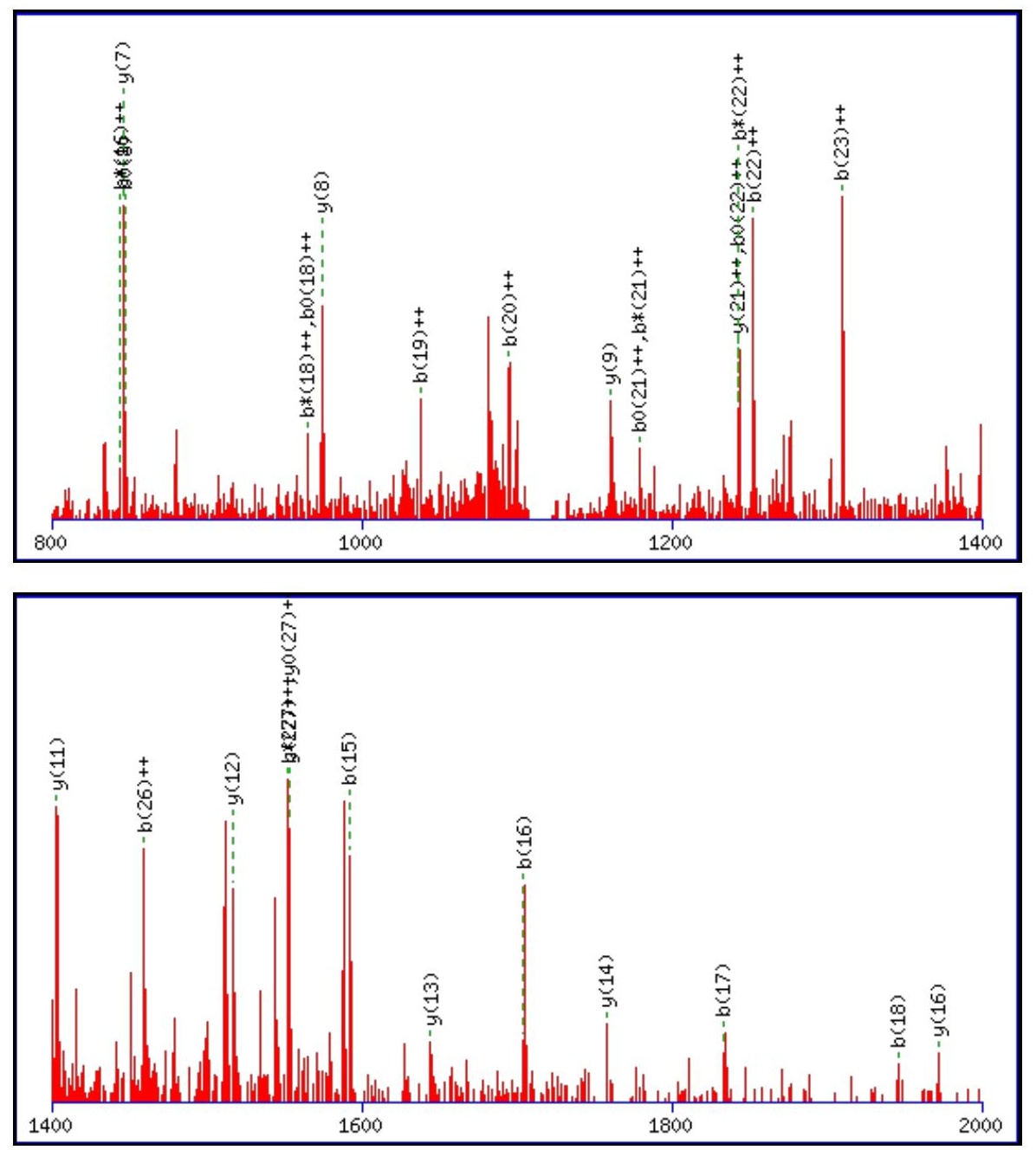

Monoisotopic mass of neutral peptide $\operatorname{Mr}$ (calc): 3347.6422

Fixed modifications: Carbamidomethyl (C)

Variable modifications:

N12 : Deamidated_N (N)

Ions Score: 45 Expect: 0.0076

Matches (Bold Red): 45/306 fragment ions using 72 most intense peaks

\begin{tabular}{|c|c|c|c|c|c|c|c|c|c|c|c|c|c|c|}
\hline$\#$ & b & $\mathbf{b}^{++}$ & $\mathbf{b}^{*}$ & $\mathbf{b}^{*^{++}}$ & $\mathbf{b}^{0}$ & $\mathbf{b}^{0++}$ & Seq. & $\mathbf{y}$ & $y^{++}$ & $\mathrm{y}^{*}$ & $\mathrm{y}^{\mathrm{*}^{++}}$ & $y^{0}$ & $y^{0++}$ & \# \\
\hline 1 & 114.0913 & 57.5493 & & & & & $\mathbf{L}$ & & & & & & & 29 \\
\hline 2 & 227.1754 & 114.0913 & & & & & L & 3235.5654 & 1618.2864 & 3218.5389 & 1609.7731 & 3217.5549 & 1609.2811 & 28 \\
\hline 3 & 328.2231 & 164.6152 & & & 310.2125 & 155.6099 & $\mathbf{T}$ & 3122.4814 & 1561.7443 & 3105.4548 & 1553.2311 & 3104.4708 & 1552.7390 & 27 \\
\hline 4 & 465.2820 & 233.1446 & & & 447.2714 & 224.1394 & $\mathbf{H}$ & 3021.4337 & 1511.2205 & 3004.4071 & 1502.7072 & 3003.4231 & 1502.2152 & 26 \\
\hline 5 & 566.3297 & 283.6685 & & & 548.3191 & 274.6632 & $T$ & 2884.3748 & 1442.6910 & 2867.3482 & 1434.1778 & 2866.3642 & 1433.6857 & 25 \\
\hline 6 & 679.4137 & 340.2105 & & & 661.4032 & 331.2052 & L & 2783.3271 & 1392.1672 & 2766.3006 & 1383.6539 & 2765.3165 & 1383.1619 & 24 \\
\hline 7 & 808.4563 & 404.7318 & & & 790.4458 & 395.7265 & $\mathbf{E}$ & 2670.2430 & 1335.6252 & 2653.2165 & 1327.1119 & 2652.2325 & 1326.6199 & 23 \\
\hline 8 & 865.4778 & 433.2425 & & & 847.4672 & 424.2373 & G & 2541.2005 & 1271.1039 & 2524.1739 & 1262.5906 & 2523.1899 & 1262.0986 & 22 \\
\hline 9 & 962.5306 & 481.7689 & & & 944.5200 & 472.7636 & $\mathbf{P}$ & 2484.1790 & 1242.5931 & 2467.1524 & 1234.0799 & 2466.1684 & 1233.5878 & 21 \\
\hline 10 & 1049.5626 & 525.2849 & & & 1031.5520 & 516.2796 & $S$ & 2387.1262 & 1194.0667 & 2370.0997 & 1185.5535 & 2369.1157 & 1185.0615 & 20 \\
\hline 11 & 1163.6055 & 582.3064 & 1146.5790 & 573.7931 & 1145.5949 & 573.3011 & $\mathbf{N}$ & 2300.0942 & 1150.5507 & 2283.0676 & 1142.0375 & 2282.0836 & 1141.5455 & 19 \\
\hline 12 & 1278.6325 & 639.8199 & 1261.6059 & 631.3066 & 1260.6219 & 630.8146 & $\mathbf{N}$ & 2186.0513 & 1093.5293 & 2169.0247 & 1085.0160 & 2168.0407 & 1084.5240 & 18 \\
\hline 13 & 1377.7009 & 689.3541 & 1360.6743 & 680.8408 & 1359.6903 & 680.3488 & $\mathbf{V}$ & 2071.0243 & 1036.0158 & 2053.9978 & 1027.5025 & 2053.0138 & 1027.0105 & 17 \\
\hline 14 & 1478.7485 & 739.8779 & 1461.7220 & 731.3646 & 1460.7380 & 730.8726 & $T$ & 1971.9559 & 986.4816 & 1954.9294 & 977.9683 & 1953.9454 & 977.4763 & 16 \\
\hline 15 & 1591.8326 & 796.4199 & 1574.8061 & 787.9067 & 1573.8220 & 787.4147 & I & 1870.9082 & 935.9578 & 1853.8817 & 927.4445 & 1852.8977 & 926.9525 & 15 \\
\hline 16 & 1704.9167 & 852.9620 & 1687.8901 & 844.4487 & 1686.9061 & 843.9567 & $\mathbf{L}$ & 1757.8242 & 879.4157 & 1740.7976 & 870.9025 & 1739.8136 & 870.4104 & 14 \\
\hline 17 & 1832.9752 & 916.9913 & 1815.9487 & 908.4780 & 1814.9647 & 907.9860 & $\mathbf{Q}$ & 1644.7401 & 822.8737 & 1627.7136 & 814.3604 & 1626.7295 & 813.8684 & 13 \\
\hline 18 & 1946.0593 & 973.5333 & 1929.0328 & 965.0200 & 1928.0487 & 964.5280 & $\mathbf{L}$ & 1516.6815 & 758.8444 & 1499.6550 & 750.3311 & 1498.6710 & 749.8391 & 12 \\
\hline
\end{tabular}




\begin{tabular}{|c|c|c|c|c|c|c|c|c|c|c|c|c|c|c|}
\hline 19 & 2074.1179 & |1037.5626 & |2057.0913 & 1029.0493 & |2056.1073 & |1028.5573 & $\mathbf{Q}$ & 1403.5975 & 702.3024 & |1386.5709 & 693.7891 & |1385.5869| & 693.2971 & 11 \\
\hline 20 & 2189.1448 & 1095.0761 & 2172.1183 & 1086.5628 & 2171.1343 & 1086.0708 & D & 1275.5389 & 638.2731 & 1258.5123 & 629.7598 & 1257.5283 & 629.2678 & 10 \\
\hline 21 & 2375.2241 & 1188.1157 & 2358.1976 & 1179.6024 & 2357.2136 & \begin{tabular}{|l|}
1179.1104 \\
\end{tabular} & W & 1160.5119 & 580.7596 & 1143.4854 & 572.2463 & 1142.5014 & 571.7543 & 9 \\
\hline 22 & 2503.2827 & 1252.1450 & 2486.2562 & 1243.6317 & 2485.2722 & 1243.1397 & $\mathbf{Q}$ & 974.4326 & 487.7200 & 957.4061 & 479.2067 & 956.4221 & 478.7147 & 8 \\
\hline 23 & 2618.3097 & 1309.6585 & 2601.2831 & 1301.1452 & 2600.2991 & 1300.6532 & D & 846.3741 & 423.6907 & 829.3475 & 415.1774 & 828.3635 & 414.6854 & 7 \\
\hline 24 & 2715.3624 & 1358.1849 & 2698.3359 & 1349.6716 & 2697.3519 & 1349.1796 & $\mathbf{P}$ & 731.3471 & 366.1772 & 714.3206 & 357.6639 & 713.3365 & 357.1719 & 6 \\
\hline 25 & 2830.3894 & 1415.6983 & 2813.3628 & 1407.1851 & 2812.3788 & 1406.6930 & D & 634.2944 & 317.6508 & 617.2678 & 309.1375 & 616.2838 & 308.6455 & 5 \\
\hline 26 & 2917.4214 & 1459.2143 & 2900.3949 & 1450.7011 & 2899.4108 & 1450.2091 & $\mathrm{~S}$ & 519.2674 & 260.1373 & 502.2409 & 251.6241 & 501.2568 & 251.1321 & 4 \\
\hline 27 & 3103.5007 & 1552.2540 & 3086.4742 & 1543.7407 & 3085.4902 & 1543.2487 & W & 432.2354 & 216.6213 & 415.2088 & 208.1081 & & & 3 \\
\hline 28 & 3174.5378 & 1587.7726 & 3157.5113 & 1579.2593 & 3156.5273 & 1578.7673 & A & 246.1561 & 123.5817 & 229.1295 & 115.0684 & & & 2 \\
\hline 29 & & & & & & & $\mathbf{R}$ & 175.1190 & 88.0631 & 158.0924 & 79.5498 & & & 1 \\
\hline
\end{tabular}
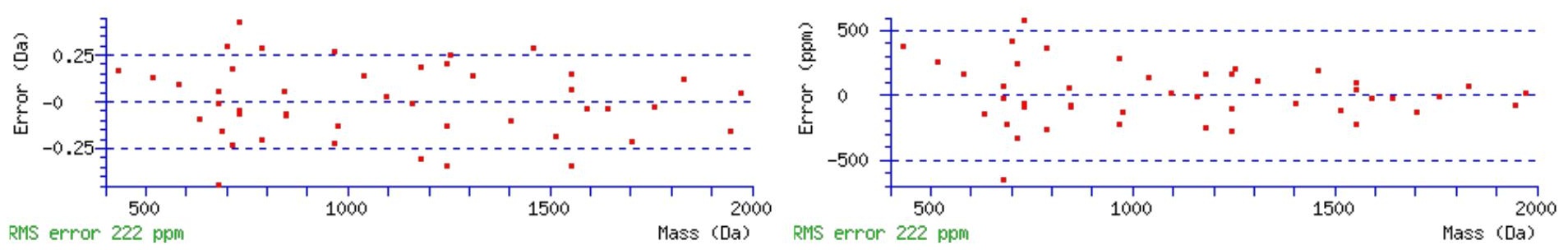

\section{All matches to this query}

\begin{tabular}{|l|l|l|l|}
\hline Score & Mr(calc): & Delta & \multicolumn{1}{|c|}{ Sequence } \\
\hline 45.3 & 3347.6422 & 0.0068 & LLTHTLEGPSNNVTILQLQDWQDPDSWAR \\
\hline 45.3 & 3347.6422 & 0.0068 & LLTHTLEGPSNNVTILQLQDWQDPDSWAR \\
\hline 25.5 & 3346.6582 & 0.9908 & LLTHTLEGPSNNVTILQLQDWQDPDSWAR \\
\hline 6.8 & 3347.6304 & 0.0186 & VISQSFPSKQILTSKNVSDSVDEQGGADHMK \\
\hline 6.4 & 3345.6162 & 2.0328 & HIVSMGYQHDMVLNVWDWKKDIVVASNK \\
\hline 4.8 & 3345.6421 & 2.0069 & LKDKPFSLVLEEDGTIVETEEYFQALPR \\
\hline 2.6 & 3346.6575 & 0.9915 & KAPEISTAVVQRSATIGSSPVLYSQSAIAAGK \\
\hline 2.1 & 3345.6173 & 2.0317 & WTLHLDKNIVPDGAALGWRQQQQTVVGR \\
\hline 1.8 & 3345.6217 & 2.0273 & IRSISYTPYDLPGNLLRYLGSASNCPNPK \\
\hline 1.7 & 3347.6230 & 0.0259 & ILYLEAGVYDVPIIVTDSGNPPLSNTSVIK \\
\hline
\end{tabular}

Spectrum No: 743; Query: 492; Rank: 1

\section{Peptide View}

MS/MS Fragmentation of GECYFYNGTQR

Found in IPI00785570, Tax_Id=10116 Gene_Symbol=- MHC class II antigen

Match to Query 492: 1394.560248 from(698.287400,2+)

Title: 100101RatKid_NS_deglyco_23.1712.1712.2.dta

Data file K:INewmanPaper|Piliang\3SubProteomes\Piliang3SP\mgf5ppm\ERLIC_3SubProteomes5ppm.mgf 

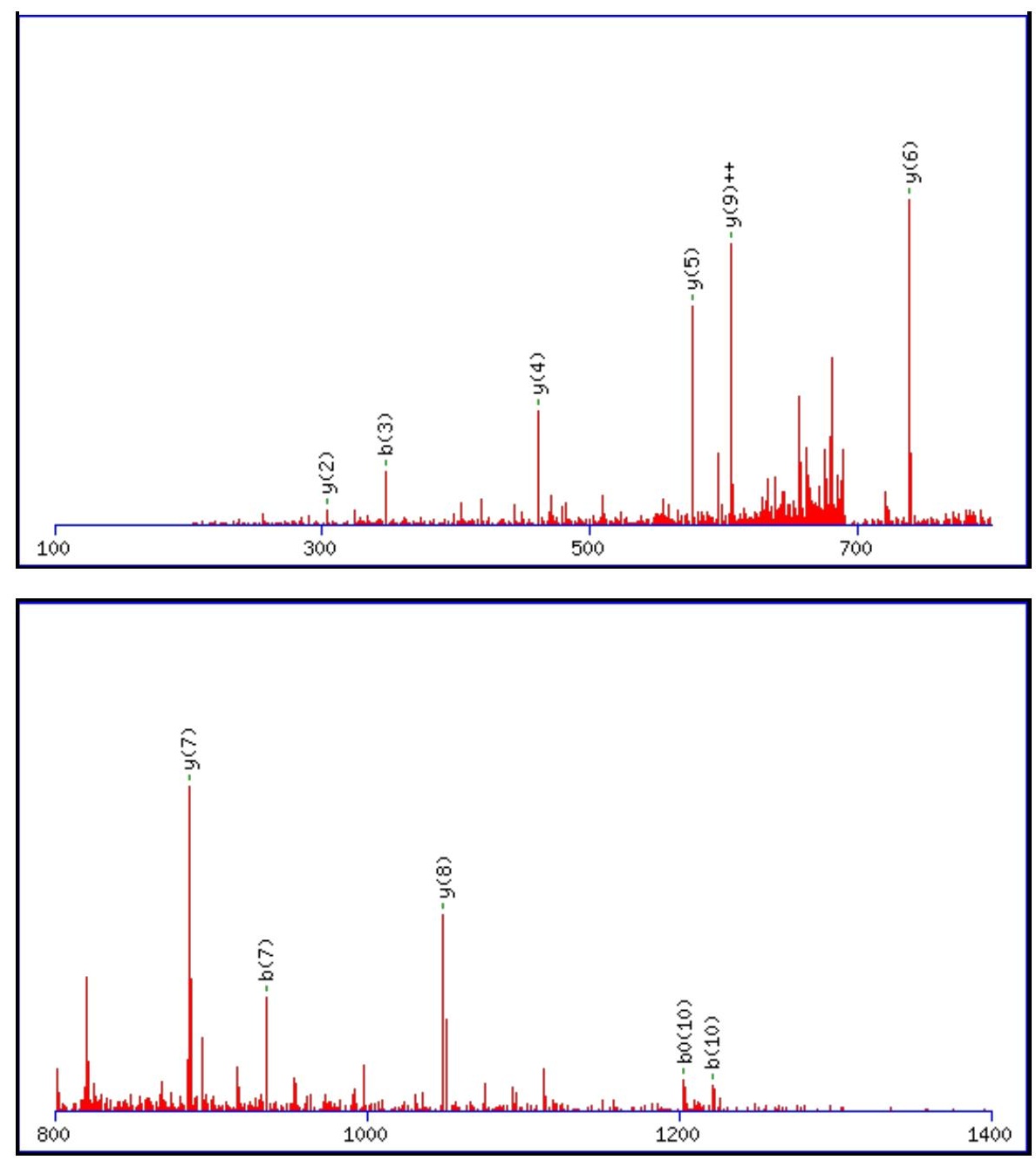

\begin{tabular}{rrrr}
\hline 00 & 1600 & 1800 & 2000 \\
\hline
\end{tabular}

Monoisotopic mass of neutral peptide $\operatorname{Mr}($ calc): 1394.5561

Fixed modifications: Carbamidomethyl (C)

Variable modifications:

N7 : Deamidated_N (N)

Ions Score: 45 Expect : 0.003

Matches (Bold Red): 11/102 fragment ions using 29 most intense peaks

\begin{tabular}{|r|c|c|c|c|c|c|c|c|c|c|c|c|c|c|}
\hline$\#$ & $\mathbf{b}$ & $\mathbf{b}^{++}$ & $\mathbf{b}^{*}$ & $\mathbf{b}^{\mathbf{*}^{++}}$ & $\mathbf{b}^{\mathbf{0}}$ & $\mathbf{b}^{\mathbf{0}++}$ & Seq. & $\mathbf{y}$ & $\mathbf{y}^{++}$ & $\mathbf{y}^{\mathbf{*}}$ & $\mathbf{y}^{\mathbf{*}^{++}}$ & $\mathbf{y}^{\mathbf{0}}$ & $\mathbf{y}^{\mathbf{0 + +}}$ & $\#$ \\
\hline $\mathbf{1}$ & 58.0287 & 29.5180 & & & & & $\mathbf{G}$ & & & & & & & $\mathbf{1 1}$ \\
\hline $\mathbf{2}$ & 187.0713 & 94.0393 & & & 169.0608 & 85.0340 & $\mathbf{E}$ & 1338.5419 & 669.7746 & 1321.5154 & 661.2613 & 1320.5314 & 660.7693 & $\mathbf{1 0}$ \\
\hline $\mathbf{3}$ & 347.1020 & 174.0546 & & & 329.0914 & 165.0493 & $\mathbf{C}$ & 1209.4993 & $\mathbf{6 0 5 . 2 5 3 3}$ & 1192.4728 & 596.7400 & 1191.4888 & 596.2480 & $\mathbf{9}$ \\
\hline $\mathbf{4}$ & 510.1653 & 255.5863 & & & 492.1547 & 246.5810 & $\mathbf{Y}$ & $\mathbf{1 0 4 9 . 4 6 8 7}$ & 525.2380 & 1032.4421 & 516.7247 & 1031.4581 & 516.2327 & $\mathbf{8}$ \\
\hline $\mathbf{5}$ & 657.2337 & 329.1205 & & & 639.2232 & 320.1152 & $\mathbf{F}$ & $\mathbf{8 8 6 . 4 0 5 4}$ & 443.7063 & 869.3788 & 435.1930 & 868.3948 & 434.7010 & $\mathbf{7}$ \\
\hline
\end{tabular}




\begin{tabular}{|r|r|r|r|r|r|r|r|r|r|r|r|r|r|r|r|r|r|}
$\mathbf{6}$ & 820.2971 & 410.6522 & & & 802.2865 & 401.6469 & $\mathbf{Y}$ & 739.3369 & 370.1721 & 722.3104 & 361.6588 & 721.3264 & 361.1668 & $\mathbf{6}$ \\
\hline $\mathbf{7}$ & $\mathbf{9 3 5 . 3 2 4 0}$ & 468.1656 & 918.2974 & 459.6524 & 917.3134 & 459.1604 & $\mathbf{N}$ & $\mathbf{5 7 6 . 2 7 3 6}$ & 288.6404 & 559.2471 & 280.1272 & 558.2630 & 279.6352 & $\mathbf{5}$ \\
\hline $\mathbf{8}$ & 992.3455 & 496.6764 & 975.3189 & 488.1631 & 974.3349 & 487.6711 & $\mathbf{G}$ & $\mathbf{4 6 1 . 2 4 6 7}$ & 231.1270 & 444.2201 & 222.6137 & 443.2361 & 222.1217 & $\mathbf{4}$ \\
\hline $\mathbf{9}$ & 1093.3931 & 547.2002 & 1076.3666 & 538.6869 & 1075.3826 & 538.1949 & $\mathbf{T}$ & 404.2252 & 202.6162 & 387.1987 & 194.1030 & 386.2146 & 193.6110 & $\mathbf{3}$ \\
\hline $\mathbf{1 0}$ & $\mathbf{1 2 2 1 . 4 5 1 7}$ & 611.2295 & 1204.4252 & 602.7162 & $\mathbf{1 2 0 3 . 4 4 1 1}$ & 602.2242 & $\mathbf{Q}$ & 303.1775 & 152.0924 & 286.1510 & 143.5791 & & & & $\mathbf{2}$ \\
\hline $\mathbf{1 1}$ & & & & & & & $\mathbf{R}$ & 175.1190 & 88.0631 & 158.0924 & 79.5498 & & & \\
\hline
\end{tabular}
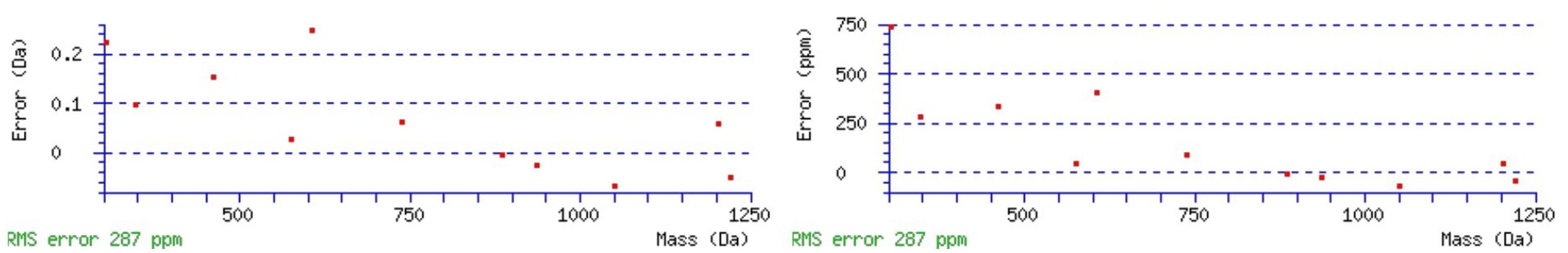

RMS error $287 \mathrm{ppm}$

Mass (Da) RMS error 287 ppm

\section{All matches to this query}

\begin{tabular}{|l|l|l|l|}
\hline Score & Mr(calc): & Delta & \multicolumn{1}{c|}{ Sequence } \\
\hline 45.3 & 1394.5561 & 0.0041 & GECYFYNGTQR \\
\hline 13.8 & 1394.5645 & -0.0042 & AKTMKMMDDTK \\
\hline 2.8 & 1394.5527 & 0.0076 & MEASGSRGRLR \\
\hline 1.0 & 1392.5632 & 1.9970 & DIMNDSNYVVK \\
\hline 0.8 & 1392.5662 & 1.9940 & SMVKFPGGTPGR \\
\hline 0.1 & 1394.5715 & -0.0112 & GVSNESVPDSPAR \\
\hline
\end{tabular}

Spectrum No: 744; Query: 482; Rank: 1

\section{Peptide View}

MS/MS Fragmentation of KATYNISLIHPK

Found in IPI00327398, Tax_Id=10116 Gene_Symbol=Enpep Isoform 1 of Glutamyl aminopeptidase

Match to Query 482: 1384.778142 from(462.599990,3+)

Title: 091008RatKidney_NH4Format01_24.1471.1471.3.dta

Data file K:INewmanPaper|Piliangl3SubProteomes\Piliang3SP\mgf5ppm\ERLIC_3SubProteomes5ppm.mgf

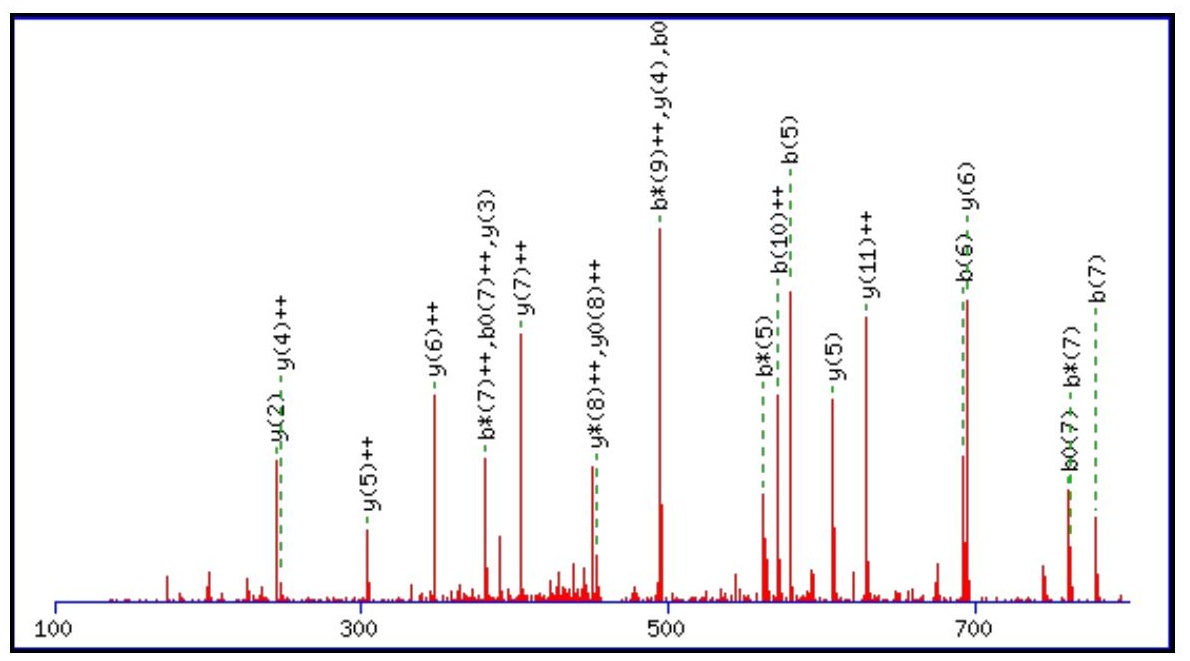



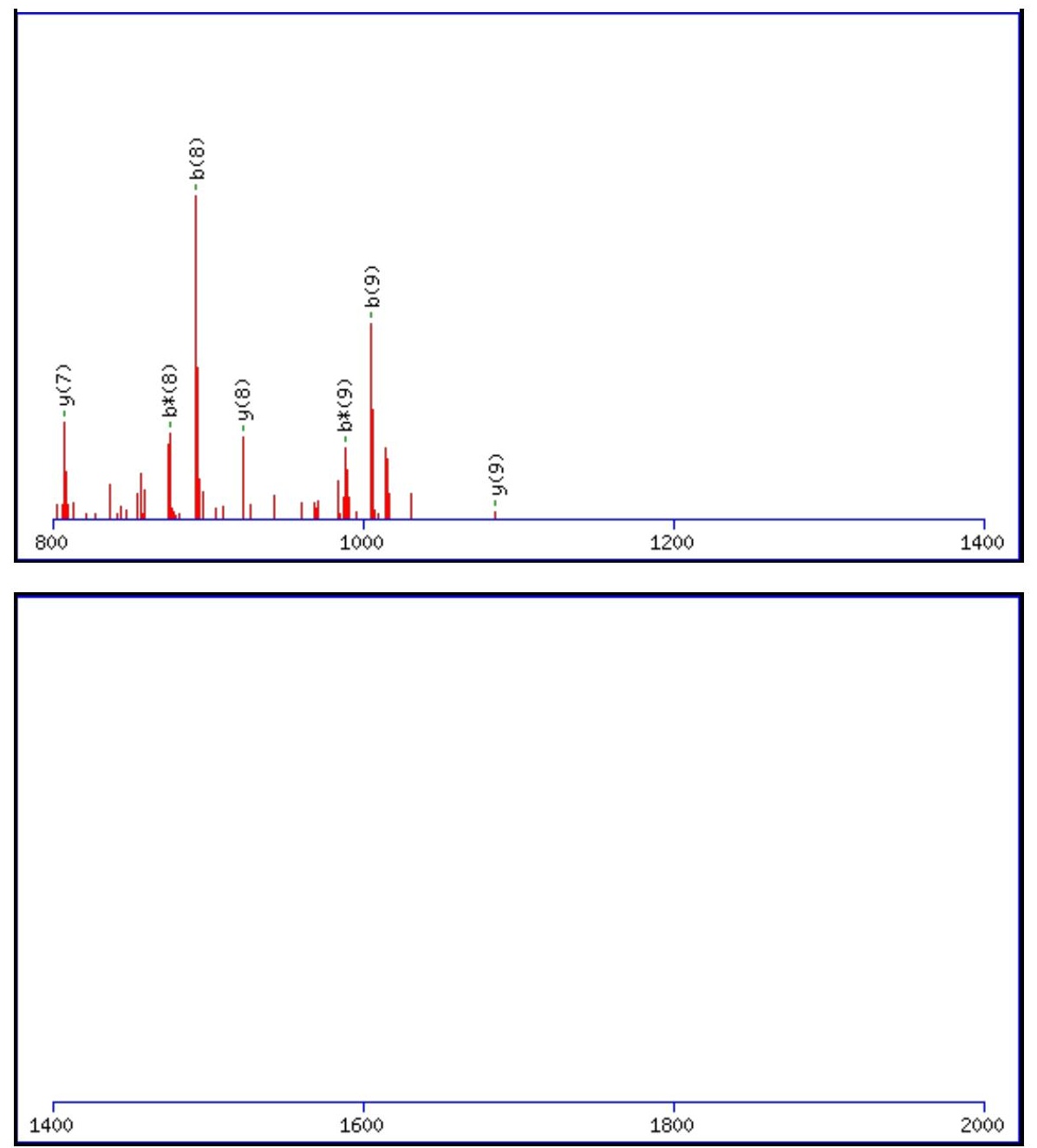

Monoisotopic mass of neutral peptide $\operatorname{Mr}($ calc): 1384.7714

Fixed modifications: Carbamidomethyl (C)

Variable modifications:

N5: Deamidated $\mathrm{N}(\mathrm{N})$

Ions Score: 45 Expect: 0.0018

Matches (Bold Red): 30/118 fragment ions using 44 most intense peaks

\begin{tabular}{|c|c|c|c|c|c|c|c|c|c|c|c|c|c|c|}
\hline$\#$ & b & $\mathbf{b}^{++}$ & b* & $\mathbf{b}^{*^{++}}$ & $\mathbf{b}^{0}$ & $\mathbf{b}^{0++}$ & Seq. & $\mathbf{y}$ & $y^{++}$ & $\mathbf{y}^{*}$ & $\mathrm{y}^{*^{++}}$ & $\mathbf{y}^{0}$ & $\mathbf{y}^{\mathbf{0 + +}}$ & \# \\
\hline 1 & 129.1022 & 65.0548 & 112.0757 & 56.5415 & & & $\mathbf{K}$ & & & & & & & 12 \\
\hline 2 & 200.1394 & 100.5733 & 183.1128 & 92.0600 & & & A & 1257.6838 & 629.3455 & 1240.6572 & 620.8322 & 1239.6732 & 620.3402 & 11 \\
\hline 3 & 301.1870 & 151.0972 & 284.1605 & 142.5839 & 283.1765 & 142.0919 & $\mathbf{T}$ & 1186.6466 & 593.8270 & 1169.6201 & 585.3137 & 1168.6361 & 584.8217 & 10 \\
\hline 4 & 464.2504 & 232.6288 & 447.2238 & 224.1155 & 446.2398 & 223.6235 & $\mathbf{Y}$ & 1085.5990 & 543.3031 & 1068.5724 & 534.7898 & 1067.5884 & 534.2978 & 9 \\
\hline 5 & 579.2773 & 290.1423 & 562.2507 & 281.6290 & 561.2667 & 281.1370 & $\mathbf{N}$ & 922.5356 & 461.7715 & 905.5091 & 453.2582 & 904.5251 & 452.7662 & 8 \\
\hline 6 & 692.3614 & 346.6843 & 675.3348 & 338.1710 & 674.3508 & 337.6790 & I & 807.5087 & 404.2580 & 790.4822 & 395.7447 & 789.4981 & 395.2527 & 7 \\
\hline 7 & 779.3934 & 390.2003 & 762.3668 & 381.6871 & 761.3828 & 381.1951 & S & 694.4246 & 347.7160 & 677.3981 & 339.2027 & 676.4141 & 338.7107 & 6 \\
\hline 8 & 892.4775 & 446.7424 & 875.4509 & 438.2291 & 874.4669 & 437.7371 & $\mathbf{L}$ & 607.3926 & 304.1999 & 590.3661 & 295.6867 & & & 5 \\
\hline 9 & 1005.5615 & 503.2844 & 988.5350 & 494.7711 & 987.5510 & 494.2791 & I & 494.3085 & 247.6579 & 477.2820 & 239.1446 & & & 4 \\
\hline 10 & 1142.6204 & 571.8139 & 1125.5939 & 563.3006 & 1124.6099 & 562.8086 & $\mathbf{H}$ & 381.2245 & 191.1159 & 364.1979 & 182.6026 & & & 3 \\
\hline 11 & 1239.6732 & 620.3402 & 1222.6466 & 611.8270 & 1221.6626 & 611.3350 & $\mathbf{P}$ & 244.1656 & 122.5864 & 227.1390 & 114.0731 & & & 2 \\
\hline 12 & & & & & & & $\mathbf{K}$ & 147.1128 & 74.0600 & 130.0863 & 65.5468 & & & 1 \\
\hline
\end{tabular}



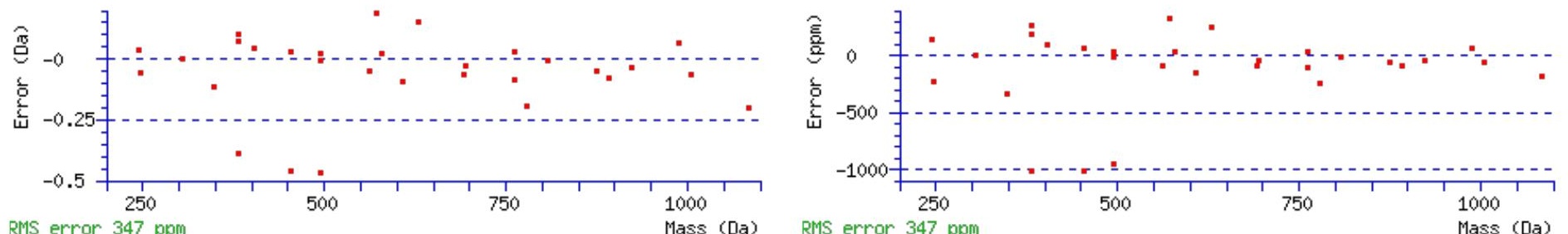

All matches to this query

\begin{tabular}{|l|l|l|l|}
\hline Score & Mr(calc): & Delta & Sequence \\
\hline 45.2 & 1384.7714 & 0.0067 & KATYNISLIHPK \\
\hline 12.8 & 1383.7874 & 0.9907 & KATYNISLIHPK \\
\hline 1.8 & 1383.7623 & 1.0159 & KVLDKHGDIFGR \\
\hline
\end{tabular}

Spectrum No: 745; Query: 2227; Rank: 1

\section{Peptide View}

MS/MS Fragmentation of TEAGAFEYVADPTFENFTGGVKK

Found in IPI00370450, Tax_Id=10116 Gene_Symbol=Plxnb2 plexin B2

Match to Query 2227: 2478.169452 from(827.063760,3+)

Title: 100101RatKid_NS_deglyco_19.4097.4097.3.dta

Data file K:INewmanPaper|Piliang|3SubProteomes\Piliang3SP\mgf5ppm\ERLIC_3SubProteomes5ppm.mgf
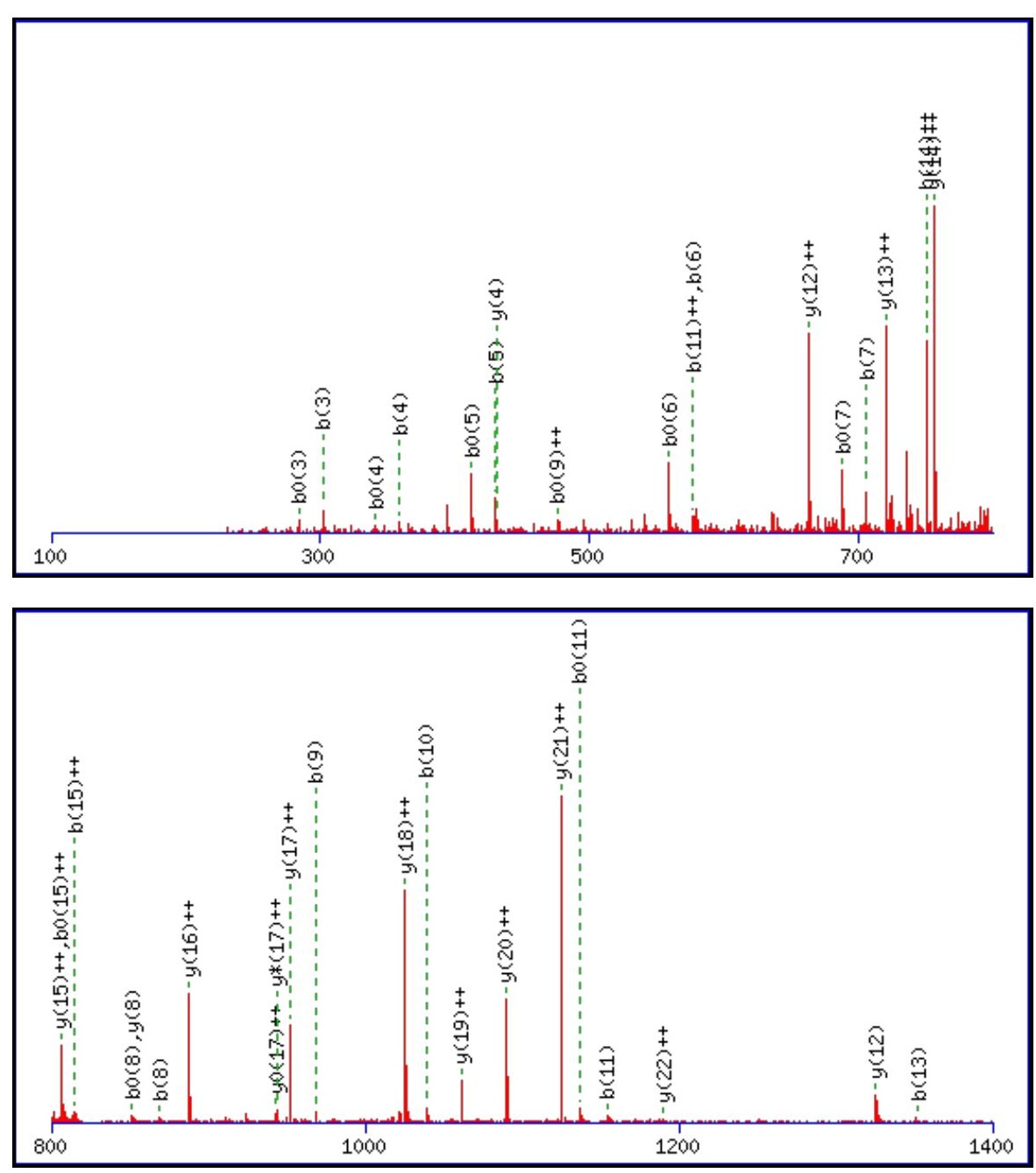


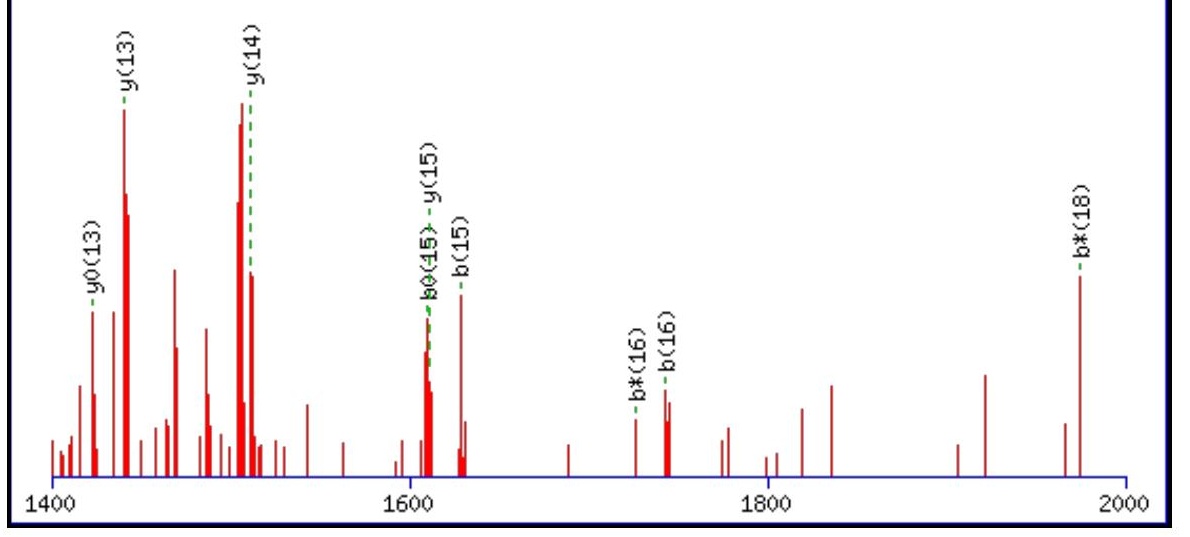

Monoisotopic mass of neutral peptide $\operatorname{Mr}($ calc): 2478.1591

Fixed modifications: Carbamidomethyl (C)

Variable modifications:

N16 : Deamidated_N (N)

Ions Score: 45 Expect : 0.0098

Matches (Bold Red): 47/224 fragment ions using 108 most intense peaks

\begin{tabular}{|c|c|c|c|c|c|c|c|c|c|c|c|c|c|c|}
\hline \# & b & $\mathbf{b}^{++}$ & $\mathbf{b}^{*}$ & $\mathbf{b}^{*^{++}}$ & $\mathbf{b}^{\mathbf{0}}$ & $\mathbf{b}^{\mathbf{0 + +}}$ & Seq. & $\mathbf{y}$ & $\mathbf{y}^{++}$ & $\mathbf{y}^{*}$ & $\mathrm{y}^{\mathrm{*}^{++}}$ & $\mathbf{y}^{\mathbf{0}}$ & $\mathbf{y}^{\mathbf{0 + +}}$ & \# \\
\hline 1 & 102.0550 & 51.5311 & & & 84.0444 & 42.5258 & $\mathbf{T}$ & & & & & & & 23 \\
\hline 2 & 231.0975 & 116.0524 & & & 213.0870 & 107.0471 & $\mathbf{E}$ & 2378.1187 & 1189.5630 & 2361.0921 & 1181.0497 & 2360.1081 & 1180.5577 & 22 \\
\hline 4 & 359.1561 & 180.0817 & & & 341.1456 & 171.0764 & G & 2178.0390 & 1089.5231 & 2161.0124 & 1081.0098 & 2160.0284 & 1080.5178 & 20 \\
\hline 5 & 430.1932 & 215.6003 & & & 412.1827 & 206.5950 & A & 2121.0175 & 1061.0124 & 2103.9910 & 1052.4991 & 2103.0069 & 1052.0071 & 19 \\
\hline 6 & 577.2617 & 289.1345 & & & 559.2511 & 280.1292 & $\mathbf{F}$ & 2049.9804 & 1025.4938 & 2032.9538 & 1016.9806 & 2031.9698 & 1016.4886 & 18 \\
\hline 7 & 706.3042 & 353.6558 & & & 688.2937 & 344.6505 & $\mathbf{E}$ & 1902.9120 & 951.9596 & 1885.8854 & 943.4464 & 1884.9014 & 942.9543 & 17 \\
\hline 8 & 869.3676 & 435.1874 & & & 851.3570 & 426.1821 & $\mathbf{Y}$ & 1773.8694 & 887.4383 & 1756.8428 & 878.9251 & 1755.8588 & 878.4330 & 16 \\
\hline 9 & 968.4360 & 484.7216 & & & 950.4254 & 475.7164 & V & 1610.8061 & 805.9067 & 1593.7795 & 797.3934 & 1592.7955 & 796.9014 & 15 \\
\hline 10 & 1039.4731 & 520.2402 & & & 1021.4625 & 511.2349 & A & 1511.7376 & 756.3725 & 1494.7111 & 747.8592 & 1493.7271 & 747.3672 & 14 \\
\hline 11 & 1154.5000 & 577.7537 & & & 1136.4895 & 568.7484 & D & 1440.7005 & 720.8539 & 1423.6740 & 712.3406 & 1422.6900 & 711.8486 & 13 \\
\hline 12 & 1251.5528 & 626.2800 & & & 1233.5422 & 617.2748 & $\mathbf{P}$ & 1325.6736 & 663.3404 & 1308.6470 & 654.8272 & 1307.6630 & 654.3351 & 12 \\
\hline 14 & 1499.6689 & 750.3381 & & & 1481.6583 & 741.3328 & $\mathbf{F}$ & 1127.5731 & 564.2902 & 1110.5466 & 555.7769 & 1109.5626 & 555.2849 & 10 \\
\hline 15 & 1628.7115 & 814.8594 & & & 1610.7009 & 805.8541 & $\mathbf{E}$ & 980.5047 & 490.7560 & 963.4782 & 482.2427 & 962.4942 & 481.7507 & 9 \\
\hline 16 & 1743.7384 & 872.3729 & 1726.7119 & 863.8596 & 1725.7279 & 863.3676 & $\mathbf{N}$ & 851.4621 & 426.2347 & 834.4356 & 417.7214 & 833.4516 & 417.2294 & 8 \\
\hline 17 & 1890.8068 & 945.9071 & 1873.7803 & 937.3938 & 1872.7963 & 936.9018 & $\mathbf{F}$ & 736.4352 & 368.7212 & 719.4087 & 360.2080 & 718.4246 & 359.7160 & 7 \\
\hline 18 & 1991.8545 & 996.4309 & 1974.8280 & 987.9176 & 1973.8440 & 987.4256 & $\mathbf{T}$ & 589.3668 & 295.1870 & 572.3402 & 286.6738 & 571.3562 & 286.1817 & 6 \\
\hline 19 & 2048.8760 & 1024.9416 & 2031.8494 & 1016.4284 & 2030.8654 & 1015.9364 & G & 488.3191 & 244.6632 & 471.2926 & 236.1499 & & & 5 \\
\hline 20 & 2105.8975 & 1053.4524 & 2088.8709 & 1044.9391 & 2087.8869 & 1044.4471 & G & 431.2976 & 216.1525 & 414.2711 & 207.6392 & & & 4 \\
\hline 21 & 2204.9659 & 1102.9866 & 2187.9393 & 1094.4733 & 2186.9553 & 1093.9813 & $\mathbf{V}$ & 374.2762 & 187.6417 & 357.2496 & 179.1285 & & & 3 \\
\hline 22 & 2333.0608 & 1167.0341 & 2316.0343 & 1158.5208 & 2315.0503 & 1158.0288 & $\mathbf{K}$ & 275.2078 & 138.1075 & 258.1812 & 129.5942 & & & 2 \\
\hline 23 & & & & & & & $\mathbf{K}$ & 147.1128 & 74.0600 & 130.0863 & 65.5468 & & & 1 \\
\hline
\end{tabular}
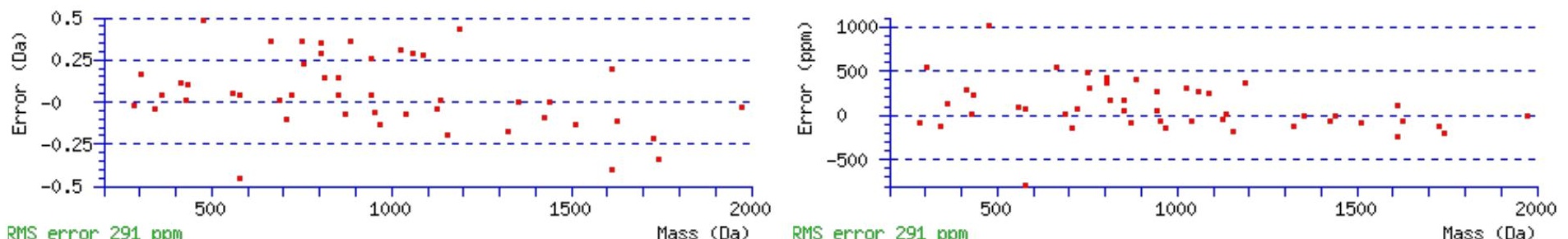

All matches to this query 


\begin{tabular}{|l|l|r|l|}
\hline Score & Mr(calc): & Delta & \multicolumn{1}{|c|}{ Sequence } \\
\hline 45.2 & 2478.1591 & 0.0104 & TEAGAFEYVADPTFENFTGGVKK \\
\hline 17.0 & 2477.1751 & 0.9944 & TEAGAFEYVADPTFENFTGGVKK \\
\hline 3.3 & 2478.1650 & 0.0045 & MVSLDIPYFADVVVPINMTMK \\
\hline 3.3 & 2478.1650 & 0.0045 & MVSLDIPYFADVVVPINMTMK \\
\hline 1.8 & 2476.1613 & 2.0081 & LIDMLSEAGLPVIEATSFVSPK \\
\hline 1.8 & 2476.1613 & 2.0081 & LIDMLSEAGLPVIEATSFVSPK \\
\hline 1.6 & 2476.1709 & 1.9986 & LLGYSEKPINLQMFIGTADDR \\
\hline 1.5 & 2478.1558 & 0.0136 & LGYSYAIVGINLTEMAYSLLK \\
\hline 1.1 & 2478.1793 & -0.0099 & CLNLPYYYLDPVLIENSISI \\
\hline 0.7 & 2476.1518 & 2.0177 & ELSVMNSEELISFPLTGLLCK \\
\hline
\end{tabular}

Spectrum No: 746; Query: 2773; Rank: 1

\section{Peptide View}

MS/MS Fragmentation of LAEGEEEKPEPDGSSEESISTVEEPENETPPAPSR

Found in IPI00400578, Tax_Id=10116 Gene_Symbol=Hp1bp3 Heterochromatin protein 1, binding protein 3

Match to Query 2773: 3752.669136 from(939.174560,4+)

Title: 091008RatKidney_NoSalt_22.1751.1751.4.dta

Data file K:INewmanPaper|Piliang|3SubProteomes\Piliang3SP\mgf5ppm\ERLIC_3SubProteomes5ppm.mgf
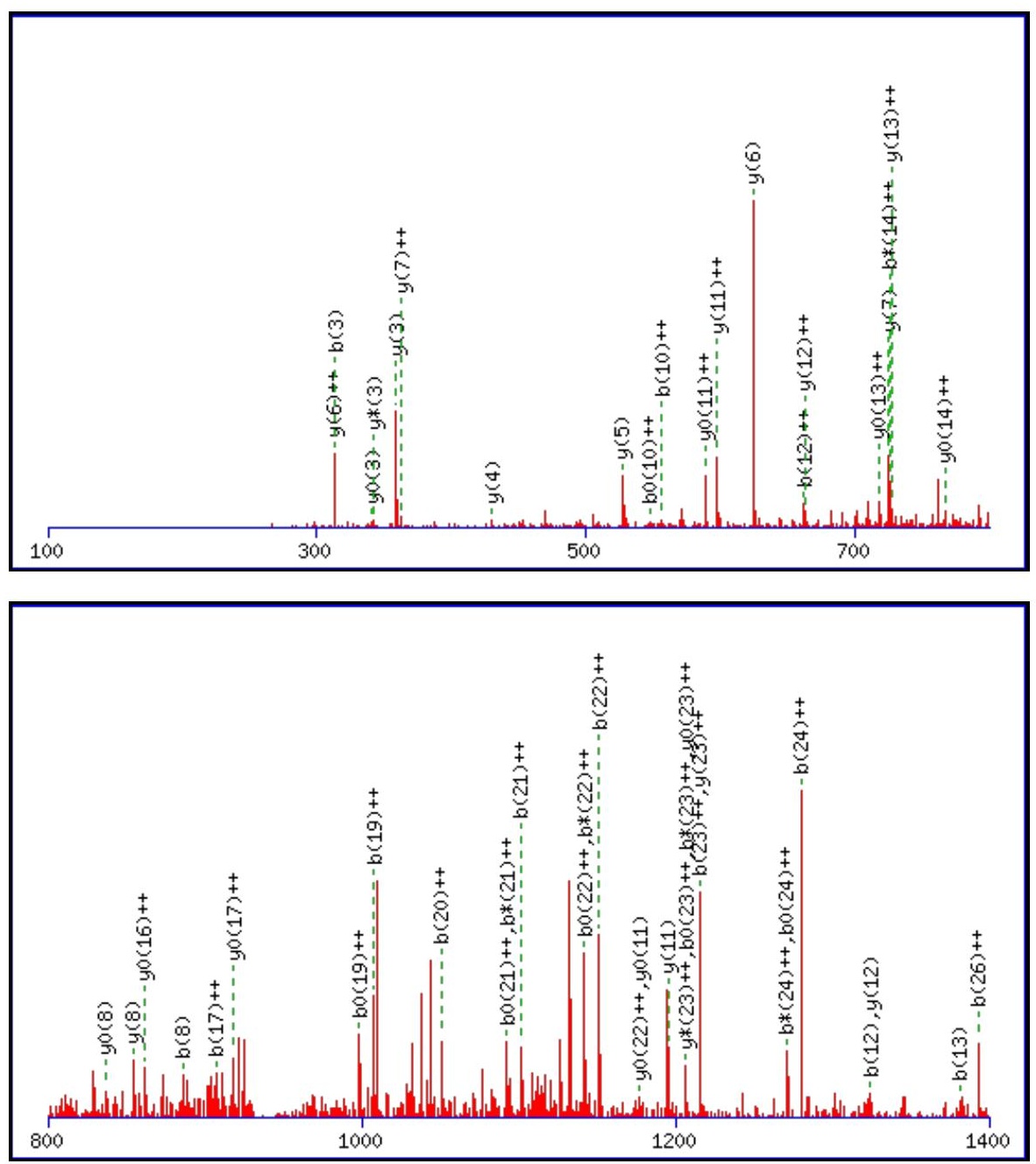


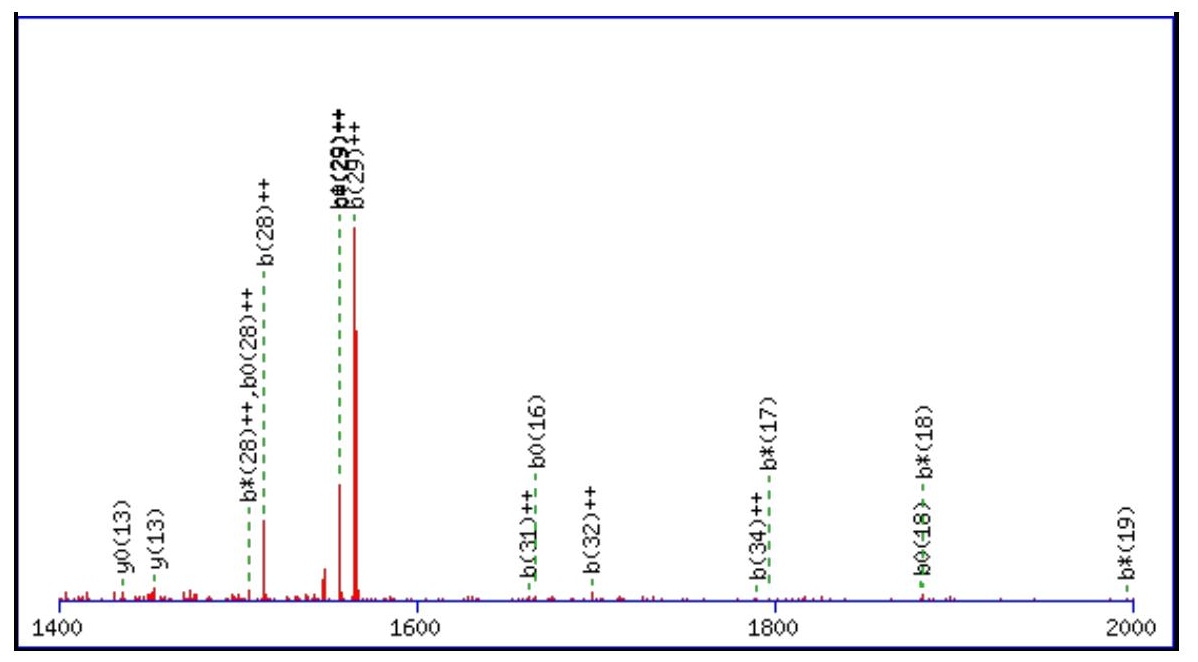

Monoisotopic mass of neutral peptide $\operatorname{Mr}($ calc): 3752.6548

Fixed modifications: Carbamidomethyl (C)

Variable modifications:

N27 : Deamidated_N (N)

Ions Score: 45 Expect : 0.015

Matches (Bold Red): 67/388 fragment ions using 143 most intense peaks

\begin{tabular}{|c|c|c|c|c|c|c|c|c|c|c|c|c|c|c|}
\hline \# & b & $\mathbf{b}^{++}$ & $\mathbf{b}^{*}$ & $\mathbf{b}^{*^{++}}$ & $\mathbf{b}^{0}$ & $\mathbf{b}^{0++}$ & Seq. & $\mathbf{y}$ & $\mathbf{y}^{++}$ & $\mathbf{y}^{*}$ & $\mathrm{y}^{\mathrm{*}^{++}}$ & $\mathbf{y}^{0}$ & $y^{0++}$ & \# \\
\hline 1 & 114.0913 & 57.5493 & & & & & $\mathbf{L}$ & & & & & & & 35 \\
\hline 2 & 185.1285 & 93.0679 & & & & & A & 3640.5780 & 1820.7927 & 3623.5515 & 1812.2794 & 3622.5675 & 1811.7874 & 34 \\
\hline 3 & 314.1710 & 157.5892 & & & 296.1605 & 148.5839 & E & 3569.5409 & 1785.2741 & 3552.5144 & 1776.7608 & 3551.5304 & 1776.2688 & 33 \\
\hline 4 & 371.1925 & 186.0999 & & & 353.1819 & 177.0946 & G & 3440.4983 & 1720.7528 & 3423.4718 & 1712.2395 & 3422.4878 & 1711.7475 & 32 \\
\hline 5 & 500.2351 & 250.6212 & & & 482.2245 & 241.6159 & $\mathbf{E}$ & 3383.4769 & 1692.2421 & 3366.4503 & 1683.7288 & 3365.4663 & 1683.2368 & 31 \\
\hline 6 & 629.2777 & 315.1425 & & & 611.2671 & 306.1372 & $\mathbf{E}$ & 3254.4343 & 1627.7208 & 3237.4077 & 1619.2075 & 3236.4237 & 1618.7155 & 30 \\
\hline 7 & 758.3203 & 379.6638 & & & 740.3097 & 370.6585 & $\mathbf{E}$ & 3125.3917 & 1563.1995 & 3108.3651 & 1554.6862 & 3107.3811 & 1554.1942 & 29 \\
\hline 8 & 886.4153 & 443.7113 & 869.3887 & 435.1980 & 868.4047 & 434.7060 & $\mathbf{K}$ & 2996.3491 & 1498.6782 & 2979.3225 & 1490.1649 & 2978.3385 & 1489.6729 & 28 \\
\hline 9 & 983.4680 & 492.2376 & 966.4415 & 483.7244 & 965.4575 & 483.2324 & $\mathbf{P}$ & 2868.2541 & 1434.6307 & 2851.2276 & 1426.1174 & 2850.2436 & 1425.6254 & 27 \\
\hline 10 & 1112.5106 & 556.7589 & 1095.4841 & 548.2457 & 1094.5000 & 547.7537 & $\mathbf{E}$ & 2771.2014 & 1386.1043 & 2754.1748 & 1377.5910 & 2753.1908 & 1377.0990 & 26 \\
\hline 11 & 1209.5634 & 605.2853 & 1192.5368 & 596.7721 & 1191.5528 & 596.2800 & $\mathbf{P}$ & 2642.1588 & 1321.5830 & 2625.1322 & 1313.0697 & 2624.1482 & 1312.5777 & 25 \\
\hline 12 & 1324.5903 & 662.7988 & 1307.5638 & 654.2855 & 1306.5798 & 653.7935 & D & 2545.1060 & 1273.0566 & 2528.0794 & 1264.5434 & 2527.0954 & 1264.0514 & 24 \\
\hline 13 & 1381.6118 & 691.3095 & 1364.5852 & 682.7963 & 1363.6012 & 682.3042 & G & 2430.0791 & 1215.5432 & 2413.0525 & \begin{tabular}{|l|}
1207.0299 \\
\end{tabular} & 2412.0685 & \begin{tabular}{|l|}
1206.5379 \\
\end{tabular} & 23 \\
\hline 14 & 1468.6438 & 734.8255 & 1451.6173 & 726.3123 & 1450.6332 & 725.8203 & $S$ & 2373.0576 & 1187.0324 & 2356.0310 & 1178.5192 & 2355.0470 & 1178.0272 & 22 \\
\hline 15 & 1555.6758 & 778.3416 & 1538.6493 & 769.8283 & 1537.6653 & 769.3363 & $S$ & 2286.0256 & 1143.5164 & 2268.9990 & 1135.0031 & 2268.0150 & 1134.5111 & 21 \\
\hline 16 & 1684.7184 & 842.8629 & 1667.6919 & 834.3496 & 1666.7079 & 833.8576 & $\mathbf{E}$ & 2198.9935 & 1100.0004 & 2181.9670 & 1091.4871 & 2180.9830 & 1090.9951 & 20 \\
\hline 17 & 1813.7610 & 907.3841 & 1796.7345 & 898.8709 & 1795.7505 & 898.3789 & $\mathbf{E}$ & 2069.9509 & 1035.4791 & 2052.9244 & 1026.9658 & 2051.9404 & 1026.4738 & 19 \\
\hline 18 & 1900.7931 & 950.9002 & 1883.7665 & 942.3869 & 1882.7825 & 941.8949 & $S$ & 1940.9083 & 578 & 1923. & 962.4445 & 1922.8978 & 961.9525 & 18 \\
\hline 19 & 2013.8771 & 1007.4422 & \begin{tabular}{|l|}
1996.8506 \\
\end{tabular} & 998.9289 & 1995.8666 & 998.4369 & I & 1853.8763 & 927.4418 & 1836.8498 & 918.9285 & 1835.8658 & 918.4365 & 17 \\
\hline 20 & 2100.9091 & 1050.9582 & 2083.8826 & 1042.4449 & 2082.8986 & 1041.9529 & $\mathrm{~S}$ & 1740.7923 & 870.8998 & 1723.7657 & 862.3865 & 1722.7817 & 861.8945 & 16 \\
\hline 21 & 2201.9568 & \begin{tabular}{|l|}
1101.4820 \\
\end{tabular} & 2184.9303 & 1092.9688 & 2183.9463 & \begin{tabular}{|l|}
1092.4768 \\
\end{tabular} & $T$ & 1653.7602 & 827.3838 & 1636.7337 & 818.8705 & 1635.7497 & 818.3785 & 15 \\
\hline 22 & 2301.0252 & \begin{tabular}{|l|}
1151.0163 \\
\end{tabular} & 2283.9987 & 1142.5030 & 2283.0147 & 1142.0110 & $\mathbf{V}$ & 1552.7126 & 776.8599 & 1535.6860 & 768.3466 & 1534.7020 & 767.8546 & 14 \\
\hline 23 & 2430.0678 & 1215.5376 & 2413.0413 & 1207.0243 & 2412.0573 & 1206.5323 & E & 1453.6441 & 727.3257 & 1436.6176 & 718.8124 & 1435.6336 & 718.3204 & 13 \\
\hline 24 & 2559.1104 & \begin{tabular}{|l|}
1280.0588 \\
\end{tabular} & 2542.0839 & 1271.5456 & 2541.0999 & 1271.0536 & $\mathbf{E}$ & \begin{tabular}{|l|}
1324.6015 \\
\end{tabular} & 662.8044 & 1307.5750 & 654.2911 & 1306.5910 & 653.7991 & 12 \\
\hline 25 & 2656.1632 & 1328.5852 & 2639.1366 & 1320.0720 & 2638.1526 & 1319.5799 & $\mathbf{P}$ & 1195.5590 & 598.2831 & 1178.5324 & 589.7698 & 1177.5484 & 589.2778 & 11 \\
\hline 26 & 2785.2058 & 1393.1065 & 2768.1792 & 1384.5933 & 2767.1952 & 1384.1012 & $\mathbf{E}$ & 1098.5062 & 549.7567 & 1081.4796 & 541.2435 & 1080.4956 & 540.7514 & 10 \\
\hline 27 & 2900.2327 & 1450.6200 & 2883.2062 & 1442.1067 & 2882.2221 & 1441.6147 & $\mathbf{N}$ & 969.4636 & 485.2354 & 952.4370 & 476.7222 & 951.4530 & 476.2302 & 9 \\
\hline 28 & 3029.2753 & 1515.1413 & 3012.2488 & 1506.6280 & 3011.2647 & 1506.1360 & $\mathbf{E}$ & 854.4367 & 427.7220 & 837.4101 & 419.2087 & 836.4261 & 418.7167 & 8 \\
\hline 29 & 3130.3230 & 1565.6651 & 3113.2964 & 1557.1519 & 3112.3124 & 1556.6598 & $T$ & 725.3941 & 363.2007 & 708.3675 & 354.6874 & 707.3835 & 354.1954 & 7 \\
\hline 30 & 3227.3758 & 1614.1915 & 3210.3492 & 1605.6782 & 3209.3652 & 1605.1862 & $\mathbf{P}$ & 624.3464 & 312.6768 & 607.3198 & 304.1636 & 606.3358 & 303.6715 & 6 \\
\hline 31 & 3324.4285 & 1662.7179 & 3307.4020 & 1654.2046 & 3306.4179 & 1653.7126 & $\mathbf{P}$ & 527.2936 & 264.1504 & 510.2671 & 255.6372 & 509.2831 & 255.1452 & 5 \\
\hline 32 & |3395.4656 & 1698.2365 & |3378.4391| & 1689.7232 & 3377.4551 & 1689.2312 & A & 430.2409 & 215.6241 & 413.2143 & 207.1108 & 412.2303 & 206.6188 & 4 \\
\hline
\end{tabular}




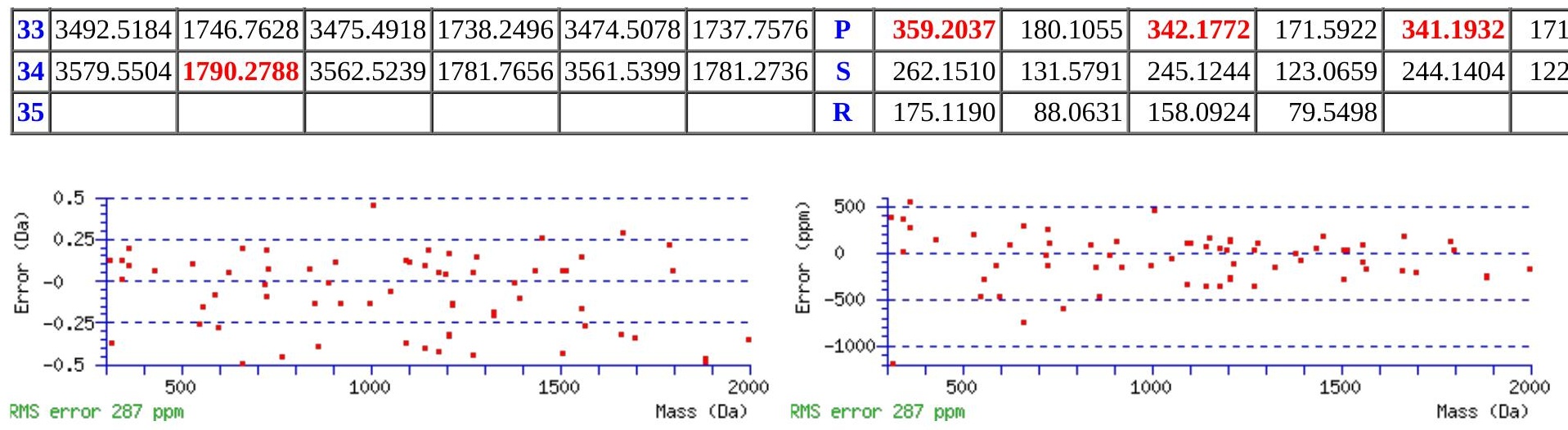

\section{All matches to this query}

\begin{tabular}{|l|c|c|l|}
\hline Score & Mr(calc): & Delta & \multicolumn{1}{c|}{ Sequence } \\
\hline 45.2 & 3752.6548 & 0.0144 & LAEGEEEKPEPDGSSEESISTVEEPENETPPAPSR \\
\hline 31.5 & 3751.6707 & 0.9984 & LAEGEEEKPEPDGSSEESISTVEEPENETPPAPSR \\
\hline 3.3 & 3752.6848 & -0.0156 & ADLEWLRGIGWMPNDSVSVNHAKHAADIFSEK \\
\hline 3.0 & 3750.6285 & 2.0406 & AMIYDKQIMTSSAKVVIIYGEMNSTLEVTSR \\
\hline 3.0 & 3750.6285 & 2.0406 & AMIYDKQIMTSSAKVVIIYGEMNSTLEVTSR \\
\hline 3.0 & 3750.6991 & 1.9700 & AGALPLPPRHASTGAMHPLRSGLLNSTSDSDLVR \\
\hline 2.5 & 3752.6389 & 0.0303 & SSENGYSVAGILSSPNIQKLLANTSLNLEQKR \\
\hline 2.1 & 3751.6736 & 0.9955 & LAEQQVTXLSLGMHSLNRQGLVNTCPLHDSNK \\
\hline 2.0 & 3750.6809 & 1.9882 & NITPELMTYVVSASISNASIENLADPVIIVLK \\
\hline 1.9 & 3750.6751 & 1.9940 & WVYYCLLNITLSDLLTGLAYVVNVLLSGTR \\
\hline
\end{tabular}

Spectrum No: 747; Query: 7; Rank: 1

\section{Peptide View}

MS/MS Fragmentation of SNLTVLR

Found in IPI00193490, Tax_Id=10116 Gene_Symbol=Itgb5 89 kDa protein

Match to Query 7: 802.452988 from(402.233770,2+)

Title: 091008RatKidney_NH4Format01_30.863.863.2.dta

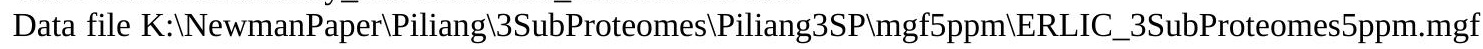

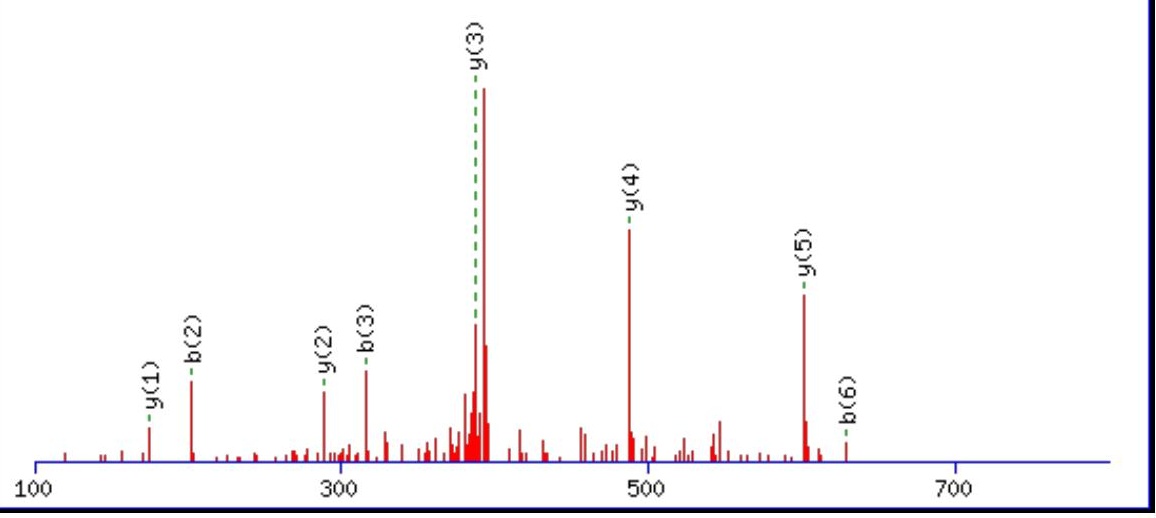




$\begin{array}{rrrr}800 & 1000 & 1200 & 1400\end{array}$

\begin{tabular}{rrr}
\hline 1600 & 1800 & 2000
\end{tabular}

Monoisotopic mass of neutral peptide $\operatorname{Mr}($ calc): 802.4548

Fixed modifications: Carbamidomethyl (C)

Variable modifications:

N2 : Deamidated $\mathrm{N}(\mathrm{N})$

Ions Score: 45 Expect : 0.003

Matches (Bold Red): 8/64 fragment ions using 14 most intense peaks

\begin{tabular}{|c|c|c|c|c|c|c|c|c|c|c|c|c|c|c|}
\hline \# & b & $\mathbf{b}^{++}$ & b* & $\mathbf{b}^{*++}$ & $\mathbf{b}^{0}$ & $\mathbf{b}^{\mathbf{0 + +}}$ & Seq. & $\mathbf{y}$ & $y^{++}$ & $\mathbf{y}^{*}$ & $\mathbf{y}^{*^{++}}$ & $\mathbf{y}^{0}$ & $\mathbf{y}^{0++}$ & \# \\
\hline 1 & 88.0393 & 44.5233 & & & 70.0287 & 35.5180 & $\mathrm{~S}$ & & & & & & & 7 \\
\hline 2 & 203.0662 & 102.0368 & 186.0397 & 93.5235 & 185.0557 & 93.0315 & $\mathbf{N}$ & 716.4301 & 358.7187 & 699.4036 & 350.2054 & 698.4195 & 349.7134 & 6 \\
\hline 4 & 417.1980 & 209.1026 & 400.1714 & 200.5894 & 399.1874 & 200.0973 & $\mathbf{T}$ & 488.3191 & 244.6632 & 471.2926 & 236.1499 & 470.3085 & 235.6579 & 4 \\
\hline 5 & 516.2664 & 258.6368 & 499.2398 & 250.1236 & 498.2558 & 249.6316 & $\mathbf{V}$ & 387.2714 & 194.1394 & 370.2449 & 185.6261 & & & 3 \\
\hline 7 & & & & & & & $\mathbf{R}$ & 175.1190 & 88.0631 & 158.0924 & 79.5498 & & & 1 \\
\hline
\end{tabular}$$
\text { 寊 }
$$

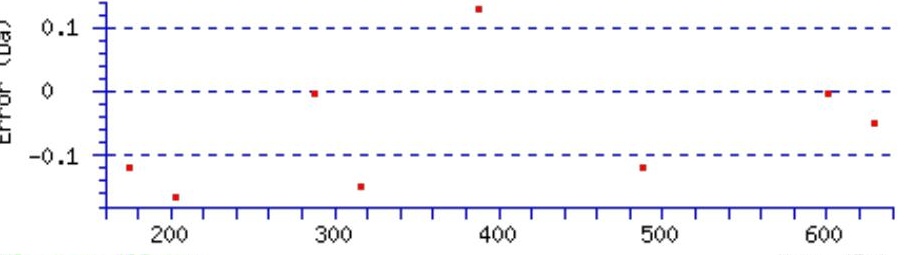

RMS error 438 ppm

Mass (Da)

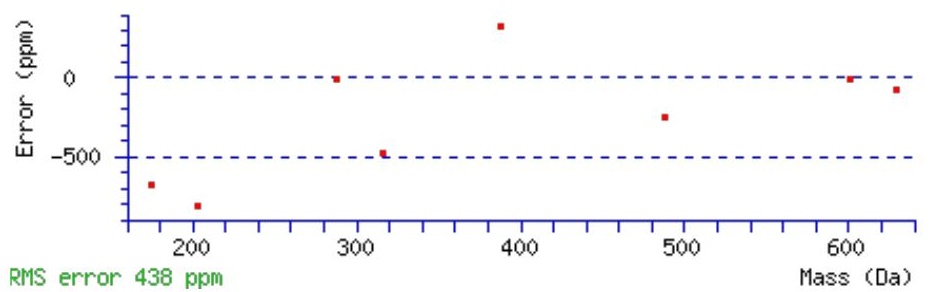

All matches to this query

\begin{tabular}{|l|l|l|c|}
\hline Score & Mr(calc): & Delta & Sequence \\
\hline 45.2 & 802.4548 & -0.0019 & SNLTVLR \\
\hline
\end{tabular}




\begin{tabular}{|l|l|l|l|}
29.9 & 802.4548 & -0.0019 & DSLISIR \\
\hline 29.9 & 802.4548 & -0.0018 & SNISILR \\
\hline 27.1 & 802.4549 & -0.0019 & DSLLTVR \\
\hline 21.8 & 800.4392 & 2.0138 & $\underline{\text { AELAELR }}$ \\
\hline 14.8 & 802.4548 & -0.0019 & $\underline{\text { LDSISLR }}$ \\
\hline 14.8 & 802.4548 & -0.0019 & VESLSIR \\
\hline 14.7 & 802.4548 & -0.0019 & TTAILER \\
\hline 14.3 & 802.4548 & -0.0018 & NSSLILR \\
\hline 13.0 & 802.4483 & 0.0047 & $\underline{\text { AMALKGGR }}$ \\
\hline
\end{tabular}

Spectrum No: 748; Query: 1386; Rank: 1

\section{Peptide View}

MS/MS Fragmentation of RIQNYSVMAICDYDK

Found in IPI00208422, Tax_Id=10116 Gene_Symbol=Dpp4 Dipeptidyl peptidase 4

Match to Query 1386: 1875.854412 from(626.292080,3+)

Title: 100101RatKid_NS_deglyco_20.3001.3001.3.dta

Data file K:\NewmanPaper|Piliang|3SubProteomes\Piliang3SP\mgf5ppm\ERLIC_3SubProteomes5ppm.mgf
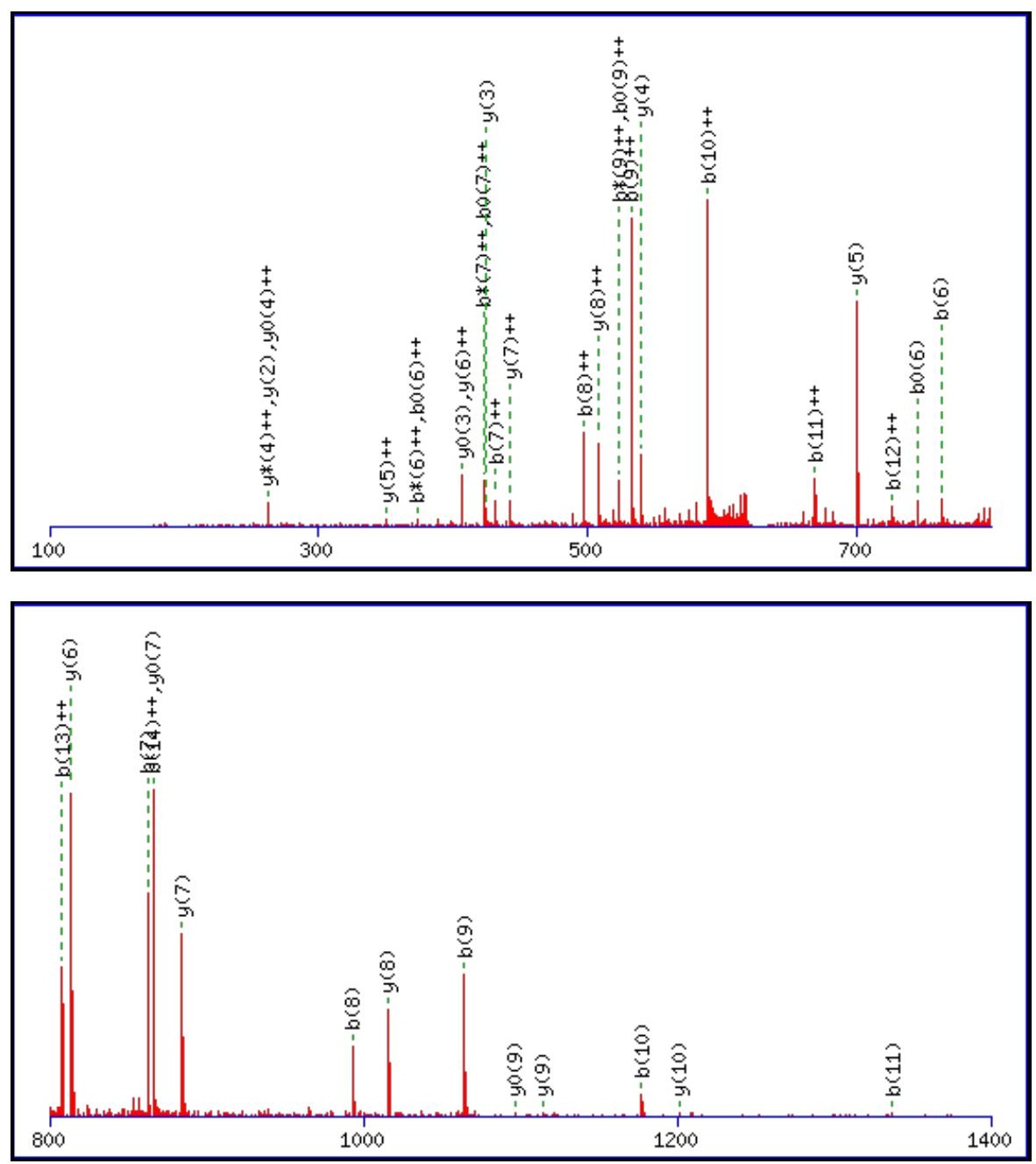


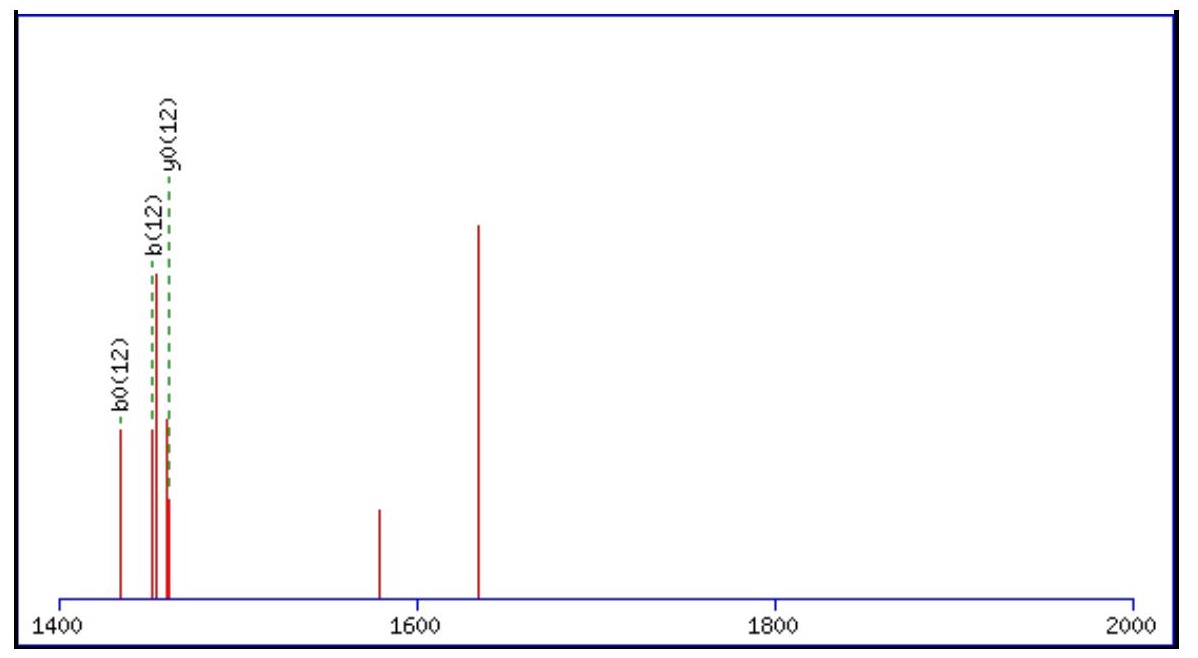

Monoisotopic mass of neutral peptide $\operatorname{Mr}($ calc): 1875.8495

Fixed modifications: Carbamidomethyl (C)

Variable modifications:

N4 : Deamidated $\mathrm{N}(\mathrm{N})$

Ions Score: 45 Expect: 0.0069

Matches (Bold Red): 42/156 fragment ions using 73 most intense peaks

\begin{tabular}{|c|c|c|c|c|c|c|c|c|c|c|c|c|c|c|}
\hline \# & b & $\mathbf{b}^{++}$ & $\mathbf{b}^{*}$ & $\mathbf{b}^{*^{++}}$ & $\mathbf{b}^{0}$ & $\mathbf{b}^{\mathbf{0 + +}}$ & Seq. & $\mathbf{y}$ & $\mathbf{y}^{++}$ & $\mathrm{y}^{*}$ & $\mathbf{y}^{*^{++}}$ & $\mathbf{y}^{0}$ & $\mathbf{y}^{0++}$ & \# \\
\hline 1 & 157.1084 & 79.0578 & 140.0818 & 70.5446 & & & $\mathbf{R}$ & & & & & & & 15 \\
\hline 2 & 270.1925 & 135.5999 & 253.1659 & 127.0866 & & & I & 1720.7557 & 860.8815 & 1703.7291 & 852.3682 & 1702.7451 & 851.8762 & 14 \\
\hline 3 & 398.2510 & 199.6292 & 381.2245 & 191.1159 & & & $\mathbf{Q}$ & 1607.6716 & 804.3394 & 1590.6451 & 795.8262 & 1589.6611 & 795.3342 & 13 \\
\hline 4 & 513.2780 & 257.1426 & 496.2514 & 248.6293 & & & $\mathbf{N}$ & 1479.6130 & 740.3102 & 1462.5865 & 731.7969 & 1461.6025 & 731.3049 & 12 \\
\hline 5 & 676.3413 & 338.6743 & 659.3147 & 330.1610 & & & $\mathbf{Y}$ & 1364.5861 & 682.7967 & 1347.5596 & 674.2834 & 1346.5755 & 673.7914 & 11 \\
\hline 6 & 763.3733 & 382.1903 & 746.3468 & 373.6770 & 745.3628 & 373.1850 & $\mathbf{S}$ & 1201.5228 & 601.2650 & 1184.4962 & 592.7518 & 1183.5122 & 592.2597 & 10 \\
\hline 7 & 862.4417 & 431.7245 & 845.4152 & 423.2112 & 844.4312 & 422.7192 & $\mathbf{V}$ & 1114.4907 & 557.7490 & 1097.4642 & 549.2357 & 1096.4802 & 548.7437 & 9 \\
\hline 8 & 993.4822 & 497.2447 & 976.4557 & 488.7315 & 975.4717 & 488.2395 & $\mathbf{M}$ & 1015.4223 & 508.2148 & 998.3958 & 499.7015 & 997.4118 & 499.2095 & 8 \\
\hline 9 & 1064.5193 & 532.7633 & 1047.4928 & 524.2500 & 1046.5088 & 523.7580 & A & 884.3818 & 442.6946 & 867.3553 & 434.1813 & 866.3713 & 433.6893 & 7 \\
\hline 10 & 1177.6034 & 589.3053 & 1160.5768 & 580.7921 & 1159.5928 & 580.3001 & I & 813.3447 & 407.1760 & 796.3182 & 398.6627 & 795.3342 & 398.1707 & 6 \\
\hline 11 & 1337.6340 & 669.3207 & 1320.6075 & 660.8074 & 1319.6235 & 660.3154 & $\mathrm{C}$ & 700.2607 & 350.6340 & 683.2341 & 342.1207 & 682.2501 & 341.6287 & 5 \\
\hline 12 & 1452.6610 & 726.8341 & 1435.6344 & 718.3209 & 1434.6504 & 717.8289 & D & 540.2300 & 270.6186 & 523.2035 & 262.1054 & 522.2195 & 261.6134 & 4 \\
\hline 13 & 1615.7243 & 808.3658 & 1598.6978 & 799.8525 & 1597.7138 & 799.3605 & $\mathbf{Y}$ & 425.2031 & 213.1052 & 408.1765 & 204.5919 & 407.1925 & 204.0999 & 3 \\
\hline 14 & 1730.7513 & 865.8793 & 1713.7247 & 857.3660 & 1712.7407 & 856.8740 & D & 262.1397 & 131.5735 & 245.1132 & 123.0602 & 244.1292 & 122.5682 & 2 \\
\hline 15 & & & & & & & $\mathbf{K}$ & 147.1128 & 74.0600 & 130.0863 & 65.5468 & & & 1 \\
\hline
\end{tabular}
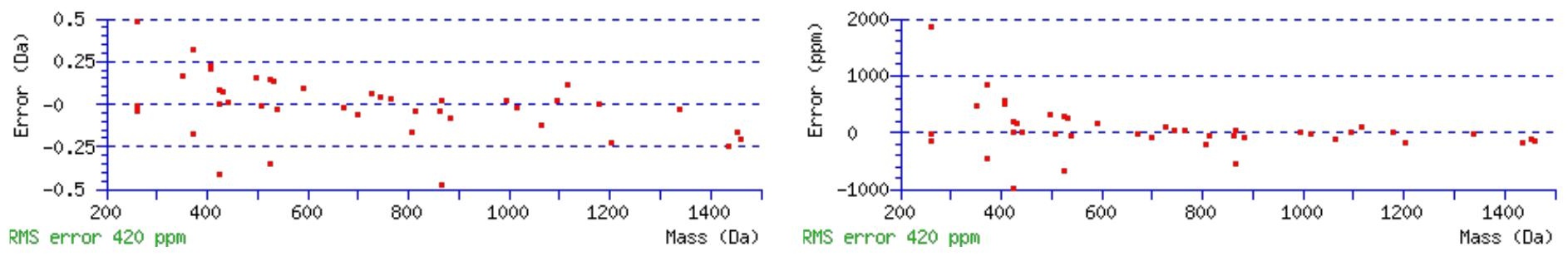

\section{All matches to this query}

\begin{tabular}{|l|l|l|l|}
\hline Score & Mr(calc): & Delta & \multicolumn{1}{c|}{ Sequence } \\
\hline 45.1 & 1875.8495 & 0.0049 & $\underline{\text { RIQNYSVMAICDYDK }}$ \\
\hline 23.7 & 1874.8655 & 0.9889 & $\underline{\text { RIQNYSVMAICDYDK }}$ \\
\hline 14.1 & 1875.8590 & -0.0046 & $\underline{\text { MNNGLGLIFSNGTKWK }}$ \\
\hline 12.7 & 1875.8689 & -0.0145 & $\underline{\text { LKENLFSACISEKEK }}$ \\
\hline 10.8 & 1875.8380 & 0.0164 & $\underline{\text { KLTDVEAQVLNQTTR }}$ \\
\hline 10.5 & 1875.8590 & -0.0046 & $\underline{\text { MNNGLGLIFSNGTKWK }}$ \\
\hline & & & \\
\hline
\end{tabular}




\begin{tabular}{|l|l|l|l|}
9.5 & 1875.8438 & 0.0106 & TQYNITITVSDMGTPR \\
\hline 9.5 & 1875.8438 & 0.0106 & TQYNITITVSDMGTPR \\
\hline 9.4 & 1875.8380 & 0.0164 & VSPSIPSSTQIRDTTK \\
\hline 9.4 & 1875.8380 & 0.0164 & VSPSIPSSTQIRDTTK \\
\hline
\end{tabular}

Spectrum No: 749; Query: 1892; Rank: 1

\section{Peptide View}

MS/MS Fragmentation of RSGPSDEGEEEMEDGTVTNGS

Found in IPI00192279, Tax_Id=10116 Gene_Symbol=- $28 \mathrm{kDa}$ protein

Match to Query 1892: 2198.836288 from(1100.425420,2+)

Title: 091008RatKidney_NoSalt_27.499.499.2.dta

Data file K:INewmanPaper|Piliangl3SubProteomes\Piliang3SP\mgf5ppm\ERLIC_3SubProteomes5ppm.mgf
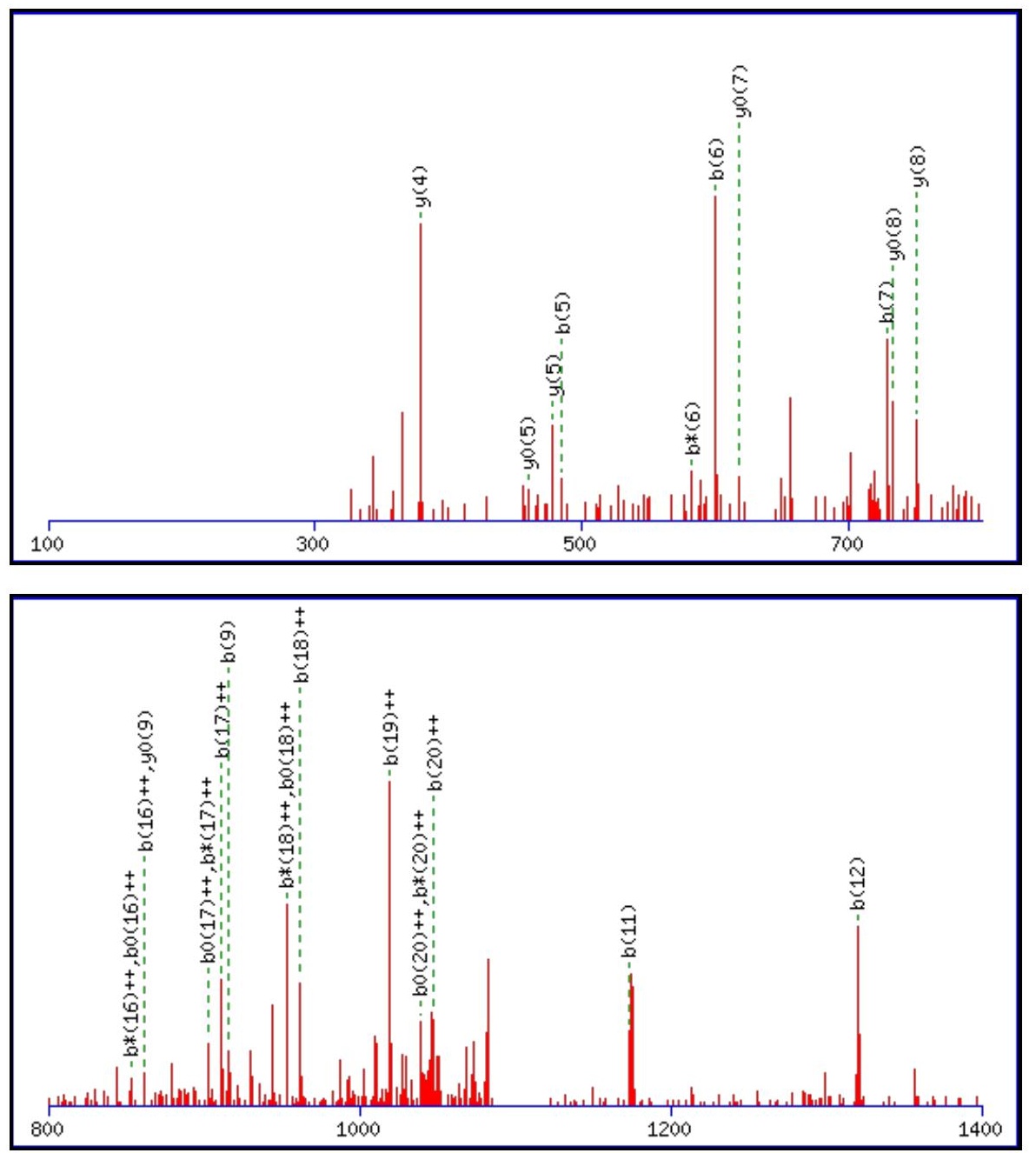


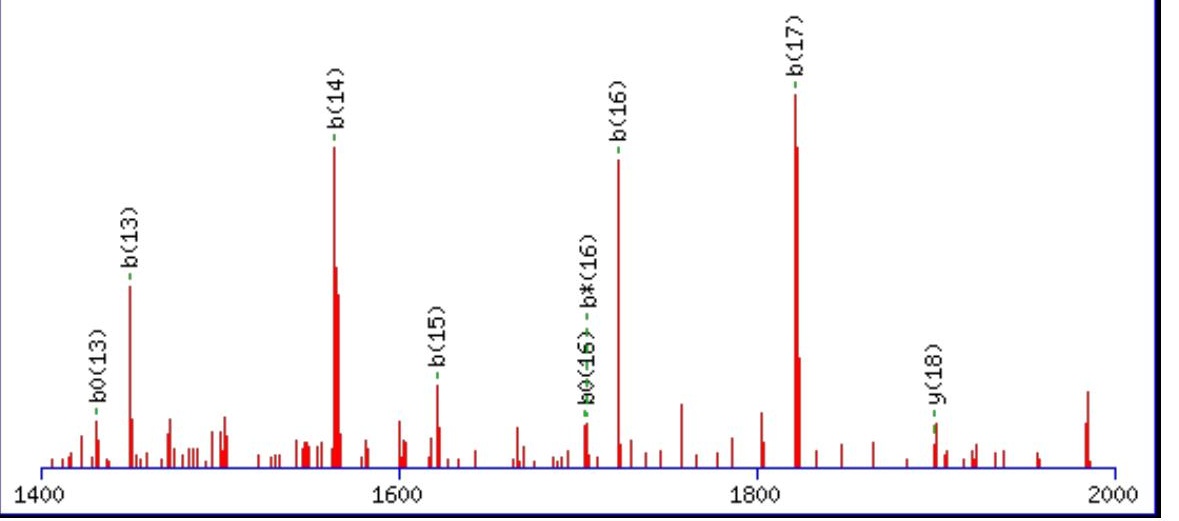

Monoisotopic mass of neutral peptide $\operatorname{Mr}($ calc): 2198.8393

Fixed modifications: Carbamidomethyl (C)

Variable modifications:

M12 : Oxidation (M)

N19 : Deamidated_N (N)

Ions Score: 45 Expect: 0.0048

Matches (Bold Red): 36/234 fragment ions using 82 most intense peaks

\begin{tabular}{|c|c|c|c|c|c|c|c|c|c|c|c|c|c|c|}
\hline \# & b & $\mathbf{b}^{++}$ & $\mathbf{b}^{*}$ & $\mathbf{b}^{*^{++}}$ & $\mathbf{b}^{\mathbf{0}}$ & $\mathbf{b}^{0++}$ & Seq. & $\mathbf{y}$ & $y^{++}$ & $\mathbf{y}^{*}$ & $\mathbf{y}^{*^{++}}$ & $\mathbf{y}^{0}$ & $y^{0++}$ & \# \\
\hline 1 & 157.1084 & 79.0578 & 140.0818 & 70.5446 & & & $\mathbf{R}$ & & & & & & & 21 \\
\hline 2 & 244.1404 & 122.5738 & 227.1139 & 114.0606 & 226.1298 & 113.5686 & S & 2043.7455 & 1022.3764 & 2026.7190 & 1013.8631 & 2025.7349 & 1013.3711 & 20 \\
\hline 3 & 301.1619 & 151.0846 & 284.1353 & 142.5713 & 283.1513 & 142.0793 & G & 1956.7135 & 978.8604 & 1939.6869 & 970.3471 & 1938.7029 & 969.8551 & 19 \\
\hline 4 & 398.2146 & 199.6110 & 381.1881 & 191.0977 & 380.2041 & 190.6057 & $\mathbf{P}$ & 1899.6920 & 950.3496 & 1882.6655 & 941.8364 & 1881.6815 & 941.3444 & 18 \\
\hline 5 & 485.2467 & 243.1270 & 468.2201 & 234.6137 & 467.2361 & 234.1217 & $\mathrm{~S}$ & 1802.6393 & 901.8233 & 1785.6127 & 893.3100 & 1784.6287 & 892.8180 & 17 \\
\hline 6 & 600.2736 & 300.6404 & 583.2471 & 292.1272 & 582.2630 & 291.6352 & D & 1715.6072 & 858.3073 & 1698.5807 & 849.7940 & 1697.5967 & 849.3020 & 16 \\
\hline 7 & 729.3162 & 365.1617 & 712.2897 & 356.6485 & 711.3056 & 356.1565 & $\mathbf{E}$ & 1600.5803 & 800.7938 & 1583.5537 & 792.2805 & 1582.5697 & 791.7885 & 15 \\
\hline 8 & 786.3377 & 393.6725 & 769.3111 & 385.1592 & 768.3271 & 384.6672 & G & 1471.5377 & 736.2725 & 1454.5111 & 727.7592 & 1453.5271 & 727.2672 & 14 \\
\hline 9 & 915.3803 & 458.1938 & 898.3537 & 449.6805 & 897.3697 & 449.1885 & $\mathbf{E}$ & 1414.5162 & 707.7618 & 1397.4897 & 699.2485 & 1396.5057 & 698.7565 & 13 \\
\hline 10 & 1044.4229 & 522.7151 & 1027.3963 & 514.2018 & 1026.4123 & 513.7098 & $\mathbf{E}$ & 1285.4736 & 643.2405 & 1268.4471 & 634.7272 & 1267.4631 & 634.2352 & 12 \\
\hline 11 & 1173.4654 & 587.2364 & 1156.4389 & 578.7231 & 1155.4549 & 578.2311 & $E$ & 1156.4310 & 578.7192 & 1139.4045 & 570.2059 & 1138.4205 & 569.7139 & 11 \\
\hline 12 & 1320.5009 & 660.7541 & 1303.4743 & 652.2408 & 1302.4903 & 651.7488 & $\mathbf{M}$ & 1027.3884 & 514.1979 & 1010.3619 & 505.6846 & 1009.3779 & 505.1926 & 10 \\
\hline 13 & 1449.5434 & 725.2754 & 1432.5169 & 716.7621 & 1431.5329 & 716.2701 & $\mathbf{E}$ & 880.3530 & 440.6802 & 863.3265 & 432.1669 & 862.3425 & 431.6749 & 9 \\
\hline 14 & 1564.5704 & 782.7888 & 1547.5438 & 774.2756 & 1546.5598 & 773.7836 & D & 751.3104 & 376.1589 & 734.2839 & 367.6456 & 733.2999 & 367.1536 & 8 \\
\hline 15 & 1621.5919 & 811.2996 & 1604.5653 & 802.7863 & 1603.5813 & 802.2943 & G & 636.2835 & 318.6454 & 619.2570 & 310.1321 & 618.2729 & 309.6401 & 7 \\
\hline 16 & 1722.6395 & 861.8234 & 1705.6130 & 853.3101 & 1704.6290 & 852.8181 & $\mathbf{T}$ & 579.2620 & 290.1347 & 562.2355 & 281.6214 & 561.2515 & 281.1294 & 6 \\
\hline 17 & 1821.7079 & 911.3576 & 1804.6814 & 902.8443 & 1803.6974 & 902.3523 & V & 478.2144 & 239.6108 & 461.1878 & 231.0975 & 460.2038 & 230.6055 & 5 \\
\hline 18 & 1922.7556 & 961.8815 & 1905.7291 & 953.3682 & 1904.7451 & 952.8762 & $\mathbf{T}$ & 379.1459 & 190.0766 & 362.1194 & 181.5633 & 361.1354 & 181.0713 & 4 \\
\hline 19 & 2037.7826 & 1019.3949 & 2020.7560 & 1010.8816 & 2019.7720 & 1010.3896 & $\mathbf{N}$ & 278.0983 & 139.5528 & 261.0717 & 131.0395 & 260.0877 & 130.5475 & 3 \\
\hline 20 & 2094.8040 & 1047.9057 & 2077.7775 & 1039.3924 & 2076.7935 & 1038.9004 & G & 163.0713 & 82.0393 & & & 145.0608 & 73.0340 & 2 \\
\hline 21 & & & & & & & S & 106.0499 & 53.5286 & & & 88.0393 & 44.5233 & 1 \\
\hline
\end{tabular}
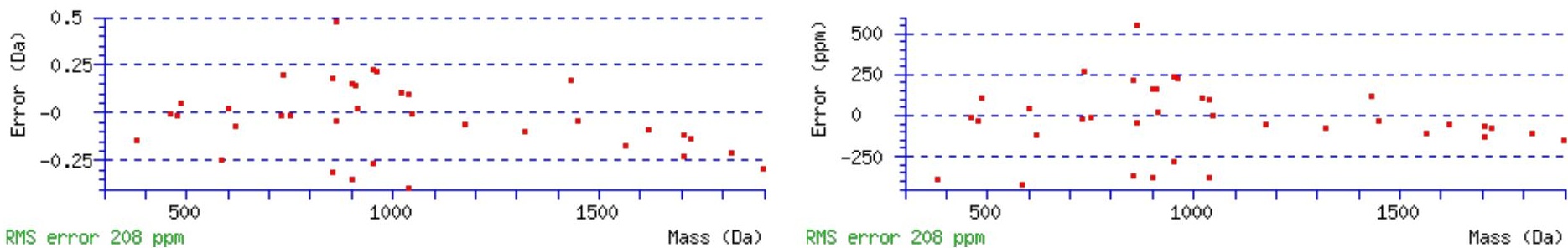

All matches to this query 


\section{|45.0|2198.8393|-0.0031|RSGPSDEGEEEMEDGTVTNGS}

Spectrum No: 750; Query: 1350; Rank: 1

\section{Peptide View}

MS/MS Fragmentation of LSHDANETLPLHLYVK

Found in IPI00370450, Tax_Id=10116 Gene_Symbol=Plxnb2 plexin B2

Match to Query 1350: 1849.960736 from(463.497460,4+)

Title: 091008RatKidney_NH4Format01_26.2391.2391.4.dta

Data file K:INewmanPaper|Piliang\3SubProteomes\Piliang3SP\mgf5ppm\ERLIC_3SubProteomes5ppm.mgf
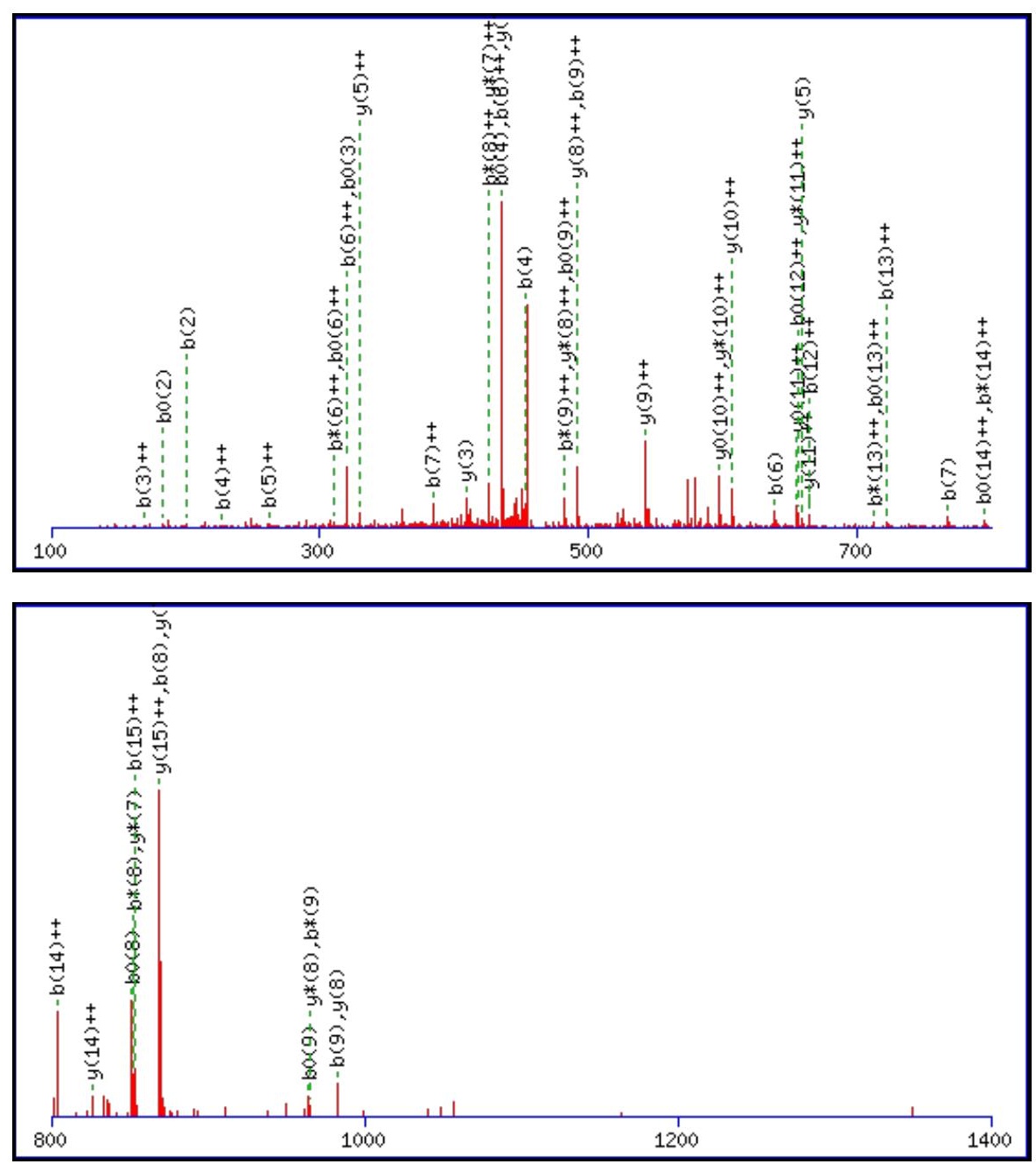
Monoisotopic mass of neutral peptide $\operatorname{Mr}($ calc): 1849.9574

Fixed modifications: Carbamidomethyl (C)

Variable modifications:

N6 : Deamidated $\mathrm{N}(\mathrm{N})$

Ions Score: 45 Expect: 0.0047

Matches (Bold Red): 55/152 fragment ions using 94 most intense peaks

\begin{tabular}{|c|c|c|c|c|c|c|c|c|c|c|c|c|c|c|}
\hline \# & b & $\mathbf{b}^{++}$ & $\mathbf{b}^{*}$ & $\mathbf{b}^{*^{++}}$ & $\mathbf{b}^{0}$ & $\mathbf{b}^{0++}$ & Seq. & $\mathbf{y}$ & $\mathbf{y}^{++}$ & $\mathbf{y}^{*}$ & $\mathbf{y}^{*^{++}}$ & $\mathbf{y}^{0}$ & $\mathbf{y}^{0++}$ & \# \\
\hline 1 & 114.0913 & 57.5493 & & & & & $\mathbf{L}$ & & & & & & & 16 \\
\hline 2 & 201.1234 & 101.0653 & & & 183.1128 & 92.0600 & S & 1737.8806 & 869.4439 & 1720.8541 & 860.9307 & 1719.8701 & 860.4387 & 15 \\
\hline 3 & 338.1823 & 169.5948 & & & 320.1717 & 160.5895 & $\mathbf{H}$ & 1650.8486 & 825.9279 & 1633.8220 & 817.4147 & 1632.8380 & 816.9227 & 14 \\
\hline 4 & 453.2092 & 227.1082 & & & 435.1987 & 218.1030 & D & 1513.7897 & 757.3985 & 1496.7631 & 748.8852 & 1495.7791 & 748.3932 & 13 \\
\hline 5 & 524.2463 & 262.6268 & & & 506.2358 & 253.6215 & A & 1398.7627 & 699.8850 & 1381.7362 & 691.3717 & 1380.7522 & 690.8797 & 12 \\
\hline 6 & 639.2733 & 320.1403 & 622.2467 & 311.6270 & 621.2627 & 311.1350 & $\mathbf{N}$ & 1327.7256 & 664.3664 & 1310.6991 & 655.8532 & 1309.7151 & 655.3612 & 11 \\
\hline 7 & 768.3159 & 384.6616 & 751.2893 & 376.1483 & 750.3053 & 375.6563 & $\mathbf{E}$ & 1212.6987 & 606.8530 & 1195.6721 & 598.3397 & 1194.6881 & 597.8477 & 10 \\
\hline 8 & 869.3635 & 435.1854 & 852.3370 & 426.6721 & 851.3530 & 426.1801 & $\mathbf{T}$ & 1083.6561 & 542.3317 & 1066.6295 & 533.8184 & 1065.6455 & 533.3264 & 9 \\
\hline 9 & 982.4476 & 491.7274 & 965.4211 & 483.2142 & 964.4370 & 482.7222 & $\mathbf{L}$ & 982.6084 & 491.8078 & 965.5819 & 483.2946 & & & 8 \\
\hline 10 & 1079.5004 & 540.2538 & 1062.4738 & 531.7406 & 1061.4898 & 531.2485 & $\mathbf{P}$ & 869.5244 & 435.2658 & 852.4978 & 426.7525 & & & 7 \\
\hline 11 & 1192.5844 & 596.7959 & 1175.5579 & 588.2826 & 1174.5739 & 587.7906 & $\mathbf{L}$ & 772.4716 & 386.7394 & 755.4450 & 378.2262 & & & 6 \\
\hline 12 & 1329.6433 & 665.3253 & 1312.6168 & 656.8120 & 1311.6328 & 656.3200 & $\mathbf{H}$ & 659.3875 & 330.1974 & 642.3610 & 321.6841 & & & 5 \\
\hline 13 & 1442.7274 & 721.8673 & 1425.7009 & 713.3541 & 1424.7168 & 712.8621 & $\mathbf{L}$ & 522.3286 & 261.6679 & 505.3021 & 253.1547 & & & 4 \\
\hline 14 & 1605.7907 & 803.3990 & 1588.7642 & 794.8857 & 1587.7802 & 794.3937 & $\mathbf{Y}$ & 409.2445 & 205.1259 & 392.2180 & 196.6126 & & & 3 \\
\hline 15 & 1704.8592 & 852.9332 & 1687.8326 & 844.4199 & 1686.8486 & 843.9279 & V & 246.1812 & 123.5942 & 229.1547 & 115.0810 & & & 2 \\
\hline 16 & & & & & & & $\mathbf{K}$ & 147.1128 & 74.0600 & 130.0863 & 65.5468 & & & 1 \\
\hline
\end{tabular}$$
\text { ( }
$$

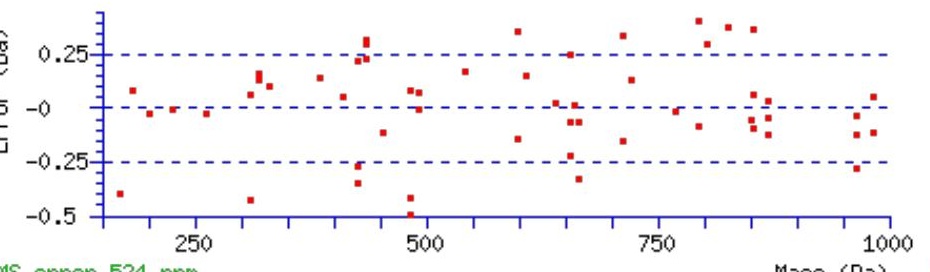

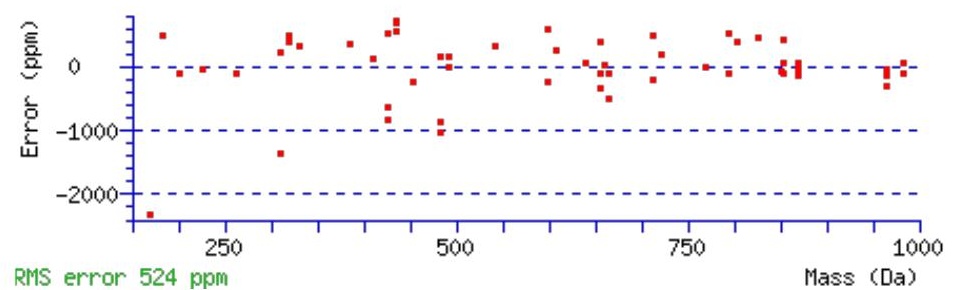

\section{All matches to this query}

\begin{tabular}{|l|l|l|l|}
\hline Score & Mr(calc): & Delta & \multicolumn{1}{c|}{ Sequence } \\
\hline 44.9 & 1849.9574 & 0.0034 & $\underline{\text { LSHDANETLPLHLYVK }}$ \\
\hline 16.9 & 1848.9734 & 0.9874 & $\underline{\text { LSHDANETLPLHLYVK }}$ \\
\hline 13.2 & 1848.9420 & 1.0187 & $\underline{\text { ISMPDVDLNLKGPKVK }}$ \\
\hline 10.0 & 1849.9662 & -0.0055 & $\underline{\text { VVRLSSLNLTNQALNK }}$ \\
\hline 7.3 & 1848.9458 & 1.0149 & $\underline{\text { TAEAVIRSGARLVPSDK }}$ \\
\hline & & & \\
\hline
\end{tabular}




\begin{tabular}{|l|l|l|l|}
6.4 & 1848.9645 & 0.9963 & VKALCSELRGTVAIPR \\
\hline 6.1 & 1848.9458 & 1.0149 & SNDGEERLAVVRLLAK \\
\hline 5.9 & 1848.9532 & 1.0075 & AHAETTKMNVLKVLAK \\
\hline 5.0 & 1849.9662 & -0.0055 & TVIIGSANINDRSLLGK \\
\hline 4.9 & 1848.9598 & 1.0010 & TVQGLEIELQSQLALK \\
\hline
\end{tabular}

Spectrum No: 751; Query: 533; Rank: 1

\section{Peptide View}

MS/MS Fragmentation of LHVQEEQNLTSK

Found in IPI00363534, Tax_Id=10116 Gene_Symbol=Lama1 laminin, alpha 1

Match to Query 533: 1425.712062 from(476.244630,3+)

Title: 100101RatKid_NS_deglyco_13.993.993.3.dta

Data file K:INewmanPaper|Piliangl3SubProteomes\Piliang3SP\mgf5ppm\ERLIC_3SubProteomes5ppm.mgf
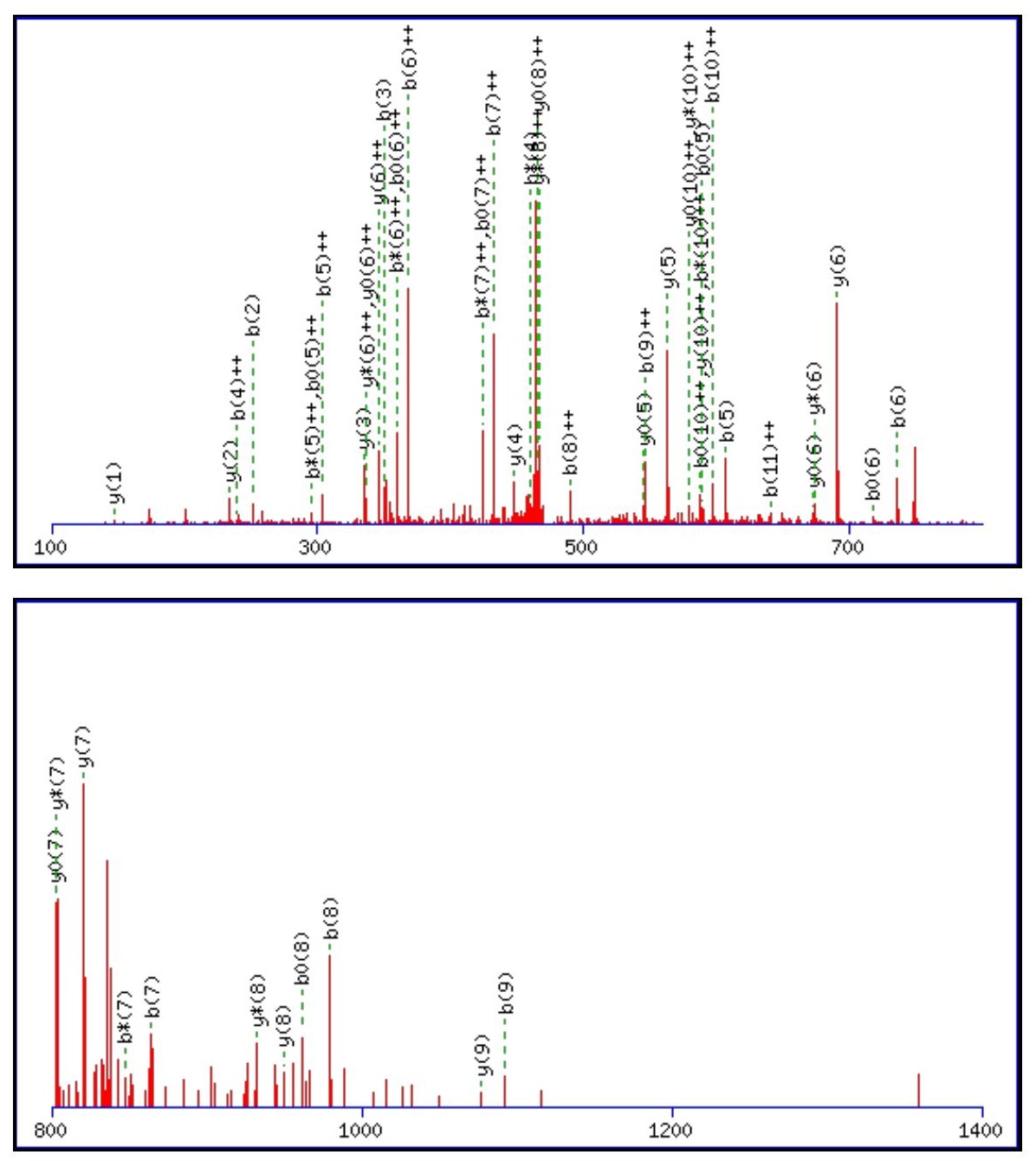


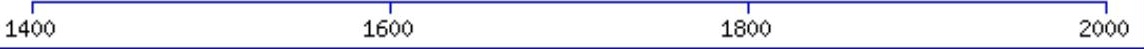

Monoisotopic mass of neutral peptide $\operatorname{Mr}($ calc): 1425.7099

Fixed modifications: Carbamidomethyl (C)

Variable modifications:

N8: : Deamidated_N (N)

Ions Score: 45 Expect: 0.0043

Matches (Bold Red): 51/116 fragment ions using 89 most intense peaks

\begin{tabular}{|c|c|c|c|c|c|c|c|c|c|c|c|c|c|c|}
\hline \# & b & $\mathbf{b}^{++}$ & $\mathbf{b}^{*}$ & $\mathbf{b}^{*^{++}}$ & $\mathbf{b}^{0}$ & $\mathbf{b}^{0++}$ & Seq. & $\mathbf{y}$ & $y^{++}$ & $\mathrm{y}^{*}$ & $y^{*^{++}}$ & $\mathbf{y}^{\mathbf{0}}$ & $y^{0++}$ & \# \\
\hline 1 & 114.0913 & 57.5493 & & & & & $\mathbf{L}$ & & & & & & & 12 \\
\hline 2 & 251.1503 & 126.0788 & & & & & $\mathbf{H}$ & 1313.6332 & 657.3202 & 1296.6066 & 648.8070 & 1295.6226 & 648.3149 & 11 \\
\hline 3 & 350.2187 & 175.6130 & & & & & V & 1176.5743 & 588.7908 & 1159.5477 & 580.2775 & 1158.5637 & 579.7855 & 10 \\
\hline 4 & 478.2772 & 239.6423 & 461.2507 & 231.1290 & & & $\mathbf{Q}$ & 1077.5059 & 539.2566 & 1060.4793 & 530.7433 & 1059.4953 & 530.2513 & 9 \\
\hline 5 & 607.3198 & 304.1636 & 590.2933 & 295.6503 & 589.3093 & 295.1583 & E & 949.4473 & 475.2273 & 932.4207 & 466.7140 & 931.4367 & 466.2220 & 8 \\
\hline 6 & 736.3624 & 368.6849 & 719.3359 & 360.1716 & 718.3519 & 359.6796 & $E$ & 820.4047 & 410.7060 & 803.3781 & 402.1927 & 802.3941 & 401.7007 & 7 \\
\hline 7 & 864.4210 & 432.7141 & 847.3945 & 424.2009 & 846.4104 & \begin{tabular}{|l|}
423.7089 \\
\end{tabular} & $\mathbf{Q}$ & 691.3621 & 346.1847 & 674.3355 & 337.6714 & 673.3515 & 337.1794 & 6 \\
\hline 8 & 979.4479 & 490.2276 & 962.4214 & 481.7143 & 961.4374 & 481.2223 & $\mathbf{N}$ & 563.3035 & 282.1554 & 546.2770 & 273.6421 & 545.2929 & 273.1501 & 5 \\
\hline 9 & 1092.5320 & 546.7696 & 1075.5055 & 538.2564 & 1074.5214 & 537.7644 & $\mathbf{L}$ & 448.2766 & 224.6419 & 431.2500 & 216.1287 & 430.2660 & 215.6366 & 4 \\
\hline 10 & 1193.5797 & 597.2935 & 1176.5531 & 588.7802 & 1175.5691 & 588.2882 & $\mathbf{T}$ & 335.1925 & 168.0999 & 318.1660 & 159.5866 & 317.1819 & 159.0946 & 3 \\
\hline 11 & 1280.6117 & 640.8095 & 1263.5852 & 632.2962 & 1262.6012 & 631.8042 & $S$ & 234.1448 & 117.5761 & 217.1183 & 109.0628 & 216.1343 & 108.5708 & 2 \\
\hline 12 & & & & & & & $\mathbf{K}$ & 147.1128 & 74.0600 & 130.0863 & 65.5468 & & & 1 \\
\hline
\end{tabular}
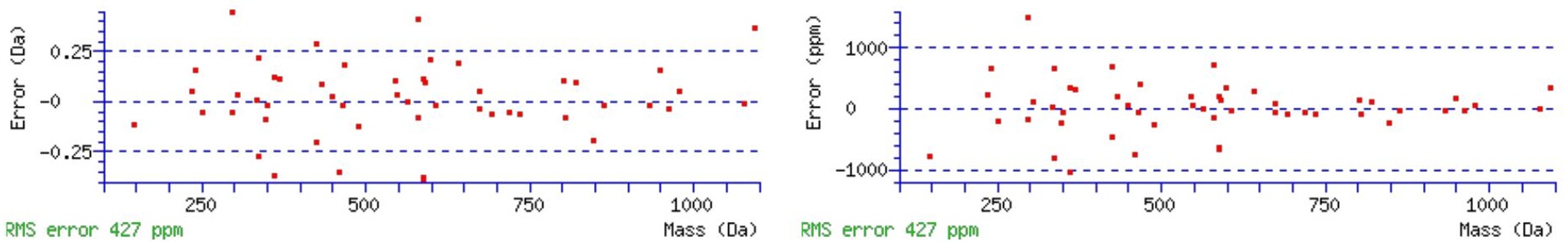

\section{All matches to this query}

\begin{tabular}{|l|l|l|l|}
\hline Score & Mr(calc): & Delta & \multicolumn{1}{|c|}{ Sequence } \\
\hline 44.9 & 1425.7099 & 0.0021 & LHVQEEQNLTSK \\
\hline 7.7 & 1424.7065 & 1.0056 & ALTAKANPDLFGK \\
\hline 4.7 & 1425.6990 & 0.0130 & NHKGGVPPQRTR \\
\hline 2.9 & 1425.7147 & -0.0026 & SLTCKTHSLSHR \\
\hline 1.6 & 1425.7017 & 0.0104 & NNSLPKKFQIR \\
\hline 0.1 & 1423.7129 & 1.9992 & QRFKEEAEMLK \\
\hline
\end{tabular}

Spectrum No: 752; Query: 2651; Rank: 1 


\section{Peptide View}

MS/MS Fragmentation of IDWEGDLGEMESTITSFPNETATIISQYK

Found in IPI00370732, Tax_Id=10116 Gene_Symbol=Pvrl3_predicted 61 kDa protein

Match to Query 2651: 3275.518482 from(1092.846770,3+)

Title: 100101RatKid_NS_deglyco_16.7078.7078.3.dta

Data file K: \NewmanPaper\Piliang \3SubProteomes \Piliang3SP $\backslash$ mgf5ppm\ERLIC_3SubProteomes5ppm.mgf
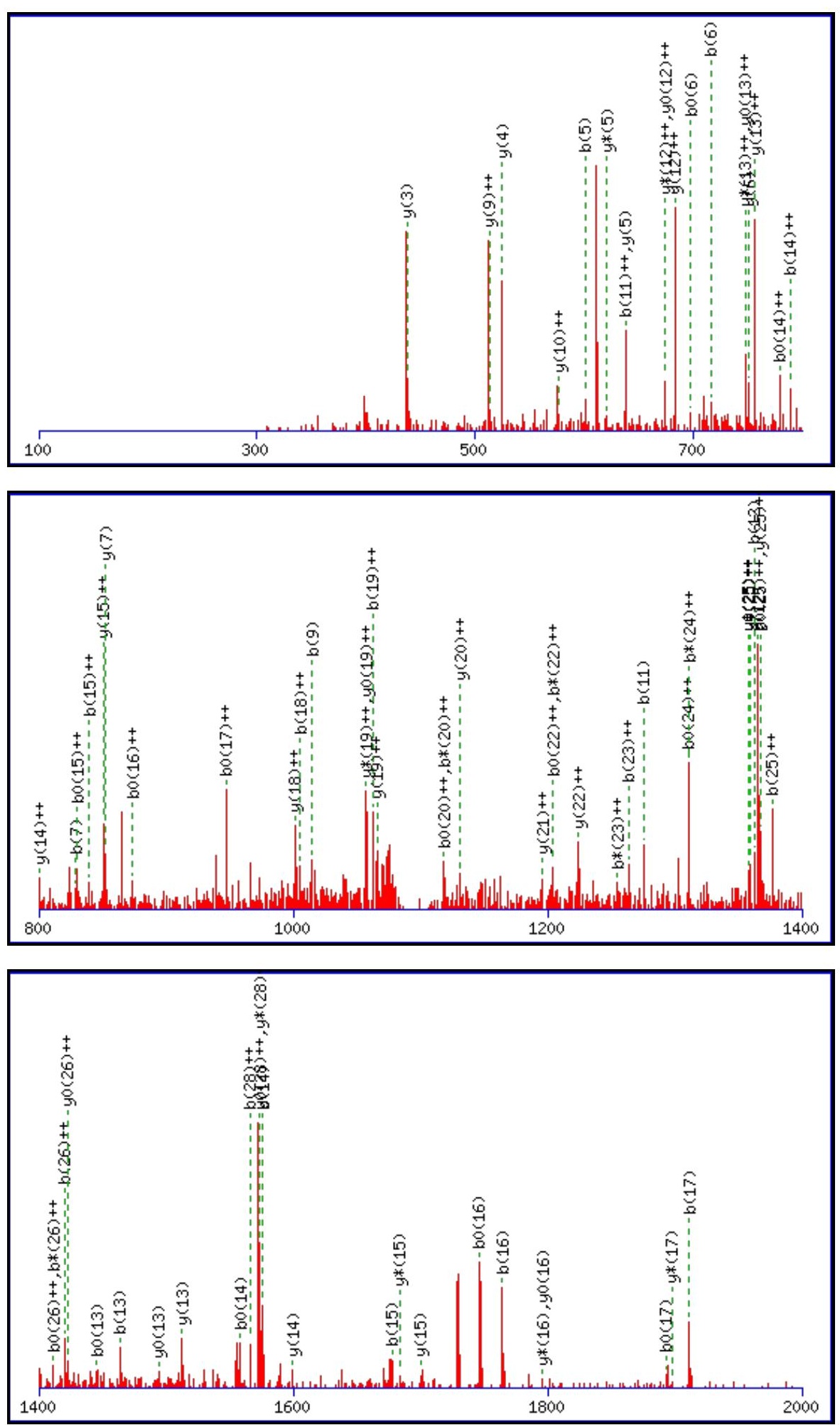

Monoisotopic mass of neutral peptide $\operatorname{Mr}($ calc): 3275.5067

Fixed modifications: Carbamidomethyl (C)

Variable modifications:

N19 : Deamidated_N (N)

Ions Score: 45 Expect: 0.013 
Matches (Bold Red): 77/292 fragment ions using 161 most intense peaks

\begin{tabular}{|c|c|c|c|c|c|c|c|c|c|c|c|c|c|c|}
\hline \# & b & $\mathbf{b}^{++}$ & $\mathbf{b}^{*}$ & $\mathbf{b}^{*^{++}}$ & $\mathbf{b}^{0}$ & $\mathbf{b}^{\mathbf{0 + +}}$ & Seq. & $\mathbf{y}$ & $y^{++}$ & $\mathbf{y}^{*}$ & $\mathrm{y}^{\mathrm{*}^{++}}$ & $\mathbf{y}^{\mathbf{0}}$ & $\mathbf{y}^{\mathbf{0 + +}}$ & \# \\
\hline 1 & 114.0913 & 57.5493 & & & & & $\mathbf{I}$ & & & & & & & 29 \\
\hline 2 & 229.1183 & 115.0628 & & & 211.1077 & 106.0575 & D & 3163.4300 & 1582.2186 & 3146.4034 & 1573.7054 & 3145.4194 & 1573.2133 & \begin{tabular}{|l|l}
28 \\
\end{tabular} \\
\hline 3 & 415.1976 & 208.1024 & & & 397.1870 & 199.0972 & W & 3048.4030 & 1524.7052 & 3031.3765 & 1516.1919 & 3030.3925 & 1515.6999 & 27 \\
\hline 4 & 544.2402 & 272.6237 & & & 526.2296 & 263.6185 & $\mathbf{E}$ & 2862.3237 & 1431.6655 & 2845.2972 & 1423.1522 & 2844.3132 & 1422.6602 & 26 \\
\hline 5 & 601.2617 & 301.1345 & & & 583.2511 & 292.1292 & G & 2733.2811 & 1367.1442 & 2716.2546 & 1358.6309 & 2715.2706 & 1358.1389 & 25 \\
\hline 6 & 716.2886 & 358.6479 & & & 698.2780 & 349.6427 & D & 2676.2597 & 1338.6335 & 2659.2331 & 1330.1202 & 2658.2491 & 1329.6282 & 24 \\
\hline 7 & 829.3727 & 415.1900 & & & 811.3621 & 406.1847 & $\mathbf{L}$ & 2561.2327 & 1281.1200 & 2544.2062 & 1272.6067 & 2543.2222 & 1272.1147 & 723 \\
\hline 8 & 886.3941 & 443.7007 & & & 868.3836 & 434.6954 & G & 2448.1487 & 1224.5780 & 2431.1221 & 1216.0647 & 2430.1381 & 1215.5727 & 722 \\
\hline 9 & 1015.4367 & 508.2220 & & & 997.4262 & 499.2167 & $\mathbf{E}$ & 2391.1272 & 1196.0672 & 2374.1006 & 1187.5540 & 2373.1166 & 1187.0620 & 21 \\
\hline 10 & 1146.4772 & 573.7422 & & & 1128.4666 & 564.7370 & $\mathbf{M}$ & 2262.0846 & 1131.5459 & 2245.0581 & 1123.0327 & 2244.0740 & 1122.5407 & 720 \\
\hline 11 & 1275.5198 & 638.2635 & & & 1257.5092 & 629.2583 & $\mathbf{E}$ & 2131.0441 & 1066.0257 & 2114.0176 & 1057.5124 & 2113.0336 & 1057.0204 & 19 \\
\hline 12 & 1362.5518 & 681.7795 & & & 1344.5413 & 672.7743 & $S$ & 2002.0015 & 1001.5044 & 1984.9750 & 992.9911 & 1983.9910 & 992.4991 & 18 \\
\hline 13 & 1463.5995 & 732.3034 & & & 1445.5889 & 723.2981 & $T$ & 1914.9695 & 957.9884 & 1897.9429 & 949.4751 & 1896.9589 & 948.9831 & 17 \\
\hline 14 & 1576.6836 & 788.8454 & & & 1558.6730 & 779.8401 & I & 1813.9218 & 907.4645 & 1796.8953 & 898.9513 & 1795.9113 & 898.4593 & 16 \\
\hline 15 & 1677.7312 & 839.3693 & & & 1659.7207 & 830.3640 & $T$ & 1700.8378 & 850.9225 & \begin{tabular}{|l|}
1683.8112 \\
\end{tabular} & 842.4092 & 1682.8272 & 841.9172 & 15 \\
\hline 16 & 1764.7633 & 882.8853 & & & 1746.7527 & 873.8800 & $\mathrm{~S}$ & 1599.7901 & 800.3987 & 1582.7635 & 791.8854 & 1581.7795 & 791.3934 & 14 \\
\hline 17 & 1911.8317 & 956.4195 & & & 1893.8211 & 947.4142 & $\mathbf{F}$ & 1512.7580 & 756.8827 & 1495.7315 & 3694 & 1494.7475 & 747.8774 & 13 \\
\hline 18 & 2008.8845 & 1004.9459 & & & 1990.8739 & 995.9406 & $\mathbf{P}$ & 1365.6896 & 683.3485 & 1348.6631 & 674.8352 & 1347.6791 & 674.3432 & 12 \\
\hline 19 & 2123.9114 & 1062.4593 & 2106.8848 & 1053.9461 & 2105.9008 & 1053.4540 & $\mathbf{N}$ & 1268.6369 & 634.8221 & 1251.6103 & 626.3088 & 1250.6263 & 625.8168 & 11 \\
\hline 20 & 2252.9540 & 1126.9806 & 2235.9274 & 1118.4674 & 2234.9434 & \begin{tabular}{|l|}
1117.9753 \\
\end{tabular} & $\mathbf{E}$ & 1153.6099 & 577.3086 & 1136.5834 & 568.7953 & 1135.5994 & 568.3033 & 10 \\
\hline 21 & 2354.0017 & 1177.5045 & 2336.9751 & 1168.9912 & 2335.9911 & 1168.4992 & $\mathbf{T}$ & 1024.5673 & 512.7873 & 1007.5408 & 504.2740 & 1006.5568 & 503.7820 & 9 \\
\hline 22 & 2425.0388 & 1213.0230 & 2408.0122 & 1204.5097 & 2407.0282 & \begin{tabular}{|l}
1204.0177 \\
\end{tabular} & $\mathbf{A}$ & 923.5197 & 462.2635 & 906.4931 & 453.7502 & 905.5091 & 453.2582 & 8 \\
\hline 23 & 2526.0865 & 1263.5469 & 2509.0599 & 1255.0336 & 2508.0759 & 1254.5416 & $\mathbf{T}$ & 852.4825 & 426.7449 & 835.4560 & 418.2316 & 834.4720 & 417.7396 & 7 \\
\hline 24 & 2639.1705 & 1320.0889 & 2622.1440 & 1311.5756 & 2621.1600 & 1311.0836 & I & 751.4349 & 376.2211 & 734.4083 & 367.7078 & 733.4243 & 367.2158 & 6 \\
\hline 25 & 2752.2546 & 1376.6309 & 2735.2280 & 1368.1177 & 2734.2440 & \begin{tabular}{|l|}
1367.6256 \\
\end{tabular} & $\mathbf{I}$ & 638.3508 & 319.6790 & 621.3243 & 311.1658 & 620.3402 & 310.6738 & 5 \\
\hline 26 & 2839.2866 & 1420.1469 & 2822.2601 & 1411.6337 & 2821.2760 & \begin{tabular}{|l|}
1411.1417 \\
\end{tabular} & $S$ & 525.2667 & 263.1370 & 508.2402 & 254.6237 & 507.2562 & 254.1317 & 4 \\
\hline 27 & 2967.3452 & 1484.1762 & 2950.3186 & 1475.6630 & 2949.3346 & 1475.1709 & $\mathbf{Q}$ & 438.2347 & 219.6210 & 421.2082 & 211.1077 & & & 3 \\
\hline 28 & 3130.4085 & 1565.7079 & 3113.3820 & 1557.1946 & 3112.3980 & 1556.7026 & $\mathbf{Y}$ & 310.1761 & 155.5917 & 293.1496 & \begin{tabular}{|l|}
147.0784 \\
\end{tabular} & & & 2 \\
\hline 29 & & & & & & & $\mathbf{K}$ & 147.1128 & 74.0600 & 130.0863 & 65.5468 & & & 1 \\
\hline
\end{tabular}
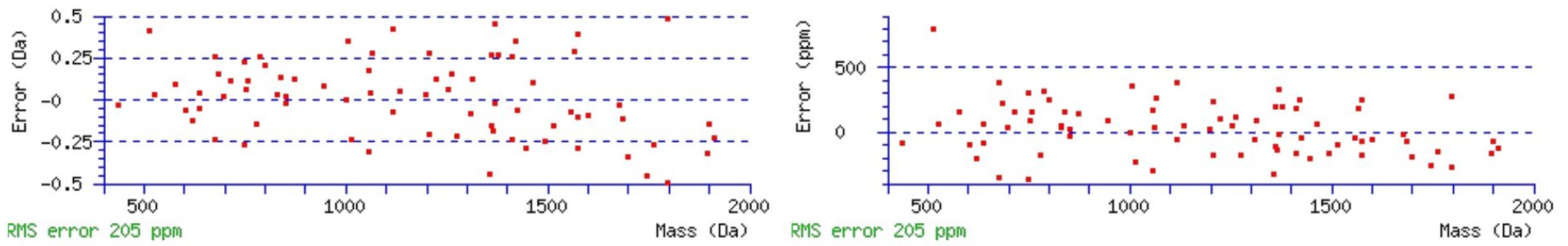

\section{All matches to this query}

\begin{tabular}{|l|l|l|l|}
\hline Score & Mr(calc): & Delta & \multicolumn{1}{|c|}{ Sequence } \\
\hline 44.8 & 3275.5067 & 0.0117 & IDWEGDLGEMESTITSFPNETATIISOYK \\
\hline 19.5 & 3274.5227 & 0.9957 & IDWEGDLGEMESTITSFPNETATIISQYK \\
\hline 1.6 & 3275.5120 & 0.0064 & ELRVLIDDSOSIIFINLDSHRNVMMR \\
\hline
\end{tabular}

Spectrum No: 753; Query: 587; Rank: 1 


\section{MS/MS Fragmentation of GTTNTTSAGVPCQR}

Found in IPI00189619, Tax_Id=10116 Gene_Symbol=Mst1 Macrophage stimulating protein precursor

Match to Query 587: 1449.657368 from(725.835960,2+)

Title: 091008RatKidney_NoSalt_30.301.301.2.dta

Data file K:INewmanPaper|Piliang|3SubProteomes\Piliang3SP\mgf5ppm\ERLIC_3SubProteomes5ppm.mgf
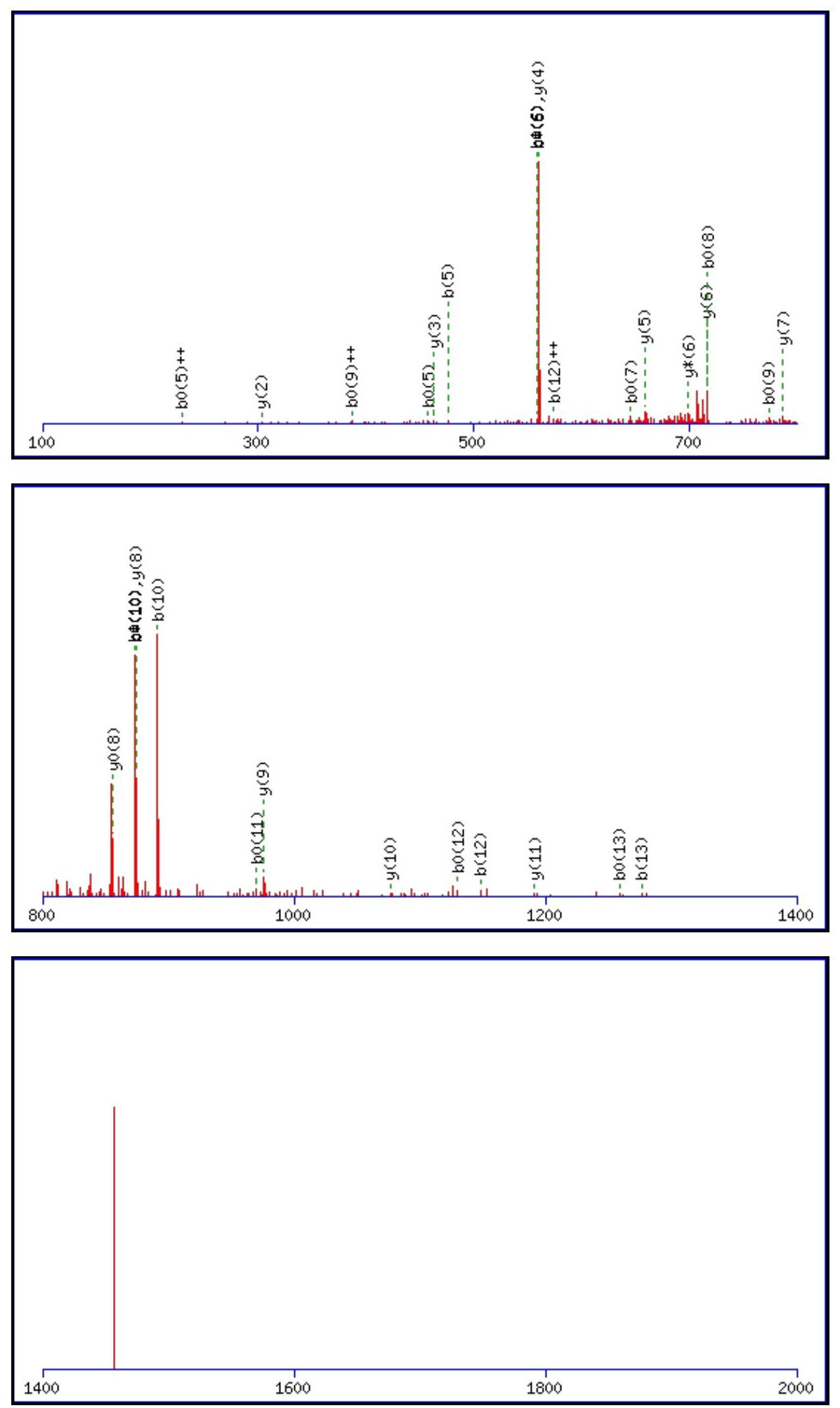

Monoisotopic mass of neutral peptide $\operatorname{Mr}($ calc): 1449.6518

Fixed modifications: Carbamidomethyl (C)

Variable modifications:

N4 : Deamidated $\mathrm{N}(\mathrm{N})$

Ions Score: 45 Expect: 0.0049

Matches (Bold Red): 30/134 fragment ions using 84 most intense peaks

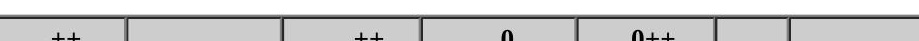




\begin{tabular}{|c|c|c|c|c|c|c|c|c|c|c|c|c|c|c|}
\hline \# & b & b & $\mathbf{b}^{*}$ & $\mathbf{b}^{*}$ & b & b & Seq. & $\mathbf{y}$ & $\mathbf{y}$ & $\mathbf{y}^{*}$ & $\mathbf{y}^{*}$ & $\mathbf{y}$ & $\mathbf{y}$ & \# \\
\hline 1 & 58.0287 & 29.5180 & & & & & G & & & & & & & 14 \\
\hline 2 & 159.0764 & 80.0418 & & & 141.0659 & 71.0366 & $\mathbf{T}$ & 1393.6376 & 697.3224 & 1376.6111 & 688.8092 & |1375.6271 & 688.3172 & 13 \\
\hline 3 & 260.1241 & 130.5657 & & & 242.1135 & 121.5604 & $\mathbf{T}$ & 1292.5899 & 646.7986 & 1275.5634 & 638.2853 & 1274.5794 & 637.7933 & 12 \\
\hline 4 & 375.1510 & 188.0792 & 358.1245 & 179.5659 & 357.1405 & 179.0739 & $\mathbf{N}$ & 1191.5423 & 596.2748 & 1174.5157 & 587.7615 & 1173.5317 & 587.2695 & 11 \\
\hline 5 & 476.1987 & 238.6030 & 459.1722 & 230.0897 & 458.1881 & 229.5977 & $\mathbf{T}$ & 1076.5153 & 538.7613 & 1059.4888 & 530.2480 & 1058.5048 & 529.7560 & 10 \\
\hline 6 & 577.2464 & 289.1268 & 560.2198 & 280.6136 & 559.2358 & 280.1216 & $T$ & 975.4676 & 488.2375 & 958.4411 & 479.7242 & 957.4571 & 479.2322 & 9 \\
\hline 7 & 664.2784 & 332.6428 & 647.2519 & 324.1296 & 646.2679 & 323.6376 & $S$ & 874.4200 & 437.7136 & 857.3934 & 429.2003 & 856.4094 & 428.7083 & 8 \\
\hline 8 & 735.3155 & 368.1614 & 718.2890 & 359.6481 & 717.3050 & 359.1561 & A & 787.3879 & 394.1976 & 770.3614 & 385.6843 & & & 7 \\
\hline 9 & 792.3370 & 396.6721 & 775.3104 & 388.1589 & 774.3264 & 387.6669 & G & 716.3508 & 358.6790 & 699.3243 & 350.1658 & & & 6 \\
\hline 10 & 891.4054 & 446.2063 & 874.3789 & 437.6931 & 873.3948 & 437.2011 & V & 659.3294 & 330.1683 & 642.3028 & 321.6550 & & & 5 \\
\hline 11 & 988.4582 & 494.7327 & 971.4316 & 486.2195 & 970.4476 & 485.7274 & $\mathbf{P}$ & 560.2609 & 280.6341 & 543.2344 & 272.1208 & & & 4 \\
\hline 12 & 1148.4888 & 574.7481 & 1131.4623 & 566.2348 & 1130.4783 & 565.7428 & C & 463.2082 & 232.1077 & 446.1816 & 223.5945 & & & 3 \\
\hline 13 & \begin{tabular}{|l|}
1276.5474 \\
\end{tabular} & 638.7773 & 1259.5209 & 630.2641 & 1258.5368 & 629.7721 & $\mathbf{Q}$ & 303.1775 & 152.0924 & 286.1510 & 143.5791 & & & 2 \\
\hline 14 & & & & & & & $\mathbf{R}$ & 175.1190 & 88.0631 & 158.0924 & \begin{tabular}{|l|}
79.5498 \\
\end{tabular} & & & 1 \\
\hline
\end{tabular}
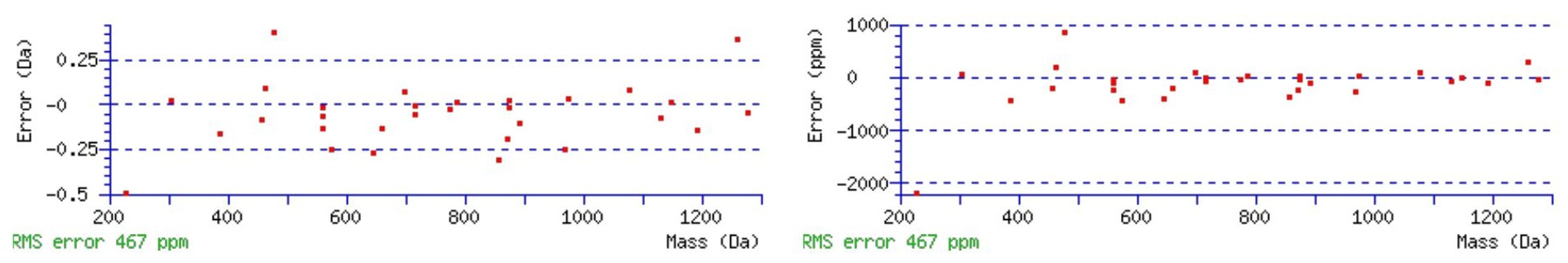

\section{All matches to this query}

\begin{tabular}{|l|l|l|l|}
\hline Score & Mr(calc): & Delta & \multicolumn{1}{|c|}{ Sequence } \\
\hline 44.8 & 1449.6518 & 0.0055 & GTTNTTSAGVPCQR \\
\hline 18.9 & 1448.6678 & 0.9896 & GTTNTTSAGVPCQR \\
\hline 4.1 & 1447.6530 & 2.0043 & MSAKDLFEQRK \\
\hline 1.3 & 1449.6709 & -0.0135 & GRGDYDGIGGRGDR \\
\hline 1.3 & 1448.6595 & 0.9979 & RMPPNPTDLXR \\
\hline 1.3 & 1449.6442 & 0.0132 & KGWLYYEAGQR \\
\hline 1.3 & 1449.6442 & 0.0132 & KGWLYYEAGQR \\
\hline 1.3 & 1449.6476 & 0.0098 & QRNCFFALSVK \\
\hline 1.2 & 1447.6579 & 1.9994 & LVTDENVSDNWR \\
\hline 0.9 & 1447.6363 & 2.0211 & MSPINMLYFNGK \\
\hline
\end{tabular}

Spectrum No: 754; Query: 2851; Rank: 1

\section{Peptide View}

MS/MS Fragmentation of YCAAPTEPVIHNGSQGTGTNGSEISDSDYQAEYPDEYHGEYPDEYPR

Found in IPI00205566, Tax_Id=10116 Gene_Symbol=Cnn3 Calponin-3

Match to Query 2851: 5267.206616 from(1317.808930,4+)

Title: 100101RatKid_NS_deglyco_16.3328.3328.4.dta

Data file K:INewmanPaper|Piliang|3SubProteomes\Piliang3SP\mgf5ppm\ERLIC_3SubProteomes5ppm.mgf 

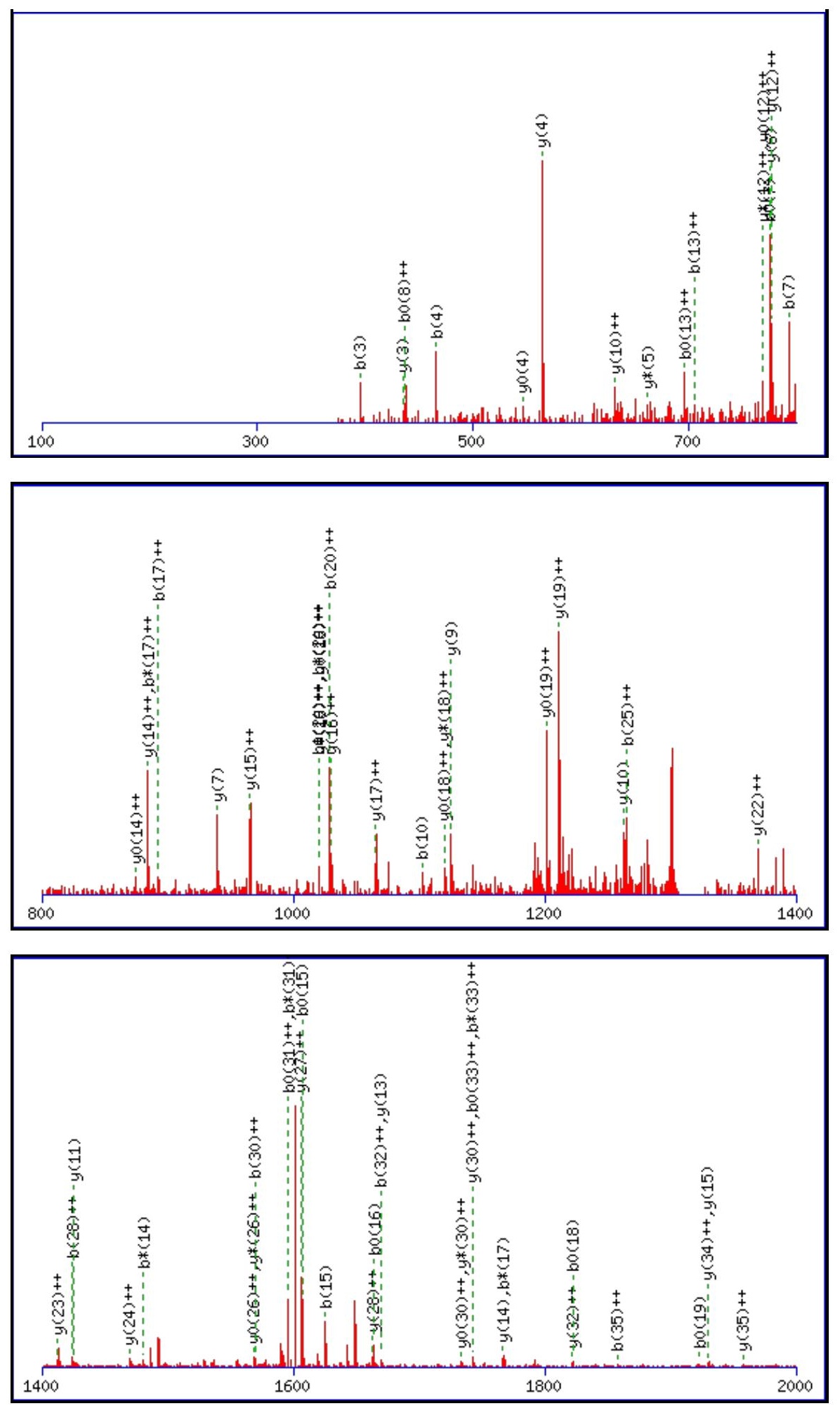

Monoisotopic mass of neutral peptide $\operatorname{Mr}($ calc): 5266.1806

Fixed modifications: Carbamidomethyl (C)

Variable modifications:

N20 : Deamidated_N (N)

Ions Score: 45 Expect: $\odot .02$

Matches (Bold Red): 69/514 fragment ions using 134 most intense peaks

\begin{tabular}{|r|c|c|c|c|c|c|c|c|c|c|c|c|c|c|}
\hline$\#$ & $\mathbf{b}$ & $\mathbf{b}^{++}$ & $\mathbf{b}^{*}$ & $\mathbf{b}^{\boldsymbol{*}^{++}}$ & $\mathbf{b}^{\mathbf{0}}$ & $\mathbf{b}^{\mathbf{0 + +}}$ & Seq. & $\mathbf{y}$ & $\mathbf{y}^{++}$ & $\mathbf{y}^{\mathbf{*}}$ & $\mathbf{y}^{\mathbf{*}^{++}}$ & $\mathbf{y}^{\mathbf{0}}$ & $\mathbf{y}^{\mathbf{0 + +}}$ & $\#$ \\
\hline $\mathbf{1}$ & 164.0706 & 82.5389 & & & & & $\mathbf{Y}$ & & & & & & & $\mathbf{4 7}$ \\
\hline $\mathbf{2}$ & 324.1013 & 162.5543 & & & & & $\mathbf{C}$ & 5104.1246 & 2552.5659 & 5087.0980 & 2544.0527 & 5086.1140 & 2543.5607 & $\mathbf{4 6}$ \\
\hline 3 & 395.1384 & 198.0728 & & & & & $\mathbf{A}$ & 4944.0939 & 2472.5506 & 4927.0674 & 2464.0373 & 4926.0834 & 2463.5453 & $\mathbf{4 5}$ \\
\hline $\mathbf{4}$ & $\mathbf{4 6 6 . 1 7 5 5}$ & 233.5914 & & & & & $\mathbf{A}$ & 4873.0568 & 2437.0321 & 4856.0303 & 2428.5188 & 4855.0463 & 2428.0268 & 44 \\
\hline $\mathbf{5}$ & 563.2282 & 282.1178 & & & & & $\mathbf{P}$ & 4802.0197 & 2401.5135 & 4784.9932 & 2393.0002 & 4784.0092 & 2392.5082 & $\mathbf{4 3}$ \\
\hline
\end{tabular}




\begin{tabular}{|c|c|c|c|c|c|c|c|c|c|c|c|c|c|c|}
\hline 6 & 664.2759 & 332.6416 & & & 646.2654 & 323.6363 & $\mathbf{T}$ & $|4704.9670|$ & |2352.9871 & 4687.9404 & |2344.4738 & |4686.9564 & |2343.9818| & |42 \\
\hline 7 & 793.3185 & 397.1629 & & & 775.3080 & 388.1576 & $E$ & 4603.9193 & 2302.4633 & 4586.8927 & 2293.9500 & 4585.9087 & 2293.4580 & 41 \\
\hline 8 & 890.3713 & 445.6893 & & & 872.3607 & 436.6840 & $\mathbf{P}$ & 4474.8767 & 2237.9420 & 4457.8501 & 2229.4287 & 4456.8661 & 2228.9367 & 40 \\
\hline 9 & 989.4397 & 495.2235 & & & 971.4291 & 486.2182 & V & 4377.8239 & 2189.4156 & 4360.7974 & 2180.9023 & 4359.8134 & 2180.4103 & 39 \\
\hline 10 & 1102.5238 & 551.7655 & & & 1084.5132 & 542.7602 & I & 4278.7555 & 2139.8814 & 4261.7290 & 2131.3681 & 4260.7449 & 2130.8761 & 38 \\
\hline 11 & 1239.5827 & 620.2950 & & & 1221.5721 & 611.2897 & $\mathbf{H}$ & 4165.6714 & 2083.3394 & 4148.6449 & 2074.8261 & 4147.6609 & 2074.3341 & 37 \\
\hline 12 & 1353.6256 & 677.3164 & 1336.5991 & 668.8032 & 1335.6150 & 668.3112 & $\mathbf{N}$ & 4028.6125 & 2014.8099 & 4011.5860 & 2006.2966 & 4010.6020 & 2005.8046 & 36 \\
\hline 13 & 1410.6471 & 705.8272 & 1393.6205 & 697.3139 & 1392.6365 & 696.8219 & G & 3914.5696 & 1957.7884 & 3897.5430 & 1949.2752 & 3896.5590 & 1948.7832 & 35 \\
\hline 14 & 1497.6791 & 749.3432 & 1480.6525 & 740.8299 & 1479.6685 & 740.3379 & $\mathrm{~S}$ & 3857.5481 & 1929.2777 & 3840.5216 & 1920.7644 & 3839.5376 & 1920.2724 & 34 \\
\hline 15 & 1625.7377 & 813.3725 & 1608.7111 & 804.8592 & 1607.7271 & 804.3672 & $\mathbf{Q}$ & 3770.5161 & 1885.7617 & 3753.4896 & 1877.2484 & 3752.5055 & 1876.7564 & 33 \\
\hline 16 & 1682.7591 & 841.8832 & 1665.7326 & 833.3699 & 1664.7486 & 832.8779 & G & 3642.4575 & 1821.7324 & 3625.4310 & 1813.2191 & 3624.4470 & 1812.7271 & 32 \\
\hline 17 & 1783.8068 & 892.4070 & 1766.7803 & 883.8938 & 1765.7962 & 883.4018 & $\mathbf{T}$ & 3585.4361 & 1793.2217 & 3568.4095 & 1784.7084 & 3567.4255 & 1784.2164 & 31 \\
\hline 18 & 1840.8283 & 920.9178 & 1823.8017 & 912.4045 & 1822.8177 & 911.9125 & G & 3484.3884 & 1742.6978 & 3467.3618 & 1734.1846 & 3466.3778 & 1733.6925 & 30 \\
\hline 19 & 1941.8760 & 971.4416 & 1924.8494 & 962.9283 & 1923.8654 & 962.4363 & $T$ & 3427.3669 & 1714.1871 & 3410.3404 & 1705.6738 & 3409.3564 & 1705.1818 & 29 \\
\hline 20 & 2056.9029 & 1028.9551 & 2039.8763 & 1020.4418 & 2038.8923 & 1019.9498 & $\mathbf{N}$ & 3326.3192 & 1663.6633 & 3309.2927 & 1655.1500 & 3308.3087 & 1654.6580 & 28 \\
\hline 21 & 2113.9244 & 1057.4658 & 2096.8978 & 1048.9525 & 2095.9138 & 1048.4605 & G & 3211.2923 & 1606.1498 & 3194.2658 & 1597.6365 & 3193.2817 & 1597.1445 & 27 \\
\hline 22 & 2200.9564 & 1100.9818 & 2183.9298 & 1092.4686 & 2182.9458 & 1091.9765 & $S$ & 3154.2708 & 1577.6391 & 3137.2443 & \begin{tabular}{|l|}
1569.1258 \\
\end{tabular} & 3136.2603 & 1568.6338 & 26 \\
\hline 23 & 2329.9990 & 1165.5031 & 2312.9724 & 1156.9899 & 2311.9884 & 1156.4978 & $E$ & 3067.2388 & 1534.1230 & 3050.2123 & 1525.6098 & 3049.2282 & 1525.1178 & 25 \\
\hline 24 & 2443.0830 & 1222.0452 & 2426.0565 & & 2425.0725 & 1213.0399 & I & 2938.1962 & 1469.6017 & 2921.1697 & 1461.0885 & 2920.1857 & 1460.5965 & 24 \\
\hline 25 & 2530.1151 & 1265.5612 & 2513.0885 & 1257.0479 & 2512.1045 & 1256.5559 & $S$ & 2825.1122 & 1413.0597 & 2808.0856 & 1404.5464 & 2807.1016 & 1404.0544 & 23 \\
\hline 26 & 2645.1420 & 1323.0746 & 2628.1155 & 1314.5614 & 2627.1314 & 1314.0694 & D & 2738.0801 & 1369.5437 & 2721.0536 & 1361.0304 & 2720.0696 & 1360.5384 & 22 \\
\hline 27 & 2732.1740 & 1366.5907 & 2715.1475 & 1358.0774 & 2714.1635 & 1357.5854 & $S$ & 2623.0532 & .0302 & 2606 & 1303.5170 & 260 & 0249 & 21 \\
\hline 28 & 2847.2010 & 1424.1041 & 2830.1744 & 1415.5909 & 2829.1904 & 1415.0988 & $\bar{D}$ & 2536.0212 & 1268.5142 & 2518.9946 & 1260.0009 & 2518.0106 & 1259.5089 & 20 \\
\hline 29 & 3010.2643 & 1505.6358 & 2993.2378 & 1497.1225 & 2992.2537 & 1496.6305 & $\mathbf{Y}$ & 2420.9942 & 1211.0007 & 2403.9677 & 1202.4875 & 2402.9836 & 1201.9955 & 19 \\
\hline 30 & 3138.3229 & 1569.6651 & 3121.2963 & 1561.1518 & 3120.3123 & 1560.6598 & $\mathbf{Q}$ & 2257.9309 & 1129.4691 & 2240.9043 & 1120.9558 & 2239.9203 & 1120.4638 & 18 \\
\hline 31 & 3209.3600 & 1605.1836 & 3192.3335 & 1596.6704 & 3191.3494 & 1596.1784 & A & 2129.8723 & 1065.4398 & 2112.8458 & 1056.9265 & 2111.8617 & 1056.4345 & 17 \\
\hline 32 & 3338.4026 & 1669.7049 & 3321.3760 & 1661.1917 & 3320.3920 & 1660.6997 & $E$ & 2058.8352 & 1029.9212 & 2041.8086 & 1021.4080 & 2040.8246 & 1020.9160 & 16 \\
\hline 33 & 3501.4659 & 1751.2366 & 3484.4394 & 1742.7233 & 3483.4554 & 1742.2313 & $\mathbf{Y}$ & 1929.7926 & 965.3999 & 1912.7661 & 956.8867 & 1911.7820 & 956.3947 & 15 \\
\hline 34 & 3598.5187 & 1799.7630 & 3581.4921 & 1791.2497 & 3580.5081 & 1790.7577 & $\mathbf{P}$ & 1766.7293 & 883.8683 & 1749.7027 & 875.3550 & 1748.7187 & 874.8630 & 14 \\
\hline 35 & 3713.5456 & 1857.2765 & 3696.5191 & 1848.7632 & 3695.5351 & 1848.2712 & D & 1669.6765 & 835.3419 & 1652.6500 & 826.8286 & 1651.6659 & 826.3366 & 13 \\
\hline 36 & 3842.5882 & 1921.7978 & 3825.5617 & 1913.2845 & 3824.5777 & 1912.7925 & $\mathbf{E}$ & 1554.6496 & 777.8284 & 1537.6230 & 769.3151 & 1536.6390 & 768.8231 & 12 \\
\hline 37 & 4005.6516 & 2003.3294 & 3988.6250 & 1994.8161 & 3987.6410 & 1994.3241 & $\mathbf{Y}$ & 1425.6070 & 713.3071 & 1408.5804 & 704.7938 & 1407.5964 & 704.3018 & 11 \\
\hline 38 & 4142.7105 & 2071.8589 & 4125.6839 & 2063.3456 & 4124.6999 & 2062.8536 & $\mathbf{H}$ & 1262.5436 & 631.7755 & 1245.5171 & 623.2622 & 1244.5331 & 622.7702 & 10 \\
\hline 39 & 4199.7319 & 2100.3696 & 4182.7054 & 2091.8563 & 4181.7214 & 2091.3643 & G & 1125.4847 & 563.2460 & 1108.4582 & 554.7327 & 1107.4742 & 554.2407 & 9 \\
\hline 40 & 4328.7745 & 2164.8909 & 4311.7480 & 2156.3776 & 4310.7640 & 2155.8856 & $\mathbf{E}$ & 1068.4633 & 534.7353 & 1051.4367 & 526.2220 & 1050.4527 & 525.7300 & 8 \\
\hline 41 & 4491.8379 & 2246.4226 & 4474.8113 & 2237.9093 & 4473.8273 & 2237.4173 & $\mathbf{Y}$ & 939.4207 & 470.2140 & 922.3941 & 461.7007 & 921.4101 & 461.2087 & 7 \\
\hline 42 & 4588.8906 & 2294.9489 & 4571.8641 & 2286.4357 & 4570.8801 & 2285.9437 & $\mathbf{P}$ & 776.3573 & 388.6823 & 759.3308 & 380.1690 & 758.3468 & 379.6770 & 6 \\
\hline 43 & 4703.9176 & 2352.4624 & 4686.8910 & 2343.9491 & 4685.9070 & 2343.4571 & D & 679.3046 & 340.1559 & 662.2780 & 331.6427 & 661.2940 & 331.1506 & 5 \\
\hline 44 & 4832.9602 & 2416.9837 & 4815.9336 & 2408.4704 & 4814.9496 & 2407.9784 & $\mathbf{E}$ & 564.2776 & 282.6425 & 547.2511 & 274.1292 & 546.2671 & 273.6372 & 4 \\
\hline 45 & 4996.0235 & 2498.5154 & 4978.9969 & 2490.0021 & 4978.0129 & 2489.5101 & $\mathbf{Y}$ & 435.2350 & 218.1212 & 418.2085 & 209.6079 & & & 3 \\
\hline 46 & 5093.0762 & 2547.0418 & 5076.0497 & 2538.5285 & 5075.0657 & 2538.0365 & $\mathbf{P}$ & 272.1717 & 136.5895 & 255.1452 & 128.0762 & & & 2 \\
\hline 47 & & & & & & & $\mathbf{R}$ & 175.1190 & 88.0631 & 158.0924 & 79.5498 & & & 1 \\
\hline
\end{tabular}
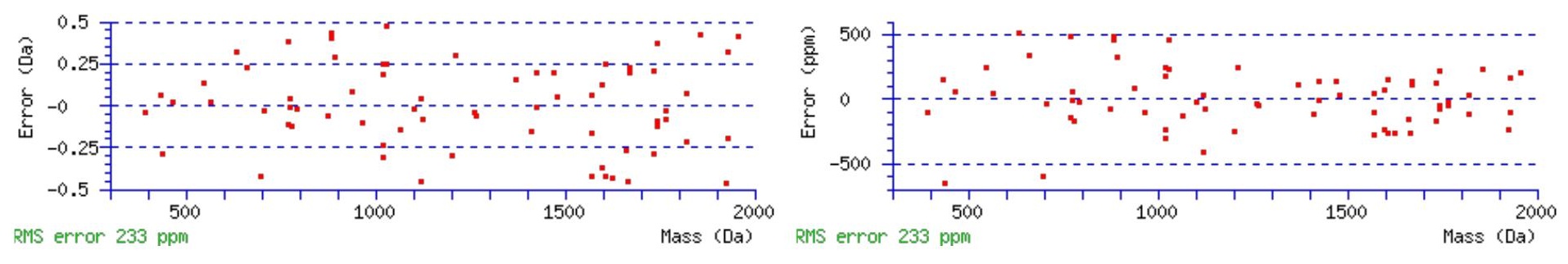


\section{All matches to this query}

\begin{tabular}{|l|l|l|l|}
\hline Score & Mr(calc): & Delta & \multicolumn{1}{c|}{ Sequence } \\
\hline 44.8 & 5266.1806 & 1.0260 & YCAAPTEPVIHNGSQGTGTNGSEISDSDYQAEYPDEYHGEYPDEYPR \\
\hline 41.5 & 5267.1646 & 0.0420 & YCAAPTEPVIHNGSQGTGTNGSEISDSDYQAEYPDEYHGEYPDEYPR \\
\hline 36.9 & 5265.1966 & 2.0100 & YCAAPTEPVIHNGSQGTGTNGSEISDSDYQAEYPDEYHGEYPDEYPR \\
\hline 32.5 & 5266.1806 & 1.0260 & YCAAPTEPVIHNGSQGTGTNGSEISDSDYQAEYPDEYHGEYPDEYPR \\
\hline 6.4 & 5266.2201 & 0.9865 & QSGTNKSTEDWVSVSVTPPVMSFQLEAMSPGYIVSFQVRVFTSK \\
\hline 5.4 & 5266.2201 & 0.9865 & QSGTNKSTEDWVSVSVTPPVMSFQLEAMSPGYIVSFQVRVFTSK \\
\hline 4.4 & 5266.2041 & 1.0025 & NQSDHTSTNNSDVSSFNMQYSVYGGGGGGGGGHPHAHVHHRGSALPKVK \\
\hline 4.2 & 5265.2047 & 2.0019 & DVLVNGDCQWITALHYAFQDENYLYLVMDYYVGGDLLTLLSK \\
\hline 4.2 & 5265.2047 & 2.0019 & DVLVNGDCOWITALHYAFODENYLYLVMDYYVGGDLLTLLSK \\
\hline 3.8 & 5266.2127 & 0.9939 & LSDEMIVITNDLGNLEIFCSVCFSSYNSAVMESSSVNVSMVHSASK \\
\hline
\end{tabular}

Spectrum No: 755; Query: 96; Rank: 1

\section{Peptide View}

MS/MS Fragmentation of KFLYNFTR

Found in IPI00190555, Tax_Id=10116 Gene_Symbol=Folh1 Glutamate carboxypeptidase 2

Match to Query 96: 1088.562808 from(545.288680,2+)

Title: 091008RatKidney_NH4Format01_23.1987.1987.2.dta

Data file K:INewmanPaper|Piliang|3SubProteomes\Piliang3SP\mgf5ppm\ERLIC_3SubProteomes5ppm.mgf
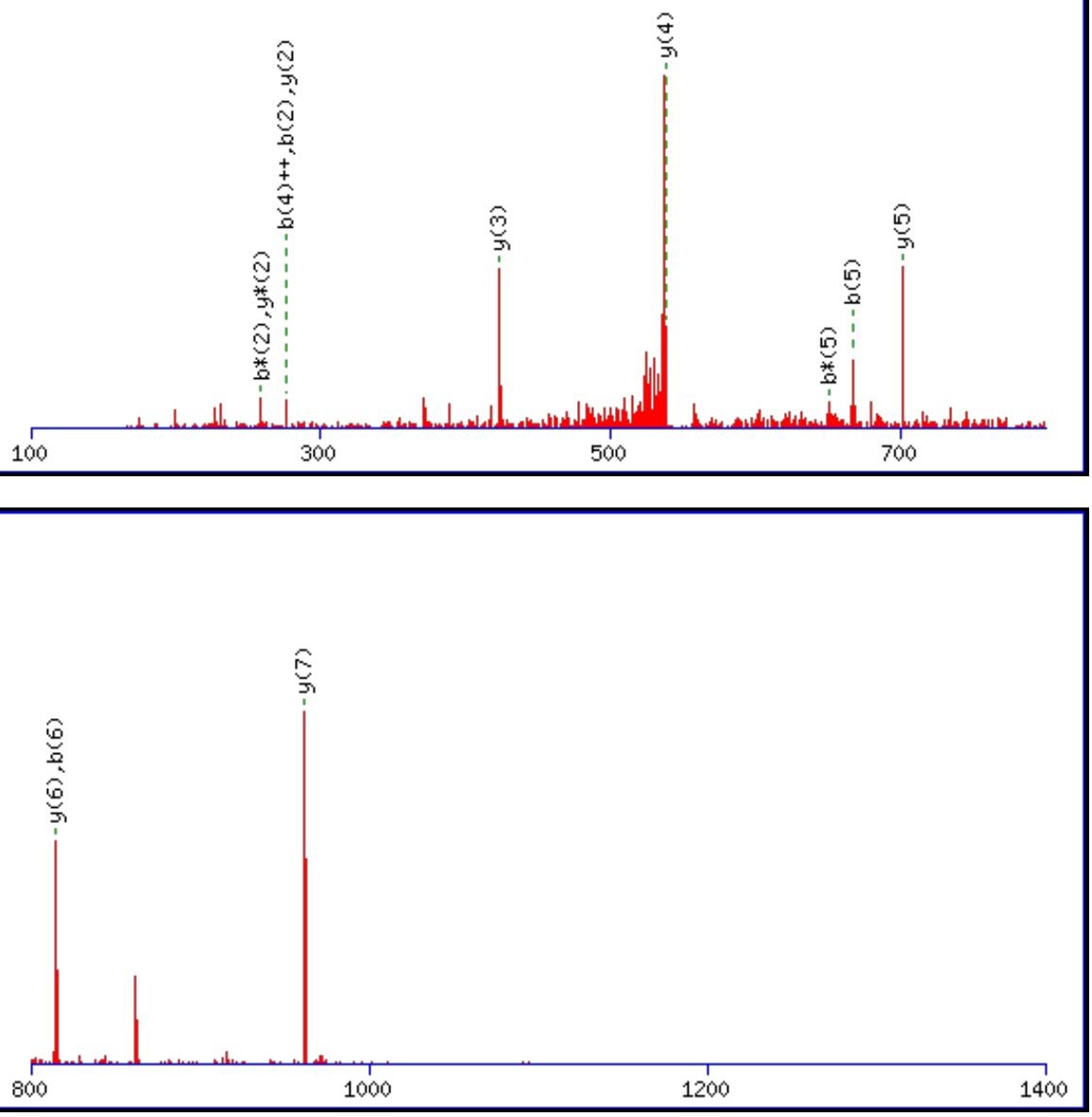


\begin{tabular}{|c|c|c|c|}
\hline 1400 & 1600 & 1800 & 2000 \\
\hline
\end{tabular}

Monoisotopic mass of neutral peptide $\operatorname{Mr}($ calc): 1088.5655

Fixed modifications: Carbamidomethyl (C)

Variable modifications:

N5 : Deamidated_N (N)

Ions Score: 44 Expect: 0.0046

Matches (Bold Red): 13/70 fragment ions using 14 most intense peaks

\begin{tabular}{|c|c|c|c|c|c|c|c|c|c|c|c|c|c|c|}
\hline \# & b & $\mathbf{b}^{++}$ & $\mathbf{b}^{*}$ & $\mathbf{b}^{*^{++}}$ & $\mathbf{b}^{0}$ & $\mathbf{b}^{\mathbf{0 + +}}$ & Seq. & $\mathbf{y}$ & $\mathbf{y}^{++}$ & $\mathbf{y}^{*}$ & $\mathbf{y}^{*^{++}}$ & $\mathbf{y}^{\mathbf{0}}$ & $\mathbf{y}^{0++}$ & $\#$ \\
\hline 1 & 129.1022 & 65.0548 & 112.0757 & 56.5415 & & & $\mathbf{K}$ & & & & & & & 8 \\
\hline 2 & 276.1707 & 138.5890 & 259.1441 & 130.0757 & & & $\mathbf{F}$ & 961.4778 & 481.2425 & 944.4512 & 472.7293 & 943.4672 & 472.2372 & 7 \\
\hline 4 & 552.3180 & 276.6627 & 535.2915 & 268.1494 & & & $\mathbf{Y}$ & 701.3253 & 351.1663 & 684.2988 & 342.6530 & 683.3147 & 342.1610 & 5 \\
\hline 5 & 667.3450 & 334.1761 & 650.3184 & 325.6629 & & & $\mathbf{N}$ & 538.2620 & 269.6346 & 521.2354 & 261.1214 & 520.2514 & 260.6293 & 4 \\
\hline 7 & 915.4611 & 458.2342 & 898.4345 & 449.7209 & 897.4505 & 449.2289 & $\mathbf{T}$ & 276.1666 & 138.5870 & 259.1401 & 130.0737 & 258.1561 & 129.5817 & 2 \\
\hline 8 & & & & & & & $\mathbf{R}$ & 175.1190 & 88.0631 & 158.0924 & 79.5498 & & & 1 \\
\hline
\end{tabular}
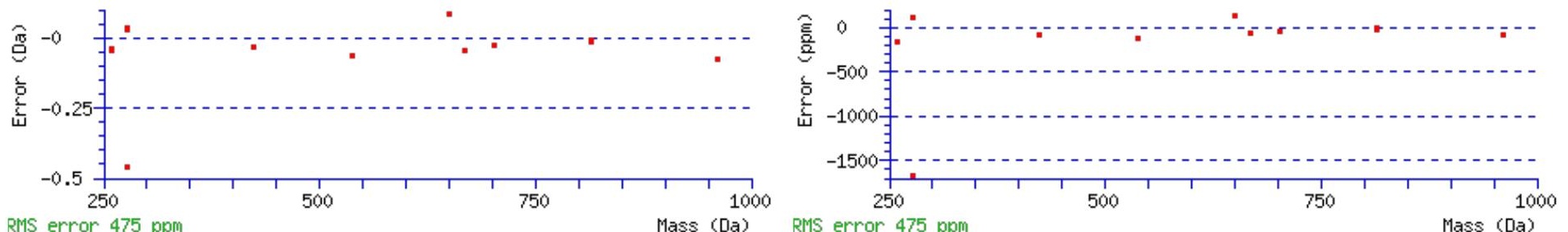

RMS error 475 ppm

Mass (Da) RMS error 475 ppm

Mass (Da)

\section{All matches to this query}

\begin{tabular}{|l|l|l|l|}
\hline Score & Mr(calc): & Delta & \multicolumn{1}{|c|}{ Sequence } \\
\hline 44.5 & 1088.5655 & -0.0027 & KFLYNFTR \\
\hline 6.9 & 1088.5648 & -0.0020 & QVIADMREK \\
\hline 6.1 & 1087.5662 & 0.9966 & EEQQWIKK \\
\hline 5.8 & 1086.5627 & 2.0001 & KFLPSFLR \\
\hline 4.6 & 1088.5713 & -0.0085 & DLQISGLSEK \\
\hline 4.5 & 1088.5614 & 0.0014 & SWAADIGLTR \\
\hline 3.6 & 1087.5509 & 1.0119 & TVGEPSELTR \\
\hline 1.9 & 1088.5713 & -0.0085 & KANKELEEK \\
\hline 1.9 & 1088.5574 & 0.0054 & KANQSAGREK \\
\hline 1.4 & 1088.5614 & 0.0014 & $\underline{\text { KFDGPEQLR }}$ \\
\hline
\end{tabular}

Spectrum No: 756; Query: 105; Rank: 1 


\section{Peptide View}

MS/MS Fragmentation of TMFNSTDIR

Found in IPI00357921, Tax_Id=10116 Gene_Symbol=RGD1307907_predicted similar to CG17660-PA

Match to Query 105: 1100.478188 from(551.246370,2+)

Title: 091008RatKidney_NoSalt_31.907.907.2.dta

Data file K:\NewmanPaper\Piliang \3SubProteomes \Piliang3SP \mgf5ppm\ERLIC_3SubProteomes5ppm.mgf
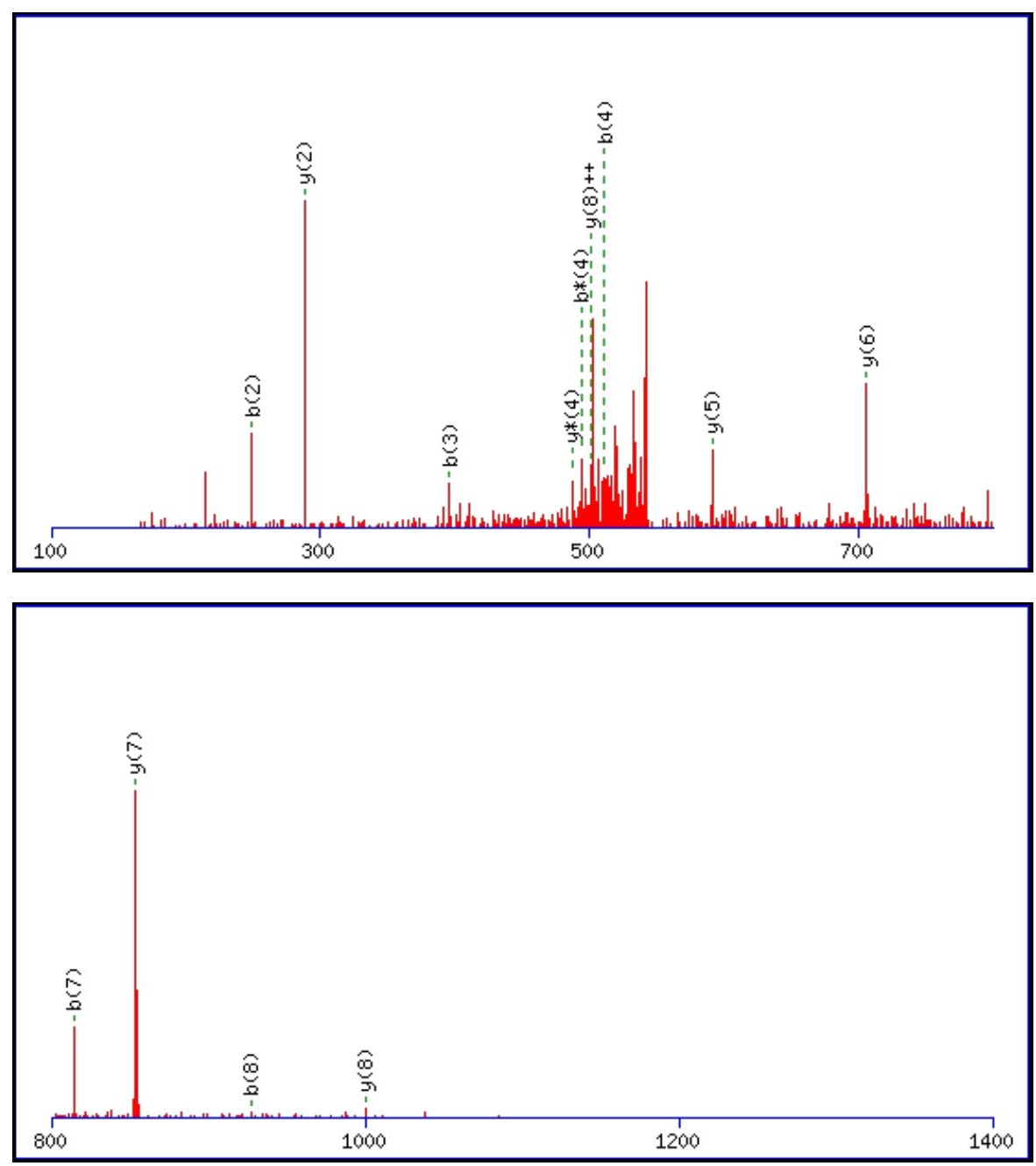

$\begin{array}{llll}1400 & 1600 & 1800 & 2000\end{array}$

Monoisotopic mass of neutral peptide $\operatorname{Mr}($ calc): 1100.4808

Fixed modifications: Carbamidomethyl (C)

Variable modifications:

M2 : Oxidation (M)

Deamidated $\mathrm{N}(\mathrm{N})$ 
N4 :

Ions Score: 44 Expect: 0.0033

Matches (Bold Red): 13/86 fragment ions using 39 most intense peaks

\begin{tabular}{|c|c|c|c|c|c|c|c|c|c|c|c|c|c|c|}
\hline \# & b & $\mathbf{b}^{++}$ & $\mathbf{b}^{*}$ & $\mathbf{b}^{*^{++}}$ & $\mathbf{b}^{\mathbf{0}}$ & $\mathbf{b}^{\mathbf{0 + +}}$ & Seq. & $\mathbf{y}$ & $y^{++}$ & $\mathbf{y}^{*}$ & $\mathrm{y}^{*^{++}}$ & $\mathbf{y}^{\mathbf{0}}$ & $\mathbf{y}^{0++}$ & $\#$ \\
\hline 1 & 102.0550 & 51.5311 & & & 84.0444 & 42.5258 & $\mathbf{T}$ & & & & & & & 9 \\
\hline 2 & 249.0904 & 125.0488 & & & 231.0798 & 116.0435 & $\mathbf{M}$ & 1000.4404 & 500.7238 & 983.4139 & 492.2106 & 982.4299 & 491.7186 & 8 \\
\hline 4 & 511.1857 & 256.0965 & 494.1592 & 247.5832 & 493.1751 & 247.0912 & $\mathbf{N}$ & 706.3366 & 353.6719 & 689.3101 & 345.1587 & 688.3260 & 344.6667 & 76 \\
\hline 5 & 598.2177 & 299.6125 & 581.1912 & 291.0992 & 580.2072 & 290.6072 & S & 591.3097 & 296.1585 & 574.2831 & 287.6452 & 573.2991 & 287.1532 & 5 \\
\hline 7 & 814.2924 & 407.6498 & 797.2658 & 399.1365 & 796.2818 & 398.6445 & D & 403.2300 & 202.1186 & 386.2034 & 193.6053 & 385.2194 & 193.1133 & 3 \\
\hline 8 & 927.3764 & 464.1918 & 910.3499 & 455.6786 & 909.3659 & 455.1866 & I & 288.2030 & 144.6051 & 271.1765 & 136.0919 & & & 2 \\
\hline 9 & & & & & & & $\mathbf{R}$ & 175.1190 & 88.0631 & 158.0924 & 79.5498 & & & 1 \\
\hline
\end{tabular}
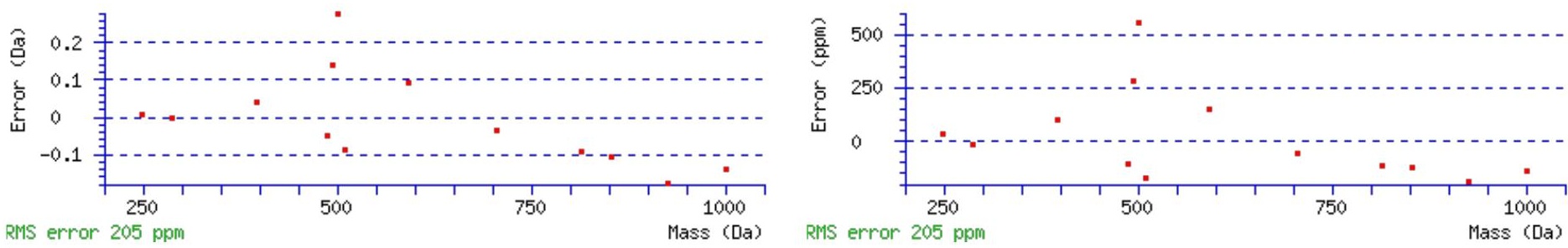

\section{All matches to this query}

\begin{tabular}{|l|l|l|l|}
\hline Score & Mr(calc): & Delta & \multicolumn{1}{c|}{ Sequence } \\
\hline 44.5 & 1100.4808 & -0.0026 & TMFNSTDIR \\
\hline 7.0 & 1099.4699 & 1.0083 & $\underline{\text { ISWSGSNIR }}$ \\
\hline 6.5 & 1100.4734 & 0.0048 & $\underline{\text { EHENSTLDR }}$ \\
\hline 6.2 & 1100.4863 & -0.0081 & $\underline{\text { SLNTGSSSIR }}$ \\
\hline 6.0 & 1100.4798 & -0.0016 & $\underline{\text { SARMGSIQR }}$ \\
\hline 5.8 & 1099.4699 & 1.0083 & $\underline{\text { ISWSGSNIR }}$ \\
\hline 5.4 & 1100.4863 & -0.0081 & $\underline{\text { SLSSSSGLQR }}$ \\
\hline 5.4 & 1100.4863 & -0.0081 & $\underline{\text { SLSSSSGLQR }}$ \\
\hline 5.1 & 1100.4692 & 0.0090 & $\underline{\text { SPSEFWIR }}$ \\
\hline 4.8 & 1099.4699 & 1.0083 & $\underline{\text { ISWSGSNIR }}$ \\
\hline
\end{tabular}

Spectrum No: 757; Query: 1070; Rank: 1

\section{Peptide View}

MS/MS Fragmentation of DSGRGDSVSDNGSEAVR

Found in IPI00200435, Tax_Id=10116 Gene_Symbol=Pdcd4 Isoform 1 of Programmed cell death protein 4

Match to Query 1070: 1707.724122 from(570.248650,3+)

Title: 091008RatKidney_NH4Format02_26.349.349.3.dta

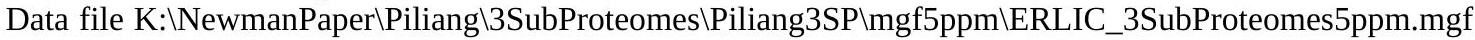



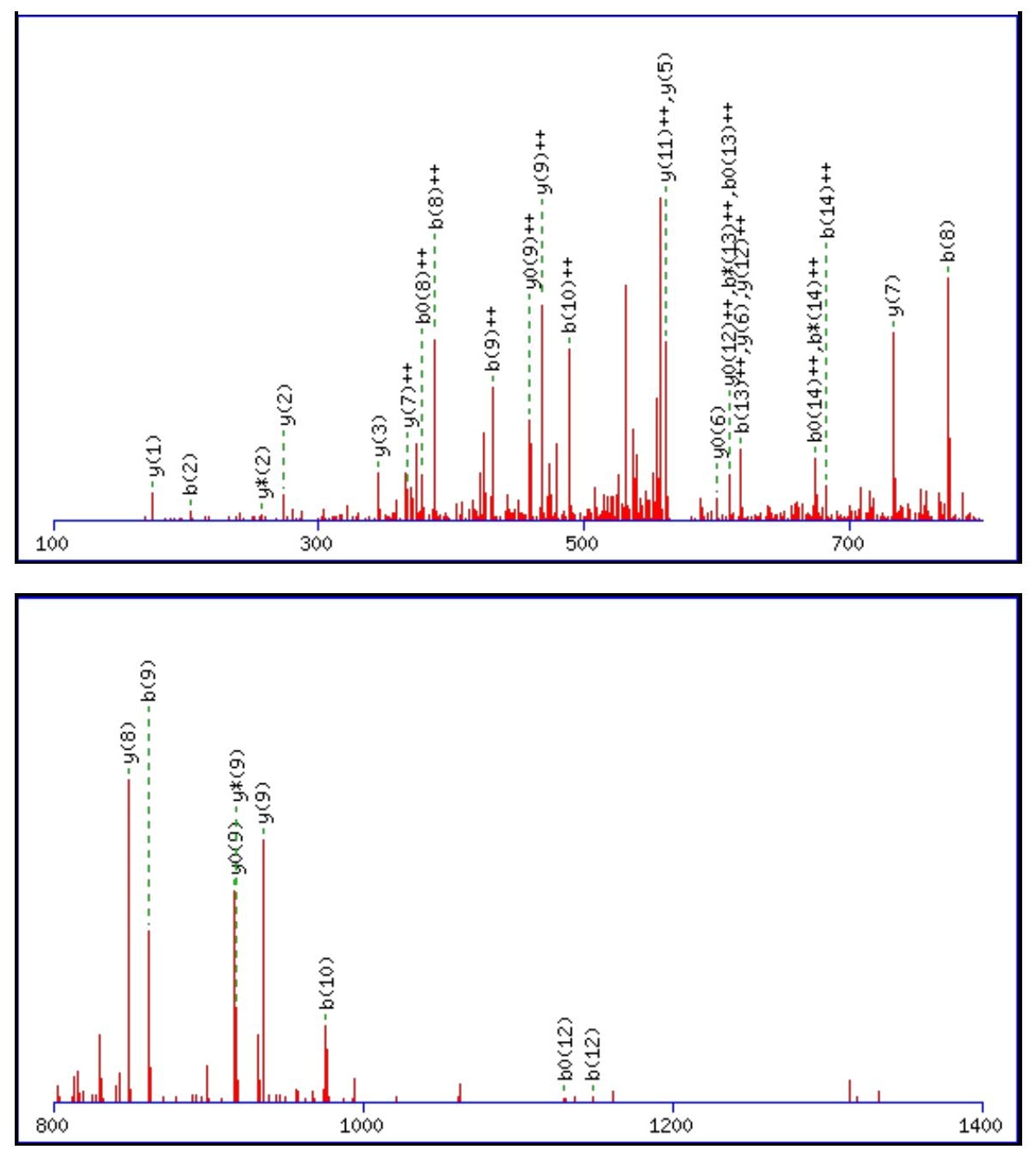

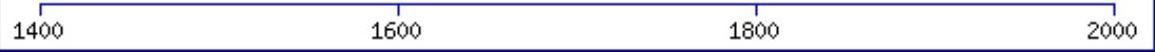

Monoisotopic mass of neutral peptide $\operatorname{Mr}($ calc): 1707.7296

Fixed modifications: Carbamidomethyl (C)

Variable modifications:

N11 : Deamidated_N (N)

Ions Score: 44 Expect: 0.0064

Matches (Bold Red): 34/180 fragment ions using 47 most intense peaks

\begin{tabular}{|r|c|c|c|c|r|r|r|r|c|c|c|c|c|c|}
\hline$\#$ & $\mathbf{b}$ & $\mathbf{b}^{++}$ & $\mathbf{b}^{*}$ & $\mathbf{b}^{\boldsymbol{*}^{++}}$ & \multicolumn{1}{|c|}{$\mathbf{b}^{\mathbf{0}}$} & $\mathbf{b}^{\mathbf{0 + +}}$ & Seq. & $\mathbf{y}$ & $\mathbf{y}^{++}$ & $\mathbf{y}^{\mathbf{*}}$ & $\mathbf{y}^{\boldsymbol{*}^{++}}$ & $\mathbf{y}^{\mathbf{0}}$ & $\mathbf{y}^{\mathbf{0 + +}}$ & $\#$ \\
\hline $\mathbf{1}$ & 116.0342 & 58.5207 & & & 98.0237 & 49.5155 & $\mathbf{D}$ & & & & & & & $\mathbf{1 7}$ \\
\hline $\mathbf{2}$ & $\mathbf{2 0 3 . 0 6 6 2}$ & 102.0368 & & & 185.0557 & 93.0315 & S & 1593.7099 & 797.3586 & 1576.6834 & 788.8453 & 1575.6994 & 788.3533 & $\mathbf{1 6}$ \\
\hline $\mathbf{3}$ & 260.0877 & 130.5475 & & & 242.0771 & 121.5422 & $\mathbf{G}$ & 1506.6779 & 753.8426 & 1489.6513 & 745.3293 & 1488.6673 & 744.8373 & $\mathbf{1 5}$ \\
\hline $\mathbf{4}$ & 416.1888 & 208.5980 & 399.1623 & 200.0848 & 398.1783 & 199.5928 & $\mathbf{R}$ & 1449.6564 & 725.3319 & 1432.6299 & 716.8186 & 1431.6459 & 716.3266 & $\mathbf{1 4}$ \\
\hline $\mathbf{5}$ & 473.2103 & 237.1088 & 456.1837 & 228.5955 & 455.1997 & 228.1035 & $\mathbf{G}$ & 1293.5553 & 647.2813 & 1276.5288 & 638.7680 & 1275.5448 & 638.2760 & $\mathbf{1 3}$ \\
\hline
\end{tabular}




\begin{tabular}{|c|c|c|c|c|c|c|c|c|c|c|c|c|c|c|}
\hline & 588.2372 & |294.6223 & 571.2107 & |286.1090 & 570.2267 & $\mid 285.6170$ & D & |1236.5339 & 618.7706 & |1219.5073| & 610.2573 & |1218.5233 & 609.7653 & 12 \\
\hline 7 & 675.2693 & 338.1383 & 658.2427 & 329.6250 & 657.2587 & 329.1330 & S & 1121.5069 & 561.2571 & 1104.4804 & 552.7438 & 1103.4963 & 552.2518 & 11 \\
\hline 8 & 774.3377 & 387.6725 & 757.3111 & 379.1592 & 756.3271 & 378.6672 & $\mathbf{V}$ & 1034.4749 & 517.7411 & 1017.4483 & 509.2278 & 1016.4643 & 508.7358 & 10 \\
\hline 9 & 861.3697 & 431.1885 & 844.3431 & 422.6752 & 843.3591 & 422.1832 & $\mathrm{~S}$ & 935.4065 & 468.2069 & 918.3799 & 459.6936 & 917.3959 & 459.2016 & 9 \\
\hline 10 & 976.3966 & 488.7020 & 959.3701 & 480.1887 & 958.3861 & 479.6967 & D & 848.3744 & 424.6909 & 831.3479 & 416.1776 & 830.3639 & 415.6856 & 8 \\
\hline 11 & 1091.4236 & 546.2154 & 1074.3970 & 537.7022 & 1073.4130 & 537.2101 & $\mathbf{N}$ & 733.3475 & 367.1774 & 716.3210 & 358.6641 & 715.3369 & 358.1721 & 7 \\
\hline 12 & 1148.4450 & 574.7262 & 1131.4185 & 566.2129 & 1130.4345 & 565.7209 & G & 618.3206 & 309.6639 & 601.2940 & 301.1506 & 600.3100 & 300.6586 & 6 \\
\hline 13 & 1235.4771 & 618.2422 & 1218.4505 & 609.7289 & 1217.4665 & 609.2369 & $\mathrm{~S}$ & 561.2991 & 281.1532 & 544.2726 & 272.6399 & 543.2885 & 272.1479 & 5 \\
\hline 14 & 1364.5197 & 682.7635 & 1347.4931 & 674.2502 & 1346.5091 & 673.7582 & $\mathbf{E}$ & 474.2671 & 237.6372 & 457.2405 & 229.1239 & 456.2565 & 228.6319 & 4 \\
\hline 15 & 1435.5568 & 718.2820 & 1418.5302 & 709.7688 & 1417.5462 & 709.2767 & A & 345.2245 & 173.1159 & 328.1979 & 164.6026 & & & 3 \\
\hline 16 & 1534.6252 & 767.8162 & 1517.5986 & 759.3030 & 1516.6146 & 758.8110 & $\mathrm{~V}$ & 274.1874 & 137.5973 & 257.1608 & 129.0840 & & & 2 \\
\hline 17 & & & & & & & $\mathbf{R}$ & 175.1190 & 88.0631 & 158.0924 & 79.5498 & & & 1 \\
\hline
\end{tabular}
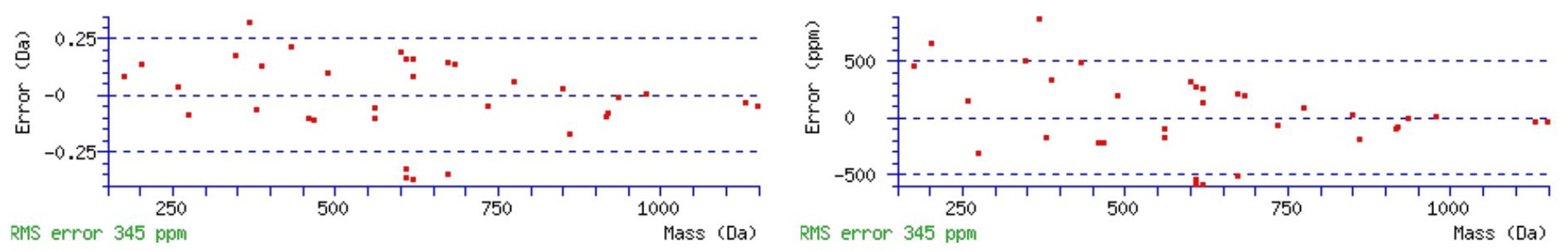

RMS error 345 ppm

\section{All matches to this query}

\begin{tabular}{|l|l|l|l|}
\hline Score & Mr(calc): & Delta & \multicolumn{1}{|c|}{ Sequence } \\
\hline 44.5 & 1707.7296 & -0.0055 & DSGRGDSVSDNGSEAVR \\
\hline 8.1 & 1707.7287 & -0.0046 & ERASSMNPSTTLYR \\
\hline 8.1 & 1707.7398 & -0.0156 & MLLVHGHFYYIR \\
\hline 7.9 & 1707.7076 & 0.0165 & GTHSFFDSLSRSCK \\
\hline 7.9 & 1707.7076 & 0.0165 & GTHSFFDSLSRSCK \\
\hline 7.0 & 1707.7304 & -0.0063 & EAITSSGQMGSLVR \\
\hline 6.5 & 1707.7361 & -0.0120 & KHTMPMPVGQNEVK \\
\hline 4.7 & 1705.7131 & 2.0110 & GLGPNMSSTPAHEVGR \\
\hline 4.3 & 1707.7171 & 0.0070 & RSDPYHVYIKNR \\
\hline 3.9 & 1707.7383 & -0.0141 & APGQKPGPDLTSAPGR \\
\hline
\end{tabular}

Spectrum No: 758; Query: 1108; Rank: 1

\section{Peptide View}

MS/MS Fragmentation of TLLNTSCDNMLMAIK

Found in IPI00561341, Tax_Id=10116 Gene_Symbol=Olfml1 26 kDa protein

Match to Query 1108: 1724.818508 from(863.416530,2+)

Title: 100101RatKid_NS_deglyco_20.4172.4172.2.dta

Data file K:\NewmanPaper|Piliangl3SubProteomes\Piliang3SP\mgf5ppm\ERLIC_3SubProteomes5ppm.mgf 

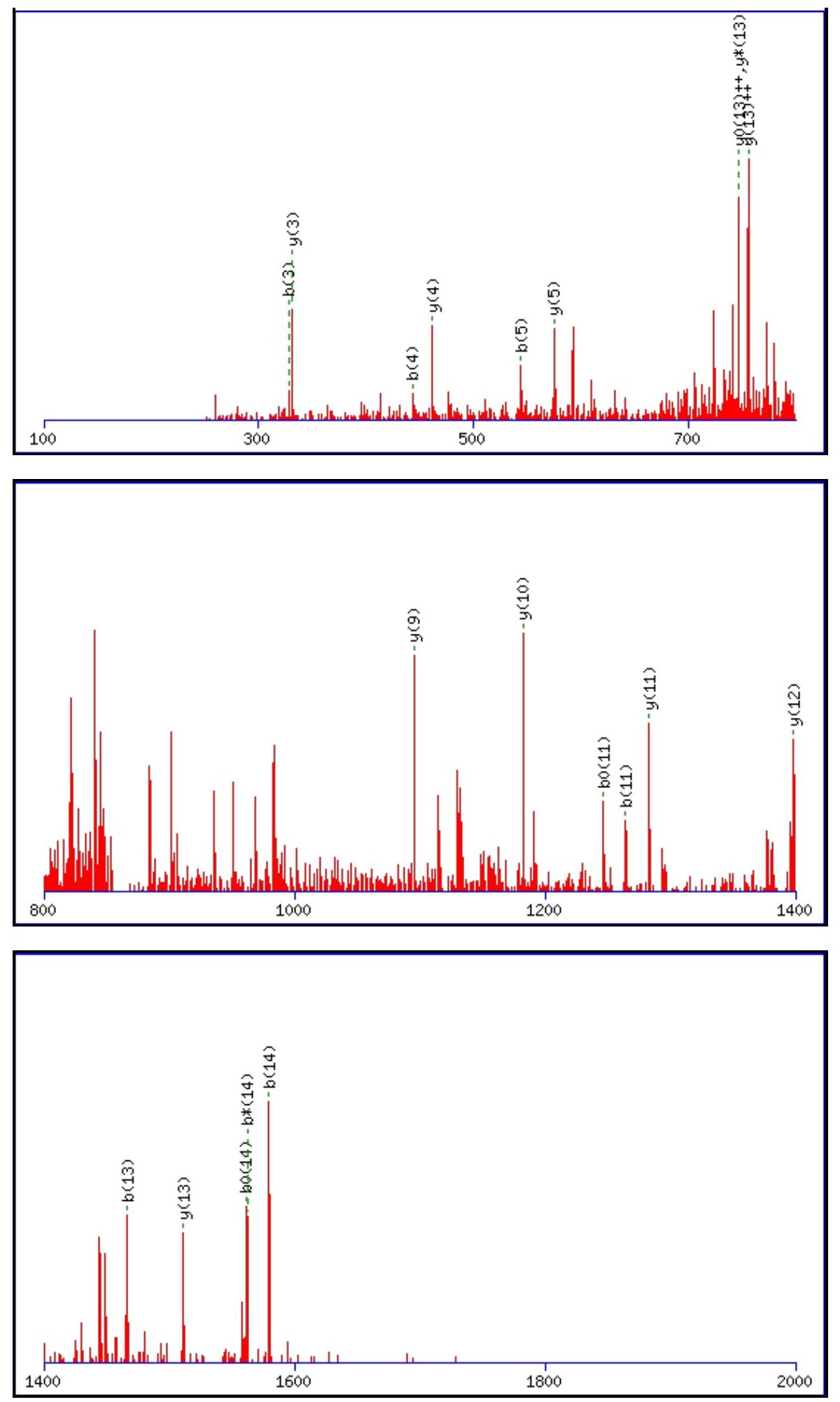

Monoisotopic mass of neutral peptide $\operatorname{Mr}($ calc): 1724.8147

Fixed modifications: Carbamidomethyl (C)

Variable modifications:

N4 : Deamidated $\mathrm{N}(\mathrm{N})$

Ions Score: 44 Expect: 0.0073

Matches (Bold Red): 20/148 fragment ions using 41 most intense peaks

\begin{tabular}{|r|c|c|c|c|c|c|c|c|c|c|c|c|c|c|}
\hline$\#$ & $\mathbf{b}$ & $\mathbf{b}^{++}$ & $\mathbf{b}^{*}$ & $\mathbf{b}^{\boldsymbol{*}^{++}}$ & $\mathbf{b}^{\mathbf{0}}$ & $\mathbf{b}^{\mathbf{0 + +}}$ & Seq. & $\mathbf{y}$ & $\mathbf{y}^{++}$ & $\mathbf{y}^{\mathbf{*}}$ & $\mathbf{y}^{\boldsymbol{*}^{++}}$ & $\mathbf{y}^{\mathbf{0}}$ & $\mathbf{y}^{\mathbf{0 + +}}$ & $\#$ \\
\hline $\mathbf{1}$ & 102.0550 & 51.5311 & & & 84.0444 & 42.5258 & $\mathbf{T}$ & & & & & & & $\mathbf{1 5}$ \\
\hline $\mathbf{2}$ & 215.1390 & 108.0731 & & & 197.1285 & 99.0679 & $\mathbf{L}$ & 1624.7743 & 812.8908 & 1607.7478 & 804.3775 & 1606.7637 & 803.8855 & $\mathbf{1 4}$ \\
\hline $\mathbf{3}$ & $\mathbf{3 2 8 . 2 2 3 1}$ & 164.6152 & & & 310.2125 & 155.6099 & $\mathbf{L}$ & $\mathbf{1 5 1 1 . 6 9 0 2}$ & 756.3488 & 1494.6637 & $\mathbf{7 4 7 . 8 3 5 5}$ & 1493.6797 & $\mathbf{7 4 7 . 3 4 3 5}$ & $\mathbf{1 3}$ \\
\hline $\mathbf{4}$ & $\mathbf{4 4 3 . 2 5 0 0}$ & 222.1286 & 426.2235 & 213.6154 & 425.2395 & 213.1234 & $\mathbf{N}$ & $\mathbf{1 3 9 8 . 6 0 6 2}$ & 699.8067 & 1381.5796 & 691.2935 & 1380.5956 & 690.8014 & $\mathbf{1 2}$ \\
\hline $\mathbf{5}$ & $\mathbf{5 4 4 . 2 9 7 7}$ & 272.6525 & 527.2711 & 264.1392 & 526.2871 & 263.6472 & $\mathbf{T}$ & $\mathbf{1 2 8 3 . 5 7 9 2}$ & 642.2933 & 1266.5527 & 633.7800 & 1265.5687 & 633.2880 & $\mathbf{1 1}$ \\
\hline
\end{tabular}




\begin{tabular}{|c|c|c|c|c|c|c|c|c|c|c|c|c|c|c|}
\hline 6 & 631.3297 & |316.1685 & 614.3032 & |307.6552 & 613.3192 & |307.1632| & $\mathbf{S}$ & |1182.5316 & |591.7694 & |1165.5050 & |583.2561 & |1164.5210| & 582.7641 & 10 \\
\hline 7 & 791.3604 & 396.1838 & 774.3338 & 387.6706 & 773.3498 & 387.1785 & C & 1095.4995 & 548.2534 & 1078.4730 & 539.7401 & 1077.4890 & 539.2481 & 9 \\
\hline 8 & 906.3873 & 453.6973 & 889.3608 & 445.1840 & 888.3768 & 444.6920 & D & 935.4689 & 468.2381 & 918.4423 & 459.7248 & 917.4583 & 459.2328 & 8 \\
\hline 9 & 1020.4302 & 510.7188 & 1003.4037 & 502.2055 & 1002.4197 & 501.7135 & $\mathbf{N}$ & 820.4419 & 410.7246 & 803.4154 & 402.2113 & & & 7 \\
\hline 10 & 1151.4707 & 576.2390 & 1134.4442 & 567.7257 & 1133.4602 & 567.2337 & $\mathbf{M}$ & 706.3990 & 353.7031 & 689.3725 & 345.1899 & & & 6 \\
\hline 11 & 1264.5548 & 632.7810 & 1247.5282 & 624.2678 & 1246.5442 & 623.7758 & L & 575.3585 & 288.1829 & 558.3320 & 279.6696 & & & 5 \\
\hline 12 & 1395.5953 & 698.3013 & 1378.5687 & 689.7880 & 1377.5847 & 689.2960 & M & 462.2745 & 231.6409 & 445.2479 & 223.1276 & & & 4 \\
\hline 13 & 1466.6324 & 733.8198 & 1449.6058 & 725.3066 & 1448.6218 & 724.8146 & A & 331.2340 & |166.1206 & 314.2074 & 157.6074 & & & 3 \\
\hline 14 & 1579.7165 & 790.3619 & 1562.6899 & 781.8486 & 1561.7059 & 781.3566 & I & 260.1969 & 130.6021 & 243.1703 & 122.0888 & & & 2 \\
\hline 15 & & & & & & & $\mathbf{K}$ & 147.1128 & 74.0600 & 130.0863 & 65.5468 & & & 1 \\
\hline
\end{tabular}
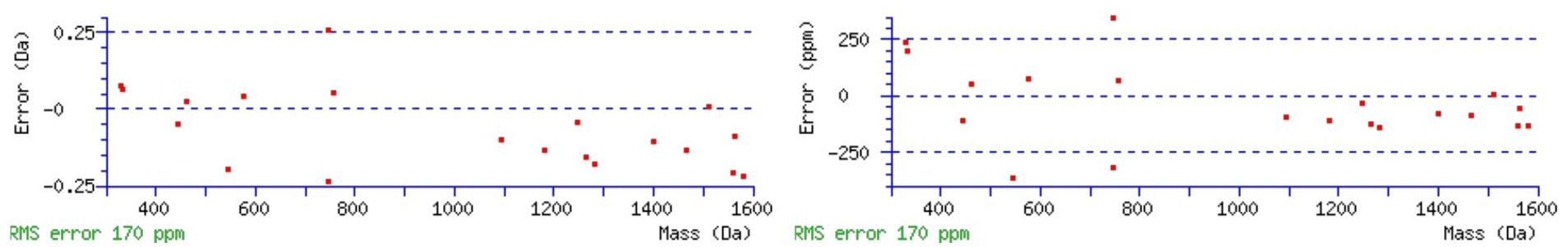

\section{All matches to this query}

\begin{tabular}{|l|l|l|l|}
\hline Score & Mr(calc): & Delta & \multicolumn{1}{|c|}{ Sequence } \\
\hline 44.4 & 1724.8147 & 0.0038 & TLLNTSCDNMLMAIK \\
\hline 28.5 & 1724.8147 & 0.0038 & TLLNTSCDNMLMAIK \\
\hline 15.2 & 1723.8307 & 0.9878 & TLLNTSCDNMLMAIK \\
\hline 5.9 & 1723.8070 & 1.0115 & DLQXEWVLPSITTK \\
\hline 5.9 & 1723.8070 & 1.0115 & DLQXEWVLPSITTK \\
\hline 5.8 & 1723.8070 & 1.0115 & DLQXEWVLPSITTK \\
\hline 5.8 & 1723.8070 & 1.0115 & DLQXEWVLPSITTK \\
\hline 4.4 & 1724.8022 & 0.0163 & LDVDWKELNELGSK \\
\hline 2.1 & 1724.8134 & 0.0051 & LRAWTNPETLTESK \\
\hline 1.8 & 1723.8216 & 0.9969 & QGKVLQEGETVTMPK \\
\hline
\end{tabular}

Spectrum No: 759; Query: 366; Rank: 1

\section{Peptide View}

\section{MS/MS Fragmentation of HAVEHFNNNTK}

Found in IPI00187799, Tax_Id=10116 Gene_Symbol=LOC25087;Kng1 Isoform HMW of Kininogen-1 precursor

Match to Query 366: 1310.602902 from(437.874910,3+)

Title: 100101RatKid_NS_deglyco_24.282.282.3.dta

Data file K:INewmanPaper|Piliangl3SubProteomes\Piliang3SP\mgf5ppm\ERLIC_3SubProteomes5ppm.mgf 

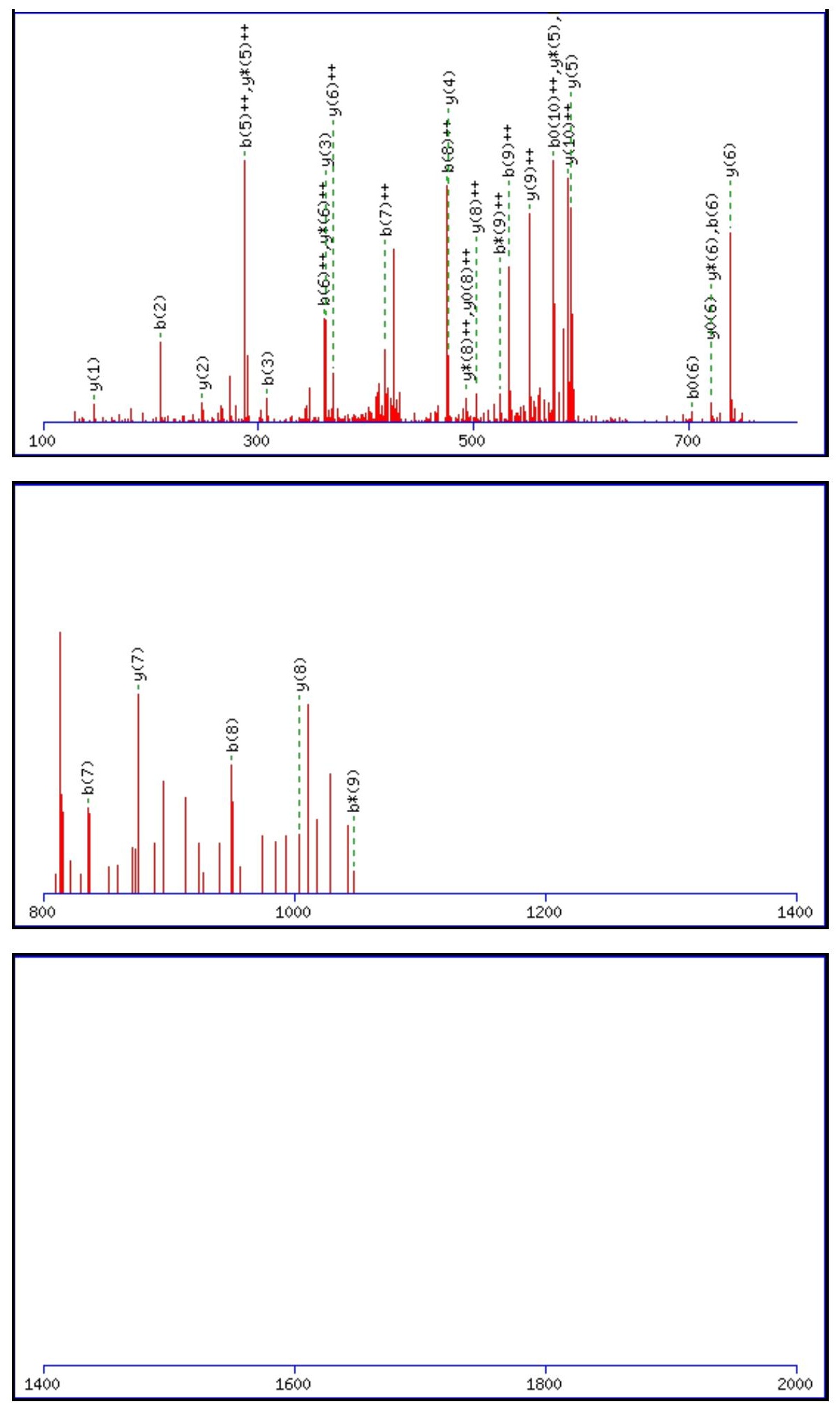

Monoisotopic mass of neutral peptide $\operatorname{Mr}($ calc): 1310.6003

Fixed modifications: Carbamidomethyl (C)

Variable modifications:

N8: Deamidated $\mathrm{N}(\mathrm{N})$

Ions Score: 44 Expect: $\odot .0053$

Matches (Bold Red): 35/100 fragment ions using 64 most intense peaks

\begin{tabular}{|r|c|c|c|c|c|c|c|c|c|c|c|c|c|c|}
\hline$\#$ & $\mathbf{b}$ & $\mathbf{b}^{++}$ & $\mathbf{b}^{*}$ & $\mathbf{b}^{\mathbf{*}^{++}}$ & $\mathbf{b}^{\mathbf{0}}$ & $\mathbf{b}^{\mathbf{0 + +}}$ & Seq. & $\mathbf{y}$ & $\mathbf{y}^{++}$ & $\mathbf{y}^{\mathbf{*}}$ & $\mathbf{y}^{\boldsymbol{*}^{++}}$ & $\mathbf{y}^{\mathbf{0}}$ & $\mathbf{y}^{\mathbf{0 + +}}$ & $\#$ \\
\hline $\mathbf{1}$ & 138.0662 & 69.5367 & & & & & $\mathbf{H}$ & & & & & & & $\mathbf{1 1}$ \\
\hline $\mathbf{2}$ & $\mathbf{2 0 9 . 1 0 3 3}$ & 105.0553 & & & & & $\mathbf{A}$ & 1174.5487 & 587.7780 & 1157.5222 & 579.2647 & 1156.5382 & 578.7727 & $\mathbf{1 0}$ \\
\hline $\mathbf{3}$ & $\mathbf{3 0 8 . 1 7 1 7}$ & 154.5895 & & & & & $\mathbf{V}$ & 1103.5116 & 552.2594 & 1086.4851 & 543.7462 & 1085.5010 & 543.2542 & $\mathbf{9}$ \\
\hline $\mathbf{4}$ & 437.2143 & 219.1108 & & & 419.2037 & 210.1055 & $\mathbf{E}$ & $\mathbf{1 0 0 4 . 4 4 3 2}$ & $\mathbf{5 0 2 . 7 2 5 2}$ & 987.4166 & $\mathbf{4 9 4 . 2 1 2 0}$ & 986.4326 & $\mathbf{4 9 3 . 7 2 0 0}$ & $\mathbf{8}$ \\
\hline $\mathbf{5}$ & $\mathbf{5 7 4 . 2 7 3 2}$ & $\mathbf{2 8 7 . 6 4 0 2}$ & & & 556.2627 & 278.6350 & $\mathbf{H}$ & $\mathbf{8 7 5 . 4 0 0 6}$ & 438.2039 & 858.3740 & 429.6907 & 857.3900 & 429.1987 & $\mathbf{7}$ \\
\hline
\end{tabular}




\begin{tabular}{|r|r|r|r|r|r|r|r|r|r|r|r|r|r|r|r|r|}
$\mathbf{6}$ & $\mathbf{7 2 1 . 3 4 1 6}$ & $\mathbf{3 6 1 . 1 7 4 5}$ & & & $\mathbf{7 0 3 . 3 3 1 1}$ & 352.1692 & $\mathbf{F}$ & $\mathbf{7 3 8 . 3 4 1 7}$ & 369.6745 & $\mathbf{7 2 1 . 3 1 5 1}$ & $\mathbf{3 6 1 . 1 6 1 2}$ & $\mathbf{7 2 0 . 3 3 1 1}$ & 360.6692 & $\mathbf{6}$ \\
\hline $\mathbf{7}$ & $\mathbf{8 3 5 . 3 8 4 6}$ & $\mathbf{4 1 8 . 1 9 5 9}$ & 818.3580 & 409.6826 & 817.3740 & 409.1906 & $\mathbf{N}$ & $\mathbf{5 9 1 . 2 7 3 3}$ & 296.1403 & 574.2467 & $\mathbf{2 8 7 . 6 2 7 0}$ & 573.2627 & 287.1350 & $\mathbf{5}$ \\
\hline $\mathbf{8}$ & $\mathbf{9 5 0 . 4 1 1 5}$ & $\mathbf{4 7 5 . 7 0 9 4}$ & 933.3849 & 467.1961 & 932.4009 & 466.7041 & $\mathbf{N}$ & $\mathbf{4 7 7 . 2 3 0 3}$ & 239.1188 & 460.2038 & 230.6055 & 459.2198 & 230.1135 & $\mathbf{4}$ \\
\hline $\mathbf{9}$ & 1064.4544 & 532.7309 & $\mathbf{1 0 4 7 . 4 2 7 9}$ & $\mathbf{5 2 4 . 2 1 7 6}$ & 1046.4439 & 523.7256 & $\mathbf{N}$ & $\mathbf{3 6 2 . 2 0 3 4}$ & 181.6053 & 345.1769 & 173.0921 & 344.1928 & 172.6001 & $\mathbf{3}$ \\
\hline $\mathbf{1 0}$ & 1165.5021 & 583.2547 & 1148.4756 & $\mathbf{5 7 4 . 7 4 1 4}$ & 1147.4915 & 574.2494 & $\mathbf{T}$ & $\mathbf{2 4 8 . 1 6 0 5}$ & 124.5839 & 231.1339 & 116.0706 & 230.1499 & 115.5786 & $\mathbf{2}$ \\
\hline $\mathbf{1 1}$ & & & & & & & $\mathbf{K}$ & $\mathbf{1 4 7 . 1 1 2 8}$ & $\mathbf{7 4 . 0 6 0 0}$ & 130.0863 & 65.5468 & & & \\
\hline
\end{tabular}
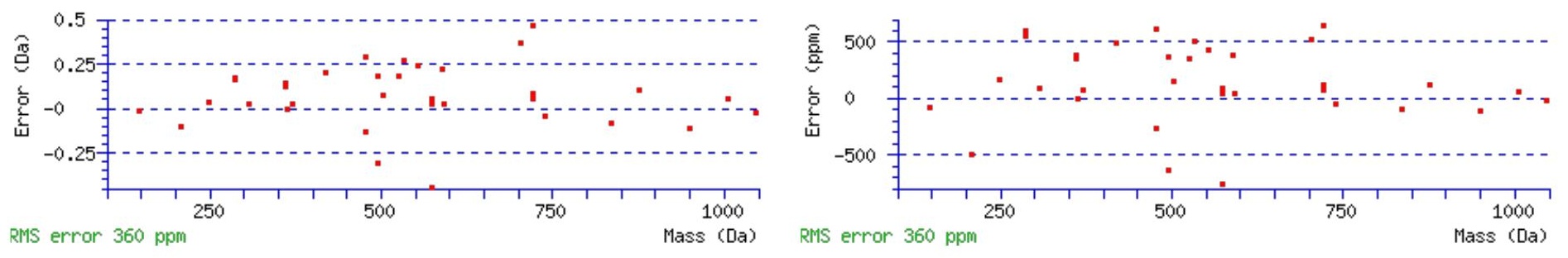

\section{All matches to this query}

\begin{tabular}{|l|c|c|l|}
\hline Score & Mr(calc): & Delta & \multicolumn{1}{|c|}{ Sequence } \\
\hline 44.1 & 1310.6003 & 0.0026 & HAVEHFNNNTK \\
\hline 43.0 & 1310.6003 & 0.0026 & HAVEHFNNNTK \\
\hline 32.8 & 1310.6003 & 0.0026 & HAVEHFNNNTK \\
\hline 10.4 & 1309.6006 & 1.0023 & DMGRPMELTTK \\
\hline 10.5 & 1310.6119 & -0.0090 & NLEQISANITK \\
\hline 7.4 & 1310.5999 & 0.0030 & SCENVXPISFK \\
\hline 7.2 & 1309.5995 & 1.0034 & YEVTFPIFSK \\
\hline 6.5 & 1308.6020 & 2.0009 & KMWSVSDNVDK \\
\hline 5.3 & 1309.5995 & 1.0034 & YEVTFPIFSK \\
\hline 4.9 & 1308.5849 & 2.0180 & HRMMMQSGRK \\
\hline
\end{tabular}

Spectrum No: 760; Query: 1846; Rank: 1

\section{Peptide View}

MS/MS Fragmentation of VNGTLTQVLLVGAPTHDDVSK

Found in IPI00325157, Tax_Id=10116 Gene_Symbol=Gpld1 Phosphatidylinositol-glycan-specific phospholipase D precursor

Match to Query 1846: 2164.144632 from(722.388820,3+)

Title: 100101RatKid_NS_deglyco_24.3449.3449.3.dta

Data file K:INewmanPaper|Piliang|3SubProteomes\Piliang3SP\mgf5ppm\ERLIC_3SubProteomes5ppm.mgf

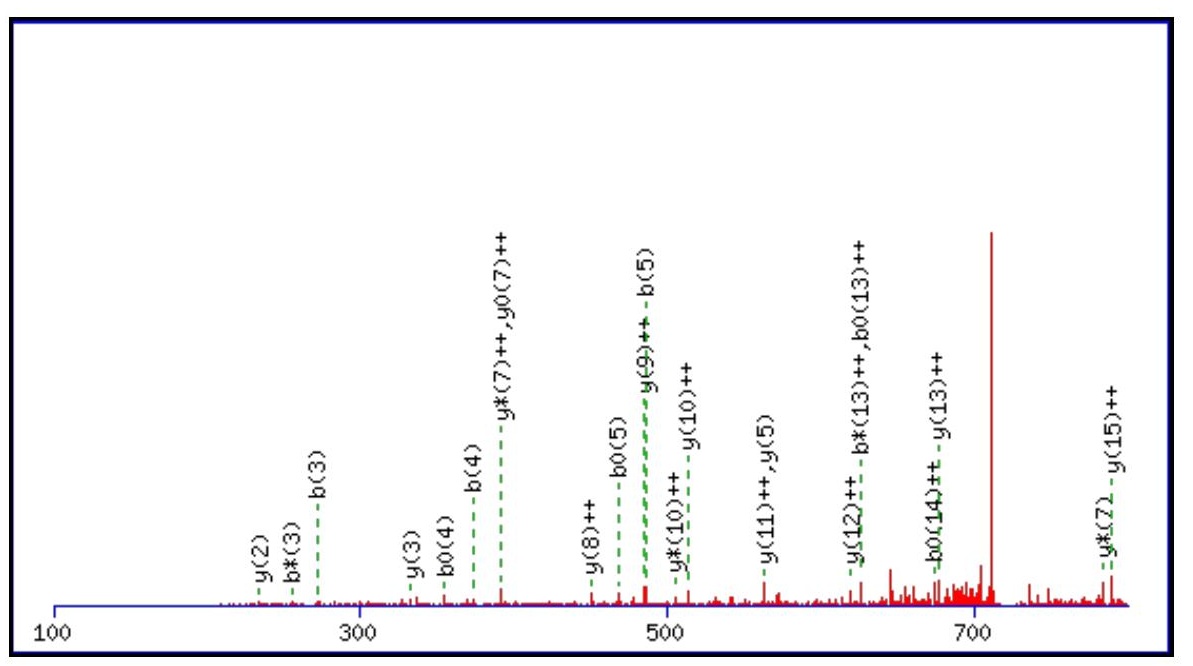



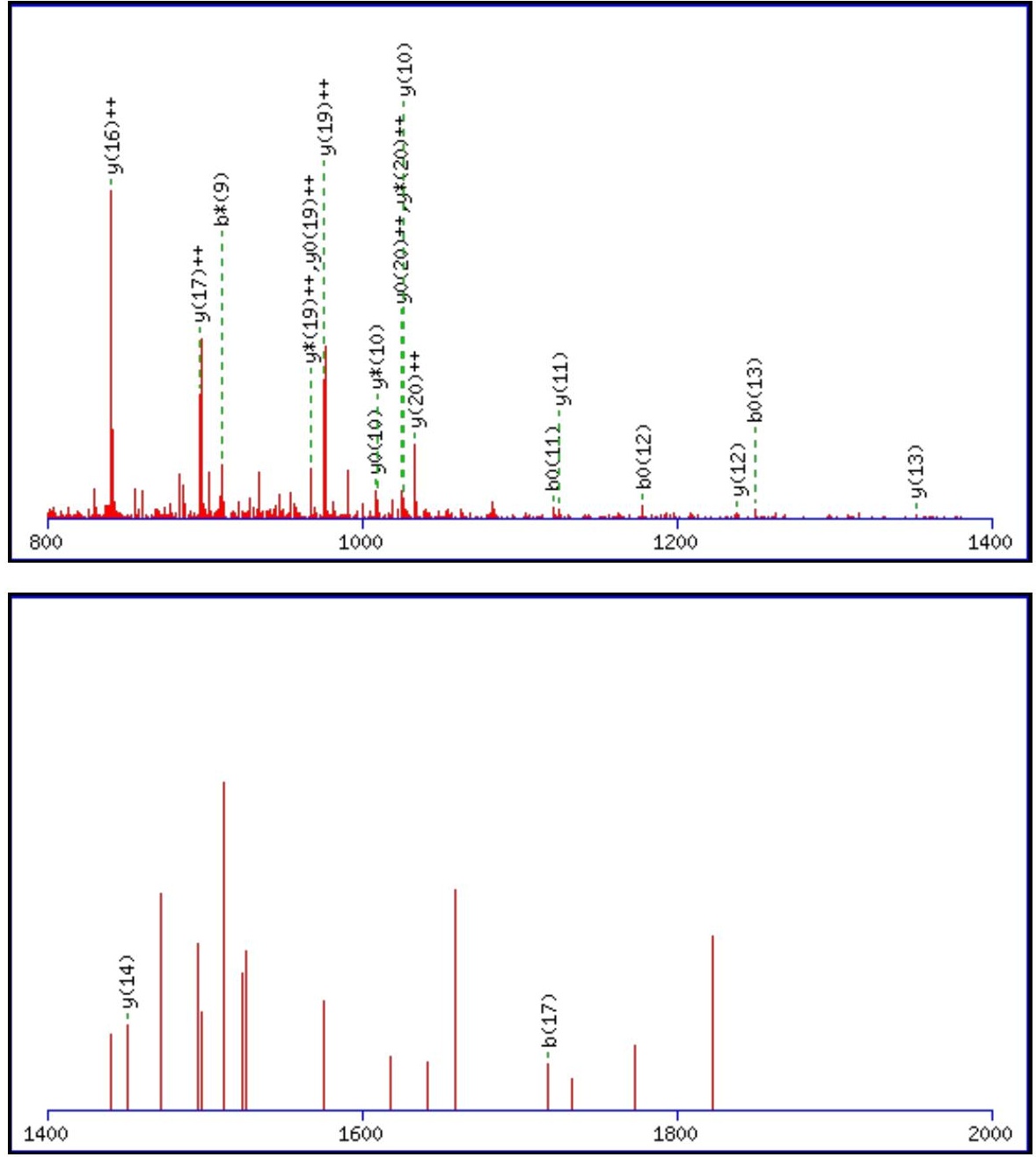

Monoisotopic mass of neutral peptide $\operatorname{Mr}$ (calc): 2164.1376

Fixed modifications: Carbamidomethyl (C)

Variable modifications:

N2 : Deamidated_N (N)

Ions Score: 44 Expect: $\odot .0 \odot 4$

Matches (Bold Red): 43/230 fragment ions using 91 most intense peaks

\begin{tabular}{|c|c|c|c|c|c|c|c|c|c|c|c|c|c|c|}
\hline \# & b & $\mathbf{b}^{++}$ & $\mathbf{b}^{*}$ & & $\mathbf{b}^{0}$ & & eq. & $\mathbf{y}$ & & $\mathbf{y}^{*}$ & & $\mathbf{y}^{0}$ & & \# \\
\hline 1 & 100.0757 & 50.5415 & & & & & $\mathbf{V}$ & & & & & & & 21 \\
\hline 2 & 215.1026 & 550 & & & & & $\mathbf{N}$ & & & & & & & 20 \\
\hline 3 & 272.1241 & 136.5657 & & & & & $\vec{G}$ & & & & & & 231 & 19 \\
\hline 4 & 373.1718 & 95 & 356.1 & & & & $\mathbf{T}$ & & & & & & & 18 \\
\hline 5 & 486.2558 & 243.6316 & 469.2293 & 235.1183 & & 234 & $\mathbf{L}$ & & 938 & 1775 & & & 887.9885 & 17 \\
\hline 6 & 587.3035 & 294.1554 & 570.2 & 285.6421 & 569. & & $\mathbf{T}$ & 3963 & & 1662 & & & 465 & 16 \\
\hline 7 & 715.3621 & 358.1847 & 698.3 & 349.6714 & 697.3 & 349 & $\mathbf{Q}$ & 486 & 279 & 1561 & 147 & 380 & 780.9227 & 15 \\
\hline 8 & 814.4305 & 407.7189 & 797.4040 & 399.2056 & 796.4199 & 398.7136 & V & & 725.8986 & 1433 & 854 & 1432 & 716.8934 & 14 \\
\hline 9 & 927.5146 & 464.2609 & 910.4880 & 455.7476 & 40 & & $\mathbf{L}$ & & 644 & 133 & & 133 & 5992 & 13 \\
\hline 10 & 1040.5986 & 520.8030 & 1023.5721 & 512.2897 & 1022.5881 & 511.7977 & $\mathbf{L}$ & 1238.6375 & 8224 & 1221 & 611.3091 & 1220 . & 610.8171 & 12 \\
\hline 11 & 1139.6670 & 570.3372 & 1122.6405 & 561.8239 & 121.6565 & 319 & $\bar{V}$ & 535 & 563.2804 & 1108 & 554.7671 & 5429 & 554.2751 & 11 \\
\hline 12 & 1196.6885 & 598.8479 & 179.6620 & 590.3346 & 178.6779 & 589.8426 & G & 1026.4851 & 513.7462 & 1009.4585 & 505.2329 & 1008.4745 & 504.7409 & 10 \\
\hline 13 & 1267.7256 & 634.3664 & 1250.6991 & 625.8532 & 1249.7151 & 625.3612 & $\mathbf{A}$ & 969.4636 & 485.2354 & 952.4371 & 476.7222 & 951.4530 & 476.2302 & 9 \\
\hline 14 & 1364.7784 & 682.8928 & 1347.7518 & 674.3796 & 1346.7678 & 673.8875 & $\mathbf{P}$ & 898.4265 & 449.7169 & 881.3999 & 441.2036 & 880.4159 & 440.7116 & 8 \\
\hline 15 & 1465.8261 & 733.4167 & 1448.7995 & 724.9034 & 1447.8155 & 724.4114 & $\mathbf{T}$ & 801.3737 & 401.1905 & 784.3472 & 392.6772 & 783.3632 & 392.1852 & 7 \\
\hline 16 & 1602.8850 & 801.9461 & 1585.8584 & 793.4329 & 1584.8744 & 792.9408 & $\mathbf{H}$ & 700.3260 & 350.6667 & 683.2995 & 342.1534 & 682.3155 & 341.6614 & 6 \\
\hline 17 & 1717.9119 & 859.4596 & 1700.8854 & 850.9463 & 1699.9014 & 850.4543 & D & 563.2671 & 282.1372 & 546.2406 & 273.6239 & 545.2566 & 273.1319 & 5 \\
\hline 18 & 1832.9389 & 916.9731 & 1815.9123 & 908.4598 & 1814.9283 & 907.9678 & D & 448.2402 & 224.6237 & 431.2136 & 216.1105 & 430.2296 & 215.6185 & 4 \\
\hline
\end{tabular}




\begin{tabular}{|c|c|c|c|c|c|c|c|c|c|c|c|c|c|c|}
\hline 19 & 1932.0073 & 966.5073 & |1914.9807 & 957.9940 & |1913.9967 & 957.5020 & $\mathbf{V}$ & 333.2132 & 167.1103 & 316.1867 & 158.5970 & 315.2027 & 158.1050 & 3 \\
\hline 20 & 2019.0393 & 1010.0233 & 2002.0128 & 1001.5100 & 2001.0287 & 1001.0180 & S & 234.1448 & 117.5761 & 217.1183 & 109.0628 & 216.1343 & 108.5708 & 2 \\
\hline 21 & & & & & & & $\mathbf{K}$ & 147.1128 & 74.0600 & 130.0863 & 65.5468 & & & 1 \\
\hline
\end{tabular}
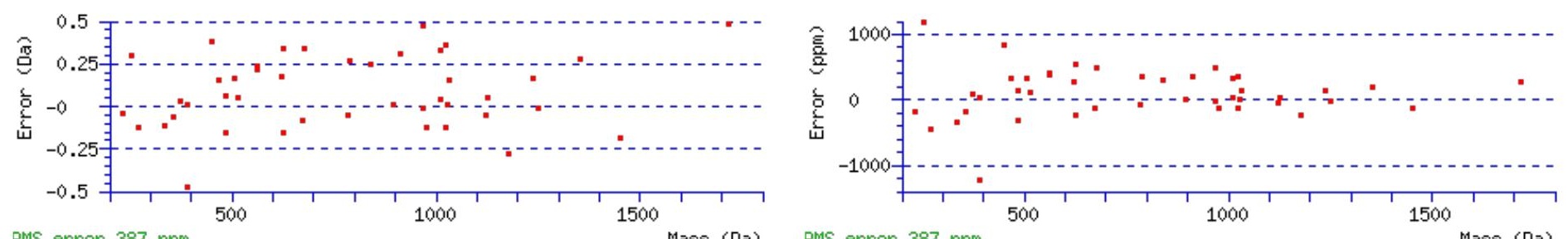

RMS error $387 \mathrm{ppm}$

\section{All matches to this query}

\begin{tabular}{|l|l|l|l|}
\hline Score & Mr(calc): & Delta & \multicolumn{1}{|c|}{ Sequence } \\
\hline 44.0 & 2164.1376 & 0.0070 & VNGTLTQVLLVGAPTHDDVSK \\
\hline 26.2 & 2163.1536 & 0.9911 & VNGTLTQVLLVGAPTHDDVSK \\
\hline 1.5 & 2162.1323 & 2.0124 & EVSSTPLHAKIPLFMIKR \\
\hline 1.5 & 2162.1323 & 2.0124 & EVSSTPLHAKIPLFMIKR \\
\hline 0.4 & 2162.1249 & 2.0198 & LATPVYASLTPQSRTRPPK \\
\hline 0.4 & 2162.1249 & 2.0198 & LATPVYASLTPQSRTRPPK \\
\hline
\end{tabular}

Spectrum No: 761; Query: 2549; Rank: 1

\section{Peptide View}

MS/MS Fragmentation of VKNMSQSIPVEEVLLQFSHYLNDSNR

Found in IPI00325312, Tax_Id=10116 Gene_Symbol=Prom1 prominin 1 isoform 1

Match to Query 2549: 3048.492776 from(763.130470,4+)

Title: 100101RatKid_NS_deglyco_23.6317.6317.4.dta

Data file K:INewmanPaper|Piliangl3SubProteomes\Piliang3SP\mgf5ppm\ERLIC_3SubProteomes5ppm.mgf

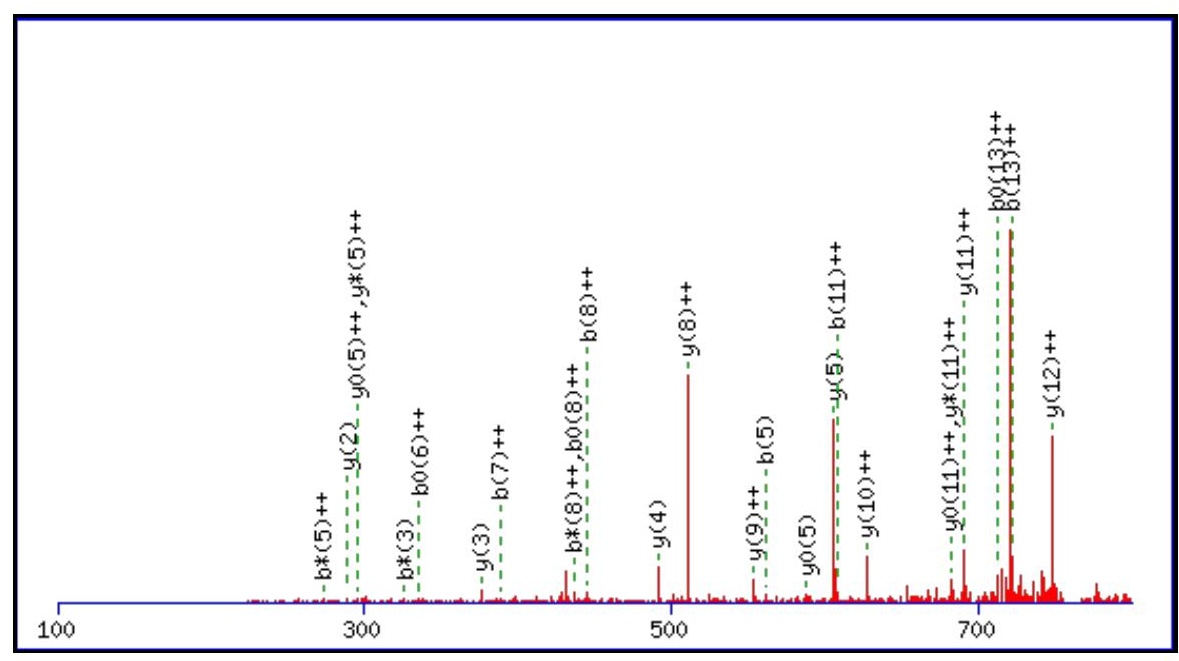



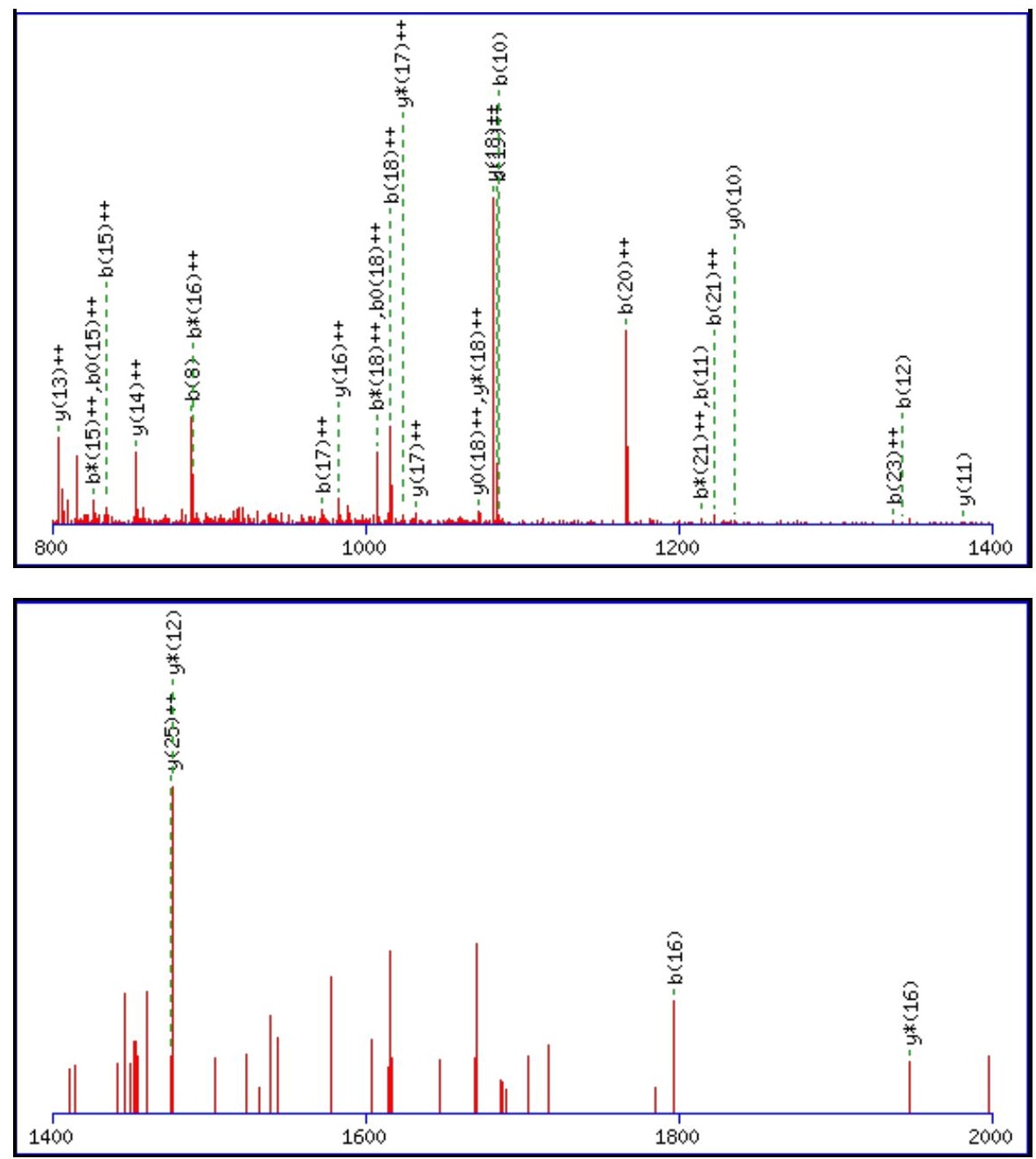

Monoisotopic mass of neutral peptide $\operatorname{Mr}($ calc): 3048.4862

Fixed modifications: Carbamidomethyl (C)

Variable modifications:

N3 : Deamidated_N (N)

N22 : Deamidated_N $(\mathrm{N})$

Ions Score: 44 Expect: 0.012

Matches (Bold Red): 56/286 fragment ions using 127 most intense peaks

\begin{tabular}{|c|c|c|c|c|c|c|c|c|c|c|c|c|c|c|}
\hline \# & b & $\mathbf{b}^{++}$ & $\mathbf{b}^{*}$ & $\mathbf{b}^{*^{++}}$ & $\mathbf{b}^{\mathbf{0}}$ & & Seq. & $\mathbf{y}$ & & $\mathbf{y}^{*}$ & & $\mathbf{y}^{0}$ & & \# \\
\hline 1 & 100.0757 & 50.5415 & & & & & V & & & & & & & 26 \\
\hline 2 & 228.1707 & & & & & & $\mathbf{K}$ & & & & & & & 25 \\
\hline 3 & 343.1976 & 172.1024 & 326.1710 & & & & $\mathbf{N}$ & & & 036 & & 3196 & 6634 & 24 \\
\hline 4 & 474.2381 & 237.6227 & 457.2115 & & & & $\mathbf{M}$ & & & & & & & 23 \\
\hline 5 & 561.2701 & 281.1387 & 544.2436 & 272. & דיב & 34 & $\mathrm{~S}$ & 2576.2627 & 350 & 362 & 217 & 2558.2521 & 6297 & 22 \\
\hline 6 & 689.3287 & 345.1680 & 672.3021 & 336. & 671. & & $\mathbf{Q}$ & 2489.2307 & 1245 & 041 & 057 & 2201 & 137 & 21 \\
\hline 7 & 776.3607 & 388.6840 & 759.3342 & 380.1707 & 758.3501 & & $S$ & 2361.1721 & 0897 & 2344. & 5764 & 2343 & 1172 & 20 \\
\hline 8 & 889.4448 & 445.2260 & 872.4 & 436. & & & I & 2274.1401 & & 2257 & 1129.0604 & 2256. & 1128.5684 & 19 \\
\hline 9 & 986.4975 & 493.7524 & 969.4710 & 485.2391 & 968.4870 & 484.7471 & $\mathbf{P}$ & 2161.0560 & 1081. & 2144. & 5184 & 2143. & 1072. & 18 \\
\hline 10 & 085.5660 & 543.2866 & 068.5394 & 534.7733 & 067.5554 & 534 & V & 2064.0033 & 1032.5053 & 2046 & 1023.9920 & 2045.9927 & 1023.5000 & 17 \\
\hline 11 & 1214.6085 & 607.8079 & 197.5820 & 599.2946 & 1196.5980 & 598.8026 & $\mathbf{E}$ & 1964.9348 & 982.9711 & 1947.9083 & 974.4578 & 1946.9243 & 973.9658 & 16 \\
\hline 12 & 343.6511 & 672.3292 & 326.6246 & 663.8159 & 1325.6406 & 663. & $\mathbf{E}$ & 1835. & 918. & 1818. & 365 & 1817. & 445 & 15 \\
\hline 13 & 1442.7196 & 721.8634 & 1425.6930 & 713.3501 & 1424.7090 & 712.8581 & $\bar{V}$ & 1706.8497 & 853.9285 & 1689.8231 & 845.4152 & 1688.8391 & 844.9232 & 14 \\
\hline 14 & 1555.8036 & 778.4054 & 1538.7771 & 769.8922 & 1537.7930 & 769.4002 & $\mathbf{L}$ & 1607.7812 & 804.3943 & 1590.7 & 795.8810 & 1589. & 3890 & 13 \\
\hline 15 & 1668.8877 & 834.9475 & 651.8611 & 826.4342 & 1650.8771 & 825.9422 & $\mathbf{L}$ & 1494.6972 & 747.8522 & 1477.6706 & 739.3390 & 1476.6866 & 738.8469 & 12 \\
\hline 16 & 1796.9463 & 898.9768 & 1779.9197 & 890.4635 & 1778.9357 & 889.9715 & $\mathbf{Q}$ & 1381.6131 & 691.3102 & 1364.5866 & 682.7969 & 1363.6025 & 682.3049 & 11 \\
\hline 17 & 1944.0147 & 972.5110 & 1926.9881 & 963.9977 & 1926.0041 & 963.5057 & $\mathbf{F}$ & 1253.5545 & 627.2809 & 1236.5280 & 618.7676 & 1235.5440 & 618.2756 & 10 \\
\hline 18 & 2031.0467 & 1016.0270 & 2014.0201 & 1007.5137 & 2013.0361 & 1007.0217 & $\mathrm{~S}$ & 1106.4861 & 553.7467 & 1089.4596 & 545.2334 & 1088.4756 & 544.7414 & 9 \\
\hline
\end{tabular}




\begin{tabular}{|c|c|c|c|c|c|c|c|c|c|c|c|c|c|c|}
\hline 19| & 2168.1056 & |1084.5564 & |2151.0791 & |1076.0432 & 2150.0950 & |1075.5512 & $\mathbf{H}$ & 1019.4541 & 510.2307 & |1002.4275 & 501.7174 & |1001.4435| & 501.2254 & 8 \\
\hline 20 & 2331.1689 & 1166.0881 & 2314.1424 & 1157.5748 & 2313.1584 & 1157.0828 & $\mathbf{Y}$ & 882.3952 & 441.7012 & 865.3686 & 433.1880 & 864.3846 & 432.6959 & 7 \\
\hline 21 & 2444.2530 & 1222.6301 & 2427.2265 & 1214.1169 & 2426.2424 & 1213.6249 & $\mathbf{L}$ & 719.3318 & 360.1696 & 702.3053 & 351.6563 & 701.3213 & 351.1643 & 6 \\
\hline 22 & 2559.2799 & 1280.1436 & 2542.2534 & 1271.6303 & 2541.2694 & 1271.1383 & $\mathbf{N}$ & 606.2478 & 303.6275 & 589.2212 & 295.1143 & 588.2372 & 294.6222 & 5 \\
\hline 23 & 2674.3069 & 1337.6571 & 2657.2803 & 1329.1438 & 2656.2963 & 1328.6518 & D & 491.2208 & 246.1141 & 474.1943 & 237.6008 & 473.2103 & 237.1088 & 4 \\
\hline 24 & 2761.3389 & 1381.1731 & 2744.3124 & 1372.6598 & 2743.3283 & 1372.1678 & S & 376.1939 & 188.6006 & 359.1674 & 180.0873 & 358.1833 & 179.5953 & 3 \\
\hline 25 & 2875.3818 & 1438.1946 & 2858.3553 & 1429.6813 & 2857.3713 & 1429.1893 & $\mathbf{N}$ & 289.1619 & 145.0846 & 272.1353 & 136.5713 & & & 2 \\
\hline 26 & & & & & & & $\mathbf{R}$ & 175.1190 & 88.0631 & 158.0924 & 79.5498 & & & 1 \\
\hline
\end{tabular}
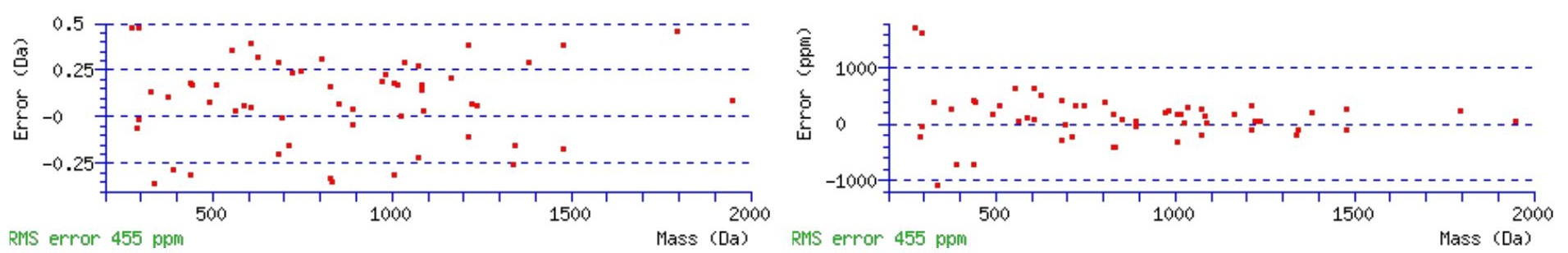

\section{All matches to this query}

\begin{tabular}{|l|l|l|l|}
\hline Score & Mr(calc): & Delta & \multicolumn{1}{|c|}{ Sequence } \\
\hline 43.8 & 3048.4862 & 0.0066 & VKNMSQSIPVEEVLLQFSHYLNDSNR \\
\hline 39.2 & 3048.4862 & 0.0066 & VKNMSQSIPVEEVLLQFSHYLNDSNR \\
\hline 22.4 & 3047.5022 & 0.9906 & VKNMSOSIPVEEVLLQFSHYLNDSNR \\
\hline 20.7 & 3047.5022 & 0.9906 & VKNMSQSIPVEEVLLQFSHYLNDSNR \\
\hline 14.1 & 3047.5022 & 0.9906 & VKNMSOSIPVEEVLLQFSHYLNDSNR \\
\hline 13.3 & 3048.4919 & 0.0009 & AALEFKDGMYANLGIGIPVLASNYISPK \\
\hline 12.6 & 3048.4919 & 0.0009 & AALEFKDGMYANLGIGIPVLASNYISPK \\
\hline 11.7 & 3048.4862 & 0.0066 & VKNMSQSIPVEEVLLQFSHYLNDSNR \\
\hline 10.9 & 3048.4919 & 0.0009 & AALEFKDGMYANLGIGIPVLASNYISPK \\
\hline 9.7 & 3047.5079 & 0.9849 & AALEFKDGMYANLGIGIPVLASNYISPK \\
\hline
\end{tabular}

Spectrum No: 762; Query: 253; Rank: 1

\section{Peptide View}

MS/MS Fragmentation of ETNVGNQTVVR

Found in IPI00200743, Tax_Id=10116 Gene_Symbol=B3gnt1_predicted UDP-GlcNAc:betaGal beta-1,3-N-acetylglucosaminyltransferase 1

Match to Query 253: 1216.602288 from(609.308420,2+)

Title: 091008RatKidney_NoSalt_31.474.474.2.dta

Data file K:INewmanPaper|Piliangl3SubProteomes\Piliang3SP\mgf5ppm\ERLIC_3SubProteomes5ppm.mgf 

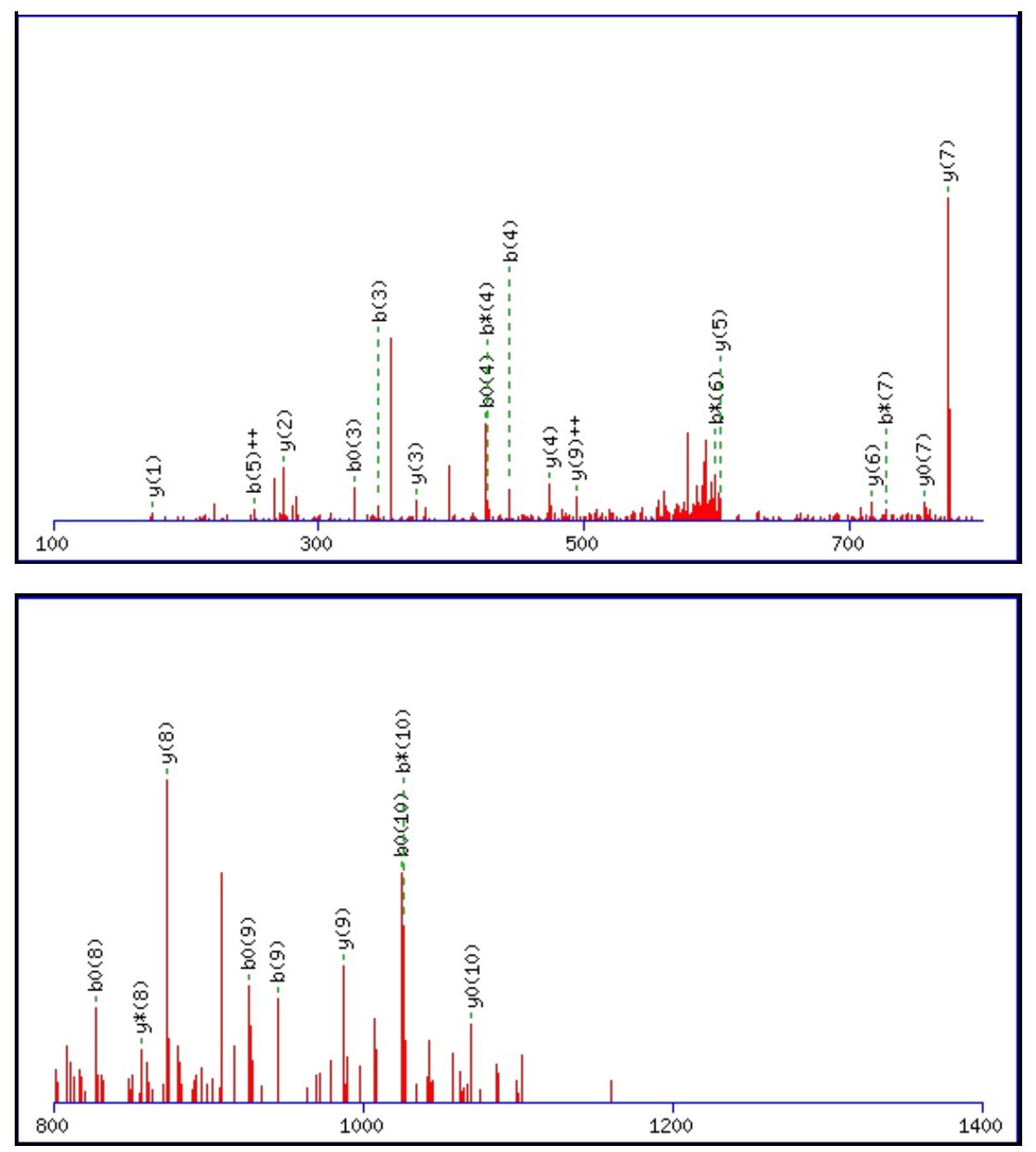

\begin{tabular}{rrrr}
\hline 00 & 1600 & 1800 & 2000 \\
\hline
\end{tabular}

Monoisotopic mass of neutral peptide $\operatorname{Mr}($ calc): 1216.6048

Fixed modifications: Carbamidomethyl (C)

Variable modifications:

N6 : Deamidated_N (N)

Ions Score: 44 Expect: 0.0057

Matches (Bold Red): 26/110 fragment ions using 54 most intense peaks

\begin{tabular}{|r|c|c|c|c|c|c|c|c|c|c|c|c|c|c|}
\hline$\#$ & $\mathbf{b}$ & $\mathbf{b}^{++}$ & $\mathbf{b}^{*}$ & $\mathbf{b}^{\mathbf{*}^{++}}$ & $\mathbf{b}^{\mathbf{0}}$ & $\mathbf{b}^{\mathbf{0 + +}}$ & Seq. & $\mathbf{y}$ & $\mathbf{y}^{++}$ & $\mathbf{y}^{\mathbf{*}}$ & $\mathbf{y}^{\boldsymbol{*}^{++}}$ & $\mathbf{y}^{\mathbf{0}}$ & $\mathbf{y}^{\mathbf{0 + +}}$ & $\#$ \\
\hline $\mathbf{1}$ & 130.0499 & 65.5286 & & & 112.0393 & 56.5233 & $\mathbf{E}$ & & & & & & & $\mathbf{1 1}$ \\
\hline $\mathbf{2}$ & 231.0975 & 116.0524 & & & 213.0870 & 107.0471 & $\mathbf{T}$ & 1088.5695 & 544.7884 & 1071.5429 & 536.2751 & $\mathbf{1 0 7 0 . 5 5 8 9}$ & 535.7831 & $\mathbf{1 0}$ \\
\hline $\mathbf{3}$ & $\mathbf{3 4 5 . 1 4 0 5}$ & 173.0739 & 328.1139 & 164.5606 & 327.1299 & 164.0686 & $\mathbf{N}$ & $\mathbf{9 8 7 . 5 2 1 8}$ & $\mathbf{4 9 4 . 2 6 4 5}$ & 970.4952 & 485.7513 & 969.5112 & 485.2592 & $\mathbf{9}$ \\
\hline $\mathbf{4}$ & $\mathbf{4 4 4 . 2 0 8 9}$ & 222.6081 & 427.1823 & 214.0948 & $\mathbf{4 2 6 . 1 9 8 3}$ & 213.6028 & $\mathbf{V}$ & $\mathbf{8 7 3 . 4 7 8 9}$ & 437.2431 & $\mathbf{8 5 6 . 4 5 2 3}$ & 428.7298 & 855.4683 & 428.2378 & $\mathbf{8}$ \\
\hline $\mathbf{5}$ & 501.2304 & $\mathbf{2 5 1 . 1 1 8 8}$ & 484.2038 & 242.6055 & 483.2198 & 242.1135 & $\mathbf{G}$ & $\mathbf{7 7 4 . 4 1 0 4}$ & 387.7089 & 757.3839 & 379.1956 & $\mathbf{7 5 6 . 3 9 9 9}$ & 378.7036 & $\mathbf{7}$ \\
\hline
\end{tabular}




\begin{tabular}{|r|r|r|r|r|r|r|r|r|r|r|r|r|r|r|r|r|r|}
$\mathbf{6}$ & 616.2573 & 308.6323 & 599.2307 & 300.1190 & 598.2467 & 299.6270 & $\mathbf{N}$ & $\mathbf{7 1 7 . 3 8 9 0}$ & 359.1981 & 700.3624 & 350.6849 & 699.3784 & 350.1928 & $\mathbf{6}$ \\
\hline $\mathbf{7}$ & 744.3159 & 372.6616 & $\mathbf{7 2 7 . 2 8 9 3}$ & 364.1483 & 726.3053 & 363.6563 & $\mathbf{Q}$ & $\mathbf{6 0 2 . 3 6 2 0}$ & 301.6847 & 585.3355 & 293.1714 & 584.3515 & 292.6794 & $\mathbf{5}$ \\
\hline $\mathbf{8}$ & 845.3635 & 423.1854 & 828.3370 & 414.6721 & $\mathbf{8 2 7 . 3 5 3 0}$ & 414.1801 & $\mathbf{T}$ & $\mathbf{4 7 4 . 3 0 3 5}$ & 237.6554 & 457.2769 & 229.1421 & 456.2929 & 228.6501 & $\mathbf{4}$ \\
\hline $\mathbf{9}$ & $\mathbf{9 4 4 . 4 3 2 0}$ & 472.7196 & 927.4054 & 464.2063 & $\mathbf{9 2 6 . 4 2 1 4}$ & 463.7143 & $\mathbf{V}$ & $\mathbf{3 7 3 . 2 5 5 8}$ & 187.1315 & 356.2292 & 178.6183 & & & \\
\hline $\mathbf{1 0}$ & 1043.5004 & 522.2538 & $\mathbf{1 0 2 6 . 4 7 3 8}$ & 513.7406 & $\mathbf{1 0 2 5 . 4 8 9 8}$ & 513.2485 & $\mathbf{V}$ & $\mathbf{2 7 4 . 1 8 7 4}$ & 137.5973 & 257.1608 & 129.0840 & & & & $\mathbf{2}$ \\
\hline $\mathbf{1 1}$ & & & & & & & $\mathbf{R}$ & $\mathbf{1 7 5 . 1 1 9 0}$ & 88.0631 & 158.0924 & 79.5498 & & & $\mathbf{1}$ \\
\hline
\end{tabular}
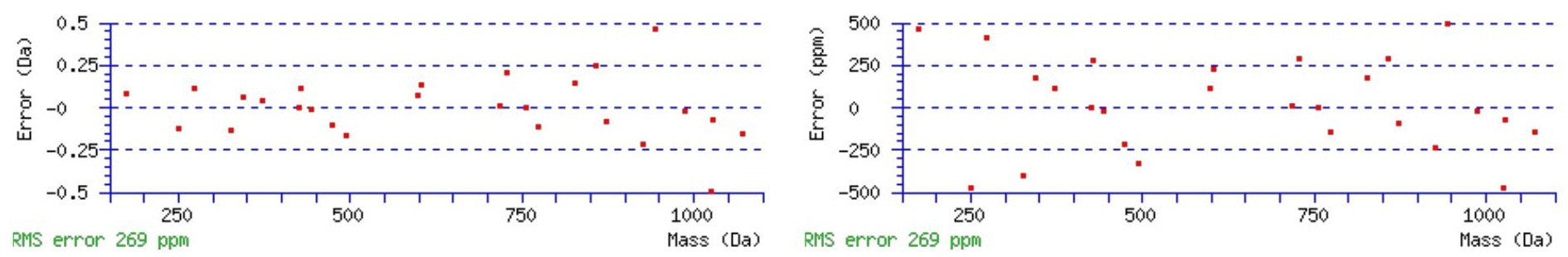

\section{All matches to this query}

\begin{tabular}{|l|l|l|l|}
\hline Score & Mr(calc): & Delta & \multicolumn{1}{|c|}{ Sequence } \\
\hline 43.8 & 1216.6048 & -0.0025 & ETNVGNQTVVR \\
\hline 9.7 & 1216.5927 & 0.0096 & VFVASCILXK \\
\hline 8.5 & 1216.6048 & -0.0025 & ETNVGNQTVVR \\
\hline 7.0 & 1216.6048 & -0.0025 & IQSNDGKELGR \\
\hline 5.6 & 1215.6013 & 1.0010 & TKNVKEYVR \\
\hline 5.5 & 1216.5965 & 0.0058 & EYATKTRIR \\
\hline 4.7 & 1215.5874 & 1.0149 & GGRGTPGKPGPR \\
\hline 3.1 & 1216.6135 & -0.0112 & MYYSRRGIR \\
\hline 3.0 & 1214.6003 & 2.0020 & DRANLQLNNR \\
\hline 2.7 & 1216.5982 & 0.0041 & MVPTRNGQSAR \\
\hline
\end{tabular}

Spectrum No: 763; Query: 6; Rank: 1

\section{Peptide View}

MS/MS Fragmentation of FGNVTVR

Found in IPI00231601, Tax_Id=10116 Gene_Symbol=Ptprc Isoform 4 of Leukocyte common antigen precursor

Match to Query 6: 792.411888 from(397.213220,2+)

Title: 091008RatKidney_NoSalt_31.657.657.2.dta

Data file K:INewmanPaper|Piliangl3SubProteomes\Piliang3SP\mgf5ppm\ERLIC_3SubProteomes5ppm.mgf

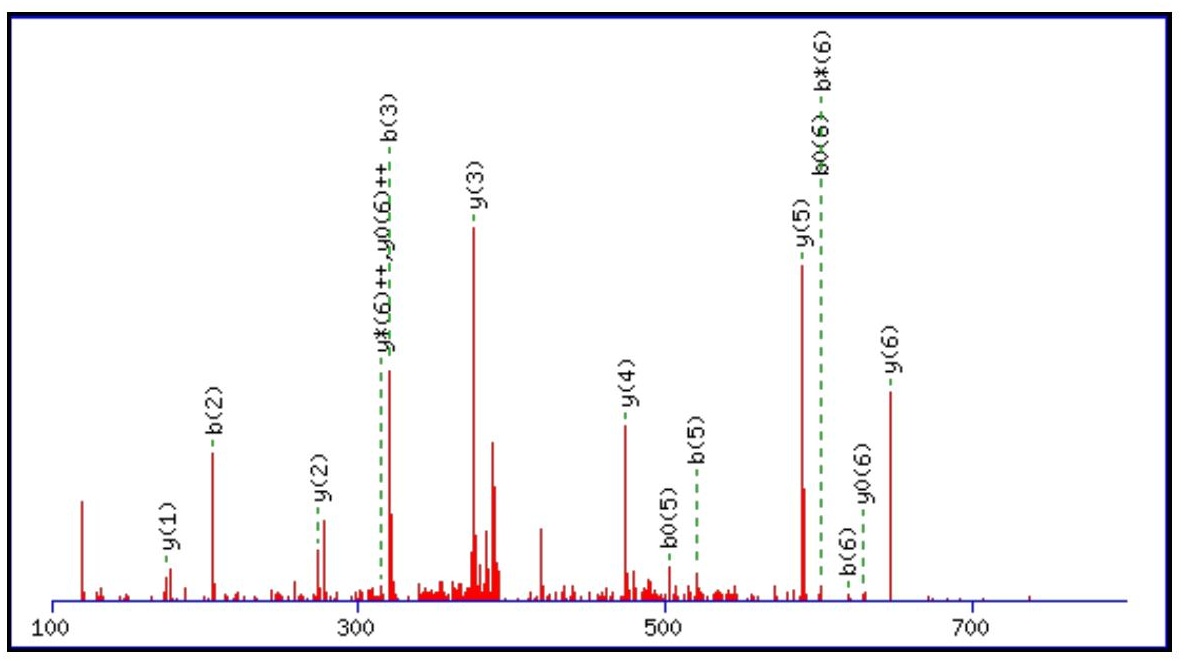




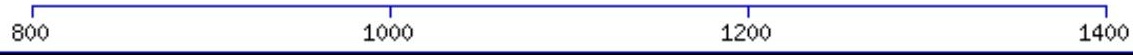

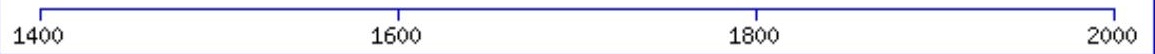

Monoisotopic mass of neutral peptide $\operatorname{Mr}($ calc): 792.4130

Fixed modifications: Carbamidomethyl (C)

Variable modifications:

N3 : Deamidated_N (N)

Ions Score: 44 Expect: 0.004

Matches (Bold Red): 16/56 fragment ions using 28 most intense peaks

\begin{tabular}{|c|c|c|c|c|c|c|c|c|c|c|c|c|c|c|}
\hline \# & b & $\mathbf{b}^{++}$ & b* & $\mathbf{b}^{*^{++}}$ & $\mathbf{b}^{\mathbf{0}}$ & $\mathbf{b}^{0++}$ & Seq. & $\mathbf{y}$ & $y^{++}$ & $\mathbf{y}^{*}$ & $\mathbf{y}^{*^{++}}$ & $\mathbf{y}^{0}$ & $\mathbf{y}^{0++}$ & $\#$ \\
\hline 1 & 148.0757 & 74.5415 & & & & & $\mathbf{F}$ & & & & & & & 7 \\
\hline 2 & 205.0972 & 103.0522 & & & & & $\mathbf{G}$ & 646.3519 & 323.6796 & 629.3253 & 315.1663 & 628.3413 & 314.6743 & 6 \\
\hline 4 & 419.1925 & 210.0999 & 402.1660 & 201.5866 & & & $\mathbf{V}$ & 474.3035 & 237.6554 & 457.2769 & 229.1421 & 456.2929 & 228.6501 & 4 \\
\hline 5 & 520.2402 & 260.6237 & 503.2136 & 252.1105 & 502.2296 & 251.6184 & $\mathbf{T}$ & 375.2350 & 188.1212 & 358.2085 & 179.6079 & 357.2245 & 179.1159 & 3 \\
\hline 7 & & & & & & & $\mathbf{R}$ & 175.1190 & 88.0631 & 158.0924 & 79.5498 & & & 1 \\
\hline
\end{tabular}
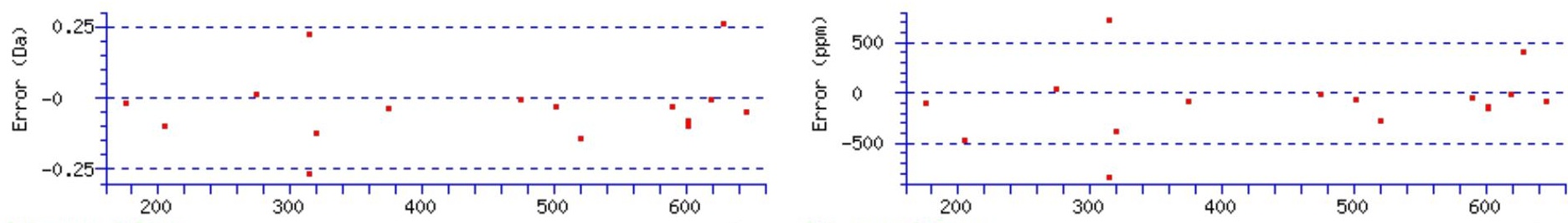

RMS error 346 ppm

Mass (Da) RMS error 346 ppm

\section{All matches to this query}

\begin{tabular}{|l|l|c|c|}
\hline Score & Mr(calc): & Delta & Sequence \\
\hline 43.7 & 792.4130 & -0.0011 & FGNVTVR \\
\hline
\end{tabular}




\begin{tabular}{|l|l|l|l|}
\hline 18.5 & 792.4164 & -0.0045 & MTAVISR \\
\hline 14.1 & 792.4164 & -0.0045 & MGTLLSR \\
\hline 13.5 & 792.4164 & -0.0045 & MATVLSR \\
\hline 8.0 & 791.4025 & 1.0094 & EGSLTGTK \\
\hline 6.3 & 792.4164 & -0.0045 & MQVKSGK \\
\hline 6.2 & 791.4072 & 1.0047 & MRNTVR \\
\hline 5.9 & 792.4065 & 0.0054 & FRAMPR \\
\hline 5.0 & 792.4164 & -0.0045 & MASILSR \\
\hline 4.8 & 791.4025 & 1.0094 & SISNVSGK \\
\hline
\end{tabular}

Spectrum No: 764; Query: 2184; Rank: 1

\section{Peptide View}

MS/MS Fragmentation of IIISPEENVTLTCTAENQLER

Found in IPI00206188, Tax_Id=10116 Gene_Symbol=Alcam CD166 antigen precursor

Match to Query 2184: 2430.196992 from(811.072940,3+)

Title: 100101RatKid_NS_deglyco_22.4104.4104.3.dta

Data file K:\NewmanPaper\Piliang \3SubProteomes $\backslash$ Piliang3SP $\backslash$ mgf5ppm\ERLIC_3SubProteomes5ppm.mgf
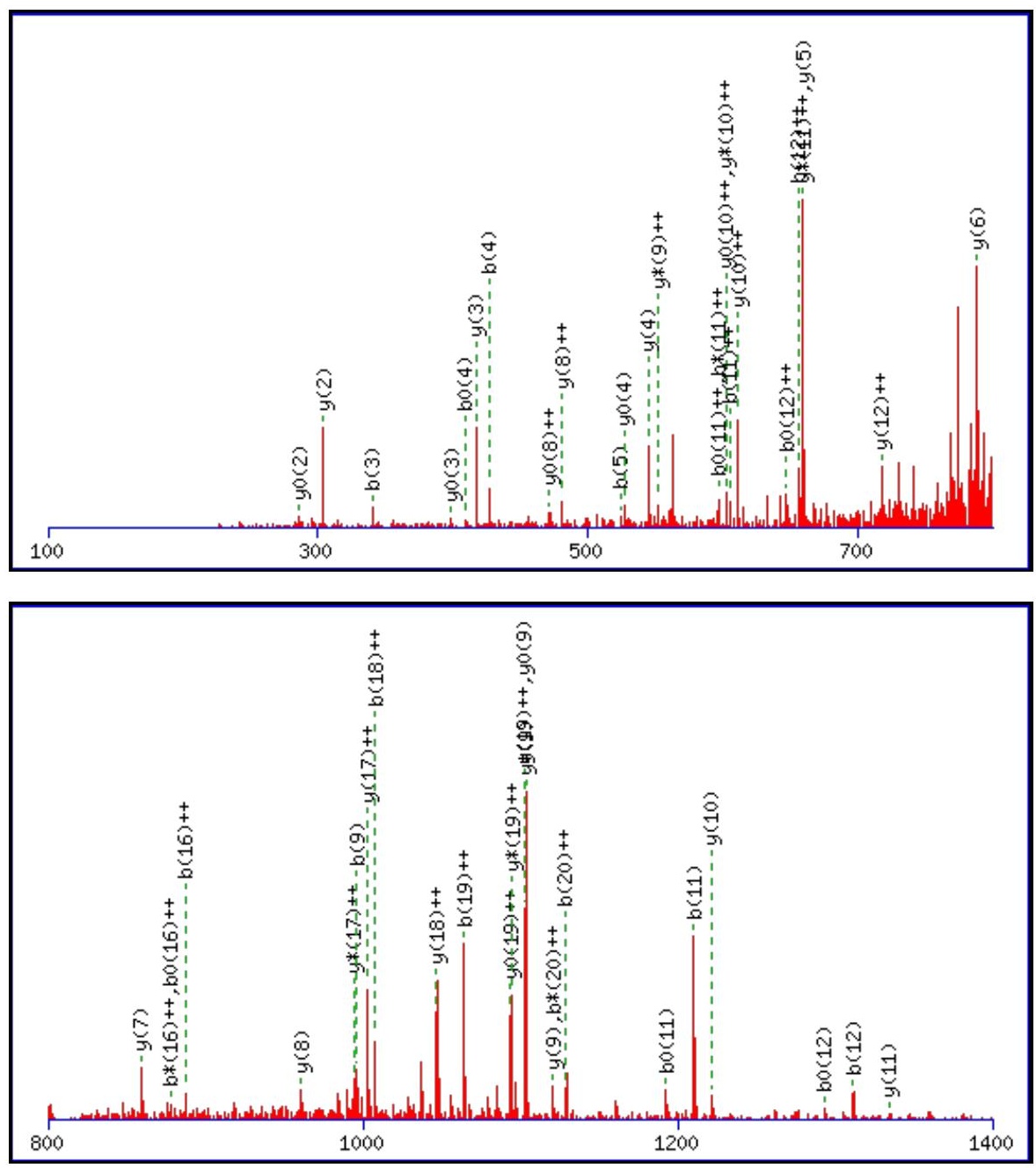


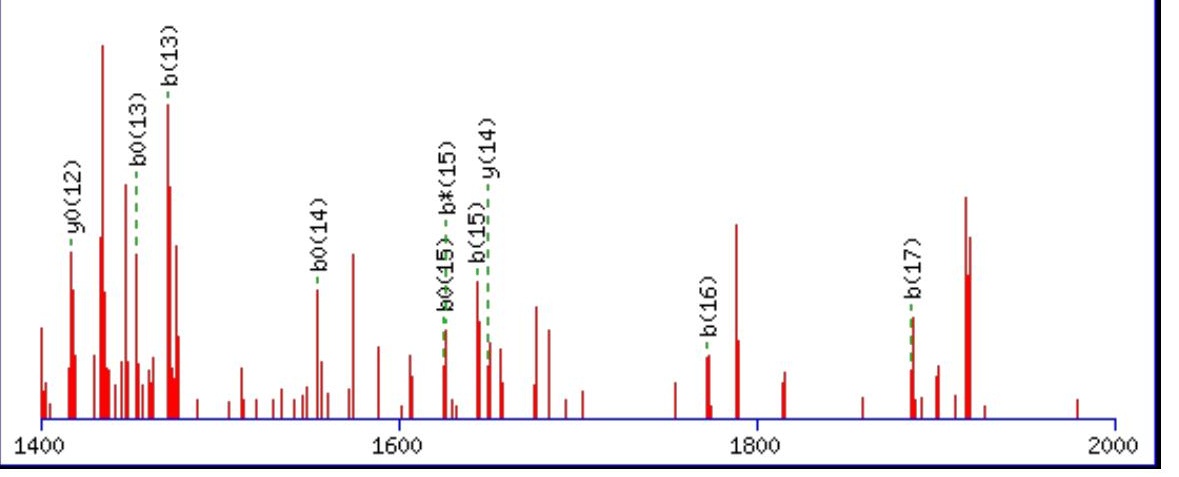

Monoisotopic mass of neutral peptide $\operatorname{Mr}($ calc): 2430.1948

Fixed modifications: Carbamidomethyl (C)

Variable modifications:

N8: : Deamidated $\mathrm{N}(\mathrm{N})$

Ions Score: 44 Expect: 0.01

Matches (Bold Red): 60/218 fragment ions using 128 most intense peaks

\begin{tabular}{|c|c|c|c|c|c|c|c|c|c|c|c|c|c|c|}
\hline \# & b & $\mathbf{b}^{++}$ & b* & $\mathbf{b}^{*^{++}}$ & $\mathbf{b}^{0}$ & $\mathbf{b}^{0++}$ & Seq. & $\mathbf{y}$ & $y^{++}$ & $\mathbf{y}^{*}$ & $\mathbf{y}^{*^{++}}$ & $\mathbf{y}^{0}$ & $y^{0++}$ & \# \\
\hline 1 & 114.0913 & 57.5493 & & & & & I & & & & & & & 21 \\
\hline 2 & 227.1754 & 114.0913 & & & & & I & 2318.1180 & 1159.5627 & 2301.0915 & 1151.0494 & 2300.1075 & 1150.5574 & 20 \\
\hline 3 & 340.2595 & 170.6334 & & & & & I & 2205.0340 & 1103.0206 & 2188.0074 & 1094.5073 & 2187.0234 & 1094.0153 & 19 \\
\hline 4 & 427.2915 & 214.1494 & & & 409.2809 & 205.1441 & S & 2091.9499 & 1046.4786 & 2074.9233 & 1037.9653 & 2073.9393 & 1037.4733 & 18 \\
\hline 5 & 524.3443 & 262.6758 & & & 506.3337 & 253.6705 & $\mathbf{P}$ & 2004.9179 & 1002.9626 & 1987.8913 & 994.4493 & 1986.9073 & 993.9573 & 17 \\
\hline 6 & 653.3869 & 327.1971 & & & 635.3763 & 318.1918 & $\mathbf{E}$ & 1907.8651 & 954.4362 & 1890.8386 & 945.9229 & 1889.8545 & 945.4309 & 16 \\
\hline 7 & 782.4294 & 391.7184 & & & 764.4189 & 382.7131 & $\mathbf{E}$ & 1778.8225 & 889.9149 & 1761.7960 & 881.4016 & 1760.8119 & 880.9096 & 15 \\
\hline 8 & 897.4564 & 449.2318 & 880.4298 & 440.7186 & 879.4458 & 440.2265 & $\mathbf{N}$ & 1649.7799 & 825.3936 & 1632.7534 & 816.8803 & 1631.7694 & 816.3883 & 14 \\
\hline 9 & 996.5248 & 498.7660 & 979.4982 & 490.2528 & 978.5142 & 489.7608 & $\mathbf{V}$ & 1534.7530 & 767.8801 & 1517.7264 & 759.3669 & 1516.7424 & 758.8748 & 13 \\
\hline 10 & 1097.5725 & 549.2899 & 1080.5459 & 540.7766 & 1079.5619 & 540.2846 & $\bar{T}$ & 1435.6846 & 718.3459 & 1418.6580 & 709.8326 & 1417.6740 & 709.3406 & 12 \\
\hline 11 & 1210.6565 & 605.8319 & 1193.6300 & 597.3186 & 1192.6460 & 596.8266 & $\mathbf{L}$ & 1334.6369 & 667.8221 & 1317.6103 & 659.3088 & 1316.6263 & 658.8168 & 11 \\
\hline 12 & 1311.7042 & 656.3557 & 1294.6777 & 647.8425 & 1293.6937 & 647.3505 & $\mathbf{T}$ & 1221.5528 & 611.2801 & 1204.5263 & 602.7668 & 1203.5423 & 602.2748 & 10 \\
\hline 13 & 1471.7349 & 736.3711 & 1454.7083 & 727.8578 & 1453.7243 & 727.3658 & C & 1120.5051 & 560.7562 & 1103.4786 & 552.2429 & 1102.4946 & 551.7509 & 9 \\
\hline 14 & 1572.7825 & 786.8949 & 1555.7560 & 778.3816 & 1554.7720 & 777.8896 & $\mathbf{T}$ & 960.4745 & 480.7409 & 943.4480 & 472.2276 & 942.4639 & 471.7356 & 8 \\
\hline 15 & 1643.8197 & 822.4135 & 1626.7931 & 813.9002 & 1625.8091 & 813.4082 & A & 859.4268 & 430.2170 & 842.4003 & 421.7038 & 841.4163 & 421.2118 & 7 \\
\hline 16 & 1772.8623 & 886.9348 & 1755.8357 & 878.4215 & 1754.8517 & 877.9295 & $\mathbf{E}$ & 788.3897 & 394.6985 & 771.3632 & 386.1852 & 770.3791 & 385.6932 & 6 \\
\hline 17 & 1886.9052 & 943.9562 & 1869.8786 & 935.4430 & 1868.8946 & 934.9509 & $\mathbf{N}$ & 659.3471 & 330.1772 & 642.3206 & 321.6639 & 641.3365 & 321.1719 & 5 \\
\hline 18 & 2014.9638 & 1007.9855 & 1997.9372 & 999.4722 & 1996.9532 & 998.9802 & $\mathbf{Q}$ & 545.3042 & 273.1557 & 528.2776 & 264.6425 & 527.2936 & 264.1504 & 4 \\
\hline 19 & 2128.0478 & 1064.5275 & 2111.0213 & 1056.0143 & 2110.0373 & 1055.5223 & $\mathbf{L}$ & 417.2456 & 209.1264 & 400.2191 & 200.6132 & 399.2350 & 200.1212 & 3 \\
\hline 20 & 2257.0904 & 1129.0488 & 2240.0639 & 1120.5356 & 2239.0799 & 1120.0436 & $\mathbf{E}$ & 304.1615 & 152.5844 & 287.1350 & 144.0711 & 286.1510 & 143.5791 & 2 \\
\hline 21 & & & & & & & $\mathbf{R}$ & 175.1190 & 88.0631 & 158.0924 & 79.5498 & & & 1 \\
\hline
\end{tabular}
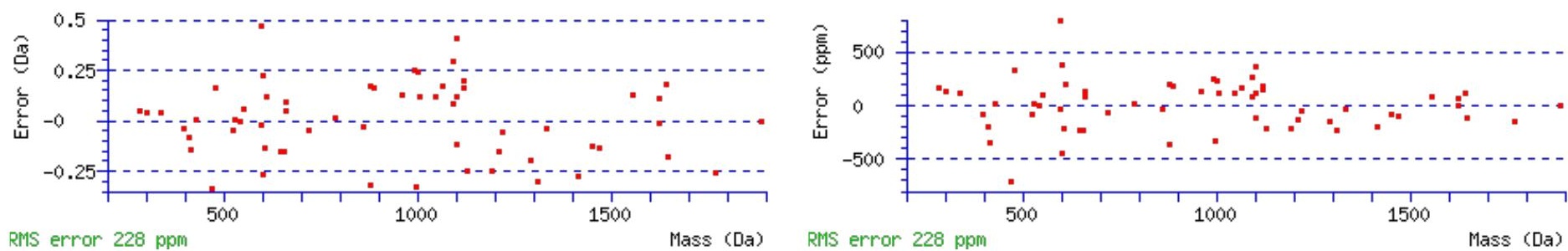

\section{All matches to this query}

\begin{tabular}{|l|l|l|l|}
\hline Score & $\operatorname{Mr}($ calc): & Delta & Sequence \\
\hline & & & \\
\hline
\end{tabular}




\begin{tabular}{|c|c|c|c|}
\hline 43.7 & 2430.1948 & 0.0022 & HOPEEINV ILICIAEN \\
\hline 20.4 & 2430.1948 & 0.0022 & IIISPEENVTLTCTAENQLER \\
\hline 12.4 & 2429.2108 & 0.9862 & IIISPEENVTLTCTAENQLER \\
\hline 0.1 & 2430.2113 & -0.0143 & VLYDPFLPPLRLSTGGEKLR \\
\hline
\end{tabular}

Spectrum No: 765; Query: 139; Rank: 1

\section{Peptide View}

MS/MS Fragmentation of FSGANDTDFR

Found in IPI00563600, Tax_Id=10116 Gene_Symbol=LOC681879 Protein O-mannosyltransferase 2

Match to Query 139: 1129.466388 from(565.740470,2+)

Title: 091008RatKidney_NH4Format01_25.1155.1155.2.dta

Data file K:INewmanPaper|Piliang|3SubProteomes\Piliang3SP\mgf5ppm\ERLIC_3SubProteomes5ppm.mgf
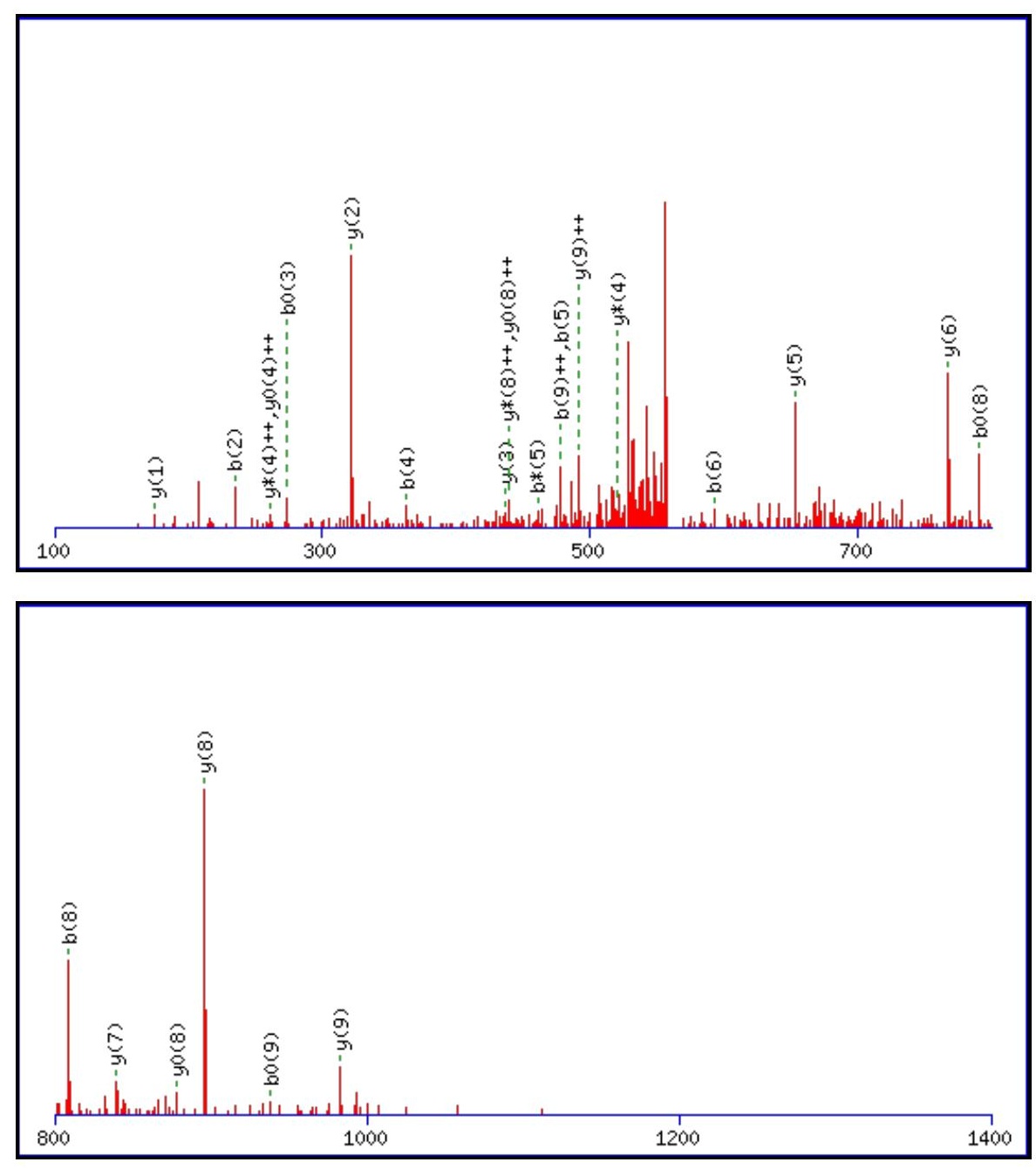


$1400 \quad 1600 \quad 1800 \quad 2000$

Monoisotopic mass of neutral peptide $\operatorname{Mr}($ calc): 1129.4676

Fixed modifications: Carbamidomethyl (C)

Variable modifications:

N5: Deamidated $\mathrm{N}(\mathrm{N})$

Ions Score: 44 Expect: 0.0038

Matches (Bold Red): 25/94 fragment ions using 68 most intense peaks

\begin{tabular}{|r|c|c|c|c|c|c|c|c|c|c|c|c|c|c|}
\hline$\#$ & $\mathbf{b}$ & $\mathbf{b}^{++}$ & $\mathbf{b}^{*}$ & $\mathbf{b}^{\mathbf{*}^{++}}$ & $\mathbf{b}^{\mathbf{0}}$ & $\mathbf{b}^{\mathbf{0 + +}}$ & $\mathbf{S e q}$ & $\mathbf{y}$ & $\mathbf{y}^{++}$ & $\mathbf{y}^{*}$ & $\mathbf{y}^{\mathbf{*}^{++}}$ & $\mathbf{y}^{\mathbf{0}}$ & $\mathbf{y}^{\mathbf{0 + +}}$ & $\#$ \\
\hline $\mathbf{1}$ & 148.0757 & 74.5415 & & & & & $\mathbf{F}$ & & & & & & & $\mathbf{1 0}$ \\
\hline $\mathbf{2}$ & 235.1077 & 118.0575 & & & 217.0972 & 109.0522 & $\mathbf{S}$ & $\mathbf{9 8 3 . 4 0 6 5}$ & $\mathbf{4 9 2 . 2 0 6 9}$ & 966.3799 & 483.6936 & 965.3959 & $\mathbf{4 8 3 . 2 0 1 6}$ & $\mathbf{9}$ \\
\hline $\mathbf{3}$ & 292.1292 & 146.5682 & & & 274.1186 & 137.5629 & $\mathbf{G}$ & $\mathbf{8 9 6 . 3 7 4 4}$ & 448.6909 & 879.3479 & $\mathbf{4 4 0 . 1 7 7 6}$ & $\mathbf{8 7 8 . 3 6 3 9}$ & $\mathbf{4 3 9 . 6 8 5 6}$ & $\mathbf{8}$ \\
\hline $\mathbf{4}$ & 363.1663 & 182.0868 & & & 345.1557 & 173.0815 & $\mathbf{A}$ & $\mathbf{8 3 9 . 3 5 3 0}$ & 420.1801 & 822.3264 & 411.6669 & 821.3424 & 411.1748 & $\mathbf{7}$ \\
\hline $\mathbf{5}$ & $\mathbf{4 7 8 . 1 9 3 2}$ & 239.6003 & $\mathbf{4 6 1 . 1 6 6 7}$ & 231.0870 & 460.1827 & 230.5950 & $\mathbf{N}$ & $\mathbf{7 6 8 . 3 1 5 9}$ & 384.6616 & 751.2893 & 376.1483 & 750.3053 & 375.6563 & $\mathbf{6}$ \\
\hline $\mathbf{6}$ & $\mathbf{5 9 3 . 2 2 0 2}$ & 297.1137 & 576.1936 & 288.6005 & 575.2096 & 288.1084 & $\mathbf{D}$ & $\mathbf{6 5 3 . 2 8 8 9}$ & 327.1481 & 636.2624 & 318.6348 & 635.2784 & 318.1428 & $\mathbf{5}$ \\
\hline $\mathbf{7}$ & 694.2679 & 347.6376 & 677.2413 & 339.1243 & 676.2573 & 338.6323 & $\mathbf{T}$ & 538.2620 & 269.6346 & $\mathbf{5 2 1 . 2 3 5 4}$ & $\mathbf{2 6 1 . 1 2 1 4}$ & 520.2514 & $\mathbf{2 6 0 . 6 2 9 3}$ & $\mathbf{4}$ \\
\hline $\mathbf{8}$ & $\mathbf{8 0 9 . 2 9 4 8}$ & 405.1510 & 792.2682 & 396.6378 & $\mathbf{7 9 1 . 2 8 4 2}$ & 396.1458 & $\mathbf{D}$ & $\mathbf{4 3 7 . 2 1 4 3}$ & 219.1108 & 420.1878 & 210.5975 & 419.2037 & 210.1055 & $\mathbf{3}$ \\
\hline $\mathbf{9}$ & 956.3632 & $\mathbf{4 7 8 . 6 8 5 2}$ & 939.3367 & 470.1720 & $\mathbf{9 3 8 . 3 5 2 6}$ & 469.6800 & $\mathbf{F}$ & $\mathbf{3 2 2 . 1 8 7 4}$ & 161.5973 & 305.1608 & 153.0840 & & & $\mathbf{2}$ \\
\hline $\mathbf{1 0}$ & & & & & & & $\mathbf{R}$ & $\mathbf{1 7 5 . 1 1 9 0}$ & 88.0631 & 158.0924 & 79.5498 & & & $\mathbf{1}$ \\
\hline
\end{tabular}
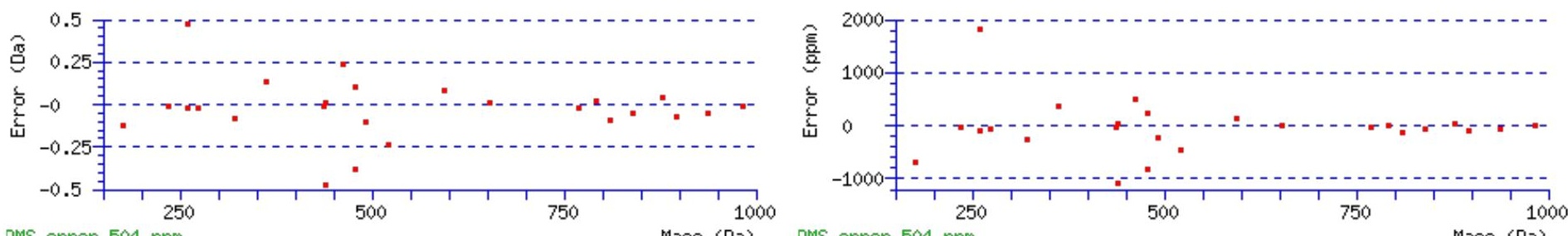

RMS error 504 ppm

Mass (Da) RMS error 504 ppm

\section{All matches to this query}

\begin{tabular}{|l|l|l|l|}
\hline Score & Mr(calc): & Delta & \multicolumn{1}{|c|}{ Sequence } \\
\hline 43.7 & 1129.4676 & -0.0012 & FSGANDTDFR \\
\hline 10.6 & 1128.4617 & 1.0047 & $\underline{\text { PSTPPQKSK }}$ \\
\hline 5.3 & 1127.4682 & 1.9982 & $\underline{\text { MTSSPSSVPR }}$ \\
\hline 4.8 & 1128.4634 & 1.0029 & $\underline{\text { RMSTPSNQK }}$ \\
\hline 4.8 & 1128.4634 & 1.0029 & $\underline{\text { RMSTPSNQK }}$ \\
\hline 4.3 & 1127.4699 & 1.9965 & QNKDSMSMR \\
\hline 4.0 & 1128.4617 & 1.0046 & GSDITVHIK \\
\hline 4.0 & 1129.4710 & -0.0046 & $\underline{\text { SSVNNMSSFR }}$ \\
\hline 3.9 & 1129.4627 & 0.0037 & $\underline{\text { CVNLPGSFR }}$ \\
\hline 3.6 & 1129.4628 & 0.0036 & $\underline{\text { CPLDSVGFR }}$ \\
\hline
\end{tabular}


Spectrum No: 766; Query: 1810; Rank: 1

\section{Peptide View}

MS/MS Fragmentation of EGNCSVQSGFAWQDCDFK

Found in IPI00187799, Tax_Id=10116 Gene_Symbol=LOC25087;Kng1 Isoform HMW of Kininogen-1 precursor

Match to Query 1810: 2134.841748 from(1068.428150,2+)

Title: 100101RatKid_NS_deglyco_25.3241.3241.2.dta

Data file K:\NewmanPaper\Piliang \3SubProteomes \Piliang3SP $\backslash$ mgf5ppm\ERLIC_3SubProteomes5ppm.mgf
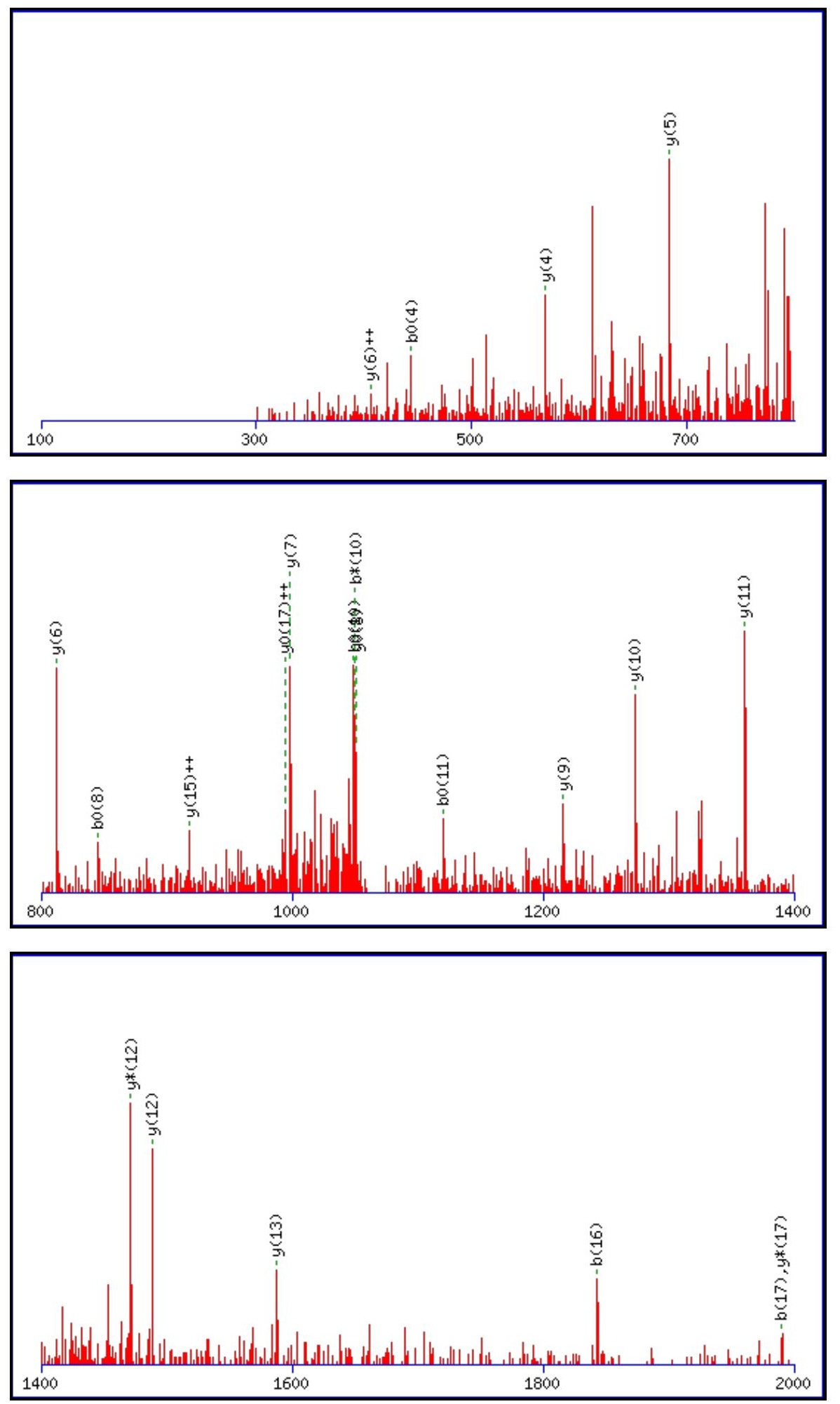

Monoisotopic mass of neutral peptide $\operatorname{Mr}($ calc): 2134.8361 
Fixed modifications: Carbamidomethyl (C)

Variable modifications:

N3 : Deamidated N ( N)

Ions Score: 44 Expect: $\odot .0079$

Matches (Bold Red): 22/196 fragment ions using 40 most intense peaks

\begin{tabular}{|c|c|c|c|c|c|c|c|c|c|c|c|c|c|c|}
\hline \# & b & $\mathbf{b}^{++}$ & $\mathbf{b}^{*}$ & $\mathbf{b}^{*^{++}}$ & $\mathbf{b}^{0}$ & $\mathbf{b}^{0++}$ & Seq. & $\mathbf{y}$ & $\mathbf{y}^{++}$ & $\mathrm{y}^{*}$ & $\mathbf{y}^{*^{++}}$ & $\mathbf{y}^{0}$ & $\mathbf{y}^{\mathbf{0 + +}}$ & \# \\
\hline 1 & 130.0499 & 65.5286 & & & 112.0393 & 56.5233 & $\mathbf{E}$ & & & & & & & 18 \\
\hline 2 & 187.0713 & 94.0393 & & & 169.0608 & 85.0340 & G & 007 & 040 & 7742 & & 1988.7902 & 3987 & 17 \\
\hline 3 & 302.0983 & 151.5528 & 285.0717 & & 284.0877 & 142.5475 & $\mathbf{N}$ & 1949.7793 & 975.3933 & 1932.7527 & 966.8800 & 1931.7687 & 966.3880 & 16 \\
\hline 4 & 462.1289 & 31.5681 & 445. & 2 & 84 & 222.5628 & C & 1834.7523 & & 317.7258 & 909.3665 & 1816.7418 & 8.8745 & 15 \\
\hline 5 & 549.1609 & 275.0841 & 532.1344 & 266.5708 & 531.1504 & 266.0788 & $S$ & 1674.7217 & 837.8645 & 1657.6951 & 829.3512 & 1656.7111 & 828.8592 & 14 \\
\hline 6 & 648.2294 & 324.6183 & 631.2028 & 316.1050 & 630.2188 & 315.6130 & $\mathbf{V}$ & 1587.6897 & 794.3485 & 1570.6631 & 785.8352 & 1569.6791 & 785.3432 & 13 \\
\hline 7 & 776.2879 & 388.6476 & 759. & 380. & 758.2 & 379.6423 & $\mathbf{Q}$ & 1488.6212 & & & & 1470 & 090 & 12 \\
\hline 8 & 863.3200 & 432.1636 & 846.2934 & 423.6503 & 845.3094 & 423.1583 & $S$ & 1360.5627 & 680.7850 & 1343.5361 & 672.2717 & 1342.5521 & 671.7797 & 11 \\
\hline 9 & 920.3414 & 460.6744 & 903.3149 & 452.1611 & 902.3309 & 451.6691 & G & 1273.5306 & 637.2690 & 1256.5041 & 628.7557 & 1255.5201 & 628.2637 & 10 \\
\hline 10 & 67.4098 & 534.2086 & 50.3833 & 525.6953 & 1049.3993 & 525.2033 & $\mathbf{F}$ & 1216.5092 & 608.7582 & 1199.4826 & 600.2449 & 1198.4986 & 599.7529 & 9 \\
\hline 11 & 1138.4470 & 569.7271 & 121.4204 & 561.2138 & 1120.4364 & 560.7218 & A & 1069.4408 & 535.2240 & 1052.4142 & 526.7107 & 1051.4302 & 526.2187 & 8 \\
\hline 12 & 1324.5263 & 662.7668 & 1307.4997 & 654.2535 & 1306.5157 & 653.7615 & $\mathbf{W}$ & 998.4036 & 499.7055 & 981.3771 & 491.1922 & 980.3931 & 490.7002 & 7 \\
\hline 13 & 1452.5848 & 726.7961 & 435.5583 & 718.2828 & 1434.5743 & 717.7908 & $\mathbf{Q}$ & 812.3243 & 406.6658 & 795.2978 & 398.1525 & 794.3138 & 397.6605 & 6 \\
\hline 14 & 1567.6118 & 784.3095 & 550.5852 & 775.7963 & 1549.6012 & 775.3043 & D & 684.2658 & 342.6365 & 667.2392 & 334.1232 & 666.2552 & 333.6312 & 5 \\
\hline 15 & 1727.6424 & 864.3249 & 1710.6159 & 855.8116 & 1709.6319 & 855.3196 & $\mathrm{C}$ & 569.2388 & 285.1230 & 552.2123 & 276.6098 & 551.2282 & 276.1178 & 4 \\
\hline 16 & 1842.6694 & 921.8383 & 1825.6428 & 913.3251 & 1824.6588 & 912.8330 & D & 409.2082 & 205.1077 & 392.1816 & 196.5944 & 391.1976 & 196.1024 & 3 \\
\hline 17 & 1989.7378 & 995.3725 & 1972.7112 & 986.8593 & 1971.7272 & 986.3673 & $\mathbf{F}$ & 294.1812 & 147.5942 & 277.1547 & 139.0810 & & & 2 \\
\hline 18 & & & & & & & K & 147.1128 & 74.0600 & 130.0863 & 65.5468 & & & 1 \\
\hline
\end{tabular}
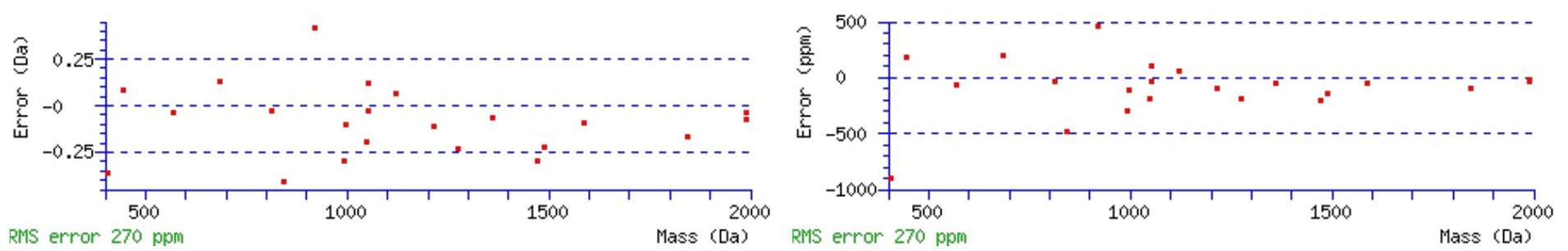

\section{All matches to this query}

\begin{tabular}{|l|l|l|l|}
\hline Score & Mr(calc): & Delta & Sequence \\
\hline 43.6 & 2134.8361 & 0.0057 & EGNCSVQSGFAWQDCDFK \\
\hline 31.3 & 2133.8521 & 0.9897 & EGNCSVQSGFAWQDCDFK \\
\hline
\end{tabular}

Spectrum No: 767; Query: 195; Rank: 1

\section{Peptide View}

MS/MS Fragmentation of HANWTLTPLK

Found in IPI00231264, Tax_Id=10116 Gene_Symbol=Pon1 Serum paraoxonase/arylesterase 1

Match to Query 195: 1180.625588 from(591.320070,2+)

Title: 100101RatKid_NS_deglyco_23.2377.2377.2.dta

Data file K:INewmanPaper|Piliangl3SubProteomes\Piliang3SP\mgf5ppm\ERLIC_3SubProteomes5ppm.mgf 

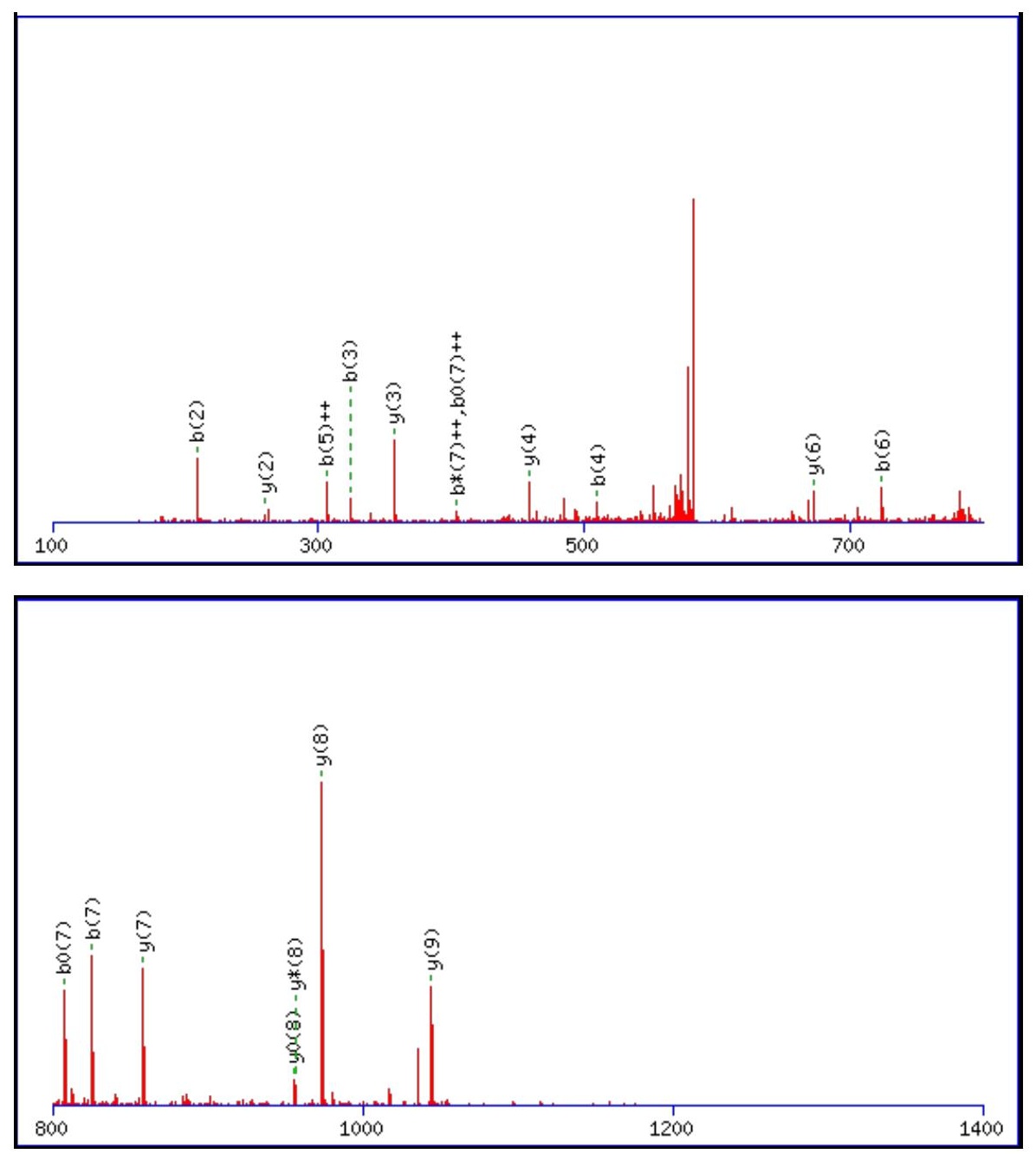

\begin{tabular}{rrrr}
\hline 1400 & 1600 & 1800 & 2000 \\
\hline
\end{tabular}

Monoisotopic mass of neutral peptide $\operatorname{Mr}($ calc): 1180.6240

Fixed modifications: Carbamidomethyl (C)

Variable modifications:

N3 : Deamidated N (N)

Ions Score: 44 Expect: 0.0043

Matches (Bold Red): 18/90 fragment ions using 36 most intense peaks

\begin{tabular}{|r|c|c|c|c|c|c|c|c|c|c|c|c|c|c|}
\hline$\#$ & $\mathbf{b}$ & $\mathbf{b}^{++}$ & $\mathbf{b}^{*}$ & $\mathbf{b}^{\boldsymbol{*}^{++}}$ & $\mathbf{b}^{\mathbf{0}}$ & $\mathbf{b}^{\mathbf{0}+}$ & $\mathbf{S e q}$ & $\mathbf{y}$ & $\mathbf{y}^{++}$ & $\mathbf{y}^{\mathbf{*}}$ & $\mathbf{y}^{\boldsymbol{*}^{++}}$ & $\mathbf{y}^{\mathbf{0}}$ & $\mathbf{y}^{\mathbf{0 + +}}$ & $\#$ \\
\hline $\mathbf{1}$ & 138.0662 & 69.5367 & & & & & $\mathbf{H}$ & & & & & & & $\mathbf{1 0}$ \\
\hline $\mathbf{2}$ & $\mathbf{2 0 9 . 1 0 3 3}$ & 105.0553 & & & & & $\mathbf{A}$ & $\mathbf{1 0 4 4 . 5 7 2 4}$ & 522.7898 & 1027.5459 & 514.2766 & 1026.5619 & 513.7846 & $\mathbf{9}$ \\
\hline $\mathbf{3}$ & $\mathbf{3 2 4 . 1 3 0 2}$ & 162.5688 & 307.1037 & 154.0555 & & & $\mathbf{N}$ & $\mathbf{9 7 3 . 5 3 5 3}$ & 487.2713 & $\mathbf{9 5 6 . 5 0 8 8}$ & 478.7580 & $\mathbf{9 5 5 . 5 2 4 7}$ & 478.2660 & $\mathbf{8}$ \\
\hline $\mathbf{4}$ & $\mathbf{5 1 0 . 2 0 9 6}$ & 255.6084 & 493.1830 & 247.0951 & & & $\mathbf{W}$ & $\mathbf{8 5 8 . 5 0 8 4}$ & 429.7578 & 841.4818 & 421.2445 & 840.4978 & 420.7525 & $\mathbf{7}$ \\
\hline $\mathbf{5}$ & 611.2572 & $\mathbf{3 0 6 . 1 3 2 3}$ & 594.2307 & 297.6190 & 593.2467 & 297.1270 & $\mathbf{T}$ & $\mathbf{6 7 2 . 4 2 9 1}$ & 336.7182 & 655.4025 & 328.2049 & 654.4185 & 327.7129 & $\mathbf{6}$ \\
\hline
\end{tabular}




\begin{tabular}{|r|r|r|r|r|r|r|r|r|r|r|r|r|r|r|r|r|r|}
$\mathbf{6}$ & $\mathbf{7 2 4 . 3 4 1 3}$ & 362.6743 & 707.3147 & 354.1610 & 706.3307 & 353.6690 & L & 571.3814 & 286.1943 & 554.3548 & 277.6811 & 553.3708 & 277.1890 & $\mathbf{5}$ \\
\hline $\mathbf{7}$ & $\mathbf{8 2 5 . 3 8 9 0}$ & 413.1981 & 808.3624 & $\mathbf{4 0 4 . 6 8 4 9}$ & $\mathbf{8 0 7 . 3 7 8 4}$ & $\mathbf{4 0 4 . 1 9 2 8}$ & T & $\mathbf{4 5 8 . 2 9 7 3}$ & 229.6523 & 441.2708 & 221.1390 & 440.2867 & 220.6470 & $\mathbf{4}$ \\
\hline $\mathbf{8}$ & 922.4417 & 461.7245 & 905.4152 & 453.2112 & 904.4312 & $\mathbf{4 5 2 . 7 1 9 2}$ & $\mathbf{P}$ & $\mathbf{3 5 7 . 2 4 9 6}$ & 179.1285 & 340.2231 & 170.6152 & & & $\mathbf{3}$ \\
\hline $\mathbf{9}$ & 1035.5258 & 518.2665 & 1018.4993 & 509.7533 & 1017.5152 & 509.2613 & $\mathbf{L}$ & $\mathbf{2 6 0 . 1 9 6 9}$ & 130.6021 & 243.1703 & 122.0888 & & & \\
\hline $\mathbf{1 0}$ & & & & & & & K & 147.1128 & 74.0600 & 130.0863 & 65.5468 & & & $\mathbf{1}$ \\
\hline
\end{tabular}
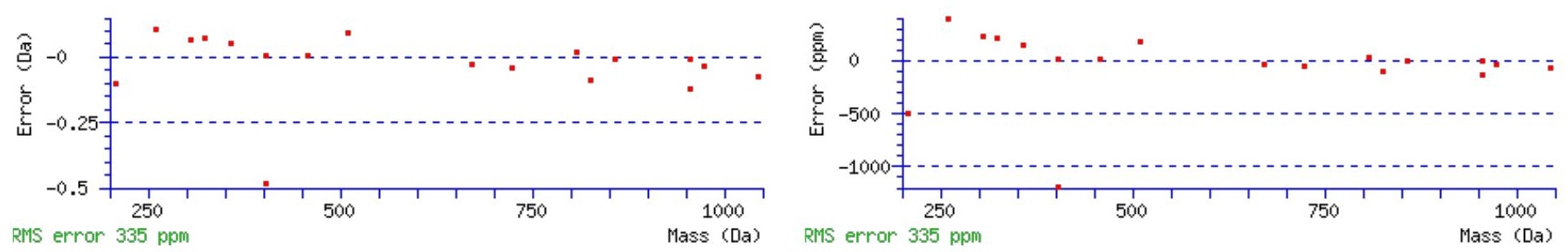

\section{All matches to this query}

\begin{tabular}{|l|c|c|c|}
\hline Score & Mr(calc): & Delta & Sequence \\
\hline 43.6 & 1180.6240 & 0.0015 & HANWTLTPLK \\
\hline 4.5 & 1180.6151 & 0.0105 & KPRIMTLSR \\
\hline 3.0 & 1180.6151 & 0.0105 & $\underline{\text { KPRIMTLSR }}$ \\
\hline
\end{tabular}

Spectrum No: 768; Query: 1910; Rank: 1

\section{Peptide View}

MS/MS Fragmentation of MQNNSAENETAEGEEKGESR

Found in IPI00208118, Tax_Id=10116 Gene_Symbol=Cald1 Non-muscle caldesmon

Match to Query 1910: 2209.913982 from(737.645270,3+)

Title: 091008RatKidney_NoSalt_21.382.382.3.dta

Data file K:INewmanPaper|Piliang\3SubProteomes\Piliang3SP\mgf5ppm\ERLIC_3SubProteomes5ppm.mgf

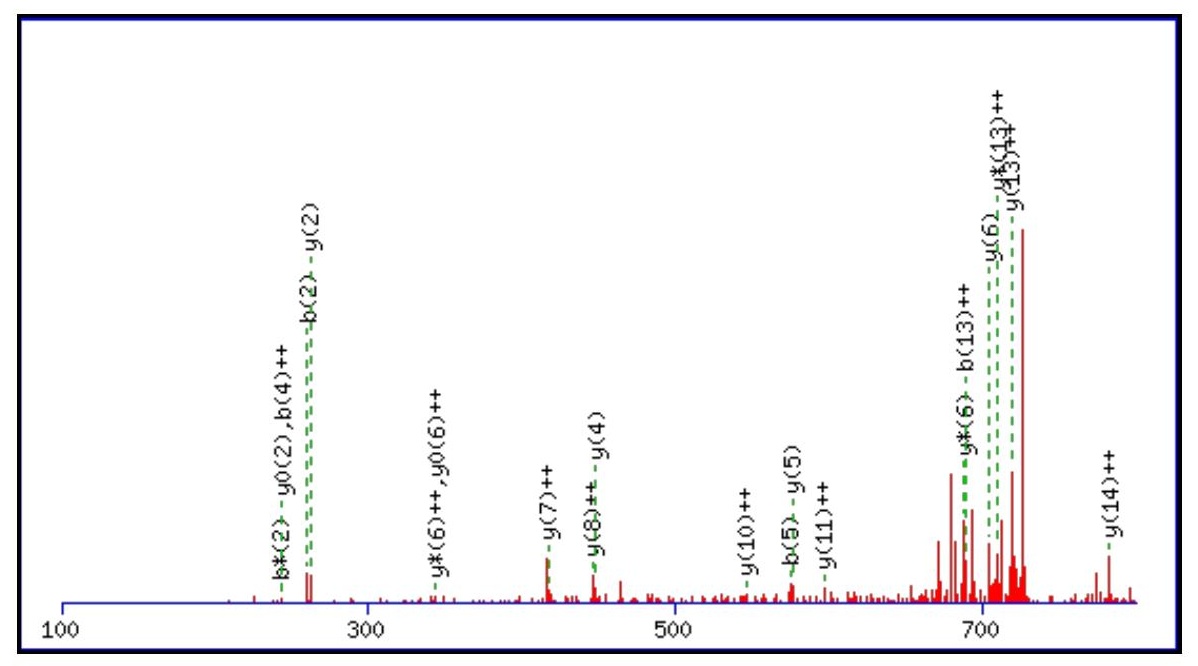



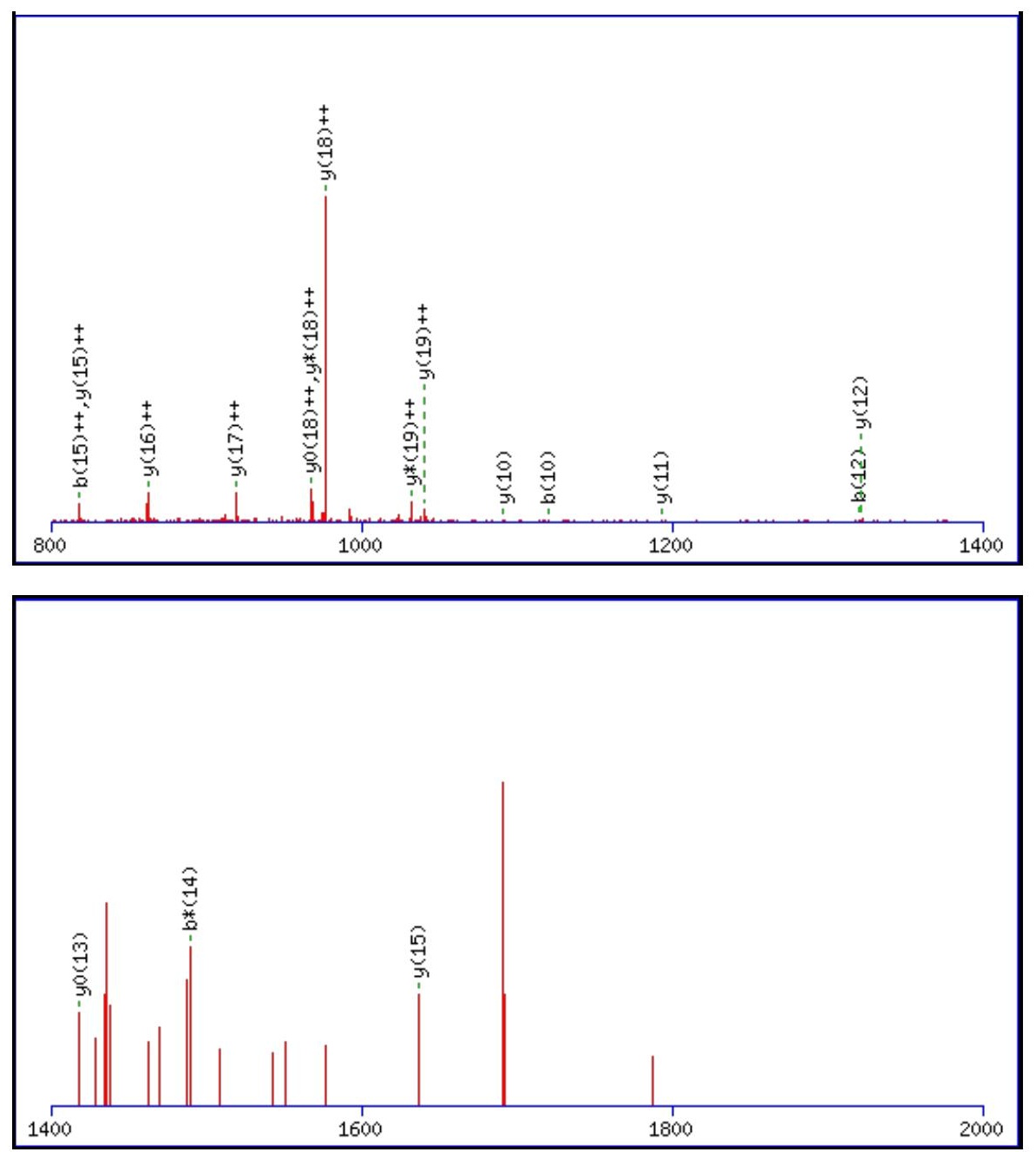

Monoisotopic mass of neutral peptide $\operatorname{Mr}($ calc): 2209.9029

Fixed modifications: Carbamidomethyl (C)

Variable modifications:

N8: : Deamidated $\mathrm{N}(\mathrm{N})$

Ions Score: 44 Expect: 0.0099

Matches (Bold Red): 37/216 fragment ions using 111 most intense peaks

\begin{tabular}{|c|c|c|c|c|c|c|c|c|c|c|c|c|c|c|}
\hline \# & b & $\mathbf{b}^{++}$ & $\mathbf{b}^{*}$ & $\mathbf{b}^{*^{++}}$ & $\mathbf{b}^{\mathbf{0}}$ & $\mathbf{b}^{0++}$ & Seq. & $\mathbf{y}$ & $y^{++}$ & $\mathbf{y}^{*}$ & $\mathrm{y}^{\mathrm{*}^{++}}$ & $\mathbf{y}^{\mathbf{0}}$ & $y^{0++}$ & \# \\
\hline 1 & 132.0478 & 66.5275 & & & & & $\mathbf{M}$ & & & & & & & 20 \\
\hline 2 & 260.1063 & 130.5568 & 243.0798 & 122.0435 & & & $\mathbf{Q}$ & 2079.8697 & 1040.4385 & 2062.8432 & 1031.9252 & 2061.8592 & 1031.4332 & 19 \\
\hline 3 & 374.1493 & 187.5783 & 357.1227 & 179.0650 & & & $\mathbf{N}$ & 1951.8112 & 976.4092 & 1934.7846 & 967.8959 & 1933.8006 & 967.4039 & 18 \\
\hline 4 & 488.1922 & 244.5997 & 471.1656 & 236.0865 & & & $\mathbf{N}$ & 1837.7682 & 919.3878 & 1820.7417 & 910.8745 & 1819.7577 & 910.3825 & 17 \\
\hline 5 & 575.2242 & 288.1157 & 558.1977 & 279.6025 & 557.2137 & & $S$ & 1723.7253 & 862.3663 & 1706.6988 & 853.8530 & 1705.7147 & 853.3610 & 16 \\
\hline 6 & 646.2613 & 323.6343 & 629.2348 & 315.1210 & 628.2508 & 314.6290 & A & 1636.6933 & 818.8503 & 1619.6667 & 810.3370 & 1618.6827 & 809.8450 & 15 \\
\hline 7 & 775.3039 & 388.1556 & 758.2774 & 379.6423 & 757.2934 & 379.1503 & $\mathbf{E}$ & 1565.6562 & 783.3317 & 1548.6296 & 774.8184 & 1547.6456 & 774.3264 & 14 \\
\hline 8 & 890.3309 & 445.6691 & 873.3043 & 437.1558 & 872.3203 & 436.6638 & $\mathbf{N}$ & 1436.6136 & 718.8104 & 1419.5870 & 710.2971 & 1418.6030 & 709.8051 & 13 \\
\hline 9 & 1019.3735 & 510.1904 & 1002.3469 & 501.6771 & 1001.3629 & 501.1851 & $\mathbf{E}$ & 1321.5866 & 661.2970 & 1304.5601 & 652.7837 & 1303.5761 & 652.2917 & 12 \\
\hline 10 & 1120.4211 & 560.7142 & 1103.3946 & 552.2009 & 1102.4106 & 551.7089 & $\mathbf{T}$ & 1192.5440 & 596.7757 & 1175.5175 & 588.2624 & 1174.5335 & 587.7704 & 11 \\
\hline 11 & 1191.4582 & 596.2328 & 1174.4317 & 587.7195 & 1173.4477 & 587.2275 & A & 1091.4964 & 546.2518 & 1074.4698 & 537.7385 & 1073.4858 & 537.2465 & 10 \\
\hline 12 & 1320.5008 & 660.7541 & 1303.4743 & 652.2408 & 1302.4903 & 651.7488 & $\mathbf{E}$ & 1020.4592 & 510.7333 & 1003.4327 & 502.2200 & 1002.4487 & 501.7280 & 9 \\
\hline 13 & 1377.5223 & 689.2648 & 1360.4958 & 680.7515 & 1359.5117 & 680.2595 & G & 891.4167 & 446.2120 & 874.3901 & 437.6987 & 873.4061 & 437.2067 & 8 \\
\hline 14 & 1506.5649 & 753.7861 & 1489.5384 & 745.2728 & 1488.5543 & 744.7808 & $\mathbf{E}$ & 834.3952 & 417.7012 & 817.3686 & 409.1880 & 816.3846 & 408.6959 & 7 \\
\hline 15 & 1635.6075 & 818.3074 & 1618.5809 & 809.7941 & 1617.5969 & 809.3021 & $\mathbf{E}$ & 705.3526 & 353.1799 & 688.3260 & 344.6667 & 687.3420 & 344.1747 & 6 \\
\hline 16 & 1763.7025 & 882.3549 & 1746.6759 & 873.8416 & 1745.6919 & 873.3496 & $\mathbf{K}$ & 576.3100 & 288.6586 & 559.2835 & 280.1454 & 558.2994 & 279.6534 & 5 \\
\hline 17 & 1820.7239 & 910.8656 & 1803.6974 & 902.3523 & 1802.7134 & 901.8603 & G & 448.2150 & 224.6112 & 431.1885 & 216.0979 & 430.2045 & 215.6059 & 4 \\
\hline 18 & 1949.7665 & 975.3869 & 1932.7400 & 966.8736 & 1931.7559 & 966.3816 & $\mathbf{E}$ & 391.1936 & 196.1004 & 374.1670 & 187.5872 & 373.1830 & 187.0951 & 3 \\
\hline
\end{tabular}




\begin{tabular}{|l|r|r|r|r|r|r|r|r|r|r|r|r|r|r|}
$\mathbf{1 9}$ & 2036.7985 & 1018.9029 & 2019.7720 & 1010.3896 & 2018.7880 & 1009.8976 & S & 262.1510 & 131.5791 & 245.1244 & 123.0659 & 244.1404 & 122.5738 & $\mathbf{2}$ \\
\hline $\mathbf{2 0}$ & & & & & & & $\mathbf{R}$ & 175.1190 & 88.0631 & 158.0924 & 79.5498 & & & $\mathbf{1}$ \\
\hline
\end{tabular}
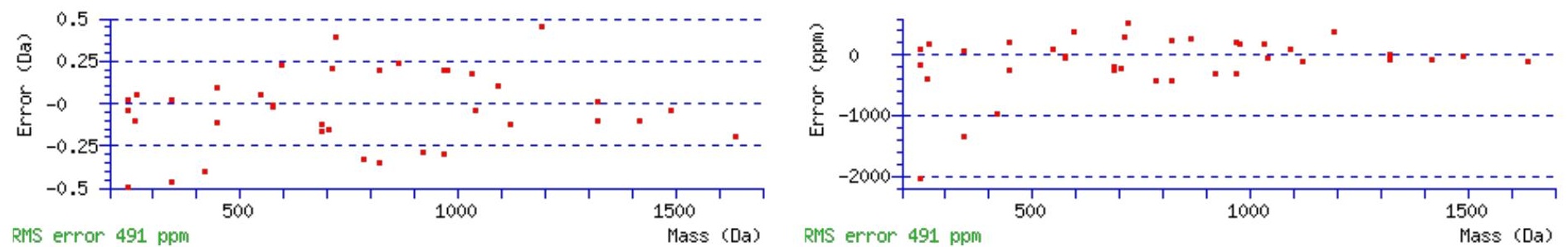

\section{All matches to this query}

\begin{tabular}{|l|l|c|l|}
\hline Score & Mr(calc): & Delta & \multicolumn{1}{|c|}{ Sequence } \\
\hline 43.6 & 2209.9029 & 0.0111 & MQNNSAENETAEGEEKGESR \\
\hline 31.4 & 2209.9029 & 0.0111 & MQNNSAENETAEGEEKGESR \\
\hline 30.6 & 2209.9029 & 0.0111 & MQNNSAENETAEGEEKGESR \\
\hline 26.6 & 2208.9189 & 0.9951 & MQNNSAENETAEGEEKGESR \\
\hline 9.5 & 2209.9132 & 0.0008 & YISTKAVGSNSRMDVTIK \\
\hline 7.5 & 2209.9083 & 0.0057 & FSGVSNRFSGSGSGTDFTLK \\
\hline 7.5 & 2209.9083 & 0.0057 & FSGVSNRFSGSGSGTDFTLK \\
\hline 4.0 & 2209.8922 & 0.0218 & MKLMADNYEDDHFRSSR \\
\hline 3.0 & 2209.9132 & 0.0008 & YISTKAVGSNSRMDVTIK \\
\hline 2.8 & 2209.9083 & 0.0057 & FSGVSNRFSGSGSGTDFTLK \\
\hline
\end{tabular}

Spectrum No: 769; Query: 2368; Rank: 1

\section{Peptide View}

MS/MS Fragmentation of VVLDKDPCSVLPSDYDLFINLSR

Found in IPI00204984, Tax_Id=10116 Gene_Symbol=Bst1 ADP-ribosyl cyclase 2 precursor

Match to Query 2368: 2665.341252 from(889.454360,3+)

Title: 100101RatKid_NS_deglyco_19.5307.5307.3.dta

Data file K:INewmanPaper|Piliang 13 SubProteomes\Piliang3SP\mgf5ppm\ERLIC_3SubProteomes5ppm.mgf

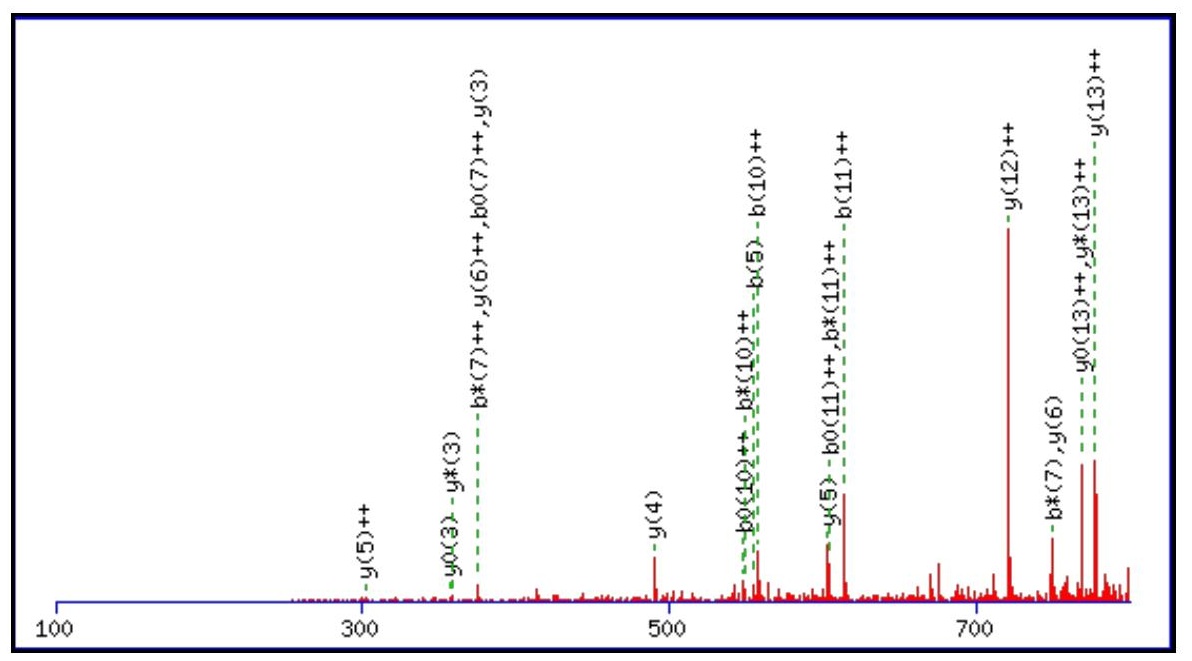



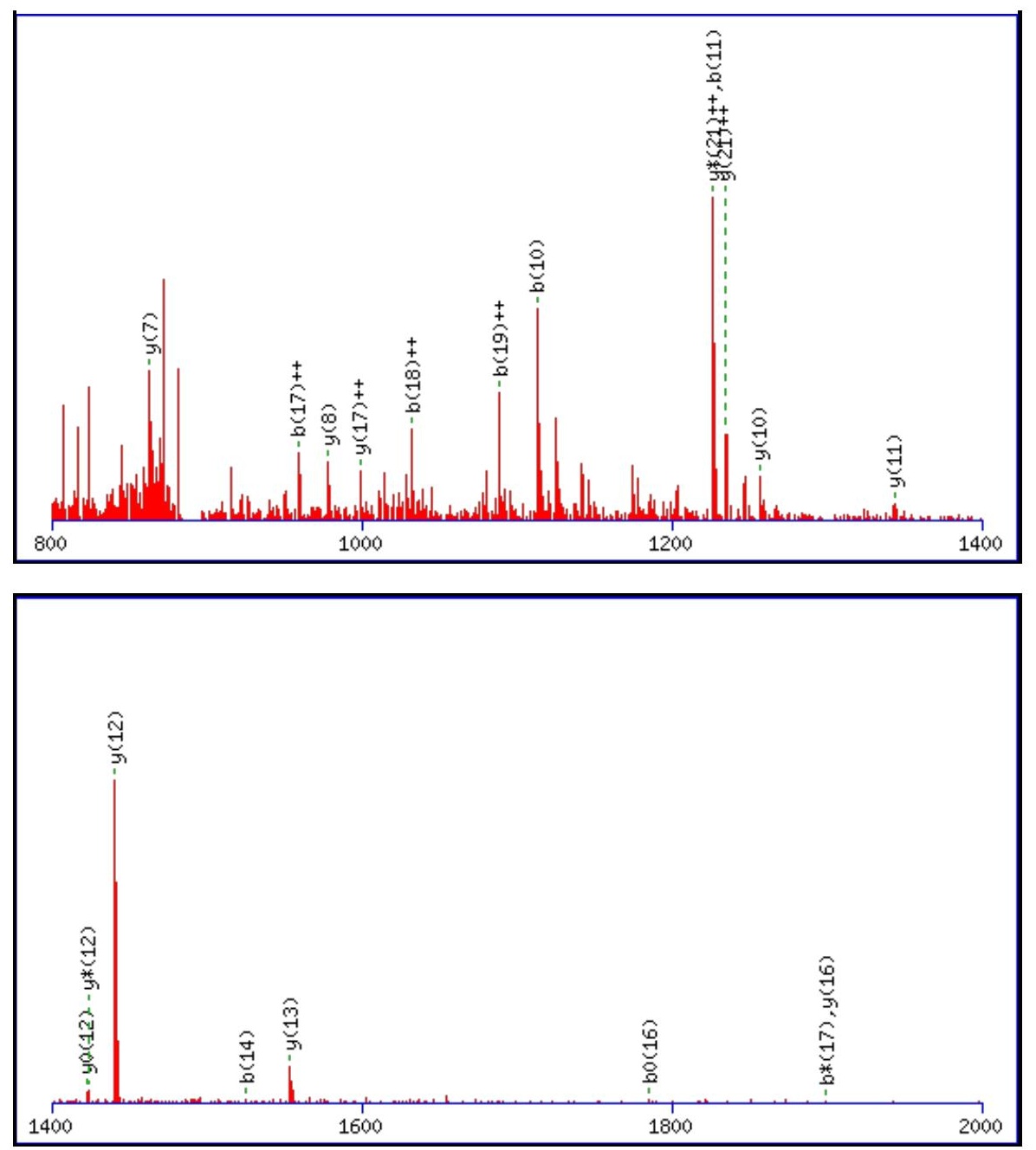

Monoisotopic mass of neutral peptide $\operatorname{Mr}($ calc): 2665.3309

Fixed modifications: Carbamidomethyl (C)

Variable modifications:

N20 : Deamidated_N (N)

Ions Score: 43 Expect: 0.0085

Matches (Bold Red): 42/248 fragment ions using 74 most intense peaks

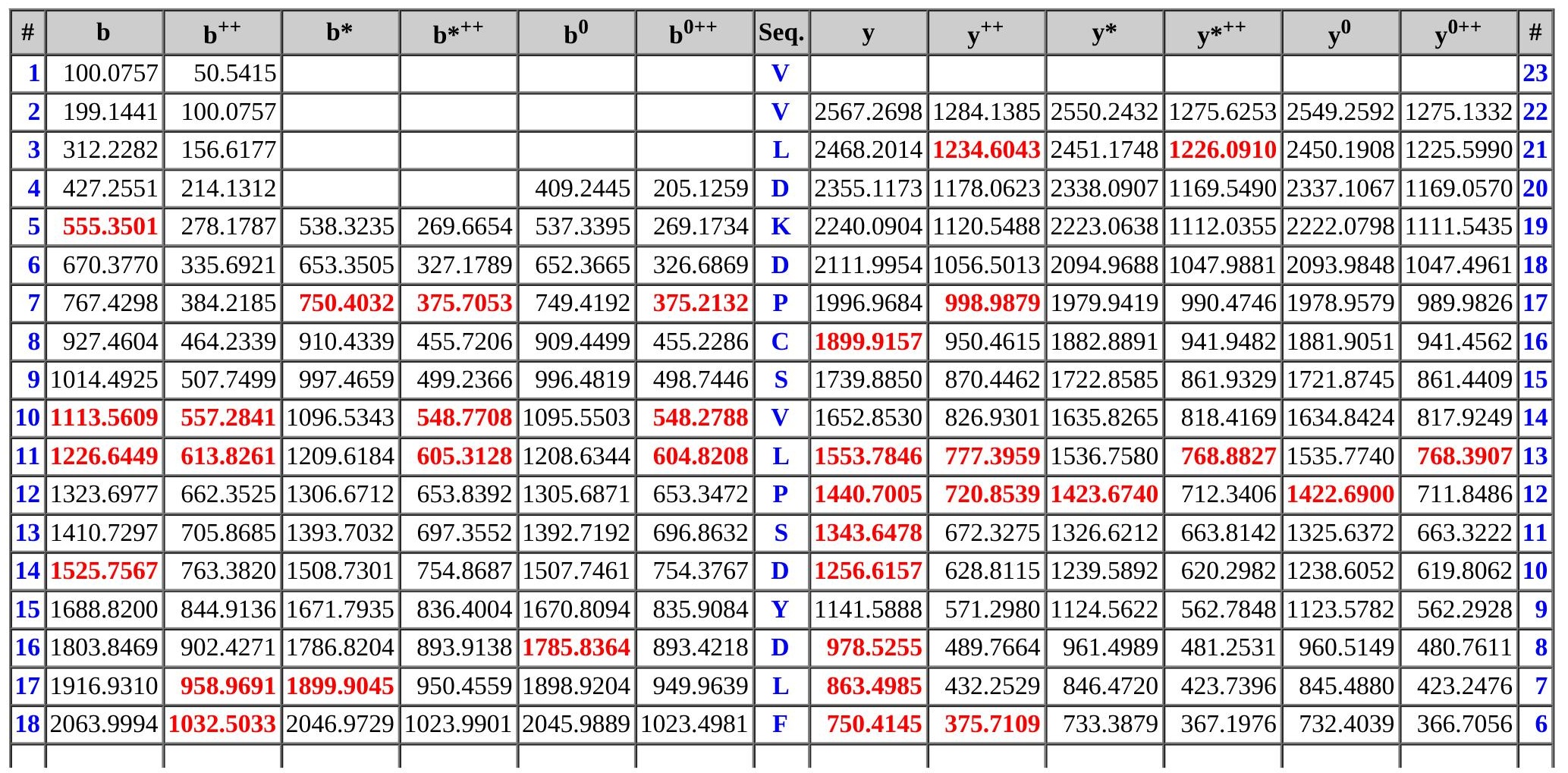




\begin{tabular}{|l|r|r|r|r|r|r|r|r|r|r|r|r|r|r|}
$\mathbf{1 9}$ & 2177.0835 & $\mathbf{1 0 8 9 . 0 4 5 4}$ & 2160.0569 & 1080.5321 & 2159.0729 & 1080.0401 & $\mathbf{I}$ & $\mathbf{6 0 3 . 3 4 6 0}$ & $\mathbf{3 0 2 . 1 7 6 7}$ & 586.3195 & 293.6634 & 585.3355 & 293.1714 & $\mathbf{5}$ \\
\hline $\mathbf{2 0}$ & 2292.1104 & 1146.5588 & 2275.0839 & 1138.0456 & 2274.0999 & 1137.5536 & $\mathbf{N}$ & $\mathbf{4 9 0 . 2 6 2 0}$ & 245.6346 & 473.2354 & 237.1214 & 472.2514 & 236.6293 & $\mathbf{4}$ \\
\hline $\mathbf{2 1}$ & 2405.1945 & 1203.1009 & 2388.1679 & 1194.5876 & 2387.1839 & 1194.0956 & $\mathbf{L}$ & $\mathbf{3 7 5 . 2 3 5 0}$ & 188.1212 & $\mathbf{3 5 8 . 2 0 8 5}$ & 179.6079 & $\mathbf{3 5 7 . 2 2 4 5}$ & 179.1159 & $\mathbf{3}$ \\
\hline $\mathbf{2 2}$ & 2492.2265 & 1246.6169 & 2475.2000 & 1238.1036 & 2474.2159 & 1237.6116 & S & 262.1510 & 131.5791 & 245.1244 & 123.0659 & 244.1404 & 122.5738 & $\mathbf{2}$ \\
\hline $\mathbf{2 3}$ & & & & & & & R & 175.1190 & 88.0631 & 158.0924 & 79.5498 & & & $\mathbf{1}$ \\
\hline
\end{tabular}
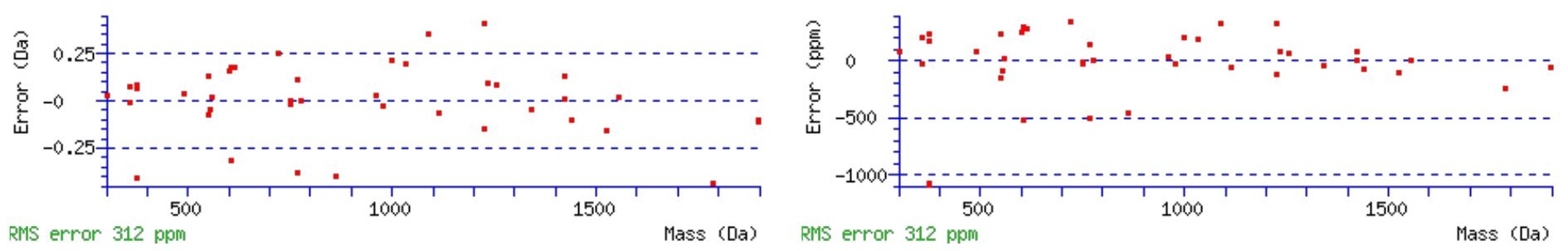

\section{All matches to this query}

\begin{tabular}{|l|c|c|l|}
\hline Score & Mr(calc): & Delta & \multicolumn{1}{c|}{ Sequence } \\
\hline 43.5 & 2665.3309 & 0.0103 & VVLDKDPCSVLPSDYDLFINLSR \\
\hline 8.9 & 2664.3469 & 0.9943 & VVLDKDPCSVLPSDYDLFINLSR \\
\hline 4.1 & 2665.3146 & 0.0266 & KMLPTTVGANSGTSLLGPSLLDGNSR \\
\hline 2.7 & 2665.3242 & 0.0171 & QPSASAPAQSSTAPCLSSXPALPRQR \\
\hline 2.1 & 2665.3186 & 0.0227 & ILENNTQWLLKLEQSIQMNLR \\
\hline 2.1 & 2665.3186 & 0.0227 & ILENNTOWLLKLEQSIOMNLR \\
\hline 1.5 & 2664.3242 & 1.0170 & GPMCRLLVLSTLGFYLMLQASAK \\
\hline 1.2 & 2665.3455 & -0.0043 & MSSLNNTAVMDFILVGLTDSPVLGR \\
\hline 1.2 & 2665.3455 & -0.0043 & MSSLNNTAVMDFILVGLTDSPVLGR \\
\hline 0.9 & 2664.3420 & 0.9993 & VILPVLAFKMLNNGSHAGNKLAMK \\
\hline
\end{tabular}

Spectrum No: 770; Query: 2323; Rank: 1

\section{Peptide View}

MS/MS Fragmentation of ILDVTETGFPGPSGPTFNATEDHSK

Found in IPI00215103, Tax_Id=10116 Gene_Symbol=Dnase2 Deoxyribonuclease-2-alpha precursor

Match to Query 2323: 2617.220952 from(873.414260,3+)

Title: 100101RatKid_NS_deglyco_12.3844.3844.3.dta

Data file K:INewmanPaper|Piliang|3SubProteomes\Piliang3SP\mgf5ppm\ERLIC_3SubProteomes5ppm.mgf

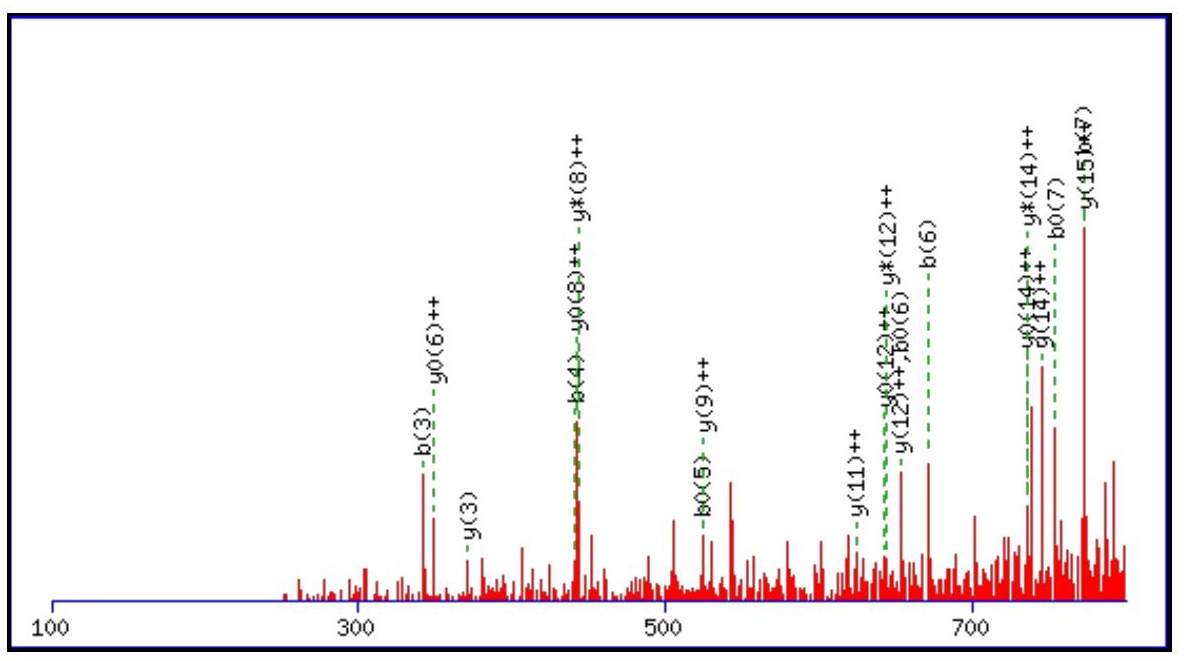



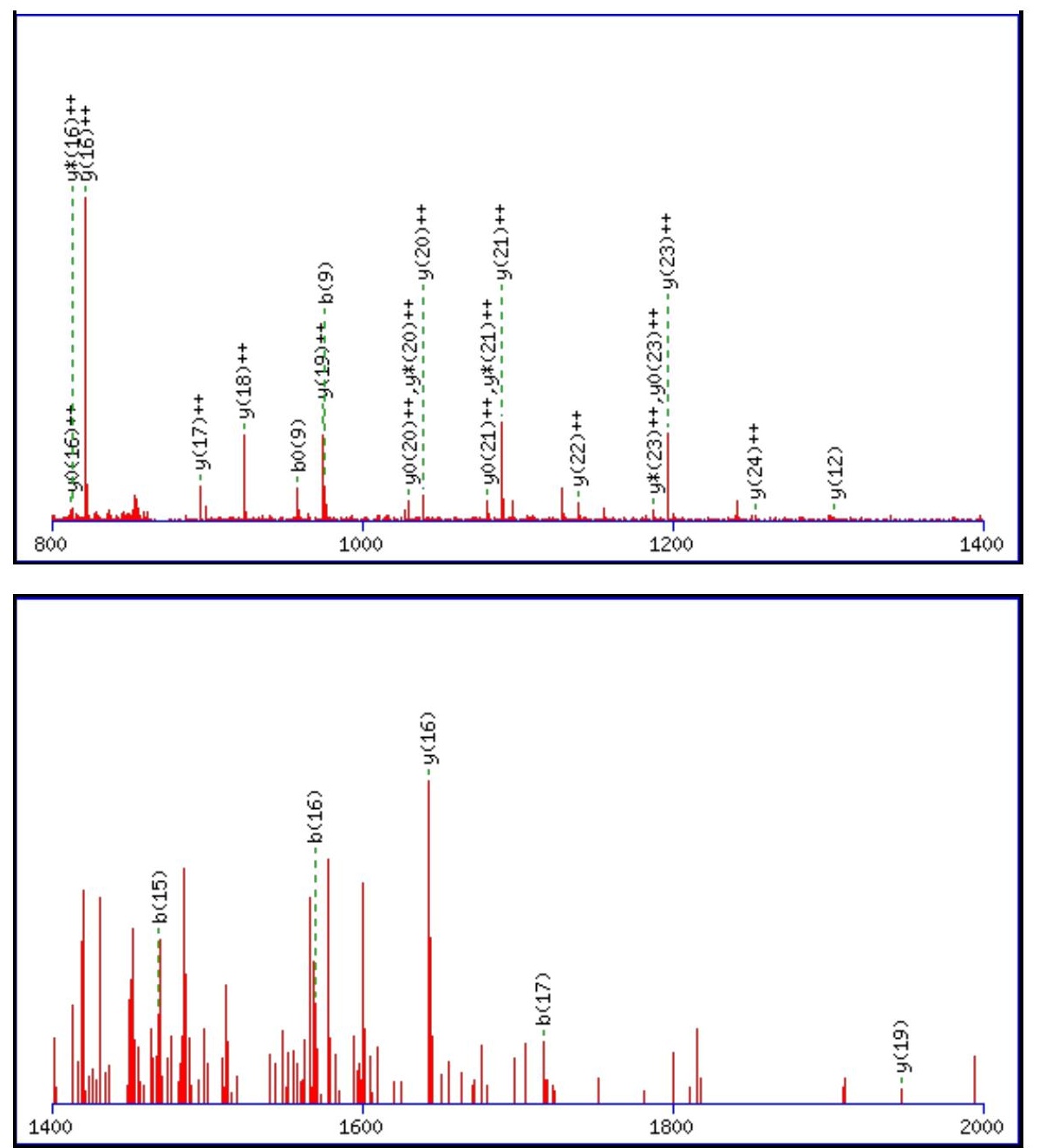

Monoisotopic mass of neutral peptide $\operatorname{Mr}($ calc): 2617.2184

Fixed modifications: Carbamidomethyl (C)

Variable modifications:

N18 : Deamidated_N (N)

Ions Score: 44 Expect: 0.015

Matches (Bold Red): 45/248 fragment ions using 131 most intense peaks

\begin{tabular}{|c|c|c|c|c|c|c|c|c|c|c|c|c|c|c|}
\hline \# & b & $\mathbf{b}^{++}$ & $\mathbf{b}^{*}$ & $\mathbf{b}^{*^{++}}$ & $\mathbf{b}^{\mathbf{0}}$ & $\mathbf{b}^{0++}$ & Seq. & $\mathbf{y}$ & $\mathbf{y}^{++}$ & $\mathbf{y}^{*}$ & $\mathrm{y}^{*^{++}}$ & $\mathbf{y}^{\mathbf{0}}$ & $\mathbf{y}^{0++}$ & \# \\
\hline 1 & 114.0913 & 57.5493 & & & & & I & & & & & & & 25 \\
\hline 2 & 227.1754 & 114.0913 & & & & & $\mathbf{L}$ & 2505.1416 & 1253.0744 & 2488.1151 & 1244.5612 & 2487.1310 & 1244.0692 & 24 \\
\hline 3 & 342.2023 & 171.6048 & & & 324.1918 & 162.5995 & D & 2392.0575 & 1196.5324 & 2375.0310 & 1188.0191 & 2374.0470 & 1187.5271 & 23 \\
\hline 4 & 441.2708 & 221.1390 & & & 423.2602 & 212.1337 & V & 2277.0306 & 1139.0189 & 2260.0040 & 1130.5057 & 2259.0200 & 1130.0137 & 22 \\
\hline 5 & 542.3184 & 271.6629 & & & 524.3079 & 262.6576 & $\mathbf{T}$ & 2177.9622 & 1089.4847 & 2160.9356 & 1080.9715 & 2159.9516 & 1080.4794 & 21 \\
\hline 6 & 671.3610 & 336.1842 & & & 653.3505 & 327.1789 & $\mathbf{E}$ & 2076.9145 & 1038.9609 & 2059.8880 & 1030.4476 & 2058.9039 & 1029.9556 & 20 \\
\hline 7 & 772.4087 & 386.7080 & & & 754.3981 & 377.7027 & $\mathbf{T}$ & 1947.8719 & 974.4396 & 1930.8454 & 965.9263 & 1929.8613 & 965.4343 & 19 \\
\hline 8 & 829.4302 & 415.2187 & & & 811.4196 & 406.2134 & G & 1846.8242 & 923.9158 & 1829.7977 & 915.4025 & 1828.8137 & 914.9105 & 18 \\
\hline 9 & 976.4986 & 488.7529 & & & 958.4880 & 479.7477 & $\mathbf{F}$ & 1789.8028 & 895.4050 & 1772.7762 & 886.8917 & 1771.7922 & 886.3997 & 17 \\
\hline 10 & 1073.5514 & 537.2793 & & & 1055.5408 & 528.2740 & $\mathbf{P}$ & 1642.7343 & 821.8708 & 1625.7078 & 813.3575 & 1624.7238 & 812.8655 & 16 \\
\hline 11 & 1130.5728 & 565.7900 & & & 1112.5623 & 556.7848 & G & 1545.6816 & 773.3444 & 1528.6550 & 764.8312 & 1527.6710 & 764.3391 & 15 \\
\hline 12 & 1227.6256 & 614.3164 & & & 1209.6150 & 605.3111 & $\mathbf{P}$ & 1488.6601 & 744.8337 & 1471.6336 & 736.3204 & 1470.6496 & 735.8284 & 14 \\
\hline 13 & 1314.6576 & 657.8324 & & & 1296.6470 & 648.8272 & S & 1391.6074 & 696.3073 & 1374.5808 & 687.7940 & 1373.5968 & 687.3020 & 13 \\
\hline 14 & 1371.6791 & 686.3432 & & & 1353.6685 & 677.3379 & G & 1304.5753 & 652.7913 & 1287.5488 & 644.2780 & 1286.5648 & 643.7860 & 12 \\
\hline 15 & 1468.7318 & 734.8696 & & & 1450.7213 & 725.8643 & $\mathbf{P}$ & 1247.5539 & 624.2806 & 1230.5273 & 615.7673 & 1229.5433 & 615.2753 & 11 \\
\hline 16 & 1569.7795 & 785.3934 & & & 1551.7690 & 776.3881 & $\mathbf{T}$ & 1150.5011 & 575.7542 & 1133.4746 & 567.2409 & 1132.4905 & 566.7489 & 10 \\
\hline 17 & 1716.8479 & 858.9276 & & & 1698.8374 & 849.9223 & $\mathbf{F}$ & 1049.4534 & 525.2303 & 1032.4269 & 516.7171 & 1031.4429 & 516.2251 & 9 \\
\hline 18 & 1831.8749 & 916.4411 & 1814.8483 & 907.9278 & 1813.8643 & 907.4358 & $\mathbf{N}$ & 902.3850 & 451.6961 & 885.3585 & 443.1829 & 884.3744 & 442.6909 & 8 \\
\hline
\end{tabular}




\begin{tabular}{|l|r|r|r|r|r|r|r|r|r|r|r|r|r|r|r|}
$\mathbf{1 9}$ & 1902.9120 & 951.9596 & 1885.8854 & 943.4464 & 1884.9014 & 942.9543 & A & 787.3581 & 394.1827 & 770.3315 & 385.6694 & 769.3475 & 385.1774 & $\mathbf{7}$ \\
\hline $\mathbf{2 0}$ & 2003.9597 & 1002.4835 & 1986.9331 & 993.9702 & 1985.9491 & 993.4782 & T & 716.3210 & 358.6641 & 699.2944 & 350.1508 & 698.3104 & 349.6588 & $\mathbf{6}$ \\
\hline $\mathbf{2 1}$ & 2133.0023 & 1067.0048 & 2115.9757 & 1058.4915 & 2114.9917 & 1057.9995 & E & 615.2733 & 308.1403 & 598.2467 & 299.6270 & 597.2627 & 299.1350 & $\mathbf{5}$ \\
\hline $\mathbf{2 2}$ & 2248.0292 & 1124.5182 & 2231.0026 & 1116.0050 & 2230.0186 & 1115.5130 & D & 486.2307 & 243.6190 & 469.2041 & 235.1057 & 468.2201 & 234.6137 & $\mathbf{4}$ \\
\hline $\mathbf{2 3}$ & 2385.0881 & 1193.0477 & 2368.0616 & 1184.5344 & 2367.0775 & 1184.0424 & H & 371.2037 & 186.1055 & 354.1772 & 177.5922 & 353.1932 & 177.1002 & $\mathbf{3}$ \\
\hline $\mathbf{2 4}$ & 2472.1201 & 1236.5637 & 2455.0936 & 1228.0504 & 2454.1096 & 1227.5584 & S & 234.1448 & 117.5761 & 217.1183 & 109.0628 & 216.1343 & 108.5708 & $\mathbf{2}$ \\
\hline $\mathbf{2 5}$ & & & & & & & K & 147.1128 & 74.0600 & 130.0863 & 65.5468 & & & $\mathbf{1}$ \\
\hline
\end{tabular}
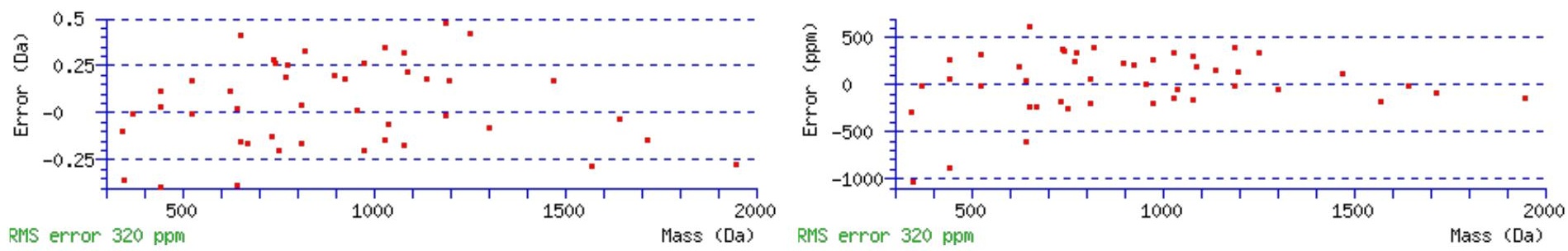

\section{All matches to this query}

\begin{tabular}{|l|l|l|l|}
\hline Score & Mr(calc): & Delta & \multicolumn{1}{|c|}{ Sequence } \\
\hline 43.5 & 2617.2184 & 0.0025 & ILDVTETGFPGPSGPTFNATEDHSK \\
\hline 3.4 & 2615.1948 & 2.0261 & EEQNFVVQNEINNMSFLITDTK \\
\hline 1.9 & 2615.2173 & 2.0036 & IANSLTGSSAAPGQFLFSSCGQNTAK \\
\hline 1.7 & 2616.2333 & 0.9876 & TLEEVTOGHSLKDGLGHSSLWSR \\
\hline 1.6 & 2617.2378 & -0.0168 & MASVFYTLVIPMLNPLIYSLR \\
\hline 1.6 & 2617.2378 & -0.0168 & MASVFYTLVIPMLNPLIYSLR \\
\hline 1.6 & 2617.2378 & -0.0168 & MASVFYTLVIPMLNPLIYSLR \\
\hline 1.5 & 2617.2011 & 0.0199 & KAISGYAGFVPRFAWVMGMNYR \\
\hline 1.5 & 2617.2011 & 0.0199 & KAISGYAGFVPRFAWVMGMNYR \\
\hline 1.0 & 2617.2376 & -0.0167 & VVLYPTSNSSKSAELHRMVVPK \\
\hline
\end{tabular}

Spectrum No: 771; Query: 1888; Rank: 1

\section{Peptide View}

MS/MS Fragmentation of GALDLMLQVNMTPGHSSAPPK

Found in IPI00388393, Tax_Id=10116 Gene_Symbol=RGD1564063_predicted 30 kDa protein

Match to Query 1888: 2196.059382 from(733.027070,3+)

Title: 091008RatKidney_NH4Format01_23.2143.2143.3.dta

Data file K:INewmanPaper|Piliang|3SubProteomes\Piliang3SP\mgf5ppm\ERLIC_3SubProteomes5ppm.mgf 

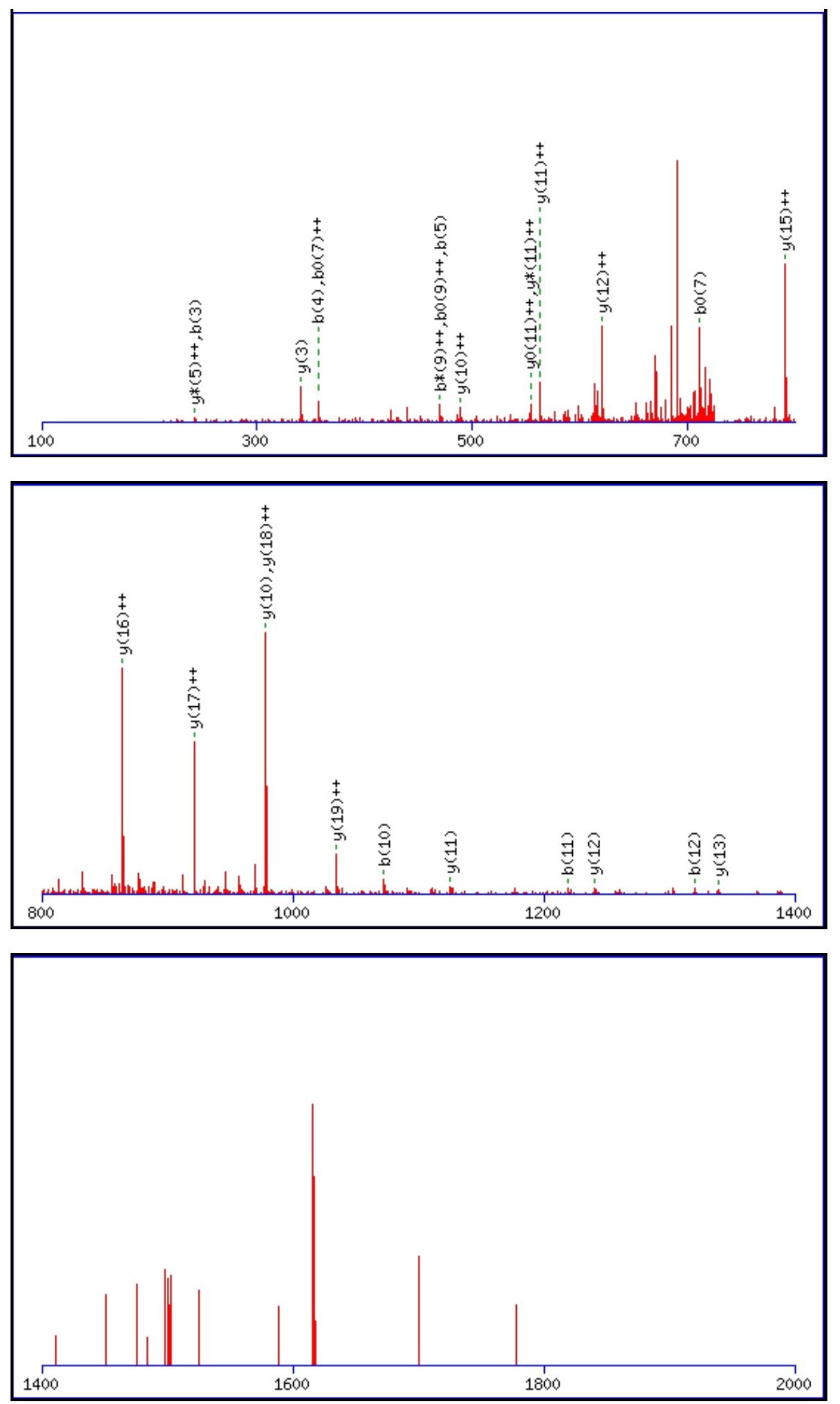

Monoisotopic mass of neutral peptide $\operatorname{Mr}($ calc): 2196.0555

Fixed modifications: Carbamidomethyl (C)

Variable modifications:

M6 : Oxidation (M)

N10 : Deamidated_N (N)

M11 : Oxidation (M)

Ions Score: 43 Expect: 0.011

Matches (Bold Red): 26/212 fragment ions using 40 most intense peaks

\begin{tabular}{|c|c|c|c|c|c|c|c|c|c|c|c|c|c|c|}
\hline \# & b & $\mathbf{b}^{++}$ & $\mathbf{b}^{*}$ & $\mathbf{b}^{*^{++}}$ & $\mathbf{b}^{\mathbf{0}}$ & $\mathbf{b}^{\mathbf{0 + +}}$ & Seq. & $\mathbf{y}$ & $\mathbf{y}^{++}$ & $\mathrm{y}^{*}$ & $\mathrm{y}^{\mathrm{*}^{++}}$ & $\mathbf{y}^{0}$ & $y^{0++}$ & $\#$ \\
\hline 1 & 58.0287 & 29.5180 & & & & & G & & & & & & & 21 \\
\hline 2 & 129.0659 & 65.0366 & & & & & A & 2140.0413 & 1070.5243 & 2123.0148 & 1062.0110 & 2122.0307 & 1061.5190 & 20 \\
\hline 3 & 242.1499 & 121.5786 & & & & & $\mathbf{L}$ & 2069.0042 & 1035.0057 & 2051.9776 & 1026.4925 & 2050.9936 & 1026.0005 & 19 \\
\hline 4 & 357.1769 & 179.0921 & & & 339.1663 & 170.0868 & D & 1955.9201 & \begin{tabular}{|c|}
$\mathbf{9 7 8 . 4 6 3 7}$ \\
\end{tabular} & 1938.8936 & 969.9504 & 1937.9096 & 969.4584 & 18 \\
\hline
\end{tabular}




\begin{tabular}{|c|c|c|c|c|c|c|c|c|c|c|c|c|c|c|}
\hline & 470.2609 & 235.6341 & & & 452.2504 & 226.6288 & L & 1840.8932 & 920.9502 & |1823.8666| & 912.4370 & 1822.8826 & 911.9449| & |17| \\
\hline 6 & 617.2963 & 309.1518 & & & 599.2858 & 300.1465 & $\mathbf{M}$ & 1727.8091 & 864.4082 & 1710.7826 & 855.8949 & 1709.7986 & 855.4029 & 16 \\
\hline 7 & 730.3804 & 365.6938 & & & 712.3698 & 356.6886 & $\mathbf{L}$ & 1580.7737 & 790.8905 & 1563.7472 & 782.3772 & 1562.7632 & 781.8852 & 15 \\
\hline 8 & 858.4390 & 429.7231 & 841.4124 & 421.2098 & 840.4284 & 420.7178 & $\mathbf{Q}$ & 1467.6897 & 734.3485 & 1450.6631 & 725.8352 & 1449.6791 & 725.3432 & 14 \\
\hline 9 & 957.5074 & 479.2573 & 940.4808 & 470.7441 & 939.4968 & 470.2520 & V & 1339.6311 & 670.3192 & 1322.6045 & 661.8059 & 1321.6205 & 661.3139 & 13 \\
\hline 10 & \begin{tabular}{|l|}
1072.5343 \\
\end{tabular} & 536.7708 & 1055.5078 & 528.2575 & 1054.5238 & 527.7655 & $\mathbf{N}$ & 1240.5627 & 620.7850 & 1223.5361 & 612.2717 & 1222.5521 & 611.7797 & 12 \\
\hline 11 & 1219.5697 & 610.2885 & 1202.5432 & 601.7752 & 1201.5592 & 601.2832 & M & 1125.5357 & 563.2715 & 1108.5092 & 554.7582 & 1107.5252 & 554.2662 & 11 \\
\hline 12 & 1320.6174 & 660.8123 & 1303.5909 & 652.2991 & 1302.6068 & 651.8071 & $\mathbf{T}$ & 978.5003 & 489.7538 & 961.4738 & 481.2405 & 960.4898 & 480.7485 & 10 \\
\hline 13 & 1417.6702 & 709.3387 & 1400.6436 & 700.8254 & 1399.6596 & 700.3334 & $\mathbf{P}$ & 877.4526 & 439.2300 & 860.4261 & 430.7167 & 859.4421 & 430.2247 & 9 \\
\hline 14 & 1474.6916 & 737.8495 & 1457.6651 & 729.3362 & 1456.6811 & 728.8442 & G & 780.3999 & 390.7036 & 763.3733 & 382.1903 & 762.3893 & 381.6983 & 8 \\
\hline 15 & 1611.7505 & 806.3789 & 1594.7240 & 797.8656 & 1593.7400 & 797.3736 & H & 723.3784 & 362.1928 & 706.3519 & 353.6796 & 705.3678 & 353.1876 & 7 \\
\hline 16 & 1698.7826 & 849.8949 & 1681.7560 & 841.3816 & 1680.7720 & 840.8896 & $S$ & 586.3195 & 293.6634 & 569.2930 & 285.1501 & 568.3089 & 284.6581 & 6 \\
\hline 17 & 1785.8146 & 893.4109 & 1768.7881 & 884.8977 & 1767.8040 & 884.4057 & $\mathrm{~S}$ & 499.2875 & 250.1474 & 482.2609 & 241.6341 & 481.2769 & 241.1421 & 5 \\
\hline 18 & 1856.8517 & 928.9295 & 1839.8252 & 920.4162 & 1838.8411 & 919.9242 & A & 412.2554 & 206.6314 & 395.2289 & 198.1181 & & & 4 \\
\hline 19 & 1953.9045 & 977.4559 & 1936.8779 & 968.9426 & 1935.8939 & 968.4506 & $\mathbf{P}$ & 341.2183 & 171.1128 & 324.1918 & 162.5995 & & & 3 \\
\hline 20 & 2050.9572 & 1025.9823 & 2033.9307 & 1017.4690 & 2032.9467 & 1016.9770 & $\mathbf{P}$ & 244.1656 & 122.5864 & 227.1390 & 114.0731 & & & 2 \\
\hline 21 & & & & & & & $\mathbf{K}$ & 147.1128 & 74.0600 & 130.0863 & 65.5468 & & & 1 \\
\hline
\end{tabular}
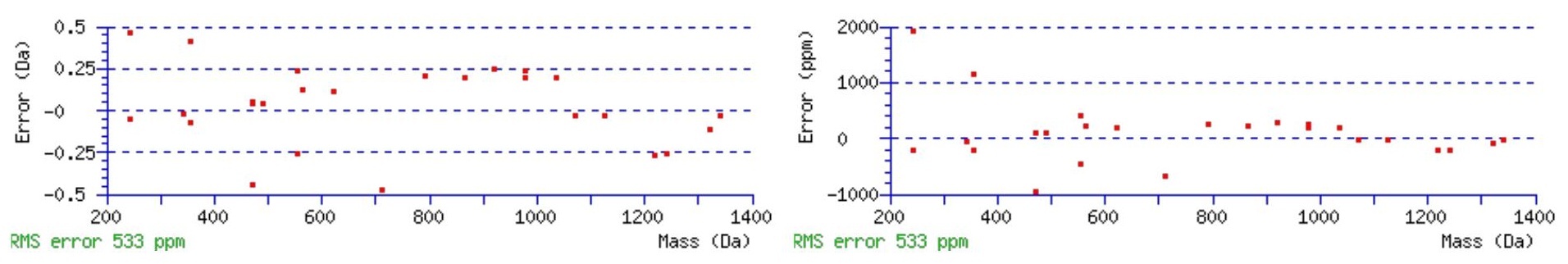

\section{All matches to this query}

\begin{tabular}{|l|l|l|l|}
\hline Score & Mr(calc): & Delta & \multicolumn{1}{|c|}{ Sequence } \\
\hline 43.4 & 2196.0555 & 0.0039 & GALDLMLQVNMTPGHSSAPPK \\
\hline 19.4 & 2196.0576 & 0.0018 & QLDIFDRQQNEILSAAQK \\
\hline 5.7 & 2195.0657 & 0.9937 & SEPSVTSPIGSPTMVKKSQR \\
\hline 5.2 & 2196.0415 & 0.0179 & NLGTLLGLGLALNSSMYRK \\
\hline 4.5 & 2195.0715 & 0.9879 & GALDLMLQVNMTPGHSSAPPK \\
\hline 4.1 & 2196.0385 & 0.0209 & KELAKLQTVQLDEDMQDL \\
\hline 1.5 & 2195.0657 & 0.9937 & SEPSVTSPIGSPTMVKKSQR \\
\hline 1.5 & 2195.0657 & 0.9937 & SEPSVTSPIGSPTMVKKSQR \\
\hline 1.4 & 2196.0432 & 0.0162 & QLLDTIAACEEMLROLGR \\
\hline 1.2 & 2195.0657 & 0.9937 & SEPSVTSPIGSPTMVKKSQR \\
\hline
\end{tabular}

Spectrum No: 772; Query: 2225; Rank: 1

\section{Peptide View}

MS/MS Fragmentation of ENYFEQYMDHFNFESFSNK

Found in IPI00230946, Tax_Id=10116 Gene_Symbol=Dpp7 Dipeptidyl-peptidase 2 precursor

Match to Query 2225: 2476.004352 from(826.342060,3+)

Title: 100101RatKid_NS_deglyco_11.6129.6129.3.dta

Data file K:INewmanPaper|Piliang\3SubProteomes\Piliang3SP\mgf5ppm\ERLIC_3SubProteomes5ppm.mgf 

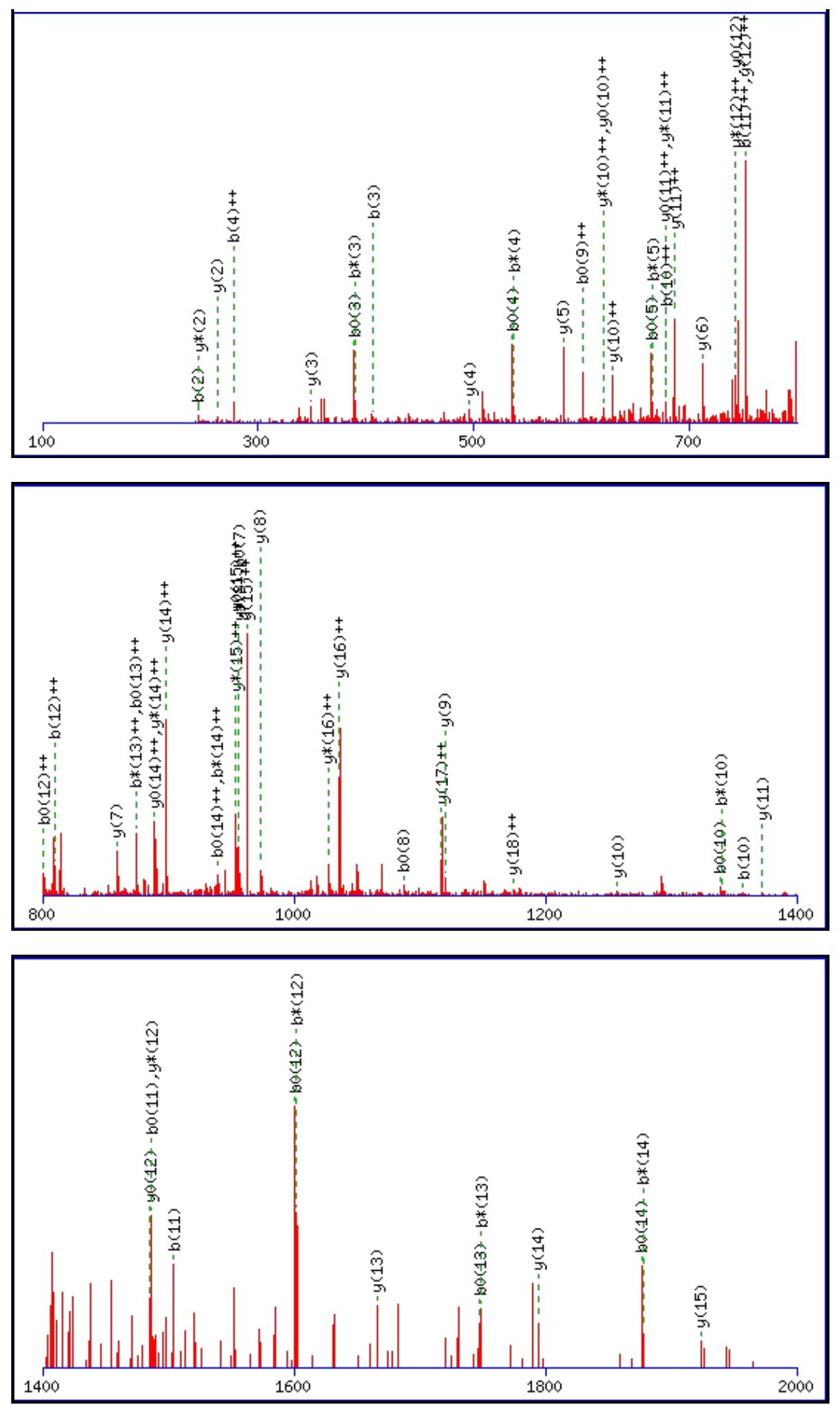

Monoisotopic mass of neutral peptide $\operatorname{Mr}($ calc): 2475.9954

Fixed modifications: Carbamidomethyl (C)

Variable modifications:

N18 : Deamidated_N (N)

Ions Score: 43 Expect: 0.012

Matches (Bold Red): 68/210 fragment ions using 163 most intense peaks

\begin{tabular}{|c|c|c|c|c|c|c|c|c|c|c|c|c|c|c|}
\hline \# & b & $\mathbf{b}^{++}$ & $\mathbf{b}^{*}$ & $\mathbf{b}^{*^{++}}$ & $\mathbf{b}^{0}$ & $\mathbf{b}^{0++}$ & Seq. & $\mathbf{y}$ & $y^{++}$ & $\mathbf{y}^{*}$ & $\mathrm{y}^{*^{++}}$ & $\mathbf{y}^{0}$ & $y^{0++}$ & \# \\
\hline 1 & 130.0499 & 65.5286 & & & 112.0393 & 56.5233 & E & & & & & & & 19 \\
\hline 2 & 244.0928 & 122.5500 & 227.0662 & 114.0368 & 226.0822 & 113.5448 & $\mathbf{N}$ & 2347.9601 & 1174.4837 & 2330.9335 & 1165.9704 & 2329.9495 & 1165.4784 & 18 \\
\hline 3 & 407.1561 & 204.0817 & 390.1296 & 195.5684 & 389.1456 & 195.0764 & $\mathbf{Y}$ & 2233.9171 & 1117.4622 & 2216.8906 & 1108.9489 & 2215.9066 & 1108.4569 & 17 \\
\hline 4 & 554.2245 & 277.6159 & 537.1980 & 269.1026 & 536.2140 & 268.6106 & $\mathbf{F}$ & 2070.8538 & 1035.9305 & 2053.8273 & 1027.4173 & 2052.8432 & 1026.9253 & 16 \\
\hline 5 & 683.2671 & 342.1372 & 666.2406 & 333.6239 & 665.2566 & 333.1319 & $\mathbf{E}$ & 1923.7854 & 962.3963 & 1906.7588 & 953.8831 & 1905.7748 & 953.3911 & 15 \\
\hline
\end{tabular}




\begin{tabular}{|c|c|c|c|c|c|c|c|c|c|c|c|c|c|c|}
\hline 6 & 811.3257 & 406.1665 & 794.2992 & 397.6532 & 793.3151 & 397.1612 & $\mathbf{Q}$ & 1794.7428 & 897.8750 & |1777.7163 & 889.3618 & |1776.7322 & 888.8698 & 14 \\
\hline 7 & 974.3890 & 487.6982 & 957.3625 & 479.1849 & 956.3785 & 478.6929 & $\mathbf{Y}$ & 1666.6842 & 833.8458 & 1649.6577 & 825.3325 & 1648.6737 & 824.8405 & 13 \\
\hline 8 & 1105.4295 & 553.2184 & 1088.4030 & 544.7051 & 1087.4190 & 544.2131 & $\mathbf{M}$ & 1503.6209 & 752.3141 & 1486.5943 & 743.8008 & 1485.6103 & 743.3088 & 12 \\
\hline 9 & 1220.4565 & 610.7319 & 1203.4299 & 602.2186 & 1202.4459 & 601.7266 & D & 1372.5804 & 686.7938 & 1355.5539 & 678.2806 & 1354.5698 & 677.7886 & 11 \\
\hline 10 & 1357.5154 & 679.2613 & 1340.4888 & 670.7481 & 1339.5048 & 670.2560 & $\mathbf{H}$ & 1257.5535 & 629.2804 & 1240.5269 & 620.7671 & 1239.5429 & 620.2751 & 10 \\
\hline 11 & 1504.5838 & 752.7955 & 1487.5572 & 744.2823 & 1486.5732 & 743.7903 & $\mathbf{F}$ & 1120.4946 & 560.7509 & 1103.4680 & 552.2376 & 1102.4840 & 551.7456 & 9 \\
\hline 12 & 1618.6267 & 809.8170 & 1601.6002 & 801.3037 & 1600.6162 & 800.8117 & $\mathbf{N}$ & 973.4261 & 487.2167 & 956.3996 & 478.7034 & 955.4156 & 478.2114 & 8 \\
\hline 14 & 1894.7377 & 947.8725 & 1877.7112 & 939.3592 & 1876.7272 & 938.8672 & $\mathbf{E}$ & 712.3148 & 356.6610 & 695.2883 & 348.1478 & 694.3042 & 347.6558 & 6 \\
\hline 15 & 1981.7698 & 991.3885 & 1964.7432 & 982.8752 & 1963.7592 & 982.3832 & S & 583.2722 & 292.1397 & 566.2457 & 283.6265 & 565.2616 & 283.1345 & 5 \\
\hline 16 & 2128.8382 & 1064.9227 & 2111.8116 & 1056.4094 & 2110.8276 & 1055.9174 & $\mathbf{F}$ & 496.2402 & 248.6237 & 479.2136 & 240.1105 & 478.2296 & 239.6184 & 4 \\
\hline 17 & 2215.8702 & 1108.4387 & 2198.8436 & 1099.9255 & 2197.8596 & 1099.4335 & S & 349.1718 & 175.0895 & 332.1452 & 166.5762 & 331.1612 & 166.0842 & 3 \\
\hline 18 & 2330.8971 & 1165.9522 & 2313.8706 & 1157.4389 & 2312.8866 & 1156.9469 & $\mathbf{N}$ & 262.1397 & 131.5735 & 245.1132 & 123.0602 & & & 2 \\
\hline 19 & & & & & & & $\mathbf{K}$ & 147.1128 & 74.0600 & 130.0863 & 65.5468 & & & 1 \\
\hline
\end{tabular}
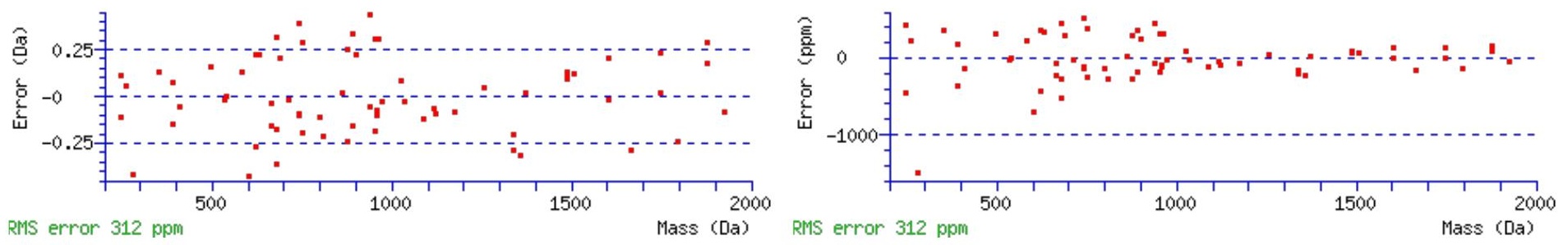

\section{All matches to this query}

\begin{tabular}{|l|l|c|l|}
\hline Score & Mr(calc): & Delta & \multicolumn{1}{|c|}{ Sequence } \\
\hline 43.4 & 2475.9954 & 0.0090 & ENYFEQYMDHFNFESFSNK \\
\hline 14.0 & 2475.9954 & 0.0090 & ENYFEQYMDHFNFESFSNK \\
\hline 1.8 & 2475.9931 & 0.0113 & YKLVSEGNSTASENTEITSER \\
\hline 0.6 & 2474.0001 & 2.0042 & SSDDFEALVASLTCLFAEDPK \\
\hline 0.2 & 2474.0029 & 2.0014 & AGTLRALSRODTFDADTPGSR \\
\hline
\end{tabular}

Spectrum No: 773; Query: 451; Rank: 1

\section{Peptide View}

MS/MS Fragmentation of VVNSTTGPGEHLR

Found in IPI00422076, Tax_Id=10116 Gene_Symbol=Thbs1 Thrombospondin 1

Match to Query 451: 1366.684182 from(456.568670,3+)

Title: 091008RatKidney_NH4Format01_24.515.515.3.dta

Data file K:INewmanPaper|Piliang|3SubProteomes\Piliang3SP\mgf5ppm\ERLIC_3SubProteomes5ppm.mgf 

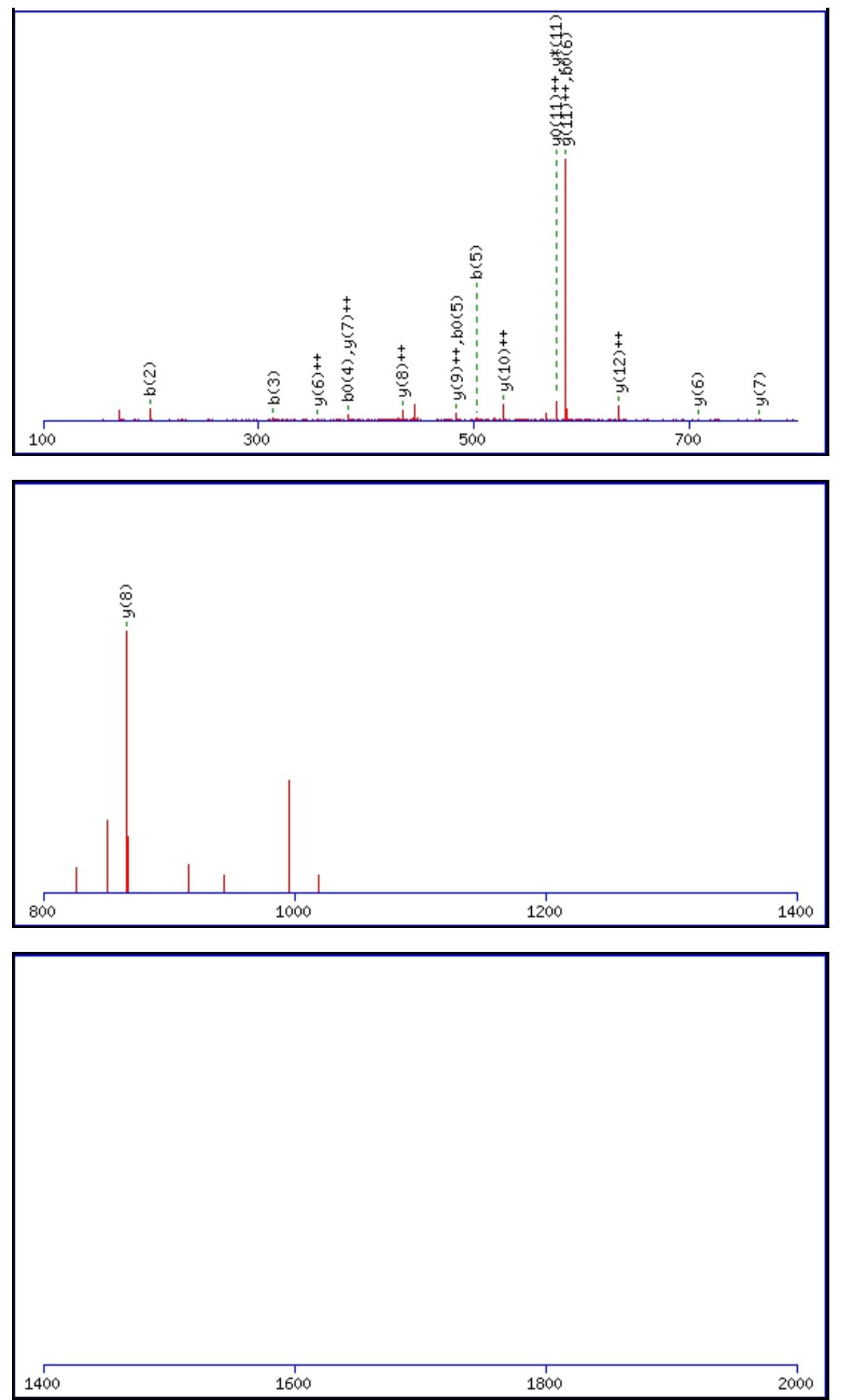

Monoisotopic mass of neutral peptide $\operatorname{Mr}($ calc): 1366.6841

Fixed modifications: Carbamidomethyl (C)

Variable modifications:

N3 : Deamidated_N (N)

Ions Score: 43 Expect: 0.0064

Matches (Bold Red): 18/128 fragment ions using 28 most intense peaks

\begin{tabular}{|r|c|c|c|c|c|c|c|c|c|c|c|c|c|c|}
\hline$\#$ & $\mathbf{b}$ & $\mathbf{b}^{++}$ & $\mathbf{b}^{*}$ & $\mathbf{b}^{\boldsymbol{*}^{++}}$ & $\mathbf{b}^{\mathbf{0}}$ & $\mathbf{b}^{\mathbf{0 + +}}$ & $\mathbf{S e q}$ & $\mathbf{y}$ & $\mathbf{y}^{++}$ & $\mathbf{y}^{\mathbf{*}}$ & $\mathbf{y}^{\boldsymbol{*}^{++}}$ & $\mathbf{y}^{\mathbf{0}}$ & $\mathbf{y}^{\mathbf{0 + +}}$ & $\#$ \\
\hline $\mathbf{1}$ & 100.0757 & 50.5415 & & & & & $\mathbf{V}$ & & & & & & & $\mathbf{1 3}$ \\
\hline $\mathbf{2}$ & $\mathbf{1 9 9 . 1 4 4 1}$ & 100.0757 & & & & & $\mathbf{V}$ & 1268.6229 & $\mathbf{6 3 4 . 8 1 5 1}$ & 1251.5964 & 626.3018 & 1250.6124 & 625.8098 & $\mathbf{1 2}$ \\
\hline $\mathbf{3}$ & $\mathbf{3 1 4 . 1 7 1 0}$ & 157.5892 & 297.1445 & 149.0759 & & & $\mathbf{N}$ & 1169.5545 & 585.2809 & 1152.5280 & 576.7676 & 1151.5440 & 576.2756 & $\mathbf{1 1}$ \\
\hline $\mathbf{4}$ & 401.2031 & 201.1052 & 384.1765 & 192.5919 & $\mathbf{3 8 3 . 1 9 2 5}$ & 192.0999 & S & 1054.5276 & 527.7674 & 1037.5011 & 519.2542 & 1036.5170 & 518.7622 & $\mathbf{1 0}$ \\
\hline $\mathbf{5}$ & $\mathbf{5 0 2 . 2 5 0 7}$ & 251.6290 & 485.2242 & 243.1157 & $\mathbf{4 8 4 . 2 4 0 2}$ & 242.6237 & $\mathbf{T}$ & 967.4956 & $\mathbf{4 8 4 . 2 5 1 4}$ & 950.4690 & 475.7381 & 949.4850 & 475.2461 & $\mathbf{9}$ \\
\hline
\end{tabular}




\begin{tabular}{|r|r|r|r|r|r|r|r|r|r|r|r|r|r|r|}
$\mathbf{6}$ & 603.2984 & 302.1529 & 586.2719 & 293.6396 & 585.2879 & 293.1476 & $\mathbf{T}$ & $\mathbf{8 6 6 . 4 4 7 9}$ & $\mathbf{4 3 3 . 7 2 7 6}$ & $\mathbf{8 4 9 . 4 2 1 3}$ & $\mathbf{4 2 5 . 2 1 4 3}$ & $\mathbf{8 4 8 . 4 3 7 3}$ & $\mathbf{4 2 4 . 7 2 2 3}$ & $\mathbf{8}$ \\
\hline $\mathbf{7}$ & 660.3199 & 330.6636 & 643.2933 & 322.1503 & 642.3093 & 321.6583 & $\mathbf{G}$ & $\mathbf{7 6 5 . 4 0 0 2}$ & $\mathbf{3 8 3 . 2 0 3 7}$ & 748.3737 & 374.6905 & 747.3896 & 374.1985 & $\mathbf{7}$ \\
\hline $\mathbf{8}$ & 757.3727 & 379.1900 & 740.3461 & 370.6767 & 739.3621 & 370.1847 & $\mathbf{P}$ & $\mathbf{7 0 8 . 3 7 8 7}$ & $\mathbf{3 5 4 . 6 9 3 0}$ & 691.3522 & 346.1797 & 690.3682 & 345.6877 & $\mathbf{6}$ \\
\hline $\mathbf{9}$ & 814.3941 & 407.7007 & 797.3676 & 399.1874 & 796.3836 & 398.6954 & $\mathbf{G}$ & 611.3260 & 306.1666 & 594.2994 & 297.6534 & 593.3154 & 297.1613 & $\mathbf{5}$ \\
\hline $\mathbf{1 0}$ & 943.4367 & 472.2220 & 926.4102 & 463.7087 & 925.4261 & 463.2167 & $\mathbf{E}$ & 554.3045 & 277.6559 & 537.2780 & 269.1426 & 536.2940 & 268.6506 & $\mathbf{4}$ \\
\hline $\mathbf{1 1}$ & 1080.4956 & 540.7514 & 1063.4691 & 532.2382 & 1062.4851 & 531.7462 & $\mathbf{H}$ & 425.2619 & 213.1346 & 408.2354 & 204.6213 & & \\
\hline $\mathbf{1 2}$ & 1193.5797 & 597.2935 & 1176.5531 & 588.7802 & 1175.5691 & 588.2882 & $\mathbf{L}$ & 288.2030 & 144.6051 & 271.1765 & 136.0919 & & $\mathbf{3}$ \\
\hline $\mathbf{1 3}$ & & & & & & & $\mathbf{R}$ & 175.1190 & 88.0631 & 158.0924 & 79.5498 & & $\mathbf{2}$ \\
\hline
\end{tabular}
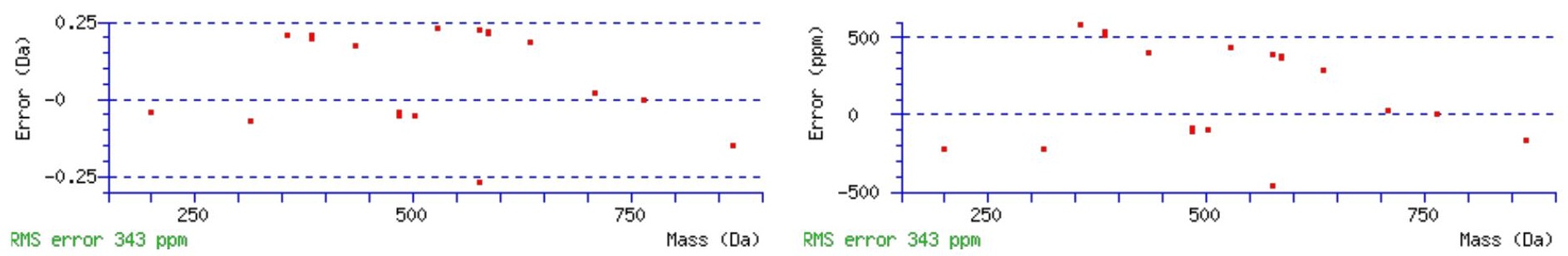

\section{All matches to this query}

\begin{tabular}{|l|l|l|l|}
\hline Score & Mr(calc): & Delta & \multicolumn{1}{|c|}{ Sequence } \\
\hline 43.3 & 1366.6841 & 0.0001 & VVNSTTGPGEHLR \\
\hline 12.4 & 1365.6765 & 1.0076 & VDENGKISRLR \\
\hline 12.4 & 1366.6857 & -0.0015 & VDVSNLKDKIR \\
\hline 12.1 & 1366.6881 & -0.0039 & TPWDIETQIHK \\
\hline 8.2 & 1364.6909 & 1.9933 & EPGSQASAHGLRR \\
\hline 5.0 & 1365.6711 & 1.0131 & RTKDTYGPPMGK \\
\hline 4.6 & 1365.6905 & 0.9937 & LSENTVIVGVVR \\
\hline 4.3 & 1365.6766 & 1.0076 & VDKGSIADGRLR \\
\hline 4.2 & 1366.6955 & -0.0113 & TPNFPSSVLFMK \\
\hline 4.1 & 1366.6969 & -0.0127 & VTAKALQKNASR \\
\hline
\end{tabular}

Spectrum No: 774; Query: 48; Rank: 1

\section{Peptide View}

MS/MS Fragmentation of FLYNFTR

Found in IPI00190555, Tax_Id=10116 Gene_Symbol=Folh1 Glutamate carboxypeptidase 2

Match to Query 48: 960.469748 from(481.242150,2+)

Title: 091008RatKidney_NH4Format01_23.2369.2369.2.dta

Data file K:INewmanPaper|Piliangl3SubProteomes\Piliang3SP\mgf5ppm\ERLIC_3SubProteomes5ppm.mgf 

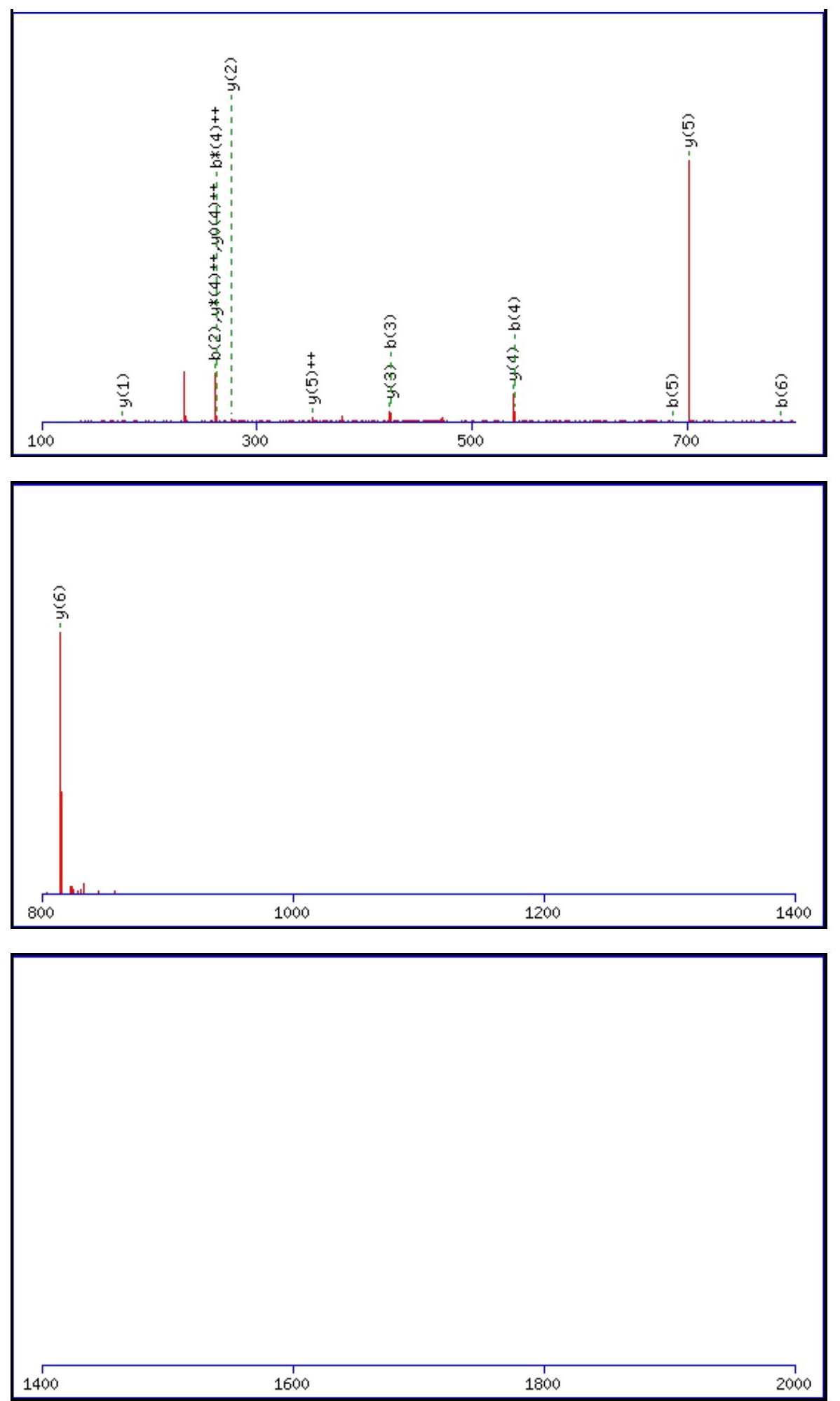

Monoisotopic mass of neutral peptide $\operatorname{Mr}($ calc): 960.4705

Fixed modifications: Carbamidomethyl (C)

Variable modifications:

N4 : Deamidated N (N)

Ions Score: 43 Expect: 0.0045

Matches (Bold Red): 15/54 fragment ions using 29 most intense peaks

\begin{tabular}{|c|c|c|c|c|c|c|c|c|c|c|c|c|c|c|}
\hline$\#$ & $\mathbf{b}$ & $\mathbf{b}^{++}$ & $\mathbf{b}^{*}$ & $\mathbf{b}^{*_{+}^{++}}$ & $\mathbf{b}^{\mathbf{0}}$ & $\mathbf{b}^{\mathbf{0}+}$ & Seq. & $\mathbf{y}$ & $\mathbf{y}^{++}$ & $\mathbf{y}^{*}$ & $\mathbf{y}^{\mathbf{*}^{++}}$ & $\mathbf{y}^{\mathbf{0}}$ & $\mathbf{y}^{\mathbf{0}++}$ & $\#$ \\
\hline $\mathbf{1}$ & 148.0757 & 74.5415 & & & & & $\mathbf{F}$ & & & & & & & $\mathbf{7}$ \\
\hline $\mathbf{2}$ & $\mathbf{2 6 1 . 1 5 9 8}$ & 131.0835 & & & & & $\mathbf{L}$ & $\mathbf{8 1 4 . 4 0 9 4}$ & 407.7083 & 797.3828 & 399.1951 & 796.3988 & 398.7030 & $\mathbf{6}$ \\
\hline $\mathbf{3}$ & $\mathbf{4 2 4 . 2 2 3 1}$ & 212.6152 & & & & & $\mathbf{Y}$ & $\mathbf{7 0 1 . 3 2 5 3}$ & 351.1663 & 684.2988 & 342.6530 & 683.3147 & 342.1610 & 5 \\
\hline $\mathbf{4}$ & 539.2500 & 270.1286 & 522.2235 & $\mathbf{2 6 1 . 6 1 5 4}$ & & & $\mathbf{N}$ & 538.2620 & 269.6346 & 521.2354 & $\mathbf{2 6 1 . 1 2 1 4}$ & 520.2514 & $\mathbf{2 6 0 . 6 2 9 3}$ & $\mathbf{4}$ \\
\hline $\mathbf{5}$ & $\mathbf{6 8 6 . 3 1 8 4}$ & 343.6629 & 669.2919 & 335.1496 & & & $\mathbf{F}$ & $\mathbf{4 2 3 . 2 3 5 0}$ & 212.1212 & 406.2085 & 203.6079 & 405.2245 & 203.1159 & 3 \\
\hline
\end{tabular}




\begin{tabular}{|l|l|l|l|l|l|l|l|l|l|l|l|l|l|l|l|l|}
$\mathbf{6}$ & $\mathbf{7 8 7 . 3 6 6 1}$ & 394.1867 & 770.3396 & 385.6734 & 769.3555 & 385.1814 & $\mathbf{T}$ & 276.1666 & 138.5870 & 259.1401 & 130.0737 & 258.1561 & 129.5817 & $\mathbf{2}$ \\
\hline 7 & & & & & & & $\mathbf{R}$ & $\mathbf{1 7 5 . 1 1 9 0}$ & 88.0631 & 158.0924 & 79.5498 & & & $\mathbf{1}$ \\
\hline
\end{tabular}
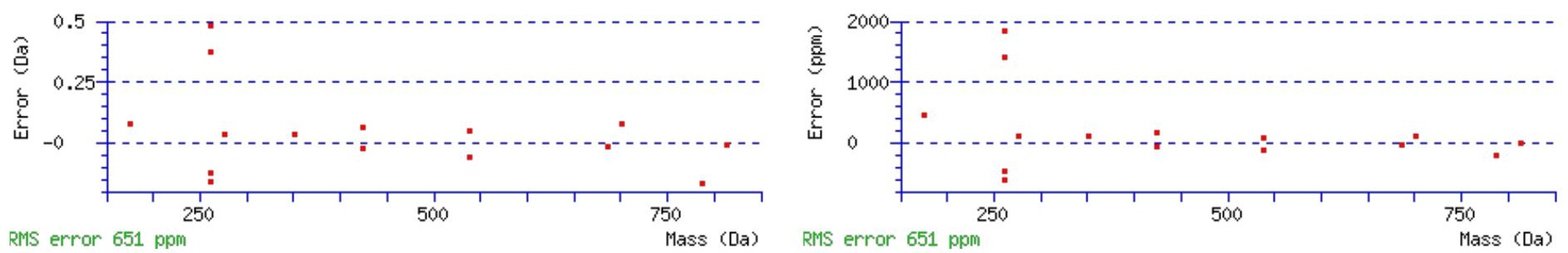

\section{All matches to this query}

\begin{tabular}{|l|l|l|l|}
\hline Score & Mr(calc): & Delta & \multicolumn{1}{|c|}{ Sequence } \\
\hline 43.2 & 960.4705 & -0.0008 & FLYNFTR \\
\hline 19.3 & 959.4729 & 0.9969 & EIKSYLK \\
\hline 13.7 & 959.4729 & 0.9969 & $\underline{\text { NYLXKIK }}$ \\
\hline 13.5 & 959.4712 & 0.9985 & EGWVGNGIK \\
\hline 13.5 & 959.4729 & 0.9969 & $\underline{\text { IYDTKIK }}$ \\
\hline 13.5 & 959.4746 & 0.9952 & MKNPGGDLK \\
\hline 9.0 & 959.4729 & 0.9969 & $\underline{\text { SYLGLSLK }}$ \\
\hline 9.0 & 959.4729 & 0.9969 & YDTIKLK \\
\hline 8.5 & 960.4698 & -0.0001 & MIEDKGPR \\
\hline 7.7 & 960.4624 & 0.0073 & EQKNNSNK \\
\hline
\end{tabular}

Spectrum No: 775; Query: 1712; Rank: 1

\section{Peptide View}

MS/MS Fragmentation of QAQQERDELADEIANSSGK

Found in IPI00209113, Tax_Id=10116 Gene_Symbol=Myh9 Myosin-9

Match to Query 1712: 2088.960972 from(697.327600,3+)

Title: 100101RatKid_NS_deglyco_15.1707.1707.3.dta

Data file K:INewmanPaper|Piliangl3SubProteomes\Piliang3SP\mgf5ppm\ERLIC_3SubProteomes5ppm.mgf

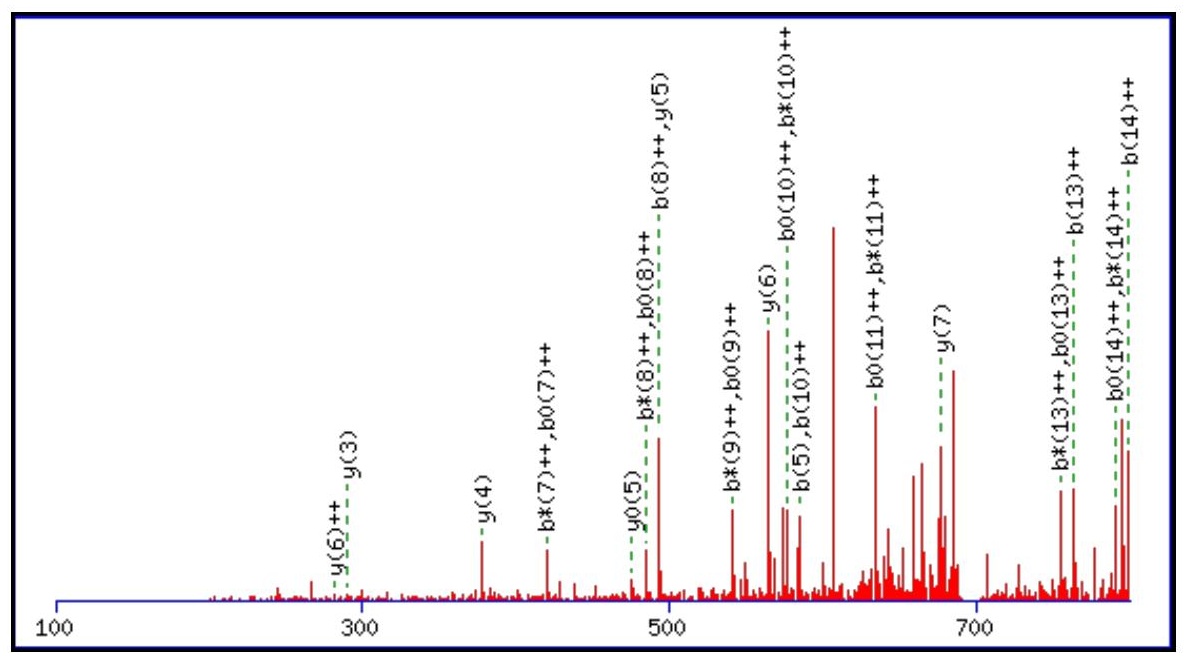



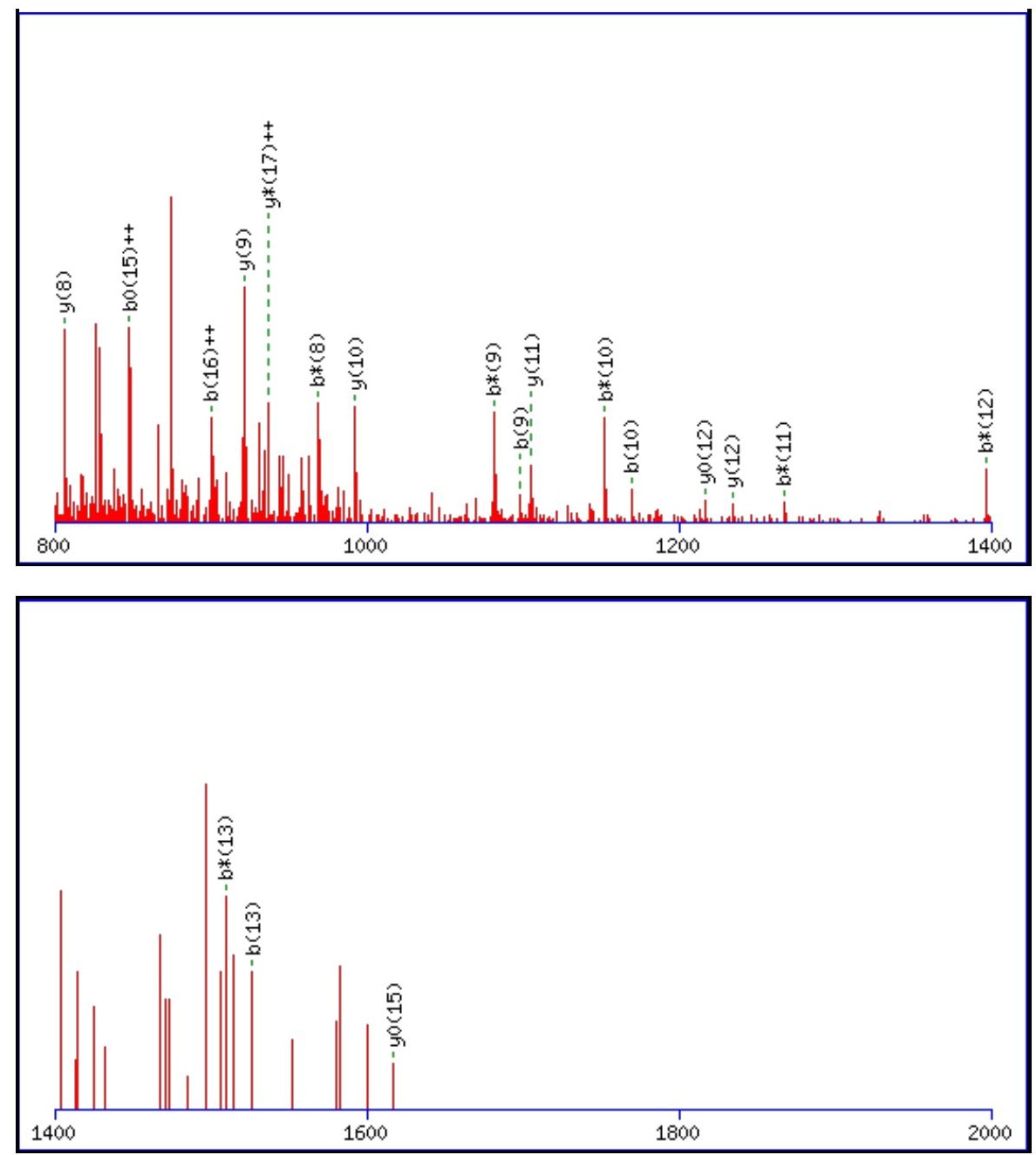

Monoisotopic mass of neutral peptide $\operatorname{Mr}($ calc): 2088.9559

Fixed modifications: Carbamidomethyl (C)

Variable modifications:

N15 : Deamidated_N (N)

Ions Score: 43 Expect: 0.012

Matches (Bold Red): 45/204 fragment ions using 74 most intense peaks

\begin{tabular}{|c|c|c|c|c|c|c|c|c|c|c|c|c|c|c|}
\hline \# & b & $\mathbf{b}^{++}$ & $\mathbf{b}^{*}$ & $\mathbf{b}^{*^{++}}$ & $\mathbf{b}^{0}$ & $\mathbf{b}^{\mathbf{0 + +}}$ & Seq. & $\mathbf{y}$ & $y^{++}$ & $\mathbf{y}^{*}$ & $\mathrm{y}^{*^{++}}$ & $\mathbf{y}^{\mathbf{0}}$ & $y^{0++}$ & \# \\
\hline 1 & 129.0659 & 65.0366 & 112.0393 & 56.5233 & & & $\mathbf{Q}$ & & & & & & & 19 \\
\hline 2 & 200.1030 & 100.5551 & 183.0764 & 92.0418 & & & A & 1961.9047 & 981.4560 & 1944.8781 & 972.9427 & 1943.8941 & 972.4507 & 18 \\
\hline 3 & 328.1615 & 164.5844 & 311.1350 & 156.0711 & & & $\mathbf{Q}$ & 1890.8675 & 945.9374 & 1873.8410 & 937.4241 & 1872.8570 & 936.9321 & 17 \\
\hline 4 & 456.2201 & 228.6137 & 439.1936 & 220.1004 & & & $\mathbf{Q}$ & 1762.8090 & 881.9081 & 1745.7824 & 873.3948 & 1744.7984 & 872.9028 & 16 \\
\hline 5 & 585.2627 & 293.1350 & 568.2362 & 284.6217 & 567.2522 & 284.1297 & $\mathbf{E}$ & 1634.7504 & 817.8788 & 1617.7238 & 809.3656 & 1616.7398 & 808.8736 & 15 \\
\hline 6 & 741.3638 & 371.1856 & 724.3373 & 362.6723 & 723.3533 & 362.1803 & $\mathbf{R}$ & 1505.7078 & 753.3575 & 1488.6813 & 744.8443 & 1487.6972 & 744.3523 & 14 \\
\hline 7 & 856.3908 & 428.6990 & 839.3642 & 420.1857 & 838.3802 & 419.6937 & D & 1349.6067 & 675.3070 & 1332.5801 & 666.7937 & 1331.5961 & 666.3017 & 13 \\
\hline 8 & 985.4334 & 493.2203 & 968.4068 & 484.7070 & 967.4228 & 484.2150 & $\mathbf{E}$ & 1234.5797 & 617.7935 & 1217.5532 & 609.2802 & 1216.5692 & 608.7882 & 12 \\
\hline 9 & 1098.5174 & 549.7624 & 1081.4909 & 541.2491 & 1080.5069 & 540.7571 & $\mathbf{L}$ & 1105.5372 & 553.2722 & 1088.5106 & 544.7589 & 1087.5266 & 544.2669 & 11 \\
\hline 10 & 1169.5545 & 585.2809 & 1152.5280 & 576.7676 & 1151.5440 & 576.2756 & A & 992.4531 & 496.7302 & 975.4265 & 488.2169 & 974.4425 & 487.7249 & 10 \\
\hline 11 & 1284.5815 & 642.7944 & 1267.5549 & 634.2811 & 1266.5709 & 633.7891 & D & 921.4160 & 461.2116 & 904.3894 & 452.6984 & 903.4054 & 452.2063 & 9 \\
\hline 12 & 1413.6241 & 707.3157 & 1396.5975 & 698.8024 & 1395.6135 & 698.3104 & $\mathbf{E}$ & 806.3890 & 403.6982 & 789.3625 & 395.1849 & 788.3785 & 394.6929 & 8 \\
\hline 13 & 1526.7081 & 763.8577 & 1509.6816 & 755.3444 & 1508.6976 & 754.8524 & I & 677.3464 & 339.1769 & 660.3199 & 330.6636 & 659.3359 & 330.1716 & 7 \\
\hline 14 & 1597.7453 & \begin{tabular}{|l|}
799.3763 \\
\end{tabular} & 1580.7187 & 790.8630 & 1579.7347 & 790.3710 & A & 564.2624 & 282.6348 & 547.2358 & 274.1216 & 546.2518 & 273.6295 & 6 \\
\hline 15 & 1712.7722 & 856.8897 & 1695.7456 & 848.3765 & 1694.7616 & 847.8845 & $\mathbf{N}$ & 493.2253 & 247.1163 & 476.1987 & 238.6030 & 475.2147 & 238.1110 & 5 \\
\hline 16 & 1799.8042 & 900.4057 & 1782.7777 & 891.8925 & 1781.7937 & 891.4005 & S & 378.1983 & 189.6028 & 361.1718 & 181.0895 & 360.1878 & 180.5975 & 4 \\
\hline 17 & 1886.8362 & 943.9218 & 1869.8097 & 935.4085 & 1868.8257 & 934.9165 & S & 291.1663 & 146.0868 & 274.1397 & 137.5735 & 273.1557 & 137.0815 & 3 \\
\hline 18 & 1943.8577 & 972.4325 & 1926.8312 & 963.9192 & 1925.8471 & 963.4272 & G & 204.1343 & 102.5708 & 187.1077 & 94.0575 & & & 2 \\
\hline
\end{tabular}




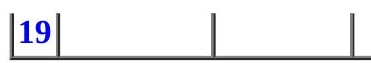
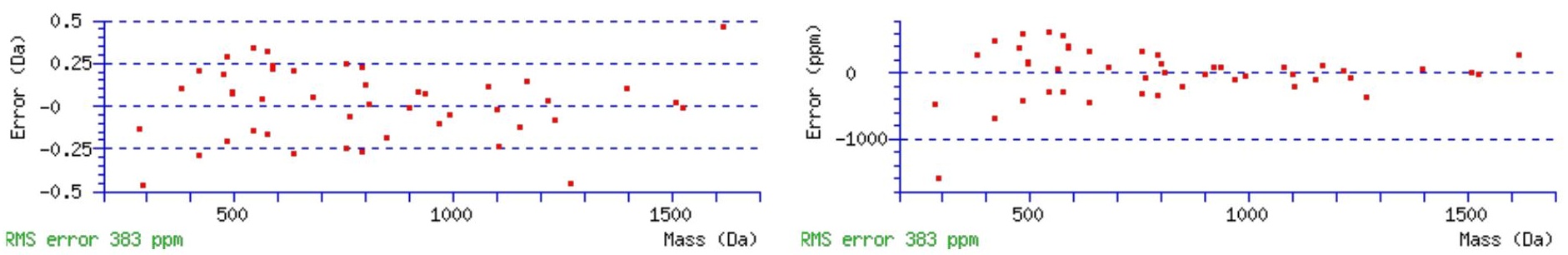

\section{All matches to this query}

\begin{tabular}{|l|l|l|l|}
\hline Score & Mr(calc): & Delta & \multicolumn{1}{c|}{ Sequence } \\
\hline 43.1 & 2088.9559 & 0.0051 & QAQQERDELADEIANSSGK \\
\hline 18.0 & 2087.9719 & 0.9891 & QAQQERDELADEIANSSGK \\
\hline 2.3 & 2087.9637 & 0.9973 & NLHDNLSDLRAQVAANQK \\
\hline 2.0 & 2088.9405 & 0.0205 & VSAENAAGVGEPSPATVYYK \\
\hline 1.6 & 2088.9405 & 0.0205 & VSAENAAGVGEPSPATVYYK \\
\hline 1.3 & 2088.9405 & 0.0205 & VSAENAAGVGEPSPATVYYK \\
\hline 1.3 & 2087.9555 & 1.0055 & $\underline{\text { RNHXPDQYASNNLSASCR }}$ \\
\hline 1.1 & 2088.9758 & -0.0149 & WTVELIVANTKTGRNAR \\
\hline 0.6 & 2086.9433 & 2.0177 & $\underline{\text { SALESHNLGIQEDHSSRK }}$ \\
\hline
\end{tabular}

Spectrum No: 776; Query: 1413; Rank: 1

\section{Peptide View}

MS/MS Fragmentation of VLYLPAYNCTLRPVSK

Found in IPI00212708, Tax_Id=10116 Gene_Symbol=Fetub Fetub protein

Match to Query 1413: 1894.000148 from(948.007350,2+)

Title: 091008RatKidney_NoSalt_25.2989.2989.2.dta

Data file K:INewmanPaper|Piliang|3SubProteomes\Piliang3SP\mgf5ppm|ERLIC_3SubProteomes5ppm.mgf

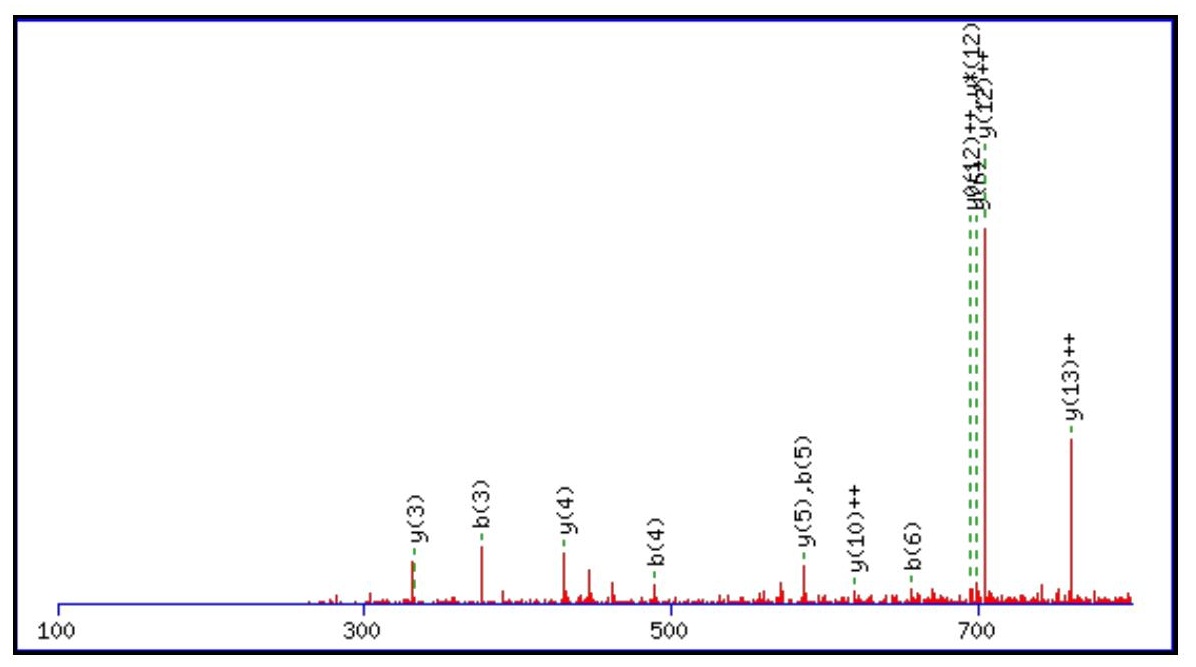



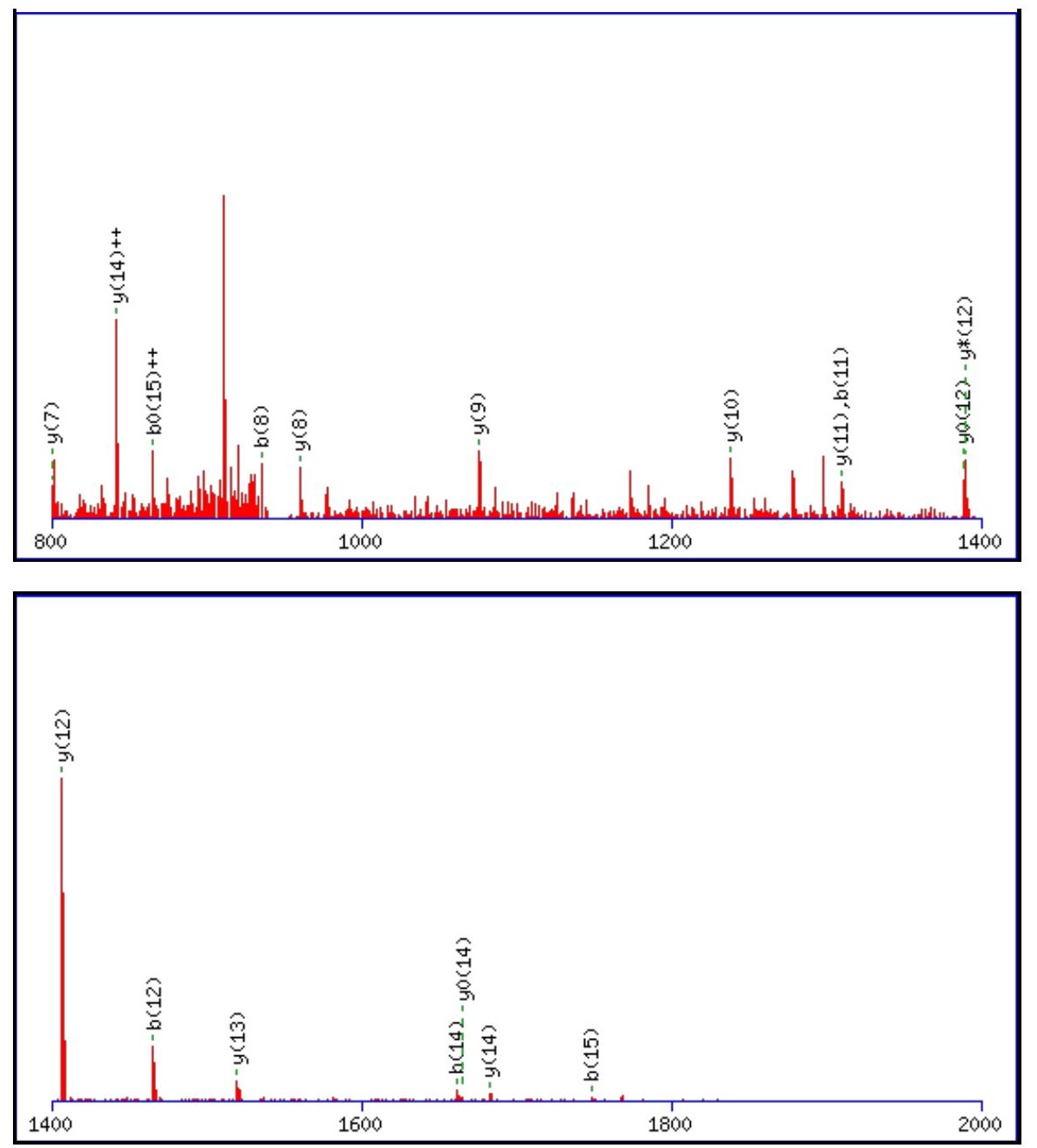

Monoisotopic mass of neutral peptide $\operatorname{Mr}($ calc): 1894.0022

Fixed modifications: Carbamidomethyl (C)

Variable modifications:

N8 : Deamidated_N (N)

Ions Score: 43 Expect: 0.0053

Matches (Bold Red): 31/146 fragment ions using 86 most intense peaks

\begin{tabular}{|c|c|c|c|c|c|c|c|c|c|c|c|c|c|c|}
\hline \# & b & $\mathbf{b}^{++}$ & b* & $\mathbf{b}^{*^{++}}$ & $\mathbf{b}^{0}$ & $\mathbf{b}^{0++}$ & Seq. & $\mathbf{y}$ & $y^{++}$ & $\mathbf{y}^{*}$ & $\mathbf{y}^{*^{++}}$ & $\mathbf{y}^{\mathbf{0}}$ & $\mathbf{y}^{0++}$ & \# \\
\hline 1 & 100.0757 & 50.5415 & & & & & $\mathbf{V}$ & & & & & & & 16 \\
\hline 2 & 213.1598 & 107.0835 & & & & & $\mathbf{L}$ & 1795.9411 & 898.4742 & 1778.9146 & 889.9609 & 1777.9305 & 889.4689 & 15 \\
\hline 3 & 376.2231 & 188.6152 & & & & & $\mathbf{Y}$ & 1682.8570 & 841.9322 & 1665.8305 & 833.4189 & 1664.8465 & 832.9269 & 14 \\
\hline 4 & 489.3071 & 245.1572 & & & & & $\mathbf{L}$ & 1519.7937 & 760.4005 & 1502.7672 & 751.8872 & 1501.7832 & 751.3952 & 13 \\
\hline 5 & 586.3599 & 293.6836 & & & & & $\mathbf{P}$ & 1406.7097 & 703.8585 & 1389.6831 & 695.3452 & 1388.6991 & 694.8532 & 12 \\
\hline 6 & 657.3970 & 329.2022 & & & & & A & 1309.6569 & 655.3321 & 1292.6303 & 646.8188 & 1291.6463 & 646.3268 & 11 \\
\hline 7 & 820.4604 & 410.7338 & & & & & $\mathbf{Y}$ & 1238.6198 & 619.8135 & 1221.5932 & 611.3003 & 1220.6092 & 610.8082 & 10 \\
\hline 8 & 935.4873 & 468.2473 & 918.4607 & 459.7340 & & & $\mathbf{N}$ & 1075.5564 & 538.2819 & 1058.5299 & 529.7686 & 1057.5459 & 529.2766 & 9 \\
\hline 9 & 1095.5179 & 548.2626 & 1078.4914 & 539.7493 & & & $\mathbf{C}$ & 960.5295 & 480.7684 & 943.5030 & 472.2551 & 942.5189 & 471.7631 & 8 \\
\hline 10 & 1196.5656 & 598.7864 & 1179.5391 & 590.2732 & 1178.5551 & 589.7812 & $\mathbf{T}$ & 800.4989 & 400.7531 & 783.4723 & 392.2398 & 782.4883 & 391.7478 & 7 \\
\hline 11 & 1309.6497 & 655.3285 & 1292.6231 & 646.8152 & 1291.6391 & 646.3232 & $\mathbf{L}$ & 699.4512 & 350.2292 & 682.4246 & 341.7160 & 681.4406 & 341.2239 & 6 \\
\hline 12 & 1465.7508 & 733.3790 & 1448.7242 & 724.8658 & 1447.7402 & 724.3738 & $\mathbf{R}$ & 586.3671 & 293.6872 & 569.3406 & 285.1739 & 568.3566 & 284.6819 & 5 \\
\hline 13 & 1562.8036 & 781.9054 & 1545.7770 & 773.3921 & 1544.7930 & 772.9001 & $\mathbf{P}$ & 430.2660 & 215.6366 & 413.2395 & 207.1234 & 412.2554 & 206.6314 & 4 \\
\hline 14 & 1661.8720 & 831.4396 & 1644.8454 & 822.9263 & 1643.8614 & 822.4343 & $\mathbf{V}$ & 333.2132 & 167.1103 & 316.1867 & 158.5970 & 315.2027 & 158.1050 & 3 \\
\hline 15 & 1748.9040 & 874.9556 & 1731.8775 & 866.4424 & 1730.8934 & 865.9504 & S & 234.1448 & 117.5761 & 217.1183 & 109.0628 & 216.1343 & 108.5708 & 2 \\
\hline 16 & & & & & & & $\mathbf{K}$ & 147.1128 & 74.0600 & 130.0863 & 65.5468 & & & 1 \\
\hline
\end{tabular}



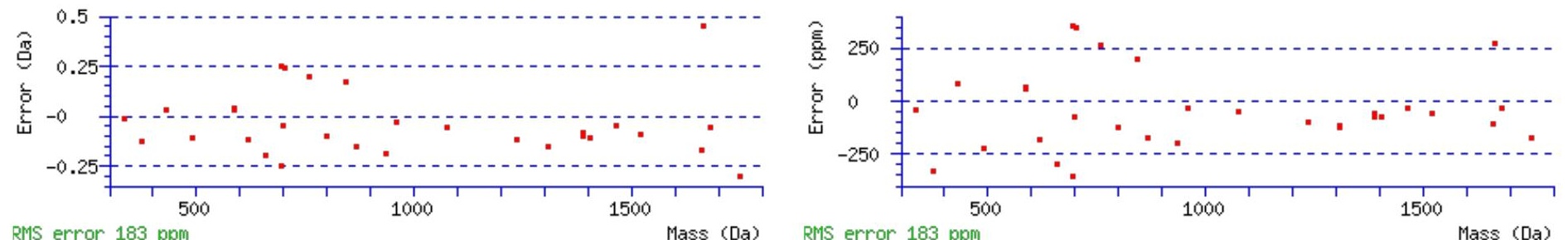

\section{All matches to this query}

\begin{tabular}{|l|l|l|l|}
\hline Score & Mr(calc): & Delta & \multicolumn{1}{c|}{ Sequence } \\
\hline 43.2 & 1894.0022 & -0.0021 & VLYLPAYNCTLRPVSK \\
\hline 11.5 & 1893.9866 & 0.0135 & KVFLEPFHKVTLSNR \\
\hline 0.8 & 1894.0061 & -0.0059 & TGSELLPAKAFGSFRASR \\
\hline 0.1 & 1892.9801 & 1.0200 & IDFIFSLLSKYAAGIR \\
\hline 0.1 & 1893.9812 & 0.0189 & TLKSGLGDDLVQALGLSK \\
\hline 0.1 & 1893.0026 & 0.9976 & LSPWGLAPLFRSVRSK \\
\hline
\end{tabular}

Spectrum No: 777; Query: 1426; Rank: 1

\section{Peptide View}

MS/MS Fragmentation of VDCTPLMHSLVYNLTK

Found in IPI00190555, Tax_Id=10116 Gene_Symbol=Folh1 Glutamate carboxypeptidase 2

Match to Query 1426: 1906.916488 from(954.465520,2+)

Title: 091008RatKidney_NH4Format01_23.2825.2825.2.dta

Data file K:INewmanPaper|Piliangl3SubProteomes\Piliang3SP\mgf5ppm\ERLIC_3SubProteomes5ppm.mgf

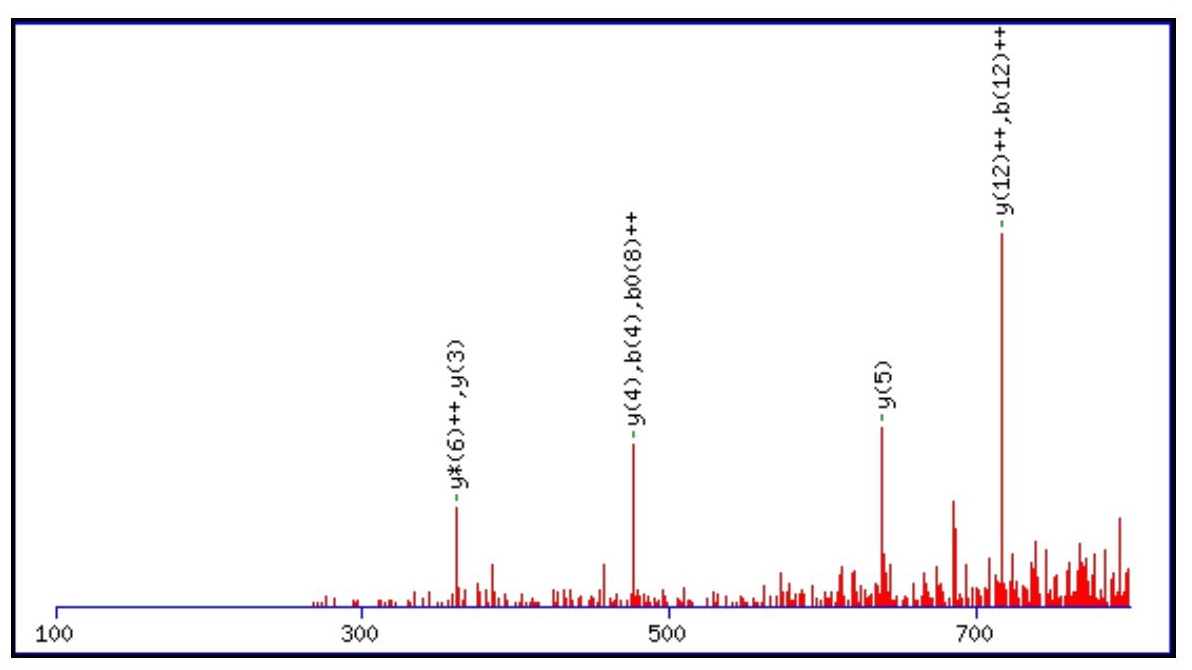




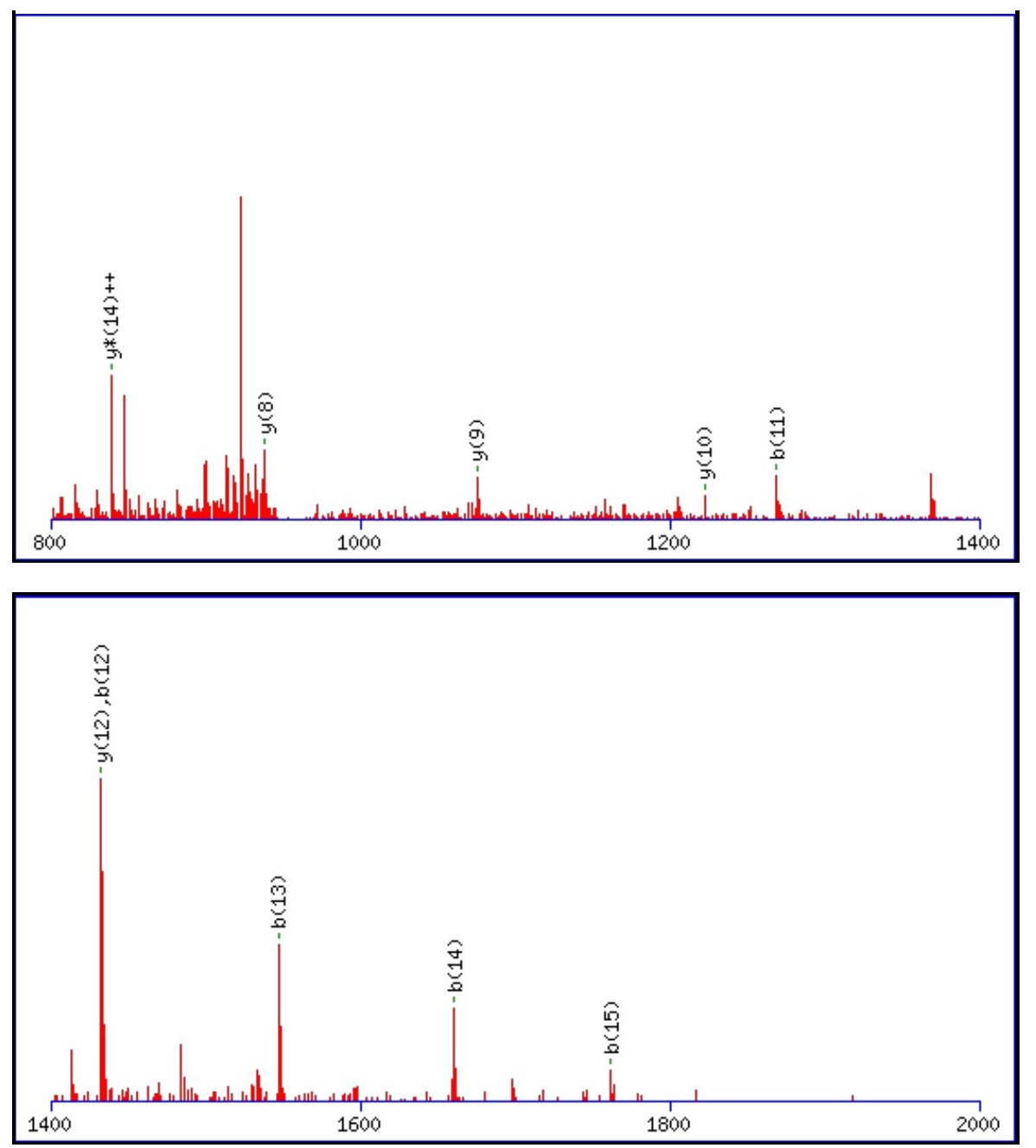

Monoisotopic mass of neutral peptide $\operatorname{Mr}($ calc): 1906.9169

Fixed modifications: Carbamidomethyl (C)

Variable modifications:

M7 : Oxidation (M)

N13 : Deamidated_N (N)

Ions Score: 43 Expect : 0.011

Matches (Bold Red): 18/152 fragment ions using 38 most intense peaks

\begin{tabular}{|c|c|c|c|c|c|c|c|c|c|c|c|c|c|c|}
\hline \# & b & $\mathbf{b}^{++}$ & b* & $\mathbf{b}^{*^{++}}$ & $\mathbf{b}^{\mathbf{0}}$ & $\mathbf{b}^{0++}$ & Seq. & $\mathbf{y}$ & $\mathbf{y}^{++}$ & $\mathbf{y}^{*}$ & $\mathbf{y}^{*^{++}}$ & $\mathbf{y}^{0}$ & $\mathbf{y}^{0++}$ & \# \\
\hline 1 & 100.0757 & 50.5415 & & & & & $\mathbf{V}$ & & & & & & & 16 \\
\hline 2 & 215.1026 & 108.0550 & & & 197.0921 & 99.0497 & D & 1808.8557 & 904.9315 & 1791.8292 & 896.4182 & 1790.8452 & 895.9262 & 15 \\
\hline 3 & 375.1333 & 188.0703 & & & 357.1227 & 179.0650 & C & 1693.8288 & 847.4180 & 1676.8022 & 838.9048 & 1675.8182 & 838.4128 & 14 \\
\hline 4 & 476.1810 & 238.5941 & & & 458.1704 & 229.5888 & $\mathbf{T}$ & 1533.7981 & 767.4027 & 1516.7716 & 758.8894 & 1515.7876 & 758.3974 & 13 \\
\hline 5 & 573.2337 & 287.1205 & & & 555.2232 & 278.1152 & $\mathbf{P}$ & 1432.7505 & 716.8789 & 1415.7239 & 708.3656 & 1414.7399 & 707.8736 & 12 \\
\hline 6 & 686.3178 & 343.6625 & & & 668.3072 & 334.6573 & $\mathbf{L}$ & 1335.6977 & 668.3525 & 1318.6711 & 659.8392 & 1317.6871 & 659.3472 & 11 \\
\hline 7 & 833.3532 & 417.1802 & & & 815.3426 & 408.1750 & $\mathbf{M}$ & 1222.6136 & 611.8105 & 1205.5871 & 603.2972 & 1204.6031 & 602.8052 & 10 \\
\hline 8 & 970.4121 & 485.7097 & & & 952.4015 & 476.7044 & $\mathbf{H}$ & 1075.5782 & 538.2928 & 1058.5517 & 529.7795 & 1057.5677 & 529.2875 & 9 \\
\hline 9 & 1057.4441 & 529.2257 & & & 1039.4336 & 520.2204 & S & 938.5193 & 469.7633 & 921.4928 & 461.2500 & 920.5088 & 460.7580 & 8 \\
\hline 10 & 1170.5282 & 585.7677 & & & 1152.5176 & 576.7625 & $\mathbf{L}$ & 851.4873 & 426.2473 & 834.4607 & 417.7340 & 833.4767 & 417.2420 & 7 \\
\hline 11 & 1269.5966 & 635.3019 & & & 1251.5860 & 626.2967 & $\mathbf{V}$ & 738.4032 & 369.7053 & 721.3767 & 361.1920 & 720.3927 & 360.7000 & 6 \\
\hline 12 & 1432.6599 & 716.8336 & & & 1414.6494 & 707.8283 & $\mathbf{Y}$ & 639.3348 & 320.1710 & 622.3083 & 311.6578 & 621.3242 & 311.1658 & 5 \\
\hline 13 & 1547.6869 & 774.3471 & 1530.6603 & 765.8338 & 1529.6763 & 765.3418 & $\mathbf{N}$ & 476.2715 & 238.6394 & 459.2449 & 230.1261 & 458.2609 & 229.6341 & 4 \\
\hline 14 & 1660.7709 & 830.8891 & 1643.7444 & 822.3758 & 1642.7604 & 821.8838 & $\mathbf{L}$ & 361.2445 & 181.1259 & 344.2180 & 172.6126 & 343.2340 & 172.1206 & 3 \\
\hline 15 & 1761.8186 & 881.4129 & 1744.7921 & 872.8997 & 1743.8081 & 872.4077 & $\mathbf{T}$ & 248.1605 & 124.5839 & 231.1339 & 116.0706 & 230.1499 & 115.5786 & 2 \\
\hline 16 & & & & & & & $\mathbf{K}$ & 147.1128 & 74.0600 & 130.0863 & 65.5468 & & & 1 \\
\hline
\end{tabular}



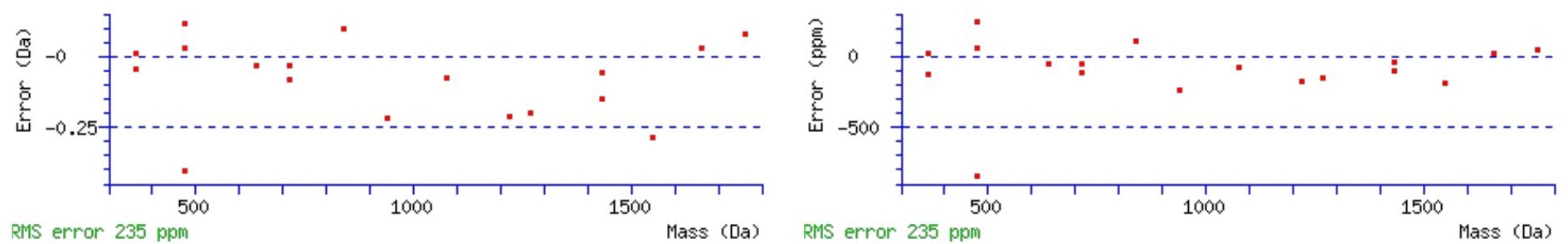

\section{All matches to this query}

\begin{tabular}{|l|l|l|l|}
\hline Score & Mr(calc): & Delta & \multicolumn{1}{c|}{ Sequence } \\
\hline 43.2 & 1906.9169 & -0.0004 & VDCTPLMHSLVYNLTK \\
\hline 9.1 & 1906.9207 & -0.0042 & IFVPARNNMQSGVNNTK \\
\hline 6.2 & 1906.9094 & 0.0070 & CSINGRVYGNEIPEVAK \\
\hline 5.3 & 1906.9054 & 0.0111 & QASVSTITEKVDKLTK \\
\hline 5.3 & 1906.9054 & 0.0111 & QASVSTITEKVDKLTK \\
\hline 5.3 & 1906.9054 & 0.0111 & QASVSTITEKVDKLTK \\
\hline 4.7 & 1906.9223 & -0.0058 & DRIVLNQEVMAPDSIK \\
\hline 2.4 & 1906.8989 & 0.0176 & GLMQRGVTGVTVTSITK \\
\hline 2.4 & 1906.8989 & 0.0176 & GLMQRGVTGVTVTSITK \\
\hline 2.4 & 1906.8989 & 0.0176 & GLMQRGVTGVTVTSITK \\
\hline
\end{tabular}

Spectrum No: 778; Query: 136; Rank: 1

\section{Peptide View}

MS/MS Fragmentation of NYTDNELEK

Found in IPI00324633, Tax_Id=10116 Gene_Symbol=Glud1 Glutamate dehydrogenase 1, mitochondrial precursor

Match to Query 136: 1125.486874 from(1126.494150,1+)

Title: 091008RatKidney_NH4Format02_25.1120.1120.1.dta

Data file K:INewmanPaper|Piliangl3SubProteomes\Piliang3SP\mgf5ppm\ERLIC_3SubProteomes5ppm.mgf

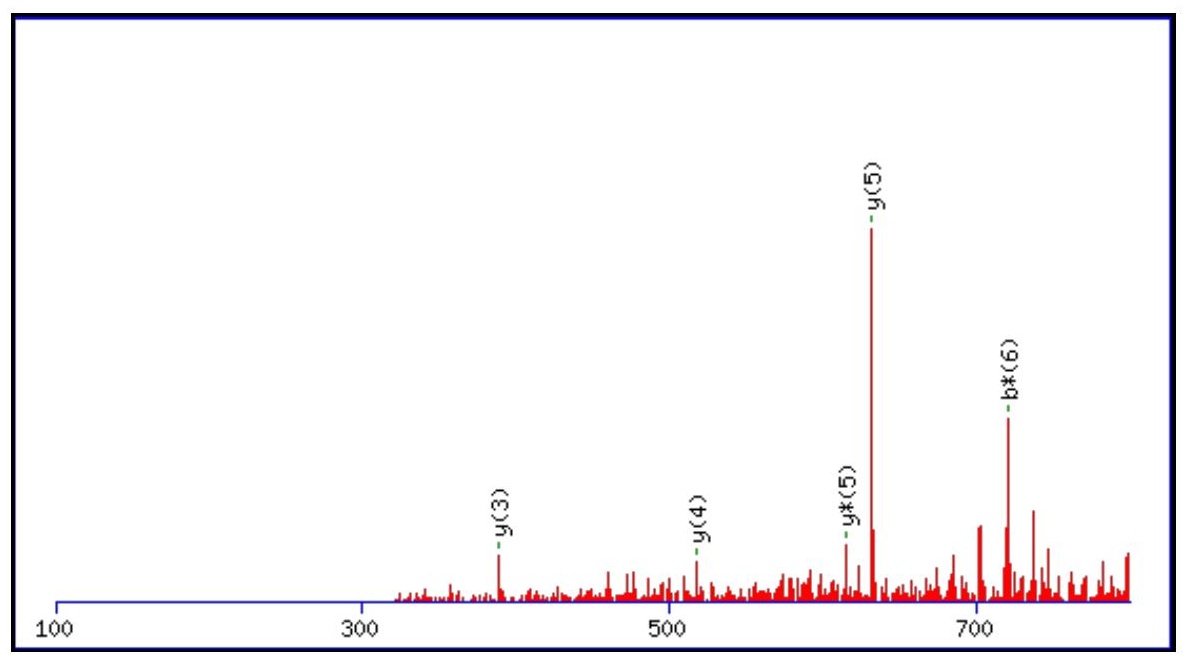




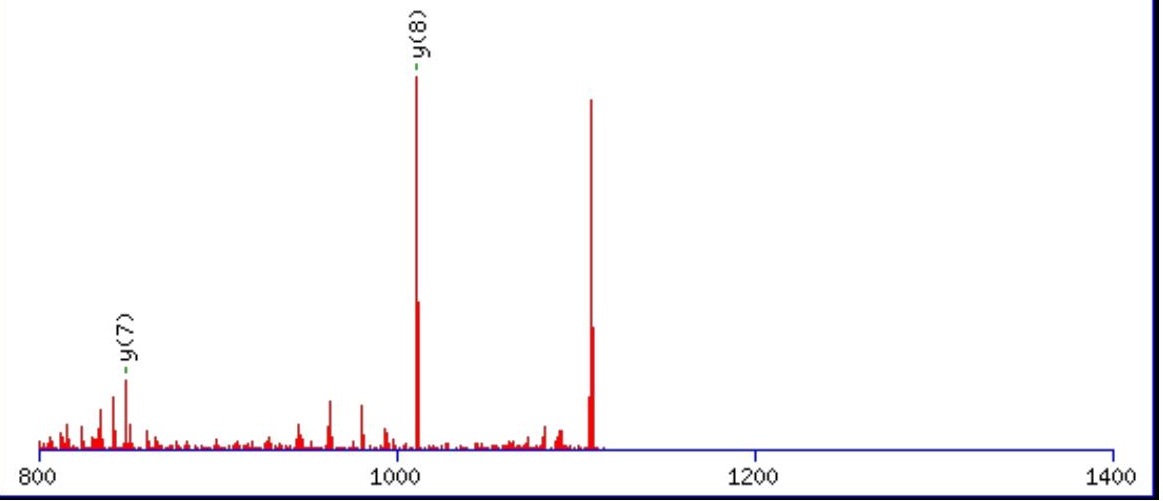

\begin{tabular}{rrrr}
\hline 1400 & 1600 & 1800 & 2000 \\
\hline
\end{tabular}

Monoisotopic mass of neutral peptide $\operatorname{Mr}($ calc): 1125.4826

Fixed modifications: Carbamidomethyl (C)

Variable modifications:

N1 : Deamidated $\mathrm{N}(\mathrm{N})$

Ions Score: 43 Expect: 0.0049

Matches (Bold Red): 7/45 fragment ions using 8 most intense peaks

\begin{tabular}{|c|c|c|c|c|c|c|c|c|}
\hline$\#$ & $\mathbf{b}$ & $\mathbf{b}^{*}$ & $\mathbf{b}^{\mathbf{0}}$ & $\mathbf{S e q} \cdot$ & $\mathbf{y}$ & $\mathbf{y}^{*}$ & $\mathbf{y}^{\mathbf{0}}$ & $\#$ \\
\hline $\mathbf{1}$ & 116.0342 & 99.0077 & & $\mathbf{N}$ & & & & $\mathbf{9}$ \\
\hline $\mathbf{2}$ & 279.0975 & 262.0710 & & $\mathbf{Y}$ & $\mathbf{1 0 1 1 . 4 6 2 9}$ & 994.4364 & 993.4524 & $\mathbf{8}$ \\
\hline $\mathbf{3}$ & 380.1452 & 363.1187 & 362.1347 & $\mathbf{T}$ & $\mathbf{8 4 8 . 3 9 9 6}$ & 831.3731 & 830.3890 & $\mathbf{7}$ \\
\hline $\mathbf{4}$ & 495.1722 & 478.1456 & 477.1616 & $\mathbf{D}$ & 747.3519 & 730.3254 & 729.3414 & $\mathbf{6}$ \\
\hline $\mathbf{5}$ & 609.2151 & 592.1885 & 591.2045 & $\mathbf{N}$ & $\mathbf{6 3 2 . 3 2 5 0}$ & $\mathbf{6 1 5 . 2 9 8 4}$ & $\mathbf{6 1 4 . 3 1 4 4}$ & $\mathbf{5}$ \\
\hline $\mathbf{6}$ & 738.2577 & $\mathbf{7 2 1 . 2 3 1 1}$ & 720.2471 & $\mathbf{E}$ & $\mathbf{5 1 8 . 2 8 2 1}$ & 501.2555 & 500.2715 & $\mathbf{4}$ \\
\hline $\mathbf{7}$ & 851.3417 & 834.3152 & 833.3312 & $\mathbf{L}$ & $\mathbf{3 8 9 . 2 3 9 5}$ & 372.2129 & 371.2289 & $\mathbf{3}$ \\
\hline $\mathbf{8}$ & 980.3843 & 963.3578 & 962.3738 & $\mathbf{E}$ & 276.1554 & 259.1288 & 258.1448 & $\mathbf{2}$ \\
\hline $\mathbf{9}$ & & & & $\mathbf{K}$ & 147.1128 & 130.0863 & & $\mathbf{1}$ \\
\hline
\end{tabular}
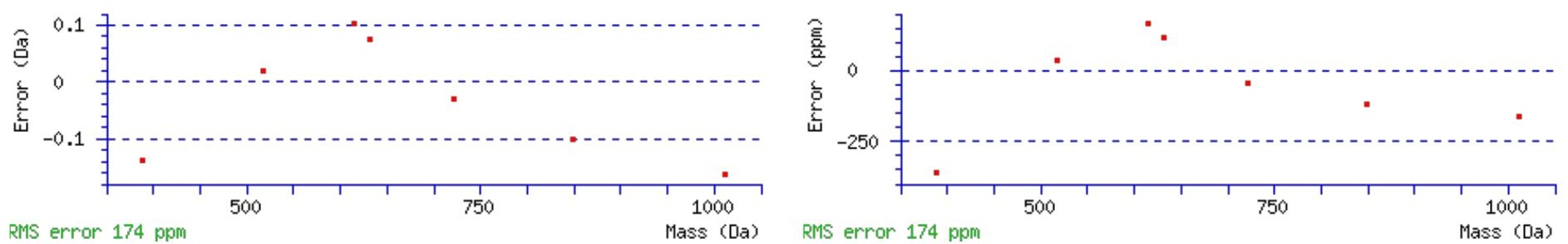

\section{All matches to this query}




\begin{tabular}{|l|l|l|l|} 
Score & $\mathbf{M r}($ calc): & Delta & \multicolumn{1}{|c|}{ Sequence } \\
\hline 43.1 & 1125.4826 & 0.0043 & $\underline{\text { NYTDNELEK }}$ \\
\hline 19.9 & 1125.4826 & 0.0043 & $\underline{\text { NYTDNELEK }}$ \\
\hline 4.8 & 1125.4760 & 0.0108 & $\underline{\text { YEEEVAACR }}$ \\
\hline 4.8 & 1124.4842 & 1.0027 & $\underline{\text { NCLLTDDMK }}$ \\
\hline 4.4 & 1125.4955 & -0.0086 & $\underline{\text { TPSKEEVEK }}$ \\
\hline 4.1 & 1124.4780 & 1.0088 & $\underline{\text { LRGSYTLR }}$ \\
\hline 3.7 & 1125.4938 & -0.0069 & $\underline{\text { SEYNDQLTR }}$ \\
\hline 3.3 & 1125.4856 & 0.0013 & $\underline{\text { THSGEKPYK }}$ \\
\hline 2.0 & 1125.4954 & -0.0086 & $\underline{\text { NEVNLSLEK }}$ \\
\hline 2.0 & 1125.4896 & -0.0027 & $\underline{\text { NYLSVFFR }}$ \\
\hline
\end{tabular}

Spectrum No: 779; Query: 2187; Rank: 1

\section{Peptide View}

MS/MS Fragmentation of VVLVNTTHKPFDIHVYHPYR

Found in IPI00205325, Tax_Id=10116 Gene_Symbol=Lrp2 Low-density lipoprotein receptor-related protein 2 precursor

Match to Query 2187: 2435.277042 from(812.766290,3+)

Title: 091008RatKidney_NH4Format01_24.2403.2403.3.dta

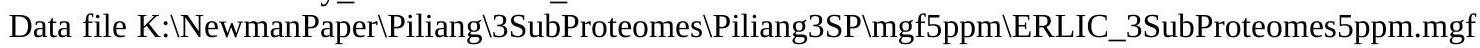
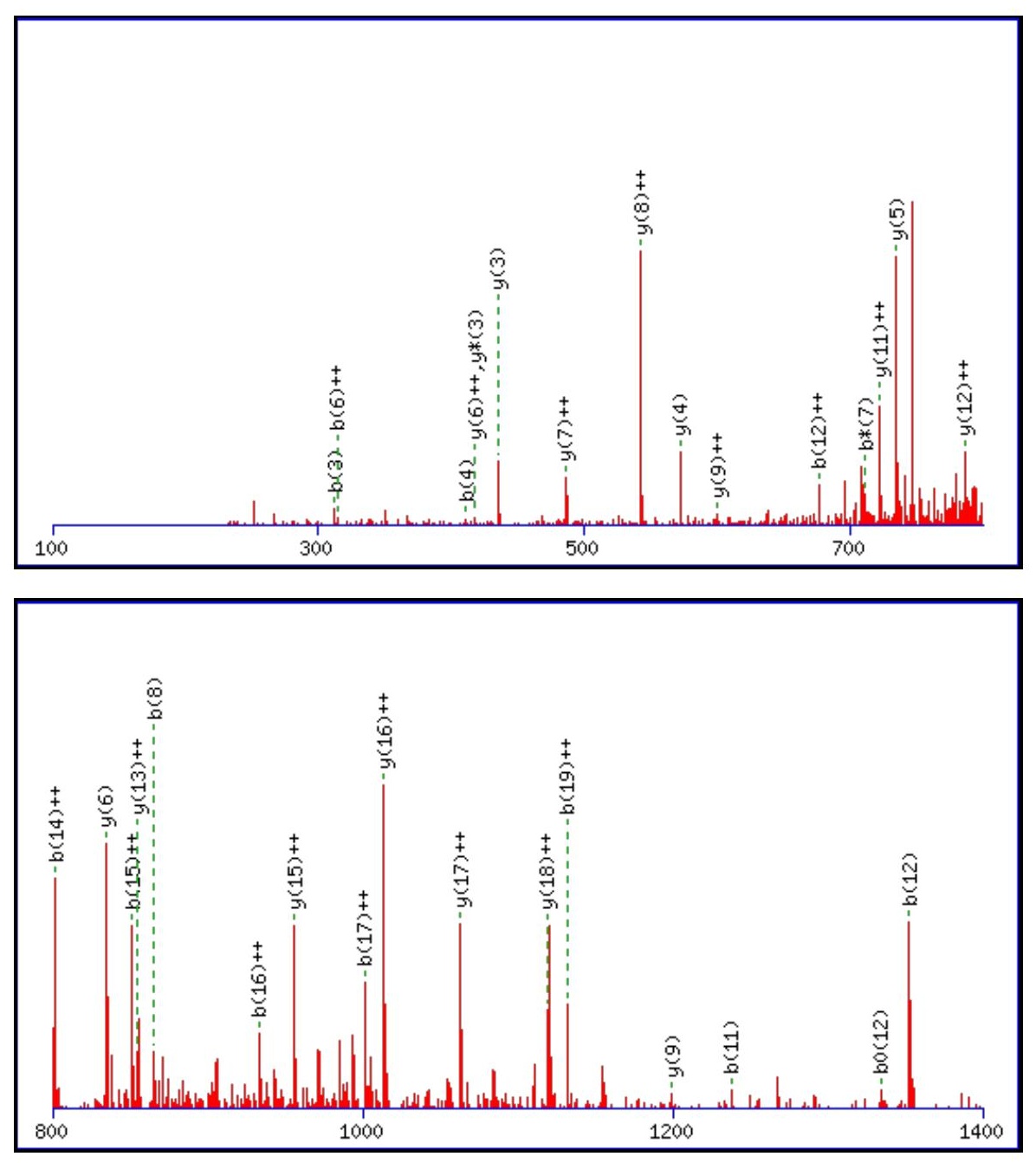


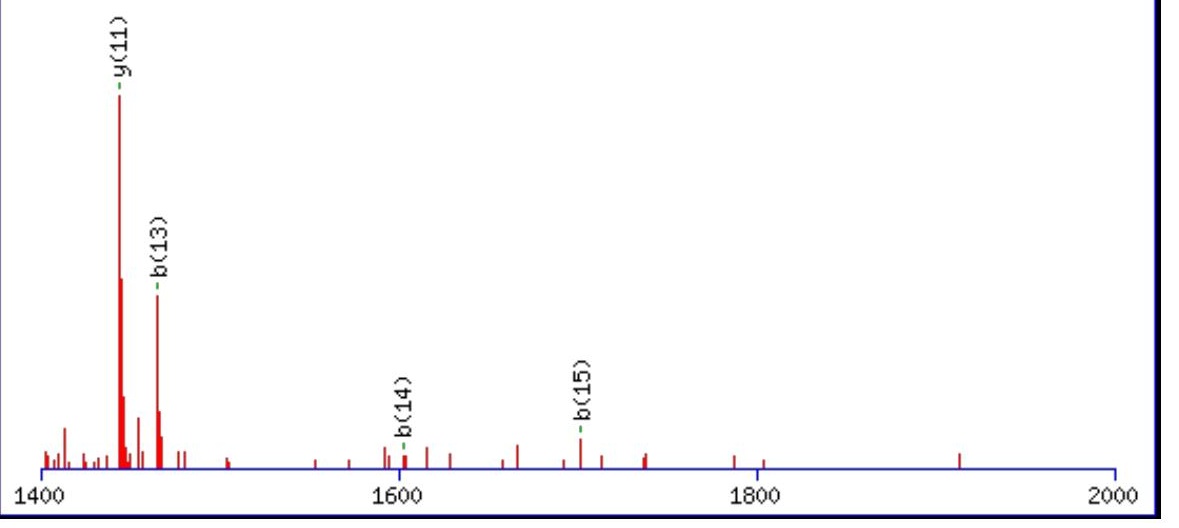

Monoisotopic mass of neutral peptide $\operatorname{Mr}($ calc): 2435.2750

Fixed modifications: Carbamidomethyl (C)

Variable modifications:

N5: : Deamidated $\mathrm{N}(\mathrm{N})$

Ions Score: 43 Expect: 0.0051

Matches (Bold Red): 35/194 fragment ions using 96 most intense peaks

\begin{tabular}{|c|c|c|c|c|c|c|c|c|c|c|c|c|c|c|}
\hline \# & b & $\mathbf{b}^{++}$ & $\mathbf{b}^{*}$ & $\mathbf{b}^{*^{++}}$ & $\mathbf{b}^{\mathbf{0}}$ & $\mathbf{b}^{0++}$ & Seq. & $\mathbf{y}$ & $y^{++}$ & $\mathbf{y}^{*}$ & $\mathrm{y}^{\mathrm{*}^{++}}$ & $\mathbf{y}^{\mathbf{0}}$ & $\mathbf{y}^{0++}$ & \# \\
\hline 1 & 100.0757 & 50.5415 & & & & & $\mathbf{V}$ & & & & & & & 20 \\
\hline 2 & 199.1441 & 100.0757 & & & & & $\mathbf{V}$ & 2337.2139 & 1169.1106 & 2320.1873 & 1160.5973 & 2319.2033 & 1160.1053 & 19 \\
\hline 3 & 312.2282 & 156.6177 & & & & & $\mathbf{L}$ & 2238.1454 & 1119.5764 & 2221.1189 & 1111.0631 & 2220.1349 & 1110.5711 & 18 \\
\hline 4 & 411.2966 & 206.1519 & & & & & $\mathbf{V}$ & 2125.0614 & 1063.0343 & 2108.0348 & 1054.5211 & 2107.0508 & 1054.0290 & 17 \\
\hline 5 & 526.3235 & 263.6654 & 509.2970 & 255.1521 & & & $\mathbf{N}$ & 2025.9930 & 1013.5001 & 2008.9664 & 1004.9868 & 2007.9824 & 1004.4948 & 16 \\
\hline 6 & 627.3712 & 314.1892 & 610.3446 & 305.6760 & 609.3606 & 305.1840 & $\mathbf{T}$ & 1910.9660 & 955.9867 & 1893.9395 & 947.4734 & 1892.9555 & 946.9814 & 15 \\
\hline 7 & 728.4189 & 364.7131 & 711.3923 & 356.1998 & 710.4083 & 355.7078 & $\mathbf{T}$ & 1809.9184 & 905.4628 & 1792.8918 & 896.9495 & 1791.9078 & 896.4575 & 14 \\
\hline 8 & 865.4778 & 433.2425 & 848.4512 & 424.7293 & 847.4672 & 424.2372 & $\mathbf{H}$ & 1708.8707 & 854.9390 & 1691.8441 & 846.4257 & 1690.8601 & 845.9337 & 13 \\
\hline 9 & 993.5728 & 497.2900 & 976.5462 & 488.7767 & 975.5622 & 488.2847 & $\mathbf{K}$ & 1571.8118 & 786.4095 & 1554.7852 & 777.8962 & 1553.8012 & 777.4042 & 12 \\
\hline 10 & 1090.6255 & 545.8164 & 1073.5990 & 537.3031 & 1072.6149 & 536.8111 & $\mathbf{P}$ & 1443.7168 & 722.3620 & 1426.6902 & 713.8488 & 1425.7062 & 713.3568 & 11 \\
\hline 11 & 1237.6939 & 619.3506 & 1220.6674 & 610.8373 & 1219.6834 & 610.3453 & $\mathbf{F}$ & 1346.6640 & 673.8357 & 1329.6375 & 665.3224 & 1328.6535 & 664.8304 & 10 \\
\hline 12 & 1352.7209 & 676.8641 & 1335.6943 & 668.3508 & 1334.7103 & 667.8588 & D & 1199.5956 & 600.3014 & 1182.5691 & 591.7882 & 1181.5851 & 591.2962 & 9 \\
\hline 13 & 1465.8049 & 733.4061 & 1448.7784 & 724.8928 & 1447.7944 & 724.4008 & I & 1084.5687 & 542.7880 & 1067.5421 & 534.2747 & & & 8 \\
\hline 14 & 1602.8638 & 801.9356 & 1585.8373 & 793.4223 & 1584.8533 & 792.9303 & $\mathbf{H}$ & 971.4846 & 486.2459 & 954.4581 & 477.7327 & & & 7 \\
\hline 15 & 1701.9323 & 851.4698 & 1684.9057 & 842.9565 & 1683.9217 & 842.4645 & V & 834.4257 & 417.7165 & 817.3992 & 409.2032 & & & 6 \\
\hline 16 & 1864.9956 & 933.0014 & 1847.9690 & 924.4882 & 1846.9850 & 923.9962 & $\mathbf{Y}$ & 735.3573 & 368.1823 & 718.3307 & 359.6690 & & & 5 \\
\hline 17 & 2002.0545 & 1001.5309 & 1985.0280 & 993.0176 & 1984.0439 & 992.5256 & $\mathbf{H}$ & 572.2940 & 286.6506 & 555.2674 & 278.1373 & & & 4 \\
\hline 18 & 2099.1073 & 1050.0573 & 2082.0807 & 1041.5440 & 2081.0967 & 1041.0520 & $\mathbf{P}$ & 435.2350 & 218.1212 & 418.2085 & 209.6079 & & & 3 \\
\hline 19 & 2262.1706 & 1131.5889 & 2245.1440 & 1123.0757 & 2244.1600 & 1122.5837 & $\mathbf{Y}$ & 338.1823 & 169.5948 & 321.1557 & 161.0815 & & & 2 \\
\hline 20 & & & & & & & $\mathbf{R}$ & 175.1190 & 88.0631 & 158.0924 & 79.5498 & & & 1 \\
\hline
\end{tabular}
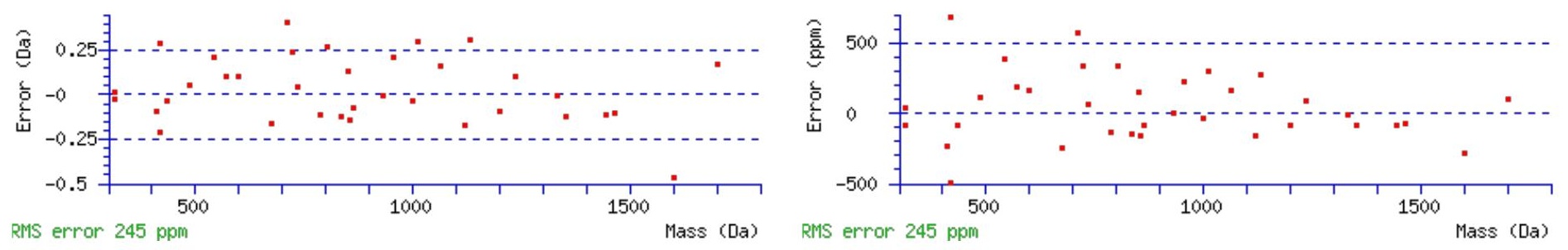

\section{All matches to this query}

\begin{tabular}{|l|l|l|l|}
\hline Score & Mr(calc): & Delta & Sequence \\
\hline 43.1 & 2435.2750 & 0.0020 & VVLVNTTHKPFDIHVYHPYR \\
\hline & & &
\end{tabular}




\section{$12.2 \quad 2434.2910|0.9860|$ VVLVNTTHKPFDIHVYHPYR}

Spectrum No: 780; Query: 2145; Rank: 1

\section{Peptide View}

MS/MS Fragmentation of EALGHSIAQLNAENNHTFYFK

Found in IPI00187799, Tax_Id=10116 Gene_Symbol=LOC25087;Kng1 Isoform HMW of Kininogen-1 precursor

Match to Query 2145: 2404.152102 from(802.391310,3+)

Title: 100101RatKid_NS_deglyco_18.3473.3473.3.dta

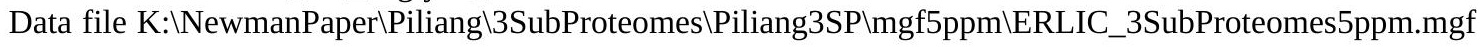
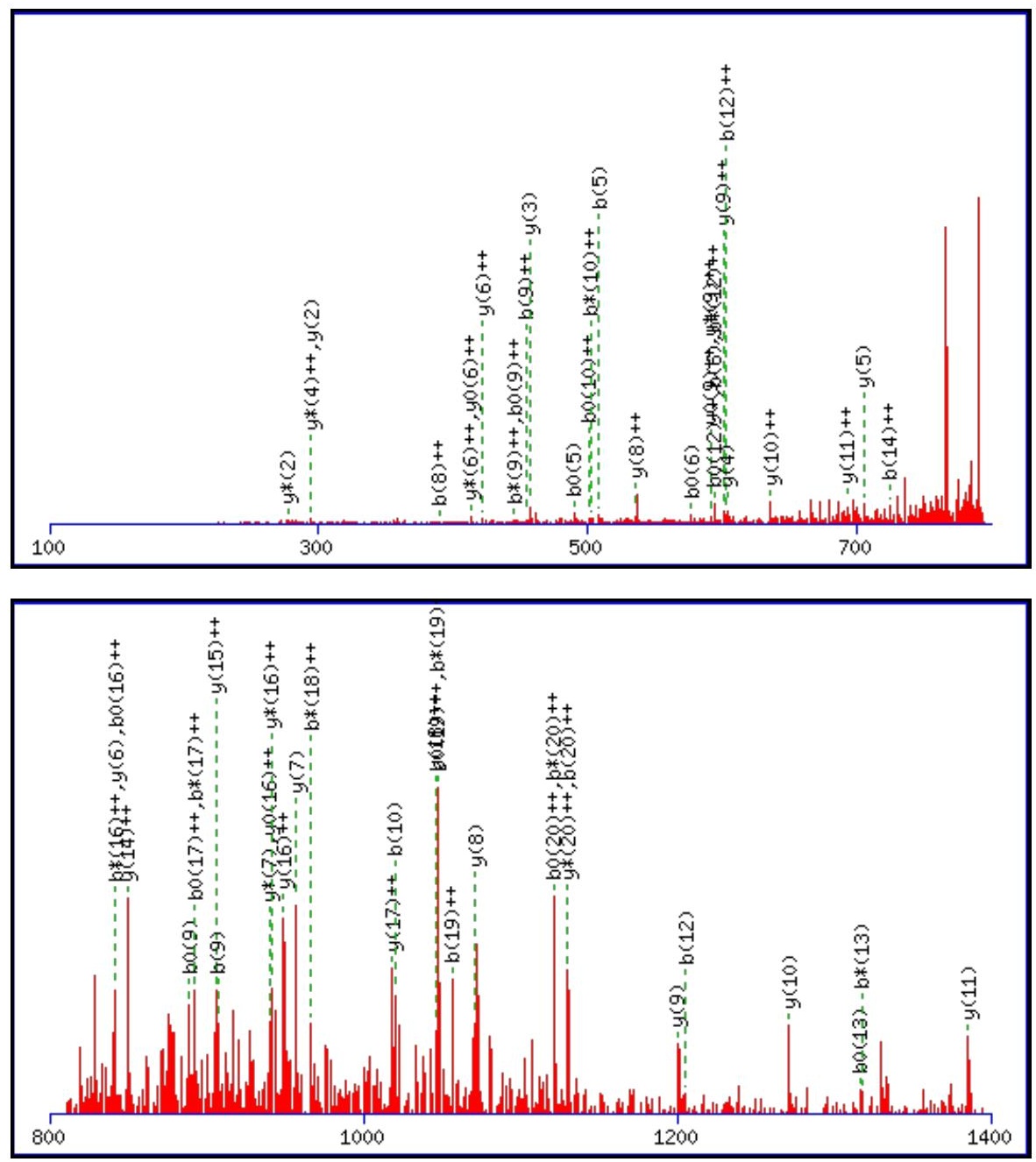


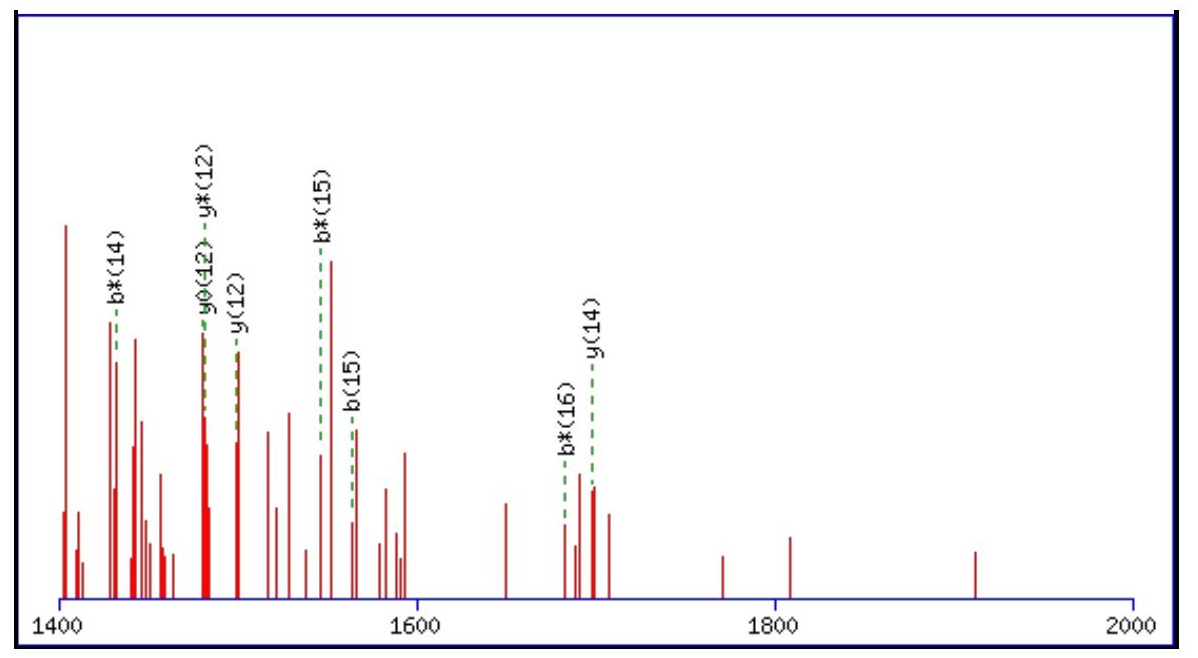

Monoisotopic mass of neutral peptide $\operatorname{Mr}($ calc): 2404.1447

Fixed modifications: Carbamidomethyl (C)

Variable modifications:

N15 : Deamidated_N (N)

Ions Score: 43 Expect: 0.014

Matches (Bold Red): 69/216 fragment ions using 149 most intense peaks

\begin{tabular}{|c|c|c|c|c|c|c|c|c|c|c|c|c|c|c|}
\hline \# & b & $\mathbf{b}^{++}$ & b* & $\mathbf{b}^{*^{++}}$ & $\mathbf{b}^{0}$ & $\mathbf{b}^{\mathbf{0 + +}}$ & Seq. & $\mathbf{y}$ & $y^{++}$ & $\mathbf{y}^{*}$ & $\mathrm{y}^{\mathrm{*}^{++}}$ & $\mathbf{y}^{\mathbf{0}}$ & $y^{0++}$ & \# \\
\hline 1 & 130.0499 & 65.5286 & & & 112.0393 & 56.5233 & $\mathbf{E}$ & & & & & & & 21 \\
\hline 2 & 201.0870 & 101.0471 & & & 183.0764 & 92.0418 & A & 2276.1095 & 1138.5584 & 2259.0829 & 1130.0451 & 2258.0989 & 1129.5531 & 20 \\
\hline 4 & 371.1925 & 186.0999 & & & 353.1819 & 177.0946 & G & 2091.9883 & 1046.4978 & 2074.9617 & 1037.9845 & 2073.9777 & 1037.4925 & 18 \\
\hline 5 & 508.2514 & 254.6293 & & & 490.2409 & 245.6241 & $\mathbf{H}$ & 2034.9668 & 1017.9870 & 2017.9403 & 1009.4738 & 2016.9562 & 1008.9818 & 17 \\
\hline 6 & 595.2835 & 298.1454 & & & 577.2729 & 289.1401 & S & 1897.9079 & 949.4576 & 1880.8813 & 940.9443 & 1879.8973 & 940.4523 & 16 \\
\hline 7 & 708.3675 & 354.6874 & & & 690.3569 & 345.6821 & I & 1810.8759 & 905.9416 & 1793.8493 & 897.4283 & 1792.8653 & 896.9363 & 15 \\
\hline 8 & 779.4046 & 390.2060 & & & 761.3941 & 381.2007 & A & 1697.7918 & 849.3995 & 1680.7653 & 840.8863 & 1679.7812 & 840.3943 & 14 \\
\hline 9 & 907.4632 & 454.2352 & 890.4367 & 445.7220 & 889.4526 & 445.2300 & Q & 1626.7547 & 813.8810 & 1609.7281 & 805.3677 & 1608.7441 & 804.8757 & 13 \\
\hline 10 & 1020.5473 & 510.7773 & 1003.5207 & 502.2640 & 1002.5367 & 501.7720 & $\mathbf{L}$ & 1498.6961 & 749.8517 & 1481.6696 & 741.3384 & 1480.6855 & 740.8464 & 12 \\
\hline 11 & 1134.5902 & 567.7987 & 1117.5636 & 559.2855 & 1116.5796 & 558.7935 & $\mathbf{N}$ & 1385.6120 & 693.3097 & 1368.5855 & 684.7964 & 1367.6015 & 684.3044 & 11 \\
\hline 12 & 1205.6273 & 603.3173 & 1188.6008 & 594.8040 & 1187.6167 & 594.3120 & A & 1271.5691 & 636.2882 & 1254.5426 & 627.7749 & 1253.5586 & 627.2829 & 10 \\
\hline 14 & 1448.7128 & 724.8601 & 1431.6863 & 716.3468 & 1430.7023 & 715.8548 & $\mathbf{N}$ & 1071.4894 & 536.2483 & 1054.4629 & 527.7351 & 1053.4788 & 527.2431 & 8 \\
\hline 15 & 1563.7398 & 782.3735 & 1546.7132 & 773.8602 & 1545.7292 & 773.3682 & $\mathbf{N}$ & 957.4465 & 479.2269 & 940.4199 & 470.7136 & 939.4359 & 470.2216 & 7 \\
\hline 16 & 1700.7987 & 850.9030 & 1683.7721 & 842.3897 & 1682.7881 & 841.8977 & $\mathbf{H}$ & 842.4196 & 421.7134 & 825.3930 & 413.2001 & 824.4090 & 412.7081 & 6 \\
\hline 17 & 1801.8464 & 901.4268 & 1784.8198 & 892.9135 & 1783.8358 & 892.4215 & $\mathbf{T}$ & 705.3606 & 353.1840 & 688.3341 & 344.6707 & 687.3501 & 344.1787 & 5 \\
\hline 18 & 1948.9148 & 974.9610 & 1931.8882 & 966.4478 & 1930.9042 & 965.9557 & $\mathbf{F}$ & 604.3130 & 302.6601 & 587.2864 & 294.1468 & & & 4 \\
\hline 19 & 2111.9781 & 1056.4927 & 2094.9516 & 1047.9794 & 2093.9675 & 1047.4874 & $\mathbf{Y}$ & 457.2445 & 229.1259 & 440.2180 & 220.6126 & & & 3 \\
\hline 20 & 2259.0465 & 1130.0269 & 2242.0200 & 1121.5136 & 2241.0360 & 1121.0216 & $\mathbf{F}$ & 294.1812 & 147.5942 & 277.1547 & 139.0810 & & & 2 \\
\hline 21 & & & & & & & $\mathbf{K}$ & 147.1128 & 74.0600 & 130.0863 & 65.5468 & & & 1 \\
\hline
\end{tabular}
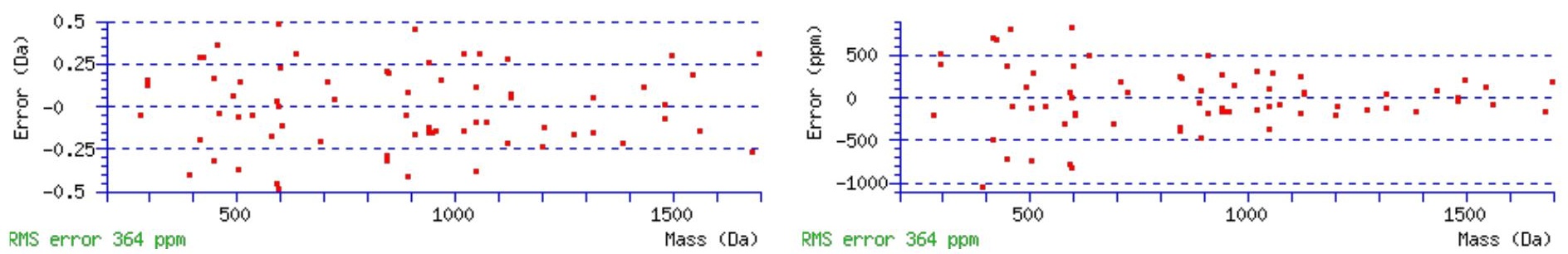

\section{All matches to this query}

\begin{tabular}{|l|l|l|l|}
\hline Score & Mr(calc): & Delta & Sequence \\
\hline
\end{tabular}




\begin{tabular}{|l|l|l|l|}
\hline 43.1 & 2404.1447 & 0.0074 & EALGHSIAQLNAENNHTFYFK \\
\hline 37.0 & 2404.1447 & 0.0074 & EALGHSIAQLNAENNHTFYFK \\
\hline 19.0 & 2404.1447 & 0.0074 & EALGHSIAQLNAENNHTFYFK \\
\hline 1.6 & 2402.1227 & 2.0294 & ASQGSNSLPSSARLGSSSNLQFK \\
\hline 1.2 & 2402.1240 & 2.0281 & NRHSQNPNLHKSLASSPGHLQ \\
\hline 0.5 & 2403.1373 & 1.0148 & tLLLKHSHPNDDKDSGFFPR \\
\hline 0.4 & 2404.1293 & 0.0228 & FPVKWSPPEVFHFNKYSSK \\
\hline
\end{tabular}

Spectrum No: 781; Query: 961; Rank: 1

\section{Peptide View}

MS/MS Fragmentation of LLPAFNTTSGLPYPR

Found in IPI00372145, Tax_Id=10116 Gene_Symbol=RGD1561496_predicted similar to Putative alpha-mannosidase C1orf22

Match to Query 961: 1646.865008 from(824.439780,2+)

Title: 091008RatKidney_NH4Format01_24.3367.3367.2.dta

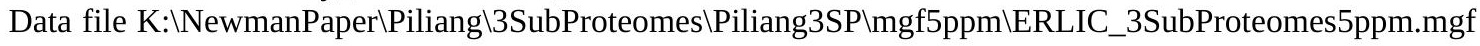
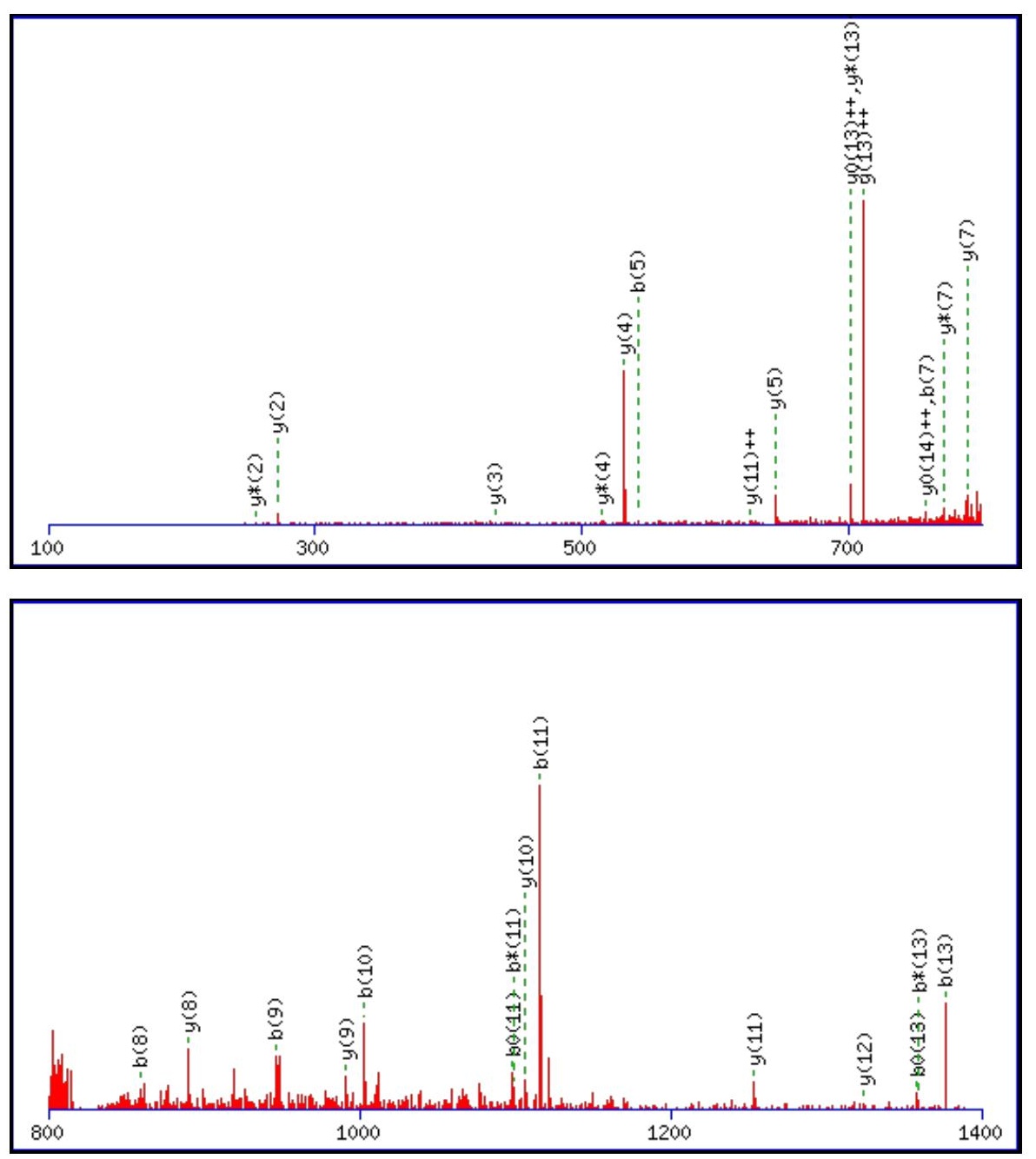


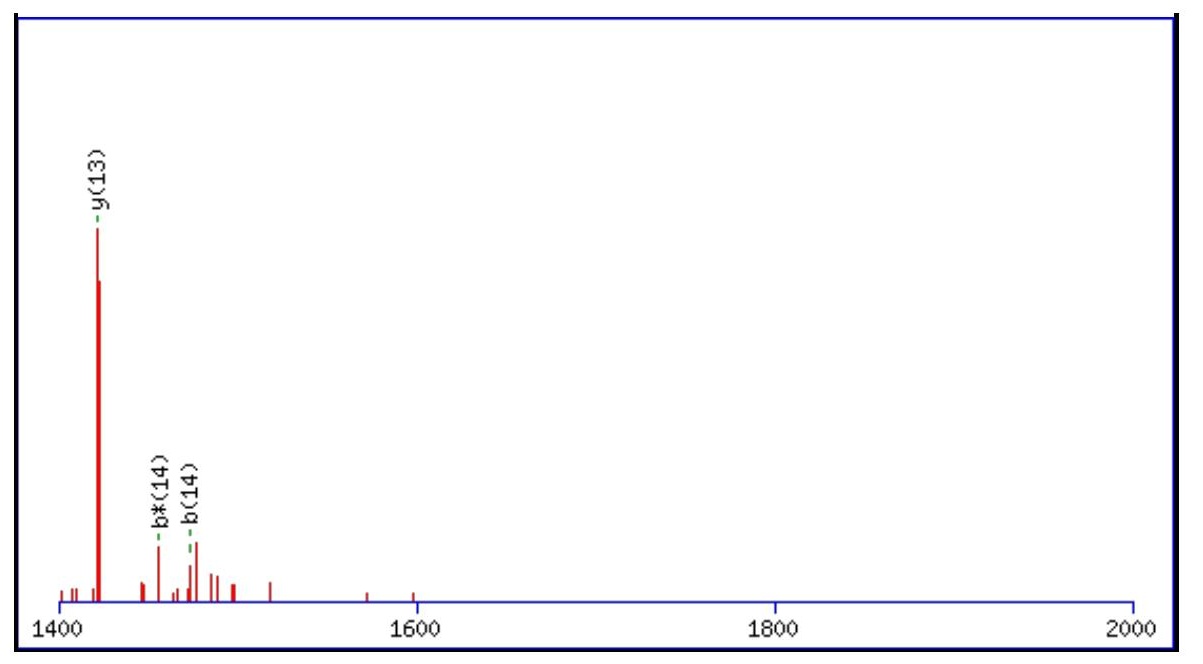

Monoisotopic mass of neutral peptide $\operatorname{Mr}($ calc): 1646.8668

Fixed modifications: Carbamidomethyl (C)

Variable modifications:

N6: Deamidated $\mathrm{N}(\mathrm{N})$

Ions Score: 43 Expect: 0.0061

Matches (Bold Red): 33/134 fragment ions using 122 most intense peaks

\begin{tabular}{|c|c|c|c|c|c|c|c|c|c|c|c|c|c|c|}
\hline \# & b & $\mathbf{b}^{++}$ & $\mathbf{b}^{*}$ & $\mathbf{b}^{*^{++}}$ & $\mathbf{b}^{\mathbf{0}}$ & $\mathbf{b}^{0++}$ & Seq. & $\mathbf{y}$ & $y^{++}$ & $\mathbf{y}^{*}$ & $\mathrm{y}^{*^{++}}$ & $\mathbf{y}^{0}$ & $y^{0++}$ & \# \\
\hline 1 & 114.0913 & 57.5493 & & & & & $\mathbf{L}$ & & & & & & & 15 \\
\hline 2 & 227.1754 & 114.0913 & & & & & $\mathbf{L}$ & 1534.7900 & 767.8986 & 1517.7635 & 759.3854 & 1516.7795 & 758.8934 & 14 \\
\hline 3 & 324.2282 & 162.6177 & & & & & $\mathbf{P}$ & 1421.7060 & 711.3566 & 1404.6794 & 702.8433 & 1403.6954 & 702.3513 & 13 \\
\hline 4 & 395.2653 & 198.1363 & & & & & A & 1324.6532 & 662.8302 & 1307.6266 & 654.3170 & 1306.6426 & 653.8249 & 12 \\
\hline 5 & 542.3337 & 271.6705 & & & & & $\mathbf{F}$ & 1253.6161 & 627.3117 & 1236.5895 & 618.7984 & 1235.6055 & 618.3064 & 11 \\
\hline 6 & 657.3606 & 329.1840 & 640.3341 & 320.6707 & & & $\mathbf{N}$ & 1106.5477 & 553.7775 & 1089.5211 & 545.2642 & 1088.5371 & 544.7722 & 10 \\
\hline 7 & 758.4083 & 379.7078 & 741.3818 & 371.1945 & 740.3977 & 370.7025 & $\mathbf{T}$ & 991.5207 & 496.2640 & 974.4942 & 487.7507 & 973.5102 & 487.2587 & 9 \\
\hline 8 & 859.4560 & 430.2316 & 842.4294 & 421.7184 & 841.4454 & 421.2264 & $\mathbf{T}$ & 890.4730 & 445.7402 & 873.4465 & 437.2269 & 872.4625 & 436.7349 & 8 \\
\hline 9 & 946.4880 & 473.7476 & 929.4615 & 465.2344 & 928.4775 & 464.7424 & $S$ & 789.4254 & 395.2163 & 772.3988 & 386.7030 & 771.4148 & 386.2110 & 7 \\
\hline 10 & 1003.5095 & 502.2584 & 986.4829 & 493.7451 & 985.4989 & 493.2531 & G & 702.3933 & 351.7003 & 685.3668 & 343.1870 & & & 6 \\
\hline 11 & 1116.5935 & 558.8004 & 1099.5670 & 550.2871 & 1098.5830 & 549.7951 & $\mathbf{L}$ & 645.3719 & 323.1896 & 628.3453 & 314.6763 & & & 5 \\
\hline 12 & 1213.6463 & 607.3268 & 1196.6198 & 598.8135 & 1195.6357 & 598.3215 & $\mathbf{P}$ & 532.2878 & 266.6475 & 515.2613 & 258.1343 & & & 4 \\
\hline 13 & 1376.7096 & 688.8585 & 1359.6831 & 680.3452 & 1358.6991 & 679.8532 & $\mathbf{Y}$ & 435.2350 & 218.1212 & 418.2085 & 209.6079 & & & 3 \\
\hline 14 & 1473.7624 & 737.3848 & 1456.7359 & 728.8716 & 1455.7518 & 728.3796 & $\mathbf{P}$ & 272.1717 & 136.5895 & 255.1452 & 128.0762 & & & 2 \\
\hline 15 & & & & & & & $\mathbf{R}$ & 175.1190 & 88.0631 & 158.0924 & 79.5498 & & & 1 \\
\hline
\end{tabular}
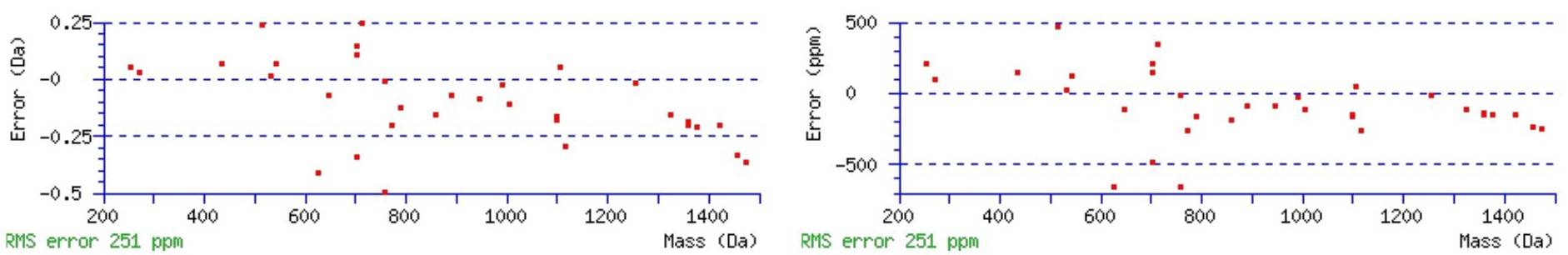

\section{All matches to this query}

\begin{tabular}{|l|l|l|l|}
\hline Score & Mr(calc): & Delta & \multicolumn{1}{c|}{ Sequence } \\
\hline 43.1 & 1646.8668 & -0.0018 & LLPAFNTTSGLPYPR \\
\hline 1.4 & 1646.8756 & -0.0106 & ENIKPQILSKLQR \\
\hline 1.0 & 1646.8661 & -0.0011 & IKMKQGETLALNER \\
\hline 0.5 & 1646.8740 & -0.0090 & QVTSALAGQLFSAQAR \\
\hline
\end{tabular}


Spectrum No: 782; Query: 2183; Rank: 1

\section{Peptide View}

MS/MS Fragmentation of FTFTSHTPGEHQICLHSNSTK

Found in IPI00364707, Tax_Id=10116 Gene_Symbol=Tmed9 Transmembrane emp24 protein transport domain containing 9

Match to Query 2183: 2430.118962 from(811.046930,3+)

Title: 100101RatKid_NS_deglyco_08.1945.1945.3.dta

Data file K:\NewmanPaper\Piliang \3SubProteomes \Piliang3SP \mgf5ppm\ERLIC_3SubProteomes5ppm.mgf
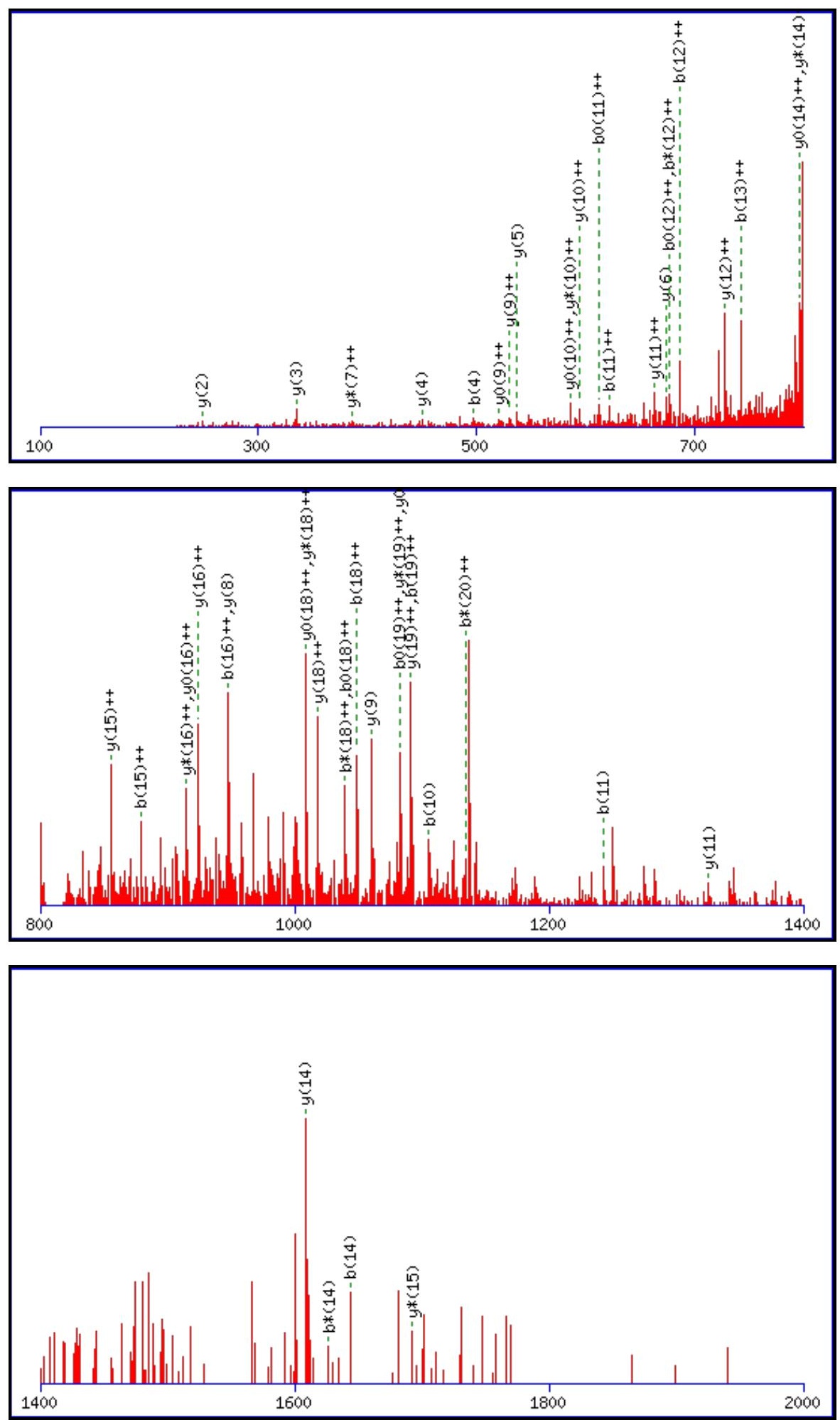

Monoisotopic mass of neutral peptide $\operatorname{Mr}($ calc): 2429.1070

Fixed modifications: Carbamidomethyl (C)

Variable modifications: 


\begin{tabular}{|c|c|c|c|c|c|c|c|c|c|c|c|c|c|c|}
\hline \# & b & $\mathbf{b}^{++}$ & $\mathbf{b}^{*}$ & $\mathbf{b}^{*^{++}}$ & $\mathbf{b}^{\mathbf{0}}$ & $\mathbf{b}^{\mathbf{0 + +}}$ & Seq. & $\mathbf{y}$ & $\mathbf{y}^{++}$ & $\mathbf{y}^{*}$ & $\mathbf{y}^{*^{++}}$ & $\mathbf{y}^{0}$ & $\mathbf{y}^{\mathbf{0}^{++}}$ & $\#$ \\
\hline 1 & 148.0757 & 74.5415 & & & & & $\mathbf{F}$ & & & & & & & 21 \\
\hline 2 & 249.1234 & 125.0653 & & & 231.1128 & 116.0600 & $T$ & 2283.0459 & 1142.0266 & 2266.0193 & 1133.5133 & 2265.0353 & 1133.0213 & 20 \\
\hline 3 & 396.1918 & 198.5995 & & & 378.1812 & 189.5942 & $\mathbf{F}$ & 2181.9982 & 1091.5027 & 2164.9716 & 1082.9895 & 2163.9876 & 1082.4974 & 19 \\
\hline 4 & 497.2395 & 249.1234 & & & 479.2289 & 240.1181 & $\mathbf{T}$ & 2034.9298 & 1017.9685 & |2017.9032 & 1009.4553 & 2016.9192 & 1008.9632 & 18 \\
\hline 5 & 584.2715 & 292.6394 & & & 566.2609 & 283.6341 & $\mathrm{~S}$ & 1933.8821 & 967.4447 & 1916.8555 & 958.9314 & 1915.8715 & 958.4394 & 17 \\
\hline 6 & 721.3304 & 361.1688 & & & 703.3198 & 352.1636 & $\mathbf{H}$ & 1846.8501 & 923.9287 & 1829.8235 & 915.4154 & 1828.8395 & 914.9234 & 16 \\
\hline 7 & 822.3781 & 411.6927 & & & 804.3675 & 402.6874 & $T$ & 1709.7912 & 855.3992 & 1692.7646 & 846.8859 & 1691.7806 & 846.3939 & 15 \\
\hline 8 & 919.4308 & 460.2191 & & & 901.4203 & 451.2138 & $\mathbf{P}$ & \begin{tabular}{|l|}
1608.7435 \\
\end{tabular} & 804.8754 & 1591.7169 & 796.3621 & 1590.7329 & 795.8701 & 14 \\
\hline 9 & 976.4523 & 488.7298 & & & 958.4417 & 479.7245 & G & 1511.6907 & 756.3490 & 1494.6642 & 747.8357 & 1493.6801 & 747.3437 & 13 \\
\hline 10 & 1105.4949 & 553.2511 & & & 1087.4843 & 544.2458 & $\mathbf{E}$ & 1454.6692 & 727.8383 & 1437.6427 & 719.3250 & 1436.6587 & 718.8330 & 12 \\
\hline 11 & 1242.5538 & 621.7805 & & & 1224.5432 & 612.7753 & $\mathbf{H}$ & 1325.6267 & 663.3170 & 1308.6001 & 654.8037 & 1307.6161 & 654.3117 & 11 \\
\hline 12 & 1370.6124 & 685.8098 & 1353.5858 & 677.2966 & 1352.6018 & 676.8046 & $\mathbf{Q}$ & 1188.5677 & 594.7875 & 1171.5412 & 586.2742 & 1170.5572 & 585.7822 & 10 \\
\hline 13 & 1483.6965 & 742.3519 & 1466.6699 & 733.8386 & 1465.6859 & 733.3466 & I & 1060.5092 & 530.7582 & 1043.4826 & 522.2449 & 1042.4986 & 521.7529 & 9 \\
\hline 14 & 1643.7271 & 822.3672 & 1626.7006 & 813.8539 & 1625.7165 & 813.3619 & C & 947.4251 & 474.2162 & 930.3986 & 465.7029 & 929.4145 & 465.2109 & 8 \\
\hline 15 & 1756.8112 & 878.9092 & 1739.7846 & 870.3959 & 1738.8006 & 869.9039 & $\mathbf{L}$ & 787.3945 & 394.2009 & 770.3679 & 385.6876 & 769.3839 & 385.1956 & 7 \\
\hline 16 & 1893.8701 & 947.4387 & 1876.8435 & 938. & 1875.8595 & 938.4334 & $\mathbf{H}$ & 674.3104 & 337.6588 & 657.2838 & 329.1456 & 656.2998 & 328.6535 & 6 \\
\hline 17 & \begin{tabular}{|l|}
1980.9021 \\
\end{tabular} & 990.9547 & 1963.8756 & 982.4414 & 1962.8915 & 981.9494 & $\mathrm{~S}$ & 537.2515 & 269.1294 & 520.2249 & 260.6161 & 519.2409 & 260.1241 & 5 \\
\hline 18 & 2095.9290 & \begin{tabular}{|l|l|}
1048.4682 \\
\end{tabular} & 2078.9025 & 1039.9549 & 2077.9185 & 1039.4629 & $\mathbf{N}$ & 450.2194 & 225.6134 & 433.1929 & 217.1001 & 432.2089 & 216.6081 & 4 \\
\hline 19 & 2182.9611 & 1091.9842 & 2165.9345 & 1083.4709 & 2164.9505 & \begin{tabular}{|l|}
1082.9789 \\
\end{tabular} & $\mathrm{~S}$ & 335.1925 & 168.0999 & 318.1660 & 159.5866 & 317.1819 & 159.0946 & 3 \\
\hline 20 & 2284.0088 & 1142.5080 & 2266.9822 & 1133.9947 & 2265.9982 & 1133.5027 & $\mathbf{T}$ & 248.1605 & 124.5839 & 231.1339 & 116.0706 & 230.1499 & 115.5786 & 2 \\
\hline 21 & & & & & & & $\mathbf{K}$ & 147.1128 & 74.0600 & 130.0863 & 65.5468 & & & \\
\hline
\end{tabular}
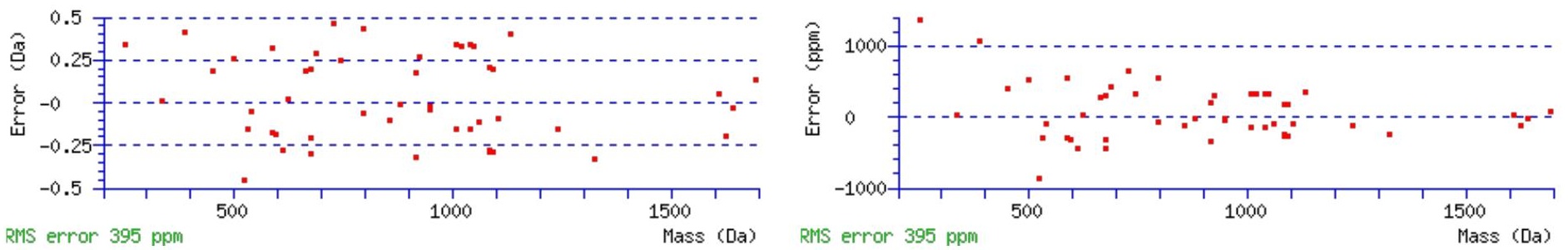

\section{All matches to this query}

\begin{tabular}{|l|l|c|l|}
\hline Score & Mr(calc): & Delta & \multicolumn{1}{|c|}{ Sequence } \\
\hline 43.0 & 2429.1070 & 1.0119 & FTFTSHTPGEHQICLHSNSTK \\
\hline 4.2 & 2428.1230 & 1.9959 & FTFTSHTPGEHQICLHSNSTK \\
\hline 1.9 & 2430.0968 & 0.0222 & VLNTGSDVEEAVADALLLGDIR \\
\hline 0.5 & 2430.1284 & -0.0094 & AEASRDIAKLTCAVESSALCAK \\
\hline
\end{tabular}

Spectrum No: 783; Query: 2235; Rank: 1

\section{Peptide View}

MS/MS Fragmentation of WPAEKENDTGVVQQLEECQVK

Found in IPI00780523, Tax_Id=10116 Gene_Symbol=Entpd1 56 kDa protein

Match to Query 2235: 2487.164652 from(830.062160,3+)

Title: 100101RatKid_NS_deglyco_20.3103.3103.3.dta

Data file K:INewmanPaper|Piliang|3SubProteomes\Piliang3SP\mgf5ppm\ERLIC_3SubProteomes5ppm.mgf 

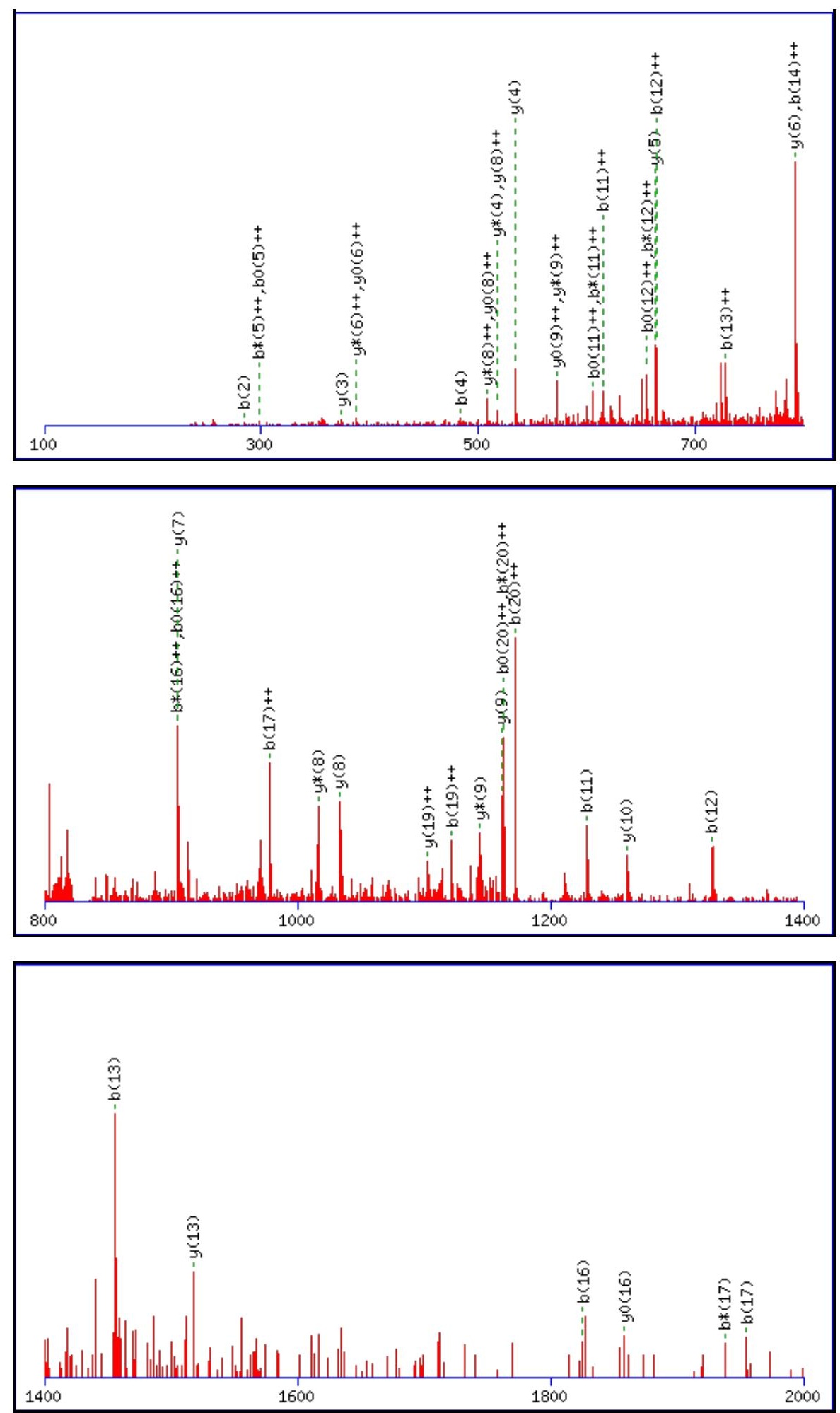

Monoisotopic mass of neutral peptide $\operatorname{Mr}($ calc): 2487.1588

Fixed modifications: Carbamidomethyl (C)

Variable modifications:

N7 : Deamidated_N (N)

Ions Score: 43 Expect: 0.016

Matches (Bold Red): 46/218 fragment ions using 64 most intense peaks

\begin{tabular}{|r|c|c|c|c|c|c|c|c|c|c|c|c|c|c|}
\hline$\#$ & $\mathbf{b}$ & $\mathbf{b}^{++}$ & $\mathbf{b}^{*}$ & $\mathbf{b}^{\boldsymbol{*}^{++}}$ & $\mathbf{b}^{\mathbf{0}}$ & $\mathbf{b}^{\mathbf{0 + +}}$ & $\mathbf{S e q}$ & $\mathbf{y}$ & $\mathbf{y}^{++}$ & $\mathbf{y}^{\mathbf{*}}$ & $\mathbf{y}^{\mathbf{*}^{++}}$ & $\mathbf{y}^{\mathbf{0}}$ & $\mathbf{y}^{\mathbf{0 + +}}$ & $\#$ \\
\hline $\mathbf{1}$ & $\mathbf{1 8 7 . 0 8 6 6}$ & 94.0469 & & & & & $\mathbf{W}$ & & & & & & & $\mathbf{2 1}$ \\
\hline $\mathbf{2}$ & $\mathbf{2 8 4 . 1 3 9 4}$ & 142.5733 & & & & & $\mathbf{P}$ & 2302.0867 & 1151.5470 & 2285.0602 & 1143.0337 & 2284.0762 & 1142.5417 & $\mathbf{2 0}$ \\
\hline $\mathbf{3}$ & 355.1765 & 178.0919 & & & & & $\mathbf{A}$ & 2205.0340 & $\mathbf{1 1 0 3 . 0 2 0 6}$ & 2188.0074 & 1094.5073 & 2187.0234 & 1094.0153 & $\mathbf{1 9}$ \\
\hline $\mathbf{4}$ & $\mathbf{4 8 4 . 2 1 9 1}$ & 242.6132 & & & 466.2085 & 233.6079 & $\mathbf{E}$ & 2133.9968 & 1067.5021 & 2116.9703 & 1058.9888 & 2115.9863 & 1058.4968 & $\mathbf{1 8}$ \\
\hline $\mathbf{5}$ & 612.3140 & 306.6606 & 595.2875 & $\mathbf{2 9 8 . 1 4 7 4}$ & 594.3035 & $\mathbf{2 9 7 . 6 5 5 4}$ & $\mathbf{K}$ & 2004.9543 & 1002.9808 & 1987.9277 & 994.4675 & 1986.9437 & 993.9755 & $\mathbf{1 7}$ \\
\hline
\end{tabular}




\begin{tabular}{|c|c|c|c|c|c|c|c|c|c|c|c|c|c|c|}
\hline & 741.3566 & 371.1819 & 724.3301| & 362.6687 & 723.3461| & 362.1767 & $\mathbf{E}$ & 1876.8593 & 938.9333 & 1859.8327 & 930.4200 & | 1858.8487 & 929.9280 & 16 \\
\hline 7 & 856.3836 & 428.6954 & 839.3570 & 420.1821 & 838.3730 & 419.6901 & $\mathbf{N}$ & 1747.8167 & 874.4120 & 1730.7902 & 865.8987 & 1729.8061 & 865.4067 & 15 \\
\hline 8 & 971.4105 & 486.2089 & 954.3839 & 477.6956 & 953.3999 & 477.2036 & D & 1632.7898 & 816.8985 & 1615.7632 & 808.3852 & 1614.7792 & 807.8932 & 14 \\
\hline 9 & 1072.4582 & 536.7327 & 1055.4316 & 528.2195 & 1054.4476 & 527.7274 & $T$ & 1517.7628 & 759.3850 & 1500.7363 & 750.8718 & 1499.7523 & 750.3798 & 13 \\
\hline 10 & 29.4796 & 565.2435 & 1112.4531 & 556.7302 & 1111.4691 & 556.2382 & G & 1416.7151 & 708.8612 & 1399.6886 & 700.3479 & 1398.7046 & 699.8559 & 12 \\
\hline 11 & 1228.5481 & 614.7777 & 1211.5215 & 606.2644 & 1210.5375 & 605.7724 & V & 1359.6937 & 680.3505 & 1342.6671 & 671.8372 & 1341.6831 & 671.3452 & 11 \\
\hline 12 & 1327.6165 & 664.3119 & 1310.5899 & 655.7986 & 1309.6059 & 655.3066 & V & 1260.6253 & 630.8163 & 1243.5987 & 622.3030 & 1242.6147 & 621.8110 & 10 \\
\hline 13 & 1455.6750 & 728.3412 & 1438.6485 & 719.8279 & 1437.6645 & 719.3359 & $\mathbf{Q}$ & 1161.5569 & 581.2821 & 1144.5303 & 572.7688 & 1143.5463 & 572.2768 & 9 \\
\hline 14 & 1583.7336 & 792.3704 & 1566.7071 & 783.8572 & 1565.7231 & 783.3652 & $\mathbf{Q}$ & 1033.4983 & 517.2528 & 1016.4717 & 508.7395 & 1015.4877 & 508.2475 & 8 \\
\hline 15 & 1696.8177 & 848.9125 & 1679.7911 & 840.3992 & 1678.8071 & 839.9072 & $\mathbf{L}$ & 905.4397 & 453.2235 & 888.4131 & 444.7102 & 887.4291 & 444.2182 & 7 \\
\hline 16 & 1825.8603 & 913.4338 & 1808.8337 & 904.9205 & 1807.8497 & 904.4285 & E & 792.3556 & 396.6815 & 775.3291 & 388.1682 & 774.3451 & 387.6762 & 6 \\
\hline 17 & \begin{tabular}{|l|l|}
1954.9029 \\
\end{tabular} & 977.9551 & \begin{tabular}{|l|}
1937.8763 \\
\end{tabular} & 969.4418 & 1936.8923 & 968.9498 & $\mathbf{E}$ & 663.3130 & 332.1602 & 646.2865 & 323.6469 & 645.3025 & 323.1549 & 5 \\
\hline 18 & 2114.9335 & 1057.9704 & 2097.9070 & 1049.4571 & 2096.9230 & 1048.9651 & C & 534.2704 & 267.6389 & 517.2439 & 259.1256 & & & 4 \\
\hline 19 & 2242.9921 & \begin{tabular}{|l|}
1121.9997 \\
\end{tabular} & 2225.9655 & 1113.4864 & 2224.9815 & 1112.9944 & $\mathbf{Q}$ & 374.2398 & 187.6235 & 357.2132 & 179.1103 & & & 3 \\
\hline 20 & 2342.0605 & 1171.5339 & 2325.0340 & 1163.0206 & 2324.0499 & 1162.5286 & $\mathbf{V}$ & 246.1812 & 123.5942 & 229.1547 & 115.0810 & & & 2 \\
\hline 21 & & & & & & & K & 147.1128 & 74.0600 & 130.0863 & 65.5468 & & & 1 \\
\hline
\end{tabular}
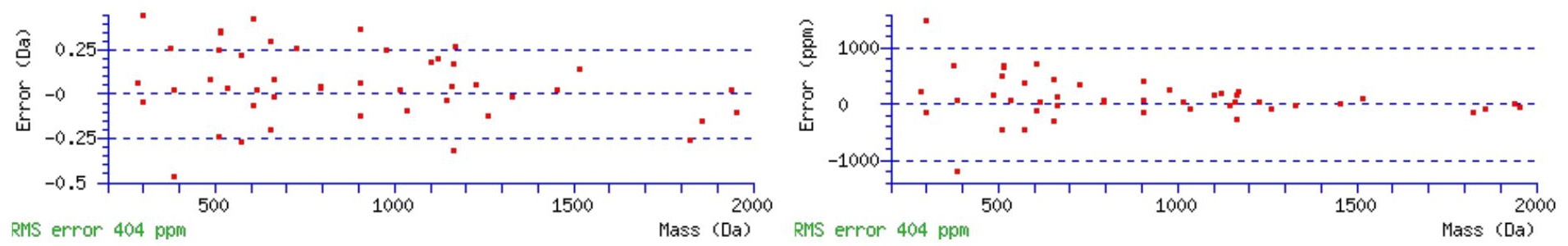

\section{All matches to this query}

\begin{tabular}{|l|l|c|l|}
\hline Score & Mr(calc): & Delta & \multicolumn{1}{|c|}{ Sequence } \\
\hline 43.0 & 2487.1588 & 0.0059 & WPAEKENDTGVVQQLEECQVK \\
\hline 21.8 & 2486.1747 & 0.9899 & WPAEKENDTGVVQQLEECQVK \\
\hline 5.7 & 2487.1489 & 0.0158 & RYAEVVDAGSIFMDHFTDRDK \\
\hline 0.8 & 2485.1568 & 2.0078 & TVTQVVPAEGQENGQREEEEEK \\
\hline 0.7 & 2487.1531 & 0.0116 & MDRMMVEHLLRCGYYNTAVK \\
\hline 0.3 & 2487.1733 & -0.0087 & QNGVQPSMPTAAERDEILGAMQK \\
\hline 0.3 & 2487.1695 & -0.0049 & EIDVIDVACQASSKMMLGDFVK \\
\hline 0.3 & 2487.1824 & -0.0177 & SLSLLAKAMDPKQPSMMADVVK \\
\hline
\end{tabular}

Spectrum No: 784; Query: 2543; Rank: 1

\section{Peptide View}

MS/MS Fragmentation of HFQIDYDEDGNCSLIISDVCGDDDAK

Found in IPI00869592, Tax_Id=10116 Gene_Symbol=Mylk_predicted myosin, light polypeptide kinase

Match to Query 2543: 3001.231302 from(1001.417710,3+)

Title: 091008RatKidney_NoSalt_22.6084.6084.3.dta

Data file K:INewmanPaper|Piliang \3SubProteomes\Piliang3SP\mgf5ppm\ERLIC_3SubProteomes5ppm.mgf 

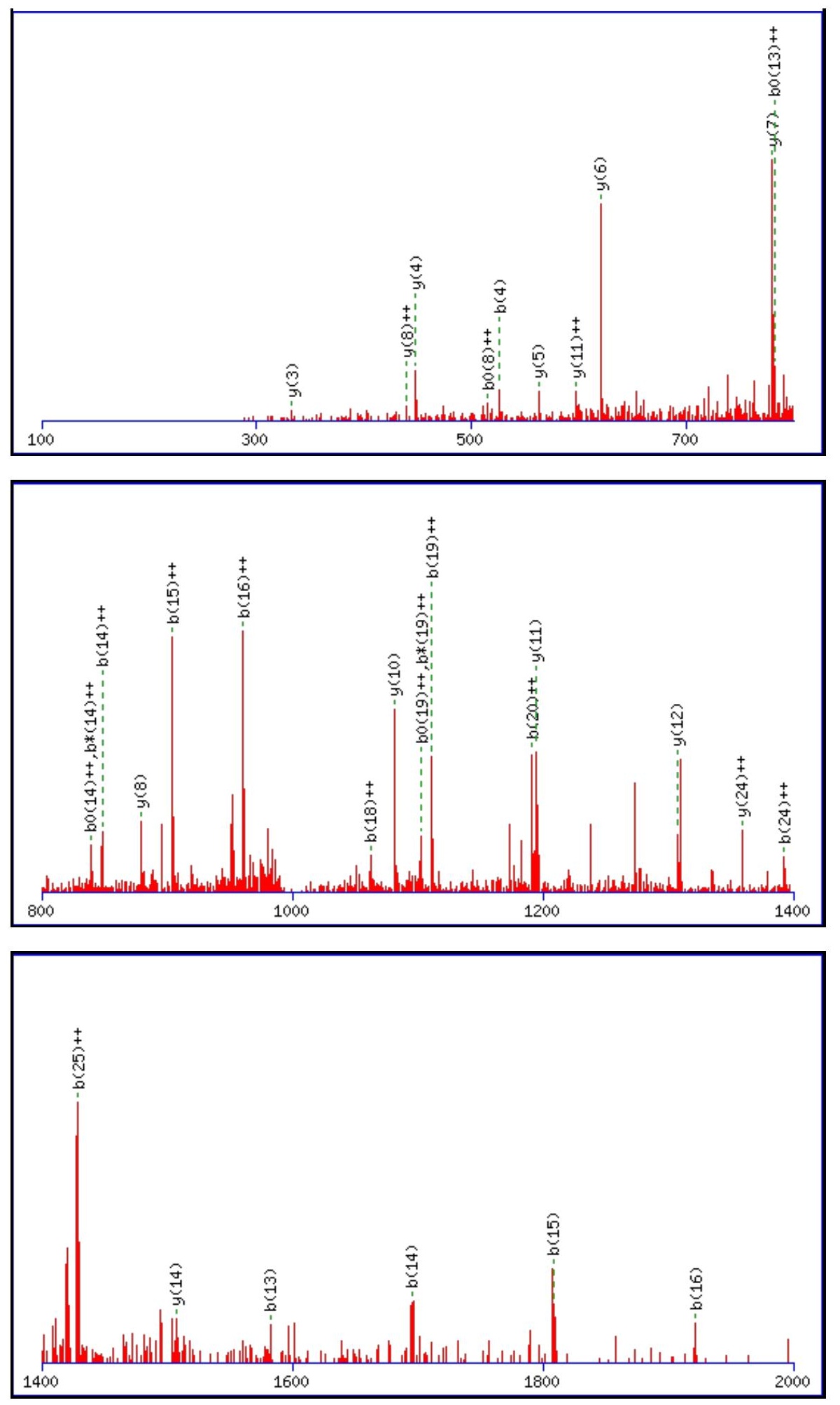

Monoisotopic mass of neutral peptide $\operatorname{Mr}($ calc): 3001.2230

Fixed modifications: Carbamidomethyl (C)

Variable modifications:

N11 : Deamidated N (N)

Ions Score: 43 Expect: 0.018

Matches (Bold Red): 32/284 fragment ions using 64 most intense peaks

\begin{tabular}{|c|c|c|c|c|c|c|c|c|c|c|c|c|c|c|}
\hline$\#$ & $\mathbf{b}$ & $\mathbf{b}^{++}$ & $\mathbf{b}^{*}$ & $\mathbf{b}^{\boldsymbol{*}^{++}}$ & $\mathbf{b}^{\mathbf{0}}$ & $\mathbf{b}^{\mathbf{0 + +}}$ & Seq. & $\mathbf{y}$ & $\mathbf{y}^{++}$ & $\mathbf{y}^{*}$ & $\mathbf{y}^{\boldsymbol{*}^{++}}$ & $\mathbf{y}^{\mathbf{0}}$ & $\mathbf{y}^{\mathbf{0 + +}}$ & $\#$ \\
\hline $\mathbf{1}$ & 138.0662 & 69.5367 & & & & & $\mathbf{H}$ & & & & & & & $\mathbf{2 6}$ \\
\hline $\mathbf{2}$ & 285.1346 & 143.0709 & & & & & $\mathbf{F}$ & 2865.1713 & 1433.0893 & 2848.1448 & 1424.5760 & 2847.1608 & 1424.0840 & $\mathbf{2 5}$ \\
\hline $\mathbf{3}$ & 413.1932 & 207.1002 & 396.1666 & 198.5870 & & & $\mathbf{Q}$ & 2718.1029 & 1359.5551 & 2701.0764 & 1351.0418 & 2700.0923 & 1350.5498 & $\mathbf{2 4}$ \\
\hline $\mathbf{4}$ & 526.2772 & 263.6423 & 509.2507 & 255.1290 & & & $\mathbf{I}$ & 2590.0443 & 1295.5258 & 2573.0178 & 1287.0125 & 2572.0338 & 1286.5205 & 23 \\
\hline $\mathbf{5}$ & 641.3042 & 321.1557 & 624.2776 & 312.6425 & 623.2936 & 312.1504 & $\mathbf{D}$ & 2476.9603 & 1238.9838 & 2459.9337 & 1230.4705 & 2458.9497 & 1229.9785 & 22 \\
\hline
\end{tabular}




\begin{tabular}{|c|c|c|c|c|c|c|c|c|c|c|c|c|c|c|}
\hline 6 & 804.3675 & 402.6874 & 787.3410 & 394.1741 & 786.3570 & 393.6821 & $\mathbf{Y}$ & |2361.9333 & |1181.4703| & 2344.9068 & $|1172.9570|$ & |2343.9228 & |1172.4650 & 21 \\
\hline 7 & 919.3945 & 460.2009 & 902.3679 & 451.6876 & 901.3839 & 451.1956 & D & 2198.8700 & 1099.9386 & 2181.8434 & 1091.4254 & 2180.8594 & 1090.9334 & 20 \\
\hline 8 & 1048.4371 & 524.7222 & 1031.4105 & 516.2089 & 1030.4265 & 515.7169 & $\mathbf{E}$ & 2083.8430 & 1042.4252 & 2066.8165 & 1033.9119 & 2065.8325 & 1033.4199 & 19 \\
\hline 9 & 1163.4640 & 582.2356 & 1146.4374 & 573.7224 & 1145.4534 & 573.2304 & D & 1954.8005 & 977.9039 & 1937.7739 & 969.3906 & 1936.7899 & 968.8986 & 18 \\
\hline 10 & 1220.4855 & 610.7464 & 1203.4589 & 602.2331 & 1202.4749 & 601.7411 & G & 1839.7735 & 920.3904 & 1822.7470 & 911.8771 & 1821.7629 & 911.3851 & 17 \\
\hline 11 & 1335.5124 & 668.2598 & 1318.4858 & 659.7466 & 1317.5018 & 659.2546 & $\mathbf{N}$ & 1782.7520 & 891.8797 & 1765.7255 & 883.3664 & 1764.7415 & 882.8744 & 16 \\
\hline 12 & 1495.5430 & 748.2752 & 1478.5165 & 739.7619 & 1477.5325 & 739.2699 & C & 1667.7251 & 834.3662 & 1650.6986 & 825.8529 & 1649.7145 & 825.3609 & 15 \\
\hline 13 & 1582.5751 & 791.7912 & 1565.5485 & 783.2779 & 1564.5645 & 782.7859 & S & 1507.6945 & 754.3509 & 1490.6679 & 745.8376 & 1489.6839 & 745.3456 & 14 \\
\hline 14 & 1695.6591 & 848.3332 & 1678.6326 & 839.8199 & 1677.6486 & 839.3279 & $\mathbf{L}$ & 1420.6624 & 710.8349 & 1403.6359 & 702.3216 & 1402.6519 & 701.8296 & 3 \\
\hline 15 & 1808.7432 & 904.8752 & 1791.7167 & 896.3620 & 1790.7326 & 895.8700 & I & 1307.5784 & 654.2928 & 1290.5518 & 645.7795 & 1289.5678 & 645.2875 & 12 \\
\hline 16 & 1921.8273 & 961.4173 & 1904.8007 & 952.9040 & 1903.8167 & 952.4120 & I & 1194.4943 & 597.7508 & 1177.4678 & 589.2375 & 1176.4837 & 588.7455 & 11 \\
\hline 17 & 2008.8593 & 1004.9333 & 1991.8327 & 996.4200 & 1990.8487 & 995.9280 & S & 1081.4102 & 541.2088 & 1064.3837 & 532.6955 & 1063.3997 & 532.2035 & 10 \\
\hline 18 & 2123.8862 & 1062.4468 & 2106.8597 & 1053.9335 & 2105.8757 & 1053.4415 & D & 994.3782 & 497.6927 & 977.3517 & 489.1795 & 976.3677 & 488.6875 & 9 \\
\hline 19 & 2222.9546 & 1111.9810 & 2205.9281 & 1103.4677 & 2204.9441 & 1102.9757 & $\mathbf{V}$ & 879.3513 & 440.1793 & 862.3247 & 431.6660 & 861.3407 & 431.1740 & 8 \\
\hline 21 & 2440.0068 & 1220.5070 & 2422.9802 & 1211.9937 & 2421.9962 & 1211.5017 & G & 620.2522 & 310.6297 & 603.2257 & 302.1165 & 602.2416 & 301.6245 & 6 \\
\hline 22 & 2555.0337 & 1278.0205 & 2538.0072 & 1269.5072 & 2537.0231 & 1269.0152 & D & 563.2307 & 282.1190 & 546.2042 & 273.6057 & 545.2202 & 273.1137 & 5 \\
\hline 23 & 2670.0606 & 1335.5340 & 2653.0341 & 1327.0207 & 2652.0501 & 1326.5287 & D & 448.2038 & 224.6055 & 431.1773 & 216.0923 & 430.1932 & 215.6003 & 4 \\
\hline 24 & 2785.0876 & 1393.0474 & 2768.0610 & 1384.5342 & 2767.0770 & 1384.0422 & D & 333.1769 & 167.0921 & 316.1503 & 158.5788 & 315.1663 & 158.0868 & 3 \\
\hline 25 & 2856.1247 & 1428.5660 & 2839.0982 & 1420.0527 & 2838.1141 & 1419.5607 & A & 218.1499 & 109.5786 & 201.1234 & 101.0653 & & & 2 \\
\hline 26 & & & & & & & $\mathbf{K}$ & 147.1128 & 74.0600 & 130.0863 & 65.5468 & & & 1 \\
\hline
\end{tabular}
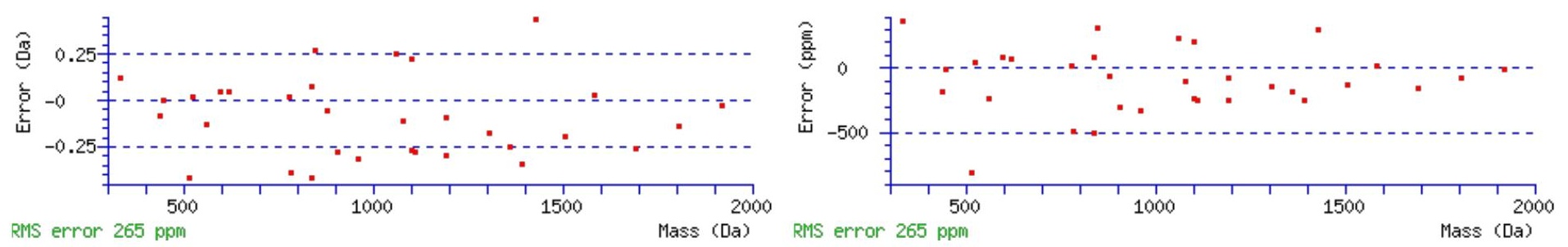

\section{All matches to this query}

\begin{tabular}{|l|l|l|l|}
\hline Score & Mr(calc): & Delta & \multicolumn{1}{|c|}{ Sequence } \\
\hline 42.9 & 3001.2230 & 0.0083 & HFQIDYDEDGNCSLIISDVCGDDDAK \\
\hline 38.7 & 3000.2390 & 0.9923 & HFQIDYDEDGNCSLIISDVCGDDDAK \\
\hline 15.9 & 3000.2524 & 0.9789 & HNCLILQXSLNNTGNLTSLTMTRK \\
\hline 15.9 & 3000.2524 & 0.9789 & HNCLILQXSLNNTGNLTSLTMTRK \\
\hline 15.5 & 3001.2364 & -0.0051 & HNCLILQXSLNNTGNLTSLTMTRK \\
\hline 15.0 & 3000.2524 & 0.9789 & HNCLILQXSLNNTGNLTSLTMTRK \\
\hline 15.0 & 3000.2524 & 0.9789 & HNCLILQXSLNNTGNLTSLTMTRK \\
\hline 14.9 & 3001.2364 & -0.0051 & HNCLILQXSLNNTGNLTSLTMTRK \\
\hline 14.9 & 3001.2364 & -0.0051 & HNCLILQXSLNNTGNLTSLTMTRK \\
\hline 13.7 & 3001.2364 & -0.0051 & HNCLILQXSLNNTGNLTSLTMTRK \\
\hline
\end{tabular}

Spectrum No: 785; Query: 311; Rank: 1

\section{Peptide View}

MS/MS Fragmentation of AVLVNNITTGEK

Found in IPI00764009, Tax_Id=10116 Gene_Symbol=LOC682869 similar to Golgi phosphoprotein 2 (Golgi membrane protein GP73) isoform 2

Match to Query 311: 1258.679568 from(630.347060,2+) 
Title: 100101RatKid_NS_deglyco_26.1792.1792.2.dta

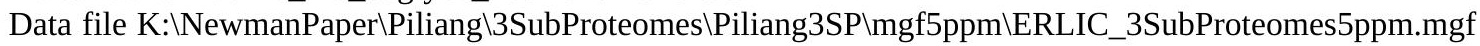
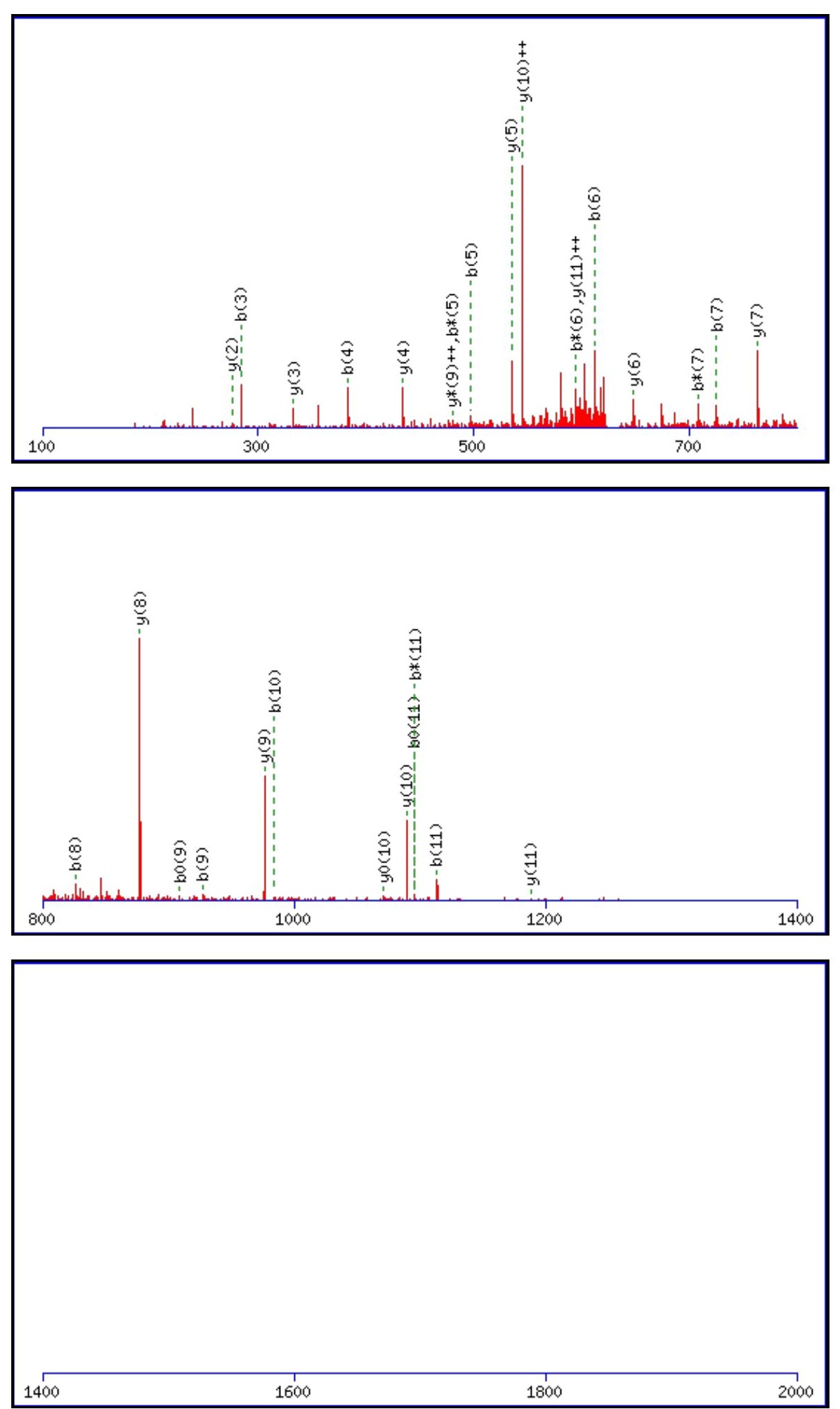

Monoisotopic mass of neutral peptide $\operatorname{Mr}($ calc): 1258.6769

Fixed modifications: Carbamidomethyl (C)

Variable modifications:

N6 : Deamidated $\mathrm{N}(\mathrm{N})$

Ions Score: 43 Expect: $\odot .0049$

Matches (Bold Red): 29/108 fragment ions using 73 most intense peaks

\begin{tabular}{|r|c|c|c|c|c|c|c|c|c|c|c|c|c|c|}
\hline$\#$ & $\mathbf{b}$ & $\mathbf{b}^{++}$ & $\mathbf{b}^{*}$ & $\mathbf{b}^{\boldsymbol{*}^{++}}$ & $\mathbf{b}^{\mathbf{0}}$ & $\mathbf{b}^{\mathbf{0 + +}}$ & Seq. & $\mathbf{y}$ & $\mathbf{y}^{++}$ & $\mathbf{y}^{\mathbf{*}}$ & $\mathbf{y}^{\boldsymbol{*}^{++}}$ & $\mathbf{y}^{\mathbf{0}}$ & $\mathbf{y}^{\mathbf{0 + +}}$ & $\#$ \\
\hline $\mathbf{1}$ & 72.0444 & 36.5258 & & & & & $\mathbf{A}$ & & & & & & & $\mathbf{1 2}$ \\
\hline $\mathbf{2}$ & 171.1128 & 86.0600 & & & & & $\mathbf{V}$ & $\mathbf{1 1 8 8 . 6 4 7 0}$ & $\mathbf{5 9 4 . 8 2 7 2}$ & 1171.6205 & 586.3139 & 1170.6365 & 585.8219 & $\mathbf{1 1}$ \\
\hline 3 & $\mathbf{2 8 4 . 1 9 6 9}$ & 142.6021 & & & & & $\mathbf{L}$ & $\mathbf{1 0 8 9 . 5 7 8 6}$ & 545.2930 & 1072.5521 & 536.7797 & $\mathbf{1 0 7 1 . 5 6 8 1}$ & 536.2877 & $\mathbf{1 0}$ \\
\hline
\end{tabular}




\begin{tabular}{|c|c|c|c|c|c|c|c|c|c|c|c|c|c|c|}
\hline 4 & 383.2653 & |192.1363 & & & & & V & 976.4946 & |488.7509 & 959.4680 & 480.2376 & 958.4840 & |479.7456 & 9 \\
\hline 5 & 497.3082 & 249.1577 & 480.2817 & 240.6445 & & & $\mathbf{N}$ & 877.4261 & |439.2167 & 860.3996 & 430.7034 & 859.4156 & 430.2114 & 8 \\
\hline 6 & 612.3351 & 306.6712 & 595.3086 & |298.1579 & & & $\mathbf{N}$ & 763.3832 & 382.1952 & 746.3567 & 373.6820 & 745.3727 & 373.1900 & 7 \\
\hline 7 & 725.4192 & 363.2132 & 708.3927 & 354.7000 & & & I & 648.3563 & 324.6818 & 631.3297 & 316.1685 & 630.3457 & |315.6765 & 6 \\
\hline 8 & 826.4669 & 413.7371 & 809.4403 & 405.2238 & 808.4563 & 404.7318 & $\mathbf{T}$ & 535.2722 & 268.1397 & 518.2457 & 259.6265 & 517.2617 & 259.1345 & 5 \\
\hline 9 & 927.5146 & 464.2609 & 910.4880 & 455.7476 & 909.5040 & 455.2556 & $T$ & 434.2245 & 217.6159 & 417.1980 & 209.1026 & 416.2140 & 208.6106 & 4 \\
\hline 10 & 984.5360 & 492.7717 & 967.5095 & 484.2584 & 966.5255 & 483.7664 & G & 333.1769 & |167.0921 & 316.1503 & 158.5788 & 315.1663 & 158.0868 & 3 \\
\hline 11 & 1113.5786 & 557.2930 & 1096.5521 & 548.7797 & 1095.5681 & 548.2877 & $\mathbf{E}$ & 276.1554 & 138.5813 & 259.1288 & 130.0681 & 258.1448 & 129.5761 & 2 \\
\hline 12 & & & & & & & $\mathbf{K}$ & 147.1128 & 74.0600 & 130.0863 & 65.5468 & & & 1 \\
\hline
\end{tabular}
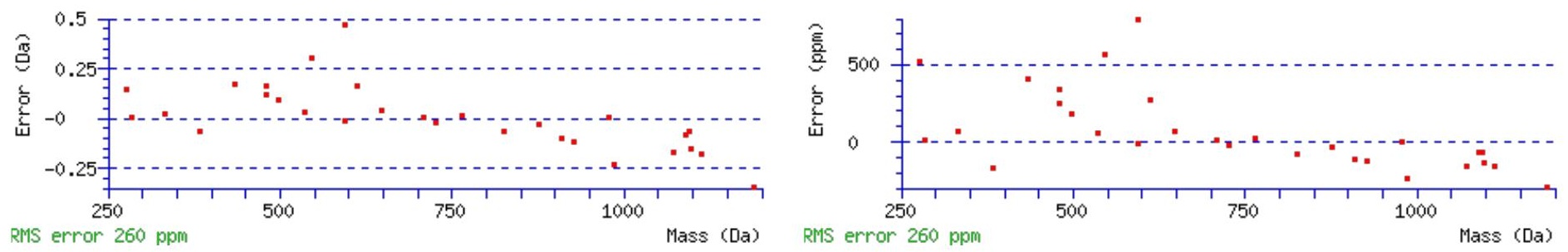

\section{All matches to this query}

\begin{tabular}{|l|l|l|l|}
\hline Score & Mr(calc): & Delta & \multicolumn{1}{|c|}{ Sequence } \\
\hline 42.9 & 1258.6769 & 0.0027 & AVLVNNITTGEK \\
\hline 34.8 & 1258.6769 & 0.0027 & AVLVNNITTGEK \\
\hline 3.8 & 1258.6703 & 0.0093 & IIRNNELMQK \\
\hline 1.7 & 1256.6612 & 2.0183 & VPAINVNDSVTK \\
\hline
\end{tabular}

Spectrum No: 786; Query: 23; Rank: 1

\section{Peptide View}

MS/MS Fragmentation of LNLTTDPK

Found in IPI00207068, Tax_Id=10116 Gene_Symbol=Glg1 Golgi apparatus protein 1 precursor

Match to Query 23: 901.474988 from(451.744770,2+)

Title: 091008RatKidney_NH4Format01_27.774.774.2.dta

Data file K:INewmanPaper|Piliang|3SubProteomes\Piliang3SP\mgf5ppm\ERLIC_3SubProteomes5ppm.mgf

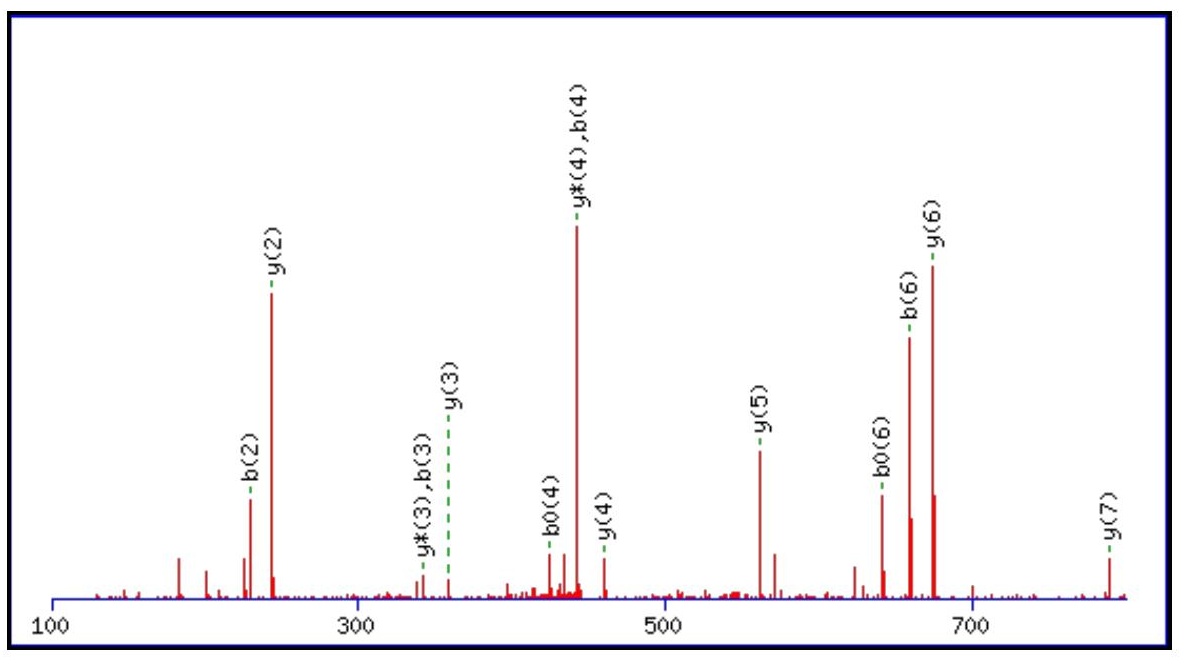



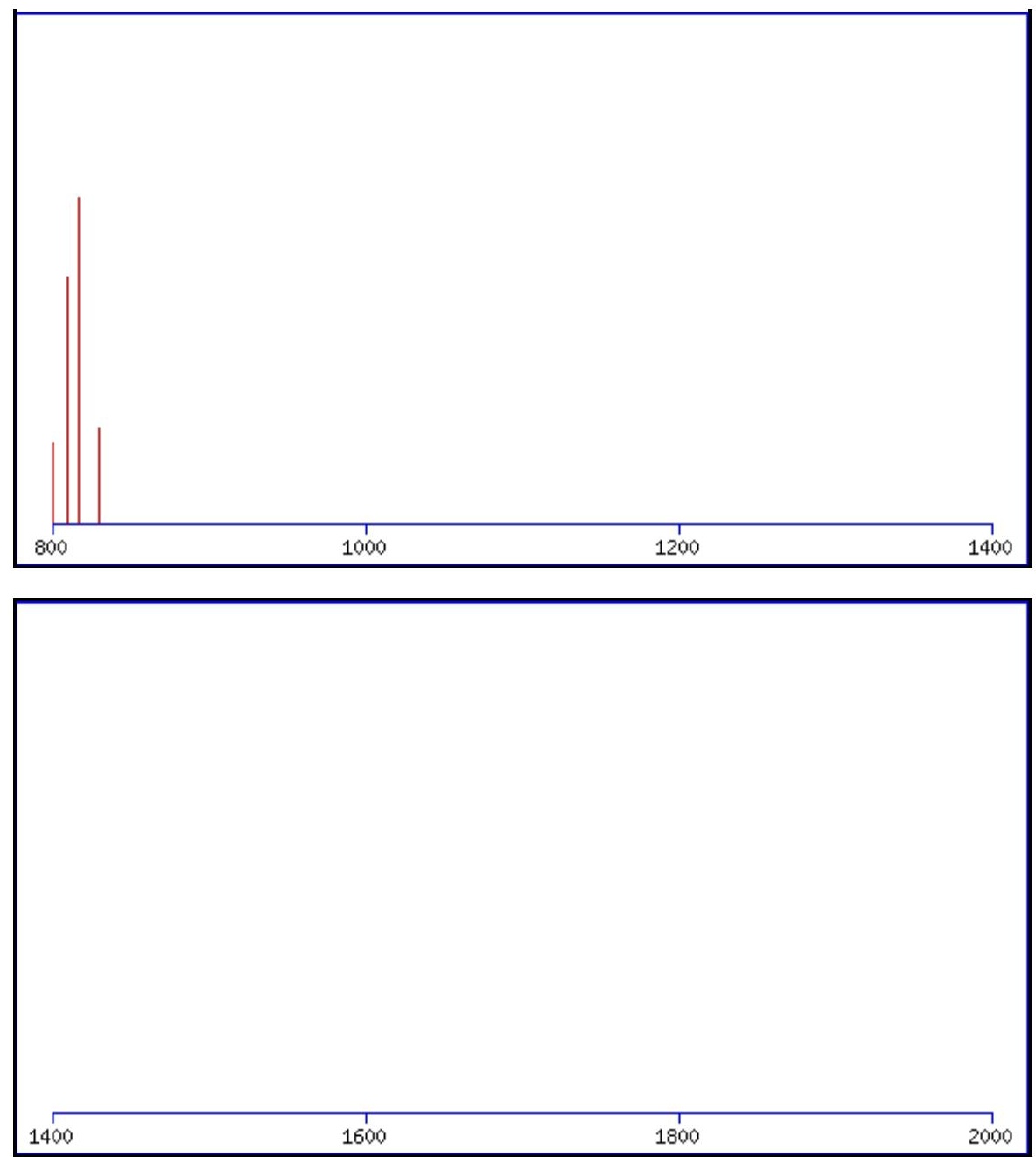

Monoisotopic mass of neutral peptide $\operatorname{Mr}($ calc): 901.4756

Fixed modifications: Carbamidomethyl (C)

Variable modifications:

N2 : Deamidated $\mathrm{N}(\mathrm{N})$

Ions Score: 43 Expect: 0.0046

Matches (Bold Red): 14/72 fragment ions using 29 most intense peaks

\begin{tabular}{|c|c|c|c|c|c|c|c|c|c|c|c|c|c|c|}
\hline$\#$ & $\mathbf{b}$ & $\mathbf{b}^{++}$ & $\mathbf{b}^{*}$ & $\mathbf{b}^{\mathbf{*}^{++}}$ & $\mathbf{b}^{\mathbf{0}}$ & $\mathbf{b}^{\mathbf{0}+}$ & $\mathbf{S e q}$ & $\mathbf{y}$ & $\mathbf{y}^{++}$ & $\mathbf{y}^{*}$ & $\mathbf{y}^{\mathbf{*}^{++}}$ & $\mathbf{y}^{\mathbf{0}}$ & $\mathbf{y}^{\mathbf{0 + +}}$ & $\#$ \\
\hline $\mathbf{1}$ & 114.0913 & 57.5493 & & & & & $\mathbf{L}$ & & & & & & & $\mathbf{8}$ \\
\hline $\mathbf{2}$ & 229.1183 & 115.0628 & 212.0917 & 106.5495 & & & $\mathbf{N}$ & $\mathbf{7 8 9 . 3 9 8 9}$ & 395.2031 & 772.3723 & 386.6898 & 771.3883 & 386.1978 & $\mathbf{7}$ \\
\hline $\mathbf{3}$ & 342.2023 & 171.6048 & 325.1758 & 163.0915 & & & $\mathbf{L}$ & $\mathbf{6 7 4 . 3 7 1 9}$ & 337.6896 & 657.3454 & 329.1763 & 656.3614 & 328.6843 & $\mathbf{6}$ \\
\hline $\mathbf{4}$ & $\mathbf{4 4 3 . 2 5 0 0}$ & 222.1286 & 426.2235 & 213.6154 & $\mathbf{4 2 5 . 2 3 9 5}$ & 213.1234 & $\mathbf{T}$ & $\mathbf{5 6 1 . 2 8 7 9}$ & 281.1476 & 544.2613 & 272.6343 & 543.2773 & 272.1423 & $\mathbf{5}$ \\
\hline $\mathbf{5}$ & 544.2977 & 272.6525 & 527.2711 & 264.1392 & 526.2871 & 263.6472 & $\mathbf{T}$ & $\mathbf{4 6 0 . 2 4 0 2}$ & 230.6237 & 443.2136 & 222.1105 & 442.2296 & 221.6185 & $\mathbf{4}$ \\
\hline $\mathbf{6}$ & $\mathbf{6 5 9 . 3 2 4 6}$ & 330.1660 & 642.2981 & 321.6527 & $\mathbf{6 4 1 . 3 1 4 1}$ & 321.1607 & $\mathbf{D}$ & $\mathbf{3 5 9 . 1 9 2 5}$ & 180.0999 & 342.1660 & 171.5866 & 341.1819 & 171.0946 & $\mathbf{3}$ \\
\hline $\mathbf{7}$ & $\mathbf{7 5 6 . 3 7 7 4}$ & 378.6923 & 739.3509 & 370.1791 & 738.3668 & 369.6871 & $\mathbf{P}$ & $\mathbf{2 4 4 . 1 6 5 6}$ & 122.5864 & 227.1390 & 114.0731 & & & $\mathbf{2}$ \\
\hline $\mathbf{8}$ & & & & & & & $\mathbf{K}$ & 147.1128 & 74.0600 & 130.0863 & 65.5468 & & & $\mathbf{1}$ \\
\hline
\end{tabular}
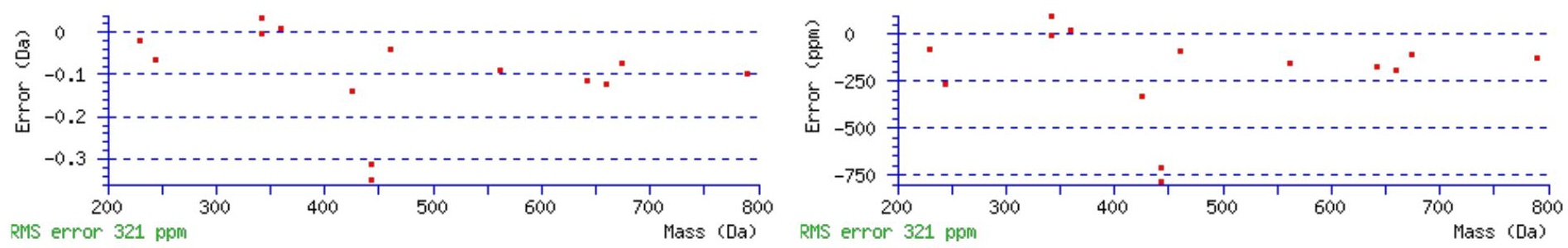

\section{All matches to this query}

\begin{tabular}{|l|l|l|l|}
\hline Score & Mr(calc): & Delta & Sequence \\
\hline
\end{tabular}




\begin{tabular}{|l|l|l|l|}
42.8 & 901.4756 & -0.0007 & $\underline{\text { LNLTTDPK }}$ \\
\hline 18.1 & 901.4756 & -0.0007 & $\underline{\text { LDLGLEDK }}$ \\
\hline 16.1 & 901.4756 & -0.0007 & $\underline{\text { LSLEDTPK }}$ \\
\hline 9.7 & 901.4691 & 0.0059 & DIAXKCPK \\
\hline 8.8 & 901.4756 & -0.0006 & $\underline{\text { IEAEEAIK }}$ \\
\hline 7.2 & 901.4756 & -0.0006 & $\underline{\text { NLIADVEK }}$ \\
\hline 7.0 & 900.4777 & 0.9973 & $\underline{\text { RALEEGAR }}$ \\
\hline 6.5 & 901.4756 & -0.0006 & $\underline{\text { ILNGDLEK }}$ \\
\hline 6.5 & 901.4674 & 0.0076 & $\underline{\text { KTFSLVK }}$ \\
\hline 5.6 & 901.4691 & 0.0059 & $\underline{\text { KXACNLPK }}$ \\
\hline
\end{tabular}

Spectrum No: 787; Query: 2261; Rank: 1

\section{Peptide View}

MS/MS Fragmentation of VETGVLKPGMVVTFAPVNVTTEVK

Found in IPI00195372, Tax_Id=10116 Gene_Symbol=Eef1a1 Elongation factor 1-alpha 1

Match to Query 2261: 2515.369768 from(1258.692160,2+)

Title: 091008RatKidney_NoSalt_07.3815.3815.2.dta

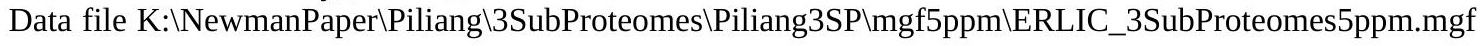
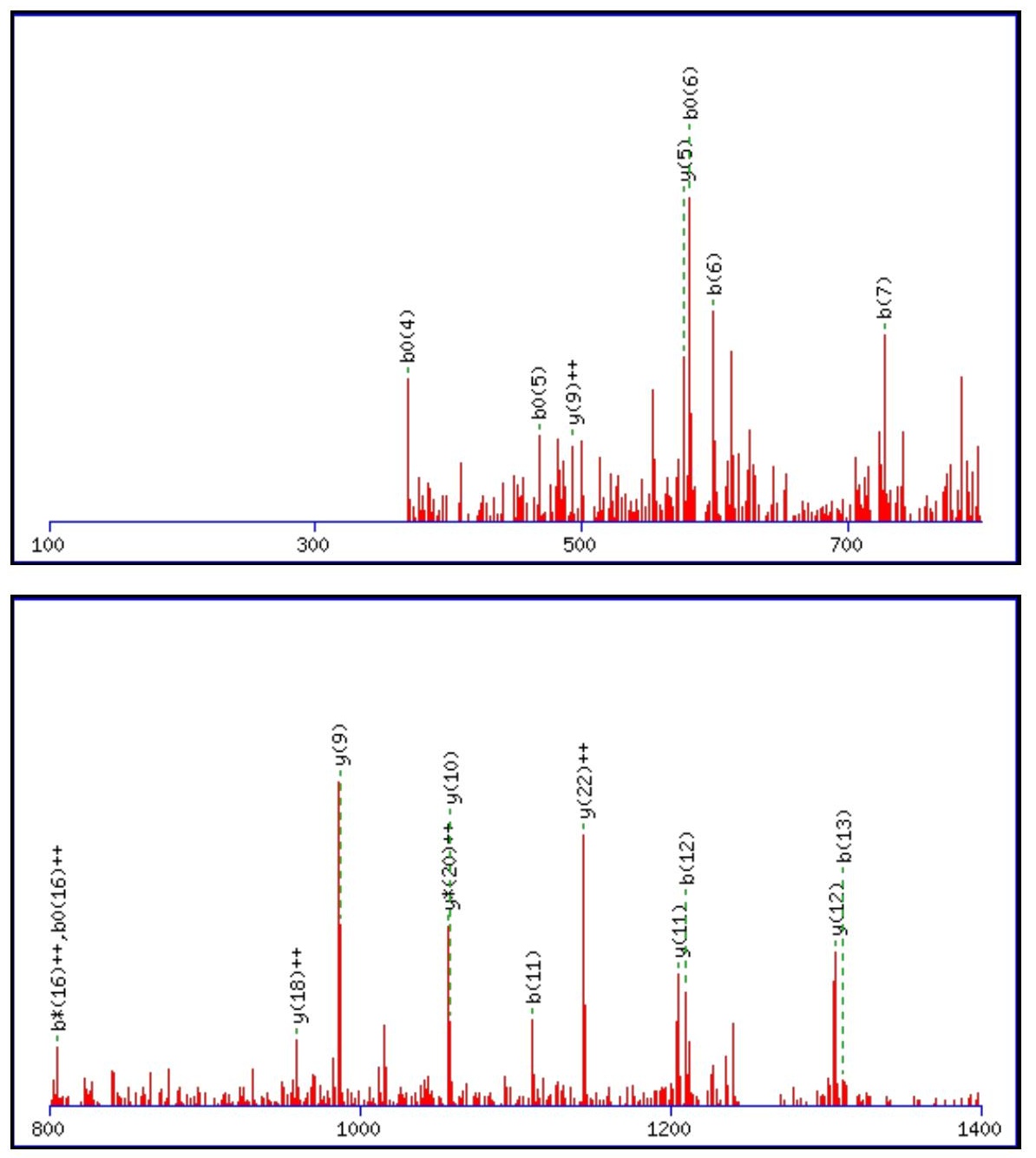


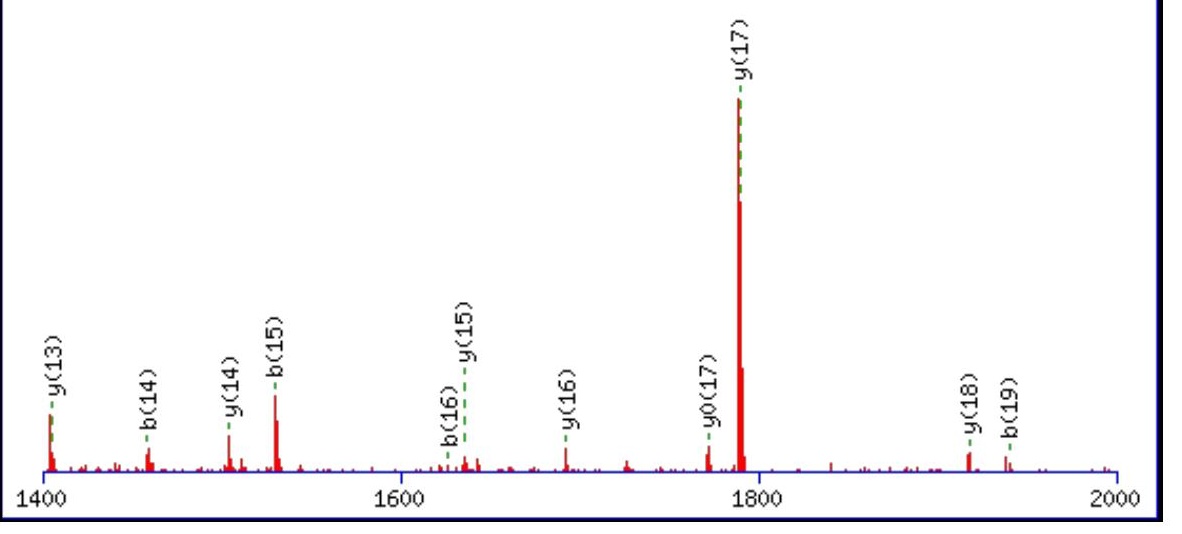

Monoisotopic mass of neutral peptide $\operatorname{Mr}($ calc): 2515.3608

Fixed modifications: Carbamidomethyl (C)

Variable modifications:

N18 : Deamidated_N (N)

Ions Score: 43 Expect: 0.0026

Matches (Bold Red): 30/258 fragment ions using 76 most intense peaks

\begin{tabular}{|c|c|c|c|c|c|c|c|c|c|c|c|c|c|c|}
\hline \# & b & $\mathbf{b}^{++}$ & $\mathbf{b}^{*}$ & $\mathbf{b}^{*^{++}}$ & $\mathbf{b}^{\mathbf{0}}$ & $\mathbf{b}^{0}$ & Seq. & $\mathbf{y}$ & $\mathbf{y}^{++}$ & $\mathbf{y}^{*}$ & $\mathrm{y}^{\mathrm{*}^{++}}$ & $\mathbf{y}^{\mathbf{0}}$ & $\mathbf{y}^{0}$ & \# \\
\hline 1 & 100.0757 & 50.5415 & & & & & V & & & & & & & 24 \\
\hline 2 & 229.1183 & 115.0628 & & & 211.1077 & 106.0575 & $\mathbf{E}$ & 2417.2996 & 1209.1534 & 2400.2731 & 1200.6402 & 2399.2891 & 1200.1482 & 23 \\
\hline 3 & 330.1660 & 165.5866 & & & 312.1554 & 156.5813 & $\mathbf{T}$ & 2288.2570 & 1144.6322 & 2271.2305 & 1136.1189 & 2270.2465 & 1135.6269 & 22 \\
\hline 4 & 387.1874 & 194.0974 & & & 369.1769 & 185.0921 & G & 2187.2093 & 1094.1083 & 2170.1828 & 1085.5950 & 2169.1988 & 1085.1030 & 21 \\
\hline 5 & 486.2558 & 243.6316 & & & 468.2453 & 234.6263 & $\mathbf{V}$ & 2130.1879 & 1065.5976 & 2113.1613 & 1057.0843 & 2112.1773 & 1056.5923 & 20 \\
\hline 6 & 599.3399 & 300.1736 & & & 581.3293 & 291.1683 & $\mathbf{L}$ & 2031.1195 & 1016.0634 & 0929 & 1007.5501 & 2013.1089 & 1007.0581 & 19 \\
\hline 7 & 727.4349 & 364.2211 & 710.4083 & 355.7078 & 709.4243 & 355.2158 & $\mathbf{K}$ & 1918.0354 & 959.5213 & 1901.0089 & 951.0081 & 1900.0248 & 950.5161 & 18 \\
\hline 8 & 824.4876 & 412.7475 & 807.4611 & 404.2342 & 806.4771 & 403.7422 & $\mathbf{P}$ & 1789.9404 & 895.4739 & 1772.9139 & 886.9606 & 1771.9299 & 886.4686 & 17 \\
\hline 9 & 881.5091 & 441.2582 & 864.4825 & 432.7449 & 863.4985 & 432.2529 & G & 1692.8877 & 846.9475 & 1675.8611 & 838.4342 & 1674.8771 & 837.9422 & 16 \\
\hline 10 & 1012.5496 & 506.7784 & 995.5230 & 498.2652 & 994.5390 & 497.7731 & $\mathbf{M}$ & 1635.8662 & 818.4367 & 1618.8397 & 809.9235 & 1617.8557 & 809.4315 & 15 \\
\hline 11 & 1111.6180 & 556.3126 & 1094.5914 & 547.7994 & 093.6074 & 547.3074 & $\mathbf{V}$ & 1504.8257 & 752.9165 & 1487.7992 & 744.4032 & 1486.8152 & 743.9112 & 14 \\
\hline 12 & 1210.6864 & 605.8468 & 1193.6599 & 597.3336 & 1192.6758 & 596.8416 & $\mathbf{V}$ & 1405.7573 & 703.3823 & 1388.7308 & 694.8690 & 1387.7468 & 694.3770 & 13 \\
\hline 13 & 1311.7341 & 656.3707 & 1294.7075 & 647.8574 & 1293.7235 & 647.3654 & $\mathbf{T}$ & 1306.6889 & 653.8481 & 1289.6624 & 645.3348 & 1288.6783 & 644.8428 & 12 \\
\hline 14 & 1458.8025 & 729.9049 & 1441.7760 & 721.3916 & 1440.7919 & 720.8996 & $\mathbf{F}$ & 1205.6412 & 603.3243 & 1188.6147 & 594.8110 & 1187.6307 & 594.3190 & 11 \\
\hline 15 & 1529.8396 & 765.4234 & 1512.8131 & 756.9102 & 1511.8291 & 756.4182 & A & 1058.5728 & 529.7900 & 1041.5463 & 521.2768 & 1040.5622 & 520.7848 & 10 \\
\hline 16 & 1626.8924 & 813.9498 & 1609.8658 & 805.4366 & 608.8818 & 804.9445 & $\mathbf{P}$ & 987.5357 & 494.2715 & 970.5091 & 485.7582 & 969.5251 & 485.2662 & 9 \\
\hline 17 & 1725.9608 & 863.4840 & 1708.9342 & 854.9708 & 1707.9502 & 854.4788 & $\mathbf{V}$ & 890.4829 & 445.7451 & 873.4564 & 437.2318 & 872.4724 & 436.7398 & 8 \\
\hline 18 & 1840.9877 & 920.9975 & 1823.9612 & 912.4842 & 1822.9772 & 911.9922 & $\mathbf{N}$ & 791.4145 & 396.2109 & 774.3880 & 387.6976 & 773.4040 & 387.2056 & 7 \\
\hline 19 & 1940.0561 & 970.5317 & 1923.0296 & 962.0184 & 1922.0456 & 961.5264 & V & 676.3876 & 338.6974 & 659.3610 & 330.1842 & 658.3770 & 329.6921 & 6 \\
\hline 20 & 2041.1038 & 1021.0555 & 2024.0773 & 1012.5423 & 2023.0933 & 1012.0503 & $\mathbf{T}$ & 577.3192 & 289.1632 & 560.2926 & 280.6499 & 559.3086 & 280.1579 & 5 \\
\hline 21 & 2142.1515 & 1071.5794 & 2125.1250 & 1063.0661 & 2124.1409 & 1062.5741 & $\mathbf{T}$ & 476.2715 & 238.6394 & 459.2449 & 230.1261 & 458.2609 & 229.6341 & 4 \\
\hline 22 & 2271.1941 & 1136.1007 & 2254.1675 & 1127.5874 & 2253.1835 & 1127.0954 & $\mathbf{E}$ & 375.2238 & 188.1155 & 358.1973 & 179.6023 & 357.2132 & 179.1103 & 3 \\
\hline 23 & 2370.2625 & 1185.6349 & 2353.2360 & 1177.1216 & 2352.2519 & 1176.6296 & $\mathbf{V}$ & 246.1812 & 123.5942 & 229.1547 & 115.0810 & & & 2 \\
\hline 24 & & & & & & & $\mathbf{K}$ & 147.1128 & 74.0600 & 130.0863 & 65.5468 & & & 1 \\
\hline
\end{tabular}
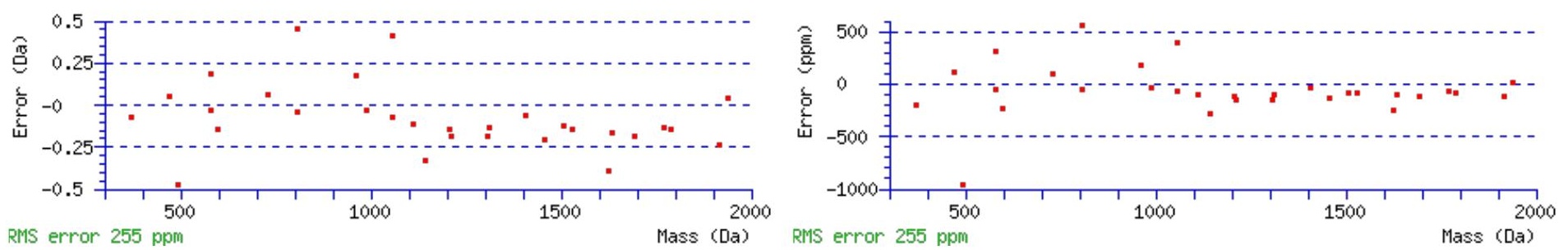


\section{All matches to this query}

\begin{tabular}{|l|l|l|l|}
\hline Score & Mr(calc): & Delta & \multicolumn{1}{|c|}{ Sequence } \\
\hline 42.8 & 2515.3608 & 0.0090 & VETGVLKPGMVVTFAPVNVTTEVK \\
\hline 32.7 & 2514.3768 & 0.9930 & VETGVLKPGMVVTFAPVNVTTEVK \\
\hline 10.9 & 2513.3815 & 1.9882 & VETGVLKPGMVVTFAPVNVTEVVK \\
\hline
\end{tabular}

Spectrum No: 788; Query: 1798; Rank: 1

\section{Peptide View}

MS/MS Fragmentation of NTDKENPYIGLGNYTLCR

Found in IPI00776785, Tax_Id=10116 Gene_Symbol=Tmem16f_predicted 94 kDa protein

Match to Query 1798: 2127.987972 from(710.336600,3+)

Title: 100101RatKid_NS_deglyco_26.2964.2964.3.dta

Data file K:INewmanPaper|Piliang|3SubProteomes\Piliang3SP\mgf5ppm\ERLIC_3SubProteomes5ppm.mgf
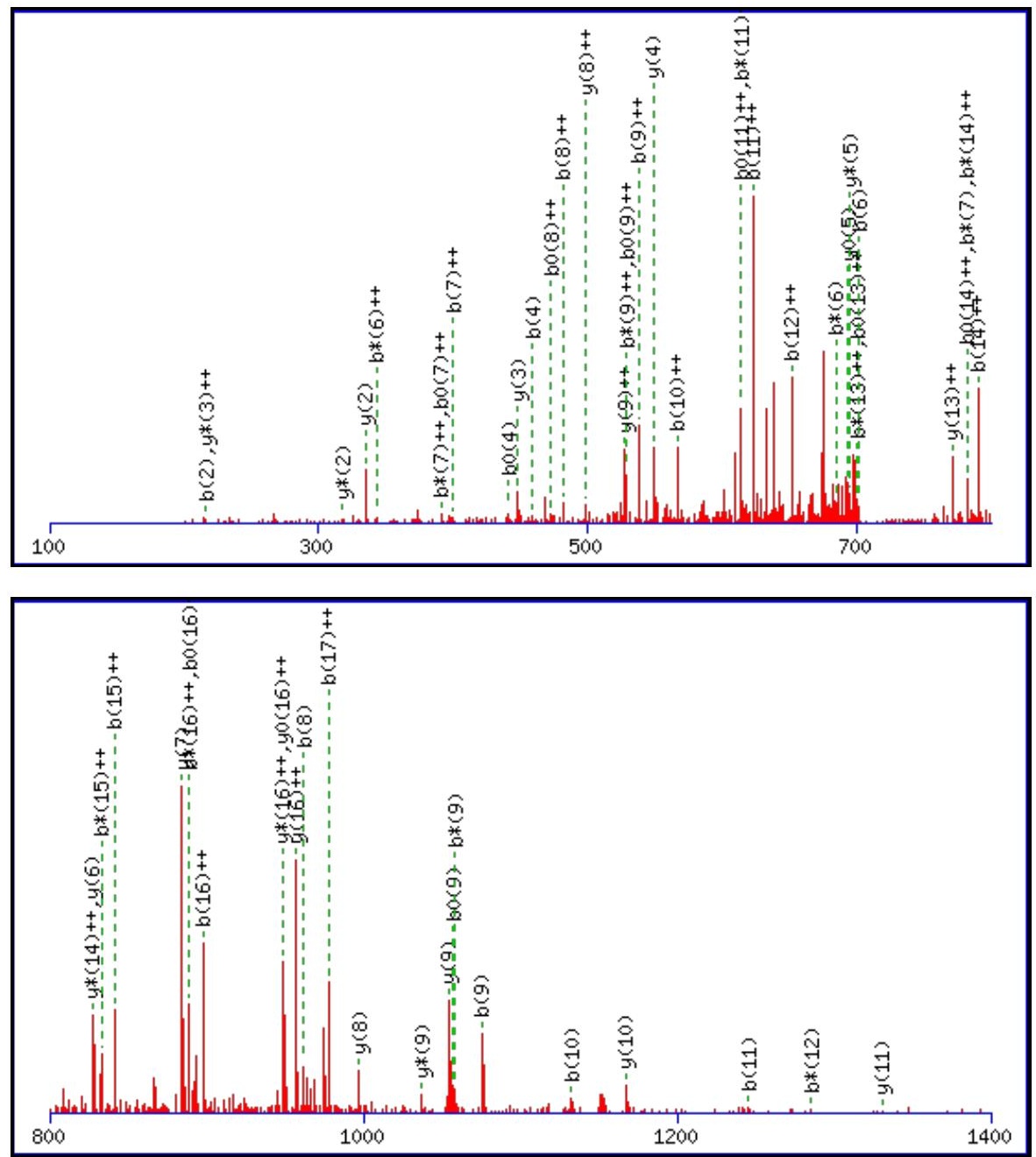


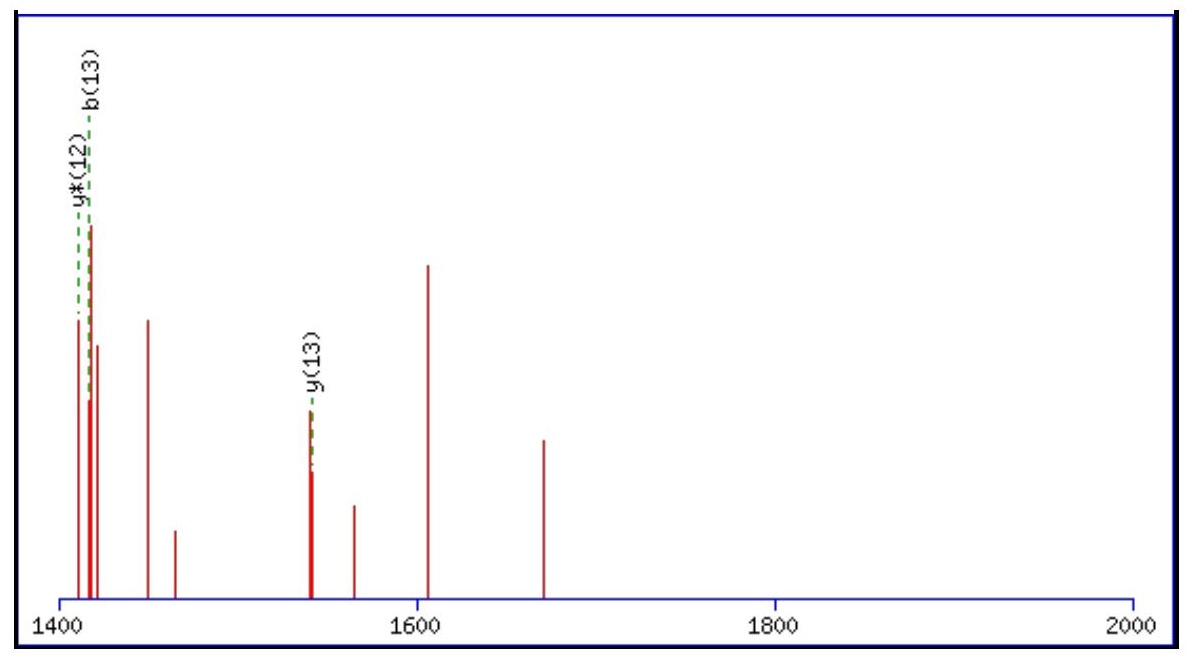

Monoisotopic mass of neutral peptide $\operatorname{Mr}($ calc): 2127.9895

Fixed modifications: Carbamidomethyl (C)

Variable modifications:

N13 : Deamidated $\mathrm{N}(\mathrm{N})$

Ions Score: 43 Expect: 0.015

Matches (Bold Red): 62/196 fragment ions using 136 most intense peaks

\begin{tabular}{|c|c|c|c|c|c|c|c|c|c|c|c|c|c|c|}
\hline \# & b & $\mathbf{b}^{++}$ & $\mathbf{b}^{*}$ & $\mathbf{b}^{*++}$ & $\mathbf{b}^{\mathbf{0}}$ & & Seq. & $\mathbf{y}$ & $\mathbf{y}^{++}$ & $\mathbf{y}^{*}$ & $\mathbf{y}^{*^{++}}$ & $\mathbf{y}^{0}$ & $\mathbf{y}^{\mathbf{0 + +}}$ & \# \\
\hline 1 & 115.0502 & 58.0287 & 98.0237 & 49.5155 & & & $\mathbf{N}$ & & & & & & & 18 \\
\hline 2 & 216.0979 & 108.5526 & 199.0713 & 100.0393 & 198.0873 & & $\mathbf{T}$ & & & & & 9433 & 998.9753 & 17 \\
\hline 3 & 331.1248 & 166.0661 & 314.0983 & 157.5528 & 313.1143 & 157.0608 & D & 1913.9062 & & 1896.8796 & & 1895.8956 & 948.4514 & 16 \\
\hline 4 & 459.2198 & 230.1135 & 442.1932 & 221.6003 & 441.2092 & 221.1082 & $\mathbf{K}$ & 1798.8792 & 9433 & 3527 & 891.4300 & 1780.8687 & 890.9380 & 15 \\
\hline 5 & 588.2624 & 294.6348 & 571.2358 & 286.1216 & 570.2518 & 285.6295 & $\mathbf{E}$ & 1670.7843 & 3958 & 7577 & 827.3825 & 1652.7737 & 826.8905 & 14 \\
\hline 6 & 702.3053 & 351.6563 & 685.2788 & 343.1430 & 684.2947 & 342.6510 & $\mathbf{N}$ & 1541.7417 & 3745 & 1524. & 762.8612 & 1523.7311 & 762.3692 & 13 \\
\hline 7 & 799.3581 & 400.1827 & 782.3315 & 391.6694 & 781.3475 & 391.1774 & $\mathbf{P}$ & 1427.6988 & 3530 & 3722 & 705.8397 & 1409.6882 & 705.3477 & 12 \\
\hline 8 & 962.4214 & 481.7143 & 945.3949 & 473.2011 & 944.4108 & 472.7091 & $\mathbf{Y}$ & 1330.6460 & 665.8266 & 1313.6194 & 657.3134 & 1312.6354 & 656.8214 & 11 \\
\hline 9 & 1075.5055 & 538.2564 & 1058.4789 & 529.7431 & 1057.4949 & 529.2511 & I & 1167.5827 & 584.2950 & 561 & 575.7817 & 1149.5721 & 575.2897 & 10 \\
\hline 10 & 1132.5269 & 566.7671 & 1115.5004 & 558.2538 & 1114.5164 & 557.7618 & $\mathbf{G}$ & 1054.4986 & 527.7529 & 1037.4721 & 519.2397 & 1036.4880 & 518.7477 & 9 \\
\hline 11 & 1245.6110 & 623.3091 & 1228.5844 & 614.7959 & 1227.6004 & 614.3039 & $\mathbf{L}$ & 997.4771 & 499.2422 & 980.4506 & 490.7289 & 979.4666 & 490.2369 & 8 \\
\hline 12 & 1302.6325 & 651.8199 & 1285.6059 & 643.3066 & 1284.6219 & 642.8146 & G & 884.3931 & 442.7002 & 867.3665 & 434.1869 & 866.3825 & 433.6949 & 7 \\
\hline 13 & 1417.6594 & 709.3333 & 1400.6328 & 700.8201 & 1399.6488 & 700.3281 & $\mathbf{N}$ & 827.3716 & 414.1894 & 810.3451 & 405.6762 & 809.3610 & 405.1842 & 6 \\
\hline 14 & 1580.7227 & 790.8650 & 1563.6962 & 782.3517 & 1562.7122 & 781.8597 & $\mathbf{Y}$ & 712.3447 & 356.6760 & 695.3181 & 348.1627 & 694.3341 & 347.6707 & 5 \\
\hline 15 & 1681.7704 & 841.3888 & 1664.7439 & 832.8756 & 1663.7598 & 832.3836 & $\mathbf{T}$ & 549.2813 & 275.1443 & 532.2548 & 266.6310 & 531.2708 & 266.1390 & 4 \\
\hline 16 & 1794.8545 & 897.9309 & 1777.8279 & 889.4176 & 1776.8439 & 888.9256 & $\mathbf{L}$ & 448.2337 & 224.6205 & 431.2071 & 216.1072 & & & 3 \\
\hline 17 & 1954.8851 & 977.9462 & 1937.8586 & 969.4329 & 1936.8745 & 968.9409 & C & 335.1496 & 168.0784 & 318.1231 & 159.5652 & & & 2 \\
\hline 18 & & & & & & & $\mathbf{R}$ & 175.1190 & 88.0631 & 158.0924 & 79.5498 & & & 1 \\
\hline
\end{tabular}

品

$$
\stackrel{20}{2}
$$

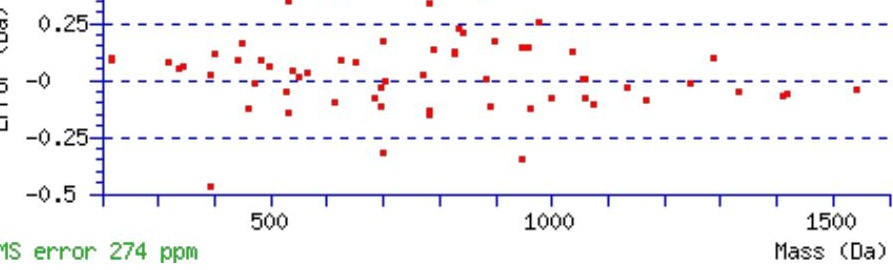

All matches to this query

\begin{tabular}{|l|l|l|l|}
\hline Score & Mr(calc): & Delta & \multicolumn{1}{|c|}{ Sequence } \\
\hline 42.7 & 2127.9895 & -0.0015 & NTDKENPYIGLGNYTLCR \\
\hline 31.7 & 2127.9895 & -0.0015 & NTDKENPYIGLGNYTLCR \\
\hline 27.5 & 2127.9895 & -0.0015 & $\underline{\text { NTDKENPYIGLGNYTLCR }}$ \\
\hline & & & \\
\hline
\end{tabular}

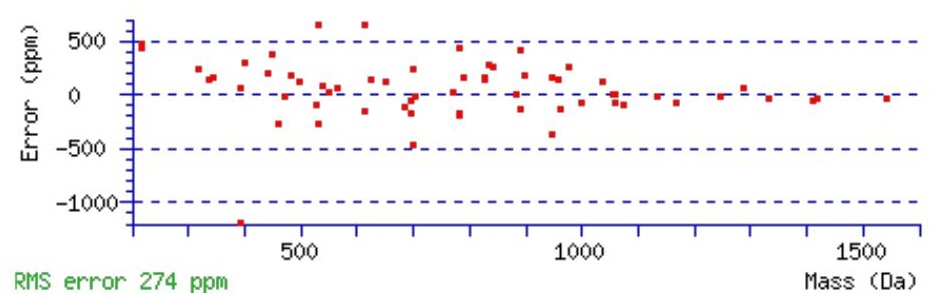




\begin{tabular}{|l|l|l|l|}
11.7 & 2127.0055 & 0.9825 & NTDKENPYIGLGNYTLCR \\
\hline 5.1 & 2127.9925 & -0.0045 & HKPLFTAAERNTFSLCR \\
\hline 3.2 & 2125.9867 & 2.0013 & IVSSKDYSLTANRYCLR \\
\hline 3.2 & 2125.9867 & 2.0013 & IVSSKDYSLTANRYCLR \\
\hline 3.2 & 2125.9867 & 2.0013 & IVSSKDYSLTANRYCLR \\
\hline 2.4 & 2127.9851 & 0.0028 & HRDTGILDSIGRFFSGDR \\
\hline 1.8 & 2127.9984 & -0.0104 & TSCGNALLVGVGGSGKQSLSR \\
\hline
\end{tabular}

Spectrum No: 789; Query: 82; Rank: 1

\section{Peptide View}

MS/MS Fragmentation of FSDGLESNR

Found in IPI00213036, Tax_Id=10116 Gene_Symbol=C4a Complement C4 precursor

Match to Query 82: 1024.445348 from(513.229950,2+)

Title: 091008RatKidney_NH4Format01_25.833.833.2.dta

Data file K:INewmanPaper|Piliang|3SubProteomes\Piliang3SP\mgf5ppm\ERLIC_3SubProteomes5ppm.mgf
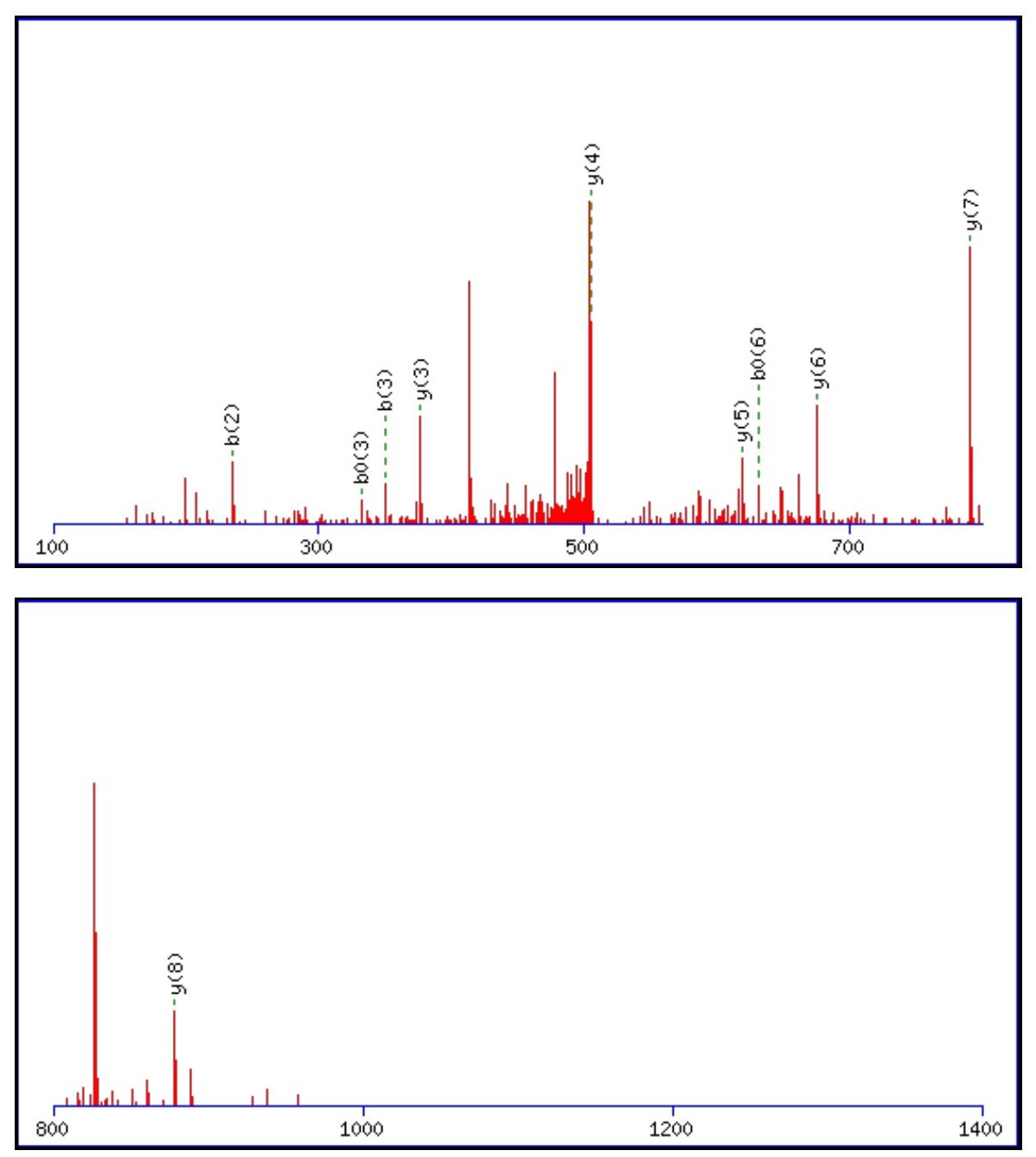


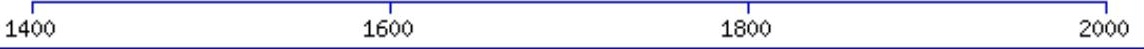

Monoisotopic mass of neutral peptide $\operatorname{Mr}($ calc): 1024.4461

Fixed modifications: Carbamidomethyl (C)

Variable modifications:

N8 : Deamidated_N (N)

Ions Score: 43 Expect: 0.0039

Matches (Bold Red): 10/76 fragment ions using 21 most intense peaks

\begin{tabular}{|c|c|c|c|c|c|c|c|c|c|c|c|c|c|c|}
\hline$\#$ & b & $\mathbf{b}^{++}$ & $\mathbf{b}^{*}$ & $\mathbf{b}^{*^{++}}$ & $\mathbf{b}^{\mathbf{0}}$ & $\mathbf{b}^{\mathbf{0 + +}}$ & Seq. & $\mathbf{y}$ & $y^{++}$ & $\mathbf{y}^{*}$ & $\mathrm{y}^{\mathrm{*}^{++}}$ & $\mathbf{y}^{\mathbf{0}}$ & $y^{0++}$ & \# \\
\hline 1 & 148.0757 & 74.5415 & & & & & $\mathbf{F}$ & & & & & & & 9 \\
\hline 2 & 235.1077 & 118.0575 & & & 217.0972 & 109.0522 & $\mathrm{~S}$ & 878.3850 & 439.6961 & 861.3585 & 431.1829 & 860.3744 & 430.6909 & 8 \\
\hline 3 & 350.1347 & 175.5710 & & & 332.1241 & 166.5657 & D & 791.3530 & 396.1801 & 774.3264 & 387.6669 & 773.3424 & 387.1748 & \\
\hline 4 & 407.1561 & 204.0817 & & & 389.1456 & 195.0764 & G & 676.3260 & 338.6667 & 659.2995 & 330.1534 & 658.3155 & 329.6614 & \\
\hline 5 & 520.2402 & 260.6237 & & & 502.2296 & 251.6185 & $\mathbf{L}$ & 619.3046 & 310.1559 & 602.2780 & 301.6427 & 601.2940 & 301.1506 & 5 \\
\hline 6 & 649.2828 & 325.1450 & & & 631.2722 & 316.1397 & E & 506.2205 & 253.6139 & 489.1940 & 245.1006 & 488.2099 & 244.6086 & 5 \\
\hline 7 & 736.3148 & 368.6610 & & & 718.3042 & 359.6558 & $\mathrm{~S}$ & 377.1779 & 189.0926 & 360.1514 & 180.5793 & 359.1674 & 180.0873 & 3 \\
\hline 8 & 851.3417 & 426.1745 & 834.3152 & 417.6612 & 833.3312 & 417.1692 & $\mathbf{N}$ & 290.1459 & 145.5766 & 273.1193 & 137.0633 & & & 2 \\
\hline & & & & & & & $\mathbf{R}$ & 175.1190 & 88.0631 & 158.0924 & 79.5498 & & & \\
\hline
\end{tabular}
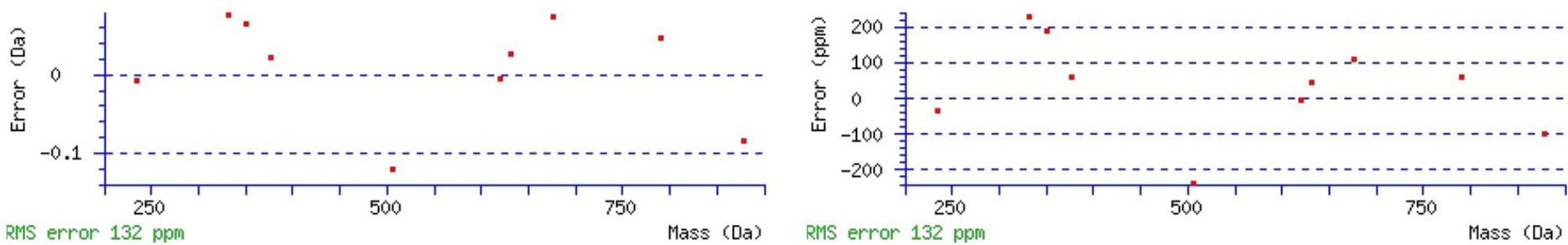

\section{All matches to this query}

\begin{tabular}{|l|l|l|l|}
\hline Score & Mr(calc): & Delta & \multicolumn{1}{|c|}{ Sequence } \\
\hline 42.7 & 1024.4461 & -0.0008 & FSDGLESNR \\
\hline 10.3 & 1023.4498 & 0.9955 & $\underline{\text { HSSKRDSK }}$ \\
\hline 10.3 & 1023.4498 & 0.9955 & HSSKRDSK \\
\hline 8.9 & 1024.4535 & -0.0082 & SMNTWIEK \\
\hline 7.8 & 1024.4379 & 0.0075 & FSSRSFSK \\
\hline 6.9 & 1024.4356 & 0.0097 & MSRSGGSGDR \\
\hline 6.4 & 1023.4509 & 0.9945 & QFNLGDSDK \\
\hline 1.9 & 1024.4379 & 0.0075 & FSSRSFSK \\
\hline 1.9 & 1024.4379 & 0.0075 & FSSRSFSK \\
\hline 1.1 & 1024.4461 & -0.0008 & $\underline{\text { DSFIGENSR }}$ \\
\hline
\end{tabular}




\section{Peptide View}

MS/MS Fragmentation of SNLSAYIIPDTDAHMSEYIGK

Found in IPI00197684, Tax_Id=10116 Gene_Symbol=Xpnpep2 Membrane-bound aminopeptidase P

Match to Query 2055: 2325.085872 from(776.035900,3+)

Title: 091008RatKidney_NoSalt_24.3877.3877.3.dta

Data file K:\NewmanPaper \Piliang \3SubProteomes \Piliang3SP \mgf5ppm\ERLIC_3SubProteomes5ppm.mgf
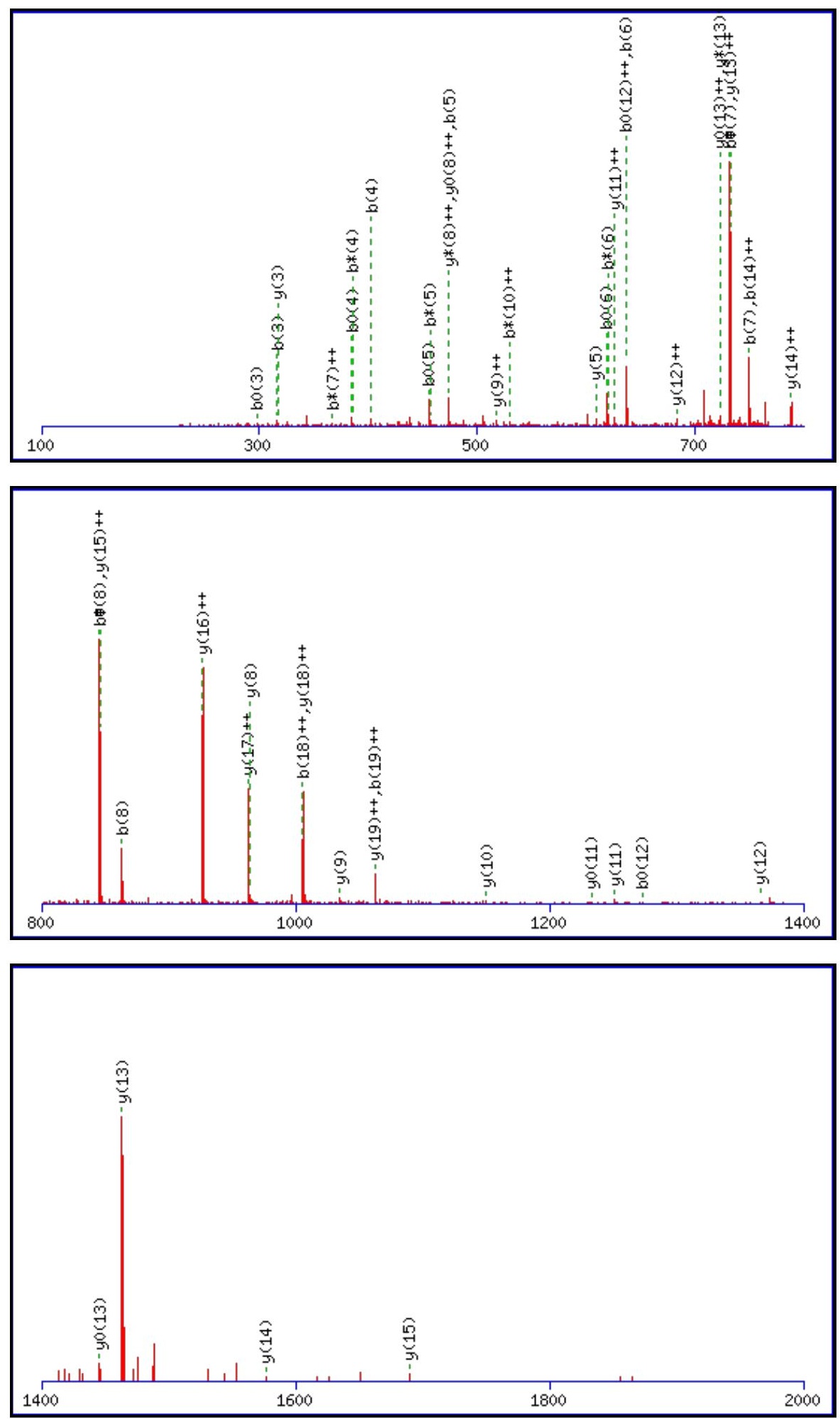

Monoisotopic mass of neutral peptide $\operatorname{Mr}($ calc): 2325.0834

Fixed modifications: Carbamidomethyl (C)

Variable modifications: 
N2 : Deamidated_N (N)

Ions Score: 43 Expect: 0.015

Matches (Bold Red): 50/230 fragment ions using 96 most intense peaks

\begin{tabular}{|c|c|c|c|c|c|c|c|c|c|c|c|c|c|c|}
\hline \# & b & $\mathbf{b}^{++}$ & $\mathbf{b}^{*}$ & $\mathbf{b}^{*^{++}}$ & $\mathbf{b}^{0}$ & $\mathbf{b}^{\mathbf{0 + +}}$ & Seq. & $\mathbf{y}$ & $\mathbf{y}^{++}$ & $\mathbf{y}^{*}$ & $\mathrm{y}^{*^{++}}$ & $\mathbf{y}^{0}$ & $\mathbf{y}^{\mathbf{0 + +}}$ & \# \\
\hline 1 & 88.0393 & 44.5233 & & & 70.0287 & 35.5180 & S & & & & & & & 21 \\
\hline 2 & 203.0662 & 102.0368 & 186.0397 & 93.5235 & 185.0557 & 93.0315 & $\mathbf{N}$ & 2239.0587 & 1120.0330 & 2222.0322 & 1111.5197 & 2221.0482 & 1111.0277 & 20 \\
\hline 3 & 316.1503 & 158.5788 & 299.1238 & 150.0655 & 298.1397 & 149.5735 & $\mathbf{L}$ & & 1062.5195 & 2107.0052 & 1054.0063 & 2106.0212 & 1053.5142 & 19 \\
\hline 4 & 403.1823 & 202.0948 & 386.1558 & 193.5815 & 385.1718 & 193.0895 & S & 2010.9477 & 1005.9775 & 1993.9212 & 997.4642 & 1992.9372 & 996.9722 & 18 \\
\hline 5 & 474.2194 & 237.6134 & 457.1929 & 229.1001 & 456.2089 & 228.6081 & A & 1923.9157 & 962.4615 & 1906.8891 & 953.9482 & 1905.9051 & 953.4562 & 17 \\
\hline 6 & 637.2828 & 319.1450 & 620.2562 & 310.6318 & 619.2722 & 310.1397 & $\mathbf{Y}$ & 1852.8786 & 926.9429 & 1835.8520 & 918.4297 & 1834.8680 & 917.9376 & 16 \\
\hline 7 & 750.3668 & 375.6871 & 733.3403 & 367.1738 & 732.3563 & 366.6818 & I & 1689.8153 & 845.4113 & 1672.7887 & 836.8980 & 1671.8047 & 836.4060 & 15 \\
\hline 8 & 863.4509 & 432.2291 & 846.4244 & 423.7158 & 845.4403 & 423.2238 & I & 1576.7312 & 788.8692 & 1559.7046 & 780.3560 & 1558.7206 & 779.8639 & 14 \\
\hline 9 & 960.5037 & 480.7555 & 943.4771 & 472.2422 & 942.4931 & 471.7502 & $\mathbf{P}$ & 1463.6471 & 732.3272 & 1446.6206 & 723.8139 & 1445.6366 & 723.3219 & 13 \\
\hline 10 & 1075.5306 & 538.2689 & 1058.5041 & 529.7557 & 1057.5200 & 529.2637 & D & 1366.5944 & 683.8008 & 1349.5678 & 675.2875 & 1348.5838 & 674.7955 & 12 \\
\hline 11 & 1176.5783 & 588.7928 & 1159.5517 & 580.2795 & 1158.5677 & 579.7875 & $\mathbf{T}$ & 1251.5674 & 626.2873 & 1234.5409 & 617.7741 & 1233.5569 & 617.2821 & 11 \\
\hline 12 & 1291.6052 & 646.3063 & 1274.5787 & 637.7930 & 1273.5947 & 637.3010 & D & 1150.5197 & 575.7635 & 1133.4932 & 567.2502 & 1132.5092 & 566.7582 & 10 \\
\hline 13 & 1362.6423 & 681.8248 & 1345.6158 & 673.3115 & 1344.6318 & 672.8195 & A & 1035.4928 & 518.2500 & 1018.4662 & 509.7368 & 1017.4822 & 509.2448 & 9 \\
\hline 14 & 1499.7013 & 750.3543 & 1482.6747 & 741.8410 & 1481.6907 & 741.3490 & H & 964.4557 & 482.7315 & 947.4291 & 474.2182 & 946.4451 & 473.7262 & 8 \\
\hline 15 & 1630.7417 & 815.8745 & 1613.7152 & 807.3612 & 1612.7312 & 806.8692 & $\mathbf{M}$ & 827.3968 & 414.2020 & 810.3702 & 405.6887 & 809.3862 & 405.1967 & 7 \\
\hline 16 & 1717.7738 & 859.3905 & 1700.7472 & 850.8772 & 1699.7632 & 850.3852 & $\mathrm{~S}$ & 696.3563 & 348.6818 & 679.3297 & 340.1685 & 678.3457 & 339.6765 & 6 \\
\hline 17 & 1846.8164 & 923.9118 & 1829.7898 & 915.3985 & 1828.8058 & 914.9065 & $\mathbf{E}$ & 609.3243 & 305.1658 & 592.2977 & 296.6525 & 591.3137 & 296.1605 & 5 \\
\hline 18 & 2009.8797 & 1005.4435 & 1992.8531 & 996.9302 & 1991.8691 & 996.4382 & $\mathbf{Y}$ & 480.2817 & 240.6445 & 463.2551 & 232.1312 & & & 4 \\
\hline 19 & 2122.9638 & 1061.9855 & 2105.9372 & 1053.4722 & 2104.9532 & 1052.9802 & I & 317.2183 & 159.1128 & 300.1918 & 150.5995 & & & 3 \\
\hline 20 & 2179.9852 & 1090.4962 & 2162.9587 & 1081.9830 & 2161.9747 & 1081.4910 & G & 204.1343 & 102.5708 & 187.1077 & 94.0575 & & & 2 \\
\hline 21 & & & & & & & $\mathbf{K}$ & 147.1128 & 74.0600 & 130.0863 & 65.5468 & & & 1 \\
\hline
\end{tabular}
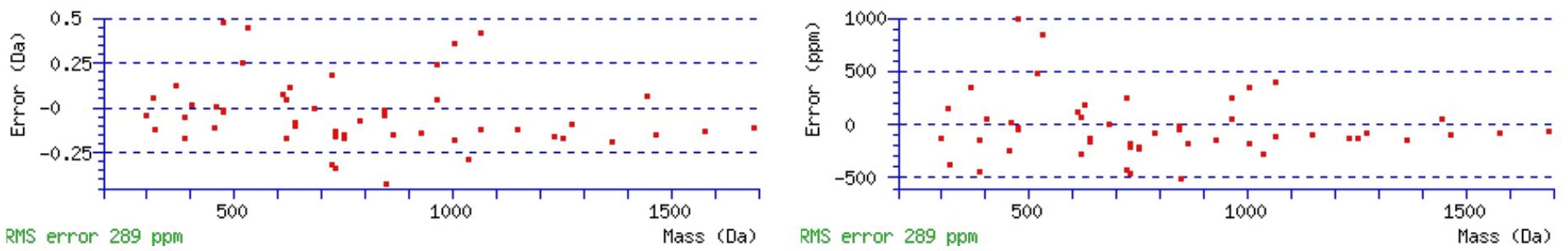

\section{All matches to this query}

\begin{tabular}{|l|l|l|l|}
\hline Score & Mr(calc): & Delta & \multicolumn{1}{|c|}{ Sequence } \\
\hline 42.6 & 2325.0834 & 0.0024 & SNLSAYIIPDTDAHMSEYIGK \\
\hline 32.7 & 2324.0994 & 0.9864 & SNLSAYIIPDTDAHMSEYIGK \\
\hline 9.3 & 2325.1035 & -0.0176 & $\underline{\text { INLSKLNVTDSVINKVTVK }}$ \\
\hline 8.4 & 2325.1035 & -0.0176 & $\underline{\text { INLSKLNVTDSVINKVTVK }}$ \\
\hline 6.5 & 2323.0871 & 1.9988 & EARIMGATTAGLFLLMDVVR \\
\hline 5.1 & 2324.0709 & 1.0150 & YIRVSDNNANISGWENGVWK \\
\hline 4.9 & 2324.0946 & 0.9913 & $\underline{\text { MALPCLSAIAGALPPDYLDTR }}$ \\
\hline 4.5 & 2324.0928 & 0.9930 & AIPTRIIYDFLMEXSPVAK \\
\hline 2.6 & 2325.0736 & 0.0123 & LNNSMASKIWTPDTFFHNGK \\
\hline 2.6 & 2325.0736 & 0.0123 & $\underline{\text { LNNSMASKIWTPDTFFHNGK }}$ \\
\hline
\end{tabular}

Spectrum No: 791; Query: 854; Rank: 1

\section{Peptide View}


MS/MS Fragmentation of DNGNGTYSCSYVPR

Found in IPI00409539, Tax_Id=10116 Gene_Symbol=Flna_predicted similar to Filamin-A

Match to Query 854: 1589.645208 from(795.829880,2+)

Title: 091008RatKidney_NH4Format01_19.1385.1385.2.dta

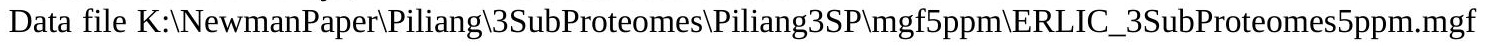
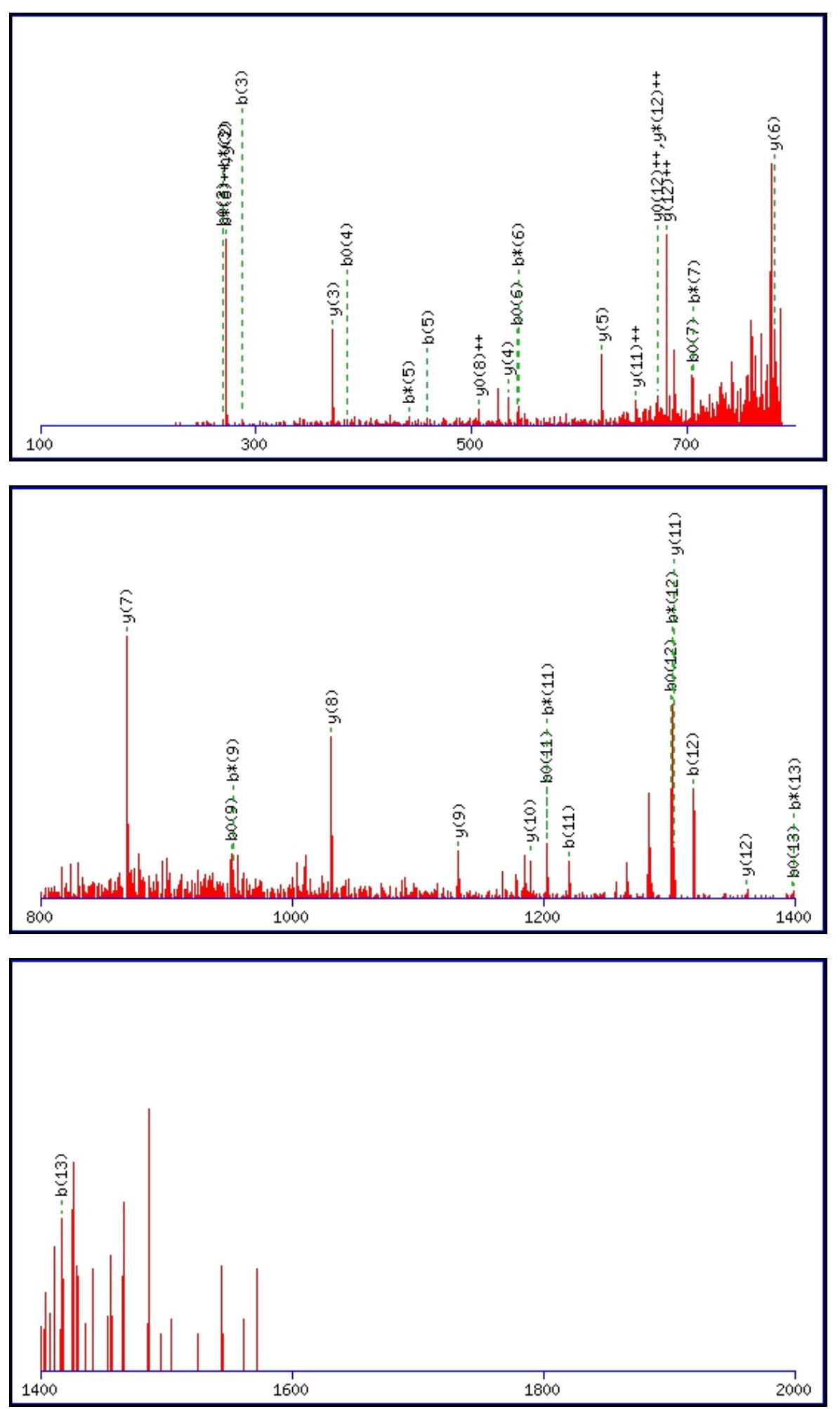

Monoisotopic mass of neutral peptide $\operatorname{Mr}$ (calc): 1589.6416

Fixed modifications: Carbamidomethyl (C)

Variable modifications:

N4

Ions Score: 43 Expect: 0.0069

Matches (Bold Red): 38/146 fragment ions using 133 most intense peaks

\begin{tabular}{|l|l|l|l|l|l|l|l|l}
\hline & ++ & & ++ & 0 & $0++$ & & & ++ \\
\hline
\end{tabular}




\begin{tabular}{|c|c|c|c|c|c|c|c|c|c|c|c|c|c|c|}
\hline \# & b & b & $\mathbf{b}^{*}$ & $\mathbf{b}^{*}$ & b & b & Seq. & $\mathbf{y}$ & $\mathbf{y}$ & $\mathbf{y}^{*}$ & $\mathbf{y}^{*}$ & $\mathbf{y}$ & $\mathbf{y}$ & \# \\
\hline 1 & 116.0342 & 58.5207 & & & 98.0237 & 49.5155 & D & & & & & & & 14 \\
\hline 2 & 230.0771 & 115.5422 & 213.0506 & 107.0289 & 212.0666 & 106.5369 & $\mathbf{N}$ & 1475.6220 & 738.3146 & |1458.5954 & 729.8013 & 1457.6114 & 729.3093 & 13 \\
\hline 3 & 287.0986 & 144.0529 & 270.0721 & 135.5397 & 269.0880 & 135.0477 & G & 1361.5790 & 681.2932 & 1344.5525 & 672.7799 & 1343.5685 & 672.2879 & 12 \\
\hline 4 & 402.1255 & 201.5664 & 385.0990 & 193.0531 & 384.1150 & 192.5611 & $\mathbf{N}$ & 1304.5576 & 652.7824 & 1287.5310 & 644.2691 & 1286.5470 & 643.7771 & 11 \\
\hline 5 & 459.1470 & 230.0771 & 442.1205 & 221.5639 & 441.1364 & 221.0719 & G & 1189.5306 & 595.2690 & 1172.5041 & 586.7557 & 1171.5201 & 586.2637 & 10 \\
\hline 6 & 560.1947 & 280.6010 & 543.1681 & 272.0877 & 542.1841 & 271.5957 & $T$ & 1132.5092 & 566.7582 & 1115.4826 & 558.2449 & 1114.4986 & 557.7529 & 9 \\
\hline 7 & 723.2580 & 362.1326 & 706.2315 & 353.6194 & 705.2475 & 353.1274 & $\mathbf{Y}$ & 1031.4615 & 516.2344 & 1014.4349 & 507.7211 & 1013.4509 & 507.2291 & 8 \\
\hline 8 & 810.2900 & 405.6487 & 793.2635 & 397.1354 & 792.2795 & 396.6434 & S & 868.3982 & 434.7027 & 851.3716 & 426.1894 & 850.3876 & 425.6974 & 7 \\
\hline 9 & 970.3207 & 485.6640 & 953.2941 & 477.1507 & 952.3101 & 476.6587 & $\mathrm{C}$ & 781.3661 & 391.1867 & 764.3396 & 382.6734 & 763.3556 & 382.1814 & 6 \\
\hline 10 & 1057.3527 & 529.1800 & 1040.3262 & 520.6667 & 1039.3422 & 520.1747 & $S$ & 621.3355 & 311.1714 & 604.3089 & 302.6581 & 603.3249 & 302.1661 & 5 \\
\hline 11 & 1220.4161 & 610.7117 & 1203.3895 & 602.1984 & 1202.4055 & 601.7064 & $\mathbf{Y}$ & 534.3035 & 267.6554 & 517.2769 & 259.1421 & & & 4 \\
\hline 12 & 1319.4845 & 660.2459 & \begin{tabular}{|l}
1302.4579 \\
\end{tabular} & 651.7326 & \begin{tabular}{|l}
1301.4739 \\
\end{tabular} & 651.2406 & V & 371.2401 & 186.1237 & 354.2136 & 177.6104 & & & 3 \\
\hline 13 & \begin{tabular}{|l|}
1416.5372 \\
\end{tabular} & 708.7723 & 1399.5107 & 700.2590 & 1398.5267 & 699.7670 & $\mathbf{P}$ & 272.1717 & 136.5895 & 255.1452 & 128.0762 & & & 2 \\
\hline 14 & & & & & & & $\mathbf{R}$ & 175.1190 & 88.0631 & 158.0924 & \begin{tabular}{|l|}
79.5498 \\
\end{tabular} & & & 1 \\
\hline
\end{tabular}
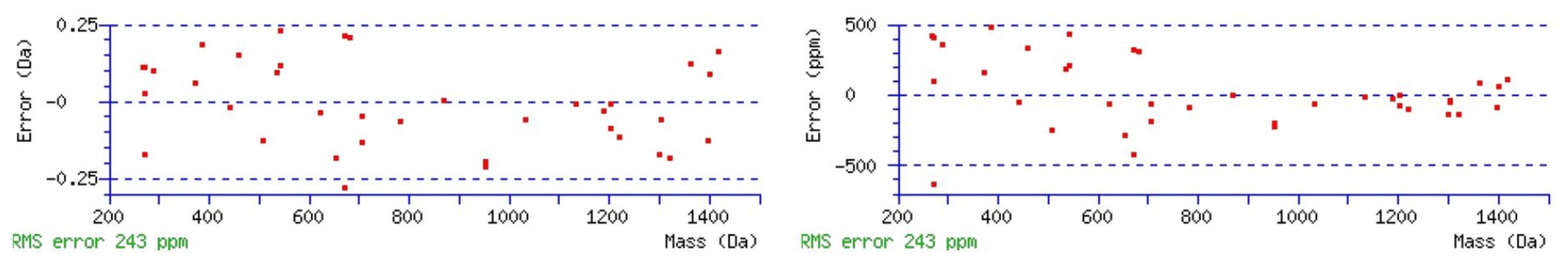

\section{All matches to this query}

\begin{tabular}{|l|c|c|c|}
\hline Score & Mr(calc): & Delta & Sequence \\
\hline 42.5 & 1589.6416 & 0.0036 & DNGNGTYSCSYVPR \\
\hline 38.9 & 1589.6416 & 0.0036 & DNGNGTYSCSYVPR \\
\hline 18.3 & 1588.6576 & 0.9876 & DNGNGTYSCSYVPR \\
\hline
\end{tabular}

Spectrum No: 792; Query: 2623; Rank: 1

\section{Peptide View}

MS/MS Fragmentation of SQSSNDTFPTAMHIAAALEVHQVLLPGLQK

Found in IPI00231611, Tax_Id=10116 Gene_Symbol=Fh1 Isoform Mitochondrial of Fumarate hydratase, mitochondrial precursor

Match to Query 2623: 3203.642232 from(1068.888020,3+)

Title: 091008RatKidney_NoSalt_06.4924.4924.3.dta

Data file K:INewmanPaper|Piliang|3SubProteomes\Piliang3SP\mgf5ppm\ERLIC_3SubProteomes5ppm.mgf 

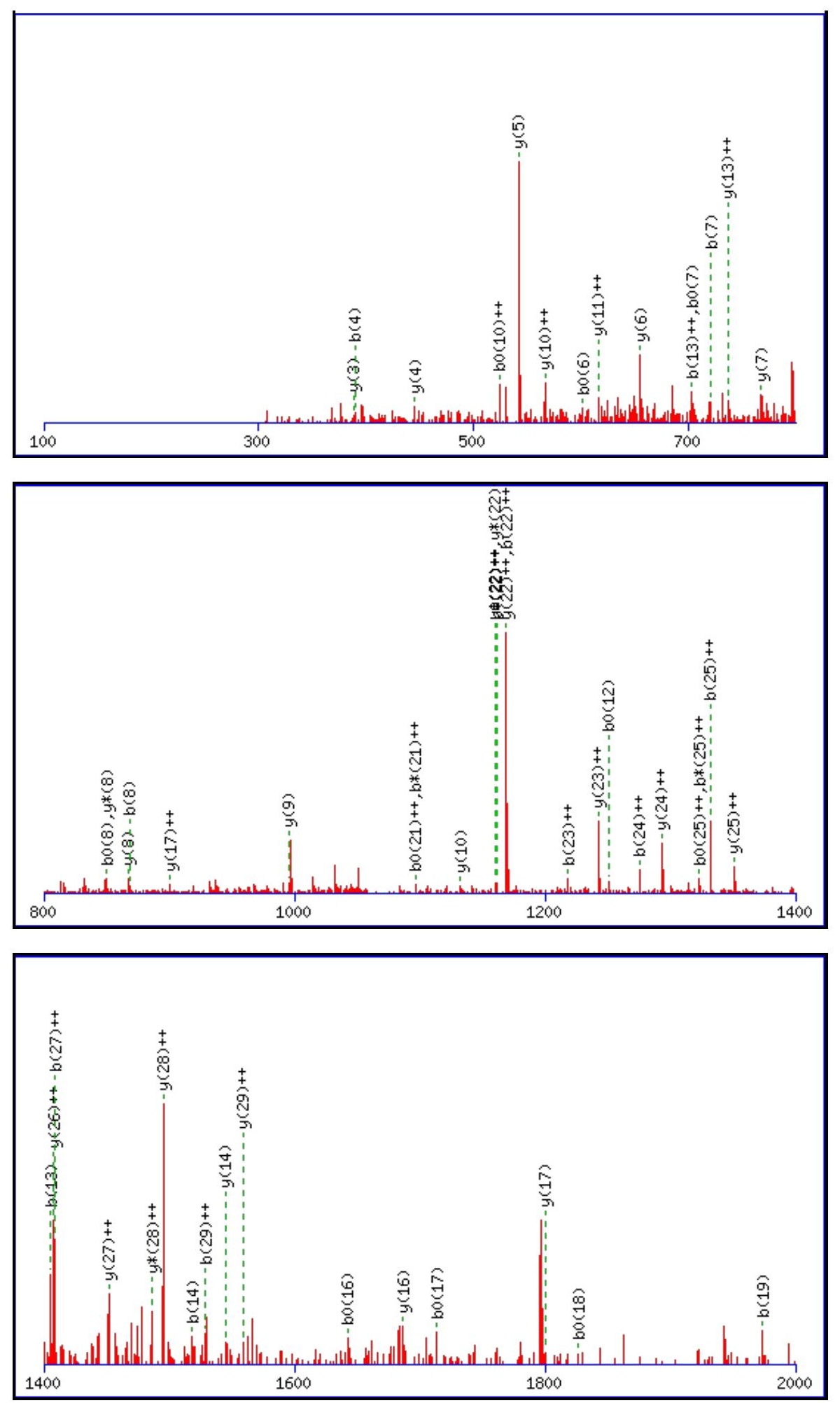

Monoisotopic mass of neutral peptide $\operatorname{Mr}($ calc): 3203.6285

Fixed modifications: Carbamidomethyl (C)

Variable modifications:

N5: Deamidated $\mathrm{N}(\mathrm{N})$

Ions Score: 43 Expect: 0.0081

Matches (Bold Red): 54/324 fragment ions using 144 most intense peaks

\begin{tabular}{|c|c|c|c|c|c|c|c|c|c|c|c|c|c|c|}
\hline \# & b & $\mathbf{b}^{++}$ & $\mathbf{b}^{*}$ & $\mathbf{b}^{*^{++}}$ & $\mathbf{b}^{0}$ & $\mathbf{b}^{\mathbf{0 + +}}$ & Seq. & $\mathbf{y}$ & $\mathbf{y}^{++}$ & $\mathbf{y}^{*}$ & $\mathrm{y}^{\mathrm{*}^{++}}$ & $\mathbf{y}^{\mathbf{0}}$ & $\mathbf{y}^{\mathbf{0 + +}}$ & \# \\
\hline 1 & 88.0393 & 44.5233 & & & 70.0287 & 35.5180 & S & & & & & & & 30 \\
\hline 2 & 216.0979 & 108.5526 & 199.0713 & 100.0393 & 198.0873 & 99.5473 & $\mathbf{Q}$ & 3117.6037 & 1559.3055 & 3100.5772 & 1550.7922 & 3099.5932 & 1550.3002 & 29 \\
\hline 3 & 303.1299 & 152.0686 & 286.1034 & 143.5553 & 285.1193 & 143.0633 & $S$ & 2989.5452 & 1495.2762 & 2972.5186 & 1486.7629 & 2971.5346 & 1486.2709 & 28 \\
\hline 4 & 390.1619 & 195.5846 & 373.1354 & 187.0713 & 372.1514 & 186.5793 & S & 2902.5131 & 1451.7602 & 2885.4866 & 1443.2469 & 2884.5026 & 1442.7549 & 27 \\
\hline 5 & 505.1889 & 253.0981 & 488.1623 & 244.5848 & 487.1783 & 244.0928 & $\mathbf{N}$ & 2815.4811 & 1408.2442 & 2798.4546 & 1399.7309 & 2797.4705 & 1399.2389 & 26 \\
\hline
\end{tabular}




\begin{tabular}{|c|c|c|c|c|c|c|c|c|c|c|c|c|c|c|}
\hline 6 & 620.2158 & 310.6115 & 603.1893 & 302.0983 & 602.2053 & 301.6063 & D & 2700.4542 & |1350.7307 & 2683.4276 & $|1342.2174|$ & 2682.4436| & 1341.7254| & 25 \\
\hline 7 & 721.2635 & 361.1354 & 704.2369 & 352.6221 & 703.2529 & 352.1301 & $\mathbf{T}$ & 2585.4272 & 1293.2173 & 2568.4007 & 1284.7040 & 2567.4167 & 1284.2120 & 24 \\
\hline 8 & 868.3319 & 434.6696 & 851.3054 & 426.1563 & 850.3213 & 425.6643 & $\mathbf{F}$ & 2484.3796 & 1242.6934 & 2467.3530 & 1234.1801 & 2466.3690 & 1233.6881 & 23 \\
\hline 9 & 965.3847 & 483.1960 & 948.3581 & 474.6827 & 947.3741 & 474.1907 & $\mathbf{P}$ & 2337.3111 & 1169.1592 & 2320.2846 & 1160.6459 & 2319.3006 & 1160.1539 & 22 \\
\hline 101 & 1066.4324 & 533.7198 & 1049.4058 & 525.2065 & 1048.4218 & 524.7145 & $T$ & 2240.2584 & 1120.6328 & 2223.2318 & 1112.1195 & 2222.2478 & 1111.6275 & 21 \\
\hline 111 & 1137.4695 & 569.2384 & 1120.4429 & 560.7251 & 1119.4589 & 560.2331 & A & 2139.2107 & 1070.1090 & 2122.1841 & 1061.5957 & 2121.2001 & 1061.1037 & 20 \\
\hline 12 & 1268.5100 & 634.7586 & 1251.4834 & 626.2453 & 1250.4994 & 625.7533 & $\mathbf{M}$ & 2068.1736 & 1034.5904 & 2051.1470 & 1026.0772 & 2050.1630 & 1025.5851 & 19 \\
\hline 131 & 1405.5689 & 703.2881 & 1388.5423 & 694.7748 & 1387.5583 & 694.2828 & $\mathbf{H}$ & 1937.1331 & 969.0702 & 1920.1065 & 960.5569 & 1919.1225 & 960.0649 & 18 \\
\hline 141 & 1518.6529 & 759.8301 & 1501.6264 & 751.3168 & 1500.6424 & 750.8248 & I & 1800.0742 & 900.5407 & 1783.0476 & 892.0275 & 1782.0636 & 891.5354 & 17 \\
\hline 151 & 1589.6900 & 795.3487 & 1572.6635 & 786.8354 & 1571.6795 & 786.3434 & A & 1686.9901 & 843.9987 & 1669.9636 & 835.4854 & 1668.9796 & 834.9934 & 16 \\
\hline 16 & 1660.7272 & 830.8672 & 1643.7006 & 822.3539 & 1642.7166 & 821.8619 & $\mathbf{A}$ & 1615.9530 & 808.4801 & 1598.9265 & 799.9669 & 1597.9424 & 799.4749 & 15 \\
\hline 171 & 1731.7643 & 866.3858 & 1714.7377 & 857.8725 & 1713.7537 & 857.3805 & A & 1544.9159 & 772.9616 & 1527.8893 & 764.4483 & 1526.9053 & 763.9563 & 14 \\
\hline 18 & 1844.8483 & 922.9278 & 1827.8218 & 914.4145 & 1826.8378 & 913.9225 & $\mathbf{L}$ & 1473.8788 & 737.4430 & 1456.8522 & 728.9298 & 1455.8682 & 728.4377 & 3 \\
\hline 191 & 1973.8909 & 987.4491 & 1956.8644 & 978.9358 & 1955.8804 & 978.4438 & $\mathbf{E}$ & 1360.7947 & 680.9010 & 1343.7682 & 672.3877 & 1342.7841 & 671.8957 & 12 \\
\hline 202 & 2072.9593 & 1036.9833 & 2055.9328 & 1028.4700 & 2054.9488 & 1027.9780 & $\mathbf{V}$ & 1231.7521 & 616.3797 & 1214.7256 & 607.8664 & & & 11 \\
\hline 21 & 2210.0183 & 1105.5128 & 2192.9917 & 1096.9995 & 2192.0077 & 1096.5075 & H & 1132.6837 & 566.8455 & 1115.6572 & 558.3322 & & & 10 \\
\hline 222 & 2338.0768 & 1169.5421 & 2321.0503 & 1161.0288 & 2320.0663 & 1160.5368 & $\mathbf{Q}$ & 995.6248 & 498.3160 & 978.5982 & 489.8028 & & & 9 \\
\hline 232 & 2437.1452 & 1219.0763 & 2420.1187 & 1210.5630 & 2419.1347 & 1210.0710 & $\mathbf{V}$ & 867.5662 & 434.2867 & 850.5397 & 425.7735 & & & 8 \\
\hline 242 & 2550.2293 & 1275.6183 & 2533.2028 & 1267.1050 & 2532.2187 & 1266.6130 & $\mathbf{L}$ & 768.4978 & 384.7525 & 751.4713 & 376.2393 & & & 7 \\
\hline $25 \sqrt{2}$ & 2663.3134 & 1332.1603 & 2646.2868 & 1323.6470 & 2645.3028 & 1323.1550 & $\mathbf{L}$ & 655.4137 & 328.2105 & 638.3872 & 319.6972 & & & 6 \\
\hline 26 & 2760.3661 & 1380.6867 & 2743.3396 & 1372.1734 & 2742.3556 & 1371.6814 & $\mathbf{P}$ & 542.3297 & 271.6685 & 525.3031 & 263.1552 & & & 5 \\
\hline 272 & 2817.3876 & 1409.1974 & 2800.3611 & 1400.6842 & 2799.3770 & 1400.1922 & G & 445.2769 & 223.1421 & 428.2504 & 214.6288 & & & 4 \\
\hline 282 & 2930.4717 & 1465.7395 & 2913.4451 & 1457.2262 & 2912.4611 & 1456.7342 & $\mathbf{L}$ & 388.2554 & 194.6314 & 371.2289 & 186.1181 & & & 3 \\
\hline 293 & 3058.5302 & 1529.7688 & 3041.5037 & 1521.2555 & 3040.5197 & 1520.7635 & $\mathbf{Q}$ & 275.1714 & 138.0893 & 258.1448 & 129.5761 & & & 2 \\
\hline 30 & & & & & & & $\mathbf{K}$ & 147.1128 & 74.0600 & 130.0863 & 65.5468 & & & 1 \\
\hline
\end{tabular}
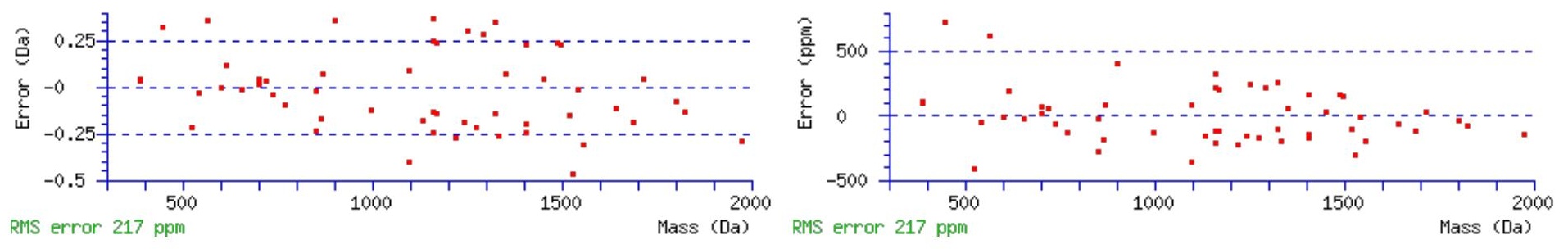

\section{All matches to this query}

\begin{tabular}{|l|l|l|l|}
\hline Score & Mr(calc): & Delta & \multicolumn{1}{c|}{ Sequence } \\
\hline 42.5 & 3203.6285 & 0.0138 & SOSSNDTFPTAMHIAAALEVHOVLLPGLQK \\
\hline 31.8 & 3202.6445 & 0.9978 & SQSSNDTFPTAMHIAAALEVHQVLLPGLQK \\
\hline 3.8 & 3203.6573 & -0.0151 & LQTGMRGAFGKPQGTVARVHIGQVIMSIR \\
\hline 0.6 & 3202.6486 & 0.9936 & VGVSGDSAGGNLTAAVTQQILQDPDVKIKLK \\
\hline 0.6 & 3202.6486 & 0.9936 & VGVSGDSAGGNLTAAVTQQILQDPDVKIKLK \\
\hline 0.3 & 3202.6162 & 1.0260 & DIEDSIKGELSGHFEDLLLAVGKPLSVNK \\
\hline 0.3 & 3203.6519 & -0.0097 & LLSGLSKPVTSYSNTFEWQSKLPAIKTK \\
\hline 0.3 & 3203.6519 & -0.0097 & LLSGLSKPVTSYSNTFEWQSKLPAIKTK \\
\hline
\end{tabular}

Spectrum No: 793; Query: 1865; Rank: 1

\section{Peptide View}

MS/MS Fragmentation of QGPQAGGTTLTINGTYLDTGSK 
Found in IPI00370450, Tax_Id=10116 Gene_Symbol=Plxnb2 plexin B2

Match to Query 1865: 2180.063648 from(1091.039100,2+)

Title: 100101RatKid_NS_deglyco_23.3059.3059.2.dta

Data file K:INewmanPaper|Piliangl3SubProteomes\Piliang3SP\mgf5ppm\ERLIC_3SubProteomes5ppm.mgf
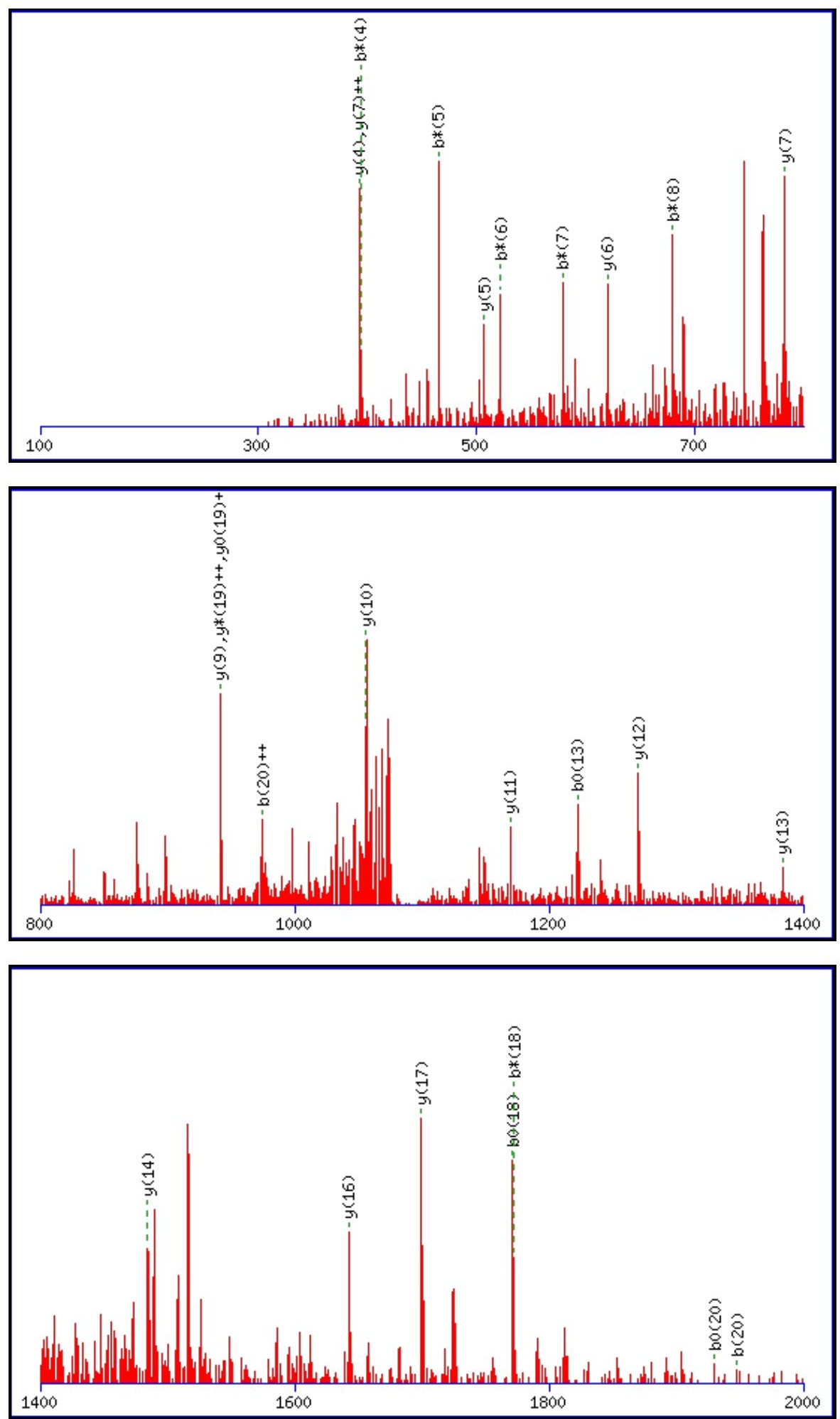

Monoisotopic mass of neutral peptide $\operatorname{Mr}($ calc): 2180.0597

Fixed modifications: Carbamidomethyl (C)

Variable modifications:

N13 : Deamidated_N (N)

Ions Score: 42 Expect: 0.013

Matches (Bold Red): 26/236 fragment ions using 40 most intense peaks

\begin{tabular}{|c|c|c|c|c|c|c|c|c|c|c|c|c|c|c|}
\hline$\#$ & $\mathbf{b}$ & $\mathbf{b}^{++}$ & $\mathbf{b}^{*}$ & $\mathbf{b}^{*_{++}}$ & $\mathbf{b}^{\mathbf{0}}$ & $\mathbf{b}^{\mathbf{0 +}}$ & Seq. & $\mathbf{y}$ & $\mathbf{y}^{++}$ & $\mathbf{y}^{*}$ & $\mathbf{y}^{*^{++}}$ & $\mathbf{y}^{\mathbf{0}}$ & $\mathbf{y}^{\mathbf{0 + +}}$ & $\#$ \\
\hline $\mathbf{1}$ & 129.0659 & 65.0366 & 112.0393 & 56.5233 & & & $\mathbf{Q}$ & & & & & & & \\
\hline
\end{tabular}




\begin{tabular}{|c|c|c|c|c|c|c|c|c|c|c|c|c|c|c|}
\hline 2 & 186.0873 & 93.5473 & 169.0608 & 85.0340 & & & G & 2053.0084 & 1027.0078 & 2035.9819 & 1018.4946 & 2034.9978 & 1018.0026 & 21 \\
\hline 3 & 283.1401 & 142.0737 & 266.1135 & 133.5604 & & & $\mathbf{P}$ & 1995.9869 & 998.4971 & 1978.9604 & 989.9838 & 1977.9764 & 989.4918 & 30 \\
\hline 4 & 411.1987 & 206.1030 & 394.1721 & 197.5897 & & & $\mathbf{Q}$ & 1898.9342 & 949.9707 & 1881.9076 & 941.4575 & 1880.9236 & 940.9654 & 19 \\
\hline 5 & 482.2358 & 241.6215 & 465.2092 & 233.1083 & & & $\mathbf{A}$ & 1770.8756 & 885.9414 & 1753.8490 & 877.4282 & 1752.8650 & 876.9362 & 18 \\
\hline 6 & 539.2572 & 270.1323 & 522.2307 & 261.6190 & & & G & 1699.8385 & 850.4229 & 1682.8119 & 841.9096 & 1681.8279 & 841.4176 & 17 \\
\hline 7 & 596.2787 & 298.6430 & 579.2522 & 290.1297 & & & G & 1642.8170 & 821.9121 & 1625.7905 & 813.3989 & 1624.8065 & 812.9069 & 16 \\
\hline 8 & 697.3264 & 349.1668 & 680.2998 & 340.6536 & 679.3158 & 340.1615 & $\mathbf{T}$ & 1585.7956 & 793.4014 & 1568.7690 & 784.8881 & 1567.7850 & 784.3961 & 15 \\
\hline 9 & 798.3741 & 399.6907 & 781.3475 & 391.1774 & 780.3635 & 390.6854 & $\mathbf{T}$ & 1484.7479 & 742.8776 & 1467.7213 & 734.3643 & 1466.7373 & 733.8723 & 14 \\
\hline 10 & 911.4581 & 456.2327 & 894.4316 & 447.7194 & 893.4476 & 447.2274 & $\mathbf{L}$ & 1383.7002 & 692.3537 & 1366.6736 & 683.8405 & 1365.6896 & 683.3485 & 13 \\
\hline 11 & 1012.5058 & 506.7565 & 995.4793 & 498.2433 & 994.4952 & 497.7513 & $T$ & 1270.6161 & 635.8117 & 1253.5896 & 627.2984 & 1252.6056 & 626.8064 & 12 \\
\hline 12 & 1125.5899 & 563.2986 & 1108.5633 & 554.7853 & 1107.5793 & 554.2933 & I & 1169.5685 & 585.2879 & 1152.5419 & 576.7746 & 1151.5579 & 576.2826 & 11 \\
\hline 13 & 1240.6168 & 620.8120 & 1223.5903 & 612.2988 & 1222.6062 & 611.8068 & $\mathbf{N}$ & 1056.4844 & 528.7458 & 1039.4578 & 520.2326 & 1038.4738 & 519.7406 & 10 \\
\hline 14 & 1297.6383 & 649.3228 & 1280.6117 & 640.8095 & 1279.6277 & 640.3175 & G & 941.4575 & 471.2324 & 924.4309 & 462.7191 & 923.4469 & 462.2271 & 9 \\
\hline 15 & 1398.6859 & 699.8466 & 1381.6594 & 691.3333 & 1380.6754 & 690.8413 & $\mathbf{T}$ & 884.4360 & 442.7216 & 867.4094 & 434.2084 & 866.4254 & 433.7164 & 8 \\
\hline 16 & 1561.7493 & 781.3783 & 1544.7227 & 772.8650 & 1543.7387 & 772.3730 & $\mathbf{Y}$ & 783.3883 & 392.1978 & 766.3618 & 383.6845 & 765.3777 & 383.1925 & 7 \\
\hline 17 & 1674.8333 & 837.9203 & 1657.8068 & 829.4070 & 1656.8228 & 828.9150 & $\mathbf{L}$ & 620.3250 & 310.6661 & 603.2984 & 302.1529 & 602.3144 & 301.6608 & 6 \\
\hline 18 & 1789.8603 & 895.4338 & \begin{tabular}{|l}
1772.8337 \\
\end{tabular} & 886.9205 & \begin{tabular}{|l}
1771.8497 \\
\end{tabular} & 886.4285 & D & 507.2409 & 254.1241 & 490.2144 & 245.6108 & 489.2304 & 245.1188 & 5 \\
\hline 19 & 1890.9080 & 945.9576 & 1873.8814 & 937.4443 & 1872.8974 & 936.9523 & $\mathbf{T}$ & 392.2140 & 196.6106 & 375.1874 & 188.0974 & 374.2034 & 187.6053 & 4 \\
\hline 20 & 1947.9294 & 974.4684 & 1930.9029 & 965.9551 & 1929.9189 & 965.4631 & G & 291.1663 & 146.0868 & 274.1397 & 137.5735 & 273.1557 & 137.0815 & 3 \\
\hline 21 & 2034.9615 & 1017.9844 & 2017.9349 & 1009.4711 & 2016.9509 & 1008.9791 & $\mathrm{~S}$ & 234.1448 & 117.5761 & 217.1183 & 109.0628 & 216.1343 & 108.5708 & 2 \\
\hline 22 & & & & & & & $\mathbf{K}$ & 147.1128 & 74.0600 & 130.0863 & 65.5468 & & & 1 \\
\hline
\end{tabular}
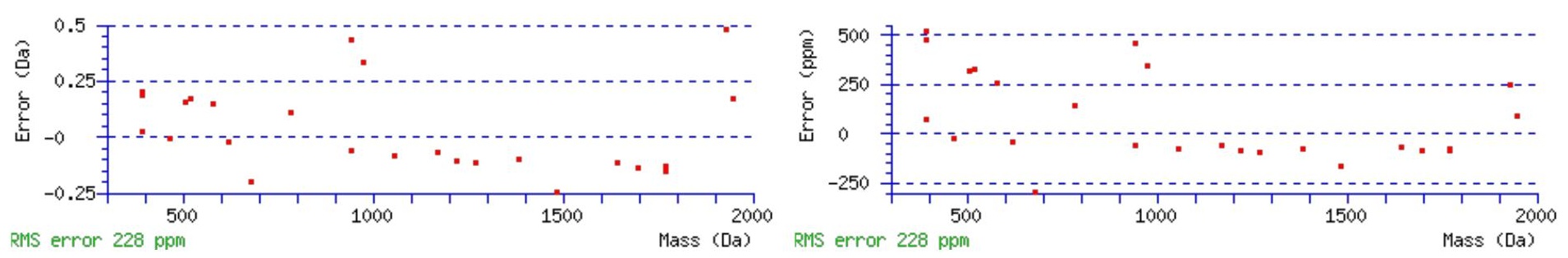

\section{All matches to this query}

\begin{tabular}{|l|l|l|c|}
\hline Score & Mr(calc): & Delta & Sequence \\
\hline 42.3 & 2180.0597 & 0.0039 & QGPQAGGTTLTINGTYLDTGSK \\
\hline
\end{tabular}

Spectrum No: 794; Query: 524; Rank: 1

\section{Peptide View}

\section{MS/MS Fragmentation of YLAKENITQDPK}

Found in IPI00231197, Tax_Id=10116 Gene_Symbol=Cd36 Platelet glycoprotein 4

Match to Query 524: 1419.725568 from(710.870060,2+)

Title: 100101RatKid_NS_deglyco_19.898.898.2.dta

Data file K:INewmanPaper|Piliangl3SubProteomes\Piliang3SP\mgf5ppm\ERLIC_3SubProteomes5ppm.mgf 

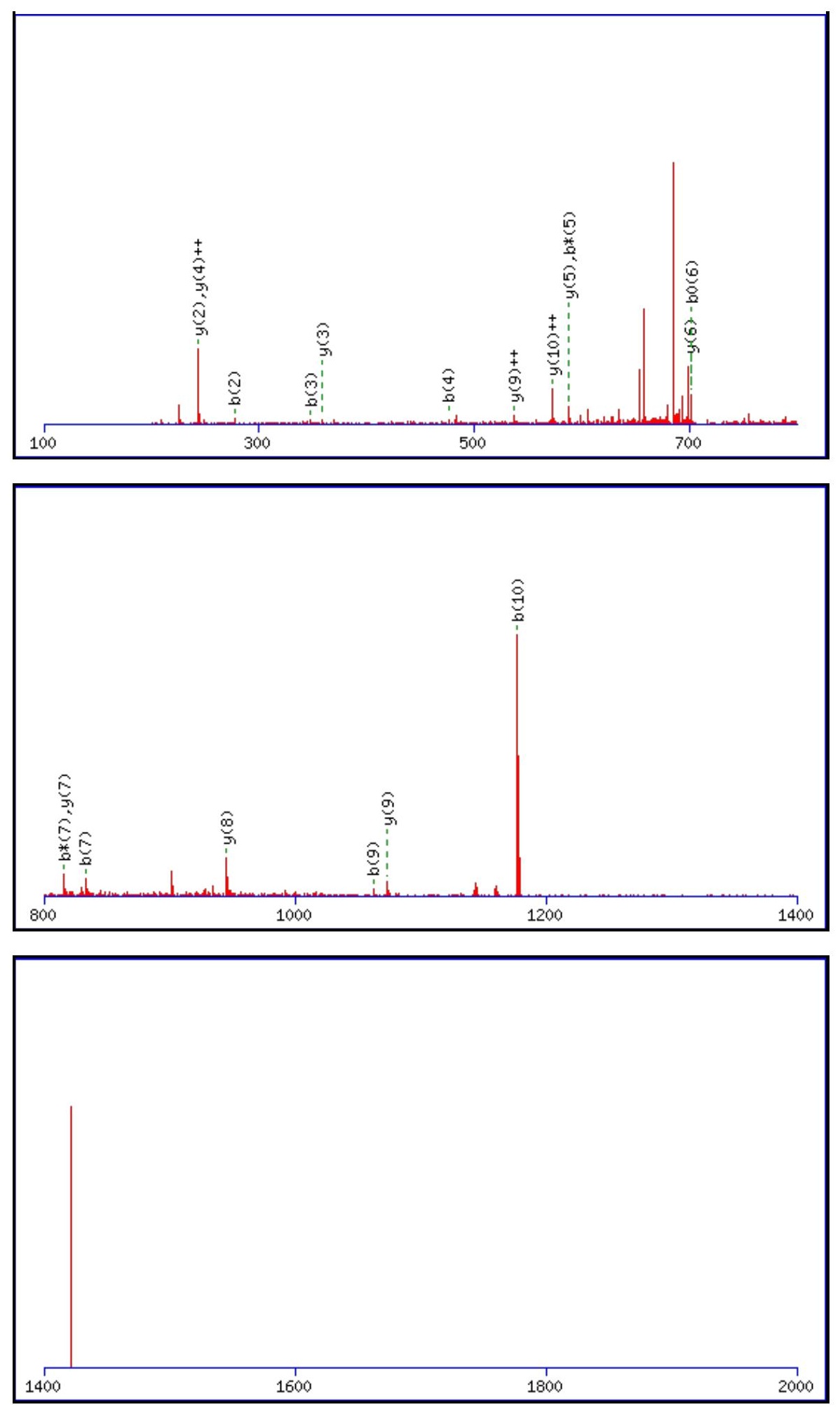

Monoisotopic mass of neutral peptide $\operatorname{Mr}($ calc): 1419.7245

Fixed modifications: Carbamidomethyl (C)

Variable modifications:

N6: Deamidated $\mathrm{N}(\mathrm{N})$

Ions Score: 42 Expect: $\odot .0086$

Matches (Bold Red): 19/114 fragment ions using 32 most intense peaks

\begin{tabular}{|r|c|c|c|c|c|c|c|c|c|c|c|c|c|c|}
\hline$\#$ & $\mathbf{b}$ & $\mathbf{b}^{++}$ & $\mathbf{b}^{*}$ & $\mathbf{b}^{\boldsymbol{*}^{++}}$ & $\mathbf{b}^{\mathbf{0}}$ & $\mathbf{b}^{\mathbf{0 + +}}$ & Seq. & $\mathbf{y}$ & $\mathbf{y}^{++}$ & $\mathbf{y}^{\mathbf{*}}$ & $\mathbf{y}^{\boldsymbol{*}^{++}}$ & $\mathbf{y}^{\mathbf{0}}$ & $\mathbf{y}^{\mathbf{0 + +}}$ & $\#$ \\
\hline $\mathbf{1}$ & 164.0706 & 82.5389 & & & & & $\mathbf{Y}$ & & & & & & & $\mathbf{1 2}$ \\
\hline $\mathbf{2}$ & $\mathbf{2 7 7 . 1 5 4 7}$ & 139.0810 & & & & & $\mathbf{L}$ & 1257.6685 & 629.3379 & 1240.6420 & 620.8246 & 1239.6579 & 620.3326 & $\mathbf{1 1}$ \\
\hline $\mathbf{3}$ & $\mathbf{3 4 8 . 1 9 1 8}$ & 174.5995 & & & & & $\mathbf{A}$ & 1144.5844 & 572.7959 & 1127.5579 & 564.2826 & 1126.5739 & 563.7906 & $\mathbf{1 0}$ \\
\hline $\mathbf{4}$ & $\mathbf{4 7 6 . 2 8 6 7}$ & 238.6470 & 459.2602 & 230.1337 & & & $\mathbf{K}$ & $\mathbf{1 0 7 3 . 5 4 7 3}$ & 537.2773 & 1056.5208 & 528.7640 & 1055.5368 & 528.2720 & $\mathbf{9}$ \\
\hline $\mathbf{5}$ & 605.3293 & 303.1683 & $\mathbf{5 8 8 . 3 0 2 8}$ & 294.6550 & 587.3188 & 294.1630 & $\mathbf{E}$ & $\mathbf{9 4 5 . 4 5 2 4}$ & 473.2298 & 928.4258 & 464.7165 & 927.4418 & 464.2245 & $\mathbf{8}$ \\
\hline
\end{tabular}




\begin{tabular}{|r|r|r|r|r|r|r|r|r|r|r|r|r|r|r|r|r|r|r|}
$\mathbf{6}$ & 720.3563 & 360.6818 & 703.3297 & 352.1685 & $\mathbf{7 0 2 . 3 4 5 7}$ & 351.6765 & $\mathbf{N}$ & $\mathbf{8 1 6 . 4 0 9 8}$ & 408.7085 & 799.3832 & 400.1952 & 798.3992 & 399.7032 & $\mathbf{7}$ \\
\hline $\mathbf{7}$ & $\mathbf{8 3 3 . 4 4 0 3}$ & 417.2238 & $\mathbf{8 1 6 . 4 1 3 8}$ & 408.7105 & 815.4298 & 408.2185 & $\mathbf{I}$ & $\mathbf{7 0 1 . 3 8 2 8}$ & 351.1951 & 684.3563 & 342.6818 & 683.3723 & 342.1898 & $\mathbf{6}$ \\
\hline $\mathbf{8}$ & 934.4880 & 467.7476 & 917.4615 & 459.2344 & 916.4775 & 458.7424 & $\mathbf{T}$ & $\mathbf{5 8 8 . 2 9 8 8}$ & 294.6530 & 571.2722 & 286.1397 & 570.2882 & 285.6477 & $\mathbf{5}$ \\
\hline $\mathbf{9}$ & $\mathbf{1 0 6 2 . 5 4 6 6}$ & 531.7769 & 1045.5200 & 523.2637 & 1044.5360 & 522.7717 & $\mathbf{Q}$ & 487.2511 & 244.1292 & 470.2245 & 235.6159 & 469.2405 & 235.1239 & $\mathbf{4}$ \\
\hline $\mathbf{1 0}$ & $\mathbf{1 1 7 7 . 5 7 3 5}$ & 589.2904 & 1160.5470 & 580.7771 & 1159.5630 & 580.2851 & $\mathbf{D}$ & 359.1925 & 180.0999 & 342.1660 & 171.5866 & 341.1819 & 171.0946 & $\mathbf{3}$ \\
\hline $\mathbf{1 1}$ & 1274.6263 & 637.8168 & 1257.5998 & 629.3035 & 1256.6157 & 628.8115 & $\mathbf{P}$ & $\mathbf{2 4 4 . 1 6 5 6}$ & 122.5864 & 227.1390 & 114.0731 & & & & $\mathbf{2}$ \\
\hline $\mathbf{1 2}$ & & & & & & & $\mathbf{K}$ & 147.1128 & 74.0600 & 130.0863 & 65.5468 & & & $\mathbf{1}$ \\
\hline
\end{tabular}
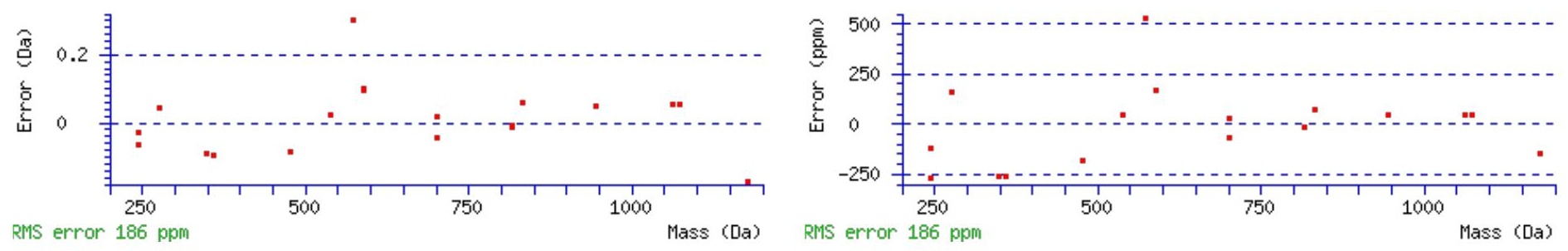

\section{All matches to this query}

\begin{tabular}{|l|l|l|l|}
\hline Score & Mr(calc): & Delta & Sequence \\
\hline 42.2 & 1419.7245 & 0.0010 & YLAKENITQDPK \\
\hline 5.9 & 1419.7149 & 0.0107 & SXMTPMRSVPMK \\
\hline 4.5 & 1419.7123 & 0.0133 & NKTAAAPVIDAIR \\
\hline 2.7 & 1419.7146 & 0.0109 & DKELSAATPWFR \\
\hline 1.9 & 1419.7123 & 0.0133 & KDSQIQKPTAPK \\
\hline 1.3 & 1418.7187 & 1.0068 & QMSEQIALDTRK \\
\hline 1.1 & 1418.7266 & 0.9990 & QNLSQFEAQARK \\
\hline 0.4 & 1417.7289 & 1.9967 & TMLAWGWRIER \\
\hline 0.4 & 1419.7123 & 0.0133 & KDSQIQKPTAPK \\
\hline
\end{tabular}

Spectrum No: 795; Query: 1058; Rank: 1

\section{Peptide View}

MS/MS Fragmentation of LRKNLSYEEAPDHK

Found in IPI00471800, Tax_Id=10116 Gene_Symbol=Cdh16 Cadherin 16

Match to Query 1058: 1699.855696 from(425.971200,4+)

Title: 100101RatKid_NS_deglyco_25.606.606.4.dta

Data file K:INewmanPaper|Piliangl3SubProteomes\Piliang3SP\mgf5ppm\ERLIC_3SubProteomes5ppm.mgf

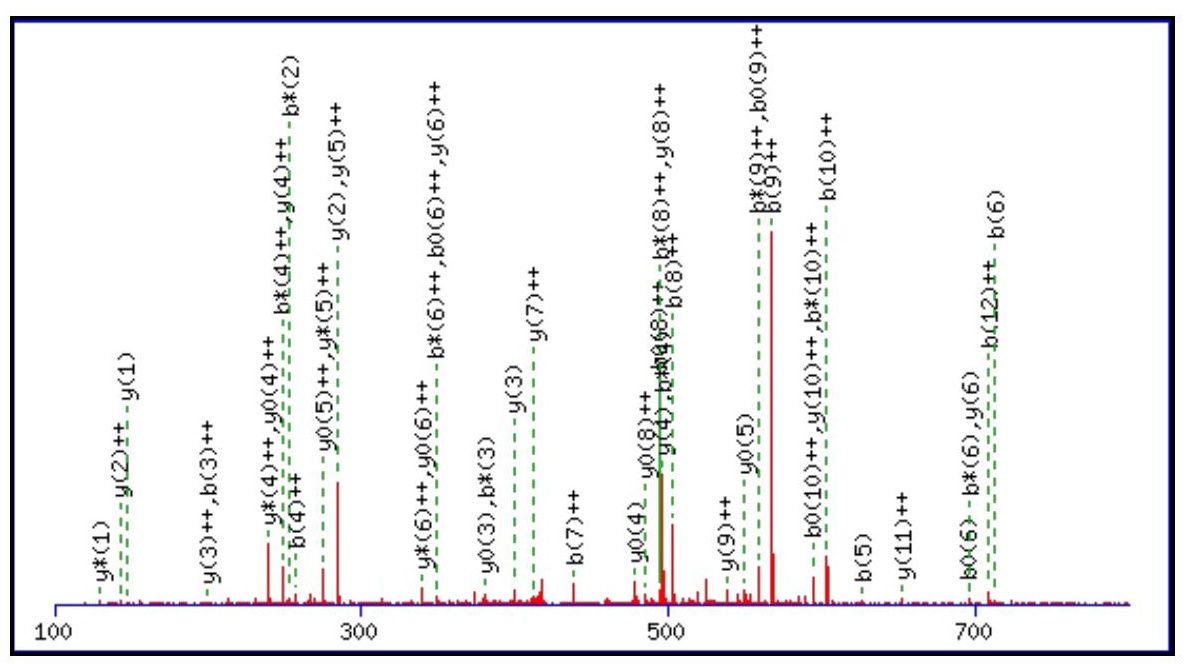




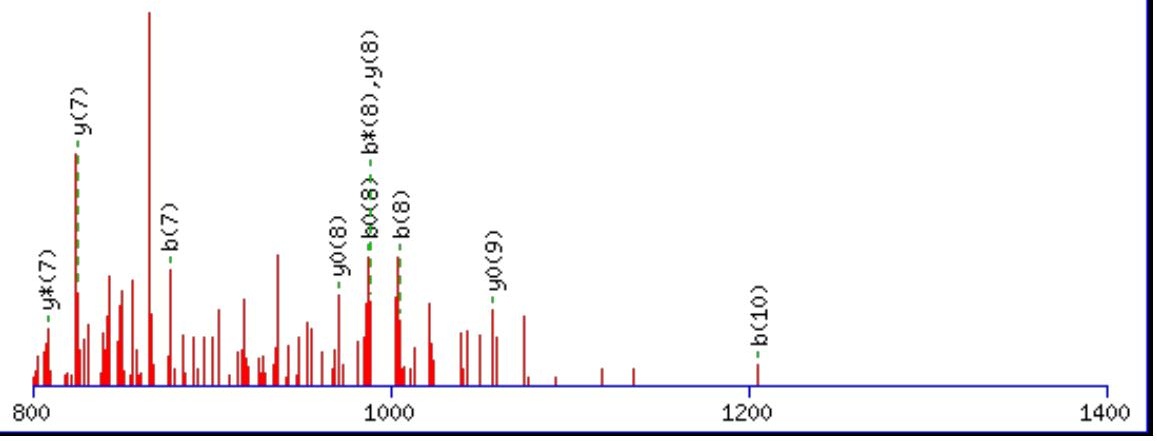

\begin{tabular}{rrrr}
\hline 1400 & 1600 & 1800 & 2000 \\
\hline
\end{tabular}

Monoisotopic mass of neutral peptide $\operatorname{Mr}$ (calc): 1699.8529

Fixed modifications: Carbamidomethyl (C)

Variable modifications:

N4 : Deamidated_N (N)

Ions Score: 42 Expect: 0.0092

Matches (Bold Red): 59/140 fragment ions using 104 most intense peaks

\begin{tabular}{|c|c|c|c|c|c|c|c|c|c|c|c|c|c|c|}
\hline \# & b & $\mathbf{b}^{++}$ & $\mathbf{b}^{*}$ & $\mathbf{b}^{*^{++}}$ & $\mathbf{b}^{\mathbf{0}}$ & $\mathbf{b}^{0++}$ & Seq. & $\mathbf{y}$ & $\mathbf{y}^{++}$ & $\mathbf{y}^{*}$ & $\mathbf{y}^{*^{++}}$ & $\mathbf{y}^{0}$ & $\mathbf{y}^{0++}$ & \# \\
\hline 1 & 114.0913 & 57.5493 & & & & & $\mathbf{L}$ & & & & & & & 14 \\
\hline 2 & 270.1925 & 135.5999 & 253.1659 & 127.0866 & & & $\mathbf{R}$ & 1587.7762 & 794.3917 & 1570.7496 & 785.8784 & 1569.7656 & 785.3864 & 13 \\
\hline 3 & 398.2874 & 199.6473 & 381.2609 & 191.1341 & & & $\mathbf{K}$ & 1431.6750 & 716.3412 & 1414.6485 & 707.8279 & 1413.6645 & 707.3359 & 12 \\
\hline 4 & 513.3144 & 257.1608 & 496.2878 & 248.6475 & & & $\mathbf{N}$ & 1303.5801 & 652.2937 & 1286.5535 & 643.7804 & 1285.5695 & 643.2884 & 11 \\
\hline 5 & 626.3984 & 313.7028 & 609.3719 & 305.1896 & & & $\mathbf{L}$ & 1188.5531 & 594.7802 & 1171.5266 & 586.2669 & 1170.5426 & 585.7749 & 10 \\
\hline 6 & 713.4304 & 357.2189 & 696.4039 & 348.7056 & 695.4199 & 348.2136 & $\mathbf{S}$ & 1075.4691 & 538.2382 & 1058.4425 & 529.7249 & 1057.4585 & 529.2329 & 9 \\
\hline 7 & 876.4938 & 438.7505 & 859.4672 & 430.2372 & 858.4832 & 429.7452 & $\mathbf{Y}$ & 988.4371 & 494.7222 & 971.4105 & 486.2089 & 970.4265 & 485.7169 & 8 \\
\hline 8 & 1005.5364 & 503.2718 & 988.5098 & 494.7585 & 987.5258 & 494.2665 & $\mathbf{E}$ & 825.3737 & 413.1905 & 808.3472 & 404.6772 & 807.3632 & 404.1852 & 7 \\
\hline 9 & 1134.5790 & 567.7931 & 1117.5524 & 559.2798 & 1116.5684 & 558.7878 & $\mathbf{E}$ & 696.3311 & 348.6692 & 679.3046 & 340.1559 & 678.3206 & 339.6639 & 6 \\
\hline 10 & 1205.6161 & 603.3117 & 1188.5895 & 594.7984 & 1187.6055 & 594.3064 & A & 567.2885 & 284.1479 & 550.2620 & 275.6346 & 549.2780 & 275.1426 & 5 \\
\hline 11 & 1302.6688 & 651.8381 & 1285.6423 & 643.3248 & 1284.6583 & 642.8328 & $\mathbf{P}$ & 496.2514 & 248.6293 & 479.2249 & 240.1161 & 478.2409 & 239.6241 & 4 \\
\hline 12 & 1417.6958 & 709.3515 & 1400.6692 & 700.8383 & 1399.6852 & 700.3462 & D & 399.1987 & 200.1030 & 382.1721 & 191.5897 & 381.1881 & 191.0977 & 3 \\
\hline 13 & 1554.7547 & 777.8810 & 1537.7281 & 769.3677 & 1536.7441 & 768.8757 & $\mathbf{H}$ & 284.1717 & 142.5895 & 267.1452 & 134.0762 & & & 2 \\
\hline 14 & & & & & & & $\mathbf{K}$ & 147.1128 & 74.0600 & 130.0863 & 65.5468 & & & 1 \\
\hline
\end{tabular}



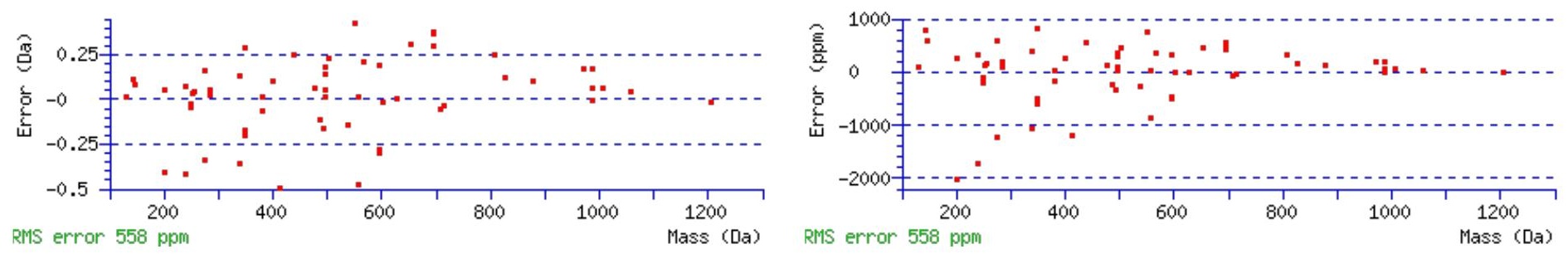

\section{All matches to this query}

\begin{tabular}{|l|l|l|l|}
\hline Score & Mr(calc): & Delta & \multicolumn{1}{|c|}{ Sequence } \\
\hline 42.2 & 1699.8529 & 0.0028 & LRKNLSYEEAPDHK \\
\hline 20.2 & 1698.8689 & 0.9868 & LRKNLSYEEAPDHK \\
\hline 9.9 & 1699.8463 & 0.0094 & IGIAIKITYLGHNK \\
\hline 7.5 & 1698.8375 & 1.0182 & KTSVTPLRENSLMK \\
\hline 6.5 & 1698.8375 & 1.0182 & KTSVTPLRENSLMK \\
\hline 5.2 & 1697.8535 & 2.0022 & AKLSGEQMLTIKQR \\
\hline 4.4 & 1697.8573 & 1.9984 & WPKRTSHGAMGLWR \\
\hline 4.2 & 1699.8546 & 0.0011 & ENQPKSSIVSLYKK \\
\hline 4.2 & 1699.8433 & 0.0124 & ALVGLLSEENTISFK \\
\hline 3.8 & 1699.8481 & 0.0076 & VGELSLKMFQKQGR \\
\hline
\end{tabular}

Spectrum No: 796; Query: 86; Rank: 1

\section{Peptide View}

MS/MS Fragmentation of CDNVVIVNK

Found in IPI00196620, Tax_Id=10116 Gene_Symbol=Cubn Cubilin precursor

Match to Query 86: 1060.519988 from(531.267270,2+)

Title: 091008RatKidney_NH4Format01_25.769.769.2.dta

Data file K:INewmanPaper|Piliang|3SubProteomes\Piliang3SP\mgf5ppm\ERLIC_3SubProteomes5ppm.mgf

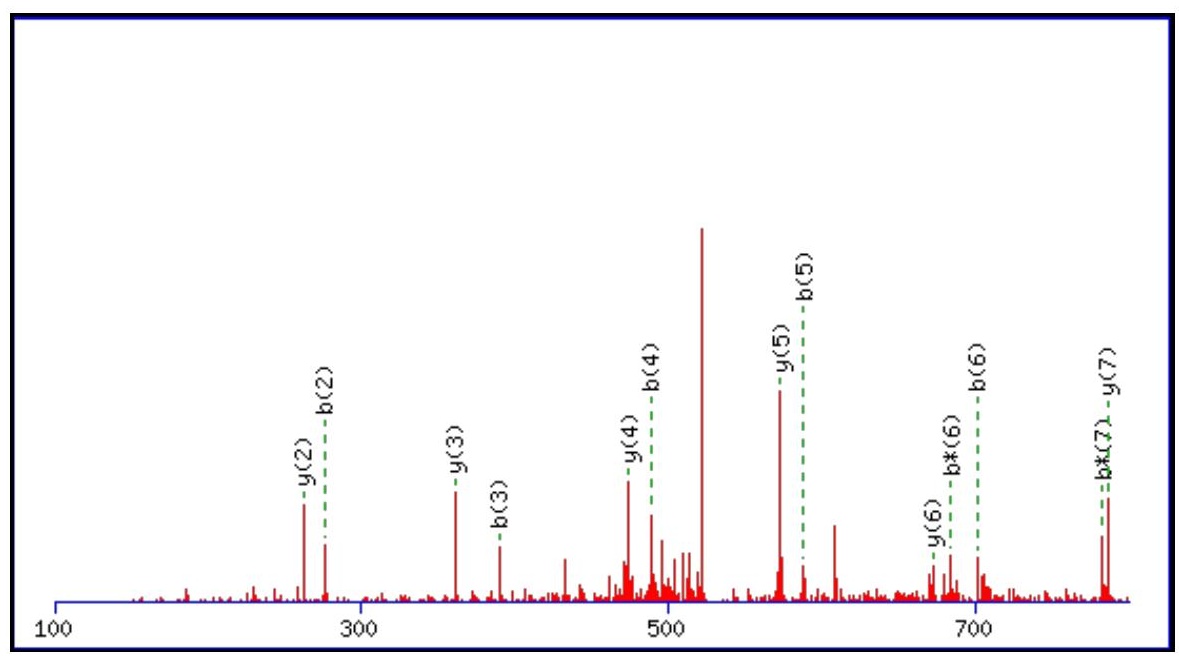



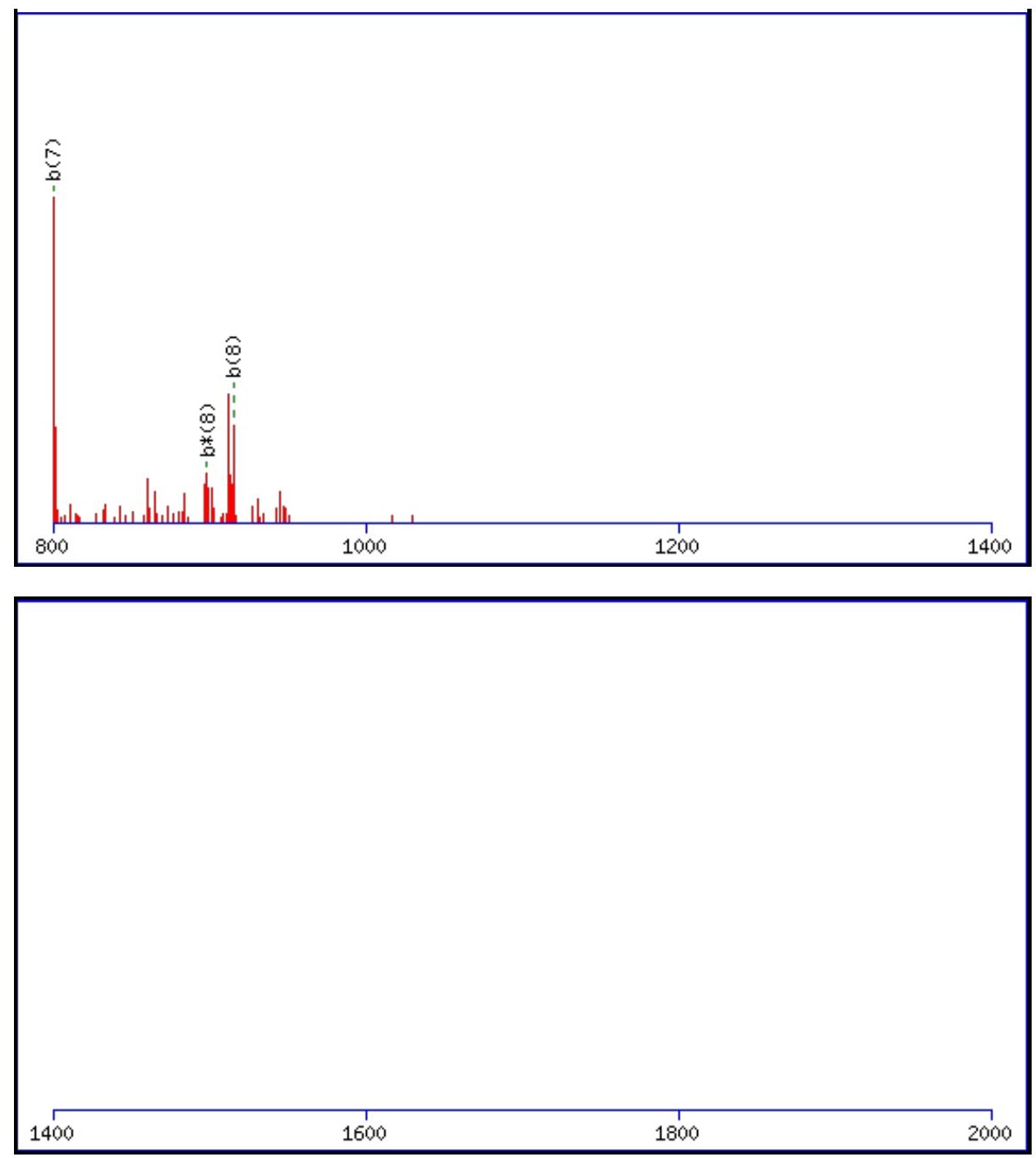

Monoisotopic mass of neutral peptide $\operatorname{Mr}($ calc): 1060.5223

Fixed modifications: Carbamidomethyl (C)

Variable modifications:

N8 : Deamidated_N (N)

Ions Score: 42 Expect: 0.0068

Matches (Bold Red): 16/76 fragment ions using 43 most intense peaks

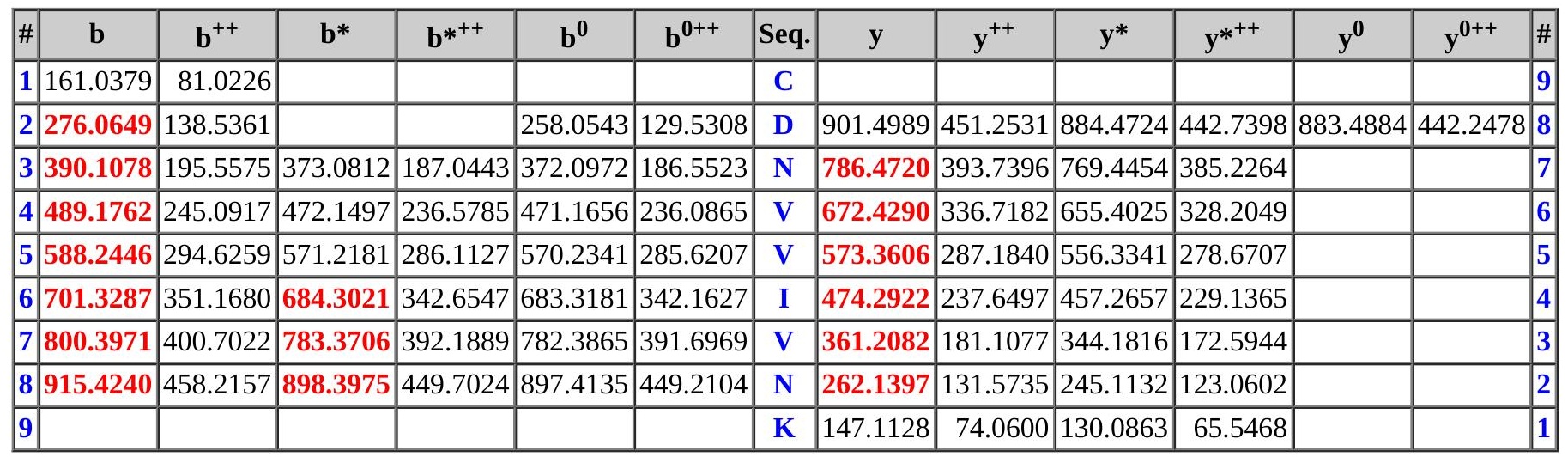

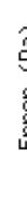$$
\begin{array}{ll}
0.1 \\
0 \\
0.2 \\
0
\end{array}
$$$$
\text { 青:- }
$$

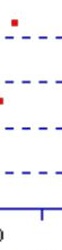

NS error 187 ppo

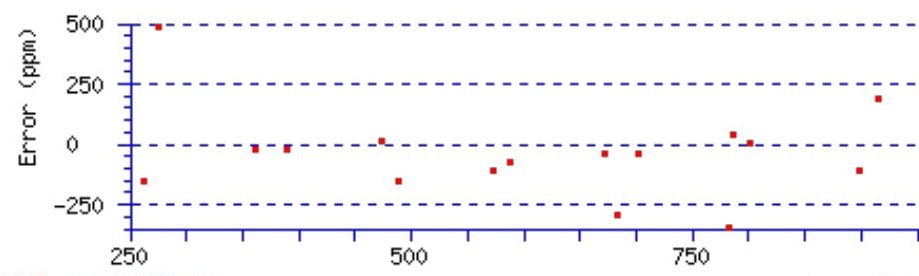

Mass (Da)

\section{All matches to this query}




\begin{tabular}{|l|c|c|l|} 
Score & Mr(calc): & Delta & \multicolumn{1}{|c|}{ Sequence } \\
\hline 42.1 & 1060.5223 & -0.0023 & CDNVVIVNK \\
\hline 10.2 & 1060.5223 & -0.0023 & CEALLAQEK \\
\hline 8.1 & 1059.5271 & 0.9929 & LMQVVPQET \\
\hline 7.5 & 1060.5149 & 0.0051 & NGGQSINKNK \\
\hline 7.3 & 1060.5149 & 0.0051 & GEGTQNQAKK \\
\hline 4.6 & 1060.5149 & 0.0051 & QETNRLGNK \\
\hline 4.1 & 1060.5261 & -0.0061 & SRAEDLNTR \\
\hline 3.9 & 1060.5189 & 0.0011 & SINAWVENK \\
\hline 3.4 & 1058.5049 & 2.0151 & YDLNTLLK \\
\hline 3.3 & 1058.5049 & 2.0151 & $\underline{\text { LFTEKVNK }}$ \\
\hline
\end{tabular}

Spectrum No: 797; Query: 1479; Rank: 1

\section{Peptide View}

MS/MS Fragmentation of IPNNTQWITWSQEGHK

Found in IPI00208422, Tax_Id=10116 Gene_Symbol=Dpp4 Dipeptidyl peptidase 4

Match to Query 1479: 1938.926292 from(647.316040,3+)

Title: 100101RatKid_NS_deglyco_26.2877.2877.3.dta

Data file K:INewmanPaper|Piliangl3SubProteomes\Piliang3SP\mgf5ppm\ERLIC_3SubProteomes5ppm.mgf
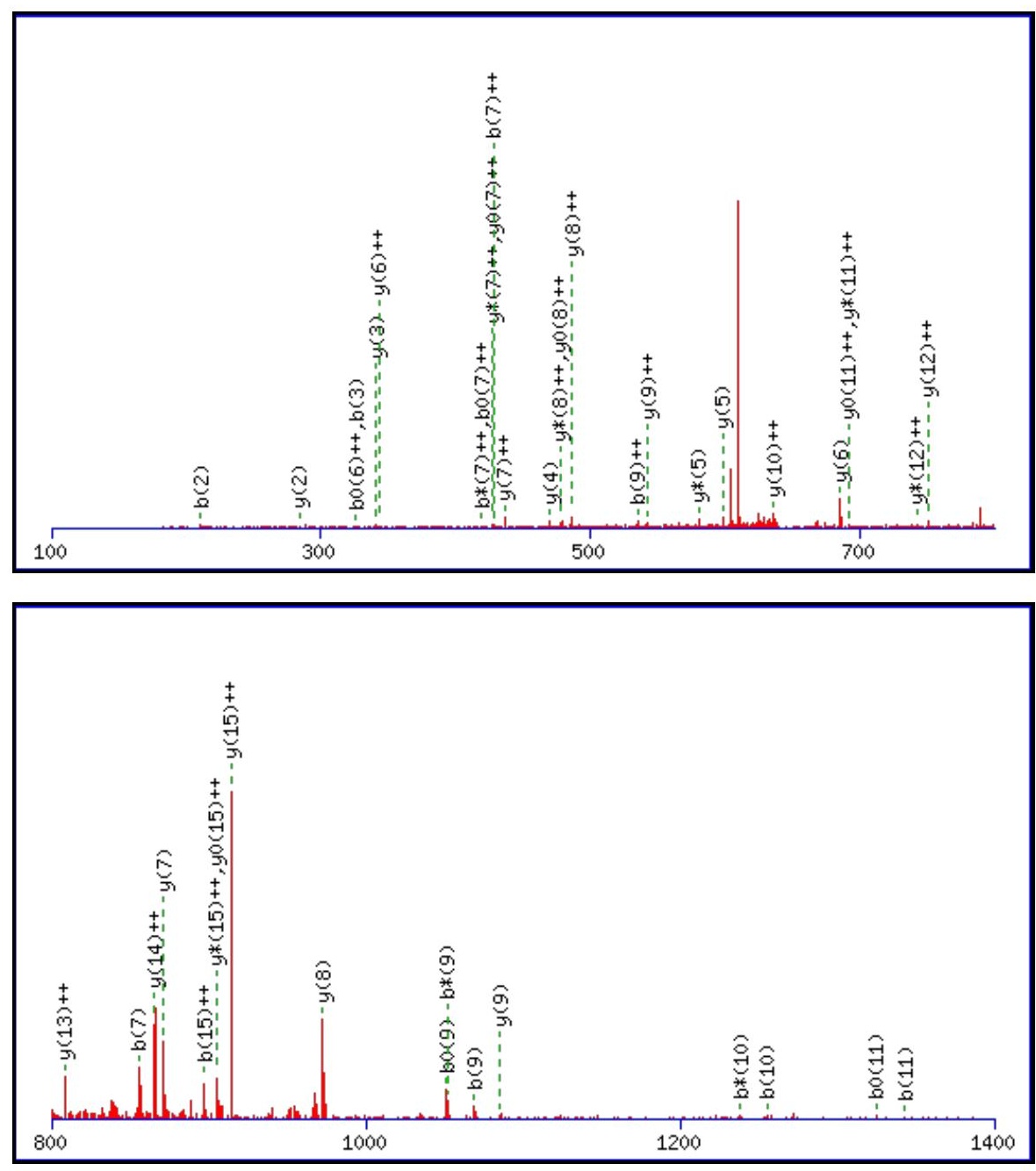


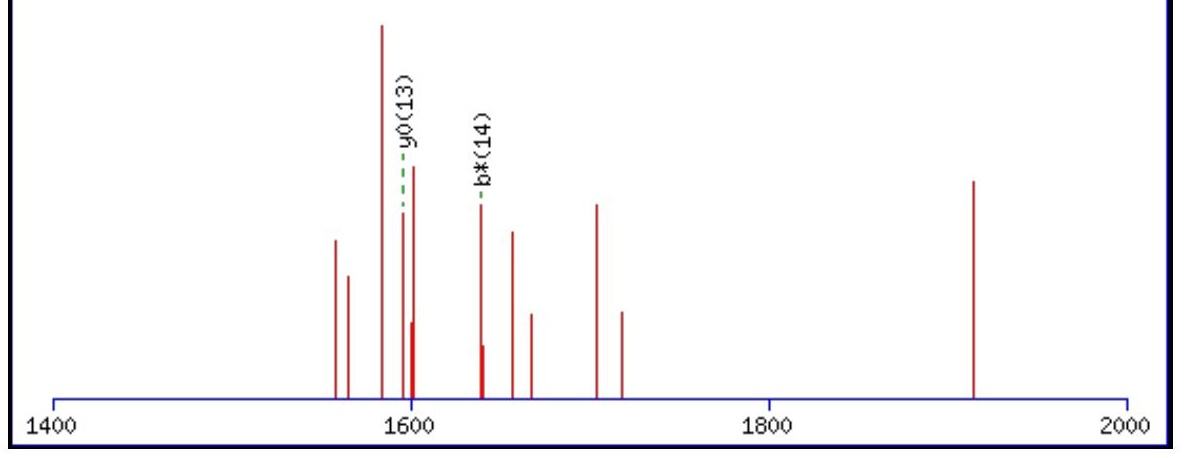

Monoisotopic mass of neutral peptide $\operatorname{Mr}($ calc): 1938.9224

Fixed modifications: Carbamidomethyl (C)

Variable modifications:

N3 : Deamidated_N (N)

Ions Score: 42 Expect: 0.016

Matches (Bold Red): 45/162 fragment ions using 87 most intense peaks

\begin{tabular}{|c|c|c|c|c|c|c|c|c|c|c|c|c|c|c|}
\hline \# & b & $\mathbf{b}^{++}$ & $\mathbf{b}^{*}$ & $\mathbf{b}^{*^{++}}$ & $\mathbf{b}^{\mathbf{0}}$ & $\mathbf{b}^{0++}$ & Seq. & $\mathbf{y}$ & $\mathbf{y}^{++}$ & $\mathrm{y}^{*}$ & $\mathrm{y}^{\mathrm{*}^{++}}$ & $\mathbf{y}^{\mathbf{0}}$ & $\mathbf{y}^{0++}$ & \# \\
\hline 1 & 114.0913 & 57.5493 & & & & & I & & & & & & & 16 \\
\hline 2 & 211.1441 & 106.0757 & & & & & $\mathbf{P}$ & 1826.8456 & 913.9265 & 1809.8191 & 905.4132 & 1808.8351 & 904.9212 & 15 \\
\hline 3 & 326.1710 & 163.5892 & 309.1445 & 155.0759 & & & $\mathbf{N}$ & 1729.7929 & 865.4001 & 1712.7663 & 856.8868 & 1711.7823 & 856.3948 & 14 \\
\hline 4 & 440.2140 & 220.6106 & 423.1874 & 212.0973 & & & $\mathbf{N}$ & 1614.7659 & 807.8866 & 1597.7394 & 799.3733 & 1596.7554 & 798.8813 & 13 \\
\hline 5 & 541.2616 & 271.1345 & 524.2351 & 262.6212 & 523.2511 & 262.1292 & $\mathbf{T}$ & 1500.7230 & 750.8651 & 1483.6965 & 742.3519 & 1482.7124 & 741.8599 & 12 \\
\hline 6 & 669.3202 & 335.1638 & 652.2937 & 326.6505 & 651.3097 & 326.1585 & $\mathbf{Q}$ & 1399.6753 & 700.3413 & 1382.6488 & 691.8280 & 1381.6648 & 691.3360 & 11 \\
\hline 7 & 855.3995 & 428.2034 & 838.3730 & 419.6901 & 837.3890 & 419.1981 & $\mathbf{W}$ & 1271.6167 & 636.3120 & 1254.5902 & 627.7987 & 1253.6062 & 627.3067 & 10 \\
\hline 8 & 968.4836 & 484.7454 & 951.4571 & 476.2322 & 950.4730 & 475.7402 & I & 1085.5374 & 543.2724 & 1068.5109 & 534.7591 & 1067.5269 & 534.2671 & 9 \\
\hline 9 & 1069.5313 & 535.2693 & 1052.5047 & 526.7560 & 1051.5207 & 526.2640 & $\mathbf{T}$ & 972.4534 & 486.7303 & 955.4268 & 478.2170 & 954.4428 & 477.7250 & 8 \\
\hline 10 & 1255.6106 & 628.3089 & 1238.5840 & 619.7957 & 1237.6000 & 619.3037 & W & 871.4057 & 436.2065 & 854.3791 & 427.6932 & 853.3951 & 427.2012 & 7 \\
\hline 11 & 1342.6426 & 671.8249 & 1325.6161 & 663.3117 & 1324.6321 & 662.8197 & $\mathrm{~S}$ & 685.3264 & 343.1668 & 668.2998 & 334.6536 & 667.3158 & 334.1615 & 6 \\
\hline 12 & 1470.7012 & 735.8542 & 1453.6746 & 727.3410 & 1452.6906 & 726.8490 & $\mathbf{Q}$ & 598.2944 & 299.6508 & 581.2678 & 291.1375 & 580.2838 & 290.6455 & 5 \\
\hline 13 & 1599.7438 & 800.3755 & 1582.7172 & 791.8623 & 1581.7332 & 791.3703 & $\mathbf{E}$ & 470.2358 & 235.6215 & 453.2092 & 227.1083 & 452.2252 & 226.6162 & 4 \\
\hline 14 & 1656.7653 & 828.8863 & 1639.7387 & 820.3730 & 1638.7547 & 819.8810 & G & 341.1932 & 171.1002 & 324.1666 & 162.5870 & & & 3 \\
\hline 15 & 1793.8242 & \begin{tabular}{|l|}
897.4157 \\
\end{tabular} & 1776.7976 & 888.9024 & 1775.8136 & 888.4104 & H & 284.1717 & 142.5895 & 267.1452 & 134.0762 & & & 2 \\
\hline \begin{tabular}{|l|l}
16 \\
\end{tabular} & & & & & & & $\mathbf{K}$ & 147.1128 & \begin{tabular}{|l|}
74.0600 \\
\end{tabular} & 130.0863 & 65.5468 & & & 1 \\
\hline
\end{tabular}

$$
\text { 奥 }
$$

璦

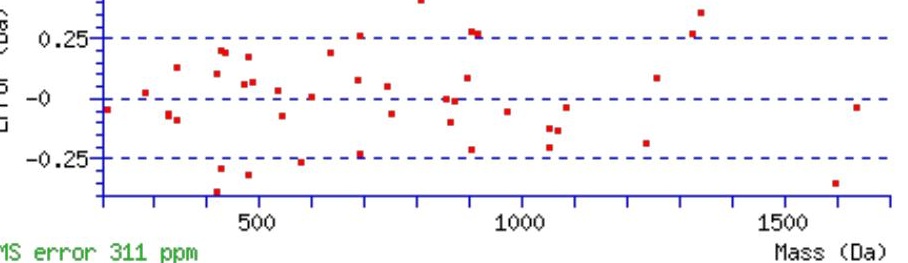

RMS error $311 \mathrm{ppm}$

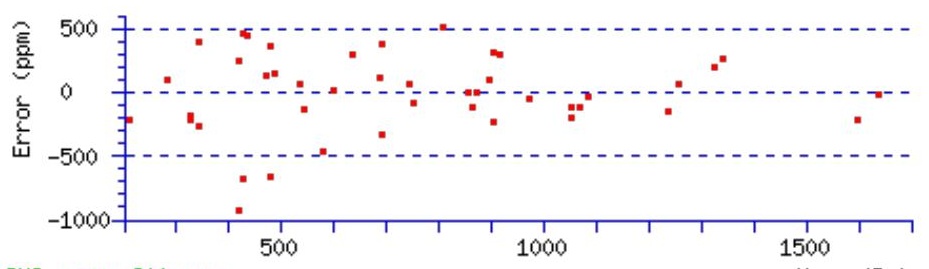

RMS error 311 ppm

All matches to this query

\begin{tabular}{|l|r|r|l|}
\hline Score & Mr(calc): & Delta & \multicolumn{1}{|c|}{ Sequence } \\
\hline 42.1 & 1938.9224 & 0.0039 & $\underline{\text { IPNNTQWITWSQEGHK }}$ \\
\hline 41.3 & 1938.9224 & 0.0039 & $\underline{\text { IPNNTQWITWSQEGHK }}$ \\
\hline 22.1 & 1937.9384 & 0.9879 & $\underline{\text { IPNNTQWITWSQEGHK }}$ \\
\hline 7.0 & 1938.9227 & 0.0036 & $\underline{\text { LYTPNSPEINEVIQK }}$ \\
\hline 3.4 & 1938.9227 & 0.0036 & $\underline{\text { ILYTPNSPEINEVIQK }}$ \\
\hline & & & \\
\hline
\end{tabular}




\begin{tabular}{|l||l|l|l|}
1.8 & 1938.9420 & -0.0157 & AEGLPRMNTSLMANVKK \\
\hline 1.6 & 1937.9248 & 1.0015 & ASLSAPTIQSLNLSWNR \\
\hline 1.6 & 1937.9248 & 1.0015 & ASLSAPTIQSLNLSWNR \\
\hline 1.6 & 1938.9088 & 0.0175 & ASLSAPTIQSLNLSWNR \\
\hline 1.6 & 1937.9248 & 1.0015 & $\underline{\text { ASLSAPTIQSLNLSWNR }}$ \\
\hline
\end{tabular}

Spectrum No: 798; Query: 1465; Rank: 1

\section{Peptide View}

MS/MS Fragmentation of ISIEMNGTLEDQLSHLK

Found in IPI00213463, Tax_Id=10116 Gene_Symbol=Actn4 Alpha-actinin-4

Match to Query 1465: 1927.953012 from(643.658280,3+)

Title: 091008RatKidney_NH4Format02_18.3823.3823.3.dta

Data file K:INewmanPaper|Piliangl3SubProteomes\Piliang3SP\mgf5ppm\ERLIC_3SubProteomes5ppm.mgf
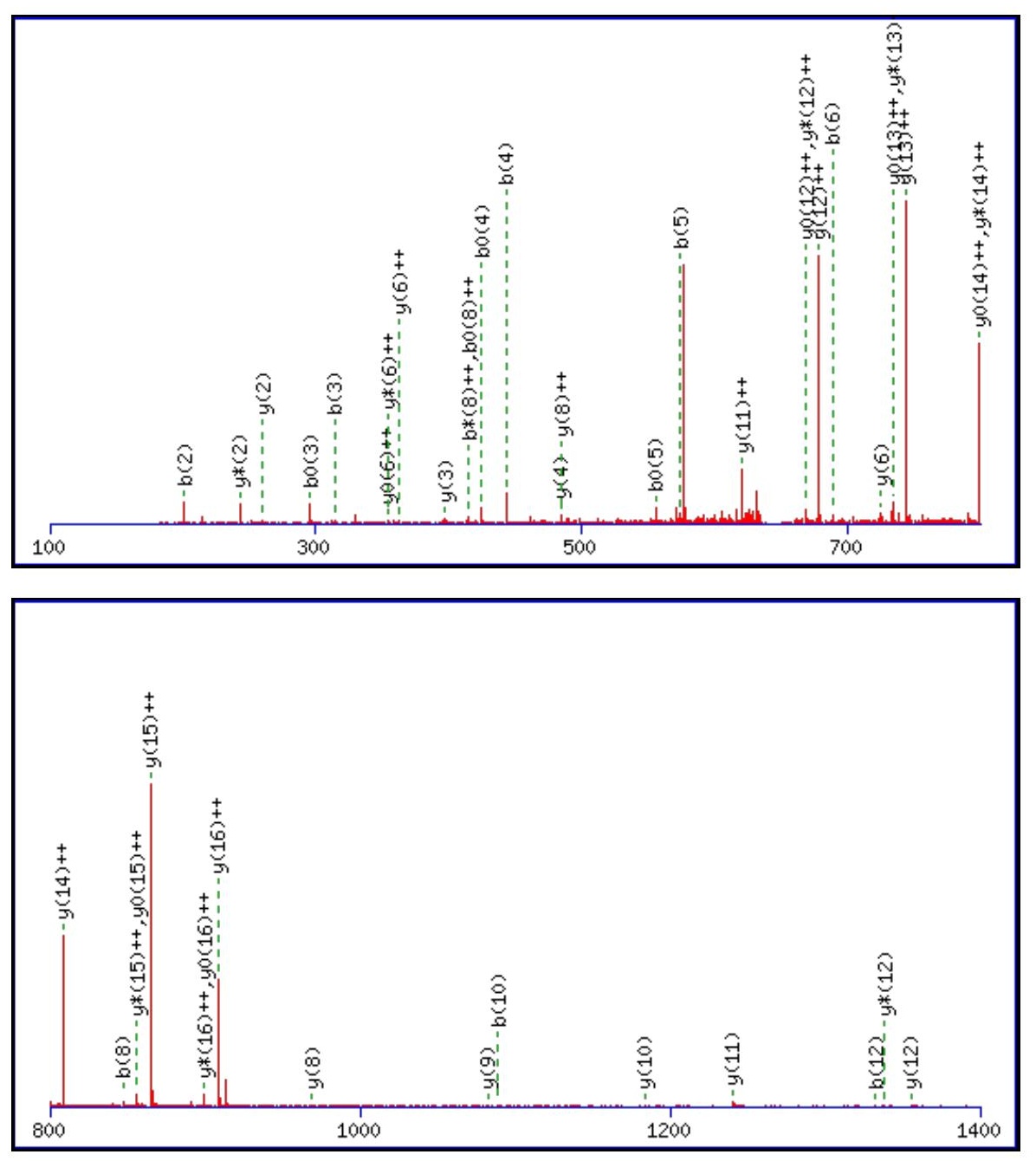


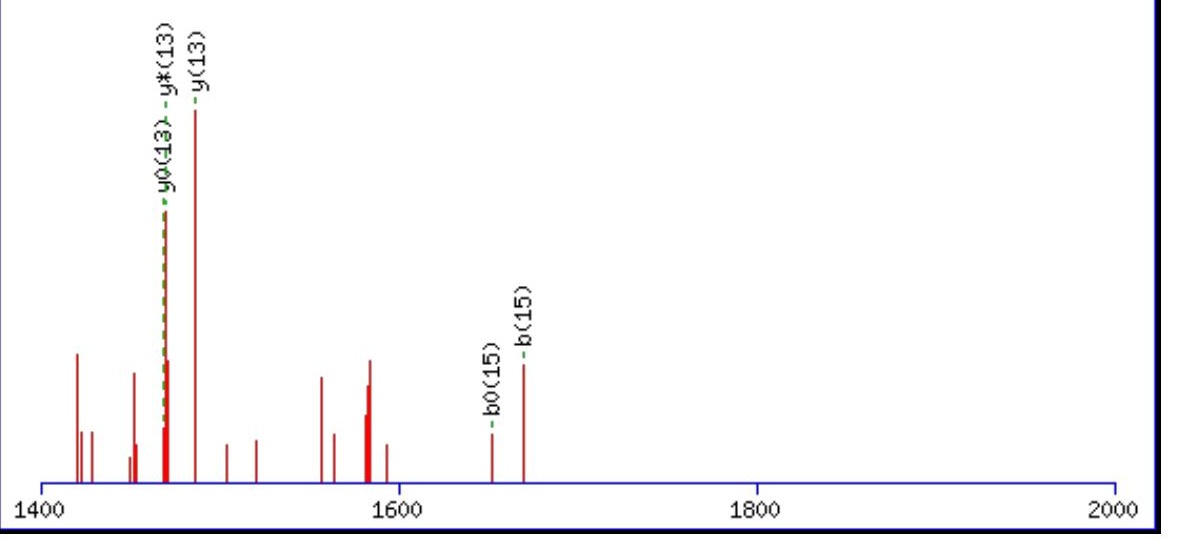

Monoisotopic mass of neutral peptide $\operatorname{Mr}($ calc): 1927.9561

Fixed modifications: Carbamidomethyl (C)

Variable modifications:

N6 : Deamidated_N (N)

Ions Score: 42 Expect: 0.012

Matches (Bold Red): 49/174 fragment ions using 106 most intense peaks

\begin{tabular}{|c|c|c|c|c|c|c|c|c|c|c|c|c|c|c|}
\hline \# & b & $\mathbf{b}^{++}$ & $\mathbf{b}^{*}$ & $\mathbf{b}^{*^{++}}$ & $\mathbf{b}^{\mathbf{0}}$ & $\mathbf{b}^{0++}$ & Seq. & $\mathbf{y}$ & $\mathbf{y}^{++}$ & $\mathbf{y}^{*}$ & $\mathbf{y}^{*^{++}}$ & $\mathbf{y}^{\mathbf{0}}$ & $\mathbf{y}^{0++}$ & \# \\
\hline 1 & 114.0913 & 57.5493 & & & & & I & & & & & & & 17 \\
\hline 2 & 201.1234 & 101.0653 & & & 183.1128 & 92.0600 & $\mathbf{S}$ & 1815.8793 & 908.4433 & 1798.8528 & 899.9300 & 1797.8687 & 899.4380 & 16 \\
\hline 3 & 314.2074 & 157.6074 & & & 296.1969 & 148.6021 & I & 1728.8473 & 864.9273 & 1711.8207 & 856.4140 & 1710.8367 & 855.9220 & 15 \\
\hline 4 & 443.2500 & 222.1287 & & & 425.2395 & 213.1234 & $\mathbf{E}$ & 1615.7632 & 808.3852 & 1598.7367 & 799.8720 & 1597.7526 & 799.3800 & 14 \\
\hline 5 & 574.2905 & 287.6489 & & & 556.2799 & 278.6436 & $\mathbf{M}$ & 1486.7206 & 743.8639 & 1469.6941 & 735.3507 & 1468.7100 & 734.8587 & 13 \\
\hline 6 & 689.3174 & 345.1624 & 672.2909 & 336.6491 & 671.3069 & 336.1571 & $\mathbf{N}$ & 1355.6801 & 678.3437 & 1338.6536 & 669.8304 & 1337.6696 & 669.3384 & 12 \\
\hline 7 & 746.3389 & 373.6731 & 729.3124 & 365.1598 & 728.3283 & 364.6678 & G & 1240.6532 & 620.8302 & 1223.6266 & 612.3170 & 1222.6426 & 611.8250 & 11 \\
\hline 8 & 847.3866 & 424.1969 & 830.3600 & 415.6837 & 829.3760 & 415.1917 & $\mathbf{T}$ & 1183.6317 & 592.3195 & 1166.6052 & 583.8062 & 1165.6212 & 583.3142 & 10 \\
\hline 9 & 960.4707 & 480.7390 & 943.4441 & 472.2257 & 942.4601 & 471.7337 & $\mathbf{L}$ & 1082.5841 & 541.7957 & 1065.5575 & 533.2824 & 1064.5735 & 532.7904 & 9 \\
\hline 10 & 1089.5132 & 545.2603 & 1072.4867 & 536.7470 & 1071.5027 & 536.2550 & $\mathbf{E}$ & 969.5000 & 485.2536 & 952.4734 & 476.7404 & 951.4894 & 476.2483 & 8 \\
\hline 11 & 1204.5402 & 602.7737 & 1187.5136 & 594.2605 & 1186.5296 & 593.7685 & D & 840.4574 & 420.7323 & 823.4308 & 412.2191 & 822.4468 & 411.7271 & 7 \\
\hline 12 & 1332.5988 & 666.8030 & 1315.5722 & 658.2897 & 1314.5882 & 657.7977 & $\mathbf{Q}$ & 725.4304 & 363.2189 & 708.4039 & 354.7056 & 707.4199 & 354.2136 & 6 \\
\hline 13 & 1445.6828 & 723.3451 & 1428.6563 & 714.8318 & 1427.6723 & 714.3398 & $\mathbf{L}$ & 597.3719 & 299.1896 & 580.3453 & 290.6763 & 579.3613 & 290.1843 & 5 \\
\hline 14 & 1532.7149 & 766.8611 & 1515.6883 & 758.3478 & 1514.7043 & 757.8558 & S & 484.2878 & 242.6475 & 467.2613 & 234.1343 & 466.2772 & 233.6423 & 4 \\
\hline 15 & 1669.7738 & 835.3905 & 1652.7472 & 826.8772 & 1651.7632 & 826.3852 & $\mathbf{H}$ & 397.2558 & 199.1315 & 380.2292 & 190.6183 & & & 3 \\
\hline 16 & 1782.8578 & 891.9326 & 1765.8313 & 883.4193 & 1764.8473 & 882.9273 & $\mathbf{L}$ & 260.1969 & 130.6021 & 243.1703 & 122.0888 & & & 2 \\
\hline 17 & & & & & & & $\mathbf{K}$ & 147.1128 & 74.0600 & 130.0863 & 65.5468 & & & 1 \\
\hline
\end{tabular}
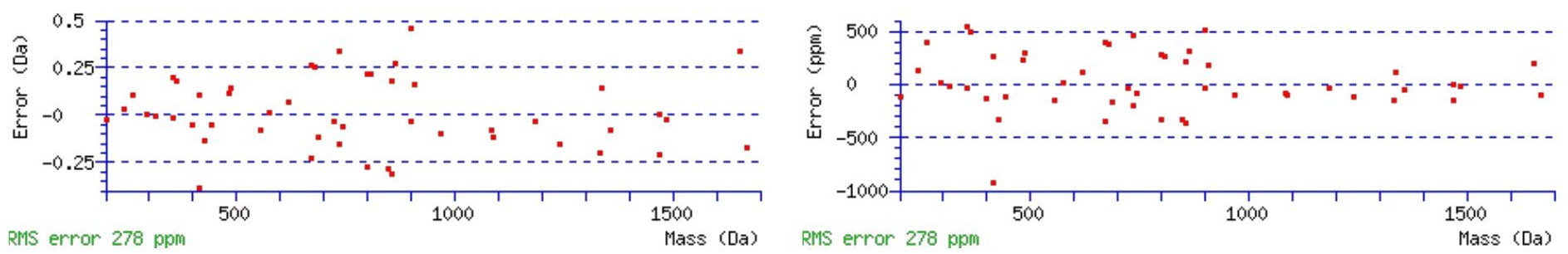

\section{All matches to this query}

\begin{tabular}{|l|l|l|l|}
\hline Score & Mr(calc): & Delta & \multicolumn{1}{c|}{ Sequence } \\
\hline 42.0 & 1927.9561 & -0.0030 & ISIEMNGTLEDQLSHLK \\
\hline 14.3 & 1927.9656 & -0.0126 & TDGLRKDFDLDIISLK \\
\hline 12.2 & 1927.9680 & -0.0149 & LSIEFHSGYSYNALTVK \\
\hline 11.4 & 1927.9478 & 0.0052 & TVIEMFKSITHSTVGAK \\
\hline & & & \\
\hline
\end{tabular}




\begin{tabular}{|l|l|l|l|}
10.9 & 1927.9478 & 0.0052 & TVIEMFKSITHSTVGAK \\
\hline 10.9 & 1927.9478 & 0.0052 & TVIEMFKSITHSTVGAK \\
\hline 10.9 & 1927.9478 & 0.0052 & TVIEMFKSITHSTVGAK \\
\hline 7.2 & 1925.9622 & 1.9908 & AEEAVVSIQDITVNYFK \\
\hline 6.5 & 1927.9599 & -0.0069 & QAEEQVEAARKDLDQAK \\
\hline 6.2 & 1927.9462 & 0.0068 & ISELSGCNPDPQIVPFR \\
\hline
\end{tabular}

Spectrum No: 799; Query: 2850; Rank: 1

\section{Peptide View}

MS/MS Fragmentation of ESYSIPGFNGSEDCAALEQLLEGYDQQDQDQVSEVCNSPLFK

Found in IPI00367524, Tax_Id=10116 Gene_Symbol=LOC682827 35 kDa protein

Match to Query 2850: 4769.101002 from(1590.707610,3+)

Title: 091008RatKidney_NoSalt_20.6451.6451.3.dta

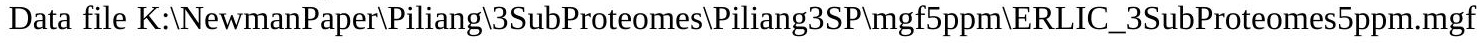
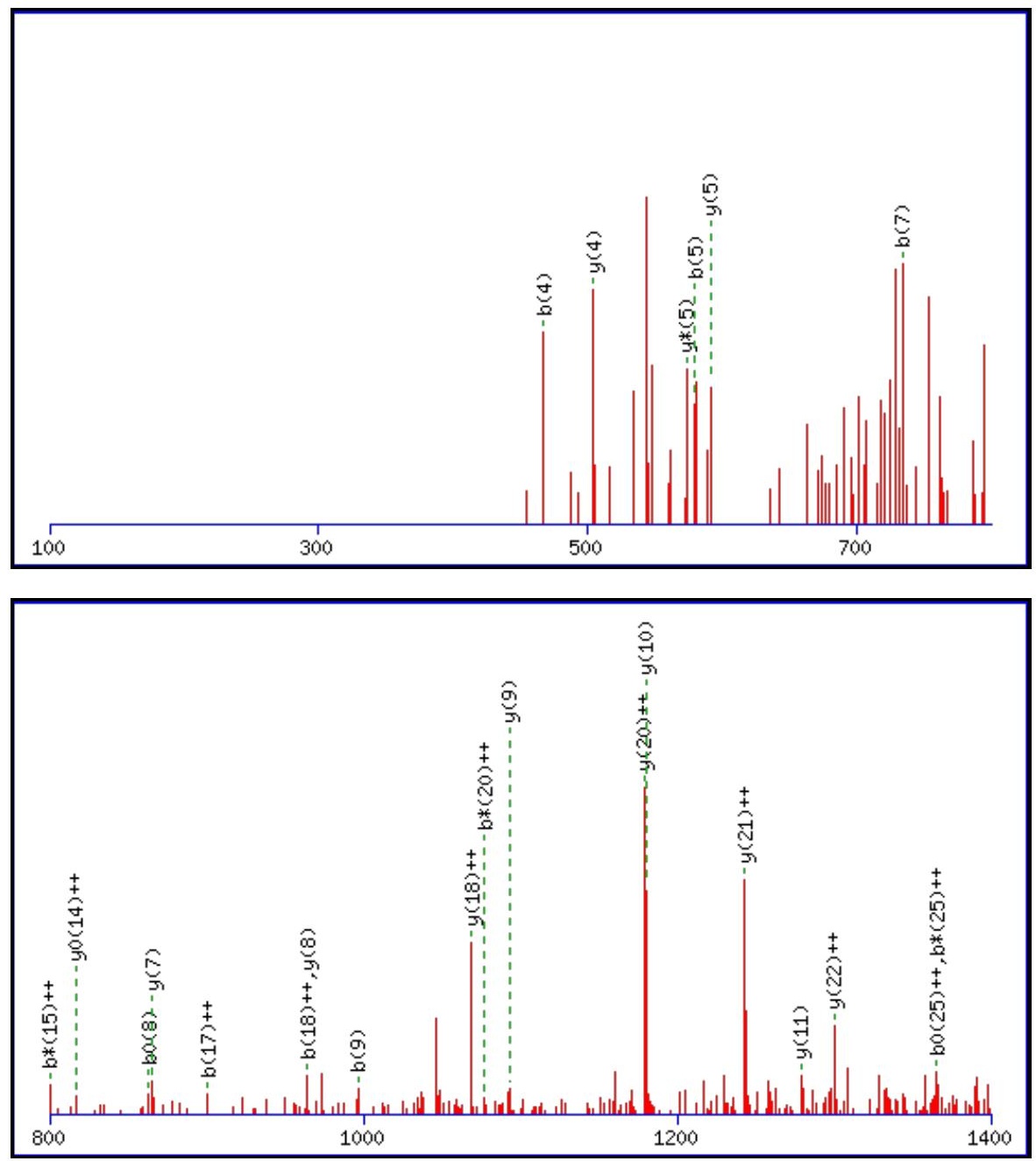


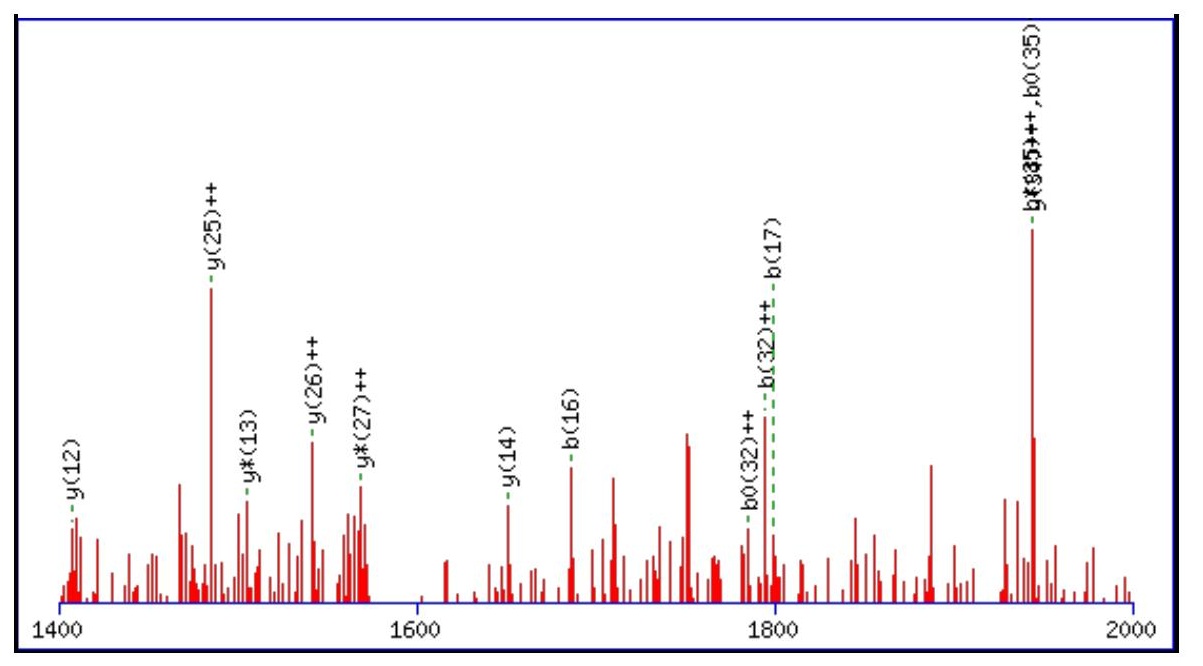

Monoisotopic mass of neutral peptide $\operatorname{Mr}($ calc): 4767.0752

Fixed modifications: Carbamidomethyl (C)

Variable modifications:

N9 : Deamidated_N (N)

Ions Score: 42 Expect: $\odot .035$

Matches (Bold Red): 37/468 fragment ions using 65 most intense peaks

\begin{tabular}{|c|c|c|c|c|c|c|c|c|c|c|c|c|c|c|}
\hline \# & b & $\mathbf{b}^{++}$ & $\mathbf{b}^{*}$ & $\mathbf{b}^{*^{++}}$ & $\mathbf{b}^{\mathbf{0}}$ & $\mathbf{b}^{0++}$ & Seq. & $\mathbf{y}$ & $\mathbf{y}^{++}$ & $\mathrm{y}^{*}$ & $\mathrm{y}^{\mathrm{*}^{++}}$ & $\mathbf{y}^{0}$ & $y^{0++}$ & \# \\
\hline 1 & 130.0499 & 65.5286 & & & 112.0393 & 56.5233 & E & & & & & & & 42 \\
\hline 2 & 217.0819 & 109.0446 & & & 199.0713 & 100.0393 & $S$ & 4639.0399 & 2320.0236 & 4622.0134 & 2311.5103 & 4621.0294 & 2311.0183 & 41 \\
\hline 3 & 380.1452 & 190.5763 & & & 362.1347 & 181.5710 & $\mathbf{Y}$ & 4552.0079 & 2276.5076 & 4534.9813 & 2267.9943 & 4533.9973 & 2267.5023 & 40 \\
\hline 4 & 467.1773 & 234.0923 & & & 449.1667 & 225.0870 & $S$ & 4388.9446 & 2194.9759 & 4371.9180 & 2186.4626 & 4370.9340 & 2185.9706 & 39 \\
\hline 5 & 580.2613 & 290.6343 & & & 562.2508 & 281.6290 & $\mathbf{I}$ & 4301.9125 & 2151.4599 & 4284.8860 & 2142.9466 & 4283.9020 & 2142.4546 & 38 \\
\hline 6 & 677.3141 & 339.1607 & & & 659.3035 & 330.1554 & $\mathbf{P}$ & 4188.8285 & 2094.9179 & 4171.8019 & 2086.4046 & 4170.8179 & 2085.9126 & 37 \\
\hline 7 & 734.3355 & 367.6714 & & & 716.3250 & 358.6661 & G & 4091.7757 & 2046.3915 & 4074.7492 & 2037.8782 & 4073.7651 & 2037.3862 & 36 \\
\hline 8 & 881.4040 & 441.2056 & & & 863.3934 & 432.2003 & $\mathbf{F}$ & 4034.7542 & 2017.8808 & 4017.7277 & 2009.3675 & 4016.7437 & 2008.8755 & 35 \\
\hline 9 & 996.4309 & 498.7191 & 979.4043 & 490.2058 & 978.4203 & 489.7138 & $\mathbf{N}$ & 3887.6858 & 1944.3466 & 3870.6593 & 1935.8333 & 3869.6753 & 1935.3413 & 34 \\
\hline 10 & 1053.4524 & 527.2298 & 1036.4258 & 518.7165 & 1035.4418 & 518.2245 & G & 3772.6589 & 1886.8331 & 3755.6323 & 1878.3198 & 3754.6483 & 1877.8278 & 33 \\
\hline 11 & 1140.4844 & 570.7458 & 1123.4578 & 562.2326 & 1122.4738 & 561.7405 & $S$ & 3715.6374 & 1858.3224 & 3698.6109 & 1849.8091 & 3697.6269 & 1849.3171 & 32 \\
\hline 12 & 1269.5270 & 635.2671 & 1252.5004 & 626.7539 & 1251.5164 & 626.2618 & $\mathbf{E}$ & 3628.6054 & 1814.8063 & 3611.5789 & 1806.2931 & 3610.5948 & 1805.8011 & 31 \\
\hline 13 & 384.5539 & 692.7806 & 1367.5274 & 684.2673 & 1366.5434 & 683.7753 & D & 3499.5628 & 1750.2850 & 3482.5363 & 1741.7718 & 3481.5522 & 1741.2798 & 30 \\
\hline 14 & 1544.5846 & 772.7959 & 1527.5580 & 764.2827 & 1526.5740 & 763.7906 & C & 3384.5359 & 1692.7716 & 3367.5093 & 1684.2583 & 3366.5253 & 1683.7663 & 29 \\
\hline 15 & 1615.6217 & 808.3145 & 1598.5951 & 799.8012 & 1597.6111 & 799.3092 & A & 3224.5052 & 1612.7562 & 3207.4787 & 1604.2430 & 3206.4947 & 1603.7510 & 28 \\
\hline 16 & 1686 & & & & 1668 & & $\mathbf{A}$ & 3153.4681 & & 3136. & & & 1568 & 27 \\
\hline 17 & 1799.7429 & 900.3751 & 1782.7163 & 891.8618 & 1781.7323 & 891.3698 & $\mathbf{L}$ & 3082.4310 & 1541.7191 & 3065.4044 & 1533.2059 & 3064.4204 & 1532.7138 & 26 \\
\hline 18 & 1928.7855 & 964.8964 & 1911.7589 & 956.3831 & 1910.7749 & 955.8911 & $\mathbf{E}$ & 2969.3469 & 1485.1771 & 2952.3204 & 1476.6638 & 2951.3364 & 1476.1718 & 25 \\
\hline 19 & 2056. & 28.9257 & 2039.8175 & 1020.41 & 2038.8335 & 1019.9204 & $\mathbf{Q}$ & 2840.3043 & 1420.6558 & 2823.2778 & 1412.1425 & 2822.2938 & 1411.6505 & \\
\hline 20 & 2169.9281 & 1085.4677 & 2152.9016 & \begin{tabular}{|l|}
1076.9544 \\
\end{tabular} & 2151.9175 & 1076.4624 & $\mathbf{L}$ & 2712.2458 & 1356.6265 & 2695.2192 & 1348.1132 & 2694.2352 & 1347.6212 & 23 \\
\hline 21 & 2283.0122 & 1142.0097 & 2265.9856 & 1133.4964 & 2265.0016 & 1133.0044 & $\mathbf{L}$ & 2599.1617 & 1300.0845 & 2582.1351 & 1291.5712 & 2581.1511 & 1291.0792 & 22 \\
\hline 22 & 2412.0548 & 1206.5310 & 2395.0282 & 1198.0177 & 2394.0442 & 1197.5257 & $\mathbf{E}$ & 2486.0776 & 1243.5425 & 2469.0511 & 1235.0292 & 2468.0671 & 1234.5372 & 21 \\
\hline 23 & 2469.0762 & 1235.0417 & 2452.0497 & 1226.5285 & 2451.0657 & 1226.0365 & G & 2357.0350 & \begin{tabular}{|l|}
1179.0212 \\
\end{tabular} & 2340.0085 & 1170.5079 & 2339.0245 & 1170.0159 & 20 \\
\hline 24 & 2632.1396 & 1316.5734 & 2615.1130 & 1308.0601 & 2614.1290 & 1307.5681 & $\mathbf{Y}$ & 2300.0136 & 1150.5104 & 2282.9870 & 1141.9971 & 2282.0030 & 1141.5051 & 19 \\
\hline 25 & 2747.1665 & 1374.0869 & 2730.1399 & 1365.5736 & 2729.1559 & 1365.0816 & D & 2136.9502 & \begin{tabular}{|l|}
1068.9788 \\
\end{tabular} & 2119.9237 & 1060.4655 & 2118.9397 & 1059.9735 & 18 \\
\hline 26 & 2875.2251 & 1438.1162 & 2858.1985 & 1429.6029 & 2857.2145 & 1429.1109 & $\bar{Q}$ & 2021.9233 & 1011.4653 & 2004.8967 & 1002.9520 & 2003.9127 & 1002.4600 & 17 \\
\hline 27 & 3003.2836 & 1502.1455 & 2986.2571 & 1493.6322 & 2985.2731 & 1493.1402 & $\mathbf{Q}$ & 1893.8647 & 947.4360 & 1876.8382 & 938.9227 & 1875.8542 & 938.4307 & 16 \\
\hline 28 & 3118.3106 & 1559.6589 & 3101.2840 & \begin{tabular}{|l|}
1551.1457 \\
\end{tabular} & 3100.3000 & 1550.6537 & D & 1765.8061 & 883.4067 & 1748.7796 & 874.8934 & 1747.7956 & 874.4014 & 15 \\
\hline 29 & 3246.3692 & 1623.6882 & 3229.3426 & 1615.1749 & 3228.3586 & 1614.6829 & $\mathbf{Q}$ & 1650.7792 & 825.8932 & 1633.7526 & 817.3800 & 1632.7686 & 816.8880 & \\
\hline 30 & 3361.3961 & \begin{tabular}{|l|}
1681.2017 \\
\end{tabular} & 3344.3696 & 1672.6884 & 3343.3855 & 1672.1964 & D & 1522.7206 & 761.8639 & \begin{tabular}{|l|}
1505.6941 \\
\end{tabular} & 753.3507 & 1504.7101 & 752.8587 & 13 \\
\hline 31 & 3489.4547 & 1745.2310 & 3472.4281 & 1736.7177 & 3471.4441 & 1736.2257 & $\mathbf{Q}$ & 1407.6937 & 704.3505 & 1390.6671 & 695.8372 & 1389.6831 & 695.3452 & 12 \\
\hline 32 & |3588.5231 & 1794.7652 & |3571.4966 & 1786.2519 & $|3570.5125|$ & 1785.7599 & $\mathbf{V}$ & 1279.6351 & 640.3212 & $\mid 1262.6086$ & 631.8079 & $\mid 1261.6245$ & 631.3159 & 11 \\
\hline
\end{tabular}




\begin{tabular}{|c|c|c|c|c|c|c|c|c|c|c|c|c|c|c|}
\hline 33 & 3675.5551 & 1838.2812 & 3658.5286 & 1829.7679 & 3657.5446 & 1829.2759 & $\mathrm{~S}$ & 1180.5667 & 590.7870 & 1163.5401 & 582.2737 & 1162.5561 & 581.7817 & 10 \\
\hline 34 & 3804.5977 & 1902.8025 & 3787.5712 & 1894.2892 & 3786.5872 & 1893.7972 & $\mathbf{E}$ & 1093.5347 & 547.2710 & 1076.5081 & 538.7577 & 1075.5241 & 538.2657 & 9 \\
\hline 35 & 3903.6661 & 1952.3367 & 3886.6396 & 1943.8234 & 3885.6556 & 1943.3314 & V & 964.4921 & 482.7497 & 947.4655 & 474.2364 & 946.4815 & 473.7444 & 8 \\
\hline 36 & 4063.6968 & 2032.3520 & 4046.6702 & 2023.8388 & 4045.6862 & 2023.3467 & C & 865.4237 & 433.2155 & 848.3971 & 424.7022 & 847.4131 & 424.2102 & 7 \\
\hline 37 & 4177.7397 & 2089.3735 & 4160.7132 & 2080.8602 & 4159.7292 & 2080.3682 & $\mathbf{N}$ & 705.3930 & 353.2001 & 688.3665 & 344.6869 & 687.3824 & 344.1949 & 6 \\
\hline 38 & 4264.7717 & 2132.8895 & 4247.7452 & 2124.3762 & 4246.7612 & 2123.8842 & $\mathrm{~S}$ & 591.3501 & 296.1787 & 574.3235 & 287.6654 & 573.3395 & 287.1734 & 5 \\
\hline 39 & 4361.8245 & 2181.4159 & 4344.7980 & 2172.9026 & 4343.8139 & 2172.4106 & $\mathbf{P}$ & 504.3180 & 252.6627 & 487.2915 & 244.1494 & & & 4 \\
\hline 40 & 4474.9086 & 2237.9579 & 4457.8820 & 2229.4446 & 4456.8980 & 2228.9526 & $\mathbf{L}$ & 407.2653 & 204.1363 & 390.2387 & 195.6230 & & & 3 \\
\hline 41 & 4621.9770 & 2311.4921 & 4604.9504 & 2302.9789 & 4603.9664 & 2302.4868 & $\mathbf{F}$ & 294.1812 & 147.5942 & 277.1547 & 139.0810 & & & 2 \\
\hline 42 & & & & & & & K & 147.1128 & 74.0600 & 130.0863 & 65.5468 & & & 1 \\
\hline
\end{tabular}
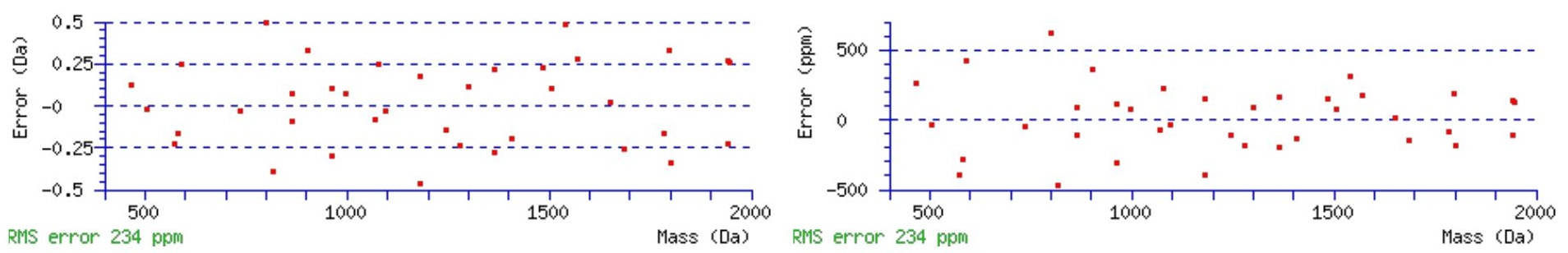

\section{All matches to this query}

\begin{tabular}{|l|c|c|l|}
\hline Score & Mr(calc): & Delta & \multicolumn{1}{c|}{ Sequence } \\
\hline 42.0 & 4767.0752 & 2.0258 & ESYSIPGFNGSEDCAALEQLLEGYDQQDQDQVSEVCNSPLFK \\
\hline 30.1 & 4768.0592 & 1.0418 & ESYSIPGFNGSEDCAALEQLLEGYDQQDQDQVSEVCNSPLFK \\
\hline 20.3 & 4767.0752 & 2.0258 & ESYSIPGFNGSEDCAALEQLLEGYDQQDQDQVSEVCNSPLFK \\
\hline 8.1 & 4769.0856 & 0.0154 & YNEWLQDGSQLSSNPLMTPDILGSSGAAVHTCVKATFHAFNK \\
\hline 7.8 & 4768.1016 & 0.9994 & YNEWLQDGSQLSSNPLMTPDILGSSGAAVHTCVKATFHAFNK \\
\hline 7.0 & 4768.1225 & 0.9786 & NTNKMENSGTCVSEMIKPCSVNLIASTSSDIQNSVDGRVTVDK \\
\hline 7.0 & 4769.1065 & -0.0055 & NTNKMENSGTCVSEMIKPCSVNLIASTSSDIQNSVDGRVTVDK \\
\hline 6.7 & 4767.0678 & 2.0332 & MEPNMDANSITITVEGMTCISCVRTIEQQIGKVNGVHHIK \\
\hline 6.7 & 4769.0911 & 0.0099 & TYSFLNLLFLCYPFGMYIPFLQLNYDLRKTNLFSHV \\
\hline 5.4 & 4769.0856 & 0.0154 & YNEWLQDGSQLSSNPLMTPDILGSSGAAVHTCVKATFHAFNK \\
\hline
\end{tabular}

Spectrum No: 800; Query: 1091; Rank: 1

\section{Peptide View}

MS/MS Fragmentation of VPGNLTSVLLNNLLPR

Found in IPI00358780, Tax_Id=10116 Gene_Symbol=Tek $119 \mathrm{kDa}$ protein

Match to Query 1091: 1719.993432 from(574.338420,3+)

Title: 091008RatKidney_NH4Format01_26.4266.4266.3.dta

Data file K:INewmanPaper|Piliangl3SubProteomes\Piliang3SP\mgf5ppm\ERLIC_3SubProteomes5ppm.mgf 

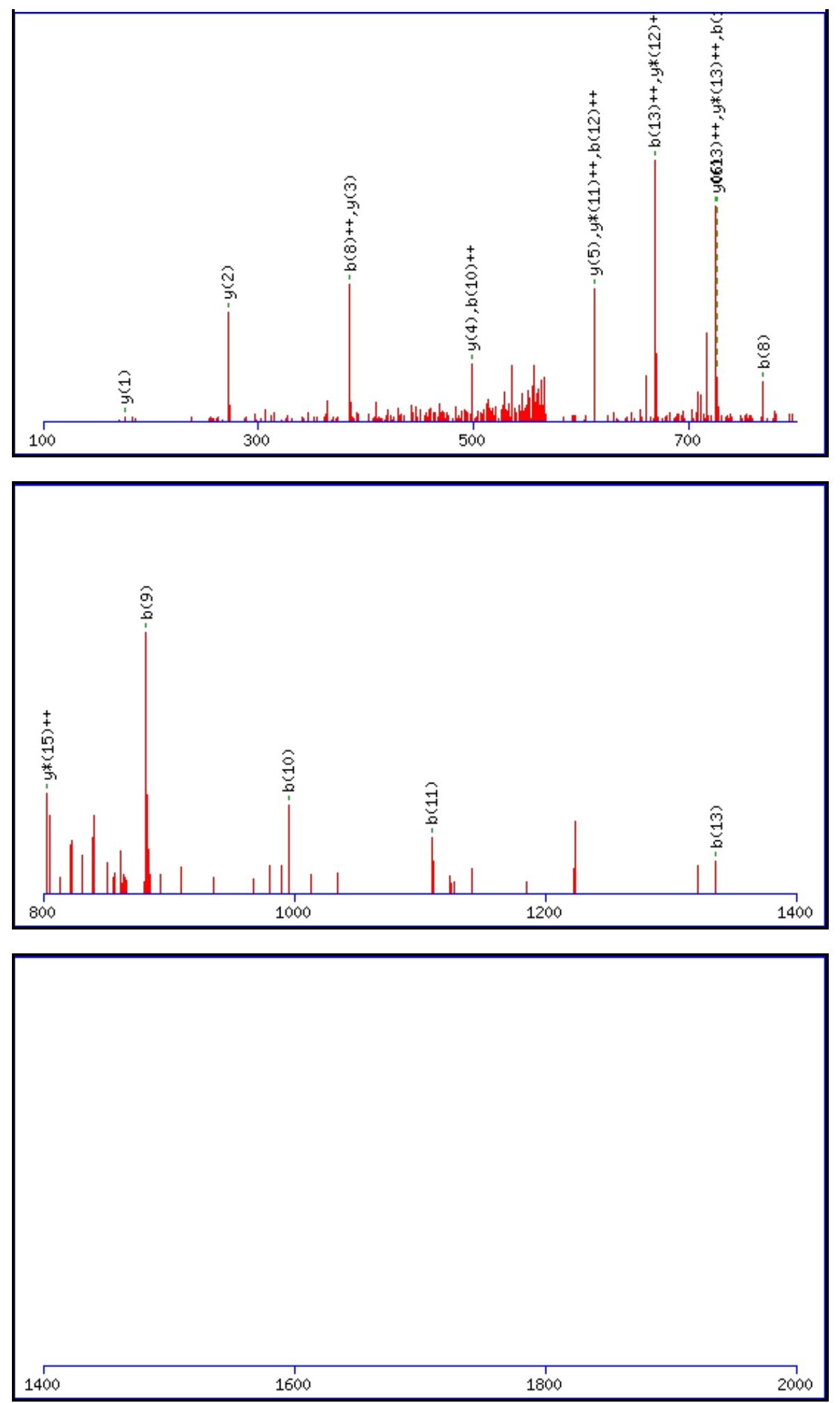

Monoisotopic mass of neutral peptide $\operatorname{Mr}($ calc): 1719.9883

Fixed modifications: Carbamidomethyl (C)

Variable modifications:

N4 : Deamidated $\mathrm{N}(\mathrm{N})$

Ions Score: 42 Expect: 0.0014

Matches (Bold Red): 21/146 fragment ions using 17 most intense peaks

\begin{tabular}{|r|c|c|c|c|c|c|c|c|c|c|c|c|c|c|}
\hline$\#$ & $\mathbf{b}$ & $\mathbf{b}^{++}$ & $\mathbf{b}^{*}$ & $\mathbf{b}^{\boldsymbol{*}^{++}}$ & $\mathbf{b}^{\mathbf{0}}$ & $\mathbf{b}^{\mathbf{0 + +}}$ & Seq. & $\mathbf{y}$ & $\mathbf{y}^{++}$ & $\mathbf{y}^{\mathbf{*}}$ & $\mathbf{y}^{\boldsymbol{*}^{++}}$ & $\mathbf{y}^{\mathbf{0}}$ & $\mathbf{y}^{\mathbf{0 + +}}$ & $\#$ \\
\hline $\mathbf{1}$ & 100.0757 & 50.5415 & & & & & $\mathbf{V}$ & & & & & & & $\mathbf{1 6}$ \\
\hline $\mathbf{2}$ & 197.1285 & 99.0679 & & & & & $\mathbf{P}$ & 1621.9272 & 811.4672 & 1604.9006 & $\mathbf{8 0 2 . 9 5 4 0}$ & 1603.9166 & 802.4619 & $\mathbf{1 5}$ \\
\hline 3 & 254.1499 & 127.5786 & & & & & $\mathbf{G}$ & 1524.8744 & 762.9408 & 1507.8479 & 754.4276 & 1506.8638 & 753.9356 & $\mathbf{1 4}$ \\
\hline $\mathbf{4}$ & 369.1769 & 185.0921 & 352.1503 & 176.5788 & & & N & 1467.8529 & 734.4301 & 1450.8264 & 725.9168 & 1449.8424 & $\mathbf{7 2 5 . 4 2 4 8}$ & $\mathbf{1 3}$ \\
\hline $\mathbf{5}$ & 482.2609 & 241.6341 & 465.2344 & 233.1208 & & & $\mathbf{L}$ & 1352.8260 & 676.9166 & 1335.7995 & $\mathbf{6 6 8 . 4 0 3 4}$ & 1334.8154 & 667.9114 & $\mathbf{1 2}$ \\
\hline
\end{tabular}




\begin{tabular}{|c|c|c|c|c|c|c|c|c|c|c|c|c|c|c|}
\hline 6 & 583.3086 & |292.1579 & 566.2820 & |283.6447 & 565.2980 & |283.1527 & $\mathbf{T}$ & |1239.7419 & |620.3746 & |1222.7154 & 611.8613 & |1221.7314 & |611.3693 & |11 \\
\hline 7 & 670.3406 & 335.6740 & 653.3141 & |327.1607 & 652.3301 & 326.6687 & $\mathbf{S}$ & |1138.6943 & 569.8508 & 1121.6677 & 561.3375 & 1120.6837 & 560.8455 & 10 \\
\hline 8 & 769.4090 & |385.2082 & 752.3825 & 376.6949 & 751.3985 & 376.2029 & V & 1051.6622 & 526.3348 & 1034.6357 & 517.8215 & & & 9 \\
\hline 9 & 882.4931 & 441.7502 & 865.4666 & 433.2369 & 864.4825 & 432.7449 & $\mathbf{L}$ & 952.5938 & 476.8006 & 935.5673 & 468.2873 & & & 8 \\
\hline 10 & 995.5772 & 498.2922 & 978.5506 & 489.7789 & 977.5666 & 489.2869 & $\mathbf{L}$ & 839.5098 & 420.2585 & 822.4832 & 411.7452 & & & 7 \\
\hline 11 & 1109.6201 & 555.3137 & 1092.5935 & 546.8004 & 1091.6095 & 546.3084 & $\mathbf{N}$ & 726.4257 & 363.7165 & 709.3991 & 355.2032 & & & 6 \\
\hline 12 & 1223.6630 & 612.3351 & 1206.6365 & 603.8219 & 1205.6525 & 603.3299 & $\mathbf{N}$ & 612.3828 & 306.6950 & 595.3562 & 298.1817 & & & 5 \\
\hline 13 & 1336.7471 & 668.8772 & 1319.7205 & 660.3639 & 1318.7365 & 659.8719 & L & 498.3398 & 249.6736 & 481.3133 & 241.1603 & & & 4 \\
\hline 14 & 1449.8311 & 725.4192 & 1432.8046 & 716.9059 & 1431.8206 & 716.4139 & L & 385.2558 & |193.1315 & 368.2292 & 184.6183 & & & 3 \\
\hline 15 & 1546.8839 & 773.9456 & 1529.8574 & 765.4323 & 1528.8733 & 764.9403 & $\mathbf{P}$ & 272.1717 & 136.5895 & 255.1452 & 128.0762 & & & 2 \\
\hline 16 & & & & & & & $\mathbf{R}$ & 175.1190 & 88.0631 & 158.0924 & 79.5498 & & & 1 \\
\hline
\end{tabular}
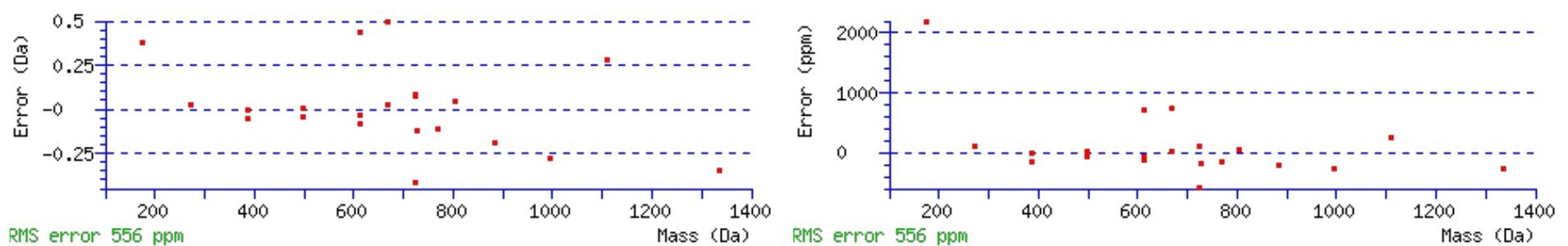

\section{All matches to this query}

\begin{tabular}{|l|l|l|l|}
\hline Score & Mr(calc): & Delta & \multicolumn{1}{|c|}{ Sequence } \\
\hline 42.0 & 1719.9883 & 0.0051 & VPGNLTSVLLNNLLPR \\
\hline 29.2 & 1719.9883 & 0.0051 & VPGNLTSVLLNNLLPR \\
\hline 26.1 & 1719.9883 & 0.0051 & VPGNLTSVLLNNLLPR \\
\hline 16.7 & 1719.0043 & 0.9891 & VPGNLTSVLLNNLLPR \\
\hline 11.3 & 1719.9856 & 0.0078 & AVTLHSGRSRLSLPAR \\
\hline 9.0 & 1717.9800 & 2.0134 & GVMLLPGIEVPNLLPR \\
\hline 7.7 & 1718.9791 & 1.0143 & RTSISSPEHLVKPLR \\
\hline 0.2 & 1719.9784 & 0.0151 & AILSPKEPPSWRLAR \\
\hline
\end{tabular}

Mascot: http://www.matrixscience.com/ 


\section{$\left.{ }_{S S C I E N C E}^{\text {MATRIX }}\right\}$ Mascot Search Results}

Results Generated by a Script Modified from Mascot Pepitde View

by Newman Sze, School of Biological Sciences, Nanyang Technological University

Spectrum No: 801; Query: 332; Rank: 1

\section{Peptide View}

MS/MS Fragmentation of TWSVFQNGTDK

Found in IPI00205325, Tax_Id=10116 Gene_Symbol=Lrp2 Low-density lipoprotein receptor-related protein 2 precursor

Match to Query 332: 1282.583328 from(642.298940,2+)

Title: 091008RatKidney_NoSalt_26.2260.2260.2.dta

Data file K:INewmanPaper|Piliang|3SubProteomes\Piliang3SP\mgf5ppm\ERLIC_3SubProteomes5ppm.mgf
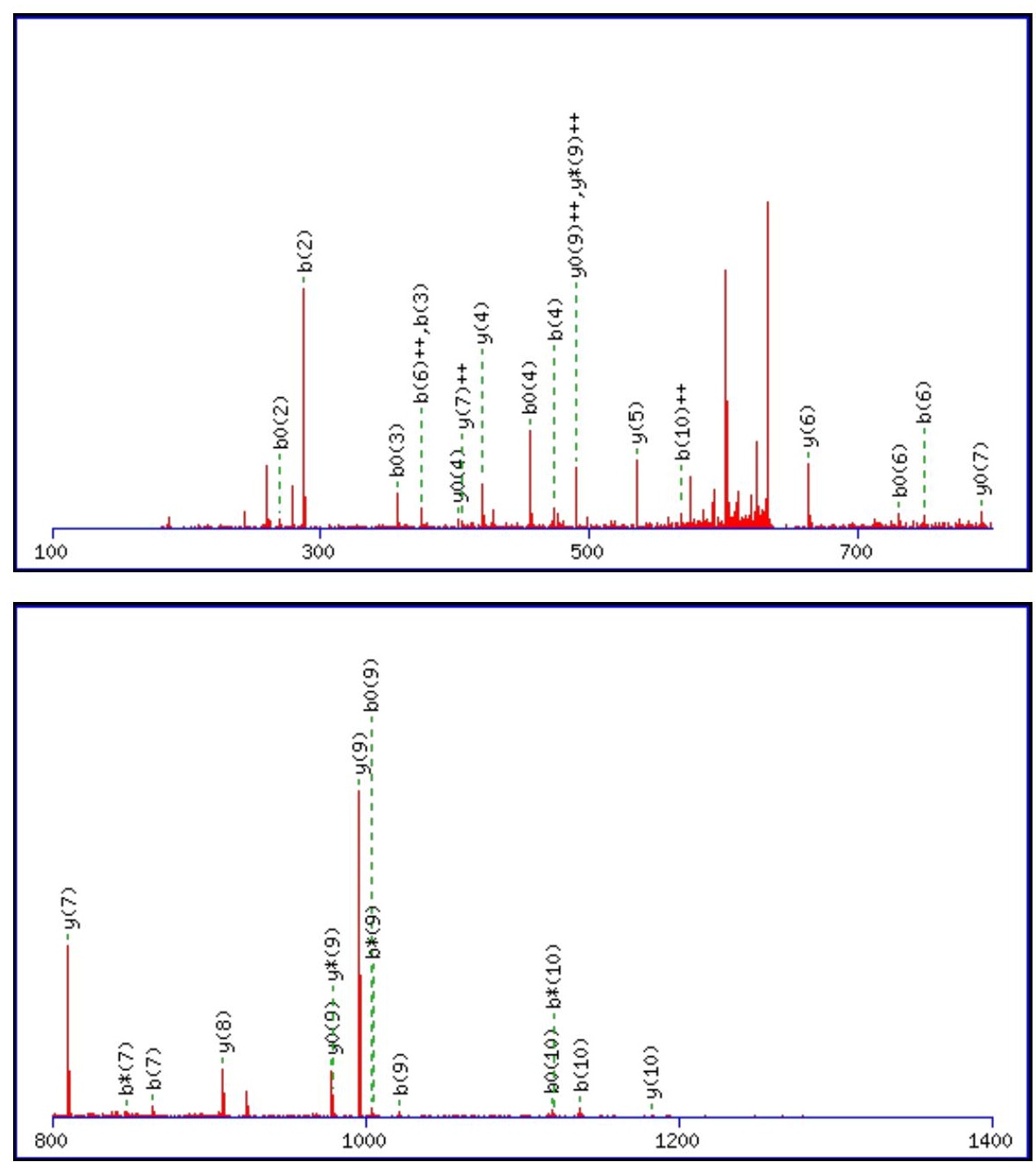


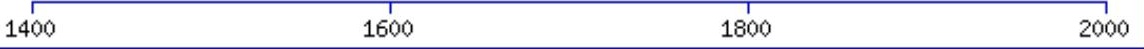

Monoisotopic mass of neutral peptide $\operatorname{Mr}($ calc): 1282.5830

Fixed modifications: Carbamidomethyl (C)

Variable modifications:

N7 : Deamidated_N (N)

Ions Score: 42 Expect: 0.0086

Matches (Bold Red): 32/108 fragment ions using 62 most intense peaks

\begin{tabular}{|c|c|c|c|c|c|c|c|c|c|c|c|c|c|c|}
\hline \# & b & $\mathbf{b}^{++}$ & $\mathbf{b}^{*}$ & $\mathbf{b}^{*^{++}}$ & $\mathbf{b}^{0}$ & $\mathbf{b}^{0++}$ & Seq. & $\mathbf{y}$ & $\mathbf{y}^{++}$ & $\mathrm{y}^{*}$ & $\mathrm{y}^{*^{++}}$ & $\mathbf{y}^{0}$ & $y^{0++}$ & \# \\
\hline 1 & 102.0550 & 51.5311 & & & 84.0444 & 42.5258 & $T$ & & & & & & & 11 \\
\hline 2 & 288.1343 & 144.5708 & & & 270.1237 & 135.5655 & W & 1182.5426 & 591.7749 & 1165.5160 & 583.2617 & 1164.5320 & 582.7696 & 10 \\
\hline 3 & 375.1663 & 188.0868 & & & 357.1557 & 179.0815 & $\mathrm{~S}$ & 996.4633 & 498.7353 & 979.4367 & 490.2220 & 978.4527 & 489.7300 & 9 \\
\hline 4 & 474.2347 & 237.6210 & & & 456.2241 & 228.6157 & V & 909.4312 & 455.2193 & 892.4047 & 446.7060 & 891.4207 & 446.2140 & 8 \\
\hline 5 & 621.3031 & 311.1552 & & & 603.2926 & 302.1499 & F & 810.3628 & 405.6850 & 793.3363 & 397.1718 & 792.3523 & 396.6798 & 7 \\
\hline 6 & 749.3617 & 375.1845 & 732.3352 & 366.6712 & 731.3511 & 366.1792 & $\mathbf{Q}$ & 663.2944 & 332.1508 & 646.2679 & 323.6376 & 645.2838 & 323.1456 & 6 \\
\hline 7 & 864.3886 & 432.6980 & 847.3621 & 424.1847 & 846.3781 & 423.6927 & $\mathbf{N}$ & 535.2358 & 268.1216 & 518.2093 & 259.6083 & 517.2253 & 259.1163 & 5 \\
\hline 8 & 921.4101 & 461.2087 & 904.3836 & 452.6954 & 903.3995 & 452.2034 & G & 420.2089 & 210.6081 & 403.1823 & 202.0948 & 402.1983 & 201.6028 & 4 \\
\hline 9 & 1022.4578 & 511.7325 & 1005.4312 & 503.2193 & 1004.4472 & 502.7272 & $T$ & 363.1874 & 182.0974 & 346.1609 & 173.5841 & 345.1769 & 173.0921 & 3 \\
\hline 10 & 1137.4847 & 569.2460 & 1120.4582 & 560.7327 & 1119.4742 & 560.2407 & D & 262.1397 & 131.5735 & 245.1132 & 123.0602 & 244.1292 & 122.5682 & 2 \\
\hline 11 & & & & & & & $\mathbf{K}$ & 147.1128 & 74.0600 & 130.0863 & 65.5468 & & & 1 \\
\hline
\end{tabular}
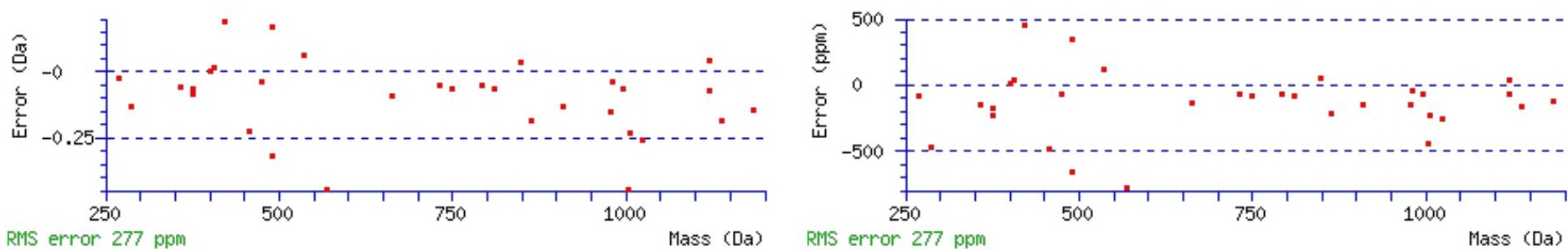

\section{All matches to this query}

\begin{tabular}{|l|l|c|l|}
\hline Score & Mr(calc): & Delta & \multicolumn{1}{|c|}{ Sequence } \\
\hline 42.0 & 1282.5830 & 0.0003 & TWSVFQNGTDK \\
\hline 10.4 & 1282.5806 & 0.0027 & DVVDDVSVSLR \\
\hline 10.3 & 1282.5918 & -0.0085 & NNTALQSVSLR \\
\hline 10.3 & 1282.5918 & -0.0085 & NNTALQSVSLR \\
\hline 10.3 & 1282.5918 & -0.0085 & NNTALQSVSLR \\
\hline 10.3 & 1282.5918 & -0.0085 & NNTALQSVSLR \\
\hline 10.0 & 1282.5806 & 0.0027 & $\underline{\text { STDAVAGPASSLK }}$ \\
\hline 8.3 & 1282.5806 & 0.0027 & STDAVAGPASSLK \\
\hline 7.2 & 1282.5918 & -0.0085 & AGESRAASVLXK \\
\hline 7.2 & 1282.5918 & -0.0085 & AGESRAASVLXK \\
\hline
\end{tabular}


Spectrum No: 802; Query: 1803; Rank: 1

\section{Peptide View}

MS/MS Fragmentation of MEMPSTPQQLQNLTEDIR

Found in IPI00365542, Tax_Id=10116 Gene_Symbol=Lamb1_predicted laminin, beta 1

Match to Query 1803: 2130.999908 from(1066.507230,2+)

Title: 100101RatKid_NS_deglyco_25.4348.4348.2.dta

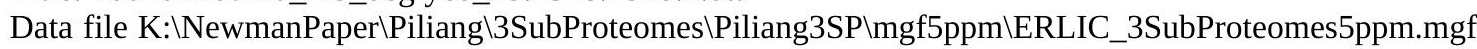
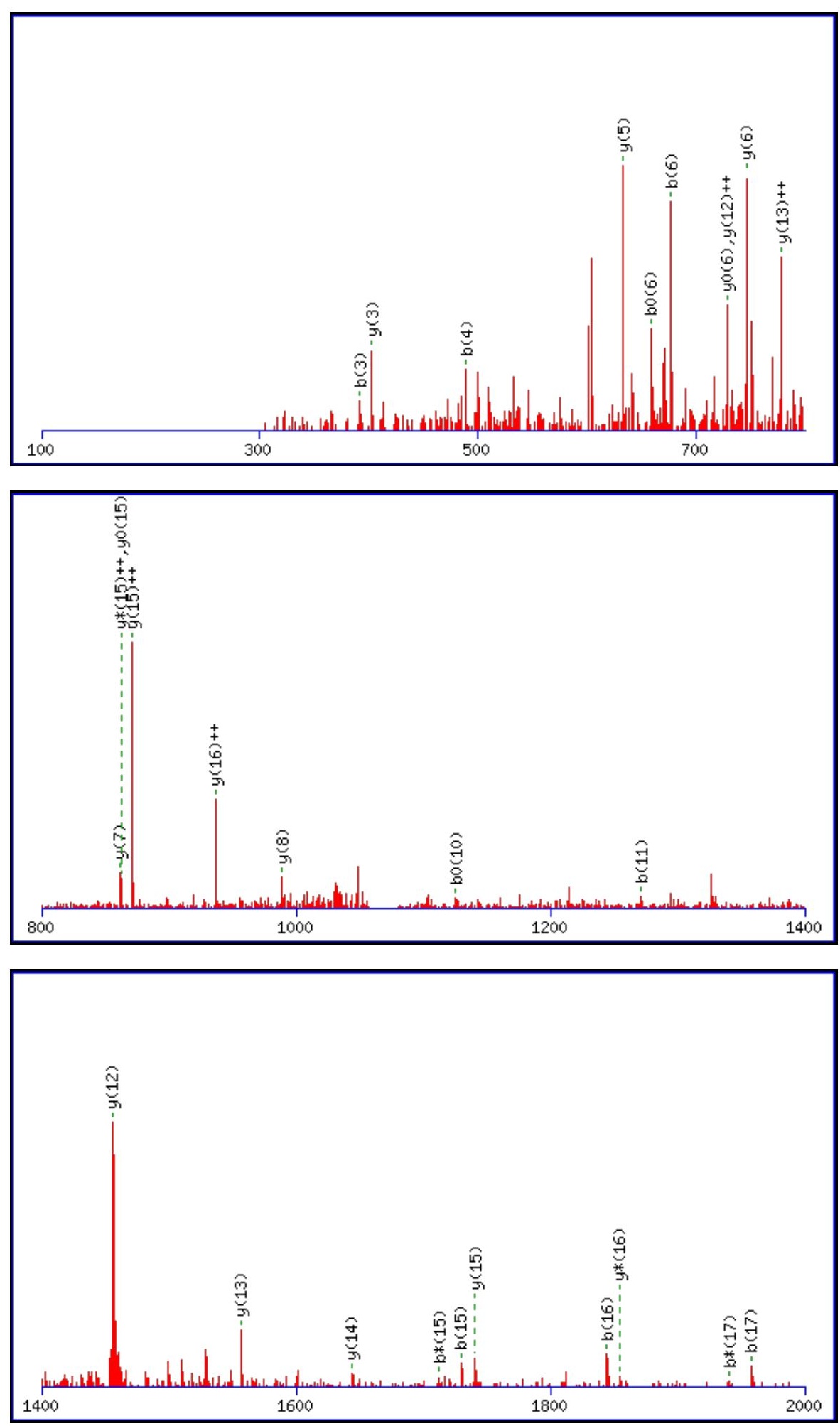
Monoisotopic mass of neutral peptide $\operatorname{Mr}($ calc): 2130.9925

Fixed modifications: Carbamidomethyl (C)

Variable modifications:

N12 : Deamidated_N (N)

Ions Score: 42 Expect: 0.016

Matches (Bold Red): 28/184 fragment ions using 61 most intense peaks

\begin{tabular}{|c|c|c|c|c|c|c|c|c|c|c|c|c|c|c|}
\hline \# & b & $\mathbf{b}^{++}$ & $\mathbf{b}^{*}$ & $\mathbf{b}^{*^{++}}$ & $\mathbf{b}^{\mathbf{0}}$ & $\mathbf{b}^{\mathbf{0 + +}}$ & Seq. & $\mathbf{y}$ & $\mathbf{y}^{++}$ & $\mathbf{y}^{*}$ & $\mathrm{y}^{*^{++}}$ & $\mathbf{y}^{\mathbf{0}}$ & $\mathbf{y}^{0++}$ & \# \\
\hline 1 & 132.0478 & 66.5275 & & & & & $\mathbf{M}$ & & & & & & & 18 \\
\hline 2 & 261.0904 & 131.0488 & & & 243.0798 & 122.0435 & $\mathbf{E}$ & 2000.9593 & 1000.9833 & 1983.9328 & 992.4700 & 1982.9488 & 991.9780 & 17 \\
\hline 3 & 392.1308 & 196.5691 & & & 374.1203 & 187.5638 & $\mathbf{M}$ & 1871.9167 & 936.4620 & 1854.8902 & 927.9487 & 1853.9062 & 927.4567 & 16 \\
\hline 4 & 489.1836 & 245.0954 & & & 471.1730 & 236.0902 & $\mathbf{P}$ & 1740.8763 & 870.9418 & 1723.8497 & 862.4285 & 1722.8657 & 861.9365 & 15 \\
\hline 5 & 576.2156 & 288.6115 & & & 558.2051 & 279.6062 & S & 1643.8235 & 822.4154 & 1626.7970 & 813.9021 & 1625.8129 & 813.4101 & 14 \\
\hline 6 & 677.2633 & 339.1353 & & & 659.2527 & 330.1300 & $\mathbf{T}$ & 1556.7915 & 778.8994 & 1539.7649 & 770.3861 & 1538.7809 & 769.8941 & 13 \\
\hline 7 & 774.3161 & 387.6617 & & & 756.3055 & 378.6564 & $\mathbf{P}$ & 1455.7438 & 728.3755 & 1438.7172 & 719.8623 & 1437.7332 & 719.3703 & 12 \\
\hline 8 & 902.3747 & 451.6910 & 885.3481 & 443.1777 & 884.3641 & 442.6857 & $\mathbf{Q}$ & 1358.6910 & 679.8492 & 1341.6645 & 671.3359 & 1340.6805 & 670.8439 & 11 \\
\hline 9 & 1030.4332 & 515.7203 & 1013.4067 & 507.2070 & 1012.4227 & 506.7150 & $\mathbf{Q}$ & 1230.6325 & 615.8199 & 1213.6059 & 607.3066 & 1212.6219 & 606.8146 & 10 \\
\hline 10 & 1143.5173 & 572.2623 & 1126.4907 & 563.7490 & 1125.5067 & 563.2570 & $\mathbf{L}$ & 1102.5739 & 551.7906 & 1085.5473 & 543.2773 & 1084.5633 & 542.7853 & 9 \\
\hline 11 & 1271.5759 & 636.2916 & 1254.5493 & 627.7783 & 1253.5653 & 627.2863 & $\mathbf{Q}$ & 989.4898 & 495.2485 & 972.4633 & 486.7353 & 971.4792 & 486.2433 & 8 \\
\hline 12 & 1386.6028 & 693.8050 & 1369.5763 & 685.2918 & 1368.5922 & 684.7998 & $\mathbf{N}$ & 861.4312 & 431.2193 & 844.4047 & 422.7060 & 843.4207 & 422.2140 & 7 \\
\hline 13 & 1499.6869 & 750.3471 & 1482.6603 & 741.8338 & 1481.6763 & 741.3418 & $\mathbf{L}$ & 746.4043 & 373.7058 & 729.3777 & 365.1925 & 728.3937 & 364.7005 & 6 \\
\hline 14 & 1600.7346 & 800.8709 & 1583.7080 & 792.3576 & 1582.7240 & 791.8656 & $\mathbf{T}$ & 633.3202 & 317.1638 & 616.2937 & 308.6505 & 615.3097 & 308.1585 & 5 \\
\hline 15 & 1729.7771 & 865.3922 & 1712.7506 & 856.8789 & 1711.7666 & 856.3869 & $\mathbf{E}$ & 532.2726 & 266.6399 & 515.2460 & 258.1266 & 514.2620 & 257.6346 & 4 \\
\hline 16 & 1844.8041 & 922.9057 & 1827.7775 & 914.3924 & 1826.7935 & 913.9004 & D & 403.2300 & 202.1186 & 386.2034 & 193.6053 & 385.2194 & 193.1133 & 3 \\
\hline 17 & 1957.8882 & 979.4477 & 1940.8616 & 970.9344 & 1939.8776 & 970.4424 & I & 288.2030 & 144.6051 & 271.1765 & 136.0919 & & & 2 \\
\hline 18 & & & & & & & $\mathbf{R}$ & 175.1190 & 88.0631 & 158.0924 & 79.5498 & & & 1 \\
\hline
\end{tabular}

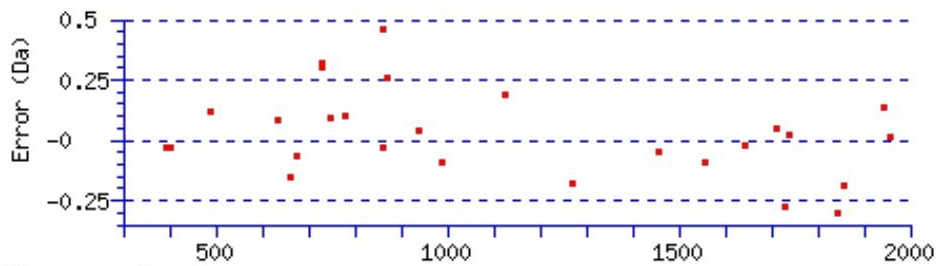

RMS error 195 ppm
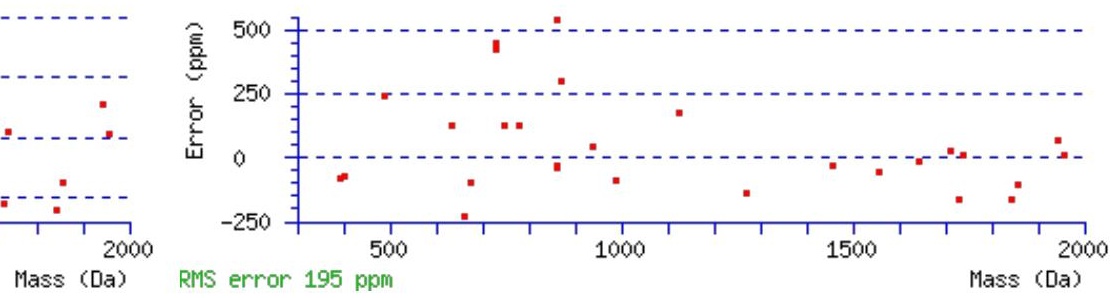

\section{All matches to this query}

\begin{tabular}{|l|l|l|l|}
\hline Score & Mr(calc): & Delta & \multicolumn{1}{c|}{ Sequence } \\
\hline 42.0 & 2130.9925 & 0.0074 & MEMPSTPQQLQNLTEDIR \\
\hline 5.7 & 2131.0103 & -0.0104 & MDVEEASPVAQSSSLTPVER \\
\hline 3.2 & 2130.0085 & 0.9914 & MEMPSTPQQLQNLTEDIR \\
\hline 2.0 & 2131.0139 & -0.0140 & NKTWKFVEGLPINDFSR \\
\hline 0.9 & 2130.9963 & 0.0036 & AALSTSSKGLLLEDNPSIR \\
\hline 0.2 & 2130.9868 & 0.0131 & TSTLSSQTNSQPLVQVSMK \\
\hline 0.1 & 2129.0116 & 1.9884 & IYLEGTNCIAGVTAPLTQK \\
\hline
\end{tabular}

Spectrum No: 803; Query: 2019; Rank: 1

\section{Peptide View}

MS/MS Fragmentation of SCDVPIFENAGTNNDSTWFK

Found in IPI00765740, Tax_Id=10116 Gene_Symbol=LOC688887 similar to complement component factor H

Match to Query 2019: 2302.998328 from(1152.506440,2+) 
Title: 100101RatKid_NS_deglyco_25.4192.4192.2.dta

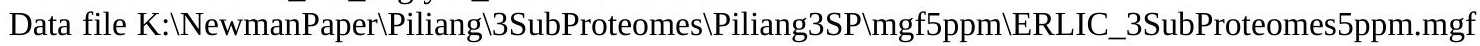
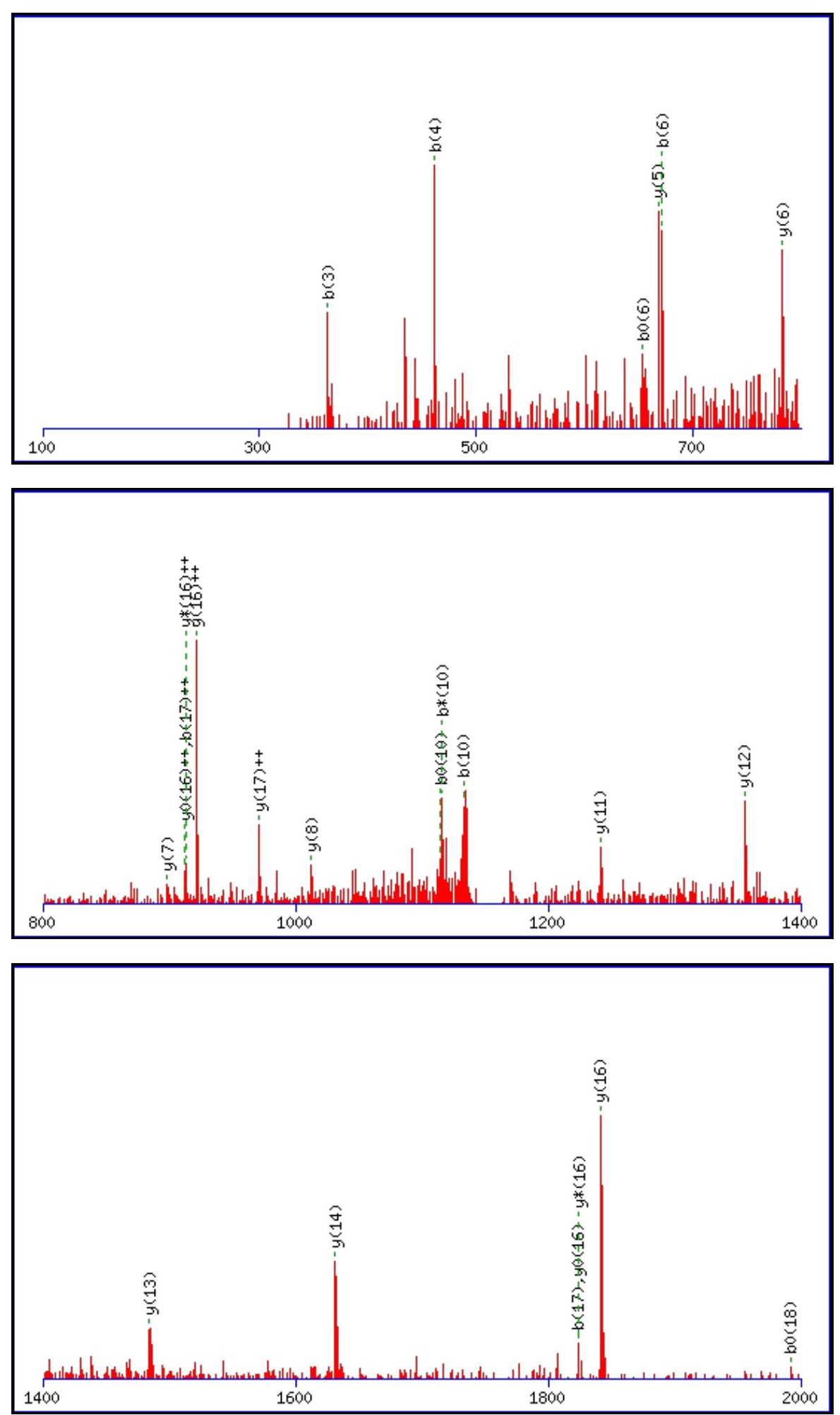

Monoisotopic mass of neutral peptide $\operatorname{Mr}($ calc): 2301.9848

Fixed modifications: Carbamidomethyl (C)

Variable modifications:

N14: Deamidated $\mathrm{N}(\mathrm{N})$

Ions Score: 42 Expect: 0.02

Matches (Bold Red): 25/206 fragment ions using 49 most intense peaks

\begin{tabular}{|c|c|c|c|c|c|c|c|c|c|c|c|c|c|c|}
\hline \# & b & $\mathbf{b}^{++}$ & b* & $\mathbf{b}^{*^{++}}$ & $\mathbf{b}^{0}$ & $\mathbf{b}^{0++}$ & Seq. & $\mathbf{y}$ & $\mathbf{y}^{++}$ & $\mathbf{y}^{*}$ & $\mathbf{y}^{*^{++}}$ & $\mathbf{y}^{\mathbf{0}}$ & $y^{0++}$ & \# \\
\hline 1 & 88.0393 & 44.5233 & & & 70.0287 & 35.5180 & $\mathrm{~S}$ & & & & & & & 20 \\
\hline 2 & 248.0700 & 124.5386 & & & 230.0594 & 115.5333 & C & 2215.9601 & 1108.4837 & 2198.9335 & 1099.9704 & 2197.9495 & 1099.4784 & 19 \\
\hline 3 & 363.0969 & 182.0521 & & & 345.0863 & 173.0468 & D & 2055.9294 & 1028.4683 & 2038.9029 & 1019.9551 & 2037.9189 & 1019.4631 & 18 \\
\hline
\end{tabular}




\begin{tabular}{|c|c|c|c|c|c|c|c|c|c|c|c|c|c|c|}
\hline & 462.1653 & 231.5863 & & & 444.1547 & 222.5810 & V & |1940.9025 & & & 962.4416 & |1922.8919| & & 17 \\
\hline 5 & 559.2181 & 280.1127 & & & 541.2075 & 271.1074 & $\mathbf{P}$ & 1841.8341 & 921.4207 & $\mid 1824.8075$ & 912.9074 & 1823.8235 & 912.4154 & 16 \\
\hline 6 & 672.3021 & 336.6547 & & & 654.2916 & 327.6494 & I & 1744.7813 & 872.8943 & 1727.7548 & 864.3810 & 1726.7707 & 863.8890 & 15 \\
\hline 7 & 819.3706 & 410.1889 & & & 801.3600 & 401.1836 & $\mathbf{F}$ & 1631.6972 & 816.3523 & 1614.6707 & 807.8390 & 1613.6867 & 807.3470 & 14 \\
\hline 8 & 948.4131 & 474.7102 & & & 930.4026 & 465.7049 & $\mathbf{E}$ & 1484.6288 & 742.8180 & 1467.6023 & 734.3048 & 1466.6183 & 733.8128 & 13 \\
\hline 9 & 1062.4561 & 531.7317 & 1045.4295 & 523.2184 & 1044.4455 & 522.7264 & $\mathbf{N}$ & 1355.5862 & 678.2968 & |1338.5597 & 669.7835 & |1337.5757 & 669.2915 & 12 \\
\hline 10 & 1133.4932 & 567.2502 & 1116.4666 & 558.7370 & 1115.4826 & 558.2449 & A & 1241.5433 & 621.2753 & 1224.5168 & 612.7620 & 1223.5327 & 612.2700 & 11 \\
\hline 11 & 1190.5147 & 595.7610 & 1173.4881 & 587.2477 & 1172.5041 & 586.7557 & G & 1170.5062 & 585.7567 & 1153.4796 & 577.2435 & 1152.4956 & 576.7514 & 10 \\
\hline 12 & 1291.5623 & 646.2848 & 1274.5358 & 637.7715 & 1273.5518 & 637.2795 & $\mathbf{T}$ & 1113.4847 & 557.2460 & 1096.4582 & 548.7327 & 1095.4742 & 548.2407 & 9 \\
\hline 13 & 1405.6053 & 703.3063 & 1388.5787 & 694.7930 & 1387.5947 & 694.3010 & $\mathbf{N}$ & 1012.4370 & 506.7222 & 995.4105 & 498.2089 & 994.4265 & 497.7169 & 8 \\
\hline 14 & 1520.6322 & 760.8197 & 1503.6056 & 752.3065 & 1502.6216 & 751.8145 & $\mathbf{N}$ & 898.3941 & 449.7007 & 881.3676 & 441.1874 & 880.3836 & 440.6954 & 7 \\
\hline 15 & 1635.6591 & 818.3332 & 1618.6326 & 809.8199 & 1617.6486 & 809.3279 & D & 783.3672 & 392.1872 & 766.3406 & 383.6740 & 765.3566 & 383.1819 & 6 \\
\hline 16 & 1722.6912 & 861.8492 & 1705.6646 & 853.3359 & 1704.6806 & 852.8439 & $\mathrm{~S}$ & 668.3402 & 334.6738 & 651.3137 & 326.1605 & 650.3297 & 325.6685 & 5 \\
\hline 17 & \begin{tabular}{|l|}
1823.7388 \\
\end{tabular} & 912.3731 & 1806.7123 & 903.8598 & 1805.7283 & 903.3678 & $T$ & 581.3082 & 291.1577 & 564.2817 & 282.6445 & 563.2976 & 282.1525 & 4 \\
\hline 18 & 2009.8182 & 1005.4127 & 1992.7916 & 996.8994 & 1991.8076 & 996.4074 & $\mathbf{W}$ & 480.2605 & 240.6339 & 463.2340 & 232.1206 & & & 3 \\
\hline 19 & 2156.8866 & 1078.9469 & 2139.8600 & 1070.4336 & 2138.8760 & 1069.9416 & $\mathbf{F}$ & 294.1812 & 147.5942 & 277.1547 & 139.0810 & & & 2 \\
\hline 20 & & & & & & & $\mathbf{K}$ & 147.1128 & 74.0600 & 130.0863 & 65.5468 & & & 1 \\
\hline
\end{tabular}
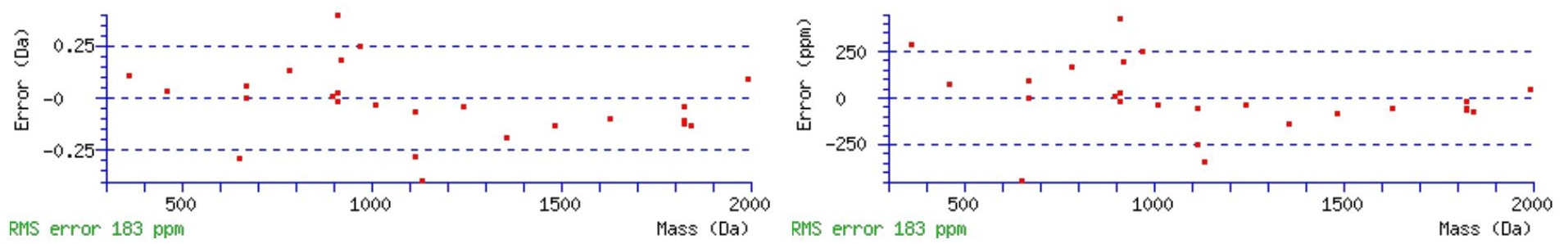

\section{All matches to this query}

\begin{tabular}{|l|l|l|l|}
\hline Score & Mr(calc): & Delta & \multicolumn{1}{|c|}{ Sequence } \\
\hline 41.8 & 2301.9848 & 1.0135 & SCDVPIFENAGTNNDSTWFK \\
\hline 41.8 & 2301.9848 & 1.0135 & SCDVPIFENAGTNNDSTWFK \\
\hline 35.6 & 2301.9848 & 1.0135 & SCDVPIFENAGTNNDSTWFK \\
\hline 4.2 & 2302.9809 & 0.0174 & TSLETQKLELMAEISNLK \\
\hline 1.1 & 2301.0008 & 1.9975 & SCDVPIFENAGTNNDSTWFK \\
\hline 0.6 & 2300.9840 & 2.0143 & KSMSVYCTPNKPSRTSMSK \\
\hline 0.4 & 2302.0083 & 0.9901 & TYVFPETDFIAVTAYQNDK \\
\hline 0.1 & 2301.9833 & 1.0151 & CMAVYQTYVNAMNDKIRK \\
\hline 0.1 & 2301.9833 & 1.0151 & CMAVYOTYVNAMNDKIRK \\
\hline 0.1 & 2301.9833 & 1.0151 & CMAVYQTYVNAMNDKIRK \\
\hline
\end{tabular}

Spectrum No: 804; Query: 2303; Rank: 1

\section{Peptide View}

MS/MS Fragmentation of VLVTGAAGQIAYSLLYSIGNGSVFGK

Found in IPI00198717, Tax_Id=10116 Gene_Symbol=Mdh1 Malate dehydrogenase, cytoplasmic

Match to Query 2303: 2585.385642 from(862.802490,3+)

Title: 091008RatKidney_NoSalt_02.6379.6379.3.dta

Data file K:INewmanPaper|Piliangl3SubProteomes\Piliang3SP\mgf5ppm\ERLIC_3SubProteomes5ppm.mgf 

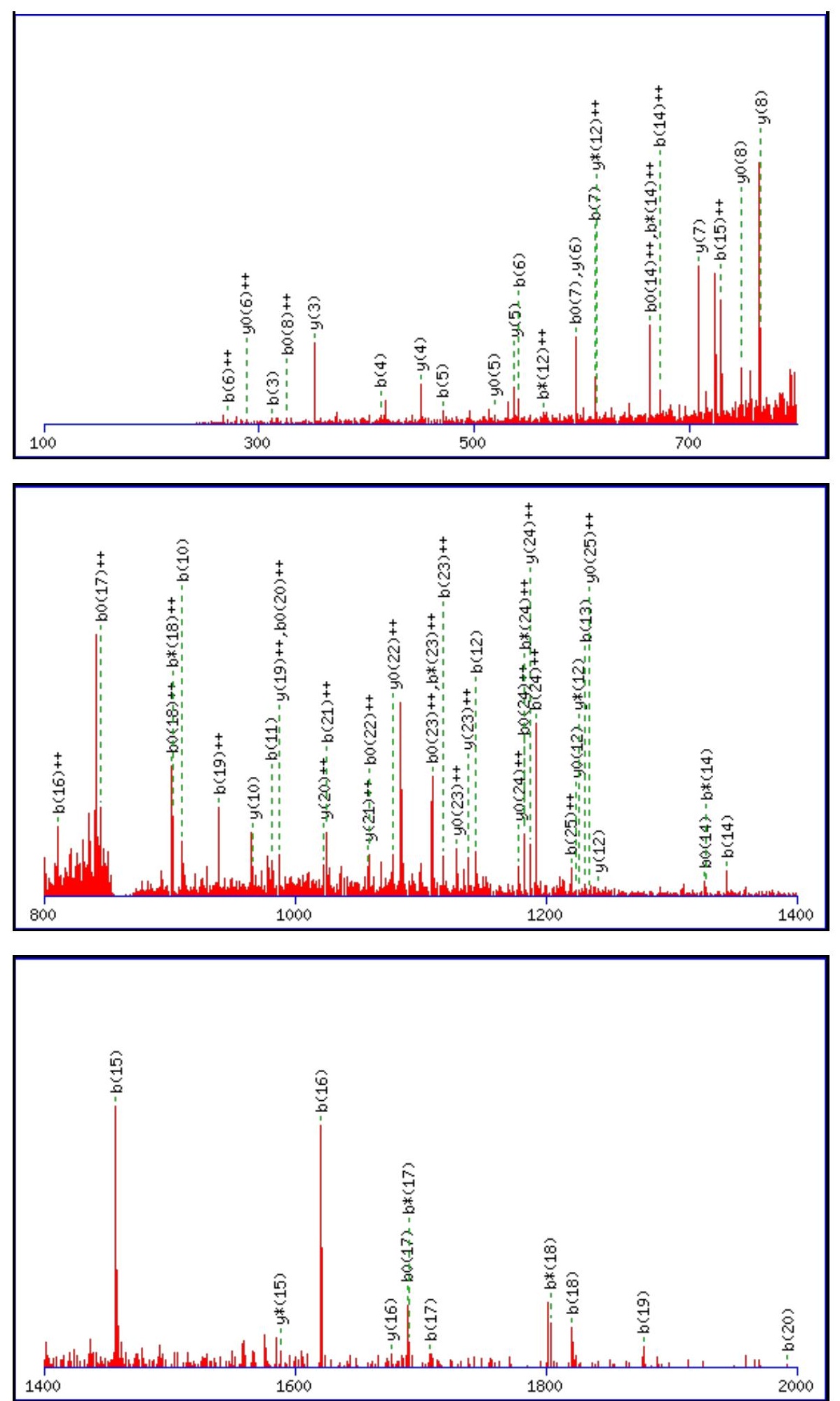

Monoisotopic mass of neutral peptide $\operatorname{Mr}($ calc): 2585.3741

Fixed modifications: Carbamidomethyl (C)

Variable modifications:

N20 : Deamidated N ( N)

Ions Score: 42 Expect: 0.0046

Matches (Bold Red): 69/270 fragment ions using 191 most intense peaks

\begin{tabular}{|c|c|c|c|c|c|c|c|c|c|c|c|c|c|c|}
\hline$\#$ & $\mathbf{b}$ & $\mathbf{b}^{++}$ & $\mathbf{b}^{*}$ & $\mathbf{b}^{\boldsymbol{*}^{++}}$ & $\mathbf{b}^{\mathbf{0}}$ & $\mathbf{b}^{\mathbf{0 + +}}$ & Seq. & $\mathbf{y}$ & $\mathbf{y}^{++}$ & $\mathbf{y}^{*}$ & $\mathbf{y}^{\mathbf{*}^{++}}$ & $\mathbf{y}^{\mathbf{0}}$ & $\mathbf{y}^{\mathbf{0 + +}}$ & $\#$ \\
\hline $\mathbf{1}$ & 100.0757 & 50.5415 & & & & & $\mathbf{V}$ & & & & & & & $\mathbf{2 6}$ \\
\hline $\mathbf{2}$ & 213.1598 & 107.0835 & & & & & $\mathbf{L}$ & 2487.3130 & 1244.1601 & 2470.2864 & 1235.6468 & 2469.3024 & $\mathbf{1 2 3 5 . 1 5 4 8}$ & $\mathbf{2 5}$ \\
\hline $\mathbf{3}$ & 312.2282 & 156.6177 & & & & & $\mathbf{V}$ & 2374.2289 & $\mathbf{1 1 8 7 . 6 1 8 1}$ & 2357.2023 & 1179.1048 & 2356.2183 & $\mathbf{1 1 7 8 . 6 1 2 8}$ & $\mathbf{2 4}$ \\
\hline $\mathbf{4}$ & $\mathbf{4 1 3 . 2 7 5 8}$ & 207.1416 & & & 395.2653 & 198.1363 & $\mathbf{T}$ & 2275.1605 & $\mathbf{1 1 3 8 . 0 8 3 9}$ & 2258.1339 & 1129.5706 & 2257.1499 & $\mathbf{1 1 2 9 . 0 7 8 6}$ & $\mathbf{2 3}$ \\
\hline $\mathbf{5}$ & $\mathbf{4 7 0 . 2 9 7 3}$ & 235.6523 & & & 452.2867 & 226.6470 & $\mathbf{G}$ & 2174.1128 & 1087.5600 & 2157.0863 & 1079.0468 & 2156.1022 & $\mathbf{1 0 7 8 . 5 5 4 8}$ & $\mathbf{2 2}$ \\
\hline
\end{tabular}




\begin{tabular}{|c|c|c|c|c|c|c|c|c|c|c|c|c|c|c|}
\hline 6] & 541.3344 & 271.1709 & & & 523.3239 & 262.1656 & A & 2117.0913 & 1059.0493 & |2100.0648| & 1050.5360 & |2099.0808 & $1050.0440 \mid$ & 21 \\
\hline 7 & 612.3715 & 306.6894 & & & 594.3610 & 297.6841 & A & 2046.0542 & 1023.5308 & 2029.0277 & 1015.0175 & 2028.0437 & 1014.5255 & 20 \\
\hline 8 & 669.3930 & 335.2001 & & & 651.3824 & 326.1949 & G & 1975.0171 & 988.0122 & 1957.9906 & 979.4989 & 1957.0065 & 979.0069 & 19 \\
\hline 9 & 797.4516 & 399.2294 & 780.4250 & 390.7162 & 779.4410 & 390.2241 & $\mathbf{Q}$ & 1917.9956 & 959.5015 & 1900.9691 & 950.9882 & 1899.9851 & 950.4962 & 18 \\
\hline 10 & 910.5356 & 455.7715 & 893.5091 & 447.2582 & 892.5251 & 446.7662 & I & 1789.9371 & 895.4722 & 1772.9105 & 886.9589 & 1771.9265 & 886.4669 & 17 \\
\hline 11 & 981.5728 & 491.2900 & 964.5462 & 482.7767 & 963.5622 & 482.2847 & A & 1676.8530 & 838.9301 & 1659.8265 & 830.4169 & 1658.8424 & 829.9249 & 16 \\
\hline $12 \mid 1$ & 1144.6361 & 572.8217 & 1127.6095 & 564.3084 & 1126.6255 & 563.8164 & $\mathbf{Y}$ & 1605.8159 & 803.4116 & \begin{tabular}{|l|}
1588.7893 \\
\end{tabular} & 794.8983 & 1587.8053 & 794.4063 & 15 \\
\hline 131 & \begin{tabular}{|l|}
1231.6681 \\
\end{tabular} & 616.3377 & 1214.6416 & 607.8244 & 1213.6576 & 607.3324 & S & 1442.7526 & 721.8799 & 1425.7260 & 713.3666 & 1424.7420 & 712.8746 & 14 \\
\hline 141 & 1344.7522 & 672.8797 & 1327.7256 & 664.3665 & 1326.7416 & 663.8744 & $\mathbf{L}$ & 1355.7205 & 678.3639 & 1338.6940 & 669.8506 & 1337.7100 & 669.3586 & 13 \\
\hline 151 & 1457.8362 & 729.4218 & 1440.8097 & 720.9085 & 1439.8257 & 720.4165 & $\mathbf{L}$ & 1242.6365 & 621.8219 & 1225.6099 & 613.3086 & 1224.6259 & 612.8166 & 12 \\
\hline 161 & 1620.8996 & 810.9534 & 1603.8730 & 802.4402 & 1602.8890 & 801.9481 & $\mathbf{Y}$ & 1129.5524 & 565.2798 & 1112.5259 & 556.7666 & 1111.5418 & 556.2746 & 11 \\
\hline 171 & 1707.9316 & 854.4694 & 1690.9051 & 845.9562 & 1689.9210 & 845.4642 & $\mathrm{~S}$ & 966.4891 & 483.7482 & 949.4625 & 475.2349 & 948.4785 & 474.7429 & 10 \\
\hline 181 & 1821.0157 & 911.0115 & 1803.9891 & 902.4982 & 1803.0051 & 902.0062 & I & 879.4571 & 440.2322 & 862.4305 & 431.7189 & 861.4465 & 431.2269 & 9 \\
\hline 191 & \begin{tabular}{|l|}
1878.0371 \\
\end{tabular} & 939.5222 & 1861.0106 & 931.0089 & 1860.0266 & 930.5169 & G & 766.3730 & 383.6901 & 749.3464 & 375.1769 & 748.3624 & 374.6848 & 8 \\
\hline 201 & 1993.0641 & 997.0357 & 1976.0375 & 988.5224 & 1975.0535 & 988.0304 & $\mathbf{N}$ & 709.3515 & 355.1794 & 692.3250 & 346.6661 & 691.3410 & 346.1741 & 7 \\
\hline 212 & 2050.0855 & 1025.5464 & 2033.0590 & 1017.0331 & 2032.0750 & 1016.5411 & G & 594.3246 & 297.6659 & 577.2980 & 289.1527 & 576.3140 & 288.6606 & 6 \\
\hline 22 & 2137.1176 & 1069.0624 & 2120.0910 & 1060.5491 & 2119.1070 & 1060.0571 & $S$ & 537.3031 & 269.1552 & 520.2766 & 260.6419 & 519.2926 & 260.1499 & 5 \\
\hline 232 & 2236.1860 & 1118.5966 & 2219.1594 & 1110.0833 & 2218.1754 & 1109.5913 & V & 450.2711 & 225.6392 & 433.2445 & 217.1259 & & & 4 \\
\hline \begin{tabular}{l|l}
24 & 2 \\
\end{tabular} & 2383.2544 & \begin{tabular}{|l|}
1192.1308 \\
\end{tabular} & 2366.2278 & 1183.6176 & 2365.2438 & 1183.1255 & $\mathbf{F}$ & 351.2027 & 176.1050 & 334.1761 & 167.5917 & & & 3 \\
\hline 252 & 2440.2758 & 1220.6416 & 2423.2493 & 1212.1283 & 2422.2653 & 1211.6363 & G & 204.1343 & 102.5708 & 187.1077 & 94.0575 & & & 2 \\
\hline 26 & & & & & & & $\mathbf{K}$ & 147.1128 & 74.0600 & 130.0863 & 65.5468 & & & 1 \\
\hline
\end{tabular}
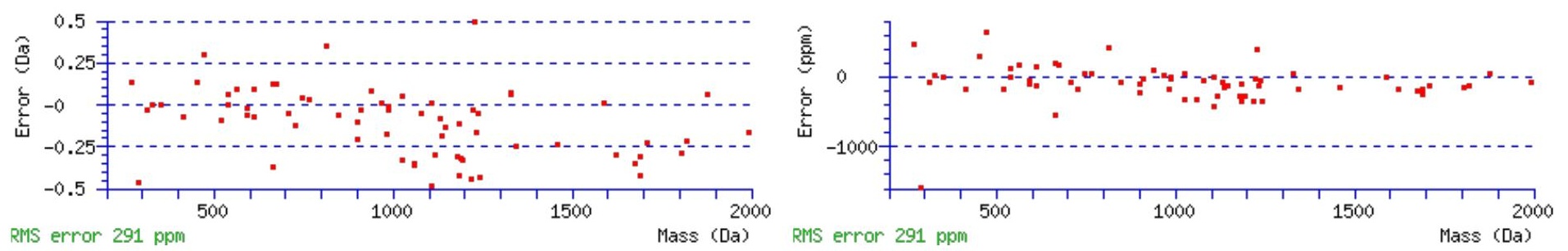

\section{All matches to this query}

\begin{tabular}{|l|c|c|c|}
\hline Score & Mr(calc): & Delta & Sequence \\
\hline 41.8 & 2585.3741 & 0.0115 & VLVTGAAGQIAYSLLYSIGNGSVFGK \\
\hline 40.4 & 2584.3901 & 0.9956 & VLVTGAAGQIAYSLLYSIGNGSVFGK \\
\hline
\end{tabular}

Spectrum No: 805; Query: 279; Rank: 1

\section{Peptide View}

MS/MS Fragmentation of LNGTDPEDVIR

Found in IPI00421625, Tax_Id=10116 Gene_Symbol=Mrlcb;RGD1565978_predicted Myosin regulatory light chain 2-B, smooth muscle isoform

Match to Query 279: 1228.597928 from(615.306240,2+)

Title: 100101RatKid_NS_deglyco_14.2004.2004.2.dta

Data file K:INewmanPaper|Piliangl3SubProteomes\Piliang3SP\mgf5ppm\ERLIC_3SubProteomes5ppm.mgf 

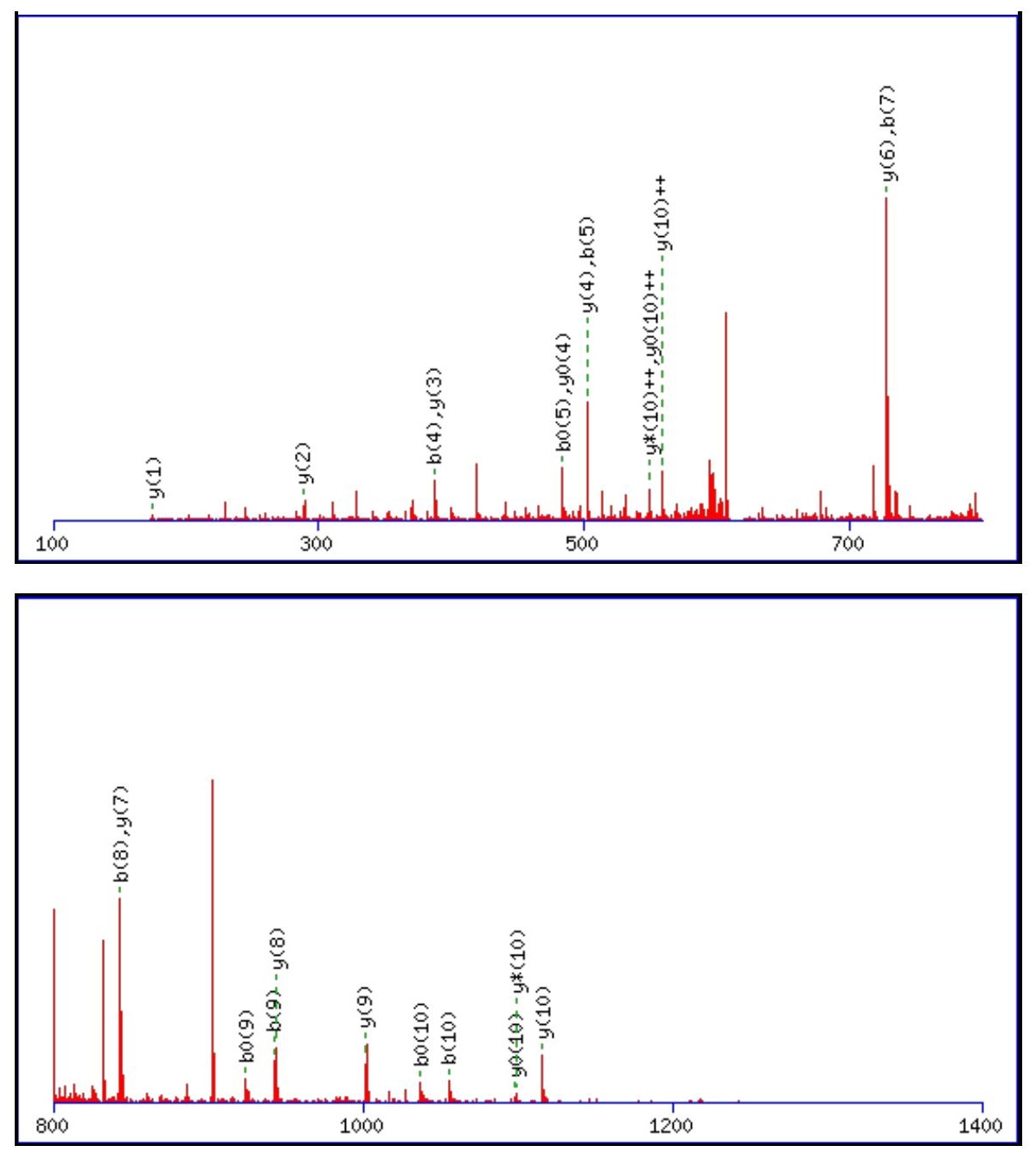

\begin{tabular}{rrrr}
\hline 00 & 1600 & 1800 & 2000 \\
\hline
\end{tabular}

Monoisotopic mass of neutral peptide $\operatorname{Mr}($ calc): 1228.5935

Fixed modifications: Carbamidomethyl (C)

Variable modifications:

N2 : Deamidated $\mathrm{N}(\mathrm{N})$

Ions Score: 42 Expect: $\odot .0076$

Matches (Bold Red): 24/106 fragment ions using 62 most intense peaks

\begin{tabular}{|r|c|c|c|c|c|c|c|c|c|c|c|c|c|c|}
\hline$\#$ & $\mathbf{b}$ & $\mathbf{b}^{++}$ & $\mathbf{b}^{*}$ & $\mathbf{b}^{\boldsymbol{*}^{++}}$ & $\mathbf{b}^{\mathbf{0}}$ & $\mathbf{b}^{\mathbf{0 + +}}$ & Seq. & $\mathbf{y}$ & $\mathbf{y}^{++}$ & $\mathbf{y}^{\mathbf{*}}$ & $\mathbf{y}^{\boldsymbol{*}^{++}}$ & $\mathbf{y}^{\mathbf{0}}$ & $\mathbf{y}^{\mathbf{0 + +}}$ & $\#$ \\
\hline $\mathbf{1}$ & 114.0913 & 57.5493 & & & & & $\mathbf{L}$ & & & & & & & $\mathbf{1 1}$ \\
\hline $\mathbf{2}$ & 229.1183 & 115.0628 & 212.0917 & 106.5495 & & & $\mathbf{N}$ & $\mathbf{1 1 1 6 . 5 1 6 8}$ & $5 \mathbf{5 8 . 7 6 2 0}$ & $\mathbf{1 0 9 9 . 4 9 0 2}$ & $\mathbf{5 5 0 . 2 4 8 7}$ & $\mathbf{1 0 9 8 . 5 0 6 2}$ & $\mathbf{5 4 9 . 7 5 6 7}$ & $\mathbf{1 0}$ \\
\hline $\mathbf{3}$ & 286.1397 & 143.5735 & 269.1132 & 135.0602 & & & $\mathbf{G}$ & $\mathbf{1 0 0 1 . 4 8 9 8}$ & 501.2485 & 984.4633 & 492.7353 & 983.4793 & 492.2433 & $\mathbf{9}$ \\
\hline $\mathbf{4}$ & $\mathbf{3 8 7 . 1 8 7 4}$ & 194.0973 & 370.1609 & 185.5841 & 369.1769 & 185.0921 & $\mathbf{T}$ & $\mathbf{9 4 4 . 4 6 8 4}$ & 472.7378 & 927.4418 & 464.2245 & 926.4578 & 463.7325 & $\mathbf{8}$ \\
\hline $\mathbf{5}$ & $\mathbf{5 0 2 . 2 1 4 4}$ & 251.6108 & 485.1878 & 243.0975 & $\mathbf{4 8 4 . 2 0 3 8}$ & 242.6055 & $\mathbf{D}$ & $\mathbf{8 4 3 . 4 2 0 7}$ & 422.2140 & 826.3941 & 413.7007 & 825.4101 & 413.2087 & $\mathbf{7}$ \\
\hline
\end{tabular}




\begin{tabular}{|r|r|r|r|r|r|r|r|r|r|r|r|r|r|r|r|r|r|r|}
$\mathbf{6}$ & 599.2671 & 300.1372 & 582.2406 & 291.6239 & 581.2566 & 291.1319 & $\mathbf{P}$ & $\mathbf{7 2 8 . 3 9 3 7}$ & 364.7005 & 711.3672 & 356.1872 & $\mathbf{7 1 0 . 3 8 3 2}$ & 355.6952 & $\mathbf{6}$ \\
\hline $\mathbf{7}$ & $\mathbf{7 2 8 . 3 0 9 7}$ & 364.6585 & 711.2832 & 356.1452 & 710.2992 & 355.6532 & $\mathbf{E}$ & 631.3410 & 316.1741 & 614.3144 & 307.6608 & 613.3304 & 307.1688 & $\mathbf{5}$ \\
\hline $\mathbf{8}$ & $\mathbf{8 4 3 . 3 3 6 7}$ & 422.1720 & 826.3101 & 413.6587 & 825.3261 & 413.1667 & $\mathbf{D}$ & $\mathbf{5 0 2 . 2 9 8 4}$ & 251.6528 & 485.2718 & 243.1395 & $\mathbf{4 8 4 . 2 8 7 8}$ & 242.6475 & $\mathbf{4}$ \\
\hline $\mathbf{9}$ & $\mathbf{9 4 2 . 4 0 5 1}$ & 471.7062 & 925.3785 & 463.1929 & $\mathbf{9 2 4 . 3 9 4 5}$ & 462.7009 & $\mathbf{V}$ & $\mathbf{3 8 7 . 2 7 1 4}$ & 194.1394 & 370.2449 & 185.6261 & & & & $\mathbf{3}$ \\
\hline $\mathbf{1 0}$ & $\mathbf{1 0 5 5 . 4 8 9 1}$ & 528.2482 & 1038.4626 & 519.7349 & $\mathbf{1 0 3 7 . 4 7 8 6}$ & 519.2429 & $\mathbf{I}$ & $\mathbf{2 8 8 . 2 0 3 0}$ & 144.6051 & 271.1765 & 136.0919 & & & & $\mathbf{2}$ \\
\hline $\mathbf{1 1}$ & & & & & & & $\mathbf{R}$ & $\mathbf{1 7 5 . 1 1 9 0}$ & 88.0631 & 158.0924 & $\mathbf{7 9 . 5 4 9 8}$ & & & & $\mathbf{1}$ \\
\hline
\end{tabular}
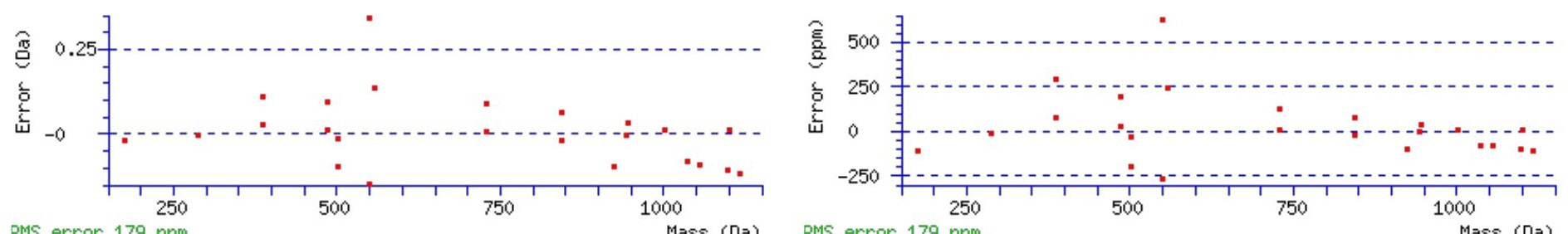

RMS error 179 ppm

\section{All matches to this query}

\begin{tabular}{|c|c|c|c|}
\hline Score & Mr(calc): & Delta & Sequence \\
\hline 41.7 & 1228.5935 & 0.0044 & LNGTDPEDVIR \\
\hline 8.1 & 1228.5965 & 0.0014 & LNVPLNVHSR \\
\hline 6.1 & 1228.5999 & \begin{tabular}{|l|}
-0.0019 \\
\end{tabular} & LASMTQKKAR \\
\hline 6.0 & 1228.5870 & 0.0109 & VQVEVSEMHR \\
\hline 5.7 & 1228.6056 & $\mid-0.0077$ & AVGNCMLHSIK \\
\hline 4.8 & 1228.5909 & 0.0071 & SSVRSAASGHDR \\
\hline 4.6 & 1228.5870 & 0.0109 & CEPPENISKR \\
\hline 3.9 & 1228.6077 & -0.0098 & RAFQKSASVR \\
\hline 3.1 & 1227.5860 & 1.0119 & LLTGSSDKTAR \\
\hline 2.2 & 1227.5900 & 1.0079 & VGDTFILNLR \\
\hline
\end{tabular}

Spectrum No: 806; Query: 224; Rank: 1

\section{Peptide View}

MS/MS Fragmentation of EKIEQNGSMR

Found in IPI00554102, Tax_Id=10116 Gene_Symbol=LOC685482;LOC259246;LOC298116;LOC259245 similar to alpha2u globulin

Match to Query 224: 1207.547288 from(604.780920,2+)

Title: 091008RatKidney_NH4Format01_11.113.113.2.dta

Data file K:INewmanPaper|Piliang|3SubProteomes\Piliang3SP\mgf5ppm\ERLIC_3SubProteomes5ppm.mgf

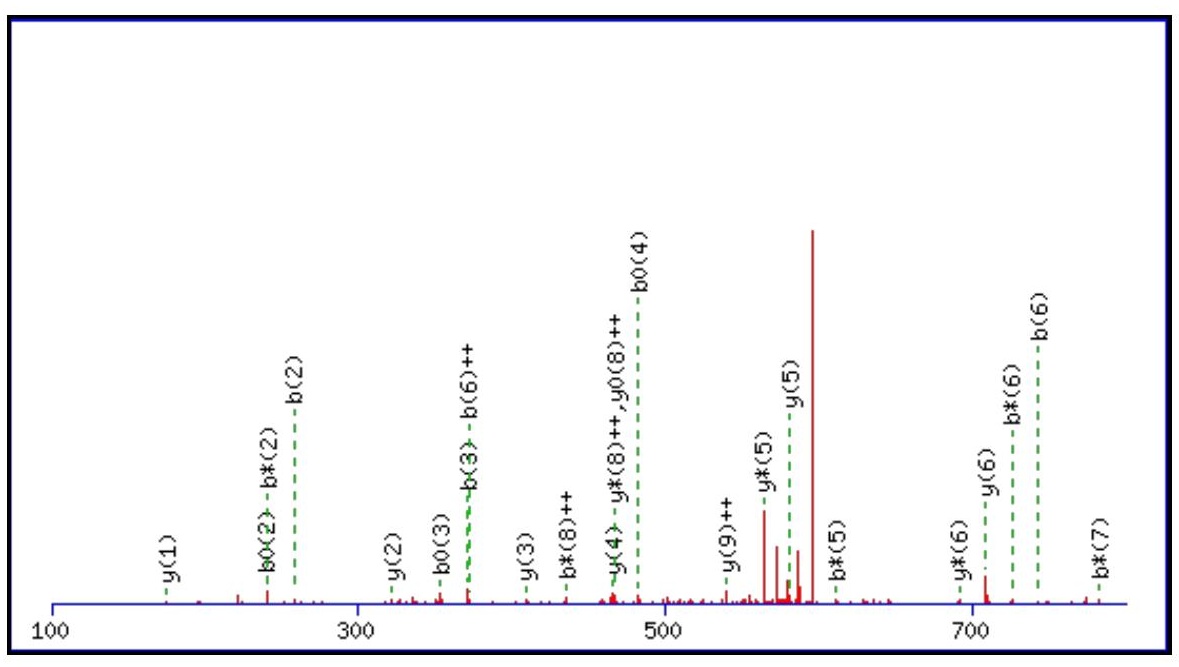




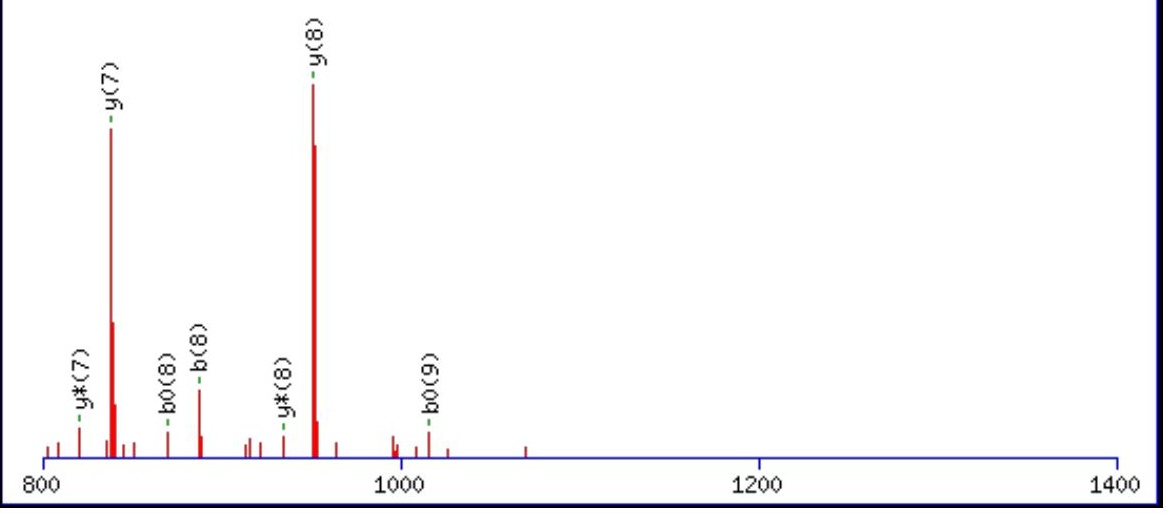

\begin{tabular}{|c|c|c|c|}
\hline 1400 & 1600 & 1800 & 2000 \\
\hline
\end{tabular}

Monoisotopic mass of neutral peptide $\operatorname{Mr}($ calc): 1207.5503

Fixed modifications: Carbamidomethyl (C)

Variable modifications:

N6 : Deamidated_N (N)

M9 : Oxidation (M)

Ions Score: 42 Expect: $\odot .008$

Matches (Bold Red): 30/102 fragment ions using 63 most intense peaks

\begin{tabular}{|r|c|c|c|c|c|c|c|c|c|c|c|c|c|c|}
\hline$\#$ & $\mathbf{b}$ & $\mathbf{b}^{++}$ & $\mathbf{b}^{*}$ & $\mathbf{b}^{\mathbf{*}^{++}}$ & $\mathbf{b}^{\mathbf{0}}$ & $\mathbf{b}^{\mathbf{0 + +}}$ & $\mathbf{S e q}$ & $\mathbf{y}$ & $\mathbf{y}^{++}$ & $\mathbf{y}^{*}$ & $\mathbf{y}^{\mathbf{*}^{++}}$ & $\mathbf{y}^{\mathbf{0}}$ & $\mathbf{y}^{\mathbf{0 + +}}$ & $\#$ \\
\hline $\mathbf{1}$ & 130.0499 & 65.5286 & & & 112.0393 & 56.5233 & $\mathbf{E}$ & & & & & & & $\mathbf{1 0}$ \\
\hline $\mathbf{2}$ & $\mathbf{2 5 8 . 1 4 4 8}$ & 129.5761 & $\mathbf{2 4 1 . 1 1 8 3}$ & 121.0628 & $\mathbf{2 4 0 . 1 3 4 3}$ & 120.5708 & $\mathbf{K}$ & 1079.5150 & 540.2611 & 1062.4884 & 531.7479 & 1061.5044 & 531.2558 & $\mathbf{9}$ \\
\hline $\mathbf{3}$ & $\mathbf{3 7 1 . 2 2 8 9}$ & 186.1181 & 354.2023 & 177.6048 & $\mathbf{3 5 3 . 2 1 8 3}$ & 177.1128 & $\mathbf{I}$ & $\mathbf{9 5 1 . 4 2 0 0}$ & 476.2136 & $\mathbf{9 3 4 . 3 9 3 5}$ & $\mathbf{4 6 7 . 7 0 0 4}$ & 933.4095 & $\mathbf{4 6 7 . 2 0 8 4}$ & $\mathbf{8}$ \\
\hline $\mathbf{4}$ & 500.2715 & 250.6394 & 483.2449 & 242.1261 & $\mathbf{4 8 2 . 2 6 0 9}$ & 241.6341 & $\mathbf{E}$ & $\mathbf{8 3 8 . 3 3 6 0}$ & 419.6716 & $\mathbf{8 2 1 . 3 0 9 4}$ & 411.1583 & 820.3254 & 410.6663 & $\mathbf{7}$ \\
\hline $\mathbf{5}$ & 628.3301 & 314.6687 & $\mathbf{6 1 1 . 3 0 3 5}$ & 306.1554 & 610.3195 & 305.6634 & $\mathbf{Q}$ & $\mathbf{7 0 9 . 2 9 3 4}$ & 355.1503 & $\mathbf{6 9 2 . 2 6 6 8}$ & 346.6370 & 691.2828 & 346.1450 & $\mathbf{6}$ \\
\hline $\mathbf{6}$ & $\mathbf{7 4 3 . 3 5 7 0}$ & $\mathbf{3 7 2 . 1 8 2 1}$ & $\mathbf{7 2 6 . 3 3 0 5}$ & 363.6689 & $\mathbf{7 2 5 . 3 4 6 4}$ & 363.1769 & $\mathbf{N}$ & $\mathbf{5 8 1 . 2 3 4 8}$ & 291.1210 & $\mathbf{5 6 4 . 2 0 8 2}$ & 282.6078 & 563.2242 & 282.1157 & $\mathbf{5}$ \\
\hline $\mathbf{7}$ & $\mathbf{8 0 0 . 3 7 8 5}$ & 400.6929 & $\mathbf{7 8 3 . 3 5 1 9}$ & 392.1796 & 782.3679 & 391.6876 & $\mathbf{G}$ & $\mathbf{4 6 6 . 2 0 7 8}$ & 233.6076 & 449.1813 & 225.0943 & 448.1973 & 224.6023 & $\mathbf{4}$ \\
\hline $\mathbf{8}$ & $\mathbf{8 8 7 . 4 1 0 5}$ & 444.2089 & 870.3839 & $\mathbf{4 3 5 . 6 9 5 6}$ & $\mathbf{8 6 9 . 3 9 9 9}$ & 435.2036 & $\mathbf{S}$ & $\mathbf{4 0 9 . 1 8 6 4}$ & 205.0968 & 392.1598 & 196.5836 & 391.1758 & 196.0915 & $\mathbf{3}$ \\
\hline $\mathbf{9}$ & 1034.4459 & 517.7266 & 1017.4194 & 509.2133 & $\mathbf{1 0 1 6 . 4 3 5 3}$ & 508.7213 & $\mathbf{M}$ & $\mathbf{3 2 2 . 1 5 4 4}$ & 161.5808 & 305.1278 & 153.0675 & & & \\
\hline $\mathbf{1 0}$ & & & & & & & $\mathbf{R}$ & $\mathbf{1 7 5 . 1 1 9 0}$ & 88.0631 & 158.0924 & 79.5498 & & & $\mathbf{1}$ \\
\hline
\end{tabular}

恶

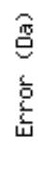

总

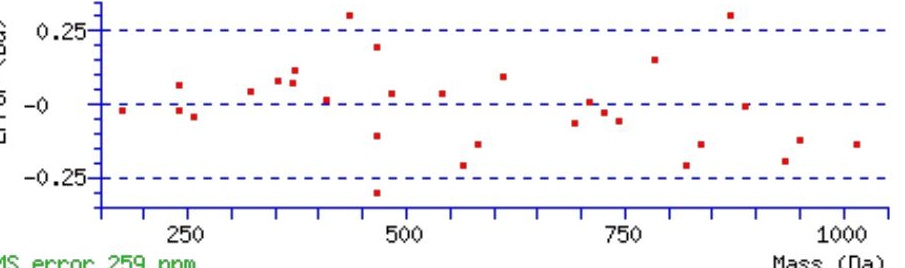

RMS error 259 ppm

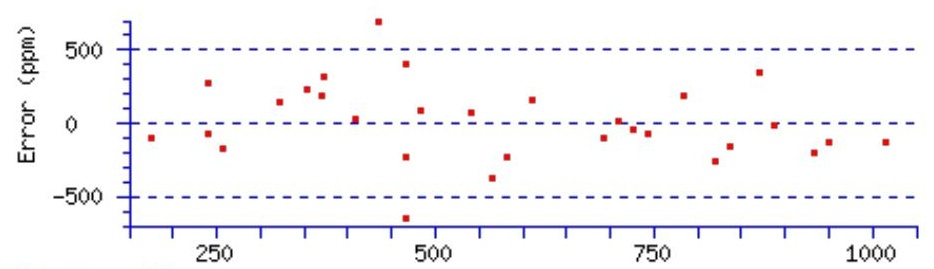

RMS error 259 ppm 


\section{All matches to this query}

\begin{tabular}{|l|l|l|l|}
\hline Score & Mr(calc): & Delta & \multicolumn{1}{|c|}{ Sequence } \\
\hline 41.7 & 1207.5503 & -0.0030 & EKIEQNGSMR \\
\hline 40.3 & 1207.5503 & -0.0030 & EKIEENGSMR \\
\hline 12.9 & 1207.5503 & -0.0030 & QELDLNSSMR \\
\hline 6.7 & 1206.5550 & 0.9922 & IEVGNLDGSMR \\
\hline 5.7 & 1205.5458 & 2.0015 & IGISLSSLTR \\
\hline 4.3 & 1206.5322 & 1.0151 & TIAVYEEFR \\
\hline 1.8 & 1206.5492 & 0.9981 & MFDWITHNK \\
\hline 1.6 & 1207.5503 & -0.0030 & EVANTDGSMR \\
\hline 1.4 & 1207.5486 & -0.0013 & DGLTPQQLEK \\
\hline 1.2 & 1205.5376 & 2.0097 & RLERSYMR \\
\hline
\end{tabular}

Spectrum No: 807; Query: 1855; Rank: 1

\section{Peptide View}

MS/MS Fragmentation of SVITSIAVGDESLRNHSLIR

Found in IPI00193397, Tax_Id=10116 Gene_Symbol=Slc34a1 Sodium-dependent phosphate transport protein 2A

Match to Query 1855: 2167.158582 from(723.393470,3+)

Title: 100101RatKid_NS_deglyco_26.3399.3399.3.dta

Data file K:INewmanPaper|Piliang|3SubProteomes\Piliang3SP\mgf5ppm\ERLIC_3SubProteomes5ppm.mgf
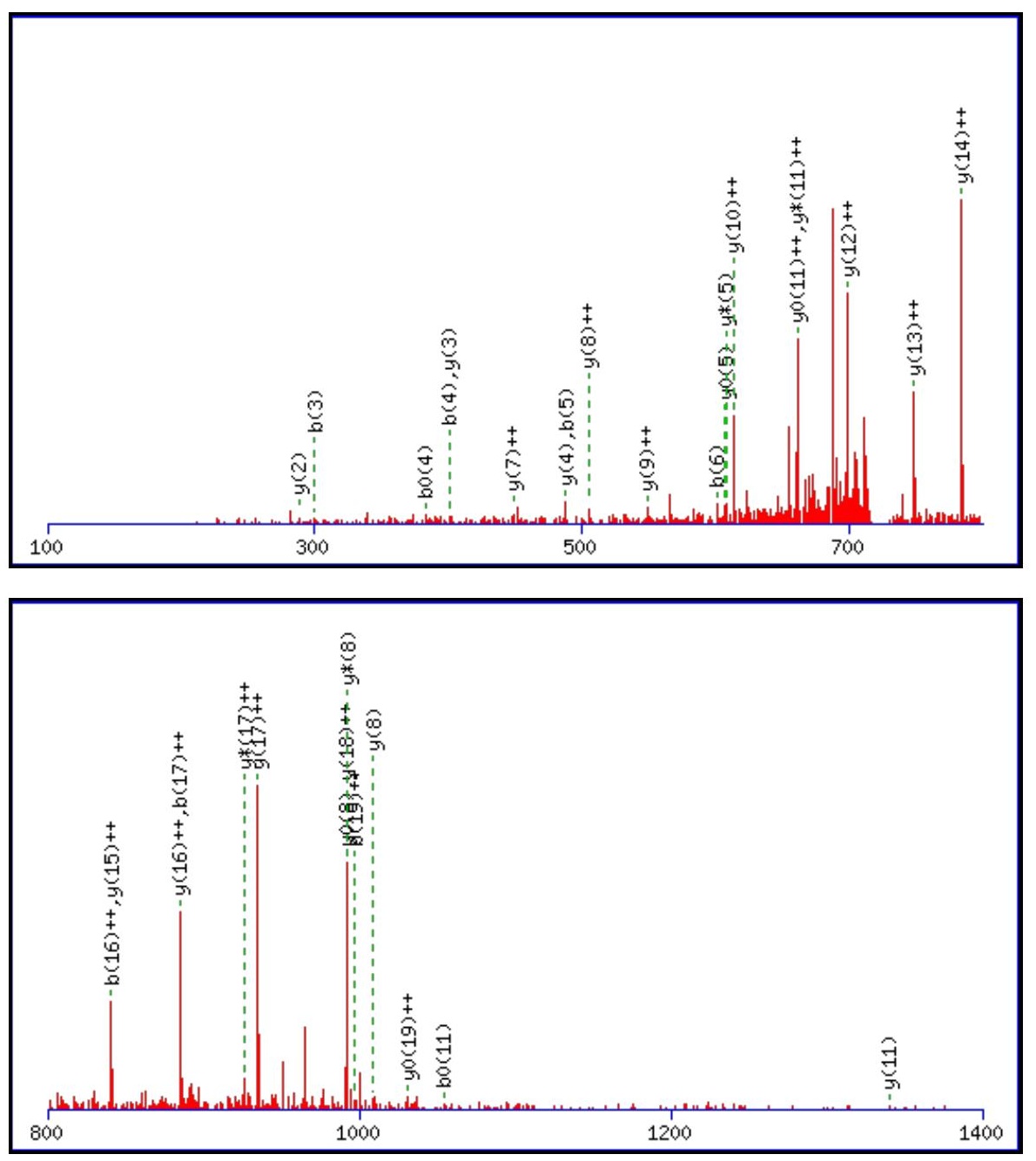


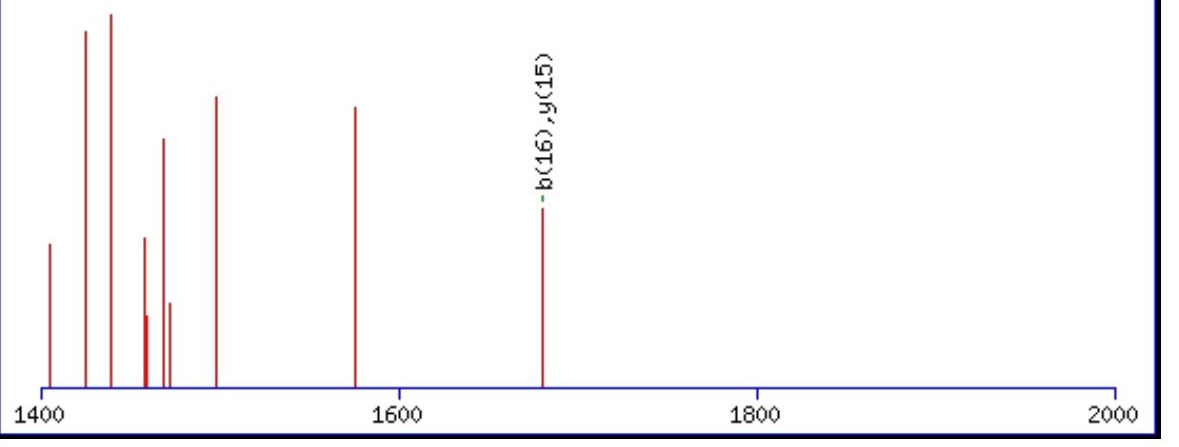

Monoisotopic mass of neutral peptide $\operatorname{Mr}($ calc): 2167.1597

Fixed modifications: Carbamidomethyl (C)

Variable modifications:

N15 : Deamidated_N (N)

Ions Score: 42 Expect: 0.0062

Matches (Bold Red): 35/196 fragment ions using 96 most intense peaks

\begin{tabular}{|c|c|c|c|c|c|c|c|c|c|c|c|c|c|c|}
\hline \# & b & $\mathbf{b}^{++}$ & b* & $\mathbf{b}^{*^{++}}$ & $\mathbf{b}^{0}$ & $\mathbf{b}^{\mathbf{0 + +}}$ & Seq. & $\mathbf{y}$ & $\mathbf{y}^{++}$ & $\mathbf{y}^{*}$ & $\mathbf{y}^{*^{++}}$ & $\mathbf{y}^{0}$ & $y^{0++}$ & \# \\
\hline 1 & 88.0393 & 44.5233 & & & 70.0287 & 35.5180 & $S$ & & & & & & & 20 \\
\hline 2 & 187.1077 & 94.0575 & & & 169.0972 & 85.0522 & $\mathbf{V}$ & 2081.1349 & 1041.0711 & 2064.1084 & 1032.5578 & 2063.1244 & 1032.0658 & 19 \\
\hline 3 & 300.1918 & 150.5995 & & & 282.1812 & 141.5942 & I & 1982.0665 & 991.5369 & 1965.0400 & 983.0236 & 1964.0560 & 982.5316 & 18 \\
\hline 4 & 401.2395 & 201.1234 & & & 383.2289 & 192.1181 & $\mathbf{T}$ & 1868.9825 & 934.9949 & 1851.9559 & 926.4816 & 1850.9719 & 925.9896 & 17 \\
\hline 5 & 488.2715 & 244.6394 & & & 470.2609 & 235.6341 & $\mathbf{S}$ & 1767.9348 & 884.4710 & 1750.9082 & 875.9578 & 1749.9242 & 875.4657 & 16 \\
\hline 6 & 601.3556 & 301.1814 & & & 583.3450 & 292.1761 & I & 1680.9028 & 840.9550 & 1663.8762 & 832.4417 & 1662.8922 & 831.9497 & 15 \\
\hline 7 & 672.3927 & 336.7000 & & & 654.3821 & 327.6947 & A & 1567.8187 & 784.4130 & 1550.7921 & 775.8997 & 1549.8081 & 775.4077 & 14 \\
\hline 8 & 771.4611 & 386.2342 & & & 753.4505 & 377.2289 & V & 1496.7816 & 748.8944 & 1479.7550 & 740.3811 & 1478.7710 & 739.8891 & 13 \\
\hline 9 & 828.4825 & 414.7449 & & & 810.4720 & 405.7396 & G & 1397.7132 & 699.3602 & 1380.6866 & 690.8469 & 1379.7026 & 690.3549 & 12 \\
\hline 10 & 943.5095 & 472.2584 & & & 925.4989 & 463.2531 & D & 1340.6917 & 670.8495 & 1323.6651 & 662.3362 & 1322.6811 & 661.8442 & 11 \\
\hline 11 & 1072.5521 & 536.7797 & & & 1054.5415 & 527.7744 & $\mathbf{E}$ & 1225.6648 & 613.3360 & 1208.6382 & 604.8227 & 1207.6542 & 604.3307 & 10 \\
\hline 12 & 1159.5841 & 580.2957 & & & 1141.5735 & 571.2904 & $\mathbf{S}$ & 1096.6222 & 548.8147 & 1079.5956 & 540.3014 & 1078.6116 & 539.8094 & 9 \\
\hline 13 & 1272.6682 & 636.8377 & & & 1254.6576 & 627.8324 & $\mathbf{L}$ & 1009.5901 & 505.2987 & 992.5636 & 496.7854 & 991.5796 & 496.2934 & 8 \\
\hline 14 & 1428.7693 & 714.8883 & 1411.7427 & 706.3750 & 1410.7587 & 705.8830 & $\mathbf{R}$ & 896.5061 & 448.7567 & 879.4795 & 440.2434 & 878.4955 & 439.7514 & 7 \\
\hline 15 & 1543.7962 & 772.4017 & 1526.7697 & 763.8885 & 1525.7857 & 763.3965 & $\mathbf{N}$ & 740.4050 & 370.7061 & 723.3784 & 362.1928 & 722.3944 & 361.7008 & 6 \\
\hline 16 & 1680.8551 & 840.9312 & 1663.8286 & 832.4179 & 1662.8446 & 831.9259 & $\mathbf{H}$ & 625.3780 & 313.1926 & 608.3515 & 304.6794 & 607.3675 & 304.1874 & 5 \\
\hline 17 & 1767.8872 & 884.4472 & 1750.8606 & 875.9339 & 1749.8766 & 875.4419 & $\mathbf{S}$ & 488.3191 & 244.6632 & 471.2926 & 236.1499 & 470.3085 & 235.6579 & 4 \\
\hline 18 & 1880.9712 & 940.9892 & 1863.9447 & 932.4760 & 1862.9607 & 931.9840 & $\mathbf{L}$ & 401.2871 & 201.1472 & 384.2605 & 192.6339 & & & 3 \\
\hline 19 & 1994.0553 & 997.5313 & 1977.0287 & 989.0180 & 1976.0447 & 988.5260 & I & 288.2030 & 144.6051 & 271.1765 & 136.0919 & & & 2 \\
\hline 20 & & & & & & & $\mathbf{R}$ & 175.1190 & 88.0631 & 158.0924 & 79.5498 & & & 1 \\
\hline
\end{tabular}
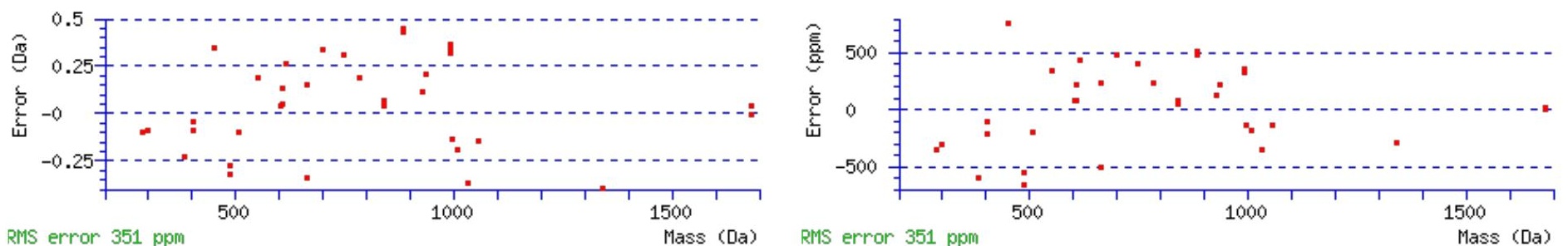

\section{All matches to this query}

\begin{tabular}{|l|c|c|c|}
\hline Score & Mr(calc): & Delta & Sequence \\
\hline 41.6 & 2167.1597 & -0.0011 & SVITSIAVGDESLRNHSLIR \\
\hline
\end{tabular}




\section{\begin{tabular}{|l|l|l|l|l|}
0.1 & 2166.1757 & 0.9829 & SVITSIAVGDESLRNHSLIR \\
\hline
\end{tabular}}

Spectrum No: 808; Query: 1539; Rank: 1

\section{Peptide View}

MS/MS Fragmentation of GRDLDTDFTSNASQPETK

Found in IPI00213182, Tax_Id=10116 Gene_Symbol=Cpd Isoform 1 of Carboxypeptidase D precursor

Match to Query 1539: 1981.892202 from(661.638010,3+)

Title: 100101RatKid_NS_deglyco_23.1509.1509.3.dta

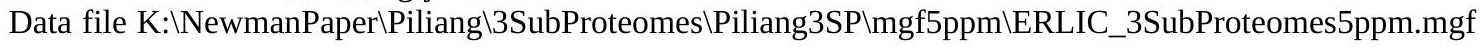
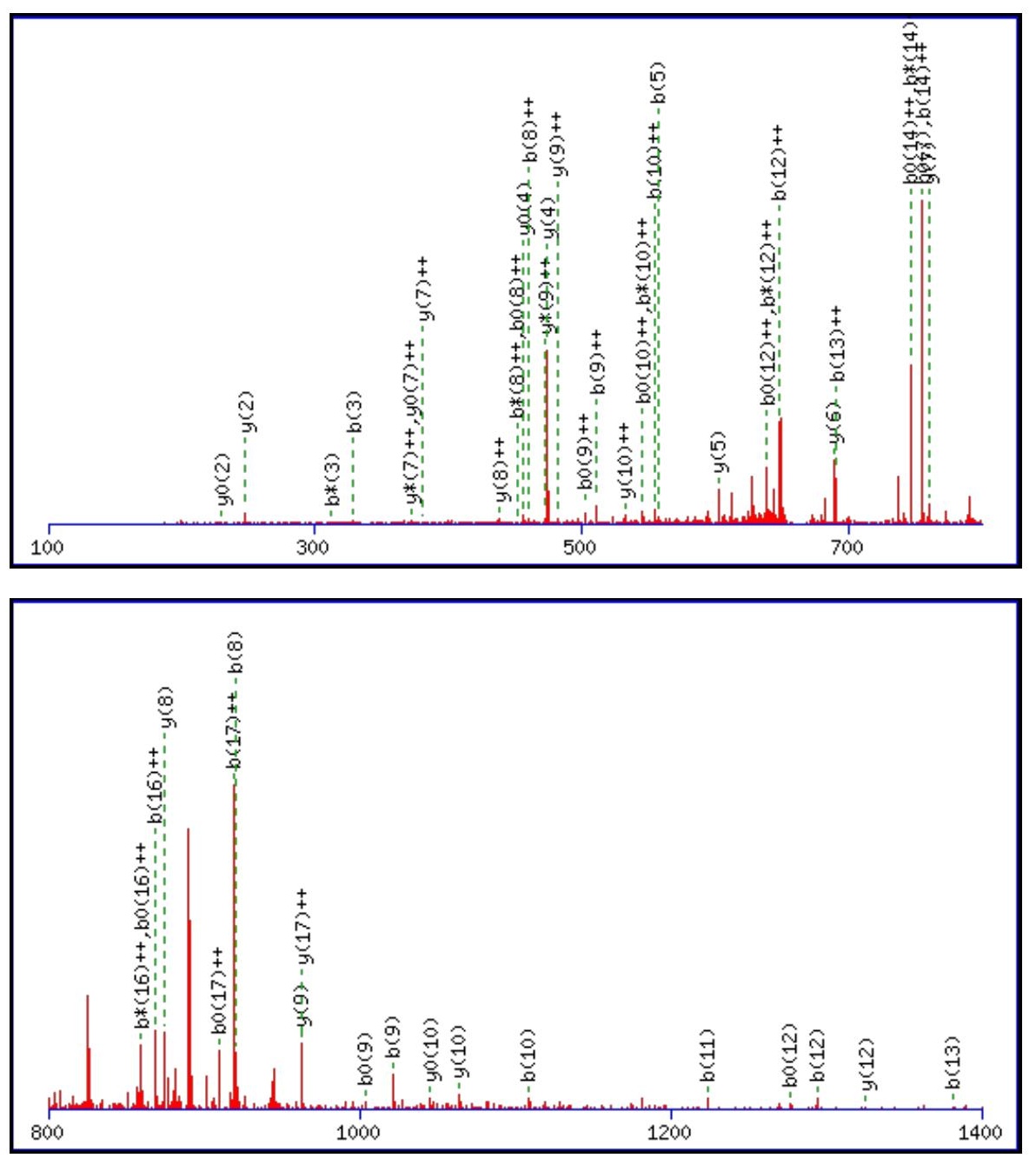


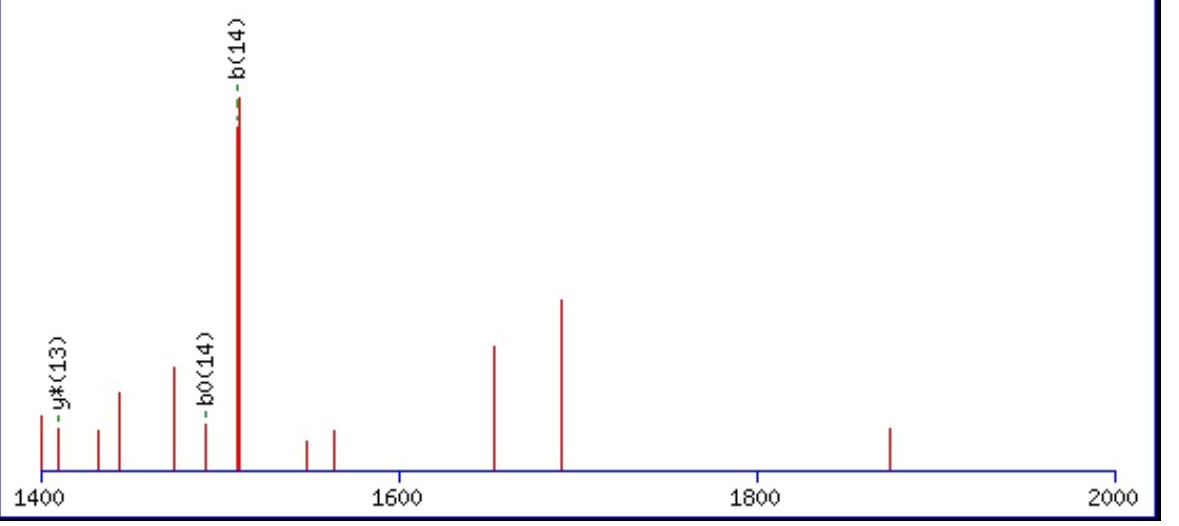

Monoisotopic mass of neutral peptide $\operatorname{Mr}($ calc): 1981.8865

Fixed modifications: Carbamidomethyl (C)

Variable modifications:

N11 : Deamidated_N (N)

Ions Score: 42 Expect: 0.018

Matches (Bold Red): 55/196 fragment ions using 98 most intense peaks

\begin{tabular}{|c|c|c|c|c|c|c|c|c|c|c|c|c|c|c|}
\hline \# & b & $\mathbf{b}^{++}$ & $\mathbf{b}^{*}$ & $\mathbf{b}^{*^{++}}$ & $\mathbf{b}^{\mathbf{0}}$ & & Seq. & $\mathbf{y}$ & $\mathbf{y}^{++}$ & $\mathrm{y}^{*}$ & $\mathbf{y}^{*^{++}}$ & $\mathbf{y}^{0}$ & $\mathbf{y}^{\mathbf{0 + +}}$ & \# \\
\hline 1 & 58.0287 & 29.5180 & & & & & G & & & & & & & 18 \\
\hline 2 & 214.1299 & 107.5686 & 197.1033 & & & & $\mathbf{R}$ & & & 1908.8458 & 954.9265 & 1907.8617 & 954.4345 & 17 \\
\hline 3 & 329.1568 & 165.0820 & 312.1302 & 156.5688 & 311.1462 & 156.0768 & D & 1769.7712 & 885.3892 & 1752.7446 & 876.8760 & 1751.7606 & 876.3840 & 16 \\
\hline 4 & 442.2409 & 221.6241 & 425.2143 & 213.1108 & 424.2303 & 212.6188 & $\mathbf{L}$ & 1654.7442 & 827.8758 & 1637.7177 & 819.3625 & 1636.7337 & 818.8705 & 15 \\
\hline 5 & 557.2678 & 279.1375 & 540.2413 & 270.6243 & 539.2572 & 270.1323 & D & 1541.6602 & 771.3337 & 1524.6336 & 762.8205 & 1523.6496 & 762.3284 & 14 \\
\hline 6 & 658.3155 & 329.6614 & 641.2889 & 321.1481 & 640.3049 & 320.6561 & $\mathbf{T}$ & 1426.6332 & 713.8203 & 1409.6067 & 705.3070 & 1408.6227 & 704.8150 & 13 \\
\hline 7 & 773.3424 & 387.1748 & 756.3159 & 378.6616 & 755.3319 & 378.1696 & D & 1325. & 663.2964 & 1308.5590 & 654.7831 & 1307.5750 & 654.2911 & 12 \\
\hline 8 & 920.4108 & 460.7091 & 903.3843 & 452.1958 & 902.4003 & 451.7038 & $\mathbf{F}$ & & 605.7829 & 1193.5321 & 597.2697 & 1192.5481 & 596.7777 & 11 \\
\hline 9 & 1021.4585 & 511.2329 & 1004.4320 & 502.7196 & 1003.4480 & 502.2276 & $\mathbf{T}$ & 1063.4902 & 532.2487 & 1046.4637 & 523.7355 & 1045.4796 & 523.2435 & 10 \\
\hline 10 & 1108.4905 & 554.7489 & 1091.4640 & 546.2356 & 1090.4800 & 545.7436 & $\mathbf{S}$ & 962.4425 & 481.7249 & 945.4160 & 473.2116 & 944.4320 & 472.7196 & 9 \\
\hline 11 & 1223.5175 & 612.2624 & 1206.4909 & 603.7491 & 1205.5069 & 603.2571 & $\mathbf{N}$ & 875.4105 & 438.2089 & 858.3839 & 429.6956 & 857.3999 & 429.2036 & 8 \\
\hline 12 & 1294.5546 & 647.7809 & 1277.5280 & 639.2677 & 1276.5440 & 638.7757 & A & 760.3836 & 380.6954 & 743.3570 & 372.1821 & 742.3730 & 371.6901 & 7 \\
\hline 13 & 1381.5866 & 691.2969 & 1364.5601 & 682.7837 & 1363.5761 & 682.2917 & S & 689.3464 & 345.1769 & 672.3199 & 336.6636 & 671.3359 & 336.1716 & 6 \\
\hline 14 & 1509.6452 & 755.3262 & 1492.6187 & 746.8130 & 1491.6346 & 746.3210 & $\mathbf{Q}$ & 602.3144 & 301.6608 & 585.2879 & 293.1476 & 584.3039 & 292.6556 & 5 \\
\hline 15 & 1606.6980 & 803.8526 & 1589.6714 & 795.3393 & 1588.6874 & 794.8473 & $\mathbf{P}$ & 474.2558 & 237.6316 & 457.2293 & 229.1183 & 456.2453 & 228.6263 & 4 \\
\hline 16 & 1735.7406 & 868.3739 & 1718.7140 & 859.8606 & 1717.7300 & 859.3686 & $\mathbf{E}$ & 377.2031 & 189.1052 & 360.1765 & 180.5919 & 359.1925 & 180.0999 & 3 \\
\hline 17 & 1836.7882 & 918.8978 & 1819.7617 & 910.3845 & 1818.7777 & 909.8925 & $\mathbf{T}$ & 248.1605 & 124.5839 & 231.1339 & 116.0706 & 230.1499 & 115.5786 & 2 \\
\hline 18 & & & & & & & $\mathbf{K}$ & 147.1128 & 74.0600 & 130.0863 & 65.5468 & & & 1 \\
\hline
\end{tabular}

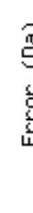

RMS error 315 ppm

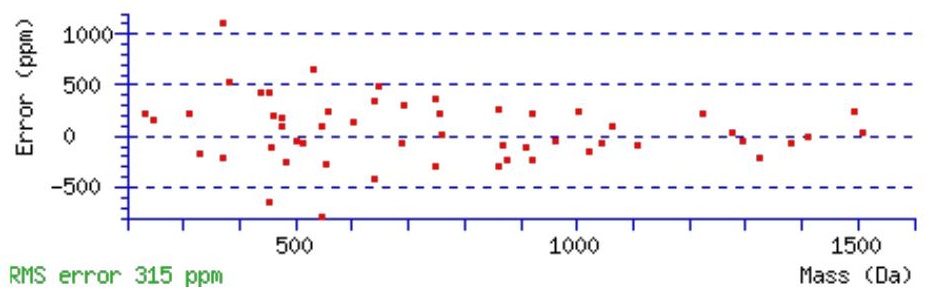

\section{All matches to this query}

\begin{tabular}{|l|l|l|l|}
\hline Score & Mr(calc): & Delta & \multicolumn{1}{c|}{ Sequence } \\
\hline 41.6 & 1981.8865 & 0.0057 & GRDLDTDFTSNASQPETK \\
\hline 18.5 & 1980.9025 & 0.9897 & GRDLDTDFTSNASQPETK \\
\hline 7.1 & 1981.8986 & -0.0064 & VSKASSELMSYCEQHAR \\
\hline & & &
\end{tabular}




\begin{tabular}{|l|l|l|l|}
6.8 & 1981.8935 & -0.0013 & GAPQHYPKTAGNSEFLGK \\
\hline 3.6 & 1981.8832 & 0.0090 & MSSVSTESKLQQAVSLK \\
\hline 3.6 & 1981.8832 & 0.0090 & MSSVSTESKLQQAVSLK \\
\hline 3.6 & 1981.8832 & 0.0090 & MSSVSTESKLQQAVSLK \\
\hline 3.3 & 1981.8939 & -0.0017 & TNMEYPNTPETQDLVSK \\
\hline 3.2 & 1981.9115 & -0.0193 & SGLRDMHLLSCLESLR \\
\hline 2.9 & 1981.8757 & 0.0165 & FLNHHSQXCPSGTLIK \\
\hline
\end{tabular}

Spectrum No: 809; Query: 2442; Rank: 1

\section{Peptide View}

MS/MS Fragmentation of GAGLENPVAVTIFFGANDSTLKDENPK

Found in IPI00421610, Tax_Id=10116 Gene_Symbol=Harpb64 Isoamyl acetate-hydrolyzing esterase 1 homolog

Match to Query 2442: 2804.400732 from(935.807520,3+)

Title: 091008RatKidney_NH4Format01_06.4179.4179.3.dta

Data file K:INewmanPaper|Piliang|3SubProteomes\Piliang3SP\mgf5ppm\ERLIC_3SubProteomes5ppm.mgf
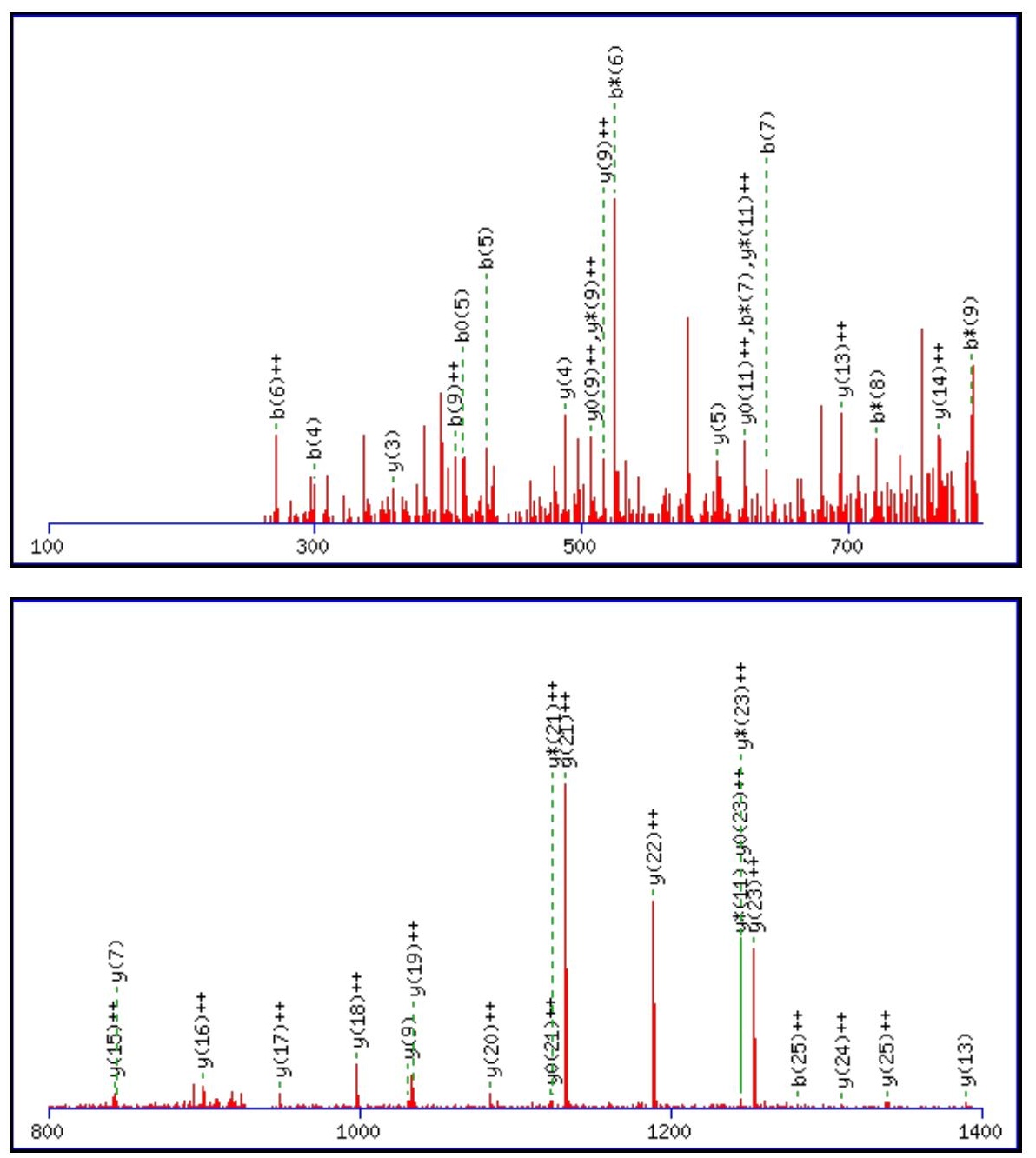


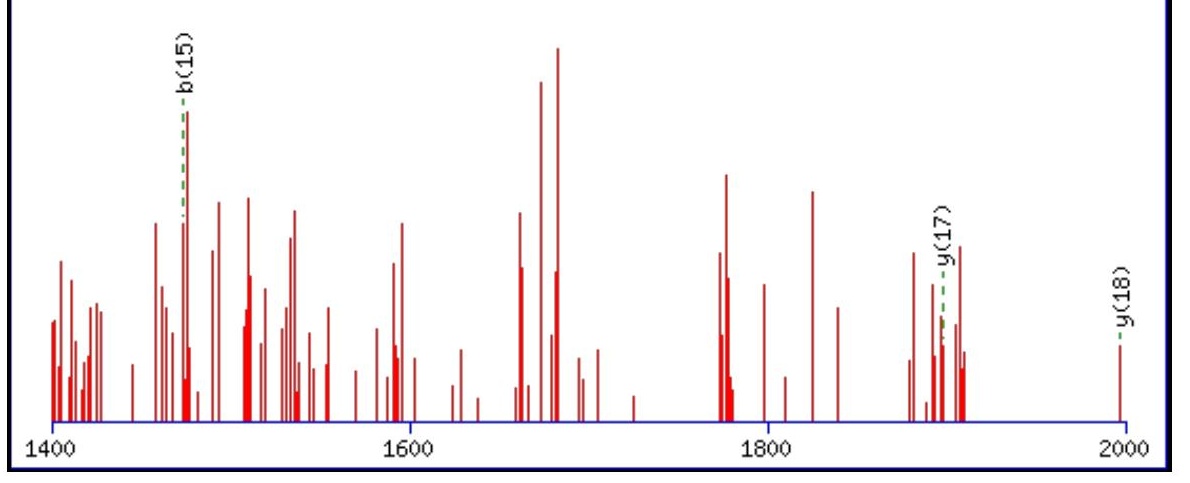

Monoisotopic mass of neutral peptide $\operatorname{Mr}($ calc): 2804.3868

Fixed modifications: Carbamidomethyl (C)

Variable modifications:

N17 : Deamidated_N (N)

Ions Score: 42 Expect: 0.015

Matches (Bold Red): 43/288 fragment ions using 124 most intense peaks

\begin{tabular}{|c|c|c|c|c|c|c|c|c|c|c|c|c|c|c|}
\hline$\pi$ & b & $\mathbf{b}^{++}$ & $\mathbf{b}^{*}$ & $\mathbf{b}^{*^{++}}$ & $\mathbf{b}^{\mathbf{0}}$ & ++ & q. & $\mathbf{y}$ & $\mathbf{y}^{++}$ & $\mathbf{y}^{*}$ & $\mathrm{y}^{*^{++}}$ & $\mathbf{y}^{0}$ & ++ & \# \\
\hline 1 & 58.0287 & 29.5180 & & & & & G & & & & & & & 27 \\
\hline 2 & 29.0659 & & & & & & A & & & & & & & 26 \\
\hline 3 & 186.0873 & 93.5 & & & & & G & 355 & 714 & 990 & & 250 & 661 & 25 \\
\hline 4 & 299.1714 & 150.0893 & & & & & $\mathbf{L}$ & 2620.3141 & 1310.6607 & 2603.2875 & 474 & 2602.3035 & 554 & 24 \\
\hline 5 & 428.2140 & 214.6106 & & & & & $\mathbf{E}$ & 2507.2300 & 1254.1186 & 2490.2035 & 6054 & 2489.2195 & 1134 & 23 \\
\hline 6 & 542.2569 & 271.6321 & 04 & & 524 & & $\mathbf{N}$ & 1874 & 974 & 609 & 841 & 2360.1769 & 621 & 22 \\
\hline 7 & 639.3097 & 320.1585 & 622.2831 & 311. & 621.2991 & 311 & $\mathbf{P}$ & 2264.1445 & 1132.5759 & 2247.1179 & 1124.0626 & 2246.1339 & 1123.5706 & 21 \\
\hline 8 & 738.3781 & 369.6927 & 721.3515 & 361. & 720.3 & 874 & $\mathbf{V}$ & 2167.0917 & 1084.0495 & 2150. & 1075 & 2149. & 0442 & 20 \\
\hline 9 & 809.4152 & 405.2112 & 792. & 396. & 791 & & $\mathbf{A}$ & 233 & 1034 & 205 & 20 & 205 & 100 & 19 \\
\hline 10 & 908.4836 & 454.7454 & 891.4571 & 446.2322 & 890.4730 & 445.7402 & $\mathbf{V}$ & 1996.9862 & 998.9967 & 1979.9597 & 4835 & 1978.9756 & 989.9915 & 18 \\
\hline 11 & 1009.5313 & 505.2693 & 47 & 496. & & & $T$ & 1897.9178 & & 1880.8 & & 1879.9072 & 4573 & 17 \\
\hline 2 & 1122.6154 & 561.8113 & 1105.5888 & 553 & 48 & 552 & I & 1796.8701 & 898.9387 & 1779.8436 & 890.4254 & 1778.8595 & 334 & 16 \\
\hline 13 & 1269.6838 & 635.3455 & 1252.6572 & 626.8322 & 1251.6732 & 626.3402 & $\mathbf{F}$ & $1683.7861 \mid$ & 842.3967 & 1666.7595 & 833.8834 & 1665.7755 & 833.3914 & 15 \\
\hline 14 & 1416.7522 & 708.8797 & 99.7256 & 700. & 416 & 699. & $\mathbf{F}$ & 1536. & 625 & 1519 & 492 & 1518 & 8572 & 14 \\
\hline 5 & 1473.7736 & 737.3905 & 1456.7471 & 728.8772 & 1455.7631 & 728.3852 & $\mathbf{G}$ & 1389.6492 & 695.3282 & 1372.6227 & & 1371.6387 & 3230 & 13 \\
\hline 16 & 1544.8108 & 772.9090 & 1527.7842 & 764.3957 & 1526.8002 & 763.9037 & A & 1332.6278 & 666.8175 & 1315.6012 & 658.3042 & 1314.6172 & 657.8122 & 12 \\
\hline 17 & 1659.8377 & 830.4225 & 1642.8111 & 821.9 & 71 & 821 & $\mathbf{N}$ & 1261.5 & 990 & 1244. & 857 & 1243 & 2937 & 11 \\
\hline 18 & 1774.8646 & 887.9360 & 1757.8381 & 879. & 1756. & 878.9307 & D & 1146. & 573.7855 & 1129. & 2722 & 1128.5 & 7802 & 10 \\
\hline 19 & 1861.8967 & 931.4520 & 1844.8701 & 922.9387 & 1843.8861 & 922.4467 & $\mathrm{~S}$ & 1031.5368 & 516.2720 & 1014.5102 & 507.7587 & 1013.5262 & 507.2667 & 9 \\
\hline 20 & 1962.9443 & 981.9758 & 1945.9178 & 973.4625 & 1944.9338 & 972.9705 & $T$ & 944.5047 & 472.7560 & 927.4782 & 464.2427 & 926.4942 & 463.7507 & 8 \\
\hline 21 & 2076.0284 & 1038.5178 & 2059.0019 & 30.0046 & 2058.0178 & 1029.5126 & $\mathbf{L}$ & 843.4571 & 422.2322 & 826.4305 & & 825.4465 & 2269 & 7 \\
\hline 22 & 2204.1234 & 1102.5653 & 2187.0968 & 1094.0520 & 2186.1128 & 1093.5600 & $\mathbf{K}$ & 730.3730 & 365.6901 & 713.3464 & 357.1769 & 712.3624 & 356.6849 & 6 \\
\hline 23 & 2319.1503 & 1160.0788 & 2302.1238 & 1151.5655 & 2301.1397 & 1151.0735 & D & 602.2780 & 301.6427 & 585.2515 & 293.1294 & 584.2675 & 292.6374 & 5 \\
\hline 24 & 2448.1929 & 1224.6001 & 2431.1664 & 1216.0868 & 2430.1823 & 1215.5948 & $\mathbf{E}$ & 487.2511 & 244.1292 & 470.2245 & 235.6159 & 469.2405 & 235.1239 & 4 \\
\hline 25 & 2562.2358 & \begin{tabular}{|l|l|}
1281.6216 \\
\end{tabular} & 2545.2093 & 1273.1083 & 2544.2253 & 1272.6163 & $\mathbf{N}$ & 358.2085 & 179.6079 & 341.1819 & & & & 3 \\
\hline 26 & 2659.2886 & 1330.1479 & 2642.2620 & 1321.6347 & 2641.2780 & 1321.1427 & $\mathbf{P}$ & 244.1656 & 122.5864 & 227.1390 & 114.0731 & & & 2 \\
\hline 27 & & & & & & & $\mathbf{K}$ & 147.1128 & 74.0600 & 130.0863 & 65.5468 & & & \\
\hline
\end{tabular}



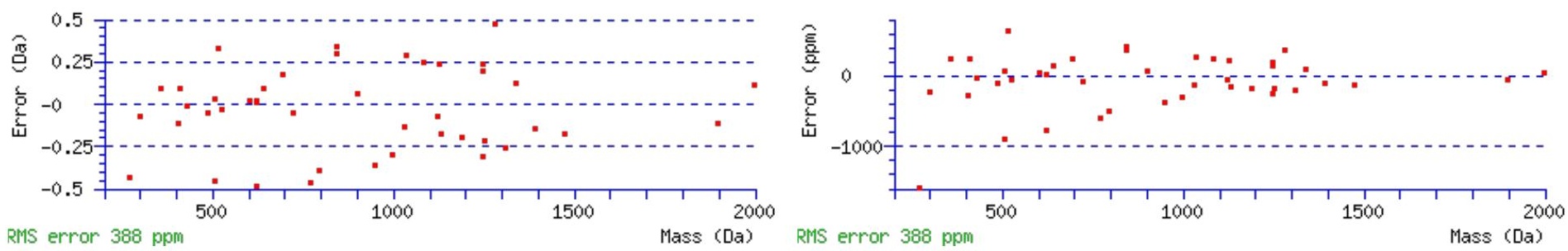

\section{All matches to this query}

\begin{tabular}{|l|l|l|l|}
\hline Score & Mr(calc): & Delta & \multicolumn{1}{|c|}{ Sequence } \\
\hline 41.6 & 2804.3868 & 0.0139 & GAGLENPVAVTIFFGANDSTLKDENPK \\
\hline 40.9 & 2803.4028 & 0.9979 & GAGLENPVAVTIFFGANDSTLKDENPK \\
\hline 38.0 & 2804.3868 & 0.0139 & GAGLENPVAVTIFFGANDSTLKDENPK \\
\hline 37.7 & 2804.3868 & 0.0139 & GAGLENPVAVTIFFGANDSTLKDENPK \\
\hline 3.0 & 2804.4110 & -0.0102 & SLELVPNNQGLTPFKLAGVEGNTVAR \\
\hline 2.4 & 2804.4110 & -0.0102 & SLELVPNNQGLTPFKLAGVEGNTVAR \\
\hline 2.4 & 2804.4110 & -0.0102 & SLELVPNNQGLTPFKLAGVEGNTVAR \\
\hline 2.3 & 2804.3954 & 0.0053 & ETTPLEGDQQQGDERVPPPRFRPR \\
\hline 2.3 & 2803.4058 & 0.9949 & SIFIDQRELLTIWGHIVQQNGQK \\
\hline 2.3 & 2803.4033 & 0.9974 & FTVPFHQALSGFHPAPLCSIRTLK \\
\hline
\end{tabular}

Spectrum No: 810; Query: 2378; Rank: 1

\section{Peptide View}

MS/MS Fragmentation of QGPQAGGTTLTINGTYLDTGSKEDVR

Found in IPI00370450, Tax_Id=10116 Gene_Symbol=Plxnb2 plexin B2

Match to Query 2378: 2679.302382 from(894.108070,3+)

Title: 100101RatKid_NS_deglyco_24.2718.2718.3.dta

Data file K:INewmanPaper|Piliang|3SubProteomes\Piliang3SP\mgf5ppm\ERLIC_3SubProteomes5ppm.mgf

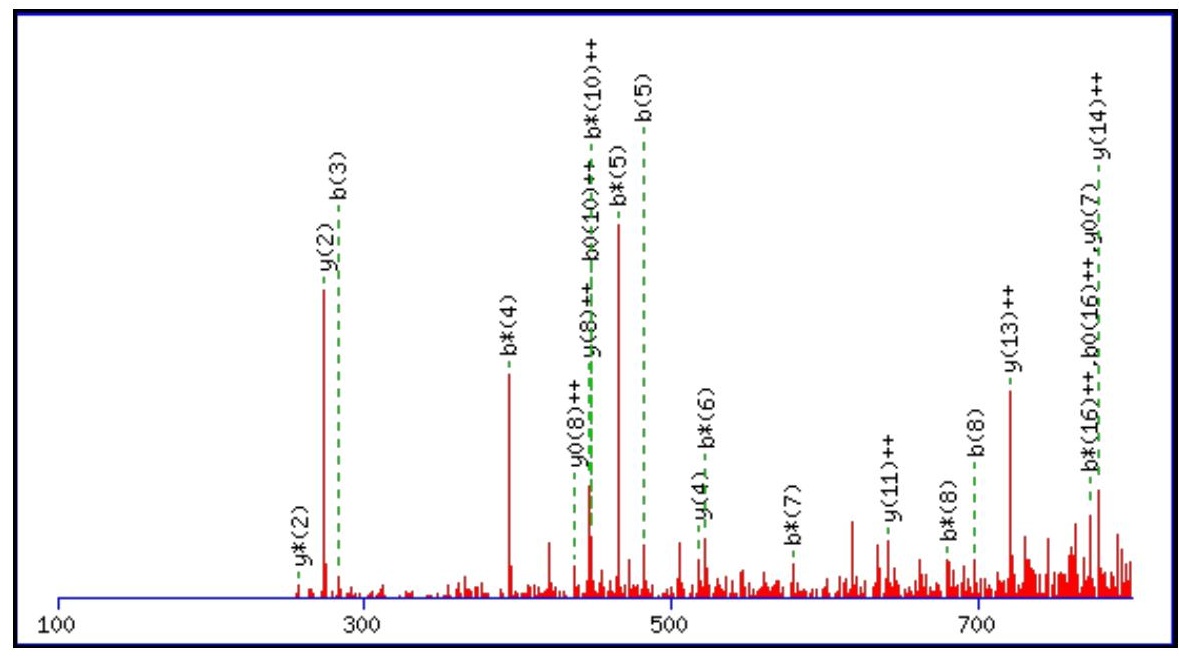



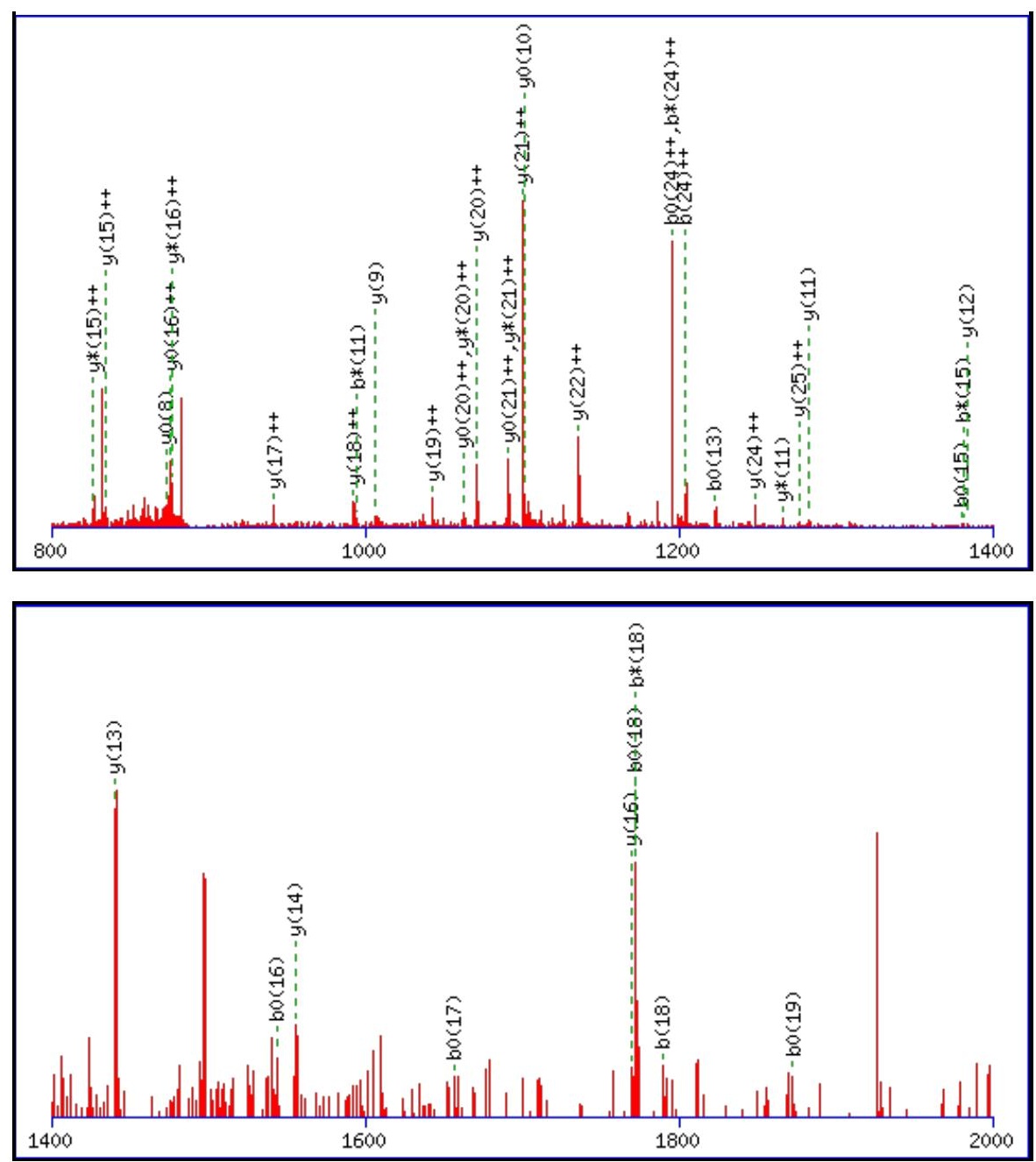

Monoisotopic mass of neutral peptide $\operatorname{Mr}($ calc): 2679.2988

Fixed modifications: Carbamidomethyl (C)

Variable modifications:

N13 : Deamidated_N (N)

Ions Score: 41 Expect: 0.019

Matches (Bold Red): 59/282 fragment ions using 158 most intense peaks

\begin{tabular}{|c|c|c|c|c|c|c|c|c|c|c|c|c|c|c|}
\hline \# & b & $\mathbf{b}^{++}$ & $\mathbf{b}^{*}$ & $\mathbf{b}^{*^{++}}$ & $\mathbf{b}^{\mathbf{0}}$ & $\mathbf{b}^{0++}$ & Seq. & $\mathbf{y}$ & $\mathbf{y}^{++}$ & $\mathbf{y}^{*}$ & $\mathbf{y}^{*^{++}}$ & $\mathbf{y}^{0}$ & $\mathbf{y}^{0++}$ & \# \\
\hline 1 & 129.0659 & 65.0366 & 112.0393 & 56.5233 & & & $\mathbf{Q}$ & & & & & & & 26 \\
\hline 2 & 186.0873 & 93.5473 & 169.0608 & 85.0340 & & & G & 2552.2475 & 1276.6274 & 2535.2209 & 1268.1141 & 2534.2369 & 1267.6221 & 25 \\
\hline 3 & 283.1401 & 142.0737 & 266.1135 & 133.5604 & & & $\mathbf{P}$ & 2495.2260 & 1248.1166 & 2478.1995 & 1239.6034 & 2477.2154 & 1239.1114 & 24 \\
\hline 4 & 411.1987 & 206.1030 & 394.1721 & 197.5897 & & & $\mathbf{Q}$ & 2398.1732 & 1199.5903 & 2381.1467 & 1191.0770 & 2380.1627 & 1190.5850 & 23 \\
\hline 5 & 482.2358 & 241.6215 & 465.2092 & 233.1083 & & & A & 2270.1147 & 1135.5610 & 2253.0881 & 1127.0477 & 2252.1041 & 1126.5557 & 22 \\
\hline 6 & 539.2572 & 270.1323 & 522.2307 & 261.6190 & & & $\mathbf{G}$ & 2199.0775 & 1100.0424 & 2182.0510 & 1091.5291 & 2181.0670 & 1091.0371 & 21 \\
\hline 7 & 596.2787 & 298.6430 & 579.2522 & 290.1297 & & & G & 2142.0561 & 1071.5317 & 2125.0295 & 1063.0184 & 2124.0455 & 1062.5264 & 20 \\
\hline 8 & 697.3264 & 349.1668 & 680.2998 & 340.6536 & 679.3158 & 340.1615 & $\mathbf{T}$ & 2085.0346 & 1043.0209 & 2068.0081 & 1034.5077 & 2067.0241 & 1034.0157 & 19 \\
\hline 9 & 798.3741 & 399.6907 & 781.3475 & 391.1774 & 780.3635 & 390.6854 & $\mathbf{T}$ & 1983.9869 & 992.4971 & 1966.9604 & 983.9838 & 1965.9764 & 983.4918 & 18 \\
\hline 10 & 911.4581 & 456.2327 & 894.4316 & 447.7194 & 893.4476 & 447.2274 & $\mathbf{L}$ & 1882.9393 & 941.9733 & 1865.9127 & 933.4600 & 1864.9287 & 932.9680 & 17 \\
\hline 11 & 1012.5058 & 506.7565 & 995.4793 & 498.2433 & 994.4952 & 497.7513 & $\mathbf{T}$ & 1769.8552 & 885.4312 & 1752.8286 & 876.9180 & 1751.8446 & 876.4260 & 16 \\
\hline 12 & 1125.5899 & 563.2986 & 1108.5633 & 554.7853 & 1107.5793 & 554.2933 & I & 1668.8075 & 834.9074 & 1651.7810 & 826.3941 & 1650.7969 & 825.9021 & 15 \\
\hline 13 & 1240.6168 & 620.8120 & 1223.5903 & 612.2988 & 1222.6062 & 611.8068 & $\mathbf{N}$ & 1555.7235 & 778.3654 & 1538.6969 & 769.8521 & 1537.7129 & 769.3601 & 14 \\
\hline 14 & 1297.6383 & 649.3228 & 1280.6117 & 640.8095 & 1279.6277 & 640.3175 & G & 1440.6965 & 720.8519 & 1423.6700 & 712.3386 & 1422.6859 & 711.8466 & 13 \\
\hline 15 & 1398.6859 & 699.8466 & 1381.6594 & 691.3333 & 1380.6754 & 690.8413 & $\mathbf{T}$ & 1383.6751 & 692.3412 & 1366.6485 & 683.8279 & 1365.6645 & 683.3359 & 12 \\
\hline 16 & 1561.7493 & 781.3783 & 1544.7227 & 772.8650 & 1543.7387 & 772.3730 & $\mathbf{Y}$ & 1282.6274 & 641.8173 & 1265.6008 & 633.3040 & 1264.6168 & 632.8120 & 11 \\
\hline 17 & 1674.8333 & 837.9203 & 1657.8068 & 829.4070 & 1656.8228 & 828.9150 & $\mathbf{L}$ & 1119.5640 & 560.2857 & 1102.5375 & 551.7724 & 1101.5535 & 551.2804 & 10 \\
\hline 18 & 1789.8603 & 895.4338 & 1772.8337 & 886.9205 & 1771.8497 & 886.4285 & D & 1006.4800 & 503.7436 & 989.4534 & 495.2304 & 988.4694 & 494.7383 & 9 \\
\hline
\end{tabular}




\begin{tabular}{|c|c|c|c|c|c|c|c|c|c|c|c|c|c|c|}
\hline 19| & |1890.9080| & 945.9576 & |1873.8814 & 937.4443 & |1872.8974 & 936.9523 & $T$ & 891.4530 & 446.2302 & 874.4265 & 437.7169 & 873.4425 & 437.2249 & 8 \\
\hline 20 & 1947.9294 & 974.4684 & 1930.9029 & 965.9551 & 1929.9189 & 965.4631 & G & 790.4054 & 395.7063 & 773.3788 & 387.1930 & 772.3948 & 386.7010 & 7 \\
\hline 21 & 2034.9615 & 1017.9844 & |2017.9349 & 1009.4711 & 2016.9509 & 1008.9791 & $\mathrm{~S}$ & 733.3839 & 367.1956 & 716.3573 & 358.6823 & 715.3733 & 358.1903 & 6 \\
\hline 22 & 2163.0564 & 1082.0318 & 2146.0299 & 1073.5186 & 2145.0459 & 1073.0266 & $\mathbf{K}$ & 646.3519 & 323.6796 & 629.3253 & 315.1663 & 628.3413 & 314.6743 & 5 \\
\hline 23 & 2292.0990 & 1146.5531 & 2275.0725 & 1138.0399 & 2274.0884 & 1137.5479 & $\mathbf{E}$ & 518.2569 & 259.6321 & 501.2304 & 251.1188 & 500.2463 & 250.6268 & 4 \\
\hline 24 & 2407.1260 & 1204.0666 & 2390.0994 & 1195.5533 & 2389.1154 & 1195.0613 & D & 389.2143 & 195.1108 & 372.1878 & 186.5975 & 371.2037 & 186.1055 & 3 \\
\hline 25 & 2506.1944 & 1253.6008 & 2489.1678 & 1245.0875 & 2488.1838 & 1244.5955 & V & 274.1874 & 137.5973 & 257.1608 & 129.0840 & & & 2 \\
\hline 26 & & & & & & & $\mathbf{R}$ & 175.1190 & 88.0631 & 158.0924 & 79.5498 & & & 1 \\
\hline
\end{tabular}
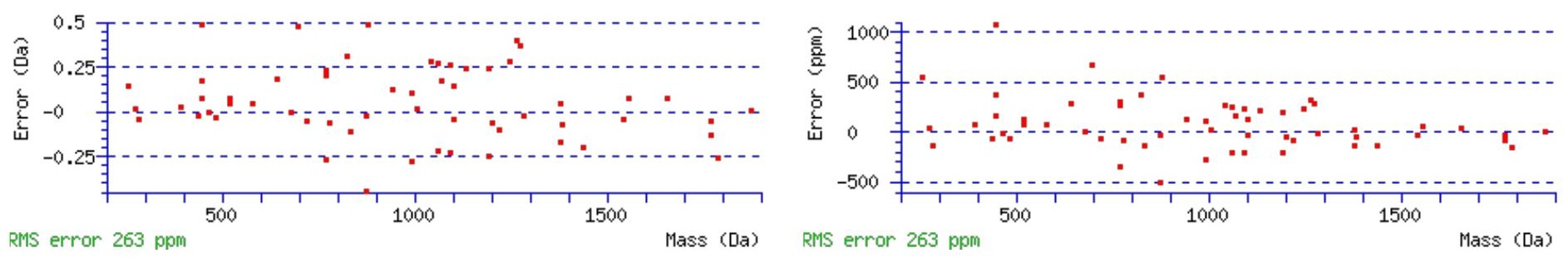

\section{All matches to this query}

\begin{tabular}{|l|l|l|l|}
\hline Score & Mr(calc): & Delta & \multicolumn{1}{c|}{ Sequence } \\
\hline 41.4 & 2679.2988 & 0.0036 & QGPQAGGTTLTINGTYLDTGSKEDVR \\
\hline 9.2 & 2678.3148 & 0.9876 & QGPQAGGTTLTINGTYLDTGSKEDVR \\
\hline 3.6 & 2677.2880 & 2.0144 & CSFATVNIQLMGTNEYVPRFVSK \\
\hline 1.1 & 2677.3096 & 1.9928 & SWHDVQGSTAYKKTQETLSQAGQK \\
\hline 0.3 & 2678.3112 & 0.9912 & ALSMHSTAEVQKTQRAPPPVASHR \\
\hline
\end{tabular}

Spectrum No: 811; Query: 849; Rank: 1

\section{Peptide View}

MS/MS Fragmentation of RVNDNKTAAEEALR

Found in IPI00363849, Tax_Id=10116 Gene_Symbol=Lamc1 similar to Laminin gamma-1 chain precursor

Match to Query 849: 1586.803722 from(529.941850,3+)

Title: 100101RatKid_NS_deglyco_18.944.944.3.dta

Data file K:INewmanPaper|Piliangl3SubProteomes\Piliang3SP\mgf5ppm\ERLIC_3SubProteomes5ppm.mgf

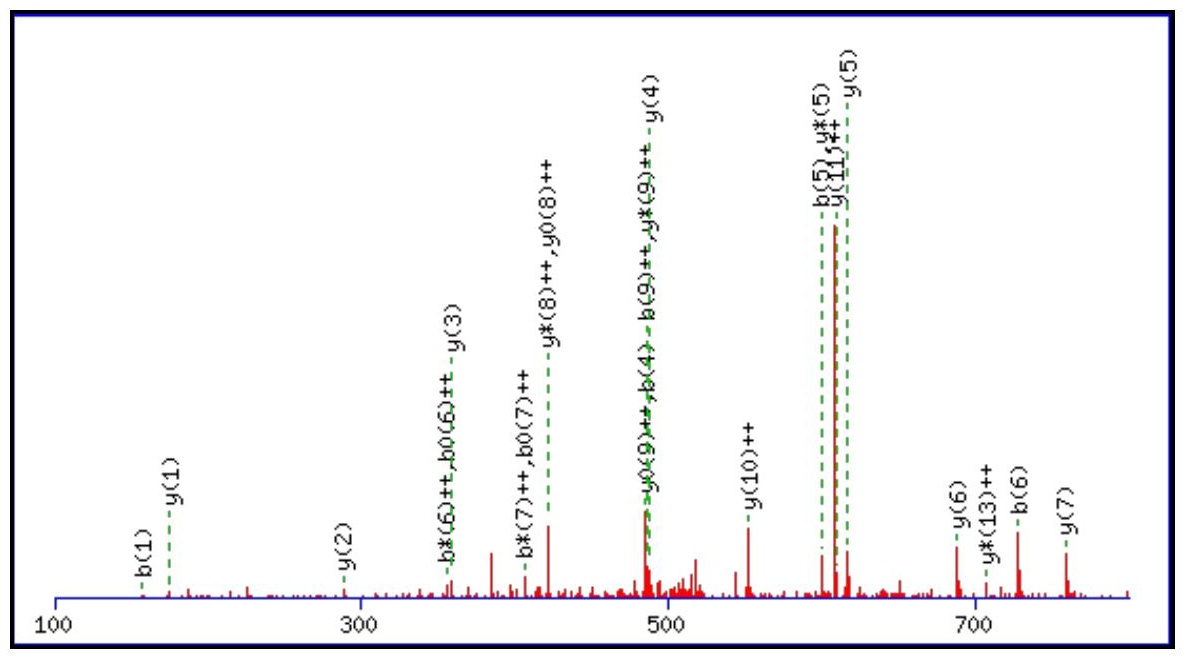



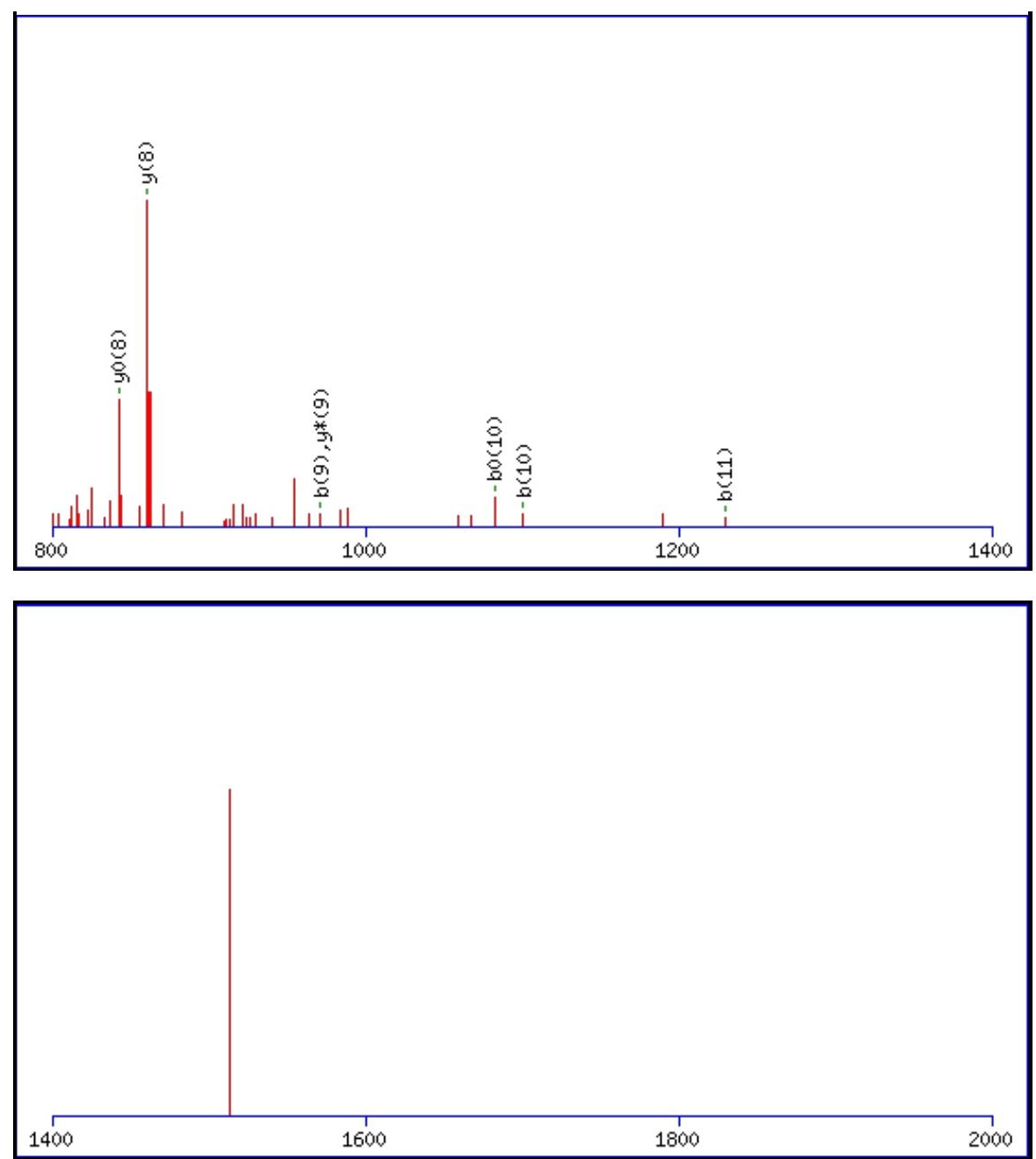

Monoisotopic mass of neutral peptide $\operatorname{Mr}($ calc): 1586.8012

Fixed modifications: Carbamidomethyl (C)

Variable modifications:

N5 : Deamidated_N (N)

Ions Score: 41 Expect: $\odot .01$

Matches (Bold Red): 31/144 fragment ions using 51 most intense peaks

\begin{tabular}{|c|c|c|c|c|c|c|c|c|c|c|c|c|c|c|}
\hline \# & b & $\mathbf{b}^{++}$ & $\mathbf{b}^{*}$ & $\mathbf{b}^{*^{++}}$ & $\mathbf{b}^{0}$ & $\mathbf{b}^{0++}$ & Seq. & $\mathbf{y}$ & $\mathbf{y}^{++}$ & $\mathbf{y}^{*}$ & $\mathrm{y}^{*^{++}}$ & $\mathbf{y}^{\mathbf{0}}$ & $\mathbf{y}^{\mathbf{0 + +}}$ & \# \\
\hline 1 & 157.1084 & 79.0578 & 140.0818 & 70.5446 & & & $\mathbf{R}$ & & & & & & & 14 \\
\hline 2 & 256.1768 & 128.5920 & 239.1503 & 120.0788 & & & $\mathbf{V}$ & 1431.7074 & 716.3573 & 1414.6809 & 707.8441 & 1413.6968 & 707.3521 & 13 \\
\hline 3 & 370.2197 & 185.6135 & 353.1932 & 177.1002 & & & $\mathbf{N}$ & 1332.6390 & 666.8231 & 1315.6124 & 658.3099 & 1314.6284 & 657.8179 & 12 \\
\hline 4 & 485.2467 & 243.1270 & 468.2201 & 234.6137 & 467.2361 & 234.1217 & D & 1218.5961 & 609.8017 & 1201.5695 & 601.2884 & 1200.5855 & 600.7964 & 11 \\
\hline 5 & 600.2736 & 300.6404 & 583.2471 & 292.1272 & 582.2630 & 291.6352 & $\mathbf{N}$ & 1103.5691 & 552.2882 & 1086.5426 & 543.7749 & 1085.5586 & 543.2829 & 10 \\
\hline 6 & 728.3686 & 364.6879 & 711.3420 & 356.1746 & 710.3580 & 355.6826 & $\mathbf{K}$ & 988.5422 & 494.7747 & 971.5156 & 486.2615 & 970.5316 & 485.7694 & 9 \\
\hline 7 & 829.4162 & 415.2118 & 812.3897 & 406.6985 & 811.4057 & 406.2065 & $\mathbf{T}$ & 860.4472 & 430.7272 & 843.4207 & 422.2140 & 842.4367 & 421.7220 & 8 \\
\hline 8 & 900.4534 & 450.7303 & 883.4268 & 442.2170 & 882.4428 & 441.7250 & A & 759.3995 & 380.2034 & 742.3730 & 371.6901 & 741.3890 & 371.1981 & 7 \\
\hline 9 & 971.4905 & 486.2489 & 954.4639 & 477.7356 & 953.4799 & 477.2436 & A & 688.3624 & 344.6849 & 671.3359 & 336.1716 & 670.3519 & 335.6796 & 6 \\
\hline 10 & 1100.5331 & 550.7702 & 1083.5065 & 542.2569 & 1082.5225 & 541.7649 & $\mathbf{E}$ & 617.3253 & 309.1663 & 600.2988 & 300.6530 & 599.3148 & 300.1610 & 5 \\
\hline 11 & 1229.5757 & 615.2915 & 1212.5491 & 606.7782 & 1211.5651 & 606.2862 & $\mathbf{E}$ & 488.2827 & 244.6450 & 471.2562 & 236.1317 & 470.2722 & 235.6397 & 4 \\
\hline 12 & 1300.6128 & 650.8100 & 1283.5862 & 642.2968 & 1282.6022 & 641.8047 & A & 359.2401 & 180.1237 & 342.2136 & 171.6104 & & & 3 \\
\hline 13 & 1413.6968 & 707.3521 & 1396.6703 & 698.8388 & 1395.6863 & 698.3468 & $\mathbf{L}$ & 288.2030 & 144.6051 & 271.1765 & 136.0919 & & & 2 \\
\hline 14 & & & & & & & $\mathbf{R}$ & 175.1190 & 88.0631 & 158.0924 & 79.5498 & & & 1 \\
\hline
\end{tabular}



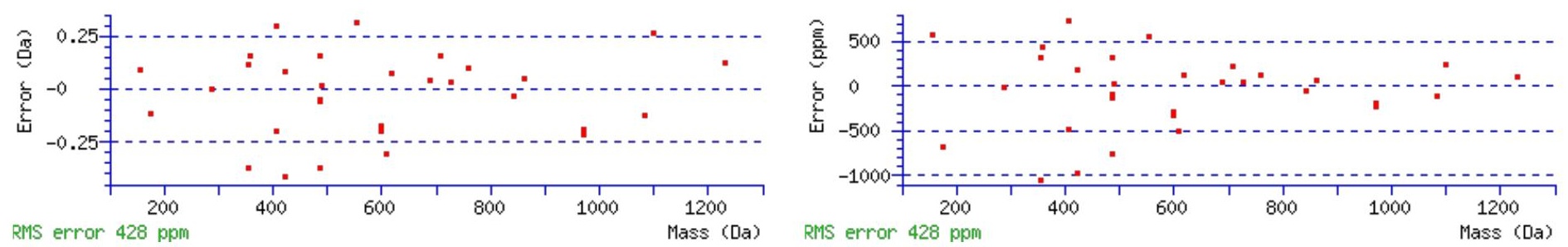

\section{All matches to this query}

\begin{tabular}{|l|l|l|l|}
\hline Score & Mr(calc): & Delta & \multicolumn{1}{|c|}{ Sequence } \\
\hline 41.4 & 1586.8012 & 0.0025 & RVNDNKTAAEEALR \\
\hline 40.9 & 1586.8012 & 0.0025 & RVNDNKTAAEEALR \\
\hline 7.5 & 1585.8134 & 0.9903 & IPVSQVSMEIQPSR \\
\hline 4.5 & 1586.7900 & 0.0137 & VSGEANETQIAEALR \\
\hline 4.5 & 1584.8058 & 1.9979 & RTSGMSSLLGKIGAK \\
\hline 3.7 & 1584.8058 & 1.9979 & RTSGMSSLLGKIGAK \\
\hline 3.6 & 1586.7930 & 0.0108 & LEQVESGLHRALR \\
\hline 2.2 & 1585.7898 & 1.0139 & QKKMSSALLNLTR \\
\hline 2.2 & 1585.7898 & 1.0139 & QKKMSSALLNLTR \\
\hline 1.8 & 1586.7974 & 0.0063 & LELEMTPQGTLAER \\
\hline
\end{tabular}

Spectrum No: 812; Query: 597; Rank: 1

\section{Peptide View}

MS/MS Fragmentation of GPNGTEGPSQTVVGR

Found in IPI00209300, Tax_Id=10116 Gene_Symbol=Ptprj Vascular protein tyrosine phosphatase 1

Match to Query 597: 1455.696148 from(728.855350,2+)

Title: 091008RatKidney_NH4Format01_25.752.752.2.dta

Data file K:INewmanPaper|Piliangl3SubProteomes\Piliang3SP\mgf5ppmlERLIC_3SubProteomes5ppm.mgf

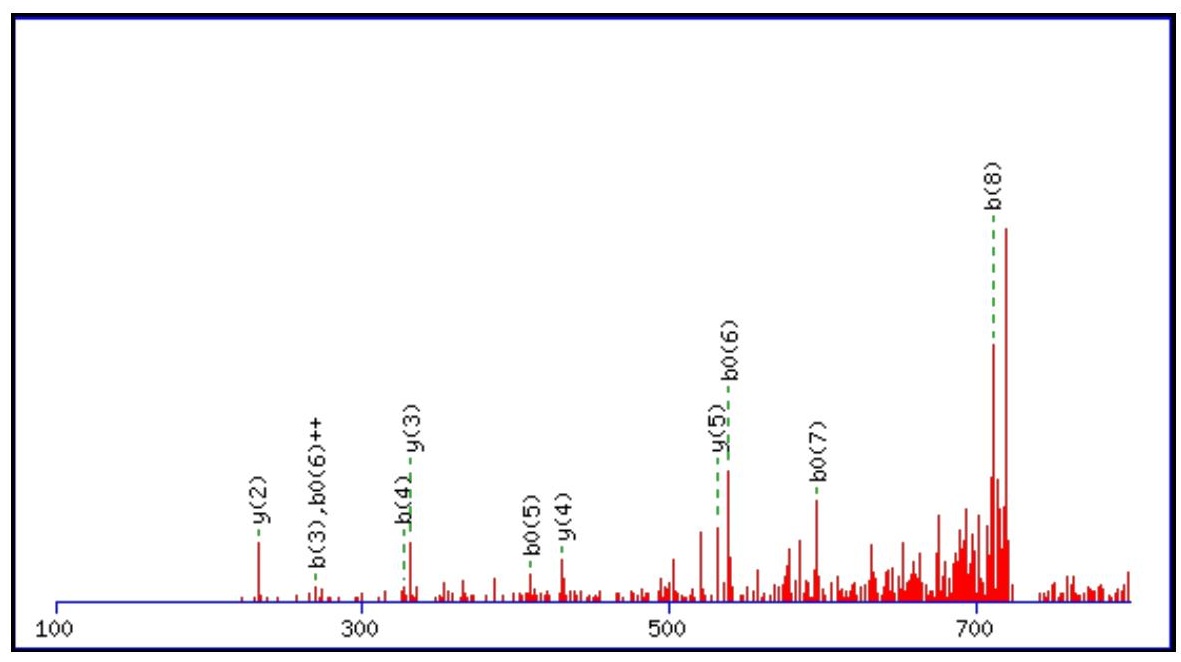



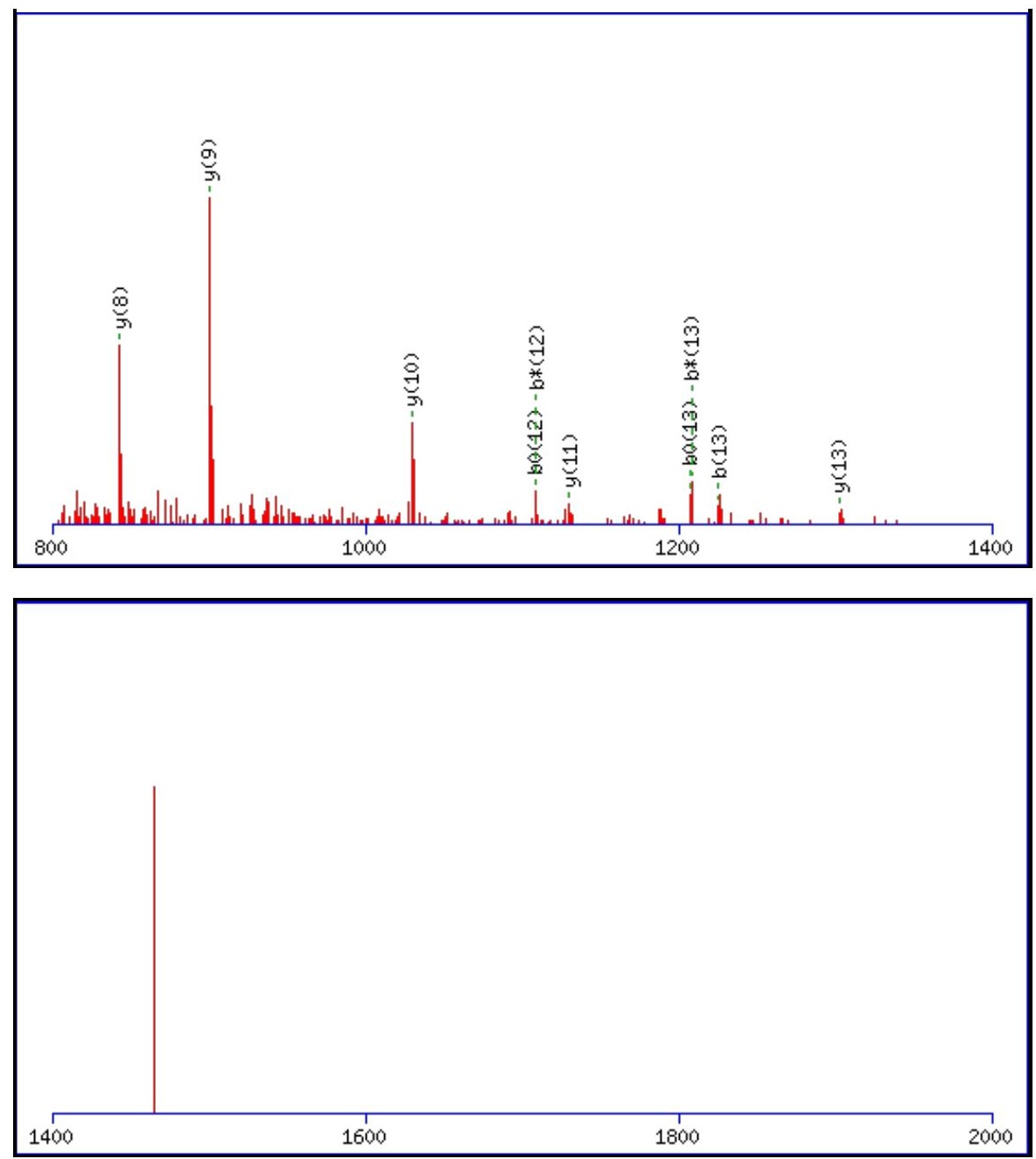

Monoisotopic mass of neutral peptide $\operatorname{Mr}($ calc): 1455.6954

Fixed modifications: Carbamidomethyl (C)

Variable modifications:

N3 : Deamidated $\mathrm{N}(\mathrm{N})$

Ions Score: 41 Expect : 0.011

Matches (Bold Red): 21/148 fragment ions using 47 most intense peaks

\begin{tabular}{|c|c|c|c|c|c|c|c|c|c|c|c|c|c|c|}
\hline$\#$ & b & $\mathbf{b}^{++}$ & $\mathbf{b}^{*}$ & $\mathbf{b}^{*^{++}}$ & $\mathbf{b}^{0}$ & $\mathbf{b}^{\mathbf{0 + +}}$ & Seq. & $\mathbf{y}$ & $\mathbf{y}^{++}$ & $\mathbf{y}^{*}$ & $\mathbf{y}^{*^{++}}$ & $\mathbf{y}^{0}$ & $\mathbf{y}^{\mathbf{0 + +}}$ & \# \\
\hline 1 & 58.0287 & 29.5180 & & & & & $\mathbf{G}$ & & & & & & & 15 \\
\hline 2 & 155.0815 & 78.0444 & & & & & $\mathbf{P}$ & 1399.6812 & 700.3442 & 1382.6546 & 691.8310 & 1381.6706 & 691.3390 & 14 \\
\hline 3 & 270.1084 & 135.5579 & 253.0819 & 127.0446 & & & $\mathbf{N}$ & 1302.6284 & 651.8179 & 1285.6019 & 643.3046 & 1284.6179 & 642.8126 & 13 \\
\hline 4 & 327.1299 & 164.0686 & 310.1034 & 155.5553 & & & G & 1187.6015 & 594.3044 & 1170.5749 & 585.7911 & 1169.5909 & 585.2991 & 12 \\
\hline 5 & 428.1776 & 214.5924 & 411.1510 & 206.0792 & 410.1670 & 205.5871 & $\mathbf{T}$ & 1130.5800 & 565.7937 & 1113.5535 & 557.2804 & 1112.5695 & 556.7884 & 11 \\
\hline 6 & 557.2202 & 279.1137 & 540.1936 & 270.6005 & 539.2096 & 270.1084 & $\mathbf{E}$ & 1029.5324 & 515.2698 & 1012.5058 & 506.7565 & 1011.5218 & 506.2645 & 10 \\
\hline 7 & 614.2416 & 307.6245 & 597.2151 & 299.1112 & 596.2311 & 298.6192 & G & 900.4898 & 450.7485 & 883.4632 & 442.2352 & 882.4792 & 441.7432 & 9 \\
\hline 8 & 711.2944 & 356.1508 & 694.2679 & 347.6376 & 693.2838 & 347.1456 & $\mathbf{P}$ & 843.4683 & 422.2378 & 826.4417 & 413.7245 & 825.4577 & 413.2325 & 8 \\
\hline 9 & 798.3264 & 399.6669 & 781.2999 & 391.1536 & 780.3159 & 390.6616 & S & 746.4155 & 373.7114 & 729.3890 & 365.1981 & 728.4050 & 364.7061 & 7 \\
\hline 10 & 926.3850 & 463.6961 & 909.3585 & 455.1829 & 908.3744 & 454.6909 & $\mathbf{Q}$ & 659.3835 & 330.1954 & 642.3570 & 321.6821 & 641.3729 & 321.1901 & 6 \\
\hline 11 & 1027.4327 & 514.2200 & 1010.4061 & 505.7067 & 1009.4221 & 505.2147 & $\mathbf{T}$ & 531.3249 & 266.1661 & 514.2984 & 257.6528 & 513.3144 & 257.1608 & 5 \\
\hline 12 & 1126.5011 & 563.7542 & 1109.4746 & 555.2409 & 1108.4905 & 554.7489 & V & 430.2772 & 215.6423 & 413.2507 & 207.1290 & & & 4 \\
\hline 13 & 1225.5695 & 613.2884 & 1208.5430 & 604.7751 & 1207.5590 & 604.2831 & V & 331.2088 & 166.1081 & 314.1823 & 157.5948 & & & 3 \\
\hline 14 & 1282.5910 & 641.7991 & 1265.5644 & 633.2859 & 1264.5804 & 632.7938 & G & 232.1404 & 116.5738 & 215.1139 & 108.0606 & & & 2 \\
\hline 15 & & & & & & & $\mathbf{R}$ & 175.1190 & 88.0631 & 158.0924 & 79.5498 & & & 1 \\
\hline
\end{tabular}



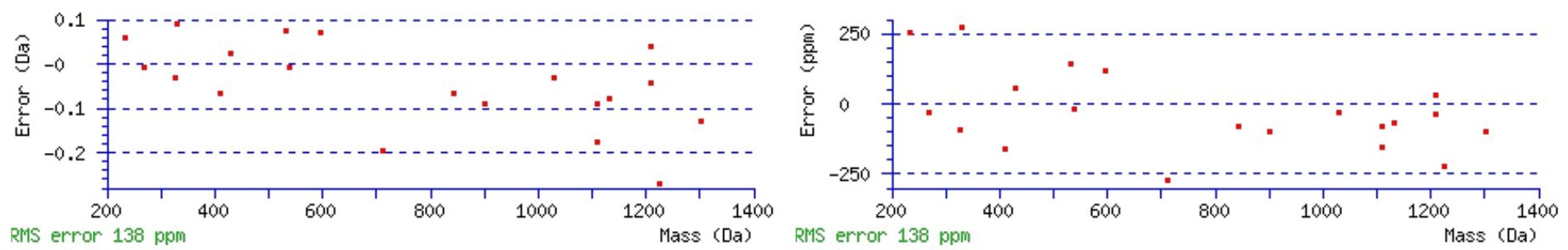

\section{All matches to this query}

\begin{tabular}{|l|l|l|c|}
\hline Score & Mr(calc): & Delta & Sequence \\
\hline 41.3 & 1455.6954 & 0.0007 & GPNGTEGPSQTVVGR \\
\hline
\end{tabular}

Spectrum No: 813; Query: 1032; Rank: 1

\section{Peptide View}

\section{MS/MS Fragmentation of NTTTYPPMCSQDAAK}

Found in IPI00558154, Tax_Id=10116 Gene_Symbol=LOC100125372 60 kDa protein

Match to Query 1032: 1684.705968 from(843.360260,2+)

Title: 091008RatKidney_NH4Format02_26.1066.1066.2.dta

Data file K:INewmanPaper|Piliangl3SubProteomes\Piliang3SP\mgf5ppm\ERLIC_3SubProteomes5ppm.mgf
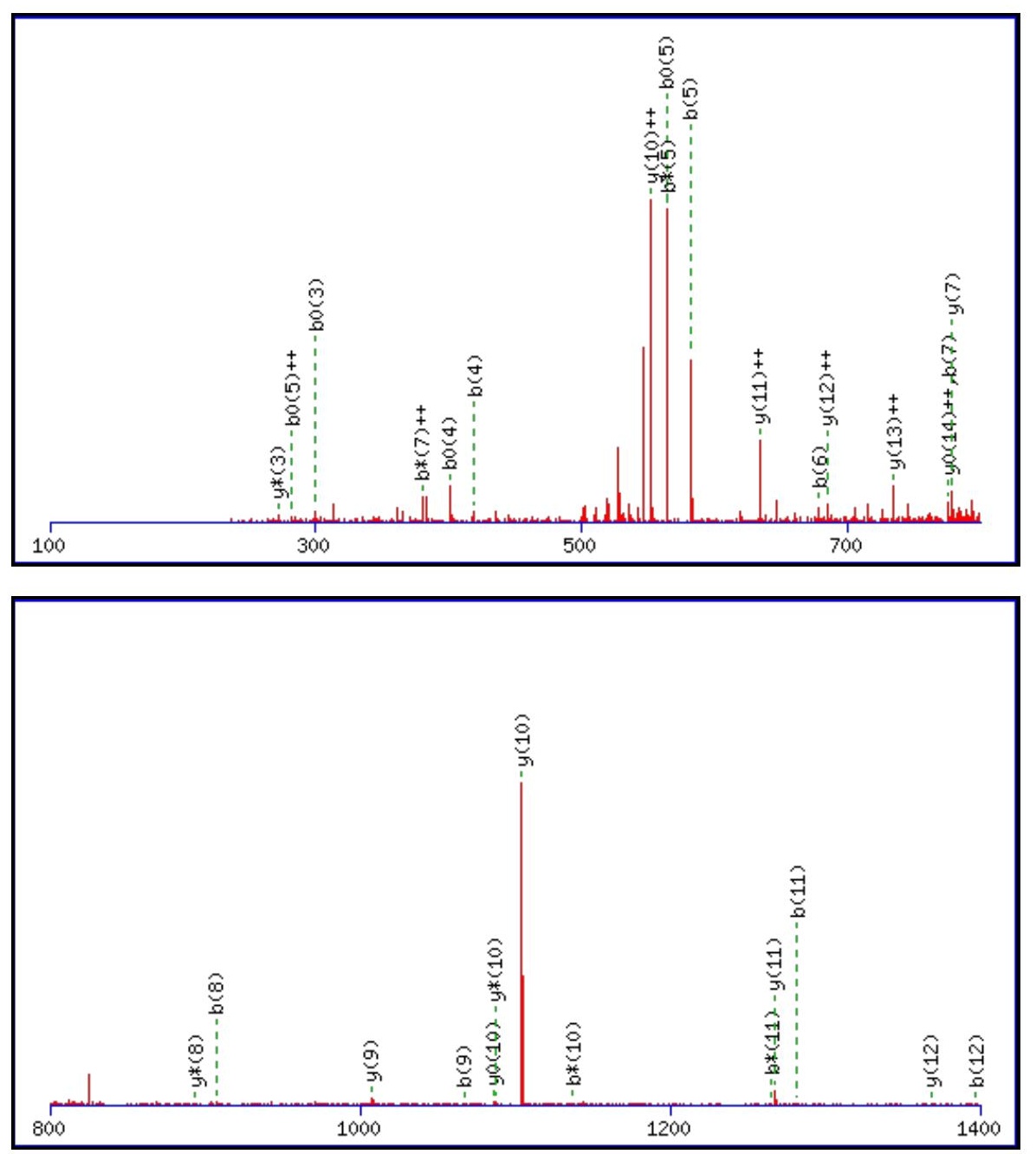


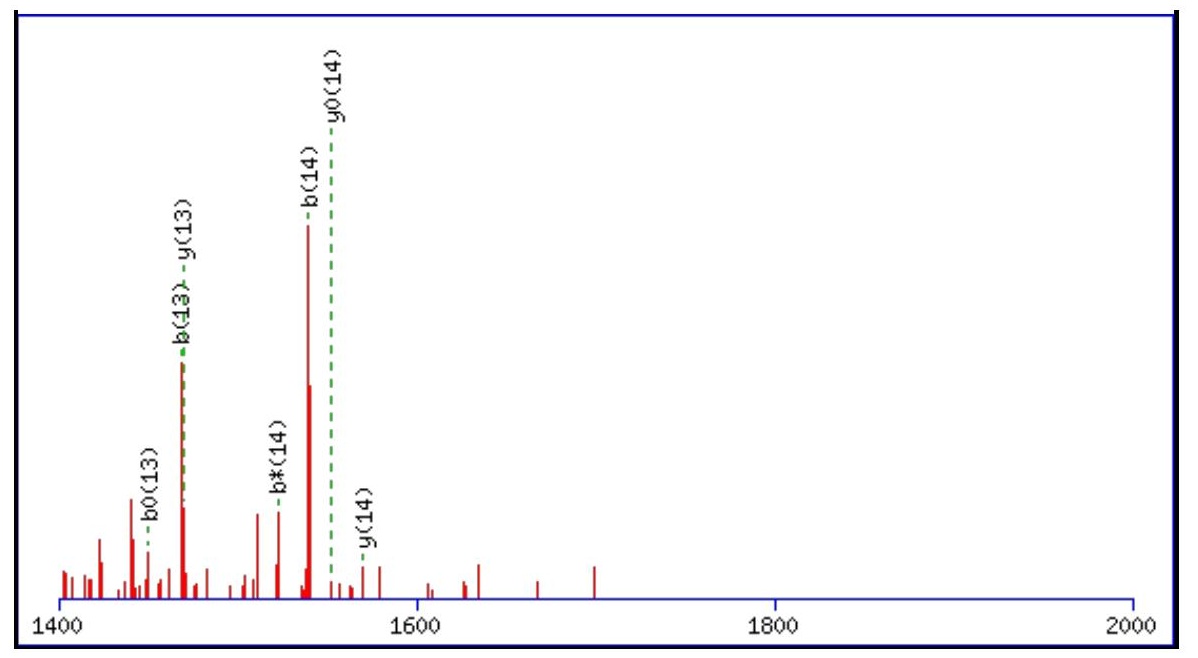

Monoisotopic mass of neutral peptide $\operatorname{Mr}($ calc): 1684.7073

Fixed modifications: Carbamidomethyl (C)

Variable modifications:

N1 : Deamidated $\mathrm{N}(\mathrm{N})$

Ions Score: 41 Expect: 0.014

Matches (Bold Red): 37/160 fragment ions using 102 most intense peaks

\begin{tabular}{|c|c|c|c|c|c|c|c|c|c|c|c|c|c|c|}
\hline \# & b & $\mathbf{b}^{++}$ & b* & $\mathbf{b}^{*^{++}}$ & $\mathbf{b}^{0}$ & $\mathbf{b}^{0++}$ & Seq. & $\mathbf{y}$ & $\mathbf{y}^{++}$ & $\mathbf{y}^{*}$ & $\mathbf{y}^{*^{++}}$ & $\mathbf{y}^{0}$ & $\mathbf{y}^{0++}$ & \# \\
\hline 1 & 116.0342 & 58.5207 & 99.0077 & 50.0075 & & & $\mathbf{N}$ & & & & & & & 15 \\
\hline 2 & 217.0819 & 109.0446 & 200.0553 & 100.5313 & 199.0713 & 100.0393 & $\mathbf{T}$ & 1570.6876 & 785.8474 & 1553.6611 & 777.3342 & 1552.6770 & 776.8422 & 14 \\
\hline 3 & 318.1296 & 159.5684 & 301.1030 & 151.0551 & 300.1190 & 150.5631 & $\mathbf{T}$ & 1469.6399 & 735.3236 & 1452.6134 & 726.8103 & 1451.6294 & 726.3183 & 13 \\
\hline 4 & 419.1772 & 210.0923 & 402.1507 & 201.5790 & 401.1667 & 201.0870 & $\mathbf{T}$ & 1368.5923 & 684.7998 & 1351.5657 & 676.2865 & 1350.5817 & 675.7945 & 12 \\
\hline 5 & 582.2406 & 291.6239 & 565.2140 & 283.1107 & 564.2300 & 282.6186 & $\mathbf{Y}$ & 1267.5446 & 634.2759 & 1250.5180 & 625.7626 & 1249.5340 & 625.2706 & 11 \\
\hline 6 & 679.2933 & 340.1503 & 662.2668 & 331.6370 & 661.2828 & 331.1450 & $\mathbf{P}$ & 1104.4812 & 552.7443 & 1087.4547 & 544.2310 & 1086.4707 & 543.7390 & 10 \\
\hline 7 & 776.3461 & 388.6767 & 759.3196 & 380.1634 & 758.3355 & 379.6714 & $\mathbf{P}$ & 1007.4285 & 504.2179 & 990.4019 & 495.7046 & 989.4179 & 495.2126 & 9 \\
\hline 8 & 907.3866 & 454.1969 & 890.3600 & 445.6837 & 889.3760 & 445.1917 & $\mathbf{M}$ & 910.3757 & 455.6915 & 893.3492 & 447.1782 & 892.3652 & 446.6862 & 8 \\
\hline 9 & 1067.4172 & 534.2123 & 1050.3907 & 525.6990 & 1049.4067 & 525.2070 & C & 779.3352 & 390.1713 & 762.3087 & 381.6580 & 761.3247 & 381.1660 & 7 \\
\hline 10 & 1154.4493 & 577.7283 & 1137.4227 & 569.2150 & 1136.4387 & 568.7230 & $\mathrm{~S}$ & 619.3046 & 310.1559 & 602.2780 & 301.6427 & 601.2940 & 301.1506 & 6 \\
\hline 11 & 1282.5078 & 641.7576 & 1265.4813 & 633.2443 & 1264.4973 & 632.7523 & $\mathbf{Q}$ & 532.2726 & 266.6399 & 515.2460 & 258.1266 & 514.2620 & 257.6346 & 5 \\
\hline 12 & 1397.5348 & 699.2710 & 1380.5082 & 690.7578 & 1379.5242 & 690.2658 & D & 404.2140 & 202.6106 & 387.1874 & 194.0974 & 386.2034 & 193.6053 & 4 \\
\hline 13 & 1468.5719 & 734.7896 & 1451.5454 & 726.2763 & 1450.5613 & 725.7843 & A & 289.1870 & 145.0972 & 272.1605 & 136.5839 & & & 3 \\
\hline 14 & 1539.6090 & 770.3081 & 1522.5825 & 761.7949 & 1521.5985 & 761.3029 & A & 218.1499 & 109.5786 & 201.1234 & 101.0653 & & & 2 \\
\hline 15 & & & & & & & $\mathbf{K}$ & 147.1128 & 74.0600 & 130.0863 & 65.5468 & & & 1 \\
\hline
\end{tabular}

$$
\text { 离 }
$$
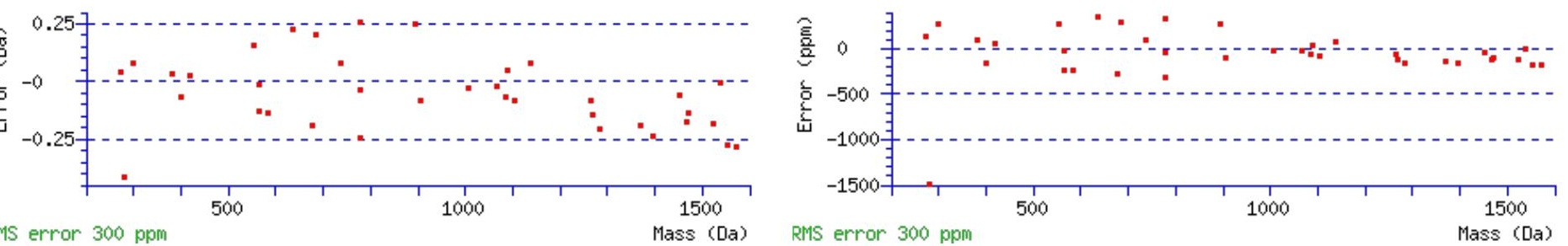

All matches to this query

\begin{tabular}{|l|l|l|l|}
\hline Score & Mr(calc): & Delta & \multicolumn{1}{c|}{ Sequence } \\
\hline 41.3 & 1684.7073 & -0.0013 & NTTTYPPMCSQDAAK \\
\hline 9.6 & 1684.7184 & -0.0124 & TLAESALQMLYAAK \\
\hline 7.9 & 1684.7184 & -0.0124 & ISTTVGKPYMETAK \\
\hline 7.9 & 1684.7184 & -0.0124 & ISTTVGKPYMETAK \\
\hline 7.9 & 1684.7184 & -0.0124 & ISTTVGKPYMETAK \\
\hline 4.6 & 1684.7184 & -0.0124 & TLAESALQMLYAAK \\
\hline & & & \\
\hline
\end{tabular}




\begin{tabular}{|l|l|l|l|}
4.6 & 1684.7184 & -0.0124 & TLAESALQMLYAAK \\
\hline 4.4 & 1683.6881 & 1.0178 & RMEFIPTTTSWR \\
\hline 4.4 & 1683.6881 & 1.0178 & RMEFIPTTTSWR \\
\hline 4.3 & 1683.6881 & 1.0178 & RMEFIPTTTSWR \\
\hline
\end{tabular}

Spectrum No: 814; Query: 527; Rank: 1

\section{Peptide View}

MS/MS Fragmentation of AYSNHTDIISFR

Found in IPI00213584, Tax_Id=10116 Gene_Symbol=Agxt2 Alanine--glyoxylate aminotransferase 2, mitochondrial precursor

Match to Query 527: 1423.671488 from(712.843020,2+)

Title: 091008RatKidney_NH4Format02_19.2021.2021.2.dta

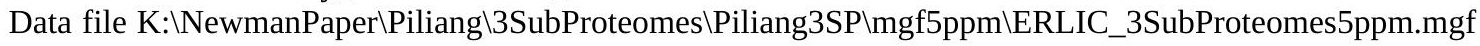
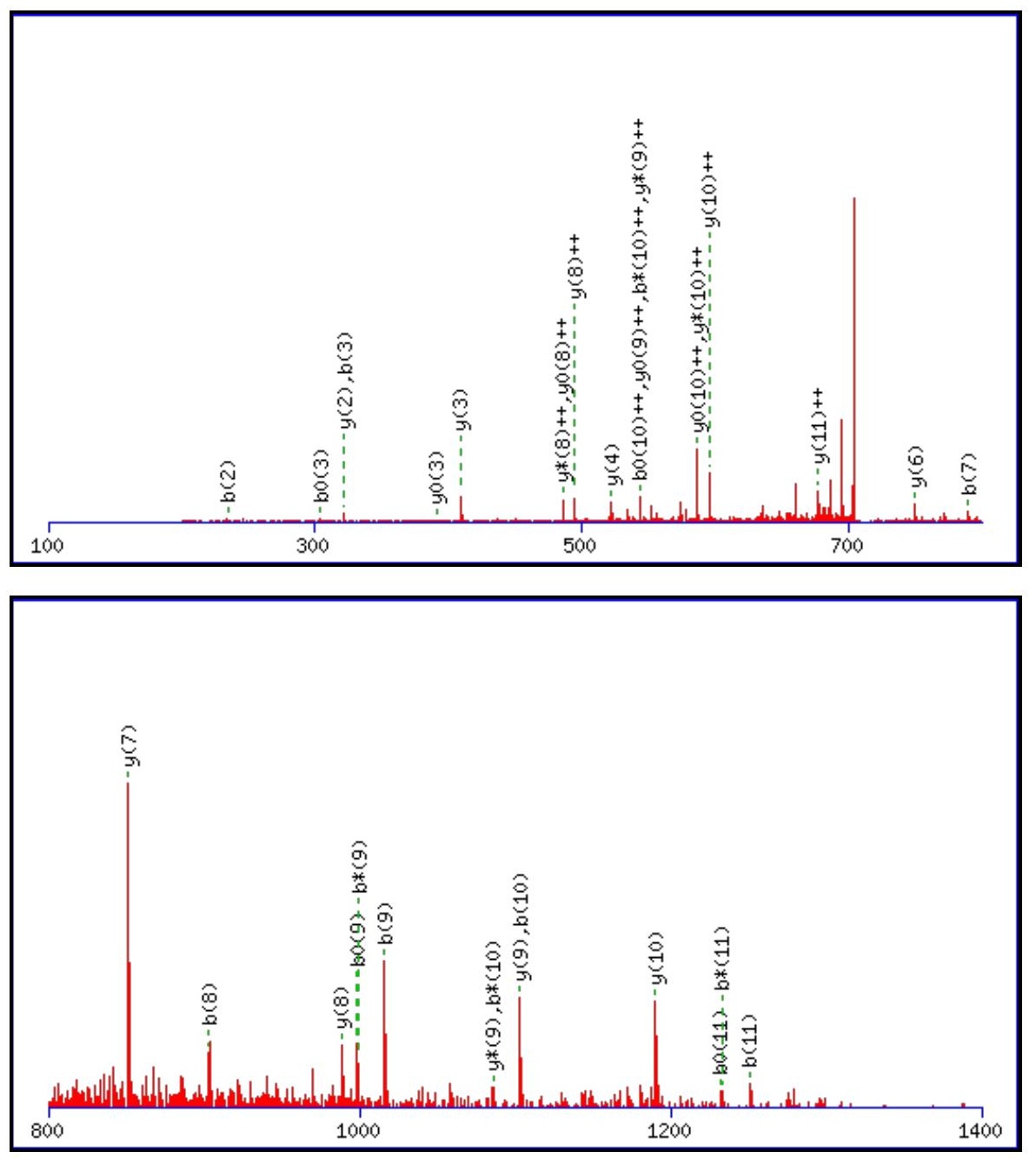


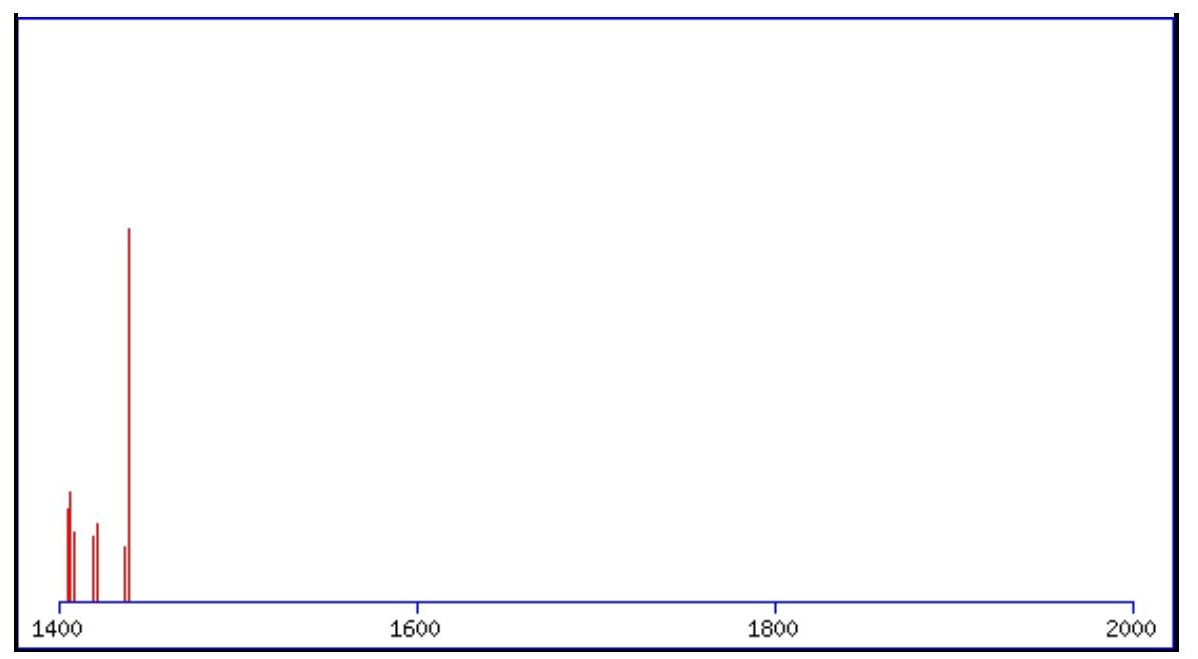

Monoisotopic mass of neutral peptide $\operatorname{Mr}($ calc): 1423.6732

Fixed modifications: Carbamidomethyl (C)

Variable modifications:

N4 : Deamidated_N (N)

Ions Score: 41 Expect: 0.012

Matches (Bold Red): 34/118 fragment ions using 73 most intense peaks

\begin{tabular}{|c|c|c|c|c|c|c|c|c|c|c|c|c|c|c|}
\hline \# & b & $\mathbf{b}^{++}$ & $\mathbf{b}^{*}$ & $\mathbf{b}^{*^{++}}$ & $\mathbf{b}^{0}$ & $\mathbf{b}^{0++}$ & Seq. & $\mathbf{y}$ & $y^{++}$ & $\mathbf{y}^{*}$ & $y^{*^{++}}$ & $\mathbf{y}^{0}$ & $y^{0++}$ & \# \\
\hline 1 & 72.0444 & 36.5258 & & & & & A & & & & & & & 12 \\
\hline 2 & 235.1077 & 118.0575 & & & & & $\mathbf{Y}$ & 1353.6433 & 677.3253 & 1336.6168 & 668.8120 & 1335.6328 & 668.3200 & 11 \\
\hline 3 & 322.1397 & 161.5735 & & & 304.1292 & 152.5682 & S & 1190.5800 & 595.7936 & 1173.5535 & 587.2804 & 1172.5695 & 586.7884 & 10 \\
\hline 4 & 437.1667 & 219.0870 & 420.1401 & 210.5737 & 419.1561 & 210.0817 & $\mathbf{N}$ & 1103.5480 & 552.2776 & 1086.5214 & 543.7644 & 1085.5374 & 543.2724 & 9 \\
\hline 5 & 574.2256 & 287.6164 & 557.1990 & 279.1032 & 556.2150 & 278.6112 & $\mathbf{H}$ & 988.5211 & 494.7642 & 971.4945 & 486.2509 & 970.5105 & 485.7589 & 8 \\
\hline 6 & 675.2733 & 338.1403 & 658.2467 & 329.6270 & 657.2627 & 329.1350 & $\mathbf{T}$ & 851.4621 & 426.2347 & 834.4356 & 417.7214 & 833.4516 & 417.2294 & 7 \\
\hline 7 & 790.3002 & 395.6537 & 773.2737 & 387.1405 & 772.2897 & 386.6485 & D & 750.4145 & 375.7109 & 733.3879 & 367.1976 & 732.4039 & 366.7056 & 6 \\
\hline 8 & 903.3843 & 452.1958 & 886.3577 & 443.6825 & 885.3737 & 443.1905 & I & 635.3875 & 318.1974 & 618.3610 & 309.6841 & 617.3770 & 309.1921 & 5 \\
\hline 9 & 1016.4683 & 508.7378 & 999.4418 & 500.2245 & 998.4578 & 499.7325 & I & 522.3035 & 261.6554 & 505.2769 & 253.1421 & 504.2929 & 252.6501 & 4 \\
\hline 10 & 1103.5004 & 552.2538 & 1086.4738 & 543.7406 & 1085.4898 & 543.2485 & $\mathrm{~S}$ & 409.2194 & 205.1133 & 392.1928 & 196.6001 & 391.2088 & 196.1081 & 3 \\
\hline 11 & 1250.5688 & 625.7880 & 1233.5422 & 617.2748 & 1232.5582 & 616.7827 & $\mathbf{F}$ & 322.1874 & 161.5973 & 305.1608 & 153.0840 & & & 2 \\
\hline 12 & & & & & & & $\mathbf{R}$ & 175.1190 & 88.0631 & 158.0924 & 79.5498 & & & 1 \\
\hline
\end{tabular}
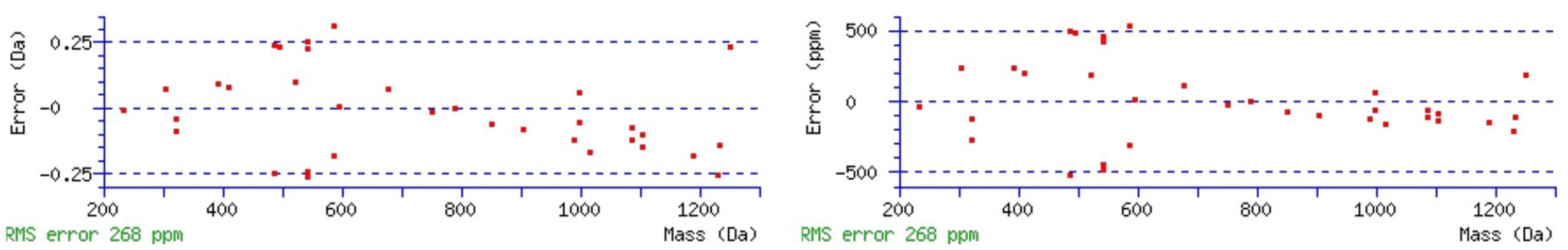

\section{All matches to this query}

\begin{tabular}{|l|l|l|l|}
\hline Score & Mr(calc): & Delta & \multicolumn{1}{c|}{ Sequence } \\
\hline 41.1 & 1423.6732 & -0.0017 & $\underline{\text { AYSNHTDIISFR }}$ \\
\hline 20.3 & 1423.6643 & 0.0072 & $\underline{\text { SGPSVARPLAMSR }}$ \\
\hline 12.8 & 1423.6596 & 0.0119 & $\underline{\text { ELSHLETTISSK }}$ \\
\hline 12.4 & 1423.6765 & -0.0050 & $\underline{\text { WILSASKDNSMR }}$ \\
\hline 11.2 & 1423.6782 & -0.0067 & $\underline{\text { TILSPKNMEPSK }}$ \\
\hline 9.6 & 1423.6643 & 0.0072 & $\underline{\text { SGPSVARPLAMSR }}$ \\
\hline 8.3 & 1423.6667 & 0.0048 & $\underline{\text { MGHNYTFTVQAR }}$ \\
\hline 8.3 & 1423.6613 & 0.0102 & $\underline{\text { AETTLSTMPSQSR }}$ \\
\hline 8.2 & 1423.6660 & 0.0055 & $\underline{\text { SCSSTRKPQAXR }}$ \\
\hline & & &
\end{tabular}




\section{|7.2 |1423.6766|-0.0051|FTCLSDLSASPAR}

Spectrum No: 815; Query: 2063; Rank: 1

\section{Peptide View}

MS/MS Fragmentation of IIYSIVQTNCSKEDFPFLR

Found in IPI00558996, Tax_Id=10116 Gene_Symbol=MGC108747 48 kDa protein

Match to Query 2063: 2330.164722 from(777.728850,3+)

Title: 100101RatKid_NS_deglyco_21.4483.4483.3.dta

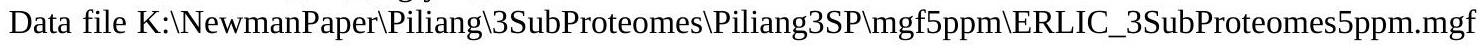
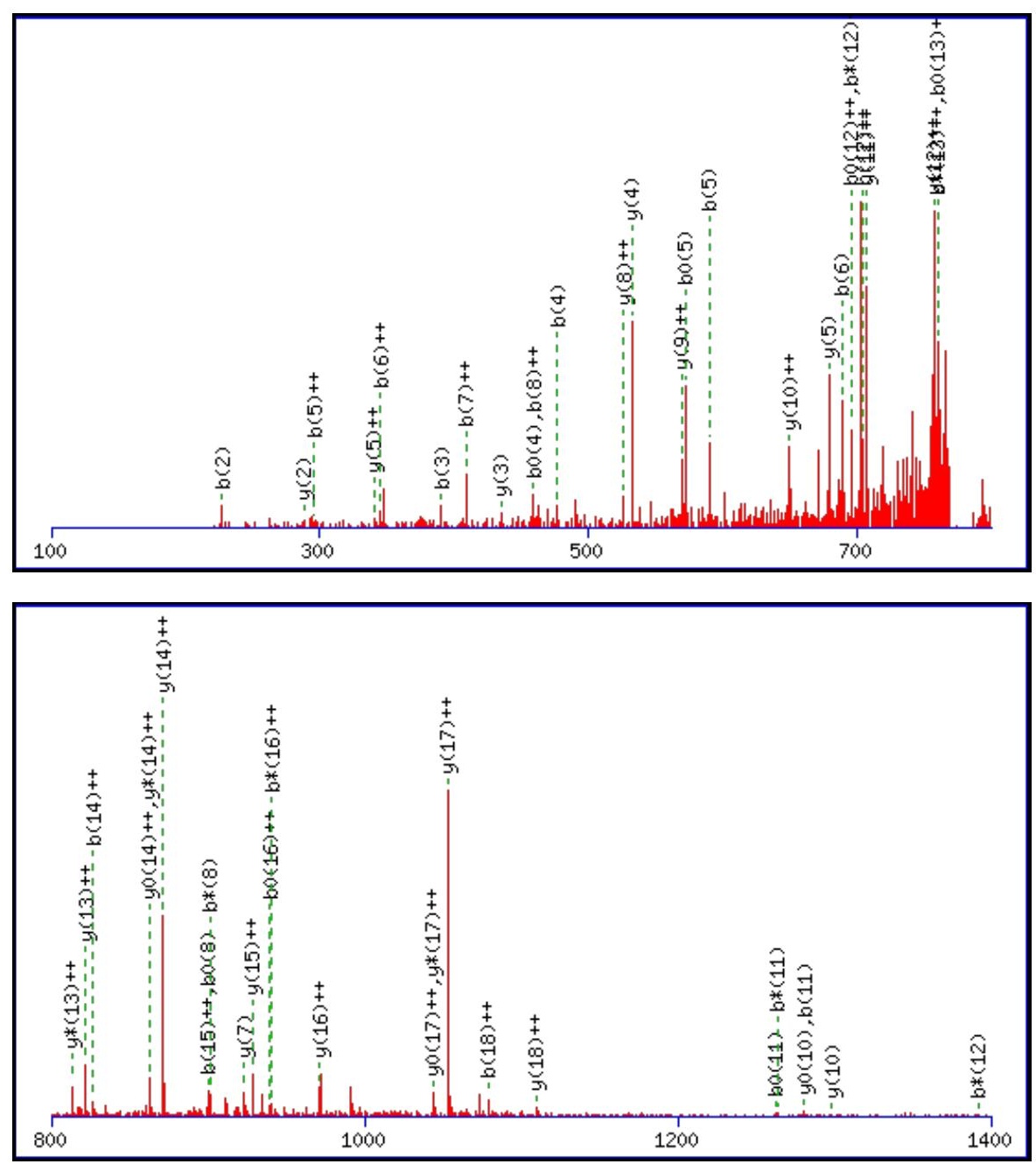


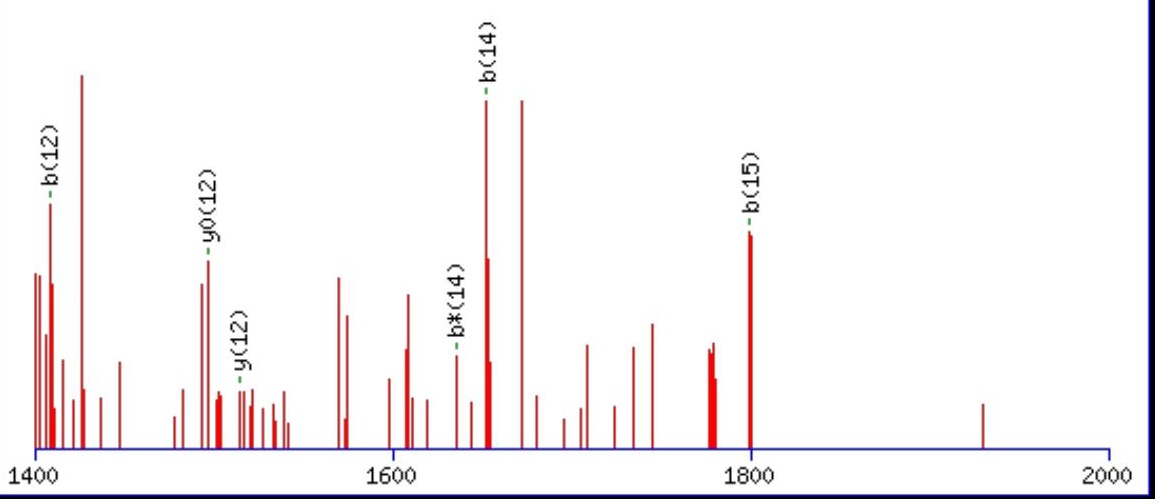

Monoisotopic mass of neutral peptide $\operatorname{Mr}($ calc): 2330.1617

Fixed modifications: Carbamidomethyl (C)

Variable modifications:

N9 : Deamidated $\mathrm{N}(\mathrm{N})$

Ions Score: 41 Expect: 0.015

Matches (Bold Red): 57/188 fragment ions using 148 most intense peaks

\begin{tabular}{|c|c|c|c|c|c|c|c|c|c|c|c|c|c|c|}
\hline \# & b & $\mathbf{b}^{++}$ & b* & $\mathbf{b}^{*^{++}}$ & $\mathbf{b}^{\mathbf{0}}$ & $\mathbf{b}^{0++}$ & Seq. & $\mathbf{y}$ & $\mathbf{y}^{++}$ & $\mathbf{y}^{*}$ & $\mathrm{y}^{\mathrm{*}^{++}}$ & $\mathbf{y}^{0}$ & $\mathbf{y}^{\mathbf{0 + +}}$ & \# \\
\hline 1 & 114.0913 & 57.5493 & & & & & I & & & & & & & 19 \\
\hline 2 & 227.1754 & 114.0913 & & & & & I & 2218.0849 & 1109.5461 & 2201.0583 & 1101.0328 & 2200.0743 & 1100.5408 & 18 \\
\hline 3 & 390.2387 & 195.6230 & & & & & $\mathbf{Y}$ & 2105.0008 & 1053.0040 & 2087.9743 & 1044.4908 & 2086.9902 & 1043.9988 & 17 \\
\hline 4 & 477.2708 & 239.1390 & & & 459.2602 & 230.1337 & $\mathbf{S}$ & 1941.9375 & 971.4724 & 1924.9109 & 962.9591 & 1923.9269 & 962.4671 & 16 \\
\hline 5 & 590.3548 & 295.6811 & & & 572.3443 & 286.6758 & I & 1854.9055 & 927.9564 & 1837.8789 & 919.4431 & 1836.8949 & 918.9511 & 15 \\
\hline 6 & 689.4232 & 345.2153 & & & 671.4127 & 336.2100 & $\mathbf{V}$ & 1741.8214 & 871.4143 & 1724.7948 & 862.9011 & 1723.8108 & 862.4091 & 14 \\
\hline 7 & 817.4818 & 409.2445 & 800.4553 & 400.7313 & 799.4713 & 400.2393 & $\mathbf{Q}$ & 1642.7530 & 821.8801 & 1625.7264 & 813.3669 & 1624.7424 & 812.8748 & 13 \\
\hline 8 & 918.5295 & 459.7684 & 901.5029 & 451.2551 & 900.5189 & 450.7631 & $\mathbf{T}$ & 1514.6944 & 757.8508 & 1497.6679 & 749.3376 & 1496.6838 & 748.8456 & 12 \\
\hline 9 & 1033.5564 & 517.2819 & 1016.5299 & 508.7686 & 1015.5459 & 508.2766 & $\mathbf{N}$ & 1413.6467 & 707.3270 & 1396.6202 & 698.8137 & 1395.6362 & 698.3217 & 11 \\
\hline 10 & 1193.5871 & 597.2972 & 1176.5605 & 588.7839 & 1175.5765 & 588.2919 & C & 1298.6198 & 649.8135 & 1281.5932 & 641.3003 & 1280.6092 & 640.8082 & 10 \\
\hline 11 & 1280.6191 & 640.8132 & 1263.5926 & 632.2999 & 1262.6085 & 631.8079 & $\mathrm{~S}$ & 1138.5891 & 569.7982 & 1121.5626 & 561.2849 & 1120.5786 & 560.7929 & 9 \\
\hline 12 & 1408.7141 & 704.8607 & 1391.6875 & 696.3474 & 1390.7035 & 695.8554 & $\mathbf{K}$ & 1051.5571 & 526.2822 & 1034.5306 & 517.7689 & 1033.5465 & 517.2769 & 8 \\
\hline 13 & 1537.7567 & 769.3820 & 1520.7301 & 760.8687 & 1519.7461 & 760.3767 & $\mathbf{E}$ & 923.4621 & 462.2347 & 906.4356 & 453.7214 & 905.4516 & 453.2294 & 7 \\
\hline 14 & 1652.7836 & 826.8954 & 1635.7571 & 818.3822 & 1634.7730 & 817.8902 & D & 794.4196 & 397.7134 & 777.3930 & 389.2001 & 776.4090 & 388.7081 & 6 \\
\hline 15 & 1799.8520 & 900.4296 & 1782.8255 & 891.9164 & 1781.8415 & 891.4244 & $\mathbf{F}$ & 679.3926 & 340.1999 & 662.3661 & 331.6867 & & & 5 \\
\hline 16 & 1896.9048 & 948.9560 & 1879.8782 & 940.4428 & 1878.8942 & 939.9507 & $\mathbf{P}$ & 532.3242 & 266.6657 & 515.2976 & 258.1525 & & & 4 \\
\hline 17 & 2043.9732 & 1022.4902 & 2026.9467 & 1013.9770 & 2025.9626 & 1013.4850 & $\mathbf{F}$ & 435.2714 & 218.1394 & 418.2449 & 209.6261 & & & 3 \\
\hline 18 & 2157.0573 & 1079.0323 & 2140.0307 & 1070.5190 & 2139.0467 & 1070.0270 & $\mathbf{L}$ & 288.2030 & 144.6051 & 271.1765 & 136.0919 & & & 2 \\
\hline 19 & & & & & & & $\mathbf{R}$ & 175.1190 & 88.0631 & 158.0924 & 79.5498 & & & 1 \\
\hline
\end{tabular}

$$
\text { 商 }
$$
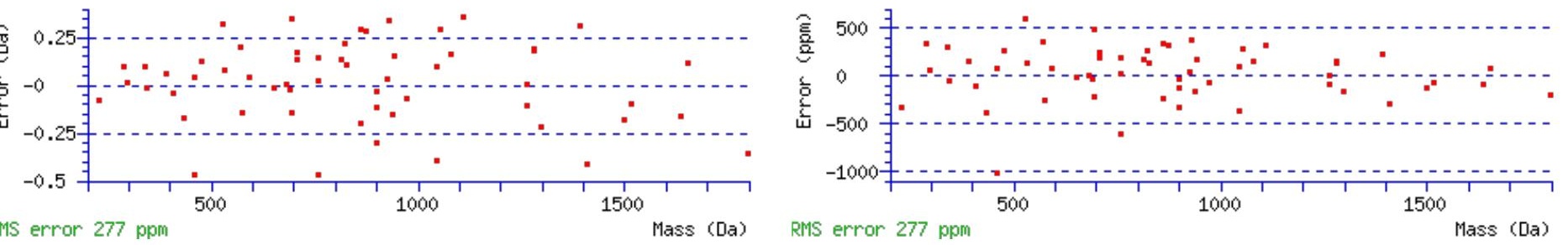

\section{All matches to this query}

\begin{tabular}{|l|l|l|l|}
\hline Score & Mr(calc): & Delta & \multicolumn{1}{c|}{ Sequence } \\
\hline 41.1 & 2330.1617 & 0.0031 & IIYSIVQTNCSKEDFPFLR \\
\hline 1.8 & 2330.1756 & -0.0108 & LIYNIVEEEPLMLFTTDFK \\
\hline & & &
\end{tabular}




\begin{tabular}{|l|l|l|l||}
1.2 & 2328.1379 & 2.0268 & LASDSLEVRMEKDNLNVDHK \\
\hline 0.9 & 2330.1767 & -0.0120 & TATVSGAHQAFAPYNKPSLSGAR \\
\hline 0.7 & 2330.1769 & -0.0122 & LISKFISSFSDFKCTFFTR \\
\hline 0.2 & 2330.1628 & 0.0019 & DANVATANLGEKRGHHLAHYR \\
\hline 0.2 & 2329.1654 & 0.9994 & YPSLGQKPGGSDFLMKRLQK \\
\hline 0.1 & 2330.1760 & -0.0113 & LILVGRTGTGKSATGNSILGQK \\
\hline 0.1 & 2330.1760 & -0.0113 & LILVGRTGTGKSATGNSILGQK \\
\hline 0.1 & 2330.1760 & -0.0113 & LILVGRTGTGKSATGNSILGQK \\
\hline
\end{tabular}

Spectrum No: 816; Query: 2555; Rank: 1

\section{Peptide View}

MS/MS Fragmentation of SLDLSHNLISEFAWSDLHNLSALQLLK

Found in IPI00765329, Tax_Id=10116 Gene_Symbol=LOC686539 similar to immunoglobulin superfamily containing leucine-rich repeat

Match to Query 2555: 3064.587936 from(767.154260,4+)

Title: 091008RatKidney_NoSalt_18.6363.6363.4.dta

Data file K:INewmanPaper|Piliang|3SubProteomes\Piliang3SP\mgf5ppm\ERLIC_3SubProteomes5ppm.mgf
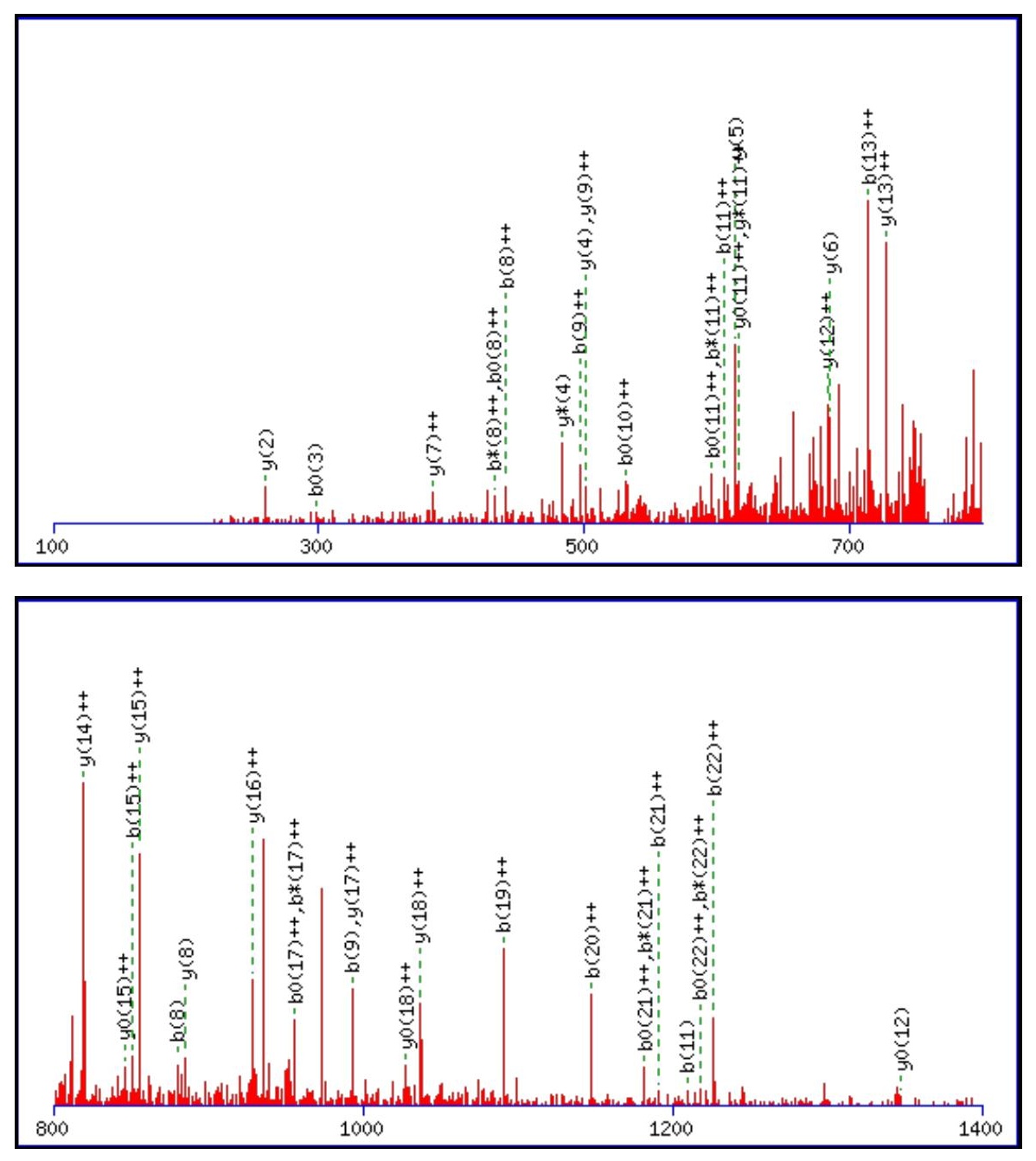


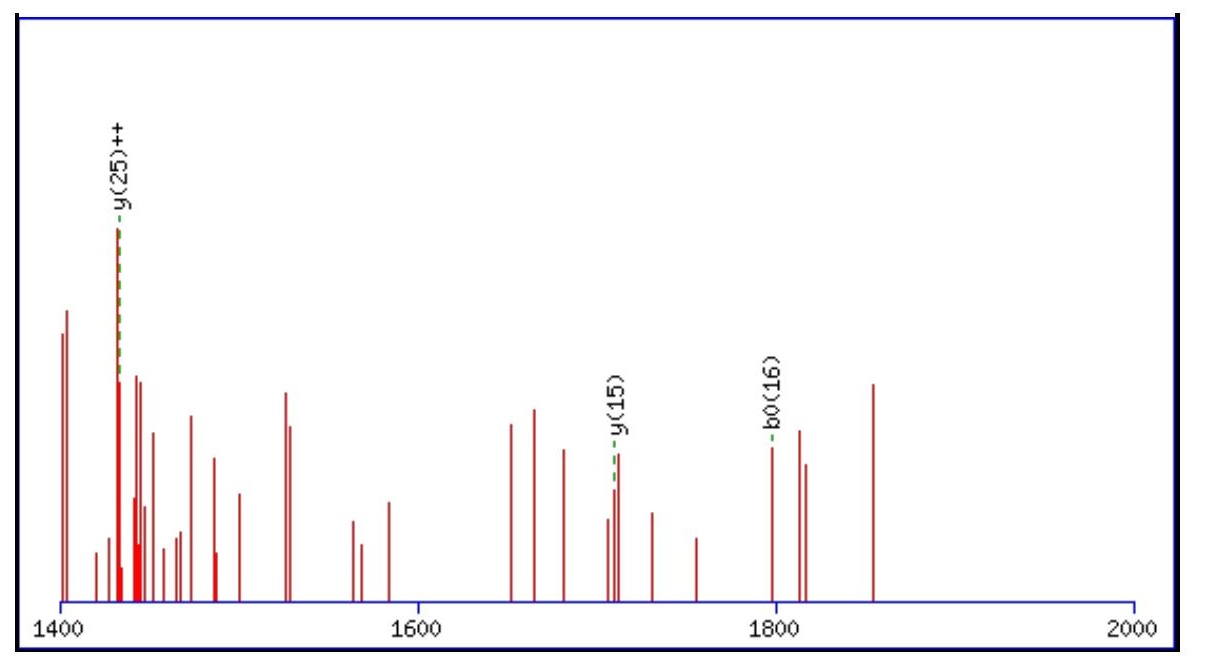

Monoisotopic mass of neutral peptide $\operatorname{Mr}($ calc): 3064.5869

Fixed modifications: Carbamidomethyl (C)

Variable modifications:

N19: Deamidated $\mathrm{N}(\mathrm{N})$

Ions Score: 41 Expect: 0.0095

Matches (Bold Red): 47/288 fragment ions using 86 most intense peaks

\begin{tabular}{|c|c|c|c|c|c|c|c|c|c|c|c|c|c|c|}
\hline \# & b & $\mathbf{b}^{++}$ & $\mathbf{b}^{*}$ & $\mathbf{b}^{*^{++}}$ & $\mathbf{b}^{0}$ & $\mathbf{b}^{\mathbf{0 + +}}$ & Seq. & $\mathbf{y}$ & $\mathbf{y}^{++}$ & $\mathbf{y}^{*}$ & $\mathrm{y}^{*^{++}}$ & $\mathbf{y}^{0}$ & $\mathbf{y}^{0++}$ & \# \\
\hline 1 & 88.0393 & 44.5233 & & & 70.0287 & 35.5180 & S & & & & & & & 27 \\
\hline 2 & 201.1234 & 101.0653 & & & 183.1128 & 92.0600 & $\mathbf{L}$ & 2978.5622 & 1489.7847 & 2961.5356 & 1481.2715 & 2960.5516 & 1480.7795 & 26 \\
\hline 3 & 316.1503 & 158.5788 & & & 298.1397 & 149.5735 & D & 2865.4781 & 1433.2427 & 2848.4516 & 1424.7294 & 2847.4676 & 1424.2374 & 25 \\
\hline 4 & 429.2344 & 215.1208 & & & 411.2238 & 206.1155 & $\mathbf{L}$ & 2750.4512 & 1375.7292 & 2733.4246 & 1367.2160 & 2732.4406 & 1366.7239 & 24 \\
\hline 5 & 516.2664 & 258.6368 & & & 498.2558 & 249.6316 & S & 2637.3671 & 1319.1872 & 2620.3406 & 1310.6739 & 2619.3566 & 1310.1819 & 23 \\
\hline 6 & 653.3253 & 327.1663 & & & 635.3148 & 318.1610 & $\mathbf{H}$ & 2550.3351 & 1275.6712 & 2533.3085 & 1267.1579 & 2532.3245 & 1266.6659 & 22 \\
\hline 7 & 767.3682 & 384.1878 & 750.3417 & 375.6745 & 749.3577 & 375.1825 & $\mathbf{N}$ & 2413.2762 & 1207.1417 & 2396.2496 & 1198.6285 & 2395.2656 & 1198.1364 & 21 \\
\hline 8 & 880.4523 & 440.7298 & 863.4258 & 432.2165 & 862.4417 & 431.7245 & $\mathbf{L}$ & 2299.2333 & 1150.1203 & 2282.2067 & 1141.6070 & 2281.2227 & 1141.1150 & 20 \\
\hline 9 & 993.5364 & 497.2718 & 976.5098 & 488.7585 & 975.5258 & 488.2665 & I & 2186.1492 & 1093.5782 & 2169.1226 & 1085.0650 & 2168.1386 & 1084.5730 & 19 \\
\hline 10 & 1080.5684 & 540.7878 & 1063.5418 & 532.2746 & 1062.5578 & 531.7826 & S & 2073.0651 & 1037.0362 & 2056.0386 & 1028.5229 & 2055.0546 & 1028.0309 & 18 \\
\hline 11 & 1209.6110 & 605.3091 & 1192.5844 & 596.7959 & 1191.6004 & 596.3039 & $\mathbf{E}$ & 1986.0331 & 993.5202 & 1969.0065 & 985.0069 & 1968.0225 & 984.5149 & 17 \\
\hline 12 & 1356.6794 & 678.8433 & 1339.6529 & 670.3301 & 1338.6688 & 669.8381 & F & 1856.9905 & 928.9989 & 1839.9640 & 920.4856 & 1838.9799 & 919.9936 & 16 \\
\hline 13 & 1427.7165 & 714.3619 & 1410.6900 & 705.8486 & 1409.7060 & 705.3566 & A & 1709.9221 & 855.4647 & 1692.8955 & 846.9514 & 1691.9115 & 846.4594 & 15 \\
\hline 14 & 1613.7958 & 807.4016 & 1596.7693 & 798.8883 & 1595.7853 & 798.3963 & $\mathbf{W}$ & 1638.8850 & 819.9461 & 1621.8584 & 811.4329 & 1620.8744 & 810.9408 & 14 \\
\hline 15 & 1700.8279 & 850.9176 & 1683.8013 & 842.4043 & 1682.8173 & 841.9123 & S & 1452.8057 & 726.9065 & 1435.7791 & 718.3932 & 1434.7951 & 717.9012 & 13 \\
\hline 16 & 1815.8548 & 908.4310 & 1798.8283 & 899.9178 & 1797.8442 & 899.4258 & D & 1365.7736 & 683.3905 & 1348.7471 & 674.8772 & 1347.7631 & 674.3852 & 12 \\
\hline 17 & 1928.9389 & 964.9731 & 1911.9123 & 956.4598 & 1910.9283 & 955.9678 & $\mathbf{L}$ & 1250.7467 & 625.8770 & 1233.7201 & 617.3637 & 1232.7361 & 616.8717 & 11 \\
\hline 18 & 2065.9978 & 1033.5025 & 2048.9712 & 1024.9893 & 2047.9872 & 1024.4972 & $\mathbf{H}$ & 1137.6626 & 569.3350 & 1120.6361 & 560.8217 & 1119.6521 & 560.3297 & 10 \\
\hline 19 & 2181.0247 & 1091.0160 & 2163.9982 & 1082.5027 & 2163.0142 & 1082.0107 & $\mathbf{N}$ & 1000.6037 & 500.8055 & 983.5772 & 492.2922 & 982.5932 & 491.8002 & 9 \\
\hline 20 & 2294.1088 & 1147.5580 & 2277.0822 & 1139.0448 & 2276.0982 & 1138.5527 & $\mathbf{L}$ & 885.5768 & 443.2920 & 868.5502 & 434.7788 & 867.5662 & 434.2867 & 8 \\
\hline 21 & 2381.1408 & 1191.0740 & 2364.1143 & 1182.5608 & 2363.1302 & 1182.0688 & $\mathbf{S}$ & 772.4927 & 386.7500 & 755.4662 & 378.2367 & 754.4822 & 377.7447 & 7 \\
\hline 22 & 2452.1779 & 1226.5926 & 2435.1514 & 1218.0793 & 2434.1674 & 1217.5873 & A & 685.4607 & 343.2340 & 668.4341 & 334.7207 & & & 6 \\
\hline 23 & 2565.2620 & 1283.1346 & 2548.2354 & 1274.6214 & 2547.2514 & 1274.1293 & $\mathbf{L}$ & 614.4236 & 307.7154 & 597.3970 & 299.2022 & & & 5 \\
\hline 24 & 2693.3206 & 1347.1639 & 2676.2940 & 1338.6506 & 2675.3100 & 1338.1586 & $\mathbf{Q}$ & 501.3395 & 251.1734 & 484.3130 & 242.6601 & & & 4 \\
\hline 25 & 2806.4046 & 1403.7060 & 2789.3781 & 1395.1927 & 2788.3941 & 1394.7007 & $\mathbf{L}$ & 373.2809 & 187.1441 & 356.2544 & 178.6308 & & & 3 \\
\hline 26 & 2919.4887 & 1460.2480 & 2902.4621 & 1451.7347 & 2901.4781 & 1451.2427 & $\mathbf{L}$ & 260.1969 & 130.6021 & 243.1703 & 122.0888 & & & 2 \\
\hline 27 & & & & & & & $\mathbf{K}$ & 147.1128 & 74.0600 & 130.0863 & 65.5468 & & & 1 \\
\hline
\end{tabular}



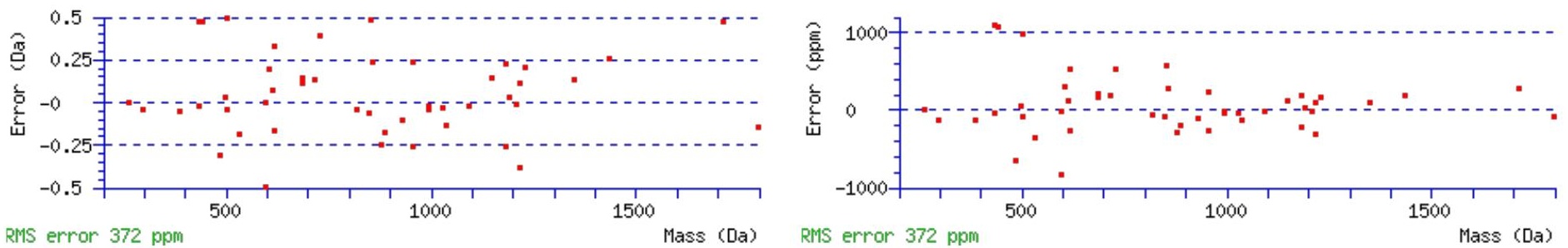

\section{All matches to this query}

\begin{tabular}{|l|l|l|l|}
\hline Score & Mr(calc): & Delta & \multicolumn{1}{|c|}{ Sequence } \\
\hline 41.1 & 3064.5869 & 0.0010 & SLDLSHNLISEFAWSDLHNLSALQLLK \\
\hline 21.1 & 3063.6029 & 0.9850 & SLDLSHNLISEFAWSDLHNLSALQLLK \\
\hline 17.0 & 3064.5869 & 0.0010 & SLDLSHNLISEFAWSDLHNLSALQLLK \\
\hline 11.0 & 3062.5512 & 2.0368 & LQTGDHIVMVNGVSVENVTSAFAIQILK \\
\hline 2.8 & 3062.5512 & 2.0368 & LQTGDHIVMVNGVSVENVTSAFAIQILK \\
\hline 0.5 & 3064.5805 & 0.0074 & LSSLIIGSSKERSLSTDASTNTAPVVVPR \\
\hline
\end{tabular}

Spectrum No: 817; Query: 221; Rank: 1

\section{Peptide View}

MS/MS Fragmentation of NGSGEVYQGPAK

Found in IPI00326948, Tax_Id=10116 Gene_Symbol=Hsd17b4 Hsd17b4 protein

Match to Query 221: 1206.546748 from(604.280650,2+)

Title: 091008RatKidney_NoSalt_19.426.426.2.dta

Data file K:INewmanPaper|Piliangl3SubProteomes\Piliang3SP\mgf5ppm\ERLIC_3SubProteomes5ppm.mgf

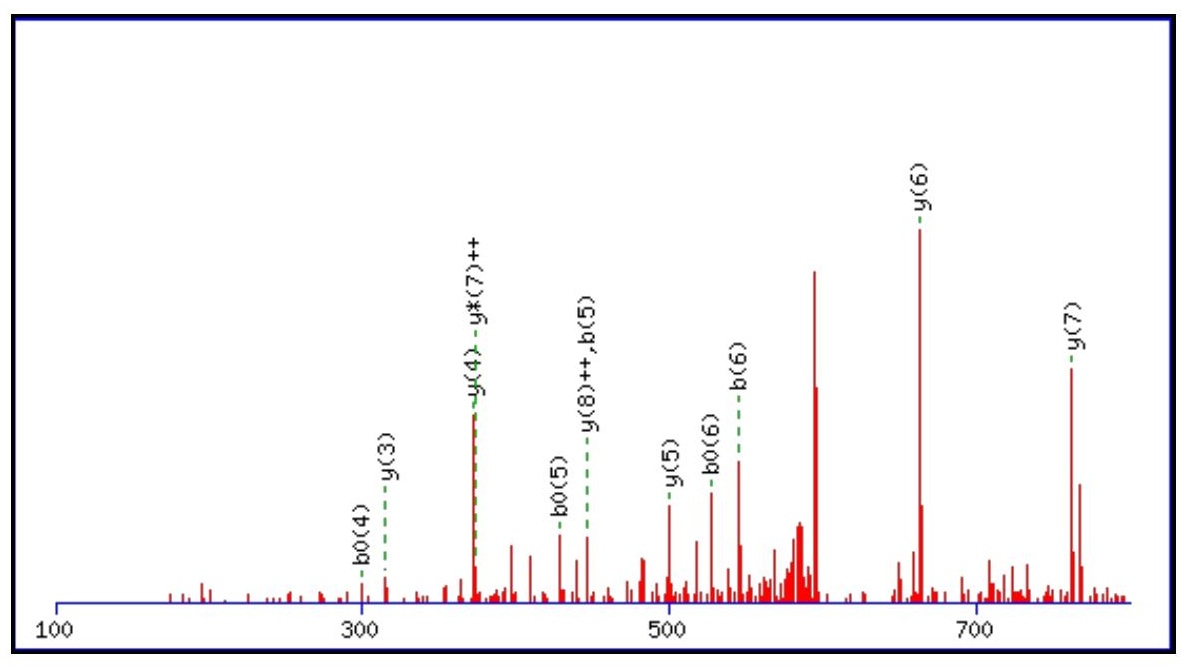



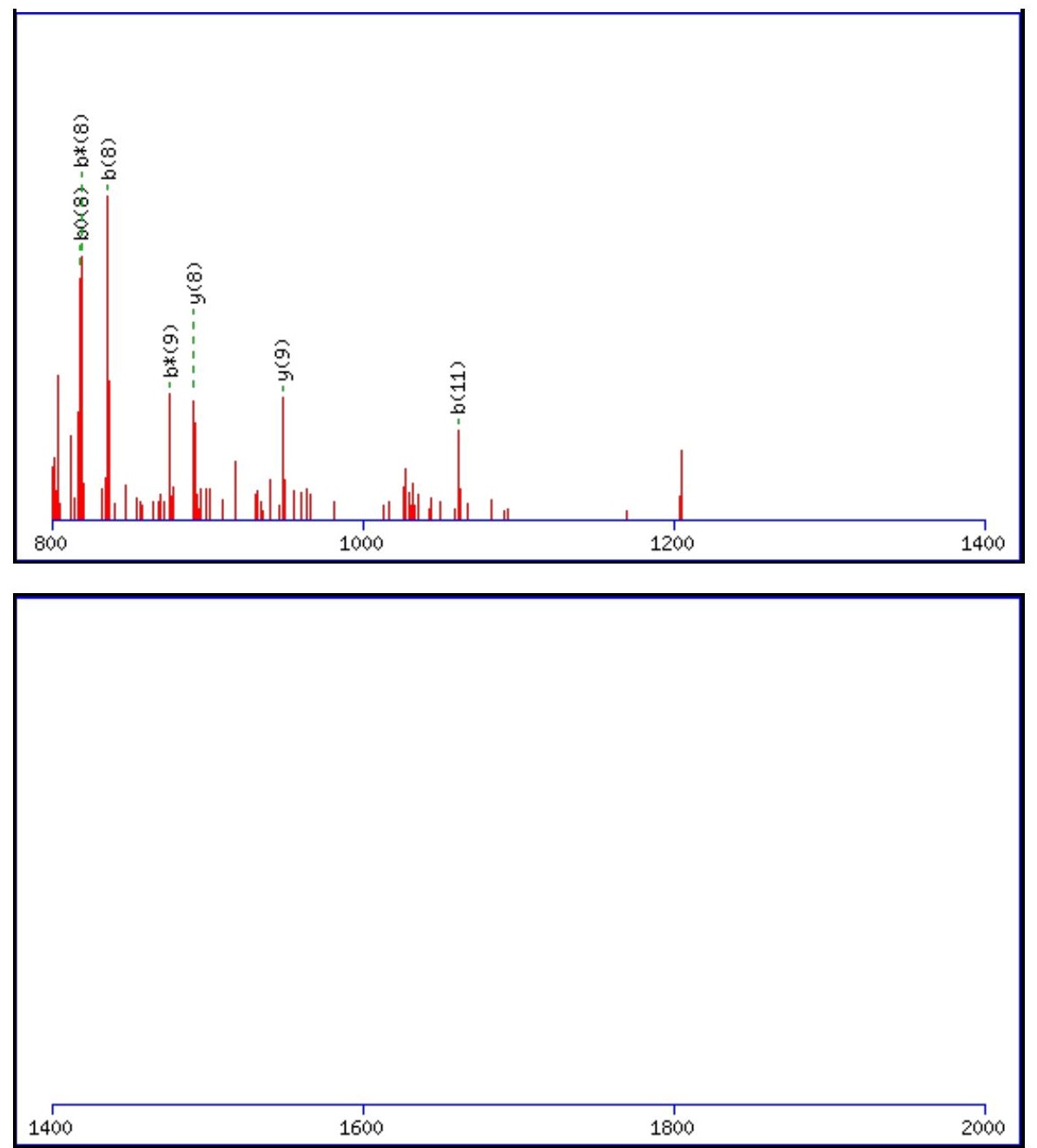

Monoisotopic mass of neutral peptide $\operatorname{Mr}($ calc): 1206.5517

Fixed modifications: Carbamidomethyl (C)

Variable modifications:

N1 : Deamidated $\mathrm{N}(\mathrm{N})$

Ions Score: 41 Expect: $\odot .0092$

Matches (Bold Red): 19/114 fragment ions using 37 most intense peaks

\begin{tabular}{|c|c|c|c|c|c|c|c|c|c|c|c|c|c|c|}
\hline \# & b & $\mathbf{b}^{++}$ & $\mathbf{b}^{*}$ & $\mathbf{b}^{*^{++}}$ & $\mathbf{b}^{0}$ & $\mathbf{b}^{0++}$ & Seq. & $\mathbf{y}$ & $\mathbf{y}^{++}$ & $\mathbf{y}^{*}$ & $\mathrm{y}^{*^{++}}$ & $\mathbf{y}^{0}$ & $\mathbf{y}^{0++}$ & \# \\
\hline 1 & 116.0342 & 58.5207 & 99.0077 & 50.0075 & & & $\mathbf{N}$ & & & & & & & 12 \\
\hline 2 & 173.0557 & 87.0315 & 156.0291 & 78.5182 & & & G & 1092.5320 & 546.7696 & 1075.5055 & 538.2564 & 1074.5215 & 537.7644 & 11 \\
\hline 3 & 260.0877 & 130.5475 & 243.0612 & 122.0342 & 242.0771 & 121.5422 & $\mathrm{~S}$ & 1035.5106 & 518.2589 & 1018.4840 & 509.7456 & 1017.5000 & 509.2536 & 10 \\
\hline 4 & 317.1092 & 159.0582 & 300.0826 & 150.5449 & 299.0986 & 150.0529 & $\mathbf{G}$ & 948.4785 & 474.7429 & 931.4520 & 466.2296 & 930.4680 & 465.7376 & 9 \\
\hline 5 & 446.1518 & 223.5795 & 429.1252 & 215.0662 & 428.1412 & 214.5742 & $\mathbf{E}$ & 891.4571 & 446.2322 & 874.4305 & 437.7189 & 873.4465 & 437.2269 & 8 \\
\hline 6 & 545.2202 & 273.1137 & 528.1936 & 264.6005 & 527.2096 & 264.1084 & V & 762.4145 & 381.7109 & 745.3879 & 373.1976 & & & 7 \\
\hline 7 & 708.2835 & 354.6454 & 691.2570 & 346.1321 & 690.2729 & 345.6401 & $\mathbf{Y}$ & 663.3461 & 332.1767 & 646.3195 & 323.6634 & & & 6 \\
\hline 8 & 836.3421 & 418.6747 & 819.3155 & 410.1614 & 818.3315 & 409.6694 & $\mathbf{Q}$ & 500.2827 & 250.6450 & 483.2562 & 242.1317 & & & 5 \\
\hline 9 & 893.3635 & 447.1854 & 876.3370 & 438.6721 & 875.3530 & 438.1801 & G & 372.2241 & 186.6157 & 355.1976 & 178.1024 & & & 4 \\
\hline 10 & 990.4163 & 495.7118 & 973.3898 & 487.1985 & 972.4057 & 486.7065 & $\mathbf{P}$ & 315.2027 & 158.1050 & 298.1761 & 149.5917 & & & 3 \\
\hline 11 & 1061.4534 & 531.2304 & 1044.4269 & 522.7171 & 1043.4429 & 522.2251 & A & 218.1499 & 109.5786 & 201.1234 & 101.0653 & & & 2 \\
\hline 12 & & & & & & & $\mathbf{K}$ & 147.1128 & 74.0600 & 130.0863 & 65.5468 & & & 1 \\
\hline
\end{tabular}



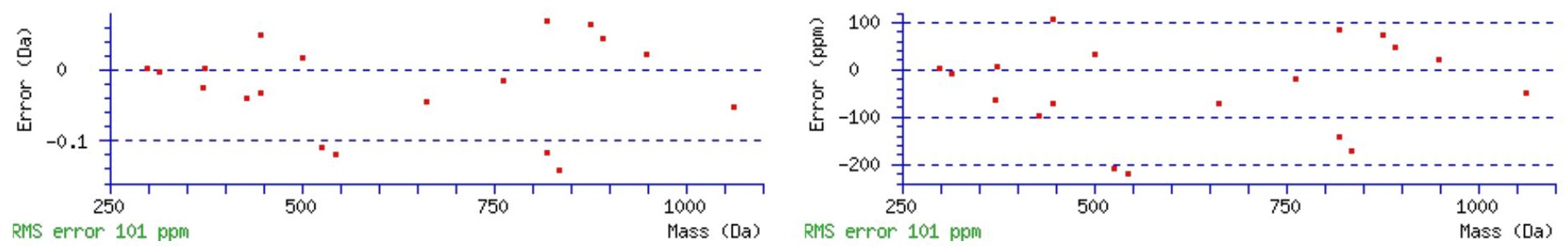

\section{All matches to this query}

\begin{tabular}{|l|l|l|l|}
\hline Score & Mr(calc): & Delta & \multicolumn{1}{|c|}{ Sequence } \\
\hline 41.1 & 1206.5517 & -0.0049 & NGSGEVYQGPAK \\
\hline 3.8 & 1204.5394 & 2.0074 & QEEGMDLINR \\
\hline 3.2 & 1205.5524 & 0.9943 & NVTASDTEDVR \\
\hline 2.9 & 1206.5451 & 0.0016 & DRCLYHNTK \\
\hline 2.8 & 1206.5580 & -0.0113 & MAAPESLRPR \\
\hline 2.5 & 1206.5362 & 0.0106 & ELEFYLWK \\
\hline 2.3 & 1206.5364 & 0.0103 & QESTNDTDGLK \\
\hline 1.9 & 1206.5394 & 0.0074 & AKNLHSNQSK \\
\hline 1.7 & 1205.5319 & 1.0149 & TSSAQRLKR \\
\hline 1.5 & 1205.5554 & 0.9914 & HTNETVLRR \\
\hline
\end{tabular}

Spectrum No: 818; Query: 954; Rank: 1

\section{Peptide View}

MS/MS Fragmentation of ENPGHIYNKNISQK

Found in IPI00194797, Tax_Id=10116 Gene_Symbol=Tmem9b_predicted TMEM9 domain family, member B

Match to Query 954: 1641.812682 from(548.278170,3+)

Title: 100101RatKid_NS_deglyco_23.535.535.3.dta

Data file K:INewmanPaper|Piliangl3SubProteomes\Piliang3SP\mgf5ppm\ERLIC_3SubProteomes5ppm.mgf

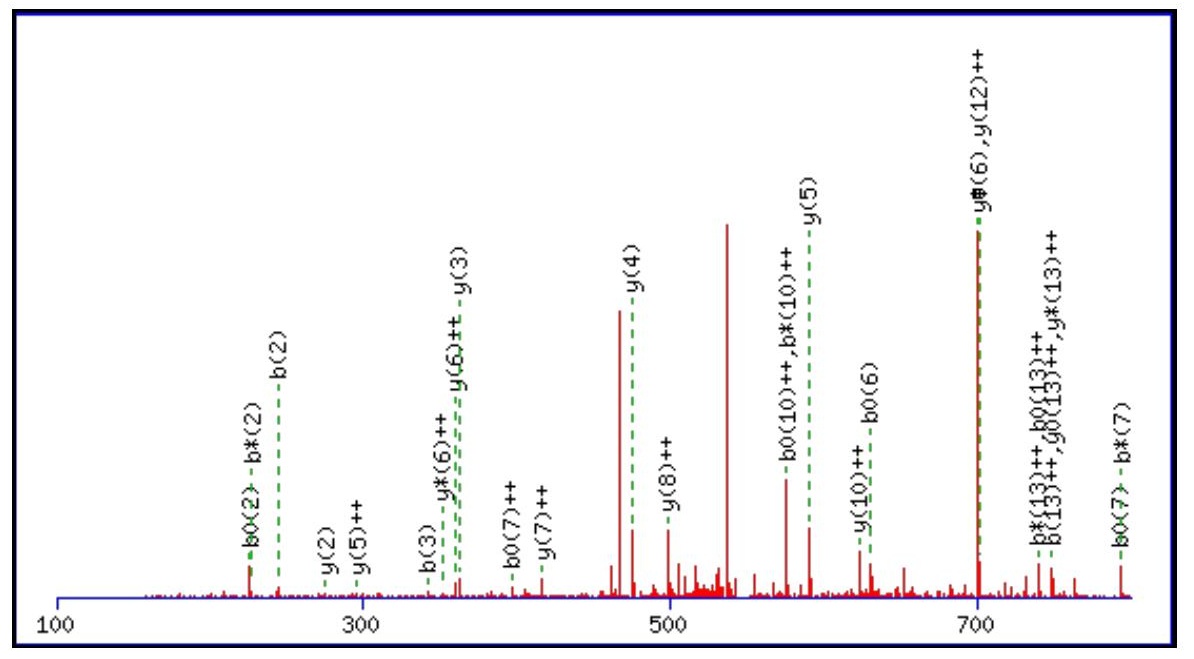



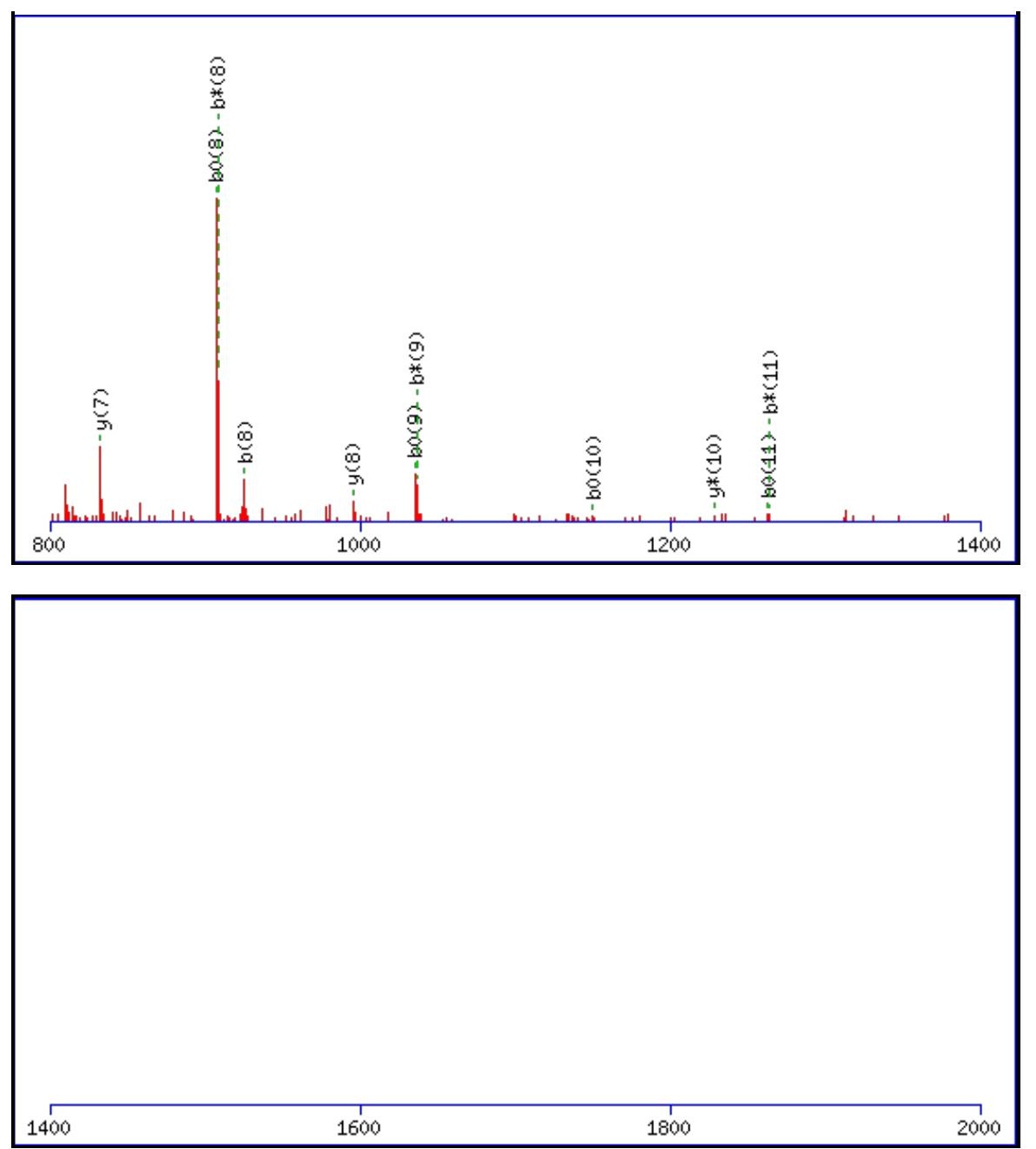

Monoisotopic mass of neutral peptide $\operatorname{Mr}($ calc): 1641.8110

Fixed modifications: Carbamidomethyl (C)

Variable modifications:

N10 : Deamidated_N (N)

Ions Score: 41 Expect : $\odot .014$

Matches (Bold Red): 39/150 fragment ions using 55 most intense peaks

\begin{tabular}{|c|c|c|c|c|c|c|c|c|c|c|c|c|c|c|}
\hline \# & b & $\mathbf{b}^{++}$ & $\mathbf{b}^{*}$ & $\mathbf{b}^{*^{++}}$ & $\mathbf{b}^{0}$ & $\mathbf{b}^{0++}$ & Seq. & $\mathbf{y}$ & $\mathbf{y}^{++}$ & $\mathbf{y}^{*}$ & $\mathrm{y}^{*^{++}}$ & $\mathbf{y}^{\mathbf{0}}$ & $\mathbf{y}^{\mathbf{0 + +}}$ & \# \\
\hline 1 & 130.0499 & 65.5286 & & & 112.0393 & 56.5233 & $\mathbf{E}$ & & & & & & & 14 \\
\hline 2 & 244.0928 & 122.5500 & 227.0662 & 114.0368 & 226.0822 & 113.5448 & $\mathbf{N}$ & 1513.7758 & 757.3915 & 1496.7492 & 748.8782 & 1495.7652 & 748.3862 & 13 \\
\hline 3 & 341.1456 & 171.0764 & 324.1190 & 162.5631 & 323.1350 & 162.0711 & $\mathbf{P}$ & 1399.7328 & 700.3701 & 1382.7063 & 691.8568 & 1381.7223 & 691.3648 & 12 \\
\hline 4 & 398.1670 & 199.5871 & 381.1405 & 191.0739 & 380.1565 & 190.5819 & $\mathbf{G}$ & 1302.6801 & 651.8437 & 1285.6535 & 643.3304 & 1284.6695 & 642.8384 & 11 \\
\hline 5 & 535.2259 & 268.1166 & 518.1994 & 259.6033 & 517.2154 & 259.1113 & $\mathbf{H}$ & 1245.6586 & 623.3329 & 1228.6321 & 614.8197 & 1227.6480 & 614.3277 & 10 \\
\hline 6 & 648.3100 & 324.6586 & 631.2835 & 316.1454 & 630.2994 & 315.6534 & I & 1108.5997 & 554.8035 & 1091.5731 & 546.2902 & 1090.5891 & 545.7982 & 9 \\
\hline 7 & 811.3733 & 406.1903 & 794.3468 & 397.6770 & 793.3628 & 397.1850 & $\mathbf{Y}$ & 995.5156 & 498.2615 & 978.4891 & 489.7482 & 977.5051 & 489.2562 & 8 \\
\hline 8 & 925.4163 & 463.2118 & 908.3897 & 454.6985 & 907.4057 & 454.2065 & $\mathbf{N}$ & 832.4523 & 416.7298 & 815.4258 & 408.2165 & 814.4417 & 407.7245 & 7 \\
\hline 9 & 1053.5112 & 527.2592 & 1036.4847 & 518.7460 & 1035.5007 & 518.2540 & $\mathbf{K}$ & 718.4094 & 359.7083 & 701.3828 & 351.1950 & 700.3988 & 350.7030 & 6 \\
\hline 10 & 1168.5382 & 584.7727 & 1151.5116 & 576.2594 & 1150.5276 & 575.7674 & $\mathbf{N}$ & 590.3144 & 295.6608 & 573.2879 & 287.1476 & 572.3038 & 286.6556 & 5 \\
\hline 11 & 1281.6222 & 641.3147 & 1264.5957 & 632.8015 & 1263.6117 & 632.3095 & I & 475.2875 & 238.1474 & 458.2609 & 229.6341 & 457.2769 & 229.1421 & 4 \\
\hline 12 & 1368.6542 & 684.8308 & 1351.6277 & 676.3175 & 1350.6437 & 675.8255 & S & 362.2034 & 181.6053 & 345.1769 & 173.0921 & 344.1928 & 172.6001 & 3 \\
\hline 13 & 1496.7128 & 748.8601 & 1479.6863 & 740.3468 & 1478.7023 & 739.8548 & $\mathbf{Q}$ & 275.1714 & 138.0893 & 258.1448 & 129.5761 & & & 2 \\
\hline 14 & & & & & & & $\mathbf{K}$ & 147.1128 & 74.0600 & 130.0863 & 65.5468 & & & 1 \\
\hline
\end{tabular}



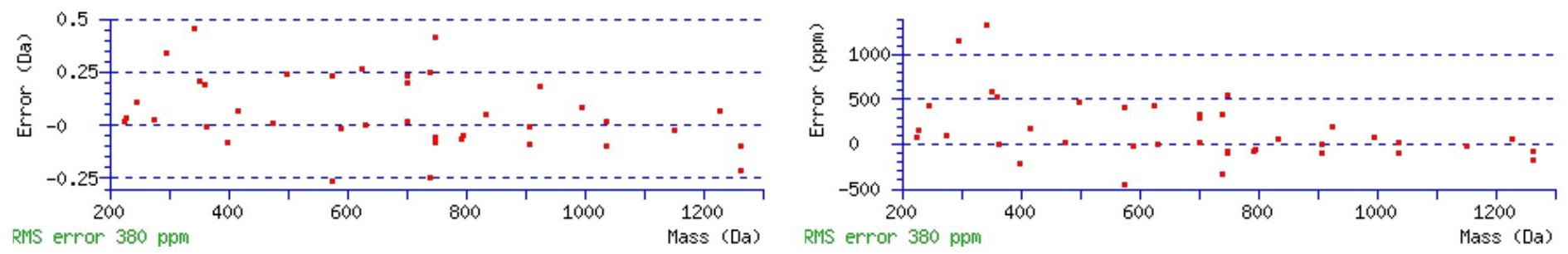

\section{All matches to this query}

\begin{tabular}{|l|l|l|l|}
\hline Score & Mr(calc): & Delta & \multicolumn{1}{|c|}{ Sequence } \\
\hline 40.9 & 1641.8110 & 0.0016 & ENPGHIYNKNISQK \\
\hline 16.8 & 1641.8110 & 0.0016 & ENPGHIYNKNISQK \\
\hline 3.8 & 1639.8094 & 2.0033 & LGAVYTEGGFVEGVNK \\
\hline 2.5 & 1639.8219 & 1.9908 & ALGVSNFNHFHIER \\
\hline 2.5 & 1640.8059 & 1.0068 & ALGVSNFNHFHIER \\
\hline 2.5 & 1640.8131 & 0.9995 & GNNGPPKSGRNFSGPR \\
\hline 0.5 & 1639.8206 & 1.9921 & ETVNSPAIYKFQSR \\
\hline 0.3 & 1639.8157 & 1.9970 & LLTWRAAMLKDNK \\
\hline 0.1 & 1639.8195 & 1.9931 & AATTGSGVKVPRNFR \\
\hline
\end{tabular}

Spectrum No: 819; Query: 81; Rank: 1

\section{Peptide View}

\section{MS/MS Fragmentation of DIWNITGAK}

Found in IPI00361798, Tax_Id=10116 Gene_Symbol=Igfbp7 Insulin-like growth factor binding protein 7

Match to Query 81: 1017.513888 from(509.764220,2+)

Title: 100101RatKid_NS_deglyco_26.3079.3079.2.dta

Data file K:INewmanPaper|Piliangl3SubProteomes\Piliang3SP\mgf5ppm\ERLIC_3SubProteomes5ppm.mgf

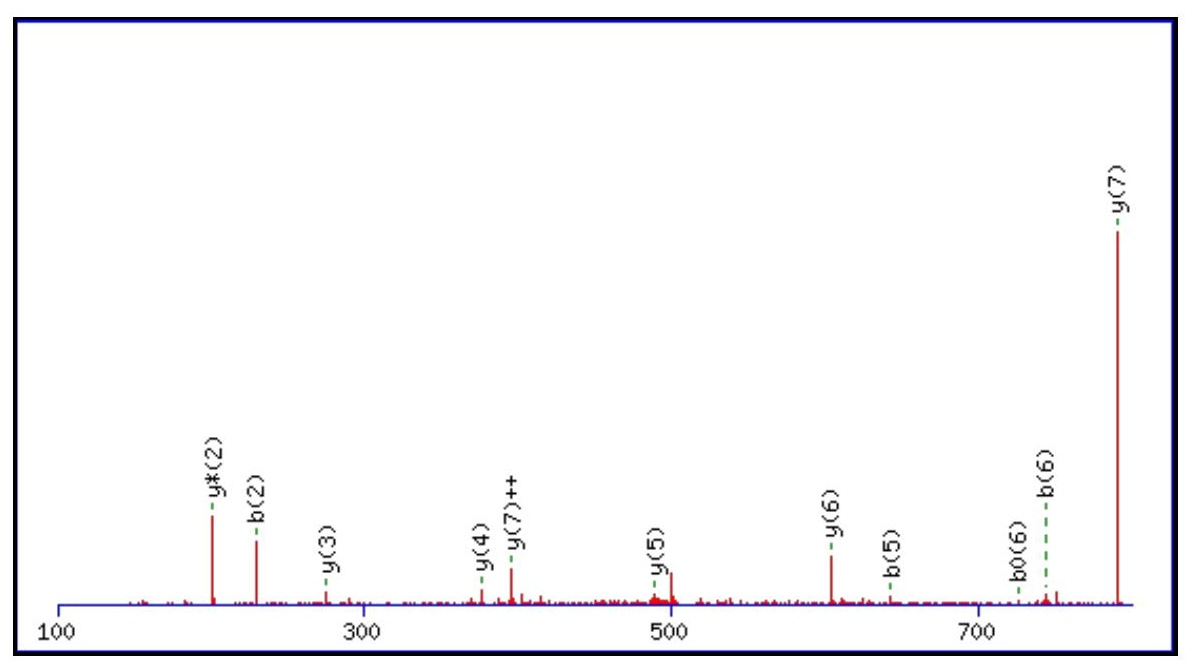



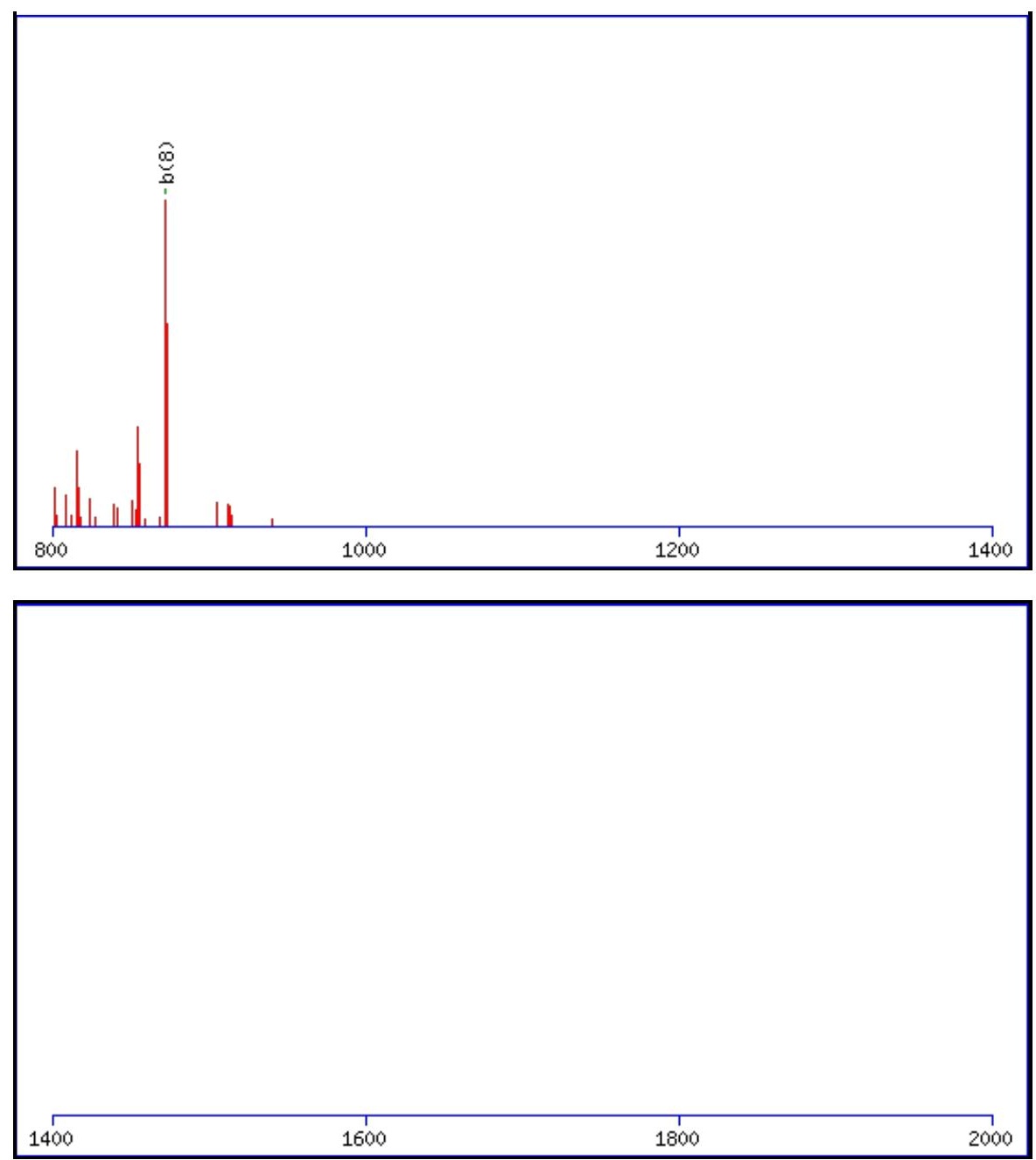

Monoisotopic mass of neutral peptide $\operatorname{Mr}($ calc): 1017.5131

Fixed modifications: Carbamidomethyl (C)

Variable modifications:

N4 : Deamidated_N (N)

Ions Score: 41 Expect: 0.0085

Matches (Bold Red): 12/84 fragment ions using 18 most intense peaks

\begin{tabular}{|l|c|c|c|c|c|c|c|c|c|c|c|c|c|c|}
\hline$\#$ & $\mathbf{b}$ & $\mathbf{b}^{++}$ & $\mathbf{b}^{*}$ & $\mathbf{b}^{\boldsymbol{*}^{++}}$ & $\mathbf{b}^{\mathbf{0}}$ & $\mathbf{b}^{\mathbf{0 + +}}$ & $\mathbf{S e q}$ & $\mathbf{y}$ & $\mathbf{y}^{++}$ & $\mathbf{y}^{*}$ & $\mathbf{y}^{\mathbf{*}^{++}}$ & $\mathbf{y}^{\mathbf{0}}$ & $\mathbf{y}^{\mathbf{0 + +}}$ & $\#$ \\
\hline $\mathbf{1}$ & 116.0342 & 58.5207 & & & 98.0237 & 49.5155 & $\mathbf{D}$ & & & & & & & $\mathbf{9}$ \\
\hline $\mathbf{2}$ & $\mathbf{2 2 9 . 1 1 8 3}$ & 115.0628 & & & 211.1077 & 106.0575 & $\mathbf{I}$ & 903.4934 & 452.2504 & 886.4669 & 443.7371 & 885.4829 & 443.2451 & $\mathbf{8}$ \\
\hline $\mathbf{3}$ & 415.1976 & 208.1024 & & & 397.1870 & 199.0972 & $\mathbf{W}$ & $\mathbf{7 9 0 . 4 0 9 4}$ & 395.7083 & 773.3828 & 387.1951 & 772.3988 & 386.7030 & $\mathbf{7}$ \\
\hline $\mathbf{4}$ & 530.2245 & 265.6159 & 513.1980 & 257.1026 & 512.2140 & 256.6106 & $\mathbf{N}$ & $\mathbf{6 0 4 . 3 3 0 1}$ & 302.6687 & 587.3035 & 294.1554 & 586.3195 & 293.6634 & $\mathbf{6}$ \\
\hline $\mathbf{5}$ & $\mathbf{6 4 3 . 3 0 8 6}$ & 322.1579 & 626.2820 & 313.6447 & 625.2980 & 313.1527 & $\mathbf{I}$ & $\mathbf{4 8 9 . 3 0 3 1}$ & 245.1552 & 472.2766 & 236.6419 & 471.2926 & 236.1499 & $\mathbf{5}$ \\
\hline $\mathbf{6}$ & $\mathbf{7 4 4 . 3 5 6 3}$ & 372.6818 & 727.3297 & 364.1685 & 726.3457 & 363.6765 & $\mathbf{T}$ & 376.2191 & 188.6132 & 359.1925 & 180.0999 & 358.2085 & 179.6079 & $\mathbf{4}$ \\
\hline $\mathbf{7}$ & 801.3777 & 401.1925 & 784.3512 & 392.6792 & 783.3672 & 392.1872 & $\mathbf{G}$ & 275.1714 & 138.0893 & 258.1448 & 129.5761 & & & 3 \\
\hline $\mathbf{8}$ & $\mathbf{8 7 2 . 4 1 4 9}$ & 436.7111 & 855.3883 & 428.1978 & 854.4043 & 427.7058 & $\mathbf{A}$ & 218.1499 & 109.5786 & $\mathbf{2 0 1 . 1 2 3 4}$ & 101.0653 & & & $\mathbf{2}$ \\
\hline $\mathbf{9}$ & & & & & & & $\mathbf{K}$ & 147.1128 & 74.0600 & 130.0863 & 65.5468 & & & $\mathbf{1}$ \\
\hline
\end{tabular}
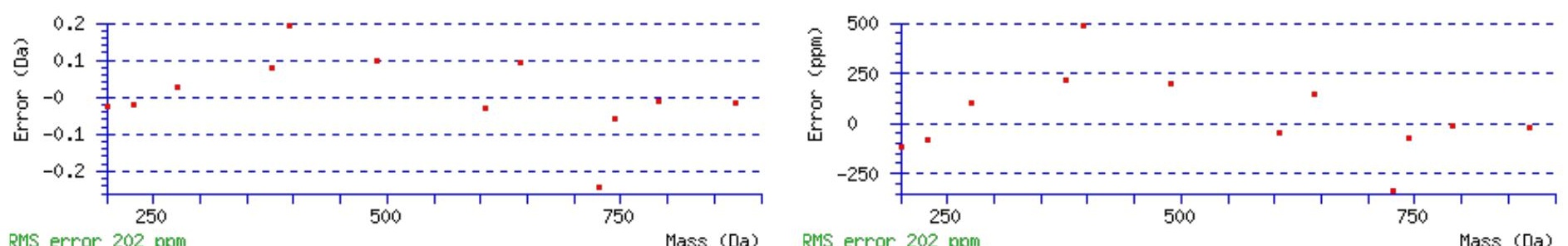

\section{All matches to this query}




\begin{tabular}{|l|l|l|l|} 
Score & Mr(calc): & Delta & \multicolumn{1}{|c|}{ Sequence } \\
\hline 40.8 & 1017.5131 & 0.0008 & DIWNITGAK \\
\hline 7.6 & 1017.5091 & 0.0048 & EVADKSQGGK \\
\hline 7.2 & 1017.5090 & 0.0048 & QEAKNATQK \\
\hline 6.8 & 1017.5131 & 0.0008 & EFVINPNGK \\
\hline 3.8 & 1017.5178 & -0.0039 & IDWICRR \\
\hline 3.8 & 1017.5164 & -0.0026 & EVEMEIIR \\
\hline 3.5 & 1017.5212 & -0.0073 & MPMPSKRR \\
\hline 3.5 & 1017.5049 & 0.0090 & FWTKTKK \\
\hline 3.5 & 1017.5203 & -0.0064 & NNKQRTQK \\
\hline 3.4 & 1016.5056 & 1.0083 & EPITPPKR \\
\hline
\end{tabular}

Spectrum No: 820; Query: 173; Rank: 1

\section{Peptide View}

MS/MS Fragmentation of FYLYPDLSR

Found in IPI00214462, Tax_Id=10116 Gene_Symbol=Slc6a11 Sodium- and chloride-dependent GABA transporter 3

Match to Query 173: 1172.583508 from(587.299030,2+)

Title: 091008RatKidney_NH4Format01_24.2811.2811.2.dta

Data file K:INewmanPaper|Piliang|3SubProteomes\Piliang3SP\mgf5ppm\ERLIC_3SubProteomes5ppm.mgf
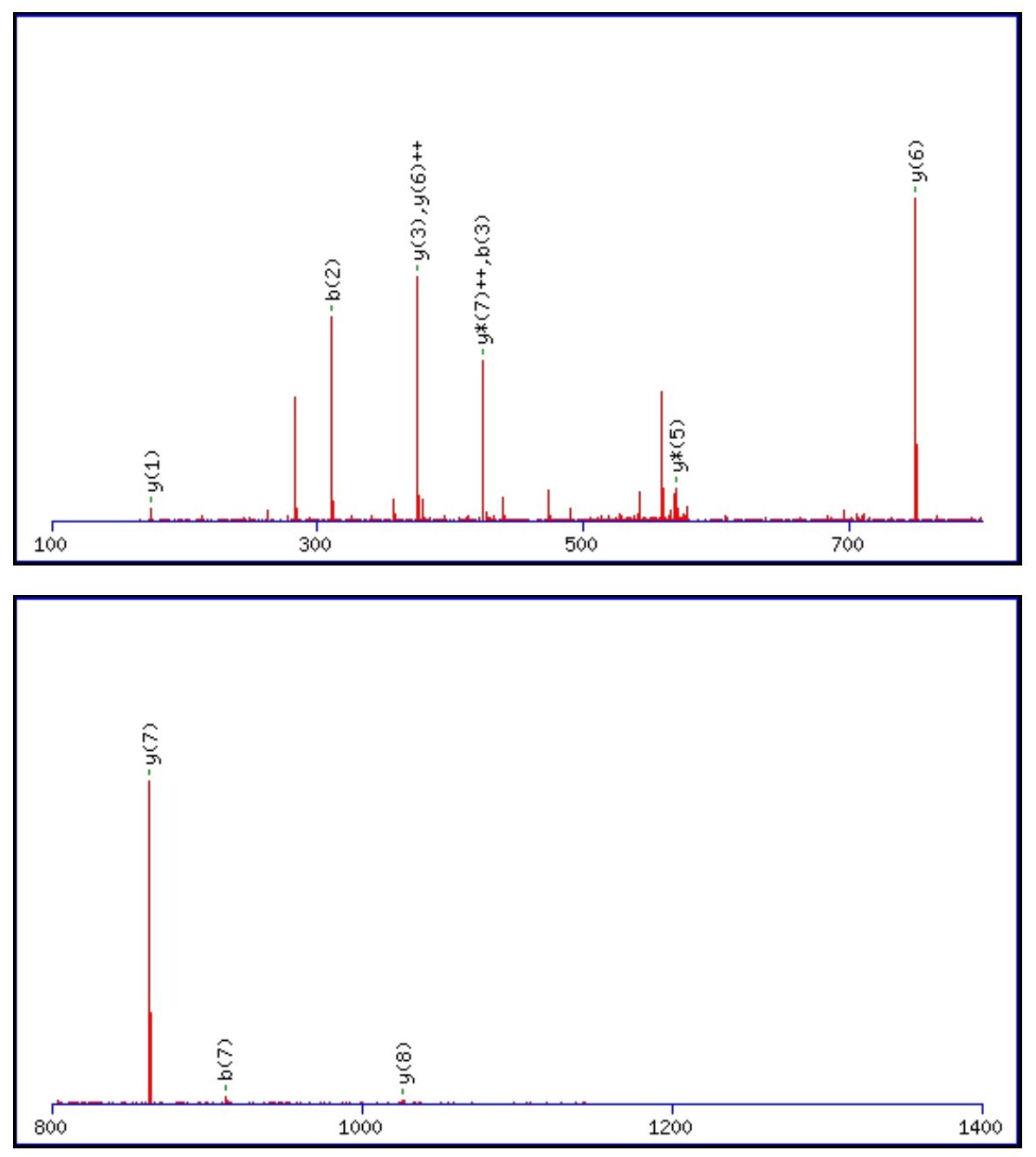
Monoisotopic mass of neutral peptide $\operatorname{Mr}($ calc): 1172.5866

Fixed modifications: Carbamidomethyl (C)

Ions Score: 41 Expect: 0.0093

Matches (Bold Red): 11/68 fragment ions using 14 most intense peaks

\begin{tabular}{|c|c|c|c|c|c|c|c|c|c|c|c|c|}
\hline$\#$ & $\mathbf{b}$ & $\mathbf{b}^{++}$ & $\mathbf{b}^{\mathbf{0}}$ & $\mathbf{b}^{\mathbf{0 + +}}$ & Seq. & $\mathbf{y}$ & $\mathbf{y}^{++}$ & $\mathbf{y}^{\mathbf{*}}$ & $\mathbf{y}^{\mathbf{*}^{++}}$ & $\mathbf{y}^{\mathbf{0}}$ & $\mathbf{y}^{\mathbf{0 + +}}$ & $\#$ \\
\hline $\mathbf{1}$ & 148.0757 & 74.5415 & & & $\mathbf{F}$ & & & & & & & $\mathbf{9}$ \\
\hline $\mathbf{2}$ & $\mathbf{3 1 1 . 1 3 9 0}$ & 156.0731 & & & $\mathbf{Y}$ & $\mathbf{1 0 2 6 . 5 2 5 5}$ & 513.7664 & 1009.4989 & 505.2531 & 1008.5149 & 504.7611 & $\mathbf{8}$ \\
\hline $\mathbf{3}$ & $\mathbf{4 2 4 . 2 2 3 1}$ & 212.6152 & & & $\mathbf{L}$ & $\mathbf{8 6 3 . 4 6 2 1}$ & 432.2347 & 846.4356 & $\mathbf{4 2 3 . 7 2 1 4}$ & 845.4516 & 423.2294 & $\mathbf{7}$ \\
\hline $\mathbf{4}$ & 587.2864 & 294.1468 & & & $\mathbf{Y}$ & 750.3781 & 375.6927 & 733.3515 & 367.1794 & 732.3675 & 366.6874 & $\mathbf{6}$ \\
\hline $\mathbf{5}$ & 684.3392 & 342.6732 & & & $\mathbf{P}$ & 587.3148 & 294.1610 & 570.2882 & 285.6477 & 569.3042 & 285.1557 & $\mathbf{5}$ \\
\hline $\mathbf{6}$ & 799.3661 & 400.1867 & 781.3556 & 391.1814 & $\mathbf{D}$ & 490.2620 & 245.6346 & 473.2354 & 237.1214 & 472.2514 & 236.6293 & $\mathbf{4}$ \\
\hline $\mathbf{7}$ & $\mathbf{9 1 2 . 4 5 0 2}$ & 456.7287 & 894.4396 & 447.7234 & $\mathbf{L}$ & 375.2350 & 188.1212 & 358.2085 & 179.6079 & 357.2245 & 179.1159 & $\mathbf{3}$ \\
\hline $\mathbf{8}$ & 999.4822 & 500.2447 & 981.4716 & 491.2395 & $\mathbf{S}$ & 262.1510 & 131.5791 & 245.1244 & 123.0659 & 244.1404 & 122.5738 & $\mathbf{2}$ \\
\hline $\mathbf{9}$ & & & & & $\mathbf{R}$ & $\mathbf{1 7 5 . 1 1 9 0}$ & 88.0631 & 158.0924 & 79.5498 & & & $\mathbf{1}$ \\
\hline
\end{tabular}
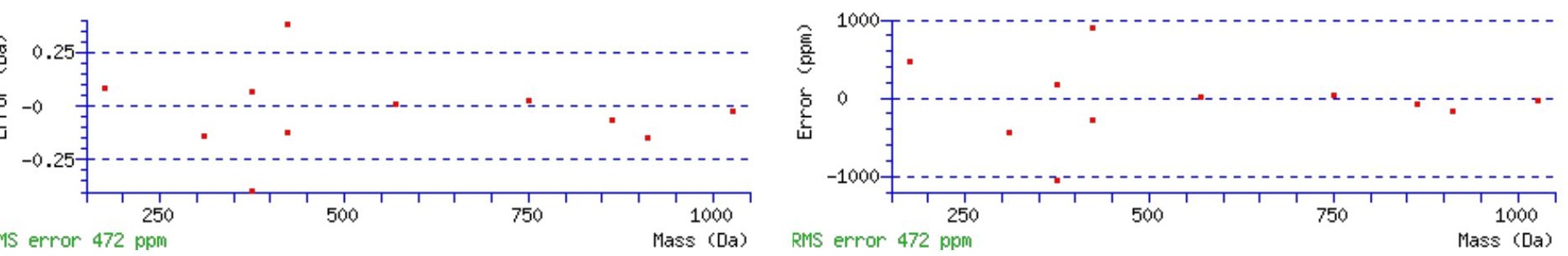

\section{All matches to this query}

\begin{tabular}{|l|l|l|l|}
\hline Score & Mr(calc): & Delta & \multicolumn{1}{|c|}{ Sequence } \\
\hline 40.7 & 1172.5866 & -0.0031 & FYLYPDLSR \\
\hline 40.7 & 1172.5866 & -0.0031 & FYLYPNISR \\
\hline 11.5 & 1172.5747 & 0.0088 & PVNENMLLNK \\
\hline 11.5 & 1171.5791 & 1.0044 & FLHSGKLYK \\
\hline 11.4 & 1171.5729 & 1.0106 & YMLRANMIK \\
\hline 9.9 & 1171.5729 & 1.0106 & DAIMRMLYK \\
\hline 8.1 & 1171.5761 & 1.0074 & TSSVPEYVYK \\
\hline 7.5 & 1170.5822 & 2.0013 & GSYLWVQYR \\
\hline 7.2 & 1172.5898 & -0.0063 & AVRPTQSGDSR \\
\hline 6.8 & 1171.5890 & 0.9945 & SLVLKEFEK \\
\hline
\end{tabular}




\section{Peptide View}

MS/MS Fragmentation of YFFLQSVDSDGRNFTSSPPGQTQFK

Found in IPI00369729, Tax_Id=10116 Gene_Symbol=Kdelc2 KDEL motif-containing protein 2 precursor

Match to Query 2467: 2854.340952 from(952.454260,3+)

Title: 100101RatKid_NS_deglyco_12.4571.4571.3.dta

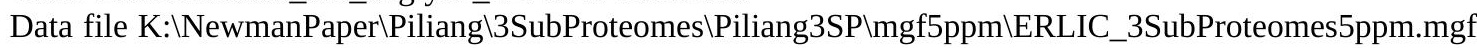
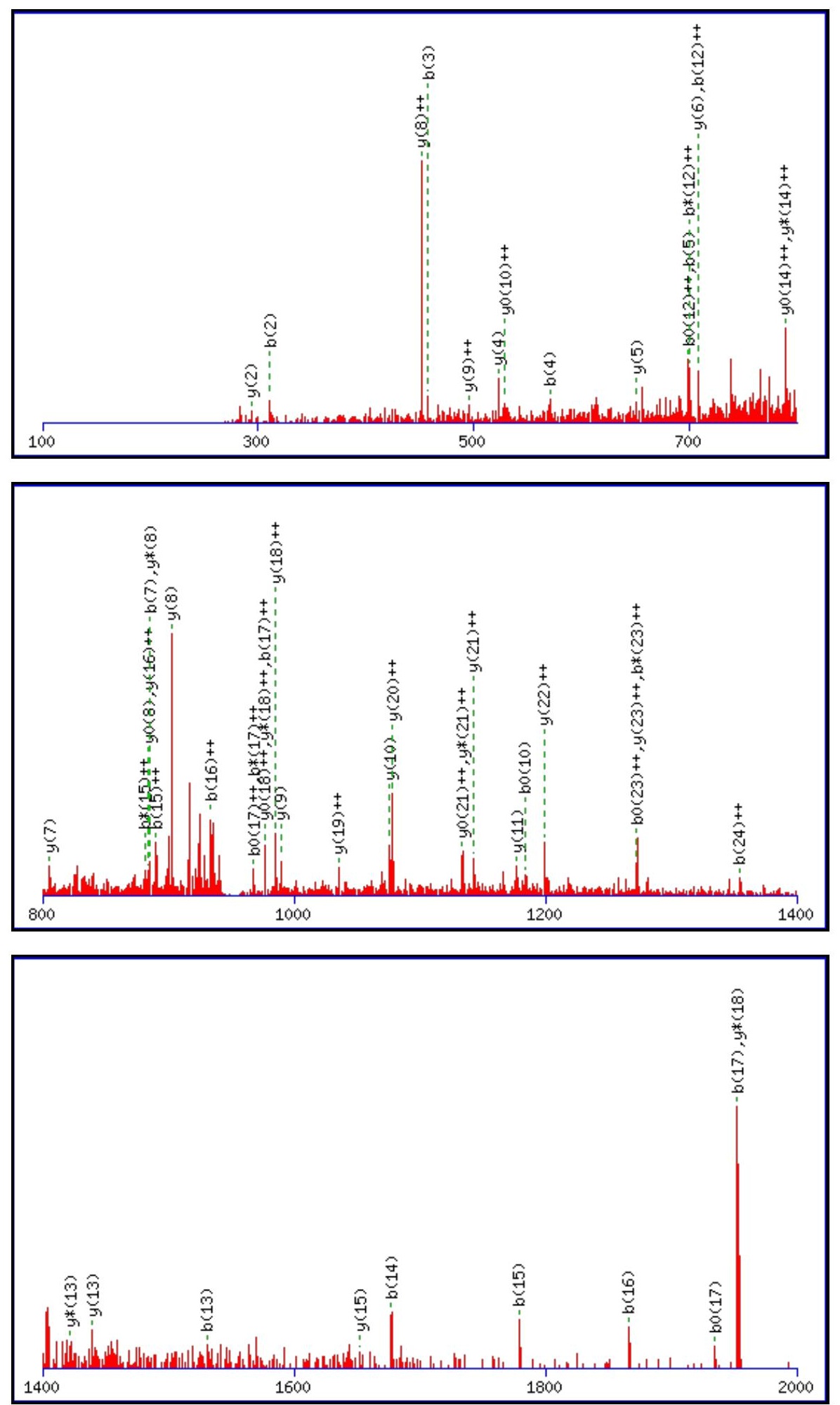

Monoisotopic mass of neutral peptide $\operatorname{Mr}($ calc): 2853.3246

Fixed modifications: Carbamidomethyl (C)

Variable modifications:

N13 : Deamidated_N (N)

Ions Score: 41 Expect: 0.03 
Matches (Bold Red): 55/264 fragment ions using 113 most intense peaks

\begin{tabular}{|c|c|c|c|c|c|c|c|c|c|c|c|c|c|c|}
\hline \# & b & $\mathbf{b}^{++}$ & b* $^{*}$ & $\mathbf{b}^{*^{++}}$ & $\mathbf{b}^{\mathbf{0}}$ & $\mathbf{b}^{0++}$ & Seq. & $\mathbf{y}$ & $\mathbf{y}^{++}$ & $\mathbf{y}^{*}$ & $\mathrm{y}^{*^{++}}$ & $y^{0}$ & $y^{0++}$ & \# \\
\hline 1 & 164.0706 & 82.5389 & & & & & $\mathbf{Y}$ & & & & & & & 25 \\
\hline 2 & 311.1390 & 156.0731 & & & & & $F$ & 2691.2685 & 1346.1379 & 2674.2420 & 1337.6246 & 2673.2580 & 1337.1326 & 24 \\
\hline 3 & 458.2074 & 229.6074 & & & & & $\mathbf{F}$ & 2544.2001 & 1272.6037 & 2527.1736 & 1264.0904 & 2526.1896 & 1263.5984 & 23 \\
\hline 4 & 571.2915 & 286.1494 & & & & & $\mathbf{L}$ & 2397.1317 & \begin{tabular}{|l|l|}
1199.0695 \\
\end{tabular} & 2380.1052 & 1190.5562 & 2379.1211 & 1190.0642 & 22 \\
\hline 5 & 699.3501 & 350.1787 & 682.3235 & 341.6654 & & & $\mathbf{Q}$ & 2284.0476 & 1142.5275 & 2267.0211 & 1134.0142 & 2266.0371 & 1133.5222 & 21 \\
\hline 6 & 786.3821 & 393.6947 & 769.3556 & 385.1814 & 768.3715 & 384.6894 & $S$ & 2155.9891 & 1078.4982 & 2138.9625 & 1069.9849 & 2137.9785 & 1069.4929 & 20 \\
\hline 7 & 885.4505 & 443.2289 & 868.4240 & 434.7156 & 867.4400 & 434.2236 & $\mathbf{V}$ & 2068.9570 & 1034.9822 & 2051.9305 & 1026.4689 & 2050.9465 & 1025.9769 & 19 \\
\hline 8 & 1000.4775 & 500.7424 & 983.4509 & 492.2291 & 982.4669 & 491.7371 & D & 1969.8886 & 985.4479 & 1952.8621 & 976.9347 & 1951.8781 & 976.4427 & 18 \\
\hline 9 & 1087.5095 & 544.2584 & 1070.4829 & 535.7451 & 1069.4989 & 535.2531 & $\mathrm{~S}$ & 1854.8617 & 927.9345 & 1837.8351 & 919.4212 & 1836.8511 & 918.9292 & 17 \\
\hline 10 & 1202.5364 & 601.7719 & 1185.5099 & 593.2586 & 1184.5259 & 592.7666 & D & 1767.8296 & 884.4185 & 1750.8031 & 875.9052 & 1749.8191 & 875.4132 & 16 \\
\hline 11 & 1259.5579 & 630.2826 & 1242.5313 & 621.7693 & 1241.5473 & 621.2773 & $\mathbf{G}$ & 1652.8027 & 826.9050 & 1635.7762 & 818.3917 & 1634.7921 & 817.8997 & 15 \\
\hline 12 & 1415.6590 & 708.3331 & 1398.6325 & 699.8199 & 1397.6484 & 699.3279 & $\mathbf{R}$ & 1595.7812 & 798.3943 & 1578.7547 & 789.8810 & 1577.7707 & 789.3890 & 14 \\
\hline 13 & 1530.6859 & 765.8466 & 1513.6594 & 757.3333 & 1512.6754 & 756.8413 & $\mathbf{N}$ & 1439.6801 & 720.3437 & 1422.6536 & 711.8304 & 1421.6696 & 711.3384 & 13 \\
\hline 14 & \begin{tabular}{|l|}
1677.7544 \\
\end{tabular} & 839.3808 & 1660.7278 & 830.8675 & 1659.7438 & 830.3755 & $\mathbf{F}$ & 1324.6532 & 662.8302 & 1307.6266 & 654.3170 & 1306.6426 & 653.8250 & 12 \\
\hline 15 & 1778.8020 & 889.9047 & 1761.7755 & 881.3914 & 1760.7915 & 880.8994 & $\mathbf{T}$ & 1177.5848 & 589.2960 & 1160.5582 & 580.7828 & 1159.5742 & 580.2907 & 11 \\
\hline 16 & \begin{tabular}{|l|}
1865.8341 \\
\end{tabular} & 933.4207 & 1848.8075 & 924.9074 & 1847.8235 & 924.4154 & $S$ & \begin{tabular}{|l|}
1076.5371 \\
\end{tabular} & 538.7722 & 1059.5106 & 530.2589 & 1058.5265 & 529.7669 & 10 \\
\hline 17 & 1952.8661 & 976.9367 & 1935.8395 & 968.4234 & 1934.8555 & 967.9314 & $\mathrm{~S}$ & 989.5051 & 495.2562 & 972.4785 & 486.7429 & 971.4945 & 486.2509 & 9 \\
\hline 18 & 2049.9189 & 1025.4631 & 2032.8923 & 1016.9498 & 2031.9083 & 1016.4578 & $\mathbf{P}$ & 902.4730 & 451.7402 & 885.4465 & 443.2269 & 884.4625 & 442.7349 & 8 \\
\hline 19 & 2146.9716 & 1073.9894 & 2129.9451 & 1065.4762 & 2128.9611 & 1064.9842 & $\mathbf{P}$ & 805.4203 & 403.2138 & 788.3937 & 394.7005 & 787.4097 & 394.2085 & 7 \\
\hline 20 & 2203.9931 & 1102.5002 & 2186.9665 & 1093.9869 & 2185.9825 & 1093.4949 & G & 708.3675 & 354.6874 & 691.3410 & 346.1741 & 690.3570 & 345.6821 & 6 \\
\hline 21 & 2332.0517 & 1166.5295 & 2315.0251 & 1158.0162 & 2314.0411 & 1157.5242 & $\bar{Q}$ & 651.3461 & 326.1767 & 634.3195 & 317.6634 & 633.3355 & 317.1714 & 5 \\
\hline 22 & 2433.0993 & 1217.0533 & 2416.0728 & 1208.5400 & 2415.0888 & 1208.0480 & $T$ & 523.2875 & 262.1474 & 506.2609 & 253.6341 & 505.2769 & 253.1421 & 4 \\
\hline 23 & 2561.1579 & 1281.0826 & 2544.1314 & 1272.5693 & 2543.1474 & 1272.0773 & $\bar{Q}$ & 422.2398 & 211.6235 & 405.2132 & 203.1103 & & & 3 \\
\hline 24 & 2708.2263 & \begin{tabular}{|l|}
1354.6168 \\
\end{tabular} & 2691.1998 & 1346.1035 & 2690.2158 & 1345.6115 & $\mathbf{F}$ & 294.1812 & 147.5942 & 277.1547 & 139.0810 & & & 2 \\
\hline 25 & & & & & & & $\mathbf{K}$ & 147.1128 & 74.0600 & 130.0863 & 65.5468 & & & 1 \\
\hline
\end{tabular}
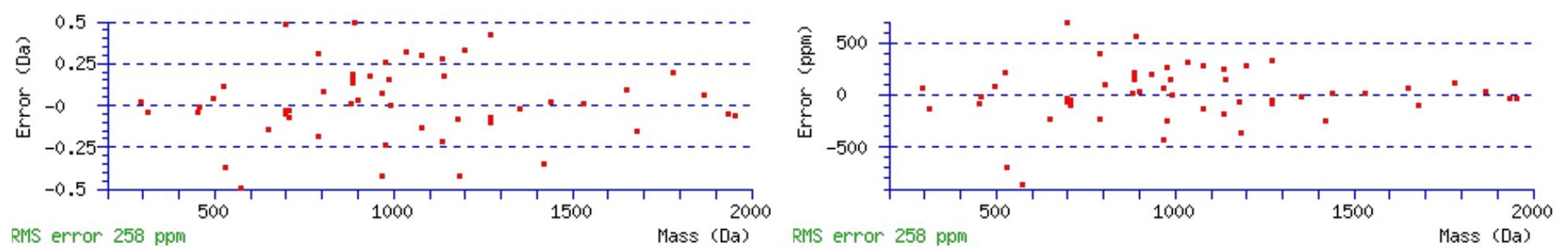

\section{All matches to this query}

\begin{tabular}{|l|c|c|l|}
\hline Score & Mr(calc): & Delta & \multicolumn{1}{c|}{ Sequence } \\
\hline 40.7 & 2853.3246 & 1.0163 & YFFLQSVDSDGRNFTSSPPGQTQFK \\
\hline 16.4 & 2852.3406 & 2.0003 & YFFLQSVDSDGRNFTSSPPGQTQFK \\
\hline 7.3 & 2853.3467 & 0.9943 & LKQSKIMGSQSHLSDELEEQTLEK \\
\hline 4.3 & 2854.3628 & -0.0219 & MTQEEYALLTAIVILSPDRQYIK \\
\hline 1.2 & 2853.3467 & 0.9943 & LKQSKIMGSOSHLSDELEEQTLEK \\
\hline 0.4 & 2854.3636 & -0.0226 & MSVLPTPASRRLSGLPLTAPQSMPR \\
\hline 0.1 & 2852.3429 & 1.9980 & IHTLSSPSPLRGHGGDCOSPKDLWK \\
\hline 0.1 & 2852.3429 & 1.9980 & $\underline{\text { IHTLSSPSPLRGHGGDCQSPKDLWK }}$ \\
\hline 0.1 & 2852.3429 & 1.9980 & $\underline{\text { IHTLSSPSPLRGHGGDCQSPKDLWK }}$ \\
\hline
\end{tabular}

Spectrum No: 822; Query: 84; Rank: 1 


\section{Peptide View}

MS/MS Fragmentation of LNITCESSK

Found in IPI00776785, Tax_Id=10116 Gene_Symbol=Tmem16f_predicted 94 kDa protein

Match to Query 84: 1051.486128 from(526.750340,2+)

Title: 091008RatKidney_NH4Format01_25.653.653.2.dta

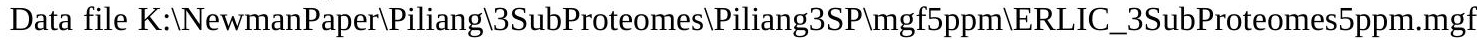
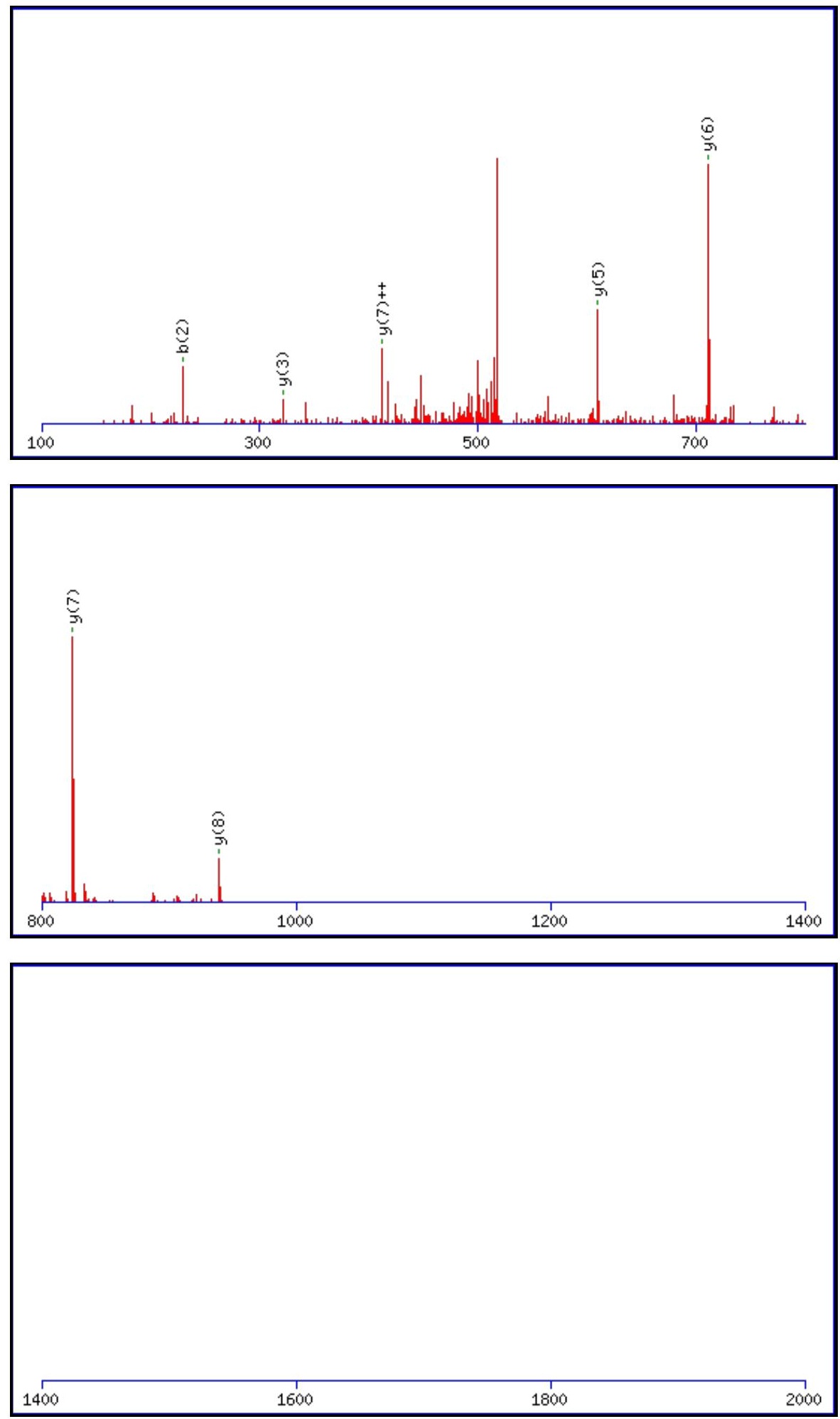

Monoisotopic mass of neutral peptide $\operatorname{Mr}($ calc): 1051.4856

Fixed modifications: Carbamidomethyl (C)

Variable modifications:

N2 : Deamidated_N (N)

Ions Score: 41 Expect: 0.0092 
Matches (Bold Red): 7/86 fragment ions using 9 most intense peaks

\begin{tabular}{|c|c|c|c|c|c|c|c|c|c|c|c|c|c|c|}
\hline$\#$ & $\mathbf{b}$ & $\mathbf{b}^{++}$ & $\mathbf{b}^{*}$ & $\mathbf{b}^{\boldsymbol{*}^{++}}$ & $\mathbf{b}^{\mathbf{0}}$ & $\mathbf{b}^{\mathbf{0 + +}}$ & $\mathbf{S e q}$ & $\mathbf{y}$ & $\mathbf{y}^{++}$ & $\mathbf{y}^{*}$ & $\mathbf{y}^{\boldsymbol{*}^{++}}$ & $\mathbf{y}^{\mathbf{0}}$ & $\mathbf{y}^{\mathbf{0 + +}}$ & $\#$ \\
\hline $\mathbf{1}$ & 114.0913 & 57.5493 & & & & & $\mathbf{L}$ & & & & & & & $\mathbf{9}$ \\
\hline $\mathbf{2}$ & $\mathbf{2 2 9 . 1 1 8 3}$ & 115.0628 & 212.0917 & 106.5495 & & & $\mathbf{N}$ & $\mathbf{9 3 9 . 4 0 8 8}$ & 470.2080 & 922.3822 & 461.6948 & 921.3982 & 461.2027 & $\mathbf{8}$ \\
\hline $\mathbf{3}$ & 342.2023 & 171.6048 & 325.1758 & 163.0915 & & & $\mathbf{I}$ & $\mathbf{8 2 4 . 3 8 1 8}$ & $\mathbf{4 1 2 . 6 9 4 6}$ & 807.3553 & 404.1813 & 806.3713 & 403.6893 & $\mathbf{7}$ \\
\hline $\mathbf{4}$ & 443.2500 & 222.1286 & 426.2235 & 213.6154 & 425.2395 & 213.1234 & $\mathbf{T}$ & $\mathbf{7 1 1 . 2 9 7 8}$ & 356.1525 & 694.2712 & 347.6393 & 693.2872 & 347.1472 & $\mathbf{6}$ \\
\hline $\mathbf{5}$ & 603.2807 & 302.1440 & 586.2541 & 293.6307 & 585.2701 & 293.1387 & $\mathbf{C}$ & $\mathbf{6 1 0 . 2 5 0 1}$ & 305.6287 & 593.2236 & 297.1154 & 592.2395 & 296.6234 & $\mathbf{5}$ \\
\hline $\mathbf{6}$ & 732.3233 & 366.6653 & 715.2967 & 358.1520 & 714.3127 & 357.6600 & $\mathbf{E}$ & 450.2195 & 225.6134 & 433.1929 & 217.1001 & 432.2089 & 216.6081 & $\mathbf{4}$ \\
\hline $\mathbf{7}$ & 819.3553 & 410.1813 & 802.3287 & 401.6680 & 801.3447 & 401.1760 & $\mathbf{S}$ & 321.1769 & 161.0921 & 304.1503 & 152.5788 & 303.1663 & 152.0868 & $\mathbf{3}$ \\
\hline $\mathbf{8}$ & $\mathbf{9 0 6 . 3 8 7 3}$ & 453.6973 & 889.3608 & 445.1840 & 888.3768 & 444.6920 & $\mathbf{S}$ & 234.1448 & 117.5761 & 217.1183 & 109.0628 & 216.1343 & 108.5708 & $\mathbf{2}$ \\
\hline $\mathbf{9}$ & & & & & & & $\mathbf{K}$ & 147.1128 & 74.0600 & 130.0863 & 65.5468 & & & $\mathbf{1}$ \\
\hline
\end{tabular}
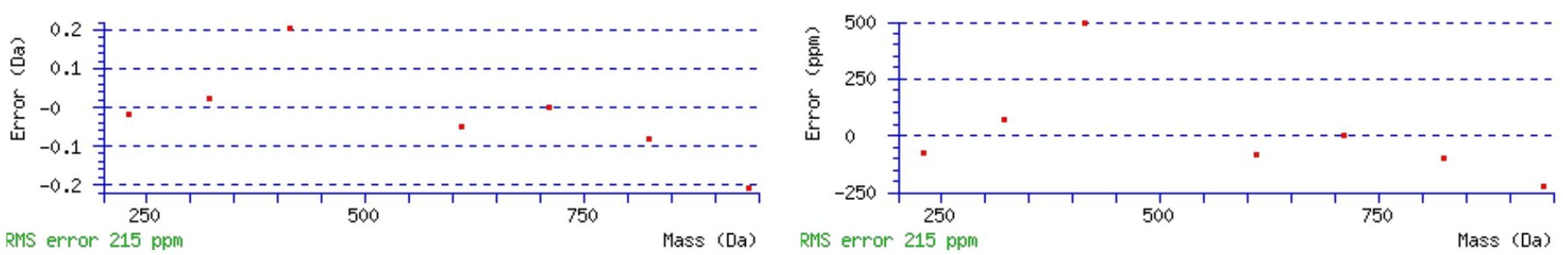

\section{All matches to this query}

\begin{tabular}{|l|l|l|l|}
\hline Score & Mr(calc): & Delta & \multicolumn{1}{|c|}{ Sequence } \\
\hline 40.7 & 1051.4856 & 0.0006 & LNITCESSK \\
\hline 9.6 & 1051.4964 & -0.0103 & LPSRWASR \\
\hline 8.3 & 1050.4747 & 1.0115 & ELSPQLER \\
\hline 7.3 & 1051.4885 & -0.0024 & LNIMTAGPR \\
\hline 7.0 & 1050.4859 & 1.0002 & AGVAHSKSSK \\
\hline 4.6 & 1051.4950 & -0.0089 & NLAPAAISSK \\
\hline 3.1 & 1050.4859 & 1.0002 & QVQSPGLSR \\
\hline 1.8 & 1050.4859 & 1.0002 & TAPAANLASR \\
\hline 1.8 & 1051.4868 & -0.0007 & YTVRLLK \\
\hline 1.7 & 1051.4934 & -0.0073 & LNYDEKNR \\
\hline
\end{tabular}

Spectrum No: 823; Query: 2226; Rank: 1

\section{Peptide View}

MS/MS Fragmentation of AMLQLDEGNVLPNSTNGAPLHLR

Found in IPI00210824, Tax_Id=10116 Gene_Symbol=Serpina6 Corticosteroid-binding globulin precursor

Match to Query 2226: 2477.250252 from(826.757360,3+)

Title: 091008RatKidney_NoSalt_30.2874.2874.3.dta

Data file K:INewmanPaper|Piliangl3SubProteomes\Piliang3SP\mgf5ppm\ERLIC_3SubProteomes5ppm.mgf 

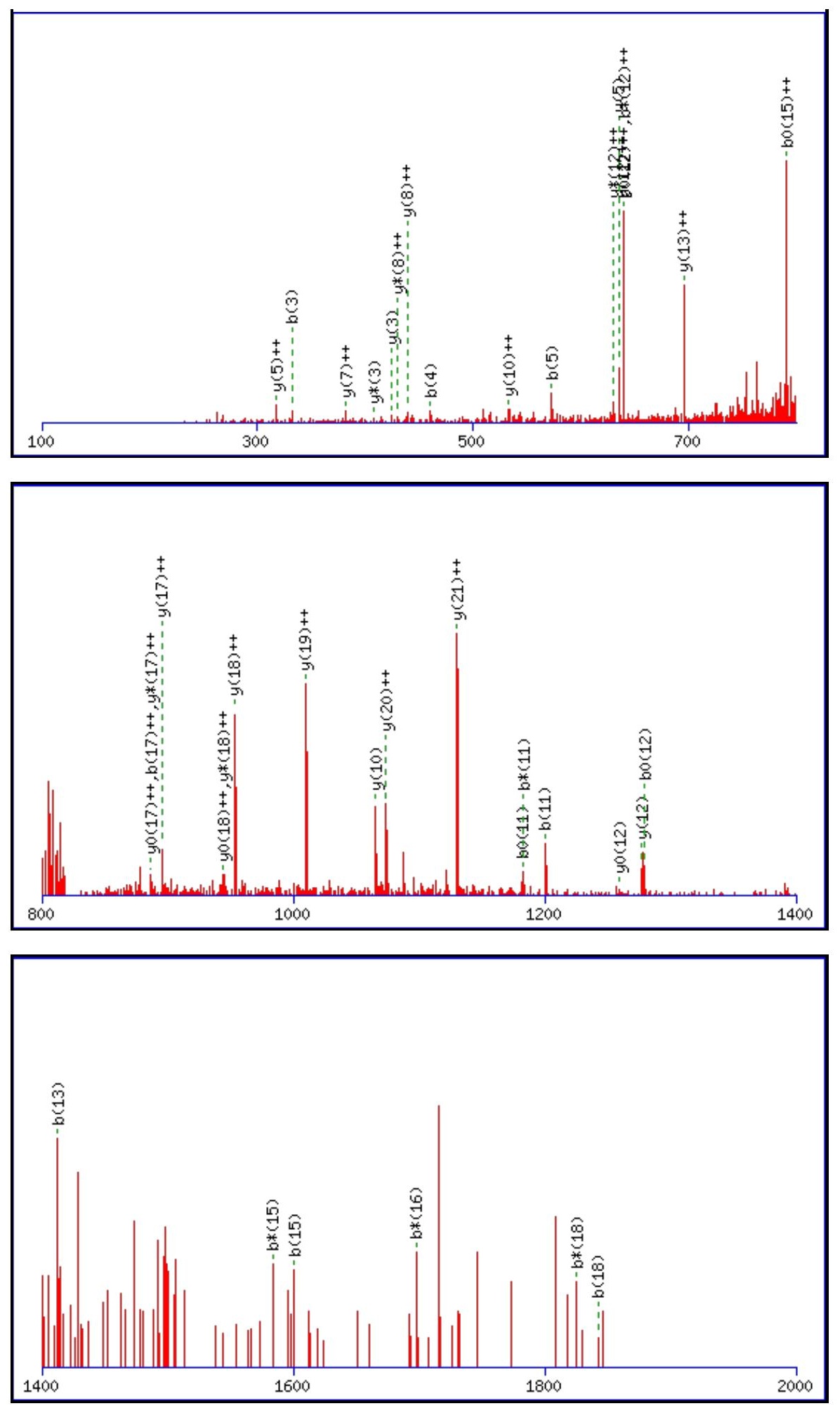

Monoisotopic mass of neutral peptide $\operatorname{Mr}($ calc): 2476.2380

Fixed modifications: Carbamidomethyl (C)

Variable modifications:

M2 : Oxidation $(\mathrm{M})$

N13 : Deamidated_N (N)

Ions Score: 41 Expect: 0.016

Matches (Bold Red): 40/232 fragment ions using 85 most intense peaks

\begin{tabular}{|r|c|c|c|c|c|c|c|c|c|c|c|c|c|c|}
\hline$\#$ & $\mathbf{b}$ & $\mathbf{b}^{++}$ & $\mathbf{b}^{*}$ & $\mathbf{b}^{*^{++}}$ & $\mathbf{b}^{\mathbf{0}}$ & $\mathbf{b}^{\mathbf{0 + +}}$ & Seq. & $\mathbf{y}$ & $\mathbf{y}^{++}$ & $\mathbf{y}^{*}$ & $\mathbf{y}^{*^{++}}$ & $\mathbf{y}^{\mathbf{0}}$ & $\mathbf{y}^{\mathbf{0}+}$ & $\#$ \\
\hline $\mathbf{1}$ & $\mathbf{7 2 . 0 4 4 4}$ & 36.5258 & & & & & $\mathbf{A}$ & & & & & & & 23 \\
\hline $\mathbf{2}$ & 219.0798 & 110.0435 & & & & & $\mathbf{M}$ & 2406.2082 & 1203.6077 & 2389.1816 & 1195.0945 & 2388.1976 & 1194.6024 & $\mathbf{2 2}$ \\
\hline $\mathbf{3}$ & 332.1639 & 166.5856 & & & & & $\mathbf{L}$ & 2259.1728 & $\mathbf{1 1 3 0 . 0 9 0 0}$ & 2242.1462 & 1121.5768 & 2241.1622 & 1121.0847 & $\mathbf{2 1}$ \\
\hline $\mathbf{4}$ & $\mathbf{4 6 0 . 2 2 2 4}$ & 230.6149 & 443.1959 & 222.1016 & & & $\mathbf{Q}$ & 2146.0887 & $\mathbf{1 0 7 3 . 5 4 8 0}$ & 2129.0622 & 1065.0347 & 2128.0781 & 1064.5427 & $\mathbf{2 0}$ \\
\hline & & & & & & & & & & & & & &
\end{tabular}




\begin{tabular}{|c|c|c|c|c|c|c|c|c|c|c|c|c|c|c|}
\hline & 573.3065 & 287.1569 & 556.2800 & 278.6436 & & & $\mathbf{L}$ & 2018.0301 & 1009.5187| & 2001.0036 & 1001.0054 & |2000.0196| & 1000.5134 & 19| \\
\hline 6 & 688.3334 & 344.6704 & 671.3069 & 336.1571 & 670.3229 & 335.6651 & D & 1904.9461 & 952.9767 & 1887.9195 & 944.4634 & 1886.9355 & 943.9714 & 18 \\
\hline 7 & 817.3760 & 409.1917 & 800.3495 & 400.6784 & 799.3655 & 400.1864 & $\mathbf{E}$ & 1789.9191 & 895.4632 & 1772.8926 & 886.9499 & 1771.9086 & 886.4579 & 17 \\
\hline 8 & 874.3975 & 437.7024 & 857.3710 & 429.1891 & 856.3869 & 428.6971 & G & 1660.8765 & 830.9419 & 1643.8500 & 822.4286 & 1642.8660 & 821.9366 & 16 \\
\hline 9 & 988.4404 & 494.7239 & 971.4139 & 486.2106 & 970.4299 & 485.7186 & $\mathbf{N}$ & 1603.8551 & 802.4312 & 1586.8285 & 793.9179 & 1585.8445 & 793.4259 & 15 \\
\hline 10 & 1087.5088 & 544.2581 & 1070.4823 & 535.7448 & 1069.4983 & 535.2528 & $\bar{V}$ & 1489.8121 & 745.4097 & 1472.7856 & 736.8964 & 1471.8016 & 736.4044 & 14 \\
\hline 11 & 1200.5929 & 600.8001 & 1183.5664 & 592.2868 & 1182.5823 & 591.7948 & $\mathbf{L}$ & 1390.7437 & 695.8755 & 1373.7172 & 687.3622 & 1372.7332 & 686.8702 & 13 \\
\hline 12 & 1297.6457 & 649.3265 & 1280.6191 & 640.8132 & 1279.6351 & 640.3212 & $\mathbf{P}$ & 1277.6597 & 639.3335 & 1260.6331 & 630.8202 & 1259.6491 & 630.3282 & 12 \\
\hline 13 & 1412.6726 & 706.8399 & 1395.6461 & 698.3267 & 1394.6620 & 697.8347 & $\mathbf{N}$ & 1180.6069 & 590.8071 & 1163.5804 & 582.2938 & 1162.5963 & 581.8018 & 11 \\
\hline 14 & 1499.7046 & 750.3560 & 1482.6781 & 741.8427 & 1481.6941 & 741.3507 & $S$ & 1065.5800 & 533.2936 & 1048.5534 & 524.7803 & 1047.5694 & 524.2883 & 10 \\
\hline 15 & 1600.7523 & 800.8798 & 1583.7258 & 792.3665 & 1582.7417 & 791.8745 & $\mathbf{T}$ & 978.5479 & 489.7776 & 961.5214 & 481.2643 & 960.5374 & 480.7723 & 9 \\
\hline 16 & 1714.7952 & 857.9013 & 1697.7687 & 849.3880 & 1696.7847 & 848.8960 & $\mathbf{N}$ & 877.5003 & 439.2538 & 860.4737 & 430.7405 & & & 8 \\
\hline 17 & 1771.8167 & 886.4120 & 1754.7902 & 877.8987 & 1753.8061 & 877.4067 & G & 763.4573 & 382.2323 & 746.4308 & 373.7190 & & & 7 \\
\hline 18 & \begin{tabular}{|l|}
1842.8538 \\
\end{tabular} & 921.9305 & 1825.8273 & 913.4173 & 1824.8433 & 912.9253 & A & 706.4359 & 353.7216 & 689.4093 & 345.2083 & & & 6 \\
\hline 19 & 1939.9066 & 970.4569 & 1922.8800 & 961.9437 & 1921.8960 & 961.4516 & $\mathbf{P}$ & 635.3988 & 318.2030 & 618.3722 & 309.6897 & & & 5 \\
\hline 20 & 2052.9906 & 1026.9990 & 2035.9641 & 1018.4857 & 2034.9801 & 1017.9937 & $\mathbf{L}$ & 538.3460 & 269.6766 & 521.3194 & 261.1634 & & & 4 \\
\hline 21 & 2190.0496 & 1095.5284 & 2173.0230 & 1087.0151 & 2172.0390 & 1086.5231 & H & 425.2619 & 213.1346 & 408.2354 & 204.6213 & & & 3 \\
\hline 22 & 2303.1336 & 1152.0704 & 2286.1071 & 1143.5572 & 2285.1231 & 1143.0652 & $\mathbf{L}$ & 288.2030 & 144.6051 & 271.1765 & 136.0919 & & & 2 \\
\hline 23 & & & & & & & $\mathbf{R}$ & 175.1190 & 88.0631 & 158.0924 & 79.5498 & & & 1 \\
\hline
\end{tabular}
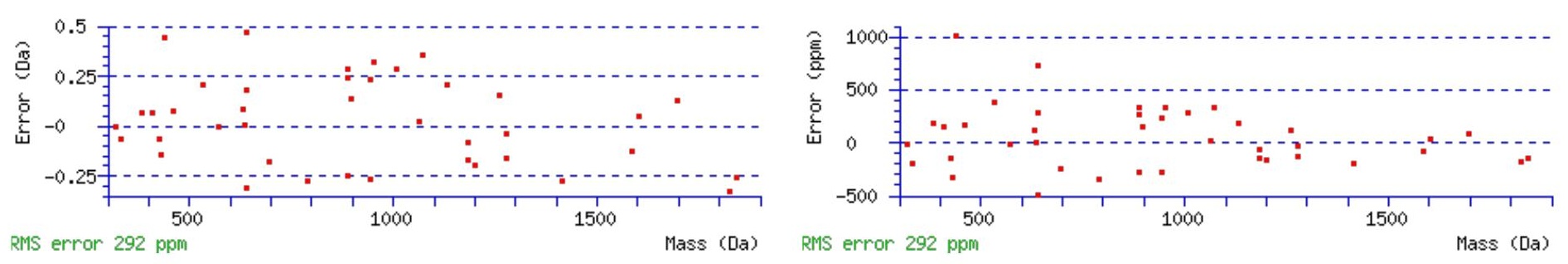

\section{All matches to this query}

\begin{tabular}{|l|l|l|l|}
\hline Score & Mr(calc): & Delta & \multicolumn{1}{|c|}{ Sequence } \\
\hline 40.7 & 2476.2380 & 1.0123 & AMLQLDEGNVLPNSTNGAPLHLR \\
\hline 39.6 & 2476.2380 & 1.0123 & AMLQLDEGNVLPNSTNGAPLHLR \\
\hline 28.8 & 2476.2380 & 1.0123 & AMLQLDEGNVLPNSTNGAPLHLR \\
\hline 9.3 & 2475.2540 & 1.9963 & AMLQLDEGNVLPNSTNGAPLHLR \\
\hline 4.1 & 2475.2419 & 2.0084 & TRELKPYVIFIKPPSMSSMR \\
\hline 4.1 & 2475.2419 & 2.0084 & TRELKPYVIFIKPPSMSSMR \\
\hline 1.6 & 2476.2556 & 0.9947 & MAAAVLLAVGMRAAHRTLAAAGSR \\
\hline 1.6 & 2476.2556 & 0.9947 & MAAAVLLAVGMRAAHRTLAAAGSR \\
\hline 1.2 & 2475.2419 & 2.0084 & TRELKPYVIFIKPPSMSSMR \\
\hline 1.2 & 2475.2419 & 2.0084 & TRELKPYVIFIKPPSMSSMR \\
\hline
\end{tabular}

Spectrum No: 824; Query: 678; Rank: 1

\section{Peptide View}

MS/MS Fragmentation of CCGWVSPSNWTR

Found in IPI00197582, Tax_Id=10116 Gene_Symbol=Cd82 CD82 antigen

Match to Query 678: 1509.615528 from(755.815040,2+)

Title: 100101RatKid_NS_deglyco_25.3014.3014.2.dta

Data file K:INewmanPaper|Piliang|3SubProteomes\Piliang3SP\mgf5ppm\ERLIC_3SubProteomes5ppm.mgf 

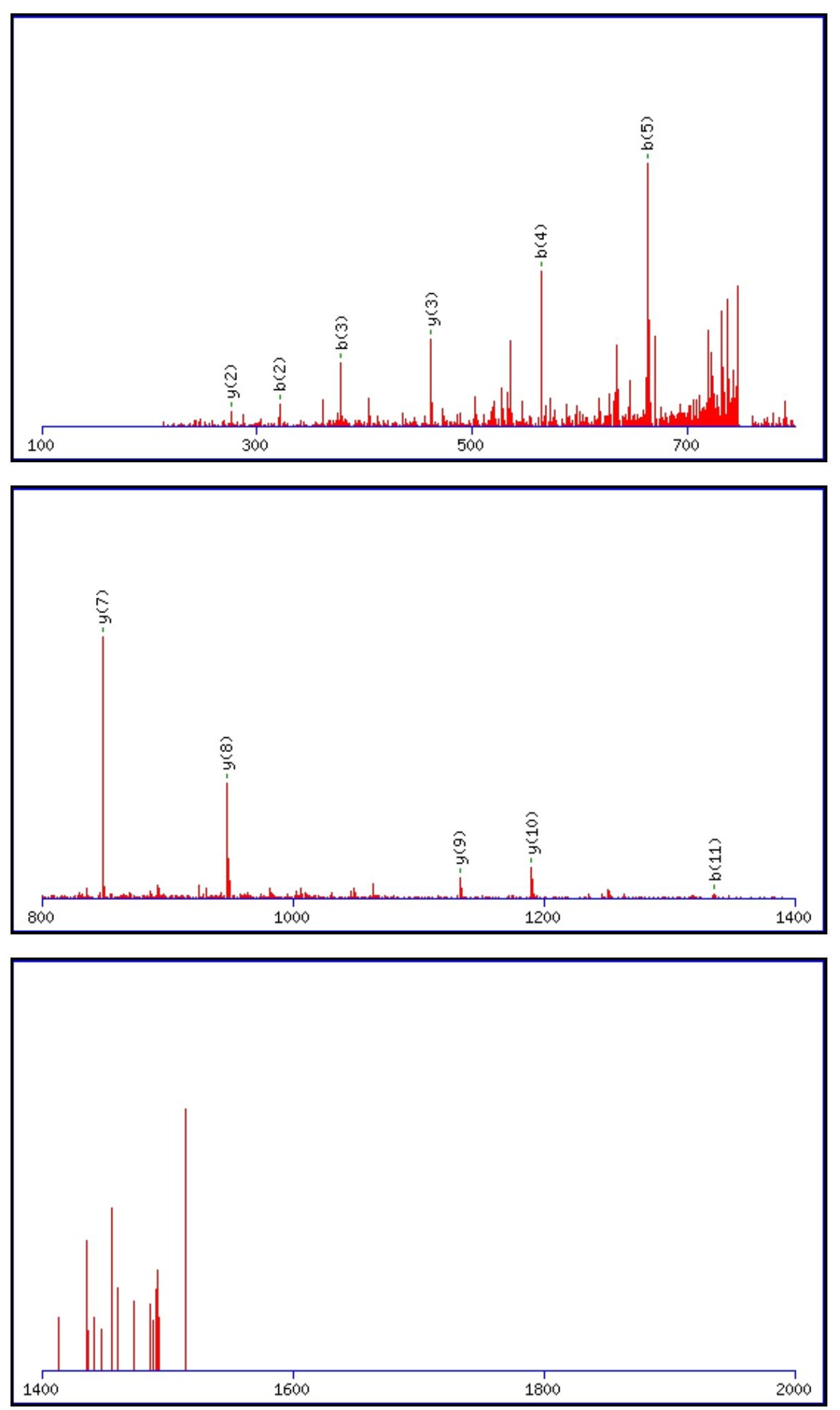

Monoisotopic mass of neutral peptide $\operatorname{Mr}($ calc): 1509.6129

Fixed modifications: Carbamidomethyl (C)

Variable modifications:

N9 : Deamidated_N (N)

Ions Score: 41 Expect: 0.01

Matches (Bold Red): 11/104 fragment ions using 27 most intense peaks

\begin{tabular}{|r|c|c|c|c|c|c|c|c|c|c|c|c|c|c|}
\hline$\#$ & $\mathbf{b}$ & $\mathbf{b}^{++}$ & $\mathbf{b}^{*}$ & $\mathbf{b}^{\mathbf{*}^{++}}$ & $\mathbf{b}^{\mathbf{0}}$ & $\mathbf{b}^{\mathbf{0 + +}}$ & Seq. & $\mathbf{y}$ & $\mathbf{y}^{++}$ & $\mathbf{y}^{\mathbf{*}}$ & $\mathbf{y}^{\boldsymbol{*}^{++}}$ & $\mathbf{y}^{\mathbf{0}}$ & $\mathbf{y}^{\mathbf{0 + +}}$ & $\#$ \\
\hline $\mathbf{1}$ & 161.0379 & 81.0226 & & & & & $\mathbf{C}$ & & & & & & & $\mathbf{1 2}$ \\
\hline $\mathbf{2}$ & $\mathbf{3 2 1 . 0 6 8 6}$ & 161.0379 & & & & & $\mathbf{C}$ & 1350.5895 & 675.7984 & 1333.5630 & 667.2851 & 1332.5790 & 666.7931 & $\mathbf{1 1}$ \\
\hline $\mathbf{3}$ & $\mathbf{3 7 8 . 0 9 0 0}$ & 189.5487 & & & & & $\mathbf{G}$ & $\mathbf{1 1 9 0 . 5 5 8 9}$ & 595.7831 & 1173.5323 & 587.2698 & 1172.5483 & 586.7778 & $\mathbf{1 0}$ \\
\hline $\mathbf{4}$ & $\mathbf{5 6 4 . 1 6 9 4}$ & 282.5883 & & & & & $\mathbf{W}$ & $\mathbf{1 1 3 3 . 5 3 7 4}$ & 567.2724 & 1116.5109 & 558.7591 & 1115.5269 & 558.2671 & $\mathbf{9}$ \\
\hline $\mathbf{5}$ & $\mathbf{6 6 3 . 2 3 7 8}$ & 332.1225 & & & & & $\mathbf{V}$ & $\mathbf{9 4 7 . 4 5 8 1}$ & 474.2327 & 930.4316 & 465.7194 & 929.4475 & 465.2274 & $\mathbf{8}$ \\
\hline
\end{tabular}




\begin{tabular}{|r|r|r|r|r|r|r|r|r|r|r|r|r|r|r|r|r|}
$\mathbf{6}$ & 750.2698 & 375.6385 & & & 732.2592 & 366.6333 & S & $\mathbf{8 4 8 . 3 8 9 7}$ & 424.6985 & 831.3632 & 416.1852 & 830.3791 & 415.6932 & $\mathbf{7}$ \\
\hline $\mathbf{7}$ & 847.3226 & 424.1649 & & & 829.3120 & 415.1596 & $\mathbf{P}$ & 761.3577 & 381.1825 & 744.3311 & 372.6692 & 743.3471 & 372.1772 & $\mathbf{6}$ \\
\hline $\mathbf{8}$ & 934.3546 & 467.6809 & & & 916.3440 & 458.6756 & S & 664.3049 & 332.6561 & 647.2784 & 324.1428 & 646.2943 & 323.6508 & $\mathbf{5}$ \\
\hline $\mathbf{9}$ & 1049.3815 & 525.1944 & 1032.3550 & 516.6811 & 1031.3710 & 516.1891 & $\mathbf{N}$ & 577.2729 & 289.1401 & 560.2463 & 280.6268 & 559.2623 & 280.1348 & $\mathbf{4}$ \\
\hline $\mathbf{1 0}$ & 1235.4608 & 618.2341 & 1218.4343 & 609.7208 & 1217.4503 & 609.2288 & $\mathbf{W}$ & $\mathbf{4 6 2 . 2 4 5 9}$ & 231.6266 & 445.2194 & 223.1133 & 444.2354 & 222.6213 & $\mathbf{3}$ \\
\hline $\mathbf{1 1}$ & $\mathbf{1 3 3 6 . 5 0 8 5}$ & 668.7579 & 1319.4820 & 660.2446 & 1318.4979 & 659.7526 & $\mathbf{T}$ & $\mathbf{2 7 6 . 1 6 6}$ & 138.5870 & 259.1401 & 130.0737 & 258.1561 & 129.5817 & $\mathbf{2}$ \\
\hline $\mathbf{1 2}$ & & & & & & & $\mathbf{R}$ & 175.1190 & 88.0631 & 158.0924 & 79.5498 & & & $\mathbf{1}$ \\
\hline
\end{tabular}
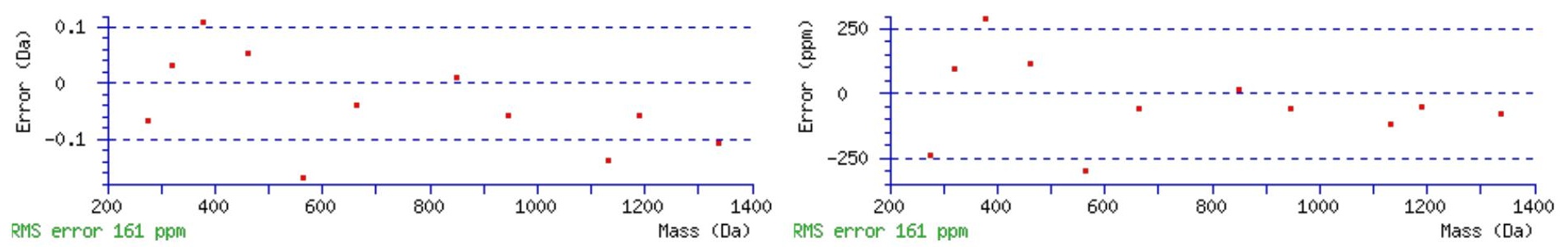

\section{All matches to this query}

\begin{tabular}{|l|c|c|c|}
\hline Score & Mr(calc): & Delta & \multicolumn{1}{c|}{ Sequence } \\
\hline 40.7 & 1509.6129 & 0.0026 & CCGWVSPSNWTR \\
\hline 0.8 & 1509.6177 & -0.0022 & NTFLWTEFTDR \\
\hline
\end{tabular}

Spectrum No: 825; Query: 2074; Rank: 1

\section{Peptide View}

MS/MS Fragmentation of MIAQHCVDANNTFCFEHPR

Found in IPI00205325, Tax_Id=10116 Gene_Symbol=Lrp2 Low-density lipoprotein receptor-related protein 2 precursor

Match to Query 2074: 2347.002492 from(783.341440,3+)

Title: 100101RatKid_NS_deglyco_09.2255.2255.3.dta

Data file K:INewmanPaper|Piliangl3SubProteomes\Piliang3SP\mgf5ppm\ERLIC_3SubProteomes5ppm.mgf

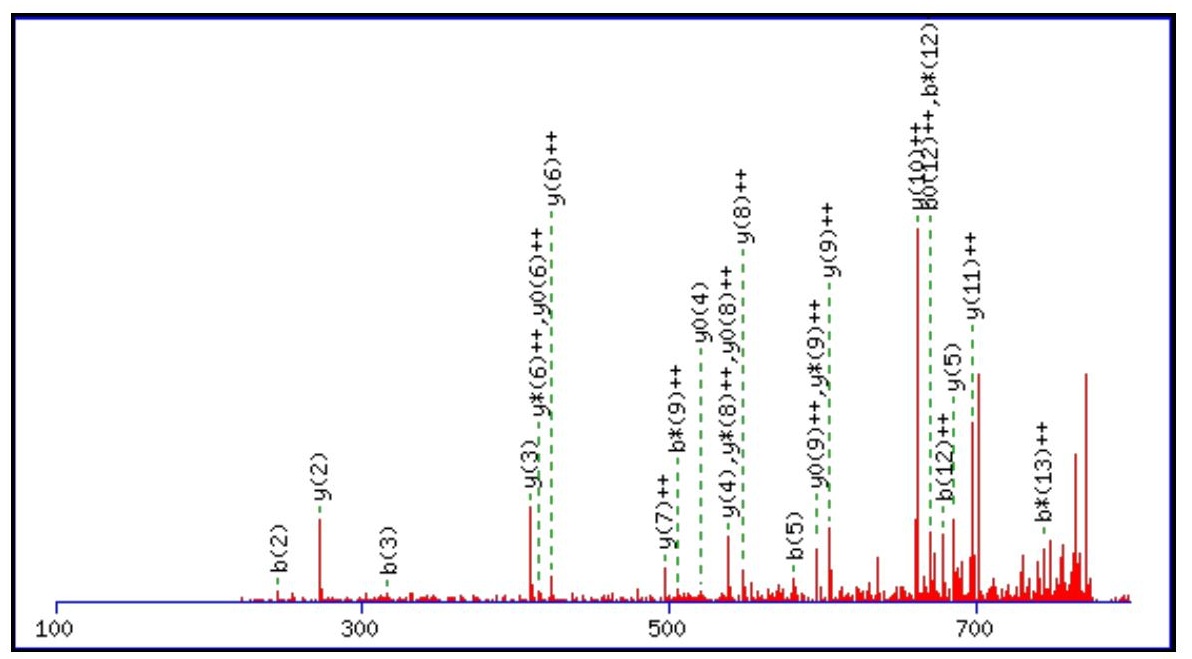



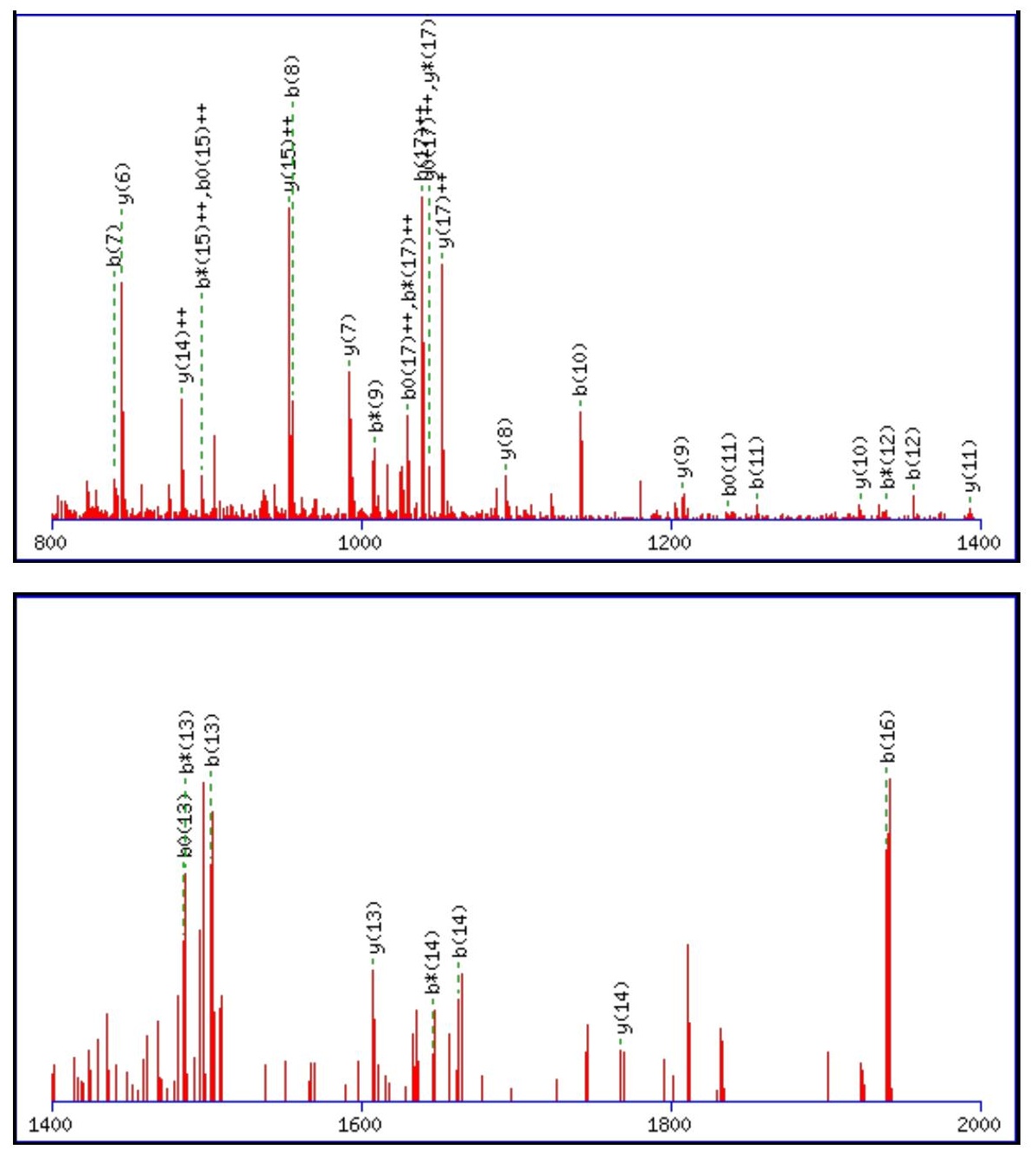

Monoisotopic mass of neutral peptide $\operatorname{Mr}($ calc): 2346.9932

Fixed modifications: Carbamidomethyl (C)

Variable modifications:

N10 : Deamidated N $(\mathrm{N})$

Ions Score: 41 Expect : $\odot .024$

Matches (Bold Red): 57/190 fragment ions using 129 most intense peaks

\begin{tabular}{|c|c|c|c|c|c|c|c|c|c|c|c|c|c|c|}
\hline \# & b & $\mathbf{b}^{++}$ & b* & $\mathbf{b}^{*^{++}}$ & $\mathbf{b}^{\mathbf{0}}$ & $\mathbf{b}^{0++}$ & Seq. & $\mathbf{y}$ & $\mathbf{y}^{++}$ & $\mathbf{y}^{*}$ & $\mathrm{y}^{*^{++}}$ & $\mathbf{y}^{\mathbf{0}}$ & $\mathbf{y}^{0++}$ & \# \\
\hline 1 & 132.0478 & 66.5275 & & & & & $\mathbf{M}$ & & & & & & & 19 \\
\hline 2 & 245.1318 & 123.0696 & & & & & I & 2216.9600 & 1108.9837 & 2199.9335 & 1100.4704 & 2198.9495 & 1099.9784 & 18 \\
\hline 3 & 316.1689 & 158.5881 & & & & & A & 2103.8760 & 1052.4416 & 2086.8494 & 1043.9283 & 2085.8654 & 1043.4363 & 17 \\
\hline 4 & 444.2275 & 222.6174 & 427.2010 & 214.1041 & & & $\mathbf{Q}$ & 2032.8389 & |1016.9231 & 2015.8123 & 1008.4098 & 2014.8283 & 1007.9178 & 16 \\
\hline 5 & 581.2864 & 291.1469 & 564.2599 & 282.6336 & & & $\mathbf{H}$ & 1904.7803 & 952.8938 & 1887.7537 & 944.3805 & 1886.7697 & 943.8885 & 15 \\
\hline 6 & 741.3171 & 371.1622 & 724.2905 & 362.6489 & & & C & 1767.7214 & 884.3643 & 1750.6948 & 875.8510 & 1749.7108 & 875.3590 & 14 \\
\hline 7 & 840.3855 & 420.6964 & 823.3589 & 412.1831 & & & $\mathbf{V}$ & 1607.6907 & 804.3490 & 1590.6642 & 795.8357 & 1589.6801 & 795.3437 & 13 \\
\hline 8 & 955.4124 & 478.2099 & 938.3859 & 469.6966 & 937.4019 & 469.2046 & D & 1508.6223 & 754.8148 & 1491.5957 & 746.3015 & 1490.6117 & 745.8095 & 12 \\
\hline 9 & 1026.4495 & 513.7284 & 1009.4230 & 505.2151 & 1008.4390 & 504.7231 & A & 1393.5954 & 697.3013 & 1376.5688 & 688.7880 & 1375.5848 & 688.2960 & 11 \\
\hline 10 & 1141.4765 & 571.2419 & 1124.4499 & 562.7286 & 1123.4659 & 562.2366 & $\mathbf{N}$ & 1322.5582 & 661.7828 & 1305.5317 & 653.2695 & 1304.5477 & 652.7775 & 10 \\
\hline 11 & 1255.5194 & 628.2633 & 1238.4929 & 619.7501 & 1237.5088 & 619.2581 & $\mathbf{N}$ & 1207.5313 & 604.2693 & 1190.5048 & 595.7560 & 1189.5207 & 595.2640 & 9 \\
\hline 12 & 1356.5671 & 678.7872 & 1339.5405 & 670.2739 & 1338.5565 & 669.7819 & $\mathbf{T}$ & 1093.4884 & 547.2478 & 1076.4618 & 538.7346 & 1075.4778 & 538.2425 & 8 \\
\hline 13 & 1503.6355 & 752.3214 & 1486.6090 & 743.8081 & 1485.6249 & 743.3161 & $\mathbf{F}$ & 992.4407 & 496.7240 & 975.4141 & 488.2107 & 974.4301 & 487.7187 & 7 \\
\hline 14 & 1663.6662 & 832.3367 & 1646.6396 & 823.8234 & 1645.6556 & 823.3314 & $\mathrm{C}$ & 845.3723 & 423.1898 & 828.3457 & 414.6765 & 827.3617 & 414.1845 & 6 \\
\hline 15 & 1810.7346 & 905.8709 & 1793.7080 & 897.3576 & 1792.7240 & 896.8656 & $\mathbf{F}$ & 685.3416 & 343.1745 & 668.3151 & 334.6612 & 667.3311 & 334.1692 & 5 \\
\hline 16 & 1939.7772 & 970.3922 & 1922.7506 & 961.8789 & 1921.7666 & 961.3869 & $\mathbf{E}$ & 538.2732 & 269.6402 & 521.2467 & 261.1270 & 520.2627 & 260.6350 & 4 \\
\hline 17 & 2076.8361 & 1038.9217 & 2059.8095 & 1030.4084 & 2058.8255 & 1029.9164 & $\mathbf{H}$ & 409.2306 & 205.1190 & 392.2041 & 196.6057 & & & 3 \\
\hline 18 & 2173.8888 & 1087.4481 & 2156.8623 & 1078.9348 & 2155.8783 & 1078.4428 & $\mathbf{P}$ & 272.1717 & 136.5895 & 255.1452 & 128.0762 & & & 2 \\
\hline
\end{tabular}




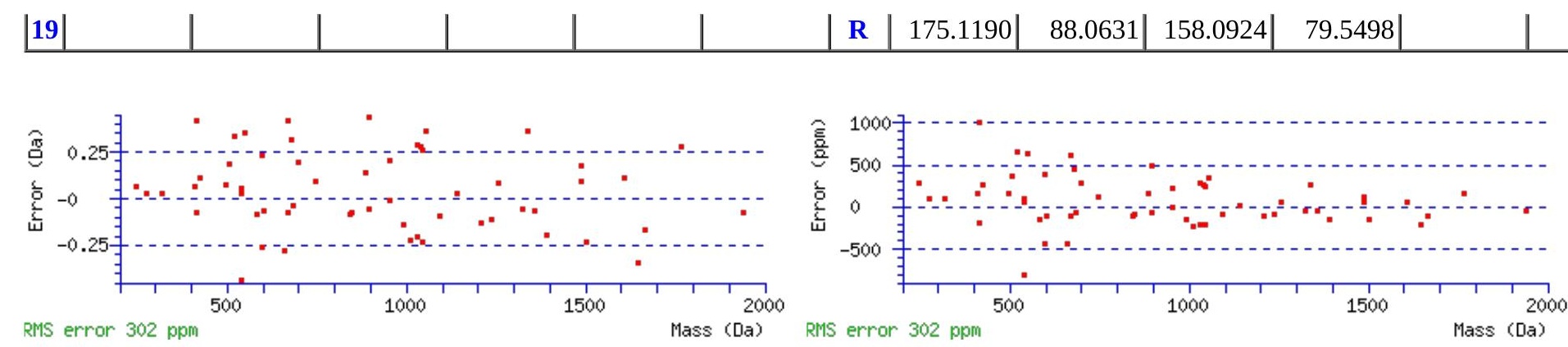

\section{All matches to this query}

\begin{tabular}{|l|l|c|c|}
\hline Score & Mr(calc): & Delta & Sequence \\
\hline 40.6 & 2346.9932 & 0.0093 & MIAQHCVDANNTFCFEHPR \\
\hline 39.2 & 2346.9932 & 0.0093 & MIAQHCVDANNTFCFEHPR \\
\hline 8.8 & 2346.0092 & 0.9933 & MIAQHCVDANNTFCFEHPR \\
\hline 1.6 & 2345.9971 & 1.0054 & LTFDTHALVQDLETHGFDK \\
\hline 0.9 & 2344.9912 & 2.0113 & LAKEIAIGRCFDGSSDGFSR \\
\hline
\end{tabular}

Spectrum No: 826; Query: 1596; Rank: 1

\section{Peptide View}

MS/MS Fragmentation of QMQNYLTDNKTATILDK

Found in IPI00231686, Tax_Id=10116 Gene_Symbol=Cd63 CD63 antigen

Match to Query 1596: 2012.974842 from(671.998890,3+)

Title: 100101RatKid_NS_deglyco_26.2207.2207.3.dta

Data file K:\NewmanPaper|Piliangl3SubProteomes\Piliang3SP\mgf5ppm\ERLIC_3SubProteomes5ppm.mgf

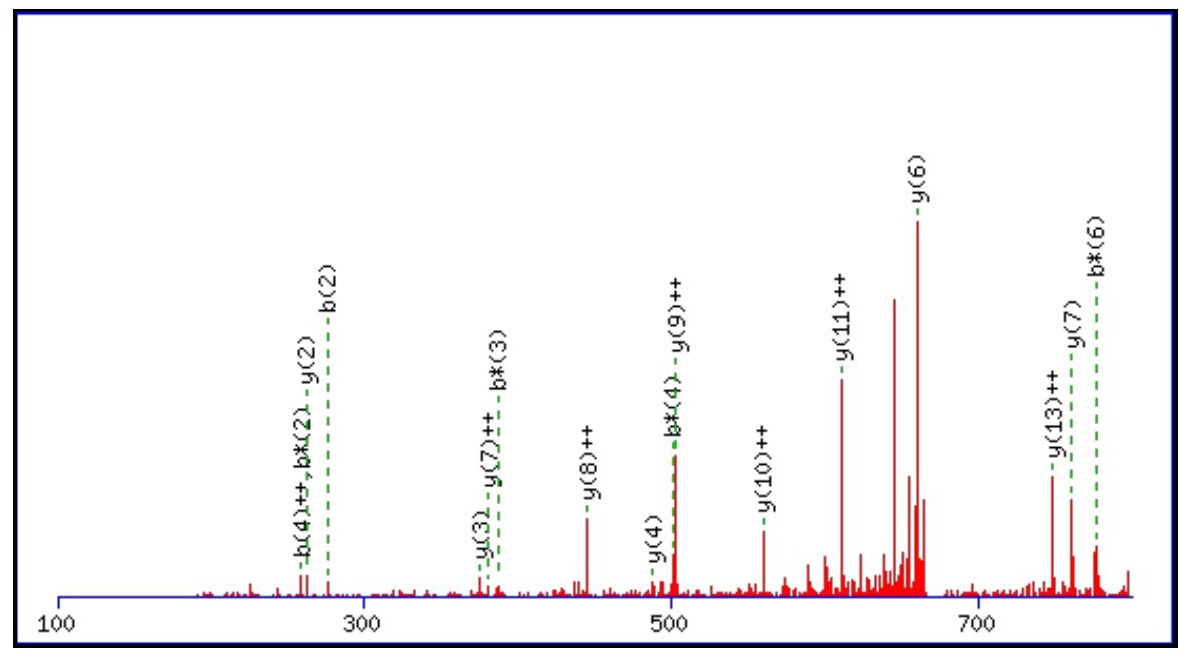



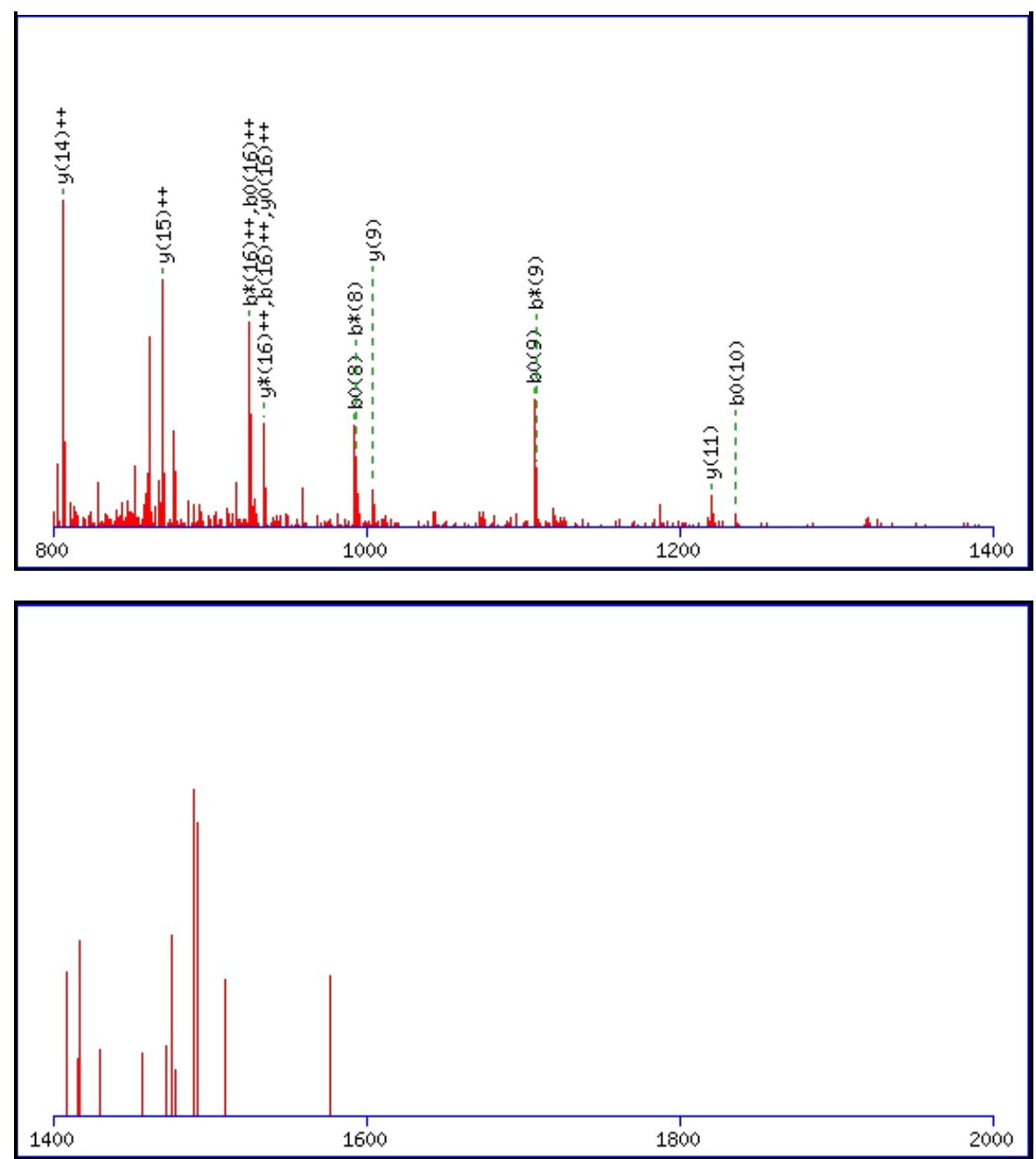

Monoisotopic mass of neutral peptide $\operatorname{Mr}($ calc): 2012.9724

Fixed modifications: Carbamidomethyl (C)

Variable modifications:

M2 : Oxidation (M)

N9 : Deamidated_N (N)

Ions Score: 41 Expect : 0.021

Matches (Bold Red): 31/178 fragment ions using 45 most intense peaks

\begin{tabular}{|c|c|c|c|c|c|c|c|c|c|c|c|c|c|c|}
\hline \# & b & $\mathbf{b}^{++}$ & $\mathbf{b}^{*}$ & $\mathbf{b}^{*^{++}}$ & $\mathbf{b}^{\mathbf{0}}$ & & Seq. & $\mathbf{y}$ & $\mathbf{y}^{++}$ & $\mathbf{y}^{*}$ & $\mathrm{y}^{*^{++}}$ & $\mathbf{y}^{\mathbf{0}}$ & & \# \\
\hline 1 & 129.0659 & 65.0366 & 112.0393 & 56.5233 & & & $\mathbf{Q}$ & & & & & & & 17 \\
\hline 2 & 276.1013 & 138.5543 & 259.0747 & 130.0410 & & & $\mathbf{M}$ & 1885.9212 & 943.4642 & 1868.8946 & & 1867.9106 & 934.4589 & 16 \\
\hline 3 & 404.1598 & 202.5836 & 387.1333 & 194.0703 & & & $\mathbf{Q}$ & 1738.8858 & 869.9465 & 1721.8592 & 861.4332 & 1720.8752 & 860.9412 & 15 \\
\hline 4 & 518.2028 & 259.6050 & 501.1762 & 251.0917 & & & $\mathbf{N}$ & 1610.8272 & 805.9172 & 1593.8006 & 797.4040 & 1592.8166 & 796.9119 & 14 \\
\hline 5 & 681.2661 & 341.1367 & 664.2395 & 332.6234 & & & $\mathbf{Y}$ & 1496.7843 & 748.8958 & 1479.7577 & 740.3825 & 1478.7737 & 739.8905 & 13 \\
\hline 6 & 794.3502 & 397.6787 & 777.3236 & 389.1654 & & & $\mathbf{L}$ & 1333.7209 & 667.3641 & 1316.6944 & 658.8508 & 1315.7104 & 658.3588 & 12 \\
\hline 7 & 895.3978 & 448.2026 & 878.3713 & 439.6893 & 877.3873 & & $\mathbf{T}$ & 1220.6369 & 610.8221 & 1203.6103 & 602.3088 & 1202.6263 & 601.8168 & 11 \\
\hline 8 & 1010.4248 & 505.7160 & 993.3982 & 497.2028 & 992.4142 & 496.7107 & D & 1119.5892 & 560.2982 & 1102.5626 & 551.7850 & 1101.5786 & 551.2930 & 10 \\
\hline 9 & 1125.4517 & 563.2295 & 1108.4252 & 554.7162 & 1107.4411 & 554.2242 & $\mathbf{N}$ & 1004.5622 & 502.7848 & 987.5357 & 494.2715 & 986.5517 & 493.7795 & 9 \\
\hline 10 & 1253.5467 & 627.2770 & 1236.5201 & 618.7637 & 1235.5361 & 618.2717 & $\mathbf{K}$ & 889.5353 & 445.2713 & 872.5088 & 436.7580 & 871.5247 & 436.2660 & 8 \\
\hline 11 & 1354.5944 & 677.8008 & 1337.5678 & 669.2875 & 1336.5838 & 668.7955 & $\mathbf{T}$ & 761.4403 & 381.2238 & 744.4138 & 372.7105 & 743.4298 & 372.2185 & 7 \\
\hline 12 & 1425.6315 & 713.3194 & 1408.6049 & 704.8061 & 1407.6209 & 704.3141 & A & 660.3927 & 330.7000 & 643.3661 & 322.1867 & 642.3821 & 321.6947 & 6 \\
\hline 13 & 1526.6791 & 763.8432 & 1509.6526 & 755.3299 & 1508.6686 & 754.8379 & $\mathbf{T}$ & 589.3556 & 295.1814 & 572.3290 & 286.6681 & 571.3450 & 286.1761 & 5 \\
\hline 14 & 1639.7632 & 820.3852 & 1622.7367 & 811.8720 & 1621.7526 & 811.3800 & I & 488.3079 & 244.6576 & 471.2813 & 236.1443 & 470.2973 & 235.6523 & 4 \\
\hline 15 & 1752.8473 & 876.9273 & 1735.8207 & 868.4140 & 1734.8367 & 867.9220 & $\mathbf{L}$ & 375.2238 & 188.1155 & 358.1973 & 179.6023 & 357.2132 & 179.1103 & 3 \\
\hline 16 & 1867.8742 & 934.4407 & 1850.8477 & 925.9275 & 1849.8637 & 925.4355 & D & 262.1397 & 131.5735 & 245.1132 & 123.0602 & 244.1292 & 122.5682 & 2 \\
\hline 17 & & & & & & & $\mathbf{K}$ & 147.1128 & 74.0600 & 130.0863 & 65.5468 & & & 1 \\
\hline
\end{tabular}




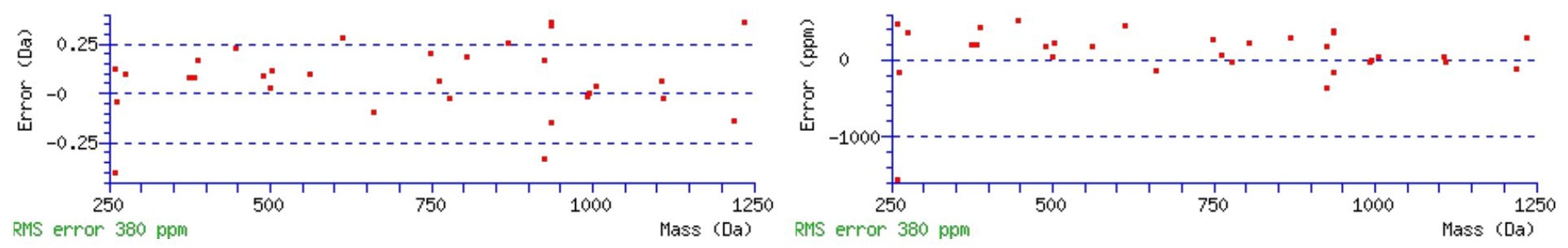

\section{All matches to this query}

\begin{tabular}{|l|l|l|l|}
\hline Score & Mr(calc): & Delta & \multicolumn{1}{|c|}{ Sequence } \\
\hline 40.6 & 2012.9724 & 0.0024 & QMQNYLTDNKTATILDK \\
\hline 14.5 & 2011.9568 & 1.0181 & LCENGSMNNVVTRRFSK \\
\hline 11.2 & 2012.9724 & 0.0024 & QMQNYLTDNKTATILDK \\
\hline 9.8 & 2011.9568 & 1.0181 & LCENGSMNNVVTRRFSK \\
\hline 8.7 & 2011.9568 & 1.0181 & LCENGSMNNVVTRRFSK \\
\hline 8.4 & 2010.9792 & 1.9956 & TITNDPSYKENAMRLSR \\
\hline 8.4 & 2011.9633 & 1.0116 & IITNDPSYKENAMRLSR \\
\hline 7.2 & 2010.9727 & 2.0021 & LCENGSMNNVVTRRFSK \\
\hline 2.8 & 2012.9932 & -0.0184 & KSALWQISNGTSSVIVSR \\
\hline 2.8 & 2012.9910 & -0.0162 & MNTSLMANVKKAFIGENK \\
\hline
\end{tabular}

Spectrum No: 827; Query: 968; Rank: 1

\section{Peptide View}

MS/MS Fragmentation of LVTNLTYFPETVPR

Found in IPI00763325, Tax_Id=10116 Gene_Symbol=LOC686310 similar to Ephrin type-B receptor 4 precursor (Tyrosine-protein kinase receptor MDK-2) (Developmental kinase 2) (Tyrosine kinase MYK-1) isoform 2

Match to Query 968: 1649.865108 from(825.939830,2+)

Title: 091008RatKidney_NoSalt_29.3146.3146.2.dta

Data file K:INewmanPaper|Piliangl3SubProteomes\Piliang3SP\mgf5ppm|ERLIC_3SubProteomes5ppm.mgf

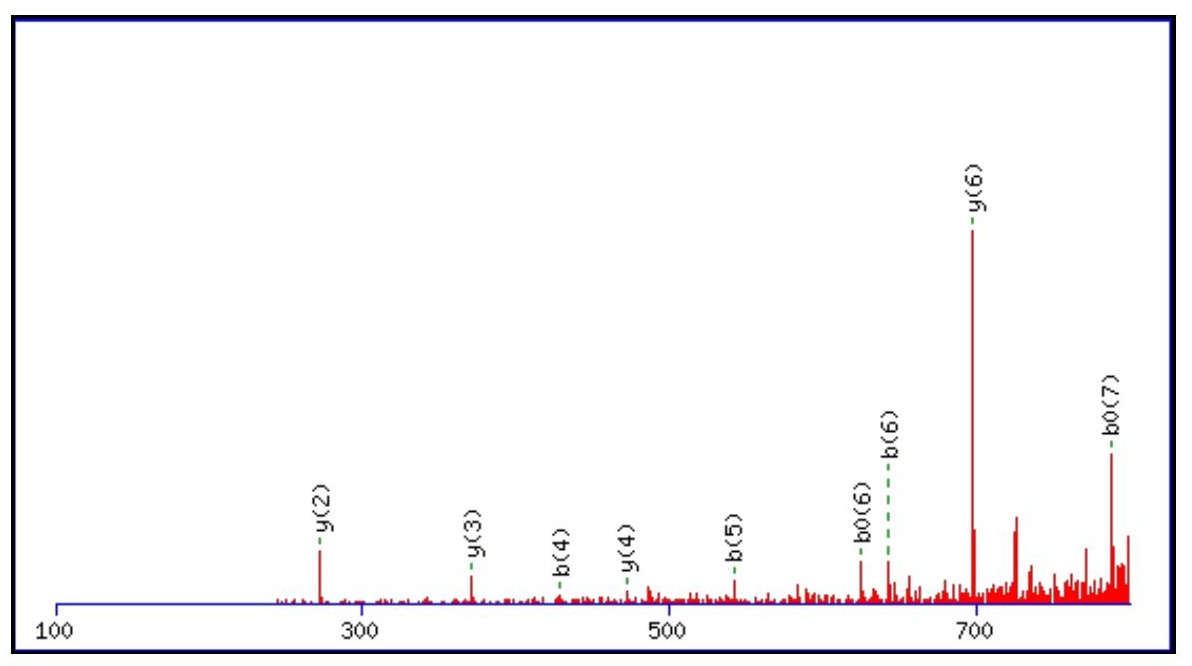



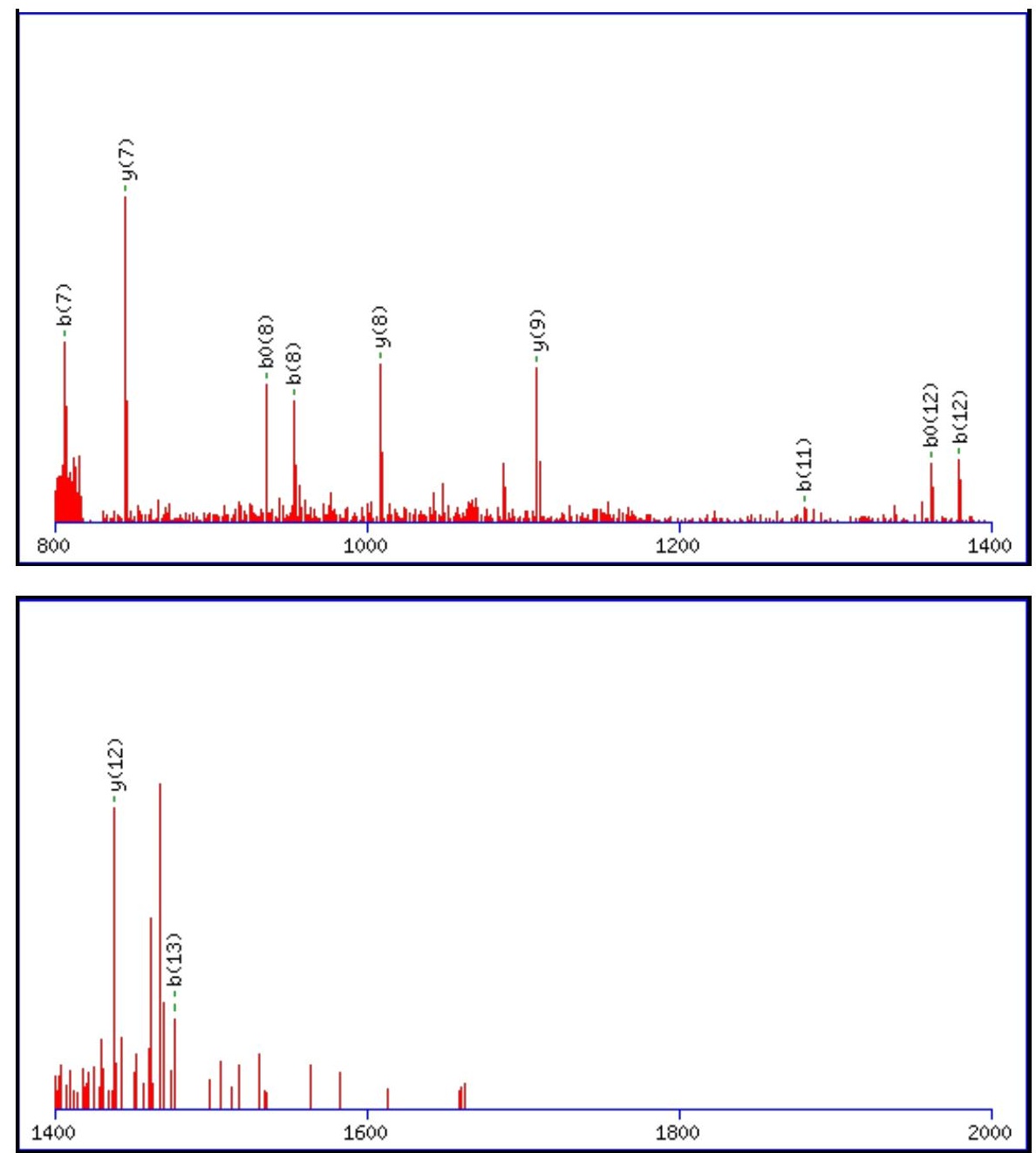

Monoisotopic mass of neutral peptide $\operatorname{Mr}($ calc): 1649.8665

Fixed modifications: Carbamidomethyl (C)

Variable modifications:

N4 : Deamidated_N (N)

Ions Score: 41 Expect: 0.01

Matches (Bold Red): 20/140 fragment ions using 38 most intense peaks

\begin{tabular}{|c|c|c|c|c|c|c|c|c|c|c|c|c|c|c|}
\hline \# & b & $\mathbf{b}^{++}$ & $\mathbf{b}^{*}$ & $\mathbf{b}^{*^{++}}$ & $\mathbf{b}^{0}$ & $\mathbf{b}^{\mathbf{0 + +}}$ & Seq. & $\mathbf{y}$ & $y^{++}$ & $\mathbf{y}^{*}$ & $\mathrm{y}^{*^{++}}$ & $\mathbf{y}^{\mathbf{0}}$ & $\mathbf{y}^{0++}$ & \# \\
\hline 1 & 114.0913 & 57.5493 & & & & & $\mathbf{L}$ & & & & & & & 14 \\
\hline 2 & 213.1598 & 107.0835 & & & & & V & 1537.7897 & 769.3985 & 1520.7631 & 760.8852 & 1519.7791 & 760.3932 & 13 \\
\hline 3 & 314.2074 & 157.6074 & & & 296.1969 & 148.6021 & $\mathbf{T}$ & 1438.7213 & 719.8643 & 1421.6947 & 711.3510 & 1420.7107 & 710.8590 & 12 \\
\hline 4 & 429.2344 & 215.1208 & 412.2078 & 206.6075 & 411.2238 & 206.1155 & $\mathbf{N}$ & 1337.6736 & 669.3404 & 1320.6470 & 660.8272 & 1319.6630 & 660.3351 & 11 \\
\hline 5 & 542.3184 & 271.6629 & 525.2919 & 263.1496 & 524.3079 & 262.6576 & $\mathbf{L}$ & 1222.6467 & 611.8270 & 1205.6201 & 603.3137 & 1204.6361 & 602.8217 & 10 \\
\hline 6 & 643.3661 & 322.1867 & 626.3396 & 313.6734 & 625.3555 & 313.1814 & $\mathbf{T}$ & 1109.5626 & 555.2849 & 1092.5360 & 546.7717 & 1091.5520 & 546.2796 & 9 \\
\hline 7 & 806.4294 & 403.7184 & 789.4029 & 395.2051 & 788.4189 & 394.7131 & $\mathbf{Y}$ & 1008.5149 & 504.7611 & 991.4884 & 496.2478 & 990.5043 & 495.7558 & 8 \\
\hline 8 & 953.4979 & 477.2526 & 936.4713 & 468.7393 & 935.4873 & 468.2473 & $\mathbf{F}$ & 845.4516 & 423.2294 & 828.4250 & 414.7162 & 827.4410 & 414.2241 & 7 \\
\hline 9 & 1050.5506 & 525.7789 & 1033.5241 & 517.2657 & 1032.5401 & 516.7737 & $\mathbf{P}$ & 698.3832 & 349.6952 & 681.3566 & 341.1819 & 680.3726 & 340.6899 & 6 \\
\hline 10 & 1179.5932 & 590.3002 & 1162.5667 & 581.7870 & 1161.5826 & 581.2950 & $\mathbf{E}$ & 601.3304 & 301.1688 & 584.3039 & 292.6556 & 583.3198 & 292.1636 & 5 \\
\hline 11 & 1280.6409 & 640.8241 & 1263.6143 & 632.3108 & 1262.6303 & 631.8188 & $\mathbf{T}$ & 472.2878 & 236.6475 & 455.2613 & 228.1343 & 454.2772 & 227.6423 & 4 \\
\hline 12 & 1379.7093 & 690.3583 & 1362.6828 & 681.8450 & 1361.6987 & 681.3530 & $\mathbf{V}$ & 371.2401 & 186.1237 & 354.2136 & 177.6104 & & & 3 \\
\hline 13 & 1476.7621 & 738.8847 & 1459.7355 & 730.3714 & 1458.7515 & 729.8794 & $\mathbf{P}$ & 272.1717 & 136.5895 & 255.1452 & 128.0762 & & & 2 \\
\hline 14 & & & & & & & $\mathbf{R}$ & 175.1190 & 88.0631 & 158.0924 & 79.5498 & & & 1 \\
\hline
\end{tabular}



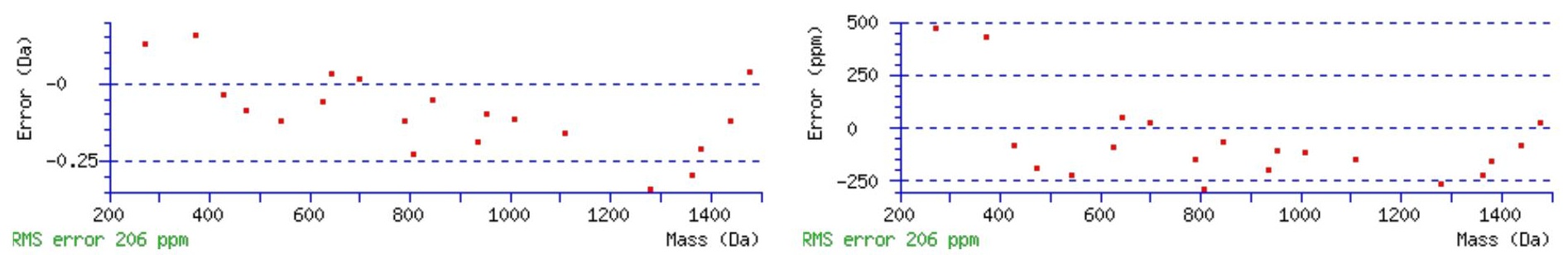

\section{All matches to this query}

\begin{tabular}{|l|l|l|l|}
\hline Score & Mr(calc): & Delta & \multicolumn{1}{c|}{ Sequence } \\
\hline 40.6 & 1649.8665 & -0.0014 & LVTNLTYFPETVPR \\
\hline 8.2 & 1649.8753 & -0.0102 & ATVVGNTEILTALIR \\
\hline 5.2 & 1649.8753 & -0.0102 & ATVVGNTEILTALIR \\
\hline 2.5 & 1649.8750 & -0.0099 & SPAFRGPDRVGAAPPR \\
\hline 0.2 & 1648.8607 & 1.0044 & LSAVLVPASPAVAACSH \\
\hline
\end{tabular}

Spectrum No: 828; Query: 8; Rank: 1

\section{Peptide View}

MS/MS Fragmentation of SNISILR

Found in IPI00362131, Tax_Id=10116 Gene_Symbol=Cdh2 Cadherin-2 precursor

Match to Query 8: 802.453908 from(402.234230,2+)

Title: 091008RatKidney_NH4Format01_24.1340.1340.2.dta

Data file K:INewmanPaper\Piliang\3SubProteomes\Piliang3SP\mgf5ppm\ERLIC_3SubProteomes5ppm.mgf

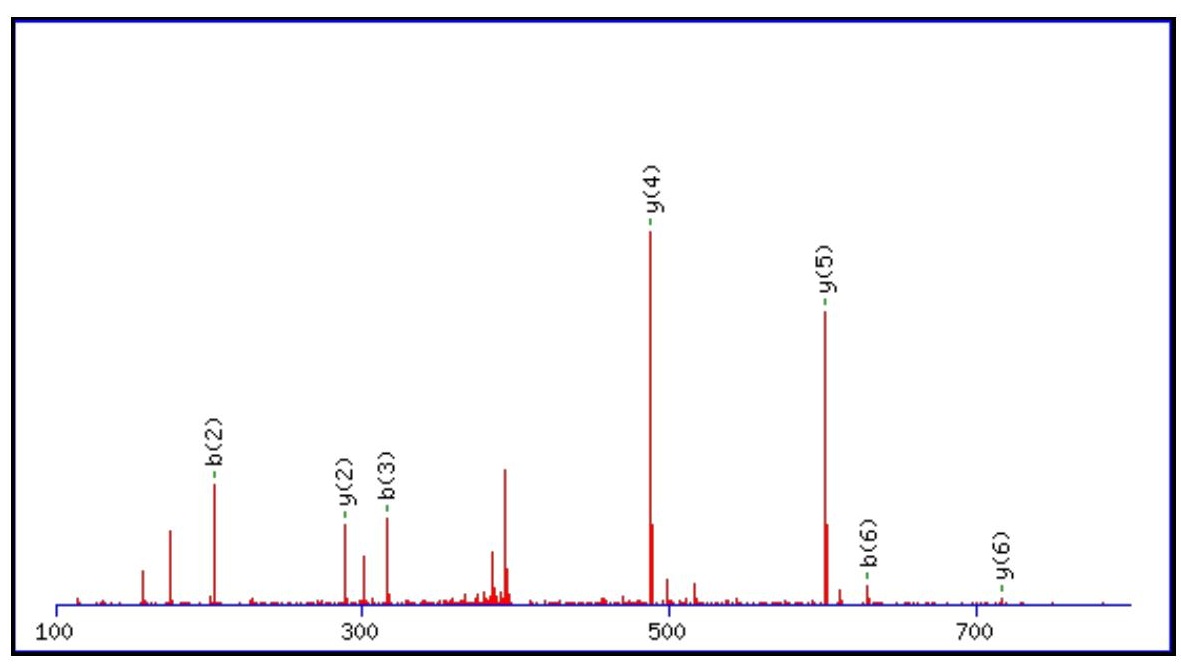




$\begin{array}{rrrr}800 & 1000 & 1200 & 1400\end{array}$

\begin{tabular}{rrr}
\hline 1600 & 1800 & 2000
\end{tabular}

Monoisotopic mass of neutral peptide $\operatorname{Mr}($ calc): 802.4548

Fixed modifications: Carbamidomethyl (C)

Variable modifications:

N2 : Deamidated $\mathrm{N}(\mathrm{N})$

Ions Score: 41 Expect: 0.0084

Matches (Bold Red): 7/64 fragment ions using 10 most intense peaks

\begin{tabular}{|c|c|c|c|c|c|c|c|c|c|c|c|c|c|c|}
\hline \# & b & $\mathbf{b}^{++}$ & b* & $\mathbf{b}^{*^{++}}$ & $\mathbf{b}^{0}$ & $\mathbf{b}^{0++}$ & Seq. & $\mathbf{y}$ & $y^{++}$ & $\mathbf{y}^{*}$ & $\mathrm{y}^{*^{++}}$ & $\mathbf{y}^{0}$ & $\mathbf{y}^{0++}$ & \# \\
\hline 1 & 88.0393 & 44.5233 & & & 70.0287 & 35.5180 & $\mathrm{~S}$ & & & & & & & 7 \\
\hline 2 & 203.0662 & 102.0368 & 186.0397 & 93.5235 & 185.0557 & 93.0315 & $\mathbf{N}$ & 716.4301 & 358.7187 & 699.4036 & 350.2054 & 698.4195 & 349.7134 & 6 \\
\hline 4 & 403.1823 & 202.0948 & 386.1558 & 193.5815 & 385.1718 & 193.0895 & S & 488.3191 & 244.6632 & 471.2926 & 236.1499 & 470.3085 & 235.6579 & 4 \\
\hline 5 & 516.2664 & 258.6368 & 499.2398 & 250.1236 & 498.2558 & 249.6316 & I & 401.2871 & 201.1472 & 384.2605 & 192.6339 & & & 3 \\
\hline 7 & & & & & & & $\mathbf{R}$ & 175.1190 & 88.0631 & 158.0924 & 79.5498 & & & 1 \\
\hline
\end{tabular}

$$
\text { 옹 }
$$
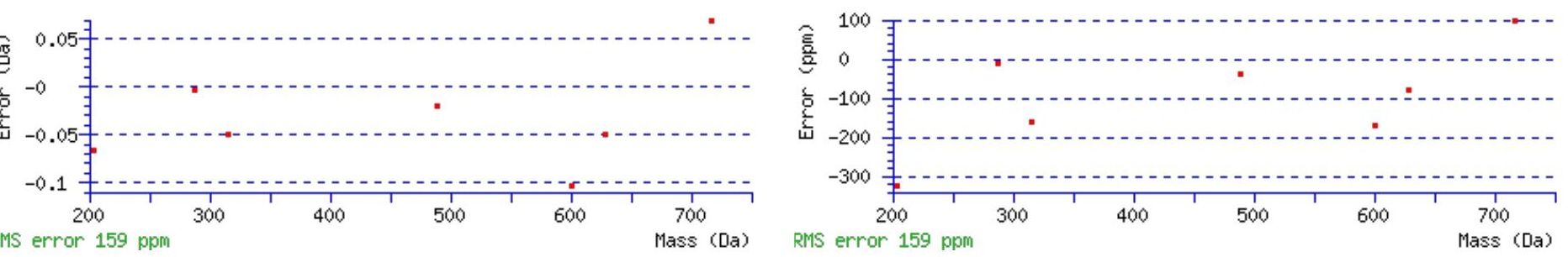

All matches to this query

\begin{tabular}{|l|l|l|l|}
\hline Score & Mr(calc): & Delta & Sequence \\
\hline 40.6 & 802.4548 & -0.0009 & $\underline{\text { SNISILR }}$ \\
\hline
\end{tabular}




\begin{tabular}{|l|l|l|l|}
440.6 & 802.4548 & -0.0009 & SNLTVLR \\
\hline 26.3 & 802.4548 & -0.0009 & DSLISIR \\
\hline 23.2 & 802.4548 & -0.0009 & LDSISLR \\
\hline 19.2 & 800.4392 & 2.0147 & $\underline{\text { AELAELR }}$ \\
\hline 17.3 & 802.4483 & 0.0056 & $\underline{\text { RMDLLR }}$ \\
\hline 16.4 & 802.4548 & -0.0009 & $\underline{\text { NSSLILR }}$ \\
\hline 16.0 & 802.4549 & -0.0010 & DSLLTVR \\
\hline 14.4 & 802.4548 & -0.0009 & $\underline{\text { VESLSIR }}$ \\
\hline 11.1 & 802.4549 & -0.0010 & $\underline{\text { EVTVSLR }}$ \\
\hline
\end{tabular}

Spectrum No: 829; Query: 2634; Rank: 1

\section{Peptide View}

MS/MS Fragmentation of TDICQGALGDCWLLAAIASLTLNETILHR

Found in IPI00231610, Tax_Id=10116 Gene_Symbol=Capn1 Calpain-1 catalytic subunit

Match to Query 2634: 3225.631272 from(1076.217700,3+)

Title: 091008RatKidney_NH4Format02_11.6676.6676.3.dta

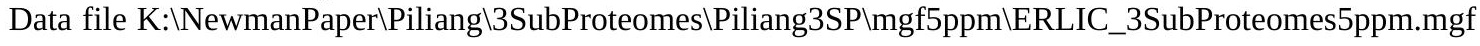
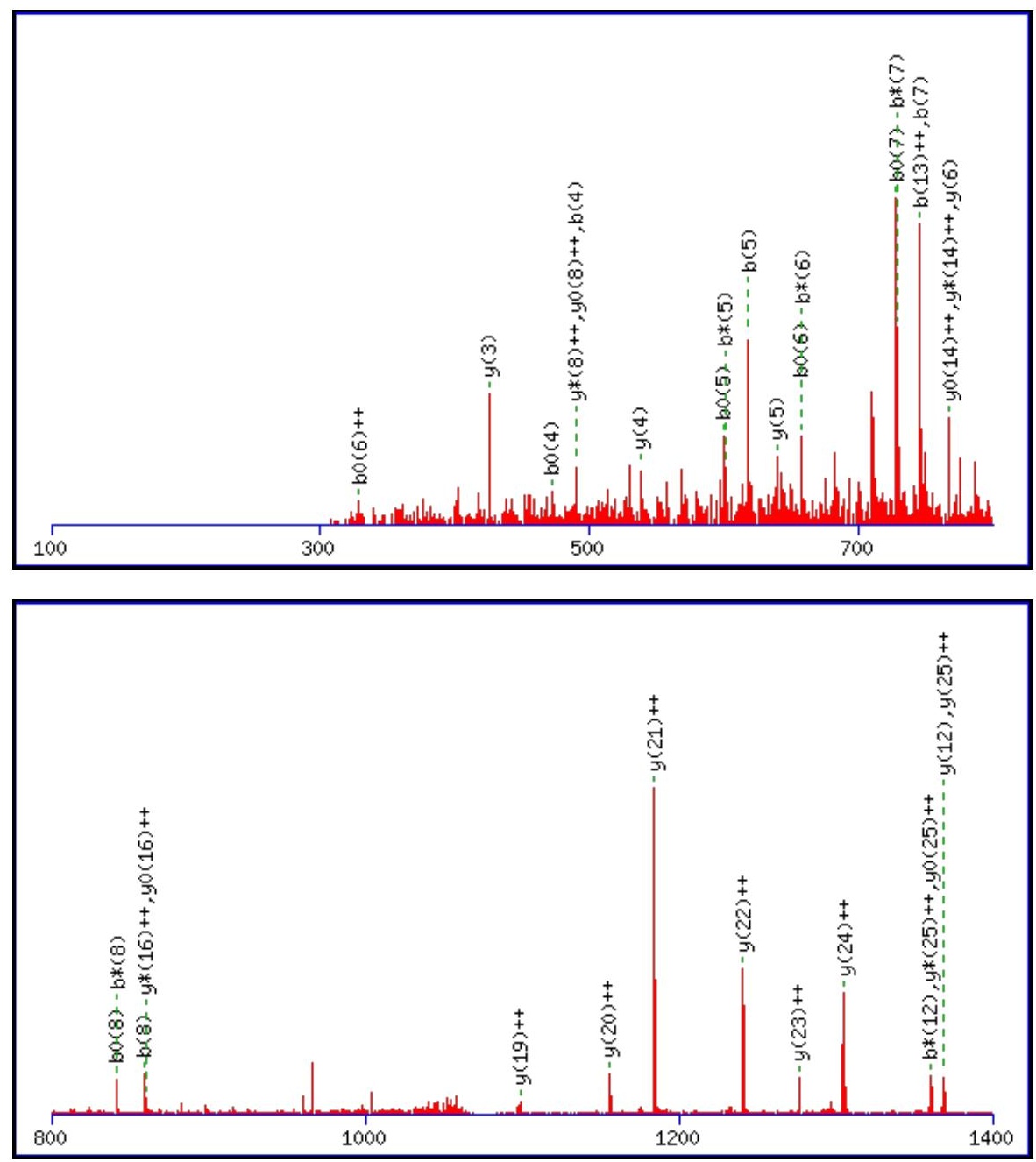


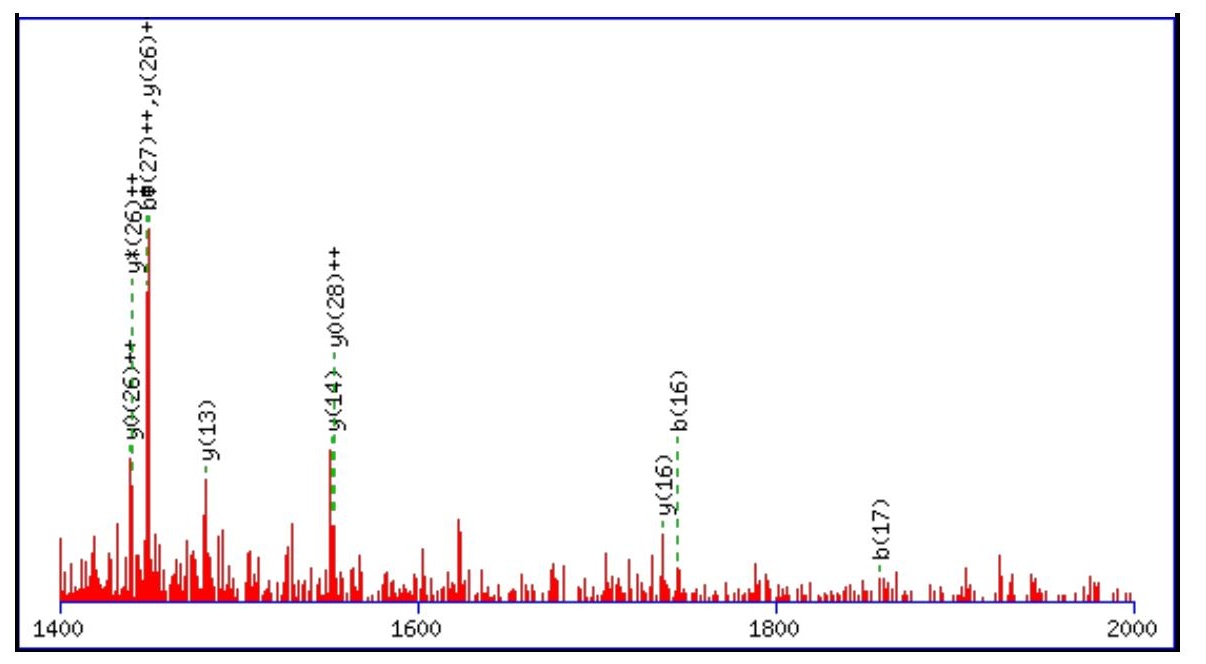

Monoisotopic mass of neutral peptide $\operatorname{Mr}($ calc): 3225.6162

Fixed modifications: Carbamidomethyl (C)

Variable modifications:

N23 : Deamidated_N (N)

Ions Score: $4 \odot$ Expect: $\odot .016$

Matches (Bold Red): 47/320 fragment ions using 91 most intense peaks

\begin{tabular}{|c|c|c|c|c|c|c|c|c|c|c|c|c|c|c|}
\hline \# & b & $\mathbf{b}^{++}$ & $\mathbf{b}^{*}$ & $\mathbf{b}^{*++}$ & $\mathbf{b}^{0}$ & p++ & Seq. & $\mathbf{y}$ & & $\mathbf{y}^{*}$ & & $\mathbf{y}^{\mathbf{0}}$ & & \# \\
\hline 1 & 102.0550 & 51.5311 & & & 4.0444 & 42.5258 & $T$ & & & & & & & 29 \\
\hline 2 & 217.0819 & 109.0446 & & & 13 & 393 & D & & & & & & & 28 \\
\hline 3 & 330.1660 & & & & & & & & & & & & & 27 \\
\hline 4 & 490.1966 & 245.6 & & & 60 & 967 & C & 648 & & 383 & & 542 & & 26 \\
\hline 5 & 618.2552 & 309.6312 & & & 600.2446 & 300.6260 & $\mathbf{Q}$ & 2737.4342 & 1369.2207 & 2720.4076 & 1360.7074 & 2719.4236 & 1360.2154 & 25 \\
\hline 6 & 675.2767 & 338.1420 & 58.2501 & & 657.2661 & 329.1367 & G & 2609.3756 & 1305 & 2592.3490 & & 650 & & 24 \\
\hline 7 & 746.3138 & 373.6605 & 729.2 & 2 & 032 & 552 & $\mathbf{A}$ & 541 & 807 & 3276 & 674 & 3436 & 754 & 23 \\
\hline 8 & 859.3978 & 430.2026 & 842.3713 & 421. & 841.3873 & 421.1973 & $\mathbf{L}$ & 2481.3170 & 1241.1621 & 2464.2905 & 1232.6489 & 2463.3064 & 1232.1569 & 22 \\
\hline 9 & 916.4193 & 458.7133 & 27 & & 87 & & G & & & & & & & 21 \\
\hline 10 & 1031.4462 & 516.2268 & 7 & 507. & $1013 .{ }^{2}$ & 507. & D & 115 & 94 & 349 & & 2293 & 041 & 20 \\
\hline 111 & 1191.4769 & 596.2421 & 74.4503 & 587. & 1173.4663 & 587.2368 & $\mathrm{C}$ & 2196.1845 & 5959 & 2179.1580 & 0826 & 2178.1740 & 1089.5906 & 19 \\
\hline 121 & 562 & 689.2817 & 97 & & 56 & & $\bar{W}$ & & 1018 & & & 2018 & 7753 & 18 \\
\hline 131 & 90.6403 & 745.8238 & 37 & 737. & 1472.6 & 185 & $\mathbf{L}$ & 1850.0746 & 5409 & 480 & 0276 & 1832 & .5356 & 17 \\
\hline 14 & 1603.7243 & 802.3658 & 86.6978 & 793.8525 & 1585.7138 & 793.3605 & $\mathbf{L}$ & 1736.9905 & 9989 & 1719.9640 & 860.4856 & 1718.9799 & .9936 & 16 \\
\hline 151 & 1674.7614 & 837.8 & 49 & 11 & 09 & & A & 1623 & & 160 & & 1605 & 516 & 15 \\
\hline 16 - & $\mathbf{1 7 4 5 . 7 9 8 6}$ & 873.4029 & 728.7720 & 864. & 1727.7880 & 864.3976 & $\mathbf{A}$ & 1552.8693 & & 1535 & & 1534 & 330 & 14 \\
\hline 17 & 1858.8826 & 929.9449 & 841.8561 & 921. & 1840.8721 & 920.9397 & I & 1481.8322 & 4197 & 1464.8057 & 9065 & 1463.8216 & 4145 & 13 \\
\hline 18 & 197 & 635 & 32 & & 92 & & A & \begin{tabular}{|l|}
1368.7481 \\
\end{tabular} & & 1351 & & 1350 & 8724 & 12 \\
\hline 192 & 2016.9518 & 1008.9795 & 999.9252 & 662 & $1998 . c$ & 999.9 & $\mathrm{~S}$ & 1297.7110 & & 1280 & & 1279 & 539 & 11 \\
\hline 202 & 2130.0358 & 1065.5216 & 2113.0093 & 1057.0083 & 2112.0253 & 1056.5163 & $\mathbf{L}$ & 1210.6790 & 605.8431 & 1193.6525 & 597.3299 & 1192.6684 & 596.8379 & 10 \\
\hline 212 & 231.0835 & 16.0454 & 2214.0570 & 321 & 2213.0729 & 1107 & $\mathbf{T}$ & 1097.5949 & 3011 & 1080.5684 & 540.7878 & 1079.5844 & 540.2958 & 9 \\
\hline 222 & 2344.1676 & 1172.5874 & 2327.1410 & 64.0741 & 2326.1570 & 1163.5821 & $\mathbf{L}$ & 996.5473 & 7773 & & & 978.5367 & 7720 & \\
\hline $23:$ & 2459.1945 & 1230.1009 & 2442.1680 & 1221.5876 & 2441.1839 & 1221.0956 & $\mathbf{N}$ & 883.4632 & 442.2352 & 866.4367 & 433.7220 & 865.4526 & 433.2300 & 7 \\
\hline 24 & 2588.2371 & 1294.6222 & 2571.2105 & 1286.1089 & 2570.2265 & 1285.6169 & $\mathbf{E}$ & 768.4363 & 384.7218 & 751.4097 & 2085 & 750.4257 & 375.7165 & $\mathbf{0}$ \\
\hline 25 & 2689.2848 & 1345.1460 & 2672.2582 & 1336.6328 & 2671.2742 & 1336.1407 & $\mathbf{T}$ & 639.3937 & 320.2005 & 622.3671 & & 3831 & 1952 & \\
\hline 26 & 2802.3688 & 1401.6881 & 2785.3423 & 1393.1748 & 2784.3583 & 1392.6828 & I & 538.3460 & 269.6766 & 521.3194 & 261.1634 & & & 4 \\
\hline 27 & 2915.4529 & 1458.2301 & 2898.4264 & \begin{tabular}{|l|l|}
1449.7168 \\
\end{tabular} & 2897.4423 & 1449.2248 & $\mathbf{L}$ & 425.2619 & 213.1346 & 408.2354 & 204.6213 & & & 2 \\
\hline 28 & 3052.5118 & 1526.7595 & 3035.4853 & 1518.2463 & 3034.5013 & 1517.7543 & $\mathbf{H}$ & 312.1779 & 156.5926 & 295.1513 & 148.0793 & & & \\
\hline 29 & & & & & & & $\mathbf{R}$ & 175.1190 & 88.0631 & 158.0924 & 79.5498 & & & \\
\hline
\end{tabular}



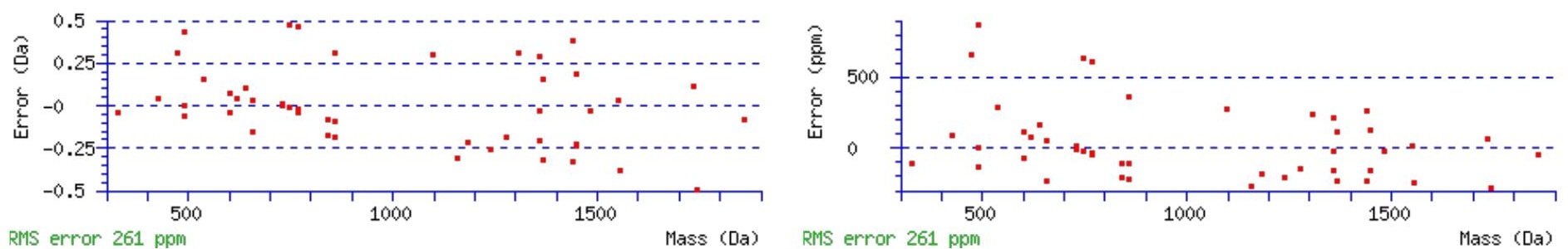

\section{All matches to this query}

\begin{tabular}{|l|l|l|l|}
\hline Score & Mr(calc): & Delta & \multicolumn{1}{|c|}{ Sequence } \\
\hline 40.5 & 3225.6162 & 0.0151 & TDICQGALGDCWLLAAIASLTLNETILHR \\
\hline 36.7 & 3224.6322 & 0.9991 & TDICQGALGDCWLLAAIASLTLNETILHR \\
\hline 3.7 & 3225.6197 & 0.0116 & RFLTGPPRPPSPAPSPSPGPPRPADSLANGR \\
\hline 2.8 & 3224.6307 & 1.0006 & MPQLDTSTWFITIISSMATLFILFQLK \\
\hline 2.8 & 3224.6307 & 1.0006 & MPQLDTSTWFITIISSMATLFILFQLK \\
\hline 1.8 & 3223.6134 & 2.0178 & VIKSASATALSVMIPAVDPHGGSPLASPMSPR \\
\hline 1.3 & 3223.6134 & 2.0178 & VIKSASATALSVMIPAVDPHGGSPLASPMSPR \\
\hline 1.3 & 3223.6134 & 2.0178 & VIKSASATALSVMIPAVDPHGGSPLASPMSPR \\
\hline 1.3 & 3223.6134 & 2.0178 & VIKSASATALSVMIPAVDPHGGSPLASPMSPR \\
\hline 1.2 & 3224.6032 & 1.0281 & LCETLKNGPGVMQVLGLVLAFGNYMNAGNK \\
\hline
\end{tabular}

Spectrum No: 830; Query: 2250; Rank: 1

\section{Peptide View}

MS/MS Fragmentation of ATDADKDDILVYTLQEVTPNASK

Found in IPI00200640, Tax_Id=10116 Gene_Symbol=Mucdhl Isoform 1 of Mucin and cadherin-like protein precursor

Match to Query 2250: 2508.237612 from(837.086480,3+)

Title: 100101RatKid_NS_deglyco_22.4521.4521.3.dta

Data file K:INewmanPaper|Piliangl3SubProteomes\Piliang3SP\mgf5ppm\ERLIC_3SubProteomes5ppm.mgf

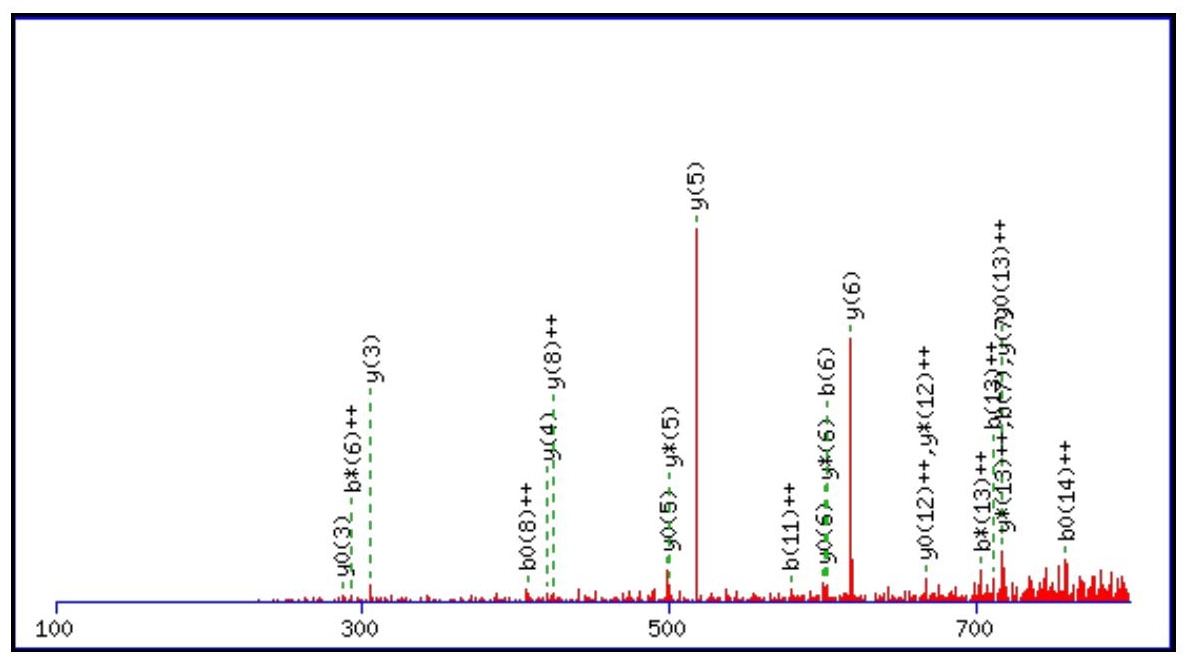



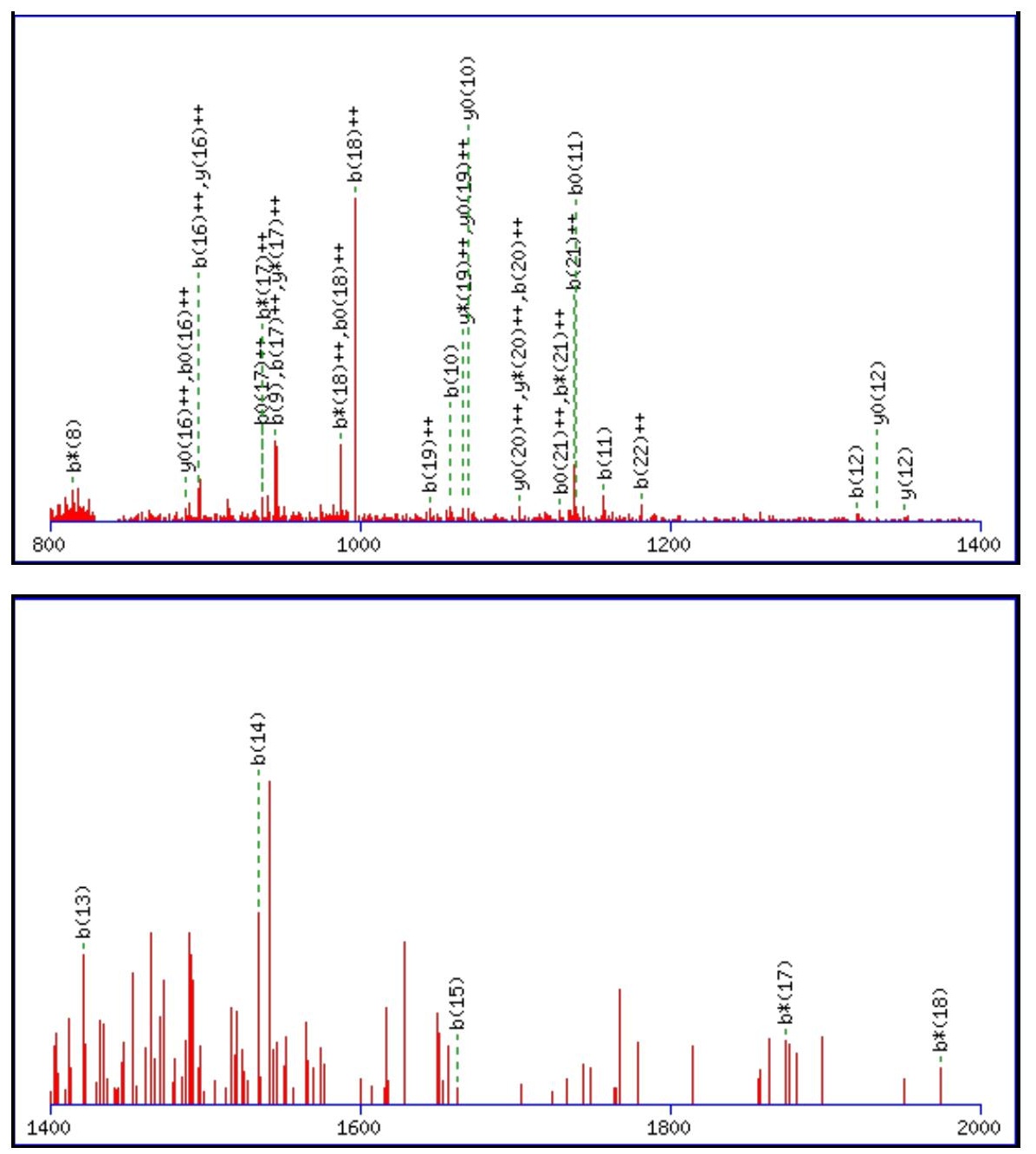

Monoisotopic mass of neutral peptide $\operatorname{Mr}($ calc): 2507.2279

Fixed modifications: Carbamidomethyl (C)

Variable modifications:

N20 : Deamidated_N (N)

Ions Score: 40 Expect : $\odot .021$

Matches (Bold Red): 58/250 fragment ions using 109 most intense peaks

\begin{tabular}{|c|c|c|c|c|c|c|c|c|c|c|c|c|c|c|}
\hline \# & b & $\mathbf{b}^{++}$ & b* & $\mathbf{b}^{*^{++}}$ & $\mathbf{b}^{0}$ & $\mathbf{b}^{0++}$ & Seq. & $\mathbf{y}$ & $y^{++}$ & $\mathbf{y}^{*}$ & $\mathbf{y}^{*^{++}}$ & $\mathbf{y}^{0}$ & $y^{0++}$ & \# \\
\hline 1 & 72.0444 & 36.5258 & & & & & A & & & & & & & 23 \\
\hline 2 & 173.0921 & 87.0497 & & & 155.0815 & 78.0444 & $\mathbf{T}$ & 2437.1981 & 1219.1027 & 2420.1715 & 1210.5894 & 2419.1875 & 1210.0974 & 22 \\
\hline 3 & 288.1190 & 144.5631 & & & 270.1084 & 135.5579 & D & 2336.1504 & 1168.5788 & 2319.1238 & 1160.0655 & 2318.1398 & 1159.5735 & 21 \\
\hline 4 & 359.1561 & 180.0817 & & & 341.1456 & 171.0764 & A & 2221.1234 & 1111.0654 & 2204.0969 & 1102.5521 & 2203.1129 & 1102.0601 & 20 \\
\hline 5 & 474.1831 & 237.5952 & & & 456.1725 & 228.5899 & D & 2150.0863 & 1075.5468 & 2133.0598 & 1067.0335 & 2132.0758 & 1066.5415 & 19 \\
\hline 6 & 602.2780 & 301.6427 & 585.2515 & & 584.2675 & 292.6374 & $\mathbf{K}$ & 2035.0594 & 1018.0333 & 2018.0328 & 1009.5201 & 2017.0488 & 1009.0280 & 18 \\
\hline 7 & 717.3050 & 359.1561 & 700.2784 & 350.6429 & 699.2944 & 350.1508 & D & 1906.9644 & 953.9858 & 1889.9379 & 945.4726 & 1888.9538 & 944.9806 & 17 \\
\hline 8 & 832.3319 & 416.6696 & 815.3054 & 408.1563 & 814.3214 & 407.6643 & D & 1791.9375 & 896.4724 & 1774.9109 & 887.9591 & 1773.9269 & 887.4671 & 16 \\
\hline 9 & 945.4160 & 473.2116 & 928.3894 & 464.6984 & 927.4054 & 464.2063 & I & 1676.9105 & 838.9589 & 1659.8840 & 830.4456 & 1658.9000 & 829.9536 & 15 \\
\hline 10 & 1058.5000 & 529.7537 & 1041.4735 & 521.2404 & 1040.4895 & 520.7484 & $\mathbf{L}$ & 1563.8265 & 782.4169 & 1546.7999 & 773.9036 & 1545.8159 & 773.4116 & 14 \\
\hline 11 & 1157.5685 & 579.2879 & 1140.5419 & 570.7746 & 1139.5579 & 570.2826 & $\bar{V}$ & 1450.7424 & 725.8748 & 1433.7158 & 717.3616 & 1432.7318 & 716.8696 & 13 \\
\hline 12 & 1320.6318 & 660.8195 & 1303.6052 & 652.3063 & 1302.6212 & 651.8143 & $\mathbf{Y}$ & 1351.6740 & 676.3406 & 1334.6474 & 667.8274 & 1333.6634 & 667.3353 & 12 \\
\hline 13 & 1421.6795 & 711.3434 & 1404.6529 & 702.8301 & 1403.6689 & 702.3381 & $\mathbf{T}$ & 1188.6107 & 594.8090 & 1171.5841 & 586.2957 & 1170.6001 & 585.8037 & 11 \\
\hline 14 & 1534.7635 & 767.8854 & 1517.7370 & 759.3721 & 1516.7530 & 758.8801 & $\mathbf{L}$ & 1087.5630 & 544.2851 & 1070.5364 & 535.7719 & 1069.5524 & 535.2798 & 10 \\
\hline 15 & 1662.8221 & 831.9147 & 1645.7956 & 823.4014 & 1644.8115 & 822.9094 & $\mathbf{Q}$ & 974.4789 & 487.7431 & 957.4524 & 479.2298 & 956.4683 & 478.7378 & 9 \\
\hline 16 & 1791.8647 & 896.4360 & 1774.8382 & 887.9227 & 1773.8541 & 887.4307 & $\mathbf{E}$ & 846.4203 & 423.7138 & 829.3938 & 415.2005 & 828.4098 & 414.7085 & 8 \\
\hline 17 & 1890.9331 & 945.9702 & 1873.9066 & 937.4569 & 1872.9226 & 936.9649 & V & 717.3777 & 359.1925 & 700.3512 & 350.6792 & 699.3672 & 350.1872 & 7 \\
\hline 18 & 1991.9808 & 996.4940 & 1974.9542 & 987.9808 & 1973.9702 & 987.4888 & $\mathbf{T}$ & 618.3093 & 309.6583 & 601.2828 & 301.1450 & 600.2988 & 300.6530 & 6 \\
\hline
\end{tabular}




\begin{tabular}{|c|c|c|c|c|c|c|c|c|c|c|c|c|c|}
\hline 19 & |2089.0336 & 1045.0204 & |2072.0070 & 1036.5 & |2071.0230| & |1036.0151 & $\mathbf{P}$ & 517.2616 & 259.1345 & 500.2351 & 250.6212 & 499.2511 & \\
\hline 20 & 2204.0605 & 1102.5339 & 2187.0339 & 1094.0206 & 2186.0499 & 1093.5286 & $\mathbf{N}$ & 420.2089 & 210.6081 & 403.1823 & 202.0948 & 402.1983 & 201.6028 \\
\hline 21 & 2275.0976 & 1138.0524 & 2258.0711 & 1129.5392 & 2257.0870 & 1129.0472 & A & 305.1819 & 153.0946 & 288.1554 & 144.5813 & 287.1714 & 144.0893 \\
\hline 22 & 2362.1296 & 1181.5685 & 2345.1031 & 1173.0552 & 2344.1191 & 1172.5632 & $S$ & 234.1448 & 117.5761 & 217.1183 & 109.0628 & 216.1343 & 108.5708 \\
\hline 23 & & & & & & & $\mathbf{K}$ & 147.1128 & 74.0600 & 130.0863 & 65.5468 & & \\
\hline
\end{tabular}
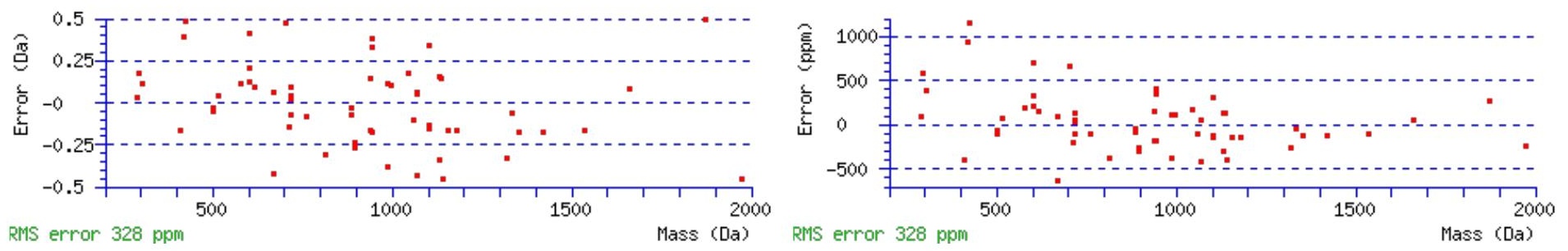

\section{All matches to this query}

\begin{tabular}{|l|c|c|l|}
\hline Score & Mr(calc): & Delta & \multicolumn{1}{c|}{ Sequence } \\
\hline 40.5 & 2507.2279 & 1.0097 & ATDADKDDILVYTLQEVTPNASK \\
\hline 20.2 & 2506.2439 & 1.9937 & ATDADKDDILVYTLQEVTPNASK \\
\hline 2.9 & 2508.2583 & -0.0206 & SIHPLALRPWDITVLVNLYK \\
\hline 2.9 & 2508.2546 & -0.0170 & LDSRVEAELOPELMSLTLAVSK \\
\hline 1.8 & 2508.2447 & -0.0071 & LEEQKPERVKPFMTGAAEQIK \\
\hline 1.8 & 2508.2546 & -0.0170 & LDSRVEAELOPELMSLTLAVSK \\
\hline 1.7 & 2507.2382 & 0.9994 & YLSAPDHLLMPQLNSLLSATVK \\
\hline 1.6 & 2508.2352 & 0.0024 & MEEPKGIFTDCQQEVSLLKTR \\
\hline 0.9 & 2508.2138 & 0.0238 & IRAHSLNTVKQSSLAEPVSPSK \\
\hline 0.8 & 2508.2505 & -0.0129 & MWSVSDNVDKIPYEQKAVLMR \\
\hline
\end{tabular}

Spectrum No: 831; Query: 178; Rank: 1

\section{Peptide View}

MS/MS Fragmentation of LVTQTIPCNK

Found in IPI00210901, Tax_Id=10116 Gene_Symbol=Cd38 ADP-ribosyl cyclase 1

Match to Query 178: 1173.607548 from(587.811050,2+)

Title: 100101RatKid_NS_deglyco_25.1050.1050.2.dta

Data file K:INewmanPaper|Piliangl3SubProteomes\Piliang3SP\mgf5ppm\ERLIC_3SubProteomes5ppm.mgf

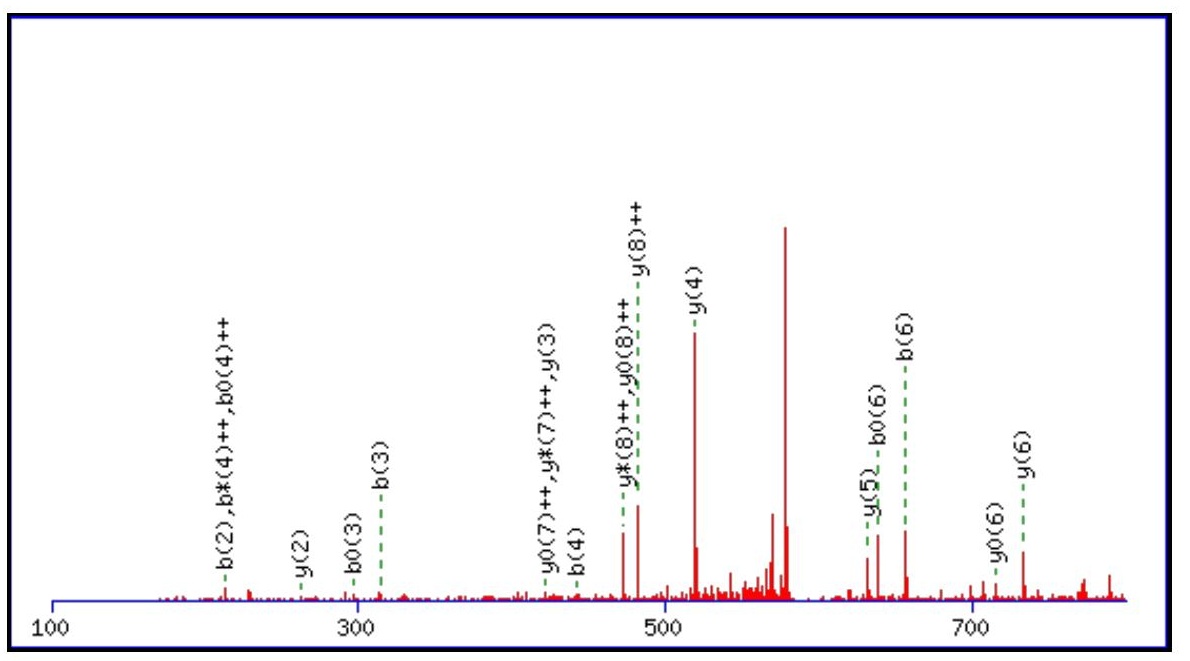



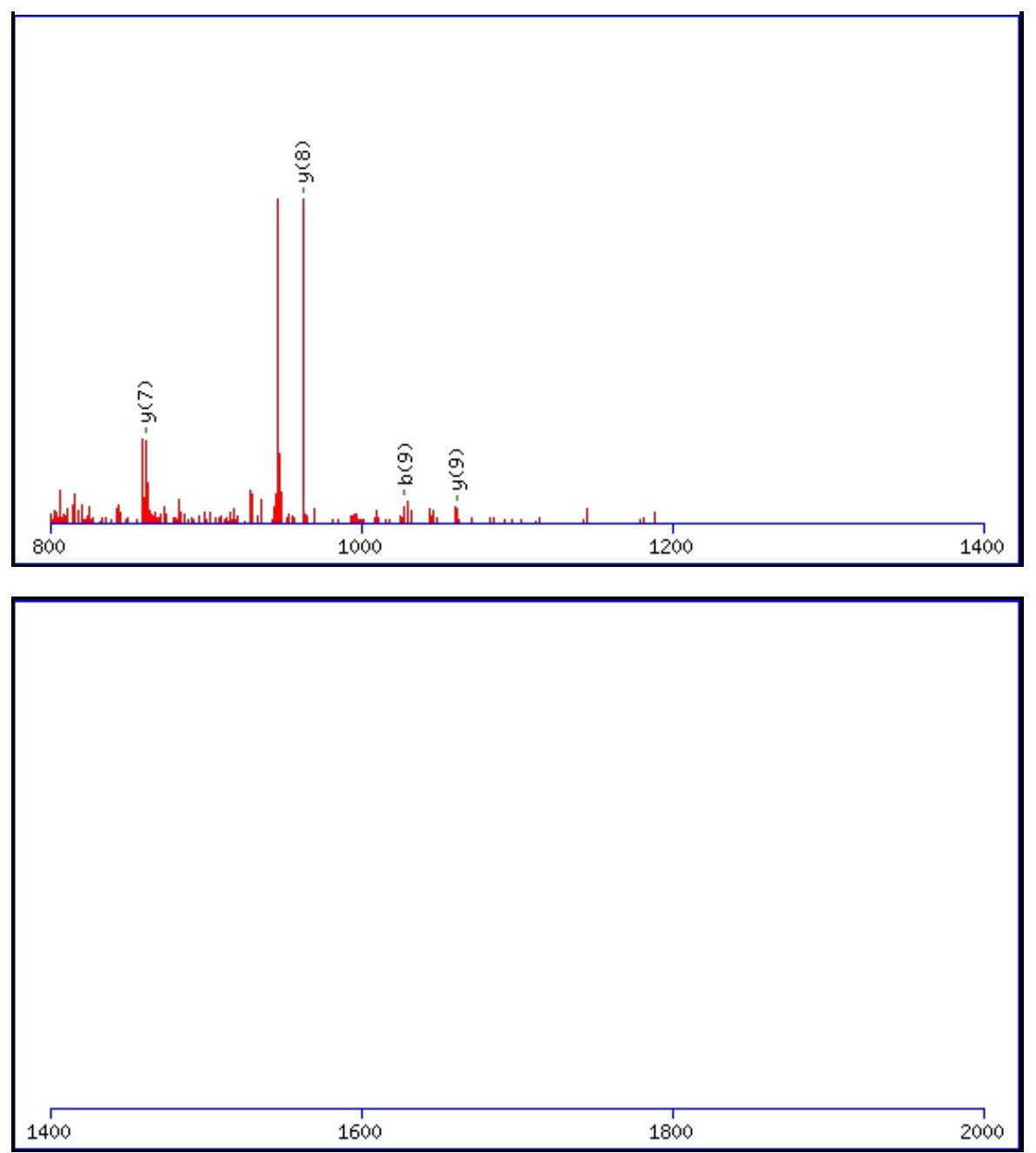

Monoisotopic mass of neutral peptide $\operatorname{Mr}($ calc): 1173.6064

Fixed modifications: Carbamidomethyl (C)

Variable modifications:

N9: Deamidated $\mathrm{N}(\mathrm{N})$

Ions Score: 40 Expect: $\odot .011$

Matches (Bold Red): 23/88 fragment ions using 52 most intense peaks

\begin{tabular}{|r|c|c|c|c|c|c|c|c|c|c|c|c|c|c|}
\hline$\#$ & $\mathbf{b}$ & $\mathbf{b}^{++}$ & $\mathbf{b}^{*}$ & $\mathbf{b}^{\mathbf{*}^{++}}$ & $\mathbf{b}^{\mathbf{0}}$ & $\mathbf{b}^{\mathbf{0 + +}}$ & $\mathbf{S e q}$ & $\mathbf{y}$ & $\mathbf{y}^{++}$ & $\mathbf{y}^{\mathbf{*}}$ & $\mathbf{y}^{\mathbf{*}^{++}}$ & $\mathbf{y}^{\mathbf{0}}$ & $\mathbf{y}^{\mathbf{0 + +}}$ & $\#$ \\
\hline $\mathbf{1}$ & 114.0913 & 57.5493 & & & & & $\mathbf{L}$ & & & & & & & $\mathbf{1 0}$ \\
\hline $\mathbf{2}$ & $\mathbf{2 1 3 . 1 5 9 8}$ & 107.0835 & & & & & $\mathbf{V}$ & $\mathbf{1 0 6 1 . 5 2 9 6}$ & 531.2684 & 1044.5030 & 522.7551 & 1043.5190 & 522.2631 & $\mathbf{9}$ \\
\hline $\mathbf{3}$ & $\mathbf{3 1 4 . 2 0 7 4}$ & 157.6074 & & & $\mathbf{2 9 6 . 1 9 6 9}$ & 148.6021 & $\mathbf{T}$ & $\mathbf{9 6 2 . 4 6 1 2}$ & $\mathbf{4 8 1 . 7 3 4 2}$ & 945.4346 & 473.2209 & 944.4506 & $\mathbf{4 7 2 . 7 2 8 9}$ & $\mathbf{8}$ \\
\hline $\mathbf{4}$ & $\mathbf{4 4 2 . 2 6 6 0}$ & 221.6366 & 425.2395 & $\mathbf{2 1 3 . 1 2 3 4}$ & 424.2554 & 212.6314 & $\mathbf{Q}$ & $\mathbf{8 6 1 . 4 1 3 5}$ & 431.2104 & 844.3869 & 422.6971 & 843.4029 & 422.2051 & $\mathbf{7}$ \\
\hline $\mathbf{5}$ & 543.3137 & 272.1605 & 526.2871 & 263.6472 & 525.3031 & 263.1552 & $\mathbf{T}$ & $\mathbf{7 3 3 . 3 5 4 9}$ & 367.1811 & 716.3283 & 358.6678 & $\mathbf{7 1 5 . 3 4 4 3}$ & 358.1758 & $\mathbf{6}$ \\
\hline $\mathbf{6}$ & $\mathbf{6 5 6 . 3 9 7 8}$ & 328.7025 & 639.3712 & 320.1892 & $\mathbf{6 3 8 . 3 8 7 2}$ & 319.6972 & $\mathbf{I}$ & $\mathbf{6 3 2 . 3 0 7 2}$ & 316.6572 & 615.2807 & 308.1440 & & & $\mathbf{5}$ \\
\hline $\mathbf{7}$ & $\mathbf{7 5 3 . 4 5 0 5}$ & 377.2289 & 736.4240 & 368.7156 & 735.4400 & 368.2236 & $\mathbf{P}$ & $\mathbf{5 1 9 . 2 2 3 2}$ & 260.1152 & 502.1966 & 251.6019 & & & $\mathbf{4}$ \\
\hline $\mathbf{8}$ & 913.4812 & 457.2442 & 896.4546 & 448.7309 & 895.4706 & 448.2389 & $\mathbf{C}$ & $\mathbf{4 2 2 . 1 7 0 4}$ & 211.5888 & 405.1438 & 203.0756 & & & $\mathbf{3}$ \\
\hline $\mathbf{9}$ & $\mathbf{1 0 2 8 . 5 0 8 1}$ & 514.7577 & 1011.4816 & 506.2444 & 1010.4975 & 505.7524 & $\mathbf{N}$ & $\mathbf{2 6 2 . 1 3 9 7}$ & 131.5735 & 245.1132 & 123.0602 & & & $\mathbf{2}$ \\
\hline $\mathbf{1 0}$ & & & & & & & $\mathbf{K}$ & 147.1128 & 74.0600 & 130.0863 & 65.5468 & & & $\mathbf{1}$ \\
\hline
\end{tabular}
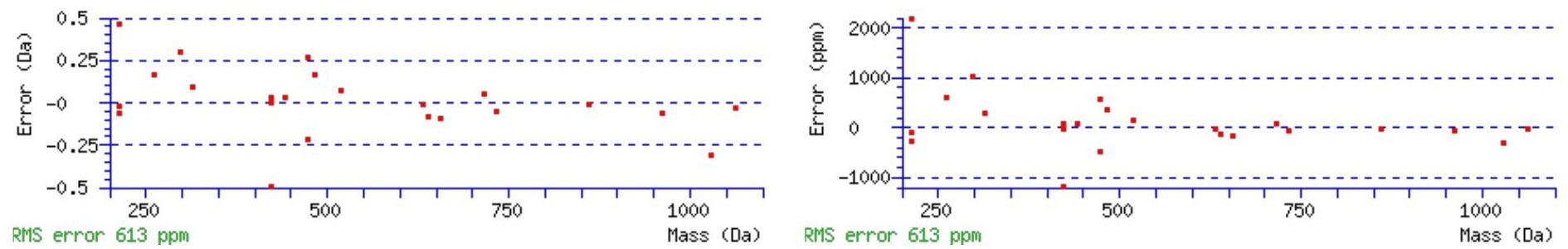

\section{All matches to this query}




\begin{tabular}{|l|l|c|l|}
\hline Score & Mr(calc): & Delta & \multicolumn{1}{|c|}{ Sequence } \\
\hline 40.5 & 1173.6064 & 0.0012 & LVTQTIPCNK \\
\hline 39.6 & 1172.6037 & 1.0038 & INSSGPVNISGK \\
\hline 7.2 & 1173.6003 & 0.0073 & NPLGRGHTGHK \\
\hline 6.1 & 1173.5989 & 0.0086 & IEAKGTQNGEK \\
\hline 5.0 & 1173.5989 & 0.0086 & IKAETAEAANR \\
\hline 4.4 & 1173.6158 & -0.0083 & LAEIAHSLLK \\
\hline 4.2 & 1173.6102 & -0.0026 & RRELSEEQK \\
\hline 3.6 & 1173.6176 & -0.0101 & VLTQMGSPSVR \\
\hline 1.0 & 1173.5989 & 0.0086 & EKLREDQEK \\
\hline 1.0 & 1172.6149 & 0.9926 & GERALKDADAK \\
\hline
\end{tabular}

Spectrum No: 832; Query: 143; Rank: 1

\section{Peptide View}

MS/MS Fragmentation of GVTHLNISGLK

Found in IPI00369995, Tax_Id=10116 Gene_Symbol=Lrp1 similar to low density lipoprotein receptor-related protein 1

Match to Query 143: 1138.632732 from(380.551520,3+)

Title: 091008RatKidney_NH4Format01_23.1569.1569.3.dta

Data file K:INewmanPaper|Piliang|3SubProteomes\Piliang3SP\mgf5ppm\ERLIC_3SubProteomes5ppm.mgf
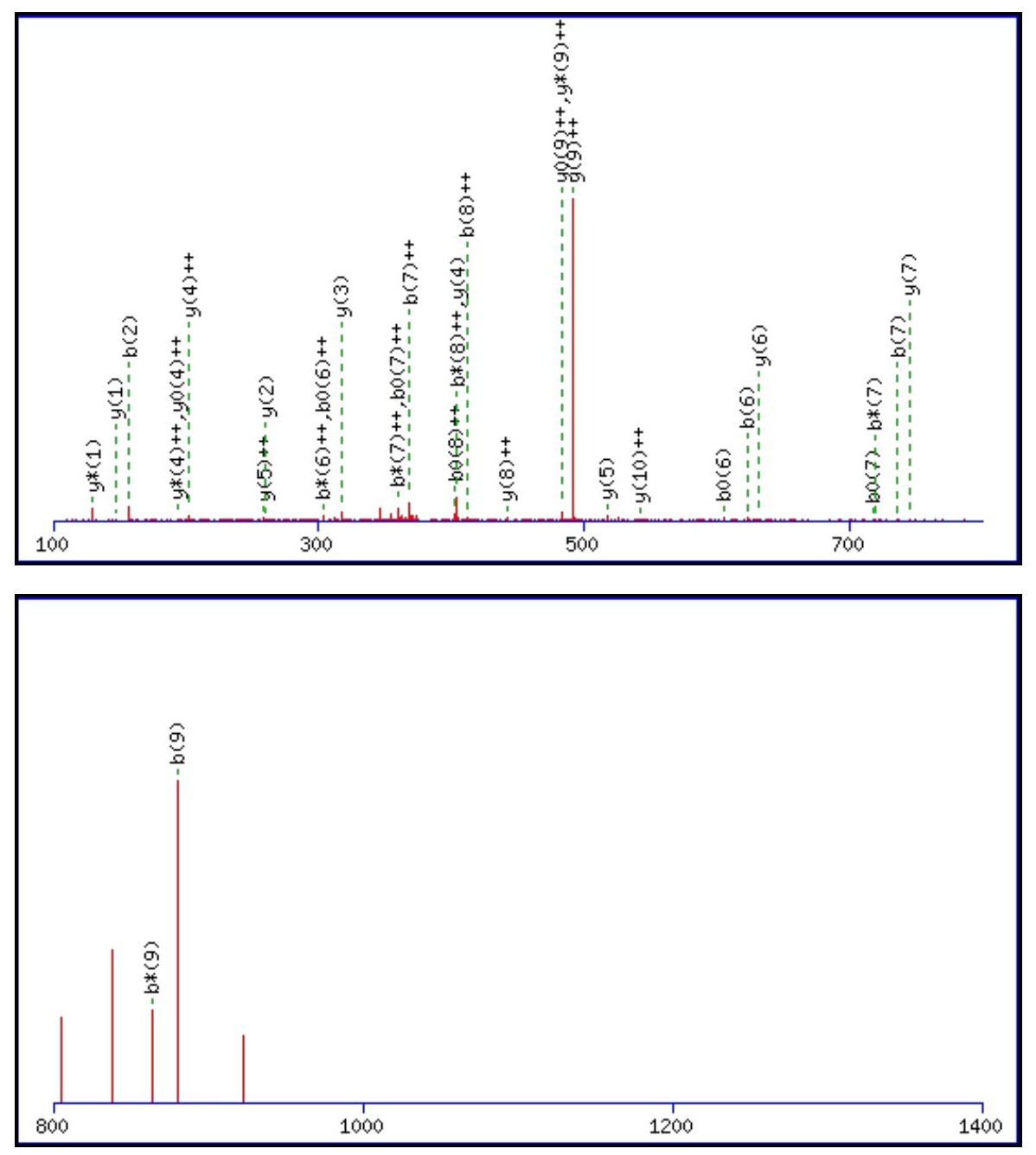


$1400 \quad 1600 \quad 1800 \quad 2000$

Monoisotopic mass of neutral peptide $\operatorname{Mr}($ calc): 1138.6346

Fixed modifications: Carbamidomethyl (C)

Variable modifications:

N6: Deamidated $\mathrm{N}(\mathrm{N})$

Ions Score: 40 Expect : $\odot .0049$

Matches (Bold Red): 33/100 fragment ions using 65 most intense peaks

\begin{tabular}{|c|c|c|c|c|c|c|c|c|c|c|c|c|c|c|}
\hline \# & b & $\mathbf{b}^{++}$ & $\mathbf{b}^{*}$ & $\mathbf{b}^{*^{++}}$ & $\mathbf{b}^{0}$ & $\mathbf{b}^{0++}$ & Seq. & $\mathbf{y}$ & $\mathbf{y}^{++}$ & $\mathbf{y}^{*}$ & $\mathbf{y}^{*^{++}}$ & $\mathbf{y}^{0}$ & $y^{0++}$ & \\
\hline 1 & 58.0287 & 29.5180 & & & & & G & & & & & & & \\
\hline 2 & 157.0972 & 79.0522 & & & & & V & 1082.6204 & 541.8139 & 1065.5939 & 533.3006 & 1064.6099 & 532.8086 & 1 \\
\hline 3 & 258.1448 & 129.5761 & & & 240.1343 & 120.5708 & $\mathbf{T}$ & 983.5520 & 492.2796 & 966.5255 & 483.7664 & 965.5415 & 483.2744 & \\
\hline 4 & 395.2037 & 198.1055 & & & 377.1932 & 189.1002 & $\mathbf{H}$ & 882.5043 & 441.7558 & 865.4778 & 433.2425 & 864.4938 & 432.7505 & \\
\hline 5 & 508.2878 & 254.6475 & & & 490.2772 & 245.6423 & $\mathbf{L}$ & 745.4454 & 373.2264 & 728.4189 & 364.7131 & 727.4349 & 364.2211 & \\
\hline 6 & 623.3147 & 312.1610 & 606.2882 & 303.6477 & 605.3042 & 303.1557 & $\mathbf{N}$ & 632.3614 & 316.6843 & 615.3348 & 308.1710 & 614.3508 & 307.6790 & \\
\hline 7 & 736.3988 & 368.7030 & 719.3723 & 360.1898 & 718.3882 & 359.6978 & I & 517.3344 & 259.1709 & 500.3079 & 250.6576 & 499.3239 & 250.1656 & \\
\hline 8 & 823.4308 & 412.2191 & 806.4043 & 403.7058 & 805.4203 & 403.2138 & $\mathrm{~S}$ & 404.2504 & 202.6288 & 387.2238 & 194.1155 & 386.2398 & 193.6235 & \\
\hline 9 & 880.4523 & 440.7298 & 863.4258 & 432.2165 & 862.4417 & 431.7245 & G & 317.2183 & 159.1128 & 300.1918 & 150.5995 & & & \\
\hline 10 & 993.5364 & 497.2718 & 976.5098 & 488.7585 & 975.5258 & 488.2665 & $\mathbf{L}$ & 260.1969 & 130.6021 & 243.1703 & 122.0888 & & & \\
\hline 11 & & & & & & & K & 147.1128 & 74.0600 & 130.0863 & 65.5468 & & & \\
\hline
\end{tabular}
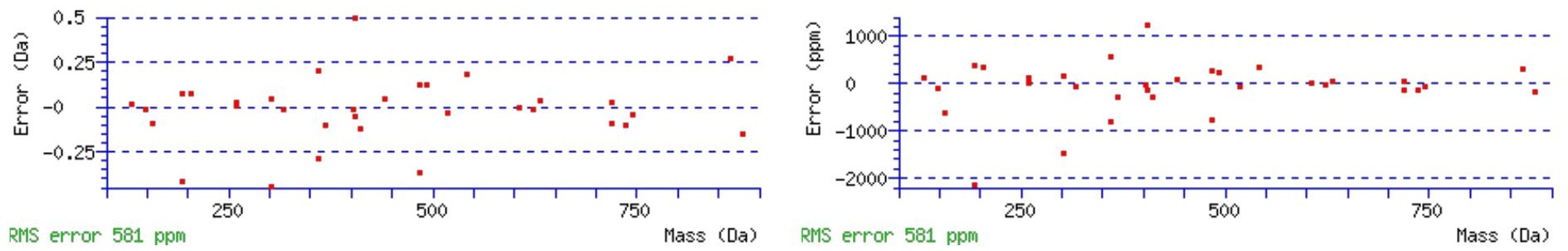

\section{All matches to this query}

\begin{tabular}{|l|l|l|l|}
\hline Score & Mr(calc): & Delta & \multicolumn{1}{|c|}{ Sequence } \\
\hline 40.3 & 1138.6346 & -0.0019 & GVTHLNISGLK \\
\hline 4.2 & 1137.6271 & 1.0056 & KVVLSQTRK \\
\hline 4.1 & 1138.6420 & -0.0093 & SFEKLMKIK \\
\hline 3.9 & 1136.6302 & 2.0025 & TRKIFTNTR \\
\hline 3.5 & 1138.6319 & 0.0008 & RNPLPSGRSR \\
\hline 2.8 & 1138.6319 & 0.0008 & RTVGKHGGTAR \\
\hline 2.7 & 1137.6328 & 0.9999 & HISPVLMGLR \\
\hline 2.6 & 1138.6346 & -0.0019 & QVSPPGSNILK \\
\hline 2.3 & 1136.6189 & 2.0138 & TAYRKELEK \\
\hline 2.2 & 1138.6319 & 0.0008 & RRQAELNPR \\
\hline
\end{tabular}


Spectrum No: 833; Query: 2793; Rank: 1

\section{Peptide View}

MS/MS Fragmentation of DAIKPNLMQTLEGTPVFVHAGPFANIAHGNSSIIADR

Found in IPI00231356, Tax_Id=10116 Gene_Symbol=Mthfd1 C-1-tetrahydrofolate synthase, cytoplasmic

Match to Query 2793: 3901.986696 from(976.503950,4+)

Title: 091008RatKidney_NoSalt_07.5371.5371.4.dta

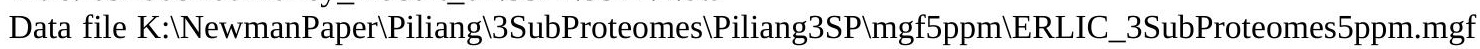
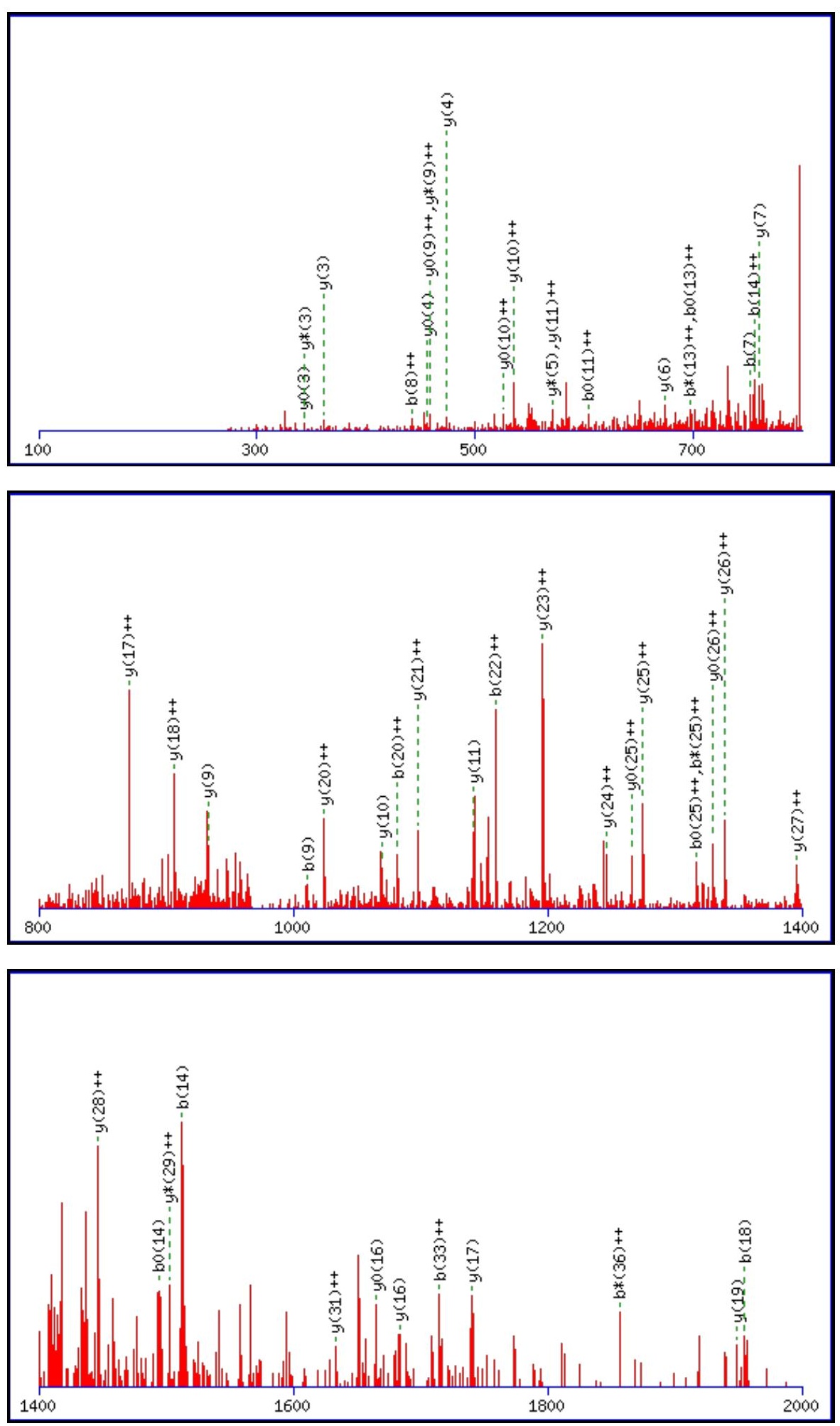
Monoisotopic mass of neutral peptide $\operatorname{Mr}($ calc): 3901.9785

Fixed modifications: Carbamidomethyl (C)

Variable modifications:

N30 : Deamidated_N ( N)

Ions Score: 40 Expect : $\odot .014$

Matches (Bold Red): 50/424 fragment ions using 125 most intense peaks

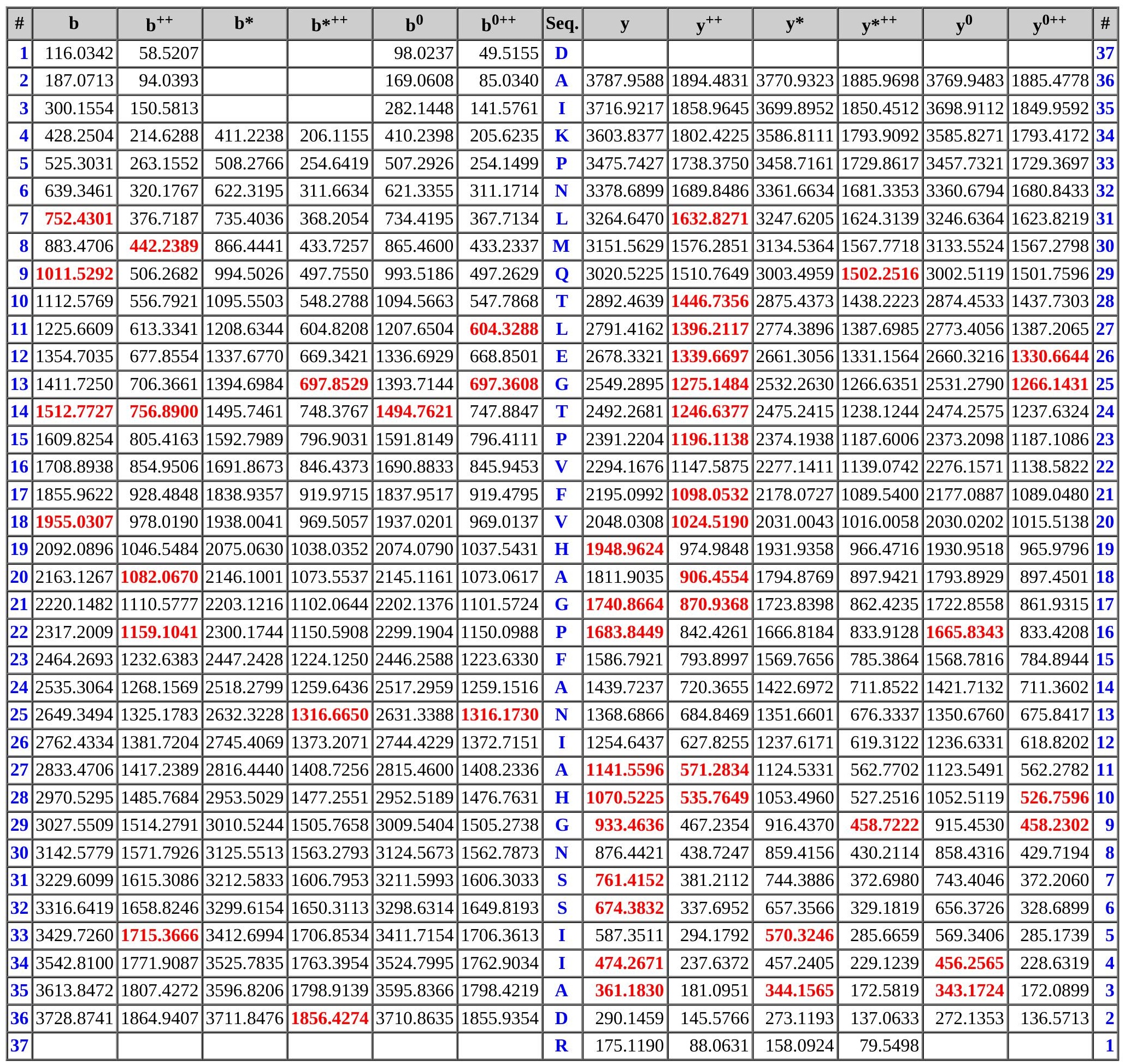
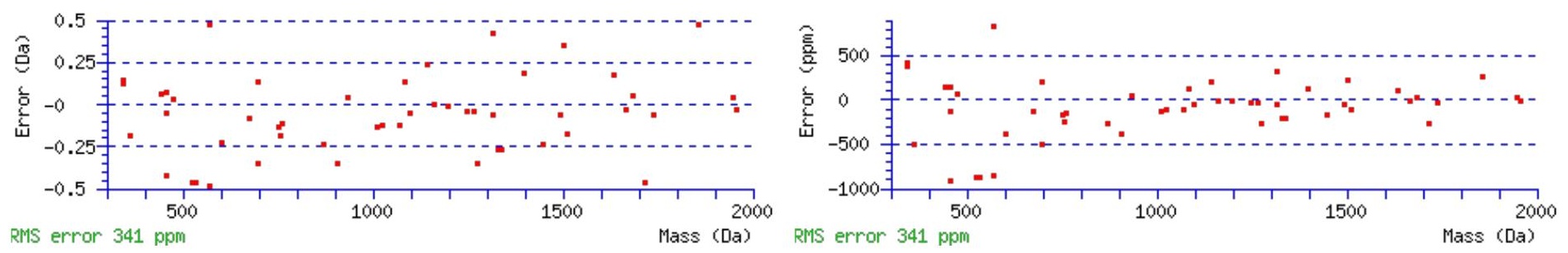
Mascot Search Results: Peptide View

\section{All matches to this query}

\begin{tabular}{|l|l|l|l|}
\hline Score & Mr(calc): & Delta & \multicolumn{1}{|c|}{ Sequence } \\
\hline 40.4 & 3901.9785 & 0.0082 & DAIKPNLMQTLEGTPVFVHAGPFANIAHGNSSIIADR \\
\hline 37.9 & 3901.9785 & 0.0082 & DAIKPNLMQTLEGTPVFVHAGPFANIAHGNSSIIADR \\
\hline 34.4 & 3900.9945 & 0.9922 & DAIKPNLMQTLEGTPVFVHAGPFANIAHGNSSIIADR \\
\hline 32.4 & 3901.9785 & 0.0082 & DAIKPNLMQTLEGTPVFVHAGPFANIAHGNSSIIADR \\
\hline 5.8 & 3900.9808 & 1.0058 & DFLVQIQKPVVKTDMGQLAKELEGLAQAHNESLR \\
\hline 3.5 & 3901.9649 & 0.0218 & DFLVQIQKPVVKTDMGQLAKELEGLAQAHNESLR \\
\hline 3.1 & 3902.0165 & -0.0298 & QPITTVVGKPIEVQMIPHPSEEEVNRLHQLYIK \\
\hline 3.1 & 3902.0165 & -0.0298 & QPITTVVGKPIEVQMIPHPSEEEVNRLHQLYIK \\
\hline 2.6 & 3901.0165 & 0.9702 & LKASLWALGNIGSSNWGLNLLQEENVIPDILKLAK \\
\hline 2.6 & 3901.0165 & 0.9702 & LKASLWALGNIGSSNWGLNLLQEENVIPDILKLAK \\
\hline
\end{tabular}

Spectrum No: 834; Query: 2796; Rank: 1

\section{Peptide View}

MS/MS Fragmentation of GVNVTMPSQPGVPPLSSTQLQIDPTLQEFQLVDLSR

Found in IPI00388323, Tax_Id=10116 Gene_Symbol=Hspg2 396 kDa protein

Match to Query 2796: 3908.971632 from(1303.997820,3+)

Title: 091008RatKidney_NH4Format01_23.4385.4385.3.dta

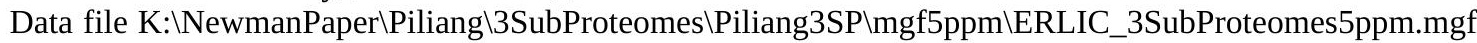
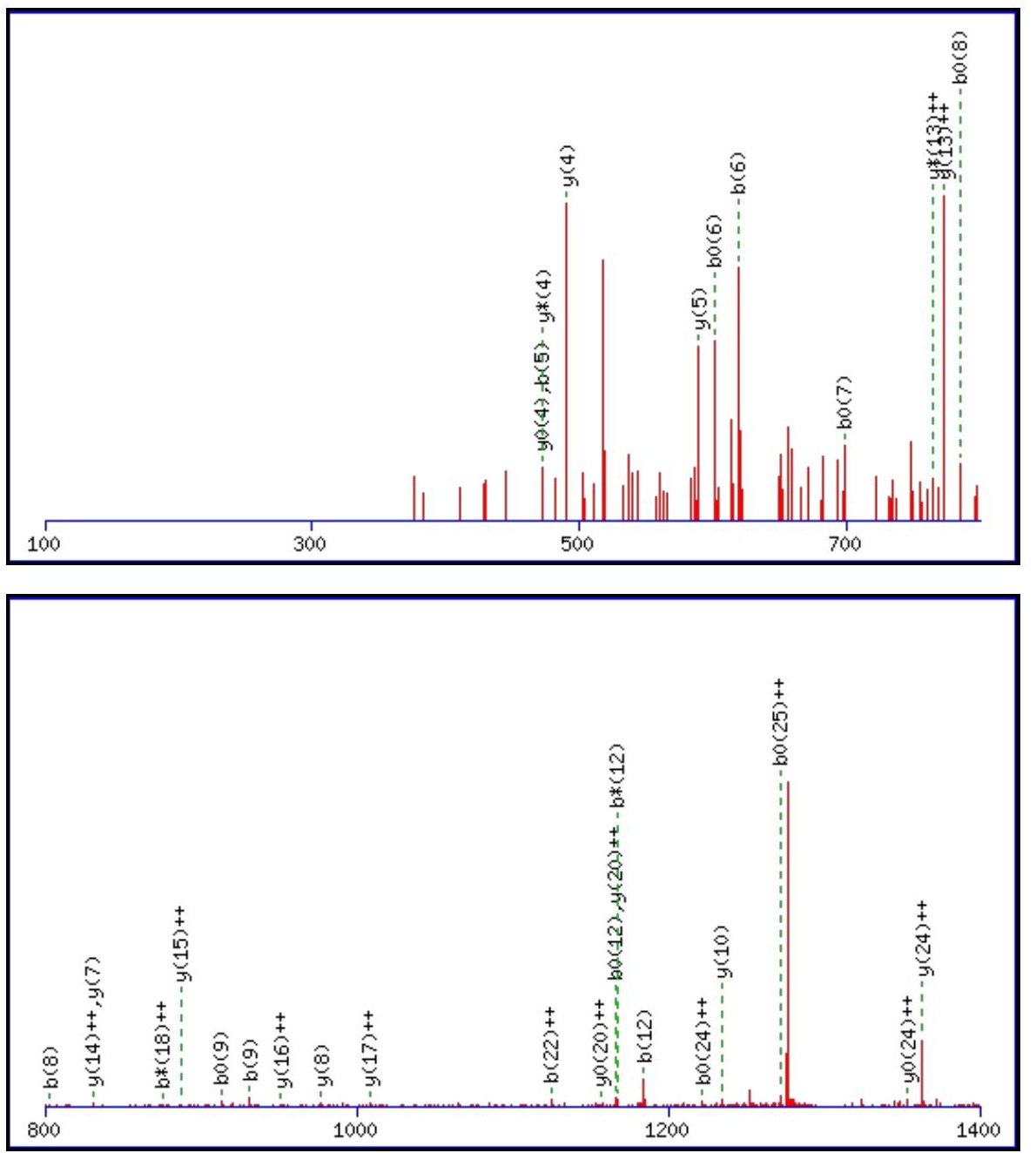


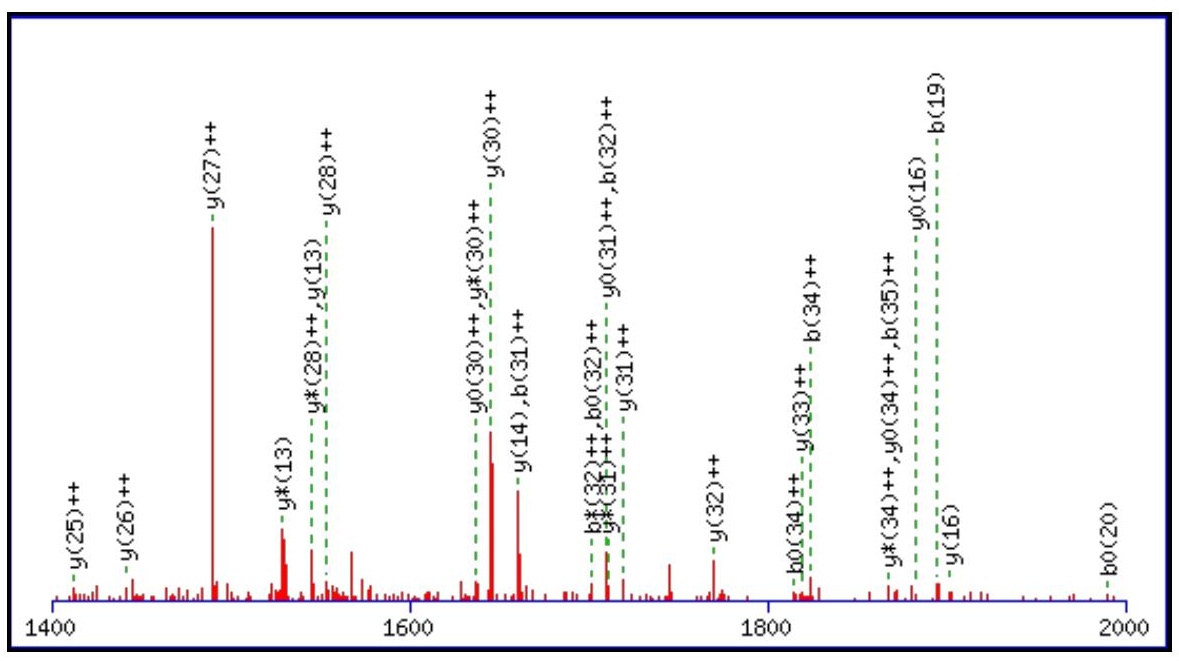

Monoisotopic mass of neutral peptide $\operatorname{Mr}($ calc): 3907.9878

Fixed modifications: Carbamidomethyl (C)

Variable modifications:

N3 : Deamidated_N (N)

M6 : oxidation (M)

Ions Score: 40 Expect : 0.017

Matches (Bold Red): 61/406 fragment ions using 142 most intense peaks

\begin{tabular}{|c|c|c|c|c|c|c|c|c|c|c|c|c|c|c|}
\hline \# & b & $\mathbf{b}^{++}$ & b* $^{*}$ & $\mathbf{b}^{*^{++}}$ & $\mathbf{b}^{\mathbf{0}}$ & $\mathbf{b}^{0++}$ & Seq. & $\mathbf{y}$ & $y^{++}$ & $\mathbf{y}^{*}$ & $\mathbf{y}^{\mathbf{*}^{++}}$ & $\mathbf{y}^{0}$ & $y^{0++}$ & \# \\
\hline 1 & 58.0287 & 29.5180 & & & & & G & & & & & & & 36 \\
\hline 2 & 157.0972 & 79.0522 & & & & & $\mathbf{V}$ & 3851.9735 & 1926.4904 & 3834.9470 & 1917.9771 & 3833.9630 & 1917.4851 & 35 \\
\hline 3 & 272.1241 & 136.5657 & 255.0975 & 128.0524 & & & $\mathbf{N}$ & 3752.9051 & 1876.9562 & 3735.8786 & \begin{tabular}{|l|}
1868.4429 \\
\end{tabular} & 3734.8946 & 1867.9509 & 34 \\
\hline 4 & 371.1925 & 186.0999 & 354.1660 & 177.5866 & & & $\mathbf{V}$ & 3637.8782 & 1819.4427 & 3620.8516 & 1810.9295 & 3619.8676 & 1810.4375 & 33 \\
\hline 5 & 472.2402 & 236.6237 & 455.2136 & 228.1105 & 454.2296 & 227.6184 & $\mathbf{T}$ & 3538.8098 & \begin{tabular}{|l|l|}
1769.9085 \\
\end{tabular} & 3521.7832 & 1761.3953 & 3520.7992 & 1760.9032 & 32 \\
\hline 6 & 619.2756 & 310.1414 & 602.2490 & 301.6282 & 601.2650 & 301.1361 & $\mathbf{M}$ & 3437.7621 & 1719.3847 & 3420.7356 & 1710.8714 & 3419.7515 & 1710.3794 & 31 \\
\hline 7 & 716.3284 & 358.6678 & 699.3018 & 350.1545 & 698.3178 & 349.6625 & $\mathbf{P}$ & 3290.7267 & 1645.8670 & 3273.7002 & 1637.3537 & 3272.7161 & 1636.8617 & 30 \\
\hline 8 & 803.3604 & 402.1838 & 786.3338 & 393.6706 & 785.3498 & 393.1785 & $S$ & 3193.6739 & 1597.3406 & 3176.6474 & 1588.8273 & 3175.6634 & 1588.3353 & 29 \\
\hline 9 & 931.4190 & 466.2131 & 914.3924 & 457.6998 & 913.4084 & 457.2078 & $\mathbf{Q}$ & 3106.6419 & 1553.8246 & 3089.6154 & 1545.3113 & 3088.6313 & 1544.8193 & 28 \\
\hline 10 & 1028.4717 & 514.7395 & 1011.4452 & 506.2262 & 1010.4612 & 505.7342 & $\mathbf{P}$ & 2978.5833 & 1489.7953 & 2961.5568 & 1481.2820 & 2960.5728 & 1480.7900 & 27 \\
\hline 11 & 1085.4932 & 543.2502 & 1068.4666 & 534.7370 & 1067.4826 & 534.2449 & G & 2881.5306 & 1441.2689 & 2864.5040 & 1432.7556 & 2863.5200 & 1432.2636 & 26 \\
\hline 12 & 1184.5616 & 592.7844 & 1167.5351 & 584.2712 & 1166.5510 & 583.7792 & $\mathbf{V}$ & 2824.5091 & 1412.7582 & 2807.4826 & 1404.2449 & 2806.4985 & 1403.7529 & 25 \\
\hline 13 & 1281.6144 & 641.3108 & 1264.5878 & 632.7975 & 1263.6038 & & $\mathbf{P}$ & 2725.4407 & 1363.2240 & 2708. & 1354.7107 & 2707.4301 & 2187 & 24 \\
\hline 14 & 1378.6671 & 689.8372 & 1361.6406 & 681.3239 & 1360.6566 & 680.8319 & $\mathbf{P}$ & 2628.3879 & 1314.6976 & 2611.3614 & 1306.1843 & 2610.3774 & 1305.6923 & 23 \\
\hline 15 & 1491.7512 & 746.3792 & 1474.7246 & 737.8660 & 1473.7406 & 737.3740 & $\mathbf{L}$ & 2531.3352 & 1266.1712 & 2514.3086 & 1257.6579 & 2513.3246 & 1257.1659 & 22 \\
\hline 16 & 1578.7 & & & & & & $S$ & & & & & & 5239 & 21 \\
\hline 17 & 1665.8152 & 833.4113 & 1648.7887 & 824.8980 & 1647.8047 & 824.4060 & $S$ & 2331.2191 & \begin{tabular}{|l|l|}
1166.1132 \\
\end{tabular} & 2314.1925 & 1157.5999 & 2313.2085 & 1157.1079 & 20 \\
\hline 18 & 1766.8629 & 883.9351 & 1749.8364 & 875.4218 & 1748.8524 & 874.9298 & $\mathbf{T}$ & 2244.1870 & 1122.5972 & 2227.1605 & 1114.0839 & 2226.1765 & 1113.5919 & 19 \\
\hline 19 & 1894.9215 & 947. & 1877. & 511 & & 938.9591 & $\mathbf{Q}$ & 2143.1394 & 1072.0733 & 2126.1128 & 5600 & 2125.1288 & 1063.0680 & 18 \\
\hline 20 & 2008.0056 & 1004.5064 & 1990.9790 & 995.9931 & 1989.9950 & 995.5011 & $\mathbf{L}$ & 2015.0808 & 1008.0440 & 1998.0542 & 999.5308 & 1997.0702 & 999.0387 & 17 \\
\hline 21 & 2136.0641 & 1068.5357 & 2119.0376 & 1060.0224 & 2118.0536 & 1059.5304 & $\mathbf{Q}$ & 1901.9967 & 951.5020 & 1884.9702 & 942.9887 & 1883.9862 & 942.4967 & 16 \\
\hline 22 & 2249.1482 & 1125.0777 & 2232.1217 & 1116.5645 & 2231.1376 & 1116.0725 & I & 1773.9381 & 887.4727 & 1756.9116 & 878.9594 & 1755.9276 & 878.4674 & 15 \\
\hline 23 & 2364.1752 & 1182.5912 & 2347.1486 & 1174.0779 & 2346.1646 & 1173.5859 & D & 1660.8541 & 830.9307 & 1643.8275 & 822.4174 & 1642.8435 & 821.9254 & 14 \\
\hline 24 & 2461.2279 & 1231.1176 & 2444.2014 & 1222.6043 & 2443.2174 & 1222.1123 & $\mathbf{P}$ & 1545.8271 & 773.4172 & 1528.8006 & 764.9039 & 1527.8166 & 764.4119 & 13 \\
\hline 25 & 2562.2756 & 1281.6414 & 2545.2490 & 1273.1282 & 2544.2650 & \begin{tabular}{|l|}
1272.6362 \\
\end{tabular} & $\mathbf{T}$ & 1448.7744 & 724.8908 & 1431.7478 & 716.3775 & 1430.7638 & 715.8855 & 12 \\
\hline 26 & 2675.3597 & 1338.1835 & 2658.3331 & 1329.6702 & 2657.3491 & 1329.1782 & $\mathbf{L}$ & 1347.7267 & 674.3670 & 1330.7001 & 665.8537 & 1329.7161 & 665.3617 & 11 \\
\hline 27 & 2803.4182 & 1402.2128 & 2786.3917 & 1393.6995 & 2785.4077 & 1393.2075 & $\mathbf{Q}$ & 1234.6426 & 617.8250 & 1217.6161 & 609.3117 & 1216.6321 & 608.8197 & 10 \\
\hline 28 & 2932.4608 & 1466.7341 & 2915.4343 & 1458.2208 & 2914.4503 & 1457.7288 & $E$ & 1106.5841 & 553.7957 & 1089.5575 & 545.2824 & 1088.5735 & 544.7904 & 9 \\
\hline 29 & 3079.5292 & 1540.2683 & 3062.5027 & 1531.7550 & 3061.5187 & 1531.2630 & $\mathbf{F}$ & \begin{tabular}{|c|}
977.5415 \\
\end{tabular} & 489.2744 & 960.5149 & 480.7611 & 959.5309 & 480.2691 & 8 \\
\hline 30 & 3207.5878 & 1604.2975 & 3190.5613 & 1595.7843 & 3189.5773 & 1595.2923 & $\mathbf{Q}$ & 830.4730 & 415.7402 & 813.4465 & 407.2269 & 812.4625 & 406.7349 & 7 \\
\hline 31 & 3320.6719 & 1660.8396 & 3303.6453 & 1652.3263 & 3302.6613 & 1651.8343 & $\mathbf{L}$ & 702.4145 & 351.7109 & 685.3879 & 343.1976 & 684.4039 & 342.7056 & 6 \\
\hline
\end{tabular}




\begin{tabular}{|r|r|r|r|r|r|r|r|r|r|r|r|r|r|r|}
$\mathbf{3 2}$ & 3419.7403 & $\mathbf{1 7 1 0 . 3 7 3 8}$ & 3402.7138 & $\mathbf{1 7 0 1 . 8 6 0 5}$ & 3401.7297 & $\mathbf{1 7 0 1 . 3 6 8 5}$ & $\mathbf{V}$ & $\mathbf{5 8 9 . 3 3 0 4}$ & $\mathbf{2 9 5 . 1 6 8 8}$ & $\mathbf{5 7 2 . 3 0 3 9}$ & $\mathbf{2 8 6 . 6 5 5 6}$ & $\mathbf{5 7 1 . 3 1 9 8}$ & $\mathbf{2 8 6 . 1 6 3 6}$ & $\mathbf{5}$ \\
\hline $\mathbf{3 3}$ & 3534.7672 & 1767.8873 & 3517.7407 & 1759.3740 & 3516.7567 & $\mathbf{1 7 5 8 . 8 8 2 0}$ & $\mathbf{D}$ & $\mathbf{4 9 0 . 2 6 2 0}$ & 245.6346 & $\mathbf{4 7 3 . 2 3 5 4}$ & 237.1214 & $\mathbf{4 7 2 . 2 5 1 4}$ & 236.6293 & $\mathbf{4}$ \\
\hline $\mathbf{3 4}$ & 3647.8513 & $\mathbf{1 8 2 4 . 4 2 9 3}$ & 3630.8248 & 1815.9160 & 3629.8407 & $\mathbf{1 8 1 5 . 4 2 4 0}$ & L & 375.2350 & 188.1212 & 358.2085 & 179.6079 & 357.2245 & 179.1159 & $\mathbf{3}$ \\
\hline $\mathbf{3 5}$ & 3734.8833 & $\mathbf{1 8 6 7 . 9 4 5 3}$ & 3717.8568 & 1859.4320 & 3716.8728 & $\mathbf{1 8 5 8 . 9 4 0 0}$ & S & 262.1510 & 131.5791 & 245.1244 & 123.0659 & 244.1404 & 122.5738 & $\mathbf{2}$ \\
\hline $\mathbf{3 6}$ & & & & & & & R & 175.1190 & $\mathbf{8 8 . 0 6 3 1}$ & 158.0924 & $\mathbf{7 9 . 5 4 9 8}$ & & & $\mathbf{1}$ \\
\hline
\end{tabular}
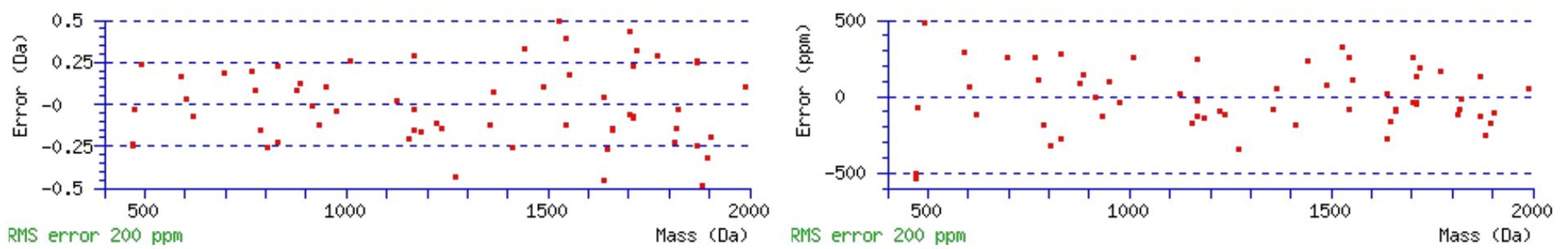

\section{All matches to this query}

\begin{tabular}{|l|c|c|l|}
\hline Score & Mr(calc): & Delta & \multicolumn{1}{c|}{ Sequence } \\
\hline 40.2 & 3907.9878 & 0.9839 & GVNVTMPSQPGVPPLSSTQLQIDPTLQEFQLVDLSR \\
\hline 27.2 & 3907.0038 & 1.9679 & GVNVTMPSOPGVPPLSSTQLQIDPTLQEFQLVDLSR \\
\hline 4.3 & 3907.9601 & 1.0116 & IIFANPCKAVSHIQYAARCGVQLLTFDSEEELTK \\
\hline 4.3 & 3906.9924 & 1.9792 & SAEFLLHMLKNAESNAELKGLDVDSLVIEHIQVNK \\
\hline 3.8 & 3908.0023 & 0.9693 & FGGIDILVNNASAISLTNTLETPTKRVDLMMSVNTR \\
\hline 3.8 & 3908.0023 & 0.9693 & FGGIDILVNNASAISLTNTLETPTKRVDLMMSVNTR \\
\hline 3.7 & 3906.9339 & 2.0377 & DAGLQVSLGAAETRSVATGPMTPQAAAPPAAPPVFPEVR \\
\hline 3.2 & 3906.9760 & 1.9956 & SIKATPMAVADLIPVDTVVNLTIAVGWYTAVHRPK \\
\hline 3.2 & 3906.9688 & 2.0028 & CHALKLDSFGQFVELLPSLEFISLDQMFREPPK \\
\hline 2.9 & 3908.9725 & -0.0009 & ADIAVAPLTITLVREEVIDFSKPFMSLGISIMIK \\
\hline
\end{tabular}

Spectrum No: 835; Query: 1391; Rank: 1

\section{Peptide View}

MS/MS Fragmentation of RGPECSQNYTAPTGVIK

Found in IPI00212307, Tax_Id=10116 Gene_Symbol=Nrp1 Neuropilin-1 precursor

Match to Query 1391: 1877.899692 from(626.973840,3+)

Title: 100101RatKid_NS_deglyco_18.1807.1807.3.dta

Data file K:INewmanPaper|Piliang|3SubProteomes\Piliang3SP\mgf5ppm\ERLIC_3SubProteomes5ppm.mgf

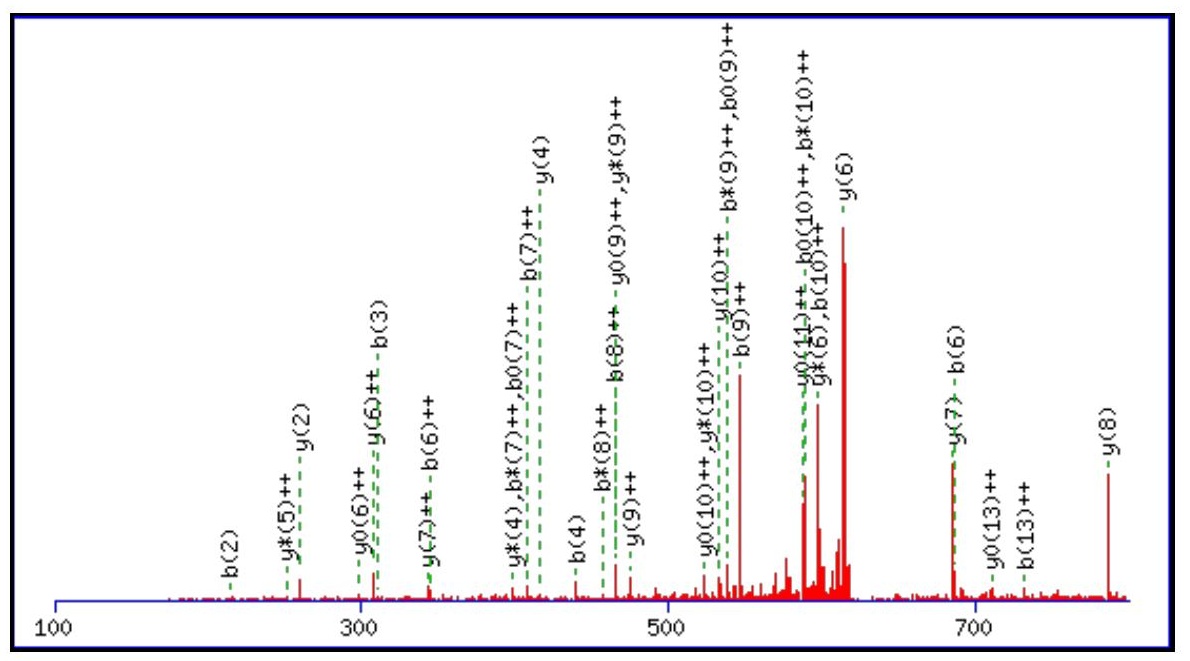



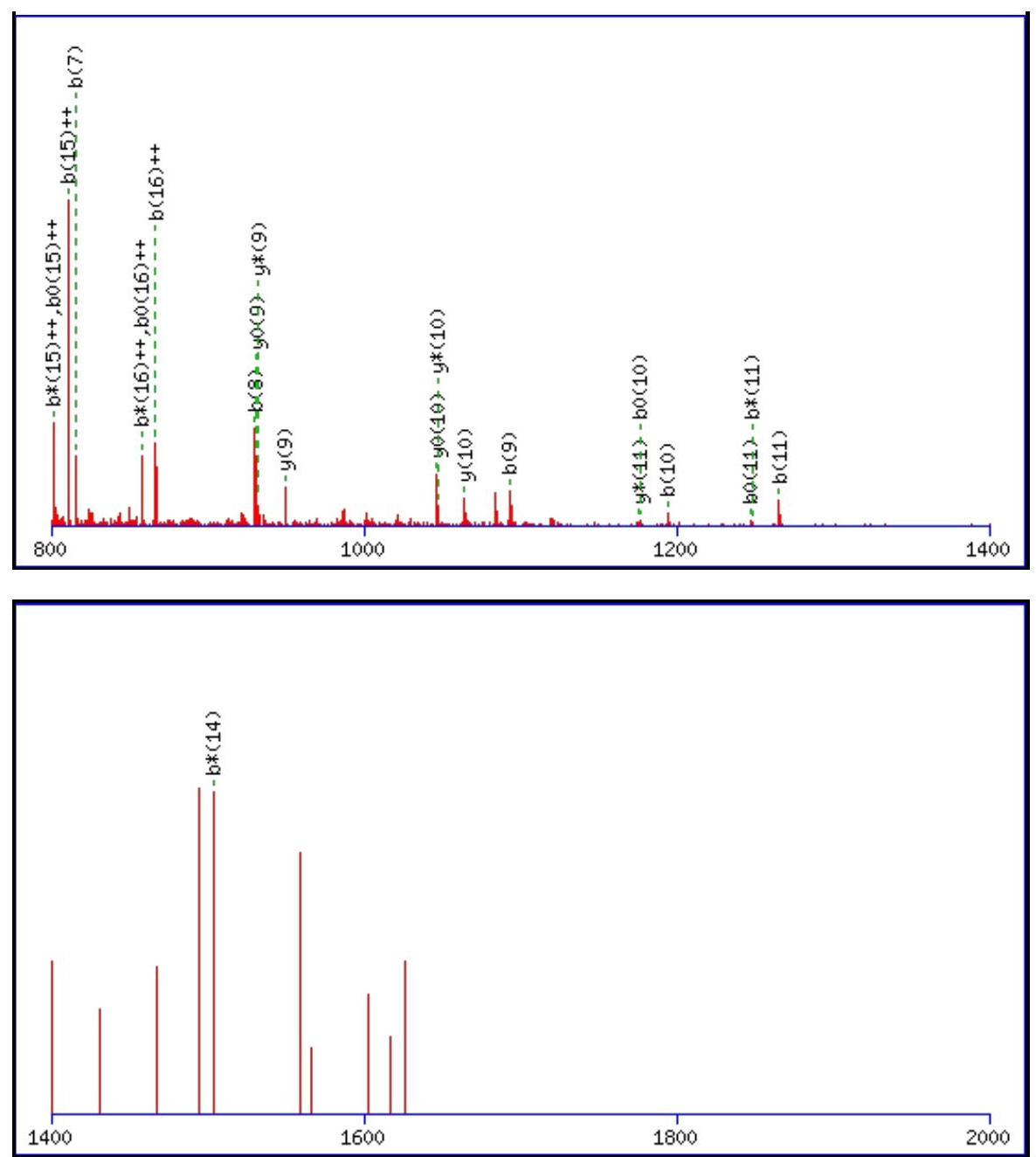

Monoisotopic mass of neutral peptide $\operatorname{Mr}($ calc): 1877.8942

Fixed modifications: Carbamidomethyl (C)

Variable modifications:

N8: : Deamidated $\mathrm{N}(\mathrm{N})$

Ions Score: 40 Expect : $\odot .022$

Matches (Bold Red): 58/178 fragment ions using 108 most intense peaks

\begin{tabular}{|c|c|c|c|c|c|c|c|c|c|c|c|c|c|c|}
\hline \# & b & $\mathbf{b}^{++}$ & b* & $\mathbf{b}^{*^{++}}$ & $\mathbf{b}^{0}$ & & Seq. & $\mathbf{y}$ & $\mathbf{y}^{++}$ & $\mathbf{y}^{*}$ & $\mathbf{y}^{*^{++}}$ & $\mathbf{y}^{\mathbf{0}}$ & $\mathbf{y}^{\mathbf{0 + +}}$ & \# \\
\hline 1 & 157.1084 & 79.0578 & 140.0818 & 70.5446 & & & $\mathbf{R}$ & & & & & & & 17 \\
\hline 2 & 214.1299 & 107.5686 & 197.1033 & 99.0553 & & & $\mathbf{G}$ & & & 1705.7738 & & 1704.7898 & 852.8985 & 16 \\
\hline 3 & 311.1826 & 156.0949 & 294.1561 & 147.5817 & & & $\mathbf{P}$ & & 833.3931 & 1648.7523 & 824.8798 & 1647.7683 & 824.3878 & 15 \\
\hline 4 & 440.2252 & 220.6162 & 423.1987 & 212.1030 & 422.2146 & 211.6110 & $\mathbf{E}$ & 1568.7261 & 784.8667 & 1551.6995 & 776.3534 & 1550.7155 & 775.8614 & 14 \\
\hline 5 & 600.2559 & 300.6316 & 583.2293 & 292.1183 & 582.2453 & 291.6263 & C & 1439.6835 & 720.3454 & 1422.6570 & 711.8321 & 1421.6729 & 711.3401 & 13 \\
\hline 6 & 687.2879 & 344.1476 & 670.2613 & 335.6343 & 669.2773 & 335.1423 & S & 1279.6529 & 640.3301 & 1262.6263 & 631.8168 & 1261.6423 & 631.3248 & 12 \\
\hline 7 & 815.3465 & 408.1769 & 798.3199 & 399.6636 & 797.3359 & 399.1716 & $\mathbf{Q}$ & 1192.6208 & 596.8141 & 1175.5943 & 588.3008 & 1174.6103 & 587.8088 & 11 \\
\hline 8 & 930.3734 & 465.6903 & 913.3469 & 457.1771 & 912.3628 & 456.6851 & $\mathbf{N}$ & 1064.5622 & 532.7848 & 1047.5357 & 524.2715 & 1046.5517 & 523.7795 & 10 \\
\hline 9 & 1093.4367 & 547.2220 & 1076.4102 & 538.7087 & 1075.4262 & 538.2167 & $\mathbf{Y}$ & 949.5353 & 475.2713 & 932.5088 & 466.7580 & 931.5247 & 466.2660 & 9 \\
\hline 10 & 1194.4844 & 597.7458 & 1177.4579 & 589.2326 & 1176.4738 & 588.7406 & $\mathbf{T}$ & 786.4720 & 393.7396 & 769.4454 & 385.2264 & 768.4614 & 384.7343 & 8 \\
\hline 11 & 265.5215 & 633.2644 & 1248.4950 & 624.7511 & 1247.5110 & 624.2591 & A & 685.4243 & 343.2158 & 668.3978 & 334.7025 & 667.4137 & 334.2105 & 7 \\
\hline 12 & 1362.5743 & 681.7908 & 1345.5477 & 673.2775 & 1344.5637 & 672.7855 & $\mathbf{P}$ & 614.3872 & 307.6972 & 597.3606 & 299.1840 & 596.3766 & 298.6920 & 6 \\
\hline 13 & 1463.6220 & 732.3146 & 1446.5954 & 723.8013 & 1445.6114 & 723.3093 & $\mathbf{T}$ & 517.3344 & 259.1709 & 500.3079 & 250.6576 & 499.3239 & 250.1656 & 5 \\
\hline 14 & 1520.6434 & 760.8254 & 1503.6169 & 752.3121 & 1502.6329 & 751.8201 & G & 416.2867 & 208.6470 & 399.2602 & 200.1337 & & & 4 \\
\hline 15 & 1619.7118 & 810.3596 & 1602.6853 & 801.8463 & 1601.7013 & 801.3543 & V & 359.2653 & 180.1363 & 342.2387 & 171.6230 & & & 3 \\
\hline 16 & 1732.7959 & 866.9016 & 1715.7694 & 858.3883 & 1714.7853 & 857.8963 & I & 260.1969 & 130.6021 & 243.1703 & 122.0888 & & & 2 \\
\hline 17 & & & & & & & $\mathbf{K}$ & 147.1128 & 74.0600 & 130.0863 & 65.5468 & & & 1 \\
\hline
\end{tabular}



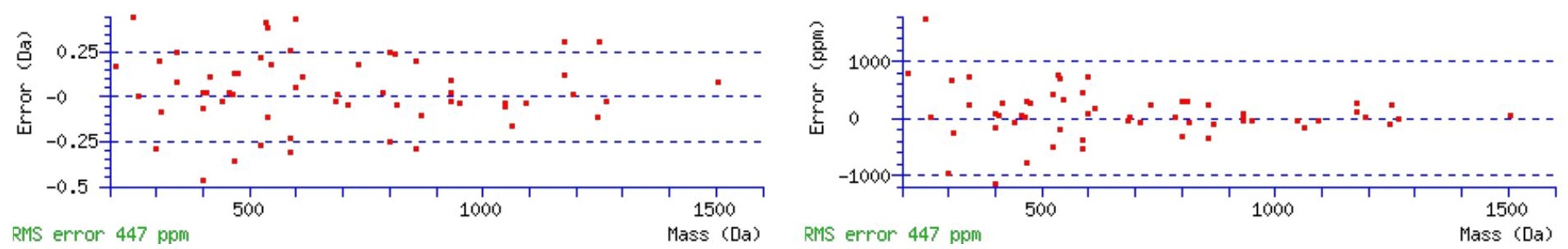

\section{All matches to this query}

\begin{tabular}{|l|l|l|l|}
\hline Score & Mr(calc): & Delta & \multicolumn{1}{|c|}{ Sequence } \\
\hline 40.2 & 1877.8942 & 0.0055 & RGPECSQNYTAPTGVIK \\
\hline 21.4 & 1876.9101 & 0.9896 & RGPECSQNYTAPTGVIK \\
\hline 7.2 & 1877.8875 & 0.0122 & AWRISVAKTSMDILK \\
\hline 2.1 & 1875.8996 & 2.0001 & SMLQATAEANNLAAVAGAR \\
\hline 1.3 & 1875.8931 & 2.0066 & NVNMRAMNVLTEAEER \\
\hline 1.3 & 1876.8961 & 1.0036 & RKTVXAKPQINSSNK \\
\hline 1.3 & 1876.8961 & 1.0036 & RKTVXAKPQINSSNK \\
\hline 1.1 & 1875.8856 & 2.0141 & NSGTSALEVLRRATIK \\
\hline 0.7 & 1875.8768 & 2.0229 & HIYNTEDIVPPLQTR \\
\hline 0.7 & 1875.8768 & 2.0229 & HIYNTEDIVPPLQTR \\
\hline
\end{tabular}

Spectrum No: 836; Query: 2; Rank: 1

\section{Peptide View}

MS/MS Fragmentation of IMNVTK

Found in IPI00480731, Tax_Id=10116 Gene_Symbol=Cntn3 Contactin-3 precursor

Match to Query 2: 705.373284 from(706.380560,1+)

Title: 091008RatKidney_NH4Format01_23.1108.1108.1.dta

Data file K:INewmanPaper|Piliang|3SubProteomes\Piliang3SP\mgf5ppm\ERLIC_3SubProteomes5ppm.mgf

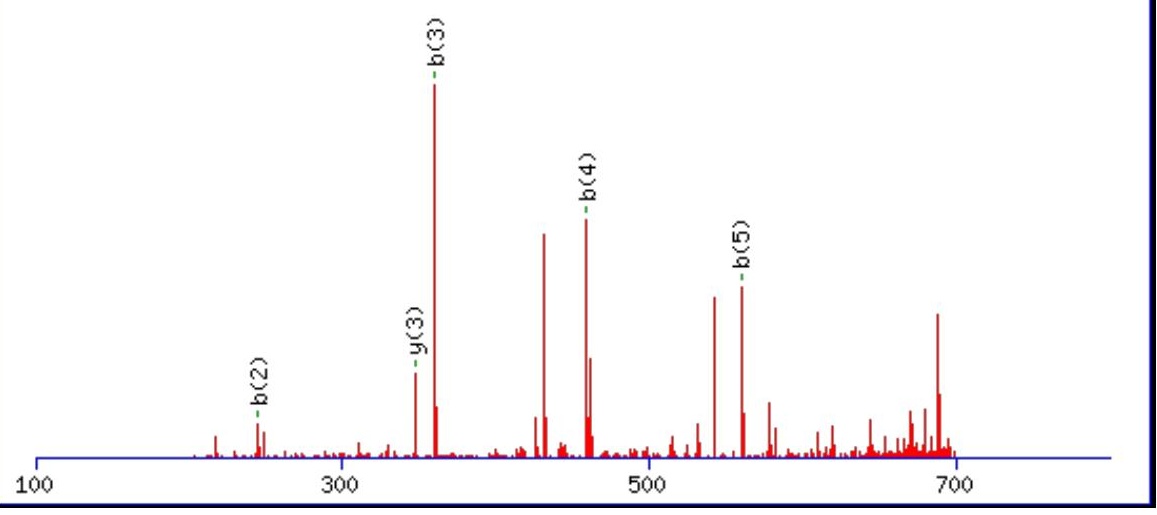




\begin{tabular}{rrrr}
800 & 1000 & 1200 & 1400 \\
\hline
\end{tabular}

\begin{tabular}{rrrr}
\hline 1400 & 1600 & 1800 & 2000 \\
\hline
\end{tabular}

Monoisotopic mass of neutral peptide $\operatorname{Mr}($ calc): 705.3731 Fixed modifications: Carbamidomethyl (C) Variable modifications:

N3: Deamidated $\mathrm{N}(\mathrm{N})$

Ions Score: 40 Expect: $\odot .0075$

Matches (Bold Red): 5/23 fragment ions using 6 most intense peaks

\begin{tabular}{|c|c|c|c|c|c|c|c|c|}
\hline$\#$ & $\mathbf{b}$ & $\mathbf{b}^{*}$ & $\mathbf{b}^{\mathbf{0}}$ & Seq. & $\mathbf{y}$ & $\mathbf{y}^{*}$ & $\mathbf{y}^{\mathbf{0}}$ & $\#$ \\
\hline $\mathbf{1}$ & $\mathbf{1 1 4 . 0 9 1 3}$ & & & $\mathbf{I}$ & & & & $\mathbf{6}$ \\
\hline $\mathbf{2}$ & $\mathbf{2 4 5 . 1 3 1 8}$ & & & $\mathbf{M}$ & 593.2963 & 576.2698 & 575.2858 & $\mathbf{5}$ \\
\hline $\mathbf{3}$ & $\mathbf{3 6 0 . 1 5 8 8}$ & 343.1322 & & $\mathbf{N}$ & 462.2558 & 445.2293 & 444.2453 & $\mathbf{4}$ \\
\hline $\mathbf{4}$ & $\mathbf{4 5 9 . 2 2 7 2}$ & 442.2006 & & $\mathbf{V}$ & 347.2289 & 330.2023 & 329.2183 & $\mathbf{3}$ \\
\hline $\mathbf{5}$ & $\mathbf{5 6 0 . 2 7 4 9}$ & 543.2483 & 542.2643 & $\mathbf{T}$ & 248.1605 & 231.1339 & 230.1499 & $\mathbf{2}$ \\
\hline $\mathbf{6}$ & & & & $\mathbf{K}$ & 147.1128 & 130.0863 & & $\mathbf{1}$ \\
\hline
\end{tabular}

要$$
\text { 它 }
$$

RMS error 152 ppm

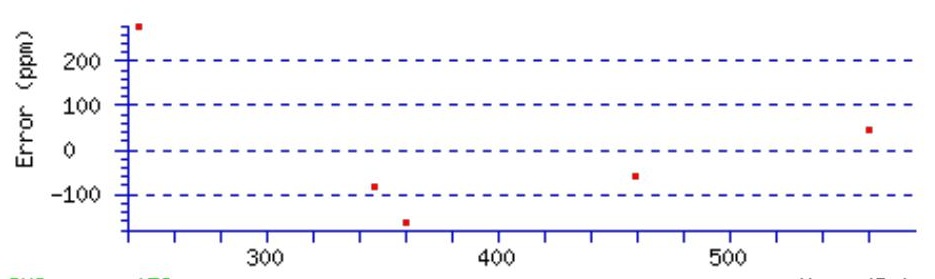

RMS error 152 ppm

\section{All matches to this query}

\begin{tabular}{|l|l|l|l|}
\hline Score & Mr(calc): & Delta & Sequence \\
\hline 40.2 & 705.3731 & 0.0002 & IMNVTK \\
\hline 21.9 & 705.3731 & 0.0002 & LMDSLK \\
\hline
\end{tabular}




\begin{tabular}{|l|l|l|l|}
21.9 & 705.3731 & 0.0002 & MLDISK \\
\hline 21.9 & 705.3731 & 0.0002 & $\underline{\text { MLDSLK }}$ \\
\hline 21.9 & 705.3731 & 0.0002 & MLDTVK \\
\hline 17.9 & 703.3687 & 2.0046 & MLNVAR \\
\hline 16.0 & 705.3731 & 0.0002 & LMVAEK \\
\hline 14.0 & 705.3731 & 0.0002 & MISVEK \\
\hline 14.0 & 705.3731 & 0.0002 & MIVSEK \\
\hline 9.2 & 704.3639 & 1.0093 & VMVANR \\
\hline
\end{tabular}

Spectrum No: 837; Query: 522; Rank: 1

\section{Peptide View}

\section{MS/MS Fragmentation of RNFTAADWGHSR}

Found in IPI00371853, Tax_Id=10116 Gene_Symbol=Col6a1_predicted similar to Collagen alpha-1(VI) chain precursor

Match to Query 522: 1417.645302 from(473.555710,3+)

Title: 091008RatKidney_NH4Format01_23.992.992.3.dta

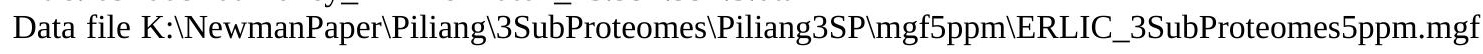
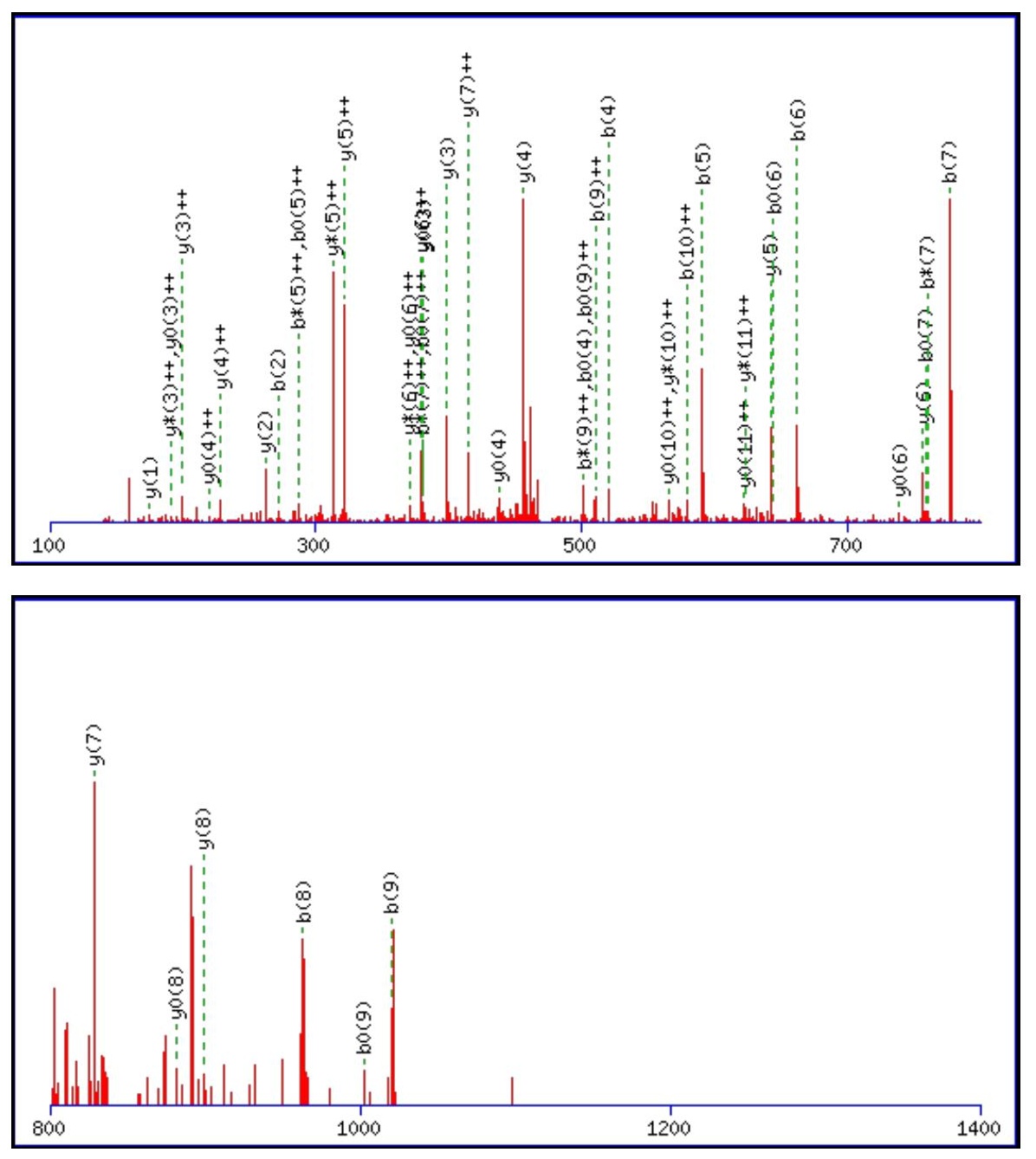


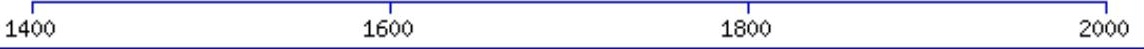

Monoisotopic mass of neutral peptide $\operatorname{Mr}($ calc): 1417.6487

Fixed modifications: Carbamidomethyl (C)

Variable modifications:

N2 : Deamidated $\mathrm{N}(\mathrm{N})$

Ions Score: 40 Expect : 0.016

Matches (Bold Red): 48/124 fragment ions using 77 most intense peaks

\begin{tabular}{|c|c|c|c|c|c|c|c|c|c|c|c|c|c|c|}
\hline \# & b & $\mathbf{b}^{++}$ & $\mathbf{b}^{*}$ & $\mathbf{b}^{*^{++}}$ & $\mathbf{b}^{0}$ & $\mathbf{b}^{0++}$ & Seq. & $\mathbf{y}$ & $y^{++}$ & $\mathrm{y}^{*}$ & $\mathrm{y}^{*^{++}}$ & $\mathbf{y}^{\mathbf{0}}$ & $y^{0++}$ & \# \\
\hline 1 & 157.1084 & 79.0578 & 140.0818 & 70.5446 & & & $\mathbf{R}$ & & & & & & & 12 \\
\hline 2 & 272.1353 & 136.5713 & 255.1088 & 128.0580 & & & $\mathbf{N}$ & 1262.5549 & 631.7811 & 1245.5283 & 623.2678 & 1244.5443 & 622.7758 & 11 \\
\hline 3 & 419.2037 & 210.1055 & 402.1772 & 201.5922 & & & $\mathbf{F}$ & 1147.5279 & 574.2676 & 1130.5014 & 565.7543 & 1129.5174 & 565.2623 & 10 \\
\hline 4 & 520.2514 & 260.6293 & 503.2249 & 252.1161 & 502.2409 & 251.6241 & $\mathbf{T}$ & 1000.4595 & 500.7334 & 983.4330 & 492.2201 & 982.4490 & 491.7281 & 9 \\
\hline 5 & 591.2885 & 296.1479 & 574.2620 & 287.6346 & 573.2780 & 287.1426 & A & 899.4118 & 450.2096 & 882.3853 & 441.6963 & 881.4013 & 441.2043 & 8 \\
\hline 6 & 662.3256 & 331.6665 & 645.2991 & 323.1532 & 644.3151 & 322.6612 & A & 828.3747 & 414.6910 & 811.3482 & 406.1777 & 810.3642 & 405.6857 & 7 \\
\hline 7 & 777.3526 & 389.1799 & 760.3260 & 380.6667 & 759.3420 & 380.1746 & D & 757.3376 & 379.1724 & 740.3111 & 370.6592 & 739.3270 & 370.1672 & 6 \\
\hline 8 & 963.4319 & 482.2196 & 946.4054 & 473.7063 & 945.4213 & 473.2143 & W & 642.3107 & 321.6590 & 625.2841 & 313.1457 & 624.3001 & 312.6537 & 5 \\
\hline 9 & 1020.4534 & 510.7303 & 1003.4268 & 502.2170 & 1002.4428 & 501.7250 & G & 456.2314 & 228.6193 & 439.2048 & 220.1060 & 438.2208 & 219.6140 & 4 \\
\hline 10 & 1157.5123 & 579.2598 & 1140.4857 & 570.7465 & 1139.5017 & 570.2545 & $\mathbf{H}$ & 399.2099 & 200.1086 & 382.1833 & 191.5953 & 381.1993 & 191.1033 & 3 \\
\hline 11 & 1244.5443 & 622.7758 & 1227.5178 & 614.2625 & 1226.5337 & 613.7705 & $S$ & 262.1510 & 131.5791 & 245.1244 & 123.0659 & 244.1404 & 122.5738 & 2 \\
\hline 12 & & & & & & & $\mathbf{R}$ & 175.1190 & 88.0631 & 158.0924 & 79.5498 & & & 1 \\
\hline
\end{tabular}
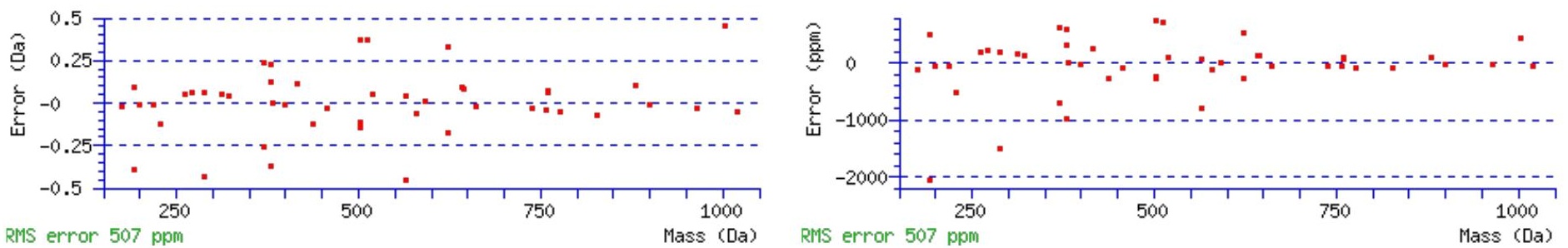

\section{All matches to this query}

\begin{tabular}{|l|l|l|l|}
\hline Score & Mr(calc): & Delta & \multicolumn{1}{c|}{ Sequence } \\
\hline 40.1 & 1417.6487 & -0.0034 & RNFTAADWGHSR \\
\hline 13.3 & 1415.6285 & 2.0168 & MTTPALLPLSGR \\
\hline 9.7 & 1416.6320 & 1.0133 & MATDELASKLSR \\
\hline 7.7 & 1415.6351 & 2.0102 & GGGPKEVPNMEER \\
\hline 6.8 & 1417.6507 & -0.0054 & EPATQDNKDICK \\
\hline 4.0 & 1417.6361 & 0.0092 & NPKGEVFDGDPDK \\
\hline 2.5 & 1417.6547 & -0.0094 & ENPNWTVVEMAK \\
\hline 2.5 & 1417.6547 & -0.0094 & ENPNWTVVEMAK \\
\hline 2.3 & 1416.6415 & 1.0038 & LERLNSTLSPK \\
\hline & & & \\
\hline
\end{tabular}




\section{$2.1 \quad|1415.6307| 2.0146 \mid \underline{\text { AKSHSPSRHDSK }}$}

Spectrum No: 838; Query: 749; Rank: 1

\section{Peptide View}

MS/MS Fragmentation of LLQTAEHNISGAER

Found in IPI00207123, Tax_Id=10116 Gene_Symbol=Tnc Tenascin (Fragment)

Match to Query 749: 1538.771052 from(513.930960,3+)

Title: 100101RatKid_NS_deglyco_27.1383.1383.3.dta

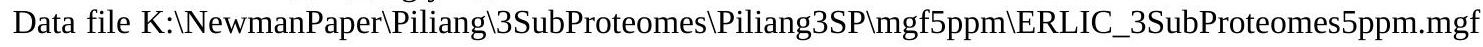
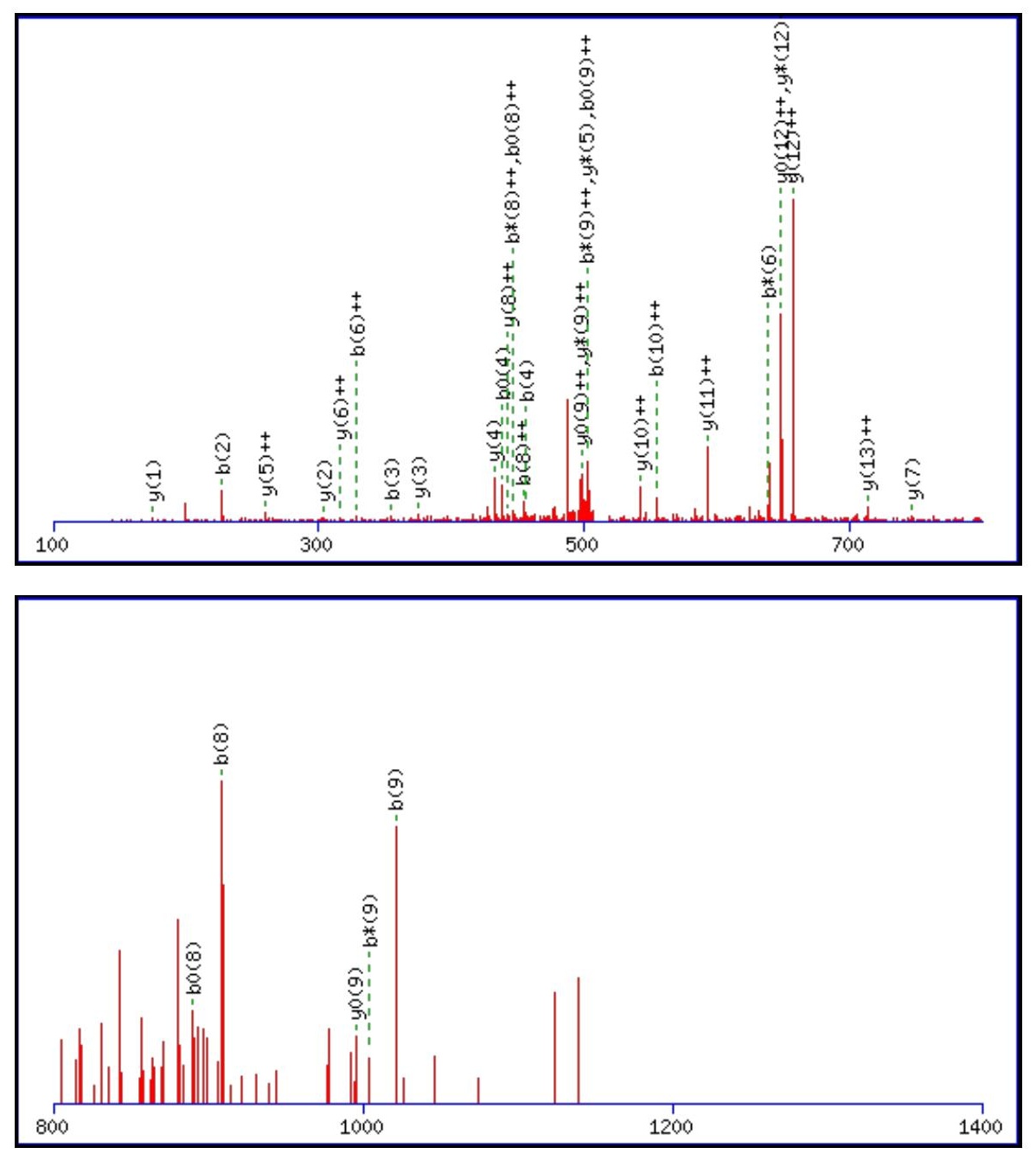
Monoisotopic mass of neutral peptide $\operatorname{Mr}($ calc): 1538.7688

Fixed modifications: Carbamidomethyl (C)

Variable modifications:

N8 : Deamidated_N (N)

Ions Score: 40 Expect: 0.016

Matches (Bold Red): 34/144 fragment ions using 68 most intense peaks

\begin{tabular}{|c|c|c|c|c|c|c|c|c|c|c|c|c|c|c|}
\hline \# & b & $\mathbf{b}^{++}$ & $\mathbf{b}^{*}$ & $\mathbf{b}^{*^{++}}$ & $\mathbf{b}^{0}$ & $\mathbf{b}^{\mathbf{0 + +}}$ & Seq. & $\mathbf{y}$ & $\mathbf{y}^{++}$ & $\mathbf{y}^{*}$ & $\mathrm{y}^{*^{++}}$ & $\mathbf{y}^{0}$ & $\mathbf{y}^{0++}$ & \# \\
\hline 1 & 114.0913 & 57.5493 & & & & & $\mathbf{L}$ & & & & & & & 14 \\
\hline 2 & 227.1754 & 114.0913 & & & & & $\mathbf{L}$ & 1426.6921 & 713.8497 & 1409.6655 & 705.3364 & 1408.6815 & 704.8444 & 13 \\
\hline 3 & 355.2340 & 178.1206 & 338.2074 & 169.6074 & & & $\mathbf{Q}$ & 1313.6080 & 657.3077 & 1296.5815 & 648.7944 & 1295.5975 & 648.3024 & 12 \\
\hline 4 & 456.2817 & 228.6445 & 439.2551 & 220.1312 & 438.2711 & 219.6392 & $\mathbf{T}$ & 1185.5494 & 593.2784 & 1168.5229 & 584.7651 & 1167.5389 & 584.2731 & 11 \\
\hline 5 & 527.3188 & 264.1630 & 510.2922 & 255.6498 & 509.3082 & 255.1577 & A & 1084.5018 & 542.7545 & 1067.4752 & 534.2412 & 1066.4912 & 533.7492 & 10 \\
\hline 6 & 656.3614 & 328.6843 & 639.3348 & 320.1710 & 638.3508 & 319.6790 & $\mathbf{E}$ & 1013.4647 & 507.2360 & 996.4381 & 498.7227 & 995.4541 & 498.2307 & 9 \\
\hline 7 & 793.4203 & 397.2138 & 776.3937 & 388.7005 & 775.4097 & 388.2085 & $\mathbf{H}$ & 884.4221 & 442.7147 & 867.3955 & 434.2014 & 866.4115 & 433.7094 & 8 \\
\hline 8 & 908.4472 & 454.7272 & 891.4207 & 446.2140 & 890.4367 & 445.7220 & $\mathbf{N}$ & 747.3632 & 374.1852 & 730.3366 & 365.6719 & 729.3526 & 365.1799 & 7 \\
\hline 9 & 1021.5313 & 511.2693 & 1004.5047 & 502.7560 & 1003.5207 & 502.2640 & I & 632.3362 & 316.6717 & 615.3097 & 308.1585 & 614.3256 & 307.6665 & 6 \\
\hline 10 & 1108.5633 & 554.7853 & 1091.5368 & 546.2720 & 1090.5527 & 545.7800 & S & 519.2522 & 260.1297 & 502.2256 & 251.6164 & 501.2416 & 251.1244 & 5 \\
\hline 11 & 1165.5848 & 583.2960 & 1148.5582 & 574.7827 & 1147.5742 & 574.2907 & G & 432.2201 & 216.6137 & 415.1936 & 208.1004 & 414.2096 & 207.6084 & 4 \\
\hline 12 & 1236.6219 & 618.8146 & 1219.5953 & 610.3013 & 1218.6113 & 609.8093 & A & 375.1987 & 188.1030 & 358.1721 & 179.5897 & 357.1881 & 179.0977 & 3 \\
\hline 13 & 1365.6645 & 683.3359 & 1348.6379 & 674.8226 & 1347.6539 & 674.3306 & $\mathbf{E}$ & 304.1615 & 152.5844 & 287.1350 & 144.0711 & 286.1510 & 143.5791 & 2 \\
\hline 14 & & & & & & & $\mathbf{R}$ & 175.1190 & 88.0631 & 158.0924 & 79.5498 & & & 1 \\
\hline
\end{tabular}

兽 RMS error 383 ppm

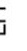

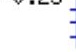

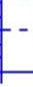

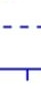

$-\cdot-\cdot$

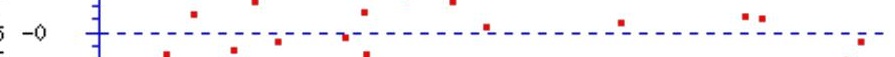<smiles>C1[C@H]2C[C@@H]1[C@@H]1C[C@H]2C1</smiles>

$$
\text { . }
$$

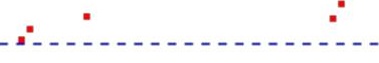

750

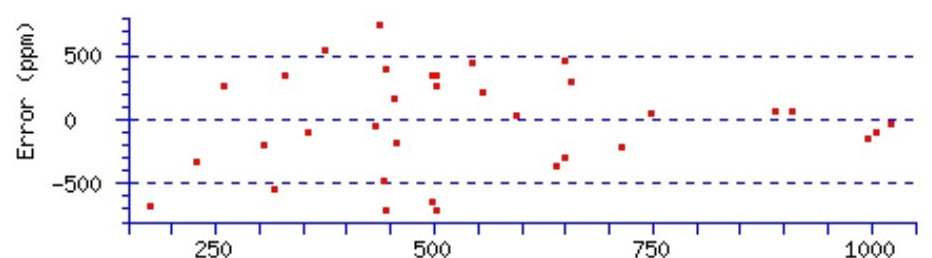

RMS error 383 ppm

\section{All matches to this query}

\begin{tabular}{|l|c|c|c|}
\hline Score & Mr(calc): & Delta & Sequence \\
\hline 40.0 & 1538.7688 & 0.0022 & LLQTAEHNISGAER \\
\hline 8.2 & 1537.7613 & 1.0097 & QLARNTVTQALSR \\
\hline 6.3 & 1537.7613 & 1.0097 & QLARNTVTQALSR \\
\hline 5.9 & 1538.7858 & -0.0147 & QQVLPLDSIYRK \\
\hline 4.9 & 1536.7548 & 2.0162 & KELNALIGLAGDSR \\
\hline 4.8 & 1537.7810 & 0.9901 & KLEMATNTTKSWK \\
\hline 3.8 & 1538.7858 & -0.0147 & VAPDEKYVAAKIR \\
\hline & & & \\
\hline
\end{tabular}




\begin{tabular}{|l|l|l|l|}
3.0 & 1538.7606 & 0.0104 & RPSPFKSPLTSSR \\
\hline 2.6 & 1538.7606 & 0.0104 & RPSPFKSPLTSSR \\
\hline 2.4 & 1537.7793 & 0.9918 & VVVNFAPTIQEIK \\
\hline
\end{tabular}

Spectrum No: 839; Query: 120; Rank: 1

\section{Peptide View}

\section{MS/MS Fragmentation of VVAVSPANISR}

Found in IPI00388914, Tax_Id=10116 Gene_Symbol=Plxnb1_predicted plexin B1

Match to Query 120: 1112.620768 from(557.317660,2+)

Title: 100101RatKid_NS_deglyco_21.1649.1649.2.dta

Data file K:INewmanPaper|Piliang|3SubProteomes\Piliang3SP\mgf5ppm\ERLIC_3SubProteomes5ppm.mgf
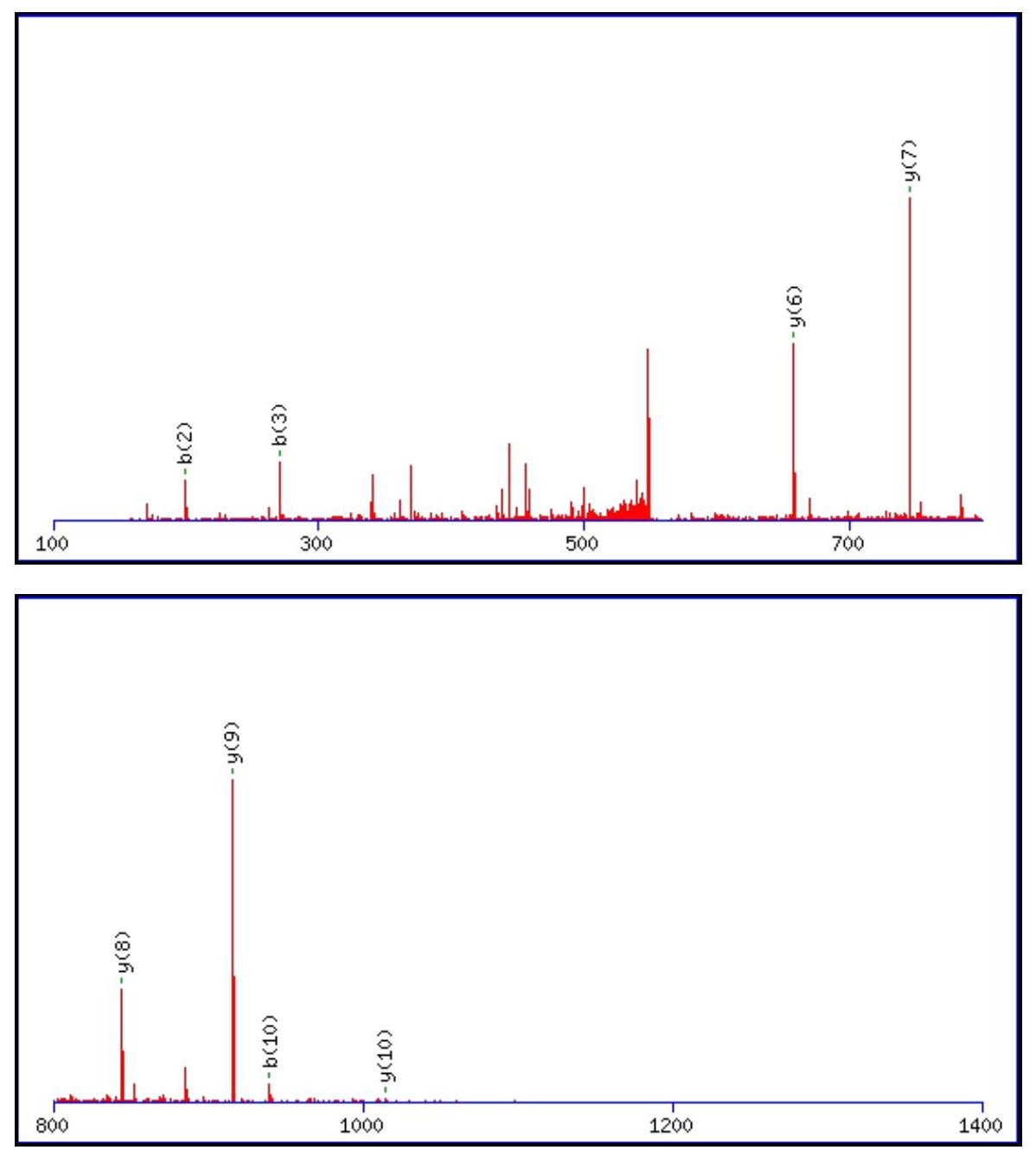


$1400 \quad 1600 \quad 1800 \quad 2000$

Monoisotopic mass of neutral peptide $\operatorname{Mr}($ calc): 1112.6190

Fixed modifications: Carbamidomethyl (C)

Variable modifications:

N8 : Deamidated_N (N)

Ions Score: 40 Expect: 0.0055

Matches (Bold Red): 8/96 fragment ions using 15 most intense peaks

\begin{tabular}{|r|c|c|c|c|c|c|c|c|c|c|c|c|c|c|}
\hline$\#$ & $\mathbf{b}$ & $\mathbf{b}^{++}$ & $\mathbf{b}^{*}$ & $\mathbf{b}^{\mathbf{*}^{++}}$ & $\mathbf{b}^{\mathbf{0}}$ & $\mathbf{b}^{\mathbf{0 + +}}$ & $\mathbf{S e q}$. & $\mathbf{y}$ & $\mathbf{y}^{++}$ & $\mathbf{y}^{\mathbf{*}}$ & $\mathbf{y}^{\mathbf{*}^{++}}$ & $\mathbf{y}^{\mathbf{0}}$ & $\mathbf{y}^{\mathbf{0 + +}}$ & $\#$ \\
\hline $\mathbf{1}$ & 100.0757 & 50.5415 & & & & & $\mathbf{V}$ & & & & & & & $\mathbf{1 1}$ \\
\hline $\mathbf{2}$ & $\mathbf{1 9 9 . 1 4 4 1}$ & 100.0757 & & & & & $\mathbf{V}$ & $\mathbf{1 0 1 4 . 5 5 7 8}$ & 507.7826 & 997.5313 & 499.2693 & 996.5473 & 498.7773 & $\mathbf{1 0}$ \\
\hline $\mathbf{3}$ & $\mathbf{2 7 0 . 1 8 1 2}$ & 135.5942 & & & & & $\mathbf{A}$ & $\mathbf{9 1 5 . 4 8 9 4}$ & 458.2483 & 898.4629 & 449.7351 & 897.4788 & 449.2431 & $\mathbf{9}$ \\
\hline $\mathbf{4}$ & 369.2496 & 185.1285 & & & & & $\mathbf{V}$ & $\mathbf{8 4 4 . 4 5 2 3}$ & 422.7298 & 827.4258 & 414.2165 & 826.4417 & 413.7245 & $\mathbf{8}$ \\
\hline $\mathbf{5}$ & 456.2817 & 228.6445 & & & 438.2711 & 219.6392 & $\mathbf{S}$ & $\mathbf{7 4 5 . 3 8 3 9}$ & 373.1956 & 728.3573 & 364.6823 & 727.3733 & 364.1903 & $\mathbf{7}$ \\
\hline $\mathbf{6}$ & 553.3344 & 277.1709 & & & 535.3239 & 268.1656 & $\mathbf{P}$ & $\mathbf{6 5 8 . 3 5 1 9}$ & 329.6796 & 641.3253 & 321.1663 & 640.3413 & 320.6743 & $\mathbf{6}$ \\
\hline $\mathbf{7}$ & 624.3715 & 312.6894 & & & 606.3610 & 303.6841 & $\mathbf{A}$ & 561.2991 & 281.1532 & 544.2725 & 272.6399 & 543.2885 & 272.1479 & $\mathbf{5}$ \\
\hline $\mathbf{8}$ & 739.3985 & 370.2029 & 722.3719 & 361.6896 & 721.3879 & 361.1976 & $\mathbf{N}$ & 490.2620 & 245.6346 & 473.2354 & 237.1214 & 472.2514 & 236.6293 & $\mathbf{4}$ \\
\hline $\mathbf{9}$ & 852.4825 & 426.7449 & 835.4560 & 418.2316 & 834.4720 & 417.7396 & $\mathbf{I}$ & 375.2350 & 188.1212 & 358.2085 & 179.6079 & 357.2245 & 179.1159 & 3 \\
\hline $\mathbf{1 0}$ & $\mathbf{9 3 9 . 5 1 4 6}$ & 470.2609 & 922.4880 & 461.7476 & 921.5040 & 461.2556 & $\mathbf{S}$ & 262.1510 & 131.5791 & 245.1244 & 123.0659 & 244.1404 & 122.5738 & $\mathbf{2}$ \\
\hline $\mathbf{1 1}$ & & & & & & & $\mathbf{R}$ & 175.1190 & 88.0631 & 158.0924 & 79.5498 & & & $\mathbf{1}$ \\
\hline
\end{tabular}
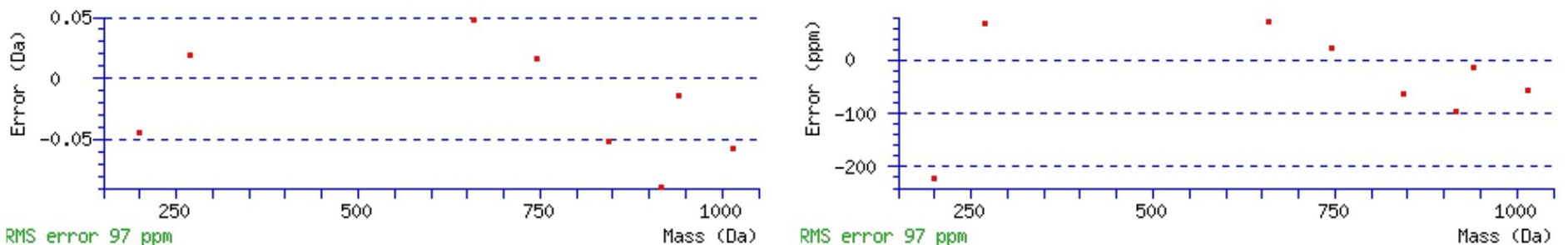

All matches to this query

\begin{tabular}{|l|l|l|l|}
\hline Score & Mr(calc): & Delta & \multicolumn{1}{|c|}{ Sequence } \\
\hline 40.0 & 1112.6190 & 0.0018 & VVAVSPANISR \\
\hline 4.8 & 1112.6189 & 0.0018 & NIAKDLVSPR \\
\hline 3.7 & 1112.6124 & 0.0083 & MTGLRVPPSR \\
\hline 3.1 & 1112.6190 & 0.0018 & LVGDATVSPVR \\
\hline 2.4 & 1112.6302 & -0.0094 & DLQIEALRR \\
\hline 2.2 & 1112.6190 & 0.0018 & LLNPTTADLR \\
\hline 1.1 & 1111.6138 & 1.0070 & HLYKLEPGR \\
\hline 0.2 & 1112.6302 & -0.0094 & RLVLDENVR \\
\hline
\end{tabular}




\section{Peptide View}

MS/MS Fragmentation of VSFWFVVTDPLKNHTLPAAEVQSAIR

Found in IPI00191929, Tax_Id=10116 Gene_Symbol=Tmem27 Collectrin precursor

Match to Query 2500: 2926.543842 from(976.521890,3+)

Title: 100101RatKid_NS_deglyco_14.5733.5733.3.dta

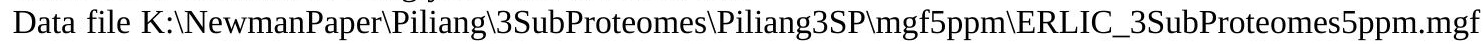
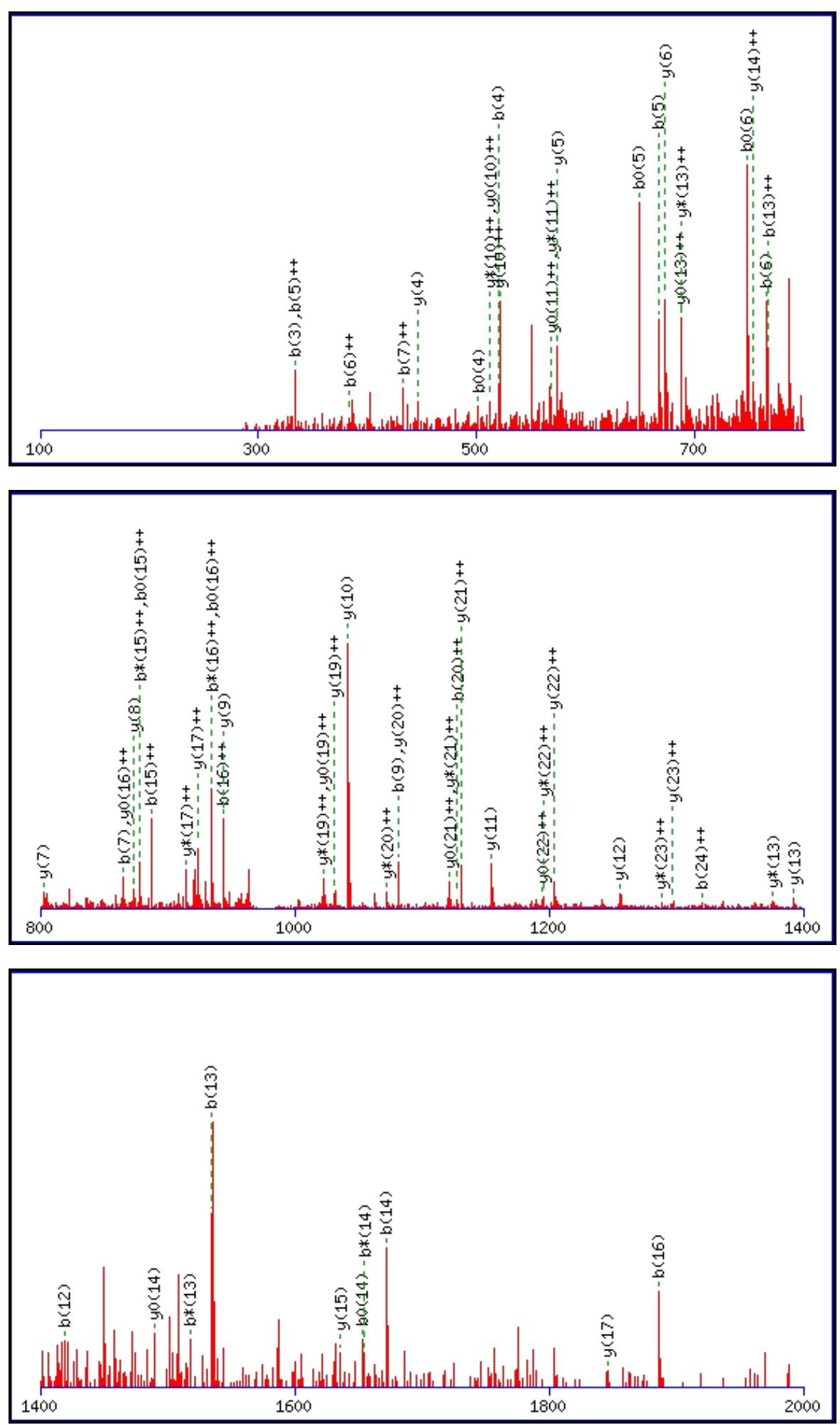

Monoisotopic mass of neutral peptide $\operatorname{Mr}($ calc): 2925.5389

Fixed modifications: Carbamidomethyl (C)

Variable modifications: 


\begin{tabular}{|c|c|c|c|c|c|c|c|c|c|c|c|c|c|c|}
\hline \# & b & $\mathbf{b}^{++}$ & b* & & $\mathbf{b}^{\mathbf{0}}$ & & Seq. & $\mathbf{y}$ & $\mathbf{y}^{++}$ & $\mathbf{y}^{*}$ & $\mathrm{y}^{*^{++}}$ & $\mathbf{y}^{0}$ & $\mathbf{y}^{0++}$ & \# \\
\hline 1 & 100.0757 & 50.5415 & & & & & $\mathbf{V}$ & & & & & & & 26 \\
\hline 2 & 187.1077 & 94.0575 & & & 169.0972 & & $\mathrm{~S}$ & 2827.4777 & & 2810.4512 & 1405.7292 & 2809.4672 & 1405.2372 & 25 \\
\hline 3 & 334.1761 & 167.5917 & & & 316.1656 & 158.5864 & $\mathbf{F}$ & 2740.4457 & 1370.7265 & 2723.4192 & 1362.2132 & 2722.4351 & 1361.7212 & 24 \\
\hline 4 & 520.2554 & 260.6314 & & & 502.2449 & 251.6261 & W & 2593.3773 & 1297.1923 & 2576.3507 & |1288.6790 & 2575.3667 & 1288.1870 & 23 \\
\hline 5 & 667.3239 & 334.1656 & & & 649.3133 & 325.1603 & $\mathbf{F}$ & 2407.2980 & 1204.1526 & 2390. & 1195.6394 & 2389.2874 & 1195.1473 & 22 \\
\hline 6 & 766.3923 & 383.6998 & & & 748.3817 & 374.6945 & $\mathbf{V}$ & 2260.2296 & 1130.6184 & 2243.2030 & 1122.1051 & 2242.2190 & 1121.6131 & 21 \\
\hline 7 & 865.4607 & 433.2340 & & & 847.4501 & 424.2287 & V & 2161.1612 & 1081.0842 & 2144.1346 & 1072.5709 & 2143.1506 & 1072.0789 & 20 \\
\hline 8 & 966.5084 & 483.7578 & & & 948.4978 & 474.7525 & $\mathbf{T}$ & 2062.0927 & 1031.5500 & 2045.0662 & 1023.0367 & 2044.0822 & 1022.5447 & 19 \\
\hline 9 & 1081.5353 & 541.2713 & & & 1063.5247 & 532.2660 & D & 1961.0451 & 981.0262 & 1944.0185 & 972.5129 & 1943.0345 & 972.0209 & 18 \\
\hline 10 & 1178.5881 & 589.7977 & & & 1160.5775 & 580.7924 & $\mathbf{P}$ & 1846.0181 & 923.5127 & 1828.9916 & 914.9994 & 1828.0076 & 914.5074 & 17 \\
\hline 11 & 1291.6721 & 646.3397 & & & 1273.6616 & 637.3344 & $\mathbf{L}$ & 1748.9654 & 874.9863 & 1731.9388 & 866.4730 & 1730.9548 & 865.9810 & 16 \\
\hline 12 & 1419.7671 & 710.3872 & 1402.7406 & 701.8739 & 1401.7565 & 701.3819 & $\mathbf{K}$ & 1635.8813 & 818.4443 & 1618.8547 & 809.9310 & 1617.8707 & 809.4390 & 15 \\
\hline 13 & 1534.7940 & 767.9007 & 1517.7675 & 759.3874 & 1516.7835 & 758.8954 & $\mathbf{N}$ & 1507.7863 & 754.3968 & 1490.7598 & 745.8835 & 1489.7758 & 745.3915 & 14 \\
\hline 14 & 1671.8529 & 836.4301 & 1654.8264 & 827.9168 & 1653.8424 & 827.4248 & $\mathbf{H}$ & 1392.7594 & 696.8833 & 1375.7328 & 688.3701 & 1374.7488 & 687.8781 & 13 \\
\hline 15 & 1772.9006 & 886.9540 & 1755.8741 & 878.4407 & 1754.8901 & 877.9487 & $\mathbf{T}$ & 1255.7005 & 628.3539 & 1238.6739 & 619.8406 & 1237.6899 & 619.3486 & 12 \\
\hline 16 & 1885.9847 & 943.4960 & 1868.9581 & 934.9827 & 1867.9741 & 934.4907 & $\mathbf{L}$ & 1154.6528 & 577.8300 & 1137.6263 & 569.3168 & 1136.6422 & 568.8248 & 11 \\
\hline 17 & 1983.0375 & 992.0224 & 1966.0109 & 983.5091 & 1965.0269 & 983.0171 & $\mathbf{P}$ & 1041.5687 & 521.2880 & 1024.5422 & 512.7747 & 1023.5582 & 512.2827 & 10 \\
\hline 18 & 2054.0746 & 1027.5409 & 2037.0480 & 1019.0276 & 2036.0640 & 1018.5356 & A & 944.5160 & 472.7616 & 927.4894 & 464.2483 & 926.5054 & 463.7563 & 9 \\
\hline 19 & 2125.1117 & 1063.0595 & 2108.0851 & 1054.5462 & 2107.1011 & 1054.0542 & A & 873.4789 & 437.2431 & 856.4523 & 428.7298 & 855.4683 & 428.2378 & 8 \\
\hline 20 & 2254.1543 & 1127.5808 & 2237.1277 & 1119.0675 & 2236.1437 & 1118.5755 & $\bar{E}$ & 802.4417 & 401.7245 & 785.4152 & 393.2112 & 784.4312 & 392.7192 & 7 \\
\hline 21 & 2353.2227 & 1177.1150 & 2336.1961 & 1168.6017 & 2335.2121 & 1168.1097 & V & 673.3992 & 337.2032 & 656.3726 & 328.6899 & 655.3886 & 328.1979 & 6 \\
\hline 22 & 2481.2813 & 1241.1443 & 2464.2547 & 1232.6310 & 2463.2707 & 1232.1390 & $\mathbf{Q}$ & 574.3307 & 287.6690 & 557.3042 & 279.1557 & 556.3202 & 278.6637 & 5 \\
\hline 23 & 2568.3133 & 1284.6603 & 2551.2867 & 1276.1470 & 2550.3027 & 1275.6550 & $\mathrm{~S}$ & 446.2722 & 223.6397 & 429.2456 & 215.1264 & 428.2616 & 214.6344 & 4 \\
\hline 24 & 2639.3504 & 1320.1788 & 2622.3239 & 1311.6656 & 2621.3398 & 1311.1736 & A & 359.2401 & 180.1237 & 342.2136 & 171.6104 & & & 3 \\
\hline 25 & 2752.4345 & 1376.7209 & 2735.4079 & 1368.2076 & 2734.4239 & 1367.7156 & I & 288.2030 & 144.6051 & 271.1765 & 136.0919 & & & 2 \\
\hline 26 & & & & & & & $\mathbf{R}$ & 175.1190 & 88.0631 & 158.0924 & 79.5498 & & & 1 \\
\hline
\end{tabular}
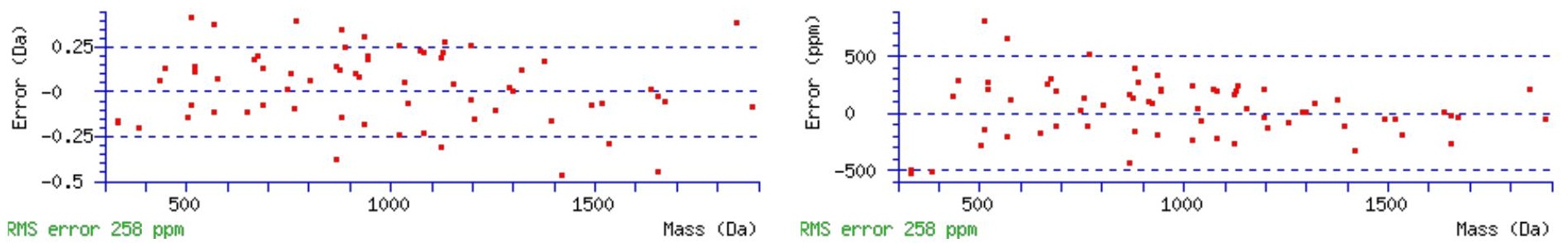

\section{All matches to this query}

\begin{tabular}{|l|l|l|l|}
\hline Score & Mr(calc): & Delta & \multicolumn{1}{|c|}{ Sequence } \\
\hline 40.0 & 2925.5389 & 1.0050 & VSFWFVVTDPLKNHTLPAAEVQSAIR \\
\hline 26.1 & 2924.5549 & 1.9890 & VSFWFVVTDPLKNHTLPAAEVQSAIR \\
\hline 3.3 & 2925.5304 & 1.0135 & XLLDMDTGVTEGGLNVTLTIRLLMQGK \\
\hline 1.6 & 2925.5607 & 0.9832 & KVISGQIVESGRPQYLDLRPAMAGGGAR \\
\hline
\end{tabular}

Spectrum No: 841; Query: 45; Rank: 1

\section{Peptide View}




\section{MS/MS Fragmentation of EQVTVINR}

Found in IPI00215135, Tax_Id=10116 Gene_Symbol=Il6st Ac1055

Match to Query 45: 959.508668 from(480.761610,2+)

Title: 091008RatKidney_NH4Format01_27.600.600.2.dta

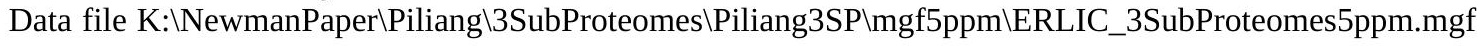
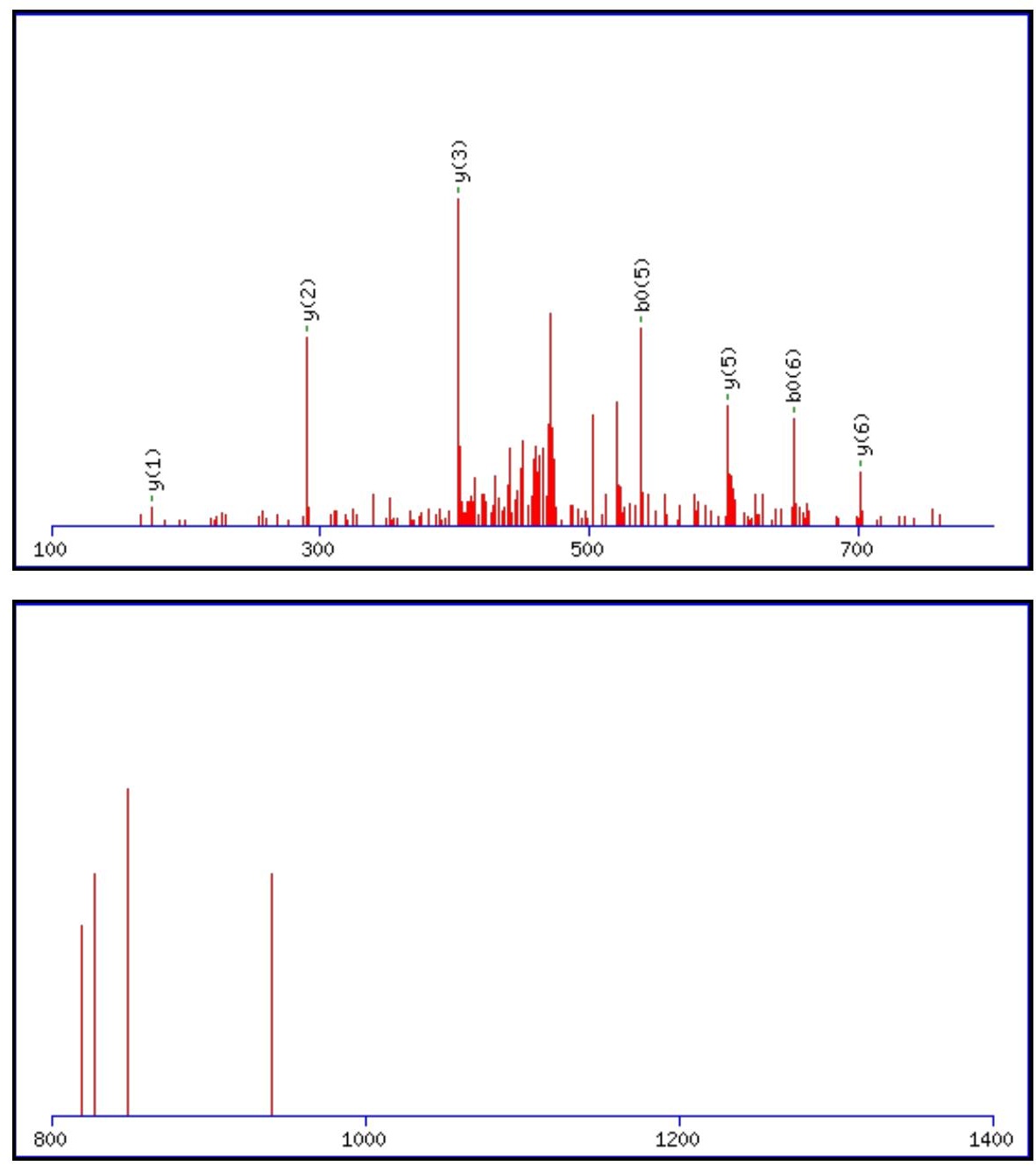

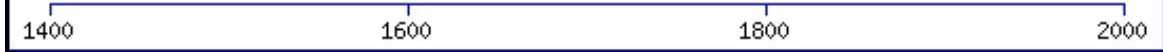

Monoisotopic mass of neutral peptide $\operatorname{Mr}($ calc): 958.5083

Fixed modifications: Carbamidomethyl (C)

Variable modifications:

N7 : Deamidated_N (N)

Ions Score: 40 Expect: 0.0092

Matches (Bold Red): 7/74 fragment ions using 17 most intense peaks

\begin{tabular}{|l|l|l|l|l|l|l|l|l|l|l|l|l|l|l|}
\hline$\#$ & $\mathbf{b}$ & $\mathbf{b}^{++}$ & $\mathbf{b}^{*}$ & $\mathbf{b}^{*++}$ & $\mathbf{b}^{\mathbf{0}}$ & $\mathbf{b}^{\mathbf{0 + +}}$ & $\mathbf{S e q} \cdot$ & $\mathbf{y}$ & $\mathbf{y}^{++}$ & $\mathbf{y}^{*}$ & $\mathbf{y}^{*^{++}}$ & $\mathbf{y}^{\mathbf{0}}$ & $\mathbf{y}^{\mathbf{0 + +}}$ & $\#$ \\
\hline
\end{tabular}


Mascot Search Results: Peptide View

\begin{tabular}{|l|l|l|l|l|l|l|l|l|l|l|l|l|l|l|}
$\mathbf{1}$ & 130.0499 & 65.5286 & & & 112.0393 & 56.5233 & $\mathbf{E}$ & & & & & & & $\mathbf{8}$ \\
\hline $\mathbf{2}$ & 258.1084 & 129.5579 & 241.0819 & 121.0446 & 240.0979 & 120.5526 & $\mathbf{Q}$ & 830.4730 & 415.7402 & 813.4465 & 407.2269 & 812.4625 & 406.7349 & $\mathbf{7}$ \\
\hline $\mathbf{3}$ & 357.1769 & 179.0921 & 340.1503 & 170.5788 & 339.1663 & 170.0868 & $\mathbf{V}$ & $\mathbf{7 0 2 . 4 1 4 5}$ & 351.7109 & 685.3879 & 343.1976 & 684.4039 & 342.7056 & $\mathbf{6}$ \\
\hline $\mathbf{4}$ & 458.2245 & 229.6159 & 441.1980 & 221.1026 & 440.2140 & 220.6106 & $\mathbf{T}$ & $\mathbf{6 0 3 . 3 4 6 0}$ & 302.1767 & 586.3195 & 293.6634 & 585.3355 & 293.1714 & $\mathbf{5}$ \\
\hline $\mathbf{5}$ & 557.2930 & 279.1501 & 540.2664 & 270.6368 & 539.2824 & 270.1448 & $\mathbf{V}$ & 502.2984 & 251.6528 & 485.2718 & 243.1395 & & & $\mathbf{4}$ \\
\hline $\mathbf{6}$ & 670.3770 & 335.6921 & 653.3505 & 327.1789 & $\mathbf{6 5 2 . 3 6 6 5}$ & 326.6869 & $\mathbf{I}$ & $\mathbf{4 0 3 . 2 3 0 0}$ & 202.1186 & 386.2034 & 193.6053 & & & $\mathbf{3}$ \\
\hline $\mathbf{7}$ & 785.4040 & 393.2056 & 768.3774 & 384.6923 & 767.3934 & 384.2003 & $\mathbf{N}$ & $\mathbf{2 9 0 . 1 4 5 9}$ & 145.5766 & 273.1193 & 137.0633 & & & $\mathbf{2}$ \\
\hline $\mathbf{8}$ & & & & & & & $\mathbf{R}$ & $\mathbf{1 7 5 . 1 1 9 0}$ & 88.0631 & 158.0924 & 79.5498 & & & $\mathbf{1}$ \\
\hline
\end{tabular}
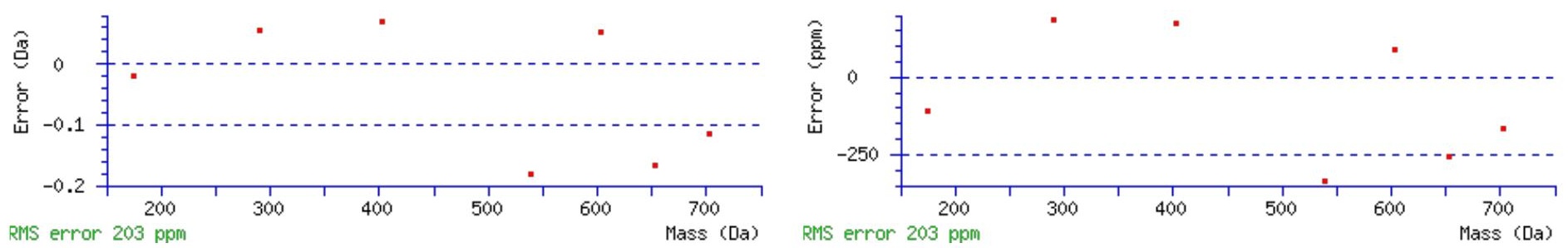

\section{All matches to this query}

\begin{tabular}{|l|l|l|l|}
\hline Score & Mr(calc): & Delta & \multicolumn{1}{|c|}{ Sequence } \\
\hline 39.9 & 958.5083 & 1.0003 & EQVTVINR \\
\hline 25.0 & 959.5148 & -0.0061 & $\underline{\text { NSRLSIDR }}$ \\
\hline 24.7 & 958.5083 & 1.0004 & EKLNAINR \\
\hline 15.0 & 959.5036 & 0.0051 & $\underline{\text { LQTQSIDR }}$ \\
\hline 12.5 & 959.5076 & 0.0011 & $\underline{\text { AFPELLDR }}$ \\
\hline 12.5 & 958.5083 & 1.0003 & $\underline{\text { ALAVSDLNR }}$ \\
\hline 12.5 & 958.5083 & 1.0004 & ENLAKLDR \\
\hline 12.5 & 958.5084 & 1.0003 & $\underline{\text { LVTDGAIDR }}$ \\
\hline 12.5 & 958.5083 & 1.0003 & $\underline{\text { NLSELIDR }}$ \\
\hline 12.5 & 958.5083 & 1.0003 & $\underline{\text { QLSLDINR }}$ \\
\hline
\end{tabular}

Spectrum No: 842; Query: 2022; Rank: 1

\section{Peptide View}

MS/MS Fragmentation of FNEVYDALANAHPNLTVYKK

Found in IPI00454558, Tax_Id=10116 Gene_Symbol=Enpp5 Ectonucleotide pyrophosphatase/phosphodiesterase family member 5 precursor

Match to Query 2022: 2307.152416 from(577.795380,4+)

Title: 091008RatKidney_NH4Format01_17.3510.3510.4.dta

Data file K:INewmanPaper|Piliang|3SubProteomes\Piliang3SP\mgf5ppm\ERLIC_3SubProteomes5ppm.mgf 

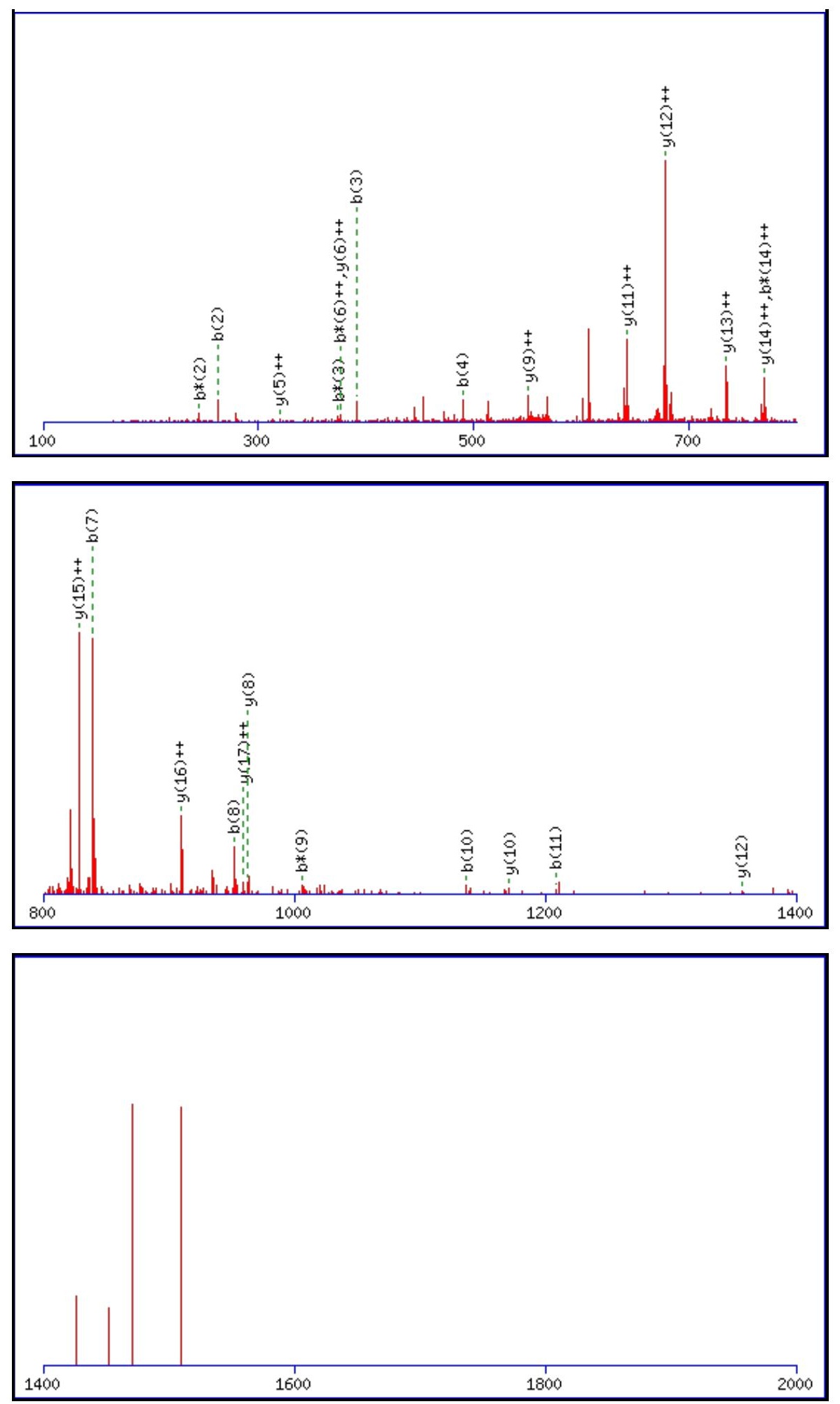

Monoisotopic mass of neutral peptide $\operatorname{Mr}($ calc): 2307.1535

Fixed modifications: Carbamidomethyl (C)

Variable modifications:

N14: Deamidated N (N)

Ions Score: 40 Expect: 0.023

Matches (Bold Red): 25/214 fragment ions using 52 most intense peaks

\begin{tabular}{|r|c|c|c|c|c|c|c|c|c|c|c|c|c|c|}
\hline$\#$ & $\mathbf{b}$ & $\mathbf{b}^{++}$ & $\mathbf{b}^{*}$ & $\mathbf{b}^{\boldsymbol{*}^{++}}$ & $\mathbf{b}^{\mathbf{0}}$ & $\mathbf{b}^{\mathbf{0 + +}}$ & $\mathbf{S e q}$ & $\mathbf{y}$ & $\mathbf{y}^{++}$ & $\mathbf{y}^{\mathbf{*}}$ & $\mathbf{y}^{\mathbf{*}^{++}}$ & $\mathbf{y}^{\mathbf{0}}$ & $\mathbf{y}^{\mathbf{0 + +}}$ & $\#$ \\
\hline $\mathbf{1}$ & 148.0757 & 74.5415 & & & & & $\mathbf{F}$ & & & & & & & $\mathbf{2 0}$ \\
\hline $\mathbf{2}$ & $\mathbf{2 6 2 . 1 1 8 6}$ & 131.5629 & $\mathbf{2 4 5 . 0 9 2 1}$ & 123.0497 & & & $\mathbf{N}$ & 2161.0924 & 1081.0498 & 2144.0659 & 1072.5366 & 2143.0818 & 1072.0446 & $\mathbf{1 9}$ \\
\hline $\mathbf{3}$ & $\mathbf{3 9 1 . 1 6 1 2}$ & 196.0842 & 374.1347 & 187.5710 & 373.1506 & 187.0790 & $\mathbf{E}$ & 2047.0495 & 1024.0284 & 2030.0229 & 1015.5151 & 2029.0389 & 1015.0231 & $\mathbf{1 8}$ \\
\hline $\mathbf{4}$ & $\mathbf{4 9 0 . 2 2 9 6}$ & 245.6185 & 473.2031 & 237.1052 & 472.2191 & 236.6132 & $\mathbf{V}$ & 1918.0069 & $\mathbf{9 5 9 . 5 0 7 1}$ & 1900.9803 & 950.9938 & 1899.9963 & 950.5018 & $\mathbf{1 7}$ \\
\hline $\mathbf{5}$ & 653.2930 & 327.1501 & 636.2664 & 318.6368 & 635.2824 & 318.1448 & $\mathbf{Y}$ & 1818.9385 & $\mathbf{9 0 9 . 9 7 2 9}$ & 1801.9119 & 901.4596 & 1800.9279 & 900.9676 & $\mathbf{1 6}$ \\
\hline
\end{tabular}




\begin{tabular}{|c|c|c|c|c|c|c|c|c|c|c|c|c|c|c|}
\hline 6 & 768.3199| & 384.6636 & 751.2933 & 376.1503 & 750.3093 & 375.6583 & D & |1655.8751 & 828.4412 & |1638.8486 & 819.9279 & |1637.8646 & 819.4359| & 15 \\
\hline 7 & 839.3570 & 420.1821 & 822.3305 & 411.6689 & 821.3464 & 411.1769 & A & 1540.8482 & 770.9277 & 1523.8216 & 762.4145 & 1522.8376 & 761.9225 & 14 \\
\hline 8 & 952.4411 & 476.7242 & 935.4145 & 468.2109 & 934.4305 & 467.7189 & $\mathbf{L}$ & 1469.8111 & 735.4092 & 1452.7845 & 726.8959 & 1451.8005 & 726.4039 & 3 \\
\hline 9 & 1023.4782 & 512.2427 & 1006.4516 & 503.7295 & 1005.4676 & 503.2374 & A & 1356.7270 & 678.8671 & 1339.7005 & 670.3539 & 1338.7165 & 669.8619 & 12 \\
\hline 10 & 1137.5211 & 569.2642 & 1120.4946 & 560.7509 & 1119.5106 & 560.2589 & $\mathbf{N}$ & 1285.6899 & 643.3486 & 1268.6634 & 634.8353 & 1267.6793 & 634.3433 & 11 \\
\hline 11 & 1208.5582 & 604.7828 & 1191.5317 & 596.2695 & 1190.5477 & 595.7775 & A & 1171.6470 & 586.3271 & 1154.6204 & 577.8139 & 1153.6364 & 577.3218 & 10 \\
\hline 12 & 1345.6171 & 673.3122 & 1328.5906 & 664.7989 & 1327.6066 & 664.3069 & $\mathbf{H}$ & 1100.6099 & 550.8086 & 1083.5833 & 542.2953 & 1082.5993 & 541.8033 & 9 \\
\hline 13 & 1442.6699 & 721.8386 & 1425.6434 & 713.3253 & 1424.6593 & 712.8333 & $\mathbf{P}$ & 963.5510 & 482.2791 & 946.5244 & 473.7658 & 945.5404 & 473.2738 & 8 \\
\hline 14 & 1557.6968 & 779.3521 & 1540.6703 & 770.8388 & 1539.6863 & 770.3468 & $\mathbf{N}$ & 866.4982 & 433.7527 & 849.4716 & 425.2395 & 848.4876 & 424.7475 & 7 \\
\hline 15 & 1670.7809 & 835.8941 & 1653.7544 & 827.3808 & 1652.7703 & 826.8888 & $\mathbf{L}$ & 751.4713 & 376.2393 & 734.4447 & 367.7260 & 733.4607 & 367.2340 & 6 \\
\hline 16 & 1771.8286 & 886.4179 & 1754.8020 & 877.9047 & 1753.8180 & 877.4126 & $\mathbf{T}$ & 638.3872 & 319.6972 & 621.3606 & 311.1840 & 620.3766 & 310.6920 & 5 \\
\hline 17 & 1870.8970 & 935.9521 & 1853.8704 & 927.4389 & 1852.8864 & 926.9469 & V & 537.3395 & 269.1734 & 520.3130 & 260.6601 & & & 4 \\
\hline 18 & 2033.9603 & 1017.4838 & 2016.9338 & 1008.9705 & 2015.9498 & 1008.4785 & $\mathbf{Y}$ & 438.2711 & 219.6392 & 421.2445 & 211.1259 & & & 3 \\
\hline 19 & 2162.0553 & 1081.5313 & 2145.0287 & 1073.0180 & 2144.0447 & 1072.5260 & $\mathbf{K}$ & 275.2078 & 138.1075 & 258.1812 & 129.5942 & & & 2 \\
\hline
\end{tabular}

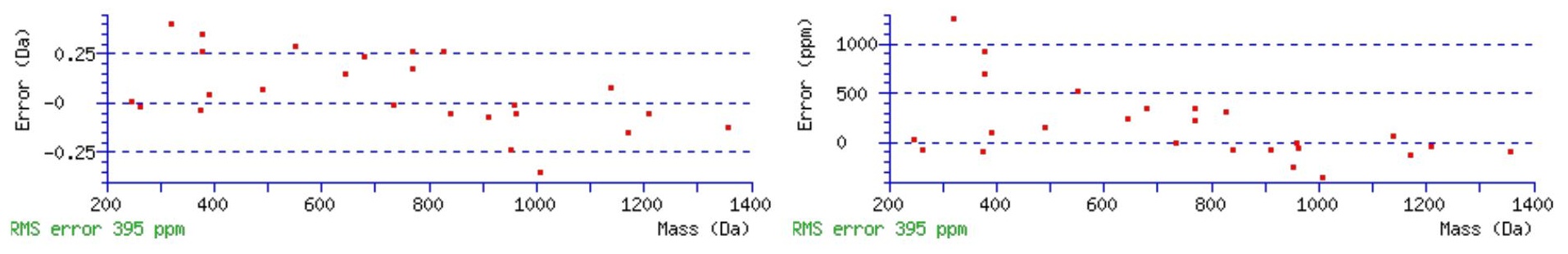

\section{All matches to this query}

\begin{tabular}{|l|l|l|l|}
\hline Score & Mr(calc): & Delta & \multicolumn{1}{c|}{ Sequence } \\
\hline 39.8 & 2307.1535 & -0.0011 & FNEVYDALANAHPNLTVYKK \\
\hline 22.8 & 2307.1535 & -0.0011 & FNEVYDALANAHPNLTVYKK \\
\hline 13.6 & 2306.1695 & 0.9829 & FNEVYDALANAHPNLTVYKK \\
\hline 7.2 & 2307.1575 & -0.0051 & MEYCLAAAALNAVDRRSLQR \\
\hline 5.1 & 2306.1337 & 1.0187 & TRPDATALPYHVALAKLGPR \\
\hline 3.5 & 2307.1535 & -0.0011 & FNEVYDALANAHPNLTVYKK \\
\hline 3.0 & 2307.1624 & -0.0100 & FPGIVTREKLAQETGIPESR \\
\hline 1.9 & 2307.1624 & -0.0100 & YVSKEQNISINVSHPKSIGK \\
\hline 1.5 & 2305.1328 & 2.0196 & YIHRDLATRNILVENENR \\
\hline
\end{tabular}

Spectrum No: 843; Query: 150; Rank: 1

\section{Peptide View}

MS/MS Fragmentation of GYNFTTTAER

Found in IPI00360356, Tax_Id=10116 Gene_Symbol=RGD1309504_predicted hypothetical protein LOC294732

Match to Query 150: 1159.514988 from(580.764770,2+)

Title: 091008RatKidney_NH4Format02_17.1609.1609.2.dta

Data file K:\NewmanPaper\Piliang \3SubProteomes\Piliang3SP \mgf5ppm\ERLIC_3SubProteomes5ppm.mgf 

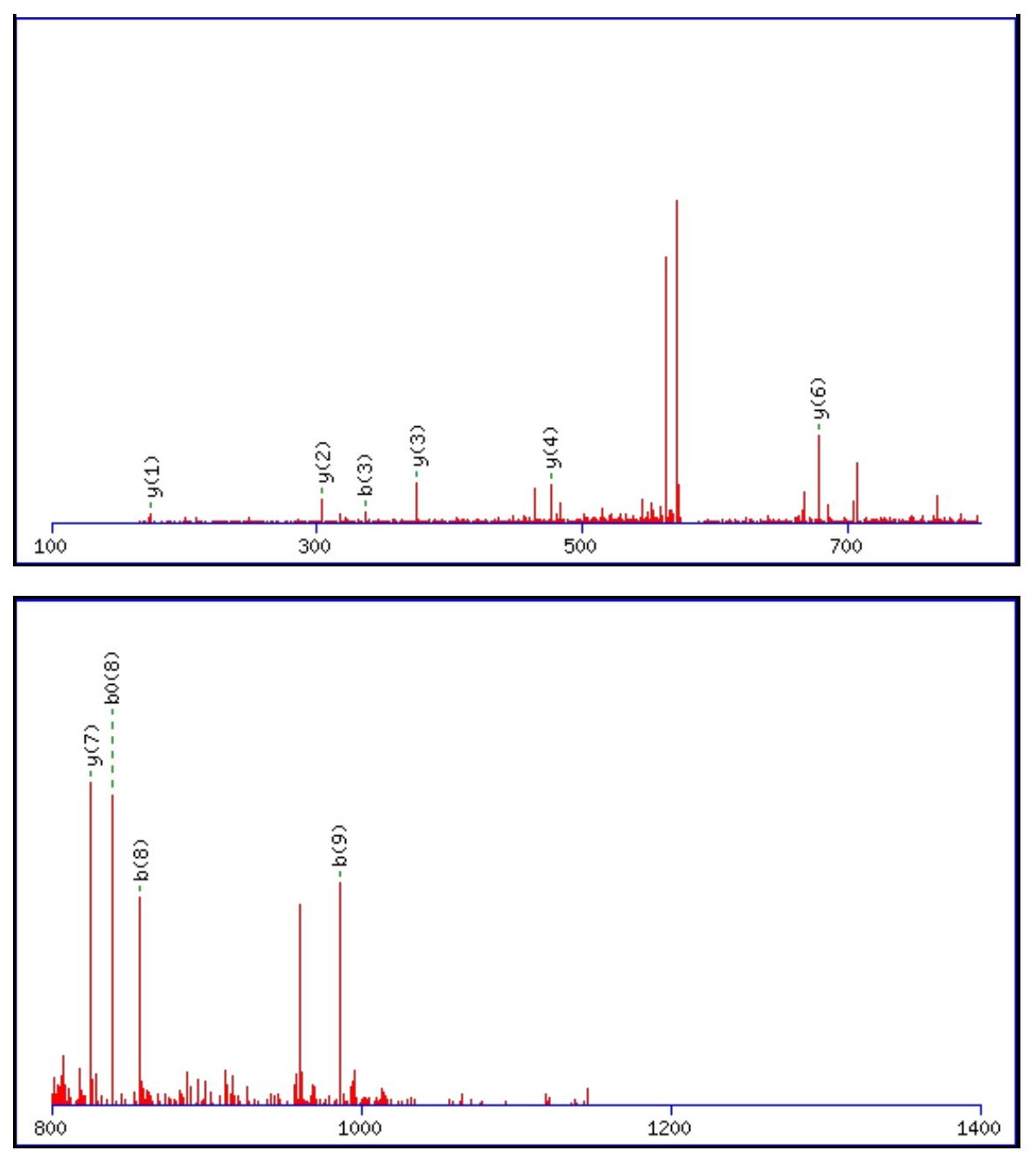

\begin{tabular}{rrrr}
\hline 1600 & 1600 & 1800 & 2000 \\
\hline
\end{tabular}

Monoisotopic mass of neutral peptide $\operatorname{Mr}($ calc): 1159.5146

Fixed modifications: Carbamidomethyl (C)

Variable modifications:

N3 : Deamidated N (N)

Ions Score: 40 Expect: $\odot .011$

Matches (Bold Red): 10/94 fragment ions using 24 most intense peaks

\begin{tabular}{|c|c|c|c|c|c|c|c|c|c|c|c|c|c|c|}
\hline$\#$ & $\mathbf{b}$ & $\mathbf{b}^{++}$ & $\mathbf{b}^{*}$ & $\mathbf{b}^{\boldsymbol{*}^{++}}$ & $\mathbf{b}^{\mathbf{0}}$ & $\mathbf{b}^{\mathbf{0}++}$ & $\mathbf{S e q} \cdot$ & $\mathbf{y}$ & $\mathbf{y}^{++}$ & $\mathbf{y}^{*}$ & $\mathbf{y}^{\boldsymbol{*}^{++}}$ & $\mathbf{y}^{\mathbf{0}}$ & $\mathbf{y}^{\mathbf{0}++}$ & $\#$ \\
\hline $\mathbf{1}$ & 58.0287 & 29.5180 & & & & & $\mathbf{G}$ & & & & & & & $\mathbf{1 0}$ \\
\hline $\mathbf{2}$ & 221.0921 & 111.0497 & & & & & $\mathbf{Y}$ & 1103.5004 & 552.2538 & 1086.4738 & 543.7406 & 1085.4898 & 543.2485 & $\mathbf{9}$ \\
\hline 3 & 336.1190 & 168.5631 & 319.0925 & 160.0499 & & & $\mathbf{N}$ & 940.4370 & 470.7222 & 923.4105 & 462.2089 & 922.4265 & 461.7169 & $\mathbf{8}$ \\
\hline $\mathbf{4}$ & 483.1874 & 242.0973 & 466.1609 & 233.5841 & & & $\mathbf{F}$ & $\mathbf{8 2 5 . 4 1 0 1}$ & 413.2087 & 808.3836 & 404.6954 & 807.3995 & 404.2034 & $\mathbf{7}$ \\
\hline $\mathbf{5}$ & 584.2351 & 292.6212 & 567.2085 & 284.1079 & 566.2245 & 283.6159 & $\mathbf{T}$ & $\mathbf{6 7 8 . 3 4 1 7}$ & 339.6745 & 661.3151 & 331.1612 & 660.3311 & 330.6692 & $\mathbf{6}$ \\
\hline
\end{tabular}




\begin{tabular}{|c|c|c|c|c|c|c|c|c|c|c|c|c|c|c|}
\hline 6 & 685.2828 & | 343.1450 & |668.2562| & |334.6318 & 667.2722 & |334.1397| & $\mathbf{T}$ & 577.2940 & |289.1506 | & 560.2675 & 280.6374 & 559.2835 & |280.1454 & 5 \\
\hline 7 & 786.3305 & 393.6689 & 769.3039 & 385.1556 & 768.3199 & 384.6636 & $\mathbf{T}$ & 476.2463 & 238.6268 & 459.2198 & 230.1135 & 458.2358 & 229.6215 & 4 \\
\hline 8 & 857.3676 & 429.1874 & 840.3410 & 420.6741 & 839.3570 & 420.1821 & A & 375.1987 & 188.1030 & 358.1721 & 179.5897 & 357.1881 & 179.0977 & 3 \\
\hline 9 & 986.4102 & 493.7087 & 969.3836 & 485.1954 & 968.3996 & 484.7034 & $\mathbf{E}$ & 304.1615 & 152.5844 & 287.1350 & 144.0711 & 286.1510 & 143.5791 & 2 \\
\hline 10 & & & & & & & $\mathbf{R}$ & 175.1190 & 88.0631 & 158.0924 & 79.5498 & & & 1 \\
\hline
\end{tabular}
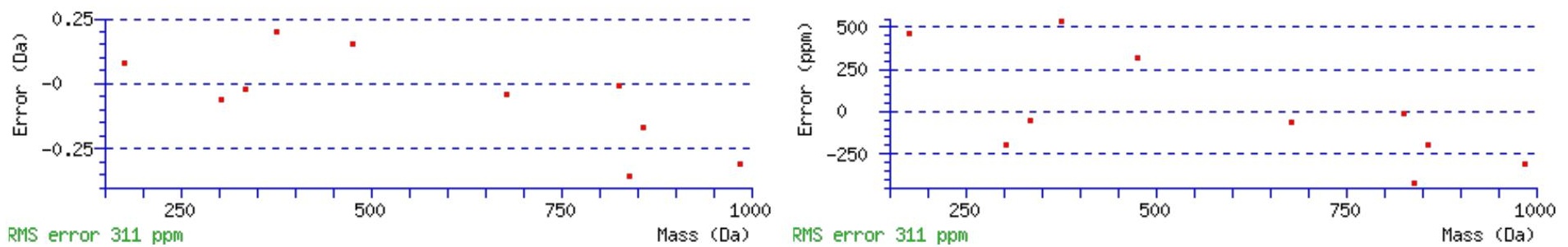

\section{All matches to this query}

\begin{tabular}{|l|l|l|l|}
\hline Score & Mr(calc): & Delta & \multicolumn{1}{c|}{ Sequence } \\
\hline 39.8 & 1159.5146 & 0.0004 & GYNFTTTAER \\
\hline 16.3 & 1157.5118 & 2.0032 & EFAAALTAER \\
\hline 11.3 & 1159.5209 & -0.0059 & $\underline{\text { XRGPITSFR }}$ \\
\hline 11.3 & 1159.5209 & -0.0059 & $\underline{\text { XRGPITSFR }}$ \\
\hline 10.7 & 1159.5105 & 0.0044 & DGDSNPTLGER \\
\hline 9.7 & 1157.5175 & 1.9974 & $\underline{\text { FGEMQLDFR }}$ \\
\hline 9.1 & 1159.5056 & 0.0093 & LSETMSRTR \\
\hline 9.1 & 1159.5056 & 0.0093 & LSETMSRTR \\
\hline 9.0 & 1157.5101 & 2.0048 & NGPWEVGGGER \\
\hline 7.5 & 1158.5104 & 1.0046 & TVTGSGMAVTR \\
\hline
\end{tabular}

Spectrum No: 844; Query: 2593; Rank: 1

\section{Peptide View}

MS/MS Fragmentation of EAMNSTDLYSLVCHLLNVTALPHNGSFR

Found in IPI00454558, Tax_Id=10116 Gene_Symbol=Enpp5 Ectonucleotide pyrophosphatase/phosphodiesterase family member 5 precursor

Match to Query 2593: 3162.483822 from(1055.168550,3+)

Title: 100101RatKid_NS_deglyco_13.7441.7441.3.dta

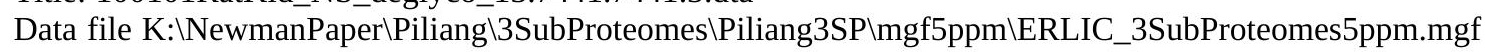

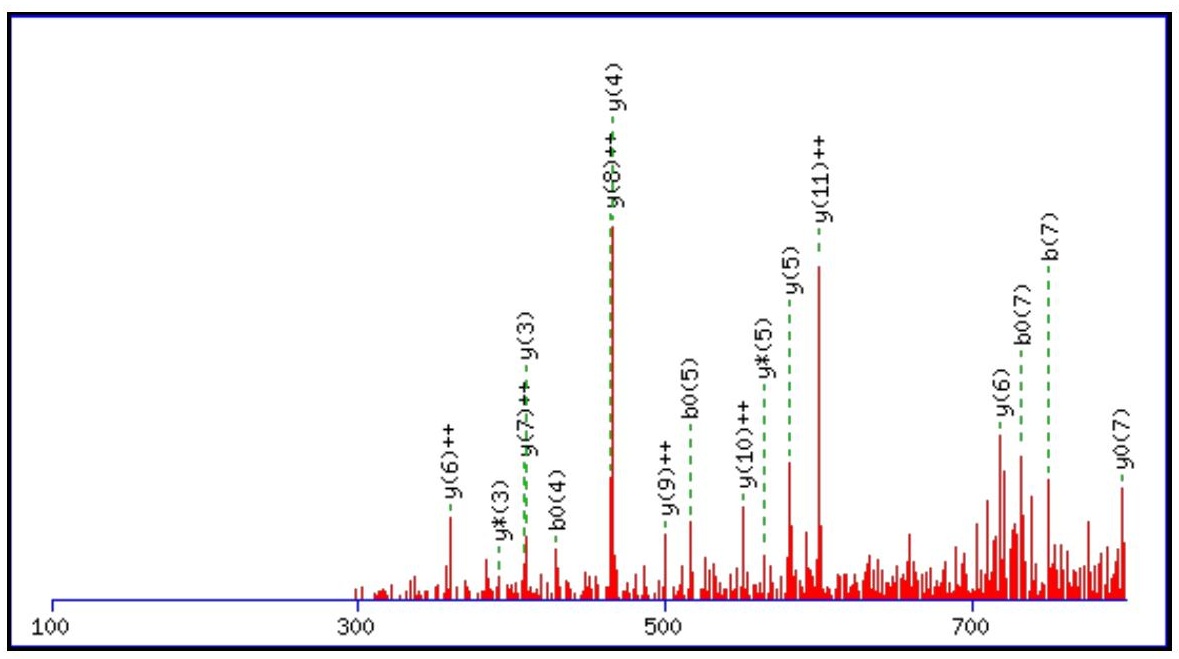



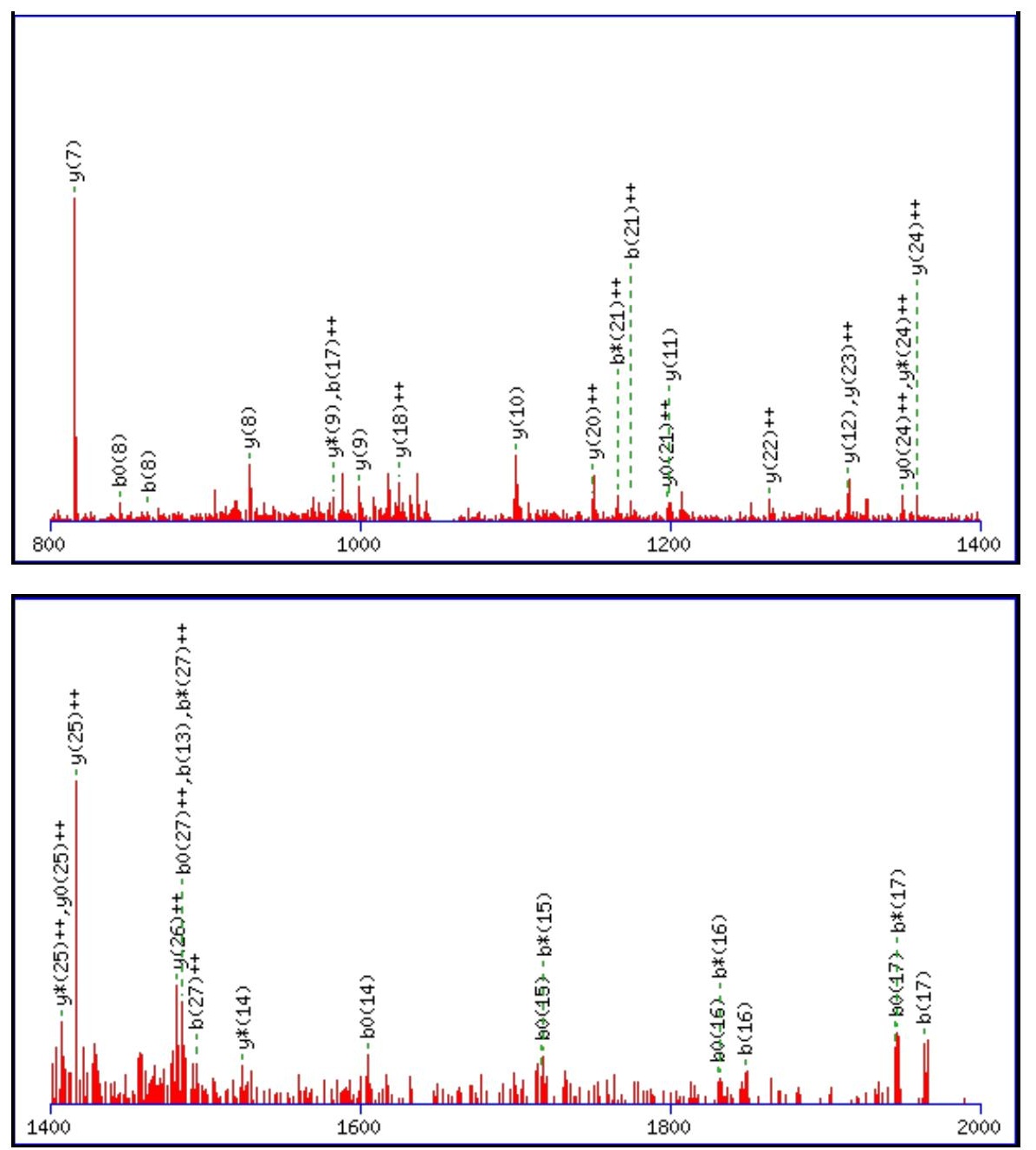

Monoisotopic mass of neutral peptide $\operatorname{Mr}($ calc): 3161.4798

Fixed modifications: Carbamidomethyl (C)

Variable modifications:

N4 : Deamidated_N (N)

N17 : Deamidated_N (N)

N24: Deamidated_N $(\mathrm{N})$

Ions Score: 40 Expect: 0.042

Matches (Bold Red): 55/314 fragment ions using 127 most intense peaks

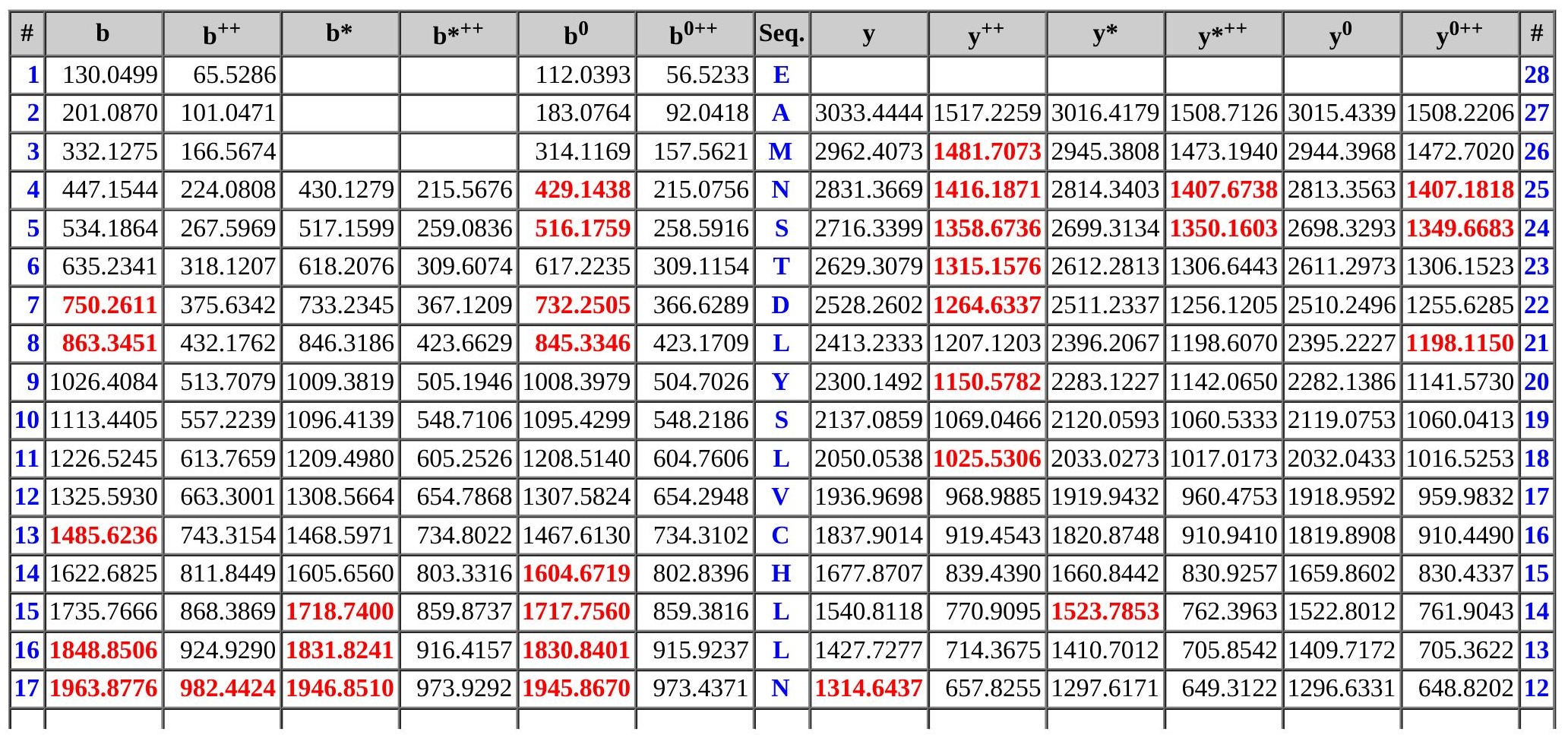




\begin{tabular}{|c|c|c|c|c|c|c|c|c|c|c|c|c|c|c|}
\hline 18 & |2062.9460| & |1031.9766 & |2045.9194 & |1023.4634 & |2044.9354 & |1022.9714 & V & 1199.6167 & 600.3120 & |1182.5902 & 591.7987 & |1181.6062| & 591.3067 & $|11|$ \\
\hline 19 & 2163.9937 & 1082.5005 & 2146.9671 & 1073.9872 & 2145.9831 & 1073.4952 & $\mathbf{T}$ & 1100.5483 & 550.7778 & 1083.5218 & 542.2645 & 1082.5378 & 541.7725 & 10 \\
\hline 20 & 2235.0308 & 1118.0190 & 2218.0042 & 1109.5058 & 2217.0202 & 1109.0137 & A & 999.5006 & 500.2540 & 982.4741 & 491.7407 & 981.4901 & 491.2487 & 9 \\
\hline 21 & 2348.1148 & 1174.5611 & 2331.0883 & 1166.0478 & 2330.1043 & 1165.5558 & $\mathbf{L}$ & 928.4635 & 464.7354 & 911.4370 & 456.2221 & 910.4530 & 455.7301 & 8 \\
\hline 22 & 2445.1676 & 1223.0874 & 2428.1411 & 1214.5742 & 2427.1570 & 1214.0822 & $\mathbf{P}$ & 815.3795 & 408.1934 & 798.3529 & 399.6801 & 797.3689 & 399.1881 & 7 \\
\hline 23 & 2582.2265 & 1291.6169 & 2565.2000 & 1283.1036 & 2564.2160 & 1282.6116 & $\mathbf{H}$ & 718.3267 & 359.6670 & 701.3002 & 351.1537 & 700.3161 & 350.6617 & 6 \\
\hline 24 & 2697.2535 & 1349.1304 & 2680.2269 & 1340.6171 & 2679.2429 & 1340.1251 & $\mathbf{N}$ & 581.2678 & 291.1375 & 564.2412 & 282.6243 & 563.2572 & 282.1323 & 5 \\
\hline 25 & 2754.2749 & 1377.6411 & 2737.2484 & 1369.1278 & 2736.2644 & 1368.6358 & $\mathbf{G}$ & 466.2409 & 233.6241 & 449.2143 & 225.1108 & 448.2303 & 224.6188 & 4 \\
\hline 26 & 2841.3070 & 1421.1571 & 2824.2804 & 1412.6438 & 2823.2964 & 1412.1518 & S & 409.2194 & 205.1133 & 392.1928 & 196.6001 & 391.2088 & 196.1081 & 3 \\
\hline 27 & 2988.3754 & 1494.6913 & 2971.3488 & 1486.1780 & 2970.3648 & 1485.6860 & $\mathbf{F}$ & 322.1874 & 161.5973 & 305.1608 & 153.0840 & & & 2 \\
\hline 28 & & & & & & & $\mathbf{R}$ & 175.1190 & 88.0631 & 158.0924 & 79.5498 & & & 1 \\
\hline
\end{tabular}
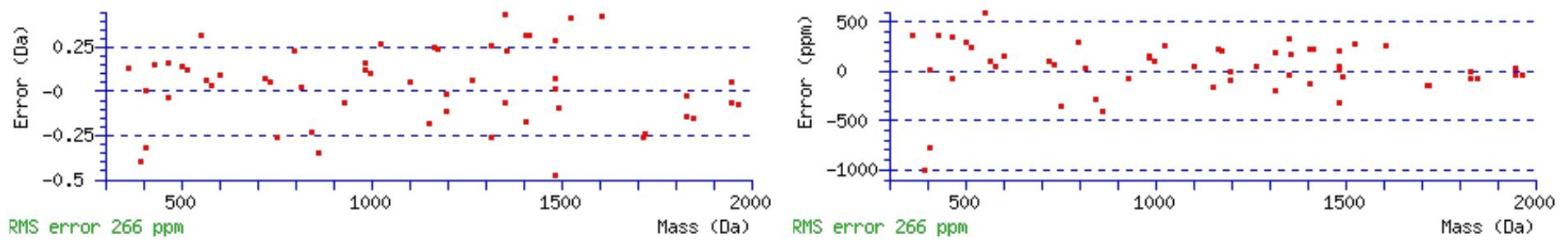

\section{All matches to this query}

\begin{tabular}{|l|l|l|l|}
\hline Score & Mr(calc): & Delta & \multicolumn{1}{|c|}{ Sequence } \\
\hline 39.8 & 3161.4798 & 1.0041 & $\underline{\text { EAMNSTDLYSLVCHLLNVTALPHNGSFR }}$ \\
\hline 23.1 & 3160.4957 & 1.9881 & EAMNSTDLYSLVCHLLNVTALPHNGSFR \\
\hline 21.3 & 3160.4957 & 1.9881 & $\underline{\text { EAMNSTDLYSLVCHLLNVTALPHNGSFR }}$ \\
\hline 7.3 & 3160.4480 & 2.0358 & $\underline{\text { YSYATLITYAINSSPAKKMTLSEIYR }}$ \\
\hline 7.3 & 3160.4480 & 2.0358 & $\underline{\text { YSYATLITYAINSSPAKKMTLSEIYR }}$ \\
\hline 4.9 & 3160.4480 & 2.0358 & $\underline{\text { YSYATLITYAINSSPAKKMTLSEIYR }}$ \\
\hline 3.9 & 3162.4996 & -0.0158 & $\underline{\text { TQHPTDANATSSRSHAIFQIFVKQQDR }}$ \\
\hline 3.7 & 3160.4970 & 1.9868 & WVMNVAILLSHANSVVNPIVYAYRNR \\
\hline 3.7 & 3160.4869 & 1.9969 & $\underline{\text { XGLLPTLPNTPMNTTLMDSCRRFTSTR }}$ \\
\hline 3.7 & 3161.4709 & 1.0129 & $\underline{\text { XGLLPTLPNTPMNTTLMDSCRRFTSTR }}$ \\
\hline
\end{tabular}

Spectrum No: 845; Query: 445; Rank: 1

\section{Peptide View}

MS/MS Fragmentation of YHGFLNASYHR

Found in IPI00201276, Tax_Id=10116 Gene_Symbol=Acp2 Lysosomal acid phosphatase precursor

Match to Query 445: 1364.623452 from(455.881760,3+)

Title: 091008RatKidney_NH4Format01_25.1316.1316.3.dta

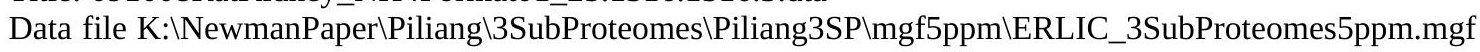



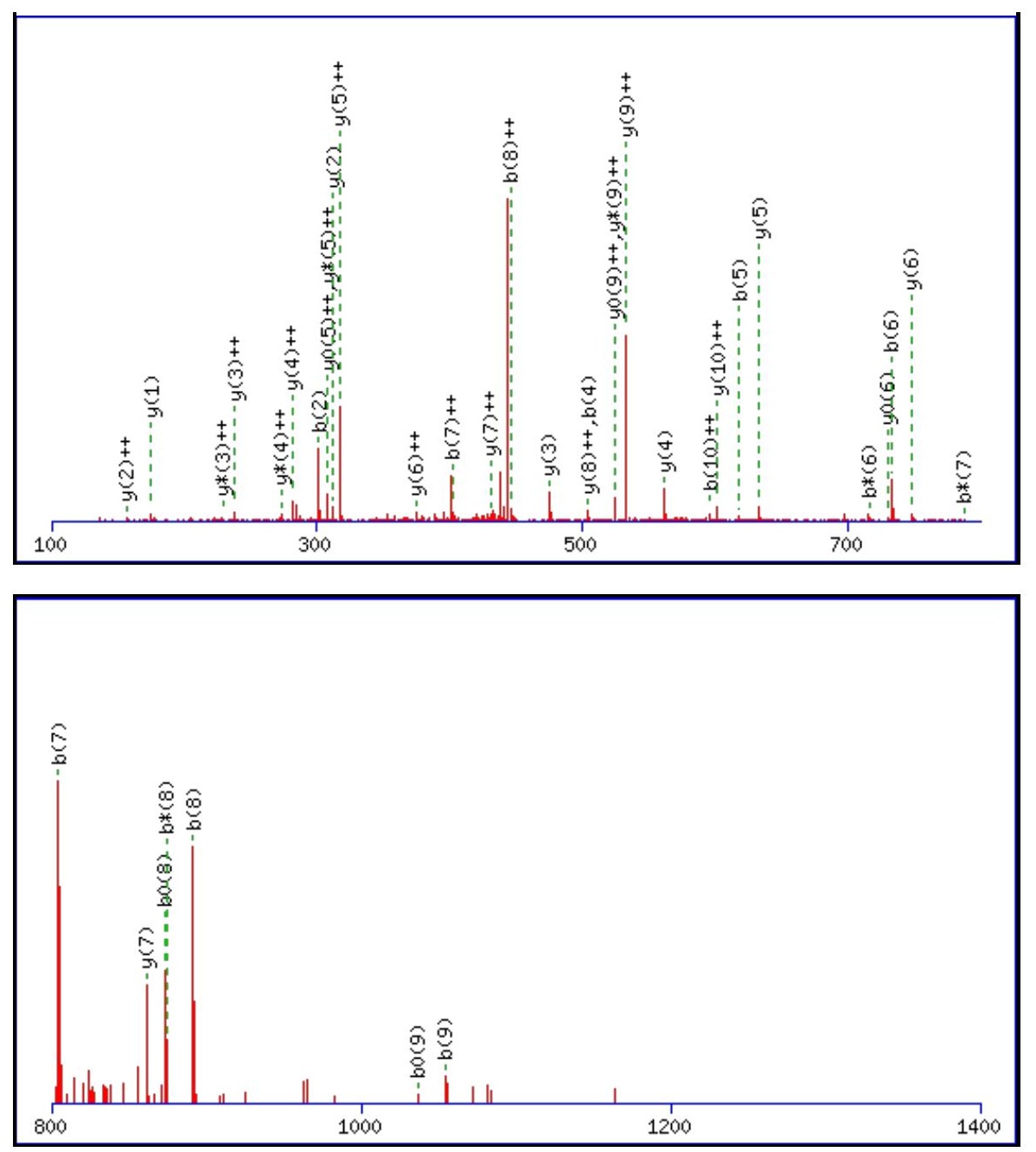

\begin{tabular}{rrrr}
\hline 00 & 1600 & 1800 & 2000 \\
\hline
\end{tabular}

Monoisotopic mass of neutral peptide $\operatorname{Mr}($ calc): 1364.6262

Fixed modifications: Carbamidomethyl (C)

Variable modifications:

N6 : Deamidated_N (N)

Ions Score: 40 Expect: 0.015

Matches (Bold Red): 38/90 fragment ions using 87 most intense peaks

\begin{tabular}{|r|c|c|c|c|c|c|c|c|c|c|c|c|c|c|}
\hline$\#$ & $\mathbf{b}$ & $\mathbf{b}^{++}$ & $\mathbf{b}^{*}$ & $\mathbf{b}^{\mathbf{*}^{++}}$ & $\mathbf{b}^{\mathbf{0}}$ & $\mathbf{b}^{\mathbf{0 + +}}$ & Seq. & $\mathbf{y}$ & $\mathbf{y}^{++}$ & $\mathbf{y}^{\mathbf{*}}$ & $\mathbf{y}^{\boldsymbol{*}^{++}}$ & $\mathbf{y}^{\mathbf{0}}$ & $\mathbf{y}^{\mathbf{0 + +}}$ & $\#$ \\
\hline $\mathbf{1}$ & 164.0706 & 82.5389 & & & & & $\mathbf{Y}$ & & & & & & & $\mathbf{1 1}$ \\
\hline $\mathbf{2}$ & $\mathbf{3 0 1 . 1 2 9 5}$ & 151.0684 & & & & & $\mathbf{H}$ & 1202.5701 & $\mathbf{6 0 1 . 7 8 8 7}$ & 1185.5436 & 593.2754 & 1184.5596 & 592.7834 & $\mathbf{1 0}$ \\
\hline $\mathbf{3}$ & 358.1510 & 179.5791 & & & & & $\mathbf{G}$ & 1065.5112 & 533.2592 & 1048.4847 & 524.7460 & 1047.5006 & 524.2540 & $\mathbf{9}$ \\
\hline $\mathbf{4}$ & $\mathbf{5 0 5 . 2 1 9 4}$ & 253.1133 & & & & & $\mathbf{F}$ & 1008.4897 & 504.7485 & 991.4632 & 496.2352 & 990.4792 & 495.7432 & $\mathbf{8}$ \\
\hline $\mathbf{5}$ & $\mathbf{6 1 8 . 3 0 3 5}$ & 309.6554 & & & & & $\mathbf{L}$ & $\mathbf{8 6 1 . 4 2 1 3}$ & $\mathbf{4 3 1 . 2 1 4 3}$ & 844.3948 & 422.7010 & 843.4108 & 422.2090 & $\mathbf{7}$ \\
\hline
\end{tabular}




\begin{tabular}{|r|r|r|r|r|r|r|r|r|r|r|r|r|r|r|r|r|r|}
$\mathbf{6}$ & $\mathbf{7 3 3 . 3 3 0 4}$ & 367.1688 & 716.3038 & 358.6556 & & & N & 748.3373 & 374.6723 & 731.3107 & 366.1590 & 730.3267 & 365.6670 & $\mathbf{6}$ \\
\hline $\mathbf{7}$ & $\mathbf{8 0 4 . 3 6 7 5}$ & $\mathbf{4 0 2 . 6 8 7 4}$ & $\mathbf{7 8 7 . 3 4 1 0}$ & 394.1741 & & & A & $\mathbf{6 3 3 . 3 1 0 3}$ & $\mathbf{3 1 7 . 1 5 8 8}$ & 616.2838 & $\mathbf{3 0 8 . 6 4 5 5}$ & 615.2998 & $\mathbf{3 0 8 . 1 5 3 5}$ & $\mathbf{5}$ \\
\hline $\mathbf{8}$ & $\mathbf{8 9 1 . 3 9 9 5}$ & $\mathbf{4 4 6 . 2 0 3 4}$ & $\mathbf{8 7 4 . 3 7 3 0}$ & 437.6901 & $\mathbf{8 7 3 . 3 8 9 0}$ & 437.1981 & S & $\mathbf{5 6 2 . 2 7 3 2}$ & $\mathbf{2 8 1 . 6 4 0 2}$ & 545.2467 & $\mathbf{2 7 3 . 1 2 7 0}$ & 544.2627 & 272.6350 & $\mathbf{4}$ \\
\hline $\mathbf{9}$ & $\mathbf{1 0 5 4 . 4 6 2 9}$ & 527.7351 & 1037.4363 & 519.2218 & $\mathbf{1 0 3 6 . 4 5 2 3}$ & 518.7298 & $\mathbf{Y}$ & $\mathbf{4 7 5 . 2 4 1 2}$ & $\mathbf{2 3 8 . 1 2 4 2}$ & $\mathbf{4 5 8 . 2 1 4 6}$ & $\mathbf{2 2 9 . 6 1 1 0}$ & & & & $\mathbf{3}$ \\
\hline $\mathbf{1 0}$ & 1191.5218 & $\mathbf{5 9 6 . 2 6 4 5}$ & 1174.4952 & 587.7513 & 1173.5112 & 587.2592 & H & $\mathbf{3 1 2 . 1 7 7 9}$ & $\mathbf{1 5 6 . 5 9 2 6}$ & 295.1513 & 148.0793 & & & & $\mathbf{2}$ \\
\hline $\mathbf{1 1}$ & & & & & & & R & $\mathbf{1 7 5 . 1 1 9 0}$ & $\mathbf{8 8 . 0 6 3 1}$ & 158.0924 & 79.5498 & & & \\
\hline
\end{tabular}
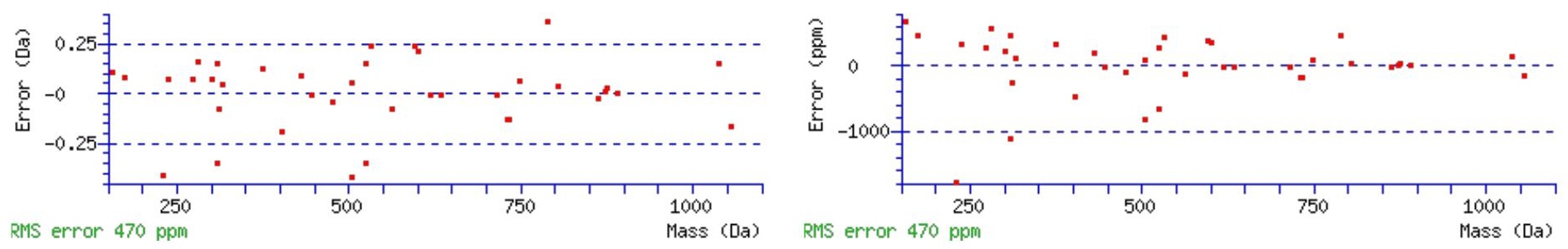

\section{All matches to this query}

\begin{tabular}{|l|l|l|l|}
\hline Score & Mr(calc): & Delta & \multicolumn{1}{c|}{ Sequence } \\
\hline 39.9 & 1364.6262 & -0.0027 & YHGFLNASYHR \\
\hline 8.1 & 1363.6261 & 0.9973 & NSKLLSTSAKR \\
\hline 8.1 & 1364.6102 & 0.0133 & NSKLLSTSAKR \\
\hline 7.7 & 1364.6199 & 0.0035 & YIYIMGIQER \\
\hline 7.2 & 1362.6037 & 2.0198 & TTCLKEMTKR \\
\hline 6.1 & 1364.6217 & 0.0017 & FSDVSPMGLPCR \\
\hline 6.1 & 1364.6199 & 0.0035 & YIYIMGIQER \\
\hline 5.7 & 1364.6159 & 0.0075 & MSLNSSLSYRK \\
\hline 5.4 & 1362.6227 & 2.0007 & ARPAASPAMNAAR \\
\hline 5.1 & 1363.6103 & 1.0131 & $\underline{\text { NTKVGDVSAASEDA }}$ \\
\hline
\end{tabular}

Spectrum No: 846; Query: 2518; Rank: 1

\section{Peptide View}

MS/MS Fragmentation of LCDLLVANNYFTHFFAPQNLTNMSK

Found in IPI00188338, Tax_Id=10116 Gene_Symbol=Itih1_predicted inter-alpha trypsin inhibitor, heavy chain 1

Match to Query 2518: 2974.398012 from(992.473280,3+)

Title: 091008RatKidney_NoSalt_25.5519.5519.3.dta

Data file K:INewmanPaper|Piliang|3SubProteomes\Piliang3SP\mgf5ppm\ERLIC_3SubProteomes5ppm.mgf

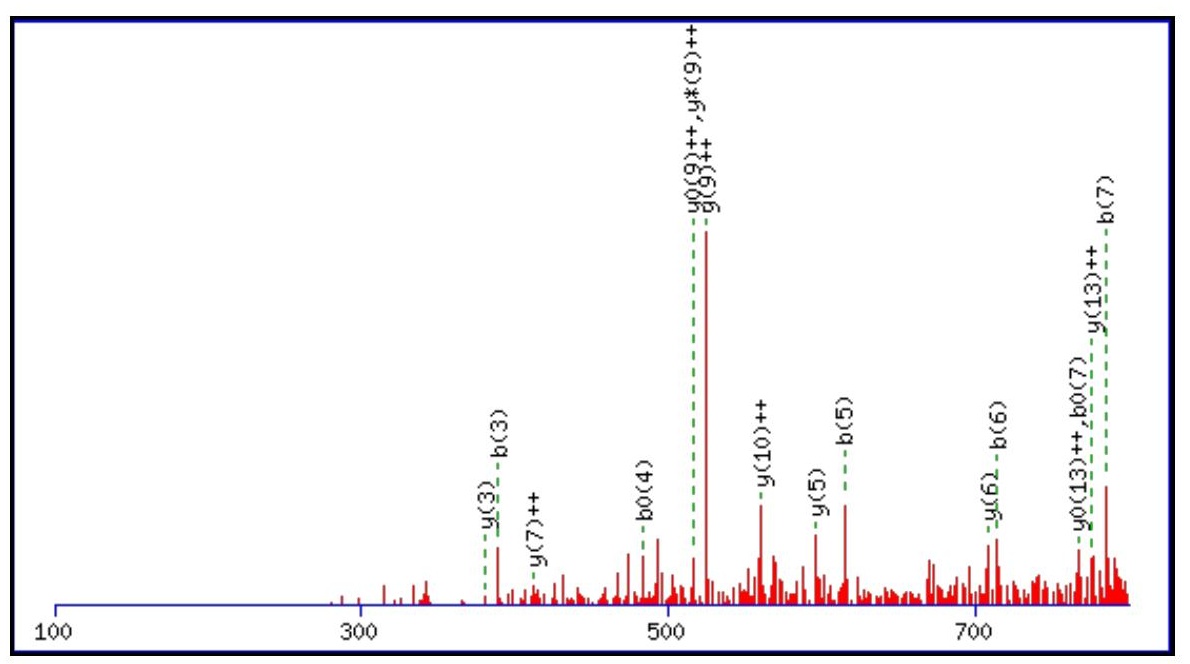



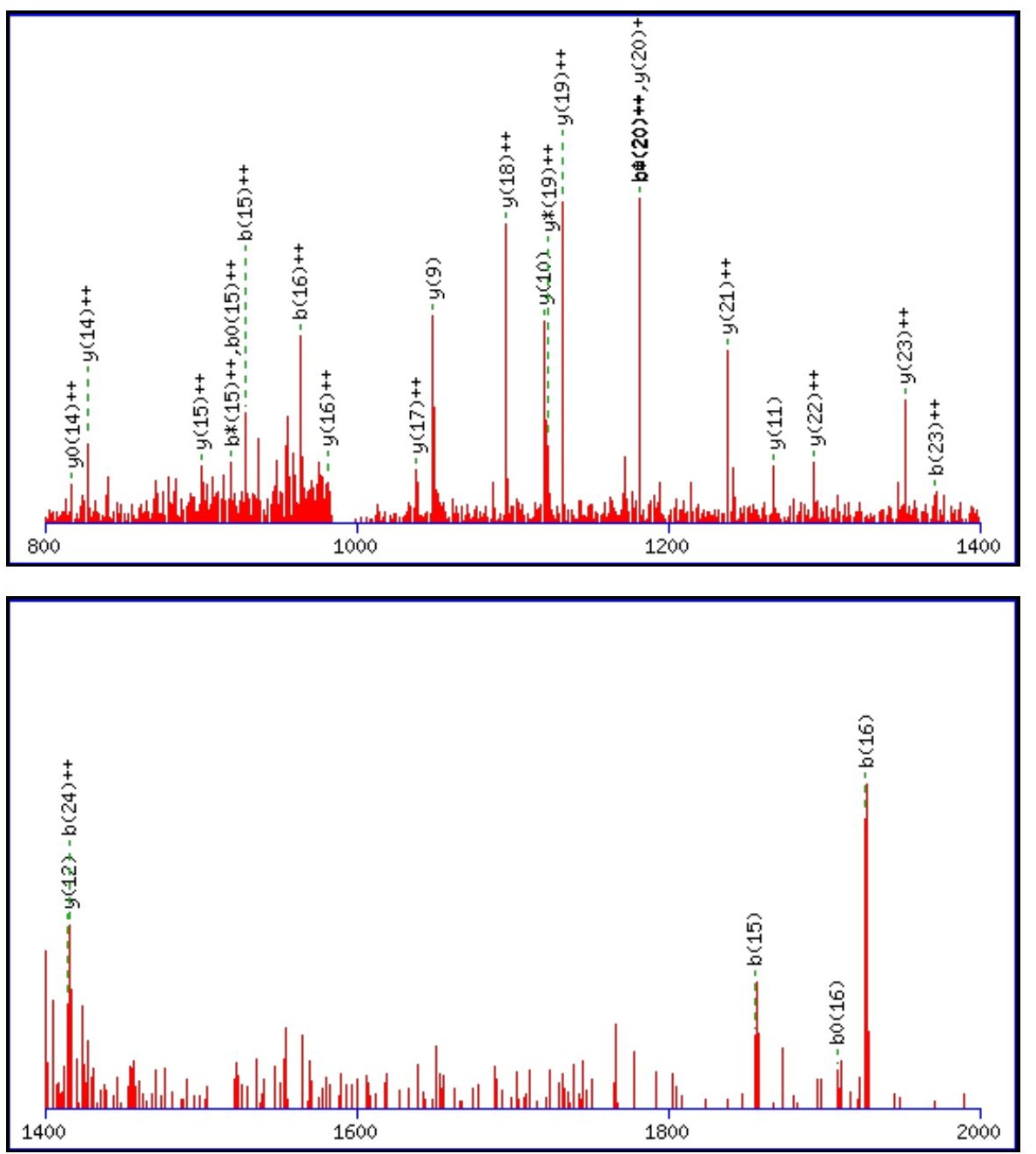

Monoisotopic mass of neutral peptide $\operatorname{Mr}($ calc): 2974.3993

Fixed modifications: Carbamidomethyl (C)

Variable modifications:

N19 : Deamidated_N (N)

M23 : Oxidation (M)

Ions Score: 40 Expect : 0.036

Matches (Bold Red): 43/268 fragment ions using 121 most intense peaks

\begin{tabular}{|c|c|c|c|c|c|c|c|c|c|c|c|c|c|c|}
\hline \# & b & $\mathbf{b}^{++}$ & $\mathbf{b}^{*}$ & $\mathbf{b}^{*++}$ & $\mathbf{b}^{0}$ & $\mathbf{b}^{0++}$ & Seq. & $\mathbf{y}$ & $\mathbf{y}^{++}$ & $\mathbf{y}^{*}$ & $\mathbf{y}^{*^{++}}$ & $\mathbf{y}^{\mathbf{0}}$ & $\mathbf{y}^{0++}$ & \# \\
\hline 1 & 114.0913 & 57.5493 & & & & & $\mathbf{L}$ & & & & & & & 25 \\
\hline 2 & 274.1220 & 137.5646 & & & & & $\mathrm{C}$ & 2862.3226 & 1431.6649 & 2845.2960 & 1423.1516 & 2844.3120 & 1422.6596 & 24 \\
\hline 3 & 389.1489 & 195.0781 & & & 371.1384 & 186.0728 & D & 2702.2919 & 1351.6496 & 2685.2654 & 1343.1363 & 2684.2813 & 1342.6443 & 23 \\
\hline 4 & 502.2330 & 251.6201 & & & 484.2224 & 242.6149 & $\mathbf{L}$ & 2587.2650 & 1294.1361 & 2570.2384 & 1285.6228 & 2569.2544 & 1285.1308 & 22 \\
\hline 5 & 615.3171 & 308.1622 & & & 597.3065 & 299.1569 & $\mathbf{L}$ & 2474.1809 & 1237.5941 & 2457.1544 & 1229.0808 & 2456.1703 & 1228.5888 & 21 \\
\hline 6 & 714.3855 & 357.6964 & & & 696.3749 & 348.6911 & $\mathbf{V}$ & 2361.0968 & 1181.0521 & 2344.0703 & 1172.5388 & 2343.0863 & 1172.0468 & 20 \\
\hline 7 & 785.4226 & 393.2149 & & & 767.4120 & 384.2096 & A & 2262.0284 & 1131.5179 & 2245.0019 & 1123.0046 & 2244.0179 & 1122.5126 & 19 \\
\hline 8 & 899.4655 & 450.2364 & 882.4390 & 441.7231 & 881.4549 & 441.2311 & $\mathbf{N}$ & 2190.9913 & 1095.9993 & 2173.9648 & 1087.4860 & 2172.9807 & 1086.9940 & 18 \\
\hline 9 & 1013.5084 & 507.2579 & 996.4819 & 498.7446 & 995.4979 & 498.2526 & $\mathbf{N}$ & 2076.9484 & 1038.9778 & 2059.9218 & 1030.4646 & 2058.9378 & 1029.9725 & 17 \\
\hline 10 & 1176.5718 & 588.7895 & 1159.5452 & 580.2762 & 1158.5612 & 579.7842 & $\mathbf{Y}$ & 1962.9055 & 981.9564 & 1945.8789 & 973.4431 & 1944.8949 & 972.9511 & 16 \\
\hline 11 & 1323.6402 & 662.3237 & 1306.6136 & 653.8105 & 1305.6296 & 653.3184 & $\mathbf{F}$ & 1799.8421 & 900.4247 & 1782.8156 & 891.9114 & 1781.8316 & 891.4194 & 15 \\
\hline 12 & 1424.6879 & 712.8476 & 1407.6613 & 704.3343 & 1406.6773 & 703.8423 & $\mathbf{T}$ & 1652.7737 & 826.8905 & 1635.7472 & 818.3772 & 1634.7632 & 817.8852 & 14 \\
\hline 13 & 1561.7468 & 781.3770 & 1544.7202 & 772.8638 & 1543.7362 & 772.3717 & $\mathbf{H}$ & 1551.7260 & 776.3667 & 1534.6995 & 767.8534 & 1533.7155 & 767.3614 & 13 \\
\hline 14 & 1708.8152 & 854.9112 & 1691.7886 & 846.3980 & 1690.8046 & 845.9060 & $\mathbf{F}$ & 1414.6671 & 707.8372 & 1397.6406 & 699.3239 & 1396.6566 & 698.8319 & 12 \\
\hline 15 & 1855.8836 & 928.4454 & 1838.8571 & 919.9322 & 1837.8730 & 919.4402 & $\mathbf{F}$ & 1267.5987 & 634.3030 & 1250.5722 & 625.7897 & 1249.5881 & 625.2977 & 11 \\
\hline 16 & 1926.9207 & 963.9640 & 1909.8942 & 955.4507 & 1908.9102 & 954.9587 & A & 1120.5303 & 560.7688 & 1103.5037 & 552.2555 & 1102.5197 & 551.7635 & 10 \\
\hline 17 & 2023.9735 & 1012.4904 & 2006.9469 & 1003.9771 & 2005.9629 & 1003.4851 & $\mathbf{P}$ & 1049.4932 & 525.2502 & 1032.4666 & 516.7370 & 1031.4826 & 516.2449 & 9 \\
\hline
\end{tabular}




\begin{tabular}{|c|c|c|c|c|c|c|c|c|c|c|c|c|c|c|}
\hline 18 & |2152.0321 | & |1076.5197 & |2135.0055 & |1068.006 & 2 & 44 & $\mathbf{Q}$ & 952.4404 & 476.7238 & 935.4139 & 468.2106 & 934.4299| & 467.7186 & 8 \\
\hline 19 & 2267.0590 & 1134.0331 & 2250.0324 & 1125.5199 & 2249.0484 & 1125.0279 & $\mathbf{N}$ & 824.3818 & 412.6946 & 807.3553 & 404.1813 & 806.3713 & 403.6893 & 7 \\
\hline 20 & 2380.1431 & 1190.5752 & 2363.1165 & 1182.0619 & 2362.1325 & 1181.5699 & $\mathbf{L}$ & 709.3549 & 355.1811 & 692.3284 & 346.6678 & 691.3443 & 346.1758 & 6 \\
\hline 21 & 2481.1907 & 1241.0990 & 2464.1642 & 1232.5857 & 2463.1802 & 1232.0937 & $\mathbf{T}$ & 596.2708 & 298.6391 & 579.2443 & 290.1258 & 578.2603 & 289.6338 & 5 \\
\hline 22 & 2595.2337 & 1298.1205 & 2578.2071 & 1289.6072 & 2577.2231 & 1289.1152 & $\mathbf{N}$ & 495.2232 & 248.1152 & 478.1966 & 239.6019 & 477.2126 & 239.1099 & 4 \\
\hline 23 & 2742.2691 & 1371.6382 & 2725.2425 & 1363.1249 & 2724.2585 & 1362.6329 & $\mathbf{M}$ & 381.1802 & 191.0938 & 364.1537 & 182.5805 & 363.1697 & 182.0885 & 3 \\
\hline 24 & 2829.3011 & 1415.1542 & 2812.2745 & 1406.6409 & 2811.2905 & 1406.1489 & S & 234.1448 & 117.5761 & 217.1183 & 109.0628 & 216.1343 & 108.5708 & 2 \\
\hline 25 & & & & & & & $\mathbf{K}$ & 147.1128 & 74.0600 & 130.0863 & 65.5468 & & & 1 \\
\hline
\end{tabular}
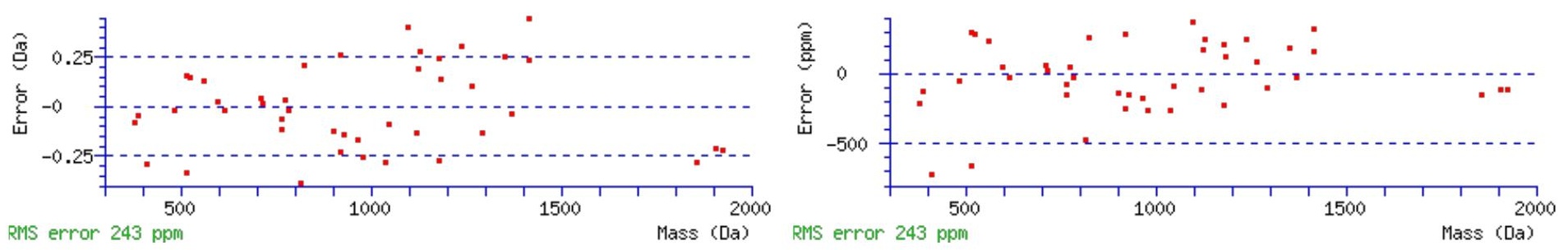

\section{All matches to this query}

\begin{tabular}{|l|l|l|l|}
\hline Score & Mr(calc): & Delta & \multicolumn{1}{|c|}{ Sequence } \\
\hline 39.8 & 2974.3993 & -0.0013 & LCDLLVANNYFTHFFAPQNLTNMSK \\
\hline 35.7 & 2974.3993 & -0.0013 & LCDLLVANNYFTHFFAPQNLTNMSK \\
\hline 21.2 & 2974.3993 & -0.0013 & LCDLLVANNYFTHFFAPQNLTNMSK \\
\hline 21.2 & 2974.3993 & -0.0013 & LCDLLVANNYFTHFFAPQNLTNMSK \\
\hline 10.1 & 2974.4221 & -0.0241 & $\underline{\text { IAPSFAVESMEDALKAADTIGYPVMIR }}$ \\
\hline 4.3 & 2973.4232 & 0.9748 & SPLSATSAFAIATAGANESPANKEIYRR \\
\hline 3.3 & 2973.4153 & 0.9827 & LCDLLVANNYFTHFFAPQNLTNMSK \\
\hline 3.3 & 2974.4221 & -0.0241 & $\underline{\text { IAPSFAVESMEDALKAADTIGYPVMIR }}$ \\
\hline 2.9 & 2973.4055 & 0.9925 & KVINMVHNLLSSHDSDPRYSDPQIK \\
\hline 2.9 & 2973.4055 & 0.9925 & KVINMVHNLLSSHDSDPRYSDPQIK \\
\hline
\end{tabular}

Spectrum No: 847; Query: 2215; Rank: 1

\section{Peptide View}

MS/MS Fragmentation of GTCEQGPSIVTPPKDIWNITGAK

Found in IPI00361798, Tax_Id=10116 Gene_Symbol=Igfbp7 Insulin-like growth factor binding protein 7

Match to Query 2215: 2469.232722 from(824.084850,3+)

Title: 100101RatKid_NS_deglyco_18.4111.4111.3.dta

Data file K:INewmanPaper|Piliangl3SubProteomes\Piliang3SP\mgf5ppm\ERLIC_3SubProteomes5ppm.mgf 

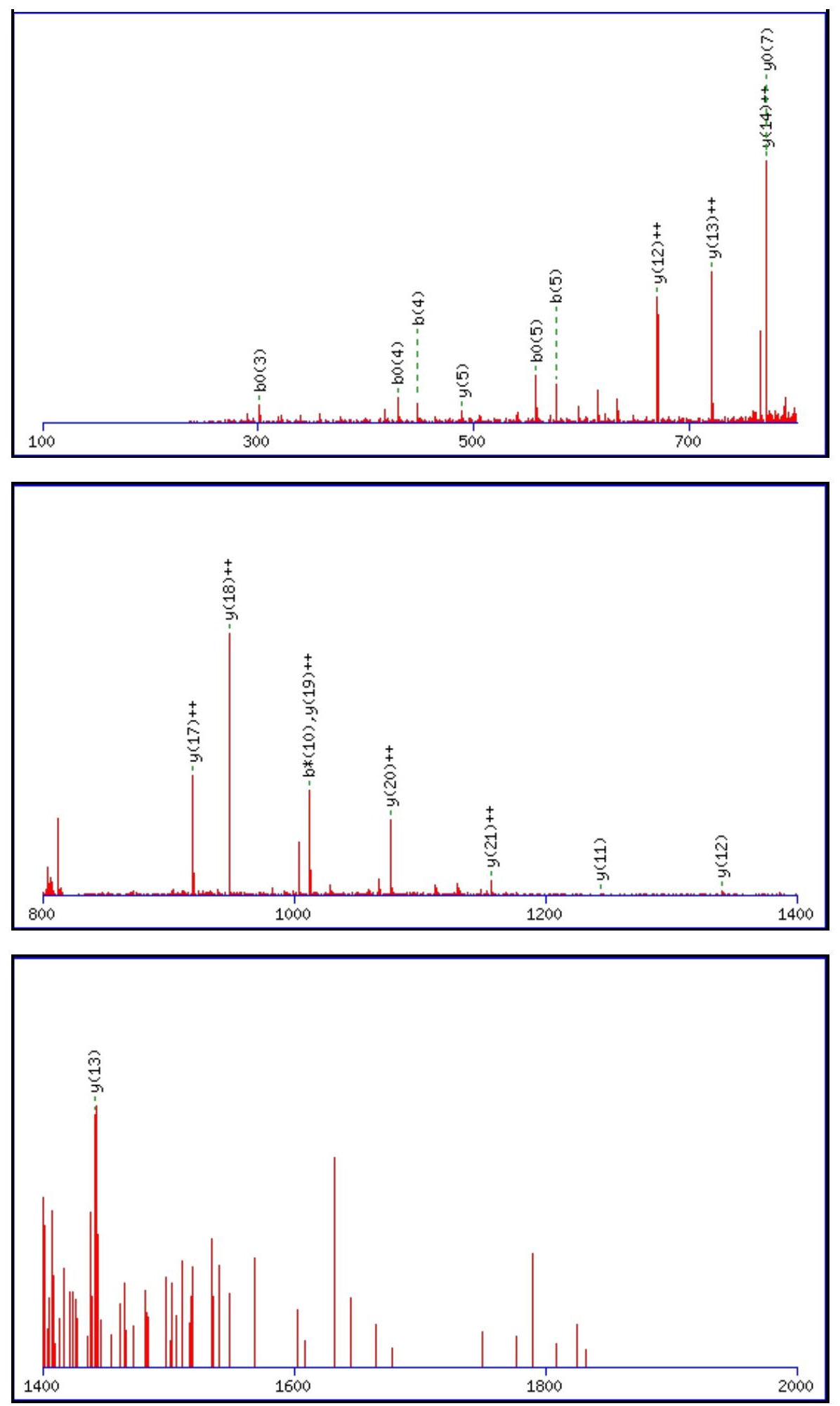

Monoisotopic mass of neutral peptide $\operatorname{Mr}($ calc): 2469.2210

Fixed modifications: Carbamidomethyl (C)

Variable modifications:

N18 : Deamidated $\mathrm{N}(\mathrm{N})$

Ions Score: 40 Expect: $\odot .023$

Matches (Bold Red): 19/248 fragment ions using 39 most intense peaks

\begin{tabular}{|c|c|c|c|c|c|c|c|c|c|c|c|c|c|c|}
\hline$\#$ & b & $\mathbf{b}^{++}$ & $\mathbf{b}^{*}$ & $\mathbf{b}^{*^{++}}$ & $\mathbf{b}^{0}$ & $\mathbf{b}^{0++}$ & Seq. & $\mathbf{y}$ & $\mathbf{y}^{++}$ & $\mathrm{y}^{*}$ & $\mathbf{y}^{*^{++}}$ & $\mathbf{y}^{0}$ & $y^{0++}$ & \# \\
\hline 1 & 58.0287 & 29.5180 & & & & & G & & & & & & & 23 \\
\hline 2 & 159.0764 & 80.0418 & & & 141.0659 & 71.0366 & $\mathbf{T}$ & 2413.2068 & 1207.1070 & 2396.1802 & 1198.5938 & 2395.1962 & 1198.1017 & 22 \\
\hline 3 & 319.1071 & 160.0572 & & & 301.0965 & 151.0519 & C & 2312.1591 & 1156.5832 & 2295.1326 & 1148.0699 & 2294.1485 & 1147.5779 & 21 \\
\hline 4 & 448.1497 & 224.5785 & & & 430.1391 & 215.5732 & $E$ & 2152.1285 & 1076.5679 & 2135.1019 & 1068.0546 & 2134.1179 & 1067.5626 & 20 \\
\hline 5 & 576.2082 & 288.6078 & 559.1817 & 280.0945 & 558.1977 & 279.6025 & $\bar{Q}$ & 2023.0859 & 1012.0466 & 2006.0593 & 1003.5333 & 2005.0753 & 1003.0413 & 19 \\
\hline
\end{tabular}




\begin{tabular}{|c|c|c|c|c|c|c|c|c|c|c|c|c|c|c|}
\hline & 633.2297 & 317. & 616. & 308 & & 132 & G & 0273 & 948.0173 & & 939.5040 & 1877.0167 & & \\
\hline 7 & 730.2825 & 365.6449 & 713.2559 & 357.1316 & 712.2719 & 356.6396 & $\mathbf{P}$ & 1838.0058 & 919.5065 & 1820.9793 & 910.9933 & 1819.9953 & 910.5013 & 17 \\
\hline 8 & 817.3145 & 409.1609 & 800.2879 & 400.6476 & 799.3039 & 400.1556 & & 1740.9531 & 870.9802 & 1723.9265 & 862.4669 & 1722.9425 & 861.9749 & \\
\hline 9 & 930.3986 & 465.7029 & 913.3720 & 457.1896 & & & & & & 1636.8945 & 818.9509 & & 818.4589 & \\
\hline 0 & 1029.4670 & 515.2371 & 1012.4404 & 506.7239 & 1011.4564 & 506.2318 & V & 370 & 770.9221 & 1523.8104 & 762.4088 & 1522.8264 & 9168 & \\
\hline 1 & 1130.5147 & 565.7610 & 1113.4881 & 557.2477 & 1112.5041 & 556.7557 & $\mathbf{T}$ & 1441.7686 & 721.3879 & 1424.7420 & 712.8746 & 1423.7580 & 712.3826 & \\
\hline 2 & 1227.5674 & 614.2 & 409 & 605.7741 & 1209.5569 & 605.2 & $\mathbf{P}$ & 1340 & & 1323.6943 & 662.3508 & & & \\
\hline 3 & 1324.6202 & $662 . \varepsilon$ & 1307.5936 & $654.3^{3}$ & 6096 & 653.8084 & D & 1243 & & 1226. & 8244 & 122 & 324 & \\
\hline 14 & 1452.7151 & 726.8612 & 1435.6886 & 718.3479 & 1434.7046 & 717.8559 & K & 1146.6153 & 573.8113 & 1129.5888 & 565.2980 & 1128.6048 & 564.8060 & 10 \\
\hline 5 & 1567.7421 & 784.3747 & 1550.7155 & 775.8614 & 1549.7315 & 775.3694 & D & 1018 & 509.7638 & 1001.4938 & 501.2506 & 1000 & 500 & \\
\hline 16 & 1680.8262 & 840.9167 & 1663.7996 & 832.4034 & 662.8156 & 831.9114 & & & & 886.4669 & 443.7371 & & 443.2451 & 8 \\
\hline 17 & 1866.9055 & 933.9564 & 1849.8789 & 925.4431 & 1848.8949 & 924.9511 & & 790.4094 & 395.7083 & 773.3828 & 387.1951 & 772.3988 & 386.7030 & 7 \\
\hline 18 & 1981.9324 & 991.4698 & 1964.9059 & 982.9566 & 1963.9218 & 982.4646 & 1 & 604.3301 & 302.6687 & 587.3035 & 294.1554 & 586.3195 & 293.6634 & 6 \\
\hline 19 & 2095.0165 & 1048.0119 & 2077.9899 & 039.4986 & 2077.0059 & 1039.0066 & & 489.3031 & 245.15 & 472.2766 & 236.6419 & 2926 & 499 & 5 \\
\hline 20 & 2196.0641 & 1098.5357 & 2179.0376 & 090.0224 & 2178.0536 & 1089.5304 & T & 376.2191 & 188.6132 & 359.1925 & 180.0999 & 358.2085 & 179.6079 & 4 \\
\hline 21 & 2253.0856 & 1127.0464 & 2236.0591 & 1118.5332 & 2235.0750 & 1118.0412 & G & 275.1714 & 138.0893 & 258.1448 & 129.5761 & & & 3 \\
\hline 22 & 2324.1227 & 1162.5650 & 2307.0962 & 1154.0517 & 2306.1122 & 1153.5597 & A & 218.1499 & 109.5786 & 201.1234 & 101.0653 & & & 2 \\
\hline & & & & & & & $\mathbf{K}$ & 147.1128 & 74.0600 & 130.0863 & 65.5468 & & & \\
\hline
\end{tabular}
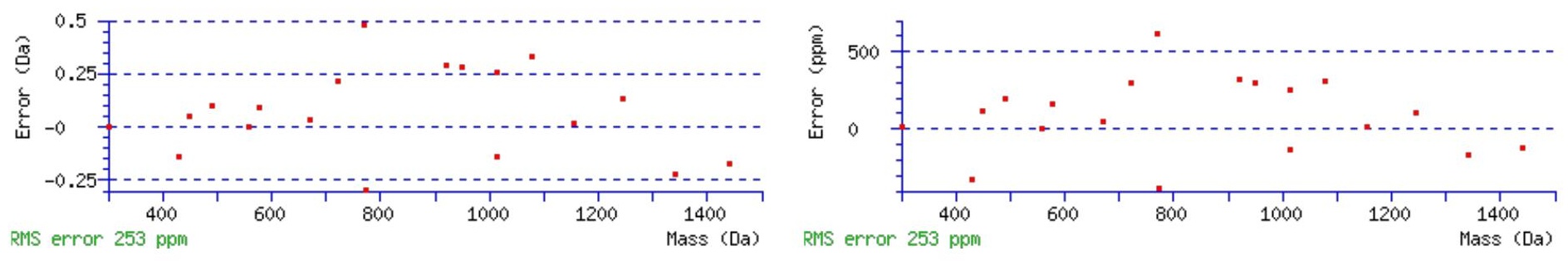

\section{All matches to this query}

\begin{tabular}{|l|l|l|l|}
\hline Score & Mr(calc): & Delta & \multicolumn{1}{|c|}{ Sequence } \\
\hline 39.7 & 2469.2210 & 0.0117 & GTCEQGPSIVTPPKDIWNITGAK \\
\hline 5.3 & 2469.2334 & -0.0007 & RFSGKAWLPLLPGLSSGSAPLR \\
\hline 5.3 & 2468.2287 & 1.0040 & IGFEAGGLAPQAFQVTMAIIQAR \\
\hline 1.9 & 2467.2488 & 1.9839 & AKTLLLVLNKNHTLLQSSEGK \\
\hline 1.9 & 2467.2488 & 1.9839 & AKTLLLVLNKNHTLLQSSEGK \\
\hline 1.7 & 2468.2424 & 0.9903 & FAEQTLRQKAQVEQELTTLR \\
\hline 1.2 & 2467.2141 & 2.0186 & EDVMETLNQALDLQSRILKR \\
\hline 1.1 & 2469.2152 & 0.0175 & LLLNAENPRGTFLVRESETTK \\
\hline 1.1 & 2468.2094 & 1.0233 & KLLVQNIQEIQDSMRSSNLR \\
\hline 1.1 & 2468.2094 & 1.0233 & KLLVQNIQEIQDSMRSSNLR \\
\hline
\end{tabular}

Spectrum No: 848; Query: 2277; Rank: 1

\section{Peptide View}

MS/MS Fragmentation of DSLNCSCLALQTSGQEEQVNQR

Found in IPI00326462, Tax_Id=10116 Gene_Symbol=Enpp3 Ectonucleotide pyrophosphatase/phosphodiesterase family member 3

Match to Query 2277: 2537.119092 from(846.713640,3+)

Title: 100101RatKid_NS_deglyco_26.2359.2359.3.dta

Data file K:INewmanPaper|Piliangl3SubProteomes\Piliang3SP\mgf5ppm\ERLIC_3SubProteomes5ppm.mgf 

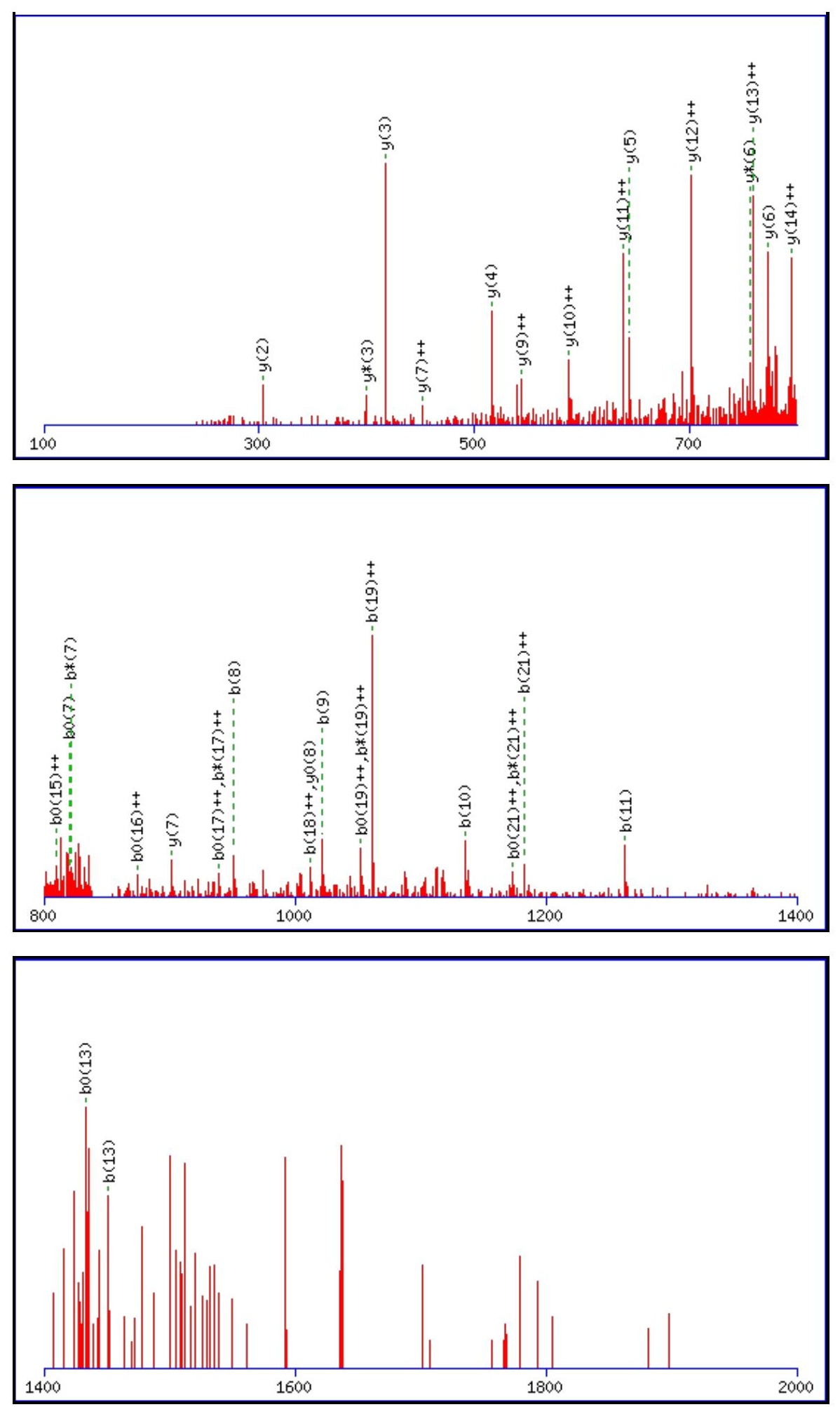

Monoisotopic mass of neutral peptide $\operatorname{Mr}($ calc): 2537.1122

Fixed modifications: Carbamidomethyl (C)

Variable modifications:

N4 : Deamidated_N (N)

Ions Score: 40 Expect: $\odot .034$

Matches (Bold Red): 35/236 fragment ions using 70 most intense peaks

\begin{tabular}{|c|c|c|c|c|c|c|c|c|c|c|c|c|c|c|}
\hline$\#$ & b & $\mathbf{b}^{++}$ & $\mathbf{b}^{*}$ & $\mathbf{b}^{*^{++}}$ & $\mathbf{b}^{\mathbf{0}}$ & $\mathbf{b}^{0++}$ & Seq. & $\mathbf{y}$ & $\mathbf{y}^{++}$ & $\mathrm{y}^{*}$ & $\mathbf{y}^{*^{++}}$ & $\mathbf{y}^{0}$ & $y^{0++}$ & \# \\
\hline 1 & 116.0342 & 58.5207 & & & 98.0237 & 49.5155 & D & & & & & & & 22 \\
\hline 2 & 203.0662 & 102.0368 & & & 185.0557 & 93.0315 & $S$ & 2423.0926 & 1212.0499 & 2406.0660 & 1203.5366 & 2405.0820 & 1203.0446 & 21 \\
\hline 3 & 316.1503 & 158.5788 & & & 298.1397 & 149.5735 & $\mathbf{L}$ & 2336.0605 & 1168.5339 & 2319.0340 & 1160.0206 & 2318.0500 & 1159.5286 & 20 \\
\hline 4 & 431.1772 & 216.0923 & 414.1507 & 207.5790 & 413.1667 & 207.0870 & $\mathbf{N}$ & 2222.9765 & 1111.9919 & 2205.9499 & 1103.4786 & 2204.9659 & 1102.9866 & 19 \\
\hline 5 & 591.2079 & 296.1076 & 574.1813 & 287.5943 & 573.1973 & 287.1023 & $\mathrm{C}$ & 2107.9495 & 1054.4784 & 2090.9230 & 1045.9651 & 2089.9390 & 1045.4731 & 18 \\
\hline
\end{tabular}




\begin{tabular}{|c|c|c|c|c|c|c|c|c|c|c|c|c|c|c|}
\hline 6 & 678.2399 & 339.6236 & 661.2134 & 331.1103 & 660.2294 & 330.6183 & S & 1947.9189 & 974.4631 & |1930.8923 & 965.9498 & 1929.9083 & 965.4578 & 17 \\
\hline 7 & 838.2706 & 419.6389 & 821.2440 & 411.1257 & 820.2600 & 410.6336 & C & 1860.8869 & 930.9471 & 1843.8603 & 922.4338 & 1842.8763 & 921.9418 & 16 \\
\hline 8 & 951.3546 & 476.1810 & 934.3281 & 467.6677 & 933.3441 & 467.1757 & L & 1700.8562 & 850.9317 & 1683.8297 & 842.4185 & 1682.8456 & 841.9265 & 15 \\
\hline 9 & 1022.3918 & 511.6995 & 1005.3652 & 503.1862 & 1004.3812 & 502.6942 & A & 1587.7721 & 794.3897 & 1570.7456 & 785.8764 & 1569.7616 & 785.3844 & 14 \\
\hline 10 & 1135.4758 & 568.2415 & 1118.4493 & 559.7283 & 1117.4653 & 559.2363 & L & 1516.7350 & 758.8712 & 1499.7085 & 750.3579 & 1498.7245 & 749.8659 & 13 \\
\hline 11 & 1263.5344 & 632.2708 & 1246.5078 & 623.7576 & 1245.5238 & 623.2656 & $\mathbf{Q}$ & 1403.6510 & 702.3291 & 1386.6244 & 693.8158 & 1385.6404 & 693.3238 & 12 \\
\hline 12 & 1364.5821 & 682.7947 & 1347.5555 & 674.2814 & 1346.5715 & 673.7894 & $\mathbf{T}$ & 1275.5924 & 638.2998 & 1258.5658 & 629.7866 & 1257.5818 & 629.2945 & 11 \\
\hline 13 & 1451.6141 & 726.3107 & 1434.5876 & 717.7974 & 1433.6035 & 717.3054 & S & 1174.5447 & 587.7760 & 1157.5182 & 579.2627 & 1156.5341 & 578.7707 & 10 \\
\hline 14 & 1508.6356 & 754.8214 & 1491.6090 & 746.3081 & 1490.6250 & 745.8161 & G & 1087.5127 & 544.2600 & 1070.4861 & 535.7467 & 1069.5021 & 535.2547 & 9 \\
\hline 15 & 1636.6941 & 818.8507 & 1619.6676 & 810.3374 & 1618.6836 & 809.8454 & $\mathbf{Q}$ & 1030.4912 & 515.7492 & 1013.4647 & 507.2360 & 1012.4806 & 506.7440 & 8 \\
\hline 16 & 1765.7367 & 883.3720 & 1748.7102 & 874.8587 & 1747.7262 & 874.3667 & $\mathbf{E}$ & 902.4326 & 451.7200 & 885.4061 & 443.2067 & 884.4221 & 442.7147 & 7 \\
\hline 17 & 1894.7793 & 947.8933 & 1877.7528 & 939.3800 & 1876.7688 & 938.8880 & $\mathbf{E}$ & 773.3900 & 387.1987 & 756.3635 & 378.6854 & 755.3795 & 378.1934 & 6 \\
\hline 18 & 2022.8379 & 1011.9226 & 2005.8114 & 1003.4093 & 2004.8273 & 1002.9173 & Q & 644.3474 & 322.6774 & 627.3209 & 314.1641 & & & 5 \\
\hline 19 & 2121.9063 & \begin{tabular}{|l}
1061.4568 \\
\end{tabular} & 2104.8798 & 1052.9435 & 2103.8958 & 1052.4515 & $\mathbf{V}$ & 516.2889 & 258.6481 & 499.2623 & 250.1348 & & & 4 \\
\hline 20 & 2235.9492 & 1118.4783 & 2218.9227 & 1109.9650 & 2217.9387 & 1109.4730 & $\mathbf{N}$ & 417.2205 & 209.1139 & 400.1939 & 200.6006 & & & 3 \\
\hline 21 & 2364.0078 & 1182.5076 & 2346.9813 & 1173.9943 & 2345.9973 & 1173.5023 & $\mathbf{Q}$ & 303.1775 & 152.0924 & 286.1510 & 143.5791 & & & 2 \\
\hline 22 & & & & & & & $\mathbf{R}$ & 175.1190 & 88.0631 & 158.0924 & 79.5498 & & & 1 \\
\hline
\end{tabular}
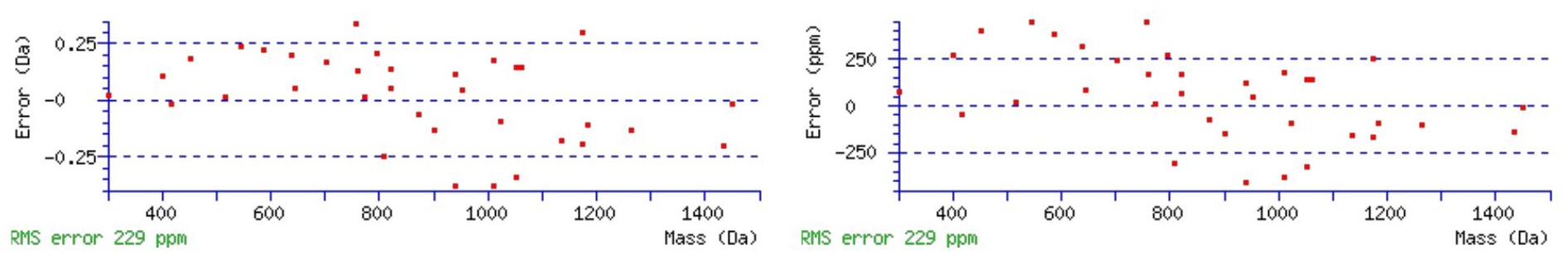

\section{All matches to this query}

\begin{tabular}{|l|c|c|l|}
\hline Score & Mr(calc): & Delta & \multicolumn{1}{|c|}{ Sequence } \\
\hline 39.7 & 2537.1122 & 0.0069 & DSLNCSCLALQTSGQEEQVNQR \\
\hline 23.0 & 2536.1282 & 0.9909 & DSLNCSCLALQTSGQEEQVNQR \\
\hline 13.7 & 2537.1122 & 0.0069 & DSLNCSCLALQTSGQEEQVNQR \\
\hline 7.8 & 2537.1314 & -0.0123 & TFLDNLVNFEKLHMIADTVR \\
\hline 4.5 & 2537.0975 & 0.0216 & ANTTGEPEEVSGALSLPSASAYVK \\
\hline 4.1 & 2537.1022 & 0.0168 & ASRTTGGATALXKMPGPQWSSTK \\
\hline 3.8 & 2537.1173 & 0.0018 & DPERAGLASSLLAGGTGHGDRGGAR \\
\hline 3.8 & 2537.1173 & 0.0018 & DPERAGLASSLLAGGTGHGDRGGAR \\
\hline 3.7 & 2537.1234 & -0.0043 & TLENGVNVTVSOINTMLGGRTR \\
\hline 3.2 & 2537.1234 & -0.0043 & TLENGVNVTVSQINTMLGGRTR \\
\hline
\end{tabular}

Spectrum No: 849; Query: 19; Rank: 1

\section{Peptide View}

MS/MS Fragmentation of LLNQTLR

Found in IPI00198080, Tax_Id=10116 Gene_Symbol=Pcyox1 Prenylcysteine oxidase precursor

Match to Query 19: 857.495488 from(429.755020,2+)

Title: 091008RatKidney_NH4Format01_23.949.949.2.dta

Data file K:INewmanPaper|Piliangl3SubProteomes\Piliang3SP\mgf5ppm\ERLIC_3SubProteomes5ppm.mgf 

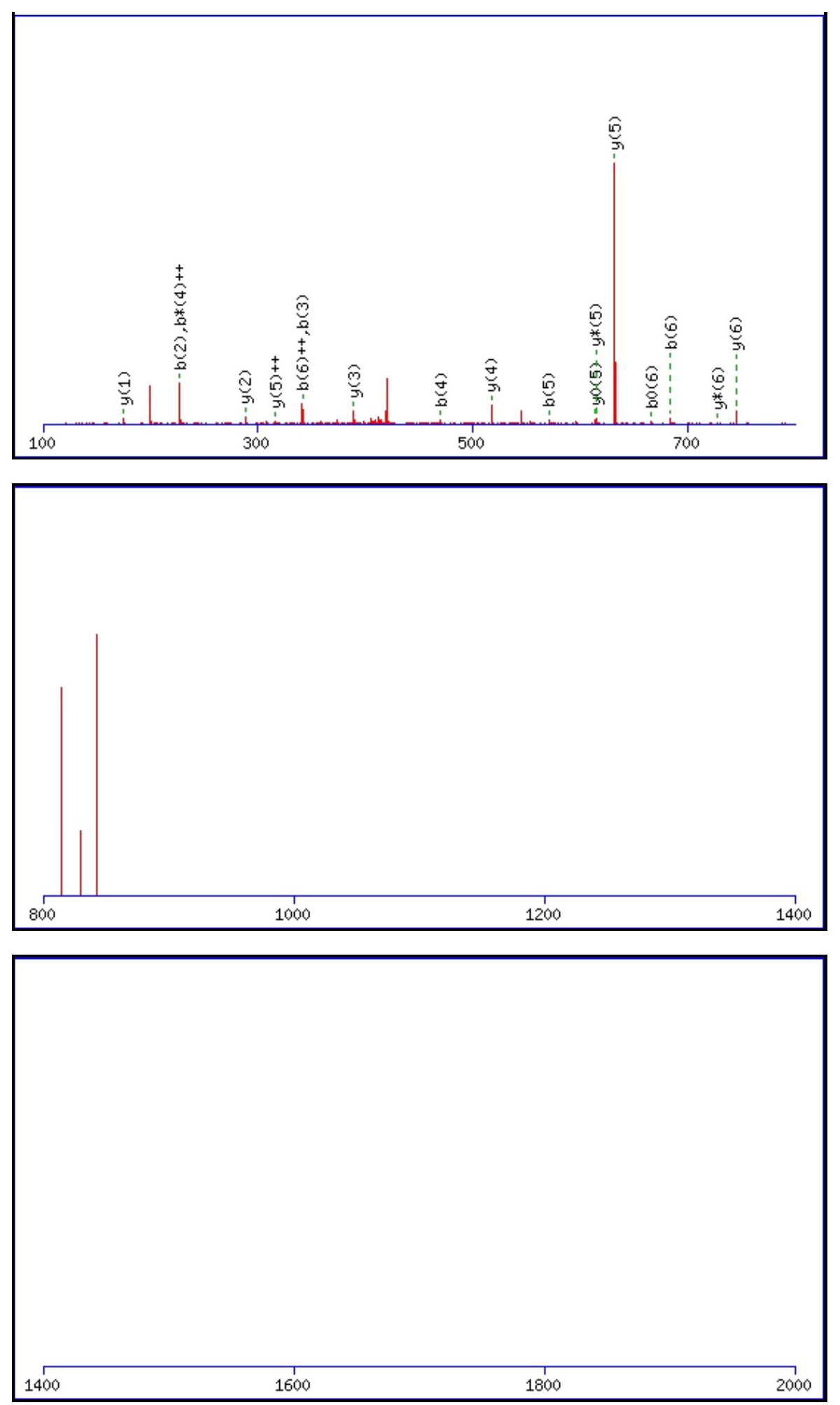

Monoisotopic mass of neutral peptide $\operatorname{Mr}($ calc): 857.4970

Fixed modifications: Carbamidomethyl (C)

Variable modifications:

N3 : Deamidated $\mathrm{N}(\mathrm{N})$

Ions Score: $4 \odot$ Expect: $\odot .0071$

Matches (Bold Red): 18/56 fragment ions using 34 most intense peaks

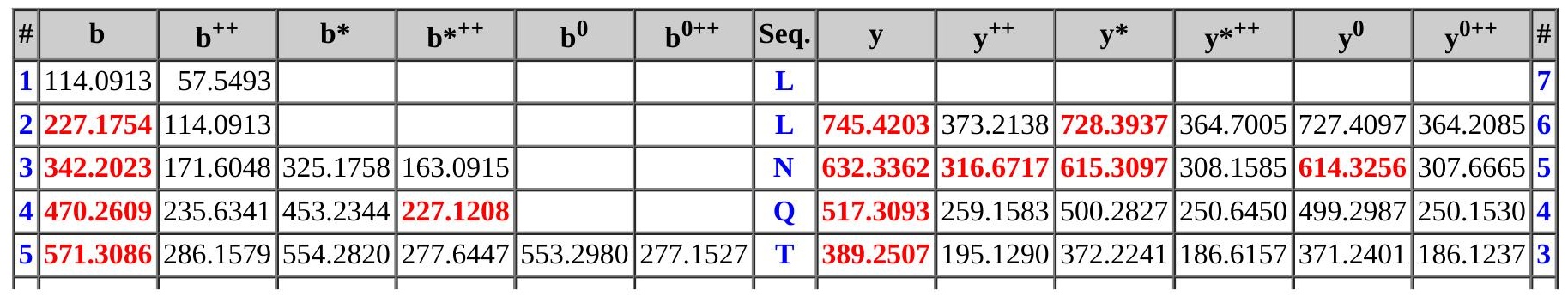




\begin{tabular}{|l|l|l|l|l|l|l|l|l|l|l|l|l|l|l|l|l||}
$\mathbf{6}$ & $\mathbf{6 8 4 . 3 9 2 7}$ & $\mathbf{3 4 2 . 7 0 0 0}$ & 667.3661 & 334.1867 & $\mathbf{6 6 6 . 3 8 2 1}$ & 333.6947 & $\mathbf{L}$ & $\mathbf{2 8 8 . 2 0 3 0}$ & $\mathbf{1 4 4 . 6 0 5 1}$ & 271.1765 & 136.0919 & & & $\mathbf{2}$ \\
\hline $\mathbf{7}$ & & & & & & & R & $\mathbf{1 7 5 . 1 1 9 0}$ & 88.0631 & 158.0924 & 79.5498 & & & $\mathbf{1}$ \\
\hline
\end{tabular}
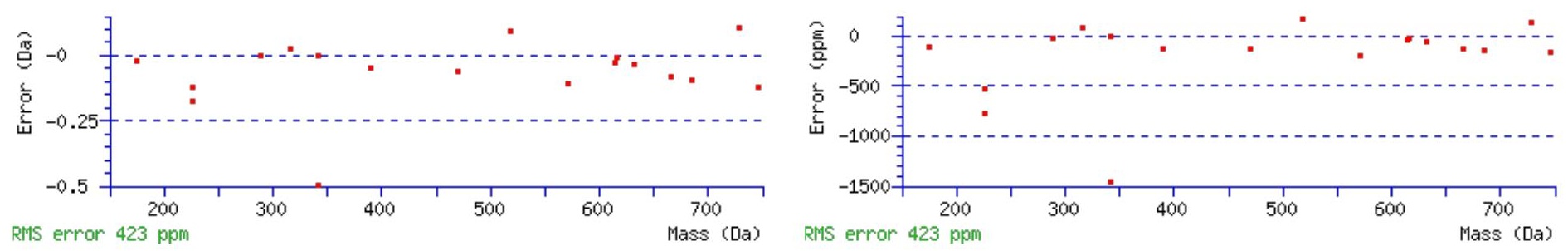

\section{All matches to this query}

\begin{tabular}{|l|l|l|l|}
\hline Score & Mr(calc): & Delta & \multicolumn{1}{|c|}{ Sequence } \\
\hline 39.6 & 857.4970 & -0.0016 & LLNQTLR \\
\hline 23.2 & 857.4971 & -0.0016 & QVIETIR \\
\hline 21.4 & 857.4970 & -0.0016 & $\underline{\text { ILNLTER }}$ \\
\hline 21.4 & 857.4970 & -0.0016 & LLNLTER \\
\hline 19.4 & 857.4971 & -0.0016 & LLGGTLER \\
\hline 19.2 & 857.4871 & 0.0084 & LINRWR \\
\hline 19.2 & 857.4971 & -0.0016 & LLDITOR \\
\hline 17.1 & 857.4970 & -0.0015 & $\underline{\text { LNKGKAN }}$ \\
\hline 17.1 & 857.4970 & -0.0016 & LINDVRK \\
\hline 13.9 & 857.4971 & -0.0016 & $\underline{\text { LLDTQR }}$ \\
\hline
\end{tabular}

Spectrum No: 850; Query: 2199; Rank: 1

\section{Peptide View}

MS/MS Fragmentation of EIANATTKPEDRNDPMLLYNK

Found in IPI00231789, Tax_Id=10116 Gene_Symbol=Mme Neprilysin

Match to Query 2199: 2449.175802 from(817.399210,3+)

Title: 091008RatKidney_NH4Format01_23.1369.1369.3.dta

Data file K:INewmanPaper|Piliangl3SubProteomes\Piliang3SP\mgf5ppm\ERLIC_3SubProteomes5ppm.mgf

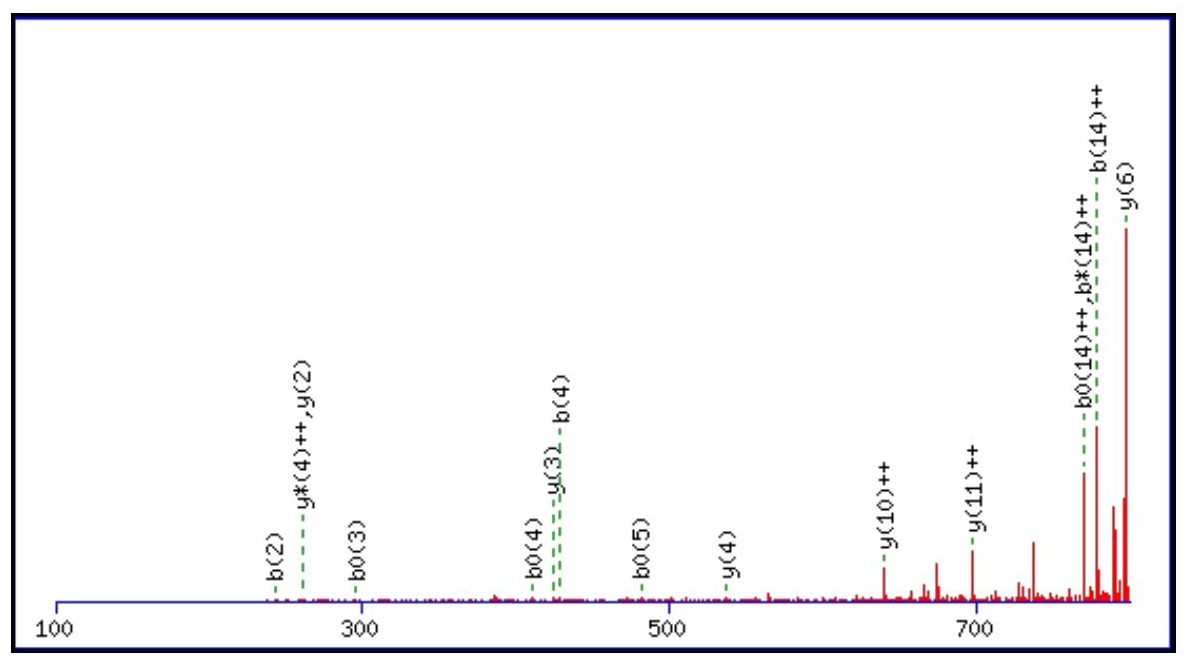



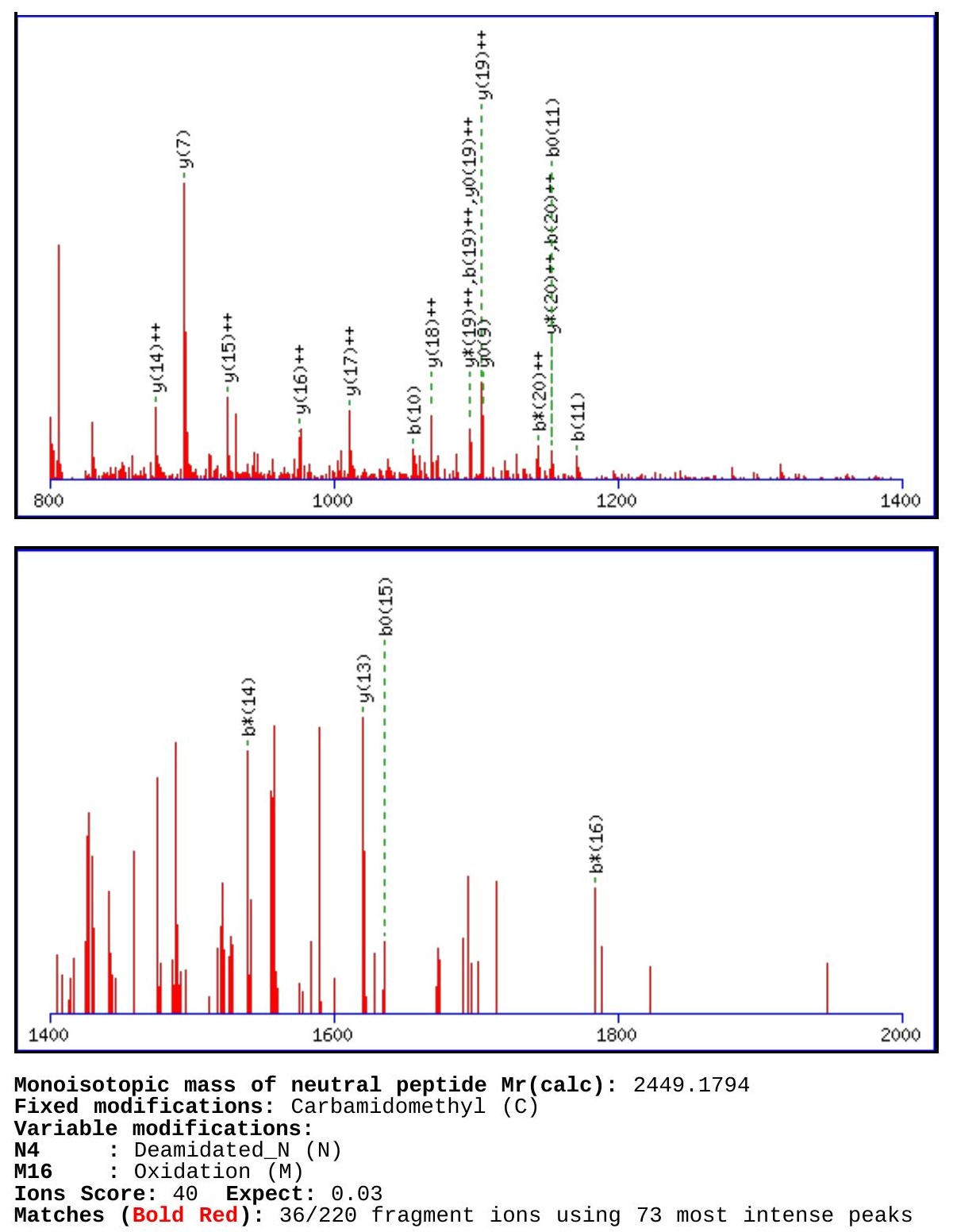

\begin{tabular}{|c|c|c|c|c|c|c|c|c|c|c|c|c|c|c|}
\hline \# & b & $\mathbf{b}^{++}$ & $\mathbf{b}^{*}$ & $\mathbf{b}^{*^{++}}$ & $\mathbf{b}^{\mathbf{0}}$ & $\mathbf{b}^{\mathbf{0 + +}}$ & Seq. & $\mathbf{y}$ & $\mathbf{y}^{++}$ & $\mathbf{y}^{*}$ & $\mathrm{y}^{\mathrm{*}^{++}}$ & $\mathbf{y}^{0}$ & $\mathbf{y}^{\mathbf{0 + +}}$ & \# \\
\hline 1 & 130.0499 & 65.5286 & & & 112.0393 & 56.5233 & $\mathbf{E}$ & & & & & & & 21 \\
\hline 2 & 243.1339 & 122.0706 & & & 225.1234 & 113.0653 & I & 2321.1442 & 1161.0757 & 2304.1176 & 1152.5625 & 2303.1336 & 1152.0704 & 20 \\
\hline 3 & 314.1710 & 157.5892 & & & 296.1605 & 148.5839 & A & 2208.0601 & 1104.5337 & 2191.0336 & 1096.0204 & 2190.0496 & 1095.5284 & 19 \\
\hline 4 & 429.1980 & 215.1026 & 412.1714 & 206.5894 & 411.1874 & 206.0973 & $\mathbf{N}$ & 2137.0230 & 1069.0151 & 2119.9965 & 1060.5019 & 2119.0124 & 1060.0099 & 18 \\
\hline 5 & 500.2351 & 250.6212 & 483.2085 & 242.1079 & 482.2245 & 241.6159 & A & 2021.9961 & 1011.5017 & 2004.9695 & 1002.9884 & 2003.9855 & 1002.4964 & 17 \\
\hline 6 & 601.2828 & 301.1450 & 584.2562 & 292.6318 & 583.2722 & 292.1397 & $T$ & 1950.9590 & 975.9831 & 1933.9324 & 967.4698 & 1932.9484 & 966.9778 & 16 \\
\hline 7 & 702.3305 & 351.6689 & 685.3039 & 343.1556 & 684.3199 & 342.6636 & $\mathbf{T}$ & 1849.9113 & 925.4593 & 1832.8847 & 916.9460 & 1831.9007 & 916.4540 & 15 \\
\hline 8 & 830.4254 & 415.7163 & 813.3989 & 407.2031 & 812.4149 & 406.7111 & $\mathbf{K}$ & 1748.8636 & 874.9354 & 1731.8371 & 866.4222 & 1730.8530 & 865.9302 & 14 \\
\hline 9 & 927.4782 & 464.2427 & 910.4516 & 455.7295 & 909.4676 & 455.2374 & $\mathbf{P}$ & 1620.7686 & 810.8880 & 1603.7421 & 802.3747 & 1602.7581 & 801.8827 & 13 \\
\hline 10 & 1056.5208 & 528.7640 & 1039.4942 & 520.2508 & 1038.5102 & 519.7587 & $\mathbf{E}$ & 1523.7159 & 762.3616 & 1506.6893 & 753.8483 & 1505.7053 & 753.3563 & 12 \\
\hline 11 & \begin{tabular}{|l|l|}
1171.5477 \\
\end{tabular} & 586.2775 & 1154.5212 & 577.7642 & 1153.5372 & 577.2722 & D & 1394.6733 & 697.8403 & 1377.6467 & 689.3270 & 1376.6627 & 688.8350 & 11 \\
\hline 12 & 1327.6488 & 664.3281 & 1310.6223 & 655.8148 & 1309.6383 & 655.3228 & $\mathbf{R}$ & 1279.6463 & 640.3268 & 1262.6198 & 631.8135 & 1261.6358 & 631.3215 & 10 \\
\hline 13 & 1441.6918 & 721.3495 & 1424.6652 & 712.8362 & 1423.6812 & 712.3442 & $\mathbf{N}$ & 1123.5452 & 562.2763 & 1106.5187 & 553.7630 & 1105.5347 & 553.2710 & 9 \\
\hline 14 & 1556.7187 & 778.8630 & 1539.6922 & 770.3497 & 1538.7081 & 769.8577 & D & 1009.5023 & 505.2548 & 992.4757 & 496.7415 & 991.4917 & 496.2495 & 8 \\
\hline 15 & 1653.7715 & 827.3894 & 1636.7449 & 818.8761 & 1635.7609 & 818.3841 & $\mathbf{P}$ & 894.4754 & 447.7413 & 877.4488 & 439.2280 & & & 7 \\
\hline 16 & 1800.8069 & 900.9071 & \begin{tabular}{|l|l|}
1783.7803 \\
\end{tabular} & 892.3938 & 1782.7963 & 891.9018 & $\mathbf{M}$ & 797.4226 & 399.2149 & 780.3960 & 390.7017 & & & 6 \\
\hline 17 & 1913.8909 & 957.4491 & 1896.8644 & 948.9358 & 1895.8804 & 948.4438 & $\mathbf{L}$ & 650.3872 & 325.6972 & 633.3606 & 317.1840 & & & 5 \\
\hline 18 & 2026.9750 & 1013.9911 & 2009.9484 & 1005.4779 & 2008.9644 & 1004.9859 & $\mathbf{L}$ & 537.3031 & 269.1552 & 520.2766 & 260.6419 & & & 4 \\
\hline
\end{tabular}




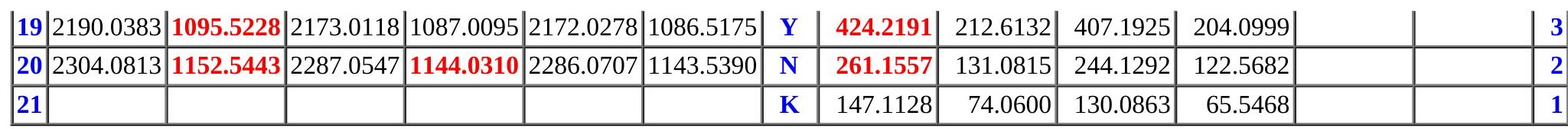
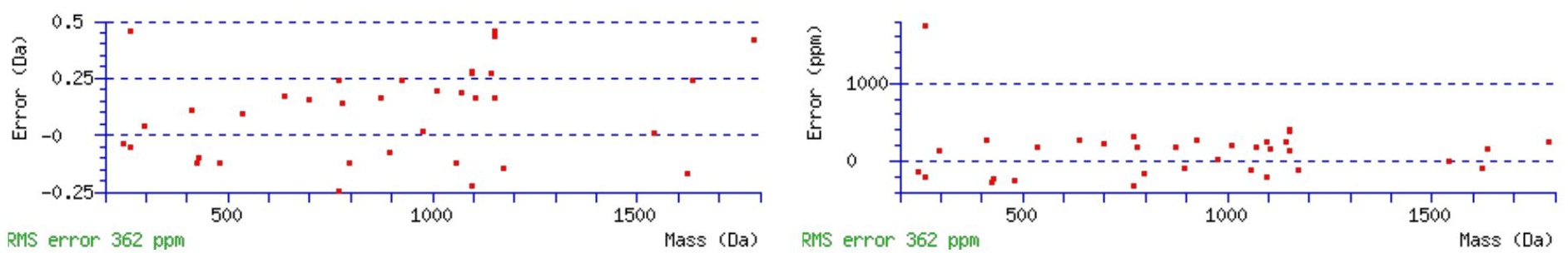

RMS error 362 ppm

\section{All matches to this query}

\begin{tabular}{|l|l|l|l|}
\hline Score & Mr(calc): & Delta & \multicolumn{1}{|c|}{ Sequence } \\
\hline 39.7 & 2449.1794 & -0.0036 & EIANATTKPEDRNDPMLLYNK \\
\hline 31.4 & 2449.1794 & -0.0036 & EIANATTKPEDRNDPMLLYNK \\
\hline 19.3 & 2448.1954 & 0.9804 & EIANATTKPEDRNDPMLLYNK \\
\hline 6.8 & 2449.1794 & -0.0036 & EIANATTKPEDRNDPMLLYNK \\
\hline 5.6 & 2449.1994 & -0.0236 & ELVYLVQIACQGKSWIVKR \\
\hline 4.4 & 2447.1464 & 2.0294 & RASQAALVPCPNCGRTFAVHR \\
\hline 3.6 & 2449.1638 & 0.0120 & QSLSNKHPELEIKSVDGFQGR \\
\hline 1.2 & 2447.1538 & 2.0220 & EKPLPYSPSVYSPENLLKAR \\
\hline 0.8 & 2447.1538 & 2.0220 & EKPLPYSPSVYSPENLLKAR \\
\hline 0.5 & 2447.1538 & 2.0220 & EKPLPYSPSVYSPENLLKAR \\
\hline
\end{tabular}

Spectrum No: 851; Query: 1226; Rank: 1

\section{Peptide View}

MS/MS Fragmentation of ASSILVNITELFPRPK

Found in IPI00206467, Tax_Id=10116 Gene_Symbol=Pecam Platelet endothelial cell adhesion molecule precursor

Match to Query 1226: 1785.001842 from(596.007890,3+)

Title: 091008RatKidney_NoSalt_21.5854.5854.3.dta

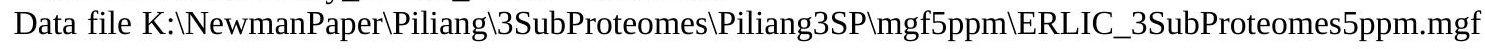

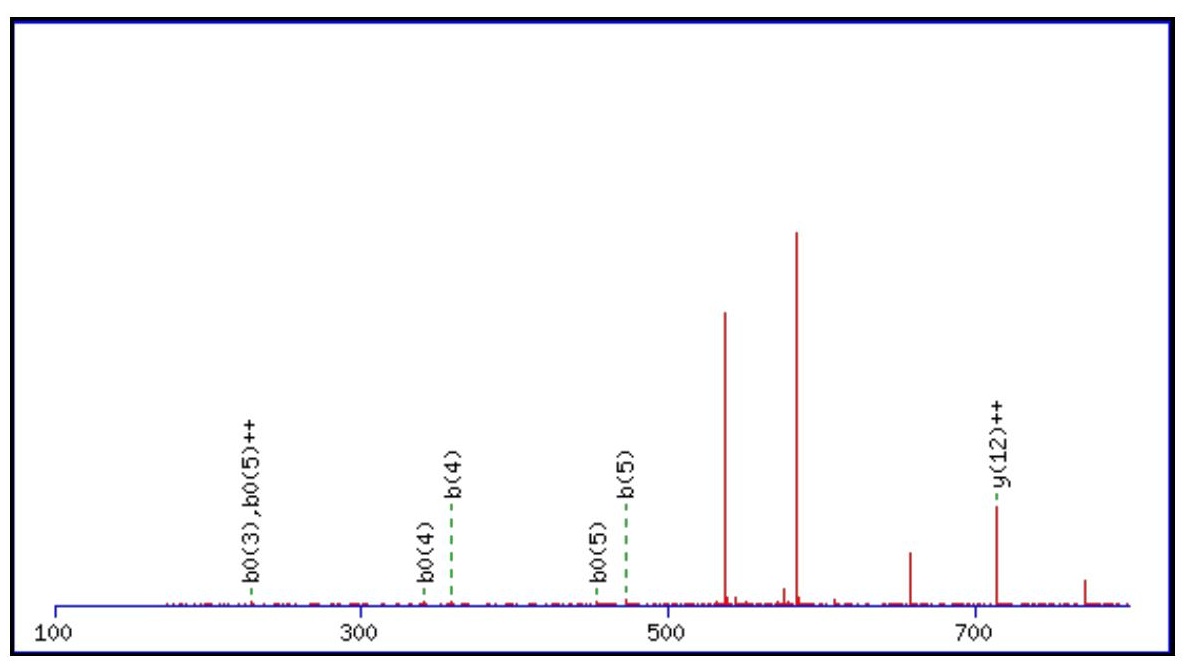



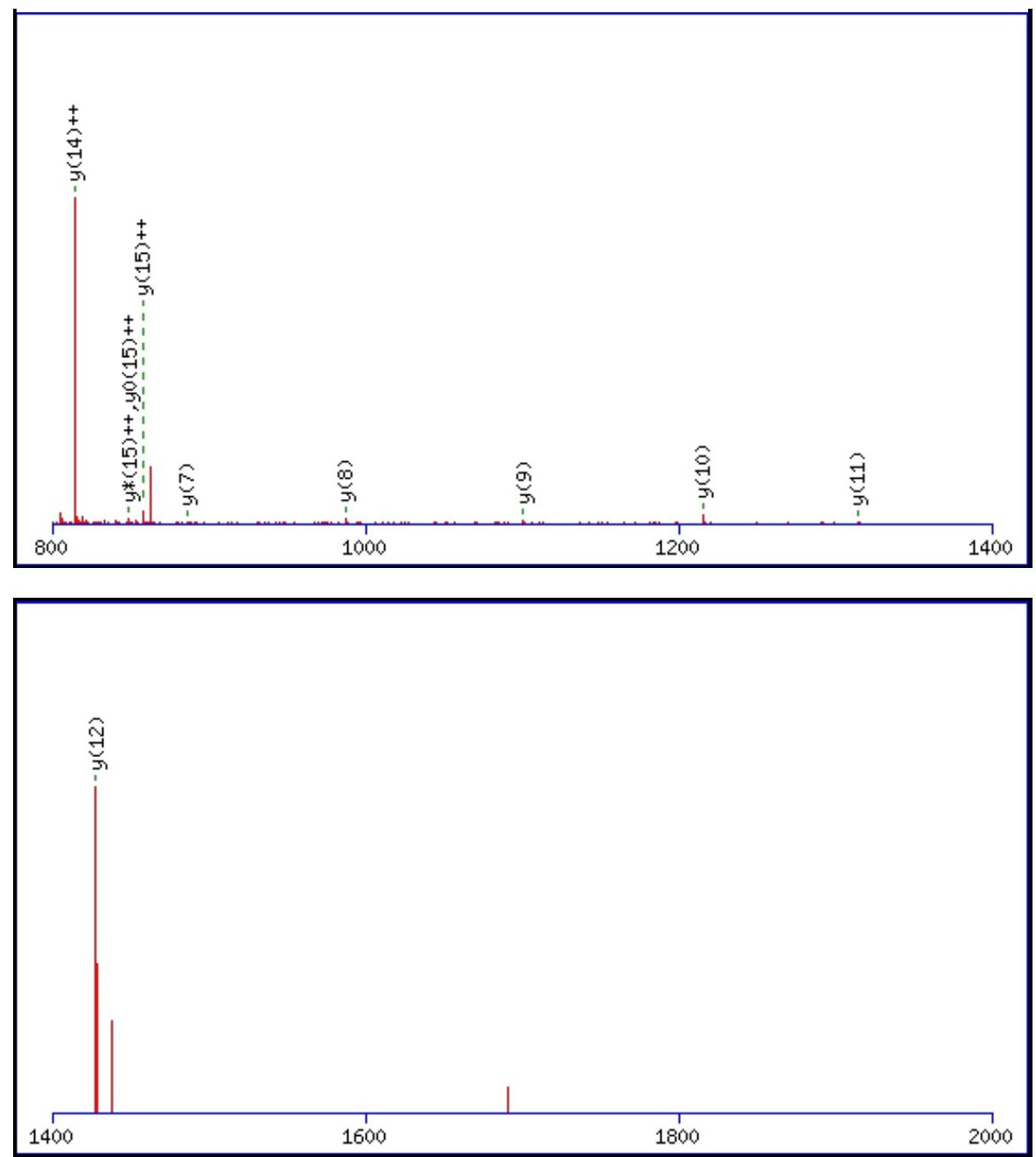

Monoisotopic mass of neutral peptide $\operatorname{Mr}($ calc): 1785.0036

Fixed modifications: Carbamidomethyl (C)

Variable modifications:

N7 : Deamidated_N (N)

Ions Score: 40 Expect: 0.0053

Matches (Bold Red): 17/154 fragment ions using 30 most intense peaks

\begin{tabular}{|c|c|c|c|c|c|c|c|c|c|c|c|c|c|c|}
\hline \# & b & $\mathbf{b}^{++}$ & $\mathbf{b}^{*}$ & $\mathbf{b}^{*^{++}}$ & $\mathbf{b}^{0}$ & $\mathbf{b}^{0++}$ & Seq. & $\mathbf{y}$ & $y^{++}$ & $\mathbf{y}^{*}$ & $\mathbf{y}^{*^{++}}$ & $\mathbf{y}^{\mathbf{0}}$ & $\mathbf{y}^{0++}$ & \# \\
\hline 1 & 72.0444 & 36.5258 & & & & & A & & & & & & & 16 \\
\hline 2 & 159.0764 & 80.0418 & & & 141.0659 & 71.0366 & S & 1714.9738 & 857.9905 & 1697.9472 & 849.4773 & 1696.9632 & 848.9853 & 15 \\
\hline 3 & 246.1084 & 123.5579 & & & 228.0979 & 114.5526 & S & 1627.9418 & 814.4745 & 1610.9152 & 805.9612 & 1609.9312 & 805.4692 & 14 \\
\hline 4 & 359.1925 & 180.0999 & & & 341.1819 & 171.0946 & I & 1540.9097 & 770.9585 & 1523.8832 & 762.4452 & 1522.8992 & 761.9532 & 13 \\
\hline 5 & 472.2766 & 236.6419 & & & 454.2660 & 227.6366 & $\mathbf{L}$ & 1427.8257 & 714.4165 & 1410.7991 & 705.9032 & 1409.8151 & 705.4112 & 12 \\
\hline 6 & 571.3450 & 286.1761 & & & 553.3344 & 277.1708 & $\mathbf{V}$ & 1314.7416 & 657.8744 & 1297.7151 & 649.3612 & 1296.7310 & 648.8692 & 11 \\
\hline 7 & 686.3719 & 343.6896 & 669.3454 & 335.1763 & 668.3614 & 334.6843 & $\mathbf{N}$ & 1215.6732 & 608.3402 & 1198.6466 & 599.8270 & 1197.6626 & 599.3350 & 10 \\
\hline 8 & 799.4560 & 400.2316 & 782.4294 & 391.7184 & 781.4454 & 391.2263 & I & 1100.6463 & 550.8268 & 1083.6197 & 542.3135 & 1082.6357 & 541.8215 & 9 \\
\hline 9 & 900.5037 & 450.7555 & 883.4771 & 442.2422 & 882.4931 & 441.7502 & $\mathbf{T}$ & 987.5622 & 494.2847 & 970.5356 & 485.7715 & 969.5516 & 485.2795 & 8 \\
\hline 10 & 1029.5463 & 515.2768 & 1012.5197 & 506.7635 & 1011.5357 & 506.2715 & $\mathbf{E}$ & 886.5145 & 443.7609 & 869.4880 & 435.2476 & 868.5039 & 434.7556 & 7 \\
\hline 11 & 1142.6303 & 571.8188 & 1125.6038 & 563.3055 & 1124.6198 & 562.8135 & $\mathbf{L}$ & 757.4719 & 379.2396 & 740.4454 & 370.7263 & & & 6 \\
\hline 12 & 1289.6987 & 645.3530 & 1272.6722 & 636.8397 & 1271.6882 & 636.3477 & $\mathbf{F}$ & 644.3879 & 322.6976 & 627.3613 & 314.1843 & & & 5 \\
\hline 13 & 1386.7515 & 693.8794 & 1369.7250 & 685.3661 & 1368.7409 & 684.8741 & $\mathbf{P}$ & 497.3194 & 249.1634 & 480.2929 & 240.6501 & & & 4 \\
\hline 14 & 1542.8526 & 771.9299 & 1525.8261 & 763.4167 & 1524.8420 & 762.9247 & $\mathbf{R}$ & 400.2667 & 200.6370 & 383.2401 & 192.1237 & & & 3 \\
\hline 15 & 1639.9054 & 820.4563 & 1622.8788 & 811.9431 & 1621.8948 & 811.4510 & $\mathbf{P}$ & 244.1656 & 122.5864 & 227.1390 & 114.0731 & & & 2 \\
\hline 16 & & & & & & & $\mathbf{K}$ & 147.1128 & 74.0600 & 130.0863 & 65.5468 & & & 1 \\
\hline
\end{tabular}



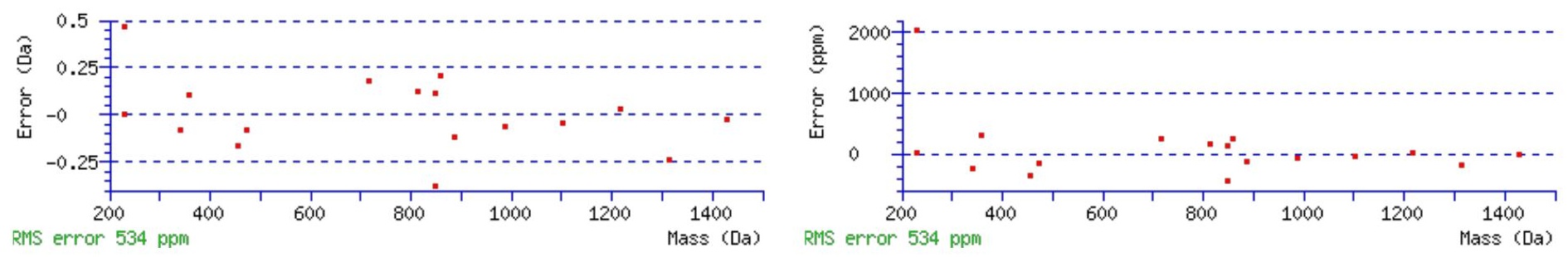

\section{All matches to this query}

\begin{tabular}{|l|l|l|l|}
\hline Score & Mr(calc): & Delta & \multicolumn{1}{c|}{ Sequence } \\
\hline 39.6 & 1785.0036 & -0.0018 & ASSILVNITELFPRPK \\
\hline 6.0 & 1783.0066 & 1.9953 & AFHLLQKLLTMDPIK \\
\hline 5.9 & 1785.0099 & -0.0081 & LRALPLPCSLLLALR \\
\hline 5.0 & 1784.9971 & 0.0047 & GILLYGPPGCGKTLLAR \\
\hline 3.9 & 1783.9822 & 1.0197 & GAGPGQLTRRPLQLLK \\
\hline 3.7 & 1784.9940 & 0.0078 & TTLLNYILTVTLIVK \\
\hline 3.7 & 1784.9940 & 0.0078 & TTLLNYILTVTLIVK \\
\hline 2.7 & 1783.9945 & 1.0074 & TGVVIVGPSGAGKSTLWR \\
\hline 2.7 & 1784.9940 & 0.0078 & TTLLNYILTVTLIVK \\
\hline 2.6 & 1784.9892 & 0.0126 & SQMKLEPKIMQLPVK \\
\hline
\end{tabular}

Spectrum No: 852; Query: 66; Rank: 1

\section{Peptide View}

MS/MS Fragmentation of DSTALWWK

Found in IPI00765413, Tax_Id=10116 Gene_Symbol=- 58 kDa protein

Match to Query 66: 1005.492948 from(503.753750,2+)

Title: 100101RatKid_NS_deglyco_21.3135.3135.2.dta

Data file K:INewmanPaper|Piliangl3SubProteomes\Piliang3SP\mgf5ppm\ERLIC_3SubProteomes5ppm.mgf

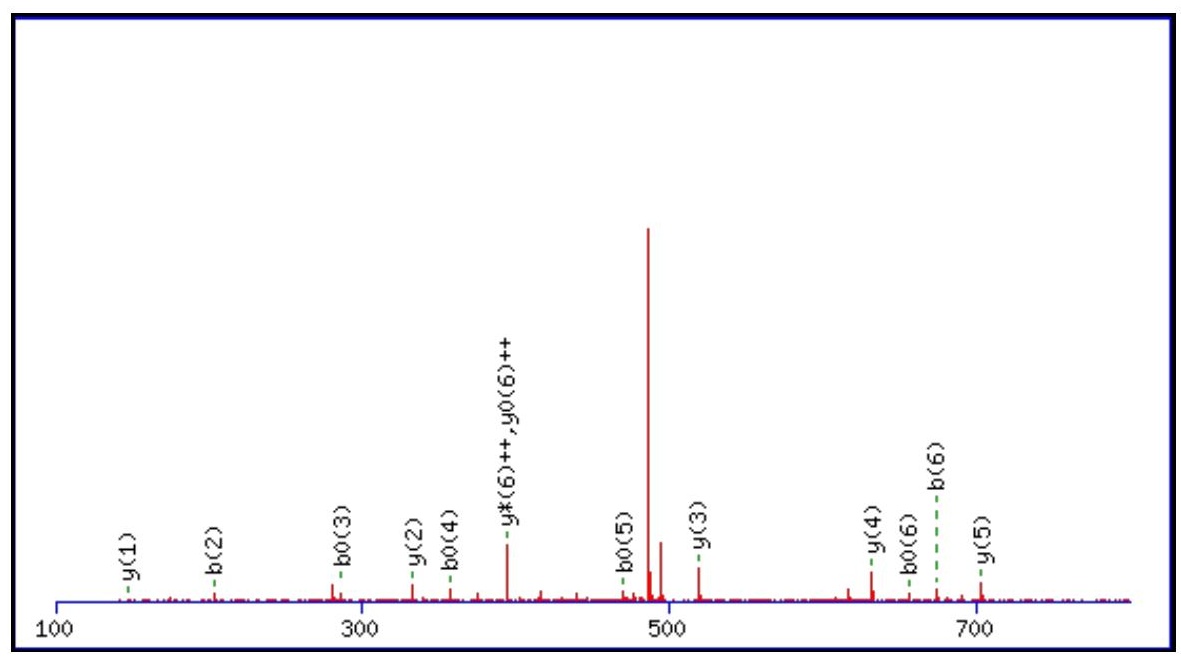



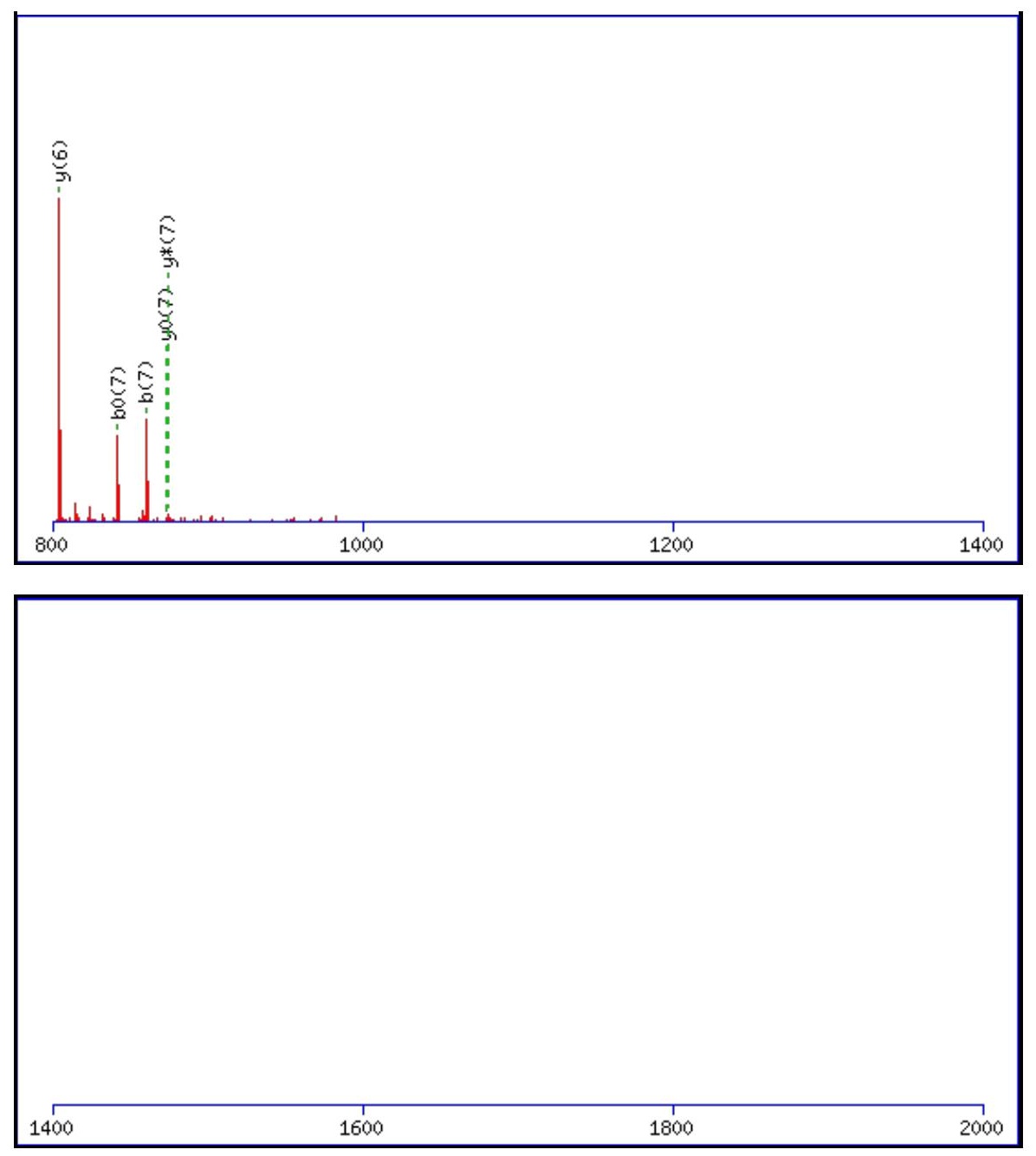

Monoisotopic mass of neutral peptide $\operatorname{Mr}($ calc): 1005.4920

Fixed modifications: Carbamidomethyl (C)

Ions Score: 40 Expect: 0.011

Matches (Bold Red): 18/60 fragment ions using 37 most intense peaks

\begin{tabular}{|c|c|c|c|c|c|c|c|c|c|c|c|c|}
\hline$\#$ & $\mathbf{b}$ & $\mathbf{b}^{++}$ & $\mathbf{b}^{\mathbf{0}}$ & $\mathbf{b}^{\mathbf{0 + +}}$ & Seq. & $\mathbf{y}$ & $\mathbf{y}^{++}$ & $\mathbf{y}^{\mathbf{*}}$ & $\mathbf{y}^{\mathbf{*}^{++}}$ & $\mathbf{y}^{\mathbf{0}}$ & $\mathbf{y}^{\mathbf{0 + +}}$ & $\#$ \\
\hline $\mathbf{1}$ & 116.0342 & 58.5207 & 98.0237 & 49.5155 & $\mathbf{D}$ & & & & & & & $\mathbf{8}$ \\
\hline $\mathbf{2}$ & $\mathbf{2 0 3 . 0 6 6 2}$ & 102.0368 & 185.0557 & 93.0315 & $\mathbf{S}$ & 891.4723 & 446.2398 & $\mathbf{8 7 4 . 4 4 5 8}$ & 437.7265 & $\mathbf{8 7 3 . 4 6 1 8}$ & 437.2345 & $\mathbf{7}$ \\
\hline $\mathbf{3}$ & 304.1139 & 152.5606 & $\mathbf{2 8 6 . 1 0 3 4}$ & 143.5553 & $\mathbf{T}$ & $\mathbf{8 0 4 . 4 4 0 3}$ & 402.7238 & 787.4137 & $\mathbf{3 9 4 . 2 1 0 5}$ & 786.4297 & 393.7185 & $\mathbf{6}$ \\
\hline $\mathbf{4}$ & 375.1510 & 188.0792 & 357.1405 & 179.0739 & $\mathbf{A}$ & $\mathbf{7 0 3 . 3 9 2 6}$ & 352.1999 & 686.3661 & 343.6867 & & & $\mathbf{5}$ \\
\hline $\mathbf{5}$ & 488.2351 & 244.6212 & $\mathbf{4 7 0 . 2 2 4 5}$ & 235.6159 & $\mathbf{L}$ & $\mathbf{6 3 2 . 3 5 5 5}$ & 316.6814 & 615.3289 & 308.1681 & & & $\mathbf{4}$ \\
\hline $\mathbf{6}$ & $\mathbf{6 7 4 . 3 1 4 4}$ & 337.6608 & $\mathbf{6 5 6 . 3 0 3 9}$ & 328.6556 & $\mathbf{W}$ & $\mathbf{5 1 9 . 2 7 1 4}$ & 260.1394 & 502.2449 & 251.6261 & & & $\mathbf{3}$ \\
\hline $\mathbf{7}$ & $\mathbf{8 6 0 . 3 9 3 7}$ & 430.7005 & $\mathbf{8 4 2 . 3 8 3 2}$ & 421.6952 & $\mathbf{W}$ & 333.1921 & 167.0997 & 316.1656 & 158.5864 & & & $\mathbf{2}$ \\
\hline $\mathbf{8}$ & & & & & $\mathbf{K}$ & $\mathbf{1 4 7 . 1 1 2 8}$ & 74.0600 & 130.0863 & 65.5468 & & & $\mathbf{1}$ \\
\hline
\end{tabular}

$$
\text { 夏 }
$$

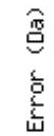

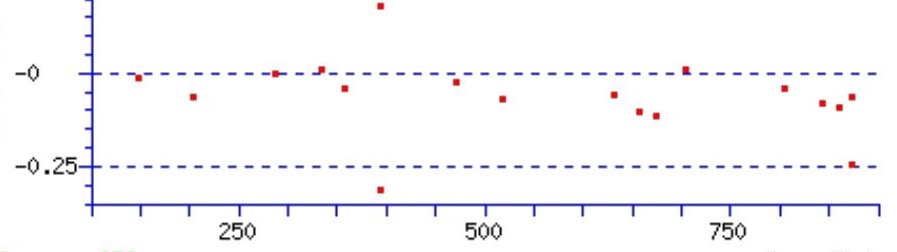

RMS error 253 ppm

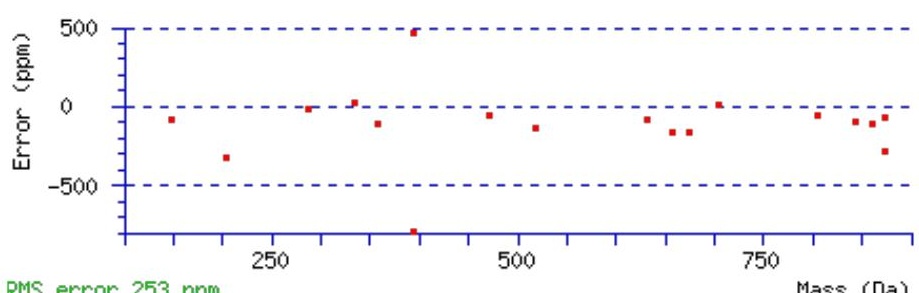

Mass (Da)

\section{All matches to this query}

\begin{tabular}{|l|c|c|c|}
\hline Score & Mr(calc): & Delta & Sequence \\
\hline 39.6 & 1005.4920 & 0.0010 & DSTALWWK \\
\hline
\end{tabular}




\begin{tabular}{|l|l|l|l|}
39.6 & 1005.4920 & 0.0010 & $\underline{\text { NSTALWWK }}$ \\
\hline 25.0 & 1005.4896 & 0.0034 & $\underline{\text { SLLTHDLK }}$ \\
\hline 13.5 & 1005.4978 & -0.0049 & $\underline{\text { SNASVLTEGK }}$ \\
\hline 13.0 & 1005.4952 & -0.0022 & DSGGGSRSKR \\
\hline 12.9 & 1005.4978 & -0.0049 & TSDSQIEVK \\
\hline 12.1 & 1005.4896 & 0.0034 & TVHTILNK \\
\hline 11.3 & 1005.4913 & 0.0016 & SNRCVLEK \\
\hline 10.3 & 1005.5025 & -0.0096 & KMGGLQNSR \\
\hline 10.0 & 1005.4879 & 0.0050 & $\underline{\text { RSINSDWK }}$ \\
\hline
\end{tabular}

Spectrum No: 853; Query: 66; Rank: 1

\section{Peptide View}

MS/MS Fragmentation of DSTALWWK

Found in IPI00765413, Tax_Id=10116 Gene_Symbol=- 58 kDa protein

Match to Query 66: 1005.492948 from(503.753750,2+)

Title: 100101RatKid_NS_deglyco_21.3135.3135.2.dta

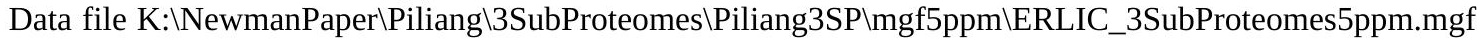
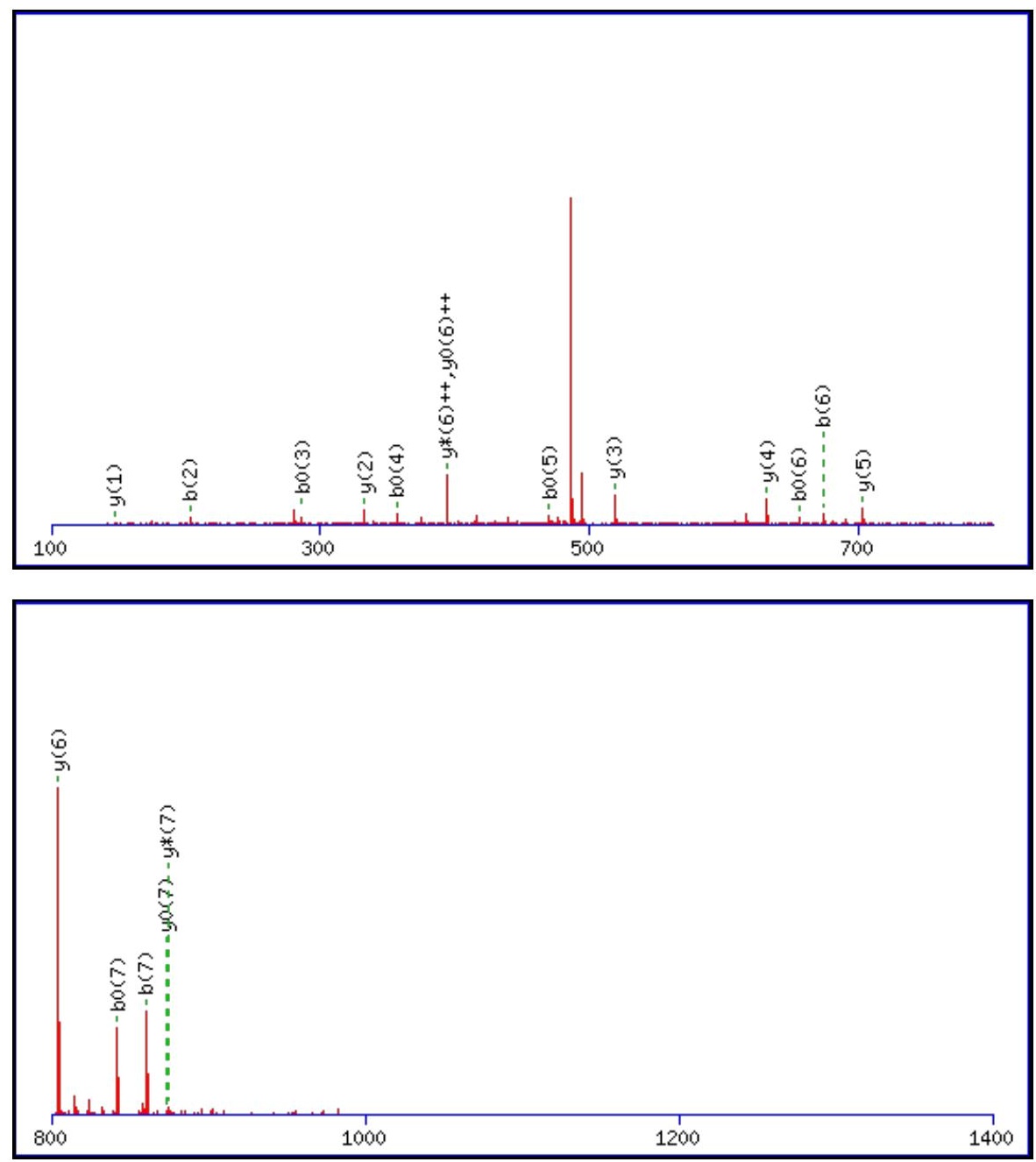
Monoisotopic mass of neutral peptide $\operatorname{Mr}($ calc): 1005.4920

Fixed modifications: Carbamidomethyl (C)

Ions Score: 40 Expect: 0.011

Matches (Bold Red): 18/60 fragment ions using 37 most intense peaks

\begin{tabular}{|c|c|c|c|c|c|c|c|c|c|c|c|c|}
\hline$\#$ & $\mathbf{b}$ & $\mathbf{b}^{++}$ & $\mathbf{b}^{\mathbf{0}}$ & $\mathbf{b}^{\mathbf{0 + +}}$ & $\mathbf{S e q}$ & $\mathbf{y}$ & $\mathbf{y}^{++}$ & $\mathbf{y}^{*}$ & $\mathbf{y}^{\mathbf{*}^{++}}$ & $\mathbf{y}^{\mathbf{0}}$ & $\mathbf{y}^{\mathbf{0 + +}}$ & $\#$ \\
\hline $\mathbf{1}$ & 116.0342 & 58.5207 & 98.0237 & 49.5155 & $\mathbf{D}$ & & & & & & & $\mathbf{8}$ \\
\hline $\mathbf{2}$ & $\mathbf{2 0 3 . 0 6 6 2}$ & 102.0368 & 185.0557 & 93.0315 & S & 891.4723 & 446.2398 & $\mathbf{8 7 4 . 4 4 5 8}$ & 437.7265 & $\mathbf{8 7 3 . 4 6 1 8}$ & 437.2345 & $\mathbf{7}$ \\
\hline $\mathbf{3}$ & 304.1139 & 152.5606 & $\mathbf{2 8 6 . 1 0 3 4}$ & 143.5553 & $\mathbf{T}$ & $\mathbf{8 0 4 . 4 4 0 3}$ & 402.7238 & 787.4137 & 394.2105 & 786.4297 & $\mathbf{3 9 3 . 7 1 8 5}$ & $\mathbf{6}$ \\
\hline $\mathbf{4}$ & 375.1510 & 188.0792 & $\mathbf{3 5 7 . 1 4 0 5}$ & 179.0739 & $\mathbf{A}$ & $\mathbf{7 0 3 . 3 9 2 6}$ & 352.1999 & 686.3661 & 343.6867 & & & $\mathbf{5}$ \\
\hline $\mathbf{5}$ & 488.2351 & 244.6212 & $\mathbf{4 7 0 . 2 2 4 5}$ & 235.6159 & $\mathbf{L}$ & $\mathbf{6 3 2 . 3 5 5 5}$ & 316.6814 & 615.3289 & 308.1681 & & & $\mathbf{4}$ \\
\hline $\mathbf{6}$ & $\mathbf{6 7 4 . 3 1 4 4}$ & 337.6608 & $\mathbf{6 5 6 . 3 0 3 9}$ & 328.6556 & $\mathbf{W}$ & $\mathbf{5 1 9 . 2 7 1 4}$ & 260.1394 & 502.2449 & 251.6261 & & & $\mathbf{3}$ \\
\hline $\mathbf{7}$ & $\mathbf{8 6 0 . 3 9 3 7}$ & 430.7005 & $\mathbf{8 4 2 . 3 8 3 2}$ & 421.6952 & $\mathbf{W}$ & $\mathbf{3 3 3 . 1 9 2 1}$ & 167.0997 & 316.1656 & 158.5864 & & & $\mathbf{2}$ \\
\hline $\mathbf{8}$ & & & & & $\mathbf{K}$ & $\mathbf{1 4 7 . 1 1 2 8}$ & 74.0600 & 130.0863 & 65.5468 & & & $\mathbf{1}$ \\
\hline
\end{tabular}

$$
\text { 営 }
$$
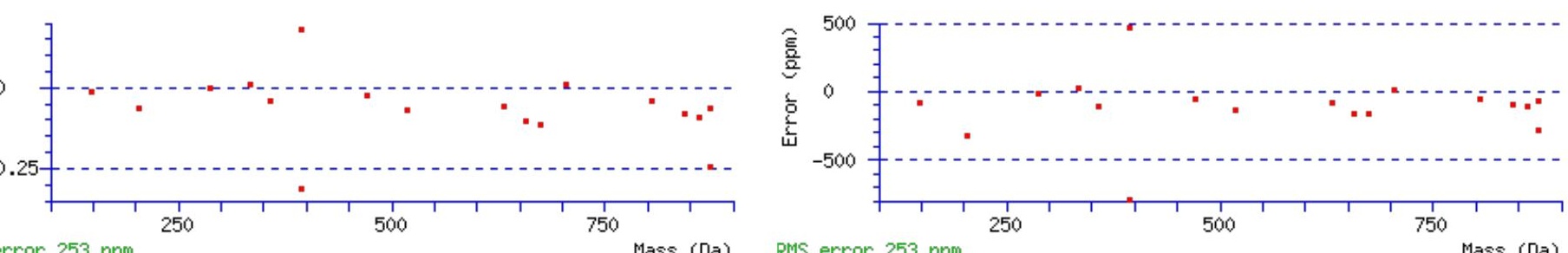

RMS error $253 \mathrm{ppm}$

Mass (Da) Rils error 253 ppm

\section{All matches to this query}

\begin{tabular}{|l|l|l|l|}
\hline Score & Mr(calc): & Delta & \multicolumn{1}{|c|}{ Sequence } \\
\hline 39.6 & 1005.4920 & 0.0010 & DSTALWWK \\
\hline 39.6 & 1005.4920 & 0.0010 & NSTALWWK \\
\hline 25.0 & 1005.4896 & 0.0034 & SLLTHDLK \\
\hline 13.5 & 1005.4978 & -0.0049 & SNASVLTEGK \\
\hline 13.0 & 1005.4952 & -0.0022 & DSGGGSRSKR \\
\hline 12.9 & 1005.4978 & -0.0049 & TSDSQIEVK \\
\hline 12.1 & 1005.4896 & 0.0034 & TVHTILNK \\
\hline 11.3 & 1005.4913 & 0.0016 & SNRCVLEK \\
\hline 10.3 & 1005.5025 & -0.0096 & KMGGLQNSR \\
\hline 10.0 & 1005.4879 & 0.0050 & RSINSDWK \\
\hline
\end{tabular}

Spectrum No: 854; Query: 493; Rank: 1 


\section{Peptide View}

\section{MS/MS Fragmentation of EALPFWMNSTGK}

Found in IPI00358524, Tax_Id=10116 Gene_Symbol=Enpp6_predicted ectonucleotide pyrophosphatase/phosphodiesterase 6

Match to Query 493: 1396.634728 from(699.324640,2+)

Title: 100101RatKid_NS_deglyco_25.3233.3233.2.dta

Data file K:\NewmanPaper $\backslash$ Piliang $\backslash 3$ SubProteomes $\backslash$ Piliang3SP $\backslash$ mgf5ppm\ERLIC_3SubProteomes5ppm.mgf
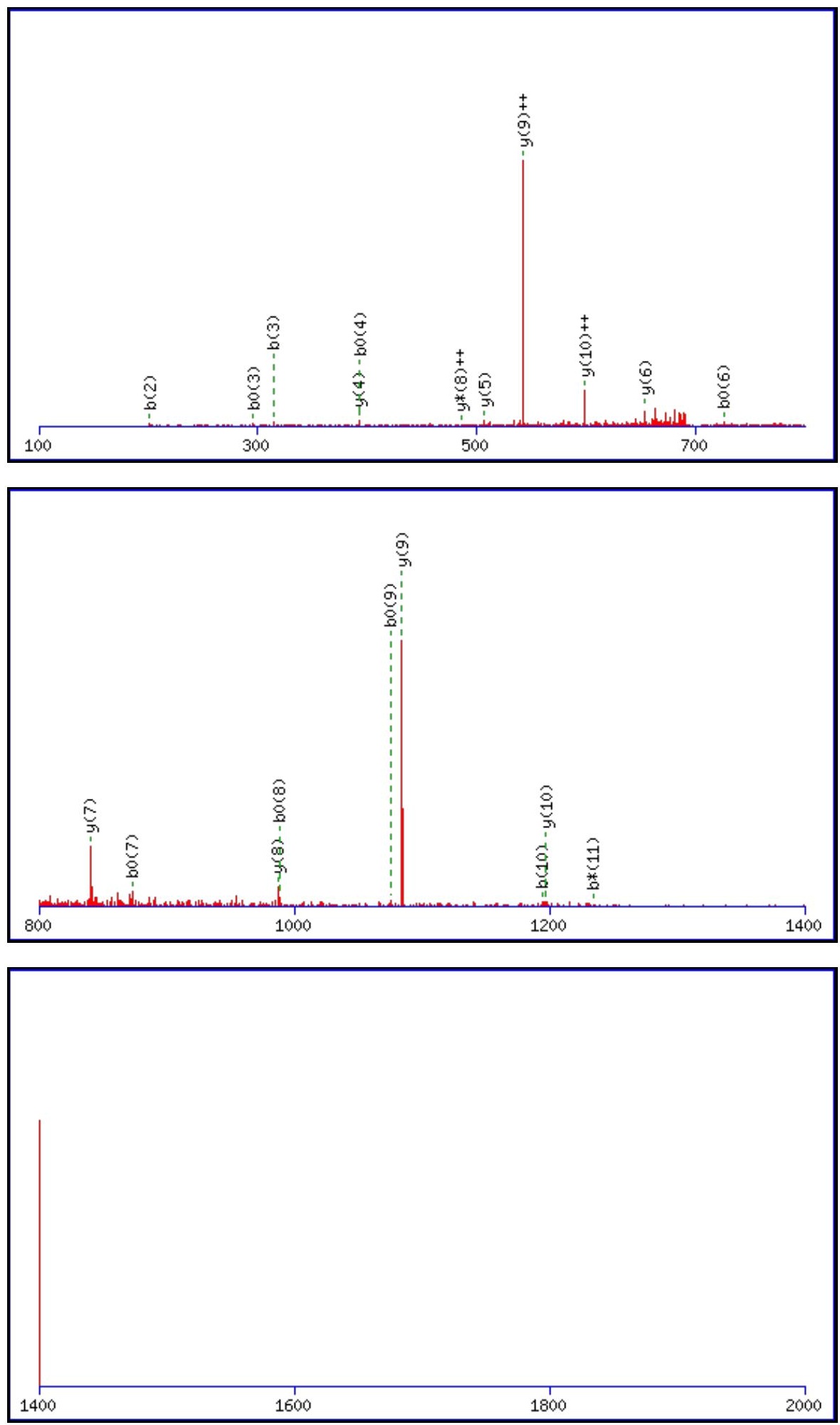

Monoisotopic mass of neutral peptide $\operatorname{Mr}($ calc): 1396.6333

Fixed modifications: Carbamidomethyl (C)

Variable modifications:

M7 : Oxidation (M)

N8 : Deamidated $\mathrm{N}(\mathrm{N})$

Ions Score: 40 Expect: 0.017 
Matches (Bold Red): 20/114 fragment ions using 50 most intense peaks

\begin{tabular}{|r|c|c|c|c|c|c|c|c|c|c|c|c|c|c|}
\hline$\#$ & $\mathbf{b}$ & $\mathbf{b}^{++}$ & $\mathbf{b}^{*}$ & $\mathbf{b}^{\mathbf{*}^{++}}$ & $\mathbf{b}^{\mathbf{0}}$ & $\mathbf{b}^{\mathbf{0 + +}}$ & $\mathbf{S e q}$ & $\mathbf{y}$ & $\mathbf{y}^{++}$ & $\mathbf{y}^{\mathbf{*}}$ & $\mathbf{y}^{\mathbf{*}^{++}}$ & $\mathbf{y}^{\mathbf{0}}$ & $\mathbf{y}^{\mathbf{0 + +}}$ & $\#$ \\
\hline $\mathbf{1}$ & 130.0499 & 65.5286 & & & 112.0393 & 56.5233 & $\mathbf{E}$ & & & & & & & $\mathbf{1 2}$ \\
\hline $\mathbf{2}$ & $\mathbf{2 0 1 . 0 8 7 0}$ & 101.0471 & & & 183.0764 & 92.0418 & $\mathbf{A}$ & 1268.5980 & 634.8026 & 1251.5714 & 626.2894 & 1250.5874 & 625.7973 & $\mathbf{1 1}$ \\
\hline $\mathbf{3}$ & $\mathbf{3 1 4 . 1 7 1 0}$ & 157.5892 & & & $\mathbf{2 9 6 . 1 6 0 5}$ & 148.5839 & $\mathbf{L}$ & $\mathbf{1 1 9 7 . 5 6 0 9}$ & 599.2841 & 1180.5343 & 590.7708 & 1179.5503 & 590.2788 & $\mathbf{1 0}$ \\
\hline $\mathbf{4}$ & 411.2238 & 206.1155 & & & 393.2132 & 197.1103 & $\mathbf{P}$ & $\mathbf{1 0 8 4 . 4 7 6 8}$ & $\mathbf{5 4 2 . 7 4 2 0}$ & 1067.4503 & 534.2288 & 1066.4662 & 533.7368 & $\mathbf{9}$ \\
\hline $\mathbf{5}$ & 558.2922 & 279.6498 & & & 540.2817 & 270.6445 & $\mathbf{F}$ & $\mathbf{9 8 7 . 4 2 4 0}$ & 494.2157 & 970.3975 & $\mathbf{4 8 5 . 7 0 2 4}$ & 969.4135 & 485.2104 & $\mathbf{8}$ \\
\hline $\mathbf{6}$ & 744.3715 & 372.6894 & & & $\mathbf{7 2 6 . 3 6 1 0}$ & 363.6841 & $\mathbf{W}$ & $\mathbf{8 4 0 . 3 5 5 6}$ & 420.6815 & 823.3291 & 412.1682 & 822.3451 & 411.6762 & $\mathbf{7}$ \\
\hline $\mathbf{7}$ & 891.4069 & 446.2071 & & & $\mathbf{8 7 3 . 3 9 6 4}$ & 437.2018 & $\mathbf{M}$ & $\mathbf{6 5 4 . 2 7 6 3}$ & 327.6418 & 637.2498 & 319.1285 & 636.2658 & 318.6365 & $\mathbf{6}$ \\
\hline $\mathbf{8}$ & 1006.4339 & 503.7206 & 989.4073 & 495.2073 & $\mathbf{9 8 8 . 4 2 3 3}$ & 494.7153 & $\mathbf{N}$ & $\mathbf{5 0 7 . 2 4 0 9}$ & 254.1241 & 490.2144 & 245.6108 & 489.2303 & 245.1188 & $\mathbf{5}$ \\
\hline $\mathbf{9}$ & 1093.4659 & 547.2366 & 1076.4394 & 538.7233 & $\mathbf{1 0 7 5 . 4 5 5 3}$ & 538.2313 & $\mathbf{S}$ & 392.2140 & 196.6106 & 375.1874 & 188.0974 & 374.2034 & 187.6053 & $\mathbf{4}$ \\
\hline $\mathbf{1 0}$ & $\mathbf{1 1 9 4 . 5 1 3 6}$ & 597.7604 & 1177.4870 & 589.2472 & 1176.5030 & 588.7551 & $\mathbf{T}$ & 305.1819 & 153.0946 & 288.1554 & 144.5813 & 287.1714 & 144.0893 & $\mathbf{3}$ \\
\hline $\mathbf{1 1}$ & 1251.5350 & 626.2712 & $\mathbf{1 2 3 4 . 5 0 8 5}$ & 617.7579 & 1233.5245 & 617.2659 & $\mathbf{G}$ & 204.1343 & 102.5708 & 187.1077 & 94.0575 & & & $\mathbf{2}$ \\
\hline $\mathbf{1 2}$ & & & & & & & $\mathbf{K}$ & 147.1128 & 74.0600 & 130.0863 & 65.5468 & & & $\mathbf{1}$ \\
\hline
\end{tabular}
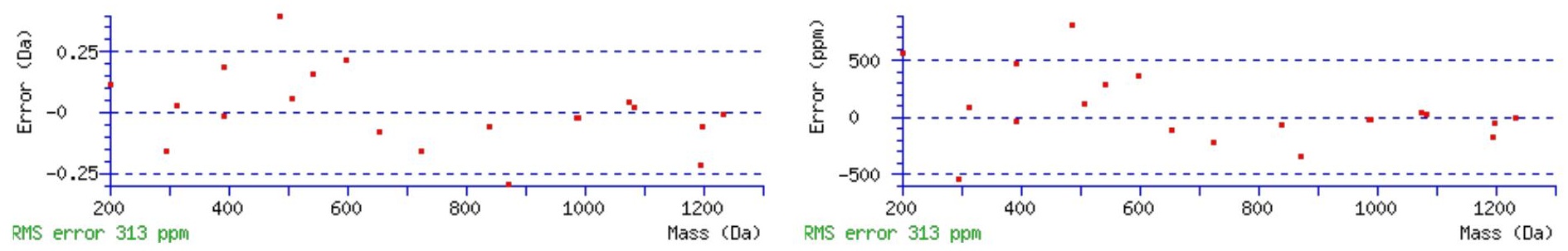

\section{All matches to this query}

\begin{tabular}{|l|l|l|l|}
\hline Score & Mr(calc): & Delta & \multicolumn{1}{c|}{ Sequence } \\
\hline 39.6 & 1396.6333 & 0.0014 & EALPFWMNSTGK \\
\hline 9.8 & 1396.6445 & -0.0097 & FLSVTLVFPSK \\
\hline 9.8 & 1396.6445 & -0.0097 & FLSVTLVFPSK \\
\hline 7.4 & 1396.6470 & -0.0123 & AEANVGPENGPDVK \\
\hline 6.5 & 1395.6291 & 1.0056 & TIMHLMINNTK \\
\hline 5.3 & 1396.6487 & -0.0139 & ELELNTVGNLSK \\
\hline 5.0 & 1396.6349 & -0.0002 & SIIYYMVTSPK \\
\hline 3.9 & 1396.6349 & -0.0002 & SIIYYMVTSPK \\
\hline 2.9 & 1396.6348 & -0.0000 & DSKVQRTPDGSK \\
\hline 2.5 & 1395.6283 & 1.0065 & TLPNATTVDNIR \\
\hline
\end{tabular}

Spectrum No: 855; Query: 851; Rank: 1

\section{Peptide View}

MS/MS Fragmentation of TANKTSLISESLAPR

Found in IPI00363534, Tax_Id=10116 Gene_Symbol=Lama1 laminin, alpha 1

Match to Query 851: 1587.849072 from(530.290300,3+)

Title: 100101RatKid_NS_deglyco_18.2366.2366.3.dta

Data file K:INewmanPaper|Piliang $\mid 3 S$ SubProteomes\Piliang3SP\mgf5ppm\ERLIC_3SubProteomes5ppm.mgf 

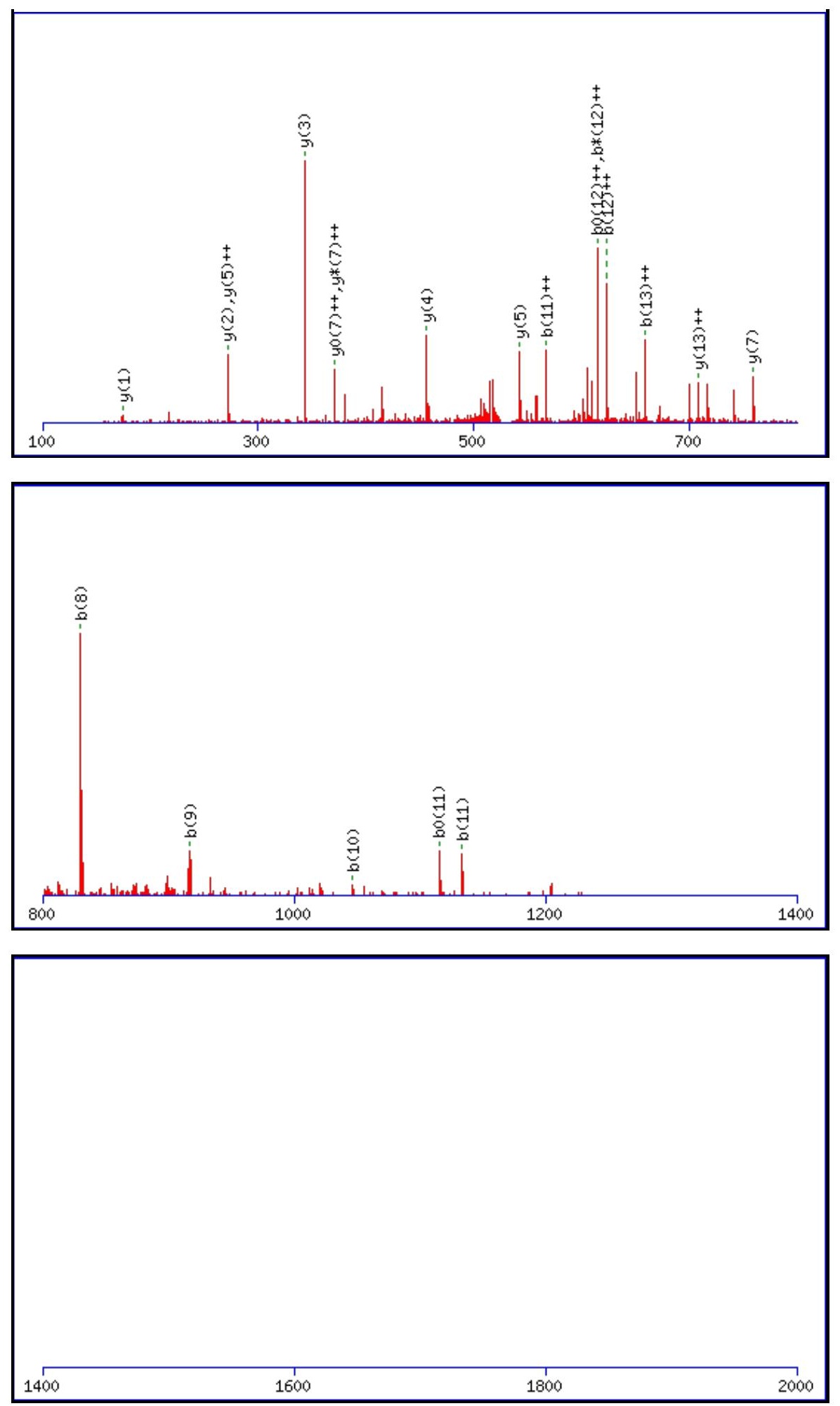

Monoisotopic mass of neutral peptide $\operatorname{Mr}($ calc): 1587.8468

Fixed modifications: Carbamidomethyl (C)

Variable modifications:

N3 : Deamidated_N (N)

Ions Score: 39 Expect: 0.012

Matches (Bold Red): 20/156 fragment ions using 26 most intense peaks

\begin{tabular}{|r|c|c|c|c|c|c|c|c|c|c|c|c|c|c|}
\hline$\#$ & $\mathbf{b}$ & $\mathbf{b}^{++}$ & $\mathbf{b}^{*}$ & $\mathbf{b}^{\boldsymbol{*}^{++}}$ & $\mathbf{b}^{\mathbf{0}}$ & $\mathbf{b}^{\mathbf{0 + +}}$ & Seq. & $\mathbf{y}$ & $\mathbf{y}^{++}$ & $\mathbf{y}^{\mathbf{*}}$ & $\mathbf{y}^{\boldsymbol{*}^{++}}$ & $\mathbf{y}^{\mathbf{0}}$ & $\mathbf{y}^{\mathbf{0 + +}}$ & $\#$ \\
\hline $\mathbf{1}$ & 102.0550 & 51.5311 & & & 84.0444 & 42.5258 & $\mathbf{T}$ & & & & & & & $\mathbf{1 5}$ \\
\hline $\mathbf{2}$ & 173.0921 & 87.0497 & & & 155.0815 & 78.0444 & $\mathbf{A}$ & 1487.8064 & 744.4068 & 1470.7798 & 735.8936 & 1469.7958 & 735.4016 & $\mathbf{1 4}$ \\
\hline $\mathbf{3}$ & 288.1190 & 144.5631 & 271.0925 & 136.0499 & 270.1084 & 135.5579 & $\mathbf{N}$ & 1416.7693 & 708.8883 & 1399.7427 & 700.3750 & 1398.7587 & 699.8830 & $\mathbf{1 3}$ \\
\hline $\mathbf{4}$ & 416.2140 & 208.6106 & 399.1874 & 200.0973 & 398.2034 & 199.6053 & $\mathbf{K}$ & 1301.7423 & 651.3748 & 1284.7158 & 642.8615 & 1283.7318 & 642.3695 & $\mathbf{1 2}$ \\
\hline $\mathbf{5}$ & 517.2616 & 259.1345 & 500.2351 & 250.6212 & 499.2511 & 250.1292 & $\mathbf{T}$ & 1173.6474 & 587.3273 & 1156.6208 & 578.8141 & 1155.6368 & 578.3220 & $\mathbf{1 1}$ \\
\hline
\end{tabular}




\begin{tabular}{|r|r|r|r|r|r|r|r|r|r|r|r|r|r|r|r|r|r|r|r|}
$\mathbf{6}$ & 604.2937 & 302.6505 & 587.2671 & 294.1372 & 586.2831 & 293.6452 & S & 1072.5997 & 536.8035 & 1055.5732 & 528.2902 & 1054.5891 & 527.7982 & $\mathbf{1 0}$ \\
\hline $\mathbf{7}$ & 717.3777 & 359.1925 & 700.3512 & 350.6792 & 699.3672 & 350.1872 & L & 985.5677 & 493.2875 & 968.5411 & 484.7742 & 967.5571 & 484.2822 & $\mathbf{9}$ \\
\hline $\mathbf{8}$ & $\mathbf{8 3 0 . 4 6 1 8}$ & 415.7345 & 813.4353 & 407.2213 & 812.4512 & 406.7293 & I & 872.4836 & 436.7454 & 855.4571 & 428.2322 & 854.4730 & 427.7402 & $\mathbf{8}$ \\
\hline $\mathbf{9}$ & $\mathbf{9 1 7 . 4 9 3 8}$ & 459.2506 & 900.4673 & 450.7373 & 899.4833 & 450.2453 & S & 759.3995 & 380.2034 & 742.3730 & 371.6901 & 741.3890 & 371.1981 & $\mathbf{7}$ \\
\hline $\mathbf{1 0}$ & $\mathbf{1 0 4 6 . 5 3 6 4}$ & 523.7719 & 1029.5099 & 515.2586 & 1028.5259 & 514.7666 & E & 672.3675 & 336.6874 & 655.3410 & 328.1741 & 654.3570 & 327.6821 & $\mathbf{6}$ \\
\hline $\mathbf{1 1}$ & $\mathbf{1 1 3 3 . 5 6 8 5}$ & $\mathbf{5 6 7 . 2 8 7 9}$ & 1116.5419 & 558.7746 & $\mathbf{1 1 1 5 . 5 5 7 9}$ & 558.2826 & S & 543.3249 & 272.1661 & 526.2984 & 263.6528 & 525.3144 & 263.1608 & $\mathbf{5}$ \\
\hline $\mathbf{1 2}$ & 1246.6525 & $\mathbf{6 2 3 . 8 2 9 9}$ & 1229.6260 & $\mathbf{6 1 5 . 3 1 6 6}$ & 1228.6420 & $\mathbf{6 1 4 . 8 2 4 6}$ & L & $\mathbf{4 5 6 . 2 9 2 9}$ & 228.6501 & 439.2663 & 220.1368 & & & & $\mathbf{4}$ \\
\hline $\mathbf{1 3}$ & 1317.6896 & $\mathbf{6 5 9 . 3 4 8 5}$ & 1300.6631 & 650.8352 & 1299.6791 & 650.3432 & A & 343.2088 & 172.1081 & 326.1823 & 163.5948 & & & & $\mathbf{3}$ \\
\hline $\mathbf{1 4}$ & $\mathbf{1 4 1 4 . 7 4 2 4}$ & $\mathbf{7 0 7 . 8 7 4 8}$ & 1397.7158 & 699.3616 & 1396.7318 & 698.8696 & $\mathbf{P}$ & $\mathbf{2 7 2 . 1 7 1 7}$ & 136.5895 & 255.1452 & 128.0762 & & & $\mathbf{2}$ \\
\hline $\mathbf{1 5}$ & & & & & & & R & $\mathbf{1 7 5 . 1 1 9 0}$ & 88.0631 & 158.0924 & 79.5498 & & & $\mathbf{1}$ \\
\hline
\end{tabular}
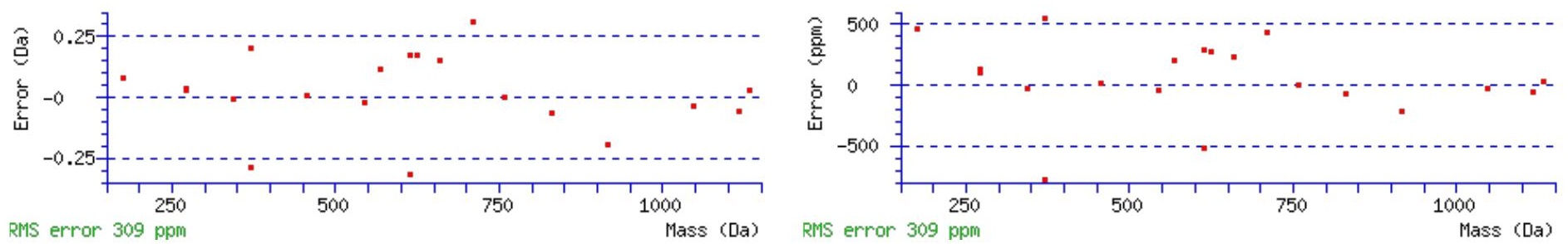

RMS error 309 ppm

Mass (Da) RMS error 309 ppm

Mass (Da)

\section{All matches to this query}

\begin{tabular}{|l|l|c|l|}
\hline Score & Mr(calc): & Delta & \multicolumn{1}{c|}{ Sequence } \\
\hline 39.4 & 1587.8468 & 0.0023 & TANKTSLISESLAPR \\
\hline 8.7 & 1587.8522 & -0.0031 & VVKFPDFDRNVPR \\
\hline 4.9 & 1586.8334 & 1.0157 & TAALPTFRKLYAR \\
\hline 4.1 & 1585.8464 & 2.0027 & GPWTVESEAIITRK \\
\hline 4.1 & 1587.8468 & 0.0023 & QTPESKSSAPTKSIK \\
\hline 3.8 & 1586.8488 & 1.0002 & TSRLGNGATLRTSPR \\
\hline 3.3 & 1586.8406 & 1.0085 & NRAPVLASRVAPTR \\
\hline 3.1 & 1587.8426 & 0.0065 & GFGFGLVKLDVKTK \\
\hline 2.4 & 1587.8498 & -0.0007 & GLSALVTRPASAPLR \\
\hline 2.4 & 1587.8498 & -0.0007 & GLSALVTRPASAPLR \\
\hline
\end{tabular}

Spectrum No: 856; Query: 2204; Rank: 1

\section{Peptide View}

MS/MS Fragmentation of VATNPQGLVGTENTTSEMDPSHR

Found in IPI00358014, Tax_Id=10116 Gene_Symbol=Btd Biotinidase precursor

Match to Query 2204: 2457.114672 from(820.045500,3+)

Title: 100101RatKid_NS_deglyco_21.1541.1541.3.dta

Data file K:INewmanPaper|Piliangl3SubProteomes\Piliang3SP\mgf5ppm\ERLIC_3SubProteomes5ppm.mgf 

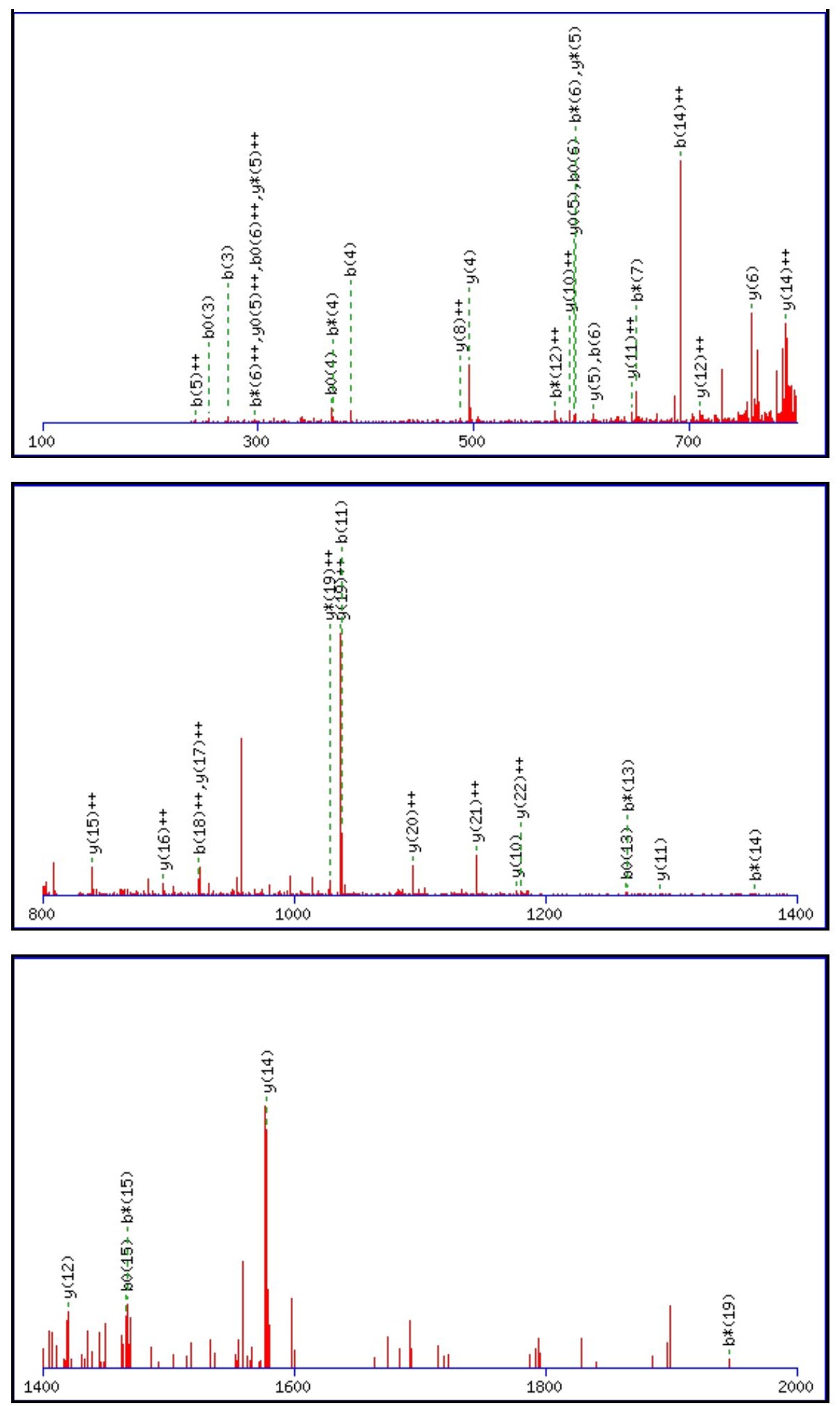

Monoisotopic mass of neutral peptide $\operatorname{Mr}($ calc): 2457.1078

Fixed modifications: Carbamidomethyl (C)

Variable modifications:

N13 : Deamidated_N (N)

M18 : Oxidation (M)

Ions Score: 39 Expect: 0.036

Matches (Bold Red): 46/250 fragment ions using 104 most intense peaks

\begin{tabular}{|c|c|c|c|c|c|c|c|c|c|c|c|c|c|c|}
\hline$\#$ & $\mathbf{b}$ & $\mathbf{b}^{++}$ & $\mathbf{b}^{*}$ & $\mathbf{b}^{*_{++}^{+}}$ & $\mathbf{b}^{\mathbf{0}}$ & $\mathbf{b}^{\mathbf{0}+}$ & $\mathbf{S e q}$ & $\mathbf{y}$ & $\mathbf{y}^{++}$ & $\mathbf{y}^{\mathbf{*}}$ & $\mathbf{y}^{\mathbf{*}^{++}}$ & $\mathbf{y}^{\mathbf{0}}$ & $\mathbf{y}^{\mathbf{0}+}$ & $\#$ \\
\hline $\mathbf{1}$ & 100.0757 & 50.5415 & & & & & $\mathbf{V}$ & & & & & & & $\mathbf{2 3}$ \\
\hline $\mathbf{2}$ & 171.1128 & 86.0600 & & & & & $\mathbf{A}$ & 2359.0467 & $\mathbf{1 1 8 0 . 0 2 7 0}$ & 2342.0201 & 1171.5137 & 2341.0361 & 1171.0217 & $\mathbf{2 2}$ \\
\hline $\mathbf{3}$ & $\mathbf{2 7 2 . 1 6 0 5}$ & 136.5839 & & & $\mathbf{2 5 4 . 1 4 9 9}$ & 127.5786 & $\mathbf{T}$ & 2288.0095 & $\mathbf{1 1 4 4 . 5 0 8 4}$ & 2270.9830 & 1135.9951 & 2269.9990 & 1135.5031 & $\mathbf{2 1}$ \\
\hline $\mathbf{4}$ & $\mathbf{3 8 6 . 2 0 3 4}$ & 193.6053 & $\mathbf{3 6 9 . 1 7 6 9}$ & 185.0921 & $\mathbf{3 6 8 . 1 9 2 8}$ & 184.6001 & $\mathbf{N}$ & 2186.9619 & $\mathbf{1 0 9 3 . 9 8 4 6}$ & 2169.9353 & 1085.4713 & 2168.9513 & 1084.9793 & $\mathbf{2 0}$ \\
\hline & & & & & & & & & & & & & &
\end{tabular}




\begin{tabular}{|c|c|c|c|c|c|c|c|c|c|c|c|c|c|c|}
\hline & 483.2562 & 242.1317 & 466.2296 & 233.6185 & 465.2456 & 233.1264 & $\mathbf{P}$ & 2072.9189 & 1036.9631 & |2055.8924| & 1028.4498 & |2054.9084 & 1027.9578 & $|19|$ \\
\hline 6 & 611.3148 & 306.1610 & 594.2882 & 297.6477 & 593.3042 & 297.1557 & $\mathbf{Q}$ & 1975.8662 & 988.4367 & 1958.8396 & 979.9235 & 1957.8556 & 979.4314 & 18 \\
\hline 7 & 668.3362 & 334.6717 & 651.3097 & 326.1585 & 650.3257 & 325.6665 & G & 1847.8076 & 924.4074 & 1830.7810 & 915.8942 & 1829.7970 & 915.4022 & 17 \\
\hline 8 & 781.4203 & 391.2138 & 764.3937 & 382.7005 & 763.4097 & 382.2085 & L & 1790.7861 & 895.8967 & 1773.7596 & 887.3834 & 1772.7756 & 886.8914 & 16 \\
\hline 9 & 880.4887 & 440.7480 & 863.4621 & 432.2347 & 862.4781 & 431.7427 & V & 1677.7021 & 839.3547 & 1660.6755 & 830.8414 & 1659.6915 & 830.3494 & 15 \\
\hline 10 & 937.5102 & 469.2587 & 920.4836 & 460.7454 & 919.4996 & 460.2534 & G & 1578.6337 & 789.8205 & 1561.6071 & 781.3072 & 1560.6231 & 780.8152 & 14 \\
\hline 11 & 1038.5578 & 519.7826 & 1021.5313 & 511.2693 & 1020.5473 & 510.7773 & $\mathbf{T}$ & 1521.6122 & 761.3097 & 1504.5856 & 752.7965 & 1503.6016 & 752.3045 & 13 \\
\hline 12 & 1167.6004 & 584.3039 & 1150.5739 & 575.7906 & 1149.5899 & 575.2986 & E & 1420.5645 & 710.7859 & 1403.5380 & 702.2726 & 1402.5539 & 701.7806 & 12 \\
\hline 13 & 1282.6274 & 641.8173 & 1265.6008 & 633.3040 & 1264.6168 & 632.8120 & $\mathbf{N}$ & 1291.5219 & 646.2646 & 1274.4954 & 637.7513 & 1273.5114 & 637.2593 & 11 \\
\hline 14 & 1383.6750 & 692.3412 & 1366.6485 & 683.8279 & 1365.6645 & 683.3359 & $T$ & 1176.4950 & 588.7511 & 1159.4684 & 580.2379 & 1158.4844 & 579.7458 & 10 \\
\hline 15 & 1484.7227 & 742.8650 & 1467.6962 & 734.3517 & 1466.7122 & 733.8597 & $T$ & 1075.4473 & 538.2273 & 1058.4208 & 529.7140 & 1057.4367 & 529.2220 & 9 \\
\hline 16 & 1571.7548 & 786.3810 & 1554.7282 & 777.8677 & 1553.7442 & 777.3757 & $S$ & 974.3996 & 487.7035 & 957.3731 & 479.1902 & 956.3891 & 478.6982 & 8 \\
\hline 17 & 1700.7973 & 850.9023 & 1683.7708 & 842.3890 & 1682.7868 & 841.8970 & $\mathbf{E}$ & 887.3676 & 444.1874 & 870.3410 & 435.6742 & 869.3570 & 435.1822 & 7 \\
\hline 18 & 1847.8327 & 924.4200 & 1830.8062 & 915.9067 & 1829.8222 & 915.4147 & $\mathbf{M}$ & 758.3250 & 379.6661 & 741.2985 & 371.1529 & 740.3144 & 370.6609 & 6 \\
\hline 19 & 1962.8597 & 981.9335 & \begin{tabular}{|l|}
1945.8331 \\
\end{tabular} & 973.4202 & 1944.8491 & 972.9282 & D & 611.2896 & 306.1484 & 594.2631 & 297.6352 & 593.2790 & 297.1432 & 5 \\
\hline 20 & 2059.9125 & 1030.4599 & 2042.8859 & 1021.9466 & 2041.9019 & 1021.4546 & $\mathbf{P}$ & 496.2627 & 248.6350 & 479.2361 & 240.1217 & 478.2521 & 239.6297 & 4 \\
\hline 21 & 2146.9445 & 1073.9759 & 2129.9179 & 1065.4626 & 2128.9339 & 1064.9706 & $S$ & 399.2099 & 200.1086 & 382.1833 & 191.5953 & 381.1993 & 191.1033 & 3 \\
\hline 22 & 2284.0034 & 1142.5053 & 2266.9768 & 1133.9921 & 2265.9928 & 1133.5001 & H & 312.1779 & 156.5926 & 295.1513 & 148.0793 & & & 2 \\
\hline 23 & & & & & & & $\mathbf{R}$ & 175.1190 & 88.0631 & 158.0924 & 79.5498 & & & 1 \\
\hline
\end{tabular}
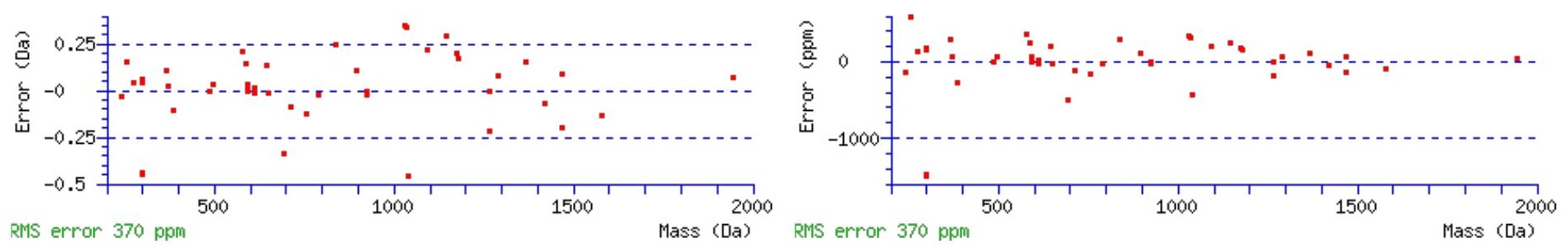

\section{All matches to this query}

\begin{tabular}{|l|l|l|l|}
\hline Score & Mr(calc): & Delta & \multicolumn{1}{|c|}{ Sequence } \\
\hline 39.3 & 2457.1078 & 0.0069 & VATNPQGLVGTENTTSEMDPSHR \\
\hline 8.7 & 2455.1199 & 1.9948 & VNSPPRFAGQRASAPLPYGGPR \\
\hline 8.2 & 2457.1078 & 0.0069 & VATNPQGLVGTENTTSEMDPSHR \\
\hline 6.2 & 2456.1238 & 0.9909 & VATNPQGLVGTENTTSEMDPSHR \\
\hline 1.7 & 2457.0994 & 0.0152 & VLDLSNKYTTALLNQTGIPR \\
\hline 1.4 & 2456.0903 & 1.0244 & ISDSEGFKANLSLLRRPGEK \\
\hline 1.0 & 2457.0994 & 0.0152 & VLDLSNKYTTALLNQTGIPR \\
\hline 0.7 & 2457.1114 & 0.0033 & AEGASLAAPTWGPEGGRYVGFER \\
\hline 0.4 & 2455.0861 & 2.0286 & REPASSNSTHSDHGRADGYTGPR \\
\hline 0.3 & 2455.0960 & 2.0186 & SSLKLLSNLFANYAGADAPVDK \\
\hline
\end{tabular}

Spectrum No: 857; Query: 392; Rank: 1

\section{Peptide View}

MS/MS Fragmentation of AYYNCSCIER

Found in IPI00393407, Tax_Id=10116 Gene_Symbol=Slco4c1 Kidney-specific organic anion transporter

Match to Query 392: 1335.524848 from(668.769700,2+)

Title: 100101RatKid_NS_deglyco_25.1396.1396.2.dta

Data file K:INewmanPaper|Piliangl3SubProteomes\Piliang3SP\mgf5ppm\ERLIC_3SubProteomes5ppm.mgf 

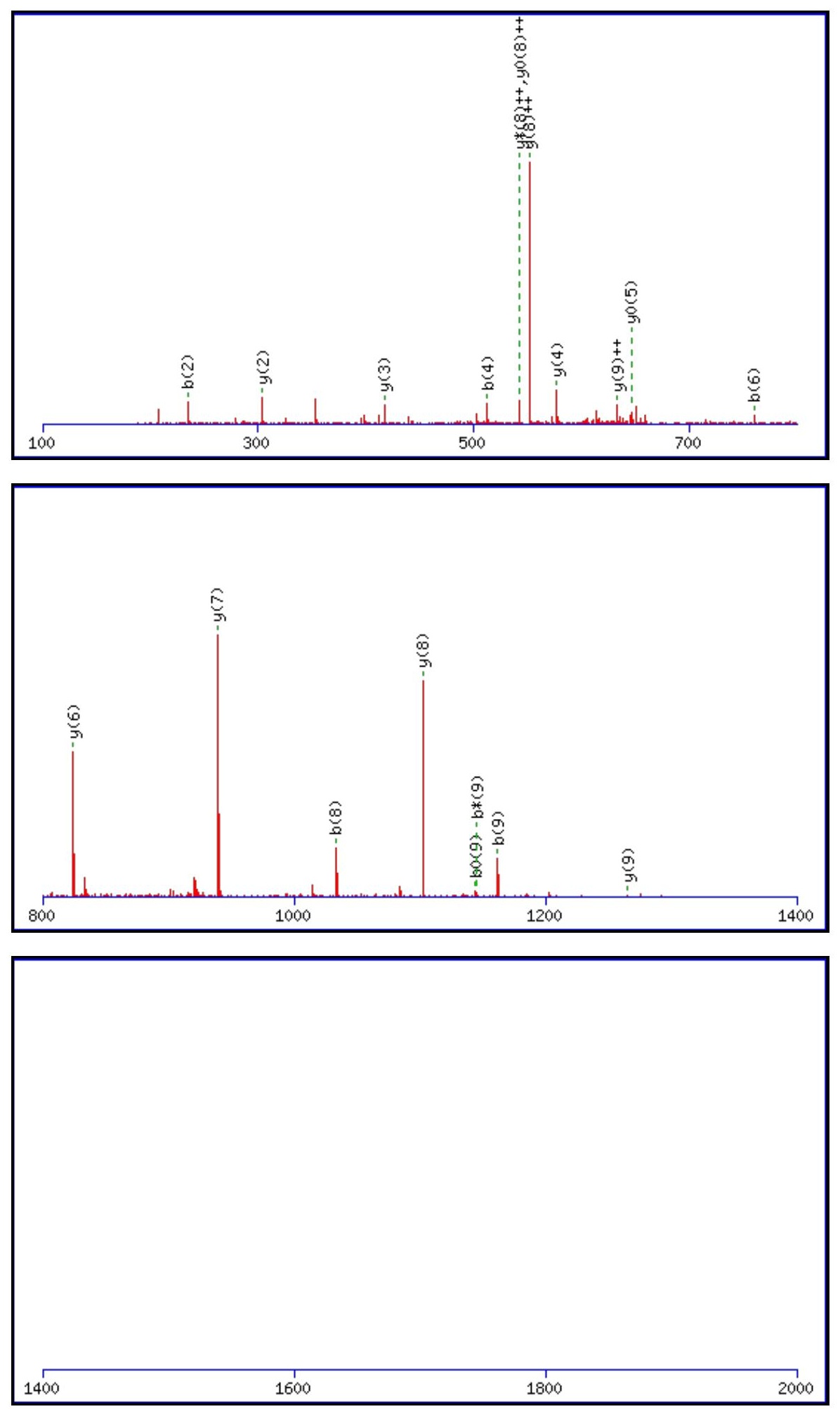

Monoisotopic mass of neutral peptide $\operatorname{Mr}$ (calc): 1335.5223

Fixed modifications: Carbamidomethyl (C)

Variable modifications:

N4 : Deamidated_N (N)

Ions Score: 39 Expect: 0.0098

Matches (Bold Red): $19 / 90$ fragment ions using 30 most intense peaks

\begin{tabular}{|r|c|c|c|c|c|c|c|c|c|c|c|c|c|c|}
\hline$\#$ & $\mathbf{b}$ & $\mathbf{b}^{++}$ & $\mathbf{b}^{*}$ & $\mathbf{b}^{\boldsymbol{*}^{++}}$ & $\mathbf{b}^{\mathbf{0}}$ & $\mathbf{b}^{\mathbf{0 + +}}$ & Seq. & $\mathbf{y}$ & $\mathbf{y}^{++}$ & $\mathbf{y}^{\mathbf{*}}$ & $\mathbf{y}^{\boldsymbol{*}^{++}}$ & $\mathbf{y}^{\mathbf{0}}$ & $\mathbf{y}^{\mathbf{0 + +}}$ & $\#$ \\
\hline $\mathbf{1}$ & $\mathbf{7 2 . 0 4 4 4}$ & 36.5258 & & & & & $\mathbf{A}$ & & & & & & & $\mathbf{1 0}$ \\
\hline $\mathbf{2}$ & $\mathbf{2 3 5 . 1 0 7 7}$ & 118.0575 & & & & & $\mathbf{Y}$ & $\mathbf{1 2 6 5 . 4 9 2 5}$ & $\mathbf{6 3 3 . 2 4 9 9}$ & 1248.4660 & 624.7366 & 1247.4820 & 624.2446 & $\mathbf{9}$ \\
\hline $\mathbf{3}$ & 398.1710 & 199.5892 & & & & & $\mathbf{Y}$ & $\mathbf{1 1 0 2 . 4 2 9 2}$ & 551.7182 & 1085.4027 & $\mathbf{5 4 3 . 2 0 5 0}$ & 1084.4186 & $\mathbf{5 4 2 . 7 1 3 0}$ & $\mathbf{8}$ \\
\hline $\mathbf{4}$ & $\mathbf{5 1 3 . 1 9 8 0}$ & 257.1026 & 496.1714 & 248.5894 & & & $\mathbf{N}$ & $\mathbf{9 3 9 . 3 6 5 9}$ & 470.1866 & 922.3393 & 461.6733 & 921.3553 & 461.1813 & $\mathbf{7}$ \\
\hline $\mathbf{5}$ & 673.2286 & 337.1180 & 656.2021 & 328.6047 & & & $\mathbf{C}$ & $\mathbf{8 2 4 . 3 3 8 9}$ & 412.6731 & 807.3124 & 404.1598 & 806.3284 & 403.6678 & $\mathbf{6}$ \\
\hline
\end{tabular}




\begin{tabular}{|r|r|r|r|r|r|r|r|r|r|r|r|r|r|r|r|r|}
$\mathbf{6}$ & $\mathbf{7 6 0 . 2 6 0 7}$ & 380.6340 & 743.2341 & 372.1207 & 742.2501 & 371.6287 & S & 664.3083 & 332.6578 & 647.2817 & 324.1445 & $\mathbf{6 4 6 . 2 9 7 7}$ & 323.6525 & $\mathbf{5}$ \\
\hline $\mathbf{7}$ & 920.2913 & 460.6493 & 903.2648 & 452.1360 & 902.2807 & 451.6440 & C & 577.2763 & 289.1418 & 560.2497 & 280.6285 & 559.2657 & 280.1365 & $\mathbf{4}$ \\
\hline $\mathbf{8}$ & $\mathbf{1 0 3 3 . 3 7 5 4}$ & 517.1913 & 1016.3488 & 508.6781 & 1015.3648 & 508.1860 & I & $\mathbf{4 1 7 . 2 4 5 6}$ & 209.1264 & 400.2191 & 200.6132 & 399.2350 & 200.1212 & $\mathbf{3}$ \\
\hline $\mathbf{9}$ & $\mathbf{1 1 6 2 . 4 1 8 0}$ & 581.7126 & $\mathbf{1 1 4 5 . 3 9 1 4}$ & 573.1993 & $\mathbf{1 1 4 4 . 4 0 7 4}$ & 572.7073 & E & $\mathbf{3 0 4 . 1 6 1 5}$ & 152.5844 & 287.1350 & 144.0711 & 286.1510 & 143.5791 & $\mathbf{2}$ \\
\hline $\mathbf{1 0}$ & & & & & & & R & 175.1190 & 88.0631 & 158.0924 & 79.5498 & & & $\mathbf{1}$ \\
\hline
\end{tabular}
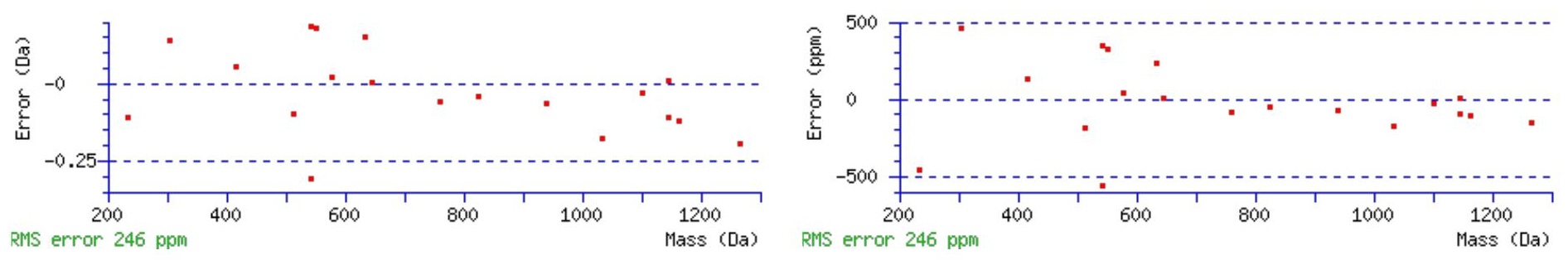

\section{All matches to this query}

\begin{tabular}{|l|l|l|l|}
\hline Score & Mr(calc): & Delta & \multicolumn{1}{c|}{ Sequence } \\
\hline 39.3 & 1335.5223 & 0.0025 & AYYNCSCIER \\
\hline 5.4 & 1335.5278 & -0.0030 & FSSREGMEASR \\
\hline 2.3 & 1335.5278 & -0.0030 & ESTQGFRMER \\
\hline 1.8 & 1333.5179 & 2.0070 & SHPXTVMNLK \\
\hline 0.3 & 1333.5179 & 2.0070 & $\underline{\text { ITNCYKFK }}$ \\
\hline 0.1 & 1335.5125 & 0.0124 & MSHWNGXWNR \\
\hline 0.1 & 1335.5261 & -0.0013 & SFSDKHGLTGK \\
\hline 0.1 & 1335.5301 & -0.0053 & AYLTYFRDK \\
\hline
\end{tabular}

Spectrum No: 858; Query: 2474; Rank: 1

\section{Peptide View}

MS/MS Fragmentation of GIASLTMDTHEAVVGNYTCEVTELSR

Found in IPI00470238, Tax_Id=10116 Gene_Symbol=Cd47 Isoform 2 of Leukocyte surface antigen CD47 precursor

Match to Query 2474: 2869.302132 from(957.441320,3+)

Title: 091008RatKidney_NH4Format01_23.3552.3552.3.dta

Data file K:\NewmanPaper|Piliang|3SubProteomes\Piliang3SP\mgf5ppm\ERLIC_3SubProteomes5ppm.mgf

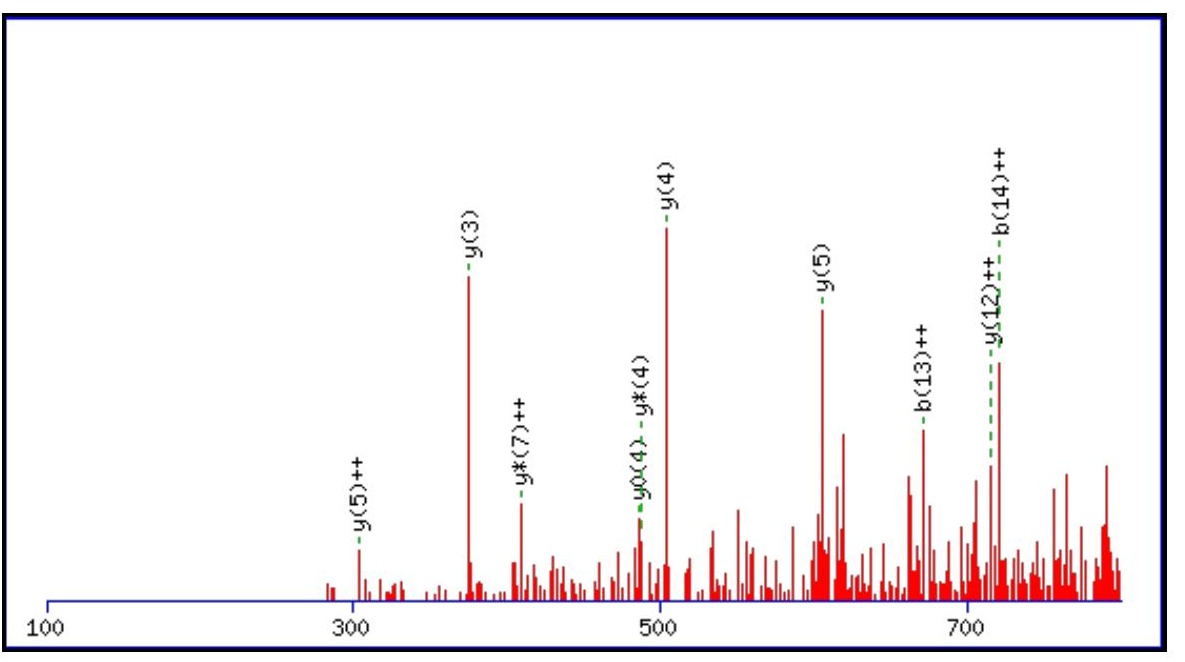



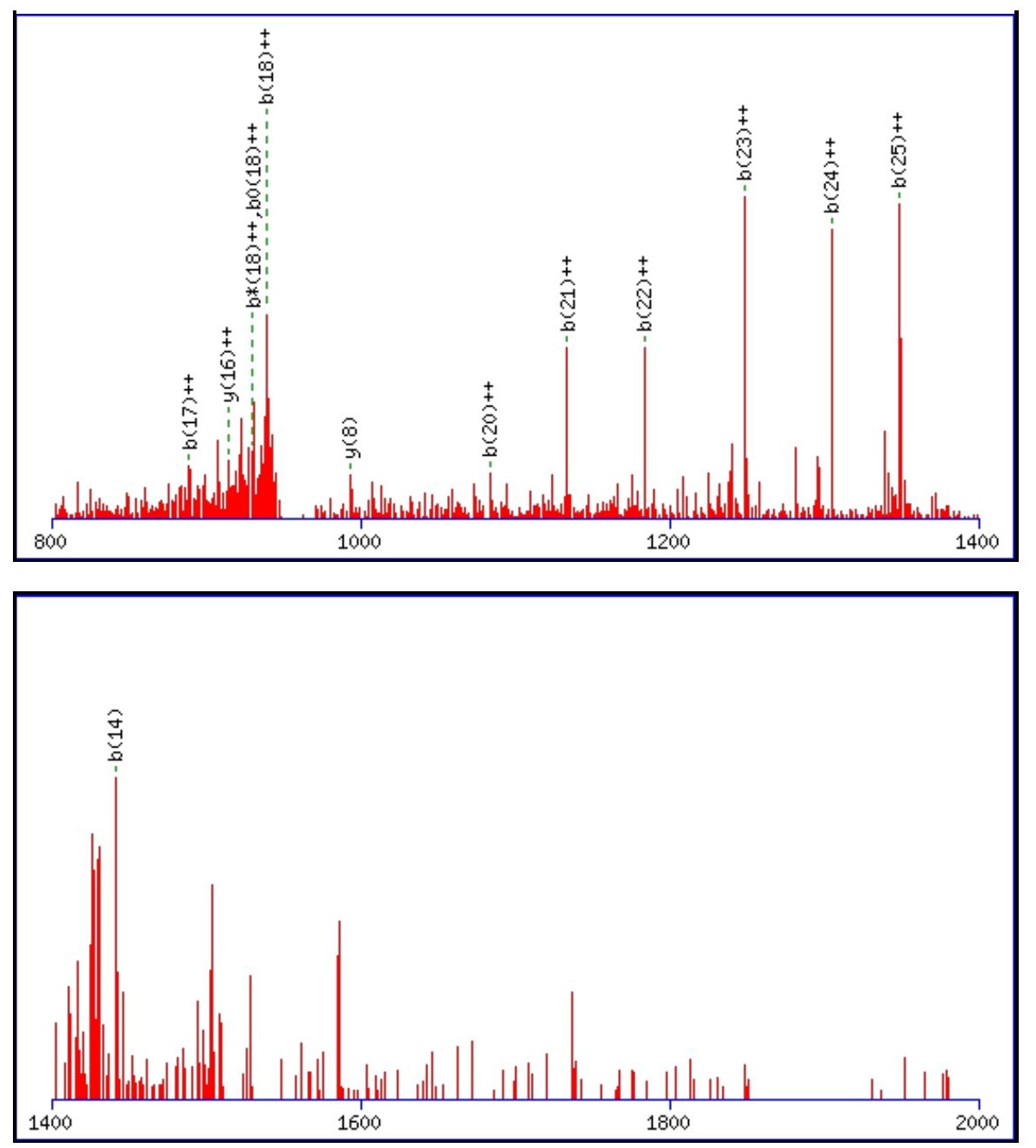

Monoisotopic mass of neutral peptide $\operatorname{Mr}($ calc): 2869.3110

Fixed modifications: Carbamidomethyl (C)

Variable modifications:

M7 : Oxidation (M)

N16 : Deamidated_N (N)

Ions Score: 39 Expect: 0.046

Matches (Bold Red): 23/262 fragment ions using 67 most intense peaks

\begin{tabular}{|c|c|c|c|c|c|c|c|c|c|c|c|c|c|c|}
\hline \# & b & $\mathbf{b}^{++}$ & $\mathbf{b}^{*}$ & $\mathbf{b}^{*^{++}}$ & $\mathbf{b}^{\mathbf{0}}$ & $\mathbf{b}^{\mathbf{0 + +}}$ & Seq. & $\mathbf{y}$ & $\mathbf{y}^{++}$ & $\mathbf{y}^{*}$ & $\mathrm{y}^{*^{++}}$ & $\mathbf{y}^{0}$ & $\mathbf{y}^{0++}$ & \# \\
\hline 1 & 58.0287 & 29.5180 & & & & & G & & & & & & & 26 \\
\hline 2 & 171.1128 & 86.0600 & & & & & I & 2813.2968 & 1407.1520 & 2796.2703 & 1398.6388 & 2795.2862 & 1398.1468 & 25 \\
\hline 3 & 242.1499 & 121.5786 & & & & & $\mathbf{A}$ & 2700.2127 & 1350.6100 & 2683.1862 & 1342.0967 & 2682.2022 & 1341.6047 & 24 \\
\hline 4 & 329.1819 & 165.0946 & & & 311.1714 & 156.0893 & $\mathbf{S}$ & 2629.1756 & 1315.0914 & 2612.1491 & 1306.5782 & 2611.1651 & 1306.0862 & 23 \\
\hline 5 & 442.2660 & 221.6366 & & & 424.2554 & 212.6314 & $\mathbf{L}$ & 2542.1436 & 1271.5754 & 2525.1170 & 1263.0622 & 2524.1330 & 1262.5702 & 22 \\
\hline 6 & 543.3137 & 272.1605 & & & 525.3031 & 263.1552 & $T$ & 2429.0595 & 1215.0334 & 2412.0330 & 1206.5201 & 2411.0490 & 1206.0281 & 21 \\
\hline 7 & 690.3491 & 345.6782 & & & 672.3385 & 336.6729 & $\mathbf{M}$ & 2328.0119 & 1164.5096 & 2310.9853 & 1155.9963 & 2310.0013 & 1155.5043 & 20 \\
\hline 8 & 805.3760 & 403.1917 & & & 787.3655 & 394.1864 & D & 2180.9764 & 1090.9919 & 2163.9499 & 1082.4786 & 2162.9659 & 1081.9866 & 19 \\
\hline 9 & 906.4237 & 453.7155 & & & 888.4131 & 444.7102 & $T$ & 2065.9495 & 1033.4784 & 2048.9230 & 1024.9651 & 2047.9389 & 1024.4731 & 18 \\
\hline 10 & 1043.4826 & 522.2450 & & & 1025.4721 & 513.2397 & $\mathbf{H}$ & 1964.9018 & 982.9546 & 1947.8753 & 974.4413 & 1946.8913 & 973.9493 & 17 \\
\hline 11 & 1172.5252 & 586.7662 & & & 1154.5147 & 577.7610 & $E$ & 1827.8429 & 914.4251 & 1810.8164 & 905.9118 & 1809.8324 & 905.4198 & 16 \\
\hline 12 & 1243.5623 & 622.2848 & & & 1225.5518 & 613.2795 & $\mathbf{A}$ & 1698.8003 & 849.9038 & 1681.7738 & 841.3905 & 1680.7898 & 840.8985 & 15 \\
\hline 13 & 1342.6307 & 671.8190 & & & 1324.6202 & 662.8137 & $\mathbf{V}$ & 1627.7632 & 814.3852 & 1610.7367 & 805.8720 & 1609.7526 & 805.3800 & 14 \\
\hline 14 & 1441.6992 & 721.3532 & & & 1423.6886 & 712.3479 & $\mathbf{V}$ & 1528.6948 & 764.8510 & 1511.6682 & 756.3378 & 1510.6842 & 755.8458 & 13 \\
\hline 15 & 1498.7206 & 749.8640 & & & 1480.7101 & 740.8587 & $G$ & 1429.6264 & 715.3168 & 1412.5998 & 706.8036 & 1411.6158 & 706.3115 & 12 \\
\hline 16 & 1613.7476 & 807.3774 & 1596.7210 & 798.8641 & 1595.7370 & 798.3721 & $\mathbf{N}$ & 1372.6049 & 686.8061 & 1355.5784 & 678.2928 & 1354.5944 & 677.8008 & 11 \\
\hline 17 & 1776.8109 & 888.9091 & 1759.7843 & 880.3958 & 1758.8003 & 879.9038 & $\mathbf{Y}$ & 1257.5780 & 629.2926 & 1240.5514 & 620.7794 & 1239.5674 & 620.2873 & 10 \\
\hline 18 & 1877.8586 & 939.4329 & 1860.8320 & 930.9196 & 1859.8480 & 930.4276 & $T$ & 1094.5147 & 547.7610 & 1077.4881 & 539.2477 & 1076.5041 & 538.7557 & 9 \\
\hline
\end{tabular}




\begin{tabular}{|c|c|c|c|c|c|c|c|c|c|c|c|c|c|c|}
\hline 19| & |2037.8892| & |1019.4482 & |2020.8627 & 1010.9350 & |2019.8787 & |1010.4430 & C & 993.4670 & 497.2371 & 976.4404 & 488.7238 & 975.4564| & 488.2318 & 8 \\
\hline 20 & 2166.9318 & 1083.9695 & 2149.9053 & 1075.4563 & 2148.9212 & 1074.9643 & E & 833.4363 & 417.2218 & 816.4098 & 408.7085 & 815.4258 & 408.2165 & 7 \\
\hline 21 & 2266.0002 & 1133.5038 & 2248.9737 & 1124.9905 & 2247.9897 & 1124.4985 & V & 704.3937 & 352.7005 & 687.3672 & 344.1872 & 686.3832 & 343.6952 & 6 \\
\hline 22 & 2367.0479 & 1184.0276 & 2350.0214 & 1175.5143 & 2349.0373 & 1175.0223 & $\mathbf{T}$ & 605.3253 & 303.1663 & 588.2988 & 294.6530 & 587.3148 & 294.1610 & 5 \\
\hline 23 & 2496.0905 & 1248.5489 & 2479.0639 & 1240.0356 & 2478.0799 & 1239.5436 & $\mathbf{E}$ & 504.2776 & 252.6425 & 487.2511 & 244.1292 & 486.2671 & 243.6372 & 4 \\
\hline 24 & 2609.1746 & 1305.0909 & 2592.1480 & 1296.5776 & 2591.1640 & 1296.0856 & $\mathbf{L}$ & 375.2350 & 188.1212 & 358.2085 & 179.6079 & 357.2245 & 179.1159 & 3 \\
\hline 25 & 2696.2066 & 1348.6069 & 2679.1800 & 1340.0937 & 2678.1960 & 1339.6017 & $\mathrm{~S}$ & 262.1510 & 131.5791 & 245.1244 & 123.0659 & 244.1404 & 122.5738 & 2 \\
\hline 26 & & & & & & & $\mathbf{R}$ & 175.1190 & 88.0631 & 158.0924 & 79.5498 & & & 1 \\
\hline
\end{tabular}
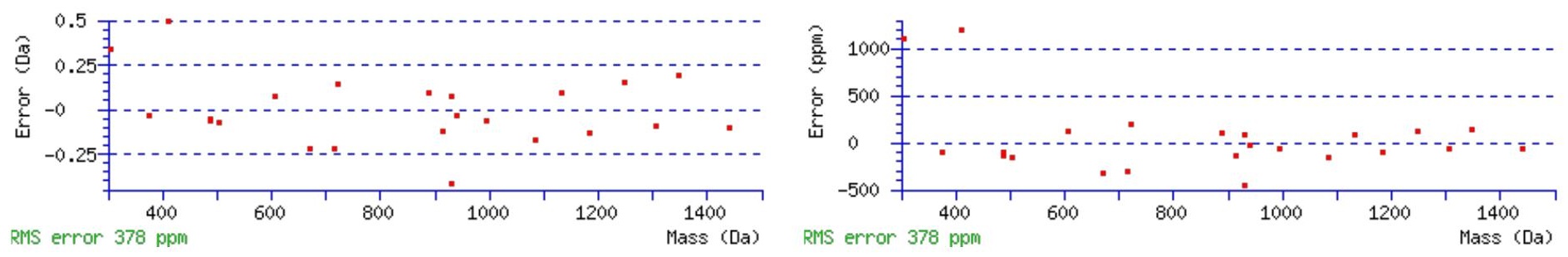

\section{All matches to this query}

\begin{tabular}{|l|l|l|l|}
\hline Score & Mr(calc): & Delta & \multicolumn{1}{|c|}{ Sequence } \\
\hline 39.2 & 2869.3110 & -0.0089 & GIASLTMDTHEAVVGNYTCEVTELSR \\
\hline 14.9 & 2868.3270 & 0.9752 & GIASLTMDTHEAVVGNYTCEVTELSR \\
\hline 4.2 & 2869.3017 & 0.0005 & LYFYLNVTGPPPPEETELQVTFR \\
\hline 4.2 & 2869.3017 & 0.0005 & LYFYLNVTGPPPPEETELQVTFR \\
\hline 3.5 & 2868.3156 & 0.9865 & TGTVNEDSSEIILDPKVIKMNYLK \\
\hline 1.4 & 2869.2814 & 0.0207 & RHNAGSNPTPPASVMGSPPSSLQEAQR \\
\hline 1.4 & 2869.2814 & 0.0207 & RHNAGSNPTPPASVMGSPPSSLQEAQR \\
\hline 0.6 & 2869.3097 & -0.0076 & YLELVMVFDLSRYVFTRGNMTR \\
\hline 0.6 & 2869.3017 & 0.0005 & LYFYLNVTGPPPPEETELQVTFR \\
\hline 0.5 & 2869.2970 & 0.0052 & DGSEDPSTNVMQKTPIILSKPPAER \\
\hline
\end{tabular}

Spectrum No: 859; Query: 1953; Rank: 1

\section{Peptide View}

MS/MS Fragmentation of LWDPYSHPGIQNISQDLLR

Found in IPI00212106, Tax_Id=10116 Gene_Symbol=Ggtla1 Gamma-glutamyltransferase 5 precursor

Match to Query 1953: 2252.127522 from(751.716450,3+)

Title: 100101RatKid_NS_deglyco_23.4699.4699.3.dta

Data file K:INewmanPaper|Piliangl3SubProteomes\Piliang3SP\mgf5ppm\ERLIC_3SubProteomes5ppm.mgf 

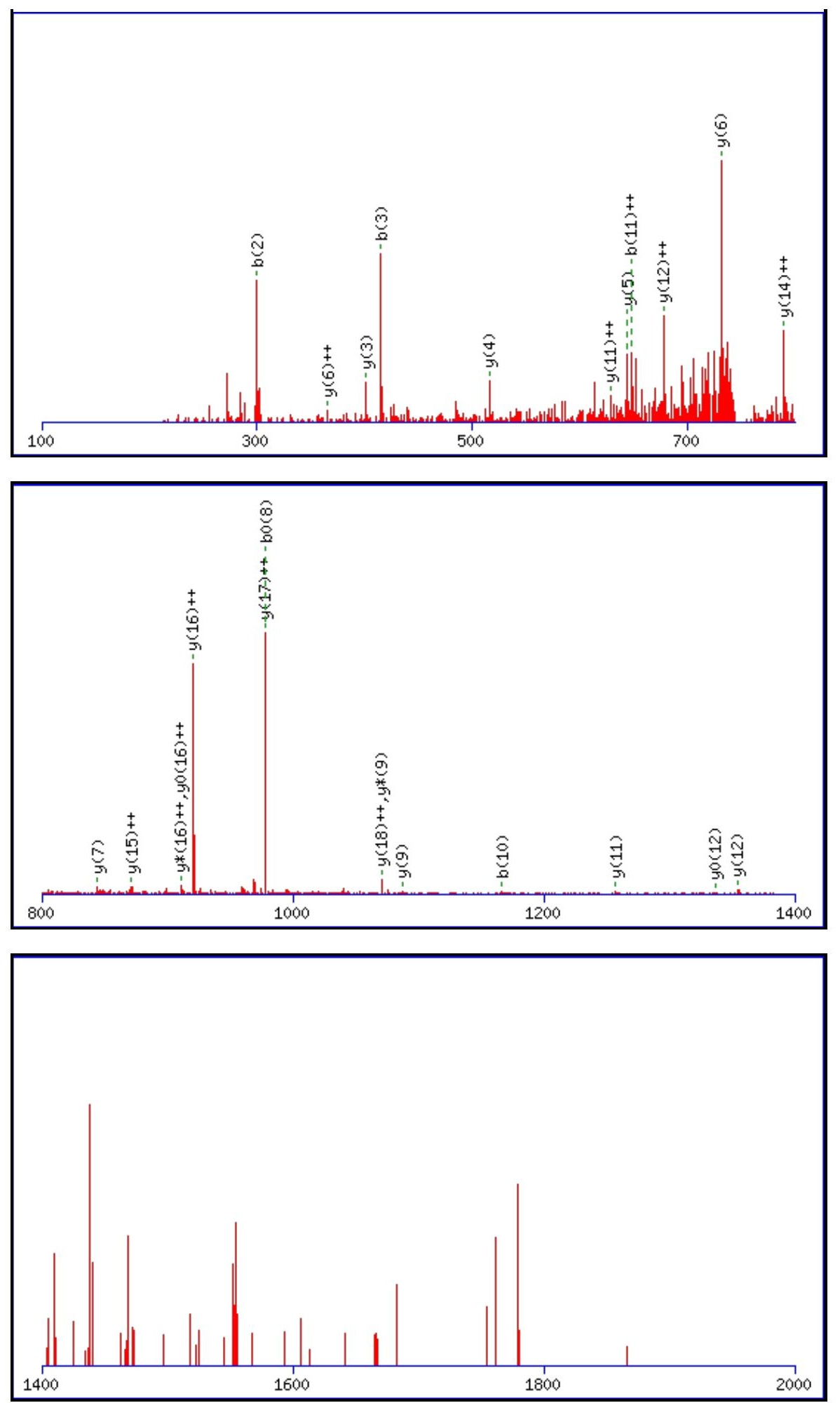

Monoisotopic mass of neutral peptide $\operatorname{Mr}($ calc): 2252.1226

Fixed modifications: Carbamidomethyl (C)

Variable modifications:

N12 : Deamidated_N (N)

Ions Score: 39 Expect: $\odot .024$

Matches (Bold Red): 25/186 fragment ions using 65 most intense peaks

\begin{tabular}{|r|c|c|c|c|c|r|r|r|r|r|r|r|r|r|}
\hline$\#$ & $\mathbf{b}$ & $\mathbf{b}^{++}$ & $\mathbf{b}^{*}$ & $\mathbf{b}^{\boldsymbol{*}^{++}}$ & $\mathbf{b}^{\mathbf{0}}$ & $\mathbf{b}^{\mathbf{0 + +}}$ & $\mathbf{S e q}$ & $\mathbf{y}$ & $\mathbf{y}^{++}$ & $\mathbf{y}^{\mathbf{*}}$ & $\mathbf{y}^{\mathbf{*}^{++}}$ & $\mathbf{y}^{\mathbf{0}}$ & $\mathbf{y}^{\mathbf{0 + +}}$ & $\#$ \\
\hline $\mathbf{1}$ & 114.0913 & 57.5493 & & & & & $\mathbf{L}$ & & & & & & & $\mathbf{1 9}$ \\
\hline $\mathbf{2}$ & $\mathbf{3 0 0 . 1 7 0 7}$ & 150.5890 & & & & & $\mathbf{W}$ & 2140.0458 & $\mathbf{1 0 7 0 . 5 2 6 5}$ & 2123.0192 & 1062.0133 & 2122.0352 & 1061.5212 & $\mathbf{1 8}$ \\
\hline $\mathbf{3}$ & $\mathbf{4 1 5 . 1 9 7 6}$ & 208.1024 & & & 397.1870 & 199.0972 & $\mathbf{D}$ & 1953.9665 & $\mathbf{9 7 7 . 4 8 6 9}$ & 1936.9399 & 968.9736 & 1935.9559 & 968.4816 & $\mathbf{1 7}$ \\
\hline $\mathbf{4}$ & 512.2504 & 256.6288 & & & 494.2398 & 247.6235 & $\mathbf{P}$ & 1838.9395 & $\mathbf{9 1 9 . 9 7 3 4}$ & 1821.9130 & $\mathbf{9 1 1 . 4 6 0 1}$ & $\mathbf{1 8 2 0 . 9 2 9 0}$ & $\mathbf{9 1 0 . 9 6 8 1}$ & $\mathbf{1 6}$ \\
\hline $\mathbf{5}$ & 675.3137 & 338.1605 & & & 657.3031 & 329.1552 & $\mathbf{Y}$ & 1741.8868 & $\mathbf{8 7 1 . 4 4 7 0}$ & $\mathbf{1 7 2 4 . 8 6 0 2}$ & 862.9337 & 1723.8762 & 862.4417 & $\mathbf{1 5}$ \\
\hline
\end{tabular}




\begin{tabular}{|c|c|c|c|c|c|c|c|c|c|c|c|c|c|c|}
\hline 6 & 762.3457 & 381.6765 & & & 744.3352 & 372.6712 & S & |1578.8234 & 789.9154 & |1561.7969 & 781.4021 & |1560.8129| & 780.9101 & $\mid 14$ \\
\hline 7 & 899.4046 & 450.2060 & & & 881.3941 & 441.2007 & $\mathbf{H}$ & 1491.7914 & 746.3993 & 1474.7649 & 737.8861 & 1473.7808 & 737.3941 & 13 \\
\hline 8 & 996.4574 & 498.7323 & & & 978.4468 & 489.7271 & $\mathbf{P}$ & 1354.7325 & 677.8699 & 1337.7060 & 669.3566 & 1336.7219 & 668.8646 & 12 \\
\hline 9 & 1053.4789 & 527.2431 & & & 1035.4683 & 518.2378 & $\mathbf{G}$ & 1257.6797 & 629.3435 & 1240.6532 & 620.8302 & 1239.6692 & 620.3382 & 11 \\
\hline 10 & 1166.5629 & 583.7851 & & & 1148.5524 & 574.7798 & I & 1200.6583 & 600.8328 & 1183.6317 & 592.3195 & 1182.6477 & 591.8275 & 10 \\
\hline 11 & 1294.6215 & 647.8144 & 1277.5950 & 639.3011 & 1276.6109 & 638.8091 & $\mathbf{Q}$ & 1087.5742 & 544.2907 & 1070.5477 & 535.7775 & 1069.5636 & 535.2855 & 9 \\
\hline 12 & 1409.6484 & 705.3279 & 1392.6219 & 696.8146 & 1391.6379 & 696.3226 & $\mathbf{N}$ & 959.5156 & 480.2615 & 942.4891 & 471.7482 & 941.5051 & 471.2562 & 8 \\
\hline 13 & 1522.7325 & 761.8699 & 1505.7060 & 753.3566 & 1504.7219 & 752.8646 & I & 844.4887 & 422.7480 & 827.4621 & 414.2347 & 826.4781 & 413.7427 & 7 \\
\hline 14 & 1609.7645 & 805.3859 & 1592.7380 & 796.8726 & 1591.7540 & 796.3806 & S & 731.4046 & 366.2060 & 714.3781 & 357.6927 & 713.3941 & 357.2007 & 6 \\
\hline 15 & 1737.8231 & 869.4152 & 1720.7966 & 860.9019 & 1719.8125 & 860.4099 & $\mathbf{Q}$ & 644.3726 & 322.6899 & 627.3461 & 314.1767 & 626.3620 & 313.6847 & 5 \\
\hline 16 & 1852.8500 & 926.9287 & 1835.8235 & 918.4154 & 1834.8395 & 917.9234 & D & 516.3140 & 258.6606 & 499.2875 & 250.1474 & 498.3035 & 249.6554 & 4 \\
\hline 17 & 1965.9341 & 983.4707 & 1948.9076 & 974.9574 & 1947.9235 & 974.4654 & $\mathbf{L}$ & 401.2871 & 201.1472 & 384.2605 & 192.6339 & & & 3 \\
\hline 18 & 2079.0182 & 1040.0127 & 2061.9916 & 1031.4995 & 2061.0076 & 1031.0074 & $\mathbf{L}$ & 288.2030 & 144.6051 & 271.1765 & 136.0919 & & & 2 \\
\hline 19 & & & & & & & $\mathbf{R}$ & 175.1190 & 88.0631 & 158.0924 & 79.5498 & & & 1 \\
\hline
\end{tabular}
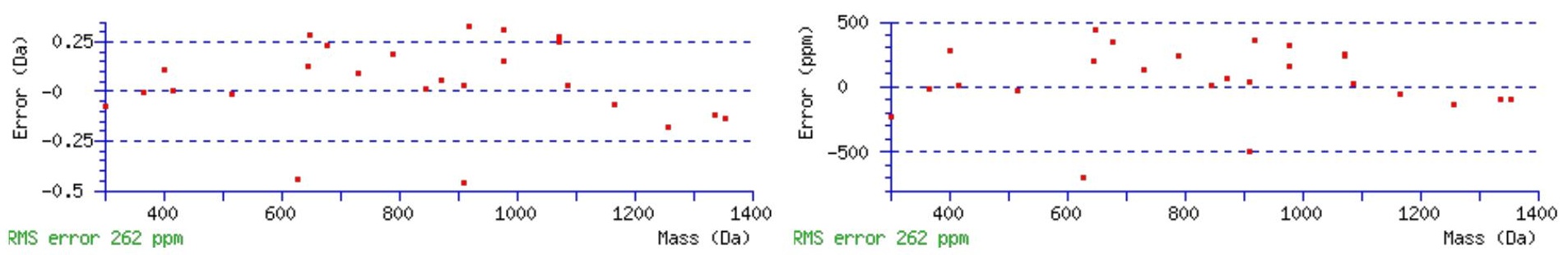

\section{All matches to this query}

\begin{tabular}{|l|l|c|l|}
\hline Score & Mr(calc): & Delta & \multicolumn{1}{|c|}{ Sequence } \\
\hline 39.2 & 2252.1226 & 0.0050 & LWDPYSHPGIQNISQDLLR \\
\hline 6.4 & 2251.1386 & 0.9890 & LWDPYSHPGIQNISQDLLR \\
\hline 3.4 & 2250.1119 & 2.0156 & ETAFIQCSPQPLSLPKQVK \\
\hline 3.3 & 2252.1181 & 0.0094 & IQGNIGVQMLVDLSQCYLGK \\
\hline 3.0 & 2250.1338 & 1.9938 & VTVTDFFQKLGPLSVFSASK \\
\hline 1.6 & 2252.1160 & 0.0115 & WLNNCVGKRNYWXGTLGVK \\
\hline 1.5 & 2252.1358 & -0.0083 & IKGDLALSGNLSNLYAMTQDK \\
\hline 1.5 & 2252.1358 & -0.0083 & IKGDLALSGNLSNLYAMTQDK \\
\hline 1.4 & 2252.1147 & 0.0128 & IPTNTWSGLGFSKSMPAETIK \\
\hline 1.4 & 2252.1160 & 0.0115 & WLNNCVGKRNYWXGTLGVK \\
\hline
\end{tabular}

Spectrum No: 860; Query: 1345; Rank: 1

\section{Peptide View}

MS/MS Fragmentation of NNLSSLLPDFTESEKR

Found in IPI00210396, Tax_Id=10116 Gene_Symbol=Kl Klotho precursor

Match to Query 1345: 1849.907652 from(617.643160,3+)

Title: 091008RatKidney_NH4Format01_24.3489.3489.3.dta

Data file K:INewmanPaper|Piliangl3SubProteomes\Piliang3SP\mgf5ppm\ERLIC_3SubProteomes5ppm.mgf 

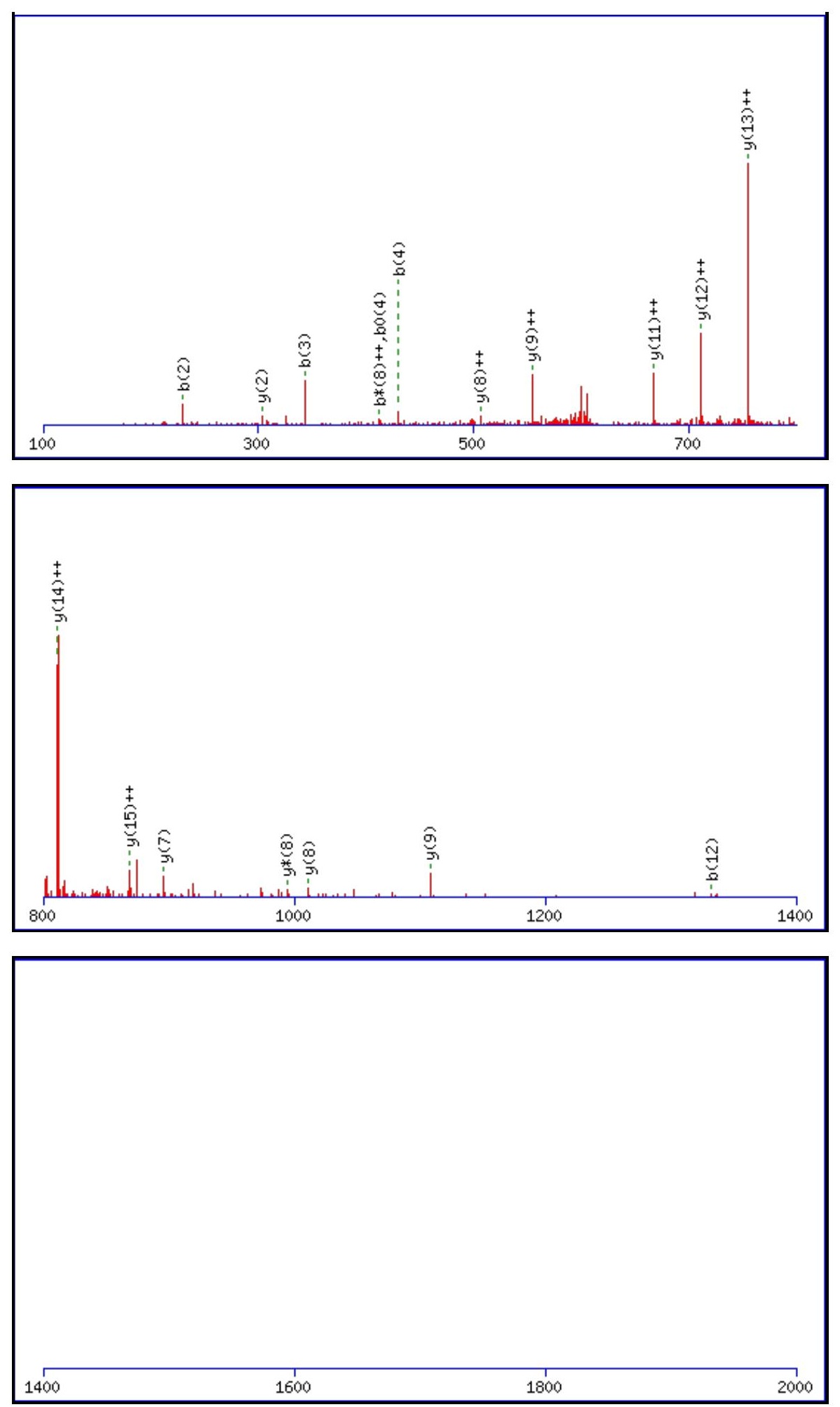

Monoisotopic mass of neutral peptide $\operatorname{Mr}($ calc): 1849.9057

Fixed modifications: Carbamidomethyl (C)

Variable modifications:

N2 : Deamidated $\mathrm{N}(\mathrm{N})$

Ions Score: 39 Expect: $\odot .026$

Matches (Bold Red): 18/170 fragment ions using 28 most intense peaks

\begin{tabular}{|r|c|c|c|c|c|c|c|c|c|c|c|c|c|c|}
\hline$\#$ & $\mathbf{b}$ & $\mathbf{b}^{++}$ & $\mathbf{b}^{*}$ & $\mathbf{b}^{\boldsymbol{*}_{++}}$ & $\mathbf{b}^{\mathbf{0}}$ & $\mathbf{b}^{\mathbf{0 + +}}$ & Seq. & $\mathbf{y}$ & $\mathbf{y}^{++}$ & $\mathbf{y}^{\mathbf{*}}$ & $\mathbf{y}^{\boldsymbol{*}^{++}}$ & $\mathbf{y}^{\mathbf{0}}$ & $\mathbf{y}^{\mathbf{0 + +}}$ & $\#$ \\
\hline $\mathbf{1}$ & 115.0502 & 58.0287 & 98.0237 & 49.5155 & & & $\mathbf{N}$ & & & & & & & $\mathbf{1 6}$ \\
\hline $\mathbf{2}$ & $\mathbf{2 3 0 . 0 7 7 1}$ & 115.5422 & 213.0506 & 107.0289 & & & $\mathbf{N}$ & 1736.8701 & $\mathbf{8 6 8 . 9 3 8 7}$ & 1719.8436 & 860.4254 & 1718.8595 & 859.9334 & $\mathbf{1 5}$ \\
\hline $\mathbf{3}$ & $\mathbf{3 4 3 . 1 6 1 2}$ & 172.0842 & 326.1347 & 163.5710 & & & $\mathbf{L}$ & 1621.8432 & $\mathbf{8 1 1 . 4 2 5 2}$ & 1604.8166 & 802.9120 & 1603.8326 & 802.4199 & $\mathbf{1 4}$ \\
\hline $\mathbf{4}$ & $\mathbf{4 3 0 . 1 9 3 2}$ & 215.6003 & 413.1667 & 207.0870 & $\mathbf{4 1 2 . 1 8 2 7}$ & 206.5950 & S & 1508.7591 & 754.8832 & 1491.7326 & 746.3699 & 1490.7485 & 745.8779 & $\mathbf{1 3}$ \\
\hline $\mathbf{5}$ & 517.2253 & 259.1163 & 500.1987 & 250.6030 & 499.2147 & 250.1110 & S & 1421.7271 & $\mathbf{7 1 1 . 3 6 7 2}$ & 1404.7005 & 702.8539 & 1403.7165 & 702.3619 & $\mathbf{1 2}$ \\
\hline
\end{tabular}




\begin{tabular}{|c|c|c|c|c|c|c|c|c|c|c|c|c|c|c|}
\hline 6 & 630.3093 & 315.6583 & 613.2828 & 307.1450 & 612.2988 & |306.6530| & $\mathbf{L}$ & 1334.6951| & 667.8512 & |1317.6685 & |659.3379| & & |658.8459| & 11 \\
\hline 7 & 743.3934 & 372.2003 & 726.3668 & 363.6871 & 725.3828 & 363.1950 & $\mathbf{L}$ & 1221.6110 & 611.3091 & 1204.5844 & 602.7959 & 1203.6004 & 602.3039 & 10 \\
\hline 8 & 840.4462 & 420.7267 & 823.4196 & 412.2134 & 822.4356 & 411.7214 & $\mathbf{P}$ & 1108.5269 & 554.7671 & 1091.5004 & 546.2538 & 1090.5164 & 545.7618 & 9 \\
\hline 9 & 955.4731 & 478.2402 & 938.4465 & 469.7 & 937.4625 & 469.2 & D & 1011.4742 & 506.2 & 994.4476 & 497.7274 & 993.4636 & 2354 & 8 \\
\hline 10 & 1102.5415 & 551.7744 & 1085.5150 & 543.2611 & 1084.5309 & 542.7691 & $\mathbf{F}$ & 896.4472 & 448.7272 & 879.4207 & 440.2140 & 878.4367 & 439.7220 & 7 \\
\hline 11 & 1203.5892 & 602.2982 & 1186.5626 & 593.7850 & 1185.5786 & 593.2929 & $\mathbf{T}$ & 749.3788 & 375.1930 & 732.3523 & 366.6798 & 731.3682 & 366.1878 & 6 \\
\hline 12 & 1332.6318 & 666.8195 & 1315.6052 & 658.3063 & 1314.6212 & 657.8142 & $\mathbf{E}$ & 648.3311 & 324.6692 & 631.3046 & 316.1559 & 0.3206 & 315.6639 & 5 \\
\hline 13 & 1419.6638 & 710.3355 & 1402.6373 & 701.8223 & 1401.6532 & 701.3303 & S & 519.2885 & 260.1479 & 502.2620 & 251.6346 & 501.2780 & 251.1426 & 4 \\
\hline 14 & 1548.7064 & 774.8568 & 1531.6799 & 766.3436 & 1530.6958 & 765.8516 & $\mathbf{E}$ & 432.2565 & 216.6319 & 415.2300 & 208.1186 & 414.2459 & 207.6266 & 3 \\
\hline 15 & 1676.8014 & 838.9043 & 1659.7748 & 830.3910 & 1658.7908 & 829.8990 & $\mathbf{K}$ & 303.2139 & 152.1106 & 286.1874 & 143.5973 & & & 2 \\
\hline 16 & & & & & & & $\mathbf{R}$ & 175.1190 & 88.0631 & 158.0924 & 79.5498 & & & a \\
\hline
\end{tabular}
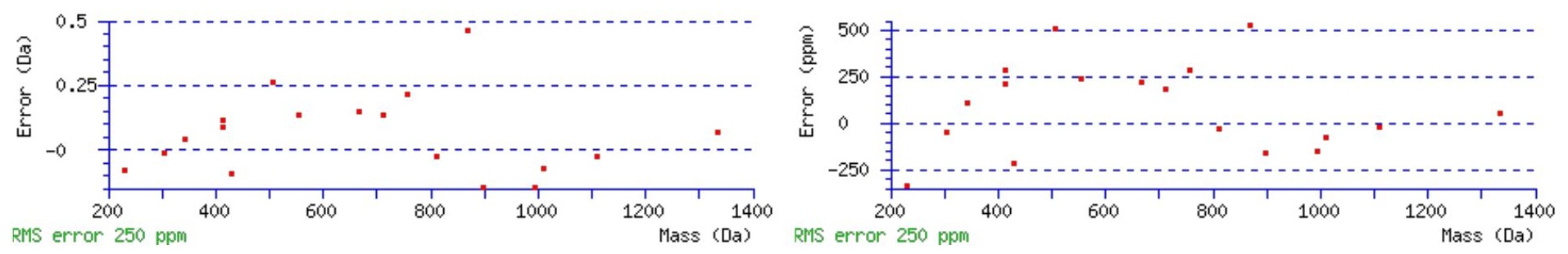

\section{All matches to this query}

\begin{tabular}{|c|c|c|c|}
\hline Score & Mr(calc): & Delta & Sequence \\
\hline 39.2 & 1849.9057 & 0.0019 & NNLSSLLPDFTESEKR \\
\hline 39.2 & 1849.9057 & 0.0019 & NNLSSLLPDFTESEKR \\
\hline 29.6 & 1848.9217 & 0.9859 & NNLSSLLPDFTESEKR \\
\hline 26.4 & 1849.9087 & -0.0011 & NNLSLPSLWSRANIGK \\
\hline 19.1 & 1849.9087 & -0.0011 & NNLSLPSLWSRANIGK \\
\hline 15.8 & 1848.8996 & 1.0081 & VIRVSINNNHGNLYR \\
\hline 12.9 & 1849.9203 & -0.0126 & TIKETLSKLTLATGSK \\
\hline 12.9 & 1849.9203 & -0.0126 & TIKETLSKLTLATGSK \\
\hline 12.9 & 1849.9203 & -0.0126 & TIKETLSKLTLATGSK \\
\hline 9.0 & 1849.9087 & \begin{tabular}{|l|}
-0.0011 \\
\end{tabular} & NNLSLPSLWSRANIGK \\
\hline
\end{tabular}

Spectrum No: 861; Query: 1186; Rank: 1

\section{Peptide View}

MS/MS Fragmentation of DLDTDFTSNASQPETK

Found in IPI00213182, Tax_Id=10116 Gene_Symbol=Cpd Isoform 1 of Carboxypeptidase D precursor

Match to Query 1186: 1768.765808 from(885.390180,2+)

Title: 100101RatKid_NS_deglyco_27.1951.1951.2.dta

Data file K:INewmanPaper|Piliangl3SubProteomes\Piliang3SP\mgf5ppm\ERLIC_3SubProteomes5ppm.mgf 

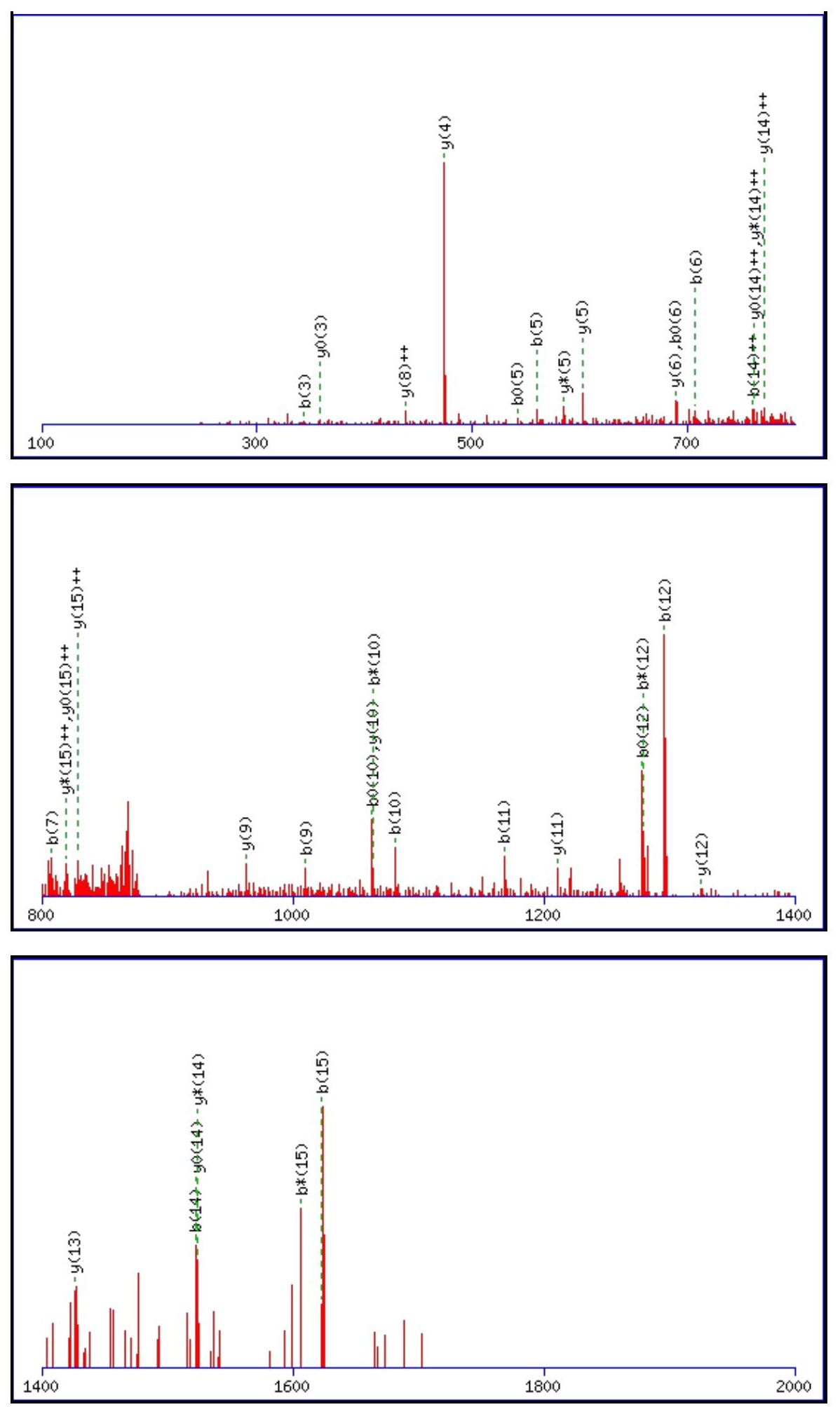

Monoisotopic mass of neutral peptide $\operatorname{Mr}($ calc): 1768.7639

Fixed modifications: Carbamidomethyl (C)

Variable modifications:

N9: Deamidated $\mathrm{N}(\mathrm{N})$

Ions Score: 39 Expect: 0.024

Matches (Bold Red): 37/162 fragment ions using 87 most intense peaks

\begin{tabular}{|r|c|c|c|c|r|r|r|r|c|c|c|c|c|c|}
\hline$\#$ & $\mathbf{b}$ & $\mathbf{b}^{++}$ & $\mathbf{b}^{*}$ & $\mathbf{b}^{\mathbf{*}^{++}}$ & $\mathbf{b}^{\mathbf{0}}$ & $\mathbf{b}^{\mathbf{0 + +}}$ & Seq. & $\mathbf{y}$ & $\mathbf{y}^{++}$ & $\mathbf{y}^{\mathbf{*}}$ & $\mathbf{y}^{\boldsymbol{*}^{++}}$ & $\mathbf{y}^{\mathbf{0}}$ & $\mathbf{y}^{\mathbf{0 + +}}$ & $\#$ \\
\hline $\mathbf{1}$ & 116.0342 & 58.5207 & & & 98.0237 & 49.5155 & $\mathbf{D}$ & & & & & & & $\mathbf{1 6}$ \\
\hline $\mathbf{2}$ & 229.1183 & 115.0628 & & & 211.1077 & 106.0575 & $\mathbf{L}$ & 1654.7442 & $\mathbf{8 2 7 . 8 7 5 8}$ & 1637.7177 & $\mathbf{8 1 9 . 3 6 2 5}$ & 1636.7337 & $\mathbf{8 1 8 . 8 7 0 5}$ & $\mathbf{1 5}$ \\
\hline $\mathbf{3}$ & $\mathbf{3 4 4 . 1 4 5 2}$ & 172.5763 & & & 326.1347 & 163.5710 & $\mathbf{D}$ & 1541.6602 & 771.3337 & $\mathbf{1 5 2 4 . 6 3 3 6}$ & $\mathbf{7 6 2 . 8 2 0 5}$ & $\mathbf{1 5 2 3 . 6 4 9 6}$ & $\mathbf{7 6 2 . 3 2 8 4}$ & $\mathbf{1 4}$ \\
\hline $\mathbf{4}$ & 445.1929 & 223.1001 & & & 427.1823 & 214.0948 & $\mathbf{T}$ & $\mathbf{1 4 2 6 . 6 3 3 2}$ & 713.8203 & 1409.6067 & 705.3070 & 1408.6227 & 704.8150 & $\mathbf{1 3}$ \\
\hline $\mathbf{5}$ & $\mathbf{5 6 0 . 2 1 9 8}$ & 280.6136 & & & 542.2093 & 271.6083 & $\mathbf{D}$ & $\mathbf{1 3 2 5 . 5 8 5 6}$ & 663.2964 & 1308.5590 & 654.7831 & 1307.5750 & 654.2911 & $\mathbf{1 2}$ \\
\hline
\end{tabular}




\begin{tabular}{|c|c|c|c|c|c|c|c|c|c|c|c|c|c|c|}
\hline 6 & 707.2883 & |354.1478 & & & 689.2777 & |345.1425 & $\mathbf{F}$ & 1210.5586 & 605.7829| & 1193.5321 & 597.2697 & 1192.5481 & 596.7777 & 11 \\
\hline 7 & 808.3359 & 404.6716 & & & 790.3254 & 395.6663 & $\mathbf{T}$ & 1063.4902 & 532.2487 & 1046.4637 & 523.7355 & 1045.4796 & 523.2435 & 10 \\
\hline 8 & 895.3680 & 448.1876 & & & 877.3574 & 439.1823 & S & 962.4425 & 481.7249 & 945.4160 & 473.2116 & 944.4320 & 472.7196 & 9 \\
\hline 9 & 1010.3949 & 505.7011 & 993.3684 & 497.1878 & 992.3843 & 496.6958 & $\mathbf{N}$ & 875.4105 & 438.2089 & 858.3839 & 429.6956 & 857.3999 & 429.2036 & 8 \\
\hline 10 & 1081.4320 & 541.2196 & 1064.4055 & 532.7064 & 1063.4215 & 532.2144 & A & 760.3836 & 380.6954 & 743.3570 & 372.1821 & 742.3730 & 371.6901 & 7 \\
\hline 11 & 1168.4640 & 584.7357 & 1151.4375 & 576.2224 & 1150.4535 & 575.7304 & S & 689.3464 & 345.1769 & 672.3199 & 336.6636 & 671.3359 & 336.1716 & 6 \\
\hline 12 & 1296.5226 & 648.7650 & 1279.4961 & 640.2517 & 1278.5121 & 639.7597 & $\mathbf{Q}$ & 602.3144 & 301.6608 & 585.2879 & 293.1476 & 584.3039 & 292.6556 & 5 \\
\hline 13 & 1393.5754 & 697.2913 & 1376.5488 & 688.7781 & 1375.5648 & 688.2861 & $\mathbf{P}$ & 474.2558 & 237.6316 & 457.2293 & 229.1183 & 456.2453 & 228.6263 & 4 \\
\hline 14 & 1522.6180 & 761.8126 & 1505.5914 & 753.2994 & 1504.6074 & 752.8073 & $\mathbf{E}$ & 377.2031 & 189.1052 & 360.1765 & 180.5919 & 359.1925 & 180.0999 & 3 \\
\hline 15 & 1623.6657 & 812.3365 & 1606.6391 & 803.8232 & 1605.6551 & 803.3312 & $\mathbf{T}$ & 248.1605 & 124.5839 & 231.1339 & 116.0706 & 230.1499 & 115.5786 & 2 \\
\hline 16 & & & & & & & $\mathbf{K}$ & 147.1128 & 74.0600 & 130.0863 & 65.5468 & & & 1 \\
\hline
\end{tabular}
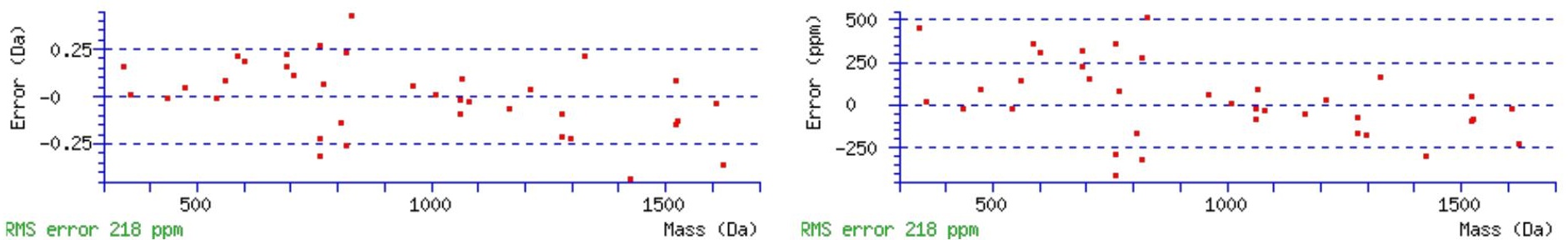

\section{All matches to this query}

\begin{tabular}{|l|l|l|c|}
\hline Score & Mr(calc): & Delta & Sequence \\
\hline 39.1 & 1768.7639 & 0.0019 & DLDTDFTSNASQPETK \\
\hline 3.5 & 1767.7799 & 0.9859 & DLDTDFTSNASQPETK \\
\hline
\end{tabular}

Spectrum No: 862; Query: 2096; Rank: 1

\section{Peptide View}

MS/MS Fragmentation of DQCIVDDITYNVNDTFHKR

Found in IPI00200757, Tax_Id=10116 Gene_Symbol=Fn1 Isoform 1 of Fibronectin precursor

Match to Query 2096: 2353.068056 from(589.274290,4+)

Title: 100101RatKid_NS_deglyco_23.4009.4009.4.dta

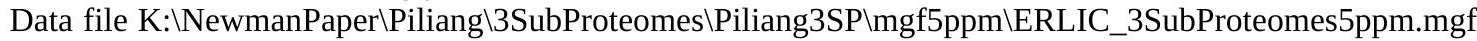

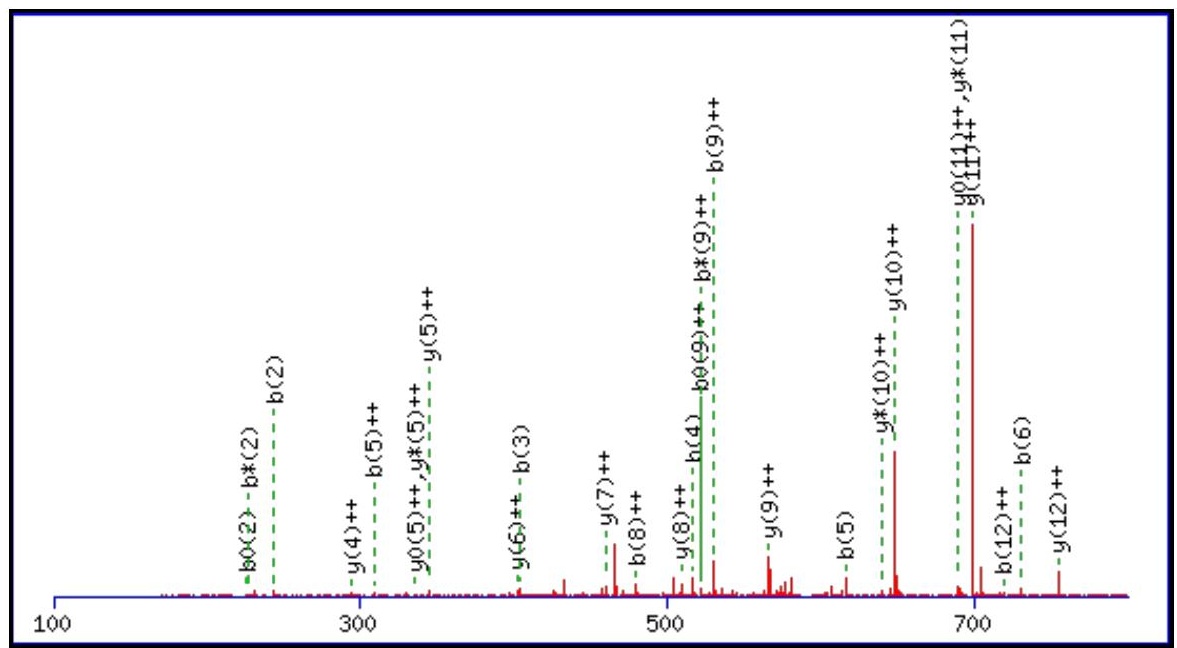



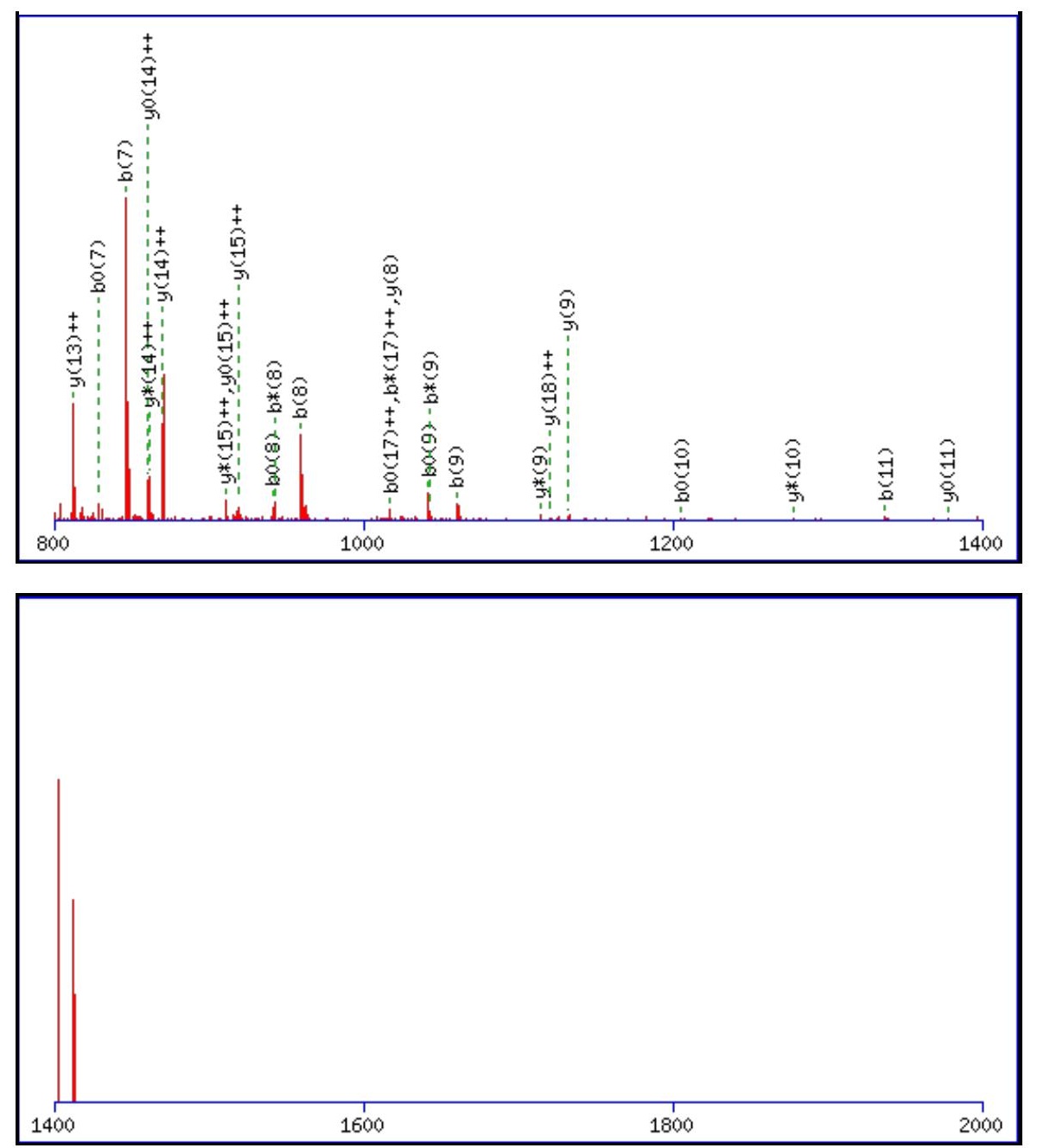

Monoisotopic mass of neutral peptide $\operatorname{Mr}($ calc): 2353.0645

Fixed modifications: Carbamidomethyl (C)

Variable modifications:

N13 : Deamidated_N (N)

Ions Score: 39 Expect: $\odot .037$

Matches (Bold Red): 52/206 fragment ions using 123 most intense peaks

\begin{tabular}{|c|c|c|c|c|c|c|c|c|c|c|c|c|c|c|}
\hline \# & b & $\mathbf{b}^{++}$ & $\mathbf{b}^{*}$ & $\mathbf{b}^{*^{++}}$ & $\mathbf{b}^{\mathbf{0}}$ & $\mathbf{b}^{\mathbf{0 + +}}$ & Seq. & $\mathbf{y}$ & $\mathbf{y}^{++}$ & $\mathbf{y}^{*}$ & $\mathrm{y}^{\mathrm{*}^{++}}$ & $\mathbf{y}^{\mathbf{0}}$ & $\mathbf{y}^{\mathbf{0 + +}}$ & \# \\
\hline 1 & 116.0342 & 58.5207 & & & 98.0237 & 49.5155 & D & & & & & & & 19 \\
\hline 2 & 244.0928 & 122.5500 & 227.0662 & 114.0368 & 226.0822 & 113.5448 & $\mathbf{Q}$ & 2239.0448 & 1120.0260 & 2222.0183 & 1111.5128 & 2221.0342 & 1111.0208 & 18 \\
\hline 3 & 404.1234 & 202.5654 & 387.0969 & 194.0521 & 386.1129 & 193.5601 & C & 2110.9862 & 1055.9968 & 2093.9597 & 1047.4835 & 2092.9757 & 1046.9915 & 17 \\
\hline 4 & 517.2075 & 259.1074 & 500.1810 & 250.5941 & 499.1969 & 250.1021 & I & 1950.9556 & 975.9814 & 1933.9290 & 967.4682 & 1932.9450 & 966.9761 & 16 \\
\hline 5 & 616.2759 & 308.6416 & 599.2494 & 300.1283 & 598.2654 & 299.6363 & V & 1837.8715 & 919.4394 & 1820.8450 & 910.9261 & 1819.8609 & 910.4341 & 15 \\
\hline 6 & 731.3029 & 366.1551 & 714.2763 & 357.6418 & 713.2923 & 357.1498 & D & 1738.8031 & 869.9052 & 1721.7765 & 861.3919 & 1720.7925 & 860.8999 & 14 \\
\hline 7 & 846.3298 & 423.6685 & 829.3033 & 415.1553 & 828.3192 & 414.6633 & D & 1623.7762 & 812.3917 & 1606.7496 & 803.8784 & 1605.7656 & 803.3864 & 13 \\
\hline 8 & 959.4139 & 480.2106 & 942.3873 & 471.6973 & 941.4033 & 471.2053 & I & 1508.7492 & 754.8782 & 1491.7227 & 746.3650 & 1490.7386 & 8730 & 12 \\
\hline 9 & 1060.4616 & 530.7344 & 1043.4350 & 522.2211 & 1042.4510 & 521.7291 & $\mathbf{T}$ & 1395.6651 & 698.3362 & 1378.6386 & 689.8229 & 1377.6546 & 689.3309 & 11 \\
\hline 10 & 1223.5249 & 612.2661 & 1206.4983 & 603.7528 & 1205.5143 & 603.2608 & $\mathbf{Y}$ & 1294.6175 & 647.8124 & 1277.5909 & 639.2991 & 1276.6069 & 638.8071 & 10 \\
\hline 11 & 1337.5678 & 669.2875 & 1320.5413 & 660.7743 & 1319.5572 & 660.2823 & $\mathbf{N}$ & 1131.5541 & 566.2807 & 1114.5276 & 557.7674 & 1113.5436 & 557.2754 & 9 \\
\hline 12 & 1436.6362 & 718.8217 & 1419.6097 & 710.3085 & 1418.6257 & 709.8165 & $\mathbf{V}$ & 1017.5112 & 509.2592 & 1000.4847 & 500.7460 & 999.5006 & 500.2540 & 8 \\
\hline 13 & 1551.6632 & 776.3352 & 1534.6366 & 767.8219 & 1533.6526 & 767.3299 & $\mathbf{N}$ & 918.4428 & 459.7250 & 901.4163 & 451.2118 & 900.4322 & 450.7198 & 7 \\
\hline 14 & 1666.6901 & 833.8487 & 1649.6636 & 825.3354 & 1648.6795 & 824.8434 & D & 803.4159 & 402.2116 & 786.3893 & 393.6983 & 785.4053 & 393.2063 & 6 \\
\hline 15 & 1767.7378 & 884.3725 & 1750.7112 & 875.8593 & 1749.7272 & 875.3672 & $\mathbf{T}$ & 688.3889 & 344.6981 & 671.3624 & 336.1848 & 670.3784 & 335.6928 & 5 \\
\hline 16 & 1914.8062 & 957.9067 & 1897.7796 & 949.3935 & 1896.7956 & 948.9015 & $\mathbf{F}$ & 587.3412 & 294.1743 & 570.3147 & 285.6610 & & & 4 \\
\hline 17 & 2051.8651 & 1026.4362 & 2034.8386 & 1017.9229 & 2033.8545 & 1017.4309 & $\mathbf{H}$ & 440.2728 & 220.6401 & 423.2463 & 212.1268 & & & 3 \\
\hline 18 & 2179.9601 & 1090.4837 & 2162.9335 & 1081.9704 & 2161.9495 & 1081.4784 & $\mathbf{K}$ & 303.2139 & 152.1106 & 286.1874 & 143.5973 & & & 2 \\
\hline
\end{tabular}




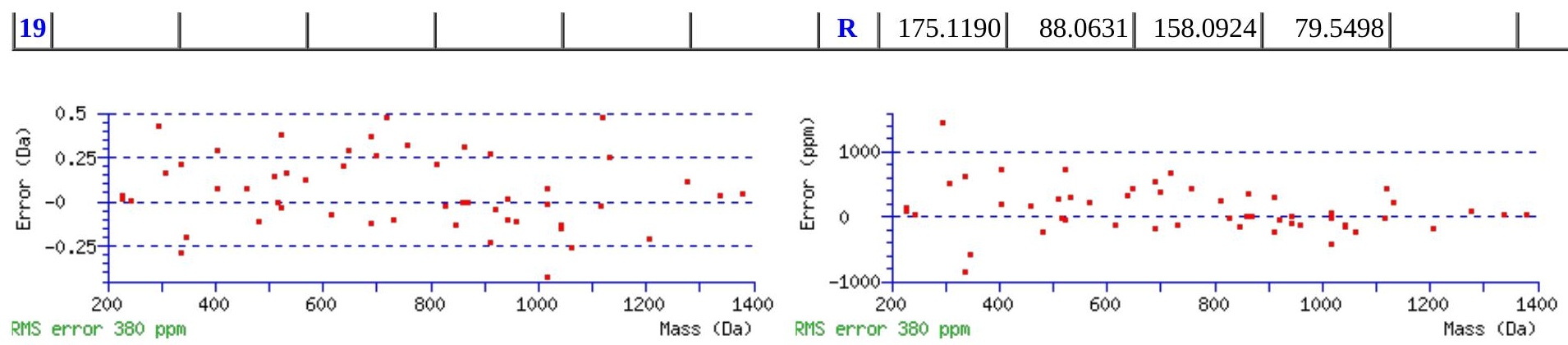

\section{All matches to this query}

\begin{tabular}{|l|l|l|l|}
\hline Score & Mr(calc): & Delta & \multicolumn{1}{|c|}{ Sequence } \\
\hline 39.1 & 2353.0645 & 0.0036 & DQCIVDDITYNVNDTFHKR \\
\hline 29.5 & 2353.0645 & 0.0036 & DQCIVDDITYNVNDTFHKR \\
\hline 11.1 & 2352.0805 & 0.9876 & DQCIVDDITYNVNDTFHKR \\
\hline 9.8 & 2353.0547 & 0.0134 & EKSRSQGPNGSEGLEVSVQER \\
\hline 9.8 & 2352.0707 & 0.9974 & EKSRSQGPNGSEGLEVSVQER \\
\hline 7.1 & 2352.0651 & 1.0030 & VENGDLSNKTLKITDFGLAR \\
\hline 5.4 & 2353.0547 & 0.0134 & EKSRSQGPNGSEGLEVSVQER \\
\hline 5.3 & 2351.0682 & 1.9998 & LSSSOVSAVKPOTAGGDSNYFK \\
\hline 5.3 & 2351.0682 & 1.9998 & LSSSQVSAVKPQTAGGDSNYFK \\
\hline 4.7 & 2351.0839 & 1.9842 & SLGLKDNNIVFSVPTDQCIDN \\
\hline
\end{tabular}

Spectrum No: 863; Query: 833; Rank: 1

\section{Peptide View}

MS/MS Fragmentation of YLWSEPQNCSATK

Found in IPI00212809, Tax_Id=10116 Gene_Symbol=Ctsh Cathepsin H precursor

Match to Query 833: 1583.695268 from(792.854910,2+)

Title: 100101RatKid_NS_deglyco_27.2044.2044.2.dta

Data file K:INewmanPaper|Piliangl3SubProteomes\Piliang3SP\mgf5ppm\ERLIC_3SubProteomes5ppm.mgf

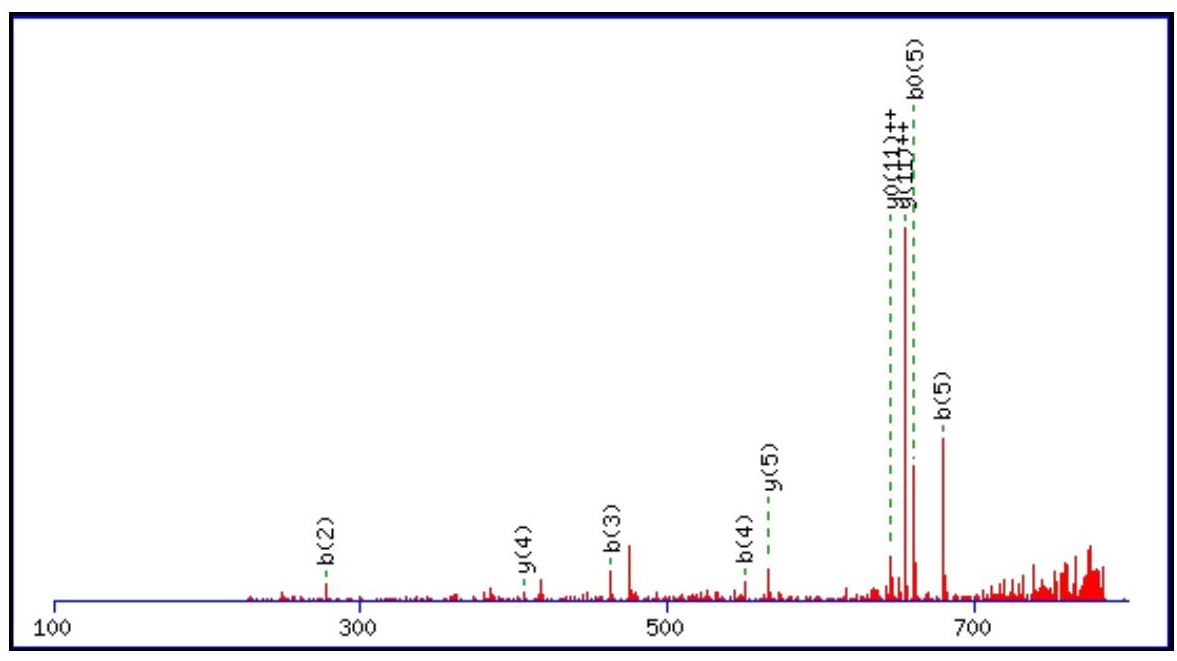



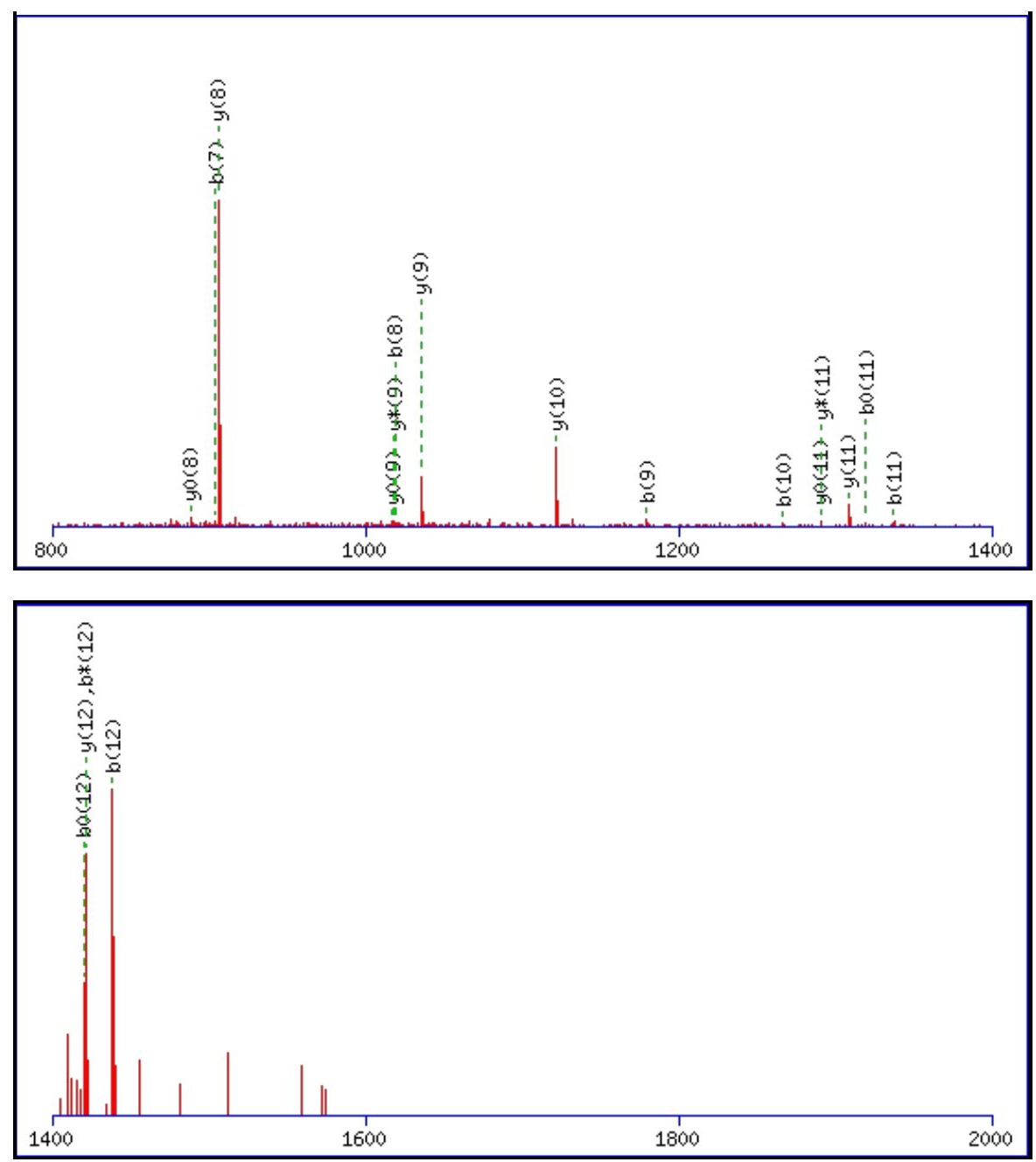

Monoisotopic mass of neutral peptide $\operatorname{Mr}($ calc): 1583.6926

Fixed modifications: Carbamidomethyl (C)

Variable modifications:

N8 : Deamidated $\mathrm{N}(\mathrm{N})$

Ions Score: 39 Expect: 0.02

Matches (Bold Red): 28/124 fragment ions using 95 most intense peaks

\begin{tabular}{|c|c|c|c|c|c|c|c|c|c|c|c|c|c|c|}
\hline \# & b & $\mathbf{b}^{++}$ & b* & $\mathbf{b}^{*^{++}}$ & $\mathbf{b}^{0}$ & $\mathbf{b}^{0++}$ & Seq. & $\mathbf{y}$ & $\mathrm{y}^{++}$ & $\mathbf{y}^{*}$ & $\mathrm{y}^{*^{++}}$ & $\mathbf{y}^{0}$ & $y^{0++}$ & \# \\
\hline 1 & 164.0706 & 82.5389 & & & & & $\mathbf{Y}$ & & & & & & & 13 \\
\hline 2 & 277.1547 & 139.0810 & & & & & $\mathbf{L}$ & 1421.6366 & 711.3219 & 1404.6100 & 702.8086 & 1403.6260 & 702.3166 & 12 \\
\hline 3 & 463.2340 & 232.1206 & & & & & $\mathbf{W}$ & 1308.5525 & 654.7799 & 1291.5259 & 646.2666 & 1290.5419 & 645.7746 & 11 \\
\hline 4 & 550.2660 & 275.6366 & & & 532.2554 & 266.6314 & S & 1122.4732 & 561.7402 & 1105.4466 & 553.2270 & 1104.4626 & 552.7349 & 10 \\
\hline 5 & 679.3086 & 340.1579 & & & 661.2980 & 331.1527 & $\mathbf{E}$ & 1035.4411 & 518.2242 & 1018.4146 & 509.7109 & 1017.4306 & 509.2189 & 9 \\
\hline 6 & 776.3614 & 388.6843 & & & 758.3508 & 379.6790 & $\mathbf{P}$ & 906.3986 & 453.7029 & 889.3720 & 445.1896 & 888.3880 & 444.6976 & 8 \\
\hline 7 & 904.4199 & 452.7136 & 887.3934 & 444.2003 & 886.4094 & 443.7083 & $\mathbf{Q}$ & 809.3458 & 405.1765 & 792.3192 & 396.6633 & 791.3352 & 396.1712 & 7 \\
\hline 8 & 1019.4469 & 510.2271 & 1002.4203 & 501.7138 & 1001.4363 & 501.2218 & $\mathbf{N}$ & 681.2872 & 341.1472 & 664.2607 & 332.6340 & 663.2766 & 332.1420 & 6 \\
\hline 9 & 1179.4775 & 590.2424 & 1162.4510 & 581.7291 & 1161.4670 & 581.2371 & C & 566.2603 & 283.6338 & 549.2337 & 275.1205 & 548.2497 & 274.6285 & 5 \\
\hline 10 & 1266.5096 & 633.7584 & 1249.4830 & 625.2451 & 1248.4990 & 624.7531 & $\mathrm{~S}$ & 406.2296 & 203.6185 & 389.2031 & 195.1052 & 388.2191 & 194.6132 & 4 \\
\hline 11 & 1337.5467 & 669.2770 & 1320.5201 & 660.7637 & 1319.5361 & 660.2717 & A & 319.1976 & 160.1024 & 302.1710 & 151.5892 & 301.1870 & 151.0972 & 3 \\
\hline 12 & 1438.5944 & 719.8008 & 1421.5678 & 711.2875 & 1420.5838 & 710.7955 & $\mathbf{T}$ & 248.1605 & 124.5839 & 231.1339 & 116.0706 & 230.1499 & 115.5786 & 2 \\
\hline 13 & & & & & & & $\mathbf{K}$ & 147.1128 & 74.0600 & 130.0863 & 65.5468 & & & 1 \\
\hline
\end{tabular}



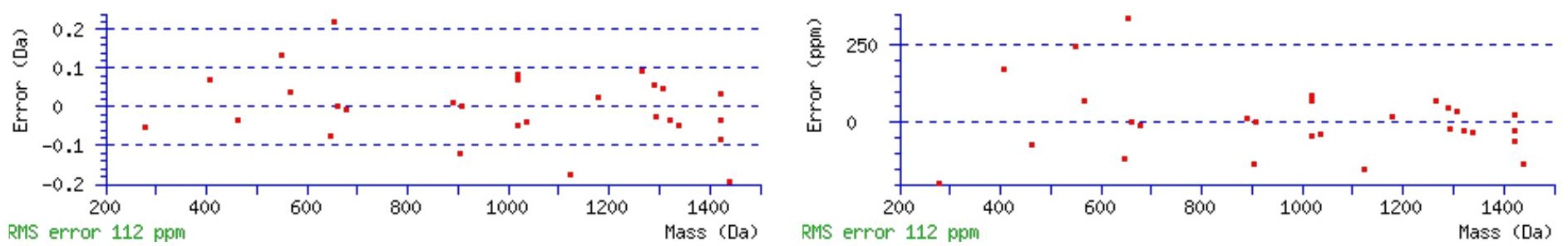

\section{All matches to this query}

\begin{tabular}{|l|l|l|l|}
\hline Score & Mr(calc): & Delta & \multicolumn{1}{|c|}{ Sequence } \\
\hline 39.0 & 1583.6926 & 0.0027 & YLWSEPQNCSATK \\
\hline 5.9 & 1582.6854 & 1.0098 & ATNMEDMLKEEEK \\
\hline 5.2 & 1582.7045 & 0.9908 & FVKTDALTSGEKK \\
\hline 4.2 & 1583.6990 & -0.0037 & FQAATMCKFTRK \\
\hline 3.4 & 1583.6942 & 0.0011 & ASNPDLMLWEISK \\
\hline 2.1 & 1583.6830 & 0.0123 & YLSMSSSIIFEDI \\
\hline 2.1 & 1583.6830 & 0.0123 & YLSMSSSIIFEDI \\
\hline 2.1 & 1583.6830 & 0.0123 & YLSMSSSIIFEDI \\
\hline 1.9 & 1583.6803 & 0.0150 & LRQSNPEFCPEK \\
\hline 1.1 & 1583.6830 & 0.0123 & YLSMSSSIIFEDI \\
\hline
\end{tabular}

Spectrum No: 864; Query: 1971; Rank: 1

\section{Peptide View}

MS/MS Fragmentation of IKGDLALSGNLSNLYAMTQDK

Found in IPI00370450, Tax_Id=10116 Gene_Symbol=Plxnb2 plexin B2

Match to Query 1971: 2268.124602 from(757.048810,3+)

Title: 091008RatKidney_NH4Format01_25.3225.3225.3.dta

Data file K:INewmanPaper|Piliang|3SubProteomes\Piliang3SP\mgf5ppm\ERLIC_3SubProteomes5ppm.mgf

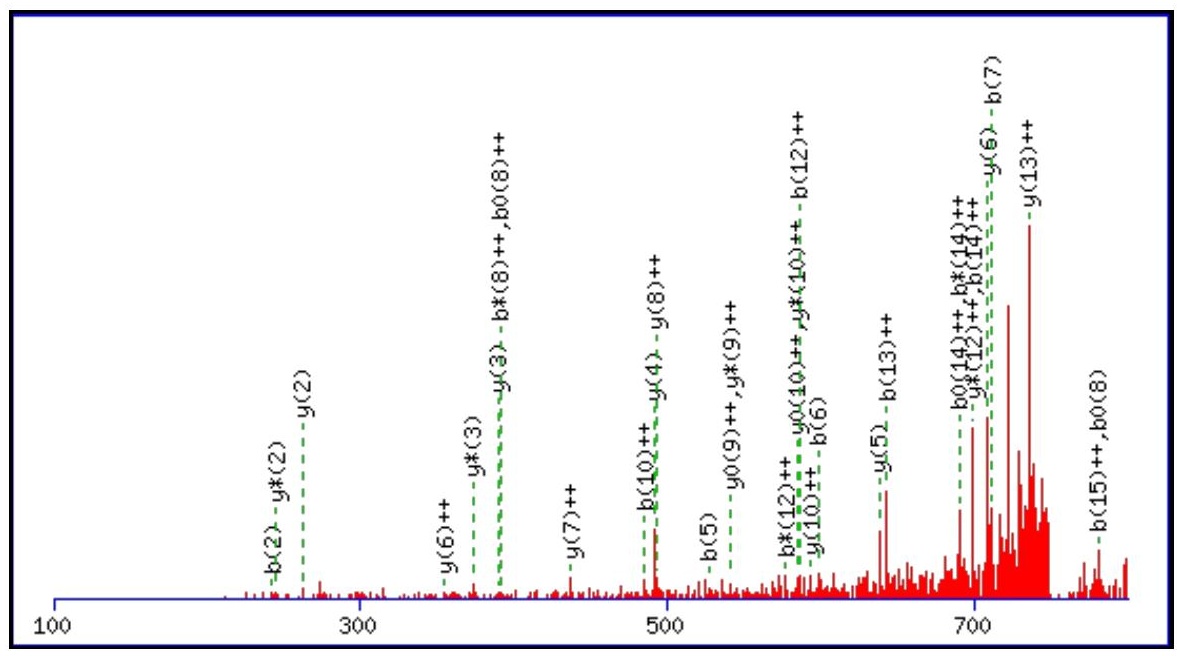



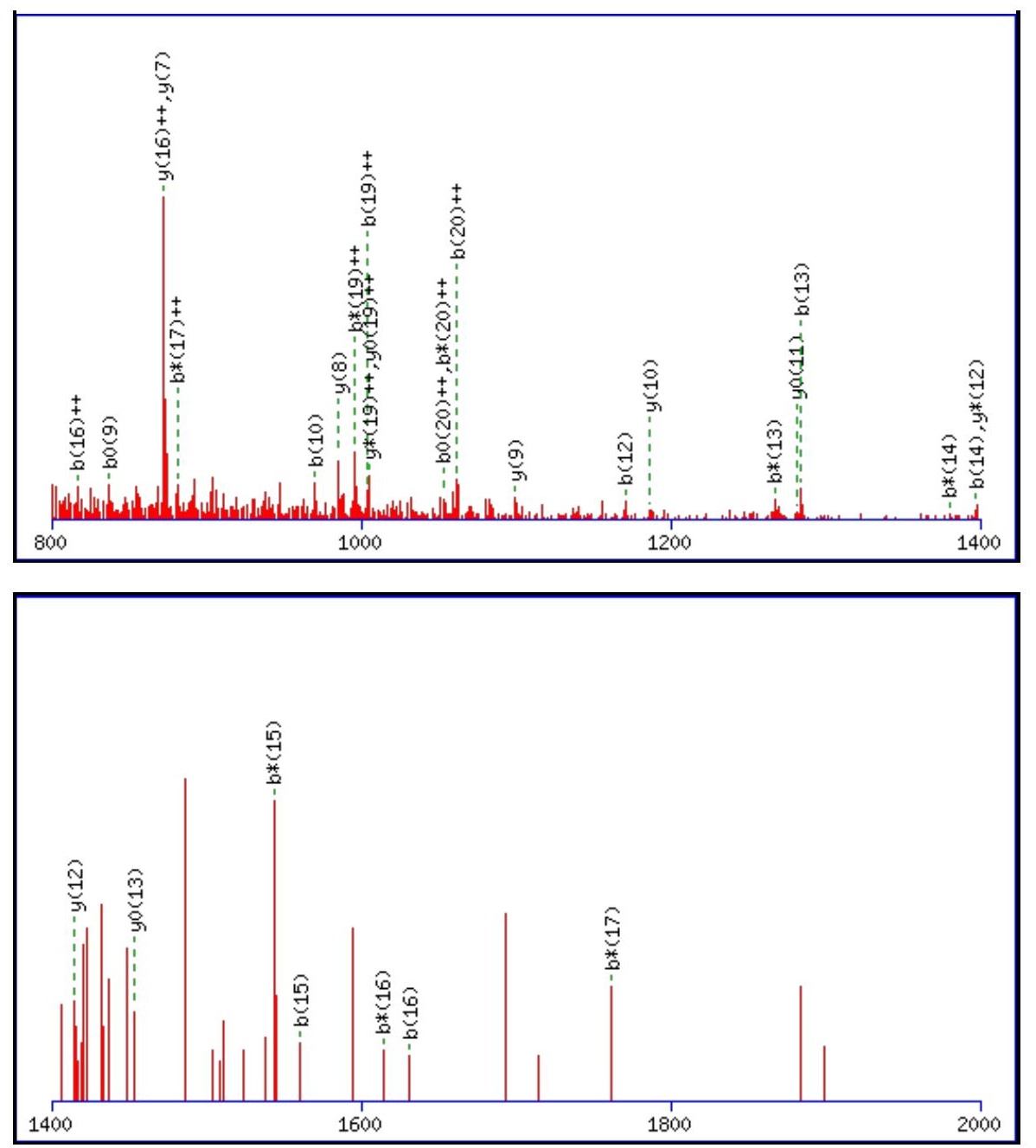

Monoisotopic mass of neutral peptide $\operatorname{Mr}($ calc): 2268.1307

Fixed modifications: Carbamidomethyl (C)

Variable modifications:

N10 : Deamidated_N (N)

M17 : 0xidation (M)

Ions Score: 39 Expect : $\odot .027$

Matches (Bold Red): 62/230 fragment ions using 143 most intense peaks

\begin{tabular}{|c|c|c|c|c|c|c|c|c|c|c|c|c|c|c|}
\hline \# & b & $\mathbf{b}^{++}$ & $\mathbf{b}^{*}$ & $b^{*++}$ & $\mathbf{b}^{\mathbf{0}}$ & & eq. & $\mathbf{y}$ & & $\mathbf{y}^{*}$ & & $\mathbf{y}^{0}$ & & \# \\
\hline 1 & 114.0913 & 57.5493 & & & & & I & & & & & & & 21 \\
\hline 2 & 242.1863 & & & & & & $\mathbf{K}$ & & & & & & & 20 \\
\hline 3 & 299.2078 & 075 & 812 & & & & G & & & & & 2009.9484 & 1005.4779 & 19 \\
\hline 4 & 414.2347 & 07.6210 & & & & & D & & & & & & & 18 \\
\hline 5 & 527.3188 & 264.1 & 510.2 & 498 & 82 & & $\mathbf{L}$ & 106 & 589 & 841 & 457 & 1837.9000 & 919.4537 & 17 \\
\hline 6 & 598.3559 & 299.6816 & 581.3 & 291.1683 & & & A & 265 & & 1725 & & 3160 & 116 & 16 \\
\hline 7 & 711.4400 & 356.2236 & 694.4134 & 347.7103 & 294 & 347.2183 & $\mathbf{L}$ & 1671.7 & 984 & 1654 & 827.8851 & 1653 & 827.3931 & 15 \\
\hline 8 & 798.4720 & 399.7396 & 781.4454 & 391.2264 & 780.4 & 390.7 & $\mathrm{~S}$ & 1558.7054 & 779.8563 & 1541 & & 1540.6948 & 770.8510 & 14 \\
\hline 9 & 855.4934 & 428.2504 & 838.4669 & 419.7371 & 837.4829 & & $\mathbf{G}$ & 1471.6733 & 736.3403 & 1454 & 727.8270 & 1453 & 727.3350 & 13 \\
\hline 10 & 970.5204 & 485.7638 & 953.4 & 477.2506 & 952.5 & 476. & $\mathbf{N}$ & 1414. & 296 & 1397 & 163 & 1396.6413 & 698.8243 & 12 \\
\hline 11 & 1083.6044 & 542.3059 & 1066.5779 & 533.7926 & 1065.5939 & 533.3006 & $\mathbf{L}$ & 1299.6249 & 650.3161 & 1282.5984 & 641.8028 & 1281.6144 & 641.3108 & 11 \\
\hline 12 & 170.6365 & 585.8219 & 153.6099 & 577.3086 & 152.6259 & 576.8 & $S$ & 1186. & 741 & 1169. & & 1168.5 & 688 & 10 \\
\hline 13 & 1284.6794 & 642.8433 & 1267.6529 & 634.3301 & 1266.6688 & 633.8381 & $\mathbf{N}$ & 1099.5088 & 550.2581 & 1082.4823 & 541.7448 & 1081.4983 & 541.2528 & 9 \\
\hline 14 & 1397.7635 & 699.3854 & 1380.7369 & 690.8721 & 1379.7 & 690.3 & $\mathbf{L}$ & & 493. & 968. & 484. & 967.4553 & 484.2313 & 8 \\
\hline 15 & 1560.8268 & 780.9170 & 1543.8002 & 772.4038 & 1542.8162 & 771.9118 & $\mathbf{Y}$ & 872.3819 & 436.6946 & 855.3553 & 428.1813 & 854.3713 & 427.6893 & 7 \\
\hline 16 & 1631.8639 & 816.4356 & 1614.8374 & 807.9223 & 1613.8533 & 807.4303 & A & 709.3185 & 355.1629 & 692.2920 & 346.6496 & 691.3080 & 346.1576 & 6 \\
\hline 17 & 1778.8993 & 889.9533 & 1761.8728 & 881.4400 & 1760.8887 & 880.9480 & $\mathbf{M}$ & 638.2814 & 319.6443 & 621.2549 & 311.1311 & 620.2708 & 310.6391 & 5 \\
\hline 18 & 1879.9470 & 940.4771 & 1862.9204 & 931.9639 & 1861.9364 & 931.4719 & $\mathbf{T}$ & 491.2460 & 246.1266 & 474.2195 & 237.6134 & 473.2354 & 237.1214 & 4 \\
\hline
\end{tabular}




\begin{tabular}{|c|c|c|c|c|c|c|c|c|c|c|c|c|c|c|}
\hline 19 & |2008.0056| & $|1004.5064|$ & | & 995.9931 & |1989.9950 & 995.5011 & $\mathbf{Q}$ & 390.1983 & 195.6028 & 373.1718 & 187.0895 & 372.1878 & 186.5975 & 3 \\
\hline 20 & 2123.0325 & 1062.0199 & 2106.0060 & 1053.5066 & 2105.0219 & 1053.0146 & D & 262.1397 & 131.5735 & 245.1132 & 123.0602 & 244.1292 & 122.5682 & 2 \\
\hline 21 & & & & & & & $\mathbf{K}$ & 147.1128 & 74.0600 & 130.0863 & 65.5468 & & & 1 \\
\hline
\end{tabular}
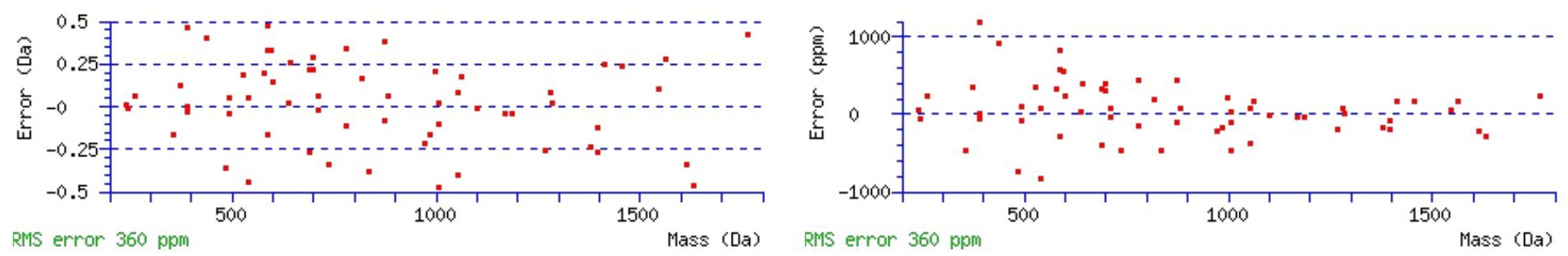

\section{All matches to this query}

\begin{tabular}{|l|l|l|l|}
\hline Score & Mr(calc): & Delta & \multicolumn{1}{|c|}{ Sequence } \\
\hline 39.0 & 2268.1307 & -0.0061 & IKGDLALSGNLSNLYAMTQDK \\
\hline 29.8 & 2268.1307 & -0.0061 & IKGDLALSGNLSNLYAMTQDK \\
\hline 8.7 & 2268.1377 & -0.0131 & GYLASGMLNGKILWENVPVK \\
\hline 3.7 & 2268.1395 & -0.0149 & KCIQTCQNYSLSFLKIHK \\
\hline 3.7 & 2268.1377 & -0.0131 & GYLASGMLNGKILWENVPVK \\
\hline 2.6 & 2268.1215 & 0.0031 & ILSRGNMSSHKGLNGACSVHK \\
\hline 1.8 & 2266.1134 & 2.0112 & LSPEEPAVLSSGFTLIGNDIK \\
\hline 1.8 & 2266.1134 & 2.0112 & LSPEEPAVLSSGFTLIGNDIK \\
\hline 1.6 & 2268.1167 & 0.0079 & ILGANALLFFGVNMYGVFVR \\
\hline 0.6 & 2266.1376 & 1.9870 & KLAAMPDHTDVSLSPEERVR \\
\hline
\end{tabular}

Spectrum No: 865; Query: 4; Rank: 1

\section{Peptide View}

MS/MS Fragmentation of LDLTLR

Found in IPI00214158, Tax_Id=10116 Gene_Symbol=Slc22a6 Renal organic anion transporter 1

Match to Query 4: 729.437088 from(365.725820,2+)

Title: 091008RatKidney_NH4Format02_09.1103.1103.2.dta

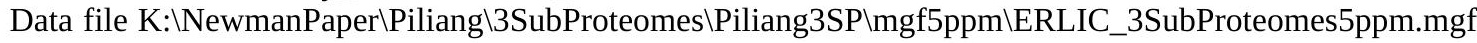

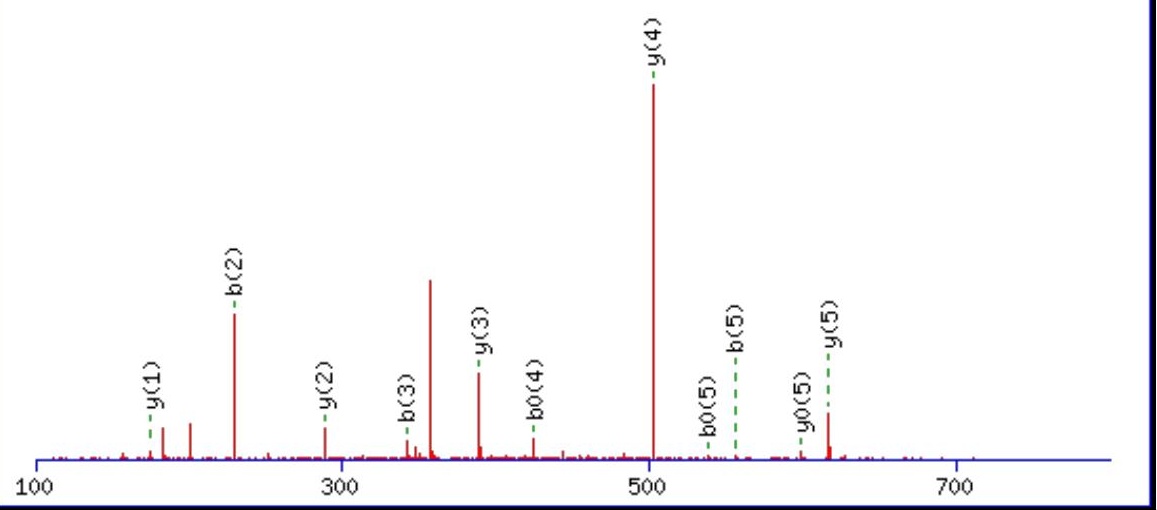




\begin{tabular}{rrrr}
800 & 1000 & 1200 & 1400 \\
\hline
\end{tabular}

\begin{tabular}{rrrr}
\hline 1400 & 1600 & 1800 & 2000 \\
\hline
\end{tabular}

Monoisotopic mass of neutral peptide $\operatorname{Mr}($ calc): 729.4385

Fixed modifications: Carbamidomethyl (C)

Ions Score: 39 Expect: 0.0073

Matches (Bold Red): 11/44 fragment ions using 22 most intense peaks

\begin{tabular}{|c|c|c|c|c|c|c|c|c|c|c|c|c|}
\hline \# & b & $\mathbf{b}^{++}$ & $\mathbf{b}^{\mathbf{0}}$ & $\mathbf{b}^{\mathbf{0 + +}}$ & Seq. & $\mathbf{y}$ & $\mathbf{y}^{++}$ & $\mathbf{y}^{*}$ & $\mathrm{y}^{*^{++}}$ & $\mathbf{y}^{\mathbf{0}}$ & $\mathbf{y}^{\mathbf{0 + +}}$ & $\#$ \\
\hline 1 & 114.0913 & 57.5493 & & & $\mathbf{L}$ & & & & & & & 6 \\
\hline 2 & 229.1183 & 115.0628 & 211.1077 & 106.0575 & D & 617.3617 & 309.1845 & 600.3352 & 300.6712 & 599.3511 & 300.1792 & 5 \\
\hline 3 & 342.2023 & 171.6048 & 324.1918 & 162.5995 & $\mathbf{L}$ & 502.3348 & 251.6710 & 485.3082 & 243.1577 & 484.3242 & 242.6657 & 4 \\
\hline 4 & 443.2500 & 222.1287 & 425.2395 & 213.1234 & $\mathbf{T}$ & 389.2507 & 195.1290 & 372.2241 & 186.6157 & 371.2401 & 186.1237 & 3 \\
\hline 5 & 556.3341 & 278.6707 & 538.3235 & 269.6654 & $\mathbf{L}$ & 288.2030 & 144.6051 & 271.1765 & 136.0919 & & & 2 \\
\hline 6 & & & & & $\mathbf{R}$ & 175.1190 & 88.0631 & 158.0924 & 79.5498 & & & 1 \\
\hline
\end{tabular}

$$
\text { 要 }
$$$$
\text { R }
$$

\section{All matches to this query}

\begin{tabular}{|l|l|l|l|}
\hline Score & Mr(calc): & Delta & Sequence \\
\hline 39.0 & 729.4385 & -0.0014 & LDLTLR \\
\hline 39.0 & 729.4385 & -0.0014 & LNLTLR \\
\hline 24.9 & 729.4385 & -0.0014 & $\underline{\text { EVLTLR }}$ \\
\hline
\end{tabular}

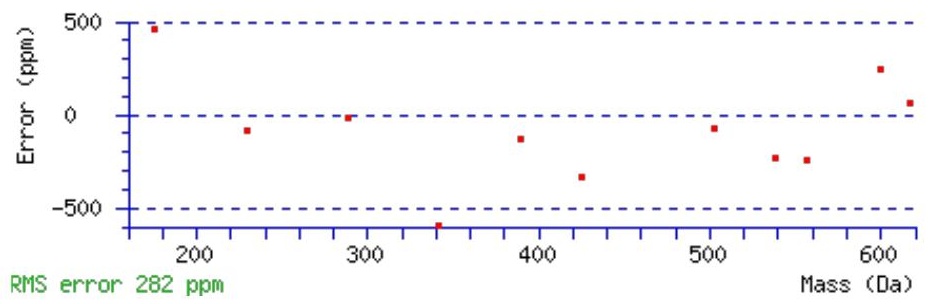

file:///R//wwwroot/SupportingData/3SubProteomes/ERLIC_3SubProteo_Glyco/ERLIC_3SubProteo_Glyco_005.html[4/27/2010 12:29:24 PM] 


\begin{tabular}{|l|l|l|l|}
24.9 & 729.4385 & -0.0014 & VEITLR \\
\hline 21.5 & 729.4385 & -0.0014 & LIDTLR \\
\hline 21.5 & 729.4385 & -0.0014 & LINTIR \\
\hline 21.5 & 729.4385 & -0.0014 & LLDTLR \\
\hline 21.5 & 729.4385 & -0.0014 & LLNTLR \\
\hline 21.5 & 729.4385 & -0.0014 & LVETIR \\
\hline 16.2 & 729.4385 & -0.0014 & NITLIR \\
\hline
\end{tabular}

Spectrum No: 866; Query: 140; Rank: 1

\section{Peptide View}

MS/MS Fragmentation of NLTVQVNSVR

Found in IPI00326140, Tax_Id=10116 Gene_Symbol=Pzp Alpha-1-macroglobulin precursor

Match to Query 140: 1129.609788 from(565.812170,2+)

Title: 091008RatKidney_NH4Format01_29.1132.1132.2.dta

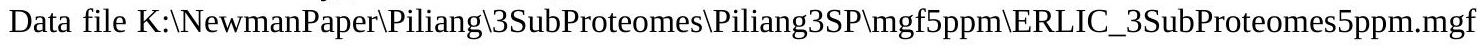
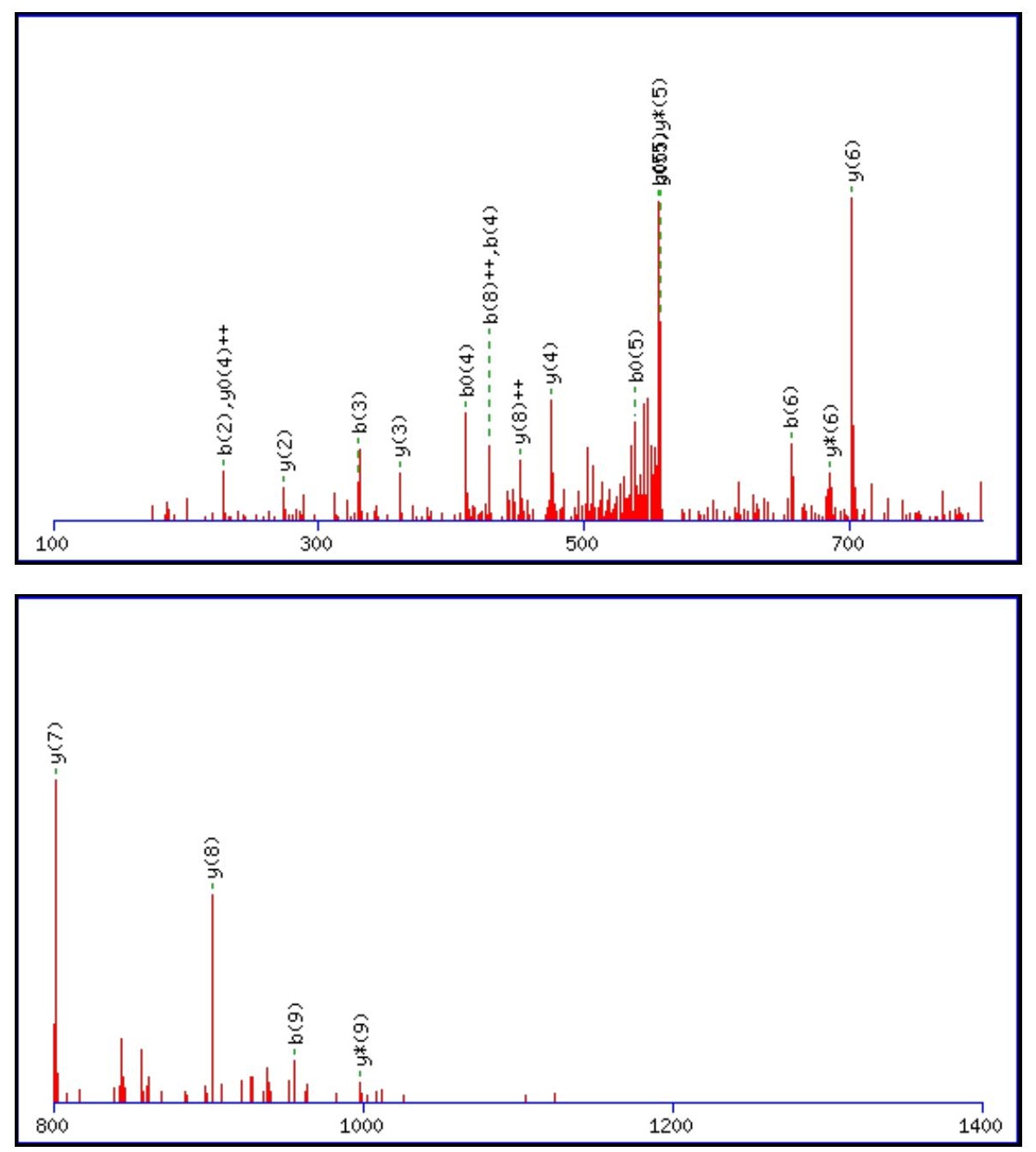
Spectrum No: 867; Query: 220; Rank: 1

\section{Peptide View}

MS/MS Fragmentation of DDNHSVVYTR

Found in IPI00211648, Tax_Id=10116 Gene_Symbol=Slc3a1 Neutral and basic amino acid transport protein rBAT

Match to Query 220: 1205.528888 from(603.771720,2+)

Title: 091008RatKidney_NH4Format01_27.386.386.2.dta

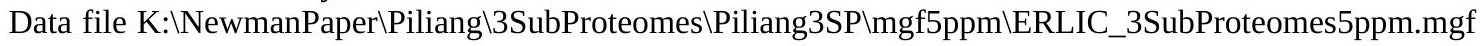
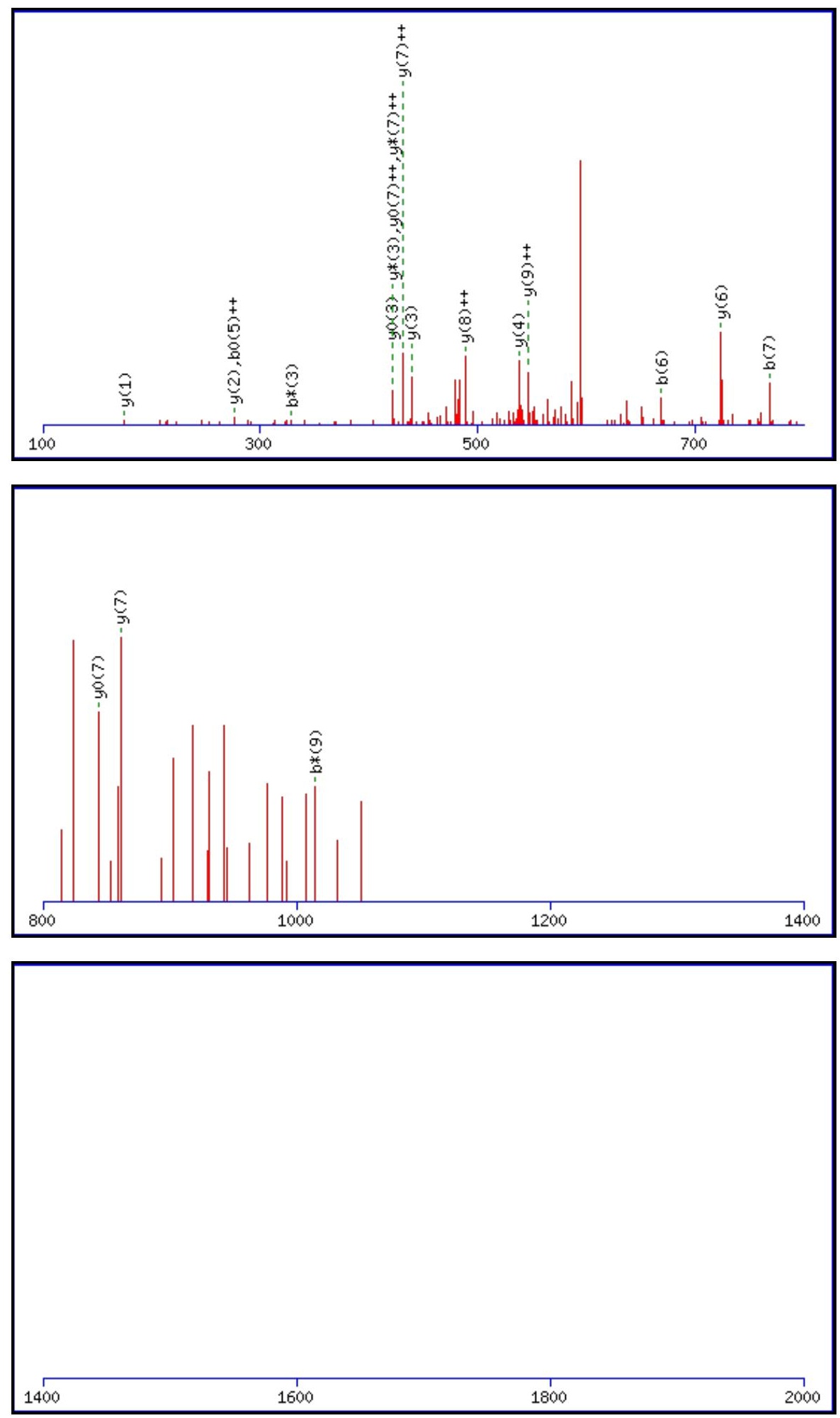

Monoisotopic mass of neutral peptide $\operatorname{Mr}($ calc): 1205.5313 
Fixed modifications: Carbamidomethyl (C)

Variable modifications:

N3 : Deamidated N ( N)

Ions Score: 39 Expect: 0.015

Matches (Bold Red): 19/102 fragment ions using 29 most intense peaks

\begin{tabular}{|c|c|c|c|c|c|c|c|c|c|c|c|c|c|c|}
\hline \# & b & $\mathbf{b}^{++}$ & b* & $\mathbf{b}^{*^{++}}$ & $\mathbf{b}^{0}$ & $\mathbf{b}^{\mathbf{0 + +}}$ & Seq. & $\mathbf{y}$ & $\mathbf{y}^{++}$ & $\mathrm{y}^{*}$ & $\mathrm{y}^{*^{++}}$ & $\mathbf{y}^{0}$ & $y^{0++}$ & $\#$ \\
\hline 1 & 116.0342 & 58.5207 & & & 98.0237 & 49.5155 & D & & & & & & & 10 \\
\hline 2 & 231.0612 & 116.0342 & & & 213.0506 & 107.0289 & D & 1091.5116 & 546.2594 & 1074.4851 & 537.7462 & 1073.5010 & 537.2542 & 9 \\
\hline 3 & 346.0881 & 173.5477 & 329.0615 & 165.0344 & 328.0775 & 164.5424 & $\mathbf{N}$ & 976.4847 & 488.7460 & 959.4581 & 480.2327 & 958.4741 & 479.7407 & 8 \\
\hline 4 & 483.1470 & 242.0771 & 466.1205 & 233.5639 & 465.1364 & 233.0719 & $\mathbf{H}$ & 861.4577 & 431.2325 & 844.4312 & 422.7192 & 843.4472 & 422.2272 & 7 \\
\hline 5 & 570.1790 & 285.5932 & 553.1525 & 277.0799 & 552.1685 & 276.5879 & $S$ & 724.3988 & 362.7030 & 707.3723 & 354.1898 & 706.3883 & 353.6978 & 6 \\
\hline 6 & 669.2475 & 335.1274 & 652.2209 & 326.6141 & 651.2369 & 326.1221 & $\mathrm{~V}$ & 637.3668 & 319.1870 & 620.3402 & 310.6738 & 619.3562 & 310.1817 & 5 \\
\hline 7 & 768.3159 & 384.6616 & 751.2893 & 376.1483 & 750.3053 & 375.6563 & $\mathbf{V}$ & 538.2984 & 269.6528 & 521.2718 & 261.1396 & 520.2878 & 260.6475 & 4 \\
\hline 8 & 931.3792 & 466.1932 & 914.3526 & 457.6800 & 913.3686 & 457.1880 & $\mathbf{Y}$ & 439.2300 & 220.1186 & 422.2034 & 211.6053 & 421.2194 & 211.1133 & 3 \\
\hline 9 & 1032.4269 & 516.7171 & 1015.4003 & 508.2038 & 1014.4163 & 507.7118 & $T$ & 276.1666 & 138.5870 & 259.1401 & 130.0737 & 258.1561 & 129.5817 & 2 \\
\hline 10 & & & & & & & $\mathbf{R}$ & 175.1190 & 88.0631 & 158.0924 & 79.5498 & & & 1 \\
\hline
\end{tabular}
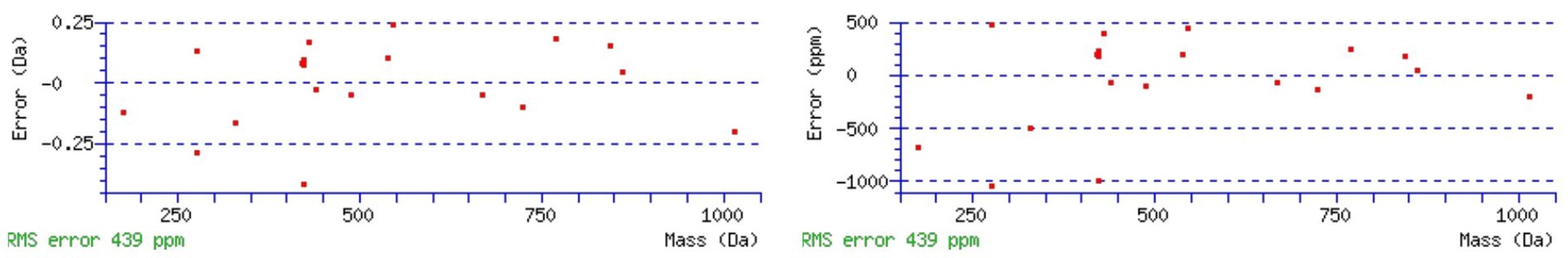

\section{All matches to this query}

\begin{tabular}{|l|l|l|l|}
\hline Score & Mr(calc): & Delta & \multicolumn{1}{c|}{ Sequence } \\
\hline 38.9 & 1205.5313 & -0.0024 & DDNHSVVYTR \\
\hline 27.1 & 1205.5264 & 0.0025 & MVDSVYRTR \\
\hline 16.3 & 1205.5264 & 0.0025 & MVDSVYRTR \\
\hline 13.1 & 1205.5329 & -0.0040 & LSSDLYATTR \\
\hline 13.1 & 1205.5329 & -0.0040 & LSSDLYATTR \\
\hline 11.4 & 1203.5301 & 1.9988 & IAVLESGSLR \\
\hline 10.1 & 1204.5311 & 0.9978 & DLAMTYKQR \\
\hline 10.1 & 1204.5311 & 0.9978 & DLAMTYKQR \\
\hline 10.1 & 1204.5237 & 1.0051 & TIASQHPNTR \\
\hline 10.1 & 1204.5237 & 1.0051 & TIASQHPNTR \\
\hline
\end{tabular}

Spectrum No: 868; Query: 2307; Rank: 1

\section{Peptide View}

MS/MS Fragmentation of CDHNCTDTITSFYCSCLPGYK

Found in IPI00205325, Tax_Id=10116 Gene_Symbol=Lrp2 Low-density lipoprotein receptor-related protein 2 precursor

Match to Query 2307: 2599.021602 from(867.347810,3+)

Title: 100101RatKid_NS_deglyco_20.3488.3488.3.dta

Data file K:INewmanPaper|Piliangl3SubProteomes\Piliang3SP\mgf5ppm\ERLIC_3SubProteomes5ppm.mgf 

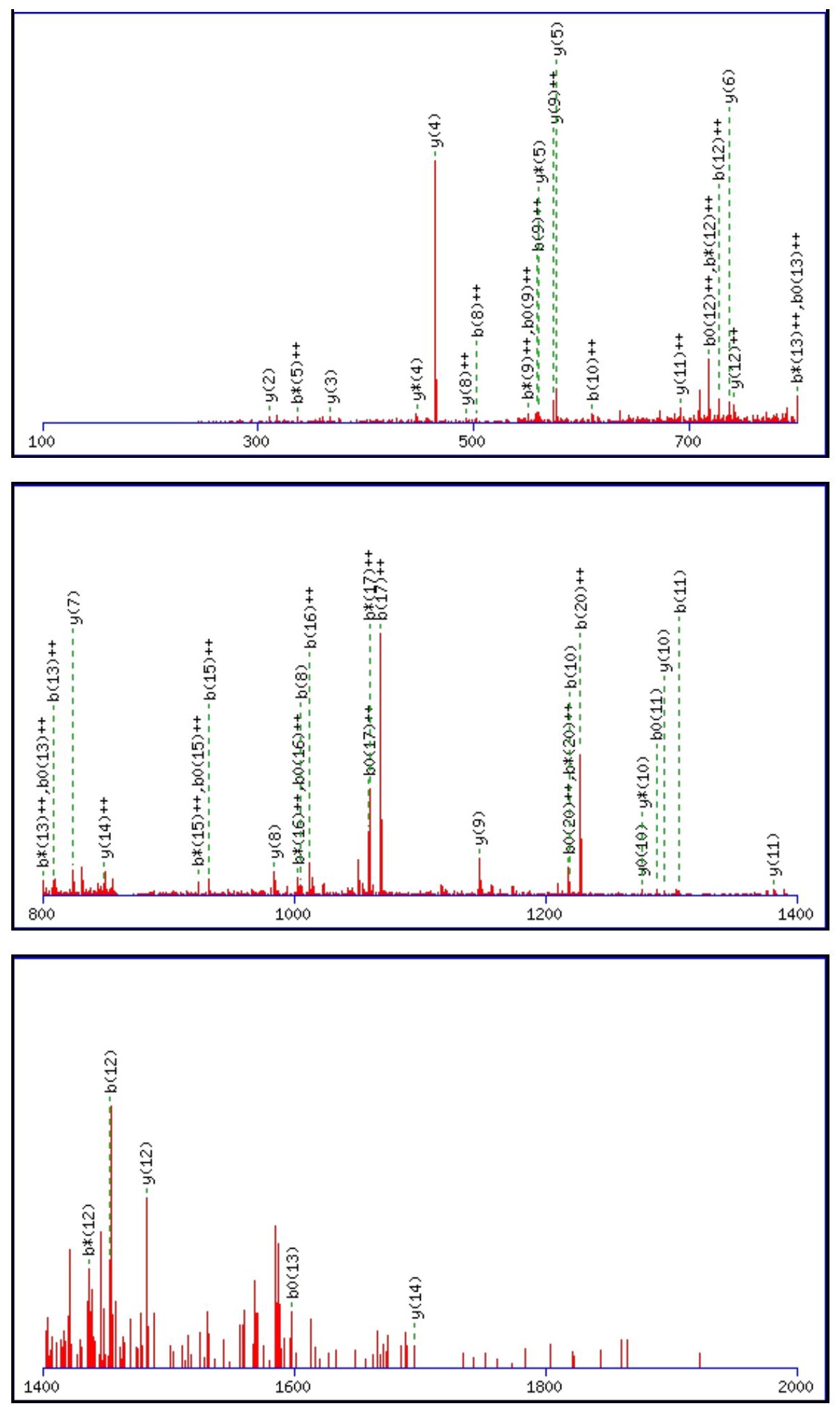

Monoisotopic mass of neutral peptide $\operatorname{Mr}($ calc): 2599.0124

Fixed modifications: Carbamidomethyl (C)

Variable modifications:

N4 : Deamidated $\mathrm{N}(\mathrm{N})$

Ions Score: 39 Expect: 0.032

Matches (Bold Red): 52/220 fragment ions using 121 most intense peaks

\begin{tabular}{|c|c|c|c|c|c|c|c|c|c|c|c|c|c|c|}
\hline$\#$ & $\mathbf{b}$ & $\mathbf{b}^{++}$ & $\mathbf{b}^{*}$ & $\mathbf{b}^{\boldsymbol{*}^{++}}$ & $\mathbf{b}^{\mathbf{0}}$ & $\mathbf{b}^{\mathbf{0 + +}}$ & Seq. & $\mathbf{y}$ & $\mathbf{y}^{++}$ & $\mathbf{y}^{\mathbf{*}}$ & $\mathbf{y}^{\mathbf{*}^{++}}$ & $\mathbf{y}^{\mathbf{0}}$ & $\mathbf{y}^{\mathbf{0 + +}}$ & $\#$ \\
\hline $\mathbf{1}$ & 161.0379 & 81.0226 & & & & & $\mathbf{C}$ & & & & & & & $\mathbf{2 1}$ \\
\hline $\mathbf{2}$ & 276.0649 & 138.5361 & & & 258.0543 & 129.5308 & $\mathbf{D}$ & 2439.9890 & 1220.4981 & 2422.9625 & 1211.9849 & 2421.9784 & 1211.4929 & $\mathbf{2 0}$ \\
\hline $\mathbf{3}$ & 413.1238 & 207.0655 & & & 395.1132 & 198.0602 & $\mathbf{H}$ & 2324.9621 & 1162.9847 & 2307.9355 & 1154.4714 & 2306.9515 & 1153.9794 & $\mathbf{1 9}$ \\
\hline $\mathbf{4}$ & 528.1507 & 264.5790 & 511.1242 & 256.0657 & 510.1402 & 255.5737 & $\mathbf{N}$ & 2187.9032 & 1094.4552 & 2170.8766 & 1085.9419 & 2169.8926 & 1085.4499 & $\mathbf{1 8}$ \\
\hline $\mathbf{5}$ & 688.1814 & 344.5943 & 671.1548 & 336.0810 & 670.1708 & 335.5890 & $\mathbf{C}$ & 2072.8762 & 1036.9417 & 2055.8497 & 1028.4285 & 2054.8656 & 1027.9365 & $\mathbf{1 7}$ \\
\hline
\end{tabular}




\begin{tabular}{|c|c|c|c|c|c|c|c|c|c|c|c|c|c|c|}
\hline 6 & 789.2290 & 395.1182 & 772.2025 & 386.6049 & 771.2185 & 386.1129 & $T$ & |1912.8456 | & 956.9264 & 1895.8190 & 948.4131 & 1894.8350 & 11 & 16 \\
\hline 7 & 904.2560 & 452.6316 & 887.2294 & 444.1184 & 886.2454 & 443.6263 & D & 1811.7979 & 906.4026 & 1794.7713 & 897.8893 & 1793.7873 & 897.3973 & 15 \\
\hline 8 & 1005.3037 & 503.1555 & 988.2771 & 494.6422 & 987.2931 & 494.1502 & $\mathbf{T}$ & 1696.7709 & 848.8891 & 1679.7444 & 840.3758 & 1678.7604 & 839.8838 & 14 \\
\hline 9 & 1118.3877 & 559.6975 & 1101.3612 & 551.1842 & 1100.3772 & 550.6922 & I & 1595.7233 & 798.3653 & 1578.6967 & 789.8520 & 1577.7127 & 789.3600 & 13 \\
\hline 10 & 1219.4354 & 610.2213 & 1202.4089 & 601.7081 & 1201.4248 & 601.2161 & $T$ & 1482.6392 & 741.8232 & 1465.6127 & 733.3100 & 1464.6286 & 732.8180 & 12 \\
\hline 11 & 1306.4674 & 653.7374 & 1289.4409 & 645.2241 & 1288.4569 & 644.7321 & S & 1381.5915 & 691.2994 & 1364.5650 & 682.7861 & 1363.5810 & 682.2941 & 11 \\
\hline 12 & 1453.5359 & 727.2716 & 1436.5093 & 718.7583 & 1435.5253 & 718.2663 & $\mathbf{F}$ & 1294.5595 & 647.7834 & 1277.5329 & 639.2701 & 1276.5489 & 638.7781 & 10 \\
\hline 13 & 1616.5992 & 808.8032 & 1599.5726 & 800.2900 & 1598.5886 & 799.7979 & $\mathbf{Y}$ & 1147.4911 & 574.2492 & 1130.4645 & 565.7359 & 1129.4805 & 565.2439 & 9 \\
\hline 14 & 1776.6298 & 888.8186 & 1759.6033 & 880.3053 & 1758.6193 & 879.8133 & C & 984.4278 & 492.7175 & 967.4012 & 484.2042 & 966.4172 & 483.7122 & 8 \\
\hline 15 & 1863.6619 & 932.3346 & 1846.6353 & 923.8213 & 1845.6513 & 923.3293 & S & 824.3971 & 412.7022 & 807.3706 & 404.1889 & 806.3865 & 403.6969 & 7 \\
\hline 16 & 2023.6925 & 1012.3499 & 2006.6660 & 1003.8366 & 2005.6819 & 1003.3446 & C & 737.3651 & 369.1862 & 720.3385 & 360.6729 & & & 6 \\
\hline 17 & 2136.7766 & 1068.8919 & 2119.7500 & 1060.3786 & 2118.7660 & 1059.8866 & $\mathbf{L}$ & 577.3344 & 289.1709 & 560.3079 & 280.6576 & & & 5 \\
\hline 18 & 2233.8293 & 1117.4183 & 2216.8028 & 1108.9050 & 2215.8188 & 1108.4130 & $\mathbf{P}$ & 464.2504 & 232.6288 & 447.2238 & 224.1155 & & & 4 \\
\hline 19 & 2290.8508 & 1145.9290 & 2273.8242 & 1137.4158 & 2272.8402 & 1136.9238 & G & 367.1976 & 184.1024 & 350.1710 & 175.5892 & & & 3 \\
\hline 20 & 2453.9141 & 1227.4607 & 2436.8876 & 1218.9474 & 2435.9036 & 1218.4554 & $\mathbf{Y}$ & 310.1761 & 155.5917 & 293.1496 & 147.0784 & & & 2 \\
\hline 21 & & & & & & & $\mathbf{K}$ & 147.1128 & 74.0600 & 130.0863 & 65.5468 & & & 1 \\
\hline
\end{tabular}
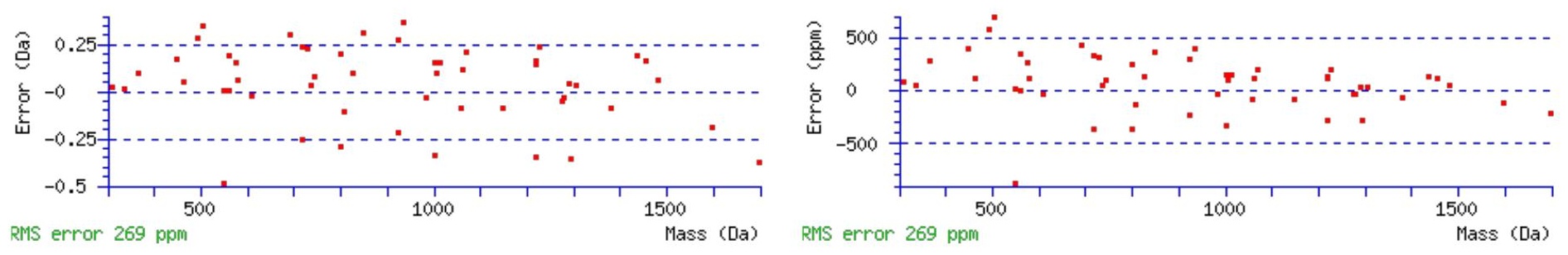

\section{All matches to this query}

\begin{tabular}{|l|l|c|l|}
\hline Score & Mr(calc): & Delta & \multicolumn{1}{|c|}{ Sequence } \\
\hline 38.9 & 2599.0124 & 0.0092 & CDHNCTDTITSFYCSCLPGYK \\
\hline 19.0 & 2598.0284 & 0.9932 & CDHNCTDTITSFYCSCLPGYK \\
\hline 5.9 & 2597.0216 & 2.0000 & GSQTSLCEQGTWSSPPVCLESK \\
\hline 5.9 & 2597.0216 & 2.0000 & GSQTSLCEQGTWSSPPVCLESK \\
\hline 3.1 & 2597.0337 & 1.9879 & TTNNPITIFSTTNTVGSSSTTGR \\
\hline 3.1 & 2599.0413 & -0.0197 & FTKSFELMSPKCSADAENSFK \\
\hline 2.9 & 2597.0337 & 1.9879 & TTNNPITIFSTTNTVGSSSTTGR \\
\hline 2.9 & 2597.0337 & 1.9879 & TTNNPITIFSTTNTVGSSSTTGR \\
\hline 2.7 & 2599.0413 & -0.0197 & FTKSFELMSPKCSADAENSFK \\
\hline 1.2 & 2599.0290 & -0.0074 & GNCSSLHEFLLLGITNNPDMK \\
\hline
\end{tabular}

Spectrum No: 869; Query: 1774; Rank: 1

\section{Peptide View}

MS/MS Fragmentation of VDKNVSGLAINWIDGEILR

Found in IPI00231848, Tax_Id=10116 Gene_Symbol=Egf Pro-epidermal growth factor precursor

Match to Query 1774: 2112.127542 from(705.049790,3+)

Title: 100101RatKid_NS_deglyco_12.5917.5917.3.dta

Data file K:INewmanPaper|Piliangl3SubProteomes\Piliang3SP\mgf5ppm\ERLIC_3SubProteomes5ppm.mgf 

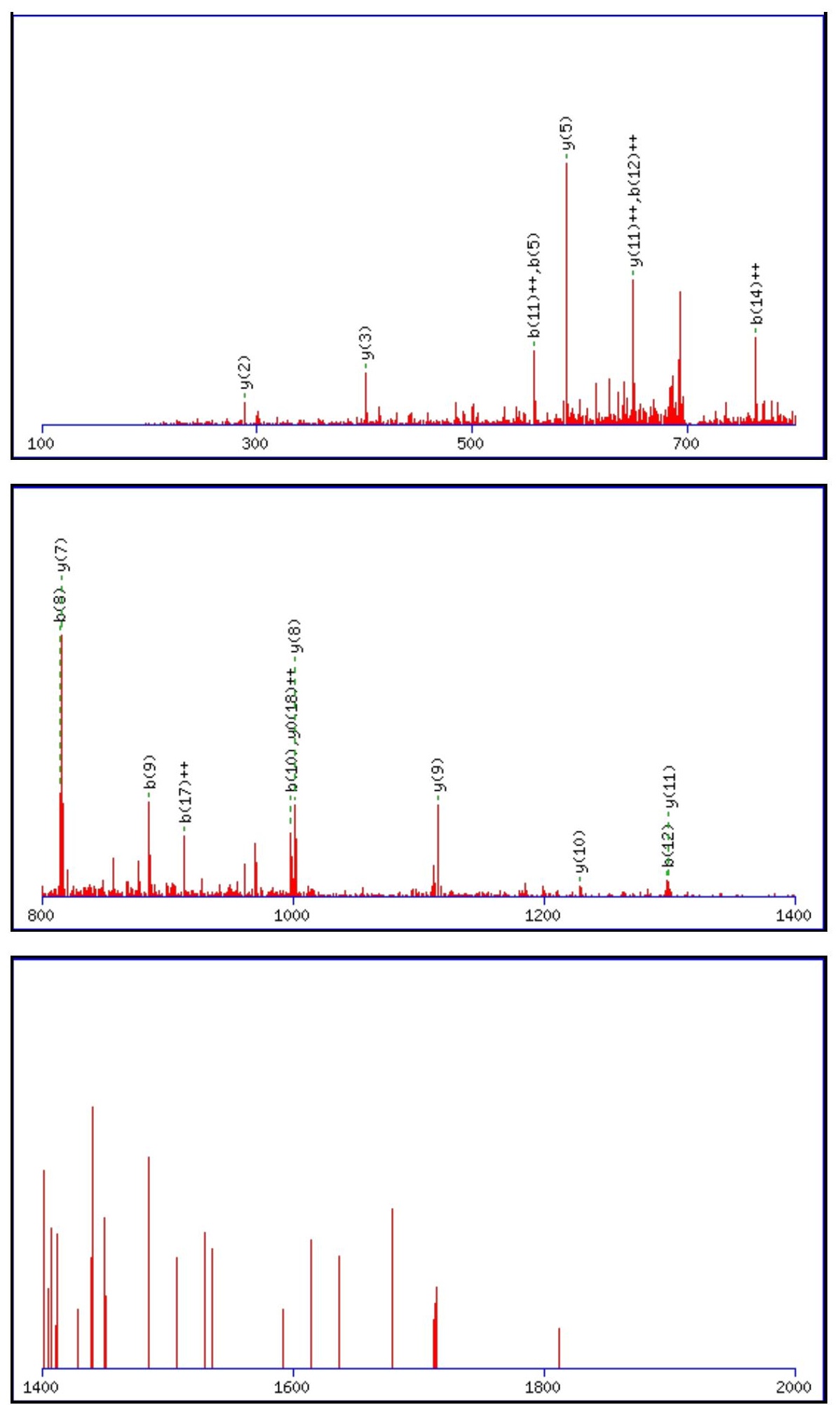

Monoisotopic mass of neutral peptide $\operatorname{Mr}($ calc): 2112.1215

Fixed modifications: Carbamidomethyl (C)

Variable modifications:

N4 : Deamidated N (N)

Ions Score: 39 Expect: 0.011

Matches (Bold Red): 19/204 fragment ions using 32 most intense peaks

\begin{tabular}{|c|c|c|c|c|c|c|c|c|c|c|c|c|c|c|}
\hline$\#$ & $\mathbf{b}$ & $\mathbf{b}^{++}$ & $\mathbf{b}^{*}$ & $\mathbf{b}^{\boldsymbol{*}^{++}}$ & $\mathbf{b}^{\mathbf{0}}$ & $\mathbf{b}^{\mathbf{0}+}$ & Seq. & $\mathbf{y}$ & $\mathbf{y}^{++}$ & $\mathbf{y}^{\mathbf{*}}$ & $\mathbf{y}^{\boldsymbol{*}^{++}}$ & $\mathbf{y}^{\mathbf{0}}$ & $\mathbf{y}^{\mathbf{0}+}$ & $\#$ \\
\hline $\mathbf{1}$ & 100.0757 & 50.5415 & & & & & $\mathbf{V}$ & & & & & & & $\mathbf{1 9}$ \\
\hline $\mathbf{2}$ & 215.1026 & 108.0550 & & & 197.0921 & 99.0497 & $\mathbf{D}$ & 2014.0604 & 1007.5338 & 1997.0338 & 999.0206 & 1996.0498 & $\mathbf{9 9 8 . 5 2 8 5}$ & $\mathbf{1 8}$ \\
\hline $\mathbf{3}$ & 343.1976 & 172.1024 & 326.1710 & 163.5892 & 325.1870 & 163.0972 & $\mathbf{K}$ & 1899.0334 & 950.0204 & 1882.0069 & 941.5071 & 1881.0229 & 941.0151 & $\mathbf{1 7}$ \\
\hline $\mathbf{4}$ & 458.2245 & 229.6159 & 441.1980 & 221.1026 & 440.2140 & 220.6106 & $\mathbf{N}$ & 1770.9385 & 885.9729 & 1753.9119 & 877.4596 & 1752.9279 & 876.9676 & $\mathbf{1 6}$ \\
\hline $\mathbf{5}$ & $\mathbf{5 5 7 . 2 9 2 9}$ & 279.1501 & 540.2664 & 270.6368 & 539.2824 & 270.1448 & $\mathbf{V}$ & 1655.9115 & 828.4594 & 1638.8850 & 819.9461 & 1637.9010 & 819.4541 & $\mathbf{1 5}$ \\
\hline
\end{tabular}




\begin{tabular}{|c|c|c|c|c|c|c|c|c|c|c|c|c|c|c|}
\hline 6 & 644.3250 & |322.6661 & 627.2984 & |314.1529 & 626.3144 & |313.6608 & $\mathrm{S}$ & |1556.8431 & 778.9252 & |1539.8166 & |770.4119 & |1538.8326 & |769.9199| & $|14|$ \\
\hline 7 & 701.3464 & 351.1769 & 684.3199 & 342.6636 & 683.3359 & 342.1716 & G & 1469.8111 & 735.4092 & 1452.7845 & 726.8959 & 1451.8005 & 726.4039 & 13 \\
\hline 8 & 814.4305 & 407.7189 & 797.4040 & 399.2056 & 796.4199 & 398.7136 & $\mathbf{L}$ & 1412.7896 & 706.8985 & 1395.7631 & 698.3852 & 1394.7791 & 697.8932 & 12 \\
\hline 9 & 885.4676 & 443.2374 & 868.4411 & 434.7242 & 867.4571 & 434.2322 & A & 1299.7056 & 650.3564 & 1282.6790 & 641.8431 & 1281.6950 & 641.3511 & 11 \\
\hline 10 & 998.5517 & 499.7795 & 981.5251 & 491.2662 & 980.5411 & 490.7742 & I & 1228.6684 & 614.8379 & 1211.6419 & 606.3246 & 1210.6579 & 605.8326 & 10 \\
\hline 11 & 1112.5946 & 556.8009 & 1095.5681 & 548.2877 & 1094.5840 & 547.7957 & $\mathbf{N}$ & 1115.5844 & 558.2958 & 1098.5578 & 549.7826 & 1097.5738 & 549.2905 & 9 \\
\hline 12 & 1298.6739 & 649.8406 & 1281.6474 & 641.3273 & 1280.6634 & 640.8353 & $\mathbf{W}$ & 1001.5415 & 501.2744 & 984.5149 & 492.7611 & 983.5309 & 492.2691 & 8 \\
\hline 13 & 1411.7580 & 706.3826 & 1394.7314 & 697.8694 & 1393.7474 & 697.3773 & I & 815.4621 & 408.2347 & 798.4356 & 399.7214 & 797.4516 & 399.2294 & 7 \\
\hline 14 & 1526.7849 & 763.8961 & 1509.7584 & 755.3828 & 1508.7744 & 754.8908 & D & 702.3781 & 351.6927 & 685.3515 & 343.1794 & 684.3675 & 342.6874 & 6 \\
\hline 15 & 1583.8064 & 792.4068 & 1566.7798 & 783.8936 & 1565.7958 & 783.4016 & $\mathbf{G}$ & 587.3511 & 294.1792 & 570.3246 & 285.6659 & 569.3406 & 285.1739 & 5 \\
\hline 16 & 1712.8490 & 856.9281 & 1695.8224 & 848.4149 & 1694.8384 & 847.9228 & $\mathbf{E}$ & 530.3297 & 265.6685 & 513.3031 & 257.1552 & 512.3191 & 256.6632 & 4 \\
\hline 17 & 1825.9330 & 913.4702 & 1808.9065 & 904.9569 & 1807.9225 & 904.4649 & I & 401.2871 & 201.1472 & 384.2605 & 192.6339 & & & 3 \\
\hline 18 & 1939.0171 & 970.0122 & 1921.9906 & 961.4989 & 1921.0065 & 961.0069 & $\mathbf{L}$ & 288.2030 & 144.6051 & 271.1765 & 136.0919 & & & 2 \\
\hline 19 & & & & & & & $\mathbf{R}$ & 175.1190 & 88.0631 & 158.0924 & 79.5498 & & & 1 \\
\hline
\end{tabular}
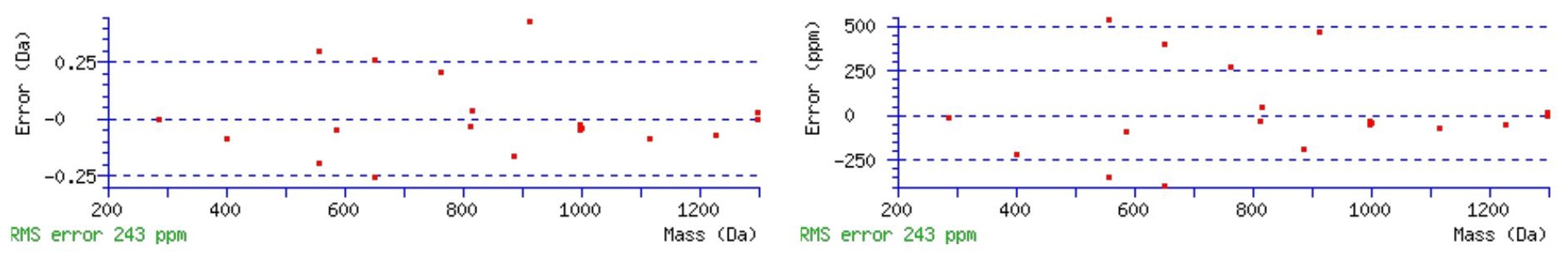

\section{All matches to this query}

\begin{tabular}{|l|l|l|l|}
\hline Score & Mr(calc): & Delta & \multicolumn{1}{|c|}{ Sequence } \\
\hline 38.8 & 2112.1215 & 0.0061 & VDKNVSGLAINWIDGEILR \\
\hline 30.4 & 2111.1375 & 0.9901 & VDKNVSGLAINWIDGEILR \\
\hline 25.0 & 2112.1215 & 0.0061 & VDKNVSGLAINWIDGEILR \\
\hline 14.2 & 2112.1467 & -0.0191 & LWVGTGNGVIISIPLTETNK \\
\hline 5.1 & 2110.1221 & 2.0055 & SREKLTAPMTISTLTLLR \\
\hline 4.6 & 2110.1221 & 2.0055 & SREKLTAPMTISTLTLLR \\
\hline 4.4 & 2110.1221 & 2.0055 & SREKLTAPMTISTLTLLR \\
\hline 4.0 & 2112.1467 & -0.0191 & LWVGTGNGVIISIPLTETNK \\
\hline 3.7 & 2112.1103 & 0.0173 & VIFPDAEVEDITDPGLKVR \\
\hline 2.5 & 2111.1045 & 1.0231 & VLVMGATNRPQELDEAVLR \\
\hline
\end{tabular}

Spectrum No: 870; Query: 167; Rank: 1

\section{Peptide View}

MS/MS Fragmentation of FQNITDLWK

Found in IPI00199778, Tax_Id=10116 Gene_Symbol=RGD1305457 IKIP1 protein

Match to Query 167: 1164.580408 from(583.297480,2+)

Title: 091008RatKidney_NH4Format01_23.3144.3144.2.dta

Data file K:\NewmanPaper\Piliang \3SubProteomes\Piliang3SP \mgf5ppm\ERLIC_3SubProteomes5ppm.mgf 

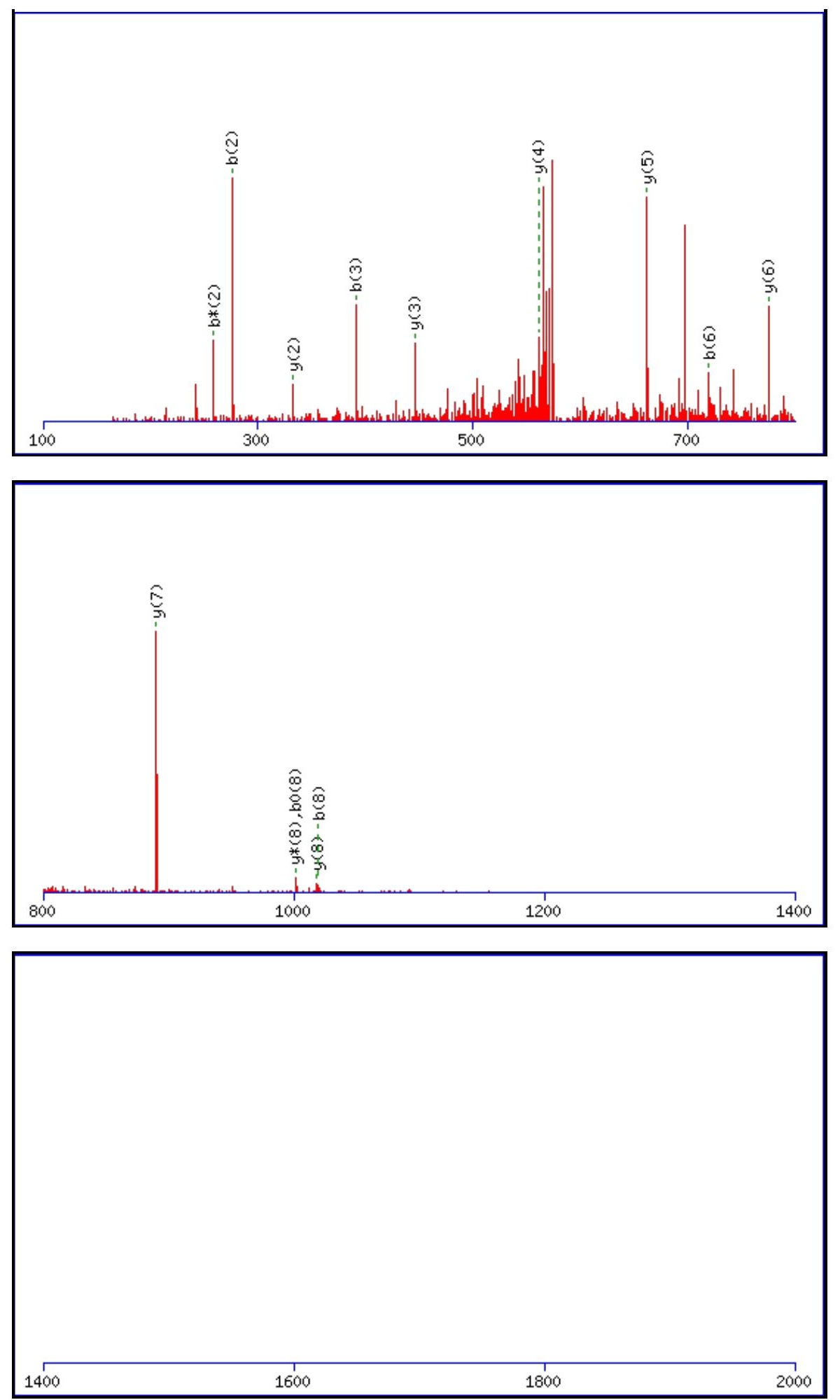

Monoisotopic mass of neutral peptide $\operatorname{Mr}($ calc): 1164.5815

Fixed modifications: Carbamidomethyl (C)

Variable modifications:

N3 : Deamidated_N (N)

Ions Score: 39 Expect: 0.015

Matches (Bold Red): 14/80 fragment ions using 32 most intense peaks

\begin{tabular}{|c|c|c|c|c|c|c|c|c|c|c|c|c|c|c|}
\hline$\#$ & $\mathbf{b}$ & $\mathbf{b}^{++}$ & $\mathbf{b}^{*}$ & $\mathbf{b}^{\boldsymbol{*}^{++}}$ & $\mathbf{b}^{\mathbf{0}}$ & $\mathbf{b}^{\mathbf{0 + +}}$ & Seq. & $\mathbf{y}$ & $\mathbf{y}^{++}$ & $\mathbf{y}^{\mathbf{*}}$ & $\mathbf{y}^{\mathbf{*}^{++}}$ & $\mathbf{y}^{\mathbf{0}}$ & $\mathbf{y}^{\mathbf{0}++}$ & $\#$ \\
\hline $\mathbf{1}$ & 148.0757 & 74.5415 & & & & & $\mathbf{F}$ & & & & & & & $\mathbf{9}$ \\
\hline $\mathbf{2}$ & $\mathbf{2 7 6 . 1 3 4 3}$ & 138.5708 & 259.1077 & 130.0575 & & & $\mathbf{Q}$ & $\mathbf{1 0 1 8 . 5 2 0 4}$ & 509.7638 & $\mathbf{1 0 0 1 . 4 9 3 8}$ & 501.2506 & 1000.5098 & 500.7585 & $\mathbf{8}$ \\
\hline $\mathbf{3}$ & $\mathbf{3 9 1 . 1 6 1 2}$ & 196.0842 & 374.1347 & 187.5710 & & & $\mathbf{N}$ & $\mathbf{8 9 0 . 4 6 1 8}$ & 445.7345 & 873.4353 & 437.2213 & 872.4512 & 436.7293 & $\mathbf{7}$ \\
\hline $\mathbf{4}$ & 504.2453 & 252.6263 & 487.2187 & 244.1130 & & & $\mathbf{I}$ & $\mathbf{7 7 5 . 4 3 4 9}$ & 388.2211 & 758.4083 & 379.7078 & $\mathbf{7 5 7 . 4 2 4 3}$ & 379.2158 & $\mathbf{6}$ \\
\hline $\mathbf{5}$ & 605.2929 & 303.1501 & 588.2664 & 294.6368 & 587.2824 & 294.1448 & $\mathbf{T}$ & $\mathbf{6 6 2 . 3 5 0 8}$ & 331.6790 & 645.3243 & 323.1658 & 644.3402 & 322.6738 & $\mathbf{5}$ \\
\hline
\end{tabular}




\begin{tabular}{|l|r|r|r|r|r|r|r|r|r|r|r|r|r|r|r|r|}
$\mathbf{6}$ & $\mathbf{7 2 0 . 3 1 9 9}$ & 360.6636 & 703.2933 & 352.1503 & 702.3093 & 351.6583 & $\mathbf{D}$ & $\mathbf{5 6 1 . 3 0 3 1}$ & 281.1552 & 544.2766 & 272.6419 & 543.2926 & 272.1499 & $\mathbf{4}$ \\
\hline $\mathbf{7}$ & 833.4040 & 417.2056 & 816.3774 & 408.6923 & 815.3934 & 408.2003 & L & $\mathbf{4 4 6 . 2 7 6 2}$ & 223.6417 & 429.2496 & 215.1285 & & & $\mathbf{3}$ \\
\hline $\mathbf{8}$ & $\mathbf{1 0 1 9 . 4 8 3 3}$ & 510.2453 & 1002.4567 & 501.7320 & $\mathbf{1 0 0 1 . 4 7 2 7}$ & 501.2400 & W & 333.1921 & 167.0997 & 316.1656 & 158.5864 & & & $\mathbf{2}$ \\
\hline $\mathbf{9}$ & & & & & & & K & 147.1128 & 74.0600 & 130.0863 & 65.5468 & & & $\mathbf{1}$ \\
\hline
\end{tabular}
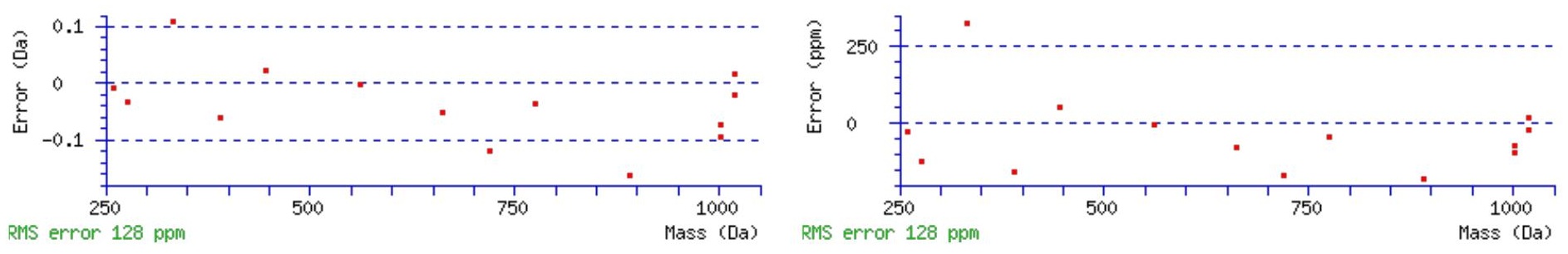

\section{All matches to this query}

\begin{tabular}{|l|l|l|l|}
\hline Score & Mr(calc): & Delta & \multicolumn{1}{|c|}{ Sequence } \\
\hline 38.8 & 1164.5815 & -0.0011 & FQNITDLWK \\
\hline 10.1 & 1163.5790 & 1.0014 & NAEMKNAMKK \\
\hline 9.3 & 1164.5791 & 0.0013 & ALTAIPKDEK \\
\hline 9.3 & 1164.5775 & 0.0029 & FNGTVKAGNEK \\
\hline 9.3 & 1164.5887 & -0.0083 & SFDIRRNEK \\
\hline 8.5 & 1163.5670 & 1.0134 & VGSTSENITQK \\
\hline 6.7 & 1163.5822 & 0.9982 & DOEAFEAVKK \\
\hline 6.3 & 1163.5678 & 1.0126 & MQLCELNKK \\
\hline 5.9 & 1163.5856 & 0.9948 & KDSNSLMIQK \\
\hline 4.9 & 1164.5791 & 0.0013 & LASLTAPVGEK \\
\hline
\end{tabular}

Spectrum No: 871; Query: 2273; Rank: 1

\section{Peptide View}

\section{MS/MS Fragmentation of EFTTTVVSCCPAELQTDGNGSKK}

Found in IPI00201819, Tax_Id=10116 Gene_Symbol=LOC619440 Alanyl-tRNA synthetase domain-containing protein 1

Match to Query 2273: 2529.145842 from(844.055890,3+)

Title: 100101RatKid_NS_deglyco_12.2395.2395.3.dta

Data file K:INewmanPaper|Piliangl3SubProteomes\Piliang3SP\mgf5ppm\ERLIC_3SubProteomes5ppm.mgf

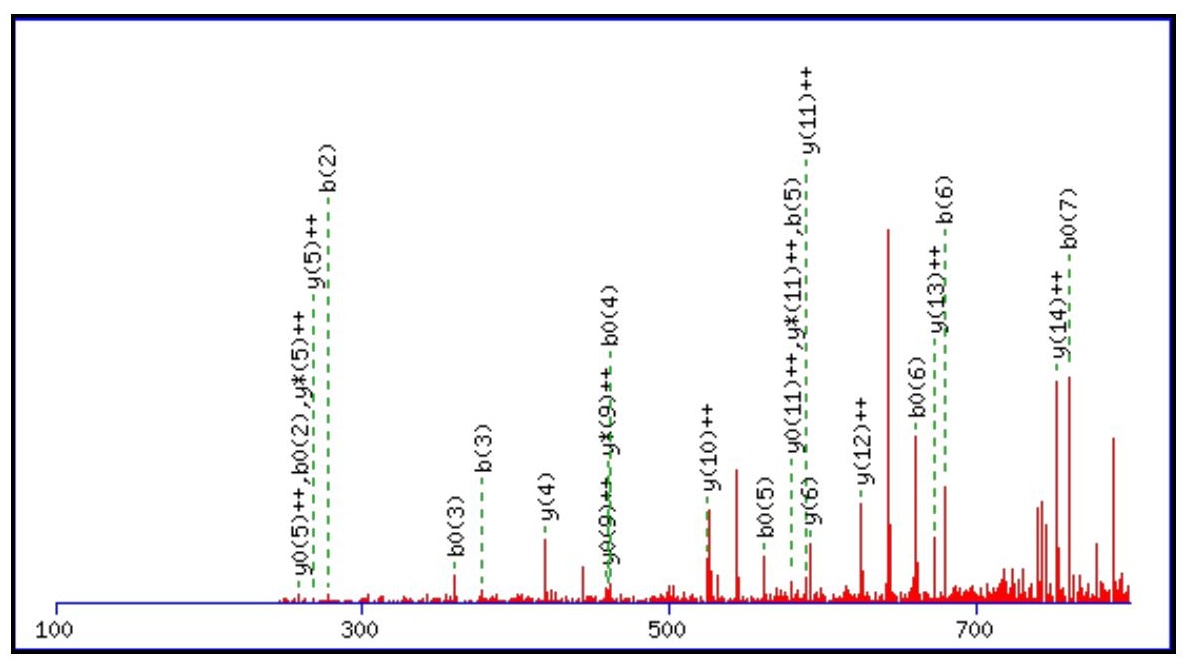



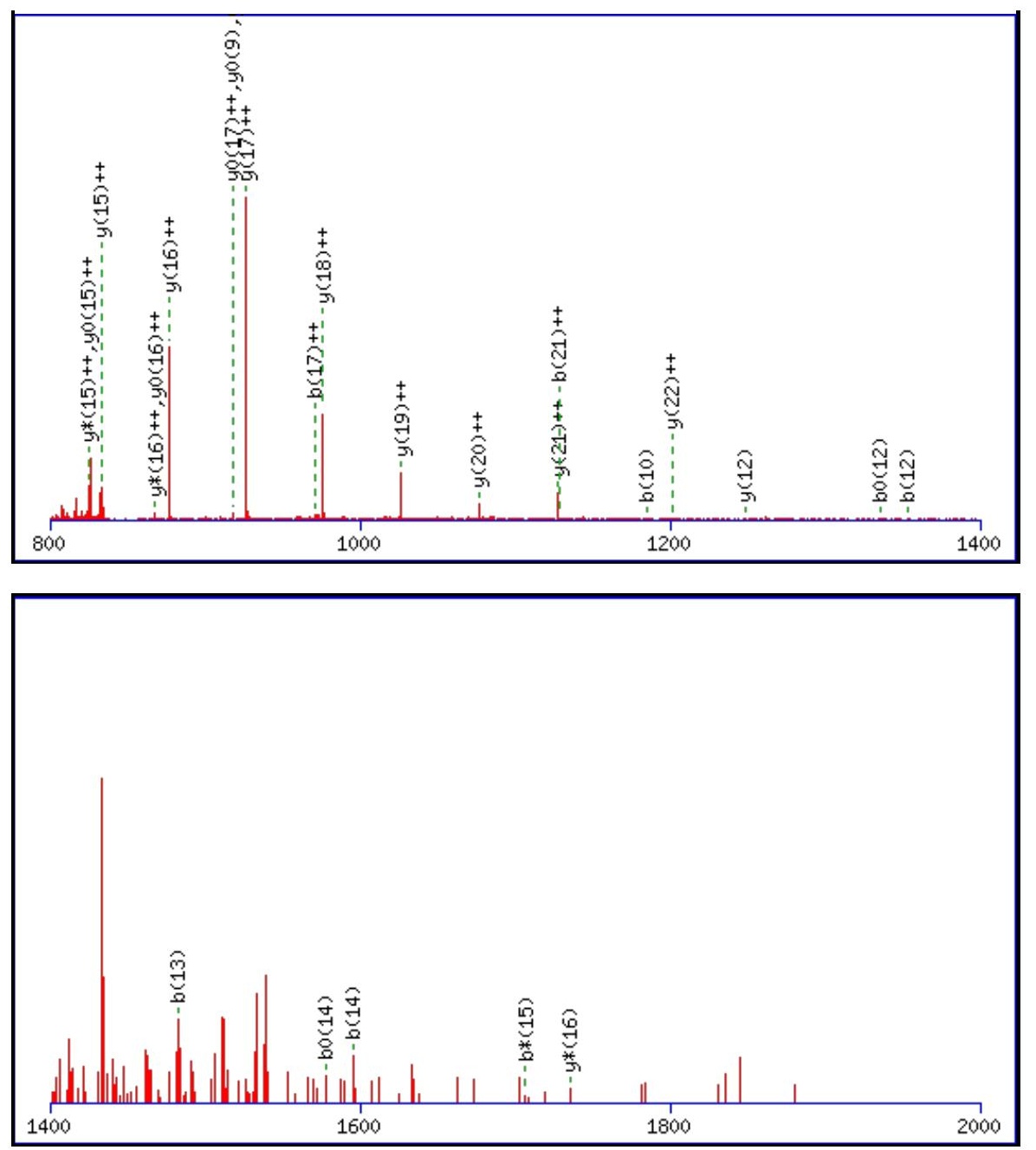

Monoisotopic mass of neutral peptide $\operatorname{Mr}($ calc): 2529.1363

Fixed modifications: Carbamidomethyl (C)

Variable modifications:

N19 : Deamidated_N (N)

Ions Score: 39 Expect: 0.046

Matches (Bold Red): 50/232 fragment ions using 132 most intense peaks

\begin{tabular}{|c|c|c|c|c|c|c|c|c|c|c|c|c|c|c|}
\hline \# & b & $\mathbf{b}^{++}$ & $\mathbf{b}^{*}$ & $\mathbf{b}^{*^{++}}$ & $\mathbf{b}^{\mathbf{0}}$ & $\mathbf{b}^{\mathbf{0 + +}}$ & Seq. & $\mathbf{y}$ & $\mathbf{y}^{++}$ & $\mathbf{y}^{*}$ & $\mathrm{y}^{*^{++}}$ & $\mathbf{y}^{\mathbf{0}}$ & $\mathbf{y}^{\mathbf{0 + +}}$ & \# \\
\hline 1 & 130.0499 & 65.5286 & & & 112.0393 & 56.5233 & $\mathbf{E}$ & & & & & & & 23 \\
\hline 2 & 277.1183 & 139.0628 & & & 259.1077 & 130.0575 & $\mathbf{F}$ & 2401.1010 & 1201.0541 & 2384.0744 & 1192.5409 & 2383.0904 & 1192.0489 & 22 \\
\hline 4 & 479.2136 & 240.1105 & & & 461.2031 & 231.1052 & $\mathbf{T}$ & 2152.9849 & 1076.9961 & 2135.9584 & 1068.4828 & 2134.9743 & 1067.9908 & 20 \\
\hline 5 & 580.2613 & 290.6343 & & & 562.2508 & 281.6290 & $\mathbf{T}$ & 2051.9372 & 1026.4723 & 2034.9107 & 1017.9590 & 2033.9267 & 1017.4670 & 19 \\
\hline 6 & 679.3297 & 340.1685 & & & 661.3192 & 331.1632 & $\mathbf{V}$ & 1950.8895 & 975.9484 & 1933.8630 & 967.4351 & 1932.8790 & 966.9431 & 18 \\
\hline 7 & 778.3981 & 389.7027 & & & 760.3876 & 380.6974 & $\mathbf{V}$ & 1851.8211 & 926.4142 & 1834.7946 & 917.9009 & 1833.8106 & 917.4089 & 17 \\
\hline 8 & 865.4302 & 433.2187 & & & 847.4196 & 424.2134 & S & 1752.7527 & 876.8800 & 1735.7262 & 868.3667 & 1734.7422 & 867.8747 & 16 \\
\hline 9 & 1025.4608 & 513.2341 & & & 1007.4503 & 504.2288 & C & 1665.7207 & 833.3640 & 1648.6941 & 824.8507 & 1647.7101 & 824.3587 & 15 \\
\hline 10 & 1185.4915 & 593.2494 & & & 1167.4809 & 584.2441 & C & 1505.6900 & 753.3487 & 1488.6635 & 744.8354 & 1487.6795 & 744.3434 & 14 \\
\hline 11 & 1282.5442 & 641.7758 & & & 1264.5337 & 632.7705 & $\mathbf{P}$ & 1345.6594 & 673.3333 & 1328.6328 & 664.8201 & 1327.6488 & 664.3281 & 13 \\
\hline 12 & 1353.5814 & 677.2943 & & & 1335.5708 & 668.2890 & A & 1248.6066 & 624.8070 & 1231.5801 & 616.2937 & 1230.5961 & 615.8017 & 12 \\
\hline 14 & 1595.7080 & 798.3576 & & & 1577.6974 & 789.3524 & $\mathbf{L}$ & 1048.5269 & 524.7671 & 1031.5004 & 516.2538 & 1030.5164 & 515.7618 & 10 \\
\hline 15 & 1723.7666 & 862.3869 & 1706.7400 & 853.8737 & 1705.7560 & 853.3816 & $\mathbf{Q}$ & 935.4429 & 468.2251 & 918.4163 & 459.7118 & 917.4323 & 459.2198 & 9 \\
\hline 16 & 1824.8143 & 912.9108 & 1807.7877 & 904.3975 & 1806.8037 & 903.9055 & $\mathbf{T}$ & 807.3843 & 404.1958 & 790.3577 & 395.6825 & 789.3737 & 395.1905 & 8 \\
\hline 17 & 1939.8412 & 970.4242 & 1922.8147 & 961.9110 & 1921.8306 & 961.4190 & D & 706.3366 & 353.6719 & 689.3101 & 345.1587 & 688.3260 & 344.6667 & 7 \\
\hline 18 & 1996.8627 & 998.9350 & 1979.8361 & 990.4217 & 1978.8521 & 989.9297 & G & 591.3097 & 296.1585 & 574.2831 & 287.6452 & 573.2991 & 287.1532 & 6 \\
\hline
\end{tabular}




\begin{tabular}{|r|r|r|r|r|r|r|r|r|r|r|r|r|r|r|r|}
$\mathbf{1 9}$ & 2111.8896 & 1056.4484 & 2094.8631 & 1047.9352 & 2093.8790 & 1047.4432 & $\mathbf{N}$ & 534.2882 & $\mathbf{2 6 7 . 6 4 7 7}$ & 517.2616 & $\mathbf{2 5 9 . 1 3 4 5}$ & $\mathbf{5 1 6 . 2 7 7 6}$ & $\mathbf{2 5 8 . 6 4 2 5}$ & $\mathbf{5}$ \\
\hline $\mathbf{2 0}$ & 2168.9111 & 1084.9592 & 2151.8845 & 1076.4459 & 2150.9005 & 1075.9539 & $\mathbf{G}$ & $\mathbf{4 1 9 . 2 6 1 3}$ & 210.1343 & 402.2347 & 201.6210 & 401.2507 & $\mathbf{2 0 1 . 1 2 9 0}$ & $\mathbf{4}$ \\
\hline $\mathbf{2 1}$ & 2255.9431 & $\mathbf{1 1 2 8 . 4 7 5 2}$ & 2238.9166 & 1119.9619 & 2237.9325 & 1119.4699 & S & 362.2398 & 181.6235 & 345.2132 & 173.1103 & 344.2292 & 172.6183 & $\mathbf{3}$ \\
\hline $\mathbf{2 2}$ & 2384.0381 & 1192.5227 & 2367.0115 & 1184.0094 & 2366.0275 & 1183.5174 & K & 275.2078 & 138.1075 & 258.1812 & 129.5942 & & & $\mathbf{2}$ \\
\hline $\mathbf{2 3}$ & & & & & & & K & 147.1128 & 74.0600 & 130.0863 & 65.5468 & & & $\mathbf{1}$ \\
\hline
\end{tabular}
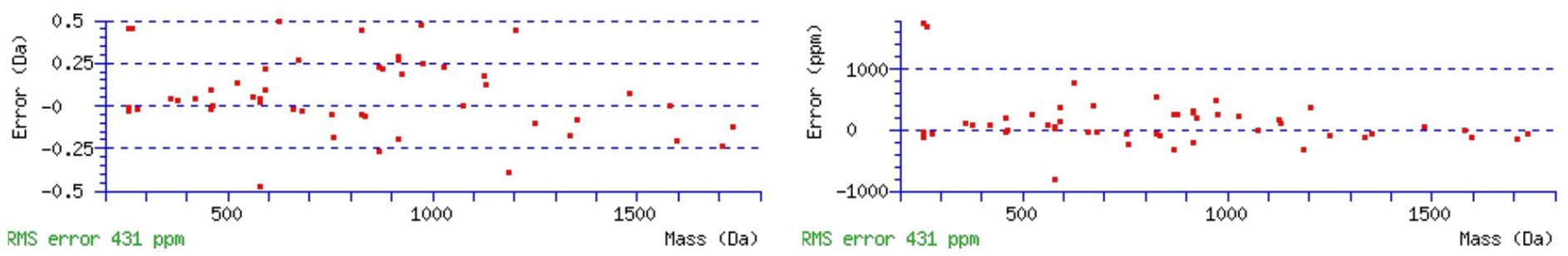

\section{All matches to this query}

\begin{tabular}{|l|l|l|l|}
\hline Score & Mr(calc): & Delta & \multicolumn{1}{|c|}{ Sequence } \\
\hline 38.8 & 2529.1363 & 0.0095 & EFTTTVVSCCPAELQTDGNGSKK \\
\hline 5.2 & 2529.1337 & 0.0121 & QIPNTAIMMATYELVVYLLNG \\
\hline 4.0 & 2528.1605 & 0.9854 & HIQTAQSRGPQGCWQPRQASR \\
\hline 3.8 & 2528.1212 & 1.0247 & LNSNMVGKIWIPDTFFRNSK \\
\hline 2.8 & 2528.1523 & 0.9935 & EFTTTVVSCCPAELQTDGNGSKK \\
\hline 2.8 & 2528.1212 & 1.0247 & LNSNMVGKIWIPDTFFRNSK \\
\hline 2.8 & 2528.1591 & 0.9868 & LTSVVTARPFGTSSRGISSLPR \\
\hline 2.7 & 2529.1337 & 0.0121 & QIPNTAIMMATYELVVYLLNG \\
\hline 2.5 & 2528.1591 & 0.9868 & LTSVVTARPFGTSSRGISSLPR \\
\hline 2.0 & 2527.1299 & 2.0159 & KQNSGNLALGSNESRNISDEASR \\
\hline
\end{tabular}

Spectrum No: 872; Query: 21; Rank: 1

\section{Peptide View}

MS/MS Fragmentation of LYNVTDR

Found in IPI00200743, Tax_Id=10116 Gene_Symbol=B3gnt1_predicted UDP-GlcNAc:betaGal beta-1,3-N-acetylglucosaminyltransferase 1

Match to Query 21: 880.427448 from(441.221000,2+)

Title: 091008RatKidney_NoSalt_31.666.666.2.dta

Data file K:INewmanPaper|Piliangl3SubProteomes\Piliang3SP\mgf5ppm\ERLIC_3SubProteomes5ppm.mgf

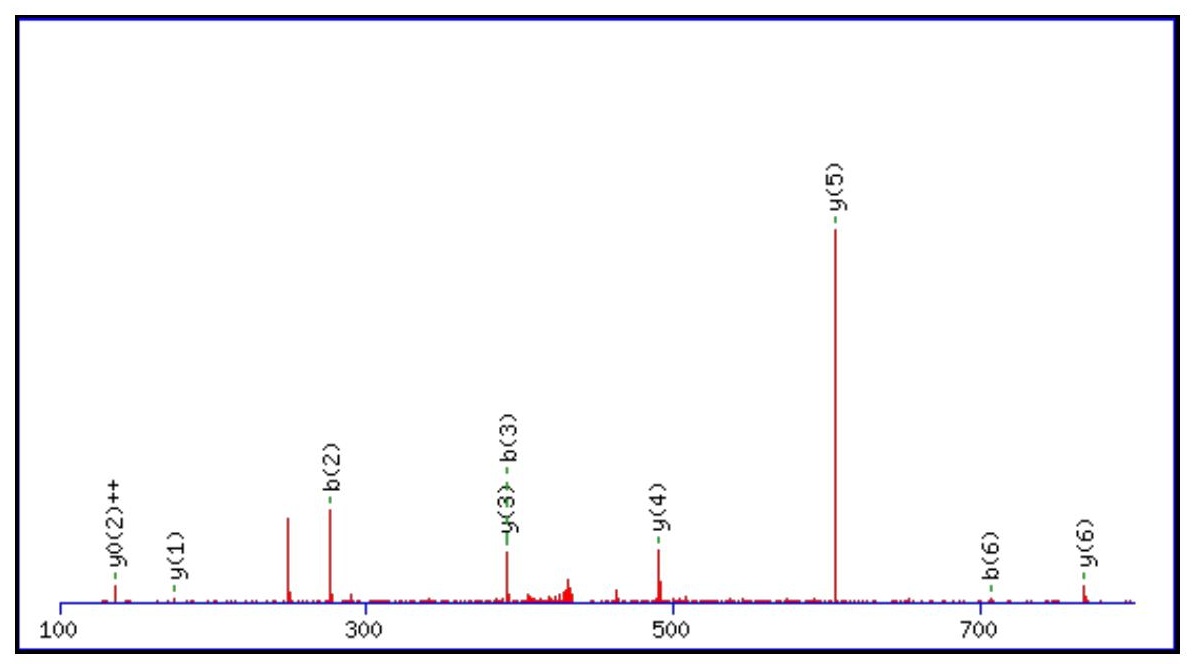



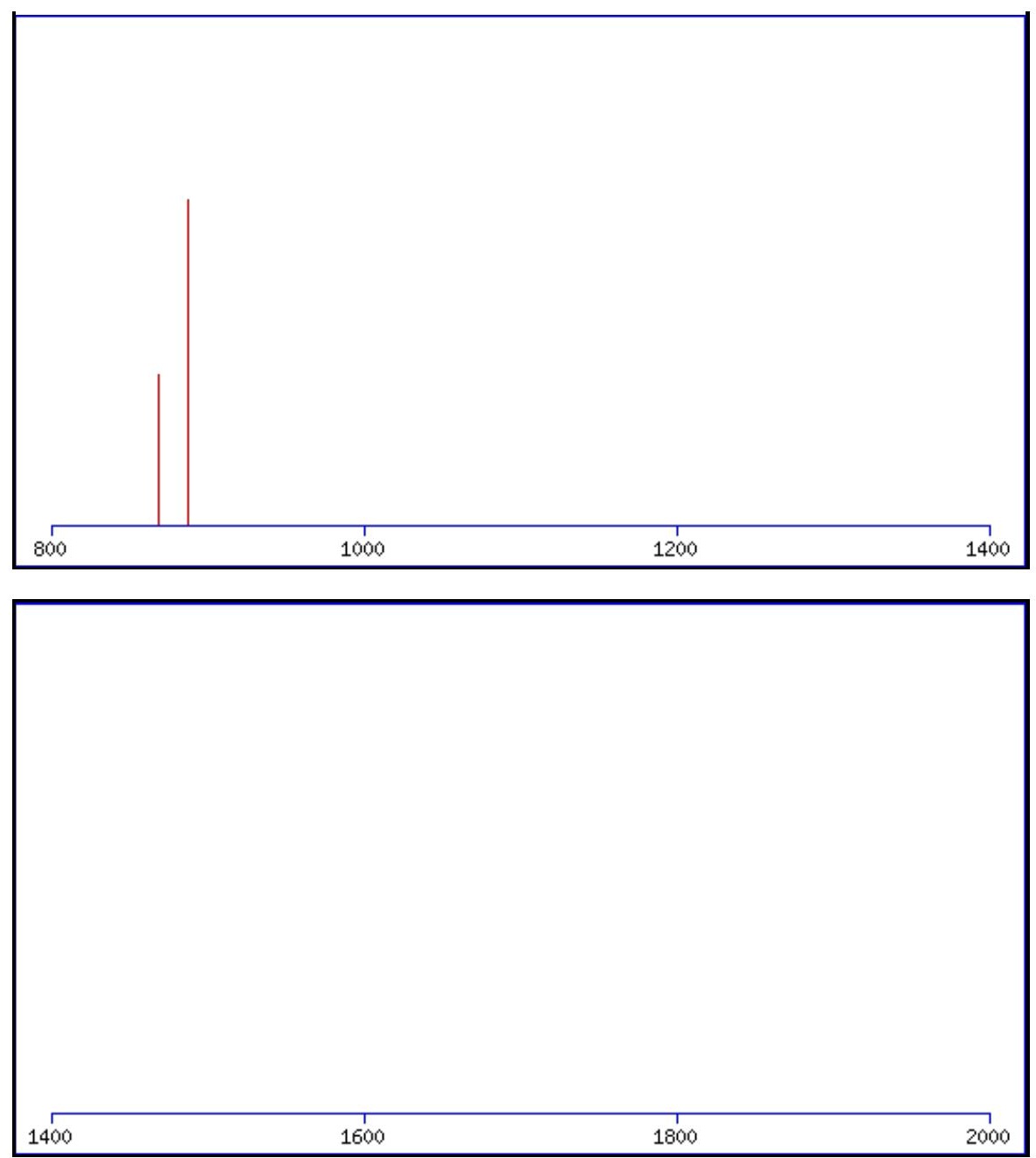

Monoisotopic mass of neutral peptide $\operatorname{Mr}($ calc): 880.4290

Fixed modifications: Carbamidomethyl (C)

Variable modifications:

N3 : Deamidated $\mathrm{N}(\mathrm{N})$

Ions Score: 39 Expect: 0.012

Matches (Bold Red): 9/58 fragment ions using 20 most intense peaks

\begin{tabular}{|c|c|c|c|c|c|c|c|c|c|c|c|c|c|c|}
\hline$\#$ & $\mathbf{b}$ & $\mathbf{b}^{++}$ & $\mathbf{b}^{*}$ & $\mathbf{b}^{\mathbf{*}^{++}}$ & $\mathbf{b}^{\mathbf{0}}$ & $\mathbf{b}^{\mathbf{0 + +}}$ & $\mathbf{S e q}$ & $\mathbf{y}$ & $\mathbf{y}^{++}$ & $\mathbf{y}^{*}$ & $\mathbf{y}^{\boldsymbol{*}^{++}}$ & $\mathbf{y}^{\mathbf{0}}$ & $\mathbf{y}^{\mathbf{0 + +}}$ & $\#$ \\
\hline $\mathbf{1}$ & 114.0913 & 57.5493 & & & & & $\mathbf{L}$ & & & & & & & $\mathbf{7}$ \\
\hline $\mathbf{2}$ & $\mathbf{2 7 7 . 1 5 4 7}$ & 139.0810 & & & & & $\mathbf{Y}$ & $\mathbf{7 6 8 . 3 5 2 3}$ & 384.6798 & 751.3257 & 376.1665 & 750.3417 & 375.6745 & $\mathbf{6}$ \\
\hline $\mathbf{3}$ & 392.1816 & 196.5944 & 375.1551 & 188.0812 & & & $\mathbf{N}$ & $\mathbf{6 0 5 . 2 8 8 9}$ & 303.1481 & 588.2624 & 294.6348 & 587.2784 & 294.1428 & $\mathbf{5}$ \\
\hline $\mathbf{4}$ & 491.2500 & 246.1286 & 474.2235 & 237.6154 & & & $\mathbf{V}$ & $\mathbf{4 9 0 . 2 6 2 0}$ & 245.6346 & 473.2354 & 237.1214 & 472.2514 & 236.6293 & $\mathbf{4}$ \\
\hline $\mathbf{5}$ & 592.2977 & 296.6525 & 575.2711 & 288.1392 & 574.2871 & 287.6472 & $\mathbf{T}$ & 391.1936 & 196.1004 & 374.1670 & 187.5872 & 373.1830 & 187.0951 & $\mathbf{3}$ \\
\hline $\mathbf{6}$ & $\mathbf{7 0 7 . 3 2 4 6}$ & 354.1660 & 690.2981 & 345.6527 & 689.3141 & 345.1607 & $\mathbf{D}$ & 290.1459 & 145.5766 & 273.1193 & 137.0633 & 272.1353 & $\mathbf{1 3 6 . 5 7 1 3}$ & $\mathbf{2}$ \\
\hline $\mathbf{7}$ & & & & & & & $\mathbf{R}$ & $\mathbf{1 7 5 . 1 1 9 0}$ & 88.0631 & 158.0924 & 79.5498 & & & $\mathbf{1}$ \\
\hline
\end{tabular}

$$
\text { 20 }
$$

\section{All matches to this query}

\begin{tabular}{|l|l|l|l|}
\hline Score & Mr(calc): & Delta & Sequence \\
\hline 38.7 & 880.4290 & -0.0016 & LYNVTDR \\
\hline
\end{tabular}

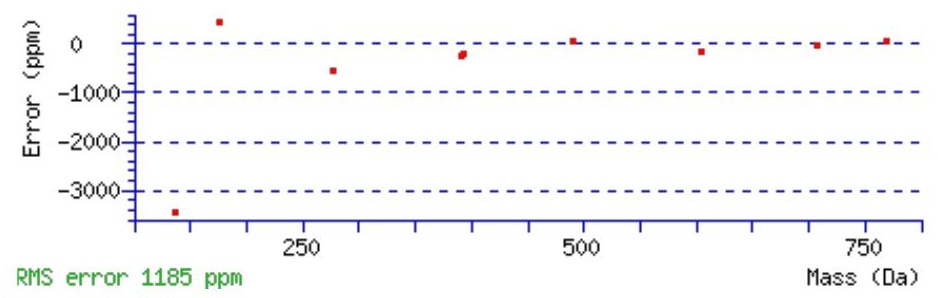

file:///R//wwwroot/SupportingData/3SubProteomes/ERLIC_3SubProteo_Glyco/ERLIC_3SubProteo_Glyco_005.html[4/27/2010 12:29:24 PM] 


\begin{tabular}{|l|l|l|l|}
16.4 & 880.4290 & -0.0016 & DLYVESR \\
\hline 15.8 & 880.4290 & -0.0016 & $\underline{\text { YLNDSLR }}$ \\
\hline 11.6 & 880.4265 & 0.0009 & $\underline{\text { IYPWMR }}$ \\
\hline 7.7 & 880.4290 & -0.0016 & $\underline{\text { IYSNAGEK }}$ \\
\hline 7.3 & 880.4290 & -0.0016 & NLYDSIR \\
\hline 5.0 & 880.4265 & 0.0009 & $\underline{\text { MYPPAFR }}$ \\
\hline 4.1 & 878.4236 & 2.0039 & QRSRPR \\
\hline 3.8 & 880.4290 & -0.0016 & $\underline{\text { LDLSYNR }}$ \\
\hline 3.4 & 879.4239 & 1.0036 & $\underline{\text { LYNEWR }}$ \\
\hline
\end{tabular}

Spectrum No: 873; Query: 1122; Rank: 1

\section{Peptide View}

\section{MS/MS Fragmentation of INTTSDEKDPTNPFR}

Found in IPI00211732, Tax_Id=10116 Gene_Symbol=Adam10 similar to ADAM 10 precursor

Match to Query 1122: 1734.802812 from(579.274880,3+)

Title: 091008RatKidney_NH4Format01_24.1369.1369.3.dta

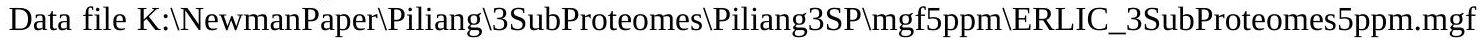
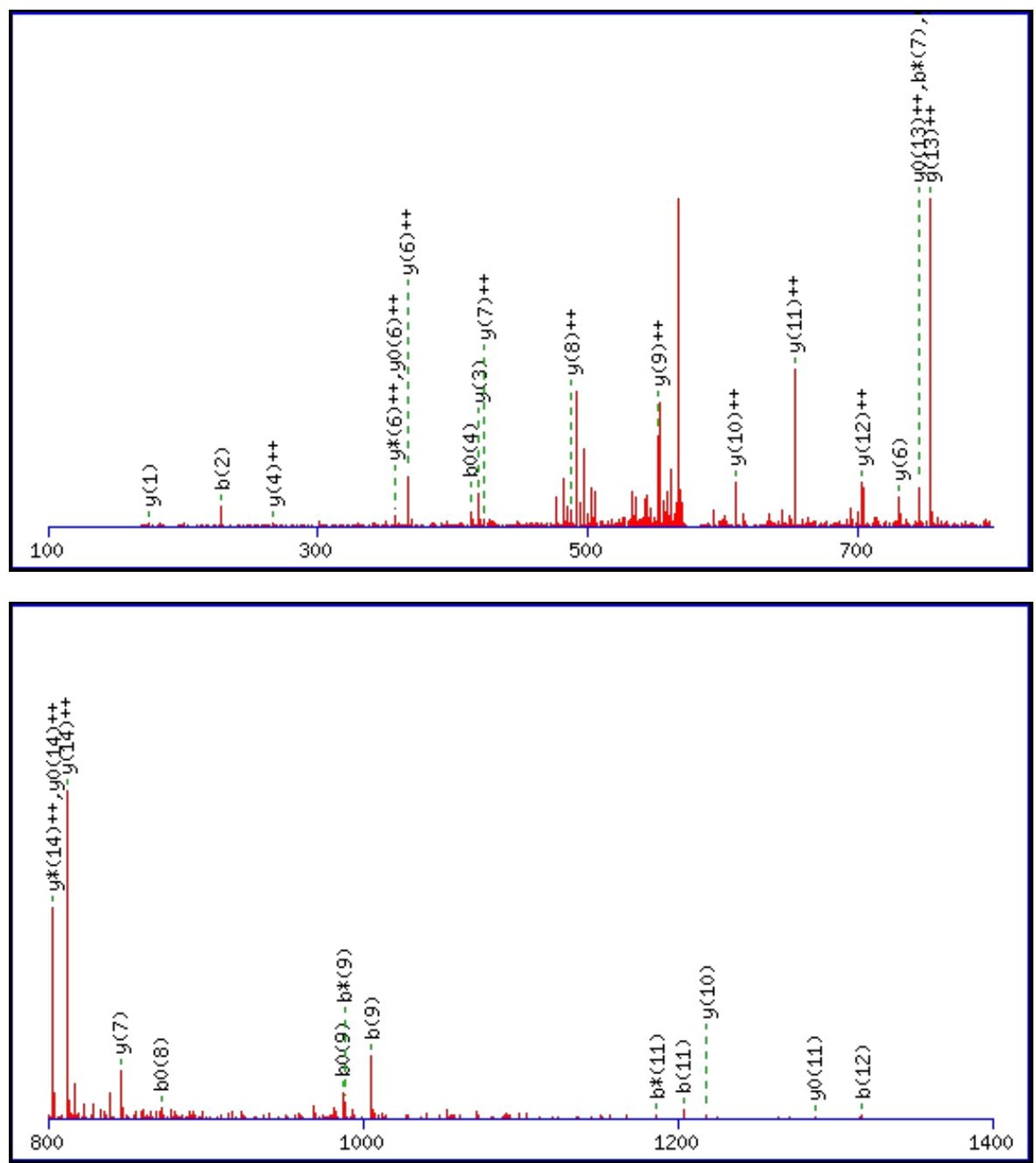


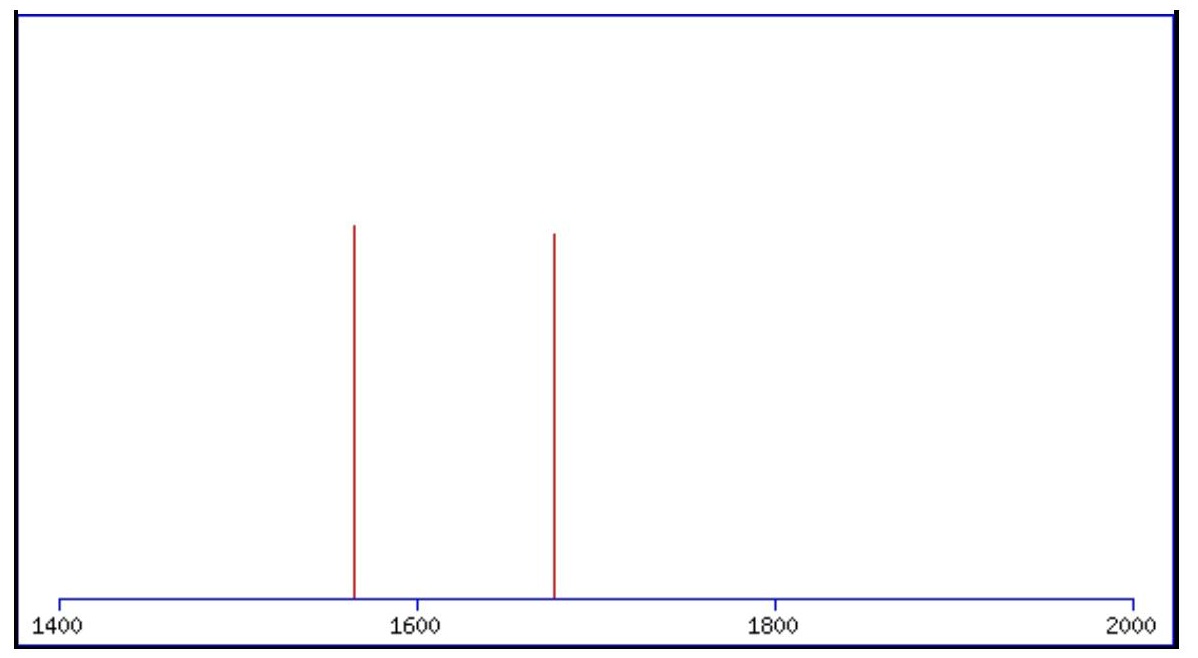

Monoisotopic mass of neutral peptide $\operatorname{Mr}($ calc): 1734.8060

Fixed modifications: Carbamidomethyl (C)

Variable modifications:

N2 : Deamidated_N (N)

Ions Score: 39 Expect: 0.027

Matches (Bold Red): 32/154 fragment ions using 69 most intense peaks

\begin{tabular}{|c|c|c|c|c|c|c|c|c|c|c|c|c|c|c|}
\hline \# & b & $\mathbf{b}^{++}$ & $\mathbf{b}^{*}$ & $\mathbf{b}^{*^{++}}$ & $\mathbf{b}^{0}$ & $\mathbf{b}^{\mathbf{0 + +}}$ & Seq. & $\mathbf{y}$ & $\mathbf{y}^{++}$ & $\mathbf{y}^{*}$ & $\mathbf{y}^{*^{++}}$ & $\mathbf{y}^{0}$ & $\mathbf{y}^{0++}$ & \# \\
\hline 1 & 114.0913 & 57.5493 & & & & & I & & & & & & & 15 \\
\hline 2 & 229.1183 & 115.0628 & 212.0917 & 106.5495 & & & $\mathbf{N}$ & 1622.7293 & 811.8683 & 1605.7027 & 803.3550 & 1604.7187 & 802.8630 & 14 \\
\hline 3 & 330.1660 & 165.5866 & 313.1394 & 157.0733 & 312.1554 & 156.5813 & $\mathbf{T}$ & 1507.7023 & 754.3548 & 1490.6758 & 745.8415 & 1489.6918 & 745.3495 & 13 \\
\hline 4 & 431.2136 & 216.1105 & 414.1871 & 207.5972 & 413.2031 & 207.1052 & $\mathbf{T}$ & 1406.6546 & 703.8310 & 1389.6281 & 695.3177 & 1388.6441 & 694.8257 & 12 \\
\hline 5 & 518.2457 & 259.6265 & 501.2191 & 251.1132 & 500.2351 & 250.6212 & S & 1305.6070 & 653.3071 & 1288.5804 & 644.7938 & 1287.5964 & 644.3018 & 11 \\
\hline 6 & 633.2726 & 317.1399 & 616.2461 & 308.6267 & 615.2620 & 308.1347 & D & 1218.5749 & 609.7911 & 1201.5484 & 601.2778 & 1200.5644 & 600.7858 & 10 \\
\hline 7 & 762.3152 & 381.6612 & 745.2886 & 373.1480 & 744.3046 & 372.6560 & $\mathbf{E}$ & 1103.5480 & 552.2776 & 1086.5215 & 543.7644 & 1085.5374 & 543.2724 & 9 \\
\hline 8 & 890.4102 & 445.7087 & 873.3836 & 437.1954 & 872.3996 & 436.7034 & $\mathbf{K}$ & 974.5054 & 487.7563 & 957.4789 & 479.2431 & 956.4948 & 478.7511 & 8 \\
\hline 9 & 1005.4371 & 503.2222 & 988.4106 & 494.7089 & 987.4265 & 494.2169 & D & 846.4104 & 423.7089 & 829.3839 & 415.1956 & 828.3999 & 414.7036 & 7 \\
\hline 10 & 1102.4899 & 551.7486 & 1085.4633 & 543.2353 & 1084.4793 & 542.7433 & $\mathbf{P}$ & 731.3835 & 366.1954 & 714.3570 & 357.6821 & 713.3729 & 357.1901 & 6 \\
\hline 11 & 1203.5375 & 602.2724 & 1186.5110 & 593.7591 & 1185.5270 & 593.2671 & $\mathbf{T}$ & 634.3307 & 317.6690 & 617.3042 & 309.1557 & 616.3202 & 308.6637 & 5 \\
\hline 12 & 1317.5805 & 659.2939 & 1300.5539 & 650.7806 & 1299.5699 & 650.2886 & $\mathbf{N}$ & 533.2831 & 267.1452 & 516.2565 & 258.6319 & & & 4 \\
\hline 13 & 1414.6332 & 707.8203 & 1397.6067 & 699.3070 & 1396.6227 & 698.8150 & $\mathbf{P}$ & 419.2401 & 210.1237 & 402.2136 & 201.6104 & & & 3 \\
\hline 14 & 1561.7017 & 781.3545 & 1544.6751 & 772.8412 & 1543.6911 & 772.3492 & $\mathbf{F}$ & 322.1874 & 161.5973 & 305.1608 & 153.0840 & & & 2 \\
\hline 15 & & & & & & & $\mathbf{R}$ & 175.1190 & 88.0631 & 158.0924 & 79.5498 & & & 1 \\
\hline
\end{tabular}
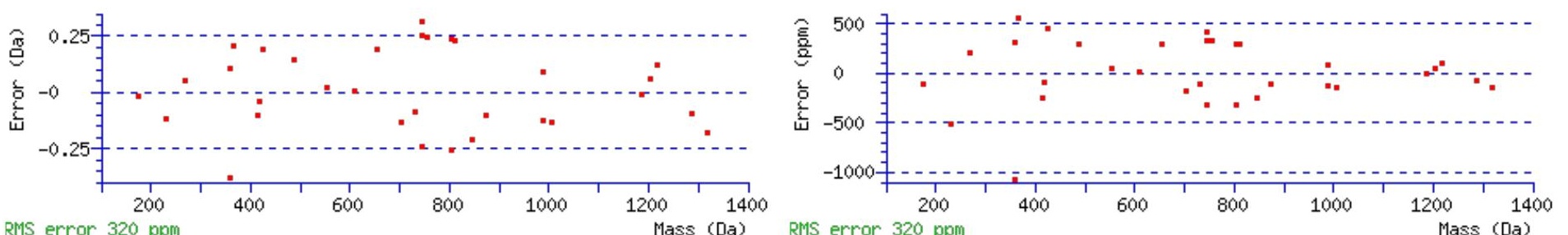

RMS error $320 \mathrm{ppm}$

\section{All matches to this query}

\begin{tabular}{|l|l|l|l|}
\hline Score & Mr(calc): & Delta & \multicolumn{1}{c|}{ Sequence } \\
\hline 38.7 & 1734.8060 & -0.0032 & $\underline{\text { INTTSDEKDPTNPFR }}$ \\
\hline 27.6 & 1734.8060 & -0.0032 & $\underline{\text { INTTSDEKDPTNPFR }}$ \\
\hline 3.2 & 1732.7941 & 2.0087 & $\underline{\text { QIQXLHRGQSDRR }}$ \\
\hline 2.7 & 1732.8008 & 2.0020 & $\underline{\text { AGFNLILSCFAIGDR }}$ \\
\hline 2.5 & 1733.7848 & 1.0180 & $\underline{\text { AGFNLILSCFAIGDR }}$ \\
\hline 0.5 & 1734.8069 & -0.0041 & $\underline{\text { IDKRVMSDMNGFYK }}$ \\
\hline & & &
\end{tabular}




\section{|0.2 |1732.8107|1.9921 |}

Spectrum No: 874; Query: 1408; Rank: 1

\section{Peptide View}

MS/MS Fragmentation of VDCTPLMHSLVYNLTK

Found in IPI00190555, Tax_Id=10116 Gene_Symbol=Folh1 Glutamate carboxypeptidase 2

Match to Query 1408: 1890.920922 from(631.314250,3+)

Title: 091008RatKidney_NH4Format02_25.4492.4492.3.dta

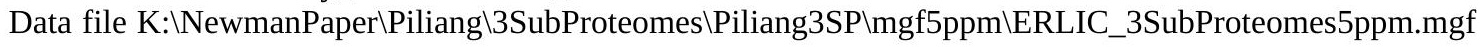
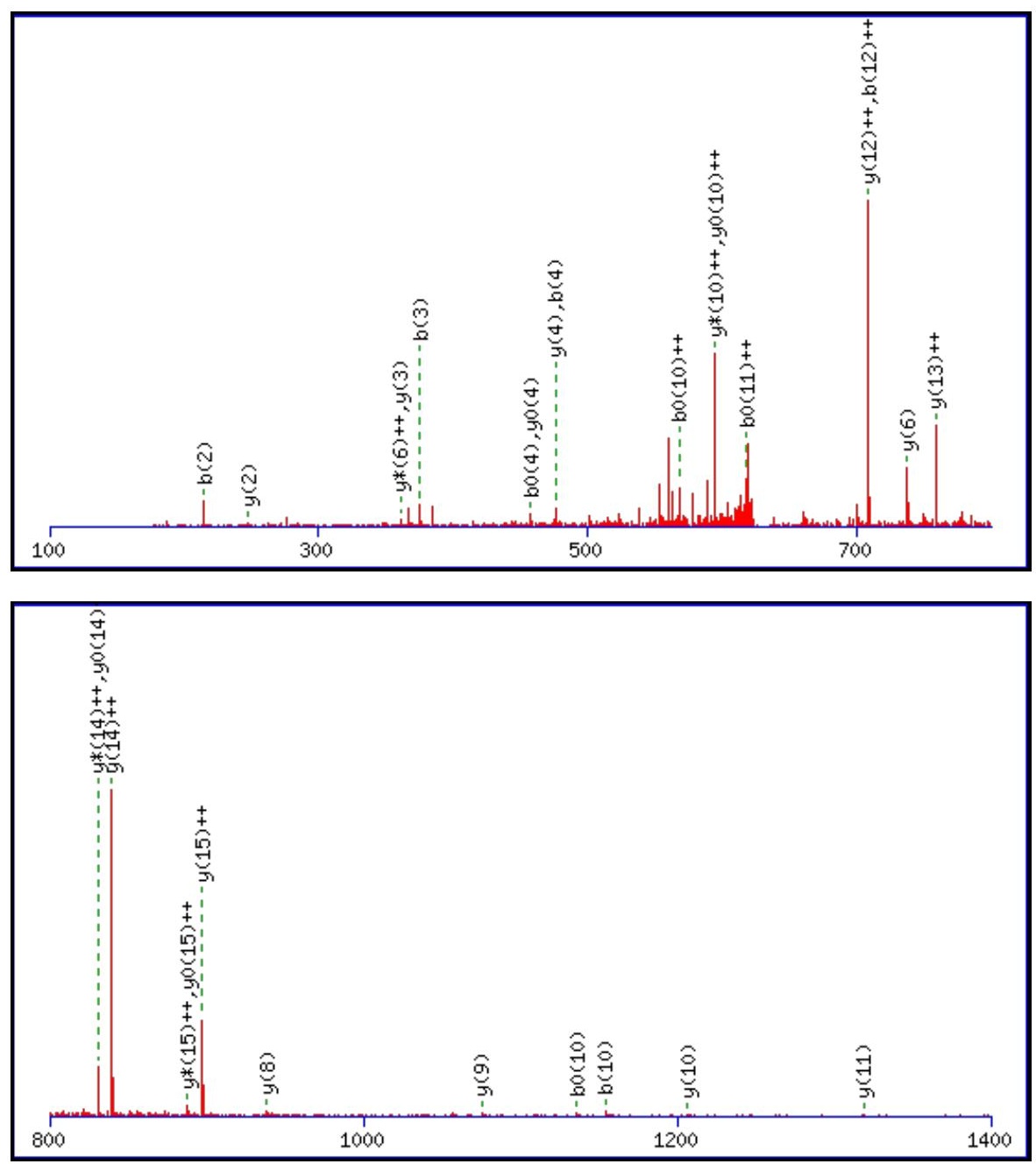


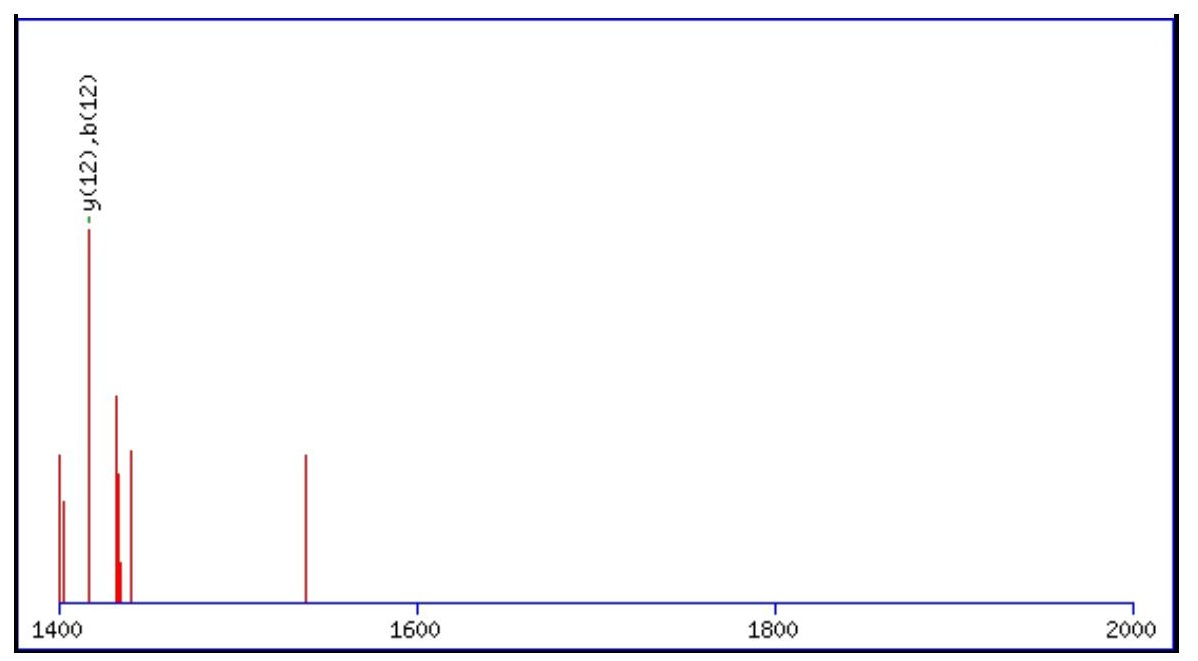

Monoisotopic mass of neutral peptide $\operatorname{Mr}($ calc): 1890.9220

Fixed modifications: Carbamidomethyl (C)

Variable modifications:

N13 : Deamidated_N (N)

Ions Score: 39 Expect : 0.027

Matches (Bold Red): 31/152 fragment ions using 58 most intense peaks

\begin{tabular}{|c|c|c|c|c|c|c|c|c|c|c|c|c|c|c|}
\hline \# & b & $\mathbf{b}^{++}$ & b* & $\mathbf{b}^{*^{++}}$ & $\mathbf{b}^{0}$ & $\mathbf{b}^{0++}$ & Seq. & $\mathbf{y}$ & $\mathbf{y}^{++}$ & $\mathbf{y}^{*}$ & $\mathbf{y}^{*^{++}}$ & $\mathbf{y}^{0}$ & $\mathbf{y}^{0++}$ & \# \\
\hline 1 & 100.0757 & 50.5415 & & & & & $\mathbf{V}$ & & & & & & & 16 \\
\hline 2 & 215.1026 & 108.0550 & & & 197.0921 & 99.0497 & D & 1792.8608 & 896.9340 & 1775.8343 & 888.4208 & 1774.8502 & 887.9288 & 15 \\
\hline 3 & 375.1333 & 188.0703 & & & 357.1227 & 179.0650 & C & 1677.8339 & 839.4206 & 1660.8073 & 830.9073 & 1659.8233 & 830.4153 & 14 \\
\hline 4 & 476.1810 & 238.5941 & & & 458.1704 & 229.5888 & $\mathbf{T}$ & 1517.8032 & 759.4052 & 1500.7767 & 750.8920 & 1499.7927 & 750.4000 & 13 \\
\hline 5 & 573.2337 & 287.1205 & & & 555.2232 & 278.1152 & $\mathbf{P}$ & 1416.7555 & 708.8814 & 1399.7290 & 700.3681 & 1398.7450 & 699.8761 & 12 \\
\hline 6 & 686.3178 & 343.6625 & & & 668.3072 & 334.6573 & $\mathbf{L}$ & 1319.7028 & 660.3550 & 1302.6762 & 651.8418 & 1301.6922 & 651.3497 & 11 \\
\hline 7 & 817.3583 & 409.1828 & & & 799.3477 & 400.1775 & $\mathbf{M}$ & 1206.6187 & 603.8130 & 1189.5922 & 595.2997 & 1188.6081 & 594.8077 & 10 \\
\hline 8 & 954.4172 & 477.7122 & & & 936.4066 & 468.7069 & $\mathbf{H}$ & 1075.5782 & 538.2928 & 1058.5517 & 529.7795 & 1057.5677 & 529.2875 & 9 \\
\hline 9 & 1041.4492 & 521.2282 & & & 1023.4386 & 512.2230 & S & 938.5193 & 469.7633 & 921.4928 & 461.2500 & 920.5088 & 460.7580 & 8 \\
\hline 10 & 1154.5333 & 577.7703 & & & 1136.5227 & 568.7650 & $\mathbf{L}$ & 851.4873 & 426.2473 & 834.4607 & 417.7340 & 833.4767 & 417.2420 & 7 \\
\hline 11 & 1253.6017 & 627.3045 & & & 1235.5911 & 618.2992 & $\mathbf{V}$ & 738.4032 & 369.7053 & 721.3767 & 361.1920 & 720.3927 & 360.7000 & 6 \\
\hline 12 & 1416.6650 & 708.8361 & & & 1398.6545 & 699.8309 & $\mathbf{Y}$ & 639.3348 & 320.1710 & 622.3083 & 311.6578 & 621.3242 & 311.1658 & 5 \\
\hline 13 & 1531.6920 & 766.3496 & 1514.6654 & 757.8363 & 1513.6814 & 757.3443 & $\mathbf{N}$ & 476.2715 & 238.6394 & 459.2449 & 230.1261 & 458.2609 & 229.6341 & 4 \\
\hline 14 & 1644.7760 & 822.8916 & 1627.7495 & 814.3784 & 1626.7655 & 813.8864 & $\mathbf{L}$ & 361.2445 & 181.1259 & 344.2180 & 172.6126 & 343.2340 & 172.1206 & 3 \\
\hline 15 & 1745.8237 & 873.4155 & 1728.7972 & 864.9022 & 1727.8131 & 864.4102 & $\mathbf{T}$ & 248.1605 & 124.5839 & 231.1339 & 116.0706 & 230.1499 & 115.5786 & 2 \\
\hline 16 & & & & & & & $\mathbf{K}$ & 147.1128 & 74.0600 & 130.0863 & 65.5468 & & & 1 \\
\hline
\end{tabular}
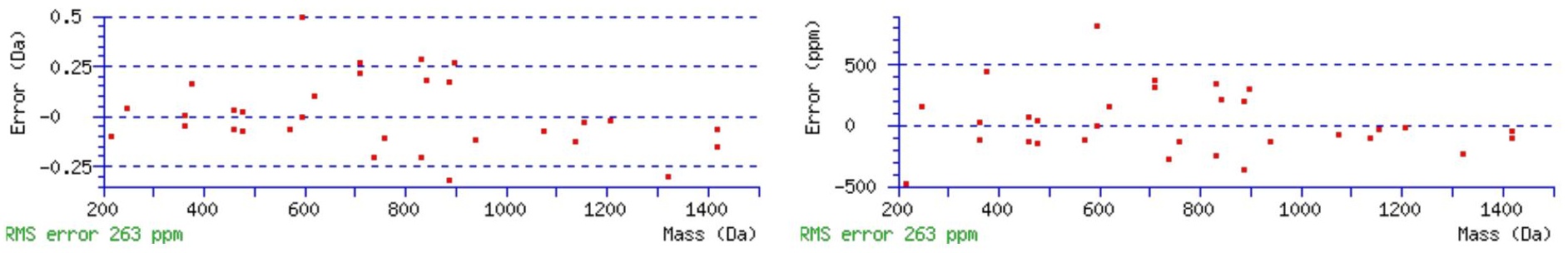

\section{All matches to this query}

\begin{tabular}{|l|l|l|l|}
\hline Score & Mr(calc): & Delta & \multicolumn{1}{c|}{ Sequence } \\
\hline 38.6 & 1890.9220 & -0.0010 & VDCTPLMHSLVYNLTK \\
\hline 7.5 & 1889.9265 & 0.9945 & VQDDTKTLIKTIVTR \\
\hline 7.3 & 1889.9362 & 0.9847 & DLFVLTYGALVAQLCK \\
\hline 5.7 & 1889.9322 & 0.9887 & ESGNSIVLLFKMISTR \\
\hline 5.3 & 1890.9162 & 0.0047 & ESGNSIVLLFKMISTR \\
\hline & & &
\end{tabular}




\begin{tabular}{|l|l|l|l|}
4.1 & 1888.9118 & 2.0091 & LRNVQPVPTTPCADIK \\
\hline 4.1 & 1888.9118 & 2.0091 & LRNVQPVPTTPCADIK \\
\hline 3.2 & 1888.9100 & 2.0109 & LYPVVPTNSRITEK \\
\hline 3.2 & 1888.9100 & 2.0109 & LYPVVPTNSRIITEK \\
\hline 2.9 & 1888.9190 & 2.0019 & LSSSRXRMTLGTGSSLR \\
\hline
\end{tabular}

Spectrum No: 875; Query: 787; Rank: 1

\section{Peptide View}

MS/MS Fragmentation of KKPNATAEPIQPDR

Found in IPI00192504, Tax_Id=10116 Gene_Symbol=Mrc2 Macrophage mannose receptor 2 precursor

Match to Query 787: 1564.823652 from(522.615160,3+)

Title: 100101RatKid_NS_deglyco_23.333.333.3.dta

Data file K:INewmanPaper|Piliangl3SubProteomes\Piliang3SP\mgf5ppm\ERLIC_3SubProteomes5ppm.mgf
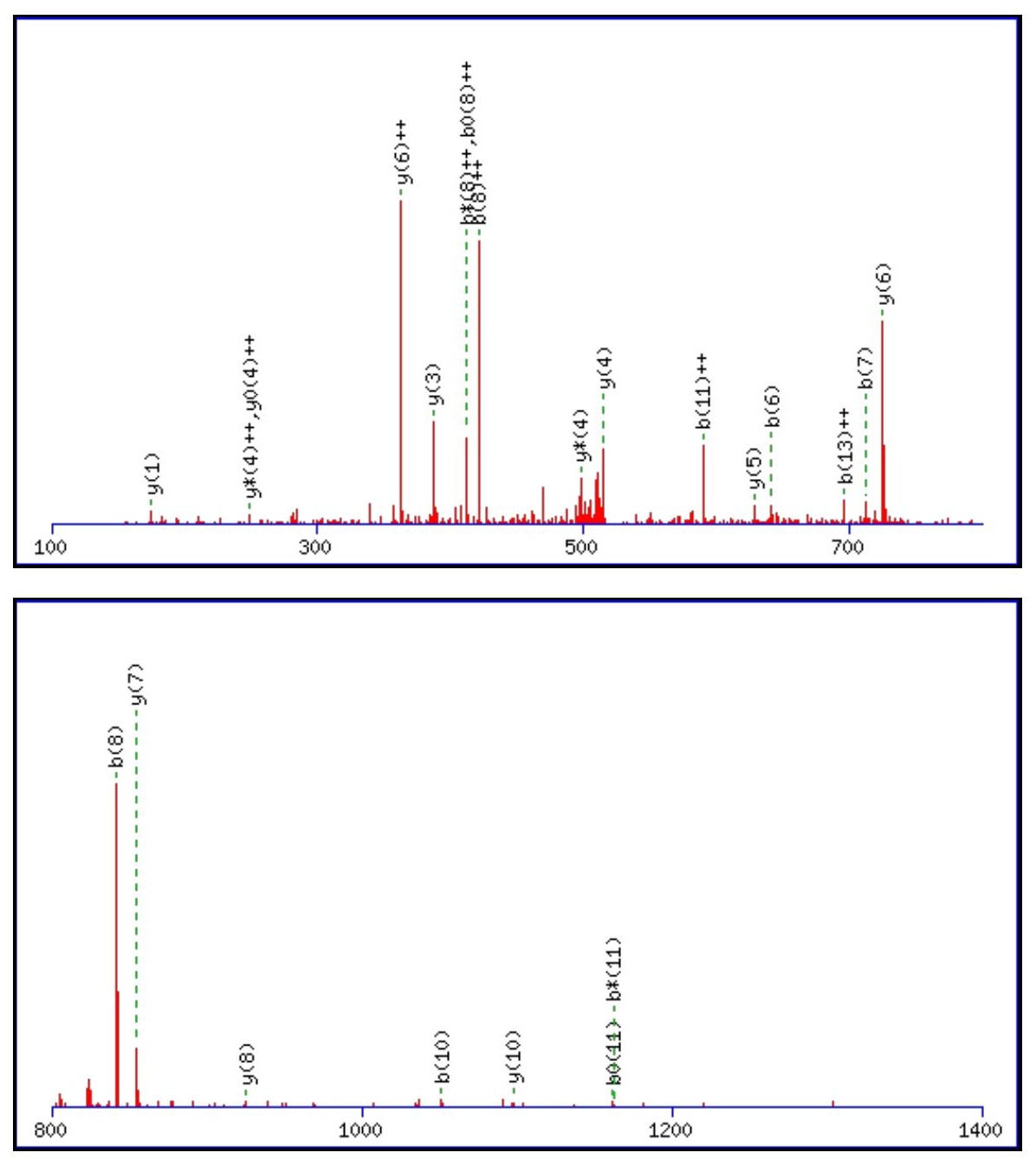
Monoisotopic mass of neutral peptide Mr(calc): 1564.8209

Fixed modifications: Carbamidomethyl (C)

Variable modifications:

N4 : Deamidated $\mathrm{N}(\mathrm{N})$

Ions Score: 39 Expect: 0.015

Matches (Bold Red): 23/144 fragment ions using 47 most intense peaks

\begin{tabular}{|c|c|c|c|c|c|c|c|c|c|c|c|c|c|c|}
\hline \# & b & $\mathbf{b}^{++}$ & b* & $\mathbf{b}^{*^{++}}$ & $\mathbf{b}^{0}$ & $\mathbf{b}^{0++}$ & Seq. & $\mathbf{y}$ & $\mathbf{y}^{++}$ & $\mathbf{y}^{*}$ & $\mathbf{y}^{*^{++}}$ & $\mathbf{y}^{0}$ & $\mathbf{y}^{0++}$ & \# \\
\hline 1 & 129.1022 & 65.0548 & 112.0757 & 56.5415 & & & $\mathbf{K}$ & & & & & & & 14 \\
\hline 2 & 257.1972 & 129.1022 & 240.1707 & 120.5890 & & & $\mathbf{K}$ & 1437.7332 & 719.3703 & 1420.7067 & 710.8570 & 1419.7227 & 710.3650 & 13 \\
\hline 3 & 354.2500 & 177.6286 & 337.2234 & 169.1153 & & & $\mathbf{P}$ & 1309.6383 & 655.3228 & 1292.6117 & 646.8095 & 1291.6277 & 646.3175 & 12 \\
\hline 4 & 469.2769 & 235.1421 & 452.2504 & 226.6288 & & & $\mathbf{N}$ & 1212.5855 & 606.7964 & 1195.5590 & 598.2831 & 1194.5749 & 597.7911 & 11 \\
\hline 5 & 540.3140 & 270.6606 & 523.2875 & 262.1474 & & & A & 1097.5586 & 549.2829 & 1080.5320 & 540.7696 & 1079.5480 & 540.2776 & 10 \\
\hline 6 & 641.3617 & 321.1845 & 624.3351 & 312.6712 & 623.3511 & 312.1792 & $\mathbf{T}$ & 1026.5215 & 513.7644 & 1009.4949 & 505.2511 & 1008.5109 & 504.7591 & 9 \\
\hline 7 & 712.3988 & 356.7030 & 695.3723 & 348.1898 & 694.3882 & 347.6978 & A & 925.4738 & 463.2405 & 908.4472 & 454.7272 & 907.4632 & 454.2352 & 8 \\
\hline 8 & 841.4414 & 421.2243 & 824.4149 & 412.7111 & 823.4308 & 412.2191 & $\mathbf{E}$ & 854.4367 & 427.7220 & 837.4101 & 419.2087 & 836.4261 & 418.7167 & 7 \\
\hline 9 & 938.4942 & 469.7507 & 921.4676 & 461.2374 & 920.4836 & 460.7454 & $\mathbf{P}$ & 725.3941 & 363.2007 & 708.3675 & 354.6874 & 707.3835 & 354.1954 & 6 \\
\hline 10 & 1051.5782 & 526.2928 & 1034.5517 & 517.7795 & 1033.5677 & 517.2875 & I & 628.3413 & 314.6743 & 611.3148 & 306.1610 & 610.3307 & 305.6690 & 5 \\
\hline 11 & 1179.6368 & 590.3220 & 1162.6103 & 581.8088 & 1161.6262 & 581.3168 & $\mathbf{Q}$ & 515.2572 & 258.1323 & 498.2307 & 249.6190 & 497.2467 & 249.1270 & 4 \\
\hline 12 & 1276.6896 & 638.8484 & 1259.6630 & 630.3351 & 1258.6790 & 629.8431 & $\mathbf{P}$ & 387.1987 & 194.1030 & 370.1721 & 185.5897 & 369.1881 & 185.0977 & 3 \\
\hline 13 & 1391.7165 & 696.3619 & 1374.6900 & 687.8486 & 1373.7060 & 687.3566 & D & 290.1459 & 145.5766 & 273.1193 & 137.0633 & 272.1353 & 136.5713 & 2 \\
\hline 14 & & & & & & & $\mathbf{R}$ & 175.1190 & 88.0631 & 158.0924 & 79.5498 & & & 1 \\
\hline
\end{tabular}
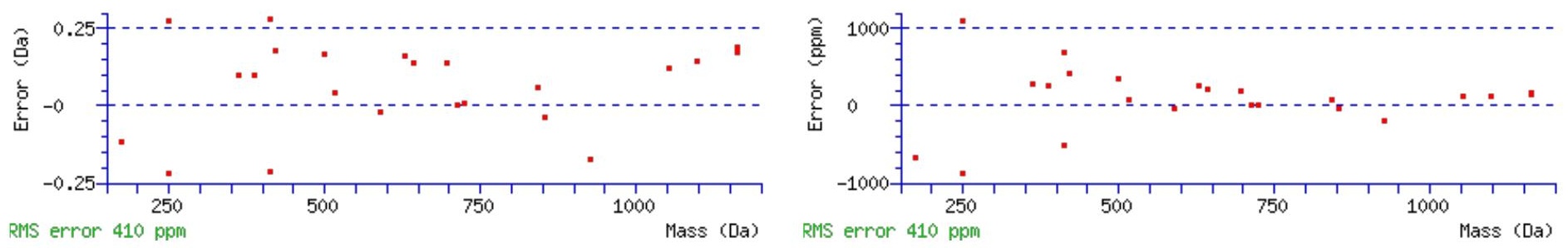

\section{All matches to this query}

\begin{tabular}{|l|l|l|l|}
\hline Score & Mr(calc): & Delta & \multicolumn{1}{c|}{ Sequence } \\
\hline 38.6 & 1564.8209 & 0.0028 & KKPNATAEPIQPDR \\
\hline 5.8 & 1564.8338 & -0.0101 & AKVVQSEVKAVTAR \\
\hline 3.0 & 1563.8147 & 1.0089 & FQKPKPNTGRRR \\
\hline 1.7 & 1564.8338 & -0.0101 & KANVTVLDTQIRK \\
\hline 1.0 & 1562.8069 & 2.0168 & ALGRLLPGLETESK \\
\hline 0.8 & 1564.8239 & -0.0002 & LPGHSGXPALPGAKR \\
\hline 0.7 & 1564.8378 & -0.0141 & LVLVIREDYLPR \\
\hline & & & \\
\hline
\end{tabular}




\begin{tabular}{|l|l|l|l|}
0.3 & 1562.8109 & 2.0127 & SLGFLPQPTPLSVK \\
\hline 0.0 & 1564.8182 & 0.0054 & ALQNALSRAGSHGQR \\
\hline 0.0 & 1564.8126 & 0.0110 & YGSPKLQRKPPSK \\
\hline
\end{tabular}

Spectrum No: 876; Query: 283; Rank: 1

\section{Peptide View}

MS/MS Fragmentation of EGNGTVMGAEIR

Found in IPI00365944, Tax_Id=10116 Gene_Symbol=Myl6_predicted Myosin light polypeptide 6

Match to Query 283: 1233.568088 from(617.791320,2+)

Title: 100101RatKid_NS_deglyco_12.1609.1609.2.dta

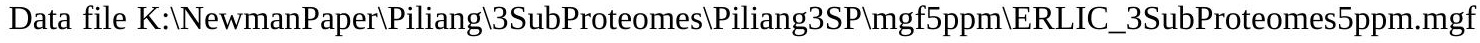
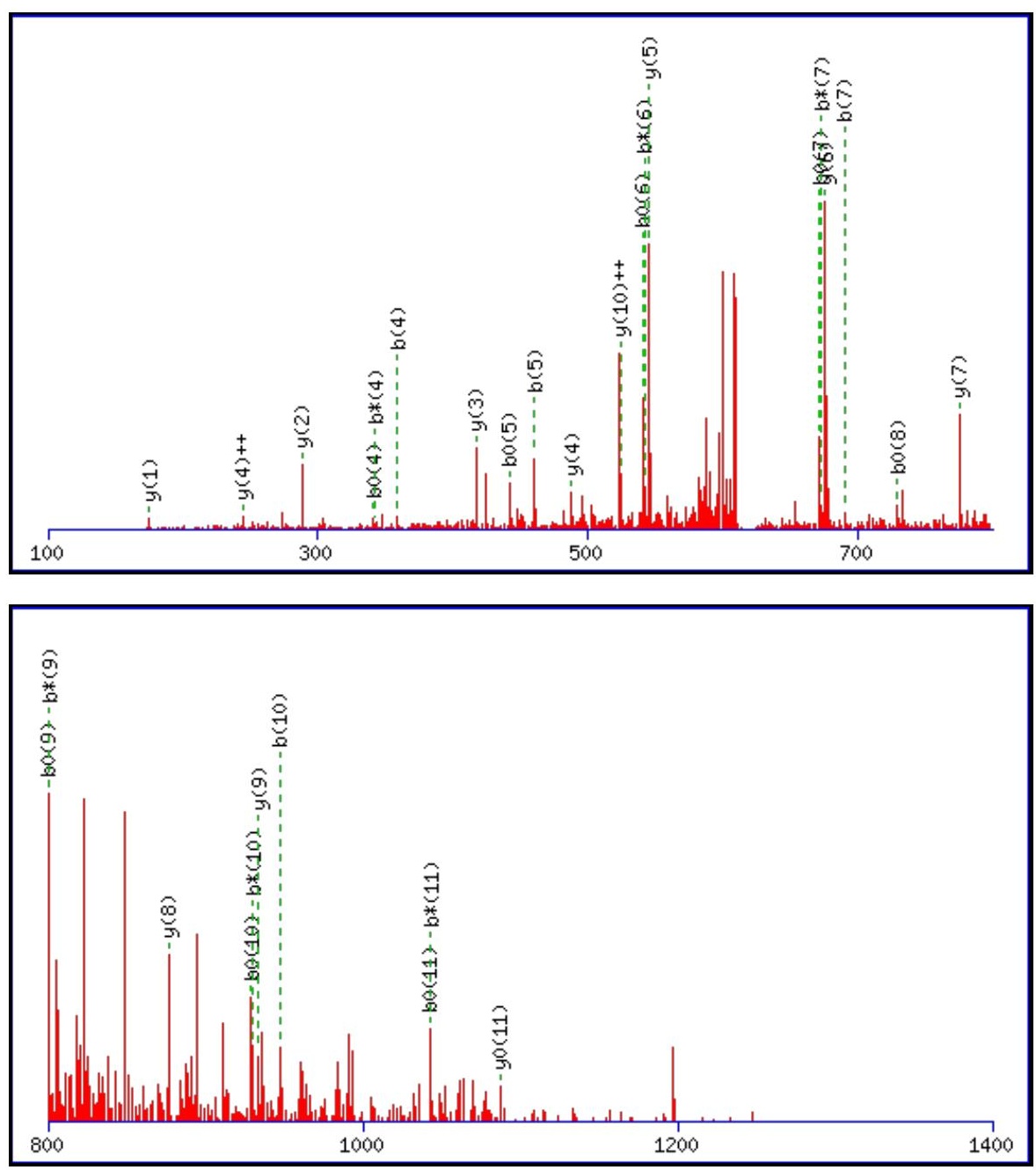


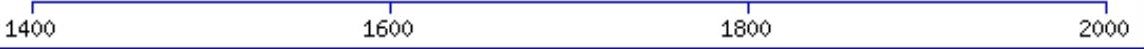

Monoisotopic mass of neutral peptide $\operatorname{Mr}($ calc): 1233.5659

Fixed modifications: Carbamidomethyl (C)

Variable modifications:

N3: Deamidated $\mathrm{N}(\mathrm{N})$

Ions Score: 39 Expect : $\odot .019$

Matches (Bold Red): 30/124 fragment ions using 94 most intense peaks

\begin{tabular}{|c|c|c|c|c|c|c|c|c|c|c|c|c|c|c|}
\hline \# & b & $\mathbf{b}^{++}$ & $\mathbf{b}^{*}$ & $\mathbf{b}^{*^{++}}$ & $\mathbf{b}^{0}$ & $\mathbf{b}^{0++}$ & Seq. & $\mathbf{y}$ & $y^{++}$ & $\mathbf{y}^{*}$ & $\mathrm{y}^{\mathrm{*}^{++}}$ & $\mathbf{y}^{0}$ & $y^{0++}$ & \# \\
\hline 1 & 130.0499 & 65.5286 & & & 112.0393 & 56.5233 & $\mathbf{E}$ & & & & & & & 12 \\
\hline 2 & 187.0713 & 94.0393 & & & 169.0608 & 85.0340 & G & 1105.5306 & 553.2690 & 1088.5041 & 544.7557 & 1087.5201 & 544.2637 & 11 \\
\hline 3 & 302.0983 & 151.5528 & 285.0717 & 143.0395 & 284.0877 & 142.5475 & $\mathbf{N}$ & 1048.5092 & 524.7582 & 1031.4826 & 516.2449 & 1030.4986 & 515.7529 & 10 \\
\hline 4 & 359.1197 & 180.0635 & 342.0932 & 171.5502 & 341.1092 & 171.0582 & G & 933.4822 & 467.2448 & 916.4557 & 458.7315 & 915.4717 & 458.2395 & 9 \\
\hline 5 & 460.1674 & 230.5873 & 443.1409 & 222.0741 & 442.1568 & 221.5821 & $\mathbf{T}$ & 876.4608 & 438.7340 & 859.4342 & 430.2207 & 858.4502 & 429.7287 & 8 \\
\hline 6 & 559.2358 & 280.1216 & 542.2093 & 271.6083 & 541.2253 & 271.1163 & V & 775.4131 & 388.2102 & 758.3865 & 379.6969 & 757.4025 & 379.2049 & 7 \\
\hline 7 & 690.2763 & 345.6418 & 673.2498 & 337.1285 & 672.2657 & 336.6365 & $\mathbf{M}$ & 676.3447 & 338.6760 & 659.3181 & 330.1627 & 658.3341 & 329.6707 & 6 \\
\hline 8 & 747.2978 & 374.1525 & 730.2712 & 365.6393 & 729.2872 & 365.1472 & G & 545.3042 & 273.1557 & 528.2776 & 264.6425 & 527.2936 & 264.1504 & 5 \\
\hline 9 & 818.3349 & 409.6711 & 801.3083 & 401.1578 & 800.3243 & 400.6658 & A & 488.2827 & 244.6450 & 471.2562 & 236.1317 & 470.2722 & 235.6397 & 4 \\
\hline 10 & 947.3775 & 474.1924 & 930.3509 & 465.6791 & 929.3669 & 465.1871 & $\mathbf{E}$ & 417.2456 & 209.1264 & 400.2191 & 200.6132 & 399.2350 & 200.1212 & 3 \\
\hline 11 & 1060.4615 & 530.7344 & 1043.4350 & 522.2211 & 1042.4510 & 521.7291 & I & 288.2030 & 144.6051 & 271.1765 & 136.0919 & & & 2 \\
\hline 12 & & & & & & & $\mathbf{R}$ & 175.1190 & 88.0631 & 158.0924 & 79.5498 & & & 1 \\
\hline
\end{tabular}
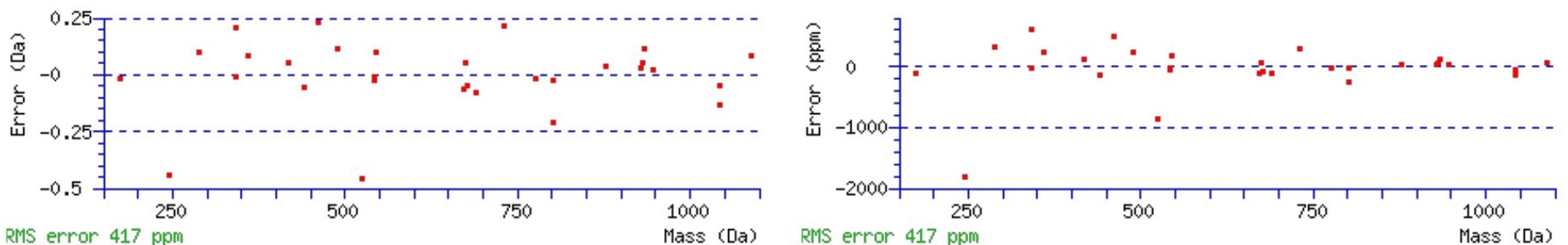

\section{All matches to this query}

\begin{tabular}{|l|l|l|l|}
\hline Score & Mr(calc): & Delta & \multicolumn{1}{|c|}{ Sequence } \\
\hline 38.5 & 1233.5659 & 0.0022 & EGNGTVMGAEIR \\
\hline 38.5 & 1233.5659 & 0.0022 & EGNGTVMGAELR \\
\hline 15.8 & 1233.5672 & 0.0009 & YPAVSLRLR \\
\hline 14.9 & 1232.5745 & 0.9936 & NQDSDNVRRK \\
\hline 14.1 & 1232.5550 & 1.0131 & HSPGTEKELR \\
\hline 10.0 & 1232.5624 & 1.0056 & GVYMTGKELR \\
\hline 9.2 & 1233.5625 & 0.0056 & GNNSSNIWALR \\
\hline 8.8 & 1232.5550 & 1.0131 & HSPGTEKELR \\
\hline 7.2 & 1232.5673 & 1.0008 & QDWDPSASLSK \\
\hline & & &
\end{tabular}




\section{|6.1 |1233.5655|0.0026|AKHLAANWSR}

Spectrum No: 877; Query: 283; Rank: 1

\section{Peptide View}

MS/MS Fragmentation of EGNGTVMGAEIR

Found in IPI00365944, Tax_Id=10116 Gene_Symbol=Myl6_predicted Myosin light polypeptide 6

Match to Query 283: 1233.568088 from(617.791320,2+)

Title: 100101RatKid_NS_deglyco_12.1609.1609.2.dta

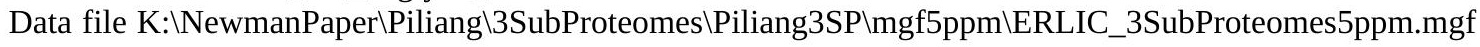
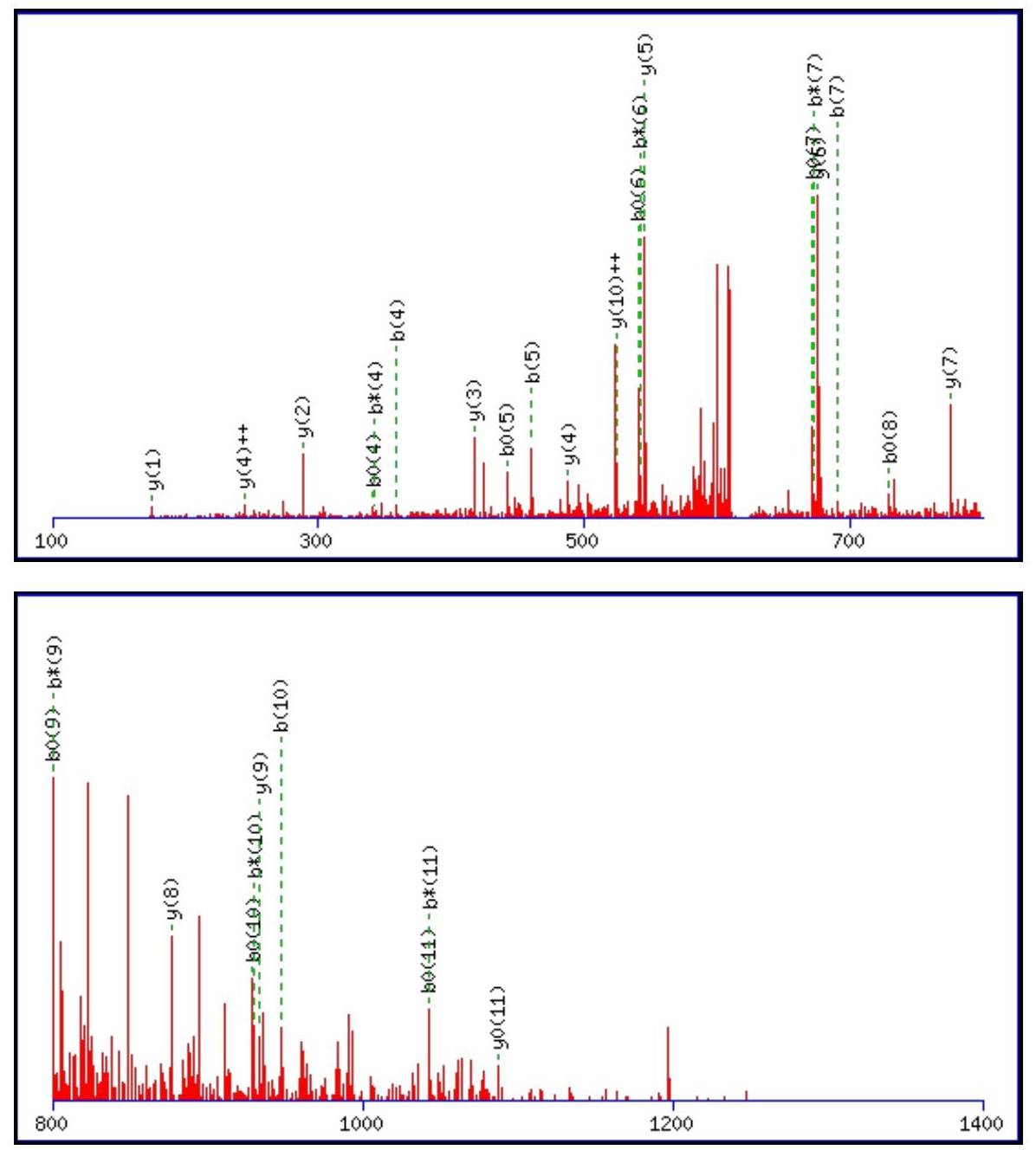


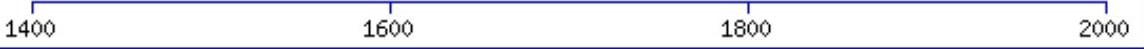

Monoisotopic mass of neutral peptide $\operatorname{Mr}($ calc): 1233.5659

Fixed modifications: Carbamidomethyl (C)

Variable modifications:

N3: Deamidated $\mathrm{N}(\mathrm{N})$

Ions Score: 39 Expect : $\odot .019$

Matches (Bold Red): 30/124 fragment ions using 94 most intense peaks

\begin{tabular}{|c|c|c|c|c|c|c|c|c|c|c|c|c|c|c|}
\hline \# & b & $\mathbf{b}^{++}$ & $\mathbf{b}^{*}$ & $\mathbf{b}^{*^{++}}$ & $\mathbf{b}^{0}$ & $\mathbf{b}^{0++}$ & Seq. & $\mathbf{y}$ & $y^{++}$ & $\mathbf{y}^{*}$ & $\mathrm{y}^{\mathrm{*}^{++}}$ & $\mathbf{y}^{0}$ & $y^{0++}$ & \# \\
\hline 1 & 130.0499 & 65.5286 & & & 112.0393 & 56.5233 & $\mathbf{E}$ & & & & & & & 12 \\
\hline 2 & 187.0713 & 94.0393 & & & 169.0608 & 85.0340 & G & 1105.5306 & 553.2690 & 1088.5041 & 544.7557 & 1087.5201 & 544.2637 & 11 \\
\hline 3 & 302.0983 & 151.5528 & 285.0717 & 143.0395 & 284.0877 & 142.5475 & $\mathbf{N}$ & 1048.5092 & 524.7582 & 1031.4826 & 516.2449 & 1030.4986 & 515.7529 & 10 \\
\hline 4 & 359.1197 & 180.0635 & 342.0932 & 171.5502 & 341.1092 & 171.0582 & G & 933.4822 & 467.2448 & 916.4557 & 458.7315 & 915.4717 & 458.2395 & 9 \\
\hline 5 & 460.1674 & 230.5873 & 443.1409 & 222.0741 & 442.1568 & 221.5821 & $\mathbf{T}$ & 876.4608 & 438.7340 & 859.4342 & 430.2207 & 858.4502 & 429.7287 & 8 \\
\hline 6 & 559.2358 & 280.1216 & 542.2093 & 271.6083 & 541.2253 & 271.1163 & V & 775.4131 & 388.2102 & 758.3865 & 379.6969 & 757.4025 & 379.2049 & 7 \\
\hline 7 & 690.2763 & 345.6418 & 673.2498 & 337.1285 & 672.2657 & 336.6365 & $\mathbf{M}$ & 676.3447 & 338.6760 & 659.3181 & 330.1627 & 658.3341 & 329.6707 & 6 \\
\hline 8 & 747.2978 & 374.1525 & 730.2712 & 365.6393 & 729.2872 & 365.1472 & G & 545.3042 & 273.1557 & 528.2776 & 264.6425 & 527.2936 & 264.1504 & 5 \\
\hline 9 & 818.3349 & 409.6711 & 801.3083 & 401.1578 & 800.3243 & 400.6658 & A & 488.2827 & 244.6450 & 471.2562 & 236.1317 & 470.2722 & 235.6397 & 4 \\
\hline 10 & 947.3775 & 474.1924 & 930.3509 & 465.6791 & 929.3669 & 465.1871 & $\mathbf{E}$ & 417.2456 & 209.1264 & 400.2191 & 200.6132 & 399.2350 & 200.1212 & 3 \\
\hline 11 & 1060.4615 & 530.7344 & 1043.4350 & 522.2211 & 1042.4510 & 521.7291 & I & 288.2030 & 144.6051 & 271.1765 & 136.0919 & & & 2 \\
\hline 12 & & & & & & & $\mathbf{R}$ & 175.1190 & 88.0631 & 158.0924 & 79.5498 & & & 1 \\
\hline
\end{tabular}
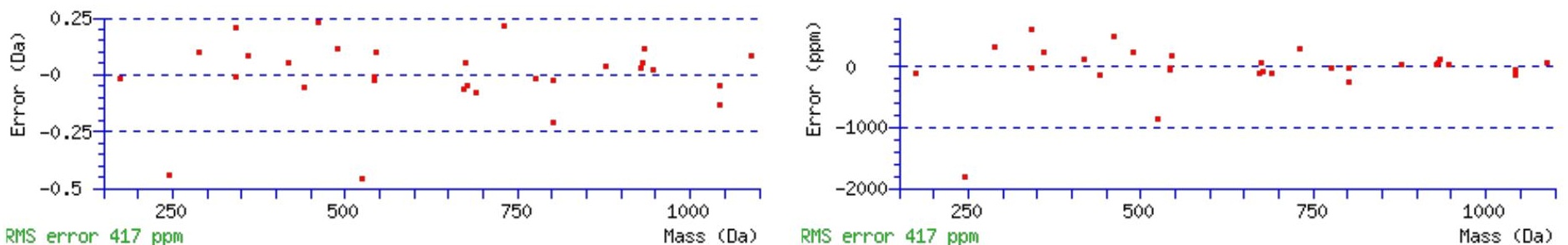

\section{All matches to this query}

\begin{tabular}{|l|l|l|l|}
\hline Score & Mr(calc): & Delta & \multicolumn{1}{|c|}{ Sequence } \\
\hline 38.5 & 1233.5659 & 0.0022 & EGNGTVMGAEIR \\
\hline 38.5 & 1233.5659 & 0.0022 & EGNGTVMGAELR \\
\hline 15.8 & 1233.5672 & 0.0009 & YPAVSLRLR \\
\hline 14.9 & 1232.5745 & 0.9936 & NQDSDNVRRK \\
\hline 14.1 & 1232.5550 & 1.0131 & HSPGTEKELR \\
\hline 10.0 & 1232.5624 & 1.0056 & GVYMTGKELR \\
\hline 9.2 & 1233.5625 & 0.0056 & GNNSSNIWALR \\
\hline 8.8 & 1232.5550 & 1.0131 & HSPGTEKELR \\
\hline 7.2 & 1232.5673 & 1.0008 & QDWDPSASLSK \\
\hline & & &
\end{tabular}




\section{|6.1 |1233.5655|0.0026|AKHLAANWSR}

Spectrum No: 878; Query: 15; Rank: 1

\section{Peptide View}

MS/MS Fragmentation of RIPAINR

Found in IPI00363849, Tax_Id=10116 Gene_Symbol=Lamc1 similar to Laminin gamma-1 chain precursor

Match to Query 15: 839.495808 from(420.755180,2+)

Title: 091008RatKidney_NH4Format01_26.395.395.2.dta

Data file K:\NewmanPaper $\backslash$ Piliang $\backslash 3$ SubProteomes $\backslash$ Piliang3SP $\backslash$ mgf5ppm\ERLIC_3SubProteomes5ppm.mgf
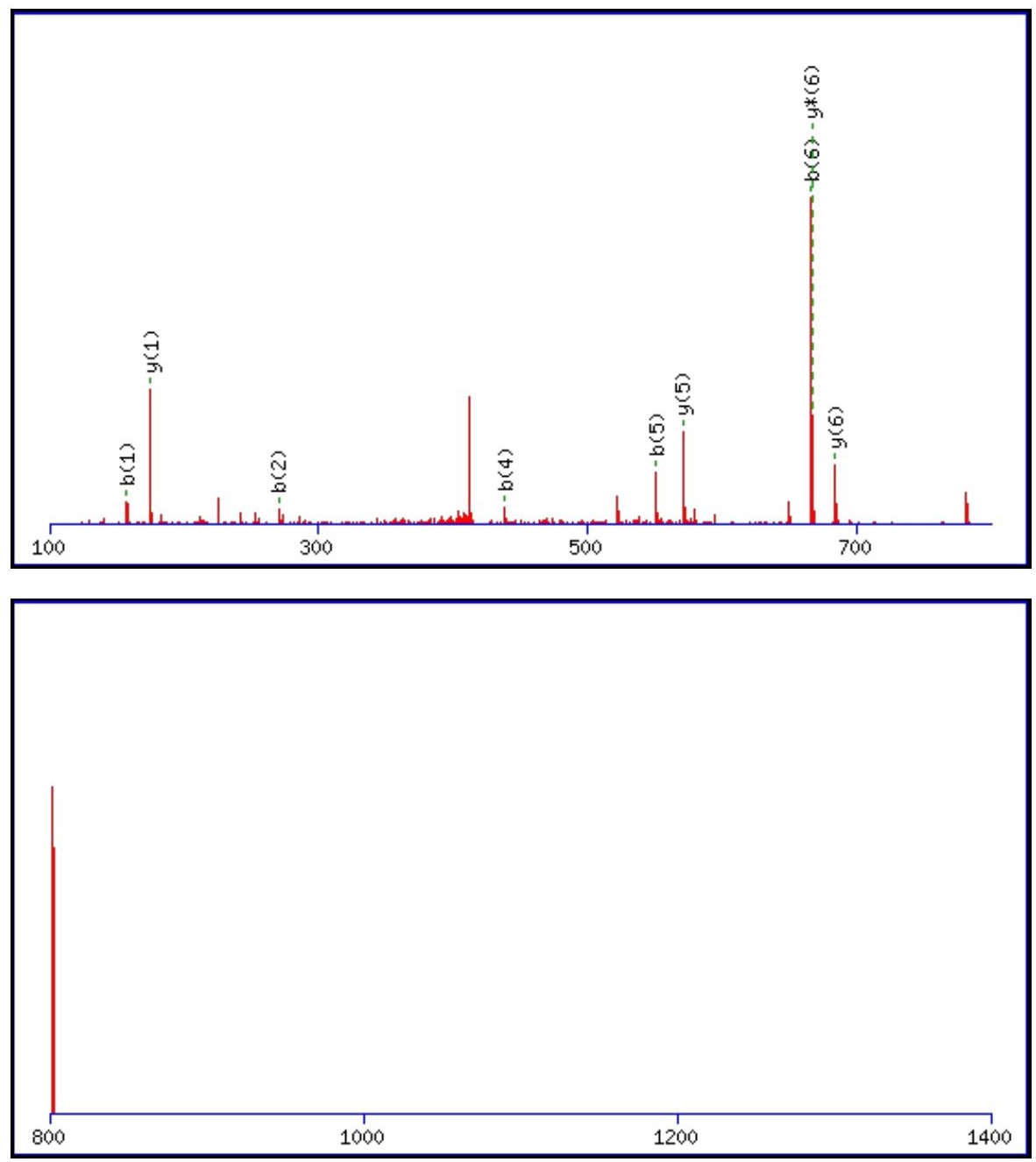


\begin{tabular}{rrrr}
\hline 1400 & 1600 & 1800 & 2000
\end{tabular}

Monoisotopic mass of neutral peptide $\operatorname{Mr}($ calc): 839.4977

Fixed modifications: Carbamidomethyl (C)

Variable modifications:

N6 : Deamidated_N (N)

Ions Score: 38 Expect: 0.0032

Matches (Bold Red): 9/48 fragment ions using 15 most intense peaks

\begin{tabular}{|c|c|c|c|c|c|c|c|c|c|c|}
\hline$\#$ & $\mathbf{b}$ & $\mathbf{b}^{++}$ & $\mathbf{b}^{*}$ & $\mathbf{b}^{\boldsymbol{*}^{++}}$ & $\mathbf{S e q} \cdot$ & $\mathbf{y}$ & $\mathbf{y}^{++}$ & $\mathbf{y}^{*}$ & $\mathbf{y}^{\boldsymbol{*}^{++}}$ & $\#$ \\
\hline $\mathbf{1}$ & $\mathbf{1 5 7 . 1 0 8 4}$ & 79.0578 & 140.0818 & 70.5446 & $\mathbf{R}$ & & & & & $\mathbf{7}$ \\
\hline $\mathbf{2}$ & $\mathbf{2 7 0 . 1 9 2 5}$ & 135.5999 & 253.1659 & 127.0866 & $\mathbf{I}$ & $\mathbf{6 8 4 . 4 0 3 9}$ & 342.7056 & $\mathbf{6 6 7 . 3 7 7 3}$ & 334.1923 & $\mathbf{6}$ \\
\hline $\mathbf{3}$ & 367.2452 & 184.1262 & 350.2187 & 175.6130 & $\mathbf{P}$ & 571.3198 & 286.1636 & 554.2933 & 277.6503 & $\mathbf{5}$ \\
\hline $\mathbf{4}$ & $\mathbf{4 3 8 . 2 8 2 3}$ & 219.6448 & 421.2558 & 211.1315 & $\mathbf{A}$ & 474.2671 & 237.6372 & 457.2405 & 229.1239 & $\mathbf{4}$ \\
\hline $\mathbf{5}$ & $\mathbf{5 5 1 . 3 6 6 4}$ & 276.1868 & 534.3398 & 267.6736 & $\mathbf{I}$ & 403.2300 & 202.1186 & 386.2034 & 193.6053 & $\mathbf{3}$ \\
\hline $\mathbf{6}$ & $\mathbf{6 6 6 . 3 9 3 3}$ & 333.7003 & 649.3668 & 325.1870 & $\mathbf{N}$ & 290.1459 & 145.5766 & 273.1193 & 137.0633 & $\mathbf{2}$ \\
\hline $\mathbf{7}$ & & & & & $\mathbf{R}$ & $\mathbf{1 7 5 . 1 1 9 0}$ & 88.0631 & 158.0924 & 79.5498 & $\mathbf{1}$ \\
\hline
\end{tabular}
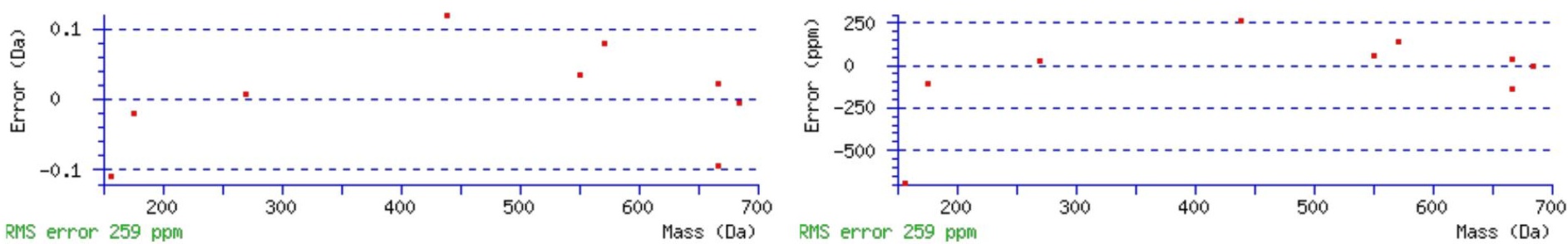

\section{All matches to this query}

\begin{tabular}{|l|l|l|l|}
\hline Score & Mr(calc): & Delta & \multicolumn{1}{|c|}{ Sequence } \\
\hline 38.3 & 839.4977 & -0.0019 & RIPAINR \\
\hline 17.9 & 839.4977 & -0.0019 & RLLEGPR \\
\hline 9.0 & 839.4977 & -0.0019 & GNPVAVKR \\
\hline 7.9 & 839.4977 & -0.0019 & LRDPLAR \\
\hline 7.9 & 839.4977 & -0.0019 & LRGIPER \\
\hline 6.8 & 839.4977 & -0.0019 & RVLPEAR \\
\hline 5.8 & 837.4821 & 2.0137 & GVEKHIR \\
\hline 5.2 & 837.4821 & 2.0137 & EGIKVHR \\
\hline 3.4 & 839.4977 & -0.0019 & RNPALIR \\
\hline 2.2 & 839.4977 & -0.0019 & APLRVER \\
\hline
\end{tabular}

Spectrum No: 879; Query: 28; Rank: 1 


\section{Peptide View}

MS/MS Fragmentation of MNASFSLK

Found in IPI00339118, Tax_Id=10116 Gene_Symbol=Enpp1 Isoform 2 of Ectonucleotide pyrophosphatase/phosphodiesterase family member 1

Match to Query 28: 913.419788 from(457.717170,2+)

Title: 091008RatKidney_NH4Format01_27.944.944.2.dta

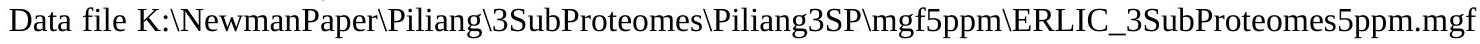
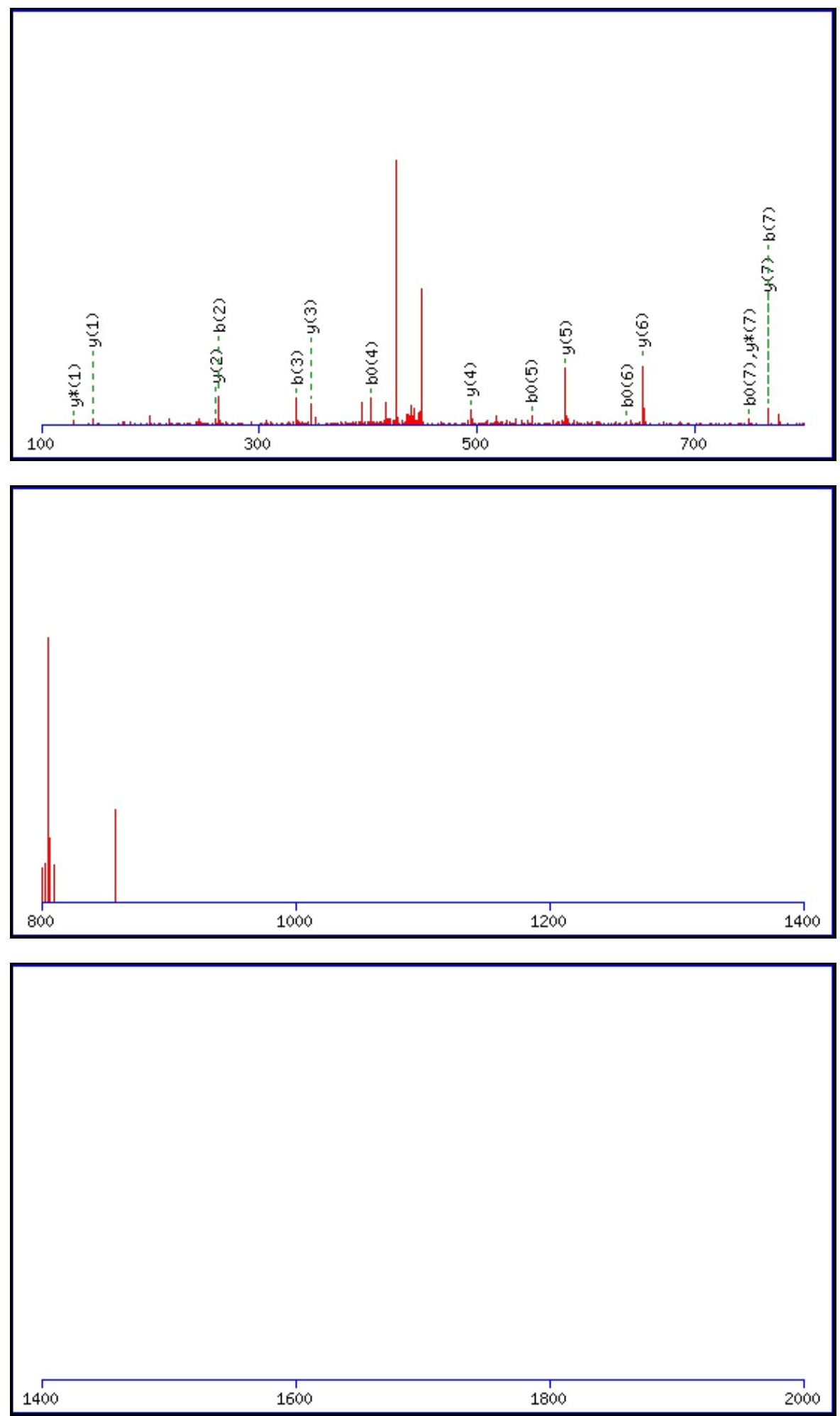

Monoisotopic mass of neutral peptide $\operatorname{Mr}($ calc): 913.4215

Fixed modifications: Carbamidomethyl (C)

Variable modifications:

M1 : Oxidation (M)

N2 : Deamidated $\mathrm{N}(\mathrm{N})$

Ions Score: 38 Expect: 0.011 
Matches (Bold Red): 16/72 fragment ions using 47 most intense peaks

\begin{tabular}{|c|c|c|c|c|c|c|c|c|c|c|c|c|c|c|}
\hline$\#$ & $\mathbf{b}$ & $\mathbf{b}^{++}$ & $\mathbf{b}^{*}$ & $\mathbf{b}^{\boldsymbol{*}^{++}}$ & $\mathbf{b}^{\mathbf{0}}$ & $\mathbf{b}^{\mathbf{0}+}$ & $\mathbf{S e q}$ & $\mathbf{y}$ & $\mathbf{y}^{++}$ & $\mathbf{y}^{*}$ & $\mathbf{y}^{\boldsymbol{*}^{++}}$ & $\mathbf{y}^{\mathbf{0}}$ & $\mathbf{y}^{\mathbf{0}++}$ & $\#$ \\
\hline $\mathbf{1}$ & 148.0427 & 74.5250 & & & & & $\mathbf{M}$ & & & & & & & $\mathbf{8}$ \\
\hline $\mathbf{2}$ & $\mathbf{2 6 3 . 0 6 9 6}$ & 132.0384 & 246.0431 & 123.5252 & & & $\mathbf{N}$ & $\mathbf{7 6 7 . 3 9 3 4}$ & 384.2003 & $\mathbf{7 5 0 . 3 6 6 8}$ & 375.6871 & 749.3828 & 375.1950 & $\mathbf{7}$ \\
\hline $\mathbf{3}$ & 334.1067 & 167.5570 & 317.0802 & 159.0437 & & & $\mathbf{A}$ & $\mathbf{6 5 2 . 3 6 6 5}$ & 326.6869 & 635.3399 & 318.1736 & 634.3559 & 317.6816 & $\mathbf{6}$ \\
\hline $\mathbf{4}$ & 421.1388 & 211.0730 & 404.1122 & 202.5597 & $\mathbf{4 0 3 . 1 2 8 2}$ & 202.0677 & $\mathbf{S}$ & $\mathbf{5 8 1 . 3 2 9 3}$ & 291.1683 & 564.3028 & 282.6550 & 563.3188 & 282.1630 & $\mathbf{5}$ \\
\hline $\mathbf{5}$ & 568.2072 & 284.6072 & 551.1806 & 276.0939 & $\mathbf{5 5 0 . 1 9 6 6}$ & 275.6019 & $\mathbf{F}$ & $\mathbf{4 9 4 . 2 9 7 3}$ & 247.6523 & 477.2708 & 239.1390 & 476.2867 & 238.6470 & $\mathbf{4}$ \\
\hline $\mathbf{6}$ & 655.2392 & 328.1232 & 638.2127 & 319.6100 & $\mathbf{6 3 7 . 2 2 8 6}$ & 319.1180 & $\mathbf{S}$ & $\mathbf{3 4 7 . 2 2 8 9}$ & 174.1181 & 330.2023 & 165.6048 & 329.2183 & 165.1128 & $\mathbf{3}$ \\
\hline $\mathbf{7}$ & $\mathbf{7 6 8 . 3 2 3 3}$ & 384.6653 & 751.2967 & 376.1520 & $\mathbf{7 5 0 . 3 1 2 7}$ & 375.6600 & $\mathbf{L}$ & $\mathbf{2 6 0 . 1 9 6 9}$ & 130.6021 & 243.1703 & 122.0888 & & & $\mathbf{2}$ \\
\hline $\mathbf{8}$ & & & & & & & $\mathbf{K}$ & $\mathbf{1 4 7 . 1 1 2 8}$ & 74.0600 & $\mathbf{1 3 0 . 0 8 6 3}$ & 65.5468 & & & $\mathbf{1}$ \\
\hline
\end{tabular}
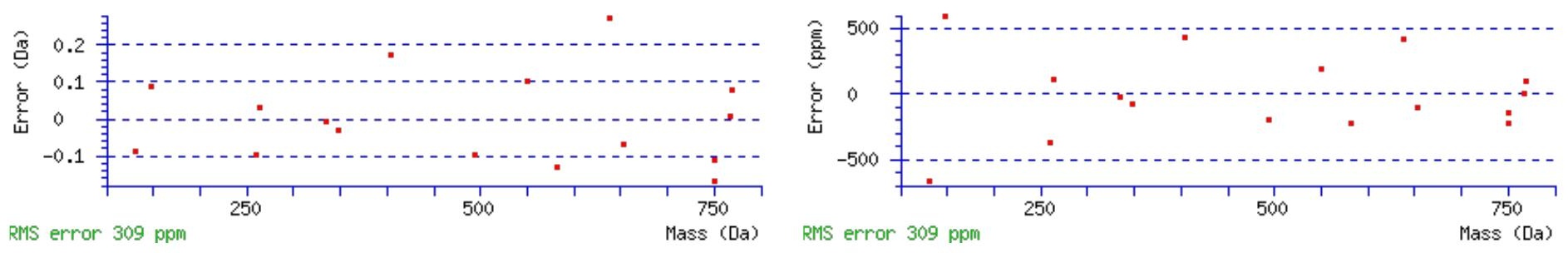

\section{All matches to this query}

\begin{tabular}{|l|l|l|l|}
\hline Score & Mr(calc): & Delta & Sequence \\
\hline 38.3 & 913.4215 & -0.0017 & MNASFSLK \\
\hline 12.5 & 913.4211 & -0.0013 & FPTWRK \\
\hline 12.5 & 913.4181 & 0.0017 & FDKFNDK \\
\hline 10.4 & 913.4156 & 0.0041 & FWMTWK \\
\hline 7.7 & 913.4181 & 0.0017 & NFFNKNK \\
\hline 7.7 & 912.4123 & 1.0075 & TYCRANK \\
\hline 6.5 & 913.4158 & 0.0040 & ETGSLSLK \\
\hline 6.3 & 913.4158 & 0.0040 & $\underline{\text { ATVSESLK }}$ \\
\hline 4.6 & 912.4106 & 1.0092 & FPSVQQK \\
\hline 4.5 & 913.4116 & 0.0082 & $\underline{\text { FGCXEVR }}$ \\
\hline
\end{tabular}

Spectrum No: 880; Query: 34; Rank: 1

\section{Peptide View}

MS/MS Fragmentation of NLTEVVPR

Found in IPI00361106, Tax_Id=10116 Gene_Symbol=RGD1560062_predicted hypothetical protein LOC309816

Match to Query 34: 927.504968 from(464.759760,2+)

Title: 091008RatKidney_NH4Format01_24.1665.1665.2.dta

Data file K:INewmanPaper|Piliang|3SubProteomes\Piliang3SP\mgf5ppm\ERLIC_3SubProteomes5ppm.mgf 

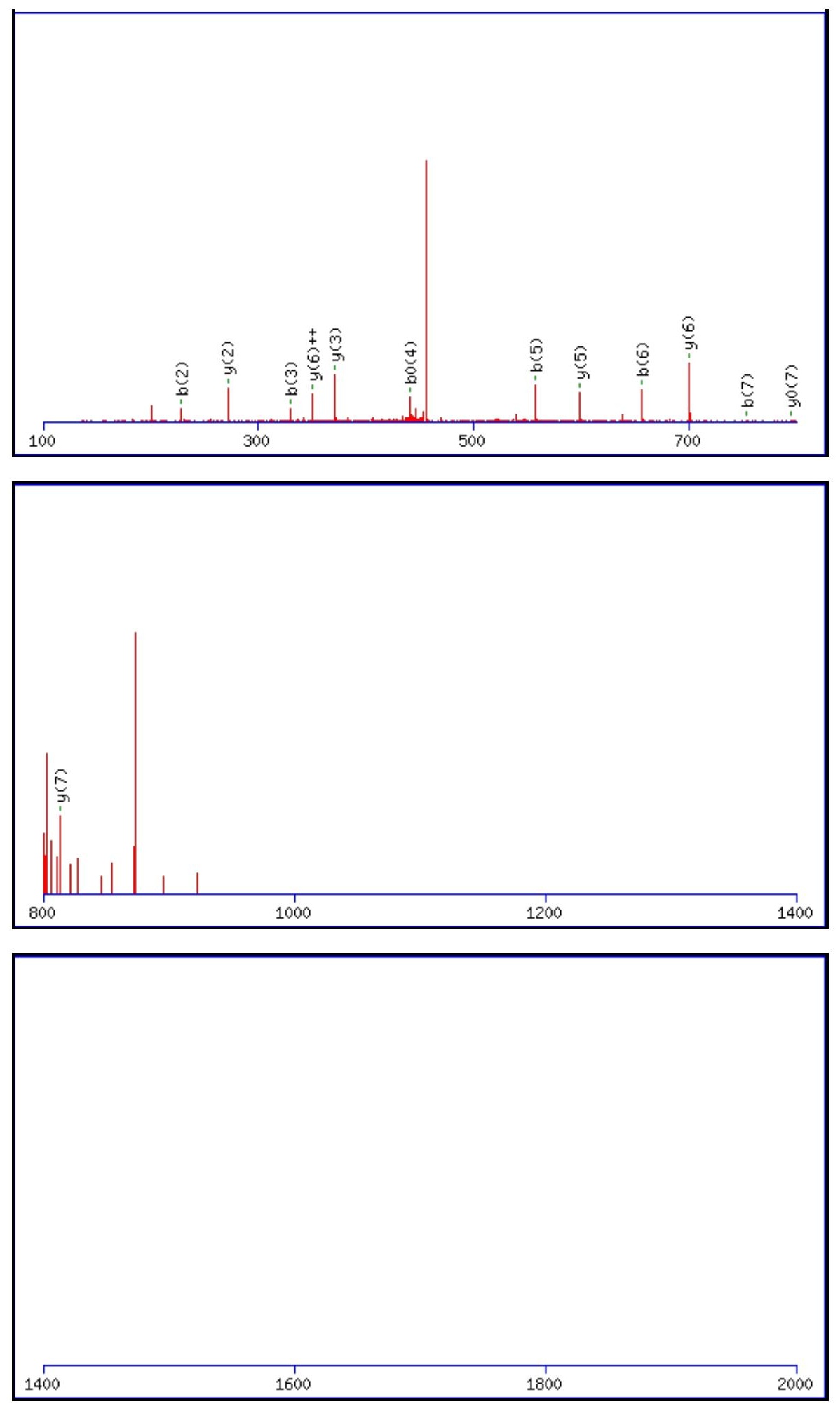

Monoisotopic mass of neutral peptide $\operatorname{Mr}($ calc): 927.5025

Fixed modifications: Carbamidomethyl (C)

Variable modifications:

N1 : Deamidated_N (N)

Ions Score: 38 Expect: 0.0084

Matches (Bold Red): 13/72 fragment ions using 27 most intense peaks

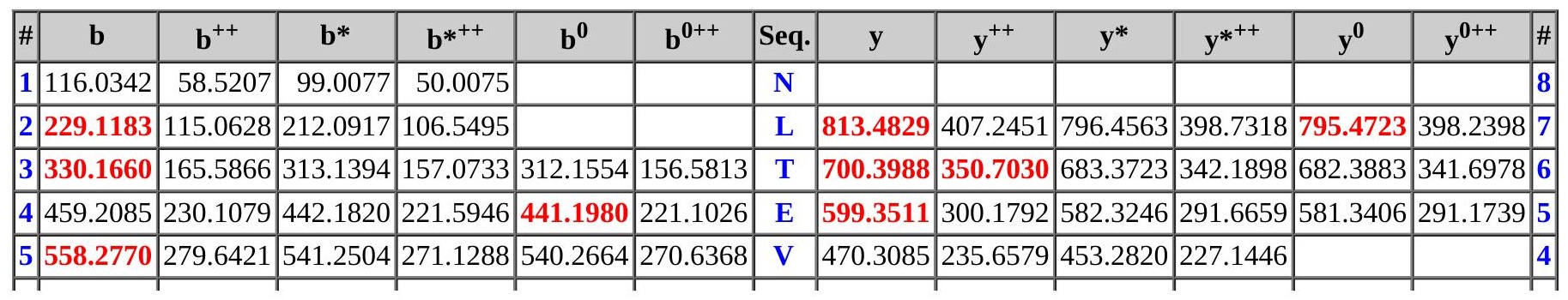




\begin{tabular}{|r|r|r|r|r|r|r|r|r|r|r|r|r|r|r|r|r|r|r|}
$\mathbf{6}$ & $\mathbf{6 5 7 . 3 4 5 4}$ & 329.1763 & 640.3188 & 320.6631 & 639.3348 & 320.1710 & $\mathbf{V}$ & 371.2401 & 186.1237 & 354.2136 & 177.6104 & & & $\mathbf{3}$ \\
\hline $\mathbf{7}$ & $\mathbf{7 5 4 . 3 9 8 1}$ & 377.7027 & 737.3716 & 369.1894 & 736.3876 & 368.6974 & $\mathbf{P}$ & 272.1717 & 136.5895 & 255.1452 & 128.0762 & & & $\mathbf{2}$ \\
\hline $\mathbf{8}$ & & & & & & & $\mathbf{R}$ & 175.1190 & 88.0631 & 158.0924 & 79.5498 & & & $\mathbf{1}$ \\
\hline
\end{tabular}
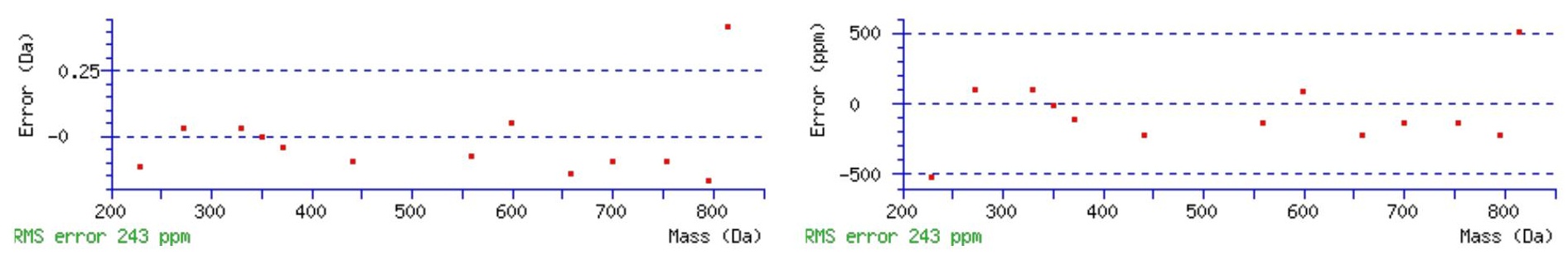

\section{All matches to this query}

\begin{tabular}{|l|l|l|l|}
\hline Score & Mr(calc): & Delta & \multicolumn{1}{|c|}{ Sequence } \\
\hline 38.3 & 927.5025 & 0.0024 & NLTEVVPR \\
\hline 13.5 & 927.5137 & -0.0088 & EAVAAAQLR \\
\hline 13.0 & 927.5138 & -0.0088 & VTGIGPSAAR \\
\hline 11.2 & 927.5025 & 0.0025 & LNNSLLPR \\
\hline 10.6 & 927.5025 & 0.0024 & VEPTIVDR \\
\hline 10.5 & 927.5039 & 0.0011 & RATWAAPR \\
\hline 8.4 & 926.4934 & 1.0116 & NLGVSPQGR \\
\hline 8.0 & 927.5025 & 0.0024 & DLTPONLK \\
\hline 7.3 & 927.5025 & 0.0024 & DLDPIVTR \\
\hline 7.2 & 927.5025 & 0.0024 & DLSLVPER \\
\hline
\end{tabular}

Spectrum No: 881; Query: 1820; Rank: 1

\section{Peptide View}

MS/MS Fragmentation of LWLPVNLTWADLEDKDGR

Found in IPI00208183, Tax_Id=10116 Gene_Symbol=Lass2 Longevity assurance homolog 2

Match to Query 1820: 2141.078472 from(714.700100,3+)

Title: 091008RatKidney_NoSalt_19.5971.5971.3.dta

Data file K:INewmanPaper|Piliang|3SubProteomes\Piliang3SP\mgf5ppm\ERLIC_3SubProteomes5ppm.mgf

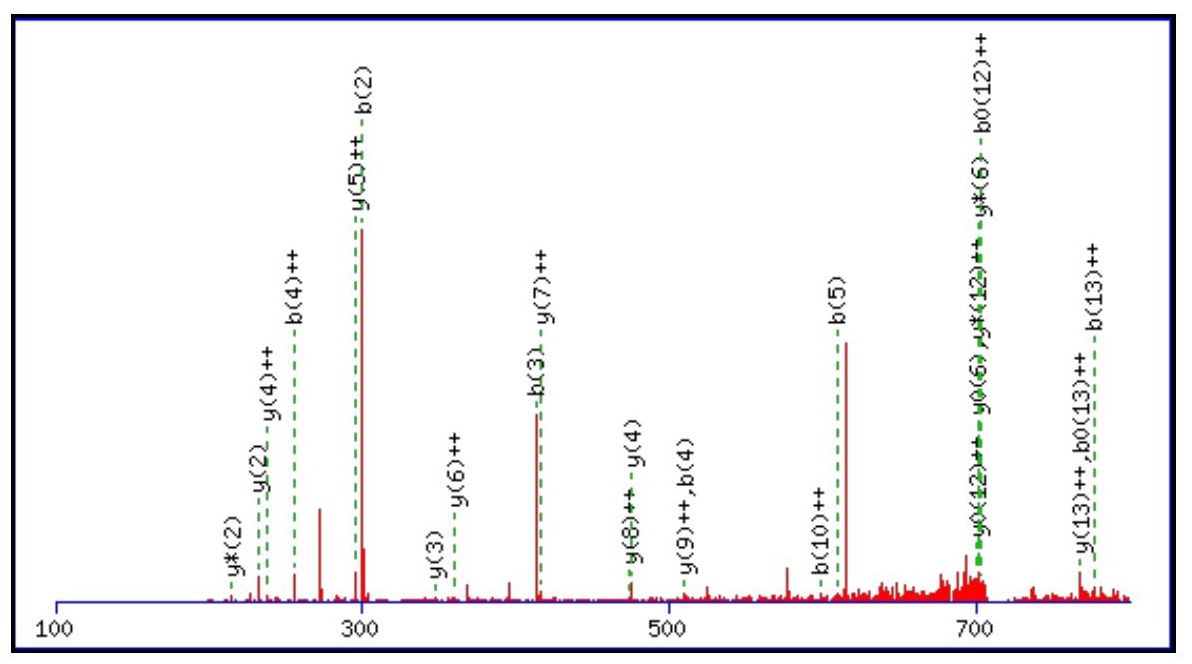



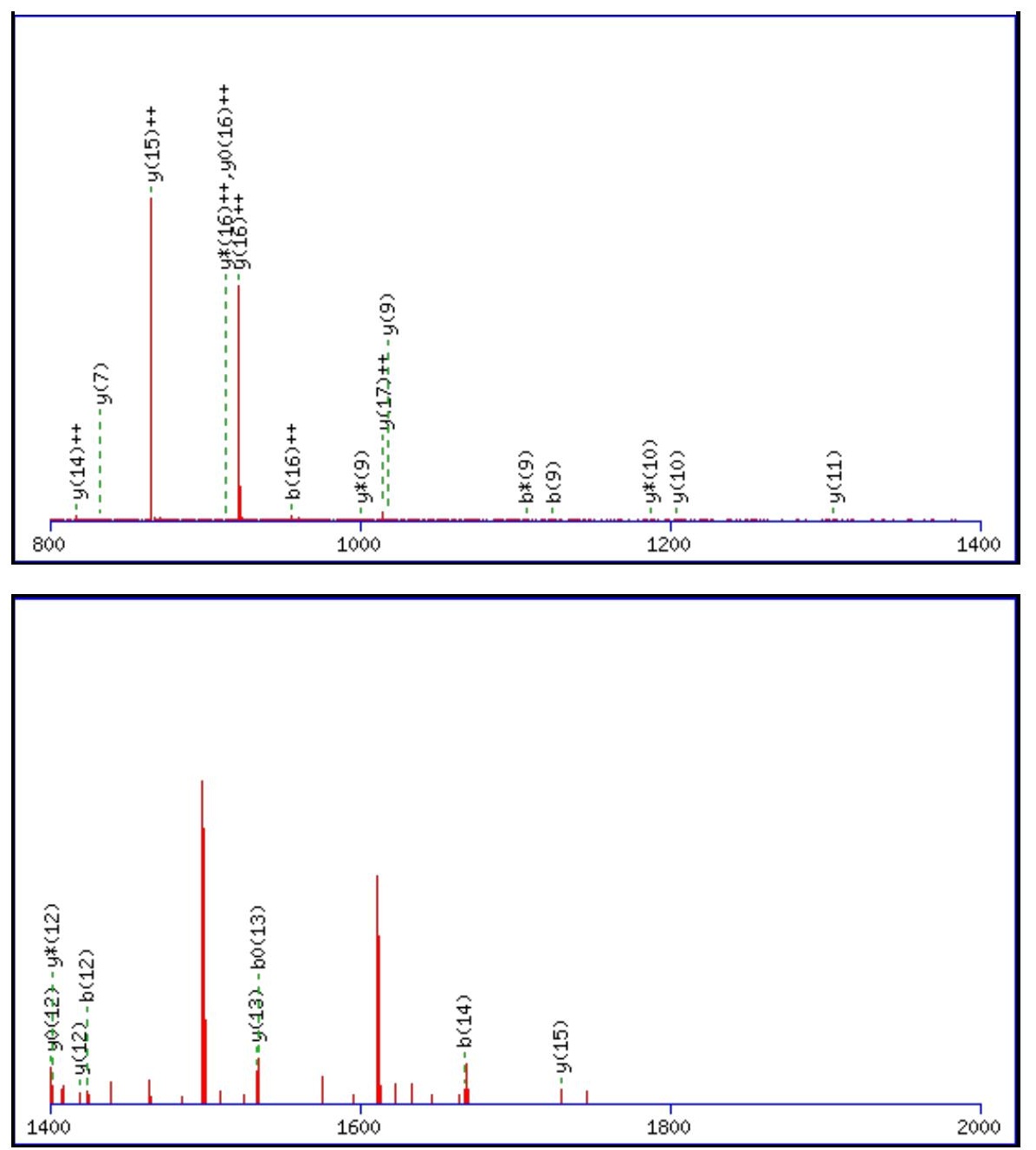

Monoisotopic mass of neutral peptide $\operatorname{Mr}($ calc): 2141.0793

Fixed modifications: Carbamidomethyl (C)

Variable modifications:

N6 : Deamidated_N (N)

Ions Score: 38 Expect: $\odot .028$

Matches (Bold Red): 47/176 fragment ions using 168 most intense peaks

\begin{tabular}{|c|c|c|c|c|c|c|c|c|c|c|c|c|c|c|}
\hline \# & b & $\mathbf{b}^{++}$ & $\mathbf{b}^{*}$ & $\mathbf{b}^{*^{++}}$ & $\mathbf{b}^{0}$ & $\mathbf{b}^{0++}$ & Seq. & $\mathbf{y}$ & $y^{++}$ & $\mathrm{y}^{*}$ & $\mathrm{y}^{*^{++}}$ & $\mathbf{y}^{0}$ & $y^{0++}$ & \\
\hline 1 & 114.0913 & 57.5493 & & & & & $\mathbf{L}$ & & & & & & & 18 \\
\hline 2 & 300.1707 & 150.5890 & & & & & $\mathbf{W}$ & 2029.0025 & 1015.0049 & 2011.9760 & 1006.4916 & 2010.9920 & 1005.9996 & 17 \\
\hline 3 & 413.2547 & 207.1310 & & & & & $\mathbf{L}$ & 1842.9232 & 921.9652 & 1825.8967 & 913.4520 & 1824.9126 & 912.9600 & 16 \\
\hline 4 & 510.3075 & 255.6574 & & & & & $\mathbf{P}$ & 1729.8391 & 865.4232 & 1712.8126 & 856.9099 & 1711.8286 & 856.4179 & 1 \\
\hline 5 & 609.3759 & 305.1916 & & & & & $\mathrm{~V}$ & 1632.7864 & 816.8968 & 1615.7598 & 808.3836 & 1614.7758 & 807.8915 & 1 \\
\hline 6 & 724.4028 & 362.7051 & 707.3763 & 354.1918 & & & $\mathbf{N}$ & 1533.7180 & 767.3626 & 1516.6914 & 758.8493 & 1515.7074 & 758.3573 & \\
\hline 7 & 837.4869 & 419.2471 & 820.4603 & 410.7338 & & & $\mathbf{L}$ & 1418.6910 & 709.8492 & 1401.6645 & 701.3359 & 1400.6805 & 700.8439 & 1 \\
\hline 8 & 938.5346 & 469.7709 & 921.5080 & 461.2577 & 920.5240 & 460.7656 & $\mathbf{T}$ & 1305.6070 & 653.3071 & 1288.5804 & 644.7938 & 1287.5964 & 644.3018 & 11 \\
\hline 9 & 1124.6139 & 562.8106 & 1107.5873 & 554.2973 & 1106.6033 & 553.8053 & $\mathbf{W}$ & 1204.5593 & 602.7833 & 1187.5327 & 594.2700 & 1186.5487 & 593.7780 & 10 \\
\hline 10 & 1195.6510 & \begin{tabular}{|l|}
598.3291 \\
\end{tabular} & 1178.6245 & 589.8159 & 1177.6404 & 589.3239 & A & 1018.4800 & 509.7436 & 1001.4534 & 501.2304 & 1000.4694 & 500.7383 & \\
\hline 11 & 1310.6779 & 655.8426 & 1293.6514 & 647.3293 & 1292.6674 & 646.8373 & D & 947.4429 & 474.2251 & 930.4163 & 465.7118 & 929.4323 & 465.2198 & \\
\hline 12 & 1423.7620 & 712.3846 & 1406.7355 & 703.8714 & 1405.7514 & 703.3794 & $\mathbf{L}$ & 832.4159 & 416.7116 & 815.3894 & 408.1983 & 814.4054 & 407.7063 & \\
\hline 13 & 1552.8046 & \begin{tabular}{|l|}
776.9059 \\
\end{tabular} & 1535.7781 & 768.3927 & \begin{tabular}{|l}
1534.7940 \\
\end{tabular} & \begin{tabular}{|l|l|}
767.9007 \\
\end{tabular} & $\mathbf{E}$ & 719.3319 & 360.1696 & 702.3053 & 351.6563 & 701.3213 & 351.1643 & \\
\hline 14 & 1667.8315 & 834.4194 & 1650.8050 & 825.9061 & 1649.8210 & 825.4141 & D & 590.2893 & 295.6483 & 573.2627 & 287.1350 & 572.2787 & 286.6430 & \\
\hline 15 & 1795.9265 & 898.4669 & 1778.9000 & 889.9536 & 1777.9159 & 889.4616 & $\mathbf{K}$ & 475.2623 & 238.1348 & 458.2358 & 229.6215 & 457.2518 & 229.1295 & 4 \\
\hline 16 & 1910.9535 & 955.9804 & 1893.9269 & 947.4671 & 1892.9429 & 946.9751 & D & 347.1674 & 174.0873 & 330.1408 & 165.5740 & 329.1568 & 165.0820 & 3 \\
\hline 17 & 1967.9749 & 984.4911 & 1950.9484 & 975.9778 & 1949.9643 & 975.4858 & G & 232.1404 & 116.5738 & 215.1139 & 108.0606 & & & \\
\hline 18 & & & & & & & $\mathbf{R}$ & 175.1190 & 88.0631 & 158.0924 & 79.5498 & & & \\
\hline
\end{tabular}



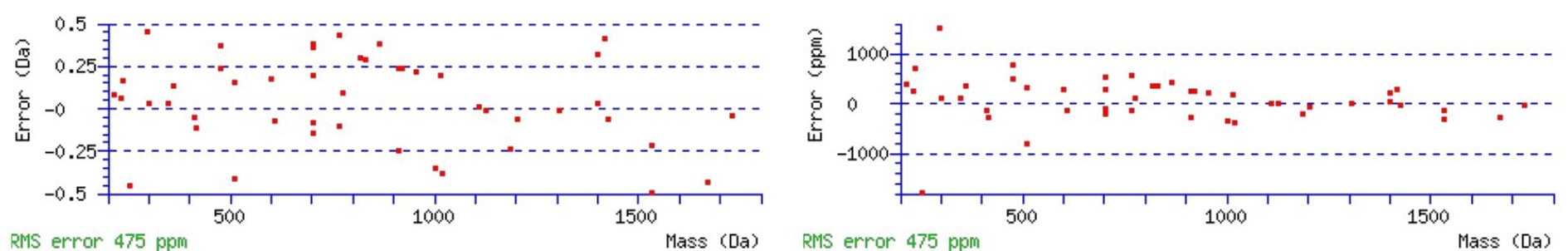

RMS error 475 ppm

\section{All matches to this query}

\begin{tabular}{|l|l|l|l|}
\hline Score & Mr(calc): & Delta & \multicolumn{1}{|c|}{ Sequence } \\
\hline 38.1 & 2141.0793 & -0.0008 & LWLPVNLTWADLEDKDGR \\
\hline 8.1 & 2141.0939 & -0.0154 & VTVLVTPFQPTVEALVLR \\
\hline 5.8 & 2141.0865 & -0.0080 & LGGAILWLSGQAETQDQGAAR \\
\hline 5.8 & 2140.0598 & 1.0186 & LSLEEELSRMKAAVLSER \\
\hline 5.8 & 2140.0598 & 1.0186 & LSLEEELSRMKAAVLSER \\
\hline 5.7 & 2141.0839 & -0.0054 & LSLLTKVWNHIKPYIR \\
\hline 5.7 & 2141.0769 & 0.0016 & LSLYVADENRELALVQTK \\
\hline 4.9 & 2139.0626 & 2.0159 & AQLLAASRELHKFFSDAR \\
\hline 4.8 & 2141.0793 & -0.0008 & DAIIAKLANQAADYFGDAFK \\
\hline 4.1 & 2141.0865 & -0.0080 & DNIKAGQQGLSVAFDLATHR \\
\hline
\end{tabular}

Spectrum No: 882; Query: 572; Rank: 1

\section{Peptide View}

MS/MS Fragmentation of NSTKEEILAALEK

Found in IPI00195160, Tax_Id=10116 Gene_Symbol=Psap Sulfated glycoprotein 1 precursor

Match to Query 572: 1445.766608 from(723.890580,2+)

Title: 091008RatKidney_NH4Format02_24.3329.3329.2.dta

Data file K:INewmanPaper|Piliangl3SubProteomes|Piliang3SP\mgf5ppm|ERLIC_3SubProteomes5ppm.mgf

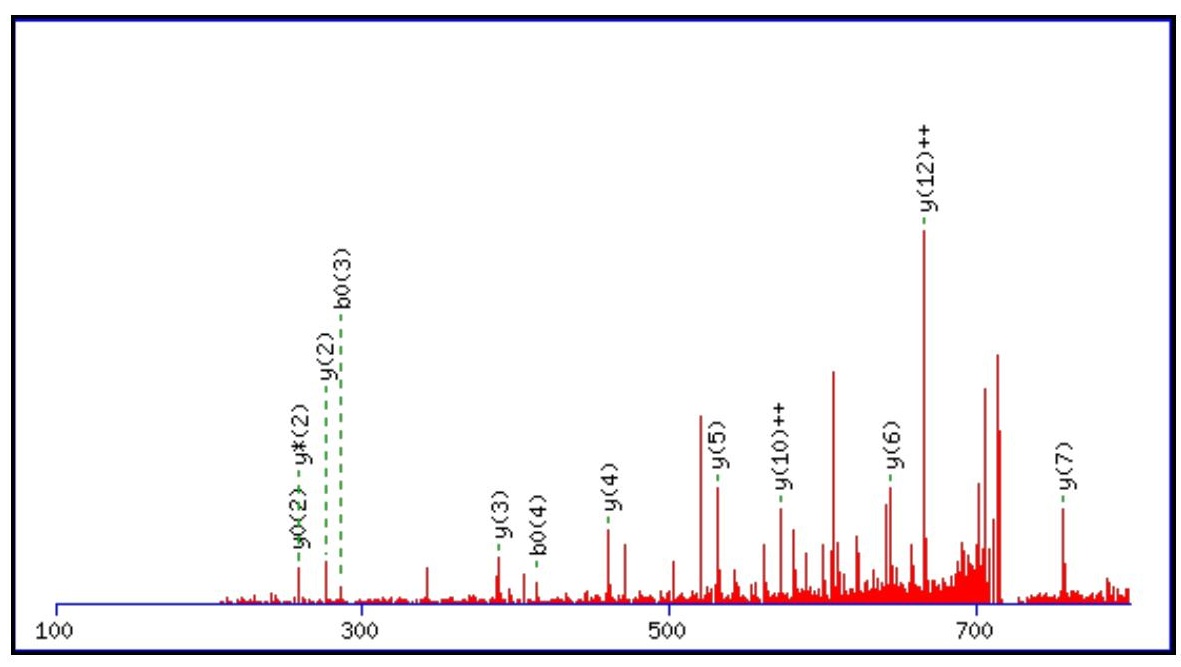



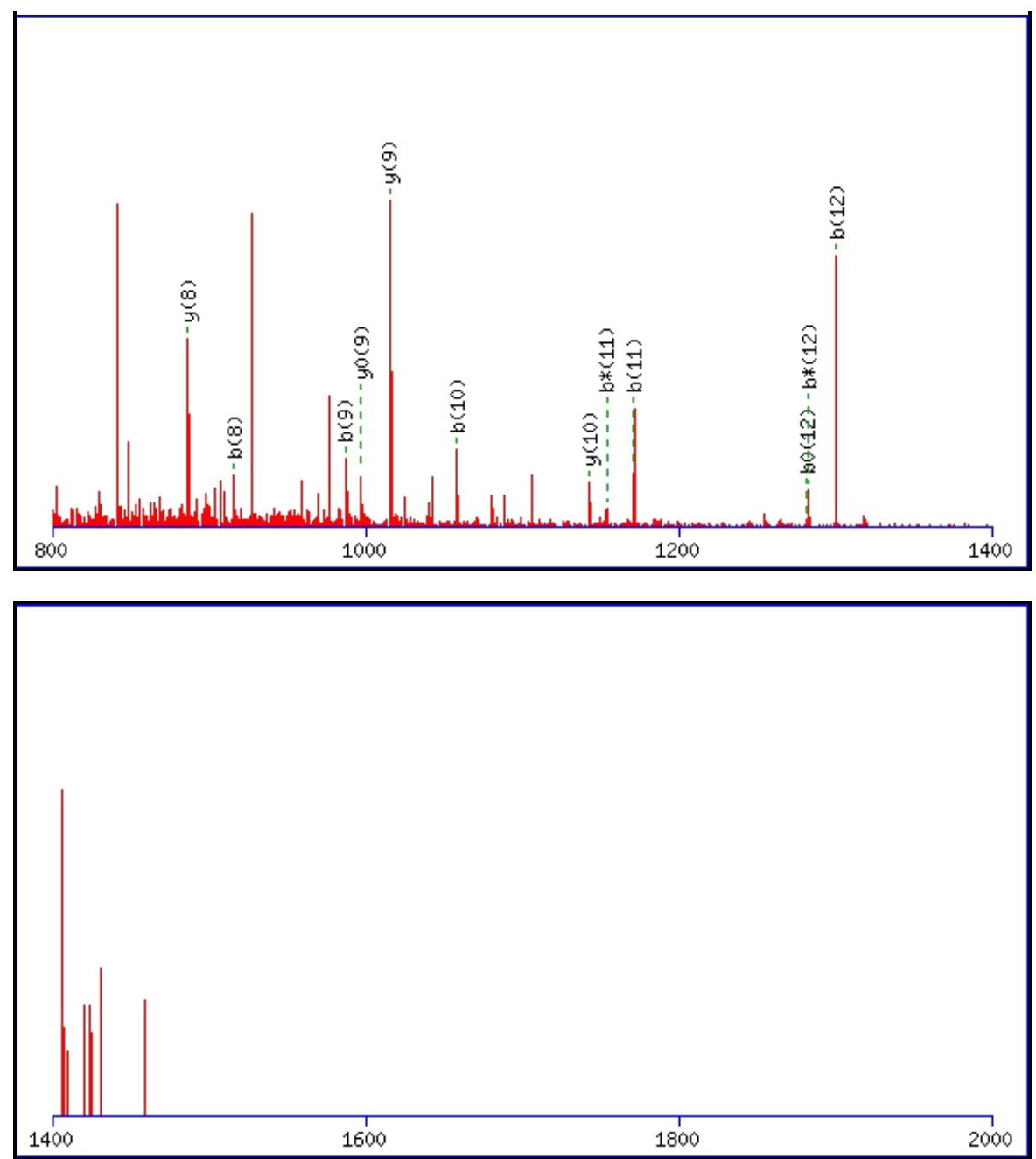

Monoisotopic mass of neutral peptide $\operatorname{Mr}($ calc): 1445.7613

Fixed modifications: Carbamidomethyl (C)

Variable modifications:

N1 : Deamidated $\mathrm{N}(\mathrm{N})$

Ions Score: 38 Expect: $\odot .018$

Matches (Bold Red): 24/140 fragment ions using 65 most intense peaks

\begin{tabular}{|c|c|c|c|c|c|c|c|c|c|c|c|c|c|c|}
\hline \# & b & $\mathbf{b}^{++}$ & $\mathbf{b}^{*}$ & $\mathbf{b}^{*^{++}}$ & $\mathbf{b}^{0}$ & $\mathbf{b}^{0++}$ & Seq. & $\mathbf{y}$ & $\mathbf{y}^{++}$ & $\mathbf{y}^{*}$ & $\mathbf{y}^{*^{++}}$ & $\mathbf{y}^{\mathbf{0}}$ & $\mathbf{y}^{\mathbf{0 + +}}$ & \# \\
\hline 1 & 116.0342 & 58.5207 & 99.0077 & 50.0075 & & & $\mathbf{N}$ & & & & & & & 13 \\
\hline 2 & 203.0662 & 102.0368 & 186.0397 & 93.5235 & 185.0557 & 93.0315 & S & 1331.7417 & 666.3745 & 1314.7151 & 657.8612 & 1313.7311 & 657.3692 & 12 \\
\hline 3 & 304.1139 & 152.5606 & 287.0874 & 144.0473 & 286.1034 & 143.5553 & $\mathbf{T}$ & 1244.7096 & 622.8585 & 1227.6831 & 614.3452 & 1226.6991 & 613.8532 & 11 \\
\hline 4 & 432.2089 & 216.6081 & 415.1823 & 208.0948 & 414.1983 & 207.6028 & $\mathbf{K}$ & 1143.6620 & 572.3346 & 1126.6354 & 563.8213 & 1125.6514 & 563.3293 & 10 \\
\hline 5 & 561.2515 & 281.1294 & 544.2249 & 272.6161 & 543.2409 & 272.1241 & $\mathbf{E}$ & 1015.5670 & 508.2871 & 998.5405 & 499.7739 & 997.5564 & 499.2819 & 9 \\
\hline 6 & 690.2941 & 345.6507 & 673.2675 & 337.1374 & 672.2835 & 336.6454 & $\mathbf{E}$ & 886.5244 & 443.7658 & 869.4979 & 435.2526 & 868.5138 & 434.7606 & 8 \\
\hline 7 & 803.3781 & 402.1927 & 786.3516 & 393.6794 & 785.3676 & 393.1874 & I & 757.4818 & 379.2445 & 740.4553 & 370.7313 & 739.4713 & 370.2393 & 7 \\
\hline 8 & 916.4622 & 458.7347 & 899.4356 & 450.2215 & 898.4516 & 449.7295 & $\mathbf{L}$ & 644.3978 & 322.7025 & 627.3712 & 314.1892 & 626.3872 & 313.6972 & 6 \\
\hline 9 & 987.4993 & 494.2533 & 970.4728 & 485.7400 & 969.4887 & 485.2480 & A & 531.3137 & 266.1605 & 514.2871 & 257.6472 & 513.3031 & 257.1552 & 5 \\
\hline 10 & 1058.5364 & 529.7719 & 1041.5099 & 521.2586 & 1040.5259 & 520.7666 & A & 460.2766 & 230.6419 & 443.2500 & 222.1287 & 442.2660 & 221.6366 & 4 \\
\hline 11 & 1171.6205 & 586.3139 & 1154.5939 & 577.8006 & 1153.6099 & 577.3086 & $\mathbf{L}$ & 389.2395 & 195.1234 & 372.2129 & 186.6101 & 371.2289 & 186.1181 & 3 \\
\hline 12 & 1300.6631 & 650.8352 & 1283.6365 & 642.3219 & 1282.6525 & 641.8299 & $\mathbf{E}$ & 276.1554 & 138.5813 & 259.1288 & 130.0681 & 258.1448 & 129.5761 & 2 \\
\hline 13 & & & & & & & $\mathbf{K}$ & 147.1128 & 74.0600 & 130.0863 & 65.5468 & & & 1 \\
\hline
\end{tabular}



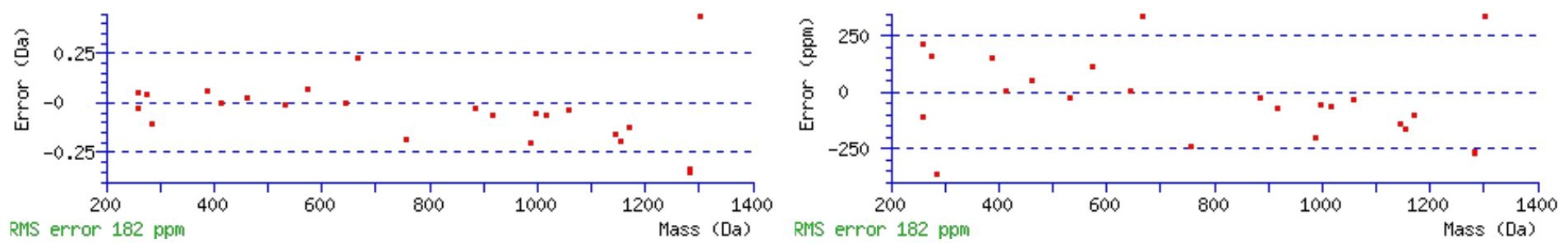

\section{All matches to this query}

\begin{tabular}{|l|l|l|l|}
\hline Score & Mr(calc): & Delta & \multicolumn{1}{|c|}{ Sequence } \\
\hline 38.1 & 1445.7613 & 0.0053 & NSTKEEILAALEK \\
\hline 20.6 & 1444.7773 & 0.9893 & NSTKEEILAALEK \\
\hline 17.4 & 1445.7766 & -0.0099 & KEILFIPSENEK \\
\hline 6.5 & 1445.7755 & -0.0089 & RPPAPLSSTGKKK \\
\hline 6.5 & 1445.7755 & -0.0089 & RPPAPLSSTGKKK \\
\hline 5.2 & 1445.7683 & -0.0017 & KFLLFTDTLLR \\
\hline 3.3 & 1445.7799 & -0.0133 & ELMGLKSNIAELK \\
\hline 3.1 & 1444.7551 & 1.0115 & LRTLERALEK \\
\hline 3.1 & 1445.7660 & 0.0006 & NCLVTEARTLLR \\
\hline 3.0 & 1445.7683 & -0.0017 & KFLLFTDTLLR \\
\hline
\end{tabular}

Spectrum No: 883; Query: 1733; Rank: 1

\section{Peptide View}

MS/MS Fragmentation of LAWGTTASAQNHTEPQLDR

Found in IPI00214328, Tax_Id=10116 Gene_Symbol=Naglt1 Sodium-dependent glucose transporter 1

Match to Query 1733: 2095.998822 from(699.673550,3+)

Title: 100101RatKid_NS_deglyco_26.2020.2020.3.dta

Data file K:INewmanPaper|Piliangl3SubProteomes\Piliang3SP\mgf5ppm|ERLIC_3SubProteomes5ppm.mgf

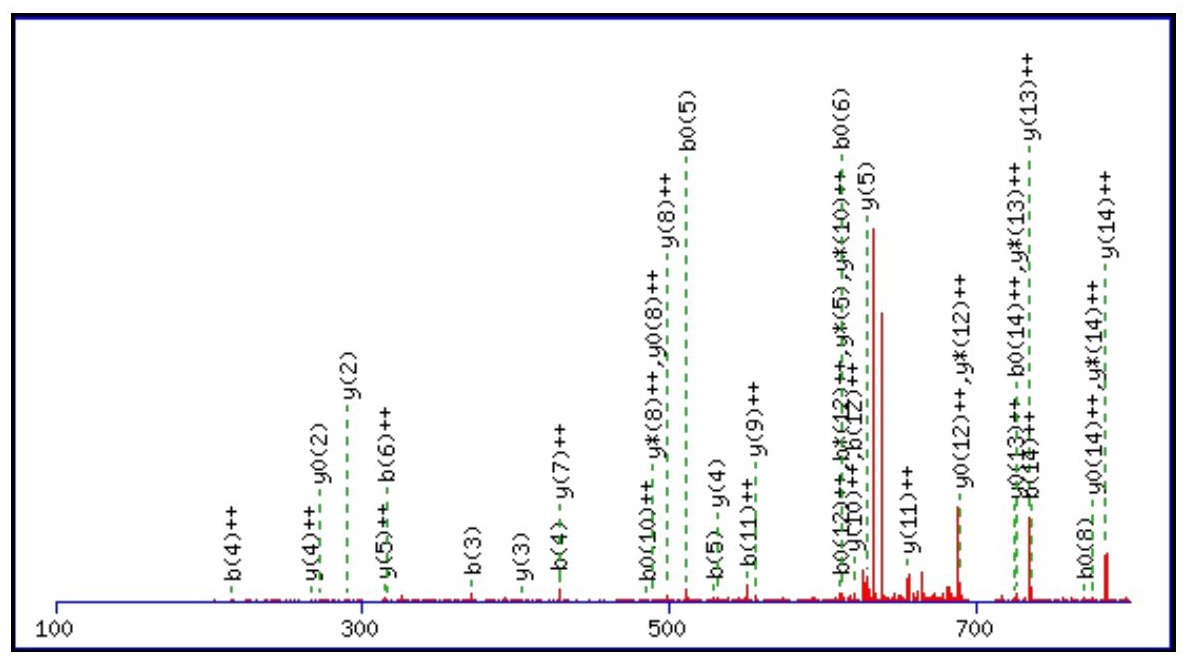



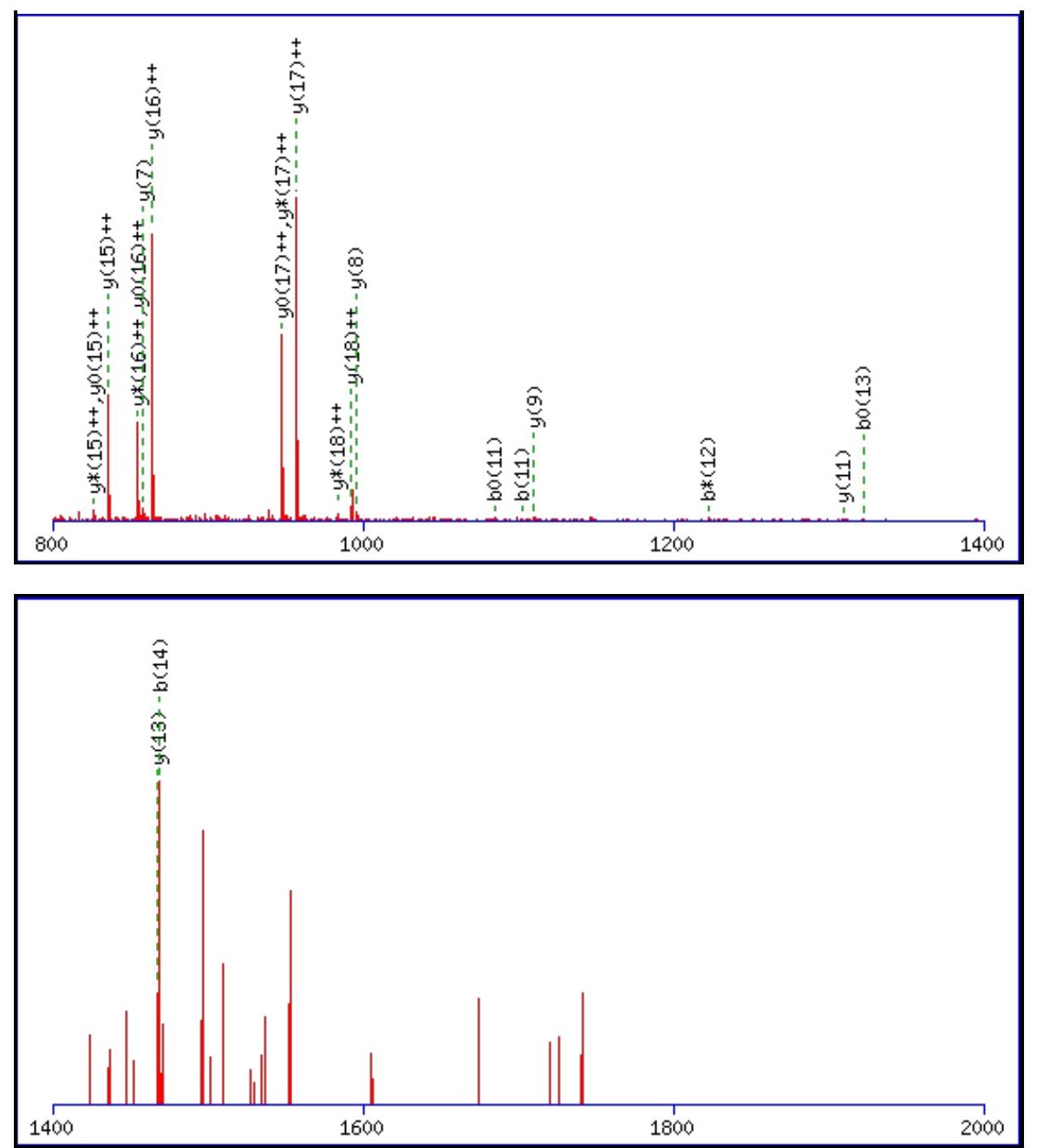

Monoisotopic mass of neutral peptide $\operatorname{Mr}($ calc): 2095.9923

Fixed modifications: Carbamidomethyl (C)

Variable modifications:

N11 : Deamidated_N (N)

Ions Score: 38 Expect: $\odot .038$

Matches (Bold Red): 60/188 fragment ions using 141 most intense peaks

\begin{tabular}{|c|c|c|c|c|c|c|c|c|c|c|c|c|c|c|}
\hline \# & b & $\mathbf{b}^{++}$ & b* & $\mathbf{b}^{*^{++}}$ & $\mathbf{b}^{0}$ & $\mathbf{b}^{0++}$ & Seq. & $\mathbf{y}$ & $\mathrm{y}^{++}$ & $\mathbf{y}^{*}$ & $\mathbf{y}^{*^{++}}$ & $\mathbf{y}^{\mathbf{0}}$ & $\mathbf{y}^{0++}$ & \# \\
\hline 1 & 114.0913 & 57.5493 & & & & & $\mathbf{L}$ & & & & & & & 19 \\
\hline 2 & 185.1285 & 93.0679 & & & & & A & 1983.9155 & 992.4614 & 1966.8890 & 983.9481 & 1965.9049 & 983.4561 & 18 \\
\hline 3 & 371.2078 & 186.1075 & & & & & $\mathbf{W}$ & 1912.8784 & 956.9428 & 1895.8518 & 948.4296 & 1894.8678 & 947.9376 & 17 \\
\hline 4 & 428.2292 & 214.6183 & & & & & G & 1726.7991 & 863.9032 & 1709.7725 & 855.3899 & 1708.7885 & 854.8979 & 16 \\
\hline 5 & 529.2769 & 265.1421 & & & 511.2663 & 256.1368 & $\mathbf{T}$ & 1669.7776 & 835.3924 & 1652.7511 & 826.8792 & 1651.7670 & 826.3872 & 15 \\
\hline 6 & 630.3246 & 315.6659 & & & 612.3140 & 306.6607 & $\mathbf{T}$ & 1568.7299 & 784.8686 & 1551.7034 & 776.3553 & 1550.7194 & 775.8633 & 14 \\
\hline 7 & 701.3617 & 351.1845 & & & 683.3511 & 342.1792 & A & 1467.6823 & 734.3448 & 1450.6557 & 725.8315 & 1449.6717 & 725.3395 & 13 \\
\hline 8 & 788.3937 & 394.7005 & & & 770.3832 & 385.6952 & S & 1396.6451 & 698.8262 & 1379.6186 & 690.3129 & 1378.6346 & 689.8209 & 12 \\
\hline 9 & 859.4308 & 430.2191 & & & 841.4203 & 421.2138 & A & 1309.6131 & 655.3102 & 1292.5866 & 646.7969 & 1291.6025 & 646.3049 & 11 \\
\hline 10 & 987.4894 & 494.2483 & 970.4629 & 485.7351 & 969.4789 & 485.2431 & $\mathbf{Q}$ & 1238.5760 & 619.7916 & 1221.5495 & 611.2784 & 1220.5654 & 610.7864 & 10 \\
\hline 11 & 1102.5164 & 551.7618 & 1085.4898 & 543.2485 & 1084.5058 & 542.7565 & $\mathbf{N}$ & 1110.5174 & 555.7623 & 1093.4909 & 547.2491 & 1092.5069 & 546.7571 & 9 \\
\hline 12 & 1239.5753 & 620.2913 & 1222.5487 & 611.7780 & 1221.5647 & 611.2860 & $\mathbf{H}$ & 995.4905 & 498.2489 & 978.4639 & 489.7356 & 977.4799 & 489.2436 & 8 \\
\hline 13 & 1340.6229 & 670.8151 & 1323.5964 & 662.3018 & 1322.6124 & 661.8098 & $\mathbf{T}$ & 858.4316 & 429.7194 & 841.4050 & 421.2062 & 840.4210 & 420.7141 & 7 \\
\hline 14 & 1469.6655 & 735.3364 & 1452.6390 & 726.8231 & 1451.6550 & 726.3311 & $\mathbf{E}$ & 757.3839 & 379.1956 & 740.3573 & 370.6823 & 739.3733 & 370.1903 & 6 \\
\hline 15 & 1566.7183 & 783.8628 & 1549.6918 & 775.3495 & 1548.7077 & 774.8575 & $\mathbf{P}$ & 628.3413 & 314.6743 & 611.3148 & 306.1610 & 610.3307 & 305.6690 & 5 \\
\hline 16 & 1694.7769 & 847.8921 & 1677.7503 & 839.3788 & 1676.7663 & 838.8868 & $\mathbf{Q}$ & 531.2885 & 266.1479 & 514.2620 & 257.6346 & 513.2780 & 257.1426 & 4 \\
\hline 17 & 1807.8609 & 904.4341 & 1790.8344 & 895.9208 & 1789.8504 & 895.4288 & $\mathbf{L}$ & 403.2300 & 202.1186 & 386.2034 & 193.6053 & 385.2194 & 193.1133 & 3 \\
\hline 18 & 1922.8879 & 961.9476 & 1905.8613 & 953.4343 & 1904.8773 & 952.9423 & D & 290.1459 & 145.5766 & 273.1193 & 137.0633 & 272.1353 & 136.5713 & 2 \\
\hline
\end{tabular}




\section{9|}

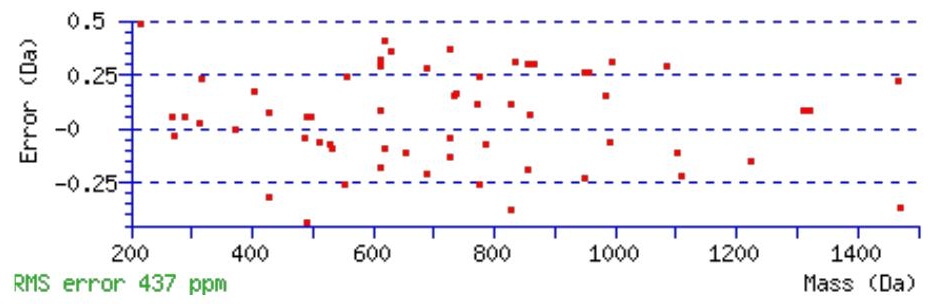

\section{All matches to this query}

\begin{tabular}{|l|l|l|l|}
\hline Score & Mr(calc): & Delta & \multicolumn{1}{|c|}{ Sequence } \\
\hline 38.0 & 2095.9923 & 0.0065 & LAWGTTASAQNHTEPQLDR \\
\hline 10.3 & 2095.0083 & 0.9906 & LAWGTTASAQNHTEPQLDR \\
\hline 3.6 & 2095.9802 & 0.0186 & HPSPEALSFPTFSLLMSR \\
\hline 1.9 & 2095.9891 & 0.0098 & VMASRVRATVLFATETGK \\
\hline 1.6 & 2094.9818 & 1.0170 & RDMCSTMFIAALFIIAR \\
\hline 0.9 & 2095.9891 & 0.0098 & VMASRVRATVLFATETGK \\
\hline 0.9 & 2096.0166 & -0.0178 & LLSGGKPPAQEWFMVQTK \\
\hline 0.8 & 2096.0109 & -0.0121 & IGTVGQLNNXDICEFRGR \\
\hline 0.8 & 2095.9779 & 0.0209 & IGTVGQLNNXDICEFRGR \\
\hline 0.7 & 2093.9813 & 2.0175 & GAVRLPXGPSGSLWGPLGGR \\
\hline
\end{tabular}

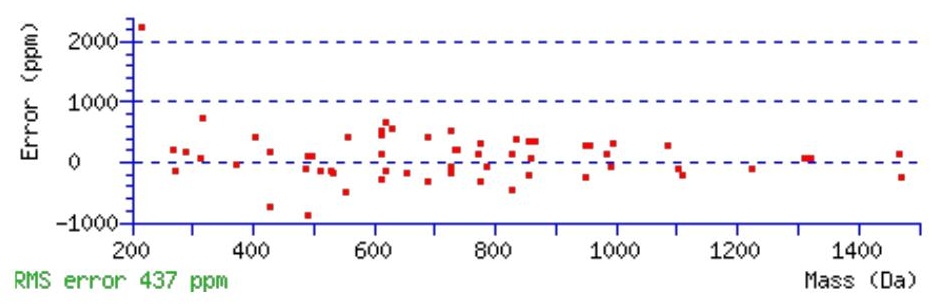

Spectrum No: 884; Query: 1284; Rank: 1

\section{Peptide View}

MS/MS Fragmentation of QDPVSWNKTFEDISR

Found in IPI00191437, Tax_Id=10116 Gene_Symbol=- 64 kDa protein

Match to Query 1284: 1821.854442 from(608.292090,3+)

Title: 100101RatKid_NS_deglyco_20.3393.3393.3.dta

Data file K:INewmanPaper|Piliangl3SubProteomes\Piliang3SP\mgf5ppm\ERLIC_3SubProteomes5ppm.mgf

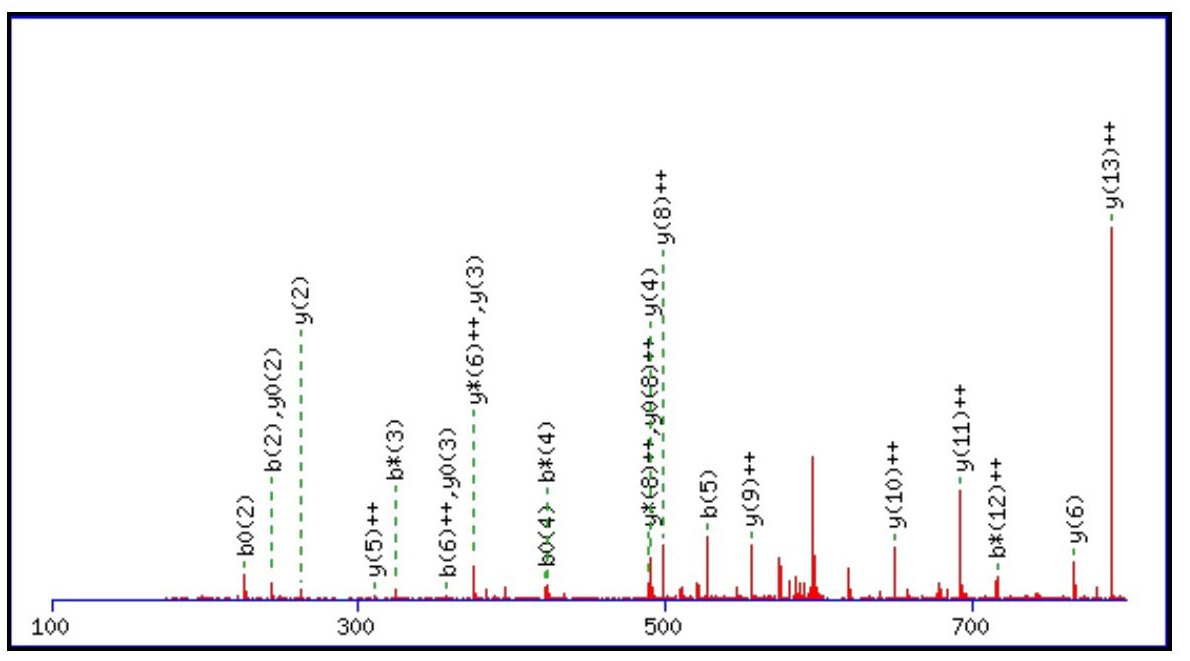



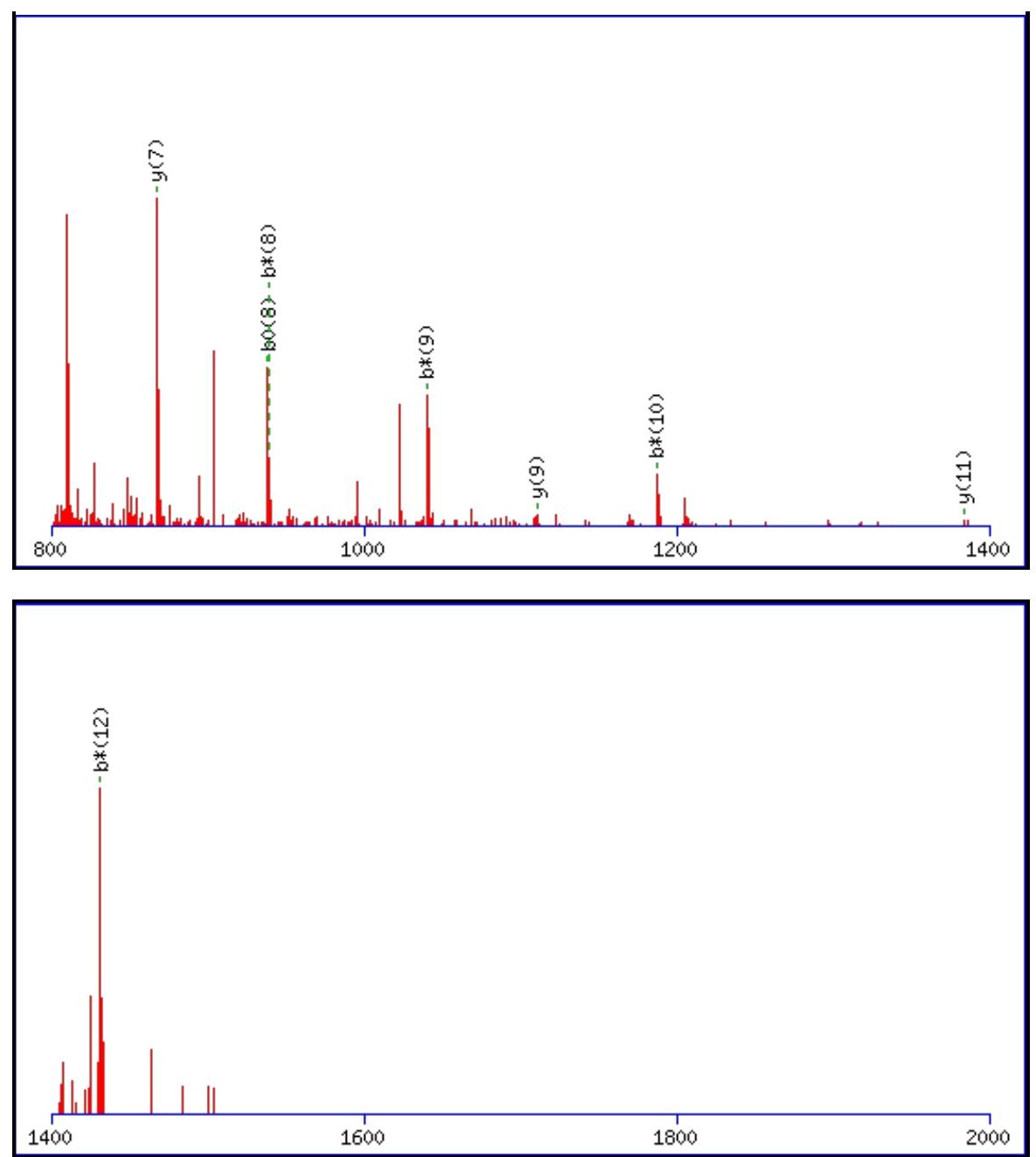

Monoisotopic mass of neutral peptide $\operatorname{Mr}($ calc): 1821.8533

Fixed modifications: Carbamidomethyl (C)

Variable modifications:

N7 : Deamidated_N (N)

Ions Score: 38 Expect : $\odot .034$

Matches (Bold Red): 31/164 fragment ions using 57 most intense peaks

\begin{tabular}{|c|c|c|c|c|c|c|c|c|c|c|c|c|c|c|}
\hline \# & b & $\mathbf{b}^{++}$ & $\mathbf{b}^{*}$ & $\mathbf{b}^{*^{++}}$ & $\mathbf{b}^{0}$ & $\mathbf{b}^{0++}$ & Seq. & $\mathbf{y}$ & $\mathbf{y}^{++}$ & $\mathbf{y}^{*}$ & $\mathbf{y}^{*^{++}}$ & $\mathbf{y}^{0}$ & & \# \\
\hline 1 & 129.0659 & 65.0366 & 112.0393 & 56.5233 & & & $\mathbf{Q}$ & & & & & & & 15 \\
\hline 2 & 244.0928 & 122.5500 & 227.0662 & 114.0368 & 226.0822 & 113.5448 & D & 1694.8020 & 847.9047 & 1677.7755 & & 1676.7915 & & 14 \\
\hline 3 & 341.1456 & 171.0764 & 324.1190 & 162.5631 & 323.1350 & 162.0711 & $\mathbf{P}$ & 751 & 790.3912 & 1562.7485 & 781.8779 & 1561.7645 & 781.3859 & 13 \\
\hline 4 & 440.2140 & 220.6106 & 423.1874 & 212.0974 & 422.2034 & 211.6053 & V & 1482.7223 & 741.8648 & 1465.6958 & 733.3515 & 1464.7118 & 732.8595 & 12 \\
\hline 5 & 527.2460 & 264.1266 & 510.2195 & 255.6134 & 509.2354 & 255.1214 & S & 1383.6539 & 692.3306 & 1366.6274 & 683.8173 & 1365.6433 & 683.3253 & 11 \\
\hline 6 & 713.3253 & 357.1663 & 696.2988 & 348.6530 & 695.3148 & 348.1610 & $\mathbf{W}$ & 1296.6219 & 648.8146 & 1279.5953 & 640.3013 & 1278.6113 & 639.8093 & 10 \\
\hline 7 & 828.3523 & 414.6798 & 811.3257 & 406.1665 & 810.3417 & 405.6745 & $\mathbf{N}$ & 1110.5426 & 555.7749 & 1093.5160 & 547.2616 & 1092.5320 & 546.7696 & 9 \\
\hline 8 & 956.4472 & 478.7272 & 939.4207 & 470.2140 & 938.4367 & 469.7220 & $\mathbf{K}$ & 995.5156 & 498.2615 & 978.4891 & 489.7482 & 977.5051 & 489.2562 & 8 \\
\hline 9 & 1057.4949 & 529.2511 & 1040.4683 & 520.7378 & 1039.4843 & 520.2458 & $\mathbf{T}$ & 867.4207 & 434.2140 & 850.3941 & 425.7007 & 849.4101 & 425.2087 & 7 \\
\hline 10 & 1204.5633 & 602.7853 & 1187.5368 & 594.2720 & 1186.5527 & 593.7800 & $\mathbf{F}$ & 766.3730 & 383.6901 & 749.3464 & 375.1769 & 748.3624 & 374.6849 & 6 \\
\hline 11 & 1333.6059 & 667.3066 & 1316.5794 & 658.7933 & 1315.5953 & 658.3013 & $\mathbf{E}$ & 619.3046 & 310.1559 & 602.2780 & 301.6427 & 601.2940 & 301.1506 & 5 \\
\hline 12 & 1448.6328 & 724.8201 & 1431.6063 & 716.3068 & 1430.6223 & 715.8148 & D & 490.2620 & 245.6346 & 473.2354 & 237.1214 & 472.2514 & 236.6293 & 4 \\
\hline 13 & 1561.7169 & 781.3621 & 1544.6904 & 772.8488 & 1543.7063 & 772.3568 & I & 375.2350 & 188.1212 & 358.2085 & 179.6079 & 357.2245 & 179.1159 & 3 \\
\hline 14 & 1648.7489 & 824.8781 & 1631.7224 & 816.3648 & 1630.7384 & 815.8728 & $\mathbf{S}$ & 262.1510 & 131.5791 & 245.1244 & 123.0659 & 244.1404 & 122.5738 & 2 \\
\hline 15 & & & & & & & $\mathbf{R}$ & 175.1190 & 88.0631 & 158.0924 & 79.5498 & & & 1 \\
\hline
\end{tabular}



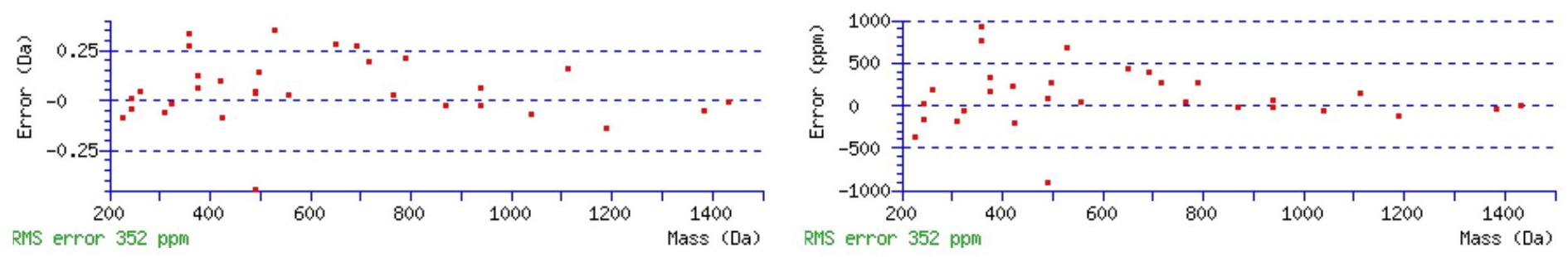

\section{All matches to this query}

\begin{tabular}{|l|l|l|l|}
\hline Score & Mr(calc): & Delta & \multicolumn{1}{|c|}{ Sequence } \\
\hline 38.0 & 1821.8533 & 0.0011 & QDPVSWNKTFEDISR \\
\hline 2.0 & 1819.8362 & 2.0183 & SMPGKPPGMDPIASALR \\
\hline 1.2 & 1819.8328 & 2.0216 & TTFMKRHLTGEFEK \\
\hline 0.8 & 1821.8662 & -0.0118 & GPSAPKSPGASNFSTLPK \\
\hline 0.7 & 1819.8383 & 2.0161 & QLDVFGQATAVLRSR \\
\hline 0.7 & 1821.8451 & 0.0093 & DPEKGPVPTFQPFQR \\
\hline 0.6 & 1821.8597 & -0.0052 & KSDFHSQMAVHKLAK \\
\hline 0.3 & 1820.8597 & 0.9947 & YSNSEESLLSKLFPK \\
\hline 0.2 & 1821.8696 & -0.0151 & DKPVAPSNVLSMAQLR \\
\hline 0.1 & 1821.8437 & 0.0107 & YSNSEESLLSKLFPK \\
\hline
\end{tabular}

Spectrum No: 885; Query: 2413; Rank: 1

\section{Peptide View}

MS/MS Fragmentation of VVSVDISFRPVNETFPVVYIENPK

Found in IPI00326140, Tax_Id=10116 Gene_Symbol=Pzp Alpha-1-macroglobulin precursor

Match to Query 2413: 2748.432642 from(917.151490,3+)

Title: 091008RatKidney_NoSalt_25.5270.5270.3.dta

Data file K:INewmanPaper|Piliang\3SubProteomes\Piliang3SP\mgf5ppm\ERLIC_3SubProteomes5ppm.mgf

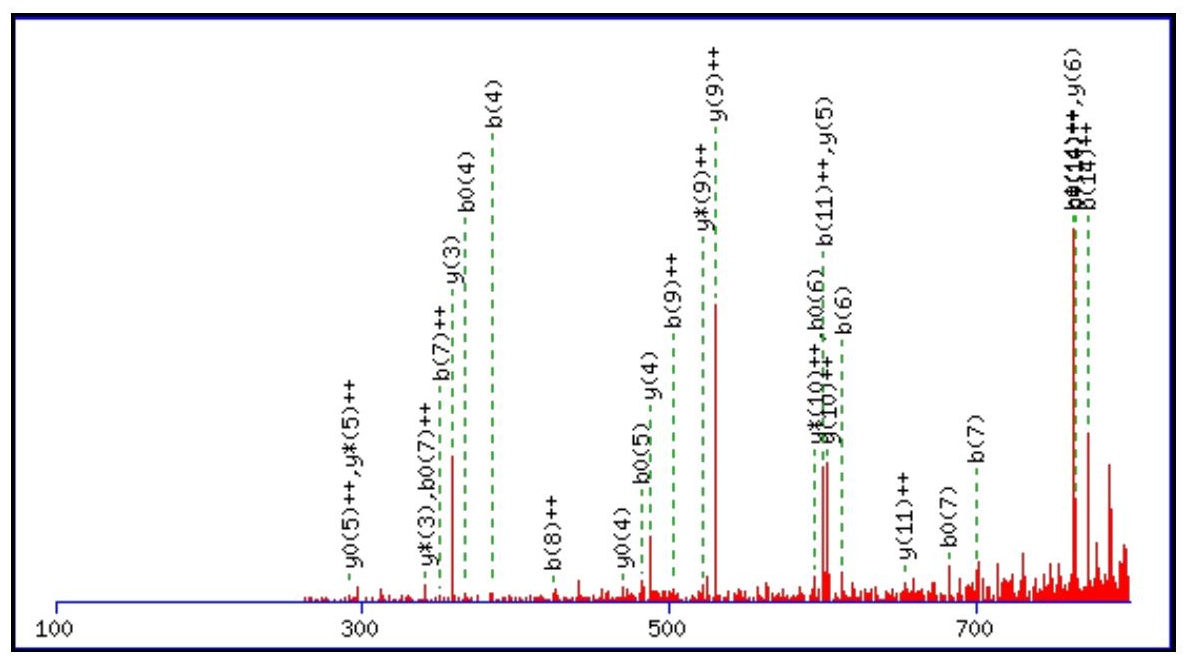



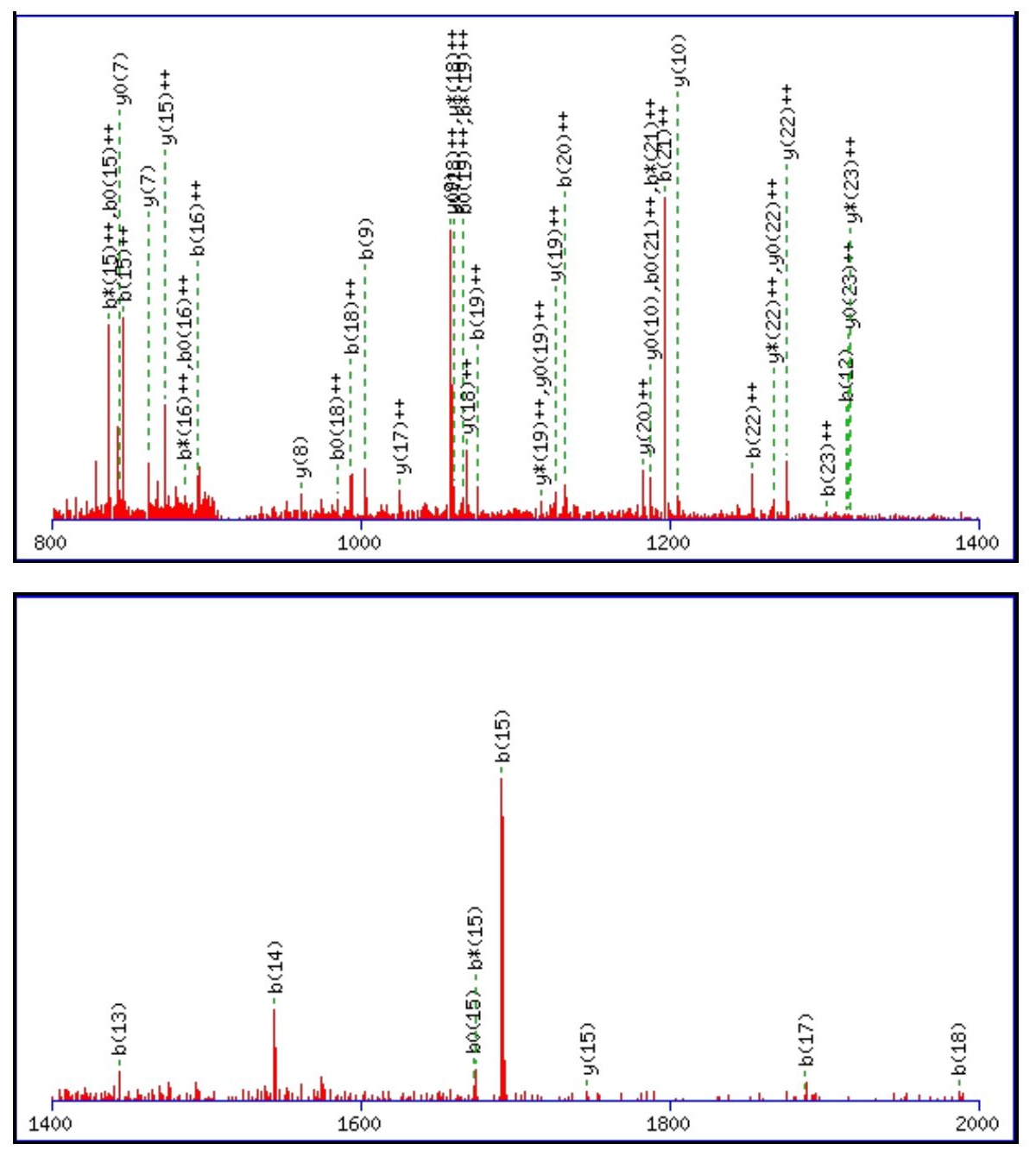

Monoisotopic mass of neutral peptide $\operatorname{Mr}($ calc): 2748.4374

Fixed modifications: Carbamidomethyl (C)

Variable modifications:

N12 : Deamidated_N (N)

Ions Score: 38 Expect: 0.02

Matches (Bold Red): 75/250 fragment ions using 195 most intense peaks

\begin{tabular}{|c|c|c|c|c|c|c|c|c|c|c|c|c|c|c|}
\hline \# & b & $\mathbf{b}^{++}$ & $\mathbf{b}^{*}$ & $\mathbf{b}^{*^{++}}$ & $\mathbf{b}^{0}$ & $\mathbf{b}^{0++}$ & Seq. & $\mathbf{y}$ & $y^{++}$ & $\mathbf{y}^{*}$ & $\mathrm{y}^{*^{++}}$ & $\mathbf{y}^{\mathbf{0}}$ & $\mathbf{y}^{0++}$ & \# \\
\hline 1 & 100.0757 & 50.5415 & & & & & V & & & & & & & 24 \\
\hline 2 & 199.1441 & 100.0757 & & & & & $\mathbf{V}$ & 2650.3763 & 1325.6918 & 2633.3497 & 1317.1785 & 2632.3657 & 1316.6865 & 23 \\
\hline 3 & 286.1761 & 143.5917 & & & 268.1656 & 134.5864 & S & 2551.3079 & 1276.1576 & 2534.2813 & 1267.6443 & 2533.2973 & 1267.1523 & 22 \\
\hline 4 & 385.2445 & 193.1259 & & & 367.2340 & 184.1206 & $\mathbf{V}$ & 2464.2758 & 1232.6416 & 2447.2493 & 1224.1283 & 2446.2653 & 1223.6363 & 21 \\
\hline 5 & 500.2715 & 250.6394 & & & 482.2609 & 241.6341 & D & 2365.2074 & 1183.1074 & 2348.1809 & 1174.5941 & 2347.1969 & 1174.1021 & 20 \\
\hline 6 & 613.3556 & 307.1814 & & & 595.3450 & 298.1761 & I & 2250.1805 & 1125.5939 & 2233.1539 & 1117.0806 & 2232.1699 & 1116.5886 & 19 \\
\hline 7 & 700.3876 & 350.6974 & & & 682.3770 & 341.6921 & $\mathrm{~S}$ & 2137.0964 & 1069.0519 & 2120.0699 & 1060.5386 & 2119.0859 & 1060.0466 & 18 \\
\hline 8 & 847.4560 & 424.2316 & & & 829.4454 & 415.2264 & $\mathbf{F}$ & 2050.0644 & 1025.5358 & 2033.0378 & 1017.0226 & 2032.0538 & 1016.5306 & 17 \\
\hline 9 & 1003.5571 & 502.2822 & 986.5306 & 493.7689 & 985.5465 & 493.2769 & $\mathbf{R}$ & 1902.9960 & 952.0016 & 1885.9694 & 943.4884 & 1884.9854 & 942.9963 & 16 \\
\hline 10 & 1100.6099 & 550.8086 & 1083.5833 & 542.2953 & 1082.5993 & 541.8033 & $\mathbf{P}$ & 1746.8949 & 873.9511 & 1729.8683 & 865.4378 & 1728.8843 & 864.9458 & 15 \\
\hline 11 & 1199.6783 & 600.3428 & 1182.6517 & 591.8295 & 1181.6677 & 591.3375 & $\bar{V}$ & 1649.8421 & 825.4247 & 1632.8156 & 816.9114 & 1631.8315 & 816.4194 & 14 \\
\hline 12 & 1314.7052 & 657.8562 & 1297.6787 & 649.3430 & 1296.6947 & 648.8510 & $\mathbf{N}$ & 1550.7737 & 775.8905 & 1533.7471 & 767.3772 & 1532.7631 & 766.8852 & 13 \\
\hline 13 & 1443.7478 & 722.3775 & 1426.7213 & 713.8643 & 1425.7372 & 713.3723 & $\mathbf{E}$ & 1435.7468 & 718.3770 & 1418.7202 & 709.8637 & 1417.7362 & 709.3717 & 12 \\
\hline 14 & 1544.7955 & 772.9014 & 1527.7689 & 764.3881 & 1526.7849 & 763.8961 & $\mathbf{T}$ & 1306.7042 & 653.8557 & 1289.6776 & 645.3424 & 1288.6936 & 644.8504 & 11 \\
\hline 15 & 1691.8639 & 846.4356 & 1674.8374 & 837.9223 & 1673.8533 & 837.4303 & $\mathbf{F}$ & 1205.6565 & 603.3319 & 1188.6299 & 594.8186 & 1187.6459 & 594.3266 & 10 \\
\hline 16 & 1788.9167 & 894.9620 & 1771.8901 & 886.4487 & 1770.9061 & 885.9567 & $\mathbf{P}$ & 1058.5881 & 529.7977 & 1041.5615 & 521.2844 & 1040.5775 & 520.7924 & 9 \\
\hline 17 & 1887.9851 & 944.4962 & 1870.9585 & 935.9829 & 1869.9745 & 935.4909 & V & 961.5353 & 481.2713 & 944.5088 & 472.7580 & 943.5247 & 472.2660 & 8 \\
\hline 18 & 1987.0535 & 994.0304 & 1970.0269 & 985.5171 & 1969.0429 & 985.0251 & $\mathbf{V}$ & 862.4669 & 431.7371 & 845.4403 & 423.2238 & 844.4563 & 422.7318 & 7 \\
\hline
\end{tabular}




\begin{tabular}{|c|c|c|c|c|c|c|c|c|c|c|c|c|c|c|}
\hline 19 & |2150.1168 & |1075.5621 & |2133.0903 & $\mid 1067.0488$ & 2132.1063 & |1066.5568| & $\mathbf{Y}$ & 763.3985 & 382.2029 & 746.3719 & 373.6896 & 745.3879 & 373.1976 & 6 \\
\hline 20 & 2263.2009 & 1132.1041 & 2246.1743 & 1123.5908 & 2245.1903 & 1123.0988 & I & 600.3352 & 300.6712 & 583.3086 & 292.1579 & 582.3246 & 291.6659 & 5 \\
\hline 21 & 2392.2435 & 1196.6254 & 2375.2169 & 1188.1121 & 2374.2329 & 1187.6201 & $\mathbf{E}$ & 487.2511 & 244.1292 & 470.2245 & 235.6159 & 469.2405 & 235.1239 & 4 \\
\hline 22 & 2506.2864 & 1253.6468 & 2489.2599 & 1245.1336 & 2488.2758 & 1244.6416 & $\mathbf{N}$ & 358.2085 & 179.6079 & 341.1819 & 171.0946 & & & 3 \\
\hline 23 & 2603.3392 & 1302.1732 & 2586.3126 & 1293.6600 & 2585.3286 & 1293.1679 & $\mathbf{P}$ & 244.1656 & 122.5864 & 227.1390 & 114.0731 & & & 2 \\
\hline 24 & & & & & & & $\mathbf{K}$ & 147.1128 & 74.0600 & 130.0863 & 65.5468 & & & 1 \\
\hline
\end{tabular}
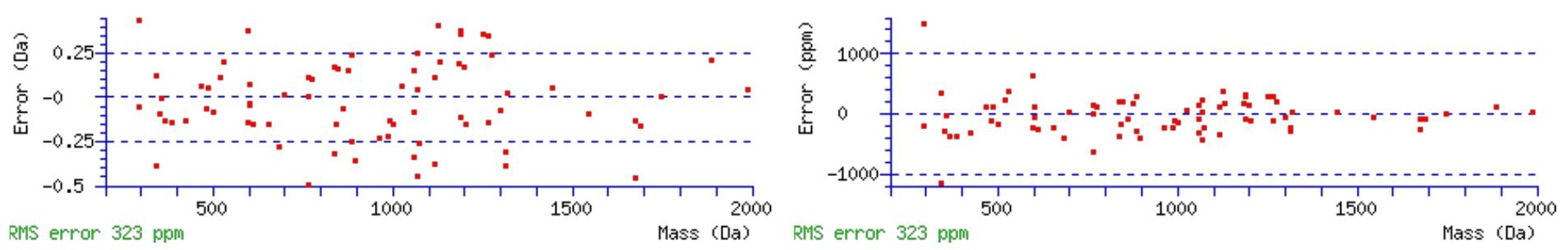

\section{All matches to this query}

\begin{tabular}{|l|l|l|l|}
\hline Score & Mr(calc): & Delta & \multicolumn{1}{|c|}{ Sequence } \\
\hline 38.0 & 2748.4374 & -0.0048 & VVSVDISFRPVNETFPVVYIENPK \\
\hline 19.8 & 2748.4374 & -0.0048 & VVSVDISFRPVNETFPVVYIENPK \\
\hline 7.0 & 2747.4534 & 0.9792 & VVSVDISFRPVNETFPVVYIENPK \\
\hline 0.9 & 2746.4471 & 1.9855 & MMEQGPVLIVTFQAQLVMVIKNPK \\
\hline 0.9 & 2746.4471 & 1.9855 & MMEQGPVLIVTFQAOLVMVIKNPK \\
\hline 0.6 & 2748.4268 & 0.0059 & VIAVLYTTVTPSLNPLIYTLRNK \\
\hline 0.6 & 2748.4268 & 0.0059 & VIAVLYTTVTPSLNPLIYTLRNK \\
\hline 0.1 & 2747.4470 & 0.9856 & VTITDLGVQLVTGLSLSLQLSPGSNR \\
\hline 0.1 & 2747.4470 & 0.9856 & VTITDLGVQLVTGLSLSLQLSPGSNR \\
\hline
\end{tabular}

Spectrum No: 886; Query: 1593; Rank: 1

\section{Peptide View}

MS/MS Fragmentation of VNLTMFFNITADGEPLGR

Found in IPI00364439, Tax_Id=10116 Gene_Symbol=RGD1559682_predicted similar to Peptidyl-prolyl cis-trans isomerase A

Match to Query 1593: 2010.968588 from(1006.491570,2+)

Title: 091008RatKidney_NoSalt_15.7083.7083.2.dta

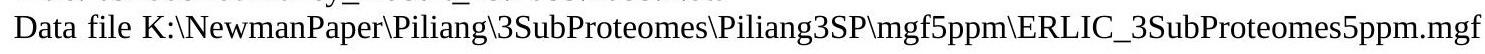

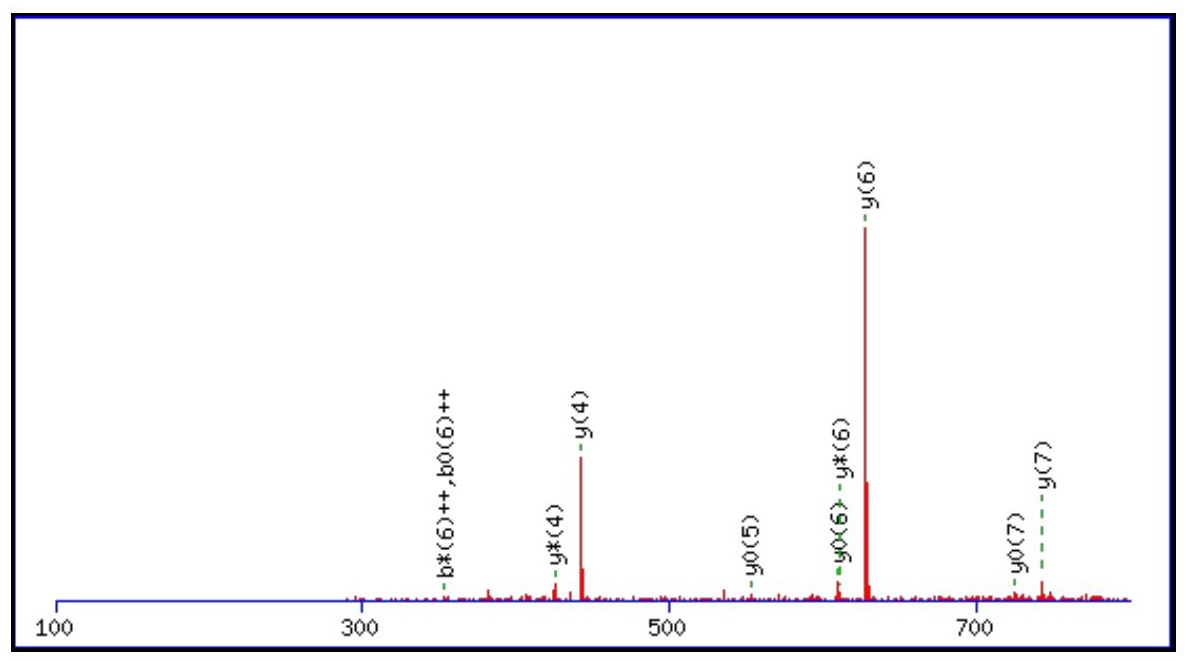



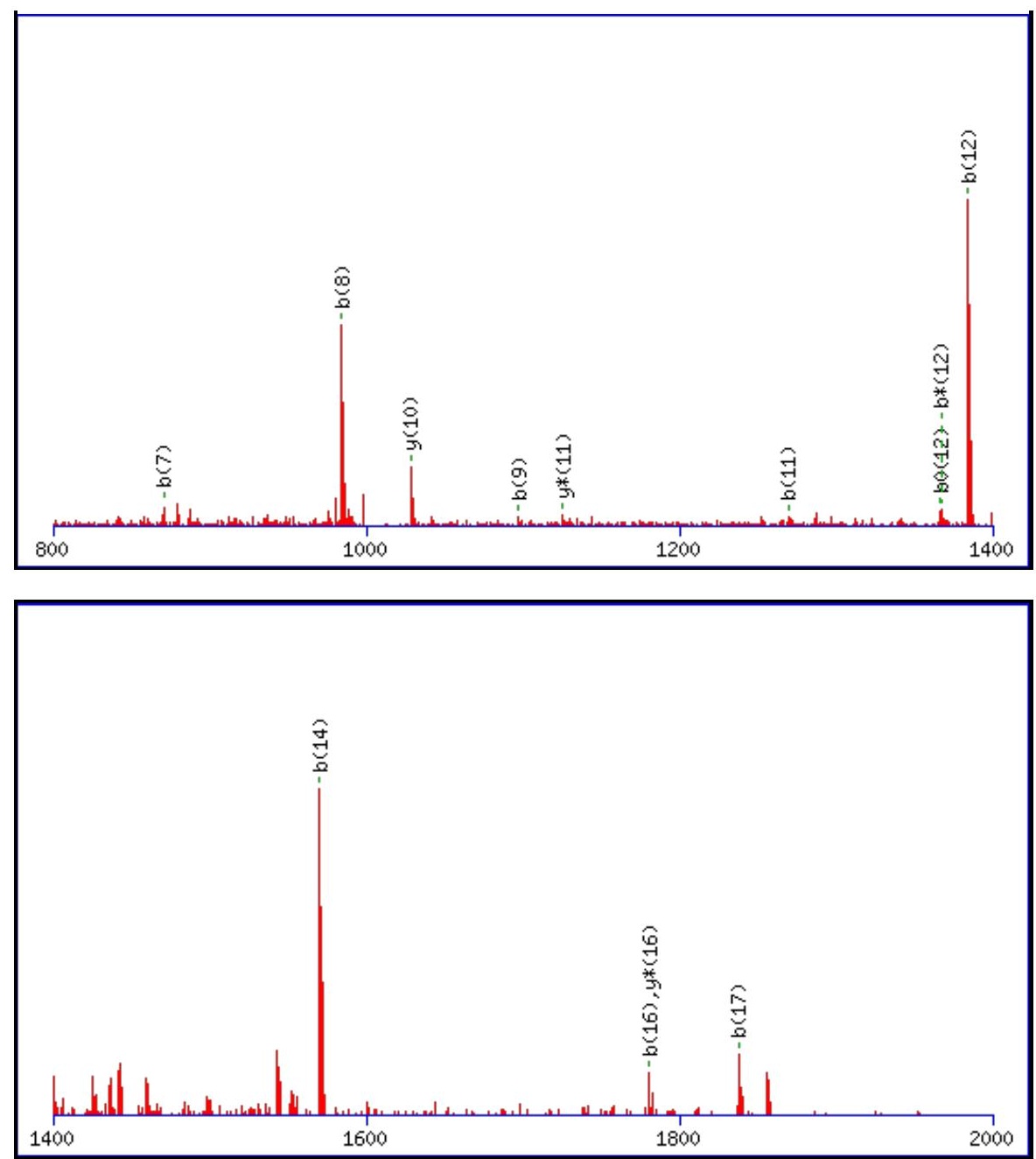

Monoisotopic mass of neutral peptide $\operatorname{Mr}($ calc): 2010.9721

Fixed modifications: Carbamidomethyl (C)

Variable modifications:

N2 : Deamidated_N (N)

M5 : Oxidation (M)

Ions Score: 38 Expect: 0.04

Matches (Bold Red): 23/188 fragment ions using 51 most intense peaks

\begin{tabular}{|c|c|c|c|c|c|c|c|c|c|c|c|c|c|c|}
\hline \# & b & $\mathbf{b}^{++}$ & $\mathbf{b}^{*}$ & $\mathbf{b}^{*^{++}}$ & $\mathbf{b}^{\mathbf{0}}$ & & Seq. & $\mathbf{y}$ & $\mathbf{y}^{++}$ & $\mathbf{y}^{*}$ & $\mathbf{y}^{*^{++}}$ & $\mathbf{y}^{\mathbf{0}}$ & $\mathbf{y}^{0++}$ & \# \\
\hline 1 & 100.0757 & 50.5415 & & & & & $\mathbf{V}$ & & & & & & & 18 \\
\hline 2 & 215.1026 & 108.0550 & 198.0761 & 99.5417 & & & $\mathbf{N}$ & & 956.9591 & 1895.8844 & & 1894.9004 & 947.9538 & 17 \\
\hline 3 & 328.1867 & 164.5970 & 311.1601 & 156.0837 & & & $\mathbf{L}$ & 1797.8840 & 899.4456 & 1780.8575 & 890.9324 & 1779.8734 & 890.4404 & 16 \\
\hline 4 & 429.2344 & 215.1208 & 412.2078 & 206.6075 & 411.2238 & & $\mathbf{T}$ & 1684.7999 & 842.9036 & 1667.7734 & 834.3903 & 1666.7894 & 833.8983 & 15 \\
\hline 5 & 576.2698 & 288.6385 & 559.2432 & 280.1252 & 558.2592 & 279.6332 & $\mathbf{M}$ & 1583.7523 & 3798 & 1566.7257 & 783.8665 & 1565.7417 & 783.3745 & 14 \\
\hline 6 & 723.3382 & 362.1727 & 706.3116 & 353.6595 & 705.3276 & 353.1674 & $\mathbf{F}$ & 1436.7169 & 718.8621 & 1419.6903 & 710.3488 & 1418.7063 & 709.8568 & 13 \\
\hline 7 & 870.4066 & 435.7069 & 853.3801 & 427.1937 & 852.3960 & 426.7017 & $\mathbf{F}$ & 1289.6484 & 645.3279 & 1272.6219 & 636.8146 & 1271.6379 & 636.3226 & 12 \\
\hline 8 & 984.4495 & 492.7284 & 967.4230 & 484.2151 & 966.4390 & 483.7231 & $\mathbf{N}$ & 1142.5800 & 571.7937 & 1125.5535 & 563.2804 & 1124.5695 & 562.7884 & 11 \\
\hline 9 & 1097.5336 & 549.2704 & 1080.5070 & 540.7572 & 1079.5230 & 540.2652 & I & 1028.5371 & 514.7722 & 1011.5106 & 506.2589 & 1010.5265 & 505.7669 & 10 \\
\hline 10 & 1198.5813 & 599.7943 & 1181.5547 & 591.2810 & 1180.5707 & 590.7890 & $\mathbf{T}$ & 915.4530 & 458.2302 & 898.4265 & 449.7169 & 897.4425 & 449.2249 & 9 \\
\hline 11 & 1269.6184 & 635.3128 & 1252.5918 & 626.7996 & 1251.6078 & 626.3075 & A & 814.4054 & 407.7063 & 797.3788 & 399.1930 & 796.3948 & 398.7010 & 8 \\
\hline 12 & 1384.6453 & 692.8263 & 1367.6188 & 684.3130 & 1366.6348 & 683.8210 & D & 743.3682 & 372.1878 & 726.3417 & 363.6745 & 725.3577 & 363.1825 & 7 \\
\hline 13 & 1441.6668 & 721.3370 & 1424.6402 & 712.8238 & 1423.6562 & 712.3318 & G & 628.3413 & 314.6743 & 611.3148 & 306.1610 & 610.3307 & 305.6690 & 6 \\
\hline 14 & 1570.7094 & 785.8583 & 1553.6828 & 777.3451 & 1552.6988 & 776.8530 & $\mathbf{E}$ & 571.3198 & 286.1636 & 554.2933 & 277.6503 & 553.3093 & 277.1583 & 5 \\
\hline 15 & 1667.7621 & 834.3847 & 1650.7356 & 825.8714 & 1649.7516 & 825.3794 & $\mathbf{P}$ & 442.2772 & 221.6423 & 425.2507 & 213.1290 & & & 4 \\
\hline 16 & $\mathbf{1 7 8 0 . 8 4 6 2}$ & 890.9267 & 1763.8197 & 882.4135 & 1762.8356 & 881.9215 & $\mathbf{L}$ & 345.2245 & 173.1159 & 328.1979 & 164.6026 & & & 3 \\
\hline 17 & 1837.8677 & 919.4375 & 1820.8411 & 910.9242 & 1819.8571 & 910.4322 & G & 232.1404 & 116.5738 & 215.1139 & 108.0606 & & & 2 \\
\hline 18 & & & & & & & $\mathbf{R}$ & 175.1190 & 88.0631 & 158.0924 & 79.5498 & & & 1 \\
\hline
\end{tabular}



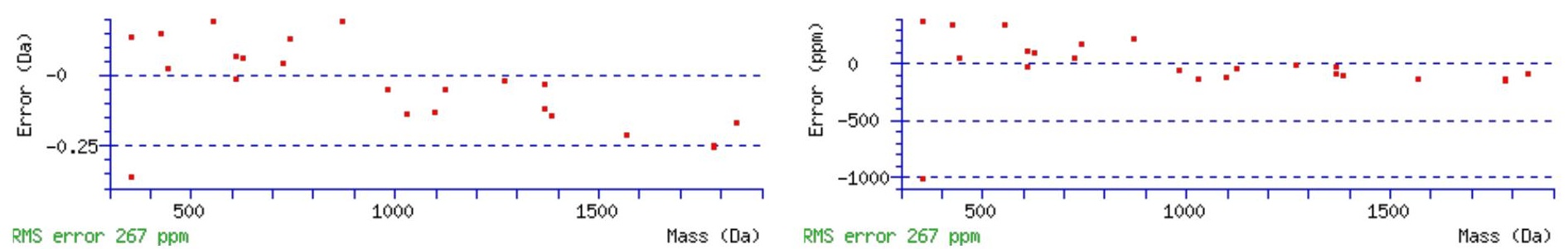

\section{All matches to this query}

\begin{tabular}{|l|l|l|l|}
\hline Score & Mr(calc): & Delta & \multicolumn{1}{|c|}{ Sequence } \\
\hline 37.9 & 2010.9721 & -0.0035 & VNLTMFFNITADGEPLGR \\
\hline 29.5 & 2010.9721 & -0.0035 & VNLTMFFNITADGEPLGR \\
\hline 12.6 & 2010.9832 & -0.0146 & YTPVSIYEEKLLLKR \\
\hline 12.6 & 2010.9832 & -0.0146 & YTPVSIYEEKLLLKR \\
\hline 11.3 & 2010.9637 & 0.0049 & SSGLPDASLPGRPLGTGGHR \\
\hline 11.2 & 2010.9637 & 0.0049 & SSGLPDASLPGRPLGTGGHR \\
\hline 3.4 & 2010.9832 & -0.0146 & YTPVSIYEEKLLLKR \\
\hline 2.3 & 2010.9557 & 0.0129 & NRVSAVDELAMATERLR \\
\hline 2.2 & 2008.9805 & 1.9881 & EVIKWRATNGQGMTPLK \\
\hline 1.6 & 2010.9693 & -0.0007 & TLKVLDQVFYQRVSR \\
\hline
\end{tabular}

Spectrum No: 887; Query: 682; Rank: 1

\section{Peptide View}

MS/MS Fragmentation of GFNFSAETVAGPEGK

Found in IPI00212106, Tax_Id=10116 Gene_Symbol=Ggtla1 Gamma-glutamyltransferase 5 precursor

Match to Query 682: 1510.697688 from(756.356120,2+)

Title: 100101RatKid_NS_deglyco_23.2917.2917.2.dta

Data file K:INewmanPaper|Piliang|3SubProteomes\Piliang3SP\mgf5ppm\ERLIC_3SubProteomes5ppm.mgf

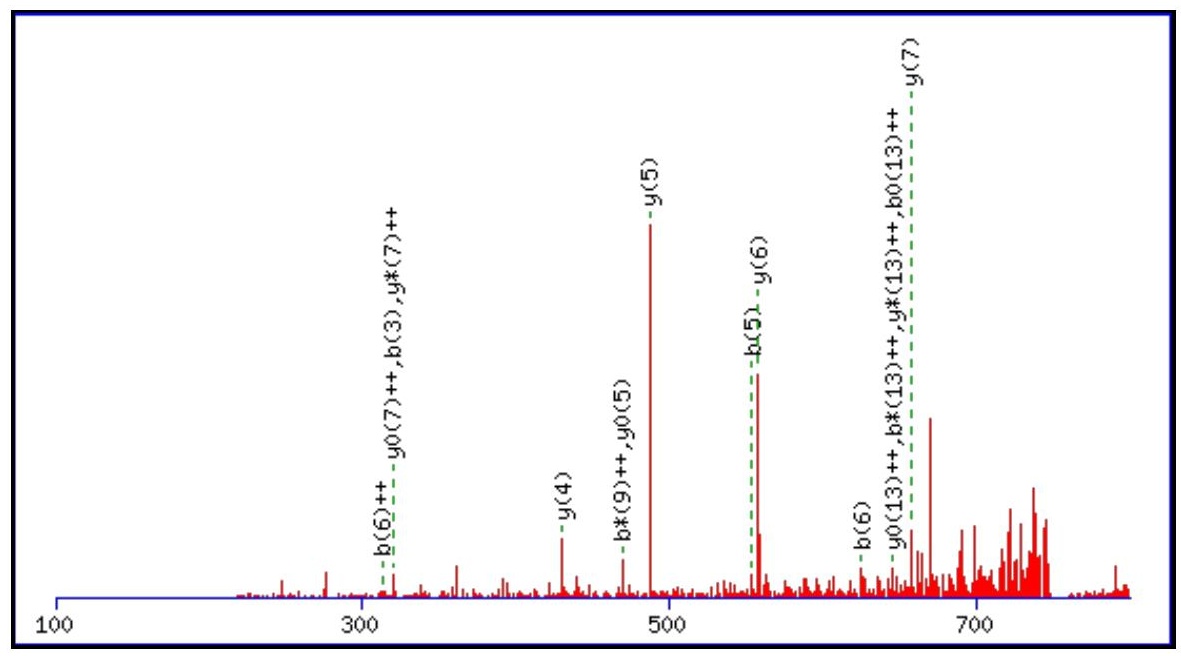



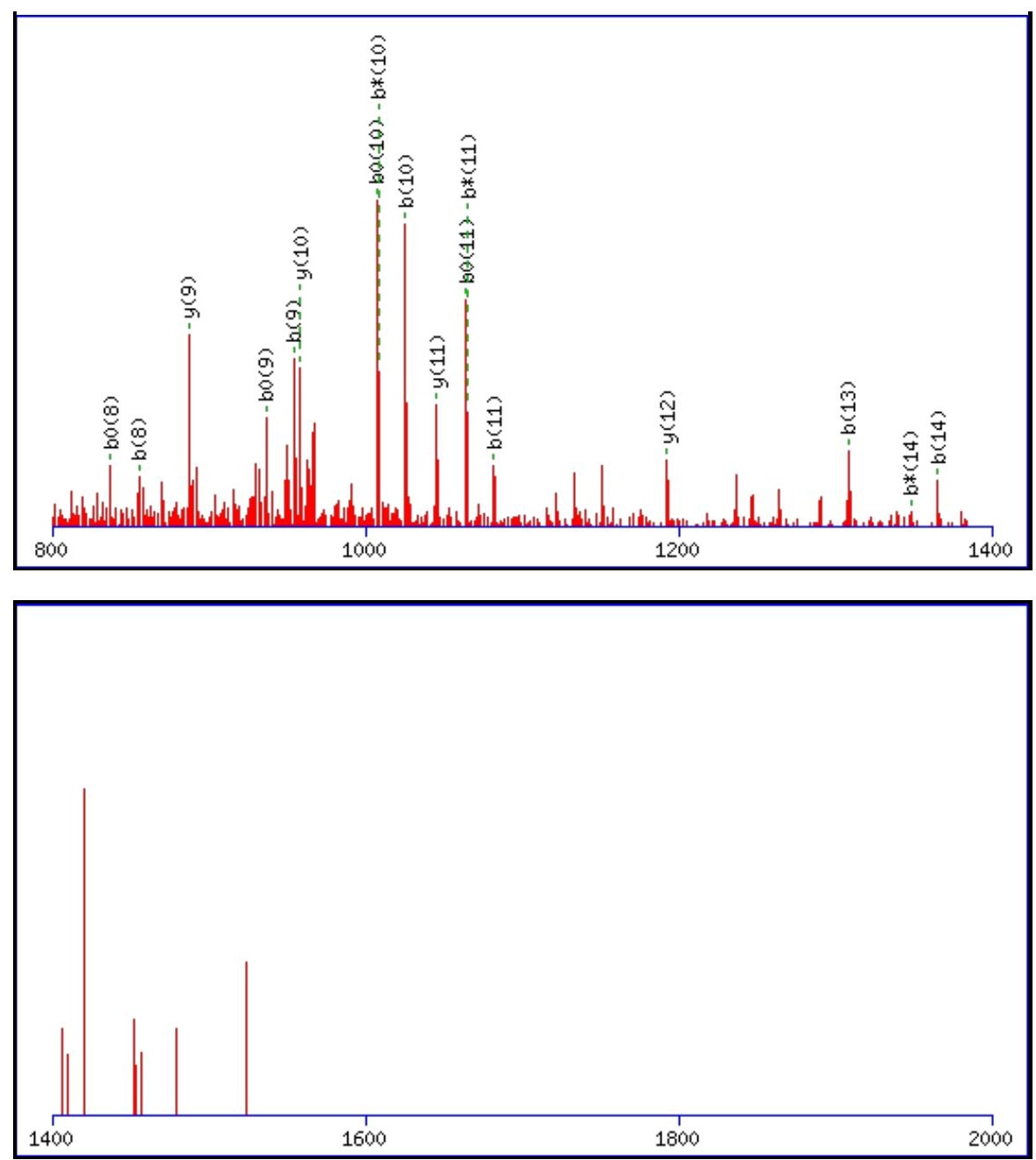

Monoisotopic mass of neutral peptide $\operatorname{Mr}($ calc): 1510.6940

Fixed modifications: Carbamidomethyl (C)

Variable modifications:

N3 : Deamidated_N (N)

Ions Score: 38 Expect : 0.028

Matches (Bold Red): 33/152 fragment ions using 61 most intense peaks

\begin{tabular}{|c|c|c|c|c|c|c|c|c|c|c|c|c|c|c|}
\hline \# & b & $\mathbf{b}^{++}$ & $\mathbf{b}^{*}$ & $\mathbf{b}^{*^{++}}$ & $\mathbf{b}^{0}$ & $\mathbf{b}^{\mathbf{0 + +}}$ & Seq. & $\mathbf{y}$ & $\mathbf{y}^{++}$ & $\mathbf{y}^{*}$ & $\mathrm{y}^{*^{++}}$ & $\mathbf{y}^{0}$ & $\mathbf{y}^{\mathbf{0 + +}}$ & \# \\
\hline 1 & 58.0287 & 29.5180 & & & & & $\mathbf{G}$ & & & & & & & 15 \\
\hline 2 & 205.0972 & 103.0522 & & & & & $\mathbf{F}$ & 1454.6798 & 727.8435 & 1437.6532 & 719.3303 & 1436.6692 & 718.8383 & 14 \\
\hline 3 & 320.1241 & 160.5657 & 303.0975 & 152.0524 & & & $\mathbf{N}$ & 1307.6114 & 654.3093 & 1290.5848 & 645.7961 & 1289.6008 & 645.3040 & 13 \\
\hline 4 & 467.1925 & 234.0999 & 450.1660 & 225.5866 & & & $\mathbf{F}$ & 1192.5844 & 596.7959 & 1175.5579 & 588.2826 & 1174.5739 & 587.7906 & 12 \\
\hline 5 & 554.2245 & 277.6159 & 537.1980 & 269.1026 & 536.2140 & 268.6106 & S & 1045.5160 & 523.2617 & 1028.4895 & 514.7484 & 1027.5055 & 514.2564 & 11 \\
\hline 6 & 625.2616 & 313.1345 & 608.2351 & 304.6212 & 607.2511 & 304.1292 & A & 958.4840 & 479.7456 & 941.4575 & 471.2324 & 940.4734 & 470.7404 & 10 \\
\hline 7 & 754.3042 & 377.6558 & 737.2777 & 369.1425 & 736.2937 & 368.6505 & $\mathbf{E}$ & 887.4469 & 444.2271 & 870.4203 & 435.7138 & 869.4363 & 435.2218 & 9 \\
\hline 8 & 855.3519 & 428.1796 & 838.3254 & 419.6663 & 837.3414 & 419.1743 & $T$ & 758.4043 & 379.7058 & 741.3777 & 371.1925 & 740.3937 & 370.7005 & 8 \\
\hline 9 & 954.4203 & 477.7138 & 937.3938 & 469.2005 & 936.4098 & 468.7085 & V & 657.3566 & 329.1819 & 640.3301 & 320.6687 & 639.3461 & 320.1767 & 7 \\
\hline 10 & 1025.4574 & 513.2324 & 1008.4309 & 504.7191 & 1007.4469 & 504.2271 & A & 558.2882 & 279.6477 & 541.2617 & 271.1345 & 540.2776 & 270.6425 & 6 \\
\hline 11 & 1082.4789 & 541.7431 & 1065.4524 & 533.2298 & 1064.4683 & 532.7378 & G & 487.2511 & 244.1292 & 470.2245 & 235.6159 & 469.2405 & 235.1239 & 5 \\
\hline 12 & 1179.5317 & 590.2695 & 1162.5051 & 581.7562 & 1161.5211 & 581.2642 & $\mathbf{P}$ & 430.2296 & 215.6185 & 413.2031 & 207.1052 & 412.2191 & 206.6132 & 4 \\
\hline 13 & 1308.5743 & 654.7908 & 1291.5477 & 646.2775 & 1290.5637 & 645.7855 & $\mathbf{E}$ & 333.1769 & 167.0921 & 316.1503 & 158.5788 & 315.1663 & 158.0868 & 3 \\
\hline 14 & 1365.5957 & 683.3015 & 1348.5692 & 674.7882 & 1347.5852 & 674.2962 & G & 204.1343 & 102.5708 & 187.1077 & 94.0575 & & & 2 \\
\hline 15 & & & & & & & $\mathbf{K}$ & 147.1128 & 74.0600 & 130.0863 & 65.5468 & & & 1 \\
\hline
\end{tabular}



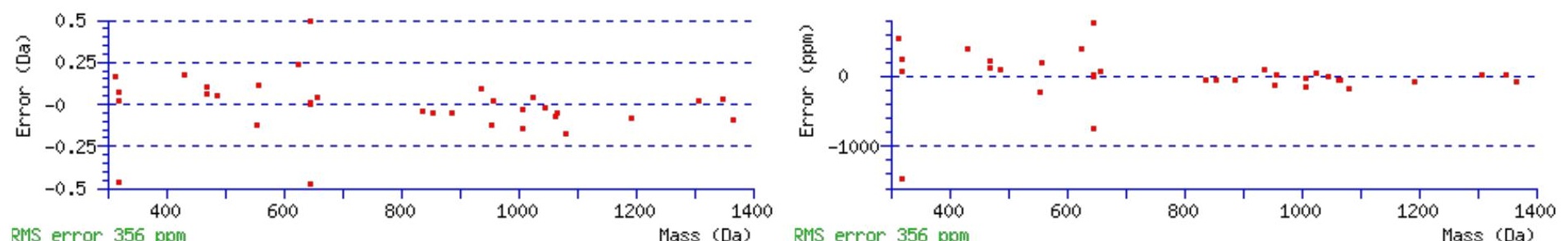

RIS error 356 ppm Mass (Da) RMS error 356 ppm

\section{All matches to this query}

\begin{tabular}{|l|l|l|l|}
\hline Score & Mr(calc): & Delta & \multicolumn{1}{c|}{ Sequence } \\
\hline 37.8 & 1510.6940 & 0.0037 & GFNFSAETVAGPEGK \\
\hline 6.8 & 1510.7028 & -0.0051 & GGAGNEKKSGPTVTK \\
\hline 5.3 & 1510.7028 & -0.0051 & $\underline{\text { GGAGNEKKSGPTVTK }}$ \\
\hline 4.7 & 1510.6916 & 0.0061 & $\underline{\text { TSKNLIDPNTAEK }}$ \\
\hline 4.7 & 1510.6916 & 0.0061 & $\underline{\text { TSKNLIDPNTAEK }}$ \\
\hline 3.6 & 1510.6857 & 0.0120 & $\underline{\text { KPYNSNIGFYTK }}$ \\
\hline 3.5 & 1510.7111 & -0.0134 & TGTVQSTNDSSSLSK \\
\hline 2.0 & 1510.6857 & 0.0120 & $\underline{\text { KPYNSNIGFYTK }}$ \\
\hline 0.8 & 1510.7028 & -0.0051 & GGAGNEKKSGPTVTK \\
\hline 0.7 & 1508.7054 & 1.9923 & LGFSPYVVRRR \\
\hline
\end{tabular}

Spectrum No: 888; Query: 2829; Rank: 1

\section{Peptide View}

MS/MS Fragmentation of IDDMLNVSGHLLSTAEVESALVEHEAVAEAAVVGHPHPVK

Found in IPI00869599, Tax_Id=10116 Gene_Symbol=Acss2_predicted acyl-CoA synthetase short-chain family member 2

Match to Query 2829: 4171.104984 from(696.191440,6+)

Title: 091008RatKidney_NH4Format02_11.5660.5660.6.dta

Data file K:INewmanPaper|Piliang|3SubProteomes\Piliang3SP\mgf5ppm\ERLIC_3SubProteomes5ppm.mgf

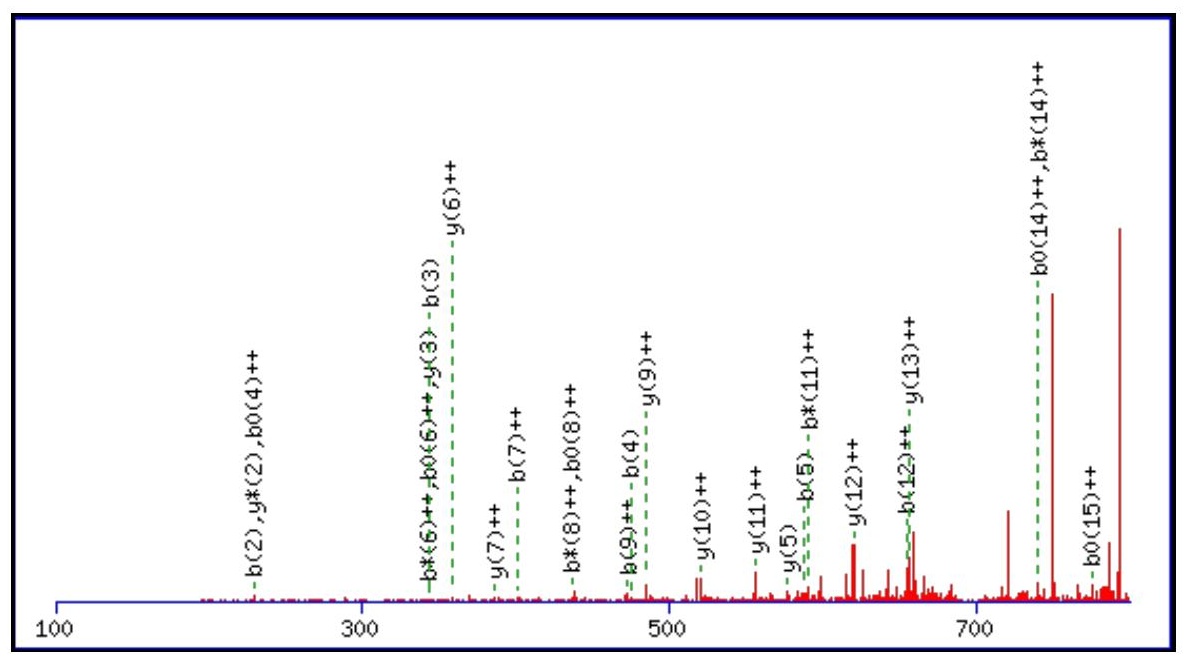



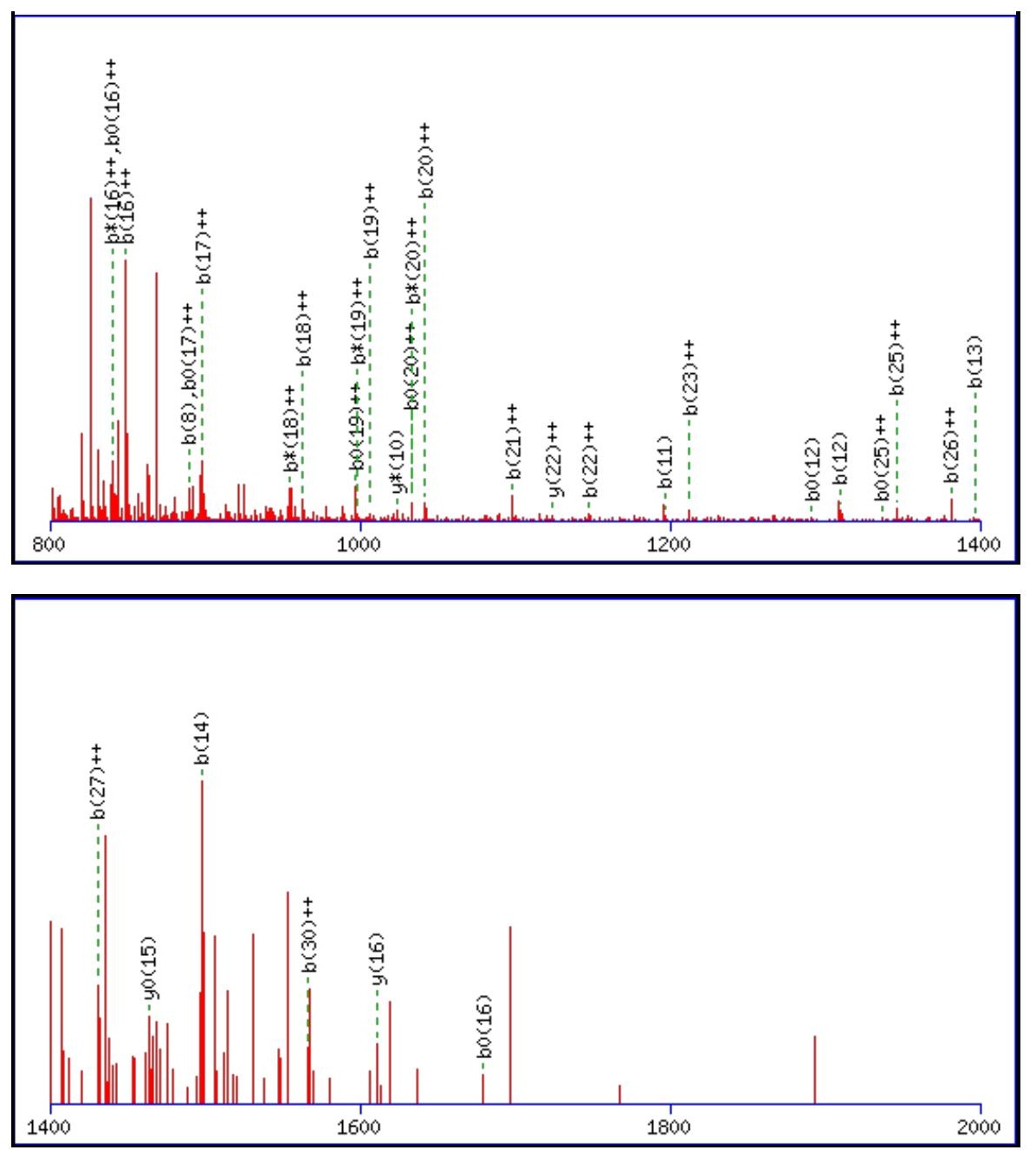

Monoisotopic mass of neutral peptide $\operatorname{Mr}($ calc): 4171.0896

Fixed modifications: Carbamidomethyl (C)

Variable modifications:

N6 : Deamidated_N (N)

Ions Score: 38 Expect: 0.032

Matches (Bold Red): 58/434 fragment ions using 153 most intense peaks

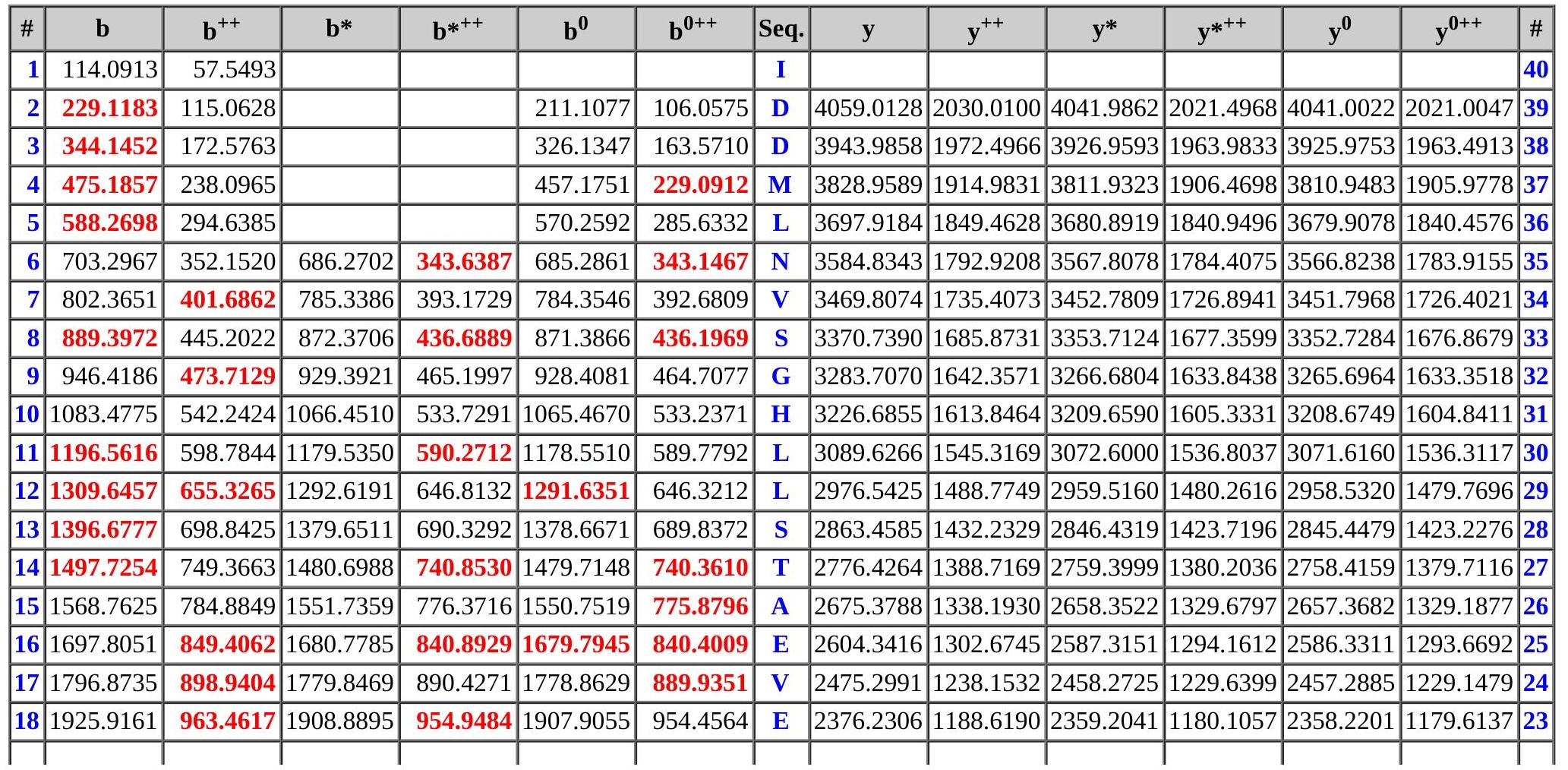




\begin{tabular}{|c|c|c|c|c|c|c|c|c|c|c|c|c|c|c|}
\hline 19 & 2012.9481 & |1006.9777 & 1995.9216 & 998.4644 & |1994.9375 & 997.9724 & $\mathrm{S}$ & 2247.1880 & 1124.0977 & |2230.1615 & 1115.5844 & |2229.1775 & 1115.0924 & $+22 \mid$ \\
\hline 20 & 2083.9852 & 1042.4962 & 2066.9587 & 1033.9830 & 2065.9747 & 1033.4910 & A & 2160.1560 & 1080.5816 & 2143.1295 & 1072.0684 & 2142.1455 & 1071.5764 & 21 \\
\hline 21 & 2197.0693 & 1099.0383 & 2180.0427 & 1090.5250 & 2179.0587 & 1090.0330 & $\mathbf{L}$ & 2089.1189 & 1045.0631 & $\mid 2072.0924$ & 1036.5498 & 2071.1083 & 1036.0578 & 20 \\
\hline 22 & 2296.1377 & 1148.5725 & 2279.1111 & 1140.0592 & 2278.1271 & 1139.5672 & $\mathbf{V}$ & 1976.0348 & 988.5211 & 1959.0083 & 980.0078 & 1958.0243 & 979.5158 & 19 \\
\hline 23 & 2425.1803 & 1213.0938 & 2408.1537 & 1204.5805 & 2407.1697 & 1204.0885 & $\mathbf{E}$ & 1876.9664 & 938.9868 & 1859.9399 & 930.4736 & 1858.9559 & 929.9816 & 18 \\
\hline 24 & 2562.2392 & 1281.6232 & 2545.2127 & 1273.1100 & 2544.2286 & 1272.6180 & H & 1747.9238 & 874.4656 & 1730.8973 & 865.9523 & 1729.9133 & 865.4603 & 17 \\
\hline 25 & 2691.2818 & 1346.1445 & 2674.2552 & 1337.6313 & 2673.2712 & 1337.1393 & $\mathbf{E}$ & 1610.8649 & 805.9361 & 1593.8384 & 797.4228 & 1592.8544 & 796.9308 & 16 \\
\hline 26 & 2762.3189 & 1381.6631 & 2745.2924 & 1373.1498 & 2744.3083 & 1372.6578 & A & 1481.8223 & 741.4148 & 1464.7958 & 732.9015 & 1463.8118 & 732.4095 & 15 \\
\hline 27 & 2861.3873 & 1431.1973 & 2844.3608 & 1422.6840 & 2843.3768 & 1422.1920 & V & 1410.7852 & 705.8962 & 1393.7587 & 697.3830 & 1392.7746 & 696.8910 & 14 \\
\hline 28 & 2932.4244 & 1466.7159 & 2915.3979 & 1458.2026 & 2914.4139 & 1457.7106 & A & 1311.7168 & 656.3620 & 1294.6902 & 647.8488 & 1293.7062 & 647.3568 & 13 \\
\hline 29 & 3061.4670 & 1531.2372 & 3044.4405 & 1522.7239 & 3043.4565 & 1522.2319 & $\mathbf{E}$ & 1240.6797 & 620.8435 & 1223.6531 & 612.3302 & 1222.6691 & 611.8382 & 12 \\
\hline 30 & 3132.5041 & 1566.7557 & 3115.4776 & 1558. & 3114.4936 & 1557.7504 & A & 1111.6371 & 556.3222 & 1094.6105 & 547.8089 & & & 11 \\
\hline 31 & 3203.5413 & 1602.2743 & 3186.5147 & 1593.7610 & 3185.5307 & 1593.2690 & A & 1040.6000 & 520.8036 & $\mid 1023.5734$ & 512.2904 & & & 10 \\
\hline 32 & 3302.6097 & 1651.8085 & 3285.5831 & 1643.2952 & 3284.5991 & 1642.8032 & $\mathbf{V}$ & 969.5629 & 485.2851 & 952.5363 & 476.7718 & & & 9 \\
\hline 33 & 3401.6781 & 1701.3427 & 3384.6515 & 1692.8294 & 3383.6675 & 1692.3374 & $\mathbf{V}$ & 870.4944 & 435.7509 & 853.4679 & 427.2376 & & & 8 \\
\hline 34 & 3458.6996 & 1729.8534 & 3441.6730 & 1721.3401 & 3440.6890 & 1720.8481 & G & 771.4260 & 386.2167 & 754.3995 & 377.7034 & & & 7 \\
\hline 35 & 3595.7585 & 1798.3829 & 3578.7319 & 1789.8696 & 3577.7479 & 1789.3776 & $\mathbf{H}$ & 714.4046 & 357.7059 & 697.3780 & 349.1926 & & & 6 \\
\hline 36 & 3692.8112 & 1846.9093 & 3675.7847 & 1838.3960 & 3674.8007 & 1837.9040 & $\mathbf{P}$ & 577.3457 & 289.1765 & 560.3191 & 280.6632 & & & 5 \\
\hline 37 & 3829.8701 & 1915.4387 & 3812.8436 & 1906.9254 & 3811.8596 & 1906.4334 & $\mathbf{H}$ & 480.2929 & 240.6501 & 463.2663 & 232.1368 & & & 4 \\
\hline 38 & 3926.9229 & 1963.9651 & 3909.8964 & 1955.4518 & 3908.9123 & 1954.9598 & $\mathbf{P}$ & 343.2340 & 172.1206 & 326.2074 & 163.6074 & & & 3 \\
\hline 39 & 4025.9913 & 2013.4993 & 4008.9648 & 2004.9860 & 4007.9808 & 2004.4940 & $\mathrm{~V}$ & 246.1812 & 123.5942 & 229.1547 & 115.0810 & & & 2 \\
\hline 40 & & & & & & & $\mathbf{K}$ & 147.1128 & 74.0600 & 130.0863 & 65.5468 & & & 1 \\
\hline
\end{tabular}
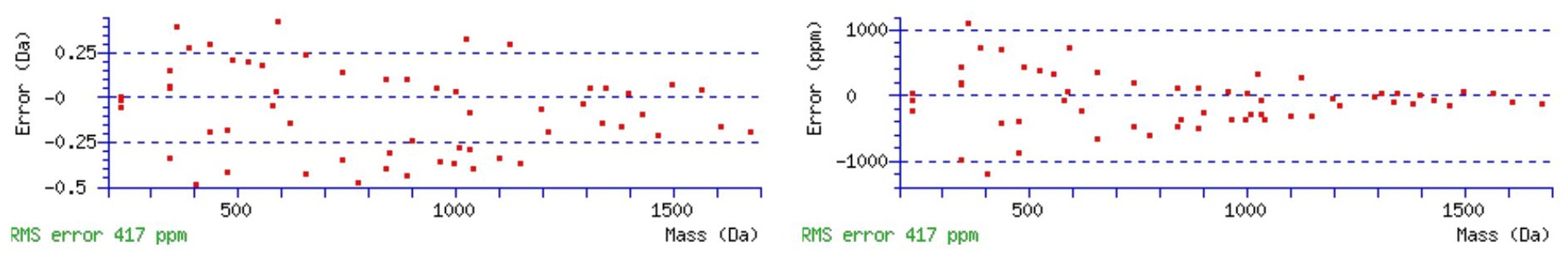

\section{All matches to this query}

\begin{tabular}{|l|l|l|l|}
\hline Score & Mr(calc): & Delta & \multicolumn{1}{|c|}{ Sequence } \\
\hline 37.8 & 4171.0896 & 0.0154 & IDDMLNVSGHLLSTAEVESALVEHEAVAEAAVVGHPHPVK \\
\hline 36.2 & 4170.1055 & 0.9994 & IDDMLNVSGHLLSTAEVESALVEHEAVAEAAVVGHPHPVK \\
\hline 3.3 & 4169.0577 & 2.0473 & MLSLSNLQNIIYNPTIPYVSTITEQLKPGSLIVIR \\
\hline 3.3 & 4169.0577 & 2.0473 & MLSLSNLQNIIYNPTIPYVSTITEQLKPGSLIVIR \\
\hline 3.3 & 4169.0577 & 2.0473 & MLSLSNLQNIIYNPTIPYVSTITEQLKPGSLIVIR \\
\hline 2.9 & 4171.0705 & 0.0344 & AYYPSIVANRWLAVRLECVGNCIVLFAALFAVISR \\
\hline 2.9 & 4171.0705 & 0.0344 & AYYPSIVANRWLAVRLECVGNCIVLFAALFAVISR \\
\hline 1.8 & 4169.1355 & 1.9695 & KGTVWSLSGSDWLGTVAAGDISGELIAAILPDMASNPINVK \\
\hline 1.8 & 4169.0923 & 2.0127 & LIAQSTSSAAPAAGPSSSILALRGAFTXAVTTAIASSVASLR \\
\hline 1.5 & 4169.0923 & 2.0127 & LIAQSTSSAAPAAGPSSSILALRGAFTXAVTTAIASSVASLR \\
\hline
\end{tabular}

Spectrum No: 889; Query: 1293; Rank: 1

\section{Peptide View}

MS/MS Fragmentation of HTDDLTSLNNTLVNIR

Found in IPI00364943, Tax_Id=10116 Gene_Symbol=Colec12 Collectin sub-family member 12 
Match to Query 1293: 1825.919622 from(609.647150,3+)

Title: 100101RatKid_NS_deglyco_20.3527.3527.3.dta

Data file K:INewmanPaper|Piliang|3SubProteomes\Piliang3SP\mgf5ppm\ERLIC_3SubProteomes5ppm.mgf
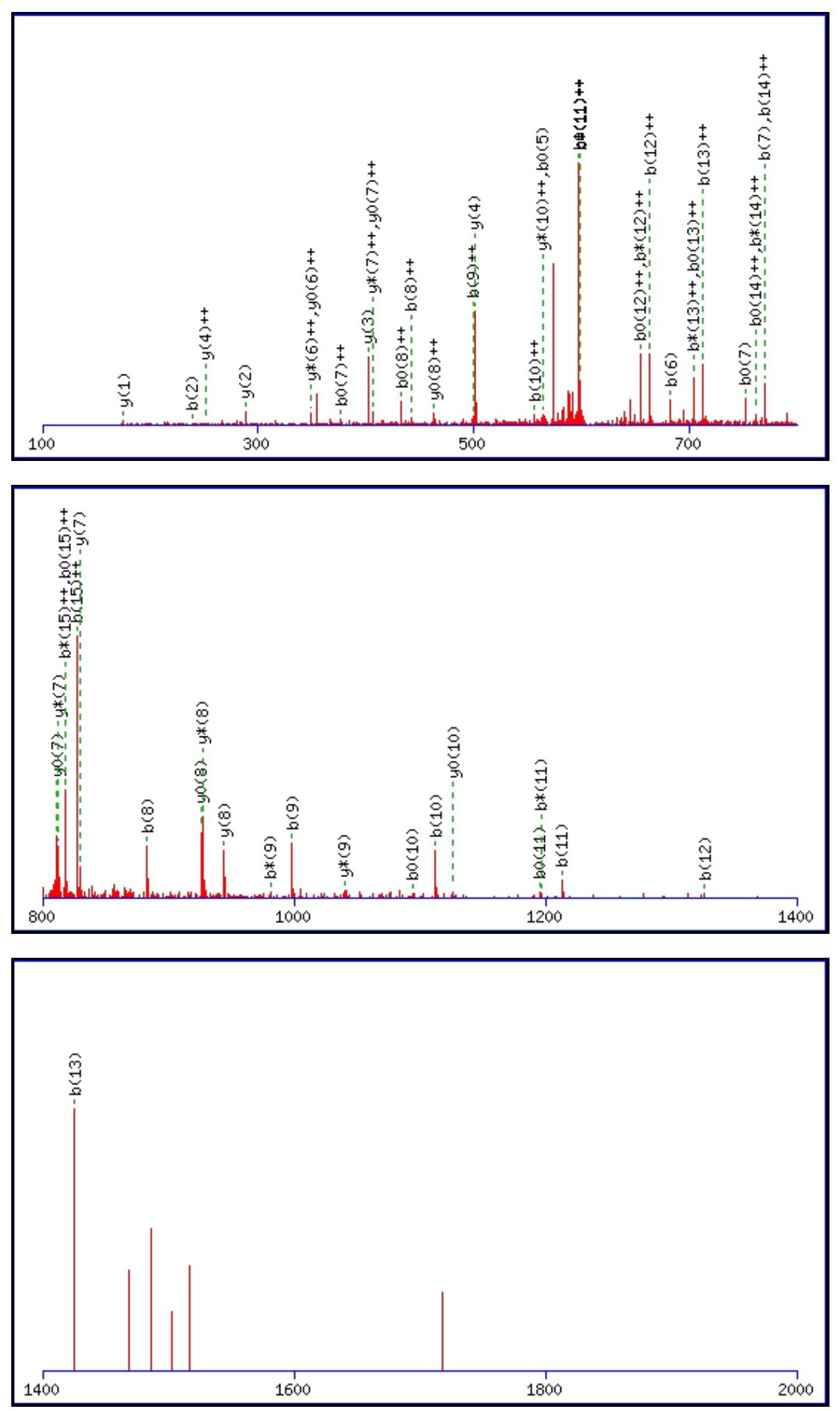

Monoisotopic mass of neutral peptide $\operatorname{Mr}($ calc): 1825.9170

Fixed modifications: Carbamidomethyl (C)

Variable modifications:

N9 : Deamidated_N (N)

Ions Score: 38 Expect: 0.029

Matches (Bold Red): 53/152 fragment ions using 91 most intense peaks

\begin{tabular}{|c|c|c|c|c|c|c|c|c|c|c|c|c|c|c|}
\hline$\#$ & $\mathbf{b}$ & $\mathbf{b}^{++}$ & $\mathbf{b}^{*}$ & $\mathbf{b}^{\mathbf{*}^{++}}$ & $\mathbf{b}^{\mathbf{0}}$ & $\mathbf{b}^{\mathbf{0}++}$ & Seq. & $\mathbf{y}$ & $\mathbf{y}^{++}$ & $\mathbf{y}^{\mathbf{*}}$ & $\mathbf{y}^{\mathbf{*}^{++}}$ & $\mathbf{y}^{\mathbf{0}}$ & $\mathbf{y}^{\mathbf{0 + +}}$ & $\#$ \\
\hline $\mathbf{1}$ & 138.0662 & 69.5367 & & & & & $\mathbf{H}$ & & & & & & & $\mathbf{1 6}$ \\
\hline $\mathbf{2}$ & 239.1139 & 120.0606 & & & 221.1033 & 111.0553 & $\mathbf{T}$ & 1689.8654 & 845.4363 & 1672.8388 & 836.9230 & 1671.8548 & 836.4310 & $\mathbf{1 5}$ \\
\hline
\end{tabular}




\begin{tabular}{|c|c|c|c|c|c|c|c|c|c|c|c|c|c|c|}
\hline & 354.1408 & | 177.5740 & & & 336.1302 & |168.5688 & D & 1588.8177 & |794.9125 & |1571.7911 & 786.3992 & 1570.8071 & 785.9072| & 14 \\
\hline 4 & 469.1678 & 235.0875 & & & 451.1572 & 226.0822 & D & 1473.7907 & 737.3990 & 1456.7642 & 728.8857 & 1455.7802 & 728.3937 & 13 \\
\hline 5 & 582.2518 & 291.6295 & & & 564.2413 & 282.6243 & $\mathbf{L}$ & 1358.7638 & 679.8855 & 1341.7372 & 671.3723 & 1340.7532 & 670.8803 & 12 \\
\hline 6 & 683.2995 & 342.1534 & & & 665.2889 & 333.1481 & $\mathrm{~T}$ & 1245.6797 & 623.3435 & 1228.6532 & 614.8302 & 1227.6692 & 614.3382 & 11 \\
\hline 7 & 770.3315 & 385.6694 & & & 752.3210 & 376.6641 & S & 1144.6321 & 572.8197 & 1127.6055 & 564.3064 & 1126.6215 & 563.8144 & 10 \\
\hline 8 & 883.4156 & 442.2114 & & & 865.4050 & 433.2061 & $\mathbf{L}$ & 1057.6000 & 529.3037 & 1040.5735 & 520.7904 & 1039.5895 & 520.2984 & 9 \\
\hline 9 & 998.4425 & 499.7249 & 981.4160 & 491.2116 & 980.4320 & 490.7196 & $\mathbf{N}$ & 944.5160 & 472.7616 & 927.4894 & 464.2483 & 926.5054 & 463.7563 & 8 \\
\hline 10 & 1112.4855 & 556.7464 & 1095.4589 & 548.2331 & 1094.4749 & 547.7411 & $\mathbf{N}$ & 829.4890 & 415.2482 & 812.4625 & 406.7349 & 811.4785 & 406.2429 & 7 \\
\hline 11 & 1213.5331 & 607.2702 & 1196.5066 & 598.7569 & 1195.5226 & 598.2649 & $\mathbf{T}$ & 715.4461 & 358.2267 & 698.4196 & 349.7134 & 697.4355 & 349.2214 & 6 \\
\hline 12 & 1326.6172 & 663.8122 & 1309.5906 & 655.2990 & 1308.6066 & 654.8070 & L & 614.3984 & 307.7028 & 597.3719 & 299.1896 & & & 5 \\
\hline 13 & 1425.6856 & 713.3464 & 1408.6591 & 704.8332 & 1407.6750 & 704.3412 & $\mathrm{~V}$ & 501.3144 & 251.1608 & 484.2878 & 242.6475 & & & 4 \\
\hline 14 & 1539.7285 & 770.3679 & 1522.7020 & 761.8546 & 1521.7180 & 761.3626 & $\mathbf{N}$ & 402.2459 & 201.6266 & 385.2194 & 193.1133 & & & 3 \\
\hline 15 & 1652.8126 & 826.9099 & 1635.7860 & 818.3967 & 1634.8020 & 817.9047 & I & 288.2030 & 144.6051 & 271.1765 & 136.0919 & & & 2 \\
\hline 16 & & & & & & & $\mathbf{R}$ & 175.1190 & 88.0631 & 158.0924 & 79.5498 & & & 1 \\
\hline
\end{tabular}
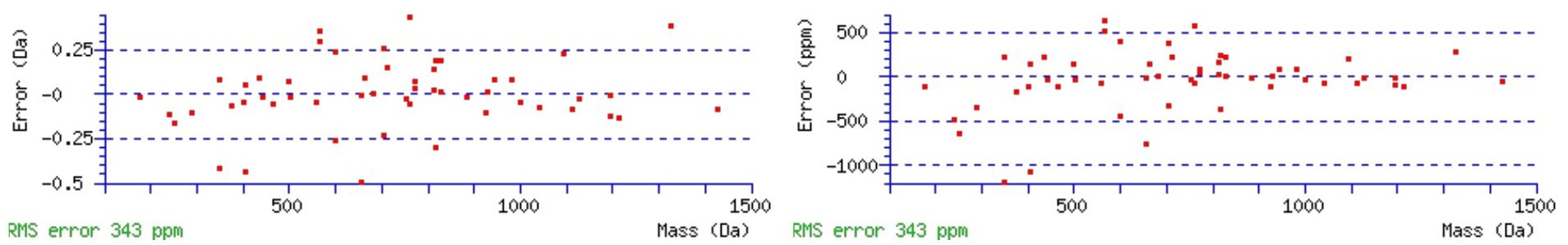

\section{All matches to this query}

\begin{tabular}{|l|l|c|l|}
\hline Score & Mr(calc): & Delta & \multicolumn{1}{c|}{ Sequence } \\
\hline 37.8 & 1825.9170 & 0.0026 & HTDDLTSLNNTLVNIR \\
\hline 28.6 & 1825.9170 & 0.0026 & HTDDLTSLNNTLVNIR \\
\hline 11.3 & 1824.9209 & 0.9987 & LSAMPQQFNVIVALAK \\
\hline 8.7 & 1824.9330 & 0.9866 & $\underline{\text { HTDDLTSLNNTLVNIR }}$ \\
\hline 8.4 & 1825.9170 & 0.0026 & $\underline{\text { HTDDLTSLNNTLVNIR }}$ \\
\hline 8.2 & 1825.9087 & 0.0109 & $\underline{\text { HDEHITAVLSKIVER }}$ \\
\hline 7.0 & 1825.9049 & 0.0147 & LSAMPQQFNVIVALAK \\
\hline 6.7 & 1825.9170 & 0.0027 & ELEEQLGPVAEETRAR \\
\hline 5.5 & 1825.9210 & -0.0014 & SSDVWGPPLLLEKDDR \\
\hline 4.7 & 1825.9031 & 0.0165 & $\underline{\text { SAALYPSIYLSKILK }}$ \\
\hline
\end{tabular}

Spectrum No: 890; Query: 174; Rank: 1

\section{Peptide View}

MS/MS Fragmentation of INSSGPVNISGK

Found in IPI00209975, Tax_Id=10116 Gene_Symbol=C4bpb C4b-binding protein beta chain precursor

Match to Query 174: 1172.604048 from(587.309300,2+)

Title: 100101RatKid_NS_deglyco_25.1067.1067.2.dta

Data file K:INewmanPaper|Piliangl3SubProteomes\Piliang3SP\mgf5ppm\ERLIC_3SubProteomes5ppm.mgf 

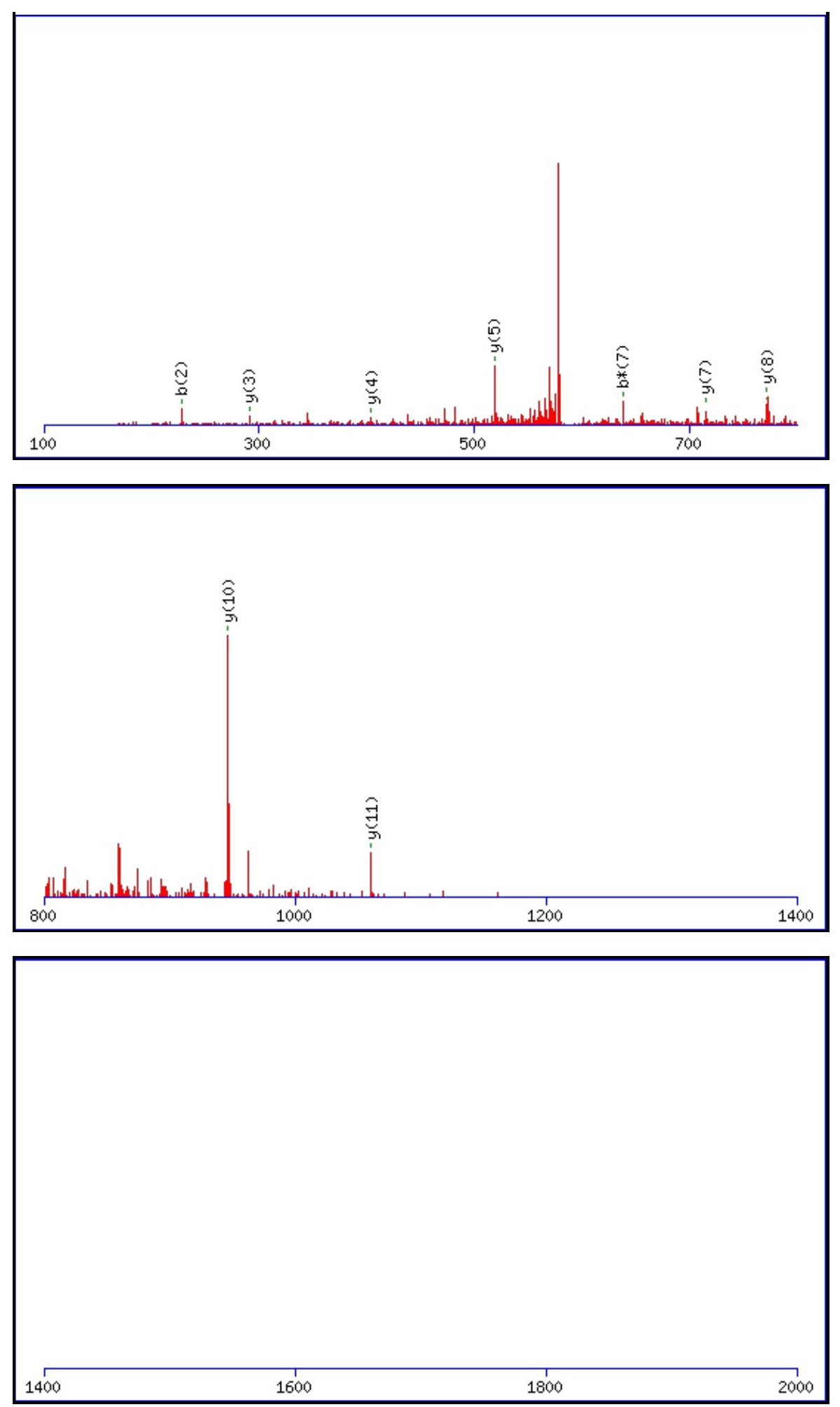

Monoisotopic mass of neutral peptide $\operatorname{Mr}($ calc): 1172.6037

Fixed modifications: Carbamidomethyl (C)

Variable modifications:

N8: Deamidated_N (N)

Ions Score: 38 Expect: $\odot .019$

Matches (Bold Red): 9/122 fragment ions using 27 most intense peaks

\begin{tabular}{|r|c|c|c|c|c|c|c|c|c|c|c|c|c|c|}
\hline$\#$ & $\mathbf{b}$ & $\mathbf{b}^{++}$ & $\mathbf{b}^{*}$ & $\mathbf{b}^{\boldsymbol{*}^{++}}$ & $\mathbf{b}^{\mathbf{0}}$ & $\mathbf{b}^{\mathbf{0 + +}}$ & Seq. & $\mathbf{y}$ & $\mathbf{y}^{++}$ & $\mathbf{y}^{\mathbf{*}}$ & $\mathbf{y}^{\boldsymbol{*}^{++}}$ & $\mathbf{y}^{\mathbf{0}}$ & $\mathbf{y}^{\mathbf{0 + +}}$ & $\#$ \\
\hline $\mathbf{1}$ & 114.0913 & 57.5493 & & & & & $\mathbf{I}$ & & & & & & & $\mathbf{1 2}$ \\
\hline $\mathbf{2}$ & $\mathbf{2 2 8 . 1 3 4 3}$ & 114.5708 & 211.1077 & 106.0575 & & & $\mathbf{N}$ & $\mathbf{1 0 6 0 . 5 2 6 9}$ & 530.7671 & 1043.5004 & 522.2538 & 1042.5164 & 521.7618 & $\mathbf{1 1}$ \\
\hline $\mathbf{3}$ & 315.1663 & 158.0868 & 298.1397 & 149.5735 & 297.1557 & 149.0815 & S & $\mathbf{9 4 6 . 4 8 4 0}$ & 473.7456 & 929.4574 & 465.2324 & 928.4734 & 464.7404 & $\mathbf{1 0}$ \\
\hline $\mathbf{4}$ & 402.1983 & 201.6028 & 385.1718 & 193.0895 & 384.1878 & 192.5975 & S & 859.4520 & 430.2296 & 842.4254 & 421.7163 & 841.4414 & 421.2243 & $\mathbf{9}$ \\
\hline $\mathbf{5}$ & 459.2198 & 230.1135 & 442.1932 & 221.6003 & 441.2092 & 221.1082 & $\mathbf{G}$ & 772.4199 & 386.7136 & 755.3934 & 378.2003 & 754.4094 & 377.7083 & $\mathbf{8}$ \\
\hline
\end{tabular}




\begin{tabular}{|c|c|c|c|c|c|c|c|c|c|c|c|c|c|c|}
\hline & 556.2726 & 278.6399 & 539.2460 & 270.1266 & 538.2620 & 269.6346 & $\mathbf{P}$ & 715.3985 & 358.2029 & 698.3719 & 349.6896 & 697.3879 & \begin{tabular}{|l|l|}
349.1976 \\
\end{tabular} & 7 \\
\hline 7 & 655.3410 & |328.1741 & 638.3144 & 319.6608 & 637.3304 & 319.1688 & V & 618.3457 & 309.6765 & 601.3192 & 301.1632 & 600.3351 & |300.6712 & 6 \\
\hline 8 & 770.3679 & 385.6876 & 753.3414 & 377.1743 & 752.3573 & 376.6823 & $\mathbf{N}$ & 519.2773 & 260.1423 & 502.2507 & 251.6290 & 501.2667 & 251.1370 & 5 \\
\hline 9 & 883.4520 & 442.2296 & 866.4254 & 433.7163 & 865.4414 & 433.2243 & I & 404.2504 & 202.6288 & 387.2238 & 194.1155 & 386.2398 & 193.6235 & 4 \\
\hline 10 & 970.4840 & 485.7456 & 953.4574 & 477.2324 & 952.4734 & 476.7404 & $\mathrm{~S}$ & 291.1663 & 146.0868 & 274.1397 & 137.5735 & 273.1557 & 137.0815 & 3 \\
\hline 11 & 1027.5055 & 514.2564 & 1010.4789 & 505.7431 & 1009.4949 & 505.2511 & G & 204.1343 & 102.5708 & 187.1077 & 94.0575 & & & 2 \\
\hline 12 & & & & & & & K & 147.1128 & 74.0600 & 130.0863 & 65.5468 & & & 1 \\
\hline
\end{tabular}
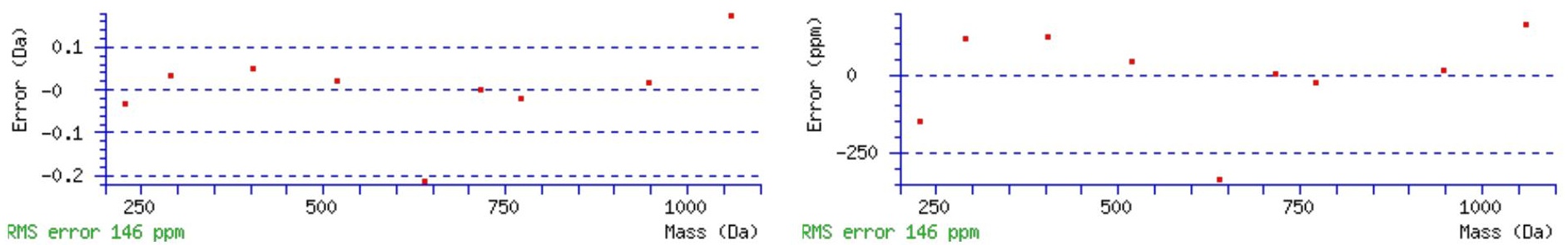

All matches to this query

\begin{tabular}{|l|l|l|l|}
\hline Score & Mr(calc): & Delta & \multicolumn{1}{|c|}{ Sequence } \\
\hline 37.7 & 1172.6037 & 0.0003 & INSSGPVNISGK \\
\hline 9.9 & 1172.6050 & -0.0010 & VKHRGEYER \\
\hline 1.4 & 1171.6019 & 1.0021 & SPCESLVPKR \\
\hline 0.9 & 1172.6037 & 0.0004 & LANNQLDKEK \\
\hline 0.9 & 1172.6037 & 0.0004 & LANNQLDKEK \\
\hline 0.9 & 1172.6037 & 0.0003 & INSSGPVNISGK \\
\hline 0.8 & 1172.6078 & -0.0037 & VFDPVPDGVTK \\
\hline 0.6 & 1172.6149 & -0.0109 & LNQEVSQLSR \\
\hline 0.4 & 1172.6037 & 0.0004 & DAPNKKSSTPK \\
\hline 0.4 & 1172.6149 & -0.0109 & KDKDPASSGLR \\
\hline
\end{tabular}

Spectrum No: 891; Query: 555; Rank: 1

\section{Peptide View}

MS/MS Fragmentation of GCKDNATDSVPLR

Found in IPI00369995, Tax_Id=10116 Gene_Symbol=Lrp1 similar to low density lipoprotein receptor-related protein 1

Match to Query 555: 1432.662072 from(478.561300,3+)

Title: 100101RatKid_NS_deglyco_21.888.888.3.dta

Data file K:INewmanPaper|Piliang|3SubProteomes\Piliang3SP\mgf5ppm\ERLIC_3SubProteomes5ppm.mgf 

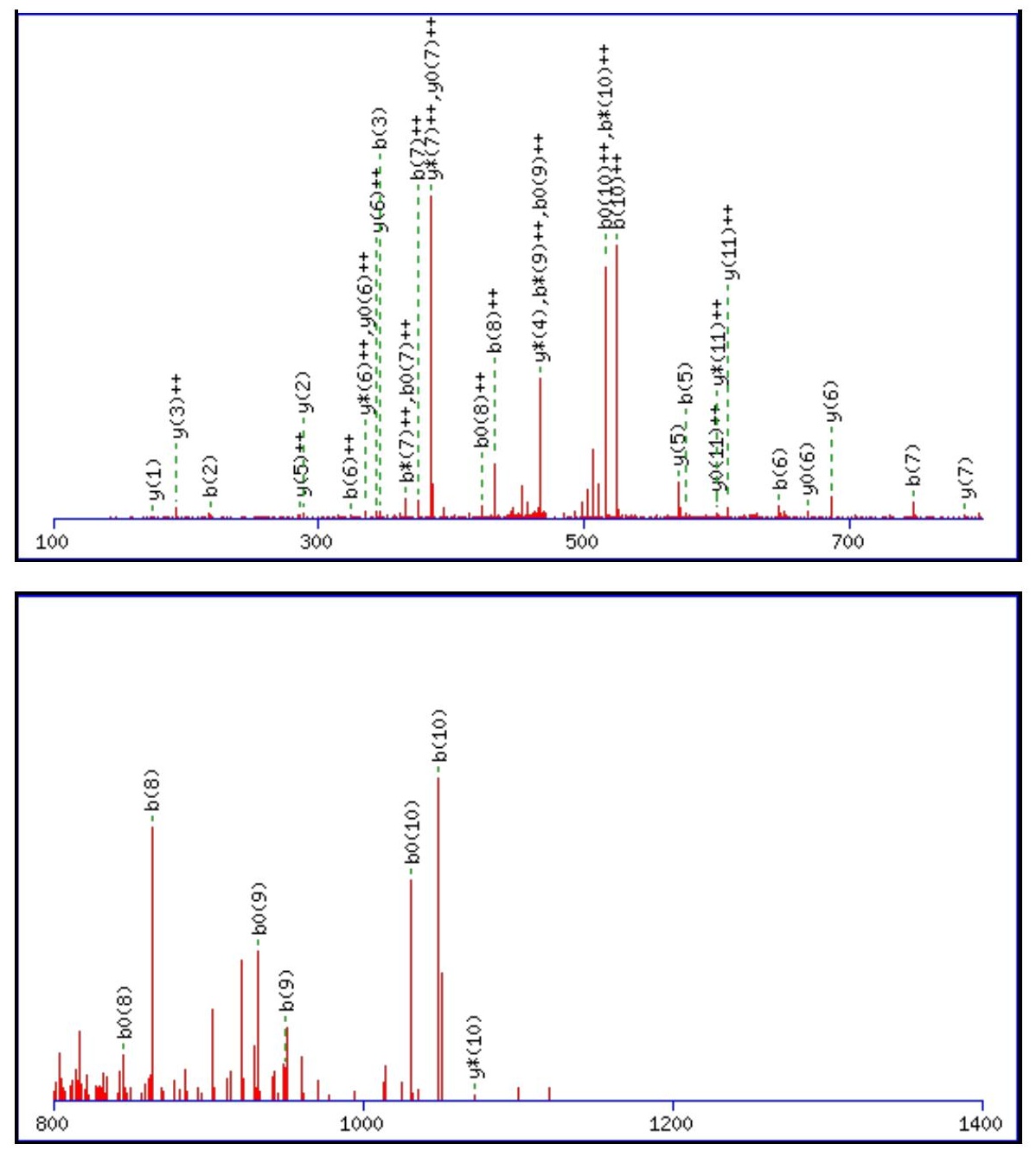

\begin{tabular}{rrrr}
\hline 1 & 1 & 1800 & 2000 \\
1400 & 1600 & 1800
\end{tabular}

Monoisotopic mass of neutral peptide $\operatorname{Mr}($ calc): 1432.6616

Fixed modifications: Carbamidomethyl (C)

Variable modifications:

N5 : Deamidated_N (N)

Ions Score: 38 Expect: 0.028

Matches (Bold Red): 41/126 fragment ions using 64 most intense peaks

\begin{tabular}{|r|c|c|c|c|c|c|c|c|c|c|c|c|c|c|}
\hline$\#$ & $\mathbf{b}$ & $\mathbf{b}^{++}$ & $\mathbf{b}^{*}$ & $\mathbf{b}^{\boldsymbol{*}^{++}}$ & $\mathbf{b}^{\mathbf{0}}$ & $\mathbf{b}^{\mathbf{0 + +}}$ & Seq. & $\mathbf{y}$ & $\mathbf{y}^{++}$ & $\mathbf{y}^{\mathbf{*}}$ & $\mathbf{y}^{\boldsymbol{*}^{++}}$ & $\mathbf{y}^{\mathbf{0}}$ & $\mathbf{y}^{\mathbf{0 + +}}$ & $\#$ \\
\hline $\mathbf{1}$ & $\mathbf{5 8 . 0 2 8 7}$ & 29.5180 & & & & & $\mathbf{G}$ & & & & & & & $\mathbf{1 3}$ \\
\hline $\mathbf{2}$ & $\mathbf{2 1 8 . 0 5 9 4}$ & 109.5333 & & & & & $\mathbf{C}$ & 1376.6474 & 688.8274 & 1359.6209 & 680.3141 & 1358.6369 & 679.8221 & $\mathbf{1 2}$ \\
\hline $\mathbf{3}$ & $\mathbf{3 4 6 . 1 5 4 4}$ & 173.5808 & 329.1278 & 165.0675 & & & $\mathbf{K}$ & 1216.6168 & $\mathbf{6 0 8 . 8 1 2 0}$ & 1199.5903 & $\mathbf{6 0 0 . 2 9 8 8}$ & 1198.6062 & 599.8068 & $\mathbf{1 1}$ \\
\hline $\mathbf{4}$ & 461.1813 & 231.0943 & 444.1547 & 222.5810 & 443.1707 & 222.0890 & $\mathbf{D}$ & 1088.5218 & 544.7646 & $\mathbf{1 0 7 1 . 4 9 5 3}$ & 536.2513 & 1070.5113 & 535.7593 & $\mathbf{1 0}$ \\
\hline $\mathbf{5}$ & $\mathbf{5 7 6 . 2 0 8 2}$ & 288.6078 & 559.1817 & 280.0945 & 558.1977 & 279.6025 & $\mathbf{N}$ & 973.4949 & 487.2511 & 956.4683 & 478.7378 & 955.4843 & 478.2458 & $\mathbf{9}$ \\
\hline
\end{tabular}




\begin{tabular}{|c|c|c|c|c|c|c|c|c|c|c|c|c|c|c|}
\hline 6 & 647.2453 & 324.1263 & 630.2188 & |315.6130 & 629.2348 & 315.1210 & A & 858.4680 & |429.7376 & 841.4414 & 421.2243 & 840.4574 & |420.7323 & 8 \\
\hline 7 & 748.2930 & 374.6502 & 731.2665 & |366.1369 & 730.2825 & 365.6449 & $\mathbf{T}$ & 787.4308 & |394.2191 & 770.4043 & 385.7058 & 769.4203 & |385.2138 & 7 \\
\hline 8 & 863.3200 & 432.1636 & 846.2934 & 423.6503 & 845.3094 & 423.1583 & D & 686.3832 & 343.6952 & 669.3566 & 335.1819 & 668.3726 & 334.6899 & 6 \\
\hline 9 & 950.3520 & 475.6796 & 933.3254 & 467.1664 & 932.3414 & 466.6744 & $\mathbf{S}$ & 571.3562 & 286.1817 & 554.3297 & 277.6685 & 553.3457 & 277.1765 & 5 \\
\hline 10 & 1049.4204 & 525.2138 & 1032.3939 & 516.7006 & 1031.4098 & 516.2086 & V & 484.3242 & 242.6657 & 467.2976 & 234.1525 & & & 4 \\
\hline 11 & 1146.4732 & 573.7402 & 1129.4466 & 565.2270 & 1128.4626 & 564.7349 & $\mathbf{P}$ & 385.2558 & 193.1315 & 368.2292 & 184.6183 & & & 3 \\
\hline 12 & 1259.5572 & 630.2823 & 1242.5307 & 621.7690 & 1241.5467 & 621.2770 & $\mathbf{L}$ & 288.2030 & |144.6051 & 271.1765 & 136.0919 & & & 2 \\
\hline 13 & & & & & & & $\mathbf{R}$ & 175.1190 & 88.0631 & 158.0924 & 79.5498 & & & 1 \\
\hline
\end{tabular}
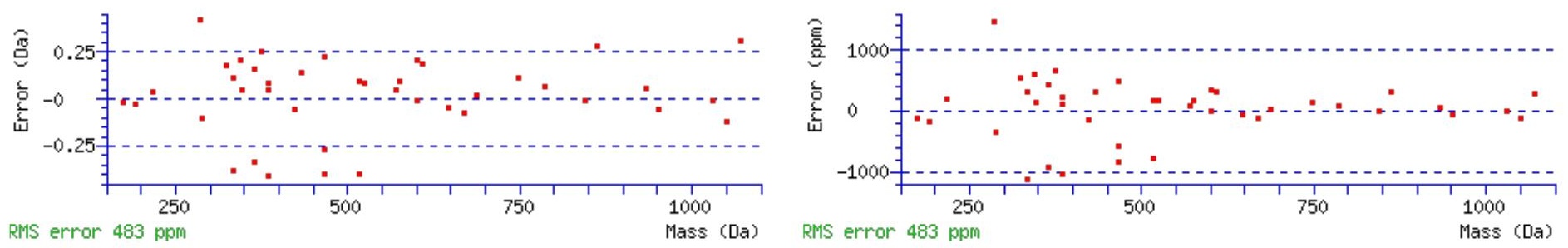

\section{All matches to this query}

\begin{tabular}{|l|l|c|l|}
\hline Score & Mr(calc): & Delta & \multicolumn{1}{c|}{ Sequence } \\
\hline 37.7 & 1432.6616 & 0.0004 & GCKDNATDSVPLR \\
\hline 9.3 & 1430.6443 & 2.0178 & LTDTSKYTGTHK \\
\hline 9.1 & 1430.6555 & 2.0066 & SSLLAHQETHTK \\
\hline 9.1 & 1431.6582 & 1.0039 & LAGCTVFITGASR \\
\hline 8.2 & 1431.6606 & 1.0014 & TLSTASSVSTNKR \\
\hline 7.3 & 1432.6654 & -0.0034 & NNRNEGTLDSRR \\
\hline 7.0 & 1432.6728 & -0.0107 & TVIAEGILKSSR \\
\hline 6.9 & 1432.6654 & -0.0034 & NNRNEGTLDSRR \\
\hline 6.4 & 1432.6615 & 0.0005 & GLIDLTLDKSAK \\
\hline 5.6 & 1432.6663 & -0.0042 & CGSRTVVLLLR \\
\hline
\end{tabular}

Spectrum No: 892; Query: 335; Rank: 1

\section{Peptide View}

MS/MS Fragmentation of RPASNVSASIQR

Found in IPI00361106, Tax_Id=10116 Gene_Symbol=RGD1560062_predicted hypothetical protein LOC309816

Match to Query 335: 1285.671102 from(429.564310,3+)

Title: 091008RatKidney_NH4Format01_26.354.354.3.dta

Data file K:INewmanPaper|Piliangl3SubProteomes\Piliang3SP\mgf5ppm\ERLIC_3SubProteomes5ppm.mgf 

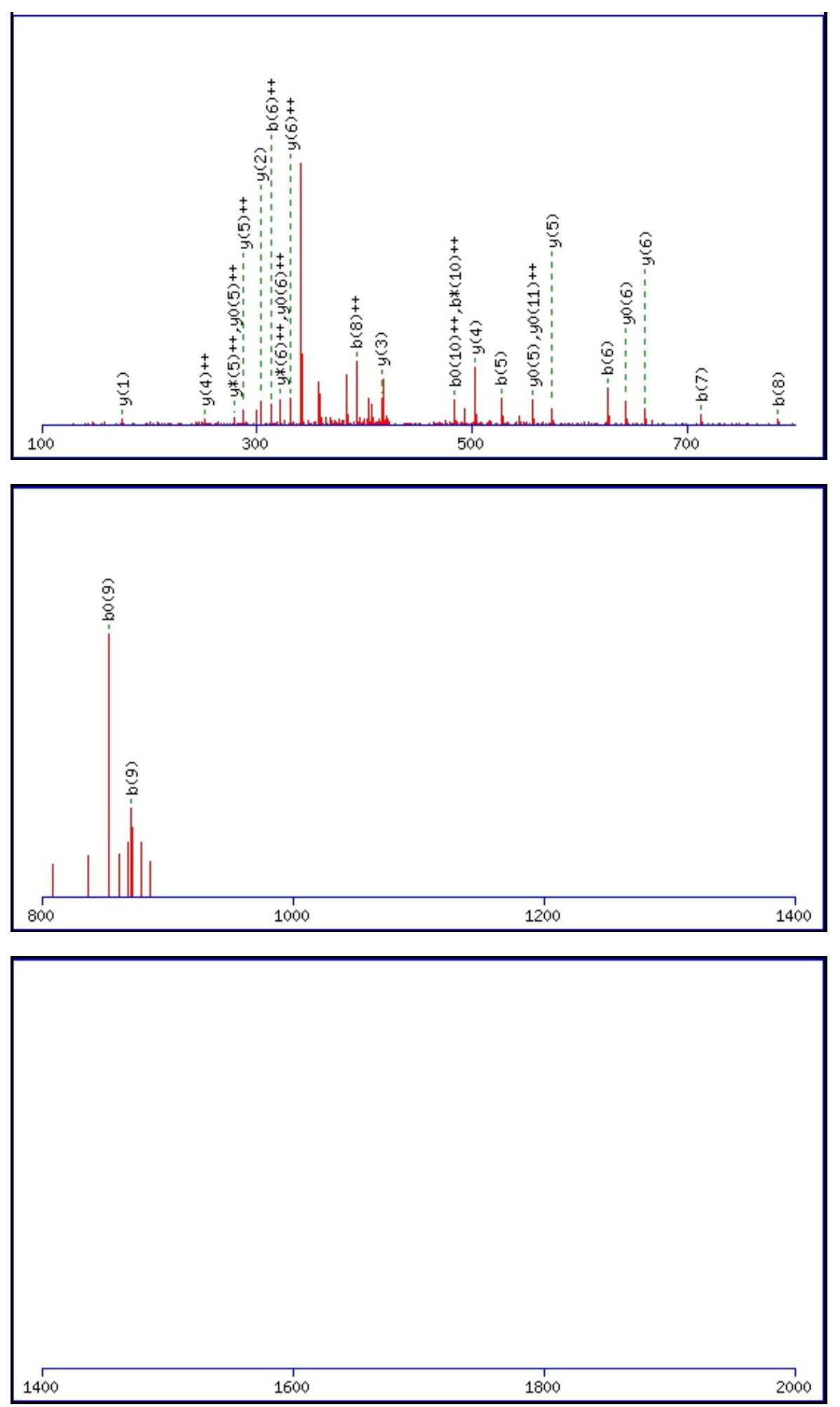

Monoisotopic mass of neutral peptide $\operatorname{Mr}($ calc): 1285.6738

Fixed modifications: Carbamidomethyl (C)

Variable modifications:

N5 : Deamidated_N (N)

Ions Score: 38 Expect: 0.018

Matches (Bold Red): 27/120 fragment ions using 32 most intense peaks

\begin{tabular}{|r|c|c|c|c|c|c|c|c|c|c|c|c|c|c|}
\hline$\#$ & $\mathbf{b}$ & $\mathbf{b}^{++}$ & $\mathbf{b}^{*}$ & $\mathbf{b}^{\boldsymbol{*}_{++}}$ & $\mathbf{b}^{\mathbf{0}}$ & $\mathbf{b}^{\mathbf{0 + +}}$ & Seq. & $\mathbf{y}$ & $\mathbf{y}^{++}$ & $\mathbf{y}^{\mathbf{*}}$ & $\mathbf{y}^{\boldsymbol{*}^{++}}$ & $\mathbf{y}^{\mathbf{0}}$ & $\mathbf{y}^{\mathbf{0 + +}}$ & $\#$ \\
\hline $\mathbf{1}$ & 157.1084 & 79.0578 & 140.0818 & 70.5446 & & & $\mathbf{R}$ & & & & & & & $\mathbf{1 2}$ \\
\hline $\mathbf{2}$ & 254.1612 & 127.5842 & 237.1346 & 119.0709 & & & $\mathbf{P}$ & 1130.5800 & 565.7936 & 1113.5535 & 557.2804 & 1112.5695 & $\mathbf{5 5 6 . 7 8 8 4}$ & $\mathbf{1 1}$ \\
\hline $\mathbf{3}$ & 325.1983 & 163.1028 & 308.1717 & 154.5895 & & & $\mathbf{A}$ & 1033.5273 & 517.2673 & 1016.5007 & 508.7540 & 1015.5167 & 508.2620 & $\mathbf{1 0}$ \\
\hline $\mathbf{4}$ & 412.2303 & 206.6188 & 395.2037 & 198.1055 & 394.2197 & 197.6135 & S & 962.4901 & 481.7487 & 945.4636 & 473.2354 & 944.4796 & 472.7434 & $\mathbf{9}$ \\
\hline $\mathbf{5}$ & $\mathbf{5 2 7 . 2 5 7 2}$ & 264.1323 & 510.2307 & 255.6190 & 509.2467 & 255.1270 & $\mathbf{N}$ & 875.4581 & 438.2327 & 858.4316 & 429.7194 & 857.4475 & 429.2274 & $\mathbf{8}$ \\
\hline
\end{tabular}




\begin{tabular}{|c|c|c|c|c|c|c|c|c|c|c|c|c|c|c|}
\hline 6 & 626.3256 & |313.6665 & 609.2991 & |305.1532 & 608.3151 & |304.6612 & V & 760.4312 & |380.7192 & 743.4046 & |372.2060 & 742.4206 & |371.7139| & 7 \\
\hline 7 & 713.3577 & 357.1825 & 696.3311 & 348.6692 & 695.3471 & 348.1772 & $\mathbf{S}$ & 661.3628 & 331.1850 & 644.3362 & 322.6717 & 643.3522 & 322.1797 & 6 \\
\hline 8 & 784.3948 & 392.7010 & 767.3682 & 384.1878 & 766.3842 & 383.6957 & A & 574.3307 & 287.6690 & 557.3042 & 279.1557 & 556.3202 & 278.6637 & 5 \\
\hline 9 & 871.4268 & 436.2170 & 854.4003 & 427.7038 & 853.4162 & 427.2118 & S & 503.2936 & 252.1504 & 486.2671 & 243.6372 & 485.2831 & 243.1452 & 4 \\
\hline 10 & 984.5109 & 492.7591 & 967.4843 & 484.2458 & 966.5003 & 483.7538 & I & 416.2616 & 208.6344 & 399.2350 & 200.1212 & & & 3 \\
\hline 11 & 1112.5695 & 556.7884 & 1095.5429 & 548.2751 & 1094.5589 & 547.7831 & $\mathbf{Q}$ & 303.1775 & 152.0924 & 286.1510 & 143.5791 & & & 2 \\
\hline 12 & & & & & & & $\mathbf{R}$ & 175.1190 & 88.0631 & 158.0924 & 79.5498 & & & 1 \\
\hline
\end{tabular}
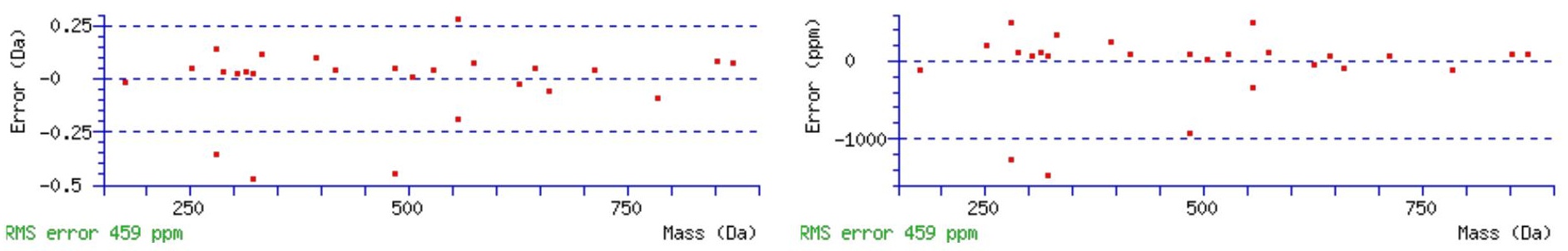

\section{All matches to this query}

\begin{tabular}{|l|l|l|l|}
\hline Score & Mr(calc): & Delta & \multicolumn{1}{c|}{ Sequence } \\
\hline 37.7 & 1285.6738 & -0.0027 & RPASNVSASIQR \\
\hline 11.9 & 1284.6786 & 0.9925 & $\underline{\text { ASELEPALGSRR }}$ \\
\hline 10.6 & 1285.6813 & -0.0102 & QLVPSCVTVQR \\
\hline 8.0 & 1285.6626 & 0.0085 & LVEEQNAEKAR \\
\hline 7.1 & 1284.6591 & 1.0120 & GGLIGFSRSLAK \\
\hline 6.9 & 1284.6551 & 1.0160 & RLSSLRASTSK \\
\hline 6.5 & 1284.6786 & 0.9925 & LLRDPSDASVGR \\
\hline 6.4 & 1284.6591 & 1.0120 & FKLINSTNIR \\
\hline 6.3 & 1283.6599 & 2.0113 & VKSSGIRVGSSK \\
\hline 6.2 & 1285.6601 & 0.0110 & $\underline{\text { RMVVYQTAFR }}$ \\
\hline
\end{tabular}

Spectrum No: 893; Query: 35; Rank: 1

\section{Peptide View}

MS/MS Fragmentation of VQPFNVTK

Found in IPI00212730, Tax_Id=10116 Gene_Symbol=Lamp2 Lysosome-associated membrane glycoprotein 2 precursor

Match to Query 35: 932.495148 from(467.254850,2+)

Title: 091008RatKidney_NH4Format01_23.1094.1094.2.dta

Data file K:INewmanPaper|Piliang|3SubProteomes\Piliang3SP\mgf5ppm\ERLIC_3SubProteomes5ppm.mgf 

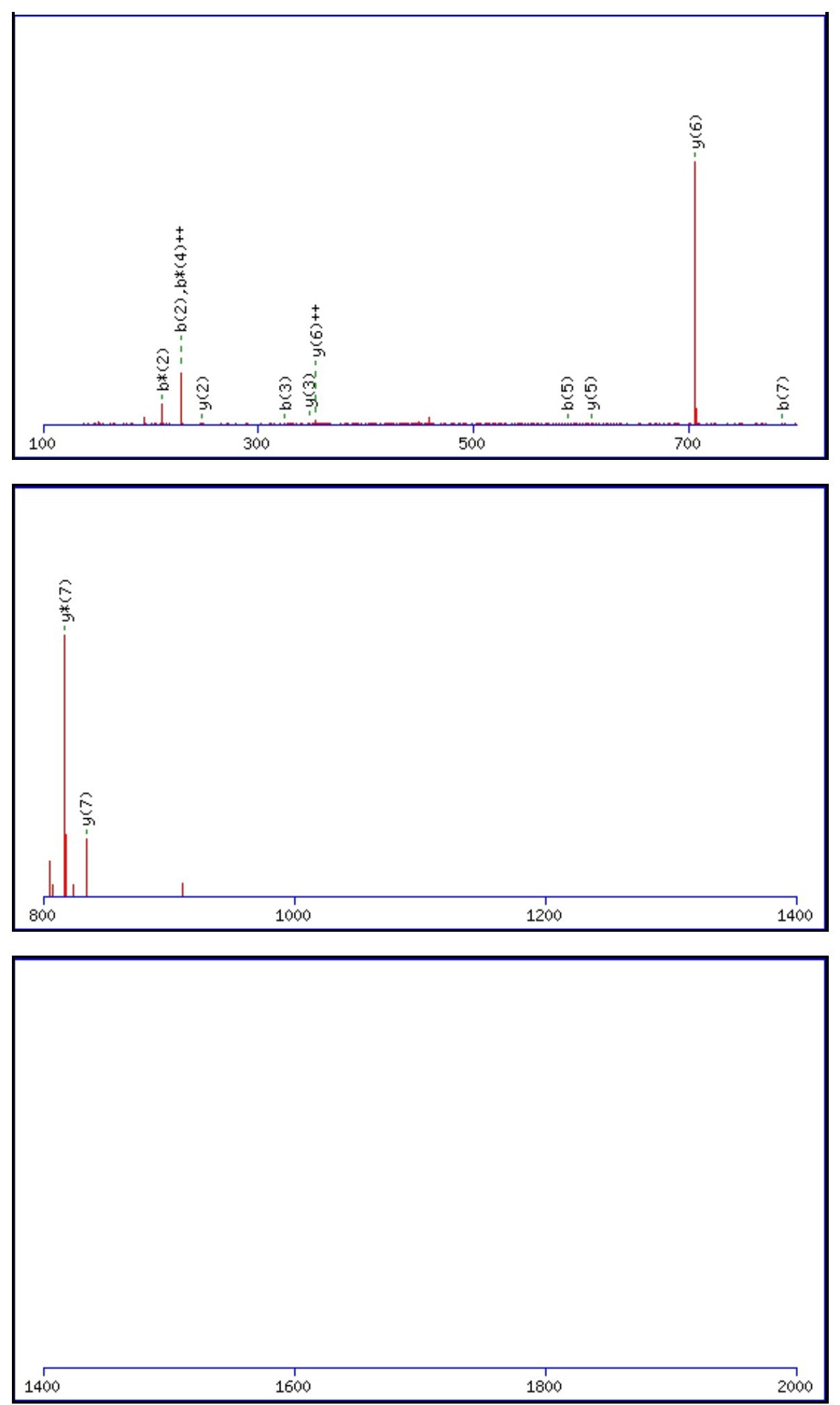

Monoisotopic mass of neutral peptide $\operatorname{Mr}($ calc): 932.4967

Fixed modifications: Carbamidomethyl (C)

Variable modifications:

N5: Deamidated $\mathrm{N}(\mathrm{N})$

Ions Score: 38 Expect: 0.018

Matches (Bold Red): 13/68 fragment ions using 18 most intense peaks

\begin{tabular}{|c|c|c|c|c|c|c|c|c|c|c|c|c|c|c|}
\hline$\#$ & $\mathbf{b}$ & $\mathbf{b}^{++}$ & $\mathbf{b}^{*}$ & $\mathbf{b}^{*^{++}}$ & $\mathbf{b}^{\mathbf{0}}$ & $\mathbf{b}^{\mathbf{0}+}$ & $\mathbf{S e q}$ & $\mathbf{y}$ & $\mathbf{y}^{++}$ & $\mathbf{y}^{*}$ & $\mathbf{y}^{\boldsymbol{*}^{++}}$ & $\mathbf{y}^{\mathbf{0}}$ & $\mathbf{y}^{\mathbf{0}++}$ & $\#$ \\
\hline $\mathbf{1}$ & 100.0757 & 50.5415 & & & & & $\mathbf{V}$ & & & & & & & $\mathbf{8}$ \\
\hline $\mathbf{2}$ & 228.1343 & 114.5708 & 211.1077 & 106.0575 & & & $\mathbf{Q}$ & $\mathbf{8 3 4 . 4 3 5 6}$ & 417.7214 & $\mathbf{8 1 7 . 4 0 9 0}$ & 409.2082 & 816.4250 & 408.7162 & $\mathbf{7}$ \\
\hline $\mathbf{3}$ & 325.1870 & 163.0972 & 308.1605 & 154.5839 & & & $\mathbf{P}$ & $\mathbf{7 0 6 . 3 7 7 0}$ & 353.6921 & 689.3505 & 345.1789 & 688.3664 & 344.6869 & $\mathbf{6}$ \\
\hline $\mathbf{4}$ & 472.2554 & 236.6314 & 455.2289 & $\mathbf{2 2 8 . 1 1 8 1}$ & & & $\mathbf{F}$ & $\mathbf{6 0 9 . 3 2 4 2}$ & 305.1658 & 592.2977 & 296.6525 & 591.3137 & 296.1605 & $\mathbf{5}$ \\
\hline $\mathbf{5}$ & $\mathbf{5 8 7 . 2 8 2 4}$ & 294.1448 & 570.2558 & 285.6316 & & & $\mathbf{N}$ & 462.2558 & 231.6316 & 445.2293 & 223.1183 & 444.2453 & 222.6263 & $\mathbf{4}$ \\
\hline
\end{tabular}




\begin{tabular}{|l|l|l|l|l|l|l|l|l|l|l|l|l|l|l|l|l|l|l|}
$\mathbf{6}$ & 686.3508 & 343.6790 & 669.3242 & 335.1658 & & & $\mathbf{V}$ & 347.2289 & 174.1181 & 330.2023 & 165.6048 & 329.2183 & 165.1128 & 3 \\
\hline $\mathbf{7}$ & $\mathbf{7 8 7 . 3 9 8 5}$ & 394.2029 & 770.3719 & 385.6896 & 769.3879 & 385.1976 & $\mathbf{T}$ & $\mathbf{2 4 8 . 1 6 0 5}$ & 124.5839 & 231.1339 & 116.0706 & 230.1499 & 115.5786 & 2 \\
\hline $\mathbf{8}$ & & & & & & & K & 147.1128 & 74.0600 & 130.0863 & 65.5468 & & & $\mathbf{1}$ \\
\hline
\end{tabular}
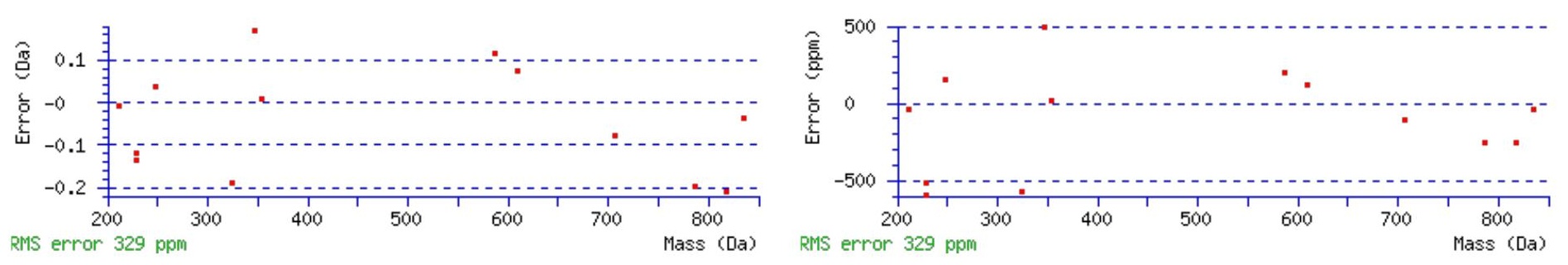

\section{All matches to this query}

\begin{tabular}{|l|l|l|l|}
\hline Score & Mr(calc): & Delta & \multicolumn{1}{|c|}{ Sequence } \\
\hline 37.6 & 932.4967 & -0.0016 & VQPFNVTK \\
\hline 35.1 & 932.4967 & -0.0016 & NLPFDVTK \\
\hline 10.2 & 932.5039 & -0.0088 & ARASKDASK \\
\hline 7.3 & 932.4902 & 0.0050 & VOKMNWK \\
\hline 5.5 & 932.4927 & 0.0025 & NLLNSSTGK \\
\hline 4.7 & 932.4862 & 0.0090 & LNDRKCK \\
\hline 4.2 & 932.5039 & -0.0088 & INSTAGRSK \\
\hline 4.2 & 932.4927 & 0.0025 & LNSSKOEK \\
\hline 3.7 & 932.5001 & -0.0049 & AGVLEMGLK \\
\hline 3.7 & 932.4862 & 0.0090 & GGLRSAXGGK \\
\hline
\end{tabular}

Spectrum No: 894; Query: 132; Rank: 1

\section{Peptide View}

MS/MS Fragmentation of IYNVSKEDR

Found in IPI00188731, Tax_Id=10116 Gene_Symbol=Cd48 CD48 antigen precursor

Match to Query 132: 1123.548288 from(562.781420,2+)

Title: 091008RatKidney_NH4Format01_28.391.391.2.dta

Data file K:INewmanPaper|Piliang|3SubProteomes\Piliang3SP\mgf5ppm\ERLIC_3SubProteomes5ppm.mgf

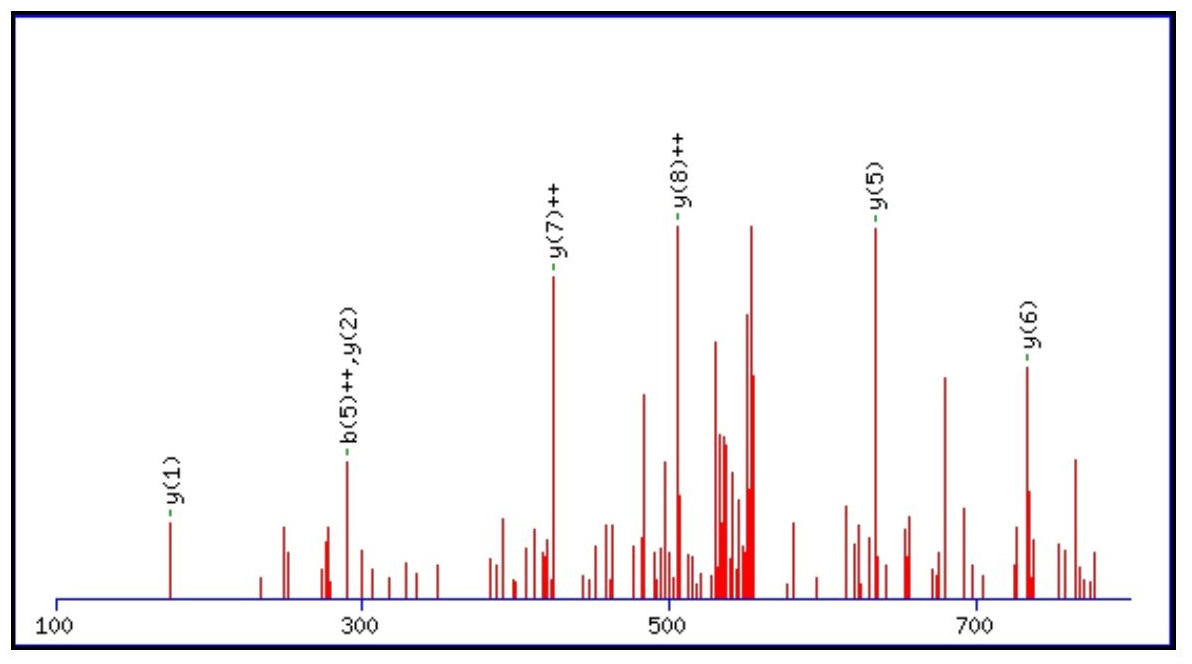



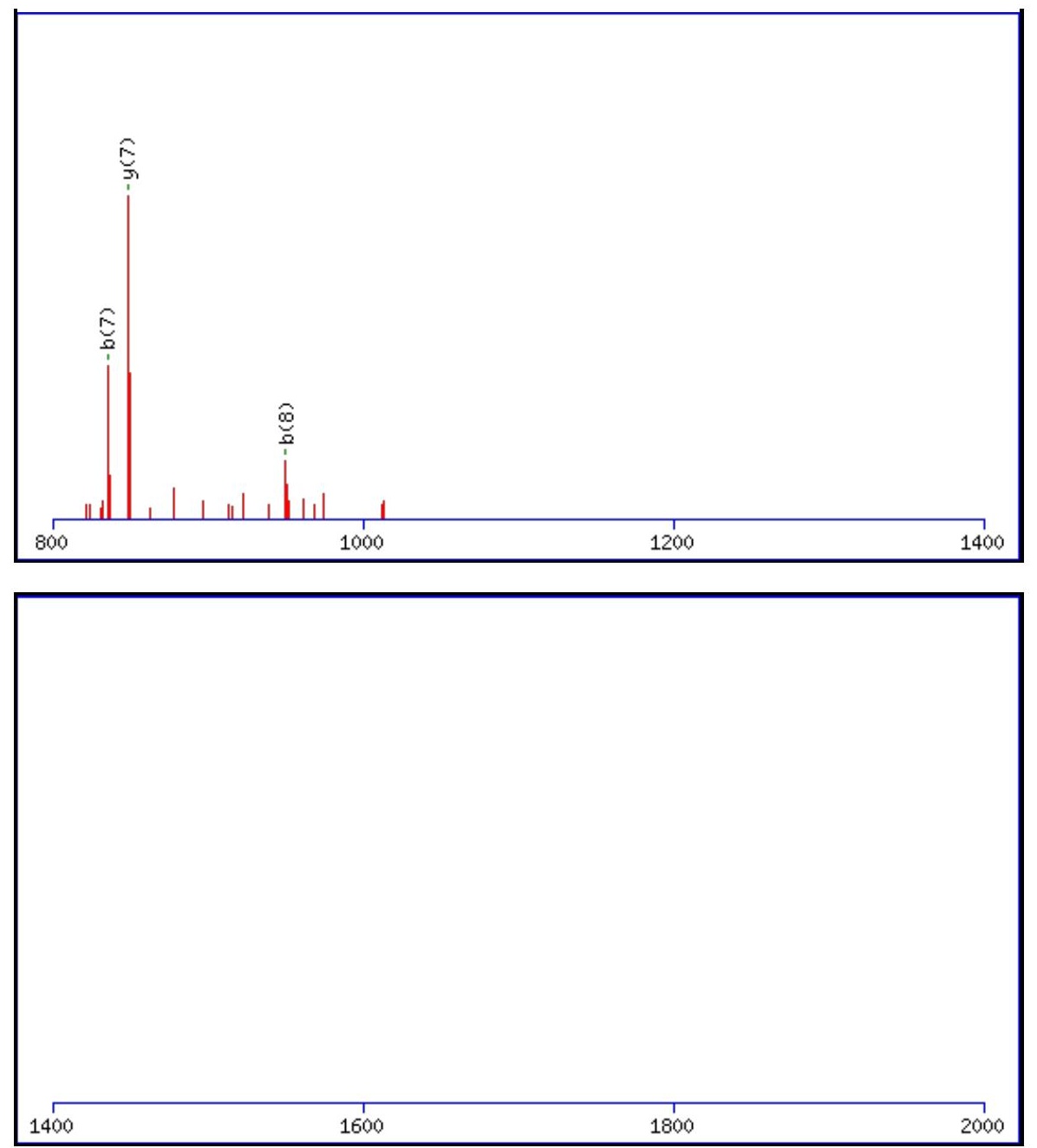

Monoisotopic mass of neutral peptide $\operatorname{Mr}($ calc): 1123.5509

Fixed modifications: Carbamidomethyl (C)

Variable modifications:

N3 : Deamidated $\mathrm{N}(\mathrm{N})$

Ions Score: 38 Expect: 0.019

Matches (Bold Red): 10/82 fragment ions using 15 most intense peaks

\begin{tabular}{|c|c|c|c|c|c|c|c|c|c|c|c|c|c|c|}
\hline$\#$ & $\mathbf{b}$ & $\mathbf{b}^{++}$ & $\mathbf{b}^{*}$ & $\mathbf{b}^{\boldsymbol{*}^{++}}$ & $\mathbf{b}^{\mathbf{0}}$ & $\mathbf{b}^{\mathbf{0 + +}}$ & $\mathbf{S e q} \cdot$ & $\mathbf{y}$ & $\mathbf{y}^{++}$ & $\mathbf{y}^{*}$ & $\mathbf{y}^{\boldsymbol{*}^{++}}$ & $\mathbf{y}^{\mathbf{0}}$ & $\mathbf{y}^{\mathbf{0 + +}}$ & $\#$ \\
\hline $\mathbf{1}$ & 114.0913 & 57.5493 & & & & & $\mathbf{I}$ & & & & & & & $\mathbf{9}$ \\
\hline $\mathbf{2}$ & 277.1547 & 139.0810 & & & & & $\mathbf{Y}$ & 1011.4742 & $\mathbf{5 0 6 . 2 4 0 7}$ & 994.4476 & 497.7274 & 993.4636 & 497.2354 & $\mathbf{8}$ \\
\hline $\mathbf{3}$ & 392.1816 & 196.5944 & 375.1551 & 188.0812 & & & $\mathbf{N}$ & $\mathbf{8 4 8 . 4 1 0 8}$ & $\mathbf{4 2 4 . 7 0 9 1}$ & 831.3843 & 416.1958 & 830.4003 & 415.7038 & $\mathbf{7}$ \\
\hline $\mathbf{4}$ & 491.2500 & 246.1286 & 474.2235 & 237.6154 & & & $\mathbf{V}$ & 733.3839 & 367.1956 & 716.3573 & 358.6823 & 715.3733 & 358.1903 & $\mathbf{6}$ \\
\hline $\mathbf{5}$ & 578.2820 & 289.6447 & 561.2555 & 281.1314 & 560.2715 & 280.6394 & $\mathbf{S}$ & $\mathbf{6 3 4 . 3 1 5 5}$ & 317.6614 & 617.2889 & 309.1481 & 616.3049 & 308.6561 & $\mathbf{5}$ \\
\hline $\mathbf{6}$ & 706.3770 & 353.6921 & 689.3505 & 345.1789 & 688.3664 & 344.6869 & $\mathbf{K}$ & 547.2835 & 274.1454 & 530.2569 & 265.6321 & 529.2729 & 265.1401 & $\mathbf{4}$ \\
\hline $\mathbf{7}$ & $\mathbf{8 3 5 . 4 1 9 6}$ & 418.2134 & 818.3931 & 409.7002 & 817.4090 & 409.2082 & $\mathbf{E}$ & 419.1885 & 210.0979 & 402.1619 & 201.5846 & 401.1779 & 201.0926 & $\mathbf{3}$ \\
\hline $\mathbf{8}$ & $\mathbf{9 5 0 . 4 4 6 5}$ & 475.7269 & 933.4200 & 467.2136 & 932.4360 & 466.7216 & $\mathbf{D}$ & $\mathbf{2 9 0 . 1 4 5 9}$ & 145.5766 & 273.1193 & 137.0633 & 272.1353 & 136.5713 & $\mathbf{2}$ \\
\hline $\mathbf{9}$ & & & & & & & $\mathbf{R}$ & $\mathbf{1 7 5 . 1 1 9 0}$ & 88.0631 & 158.0924 & 79.5498 & & & $\mathbf{1}$ \\
\hline
\end{tabular}
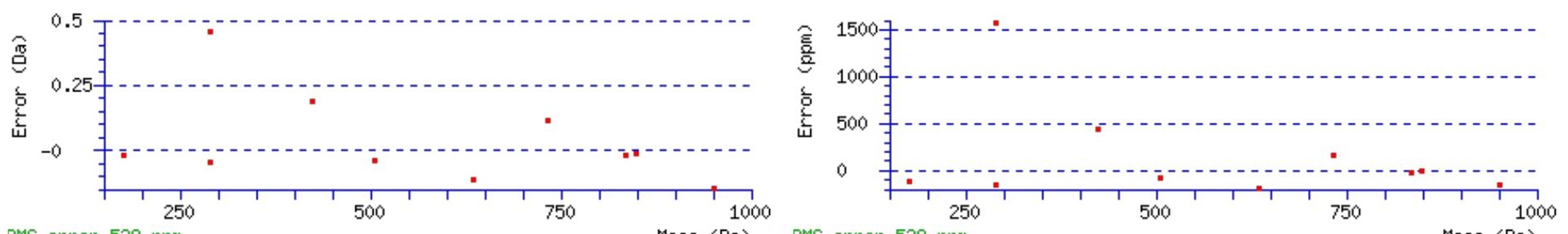

RMS error 528 ppm Mass (Da) RMS error 528 ppm

\section{All matches to this query}




\begin{tabular}{|l|l|l|l|} 
Score & Mr(calc): & Delta & \multicolumn{1}{|c|}{ Sequence } \\
\hline 37.6 & 1123.5509 & -0.0026 & $\underline{\text { IYNVSKEDR }}$ \\
\hline 5.9 & 1122.5417 & 1.0065 & AFGRSSNLNR \\
\hline 5.5 & 1122.5413 & 1.0070 & MMKNKVENK \\
\hline 5.5 & 1121.5304 & 2.0179 & MRTTKVYK \\
\hline 5.4 & 1123.5397 & 0.0086 & LYDLDKDDK \\
\hline 5.2 & 1123.5444 & 0.0039 & LYGNTPMRR \\
\hline 4.8 & 1121.5321 & 2.0162 & NKDMKEAMR \\
\hline 4.8 & 1121.5383 & 2.0100 & RAYKSYVR \\
\hline 4.4 & 1123.5387 & 0.0096 & GSKGTPAAVTR \\
\hline 3.9 & 1123.5557 & -0.0074 & AAFSVTGRCR \\
\hline
\end{tabular}

Spectrum No: 895; Query: 1400; Rank: 1

\section{Peptide View}

MS/MS Fragmentation of CNTAAPMWLNGSHPSSR

Found in IPI00198021, Tax_Id=10116 Gene_Symbol=Umod Uromodulin precursor

Match to Query 1400: 1885.823082 from(629.614970,3+)

Title: 100101RatKid_NS_deglyco_24.1953.1953.3.dta

Data file K:INewmanPaper|Piliangl3SubProteomes\Piliang3SP\mgf5ppm\ERLIC_3SubProteomes5ppm.mgf
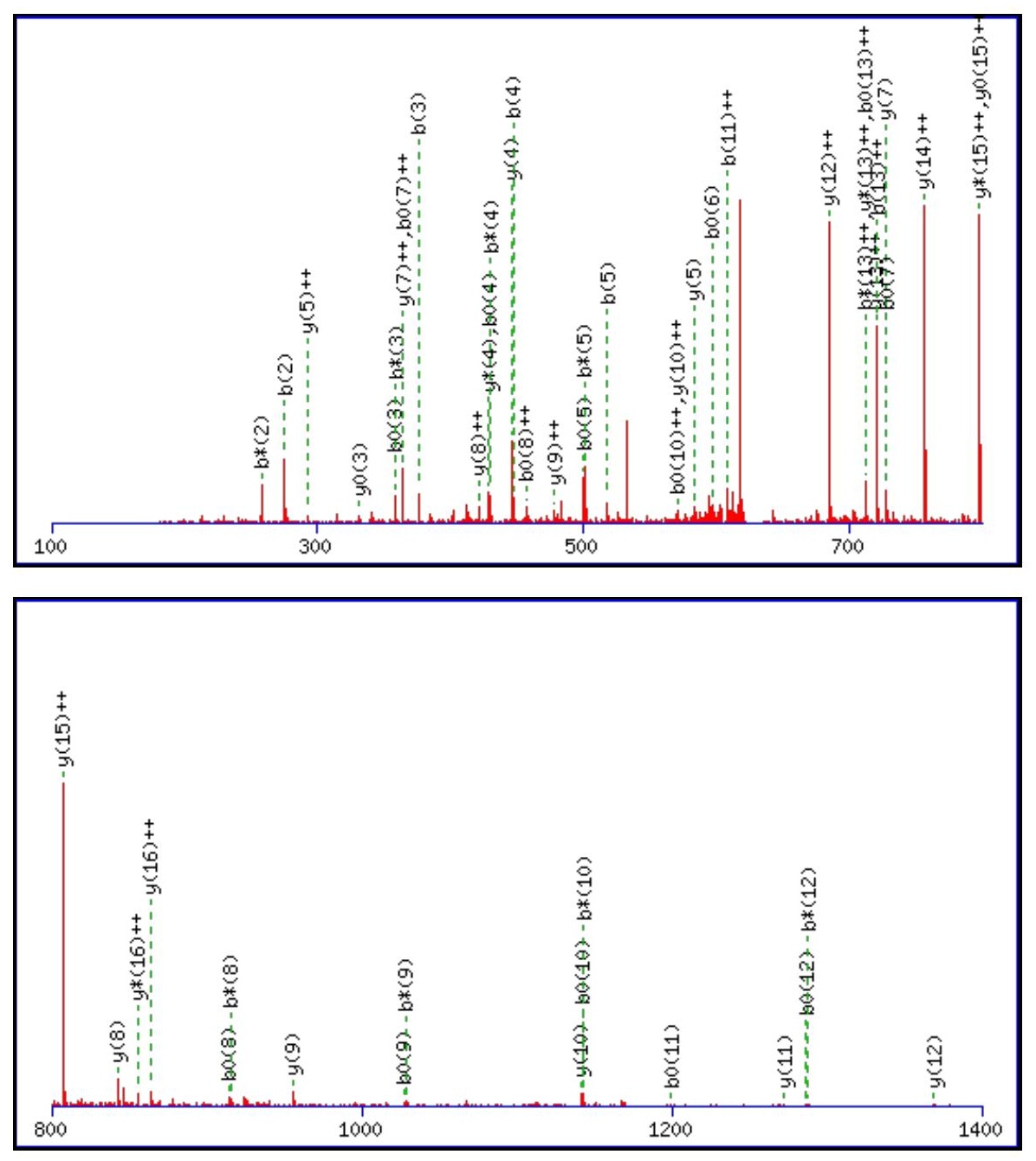


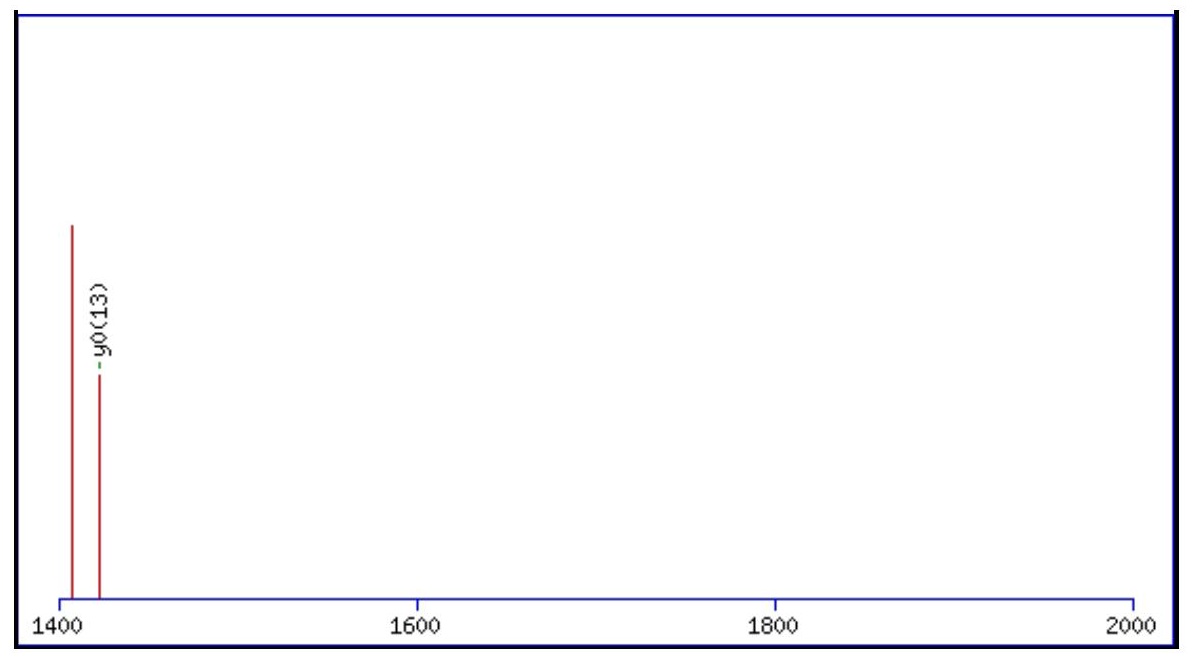

Monoisotopic mass of neutral peptide $\operatorname{Mr}($ calc): 1885.8199

Fixed modifications: Carbamidomethyl (C)

Variable modifications:

N10 : Deamidated_N ( N )

Ions Score: 37 Expect: 0.04

Matches (Bold Red): 54/184 fragment ions using 111 most intense peaks

\begin{tabular}{|c|c|c|c|c|c|c|c|c|c|c|c|c|c|c|}
\hline \# & b & $\mathbf{b}^{++}$ & $\mathbf{b}^{*}$ & $\mathbf{b}^{*^{++}}$ & $\mathbf{b}^{\mathbf{0}}$ & $\mathbf{b}^{\mathbf{0 + +}}$ & Seq. & $\mathbf{y}$ & $\mathbf{y}^{++}$ & $\mathbf{y}^{*}$ & $\mathbf{y}^{*^{++}}$ & $\mathbf{y}^{\mathbf{0}}$ & $\mathbf{y}^{0++}$ & \# \\
\hline 1 & 161.0379 & 81.0226 & & & & & C & & & & & & & 17 \\
\hline 2 & 275.0809 & 138.0441 & 258.0543 & 129.5308 & & & $\mathbf{N}$ & 1726.7966 & & 1709.7700 & 855.3886 & 1708.7860 & 854.8966 & 16 \\
\hline 3 & 376.1285 & 188.5679 & 359.1020 & 180.0546 & 358.1180 & 179.5626 & $\mathbf{T}$ & 1612.7536 & 806.8805 & 1595.7271 & 798.3672 & 1594.7431 & 797.8752 & 15 \\
\hline 4 & 447.1656 & 224.0865 & 430.1391 & 215.5732 & 429.1551 & 215.0812 & A & 1511.7060 & 756.3566 & 1494.6794 & 747.8433 & 1493.6954 & 747.3513 & 14 \\
\hline 5 & 518.2028 & 259.6050 & 501.1762 & 251.0917 & 500.1922 & 250.5997 & A & 1440.6689 & 720.8381 & 1423.6423 & 712.3248 & 1422.6583 & 711.8328 & 13 \\
\hline 6 & 615.2555 & 308.1314 & 598.2290 & 299.6181 & 597.2450 & 299.1261 & $\mathbf{P}$ & 1369.6317 & 685.3195 & 1352.6052 & 676.8062 & 1351.6212 & 676.3142 & 12 \\
\hline 7 & 746.2960 & 373.6516 & 729.2695 & 365.1384 & 728.2854 & 364.6464 & M & 1272.5790 & 636.7931 & 1255.5524 & 628.2799 & 1254.5684 & 627.7878 & 11 \\
\hline 8 & 932.3753 & 466.6913 & 915.3488 & 458.1780 & 914.3648 & 457.6860 & $\mathbf{W}$ & 1141.5385 & 571.2729 & 1124.5119 & 562.7596 & 1123.5279 & 562.2676 & 10 \\
\hline 9 & 1045.4594 & 523.2333 & 1028.4328 & 514.7201 & 1027.4488 & 514.2280 & $\mathbf{L}$ & 955.4592 & 478.2332 & 938.4326 & 469.7200 & 937.4486 & 469.2279 & 9 \\
\hline 10 & 1160.4863 & 580.7468 & 1143.4598 & 572.2335 & 1142.4758 & 571.7415 & $\mathbf{N}$ & 842.3751 & 421.6912 & 825.3486 & 413.1779 & 824.3645 & 412.6859 & 8 \\
\hline 11 & 1217.5078 & 609.2575 & 1200.4812 & 600.7443 & 1199.4972 & 600.2522 & $\mathbf{G}$ & 727.3482 & 364.1777 & 710.3216 & 355.6645 & 709.3376 & 355.1724 & 7 \\
\hline 12 & 1304.5398 & 652.7735 & 1287.5133 & 644.2603 & 1286.5292 & 643.7683 & S & 670.3267 & 335.6670 & 653.3002 & 327.1537 & 652.3161 & 326.6617 & 6 \\
\hline 13 & 1441.5987 & 721.3030 & 1424.5722 & 712.7897 & 1423.5882 & 712.2977 & $\mathbf{H}$ & 583.2947 & 292.1510 & 566.2681 & 283.6377 & 565.2841 & 283.1457 & 5 \\
\hline 14 & 1538.6515 & 769.8294 & 1521.6249 & 761.3161 & 1520.6409 & 760.8241 & $\mathbf{P}$ & 446.2358 & 223.6215 & 429.2092 & 215.1082 & 428.2252 & 214.6162 & 4 \\
\hline 15 & 1625.6835 & 813.3454 & 1608.6570 & 804.8321 & 1607.6730 & 804.3401 & S & 349.1830 & 175.0951 & 332.1565 & 166.5819 & 331.1724 & 166.0899 & 3 \\
\hline 16 & 1712.7155 & 856.8614 & 1695.6890 & 848.3481 & 1694.7050 & 847.8561 & $\mathbf{S}$ & 262.1510 & 131.5791 & 245.1244 & 123.0659 & 244.1404 & 122.5738 & 2 \\
\hline 17 & & & & & & & $\mathbf{R}$ & 175.1190 & 88.0631 & 158.0924 & 79.5498 & & & 1 \\
\hline
\end{tabular}

.

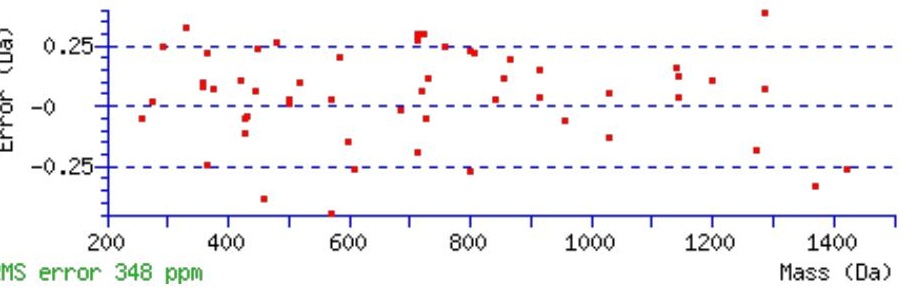

\section{All matches to this query}

\begin{tabular}{|l|l|l|l|}
\hline Score & Mr(calc): & Delta & \multicolumn{1}{c|}{ Sequence } \\
\hline 37.5 & 1885.8199 & 0.0032 & CNTAAPMWLNGSHPSSR \\
\hline 7.8 & 1885.8151 & 0.0079 & VVFAISELEEATYQK \\
\hline 5.8 & 1883.8302 & 1.9929 & SHEFTNSENLEKLEK \\
\hline 4.9 & 1883.8343 & 1.9888 & $\underline{\text { LLTGPGDYYTDYVATR }}$ \\
\hline & & & \\
\hline
\end{tabular}

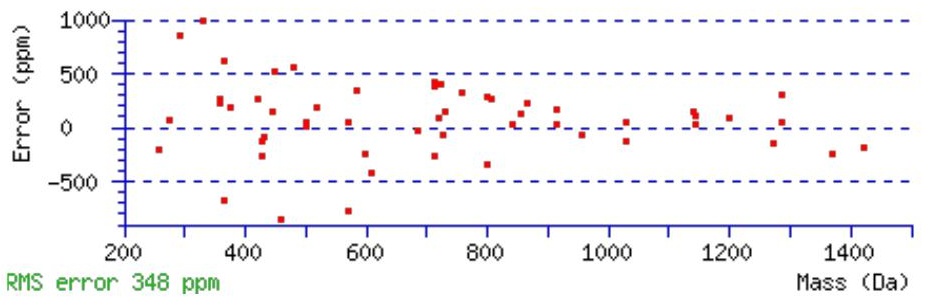

RMS error 348 ppm 


\begin{tabular}{|l||l|l|l|}
4.2 & 1883.8302 & 1.9929 & SHEFTNSENLEKLEK \\
\hline 4.0 & 1883.8104 & 2.0127 & AWENGEGGSWIWXTR \\
\hline 3.6 & 1883.7986 & 2.0245 & SCFRDTESYRGXGTR \\
\hline 3.6 & 1883.7986 & 2.0245 & SCFRDTESYRGXGTR \\
\hline 3.3 & 1883.8220 & 2.0011 & FRIDPSTGVLYTAER \\
\hline 3.2 & 1884.8359 & 0.9872 & CNTAAPMWLNGSHPSSR \\
\hline
\end{tabular}

Spectrum No: 896; Query: 742; Rank: 1

\section{Peptide View}

MS/MS Fragmentation of DGQLLPSSNYSNIK

Found in IPI00476991, Tax_Id=10116 Gene_Symbol=Ncam1 Neural cell adhesion molecule 1, 140 kDa isoform precursor

Match to Query 742: 1535.749928 from(768.882240,2+)

Title: 100101RatKid_NS_deglyco_26.2354.2354.2.dta

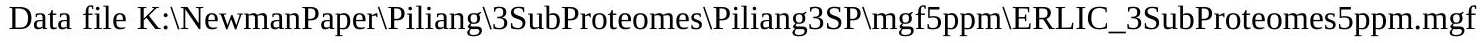
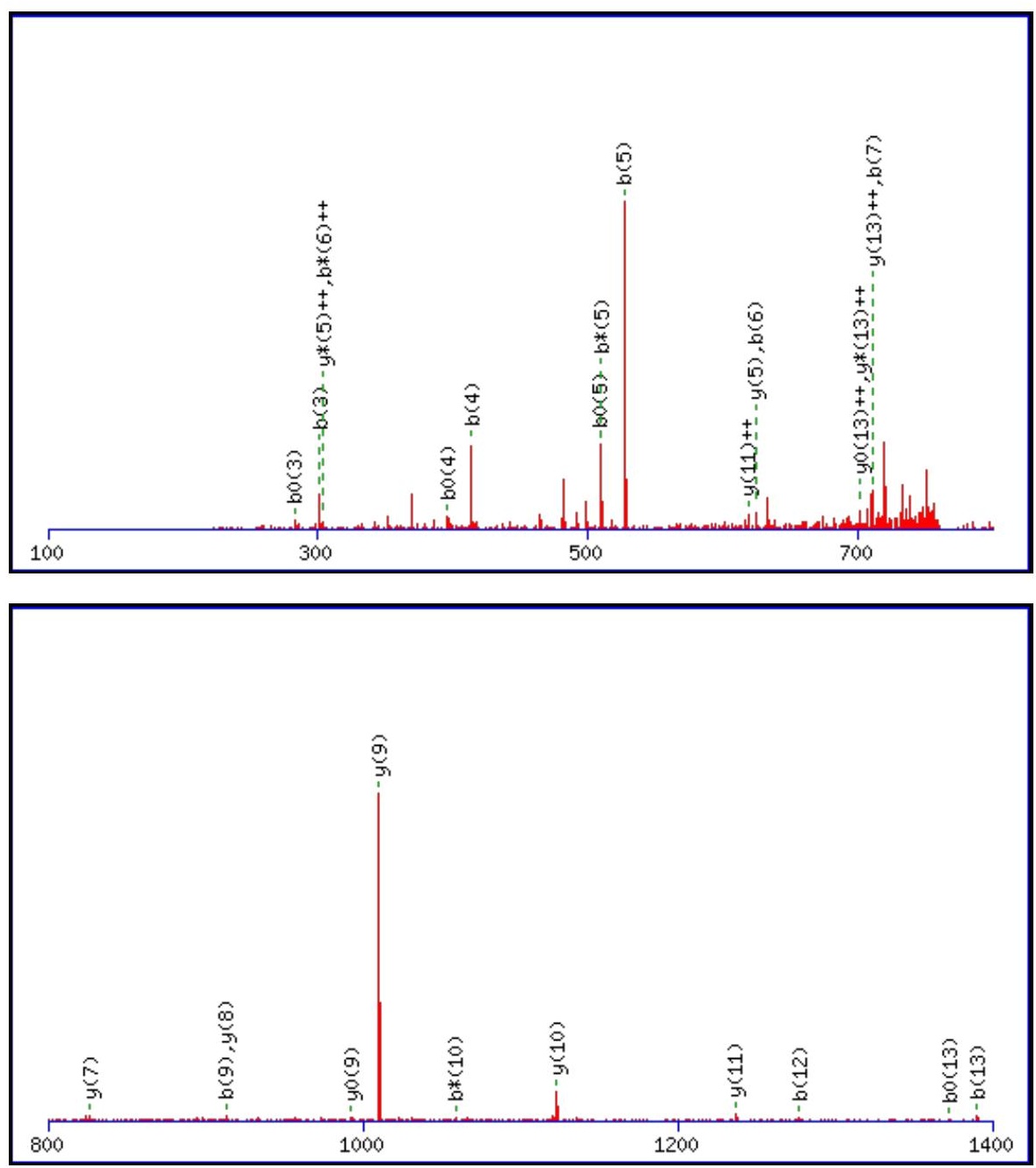


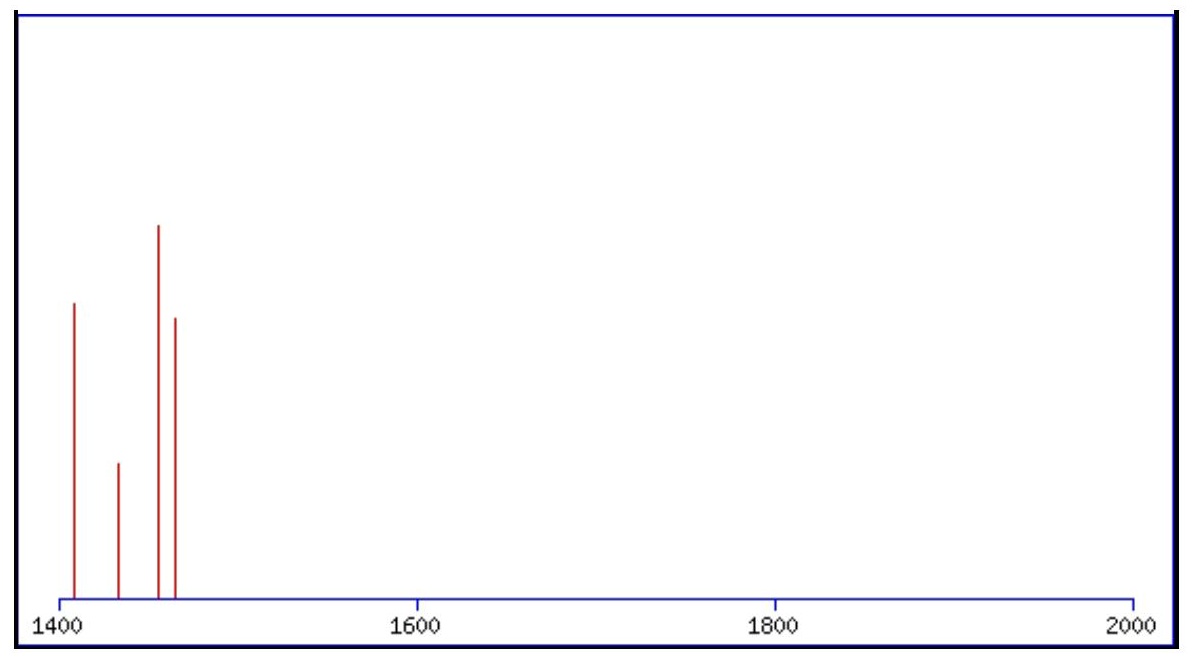

Monoisotopic mass of neutral peptide $\operatorname{Mr}($ calc): 1535.7467

Fixed modifications: Carbamidomethyl (C)

Variable modifications:

N9 : Deamidated_N (N)

Ions Score: 37 Expect : 0.027

Matches (Bold Red): 27/146 fragment ions using 52 most intense peaks

\begin{tabular}{|c|c|c|c|c|c|c|c|c|c|c|c|c|c|c|}
\hline \# & b & $\mathbf{b}^{++}$ & b* & $\mathbf{b}^{*++}$ & $\mathbf{b}^{\mathbf{0}}$ & $\mathbf{b}^{0++}$ & Seq. & $\mathbf{y}$ & $y^{++}$ & $\mathbf{y}^{*}$ & $\mathbf{y}^{*^{++}}$ & $\mathbf{y}^{\mathbf{0}}$ & $\mathbf{y}^{0++}$ & \# \\
\hline 1 & 116.0342 & 58.5207 & & & 98.0237 & 49.5155 & D & & & & & & & 14 \\
\hline 2 & 173.0557 & 87.0315 & & & 155.0451 & 78.0262 & G & 1421.7271 & 711.3672 & 1404.7005 & 702.8539 & 1403.7165 & 702.3619 & 13 \\
\hline 3 & 301.1143 & 151.0608 & 284.0877 & 142.5475 & 283.1037 & 142.0555 & $\mathbf{Q}$ & 1364.7056 & 682.8564 & 1347.6791 & 674.3432 & 1346.6950 & 673.8512 & 12 \\
\hline 4 & 414.1983 & 207.6028 & 397.1718 & 199.0895 & 396.1878 & 198.5975 & $\mathbf{L}$ & 1236.6470 & 618.8272 & 1219.6205 & 610.3139 & 1218.6365 & 609.8219 & 11 \\
\hline 5 & 527.2824 & 264.1448 & 510.2558 & 255.6316 & 509.2718 & 255.1396 & $\mathbf{L}$ & 1123.5630 & 562.2851 & 1106.5364 & 553.7718 & 1105.5524 & 553.2798 & 10 \\
\hline 6 & 624.3352 & 312.6712 & 607.3086 & 304.1579 & 606.3246 & 303.6659 & $\mathbf{P}$ & 1010.4789 & 505.7431 & 993.4524 & 497.2298 & 992.4683 & 496.7378 & 9 \\
\hline 7 & 711.3672 & 356.1872 & 694.3406 & 347.6740 & 693.3566 & 347.1819 & S & 913.4261 & 457.2167 & 896.3996 & 448.7034 & 895.4156 & 448.2114 & 8 \\
\hline 8 & 798.3992 & 399.7032 & 781.3727 & 391.1900 & 780.3886 & 390.6980 & S & 826.3941 & 413.7007 & 809.3676 & 405.1874 & 808.3836 & 404.6954 & 7 \\
\hline 9 & 913.4261 & 457.2167 & 896.3996 & 448.7034 & 895.4156 & 448.2114 & $\mathbf{N}$ & 739.3621 & 370.1847 & 722.3355 & 361.6714 & 721.3515 & 361.1794 & 6 \\
\hline 10 & 1076.4895 & 538.7484 & 1059.4629 & 530.2351 & 1058.4789 & 529.7431 & $\mathbf{Y}$ & 624.3352 & 312.6712 & 607.3086 & 304.1579 & 606.3246 & 303.6659 & 5 \\
\hline 11 & 1163.5215 & 582.2644 & 1146.4950 & 573.7511 & 1145.5109 & 573.2591 & S & 461.2718 & 231.1395 & 444.2453 & 222.6263 & 443.2613 & 222.1343 & 4 \\
\hline 12 & 1277.5644 & 639.2859 & 1260.5379 & 630.7726 & 1259.5539 & 630.2806 & $\mathbf{N}$ & 374.2398 & 187.6235 & 357.2132 & 179.1103 & & & 3 \\
\hline 13 & 1390.6485 & 695.8279 & 1373.6219 & 687.3146 & 1372.6379 & 686.8226 & I & 260.1969 & 130.6021 & 243.1703 & 122.0888 & & & 2 \\
\hline 14 & & & & & & & $\mathbf{K}$ & 147.1128 & 74.0600 & 130.0863 & 65.5468 & & & 1 \\
\hline
\end{tabular}
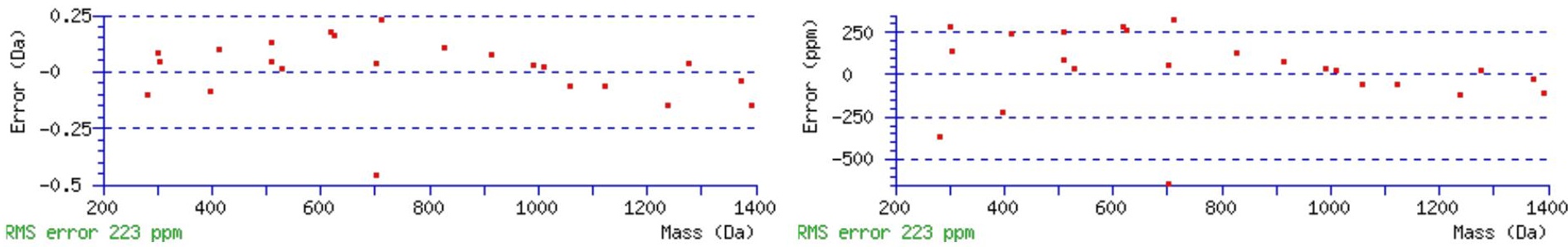

\section{All matches to this query}

\begin{tabular}{|l|l|l|l|}
\hline Score & Mr(calc): & Delta & \multicolumn{1}{c|}{ Sequence } \\
\hline 37.5 & 1535.7467 & 0.0032 & DGQLLPSSNYSNIK \\
\hline 31.3 & 1535.7467 & 0.0032 & DGQLLPSSNYSNIK \\
\hline 17.0 & 1535.7467 & 0.0032 & NGEPEPGIPVVNGEK \\
\hline 13.9 & 1535.7596 & -0.0097 & VEGKLLDDPSKQK \\
\hline 12.8 & 1534.7585 & 0.9914 & LWILRTYFTNK \\
\hline 12.8 & 1534.7585 & 0.9914 & LWILRTYFTNK \\
\hline 12.3 & 1535.7409 & 0.0091 & LNWLSVDFNNWK \\
\hline & & & \\
\hline
\end{tabular}




\begin{tabular}{|l|l|l|l|}
12.3 & 1535.7409 & 0.0091 & LNWLSVDFNNWK \\
\hline 7.5 & 1535.7362 & 0.0138 & EEASKARAQGSMQK \\
\hline 7.1 & 1535.7457 & 0.0042 & DLGLGAGELAARSAR \\
\hline
\end{tabular}

Spectrum No: 897; Query: 24; Rank: 1

\section{Peptide View}

MS/MS Fragmentation of LNLTEGEK

Found in IPI00359436, Tax_Id=10116 Gene_Symbol=RGD1308358_predicted hypothetical protein LOC291952

Match to Query 24: 903.453328 from(452.733940,2+)

Title: 091008RatKidney_NH4Format01_28.652.652.2.dta

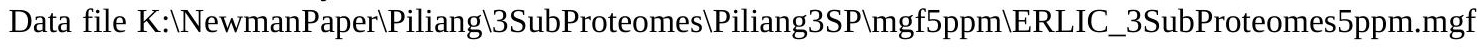
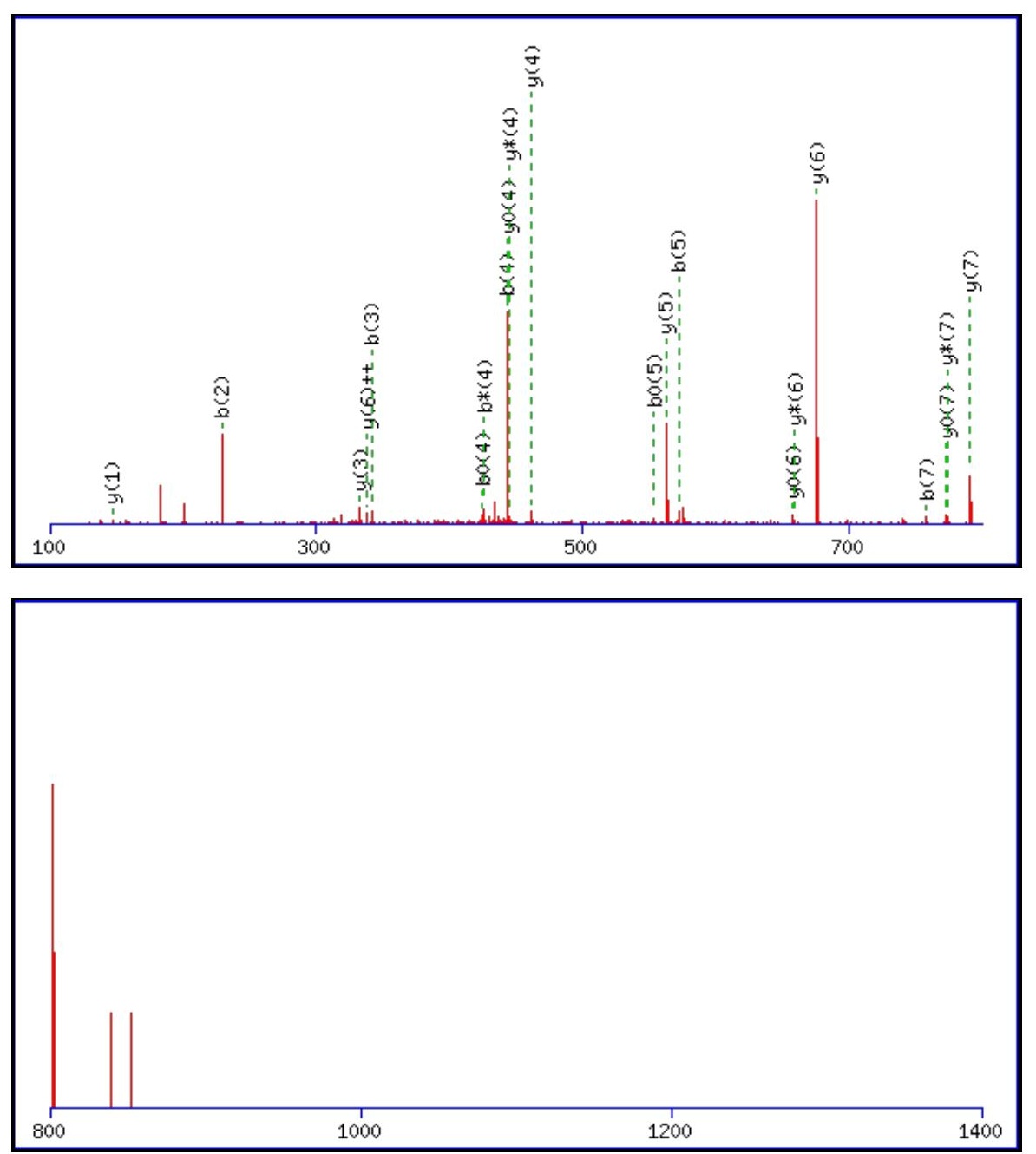


$1400 \quad 1600 \quad 1800 \quad 2000$

Monoisotopic mass of neutral peptide $\operatorname{Mr}($ calc): 903.4549

Fixed modifications: Carbamidomethyl (C)

Variable modifications:

N2 : Deamidated $\mathrm{N}(\mathrm{N})$

Ions Score: 37 Expect : 0.017

Matches (Bold Red): 21/74 fragment ions using 41 most intense peaks

\begin{tabular}{|c|c|c|c|c|c|c|c|c|c|c|c|c|c|c|}
\hline & b & $\mathbf{b}^{++}$ & $\mathbf{b}^{*}$ & $\mathbf{b}^{*^{++}}$ & $\mathbf{b}^{0}$ & $\mathbf{b}^{\mathbf{0 + +}}$ & Seq. & $\mathbf{y}$ & $\mathbf{y}^{++}$ & $\mathbf{y}^{*}$ & $\mathrm{y}^{\mathrm{*}^{++}}$ & $\mathbf{y}^{\mathbf{0}}$ & $y^{0++}$ & $\#$ \\
\hline 1 & 114.0913 & 57.5493 & & & & & $\mathbf{L}$ & & & & & & & 8 \\
\hline 2 & 229.1183 & 115.0628 & 212.0917 & 106.5495 & & & $\mathbf{N}$ & 791.3781 & 396.1927 & 774.3516 & 387.6794 & 773.3676 & 387.1874 & \\
\hline 3 & 342.2023 & 171.6048 & 325.1758 & 163.0915 & & & $\mathbf{L}$ & 676.3512 & 338.6792 & 659.3246 & 330.1660 & 658.3406 & 329.6740 & \\
\hline 4 & 443.2500 & 222.1286 & 426.2235 & 213.6154 & 425.2395 & 213.1234 & $\mathbf{T}$ & 563.2671 & 282.1372 & 546.2406 & 273.6239 & 545.2566 & 273.1319 & \\
\hline 5 & 572.2926 & 286.6499 & 555.2661 & 278.1367 & 554.2820 & 277.6447 & $E$ & 462.2195 & 231.6134 & 445.1929 & 223.1001 & 444.2089 & 222.6081 & 1 \\
\hline 6 & 629.3141 & 315.1607 & 612.2875 & 306.6474 & 611.3035 & 306.1554 & G & 333.1769 & 167.0921 & 316.1503 & 158.5788 & 315.1663 & 158.0868 & \\
\hline 7 & 758.3567 & 379.6820 & 741.3301 & 371.1687 & 740.3461 & 370.6767 & $\mathbf{E}$ & 276.1554 & 138.5813 & 259.1288 & 130.0681 & 258.1448 & 129.5761 & 2 \\
\hline & & & & & & & $\mathbf{K}$ & 147.1128 & 74.0600 & 130.0863 & 65.5468 & & & \\
\hline
\end{tabular}

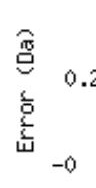

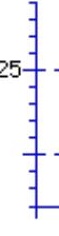

RMS error $315 \mathrm{ppm}$

\section{All matches to this query}

\begin{tabular}{|l|l|l|l|}
\hline Score & Mr(calc): & Delta & Sequence \\
\hline 37.5 & 903.4549 & -0.0016 & $\underline{\text { LNLTEGEK }}$ \\
\hline 14.5 & 903.4549 & -0.0016 & $\underline{\text { LDTLNAEK }}$ \\
\hline 12.5 & 903.4549 & -0.0016 & $\underline{\text { TVEIEGEK }}$ \\
\hline 11.6 & 903.4484 & 0.0049 & $\underline{\text { IDCPSGKK }}$ \\
\hline 10.2 & 903.4549 & -0.0016 & $\underline{\text { LDSNVEVK }}$ \\
\hline 8.9 & 903.4524 & 0.0009 & $\underline{\text { MLTPEWK }}$ \\
\hline 7.1 & 903.4562 & -0.0029 & $\underline{\text { LNNXPQR }}$ \\
\hline 7.0 & 903.4558 & -0.0025 & $\underline{\text { MPSLTMPK }}$ \\
\hline 6.0 & 902.4458 & 1.0076 & $\underline{\text { QVDVETGR }}$ \\
\hline 5.8 & 901.4422 & 2.0111 & $\underline{\text { QIHPSLK }}$ \\
\hline
\end{tabular}

Spectrum No: 898; Query: 83; Rank: 1 


\section{Peptide View}

MS/MS Fragmentation of YFANCTVR

Found in IPI00211230, Tax_Id=10116 Gene_Symbol=Tm2d3_predicted 26 kDa protein

Match to Query 83: 1030.455988 from(516.235270,2+)

Title: 091008RatKidney_NH4Format01_25.954.954.2.dta

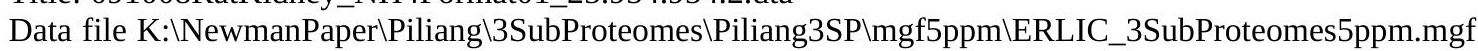
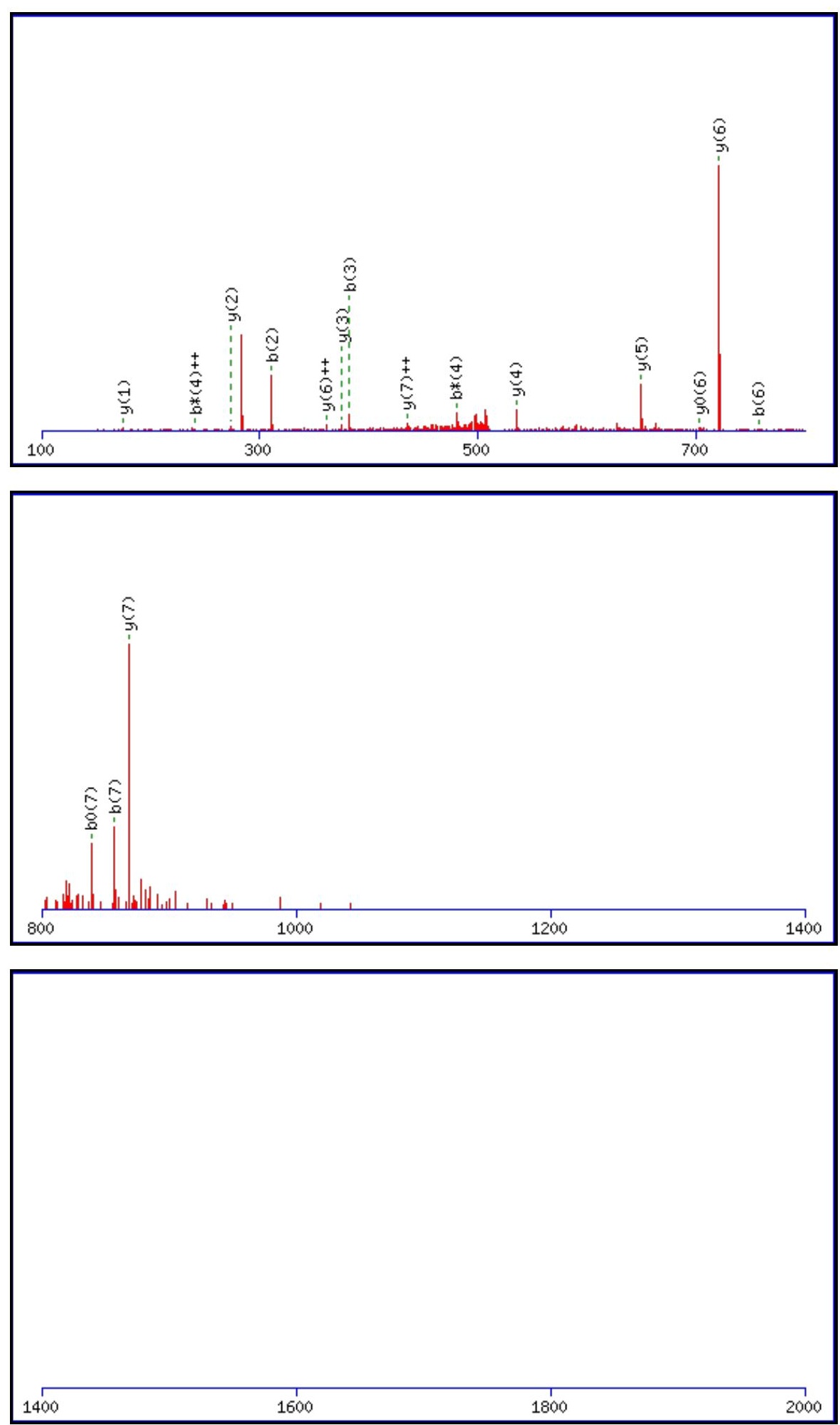

Monoisotopic mass of neutral peptide $\operatorname{Mr}($ calc): 1030.4542

Fixed modifications: Carbamidomethyl (C)

Variable modifications:

N4 : Deamidated_N (N)

Ions Score: 38 Expect: 0.016 
Matches (Bold Red): 17/64 fragment ions using 52 most intense peaks

\begin{tabular}{|c|c|c|c|c|c|c|c|c|c|c|c|c|c|c|}
\hline \# & b & $\mathbf{b}^{++}$ & $\mathbf{b}^{*}$ & $\mathbf{b}^{*^{++}}$ & $\mathbf{b}^{0}$ & $\mathbf{b}^{\mathbf{0 + +}}$ & Seq. & $\mathbf{y}$ & $\mathbf{y}^{++}$ & $\mathbf{y}^{*}$ & $\mathrm{y}^{\mathrm{*}^{++}}$ & $\mathbf{y}^{\mathbf{0}}$ & $\mathbf{y}^{\mathbf{0 + +}^{++}}$ & $\#$ \\
\hline 1 & 164.0706 & 82.5389 & & & & & $\mathbf{Y}$ & & & & & & & 8 \\
\hline 2 & 311.1390 & 156.0731 & & & & & $\mathbf{F}$ & 868.3982 & 434.7027 & 851.3716 & 426.1894 & 850.3876 & 425.6974 & 47 \\
\hline $3:$ & 382.1761 & 191.5917 & & & & & A & \begin{tabular}{|l|l|}
721.3297 \\
\end{tabular} & 361.1685 & 704.3032 & 352.6552 & 703.3192 & 352.1632 & 26 \\
\hline 4 & 497.2031 & 249.1052 & 480.1765 & 240.5919 & & & $\mathbf{N}$ & 650.2926 & 325.6500 & 633.2661 & 317.1367 & 632.2821 & 316.6447 & 75 \\
\hline 5 & 657.2337 & 329.1205 & 640.2072 & 320.6072 & & & $\mathrm{C}$ & 535.2657 & 268.1365 & 518.2391 & 259.6232 & 517.2551 & 259.1312 & 24 \\
\hline 6 & 758.2814 & 379.6443 & 741.2548 & 371.1311 & 740.2708 & 370.6391 & $T$ & 375.2350 & 188.1212 & 358.2085 & 179.6079 & 357.2245 & 179.1159 & 93 \\
\hline \begin{tabular}{|l|}
7 \\
\end{tabular} & 857.3498 & 429.1785 & 840.3233 & 420.6653 & 839.3392 & 420.1733 & V & 274.1874 & 137.5973 & 257.1608 & 129.0840 & & & 2 \\
\hline 8 & & & & & & & $\mathbf{R}$ & 175.1190 & 88.0631 & 158.0924 & 79.5498 & & & 1 \\
\hline
\end{tabular}
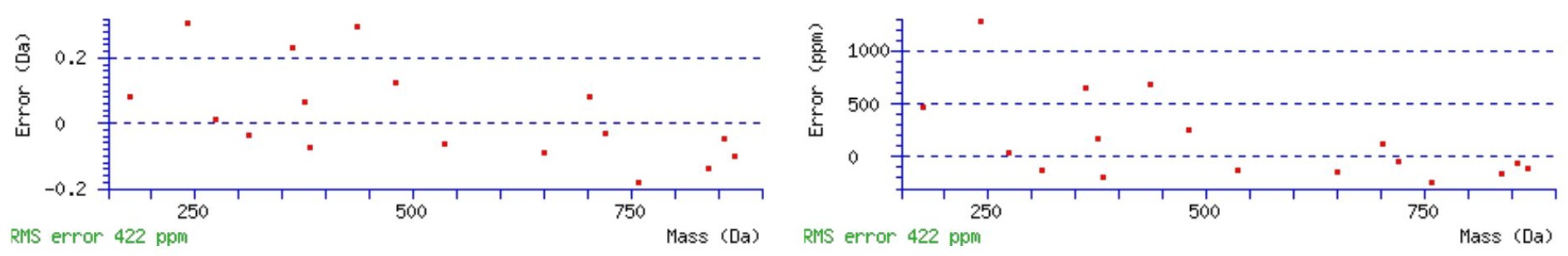

\section{All matches to this query}

\begin{tabular}{|l|l|c|l|}
\hline Score & Mr(calc): & Delta & \multicolumn{1}{|c|}{ Sequence } \\
\hline 37.5 & 1030.4542 & 0.0018 & YFANCTVR \\
\hline 10.2 & 1030.4518 & 0.0042 & KTASEMIR \\
\hline 5.4 & 1030.4485 & 0.0075 & TESAQLFR \\
\hline 3.3 & 1029.4532 & 1.0028 & $\underline{\text { AASAPPPPDK }}$ \\
\hline 0.9 & 1030.4597 & -0.0037 & DGIGHTPVR \\
\hline 0.8 & 1028.4441 & 2.0119 & HNGVGGSPPK \\
\hline 0.8 & 1029.4539 & 1.0021 & QTRSMRR \\
\hline 0.2 & 1030.4597 & -0.0037 & TEIRNYR \\
\hline 0.1 & 1030.4654 & -0.0095 & YRMFDQR \\
\hline
\end{tabular}

Spectrum No: 899; Query: 1239; Rank: 1

\section{Peptide View}

MS/MS Fragmentation of INNTHALVSLLQNLNK

Found in IPI00200257, Tax_Id=10116 Gene_Symbol=Cdh13 T-cadherin

Match to Query 1239: 1791.981192 from(598.334340,3+)

Title: 091008RatKidney_NoSalt_21.4583.4583.3.dta

Data file K:INewmanPaper|Piliang|3SubProteomes\Piliang3SP\mgf5ppm|ERLIC_3SubProteomes5ppm.mgf 

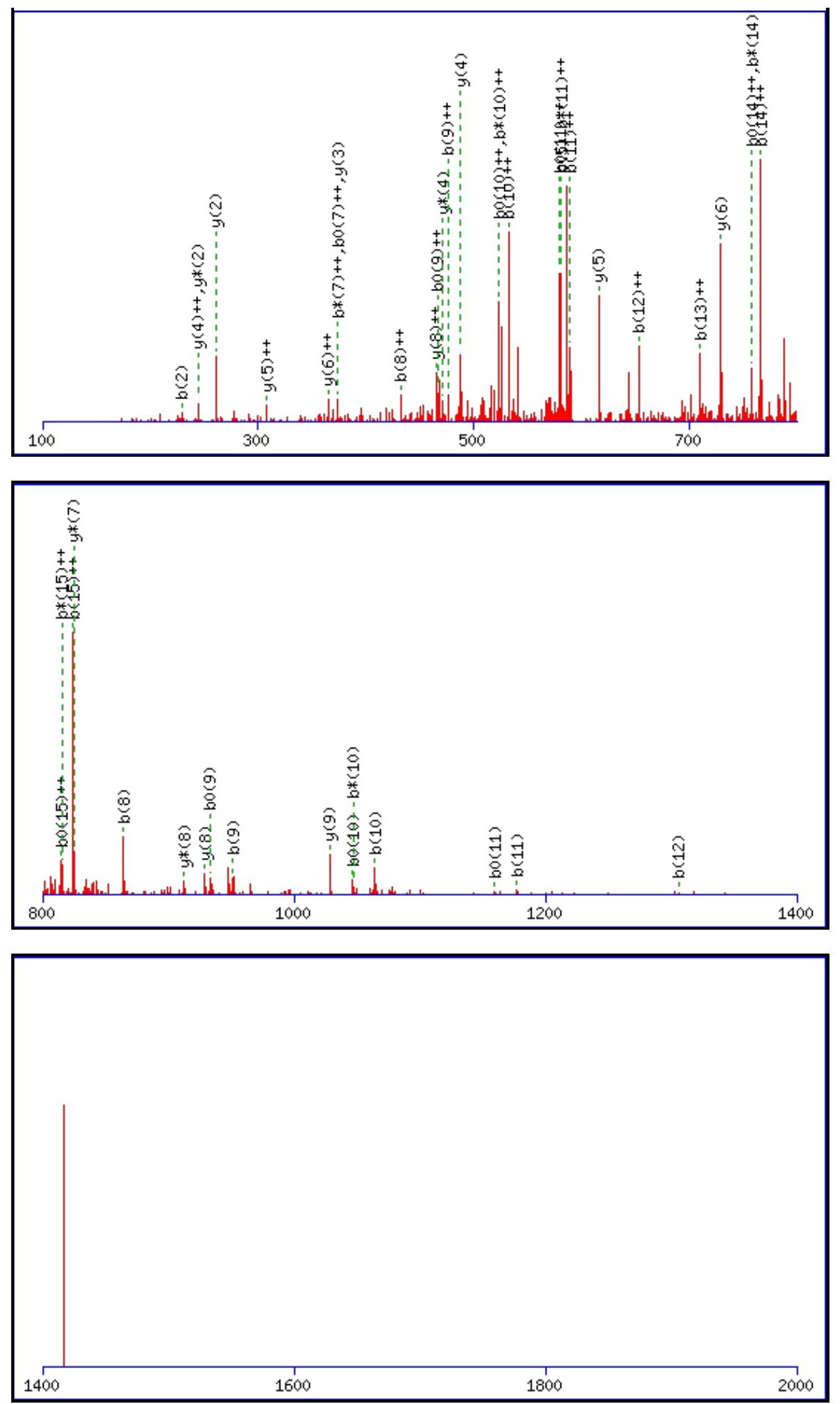

Monoisotopic mass of neutral peptide $\operatorname{Mr}($ calc): 1791.9843

Fixed modifications: Carbamidomethyl (C)

Variable modifications:

N2 : Deamidated_N (N)

Ions Score: 37 Expect : 0.012

Matches (Bold Red): 45/158 fragment ions using 72 most intense peaks

\begin{tabular}{|r|c|c|c|c|c|c|c|c|c|c|c|c|c|c|}
\hline$\#$ & $\mathbf{b}$ & $\mathbf{b}^{++}$ & $\mathbf{b}^{*}$ & $\mathbf{b}^{\boldsymbol{*}^{++}}$ & $\mathbf{b}^{\mathbf{0}}$ & $\mathbf{b}^{\mathbf{0 + +}}$ & $\mathbf{S e q}$ & $\mathbf{y}$ & $\mathbf{y}^{++}$ & $\mathbf{y}^{\mathbf{*}}$ & $\mathbf{y}^{\boldsymbol{*}^{++}}$ & $\mathbf{y}^{\mathbf{0}}$ & $\mathbf{y}^{\mathbf{0 + +}}$ & $\#$ \\
\hline $\mathbf{1}$ & 114.0913 & 57.5493 & & & & & $\mathbf{I}$ & & & & & & & $\mathbf{1 6}$ \\
\hline $\mathbf{2}$ & $\mathbf{2 2 9 . 1 1 8 3}$ & 115.0628 & 212.0917 & 106.5495 & & & $\mathbf{N}$ & 1679.9075 & 840.4574 & 1662.8810 & 831.9441 & 1661.8969 & 831.4521 & $\mathbf{1 5}$ \\
\hline $\mathbf{3}$ & 343.1612 & 172.0842 & 326.1347 & 163.5710 & & & $\mathbf{N}$ & 1564.8806 & 782.9439 & 1547.8540 & 774.4306 & 1546.8700 & 773.9386 & $\mathbf{1 4}$ \\
\hline $\mathbf{4}$ & 444.2089 & 222.6081 & 427.1823 & 214.0948 & 426.1983 & 213.6028 & $\mathbf{T}$ & 1450.8376 & 725.9225 & 1433.8111 & 717.4092 & 1432.8271 & 716.9172 & $\mathbf{1 3}$ \\
\hline $\mathbf{5}$ & $\mathbf{5 8 1 . 2 6 7 8}$ & 291.1375 & 564.2412 & 282.6243 & 563.2572 & 282.1323 & $\mathbf{H}$ & 1349.7900 & 675.3986 & 1332.7634 & 666.8853 & 1331.7794 & 666.3933 & $\mathbf{1 2}$ \\
\hline
\end{tabular}




\begin{tabular}{|c|c|c|c|c|c|c|c|c|c|c|c|c|c|c|}
\hline 6 & 652.3049 & |326.6561 & 635.2784 & 318.1428 & 634.2943 & |317.6508 & $\mathbf{A}$ & |1212.7310 & |606.8692 & |1195.7045 & |598.3559 & |1194.7205 & |597.8639 & 11 \\
\hline 7 & 765.3890 & 383.1981 & 748.3624 & 374.6848 & 747.3784 & 374.1928 & $\mathbf{L}$ & 1141.6939 & 571.3506 & 1124.6674 & 562.8373 & 1123.6834 & 562.3453 & 10 \\
\hline 8 & 864.4574 & 432.7323 & 847.4308 & 424.2191 & 846.4468 & 423.7270 & V & 1028.6099 & 514.8086 & 1011.5833 & 506.2953 & 1010.5993 & 505.8033 & 9 \\
\hline 9 & 951.4894 & 476.2483 & 934.4629 & 467.7351 & 933.4788 & 467.2431 & $\mathbf{S}$ & 929.5415 & 465.2744 & 912.5149 & 456.7611 & 911.5309 & 456.2691 & 8 \\
\hline 10 & 1064.5735 & 532.7904 & 1047.5469 & 524.2771 & 1046.5629 & 523.7851 & $\mathbf{L}$ & 842.5094 & 421.7584 & 825.4829 & 413.2451 & & & 7 \\
\hline 11 & 1177.6575 & 589.3324 & 1160.6310 & 580.8191 & 1159.6470 & 580.3271 & $\mathbf{L}$ & 729.4254 & 365.2163 & 712.3988 & 356.7030 & & & 6 \\
\hline 12 & 1305.7161 & 653.3617 & 1288.6896 & 644.8484 & 1287.7056 & 644.3564 & $\mathbf{Q}$ & 616.3413 & 308.6743 & 599.3148 & 300.1610 & & & 5 \\
\hline 13 & 1419.7590 & 710.3832 & 1402.7325 & 701.8699 & 1401.7485 & 701.3779 & $\mathbf{N}$ & 488.2827 & 244.6450 & 471.2562 & 236.1317 & & & 4 \\
\hline 14 & 1532.8431 & 766.9252 & 1515.8166 & 758.4119 & 1514.8325 & 757.9199 & $\mathbf{L}$ & 374.2398 & 187.6235 & 357.2132 & 179.1103 & & & 3 \\
\hline 15 & 1646.8860 & 823.9467 & 1629.8595 & 815.4334 & 1628.8755 & 814.9414 & $\mathbf{N}$ & 261.1557 & 131.0815 & 244.1292 & 122.5682 & & & 2 \\
\hline 16 & & & & & & & $\mathbf{K}$ & 147.1128 & 74.0600 & 130.0863 & 65.5468 & & & 1 \\
\hline
\end{tabular}
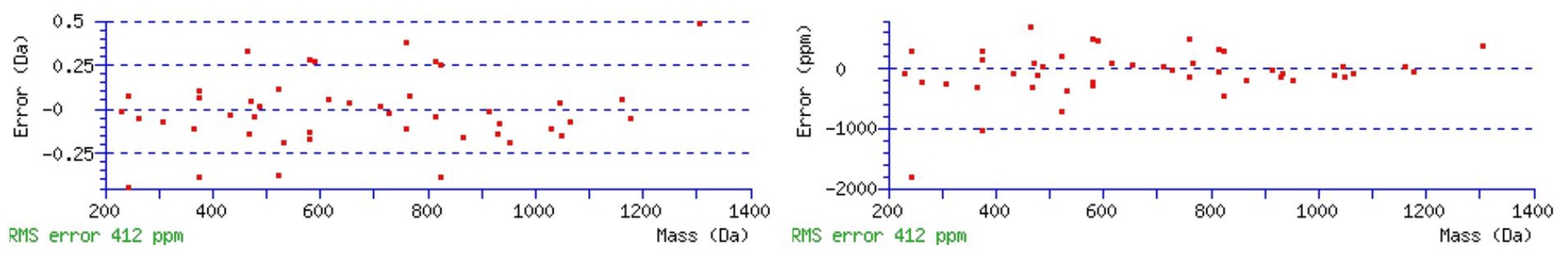

\section{All matches to this query}

\begin{tabular}{|l|l|l|l|}
\hline Score & Mr(calc): & Delta & \multicolumn{1}{|c|}{ Sequence } \\
\hline 37.3 & 1791.9843 & -0.0031 & INNTHALVSLLQNLNK \\
\hline 32.0 & 1791.9843 & -0.0031 & INNTHALVSLLQNLNK \\
\hline 9.7 & 1791.9665 & 0.0147 & QRKYCVVLLTAEANK \\
\hline 8.2 & 1791.9648 & 0.0164 & LKNSKIVISDFHLAK \\
\hline 6.7 & 1790.9655 & 1.0157 & VRNATDAVGVVLKELK \\
\hline 5.7 & 1791.9843 & -0.0031 & INNTHALVSLLQNLNK \\
\hline 5.4 & 1791.9804 & 0.0008 & MLEGKGKISVNDFIIK \\
\hline 5.4 & 1791.9706 & 0.0106 & GKIGIFPKAFVEGCAAK \\
\hline 3.0 & 1791.9764 & 0.0048 & TASDMVSTSRILVAVVK \\
\hline 1.8 & 1791.9876 & -0.0064 & SSLLSKRIASSMVGVNK \\
\hline
\end{tabular}

Spectrum No: 900; Query: 2278; Rank: 1

\section{Peptide View}

MS/MS Fragmentation of DLHVPIPAVVEMNPNVSVFIPPR

Found in IPI00368874, Tax_Id=10116 Gene_Symbol=LOC299458 LOC299458 protein

Match to Query 2278: 2541.350592 from(848.124140,3+)

Title: 100101RatKid_NS_deglyco_24.5381.5381.3.dta

Data file K:INewmanPaper|Piliang 3 SubProteomes\Piliang3SP\mgf5ppm\ERLIC_3SubProteomes5ppm.mgf 

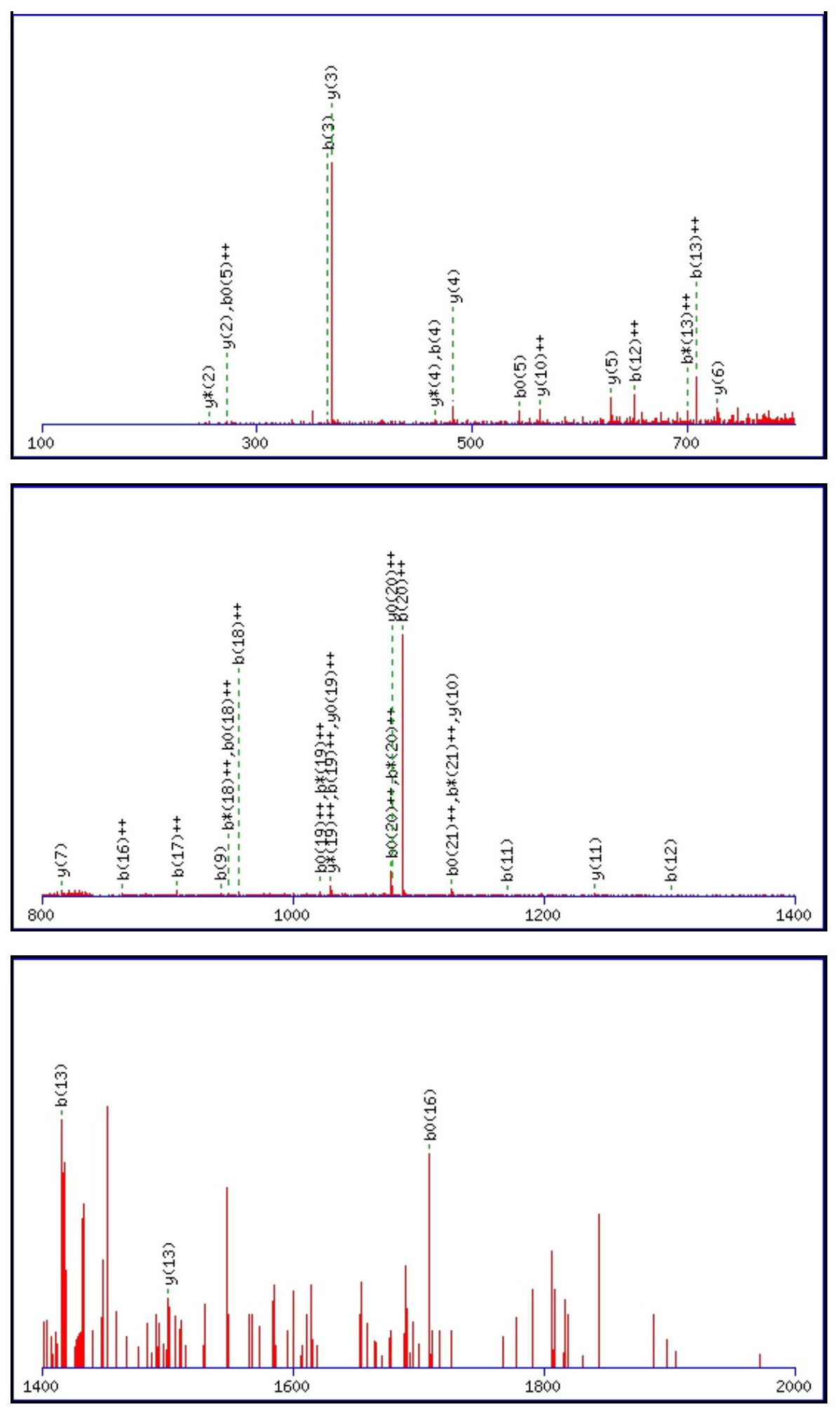

Monoisotopic mass of neutral peptide $\operatorname{Mr}($ calc): 2540.3461

Fixed modifications: Carbamidomethyl (C)

Variable modifications:

N15 : Deamidated_N (N)

Ions Score: 37 Expect: 0.016

Matches (Bold Red): 40/228 fragment ions using 77 most intense peaks

\begin{tabular}{|c|c|c|c|c|c|c|c|c|c|c|c|c|c|c|}
\hline$\#$ & b & $\mathbf{b}^{++}$ & $\mathbf{b}^{*}$ & $\mathbf{b}^{*^{++}}$ & $\mathbf{b}^{\mathbf{0}}$ & $\mathbf{b}^{0++}$ & Seq. & $\mathbf{y}$ & $y^{++}$ & $\mathbf{y}^{*}$ & $y^{*^{++}}$ & $\mathbf{y}^{0}$ & $y^{0++}$ & $\#$ \\
\hline 1 & 116.0342 & 58.5207 & & & 98.0237 & 49.5155 & D & & & & & & & 23 \\
\hline 2 & 229.1183 & 115.0628 & & & 211.1077 & 106.0575 & L & 2426.3264 & 1213.6669 & 2409.2999 & 1205.1536 & 2408.3159 & 1204.6616 & 22 \\
\hline 3 & 366.1772 & 183.5922 & & & 348.1666 & 174.5870 & $\mathbf{H}$ & 2313.2424 & 1157.1248 & 2296.2158 & 1148.6116 & 2295.2318 & 1148.1195 & 21 \\
\hline 4 & 465.2456 & 233.1264 & & & 447.2350 & 224.1212 & V & 2176.1835 & 1088.5954 & |2159.1569 & 1080.0821 & 2158.1729 & 1079.5901 & 20 \\
\hline 5 & 562.2984 & 281.6528 & & & 544.2878 & 272.6475 & $\mathbf{P}$ & 2077.1151 & 1039.0612 & 2060.0885 & 1030.5479 & 2059.1045 & 1030.0559 & 19 \\
\hline
\end{tabular}




\begin{tabular}{|c|c|c|c|c|c|c|c|c|c|c|c|c|c|c|}
\hline 6 & 675.3824 & 338.1949 & & & 657.3719 & 329.1896 & I & |1980.0623 & 990.5348 & |1963.0357 & 982.0215 & | 1962.0517 | & 981.5295 & 18 \\
\hline 7 & 772.4352 & 386.7212 & & & 754.4246 & 377.7160 & $\mathbf{P}$ & 1866.9782 & 933.9928 & 1849.9517 & 925.4795 & 1848.9677 & 924.9875 & 17 \\
\hline 8 & 843.4723 & 422.2398 & & & 825.4618 & 413.2345 & A & 1769.9255 & 885.4664 & 1752.8989 & 876.9531 & 1751.9149 & 876.4611 & 16 \\
\hline 9 & 942.5407 & 471.7740 & & & 924.5302 & 462.7687 & V & 1698.8883 & 849.9478 & 1681.8618 & 841.4345 & 1680.8778 & 840.9425 & 15 \\
\hline 10 & 1041.6091 & 521.3082 & & & 1023.5986 & 512.3029 & $\mathbf{V}$ & 1599.8199 & 800.4136 & 1582.7934 & 791.9003 & 1581.8094 & 791.4083 & 14 \\
\hline 11 & 1170.6517 & 585.8295 & & & 1152.6412 & 576.8242 & $\mathbf{E}$ & 1500.7515 & 750.8794 & 1483.7250 & 742.3661 & 1482.7410 & 741.8741 & 13 \\
\hline 12 & 1301.6922 & 651.3497 & & & 1283.6817 & 642.3445 & M & 1371.7089 & 686.3581 & 1354.6824 & 677.8448 & 1353.6984 & 677.3528 & 12 \\
\hline 13 & \begin{tabular}{|l|}
1415.7351 \\
\end{tabular} & 708.3712 & 1398.7086 & 699.8579 & 1397.7246 & 699.3659 & $\mathbf{N}$ & 1240.6684 & 620.8379 & 1223.6419 & 612.3246 & 1222.6579 & 611.8326 & 11 \\
\hline 14 & 1512.7879 & 756.8976 & 1495.7614 & 748.3843 & 1494.7773 & 747.8923 & $\mathbf{P}$ & 1126.6255 & 563.8164 & 1109.5990 & 555.3031 & 1108.6149 & 554.8111 & 10 \\
\hline 15 & 1627.8148 & 814.4111 & 1610.7883 & 805.8978 & 1609.8043 & 805.4058 & $\mathbf{N}$ & 1029.5728 & 515.2900 & 1012.5462 & 506.7767 & 1011.5622 & 506.2847 & 9 \\
\hline 16 & 1726.8833 & 863.9453 & 1709.8567 & 855.4320 & 1708.8727 & 854.9400 & V & 914.5458 & 457.7765 & 897.5193 & 449.2633 & 896.5352 & 448.7713 & 8 \\
\hline 17 & 1813.9153 & 907.4613 & 1796.8887 & 898.9480 & 1795.9047 & 898.4560 & $\mathrm{~S}$ & 815.4774 & 408.2423 & 798.4509 & 399.7291 & 797.4668 & 399.2371 & 7 \\
\hline 18 & 1912.9837 & 956.9955 & 1895.9572 & 948.4822 & 1894.9731 & 947.9902 & $\bar{V}$ & 728.4454 & 364.7263 & 711.4188 & 356.2130 & & & 6 \\
\hline 19 & 2060.0521 & 1030.5297 & 2043.0256 & 1022.0164 & 2042.0416 & 1021.5244 & $\mathbf{F}$ & 629.3770 & 315.1921 & 612.3504 & 306.6788 & & & 5 \\
\hline 20 & 2173.1362 & 1087.0717 & 2156.1096 & 1078.5585 & 2155.1256 & 1078.0664 & I & 482.3085 & 241.6579 & 465.2820 & 233.1446 & & & 4 \\
\hline 21 & 2270.1889 & 1135.5981 & 2253.1624 & \begin{tabular}{|l|}
1127.0848 \\
\end{tabular} & 2252.1784 & 1126.5928 & $\mathbf{P}$ & 369.2245 & 185.1159 & 352.1979 & 176.6026 & & & 3 \\
\hline 22 & 2367.2417 & 1184.1245 & 2350.2152 & 1175.6112 & 2349.2311 & 1175.1192 & $\mathbf{P}$ & 272.1717 & 136.5895 & 255.1452 & 128.0762 & & & 2 \\
\hline 23 & & & & & & & $\mathbf{R}$ & 175.1190 & 88.0631 & 158.0924 & 79.5498 & & & 1 \\
\hline
\end{tabular}
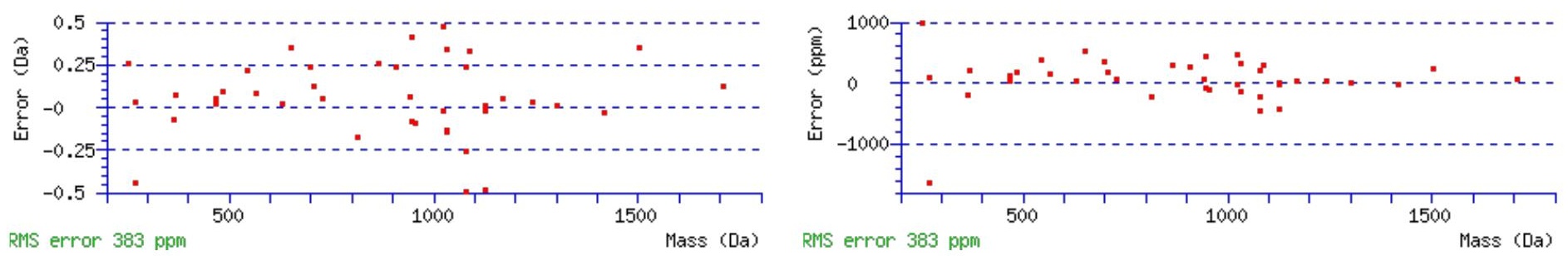

\section{All matches to this query}

\begin{tabular}{|l|r|r|l|}
\hline Score & Mr(calc): & Delta & \multicolumn{1}{|c|}{ Sequence } \\
\hline 37.2 & 2540.3461 & 1.0045 & DLHVPIPAVVEMNPNVSVFIPPR \\
\hline 35.3 & 2540.3461 & 1.0045 & DLHVPIPAVVEMNPNVSVFIPPR \\
\hline 31.0 & 2541.3301 & 0.0205 & DLHVPIPAVVEMNPNVSVFIPPR \\
\hline 20.2 & 2539.3621 & 1.9885 & DLHVPIPAVVEMNPNVSVFIPPR \\
\hline 7.6 & 2539.3376 & 2.0130 & YFGLRFYAYNPLAGGLLTGKYR \\
\hline 4.2 & 2541.3576 & -0.0070 & AKPLMLIAPKPQEKKSMGLPPR \\
\hline 3.3 & 2540.3421 & 1.0085 & LRVLEVQGLGWEGQEAEKVLCK \\
\hline 3.3 & 2540.3421 & 1.0085 & LVRLTGDLIVWSDEMNPAOVIR \\
\hline 3.0 & 2540.3322 & 1.0184 & TGLPIHINGCFAVTSNRKEIWK \\
\hline 3.0 & 2541.3567 & -0.0061 & QAQTEVVPSASSLIIKALKEPPR \\
\hline
\end{tabular}

Spectrum No: 901; Query: 2098; Rank: 1

\section{Peptide View}

MS/MS Fragmentation of NLPEELLNSLASENLTANFHK

Found in IPI00209265, Tax_Id=10116 Gene_Symbol=Ggh Gamma-glutamyl hydrolase precursor

Match to Query 2098: 2354.175402 from(785.732410,3+)

Title: 091008RatKidney_NH4Format02_24.5573.5573.3.dta

Data file K:INewmanPaper|Piliang\3SubProteomes\Piliang3SP\mgf5ppm\ERLIC_3SubProteomes5ppm.mgf 

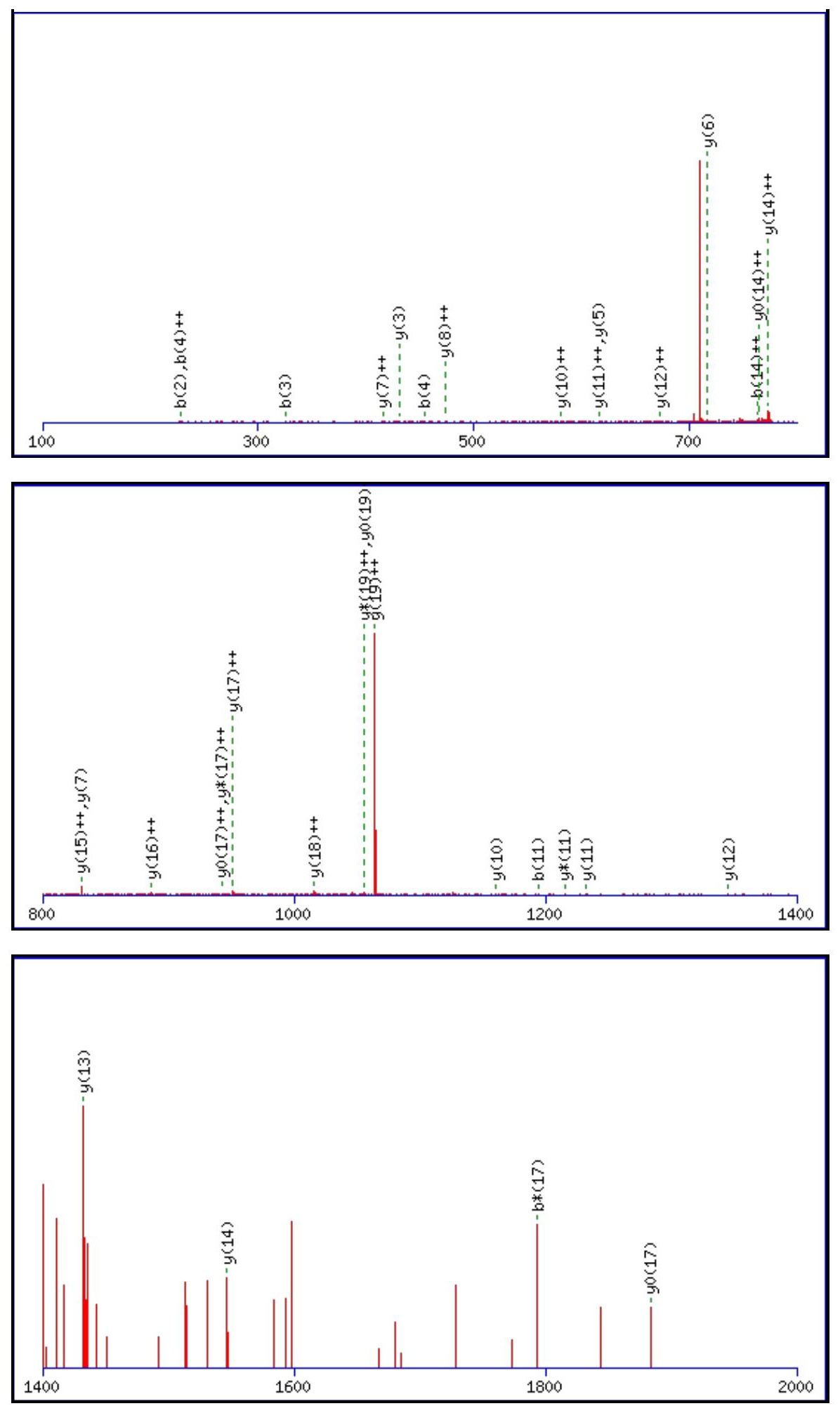

Monoisotopic mass of neutral peptide $\operatorname{Mr}($ calc): 2354.1753

Fixed modifications: Carbamidomethyl (C)

Variable modifications:

N14 : Deamidated_N (N)

Ions Score: 37 Expect: 0.045

Matches (Bold Red): 34/224 fragment ions using 96 most intense peaks

\begin{tabular}{|r|c|r|r|r|r|r|r|r|r|r|r|r|r|r|}
\hline$\#$ & $\mathbf{b}$ & \multicolumn{1}{|c|}{$\mathbf{b}^{++}$} & $\mathbf{b}^{*}$ & $\mathbf{b}^{\boldsymbol{*}^{++}}$ & $\mathbf{b}^{\mathbf{0}}$ & $\mathbf{b}^{\mathbf{0 + +}}$ & $\mathbf{S e q}$ & $\mathbf{y}$ & $\mathbf{y}^{++}$ & $\mathbf{y}^{\mathbf{*}}$ & $\mathbf{y}^{\mathbf{*}^{++}}$ & $\mathbf{y}^{\mathbf{0}}$ & $\mathbf{y}^{\mathbf{0 + +}}$ & $\#$ \\
\hline $\mathbf{1}$ & 115.0502 & 58.0287 & 98.0237 & 49.5155 & & & $\mathbf{N}$ & & & & & & & $\mathbf{2 1}$ \\
\hline $\mathbf{2}$ & $\mathbf{2 2 8 . 1 3 4 3}$ & 114.5708 & 211.1077 & 106.0575 & & & $\mathbf{L}$ & 2241.1397 & 1121.0735 & 2224.1132 & 1112.5602 & 2223.1292 & 1112.0682 & $\mathbf{2 0}$ \\
\hline $\mathbf{3}$ & $\mathbf{3 2 5 . 1 8 7 0}$ & 163.0972 & 308.1605 & 154.5839 & & & $\mathbf{P}$ & 2128.0557 & $\mathbf{1 0 6 4 . 5 3 1 5}$ & 2111.0291 & $\mathbf{1 0 5 6 . 0 1 8 2}$ & 2110.0451 & $\mathbf{1 0 5 5 . 5 2 6 2}$ & $\mathbf{1 9}$ \\
\hline $\mathbf{4}$ & $\mathbf{4 5 4 . 2 2 9 6}$ & $\mathbf{2 2 7 . 6 1 8 5}$ & 437.2031 & 219.1052 & 436.2191 & 218.6132 & $\mathbf{E}$ & 2031.0029 & $\mathbf{1 0 1 6 . 0 0 5 1}$ & 2013.9764 & 1007.4918 & 2012.9924 & 1006.9998 & $\mathbf{1 8}$ \\
\hline $\mathbf{5}$ & 583.2722 & 292.1397 & 566.2457 & 283.6265 & 565.2617 & 283.1345 & $\mathbf{E}$ & 1901.9603 & $\mathbf{9 5 1 . 4 8 3 8}$ & 1884.9338 & $\mathbf{9 4 2 . 9 7 0 5}$ & $\mathbf{1 8 8 3 . 9 4 9 8}$ & $\mathbf{9 4 2 . 4 7 8 5}$ & $\mathbf{1 7}$ \\
\hline
\end{tabular}




\begin{tabular}{|c|c|c|c|c|c|c|c|c|c|c|c|c|c|c|}
\hline & 696.3563 & 348.6818 & 679.3297 & 340.1685 & 678.3457| & 339.6765 & $\mathbf{L}$ & |1772.9177 & 886.9625 & 1755.8912 & 878.4492 & |1754.9072 & 877.9572 & 16 \\
\hline 7 & 809.4403 & 405.2238 & 792.4138 & 396.7105 & 791.4298 & 396.2185 & $\mathbf{L}$ & 1659.8337 & 830.4205 & 1642.8071 & 821.9072 & 1641.8231 & 821.4152 & 15 \\
\hline 8 & 923.4833 & 462.2453 & 906.4567 & 453.7320 & 905.4727 & 453.2400 & $\mathbf{N}$ & 1546.7496 & 773.8784 & 1529.7231 & 765.3652 & 1528.7390 & 764.8732 & 14 \\
\hline 9 & 1010.5153 & 505.7613 & 993.4888 & 497.2480 & 992.5047 & 496.7560 & $S$ & 1432.7067 & 716.8570 & 1415.6801 & 708.3437 & 1414.6961 & 707.8517 & 13 \\
\hline 10 & 1123.5994 & 562.3033 & 1106.5728 & 553.7900 & 1105.5888 & 553.2980 & $\mathbf{L}$ & 1345.6746 & 673.3410 & 1328.6481 & 664.8277 & 1327.6641 & 664.3357 & 12 \\
\hline 11 & \begin{tabular}{|l|}
1194.6365 \\
\end{tabular} & 597.8219 & 1177.6099 & 589.3086 & 1176.6259 & 588.8166 & A & 1232.5906 & 616.7989 & 1215.5640 & 608.2857 & 1214.5800 & 607.7936 & 11 \\
\hline 12 & 1281.6685 & 641.3379 & 1264.6420 & 632.8246 & 1263.6579 & 632.3326 & $\mathrm{~S}$ & 1161.5535 & 581.2804 & 1144.5269 & 572.7671 & 1143.5429 & 572.2751 & 10 \\
\hline 13 & 1410.7111 & 705.8592 & 1393.6845 & 697.3459 & 1392.7005 & 696.8539 & E & 1074.5214 & 537.7644 & 1057.4949 & 529.2511 & 1056.5109 & 528.7591 & 9 \\
\hline 14 & 1525.7380 & 763.3727 & 1508.7115 & 754.8594 & 1507.7275 & 754.3674 & $\mathbf{N}$ & 945.4788 & 473.2431 & 928.4523 & 464.7298 & 927.4683 & 464.2378 & 8 \\
\hline 15 & 1638.8221 & 819.9147 & 1621.7955 & 811.4014 & 1620.8115 & 810.9094 & $\mathbf{L}$ & 830.4519 & 415.7296 & 813.4254 & 407.2163 & 812.4413 & 406.7243 & 7 \\
\hline 16 & 1739.8698 & 870.4385 & 1722.8432 & 861.9253 & 1721.8592 & 861.4332 & $\mathbf{T}$ & 717.3678 & 359.1876 & 700.3413 & 350.6743 & 699.3573 & 350.1823 & 6 \\
\hline 17 & 1810.9069 & 905.9571 & 1793.8803 & 897.4438 & 1792.8963 & 896.9518 & A & 616.3202 & 308.6637 & 599.2936 & 300.1504 & & & 5 \\
\hline 18 & 1924.9498 & 962.9785 & 1907.9233 & 954.4653 & 1906.9393 & 953.9733 & $\mathbf{N}$ & 545.2831 & 273.1452 & 528.2565 & 264.6319 & & & 4 \\
\hline 19 & 2072.0182 & 1036.5128 & 2054.9917 & 1027.9995 & 2054.0077 & 1027.5075 & $\mathbf{F}$ & 431.2401 & 216.1237 & 414.2136 & 207.6104 & & & 3 \\
\hline 20 & 2209.0771 & 1105.0422 & 2192.0506 & 1096.5289 & 2191.0666 & 1096.0369 & $\mathbf{H}$ & 284.1717 & 142.5895 & 267.1452 & 134.0762 & & & 2 \\
\hline 21 & & & & & & & K & 147.1128 & 74.0600 & 130.0863 & 65.5468 & & & 1 \\
\hline
\end{tabular}
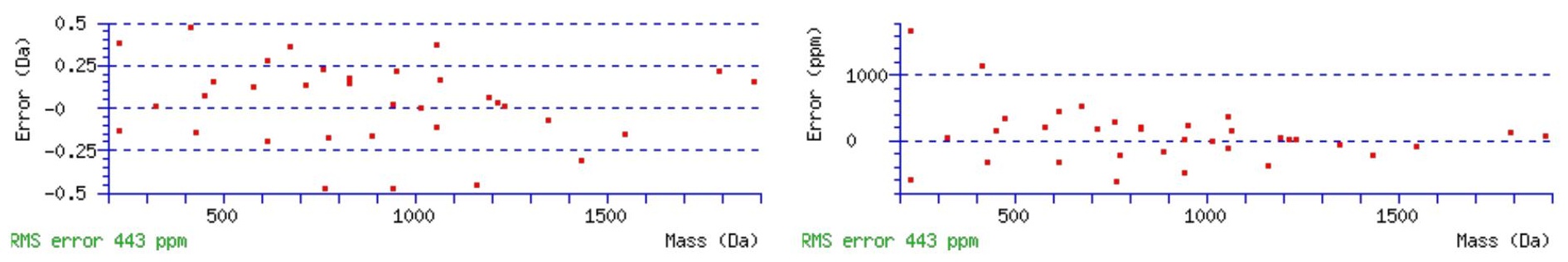

\section{All matches to this query}

\begin{tabular}{|l|l|l|l|}
\hline Score & Mr(calc): & Delta & \multicolumn{1}{|c|}{ Sequence } \\
\hline 36.7 & 2354.1753 & 0.0001 & NLPEELLNSLASENLTANFHK \\
\hline 32.2 & 2354.1753 & 0.0001 & NLPEELLNSLASENLTANFHK \\
\hline 16.4 & 2354.1753 & 0.0001 & NLPEELLNSLASENLTANFHK \\
\hline 8.3 & 2354.1701 & 0.0053 & LISLIRIIWVNSPHYNTR \\
\hline 7.4 & 2354.1851 & -0.0097 & QLIQLSVANNRLVRMMGVAK \\
\hline 6.2 & 2354.1688 & 0.0066 & FLPPSVAPIGQNLTKIETLR \\
\hline 6.2 & 2354.1688 & 0.0066 & FLPPSVAPIGQNLTKIETLR \\
\hline 6.0 & 2354.1701 & 0.0053 & LISLIRIIWVNSPHYNTR \\
\hline 5.0 & 2354.1883 & -0.0129 & LNSLTTVLGQEVDRFNNLLK \\
\hline 5.0 & 2354.1883 & -0.0129 & LNSLTTVLGOEVDRFNNLLK \\
\hline
\end{tabular}

Spectrum No: 902; Query: 3; Rank: 1

\section{Peptide View}

MS/MS Fragmentation of INASFR

Found in IPI00204504, Tax_Id=10116 Gene_Symbol=Plvap Plasmalemma vesicle-associated protein

Match to Query 3: 707.359768 from(354.687160,2+)

Title: 091008RatKidney_NH4Format01_27.637.637.2.dta

Data file K:\NewmanPaper|Piliang|3SubProteomes\Piliang3SP\mgf5ppm\ERLIC_3SubProteomes5ppm.mgf 

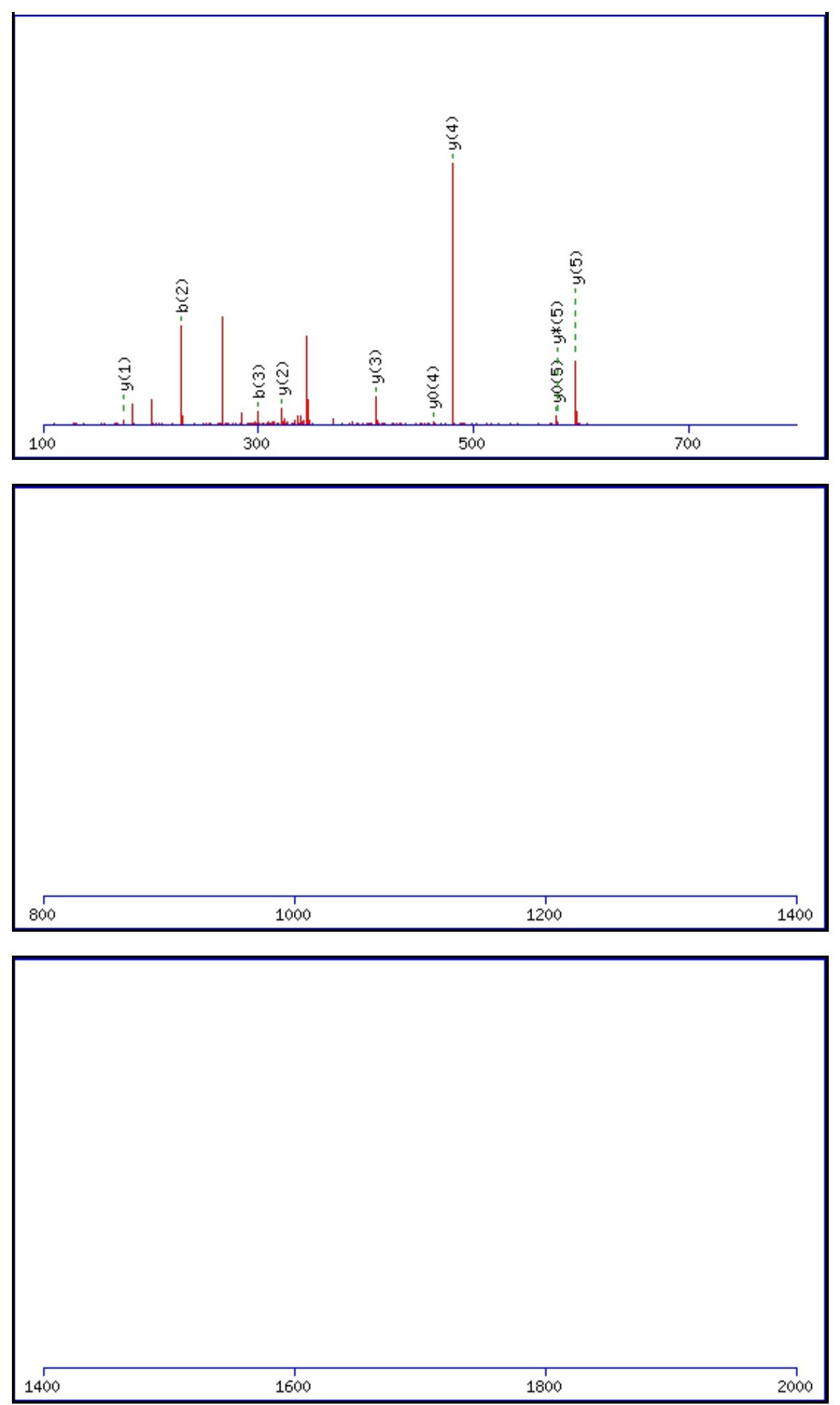

Monoisotopic mass of neutral peptide $\operatorname{Mr}($ calc): 707.3602

Fixed modifications: Carbamidomethyl (C)

Variable modifications:

N2 : Deamidated $\mathrm{N}(\mathrm{N})$

Ions Score: 37 Expect: $\odot .017$

Matches (Bold Red): 10/48 fragment ions using 20 most intense peaks

\begin{tabular}{|c|c|c|c|c|c|c|c|c|c|c|c|c|c|c|}
\hline$\#$ & $\mathbf{b}$ & $\mathbf{b}^{++}$ & $\mathbf{b}^{*}$ & $\mathbf{b}^{*^{++}}$ & $\mathbf{b}^{\mathbf{0}}$ & $\mathbf{b}^{\mathbf{0}+}$ & Seq. & $\mathbf{y}$ & $\mathbf{y}^{++}$ & $\mathbf{y}^{*}$ & $\mathbf{y}^{\boldsymbol{*}^{++}}$ & $\mathbf{y}^{\mathbf{0}}$ & $\mathbf{y}^{\mathbf{0}++}$ & $\#$ \\
\hline $\mathbf{1}$ & 114.0913 & 57.5493 & & & & & $\mathbf{I}$ & & & & & & & $\mathbf{6}$ \\
\hline $\mathbf{2}$ & 229.1183 & 115.0628 & 212.0917 & 106.5495 & & & $\mathbf{N}$ & $\mathbf{5 9 5 . 2 8 3 4}$ & 298.1454 & $\mathbf{5 7 8 . 2 5 6 9}$ & 289.6321 & $\mathbf{5 7 7 . 2 7 2 9}$ & 289.1401 & $\mathbf{5}$ \\
\hline $\mathbf{3}$ & $3 \mathbf{0 0 . 1 5 5 4}$ & 150.5813 & 283.1288 & 142.0681 & & & $\mathbf{A}$ & $\mathbf{4 8 0 . 2 5 6 5}$ & 240.6319 & 463.2300 & 232.1186 & $\mathbf{4 6 2 . 2 4 5 9}$ & 231.6266 & $\mathbf{4}$ \\
\hline $\mathbf{4}$ & 387.1874 & 194.0973 & 370.1609 & 185.5841 & 369.1769 & 185.0921 & $\mathbf{S}$ & $\mathbf{4 0 9 . 2 1 9 4}$ & 205.1133 & 392.1928 & 196.6001 & 391.2088 & 196.1081 & $\mathbf{3}$ \\
\hline $\mathbf{5}$ & 534.2558 & 267.6316 & 517.2293 & 259.1183 & 516.2453 & 258.6263 & $\mathbf{F}$ & $\mathbf{3 2 2 . 1 8 7 4}$ & 161.5973 & 305.1608 & 153.0840 & & & $\mathbf{2}$ \\
\hline
\end{tabular}




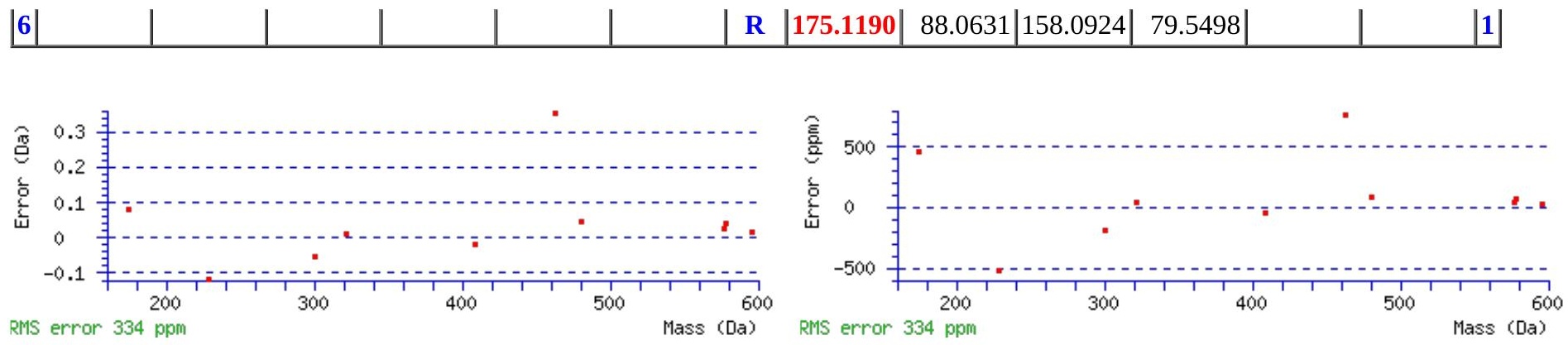

\section{All matches to this query}

\begin{tabular}{|l|l|l|l|}
\hline Score & Mr(calc): & Delta & Sequence \\
\hline 36.6 & 707.3602 & -0.0004 & $\underline{\text { INASFR }}$ \\
\hline 27.4 & 707.3602 & -0.0004 & $\underline{\text { IDAAYR }}$ \\
\hline 27.4 & 707.3602 & -0.0004 & $\underline{\text { LDAAYR }}$ \\
\hline 21.4 & 707.3602 & -0.0005 & $\underline{\text { LDTGFR }}$ \\
\hline 21.4 & 707.3602 & -0.0004 & $\underline{\text { LNSAFR }}$ \\
\hline 14.5 & 707.3602 & -0.0004 & DIAAYR \\
\hline 13.2 & 707.3602 & -0.0004 & $\underline{\text { NIAAYR }}$ \\
\hline 9.9 & 707.3602 & -0.0004 & $\underline{\text { LANYAR }}$ \\
\hline 9.6 & 707.3602 & -0.0004 & $\underline{\text { LANFSR }}$ \\
\hline 9.2 & 707.3602 & -0.0005 & $\underline{\text { LASDFR }}$ \\
\hline
\end{tabular}

Spectrum No: 903; Query: 972; Rank: 1

\section{Peptide View}

\section{MS/MS Fragmentation of CHEGNGTFECGACR}

Found in IPI00191681, Tax_Id=10116 Gene_Symbol=Itgb1 Integrin beta-1 precursor

Match to Query 972: 1654.595622 from(552.539150,3+)

Title: 100101RatKid_NS_deglyco_21.630.630.3.dta

Data file K:INewmanPaper|Piliangl3SubProteomes\Piliang3SP\mgf5ppm\ERLIC_3SubProteomes5ppm.mgf

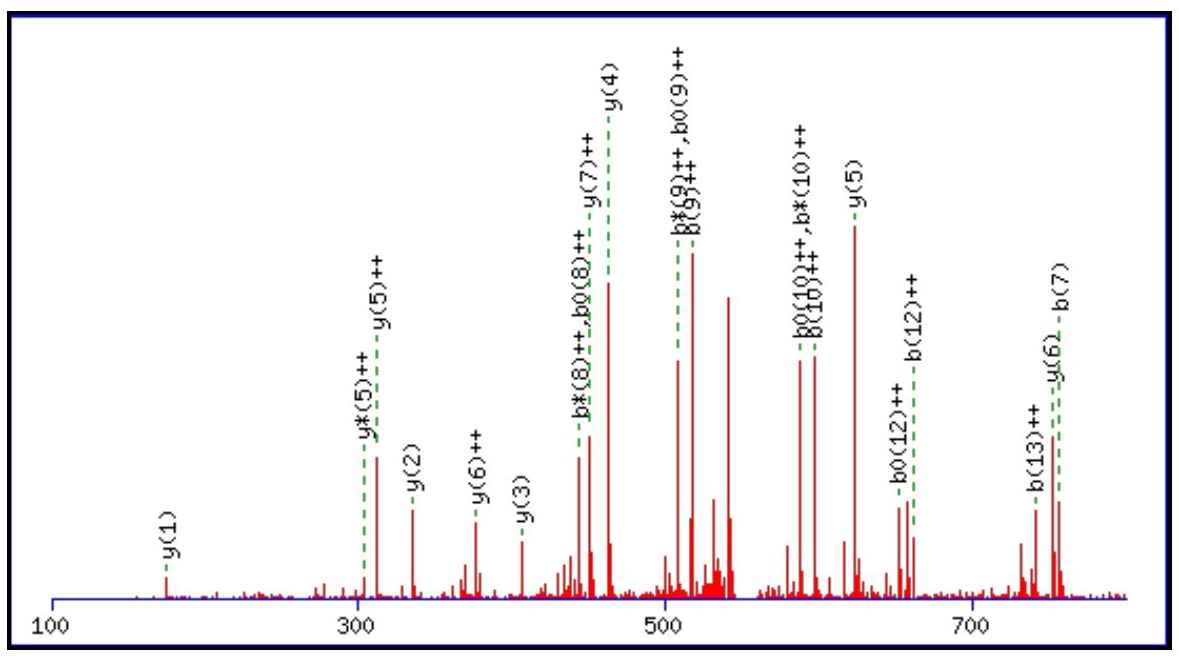



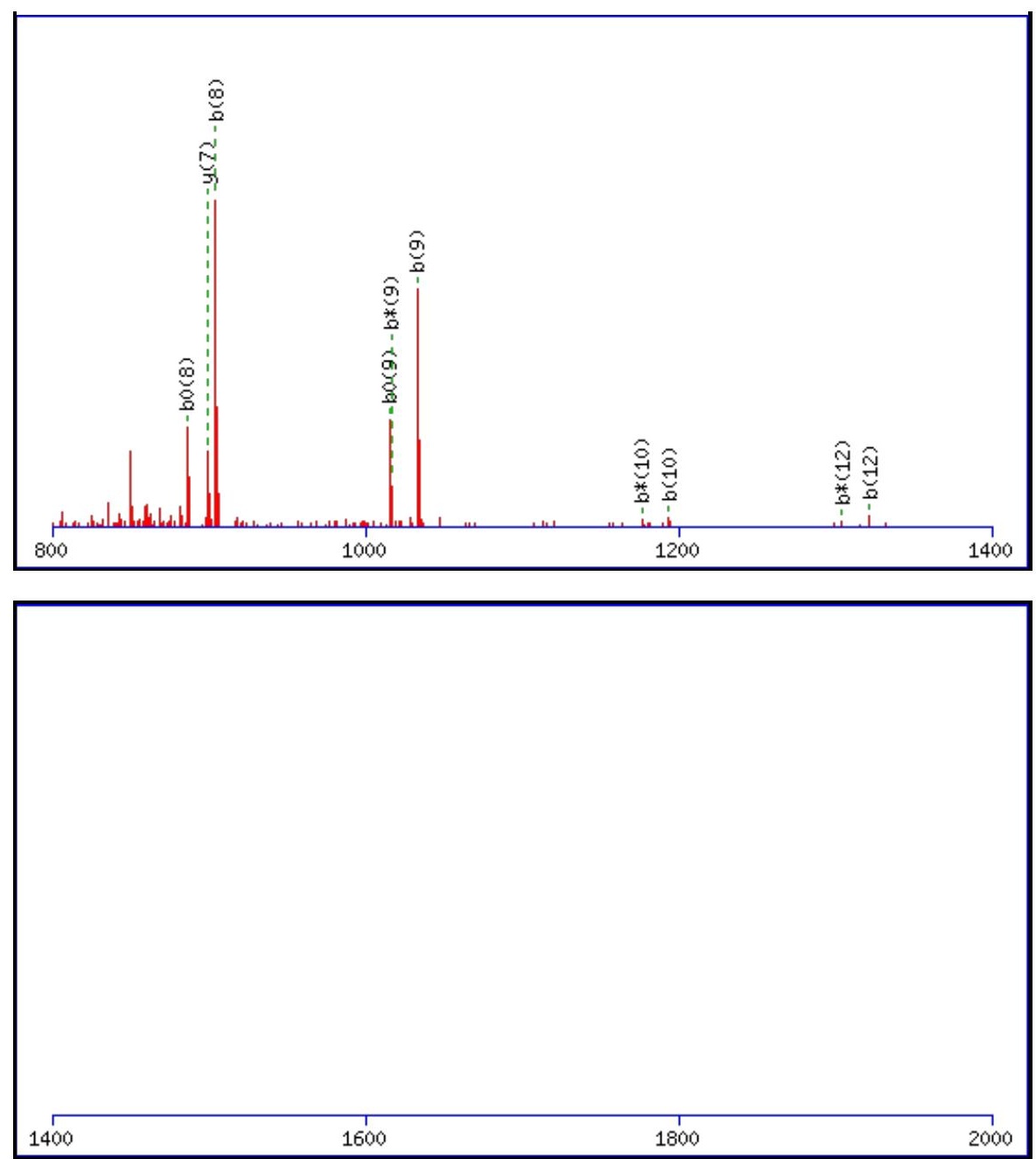

Monoisotopic mass of neutral peptide $\operatorname{Mr}($ calc): 1654.5923

Fixed modifications: Carbamidomethyl (C)

Variable modifications:

N5: Deamidated $\mathrm{N}(\mathrm{N})$

Ions Score: 37 Expect : 0.015

Matches (Bold Red): 32/134 fragment ions using 52 most intense peaks

\begin{tabular}{|c|c|c|c|c|c|c|c|c|c|c|c|c|c|c|}
\hline \# & b & $\mathbf{b}^{++}$ & b* & $\mathbf{b}^{*^{++}}$ & $\mathbf{b}^{0}$ & $\mathbf{b}^{0++}$ & Seq. & $\mathbf{y}$ & $\mathrm{y}^{++}$ & $\mathbf{y}^{*}$ & $\mathbf{y}^{*^{++}}$ & $\mathbf{y}^{\mathbf{0}}$ & $\mathbf{y}^{0++}$ & \# \\
\hline 1 & 161.0379 & 81.0226 & & & & & C & & & & & & & 14 \\
\hline 2 & 298.0968 & 149.5521 & & & & & H & 1495.5689 & 748.2881 & 1478.5423 & 739.7748 & 1477.5583 & 739.2828 & 13 \\
\hline 3 & 427.1394 & 214.0734 & & & 409.1289 & 205.0681 & $\mathbf{E}$ & 1358.5100 & 679.7586 & 1341.4834 & 671.2453 & 1340.4994 & 670.7533 & 12 \\
\hline 4 & 484.1609 & 242.5841 & & & 466.1503 & 233.5788 & $\mathbf{G}$ & 1229.4674 & 615.2373 & 1212.4408 & 606.7241 & 1211.4568 & 606.2320 & 11 \\
\hline 5 & 599.1878 & 300.0976 & 582.1613 & 291.5843 & 581.1773 & 291.0923 & $\mathbf{N}$ & 1172.4459 & 586.7266 & 1155.4194 & 578.2133 & 1154.4353 & 577.7213 & 10 \\
\hline 6 & 656.2093 & 328.6083 & 639.1827 & 320.0950 & 638.1987 & 319.6030 & G & 1057.4190 & 529.2131 & 1040.3924 & 520.6999 & 1039.4084 & 520.2078 & 9 \\
\hline 7 & 757.2570 & 379.1321 & 740.2304 & 370.6189 & 739.2464 & 370.1268 & $\mathbf{T}$ & 1000.3975 & 500.7024 & 983.3710 & 492.1891 & 982.3869 & 491.6971 & 8 \\
\hline 8 & 904.3254 & 452.6663 & 887.2988 & 444.1531 & 886.3148 & 443.6610 & $\mathbf{F}$ & 899.3498 & 450.1786 & 882.3233 & 441.6653 & 881.3393 & 441.1733 & 7 \\
\hline 9 & 1033.3680 & 517.1876 & 1016.3414 & 508.6744 & 1015.3574 & 508.1823 & $\mathbf{E}$ & 752.2814 & 376.6443 & 735.2549 & 368.1311 & 734.2709 & 367.6391 & 6 \\
\hline 10 & 1193.3986 & 597.2030 & 1176.3721 & 588.6897 & 1175.3881 & 588.1977 & C & 623.2388 & 312.1231 & 606.2123 & 303.6098 & & & 5 \\
\hline 11 & 1250.4201 & 625.7137 & 1233.3935 & 617.2004 & 1232.4095 & 616.7084 & G & 463.2082 & 232.1077 & 446.1816 & 223.5945 & & & 4 \\
\hline 12 & 1321.4572 & 661.2322 & 1304.4307 & 652.7190 & 1303.4466 & 652.2270 & A & 406.1867 & 203.5970 & 389.1602 & 195.0837 & & & 3 \\
\hline 13 & 1481.4879 & 741.2476 & 1464.4613 & 732.7343 & 1463.4773 & 732.2423 & C & 335.1496 & 168.0784 & 318.1231 & 159.5652 & & & 2 \\
\hline 14 & & & & & & & $\mathbf{R}$ & 175.1190 & 88.0631 & 158.0924 & 79.5498 & & & 1 \\
\hline
\end{tabular}



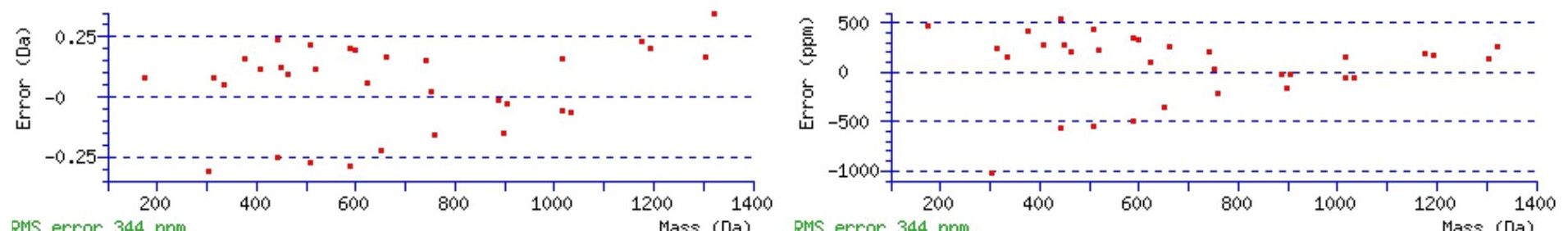

RMS error 344 ppm

Mass (Da) RMS error 344 ppm

\section{All matches to this query}

\begin{tabular}{|l|l|l|l|}
\hline Score & Mr(calc): & Delta & \multicolumn{1}{c|}{ Sequence } \\
\hline 36.5 & 1654.5923 & 0.0034 & CHEGNGTFECGACR \\
\hline 14.4 & 1653.6083 & 0.9874 & CHEGNGTFECGACR \\
\hline 7.6 & 1654.5938 & 0.0018 & MTLTAGFSMATIR \\
\hline 7.1 & 1652.5746 & 2.0210 & NSIPQXSGSSSHR \\
\hline 6.7 & 1654.5938 & 0.0018 & MTLTAGFSMATIR \\
\hline 6.2 & 1653.5854 & 1.0102 & SLETQKTTVTAR \\
\hline 3.3 & 1653.5854 & 1.0102 & SLETQKTTVTAR \\
\hline 1.2 & 1652.5746 & 2.0210 & NSIPQXSGSSSHR \\
\hline 1.2 & 1652.5746 & 2.0210 & NSIPQXSGSSSHR \\
\hline 1.2 & 1652.5746 & 2.0210 & NSIPQXSGSSSHR \\
\hline
\end{tabular}

Spectrum No: 904; Query: 1193; Rank: 1

\section{Peptide View}

MS/MS Fragmentation of LHVAQPENDSHVALLK

Found in IPI00409514, Tax_Id=10116 Gene_Symbol=Pgap1 GPI inositol-deacylase

Match to Query 1193: 1770.922842 from(591.314890,3+)

Title: 091008RatKidney_NH4Format01_23.1522.1522.3.dta

Data file K:INewmanPaper|Piliangl3SubProteomes\Piliang3SP\mgf5ppm\ERLIC_3SubProteomes5ppm.mgf

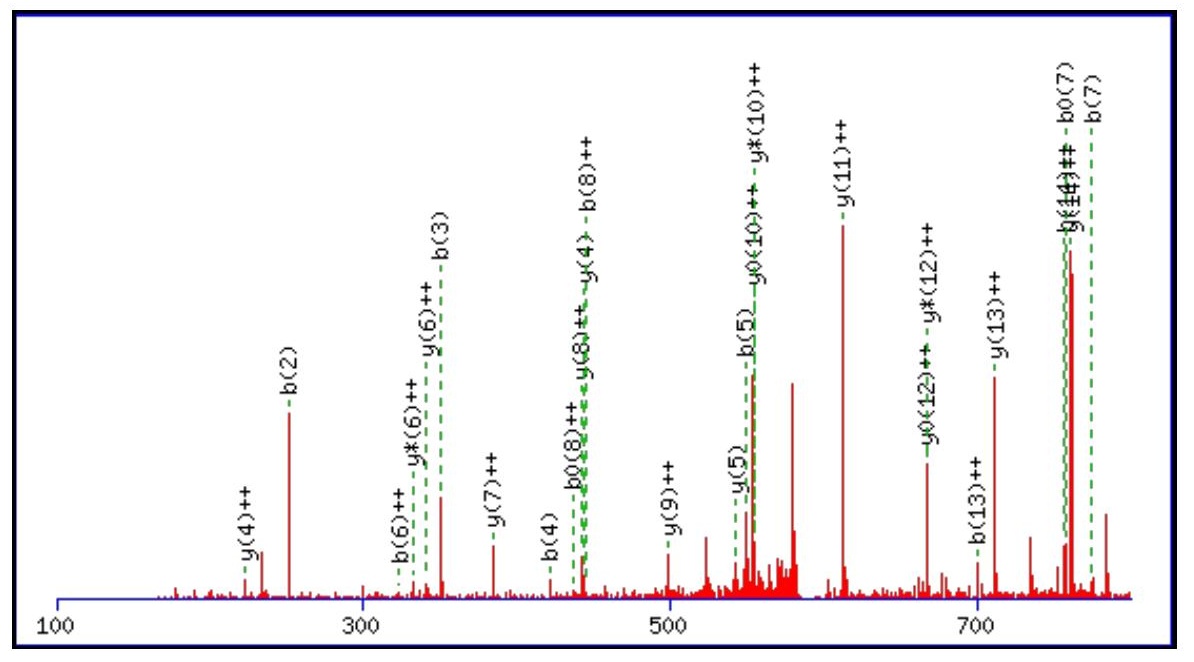



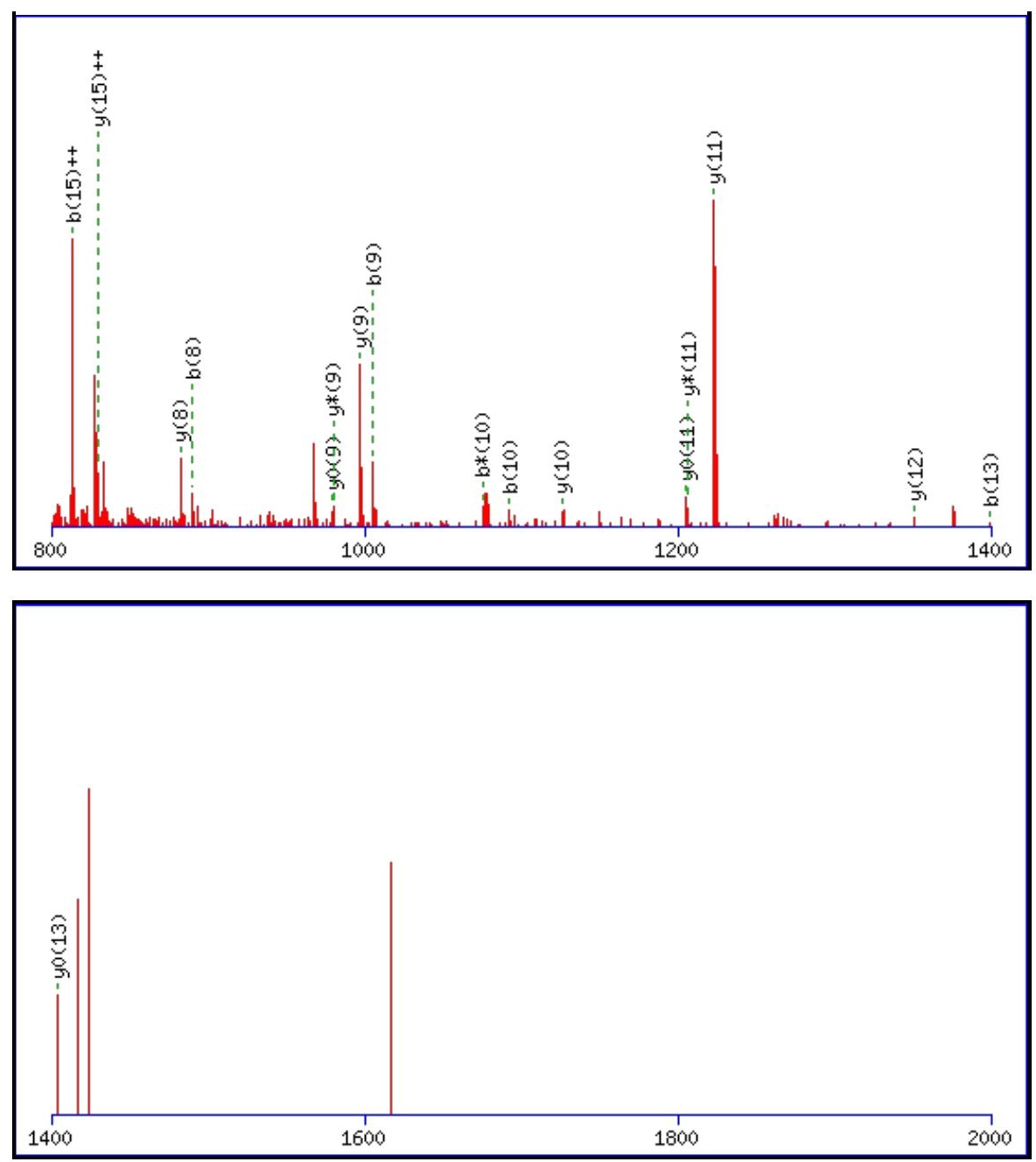

Monoisotopic mass of neutral peptide $\operatorname{Mr}($ calc): 1770.9264

Fixed modifications: Carbamidomethyl (C)

Variable modifications:

N8: : Deamidated $\mathrm{N}(\mathrm{N})$

Ions Score: 36 Expect: 0.03

Matches (Bold Red): 43/148 fragment ions using 118 most intense peaks

\begin{tabular}{|c|c|c|c|c|c|c|c|c|c|c|c|c|c|c|}
\hline \# & b & $\mathbf{b}^{++}$ & b* & $\mathbf{b}^{*^{++}}$ & $\mathbf{b}^{0}$ & $\mathbf{b}^{0++}$ & Seq. & $\mathbf{y}$ & $y^{++}$ & $\mathbf{y}^{*}$ & $\mathbf{y}^{*^{++}}$ & $\mathbf{y}^{\mathbf{0}}$ & $\mathbf{y}^{0++}$ & \# \\
\hline 1 & 114.0913 & 57.5493 & & & & & $\mathbf{L}$ & & & & & & & 16 \\
\hline 2 & 251.1503 & 126.0788 & & & & & $\mathbf{H}$ & 1658.8497 & 829.9285 & 1641.8231 & 821.4152 & 1640.8391 & 820.9232 & 15 \\
\hline 3 & 350.2187 & 175.6130 & & & & & $\mathbf{V}$ & 1521.7907 & 761.3990 & 1504.7642 & 752.8857 & 1503.7802 & 752.3937 & 14 \\
\hline 4 & 421.2558 & 211.1315 & & & & & A & 1422.7223 & 711.8648 & 1405.6958 & 703.3515 & 1404.7118 & 702.8595 & 13 \\
\hline 5 & 549.3144 & 275.1608 & 532.2878 & 266.6475 & & & $\mathbf{Q}$ & 1351.6852 & 676.3462 & 1334.6587 & 667.8330 & 1333.6746 & 667.3410 & 12 \\
\hline 6 & 646.3671 & 323.6872 & 629.3406 & 315.1739 & & & $\mathbf{P}$ & 1223.6266 & 612.3170 & 1206.6001 & 603.8037 & 1205.6161 & 603.3117 & 11 \\
\hline 7 & 775.4097 & 388.2085 & 758.3832 & 379.6952 & 757.3992 & 379.2032 & $\mathbf{E}$ & 1126.5739 & 563.7906 & 1109.5473 & 555.2773 & 1108.5633 & 554.7853 & 10 \\
\hline 8 & 890.4367 & 445.7220 & 873.4101 & 437.2087 & 872.4261 & 436.7167 & $\mathbf{N}$ & 997.5313 & 499.2693 & 980.5047 & 490.7560 & 979.5207 & 490.2640 & 9 \\
\hline 9 & 1005.4636 & 503.2354 & 988.4370 & 494.7222 & 987.4530 & 494.2302 & D & 882.5043 & 441.7558 & 865.4778 & 433.2425 & 864.4938 & 432.7505 & 8 \\
\hline 10 & 1092.4956 & 546.7514 & 1075.4691 & 538.2382 & 1074.4851 & 537.7462 & $\mathrm{~S}$ & 767.4774 & 384.2423 & 750.4509 & 375.7291 & 749.4668 & 375.2371 & 7 \\
\hline 11 & 1229.5545 & 615.2809 & 1212.5280 & 606.7676 & 1211.5440 & 606.2756 & $\mathbf{H}$ & 680.4454 & 340.7263 & 663.4188 & 332.2130 & & & 6 \\
\hline 12 & 1328.6229 & 664.8151 & 1311.5964 & 656.3018 & 1310.6124 & 655.8098 & V & 543.3865 & 272.1969 & 526.3599 & 263.6836 & & & 5 \\
\hline 13 & 1399.6601 & 700.3337 & 1382.6335 & 691.8204 & 1381.6495 & 691.3284 & A & 444.3180 & 222.6627 & 427.2915 & 214.1494 & & & 4 \\
\hline 14 & 1512.7441 & 756.8757 & 1495.7176 & 748.3624 & 1494.7336 & 747.8704 & $\mathbf{L}$ & 373.2809 & 187.1441 & 356.2544 & 178.6308 & & & 3 \\
\hline 15 & 1625.8282 & 813.4177 & 1608.8016 & 804.9045 & 1607.8176 & 804.4125 & $\mathbf{L}$ & 260.1969 & 130.6021 & 243.1703 & 122.0888 & & & 2 \\
\hline 16 & & & & & & & $\mathbf{K}$ & 147.1128 & 74.0600 & 130.0863 & 65.5468 & & & 1 \\
\hline
\end{tabular}



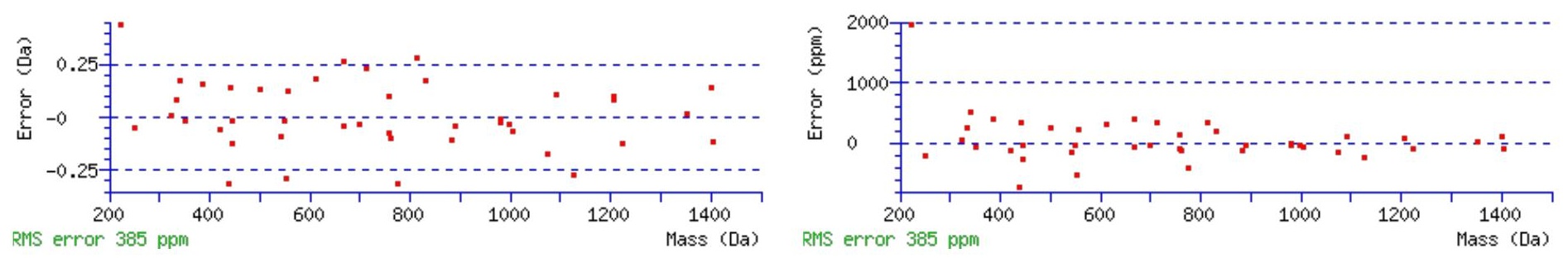

\section{All matches to this query}

\begin{tabular}{|l|l|c|l|}
\hline Score & Mr(calc): & Delta & Sequence \\
\hline 36.5 & 1770.9264 & -0.0036 & LHVAQPENDSHVALLK \\
\hline 0.1 & 1770.9305 & -0.0076 & HLVTLSNSKFTFSYK \\
\hline
\end{tabular}

Spectrum No: 905; Query: 2209; Rank: 1

\section{Peptide View}

MS/MS Fragmentation of GVTNDLLSVQGNTGPSWINKTEK

Found in IPI00369729, Tax_Id=10116 Gene_Symbol=Kdelc2 KDEL motif-containing protein 2 precursor

Match to Query 2209: 2458.231932 from(820.417920,3+)

Title: 091008RatKidney_NH4Format01_23.2872.2872.3.dta

Data file K:\NewmanPaper|Piliang|3SubProteomes\Piliang3SP\mgf5ppm\ERLIC_3SubProteomes5ppm.mgf
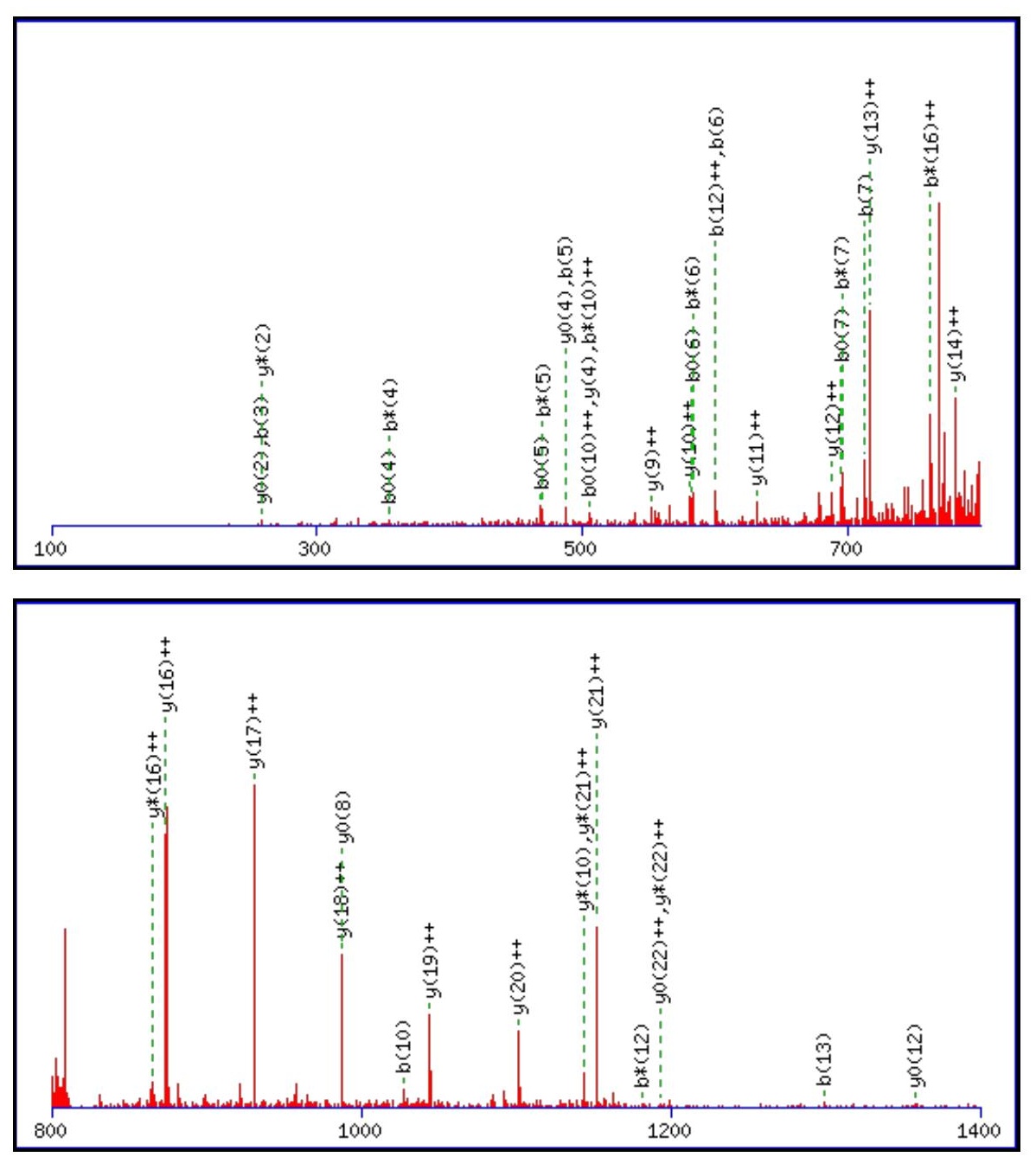


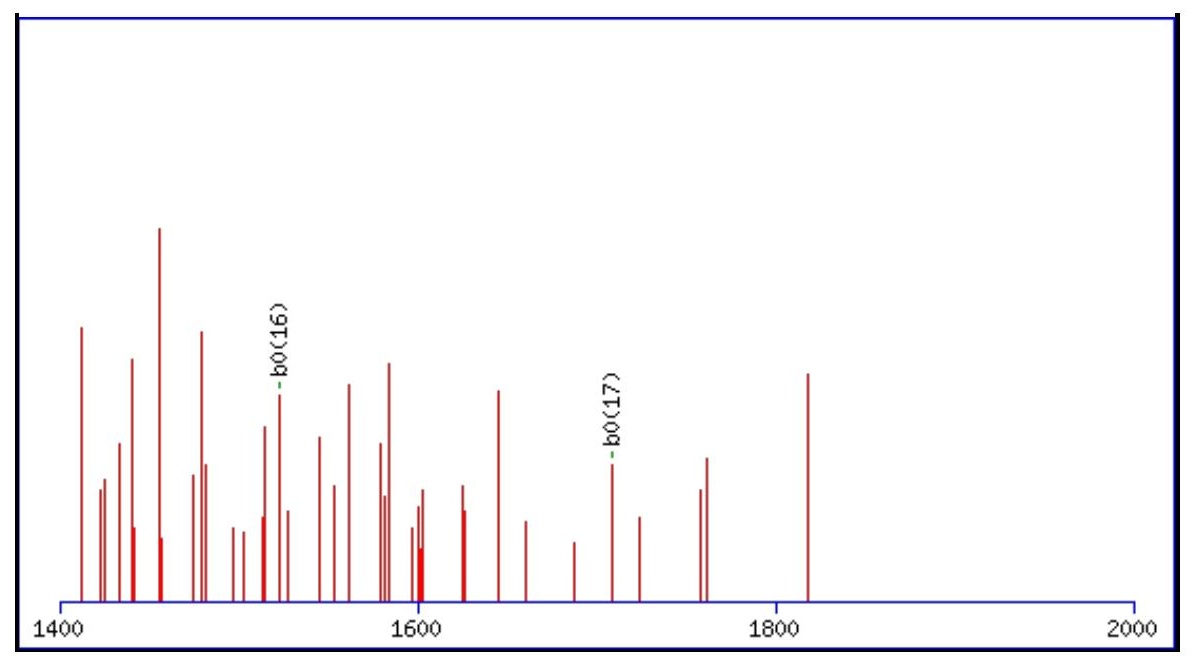

Monoisotopic mass of neutral peptide $\operatorname{Mr}($ calc): 2458.2340

Fixed modifications: Carbamidomethyl (C)

Variable modifications:

N19 : Deamidated_N (N)

Ions Score: 36 Expect: 0.045

Matches (Bold Red): 44/252 fragment ions using 95 most intense peaks

\begin{tabular}{|c|c|c|c|c|c|c|c|c|c|c|c|c|c|c|}
\hline \# & b & $\mathbf{b}^{++}$ & $\mathbf{b}^{*}$ & $\mathbf{b}^{*++}$ & $\mathbf{b}^{0}$ & $\mathbf{b}^{0++}$ & Seq. & $\mathbf{y}$ & $\mathbf{y}^{++}$ & $\mathbf{y}^{*}$ & $\mathbf{y}^{*^{++}}$ & $\mathbf{y}^{0}$ & & \# \\
\hline 1 & 58.0287 & 29.5180 & & & & & G & & & & & & & 23 \\
\hline 2 & 157.0972 & 79.0522 & & & & & $\mathbf{V}$ & 2402.2198 & 6135 & 2385.1932 & & 2384.2092 & 1192.6082 & 22 \\
\hline 3 & 258.1448 & 129.5761 & & & 240.1343 & & $T$ & 2303.1514 & 1152.0793 & 2286.1248 & 1143.5661 & 2285.1408 & .0740 & 21 \\
\hline 4 & 372.1878 & 186.5975 & 355.1612 & 178.0 & 354.1772 & 177.5922 & $\mathbf{N}$ & 2202.1037 & 1101.5555 & 2185.0771 & 1093.0422 & 2184.0931 & 1092.5502 & 20 \\
\hline 5 & 487.2147 & 244.1110 & 470.1882 & 235.5977 & 469.2041 & 235.1057 & D & 2088.0608 & 1044.5340 & 2071.0342 & 1036.0207 & 2070.0502 & 1035.5287 & 19 \\
\hline 6 & 600.2988 & 300.6530 & 583.2722 & 292. & 582.2882 & 291. & $\mathbf{L}$ & 1973.0338 & 987.0206 & 1956.0073 & 978.5073 & 1955.0233 & 978.0153 & 18 \\
\hline 7 & 713.3828 & 357.1951 & 696.3563 & 348.6818 & 695.3723 & 348.1898 & $\mathbf{L}$ & 1859.9498 & 930.4785 & 1842.9232 & 921.9652 & 1841.9392 & 921.4732 & 17 \\
\hline 8 & 800.4149 & 400.7111 & 783.3883 & 392. & 782.4043 & 391. & $S$ & 1746.8657 & 365 & 1729. & 232 & 1728.8551 & 864.9312 & 16 \\
\hline 9 & 899.4833 & 450.2453 & 882.4567 & 441.7320 & 881.4727 & 441.2400 & V & 1659.8337 & & 1642.8071 & 821.9072 & 1641.8231 & 821.4152 & 15 \\
\hline 10 & 1027.5419 & 514.2746 & 1010.5153 & 505.7613 & 1009.5313 & 505.2693 & $\mathbf{Q}$ & 1560.7653 & 780.8863 & 1543.7387 & 772.3730 & 1542.7547 & 771.8810 & 14 \\
\hline 11 & 1084.5633 & 542.7853 & 1067.5368 & 534.2720 & 1066.5528 & 533.7800 & G & 1432.7067 & 716.8570 & 1415.6801 & 708.3437 & 1414.6961 & 707.8517 & 13 \\
\hline 12 & 1198.6062 & 599.8068 & 1181.5797 & 591.2935 & 1180.5957 & 590.8015 & $\mathbf{N}$ & 1375.6852 & 688.3462 & 1358.6587 & 679.8330 & 1357.6746 & 679.3410 & 12 \\
\hline 13 & 1299.6539 & 650.3306 & 1282.6274 & 641.8173 & 281.6434 & 641.3253 & $\mathbf{T}$ & 1261.6423 & 631.3248 & 1244.6157 & 622.8115 & 1243.6317 & 622.3195 & 11 \\
\hline 14 & 1356.6754 & 678.8413 & 1339.6488 & 670.3281 & 1338.6648 & 669.8360 & G & 1160.5946 & 580.8009 & \begin{tabular}{|l|}
1143.5681 \\
\end{tabular} & 572.2877 & 1142.5840 & 571.7957 & 10 \\
\hline 15 & 1453.7281 & 727.3677 & 1436.7016 & 718.8544 & 1435.7176 & 718.3624 & $\mathbf{P}$ & 1103.5731 & 552.2902 & 1086.5466 & 543.7769 & 1085.5626 & 543.2849 & 9 \\
\hline 16 & 1540.7602 & 770.8837 & 1523.7336 & 762.3705 & 1522.7496 & 761.8784 & $S$ & 1006.5204 & 503.7638 & 989.4938 & 495.2506 & 988.5098 & 494.7585 & 8 \\
\hline 17 & 1726.8395 & 863.9234 & 1709.8129 & 855.4101 & 1708.8289 & 854.9181 & $\mathbf{W}$ & 919.4884 & 460.2478 & 902.4618 & 451.7345 & 901.4778 & 451.2425 & 7 \\
\hline 18 & 1839.9236 & 920.4654 & 1822.8970 & 911.9521 & 1821.9130 & 911.4601 & I & 733.4090 & 367.2082 & 716.3825 & 358.6949 & 715.3985 & 358.2029 & 6 \\
\hline 19 & 1954.9505 & 977.9789 & 1937.9239 & 969.4656 & 1936.9399 & 968.9736 & $\mathbf{N}$ & 620.3250 & 310.6661 & 603.2984 & 302.1529 & 602.3144 & 301.6608 & 5 \\
\hline 20 & 2083.0455 & 1042.0264 & 2066.0189 & 1033.5131 & 2065.0349 & 1033.0211 & $\mathbf{K}$ & 505.2980 & 253.1527 & 488.2715 & 244.6394 & 487.2875 & 244.1474 & 4 \\
\hline 21 & 2184.0931 & 1092.5502 & 2167.0666 & 1084.0369 & 2166.0826 & 1083.5449 & $\mathbf{T}$ & 377.2031 & 189.1052 & 360.1765 & 180.5919 & 359.1925 & 180.0999 & 3 \\
\hline 22 & 2313.1357 & 1157.0715 & 2296.1092 & 1148.5582 & 2295.1252 & 1148.0662 & $\mathbf{E}$ & 276.1554 & 138.5813 & 259.1288 & 130.0681 & 258.1448 & 129.5761 & 2 \\
\hline 23 & & & & & & & $\mathbf{K}$ & 147.1128 & 74.0600 & 130.0863 & 65.5468 & & & 1 \\
\hline
\end{tabular}
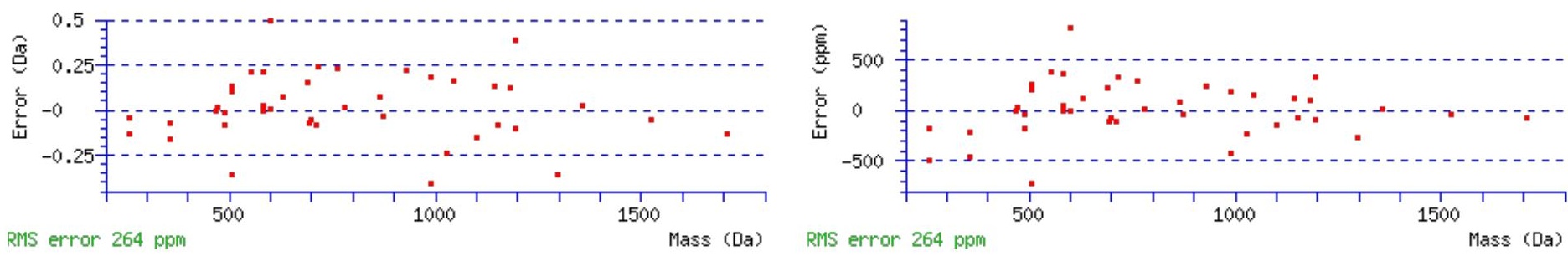

\section{All matches to this query}




\begin{tabular}{|l|l|l|l|}
\hline Score & Mr(calc): & Delta & \multicolumn{1}{|c|}{ Sequence } \\
\hline 36.2 & 2458.2340 & -0.0020 & GVTNDLLSVQGNTGPSWINKTEK \\
\hline 31.3 & 2458.2340 & -0.0020 & GVTNDLLSVQGNTGPSWINKTEK \\
\hline 6.9 & 2457.2500 & 0.9820 & GVTNDLLSVQGNTGPSWINKTEK \\
\hline 5.4 & 2456.2213 & 2.0106 & TVAGGAWAYNPTSAVTVKSAIRR \\
\hline 4.2 & 2457.2288 & 1.0031 & EQDLNLELFGEIHNLQNKFR \\
\hline 4.2 & 2457.2288 & 1.0031 & EQDLNLELFGEIHNLQNKFR \\
\hline 3.2 & 2456.2213 & 2.0106 & TVAGGAWAYNPTSAVTVKSAIRR \\
\hline 3.2 & 2458.2370 & -0.0050 & NSVGGLLLFDVTNRRSFLNVR \\
\hline 3.2 & 2458.2370 & -0.0050 & NSVGGLLLFDVTNRRSFLNVR \\
\hline 3.2 & 2458.2370 & -0.0050 & NSVGGLLLFDVTNRRSFLNVR \\
\hline
\end{tabular}

Spectrum No: 906; Query: 317; Rank: 1

\section{Peptide View}

MS/MS Fragmentation of KINSSLQLPDR

Found in IPI00371665, Tax_Id=10116 Gene_Symbol=Sema4b similar to Semaphorin-4B precursor

Match to Query 317: 1270.685922 from(424.569250,3+)

Title: 091008RatKidney_NoSalt_28.1353.1353.3.dta

Data file K:INewmanPaper|Piliang|3SubProteomes\Piliang3SP\mgf5ppm\ERLIC_3SubProteomes5ppm.mgf
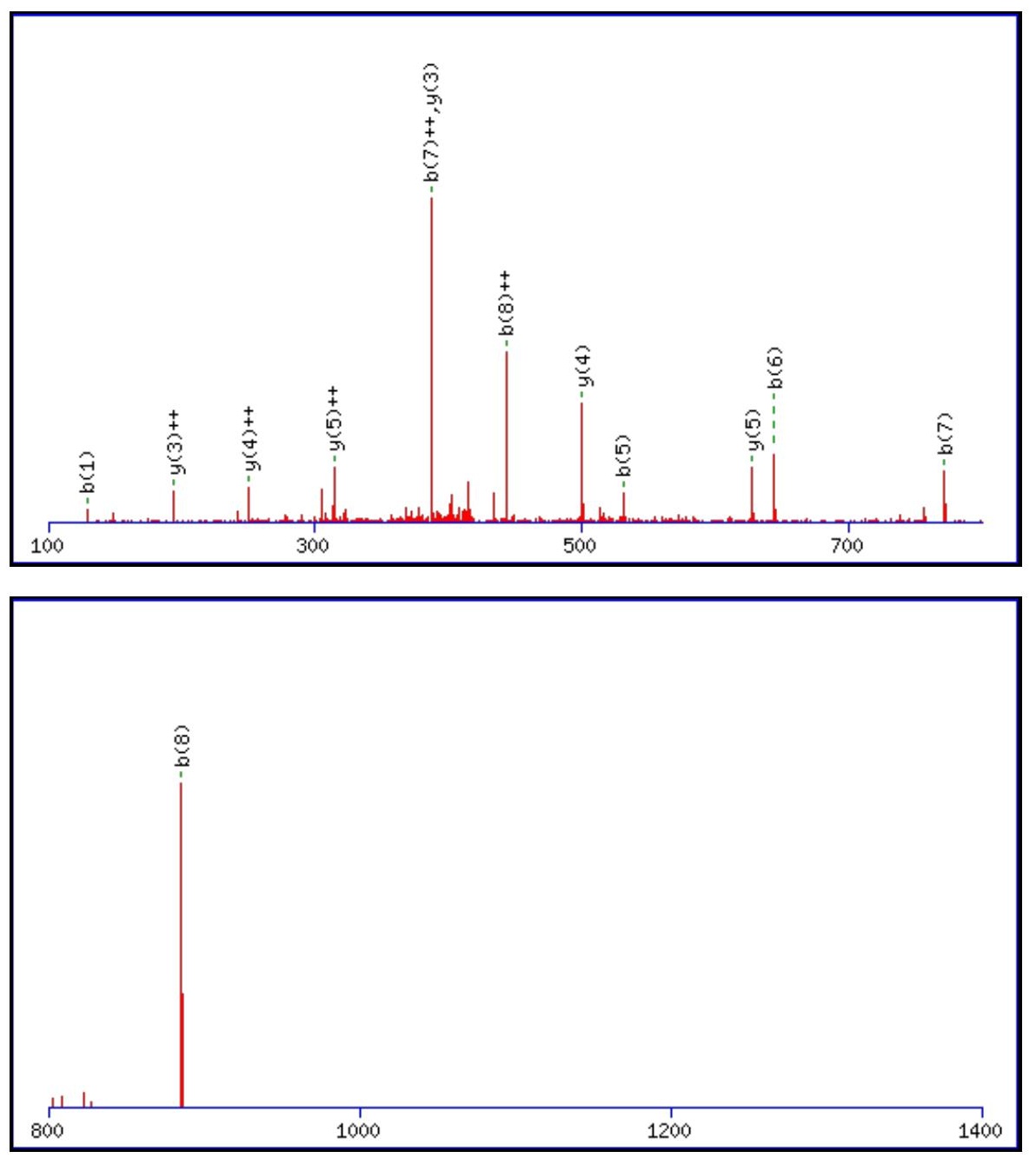


$1400 \quad 1600 \quad 1800 \quad 2000$

Monoisotopic mass of neutral peptide $\operatorname{Mr}($ calc): 1270.6881

Fixed modifications: Carbamidomethyl (C)

Variable modifications:

N3 : Deamidated $\mathrm{N}(\mathrm{N})$

Ions Score: 36 Expect: 0.019

Matches (Bold Red): 13/112 fragment ions using 17 most intense peaks

\begin{tabular}{|r|c|c|c|c|c|c|c|c|c|c|c|c|c|c|}
\hline$\#$ & $\mathbf{b}$ & $\mathbf{b}^{++}$ & $\mathbf{b}^{*}$ & $\mathbf{b}^{\mathbf{*}^{++}}$ & $\mathbf{b}^{\mathbf{0}}$ & $\mathbf{b}^{\mathbf{0 +}}$ & $\mathbf{S e q}$ & $\mathbf{y}$ & $\mathbf{y}^{++}$ & $\mathbf{y}^{*}$ & $\mathbf{y}^{\mathbf{*}^{++}}$ & $\mathbf{y}^{\mathbf{0}}$ & $\mathbf{y}^{\mathbf{0 + +}}$ & $\#$ \\
\hline $\mathbf{1}$ & $\mathbf{1 2 9 . 1 0 2 2}$ & 65.0548 & 112.0757 & 56.5415 & & & $\mathbf{K}$ & & & & & & & $\mathbf{1 1}$ \\
\hline $\mathbf{2}$ & 242.1863 & 121.5968 & 225.1598 & 113.0835 & & & $\mathbf{I}$ & 1143.6004 & 572.3038 & 1126.5739 & 563.7906 & 1125.5899 & 563.2986 & $\mathbf{1 0}$ \\
\hline $\mathbf{3}$ & 357.2132 & 179.1103 & 340.1867 & 170.5970 & & & $\mathbf{N}$ & 1030.5164 & 515.7618 & 1013.4898 & 507.2485 & 1012.5058 & 506.7565 & $\mathbf{9}$ \\
\hline $\mathbf{4}$ & 444.2453 & 222.6263 & 427.2187 & 214.1130 & 426.2347 & 213.6210 & $\mathbf{S}$ & 915.4894 & 458.2483 & 898.4629 & 449.7351 & 897.4789 & 449.2431 & $\mathbf{8}$ \\
\hline $\mathbf{5}$ & $\mathbf{5 3 1 . 2 7 7 3}$ & 266.1423 & 514.2507 & 257.6290 & 513.2667 & 257.1370 & $\mathbf{S}$ & 828.4574 & 414.7323 & 811.4308 & 406.2191 & 810.4468 & 405.7271 & $\mathbf{7}$ \\
\hline $\mathbf{6}$ & $\mathbf{6 4 4 . 3 6 1 4}$ & 322.6843 & 627.3348 & 314.1710 & 626.3508 & 313.6790 & $\mathbf{L}$ & 741.4254 & 371.2163 & 724.3988 & 362.7030 & 723.4148 & 362.2110 & $\mathbf{6}$ \\
\hline $\mathbf{7}$ & $\mathbf{7 7 2 . 4 1 9 9}$ & $\mathbf{3 8 6 . 7 1 3 6}$ & 755.3934 & 378.2003 & 754.4094 & 377.7083 & $\mathbf{Q}$ & $\mathbf{6 2 8 . 3 4 1 3}$ & 314.6743 & 611.3148 & 306.1610 & 610.3307 & 305.6690 & $\mathbf{5}$ \\
\hline $\mathbf{8}$ & $\mathbf{8 8 5 . 5 0 4 0}$ & $\mathbf{4 4 3 . 2 5 5 6}$ & 868.4775 & 434.7424 & 867.4934 & 434.2504 & $\mathbf{L}$ & $\mathbf{5 0 0 . 2 8 2 7}$ & 250.6450 & 483.2562 & 242.1317 & 482.2722 & 241.6397 & $\mathbf{4}$ \\
\hline $\mathbf{9}$ & 982.5568 & 491.7820 & 965.5302 & 483.2687 & 964.5462 & 482.7767 & $\mathbf{P}$ & 387.1987 & $\mathbf{1 9 4 . 1 0 3 0}$ & 370.1721 & 185.5897 & 369.1881 & 185.0977 & $\mathbf{3}$ \\
\hline $\mathbf{1 0}$ & 1097.5837 & 549.2955 & 1080.5572 & 540.7822 & 1079.5731 & 540.2902 & $\mathbf{D}$ & 290.1459 & 145.5766 & 273.1193 & 137.0633 & 272.1353 & 136.5713 & $\mathbf{2}$ \\
\hline $\mathbf{1 1}$ & & & & & & & $\mathbf{R}$ & 175.1190 & 88.0631 & 158.0924 & 79.5498 & & & $\mathbf{1}$ \\
\hline
\end{tabular}
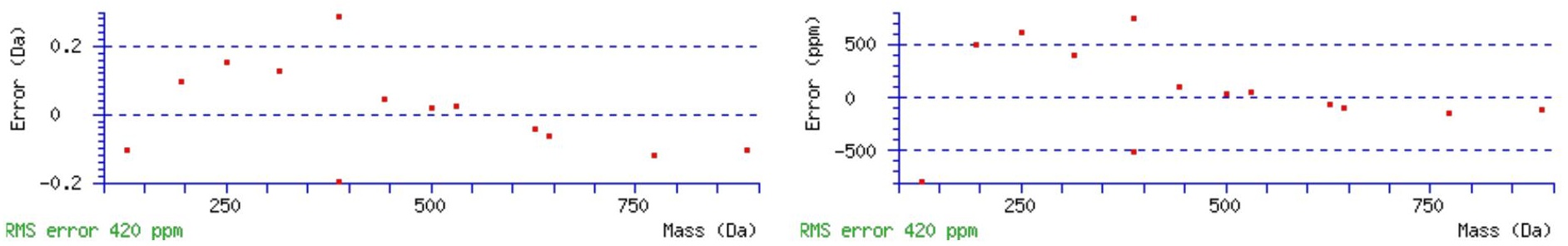

\section{All matches to this query}

\begin{tabular}{|l|l|l|l|}
\hline Score & Mr(calc): & Delta & \multicolumn{1}{|c|}{ Sequence } \\
\hline 36.2 & 1270.6881 & -0.0022 & KINSSLQLPDR \\
\hline 20.9 & 1268.6837 & 2.0023 & NPATLGSRPISR \\
\hline 16.3 & 1270.6881 & -0.0022 & QEEVSAIVIQR \\
\hline 13.7 & 1270.6881 & -0.0022 & QQIEELQKQK \\
\hline 13.1 & 1270.6856 & 0.0003 & WAEIAKMLPGR \\
\hline 12.8 & 1270.6881 & -0.0022 & QEEVSAVIIQR \\
\hline 11.9 & 1269.6764 & 1.0095 & HLXHQPILPR \\
\hline 11.7 & 1270.6816 & 0.0044 & CNRVLQPQKK \\
\hline 11.6 & 1269.6863 & 0.9996 & LRPEMLQDLR \\
\hline 11.4 & 1270.6782 & 0.0077 & NPERLYINPR \\
\hline
\end{tabular}


Spectrum No: 907; Query: 353; Rank: 1

\section{Peptide View}

MS/MS Fragmentation of LGGWNITGPWAK

Found in IPI00200747, Tax_Id=10116 Gene_Symbol=Ece1 Isoform A of Endothelin-converting enzyme 1

Match to Query 353: 1299.660648 from(650.837600,2+)

Title: 091008RatKidney_NoSalt_29.3367.3367.2.dta

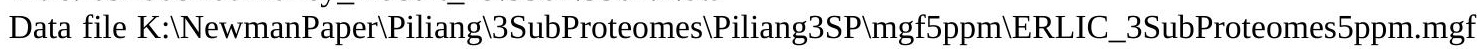
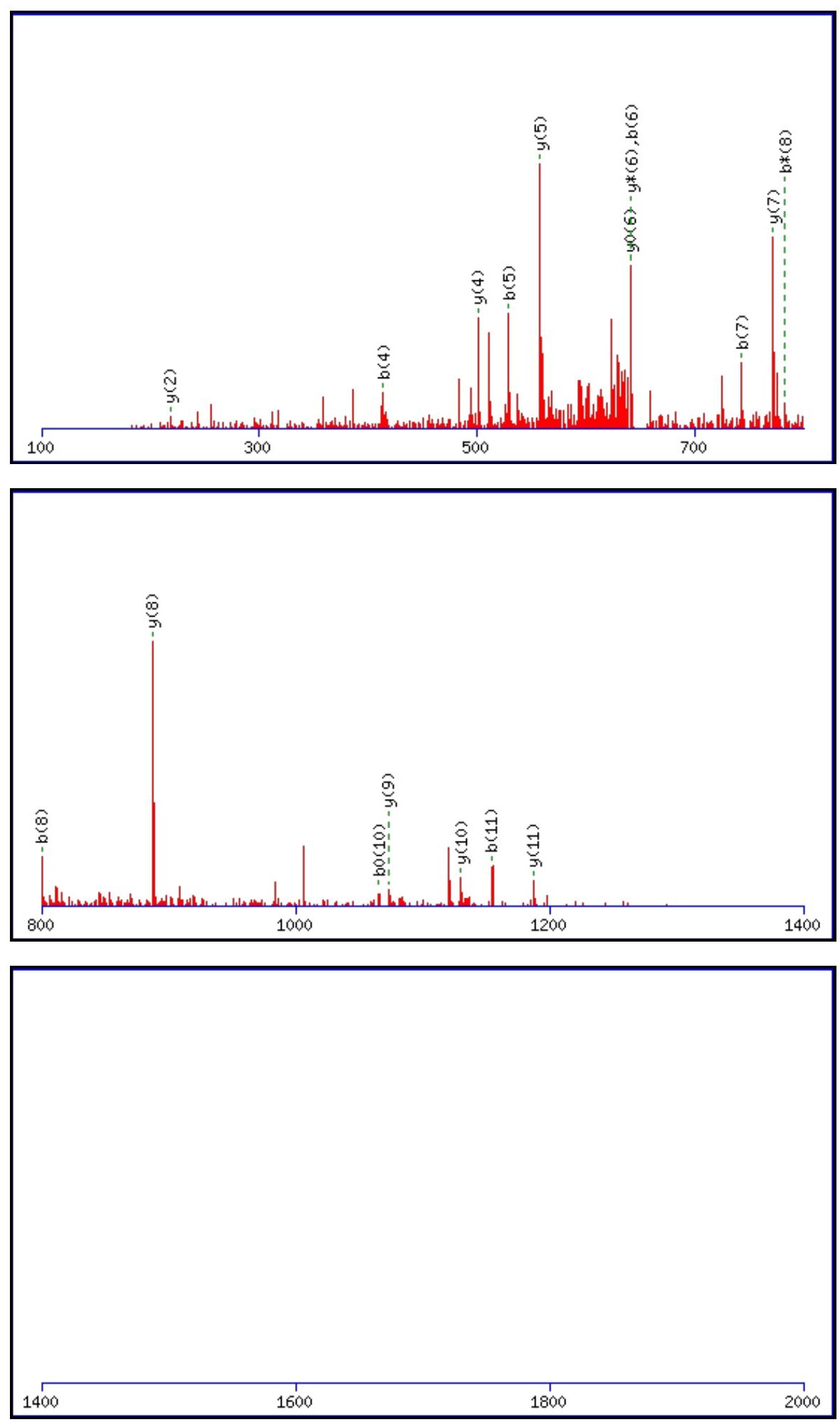
Monoisotopic mass of neutral peptide $\operatorname{Mr}($ calc): 1299.6612

Fixed modifications: Carbamidomethyl (C)

Variable modifications:

N5 : Deamidated_N (N)

Ions Score: 36 Expect: 0.029

Matches (Bold Red): 18/102 fragment ions using 56 most intense peaks

\begin{tabular}{|c|c|c|c|c|c|c|c|c|c|c|c|c|c|c|}
\hline \# & b & $\mathbf{b}^{++}$ & $\mathbf{b}^{*}$ & $\mathbf{b}^{*^{++}}$ & $\mathbf{b}^{\mathbf{0}}$ & $\mathbf{b}^{0++}$ & Seq. & $\mathbf{y}$ & $\mathbf{y}^{++}$ & $\mathrm{y}^{*}$ & $\mathrm{y}^{\mathrm{*}^{++}}$ & $\mathbf{y}^{0}$ & $y^{0++}$ & $\#$ \\
\hline 1 & 114.0913 & 57.5493 & & & & & $\mathbf{L}$ & & & & & & & 12 \\
\hline 2 & 171.1128 & 86.0600 & & & & & G & 1187.5844 & 594.2958 & 1170.5578 & 585.7826 & 1169.5738 & 585.2905 & 11 \\
\hline 3 & 228.1343 & 114.5708 & & & & & G & 1130.5629 & 565.7851 & 1113.5364 & 557.2718 & 1112.5524 & 556.7798 & 10 \\
\hline 4 & 414.2136 & 207.6104 & & & & & $\mathbf{W}$ & 1073.5415 & 537.2744 & 1056.5149 & 528.7611 & 1055.5309 & 528.2691 & 9 \\
\hline 5 & 529.2405 & 265.1239 & 512.2140 & 256.6106 & & & $\mathbf{N}$ & 887.4621 & 444.2347 & 870.4356 & 435.7214 & 869.4516 & 435.2294 & 8 \\
\hline 6 & 642.3246 & 321.6659 & 625.2980 & 313.1527 & & & $\mathbf{I}$ & 772.4352 & 386.7212 & 755.4087 & 378.2080 & 754.4246 & 377.7160 & 7 \\
\hline 7 & 743.3723 & 372.1898 & 726.3457 & 363.6765 & 725.3617 & 363.1845 & $\mathbf{T}$ & 659.3511 & 330.1792 & 642.3246 & 321.6659 & 641.3406 & 321.1739 & 6 \\
\hline 8 & 800.3937 & 400.7005 & 783.3672 & 392.1872 & 782.3832 & 391.6952 & G & 558.3035 & 279.6554 & 541.2769 & 271.1421 & & & 5 \\
\hline 9 & 897.4465 & 449.2269 & 880.4199 & 440.7136 & 879.4359 & 440.2216 & $\mathbf{P}$ & 501.2820 & 251.1446 & 484.2554 & 242.6314 & & & 4 \\
\hline 10 & 1083.5258 & 542.2665 & 1066.4993 & 533.7533 & 1065.5152 & 533.2613 & $\mathbf{W}$ & 404.2292 & 202.6183 & 387.2027 & 194.1050 & & & 3 \\
\hline 11 & \begin{tabular}{|l}
1154.5629 \\
\end{tabular} & 577.7851 & 1137.5364 & 569.2718 & 1136.5524 & 568.7798 & A & 218.1499 & 109.5786 & 201.1234 & 101.0653 & & & 2 \\
\hline 12 & & & & & & & K & 147.1128 & \begin{tabular}{|l|}
74.0600 \\
\end{tabular} & 130.0863 & 65.5468 & & & 1 \\
\hline
\end{tabular}
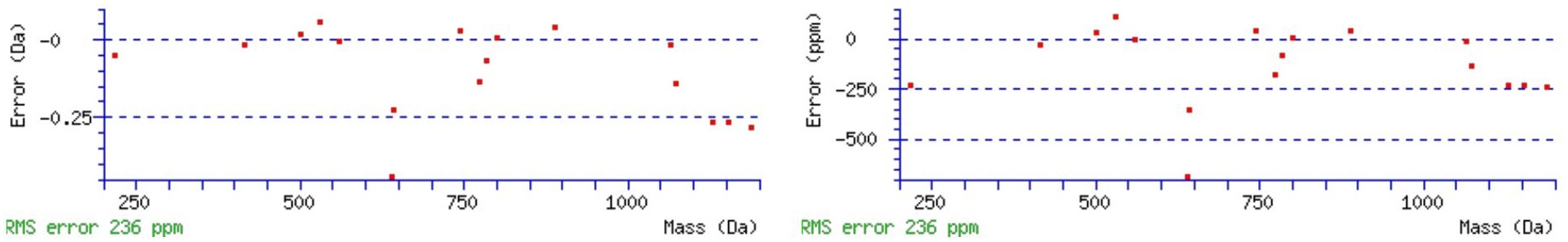

All matches to this query

\begin{tabular}{|l|l|l|l|}
\hline Score & Mr(calc): & Delta & \multicolumn{1}{c|}{ Sequence } \\
\hline 36.2 & 1299.6612 & -0.0005 & LGGWNITGPWAK \\
\hline 5.2 & 1298.6466 & 1.0140 & NLSPSQSPELAR \\
\hline 2.7 & 1299.6588 & 0.0019 & IHLPGSSTPLAK \\
\hline 2.7 & 1299.6588 & 0.0019 & IHLPGSSTPLAK \\
\hline 2.7 & 1297.6626 & 1.9981 & NLSPSOSPELAR \\
\hline
\end{tabular}

Spectrum No: 908; Query: 726; Rank: 1

\section{Peptide View}

MS/MS Fragmentation of HVTDMNSTIHLLK

Found in IPI00361106, Tax_Id=10116 Gene_Symbol=RGD1560062_predicted hypothetical protein LOC309816

Match to Query 726: 1524.756702 from(509.259510,3+)

Title: 091008RatKidney_NH4Format01_25.1098.1098.3.dta

Data file K:INewmanPaper|Piliangl3SubProteomes\Piliang3SP\mgf5ppm\ERLIC_3SubProteomes5ppm.mgf 

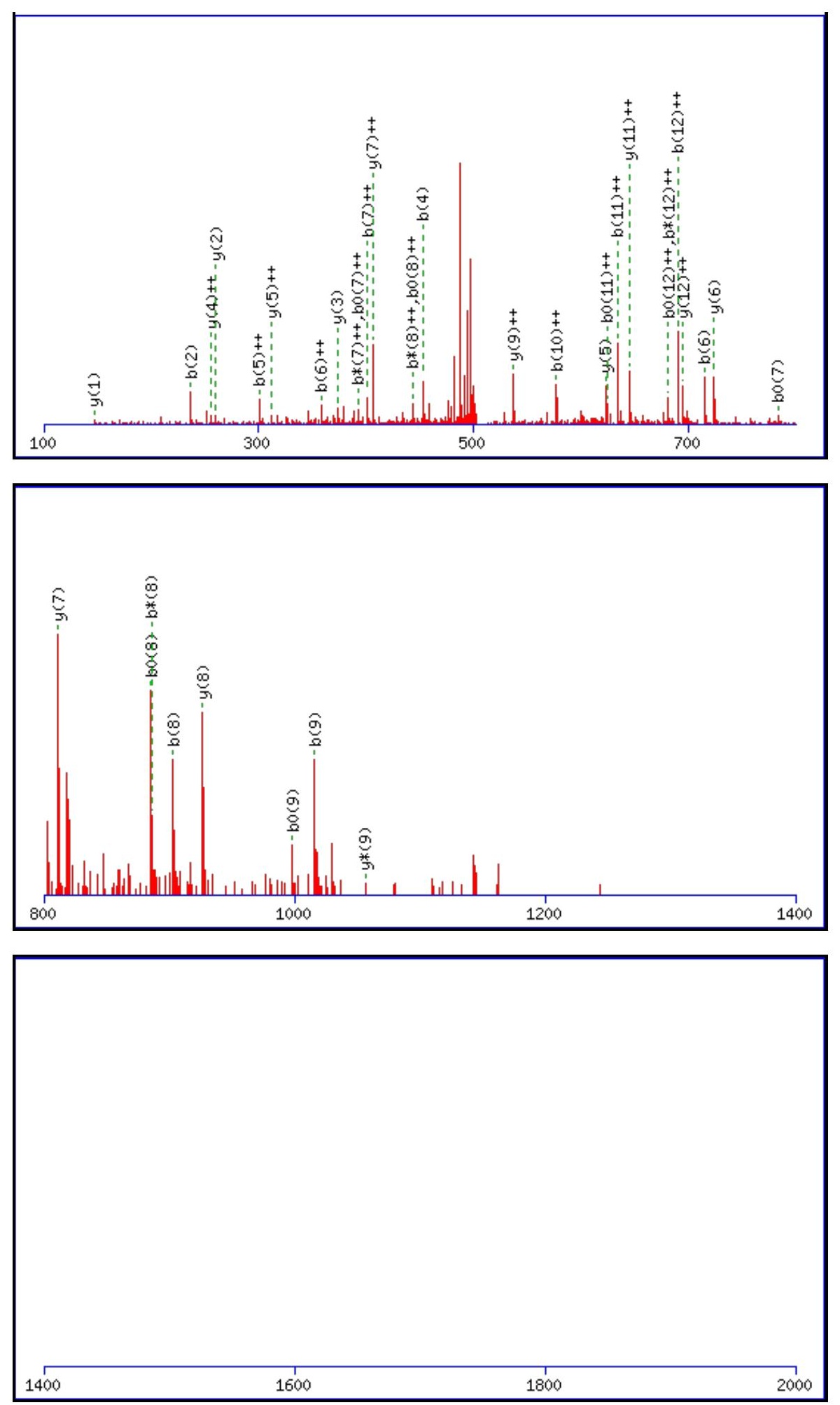

Monoisotopic mass of neutral peptide $\operatorname{Mr}($ calc): 1524.7606

Fixed modifications: Carbamidomethyl (C)

Variable modifications:

M5 : Oxidation (M)

N6 : Deamidated_N (N)

Ions Score: 36 Expect: $\odot .042$

Matches (Bold Red): 36/120 fragment ions using 80 most intense peaks

\begin{tabular}{|c|c|c|c|c|c|c|c|c|c|c|c|c|c|c|}
\hline$\#$ & $\mathbf{b}$ & $\mathbf{b}^{++}$ & $\mathbf{b}^{*}$ & $\mathbf{b}^{*^{++}}$ & $\mathbf{b}^{\mathbf{0}}$ & $\mathbf{b}^{\mathbf{0}+}$ & Seq. & $\mathbf{y}$ & $\mathbf{y}^{++}$ & $\mathbf{y}^{*}$ & $\mathbf{y}^{\boldsymbol{*}^{++}}$ & $\mathbf{y}^{\mathbf{0}}$ & $\mathbf{y}^{\mathbf{0 + +}}$ & $\#$ \\
\hline $\mathbf{1}$ & 138.0662 & 69.5367 & & & & & $\mathbf{H}$ & & & & & & & $\mathbf{1 3}$ \\
\hline $\mathbf{2}$ & 237.1346 & 119.0709 & & & & & $\mathbf{V}$ & 1388.7090 & $\mathbf{6 9 4 . 8 5 8 1}$ & 1371.6824 & 686.3449 & 1370.6984 & 685.8529 & $\mathbf{1 2}$ \\
\hline $\mathbf{3}$ & 338.1823 & 169.5948 & & & 320.1717 & 160.5895 & $\mathbf{T}$ & 1289.6406 & $\mathbf{6 4 5 . 3 2 3 9}$ & 1272.6140 & 636.8107 & 1271.6300 & 636.3186 & $\mathbf{1 1}$ \\
\hline $\mathbf{4}$ & $\mathbf{4 5 3 . 2 0 9 2}$ & 227.1083 & & & 435.1987 & 218.1030 & $\mathbf{D}$ & 1188.5929 & 594.8001 & 1171.5663 & 586.2868 & 1170.5823 & 585.7948 & $\mathbf{1 0}$ \\
\hline & & & & & & & & & & & & & &
\end{tabular}




\begin{tabular}{|c|c|c|c|c|c|c|c|c|c|c|c|c|c|c|}
\hline & 600.2446 & 300.6260 & & & 582.2341 & |291.6207 & M & 1073.5660 & |537.2866 & 1056.5394 & 528.7733 & 1055.5554 & |528.2813 & 9 \\
\hline 6 & 715.2716 & 358.1394 & 698.2450 & 349.6261 & 697.2610 & |349.1341 & $\mathbf{N}$ & 926.5306 & 463.7689 & 909.5040 & 455.2556 & 908.5200 & 454.7636 & 8 \\
\hline 7 & 802.3036 & 401.6554 & 785.2770 & 393.1422 & 784.2930 & 392.6502 & S & 811.5036 & 406.2554 & 794.4771 & 397.7422 & 793.4930 & 397.2502 & 7 \\
\hline 8 & 903.3513 & 452.1793 & 886.3247 & 443.6660 & 885.3407 & 443.1740 & $\mathbf{T}$ & 724.4716 & 362.7394 & 707.4450 & 354.2262 & 706.4610 & 353.7341 & 6 \\
\hline 9 & 1016.4353 & 508.7213 & 999.4088 & 500.2080 & 998.4248 & 499.7160 & I & 623.4239 & 312.2156 & 606.3974 & 303.7023 & & & 5 \\
\hline 10 & 1153.4942 & 577.2508 & 1136.4677 & 568.7375 & 1135.4837 & 568.2455 & $\mathbf{H}$ & 510.3398 & 255.6736 & 493.3133 & 247.1603 & & & 4 \\
\hline 11 & 1266.5783 & 633.7928 & 1249.5518 & 625.2795 & 1248.5677 & 624.7875 & $\mathbf{L}$ & 373.2809 & |187.1441 & 356.2544 & 178.6308 & & & 3 \\
\hline 12 & 1379.6624 & 690.3348 & 1362.6358 & 681.8216 & 1361.6518 & 681.3295 & $\mathbf{L}$ & 260.1969 & |130.6021 & 243.1703 & 122.0888 & & & 2 \\
\hline 13 & & & & & & & $\mathbf{K}$ & 147.1128 & 74.0600 & 130.0863 & 65.5468 & & & 1 \\
\hline
\end{tabular}
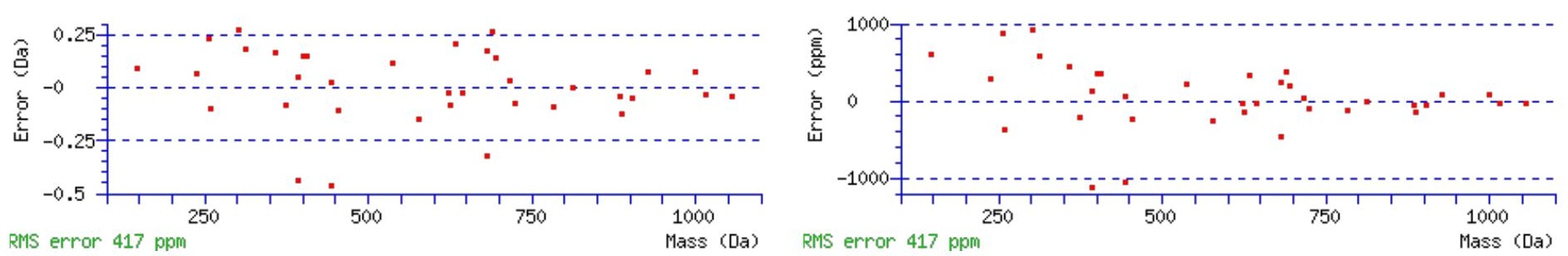

\section{All matches to this query}

\begin{tabular}{|l|l|l|l|}
\hline Score & Mr(calc): & Delta & \multicolumn{1}{|c|}{ Sequence } \\
\hline 36.1 & 1524.7606 & -0.0039 & HVTDMNSTIHLLK \\
\hline 11.1 & 1524.7483 & 0.0084 & AMDRRTGEVVAIK \\
\hline 4.9 & 1522.7644 & 1.9923 & NVTIIVIGLDNSGK \\
\hline 4.9 & 1523.7484 & 1.0083 & NVTIIVIGLDNSGK \\
\hline 4.2 & 1524.7559 & 0.0008 & NEVENTITYSLLK \\
\hline 3.3 & 1522.7505 & 2.0062 & HLSRTISVSGSTAK \\
\hline 2.9 & 1524.7671 & -0.0104 & EEDKVLAYTKDSK \\
\hline 2.0 & 1523.7476 & 1.0091 & TPVRMLPYAMADK \\
\hline 1.8 & 1522.7627 & 1.9940 & NTYNEVDSRLIAK \\
\hline 1.2 & 1523.7528 & 1.0039 & HRAWMNQKGPGSR \\
\hline
\end{tabular}

Spectrum No: 909; Query: 774; Rank: 1

\section{Peptide View}

MS/MS Fragmentation of EEDFYVNETSTVK

Found in IPI00210824, Tax_Id=10116 Gene_Symbol=Serpina6 Corticosteroid-binding globulin precursor

Match to Query 774: 1560.685588 from(781.350070,2+)

Title: 100101RatKid_NS_deglyco_27.1931.1931.2.dta

Data file K:\NewmanPaper|Piliang|3SubProteomes\Piliang3SP\mgf5ppm\ERLIC_3SubProteomes5ppm.mgf 

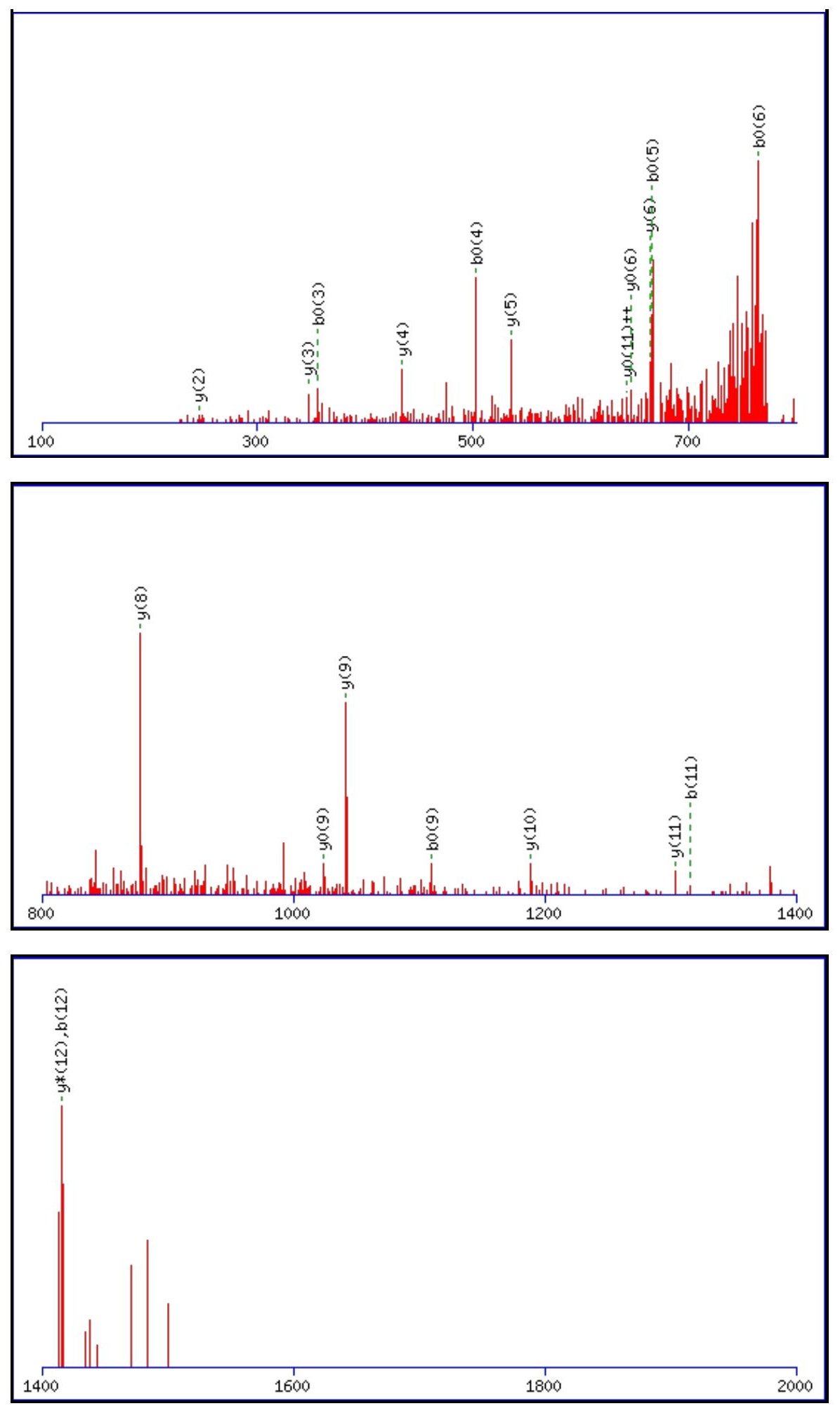

Monoisotopic mass of neutral peptide $\operatorname{Mr}($ calc): 1560.6831

Fixed modifications: Carbamidomethyl (C)

Variable modifications:

N7 : Deamidated_N (N)

Ions Score: 36 Expect: 0.04

Matches (Bold Red): 20/128 fragment ions using 62 most intense peaks

\begin{tabular}{|r|c|c|c|c|c|c|c|c|c|c|c|c|c|c|}
\hline$\#$ & $\mathbf{b}$ & $\mathbf{b}^{++}$ & $\mathbf{b}^{*}$ & $\mathbf{b}^{\boldsymbol{*}^{++}}$ & $\mathbf{b}^{\mathbf{0}}$ & $\mathbf{b}^{\mathbf{0 + +}}$ & Seq. & $\mathbf{y}$ & $\mathbf{y}^{++}$ & $\mathbf{y}^{\mathbf{*}}$ & $\mathbf{y}^{\boldsymbol{*}^{++}}$ & $\mathbf{y}^{\mathbf{0}}$ & $\mathbf{y}^{\mathbf{0 + +}}$ & $\#$ \\
\hline $\mathbf{1}$ & 130.0499 & 65.5286 & & & 112.0393 & 56.5233 & $\mathbf{E}$ & & & & & & & $\mathbf{1 3}$ \\
\hline $\mathbf{2}$ & 259.0925 & 130.0499 & & & 241.0819 & 121.0446 & $\mathbf{E}$ & 1432.6478 & 716.8276 & $\mathbf{1 4 1 5 . 6 2 1 3}$ & 708.3143 & 1414.6373 & 707.8223 & $\mathbf{1 2}$ \\
\hline 3 & 374.1194 & 187.5633 & & & 356.1088 & 178.5581 & $\mathbf{D}$ & $\mathbf{1 3 0 3 . 6 0 5 2}$ & 652.3063 & 1286.5787 & 643.7930 & 1285.5947 & $\mathbf{6 4 3 . 3 0 1 0}$ & $\mathbf{1 1}$ \\
\hline $\mathbf{4}$ & 521.1878 & 261.0975 & & & $5 \mathbf{0 3 . 1 7 7 3}$ & 252.0923 & $\mathbf{F}$ & $\mathbf{1 1 8 8 . 5 7 8 3}$ & 594.7928 & 1171.5517 & 586.2795 & 1170.5677 & 585.7875 & $\mathbf{1 0}$ \\
\hline $\mathbf{5}$ & 684.2511 & 342.6292 & & & $\mathbf{6 6 6 . 2 4 0 6}$ & 333.6239 & $\mathbf{Y}$ & $\mathbf{1 0 4 1 . 5 0 9 9}$ & 521.2586 & 1024.4833 & 512.7453 & $\mathbf{1 0 2 3 . 4 9 9 3}$ & 512.2533 & $\mathbf{9}$ \\
\hline
\end{tabular}




\begin{tabular}{|r|r|r|r|r|r|r|r|r|r|r|r|r|r|r|r|r|}
$\mathbf{6}$ & 783.3196 & 392.1634 & & & $\mathbf{7 6 5 . 3 0 9 0}$ & 383.1581 & $\mathbf{V}$ & $\mathbf{8 7 8 . 4 4 6 5}$ & 439.7269 & 861.4200 & 431.2136 & 860.4360 & 430.7216 & $\mathbf{8}$ \\
\hline $\mathbf{7}$ & 898.3465 & 449.6769 & 881.3199 & 441.1636 & 880.3359 & 440.6716 & $\mathbf{N}$ & 779.3781 & 390.1927 & 762.3516 & 381.6794 & 761.3676 & 381.1874 & $\mathbf{7}$ \\
\hline $\mathbf{8}$ & 1027.3891 & 514.1982 & 1010.3625 & 505.6849 & 1009.3785 & 505.1929 & $\mathbf{E}$ & $\mathbf{6 6 4 . 3 5 1 2}$ & 332.6792 & 647.3246 & 324.1660 & $\mathbf{6 4 6 . 3 4 0 6}$ & 323.6740 & $\mathbf{6}$ \\
\hline $\mathbf{9}$ & 1128.4368 & 564.7220 & 1111.4102 & 556.2087 & $\mathbf{1 1 1 0 . 4 2 6 2}$ & 555.7167 & $\mathbf{T}$ & 535.3086 & 268.1579 & 518.2821 & 259.6447 & 517.2980 & 259.1527 & $\mathbf{5}$ \\
\hline $\mathbf{1 0}$ & 1215.4688 & 608.2380 & 1198.4422 & 599.7248 & 1197.4582 & 599.2328 & $\mathbf{S}$ & $\mathbf{4 3 4 . 2 6 0 9}$ & 217.6341 & 417.2344 & 209.1208 & 416.2504 & 208.6288 & $\mathbf{4}$ \\
\hline $\mathbf{1 1}$ & $\mathbf{1 3 1 6 . 5 1 6 5}$ & 658.7619 & 1299.4899 & 650.2486 & 1298.5059 & 649.7566 & $\mathbf{T}$ & 347.2289 & 174.1181 & 330.2023 & 165.6048 & 329.2183 & 165.1128 & $\mathbf{3}$ \\
\hline $\mathbf{1 2}$ & $\mathbf{1 4 1 5 . 5 8 4 9}$ & 708.2961 & 1398.5583 & 699.7828 & 1397.5743 & 699.2908 & $\mathbf{V}$ & $\mathbf{2 4 6 . 1 8 1 2}$ & 123.5942 & 229.1547 & 115.0810 & & & $\mathbf{2}$ \\
\hline $\mathbf{1 3}$ & & & & & & & $\mathbf{K}$ & 147.1128 & 74.0600 & 130.0863 & 65.5468 & & & \\
\hline
\end{tabular}
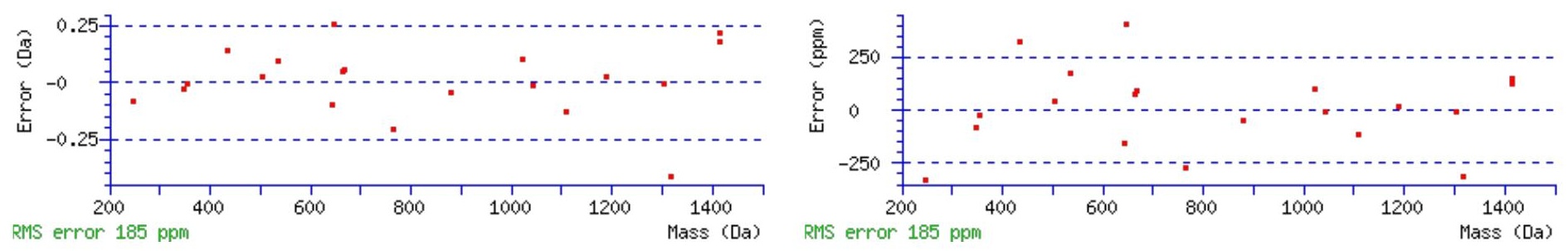

\section{All matches to this query}

\begin{tabular}{|l|l|r|l|}
\hline Score & Mr(calc): & Delta & \multicolumn{1}{c|}{ Sequence } \\
\hline 36.0 & 1560.6831 & 0.0025 & EEDFYVNETSTVK \\
\hline 3.7 & 1560.6739 & 0.0117 & SVSLTTFYRTAR \\
\hline 1.7 & 1558.6876 & 1.9980 & NTSKSVEVGGSTEGK \\
\hline 1.4 & 1559.6746 & 1.0110 & VAGQNSPSGIQSKK \\
\hline 0.9 & 1560.6830 & 0.0026 & LMHNGTILYTMR \\
\hline 0.4 & 1560.7007 & -0.0151 & MVYISNGQVLDSR \\
\hline
\end{tabular}

Spectrum No: 910; Query: 1698; Rank: 1

\section{Peptide View}

MS/MS Fragmentation of LNQLAINLSGIIQGINQDR

Found in IPI00190577, Tax_Id=10116 Gene_Symbol=Lama5 similar to Laminin alpha-5 chain precursor

Match to Query 1698: 2080.128252 from(694.383360,3+)

Title: 091008RatKidney_NH4Format01_26.4296.4296.3.dta

Data file K:INewmanPaper|Piliang|3SubProteomes\Piliang3SP\mgf5ppm\ERLIC_3SubProteomes5ppm.mgf

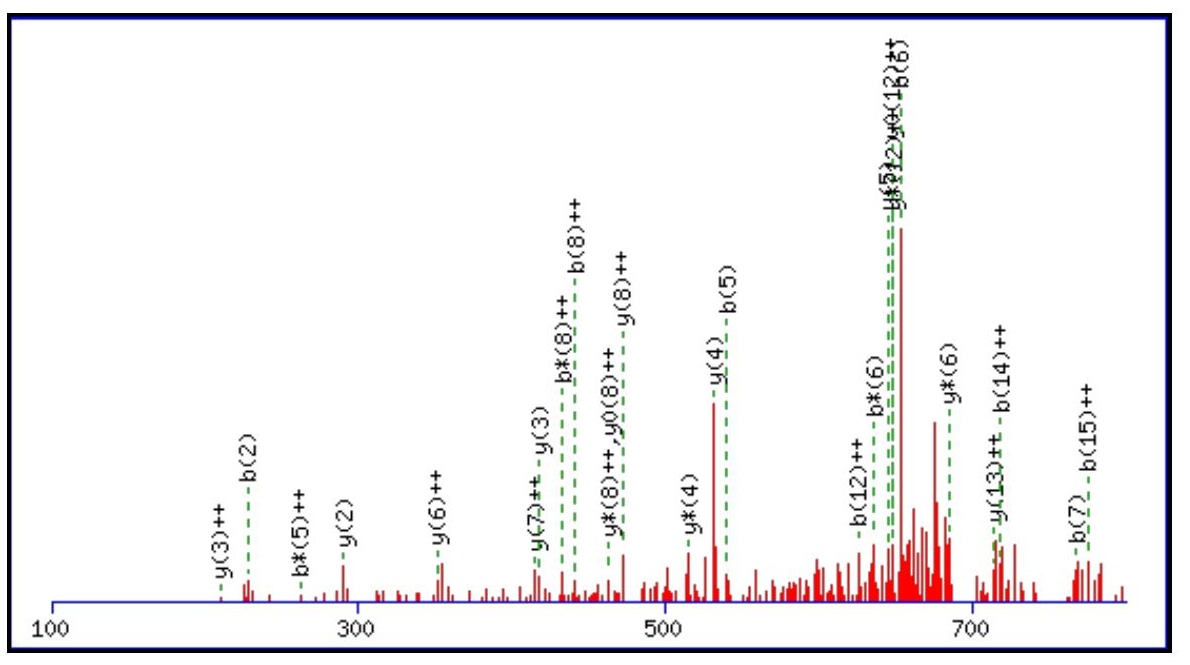



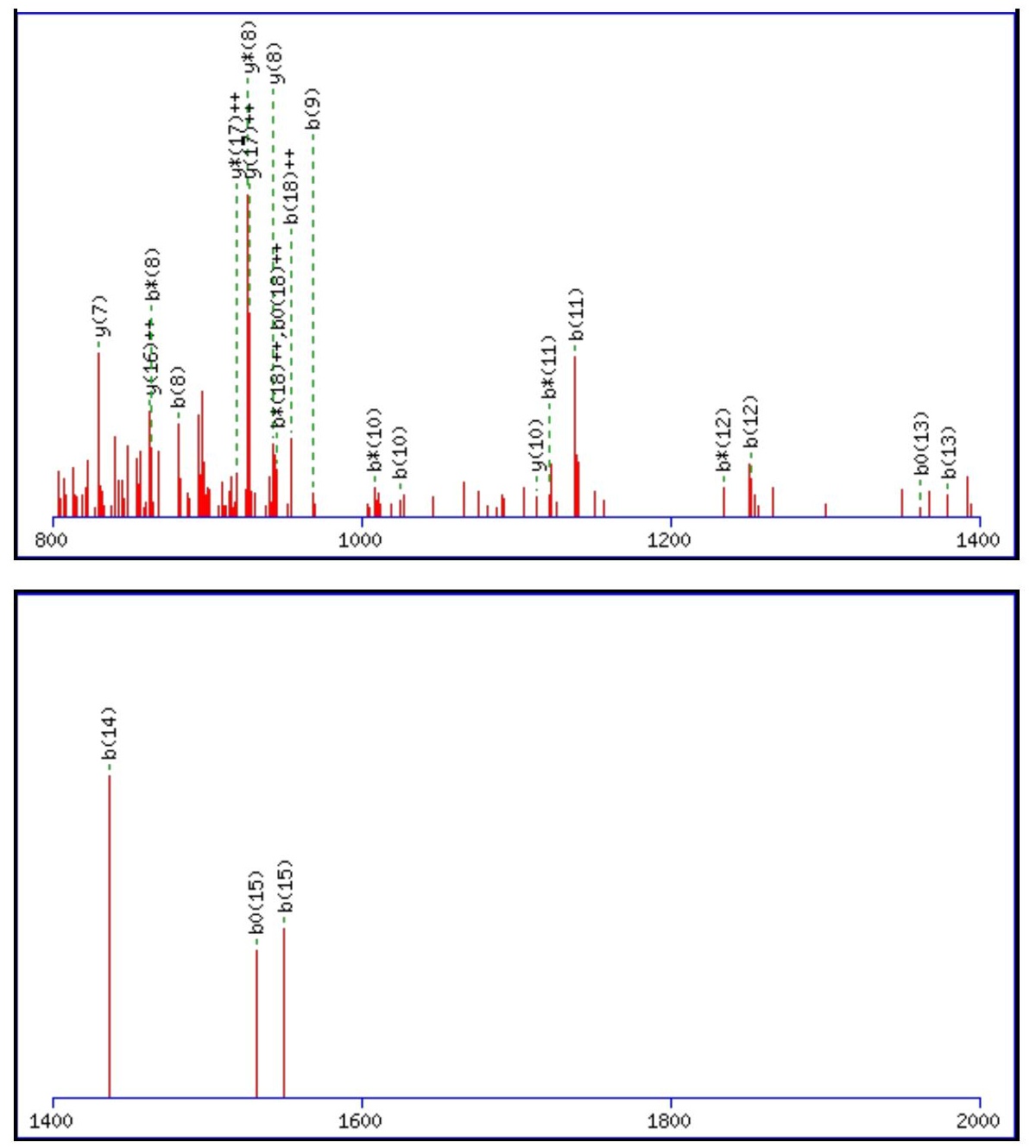

Monoisotopic mass of neutral peptide $\operatorname{Mr}($ calc): 2080.1276

Fixed modifications: Carbamidomethyl (C)

Variable modifications:

N7 : Deamidated_N (N)

Ions Score: 36 Expect : 0.016

Matches (Bold Red): 50/196 fragment ions using 129 most intense peaks

\begin{tabular}{|c|c|c|c|c|c|c|c|c|c|c|c|c|c|c|}
\hline \# & b & $\mathbf{b}^{++}$ & $\mathbf{b}^{*}$ & $\mathbf{b}^{*^{++}}$ & $\mathbf{b}^{0}$ & $\mathbf{b}^{\mathbf{0 + +}}$ & Seq. & $\mathbf{y}$ & $\mathbf{y}^{++}$ & $\mathrm{y}^{*}$ & $\mathbf{y}^{*^{++}}$ & $\mathbf{y}^{0}$ & $y^{0++}$ & \# \\
\hline 1 & 114.0913 & 57.5493 & & & & & $\mathbf{L}$ & & & & & & & 19 \\
\hline 2 & 228.1343 & 114.5708 & 211.1077 & 106.0575 & & & $\mathbf{N}$ & 1968.0509 & 984.5291 & 1951.0243 & 976.0158 & 1950.0403 & 975.5238 & 18 \\
\hline 3 & 356.1928 & 178.6001 & 339.1663 & 170.0868 & & & $\mathbf{Q}$ & 1854.0079 & 927.5076 & 1836.9814 & 918.9943 & 1835.9974 & 918.5023 & 17 \\
\hline 4 & 469.2769 & 235.1421 & 452.2504 & 226.6288 & & & $\mathbf{L}$ & 1725.9494 & 863.4783 & 1708.9228 & 854.9650 & |1707.9388 & 854.4730 & 16 \\
\hline 5 & 540.3140 & 270.6606 & 523.2875 & 262.1474 & & & $\mathbf{A}$ & 1612.8653 & 806.9363 & 1595.8388 & 798.4230 & 1594.8547 & 797.9310 & 15 \\
\hline 6 & 653.3981 & 327.2027 & 636.3715 & 318.6894 & & & I & 1541.8282 & 771.4177 & 1524.8016 & 762.9045 & 1523.8176 & 762.4125 & 14 \\
\hline 7 & 768.4250 & 384.7161 & 751.3985 & 376.2029 & & & $\mathbf{N}$ & 1428.7441 & 714.8757 & 1411.7176 & 706.3624 & 1410.7336 & 705.8704 & 13 \\
\hline 8 & 881.5091 & 441.2582 & 864.4825 & 432.7449 & & & $\mathbf{L}$ & 1313.7172 & 657.3622 & 1296.6906 & 648.8490 & 1295.7066 & 648.3570 & 12 \\
\hline 9 & 968.5411 & 484.7742 & 951.5146 & 476.2609 & 950.5305 & 475.7689 & S & 1200.6331 & 600.8202 & 1183.6066 & 592.3069 & 1182.6226 & 591.8149 & 11 \\
\hline 10 & 1025.5626 & 513.2849 & 1008.5360 & 504.7717 & 1007.5520 & 504.2796 & G & 1113.6011 & 557.3042 & 1096.5745 & 548.7909 & 1095.5905 & 548.2989 & 10 \\
\hline 11 & 1138.6466 & 569.8270 & 1121.6201 & 561.3137 & 1120.6361 & 560.8217 & I & 1056.5796 & 528.7935 & 1039.5531 & 520.2802 & 1038.5691 & 519.7882 & 9 \\
\hline 12 & 1251.7307 & 626.3690 & 1234.7042 & 617.8557 & 1233.7201 & 617.3637 & I & 943.4956 & 472.2514 & 926.4690 & 463.7381 & 925.4850 & 463.2461 & 8 \\
\hline 13 & 1379.7893 & 690.3983 & 1362.7627 & 681.8850 & \begin{tabular}{|l}
1361.7787 \\
\end{tabular} & 681.3930 & $\mathbf{Q}$ & 830.4115 & 415.7094 & 813.3850 & 407.1961 & 812.4009 & 406.7041 & 7 \\
\hline 14 & 1436.8107 & 718.9090 & 1419.7842 & 710.3957 & 1418.8002 & 709.9037 & G & 702.3529 & 351.6801 & 685.3264 & 343.1668 & 684.3424 & 342.6748 & 6 \\
\hline 15 & 1549.8948 & 775.4510 & 1532.8683 & 766.9378 & 1531.8842 & 766.4458 & I & 645.3315 & 323.1694 & 628.3049 & 314.6561 & 627.3209 & 314.1641 & 5 \\
\hline 16 & 1663.9377 & 832.4725 & 1646.9112 & 823.9592 & 1645.9272 & 823.4672 & $\mathbf{N}$ & 532.2474 & 266.6273 & 515.2209 & 258.1141 & 514.2368 & 257.6221 & 4 \\
\hline 17 & 1791.9963 & 896.5018 & 1774.9698 & 887.9885 & 1773.9858 & 887.4965 & $\mathbf{Q}$ & 418.2045 & 209.6059 & 401.1779 & 201.0926 & 400.1939 & 200.6006 & 3 \\
\hline 18 & 1907.0233 & 954.0153 & 1889.9967 & 945.5020 & 1889.0127 & 945.0100 & D & 290.1459 & 145.5766 & 273.1193 & 137.0633 & 272.1353 & 136.5713 & 2 \\
\hline
\end{tabular}




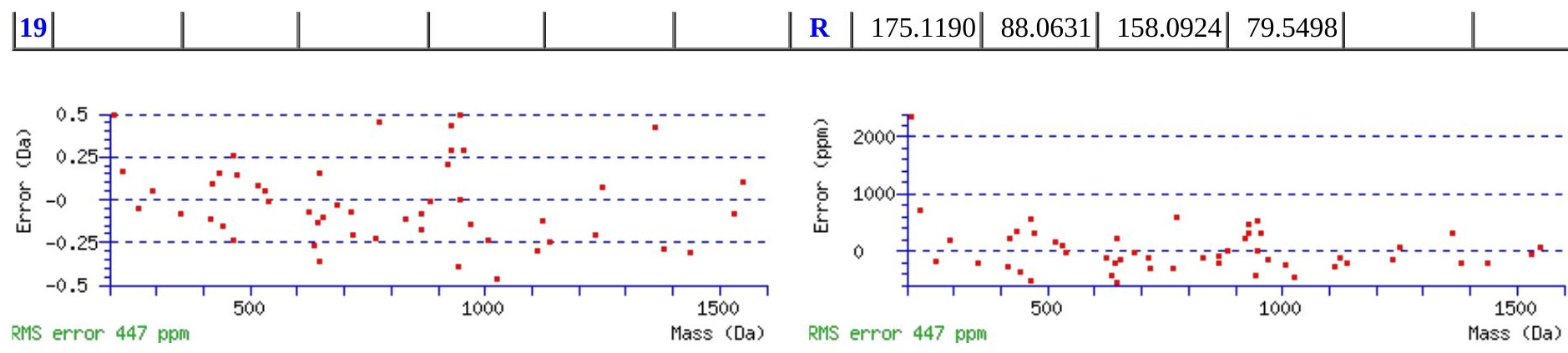

\section{All matches to this query}

\begin{tabular}{|l|l|l|l|}
\hline Score & Mr(calc): & Delta & \multicolumn{1}{c|}{ Sequence } \\
\hline 35.9 & 2080.1276 & 0.0006 & LNQLAINLSGIIQGINQDR \\
\hline 22.0 & 2080.1276 & 0.0006 & LNQLAINLSGIIQGINQDR \\
\hline 9.7 & 2079.1436 & 0.9846 & LNQLAINLSGIIQGINQDR \\
\hline 2.1 & 2080.1276 & 0.0006 & LNQLAINLSGIIQGINQDR \\
\hline 0.9 & 2079.1241 & 1.0042 & AANIYPLSLLLNLGRRSK \\
\hline 0.2 & 2079.1411 & 0.9871 & HRPIGIGVQGLADAFILMR \\
\hline
\end{tabular}

Spectrum No: 911; Query: 929; Rank: 1

\section{Peptide View}

MS/MS Fragmentation of FWLPHNVTWADLK

Found in IPI00368570, Tax_Id=10116 Gene_Symbol=RGD1561599_predicted similar to longevity assurance homolog 6

Match to Query 929: 1626.818232 from(543.280020,3+)

Title: 091008RatKidney_NH4Format02_24.4810.4810.3.dta

Data file K:INewmanPaper|Piliang|3SubProteomes\Piliang3SP\mgf5ppm\ERLIC_3SubProteomes5ppm.mgf

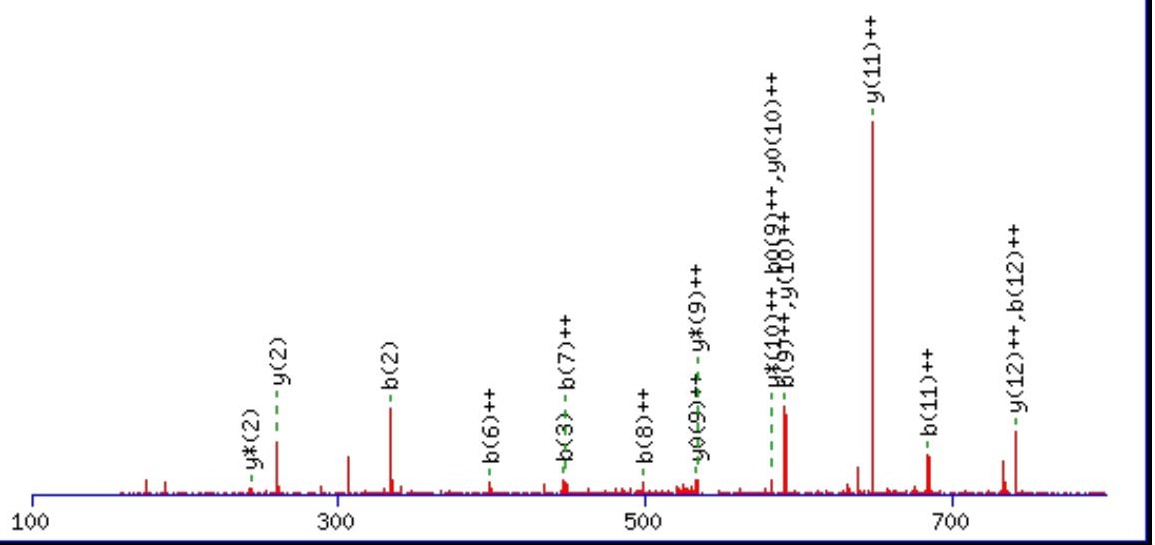



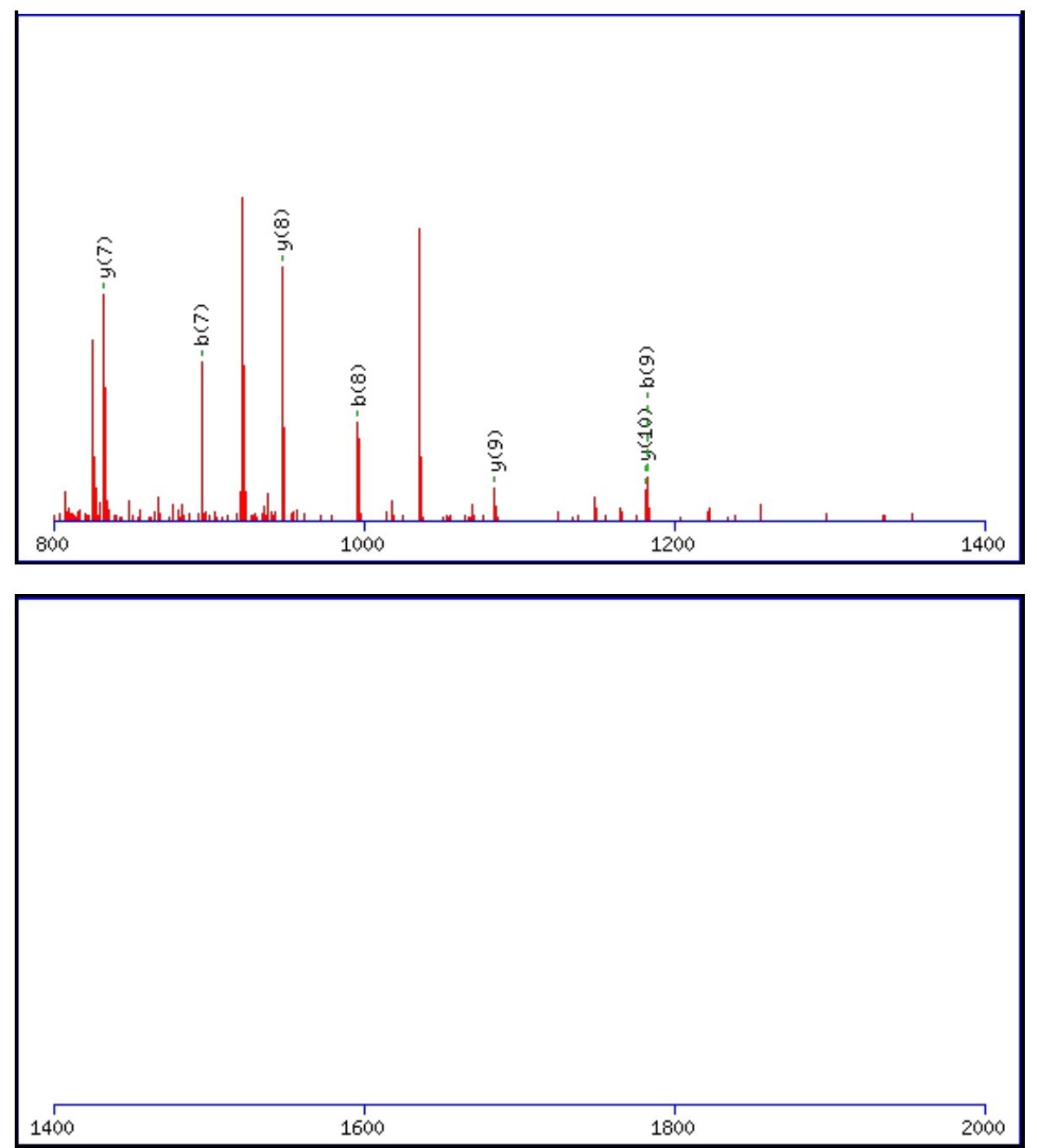

Monoisotopic mass of neutral peptide $\operatorname{Mr}($ calc): 1626.8195

Fixed modifications: Carbamidomethyl (C)

Variable modifications:

N6: Deamidated $\mathrm{N}(\mathrm{N})$

Ions Score: 36 Expect : 0.044

Matches (Bold Red): 25/116 fragment ions using 41 most intense peaks

\begin{tabular}{|r|c|c|c|c|c|c|c|c|c|c|c|c|c|c|}
\hline$\#$ & $\mathbf{b}$ & $\mathbf{b}^{++}$ & $\mathbf{b}^{*}$ & $\mathbf{b}^{\mathbf{*}^{++}}$ & $\mathbf{b}^{\mathbf{0}}$ & $\mathbf{b}^{\mathbf{0 + +}}$ & $\mathbf{S e q}$ & $\mathbf{y}$ & $\mathbf{y}^{++}$ & $\mathbf{y}^{\mathbf{*}}$ & $\mathbf{y}^{\mathbf{*}^{++}}$ & $\mathbf{y}^{\mathbf{0}}$ & $\mathbf{y}^{\mathbf{0 + +}}$ & $\#$ \\
\hline $\mathbf{1}$ & 148.0757 & 74.5415 & & & & & $\mathbf{F}$ & & & & & & & $\mathbf{1 3}$ \\
\hline $\mathbf{2}$ & 334.1550 & 167.5811 & & & & & $\mathbf{W}$ & 1480.7583 & 740.8828 & 1463.7318 & 732.3695 & 1462.7478 & 731.8775 & $\mathbf{1 2}$ \\
\hline $\mathbf{3}$ & $\mathbf{4 4 7 . 2 3 9 1}$ & 224.1232 & & & & & $\mathbf{L}$ & 1294.6790 & $\mathbf{6 4 7 . 8 4 3 1}$ & 1277.6525 & 639.3299 & 1276.6684 & 638.8379 & $\mathbf{1 1}$ \\
\hline $\mathbf{4}$ & 544.2918 & 272.6496 & & & & & $\mathbf{P}$ & $\mathbf{1 1 8 1 . 5 9 4 9}$ & 591.3011 & 1164.5684 & $\mathbf{5 8 2 . 7 8 7 8}$ & 1163.5844 & $\mathbf{5 8 2 . 2 9 5 8}$ & $\mathbf{1 0}$ \\
\hline $\mathbf{5}$ & 681.3507 & 341.1790 & & & & & $\mathbf{H}$ & $\mathbf{1 0 8 4 . 5 4 2 2}$ & 542.7747 & 1067.5156 & 534.2615 & 1066.5316 & $\mathbf{5 3 3 . 7 6 9 4}$ & $\mathbf{9}$ \\
\hline $\mathbf{6}$ & 796.3777 & $\mathbf{3 9 8 . 6 9 2 5}$ & 779.3511 & 390.1792 & & & $\mathbf{N}$ & $\mathbf{9 4 7 . 4 8 3 3}$ & 474.2453 & 930.4567 & 465.7320 & 929.4727 & 465.2400 & $\mathbf{8}$ \\
\hline $\mathbf{7}$ & $\mathbf{8 9 5 . 4 4 6 1}$ & $\mathbf{4 4 8 . 2 2 6 7}$ & 878.4195 & 439.7134 & & & $\mathbf{V}$ & $\mathbf{8 3 2 . 4 5 6 3}$ & 416.7318 & 815.4298 & 408.2185 & 814.4458 & 407.7265 & $\mathbf{7}$ \\
\hline $\mathbf{8}$ & $\mathbf{9 9 6 . 4 9 3 8}$ & $\mathbf{4 9 8 . 7 5 0 5}$ & 979.4672 & 490.2372 & 978.4832 & 489.7452 & $\mathbf{T}$ & 733.3879 & 367.1976 & 716.3614 & 358.6843 & 715.3774 & 358.1923 & $\mathbf{6}$ \\
\hline $\mathbf{9}$ & $\mathbf{1 1 8 2 . 5 7 3 1}$ & $\mathbf{5 9 1 . 7 9 0 2}$ & 1165.5465 & 583.2769 & 1164.5625 & $\mathbf{5 8 2 . 7 8 4 9}$ & $\mathbf{W}$ & 632.3402 & 316.6738 & 615.3137 & 308.1605 & 614.3297 & 307.6685 & $\mathbf{5}$ \\
\hline $\mathbf{1 0}$ & 1253.6102 & 627.3087 & 1236.5837 & 618.7955 & 1235.5996 & 618.3035 & $\mathbf{A}$ & 446.2609 & 223.6341 & 429.2344 & 215.1208 & 428.2504 & 214.6288 & $\mathbf{4}$ \\
\hline $\mathbf{1 1}$ & 1368.6371 & $\mathbf{6 8 4 . 8 2 2 2}$ & 1351.6106 & 676.3089 & 1350.6266 & 675.8169 & $\mathbf{D}$ & 375.2238 & 188.1155 & 358.1973 & 179.6023 & 357.2132 & 179.1103 & $\mathbf{3}$ \\
\hline $\mathbf{1 2}$ & 1481.7212 & $\mathbf{7 4 1 . 3 6 4 2}$ & 1464.6947 & 732.8510 & 1463.7106 & 732.3590 & $\mathbf{L}$ & $\mathbf{2 6 0 . 1 9 6 9}$ & 130.6021 & $\mathbf{2 4 3 . 1 7 0 3}$ & 122.0888 & & & $\mathbf{2}$ \\
\hline $\mathbf{1 3}$ & & & & & & & $\mathbf{K}$ & 147.1128 & 74.0600 & 130.0863 & 65.5468 & & & $\mathbf{1}$ \\
\hline
\end{tabular}



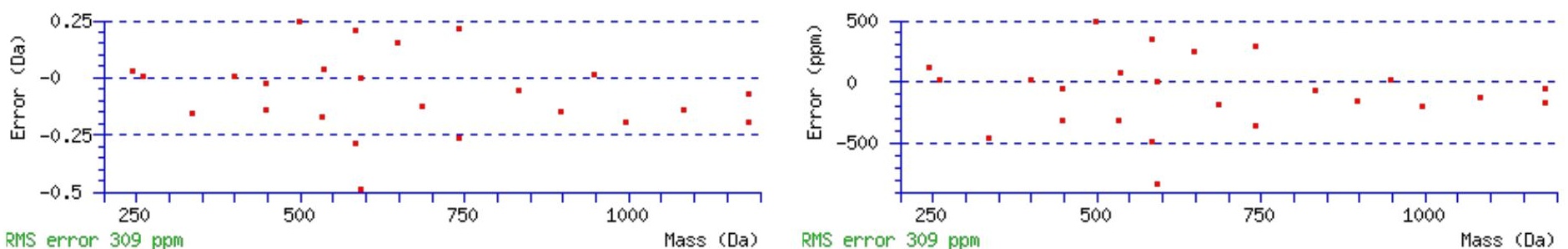

RNS error 309 ppm

Mass (Da) RNS error 309 ppm

Mass (Da)

\section{All matches to this query}

\begin{tabular}{|l|l|l|l|}
\hline Score & Mr(calc): & Delta & \multicolumn{1}{c|}{ Sequence } \\
\hline 35.6 & 1626.8195 & -0.0012 & $\underline{\text { FWLPHNVTWADLK }}$ \\
\hline 11.3 & 1624.8130 & 2.0052 & MALLNGLASALESYR \\
\hline 8.1 & 1626.8262 & -0.0080 & WLMSXMSLGKTWK \\
\hline 8.1 & 1626.8076 & 0.0106 & $\underline{\text { CVLSWLPPLDDGGAK }}$ \\
\hline 7.9 & 1624.8273 & 1.9910 & TFGRSSILKLHMR \\
\hline 7.8 & 1626.8243 & -0.0061 & DALTGHLRTHSVIK \\
\hline 7.7 & 1625.8007 & 1.0175 & $\underline{\text { IATFSHPSFLWLK }}$ \\
\hline 6.9 & 1626.8275 & -0.0093 & $\underline{\text { FRAAFLKLCWCR }}$ \\
\hline 6.5 & 1624.8086 & 2.0096 & LLRVNSNFRPTAR \\
\hline 5.5 & 1624.8007 & 2.0175 & $\underline{\text { MEADITNLRNKLK }}$ \\
\hline
\end{tabular}

Spectrum No: 912; Query: 29; Rank: 1

\section{Peptide View}

MS/MS Fragmentation of QNATLELK

Found in IPI00363534, Tax_Id=10116 Gene_Symbol=Lama1 laminin, alpha 1

Match to Query 29: 916.487568 from(459.251060,2+)

Title: 091008RatKidney_NH4Format01_26.682.682.2.dta

Data file K:\NewmanPaper \Piliang \3SubProteomes \Piliang3SP \mgf5ppm\ERLIC_3SubProteomes5ppm.mgf

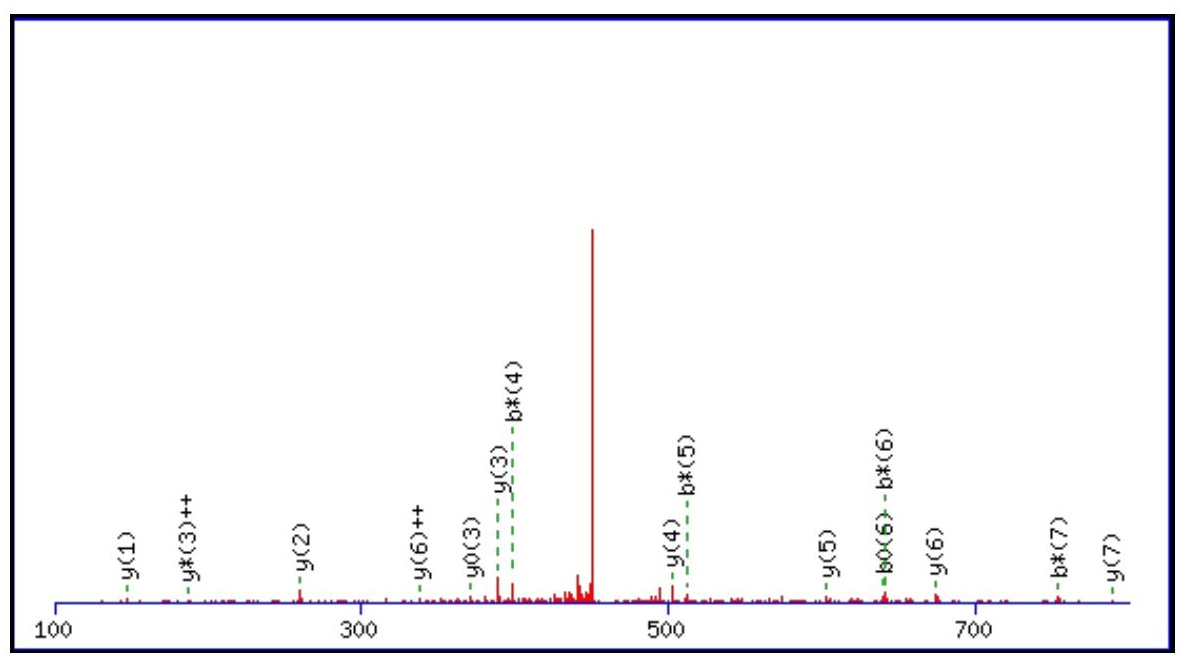



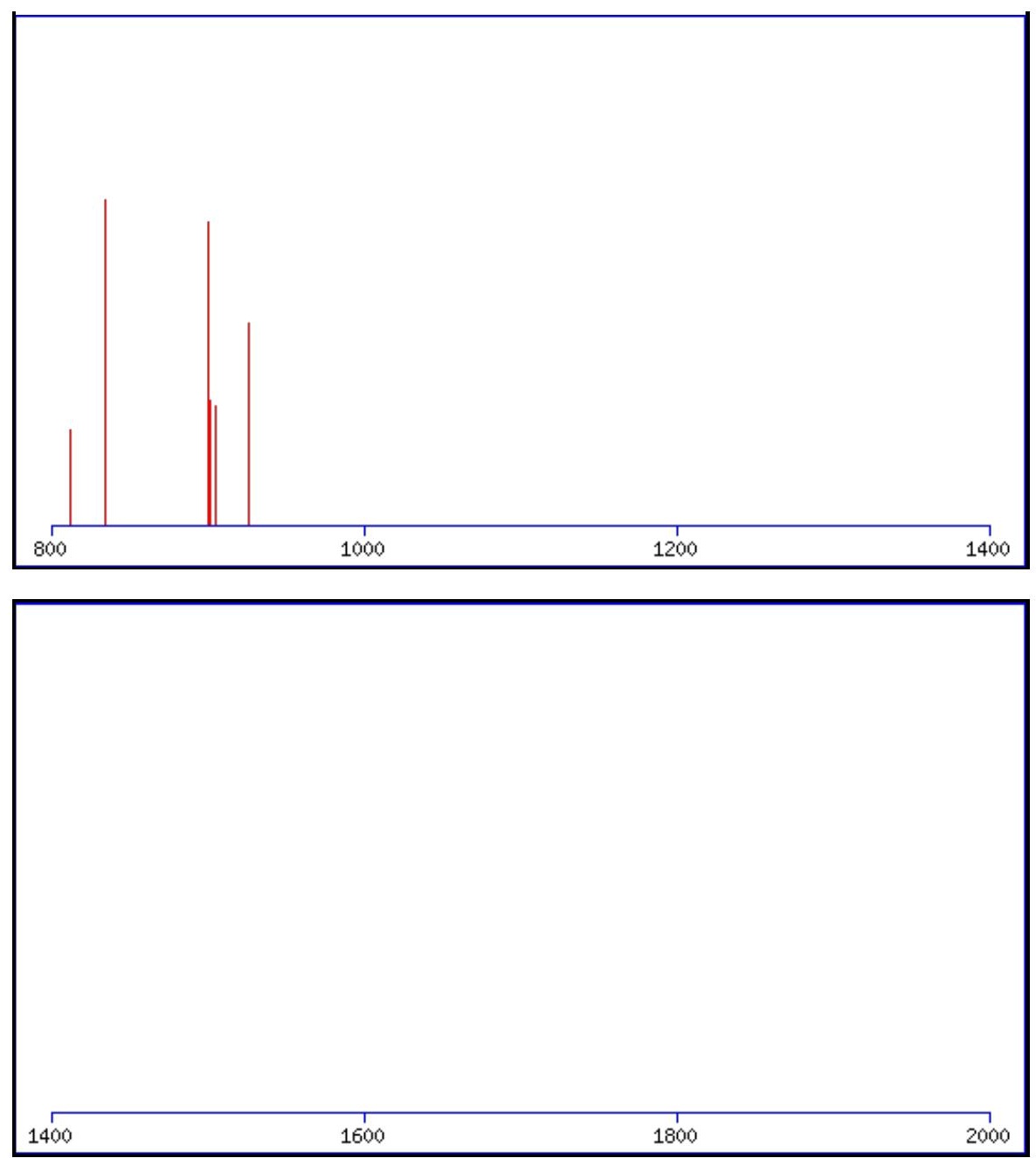

Monoisotopic mass of neutral peptide $\operatorname{Mr}($ calc): 916.4865

Fixed modifications: Carbamidomethyl (C)

Variable modifications:

N2 : Deamidated $\mathrm{N}(\mathrm{N})$

Ions Score: 35 Expect : 0.026

Matches (Bold Red): 15/74 fragment ions using 47 most intense peaks

\begin{tabular}{|c|c|c|c|c|c|c|c|c|c|c|c|c|c|c|}
\hline \# & b & $\mathbf{b}^{++}$ & $\mathbf{b}^{*}$ & $\mathbf{b}^{*^{++}}$ & $\mathbf{b}^{0}$ & $\mathbf{b}^{0++}$ & Seq. & $\mathbf{y}$ & $\mathbf{y}^{++}$ & $\mathbf{y}^{*}$ & $\mathbf{y}^{*^{++}}$ & $\mathbf{y}^{0}$ & $\mathbf{y}^{\mathbf{0 + +}}$ & $\#$ \\
\hline 1 & 129.0659 & 65.0366 & 112.0393 & 56.5233 & & & $\mathbf{Q}$ & & & & & & & 8 \\
\hline 2 & 244.0928 & 122.5500 & 227.0662 & 114.0368 & & & $\mathbf{N}$ & 789.4353 & 395.2213 & 772.4087 & 386.7080 & 771.4247 & 386.2160 & 7 \\
\hline $3:$ & 315.1299 & 158.0686 & 298.1034 & 149.5553 & & & A & 674.4083 & 337.7078 & 657.3818 & 329.1945 & 656.3978 & 328.7025 & 5 \\
\hline 4 & 416.1776 & 208.5924 & 399.1510 & 200.0792 & 398.1670 & 199.5871 & $\mathbf{T}$ & 603.3712 & 302.1892 & 586.3447 & 293.6760 & 585.3606 & 293.1840 & \\
\hline 5 & 529.2616 & 265.1345 & 512.2351 & 256.6212 & 511.2511 & 256.1292 & $\mathbf{L}$ & 502.3235 & 251.6654 & 485.2970 & 243.1521 & 484.3130 & 242.6601 & 14 \\
\hline 6 & 658.3042 & 329.6558 & 641.2777 & 321.1425 & 640.2937 & 320.6505 & $\mathbf{E}$ & 389.2395 & 195.1234 & 372.2129 & |186.6101 & 371.2289 & 186.1181 & 3 \\
\hline 7 & 771.3883 & 386.1978 & 754.3618 & 377.6845 & 753.3777 & 377.1925 & $\mathbf{L}$ & 260.1969 & 130.6021 & 243.1703 & 122.0888 & & & 2 \\
\hline 8 & & & & & & & $\mathbf{K}$ & 147.1128 & 74.0600 & 130.0863 & 65.5468 & & & 1 \\
\hline
\end{tabular}
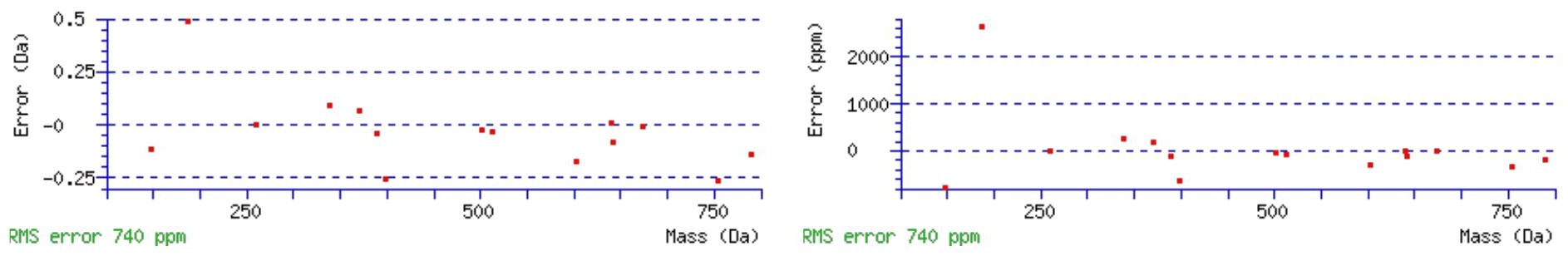

\section{All matches to this query}

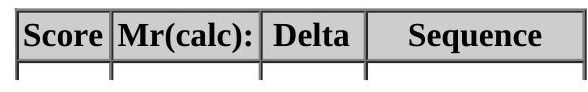




\begin{tabular}{|l|l|l|l|}
\hline 35.5 & 916.4865 & 0.0010 & QNATLELK \\
\hline 16.6 & 915.4913 & 0.9963 & DNLAILEK \\
\hline 16.5 & 916.4865 & 0.0010 & DATIQELK \\
\hline 16.5 & 915.4913 & 0.9963 & GNEILELK \\
\hline 16.5 & 915.4774 & 1.0102 & QQDRELK \\
\hline 16.5 & 915.4774 & 1.0102 & RANEGELK \\
\hline 16.5 & 916.4865 & 0.0010 & TQAVEELK \\
\hline 16.3 & 915.4913 & 0.9963 & EDVIEAIK \\
\hline 16.3 & 916.4866 & 0.0010 & GEASVVDLK \\
\hline 16.3 & 916.4840 & 0.0035 & YIIHMPK \\
\hline
\end{tabular}

Spectrum No: 913; Query: 384; Rank: 1

\section{Peptide View}

MS/MS Fragmentation of TQLEENISQLR

Found in IPI00566692, Tax_Id=10116 Gene_Symbol=- 20 kDa protein

Match to Query 384: 1330.676688 from(666.345620,2+)

Title: 100101RatKid_NS_deglyco_07.2531.2531.2.dta

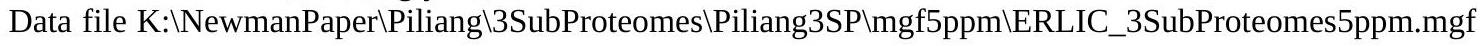
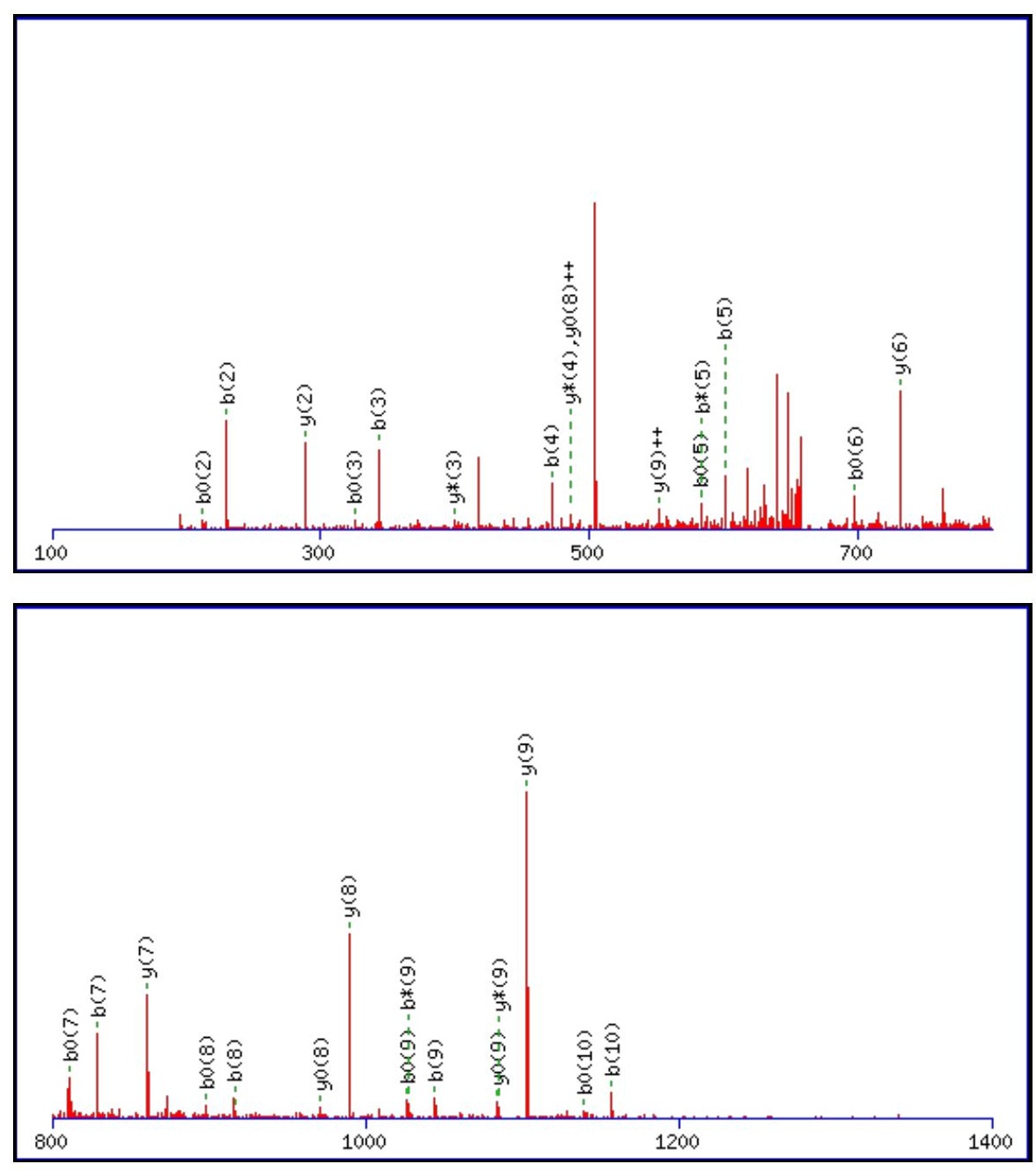


$1400 \quad 1600 \quad 1800 \quad 2000$

Monoisotopic mass of neutral peptide $\operatorname{Mr}($ calc): 1330.6728

Fixed modifications: Carbamidomethyl (C)

Variable modifications:

N6: Deamidated $\mathrm{N}(\mathrm{N})$

Ions Score: 35 Expect: 0.036

Matches (Bold Red): 30/112 fragment ions using 76 most intense peaks

\begin{tabular}{|c|c|c|c|c|c|c|c|c|c|c|c|c|c|c|}
\hline \# & b & $\mathbf{b}^{++}$ & $\mathbf{b}^{*}$ & $\mathbf{b}^{*^{++}}$ & $\mathbf{b}^{0}$ & $\mathbf{b}^{0++}$ & Seq. & $\mathbf{y}$ & $y^{++}$ & $\mathrm{y}^{*}$ & $y^{*^{++}}$ & $\mathbf{y}^{\mathbf{0}}$ & $y^{0++}$ & $\#$ \\
\hline 1 & 102.0550 & 51.5311 & & & 84.0444 & 42.5258 & $T$ & & & & & & & 11 \\
\hline 2 & 230.1135 & 115.5604 & 213.0870 & 107.0471 & 212.1030 & 106.5551 & $\mathbf{Q}$ & 1230.6324 & 615.8199 & 1213.6059 & 607.3066 & 1212.6219 & 606.8146 & 10 \\
\hline 3 & 343.1976 & 172.1024 & 326.1710 & 163.5892 & 325.1870 & 163.0972 & $\mathbf{L}$ & 1102.5739 & 551.7906 & 1085.5473 & 543.2773 & 1084.5633 & 542.7853 & 9 \\
\hline 4 & 472.2402 & 236.6237 & 455.2136 & 228.1105 & 454.2296 & 227.6185 & $\mathbf{E}$ & 989.4898 & 495.2485 & 972.4633 & 486.7353 & 971.4792 & 486.2433 & 8 \\
\hline 5 & 601.2828 & 301.1450 & 584.2562 & 292.6318 & 583.2722 & 292.1397 & $\mathbf{E}$ & 860.4472 & 430.7272 & 843.4207 & 422.2140 & 842.4366 & 421.7220 & 7 \\
\hline 6 & 716.3097 & 358.6585 & 699.2832 & 350.1452 & 698.2992 & 349.6532 & $\mathbf{N}$ & 731.4046 & 366.2059 & 714.3781 & 357.6927 & 713.3941 & 357.2007 & 6 \\
\hline 7 & 829.3938 & 415.2005 & 812.3672 & 406.6873 & 811.3832 & 406.1952 & I & 616.3777 & 308.6925 & 599.3511 & 300.1792 & 598.3671 & 299.6872 & 5 \\
\hline 8 & 916.4258 & 458.7165 & 899.3993 & 450.2033 & 898.4152 & 449.7113 & $S$ & 503.2936 & 252.1504 & 486.2671 & 243.6372 & 485.2831 & 243.1452 & 4 \\
\hline 9 & 1044.4844 & 522.7458 & 1027.4578 & 514.2326 & \begin{tabular}{|l|}
1026.4738 \\
\end{tabular} & 513.7405 & $\mathbf{Q}$ & 416.2616 & 208.6344 & 399.2350 & 200.1212 & & & 3 \\
\hline 10 & 1157.5685 & 579.2879 & 1140.5419 & 570.7746 & 1139.5579 & 570.2826 & $\mathbf{L}$ & 288.2030 & 144.6051 & 271.1765 & 136.0919 & & & 2 \\
\hline 11 & & & & & & & $\mathbf{R}$ & 175.1190 & 88.0631 & 158.0924 & 79.5498 & & & 1 \\
\hline
\end{tabular}
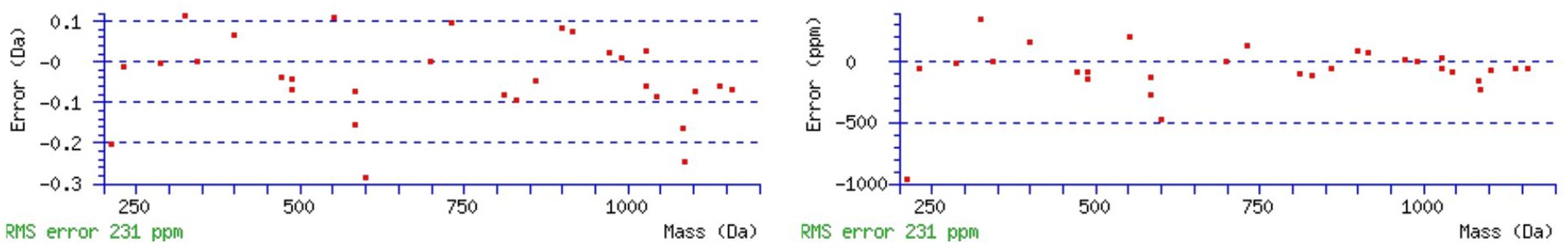

\section{All matches to this query}

\begin{tabular}{|l|l|l|l|}
\hline Score & Mr(calc): & Delta & \multicolumn{1}{|c|}{ Sequence } \\
\hline 35.4 & 1330.6728 & 0.0039 & TQLEENISQLR \\
\hline 8.9 & 1330.6898 & -0.0131 & TKIEFVTGTKK \\
\hline 8.9 & 1330.6898 & -0.0131 & TKIEFVTGTKK \\
\hline 7.7 & 1330.6841 & -0.0074 & QTNKRTEPETK \\
\hline 5.7 & 1330.6646 & 0.0121 & SPAAPVEQVVVR \\
\hline 5.3 & 1330.6798 & -0.0032 & FRSLIPSYIR \\
\hline 5.3 & 1330.6798 & -0.0032 & FRSLIPSYIR \\
\hline 4.3 & 1330.6841 & -0.0074 & DKLNDTARELR \\
\hline 3.5 & 1330.6881 & -0.0114 & NLNPIFNETLR \\
\hline 2.8 & 1328.6709 & 2.0057 & VKICMVSKIR \\
\hline
\end{tabular}


Spectrum No: 914; Query: 14; Rank: 1

\section{Peptide View}

MS/MS Fragmentation of GFNASWR

Found in IPI00196620, Tax_Id=10116 Gene_Symbol=Cubn Cubilin precursor

Match to Query 14: 837.375768 from(419.695160,2+)

Title: 091008RatKidney_NH4Format01_24.1508.1508.2.dta

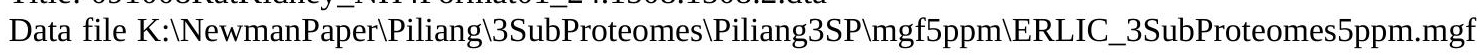
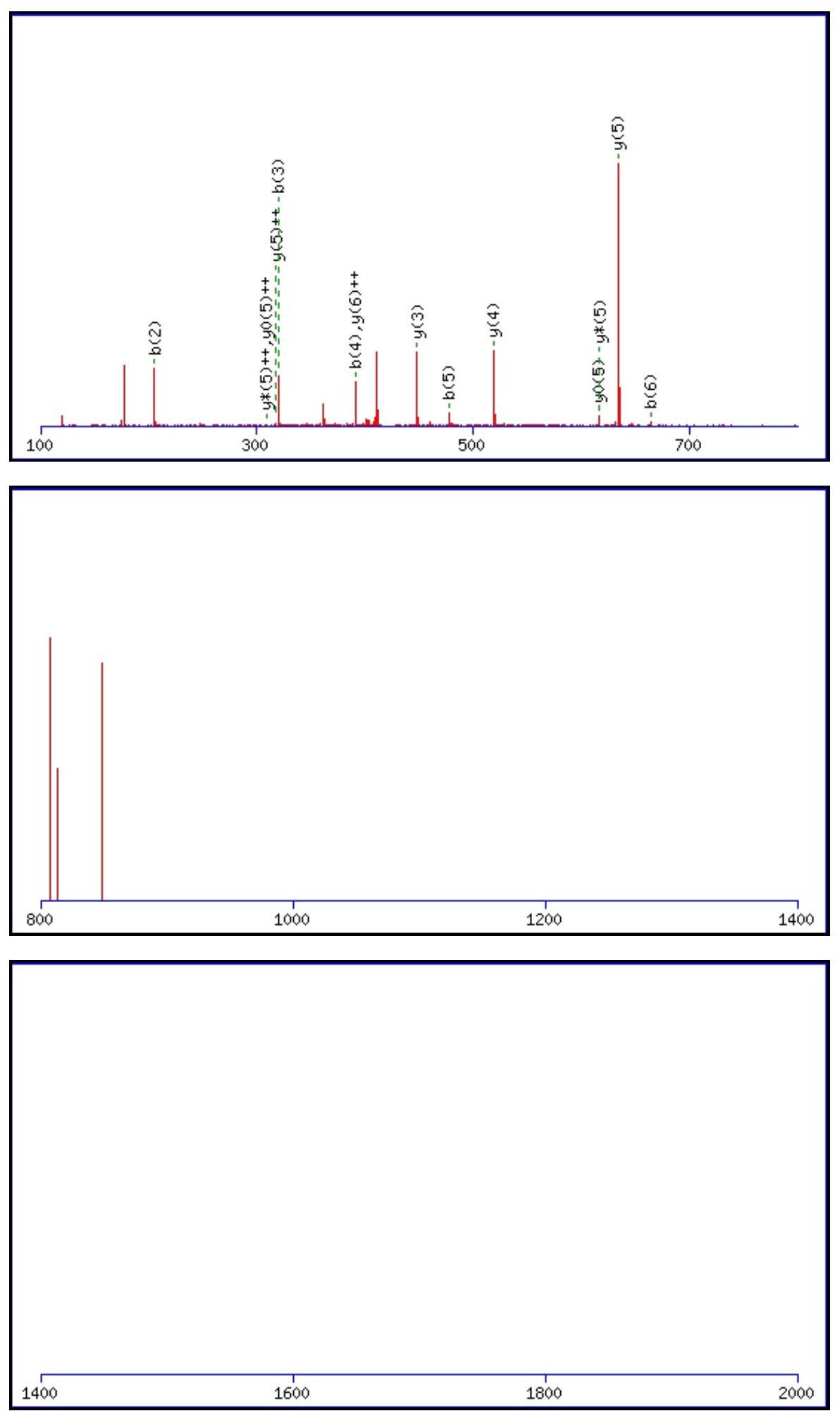
Monoisotopic mass of neutral peptide $\operatorname{Mr}(\mathbf{c a l c}): 837.3769$

Fixed modifications: Carbamidomethyl (C)

Variable modifications:

N3 : Deamidated_N ( N )

Ions Score: 35 Expect: 0.015

Matches (Bold Red): 14/56 fragment ions using 24 most intense peaks

\begin{tabular}{|c|c|c|c|c|c|c|c|c|c|c|c|c|c|c|}
\hline \# & b & $\mathbf{b}^{++}$ & $\mathbf{b}^{*}$ & $\mathbf{b}^{*^{++}}$ & $\mathbf{b}^{\mathbf{0}}$ & $\mathbf{b}^{\mathbf{0 + +}}$ & Seq. & $\mathbf{y}$ & $\mathbf{y}^{++}$ & $\mathbf{y}^{*}$ & $\mathrm{y}^{*^{++}}$ & $\mathbf{y}^{\mathbf{0}}$ & $y^{0++}$ & \# \\
\hline 1 & 58.0287 & 29.5180 & & & & & G & & & & & & & 7 \\
\hline 2 & 205.0972 & 103.0522 & & & & & $\mathbf{F}$ & 781.3628 & 391.1850 & 764.3362 & 382.6717 & 763.3522 & 382.1797 & 76 \\
\hline 3 & 320.1241 & 160.5657 & 303.0975 & 152.0524 & & & $\mathbf{N}$ & 634.2943 & 317.6508 & 617.2678 & 309.1375 & 616.2838 & 308.6455 & 5 \\
\hline 4 & 391.1612 & 196.0842 & 374.1347 & 187.5710 & & & A & 519.2674 & 260.1373 & 502.2409 & 251.6241 & 501.2568 & 251.1321 & 4 \\
\hline 5 & 478.1932 & 239.6003 & 461.1667 & 231.0870 & 460.1827 & 230.5950 & $\mathrm{~S}$ & 448.2303 & 224.6188 & 431.2037 & 216.1055 & 430.2197 & 215.6135 & 5 \\
\hline 6 & 664.2725 & 332.6399 & 647.2460 & 324.1266 & 646.2620 & 323.6346 & $\mathbf{W}$ & 361.1983 & 181.1028 & 344.1717 & 172.5895 & & & 2 \\
\hline & & & & & & & $\mathbf{R}$ & 175.1190 & 88.0631 & 158.0924 & 79.5498 & & & \\
\hline
\end{tabular}
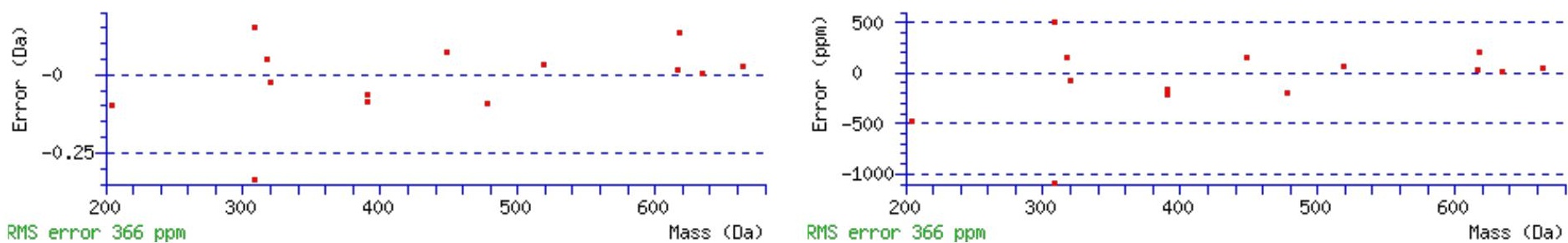

All matches to this query

\begin{tabular}{|l|l|l|l|}
\hline Score & Mr(calc): & Delta & \multicolumn{1}{|c|}{ Sequence } \\
\hline 35.2 & 837.3769 & -0.0012 & GFNASWR \\
\hline 16.6 & 837.3770 & -0.0012 & GFNGWTR \\
\hline 9.7 & 837.3770 & -0.0012 & FQDSWR \\
\hline 8.2 & 837.3803 & -0.0046 & FGCXEVR \\
\hline 7.6 & 835.3672 & 2.0086 & $\underline{\text { NSDSVGXR }}$ \\
\hline 7.5 & 836.3793 & 0.9964 & $\underline{\text { ALSQPGGK }}$ \\
\hline 7.1 & 836.3698 & 1.0060 & $\underline{\text { NQMKNGK }}$ \\
\hline 5.9 & 836.3698 & 1.0059 & $\underline{\text { TTDDMVR }}$ \\
\hline 5.4 & 837.3828 & -0.0070 & $\underline{\text { SSSNTSVR }}$ \\
\hline 4.3 & 836.3793 & 0.9964 & $\underline{\text { GGAOIPSK }}$ \\
\hline
\end{tabular}

Spectrum No: 915; Query: 494; Rank: 1

\section{Peptide View}

MS/MS Fragmentation of SYRIDQNITYK

Found in IPI00372786, Tax_Id=10116 Gene_Symbol=Nid2 similar to nidogen 2 isoform 2

Match to Query 494: 1400.695692 from(467.905840,3+)

Title: 100101RatKid_NS_deglyco_19.1753.1753.3.dta

Data file K:INewmanPaper|Piliang|3SubProteomes\Piliang3SP\mgf5ppm\ERLIC_3SubProteomes5ppm.mgf 

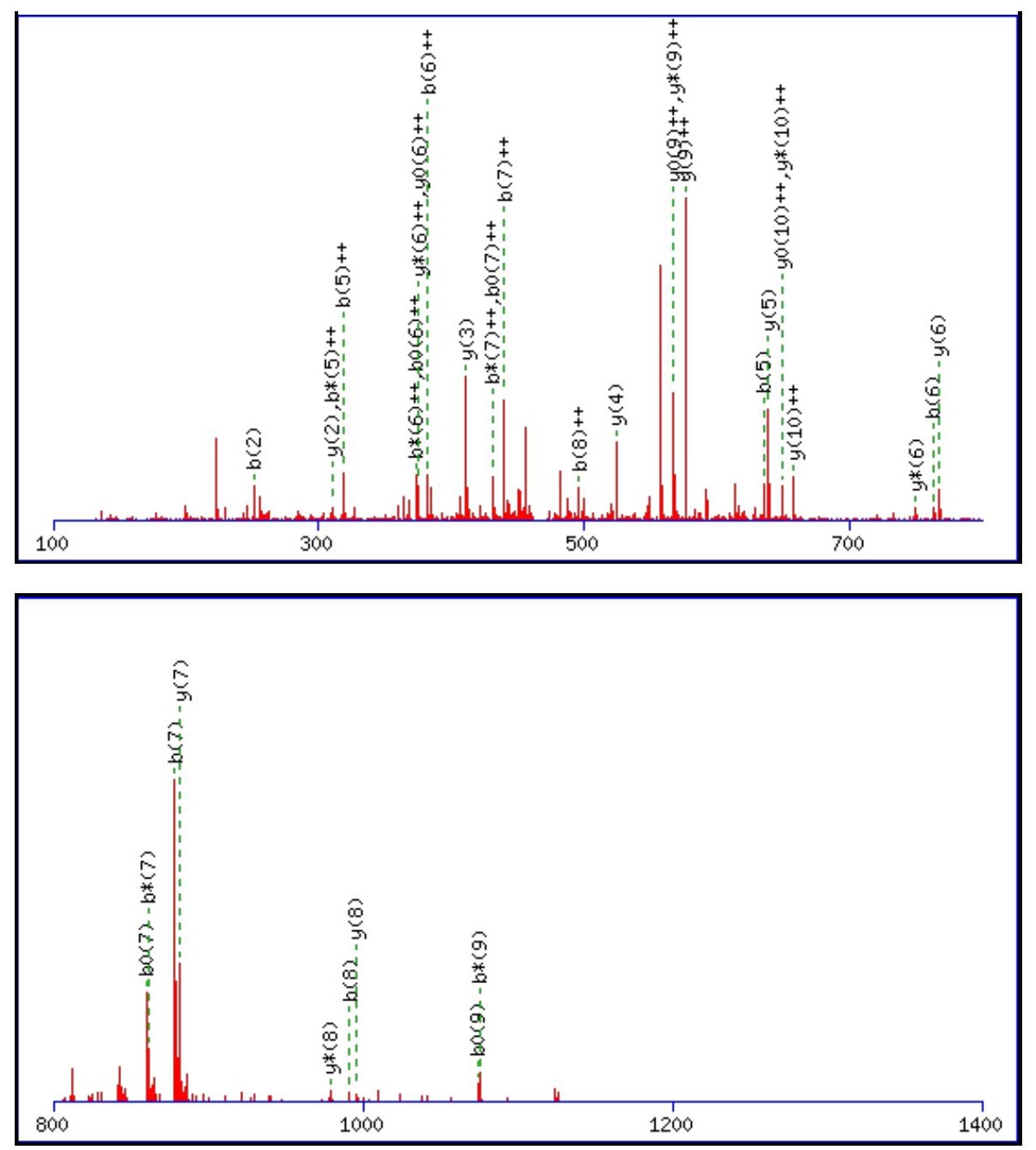

\begin{tabular}{rrrr}
\hline 1 & 1 & 1800 & 2000 \\
1400 & 1600 & 1800
\end{tabular}

Monoisotopic mass of neutral peptide $\operatorname{Mr}($ calc): 1400.6936

Fixed modifications: Carbamidomethyl (C)

Variable modifications:

N7 : Deamidated N (N)

Ions Score: 35 Expect: $\odot .047$

Matches (Bold Red): 35/112 fragment ions using 54 most intense peaks

\begin{tabular}{|r|c|c|c|c|r|r|r|r|c|c|c|c|c|c|}
\hline$\#$ & $\mathbf{b}$ & $\mathbf{b}^{++}$ & $\mathbf{b}^{*}$ & $\mathbf{b}^{\boldsymbol{*}^{++}}$ & $\mathbf{b}^{\mathbf{0}}$ & \multicolumn{1}{|c|}{$\mathbf{b}^{\mathbf{0 + +}}$} & Seq. & $\mathbf{y}$ & $\mathbf{y}^{++}$ & $\mathbf{y}^{\mathbf{*}}$ & $\mathbf{y}^{\boldsymbol{*}^{++}}$ & $\mathbf{y}^{\mathbf{0}}$ & $\mathbf{y}^{\mathbf{0 + +}}$ & $\#$ \\
\hline $\mathbf{1}$ & $\mathbf{8 8 . 0 3 9 3}$ & 44.5233 & & & 70.0287 & 35.5180 & $\mathbf{S}$ & & & & & & & $\mathbf{1 1}$ \\
\hline $\mathbf{2}$ & $\mathbf{2 5 1 . 1 0 2 6}$ & 126.0550 & & & 233.0921 & 117.0497 & $\mathbf{Y}$ & 1314.6688 & $\mathbf{6 5 7 . 8 3 8 1}$ & 1297.6423 & $\mathbf{6 4 9 . 3 2 4 8}$ & 1296.6583 & $\mathbf{6 4 8 . 8 3 2 8}$ & $\mathbf{1 0}$ \\
\hline $\mathbf{3}$ & 407.2037 & 204.1055 & 390.1772 & 195.5922 & 389.1932 & 195.1002 & $\mathbf{R}$ & 1151.6055 & 576.3064 & 1134.5790 & $\mathbf{5 6 7 . 7 9 3 1}$ & 1133.5949 & $\mathbf{5 6 7 . 3 0 1 1}$ & $\mathbf{9}$ \\
\hline $\mathbf{4}$ & 520.2878 & 260.6475 & 503.2613 & 252.1343 & 502.2772 & 251.6423 & $\mathbf{I}$ & $\mathbf{9 9 5 . 5 0 4 4}$ & 498.2558 & $\mathbf{9 7 8 . 4 7 7 8}$ & 489.7426 & 977.4938 & 489.2506 & $\mathbf{8}$ \\
\hline $\mathbf{5}$ & $\mathbf{6 3 5 . 3 1 4 8}$ & $\mathbf{3 1 8 . 1 6 1 0}$ & 618.2882 & 309.6477 & 617.3042 & 309.1557 & $\mathbf{D}$ & $\mathbf{8 8 2 . 4 2 0 3}$ & 441.7138 & 865.3938 & 433.2005 & 864.4098 & 432.7085 & $\mathbf{7}$ \\
\hline
\end{tabular}




\begin{tabular}{|r|r|r|r|r|r|r|r|r|r|r|r|r|r|r|r|r|r|}
$\mathbf{6}$ & $\mathbf{7 6 3 . 3 7 3 3}$ & $\mathbf{3 8 2 . 1 9 0 3}$ & 746.3468 & $\mathbf{3 7 3 . 6 7 7 0}$ & $\mathbf{7 4 5 . 3 6 2 8}$ & $\mathbf{3 7 3 . 1 8 5 0}$ & $\mathbf{Q}$ & $\mathbf{7 6 7 . 3 9 3 4}$ & 384.2003 & $\mathbf{7 5 0 . 3 6 6 8}$ & $\mathbf{3 7 5 . 6 8 7 1}$ & $\mathbf{7 4 9 . 3 8 2 8}$ & $\mathbf{3 7 5 . 1 9 5 1}$ & $\mathbf{6}$ \\
\hline $\mathbf{7}$ & $\mathbf{8 7 8 . 4 0 0 3}$ & $\mathbf{4 3 9 . 7 0 3 8}$ & $\mathbf{8 6 1 . 3 7 3 7}$ & $\mathbf{4 3 1 . 1 9 0 5}$ & $\mathbf{8 6 0 . 3 8 9 7}$ & $\mathbf{4 3 0 . 6 9 8 5}$ & $\mathbf{N}$ & $\mathbf{6 3 9 . 3 3 4 8}$ & 320.1710 & 622.3083 & 311.6578 & 621.3242 & 311.1658 & $\mathbf{5}$ \\
\hline $\mathbf{8}$ & $\mathbf{9 9 1 . 4 8 4 3}$ & $\mathbf{4 9 6 . 2 4 5 8}$ & 974.4578 & 487.7325 & $\mathbf{9 7 3 . 4 7 3 8}$ & $\mathbf{4 8 7 . 2 4 0 5}$ & $\mathbf{I}$ & $\mathbf{5 2 4 . 3 0 7 9}$ & 262.6576 & 507.2813 & 254.1443 & 506.2973 & 253.6523 & $\mathbf{4}$ \\
\hline $\mathbf{9}$ & 1092.5320 & 546.7696 & $\mathbf{1 0 7 5 . 5 0 5 5}$ & 538.2564 & $\mathbf{1 0 7 4 . 5 2 1 4}$ & 537.7644 & $\mathbf{T}$ & $\mathbf{4 1 1 . 2 2 3 8}$ & 206.1155 & 394.1973 & 197.6023 & 393.2132 & 197.1103 & $\mathbf{3}$ \\
\hline $\mathbf{1 0}$ & 1255.5953 & 628.3013 & 1238.5688 & 619.7880 & 1237.5848 & 619.2960 & $\mathbf{Y}$ & $\mathbf{3 1 0 . 1 7 6 1}$ & 155.5917 & 293.1496 & 147.0784 & & & \\
\hline $\mathbf{1 1}$ & & & & & & & $\mathbf{K}$ & 147.1128 & 74.0600 & 130.0863 & 65.5468 & & & & $\mathbf{1}$ \\
\hline
\end{tabular}
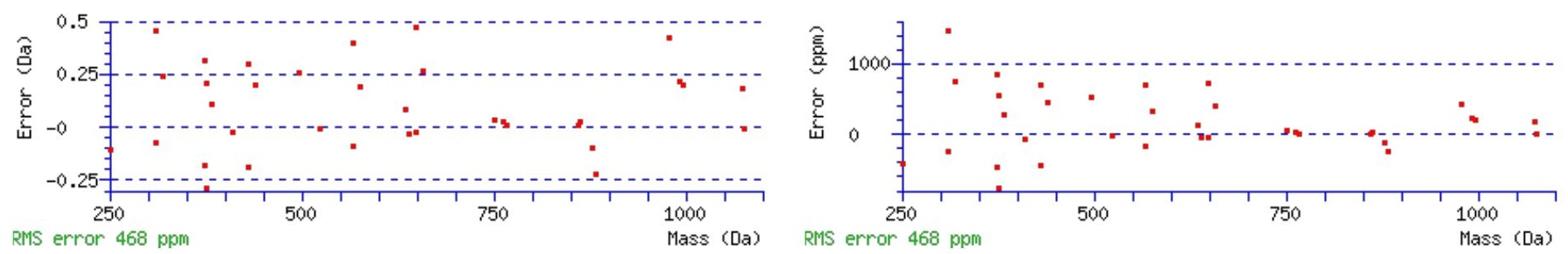

\section{All matches to this query}

\begin{tabular}{|l|l|c|l|}
\hline Score & Mr(calc): & Delta & \multicolumn{1}{c|}{ Sequence } \\
\hline 34.7 & 1400.6936 & 0.0021 & SYRIDQNITYK \\
\hline 7.0 & 1400.6926 & 0.0031 & SAPSTGGVKKPHR \\
\hline 7.0 & 1400.6926 & 0.0031 & SAPSTGGVKKPHR \\
\hline 6.5 & 1400.6926 & 0.0031 & SAPSTGGVKKPHR \\
\hline 3.5 & 1398.7020 & 1.9937 & RKPLPGDPQSPK \\
\hline 2.5 & 1400.7082 & -0.0125 & TPAGQLEAALGMSR \\
\hline 1.1 & 1398.6769 & 2.0188 & DRHIKLHTGDK \\
\hline 0.1 & 1400.6870 & 0.0086 & NYKPDMEVHIR \\
\hline
\end{tabular}

Spectrum No: 916; Query: 2485; Rank: 1

\section{Peptide View}

\section{MS/MS Fragmentation of VVSVDISFRPVNETFPVVYIENPKR}

Found in IPI00326140, Tax_Id=10116 Gene_Symbol=Pzp Alpha-1-macroglobulin precursor

Match to Query 2485: 2905.548792 from(969.523540,3+)

Title: 091008RatKidney_NH4Format01_25.3860.3860.3.dta

Data file K:INewmanPaper\Piliang $\mid 3 S$ ubProteomes\Piliang3SP\mgf5ppm\ERLIC_3SubProteomes5ppm.mgf

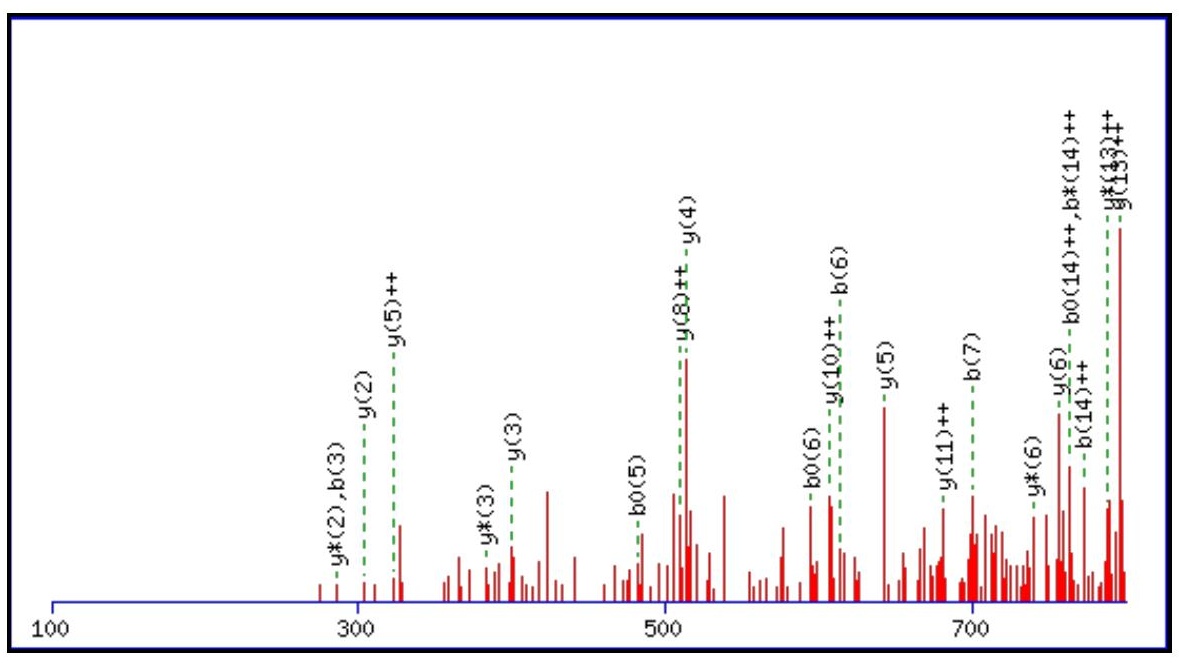




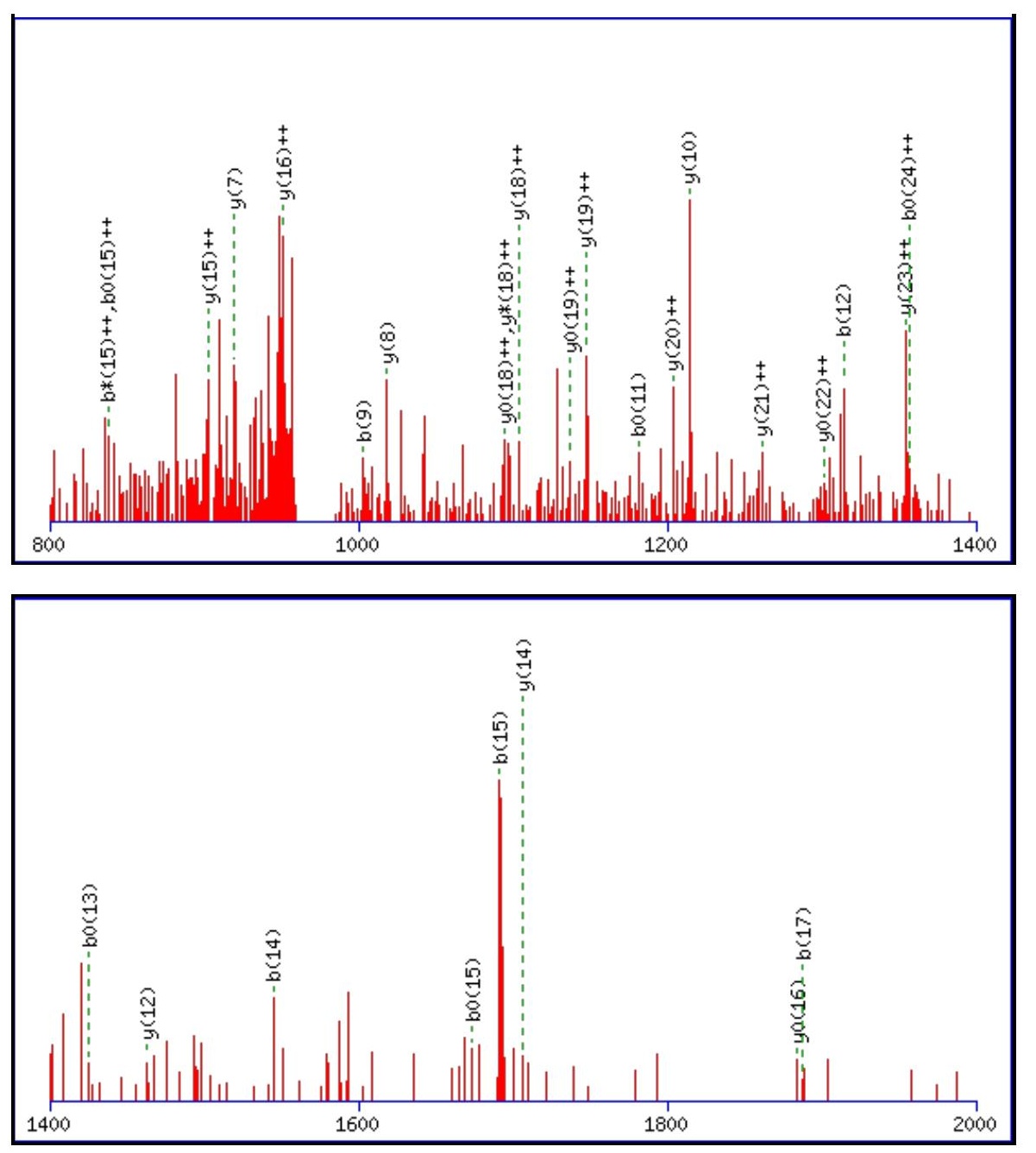

Monoisotopic mass of neutral peptide $\operatorname{Mr}($ calc): 2904.5385

Fixed modifications: Carbamidomethyl (C)

Variable modifications:

N12 : Deamidated $\mathrm{N}(\mathrm{N})$

Ions Score: 34 Expect : 0.027

Matches (Bold Red): 50/260 fragment ions using 161 most intense peaks

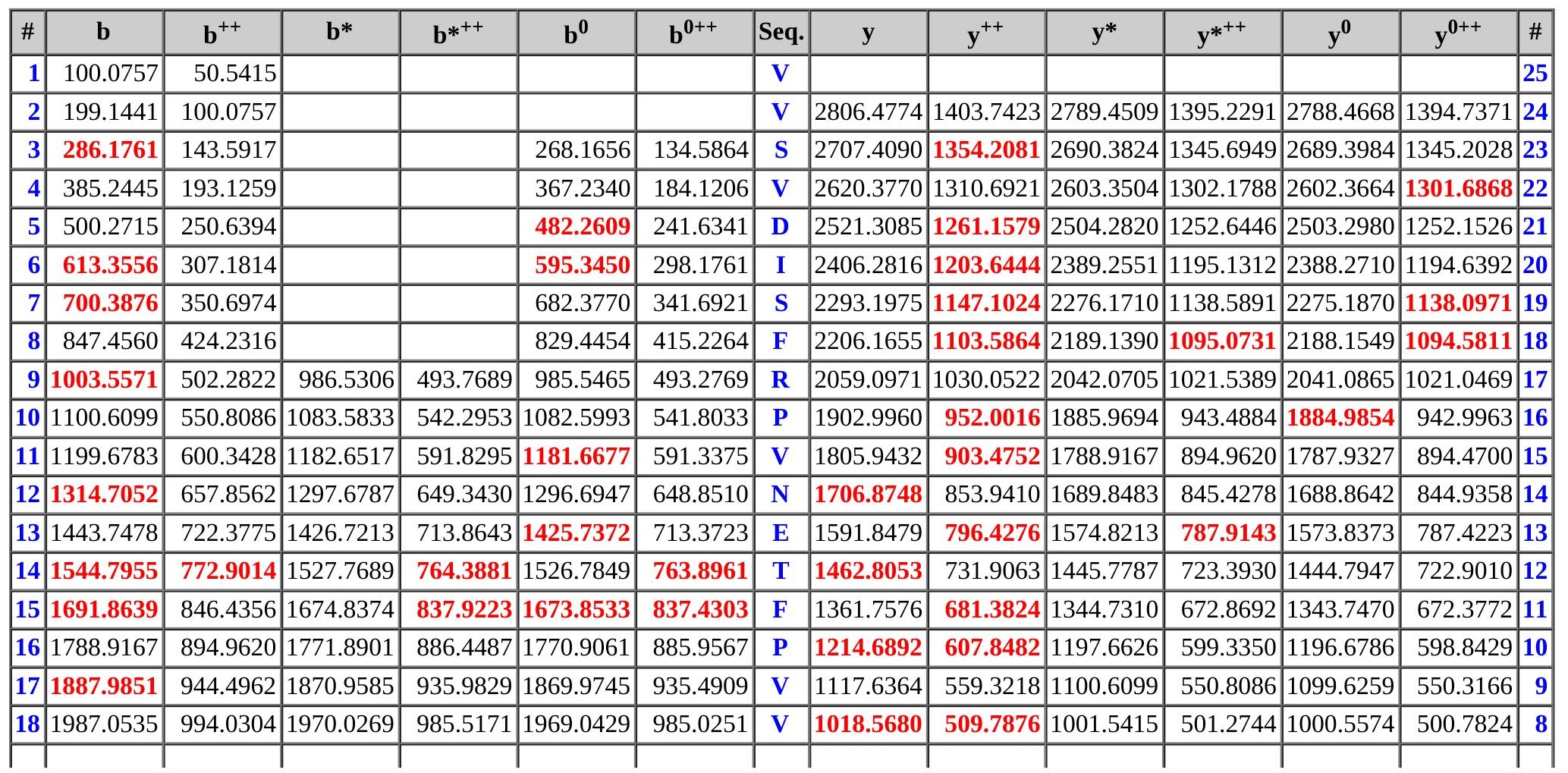




\begin{tabular}{|c|c|c|c|c|c|c|c|c|c|c|c|c|c|c|}
\hline 19 & |2150.1168 & 1075.5621 & |2133.0903 & |1067.0488 & 2132.1063 & |1066.5568| & $\mathbf{Y}$ & 919.4996 & 460.2534 & 902.4730 & 451.7402 & 901.4890 & 451.2482 & 7 \\
\hline 20 & 2263.2009 & 1132.1041 & 2246.1743 & 1123.5908 & 2245.1903 & 1123.0988 & I & 756.4363 & 378.7218 & 739.4097 & 370.2085 & 738.4257 & 369.7165 & 6 \\
\hline 21 & 2392.2435 & 1196.6254 & 2375.2169 & 1188.1121 & 2374.2329 & 1187.6201 & $\mathbf{E}$ & 643.3522 & 322.1797 & 626.3257 & 313.6665 & 625.3416 & 313.1745 & 5 \\
\hline 22 & 2506.2864 & 1253.6468 & 2489.2599 & 1245.1336 & 2488.2758 & 1244.6416 & $\mathbf{N}$ & 514.3096 & 257.6584 & 497.2831 & 249.1452 & & & 4 \\
\hline 23 & 2603.3392 & 1302.1732 & 2586.3126 & 1293.6600 & 2585.3286 & 1293.1679 & $\mathbf{P}$ & 400.2667 & 200.6370 & 383.2401 & 192.1237 & & & 3 \\
\hline 24 & 2731.4341 & 1366.2207 & 2714.4076 & 1357.7074 & 2713.4236 & 1357.2154 & $\mathbf{K}$ & 303.2139 & 152.1106 & 286.1874 & 143.5973 & & & 2 \\
\hline 25 & & & & & & & $\mathbf{R}$ & 175.1190 & 88.0631 & 158.0924 & 79.5498 & & & 1 \\
\hline
\end{tabular}
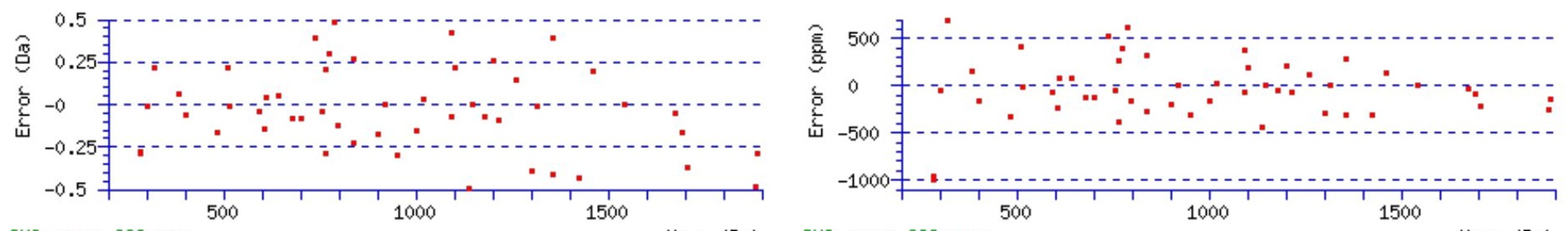

RMS error 322 ppm

Mass (Da) RMS error 322 ppm

Mass (Da)

\section{All matches to this query}

\begin{tabular}{|l|c|c|c|}
\hline Score & Mr(calc): & Delta & Sequence \\
\hline 33.7 & 2904.5385 & 1.0102 & VVSVDISFRPVNETFPVVYIENPKR \\
\hline 11.7 & 2903.5545 & 1.9943 & VVSVDISFRPVNETFPVVYIENPKR \\
\hline 6.6 & 2905.5226 & 0.0262 & VVSVDISFRPVNETFPVVYIENPKR \\
\hline 6.0 & 2904.5385 & 1.0102 & VVSVDISFRPVNETFPVVYIENPKR \\
\hline
\end{tabular}

Mascot: http://www.matrixscience.com/ 